\title{
CROSSCUTTING TECHNOLOGY DEVELOPMENT AT THE CENTER FOR ADVANCED SEPARATION TECHNOLOGIES
}

FINAL TECHNICAL REPORT

\author{
Report Period \\ October 1, 2002 to September 30, 2009 \\ Compiled by \\ Christopher E Hull
}

Issued November 23, 2010

DOE Award Number:

DE-FC26-02NT41607

Center for Advanced Separation Technologies

Virginia Polytechnic Institute \& State University

Blacksburg, Virginia 24061-0258

National Research Center for Coal \& Energy

West Virginia University

Morgantown, WV 26506-6064

Dept. of Metallurgical Engineering

University of Utah

Salt Lake City, UT 84112-0114

NMBGR

New Mexico Inst. of Mining \& Technology

Socorro, NM 87801
Department of Mining Engineering University of Kentucky

Lexington, KY 40506-0107

School of Mines \& Engineering Montana Tech of The Univ. of Montana Butte, MT 59701

Metallurgical \& Materials Engineering University of Nevada, Reno Reno, NV 89557-0042 


\section{DISCLAIMER}

This report was prepared as an account of work sponsored by an agency of the United States Government. Neither the United States Government nor any agency thereof, nor any of their employees, makes any warranty, express or implied, or assumes any legal liability or responsibility for the accuracy, completeness, or usefulness of any information, apparatus, product, or process disclosed, or represents that its use would not infringe privately owned rights. Reference herein to any specific commercial product, process, or service by trade name, trademark, manufacture, or otherwise does not necessarily constitute or imply its endorsement, recommendation, or favoring by the United States Government or any agency thereof. The views and opinions of authors expressed herein do not necessarily state or reflect those of the United States Government or any agency thereof. 


\section{Table of Contents}

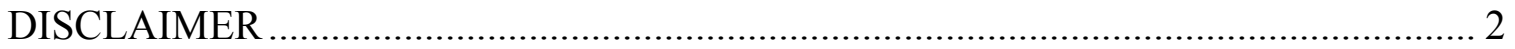

ABSTRACT

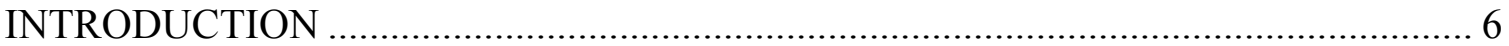

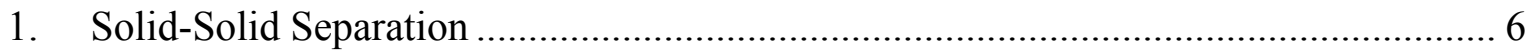

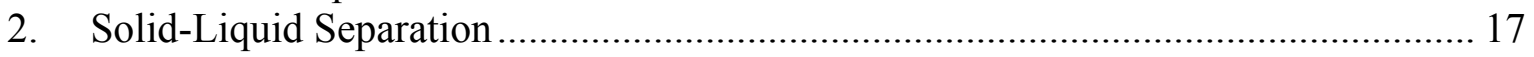

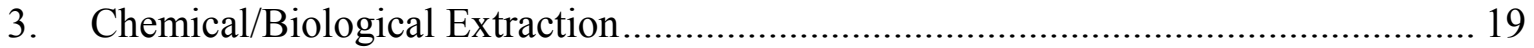

4. Modeling and Control ...................................................................................... 27

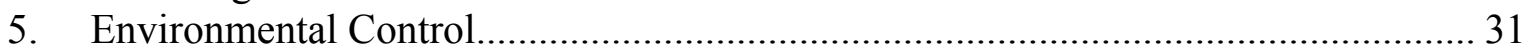

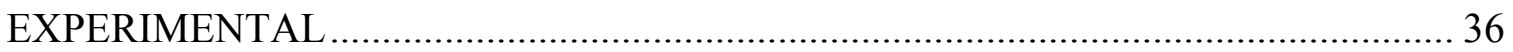

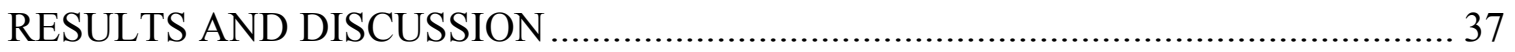

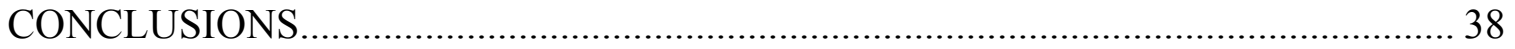

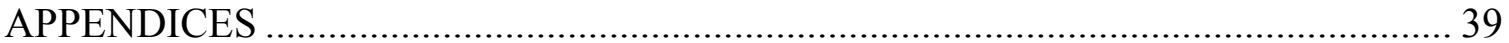

Appendix 1: Development of Novel Ultrafine Sizing Methods (KY001/VA008)

Appendix 2: Alternative Materials for Dense Medium Separation (KY004)

Appendix 3: Enhanced Flotation Performance Through Column Froth Enrichment (KY006)

Appendix 4: Dispersion of Flotation Clays from New Mexico Potash Ores (NM001)

Appendix 5: Development of New Reagents for the Flotation of Dolomite from Phosphate

Ore (NV002)

Appendix 6: Flotation Technology for the Trona Industry (UT001)

Appendix 7: High Frequency Eddy Current Separation of Metallic Residue from Slags,

Sands, Electronic Scraps and Other Wastes (UT004)

Appendix 8: Direct Measurement of Forces in Flotation (VA004)

Appendix 9: Flotation Processes, Experiments and analysis (VA009)

Appendix 10: Engineering Development of a Fine Particle Heavy Medium Separator

(VA012)

Appendix 11: Development of a Turbulent Flotation Model and a Computer Simulator (VA009)

Appendix 12: Measurement of Surface Forces Between Hydrophobic Surfaces (VA016)

Appendix 13 Novel Surfactants as Collectors for Froth Flotation (VA017)

Appendix 14: Continuation of Project-Dry Particle Separation in a CFB Rise System

(WV009)

Appendix 15: Development of an Optimized Control System for HMC Circuits (VA021)

Appendix 16: Densification of Fine Coal Refuse Slurries to Eliminate Slurry Ponds (KY002)

Appendix 17: Development of a Fine Particle centrifuge (VA006)

Appendix 18: Development and Testing of a Horizantal Belt Filter (VA010)

Appendix 19: Improvements in Screen Bowl Centrifuge Performance (VA013)

Appendix 20: Development of Alkaline Sulphide Leaching and Recovery of Gold (MT001)

Appendix 21: Simultaneous Copper Cathode Production and Ferric Sulfate Regeneration (MT002)

Appendix 22: Recovery of Gold From Thiosulfate Leach Liquor Using Activated Carbon (MT005)

Appendix 23: Ion Exchange Recovery of Cobalt from Copper Leach Solutions (NM002)

Appendix 24: Development of Enhanced Surfactants for the Potash Industry (NM003) 
Appendix 25: The Effect of Alkyl Diphenyl Oxide and Sulfonated Oleic Acid Surfactants on Nucleation and Growth of Potassium Sulfate Crystals: Optimization of Surfactants for the Potash Industry (NM005)

Appendix 26: Hydrometallurgical Processing of Chalcopyrite Concentrates (NV001) Appendix 27: Thiosulfate as a Replacement For Cyanide In The Presence of Activators (NV003)

Appendix 28: Bio-Assisted Heap Leaching of Nickel Laterites (UT002)

Appendix 29: Overcoming Technological Barriers to More Efficient Recovery of Copper from Chalcopyrite (UT005)

Appendix 30: Development of Chemical and Biochemical Techniques for the Extraction of Mercury from Fine Coal Particle Solutions (WV010)

Appendix 31: Coal Desulfurization with Hypochlorite (WV011)

Appendix 32: Phyto-Extraction / Fabrication of Gold Nanoparticles (WV012)

Appendix 33: Phytomining for Nickel and Silver Nanoparticles (WV016)

Appendix 34: Development of a Novel Optical Radiation Depolarization Technique for OnLine Measurements of Particle and Bubble Sizes (KY003)

Appendix 35: Mineral Liberation Analysis in 3D by X-Ray MicroCT for the Evaluation of Particle Separation Efficiency (UT006)

Appendix 36: Development of a 3D Lattice-Boltzmann Model for Fluid Flow Simulation under Partially-Saturated Conditions in Packed Particle Beds (UT007)

Appendix 37: On-Line Monitoring and Diagnosis of Coal Fines During Separation Process (WV008)

Appendix 38: Determination of Factors Affecting the Separation of Potentially Hazardous Trace Elements and their Behavior in Coal Tailings Impoundments (KY005)

Appendix 39: Portable Sensor for Detecting Mercury and other Heavy Metals Encountered in Coal Processing and Utilization (WV013)

Appendix 40: Gold Filters for Removing Mercury from Coal Fired Power Stations (MT003) Appendix 41: Development of Metallic Filters to Control Mercury From Coal Fired Power Plant Flue Gas (MT004)

Appendix: 42 Electrolytic Solution Purification and Metal Recovery from Toxic Waste Streams (UT003)

Appendix 43: Recovery of Chromium and Arsenic from Toxic Waste Streams by Reactive Polymer-Coated Absorbents (WV014)

Appendix 44: Mercury Reduction From Coal Power Plant Emission Using Functionalized Ordered Mesoporous Carbons (FOMCs) (WV015)

Appendix 45: : Removal of Metal Ions from Acid Mine Drainage using a Novel Low-Cost, Low Technology (WV017) 


\section{ABSTRACT}

This FinalTechnical Report describes progress made on the forty nine sub-projects awarded in the s Cooperative Agreement DE-FC26-02NT41607: Crosscutting Technology Development at the Center for Advanced Separation Technologies.

Note: SI is an abbreviation for "Le Systeme International d'Unites." 


\section{INTRODUCTION}

The U.S. is the largest producer of mining products in the world. In 2003, U.S. mining operations produced $\$ 57$ billion worth of raw materials that contributed a total of $\$ 564$ billion to the nation's wealth. Despite these contributions, the mining industry has not been well supported with research and development funds as compared to mining industries in other countries. To overcome this problem, the Center for Advanced Separation Technologies (CAST) was established to develop technologies that can be used by the U.S. mining industry to create new products, reduce production costs, and meet environmental regulations. Originally set up by Virginia Tech and West Virginia University, this endeavor has been expanded into a seven-university consortium - Virginia Tech, West Virginia University, University of Kentucky, University of Utah, Montana Tech, New Mexico Tech and University of Nevada, Reno - that is supported through U.S. DOE Cooperative Agreement No. DE-FC26-02NT41607: Crosscutting Technology Development at the Center for Advanced Separation Technologies.

Much of the research to be conducted with Cooperative Agreement funds will be longerterm, high-risk, basic research and will be carried out in five broad areas:

1) Solid-solid separation

2) Solid-liquid separation

3) Chemical/Biological Extraction

4) Modeling and Control, and

5) Environmental Control.

Distribution of funds is handled via competitive solicitation of research proposals through Site Coordinators at the seven member universities. These were first reviewed and ranked by a group of technical reviewers (selected primarily from industry). Based on these reviews, and an assessment of overall program requirements, the CAST Technical Committee made an initial selection/ranking of proposals and forwarded these to the DOE/NETL Project Officer for final review and approval. The successful projects are listed below by category, along with brief abstracts of their aims and objectives.

\section{Solid-Solid Separation}

\subsection{Development of Novel Ultrafine Sizing Methods (Joint UK/VT Project) (KY001/VA008)}

Principal Investigators: R.-H. Yoon and G.H. Luttrell, Virginia Tech

Principal Investigators: R.Q. Honaker and BK. Parekh, University of Kentucky

Period of Performance: May 1, 2003- October 31, 2008 (2-Year Project)

The conventional techniques employed for sizing ultrafine particles in the coal and mineral processing industries have inherent inefficiencies that negatively impact on separation performance and production costs. In light of this problem, a broad based $\mathrm{R} \& \mathrm{D}$ program is proposed to investigate several innovative techniques for fine particle 
sizing. The processes to be evaluated will include a wide array of mechanical, hydraulic, and novel approaches for fine particle sizing. For each process, detailed tests programs will be conducted to optimize operating parameters so that maximum efficiency and capacity can be achieved while maintaining particle size cuts in the $25-50 \mu \mathrm{m}$ size range. The resultant test data will be used to mathematically simulate different circuit arrangements for the most promising technologies. A detailed economic study will be performed for those circuits that have the greatest potential for commercialization and industrial implementation. Due to the large scope of this project, the proposed work will be conducted as a joint effort between researchers at the University of Kentucky and Virginia Tech.

\subsection{Alternative materials for Dense Medium Separation (KY004)}

Principal Investigator: Rick Honaker, University of Kentucky

Period of Performance: June 1, 2005-October 31, 2008 (2-Year Project)

Approximately 640 million tons of coal are treated annually in the U. S. to remove impurities that could cause negative environmental effects during combustion and to upgrade heating value. Dense medium processes, which are used to clean approximately $65 \%$ of the total processed coal in the U. S., employ ultrafine magnetite suspensions to separate the coal from the mineral matter. As a result of a recent closure of the only domestic magnetite source and a growing demand from the Chinese coal industry that is exhausting supplies on the international market, the U.S. coal industry is facing increased magnetite costs and a lack of confidence in future supplies. The proposed 2year project will evaluate and develop alternative materials that can be used in the dense medium process.

Alternatives to ultrafine magnetite used in dense medium separators include a vast source of coarse magnetite, steel production slag, magnetic material from combustion residue and fine reject material that comprises the feed coal. Coarse magnetite is generally perceived as providing inferior separation performances. However, recent research indicates that coarse magnetite containing greater than $30 \%+100 \mathrm{~m}$ particles provides excellent separation performances in dense medium cyclones. Similar performances were also reported from a full-scale study conducted in Australia involving the use of magnetic material from combustion residue.

The associated fine mineral matter that exists within the raw coal may also provide a material that can be successfully used in formulating dense medium as a sole source or as a supplement to magnetite. The findings of previous research concluded that the use of fine coal rejects results in inferior separation performance due to an elevated medium viscosity. However, it is proposed to investigate a nominal $150 \times 25 \mathrm{~m}$ fraction in the fine reject stream of an operating plant. Varying amounts of $-25 \mathrm{~m}$ coal reject and magnetite will be added to the coarser fraction in an effort to identify an optimum particle size distribution and blend, respectively.

The proposal requests funds for a two-year study that will investigate the aforementioned alternatives for dense medium material. The test program will be 
conducted using a 152-mm (6-in) dense medium cyclone to treat $10 \times 1 \mathrm{~mm}$ coal in a closed loop system that exists in the University of Kentucky processing laboratory. The criteria used to determine adequacy of each alternative include separation performance, efficiency and economics.

\subsection{Enhanced Flotation Performance Through Column Froth Enrichment (KY006)}

Principal Investigators: Rick Q. Honaker and Daniel Tao, University of Kentucky Period of Performance: June 1, 2005-May 31, 2008 (2-Year Project)

Froth flotation is a process that separates particles based on their differences in physical and surface chemistry properties. For fine feed material that is comprised of particles having wide degrees of floatability, selectivity is often optimized by maximizing the differences in the flotation rates between the particles targeted for recovery in the froth concentrate and those needed to report to the underflow stream. The benefits of differential flotation rates are realized in the collection zone of a flotation system where bubble-particle collision and attachment occurs. However, selectivity between particles of varying floatability can be significantly enhanced in the froth zone through a reflux mechanism that circulates detached particles back to the collection zone. Particle detachment in the froth zone occurs due to bubble coalescence, which leads to an insufficient amount of bubble surface area to carry all of the material recovered in the collection zone. Based on previous research, the detachment process is selective in that particles having a lower degree of floatability (or hydrophobicity) are preferentially released from the bubble surfaces. Selectivity through the detachment process can be improved by the addition of a more hydrophobic material into the froth zone and possibly by recycling a portion of the flotation concentrate, thereby enriching the flotation froth.

In a recent study, froth zone and overall flotation recovery values were quantified for particles in an anthracite coal that were characterized as having wide differences in floatability potential. The unique aspect of the coal was the presence of 'bone' material in the high density fractions which had a relatively high degree of floatability. As a result, the minimum product grade achieved by froth flotation was about $15 \%$ despite washability data indicating the potential for achieving a product containing nearly $3 \%$ ash. Highly floatable material was added directly into the froth zone while treating the anthracite coal and then removed from the product and tailing samples using density fractionation. The enriched froth phase reduced the product ash content of the anthracite product by 5 absolute percentage points while maintaining coal recovery at the same level. These results support the findings of previous fundamental research conducted on hematite flotation with the addition of hydrophobic silica directly into the froth phase.

The proposed two-year project will further investigate the fundamentals of the detachment process as well as develop unique methods of commercial application that may be more useful for sulfide and precious mineral industries. Plastic material that contains an amount of magnetite that makes the plastic easily recoverable by a lowintensity magnetic separator will be directly added in the flotation froth through the wash water distributor of a flotation column. By varying the formulations of the plastic, 
surface hydrophobicity and thus floatability can be controlled. Initial tests will involve the flotation of silica that will be methylated to achieve varying degrees of floatability. External refluxing of a portion of the flotation froth will also be investigated using the methylated silica. To evaluate the commercial benefits, the improved selectivity achieved by froth enrichment using external refluxing and the addition of the magnetic material will be accessed for the flotation of coal, sulfide minerals and phosphate.

\subsection{Dispersion and Flotation of Clays from New Mexico Potash Ores (NM001)}

Principal Investigators: I. Gundiler, S. Titkov, and M. Yekeler, New Mexico Tech Period of Performance: May 1, 2003-October 31, 2008 (1-Year Project)

New Mexico is the largest potash producer in the United States, supplying $70 \%$ of the domestic consumption of agricultural fertilizers. Potash mining began in the Carlsbad potash district in the early 1940s and while there are still vast reserves of potash minerals, producers are now dealing with low grade ores contaminated with clays and water-soluble magnesium minerals, which adversely affect the flotation of sylvite ( $\mathrm{KCl})$. Sylvite is floated from saturated brines with cationic collectors. The clays present in these brines absorb flotation reagents (thus increasing reagent costs), decrease recoveries of sylvite, contaminate the product and increase energy consumption for dewatering and drying. These clays are dispersed during grinding and/or attrition scrubbing and are then removed by hydrocyclones ahead of flotation. However, significant amounts of clay are carried over into flotation, where they are further dispersed by the mechanical action of impellers, thus hindering flotation. Furthermore, elevated brine temperatures during the summer affect collector adsorption on clays, depressing sylvite flotation. The presence of high concentrations of magnesium ions in the brine, which is peculiar to this district, also affects recoveries.

These technological problems must be solved for the state potash industry to remain viable and competitive. This study will investigate means of improving clay dispersion using organic and inorganic dispersants to increase the efficiency of slimes removal in existing facilities, and will study the effects of elevated magnesium ion concentrations and elevated temperatures on the flotation of sylvite. Clay flotation, which has been shown to be superior to hydroseparators for clay removal in foreign operations, will also be investigated.

\subsection{Beneficiation of Mixed Potash Ores From New Mexico (NM004)}

Principal Investigator: Ibrahim Gundiler, Lynn Brandvold, Tanja Pietraß, Stanislav

Titkov, New Mexico Tech

Period of Performance: May 1, 2004- October 31, 2008 (2-Year Project)

New Mexico is the largest potash producer in the United States, which supplies $70 \%$ of the domestic production for agricultural fertilizers. Potash mining began in the Carlsbad potash district, in the southeastern corner of the state, in early 1940s. Although, there are still vast reserves of potash minerals, clean, high-grade ores are depleted. Sylvite (KCL) and langbeinite are the major minerals mined in the district, and New Mexico is the only location in the world where langbeinite concentrates are produced. Langbeinite, a double 
salt of potassium and magnesium, is the preferred form of potassium fertilizers for plants, which can not tolerate chloride ions.

Sylvite flotation in carried out from saturated brines with cationic collectors. Clay minerals adsorb flotation reagents, thus increase reagent costs and reduce recovery of sylvite. Therefore, clays are dispersed during grinding and in attrition scrubbers, and removed by hydrocyclones. Furthermore, elevated brine temperatures during the summer also affect collector adsorption on clays, depressing sylvite flotation. Presence of high concentrations of magnesium ions, which is peculiar to this district, also affects the recovery.

Langbeinite ores are concentrated by gravity separation in coarse sizes and further cleaned by leaching halite $(\mathrm{NaCl})$ with water. Some impurity minerals, which have similar specific gravity and solubility in water, however, contaminate the product. The present concentration process is water-intensive and availability of fresh water in drought-stricken southwest is becoming increasingly sensitive issue. Furthermore, as the high grade ores are depleted, mixed sylvite-langbeinite ores and langbeinite- kieserite ores have to mined and processed. Technology for efficient separation of these minerals is not available, and, usually, one or more of the potash minerals are lost to the tailings.

For the potash industry of the state to remain viable and competitive, these technological problems have to be solved. This study will address to improve sylvite flotation from mixed ores in brines containing high concentrations of magnesium ions, and develop new flotation method for separation of sylvite, halite, and kieserite from mixed langbeinite ores.

Finally, the effect of elevated magnesium ion concentrations and elevated temperatures on the flotation of sylvite will be investigated. Flotation studies will be augmented with fundamental studies to investigate the hydration of $\mathrm{KCl}$ surfaces in $\mathrm{Mg}$-containing brines using the Nuclear Magnetic Resonance, proton relaxation techniques, and changes in morphology and surface composition will be investigated using Atomic Force Microscopy and Electron Microprobe techniques. This study is expected to produce tangible results to improve the efficiency of the existing plants and contribute to the understanding of sylvite flotation from magnesium bearing brines, and soluble salt flotation in general.

\subsection{Development of New Reagents for the Flotation of Dolomite from Phosphate Ore (NV002)}

Principal Investigators: Maurice C. Fuerstenau, Manoranjan Misra and Thomas W. Bell, University of Nevada, Reno

Period of Performance: May 1, 2004- October 31, 2008 (2-Year Project)

The United States is the largest producer of phosphate rock in the world, which corresponds to $30 \%$ of the world production. Florida accounts for $80 \%$ of the U.S. phosphate production. During the past century, the Florida phosphate industry has produced high quality phosphate with low $\mathrm{MgO}(<0.5 \%)$ content. As low dolomitic 
phosphate reserves become exhausted, the remaining deposits contain lower amounts of phosphate with significantly higher dolomite $(\mathrm{MgO})$ content. It is generally difficult to obtain a phosphate concentrate from such materials containing less than the desired $\mathrm{MgO}$ content of $1 \% \mathrm{MgO}$. The objective of the research work is to evaluate the effectiveness of new synthesized collectors for the selective flotation of dolomite from phosphate rock. The experimentation will include evaluating the applicability of the new collectors through adsorption, electrokinetic and microflotation studies, and the flotation of dolomitic phosphate ores from Florida, Utah and Idaho

\subsection{Flotation Technology for the Trona Industry (UT001)}

Principal Investigator: Jan D. Miller, University of Utah

Period of Performance: May 1, 2003 - October 31, 2008 (2-Year Project)

Soda ash $(\mathrm{Na} 2 \mathrm{CO} 3)$ produced from the trona deposits of the Green River Basin in Wyoming by chemical treatment is valued at approximately $\$ 800$ million per annum. Existing process technologies for the production of soda ash from trona involve dissolution in hot brine, drying, sedimentation and filtration for the removal of impurities, and subsequent crystallization and calcination for the recovery of soda ash. In this regard, mining and operating costs, particularly energy costs, are higher than desired. A preferred processing strategy might be to remove gangue mineral contaminants from the plant feed prior to dissolution in hot brine. It is expected that in this way improved productivity can be achieved in addition to significant savings in energy. Such a preprocessing strategy at ambient temperature and pressure has been limited by the lack of satisfactory process technology. Now based on recent results from laboratory research at the University of Utah it seems that the run-of-mine trona ore can be treated at ambient temperature and pressure using a special flotation procedure to separate the gangue minerals and make a trona concentrate with a purity of almost $99 \%$ trona at a recovery of more than 97\%. Development and utilization of this new technology will allow for energy conservation, improved resource utilization, increased productivity and the development of a new product for the marketplace. In this regard a two-year research program involving industrial participation is proposed to develop the technology and demonstrate its effectiveness at a plant site.

\subsection{High Frequency Eddy Current Separation of Metallicic Residue from Slags, Sands, Electronic Scrap and Other Wastes (UT004)}

Principal Investigators: Raj K. Rajamani, University of Utah

Period of Performance: May 1, 2004- October 31, 2008 (2-Year Project)

The proposed work is to separate metallic grains from sands, slags or electronic scrap by means of a novel device called High Frequency Eddy Current Separator. The dry mixture of foundry sands is passed through the gap between the poles of a magnet. The metallic granules are repelled from the falling stream of sand, and hence are separated. This device has many technical and commercial advantages over the existing technologies (i.e. lower cost, no use of water or chemicals, absence of polluting gases, etc.). 
A high frequency magnetic field is generated in the gap when radio-frequency current is passed through windings on a ferrite core. As the electrically conducting particle enters the field, an electromotive force (emf) is generated on the particle itself. Due to the emf, an eddy current flows on the particle. These currents, in turn, induce their own magnetic field around the particle. Lenz rule of physics states that the induced magnetic field is directly opposed to the original or imposed oscillating field. Hence, the particle experiences a repelling force. The repelling force depends on the strength of the magnetic field, the frequency of oscillations, and the cross-sectional area and electrical conductivity of the particle.

The object of this program is to develop third prototype for testing. The prototype engineering is based on the first lab unit developed at the University of Utah and the second lab unit built by EMPS Corporation, Salt Lake City, Utah. These two units used a ferrite toroid and a bi-polar amplifier to generate the high frequency magnetic field in the gap of the toroid. Invariably the choice of ferrite core limited the gap dimensions to about 10X10 mm. The unit proposed here is a larger toroid with $125 \mathrm{X} 125 \mathrm{~mm}$ gap to be driven by a direct drive electronic circuitry. This unit would be capable of processing $100 \mathrm{lb} . / \mathrm{hr}$ of solid stream and hence suitable for process optimization and operating parameter study.

Present eddy-current devices, have been confined to the recovery of large aluminum cans from municipal wastes. For example Eriez Magnetics has a line of eddy current separators, which uses permanent magnets of alternating polarity spinning at high speeds under a belt conveyor. Hence this unit can only produce about $1 \mathrm{kHz}$ frequency. Low frequency devices can separate only larger pieces, of size $1 / 2$ inch, of metal. The proposed high-frequency separator uses magnetic-field frequencies of 50 to $100 \mathrm{kHz}$ that enhances its ability to separate much smaller grains of size larger than $0.2 \mathrm{~mm}$.

In copper production, both a copper-sulfur matte and a blister copper are formed, which are covered by a slag layer. In the production of aluminum and magnesium, electrolytic cells are used to electrowin the liquid metal at the cathode. A slag layer is also present in this process. Typically, there is mixing at the liquid metal/slag interface, which results in droplets or prills of metal suspended in the slag phase. Approximately 3.6 million metric tons each of copper and phosphorous slag are produced each year in the U.S. The annual production of nickel, lead and zinc slag is estimated at 0.45 to 0.9 million metric tons.

Electric and electronic goods comprise a vast and diverse spectrum of items that are sent to the landfills each year. A 1991 study estimates that 150 million personal computers and workstations will have been sent to landfills by the year 2005 . The recovery of metallic copper, gold, palladium and aluminum from scrap is in the best interest of environmental protection. These waste streams are typical application targets for the high frequency separator proposed here.

\subsection{Studies of Froth Stability and Model Development (VA002)}

(Continuation from CAST I)

Principal Investigator: Roe-Hoan Yoon, Virginia Tech

Period of Performance: May 1, 2004- October 31, 2008 (2-Year Project) 
Froth plays an important role in flotation. It determines the final grade of the product and the maximum carrying capacity (or throughput) of a flotation machine. Also, many operators use stronger frothers to produce smaller air bubbles and, hence, higher recovery and throughput. Despite its importance, little is known of the fundamentals of foam and froth stability. It is, therefore, proposed to study the various factors affecting the stability of flotation froth. This will be accomplished by using the thin film balance (TFB) technique of Scheludko and Exerowa (1959) and by monitoring the stability of froth in a bubble column using a video camera. The results will be used for developing a froth model and also for developing effective defoamers.

\subsection{Direct Measurement of Forces in Flotation (VA004)}

(Continuation from CAST I)

Principal Investigator: Roe-Hoan Yoon, Virginia Tech

Period of Performance: May 1, 2004- October 31, 2008 (2-Year Project)

The objective of this project is to directly measure the interaction force between a particle and bubbles as a function of separation distance. This force controls the attachment and detachment of particles to bubbles, which is an essential step in determining the efficiency of a flotation process. A specially designed device will be fabricated for these measurements. The device will use the force-detection method employed by an Atomic Force Microscope and the separation-detection method employed by a Surface Forces Apparatus. The key advances are to explicitly measure both the separation between the particle and the bubble and the shape of the bubble at all times. Without this separation and shape data, it would be difficult to relate the measured forces to flotation results. After fabrication of the device, measurements of the interaction forces acting on hydrophilic, hydrophobic, and charged particles in aqueous solutions of surfactant molecules will be obtained.

\subsection{Flotation Processes/Experiments and Analysis (VA009)}

Principal Investigators: D. Telionis and P. Vlachos, Virginia Tech

Period of Performance: May 1, 2003- October 31, 2008 (2-Year Project)

Flotation processes involve complex, three-phase flow interactions between a liquid, air bubbles and solid particles. For decades, engineers and researchers based their calculations on algebraic formulas that model these interactions. These formulas were derived from simple models, experimental data and/or arbitrary assumptions. Considerable progress has been made but this approach is still far from providing a reliable tool for the design of flotation machines.

We will take a more rigorous approach to the analysis and modeling of the flotation process. The proposed effort will combine detailed theoretical analysis and modeling with state-of-the-art, global, multi-phase flow measurements to quantify the effects of the various hydrodynamic parameters on the flotation process. We will employ a Digital Particle Image Velocimeter (DPIV) that can record velocity vectors of all three phases. We will measure three-phase flow interactions of bubbles and model particles of different hydrophobicity with a turbulent flow field. We will deliver global, time- 
resolved velocity distributions and turbulence characteristics for each phase. Our modeling approach will incorporate all physical parameters that affect the collision efficiency of coal particles and flotation bubbles and the probabilities of attachment and detachment. An advanced model for predicting and quantifying the efficiency of the flotation process will be the final deliverable of this two-year effort. Such a tool will improve the design of flotation equipment and/or enhance the performance of existing systems.

\subsection{Engineering Development of a Fine Particle Heavy Medium Separator (VA012)}

Principal Investigators: Gerald H. Luttrell, Virginia Tech and Robert Moorhead, Krebs Engineers, Inc

Period of Performance: June 1, 2005-October 31, 2008 (2-Year Project)

The objective of this project is to develop an innovative heavy medium separator that can reduce the cost and improve the efficiency of fine coal cleaning. The technology is designed to replace inefficient water-based separators such as spirals and water-only cyclones that are currently used by industry to upgrade 1 x $0.15 \mathrm{~mm}$ run-of-mine coal. The new heavy medium separator incorporates novel design features that allow it make sharp separations without the need for costly micronized magnetite. As a result, the new technology can share circulating medium from other circuits, thereby avoiding the expense of installing and maintaining an additional medium circuit. These advantages, together with the low headroom design of the module, make it possible to integrate this technology into an existing plant with minimal retrofit costs. The project tasks will include (i) design and construction, (ii) pilot-scale testing, (iii) field testing, and (iv) flowsheet development

\subsection{Improved Destruction and Control of Residual Flotation Froths (VA014)}

Principal Investigators: Gerald H. Luttrell and Roe-Hoan Yoon, Virginia Tech Period of Performance: June 1, 2005-October 31, 2008 (2-Year Project)

Flotation froths containing large amounts of ultrafine particles can become excessively stable and create serious handling problems for coal preparation plants. Steps taken by operators to combat these problems, such as lowering the frother dosage, have resulted in large reductions in fine coal recovery and plant profitability. The objective of this project is to develop and evaluate several improved methods for the control and destruction of residual flotation froths. The first phase of the proposed work will focus on a detailed laboratory study of the physical and chemical parameters that impact froth stability. These studies will be followed by in-plant sampling campaigns at several coal plants to establish how frothing agents partition within different circuits and to determine whether modifications to the layout or dilution practices can minimize handling problems. The data obtained from these investigations will be used to develop improved mechanical and chemical methods for froth control/destruction at an industrial plant site 


\subsection{Development of a Turbulent Flotation Model and a Computer Simulator (VA015)}

Principal Investigators: Roe-Hoan Yoon and Gerald H. Luttrell, Virginia Tech

Period of Performance: June 1, 2005-October 31, 2008 (2-Year Project)

Flotation is the most widely used method of separating fine particles in the mining industry. The method of using air bubbles was awarded a U.S. patent in 1905, exactly 100 years ago. Yet, there are no flotation models incorporating both surface chemistry (e.g., hydrophobicity, -potential, and surface tension) and hydrodynamic (e.g., bubble size, particle size, energy dissipation) parameters under turbulent flow conditions. It is, therefore, proposed to develop a comprehensive flotation model incorporating practically all of the process variables employed in flotation practice. The turbulent flotation model will be able to describe the events taking place in both the pulp and froth phases. Laboratory experiments will be carried out to verify the model, and model will be transformed into a compute simulator that can predict complicated flotation circuits incorporating rougher, scavenger and cleaner operations.

\subsection{Measurement of Surface Forces Between Hydrophobic Surfaces (VA016)}

Principal Investigators: Roe-Hoan Yoon, Virginia Tech

Period of Performance: June 1, 2005-October 31, 2008 (2-Year Project)

Froth flotation is the most widely used method of separating fine particles in the mining industry. The process is based on rendering a selected mineral hydrophobic, so that it can be separated from other minerals that are hydrophilic. Thus, control of particle hydrophobicity is critically important in flotation. However, the nature of the hydrophobicy is not fully understood, particularly in view of the forces acting between two hydrophobic particles suspended in water. In the proposed work, surface force measurements will be conducted using an atomic force microscope (AFM). The force measurements will be conducted with gold, sulfide minerals, and rutile after coating them with thiols, xanthates, and anionic and cationic surfactants. In addition, the surfaces will be characterized in terms of their acid-base properties, infrared spectra, and AFM images. The results will be useful for better understanding the nature of hydrophobicity and, hence, furthering the flotation technology.

\subsection{Novel Surfactants as Collectors for Froth Flotation (VA015)}

Principal Investigators: Richard D. Gandour, Virginia Tech

Period of Performance: June 1, 2005-October 31, 2008 (2-Year Project)

Our goals are to synthesize and study novel, inexpensive amphiphiles that can perform as co-surfactant collectors in mineral flotation. These surfactants will selectively bind to particles and create a hydrophobic coating on a particle. These novel surfactants will bind more selectively and provide hydrophobic sites where cheaper, less-selective collectors can bind. These novel surfactants have very hydrophobic, long chains, yet are sufficiently water soluble for mineral processing and coal-fines processing. These amphiphiles, which we call 'hydra surfactants', will have two tails and three heads. 
These novel molecules will be useful for many different separations in mineral processing.

\subsection{Column Flotation of Relative Coarse and Fine Dolomitic Phosphate Pebbles (WV009)}

Principal Investigator: Felicia F. Peng, West Virginia University

Period of Performance: May 1, 2003- October 31, 2008 (2-Year Project)

Dolomite in phosphate flotation concentrates is troublesome for down-stream operations. High dolomite contents cause higher consumption of sulfuric acid, reduce filtration rates and lower P205 content in the fertilizer manufacturing process. However, the separation of dolomite particles from phosphate minerals is difficult because the dolomite is finely disseminated throughout the phosphatic pebbles and both are oxide type minerals with the same cationic component. Thus, they show similar electrokinetic, adsorptive and desorptive behavior in physical separation process such as flotation. Various flotation processes have been developed in the past four decades, but none is satisfactory due to high $\mathrm{MgO}$ content and/or low overall P205 recovery in the final phosphate concentrate. In this research project, dolomitic phosphate pebbles from Florida will be treated by column flotation. A liberation analysis of the dolomite-phosphate matrix will be conducted to determine optimum grinding conditions; new selective mixtures of fatty acid collectors and non-ionic surfactants will be evaluated on relatively coarse (minus 300 microns) and fine (minus 150 microns) dolomite particles; appropriate mixtures of phosphoric acid/sulfuric acid will be used to depress phosphate particles; and the effect of addition of non-ionic surfactants on the separation performance will be determined. The goal is to produce a phosphate concentrate containing 30\% P205 and less than $1.0 \%$ $\mathrm{MgO}$ contents at high $\mathrm{P} 205$ recoveries from low grade phosphatic pebbles stockpiled at plant sites and from pebbles generated from mining lower grade reserves.

\subsection{Dry Particle Separation in a CFB Riser System (WV009)}

Continuation of Project WV002

Principal Investigator: Eric Johnson and Bruce W. Kang, West Virginia University

Period of Performance: May 1, 2004- October 31, 2008 (2-Year Project)

The proposed project is a continuation of the study to determine the potential for separating small dense particles from small light particles in a CFB riser system. This exploratory work led to many interesting and practical results. It is proposed to now refine and expand our test conditions and develop a more comprehensive analysis of the results. The experimental conditions planned for this continuation project are 1) more closely matched particle size distributions for the heavy and light particles in the mixture, 2) smaller density difference between the heavy and light particles, 3) separation of particles based only on size differences, and 4) developing and employing a larger diameter riser to study the scaling of the separation processes in a riser. 


\subsection{Development of an Optimized Control System for Heavy Medium Control Circuits (VA021)}

Principal Investigators: Robert Bratton, Gerald H. Luttrell and Roe-Hoan Yoon, Virginia Tech

Period of Performance: August 17, 2007-October 31, 2008 (2-Year Project)

Heavy medium cyclones (HMCs) are widely recognized as the workhorse of the coal preparation industry. This high tonnage separator is used in more than $80 \%$ of U.S. plants and represents an installed capacity approaching 75,000 t/hr. Despite this level of popularity, recent in-plant studies indicate that many industrial HMC circuits suffer from poor control due to difficulties associated with the accurate monitoring of the specific gravity cutpoint. This shortcoming makes it impossible to globally optimize plant performance in cases where the consistency of the feed coals to the heavy medium circuits vary considerably over time due to changes in mining conditions of feed blends. To address this problem, a multi-stream on-line control system is proposed to monitor the circulating medium within the HMC circuits in real time so that the cutpoints can be accurately predicted and optimized. The specific tasks to be completed in this project will include (i) in-plant sampling, (ii) algorithm development, (iii) equipment installation, (iv) control system testing, and (v) technical and economic evaluation. All of the proposed experimental work will be performed on-site at an industrial coal preparation plant in order to promote the rapid commercialization of this technology.

\section{Solid-Liquid Separation}

\subsection{Improving Densification of Fine Coal Refuse Slurries to Eliminate Slurry Ponds (KY002)}

Principal Investigators: B.K. Parekh and R.Q. Honaker, University of Kentucky Period of Performance: May 1, 2003-May 31, 2008 (2-Year Project)

Increased mechanization in underground coal mining has increased the volume of refuse generated by coal preparation plants. The fine refuse slurry, composed of coal and mineral matter, is usually disposed of in a holding pond (impoundment), but incidents of impoundment breakthrough have forced the industry to look for alternative methods for fine refuse storage in the future. The main objective of the proposed program is to evaluate a new technique known as "Paste Thickening Technology," which utilizes a DORR-OLIVER EIMCO DEEP CONE Thickener to discharge the waste slurry as a paste. The paste material should be stackable at low repose angles and would dry over a period of time, thus avoiding the storage of slurries in ponds. The proposed study will be conducted on coal waste slurries obtained from two different preparation plants. Laboratory studies will involve the characterization of the slurries, bench-scale flocculation studies and rheological analysis of the flocculated materials to identify yield stresses at various solid concentrations. This information will be used to establish optimum conditions for the production of a thickened paste product. A pilot-scale study will then be conducted at one of the coal preparation sites to obtain technical and economic data for a commercial installation and operation. It is anticipated that the 
outcome of the study will be an effective and economical process for the safe disposal of fine coal refuse.

\subsection{Development of a Fine Particle Centrifuge (VA006)}

(Continuation from CAST I)

Principal Investigators: G.H. Luttrell \& R.-H. Yoon, Virginia Tech

Period of Performance: May 1, 2004- October 31, 2008 (2-Year Project)

The solid-solid separation processes employed by modern coal preparation plants require large amounts of process water. After cleaning, the unwanted water must be removed from the surfaces of the particles using mechanical dewatering equipment. Coarse particles can be readily dewatered using simple screening systems, while finer particles require more complicated unit operations such as centrifuges and filters. Unfortunately, the processes used to dewater fine particles are inherently inefficient and expensive to operate and maintain. To overcome these problems, a novel centrifugal filter has been developed by researchers at Virginia Tech. Preliminary test data suggest that this new technology can reduce the moisture content of fine coal products by approximately 30$50 \%$ compared to existing dewatering processes. The objective of this project will be to construct a continuous prototype unit and to conduct a detailed experimental investigation of this new technology.

\subsection{Development and Testing of a Horizontal Pressure Belt Filter (VA010)}

Principal Investigators: R.-H. Yoon \& G.H. Luttrell, Virginia Tech

Period of Performance: May 1, 2003 - October 31, 2008 (2-Year Project)

A variety of mechanical processes are available for dewatering fine particles in the coal and mineral processing industries. Unfortunately, many of these processes suffer from major shortcomings such as poor dewatering performance, low throughput capacity, and high capital and operating costs. This project seeks to overcome these problems by developing a new type of dewatering process that combines the operational flexibility of a continuous belt filter with the dewatering efficiency of a batch pressure filter. The proposed project involves the design, construction, testing, and evaluation of a prototype unit and pilot-scale test circuit having a production capacity of approximately $100 \mathrm{lb} / \mathrm{hr}$. Test data obtained from the project will be used to promote the engineering development of a full-scale commercial unit.

\subsection{Improvements in Screen Bowl Centrifuge Performance (VA013)}

Principal Investigators: Robert C. Bratton \& G.H. Luttrell, Virginia Tech Period of Performance: May 1, 2004- October 31, 2008 (2-Year Project)

Screen-bowl centrifuges are the most commonly used method for dewatering fine coal in the United States. Unfortunately, this process is the least efficient and most costly dewatering operation in the preparation plant. The work outlined in this proposal will seek to develop an improved understanding of the operating characteristics and technical capabilities of screen-bowl centrifuges. Plant operators will be able to use this fundamental knowledge to improve the performance of their dewatering circuits and 
improve plant profitability. In addition, several new screen-bowl centrifuge features will be developed and evaluated as part of the proposed work. The innovations will include the development and testing of internal injection ports for (i) adding flocculant to the low-solids pool and (ii) adding dewatering aids and surface tension modifiers to the dewater screen solids. These technological enhancements are expected to improve moisture reduction and increase fine coal recovery

\section{Chemical/Biological Extraction}

\subsection{The Development and Utilization of Alkaline Sulfide Leaching and Recovery of Gold (MT001)}

Principal Investigator: C. Anderson and L.G. Twidwell, Montana Tech

Period of Performance: May 1, 2003- October 31, 2008 (2-Year Project)

Due to the increasing concerns over the emission of sulfur dioxide from roasting and smelting, there has been an increased interest in pressure oxidation as a means of treating gold bearing ores and concentrates. One of the problems with the partial oxidation of the sulfide host matrix to form elemental sulfur is that sulfur containing streams are difficult to treat using cyanidation. In the present study, the alkaline sulfide system will be studied as an alternative to cyanide for recovering gold from elemental sulfur. A complete thermodynamic model of the alkaline sulfide gold leaching system will be developed using Stab-Cal software and the leaching system and its kinetics will be optimized.

\subsection{Simultaneous Electrolysis of Copper and Ferrous Ions to Produce Copper Cathode and to Regenerate Ferric Sulfate - The Lixiviant to Dissolve Copper Sulfide Minerals (MT002)}

Principal Investigator: C. Young, H.-H. Huang and C. Fabian, Montana Tech

Period of Performance: May 1, 2003- October 31, 2008 (2-Year Project)

Copper leaching is primarily conducted on oxidized ore bodies at low $\mathrm{pH}$ by using sulfuric acid (H2SO4). The sulfate acts as the lixivant to solubilize the copper as copper sulfate (CuSO4). An oxidant is also needed to enhance reaction rates - typically this is dissolved oxygen $(\mathrm{O} 2)$ and/or ferric $(\mathrm{Fe} 3+)$ ion. The dissolved copper must then be extracted from the water to separate it from other dissolved constituents including iron. Traditional solvent extraction and electrowinning (SX/EW) procedures do this and ultimately yield quality copper cathode as product. After SX/EW, leach solution is recycled to the leaching process and replenished with oxidant as needed. For example, biological activity can regenerate ferric ions or mechanical agitation can produce dissolved oxygen (which in turn can regenerate ferric ions).

Ferric ions can also be regenerated electrolytically and simultaneously to copper cathode production. This is the basis of the proposed research. To accomplish this, a porous membrane will be used to keep the anode and cathode compartments separated and modern rotating cylinder electrodes will be used to stir solutions, obtain high current densities and ultimately produce superior grade copper cathode. Although it is possible 
to avoid the SX step, it will be used to produce two streams: raffinate, which will go to the anode compartment where ferric is regenerated, and pregnant or advance solution, which will be go to the cathode compartment where copper metal is produced. Various chemicals and additives may have to be added to control copper cathode formation and thereby avoid, for example, dendritic growth. Preliminary cost analyses show that copper can be produced for approximately $\$ 0.30 / 1 \mathrm{~b}$ with this new, more energy efficient, technique.

\subsection{Recovery of Gold from Thiosulfate Leach Liquor Using Activated Carbon (MT005)}

Principal Investigator: Courtney A. Young and Larry G. Twidwell, Montana Tech Period of Performance: June 1, 2005-October 31, 2008 (2-Year Project)

Gold is generally recovered from the ore by a cyanide leaching process. This procedure is used because of its simplicity and ability to work effectively at low concentrations. However, cyanide itself is highly toxic. Furthermore, it also leaches relatively nonspecifically causing other metals to go into solution as well. These metal cyanide complexes can also be toxic. Resulting leach solutions have been accidentally released from various process facilities causing environmental damage. Consequently, particularly over the last decade, alternatives to cyanide leaching have been sought.

Particular attention has been given to thiosulfate; however, recovery of the gold from the thiosulfate leach presents a problem. Conventional carbon adsorption does not work so more expensive recovery processes have been investigated with resin adsorption being the most common. The research we propose to undertake investigates a novel use of activated carbon by pre-adsorbing cuprous cyanide at its surface and using it to exchange with gold in a metal-exchange, cementation-type reaction:

$$
\mathrm{Au}(\mathrm{S} 2 \mathrm{O} 3) 23-+\mathrm{Cu}(\mathrm{CN}) 2-\text { ads } \rightarrow \mathrm{Au}(\mathrm{CN}) 2-\mathrm{ads}+\mathrm{Cu}(\mathrm{S} 2 \mathrm{O} 3) 23-
$$

Resulting cuprous thiosulfate is recycled for further gold leaching with the copper serving as catalyst to the leaching process. Likewise, the adsorbed gold cyanide product can be eluted using a subsequent conventional gold recovery process common in traditional cyanide operations:

$$
\mathrm{Au}(\mathrm{CN}) 2-\text { ads }+\mathrm{CN}-\rightarrow \text { Csite }+\mathrm{Au}(\mathrm{CN}) 2-+\mathrm{CN}-
$$

Consequently, the novel process is believed to be cost-effective and will ultimately allow thiosulfate leaching to be a viable alternative to cyanide. Because direct investigation of these adsorption reactions are masked by the location of the carbon adsorption sites in commercial activated carbons, we propose to study them in-situ using Raman spectroscopy.

We also propose to investigate using coal-based metathesis technologies to generate carbon alternatives to activated carbon. In this regard, carbon nanotubes, because of their graphite- like structure, is expected to have similar adsorption capacities and could therefore prove to be an extremely effective high surface-area alternative with high 
adsorptive capacity. Although nanotubes are currently expensive, their production from coal and other cheap carbon sources could be economically equivalent to coconut shellbased activated carbons.

\subsection{Ion Exchange Recovery of Cobalt from Copper Leach Solutions (NM002)}

Principal Investigator: I. Gundiler and M. Hatch, New Mexico Tech

Period of Performance: May 1, 2003- October 31, 2008 (2-Year Project)

Cobalt is a strategic and critical metal which is used in production of super alloys for use in jet engine turbines, wear and corrosion resistant alloys, cutting tools, magnets and various other alloys and chemicals. It is not mined or refined in the United States; therefore, $80 \%$ of the cobalt consumed in the U.S. is imported. The remaining balance is met with recycled alloys and supplies from stockpiles. Although there is historical production and known reserves, these are not economical to exploit at the present. However, there is a readily available source of cobalt in the leach solutions generated in large copper mining operations in the Southwestern U.S. If only a fraction of this cobalt could be recovered, New Mexico and Eastern Arizona copper leach operations could potentially supply $20 \%$ of U.S. imports. Currently, the technology to recover the metal from these solutions economically is not available.

Ion exchange processes could recover this cobalt. However, commercially available resins are either too expensive, or do not function in acidic solutions. Absorption kinetics of these resins are also fairly slow. Three new resins will be synthesized at New Mexico Tech for copper and cobalt recovery from acidic solutions. The resins will be characterized and tested in the laboratory using pure chemicals, as well as process solutions to be obtained from the Phelps Dodge Mining Company, New Mexico operations. The performance of the synthesized resins will also be compared with that of commercially available resins.

\subsection{The Effect of Diphenyl Oxide Surfactants on Nucleation and Growth of Potassium Sulfate Crystals: Development of Enhanced Surfactants for the Potash Industry (NM003)}

Principal Investigator: G. Bond and C. Hockensmith, New Mexico Tech

Period of Performance: May 1, 2003- October 31, 2008 (2-Year Project)

Surfactant-controlled crystallization leads to an increase in the efficiency of potassium sulfate production, particularly when alkyl diphenyl oxide-type (ADO) surfactants are employed in synthesis. Improved control of crystallization in both the initiation and termination phases can increase the ratio of granular particles to fines, decreasing costs associated with production and facilitating the utilization of lower-grade ores. Mother liquors in potash extraction contain high percentages of magnesium as well as other ions including chloride and impurities such as clay and silica. Potassium sulfate crystals will be produced in the presence of different ADO surfactants, and evaluated by SEM, TEM, XRD, FTIR and AFM. Crystallization processes, particularly those that affect crystal morphology, size, initiation and termination, will be studied in relation to the surfactant 
effect. This information will be used to improve control of potassium sulfate precipitation and develop the next generation of surfactants to facilitate extraction of granular potassium sulfate.

\subsection{The Effect of Alkyl Diphenyl Oxide and Sulfonated Oleic Acid Surfactants on Nucleation and Growth of Potassium Sulfate Crystals: Optimization of Surfactants for the Potash Industry (NM005)}

Principal Investigator: Gillian Bond and Christa Hockensmith, New Mexico Tech

Period of Performance: June 1, 2005-October 31, 2008 (2-Year Project)

We have shown that significant improvements occur in the crystal size distribution (CSD) of potassium sulfate crystallized from potassium sulfate/magnesium sulfate brine at $40 \mathrm{C}$ in the presence of concentrations of Calfax 16L-35 a linear, alkyl diphenyl oxide (ADO) disulfonate surfactant\} well below the critical micelle concentration $(\mathrm{CMC})$. The ratio of granular particles to fines is very greatly improved, with essentially all the crystals having sizes between 2 and $3 \mathrm{~mm}$. This size range is very desirable for potassium sulfate crystals that will be supplied for agricultural use as a spreadable fertilizer. However, the yield, at present, is too low for commercial application.

We now propose:

To optimize the yield obtainable with ADO disulfonate surfactants, while maintaining the desirable CSD.

To study the performance of sulfonated oleic acid surfactants with a view to improving CSD and yield.

The Carlsbad potash basin accounts for $85 \%$ of mined potash production in the United States and it is for the potash industry that this work's goals are set. A successful outcome to this work will benefit a critical industry in New Mexico, and help to ensure the viability of the new potassium sulfate production facility that is under construction near Carlsbad.

\subsection{Hydrometallurgical Processing of Chalcopyrite Concentrates (NV001)}

Principal Investigator: M. Misra and M. Fuerstenau, University of Nevada, Reno Period of Performance: May 1, 2003- October 31, 2008 (2-Year Project)

Hydrometallurgical processing of chalcopyrite at moderate temperature and pressure has been a goal of the copper industry for decades. Research has shown that a protective sulfur layer forms on chalcopyrite when leached with ferric salts in acidic medium. This layer limits the transport of electrons from the mineral surface to the oxidant and precludes the use of this technology for chalcopyrite processing. Research at the University of Nevada, Reno has shown that the introduction of finely-divided silica improves the kinetics of leaching dramatically. Silica adsorbs on the surface of chalcopyrite, and since it is an n-type conductor, conduction of electrons from the mineral surface to the oxidant occurs by photocatalysis of the sulfur product layer. Dissolution amounts of as much as $80 \%$ have been achieved under the limited conditions examined, namely at atmospheric pressure and $50^{\circ} \mathrm{C}$. 


\subsection{Thiosulfate as a Replacement for Cyanide in the Presence of Activators (NV003)}

Principal Investigator: Maurice C. Fuerstenau, University of Nevada, Reno

Period of Performance: June 1, 2005-October 31, 2008 (2-Year Project)

The United States is the third largest producer of gold in the world, and Nevada is by far the largest gold-producing state in the country. In processing gold and other precious metals, cyanide is used universally as a lixiviant. It is effective and reasonably low cost and has been used for greater than a century commercially. It is an extremely toxic reagent, however, and considerable care must be exercised to prevent personal injury and harm to the environment during and after metal processing. To obviate the downsides of the use of cyanide, considerable research has been conducted with other lixiviants that are not as toxic as cyanide to establish if a replacement for cyanide can be found. These include the halides (chlorine, iodine and bromine), thiourea, thiosulfate and polysulfides. Of these reagents thiosulfate is the most promising. The problem with this reagent, however, is the stability of thiosulfate in solution. Oxidative conditions are essential for the dissolution of gold and under these conditions, some thiosulfate is oxidized to tetrathionate, and its effectiveness is greatly reduced. The development of reaction conditions is needed in which the kinetics of gold dissolution is increased, while the losses of thiosulfate by oxidation are minimized. In this investigation the use of activators in gold dissolution will be studied, and the interaction between the various parameters involved in thiosulfate leaching of gold will be carefully investigated.

\subsection{Bio-Assisted Heap Leaching of Nickel Laterites for the Development of a Domestic Nickel Industry (UT002)}

Principal Investigator: S. Duyvesteyn, University of Utah

Period of Performance: May 1, 2003- October 31, 2008 (2-Year Project)

The U.S. currently imports all of its nickel, which is a strategic material used in stainless steels and other corrosion-resistant materials. Existing U.S. resources are worth around $\$ 10$ billion at current metal prices, but occur mostly in low-grade laterite deposits, for which conventional hydrometallurgical processes are not economically feasible production routes. These processes typically utilize mineral acids that cannot be recycled economically and can cause significant environmental problems. The solubilization of metal ions from ores by organic acids produced by microorganisms has been demonstrated to be a viable alternative for metal production with reduced environmental and remediation issues, as organic acids are readily biodegradable. The fact that many of these organic acids and other metabolites form strong chelating complexes with the base metal ions also results in a reduction in the acid requirements to achieve complete solubilization.

This research proposal involves a biotechnological approach, called bio-assisted heap leaching (BAHL), for the production of nickel metal from low-grade ore synergistically with the co-production of citric acid. The BAHL process can be described as follows: laterite nickel ore is combined with an organic nutrient and put on heaps where fungi, such as Aspergillus niger, produce organic acids during their natural metabolic cycle. Recycle solution is sprayed over the heaps and its percolation through the heaps results 
in the dissolution of nickel. The resulting nickel-containing leach solution is collected and the nickel and citrate values are recovered.

\subsection{Overcoming Technological Barriers to More Efficient Recovery of Copper from Chalcopyrite (UT005)}

Principal Investigator: Michael L. Free, University of Utah

Period of Performance: May 1, 2004- October 31, 2008 (2-Year Project)

Extraction and recovery of copper from chalcopyrite is one of the most important processes in the nonferrous metals industry. It is currently performed using smelting, converting, and electrorefining, yet it also necessitates by-product sulfuric acid production at a rate of three tons of acid per ton of copper. Efforts to convert this process to leaching, solvent extraction, and electrowinning, which is a lower cost alternative to the smelting, converting, and electrorefining process that is practiced for most other copper minerals, have been unsuccessful. However, new technologies, such as halide leaching and electrowinning offer significant economic and environmental advantages over the current industrial practices.

Halide leaching and electrowinning requires only $25 \%$ of the energy needed to electrowin copper using traditional sulfate-based solution technology. The halide-based technology, which is also compatible with precious-metal recovery from sulfide minerals, also eliminates acid misting and solvent extraction, and it results primarily in the production of by-product elemental sulfur rather than sulfuric acid.

The halide leaching and electrowinning process has not been adopted by industry due to large capital investment in current smelters, the risk of new technology that has not been proven on a large scale, and the incompatibility with existing electrowinning operations due to the morphology of the deposit, which is granular, rather than smooth using current technology. This project is designed to modify the morphology of the resulting copper deposit to make it compatible with existing electrowinning processes.

\subsection{Development of Chemical and Biochemical Techniques for the Extraction of Mercury from Fine Coal Particle Solutions. (WV010)}

Principal Investigator: Scott M. Hurst, Anthony G. Abatjoglou and Jay M. Weidermann, West Virginia University

Period of Performance: May 1, 2004- October 31, 2008 (2-Year Project)

The WVU Tech Project, "Development of Chemical and Biochemical Techniques for the Extraction of Mercury from Fine Coal Particle Solutions", includes three objectives: development of a biochemical technique to facilitate the conversion of organically bound forms of mercury to water soluble mercury, development of a chemical technique using physically adsorbed ligands that selectively removes mercury from an aqueous solution that contains relatively large concentrations of other metals, and the development of a biochemical process for extracting liquid mercury from the ligands. The results of this work will be the bases for the development of a novel process for extracting mercury from coal solutions in a "zero waste" manner. 


\subsection{Coal Desulfurization with Hypochlorite (WV011)}

Principal Investigator: Ray K. Yang \& Eung Ha Cho, West Virginia University

Period of Performance: May 1, 2004- October 31, 2008 (2-Year Project)

The utilization of coal as a feedstock to produce carbon materials is very much hampered by the presence of its sulfur content particularly organic sulfur content. However, the organic sulfur is extremely difficult to remove because much of its sulfur atoms are "inserted" into the matrix of the coal structure. Thus, unless the coal structure is "destroyed", the sulfur atoms can not be removed. Chlorine is one of a few chemicals known today that have capability to reduce organic sulfur in a practical sense. However, this chemical chlorinates the coal structure especially at its low $\mathrm{pHs}$, which becomes a roadblock for commercialization of a process utilizing this chemical. We observed through preliminary experiments that hypochlorite has a capability to reduce pyritic sulfur and organic sulfur at high temperatures in a solution. Hypochlorite is the predominant chlorine derivative at high $\mathrm{pHs}$.

This proposal is for continuation of a current project entitled "Coal Desulfurization Using Hypochlorite and Cupric ion As a Catalyst - Feasibility Study," funded by CAST for 2003 - 2004. During the past 6 months, more than 30 experiments were conducted to explore the feasibility of desulfurization of Pittsburgh No. 8 coal with hypochlorite and cupric ammine. It was found that cupric ammine and hypochlorite could not be prepared in the same solution because ammonia used to solubilize cupric reacts with hypochlorite to hydrazine $(\mathrm{N} 2 \mathrm{H} \mathrm{4})$. It was also found that cupric ammine used in a pretreatment of coal did not catalyze the desulfurization of coal with hypochlorite in the next step. The catalysis is the major theme of the current project.

Thus, a new leaching scheme was used, and a couple of experiments were conducted. The coal was leached with hypochlorite and then the leach coal was hydrolyzed in a sodium hydroxide solution at high temperatures without having a pretreatment step. It was found that this desulfurization method was better than the proposed method which had an additional pretreatment step. The results of an experiment with the new method showed that the total sulfur was reduced from $3 \%$ to $1.8 \%$ or $40 \%$ reduction at 0.68 molar hypochlorite and 0.2 molar sodium hydroxide at $90 \mathrm{0C}$. This reduction is translated into $80 \%$ reduction of pyritic sulfur and $20 \%$ reduction of organic sulfur. The chlorine content of the hydrolyzed coal was $0.6 \%$ which is much lower than any other reported values in the literature for chlorine leaching. It is felt that the sulfur reduction could be improved if more rigorous conditions such as higher temperatures above $900 \mathrm{C}$ (but lower than $1000 \mathrm{C}$ ) and higher concentrations of sodium hydroxide above 0.2 molar in the hydrolysis step were applied.

It is proposed in the continuation project that the coal desulfurization process with hypochlorite leaching and then hydrolysis with sodium hydroxide be optimized in terms of particularly hypochlorite concentration and temperature. The optimization will be based on a compromise between maximum sulfur reduction and minimum hypochlorite consumption. High-sulfur coals such as Illinois No. 6 and Upper Freeport coal will be used particularly in the continuation project. The desulfurization behaviors of these coals 
will be compared to that of Pittsburgh No. 8 coal which will be characterized for the rest of the current desulfurization project.

\title{
3.13. Phyto-Extraction / Fabrication of Gold and Silver Nanoparticles (WV012)
}

\author{
Principal Investigator: Ray K. Yang \& Eung Ha Cho, West Virginia University
}

Period of Performance: May 1, 2004- October 31, 2008 (2-Year Project)

In the traditional cyanidation process, cyanide solutions are used extensively to leach precious metals from crushed ores, concentrates, and enriched tailings. Being extremely poisonous, cyanide solutions are heavily regulated, if not outright banned, in many regions around the world. One of the mid- to long-term research priorities of the Center for Advanced Separation Technologies is to develop such an alternative for precious metal extraction. An emerging alternative with great potential is phytomining i.e., growing plants that hyper-accumulate high concentrations of a target metal. A phytomining operation would entail planting a hyperaccumlator crop over a low-grade ore body or mineralized soil, followed by harvesting and incineration of the biomass to ash to produce "bio-ore". Phytomining has several advantages over conventional mining. It offers the possibility of utilizing ore bodies or mineralized soils that are otherwise uneconomical, and its effect on the environment is minimal; the area to be mined may be "ready-vegetated"; a "bio-ore" has a higher metal content than a conventional ore and needs far less space for storage. Being in its infancy, phytomining is not yet commercialized, and more research and development are still needed to make this technology cost effective. One way to make its commercialization a reality is to produce products, such as gold nanoparticles which are highly valuable in today's marketplace and are expected to be in ever increasing demands in the future.

The long-term goal of our phytomining program is to bridge together future mining industry with nanoparticle market via phytomining. This is to be done by re-focusing phytomining at producing, not just gold ingots, but more importantly, gold nanoparticles (crystallite or primary particles measuring less than $100 \mathrm{~nm}$ in size) for the rapidly expanding nanoparticle market. This new and re-oriented phytomining, which may gradually start to take shape as a result of successfully conducting the proposed research, has the potential of becoming a promising method to mass produce gold and other metallic nanoparticles, making the nanoparticles produced by future mining industry competitive in the rapidly expanding nano-product market.

The proposed tasks of this proposal cover a broad front of experimentation in order to eventually achieve the long-term goal envisioned. The experimental studies, due to the exploratory nature of the proposed research, will focus on phyto-extraction/fabrication of gold nanoparticles from hyperaccumulator plants, bean sprouts, and plant cells grown in callus and suspension cultures. The research tasks aim at providing a better understanding of the gold nanoparticles produced by the various plants selected, the external factors that impact the forms and sizes of the nanoparticles produced in those plants, and the fundamental phyto-fabrication processes leading to their formations. One may term the proposed tasks as high risk exploratory research, but even if only one of tasks leads to a profound finding the pay-off could be reasonably high. 


\subsection{Phytomining for Nickel and Silver Nanoparticles (WV016)}

Principal Investigator: Ray K. Yang \& Eung Ha Cho, West Virginia University

Period of Performance: June 1, 2005- October 31, 2008 (2-Year Project)

Phytomining is the production of a metal by growing high-biomass plants that hyperaccumulate high concentrations of a target metal. A phytomining operation would entail planting a hyperaccumlator crop over a low-grade ore body or mineralized soil, followed by harvesting and incineration of the biomass to ash to produce "bio-ore". Some plants are natural hyperaccumulators, while others require induction for hyperaccumulation. Phytomining has several advantages over conventional mining. It offers the possibility of utilizing ore bodies or mineralized soils that are otherwise uneconomical, and its effect on the environment is minimal; the area to be mined may be "ready-vegetated"; a "bio-ore" has a higher metal content than a conventional ore and needs far less space for storage.

The proposed research may eventually be able to bridge together future mining industry with nanoparticle market via phytomining using the nanobiotechnology techniques currently being developed at a fast pace. This may be done by re-focusing phytomming aiming to produce, not just metals, but more importantly, metallic nanoparticles for the rapidly expanding nanoparticle market. The nano-product market, on the other hand, may just as well find that the re-oriented phytomining has the potential of becoming a promising approach to mass produce nickel, silver and other metallic nanoparticles, with advantages similar to that of producing human and veterinary vaccines from plants.

The long-term goal of the proposed research is to tailor-make in large scale and at much reduced costs nickel and silver nanoparticles in the sizes and shapes suitable for use in a variety of industries with high demands for such nanoparticles. Exploratory research tasks, including investigations on nickel and silver nanoparticles in growing plants, as well as silver nanoparticles in bean sprouts and plant cells, will be conducted simultaneously through this two-year project using a scanning probe microscopy, aiming to achieve the following two objectives: (1) to show the existence of nickel and silver nanoparticles in a few carefully selected species of plants in various forms including their ashes, and (2) to study the sizes, shapes, surface characteristics, and other pertinent features of the nickel and silver nanoparticles formed therein, and investigate the factors that influence their relevant properties.

\section{Modeling and Control}

\subsection{Development of a Novel Optical Radiation Depolarization Technique for On-Line Measurements of Particle and Bubble Sizes (KY003)}

Principal Investigators: D. Tao, M.P. Menguc and C. Crofcheck, University of Kentucky Period of Performance: May 1, 2003- October 31, 2008 (2-Year Project)

Grinding and froth flotation are the two most important processes for mineral beneficiation. The importance of grinding is well reflected in the fact that approximately $80 \%$ of beneficiation costs are for grinding, mainly due to high energy consumption. To 
reduce energy consumed by grinding, fines should be removed quickly from the grinding circuit. This requires a reliable on-line particle size analysis technique. Similarly, froth flotation is the most widely used solid-solid separation process for coal and minerals beneficiation and about $90 \%$ of mineral concentrates are produced by froth flotation. Air bubble size distribution plays an important role in flotation separation performance but optimization of bubble size distributions will only be possible if bubble size can be monitored on-line.

The proposed project is aimed at developing an optical radiation depolarization technique for on-line size measurement of particle sizes in grinding and bubble sizes in flotation. The technique is based on the analysis of angular and radial profiles of reflection and transmittance of an object subjected to a collimated, polarized light beam. A hybrid Monte Carlo/Ray Tracing method will be used to simulate the depolarization of radiation by particles or air bubbles and size distributions will be determined by best fitting experimentally determined vertical and horizontal polarization components of the radial and angular profiles of reflection and transmission. The proposed technique should also be able to determine water film thicknesses and bubble separation distance distributions in foams.

\subsection{Mineral Liberation Analysis in 3D by X-Ray MicroCT for the Evaluation of Particle Separation Efficiency. (UT006)}

Principal Investigators: C. L. Lin, University of Utah

Period of Performance: May 1, 2004- October 31, 2008 (2-Year Project)

New processing technology for improved productivity and efficiency is the key to success in today's highly competitive market place. It has been well established that appropriate analytical control systems can provide for such improvements. This is especially true of the particle separation processes used in the coal and mineral industries. In general, the separation efficiency for multiphase particulate systems depends on the statistical characteristics of particle microstructures, such as composition distribution, surface exposure of mineral grains, etc. For continued technological progress in the field of multiphase particulate separation processes, the need for quantitative spatial analysis of multiphase particles in three-dimensions has increased significantly. Such quantitative information must be accurate enough so that the measured values can be used as parameters for simulation models, process design procedures, and control strategies. Cone-beam X-ray microtomography technology (XMT) offers a unique imaging capability that can produce high-resolution (a few micrometers) three-dimensional images of the internal structure of multiphase samples. We propose to adapt this new technology to the mining industry to provide for the advanced analysis of coal and ore samples; such analysis of on-line plant samples will allow continuous control of separation processes. In this regard, The primary objective of the proposed project is the development of x-ray micorotomography technology (XMT) for detailed 3D liberation/exposure analysis of coal and mineral samples. The secondary objective will be to evaluate the feasibility of the XMT technology for the control of separation processes. At present, results from traditional sink/float and polished-section analysis cannot be obtained in a reasonable amount of time to provide a 
satisfactory feedback mechanism for the control of various unit operations in coal preparation and mineral processing plants. This new XMT will combine the highresolution 3-D XMT instrument and a classification algorithm in order to determine the sample composition and particle characteristics of plant samples. XMT will permit a fast, direct, and detailed 3-D analysis without sample preparation. Reconstruction of 3-D particle populations from XMT images will not only provide sufficient information to construct the true liberation spectrum for the coal and ore samples in question, but also provide information on the particle size distribution, grain size distribution, and exposed surface area of mineral matter grains contained in each particle of the population sample.

Results from the proposed research will improve the efficiency of valuable mineral recovery using direct measurement of liberation information in order to optimize the separation efficiency. The XMT technique will be of great utility for the liberation analysis of a wide variety of mineral resources, including coal, industrial minerals, and metallic ores. In this regard, the XMT technology for coal washability analysis will allow for blending control to maximize coal recovery from coal preparation plants at a specified product quality. The results from the proposed research can be easily incorporated into existing simulation and estimation software systems for convenient application in industrial practice, both in the coal industry and in the mineral industry. Development of the XMT technology will allow for more rapid, detailed, and accurate analysis than previously thought possible.

\subsection{Development of a 3D Lattice-Boltzmann Model for Fluid Flow Simulation under Partially-Saturated Conditions in Packed Particle Beds}

Principal Investigators: J. Miller and M. Sukop, University of Utah

Period of Performance: May 1, 2004- October 31, 2008 (2-Year Project)

Heap leaching is a process extensively used by the mining industry to recover metals from large quantities of marginal grade materials. Currently, heap leaching finds important applications in the recovery of copper and gold from their ores because of its low cost, short construction time, operational simplicity, good performance, and environmental advantages. Characterization and analysis of heap leaching systems should involve the study of both the chemistry/biochemistry and mechanics of the heap leaching system. Optimum design of the chemistry, utilization of reactants (acids, air, microorganisms, bacteria population, iron), is limited by our current understanding of the mechanics of the system. Mechanics of the heap leaching system include such issues as particle breakage/exposure/liberation, particle agglomeration/pretreatment, and fluid flow phenomena inside the packed bed of particles which constitute the heap.

The proposed research has been designed to develop software capable of simulating the fluid flow of a leaching solution through a packed bed of particles under partiallysaturated conditions. The software will use the Lattice Boltzmann Method (LBM), which has numerous advantages over other approaches. The proposed research involves consideration of the mechanics of the heap leaching system as influenced by particle size distribution, pore-network structures, solution flow rate, oxygen flow rate, and moisture content. In a broader sense, this research is part of a long range program using X-Ray 
Microtomography (XMT) to determine to what extent the rate and practical recovery from heap leaching is limited by: (1) mineral exposure, (2) fluid flow/transport phenomena and (3) chemistry. It is expected that the results from the proposed research will help to design heap leaching operations for more effective utilization of our mineral resources.

\subsection{A Comprehensive Study of Froth Behavior. (VT011)}

Principal Investigators: Roe-Hoan Yoon, Demetri Telionis and Pavlos Vlachos, Virginia Tech

Period of Performance: May 1, 2004- October 31, 2008 (2-Year Project)

Froth flotation is the most widely used and versatile method of separating one solid from another in the coal and minerals industries. In this process, air bubbles are used to selectively collect hydrophobic particles and rise to the surface of a flotation pulp, forming a froth phase. The stability of the froth plays an important role, as it determines the final grade, recovery and throughput. Despite its importance, little is known of the mechanisms involved in the stability of foam and, particularly, of flotation froth, as most of the information available in the literature is on dry foam without particles.

It is, therefore, proposed to conduct a comprehensive study on both dry and wet foams in the presence of particles. The proposed work will include determination of bubble size distribution, particle size distribution, liquid fraction (or air-hold-up), grade of hydrophobic particles along the heights of froth columns. The results will give information on the effects of both hydrodynamic and surface chemistry parameters on froth stability. The new information obtained in the present work will be used to develop a froth model. It will be combined with the model describing the bubble-particle interactions occurring in the pulp phase, so that a comprehensive flotation model will be developed. Since the combined model will be based on first principles, it will have predictive and diagnostic capabilities, and can be used for developing methods of improving flotation. The basic information obtained in the present work will be particularly useful for developing effective deformers, maximizing carrying capacity, and improving coarse particle flotation.

\subsection{Online Monitoring and Diagnosing of Coal Fines During Separation Process (WV008)}

Principal Investigator: B.S. Kang and E.K. Johnson, West Virginia University

Period of Performance: May 1, 2003- October 31, 2008 (2-Year Project)

The goal of this research program is to develop an on-line, non-contact, elemental analysis of coal fines during solid-solid separation process through the use of LaserInduced Breakdown Spectroscopy (LIBS). Of particular interest is the detection and quantitative measurement of the amount of carbon, sulfur, mercury, and other trace elements in the separated coal fines. The proposed experimental technique will be applied to a circulating fluidized bed (CFB) riser system for determination of coal fines separation efficiency as well as optimization of the separation process variables using a fuzzy logic control approach. 


\subsection{Portable Sensor for Detecting Mercury and other Heavy Metals Encountered in Coal Processing and Utilization (WV013)}

Principal Investigators: A. Manivannan and Mohindar S. Seehra, West Virginia University

Period of Performance: May 1, 2004- October 31, 2008 (2-Year Project)

A two year research program is proposed to develop a portable and reliable sensor for on-site detection of mercury in solutions in the ppb range using boron-doped diamond (BDD) electrodes. This proposal is based on our demonstrated feasibility of detecting mercury in laboratory solutions in the ppb range using the BDD electrodes. Similar success has been accomplished for detecting $\mathrm{Pb}, \mathrm{Cd}$, and $\mathrm{Cu}$ in solutions. For mercury detection, the media used so far have been $\mathrm{KCl}$, and $\mathrm{KNO} 3$. Proposed research include developing the necessary calibration curves for $\mathrm{Hg}$ in other media such as thiocyante (KSCN), perchlorate, and EDTA, the use of a rotating disk electrode to improve sensitivity and reproducibility of results, and testing of the flue gas samples obtained from coal-fired plant at NETL (Pittsburgh). The tasks needed to develop a suitable portable unit are appropriately addressed. Feasibilty of similar portable system for detecting other heavy metal ions encountered in coal processing and utilization is described.

\section{Environmental Control}

\subsection{Determination of Factors Affecting the Separation of Potentially Hazardous Trace Elements and their Behavior in Coal Tailings Impoundments (KY005)}

Principal Investigators: Frank E. Huggins, Naresh Ahah, Gerald P. Huffman, University of Kentucky

Period of Performance: June 1, 2005- October 31, 2008 (2-Year Project)

A number of trace elements in coal are of significant environmental concern. Such potentially hazardous elements include $\mathrm{As}, \mathrm{Cr}, \mathrm{Se}$, and $\mathrm{Hg}$, and are regulated under either the 1990 Clean Air Act Amendments or the 1976 Resource Conservation and Recovery Act or both. Coal cleaning is performed principally to minimize the amount of pyrite and other mineral matter entering pulverized coal combustion. At the same time, hazardous trace elements associated with these minerals are also removed from coal combustion and hence their environmental impact during combustion is thereby reduced. However, such trace elements tend to be concentrated in the waste tailings from coal separation technologies, which normally are disposed of under water in coal waste impoundments. Here, trace elements may pose other environmental problems because both major and trace elements may be leached and mobilized. Consequently, the waters in such impoundments have to be isolated from the local groundwater in order to avoid possible contamination of local drinking water supplies. Despite concerns about solubilized trace elements in coal impoundments, relatively little is known about the concentrations and specific chemical forms of such trace elements that can be leached from coal minerals and mobilized in these waters. More data are clearly needed to assess the environmental hazard from mobilized ions in coal tailings impoundments. 
The behavior of trace elements in both coal separation technologies and tailings disposal scenarios depends on their modes of occurrence and associations with major minerals or macerals in the coal or coal fractions. Such information is rarely determined, but is of vital importance to our understanding of trace elements in these processes. In this proposal, we intend to determine the mineral or chemical forms (mode of occurrence) and association of a number of critical trace elements (As, $\mathrm{Cr}, \mathrm{Se}, \mathrm{Hg}$, etc.) in coals and coal fractions using a comprehensive array of conventional and advanced characterization methods. Our intention is to collaborate with other CAST-supported investigations of advanced coal separation technologies that can supply the clean coal and tailings fractions needed for this investigation. The information obtained will then be used (i) to determine the efficiency of various coal cleaning methods for specific trace element removal; (ii) to interpret the behavior of specific elemental forms and associations in coal tailings exposed to water in laboratory simulations of storage ponds; and (iii) to assess the actual environmental impact posed by specific trace elements in coal tailings impoundments.

\subsection{Determining the Effectiveness of Gold Filters for Removing Mercury from Coal Fired Power Plants (MT003)}

Principal Investigator: K. Ganesan, Montana Tech

Period of Performance: May 1, 2003- October 31, 2008 (1-Year Project)

Because the US EPA is planning to regulate mercury emissions from power plants, there is a clear need to develop devices that can cost effectively remove mercury from power plant flue gases. Most current research has focused on transferring mercury from the air stream to fly ash or converting elemental mercury to divalent mercury to absorb it in wet scrubbers. However, when low chlorine content coal is combusted most of the mercury in the flue gas exists as elemental mercury, which is very hard to remove. Thus, to comply with the potential $90 \%$ mercury reduction proposed by EPA, one must oxidize the elemental mercury to either mercuric chloride or oxide as a way to collect particulates. In either case, mercury is merely converted to a form that is also difficult and expensive to recover. Therefore, it appears that directly removing mercury from the flue gas will be the most cost effective and environmentally friendly approach. This proposal is focused on removing elemental mercury from flue gas using gold filters. The main objective of this research is to evaluate the effectiveness of gold wire mesh in removing mercury vapor from flue gas. A wire mesh made of thin $(<0.1 \mathrm{~mm}$ diameter $)$ gold wires will be tested in the laboratory, initially with a synthetic gas stream

containing mercury vapor in the range of $1-300 \mu \mathrm{g} / \mathrm{m} 3$. Similar tests will be conducted in combustion gases by burning six types of western and eastern coals. The study will be conducted for six concentration levels of mercury; 5, 10, 25, 50, 100 and $300 \mu \mathrm{g} / \mathrm{m} 3$.

\subsection{Development of Metallic Filters to Control Mercury From Coal Fired Power Plant Flue Gas (MT004)}

Principal Investigator: K. Ganesan, Montana Tech

Period of Performance: May 1, 2004- October 31, 2008 (1-Year Project) 
The focus of this research is to develop metallic filters to remove vapor phase mercury from coal fired flue gas. Three types of metallic filters will be tested for its effectiveness in removing mercury vapor from gas streams. Based on the results, one of them will be tested for its performance in a flue gas stream of a coal fired power plant. The previous funding from CAST enabled the PI to investigate the potential of gold filters to remove mercury vapor from gas streams. The results indicate that the removal efficiency is over $90 \%$ with gold filters. In addition, preliminary tests with silver filters showed promising results. Therefore, this proposal is to test three metallic filters: copper wire with gold plating, copper wire with silver plating and copper wire mesh. These filters initially will be made in the laboratory and tests will be performed to compare their performance. Tests will be performed at concentration ranges of $1-100 \mu \mathrm{g} / \mathrm{m} 3$ of vapor phase mercury. Tests also will be performed at elevated temperatures by heating the incoming air stream up to $250^{\circ} \mathrm{F}$. Two bench scale systems will be designed based on the results of the laboratory and field study. These filters then will be tested in the field in a coal fired power plant flue gas stream. This field work will be conducted in collaboration with PPL Montana, in Colstrip where four coal fired power plants are in operation. The results of this field tests will provide valuable information of the performance of these filters in real world conditions. The data then will be used to design a pilot scale system for further testing.

\subsection{Electrolytic Solution Purification and Metal Recovery from Metal-Bearing Toxic Waste Streams (UT003)}

Principal Investigator: Michael L. Free, University of Utah

Period of Performance: May 1, 2003- October 31, 2008 (1-Year Project)

Industry creates numerous waste streams, many of which contain dissolved metal ions that must be removed to preserve the environment. Many of these waste streams can be created by natural processes that are accelerated by industry such as acid mine drainage, made as byproducts of chemical processing such as metal extraction, or as the result of manufacturing processes such as electronic component manufacturing. Often such wastes are complex and involve multiple metal ions. Regardless of the waste origin, toxic metal ions must be removed.

In the proposed project a novel method of removing multiple toxic metals from aqueous media by selective pulse-plating with high surface area electrodes will be evaluated and developed in a manner that will allow the metals to be recovered individually as purified metals in an environmentally sound way. The direct production of metal as a byproduct, rather than as a toxic waste, will contribute to better resource utilization as well as a reduction in toxic waste generation.

\subsection{Recovery of Chromium and Arsenic From Toxic Waste Streams by Reactive Polymer-Coated Absorbents (WV014)}

Principal Investigators: Dianchen Gang \& Baolin Deng, West Virginia University Period of Performance: May 1, 2004- October 31, 2008 (2-Year Project) 
One of the major challenges facing coal and metal mining industries today is to address public concerns of environmental damage associated with the mining activities. Oxidation of pyrites and other metal sulfides in coal and metal mine tailings can generate high concentration of sulfuric acid, forming acid mine drainage (AMD) that may contain high concentrations of sulfate, iron, and many toxic elements suc $h$ as arsenic (As) and chromium $(\mathrm{Cr})$. The objective of this project is to develop a novel method of removing and recovering $\mathrm{As}$ and $\mathrm{Cr}$ from wastewater including AMD. Reactive polymers will be synthesized under defined conditions and their structure be optimized according to their capability for As and $\mathrm{Cr}$ removal and recovery. This study focuses on these anionic contaminants (i.e., AsO 4 3-/AsO 3 3- and $\mathrm{CrO} 4$ 2-) because they are highly soluble and normally not removed by many of the treatment processes designed for divalent heavy metals such as lime neutralization and precipitation. The research objective is consistent with the research roadmap developed by CAST on the chemical separations and environmental control issues. The development and successful testing of this material could potentially lead to a patent application.

\subsection{Mercury Reduction From Coal Power Plant Emission Using Functionalized Ordered Mesoporous Carbons (FOMCs) (WV015)}

Principal Investigators: Dianchen Gang \& Baolin Deng, West Virginia University Period of Performance: June 1, 2005- October 31, 2008 (2-Year Project)

One of the major challenges facing coal-fired power plants (CFPPs) today is to address the public concerns of mercury emissions. Mercury emitted from CFPPs exists in two valence states: elemental mercury $(\mathrm{Hg} 0)$ and oxidized forms $(\mathrm{Hg} 2+)$. Elemental mercury is normally dominant, accounting for $92 \%$ to $99 \%$ of the total mercury in the flue gas. The effectiveness of an individual control technology for $\mathrm{Hg}$ emission depends largely on its chemical speciation. For example, $\mathrm{Hg} 0$ is highly volatile with low water solubility, therefore, it is not absorbed significantly by water-based scrubbing processes. The objective of this project is to develop and evaluate functionalized ordered mesoporous carbons (FOMCs) for the maximum $\mathrm{Hg}$ removal from flue gas emissions at high temperature $(80-200 \mathrm{oC})$. Highly ordered mesoporous carbons (OMCs) with uniform nanopore size will be synthesized under defined conditions and their structure optimized according to their capability toward $\mathrm{Hg}$ removal in the high particulate environment. OMCs will be functionalized by elemental sulfur and other chemicals to enhance $\mathrm{Hg}$ uptake. The research objective is consistent with the research roadmap developed by CAST on the chemical separations and environmental control issues. The development and successful testing of this material could potentially lead to a patent application.

\subsection{Removal of Metal Ions from Acid Mine Drainage using a Novel Low-Cost, Low Technology (WV017)}

Principal Investigators: Benjamin Dawson-Andoh, West Virginia University

Period of Performance: June 1, 2005- October 31, 2008 (2-Year Project)

Acid Mine Drainage (AMD) produced by active and dormant coal mines continues to pose a major threat to the environment. One of the goals of the National Energy Policy 
developed by President Bush in 2001 is "to ensure the steady supply of affordable energy in environmentally responsible and sustaining manner." Current technologies employed to remove toxic environmental polluting metals from AMD are expensive and economically challenging to the Coal Mining Industry. The work proposed here seeks to evaluate a low-cost, low technology that employs renewable biomaterials: wood and fungal biomass, to remove toxic metals that occur in AMD. Additionally, the proposed work also seeks to determine the recovery potential of toxic metals from the biomaterials and the regeneration and re-use of the biomaterials. West Virginia is one of the premier hardwood States in the US and tremendous amount of wood biomass is available as residue. The proposed study if successful will provide an avenue for the use of the tremendous wood biomass generated by both the primary and secondary forestry operations in West Virginia. 


\section{EXPERIMENTAL}

Experimental work has been completed and the Final Reports are attached. 


\section{RESULTS AND DISCUSSION}

The project closed date on October 31, 2009, and the Final Reports on the sub-projects are attached 


\section{CONCLUSIONS}

Conclusions for each of the sub-projects are included in the reports attached as appendices. 
APPENDICES 
Appendix 1: Development of Novel Ultrafine Sizing Methods (KY001/VA008) 


\section{FINAL TECHNICAL REPORT}

Contract Title and Number:

Crosscutting Technology Development at the Center for Advanced Separation Technologies

(DE-FC26-02NT41607)

Sub-Recipient Project Title:

Development Of Novel Ultrafine Sizing Methods

Principal Investigators:

Roe-Hoan Yoon and Gerald H. Luttrell

Contact Address:

146 Holden Hall

Virginia Tech, Blacksburg, VA 24061

Subcontractor Address:

No subcontracts issued.
Period of Performance:

Starting Date: 10/1/2002

Ending Date: 10/31/2009
Report Information:

Type: FINAL

Number:

Period:

Date: $\quad$ 10/22/08

Code: VA008-FINAL

Contact Information:

Phone: (540) 231-4508

Fax: $\quad$ (540) 231-3948

E-Mail: cast@vt.edu

Subcontractor Information:

Phone:

Fax:

E-Mail: 


\begin{abstract}
Most mineral and coal processing plants are forced to size their particulate streams in order to maximize the efficiency of their unit operations. Classifiers are generally considered to be more practical than screens for fine sizing, but the separation efficiency decreases dramatically for particles smaller than approximately $150 \mu \mathrm{m}$. In addition, classifiers commonly suffer from bypass, which occurs when a portion of the ultrafine particles (slimes) are misplaced by hydraulic carryover into the oversize product. The unwanted misplacement can have a large adverse impact on downstream separation processes. One method of reducing bypass is to inject water into the cyclone apex. Unfortunately, existing water injection systems tend to substantially increase the particle cut size, which makes it unacceptable for ultrafine sizing applications. A new apex washing technology was developed to reduce the bypass of ultrafine material to the hydrocyclone underflow while maintaining particle size cuts in the $25-50 \mu \mathrm{m}$ size range.

Another method of reducing bypass is to retreat the cyclone underflow using multiple stages of classifiers. However, natural variations in the physical properties of the feed make it difficult to calculate the exact improvement offered by multistage classification in experimental studies. Therefore, several mathematical equations for multistage classification circuits were evaluated using mathematical tools to calculate the expected impact of multistage hydrocyclone circuits on overall cut size, separation efficiency and bypass. These studies suggest that a twostage circuit which retreats primary underflow and recycles secondary overflow offers the best balance between reducing bypass and maintaining a small cut size and high efficiency.
\end{abstract}




\section{TABLE OF CONTENTS}

ABSTRACT iii

ACKNOWLEDGMENTS

Error! Bookmark not defined.

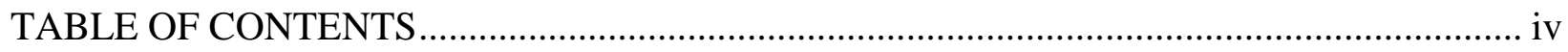

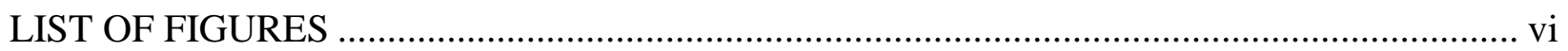

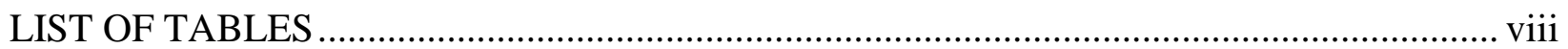

CHAPTER 1 - DEVELOPMENT OF A NEW WATER-INJECTION SYSTEM FOR

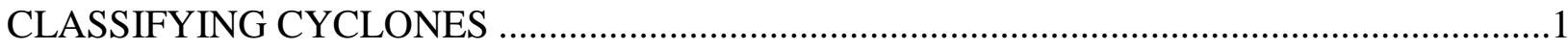

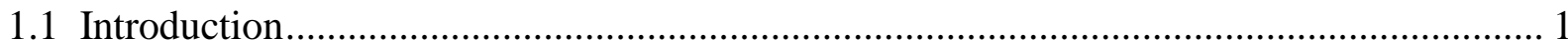

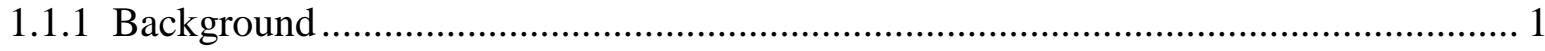

1.1.2 Objectives ……………………………………….......................................... 1

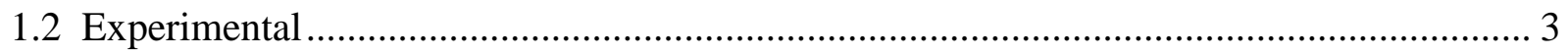

1.2.1 Description of the Water-Injection System............................................................... 3

1.2.2 Test Circuit Setup ....................................................................................... 4

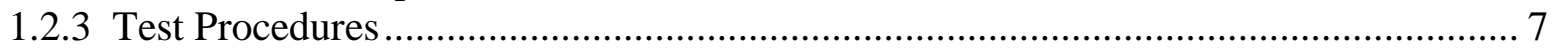

1.2.3.1 Test Sample

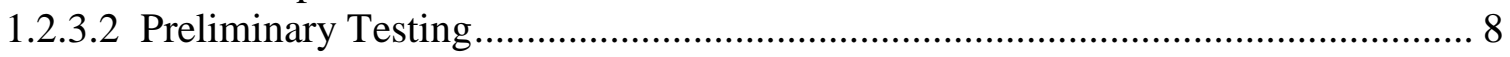

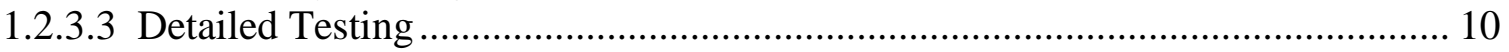

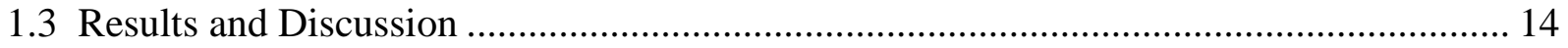

1.3.1 Parametric Study Results ................................................................................... 14

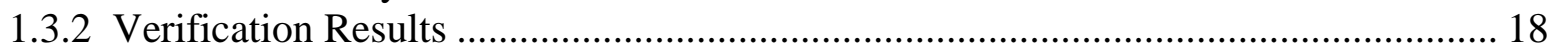

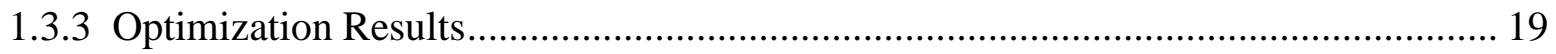

1.3.3.1 Optimum Conditions for the 20-25 $\mu \mathrm{m}$ Size Range ………................................ 25

1.3.3.2 Optimum Conditions for the 25-30 $\mu \mathrm{m}$ Size Range ……………......................... 25

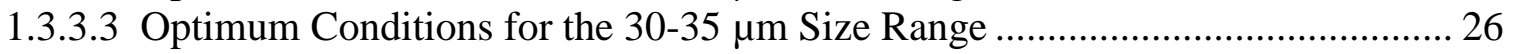

1.3.3.4 Optimum Conditions for the $35-40 \mu \mathrm{m}$ Size Range ………............................... 26

1.3.3.5 Optimum Conditions for the 40-45 $\mu \mathrm{m}$ Size Range ………………….................. 27

1.3.3.6 Bypass and Cutsize Correlation Under Optimum Conditions .............................. 27

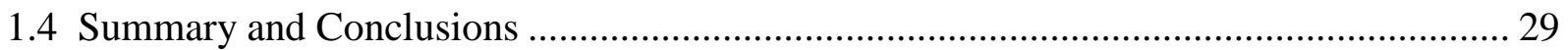

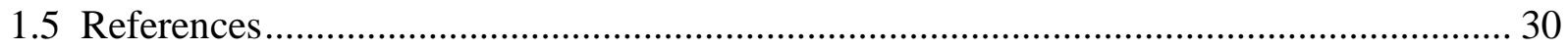

CHAPTER 2 - MATHEMATICAL SOLUTIONS TO PARITIONING EQUATIONS FOR

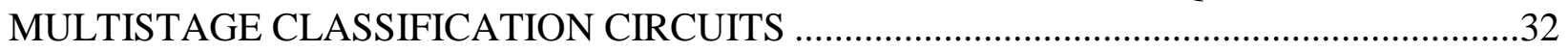

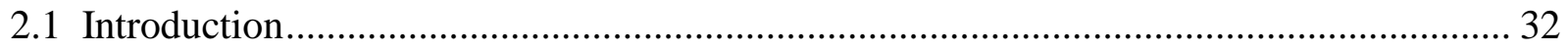




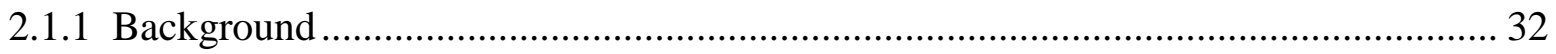

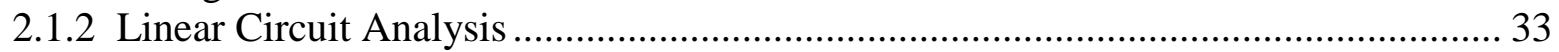

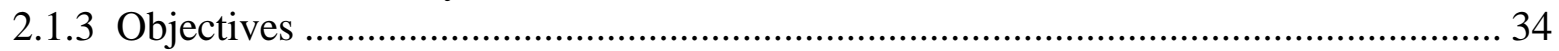

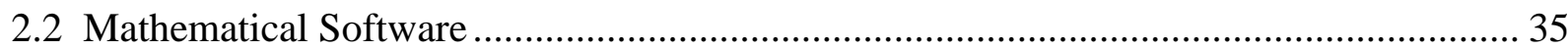

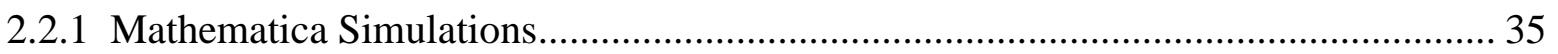

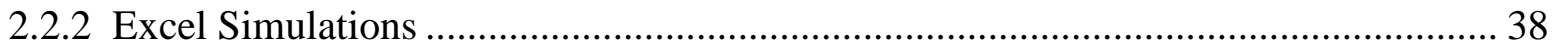

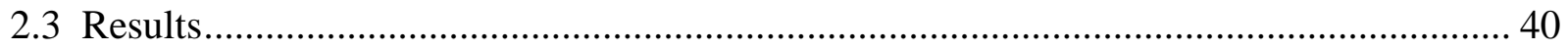

2.3.1 Underflow Reprocessing Circuit Without Recycle.................................................... 40

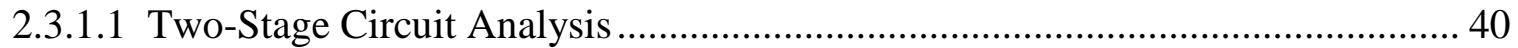

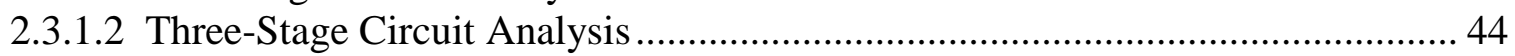

3.3.2 Underflow Reprocessing Circuit With Recycle....................................................... 45

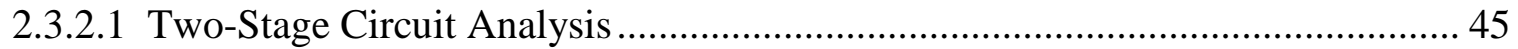

2.3.2.2 Three-Stage Circuit Analysis ........................................................................... 46

2.3.3 Overflow Reprocessing Circuit Without Recycle...................................................... 48

2.3.3.1 Two-Stage Circuit Analysis ............................................................................. 48

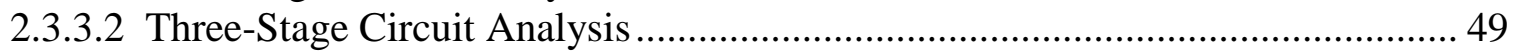

2.3.4 Overflow Reprocessing Circuit With Recycle............................................................ 50

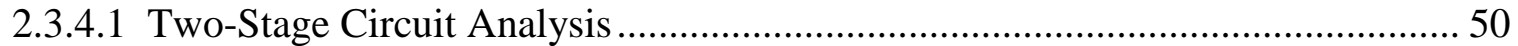

2.3.4.2 Three-Stage Circuit Analysis ......................................................................... 52

2.4 Generalized Partitioning Expressions .............................................................................. 54

2.4.1 Underflow Reprocessing Circuit Without Recycle................................................... 54

2.4.2 Underflow Reprocessing Circuit With Recycle............................................................ 55

2.4.3 Overflow Reprocessing Circuit Without Recycle...................................................... 57

2.4.4 Overflow Reprocessing Circuit With Recycle ........................................................... 58

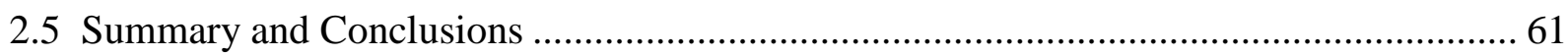

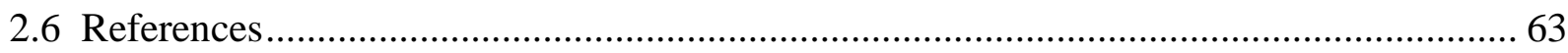

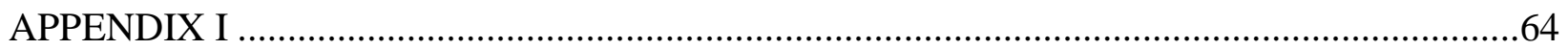

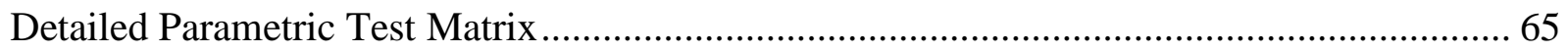

Tabular Summary of Parametric Test Results ...................................................................... 167 


\section{LIST OF FIGURES}

Figure 1.1 - Cutaway view and photograph of the water-injected apex. .................................. 4

Figure 1.2 - Test circuit used to evaluate the water injection apex...................................... 5

Figure 1.3 - Control and metering systems used with the water injected apex........................ 6

Figure 1.4 - Operating principle for the proportional sampler.......................................... 7

Figure 1.5 - Flow and pressure response as a function of apex and vortex sizes for the 6-inch $(15.2-\mathrm{cm})$ diameter Krebs G-Max cyclone used in the test program....................... 8

Figure 1.6 - Effect of water injection on hydrocyclone partitioning performance. ................... 10

Figure 1.7 - Partitioning data from replicate tests conducted under identical test conditions (0 level settings) for the water-injected apex (with 10\% error bars) ........................ 13

Figure 1.8 - Correlation between actual and predicted cut size and bypass........................... 15

Figure 1.9 - Effect of water injection rate on cutsize and bypass under central point conditions ( 0.75 inch apex inlet, 4 inch chamber, 1 inch outlet)........................................ 16

Figure 1.10 - Effect of apex outlet diameter on cutsize and bypass under central point conditions (0.75 inch apex inlet, 4 inch chamber, 20 GPM water rate).

Figure 1.11 - Effect of apex inlet diameter (top two plots) and chamber diameter (bottom two plots) on cutsize and bypass.

Figure 1.12 - Partitioning performance as a function of water injection rate under central point conditions ( 0.75 inch apex inlet, 4 inch chamber, 1 inch outlet)........................... 18

Figure 1.13 - Cutsize response at the optimum point for $20 \sim 25 \mu \mathrm{m}$ classification. .................. 20

Figure 1.14 - Bypass response at the optimum point for $20 \sim 25 \mu \mathrm{m}$ classification..................... 20

Figure 1.15 - Cutsize response at the optimum point for $25 \sim 30 \mu \mathrm{m}$ classification.................. 21

Figure 1.16 - Bypass response at the optimum point for $25 \sim 30 \mu \mathrm{m}$ classification.................... 21

Figure 1.17 - Cutsize response at the optimum point for $30 \sim 35 \mu \mathrm{m}$ classification................... 22

Figure 1.18 - Bypass response at the optimum point for $30 \sim 35 \mu$ m classification.................... 22

Figure 1.19 - Cutsize response at the optimum point for $35 \sim 40 \mu \mathrm{m}$ classification.................. 23

Figure 1.20 - Bypass response at the optimum point for $35 \sim 40 \mu \mathrm{m}$ classification.................... 23 
Figure 1.21 - Cutsize response at the optimum point for $40 \sim 45 \mu \mathrm{m}$ classification.

Figure 1.22 - Bypass response at the optimum point for $40 \sim 45 \mu \mathrm{m}$ classification.................... 24

Figure 1.23 - Correlation between predicted cutsize and bypass. .......................................... 28

Figure 2.1 - Multistage classifier simulation using a spreadsheet-based model (example shows three-stage overflow reprocessing circuit with recycle). .................................. 38

Figure 2.2 - Schematic of a two-stage underflow reprocessing circuit without recycle............. 40

Figure 2.3 - Example of the partitioning response of the two-stage circuit underflow reprocessing circuit without recycle. ....................................................... 42

Figure 2.4 - Schematic of a three-stage underflow reprocessing circuit without recycle........... 44

Figure 2.5 - Schematic of a two-stage underflow reprocessing circuit with recycle. ................ 46

Figure 2.6 - Schematic of a three-stage underflow reprocessing circuit with recycle.............. 47

Figure 2.7 - Schematic of a two-stage overflow reprocessing circuit without recycle. .............. 48

Figure 2.8 - Schematic of a three-stage overflow reprocessing circuit without recycle............. 50

Figure 2.9 - Schematic of a two-stage overflow reprocessing circuit with recycle.................. 51

Figure 2.10 - Schematic of a three-stage overflow reprocessing circuit with recycle............... 52

Figure 2.11 - Generic configurations of underflow reprocessing circuits without recycle......... 54

Figure 2.12 - Generic configurations of underflow reprocessing circuits with recycle.............. 56

Figure 2.13 - Generic configurations of overflow reprocessing circuits without recycle........... 57

Figure 2.14 - Generic configurations of overflow reprocessing circuits with recycle............... 59 


\section{LIST OF TABLES}

Table 1.1 - Variables used in the parametric study to evaluate the effects of various operating and geometric variables on apex washing performance. .................................. 12

Table 1.2 - Regression expressions obtained from the Box-Behnken parametric study............. 14

Table 1.3 - Optimized conditions for the $20 \sim 25 \mu$ m cutsize range......................................... 20

Table 1.4 - Optimized conditions for the $25 \sim 30 \mu \mathrm{m}$ cutsize range...................................... 21

Table 1.5 - Optimized conditions for the $30 \sim 35 \mu \mathrm{m}$ cutsize range ........................................ 22

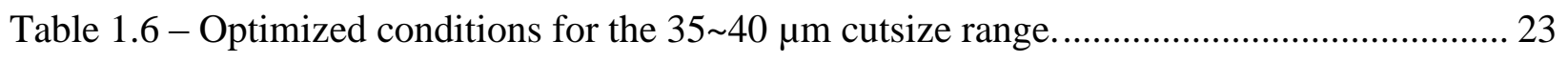

Table 1.7 - Optimized conditions for the $40 \sim 45 \mu \mathrm{m}$ cutsize range.......................................... 24

Table 1.8 - Summary of optimal conditions needed for different cutsize ranges. .................... 25

Table 2.1 - Comparison of Mathematica and Excel determined partition factors for the two-stage underflow reprocessing circuit without recycle.............................................. 43 


\section{CHAPTER 1 - DEVELOPMENT OF A NEW WATER-INJECTION SYSTEM FOR CLASSIFYING CYCLONES}

\subsection{Introduction}

\section{$\underline{1.1 .1 \text { Background }}$}

Most mineral and coal processing plants are forced to size their particulate streams in order to maximize the efficiency of their unit operations. These sizing techniques commonly include various types of screens and classifiers. Screens exploit differences in the physical dimensions of particles by allowing fines to pass through a perforated plate or open mesh while coarser solids are retained. Unfortunately, screening systems are generally limited to particle size separations coarser than approximately $250 \mu \mathrm{m}$ due to limitations associated with capacity and blinding. Hydraulic classifiers, which include both static and centrifugal devices, are generally employed for finer size separations. Hydraulic classifiers exploit differences in the settling rates of particles and are influenced by factors such as particle shape and density as well as particle size. Classifiers are generally considered to be more practical than screens for fine sizing, but the separation efficiency decreases dramatically for particles smaller than approximately $150 \mu \mathrm{m}$ (Heiskanen, 1993). In addition, classifiers commonly suffer from bypass, which occurs when a portion of the ultrafine particles (slimes) are misplaced by hydraulic carryover into the oversize product. The unwanted misplacement can have a large adverse impact on downstream separation processes.

\section{$\underline{1.1 .2 \text { Objectives }}$}

The primary objective of the work outlined in this chapter is to evaluate a new apex washing system for hydrocyclone classification. The new technology is designed to reduce the 
bypass of ultrafine material to the hydrocyclone underflow while maintaining particle size separations in the $25-50 \mu \mathrm{m}$ size range. The work required the construction and testing of a 6inch $(15.2-\mathrm{cm})$ diameter hydrocyclone in a closed-loop test circuit. To optimize the operating and design parameters associated with the water-injected apex, an optimization study was carried out using the Box-Behnken method statistical design of experiments. Analysis of the data from this parametric study was performed using a statistics program known as "Design Expert". The optimization was performed in order to determine the conditions that would provide the smallest amount of bypass while maintaining the size of separation (i.e., cut size) within a fixed narrow range. In this particular study, the optimum injection water flow rate and combination of dimensions for the water injection apex were established for five different size ranges (i.e., 20-25, $25-30,30-35,35-40$ and $40-45 \mu \mathrm{m})$. 


\subsection{Experimental}

\subsubsection{Description of the Water-Injection System}

Water-injected apex systems have been shown to be capable of reducing the bypass of ultrafine particles that are misplaced to the hydrocyclone underflow. Unfortunately, past studies have shown that existing water injection systems tend to substantially increase the particle cutsize, which makes these systems unacceptable for many ultrafine sizing applications. In addition, existing systems typically require large amounts of clarified injection water that may not be readily available in industrial plants. In light of these problems, a new type of waterinjected cyclone technology was designed by Krebs Engineers to overcome some of the inherent limitations associated with existing apex washing systems. In particular, the system was designed to efficiently reduce the bypass of ultrafine particles to the underflow while maintaining a relatively small particle cutsize.

The new water-injected apex consists of three parts (see Figure 1.1). The top section consists of a grooved flange that is used to attach the apex to the bottom of a 6-inch $(15.2-\mathrm{cm})$ diameter Krebs G-Max hydrocyclone. The middle portion consists of interchangeable chambers that serve as the body of the water-injected apex. Finally, the bottom part consists of the underflow port (apex finder) and a tangential wash water inlet port. The cutaway view provided in Figure 1.1 provides an example of the dimensions used for one possible combination of these various components. For testing purposes, three interchangeable sections for Chamber A were constructed with a height of 2.5 inches and three different inner diameters of 3.5, 4.0 and 4.5 inches. Likewise, nine different components for Chamber B were constructed with three different inner diameters (i.e., 3.0, 3.5 and 4.0 inches) and three different inlet diameters (i.e., 0.50, 0.75 and 1.00 inches) so that all possible combinations of diameters and inlets could be evaluated. 

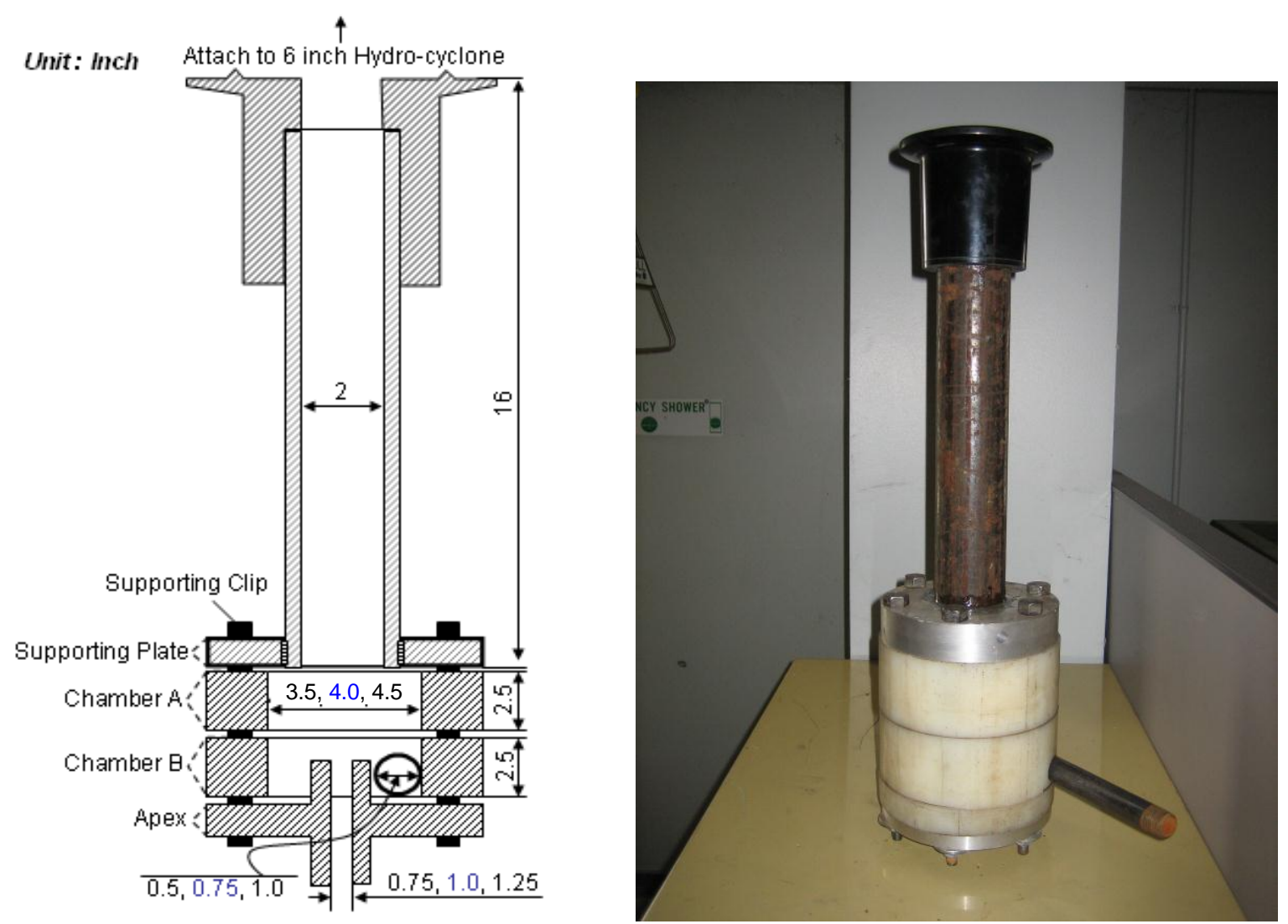

Figure 1.1 - Cutaway view and photograph of the water-injected apex.

Finally, three interchangeable apexes were constructed with outlet diameters of $0.75,1.00$ and 1.25 inches. The height of the top section, which attached to the bottom of the hydrocyclone, was kept constant at 16 inches (including the thickness of the supporting plate). The relatively long height of the cylinder of the topmost section was specifically chosen to increase the retention time of slurry and water within the apex. Six supporting clips were used to hold the various sections together and to prevent leaking problems between the joints.

\subsubsection{Test Circuit Setup}

The experimental test program required the construction of a complete closed-loop test circuit. A schematic of the test circuit is provided in Figure 1.2. The circuit, which was designed to be extremely flexible, incorporated a 6-inch (15.2-cm) diameter Krebs G-Max classifying 


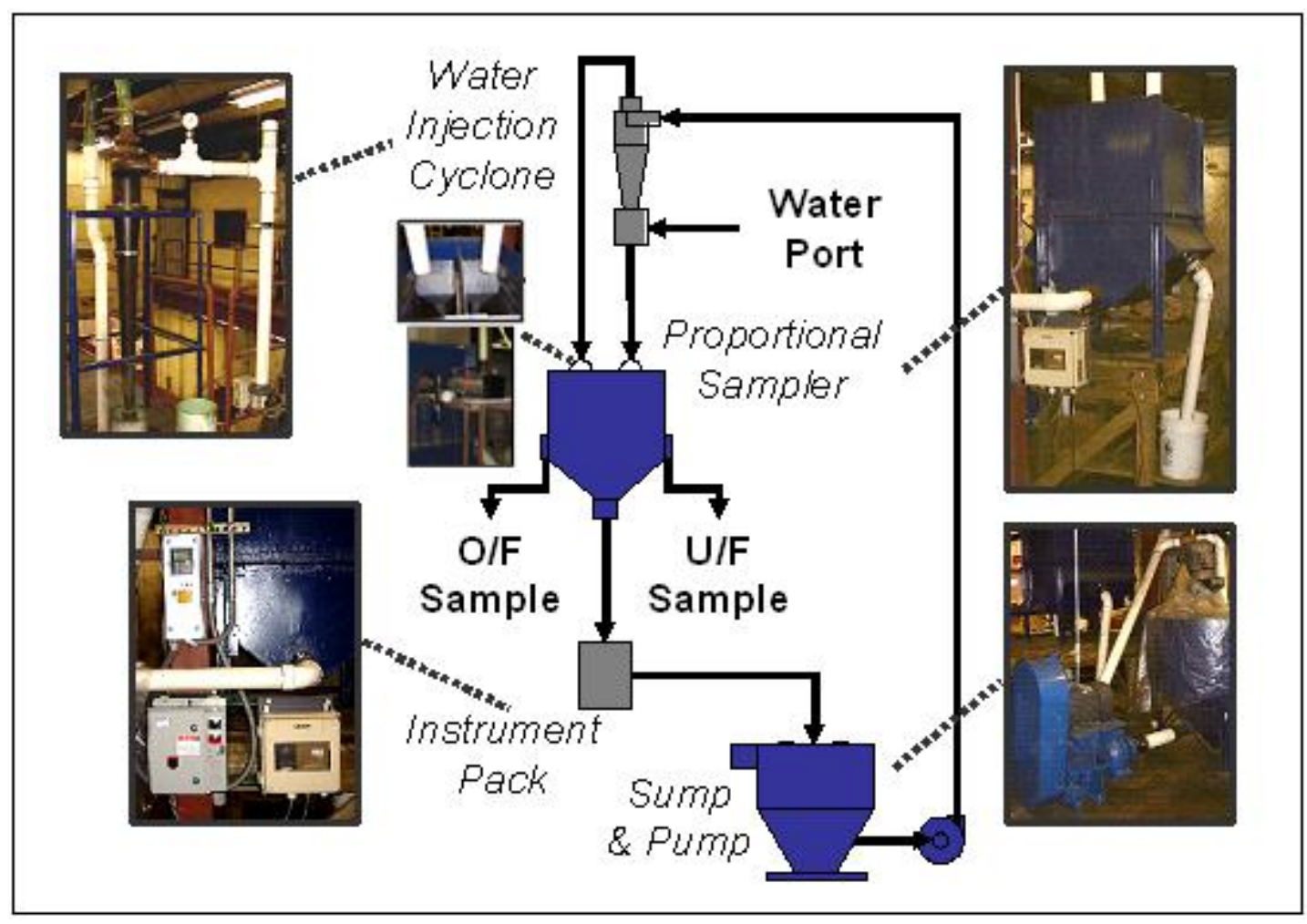

Figure 1.2 - Test circuit used to evaluate the water injection apex.

cyclone with interchangeable components, an electronically controlled variable speed circulation pump, and an integrated linear-pass proportional sample cutter. The hydrocyclone and slurry pump equipment were donated by Krebs Engineers and Morris Coker Equipment, respectively, as a cost-sharing contribution to the project. The pressure and volumetric flow rate of both the feed slurry and injection water were monitored by an instrument pack. The flow rate and pressure of the injection water was monitored using a mechanical flow meter and a rotary pressure gauge, respectively. A photograph showing the valve configuration and metering system is provided in Figure 1.3. A centrifugal pumping system connected to the town municipal water supply was used to provide a flow rate of up to $30 \mathrm{GPM}$ of fresh water to the waterinjected apex. 


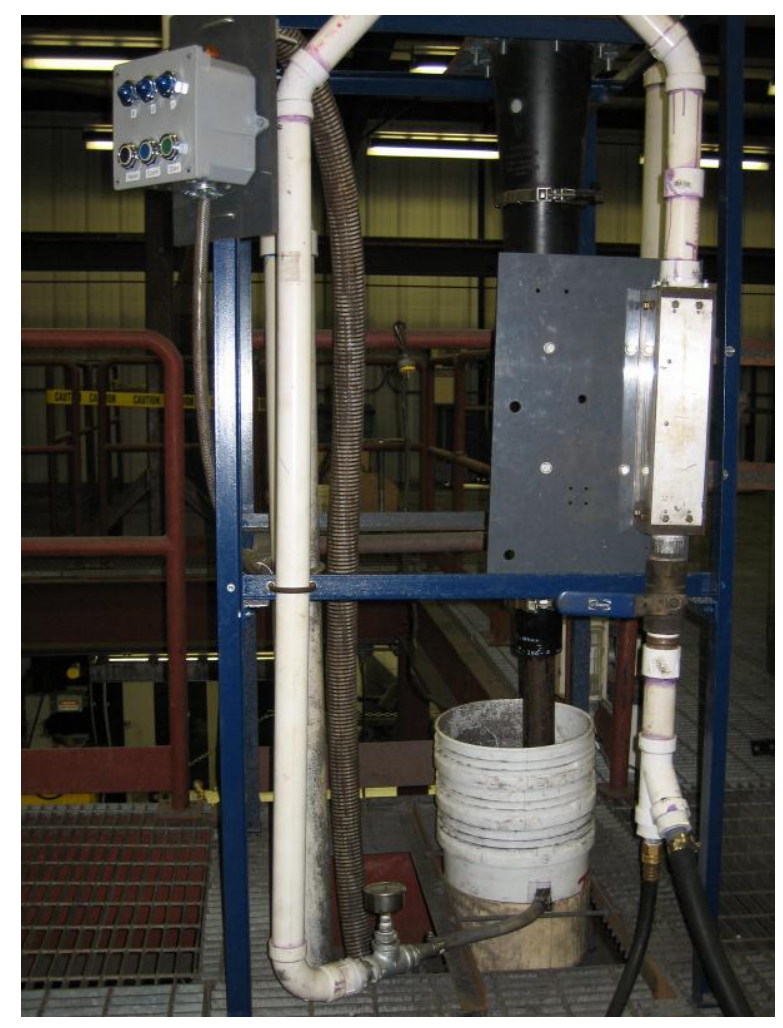

Figure 1.3 - Control and metering systems used with the water injected apex.

Samples of the overflow and underflow streams from the hydrocyclone were obtained using a linear-action proportional sampler (see Figure 1.4). The proportional sampler made it possible to reconstitute the feed stream from the weights of the two product samples. To ensure the samples were reliable, the reconstituted feed stream was compared with an independent sample of feed slurry taken from the return pipe that discharged into the sump. To reduce manpower and reduce operator bias, the motion of the proportional sample cutters was automated using a pneumatic ram and microprocessor. The automated system made it possible to accurately input and control both the number of sample cuts (which was input via pushbuttons on the circuit control panel) and linear velocity of the cutters (which was adjusted by changing the air pressure on the pneumatic ram). 

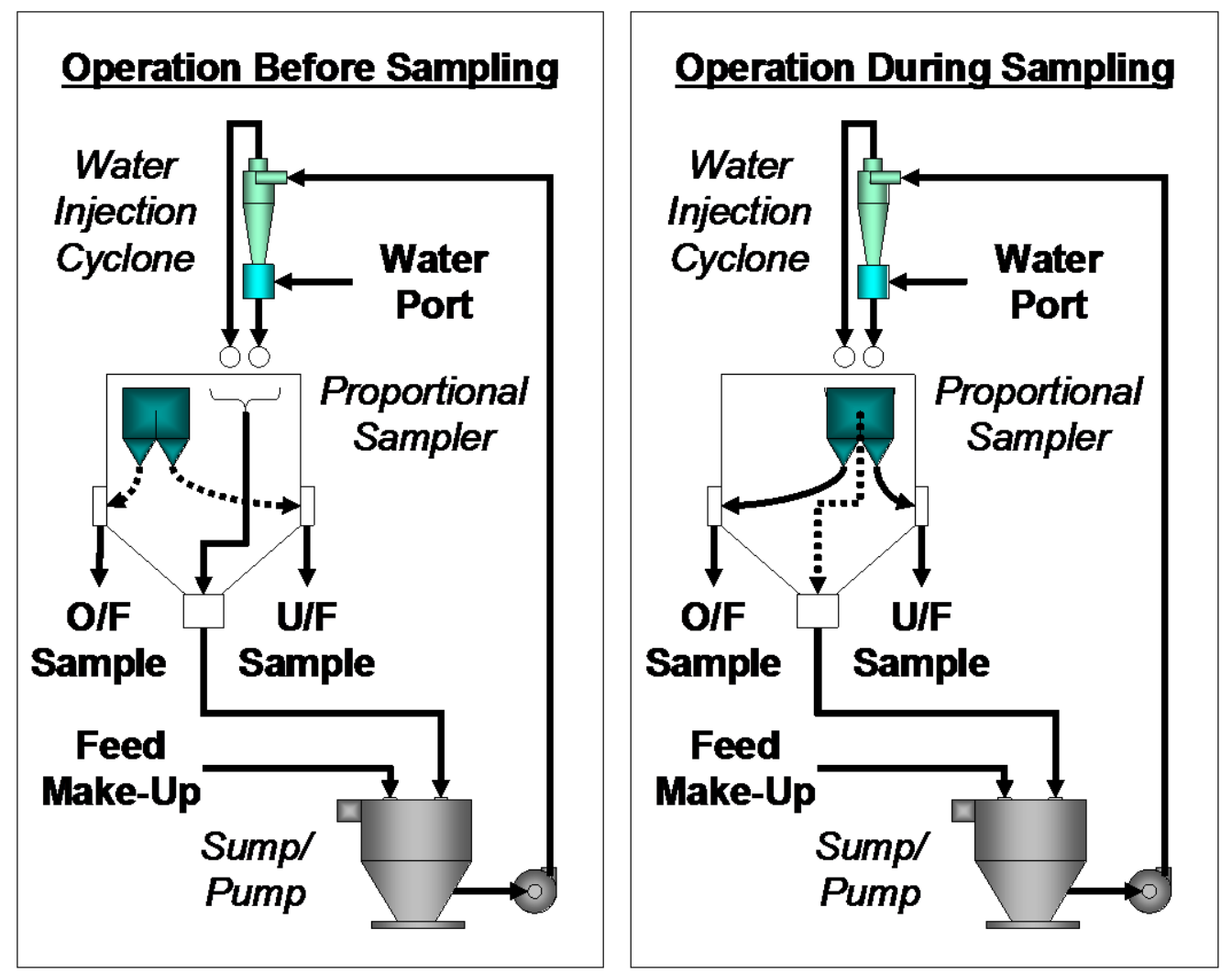

Figure 1.4 - Operating principle for the proportional sampler.

\subsubsection{Test Procedures}

\subsubsection{Test Sample}

During the process of collecting data, bench-scale 6-inch $(15.2-\mathrm{cm})$ hydrocyclone tests were conducted using flotation feed samples from the Toms Creek coal preparation plant. The plant is owned and operated by Alpha Natural Resources. The particle size distribution of this sample was nominally $0.15 \mathrm{~mm} \times 0$ (100 mesh $\mathrm{x} 0)$. Wet sieving indicated that the sample was a good candidate for ultrafine particle separation since about $70 \%$ of the sample by weight was finer than $45 \mu \mathrm{m}$ (325 mesh). The as-received solids content of the sample was found be about $6.5 \%$ by weight. Therefore, the solids content was diluted down to the $4.5 \sim 5.0 \%$ range prior to 
testing to better match the feed solids content of slurry typically treated by desliming cyclones at operating plant sites in the coal industry.

\subsubsection{Preliminary Testing}

Several preliminary test runs were conducted to determine the appropriate vortex finder size for the test program. In these tests, the effects of vortex finder and apex geometries on pressure drop and volumetric flow rate were evaluated. These initial experiments were carried out using water and minus 100 mesh coal slurry having a solids content of approximately $4.6 \%$ solids by weight. The pressure drop across the cyclone was measured by taking the difference between the feed pressure at the cyclone inlet and the overflow pressure at the vortex outlet. The effects of changes to the hydrocyclone geometry on pressure drop and volumetric flow rate are summarized in Figure 1.5.

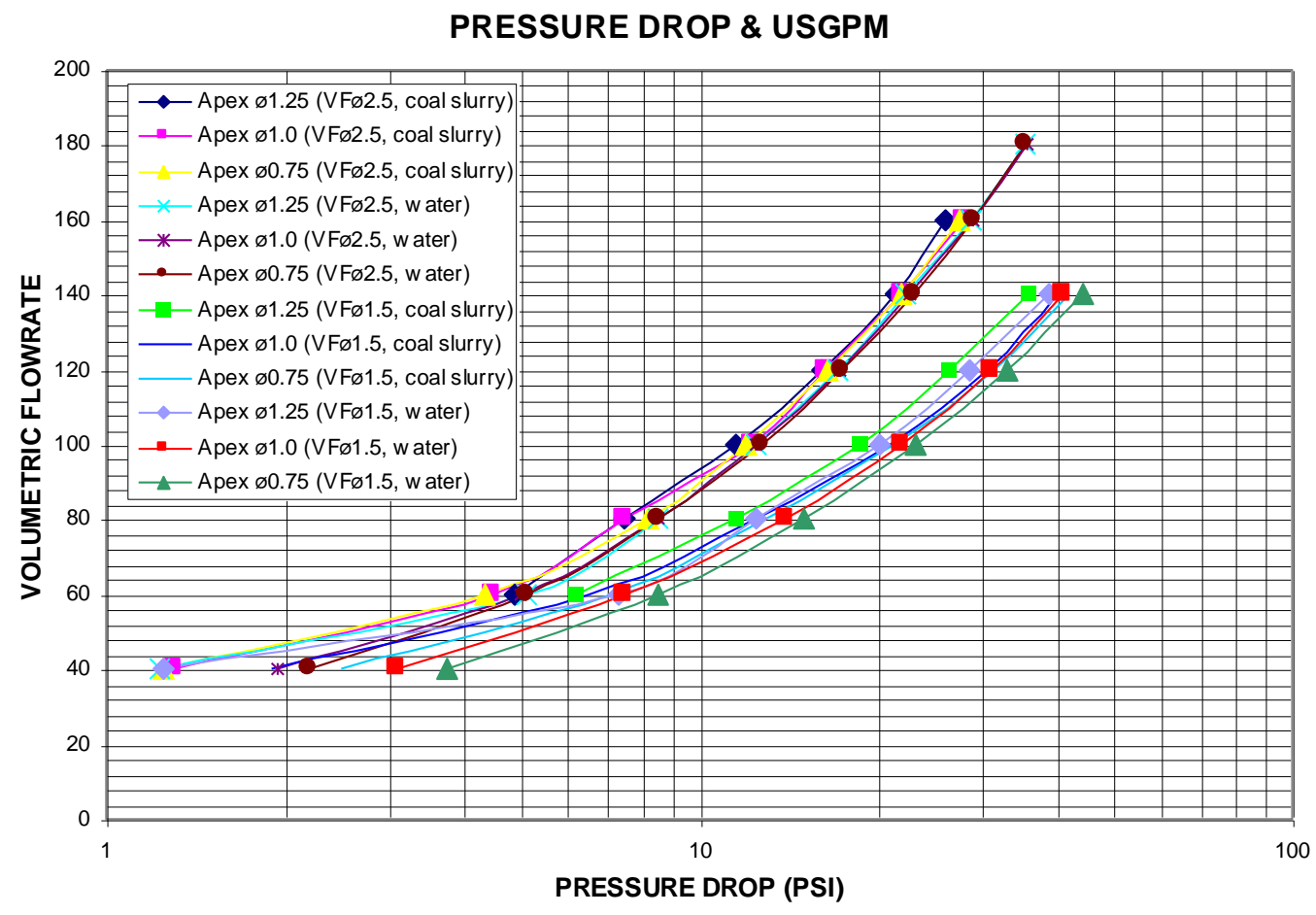

Figure 1.5 - Flow and pressure response as a function of apex and vortex sizes for the 6-inch $(15.2-\mathrm{cm})$ diameter Krebs G-Max cyclone used in the test program. 
The data provided in Figure 1.5 show that the size of the vortex finder and pressure drop is interdependent. In addition, the data demonstrate that the hydrocyclone can pass more slurry at a given pressure than water. A larger vortex finder results in a lower pressure drop for the same volume or a greater capacity for the same pressure drop. Conversely, a small diameter vortex finder result in a larger pressure drop for the same volume. Although particle size analyses were not conducted on these particular samples, an increase in cyclone pressure drop usually leads to a higher volumetric throughput and a finer particle cut size (Svarovsky, 1984). Therefore, based on these results, a 1.5-inch $(3.8-\mathrm{cm})$ diameter vortex finder was selected for the test program since it could provide a higher pressure drop at given volumetric flow rate for the new water-injected apex system.

A few preliminary test runs were also performed to compare the performance of the standard conventional apex and the water-injected apex prior to the initiation of extensive detailed testing. These initial tests were conducted using a 1.5-inch $(3.8-\mathrm{cm})$ diameter vortex finer and a constant 120 GPM volumetric slurry flow rate. The experiments were carried out using water and minus 100 mesh coal slurry having a solids content of approximately $4.5 \%$ solids by weight. The resultant test data shown in Figure 1.6 indicate that the new water-injected apex has the ability to achieve much better classification performance with a low pressure drop. In fact, the particle size partition curve obtained at a pressure drop of 26 PSI with a 1 -inch diameter water-washed apex was nearly identical to the curve obtained at a much higher pressure drop of 32 PSI with a smaller 0.75 -inch diameter conventional apex. The ability to operate with a larger apex has substantial advantages in term of being less prone to plugging. In addition, these results suggest that the new water-injection apex may make it possible to achieve finer particle size separations with larger diameter (higher capacity) cyclones in the future. 


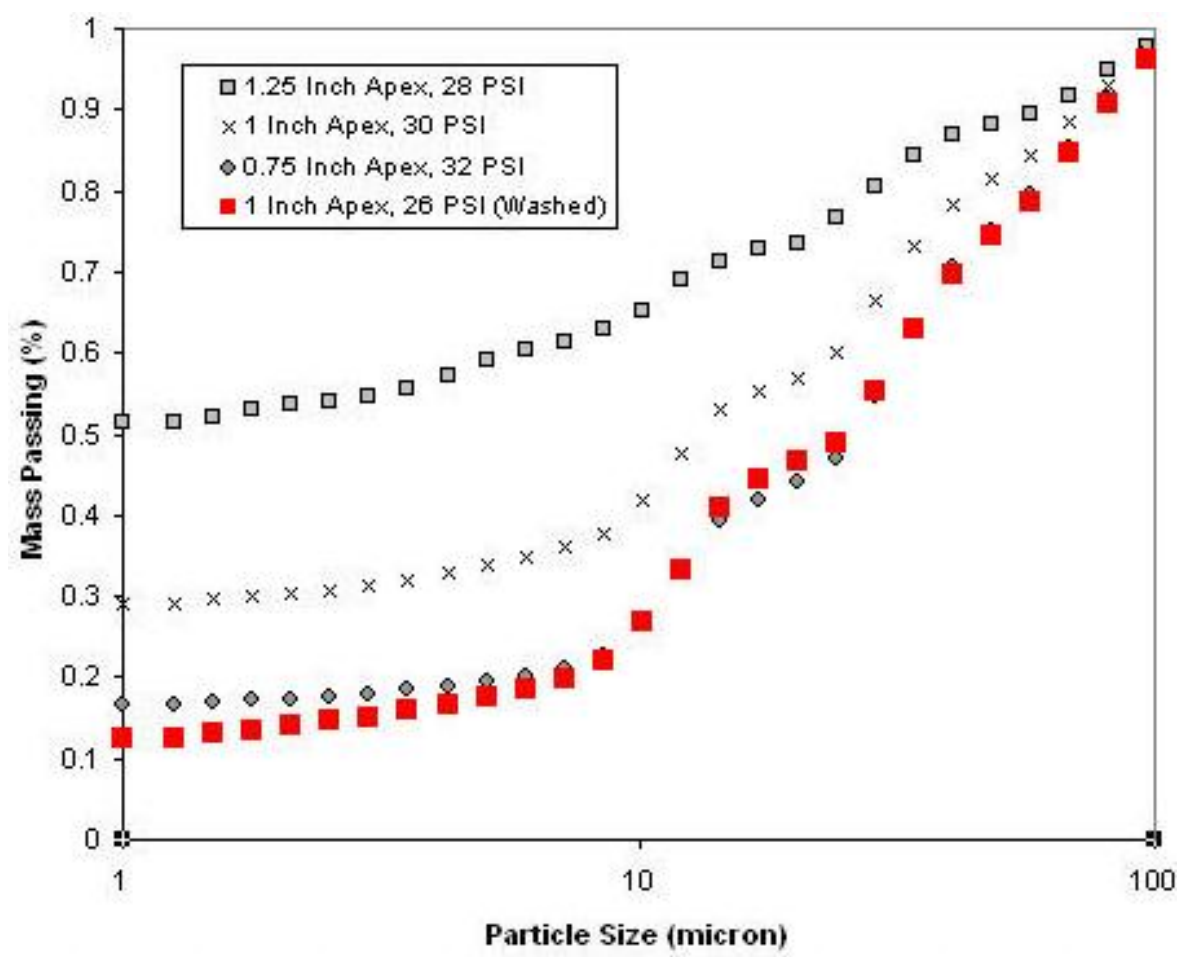

Figure 1.6 - Effect of water injection on hydrocyclone partitioning performance.

\subsubsection{Detailed Testing}

After completing the preliminary tests, a detailed test program was undertaken to better identify the operational capabilities of the new water-injection apex. All of the detailed tests were conducted using the 6-inch $(15.2-\mathrm{cm})$ diameter classifying cyclone equipped with a 1.5inch $(3.8-\mathrm{cm})$ diameter vortex finder. Different geometrical combinations of apex inlet, outlet and diameter were examined for the water-injected system. In each test run, two sets of samples were collected under steady-state conditions for the same performance. The first sample was used to determine the total solid contents and head ash contents of the feed, overflow and underflow streams. The second identical sample was subjected to laboratory particle size 
analysis. Particle size analysis was performed by wet sieving particles larger than $45 \mu \mathrm{m}$ (325 mesh) and by laser analysis (Microtrac) of particles finer than $45 \mu \mathrm{m}$ (325 mesh).

The sample point for the feed stream was the discharge of slurry from the return line that circulated back to the slurry sump. Sample points for the underflow and overflow streams were cut by the linear proportional cutter located inside the automated sampler. The total volume of each slurry sample was reduced to a manageable volume by representatively subdividing the slurry into smaller lots using a wet rotary slurry splitter. Because of the use of a closed-loop system, the addition of injection water increased the volume of circulating slurry which, in turn, raised the sump level and reduced the feed solids content. To overcome this problem, a small dosage of flocculant was added to the circulating feed sump after each series of test runs to quickly aggregate and settle coal particles. Once settled, some of the clarified water at the top of the sump was pumped out to restore the solids content of the feed slurry back to the desired range of $4.5-5.0 \%$ by weight. Comparison studies showed that the required flocculant dosage was too low to impact the sizing performance of the hydrocyclone due to the high levels of shear within the centrifugal feed pump, piping network and cyclone.

All of the detailed tests were conducted using a volumetric feed slurry flow rate of 100 GPM, which typically provided a pressure drop across the cyclone of 21 PSI. Figure 1.5 indicates that this pressure drop is appropriate for the 6-inch $(15.2-\mathrm{cm})$ diameter hydrocyclone used in this study. The detailed tests were run in accordance with a Box-Behnken parametric test matrix developed using the Design Expert ${ }^{\mathrm{TM}}$ software package. Four parameters were varied (i.e., water injection flow rate, apex outlet diameter, apex inlet diameter and apex chamber diameter) to create a 30 point test matrix for the water-injected apex system. The lower, middle and upper settings for each of these parameters are summarized in Table 1.1. As shown, the water injection 
Table 1.1 - Variables used in the parametric study to evaluate the effects of various operating and geometric variables on apex washing performance.

\begin{tabular}{cccc}
\hline Parameter & Lower (-1) Level & Central (0) Level & Upper (+1) Level \\
\hline $\begin{array}{c}\text { Apex Chamber } \\
\text { Diameter }\left(\mathrm{D}_{\mathrm{c}} \text {, inch) }\right.\end{array}$ & 3.50 & 4.00 & 4.50 \\
\hline $\begin{array}{c}\text { Apex Water Inlet } \\
\text { Diameter }\left(\mathrm{D}_{\mathrm{i}} \text {, inch) }\right.\end{array}$ & 0.50 & 0.75 & 1.00 \\
\hline $\begin{array}{c}\text { Apex Water Outlet } \\
\text { Diameter ( } \mathrm{D}_{\mathrm{a}} \text {, inch) }\end{array}$ & 0.75 & 1.00 & 1.25 \\
\hline $\begin{array}{c}\text { Apex Water Flow } \\
\text { Rate }(\mathrm{Q}, \mathrm{GPM})\end{array}$ & 0.00 & 15.00 & 30.00 \\
\hline
\end{tabular}

rates was varied over three levels (i.e., 0,15 and $30 \mathrm{GPM}$ ), which included the baseline condition of adding no water (0 GPM). Water flow rate to a water-injected apex was controlled using a combination of a flow control valve and centrifugal booster pump. The remaining variables evaluated in the test program focused on modifications to the internal dimensions of the waterinjected apex. These changes included three levels for the diameters for the apex chamber (3.5, 4.0 and 4.5 inches), apex water inlet (0.50, 0.75 and 1.00 inches), and apex outlet $(0.75,1.00$ and 1.25 inches). Details related to the text matrix and a summary of the detailed test data are provided in tabular form in Appendix I.

Several series of replicate tests were included in the test matrix. These replicate tests were randomly run within the testing sequence so that the impact of natural variations (random error) in the experimental test data could be quantified and compared to the response changes attributed to the input variables. These "normal" tests were all run at the middle setting for each of the four test variables (i.e., 15 GPM injection water, 4.0-inch diameter apex chamber, 0.75inch diameter apex inlet, 1.00-inch diameter apex outlet). Figure 1.7 provides a comparison of the partitioning data from each of these four normal tests. The data indicate that the cyclone 


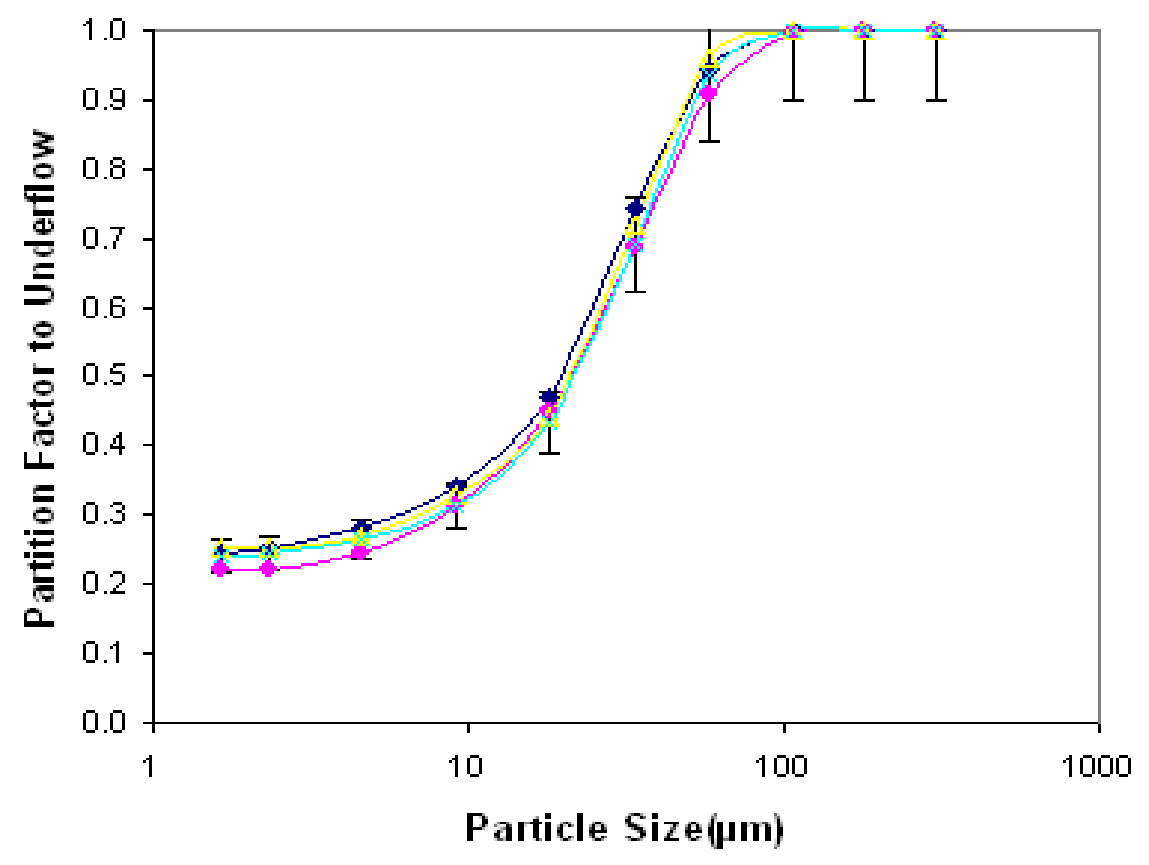

Figure 1.7 - Partitioning data from replicate tests conducted under identical test conditions $(0$ level settings) for the water-injected apex (with 10\% error bars).

performance was very stable with an overall error of less than $10 \%$ error in the partitioning curves. The particle cutsize determined from the partition curves varied from just 26 to $29 \mu \mathrm{m}$, while the ultrafine bypass varied between 20 to $25 \%$ for all replicate tests. 


\subsection{Results and Discussion}

\subsubsection{Parametric Study Results}

To fully investigate the effects of the operating and geometric parameters on sizing performance, regression equations for cutsize and bypass were obtained from the Box-Behnken parametric study using the Design-Expert ${ }^{\mathrm{TM}}$ software. The resultant linear regression equations obtained for cutsize and bypass are shown in Table 1.2. The input variables used in the uncoded expressions are entered as true units of measure (GPM and inches), while the coded values are entered as normalized units ranging between -1 and +1 (see Table 1.2).

The overall data analysis suggested that the effects of water injection flow rate, apex inlet diameter, apex chamber diameter and apex outlet diameter were all interrelated. Nevertheless, a simple linear model was selected for the regression analysis since it still provided a relatively good fit to the experimental data. As shown in Figure 1.8, the cutsize and bypass values predicted by the linear model were in reasonably good agreement with the experimentally determined values. More importantly, the linear model maximizes the numerical significance of

Table 1.2 - Regression expressions obtained from the Box-Behnken parametric study.

\begin{tabular}{lc}
\hline \multicolumn{1}{c}{ Uncoded Expressions } & \multicolumn{1}{c}{ Coded Expressions } \\
\hline Cut Size $=$ & Cut size $=$ \\
+61.84083 & +30.43 \\
+0.32717 Water Injection Rate & +4.91 Water Injection Rate \\
-1.66000 Apex Inlet & -0.42 Apex Inlet \\
-3.15667 Apex Chamber & -1.58 Apex Chamber \\
-22.44333 Apex Outlet & -5.61 Apex Outlet \\
\hline Bypass $=$ & Bypass $=$ \\
-0.43483 & +0.26 \\
-0.0062222 Water Injection Rate & -0.093 Water Injection Rate \\
-0.02333 Apex Inlet & -0.005833 Apex Inlet \\
+0.055000 Apex Chamber & +0.027 Apex Chamber \\
+0.58667 Apex Outlet & +0.15 Apex Outlet \\
\hline
\end{tabular}



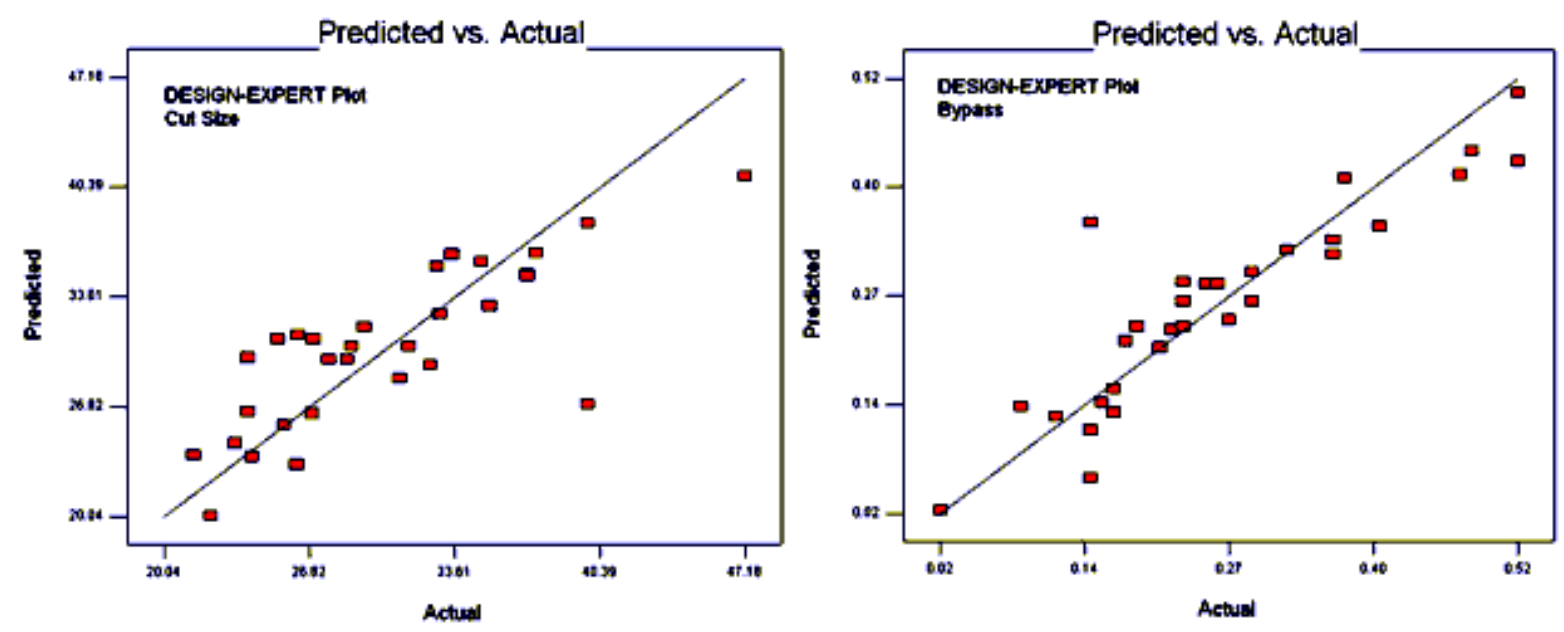

Figure 1.8 - Correlation between actual and predicted cut size and bypass.

the main effect for each variable. As such, the coefficients in the regression equations are more meaningful for a linear model than for other higher term (quadratic or cubic) models that could have been used in the statistical analysis.

The regression expressions indicate that both the water injection flow rate and apex outlet diameter are significant terms in the linear models for cutsize and bypass since the coded coefficients are relatively large. The expressions show that a smaller apex outlet diameter and higher water injection rate produce a larger particle cutsize and smaller bypass. On the other hand, the coded expressions show that the apex inlet diameter and chamber diameter are not significant in either of the linear models. As such, these parameters do not have a significant influence on either the cutsize or bypass obtained using the water-injected apex system.

To further illustrate the effects described above, the linear regression data was plotted to show the correlations between the water injection rate and the two responses of primary interest (i.e., cutsize and bypass). The plots for water injection rate and apex outlet diameter are shown in Figures 1.9 and 1.10, respectively. The large value for Pearson's correlation coefficient $\left(\mathrm{R}^{2}\right)$ 

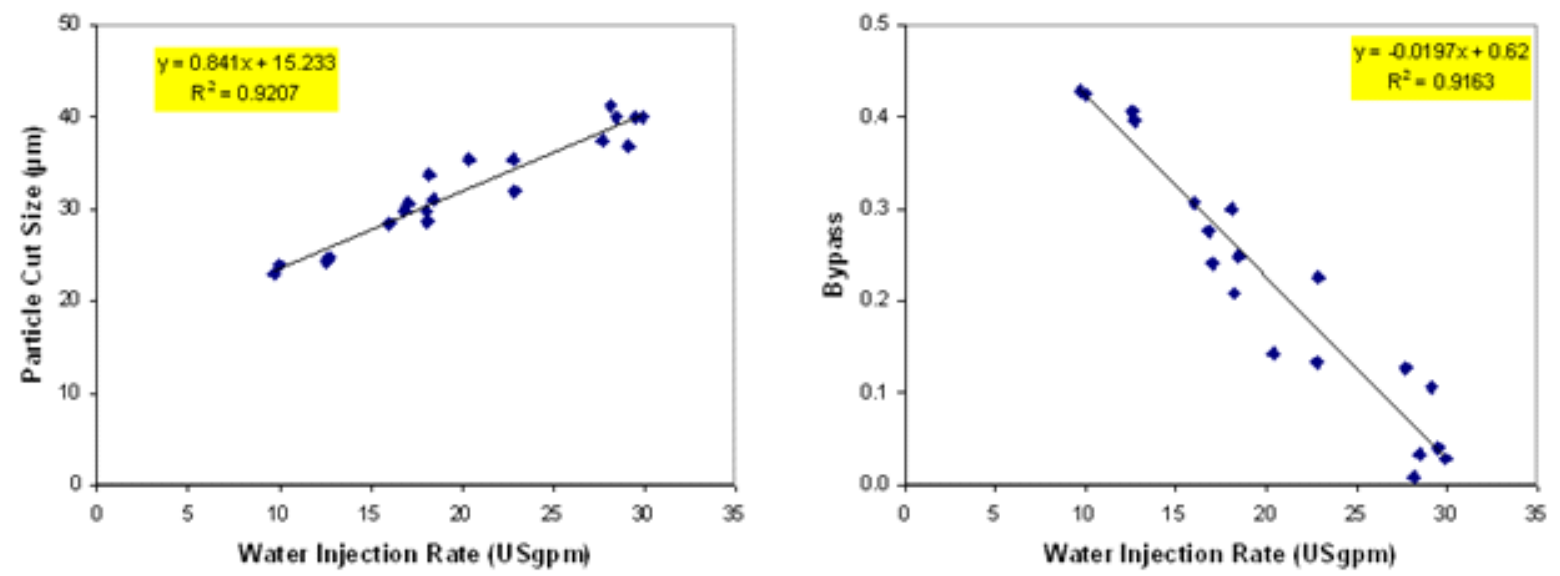

Figure 1.9 - Effect of water injection rate on cutsize and bypass under central point conditions ( 0.75 inch apex inlet, 4 inch chamber, 1 inch outlet).
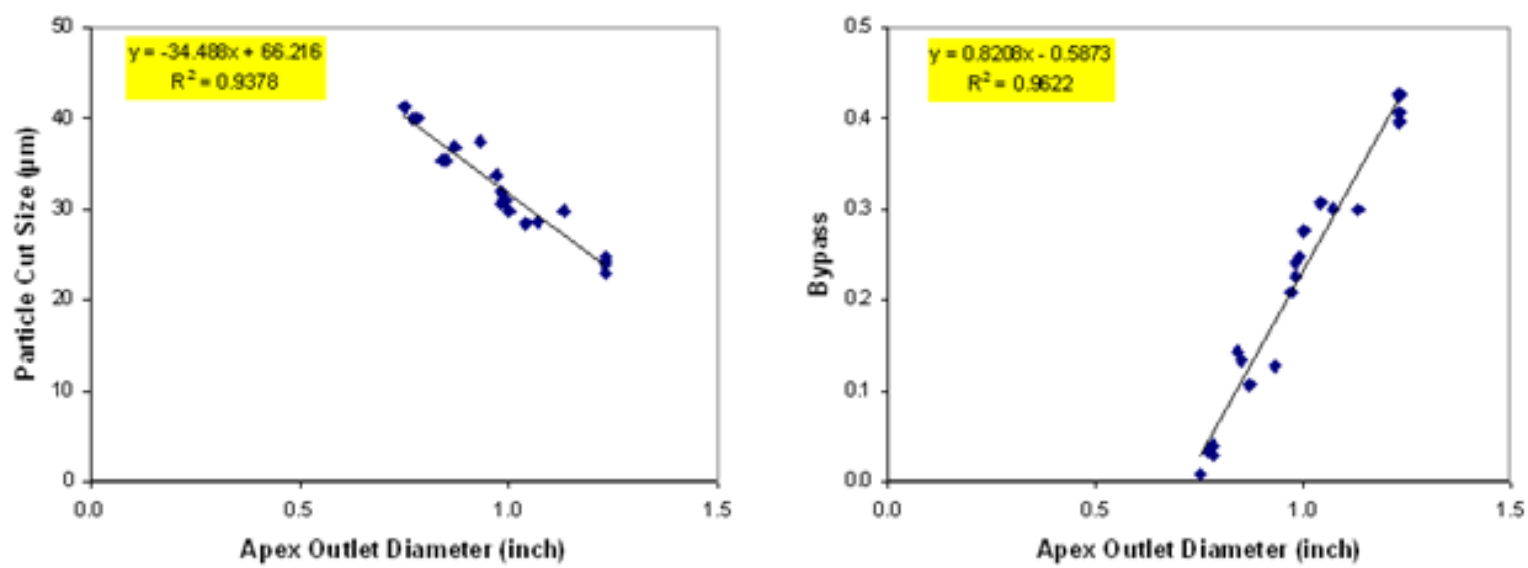

Figure 1.10 - Effect of apex outlet diameter on cutsize and bypass under central point conditions ( 0.75 inch apex inlet, 4 inch chamber, 20 GPM water rate).

indicates that the water injection rate and two responses are statistically highly correlated. In other words, a higher water injection rate produces a strong increase in cutsize and strong decrease in bypass. Likewise, in the case of apex outlet diameter, the linear regression plots again show a large $\mathrm{R}^{2}$ value. This statistical correlation indicates as that an increase in apex outlet diameter strongly decreases particle cutsize and increases ultrafine bypass. 

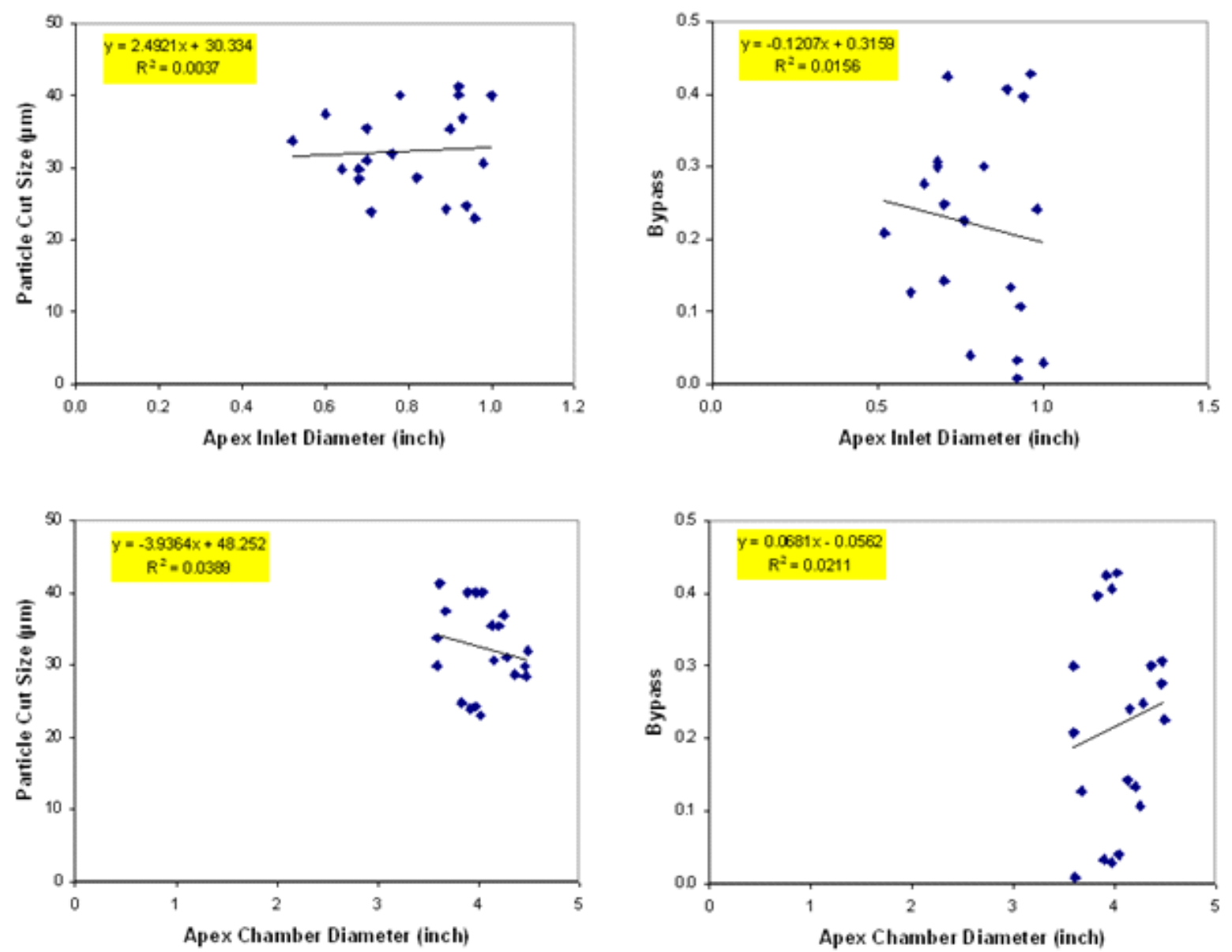

Figure 1.11 - Effect of apex inlet diameter (top two plots) and chamber diameter (bottom two plots) on cutsize and bypass.

The correlations plotted in Figure 1.11, however, show that apex inlet diameter and chamber diameter do not significantly affect either the cutsize or bypass. The $\mathrm{R}^{2}$ value was less than 0.01 when comparing the effect of apex inlet diameter on cutsize and bypass. Similarly, poor $\mathrm{R}^{2}$ values of less than 0.03 were obtained when comparing the effect of apex chamber diameter on cutsize and bypass. Thus, neither of these parameters was found to have a statistically significant impact on the performance of the new water-injected apex. 


\subsubsection{Verification Results}

The results of the parametric study indicate that water injection rate has the largest overall impact on the partitioning performance provided by the water injected apex. Therefore, to better examine the influence of this parameter on the two significant responses (i.e. cutsize and bypass), an additional set of four tests were conducted at water injection rates of $0,10,20$ and 30 GPM. All other geometric variables were held constant at their central point as specified in the detailed test matrix. The resultant data, which are plotted in Figure 1.12, shows that increasing the water injection rate produces a larger particle cutsize and lower bypass. Particle cutsize increased steadily from $23.20,25.41,30.41$ and $38.07 \mu \mathrm{m}$ as the injection water flow rate increased. Likewise, the bypass of ultrafines to the underflow decreased steadily from $0.32,0.24$,

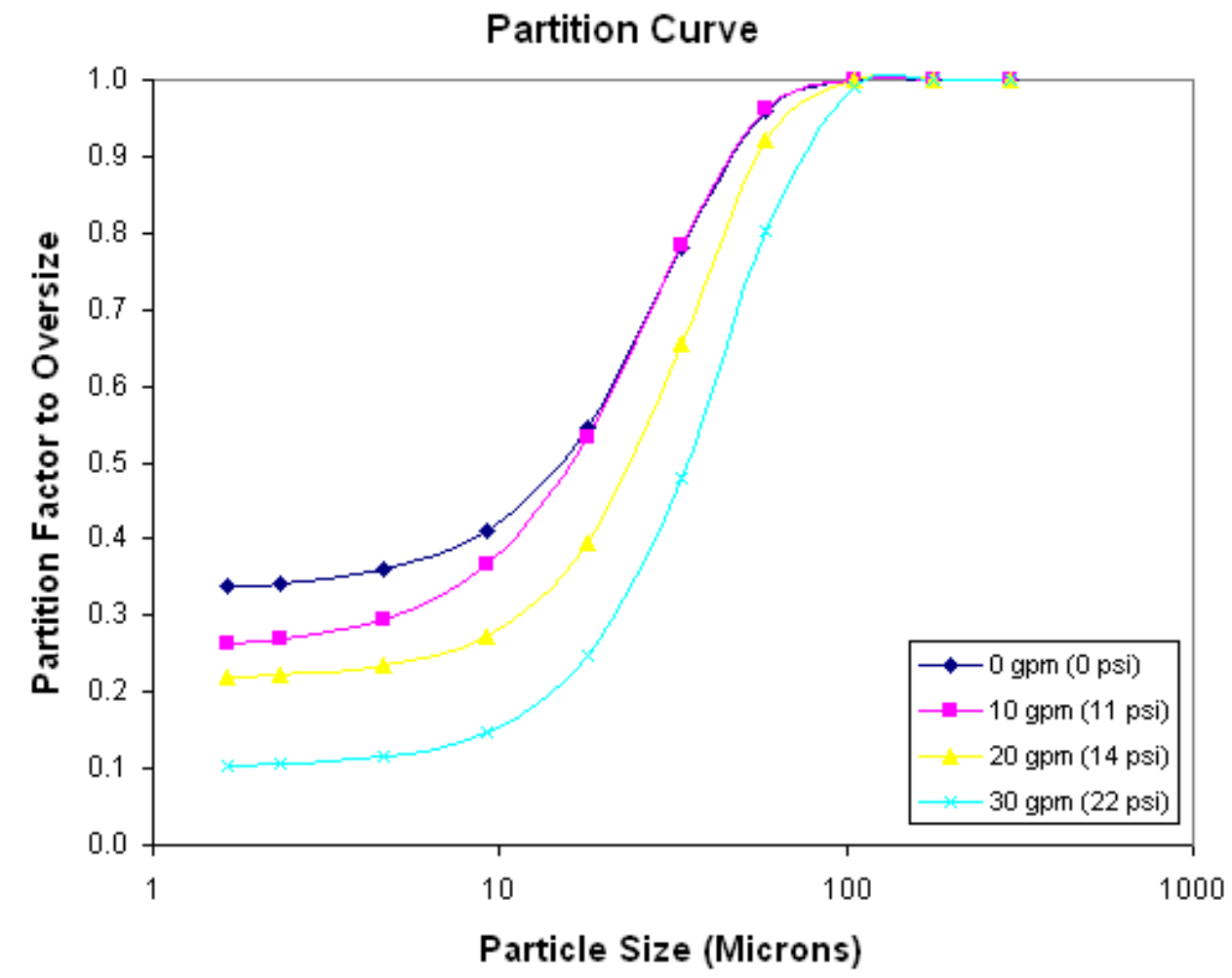

Figure 1.12 - Partitioning performance as a function of water injection rate under central point conditions ( 0.75 inch apex inlet, 4 inch chamber, 1 inch outlet). 
0.20 and 0.09 as the water injection rate increased. These opposing trends demonstrate the tradeoff between cutsize and bypass that must be considered when using a water-injected apex.

\subsubsection{Optimization Results}

Several series of statistical analyses were conducted using the Design-Expert ${ }^{\mathrm{TM}}$ software to identify the optimum settings of controllable variables that minimize bypass for a desired cutsize range. The optimization was carried out over the same range of controllable variables as used in the detailed test matrix. As such, the water injection flow rate was varied from 0 to 30 GPM, apex inlet diameter was varied from 0.5 to 1 inch, apex chamber diameter was varied from 3.5 to 4.5 inches, and apex outlet diameter was varied from 0.75 to 1.25 inches. Five different cutsize ranges were considered in the optimization, i.e., $20 \sim 25 \mu \mathrm{m}, 25 \sim 30 \mu \mathrm{m}, 30 \sim 35 \mu \mathrm{m}, 35 \sim 40$ $\mu \mathrm{m}$ and $40 \sim 45 \mu \mathrm{m}$. The combination of variables that provided the smallest bypass was considered the best solution among several optimized solutions obtained for each cutsize range. For cases in which many optimized solutions were found, a secondary objective of a lower water injection rate was used to select the best combination of controllable variables. Once the optimum solution was identified for each cutsize range, three-dimensional (3D) response surface plots were created so that the influence of the two most significant variables (i.e., water-injection rate and apex outlet diameter) could be visualized at the optimum settings for the two remaining variables (i.e., apex inlet diameter and chamber diameter).

The results of the optimization runs are summarized in Tables 1.3-1.7 for each of the five size ranges examined in this study. The corresponding response surface plots for each of these tables are also provided in Figures 1.13-1.22. For ease of comparison, the optimum values are also summarized in Table 1.8. 
Table 1.3 - Optimized conditions for the $20 \sim 25 \mu \mathrm{m}$ cutsize range.

\begin{tabular}{cccccc}
\hline $\begin{array}{c}\text { Injection } \\
\text { Rate } \\
(\mathrm{GPM})\end{array}$ & $\begin{array}{c}\text { Apex } \\
\text { Outlet } \\
\text { (Inch) }\end{array}$ & $\begin{array}{c}\text { Apex Inlet } \\
\text { Diameter } \\
\text { (Inch) }\end{array}$ & $\begin{array}{c}\text { Apex } \\
\text { Chamber } \\
\text { Diameter } \\
\text { (Inch) }\end{array}$ & $\begin{array}{c}\text { Cut Size } \\
\text { (Microns) }\end{array}$ & Bypass \\
\hline 11.78 & 1.25 & 0.87 & 4.02 & 23.56 & 0.4247 \\
\hline
\end{tabular}

DESIGN-EXPERT Plot

Cutsize

$\mathrm{X}=\mathrm{A}$ : Water Injection Rate

$\mathrm{Y}=\mathrm{B}$. Apex Outlet

Actual Factors

C: Apex Inlet $=0.87$

D: Apex Chamber $=4.02$

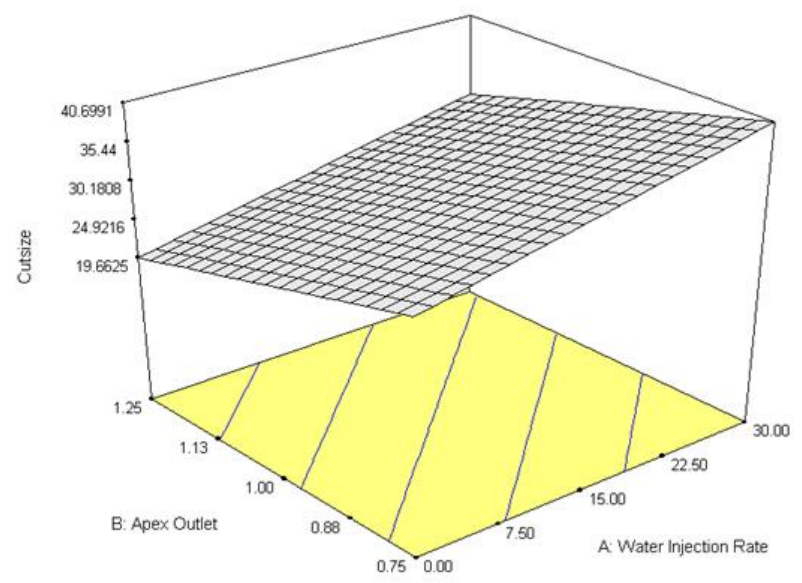

Figure 1.13 - Cutsize response at the optimum point for $20 \sim 25 \mu \mathrm{m}$ classification.
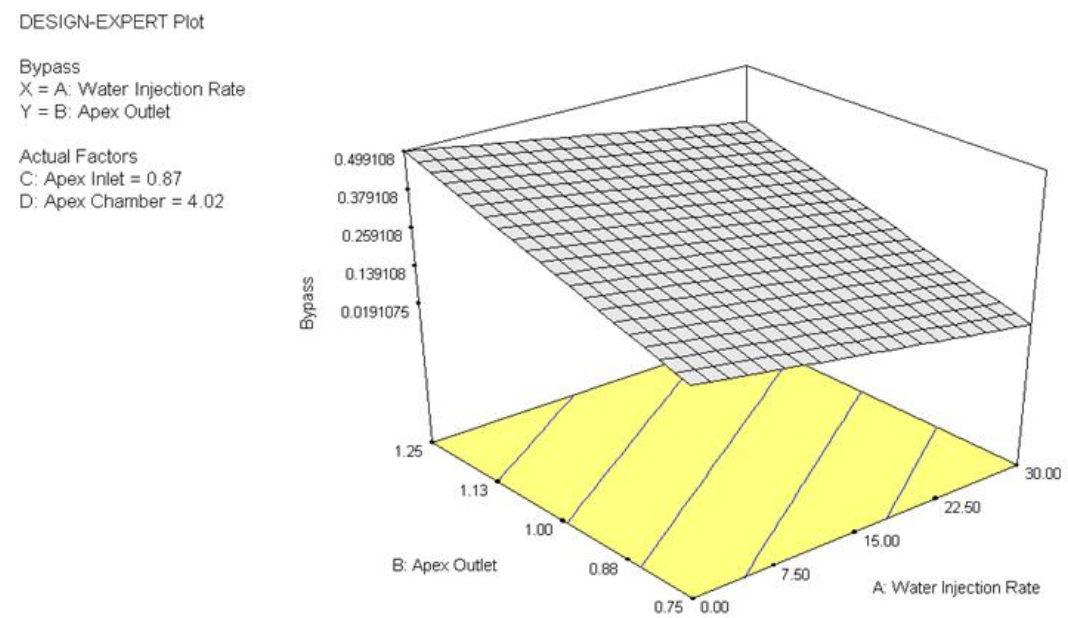

Figure 1.14 - Bypass response at the optimum point for $20 \sim 25 \mu \mathrm{m}$ classification. 
Table 1.4 - Optimized conditions for the $25 \sim 30 \mu \mathrm{m}$ cutsize range.

\begin{tabular}{cccccc}
\hline $\begin{array}{c}\text { Injection } \\
\text { Rate } \\
(\text { GPM })\end{array}$ & $\begin{array}{c}\text { Apex } \\
\text { Outlet } \\
\text { (Inch) }\end{array}$ & $\begin{array}{c}\text { Apex Inlet } \\
\text { Diameter } \\
\text { (Inch) }\end{array}$ & $\begin{array}{c}\text { Apex } \\
\text { Chamber } \\
\text { Diameter } \\
\text { (Inch) }\end{array}$ & $\begin{array}{c}\text { Cut Size } \\
\text { (Microns) }\end{array}$ & Bypass \\
\hline 13.58 & 1.03 & 0.78 & 4.11 & 28.98 & 0.2908 \\
\hline
\end{tabular}

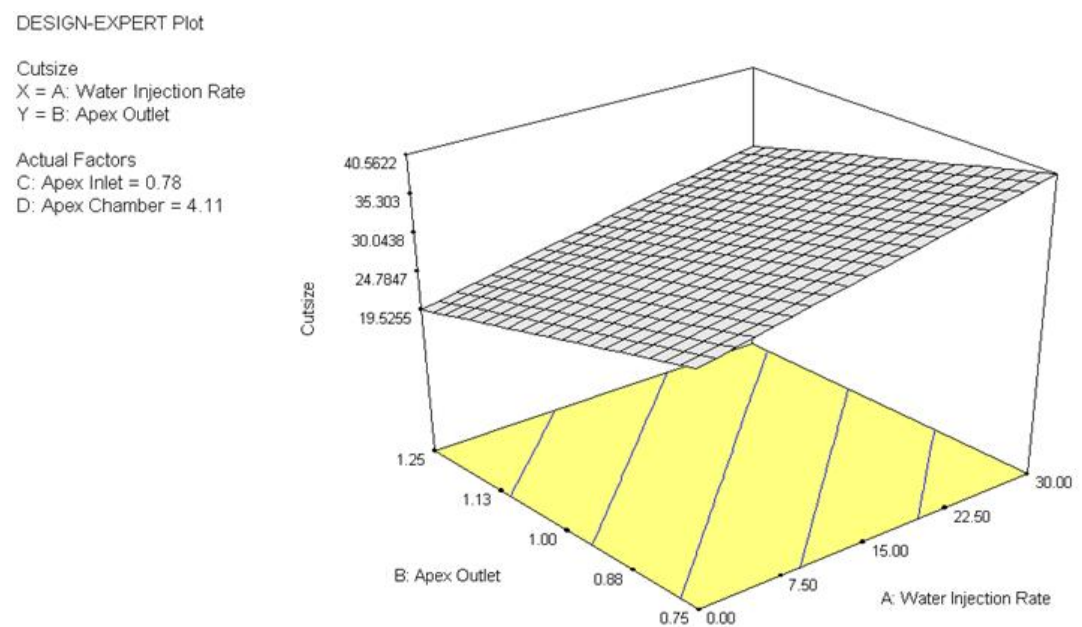

Figure 1.15 - Cutsize response at the optimum point for $25 \sim 30 \mu \mathrm{m}$ classification.
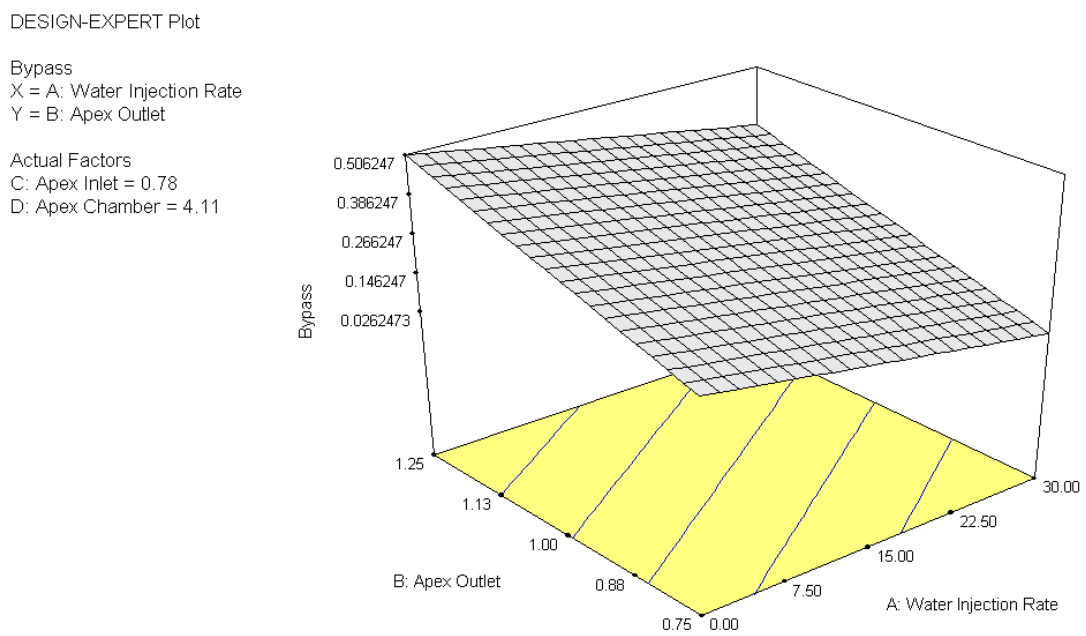

Figure 1.16 - Bypass response at the optimum point for $25 \sim 30 \mu \mathrm{m}$ classification. 
Table 1.5 - Optimized conditions for the $30 \sim 35 \mu \mathrm{m}$ cutsize range.

\begin{tabular}{cccccc}
\hline $\begin{array}{c}\text { Injection } \\
\text { Rate } \\
(\mathrm{GPM})\end{array}$ & $\begin{array}{c}\text { Apex } \\
\text { Outlet } \\
\text { (Inch) }\end{array}$ & $\begin{array}{c}\text { Apex Inlet } \\
\text { Diameter } \\
\text { (Inch) }\end{array}$ & $\begin{array}{c}\text { Apex } \\
\text { Chamber } \\
\text { Diameter } \\
\text { (Inch) }\end{array}$ & $\begin{array}{c}\text { Cut Size } \\
\text { (Microns) }\end{array}$ & Bypass \\
\hline 18.50 & 0.99 & 0.96 & 4.24 & 30.71 & 0.2416 \\
\hline
\end{tabular}

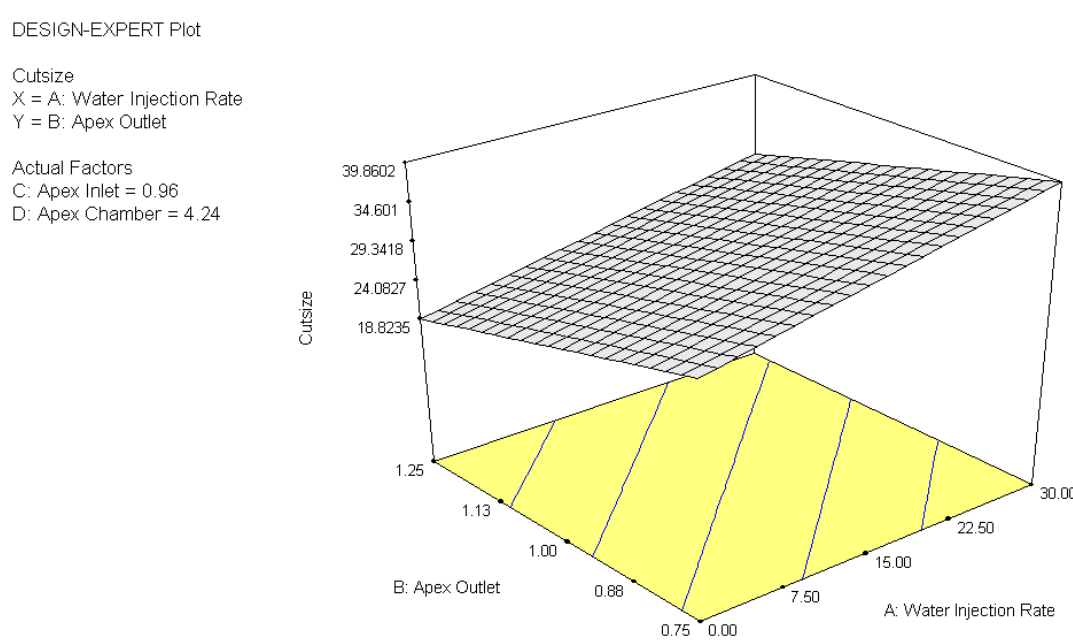

Figure 1.17 - Cutsize response at the optimum point for 30 35 $\mu \mathrm{m}$ classification.

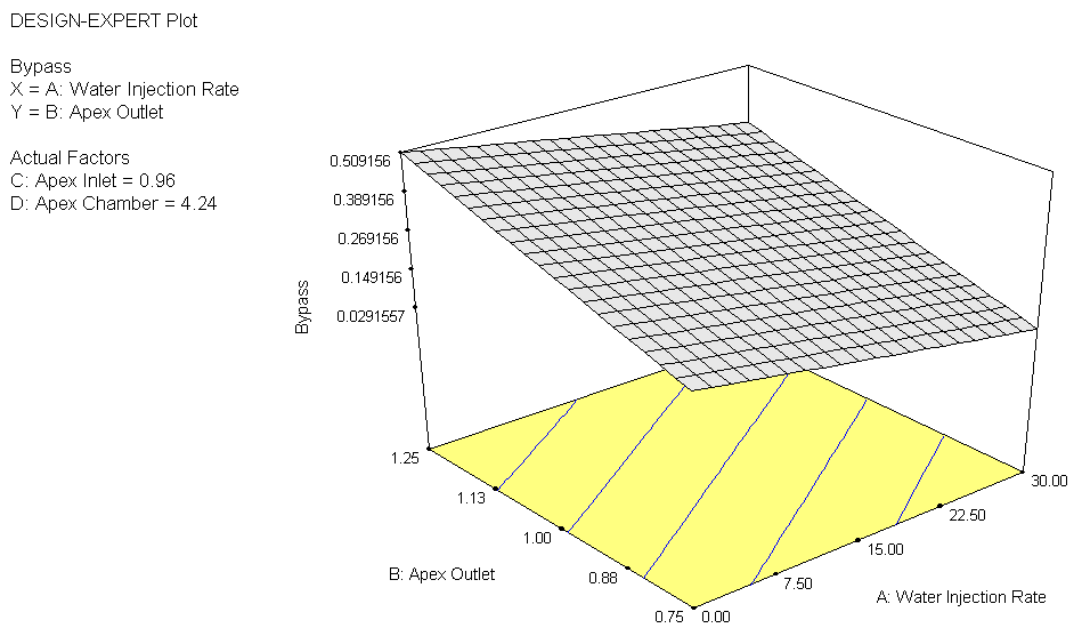

Figure 1.18 - Bypass response at the optimum point for 30 35 $\mu \mathrm{m}$ classification. 
Table 1.6 - Optimized conditions for the $35 \sim 40 \mu \mathrm{m}$ cutsize range.

\begin{tabular}{cccccc}
\hline $\begin{array}{c}\text { Injection } \\
\text { Rate } \\
(\mathrm{GPM})\end{array}$ & $\begin{array}{c}\text { Apex } \\
\text { Outlet } \\
(\text { Inch) }\end{array}$ & $\begin{array}{c}\text { Apex Inlet } \\
\text { Diameter } \\
\text { (Inch) }\end{array}$ & $\begin{array}{c}\text { Apex } \\
\text { Chamber } \\
\text { Diameter } \\
\text { (Inch) }\end{array}$ & $\begin{array}{c}\text { Cut Size } \\
\text { (Microns) }\end{array}$ & Bypass \\
\hline 25.40 & 0.85 & 0.70 & 4.36 & 36.09 & 0.1309 \\
\hline
\end{tabular}

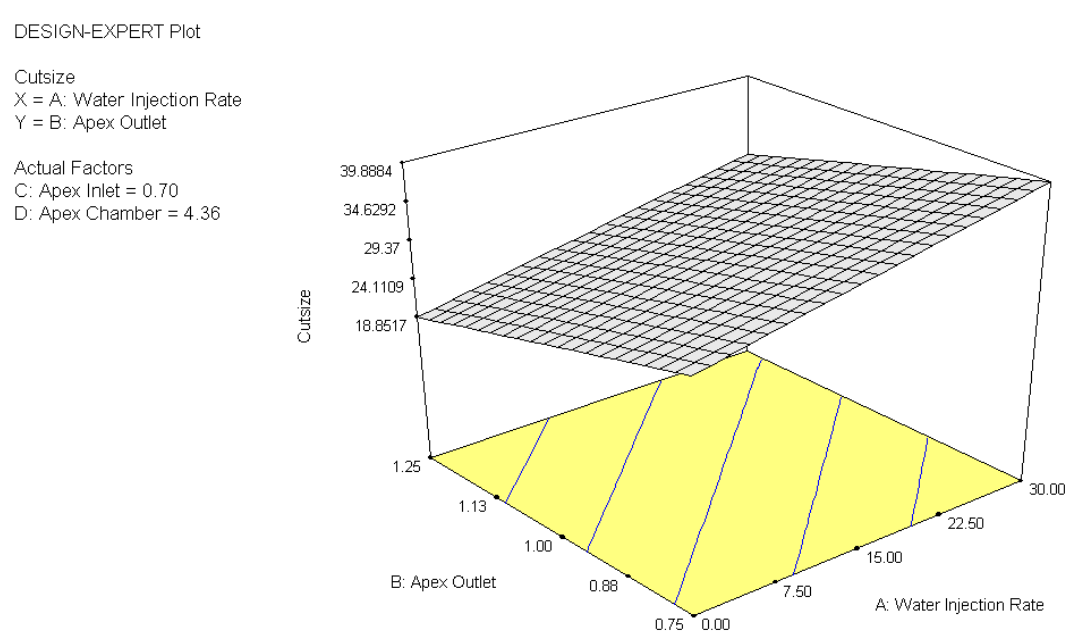

Figure 1.19 - Cutsize response at the optimum point for $35 \sim 40 \mu \mathrm{m}$ classification.
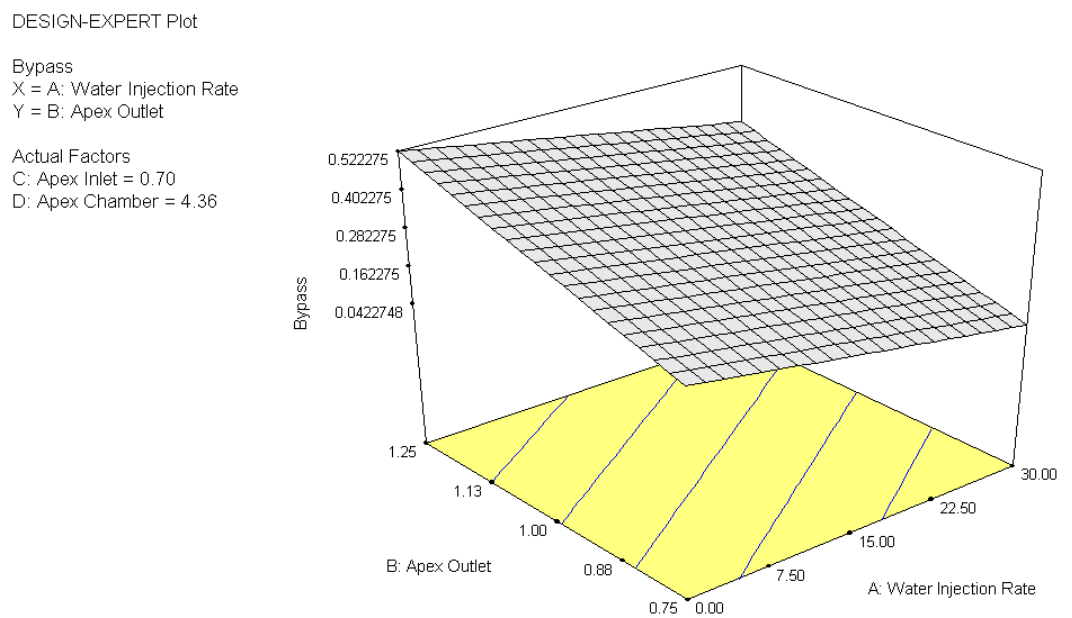

Figure 1.20 - Bypass response at the optimum point for $35 \sim 40 \mu \mathrm{m}$ classification. 
Table 1.7 - Optimized conditions for the $40 \sim 45 \mu \mathrm{m}$ cutsize range.

\begin{tabular}{cccccc}
\hline $\begin{array}{c}\text { Injection } \\
\text { Rate } \\
(\mathrm{GPM})\end{array}$ & $\begin{array}{c}\text { Apex } \\
\text { Outlet } \\
\text { (Inch) }\end{array}$ & $\begin{array}{c}\text { Apex Inlet } \\
\text { Diameter } \\
\text { (Inch) }\end{array}$ & $\begin{array}{c}\text { Apex } \\
\text { Chamber } \\
\text { Diameter } \\
\text { (Inch) }\end{array}$ & $\begin{array}{c}\text { Cut Size } \\
\text { (Microns) }\end{array}$ & Bypass \\
\hline 29.77 & 0.76 & 0.81 & 3.94 & 40.77 & 0.0231 \\
\hline
\end{tabular}
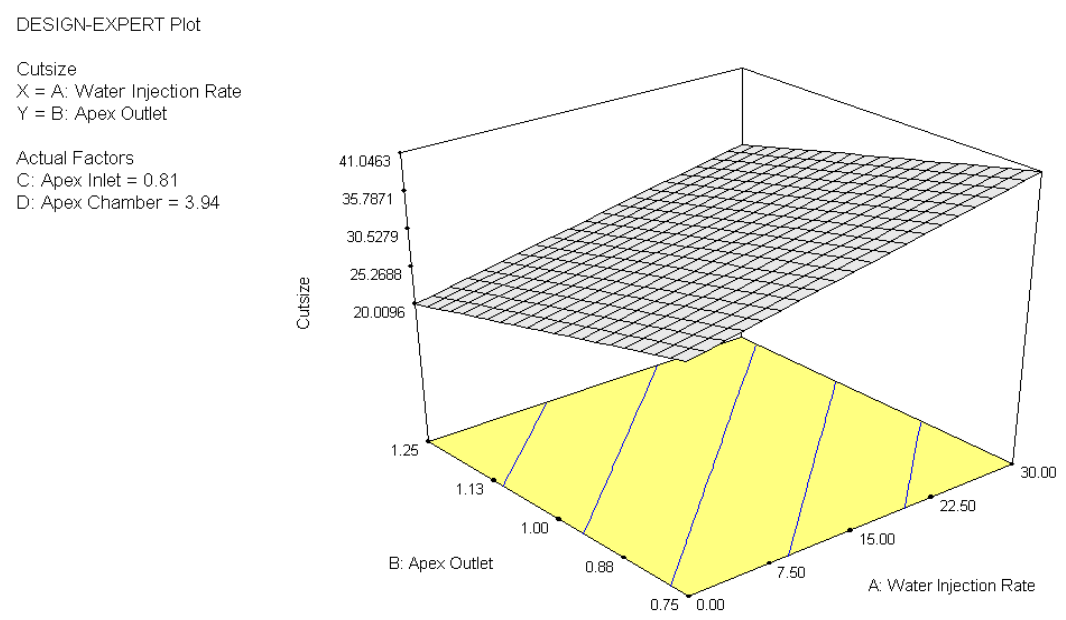

Figure 1.21 - Cutsize response at the optimum point for $40 \sim 45 \mu \mathrm{m}$ classification.

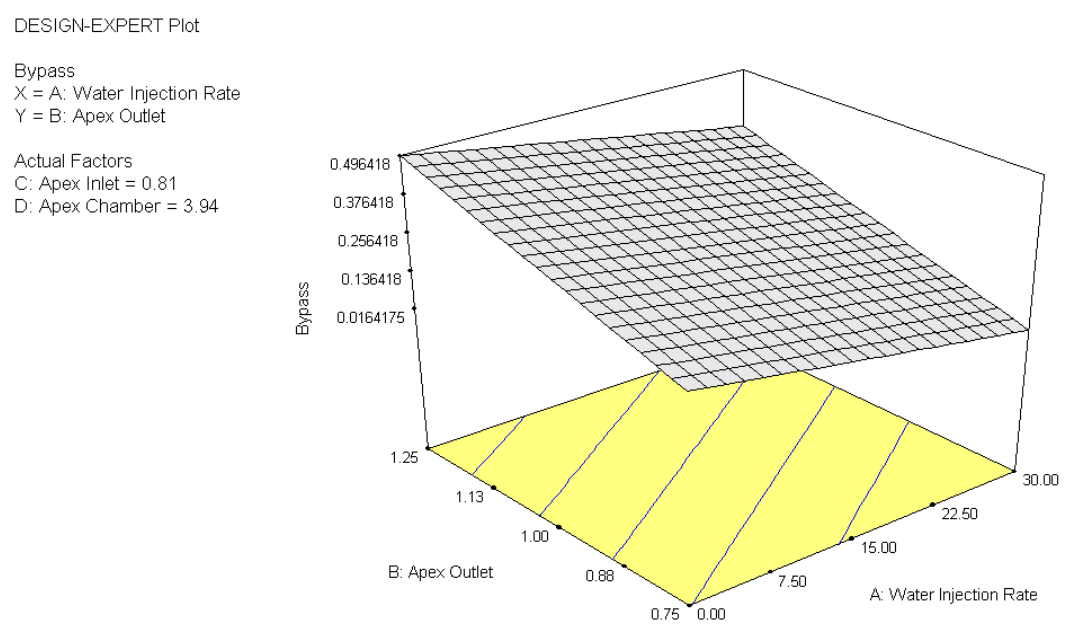

Figure 1.22 - Bypass response at the optimum point for $40 \sim 45 \mu \mathrm{m}$ classification. 
Table 1.8 - Summary of optimal conditions needed for different cutsize ranges.

\begin{tabular}{ccccccc}
\hline $\begin{array}{c}\text { Desired } \\
\begin{array}{c}\text { Size Range } \\
\text { (Microns }\end{array}\end{array}$ & $\begin{array}{c}\text { Injection } \\
\text { Rate } \\
\text { (GPM) }\end{array}$ & $\begin{array}{c}\text { Apex } \\
\text { Outlet } \\
\text { (Inch) }\end{array}$ & $\begin{array}{c}\text { Apex Inlet } \\
\text { Diameter } \\
\text { (Inch) }\end{array}$ & $\begin{array}{c}\text { Apex } \\
\text { Chamber } \\
\text { Diameter } \\
\text { Inch) }\end{array}$ & $\begin{array}{c}\text { Expected } \\
\text { Cut Size } \\
\text { (Microns) }\end{array}$ & $\begin{array}{c}\text { Expected } \\
\text { Bypass } \\
--\end{array}$ \\
\hline $20-25$ & 11.78 & 1.25 & 0.87 & 4.02 & 23.56 & 0.4247 \\
\hline $25-30$ & 13.58 & 1.03 & 0.78 & 4.11 & 28.98 & 0.2908 \\
\hline $30-35$ & 18.50 & 0.99 & 0.96 & 4.24 & 30.71 & 0.2416 \\
\hline $35-40$ & 25.40 & 0.85 & 0.70 & 4.36 & 36.09 & 0.1309 \\
\hline $40-45$ & 29.77 & 0.76 & 0.81 & 3.94 & 40.77 & 0.0231 \\
\hline
\end{tabular}

\subsubsection{Optimum Conditions for the 20-25 $\mu \mathrm{m}$ Size Range}

The best solution (i.e., combination of water injection rate, apex outlet diameter, apex inlet diameter and apex chamber diameter) for the 20-25 $\mu \mathrm{m}$ size range is shown in Table 1.8. For this very small cutsize, it was necessary to employ a low water injection rate and large apex outlet diameter. As a result, the bypass value was very high at nearly 0.42 . These results suggest that it is not possible to achieve such a small cutsize with this technology unless other operational parameters not examined in the study are changed (i.e., hydrocyclone geometry, feed inlet pressure, feed inlet diameter, etc.).

\subsubsection{Optimum Conditions for the 25-30 $\mu \mathrm{m}$ Size Range}

The best solution (i.e., combination of water injection rate, apex outlet diameter, apex inlet diameter and apex chamber diameter) for the $25-30 \mu \mathrm{m}$ size range is shown in Table 1.8. Again, the only way to achieve the small cutsize range was to operate with a relatively low water injection rate (13.6 GPM) and relatively large apex outlet (1.03 inches). The amount of bypass 
was somewhat lower for this case compared to finer 20-25 $\mu \mathrm{m}$ size range (i.e., 0.29 versus 0.42 ); however, the bypass was still relatively large compared to the project goal of achieving bypass values of less than $0.10-0.15$. Nonetheless, these results are considered an improvement over those typically provided by hydrocyclone deslime circuits that currently operate in the coal industry. These industrial circuits typically provide cutsizes in the $40-45 \mu \mathrm{m}$ size range with bypass values of $0.30-0.35$. Thus, the water-injected apex makes it possible to attain a smaller cutsize with a similar bypass to that of current industrial circuits.

\subsubsection{Optimum Conditions for the 30-35 $\mu \mathrm{m}$ Size Range}

The best solution (i.e., combination of water injection rate, apex outlet diameter, apex inlet diameter and apex chamber diameter) for the $30-35 \mu \mathrm{m}$ size range is shown in Table 1.8. In this case, the somewhat larger cutsize range made it possible to operate with a higher water flow rate so that the bypass could be reduced below 0.25 . This operating range is attractive since it provides a smaller cutsize that typically found in industrial plants (i.e., normally $40-45 \mu \mathrm{m}$ ) with significantly less bypass (i.e., normally 30-35\%).

\subsubsection{Optimum Conditions for the 35-40 $\mu \mathrm{m}$ Size Range}

The best solution (i.e., combination of water injection rate, apex outlet diameter, apex inlet diameter and apex chamber diameter) for the $35-40 \mu \mathrm{m}$ size range is shown in Table 1.8. The performance obtained in this particular operating range represents a considerable improvement over that normally achieved in industrial plants. The low bypass of 0.13 for this case can be attributed to the use of a higher water injection rate (25.4 GPM) and smaller apex outlet diameter (0.85 inches). Furthermore, the cutsize is smaller (by about $5 \mu \mathrm{m}$ ) than that 
typically obtained in industrial circuits which utilize conventional hydrocyclones that do not employ the water injected apex technologies. Therefore, this operating point is considered to be a very attractive for many of the deslime cyclone circuits that are currently operating in the coal industry. The use of the new apex washing system would be expected to improve product quality (due to less bypass) and improve coal recovery (due to the smaller cutsize).

\subsubsection{Optimum Conditions for the 40-45 $\mu \mathrm{m}$ Size Range}

The best solution (i.e., combination of water injection rate, apex outlet diameter, apex inlet diameter and apex chamber diameter) for the $40-45 \mu \mathrm{m}$ size range is shown in Table 1.8. This particular range of cutsize values represents the range that is typically achieved in industrial deslime cyclone circuits. For this practical range, a very low bypass of just over 0.02 could be realized by using a water injection flow rate approaching the maximum tested value of 30 GPM. To achieve the low bypass, a relatively small apex outlet diameter of 0.76 inches had to be used. The exceptionally low bypass makes this operating point attractive for cases in which the misplacement of slimes must be avoided in order to make the best possible quality for the final product. Such applications would include deslime circuits ahead of flotation and product sizing cyclones installed downstream of fine (100x325 mesh) spirals utilized in some industrial plants.

\subsubsection{Bypass and Cutsize Correlation Under Optimum Conditions}

Several important observations can be made based on the information gathered from the optimization study. The study indicates that there are no solutions (i.e., no combination of controllable variables) that provide cutsize values below $20 \mu \mathrm{m}$ or above $50 \mu \mathrm{m}$. This finding should be expected since none of the cutsize values determined experimentally was found to fall 
in these ranges. More importantly, the study showed a very strong negative correlation between cutsize and bypass when operating under optimal conditions. This trend can be seen by the data plotted in Figure 1.23 for the entire set of optimized test runs conducted in the parametric study.

The Pearson correlation coefficient $\left(\mathrm{R}^{2}\right)$ value of near unity $\left(\mathrm{R}^{2}=0.99\right)$ shows an almost perfect correlation between cutsize and bypass. As such, this plot can be used to estimate the minimum amount of bypass that can be achieved for a target cutsize. As shown, a reduction in bypass to 0.1 or lower using the water-injected apex will force the cutsize to increase to approximately $37 \mu \mathrm{m}$ or larger. This operating point will likely require a modestly high water rate (e.g., 26 GPM) and small apex outlet diameter (e.g., 0.8 inches). Likewise, the regression line shows that a bypass of less than 0.05 dictates a larger cutsize of $40 \mu \mathrm{m}$ or larger. This requires a higher water flow rate approaching 30 GPM and relatively small apex approaching 0.75 inches.

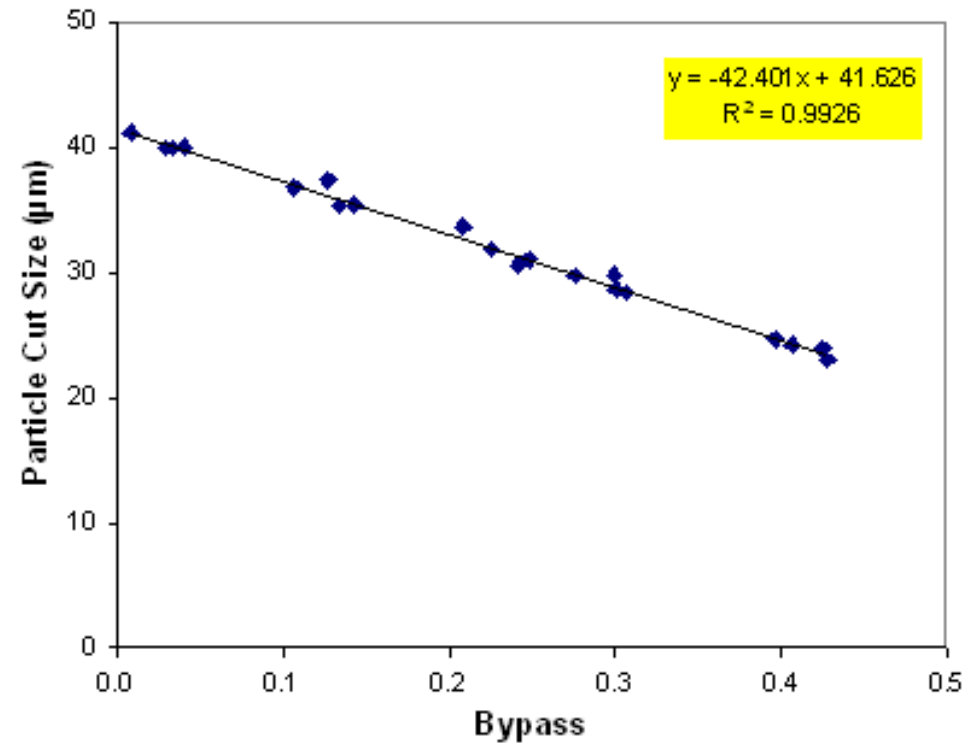

Figure 1.23 - Correlation between predicted cutsize and bypass. 


\subsection{Summary and Conclusions}

A parametric study was performed to evaluate a new water-injected apex system. The study indicated that apex outlet diameter and water injection flow rate have the main effect on minimizing the bypass of ultrafine particles to the underflow. The effects of apex inlet diameter and apex chamber diameter where not found to be important variables for the range of dimensions examined in this study. When operated under optimum conditions, the new apex washing system makes it possible to reduce ultrafine bypass from a typical range of 30 35\% down to approximately $2 \%$ when operated within a cutsize range of $40-45 \mathrm{um}$. A smaller cutsize range was possible when using less injection water and larger apex outlets, but these changes tended to rapidly increase the amount of bypass. In fact, a near perfect linear correlation was observed between cutsize and bypass when operating under the optimum settings of apex geometry and water flow rate that were needed to minimize bypass. 


\subsection{References}

1. K. Heiskanen, 1993. Particle Classification, Chapman \& Hall.

2. L. Svarovsky, 1984. Hydrocyclones, Technomic publishing.

3. Stat-Ease Inc., 2002. Design-Expert 6 User's Guide.

4. M.K. Mohanty, A. Palit and B. Dube, 2002. A comparative evaluation of new fine particle size separation technologies, Minerals Engineering, Vol. 15, pp. 727-736.

5. M. Frachon and J.J. Cilliers, 1999. A general model for hydrocyclone partition curves, Chemical Engineering Journal, Vol. 73, pp. 53-59.

6. M.A.Z. Coelho and R.A. Medronho, 2001. A model for performance prediction of hydrocyclones, Chemical Engineering Journal, Vol. 84, pp. 7-14.

7. Bangxian $\mathrm{Wu}$ et al., 2002. A study on advanced concept for fine particle separation, Experimental Thermal and Fluid Science, Vol. 26, pp. 723-730.

8. F. Concha et al., 1996. Air core and roping in hydrocyclones, Int. J. Miner. Process., Vol. 4445, pp. 743-749.

9. R.Q. Honaker et al., 2001. Apex water injection for improved hydrocyclone classification efficiency, Minerals Engineering, Vol. 14, No. 11, pp. 1445-1457.

10. D.D. Patil and T.C. Rao, 1999. Classification evaluation of water injected hydrocyclone (Technical note), Minerals Engineering, Vol. 12, No. 12, pp. 1527-1532.

11. A.K. Mukherjee et al., 2003. Effect of increase in feed inlet pressure on feed rate of dense media cyclone, Int. J. Miner. Process. Vol. 69, pp. 259-274.

12. Atakan Avci and Irfan Karagoz, 2003. Effects of flow and geometrical parameters on the collection efficiency in cyclone separators, Aerosol Science, Vol. 34, pp. 937-955. 
13. G. Vallebuona, A. Casali, G Ferrara, O. Leal and P. Bevilacqua, 1995. Modeling for small diameter hydrocyclones (Technical note), Minerals Engineering, Vol. 8, No. 3, pp. 321-327.

14. K. Nageswararao, 1999. Normalization of the efficiency curves of hydrocyclone classifiers, Minerals Engineering, Vol. 12, No. 1, pp. 107-118.

15. T. Neesse, J. Dueck and L. Minkov, 2004. Separation of finest particles in hydrocyclones, Minerals Engineering, Vol. 17, pp. 689-696.

16. A.J. Lynch and Rao T. C., 1975. Modelling and scale-up of hydrocyclone classifiers, Proceedings, XI Inter. Miner. Proc. Congress (IMPC), Cagliari, pp. 245-269.

17. L. R. Plitt, 1976. Mathematical Modelling of the hydrocyclone classifier, CIM Bulletin 69, Vol. 776, pp. 114-123. 


\section{CHAPTER 2 - MATHEMATICAL SOLUTIONS TO PARITIONING EQUATIONS FOR MULTISTAGE CLASSIFICATION CIRCUITS}

\subsection{Introduction}

\subsubsection{Background}

Classification processes are used in a wide variety of applications in both the mineral processing and coal preparation industries. Both static tank and centrifugal separators are used primarily for the purpose of sorting particles according to size based on differences in settling rates. In some applications, the classification processes may be used in multistage circuits that are specifically designed to minimize the misplacement of particles and improve separation efficiency. For hydraulic classifiers, scavenging circuits can be used to reduce unwanted losses of coarse particles by retreating the undersize stream using one or more additional stages of separation. Likewise, cleaning circuits can be used to improve the quality of the coarse product by retreating the oversize stream in one or more additional units designed to reduce the inadvertent bypass of fine materials.

In most cases, the natural variations in the physical properties of the feed particles (i.e., density, conductivity, magnetic susceptibility, washability) make it difficult to experimentally determine the extent of the improvement offered by multistage classification circuits. To overcome this problem, an evaluation of multistage separation circuits was performed in this study using a mathematical approach. An S-shaped partition function, which has been advocated for describing hydrocyclone efficiency curves (Lynch and Rao, 1975), was used for all of the performance calculations conducted in this work. According to this expression, the partition curve for a separation may be represented by the following exponential transition function: 


$$
P=\frac{\exp [\alpha Z]-1}{\exp [\alpha Z]+\exp [\alpha]-2}
$$

where $P$ is probability function to a particular stream, $\alpha$ is the sharpness of separation, and $Z$ is the ratio of the particle size $(X)$ to particle size cutpoint $\left(X_{50}\right)$ (i.e., $\left.Z=X / X_{50}\right)$. It is generally assumed that the bypass is independent of particle size and equals the water recovery from the feed to the underflow (oversize) product. This condition assumes that the fraction of the feed water recovered in the underflow stream carries an equivalent fraction of the feed solids. Austin and Klimpel (1981) argue that there is no fundamental reason why, in general, this should be so, and show data where the bypass is clearly not equal to the water recovery. Svarovsky (1992) and Braun and Bohnet (1990) assume that the bypass equals the fraction of the feed slurry reporting to the underflow. This assumption is not commonly used, but is a close approximation to the water recovery at low feed solids concentrations and is more readily measured. The generalized equation for simulating overall bypass of multistage classification circuits is obtained by using a following equation:

$$
P=\left(P^{*}-B p\right) /(1-B p)
$$

where $P$ and $P^{*}$ represent the corrected and actual probability functions, respectively, and $B p$ is the bypass of ultrafine particles to underflow. The actual probability can be obtained by simply adding water entrainment to corrected probability function.

\subsubsection{Linear Circuit Analysis}

A comparison of the performance of different configurations of multistage circuits can be accomplished using a mathematical approach called linear circuit analysis (LCA). This technique, which was first advocated by Meloy (1983), is one of the most powerful tools for 
analyzing processing circuits. The LCA approach has been used to improve the performance of processing circuits in variety of industrial applications (Luttrell et al., 1998). LCA can only be applied if particle-particle interactions do not influence the probability that a particle will report to a particular stream, i.e. the partition curve should remain unchanged in each stage of separation. This assumption is reasonably valid for most classification separators provided that the machine is functioning within its recommended operating limits (e.g., feed solids content is not too high). Based on this assumption, circuit analysis will provide not only useful insight into how unit operations should be configured in a multistage circuit, but also numerical solutions that predict overall circuit performance.

\subsubsection{Objectives}

The primary objective of the work outlined in this chapter is to use partition models and linear circuit analysis to derive analytical expressions that can be used to directly calculate key indicators that describe the separation performance of multistage classification circuits. For hydraulic classifiers, some of the specific indicators of interest include particle cutsize, bypass and separation efficiency. Due to the complexity of the mathematics involved, a commercial software package known as Mathematica was used to algebraically solve most of the performance expressions developed in this study. In addition, the accuracy of the analytical expressions was evaluated by means of direct numerical simulations conducted using iterative models developed in an Excel spreadsheet format. 


\subsection{Mathematical Software}

\subsubsection{Mathematica Simulations}

Mathematica, a powerful mathematical software package, was utilized to derive general mathematical equations for the multistage classification circuits and to calculate their overall particle cutsize and separation efficiency. For the purpose of this study, multistage classification circuits represent a combination of processing units that include two-stage and three-stage circuits that incorporate underflow reprocessing, overflow reprocessing, recycle and no recycle. The "preferred" configurations identified by circuit analysis are limited in this study to three or less units for practical reasons.

To derive the generalized equations for multistage classification circuits, the combined probability function for two-stage and three-stage circuits was calculated from the individual probability function for a single-stage unit using the linear circuit analysis (LCA) methodology. The combined probability function was entered, simplified and then generalized by the Mathematica software package. The combined probability function for the multistage circuits followed the generalized form given by:

$$
P=f\left(\alpha, X_{50}\right)
$$

where $P$ is a probability function (fraction reporting to underflow), $\alpha$ is the separation sharpness, and $X_{50}$ is the separation cutsize for each unit. All of the probability equations were found to be expressed as complex exponential functions, which are non-algebraic and non-linear. Therefore, to get a solution (i.e., to find $\alpha$ and $X_{50}$ ) from the combined equations for multistage circuits, the built-in "FindRoot" function was used in Mathematica. This function can search for a numerical solution to complex non-algebraic equations by Newton's method. To find a solution to an equation of the form $f(x)=0$ using Newton's method, the algorithm starts at $x=0$, then 
uses knowledge of the derivative $f^{\prime}$ to take a sequence of steps toward a solution. Each new calculated point $x_{n}$ that the algorithm tries is found from the previous point $x_{n-1}$ using the formula $x_{n}=x_{n-1}-f\left(x_{n-1}\right) / f^{\prime}\left(x_{n-1}\right)$.

When searching for a solution, the particle cutsize $\left(X_{50}\right)$ was represented by a value of $X$ at which $\mathrm{P}=0.5$. Likewise, the separation sharpness was expressed as follows:

$$
\alpha=1.0986 \frac{X_{50}}{E p}=1.0986 \frac{2 X_{50}}{X_{75}-X_{25}}
$$

where $E p$ is the Ecart probable error (another criterion for the separation efficiency) and $X_{25}$ and $X_{75}$ are the particle sizes defined at $\mathrm{P}=0.25$ and $\mathrm{P}=0.75$, respectively. If the values of $X_{25}$, $X_{50}$ and $X_{75}$ are known, the particle cutsize and separation sharpness (or Ecart probable error) can be determined numerically. The formations of the "FindRoot" function that are related with $X_{25}, X_{50}$ and $X_{75}$ are as follows:

$$
\text { FindRoot }\left[P==0.5,\left\{X, X_{0}\right\}\right]
$$

$$
\text { FindRoot }\left[P==0.25,\left\{X, X_{0}\right\}\right]
$$

$$
\text { FindRoot }\left[P==0.75,\left\{X, X_{0}\right\}\right]
$$

These formations instruct the program to search for an $\mathrm{X}$ value that numerically satisfies the equation " $P==0.5$ or 0.25 or 0.75 " starting with $\mathrm{X}=\mathrm{X}_{0}$.

For the cases involving the probability function with bypass, the following equation was used to calculate the overall probability function:

$$
P=\left(P^{*}-B p\right) /(1-B p)
$$


where $P^{*}$ represents the actual probability function (with entrainment), $P$ is the corrected probability function (no entrainment), and $B p$ is the ultrafine misplacement to underflow for each unit. This equation can be rearranged to provide the following expression for $P^{*}$ :

$$
P^{*}=(1-B p) P+B p
$$

In a manner similar to deriving equations for multistage circuits for overall cutsize and separation efficiency, the equations for multistage circuits can be generalized for determining the overall bypass. The combined probability function for multistage circuits that include bypass followed the generalized form given by:

$$
P^{*}=f\left(\alpha, X_{50}, \phi_{L}\right)
$$

where $P^{*}$ is probability function that includes bypass. The terms $\alpha, X_{50}$ and $\phi_{L}$ represent the separation sharpness, cutsize, and ultrafine size bypass (misplacement) to underflow for each unit, respectively. The overall bypass for a specific circuit configuration can be calculated by setting this probability function $\left(P^{*}\right)$ equal to 0 within the "FindRoot" function.

Unfortunately, the Mathematica software package had great difficulty in deriving an equation for the specific particle size of interest due to the complexity of the equations involved. The form of the exponential expressions constrained Mathematica to solve for $X$ using inverse functions. This made solutions nearly impossible to obtain. Therefore, in order to derive an equation for the specific particle size of interest, the term $X$ had to be separated from the other variables present in the partition expression. The following functions are related with the specific particle size of interest:

$$
\begin{aligned}
& X_{50}=f(P, \alpha) \\
& X_{50}=f\left(P^{*}, \alpha, \phi_{L}\right)
\end{aligned}
$$




\subsubsection{Excel Simulations}

A spreadsheet-based (Microsoft Excel) partitioning model was developed to simulate the behavior of multistage hydrocyclone circuits. The partitioning performance was calculated using the following expression (Lynch and Rao, 1975) for the probability function:

$$
P=\frac{e^{\alpha Z}-1}{e^{\alpha Z}+e^{\alpha}-2}
$$

where $Z=X / X_{50}$ and $\alpha$ is the separation sharpness. An example of the input/output screen from the Excel simulations of multistage circuits is shown in Figure 2.1. In this display screen, the units $\mathrm{A}, \mathrm{B}$ and $\mathrm{C}$ represent the primary, secondary and tertiary separators, respectively. The yellow-shaded input section (A) allows input values for the separation cutsize $\left(X_{50}\right)$ and

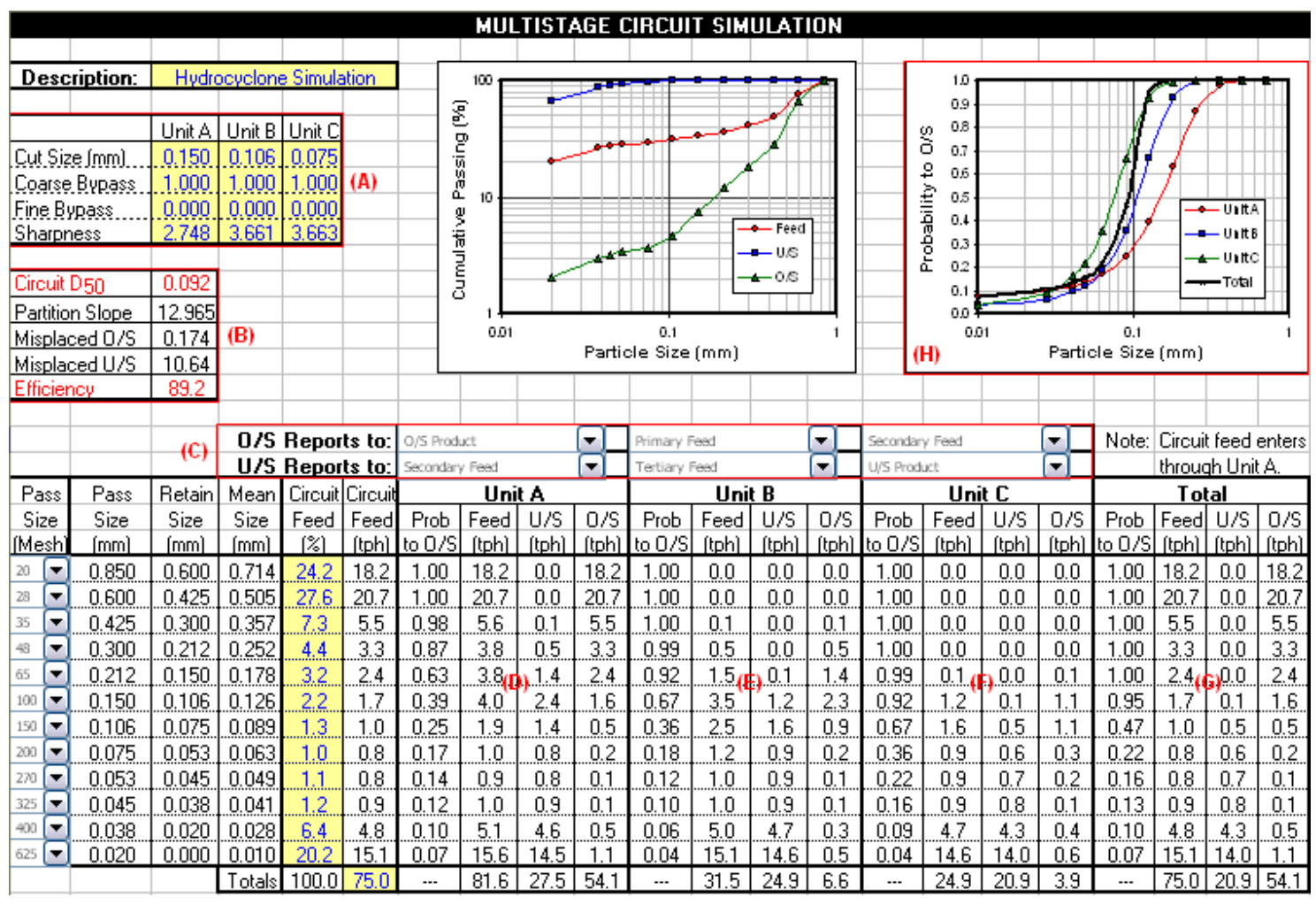

Figure 2.1 - Multistage classifier simulation using a spreadsheet-based model (example shows three-stage overflow reprocessing circuit with recycle). 
separation sharpness $(\alpha)$ to be enter for each unit in the circuit. The interconnection of the various streams between units $\mathrm{A}, \mathrm{B}$ and $\mathrm{C}$ can be varied using a series of six dropdown menus (C), which indicate where each of the two products from each separator should report. The probability to underflow for each unit is calculated from Equation [2.2.11] in the left most columns (D), (E) and (F). These probabilities are used with the feed tonnage distribution (yellow-shaded column) to calculate the tonnage entering and exiting each unit A, B and C. The calculated tonnage values are then used to determine the overall partition probabilities (G) for the combined circuitry using the simple relationship:

$$
\text { Probability to Underflow }=\frac{\text { Underflow Tonnage for ith Size Class }}{\text { Feed } \text { Tonnage for ith Size Class }}
$$

The partition curves (H) are then obtained by plotting the mean size $(\mathrm{C})$ as a function of the combined partition values (G), as well as the individual partition values (D), (E) and (F) for each unit. The overall cutsize and separation efficiency for the multistage circuit is reported as a summary output (B).

The predictions obtained from the theoretical equations derived from Mathematica for determining the circuit partition factors were found to be equivalent to the simulation results obtained from the Excel simulation spreadsheet. The exact agreement between the Mathematica and Excel partition values verifies that, for any particle cutsize and separation sharpness, a circuit partition curve can be calculated analytically from the probabilities without the need to know the feed size distribution. This finding is extremely important since most investigators do not realize that simulations based on partitioning probabilities are independent of the physical properties of the feed stream. In other words, the same cutsize and efficiency will be obtained from the simulations routines regardless of what feed size distribution is entered. Only the product size distributions will change in response to changes in the feed size distribution. 


\subsection{Results}

\subsubsection{Underflow Reprocessing Circuit Without Recycle}

\subsubsection{Two-Stage Circuit Analysis}

The underlying principle of LCA is that all particles that enter a separator as feed $(F)$ are selected to report to either the concentrate $(C)$ or tailing $(T)$ streams by a dimensionless probability function $(P)$. This can be mathematically described for a two-stage underflow reprocessing circuit without recycle as shown in Figure 2.2. In this case, $P_{0}$ and $P_{1}$ represent the partition probabilities for the primary and secondary units, respectively. By simple algebraic calculation, the oversize-to-feed ratio $\left(T / F=P_{T, \text { under }}\right)$ for this particular circuit can be represented as:

$$
P_{T, \text { under }}=P_{0} P_{1}
$$

This equation can be easily expanded using a transition function to quantify the separation probability that occurs for each separator. If a standard classification model is used (Lynch and Rao, 1977), then the partition for each unit in the circuit can be calculated using:

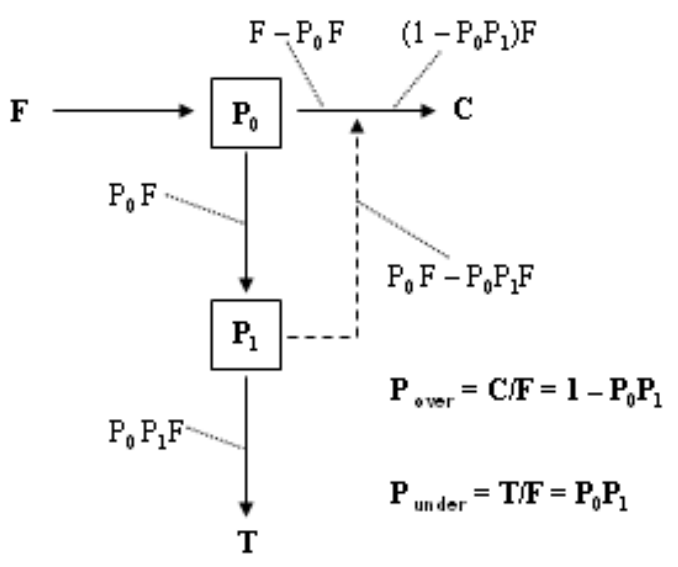

Figure 2.2 - Schematic of a two-stage underflow reprocessing circuit without recycle. 


$$
P=\frac{e^{\alpha Z}-1}{e^{\alpha Z}+e^{\alpha}-2}
$$

where $P$ is the partition factor, $\alpha$ is a sharpness value and $Z$ represent the normalized size given by $X / X_{50}$. By substituting the partition function given by Equation [2.3.2] into the separation probabilities represented in Equation [2.3.1], the overall partition expression for this circuit now becomes:

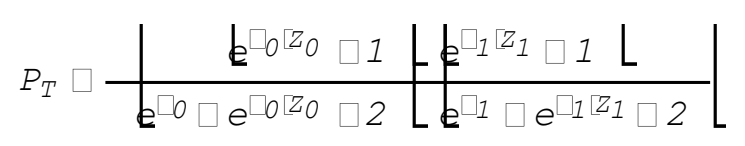

where $\alpha_{0}$ and $\alpha_{1}$ are the sharpness values and $Z_{0}$ and $Z_{1}$ are the normalized size for the primary and secondary separators, respectively. This equation represents the combined partitioning probability for a two-stage underflow reprocessing circuit without any recycle streams.

To check the validity of Equation [2.3.3], a comparison was made between the analytical solution and a simulation results obtained using the spreadsheet program described previously. The partitioning data selected for use in this validation procedure are shown in Figure 2.3. For each technique, the primary and secondary separators were set to make a respective cutsize of 150 and $106 \mu \mathrm{m}$. The separation sharpness values for the primary and secondary units were also set at different values of 2.748 and 3.661, respectively. By substituting these values into the Equation [2.3.3], the overall partition expression for this circuit becomes:

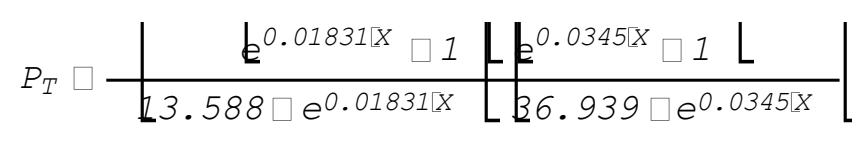

A comparison of the partitioning results obtained using this expression and those obtained from the Excel simulations are summarized in Table 2.1. As should be expected, the Mathematica solution for determining circuit partition factors is mathematically equivalent to the Excel simulation (which utilized feed properties). The good agreement between the Mathematica and 


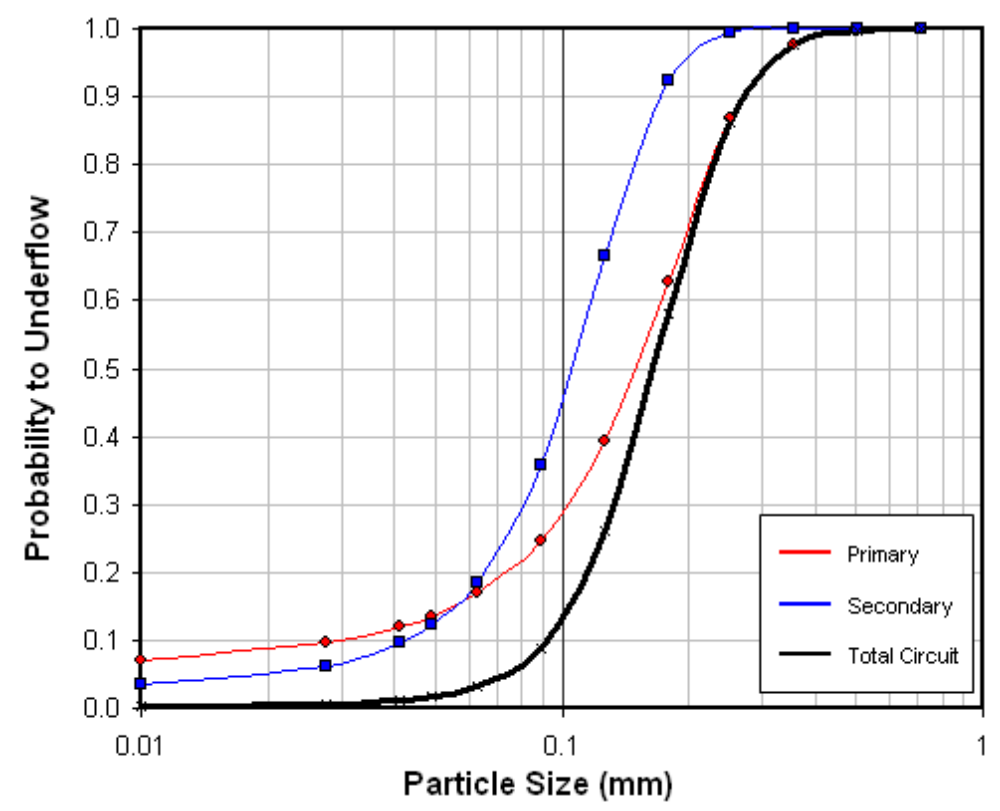

Figure 2.3 - Example of the partitioning response of the two-stage circuit underflow reprocessing circuit without recycle.

Excel partition values verifies that for any particle cutsize, a circuit partition value can be calculated. This also indicates that important size values, such as $X_{25}, X_{50}$, and $X_{75}$, can be back-calculated from Equation [2.3.4] by varying $X$ until the desired values of $P$ are found. More importantly, important performance indicators, such as the separation sharpness $(\alpha)$ and cutsize $\left(X_{50}\right)$, can be determined for the entire circuit completely independent of feed properties.

As discussed previously, Mathematica can be used to perform the calculations required to determine the important performance indicators for the two-stage circuit. In trying to find solution to this equation, Newton's method was used to determine the values of $X$ needed to identify the cutsize $\left(X_{50}\right)$ and calculate the separation sharpness $(\alpha)$. To accomplish this goal, the appropriate $X$ values were determined using the "FindRoot" function which searches for a 
Table 2.1 - Comparison of Mathematica and Excel determined partition factors for the twostage underflow reprocessing circuit without recycle.

\begin{tabular}{ccc}
\hline $\begin{array}{c}\text { Particle Size } \\
(\mathrm{mm})\end{array}$ & $\begin{array}{c}\text { Mathematica } \\
\text { Partition Factor }\end{array}$ & $\begin{array}{c}\text { Excel } \\
\text { Partition Factor }\end{array}$ \\
\hline 0.714 & 1.000 & 1.000 \\
0.505 & 0.999 & 0.999 \\
0.357 & 0.978 & 0.978 \\
0.252 & 0.861 & 0.861 \\
0.18 & 0.579 & 0.579 \\
0.126 & 0.262 & 0.262 \\
0.089 & 0.089 & 0.089 \\
0.063 & 0.031 & 0.031 \\
0.049 & 0.017 & 0.017 \\
$0 . .041$ & 0.012 & 0.012 \\
0.028 & 0.006 & 0.006 \\
0.010 & 0.003 & 0.003 \\
\hline
\end{tabular}

numerical solution to each expression using Newton's method. The form of the "FindRoot" function used to calculate these values are as follows:

$$
\begin{aligned}
& \text { FindRoot }\left[P==0.5,\left\{X, X_{0}\right\}\right] \\
& \text { FindRoot }\left[P==0.25,\left\{X, X_{0}\right\}\right] \\
& \text { FindRoot }\left[P==0.75,\left\{X, X_{0}\right\}\right]
\end{aligned}
$$

The statement represented by Equation [2.3.6] instructs Mathematica to search $X$ values for a numerical solution to the equation " $P=0.5$ " starting with $X=X_{0}$. Using this approach, the cutsize and separation sharpness for the total two-stage circuit was found to be $164.54 \mu \mathrm{m}$ and 3.971, respectively. These exact values were calculated totally independent of the feed properties using the features readily available within Mathematica. The analytical solution also indicates that the use of this particular two-stage circuit substantially increases the cutsize when compared to the cutsize values set for either of the single unit operations. 


\subsubsection{Three-Stage Circuit Analysis}

The approach described previously for the two-stage circuit was again applied to provide an analytical partitioning expression for a three-stage underflow reprocessing circuit without recycle. In this case, $P_{0}, P_{1}$ and $P_{2}$ are the dimensionless probability functions that select particles to report to a given stream for the primary, secondary and tertiary separators, respectively. As shown in Figure 2.4, the overall refuse-to-feed ratio $\left(T / F=P_{T, \text { under }}\right)$ for this circuit can be represented as:

$$
P_{T, \text { under }}=P_{0} P_{1} P_{2}
$$

By substituting Equation [2.3.2] into this expression, the overall partition function for this threestage circuit becomes:

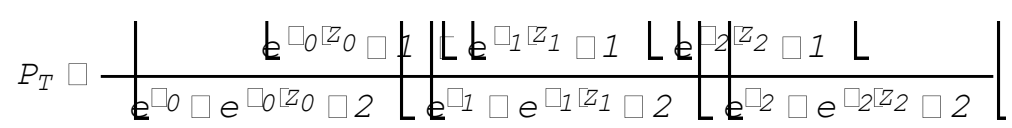

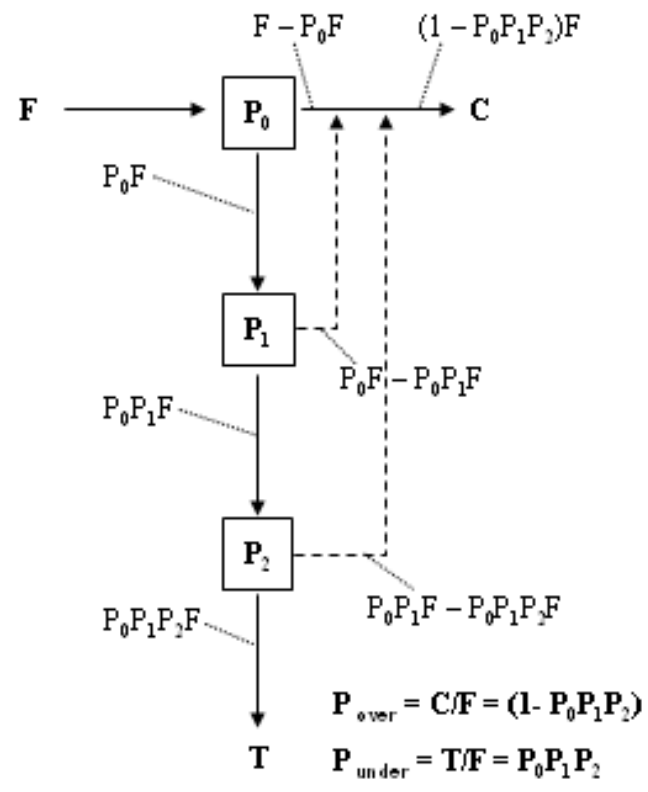

Figure 2.4 - Schematic of a three-stage underflow reprocessing circuit without recycle. 
In this case, the primary, secondary and tertiary separators were set to make separations at the particle cutsize of 150,106 and $75 \mu \mathrm{m}$ with separation sharpness values of $2.748,3.661$ and 3.663, respectively. The partition values calculated from Mathematica and Excel were again found to exactly agree for this circuit. The cutsize and separation sharpness for total circuit was found to be $165.55 \mu m$ and 4.135, respectively. Once again, the analytical solution shows that the cutsize for the combined circuit is larger than that obtained for either of the single unit operations.

\subsubsection{Underflow Reprocessing Circuit With Recycle}

\subsubsection{Two-Stage Circuit Analysis}

The approach described above can also be used to evaluate the effects of recycle streams on the performance of multistage circuits. This type of assessment is traditionally much more difficult to perform with standard simulation routines since it requires several iterations to find a stable solution. However, no such problem exists for analytical solutions obtained using linear circuit analysis.

Consider the two-stage underflow reprocessing circuit with recycle shown in Figure 2.5.

Once again, $P_{0}$ and $P_{1}$ represent the dimensionless probability functions that select particles to report to a given stream. By simple algebraic substitution, the overall oversize-to-feed ratio $\left(T / F=P_{T, \text { under }}\right)$ for this particular circuit can be calculated as:

$$
P_{T, \text { under }}=P_{0} P_{1} /\left(1-P_{0}+P_{0} P_{1}\right)
$$

By substituting Equation [2.3.2] into this expression, the overall partition expression for this circuit becomes: 


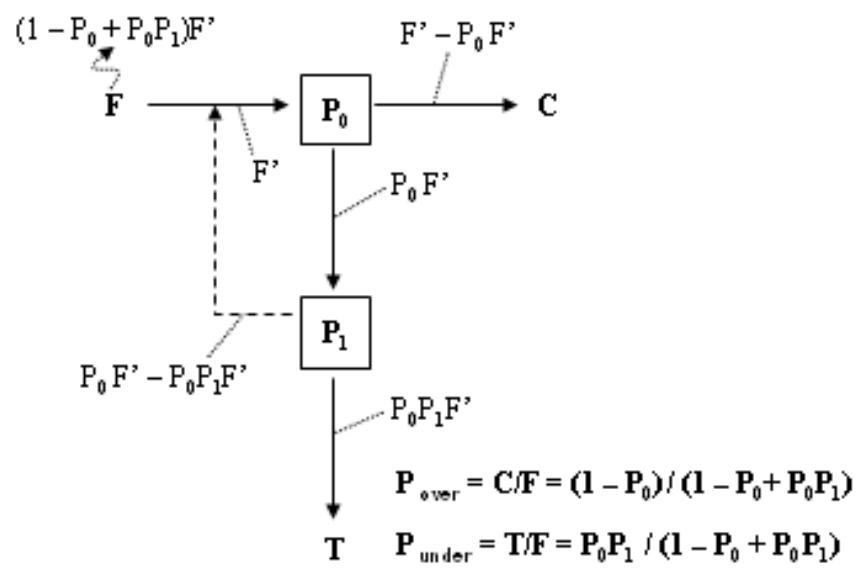

Figure 2.5 - Schematic of a two-stage underflow reprocessing circuit with recycle.

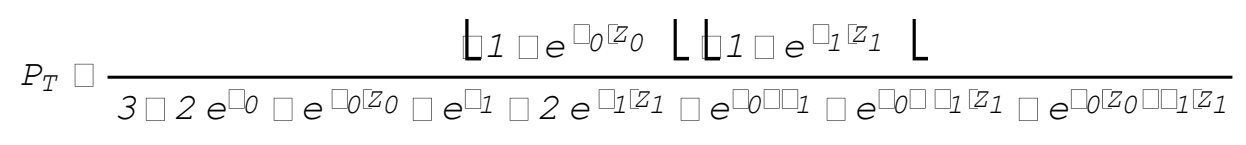

To check the validity of this expression, a comparative solution was again obtained from the Excel spreadsheet simulation routine. In this case, the primary, secondary and tertiary separators were set to make a cutsize of 150,106 and $75 \mu m$ with separation sharpness values of 2.748 , 3.661 and 3.663, respectively. As expected, both the Mathematica and Excel partition values were in perfect agreement for this three-stage circuit. The cutsize and separation sharpness for total circuit was found to be $158.30 \mu \mathrm{m}$ and 3.717 , respectively. In this case, the increase in cutsize created by the use of a two-stage circuit is less with a recycle stream than without a recycle stream (i.e., 158.30 versus $164.54 \mu m$ ).

\subsubsection{Three-Stage Circuit Analysis}

In this case, an analytical solution to the partitioning performance of a three-stage circuit with recycle was derived using linear circuit analysis. A schematic of the three-stage circuit is 


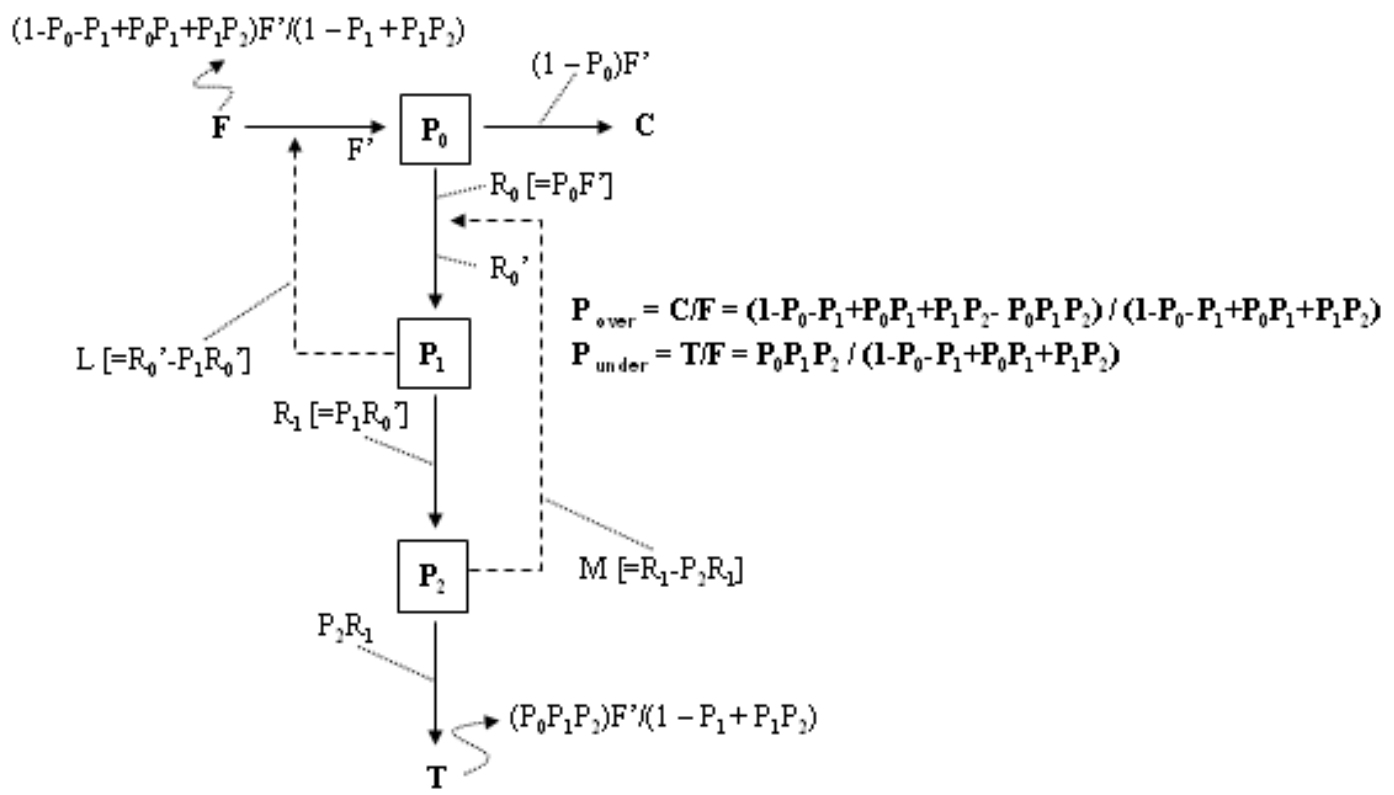

Figure 2.6 - Schematic of a three-stage underflow reprocessing circuit with recycle.

shown in Figure 2.6. $P_{0}, P_{1}$ and $P_{2}$ are again the dimensionless probability functions that select particles to report to a given stream. The overall oversize-to-feed ratio $\left(T / F=P_{T, \text { under }}\right)$ for this particular circuit can be algebraically represented as:

$$
P_{T, \text { under }}=P_{0} P_{1} P_{2} /\left(1-P_{0}-P_{1}+P_{0} P_{1}+P_{1} P_{2}\right)
$$

Likewise, the overall partition expression for this circuit becomes:

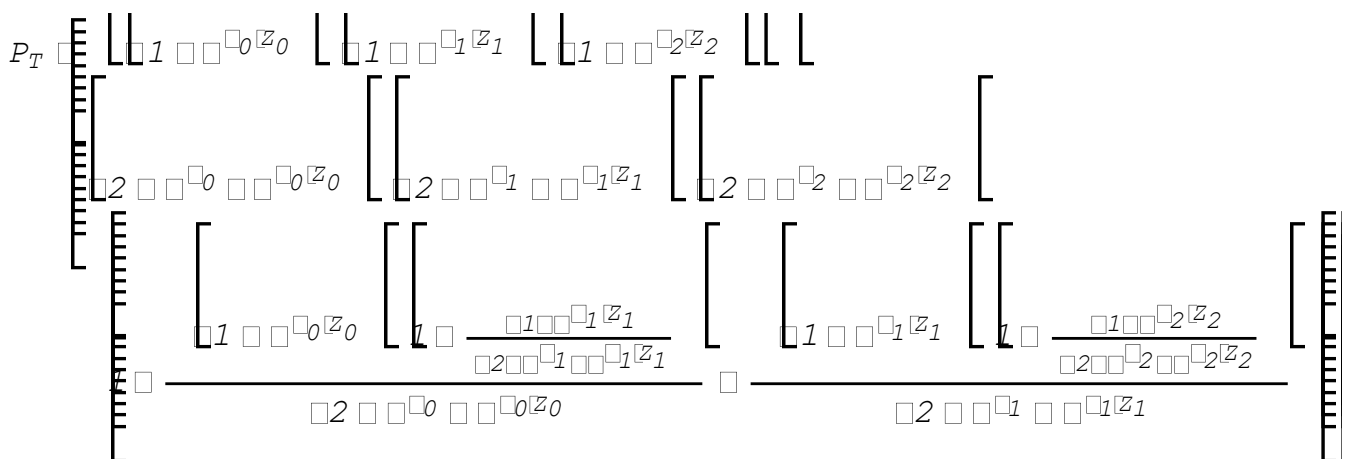


when substituting Equation [2.3.2] into the expression derived from linear circuit analysis. For verification purposes, the primary, secondary and tertiary separator were set to make separations at cutsize values of 150,106 and $75 \mu m$ with separation sharpness values of $2.748,3.661$ and 3.663, respectively. The Mathematica and Excel partition values were again found to be consistent for this circuit, indicating that the analytical solution was indeed accurate. The cutsize and separation sharpness for total circuit were found to be $158.32 \mu m$ and 3.737 , respectively, based on the input values selected for the individual units. It is important to notice that cutsize of 158.32 $\mu m$ obtained with this three-stage circuit with recycle streams was substantially smaller than the cutsize of $165.55 \mu m$ obtained for the three-stage circuit without recycle. Thus, the use of recycle streams suppresses the impact of increasing cutsize for multistage circuits in which the underflow stream is retreated to reduce bypass.

\subsubsection{Overflow Reprocessing Circuit Without Recycle}

\subsubsection{Two-Stage Circuit Analysis}

Several series of calculations were also performed to quantify the partitioning behavior of

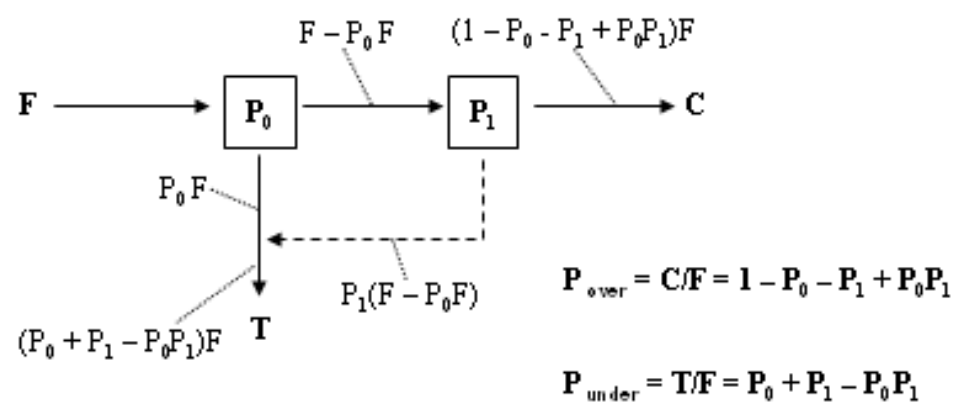

Figure 2.7 - Schematic of a two-stage overflow reprocessing circuit without recycle. 
circuits in which one or more of the overflow (undersize) streams were reprocessed. In the first example, a two-stage overflow reprocessing circuit without recycle was evaluated as shown in Figure 2.7. For this configuration, the overall oversize-to-feed ratio $\left(T / F=P_{T, \text { under }}\right)$ is:

$$
P_{T, \text { under }}=P_{0}+P_{1}-P_{0} P_{1}
$$

Substituting Equation [2.3.2] into this expression gives the overall partition expression as:

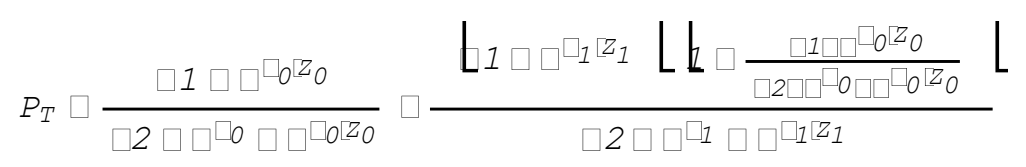

Both the Mathematica and Excel solutions for the partition values were identical, indicating again that the analytical solution was accurate. In this case, the primary and secondary separators were set to provide respective cutsize values of 150 and $106 \mu m$ and respective separation sharpness values of 2.748 and 3.661. Based on these input values, the cutsize and separation sharpness for the total circuit was found to be $87.06 \mu m$ and 2.805 , respectively. In this case, the use of a two-stage circuit to retreat the undersize product reduced the overall cutsize compared to the cutsize .

\subsubsection{Three-Stage Circuit Analysis}

Figure 2.8 shows the layout for a three-stage overflow reprocessing circuit without recycle. The overall oversize-to-feed ratio $\left(T / F=P_{T, \text { under }}\right)$ for this circuit can be calculated from linear circuit analysis as:

$$
P_{T, \text { under }}=P_{0}+P_{1}+P_{2}-P_{0} P_{1}-P_{0} P_{2}-P_{1} P_{2}+P_{0} P_{1} P_{2}
$$

Similar to the previous section, the overall partition expression for this circuit becomes: 


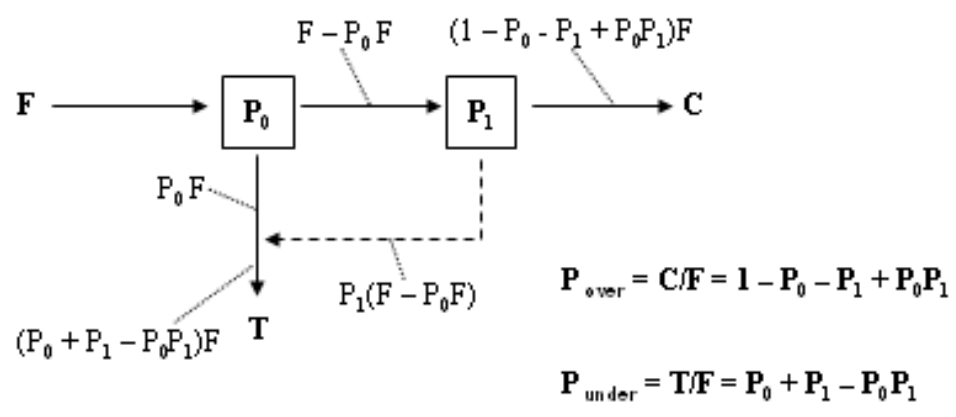

Figure 2.8 - Schematic of a three-stage overflow reprocessing circuit without recycle.

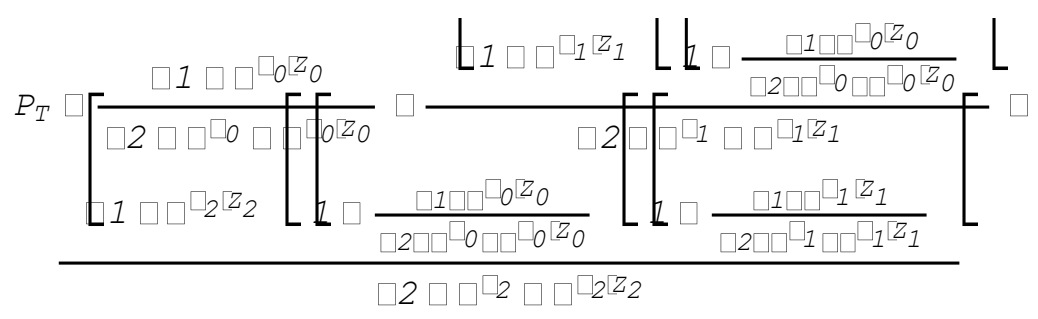

The primary, secondary and tertiary separators were set to make separations at the particle size cut-point of the 150, 106 and $75 \mu \mathrm{m}$ with sharpness values of 2.748, 3.661 and 3.663, respectively. The Mathematica and Excel partition values are also consistent in this circuit. The cutsize and separation sharpness values for total circuit were found to be $61.56 \mu \mathrm{m}$ and 3.449 , respectively. Notice that reprocessing of the overflow by three stages of operations substantially reduced the cutsize compared to the values entered for the individual unit operations.

\subsubsection{Overflow Reprocessing Circuit With Recycle}

\subsubsection{Two-Stage Circuit Analysis}

This particular analysis examined a two stage overflow reprocessing circuit with recycle. A schematic of the circuit is shown in Figure 2.9. The overall refuse-to-feed ratio and partitioning expression for this circuit is given by: 


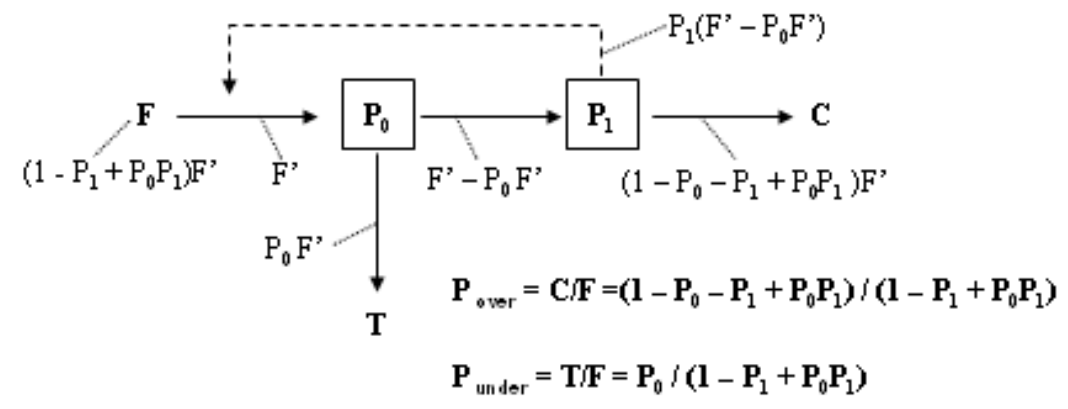

Figure 2.9 - Schematic of a two-stage overflow reprocessing circuit with recycle.

$$
P_{T, \text { under }}=P_{0} /\left(1-P_{1}+P_{0} P_{1}\right)
$$

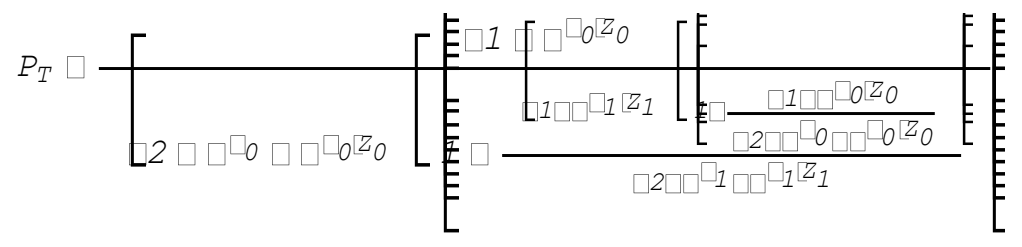

The Mathematica and Excel solutions were again found to provide identical partition values for this circuit. For validation purposes, the primary, secondary and tertiary separator were set to make separations at cutsize values of 150 and $106 \mu m$ with sharpness values of 2.748 and 3.661, respectively. Based on these values, the cutsize and separation sharpness for total circuit was found to be $109.12 \mu m$ and 3.862, respectively. When compared to the two-stage overflow reprocessing circuit without recycle, the recycle configuration provided a substantially smaller cutsize (i.e., 87.06 vs. $109.12 \mu m$ ). 


\subsubsection{Three-Stage Circuit Analysis}

The last circuit examined in detail in this study was a three-stage overflow reprocessing circuit with recycle. The layout of units for this particular circuit is shown in Figure 2.10. Based on the probability analyses for this circuit, the overall oversize-to-feed ratio $\left(T / F=P_{T, \text { under }}\right)$ was found to be given by:

$$
P_{T, \text { under }}=\left(P_{0}-P_{0} P_{2}+P_{0} P_{1} P_{2}\right) /\left(1-P_{1}-P_{2}+P_{0} P_{1}+P_{1} P_{2}\right)
$$

Once again, by substituting Equation [2.3.2] into this expression, the overall partition expression for this circuit can be directly calculated as:

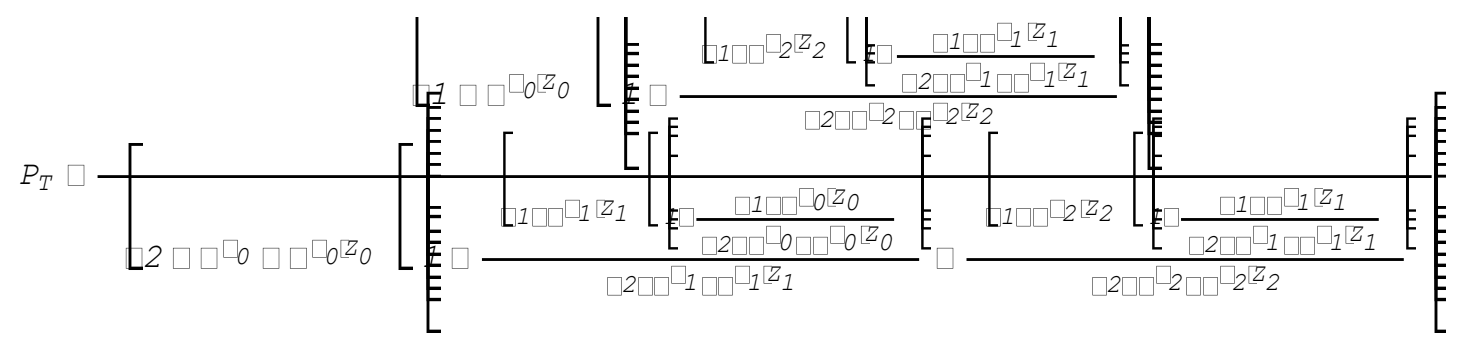

The primary, secondary and tertiary separator were set to make separations at cutsize values of

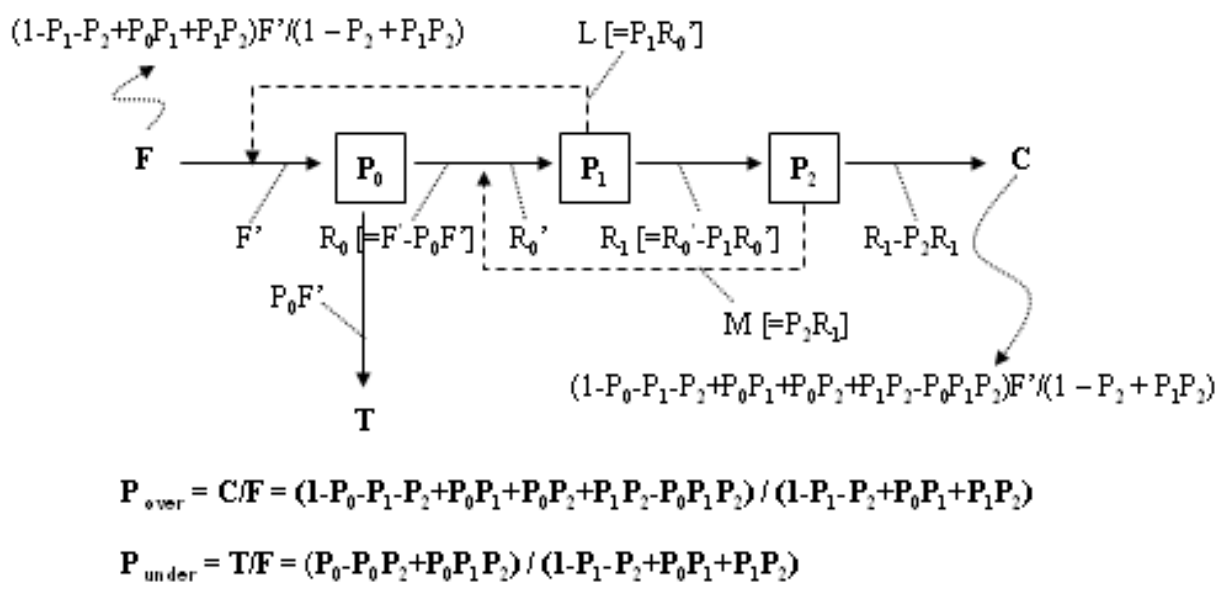

Figure 2.10 - Schematic of a three-stage overflow reprocessing circuit with recycle. 
150,106 and $75 \mu \mathrm{m}$ ) with separation sharpness values of $2.748,3.661$ and 3.663 , respectively. The Mathematica and Excel partition values were again identical, providing cutsize and sharpness values for total circuit of $88.79 \mu m$ and 6.167, respectively. 


\subsection{Generalized Partitioning Expressions}

\subsubsection{Underflow Reprocessing Circuit Without Recycle}

The generic configurations of underflow reprocessing circuits without recycle are shown in Figure 2.11. The probability functions for each circuit can be readily calculated by linear circuit analysis. The single-stage probability can be expressed as follows:

$$
P_{T}=P_{0}
$$

The two-stage probability can be expressed as follows:

$$
P_{T}=P_{0} P_{1}
$$

The three-stage probability can be expressed as follows:

$$
P_{T}=P_{0} P_{1} P_{2}
$$

From these expressions, a generalized equation of this specific type of circuit can be obtained as follows:

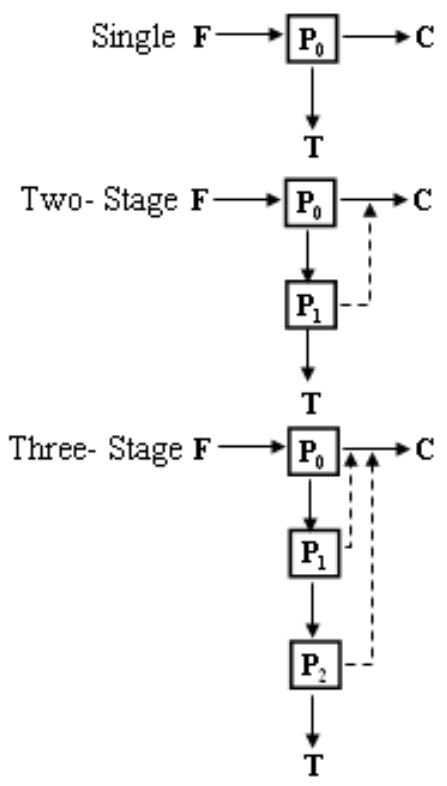

Figure 2.11 - Generic configurations of underflow reprocessing circuits without recycle. 


$$
P_{T} \square \underset{N \square 1}{\eta \mid} P_{\square \square 1}
$$

where $n$ is number of classification separators in the circuit. When considering bypass, the generalized equation for this circuit can be obtained as:

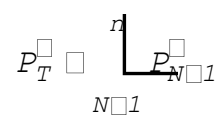

where the probability $\left(P^{*}\right)$ for single-stage, two-stage and three-stage units can be expressed as:

$$
P^{*}=(1-B p) P+B p
$$

As indicated previously, the respective values of $P$ and $P^{*}$ represent the corrected and actual probability functions for the circuit and $B p$ represents the bypass of fine particles to the underflow stream. The probability functions $(P)$ for each separator can be estimated based on the empirical formula (Lynch and Rao, 1977):

$$
P=\frac{e^{\alpha Z}-1}{e^{\alpha Z}+e^{\alpha}-2}
$$

where $\alpha$ is the separation sharpness and $\mathrm{Z}=\mathrm{X} / \mathrm{X}_{50}$ is the normalized particle size, i.e., the ratio of the actual particle size of interest $(\mathrm{X})$ divided by the cutsize $\left(\mathrm{X}_{50}\right)$.

\subsubsection{Underflow Reprocessing Circuit With Recycle}

Generic configurations of underflow reprocessing circuits without recycle are shown in Figure 2.12. The probability functions calculated by linear circuit analysis for each circuit are as follows. The single-stage probability can be expressed as follows:

$$
P_{T}=P_{0}
$$

The two-stage probability can be expressed as follows: 


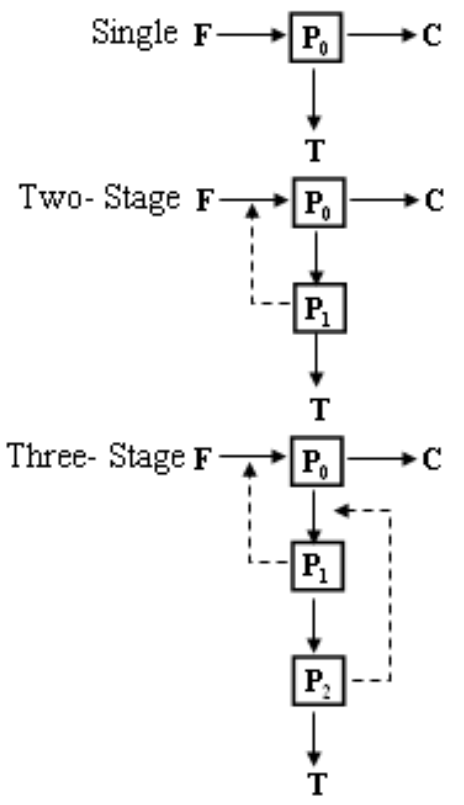

Figure 2.12 - Generic configurations of underflow reprocessing circuits with recycle.

$$
P_{T} \square \frac{P_{0} P_{1}}{1 \square P_{0} \square P_{0} \mathbb{P}_{1}}
$$

This equation can be further simplified as follows:

$$
P_{T} \square \frac{P_{0} P_{1}}{1 \square P_{0} \quad \square \square P_{1}} \mid
$$

The three-stage probability can be expressed as follows:

$$
P_{T} \square \frac{P_{0} P_{1} P_{2}}{1 \square P_{0} \square P_{1} \square P_{0} P_{1} \square P_{1} P_{2}}
$$

Likewise, this equation can be further simplified as follows:

$$
P_{T} \square \begin{array}{l|l|l|l|} 
& P_{0} P_{2} & P_{2} & \\
\hline 1 \square P_{0} & \mathbf{\square} \square P_{1} & \square P_{1} & \mathbf{L} \square P_{2}
\end{array}
$$

From the functional forms of these equations, a generalized equation for this specific type of circuit can be obtained as follows: 


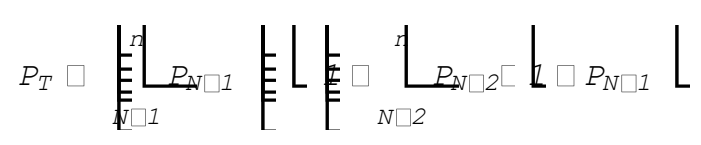

where $n$ is number of classification separators in the circuit. When considering bypass, the generalized equation for this specific circuit is simply modified to:

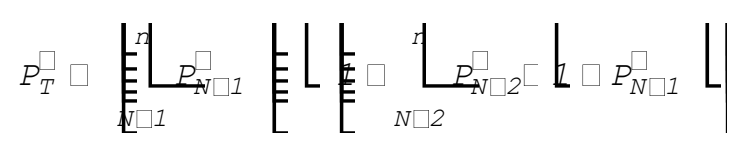

\subsubsection{Overflow Reprocessing Circuit Without Recycle}

Generic configurations of underflow reprocessing circuits without recycle are shown in Figure 2.13. From linear circuit analysis, the probability for a single stage circuit can be expressed as follows:

$$
P_{T}=P_{0}
$$

The partitioning probability for a two-stage circuit can be expressed as follows:

$$
P_{T} \square P_{0} \square P_{1} \square P_{0} \llbracket P_{1}
$$

This equation can be further simplified to:

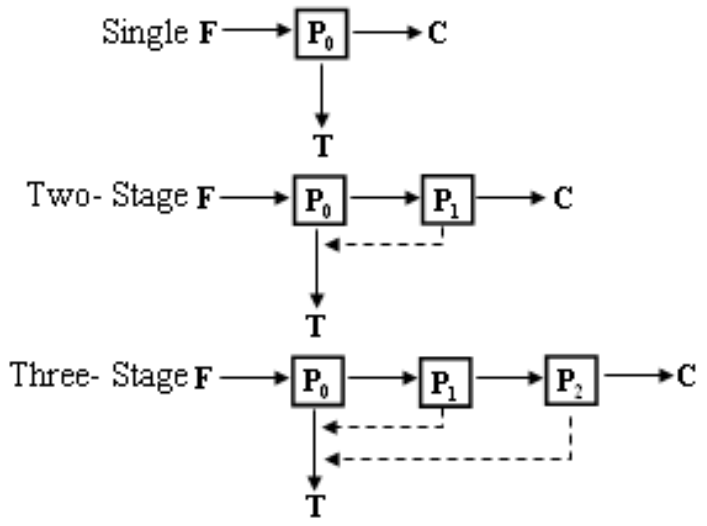

Figure 2.13 - Generic configurations of overflow reprocessing circuits without recycle. 


$$
P_{T} \square P_{0} \square \underline{\mathbf{L}} \square P_{0} \quad \underline{P}_{1}
$$

Likewise, the partitioning probability for a three-stage can be expressed as follows:

$$
P_{T} \square P_{0} \square P_{1} \square P_{2} \square P_{0} \backslash P_{1} \square P_{0} \backslash P_{2} \square P_{1} \backslash P_{2} \square P_{0} \backslash P_{1} \backslash P_{2}
$$

This equation can be further simplified to:

$$
P_{T} \square P_{0} \square \underline{k} \square P_{0} \quad \underline{P}_{1} \square \underline{\mathrm{k}} \square P_{0} \quad \mathfrak{L} \underline{\underline{L}} \square P_{1} \quad \underline{P}_{2}
$$

From these equations, a generalized expression for this specific type of circuit incorporating $n$ unit operations can be obtained by inspection. The partitioning probability for circuits without bypass is given as:

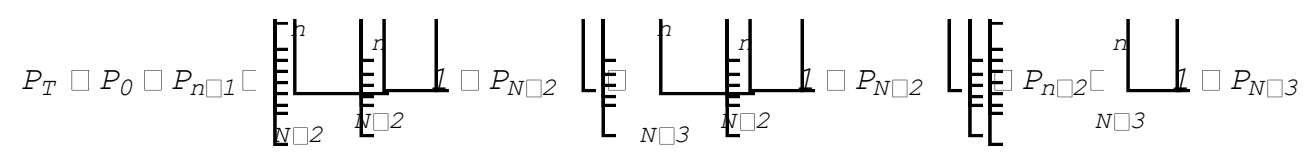

while the partitioning probability for circuits with bypass is given as:

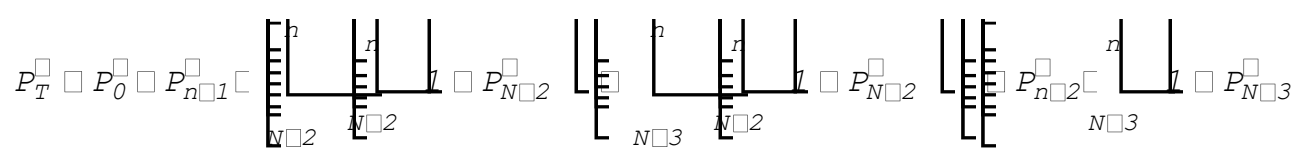

The values of $P$ and $P^{*}$ in these expression represent the partitioning probabilities for each unit operation with and without bypass, respectively.

\subsubsection{Overflow Reprocessing Circuit With Recycle}

Generic configurations of underflow reprocessing circuit without recycle are as shown in Figure 2.14. The probability functions for the single-, two- and three-stage circuits can be mathematically represented as follows. The single-stage probability can be expressed as:

$$
P_{T}=P_{0}
$$

The two-stage probability, which can be expressed as: 


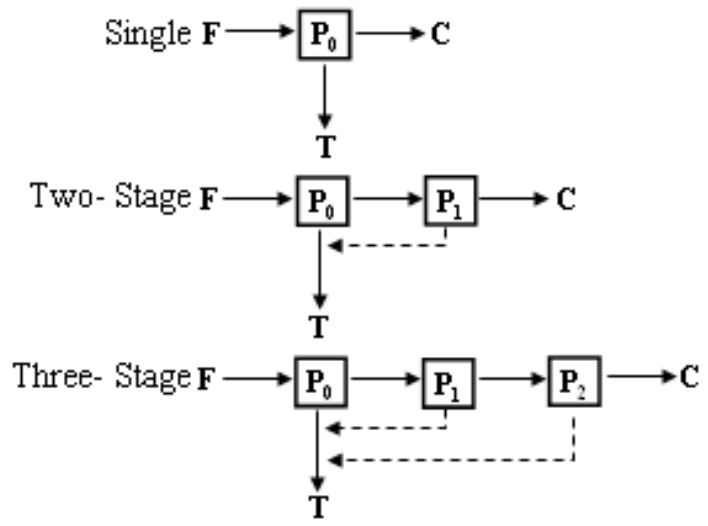

Figure 2.14 - Generic configurations of overflow reprocessing circuits with recycle.

$$
P_{T} \square \frac{P_{0}}{1 \square P_{1} \square P_{1} \mathbb{P}_{0}}
$$

This equation can be further simplified to:

$$
P_{T} \square \frac{P_{0}}{1 \square} \underline{\underline{1} \square P_{0}} \underline{P}_{1}
$$

The three-stage probability, which can be expressed as:

$$
P_{T} \square \frac{P_{0} \square P_{0} P_{2} \square P_{0}\left[P_{1} P_{2}\right.}{1 \square P_{1} \square P_{2} \square P_{0} P_{1} \square P_{1} P_{2}}
$$

This equation can also be further simplified to:

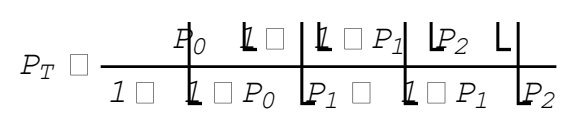

The generalized equation of this specific type of circuit can be obtained as follows for cases in which bypass is ignored.

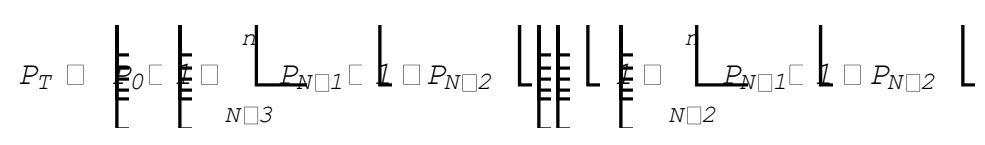

Likewise, the generalized equation of this specific type of circuit can be obtained as follows for cases in which bypass is considered. 


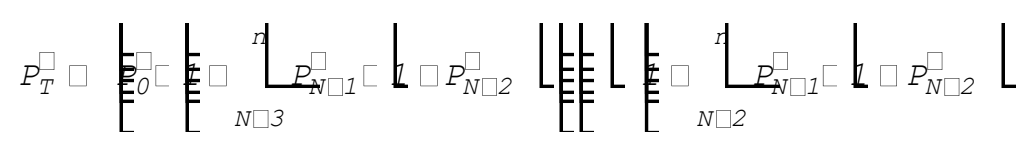

In both these expressions, $n$ represents the number of classification separators in each circuit. 


\subsection{Summary and Conclusions}

Linear circuit analysis was combined with an empirical model of particle classification to derive analytical expressions that describe the partitioning performance of multistage circuits. Due to the complexity of the mathematical functions, a software package known as Mathematica was used to perform the required computations dictated by linear circuit analysis. Although Mathematica had great difficulty in deriving analytical equations for a specific particle size of interest, this powerful tool still provided a convenient platform for searching for solutions via Newton's method. In addition, this approach provided useful insight into how unit operations should be configured in multistage circuits to reduce bypass and manipulate cutsize.

The partitioning data derived from Mathematica provided values that were identical to those obtained from simulations performed using a traditional partition model developed using an Excel spreadsheet. The perfect agreement between these two diverse approaches verifies that circuit partition values can be accurately calculated using a direct analytical approach and without the need for simulation. As such, critical values of particle size (e.g., $X_{25}, X_{50}$ and $X_{75}$ ) that are important in describing the performance of classification units, can be backcalculated from the partitioning equations derived by linear circuit analysis. This ability confirms that the two most important performance indicators, i.e., cutsize $\left(X_{50}\right)$ and separation sharpness $(\alpha)$, can be determined for the combined circuit completely independent of feed properties.

The analytical approach outlined in this study also made it possible to derive generic expressions for partitioning probability for generalized circuits that reprocess either overflow (undersize) or underflow (oversize). These expressions indicate that classification performance is indeed improved through the proper application of multistage circuits and that recycling of 
secondary streams can in many cases effectively minimize cutsize changes that inadvertently occur in multistage processing circuits. 


\subsection{References}

1. A.J. Lynch and T.C. Rao, "Modeling and Scale-Up of Hydrocyclone Classifiers." No.9, $11^{\text {th }}$ Inter Mineral Processing Congress, Cagliari, Italy, April 21-26, 1975.

2. L.G. Austin and R.R. Klimpel, 1981. An improved method for analyzing classifier data, Powder Technol., Vol. 29, pp. 277-281.

3. L. Svarovsky, Hydrocyclones, Holt, Rinehart and Winston, London, 1992, pp. 198.

4. T. Braun and M. Bohnet, 1990. Influence of feed solid concentration on the performance of hydrocyclones, Chem. Eng. Technol., Vol. 13, pp. 15-20.

5. T. P. Meloy, 1983. Analysis and optimization of mineral processing and coal-cleaning circuits-circuit analysis, International Journal of Mineral Processing, Vol. 10, pp. 61-80.

6. G. H. Luttrell, J. N. Kohmuench, F. L. Sranley, and G. D. Trump, 1998. Improving spiral performance using circuit analysis, Minerals \& Metallurgical Processing, Vol. 15, pp.16-21.

7. G. H. Luttrell, J. N. Kohmuench, and M. J. Mankosa, 2004. Optimization of magnetic separator circuit configurations, Minerals \& Metallurgical Processing, Vol. 21, pp. 153-157.

8. J. J. Cilliers and M. Franchon, 1999. A general model for hydrocyclone partition curve, Chemical Engineering Journal, Vol. 73, pp. 53-59.

9. B. Firth and M. O’Brien, 2003. Hydrocyclone Circuits, Coal Preparation, Vol. 23, pp. 167183.

10. L. R. Plitt, 1976. A mathematical model of the hydrocyclone classifier, CIM Bull., pp. 114122. 
APPENDIX I 


\section{Detailed Parametric Test Matrix}

\section{HYDROCYCLONE EVALUATION (UNBALANCED)}

\begin{tabular}{|c|c|}
\hline Test: & \\
\hline Feedflow (GPM) & 100.0 \\
\hline Feed Pressure (PSl) & 21.0 \\
\hline Water Flow (GPM) & 0.0 \\
\hline Water Pressure (PSl) & 0.0 \\
\hline Feed Rate (TPH) & 1.14 \\
\hline
\end{tabular}

\begin{tabular}{|r|c|c|c|}
\hline & OS & US & Feed \\
\hline Solids Content (\%) & 8.57 & 2.44 & 4.50 \\
\hline Ash Content (\%) & 25.88 & 49.01 & 34.62 \\
\hline Solids Yield (\%) & 64.0 & 36.0 & 100.0 \\
\hline Ash Yield (\%) & 62.2 & 37.8 & 100.0 \\
\hline Size Class Yield (\%) & 71.1 & 28.9 & 100.0 \\
\hline
\end{tabular}

\begin{tabular}{|c|c|c|c|c|c|c|c|c|}
\hline $\begin{array}{c}\text { Pass } \\
\text { Size } \\
(u m)\end{array}$ & $\begin{array}{c}\text { Retain } \\
\text { Size } \\
(u m)\end{array}$ & $\begin{array}{c}\text { O/S } \\
\text { Sample } \\
(\mathrm{gms})\end{array}$ & $\begin{array}{c}\text { U/S } \\
\text { Sample } \\
(\mathrm{gms})\end{array}$ & $\begin{array}{c}\text { OS } \\
\text { Mass } \\
(\%, \text { strm) }\end{array}$ & $\begin{array}{c}\text { U/S } \\
\text { Mass } \\
(\%, \text { strm })\end{array}$ & $\begin{array}{c}0 / S \\
\text { Mass } \\
(\%, \text { feed })\end{array}$ & $\begin{array}{c}\text { U/S } \\
\text { Mass } \\
(\%, \text { feed })\end{array}$ & $\begin{array}{c}\text { Feed } \\
\text { Mass } \\
(\%, \text { feed })\end{array}$ \\
\hline & 212 & 1.71 & 0.00 & 7.21 & 0.00 & 4.48 & 0.00 & 4.48 \\
\hline 212 & 150 & 1.86 & 0.00 & 7.84 & 0.00 & 4.88 & 0.00 & 4.88 \\
\hline 150 & 75 & 5.42 & 0.18 & 22.84 & 1.22 & 14.21 & 0.46 & 14.67 \\
\hline 75 & 45 & 4.03 & 0.35 & 16.98 & 2.38 & 10.57 & 0.90 & 11.46 \\
\hline 45 & 25 & 1.74 & 0.35 & 7.33 & 2.38 & 4.56 & 0.90 & 5.46 \\
\hline 25 & 13.1 & 1.58 & 0.94 & 6.66 & 6.42 & 4.14 & 2.42 & 6.57 \\
\hline 13.1 & 6.5 & 1.83 & 3.40 & 7.72 & 23.10 & 4.80 & 8.73 & 13.53 \\
\hline 6.5 & 3.3 & 2.26 & 5.03 & 9.53 & 34.17 & 5.93 & 12.91 & 18.84 \\
\hline 3.3 & 1.5 & 1.59 & 2.45 & 6.69 & 16.62 & 4.16 & 6.28 & 10.44 \\
\hline 1.5 & & 1.71 & 2.02 & 7.21 & 13.72 & 4.48 & 5.19 & 9.67 \\
\hline Totals & -- & 23.73 & 14.73 & 100.00 & 100.00 & 62.21 & 37.79 & 100.00 \\
\hline
\end{tabular}

\begin{tabular}{|c|c|c|c|c|c|c|c|c|}
\hline $\begin{array}{c}\text { Pass } \\
\text { Size } \\
(u m)\end{array}$ & $\begin{array}{c}\text { Retain } \\
\text { Size } \\
(u m)\end{array}$ & $\begin{array}{c}\text { O/S } \\
\text { Mass } \\
(\mathrm{TPH})\end{array}$ & $\begin{array}{c}\text { U/S } \\
\text { Mass } \\
(\mathrm{TPH})\end{array}$ & $\begin{array}{c}\text { Feed } \\
\text { Mass } \\
(\mathrm{TPH})\end{array}$ & $\begin{array}{c}\text { Delta } \\
\text { Mass } \\
(\mathrm{TPH})\end{array}$ & $\begin{array}{c}\text { Value } \\
(\mathrm{f}-\mathrm{u})\end{array}$ & $\begin{array}{c}\mathrm{X} \\
\text { Value } \\
(\mathrm{0}-\mathrm{u})\end{array}$ & $\begin{array}{c}\text { Partition } \\
\text { Factor } \\
(-)\end{array}$ \\
\hline & 212 & 0.05 & 0.00 & 0.05 & 0.00 & 4.48 & 4.48 & 1.00 \\
\hline 212 & 150 & 0.06 & 0.00 & 0.06 & 0.00 & 4.88 & 4.88 & 1.00 \\
\hline 150 & 75 & 0.16 & 0.01 & 0.17 & 0.00 & 14.21 & 13.75 & 0.97 \\
\hline 75 & 45 & 0.12 & 0.01 & 0.13 & 0.00 & 10.57 & 9.67 & 0.92 \\
\hline 45 & 25 & 0.05 & 0.01 & 0.06 & 0.00 & 4.56 & 3.66 & 0.84 \\
\hline 25 & 13.1 & 0.05 & 0.03 & 0.08 & 0.00 & 4.14 & 1.72 & 0.63 \\
\hline 13.1 & 6.5 & 0.05 & 0.10 & 0.15 & 0.00 & 4.80 & -3.93 & 0.35 \\
\hline 6.5 & 3.3 & 0.07 & 0.15 & 0.22 & 0.00 & 5.93 & -6.98 & 0.31 \\
\hline 3.3 & 1.5 & 0.05 & 0.07 & 0.12 & 0.00 & 4.16 & -2.12 & 0.40 \\
\hline 1.5 & & 0.05 & 0.06 & 0.11 & 0.00 & 4.48 & -0.70 & 0.46 \\
\hline Totals & -- & 0.71 & 0.43 & 1.14 & 0.00 & & & \\
\hline & Slurry (TPH) & 8.31 & 17.72 & 25.43 & -0.60 & & & \\
\hline
\end{tabular}




\section{HYDROCYCLONE EVALUATION (BALANCED)}

\begin{tabular}{|c|c|}
\hline $\begin{array}{ll}\text { Test: } & \text { No. } 1\end{array}$ & \\
\hline FeedFlow (GPM) & 100.0 \\
\hline Feed Pressure (PSl) & 21.0 \\
\hline Water Flow (GPM) & 0.0 \\
\hline Water Pressure (PSI) & 0.0 \\
\hline Feed Rate (TPH) & 1.13 \\
\hline
\end{tabular}

\begin{tabular}{|r|c|c|c|}
\hline & OS & U/S & Feed \\
\hline Solids Content (\%) & 8.58 & 2.50 & 4.45 \\
\hline Ash Content (\%) & 25.86 & 49.00 & 34.65 \\
\hline Solids Yield (\%) & 62.0 & 38.0 & 100.0 \\
\hline Ash Yield (\%) & 62.0 & 38.0 & 100.0 \\
\hline Size Class Yield (\%) & 62.0 & 38.0 & 100.0 \\
\hline
\end{tabular}

\begin{tabular}{|c|c|c|c|c|c|c|c|c|}
\hline $\begin{array}{l}\text { Pass } \\
\text { Size } \\
\text { (um) } \\
\end{array}$ & $\begin{array}{c}\text { Retain } \\
\text { Size } \\
\text { (um) } \\
\end{array}$ & $\begin{array}{c}\text { O/S } \\
\text { Sample } \\
\text { (gms) }\end{array}$ & $\begin{array}{c}\text { U/S } \\
\text { Sample } \\
\text { (gms) }\end{array}$ & $\begin{array}{c}\text { OS } \\
\text { Mass } \\
(\% \text {, strm })\end{array}$ & $\begin{array}{c}\text { USS } \\
\text { Mass } \\
(\% \text {, strm) }\end{array}$ & $\begin{array}{c}\text { OS } \\
\text { Mass } \\
(\% \text {, feed })\end{array}$ & $\begin{array}{c}\text { U/S } \\
\text { Mass } \\
(\%, \text { feed })\end{array}$ & $\begin{array}{c}\text { Feed } \\
\text { Mass } \\
(\% \text {, feed })\end{array}$ \\
\hline & 212 & 1.78 & 0.00 & 7.44 & 0.00 & 4.61 & 0.00 & 4.61 \\
\hline 212 & 150 & 1.92 & 0.00 & 8.04 & 0.00 & 4.98 & 0.00 & 4.98 \\
\hline 150 & 75 & 5.34 & 0.00 & 22.31 & 0.00 & 13.83 & 0.00 & 13.83 \\
\hline 75 & 45 & 4.00 & 0.06 & 16.72 & 0.42 & 10.37 & 0.16 & 10.53 \\
\hline 45 & 25 & 1.81 & 0.10 & 7.54 & 0.70 & 4.68 & 0.27 & 4.94 \\
\hline 25 & 13.1 & 1.64 & 0.74 & 6.86 & 5.41 & 4.25 & 2.06 & 6.31 \\
\hline 13.1 & 6.5 & 1.84 & 3.40 & 7.70 & 24.76 & 4.77 & 9.41 & 14.18 \\
\hline 6.5 & 3.3 & 2.23 & 5.16 & 9.31 & 37.56 & 5.77 & 14.27 & 20.04 \\
\hline 3.3 & 1.5 & 1.62 & 2.37 & 6.78 & 17.26 & 4.21 & 6.56 & 10.77 \\
\hline 1.5 & & 1.75 & 1.91 & 7.31 & 13.88 & 4.53 & 5.28 & 9.81 \\
\hline Totals & -- & 23.94 & 13.75 & 100.00 & 100.00 & 62.00 & 38.00 & 100.00 \\
\hline
\end{tabular}

\begin{tabular}{|c|c|c|c|c|c|c|c|c|}
\hline $\begin{array}{c}\text { Pass } \\
\text { Size } \\
(u m)\end{array}$ & $\begin{array}{c}\text { Retain } \\
\text { Size } \\
(u m)\end{array}$ & $\begin{array}{c}\text { O/S } \\
\text { Mass } \\
(\mathrm{TPH})\end{array}$ & $\begin{array}{c}\text { O/S } \\
\text { Mass } \\
(\mathrm{TPH})\end{array}$ & $\begin{array}{c}\text { Feed } \\
\text { Mass } \\
(\mathrm{TPH})\end{array}$ & $\begin{array}{c}\text { Delta } \\
\text { Mass } \\
(\mathrm{TPH})\end{array}$ & $\begin{array}{c}\text { Value } \\
\text { (f-u) }\end{array}$ & $\begin{array}{c}\text { X } \\
\text { Value } \\
(0-u)\end{array}$ & $\begin{array}{c}\text { Partition } \\
\text { Factor } \\
(-)\end{array}$ \\
\hline & 212 & 0.05 & 0.00 & 0.05 & 0.00 & 4.61 & 4.61 & 1.00 \\
\hline 212 & 150 & 0.06 & 0.00 & 0.06 & 0.00 & 4.98 & 4.98 & 1.00 \\
\hline 150 & 75 & 0.16 & 0.00 & 0.16 & 0.00 & 13.83 & 13.83 & 1.00 \\
\hline 75 & 45 & 0.12 & 0.00 & 0.12 & 0.00 & 10.37 & 10.21 & 0.98 \\
\hline 45 & 25 & 0.05 & 0.00 & 0.06 & 0.00 & 4.68 & 4.41 & 0.95 \\
\hline 25 & 13.1 & 0.05 & 0.02 & 0.07 & 0.00 & 4.25 & 2.20 & 0.67 \\
\hline 13.1 & 6.5 & 0.05 & 0.11 & 0.16 & 0.00 & 4.77 & -4.64 & 0.34 \\
\hline 6.5 & 3.3 & 0.07 & 0.16 & 0.23 & 0.00 & 5.77 & -8.50 & 0.29 \\
\hline 3.3 & 1.5 & 0.05 & 0.07 & 0.12 & 0.00 & 4.21 & -2.35 & 0.39 \\
\hline 1.5 & & 0.05 & 0.06 & 0.11 & 0.00 & 4.53 & -0.75 & 0.46 \\
\hline Totals & -- & 0.70 & 0.43 & 1.13 & 0.00 & & & \\
\hline & Slurry(TPH) & 8.18 & 17.24 & 25.42 & 0.00 & & & \\
\hline
\end{tabular}




\begin{tabular}{|c|c|c|c|}
\hline $\begin{array}{c}\text { Experimental } \\
\text { Partition F actor }\end{array}$ & $\begin{array}{c}\text { Mean } \\
\text { Particle } \\
\text { Size }(\mu \mathrm{m})\end{array}$ & $\begin{array}{c}\text { Predicted } \\
\text { Partition } \\
\text { Factor }\end{array}$ & $\begin{array}{c}\text { Predicted } \\
\text { Partition } \\
\text { Factor }\end{array}$ \\
\hline$(--)$ & $(--)$ & (Plitt) & (Linch) \\
\hline 1.00 & 299.81 & 1.000 & 1.000 \\
\hline 1.00 & 178.33 & 1.000 & 1.000 \\
\hline 0.97 & 106.07 & 1.000 & 1.000 \\
\hline 0.92 & 58.09 & 0.991 & 0.988 \\
\hline 0.84 & 33.54 & 0.841 & 0.845 \\
\hline 0.63 & 18.08 & 0.576 & 0.570 \\
\hline 0.35 & 9.25 & 0.433 & 0.438 \\
\hline 0.31 & 4.62 & 0.389 & 0.393 \\
\hline 0.40 & 2.32 & 0.378 & 0.377 \\
\hline 0.46 & 1.64 & 0.376 & 0.373 \\
\hline & WSSQ & 0.029 & 0.031 \\
\hline
\end{tabular}

\begin{tabular}{|c|c|}
\multicolumn{1}{c}{ (Plitt) } & Predicted \\
\hline $\mathrm{D} 50$ & 24.02 \\
\hline $\mathrm{m}$ & 2.04 \\
\hline Bypass(Rf) & 0.37 \\
\hline
\end{tabular}

(Linch) Predicted

\begin{tabular}{|c|c|}
\hline D50 & 23.94 \\
\hline Alpha & 2.70 \\
\hline Bypass(Rf) & 0.36 \\
\hline
\end{tabular}

\begin{tabular}{|l|l|}
\hline TWSSQ & 0.060 \\
\hline
\end{tabular}

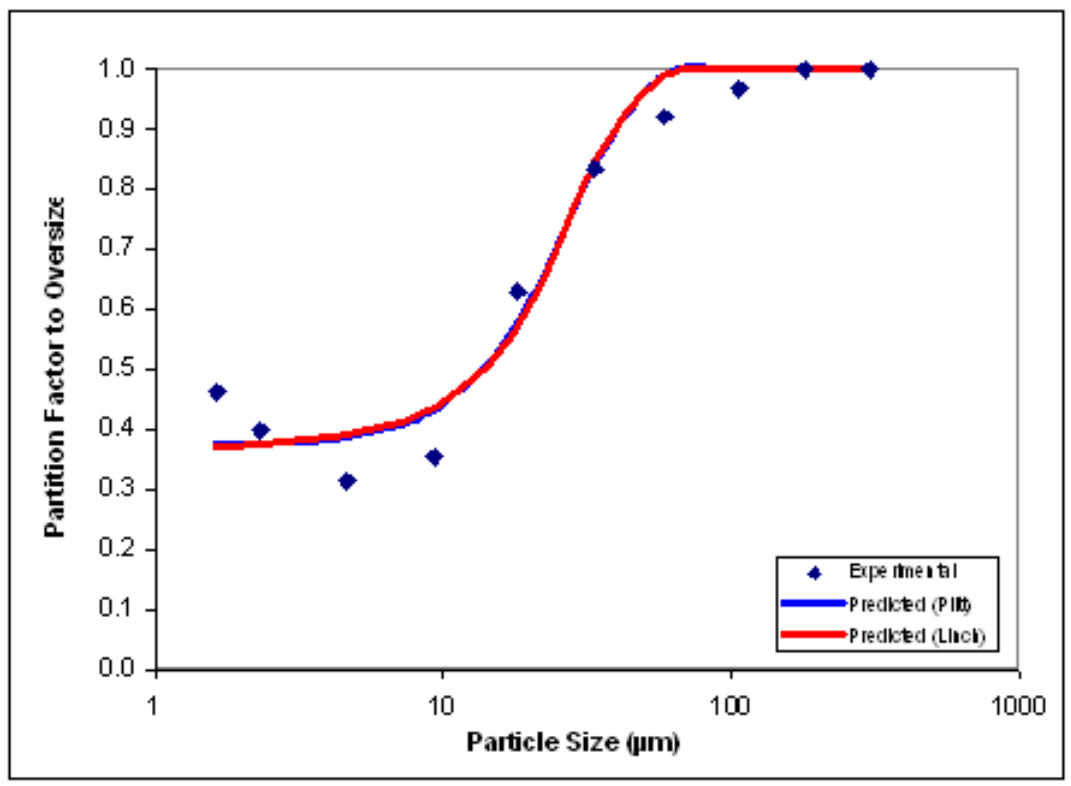




\section{HYDROCYCLONE EVALUATION (UNBALANCED)}

\begin{tabular}{|c|c|}
\hline No. 02 & \\
\hline FeedFlow (GPM) & 900.0 \\
\hline Feed Pressure (PSl) & 21.0 \\
\hline Water Flow (GPM) & 30.0 \\
\hline Water Pressure (PSl) & 28.0 \\
\hline Feed Rate (TPH) & 1.14 \\
\hline
\end{tabular}

\begin{tabular}{|r|c|c|c|}
\hline & OS & U/S & Feed \\
\hline Solids Content (\%) & 5.27 & 2.56 & 4.50 \\
\hline Ash Content (\%) & 18.57 & 47.67 & 34.55 \\
\hline Solids Yield (\%) & 51.2 & 48.8 & 100.0 \\
\hline Ash Yield (\%) & 45.1 & 54.9 & 100.0 \\
\hline Size Class Yield (\%) & 31.4 & 68.6 & 100.0 \\
\hline
\end{tabular}

\begin{tabular}{|c|c|c|c|c|c|c|c|c|}
\hline $\begin{array}{c}\text { Pass } \\
\text { Size } \\
(\mathrm{um})\end{array}$ & $\begin{array}{c}\text { Retain } \\
\text { Size } \\
(\mathrm{um})\end{array}$ & $\begin{array}{c}\mathrm{O} / \mathrm{S} \\
\text { Sample } \\
(\mathrm{gms})\end{array}$ & $\begin{array}{c}\mathrm{U} / \mathrm{S} \\
\text { Sample } \\
(\mathrm{gms})\end{array}$ & $\begin{array}{c}\text { O/S } \\
\text { Mass } \\
(\%, \text { strm) }\end{array}$ & $\begin{array}{c}\text { U/S } \\
\text { Mass } \\
(\%, \text { strm })\end{array}$ & $\begin{array}{c}\text { OS } \\
\text { Mass } \\
(\%, \text { feed })\end{array}$ & $\begin{array}{c}\text { U/S } \\
\text { Mass } \\
(\%, \text { feed })\end{array}$ & $\begin{array}{c}\text { Feed } \\
\text { Mass } \\
(\%, \text { feed })\end{array}$ \\
\hline & 212 & 1.54 & 0.00 & 8.86 & 0.00 & 3.99 & 0.00 & 3.99 \\
\hline 212 & 150 & 1.83 & 0.00 & 10.52 & 0.00 & 4.74 & 0.00 & 4.74 \\
\hline 150 & 75 & 5.08 & 0.17 & 29.21 & 0.89 & 13.17 & 0.49 & 13.66 \\
\hline 75 & 45 & 3.40 & 0.48 & 19.55 & 2.51 & 8.81 & 1.38 & 10.19 \\
\hline 45 & 25 & 1.13 & 0.85 & 6.50 & 4.44 & 2.93 & 2.44 & 5.37 \\
\hline 25 & 13.1 & 0.92 & 3.46 & 5.27 & 18.06 & 2.38 & 9.92 & 12.30 \\
\hline 13.1 & 6.5 & 0.94 & 4.01 & 5.43 & 20.96 & 2.45 & 11.51 & 13.96 \\
\hline 6.5 & 3.3 & 1.06 & 4.50 & 6.09 & 23.50 & 2.75 & 12.90 & 15.65 \\
\hline 3.3 & 1.5 & 0.71 & 2.77 & 4.06 & 14.46 & 1.83 & 7.94 & 9.77 \\
\hline 1.5 & & 0.78 & 2.91 & 4.51 & 15.18 & 2.03 & 8.34 & 10.37 \\
\hline Totals & -- & 17.39 & 19.14 & 100.00 & 100.00 & 45.09 & 54.91 & 100.00 \\
\hline
\end{tabular}

\begin{tabular}{|c|c|c|c|c|c|c|c|c|}
\hline $\begin{array}{c}\text { Pass } \\
\text { Size } \\
(u m)\end{array}$ & $\begin{array}{c}\text { Retain } \\
\text { Size } \\
(u m)\end{array}$ & $\begin{array}{c}\text { O/S } \\
\text { Mass } \\
(\mathrm{TPH})\end{array}$ & $\begin{array}{c}\text { U/S } \\
\text { Mass } \\
(\mathrm{TPH})\end{array}$ & $\begin{array}{c}\text { Feed } \\
\text { Mass } \\
(\mathrm{TPH})\end{array}$ & $\begin{array}{c}\text { Delta } \\
\text { Mass } \\
(\mathrm{TPH})\end{array}$ & $\begin{array}{c}\text { Y } \\
\text { Value } \\
(\mathrm{f}-\mathrm{u})\end{array}$ & $\begin{array}{c}\text { X } \\
\text { Value } \\
(0-\mathrm{u})\end{array}$ & $\begin{array}{c}\text { Partition } \\
\text { Factor } \\
(-)\end{array}$ \\
\hline & 212 & 0.05 & 0.00 & 0.05 & 0.00 & 3.99 & 3.99 & 1.00 \\
\hline 212 & 150 & 0.05 & 0.00 & 0.05 & 0.00 & 4.74 & 4.74 & 1.00 \\
\hline 150 & 75 & 0.15 & 0.01 & 0.16 & 0.00 & 13.17 & 12.68 & 0.96 \\
\hline 75 & 45 & 0.10 & 0.02 & 0.12 & 0.00 & 8.81 & 7.44 & 0.86 \\
\hline 45 & 25 & 0.03 & 0.03 & 0.06 & 0.00 & 2.93 & 0.49 & 0.55 \\
\hline 25 & 13.1 & 0.03 & 0.11 & 0.14 & 0.00 & 2.38 & -7.54 & 0.19 \\
\hline 13.1 & 6.5 & 0.03 & 0.13 & 0.16 & 0.00 & 2.45 & -9.06 & 0.18 \\
\hline 6.5 & 3.3 & 0.03 & 0.15 & 0.18 & 0.00 & 2.75 & -10.16 & 0.18 \\
\hline 3.3 & 1.5 & 0.02 & 0.09 & 0.11 & 0.00 & 1.83 & -6.11 & 0.19 \\
\hline 1.5 & & 0.02 & 0.10 & 0.12 & 0.00 & 2.03 & -6.30 & 0.20 \\
\hline Totals & -- & 0.52 & 0.63 & 1.14 & 0.00 & & & \\
\hline & Slurry (TPH) & 9.79 & 24.54 & 25.43 & -1.41 & & & \\
\hline
\end{tabular}




\section{HYDROCYCLONE EVALUATION (BALANCED)}

\begin{tabular}{|c|c|}
\hline No. 02 & \\
\hline Feedflow (GPF) & 100.0 \\
\hline Feed Pressure (PSl) & 21.0 \\
\hline Water Flow (GPM) & 30.0 \\
\hline Water Pressure (PSl) & 28.0 \\
\hline Feed Rate (TPH) & 1.16 \\
\hline
\end{tabular}

\begin{tabular}{|r|c|c|c|}
\hline & OrS & U/S & Feed \\
\hline Solids Content (\%) & 5.17 & 2.91 & 4.57 \\
\hline Ash Content (\%) & 19.03 & 47.90 & 34.21 \\
\hline Solids Yield (\%) & 40.3 & 59.7 & 100.0 \\
\hline Ash Yield (\%) & 47.4 & 52.6 & 100.0 \\
\hline Size Class Yield (\%) & 47.4 & 52.6 & 100.0 \\
\hline
\end{tabular}

\begin{tabular}{|c|c|c|c|c|c|c|c|c|}
\hline $\begin{array}{l}\text { Pass } \\
\text { Size } \\
\text { (um) }\end{array}$ & $\begin{array}{c}\text { Retain } \\
\text { Size } \\
\text { (um) }\end{array}$ & $\begin{array}{c}\text { O/S } \\
\text { Sample } \\
\text { (gms) }\end{array}$ & $\begin{array}{c}\text { USS } \\
\text { Sample } \\
\text { (gms) }\end{array}$ & $\begin{array}{c}\text { O/S } \\
\text { Mass } \\
(\%, \text { strm) }\end{array}$ & $\begin{array}{c}\text { USS } \\
\text { Mass } \\
(\%, \text { strm) }\end{array}$ & $\begin{array}{c}\text { OS } \\
\text { Mass } \\
(\%, \text { feed })\end{array}$ & $\begin{array}{c}\text { U/S } \\
\text { Mass } \\
(\%, \text { feed })\end{array}$ & $\begin{array}{c}\text { Feed } \\
\text { Mass } \\
(\%, \text { feed })\end{array}$ \\
\hline & 212 & 1.54 & 0.00 & 9.43 & 0.00 & 4.47 & 0.00 & 4.47 \\
\hline 212 & 150 & 1.74 & 0.00 & 10.70 & 0.00 & 5.08 & 0.00 & 5.08 \\
\hline 150 & 75 & 5.64 & 0.27 & 34.61 & 1.40 & 16.41 & 0.73 & 17.15 \\
\hline 75 & 45 & 3.46 & 0.61 & 21.27 & 3.13 & 10.09 & 1.65 & 11.73 \\
\hline 45 & 25 & 0.92 & 1.02 & 5.66 & 5.20 & 2.68 & 2.74 & 5.42 \\
\hline 25 & 13.1 & 0.65 & 3.46 & 3.97 & 17.71 & 1.88 & 9.31 & 11.19 \\
\hline 13.1 & 6.5 & 0.66 & 3.97 & 4.07 & 20.36 & 1.93 & 10.70 & 12.63 \\
\hline 6.5 & 3.3 & 0.78 & 4.42 & 4.77 & 22.67 & 2.26 & 11.92 & 14.18 \\
\hline 3.3 & 1.5 & 0.43 & 2.82 & 2.65 & 14.44 & 1.26 & 7.59 & 8.85 \\
\hline 1.5 & & 0.47 & 2.95 & 2.88 & 15.09 & 1.37 & 7.93 & 9.30 \\
\hline Totals & -- & 16.29 & 19.52 & 100.00 & 100.00 & 47.43 & 52.57 & 100.00 \\
\hline
\end{tabular}

\begin{tabular}{|c|c|c|c|c|c|c|c|c|}
\hline $\begin{array}{l}\text { Pass } \\
\text { Size } \\
\text { (um) }\end{array}$ & $\begin{array}{c}\text { Retain } \\
\text { Size } \\
\text { (um) }\end{array}$ & $\begin{array}{c}\mathrm{O} / \mathrm{S} \\
\mathrm{Mass} \\
(\mathrm{TPH})\end{array}$ & $\begin{array}{c}\text { U/S } \\
\text { Mass } \\
(\mathrm{TPH})\end{array}$ & $\begin{array}{l}\text { Feed } \\
\text { Mass } \\
(\mathrm{TPH})\end{array}$ & $\begin{array}{l}\text { Delta } \\
\text { Mass } \\
(\mathrm{TPH})\end{array}$ & $\begin{array}{c}Y \\
\text { Value } \\
(f-u)\end{array}$ & $\begin{array}{c}X \\
\text { Value } \\
\text { (0-u) }\end{array}$ & $\begin{array}{c}\text { Partition } \\
\text { Factor } \\
(--)\end{array}$ \\
\hline & 212 & 0.05 & 0.00 & 0.05 & 0.00 & 4.47 & 4.47 & 1.00 \\
\hline 212 & 150 & 0.06 & 0.00 & 0.06 & 0.00 & 5.08 & 5.08 & 1.00 \\
\hline 150 & 75 & 0.19 & 0.01 & 0.20 & 0.00 & 16.41 & 15.68 & 0.96 \\
\hline 75 & 45 & 0.12 & 0.02 & 0.14 & 0.00 & 10.09 & 8.44 & 0.86 \\
\hline 45 & 25 & 0.03 & 0.03 & 0.06 & 0.00 & 2.68 & -0.05 & 0.50 \\
\hline 25 & 13.1 & 0.02 & 0.11 & 0.13 & 0.00 & 1.88 & -7.43 & 0.17 \\
\hline 13.1 & 6.5 & 0.02 & 0.12 & 0.15 & 0.00 & 1.93 & -8.77 & 0.15 \\
\hline 6.5 & 3.3 & 0.03 & 0.14 & 0.16 & 0.00 & 2.26 & -9.65 & 0.16 \\
\hline 3.3 & 1.5 & 0.01 & 0.09 & 0.10 & 0.00 & 1.26 & -6.33 & 0.14 \\
\hline 1.5 & & 0.02 & 0.09 & 0.11 & 0.00 & 1.37 & -6.57 & 0.15 \\
\hline Totals & -- & 0.55 & 0.61 & 1.16 & 0.00 & & & \\
\hline \multicolumn{2}{|c|}{ Slurry (TPH) } & 10.67 & 21.01 & 25.43 & 1.25 & & & \\
\hline
\end{tabular}




\begin{tabular}{|c|c|c|c|c|c|c|c|}
\hline \multirow{3}{*}{$\begin{array}{l}\text { Experimental } \\
\text { Partition F actor }\end{array}$} & \multirow{3}{*}{$\begin{array}{c}\text { Mean } \\
\text { Particle } \\
\text { Size ( } \mu \mathrm{m})\end{array}$} & \multirow{3}{*}{$\begin{array}{c}\text { Predicted } \\
\text { Partition } \\
\text { Factor }\end{array}$} & \multirow{3}{*}{$\begin{array}{c}\text { Predicted } \\
\text { Partition } \\
\text { Factor }\end{array}$} & \multirow{2}{*}{$\frac{\text { (Plitt) }}{\mathrm{D} 50}$} & Predicted & (Linch) & Predicted \\
\hline & & & & & 37.86 & D50 & 37.42 \\
\hline & & & & $m$ & 2.52 & Alpha & 3.61 \\
\hline$(-)$ & $(-)$ & (Plitt) & (Linch) & Bypass(Rf) & 0.17 & Bypass(Rt) & 0.16 \\
\hline 1.00 & 299.81 & 1.000 & 1.000 & & & & \\
\hline 1.00 & 178.33 & 1.000 & 1.000 & & & & \\
\hline 0.96 & 106.07 & 1.000 & 0.999 & & & & \\
\hline 0.86 & 58.09 & 0.892 & 0.902 & & & & \\
\hline 0.55 & 33.54 & 0.502 & 0.500 & & & & \\
\hline 0.19 & 18.08 & 0.255 & 0.258 & & & & \\
\hline 0.18 & 9.25 & 0.187 & 0.193 & & & & \\
\hline 0.18 & 4.62 & 0.173 & 0.174 & & & & \\
\hline 0.19 & 2.32 & 0.171 & 0.167 & & & & \\
\hline \multirow[t]{3}{*}{0.20} & 1.64 & 0.171 & 0.165 & & & & \\
\hline & WSSQ & 0.009 & 0.011 & & & & \\
\hline & TWSSQ & 0.019 & & & & & \\
\hline
\end{tabular}

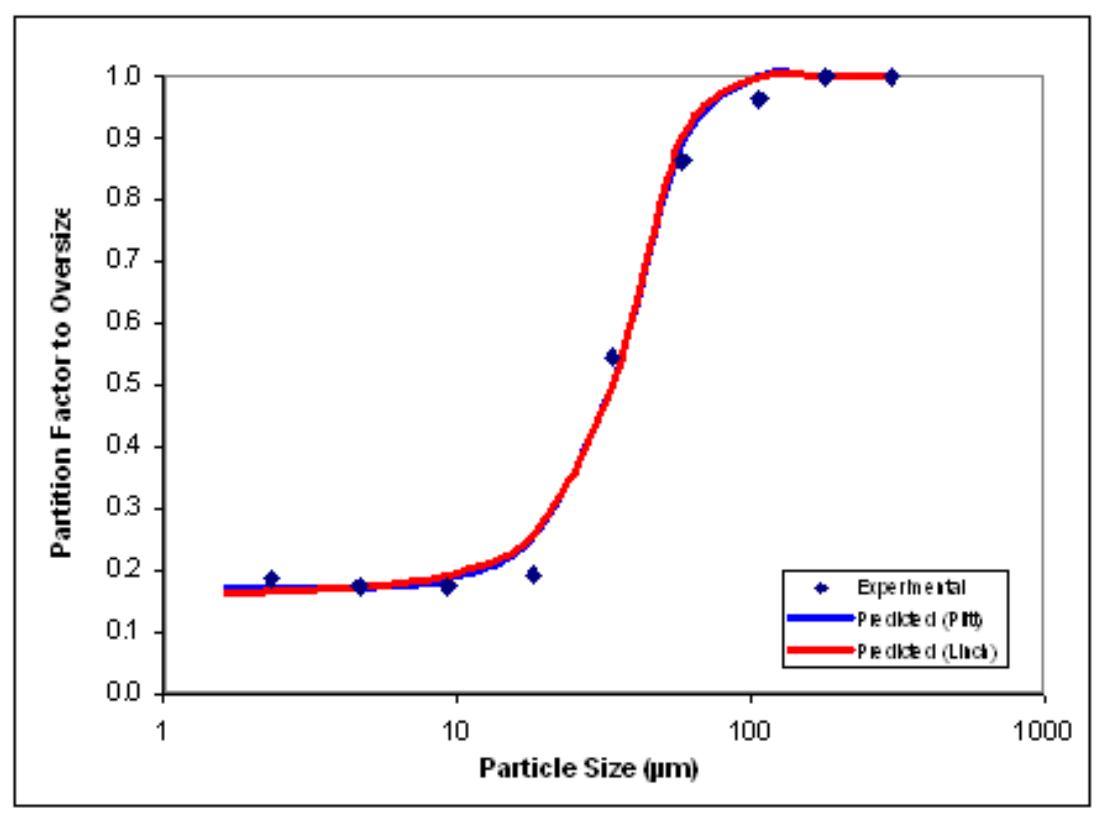




\section{HYDROCYCLONE EVALUATION (UNBALANCED)}

\begin{tabular}{|c|c|}
\hline No. 03 & \\
\hline Feedflow (GPV) & 100.0 \\
\hline Feed Pressure (PSl) & 21.0 \\
\hline Water Flow (GPM) & 15.0 \\
\hline Water Pressure (PSl) & 15.0 \\
\hline Feed Rate (TPH) & 1.18 \\
\hline
\end{tabular}

\begin{tabular}{|r|c|c|c|}
\hline & OS & U/S & Feed \\
\hline Solids Content (\%) & 6.61 & 2.75 & 4.63 \\
\hline Ash Content (\%) & 22.47 & 48.29 & 34.55 \\
\hline Solids Yield (\%) & 54.5 & 45.5 & 100.0 \\
\hline Ash Yield (\%) & 53.2 & 46.8 & 100.0 \\
\hline Size Class Yield (\%) & 49.7 & 50.3 & 100.0 \\
\hline
\end{tabular}

\begin{tabular}{|c|c|c|c|c|c|c|c|c|}
\hline $\begin{array}{c}\text { Pass } \\
\text { Size } \\
(u m)\end{array}$ & $\begin{array}{c}\text { Retain } \\
\text { Size } \\
(u m)\end{array}$ & $\begin{array}{c}\text { O/S } \\
\text { Sample } \\
\text { (gms) }\end{array}$ & $\begin{array}{c}\text { U/S } \\
\text { Sample } \\
(\mathrm{gms})\end{array}$ & $\begin{array}{c}\text { OS } \\
\text { Mass } \\
(\%, \text { strm) }\end{array}$ & $\begin{array}{c}\text { U/S } \\
\text { Mass } \\
(\%, \text { strm) }\end{array}$ & $\begin{array}{c}\text { OSS } \\
\text { Mass } \\
(\%, \text { feed) })\end{array}$ & $\begin{array}{c}\text { U/S } \\
\text { Mass } \\
(\%, \text { feed) }\end{array}$ & $\begin{array}{c}\text { Feed } \\
\text { Mass } \\
(\%, \text { feed) }\end{array}$ \\
\hline & 212 & 1.52 & 0.00 & 8.15 & 0.00 & 4.34 & 0.00 & 4.34 \\
\hline 212 & 150 & 1.61 & 0.00 & 8.63 & 0.00 & 4.59 & 0.00 & 4.59 \\
\hline 150 & 75 & 4.81 & 0.16 & 25.79 & 0.91 & 13.72 & 0.43 & 14.15 \\
\hline 75 & 45 & 3.12 & 0.38 & 16.73 & 2.16 & 8.90 & 1.01 & 9.91 \\
\hline 45 & 25 & 1.30 & 0.62 & 6.97 & 3.52 & 3.71 & 1.65 & 5.36 \\
\hline 25 & 13.1 & 0.70 & 2.59 & 3.73 & 14.71 & 1.99 & 6.88 & 8.87 \\
\hline 13.1 & 6.5 & 0.80 & 3.88 & 4.27 & 22.05 & 2.27 & 10.32 & 12.59 \\
\hline 6.5 & 3.3 & 1.33 & 4.13 & 7.12 & 23.51 & 3.79 & 11.00 & 14.78 \\
\hline 3.3 & 1.5 & 1.37 & 2.91 & 7.34 & 16.54 & 3.90 & 7.74 & 11.64 \\
\hline 1.5 & & 2.10 & 2.92 & 11.27 & 16.60 & 6.00 & 7.77 & 13.76 \\
\hline Totals & -- & 18.65 & 17.59 & 100.00 & 100.00 & 53.21 & 46.79 & 100.00 \\
\hline
\end{tabular}

\begin{tabular}{|c|c|c|c|c|c|c|c|c|}
\hline $\begin{array}{c}\text { Pass } \\
\text { Size } \\
(\mathrm{um})\end{array}$ & $\begin{array}{c}\text { Retain } \\
\text { Size } \\
(\mathrm{um})\end{array}$ & $\begin{array}{c}\text { O/S } \\
\text { Mass } \\
(\mathrm{TPH})\end{array}$ & $\begin{array}{c}\text { U/S } \\
\text { Mass } \\
(\mathrm{TPH})\end{array}$ & $\begin{array}{c}\text { Feed } \\
\text { Mass } \\
(\mathrm{TPH})\end{array}$ & $\begin{array}{c}\text { Delta } \\
\text { Mass } \\
(\mathrm{TPH})\end{array}$ & $\begin{array}{c}\text { Y } \\
\text { Value } \\
(\mathrm{f}-\mathrm{u})\end{array}$ & $\begin{array}{c}\text { Xalue } \\
\text { (o-u) }\end{array}$ & $\begin{array}{c}\text { Partition } \\
\text { Factor } \\
(--)\end{array}$ \\
\hline & 212 & 0.05 & 0.00 & 0.05 & 0.00 & 4.34 & 4.34 & 1.00 \\
\hline 212 & 150 & 0.05 & 0.00 & 0.05 & 0.00 & 4.59 & 4.59 & 1.00 \\
\hline 150 & 75 & 0.16 & 0.01 & 0.17 & 0.00 & 13.72 & 13.30 & 0.97 \\
\hline 75 & 45 & 0.10 & 0.01 & 0.12 & 0.00 & 8.90 & 7.89 & 0.90 \\
\hline 45 & 25 & 0.04 & 0.02 & 0.06 & 0.00 & 3.71 & 2.06 & 0.69 \\
\hline 25 & 13.1 & 0.02 & 0.08 & 0.10 & 0.00 & 1.99 & -4.89 & 0.22 \\
\hline 13.1 & 6.5 & 0.03 & 0.12 & 0.15 & 0.00 & 2.27 & -8.04 & 0.18 \\
\hline 6.5 & 3.3 & 0.04 & 0.13 & 0.17 & 0.00 & 3.79 & -7.21 & 0.26 \\
\hline 3.3 & 1.5 & 0.05 & 0.09 & 0.14 & 0.00 & 3.90 & -3.83 & 0.34 \\
\hline 1.5 & & 0.07 & 0.09 & 0.16 & 0.00 & 6.00 & -1.77 & 0.44 \\
\hline Totals & -- & 0.63 & 0.55 & 1.18 & 0.00 & & & \\
\hline
\end{tabular}




\section{HYDROCYCLONE EVALUATION (BALANCED)}

\begin{tabular}{|c|c|}
\hline No. 03 & \\
\hline Feedflow (GPV) & 100.0 \\
\hline Feed Pressure (PSl) & 21.0 \\
\hline Water Flow (GPM) & 15.0 \\
\hline Water Pressure (PSl) & 15.0 \\
\hline Feed Rate (TPH) & 1.20 \\
\hline
\end{tabular}

\begin{tabular}{|r|c|c|c|}
\hline & OS & US & Feed \\
\hline Solids Content (\%) & 6.50 & 2.88 & 4.71 \\
\hline Ash Content (\%) & 22.54 & 48.35 & 34.48 \\
\hline Solids Yield (\%) & 53.7 & 46.3 & 100.0 \\
\hline Ash Yield (\%) & 53.7 & 46.3 & 100.0 \\
\hline Size Class Yield (\%) & 53.7 & 46.3 & 100.0 \\
\hline
\end{tabular}

\begin{tabular}{|c|c|c|c|c|c|c|c|c|}
\hline $\begin{array}{c}\text { Pass } \\
\text { Size } \\
(u m)\end{array}$ & $\begin{array}{c}\text { Retain } \\
\text { Size } \\
(u m)\end{array}$ & $\begin{array}{c}\text { O/S } \\
\text { Sample } \\
\text { (gms) }\end{array}$ & $\begin{array}{c}\text { U/S } \\
\text { Sample } \\
(\mathrm{gmm})\end{array}$ & $\begin{array}{c}\text { OS } \\
\text { Mass } \\
(\%, \text { strm) }\end{array}$ & $\begin{array}{c}\text { U/S } \\
\text { Mass } \\
(\%, \text { strm) }\end{array}$ & $\begin{array}{c}\text { OSS } \\
\text { Mass } \\
(\%, \text { feed) })\end{array}$ & $\begin{array}{c}\text { U/S } \\
\text { Mass } \\
(\%, \text { feed) }\end{array}$ & $\begin{array}{c}\text { Feed } \\
\text { Mass } \\
(\%, \text { feed) }\end{array}$ \\
\hline & 212 & 1.49 & 0.00 & 8.06 & 0.00 & 4.33 & 0.00 & 4.33 \\
\hline 212 & 150 & 1.58 & 0.00 & 8.57 & 0.00 & 4.60 & 0.00 & 4.60 \\
\hline 150 & 75 & 4.88 & 0.24 & 26.47 & 1.36 & 14.22 & 0.63 & 14.85 \\
\hline 75 & 45 & 3.14 & 0.46 & 17.02 & 2.56 & 9.14 & 1.19 & 10.33 \\
\hline 45 & 25 & 1.26 & 0.69 & 6.84 & 3.88 & 3.67 & 1.79 & 5.47 \\
\hline 25 & 13.1 & 0.64 & 2.60 & 3.46 & 14.62 & 1.86 & 6.77 & 8.63 \\
\hline 13.1 & 6.5 & 0.74 & 3.85 & 4.03 & 21.64 & 2.16 & 10.02 & 12.18 \\
\hline 6.5 & 3.3 & 1.29 & 4.11 & 7.00 & 23.09 & 3.76 & 10.69 & 14.45 \\
\hline 3.3 & 1.5 & 1.33 & 2.92 & 7.23 & 16.39 & 3.88 & 7.58 & 11.46 \\
\hline 1.5 & & 2.09 & 2.93 & 11.33 & 16.45 & 6.09 & 7.61 & 13.70 \\
\hline Totals & -- & 18.42 & 17.80 & 100.00 & 100.00 & 53.72 & 46.28 & 100.00 \\
\hline
\end{tabular}

\begin{tabular}{|c|c|c|c|c|c|c|c|c|}
\hline $\begin{array}{l}\text { Pass } \\
\text { Size } \\
\text { (um) }\end{array}$ & $\begin{array}{c}\text { Retain } \\
\text { Size } \\
\text { (um) }\end{array}$ & $\begin{array}{c}\mathrm{O} / \mathrm{S} \\
\text { Mass } \\
(\mathrm{TPH})\end{array}$ & $\begin{array}{c}\text { USS } \\
\text { Mass } \\
(\mathrm{TPH})\end{array}$ & $\begin{array}{l}\text { Feed } \\
\text { Mass } \\
(\mathrm{TPH})\end{array}$ & $\begin{array}{l}\text { Delta } \\
\text { Mass } \\
(\mathrm{TPH}) \\
\end{array}$ & $\begin{array}{c}\text { Y } \\
\text { Value } \\
(\mathrm{f}-\mathrm{u})\end{array}$ & $\begin{array}{c}X \\
\text { Value } \\
(0-4)\end{array}$ & $\begin{array}{c}\text { Partition } \\
\text { Factor } \\
(--)\end{array}$ \\
\hline & 212 & 0.05 & 0.00 & 0.05 & 0.00 & 4.33 & 4.33 & 1.00 \\
\hline 212 & 150 & 0.06 & 0.00 & 0.06 & 0.00 & 4.60 & 4.60 & 1.00 \\
\hline 150 & 75 & 0.17 & 0.01 & 0.18 & 0.00 & 14.22 & 13.59 & 0.96 \\
\hline 75 & 45 & 0.11 & 0.01 & 0.12 & 0.00 & 9.14 & 7.96 & 0.89 \\
\hline 45 & 25 & 0.04 & 0.02 & 0.07 & 0.00 & 3.67 & 1.88 & 0.67 \\
\hline 25 & 13.1 & 0.02 & 0.08 & 0.10 & 0.00 & 1.86 & -4.91 & 0.22 \\
\hline 13.1 & 6.5 & 0.03 & 0.12 & 0.15 & 0.00 & 2.16 & -7.85 & 0.18 \\
\hline 6.5 & 3.3 & 0.05 & 0.13 & 0.17 & 0.00 & 3.76 & -6.93 & 0.26 \\
\hline 3.3 & 1.5 & 0.05 & 0.09 & 0.14 & 0.00 & 3.88 & -3.70 & 0.34 \\
\hline 1.5 & & 0.07 & 0.09 & 0.16 & 0.00 & 6.09 & -1.53 & 0.44 \\
\hline Totals & -- & 0.64 & 0.55 & 1.20 & 0.00 & & & \\
\hline \multicolumn{2}{|c|}{ Slurry (TPH) } & 9.91 & 19.29 & 25.45 & 0.00 & & & \\
\hline
\end{tabular}




\begin{tabular}{|c|c|c|c|c|c|c|c|}
\hline \multirow{3}{*}{$\begin{array}{l}\text { Experimental } \\
\text { Partition } F \text { actor }\end{array}$} & \multirow{3}{*}{$\begin{array}{c}\text { Mean } \\
\text { Particle } \\
\text { Sze ( } \mu \mathrm{m})\end{array}$} & \multirow{3}{*}{$\begin{array}{c}\text { Predicted } \\
\text { Partition } \\
\text { Factor }\end{array}$} & \multirow{3}{*}{$\begin{array}{c}\text { Predicted } \\
\text { Partition } \\
\text { Factor }\end{array}$} & (Plitt) & Predicted & (Linch) & Predicted \\
\hline & & & & D50 & 32.43 & D50 & 32.89 \\
\hline & & & & $m$ & 4.80 & Alpha & 5.00 \\
\hline$(-)$ & $(-)$ & (Plitt) & (Linch) & Bypass(R) & 0.28 & Bypass(R0) & 0.27 \\
\hline 1.00 & 299.81 & 1.000 & 1.000 & & & & \\
\hline 1.00 & 178.33 & 1.000 & 1.000 & & & & \\
\hline 0.97 & 106.07 & 1.000 & 1.000 & & & & \\
\hline 0.90 & 58.09 & 1.000 & 0.985 & & & & \\
\hline 0.69 & 33.54 & 0.682 & 0.655 & & & & \\
\hline 0.22 & 18.08 & 0.311 & 0.340 & & & & \\
\hline 0.18 & 9.25 & 0.283 & 0.289 & & & & \\
\hline 0.26 & 4.62 & 0.282 & 0.279 & & & & \\
\hline 0.34 & 2.32 & 0.282 & 0.276 & & & & \\
\hline 0.44 & 1.64 & 0.282 & 0.276 & & & & \\
\hline & WSSQ & 0.057 & 0.065 & & & & \\
\hline & TWSSQ & 0.121 & & & & & \\
\hline
\end{tabular}

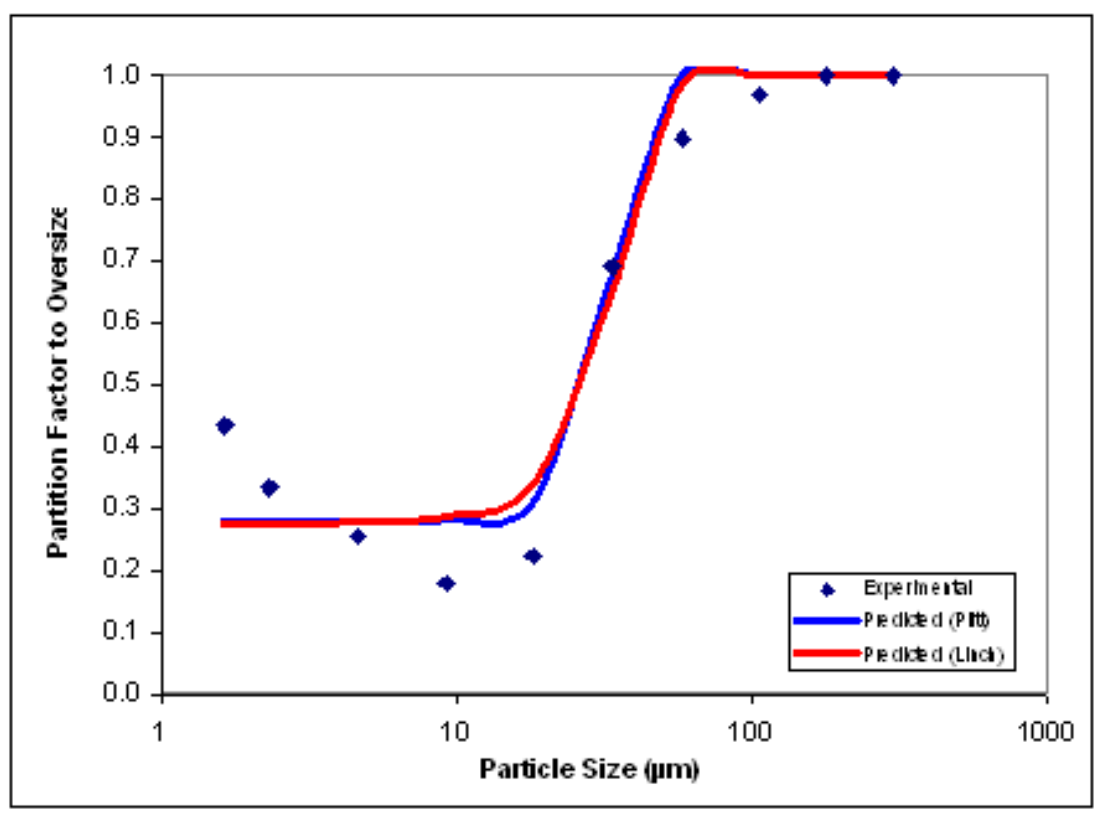




\section{HYDROCYCLONE EVALUATION (UNBALANCED)}

\begin{tabular}{|c|c|}
\hline No. 04 & \\
\hline Feedflow (GPV) & 100.0 \\
\hline Feed Pressure (PSl) & 21.0 \\
\hline Water Flow (GPM) & 15.0 \\
\hline Water Pressure (PSl) & 15.0 \\
\hline Feed Rate (TPH) & 1.18 \\
\hline
\end{tabular}

\begin{tabular}{|r|c|c|c|}
\hline & OS & U/S & Feed \\
\hline Solids Content (\%) & 6.73 & 2.80 & 4.63 \\
\hline Ash Content (\%) & 23.87 & 48.10 & 34.97 \\
\hline Solids Yield (\%) & 52.4 & 47.6 & 100.0 \\
\hline Ash Yield (\%) & 54.2 & 45.8 & 100.0 \\
\hline Size Class Yield (\%) & 53.3 & 46.7 & 100.0 \\
\hline
\end{tabular}

\begin{tabular}{|c|c|c|c|c|c|c|c|c|}
\hline $\begin{array}{c}\text { Pass } \\
\text { Size } \\
(u m)\end{array}$ & $\begin{array}{c}\text { Retain } \\
\text { Size } \\
(u m)\end{array}$ & $\begin{array}{c}\text { O/S } \\
\text { Sample } \\
\text { (gms) }\end{array}$ & $\begin{array}{c}\text { U/S } \\
\text { Sample } \\
(\mathrm{gms})\end{array}$ & $\begin{array}{c}\text { OS } \\
\text { Mass } \\
(\%, \text { strm) }\end{array}$ & $\begin{array}{c}\text { U/S } \\
\text { Mass } \\
(\%, \text { strm) }\end{array}$ & $\begin{array}{c}\text { OSS } \\
\text { Mass } \\
(\%, \text { feed) })\end{array}$ & $\begin{array}{c}\text { U/S } \\
\text { Mass } \\
(\%, \text { feed) }\end{array}$ & $\begin{array}{c}\text { Feed } \\
\text { Mass } \\
(\%, \text { feed) }\end{array}$ \\
\hline & 212 & 1.61 & 0.00 & 7.86 & 0.00 & 4.26 & 0.00 & 4.26 \\
\hline 212 & 150 & 1.67 & 0.00 & 8.15 & 0.00 & 4.42 & 0.00 & 4.42 \\
\hline 150 & 75 & 5.45 & 0.19 & 26.60 & 1.02 & 14.41 & 0.47 & 14.88 \\
\hline 75 & 45 & 3.46 & 0.36 & 16.89 & 1.93 & 9.15 & 0.89 & 10.04 \\
\hline 45 & 25 & 1.33 & 0.65 & 6.49 & 3.49 & 3.52 & 1.60 & 5.12 \\
\hline 25 & 13.1 & 1.60 & 3.53 & 7.79 & 18.96 & 4.22 & 8.69 & 12.91 \\
\hline 13.1 & 6.5 & 1.55 & 4.09 & 7.55 & 21.98 & 4.09 & 10.07 & 14.16 \\
\hline 6.5 & 3.3 & 1.53 & 4.26 & 7.48 & 22.87 & 4.05 & 10.47 & 14.53 \\
\hline 3.3 & 1.5 & 1.07 & 2.75 & 5.24 & 14.79 & 2.84 & 6.78 & 9.61 \\
\hline 1.5 & & 1.22 & 2.78 & 5.96 & 14.95 & 3.23 & 6.85 & 10.08 \\
\hline Totals & -- & 20.49 & 18.61 & 100.00 & 100.00 & 54.19 & 45.81 & 100.00 \\
\hline
\end{tabular}

\begin{tabular}{|c|c|c|c|c|c|c|c|c|}
\hline $\begin{array}{c}\text { Pass } \\
\begin{array}{c}\text { Size } \\
(u m)\end{array}\end{array}$ & $\begin{array}{c}\text { Retain } \\
\text { Size } \\
(u m)\end{array}$ & $\begin{array}{c}\text { O/S } \\
\text { Mass } \\
(\mathrm{TPH})\end{array}$ & $\begin{array}{c}\text { USS } \\
\text { Mass } \\
(\mathrm{TPH})\end{array}$ & $\begin{array}{c}\text { Feed } \\
\text { Mass } \\
(\mathrm{TPH})\end{array}$ & $\begin{array}{c}\text { Delta } \\
\text { Mass } \\
(\mathrm{TPH})\end{array}$ & $\begin{array}{c}\text { Y } \\
\text { Value } \\
(\mathrm{f}-\mathrm{u})\end{array}$ & $\begin{array}{c}\text { Xalue } \\
\text { Vartition } \\
(0-u)\end{array}$ & $\begin{array}{c}\text { Pactor } \\
\text { Far } \\
(--)\end{array}$ \\
\hline & 212 & 0.05 & 0.00 & 0.05 & 0.00 & 4.26 & 4.26 & 1.00 \\
\hline 212 & 150 & 0.05 & 0.00 & 0.05 & 0.00 & 4.42 & 4.42 & 1.00 \\
\hline 150 & 75 & 0.17 & 0.01 & 0.18 & 0.00 & 14.41 & 13.95 & 0.97 \\
\hline 75 & 45 & 0.11 & 0.01 & 0.12 & 0.00 & 9.15 & 8.26 & 0.91 \\
\hline 45 & 25 & 0.04 & 0.02 & 0.06 & 0.00 & 3.52 & 1.92 & 0.69 \\
\hline 25 & 13.1 & 0.05 & 0.10 & 0.15 & 0.00 & 4.22 & -4.47 & 0.33 \\
\hline 13.1 & 6.5 & 0.05 & 0.12 & 0.17 & 0.00 & 4.09 & -5.98 & 0.29 \\
\hline 6.5 & 3.3 & 0.05 & 0.12 & 0.17 & 0.00 & 4.05 & -6.42 & 0.28 \\
\hline 3.3 & 1.5 & 0.03 & 0.08 & 0.11 & 0.00 & 2.84 & -3.94 & 0.30 \\
\hline 1.5 & & 0.04 & 0.08 & 0.12 & 0.00 & 3.23 & -3.62 & 0.32 \\
\hline Totals & -- & 0.64 & 0.54 & 1.18 & 0.00 & & & \\
\hline
\end{tabular}


HYDROCYCLONE EVALUATION (BALANCED)

\begin{tabular}{|c|c|}
\hline No. 04 & \\
\hline Feedflow (GPF) & 100.0 \\
\hline Feed Pressure (PSl) & 21.0 \\
\hline Water Flow (GPM) & 15.0 \\
\hline Water Pressure (PSl) & 15.0 \\
\hline Feed Rate (TPH) & 1.16 \\
\hline
\end{tabular}

\begin{tabular}{|r|c|c|c|}
\hline & OS & U/S & Feed \\
\hline Solids Content (\%) & 6.79 & 2.65 & 4.55 \\
\hline Ash Content (\%) & 23.89 & 48.12 & 34.91 \\
\hline Solids Yield (\%) & 54.5 & 45.5 & 100.0 \\
\hline Ash Yield (\%) & 54.5 & 45.5 & 100.0 \\
\hline Size Class Yield (\%) & 54.5 & 45.5 & 100.0 \\
\hline
\end{tabular}

\begin{tabular}{|c|c|c|c|c|c|c|c|c|}
\hline $\begin{array}{c}\text { Pass } \\
\begin{array}{c}\text { Size } \\
(u m)\end{array}\end{array}$ & $\begin{array}{c}\text { Retain } \\
\text { Size } \\
(u m)\end{array}$ & $\begin{array}{c}\text { O/S } \\
\text { Sample } \\
(\mathrm{gms})\end{array}$ & $\begin{array}{c}\text { U/S } \\
\text { Sample } \\
(\mathrm{gms})\end{array}$ & $\begin{array}{c}\text { OS } \\
\text { Mass } \\
(\%, \text { strm) }\end{array}$ & $\begin{array}{c}\text { U/S } \\
\text { Mass } \\
(\%, \text { strm) }\end{array}$ & $\begin{array}{c}\text { OSS } \\
\text { Mass } \\
(\%, \text { feed) })\end{array}$ & $\begin{array}{c}\text { U/S } \\
\text { Mass } \\
(\%, \text { feed) })\end{array}$ & $\begin{array}{c}\text { Feed } \\
\text { Mass } \\
(\%, \text { feed) })\end{array}$ \\
\hline & 212 & 1.61 & 0.00 & 7.85 & 0.00 & 4.28 & 0.00 & 4.28 \\
\hline 212 & 150 & 1.67 & 0.00 & 8.15 & 0.00 & 4.44 & 0.00 & 4.44 \\
\hline 150 & 75 & 5.45 & 0.19 & 26.63 & 1.04 & 14.51 & 0.47 & 14.98 \\
\hline 75 & 45 & 3.46 & 0.36 & 16.90 & 1.95 & 9.21 & 0.89 & 10.10 \\
\hline 45 & 25 & 1.33 & 0.65 & 6.49 & 3.51 & 3.53 & 1.60 & 5.13 \\
\hline 25 & 13.1 & 1.59 & 3.53 & 7.78 & 18.95 & 4.24 & 8.62 & 12.87 \\
\hline 13.1 & 6.5 & 1.55 & 4.09 & 7.55 & 21.97 & 4.11 & 10.00 & 14.11 \\
\hline 6.5 & 3.3 & 1.53 & 4.25 & 7.47 & 22.85 & 4.07 & 10.40 & 14.47 \\
\hline 3.3 & 1.5 & 1.07 & 2.75 & 5.23 & 14.79 & 2.85 & 6.73 & 9.58 \\
\hline 1.5 & & 1.22 & 2.78 & 5.95 & 14.95 & 3.24 & 6.80 & 10.04 \\
\hline Totals & -- & 20.48 & 18.62 & 100.00 & 100.00 & 54.50 & 45.50 & 100.00 \\
\hline
\end{tabular}

\begin{tabular}{|c|c|c|c|c|c|c|c|c|}
\hline $\begin{array}{c}\text { Pass } \\
\text { Size } \\
\text { (um) }\end{array}$ & $\begin{array}{c}\text { Retain } \\
\text { Size } \\
\text { (um) }\end{array}$ & $\begin{array}{c}\mathrm{O} / \mathrm{S} \\
\text { Mass } \\
(\mathrm{TPH})\end{array}$ & $\begin{array}{c}\text { U/S } \\
\text { Mass } \\
(\mathrm{TPH})\end{array}$ & $\begin{array}{l}\text { Feed } \\
\text { Mass } \\
(\mathrm{TPH})\end{array}$ & $\begin{array}{l}\text { Delta } \\
\text { Mass } \\
(\mathrm{TPH})\end{array}$ & $\begin{array}{c}\mathrm{Y} \\
\text { Value } \\
(\mathrm{f}-\mathrm{u})\end{array}$ & $\begin{array}{c}X \\
\text { Value } \\
(0-u)\end{array}$ & $\begin{array}{c}\text { Partition } \\
\text { Factor } \\
(--)\end{array}$ \\
\hline & 212 & 0.05 & 0.00 & 0.05 & 0.00 & 4.28 & 4.28 & 1.00 \\
\hline 212 & 150 & 0.05 & 0.00 & 0.05 & 0.00 & 4.44 & 4.44 & 1.00 \\
\hline 150 & 75 & 0.17 & 0.01 & 0.17 & 0.00 & 14.51 & 14.04 & 0.97 \\
\hline 75 & 45 & 0.11 & 0.01 & 0.12 & 0.00 & 9.21 & 8.32 & 0.91 \\
\hline 45 & 25 & 0.04 & 0.02 & 0.06 & 0.00 & 3.53 & 1.94 & 0.69 \\
\hline 25 & 13.1 & 0.05 & 0.10 & 0.15 & 0.00 & 4.24 & -4.38 & 0.33 \\
\hline 13.1 & 6.5 & 0.05 & 0.12 & 0.16 & 0.00 & 4.11 & -5.88 & 0.29 \\
\hline 6.5 & 3.3 & 0.05 & 0.12 & 0.17 & 0.00 & 4.07 & -6.32 & 0.28 \\
\hline 3.3 & 1.5 & 0.03 & 0.08 & 0.11 & 0.00 & 2.85 & -3.88 & 0.30 \\
\hline 1.5 & & 0.04 & 0.08 & 0.12 & 0.00 & 3.24 & -3.56 & 0.32 \\
\hline Totals & -- & 0.63 & 0.53 & 1.16 & 0.00 & & & \\
\hline & $\operatorname{rry}(\mathrm{TPH})$ & 9.29 & 19.90 & 25.43 & 0.00 & & & \\
\hline
\end{tabular}




\begin{tabular}{|c|c|c|c|}
\hline $\begin{array}{c}\text { Experimental } \\
\text { Partition Factor }\end{array}$ & $\begin{array}{c}\text { Mean } \\
\text { Particle } \\
\text { Sze }(\mu \mathrm{m})\end{array}$ & $\begin{array}{c}\text { Predicted } \\
\text { Partition } \\
\text { Factor }\end{array}$ & $\begin{array}{c}\text { Predicted } \\
\text { Partition } \\
\text { Factor }\end{array}$ \\
\hline$(--)$ & $(--)$ & (Plitt) & (Linch) \\
\hline 1.00 & 299.81 & 1.000 & 1.000 \\
\hline 1.00 & 178.33 & 1.000 & 1.000 \\
\hline 0.97 & 106.07 & 1.000 & 1.000 \\
\hline 0.91 & 58.09 & 0.962 & 0.988 \\
\hline 0.69 & 33.54 & 0.646 & 0.675 \\
\hline 0.33 & 18.08 & 0.378 & 0.349 \\
\hline 0.29 & 9.25 & 0.302 & 0.300 \\
\hline 0.28 & 4.62 & 0.287 & 0.291 \\
\hline 0.30 & 2.32 & 0.284 & 0.289 \\
\hline 0.32 & 1.64 & 0.284 & 0.288 \\
\hline & $W S S \mathrm{Q}$ & 0.010 & 0.009 \\
\hline
\end{tabular}

\begin{tabular}{|c|c|c|c|}
\multicolumn{1}{c}{ (Plitt) } & Predicted & \multicolumn{1}{c}{ ( inch $)$} & Predicted \\
\hline $\mathrm{D} 50$ & 33.34 \\
\hline$m$ & 2.60 \\
\hline Bypass(R0) & 0.28 \\
\hline Alpha & 32.45 \\
\hline Bypass(R0) & 0.19 \\
\hline
\end{tabular}

\begin{tabular}{l|l}
\hline TWSSQ & 0.018
\end{tabular}

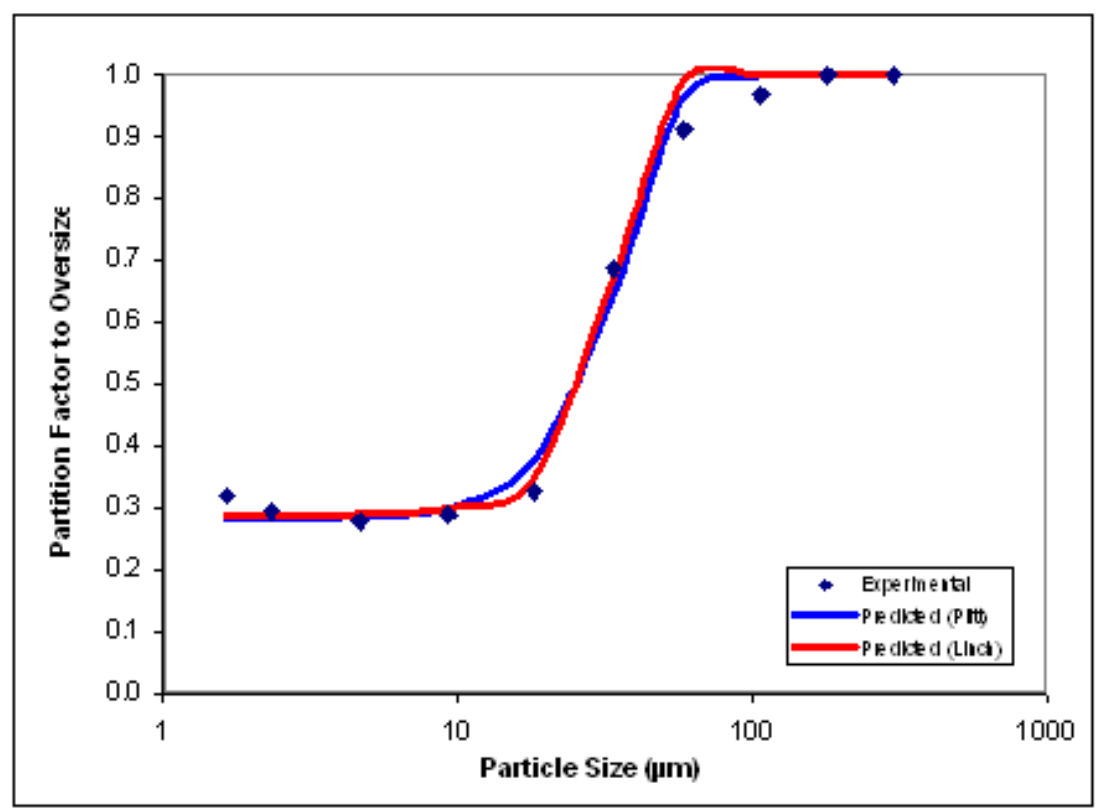




\section{HYDROCYCLONE EVALUATION (UNBALANCED)}

\begin{tabular}{|c|c|}
\hline No. 05 & \\
\hline Feedflow (GPM) & 100.0 \\
\hline Feed Pressure (PSl) & 21.0 \\
\hline Water Flow (GPM) & 15.0 \\
\hline Water Pressure (PSl) & 16.0 \\
\hline Feed Rate (TPH) & 1.20 \\
\hline
\end{tabular}

\begin{tabular}{|r|c|c|c|}
\hline & OS & US & Feed \\
\hline Solids Content (\%) & 8.42 & 2.86 & 4.73 \\
\hline Ash Content (\%) & 18.86 & 47.00 & 34.24 \\
\hline Solids Yield (\%) & 46.4 & 53.6 & 100.0 \\
\hline Ash Yield (\%) & 45.3 & 54.7 & 100.0 \\
\hline Size Class Yield (\%) & 35.1 & 64.9 & 100.0 \\
\hline
\end{tabular}

\begin{tabular}{|c|c|c|c|c|c|c|c|c|}
\hline $\begin{array}{c}\text { Pass } \\
\begin{array}{c}\text { Size } \\
(\mathrm{um})\end{array}\end{array}$ & $\begin{array}{c}\text { Retain } \\
\text { Size } \\
(\mathrm{um})\end{array}$ & $\begin{array}{c}\text { OSS } \\
\text { Sample } \\
(\mathrm{gms})\end{array}$ & $\begin{array}{c}\text { U/S } \\
\text { Sample } \\
(\mathrm{gms})\end{array}$ & $\begin{array}{c}\text { O/S } \\
\text { Mass } \\
(\%, \text { strm) }\end{array}$ & $\begin{array}{c}\text { U/S } \\
\text { Mass } \\
(\%, \text { strm) }\end{array}$ & $\begin{array}{c}\text { O/S } \\
\text { Mass } \\
(\%, \text { feed) })\end{array}$ & $\begin{array}{c}\text { U/S } \\
\text { Mass } \\
(\%, \text { feed) }\end{array}$ & $\begin{array}{c}\text { Feed } \\
\text { Mass } \\
(\%, \text { feed) }\end{array}$ \\
\hline & 212 & 1.75 & 0.00 & 10.21 & 0.00 & 4.63 & 0.00 & 4.63 \\
\hline 212 & 150 & 2.08 & 0.00 & 12.14 & 0.00 & 5.50 & 0.00 & 5.50 \\
\hline 150 & 75 & 4.97 & 0.24 & 29.00 & 1.05 & 13.15 & 0.58 & 13.72 \\
\hline 75 & 45 & 3.25 & 0.73 & 18.96 & 3.21 & 8.60 & 1.75 & 10.35 \\
\hline 45 & 25 & 1.10 & 1.24 & 6.42 & 5.45 & 2.91 & 2.98 & 5.89 \\
\hline 25 & 13.1 & 1.48 & 3.36 & 8.65 & 14.76 & 3.92 & 8.07 & 11.99 \\
\hline 13.1 & 6.5 & 0.93 & 4.13 & 5.41 & 18.16 & 2.45 & 9.92 & 12.38 \\
\hline 6.5 & 3.3 & 0.74 & 4.98 & 4.32 & 21.89 & 1.96 & 11.97 & 13.93 \\
\hline 3.3 & 1.5 & 0.45 & 3.93 & 2.64 & 17.27 & 1.20 & 9.44 & 10.64 \\
\hline 1.5 & & 0.39 & 4.14 & 2.25 & 18.21 & 1.02 & 9.95 & 10.97 \\
\hline Totals & -- & 17.14 & 22.76 & 100.00 & 100.00 & 45.34 & 54.66 & 100.00 \\
\hline
\end{tabular}

\begin{tabular}{|c|c|c|c|c|c|c|c|c|}
\hline $\begin{array}{l}\text { Pass } \\
\text { Size } \\
\text { (um) }\end{array}$ & $\begin{array}{c}\text { Retain } \\
\text { Size } \\
\text { (um) }\end{array}$ & $\begin{array}{c}\text { O/S } \\
\text { Mass } \\
(\mathrm{TPH})\end{array}$ & $\begin{array}{c}\text { U/S } \\
\text { Mass } \\
(\mathrm{TPH})\end{array}$ & $\begin{array}{l}\text { Feed } \\
\text { Mass } \\
(\mathrm{TPH})\end{array}$ & $\begin{array}{l}\text { Delta } \\
\text { Mass } \\
(\mathrm{TPH})\end{array}$ & $\begin{array}{c} \\
\text { Value } \\
(f-u)\end{array}$ & $\begin{array}{c}X \\
\text { Value } \\
(0-1)\end{array}$ & $\begin{array}{c}\text { Partition } \\
\text { Factor } \\
(--)\end{array}$ \\
\hline & 212 & 0.06 & 0.00 & 0.06 & 0.00 & 4.63 & 4.63 & 1.00 \\
\hline 212 & 150 & 0.07 & 0.00 & 0.07 & 0.00 & 5.50 & 5.50 & 1.00 \\
\hline 150 & 75 & 0.16 & 0.01 & 0.17 & 0.00 & 13.15 & 12.57 & 0.96 \\
\hline 75 & 45 & 0.10 & 0.02 & 0.12 & 0.00 & 8.60 & 6.85 & 0.83 \\
\hline 45 & 25 & 0.04 & 0.04 & 0.07 & 0.00 & 2.91 & -0.07 & 0.49 \\
\hline 25 & 13.1 & 0.05 & 0.10 & 0.14 & 0.00 & 3.92 & -4.14 & 0.33 \\
\hline 13.1 & 6.5 & 0.03 & 0.12 & 0.15 & 0.00 & 2.45 & -7.47 & 0.20 \\
\hline 6.5 & 3.3 & 0.02 & 0.14 & 0.17 & 0.00 & 1.96 & -10.01 & 0.14 \\
\hline 3.3 & 1.5 & 0.01 & 0.11 & 0.13 & 0.00 & 1.20 & -8.24 & 0.11 \\
\hline 1.5 & & 0.01 & 0.12 & $\overline{0.13}$ & 0.00 & 1.02 & -8.93 & 0.09 \\
\hline Totals & -- & 0.55 & 0.66 & 1.20 & 0.00 & & & \\
\hline \multicolumn{2}{|c|}{ Slurry (TPH) } & 6.48 & 23.00 & 25.45 & -0.29 & & & \\
\hline
\end{tabular}


HYDROCYCLONE EVALUATION (BALANCED)

\begin{tabular}{|c|c|}
\hline No. 05 & \\
\hline Feedflow (GPM) & 100.0 \\
\hline Feed Pressure (PSI) & 21.0 \\
\hline Water Flow (GPM) & 15.0 \\
\hline Water Pressure (PSl) & 16.0 \\
\hline Feed Rate (TPH) & 1.20 \\
\hline
\end{tabular}

\begin{tabular}{|r|c|c|c|}
\hline & OS & U/S & Feed \\
\hline Solids Content (\%) & 8.41 & 2.82 & 4.71 \\
\hline Ash Content (\%) & 19.03 & 47.20 & 33.89 \\
\hline Solids Yield (\%) & 47.3 & 52.7 & 100.0 \\
\hline Ash Yield (\%) & 47.3 & 52.7 & 100.0 \\
\hline Size Class Yield (\%) & 47.3 & 52.7 & 100.0 \\
\hline
\end{tabular}

\begin{tabular}{|c|c|c|c|c|c|c|c|c|}
\hline $\begin{array}{l}\text { Pass } \\
\text { Size } \\
\text { (um) }\end{array}$ & $\begin{array}{l}\text { Retain } \\
\text { Size } \\
\text { (um) }\end{array}$ & $\begin{array}{c}\text { O/S } \\
\text { Sample } \\
\text { (gms) }\end{array}$ & $\begin{array}{c}\text { U/S } \\
\text { Sample } \\
\text { (gms) }\end{array}$ & $\begin{array}{c}\text { orS } \\
\text { Mass } \\
(\% \text {, strm })\end{array}$ & $\begin{array}{c}\text { USS } \\
\text { Mass } \\
(\% \text {, strm) }\end{array}$ & $\begin{array}{c}\text { OS } \\
\text { Mass } \\
(\% \text {, feed })\end{array}$ & $\begin{array}{c}\text { U/S } \\
\text { Mass } \\
(\%, \text { feed })\end{array}$ & $\begin{array}{c}\text { Feed } \\
\text { Mass } \\
(\% \text {, feed })\end{array}$ \\
\hline & 212 & 1.67 & 0.00 & 10.54 & 0.00 & 4.98 & 0.00 & 4.98 \\
\hline 212 & 150 & 2.04 & 0.00 & 12.80 & 0.00 & 6.05 & 0.00 & 6.05 \\
\hline 150 & 75 & 5.20 & 0.32 & 32.70 & 1.37 & 15.46 & 0.72 & 16.18 \\
\hline 75 & 45 & 3.30 & 0.82 & 20.78 & 3.55 & 9.82 & 1.87 & 11.70 \\
\hline 45 & 25 & 0.94 & 1.35 & 5.89 & 5.87 & 2.79 & 3.10 & 5.88 \\
\hline 25 & 13.1 & 1.32 & 3.37 & 8.27 & 14.65 & 3.91 & 7.73 & 11.64 \\
\hline 13.1 & 6.5 & 0.69 & 4.12 & 4.35 & 17.93 & 2.05 & 9.45 & 11.51 \\
\hline 6.5 & 3.3 & 0.47 & 4.94 & 2.95 & 21.48 & 1.39 & 11.33 & 12.72 \\
\hline 3.3 & 1.5 & 0.17 & 3.94 & 1.09 & 17.13 & 0.51 & 9.03 & 9.55 \\
\hline 1.5 & & 0.10 & 4.14 & 0.62 & 18.02 & 0.30 & 9.50 & 9.80 \\
\hline Totals & -- & 15.90 & 22.99 & 100.00 & 100.00 & 47.26 & 52.74 & 100.00 \\
\hline
\end{tabular}

\begin{tabular}{|c|c|c|c|c|c|c|c|c|}
\hline $\begin{array}{l}\text { Pass } \\
\text { Size } \\
\text { (um) }\end{array}$ & $\begin{array}{c}\text { Retain } \\
\text { Size } \\
\text { (um) }\end{array}$ & $\begin{array}{c}\mathrm{O} / \mathrm{S} \\
\text { Mass } \\
(\mathrm{TPH})\end{array}$ & $\begin{array}{c}\text { U/S } \\
\text { Mass } \\
\text { (TPH) }\end{array}$ & $\begin{array}{l}\text { Feed } \\
\text { Mass } \\
\text { (TPH) }\end{array}$ & $\begin{array}{l}\text { Delta } \\
\text { Mass } \\
\text { (TPH) }\end{array}$ & $\begin{array}{c}Y \\
\text { Value } \\
(f-u)\end{array}$ & $\begin{array}{c}X \\
\text { Value } \\
(0-u)\end{array}$ & $\begin{array}{c}\text { Partition } \\
\text { Factor } \\
(--)\end{array}$ \\
\hline & 212 & 0.06 & 0.00 & 0.06 & 0.00 & 4.98 & 4.98 & 1.00 \\
\hline 212 & 150 & 0.07 & 0.00 & 0.07 & 0.00 & 6.05 & 6.05 & 1.00 \\
\hline 150 & 75 & 0.19 & 0.01 & 0.19 & 0.00 & 15.46 & 14.73 & 0.96 \\
\hline 75 & 45 & 0.12 & 0.02 & 0.14 & 0.00 & 9.82 & 7.95 & 0.84 \\
\hline 45 & 25 & 0.03 & 0.04 & 0.07 & 0.00 & 2.79 & -0.31 & 0.47 \\
\hline 25 & 13.1 & 0.05 & 0.09 & 0.14 & 0.00 & 3.91 & -3.82 & 0.34 \\
\hline 13.1 & 6.5 & 0.02 & 0.11 & 0.14 & 0.00 & 2.05 & -7.40 & 0.18 \\
\hline 6.5 & 3.3 & 0.02 & 0.14 & 0.15 & 0.00 & 1.39 & -9.94 & 0.11 \\
\hline 3.3 & 1.5 & 0.01 & 0.11 & 0.11 & 0.00 & 0.51 & -8.52 & 0.05 \\
\hline 1.5 & & 0.00 & 0.11 & 0.12 & 0.00 & 0.30 & -9.21 & 0.03 \\
\hline Totals & $\begin{array}{ll} & -- \\
\end{array}$ & 0.57 & 0.63 & 1.20 & 0.00 & & & \\
\hline & Slurry (TPH) & 6.74 & 22.45 & 25.44 & 0.00 & & & \\
\hline
\end{tabular}




\begin{tabular}{|c|c|c|c|c|c|c|c|}
\hline \multirow{3}{*}{$\begin{array}{l}\text { Experimental } \\
\text { Partition F actor }\end{array}$} & \multirow{3}{*}{$\begin{array}{c}\text { Mean } \\
\text { Particle } \\
\text { Size }(\mu \mathrm{m})\end{array}$} & \multirow{3}{*}{$\begin{array}{c}\text { Predicted } \\
\text { Partition } \\
\text { Factor }\end{array}$} & \multirow{3}{*}{$\begin{array}{c}\text { Predicted } \\
\text { Partition } \\
\text { Factor }\end{array}$} & \multirow{2}{*}{$\frac{\text { (Plitt) }}{\text { D50 }}$} & Predicted & (Linch) & Predicted \\
\hline & & & & & 34.80 & D50 & 34.85 \\
\hline & & & & $m$ & 1.50 & Alpha & 1.76 \\
\hline$(-)$ & $(-)$ & (Plitt) & (Linch) & Bypass(R) & 0.10 & Bypass(Rf) & 0.09 \\
\hline 1.00 & 299.81 & 1.000 & 1.000 & & & & \\
\hline 1.00 & 178.33 & 1.000 & 0.999 & & & & \\
\hline 0.96 & 106.07 & 0.977 & 0.980 & & & & \\
\hline 0.83 & 58.09 & 0.799 & 0.806 & & & & \\
\hline 0.49 & 33.54 & 0.535 & 0.527 & & & & \\
\hline 0.33 & 18.08 & 0.309 & 0.306 & & & & \\
\hline 0.20 & 9.25 & 0.185 & 0.191 & & & & \\
\hline 0.14 & 4.62 & 0.133 & 0.138 & & & & \\
\hline 0.11 & 2.32 & 0.114 & 0.113 & & & & \\
\hline 0.09 & 1.64 & 0.110 & 0.106 & & & & \\
\hline & WSSQ & 0.004 & 0.003 & & & & \\
\hline & TWSSQ & 0.007 & & & & & \\
\hline
\end{tabular}

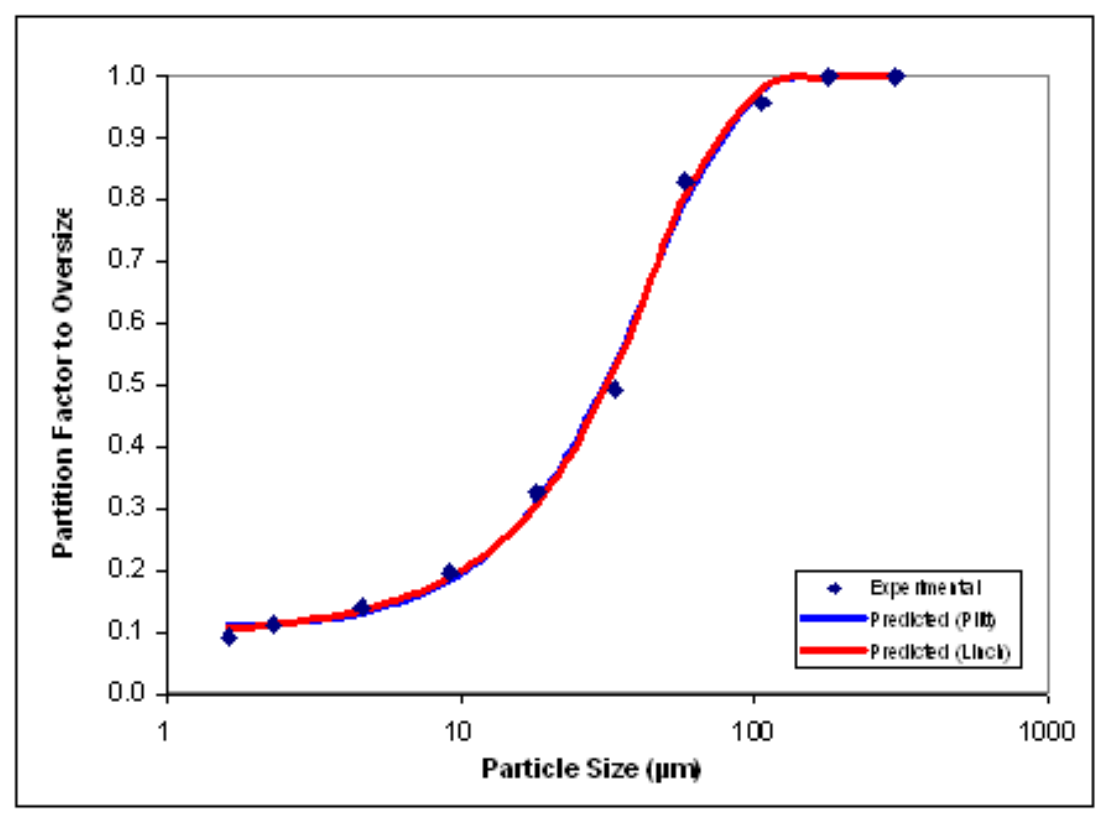




\section{HYDROCYCLONE EVALUATION (UNBALANCED)}

\begin{tabular}{|c|c|}
\hline No. 06 & \\
\hline Feedflow (GPM) & 1000 \\
\hline Feed Pressure (PSI) & 21.0 \\
\hline Water Flow (GPM) & 15.0 \\
\hline Water Pressure (PSl) & 13.0 \\
\hline Feed Rate (TPH) & 1.17 \\
\hline
\end{tabular}

\begin{tabular}{|r|c|c|c|}
\hline & OS & U/S & Feed \\
\hline Solids Content (\%) & 5.69 & 2.43 & 4.59 \\
\hline Ash Content (\%) & 28.07 & 49.25 & 33.96 \\
\hline Solids Yield (\%) & 68.5 & 31.5 & 100.0 \\
\hline Ash Yield (\%) & 72.2 & 27.8 & 100.0 \\
\hline Size Class Yield (\%) & 112.2 & -12.2 & 100.0 \\
\hline
\end{tabular}

\begin{tabular}{|c|c|c|c|c|c|c|c|c|}
\hline $\begin{array}{c}\text { Pass } \\
\text { Size } \\
(u m)\end{array}$ & $\begin{array}{c}\text { Retain } \\
\text { Size } \\
(u m)\end{array}$ & $\begin{array}{c}\text { O/S } \\
\text { Sample } \\
(\mathrm{gms})\end{array}$ & $\begin{array}{c}\text { U/S } \\
\text { Sample } \\
(\mathrm{gms})\end{array}$ & $\begin{array}{c}\text { O/S } \\
\text { Mass } \\
(\%, \text { strm) }\end{array}$ & $\begin{array}{c}\text { U/S } \\
\text { Mass } \\
(\%, \text { strm) }\end{array}$ & $\begin{array}{c}0 / S \\
\text { Mass } \\
(\%, \text { feed })\end{array}$ & $\begin{array}{c}\text { U/S } \\
\text { Mass } \\
(\%, \text { feed })\end{array}$ & $\begin{array}{c}\text { Feed } \\
\text { Mass } \\
(\%, \text { feed })\end{array}$ \\
\hline & 212 & 2.08 & 0.00 & 6.66 & 0.00 & 4.81 & 0.00 & 4.81 \\
\hline 212 & 150 & 2.48 & 0.00 & 7.94 & 0.00 & 5.73 & 0.00 & 5.73 \\
\hline 150 & 75 & 6.25 & 0.16 & 20.01 & 1.47 & 14.45 & 0.41 & 14.86 \\
\hline 75 & 45 & 4.34 & 0.31 & 13.90 & 2.85 & 10.03 & 0.79 & 10.82 \\
\hline 45 & 25 & 2.15 & 0.26 & 6.88 & 2.39 & 4.97 & 0.66 & 5.63 \\
\hline 25 & 13.1 & 3.64 & 1.66 & 11.67 & 15.22 & 8.42 & 4.23 & 12.65 \\
\hline 13.1 & 6.5 & 3.23 & 2.37 & 10.35 & 21.77 & 7.47 & 6.05 & 13.53 \\
\hline 6.5 & 3.3 & 3.01 & 2.53 & 9.63 & 23.27 & 6.95 & 6.47 & 13.42 \\
\hline 3.3 & 1.5 & 2.07 & 1.71 & 6.63 & 15.66 & 4.79 & 4.36 & 9.14 \\
\hline 1.5 & & 1.97 & 1.89 & 6.32 & 17.38 & 4.56 & 4.83 & 9.40 \\
\hline Totals & -- & 31.23 & 10.89 & 100.00 & 100.00 & 72.19 & 27.81 & 100.00 \\
\hline
\end{tabular}

\begin{tabular}{|c|c|c|c|c|c|c|c|c|}
\hline $\begin{array}{c}\text { Pass } \\
\text { Size } \\
(u m)\end{array}$ & $\begin{array}{c}\text { Retain } \\
\text { Size } \\
(u m)\end{array}$ & $\begin{array}{c}0 / 5 \\
\text { Mass } \\
(\mathrm{TPH})\end{array}$ & $\begin{array}{c}\text { U/S } \\
\text { Mass } \\
(\mathrm{TPH})\end{array}$ & $\begin{array}{c}\text { Feed } \\
\text { Mass } \\
(\mathrm{TPH})\end{array}$ & $\begin{array}{c}\text { Delta } \\
\text { Mass } \\
(\mathrm{TPH})\end{array}$ & $\begin{array}{c}\text { Value } \\
\text { (f-u) }\end{array}$ & $\begin{array}{c}\text { X } \\
\text { Value } \\
(\mathrm{0}-\mathrm{u})\end{array}$ & $\begin{array}{c}\text { Partition } \\
\text { Factor } \\
(-)\end{array}$ \\
\hline & 212 & 0.06 & 0.00 & 0.06 & 0.00 & 4.81 & 4.81 & 1.00 \\
\hline 212 & 150 & 0.07 & 0.00 & 0.07 & 0.00 & 5.73 & 5.73 & 1.00 \\
\hline 150 & 75 & 0.17 & 0.00 & 0.17 & 0.00 & 14.45 & 14.04 & 0.97 \\
\hline 75 & 45 & 0.12 & 0.01 & 0.13 & 0.00 & 10.03 & 9.24 & 0.93 \\
\hline 45 & 25 & 0.06 & 0.01 & 0.07 & 0.00 & 4.97 & 4.31 & 0.88 \\
\hline 25 & 13.1 & 0.10 & 0.05 & 0.15 & 0.00 & 8.42 & 4.19 & 0.67 \\
\hline 13.1 & 6.5 & 0.09 & 0.07 & 0.16 & 0.00 & 7.47 & 1.42 & 0.55 \\
\hline 6.5 & 3.3 & 0.08 & 0.08 & 0.16 & 0.00 & 6.95 & 0.48 & 0.52 \\
\hline 3.3 & 1.5 & 0.06 & 0.05 & 0.11 & 0.00 & 4.79 & 0.43 & 0.52 \\
\hline 1.5 & & 0.05 & 0.06 & 0.11 & 0.00 & 4.56 & -0.27 & 0.49 \\
\hline Totals & -- & 0.84 & 0.32 & 1.17 & 0.00 & & & \\
\hline & Slurry (TPH) & 14.81 & 13.36 & 25.43 & 1.01 & & & \\
\hline
\end{tabular}




\section{HYDROCYCLONE EVALUATION (BALANCED)}

\begin{tabular}{|c|c|}
\hline No. 06 & \\
\hline Feedflow (GPF) & 100.0 \\
\hline Feed Pressure (PSl) & 21.0 \\
\hline Water Flow (GPM) & 15.0 \\
\hline Water Pressure (PSl) & 13.0 \\
\hline Feed Rate (TPH) & 1.07 \\
\hline
\end{tabular}

\begin{tabular}{|r|c|c|c|}
\hline & OS & U/S & Feed \\
\hline Solids Content (\%) & 5.68 & 2.33 & 4.21 \\
\hline Ash Content (\%) & 27.06 & 48.85 & 35.37 \\
\hline Solids Yield (\%) & 61.8 & 38.2 & 100.0 \\
\hline Ash Yield (\%) & 61.8 & 38.2 & 100.0 \\
\hline Size Class Yield (\%) & 61.8 & 38.2 & 100.0 \\
\hline
\end{tabular}

\begin{tabular}{|c|c|c|c|c|c|c|c|c|}
\hline $\begin{array}{c}\text { Pass } \\
\text { Size } \\
(u m)\end{array}$ & $\begin{array}{c}\text { Retain } \\
\text { Size } \\
(u m)\end{array}$ & $\begin{array}{c}\text { O/S } \\
\text { Sample } \\
(\mathrm{gms})\end{array}$ & $\begin{array}{c}\text { U/S } \\
\text { Sample } \\
(\mathrm{gmm})\end{array}$ & $\begin{array}{c}\text { O/S } \\
\text { Mass } \\
(\%, \text { strm) }\end{array}$ & $\begin{array}{c}\text { U/S } \\
\text { Mass } \\
(\%, \text { strm) }\end{array}$ & $\begin{array}{c}\text { O/S } \\
\text { Mass } \\
(\%, \text { feed) })\end{array}$ & $\begin{array}{c}\text { U/S } \\
\text { Mass } \\
(\%, \text { feed) }\end{array}$ & $\begin{array}{c}\text { Feed } \\
\text { Mass, feed) }\end{array}$ \\
\hline & 212 & 2.55 & 0.00 & 8.38 & 0.00 & 5.19 & 0.00 & 5.19 \\
\hline 212 & 150 & 2.96 & 0.00 & 9.73 & 0.00 & 6.02 & 0.00 & 6.02 \\
\hline 150 & 75 & 6.81 & 0.00 & 22.40 & 0.00 & 13.85 & 0.00 & 13.85 \\
\hline 75 & 45 & 4.84 & 0.00 & 15.94 & 0.00 & 9.86 & 0.00 & 9.86 \\
\hline 45 & 25 & 2.60 & 0.00 & 8.57 & 0.00 & 5.30 & 0.00 & 5.30 \\
\hline 25 & 13.1 & 3.28 & 0.40 & 10.81 & 4.09 & 6.68 & 1.56 & 8.24 \\
\hline 13.1 & 6.5 & 2.38 & 2.52 & 7.83 & 25.97 & 4.84 & 9.91 & 14.75 \\
\hline 6.5 & 3.3 & 2.03 & 3.10 & 6.69 & 31.88 & 4.14 & 12.16 & 16.30 \\
\hline 3.3 & 1.5 & 1.58 & 1.57 & 5.20 & 16.19 & 3.21 & 6.18 & 9.39 \\
\hline 1.5 & & 1.35 & 2.13 & 4.45 & 21.87 & 2.75 & 8.34 & 11.10 \\
\hline Totals & -- & 30.39 & 9.72 & 100.00 & 100.00 & 61.85 & 38.15 & 100.00 \\
\hline
\end{tabular}

\begin{tabular}{|c|c|c|c|c|c|c|c|c|}
\hline $\begin{array}{c}\text { Pass } \\
\begin{array}{c}\text { Size } \\
(\mathrm{um})\end{array}\end{array}$ & $\begin{array}{c}\text { Retain } \\
\text { Size } \\
(\mathrm{um})\end{array}$ & $\begin{array}{c}\text { O/S } \\
\text { Mass } \\
(\mathrm{TPH})\end{array}$ & $\begin{array}{c}\text { U/S } \\
\text { Mass } \\
(\mathrm{TPH})\end{array}$ & $\begin{array}{c}\text { Feed } \\
\text { Mass } \\
(\mathrm{TPH})\end{array}$ & $\begin{array}{c}\text { Delta } \\
\text { Mass } \\
(\mathrm{TPH})\end{array}$ & $\begin{array}{c}\text { Y } \\
\text { Value } \\
(\mathrm{f}-\mathrm{u})\end{array}$ & $\begin{array}{c}\text { Xalue } \\
\text { (o-u) }\end{array}$ & $\begin{array}{c}\text { Partition } \\
\text { Factor } \\
(--)\end{array}$ \\
\hline & 212 & 0.06 & 0.00 & 0.06 & 0.00 & 5.19 & 5.19 & 1.00 \\
\hline 212 & 150 & 0.06 & 0.00 & 0.06 & 0.00 & 6.02 & 6.02 & 1.00 \\
\hline 150 & 75 & 0.15 & 0.00 & 0.15 & 0.00 & 13.85 & 13.85 & 1.00 \\
\hline 75 & 45 & 0.11 & 0.00 & 0.11 & 0.00 & 9.86 & 9.86 & 1.00 \\
\hline 45 & 25 & 0.06 & 0.00 & 0.06 & 0.00 & 5.30 & 5.30 & 1.00 \\
\hline 25 & 13.1 & 0.07 & 0.02 & 0.09 & 0.00 & 6.68 & 5.12 & 0.81 \\
\hline 13.1 & 6.5 & 0.05 & 0.11 & 0.16 & 0.00 & 4.84 & -5.06 & 0.33 \\
\hline 6.5 & 3.3 & 0.04 & 0.13 & 0.17 & 0.00 & 4.14 & -8.02 & 0.25 \\
\hline 3.3 & 1.5 & 0.03 & 0.07 & 0.10 & 0.00 & 3.21 & -2.96 & 0.34 \\
\hline 1.5 & & 0.03 & 0.09 & 0.12 & 0.00 & 2.75 & -5.59 & 0.25 \\
\hline Totals & -- & 0.66 & 0.41 & 1.07 & 0.00 & & & \\
\hline
\end{tabular}




\begin{tabular}{|c|c|c|c|c|c|c|c|}
\hline \multirow{3}{*}{$\begin{array}{c}\text { Experimental } \\
\text { Partition F actor }\end{array}$} & \multirow{3}{*}{$\begin{array}{c}\text { Mean } \\
\text { Particle } \\
\text { Size ( } \mu \mathrm{m})\end{array}$} & \multirow{3}{*}{$\begin{array}{c}\text { Predicted } \\
\text { Partition } \\
\text { Factor }\end{array}$} & \multirow{3}{*}{$\begin{array}{c}\text { Predicted } \\
\text { Partition } \\
\text { Factor }\end{array}$} & \multirow{2}{*}{$\frac{\text { (Plitt) }}{\text { D50 }}$} & Predicted & $($ Linch $)$ & Predicted \\
\hline & & & & & 23.53 & $\mathrm{D} 50$ & 23.34 \\
\hline & & & & $m$ & 1.55 & Alpha & 1.92 \\
\hline$(--)$ & $(--)$ & (Plitt) & (Linch) & Bypass(Rf) & 0.49 & Bypass(Rf) & 0.48 \\
\hline 1.00 & 299.81 & 1.000 & 1.000 & & & & \\
\hline 1.00 & 178.33 & 1.000 & 1.000 & & & & \\
\hline 0.97 & 106.07 & 1.000 & 1.000 & & & & \\
\hline 0.93 & 58.09 & 0.970 & 0.976 & & & & \\
\hline 0.88 & 33.54 & 0.847 & 0.854 & & & & \\
\hline 0.67 & 18.08 & 0.679 & 0.674 & & & & \\
\hline 0.55 & 9.25 & 0.567 & 0.567 & & & & \\
\hline 0.52 & 4.62 & 0.518 & 0.520 & & & & \\
\hline 0.52 & 2.32 & 0.500 & 0.500 & & & & \\
\hline 0.49 & 1.64 & 0.496 & 0.495 & & & & \\
\hline & WSSQ & 0.005 & 0.005 & & & & \\
\hline & TWSSQ & 0.010 & & & & & \\
\hline
\end{tabular}

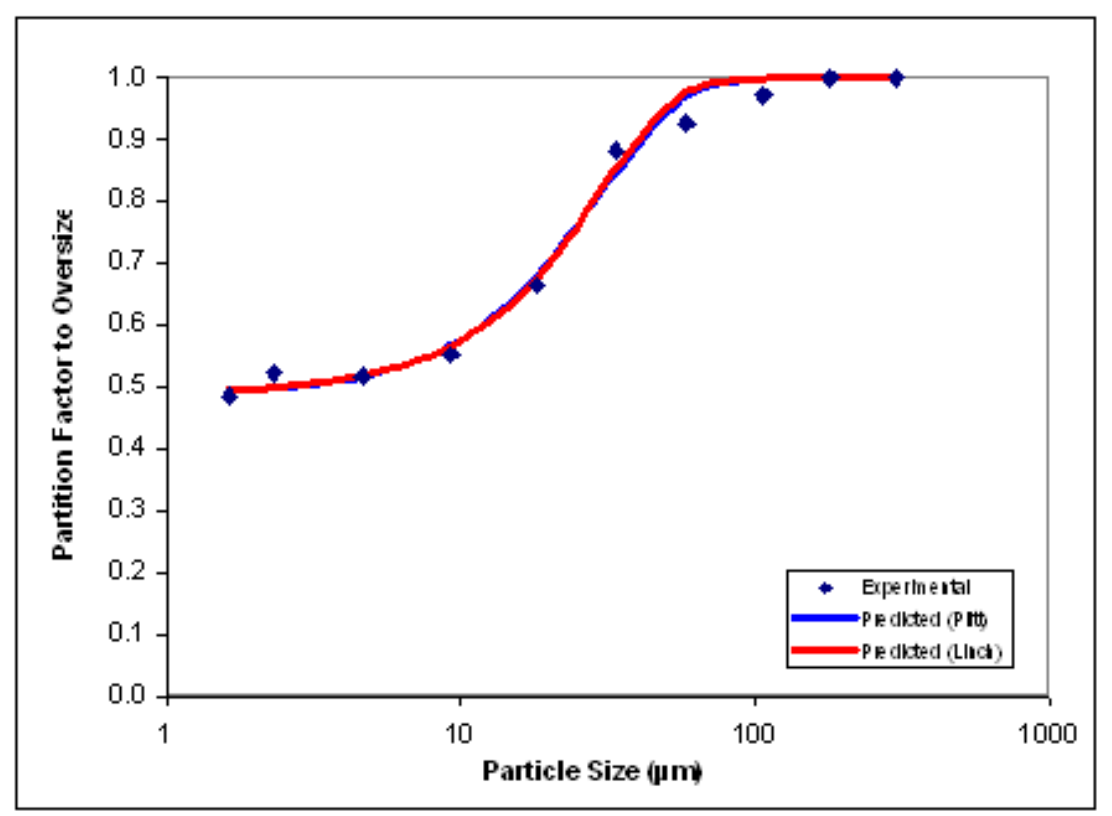


HYDROCYCLONE EVALUATION (UNBALANCED)

\begin{tabular}{|c|c|}
\hline No. 07 & \\
\hline FeedFlow (GPM) & 10000 \\
\hline Feed Pressure (PSl) & 21.0 \\
\hline Water Flow (GPM) & 15.0 \\
\hline Water Pressure (PSI) & 13.0 \\
\hline Feed Rate (TPH) & 1.14 \\
\hline
\end{tabular}

\begin{tabular}{|r|c|c|c|}
\hline & OS & U/S & Feed \\
\hline Solids Content (\%) & 7.50 & 2.70 & 4.50 \\
\hline Ash Content (\%) & 19.60 & 47.33 & 34.90 \\
\hline Solids Yield (\%) & 48.7 & 51.3 & 100.0 \\
\hline Ash Yield (\%) & 44.8 & 55.2 & 100.0 \\
\hline Size Class Yield (\%) & 33.0 & 67.0 & 100.0 \\
\hline
\end{tabular}

\begin{tabular}{|c|c|c|c|c|c|c|c|c|}
\hline $\begin{array}{c}\text { Pass } \\
\text { Size } \\
(u m)\end{array}$ & $\begin{array}{c}\text { Retain } \\
\text { Size } \\
(u m)\end{array}$ & $\begin{array}{c}0 / S \\
\text { Sample } \\
(\mathrm{gms})\end{array}$ & $\begin{array}{c}\mathrm{U} / \mathrm{S} \\
\text { Sample } \\
(\mathrm{gms})\end{array}$ & $\begin{array}{c}\mathrm{O} / \mathrm{S} \\
\text { Mass } \\
(\%, \text { strm) }\end{array}$ & $\begin{array}{c}\mathrm{U} / \mathrm{S} \\
\text { Mass } \\
(\%, \text { strm) }\end{array}$ & $\begin{array}{c}\text { OS } \\
\text { Mass } \\
(\%, \text { feed })\end{array}$ & $\begin{array}{c}\text { U/S } \\
\text { Mass } \\
(\%, \text { feed })\end{array}$ & $\begin{array}{c}\text { Feed } \\
\text { Mass } \\
(\%, \text { feed })\end{array}$ \\
\hline & 212 & 1.50 & 0.00 & 10.52 & 0.00 & 4.72 & 0.00 & 4.72 \\
\hline 212 & 150 & 1.60 & 0.00 & 11.22 & 0.00 & 5.03 & 0.00 & 5.03 \\
\hline 150 & 75 & 4.36 & 0.13 & 30.58 & 0.64 & 13.71 & 0.35 & 14.06 \\
\hline 75 & 45 & 2.62 & 0.54 & 18.37 & 2.65 & 8.24 & 1.46 & 9.70 \\
\hline 45 & 25 & 0.82 & 0.97 & 5.75 & 4.76 & 2.58 & 2.63 & 5.21 \\
\hline 25 & 13.1 & 0.49 & 3.00 & 3.45 & 14.75 & 1.55 & 8.14 & 9.69 \\
\hline 13.1 & 6.5 & 0.68 & 4.29 & 4.76 & 21.05 & 2.14 & 11.61 & 13.75 \\
\hline 6.5 & 3.3 & 0.81 & 4.64 & 5.70 & 22.77 & 2.55 & 12.56 & 15.12 \\
\hline 3.3 & 1.5 & 0.61 & 3.24 & 4.30 & 15.90 & 1.93 & 8.77 & 10.70 \\
\hline 1.5 & & 0.76 & 3.56 & 5.35 & 17.48 & 2.40 & 9.64 & 12.04 \\
\hline Totals & -- & 14.26 & 20.36 & 100.00 & 100.00 & 44.83 & 55.17 & 100.00 \\
\hline
\end{tabular}

\begin{tabular}{|c|c|c|c|c|c|c|c|c|}
\hline $\begin{array}{c}\text { Pass } \\
\text { Size } \\
(u m)\end{array}$ & $\begin{array}{c}\text { Retain } \\
\text { Size } \\
(u m)\end{array}$ & $\begin{array}{c}0 / 5 \\
\text { Mass } \\
(\mathrm{TPH})\end{array}$ & $\begin{array}{c}\text { U/S } \\
\text { Mass } \\
(\mathrm{TPH})\end{array}$ & $\begin{array}{c}\text { Feed } \\
\text { Mass } \\
(\mathrm{TPH})\end{array}$ & $\begin{array}{c}\text { Delta } \\
\text { Mass } \\
(\mathrm{TPH})\end{array}$ & $\begin{array}{c}\text { Value } \\
\text { (f-u) }\end{array}$ & $\begin{array}{c}\text { X } \\
\text { Value } \\
(\mathrm{0}-\mathrm{u})\end{array}$ & $\begin{array}{c}\text { Partition } \\
\text { Factor } \\
(-)\end{array}$ \\
\hline & 212 & 0.05 & 0.00 & 0.05 & 0.00 & 4.72 & 4.72 & 1.00 \\
\hline 212 & 150 & 0.06 & 0.00 & 0.06 & 0.00 & 5.03 & 5.03 & 1.00 \\
\hline 150 & 75 & 0.16 & 0.00 & 0.16 & 0.00 & 13.71 & 13.35 & 0.97 \\
\hline 75 & 45 & 0.09 & 0.02 & 0.11 & 0.00 & 8.24 & 6.77 & 0.85 \\
\hline 45 & 25 & 0.03 & 0.03 & 0.06 & 0.00 & 2.58 & -0.05 & 0.50 \\
\hline 25 & 13.1 & 0.02 & 0.09 & 0.11 & 0.00 & 1.55 & -6.59 & 0.16 \\
\hline 13.1 & 6.5 & 0.02 & 0.13 & 0.16 & 0.00 & 2.14 & -9.48 & 0.16 \\
\hline 6.5 & 3.3 & 0.03 & 0.14 & 0.17 & 0.00 & 2.55 & -10.01 & 0.17 \\
\hline 3.3 & 1.5 & 0.02 & 0.10 & 0.12 & 0.00 & 1.93 & -6.84 & 0.18 \\
\hline 1.5 & & 0.03 & 0.11 & 0.14 & 0.00 & 2.40 & -7.25 & 0.20 \\
\hline Totals & -- & 0.51 & 0.63 & 1.14 & 0.00 & & & \\
\hline & Slurry(TPH) & 6.84 & 23.38 & 25.43 & -1.05 & & & \\
\hline
\end{tabular}


HYDROCYCLONE EVALUATION (BALANCED)

\begin{tabular}{|c|c|}
\hline No. 07 & \\
\hline Feedflow (GPM) & 900.0 \\
\hline Feed Pressure (PSl) & 21.0 \\
\hline Water Flow (GPM) & 15.0 \\
\hline Water Pressure (PSl) & 13.0 \\
\hline Feed Rate (TPH) & 1.15 \\
\hline
\end{tabular}

\begin{tabular}{|r|c|c|c|}
\hline & OS & U/S & Feed \\
\hline Solids Content (\%) & 7.39 & 2.83 & 4.51 \\
\hline Ash Content (\%) & 19.75 & 47.51 & 34.89 \\
\hline Solids Yield (\%) & 45.5 & 54.5 & 100.0 \\
\hline Ash Yield (\%) & 45.5 & 54.5 & 100.0 \\
\hline Size Class Yield (\%) & 45.5 & 54.5 & 100.0 \\
\hline
\end{tabular}

\begin{tabular}{|c|c|c|c|c|c|c|c|c|}
\hline $\begin{array}{l}\text { Pass } \\
\text { Size } \\
\text { (um) }\end{array}$ & $\begin{array}{l}\text { Retain } \\
\text { Size } \\
\text { (um) }\end{array}$ & $\begin{array}{c}\text { O/S } \\
\text { Sample } \\
\text { (gms) }\end{array}$ & $\begin{array}{c}\text { U/S } \\
\text { Sample } \\
\text { (gms) }\end{array}$ & $\begin{array}{c}\text { orS } \\
\text { Mass } \\
(\% \text {, strm })\end{array}$ & $\begin{array}{c}\text { USS } \\
\text { Mass } \\
(\% \text {, strm) }\end{array}$ & $\begin{array}{c}\text { OS } \\
\text { Mass } \\
(\% \text {, feed })\end{array}$ & $\begin{array}{c}\text { U/S } \\
\text { Mass } \\
(\%, \text { feed })\end{array}$ & $\begin{array}{c}\text { Feed } \\
\text { Mass } \\
(\% \text {, feed })\end{array}$ \\
\hline & 212 & 1.40 & 0.00 & 11.14 & 0.00 & 5.06 & 0.00 & 5.06 \\
\hline 212 & 150 & 1.49 & 0.00 & 11.84 & 0.00 & 5.38 & 0.00 & 5.38 \\
\hline 150 & 75 & 4.65 & 0.21 & 36.89 & 1.04 & 16.77 & 0.57 & 17.34 \\
\hline 75 & 45 & 2.66 & 0.65 & 21.12 & 3.13 & 9.60 & 1.71 & 11.31 \\
\hline 45 & 25 & 0.61 & 1.10 & 4.81 & 5.33 & 2.19 & 2.91 & 5.09 \\
\hline 25 & 13.1 & 0.18 & 3.04 & 1.44 & 14.72 & 0.65 & 8.03 & 8.68 \\
\hline 13.1 & 6.5 & 0.32 & 4.25 & 2.54 & 20.59 & 1.16 & 11.23 & 12.38 \\
\hline 6.5 & 3.3 & 0.50 & 4.58 & 3.98 & 22.18 & 1.81 & 12.10 & 13.91 \\
\hline 3.3 & 1.5 & 0.31 & 3.26 & 2.48 & 15.78 & 1.13 & 8.60 & 9.73 \\
\hline 1.5 & & 0.47 & 3.56 & 3.76 & 17.24 & 1.71 & 9.40 & 11.11 \\
\hline Totals & -- & 12.61 & 20.66 & 100.00 & 100.00 & 45.47 & 54.53 & 100.00 \\
\hline
\end{tabular}

\begin{tabular}{|c|c|c|c|c|c|c|c|c|}
\hline $\begin{array}{c}\text { Pass } \\
\begin{array}{c}\text { Size } \\
(u m)\end{array}\end{array}$ & $\begin{array}{c}\text { Retain } \\
\text { Size } \\
(u m)\end{array}$ & $\begin{array}{c}0 / S \\
\text { Mass } \\
(\mathrm{TPH})\end{array}$ & $\begin{array}{c}\mathrm{U} / \mathrm{S} \\
\text { Mass } \\
(\mathrm{TPH})\end{array}$ & $\begin{array}{c}\text { Feed } \\
\text { Mass } \\
(\mathrm{TPH})\end{array}$ & $\begin{array}{c}\text { Delta } \\
\text { Mass } \\
(\mathrm{TPH})\end{array}$ & $\begin{array}{c}\text { Value } \\
\text { (f-u) }\end{array}$ & $\begin{array}{c}\text { X } \\
\text { Value } \\
(0-\mathrm{u})\end{array}$ & $\begin{array}{c}\text { Partition } \\
\text { Factor } \\
(-)\end{array}$ \\
\hline & 212 & 0.06 & 0.00 & 0.06 & 0.00 & 5.06 & 5.06 & 1.00 \\
\hline 212 & 150 & 0.06 & 0.00 & 0.06 & 0.00 & 5.38 & 5.38 & 1.00 \\
\hline 150 & 75 & 0.19 & 0.01 & 0.20 & 0.00 & 16.77 & 16.21 & 0.97 \\
\hline 75 & 45 & 0.11 & 0.02 & 0.13 & 0.00 & 9.60 & 7.89 & 0.85 \\
\hline 45 & 25 & 0.03 & 0.03 & 0.06 & 0.00 & 2.19 & -0.72 & 0.43 \\
\hline 25 & 13.1 & 0.01 & 0.09 & 0.10 & 0.00 & 0.65 & -7.37 & 0.08 \\
\hline 13.1 & 6.5 & 0.01 & 0.13 & 0.14 & 0.00 & 1.16 & -10.07 & 0.09 \\
\hline 6.5 & 3.3 & 0.02 & 0.14 & 0.16 & 0.00 & 1.81 & -10.29 & 0.13 \\
\hline 3.3 & 1.5 & 0.01 & 0.10 & 0.11 & 0.00 & 1.13 & -7.47 & 0.12 \\
\hline 1.5 & & 0.02 & 0.11 & 0.13 & 0.00 & 1.71 & -7.69 & 0.15 \\
\hline Totals & -- & 0.52 & 0.63 & 1.15 & 0.00 & & & \\
\hline & Slurry(TPH) & 7.06 & 22.12 & 25.43 & 0.00 & & & \\
\hline
\end{tabular}




\begin{tabular}{|c|c|c|c|c|c|c|c|}
\hline \multirow{3}{*}{$\begin{array}{l}\text { Experimental } \\
\text { Partition } \mathrm{F} \text { actor }\end{array}$} & \multirow{3}{*}{$\begin{array}{c}\text { Mean } \\
\text { Particle } \\
\text { Size ( } \mu \mathrm{m})\end{array}$} & \multirow{3}{*}{$\begin{array}{c}\text { Predicted } \\
\text { Partition } \\
\text { Factor }\end{array}$} & \multirow{3}{*}{$\begin{array}{c}\text { Predicted } \\
\text { Partition } \\
\text { Factor }\end{array}$} & \multirow{2}{*}{$\frac{\text { (Plitt) }}{\mathrm{D} 50}$} & Predicted & (Linch) & Predicted \\
\hline & & & & & 40.17 & D50 & 39.81 \\
\hline & & & & $m$ & 2.69 & Alpha & 3.86 \\
\hline$(-)$ & $(--)$ & (Plitt) & (Linch) & Bypass(Rf) & 0.16 & Bypass(Rf) & 0.15 \\
\hline 1.00 & 299.81 & 1.000 & 1.000 & & & & \\
\hline 1.00 & 178.33 & 1.000 & 1.000 & & & & \\
\hline 0.97 & 106.07 & 1.000 & 0.999 & & & & \\
\hline 0.85 & 58.09 & 0.871 & 0.879 & & & & \\
\hline 0.50 & 33.54 & 0.454 & 0.449 & & & & \\
\hline 0.16 & 18.08 & 0.228 & 0.234 & & & & \\
\hline 0.16 & 9.25 & 0.174 & 0.180 & & & & \\
\hline 0.17 & 4.62 & 0.165 & 0.165 & & & & \\
\hline 0.18 & 2.32 & 0.163 & 0.159 & & & & \\
\hline 0.20 & 1.64 & 0.163 & 0.158 & & & & \\
\hline & WSSQ & 0.009 & 0.012 & & & & \\
\hline & TWSSQ & 0.021 & & & & & \\
\hline
\end{tabular}

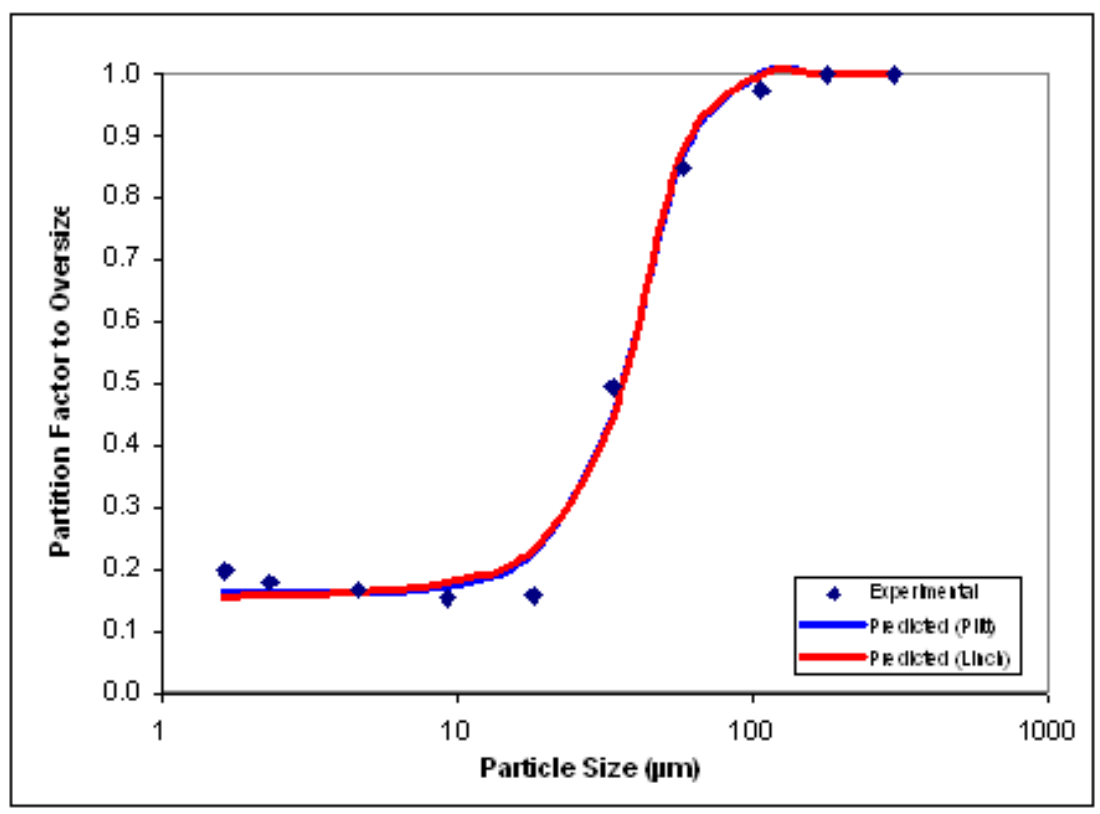




\section{HYDROCYCLONE EVALUATION (UNBALANCED)}

\begin{tabular}{|c|c|}
\hline No. 08 & \\
\hline Feedflow (GPM) & 1000 \\
\hline Feed Pressure (PSI) & 21.0 \\
\hline Water Flow (GPM) & 15.0 \\
\hline Water Pressure (PSl) & 14.0 \\
\hline Feed Rate (TPH) & 1.14 \\
\hline
\end{tabular}

\begin{tabular}{|r|c|c|c|}
\hline & OS & U/S & Feed \\
\hline Solids Content (\%) & 7.75 & 2.68 & 4.50 \\
\hline Ash Content (\%) & 20.49 & 47.32 & 34.60 \\
\hline Solids Yield (\%) & 48.4 & 51.6 & 100.0 \\
\hline Ash Yield (\%) & 47.4 & 52.6 & 100.0 \\
\hline Size Class Yield (\%) & 41.9 & 58.1 & 100.0 \\
\hline
\end{tabular}

\begin{tabular}{|c|c|c|c|c|c|c|c|c|}
\hline $\begin{array}{l}\text { Pass } \\
\text { Size } \\
\text { (um) }\end{array}$ & $\begin{array}{l}\text { Retain } \\
\text { Size } \\
\text { (um) }\end{array}$ & $\begin{array}{c}\text { O/S } \\
\text { Sample } \\
\text { (gms) }\end{array}$ & $\begin{array}{c}\text { U/S } \\
\text { Sample } \\
\text { (gms) }\end{array}$ & $\begin{array}{c}\text { orS } \\
\text { Mass } \\
(\% \text {, strm })\end{array}$ & $\begin{array}{c}\text { U/S } \\
\text { Mass } \\
(\% \text {, strm) }\end{array}$ & $\begin{array}{c}\text { OS } \\
\text { Mass } \\
(\% \text {, feed })\end{array}$ & $\begin{array}{c}\text { U/S } \\
\text { Mass } \\
(\%, \text { feed })\end{array}$ & $\begin{array}{c}\text { Feed } \\
\text { Mass } \\
(\% \text {, feed })\end{array}$ \\
\hline & 212 & 1.59 & 0.00 & 9.83 & 0.00 & 4.66 & 0.00 & 4.66 \\
\hline 212 & 150 & 1.70 & 0.00 & 10.51 & 0.00 & 4.98 & 0.00 & 4.98 \\
\hline 150 & 75 & 5.23 & 0.21 & 32.32 & 0.97 & 15.32 & 0.51 & 15.83 \\
\hline 75 & 45 & 2.87 & 0.61 & 17.74 & 2.80 & 8.41 & 1.47 & 9.88 \\
\hline 45 & 25 & 0.95 & 0.98 & 5.87 & 4.50 & 2.78 & 2.37 & 5.15 \\
\hline 25 & 13.1 & 1.05 & 3.76 & 6.46 & 17.27 & 3.06 & 9.08 & 12.14 \\
\hline 13.1 & 6.5 & 0.82 & 4.51 & 5.04 & 20.71 & 2.39 & 10.89 & 13.28 \\
\hline 6.5 & 3.3 & 0.81 & 4.77 & 5.01 & 21.91 & 2.37 & 11.52 & 13.89 \\
\hline 3.3 & 1.5 & 0.54 & 3.31 & 3.35 & 15.22 & 1.59 & 8.00 & 9.59 \\
\hline 1.5 & & 0.63 & 3.62 & 3.88 & 16.62 & 1.84 & 8.74 & 10.58 \\
\hline Totals & -- & 16.18 & 21.76 & 100.00 & 100.00 & 47.41 & 52.59 & 100.00 \\
\hline
\end{tabular}

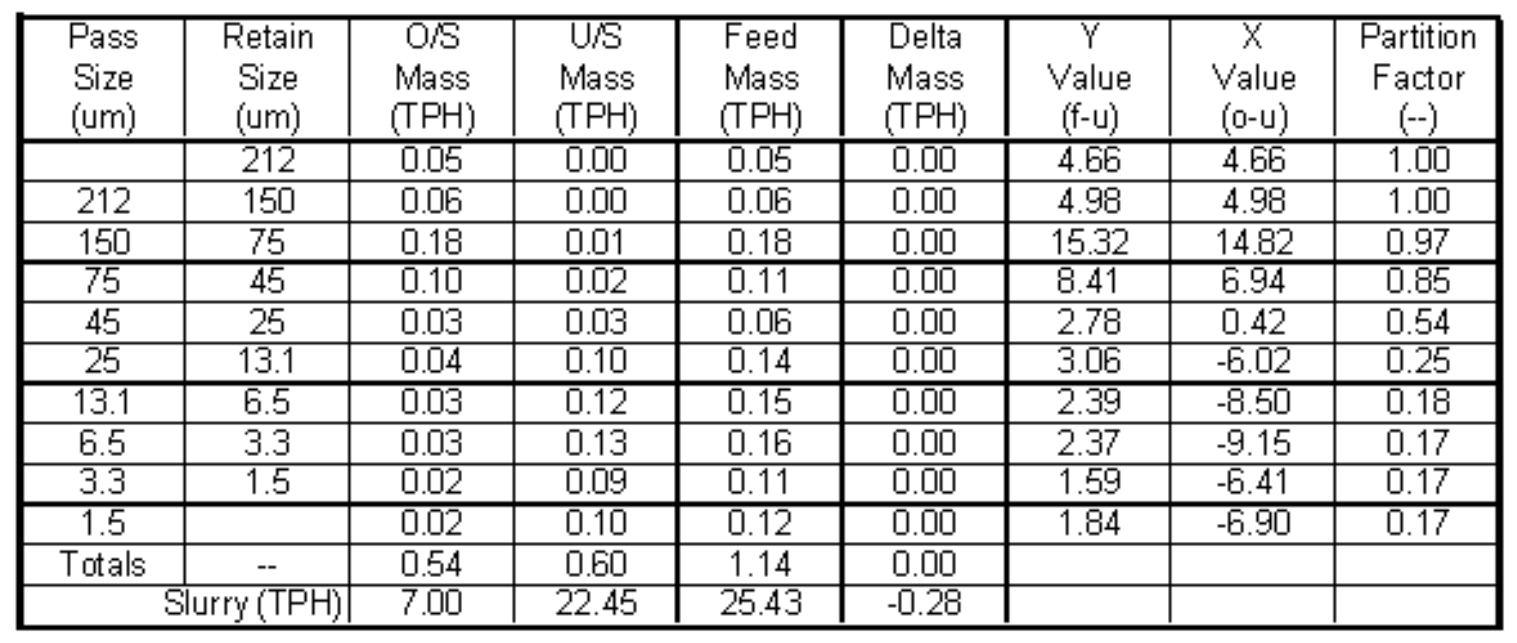


HYDROCYCLONE EVALUATION (BALANCED)

\begin{tabular}{|c|c|}
\hline No. 08 & \\
\hline Feedflow (GPM) & 10000 \\
\hline Feed Pressure (PSl) & 21.0 \\
\hline Water Flow (GPM) & 15.0 \\
\hline Water Pressure (PSI) & 14.0 \\
\hline Feed Rate (TPH) & 1.18 \\
\hline
\end{tabular}

\begin{tabular}{|r|c|c|c|}
\hline & OS & U/S & Feed \\
\hline Solids Content (\%) & 7.65 & 2.83 & 4.66 \\
\hline Ash Content (\%) & 20.58 & 47.42 & 34.55 \\
\hline Solids Yield (\%) & 48.0 & 52.0 & 100.0 \\
\hline Ash Yield (\%) & 48.0 & 52.0 & 100.0 \\
\hline Size Class Yield (\%) & 48.0 & 52.0 & 100.0 \\
\hline
\end{tabular}

\begin{tabular}{|c|c|c|c|c|c|c|c|c|}
\hline $\begin{array}{l}\text { Pass } \\
\text { Size } \\
\text { (um) }\end{array}$ & $\begin{array}{l}\text { Retain } \\
\text { Size } \\
\text { (um) }\end{array}$ & $\begin{array}{c}\text { O/S } \\
\text { Sample } \\
\text { (gms) }\end{array}$ & $\begin{array}{c}\text { U/S } \\
\text { Sample } \\
\text { (gms) }\end{array}$ & $\begin{array}{c}\text { orS } \\
\text { Mass } \\
(\% \text {, strm })\end{array}$ & $\begin{array}{c}\text { U/S } \\
\text { Mass } \\
(\% \text {, strm) }\end{array}$ & $\begin{array}{c}\text { OS } \\
\text { Mass } \\
(\% \text {, feed })\end{array}$ & $\begin{array}{c}\text { U/S } \\
\text { Mass } \\
(\%, \text { feed })\end{array}$ & $\begin{array}{c}\text { Feed } \\
\text { Mass } \\
(\% \text {, feed })\end{array}$ \\
\hline & 212 & 1.52 & 0.00 & 9.87 & 0.00 & 4.73 & 0.00 & 4.73 \\
\hline 212 & 150 & 1.64 & 0.00 & 10.64 & 0.00 & 5.10 & 0.00 & 5.10 \\
\hline 150 & 75 & 5.38 & 0.29 & 34.90 & 1.33 & 16.73 & 0.69 & 17.43 \\
\hline 75 & 45 & 2.88 & 0.68 & 18.67 & 3.12 & 8.95 & 1.62 & 10.57 \\
\hline 45 & 25 & 0.84 & 1.06 & 5.45 & 4.85 & 2.62 & 2.52 & 5.14 \\
\hline 25 & 13.1 & 0.92 & 3.76 & 6.00 & 17.16 & 2.88 & 8.93 & 11.81 \\
\hline 13.1 & 6.5 & 0.68 & 4.49 & 4.38 & 20.48 & 2.10 & 10.66 & 12.76 \\
\hline 6.5 & 3.3 & 0.67 & 4.67 & 4.36 & 21.33 & 2.09 & 11.10 & 13.19 \\
\hline 3.3 & 1.5 & 0.40 & 3.33 & 2.57 & 15.19 & 1.23 & 7.91 & 9.14 \\
\hline 1.5 & & 0.49 & 3.62 & 3.16 & 16.55 & 1.52 & 8.61 & 10.13 \\
\hline Totals & -- & 15.41 & 21.90 & 100.00 & 100.00 & 47.95 & 52.05 & 100.00 \\
\hline
\end{tabular}

\begin{tabular}{|c|c|c|c|c|c|c|c|c|}
\hline $\begin{array}{c}\text { Pass } \\
\text { Size } \\
(u m)\end{array}$ & $\begin{array}{c}\text { Retain } \\
\text { Size } \\
(u m)\end{array}$ & $\begin{array}{c}0 / S \\
\text { Mass } \\
(\mathrm{TPH})\end{array}$ & $\begin{array}{c}\text { U/S } \\
\text { Mass } \\
(\mathrm{TPH})\end{array}$ & $\begin{array}{c}\text { Feed } \\
\text { Mass } \\
(\mathrm{TPH})\end{array}$ & $\begin{array}{c}\text { Delta } \\
\text { Mass } \\
(\mathrm{TPH})\end{array}$ & $\begin{array}{c}\text { Value } \\
\text { (f-u) }\end{array}$ & $\begin{array}{c}\text { X } \\
\text { Value } \\
(0-\mathrm{u})\end{array}$ & $\begin{array}{c}\text { Partition } \\
\text { Factor } \\
(-)\end{array}$ \\
\hline & 212 & 0.06 & 0.00 & 0.06 & 0.00 & 4.73 & 4.73 & 1.00 \\
\hline 212 & 150 & 0.06 & 0.00 & 0.06 & 0.00 & 5.10 & 5.10 & 1.00 \\
\hline 150 & 75 & 0.20 & 0.01 & 0.21 & 0.00 & 16.73 & 16.04 & 0.96 \\
\hline 75 & 45 & 0.11 & 0.02 & 0.13 & 0.00 & 8.95 & 7.33 & 0.85 \\
\hline 45 & 25 & 0.03 & 0.03 & 0.06 & 0.00 & 2.62 & 0.09 & 0.51 \\
\hline 25 & 13.1 & 0.03 & 0.11 & 0.14 & 0.00 & 2.88 & -6.05 & 0.24 \\
\hline 13.1 & 6.5 & 0.02 & 0.13 & 0.15 & 0.00 & 2.10 & -8.56 & 0.16 \\
\hline 6.5 & 3.3 & 0.02 & 0.13 & 0.16 & 0.00 & 2.09 & -9.01 & 0.16 \\
\hline 3.3 & 1.5 & 0.01 & 0.09 & 0.11 & 0.00 & 1.23 & -6.68 & 0.13 \\
\hline 1.5 & & 0.02 & 0.10 & 0.12 & 0.00 & 1.52 & -7.10 & 0.15 \\
\hline Totals & -- & 0.57 & 0.62 & 1.18 & 0.00 & & & \\
\hline & Slurry (TPH) & 7.42 & 21.77 & 25.44 & 0.00 & & & \\
\hline
\end{tabular}




\begin{tabular}{|c|c|c|c|c|c|c|c|}
\hline \multirow{3}{*}{$\begin{array}{l}\text { Experimental } \\
\text { Partition } \mathrm{F} \text { actor }\end{array}$} & \multirow{3}{*}{$\begin{array}{c}\text { Mean } \\
\text { Particle } \\
\text { Size }(\mu \mathrm{m})\end{array}$} & \multirow{3}{*}{$\begin{array}{c}\text { Predicted } \\
\text { Partition } \\
\text { Factor }\end{array}$} & \multirow{3}{*}{$\begin{array}{c}\text { Predicted } \\
\text { Partition } \\
\text { Factor }\end{array}$} & \multirow{2}{*}{$\frac{\text { (Plitt) }}{\mathrm{D} 50}$} & Predicted & (Linch) & Predicted \\
\hline & & & & & 37.18 & D50 & 37.00 \\
\hline & & & & $m$ & 2.15 & Alpha & 2.88 \\
\hline$(-)$ & $(-)$ & (Plitt) & (Linch) & Bypass(Rf) & 0.16 & Bypass(Rf) & 0.15 \\
\hline 1.00 & 299.81 & 1.000 & 1.000 & & & & \\
\hline 1.00 & 178.33 & 1.000 & 1.000 & & & & \\
\hline 0.97 & 106.07 & 0.999 & 0.996 & & & & \\
\hline 0.85 & 58.09 & 0.862 & 0.867 & & & & \\
\hline 0.54 & 33.54 & 0.518 & 0.513 & & & & \\
\hline 0.25 & 18.08 & 0.276 & 0.280 & & & & \\
\hline 0.18 & 9.25 & 0.190 & 0.198 & & & & \\
\hline 0.17 & 4.62 & 0.167 & 0.169 & & & & \\
\hline 0.17 & 2.32 & 0.162 & 0.158 & & & & \\
\hline 0.17 & 1.64 & 0.161 & 0.155 & & & & \\
\hline & WSSQ & 0.002 & 0.003 & & & & \\
\hline & TWSSQ & 0.006 & & & & & \\
\hline
\end{tabular}

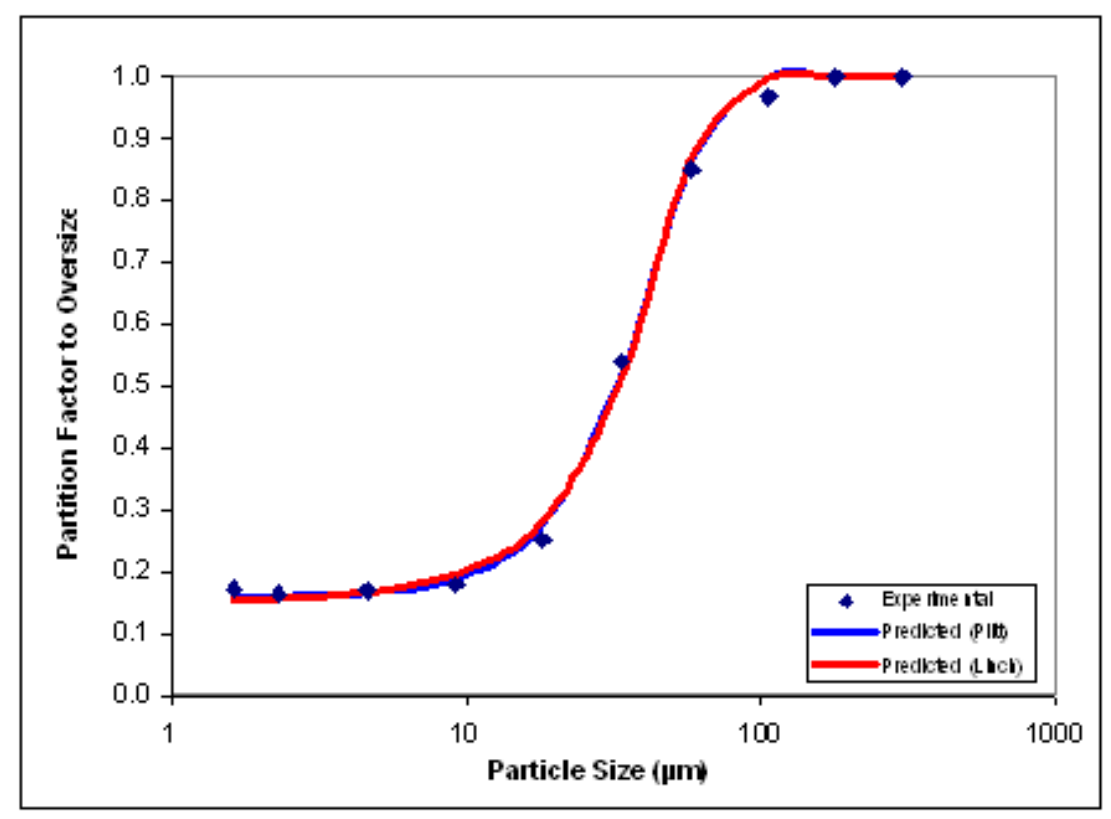




\section{HYDROCYCLONE EVALUATION (UNBALANCED)}

\begin{tabular}{|c|c|}
\hline No. 09 & \\
\hline Feedflow (GPM) & 1000 \\
\hline Feed Pressure (PSl) & 21.0 \\
\hline Water Flow (GPM) & 0.0 \\
\hline Water Pressure (PSl) & 0.0 \\
\hline Feed Rate (TPH) & 1.17 \\
\hline
\end{tabular}

\begin{tabular}{|r|c|c|c|}
\hline & OS & U/S & Feed \\
\hline Solids Content (\%) & 8.32 & 2.68 & 4.59 \\
\hline Ash Content (\%) & 27.01 & 49.03 & 34.63 \\
\hline Solids Yield (\%) & 61.4 & 38.6 & 100.0 \\
\hline Ash Yield (\%) & 65.4 & 34.6 & 100.0 \\
\hline Size Class Yield (\%) & 92.2 & 7.8 & 100.0 \\
\hline
\end{tabular}

\begin{tabular}{|c|c|c|c|c|c|c|c|c|}
\hline $\begin{array}{l}\text { Pass } \\
\text { Size } \\
\text { (um) }\end{array}$ & $\begin{array}{l}\text { Retain } \\
\text { Size } \\
\text { (um) }\end{array}$ & $\begin{array}{c}\text { O/S } \\
\text { Sample } \\
\text { (gms) }\end{array}$ & $\begin{array}{c}\text { U/S } \\
\text { Sample } \\
\text { (gms) }\end{array}$ & $\begin{array}{c}\text { orS } \\
\text { Mass } \\
(\% \text {, strm })\end{array}$ & $\begin{array}{c}\text { U/S } \\
\text { Mass } \\
(\% \text {, strm) }\end{array}$ & $\begin{array}{c}\text { OS } \\
\text { Mass } \\
(\% \text {, feed })\end{array}$ & $\begin{array}{c}\text { U/S } \\
\text { Mass } \\
(\%, \text { feed })\end{array}$ & $\begin{array}{c}\text { Feed } \\
\text { Mass } \\
(\% \text {, feed })\end{array}$ \\
\hline & 212 & 1.65 & 0.00 & 7.29 & 0.00 & 4.77 & 0.00 & 4.77 \\
\hline 212 & 150 & 1.66 & 0.00 & 7.33 & 0.00 & 4.79 & 0.00 & 4.79 \\
\hline 150 & 75 & 5.27 & 0.16 & 23.28 & 1.01 & 15.22 & 0.35 & 15.57 \\
\hline 75 & 45 & 3.59 & 0.36 & 15.86 & 2.26 & 10.37 & 0.78 & 11.15 \\
\hline 45 & 25 & 1.50 & 0.39 & 6.63 & 2.45 & 4.33 & 0.85 & 5.18 \\
\hline 25 & 13.1 & 2.52 & 2.97 & 11.15 & 18.69 & 7.29 & 6.47 & 13.76 \\
\hline 13.1 & 6.5 & 2.01 & 3.57 & 8.89 & 22.47 & 5.81 & 7.77 & 13.59 \\
\hline 6.5 & 3.3 & 1.92 & 3.67 & 8.46 & 23.07 & 5.53 & 7.98 & 13.52 \\
\hline 3.3 & 1.5 & 1.31 & 2.41 & 5.79 & 15.13 & 3.79 & 5.23 & 9.02 \\
\hline 1.5 & & 1.21 & 2.38 & 5.33 & 14.93 & 3.48 & 5.17 & 8.65 \\
\hline Totals & -- & 22.64 & 15.91 & 100.00 & 100.00 & 65.40 & 34.60 & 100.00 \\
\hline
\end{tabular}

\begin{tabular}{|c|c|c|c|c|c|c|c|c|}
\hline $\begin{array}{c}\text { Pass } \\
\text { Size } \\
(u m)\end{array}$ & $\begin{array}{c}\text { Retain } \\
\text { Size } \\
(u m)\end{array}$ & $\begin{array}{c}0 / 5 \\
\text { Mass } \\
(\mathrm{TPH})\end{array}$ & $\begin{array}{c}\text { U/S } \\
\text { Mass } \\
(\mathrm{TPH})\end{array}$ & $\begin{array}{c}\text { Feed } \\
\text { Mass } \\
(\mathrm{TPH})\end{array}$ & $\begin{array}{c}\text { Delta } \\
\text { Mass } \\
(\mathrm{TPH})\end{array}$ & $\begin{array}{c}\text { Value } \\
\text { (f-u) }\end{array}$ & $\begin{array}{c}\text { X } \\
\text { Value } \\
(\mathrm{0}-\mathrm{u})\end{array}$ & $\begin{array}{c}\text { Partition } \\
\text { Factor } \\
(-)\end{array}$ \\
\hline & 212 & 0.06 & 0.00 & 0.06 & 0.00 & 4.77 & 4.77 & 1.00 \\
\hline 212 & 150 & 0.06 & 0.00 & 0.06 & 0.00 & 4.79 & 4.79 & 1.00 \\
\hline 150 & 75 & 0.18 & 0.00 & 0.18 & 0.00 & 15.22 & 14.87 & 0.98 \\
\hline 75 & 45 & 0.12 & 0.01 & 0.13 & 0.00 & 10.37 & 9.59 & 0.93 \\
\hline 45 & 25 & 0.05 & 0.01 & 0.06 & 0.00 & 4.33 & 3.48 & 0.84 \\
\hline 25 & 13.1 & 0.09 & 0.08 & 0.16 & 0.00 & 7.29 & 0.82 & 0.53 \\
\hline 13.1 & 6.5 & 0.07 & 0.09 & 0.16 & 0.00 & 5.81 & -1.96 & 0.43 \\
\hline 6.5 & 3.3 & 0.06 & 0.09 & 0.16 & 0.00 & 5.53 & -2.45 & 0.41 \\
\hline 3.3 & 1.5 & 0.04 & 0.06 & 0.11 & 0.00 & 3.79 & -1.45 & 0.42 \\
\hline 1.5 & & 0.04 & 0.06 & 0.10 & 0.00 & 3.48 & -1.68 & 0.40 \\
\hline Totals & -- & 0.76 & 0.40 & 1.17 & 0.00 & & & \\
\hline & Slurry (TPH) & 9.18 & 15.08 & 25.44 & 1.18 & & & \\
\hline
\end{tabular}




\section{HYDROCYCLONE EVALUATION (BALANCED)}

\begin{tabular}{|c|c|}
\hline No. 09 & \\
\hline Feedflow (GPM) & 900.0 \\
\hline Feed Pressure (PSl) & 21.0 \\
\hline Water Flow (GPM) & 0.0 \\
\hline Water Pressure (PSl) & 0.0 \\
\hline Feed Rate (TPH) & 1.18 \\
\hline
\end{tabular}

\begin{tabular}{|r|c|c|c|}
\hline & OS & U/S & Feed \\
\hline Solids Content (\%) & 8.31 & 2.63 & 4.63 \\
\hline Ash Content (\%) & 26.80 & 48.90 & 34.97 \\
\hline Solids Yield (\%) & 63.0 & 37.0 & 100.0 \\
\hline Ash Yield (\%) & 63.0 & 37.0 & 100.0 \\
\hline Size Class Yield (\%) & 63.0 & 37.0 & 100.0 \\
\hline
\end{tabular}

\begin{tabular}{|c|c|c|c|c|c|c|c|c|}
\hline $\begin{array}{c}\text { Pass } \\
\text { Size } \\
(u m)\end{array}$ & $\begin{array}{c}\text { Retain } \\
\text { Size } \\
(u m)\end{array}$ & $\begin{array}{c}\text { O/S } \\
\text { Sample } \\
(\mathrm{gms})\end{array}$ & $\begin{array}{c}\text { U/S } \\
\text { Sample } \\
(\mathrm{gms})\end{array}$ & $\begin{array}{c}\text { O/S } \\
\text { Mass } \\
(\%, \text { strm) }\end{array}$ & $\begin{array}{c}\text { U/S } \\
\text { Mass } \\
(\%, \text { strm) }\end{array}$ & $\begin{array}{c}0 / S \\
\text { Mass } \\
(\%, \text { feed })\end{array}$ & $\begin{array}{c}\text { U/S } \\
\text { Mass } \\
(\%, \text { feed })\end{array}$ & $\begin{array}{c}\text { Feed } \\
\text { Mass } \\
(\%, \text { feed })\end{array}$ \\
\hline & 212 & 2.00 & 0.00 & 8.48 & 0.00 & 5.34 & 0.00 & 5.34 \\
\hline 212 & 150 & 2.00 & 0.00 & 8.51 & 0.00 & 5.36 & 0.00 & 5.36 \\
\hline 150 & 75 & 4.77 & 0.00 & 20.26 & 0.00 & 12.77 & 0.00 & 12.77 \\
\hline 75 & 45 & 3.49 & 0.00 & 14.79 & 0.00 & 9.32 & 0.00 & 9.32 \\
\hline 45 & 25 & 1.88 & 0.00 & 7.99 & 0.00 & 5.03 & 0.00 & 5.03 \\
\hline 25 & 13.1 & 2.46 & 2.34 & 10.46 & 18.22 & 6.59 & 6.74 & 13.33 \\
\hline 13.1 & 6.5 & 1.92 & 4.01 & 8.16 & 31.22 & 5.14 & 11.54 & 16.68 \\
\hline 6.5 & 3.3 & 1.82 & 4.28 & 7.74 & 33.32 & 4.88 & 12.32 & 17.20 \\
\hline 3.3 & 1.5 & 1.64 & 1.14 & 6.97 & 8.85 & 4.39 & 3.27 & 7.66 \\
\hline 1.5 & & 1.57 & 1.08 & 6.65 & 8.39 & 4.19 & 3.10 & 7.29 \\
\hline Totals & -- & 23.56 & 12.86 & 100.00 & 100.00 & 63.03 & 36.97 & 100.00 \\
\hline
\end{tabular}

\begin{tabular}{|c|c|c|c|c|c|c|c|c|}
\hline $\begin{array}{c}\text { Pass } \\
\text { Size } \\
(u m)\end{array}$ & $\begin{array}{c}\text { Retain } \\
\text { Size } \\
(u m)\end{array}$ & $\begin{array}{c}0 / 5 \\
\text { Mass } \\
(\mathrm{TPH})\end{array}$ & $\begin{array}{c}\text { U/S } \\
\text { Mass } \\
(\mathrm{TPH})\end{array}$ & $\begin{array}{c}\text { Feed } \\
\text { Mass } \\
(\mathrm{TPH})\end{array}$ & $\begin{array}{c}\text { Delta } \\
\text { Mass } \\
(\mathrm{TPH})\end{array}$ & $\begin{array}{c}\text { V } \\
\text { alue } \\
(\mathrm{f}-\mathrm{u})\end{array}$ & $\begin{array}{c}\mathrm{X} \\
\text { Value } \\
(\mathrm{0}-\mathrm{u})\end{array}$ & $\begin{array}{c}\text { Partition } \\
\text { Factor } \\
(-)\end{array}$ \\
\hline & 212 & 0.06 & 0.00 & 0.06 & 0.00 & 5.34 & 5.34 & 1.00 \\
\hline 212 & 150 & 0.06 & 0.00 & 0.06 & 0.00 & 5.36 & 5.36 & 1.00 \\
\hline 150 & 75 & 0.15 & 0.00 & 0.15 & 0.00 & 12.77 & 12.77 & 1.00 \\
\hline 75 & 45 & 0.11 & 0.00 & 0.11 & 0.00 & 9.32 & 9.32 & 1.00 \\
\hline 45 & 25 & 0.06 & 0.00 & 0.06 & 0.00 & 5.03 & 5.03 & 1.00 \\
\hline 25 & 13.1 & 0.08 & 0.08 & 0.16 & 0.00 & 6.59 & -0.14 & 0.49 \\
\hline 13.1 & 6.5 & 0.06 & 0.14 & 0.20 & 0.00 & 5.14 & -6.40 & 0.31 \\
\hline 6.5 & 3.3 & 0.06 & 0.15 & 0.20 & 0.00 & 4.80 & -7.44 & 0.28 \\
\hline 3.3 & 1.5 & 0.05 & 0.04 & 0.09 & 0.00 & 4.39 & 1.12 & 0.57 \\
\hline 1.5 & & 0.05 & 0.04 & 0.09 & 0.00 & 4.19 & 1.09 & 0.57 \\
\hline Totals & -- & 0.74 & 0.44 & 1.18 & 0.00 & & & \\
\hline & Slurry (TPH) & 8.92 & 16.52 & 25.44 & 0.00 & & & \\
\hline
\end{tabular}




\begin{tabular}{|c|c|c|c|}
\hline $\begin{array}{c}\text { Experimental } \\
\text { Partition Factor }\end{array}$ & $\begin{array}{c}\text { Mean } \\
\text { Particle } \\
\text { Size }(\mu \mathrm{m})\end{array}$ & $\begin{array}{c}\text { Predicted } \\
\text { Partition } \\
\text { Factor }\end{array}$ & $\begin{array}{c}\text { Predicted } \\
\text { Partition } \\
\text { Factor }\end{array}$ \\
\hline$(--)$ & $(--)$ & (Plitt) & (Linch) \\
\hline 1.00 & 299.81 & 1.000 & 1.000 \\
\hline 1.00 & 178.33 & 1.000 & 1.000 \\
\hline 0.98 & 106.07 & 1.000 & 1.000 \\
\hline 0.93 & 58.09 & 0.996 & 0.991 \\
\hline 0.84 & 33.54 & 0.825 & 0.825 \\
\hline 0.53 & 18.08 & 0.538 & 0.534 \\
\hline 0.43 & 9.25 & 0.432 & 0.438 \\
\hline 0.41 & 4.62 & 0.411 & 0.413 \\
\hline 0.42 & 2.32 & 0.407 & 0.405 \\
\hline 0.40 & 1.64 & 0.406 & 0.403 \\
\hline & $W S S Q$ & 0.005 & 0.005 \\
\hline
\end{tabular}

\begin{tabular}{|c|c|c|c|}
\multicolumn{1}{c}{ (Plitt) } & Predicted & $($ Linch $)$ & Predicted \\
\hline $\mathrm{D} 50$ & 26.87 \\
\hline$m$ & 2.55 \\
\hline Bypass(Rf) & 0.41 \\
\hline
\end{tabular}$\quad$\begin{tabular}{|c|c|}
\hline D 50 & 26.91 \\
\hline Bypass(Rf) & 0.40 \\
\hline
\end{tabular}

\begin{tabular}{|l|l|}
\hline TWSSQ & 0.010 \\
\hline
\end{tabular}

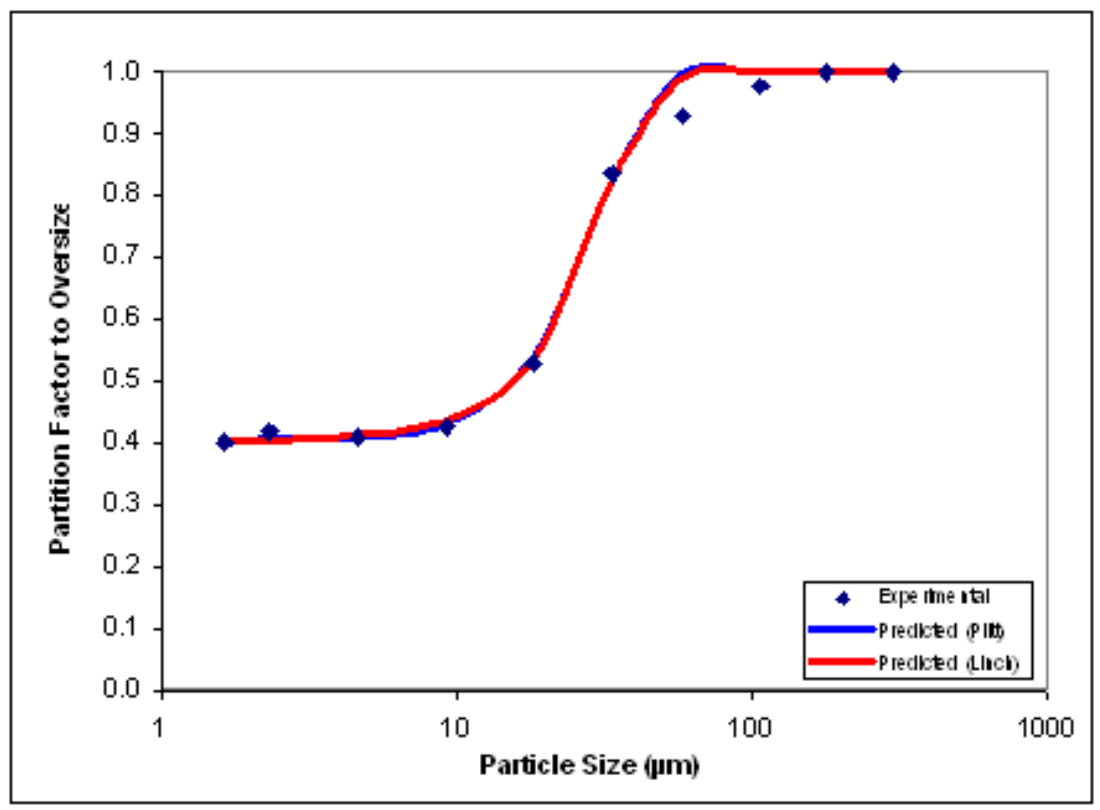




\section{HYDROCYCLONE EVALUATION (UNBALANCED)}

\begin{tabular}{|c|c|}
\hline No. 10 & \\
\hline Feedflow (GPM) & 1000 \\
\hline Feed Pressure (PSl) & 21.0 \\
\hline Water Flow (GPM) & 15.0 \\
\hline Water Pressure (PSl) & 12.0 \\
\hline Feed Rate (TPH) & 1.14 \\
\hline
\end{tabular}

\begin{tabular}{|r|c|c|c|}
\hline & OS & U/S & Feed \\
\hline Solids Content (\%) & 5.18 & 2.31 & 4.50 \\
\hline Ash Content (\%) & 29.20 & 49.41 & 34.98 \\
\hline Solids Yield (\%) & 74.2 & 25.8 & 100.0 \\
\hline Ash Yield (\%) & 71.4 & 28.6 & 100.0 \\
\hline Size Class Yield (\%) & 110.1 & -10.1 & 100.0 \\
\hline
\end{tabular}

\begin{tabular}{|c|c|c|c|c|c|c|c|c|}
\hline $\begin{array}{l}\text { Pass } \\
\text { Size } \\
\text { (um) }\end{array}$ & $\begin{array}{l}\text { Retain } \\
\text { Size } \\
\text { (um) }\end{array}$ & $\begin{array}{c}\text { O/S } \\
\text { Sample } \\
\text { (gms) }\end{array}$ & $\begin{array}{c}\text { U/S } \\
\text { Sample } \\
\text { (gms) }\end{array}$ & $\begin{array}{c}\text { orS } \\
\text { Mass } \\
(\% \text {, strm })\end{array}$ & $\begin{array}{c}\text { U/S } \\
\text { Mass } \\
(\% \text {, strm) }\end{array}$ & $\begin{array}{c}\text { OS } \\
\text { Mass } \\
(\% \text {, feed })\end{array}$ & $\begin{array}{c}\text { U/S } \\
\text { Mass } \\
(\%, \text { feed })\end{array}$ & $\begin{array}{c}\text { Feed } \\
\text { Mass } \\
(\% \text {, feed })\end{array}$ \\
\hline & 212 & 2.21 & 0.00 & 7.40 & 0.00 & 5.28 & 0.00 & 5.28 \\
\hline 212 & 150 & 2.13 & 0.00 & 7.13 & 0.00 & 5.09 & 0.00 & 5.09 \\
\hline 150 & 75 & 5.87 & 0.17 & 19.66 & 1.52 & 14.04 & 0.44 & 14.47 \\
\hline 75 & 45 & 4.20 & 0.23 & 14.07 & 2.06 & 10.04 & 0.59 & 10.63 \\
\hline 45 & 25 & 2.05 & 0.15 & 6.87 & 1.35 & 4.90 & 0.38 & 5.29 \\
\hline 25 & 13.1 & 3.68 & 1.73 & 12.33 & 15.54 & 8.80 & 4.44 & 13.25 \\
\hline 13.1 & 6.5 & 3.23 & 2.52 & 10.81 & 22.56 & 7.71 & 6.45 & 14.17 \\
\hline 6.5 & 3.3 & 2.84 & 2.58 & 9.51 & 23.15 & 6.79 & 6.62 & 13.41 \\
\hline 3.3 & 1.5 & 1.79 & 1.81 & 6.00 & 16.24 & 4.28 & $\overline{4.64}$ & 8.93 \\
\hline 1.5 & & 1.86 & 1.96 & 6.23 & 17.57 & 4.45 & 5.03 & 9.47 \\
\hline Totals & -- & 29.86 & 11.15 & 100.00 & 100.00 & 71.40 & 28.60 & 100.00 \\
\hline
\end{tabular}

\begin{tabular}{|c|c|c|c|c|c|c|c|c|}
\hline $\begin{array}{c}\text { Pass } \\
\text { Size } \\
(u m)\end{array}$ & $\begin{array}{c}\text { Retain } \\
\text { Size } \\
(u m)\end{array}$ & $\begin{array}{c}0 / 5 \\
\text { Mass } \\
(\mathrm{TPH})\end{array}$ & $\begin{array}{c}\text { U/S } \\
\text { Mass } \\
(\mathrm{TPH})\end{array}$ & $\begin{array}{c}\text { Feed } \\
\text { Mass } \\
(\mathrm{TPH})\end{array}$ & $\begin{array}{c}\text { Delta } \\
\text { Mass } \\
(\mathrm{TPH})\end{array}$ & $\begin{array}{c}\text { Value } \\
\text { (f-u) }\end{array}$ & $\begin{array}{c}\text { X } \\
\text { Value } \\
(0-\mathrm{u})\end{array}$ & $\begin{array}{c}\text { Partition } \\
\text { Factor } \\
(-)\end{array}$ \\
\hline & 212 & 0.06 & 0.00 & 0.06 & 0.00 & 5.28 & 5.28 & 1.00 \\
\hline 212 & 150 & 0.06 & 0.00 & 0.06 & 0.00 & 5.09 & 5.09 & 1.00 \\
\hline 150 & 75 & 0.16 & 0.00 & 0.17 & 0.00 & 14.04 & 13.60 & 0.97 \\
\hline 75 & 45 & 0.11 & 0.01 & 0.12 & 0.00 & 10.04 & 9.45 & 0.94 \\
\hline 45 & 25 & 0.06 & 0.00 & 0.06 & 0.00 & 4.90 & 4.52 & 0.93 \\
\hline 25 & 13.1 & 0.10 & 0.05 & 0.15 & 0.00 & 8.80 & 4.36 & 0.66 \\
\hline 13.1 & 6.5 & 0.09 & 0.07 & 0.16 & 0.00 & 7.71 & 1.26 & 0.54 \\
\hline 6.5 & 3.3 & 0.08 & 0.08 & 0.15 & 0.00 & 6.79 & 0.17 & 0.51 \\
\hline 3.3 & 1.5 & 0.05 & 0.05 & 0.10 & 0.00 & 4.28 & -0.36 & 0.48 \\
\hline 1.5 & & 0.05 & 0.06 & 0.11 & 0.00 & 4.45 & -0.58 & 0.47 \\
\hline Totals & -- & 0.82 & 0.33 & 1.14 & 0.00 & & & \\
\hline & Slurry (TPH) & 15.77 & 14.17 & 25.43 & -0.76 & & & \\
\hline
\end{tabular}




\section{HYDROCYCLONE EVALUATION (BALANCED)}

\begin{tabular}{|c|c|}
\hline No. 10 & \\
\hline Feedflow (GPV) & 100.0 \\
\hline Feed Pressure (PSl) & 21.0 \\
\hline Water Flow (GPM) & 15.0 \\
\hline Water Pressure (PSl) & 12.0 \\
\hline Feed Rate (TPH) & 1.05 \\
\hline
\end{tabular}

\begin{tabular}{|r|c|c|c|}
\hline & OS & US & Feed \\
\hline Solids Content (\%) & 5.13 & 2.49 & 4.15 \\
\hline Ash Content (\%) & 28.21 & 49.00 & 36.43 \\
\hline Solids Yield (\%) & 60.5 & 39.5 & 100.0 \\
\hline Ash Yield (\%) & 60.5 & 39.5 & 100.0 \\
\hline Size Class Yield (\%) & 60.5 & 39.5 & 100.0 \\
\hline
\end{tabular}

\begin{tabular}{|c|c|c|c|c|c|c|c|c|}
\hline $\begin{array}{l}\text { Pass } \\
\text { Size } \\
\text { (um) } \\
\end{array}$ & $\begin{array}{c}\text { Retain } \\
\text { Size } \\
\text { (um) }\end{array}$ & $\begin{array}{c}\text { O/S } \\
\text { Sample } \\
\text { (gms) }\end{array}$ & $\begin{array}{c}\text { U/S } \\
\text { Sample } \\
\text { (gms) }\end{array}$ & $\begin{array}{c}\text { OSS } \\
\text { Mass } \\
(\%, \text { strm) }\end{array}$ & $\begin{array}{c}\text { US } \\
\text { Mass } \\
(\% \text {, strm) }\end{array}$ & $\begin{array}{c}\text { OS } \\
\text { Mass } \\
(\%, \text { feed })\end{array}$ & $\begin{array}{c}\text { U/S } \\
\text { Mass } \\
(\%, \text { feed })\end{array}$ & $\begin{array}{c}\text { Feed } \\
\text { Mass } \\
(\%, \text { feed })\end{array}$ \\
\hline & 212 & 2.65 & 0.00 & 9.09 & 0.00 & 5.50 & 0.00 & 5.50 \\
\hline 212 & 150 & 2.57 & 0.00 & 8.81 & 0.00 & 5.33 & 0.00 & 5.33 \\
\hline 150 & 75 & 6.39 & 0.02 & 21.91 & 0.19 & 13.25 & 0.07 & 13.33 \\
\hline 75 & 45 & 4.68 & 0.02 & 16.05 & 0.18 & 9.70 & 0.07 & 9.77 \\
\hline 45 & 25 & 2.48 & 0.02 & 8.52 & 0.16 & 5.15 & 0.06 & 5.22 \\
\hline 25 & 13.1 & 3.33 & $\overline{0.66}$ & 11.42 & 6.39 & 6.91 & 2.53 & 9.43 \\
\hline 13.1 & 6.5 & 2.43 & 2.64 & 8.35 & 25.49 & 5.05 & 10.07 & 15.12 \\
\hline 6.5 & 3.3 & 2.00 & 3.00 & 6.85 & 28.91 & 4.14 & 11.43 & 15.57 \\
\hline 3.3 & 1.5 & 1.32 & 1.86 & 4.52 & 17.94 & 2.73 & 7.09 & 9.82 \\
\hline 1.5 & & 1.31 & 2.15 & 4.49 & 20.75 & 2.72 & 8.20 & 10.91 \\
\hline Totals & -- & 29.16 & 10.36 & 100.00 & 100.00 & 60.48 & 39.52 & 100.00 \\
\hline
\end{tabular}

\begin{tabular}{|c|c|c|c|c|c|c|c|c|}
\hline $\begin{array}{l}\text { Pass } \\
\text { Size } \\
\text { (um) }\end{array}$ & $\begin{array}{c}\text { Retain } \\
\text { Size } \\
\text { (um) }\end{array}$ & $\begin{array}{c}\text { O/S } \\
\text { Mass } \\
(\mathrm{TPH})\end{array}$ & $\begin{array}{c}\text { USS } \\
\text { Mass } \\
(\mathrm{TPH})\end{array}$ & $\begin{array}{l}\text { Feed } \\
\text { Mass } \\
(\mathrm{TPH})\end{array}$ & $\begin{array}{l}\text { Delta } \\
\text { Mass } \\
(\mathrm{TPH}) \\
\end{array}$ & $\begin{array}{c}\text { Y } \\
\text { Value } \\
(\mathrm{f}-\mathrm{u})\end{array}$ & $\begin{array}{c}X \\
\text { Value } \\
(0-4)\end{array}$ & $\begin{array}{c}\text { Partition } \\
\text { Factor } \\
(--)\end{array}$ \\
\hline & 212 & 0.06 & 0.00 & 0.06 & 0.00 & 5.50 & 5.50 & 1.00 \\
\hline 212 & 150 & 0.06 & 0.00 & 0.06 & 0.00 & 5.33 & 5.33 & 1.00 \\
\hline 150 & 75 & 0.14 & 0.00 & 0.14 & 0.00 & 13.25 & 13.18 & 0.99 \\
\hline 75 & 45 & 0.10 & 0.00 & 0.10 & 0.00 & 9.70 & 9.63 & 0.99 \\
\hline 45 & 25 & 0.05 & 0.00 & 0.05 & 0.00 & 5.15 & 5.09 & 0.99 \\
\hline 25 & 13.1 & 0.07 & 0.03 & 0.10 & 0.00 & 6.91 & 4.38 & 0.73 \\
\hline 13.1 & 6.5 & 0.05 & 0.11 & 0.16 & 0.00 & 5.05 & -5.02 & 0.33 \\
\hline 6.5 & 3.3 & 0.04 & 0.12 & 0.16 & 0.00 & 4.14 & -7.29 & 0.27 \\
\hline 3.3 & 1.5 & 0.03 & 0.07 & 0.10 & 0.00 & 2.73 & -4.36 & 0.28 \\
\hline 1.5 & & 0.03 & 0.09 & 0.12 & 0.00 & 2.72 & -5.48 & 0.25 \\
\hline Totals & -- & 0.64 & 0.42 & 1.05 & 0.00 & & & \\
\hline \multicolumn{2}{|c|}{ Slurry (TPH) } & 12.42 & 16.73 & 25.40 & 0.00 & & & \\
\hline
\end{tabular}




\begin{tabular}{|c|c|c|c|c|c|c|c|}
\hline \multirow{3}{*}{$\begin{array}{l}\text { Experimental } \\
\text { Partition F actor }\end{array}$} & \multirow{3}{*}{$\begin{array}{c}\text { Mean } \\
\text { Particle } \\
\text { Size ( } \mu \mathrm{m})\end{array}$} & \multirow{3}{*}{$\begin{array}{c}\text { Predicted } \\
\text { Partition } \\
\text { Factor }\end{array}$} & \multirow{3}{*}{$\begin{array}{c}\text { Predicted } \\
\text { Partition } \\
\text { Factor }\end{array}$} & (Plitt) & Predicted & (Linch) & Predicted \\
\hline & & & & D50 & 21.53 & D50 & 21.39 \\
\hline & & & & $m$ & 2.20 & Alpha & 2.86 \\
\hline$(-)$ & $(-)$ & (Plitt) & (Linch) & Bypass(Rt) & 0.48 & Bypass(Rf) & 0.47 \\
\hline 1.00 & 299.81 & 1.000 & 1.000 & & & & \\
\hline 1.00 & 178.33 & 1.000 & 1.000 & & & & \\
\hline 0.97 & 106.07 & 1.000 & 1.000 & & & & \\
\hline 0.94 & 58.09 & 0.999 & 0.996 & & & & \\
\hline 0.93 & 33.54 & 0.917 & 0.916 & & & & \\
\hline 0.66 & 18.08 & 0.676 & 0.673 & & & & \\
\hline 0.54 & 9.25 & 0.534 & 0.539 & & & & \\
\hline 0.51 & 4.62 & 0.493 & 0.496 & & & & \\
\hline 0.48 & 2.32 & 0.484 & 0.482 & & & & \\
\hline 0.47 & 1.64 & 0.482 & 0.478 & & & & \\
\hline & WSSQ & 0.005 & 0.004 & & & & \\
\hline & TWSSQ & 0.009 & & & & & \\
\hline
\end{tabular}

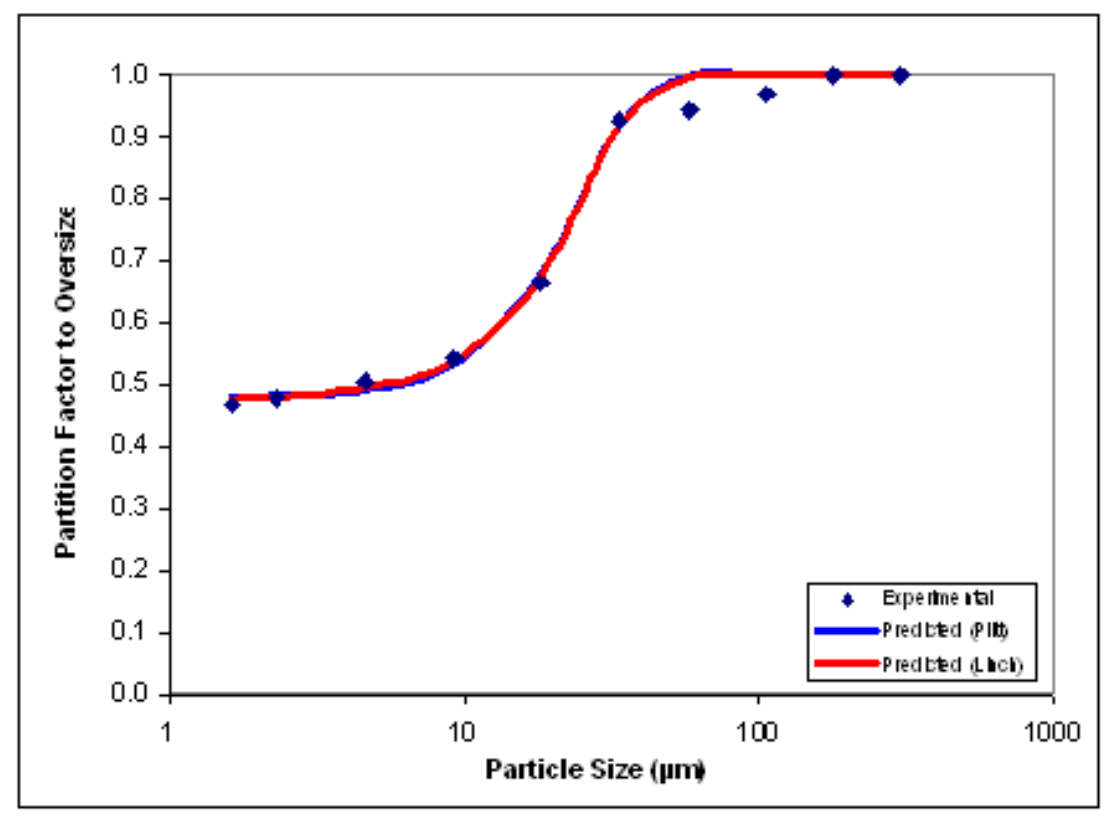




\section{HYDROCYCLONE EVALUATION (UNBALANCED)}

\begin{tabular}{|c|c|}
\hline No. 11 & \\
\hline Feedflow (GPM) & 100.0 \\
\hline Feed Pressure (PSl) & 21.0 \\
\hline Water Flow (GPM) & 15.0 \\
\hline Water Pressure (PSl) & 12.0 \\
\hline Feed Rate (TPH) & 1.21 \\
\hline
\end{tabular}

\begin{tabular}{|r|c|c|c|}
\hline & OS & U/S & Feed \\
\hline Solids Content (\%) & 7.02 & 2.66 & 4.74 \\
\hline Ash Content (\%) & 23.18 & 48.59 & 33.80 \\
\hline Solids Yield (\%) & 57.3 & 42.7 & 100.0 \\
\hline Ash Yield (\%) & 58.2 & 41.8 & 100.0 \\
\hline Size Class Yield (\%) & 66.2 & 33.8 & 100.0 \\
\hline
\end{tabular}

\begin{tabular}{|c|c|c|c|c|c|c|c|c|}
\hline $\begin{array}{c}\text { Pass } \\
\text { Size } \\
(u m)\end{array}$ & $\begin{array}{c}\text { Retain } \\
\text { Size } \\
(u m)\end{array}$ & $\begin{array}{c}\text { O/S } \\
\text { Sample } \\
(\mathrm{gms})\end{array}$ & $\begin{array}{c}\text { U/S } \\
\text { Sample } \\
(\mathrm{gms})\end{array}$ & $\begin{array}{c}\text { O/S } \\
\text { Mass } \\
(\%, \text { strm) }\end{array}$ & $\begin{array}{c}\text { U/S } \\
\text { Mass } \\
(\%, \text { strm) }\end{array}$ & $\begin{array}{c}\text { OS } \\
\text { Mass } \\
(\%, \text { feed })\end{array}$ & $\begin{array}{c}\text { U/S } \\
\text { Mass } \\
(\%, \text { feed })\end{array}$ & $\begin{array}{c}\text { Feed } \\
\text { Mass } \\
(\%, \text { feed })\end{array}$ \\
\hline & 212 & 1.95 & 0.00 & 9.31 & 0.00 & 5.42 & 0.00 & 5.42 \\
\hline 212 & 150 & 1.96 & 0.00 & 9.36 & 0.00 & 5.45 & 0.00 & 5.45 \\
\hline 150 & 75 & 4.99 & 0.18 & 23.82 & 1.00 & 13.86 & 0.42 & 14.28 \\
\hline 75 & 45 & 3.69 & 0.42 & 17.61 & 2.34 & 10.25 & 0.98 & 11.23 \\
\hline 45 & 25 & 1.49 & 0.54 & 7.11 & 3.01 & 4.14 & 1.26 & 5.40 \\
\hline 25 & 13.1 & 2.22 & 2.88 & 10.59 & 16.03 & 6.16 & 6.70 & 12.86 \\
\hline 13.1 & 6.5 & 1.61 & 3.96 & 7.68 & 22.07 & 4.47 & 9.22 & 13.69 \\
\hline 6.5 & 3.3 & 1.37 & 4.06 & 6.54 & 22.61 & 3.81 & 9.45 & 13.26 \\
\hline 3.3 & 1.5 & 0.86 & 3.07 & 4.11 & 17.09 & 2.39 & 7.14 & 9.54 \\
\hline 1.5 & & 0.81 & 2.85 & 3.87 & 15.85 & 2.25 & 6.63 & 8.88 \\
\hline Totals & -- & 20.95 & 17.96 & 100.00 & 100.00 & 58.21 & 41.79 & 100.00 \\
\hline
\end{tabular}

\begin{tabular}{|c|c|c|c|c|c|c|c|c|}
\hline $\begin{array}{c}\text { Pass } \\
\text { Size } \\
\text { (um) }\end{array}$ & $\begin{array}{c}\text { Retain } \\
\text { Size } \\
\text { (um) }\end{array}$ & $\begin{array}{c}\text { OSS } \\
\text { Mass } \\
(\mathrm{TPH})\end{array}$ & $\begin{array}{c}\text { U/S } \\
\text { Mass } \\
\text { (TPH) }\end{array}$ & $\begin{array}{l}\text { Feed } \\
\text { Mass } \\
\text { (TPH) }\end{array}$ & $\begin{array}{l}\text { Delta } \\
\text { Mass } \\
\text { (TPH) }\end{array}$ & $\begin{array}{c}Y \\
\text { Value } \\
(f-u)\end{array}$ & $\begin{array}{c}X \\
\text { Value } \\
(0-u)\end{array}$ & $\begin{array}{c}\text { Partition } \\
\text { Factor } \\
(--)\end{array}$ \\
\hline & 212 & 0.07 & 0.00 & 0.07 & 0.00 & 5.42 & 5.42 & 1.00 \\
\hline 212 & 150 & 0.07 & 0.00 & 0.07 & 0.00 & 5.45 & 5.45 & 1.00 \\
\hline 150 & 75 & 0.17 & 0.01 & 0.17 & 0.00 & 13.86 & 13.44 & 0.97 \\
\hline 75 & 45 & 0.12 & 0.01 & $\overline{0.14}$ & 0.00 & 10.25 & 9.27 & 0.91 \\
\hline 45 & 25 & 0.05 & 0.02 & 0.07 & 0.00 & 4.14 & 2.88 & 0.77 \\
\hline 25 & 13.1 & 0.07 & 0.08 & 0.16 & 0.00 & 6.16 & -0.53 & 0.48 \\
\hline 13.1 & 6.5 & 0.05 & 0.11 & 0.17 & 0.00 & 4.47 & -4.76 & 0.33 \\
\hline 6.5 & 3.3 & 0.05 & 0.11 & 0.16 & 0.00 & 3.81 & -5.64 & 0.29 \\
\hline 3.3 & 1.5 & 0.03 & 0.09 & 0.12 & 0.00 & 2.39 & -4.75 & 0.25 \\
\hline 1.5 & & 0.03 & 0.08 & 0.11 & 0.00 & 2.25 & -4.37 & 0.25 \\
\hline Totals & -- & 0.70 & 0.50 & 1.21 & 0.00 & & & \\
\hline \multicolumn{2}{|c|}{ Slurry (TPH) } & 10.00 & 18.95 & 25.45 & 0.24 & & & \\
\hline
\end{tabular}


HYDROCYCLONE EVALUATION (BALANCED)

\begin{tabular}{|c|c|}
\hline No. 11 & \\
\hline Feedflow (GPM) & 1000 \\
\hline Feed Pressure (PSI) & 21.0 \\
\hline Water Flow (GPM) & 15.0 \\
\hline Water Pressure (PSl) & 12.0 \\
\hline Feed Rate (TPH) & 1.16 \\
\hline
\end{tabular}

\begin{tabular}{|r|c|c|c|}
\hline & OS & U/S & Feed \\
\hline Solids Content (\%) & 7.16 & 2.51 & 4.55 \\
\hline Ash Content (\%) & 23.01 & 48.46 & 34.05 \\
\hline Solids Yield (\%) & 56.6 & 43.4 & 100.0 \\
\hline Ash Yield (\%) & 56.6 & 43.4 & 100.0 \\
\hline Size Class Yield (\%) & 56.6 & 43.4 & 100.0 \\
\hline
\end{tabular}

\begin{tabular}{|c|c|c|c|c|c|c|c|c|}
\hline $\begin{array}{l}\text { Pass } \\
\text { Size } \\
\text { (um) }\end{array}$ & $\begin{array}{l}\text { Retain } \\
\text { Size } \\
\text { (um) }\end{array}$ & $\begin{array}{c}\text { O/S } \\
\text { Sample } \\
\text { (gms) }\end{array}$ & $\begin{array}{c}\text { U/S } \\
\text { Sample } \\
\text { (gms) }\end{array}$ & $\begin{array}{c}\text { orS } \\
\text { Mass } \\
(\% \text {, strm })\end{array}$ & $\begin{array}{c}\text { U/S } \\
\text { Mass } \\
(\% \text {, strm) }\end{array}$ & $\begin{array}{c}\text { OS } \\
\text { Mass } \\
(\% \text {, feed })\end{array}$ & $\begin{array}{c}\text { U/S } \\
\text { Mass } \\
(\%, \text { feed })\end{array}$ & $\begin{array}{c}\text { Feed } \\
\text { Mass } \\
(\% \text {, feed })\end{array}$ \\
\hline & 212 & 2.00 & 0.00 & 9.45 & 0.00 & 5.35 & 0.00 & 5.35 \\
\hline 212 & 150 & 2.02 & 0.00 & 9.52 & 0.00 & 5.39 & 0.00 & 5.39 \\
\hline 150 & 75 & 4.91 & 0.00 & 23.15 & 0.00 & $\overline{13.11}$ & 0.00 & 13.11 \\
\hline 75 & 45 & 3.66 & 0.21 & 17.28 & 1.22 & 9.78 & 0.53 & 10.31 \\
\hline 45 & 25 & 1.56 & 0.37 & 7.36 & 2.14 & 4.17 & 0.93 & 5.10 \\
\hline 25 & 13.1 & 2.22 & 2.84 & 10.49 & 16.30 & 5.94 & 7.07 & 13.01 \\
\hline 13.1 & 6.5 & 1.62 & 4.00 & 7.66 & 22.96 & 4.34 & 9.96 & 14.30 \\
\hline 6.5 & 3.3 & 1.40 & 4.11 & 6.58 & 23.57 & 3.73 & 10.22 & 13.95 \\
\hline 3.3 & 1.5 & 0.92 & 3.07 & 4.36 & 17.58 & 2.47 & 7.63 & 10.10 \\
\hline 1.5 & & 0.88 & 2.83 & 4.15 & 16.23 & 2.35 & 7.04 & 9.39 \\
\hline Totals & -- & 21.20 & 17.44 & 100.00 & 100.00 & 56.62 & 43.38 & 100.00 \\
\hline
\end{tabular}

\begin{tabular}{|c|c|c|c|c|c|c|c|c|}
\hline $\begin{array}{c}\text { Pass } \\
\text { Size } \\
(u m)\end{array}$ & $\begin{array}{c}\text { Retain } \\
\text { Size } \\
(u m)\end{array}$ & $\begin{array}{c}0 / S \\
\text { Mass } \\
(\mathrm{TPH})\end{array}$ & $\begin{array}{c}\text { U/S } \\
\text { Mass } \\
(\mathrm{TPH})\end{array}$ & $\begin{array}{c}\text { Feed } \\
\text { Mass } \\
(\mathrm{TPH})\end{array}$ & $\begin{array}{c}\text { Delta } \\
\text { Mass } \\
(\mathrm{TPH})\end{array}$ & $\begin{array}{c}\text { Value } \\
\text { (f-u) }\end{array}$ & $\begin{array}{c}\text { X } \\
\text { Value } \\
(0-\mathrm{u})\end{array}$ & $\begin{array}{c}\text { Partition } \\
\text { Factor } \\
(-)\end{array}$ \\
\hline & 212 & 0.06 & 0.00 & 0.06 & 0.00 & 5.35 & 5.35 & 1.00 \\
\hline 212 & 150 & 0.06 & 0.00 & 0.06 & 0.00 & 5.39 & 5.39 & 1.00 \\
\hline 150 & 75 & 0.15 & 0.00 & 0.15 & 0.00 & 13.11 & 13.11 & 1.00 \\
\hline 75 & 45 & 0.11 & 0.01 & 0.12 & 0.00 & 9.78 & 9.26 & 0.95 \\
\hline 45 & 25 & 0.05 & 0.01 & 0.06 & 0.00 & 4.17 & 3.24 & 0.82 \\
\hline 25 & 13.1 & 0.07 & 0.08 & 0.15 & 0.00 & 5.94 & -1.14 & 0.46 \\
\hline 13.1 & 6.5 & 0.05 & 0.12 & 0.17 & 0.00 & 4.34 & -5.62 & 0.30 \\
\hline 6.5 & 3.3 & 0.04 & 0.12 & 0.16 & 0.00 & 3.73 & -6.50 & 0.27 \\
\hline 3.3 & 1.5 & 0.03 & 0.09 & 0.12 & 0.00 & 2.47 & -5.16 & 0.24 \\
\hline 1.5 & & 0.03 & 0.08 & 0.11 & 0.00 & 2.35 & -4.69 & 0.25 \\
\hline Totals & -- & 0.66 & 0.50 & 1.16 & 0.00 & & & \\
\hline & Slurry (TPH) & 9.16 & 20.02 & 25.43 & 0.00 & & & \\
\hline
\end{tabular}




\begin{tabular}{|c|c|c|c|}
\hline $\begin{array}{c}\text { Experimental } \\
\text { Partition Factor }\end{array}$ & $\begin{array}{c}\text { Mean } \\
\text { Particle } \\
\text { Size }(\mu \mathrm{m})\end{array}$ & $\begin{array}{c}\text { Predicted } \\
\text { Partition } \\
\text { Factor }\end{array}$ & $\begin{array}{c}\text { Predicted } \\
\text { Partition } \\
\text { Factor }\end{array}$ \\
\hline$(--)$ & $(--)$ & (Plitt) & (Linch) \\
\hline 1.00 & 299.81 & 1.000 & 1.000 \\
\hline 1.00 & 178.33 & 1.000 & 1.000 \\
\hline 0.97 & 106.07 & 0.999 & 0.998 \\
\hline 0.91 & 58.09 & 0.940 & 0.945 \\
\hline 0.77 & 33.54 & 0.739 & 0.741 \\
\hline 0.48 & 18.08 & 0.492 & 0.488 \\
\hline 0.33 & 9.25 & 0.340 & 0.344 \\
\hline 0.29 & 4.62 & 0.276 & 0.280 \\
\hline 0.25 & 2.32 & 0.254 & 0.252 \\
\hline 0.25 & 1.64 & 0.249 & 0.245 \\
\hline & $W S S Q$ & 0.003 & 0.003 \\
\hline
\end{tabular}

\begin{tabular}{|c|c|c|c|}
\hline (Plitt) & Predicted & (Linch) & Predicted \\
\hline D50 & 25.55 & D50 & 25.33 \\
\hline$m$ & 1.58 & Alpha & 1.87 \\
\hline Bypass(Rf) & 0.24 & Bypass(Rf) & 0.23 \\
\hline
\end{tabular}

\begin{tabular}{l|l}
\hline TWSSQ & 0.006
\end{tabular}

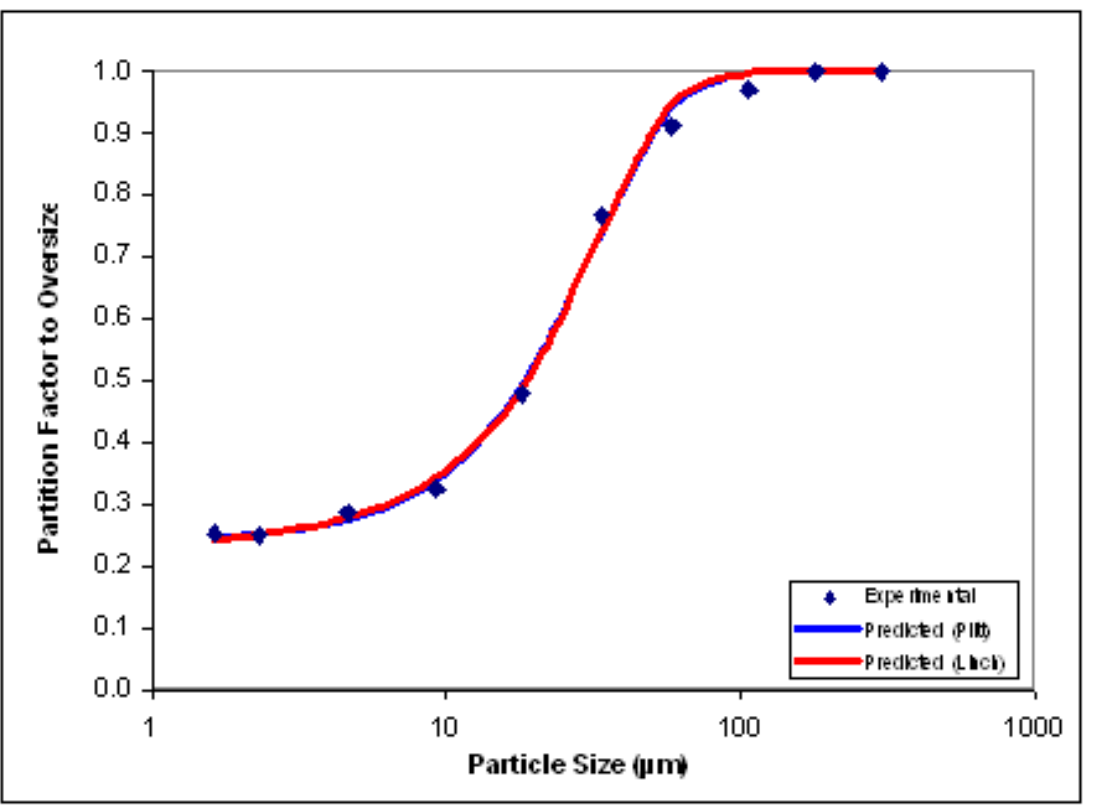


HYDROCYCLONE EVALUATION (UNBALANCED)

\begin{tabular}{|c|c|}
\hline No. 12 & \\
\hline FeedFlow (GPM) & 100.0 \\
\hline Feed Pressure (PSI) & 21.0 \\
\hline Water Flow (GPM) & 15.0 \\
\hline Water Pressure (PSl) & 12.0 \\
\hline Feed Rate (TPH) & 1.18 \\
\hline
\end{tabular}

\begin{tabular}{|r|c|c|c|}
\hline & OS & U/S & Feed \\
\hline Solids Content (\%) & 7.34 & 2.65 & 4.63 \\
\hline Ash Content (\%) & 23.26 & 48.38 & 34.37 \\
\hline Solids Yield (\%) & 53.7 & 46.3 & 100.0 \\
\hline Ash Yield (\%) & 55.8 & 44.2 & 100.0 \\
\hline Size Class Yield (\%) & 60.7 & 39.3 & 100.0 \\
\hline
\end{tabular}

\begin{tabular}{|c|c|c|c|c|c|c|c|c|}
\hline $\begin{array}{c}\text { Pass } \\
\text { Size } \\
(u m)\end{array}$ & $\begin{array}{c}\text { Retain } \\
\text { Size } \\
(\mathrm{um})\end{array}$ & $\begin{array}{c}\mathrm{O} / \mathrm{S} \\
\text { Sample } \\
(\mathrm{gms})\end{array}$ & $\begin{array}{c}\mathrm{U} / \mathrm{S} \\
\text { Sample } \\
(\mathrm{gms})\end{array}$ & $\begin{array}{c}\mathrm{O} / \mathrm{S} \\
\text { Mass } \\
(\%, \text { strm) }\end{array}$ & $\begin{array}{c}\text { U/S } \\
\text { Mass } \\
(\%, \text { strm) }\end{array}$ & $\begin{array}{c}\text { OS } \\
\text { Mass } \\
(\%, \text { feed })\end{array}$ & $\begin{array}{c}\text { U/S } \\
\text { Mass } \\
(\%, \text { feed })\end{array}$ & $\begin{array}{c}\text { Feed } \\
\text { Mass } \\
(\%, \text { feed })\end{array}$ \\
\hline & 212 & 1.82 & 0.00 & 8.78 & 0.00 & 4.90 & 0.00 & 4.90 \\
\hline 212 & 150 & 1.88 & 0.00 & 9.07 & 0.00 & 5.06 & 0.00 & 5.06 \\
\hline 150 & 75 & 5.24 & 0.20 & 25.29 & 1.12 & 14.10 & 0.50 & 14.60 \\
\hline 75 & 45 & 3.78 & 0.41 & 18.24 & 2.31 & 10.17 & 1.02 & 11.19 \\
\hline 45 & 25 & 1.51 & 0.72 & 7.29 & 4.05 & 4.06 & 1.79 & 5.86 \\
\hline 25 & 13.1 & 2.28 & 3.15 & 10.98 & 17.74 & 6.12 & 7.85 & 13.97 \\
\hline 13.1 & 6.5 & 1.64 & 3.86 & 7.92 & 21.71 & 4.42 & 9.60 & 14.02 \\
\hline 6.5 & 3.3 & 1.26 & 3.95 & 6.06 & 22.19 & 3.38 & 9.81 & 13.19 \\
\hline 3.3 & 1.5 & 0.72 & 2.72 & 3.49 & 15.30 & 1.95 & 6.77 & 8.71 \\
\hline 1.5 & & 0.60 & 2.77 & 2.87 & 15.58 & 1.60 & 6.89 & 8.49 \\
\hline Totals & -- & 20.72 & 17.78 & 100.00 & 100.00 & 55.77 & 44.23 & 100.00 \\
\hline
\end{tabular}

\begin{tabular}{|c|c|c|c|c|c|c|c|c|}
\hline $\begin{array}{c}\text { Pass } \\
\text { Size } \\
(u m)\end{array}$ & $\begin{array}{c}\text { Retain } \\
\text { Size } \\
(u m)\end{array}$ & $\begin{array}{c}0 / 5 \\
\text { Mass } \\
(\mathrm{TPH})\end{array}$ & $\begin{array}{c}\text { U/S } \\
\text { Mass } \\
(\mathrm{TPH})\end{array}$ & $\begin{array}{c}\text { Feed } \\
\text { Mass } \\
(\mathrm{TPH})\end{array}$ & $\begin{array}{c}\text { Delta } \\
\text { Mass } \\
(\mathrm{TPH})\end{array}$ & $\begin{array}{c}\text { Value } \\
\text { (f-u) }\end{array}$ & $\begin{array}{c}\text { X } \\
\text { Value } \\
(\mathrm{0}-\mathrm{u})\end{array}$ & $\begin{array}{c}\text { Partition } \\
\text { Factor } \\
(-)\end{array}$ \\
\hline & 212 & 0.06 & 0.00 & 0.06 & 0.00 & 4.90 & 4.90 & 1.00 \\
\hline 212 & 150 & 0.06 & 0.00 & 0.06 & 0.00 & 5.06 & 5.06 & 1.00 \\
\hline 150 & 75 & 0.17 & 0.01 & 0.17 & 0.00 & 14.10 & 13.61 & 0.97 \\
\hline 75 & 45 & 0.12 & 0.01 & 0.13 & 0.00 & 10.17 & 9.15 & 0.91 \\
\hline 45 & 25 & 0.05 & 0.02 & 0.07 & 0.00 & 4.06 & 2.27 & 0.69 \\
\hline 25 & 13.1 & 0.07 & 0.09 & 0.16 & 0.00 & 6.12 & -1.72 & 0.44 \\
\hline 13.1 & 6.5 & 0.05 & 0.11 & 0.17 & 0.00 & 4.42 & -5.19 & 0.31 \\
\hline 6.5 & 3.3 & 0.04 & 0.12 & 0.16 & 0.00 & 3.38 & -6.44 & 0.26 \\
\hline 3.3 & 1.5 & 0.02 & 0.08 & 0.10 & 0.00 & 1.95 & -4.82 & 0.22 \\
\hline 1.5 & & 0.02 & 0.08 & 0.10 & 0.00 & 1.60 & -5.29 & 0.19 \\
\hline Totals & -- & 0.66 & 0.52 & 1.18 & 0.00 & & & \\
\hline & Slurry (TPH) & 8.95 & 19.66 & 25.44 & 0.58 & & & \\
\hline
\end{tabular}




\section{HYDROCYCLONE EVALUATION (BALANCED)}

\begin{tabular}{|c|c|}
\hline No. 12 & \\
\hline FeedFlow (GPM) & 100.0 \\
\hline Feed Pressure (PSl) & 21.0 \\
\hline Water Flow (GPM) & 15.0 \\
\hline Water Pressure (PSl) & 12.0 \\
\hline Feed Rate (TPH) & 1.15 \\
\hline
\end{tabular}

\begin{tabular}{|r|c|c|c|}
\hline & OS & U/S & Feed \\
\hline Solids Content (\%) & 7.43 & 2.51 & 4.52 \\
\hline Ash Content (\%) & 23.15 & 48.37 & 34.51 \\
\hline Solids Yield (\%) & 54.9 & 45.1 & 100.0 \\
\hline Ash Yield (\%) & 54.9 & 45.1 & 100.0 \\
\hline Size Class Yield (\%) & 54.9 & 45.1 & 100.0 \\
\hline
\end{tabular}

\begin{tabular}{|c|c|c|c|c|c|c|c|c|}
\hline $\begin{array}{l}\text { Pass } \\
\text { Size } \\
\text { (um) }\end{array}$ & $\begin{array}{c}\text { Retain } \\
\text { Size } \\
\text { (um) }\end{array}$ & $\begin{array}{c}\text { O/S } \\
\text { Sample } \\
\text { (gms) }\end{array}$ & $\begin{array}{c}\text { U/S } \\
\text { Sample } \\
\text { (gms) }\end{array}$ & $\begin{array}{c}\text { orS } \\
\text { Mass } \\
(\% \text {, strm })\end{array}$ & $\begin{array}{c}\text { U/S } \\
\text { Mass } \\
(\% \text {, strm) }\end{array}$ & $\begin{array}{c}\text { OS } \\
\text { Mass } \\
(\% \text {, feed })\end{array}$ & $\begin{array}{c}\text { U/S } \\
\text { Mass } \\
(\%, \text { feed })\end{array}$ & $\begin{array}{c}\text { Feed } \\
\text { Mass } \\
(\% \text {, feed })\end{array}$ \\
\hline & 212 & 1.86 & 0.00 & 8.89 & 0.00 & 4.89 & 0.00 & 4.89 \\
\hline 212 & 150 & 1.92 & 0.00 & 9.17 & 0.00 & 5.04 & 0.00 & 5.04 \\
\hline 150 & 75 & 5.18 & 0.05 & 24.70 & 0.31 & 13.57 & 0.14 & 13.71 \\
\hline 75 & 45 & 3.76 & 0.28 & 17.94 & 1.62 & 9.86 & 0.73 & 10.59 \\
\hline 45 & 25 & 1.56 & 0.62 & 7.44 & 3.56 & 4.09 & 1.60 & 5.69 \\
\hline 25 & 13.1 & 2.28 & 3.15 & 10.90 & 18.05 & 5.99 & 8.13 & 14.12 \\
\hline 13.1 & 6.5 & 1.66 & 3.89 & 7.94 & 22.29 & 4.36 & 10.04 & 14.41 \\
\hline 6.5 & 3.3 & 1.29 & 3.98 & 6.16 & 22.82 & 3.38 & 10.28 & 13.66 \\
\hline 3.3 & 1.5 & 0.78 & 2.71 & 3.74 & 15.52 & 2.05 & 6.99 & 9.05 \\
\hline 1.5 & & 0.66 & 2.76 & 3.14 & 15.83 & 1.73 & 7.13 & 8.86 \\
\hline Totals & -- & 20.96 & 17.44 & 100.00 & 100.00 & 54.95 & 45.05 & 100.00 \\
\hline
\end{tabular}

\begin{tabular}{|c|c|c|c|c|c|c|c|c|}
\hline $\begin{array}{c}\text { Pass } \\
\text { Size } \\
(u m)\end{array}$ & $\begin{array}{c}\text { Retain } \\
\text { Size } \\
(u m)\end{array}$ & $\begin{array}{c}0 / S \\
\text { Mass } \\
(\mathrm{TPH})\end{array}$ & $\begin{array}{c}\text { U/S } \\
\text { Mass } \\
(\mathrm{TPH})\end{array}$ & $\begin{array}{c}\text { Feed } \\
\text { Mass } \\
(\mathrm{TPH})\end{array}$ & $\begin{array}{c}\text { Delta } \\
\text { Mass } \\
(\mathrm{TPH})\end{array}$ & $\begin{array}{c}\text { Value } \\
\text { (f-u) }\end{array}$ & $\begin{array}{c}\text { X } \\
\text { Value } \\
(\mathrm{0}-\mathrm{u})\end{array}$ & $\begin{array}{c}\text { Partition } \\
\text { Factor } \\
(-)\end{array}$ \\
\hline & 212 & 0.06 & 0.00 & 0.06 & 0.00 & 4.89 & 4.89 & 1.00 \\
\hline 212 & 150 & 0.06 & 0.00 & 0.06 & 0.00 & 5.04 & 5.04 & 1.00 \\
\hline 150 & 75 & 0.16 & 0.00 & 0.16 & 0.00 & 13.57 & 13.43 & 0.99 \\
\hline 75 & 45 & 0.11 & 0.01 & 0.12 & 0.00 & 9.86 & 9.13 & 0.93 \\
\hline 45 & 25 & 0.05 & 0.02 & 0.07 & 0.00 & 4.09 & 2.48 & 0.72 \\
\hline 25 & 13.1 & 0.07 & 0.09 & 0.16 & 0.00 & 5.99 & -2.15 & 0.42 \\
\hline 13.1 & 6.5 & 0.05 & 0.12 & 0.17 & 0.00 & 4.36 & -5.68 & 0.30 \\
\hline 6.5 & 3.3 & 0.04 & 0.12 & 0.16 & 0.00 & 3.38 & -6.90 & 0.25 \\
\hline 3.3 & 1.5 & 0.02 & 0.08 & 0.10 & 0.00 & 2.05 & -4.94 & 0.23 \\
\hline 1.5 & & 0.02 & 0.08 & 0.10 & 0.00 & 1.73 & -5.40 & 0.19 \\
\hline Totals & -- & 0.63 & 0.52 & 1.15 & 0.00 & & & \\
\hline & Slurry (TPH) & 8.51 & 20.67 & 25.43 & 0.00 & & & \\
\hline
\end{tabular}




\begin{tabular}{|c|c|c|c|}
\hline $\begin{array}{c}\text { Experimental } \\
\text { Partition F actor }\end{array}$ & $\begin{array}{c}\text { Mean } \\
\text { Particle } \\
\text { Size }(\mu \mathrm{m})\end{array}$ & $\begin{array}{c}\text { Predicted } \\
\text { Partition } \\
\text { Factor }\end{array}$ & $\begin{array}{c}\text { Predicted } \\
\text { Partition } \\
\text { Factor }\end{array}$ \\
\hline$(-)$ & $(--)$ & (Plitt) & (Linch) \\
\hline 1.00 & 299.81 & 1.000 & 1.000 \\
\hline 1.00 & 178.33 & 1.000 & 1.000 \\
\hline 0.97 & 106.07 & 0.995 & 0.994 \\
\hline 0.91 & 58.09 & 0.903 & 0.908 \\
\hline 0.69 & 33.54 & 0.689 & 0.687 \\
\hline 0.44 & 18.08 & 0.455 & 0.451 \\
\hline 0.31 & 9.25 & 0.307 & 0.311 \\
\hline 0.26 & 4.62 & 0.241 & 0.245 \\
\hline 0.22 & 2.32 & 0.215 & 0.214 \\
\hline 0.19 & 1.64 & 0.210 & 0.206 \\
\hline & MSSQ & 0.002 & 0.002 \\
\hline
\end{tabular}

\begin{tabular}{|c|c|c|c|}
\hline (Plitt) & Predicted & (Linch) & Predicted \\
\hline D50 & 27.15 & D50 & 26.99 \\
\hline$m$ & 1.46 & Alpha & 1.62 \\
\hline Bypass(Rt) & 0.20 & Bypass(Rf) & 0.19 \\
\hline
\end{tabular}

\begin{tabular}{l|l}
\hline TWSSQ & 0.003 \\
\hline
\end{tabular}

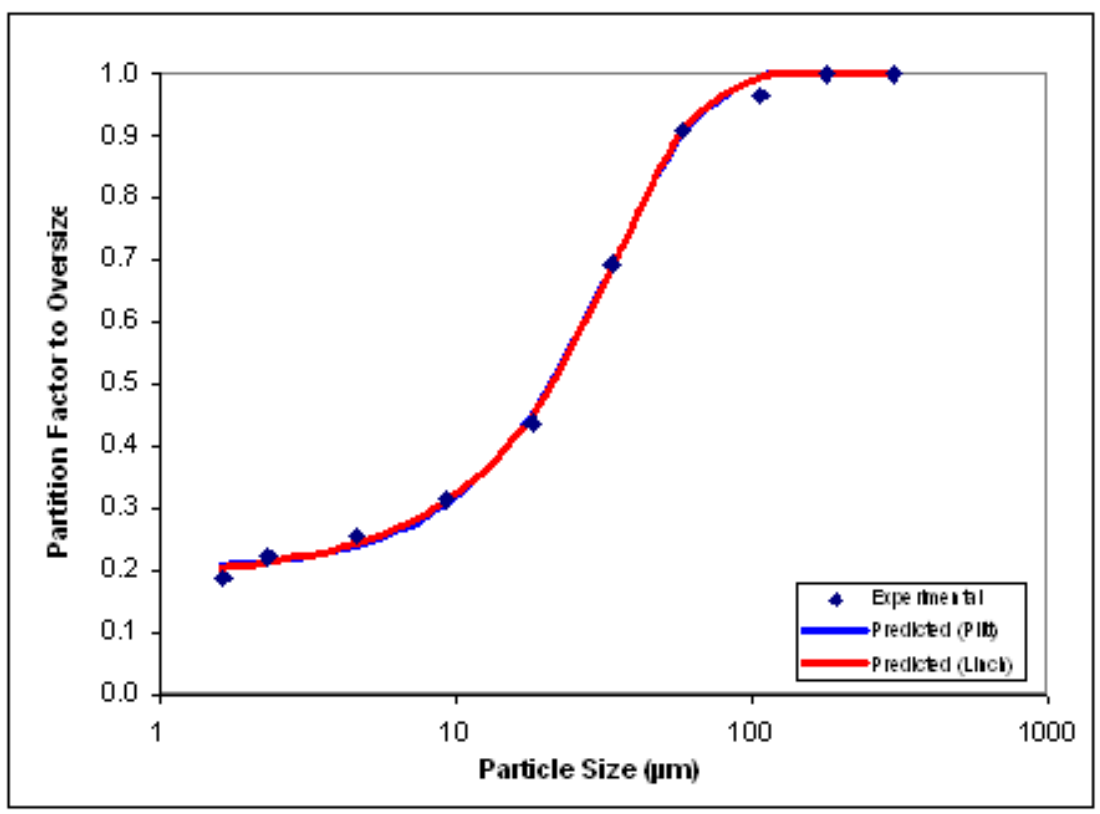




\section{HYDROCYCLONE EVALUATION (UNBALANCED)}

\begin{tabular}{|c|c|}
\hline No.13 & \\
\hline Feedflow (GPM) & 100.0 \\
\hline Feed Pressure (PSl) & 21.0 \\
\hline Water Flow (GPM) & 0.0 \\
\hline Water Pressure (PSl) & 0.0 \\
\hline Feed Rate (TPH) & 1.18 \\
\hline
\end{tabular}

\begin{tabular}{|r|c|c|c|}
\hline & OS & US & Feed \\
\hline Solids Content (\%) & 11.23 & 2.73 & 4.65 \\
\hline Ash Content (\%) & 23.84 & 48.60 & 34.28 \\
\hline Solids Yield (\%) & 54.6 & 45.4 & 100.0 \\
\hline Ash Yield (\%) & 57.8 & 42.2 & 100.0 \\
\hline Size Class Yield (\%) & 67.2 & 32.8 & 100.0 \\
\hline
\end{tabular}

\begin{tabular}{|c|c|c|c|c|c|c|c|c|}
\hline $\begin{array}{c}\text { Pass } \\
\text { Size } \\
(u m)\end{array}$ & $\begin{array}{c}\text { Retain } \\
\text { Size } \\
(u m)\end{array}$ & $\begin{array}{c}\text { O/S } \\
\text { Sample } \\
(\mathrm{gms})\end{array}$ & $\begin{array}{c}\text { U/S } \\
\text { Sample } \\
(\mathrm{gms})\end{array}$ & $\begin{array}{c}\text { O/S } \\
\text { Mass } \\
(\%, \text { strm) }\end{array}$ & $\begin{array}{c}\text { U/S } \\
\text { Mass } \\
(\%, \text { strm) }\end{array}$ & $\begin{array}{c}\text { OSS } \\
\text { Mass } \\
(\%, \text { feed) })\end{array}$ & $\begin{array}{c}\text { U/S } \\
\text { Mass } \\
(\%, \text { feed) })\end{array}$ & $\begin{array}{c}\text { Feed } \\
\text { Mass } \\
(\%, \text { feed })\end{array}$ \\
\hline & 212 & 1.25 & 0.00 & 7.79 & 0.00 & 4.50 & 0.00 & 4.50 \\
\hline 212 & 150 & 1.42 & 0.00 & 8.85 & 0.00 & 5.12 & 0.00 & 5.12 \\
\hline 150 & 75 & 4.18 & 0.16 & 26.04 & 0.86 & 15.06 & 0.36 & 15.43 \\
\hline 75 & 45 & 2.91 & 0.39 & 18.13 & 2.10 & 10.49 & 0.89 & 11.37 \\
\hline 45 & 25 & 1.21 & 0.67 & 7.54 & 3.62 & 4.36 & 1.52 & 5.88 \\
\hline 25 & 13.1 & 1.77 & 3.38 & 11.02 & 18.24 & 6.37 & 7.69 & 14.07 \\
\hline 13.1 & 6.5 & 1.24 & 4.18 & 7.75 & 22.56 & 4.48 & 9.51 & 14.00 \\
\hline 6.5 & 3.3 & 0.98 & 4.15 & 6.10 & 22.40 & 3.53 & 9.45 & 12.97 \\
\hline 3.3 & 1.5 & 0.57 & 2.72 & 3.54 & 14.67 & 2.05 & 6.19 & 8.23 \\
\hline 1.5 & & 0.52 & 2.88 & 3.24 & 15.54 & 1.87 & 6.55 & 8.43 \\
\hline Totals & -- & 16.05 & 18.53 & 100.00 & 100.00 & 57.84 & 42.16 & 100.00 \\
\hline
\end{tabular}

\begin{tabular}{|c|c|c|c|c|c|c|c|c|}
\hline $\begin{array}{l}\text { Pass } \\
\text { Size } \\
\text { (um) }\end{array}$ & $\begin{array}{c}\text { Retain } \\
\text { Size } \\
\text { (um) }\end{array}$ & $\begin{array}{c}\text { O/S } \\
\text { Mass } \\
(\mathrm{TPH})\end{array}$ & $\begin{array}{c}\text { U/S } \\
\text { Mass } \\
(\mathrm{TPH})\end{array}$ & $\begin{array}{l}\text { Feed } \\
\text { Mass } \\
\text { (TPH) }\end{array}$ & $\begin{array}{l}\text { Delta } \\
\text { Mass } \\
(\mathrm{TPH})\end{array}$ & $\begin{array}{c} \\
\text { Value } \\
(f-u)\end{array}$ & $\begin{array}{c}X \\
\text { Value } \\
(0-1)\end{array}$ & $\begin{array}{c}\text { Partition } \\
\text { Factor } \\
(--)\end{array}$ \\
\hline & 212 & 0.05 & 0.00 & 0.05 & 0.00 & 4.50 & 4.50 & 1.00 \\
\hline 212 & 150 & 0.06 & 0.00 & 0.06 & 0.00 & 5.12 & 5.12 & 1.00 \\
\hline 150 & 75 & 0.18 & 0.00 & 0.18 & 0.00 & 15.06 & 14.70 & 0.98 \\
\hline 75 & 45 & 0.12 & 0.01 & 0.13 & 0.00 & 10.49 & 9.60 & 0.92 \\
\hline 45 & 25 & 0.05 & 0.02 & 0.07 & 0.00 & 4.36 & 2.84 & 0.74 \\
\hline 25 & 13.1 & 0.08 & 0.09 & 0.17 & 0.00 & 6.37 & -1.32 & 0.45 \\
\hline 13.1 & 6.5 & 0.05 & $\overline{0.11}$ & 0.17 & 0.00 & 4.48 & -5.03 & 0.32 \\
\hline 6.5 & 3.3 & 0.04 & 0.11 & 0.15 & 0.00 & 3.53 & -5.92 & 0.27 \\
\hline 3.3 & 1.5 & 0.02 & 0.07 & 0.10 & 0.00 & 2.05 & -4.14 & 0.25 \\
\hline 1.5 & & 0.02 & 0.08 & 0.10 & 0.00 & 1.87 & -4.68 & 0.22 \\
\hline Totals & -- & 0.68 & 0.50 & 1.18 & 0.00 & & & \\
\hline \multicolumn{2}{|c|}{ Slurry (TPH) } & 6.09 & 18.27 & 25.44 & 1.08 & & & \\
\hline
\end{tabular}




\section{HYDROCYCLONE EVALUATION (BALANCED)}

\begin{tabular}{|c|c|}
\hline No.13 & \\
\hline Feedflow (GPF) & 100.0 \\
\hline Feed Pressure (PSl) & 21.0 \\
\hline Water Flow (GPM) & 0.0 \\
\hline Water Pressure (PSl) & 0.0 \\
\hline Feed Rate (TPH) & 1.20 \\
\hline
\end{tabular}

\begin{tabular}{|r|c|c|c|}
\hline & OS & US & Feed \\
\hline Solids Content (\%) & 11.22 & 2.65 & 4.71 \\
\hline Ash Content (\%) & 23.79 & 48.56 & 34.37 \\
\hline Solids Yield (\%) & 57.3 & 42.7 & 100.0 \\
\hline Ash Yield (\%) & 57.3 & 42.7 & 100.0 \\
\hline Size Class Yield (\%) & 57.3 & 42.7 & 100.0 \\
\hline
\end{tabular}

\begin{tabular}{|c|c|c|c|c|c|c|c|c|}
\hline $\begin{array}{c}\text { Pass } \\
\text { Size } \\
(u m)\end{array}$ & $\begin{array}{c}\text { Retain } \\
\text { Size } \\
(u m)\end{array}$ & $\begin{array}{c}\text { O/S } \\
\text { Sample } \\
(\mathrm{gms})\end{array}$ & $\begin{array}{c}\text { U/S } \\
\text { Sample } \\
(\mathrm{gms})\end{array}$ & $\begin{array}{c}\text { O/S } \\
\text { Mass } \\
(\%, \text { strm) }\end{array}$ & $\begin{array}{c}\text { U/S } \\
\text { Mass } \\
(\%, \text { strm) }\end{array}$ & $\begin{array}{c}\text { OSS } \\
\text { Mass } \\
(\%, \text { feed) })\end{array}$ & $\begin{array}{c}\text { U/S } \\
\text { Mass } \\
(\%, \text { feed) })\end{array}$ & $\begin{array}{c}\text { Feed } \\
\text { Mass } \\
(\%, \text { feed })\end{array}$ \\
\hline & 212 & 1.40 & 0.00 & 8.36 & 0.00 & 4.79 & 0.00 & 4.79 \\
\hline 212 & 150 & 1.55 & 0.00 & 9.23 & 0.00 & 5.29 & 0.00 & 5.29 \\
\hline 150 & 75 & 3.94 & 0.00 & 23.44 & 0.00 & 13.43 & 0.00 & 13.43 \\
\hline 75 & 45 & 2.84 & 0.00 & 16.90 & 0.00 & 9.68 & 0.00 & 9.68 \\
\hline 45 & 25 & 1.36 & 0.34 & 8.11 & 1.94 & 4.64 & 0.83 & 5.47 \\
\hline 25 & 13.1 & 1.79 & 3.35 & 10.68 & 19.01 & 6.12 & 8.12 & 14.24 \\
\hline 13.1 & 6.5 & 1.32 & 4.25 & 7.89 & 24.12 & 4.52 & 10.31 & 14.82 \\
\hline 6.5 & 3.3 & 1.10 & 4.23 & 6.52 & 23.97 & 3.74 & 10.24 & 13.98 \\
\hline 3.3 & 1.5 & 0.77 & 2.64 & 4.57 & 14.97 & 2.62 & 6.40 & 9.01 \\
\hline 1.5 & & 0.72 & 2.82 & 4.30 & 15.98 & 2.46 & 6.83 & 9.29 \\
\hline Totals & -- & 16.79 & 17.64 & 100.00 & 100.00 & 57.28 & 42.72 & 100.00 \\
\hline
\end{tabular}

\begin{tabular}{|c|c|c|c|c|c|c|c|c|}
\hline $\begin{array}{c}\text { Pass } \\
\begin{array}{c}\text { Size } \\
(\mathrm{um})\end{array}\end{array}$ & $\begin{array}{c}\text { Retain } \\
\text { Size } \\
(\mathrm{um})\end{array}$ & $\begin{array}{c}\mathrm{O} / \mathrm{S} \\
\text { Mass } \\
(\mathrm{TPH})\end{array}$ & $\begin{array}{c}\text { U/S } \\
\text { Mass } \\
(\mathrm{TPH})\end{array}$ & $\begin{array}{c}\text { Feed } \\
\text { Mass } \\
(\mathrm{TPH})\end{array}$ & $\begin{array}{c}\text { Delta } \\
\text { Mass } \\
(\mathrm{TPH})\end{array}$ & $\begin{array}{c}\text { Y } \\
\text { Value } \\
(\mathrm{f}-\mathrm{u})\end{array}$ & $\begin{array}{c}\text { X } \\
\text { Value } \\
(0-u)\end{array}$ & $\begin{array}{c}\text { Partition } \\
\text { Factor } \\
(--)\end{array}$ \\
\hline & 212 & 0.06 & 0.00 & 0.06 & 0.00 & 4.79 & 4.79 & 1.00 \\
\hline 212 & 150 & 0.06 & 0.00 & 0.06 & 0.00 & 5.29 & 5.29 & 1.00 \\
\hline 150 & 75 & 0.16 & 0.00 & 0.16 & 0.00 & 13.43 & 13.43 & 1.00 \\
\hline 75 & 45 & 0.12 & 0.00 & 0.12 & 0.00 & 9.68 & 9.68 & 1.00 \\
\hline 45 & 25 & 0.06 & 0.01 & 0.07 & 0.00 & 4.64 & 3.81 & 0.85 \\
\hline 25 & 13.1 & 0.07 & 0.10 & 0.17 & 0.00 & 6.12 & -2.00 & 0.43 \\
\hline 13.1 & 6.5 & 0.05 & 0.12 & 0.18 & 0.00 & 4.52 & -5.79 & 0.30 \\
\hline 6.5 & 3.3 & 0.04 & 0.12 & 0.17 & 0.00 & 3.74 & -6.50 & 0.27 \\
\hline 3.3 & 1.5 & 0.03 & 0.08 & 0.11 & 0.00 & 2.62 & -3.78 & 0.29 \\
\hline 1.5 & & 0.03 & 0.08 & 0.11 & 0.00 & 2.46 & -4.36 & 0.27 \\
\hline Totals & -- & 0.69 & 0.51 & 1.20 & 0.00 & & & \\
\hline
\end{tabular}




\begin{tabular}{|c|c|c|c|c|c|c|c|}
\hline \multirow{3}{*}{$\begin{array}{l}\text { Experimental } \\
\text { Partition } F \text { actor }\end{array}$} & \multirow{3}{*}{$\begin{array}{c}\text { Mean } \\
\text { Particle } \\
\text { Size }(\mu \mathrm{m})\end{array}$} & \multirow{3}{*}{$\begin{array}{c}\text { Predicted } \\
\text { Partition } \\
\text { Factor }\end{array}$} & \multirow{3}{*}{$\begin{array}{c}\text { Predicted } \\
\text { Partition } \\
\text { Factor }\end{array}$} & (Plitt) & Predicted & (Linch) & Predicted \\
\hline & & & & D50 & 26.46 & D50 & 26.26 \\
\hline & & & & $m$ & 1.63 & Alpha & 1.95 \\
\hline$(-)$ & $(-)$ & (Plitt) & (Linch) & Bypass(Rf) & 0.23 & Bypass(Rf) & 0.21 \\
\hline 1.00 & 299.81 & 1.000 & 1.000 & & & & \\
\hline 1.00 & 178.33 & 1.000 & 1.000 & & & & \\
\hline 0.98 & 106.07 & 0.999 & 0.998 & & & & \\
\hline 0.92 & 58.09 & 0.936 & 0.940 & & & & \\
\hline 0.74 & 33.54 & 0.722 & 0.723 & & & & \\
\hline 0.45 & 18.08 & 0.470 & 0.466 & & & & \\
\hline 0.32 & 9.25 & 0.321 & 0.325 & & & & \\
\hline 0.27 & 4.62 & 0.261 & 0.265 & & & & \\
\hline 0.25 & 2.32 & 0.240 & 0.238 & & & & \\
\hline 0.22 & 1.64 & 0.236 & 0.231 & & & & \\
\hline & WSSQ & 0.002 & 0.002 & & & & \\
\hline & TWSSQ & 0.003 & & & & & \\
\hline
\end{tabular}

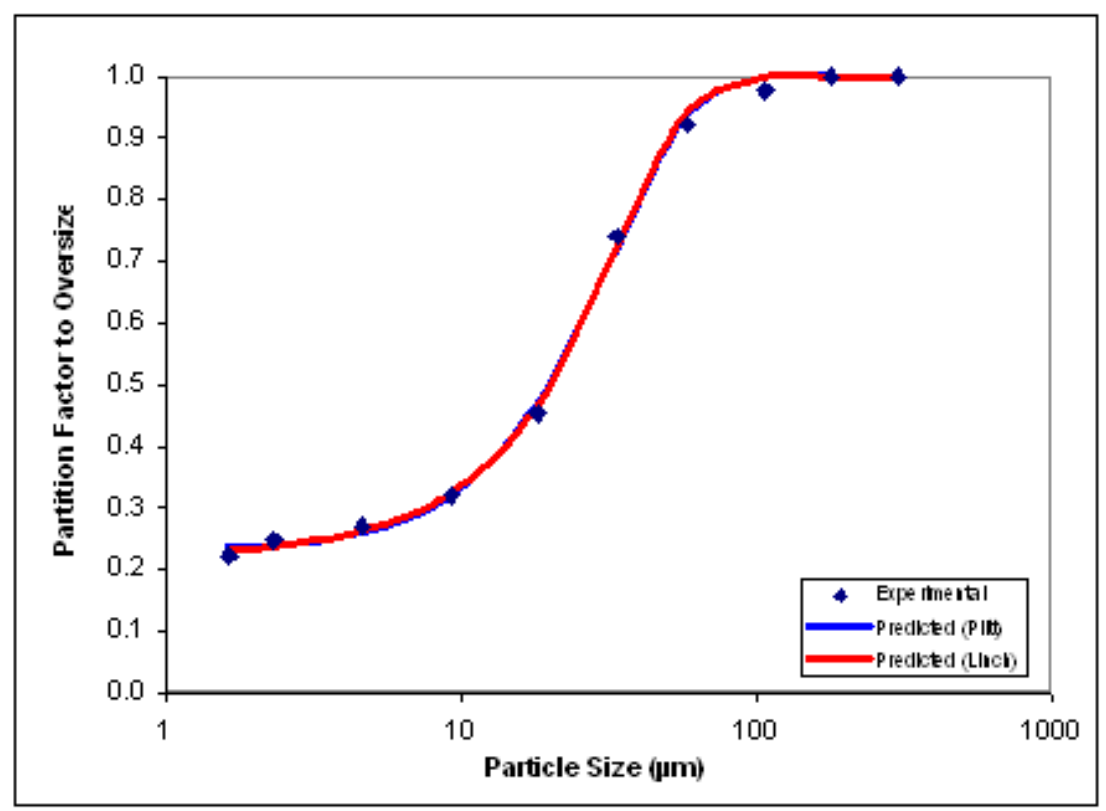


HYDROCYCLONE EVALUATION (UNBALANCED)

\begin{tabular}{|c|c|}
\hline No. 14 & \\
\hline Feedflow (GPM) & 100.0 \\
\hline Feed Pressure (PSl) & 21.0 \\
\hline Water Flow (GPM) & 30.0 \\
\hline Water Pressure (PSI) & 18.0 \\
\hline Feed Rate (TPH) & 1.18 \\
\hline
\end{tabular}

\begin{tabular}{|r|c|c|c|}
\hline & OS & U/S & Feed \\
\hline Solids Content (\%) & 6.05 & 3.00 & 4.63 \\
\hline Ash Content (\%) & 10.52 & 45.61 & 34.11 \\
\hline Solids Yield (\%) & 31.9 & 68.1 & 100.0 \\
\hline Ash Yield (\%) & 32.8 & 67.2 & 100.0 \\
\hline Size Class Yield (\%) & 19.8 & 80.2 & 100.0 \\
\hline
\end{tabular}

\begin{tabular}{|c|c|c|c|c|c|c|c|c|}
\hline $\begin{array}{c}\text { Pass } \\
\text { Size } \\
(u m)\end{array}$ & $\begin{array}{c}\text { Retain } \\
\text { Size } \\
(\mathrm{um})\end{array}$ & $\begin{array}{c}\text { O/S } \\
\text { Sample } \\
(\mathrm{gms})\end{array}$ & $\begin{array}{c}\text { U/S } \\
\text { Sample } \\
(\mathrm{gms})\end{array}$ & $\begin{array}{c}\text { O/S } \\
\text { Mass } \\
(\%, \text { strm) }\end{array}$ & $\begin{array}{c}\text { U/S } \\
\text { Mass } \\
(\%, \text { strm) }\end{array}$ & $\begin{array}{c}\text { OS } \\
\text { Mass } \\
(\%, \text { feed })\end{array}$ & $\begin{array}{c}\text { U/S } \\
\text { Mass } \\
(\%, \text { feed })\end{array}$ & $\begin{array}{c}\text { Feed } \\
\text { Mass } \\
(\%, \text { feed })\end{array}$ \\
\hline & 212 & 1.36 & 0.00 & 10.95 & 0.00 & 3.59 & 0.00 & 3.59 \\
\hline 212 & 150 & 1.73 & 0.00 & 13.93 & 0.00 & 4.56 & 0.00 & 4.56 \\
\hline 150 & 75 & 4.79 & 0.20 & 38.57 & 0.78 & 12.64 & 0.53 & 13.17 \\
\hline 75 & 45 & 2.61 & 1.18 & 21.01 & 4.62 & 6.89 & 3.11 & 9.99 \\
\hline 45 & 25 & 0.62 & 1.62 & 4.99 & 6.35 & 1.64 & 4.27 & 5.90 \\
\hline 25 & 13.1 & 0.69 & 4.98 & 5.57 & 19.50 & 1.82 & 13.11 & 14.93 \\
\hline 13.1 & 6.5 & 0.29 & 5.44 & 2.34 & 21.30 & 0.77 & 14.32 & 15.08 \\
\hline 6.5 & 3.3 & 0.18 & 5.19 & 1.46 & 20.32 & 0.48 & 13.66 & 14.14 \\
\hline 3.3 & 1.5 & 0.09 & 3.44 & 0.74 & 13.48 & 0.24 & 9.06 & 9.31 \\
\hline 1.5 & & 0.05 & 3.48 & 0.44 & 13.65 & 0.14 & 9.18 & 9.32 \\
\hline Totals & -- & 12.42 & 25.53 & 100.00 & 100.00 & 32.77 & 67.23 & 100.00 \\
\hline
\end{tabular}

\begin{tabular}{|c|c|c|c|c|c|c|c|c|}
\hline $\begin{array}{c}\text { Pass } \\
\text { Size } \\
(u m)\end{array}$ & $\begin{array}{c}\text { Retain } \\
\text { Size } \\
(u m)\end{array}$ & $\begin{array}{c}\text { O/S } \\
\text { Mass } \\
(\mathrm{TPH})\end{array}$ & $\begin{array}{c}\text { U/S } \\
\text { Mass } \\
(\mathrm{TPH})\end{array}$ & $\begin{array}{c}\text { Feed } \\
\text { Mass } \\
(\mathrm{TPH})\end{array}$ & $\begin{array}{c}\text { Delta } \\
\text { Mass } \\
(\mathrm{TPH})\end{array}$ & $\begin{array}{c}\text { Y } \\
\text { Value } \\
(\mathrm{f}-\mathrm{u})\end{array}$ & $\begin{array}{c}\text { X } \\
\text { Value } \\
(0-\mathrm{u})\end{array}$ & $\begin{array}{c}\text { Partition } \\
\text { Factor } \\
(-)\end{array}$ \\
\hline & 212 & 0.04 & 0.00 & 0.04 & 0.00 & 3.59 & 3.59 & 1.00 \\
\hline 212 & 150 & 0.05 & 0.00 & 0.05 & 0.00 & 4.56 & 4.56 & 1.00 \\
\hline 150 & 75 & 0.15 & 0.01 & 0.16 & 0.00 & 12.64 & 12.11 & 0.96 \\
\hline 75 & 45 & 0.08 & 0.04 & 0.12 & 0.00 & 6.89 & 3.78 & 0.69 \\
\hline 45 & 25 & 0.02 & 0.05 & 0.07 & 0.00 & 1.64 & -2.63 & 0.28 \\
\hline 25 & 13.1 & 0.02 & 0.15 & 0.18 & 0.00 & 1.82 & -11.28 & 0.12 \\
\hline 13.1 & 6.5 & 0.01 & 0.17 & 0.18 & 0.00 & 0.77 & -13.55 & 0.05 \\
\hline 6.5 & 3.3 & 0.01 & 0.16 & 0.17 & 0.00 & 0.48 & -13.18 & 0.03 \\
\hline 3.3 & 1.5 & 0.00 & 0.11 & 0.11 & 0.00 & 0.24 & -8.82 & 0.03 \\
\hline 1.5 & & 0.00 & 0.11 & 0.11 & 0.00 & 0.14 & -9.03 & 0.02 \\
\hline Totals & -- & 0.39 & 0.79 & 1.18 & 0.00 & & & \\
\hline & Slurry (TPH) & 6.38 & 26.39 & 25.44 & 0.16 & & & \\
\hline
\end{tabular}


HYDROCYCLONE EVALUATION (BALANCED)

\begin{tabular}{|c|c|}
\hline No. 14 & \\
\hline Feedflow (GPM) & 100.0 \\
\hline Feed Pressure (PSl) & 21.0 \\
\hline Water Flow (GPM) & 30.0 \\
\hline Water Pressure (PSI) & 18.0 \\
\hline Feed Rate (TPH) & 1.21 \\
\hline
\end{tabular}

\begin{tabular}{|r|c|c|c|}
\hline & OSS & U/S & Feed \\
\hline Solids Content (\%) & 6.05 & 3.19 & 4.74 \\
\hline Ash Content (\%) & 10.63 & 45.85 & 33.79 \\
\hline Solids Yield (\%) & 27.3 & 72.7 & 100.0 \\
\hline Ash Yield (\%) & 34.2 & 65.8 & 100.0 \\
\hline Size Class Yield (\%) & 34.2 & 65.8 & 100.0 \\
\hline
\end{tabular}

\begin{tabular}{|c|c|c|c|c|c|c|c|c|}
\hline $\begin{array}{c}\text { Pass } \\
\text { Size } \\
(\mathrm{um})\end{array}$ & $\begin{array}{c}\text { Retain } \\
\text { Size } \\
(\mathrm{um})\end{array}$ & $\begin{array}{c}\mathrm{O} / \mathrm{S} \\
\text { Sample } \\
(\mathrm{gms})\end{array}$ & $\begin{array}{c}\text { U/S } \\
\text { Sample } \\
(\mathrm{gms})\end{array}$ & $\begin{array}{c}\text { O/S } \\
\text { Mass } \\
(\%, \text { strm) }\end{array}$ & $\begin{array}{c}\text { U/S } \\
\text { Mass } \\
(\%, \text { strm) }\end{array}$ & $\begin{array}{c}\text { OS } \\
\text { Mass } \\
(\%, \text { feed })\end{array}$ & $\begin{array}{c}\text { U/S } \\
\text { Mass } \\
(\%, \text { feed })\end{array}$ & $\begin{array}{c}\text { Feed } \\
\text { Mass } \\
(\%, \text { feed })\end{array}$ \\
\hline & 212 & 1.09 & 0.00 & 10.51 & 0.00 & 3.60 & 0.00 & 3.60 \\
\hline 212 & 150 & 1.53 & 0.00 & 14.71 & 0.00 & 5.04 & 0.00 & 5.04 \\
\hline 150 & 75 & 5.12 & 0.17 & 49.27 & 0.65 & 16.87 & 0.42 & 17.30 \\
\hline 75 & 45 & 2.50 & 1.21 & 24.07 & 4.74 & 8.24 & 3.12 & 11.36 \\
\hline 45 & 25 & 0.14 & 1.74 & 1.31 & 6.79 & 0.45 & 4.46 & 4.91 \\
\hline 25 & 13.1 & 0.00 & 4.93 & 0.00 & 19.30 & 0.00 & 12.69 & 12.69 \\
\hline 13.1 & 6.5 & 0.00 & 5.33 & 0.03 & 20.85 & 0.01 & 13.71 & 13.73 \\
\hline 6.5 & 3.3 & 0.00 & 5.16 & 0.03 & 20.17 & 0.01 & 13.27 & 13.28 \\
\hline 3.3 & 1.5 & 0.00 & 3.50 & 0.03 & 13.67 & 0.01 & 8.99 & 9.00 \\
\hline 1.5 & & 0.00 & 3.54 & 0.03 & 13.83 & 0.01 & 9.09 & 9.11 \\
\hline Totals & -- & 10.39 & 25.57 & 100.00 & 100.00 & 34.24 & 65.76 & 100.00 \\
\hline
\end{tabular}

\begin{tabular}{|c|c|c|c|c|c|c|c|c|}
\hline $\begin{array}{l}\text { Pass } \\
\text { Size } \\
\text { (um) }\end{array}$ & $\begin{array}{l}\text { Retain } \\
\text { Size } \\
\text { (um) }\end{array}$ & $\begin{array}{l}\text { OSS } \\
\text { Mass } \\
\text { (TPH) }\end{array}$ & $\begin{array}{l}\text { U/S } \\
\text { Mass } \\
\text { (TPH) }\end{array}$ & $\begin{array}{l}\text { Feed } \\
\text { Mass } \\
\text { (TPH) }\end{array}$ & $\begin{array}{l}\text { Delta } \\
\text { Mass } \\
\text { (TPH) }\end{array}$ & $\begin{array}{c}Y \\
\text { Value } \\
\text { (f-u) }\end{array}$ & $\begin{array}{c}X \\
\text { Value } \\
\text { (0-u) }\end{array}$ & $\begin{array}{c}\text { Partition } \\
\text { Factor } \\
(--)\end{array}$ \\
\hline & 212 & 0.04 & 0.00 & 0.04 & 0.00 & 3.60 & 3.60 & 1.00 \\
\hline 212 & 150 & 0.06 & 0.00 & 0.06 & 0.00 & 5.04 & 5.04 & 1.00 \\
\hline 150 & 75 & 0.20 & 0.01 & 0.21 & 0.00 & 16.87 & 16.45 & 0.98 \\
\hline 75 & 45 & 0.10 & 0.04 & 0.14 & 0.00 & 8.24 & 5.12 & 0.73 \\
\hline 45 & 25 & 0.01 & 0.05 & 0.06 & 0.00 & 0.45 & -4.02 & 0.09 \\
\hline 25 & 13.1 & 0.00 & 0.15 & 0.15 & 0.00 & 0.00 & -12.69 & 0.00 \\
\hline 13.1 & 6.5 & 0.00 & 0.17 & 0.17 & 0.00 & 0.01 & -13.70 & 0.00 \\
\hline 6.5 & 3.3 & 0.00 & 0.16 & 0.16 & 0.00 & 0.01 & -13.25 & 0.00 \\
\hline 3.3 & 1.5 & 0.00 & 0.11 & 0.11 & 0.00 & 0.01 & -8.98 & 0.00 \\
\hline 1.5 & & 0.00 & 0.11 & 0.11 & 0.00 & 0.01 & -9.08 & 0.00 \\
\hline Totals & -- & 0.41 & 0.79 & 1.21 & 0.00 & & & \\
\hline & Jrry (TPH) & 6.82 & 24.87 & 25.45 & 1.25 & & & \\
\hline
\end{tabular}




\begin{tabular}{|c|c|c|c|}
\hline $\begin{array}{c}\text { Experimental } \\
\text { Partition F actor }\end{array}$ & $\begin{array}{c}\text { Mean } \\
\text { Particle } \\
\text { Size ( } \mu \mathrm{m})\end{array}$ & $\begin{array}{c}\text { Predicted } \\
\text { Partition } \\
\text { Factor }\end{array}$ & $\begin{array}{c}\text { Predicted } \\
\text { Partition } \\
\text { Factor }\end{array}$ \\
\hline$(--)$ & $(--)$ & (Plitt) & (Linch) \\
\hline 1.00 & 299.81 & 1.000 & 1.000 \\
\hline 1.00 & 178.33 & 1.000 & 1.000 \\
\hline 0.96 & 106.07 & 0.984 & 0.980 \\
\hline 0.69 & 58.09 & 0.675 & 0.681 \\
\hline 0.28 & 33.54 & 0.298 & 0.288 \\
\hline 0.12 & 18.08 & 0.105 & 0.112 \\
\hline 0.05 & 9.25 & 0.045 & 0.053 \\
\hline 0.03 & 4.62 & 0.031 & 0.032 \\
\hline 0.03 & 2.32 & 0.028 & 0.023 \\
\hline 0.02 & 1.64 & 0.027 & 0.021 \\
\hline & WSSQ & 0.002 & 0.001 \\
\hline
\end{tabular}

\begin{tabular}{|c|c|}
\multicolumn{1}{c}{ (Plitt) } & Predicted \\
\hline D50 & 47.17 \\
\hline$m$ & 2.20 \\
\hline Bypass(Rf) & 0.03 \\
\hline
\end{tabular}

(Linch) Predicted

\begin{tabular}{|c|c|}
\hline D50 & 47.18 \\
\hline Alpha & 3.08 \\
\hline Bypass(Rf) & 0.02 \\
\hline
\end{tabular}

\begin{tabular}{l|l}
\hline TWSSQ & 0.002 \\
\hline
\end{tabular}

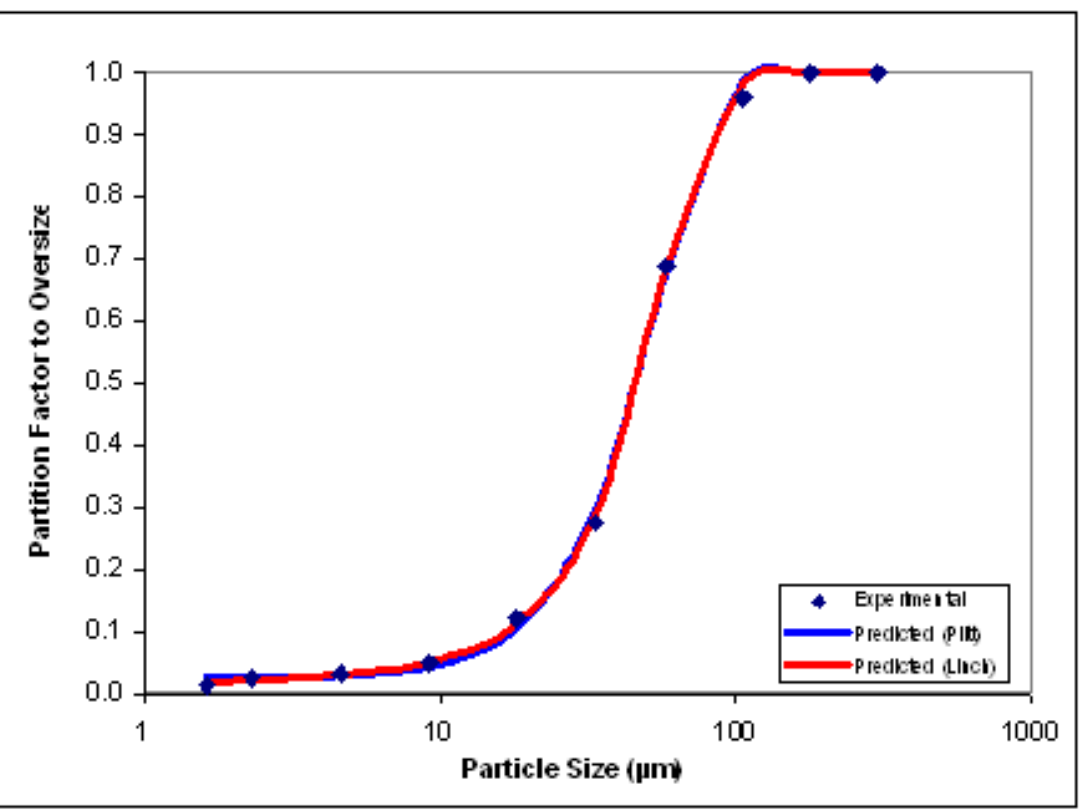




\section{HYDROCYCLONE EVALUATION (UNBALANCED)}

\begin{tabular}{|c|c|}
\hline No. 15 & \\
\hline Feedflow (GPM) & 100.0 \\
\hline Feed Pressure (PSl) & 21.0 \\
\hline Water Flow (GPM) & 0.0 \\
\hline Water Pressure (PSl) & 0.0 \\
\hline Feed Rate (TPH) & 1.16 \\
\hline
\end{tabular}

\begin{tabular}{|r|c|c|c|}
\hline & OS & US & Feed \\
\hline Solids Content (\%) & 6.52 & 2.49 & 4.55 \\
\hline Ash Content (\%) & 29.38 & 48.70 & 34.42 \\
\hline Solids Yield (\%) & 73.2 & 26.8 & 100.0 \\
\hline Ash Yield (\%) & 73.9 & 26.1 & 100.0 \\
\hline Size Class Yield (\%) & 116.6 & -16.6 & 100.0 \\
\hline
\end{tabular}

\begin{tabular}{|c|c|c|c|c|c|c|c|c|}
\hline $\begin{array}{c}\text { Pass } \\
\text { Size } \\
(u m)\end{array}$ & $\begin{array}{c}\text { Retain } \\
\text { Size } \\
(u m)\end{array}$ & $\begin{array}{c}\text { O/S } \\
\text { Sample } \\
(\mathrm{gms})\end{array}$ & $\begin{array}{c}\text { U/S } \\
\text { Sample } \\
(\mathrm{gms})\end{array}$ & $\begin{array}{c}\text { O/S } \\
\text { Mass } \\
(\%, \text { strm) }\end{array}$ & $\begin{array}{c}\text { U/S } \\
\text { Mass } \\
(\%, \text { strm) }\end{array}$ & $\begin{array}{c}\text { OSS } \\
\text { Mass } \\
(\%, \text { feed) })\end{array}$ & $\begin{array}{c}\text { U/S } \\
\text { Mass } \\
(\%, \text { feed) })\end{array}$ & $\begin{array}{c}\text { Feed } \\
\text { Mass } \\
(\%, \text { feed })\end{array}$ \\
\hline & 212 & 2.20 & 0.00 & 6.64 & 0.00 & 4.91 & 0.00 & 4.91 \\
\hline 212 & 150 & 2.00 & 0.00 & 6.04 & 0.00 & 4.46 & 0.00 & 4.46 \\
\hline 150 & 75 & 6.45 & 0.17 & 19.48 & 1.86 & 14.40 & 0.49 & 14.88 \\
\hline 75 & 45 & 4.73 & 0.27 & 14.29 & 2.95 & 10.56 & 0.77 & 11.33 \\
\hline 45 & 25 & 2.27 & 0.15 & 6.86 & 1.64 & 5.07 & 0.43 & 5.50 \\
\hline 25 & 13.1 & 4.06 & 1.41 & 12.27 & 15.39 & 9.07 & 4.02 & 13.08 \\
\hline 13.1 & 6.5 & 3.72 & 2.05 & 11.23 & 22.39 & 8.30 & 5.84 & 14.14 \\
\hline 6.5 & 3.3 & 3.34 & 2.12 & 10.08 & 23.15 & 7.45 & 6.04 & 13.49 \\
\hline 3.3 & 1.5 & 2.20 & 1.51 & 6.64 & 16.56 & 4.91 & 4.32 & 9.23 \\
\hline 1.5 & & 2.14 & 1.47 & 6.48 & 16.06 & 4.79 & 4.19 & 8.98 \\
\hline Totals & -- & 33.11 & 9.14 & 100.00 & 100.00 & 73.91 & 26.09 & 100.00 \\
\hline
\end{tabular}

\begin{tabular}{|c|c|c|c|c|c|c|c|c|}
\hline $\begin{array}{c}\text { Pass } \\
\text { Size } \\
(\mathrm{um})\end{array}$ & $\begin{array}{c}\text { Retain } \\
\text { Size } \\
(\mathrm{um})\end{array}$ & $\begin{array}{c}\text { O/S } \\
\text { Mass } \\
(\mathrm{TPH})\end{array}$ & $\begin{array}{c}\text { U/S } \\
\text { Mass } \\
(\mathrm{TPH})\end{array}$ & $\begin{array}{c}\text { Feed } \\
\text { Mass } \\
(\mathrm{TPH})\end{array}$ & $\begin{array}{c}\text { Delta } \\
\text { Mass } \\
(\mathrm{TPH})\end{array}$ & $\begin{array}{c}\text { Y } \\
\text { Value } \\
(\mathrm{f}-\mathrm{u})\end{array}$ & $\begin{array}{c}\text { X } \\
\text { Value } \\
(0-u)\end{array}$ & $\begin{array}{c}\text { Partition } \\
\text { Factor } \\
(--)\end{array}$ \\
\hline & 212 & 0.06 & 0.00 & 0.06 & 0.00 & 4.91 & 4.91 & 1.00 \\
\hline 212 & 150 & 0.05 & 0.00 & 0.05 & 0.00 & 4.46 & 4.46 & 1.00 \\
\hline 150 & 75 & 0.17 & 0.01 & 0.17 & 0.00 & 14.40 & 13.91 & 0.97 \\
\hline 75 & 45 & 0.12 & 0.01 & 0.13 & 0.00 & 10.56 & 9.79 & 0.93 \\
\hline 45 & 25 & 0.06 & 0.00 & 0.06 & 0.00 & 5.07 & 4.64 & 0.92 \\
\hline 25 & 13.1 & 0.10 & 0.05 & 0.15 & 0.00 & 9.07 & 5.05 & 0.69 \\
\hline 13.1 & 6.5 & 0.10 & 0.07 & 0.16 & 0.00 & 0.30 & 2.46 & 0.59 \\
\hline 6.5 & 3.3 & 0.09 & 0.07 & 0.16 & 0.00 & 7.45 & 1.41 & 0.55 \\
\hline 3.3 & 1.5 & 0.06 & 0.05 & 0.11 & 0.00 & 4.91 & 0.59 & 0.53 \\
\hline 1.5 & & 0.06 & 0.05 & 0.10 & 0.00 & 4.79 & 0.60 & 0.53 \\
\hline Totals & -- & 0.86 & 0.30 & 1.16 & 0.00 & & & \\
\hline
\end{tabular}


HYDROCYCLONE EVALUATION (BALANCED)

\begin{tabular}{|c|c|}
\hline No. 15 & \\
\hline Feedflow (GPM) & 100.0 \\
\hline Feed Pressure (PSl) & 21.0 \\
\hline Water Flow (GPM) & 0.0 \\
\hline Water Pressure (PSl) & 0.0 \\
\hline Feed Rate (TPH) & 1.16 \\
\hline
\end{tabular}

\begin{tabular}{|r|c|c|c|}
\hline & OS & U/S & Feed \\
\hline Solids Content (\%) & 6.52 & 2.49 & 4.55 \\
\hline Ash Content (\%) & 29.38 & 48.70 & 34.42 \\
\hline Solids Yield (\%) & 73.2 & 26.8 & 100.0 \\
\hline Ash Yield (\%) & 73.9 & 26.1 & 100.0 \\
\hline Size Class Yield (\%) & 116.6 & -16.6 & 100.0 \\
\hline
\end{tabular}

\begin{tabular}{|c|c|c|c|c|c|c|c|c|}
\hline Pass & Retain & $0 / 5$ & U/S & o/S & U/S & OS & U/S & Feed \\
\hline $\begin{array}{l}\text { Size } \\
\text { (um) }\end{array}$ & $\begin{array}{l}\text { Size } \\
\text { (um) }\end{array}$ & $\begin{array}{c}\text { Sample } \\
\text { (gms) }\end{array}$ & $\begin{array}{c}\text { Sample } \\
\text { (gms) }\end{array}$ & $\begin{array}{c}\text { Mass } \\
(\%, \text { strm) }\end{array}$ & $\begin{array}{c}\text { Mass } \\
(\%, \text { strm })\end{array}$ & $\begin{array}{c}\text { Mass } \\
(\% \text {, feed })\end{array}$ & $\begin{array}{c}\text { Mass } \\
(\%, \text { feed })\end{array}$ & $\begin{array}{c}\text { Mass } \\
(\%, \text { feed })\end{array}$ \\
\hline & 212 & 2.20 & 0.00 & 6.64 & 0.00 & 4.91 & 0.00 & 4.91 \\
\hline 212 & 150 & 2.00 & 0.00 & 6.04 & 0.00 & 4.46 & 0.00 & 4.46 \\
\hline 150 & 75 & 6.45 & 0.17 & 19.48 & 1.86 & 14.40 & 0.49 & 14.88 \\
\hline 75 & 45 & 4.73 & 0.27 & 14.29 & 2.95 & 10.56 & 0.77 & 11.33 \\
\hline 45 & 25 & 2.27 & 0.15 & 6.86 & 1.64 & 5.07 & 0.43 & 5.50 \\
\hline 25 & 13.1 & 4.06 & 1.41 & 12.27 & 15.39 & 9.07 & 4.02 & 13.08 \\
\hline 13.1 & 6.5 & 3.72 & 2.05 & 11.23 & 22.39 & 8.30 & 5.84 & 14.14 \\
\hline 6.5 & 3.3 & 3.34 & 2.12 & 10.08 & 23.15 & 7.45 & 6.04 & 13.49 \\
\hline 3.3 & 1.5 & 2.20 & 1.51 & 6.64 & 16.56 & 4.91 & 4.32 & 9.23 \\
\hline 1.5 & & 2.14 & 1.47 & 6.48 & 16.06 & 4.79 & 4.19 & 8.98 \\
\hline Totals & -- & 33.11 & 9.14 & 100.00 & 100.00 & 73.91 & 26.09 & 100.00 \\
\hline
\end{tabular}

\begin{tabular}{|c|c|c|c|c|c|c|c|c|}
\hline $\begin{array}{c}\text { Pass } \\
\text { Size } \\
(u m)\end{array}$ & $\begin{array}{c}\text { Retain } \\
\text { Size } \\
(u m)\end{array}$ & $\begin{array}{c}\text { O/S } \\
\text { Mass } \\
(\mathrm{TPH})\end{array}$ & $\begin{array}{c}\text { U/S } \\
\text { Mass } \\
(\mathrm{TPH})\end{array}$ & $\begin{array}{c}\text { Feed } \\
\text { Mass } \\
(\mathrm{TPH})\end{array}$ & $\begin{array}{c}\text { Delta } \\
\text { Mass } \\
(\mathrm{TPH})\end{array}$ & $\begin{array}{c}\text { V } \\
\text { Value } \\
(\mathrm{f}-\mathrm{u})\end{array}$ & $\begin{array}{c}\text { X } \\
\text { Value } \\
(0-\mathrm{u})\end{array}$ & $\begin{array}{c}\text { Partition } \\
\text { Factor } \\
(-)\end{array}$ \\
\hline & 212 & 0.06 & 0.00 & 0.06 & 0.00 & 4.91 & 4.91 & 1.00 \\
\hline 212 & 150 & 0.05 & 0.00 & 0.05 & 0.00 & 4.46 & 4.46 & 1.00 \\
\hline 150 & 75 & 0.17 & 0.01 & 0.17 & 0.00 & 14.40 & 13.91 & 0.97 \\
\hline 75 & 45 & 0.12 & 0.01 & 0.13 & 0.00 & 10.56 & 9.79 & 0.93 \\
\hline 45 & 25 & 0.06 & 0.00 & 0.06 & 0.00 & 5.07 & 4.64 & 0.92 \\
\hline 25 & 13.1 & 0.10 & 0.05 & 0.15 & 0.00 & 9.07 & 5.05 & 0.69 \\
\hline 13.1 & 6.5 & 0.10 & 0.07 & 0.16 & 0.00 & 8.30 & 2.46 & 0.59 \\
\hline 6.5 & 3.3 & 0.09 & 0.07 & 0.16 & 0.00 & 7.45 & 1.41 & 0.55 \\
\hline 3.3 & 1.5 & 0.06 & 0.05 & 0.11 & 0.00 & 4.91 & 0.59 & 0.53 \\
\hline 1.5 & & 0.06 & 0.05 & 0.10 & 0.00 & 4.79 & 0.60 & 0.53 \\
\hline Totals & -- & 0.86 & 0.30 & 1.16 & 0.00 & & & \\
\hline \multicolumn{2}{|c|}{ Slurry (TPH) } & 13.12 & 12.12 & 25.43 & 0.19 & & & \\
\hline
\end{tabular}




\begin{tabular}{|c|c|c|c|c|c|c|c|}
\hline \multirow{3}{*}{$\begin{array}{l}\text { Experimental } \\
\text { Partition } \mathrm{F} \text { actor }\end{array}$} & \multirow{3}{*}{$\begin{array}{c}\text { Mean } \\
\text { Particle } \\
\text { Size }(\mu \mathrm{m})\end{array}$} & \multirow{3}{*}{$\begin{array}{c}\text { Predicted } \\
\text { Partition } \\
\text { Factor }\end{array}$} & \multirow{3}{*}{$\begin{array}{c}\text { Predicted } \\
\text { Partition } \\
\text { Factor }\end{array}$} & (Plitt) & Predicted & (Linch) & Predicted \\
\hline & & & & D50 & 22.11 & D50 & 22.19 \\
\hline & & & & $m$ & 2.02 & Alpha & 2.70 \\
\hline$(-)$ & $(-)$ & (Plitt) & (Linch) & Bypass(Rf) & 0.53 & Bypass(Rf) & 0.52 \\
\hline 1.00 & 299.81 & 1.000 & 1.000 & & & & \\
\hline 1.00 & 178.33 & 1.000 & 1.000 & & & & \\
\hline 0.97 & 106.07 & 1.000 & 1.000 & & & & \\
\hline 0.93 & 58.09 & 0.996 & 0.994 & & & & \\
\hline 0.92 & 33.54 & 0.906 & 0.908 & & & & \\
\hline 0.69 & 18.08 & 0.705 & 0.699 & & & & \\
\hline 0.59 & 9.25 & 0.584 & 0.587 & & & & \\
\hline 0.55 & 4.62 & 0.545 & 0.549 & & & & \\
\hline 0.53 & 2.32 & 0.535 & 0.535 & & & & \\
\hline 0.53 & 1.64 & 0.534 & 0.532 & & & & \\
\hline & WSSQ & 0.006 & 0.005 & & & & \\
\hline & TWSSQ & 0.011 & & & & & \\
\hline
\end{tabular}

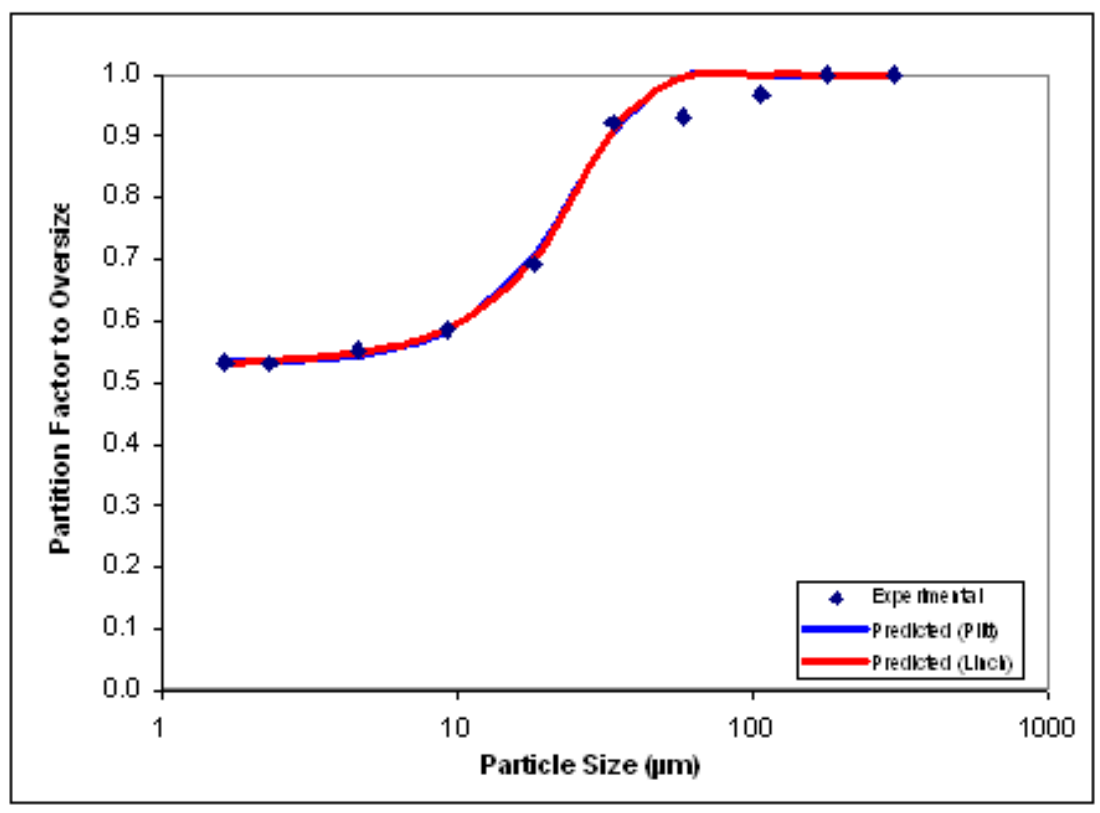




\section{HYDROCYCLONE EVALUATION (UNBALANCED)}

\begin{tabular}{|c|c|}
\hline No.16 & \\
\hline FeedFlow (GPM) & 900.0 \\
\hline Feed Pressure (PSl) & 21.0 \\
\hline Water Flow (GPM) & 30.0 \\
\hline Water Pressure (PSl) & 14.0 \\
\hline Feed Rate (TPH) & 1.16 \\
\hline
\end{tabular}

\begin{tabular}{|r|c|c|c|}
\hline & OS & U/S & Feed \\
\hline Solids Content (\%) & 6.41 & 2.40 & 4.57 \\
\hline Ash Content (\%) & 26.16 & 49.30 & 34.56 \\
\hline Solids Yield (\%) & 51.1 & 48.9 & 100.0 \\
\hline Ash Yield (\%) & 63.7 & 36.3 & 100.0 \\
\hline Size Class Yield (\%) & 88.8 & 11.2 & 100.0 \\
\hline
\end{tabular}

\begin{tabular}{|c|c|c|c|c|c|c|c|c|}
\hline $\begin{array}{c}\text { Pass } \\
\text { Size } \\
(\mathrm{um})\end{array}$ & $\begin{array}{c}\text { Retain } \\
\text { Size } \\
(\mathrm{um})\end{array}$ & $\begin{array}{c}\mathrm{O} / \mathrm{S} \\
\text { Sample } \\
(\mathrm{gms})\end{array}$ & $\begin{array}{c}\mathrm{U} / \mathrm{S} \\
\text { Sample } \\
(\mathrm{gms})\end{array}$ & $\begin{array}{c}\text { O/S } \\
\text { Mass } \\
(\%, \text { strm) }\end{array}$ & $\begin{array}{c}\text { U/S } \\
\text { Mass } \\
(\%, \text { strm })\end{array}$ & $\begin{array}{c}\text { OS } \\
\text { Mass } \\
(\%, \text { feed })\end{array}$ & $\begin{array}{c}\text { U/S } \\
\text { Mass } \\
(\%, \text { feed })\end{array}$ & $\begin{array}{c}\text { Feed } \\
\text { Mass } \\
(\%, \text { feed })\end{array}$ \\
\hline & 212 & 2.04 & 0.00 & 6.93 & 0.00 & 4.41 & 0.00 & 4.41 \\
\hline 212 & 150 & 2.20 & 0.00 & 7.47 & 0.00 & 4.76 & 0.00 & 4.76 \\
\hline 150 & 75 & 6.24 & 0.18 & 21.19 & 1.39 & 13.50 & 0.50 & 14.00 \\
\hline 75 & 45 & 4.48 & 0.34 & 15.21 & 2.62 & 9.69 & 0.95 & 10.64 \\
\hline 45 & 25 & 2.27 & 0.28 & 7.71 & 2.16 & 4.91 & 0.78 & 5.69 \\
\hline 25 & 13.1 & 3.52 & 2.18 & 11.97 & 16.77 & 7.62 & 6.09 & 13.71 \\
\hline 13.1 & 6.5 & 2.95 & 2.91 & 10.01 & 22.44 & 6.38 & 8.15 & 14.53 \\
\hline 6.5 & 3.3 & 2.58 & 2.95 & 8.74 & 22.76 & 5.57 & 8.26 & 13.83 \\
\hline 3.3 & 1.5 & 1.66 & 1.98 & 5.65 & 15.27 & 3.60 & 5.54 & 9.14 \\
\hline 1.5 & & 1.51 & 2.15 & 5.12 & 16.59 & 3.26 & 6.02 & 9.29 \\
\hline Totals & -- & 29.45 & 12.98 & 100.00 & 100.00 & 63.70 & 36.30 & 100.00 \\
\hline
\end{tabular}

\begin{tabular}{|c|c|c|c|c|c|c|c|c|}
\hline $\begin{array}{c}\text { Pass } \\
\text { Size } \\
(u m)\end{array}$ & $\begin{array}{c}\text { Retain } \\
\text { Size } \\
(u m)\end{array}$ & $\begin{array}{c}\text { O/S } \\
\text { Mass } \\
(\mathrm{TPH})\end{array}$ & $\begin{array}{c}\text { U/S } \\
\text { Mass } \\
(\mathrm{TPH})\end{array}$ & $\begin{array}{c}\text { Feed } \\
\text { Mass } \\
(\mathrm{TPH})\end{array}$ & $\begin{array}{c}\text { Delta } \\
\text { Mass } \\
(\mathrm{TPH})\end{array}$ & $\begin{array}{c}\text { Y } \\
\text { Value } \\
(\mathrm{f}-\mathrm{u})\end{array}$ & $\begin{array}{c}\text { X } \\
\text { Value } \\
(0-\mathrm{u})\end{array}$ & $\begin{array}{c}\text { Partition } \\
\text { Factor } \\
(-)\end{array}$ \\
\hline & 212 & 0.05 & 0.00 & 0.05 & 0.00 & 4.41 & 4.41 & 1.00 \\
\hline 212 & 150 & 0.06 & 0.00 & 0.06 & 0.00 & 4.76 & 4.76 & 1.00 \\
\hline 150 & 75 & 0.16 & 0.01 & 0.16 & 0.00 & 13.50 & 12.99 & 0.96 \\
\hline 75 & 45 & 0.11 & 0.01 & 0.12 & 0.00 & 9.69 & 8.74 & 0.91 \\
\hline 45 & 25 & 0.06 & 0.01 & 0.07 & 0.00 & 4.91 & 4.13 & 0.86 \\
\hline 25 & 13.1 & 0.09 & 0.07 & 0.16 & 0.00 & 7.62 & 1.54 & 0.56 \\
\hline 13.1 & 6.5 & 0.07 & 0.09 & 0.17 & 0.00 & 6.38 & -1.77 & 0.44 \\
\hline 6.5 & 3.3 & 0.06 & 0.10 & 0.16 & 0.00 & 5.57 & -2.69 & 0.40 \\
\hline 3.3 & 1.5 & 0.04 & 0.06 & 0.11 & 0.00 & 3.60 & -1.95 & 0.39 \\
\hline 1.5 & & 0.04 & 0.07 & 0.11 & 0.00 & 3.26 & -2.76 & 0.35 \\
\hline Totals & -- & 0.74 & 0.42 & 1.16 & 0.00 & & & \\
\hline & Slurry (TPH) & 11.55 & 17.58 & 25.43 & 3.80 & & & \\
\hline
\end{tabular}




\section{HYDROCYCLONE EVALUATION (BALANCED)}

\begin{tabular}{|c|c|}
\hline No.16 & \\
\hline Feedflow (GPM) & 100.0 \\
\hline Feed Pressure (PSl) & 21.0 \\
\hline Water Flow (GPM) & 30.0 \\
\hline Water Pressure (PSl) & 14.0 \\
\hline Feed Rate (TPH) & 1.15 \\
\hline
\end{tabular}

\begin{tabular}{|r|c|c|c|}
\hline & OSS & U/S & Feed \\
\hline Solids Content (\%) & 6.79 & 2.57 & 4.51 \\
\hline Ash Content (\%) & 23.74 & 48.18 & 34.58 \\
\hline Solids Yield (\%) & 42.1 & 57.9 & 100.0 \\
\hline Ash Yield (\%) & 55.6 & 44.4 & 100.0 \\
\hline Size Class Yield (\%) & 55.6 & 44.4 & 100.0 \\
\hline
\end{tabular}

\begin{tabular}{|c|c|c|c|c|c|c|c|c|}
\hline $\begin{array}{l}\text { Pass } \\
\text { Size } \\
\text { (um) }\end{array}$ & $\begin{array}{c}\text { Retain } \\
\text { Size } \\
\text { (um) }\end{array}$ & $\begin{array}{c}\text { OSS } \\
\text { Sample } \\
\text { (gms) }\end{array}$ & $\begin{array}{c}\text { US } \\
\text { Sample } \\
\text { (gms) }\end{array}$ & $\begin{array}{c}\mathrm{OS} \\
\text { Mass } \\
(\% \text {, strm) }\end{array}$ & $\begin{array}{c}\text { USS } \\
\text { Mass } \\
(\%, \text { strm) }\end{array}$ & $\begin{array}{c}\text { OSS } \\
\text { Mass } \\
(\%, \text { feed })\end{array}$ & $\begin{array}{c}\text { USS } \\
\text { Mass } \\
(\%, \text { feed })\end{array}$ & $\begin{array}{c}\text { Feed } \\
\text { Mass } \\
(\%, \text { feed })\end{array}$ \\
\hline & 212 & 1.63 & 0.00 & 7.75 & 0.00 & 4.31 & 0.00 & 4.31 \\
\hline 212 & 150 & 1.89 & 0.00 & 8.99 & 0.00 & 5.00 & 0.00 & 5.00 \\
\hline 150 & 75 & 5.19 & 0.02 & 24.64 & 0.12 & 13.71 & 0.05 & 13.76 \\
\hline 75 & 45 & 3.83 & 0.27 & 18.18 & 1.59 & 10.12 & 0.71 & 10.82 \\
\hline 45 & 25 & 1.45 & 0.40 & 6.89 & 2.37 & 3.83 & 1.05 & 4.89 \\
\hline 25 & 13.1 & 1.98 & 2.94 & 9.42 & 17.60 & 5.24 & 7.81 & 13.05 \\
\hline 13.1 & 6.5 & 1.60 & 3.76 & 7.59 & 22.48 & 4.22 & 9.98 & 14.20 \\
\hline 6.5 & 3.3 & 1.44 & 3.80 & 6.82 & 22.76 & 3.80 & 10.10 & 13.90 \\
\hline 3.3 & 1.5 & 0.95 & 2.55 & 4.50 & 15.27 & 2.50 & 6.77 & 9.28 \\
\hline 1.5 & & 1.10 & 2.97 & 5.22 & 17.80 & 2.90 & 7.90 & 10.80 \\
\hline Totals & -- & 21.07 & 16.70 & 100.00 & 100.00 & 55.63 & 44.37 & 100.00 \\
\hline
\end{tabular}

\begin{tabular}{|c|c|c|c|c|c|c|c|c|}
\hline $\begin{array}{l}\text { Pass } \\
\text { Size } \\
\text { (um) }\end{array}$ & $\begin{array}{c}\text { Retain } \\
\text { Size } \\
\text { (um) }\end{array}$ & $\begin{array}{c}\text { O/S } \\
\text { Mass } \\
(\mathrm{TPH})\end{array}$ & $\begin{array}{c}\text { U/S } \\
\text { Mass } \\
(\mathrm{TPH})\end{array}$ & $\begin{array}{l}\text { Feed } \\
\text { Mass } \\
(\mathrm{TPH})\end{array}$ & $\begin{array}{l}\text { Delta } \\
\text { Mass } \\
(\mathrm{TPH})\end{array}$ & $\begin{array}{c}\mathrm{Y} \\
\text { Value } \\
(\mathrm{f}-\mathrm{u})\end{array}$ & $\begin{array}{c}x \\
\text { Value } \\
(0-u)\end{array}$ & $\begin{array}{c}\text { Partition } \\
\text { Factor } \\
(--)\end{array}$ \\
\hline & 212 & 0.05 & 0.00 & 0.05 & 0.00 & 4.31 & 4.31 & 1.00 \\
\hline 212 & 150 & 0.06 & 0.00 & 0.06 & 0.00 & 5.00 & 5.00 & 1.00 \\
\hline 150 & 75 & 0.16 & 0.00 & 0.16 & 0.00 & 13.71 & 13.65 & 1.00 \\
\hline 75 & 45 & 0.12 & 0.01 & 0.12 & 0.00 & 10.12 & 9.41 & 0.93 \\
\hline 45 & 25 & 0.04 & 0.01 & 0.06 & 0.00 & 3.83 & 2.78 & 0.78 \\
\hline 25 & 13.1 & 0.06 & 0.09 & 0.15 & 0.00 & 5.24 & -2.57 & 0.40 \\
\hline 13.1 & 6.5 & 0.05 & 0.11 & 0.16 & 0.00 & 4.22 & -5.75 & 0.30 \\
\hline 6.5 & 3.3 & 0.04 & 0.12 & 0.16 & 0.00 & 3.80 & -6.30 & 0.27 \\
\hline 3.3 & 1.5 & 0.03 & 0.08 & 0.11 & 0.00 & 2.50 & -4.27 & 0.27 \\
\hline 1.5 & & 0.03 & 0.09 & 0.12 & 0.00 & 2.90 & -5.00 & 0.27 \\
\hline Totals & -- & 0.64 & 0.51 & 1.15 & 0.00 & & & \\
\hline & Irry (TPH) & 9.39 & 19.79 & 25.43 & 3.75 & & & \\
\hline
\end{tabular}




\begin{tabular}{|c|c|c|c|}
\hline $\begin{array}{c}\text { Experimental } \\
\text { Partition Factor }\end{array}$ & $\begin{array}{c}\text { Mean } \\
\text { Particle } \\
\text { Size ( } \mathrm{\mu m})\end{array}$ & $\begin{array}{c}\text { Predicted } \\
\text { Partition } \\
\text { Factor }\end{array}$ & $\begin{array}{c}\text { Predicted } \\
\text { Partition } \\
\text { Factor }\end{array}$ \\
\hline$(--)$ & $(--)$ & (Plitt) & (Linch) \\
\hline 1.00 & 299.81 & 1.000 & 1.000 \\
\hline 1.00 & 178.33 & 1.000 & 1.000 \\
\hline 0.96 & 106.07 & 1.000 & 1.000 \\
\hline 0.91 & 58.09 & 0.975 & 0.983 \\
\hline 0.86 & 33.54 & 0.817 & 0.834 \\
\hline 0.56 & 18.08 & 0.585 & 0.574 \\
\hline 0.44 & 9.25 & 0.444 & 0.441 \\
\hline 0.40 & 4.62 & 0.390 & 0.393 \\
\hline 0.39 & 2.32 & 0.373 & 0.375 \\
\hline 0.35 & 1.64 & 0.370 & 0.370 \\
\hline & $W S S \mathrm{Q}$ & 0.009 & 0.008 \\
\hline
\end{tabular}

\begin{tabular}{|c|c|c|c|}
\hline (Plitt) & Predicted & (Linch) & Predicted \\
\hline D50 & 23.99 & D50 & 23.94 \\
\hline$m$ & 1.74 & Alpha & 2.48 \\
\hline Bypass(Rf) & 0.37 & Bypass(Rf) & 0.36 \\
\hline
\end{tabular}

\begin{tabular}{|l|l|}
\hline TWSSQ & 0.018 \\
\hline
\end{tabular}

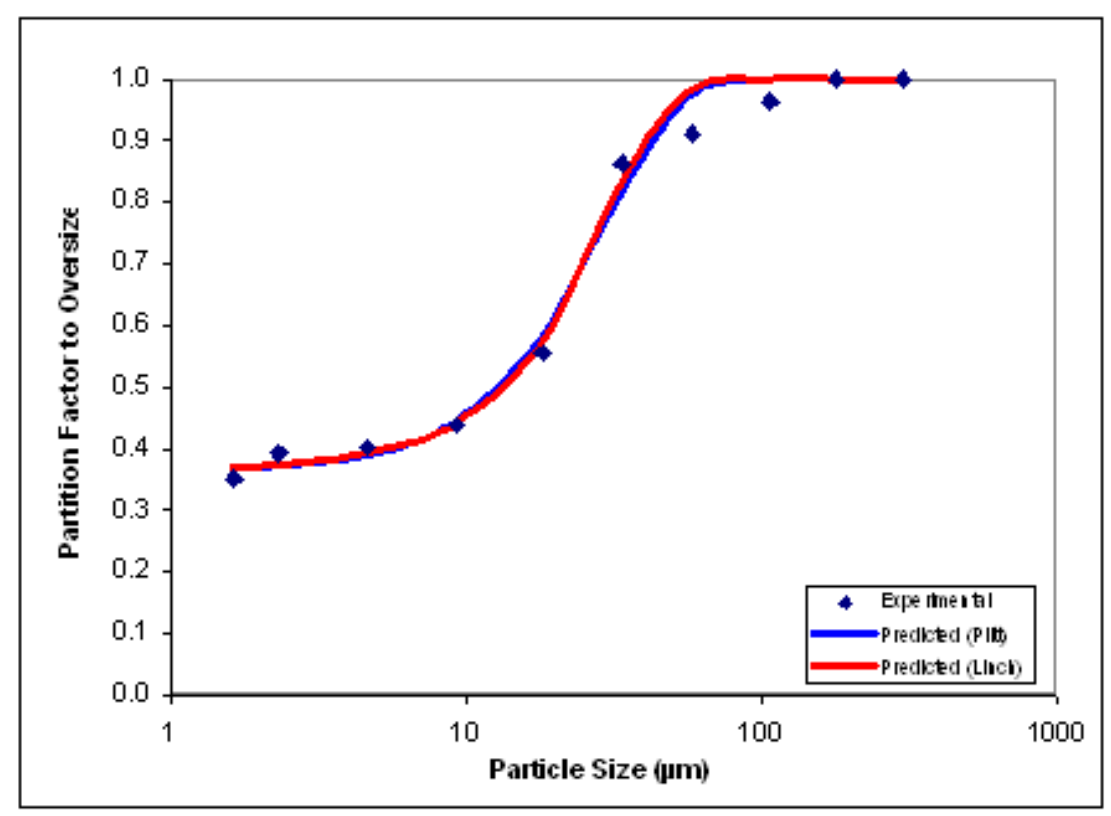


HYDROCYCLONE EVALUATION (UNBALANCED)

\begin{tabular}{|c|c|}
\hline No. 17 & \\
\hline Feedflow (GPM) & 100.0 \\
\hline Feed Pressure (PSl) & 21.0 \\
\hline Water Flow (GPM) & 15.0 \\
\hline Water Pressure (PSl) & 12.0 \\
\hline Feed Rate (TPH) & 1.16 \\
\hline
\end{tabular}

\begin{tabular}{|r|c|c|c|}
\hline & OS & U/S & Feed \\
\hline Solids Content (\%) & 6.95 & 2.61 & 4.57 \\
\hline Ash Content (\%) & 23.16 & 48.23 & 34.34 \\
\hline Solids Yield (\%) & 55.2 & 44.8 & 100.0 \\
\hline Ash Yield (\%) & 55.4 & 44.6 & 100.0 \\
\hline Size Class Yield (\%) & 58.0 & 42.0 & 100.0 \\
\hline
\end{tabular}

\begin{tabular}{|c|c|c|c|c|c|c|c|c|}
\hline Pass & Retain & $0 / 5$ & U/S & o/S & U/S & OS & U/S & Feed \\
\hline $\begin{array}{l}\text { Size } \\
\text { (um) }\end{array}$ & $\begin{array}{l}\text { Size } \\
\text { (um) }\end{array}$ & $\begin{array}{c}\text { Sample } \\
\text { (gms) }\end{array}$ & $\begin{array}{c}\text { Sample } \\
\text { (gms) }\end{array}$ & $\begin{array}{c}\text { Mass } \\
(\%, \text { strm) }\end{array}$ & $\begin{array}{c}\text { Mass } \\
(\%, \text { strm })\end{array}$ & $\begin{array}{c}\text { Mass } \\
(\% \text {, feed })\end{array}$ & $\begin{array}{c}\text { Mass } \\
(\%, \text { feed })\end{array}$ & $\begin{array}{c}\text { Mass } \\
(\%, \text { feed })\end{array}$ \\
\hline & 212 & 1.76 & 0.00 & 8.67 & 0.00 & 4.80 & 0.00 & 4.80 \\
\hline 212 & 150 & 1.58 & 0.00 & 7.78 & 0.00 & 4.31 & 0.00 & 4.31 \\
\hline 150 & 75 & 5.26 & 0.19 & 25.90 & 1.03 & 14.35 & 0.46 & 14.81 \\
\hline 75 & 45 & 3.60 & 0.44 & 17.73 & 2.38 & 9.82 & 1.06 & 10.88 \\
\hline 45 & 25 & 1.36 & 0.60 & 6.70 & 3.25 & 3.71 & 1.45 & 5.16 \\
\hline 25 & 13.1 & 1.48 & 3.37 & 7.30 & 18.25 & 4.05 & 8.14 & 12.19 \\
\hline 13.1 & 6.5 & 1.62 & 4.16 & 7.96 & 22.52 & 4.41 & 10.04 & 14.45 \\
\hline 6.5 & 3.3 & 1.57 & 4.07 & 7.75 & 22.02 & 4.30 & 9.82 & 14.12 \\
\hline 3.3 & 1.5 & 0.99 & 2.79 & 4.89 & 15.09 & 2.71 & 6.73 & 9.44 \\
\hline 1.5 & & 1.08 & 2.86 & 5.32 & 15.46 & 2.95 & 6.89 & 9.84 \\
\hline Totals & -- & 20.31 & 18.47 & 100.00 & 100.00 & 55.40 & 44.60 & 100.00 \\
\hline
\end{tabular}

\begin{tabular}{|c|c|c|c|c|c|c|c|c|}
\hline $\begin{array}{c}\text { Pass } \\
\text { Size } \\
(u m)\end{array}$ & $\begin{array}{c}\text { Retain } \\
\text { Size } \\
(u m)\end{array}$ & $\begin{array}{c}0 / 5 \\
\text { Mass } \\
(\mathrm{TPH})\end{array}$ & $\begin{array}{c}\text { U/S } \\
\text { Mass } \\
(\mathrm{TPH})\end{array}$ & $\begin{array}{c}\text { Feed } \\
\text { Mass } \\
(\mathrm{TPH})\end{array}$ & $\begin{array}{c}\text { Delta } \\
\text { Mass } \\
(\mathrm{TPH})\end{array}$ & $\begin{array}{c}\text { Value } \\
\text { (f-u) }\end{array}$ & $\begin{array}{c}\text { X } \\
\text { Value } \\
(\mathrm{0}-\mathrm{u})\end{array}$ & $\begin{array}{c}\text { Partition } \\
\text { Factor } \\
(-)\end{array}$ \\
\hline & 212 & 0.06 & 0.00 & 0.06 & 0.00 & 4.80 & 4.80 & 1.00 \\
\hline 212 & 150 & 0.05 & 0.00 & 0.05 & 0.00 & 4.31 & 4.31 & 1.00 \\
\hline 150 & 75 & 0.17 & 0.01 & 0.17 & 0.00 & 14.35 & 13.89 & 0.97 \\
\hline 75 & 45 & 0.11 & 0.01 & 0.13 & 0.00 & 9.82 & 8.76 & 0.90 \\
\hline 45 & 25 & 0.04 & 0.02 & 0.06 & 0.00 & 3.71 & 2.26 & 0.72 \\
\hline 25 & 13.1 & 0.05 & 0.09 & 0.14 & 0.00 & 4.05 & -4.09 & 0.33 \\
\hline 13.1 & 6.5 & 0.05 & 0.12 & 0.17 & 0.00 & 4.41 & -5.63 & 0.31 \\
\hline 6.5 & 3.3 & 0.05 & 0.11 & 0.16 & 0.00 & 4.30 & -5.52 & 0.30 \\
\hline 3.3 & 1.5 & 0.03 & 0.08 & 0.11 & 0.00 & 2.71 & -4.02 & 0.29 \\
\hline 1.5 & & 0.03 & 0.08 & 0.11 & 0.00 & 2.95 & -3.95 & 0.30 \\
\hline Totals & -- & 0.64 & 0.52 & 1.16 & 0.00 & & & \\
\hline & Slurry (TPH) & 9.27 & 19.86 & 25.43 & 0.06 & & & \\
\hline
\end{tabular}


HYDROCYCLONE EVALUATION (BALANCED)

\begin{tabular}{|c|c|}
\hline No. 17 & \\
\hline Feedflow (GPM) & 100.0 \\
\hline Feed Pressure (PSl) & 21.0 \\
\hline Water Flow (GPM) & 15.0 \\
\hline Water Pressure (PSI) & 12.0 \\
\hline Feed Rate (TPH) & 1.15 \\
\hline
\end{tabular}

\begin{tabular}{|r|c|c|c|}
\hline & OS & U/S & Feed \\
\hline Solids Content (\%) & 6.79 & 2.57 & 4.51 \\
\hline Ash Content (\%) & 23.74 & 48.18 & 34.58 \\
\hline Solids Yield (\%) & 55.6 & 44.4 & 100.0 \\
\hline Ash Yield (\%) & 55.6 & 44.4 & 100.0 \\
\hline Size Class Yield (\%) & 55.6 & 44.4 & 100.0 \\
\hline
\end{tabular}

\begin{tabular}{|c|c|c|c|c|c|c|c|c|}
\hline Pass & Retain & $0 / 5$ & U/S & o/s & U/S & OS & U/S & Feed \\
\hline $\begin{array}{l}\text { Size } \\
\text { (um) }\end{array}$ & $\begin{array}{l}\text { Size } \\
\text { (um) }\end{array}$ & $\begin{array}{c}\text { Sample } \\
\text { (gms) }\end{array}$ & $\begin{array}{c}\text { Sample } \\
\text { (gms) }\end{array}$ & $\begin{array}{c}\text { Mass } \\
(\%, \text { strm) }\end{array}$ & $\begin{array}{c}\text { Mass } \\
(\%, \text { strm })\end{array}$ & $\begin{array}{c}\text { Mass } \\
(\% \text {, feed })\end{array}$ & $\begin{array}{c}\text { Mass } \\
(\%, \text { feed })\end{array}$ & $\begin{array}{c}\text { Mass } \\
(\%, \text { feed })\end{array}$ \\
\hline & 212 & 1.63 & 0.00 & 7.75 & 0.00 & 4.31 & 0.00 & 4.31 \\
\hline 212 & 150 & 1.89 & 0.00 & 8.99 & 0.00 & 5.00 & 0.00 & 5.00 \\
\hline 150 & 75 & 5.19 & 0.02 & 24.64 & 0.12 & 13.71 & 0.05 & 13.76 \\
\hline 75 & 45 & 3.83 & 0.27 & 18.18 & 1.59 & 10.12 & 0.71 & 10.82 \\
\hline 45 & 25 & 1.45 & 0.40 & 6.89 & 2.37 & 3.83 & 1.05 & 4.89 \\
\hline 25 & 13.1 & 1.98 & 2.94 & 9.42 & 17.60 & 5.24 & 7.81 & 13.05 \\
\hline 13.1 & 6.5 & 1.60 & 3.76 & 7.59 & 22.48 & 4.22 & 9.98 & 14.20 \\
\hline 6.5 & 3.3 & 1.44 & 3.80 & 6.82 & 22.76 & 3.80 & 10.10 & 13.90 \\
\hline 3.3 & 1.5 & 0.95 & 2.55 & 4.50 & 15.27 & 2.50 & 6.77 & 9.28 \\
\hline 1.5 & & 1.10 & 2.97 & 5.22 & 17.80 & 2.90 & 7.90 & 10.80 \\
\hline Totals & -- & 21.07 & 16.70 & 100.00 & 100.00 & 55.63 & 44.37 & 100.00 \\
\hline
\end{tabular}

\begin{tabular}{|c|c|c|c|c|c|c|c|c|}
\hline $\begin{array}{c}\text { Pass } \\
\text { Size } \\
(u m)\end{array}$ & $\begin{array}{c}\text { Retain } \\
\text { Size } \\
(u m)\end{array}$ & $\begin{array}{c}0 / S \\
\text { Mass } \\
(\mathrm{TPH})\end{array}$ & $\begin{array}{c}\text { U/S } \\
\text { Mass } \\
(\mathrm{TPH})\end{array}$ & $\begin{array}{c}\text { Feed } \\
\text { Mass } \\
(\mathrm{TPH})\end{array}$ & $\begin{array}{c}\text { Delta } \\
\text { Mass } \\
(\mathrm{TPH})\end{array}$ & $\begin{array}{c}\text { Value } \\
(\mathrm{f}-\mathrm{u})\end{array}$ & $\begin{array}{c}\mathrm{X} \\
\text { Value } \\
(\mathrm{0}-\mathrm{u})\end{array}$ & $\begin{array}{c}\text { Partition } \\
\text { Factor } \\
(-)\end{array}$ \\
\hline & 212 & 0.05 & 0.00 & 0.05 & 0.00 & 4.31 & 4.31 & 1.00 \\
\hline 212 & 150 & 0.06 & 0.00 & 0.06 & 0.00 & 5.00 & 5.00 & 1.00 \\
\hline 150 & 75 & 0.16 & 0.00 & 0.16 & 0.00 & 13.71 & 13.65 & 1.00 \\
\hline 75 & 45 & 0.12 & 0.01 & 0.12 & 0.00 & 10.12 & 9.41 & 0.93 \\
\hline 45 & 25 & 0.04 & 0.01 & 0.06 & 0.00 & 3.83 & 2.78 & 0.78 \\
\hline 25 & 13.1 & 0.06 & 0.09 & 0.15 & 0.00 & 5.24 & -2.57 & 0.40 \\
\hline 13.1 & 6.5 & 0.05 & 0.11 & 0.16 & 0.00 & 4.22 & -5.75 & 0.30 \\
\hline 6.5 & 3.3 & 0.04 & 0.12 & 0.16 & 0.00 & 3.80 & -6.30 & 0.27 \\
\hline 3.3 & 1.5 & 0.03 & 0.08 & 0.11 & 0.00 & 2.50 & -4.27 & 0.27 \\
\hline 1.5 & & 0.03 & 0.09 & 0.12 & 0.00 & 2.90 & -5.00 & 0.27 \\
\hline Totals & -- & 0.64 & 0.51 & 1.15 & 0.00 & & & \\
\hline \multicolumn{2}{c}{ Slurry (TPH) } & 9.39 & 19.79 & 25.43 & 0.00 & & & \\
\hline
\end{tabular}




\begin{tabular}{|c|c|c|c|c|c|c|c|}
\hline \multirow{3}{*}{$\begin{array}{l}\text { Experimental } \\
\text { Partition } F \text { actor }\end{array}$} & \multirow{3}{*}{$\begin{array}{c}\text { Mean } \\
\text { Particle } \\
\text { Size ( } \mu \mathrm{m})\end{array}$} & \multirow{3}{*}{$\begin{array}{c}\text { Predicted } \\
\text { Partition } \\
\text { Factor }\end{array}$} & \multirow{3}{*}{$\begin{array}{c}\text { Predicted } \\
\text { Partition } \\
\text { Factor }\end{array}$} & (Plitt) & Predicted & (Linch) & Predicted \\
\hline & & & & D50 & 31.62 & D50 & 31.44 \\
\hline & & & & $m$ & 4.71 & Alpha & 5.43 \\
\hline$(-)$ & $(-)$ & (Plitt) & (Linch) & Bypass(Rf) & 0.30 & Bypass(Rf) & 0.29 \\
\hline 1.00 & 299.81 & 1.000 & 1.000 & & & & \\
\hline 1.00 & 178.33 & 1.000 & 1.000 & & & & \\
\hline 0.97 & 106.07 & 1.000 & 1.000 & & & & \\
\hline 0.90 & 58.09 & 1.000 & 0.993 & & & & \\
\hline 0.72 & 33.54 & 0.719 & 0.709 & & & & \\
\hline 0.33 & 18.08 & 0.333 & 0.352 & & & & \\
\hline 0.31 & 9.25 & 0.300 & 0.303 & & & & \\
\hline 0.30 & 4.62 & 0.299 & 0.294 & & & & \\
\hline 0.29 & 2.32 & 0.299 & 0.292 & & & & \\
\hline 0.30 & 1.64 & 0.299 & 0.292 & & & & \\
\hline & WSSQ & 0.011 & 0.010 & & & & \\
\hline & TWSSQ & 0.021 & & & & & \\
\hline
\end{tabular}

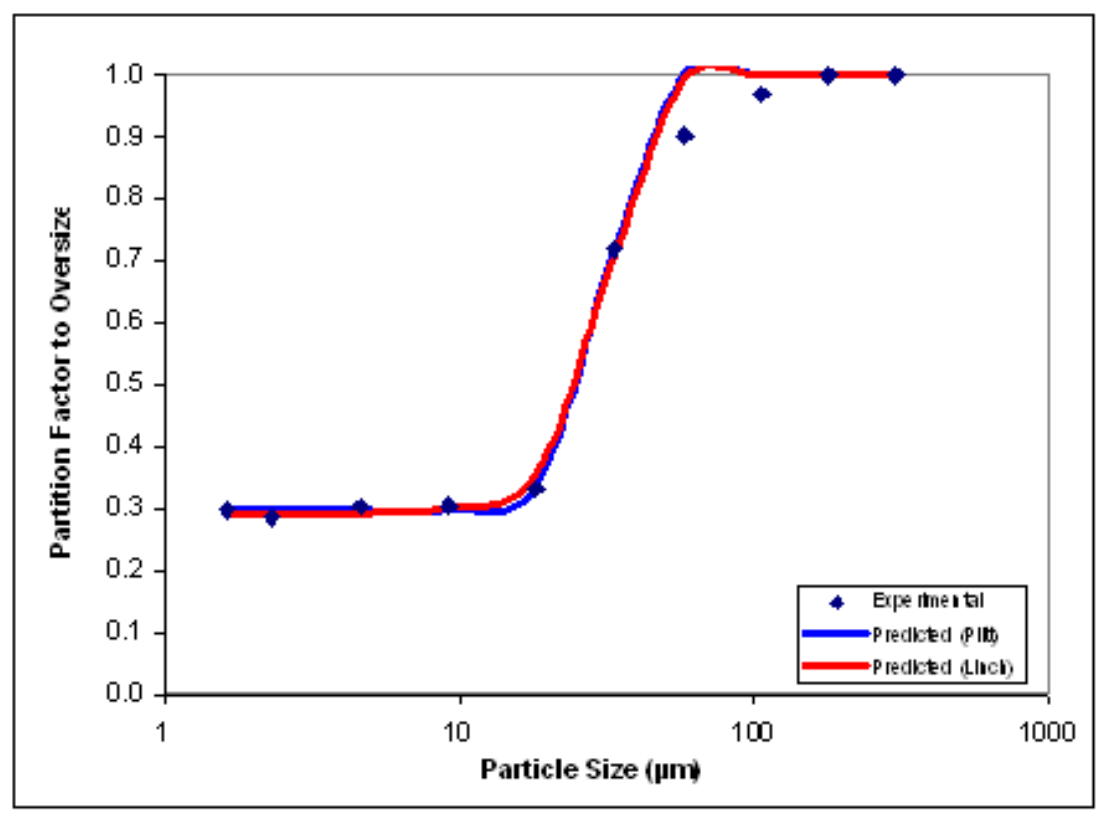


HYDROCYCLONE EVALUATION (UNBALANCED)

\begin{tabular}{l|c|}
\hline Test: No. 18 & \\
\hline \multicolumn{2}{|c|}{} \\
\hline Feed Flow (GPM) & 100.0 \\
\hline Feed Pressure (PSI) & 21.0 \\
\hline Water Flow (GPM) & 15.0 \\
\hline Water Pressure (PSI) & 12.0 \\
\hline Feed Rate (TPH) & 1.17 \\
\hline
\end{tabular}

\begin{tabular}{|r|c|c|c|}
\hline & OS & U/S & Feed \\
\hline Solids Content (\%) & 6.83 & 2.59 & 4.59 \\
\hline Ash Content (\%) & 23.72 & 48.49 & 33.95 \\
\hline Solids Yield (\%) & 56.8 & 43.2 & 100.0 \\
\hline Ash Yield (\%) & 58.7 & 41.3 & 100.0 \\
\hline Size Class Yield (\%) & 69.5 & 30.5 & 100.0 \\
\hline
\end{tabular}

\begin{tabular}{|c|c|c|c|c|c|c|c|c|}
\hline $\begin{array}{c}\text { Pass } \\
\text { Size } \\
(\text { um) }\end{array}$ & $\begin{array}{c}\text { Retain } \\
\text { Size } \\
(u m)\end{array}$ & $\begin{array}{c}\text { O/S } \\
\text { Sample } \\
(\mathrm{gms})\end{array}$ & $\begin{array}{c}\text { U/S } \\
\text { Sample } \\
(\mathrm{gms})\end{array}$ & $\begin{array}{c}\text { O/S } \\
\text { Mass } \\
(\%, \text { strm) }\end{array}$ & $\begin{array}{c}\text { U/S } \\
\text { Mass } \\
(\%, \text { strm) }\end{array}$ & $\begin{array}{c}\text { OS } \\
\text { Mass } \\
(\%, \text { feed })\end{array}$ & $\begin{array}{c}\text { U/S } \\
\text { Mass } \\
(\%, \text { feed })\end{array}$ & $\begin{array}{c}\text { Feed } \\
\text { Mass } \\
(\%, \text { feed })\end{array}$ \\
\hline & 212 & 1.57 & 0.00 & 7.93 & 0.00 & 4.66 & 0.00 & 4.66 \\
\hline 212 & 150 & 1.78 & 0.00 & 8.99 & 0.00 & 5.28 & 0.00 & 5.28 \\
\hline 150 & 75 & 5.17 & 0.12 & 26.12 & 0.71 & 15.33 & 0.30 & 15.63 \\
\hline 75 & 45 & 3.53 & 0.35 & 17.84 & 2.08 & 10.47 & 0.86 & 11.33 \\
\hline 45 & 25 & 1.33 & 0.55 & 6.72 & 3.28 & 3.94 & 1.35 & 5.30 \\
\hline 25 & 13.1 & 2.05 & 3.07 & 10.35 & 18.29 & 6.07 & 7.55 & 13.63 \\
\hline 13.1 & 6.5 & 1.50 & 3.76 & 7.58 & 22.37 & 4.45 & 9.24 & 13.68 \\
\hline 6.5 & 3.3 & 1.28 & 3.75 & 6.45 & 22.32 & 3.78 & 9.22 & 13.00 \\
\hline 3.3 & 1.5 & 0.78 & 2.49 & 3.94 & 14.83 & 2.31 & 6.12 & 8.44 \\
\hline 1.5 & & 0.81 & 2.71 & 4.08 & 16.13 & 2.40 & 6.66 & 9.06 \\
\hline Totals & --- & 19.79 & 16.79 & 100.00 & 100.00 & 58.70 & 41.30 & 100.00 \\
\hline
\end{tabular}

\begin{tabular}{|c|c|c|c|c|c|c|c|c|}
\hline $\begin{array}{c}\text { Pass } \\
\text { Size } \\
(u m)\end{array}$ & $\begin{array}{c}\text { Retain } \\
\text { Size } \\
(u m)\end{array}$ & $\begin{array}{c}0 / S \\
\text { Mass } \\
(\mathrm{TPH})\end{array}$ & $\begin{array}{c}\text { U/S } \\
\text { Mass } \\
(\mathrm{TPH})\end{array}$ & $\begin{array}{c}\text { Feed } \\
\text { Mass } \\
(\mathrm{TPH})\end{array}$ & $\begin{array}{c}\text { Delta } \\
\text { Mass } \\
(\mathrm{TPH})\end{array}$ & $\begin{array}{c}\text { Value } \\
(\mathrm{f}-\mathrm{u})\end{array}$ & $\begin{array}{c}\text { X } \\
\text { Value } \\
(0-\mathrm{u})\end{array}$ & $\begin{array}{c}\text { Partition } \\
\text { Factor } \\
(--)\end{array}$ \\
\hline & 212 & 0.05 & 0.00 & 0.05 & 0.00 & 4.66 & 4.66 & 1.00 \\
\hline 212 & 150 & 0.06 & 0.00 & 0.06 & 0.00 & 5.28 & 5.28 & 1.00 \\
\hline 150 & 75 & 0.18 & 0.00 & 0.18 & 0.00 & 15.33 & 15.04 & 0.98 \\
\hline 75 & 45 & 0.12 & 0.01 & 0.13 & 0.00 & 10.47 & 9.61 & 0.92 \\
\hline 45 & 25 & 0.05 & 0.02 & 0.06 & 0.00 & 3.94 & 2.59 & 0.74 \\
\hline 25 & 13.1 & 0.07 & 0.09 & 0.16 & 0.00 & 6.07 & -1.48 & 0.45 \\
\hline 13.1 & 6.5 & 0.05 & 0.11 & 0.16 & 0.00 & 4.45 & -4.79 & 0.32 \\
\hline 6.5 & 3.3 & 0.04 & 0.11 & 0.15 & 0.00 & 3.78 & -5.43 & 0.29 \\
\hline 3.3 & 1.5 & 0.03 & 0.07 & 0.10 & 0.00 & 2.31 & -3.81 & 0.27 \\
\hline 1.5 & & 0.03 & 0.08 & 0.11 & 0.00 & 2.40 & -4.27 & 0.26 \\
\hline Totals & -- & 0.69 & 0.48 & 1.17 & 0.00 & & & \\
\hline & Slurry (TPH) & 10.03 & 18.61 & 25.43 & 0.53 & & & \\
\hline
\end{tabular}


HYDROCYCLONE EVALUATION (BALANCED)

\begin{tabular}{l|c|}
\hline Test: No. 18 & \\
\hline \multicolumn{2}{|c|}{} \\
\hline Feed Flow (GPM) & 100.0 \\
\hline Feed Pressure (PSI) & 21.0 \\
\hline Water Flow (GPM) & 15.0 \\
\hline Water Pressure (PSI) & 12.0 \\
\hline Feed Rate (TPH) & 1.15 \\
\hline
\end{tabular}

\begin{tabular}{|r|c|c|c|}
\hline & OS & U/S & Feed \\
\hline Solids Content (\%) & 6.79 & 2.57 & 4.51 \\
\hline Ash Content (\%) & 23.74 & 48.18 & 34.58 \\
\hline Solids Yield (\%) & 55.6 & 44.4 & 100.0 \\
\hline Ash Yield (\%) & 55.6 & 44.4 & 100.0 \\
\hline Size Class Yield (\%) & 55.6 & 44.4 & 100.0 \\
\hline
\end{tabular}

\begin{tabular}{|c|c|c|c|c|c|c|c|c|}
\hline Pass & Retain & $0 / 5$ & U/S & o/s & U/S & OS & U/S & Feed \\
\hline $\begin{array}{l}\text { Size } \\
\text { (um) }\end{array}$ & $\begin{array}{l}\text { Size } \\
\text { (um) }\end{array}$ & $\begin{array}{c}\text { Sample } \\
\text { (gms) }\end{array}$ & $\begin{array}{c}\text { Sample } \\
\text { (gms) }\end{array}$ & $\begin{array}{c}\text { Mass } \\
(\%, \text { strm) }\end{array}$ & $\begin{array}{c}\text { Mass } \\
(\%, \text { strm })\end{array}$ & $\begin{array}{c}\text { Mass } \\
(\% \text {, feed })\end{array}$ & $\begin{array}{c}\text { Mass } \\
(\%, \text { feed })\end{array}$ & $\begin{array}{c}\text { Mass } \\
(\%, \text { feed })\end{array}$ \\
\hline & 212 & 1.63 & 0.00 & 7.75 & 0.00 & 4.31 & 0.00 & 4.31 \\
\hline 212 & 150 & 1.89 & 0.00 & 8.99 & 0.00 & 5.00 & 0.00 & 5.00 \\
\hline 150 & 75 & 5.19 & 0.02 & 24.64 & 0.12 & 13.71 & 0.05 & 13.76 \\
\hline 75 & 45 & 3.83 & 0.27 & 18.18 & 1.59 & 10.12 & 0.71 & 10.82 \\
\hline 45 & 25 & 1.45 & 0.40 & 6.89 & 2.37 & 3.83 & 1.05 & 4.89 \\
\hline 25 & 13.1 & 1.98 & 2.94 & 9.42 & 17.60 & 5.24 & 7.81 & 13.05 \\
\hline 13.1 & 6.5 & 1.60 & 3.76 & 7.59 & 22.48 & 4.22 & 9.98 & 14.20 \\
\hline 6.5 & 3.3 & 1.44 & 3.80 & 6.82 & 22.76 & 3.80 & 10.10 & 13.90 \\
\hline 3.3 & 1.5 & 0.95 & 2.55 & 4.50 & 15.27 & 2.50 & 6.77 & 9.28 \\
\hline 1.5 & & 1.10 & 2.97 & 5.22 & 17.80 & 2.90 & 7.90 & 10.80 \\
\hline Totals & -- & 21.07 & 16.70 & 100.00 & 100.00 & 55.63 & 44.37 & 100.00 \\
\hline
\end{tabular}

\begin{tabular}{|c|c|c|c|c|c|c|c|c|}
\hline $\begin{array}{c}\text { Pass } \\
\text { Size } \\
(u m)\end{array}$ & $\begin{array}{c}\text { Retain } \\
\text { Size } \\
(u m)\end{array}$ & $\begin{array}{c}0 / S \\
\text { Mass } \\
(\mathrm{TPH})\end{array}$ & $\begin{array}{c}\text { U/S } \\
\text { Mass } \\
(\mathrm{TPH})\end{array}$ & $\begin{array}{c}\text { Feed } \\
\text { Mass } \\
(\mathrm{TPH})\end{array}$ & $\begin{array}{c}\text { Delta } \\
\text { Mass } \\
(\mathrm{TPH})\end{array}$ & $\begin{array}{c}\text { Value } \\
(\mathrm{f}-\mathrm{u})\end{array}$ & $\begin{array}{c}\mathrm{X} \\
\text { Value } \\
(\mathrm{0}-\mathrm{u})\end{array}$ & $\begin{array}{c}\text { Partition } \\
\text { Factor } \\
(-)\end{array}$ \\
\hline & 212 & 0.05 & 0.00 & 0.05 & 0.00 & 4.31 & 4.31 & 1.00 \\
\hline 212 & 150 & 0.06 & 0.00 & 0.06 & 0.00 & 5.00 & 5.00 & 1.00 \\
\hline 150 & 75 & 0.16 & 0.00 & 0.16 & 0.00 & 13.71 & 13.65 & 1.00 \\
\hline 75 & 45 & 0.12 & 0.01 & 0.12 & 0.00 & 10.12 & 9.41 & 0.93 \\
\hline 45 & 25 & 0.04 & 0.01 & 0.06 & 0.00 & 3.83 & 2.78 & 0.78 \\
\hline 25 & 13.1 & 0.06 & 0.09 & 0.15 & 0.00 & 5.24 & -2.57 & 0.40 \\
\hline 13.1 & 6.5 & 0.05 & 0.11 & 0.16 & 0.00 & 4.22 & -5.75 & 0.30 \\
\hline 6.5 & 3.3 & 0.04 & 0.12 & 0.16 & 0.00 & 3.80 & -6.30 & 0.27 \\
\hline 3.3 & 1.5 & 0.03 & 0.08 & 0.11 & 0.00 & 2.50 & -4.27 & 0.27 \\
\hline 1.5 & & 0.03 & 0.09 & 0.12 & 0.00 & 2.90 & -5.00 & 0.27 \\
\hline Totals & -- & 0.64 & 0.51 & 1.15 & 0.00 & & & \\
\hline \multicolumn{2}{c}{ Slurry (TPH) } & 9.39 & 19.79 & 25.43 & 0.00 & & & \\
\hline
\end{tabular}




\begin{tabular}{|c|c|c|c|}
\hline $\begin{array}{c}\text { Experimental } \\
\text { Partition Factor }\end{array}$ & $\begin{array}{c}\text { Mean } \\
\text { Particle } \\
\text { Size }(\mu \mathrm{m})\end{array}$ & $\begin{array}{c}\text { Predicted } \\
\text { Partition } \\
\text { Factor }\end{array}$ & $\begin{array}{c}\text { Predicted } \\
\text { Partition } \\
\text { Factor }\end{array}$ \\
\hline$(-)$ & $(--)$ & (Plitt) & (Linch) \\
\hline 1.00 & 299.81 & 1.000 & 1.000 \\
\hline 1.00 & 178.33 & 1.000 & 1.000 \\
\hline 0.98 & 106.07 & 1.000 & 0.999 \\
\hline 0.92 & 58.09 & 0.947 & 0.953 \\
\hline 0.74 & 33.54 & 0.721 & 0.724 \\
\hline 0.45 & 18.08 & 0.463 & 0.458 \\
\hline 0.32 & 9.25 & 0.329 & 0.334 \\
\hline 0.29 & 4.62 & 0.283 & 0.287 \\
\hline 0.27 & 2.32 & 0.270 & 0.268 \\
\hline 0.26 & 1.64 & 0.267 & 0.263 \\
\hline & WSSQ & 0.002 & 0.002 \\
\hline
\end{tabular}

\begin{tabular}{|c|c|}
\multicolumn{1}{c}{ (Plitt) } & Predicted \\
\hline $\mathrm{D} 50$ & 27.88 \\
\hline $\mathrm{m}$ & 1.82 \\
\hline Bypass(R0) & 0.26 \\
\hline
\end{tabular}

(Linch) Predicted

\begin{tabular}{|c|c|}
\hline D50 & 27.70 \\
\hline Alpha & 2.37 \\
\hline Bypass(Rf) & 0.25 \\
\hline
\end{tabular}

\begin{tabular}{|l|l|}
\hline TWSSQ & 0.004 \\
\hline
\end{tabular}

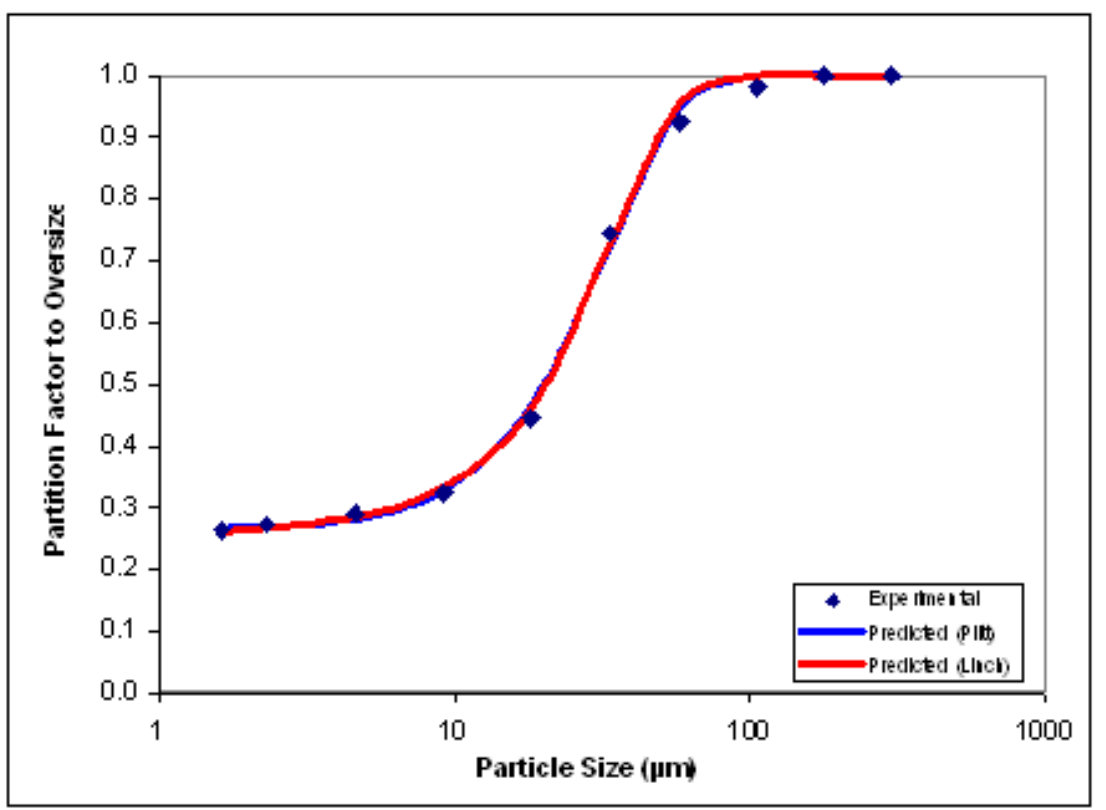


HYDROCYCLONE EVALUATION (UNBALANCED)

\begin{tabular}{|c|c|}
\hline No. 19 & \\
\hline Feedflow (GPM) & 100.0 \\
\hline Feed Pressure (PSl) & 21.0 \\
\hline Water Flow (GPM) & 30.0 \\
\hline Water Pressure (PSI) & 17.0 \\
\hline Feed Rate (TPH) & 1.17 \\
\hline
\end{tabular}

\begin{tabular}{|r|c|c|c|}
\hline & O/S & U/S & Feed \\
\hline Solids Content (\%) & 5.34 & 2.78 & 4.61 \\
\hline Ash Content (\%) & 18.60 & 47.65 & 33.90 \\
\hline Solids Yield (\%) & 45.7 & 54.3 & 100.0 \\
\hline Ash Yield (\%) & 47.3 & 52.7 & 100.0 \\
\hline Size Class Yield (\%) & 35.0 & 65.0 & 100.0 \\
\hline
\end{tabular}

\begin{tabular}{|c|c|c|c|c|c|c|c|c|}
\hline $\begin{array}{c}\text { Pass } \\
\text { Size } \\
(\mathrm{um})\end{array}$ & $\begin{array}{c}\text { Retain } \\
\text { Size } \\
(\mathrm{um})\end{array}$ & $\begin{array}{c}\text { O/S } \\
\text { Sample } \\
(\mathrm{gms})\end{array}$ & $\begin{array}{c}\text { U/S } \\
\text { Sample } \\
(\mathrm{gms})\end{array}$ & $\begin{array}{c}\text { O/S } \\
\text { Mass } \\
(\%, \text { strm) }\end{array}$ & $\begin{array}{c}\text { U/S } \\
\text { Mass } \\
(\%, \text { strm) }\end{array}$ & $\begin{array}{c}\text { OS } \\
\text { Mass } \\
(\%, \text { feed })\end{array}$ & $\begin{array}{c}\text { U/S } \\
\text { Mass } \\
(\%, \text { feed })\end{array}$ & $\begin{array}{c}\text { Feed } \\
\text { Mass } \\
(\%, \text { feed })\end{array}$ \\
\hline & 212 & 1.55 & 0.00 & 9.29 & 0.00 & 4.40 & 0.00 & 4.40 \\
\hline 212 & 150 & 1.64 & 0.00 & 9.83 & 0.00 & 4.65 & 0.00 & 4.65 \\
\hline 150 & 75 & 4.80 & 0.17 & 28.78 & 0.85 & 13.62 & 0.45 & 14.07 \\
\hline 75 & 45 & 2.95 & 0.43 & 17.69 & 2.15 & 8.37 & 1.13 & 9.50 \\
\hline 45 & 25 & 1.08 & 0.69 & 6.47 & 3.45 & 3.06 & 1.82 & 4.88 \\
\hline 25 & 13.1 & 1.29 & 3.78 & 7.73 & 18.87 & 3.66 & 9.94 & 13.60 \\
\hline 13.1 & 6.5 & 1.03 & 4.46 & 6.16 & 22.29 & 2.92 & 11.74 & 14.65 \\
\hline 6.5 & 3.3 & 0.97 & 4.40 & 5.81 & 21.98 & 2.75 & 11.57 & 14.32 \\
\hline 3.3 & 1.5 & 0.63 & 2.81 & 3.79 & 14.04 & 1.79 & 7.39 & 9.19 \\
\hline 1.5 & & 0.74 & 3.28 & 4.45 & 16.38 & 2.11 & 8.63 & 10.73 \\
\hline Totals & -- & 16.68 & 20.01 & 100.00 & 100.00 & 47.33 & 52.67 & 100.00 \\
\hline
\end{tabular}

\begin{tabular}{|c|c|c|c|c|c|c|c|c|}
\hline $\begin{array}{c}\text { Pass } \\
\text { Size } \\
(u m)\end{array}$ & $\begin{array}{c}\text { Retain } \\
\text { Size } \\
(u m)\end{array}$ & $\begin{array}{c}\text { O/S } \\
\text { Mass } \\
(\mathrm{TPH})\end{array}$ & $\begin{array}{c}\text { U/S } \\
\text { Mass } \\
(\mathrm{TPH})\end{array}$ & $\begin{array}{c}\text { Feed } \\
\text { Mass } \\
(\mathrm{TPH})\end{array}$ & $\begin{array}{c}\text { Delta } \\
\text { Mass } \\
(\mathrm{TPH})\end{array}$ & $\begin{array}{c}\text { Y } \\
\text { Value } \\
(\mathrm{f}-\mathrm{u})\end{array}$ & $\begin{array}{c}\text { X } \\
\text { Value } \\
(0-\mathrm{u})\end{array}$ & $\begin{array}{c}\text { Partition } \\
\text { Factor } \\
(-)\end{array}$ \\
\hline & 212 & 0.05 & 0.00 & 0.05 & 0.00 & 4.40 & 4.40 & 1.00 \\
\hline 212 & 150 & 0.05 & 0.00 & 0.05 & 0.00 & 4.65 & 4.65 & 1.00 \\
\hline 150 & 75 & 0.16 & 0.01 & 0.16 & 0.00 & 13.62 & 13.17 & 0.97 \\
\hline 75 & 45 & 0.10 & 0.01 & 0.11 & 0.00 & 8.37 & 7.24 & 0.88 \\
\hline 45 & 25 & 0.04 & 0.02 & 0.06 & 0.00 & 3.06 & 1.25 & 0.63 \\
\hline 25 & 13.1 & 0.04 & 0.12 & 0.16 & 0.00 & 3.66 & -6.28 & 0.27 \\
\hline 13.1 & 6.5 & 0.03 & 0.14 & 0.17 & 0.00 & 2.92 & -8.82 & 0.20 \\
\hline 6.5 & 3.3 & 0.03 & 0.14 & 0.17 & 0.00 & 2.75 & -8.82 & 0.19 \\
\hline 3.3 & 1.5 & 0.02 & 0.09 & 0.11 & 0.00 & 1.79 & -5.60 & 0.20 \\
\hline 1.5 & & 0.02 & 0.10 & 0.13 & 0.00 & 2.11 & -6.52 & 0.20 \\
\hline Totals & -- & 0.55 & 0.62 & 1.17 & 0.00 & & & \\
\hline & Slurry (TPH) & 10.39 & 22.21 & 25.43 & 0.32 & & & \\
\hline
\end{tabular}


HYDROCYCLONE EVALUATION (BALANCED)

\begin{tabular}{|c|c|}
\hline No. 19 & \\
\hline Feedflow (GPM) & 100.0 \\
\hline Feed Pressure (PSl) & 21.0 \\
\hline Water Flow (GPM) & 30.0 \\
\hline Water Pressure (PSI) & 17.0 \\
\hline Feed Rate (TPH) & 1.15 \\
\hline
\end{tabular}

\begin{tabular}{|r|c|c|c|}
\hline & O/S & U/S & Feed \\
\hline Solids Content (\%) & 6.79 & 2.57 & 4.51 \\
\hline Ash Content (\%) & 23.74 & 48.18 & 34.58 \\
\hline Solids Yield (\%) & 42.1 & 57.9 & 100.0 \\
\hline Ash Yield (\%) & 55.6 & 44.4 & 100.0 \\
\hline Size Class Yield (\%) & 55.6 & 44.4 & 100.0 \\
\hline
\end{tabular}

\begin{tabular}{|c|c|c|c|c|c|c|c|c|}
\hline $\begin{array}{c}\text { Pass } \\
\text { Size } \\
(u m)\end{array}$ & $\begin{array}{c}\text { Retain } \\
\text { Size } \\
(\mathrm{um})\end{array}$ & $\begin{array}{c}\text { O/S } \\
\text { Sample } \\
(\mathrm{gms})\end{array}$ & $\begin{array}{c}\text { U/S } \\
\text { Sample } \\
(\mathrm{gms})\end{array}$ & $\begin{array}{c}\text { O/S } \\
\text { Mass } \\
(\%, \text { strm) }\end{array}$ & $\begin{array}{c}\text { U/S } \\
\text { Mass } \\
(\%, \text { strm })\end{array}$ & $\begin{array}{c}\text { OS } \\
\text { Mass } \\
(\%, \text { feed })\end{array}$ & $\begin{array}{c}\text { U/S } \\
\text { Mass } \\
(\%, \text { feed })\end{array}$ & $\begin{array}{c}\text { Feed } \\
\text { Mass } \\
(\%, \text { feed })\end{array}$ \\
\hline & 212 & 1.63 & 0.00 & 7.75 & 0.00 & 4.31 & 0.00 & 4.31 \\
\hline 212 & 150 & 1.89 & 0.00 & 8.99 & 0.00 & 5.00 & 0.00 & 5.00 \\
\hline 150 & 75 & 5.19 & 0.02 & 24.64 & 0.12 & 13.71 & 0.05 & 13.76 \\
\hline 75 & 45 & 3.83 & 0.27 & 18.18 & 1.59 & 10.12 & 0.71 & 10.82 \\
\hline 45 & 25 & 1.45 & 0.40 & 6.89 & 2.37 & 3.83 & 1.05 & 4.89 \\
\hline 25 & 13.1 & 1.98 & 2.94 & 9.42 & 17.60 & 5.24 & 7.81 & 13.05 \\
\hline 13.1 & 6.5 & 1.60 & 3.76 & 7.59 & 22.48 & 4.22 & 9.98 & 14.20 \\
\hline 6.5 & 3.3 & 1.44 & 3.80 & 6.82 & 22.76 & 3.80 & 10.10 & 13.90 \\
\hline 3.3 & 1.5 & 0.95 & 2.55 & 4.50 & 15.27 & 2.50 & 6.77 & 9.28 \\
\hline 1.5 & & 1.10 & 2.97 & 5.22 & 17.80 & 2.90 & 7.90 & 10.80 \\
\hline Totals & -- & 21.07 & 16.70 & 100.00 & 100.00 & 55.63 & 44.37 & 100.00 \\
\hline
\end{tabular}

\begin{tabular}{|c|c|c|c|c|c|c|c|c|}
\hline $\begin{array}{c}\text { Pass } \\
\text { Size } \\
(\mathrm{um})\end{array}$ & $\begin{array}{c}\text { Retain } \\
\text { Size } \\
(\mathrm{um})\end{array}$ & $\begin{array}{c}0 / \mathrm{S} \\
\text { Mass } \\
(\mathrm{TPH})\end{array}$ & $\begin{array}{c}\mathrm{U} / \mathrm{S} \\
\text { Mass } \\
(\mathrm{TPH})\end{array}$ & $\begin{array}{c}\text { Feed } \\
\text { Mass } \\
(\mathrm{TPH})\end{array}$ & $\begin{array}{c}\text { Delta } \\
\text { Mass } \\
(\mathrm{TPH})\end{array}$ & $\begin{array}{c}\text { Y } \\
\text { Value } \\
(\mathrm{f}-\mathrm{u})\end{array}$ & $\begin{array}{c}\text { X } \\
\text { Value } \\
(\mathbf{0}-\mathrm{u})\end{array}$ & $\begin{array}{c}\text { Partition } \\
\text { Factor } \\
(-)\end{array}$ \\
\hline & 212 & 0.05 & 0.00 & 0.05 & 0.00 & 4.31 & 4.31 & 1.00 \\
\hline 212 & 150 & 0.06 & 0.00 & 0.06 & 0.00 & 5.00 & 5.00 & 1.00 \\
\hline 150 & 75 & 0.16 & 0.00 & 0.16 & 0.00 & 13.71 & 13.65 & 1.00 \\
\hline 75 & 45 & 0.12 & 0.01 & 0.12 & 0.00 & 10.12 & 9.41 & 0.93 \\
\hline 45 & 25 & 0.04 & 0.01 & 0.06 & 0.00 & 3.83 & 2.78 & 0.78 \\
\hline 25 & 13.1 & 0.06 & 0.09 & 0.15 & 0.00 & 5.24 & -2.57 & 0.40 \\
\hline 13.1 & 6.5 & 0.05 & 0.11 & 0.16 & 0.00 & 4.22 & -5.75 & 0.30 \\
\hline 6.5 & 3.3 & 0.04 & 0.12 & 0.16 & 0.00 & 3.80 & -6.30 & 0.27 \\
\hline 3.3 & 1.5 & 0.03 & 0.08 & 0.11 & 0.00 & 2.50 & -4.27 & 0.27 \\
\hline 1.5 & & 0.03 & 0.09 & 0.12 & 0.00 & 2.90 & -5.00 & 0.27 \\
\hline Totals & -- & 0.64 & 0.51 & 1.15 & 0.00 & & & \\
\hline \multicolumn{2}{r}{ Slurry (TPH) } & 9.39 & 19.79 & 25.43 & 3.75 & & & \\
\hline
\end{tabular}




\begin{tabular}{|c|c|c|c|}
\hline $\begin{array}{c}\text { Experimental } \\
\text { Partition F actor }\end{array}$ & $\begin{array}{c}\text { Mean } \\
\text { Particle } \\
\text { Size }(\mu \mathrm{m})\end{array}$ & $\begin{array}{c}\text { Predicted } \\
\text { Partition } \\
\text { Factor }\end{array}$ & $\begin{array}{c}\text { Predicted } \\
\text { Partition } \\
\text { Factor }\end{array}$ \\
\hline$(-)$ & $(--)$ & (Plitt) & (Linch) \\
\hline 1.00 & 299.81 & 1.000 & 1.000 \\
\hline 1.00 & 178.33 & 1.000 & 1.000 \\
\hline 0.97 & 106.07 & 1.000 & 0.999 \\
\hline 0.88 & 58.09 & 0.915 & 0.927 \\
\hline 0.63 & 33.54 & 0.585 & 0.587 \\
\hline 0.27 & 18.08 & 0.312 & 0.310 \\
\hline 0.20 & 9.25 & 0.213 & 0.220 \\
\hline 0.19 & 4.62 & 0.188 & 0.190 \\
\hline 0.20 & 2.32 & 0.182 & 0.179 \\
\hline 0.20 & 1.64 & 0.181 & 0.176 \\
\hline & WSSQ & 0.007 & 0.008 \\
\hline
\end{tabular}

\begin{tabular}{|c|c|c|c|}
\hline (Plitt) & Predicted & (Linch) & Predicted \\
\hline D50 & 33.85 & D50 & 33.46 \\
\hline$m$ & 2.19 & Alpha & 3.12 \\
\hline Bypass(R0) & 0.18 & Bypass(Rf) & 0.17 \\
\hline
\end{tabular}

\begin{tabular}{|l|l|}
\hline TWSSQ & 0.014 \\
\hline
\end{tabular}

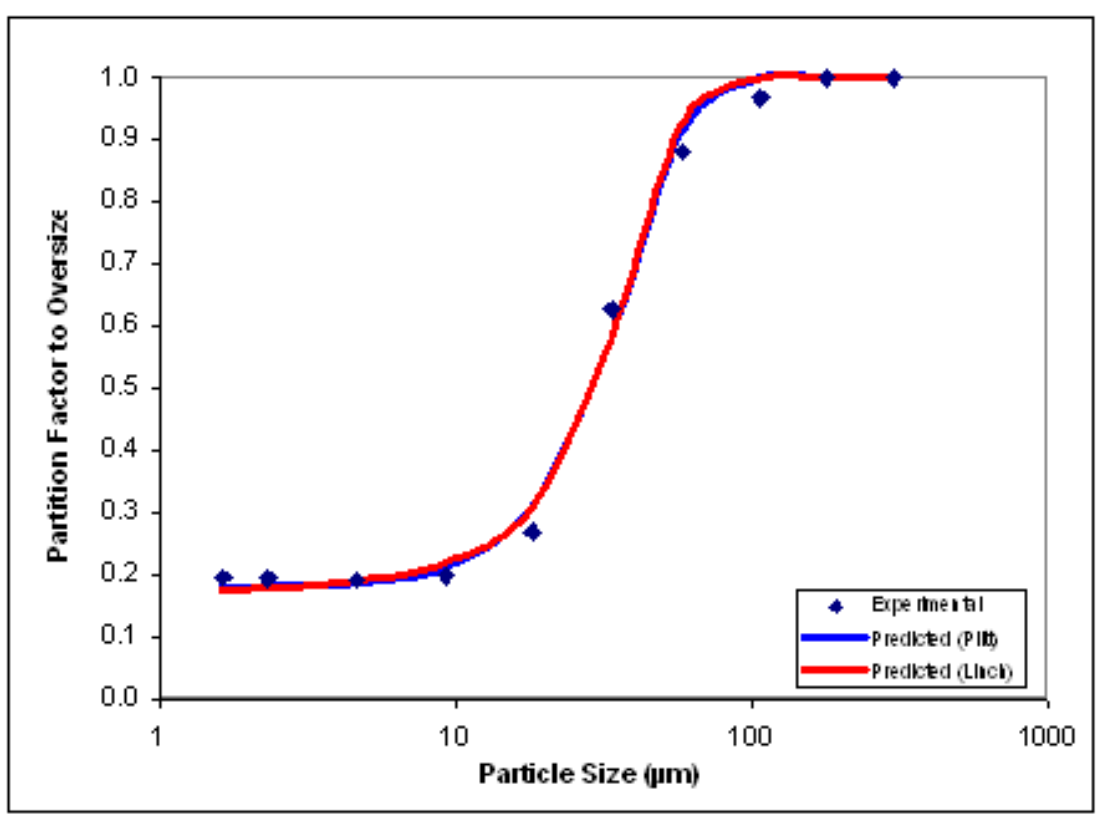


HYDROCYCLONE EVALUATION (UNBALANCED)

\begin{tabular}{|c|c|}
\hline No. 20 & \\
\hline Feedflow (GPM) & 10000 \\
\hline Feed Pressure (PSI) & 21.0 \\
\hline Water Flow (GPM) & 15.0 \\
\hline Water Pressure (PSI) & 12.0 \\
\hline Feed Rate (TPH) & 1.14 \\
\hline
\end{tabular}

\begin{tabular}{|r|c|c|c|}
\hline & OS & U/S & Feed \\
\hline Solids Content (\%) & 5.17 & 2.37 & 4.50 \\
\hline Ash Content (\%) & 29.26 & 49.49 & 34.55 \\
\hline Solids Yield (\%) & 73.1 & 26.9 & 100.0 \\
\hline Ash Yield (\%) & 73.9 & 26.1 & 100.0 \\
\hline Size Class Yield (\%) & 113.2 & -13.2 & 100.0 \\
\hline
\end{tabular}

\begin{tabular}{|c|c|c|c|c|c|c|c|c|}
\hline Pass & Retain & $0 / 5$ & U/S & o/S & U/S & OS & U/S & Feed \\
\hline $\begin{array}{l}\text { Size } \\
\text { (um) }\end{array}$ & $\begin{array}{l}\text { Size } \\
\text { (um) }\end{array}$ & $\begin{array}{c}\text { Sample } \\
\text { (gms) }\end{array}$ & $\begin{array}{c}\text { Sample } \\
\text { (gms) }\end{array}$ & $\begin{array}{c}\text { Mass } \\
(\%, \text { strm) }\end{array}$ & $\begin{array}{c}\text { Mass } \\
(\%, \text { strm })\end{array}$ & $\begin{array}{c}\text { Mass } \\
(\% \text {, feed })\end{array}$ & $\begin{array}{c}\text { Mass } \\
(\%, \text { feed })\end{array}$ & $\begin{array}{c}\text { Mass } \\
(\%, \text { feed })\end{array}$ \\
\hline & 212 & 2.12 & 0.00 & 7.26 & 0.00 & 5.36 & 0.00 & 5.36 \\
\hline 212 & 150 & 2.39 & 0.00 & 8.18 & 0.00 & 6.04 & 0.00 & 6.04 \\
\hline 150 & 75 & 5.63 & 0.11 & 19.28 & 0.98 & 14.24 & 0.26 & 14.49 \\
\hline 75 & 45 & 4.01 & 0.23 & 13.73 & 2.05 & 10.14 & 0.54 & 10.68 \\
\hline 45 & 25 & 1.91 & 0.21 & 6.54 & 1.87 & 4.83 & 0.49 & 5.32 \\
\hline 25 & 13.1 & 3.43 & 1.90 & 11.75 & 16.94 & 8.68 & 4.43 & 13.11 \\
\hline 13.1 & 6.5 & 3.05 & 2.58 & 10.44 & 22.99 & 7.71 & 6.01 & 13.72 \\
\hline 6.5 & 3.3 & 2.82 & 2.59 & 9.67 & 23.01 & 7.14 & 6.02 & 13.16 \\
\hline 3.3 & 1.5 & 1.78 & 1.67 & 6.10 & 14.85 & 4.50 & 3.88 & 8.38 \\
\hline 1.5 & & 2.06 & 1.95 & 7.05 & 17.31 & 5.20 & 4.53 & 9.73 \\
\hline Totals & -- & 29.20 & 11.24 & 100.00 & 100.00 & 73.85 & 26.15 & 100.00 \\
\hline
\end{tabular}

\begin{tabular}{|c|c|c|c|c|c|c|c|c|}
\hline $\begin{array}{c}\text { Pass } \\
\text { Size } \\
(u m)\end{array}$ & $\begin{array}{c}\text { Retain } \\
\text { Size } \\
(u m)\end{array}$ & $\begin{array}{c}0 / 5 \\
\text { Mass } \\
(\mathrm{TPH})\end{array}$ & $\begin{array}{c}\text { U/S } \\
\text { Mass } \\
(\mathrm{TPH})\end{array}$ & $\begin{array}{c}\text { Feed } \\
\text { Mass } \\
(\mathrm{TPH})\end{array}$ & $\begin{array}{c}\text { Delta } \\
\text { Mass } \\
(\mathrm{TPH})\end{array}$ & $\begin{array}{c}\text { Value } \\
(\mathrm{f}-\mathrm{u})\end{array}$ & $\begin{array}{c}\mathrm{X} \\
\text { Value } \\
(\mathrm{0}-\mathrm{u})\end{array}$ & $\begin{array}{c}\text { Partition } \\
\text { Factor } \\
(-)\end{array}$ \\
\hline & 212 & 0.06 & 0.00 & 0.06 & 0.00 & 5.36 & 5.36 & 1.00 \\
\hline 212 & 150 & 0.07 & 0.00 & 0.07 & 0.00 & 6.04 & 6.04 & 1.00 \\
\hline 150 & 75 & 0.16 & 0.00 & 0.17 & 0.00 & 14.24 & 13.98 & 0.98 \\
\hline 75 & 45 & 0.12 & 0.01 & 0.12 & 0.00 & 10.14 & 9.61 & 0.95 \\
\hline 45 & 25 & 0.06 & 0.01 & 0.06 & 0.00 & 4.83 & 4.34 & 0.91 \\
\hline 25 & 13.1 & 0.10 & 0.05 & 0.15 & 0.00 & 8.68 & 4.25 & 0.66 \\
\hline 13.1 & 6.5 & 0.09 & 0.07 & 0.16 & 0.00 & 7.71 & 1.69 & 0.56 \\
\hline 6.5 & 3.3 & 0.08 & 0.07 & 0.15 & 0.00 & 7.14 & 1.12 & 0.54 \\
\hline 3.3 & 1.5 & 0.05 & 0.04 & 0.10 & 0.00 & 4.50 & 0.62 & 0.54 \\
\hline 1.5 & & 0.06 & 0.05 & 0.11 & 0.00 & 5.20 & 0.68 & 0.53 \\
\hline Totals & -- & 0.85 & 0.30 & 1.14 & 0.00 & & & \\
\hline & Slurry (TPH) & 16.34 & 12.62 & 25.43 & 0.21 & & & \\
\hline
\end{tabular}




\section{HYDROCYCLONE EVALUATION (BALANCED)}

\begin{tabular}{|c|c|}
\hline No. 20 & \\
\hline Feedflow (GPM) & 100.0 \\
\hline Feed Pressure (PSl) & 21.0 \\
\hline Water Flow (GPM) & 15.0 \\
\hline Water Pressure (PSl) & 12.0 \\
\hline Feed Rate (TPH) & 1.15 \\
\hline
\end{tabular}

\begin{tabular}{|r|c|c|c|}
\hline & OS & U/S & Feed \\
\hline Solids Content (\%) & 6.79 & 2.57 & 4.51 \\
\hline Ash Content (\%) & 23.74 & 48.18 & 34.58 \\
\hline Solids Yield (\%) & 55.6 & 44.4 & 100.0 \\
\hline Ash Yield (\%) & 55.6 & 44.4 & 100.0 \\
\hline Size Class Yield (\%) & 55.6 & 44.4 & 100.0 \\
\hline
\end{tabular}

\begin{tabular}{|c|c|c|c|c|c|c|c|c|}
\hline $\begin{array}{l}\text { Pass } \\
\text { Size } \\
\text { (um) }\end{array}$ & $\begin{array}{l}\text { Retain } \\
\text { Size } \\
\text { (um) }\end{array}$ & $\begin{array}{c}\text { O/S } \\
\text { Sample } \\
\text { (gms) }\end{array}$ & $\begin{array}{c}\text { U/S } \\
\text { Sample } \\
\text { (gms) }\end{array}$ & $\begin{array}{c}\text { orS } \\
\text { Mass } \\
(\% \text {, strm })\end{array}$ & $\begin{array}{c}\text { U/S } \\
\text { Mass } \\
(\% \text {, strm) }\end{array}$ & $\begin{array}{c}\text { OS } \\
\text { Mass } \\
(\% \text {, feed })\end{array}$ & $\begin{array}{c}\text { U/S } \\
\text { Mass } \\
(\%, \text { feed })\end{array}$ & $\begin{array}{c}\text { Feed } \\
\text { Mass } \\
(\% \text {, feed })\end{array}$ \\
\hline & 212 & 1.63 & 0.00 & 7.75 & 0.00 & 4.31 & 0.00 & 4.31 \\
\hline 212 & 150 & 1.89 & 0.00 & 8.99 & 0.00 & 5.00 & 0.00 & 5.00 \\
\hline 150 & 75 & 5.19 & 0.02 & 24.64 & 0.12 & 13.71 & 0.05 & 13.76 \\
\hline 75 & 45 & 3.83 & 0.27 & 18.18 & 1.59 & 10.12 & 0.71 & 10.82 \\
\hline 45 & 25 & 1.45 & 0.40 & 6.89 & 2.37 & 3.83 & 1.05 & 4.89 \\
\hline 25 & 13.1 & 1.98 & 2.94 & 9.42 & 17.60 & 5.24 & 7.81 & 13.05 \\
\hline 13.1 & 6.5 & 1.60 & 3.76 & 7.59 & 22.48 & 4.22 & 9.98 & 14.20 \\
\hline 6.5 & 3.3 & 1.44 & 3.80 & 6.82 & 22.76 & 3.80 & 10.10 & 13.90 \\
\hline 3.3 & 1.5 & 0.95 & 2.55 & 4.50 & 15.27 & 2.50 & 6.77 & 9.28 \\
\hline 1.5 & & 1.10 & 2.97 & 5.22 & 17.80 & 2.90 & 7.90 & 10.80 \\
\hline Totals & -- & 21.07 & 16.70 & 100.00 & 100.00 & 55.63 & 44.37 & 100.00 \\
\hline
\end{tabular}

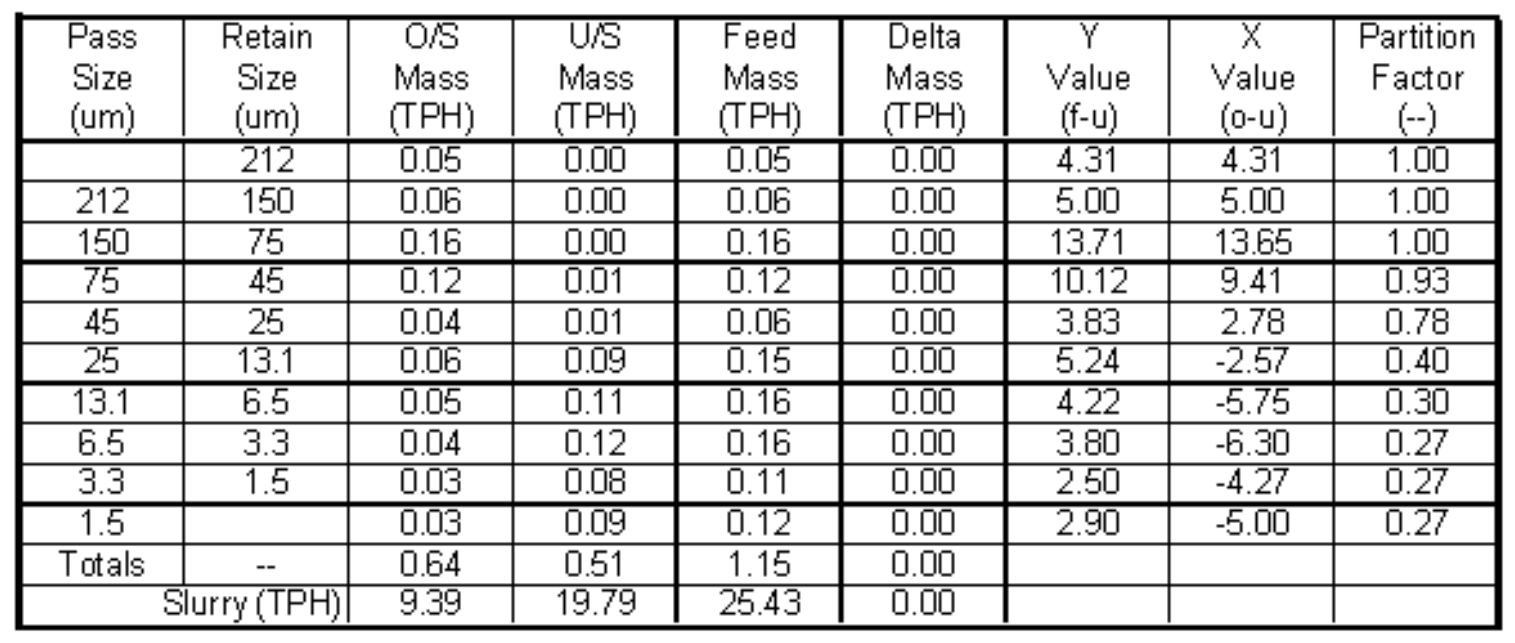




\begin{tabular}{|c|c|c|c|}
\hline $\begin{array}{c}\text { Experimental } \\
\text { Partition Factor }\end{array}$ & $\begin{array}{c}\text { Mean } \\
\text { Particle } \\
\text { Size }(\mu \mathrm{m})\end{array}$ & $\begin{array}{c}\text { Predicted } \\
\text { Partition } \\
\text { Factor }\end{array}$ & $\begin{array}{c}\text { Predicted } \\
\text { Partition } \\
\text { Factor }\end{array}$ \\
\hline$(-)$ & $(--)$ & (Plitt) & (Linch) \\
\hline 1.00 & 299.81 & 1.000 & 1.000 \\
\hline 1.00 & 178.33 & 1.000 & 1.000 \\
\hline 0.98 & 106.07 & 1.000 & 1.000 \\
\hline 0.95 & 58.09 & 0.999 & 0.996 \\
\hline 0.91 & 33.54 & 0.905 & 0.903 \\
\hline 0.66 & 18.08 & 0.664 & 0.662 \\
\hline 0.56 & 9.25 & 0.562 & 0.567 \\
\hline 0.54 & 4.62 & 0.540 & 0.543 \\
\hline 0.54 & 2.32 & 0.537 & 0.535 \\
\hline 0.53 & 1.64 & 0.536 & 0.533 \\
\hline & WSSQ & 0.003 & 0.003 \\
\hline
\end{tabular}

\begin{tabular}{|c|c|c|c|}
\hline (Plitt) & Predicted & (Linch) & Predicted \\
\hline D50 & 24.31 & $\mathrm{D} 50$ & 24.23 \\
\hline $\mathrm{m}$ & 2.58 & Alpha & 3.46 \\
\hline Bypass(R0) & 0.54 & Bypass(Rf) & 0.53 \\
\hline
\end{tabular}

\begin{tabular}{l|l}
\hline TWSSQ & 0.005 \\
\hline
\end{tabular}

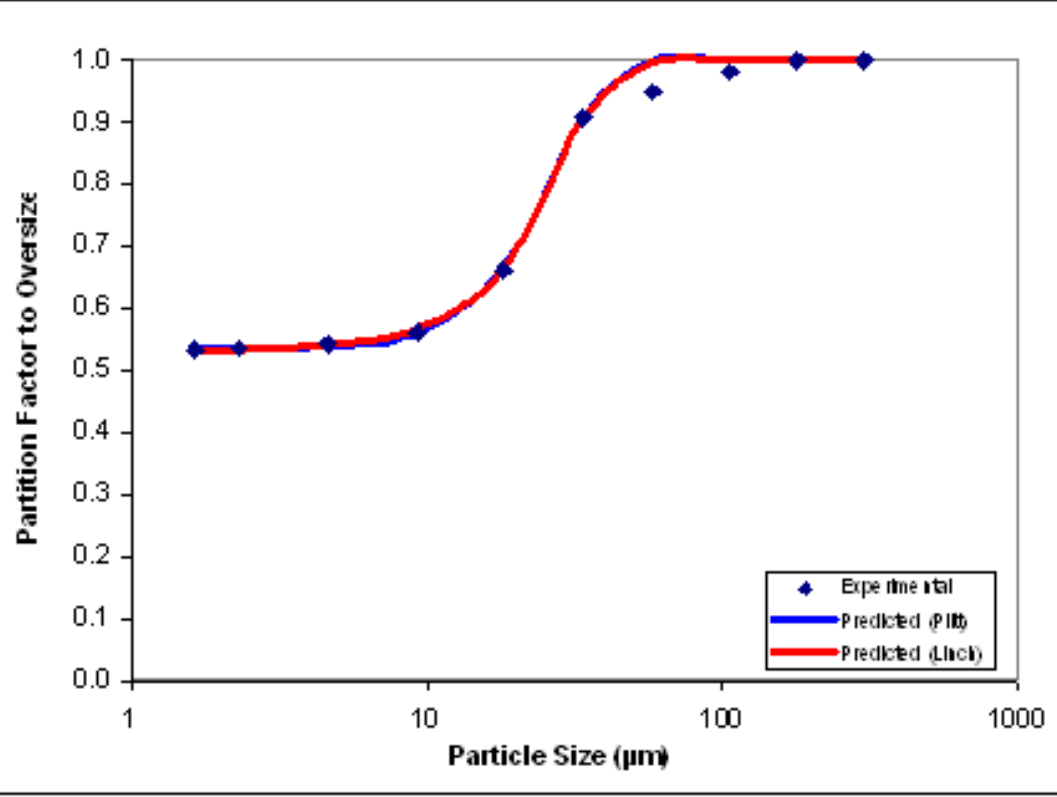




\section{HYDROCYCLONE EVALUATION (UNBALANCED)}

\begin{tabular}{l}
\hline Test: No. 21 \\
\begin{tabular}{|l|c|}
\hline \multicolumn{2}{|c|}{} \\
\hline Feed Flow (GPM) & 100.0 \\
\hline Feed Pressure (PSl) & 21.0 \\
\hline Water Flow (GPM) & 0.0 \\
\hline Water Pressure (PS) & 0.0 \\
\hline Feed Rate (TPH) & 1.14 \\
\hline
\end{tabular}
\end{tabular}

\begin{tabular}{|r|c|c|c|}
\hline & OS & U/S & Feed \\
\hline Solids Content (\%) & 7.87 & 2.48 & 4.50 \\
\hline Ash Content (\%) & 26.99 & 49.16 & 35.04 \\
\hline Solids Yield (\%) & 65.5 & 34.5 & 100.0 \\
\hline Ash Yield (\%) & 63.7 & 36.3 & 100.0 \\
\hline Size Class Yield (\%) & 84.8 & 15.2 & 100.0 \\
\hline
\end{tabular}

\begin{tabular}{|c|c|c|c|c|c|c|c|c|}
\hline $\begin{array}{c}\text { Pass } \\
\begin{array}{c}\text { Size } \\
(u m)\end{array}\end{array}$ & $\begin{array}{c}\text { Retain } \\
\text { Size } \\
(u m)\end{array}$ & $\begin{array}{c}\text { O/S } \\
\text { Sample } \\
(\mathrm{gms})\end{array}$ & $\begin{array}{c}\text { U/S } \\
\text { Sample } \\
(\mathrm{gms})\end{array}$ & $\begin{array}{c}\text { O/S } \\
\text { Mass } \\
(\%, \text { strm) }\end{array}$ & $\begin{array}{c}\text { U/S } \\
\text { Mass } \\
(\%, \text { strm) }\end{array}$ & $\begin{array}{c}\text { OS } \\
\text { Mass } \\
(\%, \text { feed })\end{array}$ & $\begin{array}{c}\text { U/S } \\
\text { Mass } \\
(\%, \text { feed })\end{array}$ & $\begin{array}{c}\text { Feed } \\
\text { Mass } \\
(\%, \text { feed })\end{array}$ \\
\hline & 212 & 1.78 & 0.00 & 8.15 & 0.00 & 5.19 & 0.00 & 5.19 \\
\hline 212 & 150 & 1.66 & 0.00 & 7.60 & 0.00 & 4.84 & 0.00 & 4.84 \\
\hline 150 & 75 & 4.99 & 0.12 & 22.86 & 0.79 & 14.56 & 0.29 & 14.84 \\
\hline 75 & 45 & 3.39 & 0.24 & 15.53 & 1.57 & 9.89 & 0.57 & 10.46 \\
\hline 45 & 25 & 1.43 & 0.35 & 6.55 & 2.29 & 4.17 & 0.83 & 5.00 \\
\hline 25 & 13.1 & 2.37 & 2.66 & 10.86 & 17.42 & 6.91 & 6.33 & 13.24 \\
\hline 13.1 & 6.5 & 1.95 & 3.42 & 8.92 & 22.36 & 5.68 & 8.12 & 13.80 \\
\hline 6.5 & 3.3 & 1.78 & 3.53 & 8.16 & 23.08 & 5.19 & 8.38 & 13.58 \\
\hline 3.3 & 1.5 & 1.12 & 2.47 & 5.12 & 16.19 & 3.26 & 5.88 & 9.14 \\
\hline 1.5 & & 1.37 & 2.49 & 6.25 & 16.30 & 3.98 & 5.92 & 9.90 \\
\hline Totals & --- & 21.83 & 15.28 & 100.00 & 100.00 & 63.69 & 36.31 & 100.00 \\
\hline
\end{tabular}

\begin{tabular}{|c|c|c|c|c|c|c|c|c|}
\hline $\begin{array}{c}\text { Pass } \\
\text { Size } \\
(u m)\end{array}$ & $\begin{array}{c}\text { Retain } \\
\text { Size } \\
(u m)\end{array}$ & $\begin{array}{c}\text { O/S } \\
\text { Mass } \\
(\mathrm{TPH})\end{array}$ & $\begin{array}{c}\text { U/S } \\
\text { Mass } \\
(\mathrm{TPH})\end{array}$ & $\begin{array}{c}\text { Feed } \\
\text { Mass } \\
(\mathrm{TPH})\end{array}$ & $\begin{array}{c}\text { Delta } \\
\text { Mass } \\
(\mathrm{TPH})\end{array}$ & $\begin{array}{c}\mathrm{V} \\
\text { Value } \\
(\mathrm{f}-\mathrm{u})\end{array}$ & $\begin{array}{c}\mathrm{X} \\
\text { Value } \\
(\mathbf{0}-\mathrm{u})\end{array}$ & $\begin{array}{c}\text { Partition } \\
\text { Factor } \\
(-)\end{array}$ \\
\hline & 212 & 0.06 & 0.00 & 0.06 & 0.00 & 5.19 & 5.19 & 1.00 \\
\hline 212 & 150 & 0.06 & 0.00 & 0.06 & 0.00 & 4.84 & 4.84 & 1.00 \\
\hline 150 & 75 & 0.17 & 0.00 & 0.17 & 0.00 & 14.56 & 14.27 & 0.98 \\
\hline 75 & 45 & 0.11 & 0.01 & 0.12 & 0.00 & 9.89 & 9.32 & 0.95 \\
\hline 45 & 25 & 0.05 & 0.01 & 0.06 & 0.00 & 4.17 & 3.34 & 0.83 \\
\hline 25 & 13.1 & 0.08 & 0.07 & 0.15 & 0.00 & 6.91 & 0.59 & 0.52 \\
\hline 13.1 & 6.5 & 0.07 & 0.09 & 0.16 & 0.00 & 5.68 & -2.44 & 0.41 \\
\hline 6.5 & 3.3 & 0.06 & 0.10 & 0.16 & 0.00 & 5.19 & -3.19 & 0.38 \\
\hline 3.3 & 1.5 & 0.04 & 0.07 & 0.10 & 0.00 & 3.26 & -2.62 & 0.36 \\
\hline 1.5 & & 0.05 & 0.07 & 0.11 & 0.00 & 3.98 & -1.94 & 0.40 \\
\hline Totals & -- & 0.73 & 0.42 & 1.14 & 0.00 & & & \\
\hline \multicolumn{2}{r}{ Slurry (TPH) } & 9.26 & 16.75 & 25.43 & -0.59 & & & \\
\hline
\end{tabular}


HYDROCYCLONE EVALUATION (BALANCED)

\begin{tabular}{|c|c|}
\hline No. 21 & \\
\hline Feed Flow (GPM) & 100.0 \\
\hline Feed Pressure (PSI) & 21.0 \\
\hline Water Flow (GPM) & 0.0 \\
\hline Water Pressure (PSl) & 0.0 \\
\hline Feed Rate (TPH) & 1.15 \\
\hline
\end{tabular}

\begin{tabular}{|r|c|c|c|}
\hline & OS & U/S & Feed \\
\hline Solids Content (\%) & 6.79 & 2.57 & 4.51 \\
\hline Ash Content (\%) & 23.74 & 48.18 & 34.58 \\
\hline Solids Yield (\%) & 69.2 & 30.8 & 100.0 \\
\hline Ash Yield (\%) & 55.6 & 44.4 & 100.0 \\
\hline Size Class Yield (\%) & 55.6 & 44.4 & 100.0 \\
\hline
\end{tabular}

\begin{tabular}{|c|c|c|c|c|c|c|c|c|}
\hline $\begin{array}{l}\text { Pass } \\
\text { Size } \\
\text { (um) }\end{array}$ & $\begin{array}{c}\text { Retain } \\
\text { Size } \\
\text { (um) }\end{array}$ & $\begin{array}{c}\text { O/S } \\
\text { Sample } \\
\text { (gms) }\end{array}$ & $\begin{array}{c}\text { U/S } \\
\text { Sample } \\
\text { (gms) }\end{array}$ & $\begin{array}{c}\text { orS } \\
\text { Mass } \\
(\% \text {, strm })\end{array}$ & $\begin{array}{c}\text { U/S } \\
\text { Mass } \\
(\% \text {, strm) }\end{array}$ & $\begin{array}{c}\text { OS } \\
\text { Mass } \\
(\% \text {, feed })\end{array}$ & $\begin{array}{c}\text { U/S } \\
\text { Mass } \\
(\%, \text { feed })\end{array}$ & $\begin{array}{c}\text { Feed } \\
\text { Mass } \\
(\% \text {, feed })\end{array}$ \\
\hline & 212 & 1.63 & 0.00 & 7.75 & 0.00 & 4.31 & 0.00 & 4.31 \\
\hline 212 & 150 & 1.89 & 0.00 & 8.99 & 0.00 & 5.00 & 0.00 & 5.00 \\
\hline 150 & 75 & 5.19 & 0.02 & 24.64 & 0.12 & 13.71 & 0.05 & 13.76 \\
\hline 75 & 45 & 3.83 & 0.27 & 18.18 & 1.59 & 10.12 & 0.71 & 10.82 \\
\hline 45 & 25 & 1.45 & 0.40 & 6.89 & 2.37 & 3.83 & 1.05 & 4.89 \\
\hline 25 & 13.1 & 1.98 & 2.94 & 9.42 & 17.60 & 5.24 & 7.81 & 13.05 \\
\hline 13.1 & 6.5 & 1.60 & 3.76 & 7.59 & 22.48 & 4.22 & 9.98 & 14.20 \\
\hline 6.5 & 3.3 & 1.44 & 3.80 & 6.82 & 22.76 & 3.80 & 10.10 & 13.90 \\
\hline 3.3 & 1.5 & 0.95 & 2.55 & 4.50 & 15.27 & 2.50 & 6.77 & 9.28 \\
\hline 1.5 & & 1.10 & 2.97 & 5.22 & 17.80 & 2.90 & 7.90 & 10.80 \\
\hline Totals & -- & 21.07 & 16.70 & 100.00 & 100.00 & 55.63 & 44.37 & 100.00 \\
\hline
\end{tabular}

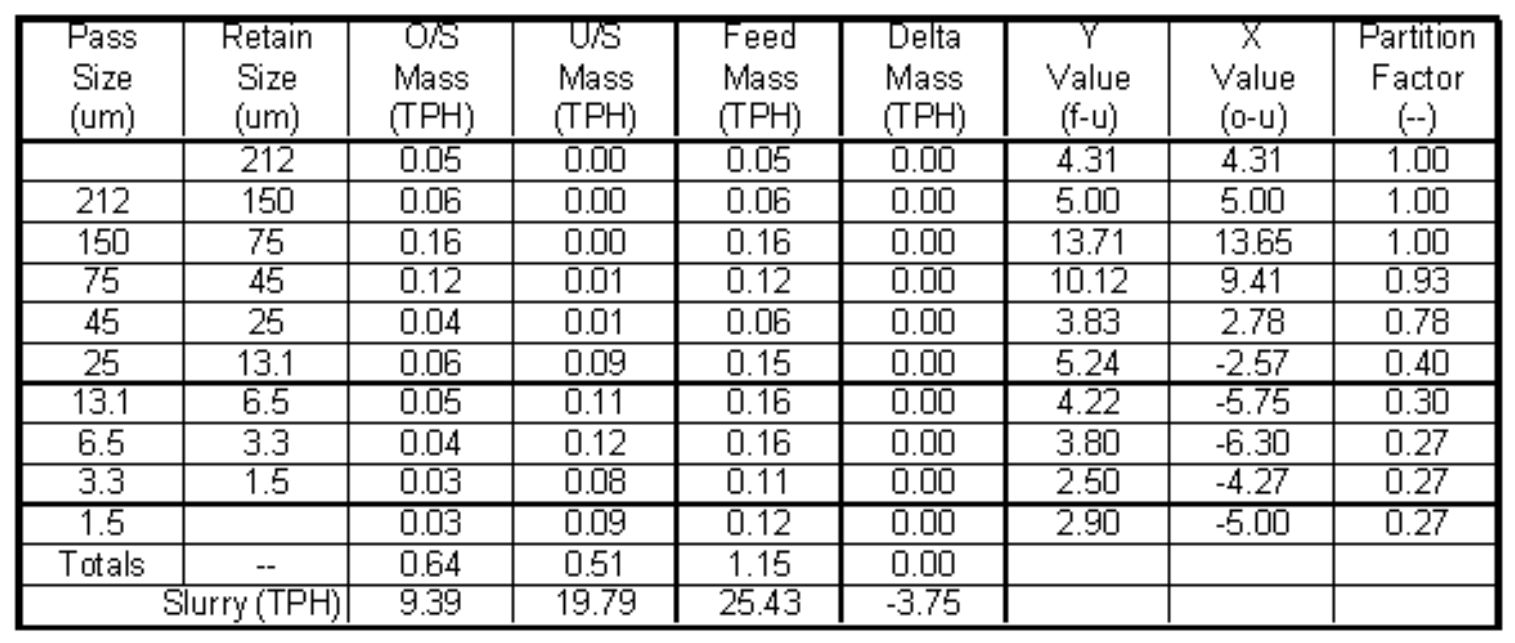




\begin{tabular}{|c|c|c|c|}
\hline $\begin{array}{c}\text { Experimental } \\
\text { Partition Factor }\end{array}$ & $\begin{array}{c}\text { Mean } \\
\text { Particle } \\
\text { Size }(\mu \mathrm{m})\end{array}$ & $\begin{array}{c}\text { Predicted } \\
\text { Partition } \\
\text { Factor }\end{array}$ & $\begin{array}{c}\text { Predicted } \\
\text { Partition } \\
\text { Factor }\end{array}$ \\
\hline$(-)$ & $(--)$ & (Plitt) & (Linch) \\
\hline 1.00 & 299.81 & 1.000 & 1.000 \\
\hline 1.00 & 178.33 & 1.000 & 1.000 \\
\hline 0.98 & 106.07 & 1.000 & 1.000 \\
\hline 0.95 & 58.09 & 0.995 & 0.990 \\
\hline 0.83 & 33.54 & 0.823 & 0.825 \\
\hline 0.52 & 18.08 & 0.531 & 0.525 \\
\hline 0.41 & 9.25 & 0.411 & 0.416 \\
\hline 0.38 & 4.62 & 0.383 & 0.387 \\
\hline 0.36 & 2.32 & 0.378 & 0.376 \\
\hline 0.40 & 1.64 & 0.377 & 0.374 \\
\hline & WSSQ & 0.004 & 0.004 \\
\hline
\end{tabular}

\begin{tabular}{|c|c|c|c|}
\hline (Plitt) & Predicted & (Linch) & Predicted \\
\hline D50 & 26.18 & D50 & 26.20 \\
\hline$m$ & 2.41 & Alpha & 3.36 \\
\hline Bypass(Rt) & 0.38 & Bypass(Rf) & 0.37 \\
\hline
\end{tabular}

\begin{tabular}{l|l}
\hline TWSSQ & 0.008 \\
\hline
\end{tabular}

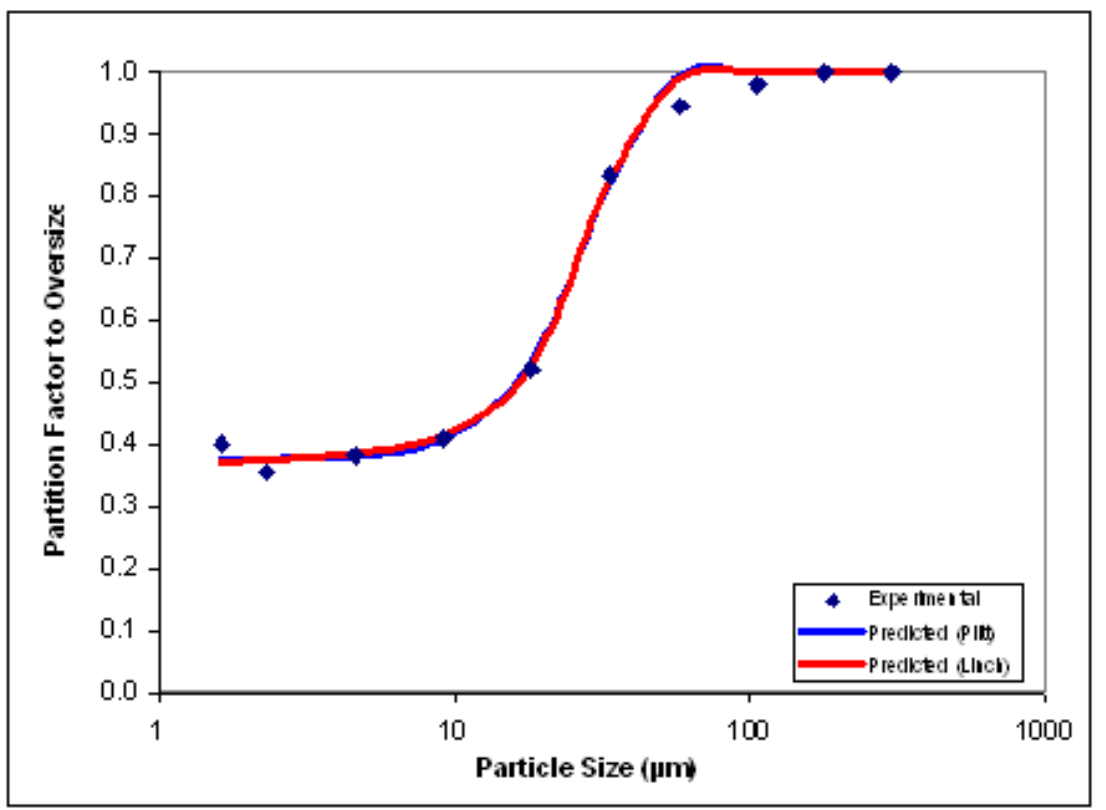


HYDROCYCLONE EVALUATION (UNBALANCED)

\begin{tabular}{|c|c|}
\hline No.22 & \\
\hline Feedflow (GPM) & 100.0 \\
\hline Feed Pressure (PSl) & 21.0 \\
\hline Water Flow (GPM) & 30.0 \\
\hline Water Pressure (PSI) & 19.0 \\
\hline Feed Rate (TPH) & 1.14 \\
\hline
\end{tabular}

\begin{tabular}{|r|c|c|c|}
\hline & OSS & U/S & Feed \\
\hline Solids Content (\%) & 5.16 & 2.58 & 4.50 \\
\hline Ash Content (\%) & 20.38 & 48.05 & 34.75 \\
\hline Solids Yield (\%) & 51.5 & 48.5 & 100.0 \\
\hline Ash Yield (\%) & 48.1 & 51.9 & 100.0 \\
\hline Size Class Yield (\%) & 37.5 & 62.5 & 100.0 \\
\hline
\end{tabular}

\begin{tabular}{|c|c|c|c|c|c|c|c|c|}
\hline $\begin{array}{c}\text { Pass } \\
\text { Size } \\
(u m)\end{array}$ & $\begin{array}{c}\text { Retain } \\
\text { Size } \\
(\mathrm{um})\end{array}$ & $\begin{array}{c}\text { O/S } \\
\text { Sample } \\
(\mathrm{gms})\end{array}$ & $\begin{array}{c}\text { U/S } \\
\text { Sample } \\
(\mathrm{gms})\end{array}$ & $\begin{array}{c}\text { O/S } \\
\text { Mass } \\
(\%, \text { strm) }\end{array}$ & $\begin{array}{c}\text { U/S } \\
\text { Mass } \\
(\%, \text { strm) }\end{array}$ & $\begin{array}{c}\text { OS } \\
\text { Mass } \\
(\%, \text { feed })\end{array}$ & $\begin{array}{c}\text { U/S } \\
\text { Mass } \\
(\%, \text { feed })\end{array}$ & $\begin{array}{c}\text { Feed } \\
\text { Mass } \\
(\%, \text { feed })\end{array}$ \\
\hline & 212 & 1.96 & 0.00 & 11.09 & 0.00 & 5.33 & 0.00 & 5.33 \\
\hline 212 & 150 & 1.61 & 0.00 & 9.11 & 0.00 & 4.38 & 0.00 & 4.38 \\
\hline 150 & 75 & 4.89 & 0.19 & 27.66 & 0.96 & 13.29 & 0.50 & 13.79 \\
\hline 75 & 45 & 3.31 & 0.42 & 18.72 & 2.12 & 9.00 & 1.10 & 10.10 \\
\hline 45 & 25 & 1.12 & 0.81 & 6.33 & 4.08 & 3.04 & 2.12 & 5.16 \\
\hline 25 & 13.1 & 1.14 & 3.59 & 6.45 & 18.07 & 3.10 & 9.39 & 12.49 \\
\hline 13.1 & 6.5 & 1.05 & 4.31 & 5.93 & 21.69 & 2.85 & 11.26 & 14.11 \\
\hline 6.5 & 3.3 & 1.04 & 4.37 & 5.90 & 22.02 & 2.83 & 11.44 & 14.27 \\
\hline 3.3 & 1.5 & 0.72 & 3.11 & 4.04 & 15.66 & 1.94 & 8.13 & 10.08 \\
\hline 1.5 & & 0.84 & 3.06 & 4.77 & 15.40 & 2.29 & 8.00 & 10.29 \\
\hline Totals & -- & 17.68 & 19.85 & 100.00 & 100.00 & 48.07 & 51.93 & 100.00 \\
\hline
\end{tabular}

\begin{tabular}{|c|c|c|c|c|c|c|c|c|}
\hline $\begin{array}{c}\text { Pass } \\
\text { Size } \\
(u m)\end{array}$ & $\begin{array}{c}\text { Retain } \\
\text { Size } \\
(u m)\end{array}$ & $\begin{array}{c}\text { O/S } \\
\text { Mass } \\
(\mathrm{TPH})\end{array}$ & $\begin{array}{c}\text { U/S } \\
\text { Mass } \\
(\mathrm{TPH})\end{array}$ & $\begin{array}{c}\text { Feed } \\
\text { Mass } \\
(\mathrm{TPH})\end{array}$ & $\begin{array}{c}\text { Delta } \\
\text { Mass } \\
(\mathrm{TPH})\end{array}$ & $\begin{array}{c}\text { Y } \\
\text { Value } \\
(\mathrm{f}-\mathrm{u})\end{array}$ & $\begin{array}{c}\text { X } \\
\text { Value } \\
(0-\mathrm{u})\end{array}$ & $\begin{array}{c}\text { Partition } \\
\text { Factor } \\
(-)\end{array}$ \\
\hline & 212 & 0.06 & 0.00 & 0.06 & 0.00 & 5.33 & 5.33 & 1.00 \\
\hline 212 & 150 & 0.05 & 0.00 & 0.05 & 0.00 & 4.38 & 4.38 & 1.00 \\
\hline 150 & 75 & 0.15 & 0.01 & 0.16 & 0.00 & 13.29 & 12.80 & 0.96 \\
\hline 75 & 45 & 0.10 & 0.01 & 0.12 & 0.00 & 9.00 & 7.90 & 0.89 \\
\hline 45 & 25 & 0.03 & 0.02 & 0.06 & 0.00 & 3.04 & 0.93 & 0.59 \\
\hline 25 & 13.1 & 0.04 & 0.11 & 0.14 & 0.00 & 3.10 & -6.29 & 0.25 \\
\hline 13.1 & 6.5 & 0.03 & 0.13 & 0.16 & 0.00 & 2.85 & -8.41 & 0.20 \\
\hline 6.5 & 3.3 & 0.03 & 0.13 & 0.16 & 0.00 & 2.83 & -8.60 & 0.20 \\
\hline 3.3 & 1.5 & 0.02 & 0.09 & 0.12 & 0.00 & 1.94 & -6.19 & 0.19 \\
\hline 1.5 & & 0.03 & 0.09 & 0.12 & 0.00 & 2.29 & -5.71 & 0.22 \\
\hline Totals & -- & 0.55 & 0.59 & 1.14 & 0.00 & & & \\
\hline & Slurry (TPH) & 10.66 & 23.03 & 25.43 & -0.77 & & & \\
\hline
\end{tabular}


HYDROCYCLONE EVALUATION (BALANCED)

\begin{tabular}{|c|c|}
\hline No. 22 & \\
\hline Feedflow (GPM) & 700.0 \\
\hline Feed Pressure (PSl) & 21.0 \\
\hline Water Flow (GPM) & 30.0 \\
\hline Water Pressure (PSI) & 19.0 \\
\hline Feed Rate (TPH) & 1.15 \\
\hline
\end{tabular}

\begin{tabular}{|r|c|c|c|}
\hline & OSS & U/S & Feed \\
\hline Solids Content (\%) & 6.79 & 2.57 & 4.51 \\
\hline Ash Content (\%) & 23.74 & 48.18 & 34.58 \\
\hline Solids Yield (\%) & 42.1 & 57.9 & 100.0 \\
\hline Ash Yield (\%) & 55.6 & 44.4 & 100.0 \\
\hline Size Class Yield (\%) & 55.6 & 44.4 & 100.0 \\
\hline
\end{tabular}

\begin{tabular}{|c|c|c|c|c|c|c|c|c|}
\hline $\begin{array}{c}\text { Pass } \\
\text { Size } \\
(u m)\end{array}$ & $\begin{array}{c}\text { Retain } \\
\text { Size } \\
(\mathrm{um})\end{array}$ & $\begin{array}{c}\text { O/S } \\
\text { Sample } \\
(\mathrm{gms})\end{array}$ & $\begin{array}{c}\text { U/S } \\
\text { Sample } \\
(\mathrm{gms})\end{array}$ & $\begin{array}{c}\text { O/S } \\
\text { Mass } \\
(\%, \text { strm) }\end{array}$ & $\begin{array}{c}\text { U/S } \\
\text { Mass } \\
(\%, \text { strm })\end{array}$ & $\begin{array}{c}\text { OS } \\
\text { Mass } \\
(\%, \text { feed })\end{array}$ & $\begin{array}{c}\text { U/S } \\
\text { Mass } \\
(\%, \text { feed })\end{array}$ & $\begin{array}{c}\text { Feed } \\
\text { Mass } \\
(\%, \text { feed })\end{array}$ \\
\hline & 212 & 1.63 & 0.00 & 7.75 & 0.00 & 4.31 & 0.00 & 4.31 \\
\hline 212 & 150 & 1.89 & 0.00 & 8.99 & 0.00 & 5.00 & 0.00 & 5.00 \\
\hline 150 & 75 & 5.19 & 0.02 & 24.64 & 0.12 & 13.71 & 0.05 & 13.76 \\
\hline 75 & 45 & 3.83 & 0.27 & 18.18 & 1.59 & 10.12 & 0.71 & 10.82 \\
\hline 45 & 25 & 1.45 & 0.40 & 6.89 & 2.37 & 3.83 & 1.05 & 4.89 \\
\hline 25 & 13.1 & 1.98 & 2.94 & 9.42 & 17.60 & 5.24 & 7.81 & 13.05 \\
\hline 13.1 & 6.5 & 1.60 & 3.76 & 7.59 & 22.48 & 4.22 & 9.98 & 14.20 \\
\hline 6.5 & 3.3 & 1.44 & 3.80 & 6.82 & 22.76 & 3.80 & 10.10 & 13.90 \\
\hline 3.3 & 1.5 & 0.95 & 2.55 & 4.50 & 15.27 & 2.50 & 6.77 & 9.28 \\
\hline 1.5 & & 1.10 & 2.97 & 5.22 & 17.80 & 2.90 & 7.90 & 10.80 \\
\hline Totals & -- & 21.07 & 16.70 & 100.00 & 100.00 & 55.63 & 44.37 & 100.00 \\
\hline
\end{tabular}

\begin{tabular}{|c|c|c|c|c|c|c|c|c|}
\hline $\begin{array}{c}\text { Pass } \\
\text { Size } \\
(\mathrm{um})\end{array}$ & $\begin{array}{c}\text { Retain } \\
\text { Size } \\
(\mathrm{um})\end{array}$ & $\begin{array}{c}0 / \mathrm{S} \\
\text { Mass } \\
(\mathrm{TPH})\end{array}$ & $\begin{array}{c}\mathrm{U} / \mathrm{S} \\
\text { Mass } \\
(\mathrm{TPH})\end{array}$ & $\begin{array}{c}\text { Feed } \\
\text { Mass } \\
(\mathrm{TPH})\end{array}$ & $\begin{array}{c}\text { Delta } \\
\text { Mass } \\
(\mathrm{TPH})\end{array}$ & $\begin{array}{c}\text { Y } \\
\text { Value } \\
(\mathrm{f}-\mathrm{u})\end{array}$ & $\begin{array}{c}\text { X } \\
\text { Value } \\
(\mathbf{0}-\mathrm{u})\end{array}$ & $\begin{array}{c}\text { Partition } \\
\text { Factor } \\
(-)\end{array}$ \\
\hline & 212 & 0.05 & 0.00 & 0.05 & 0.00 & 4.31 & 4.31 & 1.00 \\
\hline 212 & 150 & 0.06 & 0.00 & 0.06 & 0.00 & 5.00 & 5.00 & 1.00 \\
\hline 150 & 75 & 0.16 & 0.00 & 0.16 & 0.00 & 13.71 & 13.65 & 1.00 \\
\hline 75 & 45 & 0.12 & 0.01 & 0.12 & 0.00 & 10.12 & 9.41 & 0.93 \\
\hline 45 & 25 & 0.04 & 0.01 & 0.06 & 0.00 & 3.83 & 2.78 & 0.78 \\
\hline 25 & 13.1 & 0.06 & 0.09 & 0.15 & 0.00 & 5.24 & -2.57 & 0.40 \\
\hline 13.1 & 6.5 & 0.05 & 0.11 & 0.16 & 0.00 & 4.22 & -5.75 & 0.30 \\
\hline 6.5 & 3.3 & 0.04 & 0.12 & 0.16 & 0.00 & 3.80 & -6.30 & 0.27 \\
\hline 3.3 & 1.5 & 0.03 & 0.08 & 0.11 & 0.00 & 2.50 & -4.27 & 0.27 \\
\hline 1.5 & & 0.03 & 0.09 & 0.12 & 0.00 & 2.90 & -5.00 & 0.27 \\
\hline Totals & -- & 0.64 & 0.51 & 1.15 & 0.00 & & & \\
\hline \multicolumn{2}{r}{ Slurry (TPH) } & 9.39 & 19.79 & 25.43 & 3.75 & & & \\
\hline
\end{tabular}




\begin{tabular}{|c|c|c|c|}
\hline $\begin{array}{c}\text { Experimental } \\
\text { Partition F actor }\end{array}$ & $\begin{array}{c}\text { Mean } \\
\text { Particle } \\
\text { Size }(\mu \mathrm{m})\end{array}$ & $\begin{array}{c}\text { Predicted } \\
\text { Partition } \\
\text { Factor }\end{array}$ & $\begin{array}{c}\text { Predicted } \\
\text { Partition } \\
\text { Factor }\end{array}$ \\
\hline$(--)$ & $(--)$ & (Plitt) & (Linch) \\
\hline 1.00 & 299.81 & 1.000 & 1.000 \\
\hline 1.00 & 178.33 & 1.000 & 1.000 \\
\hline 0.96 & 106.07 & 1.000 & 0.999 \\
\hline 0.89 & 58.09 & 0.918 & 0.929 \\
\hline 0.59 & 33.54 & 0.555 & 0.556 \\
\hline 0.25 & 18.08 & 0.292 & 0.291 \\
\hline 0.20 & 9.25 & 0.213 & 0.219 \\
\hline 0.20 & 4.62 & 0.196 & 0.198 \\
\hline 0.19 & 2.32 & 0.193 & 0.190 \\
\hline 0.22 & 1.64 & 0.193 & 0.188 \\
\hline & WSSQ & 0.006 & 0.007 \\
\hline
\end{tabular}

\begin{tabular}{|c|c|c|c|}
\hline (Plitt) & Predicted & (Linch) & Predicted \\
\hline D50 & 35.69 & D50 & 35.23 \\
\hline$m$ & 2.45 & Alpha & 3.58 \\
\hline Bypass(Rt) & 0.19 & Bypass(Rf) & 0.18 \\
\hline
\end{tabular}

\begin{tabular}{|l|l|}
\hline TWSSQ & 0.013 \\
\hline
\end{tabular}

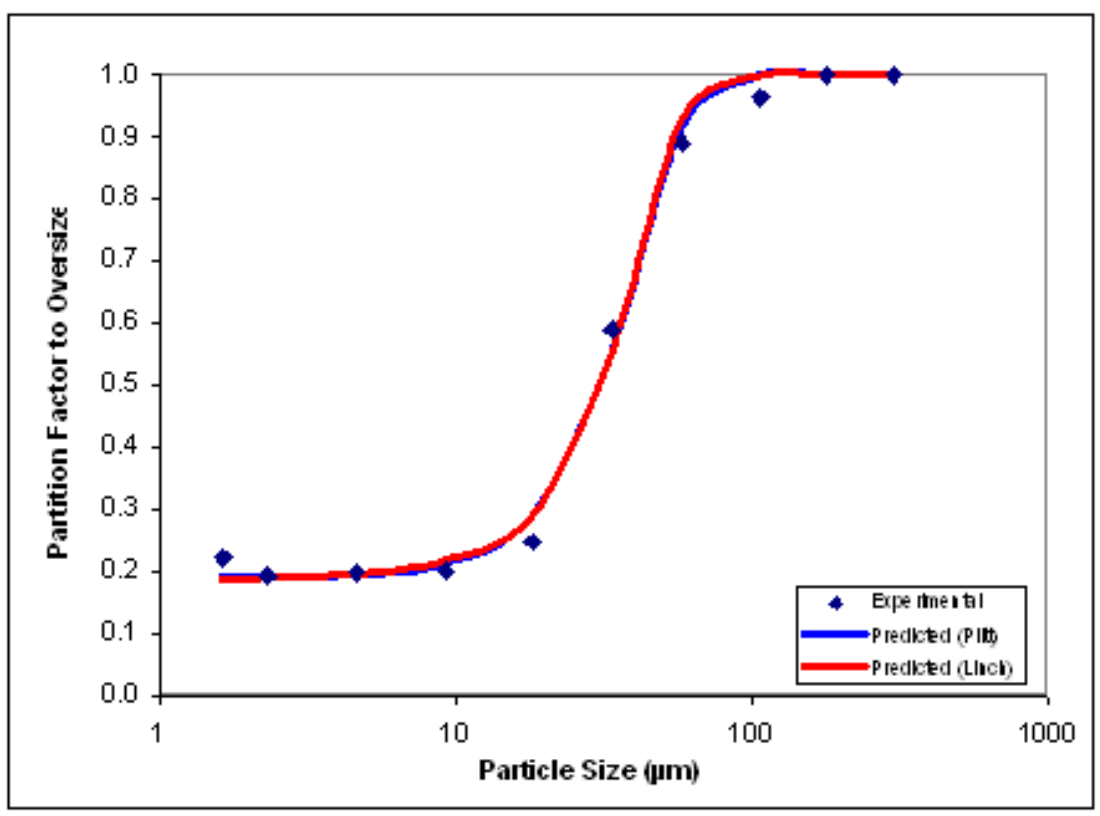


HYDROCYCLONE EVALUATION (UNBALANCED)

\begin{tabular}{|c|c|}
\hline No. 23 & \\
\hline Feed Flow (GPM) & 100.0 \\
\hline Feed Pressure (PSI) & 21.0 \\
\hline Water Flow (GPM) & 15.0 \\
\hline Water Pressure (PSI) & 13.0 \\
\hline Feed Rate (TPH) & 1.15 \\
\hline
\end{tabular}

\begin{tabular}{|r|c|c|c|}
\hline & OS & U/S & Feed \\
\hline Solids Content (\%) & 6.79 & 2.51 & 4.51 \\
\hline Ash Content (\%) & 23.88 & 48.29 & 34.34 \\
\hline Solids Yield (\%) & 57.3 & 42.7 & 100.0 \\
\hline Ash Yield (\%) & 57.1 & 42.9 & 100.0 \\
\hline Size Class Yield (\%) & 64.3 & 35.7 & 100.0 \\
\hline
\end{tabular}

\begin{tabular}{|c|c|c|c|c|c|c|c|c|}
\hline Pass & Retain & $0 / 5$ & U/S & o/S & U/S & OS & U/S & Feed \\
\hline $\begin{array}{l}\text { Size } \\
\text { (um) }\end{array}$ & $\begin{array}{l}\text { Size } \\
\text { (um) }\end{array}$ & $\begin{array}{c}\text { Sample } \\
\text { (gms) }\end{array}$ & $\begin{array}{c}\text { Sample } \\
\text { (gms) }\end{array}$ & $\begin{array}{c}\text { Mass } \\
(\%, \text { strm) }\end{array}$ & $\begin{array}{c}\text { Mass } \\
(\%, \text { strm })\end{array}$ & $\begin{array}{c}\text { Mass } \\
(\% \text {, feed })\end{array}$ & $\begin{array}{c}\text { Mass } \\
(\%, \text { feed })\end{array}$ & $\begin{array}{c}\text { Mass } \\
(\%, \text { feed })\end{array}$ \\
\hline & 212 & 1.58 & 0.00 & 7.57 & 0.00 & 4.32 & 0.00 & 4.32 \\
\hline 212 & 150 & 1.85 & 0.00 & 8.86 & 0.00 & 5.06 & 0.00 & 5.06 \\
\hline 150 & 75 & 5.25 & 0.19 & 25.14 & 1.11 & 14.37 & 0.48 & 14.85 \\
\hline 75 & 45 & 3.85 & 0.41 & 18.44 & 2.40 & 10.54 & 1.03 & 11.56 \\
\hline 45 & 25 & 1.40 & 0.51 & 6.70 & 2.98 & 3.83 & 1.28 & 5.11 \\
\hline 25 & 13.1 & 1.98 & 2.95 & 9.46 & 17.25 & 5.41 & 7.39 & 12.80 \\
\hline 13.1 & 6.5 & 1.59 & 3.73 & 7.60 & 21.80 & 4.34 & 9.34 & 13.69 \\
\hline 6.5 & 3.3 & 1.42 & 3.77 & 6.81 & 22.05 & 3.89 & 9.45 & 13.34 \\
\hline 3.3 & 1.5 & 0.90 & 2.57 & 4.32 & 15.01 & 2.47 & 6.43 & 8.90 \\
\hline 1.5 & & 1.06 & 2.97 & 5.09 & 17.39 & 2.91 & 7.45 & 10.36 \\
\hline Totals & -- & 20.88 & 17.10 & 100.00 & 100.00 & 57.15 & 42.85 & 100.00 \\
\hline
\end{tabular}

\begin{tabular}{|c|c|c|c|c|c|c|c|c|}
\hline $\begin{array}{c}\text { Pass } \\
\text { Size } \\
(u m)\end{array}$ & $\begin{array}{c}\text { Retain } \\
\text { Size } \\
(u m)\end{array}$ & $\begin{array}{c}\text { O/S } \\
\text { Mass } \\
(\mathrm{TPH})\end{array}$ & $\begin{array}{c}\text { O/S } \\
\text { Mass } \\
(\mathrm{TPH})\end{array}$ & $\begin{array}{c}\text { Feed } \\
\text { Mass } \\
(\mathrm{TPH})\end{array}$ & $\begin{array}{c}\text { Delta } \\
\text { Mass } \\
(\mathrm{TPH})\end{array}$ & $\begin{array}{c}\mathrm{V} \\
\text { Value } \\
(\mathrm{f}-\mathrm{u})\end{array}$ & $\begin{array}{c}\mathrm{X} \\
\text { Value } \\
(\mathrm{0}-\mathrm{u})\end{array}$ & $\begin{array}{c}\text { Partition } \\
\text { Factor } \\
(-)\end{array}$ \\
\hline & 212 & 0.05 & 0.00 & 0.05 & 0.00 & 4.32 & 4.32 & 1.00 \\
\hline 212 & 150 & 0.06 & 0.00 & 0.06 & 0.00 & 5.06 & 5.06 & 1.00 \\
\hline 150 & 75 & 0.16 & 0.01 & 0.17 & 0.00 & 14.37 & 13.89 & 0.97 \\
\hline 75 & 45 & 0.12 & 0.01 & 0.13 & 0.00 & 10.54 & 9.51 & 0.91 \\
\hline 45 & 25 & 0.04 & 0.01 & 0.06 & 0.00 & 3.83 & 2.55 & 0.75 \\
\hline 25 & 13.1 & 0.06 & 0.08 & 0.15 & 0.00 & 5.41 & -1.99 & 0.42 \\
\hline 13.1 & 6.5 & 0.05 & 0.11 & 0.16 & 0.00 & 4.34 & -5.00 & 0.32 \\
\hline 6.5 & 3.3 & 0.04 & 0.11 & 0.15 & 0.00 & 3.89 & -5.56 & 0.29 \\
\hline 3.3 & 1.5 & 0.03 & 0.07 & 0.10 & 0.00 & 2.47 & -3.96 & 0.28 \\
\hline 1.5 & & 0.03 & 0.09 & 0.12 & 0.00 & 2.91 & -4.54 & 0.28 \\
\hline Totals & -- & 0.66 & 0.49 & 1.15 & 0.00 & & & \\
\hline & Slurry (TPH) & 9.65 & 19.58 & 25.43 & -0.05 & & & \\
\hline
\end{tabular}




\section{HYDROCYCLONE EVALUATION (BALANCED)}

\begin{tabular}{|c|c|}
\hline No. 23 & \\
\hline Feed Flow (GPM) & 100.0 \\
\hline Feed Pressure (PSI) & 21.0 \\
\hline Water Flow (GPM) & 15.0 \\
\hline Water Pressure (PSl) & 13.0 \\
\hline Feed Rate (TPH) & 1.15 \\
\hline
\end{tabular}

\begin{tabular}{|r|c|c|c|}
\hline & OS & U/S & Feed \\
\hline Solids Content (\%) & 6.79 & 2.57 & 4.51 \\
\hline Ash Content (\%) & 23.74 & 48.18 & 34.58 \\
\hline Solids Yield (\%) & 55.6 & 44.4 & 100.0 \\
\hline Ash Yield (\%) & 55.6 & 44.4 & 100.0 \\
\hline Size Class Yield (\%) & 55.6 & 44.4 & 100.0 \\
\hline
\end{tabular}

\begin{tabular}{|c|c|c|c|c|c|c|c|c|}
\hline $\begin{array}{c}\text { Pass } \\
\text { Size } \\
(u m)\end{array}$ & $\begin{array}{c}\text { Retain } \\
\text { Size } \\
(u m)\end{array}$ & $\begin{array}{c}\text { O/S } \\
\text { Sample } \\
(\mathrm{gms})\end{array}$ & $\begin{array}{c}\text { U/S } \\
\text { Sample } \\
(\mathrm{gms})\end{array}$ & $\begin{array}{c}\text { O/S } \\
\text { Mass } \\
(\%, \text { strm) }\end{array}$ & $\begin{array}{c}\text { U/S } \\
\text { Mass } \\
(\%, \text { strm) }\end{array}$ & $\begin{array}{c}\text { OS } \\
\text { Mass } \\
(\%, \text { feed })\end{array}$ & $\begin{array}{c}\text { U/S } \\
\text { Mass } \\
(\%, \text { feed })\end{array}$ & $\begin{array}{c}\text { Feed } \\
\text { Mass } \\
(\%, \text { feed })\end{array}$ \\
\hline & 212 & 1.63 & 0.00 & 7.75 & 0.00 & 4.31 & 0.00 & 4.31 \\
\hline 212 & 150 & 1.89 & 0.00 & 8.99 & 0.00 & 5.00 & 0.00 & 5.00 \\
\hline 150 & 75 & 5.19 & 0.02 & 24.64 & 0.12 & 13.71 & 0.05 & 13.76 \\
\hline 75 & 45 & 3.83 & 0.27 & 18.18 & 1.59 & 10.12 & 0.71 & 10.82 \\
\hline 45 & 25 & 1.45 & 0.40 & 6.89 & 2.37 & 3.83 & 1.05 & 4.89 \\
\hline 25 & 13.1 & 1.98 & 2.94 & 9.42 & 17.60 & 5.24 & 7.81 & 13.05 \\
\hline 13.1 & 6.5 & 1.60 & 3.76 & 7.59 & 22.48 & 4.22 & 9.98 & 14.20 \\
\hline 6.5 & 3.3 & 1.44 & 3.80 & 6.82 & 22.76 & 3.80 & 10.10 & 13.90 \\
\hline 3.3 & 1.5 & 0.95 & 2.55 & 4.50 & 15.27 & 2.50 & 6.77 & 9.28 \\
\hline 1.5 & & 1.10 & 2.97 & 5.22 & 17.80 & 2.90 & 7.90 & 10.80 \\
\hline Totals & -- & 21.07 & 16.70 & 100.00 & 100.00 & 55.63 & 44.37 & 100.00 \\
\hline
\end{tabular}

\begin{tabular}{|c|c|c|c|c|c|c|c|c|}
\hline $\begin{array}{c}\text { Pass } \\
\text { Size } \\
(u m)\end{array}$ & $\begin{array}{c}\text { Retain } \\
\text { Size } \\
(u m)\end{array}$ & $\begin{array}{c}\text { O/S } \\
\text { Mass } \\
(\mathrm{TPH})\end{array}$ & $\begin{array}{c}\text { U/S } \\
\text { Mass } \\
(\mathrm{TPH})\end{array}$ & $\begin{array}{c}\text { Feed } \\
\text { Mass } \\
(\mathrm{TPH})\end{array}$ & $\begin{array}{c}\text { Delta } \\
\text { Mass } \\
(\mathrm{TPH})\end{array}$ & $\begin{array}{c}\text { V } \\
\text { Value } \\
(\mathrm{f}-\mathrm{u})\end{array}$ & $\begin{array}{c}\text { X } \\
\text { Value } \\
(0-\mathrm{u})\end{array}$ & $\begin{array}{c}\text { Partition } \\
\text { Factor } \\
(-)\end{array}$ \\
\hline & 212 & 0.05 & 0.00 & 0.05 & 0.00 & 4.31 & 4.31 & 1.00 \\
\hline 212 & 150 & 0.06 & 0.00 & 0.06 & 0.00 & 5.00 & 5.00 & 1.00 \\
\hline 150 & 75 & 0.16 & 0.00 & 0.16 & 0.00 & 13.71 & 13.65 & 1.00 \\
\hline 75 & 45 & 0.12 & 0.01 & 0.12 & 0.00 & 10.12 & 9.41 & 0.93 \\
\hline 45 & 25 & 0.04 & 0.01 & 0.06 & 0.00 & 3.83 & 2.78 & 0.78 \\
\hline 25 & 13.1 & 0.06 & 0.09 & 0.15 & 0.00 & 5.24 & -2.57 & 0.40 \\
\hline 13.1 & 6.5 & 0.05 & 0.11 & 0.16 & 0.00 & 4.22 & -5.75 & 0.30 \\
\hline 6.5 & 3.3 & 0.04 & 0.12 & 0.16 & 0.00 & 3.80 & -6.30 & 0.27 \\
\hline 3.3 & 1.5 & 0.03 & 0.08 & 0.11 & 0.00 & 2.50 & -4.27 & 0.27 \\
\hline 1.5 & & 0.03 & 0.09 & 0.12 & 0.00 & 2.90 & -5.00 & 0.27 \\
\hline Totals & -- & 0.64 & 0.51 & 1.15 & 0.00 & & & \\
\hline & Slurry (TPH) & 9.39 & 19.79 & 25.43 & 0.00 & & & \\
\hline
\end{tabular}




\begin{tabular}{|c|c|c|c|}
\hline $\begin{array}{c}\text { Experimental } \\
\text { Partition Factor }\end{array}$ & $\begin{array}{c}\text { Mean } \\
\text { Particle } \\
\text { Size }(\mu \mathrm{m})\end{array}$ & $\begin{array}{c}\text { Predicted } \\
\text { Partition } \\
\text { Factor }\end{array}$ & $\begin{array}{c}\text { Predicted } \\
\text { Partition } \\
\text { Factor }\end{array}$ \\
\hline$(-)$ & $(--)$ & (Plitt) & (Linch) \\
\hline 1.00 & 299.81 & 1.000 & 1.000 \\
\hline 1.00 & 178.33 & 1.000 & 1.000 \\
\hline 0.97 & 106.07 & 1.000 & 1.000 \\
\hline 0.91 & 58.09 & 0.951 & 0.960 \\
\hline 0.75 & 33.54 & 0.713 & 0.720 \\
\hline 0.42 & 18.08 & 0.450 & 0.443 \\
\hline 0.32 & 9.25 & 0.325 & 0.329 \\
\hline 0.29 & 4.62 & 0.286 & 0.289 \\
\hline 0.28 & 2.32 & 0.275 & 0.274 \\
\hline 0.28 & 1.64 & 0.273 & 0.270 \\
\hline & WSSQ & 0.005 & 0.005 \\
\hline
\end{tabular}

\begin{tabular}{|c|c|c|c|}
\hline (Plitt) & Predicted & (Linch) & Predicted \\
\hline D50 & 28.78 & D50 & 28.57 \\
\hline$m$ & 1.93 & Alpha & 2.70 \\
\hline Bypass(Rt) & 0.27 & Bypass(Rf) & 0.26 \\
\hline
\end{tabular}

\begin{tabular}{l|l}
\hline TWSSQ & 0.010
\end{tabular}

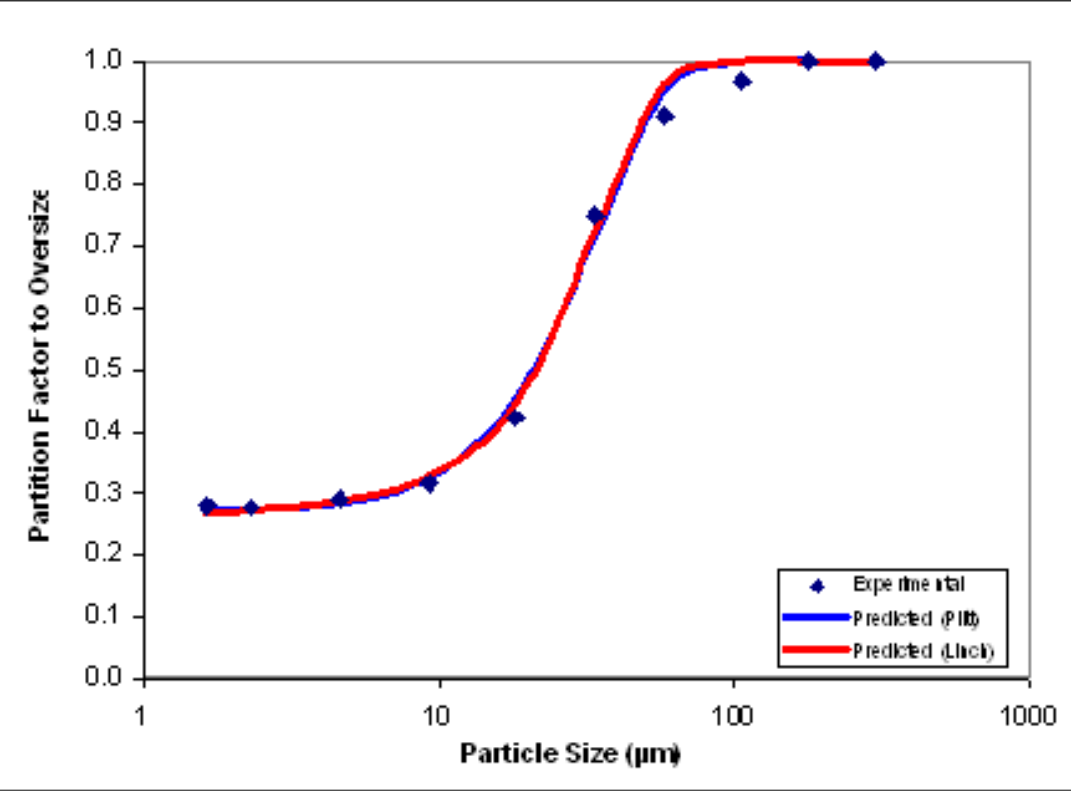


HYDROCYCLONE EVALUATION (UNBALANCED)

\begin{tabular}{|c|c|}
\hline No. 24 & \\
\hline Feed Flow (GPM) & 100.0 \\
\hline Feed Pressure (PSI) & 21.0 \\
\hline Water Flow (GPM) & 15.0 \\
\hline Water Pressure (PSl) & 12.0 \\
\hline Feed Rate (TPH) & 1.15 \\
\hline
\end{tabular}

\begin{tabular}{|r|c|c|c|}
\hline & OS & U/S & Feed \\
\hline Solids Content (\%) & 6.31 & 2.48 & 4.51 \\
\hline Ash Content (\%) & 23.23 & 48.24 & 34.73 \\
\hline Solids Yield (\%) & 60.8 & 39.2 & 100.0 \\
\hline Ash Yield (\%) & 54.0 & 46.0 & 100.0 \\
\hline Size Class Yield (\%) & 53.3 & 46.7 & 100.0 \\
\hline
\end{tabular}

\begin{tabular}{|c|c|c|c|c|c|c|c|c|}
\hline $\begin{array}{l}\text { Pass } \\
\text { Size } \\
\text { (um) }\end{array}$ & $\begin{array}{l}\text { Retain } \\
\text { Size } \\
\text { (um) }\end{array}$ & $\begin{array}{c}\text { O/S } \\
\text { Sample } \\
\text { (gms) }\end{array}$ & $\begin{array}{c}\text { U/S } \\
\text { Sample } \\
\text { (gms) }\end{array}$ & $\begin{array}{c}\text { orS } \\
\text { Mass } \\
(\% \text {, strm })\end{array}$ & $\begin{array}{c}\text { USS } \\
\text { Mass } \\
(\% \text {, strm) }\end{array}$ & $\begin{array}{c}\text { OS } \\
\text { Mass } \\
(\% \text {, feed })\end{array}$ & $\begin{array}{c}\text { U/S } \\
\text { Mass } \\
(\%, \text { feed })\end{array}$ & $\begin{array}{c}\text { Feed } \\
\text { Mass } \\
(\% \text {, feed })\end{array}$ \\
\hline & 212 & 1.34 & 0.00 & 6.92 & 0.00 & 3.74 & 0.00 & 3.74 \\
\hline 212 & 150 & 1.52 & 0.00 & 7.85 & 0.00 & 4.24 & 0.00 & 4.24 \\
\hline 150 & 75 & 5.06 & 0.16 & 26.12 & 0.94 & 14.11 & 0.43 & 14.54 \\
\hline 75 & 45 & 3.35 & 0.40 & 17.29 & 2.35 & 9.34 & 1.08 & 10.42 \\
\hline 45 & 25 & 1.38 & 0.54 & 7.12 & 3.17 & 3.85 & 1.46 & 5.31 \\
\hline 25 & 13.1 & 2.14 & 3.12 & 11.04 & 18.33 & 5.96 & 8.43 & 14.39 \\
\hline 13.1 & 6.5 & 1.58 & 3.82 & 8.14 & 22.44 & 4.40 & 10.32 & 14.71 \\
\hline 6.5 & 3.3 & 1.33 & 3.84 & 6.86 & 22.56 & 3.70 & 10.37 & 14.08 \\
\hline 3.3 & 1.5 & 0.81 & 2.49 & 4.17 & 14.61 & 2.25 & 6.72 & 8.97 \\
\hline 1.5 & & 0.87 & 2.65 & 4.49 & 15.60 & 2.43 & 7.17 & 9.60 \\
\hline Totals & -- & 19.37 & 17.02 & 100.00 & 100.00 & 54.02 & 45.98 & 100.00 \\
\hline
\end{tabular}

\begin{tabular}{|c|c|c|c|c|c|c|c|c|}
\hline $\begin{array}{c}\text { Pass } \\
\text { Size } \\
(u m)\end{array}$ & $\begin{array}{c}\text { Retain } \\
\text { Size } \\
(u m)\end{array}$ & $\begin{array}{c}\text { O/5 } \\
\text { Mass } \\
(\mathrm{TPH})\end{array}$ & $\begin{array}{c}\text { O/S } \\
\text { Mass } \\
(\mathrm{TPH})\end{array}$ & $\begin{array}{c}\text { Feed } \\
\text { Mass } \\
(\mathrm{TPH})\end{array}$ & $\begin{array}{c}\text { Delta } \\
\text { Mass } \\
(\mathrm{TPH})\end{array}$ & $\begin{array}{c}\mathrm{V} \\
\text { Value } \\
(\mathrm{f}-\mathrm{u})\end{array}$ & $\begin{array}{c}\mathrm{X} \\
\text { Value } \\
(\mathrm{0}-\mathrm{u})\end{array}$ & $\begin{array}{c}\text { Partition } \\
\text { Factor } \\
(-)\end{array}$ \\
\hline & 212 & 0.04 & 0.00 & 0.04 & 0.00 & 3.74 & 3.74 & 1.00 \\
\hline 212 & 150 & 0.05 & 0.00 & 0.05 & 0.00 & 4.24 & 4.24 & 1.00 \\
\hline 150 & 75 & 0.16 & 0.00 & 0.17 & 0.00 & 14.11 & 13.68 & 0.97 \\
\hline 75 & 45 & 0.11 & 0.01 & 0.12 & 0.00 & 9.34 & 8.26 & 0.90 \\
\hline 45 & 25 & 0.04 & 0.02 & 0.06 & 0.00 & 3.85 & 2.39 & 0.73 \\
\hline 25 & 13.1 & 0.07 & 0.10 & 0.17 & 0.00 & 5.96 & -2.47 & 0.41 \\
\hline 13.1 & 6.5 & 0.05 & 0.12 & 0.17 & 0.00 & 4.40 & -5.92 & 0.30 \\
\hline 6.5 & 3.3 & 0.04 & 0.12 & 0.16 & 0.00 & 3.70 & -6.67 & 0.26 \\
\hline 3.3 & 1.5 & 0.03 & 0.08 & 0.10 & 0.00 & 2.25 & -4.46 & 0.25 \\
\hline 1.5 & & 0.03 & 0.08 & 0.11 & 0.00 & 2.43 & -4.75 & 0.25 \\
\hline Totals & -- & 0.62 & 0.53 & 1.15 & 0.00 & & & \\
\hline & Slurry (TPH) & 9.82 & 21.26 & 25.43 & -1.90 & & & \\
\hline
\end{tabular}


HYDROCYCLONE EVALUATION (BALANCED)

\begin{tabular}{|c|c|}
\hline No. 24 & \\
\hline Feed Flow (GPM) & 100.0 \\
\hline Feed Pressure (PSI) & 21.0 \\
\hline Water Flow (GPM) & 15.0 \\
\hline Water Pressure (PSl) & 12.0 \\
\hline Feed Rate (TPH) & 1.15 \\
\hline
\end{tabular}

\begin{tabular}{|r|c|c|c|}
\hline & OS & U/S & Feed \\
\hline Solids Content (\%) & 6.79 & 2.57 & 4.51 \\
\hline Ash Content (\%) & 23.74 & 48.18 & 34.58 \\
\hline Solids Yield (\%) & 55.6 & 44.4 & 100.0 \\
\hline Ash Yield (\%) & 55.6 & 44.4 & 100.0 \\
\hline Size Class Yield (\%) & 55.6 & 44.4 & 100.0 \\
\hline
\end{tabular}

\begin{tabular}{|c|c|c|c|c|c|c|c|c|}
\hline $\begin{array}{l}\text { Pass } \\
\text { Size } \\
\text { (um) }\end{array}$ & $\begin{array}{c}\text { Retain } \\
\text { Size } \\
\text { (um) }\end{array}$ & $\begin{array}{c}\text { O/S } \\
\text { Sample } \\
\text { (gms) }\end{array}$ & $\begin{array}{c}\text { U/S } \\
\text { Sample } \\
\text { (gms) }\end{array}$ & $\begin{array}{c}\text { orS } \\
\text { Mass } \\
(\% \text {, strm })\end{array}$ & $\begin{array}{c}\text { U/S } \\
\text { Mass } \\
(\% \text {, strm) }\end{array}$ & $\begin{array}{c}\text { OS } \\
\text { Mass } \\
(\% \text {, feed })\end{array}$ & $\begin{array}{c}\text { U/S } \\
\text { Mass } \\
(\%, \text { feed })\end{array}$ & $\begin{array}{c}\text { Feed } \\
\text { Mass } \\
(\% \text {, feed })\end{array}$ \\
\hline & 212 & 1.63 & 0.00 & 7.75 & 0.00 & 4.31 & 0.00 & 4.31 \\
\hline 212 & 150 & 1.89 & 0.00 & 8.99 & 0.00 & 5.00 & 0.00 & 5.00 \\
\hline 150 & 75 & 5.19 & 0.02 & 24.64 & 0.12 & 13.71 & 0.05 & 13.76 \\
\hline 75 & 45 & 3.83 & 0.27 & 18.18 & 1.59 & 10.12 & 0.71 & 10.82 \\
\hline 45 & 25 & 1.45 & 0.40 & 6.89 & 2.37 & 3.83 & 1.05 & 4.89 \\
\hline 25 & 13.1 & 1.98 & 2.94 & 9.42 & 17.60 & 5.24 & 7.81 & 13.05 \\
\hline 13.1 & 6.5 & 1.60 & 3.76 & 7.59 & 22.48 & 4.22 & 9.98 & 14.20 \\
\hline 6.5 & 3.3 & 1.44 & 3.80 & 6.82 & 22.76 & 3.80 & 10.10 & 13.90 \\
\hline 3.3 & 1.5 & 0.95 & 2.55 & 4.50 & 15.27 & 2.50 & 6.77 & 9.28 \\
\hline 1.5 & & 1.10 & 2.97 & 5.22 & 17.80 & 2.90 & 7.90 & 10.80 \\
\hline Totals & -- & 21.07 & 16.70 & 100.00 & 100.00 & 55.63 & 44.37 & 100.00 \\
\hline
\end{tabular}

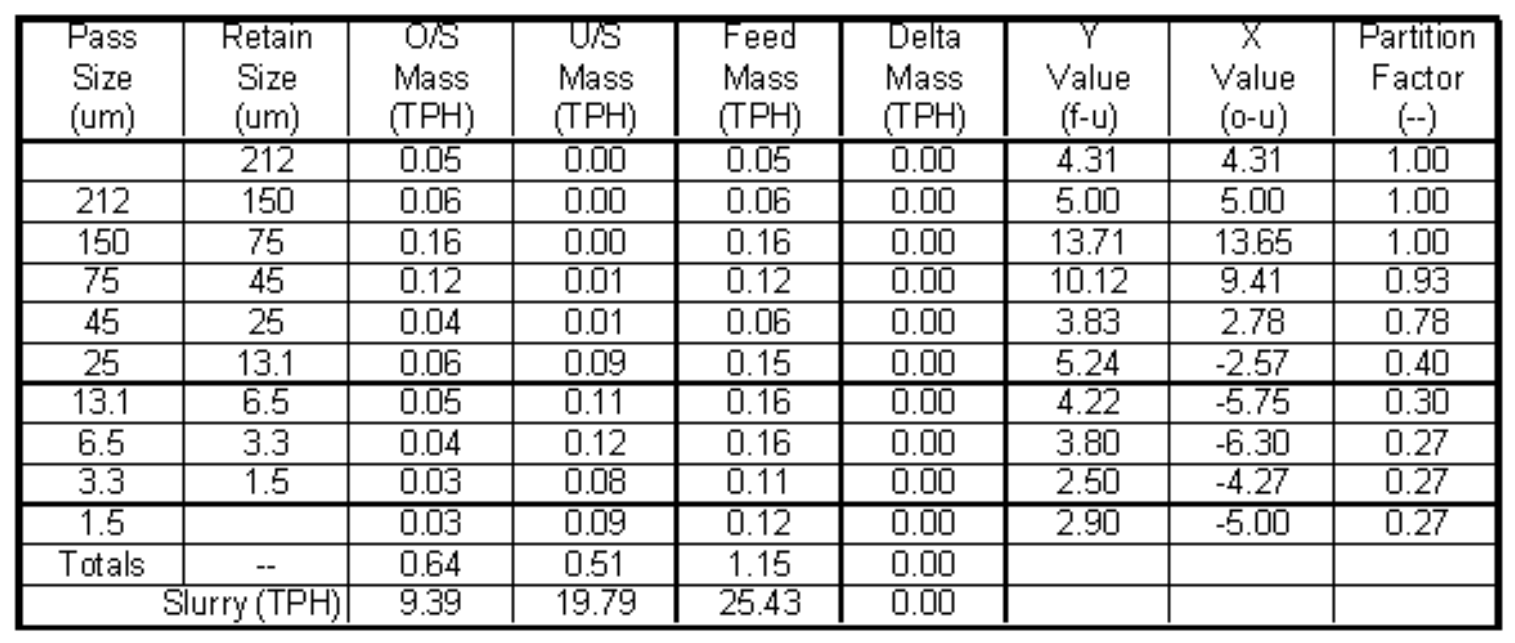




\begin{tabular}{|c|c|c|c|}
\hline $\begin{array}{c}\text { Experimental } \\
\text { Partition Factor }\end{array}$ & $\begin{array}{c}\text { Mean } \\
\text { Particle } \\
\text { Size }(\mu \mathrm{m})\end{array}$ & $\begin{array}{c}\text { Predicted } \\
\text { Partition } \\
\text { Factor }\end{array}$ & $\begin{array}{c}\text { Predicted } \\
\text { Partition } \\
\text { Factor }\end{array}$ \\
\hline$(-)$ & $(--)$ & (Plitt) & (Linch) \\
\hline 1.00 & 299.81 & 1.000 & 1.000 \\
\hline 1.00 & 178.33 & 1.000 & 1.000 \\
\hline 0.97 & 106.07 & 0.999 & 0.998 \\
\hline 0.90 & 58.09 & 0.927 & 0.934 \\
\hline 0.73 & 33.54 & 0.689 & 0.691 \\
\hline 0.41 & 18.08 & 0.438 & 0.435 \\
\hline 0.30 & 9.25 & 0.308 & 0.313 \\
\hline 0.26 & 4.62 & 0.261 & 0.265 \\
\hline 0.25 & 2.32 & 0.247 & 0.245 \\
\hline 0.25 & 1.64 & 0.244 & 0.239 \\
\hline & WSSQ & 0.004 & 0.004 \\
\hline
\end{tabular}

\begin{tabular}{|c|c|}
\multicolumn{1}{c|}{ (Plitt) } & Predicted \\
\hline $\mathrm{D} 50$ & 29.04 \\
\hline$m$ & 1.76 \\
\hline Bypass(R0) & 0.24 \\
\hline
\end{tabular}

(Linch) Predicted

\begin{tabular}{|c|c|}
\hline D50 & 28.79 \\
\hline Alpha & 2.23 \\
\hline Bypass(Rf) & 0.23 \\
\hline
\end{tabular}

\begin{tabular}{l|l}
\hline TWSSQ & 0.008 \\
\hline
\end{tabular}

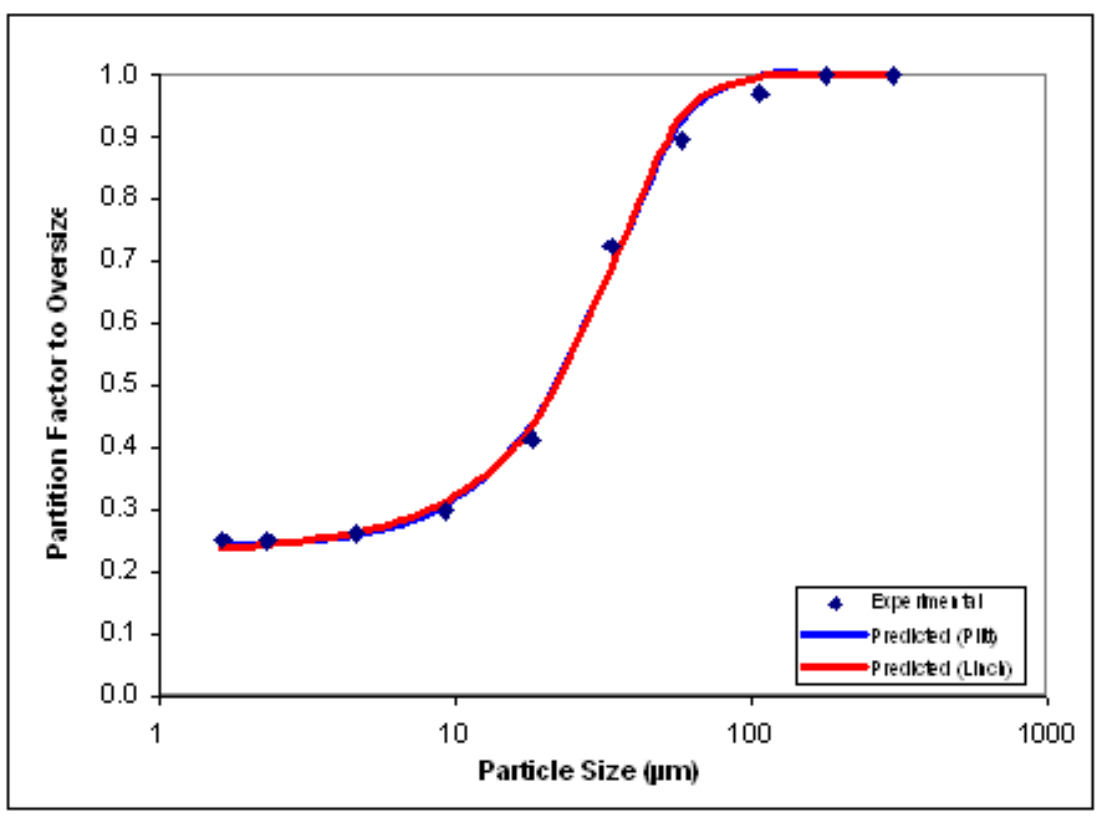


HYDROCYCLONE EVALUATION (UNBALANCED)

\begin{tabular}{|c|c|}
\hline Test: $\quad$ No. 25 & \\
\hline Feed Flow (GPM) & 100.0 \\
\hline Feed Pressure (PSl) & 21.0 \\
\hline Water Flow (GPM) & 0.0 \\
\hline Water Pressure (PSI) & 0.0 \\
\hline Feed Rate (TPH) & 1.15 \\
\hline
\end{tabular}

\begin{tabular}{|r|c|c|c|}
\hline & OS & U/S & Feed \\
\hline Solids Content (\%) & 8.10 & 2.42 & 4.51 \\
\hline Ash Content (\%) & 26.33 & 48.96 & 35.36 \\
\hline Solids Yield (\%) & 66.1 & 33.9 & 100.0 \\
\hline Ash Yield (\%) & 60.1 & 39.9 & 100.0 \\
\hline Size Class Yield (\%) & 74.3 & 25.7 & 100.0 \\
\hline
\end{tabular}

\begin{tabular}{|c|c|c|c|c|c|c|c|c|}
\hline $\begin{array}{l}\text { Pass } \\
\text { Size } \\
\text { (um) }\end{array}$ & $\begin{array}{l}\text { Retain } \\
\text { Size } \\
\text { (um) }\end{array}$ & $\begin{array}{c}\text { OS } \\
\text { Sample } \\
\text { (gms) }\end{array}$ & $\begin{array}{c}\text { US } \\
\text { Sample } \\
\text { (gms) }\end{array}$ & $\begin{array}{c}\text { o/S } \\
\text { Mass } \\
(\% \text {, strm) }\end{array}$ & $\begin{array}{c}\text { U/S } \\
\text { Mass } \\
(\% \text {, strm) }\end{array}$ & $\begin{array}{c}\text { OS } \\
\text { Mass } \\
(\% \text {, feed })\end{array}$ & $\begin{array}{c}\text { U/S } \\
\text { Mass } \\
(\%, \text { feed })\end{array}$ & $\begin{array}{c}\text { Feed } \\
\text { Mass } \\
(\% \text {, feed })\end{array}$ \\
\hline & 212 & 1.43 & 0.00 & 6.56 & 0.00 & 3.94 & 0.00 & 3.94 \\
\hline 212 & 150 & 1.62 & 0.00 & 7.43 & 0.00 & 4.47 & 0.00 & 4.47 \\
\hline 150 & 75 & 5.23 & 0.16 & 24.00 & 1.17 & 14.42 & 0.47 & 14.89 \\
\hline 75 & 45 & 3.34 & 0.25 & 15.33 & 1.83 & 9.21 & 0.73 & 9.94 \\
\hline 45 & 25 & 1.52 & 0.31 & 6.98 & 2.27 & 4.19 & 0.91 & 5.10 \\
\hline 25 & $\overline{13.1}$ & 2.58 & 2.41 & 11.85 & 17.66 & 7.12 & 7.05 & 14.17 \\
\hline 13.1 & 6.5 & 2.04 & 3.12 & 9.37 & 22.81 & 5.63 & 9.10 & 14.73 \\
\hline 6.5 & 3.3 & 1.77 & 3.07 & 8.11 & 22.46 & 4.87 & 8.96 & 13.84 \\
\hline 3.3 & 1.5 & 1.08 & 1.99 & 4.97 & 14.59 & 2.99 & 5.82 & 8.81 \\
\hline 1.5 & & 1.18 & 2.35 & 5.40 & 17.21 & 3.24 & 6.87 & 10.11 \\
\hline Totals & -- & 21.79 & 13.66 & 100.00 & 100.00 & 60.10 & 39.90 & 100.00 \\
\hline
\end{tabular}

\begin{tabular}{|c|c|c|c|c|c|c|c|c|}
\hline $\begin{array}{l}\text { Pass } \\
\text { Size } \\
\text { (um) }\end{array}$ & $\begin{array}{l}\text { Retain } \\
\text { Size } \\
\text { (um) }\end{array}$ & $\begin{array}{c}\text { O/S } \\
\text { Mass } \\
(\mathrm{TPH})\end{array}$ & $\begin{array}{l}\text { O/S } \\
\text { Mass } \\
\text { (TPH) }\end{array}$ & $\begin{array}{l}\text { Feed } \\
\text { Mass } \\
(\mathrm{TPH})\end{array}$ & $\begin{array}{l}\text { Delta } \\
\text { Mass } \\
\text { (TPH) }\end{array}$ & $\begin{array}{c}Y \\
\text { Value } \\
(\mathrm{f}-\mathrm{u})\end{array}$ & $\begin{array}{c}X \\
\text { Value } \\
\text { (0-u) }\end{array}$ & $\begin{array}{l}\text { Partition } \\
\text { Factor } \\
\text { (--) }\end{array}$ \\
\hline & 212 & 0.05 & 0.00 & 0.05 & 0.00 & 3.94 & 3.94 & $\begin{array}{l}.00 \\
\end{array}$ \\
\hline 212 & 150 & 0.05 & 0.00 & 0.05 & 0.00 & 4.47 & 4.47 & 1.00 \\
\hline 150 & 75 & 0.17 & 0.01 & 0.17 & 0.00 & 14.42 & 13.96 & 0.97 \\
\hline 75 & 45 & 0.11 & 0.01 & 0.11 & 0.00 & 9.21 & 8.48 & 0.93 \\
\hline 45 & 25 & 0.05 & 0.01 & 0.06 & 0.00 & 4.19 & 3.29 & 0.82 \\
\hline 25 & 13.1 & 0.08 & 0.08 & 0.16 & 0.00 & 7.12 & 0.07 & 0.50 \\
\hline 13.1 & 6.5 & 0.06 & 0.10 & 0.17 & 0.00 & 5.63 & -3.47 & 0.38 \\
\hline 6.5 & 3.3 & 0.06 & 0.10 & 0.16 & 0.00 & 4.87 & -4.09 & 0.35 \\
\hline 3.3 & 1.5 & 0.03 & 0.07 & 0.10 & 0.00 & 2.99 & -2.83 & 0.34 \\
\hline 1.5 & & 0.04 & 0.08 & 0.12 & 0.00 & 3.24 & -3.62 & 0.32 \\
\hline Totals & -- & 0.69 & 0.46 & 1.15 & 0.00 & & & \\
\hline & Slurry (TPH) & 8.51 & 18.91 & 25.43 & -1.99 & & & \\
\hline
\end{tabular}




\section{HYDROCYCLONE EVALUATION (BALANCED)}

\begin{tabular}{l}
\hline Test: No. 25 \\
\begin{tabular}{|l|c|}
\hline \multicolumn{2}{|c|}{} \\
\hline Feed Flow (GPM) & 100.0 \\
\hline Feed Pressure (PSl) & 21.0 \\
\hline Water Flow (GPM) & 0.0 \\
\hline Water Pressure (PS) & 0.0 \\
\hline Feed Rate (TPH) & 1.15 \\
\hline
\end{tabular}
\end{tabular}

\begin{tabular}{|r|c|c|c|}
\hline & OS & U/S & Feed \\
\hline Solids Content (\%) & 6.79 & 2.57 & 4.51 \\
\hline Ash Content (\%) & 23.74 & 48.18 & 34.58 \\
\hline Solids Yield (\%) & 69.2 & 30.8 & 100.0 \\
\hline Ash Yield (\%) & 55.6 & 44.4 & 100.0 \\
\hline Size Class Yield (\%) & 55.6 & 44.4 & 100.0 \\
\hline
\end{tabular}

\begin{tabular}{|c|c|c|c|c|c|c|c|c|}
\hline $\begin{array}{l}\text { Pass } \\
\text { Size } \\
\text { (um) } \\
\end{array}$ & $\begin{array}{c}\text { Retain } \\
\text { Size } \\
\text { (um) } \\
\end{array}$ & $\begin{array}{c}\text { OS } \\
\text { Sample } \\
\text { (gms) }\end{array}$ & $\begin{array}{c}\text { U/S } \\
\text { Sample } \\
\text { (gms) }\end{array}$ & $\begin{array}{c}\text { O/S } \\
\text { Mass } \\
(\%, \text { strm })\end{array}$ & $\begin{array}{c}\text { USS } \\
\text { Mass } \\
(\%, \text { strm })\end{array}$ & $\begin{array}{c}\text { OS } \\
\text { Mass } \\
(\%, \text { feed })\end{array}$ & $\begin{array}{c}\text { U/S } \\
\text { Mass } \\
(\%, \text { feed })\end{array}$ & $\begin{array}{c}\text { Feed } \\
\text { Mass } \\
(\%, \text { feed })\end{array}$ \\
\hline & 212 & 1.63 & 0.00 & 7.75 & 0.00 & 4.31 & 0.00 & 4.31 \\
\hline 212 & 150 & 1.89 & 0.00 & 8.99 & 0.00 & 5.00 & 0.00 & 5.00 \\
\hline 150 & 75 & 5.19 & 0.02 & 24.64 & 0.12 & 13.71 & 0.05 & 13.76 \\
\hline 75 & 45 & 3.83 & 0.27 & 18.18 & 1.59 & 10.12 & 0.71 & 10.82 \\
\hline 45 & 25 & 1.45 & 0.40 & 6.89 & 2.37 & 3.83 & 1.05 & 4.89 \\
\hline 25 & 13.1 & 1.98 & 2.94 & 9.42 & 17.60 & 5.24 & 7.81 & 13.05 \\
\hline 13.1 & 6.5 & 1.60 & 3.76 & 7.59 & 22.48 & 4.22 & 9.98 & 14.20 \\
\hline 6.5 & 3.3 & 1.44 & 3.80 & 6.82 & 22.76 & 3.80 & 10.10 & 13.90 \\
\hline 3.3 & 1.5 & 0.95 & 2.55 & 4.50 & 15.27 & 2.50 & 6.77 & 9.28 \\
\hline 1.5 & & 1.10 & 2.97 & 5.22 & 17.80 & 2.90 & 7.90 & 10.80 \\
\hline Totals & -- & 21.07 & 16.70 & 100.00 & 100.00 & 55.63 & 44.37 & 100.00 \\
\hline
\end{tabular}

\begin{tabular}{|c|c|c|c|c|c|c|c|c|}
\hline $\begin{array}{c}\text { Pass } \\
\text { Size } \\
(u m)\end{array}$ & $\begin{array}{c}\text { Retain } \\
\text { Size } \\
(u m)\end{array}$ & $\begin{array}{c}\text { O/S } \\
\text { Mass } \\
(\mathrm{TPH})\end{array}$ & $\begin{array}{c}\text { U/S } \\
\text { Mass } \\
(\mathrm{TPH})\end{array}$ & $\begin{array}{c}\text { Feed } \\
\text { Mass } \\
(\mathrm{TPH})\end{array}$ & $\begin{array}{c}\text { Delta } \\
\text { Mass } \\
(\mathrm{TPH})\end{array}$ & $\begin{array}{c}\mathrm{V} \\
\text { Value } \\
(\mathrm{f}-\mathrm{u})\end{array}$ & $\begin{array}{c}\mathrm{X} \\
\text { Value } \\
(\mathbf{0}-\mathrm{u})\end{array}$ & $\begin{array}{c}\text { Partition } \\
\text { Factor } \\
(-)\end{array}$ \\
\hline & 212 & 0.05 & 0.00 & 0.05 & 0.00 & 4.31 & 4.31 & 1.00 \\
\hline 212 & 150 & 0.06 & 0.00 & 0.06 & 0.00 & 5.00 & 5.00 & 1.00 \\
\hline 150 & 75 & 0.16 & 0.00 & 0.16 & 0.00 & 13.71 & 13.65 & 1.00 \\
\hline 75 & 45 & 0.12 & 0.01 & 0.12 & 0.00 & 10.12 & 9.41 & 0.93 \\
\hline 45 & 25 & 0.04 & 0.01 & 0.06 & 0.00 & 3.83 & 2.78 & 0.78 \\
\hline 25 & 13.1 & 0.06 & 0.09 & 0.15 & 0.00 & 5.24 & -2.57 & 0.40 \\
\hline 13.1 & 6.5 & 0.05 & 0.11 & 0.16 & 0.00 & 4.22 & -5.75 & 0.30 \\
\hline 6.5 & 3.3 & 0.04 & 0.12 & 0.16 & 0.00 & 3.80 & -6.30 & 0.27 \\
\hline 3.3 & 1.5 & 0.03 & 0.08 & 0.11 & 0.00 & 2.50 & -4.27 & 0.27 \\
\hline 1.5 & & 0.03 & 0.09 & 0.12 & 0.00 & 2.90 & -5.00 & 0.27 \\
\hline Totals & -- & 0.64 & 0.51 & 1.15 & 0.00 & & & \\
\hline \multicolumn{2}{r}{ Slurry (TPH) } & 9.39 & 19.79 & 25.43 & -3.75 & & & \\
\hline
\end{tabular}




\begin{tabular}{|c|c|c|c|c|c|c|c|}
\hline \multirow{3}{*}{$\begin{array}{l}\text { Experimental } \\
\text { Partition } \mathrm{F} \text { actor }\end{array}$} & \multirow{3}{*}{$\begin{array}{c}\text { Mean } \\
\text { Particle } \\
\text { Size ( } \mu \mathrm{m})\end{array}$} & \multirow{3}{*}{$\begin{array}{c}\text { Predicted } \\
\text { Partition } \\
\text { Factor }\end{array}$} & \multirow{3}{*}{$\begin{array}{c}\text { Predicted } \\
\text { Partition } \\
\text { Factor }\end{array}$} & (Plitt) & Predicted & (Linch) & Predicted \\
\hline & & & & D50 & 25.67 & D50 & 25.61 \\
\hline & & & & $m$ & 1.98 & Alpha & 2.79 \\
\hline$(-)$ & $(-)$ & (Plitt) & (Linch) & Bypass(Rf) & 0.33 & Bypass(Rf) & 0.32 \\
\hline 1.00 & 299.81 & 1.000 & 1.000 & & & & \\
\hline 1.00 & 178.33 & 1.000 & 1.000 & & & & \\
\hline 0.97 & 106.07 & 1.000 & 1.000 & & & & \\
\hline 0.93 & 58.09 & 0.979 & 0.982 & & & & \\
\hline 0.82 & 33.54 & 0.792 & 0.803 & & & & \\
\hline 0.50 & 18.08 & 0.524 & 0.514 & & & & \\
\hline 0.38 & 9.25 & 0.386 & 0.388 & & & & \\
\hline 0.35 & 4.62 & 0.342 & 0.346 & & & & \\
\hline 0.34 & 2.32 & 0.331 & 0.330 & & & & \\
\hline 0.32 & 1.64 & 0.329 & 0.327 & & & & \\
\hline & WSSQ & 0.005 & 0.005 & & & & \\
\hline & TWSSQ & 0.010 & & & & & \\
\hline
\end{tabular}

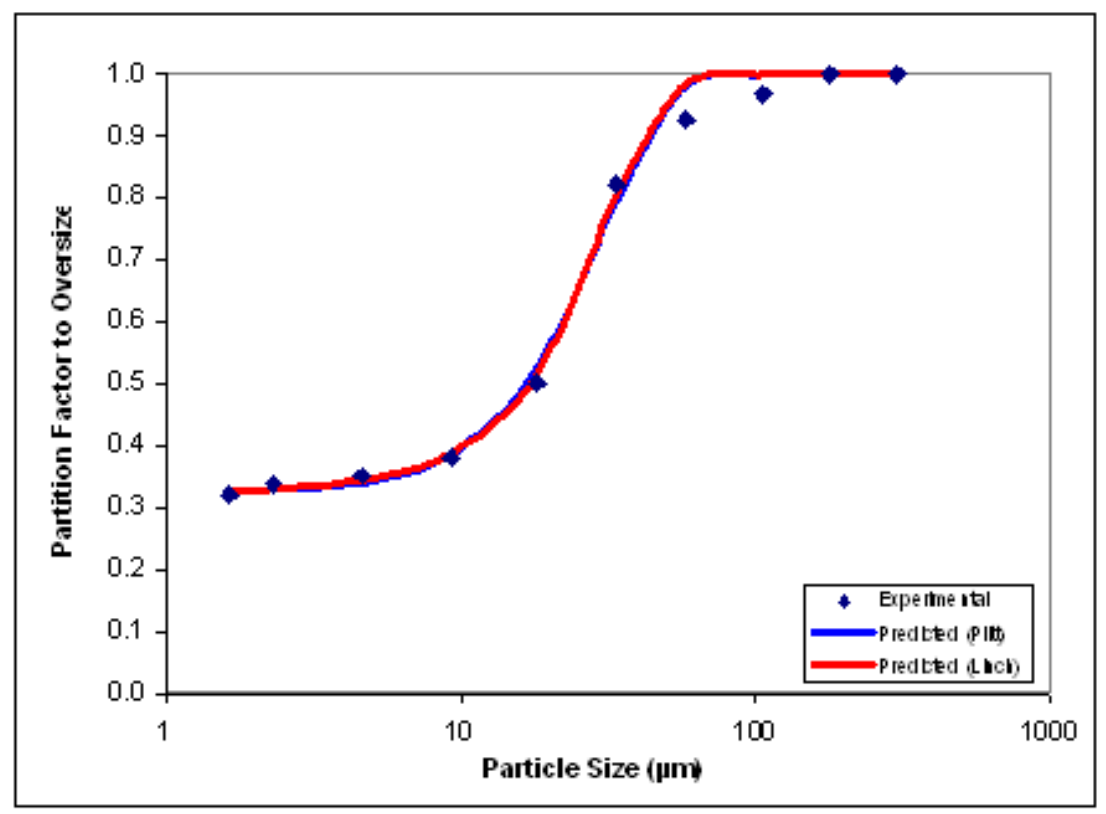


HYDROCYCLONE EVALUATION (UNBALANCED)

\begin{tabular}{|c|c|}
\hline No. 26 & \\
\hline Feedflow (GPM) & 100.0 \\
\hline Feed Pressure (PSl) & 21.0 \\
\hline Water Flow (GPM) & 30.0 \\
\hline Water Pressure (PSl) & 14.0 \\
\hline Feed Rate (TPH) & 1.15 \\
\hline
\end{tabular}

\begin{tabular}{|r|c|c|c|}
\hline & OS & U/S & Feed \\
\hline Solids Content (\%) & 5.25 & 2.51 & 4.51 \\
\hline Ash Content (\%) & 21.17 & 47.88 & 34.50 \\
\hline Solids Yield (\%) & 53.5 & 46.5 & 100.0 \\
\hline Ash Yield (\%) & 50.1 & 49.9 & 100.0 \\
\hline Size Class Yield (\%) & 45.5 & 54.5 & 100.0 \\
\hline
\end{tabular}

\begin{tabular}{|c|c|c|c|c|c|c|c|c|}
\hline $\begin{array}{c}\text { Pass } \\
\text { Size } \\
(u m)\end{array}$ & $\begin{array}{c}\text { Retain } \\
\text { Size } \\
(\mathrm{um})\end{array}$ & $\begin{array}{c}\text { O/S } \\
\text { Sample } \\
(\mathrm{gms})\end{array}$ & $\begin{array}{c}\text { U/S } \\
\text { Sample } \\
(\mathrm{gms})\end{array}$ & $\begin{array}{c}\text { O/S } \\
\text { Mass } \\
(\%, \text { strm) }\end{array}$ & $\begin{array}{c}\text { U/S } \\
\text { Mass } \\
(\%, \text { strm) }\end{array}$ & $\begin{array}{c}\text { OS } \\
\text { Mass } \\
(\%, \text { feed })\end{array}$ & $\begin{array}{c}\text { U/S } \\
\text { Mass } \\
(\%, \text { feed })\end{array}$ & $\begin{array}{c}\text { Feed } \\
\text { Mass } \\
(\%, \text { feed })\end{array}$ \\
\hline & 212 & 1.30 & 0.00 & 7.57 & 0.00 & 3.79 & 0.00 & 3.79 \\
\hline 212 & 150 & 1.84 & 0.00 & 10.72 & 0.00 & 5.37 & 0.00 & 5.37 \\
\hline 150 & 75 & 5.14 & 0.19 & 29.94 & 0.96 & 15.00 & 0.48 & 15.48 \\
\hline 75 & 45 & 2.92 & 0.48 & 17.01 & 2.43 & 8.52 & 1.21 & 9.73 \\
\hline 45 & 25 & 1.14 & 0.83 & 6.64 & 4.20 & 3.33 & 2.09 & 5.42 \\
\hline 25 & 13.1 & 1.61 & 3.61 & 9.37 & 18.24 & 4.69 & 9.10 & 13.79 \\
\hline 13.1 & 6.5 & 1.10 & 4.33 & 6.39 & 21.87 & 3.20 & 10.91 & 14.12 \\
\hline 6.5 & 3.3 & 0.93 & 4.42 & 5.41 & 22.32 & 2.71 & 11.14 & 13.85 \\
\hline 3.3 & 1.5 & 0.57 & 2.92 & 3.30 & 14.75 & 1.65 & 7.36 & 9.01 \\
\hline 1.5 & & 0.63 & 3.01 & 3.66 & 15.23 & 1.83 & 7.60 & 9.44 \\
\hline Totals & -- & 17.17 & 19.78 & 100.00 & 100.00 & 50.09 & 49.91 & 100.00 \\
\hline
\end{tabular}

\begin{tabular}{|c|c|c|c|c|c|c|c|c|}
\hline $\begin{array}{c}\text { Pass } \\
\text { Size } \\
(u m)\end{array}$ & $\begin{array}{c}\text { Retain } \\
\text { Size } \\
(u m)\end{array}$ & $\begin{array}{c}\text { O/S } \\
\text { Mass } \\
(\mathrm{TPH})\end{array}$ & $\begin{array}{c}\text { U/S } \\
\text { Mass } \\
(\mathrm{TPH})\end{array}$ & $\begin{array}{c}\text { Feed } \\
\text { Mass } \\
(\mathrm{TPH})\end{array}$ & $\begin{array}{c}\text { Delta } \\
\text { Mass } \\
(\mathrm{TPH})\end{array}$ & $\begin{array}{c}\text { Y } \\
\text { Value } \\
(\mathrm{f}-\mathrm{u})\end{array}$ & $\begin{array}{c}\text { X } \\
\text { Value } \\
(0-\mathrm{u})\end{array}$ & $\begin{array}{c}\text { Partition } \\
\text { Factor } \\
(-)\end{array}$ \\
\hline & 212 & 0.04 & 0.00 & 0.04 & 0.00 & 3.79 & 3.79 & 1.00 \\
\hline 212 & 150 & 0.06 & 0.00 & 0.06 & 0.00 & 5.37 & 5.37 & 1.00 \\
\hline 150 & 75 & 0.17 & 0.01 & 0.18 & 0.00 & 15.00 & 14.52 & 0.97 \\
\hline 75 & 45 & 0.10 & 0.01 & 0.11 & 0.00 & 8.52 & 7.31 & 0.88 \\
\hline 45 & 25 & 0.04 & 0.02 & 0.06 & 0.00 & 3.33 & 1.23 & 0.61 \\
\hline 25 & 13.1 & 0.05 & 0.10 & 0.16 & 0.00 & 4.69 & -4.41 & 0.34 \\
\hline 13.1 & 6.5 & 0.04 & 0.13 & 0.16 & 0.00 & 3.20 & -7.71 & 0.23 \\
\hline 6.5 & 3.3 & 0.03 & 0.13 & 0.16 & 0.00 & 2.71 & -8.43 & 0.20 \\
\hline 3.3 & 1.5 & 0.02 & 0.08 & 0.10 & 0.00 & 1.65 & -5.71 & 0.18 \\
\hline 1.5 & & 0.02 & 0.09 & 0.11 & 0.00 & 1.83 & -5.77 & 0.19 \\
\hline Totals & -- & 0.57 & 0.57 & 1.15 & 0.00 & & & \\
\hline & Slurry (TPH) & 10.94 & 22.80 & 25.43 & -0.82 & & & \\
\hline
\end{tabular}


HYDROCYCLONE EVALUATION (BALANCED)

\begin{tabular}{|c|c|}
\hline No. 26 & \\
\hline Feedflow (GPM) & 700.0 \\
\hline Feed Pressure (PSl) & 21.0 \\
\hline Water Flow (GPM) & 30.0 \\
\hline Water Pressure (PSI) & 14.0 \\
\hline Feed Rate (TPH) & 1.15 \\
\hline
\end{tabular}

\begin{tabular}{|r|c|c|c|}
\hline & OSS & U/S & Feed \\
\hline Solids Content (\%) & 6.79 & 2.57 & 4.51 \\
\hline Ash Content (\%) & 23.74 & 48.18 & 34.58 \\
\hline Solids Yield (\%) & 42.1 & 57.9 & 100.0 \\
\hline Ash Yield (\%) & 55.6 & 44.4 & 100.0 \\
\hline Size Class Yield (\%) & 55.6 & 44.4 & 100.0 \\
\hline
\end{tabular}

\begin{tabular}{|c|c|c|c|c|c|c|c|c|}
\hline $\begin{array}{c}\text { Pass } \\
\text { Size } \\
(u m)\end{array}$ & $\begin{array}{c}\text { Retain } \\
\text { Size } \\
(\mathrm{um})\end{array}$ & $\begin{array}{c}\text { O/S } \\
\text { Sample } \\
(\mathrm{gms})\end{array}$ & $\begin{array}{c}\text { U/S } \\
\text { Sample } \\
(\mathrm{gms})\end{array}$ & $\begin{array}{c}\text { O/S } \\
\text { Mass } \\
(\%, \text { strm) }\end{array}$ & $\begin{array}{c}\text { U/S } \\
\text { Mass } \\
(\%, \text { strm })\end{array}$ & $\begin{array}{c}\text { OS } \\
\text { Mass } \\
(\%, \text { feed })\end{array}$ & $\begin{array}{c}\text { U/S } \\
\text { Mass } \\
(\%, \text { feed })\end{array}$ & $\begin{array}{c}\text { Feed } \\
\text { Mass } \\
(\%, \text { feed })\end{array}$ \\
\hline & 212 & 1.63 & 0.00 & 7.75 & 0.00 & 4.31 & 0.00 & 4.31 \\
\hline 212 & 150 & 1.89 & 0.00 & 8.99 & 0.00 & 5.00 & 0.00 & 5.00 \\
\hline 150 & 75 & 5.19 & 0.02 & 24.64 & 0.12 & 13.71 & 0.05 & 13.76 \\
\hline 75 & 45 & 3.83 & 0.27 & 18.18 & 1.59 & 10.12 & 0.71 & 10.82 \\
\hline 45 & 25 & 1.45 & 0.40 & 6.89 & 2.37 & 3.83 & 1.05 & 4.89 \\
\hline 25 & 13.1 & 1.98 & 2.94 & 9.42 & 17.60 & 5.24 & 7.81 & 13.05 \\
\hline 13.1 & 6.5 & 1.60 & 3.76 & 7.59 & 22.48 & 4.22 & 9.98 & 14.20 \\
\hline 6.5 & 3.3 & 1.44 & 3.80 & 6.82 & 22.76 & 3.80 & 10.10 & 13.90 \\
\hline 3.3 & 1.5 & 0.95 & 2.55 & 4.50 & 15.27 & 2.50 & 6.77 & 9.28 \\
\hline 1.5 & & 1.10 & 2.97 & 5.22 & 17.80 & 2.90 & 7.90 & 10.80 \\
\hline Totals & -- & 21.07 & 16.70 & 100.00 & 100.00 & 55.63 & 44.37 & 100.00 \\
\hline
\end{tabular}

\begin{tabular}{|c|c|c|c|c|c|c|c|c|}
\hline $\begin{array}{c}\text { Pass } \\
\text { Size } \\
(\mathrm{um})\end{array}$ & $\begin{array}{c}\text { Retain } \\
\text { Size } \\
(\mathrm{um})\end{array}$ & $\begin{array}{c}0 / \mathrm{S} \\
\text { Mass } \\
(\mathrm{TPH})\end{array}$ & $\begin{array}{c}\mathrm{U} / \mathrm{S} \\
\text { Mass } \\
(\mathrm{TPH})\end{array}$ & $\begin{array}{c}\text { Feed } \\
\text { Mass } \\
(\mathrm{TPH})\end{array}$ & $\begin{array}{c}\text { Delta } \\
\text { Mass } \\
(\mathrm{TPH})\end{array}$ & $\begin{array}{c}\text { Y } \\
\text { Value } \\
(\mathrm{f}-\mathrm{u})\end{array}$ & $\begin{array}{c}\text { X } \\
\text { Value } \\
(\mathbf{0}-\mathrm{u})\end{array}$ & $\begin{array}{c}\text { Partition } \\
\text { Factor } \\
(-)\end{array}$ \\
\hline & 212 & 0.05 & 0.00 & 0.05 & 0.00 & 4.31 & 4.31 & 1.00 \\
\hline 212 & 150 & 0.06 & 0.00 & 0.06 & 0.00 & 5.00 & 5.00 & 1.00 \\
\hline 150 & 75 & 0.16 & 0.00 & 0.16 & 0.00 & 13.71 & 13.65 & 1.00 \\
\hline 75 & 45 & 0.12 & 0.01 & 0.12 & 0.00 & 10.12 & 9.41 & 0.93 \\
\hline 45 & 25 & 0.04 & 0.01 & 0.06 & 0.00 & 3.83 & 2.78 & 0.78 \\
\hline 25 & 13.1 & 0.06 & 0.09 & 0.15 & 0.00 & 5.24 & -2.57 & 0.40 \\
\hline 13.1 & 6.5 & 0.05 & 0.11 & 0.16 & 0.00 & 4.22 & -5.75 & 0.30 \\
\hline 6.5 & 3.3 & 0.04 & 0.12 & 0.16 & 0.00 & 3.80 & -6.30 & 0.27 \\
\hline 3.3 & 1.5 & 0.03 & 0.08 & 0.11 & 0.00 & 2.50 & -4.27 & 0.27 \\
\hline 1.5 & & 0.03 & 0.09 & 0.12 & 0.00 & 2.90 & -5.00 & 0.27 \\
\hline Totals & -- & 0.64 & 0.51 & 1.15 & 0.00 & & & \\
\hline \multicolumn{2}{r}{ Slurry (TPH) } & 9.39 & 19.79 & 25.43 & 3.75 & & & \\
\hline
\end{tabular}




\begin{tabular}{|c|c|c|c|}
\hline $\begin{array}{c}\text { Experimental } \\
\text { Partition F actor }\end{array}$ & $\begin{array}{c}\text { Mean } \\
\text { Particle } \\
\text { Size }(\mu \mathrm{m})\end{array}$ & $\begin{array}{c}\text { Predicted } \\
\text { Partition } \\
\text { Factor }\end{array}$ & $\begin{array}{c}\text { Predicted } \\
\text { Partition } \\
\text { Factor }\end{array}$ \\
\hline$(-)$ & $(--)$ & (Plitt) & (Linch) \\
\hline 1.00 & 299.81 & 1.000 & 1.000 \\
\hline 1.00 & 178.33 & 1.000 & 1.000 \\
\hline 0.97 & 106.07 & 0.998 & 0.996 \\
\hline 0.88 & 58.09 & 0.883 & 0.888 \\
\hline 0.61 & 33.54 & 0.599 & 0.595 \\
\hline 0.34 & 18.08 & 0.350 & 0.351 \\
\hline 0.23 & 9.25 & 0.235 & 0.243 \\
\hline 0.20 & 4.62 & 0.196 & 0.200 \\
\hline 0.18 & 2.32 & 0.185 & 0.182 \\
\hline 0.19 & 1.64 & 0.183 & 0.177 \\
\hline & MSSQ & 0.001 & 0.002 \\
\hline
\end{tabular}

\begin{tabular}{|c|c|c|c|}
\hline (Plitt) & Predicted & (Linch) & Predicted \\
\hline D50 & 32.94 & D50 & 32.77 \\
\hline$m$ & 1.82 & Alpha & 2.30 \\
\hline Bypass(Rt) & 0.18 & Bypass(Rf) & 0.17 \\
\hline
\end{tabular}

\begin{tabular}{|l|l|}
\hline TWSSQ & 0.003 \\
\hline
\end{tabular}

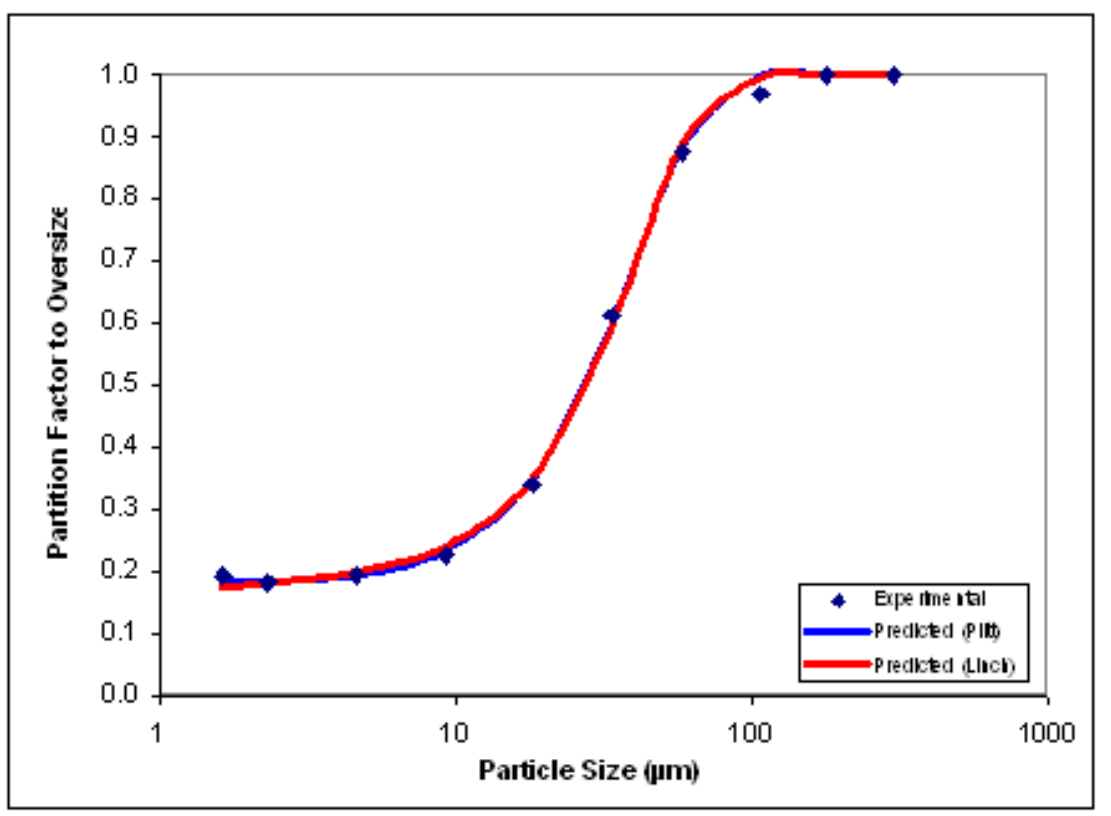


HYDROCYCLONE EVALUATION (UNBALANCED)

\begin{tabular}{|c|c|}
\hline No. 27 & \\
\hline FeedFlow (GPM) & 100.0 \\
\hline Feed Pressure (PSI) & 21.0 \\
\hline Water Flow (GPM) & 15.0 \\
\hline Water Pressure (PSI) & 12.0 \\
\hline Feed Rate (TPH) & 1.18 \\
\hline
\end{tabular}

\begin{tabular}{|r|c|c|c|}
\hline & OS & U/S & Feed \\
\hline Solids Content (\%) & 6.60 & 2.79 & 4.64 \\
\hline Ash Content (\%) & 23.68 & 48.24 & 35.15 \\
\hline Solids Yield (\%) & 53.7 & 46.3 & 100.0 \\
\hline Ash Yield (\%) & 53.3 & 46.7 & 100.0 \\
\hline Size Class Yield (\%) & 49.7 & 50.3 & 100.0 \\
\hline
\end{tabular}

\begin{tabular}{|c|c|c|c|c|c|c|c|c|}
\hline Pass & Retain & $0 / 5$ & U/S & o/S & U/S & OS & U/S & Feed \\
\hline $\begin{array}{l}\text { Size } \\
\text { (um) }\end{array}$ & $\begin{array}{l}\text { Size } \\
\text { (um) }\end{array}$ & $\begin{array}{c}\text { Sample } \\
\text { (gms) }\end{array}$ & $\begin{array}{c}\text { Sample } \\
\text { (gms) }\end{array}$ & $\begin{array}{c}\text { Mass } \\
(\%, \text { strm) }\end{array}$ & $\begin{array}{c}\text { Mass } \\
(\%, \text { strm })\end{array}$ & $\begin{array}{c}\text { Mass } \\
(\% \text {, feed })\end{array}$ & $\begin{array}{c}\text { Mass } \\
(\%, \text { feed })\end{array}$ & $\begin{array}{c}\text { Mass } \\
(\%, \text { feed })\end{array}$ \\
\hline & 212 & 1.54 & 0.00 & 7.68 & 0.00 & 4.09 & 0.00 & 4.09 \\
\hline 212 & 150 & 1.89 & 0.00 & 9.43 & 0.00 & 5.02 & 0.00 & 5.02 \\
\hline 150 & 75 & 5.14 & 0.15 & 25.64 & 0.85 & 13.66 & 0.40 & 14.06 \\
\hline 75 & 45 & 3.30 & 0.40 & 16.46 & 2.28 & 8.77 & 1.06 & 9.84 \\
\hline 45 & 25 & 1.30 & 0.55 & 6.48 & 3.13 & 3.46 & 1.46 & 4.92 \\
\hline 25 & 13.1 & 2.16 & 3.14 & 10.79 & 17.91 & 5.75 & 8.36 & 14.11 \\
\hline 13.1 & 6.5 & 1.59 & 3.80 & 7.95 & 21.63 & 4.24 & 10.10 & 14.34 \\
\hline 6.5 & 3.3 & 1.38 & 3.94 & 6.88 & 22.44 & 3.66 & 10.48 & 14.14 \\
\hline 3.3 & 1.5 & 0.84 & 2.78 & 4.18 & 15.86 & 2.23 & 7.41 & 9.64 \\
\hline 1.5 & & 0.90 & 2.79 & 4.51 & 15.89 & 2.41 & 7.42 & 9.83 \\
\hline Totals & -- & 20.05 & 17.55 & 100.00 & 100.00 & 53.30 & 46.70 & 100.00 \\
\hline
\end{tabular}

\begin{tabular}{|c|c|c|c|c|c|c|c|c|}
\hline $\begin{array}{c}\text { Pass } \\
\text { Size } \\
(u m)\end{array}$ & $\begin{array}{c}\text { Retain } \\
\text { Size } \\
(u m)\end{array}$ & $\begin{array}{c}\text { O/S } \\
\text { Mass } \\
(\mathrm{TPH})\end{array}$ & $\begin{array}{c}\text { O/S } \\
\text { Mass } \\
(\mathrm{TPH})\end{array}$ & $\begin{array}{c}\text { Feed } \\
\text { Mass } \\
(\mathrm{TPH})\end{array}$ & $\begin{array}{c}\text { Delta } \\
\text { Mass } \\
(\mathrm{TPH})\end{array}$ & $\begin{array}{c}\mathrm{V} \\
\text { Value } \\
(\mathrm{f}-\mathrm{u})\end{array}$ & $\begin{array}{c}\mathrm{X} \\
\text { Value } \\
(\mathrm{0}-\mathrm{u})\end{array}$ & $\begin{array}{c}\text { Partition } \\
\text { Factor } \\
(-)\end{array}$ \\
\hline & 212 & 0.05 & 0.00 & 0.05 & 0.00 & 4.09 & 4.09 & 1.00 \\
\hline 212 & 150 & 0.06 & 0.00 & 0.06 & 0.00 & 5.02 & 5.02 & 1.00 \\
\hline 150 & 75 & 0.16 & 0.00 & 0.17 & 0.00 & 13.66 & 13.26 & 0.97 \\
\hline 75 & 45 & 0.10 & 0.01 & 0.12 & 0.00 & 8.77 & 7.71 & 0.89 \\
\hline 45 & 25 & 0.04 & 0.02 & 0.06 & 0.00 & 3.46 & 1.99 & 0.70 \\
\hline 25 & 13.1 & 0.07 & 0.10 & 0.17 & 0.00 & 5.75 & -2.61 & 0.41 \\
\hline 13.1 & 6.5 & 0.05 & 0.12 & 0.17 & 0.00 & 4.24 & -5.86 & 0.30 \\
\hline 6.5 & 3.3 & 0.04 & 0.12 & 0.17 & 0.00 & 3.66 & -6.81 & 0.26 \\
\hline 3.3 & 1.5 & 0.03 & 0.09 & 0.11 & 0.00 & 2.23 & -5.18 & 0.23 \\
\hline 1.5 & & 0.03 & 0.09 & 0.12 & 0.00 & 2.41 & -5.02 & 0.24 \\
\hline Totals & -- & 0.63 & 0.55 & 1.18 & 0.00 & & & \\
\hline & Slurry (TPH) & 9.53 & 19.76 & 25.44 & -0.10 & & & \\
\hline
\end{tabular}


HYDROCYCLONE EVALUATION (BALANCED)

\begin{tabular}{|c|c|}
\hline No. 27 & \\
\hline Feed Flow (GPM) & 100.0 \\
\hline Feed Pressure (PSl) & 21.0 \\
\hline Water Flow (GPM) & 15.0 \\
\hline Water Pressure (PSl) & 12.0 \\
\hline Feed Rate (TPH) & 1.15 \\
\hline
\end{tabular}

\begin{tabular}{|r|c|c|c|}
\hline & OS & US & Feed \\
\hline Solids Content (\%) & 6.79 & 2.57 & 4.51 \\
\hline Ash Content (\%) & 23.74 & 48.18 & 34.58 \\
\hline Solids Yield (\%) & 55.6 & 44.4 & 100.0 \\
\hline Ash Yield (\%) & 55.6 & 44.4 & 100.0 \\
\hline Size Class Yield (\%) & 55.6 & 44.4 & 100.0 \\
\hline
\end{tabular}

\begin{tabular}{|c|c|c|c|c|c|c|c|c|}
\hline $\begin{array}{c}\text { Pass } \\
\text { Size } \\
(u m)\end{array}$ & $\begin{array}{c}\text { Retain } \\
\text { Size } \\
(\mathrm{um})\end{array}$ & $\begin{array}{c}\text { O/S } \\
\text { Sample } \\
\text { (gms) }\end{array}$ & $\begin{array}{c}\text { U/S } \\
\text { Sample } \\
(\mathrm{gms})\end{array}$ & $\begin{array}{c}\text { O/S } \\
\text { Mass } \\
(\%, \text { strm) }\end{array}$ & $\begin{array}{c}\text { U/S } \\
\text { Mass } \\
(\%, \text { strm) }\end{array}$ & $\begin{array}{c}\text { O/S } \\
\text { Mass } \\
(\%, \text { feed) })\end{array}$ & $\begin{array}{c}\text { U/S } \\
\text { Mass } \\
(\%, \text { feed) }\end{array}$ & $\begin{array}{c}\text { Feed } \\
\text { Mass } \\
(\%, \text { feed) }\end{array}$ \\
\hline & 212 & 1.63 & 0.00 & 7.75 & 0.00 & 4.31 & 0.00 & 4.31 \\
\hline 212 & 150 & 1.89 & 0.00 & 8.99 & 0.00 & 5.00 & 0.00 & 5.00 \\
\hline 150 & 75 & 5.19 & 0.02 & 24.64 & 0.12 & 13.71 & 0.05 & 13.76 \\
\hline 75 & 45 & 3.83 & 0.27 & 18.18 & 1.59 & 10.12 & 0.71 & 10.82 \\
\hline 45 & 25 & 1.45 & 0.40 & 6.89 & 2.37 & 3.83 & 1.05 & 4.89 \\
\hline 25 & 13.1 & 1.98 & 2.94 & 9.42 & 17.60 & 5.24 & 7.81 & 13.05 \\
\hline 13.1 & 6.5 & 1.60 & 3.76 & 7.59 & 22.48 & 4.22 & 9.98 & 14.20 \\
\hline 6.5 & 3.3 & 1.44 & 3.80 & 6.82 & 22.76 & 3.80 & 10.10 & 13.90 \\
\hline 3.3 & 1.5 & 0.95 & 2.55 & 4.50 & 15.27 & 2.50 & 6.77 & 9.28 \\
\hline 1.5 & & 1.10 & 2.97 & 5.22 & 17.80 & 2.90 & 7.90 & 10.80 \\
\hline Totals & -- & 21.07 & 16.70 & 100.00 & 100.00 & 55.63 & 44.37 & 100.00 \\
\hline
\end{tabular}

\begin{tabular}{|c|c|c|c|c|c|c|c|c|}
\hline $\begin{array}{c}\text { Pass } \\
\begin{array}{c}\text { Size } \\
(\mathrm{um})\end{array}\end{array}$ & $\begin{array}{c}\text { Retain } \\
\text { Size } \\
(\mathrm{um})\end{array}$ & $\begin{array}{c}\text { ORS } \\
\text { Mass } \\
(\mathrm{TPH})\end{array}$ & $\begin{array}{c}\text { OSS } \\
\text { Mass } \\
(\mathrm{TPH})\end{array}$ & $\begin{array}{c}\text { Feed } \\
\text { Mass } \\
(\mathrm{TPH})\end{array}$ & $\begin{array}{c}\text { Delta } \\
\text { Mass } \\
(\mathrm{TPH})\end{array}$ & $\begin{array}{c}\text { V } \\
\text { Value } \\
(\mathrm{f}-\mathrm{u})\end{array}$ & $\begin{array}{c}\text { X } \\
\text { Value } \\
(0-u)\end{array}$ & $\begin{array}{c}\text { Partition } \\
\text { Factor } \\
(--)\end{array}$ \\
\hline & 212 & 0.05 & 0.00 & 0.05 & 0.00 & 4.31 & 4.31 & 1.00 \\
\hline 212 & 150 & 0.06 & 0.00 & 0.06 & 0.00 & 5.00 & 5.00 & 1.00 \\
\hline 150 & 75 & 0.16 & 0.00 & 0.16 & 0.00 & 13.71 & 13.65 & 1.00 \\
\hline 75 & 45 & 0.12 & 0.01 & 0.12 & 0.00 & 10.12 & 9.41 & 0.93 \\
\hline 45 & 25 & 0.04 & 0.01 & 0.06 & 0.00 & 3.83 & 2.78 & 0.78 \\
\hline 25 & 13.1 & 0.06 & 0.09 & 0.15 & 0.00 & 5.24 & -2.57 & 0.40 \\
\hline 13.1 & 6.5 & 0.05 & 0.11 & 0.16 & 0.00 & 4.22 & -5.75 & 0.30 \\
\hline 6.5 & 3.3 & 0.04 & 0.12 & 0.16 & 0.00 & 3.80 & -6.30 & 0.27 \\
\hline 3.3 & 1.5 & 0.03 & 0.08 & 0.11 & 0.00 & 2.50 & -4.27 & 0.27 \\
\hline 1.5 & & 0.03 & 0.09 & 0.12 & 0.00 & 2.90 & -5.00 & 0.27 \\
\hline Totals & -- & 0.64 & 0.51 & 1.15 & 0.00 & & & \\
\hline
\end{tabular}




\begin{tabular}{|c|c|c|c|}
\hline $\begin{array}{c}\text { Experimental } \\
\text { Partition Factor }\end{array}$ & $\begin{array}{c}\text { Mean } \\
\text { Particle } \\
\text { Size ( } \mu \mathrm{m})\end{array}$ & $\begin{array}{c}\text { Predicted } \\
\text { Partition } \\
\text { Factor }\end{array}$ & $\begin{array}{c}\text { Predicted } \\
\text { Partition } \\
\text { Factor }\end{array}$ \\
\hline$(--)$ & $(--)$ & (Plitt) & (Linch) \\
\hline 1.00 & 299.81 & 1.000 & 1.000 \\
\hline 1.00 & 178.33 & 1.000 & 1.000 \\
\hline 0.97 & 106.07 & 0.998 & 0.997 \\
\hline 0.89 & 58.09 & 0.913 & 0.918 \\
\hline 0.70 & 33.54 & 0.673 & 0.672 \\
\hline 0.41 & 18.08 & 0.430 & 0.428 \\
\hline 0.30 & 9.25 & 0.301 & 0.307 \\
\hline 0.26 & 4.62 & 0.253 & 0.256 \\
\hline 0.23 & 2.32 & 0.237 & 0.235 \\
\hline 0.24 & 1.64 & 0.234 & 0.229 \\
\hline & WSSQ & 0.003 & 0.003 \\
\hline
\end{tabular}

\begin{tabular}{|c|c|c|c|}
\hline (Plitt) & Predicted & (Linch) & Predicted \\
\hline $\mathrm{D} 50$ & 29.61 & D50 & 29.37 \\
\hline$m$ & 1.70 & Alpha & 2.08 \\
\hline Bypass(Rf) & 0.23 & Bypass(Rf) & 0.22 \\
\hline
\end{tabular}

\begin{tabular}{|l|l|}
\hline TWSSQ & 0.006 \\
\hline
\end{tabular}

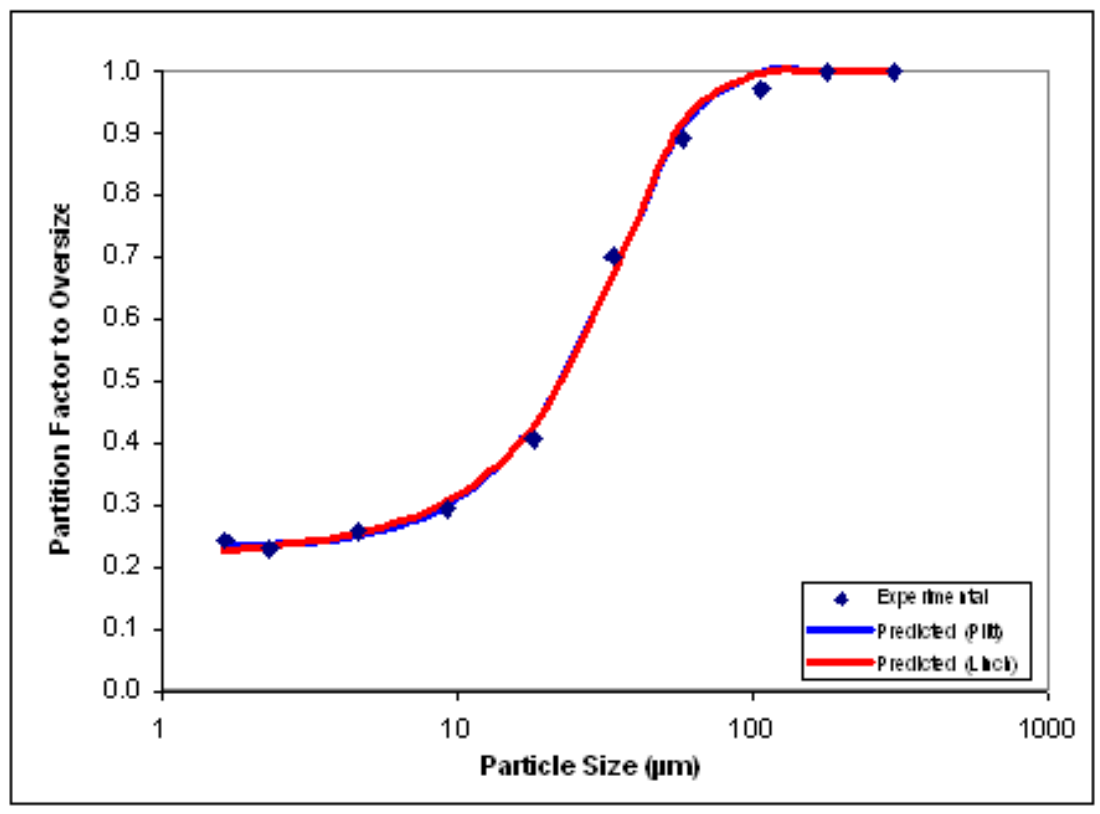


HYDROCYCLONE EVALUATION (UNBALANCED)

\begin{tabular}{|c|c|}
\hline No. 28 & \\
\hline Feed Flow (GPM) & 100.0 \\
\hline Feed Pressure (PSI) & 21.0 \\
\hline Water Flow (GPM) & 15.0 \\
\hline Water Pressure (PSI) & 12.0 \\
\hline Feed Rate (TPH) & 1.18 \\
\hline
\end{tabular}

\begin{tabular}{|r|c|c|c|}
\hline & OS & U/S & Feed \\
\hline Solids Content (\%) & 6.53 & 2.80 & 4.63 \\
\hline Ash Content (\%) & 24.36 & 47.80 & 35.30 \\
\hline Solids Yield (\%) & 53.6 & 46.4 & 100.0 \\
\hline Ash Yield (\%) & 53.3 & 46.7 & 100.0 \\
\hline Size Class Yield (\%) & 49.8 & 50.2 & 100.0 \\
\hline
\end{tabular}

\begin{tabular}{|c|c|c|c|c|c|c|c|c|}
\hline Pass & Retain & $0 / 5$ & U/S & o/S & U/S & OS & U/S & Feed \\
\hline $\begin{array}{l}\text { Size } \\
\text { (um) }\end{array}$ & $\begin{array}{l}\text { Size } \\
\text { (um) }\end{array}$ & $\begin{array}{c}\text { Sample } \\
\text { (gms) }\end{array}$ & $\begin{array}{c}\text { Sample } \\
\text { (gms) }\end{array}$ & $\begin{array}{c}\text { Mass } \\
(\%, \text { strm })\end{array}$ & $\begin{array}{c}\text { Mass } \\
(\%, \text { strm })\end{array}$ & $\begin{array}{c}\text { Mass } \\
(\% \text {, feed })\end{array}$ & $\begin{array}{c}\text { Mass } \\
(\%, \text { feed })\end{array}$ & $\begin{array}{c}\text { Mass } \\
(\%, \text { feed })\end{array}$ \\
\hline & 212 & 1.65 & 0.00 & 8.26 & 0.00 & 4.40 & 0.00 & 4.40 \\
\hline 212 & 150 & 1.65 & 0.00 & 8.26 & 0.00 & 4.40 & 0.00 & 4.40 \\
\hline 150 & 75 & 5.00 & 0.12 & 25.03 & 0.70 & 13.35 & 0.33 & 13.67 \\
\hline 75 & 45 & 3.51 & 0.37 & 17.57 & 2.15 & 9.37 & 1.00 & 10.37 \\
\hline 45 & 25 & 1.32 & 0.65 & 6.61 & 3.77 & 3.52 & 1.76 & 5.28 \\
\hline 25 & 13.1 & 2.07 & 3.04 & 10.38 & 17.67 & 5.54 & 8.25 & 13.78 \\
\hline 13.1 & 6.5 & 1.60 & 3.74 & 7.98 & 21.73 & 4.26 & 10.14 & 14.40 \\
\hline 6.5 & 3.3 & 1.40 & 3.87 & 7.03 & 22.44 & 3.75 & 10.47 & 14.22 \\
\hline 3.3 & 1.5 & 0.85 & 2.68 & 4.27 & 15.56 & 2.28 & 7.26 & 9.54 \\
\hline 1.5 & & 0.92 & 2.76 & 4.62 & 15.99 & 2.46 & 7.46 & 9.93 \\
\hline Totals & -- & 19.98 & 17.23 & 100.00 & 100.00 & 53.33 & 46.67 & 100.00 \\
\hline
\end{tabular}

\begin{tabular}{|c|c|c|c|c|c|c|c|c|}
\hline $\begin{array}{c}\text { Pass } \\
\text { Size } \\
(u m)\end{array}$ & $\begin{array}{c}\text { Retain } \\
\text { Size } \\
(u m)\end{array}$ & $\begin{array}{c}\text { O/S } \\
\text { Mass } \\
(\mathrm{TPH})\end{array}$ & $\begin{array}{c}\text { O/S } \\
\text { Mass } \\
(\mathrm{TPH})\end{array}$ & $\begin{array}{c}\text { Feed } \\
\text { Mass } \\
(\mathrm{TPH})\end{array}$ & $\begin{array}{c}\text { Delta } \\
\text { Mass } \\
(\mathrm{TPH})\end{array}$ & $\begin{array}{c}\mathrm{V} \\
\text { Value } \\
(\mathrm{f}-\mathrm{u})\end{array}$ & $\begin{array}{c}\mathrm{X} \\
\text { Value } \\
(\mathrm{0}-\mathrm{u})\end{array}$ & $\begin{array}{c}\text { Partition } \\
\text { Factor } \\
(-)\end{array}$ \\
\hline & 212 & 0.05 & 0.00 & 0.05 & 0.00 & 4.40 & 4.40 & 1.00 \\
\hline 212 & 150 & 0.05 & 0.00 & 0.05 & 0.00 & 4.40 & 4.40 & 1.00 \\
\hline 150 & 75 & 0.16 & 0.00 & 0.16 & 0.00 & 13.35 & 13.02 & 0.98 \\
\hline 75 & 45 & 0.11 & 0.01 & 0.12 & 0.00 & 9.37 & 8.37 & 0.90 \\
\hline 45 & 25 & 0.04 & 0.02 & 0.06 & 0.00 & 3.52 & 1.76 & 0.67 \\
\hline 25 & 13.1 & 0.07 & 0.10 & 0.16 & 0.00 & 5.54 & -2.71 & 0.40 \\
\hline 13.1 & 6.5 & 0.05 & 0.12 & 0.17 & 0.00 & 4.26 & -5.88 & 0.30 \\
\hline 6.5 & 3.3 & 0.04 & 0.12 & 0.17 & 0.00 & 3.75 & -6.72 & 0.26 \\
\hline 3.3 & 1.5 & 0.03 & 0.09 & 0.11 & 0.00 & 2.28 & -4.98 & 0.24 \\
\hline 1.5 & & 0.03 & 0.09 & 0.12 & 0.00 & 2.46 & -5.00 & 0.25 \\
\hline Totals & -- & 0.63 & 0.55 & 1.18 & 0.00 & & & \\
\hline & Slurry (TPH) & 9.62 & 19.64 & 25.44 & -0.06 & & & \\
\hline
\end{tabular}


HYDROCYCLONE EVALUATION (BALANCED)

\begin{tabular}{|c|c|}
\hline No. 28 & \\
\hline Feed Flow (GPM) & 100.0 \\
\hline Feed Pressure (PSl) & 21.0 \\
\hline Water Flow (GPM) & 15.0 \\
\hline Water Pressure (PSI) & 12.0 \\
\hline Feed Rate (TPH) & 1.15 \\
\hline
\end{tabular}

\begin{tabular}{|r|c|c|c|}
\hline & OS & U/S & Feed \\
\hline Solids Content (\%) & 6.79 & 2.57 & 4.51 \\
\hline Ash Content (\%) & 23.74 & 48.18 & 34.58 \\
\hline Solids Yield (\%) & 55.6 & 44.4 & 100.0 \\
\hline Ash Yield (\%) & 55.6 & 44.4 & 100.0 \\
\hline Size Class Yield (\%) & 55.6 & 44.4 & 100.0 \\
\hline
\end{tabular}

\begin{tabular}{|c|c|c|c|c|c|c|c|c|}
\hline Pass & Retain & $0 / 5$ & U/S & o/s & U/S & OS & U/S & Feed \\
\hline $\begin{array}{l}\text { Size } \\
\text { (um) }\end{array}$ & $\begin{array}{l}\text { Size } \\
\text { (um) }\end{array}$ & $\begin{array}{c}\text { Sample } \\
\text { (gms) }\end{array}$ & $\begin{array}{c}\text { Sample } \\
\text { (gms) }\end{array}$ & $\begin{array}{c}\text { Mass } \\
(\%, \text { strm) }\end{array}$ & $\begin{array}{c}\text { Mass } \\
(\%, \text { strm })\end{array}$ & $\begin{array}{c}\text { Mass } \\
(\% \text {, feed })\end{array}$ & $\begin{array}{c}\text { Mass } \\
(\%, \text { feed })\end{array}$ & $\begin{array}{c}\text { Mass } \\
(\%, \text { feed })\end{array}$ \\
\hline & 212 & 1.63 & 0.00 & 7.75 & 0.00 & 4.31 & 0.00 & 4.31 \\
\hline 212 & 150 & 1.89 & 0.00 & 8.99 & 0.00 & 5.00 & 0.00 & 5.00 \\
\hline 150 & 75 & 5.19 & 0.02 & 24.64 & 0.12 & 13.71 & 0.05 & 13.76 \\
\hline 75 & 45 & 3.83 & 0.27 & 18.18 & 1.59 & 10.12 & 0.71 & 10.82 \\
\hline 45 & 25 & 1.45 & 0.40 & 6.89 & 2.37 & 3.83 & 1.05 & 4.89 \\
\hline 25 & 13.1 & 1.98 & 2.94 & 9.42 & 17.60 & 5.24 & 7.81 & 13.05 \\
\hline 13.1 & 6.5 & 1.60 & 3.76 & 7.59 & 22.48 & 4.22 & 9.98 & 14.20 \\
\hline 6.5 & 3.3 & 1.44 & 3.80 & 6.82 & 22.76 & 3.80 & 10.10 & 13.90 \\
\hline 3.3 & 1.5 & 0.95 & 2.55 & 4.50 & 15.27 & 2.50 & 6.77 & 9.28 \\
\hline 1.5 & & 1.10 & 2.97 & 5.22 & 17.80 & 2.90 & 7.90 & 10.80 \\
\hline Totals & -- & 21.07 & 16.70 & 100.00 & 100.00 & 55.63 & 44.37 & 100.00 \\
\hline
\end{tabular}

\begin{tabular}{|c|c|c|c|c|c|c|c|c|}
\hline $\begin{array}{c}\text { Pass } \\
\text { Size } \\
(u m)\end{array}$ & $\begin{array}{c}\text { Retain } \\
\text { Size } \\
(u m)\end{array}$ & $\begin{array}{c}\text { O/S } \\
\text { Mass } \\
(\mathrm{TPH})\end{array}$ & $\begin{array}{c}\text { O/S } \\
\text { Mass } \\
(\mathrm{TPH})\end{array}$ & $\begin{array}{c}\text { Feed } \\
\text { Mass } \\
(\mathrm{TPH})\end{array}$ & $\begin{array}{c}\text { Delta } \\
\text { Mass } \\
(\mathrm{TPH})\end{array}$ & $\begin{array}{c}\mathrm{V} \\
\text { Value } \\
(\mathrm{f}-\mathrm{u})\end{array}$ & $\begin{array}{c}\mathrm{X} \\
\text { Value } \\
(\mathrm{0}-\mathrm{u})\end{array}$ & $\begin{array}{c}\text { Partition } \\
\text { Factor } \\
(-)\end{array}$ \\
\hline & 212 & 0.05 & 0.00 & 0.05 & 0.00 & 4.31 & 4.31 & 1.00 \\
\hline 212 & 150 & 0.06 & 0.00 & 0.06 & 0.00 & 5.00 & 5.00 & 1.00 \\
\hline 150 & 75 & 0.16 & 0.00 & 0.16 & 0.00 & 13.71 & 13.65 & 1.00 \\
\hline 75 & 45 & 0.12 & 0.01 & 0.12 & 0.00 & 10.12 & 9.41 & 0.93 \\
\hline 45 & 25 & 0.04 & 0.01 & 0.06 & 0.00 & 3.83 & 2.78 & 0.78 \\
\hline 25 & 13.1 & 0.06 & 0.09 & 0.15 & 0.00 & 5.24 & -2.57 & 0.40 \\
\hline 13.1 & 6.5 & 0.05 & 0.11 & 0.16 & 0.00 & 4.22 & -5.75 & 0.30 \\
\hline 6.5 & 3.3 & 0.04 & 0.12 & 0.16 & 0.00 & 3.80 & -6.30 & 0.27 \\
\hline 3.3 & 1.5 & 0.03 & 0.08 & 0.11 & 0.00 & 2.50 & -4.27 & 0.27 \\
\hline 1.5 & & 0.03 & 0.09 & 0.12 & 0.00 & 2.90 & -5.00 & 0.27 \\
\hline Totals & -- & 0.64 & 0.51 & 1.15 & 0.00 & & & \\
\hline & Slurry (TPH) & 9.39 & 19.79 & 25.43 & 0.00 & & & \\
\hline
\end{tabular}




\begin{tabular}{|c|c|c|c|}
\hline $\begin{array}{c}\text { Experimental } \\
\text { Partition Factor }\end{array}$ & $\begin{array}{c}\text { Mean } \\
\text { Particle } \\
\text { Size }(\mu \mathrm{m})\end{array}$ & $\begin{array}{c}\text { Predicted } \\
\text { Partition } \\
\text { Factor }\end{array}$ & $\begin{array}{c}\text { Predicted } \\
\text { Partition } \\
\text { Factor }\end{array}$ \\
\hline$(--)$ & $(--)$ & (Plitt) & (Linch) \\
\hline 1.00 & 299.81 & 1.000 & 1.000 \\
\hline 1.00 & 178.33 & 1.000 & 1.000 \\
\hline 0.98 & 106.07 & 0.999 & 0.997 \\
\hline 0.90 & 58.09 & 0.910 & 0.915 \\
\hline 0.67 & 33.54 & 0.655 & 0.653 \\
\hline 0.40 & 18.08 & 0.413 & 0.412 \\
\hline 0.30 & 9.25 & 0.296 & 0.303 \\
\hline 0.26 & 4.62 & 0.257 & 0.260 \\
\hline 0.24 & 2.32 & 0.245 & 0.243 \\
\hline 0.25 & 1.64 & 0.243 & 0.238 \\
\hline & $W S S \mathrm{Q}$ & 0.001 & 0.001 \\
\hline
\end{tabular}

\begin{tabular}{|c|c|c|c|}
\hline (Plitt) & Predicted & (Linch) & Predicted \\
\hline D50 & 31.22 & D50 & 31.04 \\
\hline$m$ & 1.81 & Alpha & 2.29 \\
\hline Bypass(Rf) & 0.24 & Bypass(Rf) & 0.23 \\
\hline
\end{tabular}

\begin{tabular}{l|l}
\hline TWSSQ & 0.002 \\
\hline
\end{tabular}

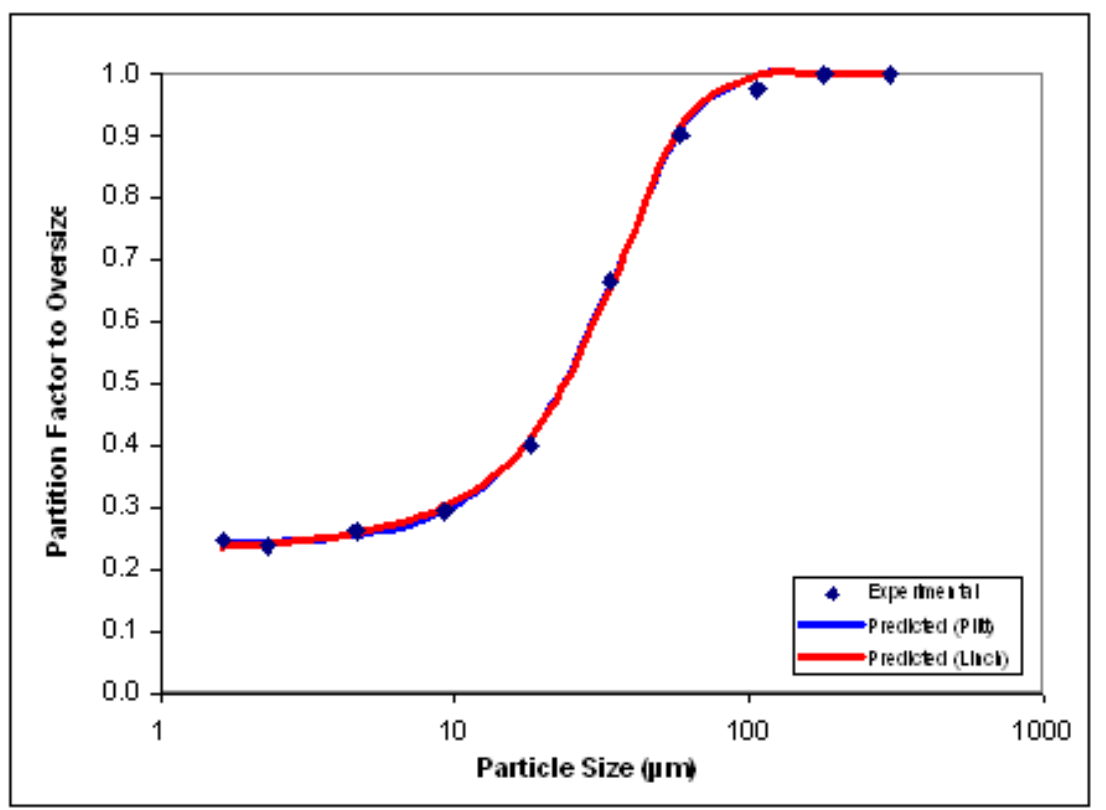


HYDROCYCLONE EVALUATION (UNBALANCED)

\begin{tabular}{|c|c|}
\hline No. 29 & \\
\hline Feed Flow (GPM) & 100.0 \\
\hline Feed Pressure (PSI) & 21.0 \\
\hline Water Flow (GPM) & 15.0 \\
\hline Water Pressure (PSl) & 12.0 \\
\hline Feed Rate (TPH) & 1.19 \\
\hline
\end{tabular}

\begin{tabular}{|r|c|c|c|}
\hline & OS & U/S & Feed \\
\hline Solids Content (\%) & 8.37 & 2.82 & 4.67 \\
\hline Ash Content (\%) & 20.01 & 47.23 & 34.52 \\
\hline Solids Yield (\%) & 46.3 & 53.7 & 100.0 \\
\hline Ash Yield (\%) & 46.7 & 53.3 & 100.0 \\
\hline Size Class Yield (\%) & 37.9 & 62.1 & 100.0 \\
\hline
\end{tabular}

\begin{tabular}{|c|c|c|c|c|c|c|c|c|}
\hline $\begin{array}{l}\text { Pass } \\
\text { Size } \\
\text { (um) }\end{array}$ & $\begin{array}{l}\text { Retain } \\
\text { Size } \\
\text { (um) }\end{array}$ & $\begin{array}{c}\text { O/S } \\
\text { Sample } \\
\text { (gms) }\end{array}$ & $\begin{array}{c}\text { U/S } \\
\text { Sample } \\
\text { (gms) }\end{array}$ & $\begin{array}{c}\text { orS } \\
\text { Mass } \\
(\% \text {, strm })\end{array}$ & $\begin{array}{c}\text { U/S } \\
\text { Mass } \\
(\% \text {, strm) }\end{array}$ & $\begin{array}{c}\text { OS } \\
\text { Mass } \\
(\% \text {, feed })\end{array}$ & $\begin{array}{c}\text { U/S } \\
\text { Mass } \\
(\%, \text { feed })\end{array}$ & $\begin{array}{c}\text { Feed } \\
\text { Mass } \\
(\% \text {, feed })\end{array}$ \\
\hline & 212 & 1.91 & 0.00 & 11.62 & 0.00 & 5.42 & 0.00 & 5.42 \\
\hline 212 & 150 & 1.90 & 0.00 & 11.56 & 0.00 & 5.40 & 0.00 & 5.40 \\
\hline 150 & 75 & 4.73 & 0.22 & 28.77 & 1.05 & 13.43 & 0.56 & 13.99 \\
\hline 75 & 45 & 3.07 & 0.65 & 18.67 & 3.10 & 8.72 & 1.65 & 10.37 \\
\hline 45 & 25 & 0.99 & 1.11 & 6.02 & 5.30 & 2.81 & 2.82 & 5.64 \\
\hline 25 & 13.1 & 1.35 & 3.69 & 8.20 & 17.61 & 3.83 & 9.39 & 13.21 \\
\hline 13.1 & 6.5 & 0.88 & 4.50 & 5.35 & 21.47 & 2.50 & 11.45 & 13.94 \\
\hline 6.5 & 3.3 & 0.74 & 4.44 & 4.49 & 21.18 & 2.10 & 11.29 & 13.39 \\
\hline 3.3 & 1.5 & 0.47 & 3.12 & 2.84 & 14.90 & 1.33 & 7.94 & 9.27 \\
\hline 1.5 & & 0.41 & 3.22 & 2.48 & 15.39 & 1.16 & 8.20 & 9.36 \\
\hline Totals & -- & 16.44 & 20.95 & 100.00 & 100.00 & 46.69 & 53.31 & 100.00 \\
\hline
\end{tabular}

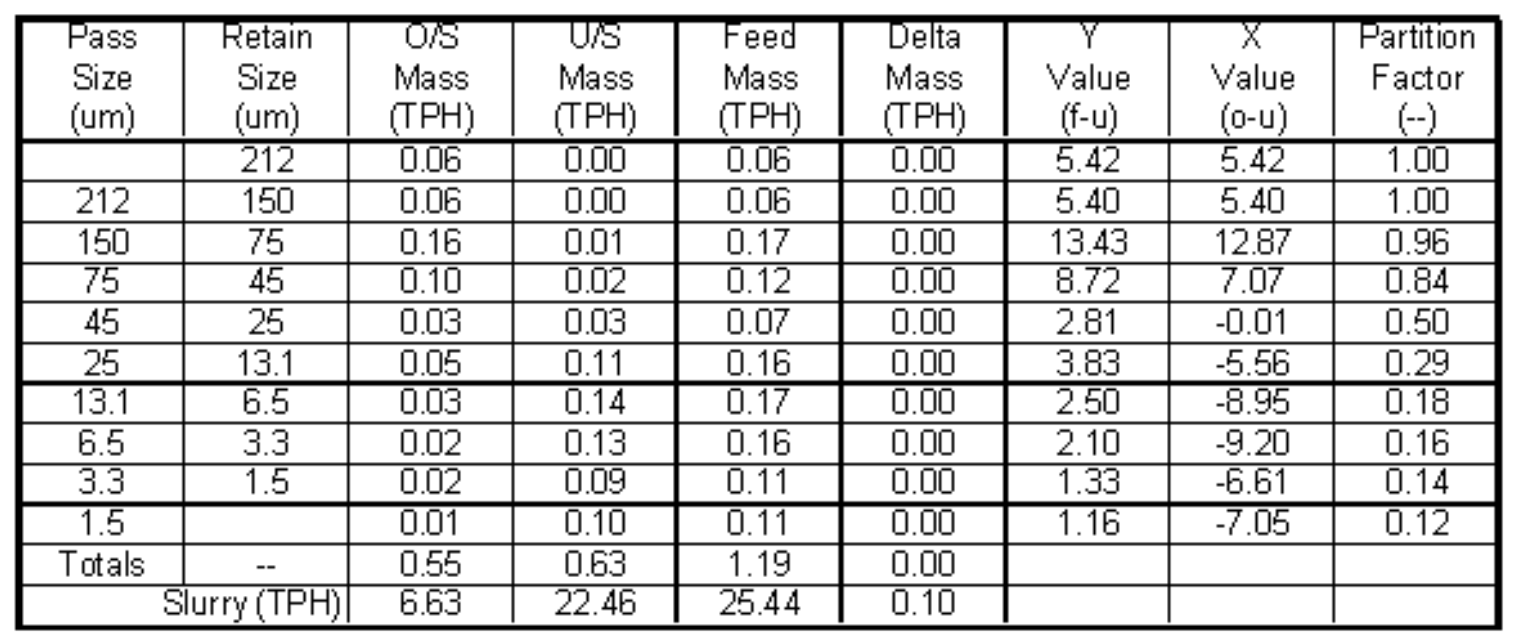




\section{HYDROCYCLONE EVALUATION (BALANCED)}

\begin{tabular}{|c|c|}
\hline No. 29 & \\
\hline Feed Flow (GPM) & 100.0 \\
\hline Feed Pressure (PSI) & 21.0 \\
\hline Water Flow (GPM) & 15.0 \\
\hline Water Pressure (PSl) & 12.0 \\
\hline Feed Rate (TPH) & 1.15 \\
\hline
\end{tabular}

\begin{tabular}{|r|c|c|c|}
\hline & OS & U/S & Feed \\
\hline Solids Content (\%) & 6.79 & 2.57 & 4.51 \\
\hline Ash Content (\%) & 23.74 & 48.18 & 34.58 \\
\hline Solids Yield (\%) & 55.6 & 44.4 & 100.0 \\
\hline Ash Yield (\%) & 55.6 & 44.4 & 100.0 \\
\hline Size Class Yield (\%) & 55.6 & 44.4 & 100.0 \\
\hline
\end{tabular}

\begin{tabular}{|c|c|c|c|c|c|c|c|c|}
\hline $\begin{array}{c}\text { Pass } \\
\text { Size } \\
(u m)\end{array}$ & $\begin{array}{c}\text { Retain } \\
\text { Size } \\
(u m)\end{array}$ & $\begin{array}{c}\text { O/S } \\
\text { Sample } \\
(\mathrm{gms})\end{array}$ & $\begin{array}{c}\text { U/S } \\
\text { Sample } \\
(\mathrm{gms})\end{array}$ & $\begin{array}{c}\text { O/S } \\
\text { Mass } \\
(\%, \text { strm) }\end{array}$ & $\begin{array}{c}\text { U/S } \\
\text { Mass } \\
(\%, \text { strm) }\end{array}$ & $\begin{array}{c}\text { OSS } \\
\text { Mass } \\
(\%, \text { feed })\end{array}$ & $\begin{array}{c}\text { U/S } \\
\text { Mass } \\
(\%, \text { feed })\end{array}$ & $\begin{array}{c}\text { Feed } \\
\text { Mass } \\
(\%, \text { feed })\end{array}$ \\
\hline & 212 & 1.63 & 0.00 & 7.75 & 0.00 & 4.31 & 0.00 & 4.31 \\
\hline 212 & 150 & 1.89 & 0.00 & 8.99 & 0.00 & 5.00 & 0.00 & 5.00 \\
\hline 150 & 75 & 5.19 & 0.02 & 24.64 & 0.12 & 13.71 & 0.05 & 13.76 \\
\hline 75 & 45 & 3.83 & 0.27 & 18.18 & 1.59 & 10.12 & 0.71 & 10.82 \\
\hline 45 & 25 & 1.45 & 0.40 & 6.89 & 2.37 & 3.83 & 1.05 & 4.89 \\
\hline 25 & 13.1 & 1.98 & 2.94 & 9.42 & 17.60 & 5.24 & 7.81 & 13.05 \\
\hline 13.1 & 6.5 & 1.60 & 3.76 & 7.59 & 22.48 & 4.22 & 9.98 & 14.20 \\
\hline 6.5 & 3.3 & 1.44 & 3.80 & 6.82 & 22.76 & 3.80 & 10.10 & 13.90 \\
\hline 3.3 & 1.5 & 0.95 & 2.55 & 4.50 & 15.27 & 2.50 & 6.77 & 9.28 \\
\hline 1.5 & & 1.10 & 2.97 & 5.22 & 17.80 & 2.90 & 7.90 & 10.80 \\
\hline Totals & -- & 21.07 & 16.70 & 100.00 & 100.00 & 55.63 & 44.37 & 100.00 \\
\hline
\end{tabular}

\begin{tabular}{|c|c|c|c|c|c|c|c|c|}
\hline $\begin{array}{c}\text { Pass } \\
\begin{array}{c}\text { Size } \\
(u m)\end{array}\end{array}$ & $\begin{array}{c}\text { Retain } \\
\text { Size } \\
(u m)\end{array}$ & $\begin{array}{c}\text { O/S } \\
\text { Mass } \\
(\mathrm{TPH})\end{array}$ & $\begin{array}{c}\text { O/S } \\
\text { Mass } \\
(\mathrm{TPH})\end{array}$ & $\begin{array}{c}\text { Feed } \\
\text { Mass } \\
(\mathrm{TPH})\end{array}$ & $\begin{array}{c}\text { Delta } \\
\text { Mass } \\
(\mathrm{TPH})\end{array}$ & $\begin{array}{c}\mathrm{V} \\
\text { Value } \\
(\mathrm{f}-\mathrm{u})\end{array}$ & $\begin{array}{c}\mathrm{X} \\
\text { Value } \\
(\mathbf{0}-\mathrm{u})\end{array}$ & $\begin{array}{c}\text { Partition } \\
\text { Factor } \\
(-)\end{array}$ \\
\hline & 212 & 0.05 & 0.00 & 0.05 & 0.00 & 4.31 & 4.31 & 1.00 \\
\hline 212 & 150 & 0.06 & 0.00 & 0.06 & 0.00 & 5.00 & 5.00 & 1.00 \\
\hline 150 & 75 & 0.16 & 0.00 & 0.16 & 0.00 & 13.71 & 13.65 & 1.00 \\
\hline 75 & 45 & 0.12 & 0.01 & 0.12 & 0.00 & 10.12 & 9.41 & 0.93 \\
\hline 45 & 25 & 0.04 & 0.01 & 0.06 & 0.00 & 3.83 & 2.78 & 0.78 \\
\hline 25 & 13.1 & 0.06 & 0.09 & 0.15 & 0.00 & 5.24 & -2.57 & 0.40 \\
\hline 13.1 & 6.5 & 0.05 & 0.11 & 0.16 & 0.00 & 4.22 & -5.75 & 0.30 \\
\hline 6.5 & 3.3 & 0.04 & 0.12 & 0.16 & 0.00 & 3.80 & -6.30 & 0.27 \\
\hline 3.3 & 1.5 & 0.03 & 0.08 & 0.11 & 0.00 & 2.50 & -4.27 & 0.27 \\
\hline 1.5 & & 0.03 & 0.09 & 0.12 & 0.00 & 2.90 & -5.00 & 0.27 \\
\hline Totals & -- & 0.64 & 0.51 & 1.15 & 0.00 & & & \\
\hline & Slurry (TPH) & 9.39 & 19.79 & 25.43 & 0.00 & & & \\
\hline
\end{tabular}




\begin{tabular}{|c|c|c|c|c|c|c|c|}
\hline \multirow{3}{*}{$\begin{array}{l}\text { Experimental } \\
\text { Partition F actor }\end{array}$} & \multirow{3}{*}{$\begin{array}{c}\text { Mean } \\
\text { Particle } \\
\text { Size ( } \mu \mathrm{m})\end{array}$} & \multirow{3}{*}{$\begin{array}{c}\text { Predicted } \\
\text { Partition } \\
\text { Factor }\end{array}$} & \multirow{3}{*}{$\begin{array}{c}\text { Predicted } \\
\text { Partition } \\
\text { Factor }\end{array}$} & (Plitt) & Predicted & (Linch) & Predicted \\
\hline & & & & D50 & 37.04 & D50 & 37.01 \\
\hline & & & & $m$ & 1.87 & Alpha & 2.41 \\
\hline$(-)$ & $(-)$ & (Plitt) & (Linch) & Bypass(Rt) & 0.14 & Bypass(Rf) & 0.12 \\
\hline 1.00 & 299.81 & 1.000 & 1.000 & & & & \\
\hline 1.00 & 178.33 & 1.000 & 1.000 & & & & \\
\hline 0.96 & 106.07 & 0.994 & 0.991 & & & & \\
\hline 0.84 & 58.09 & 0.827 & 0.832 & & & & \\
\hline 0.50 & 33.54 & 0.514 & 0.506 & & & & \\
\hline 0.29 & 18.08 & 0.279 & 0.281 & & & & \\
\hline 0.18 & 9.25 & 0.180 & 0.188 & & & & \\
\hline 0.16 & 4.62 & 0.148 & 0.151 & & & & \\
\hline 0.14 & 2.32 & 0.139 & 0.136 & & & & \\
\hline 0.12 & 1.64 & 0.138 & 0.132 & & & & \\
\hline & WSSQ & 0.002 & 0.001 & & & & \\
\hline & TWSSQ & 0.003 & & & & & \\
\hline
\end{tabular}

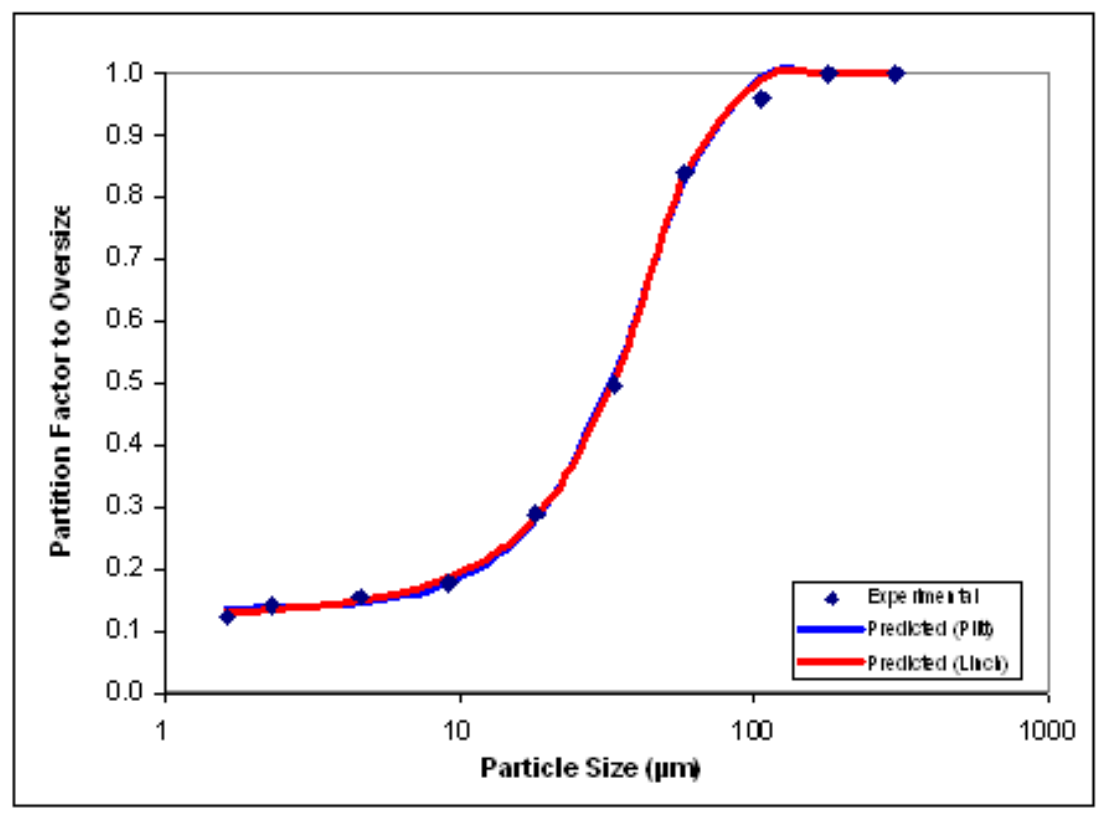


HYDROCYCLONE EVALUATION (UNBALANCED)

\begin{tabular}{|c|c|}
\hline No.30 & \\
\hline Feed Flow (GPM) & 100.0 \\
\hline Feed Pressure (PSl) & 21.0 \\
\hline Water Flow (GPM) & 15.0 \\
\hline Water Pressure ( $\mathrm{PSI})$ & 10.0 \\
\hline Feed Rate (TPH) & 1.17 \\
\hline
\end{tabular}

\begin{tabular}{|r|c|c|c|}
\hline & OS & U/S & Feed \\
\hline Solids Content (\%) & 5.54 & 2.42 & 4.59 \\
\hline Ash Content (\%) & 28.64 & 49.28 & 34.12 \\
\hline Solids Yield (\%) & 70.1 & 29.9 & 100.0 \\
\hline Ash Yield (\%) & 73.4 & 26.6 & 100.0 \\
\hline Size Class Yield (\%) & 114.5 & -14.5 & 100.0 \\
\hline
\end{tabular}

\begin{tabular}{|c|c|c|c|c|c|c|c|c|}
\hline Pass & Retain & $0 / 5$ & U/S & o/s & U/S & OS & U/S & Feed \\
\hline $\begin{array}{l}\text { Size } \\
\text { (um) }\end{array}$ & $\begin{array}{l}\text { Size } \\
\text { (um) }\end{array}$ & $\begin{array}{c}\text { Sample } \\
\text { (gms) }\end{array}$ & $\begin{array}{c}\text { Sample } \\
\text { (gms) }\end{array}$ & $\begin{array}{c}\text { Mass } \\
(\%, \text { strm) }\end{array}$ & $\begin{array}{c}\text { Mass } \\
(\%, \text { strm })\end{array}$ & $\begin{array}{c}\text { Mass } \\
(\% \text {, feed })\end{array}$ & $\begin{array}{c}\text { Mass } \\
(\%, \text { feed })\end{array}$ & $\begin{array}{c}\text { Mass } \\
(\%, \text { feed })\end{array}$ \\
\hline & 212 & 1.99 & 0.00 & 6.48 & 0.00 & 4.76 & 0.00 & 4.76 \\
\hline 212 & 150 & 2.20 & 0.00 & 7.17 & 0.00 & 5.27 & 0.00 & 5.27 \\
\hline 150 & 75 & 6.23 & 0.19 & 20.30 & 1.77 & 14.91 & 0.47 & 15.38 \\
\hline 75 & 45 & 4.32 & 0.29 & 14.08 & 2.70 & 10.34 & 0.72 & 11.06 \\
\hline 45 & 25 & 2.10 & 0.25 & 6.84 & 2.33 & 5.03 & 0.62 & 5.64 \\
\hline 25 & 13.1 & 3.64 & 1.75 & 11.87 & 16.30 & 8.72 & 4.33 & 13.05 \\
\hline 13.1 & 6.5 & 3.27 & 2.41 & 10.67 & 22.39 & 7.83 & 5.95 & 13.78 \\
\hline 6.5 & 3.3 & 3.02 & 2.50 & 9.84 & 23.25 & 7.23 & 6.17 & 13.40 \\
\hline 3.3 & 1.5 & 2.06 & 1.64 & 6.71 & 15.23 & 4.93 & 4.04 & 8.97 \\
\hline 1.5 & & 1.85 & 1.72 & 6.04 & 16.03 & 4.44 & 4.26 & 8.69 \\
\hline Totals & -- & 30.69 & 10.75 & 100.00 & 100.00 & 73.45 & 26.55 & 100.00 \\
\hline
\end{tabular}

\begin{tabular}{|c|c|c|c|c|c|c|c|c|}
\hline $\begin{array}{c}\text { Pass } \\
\text { Size } \\
(u m)\end{array}$ & $\begin{array}{c}\text { Retain } \\
\text { Size } \\
(u m)\end{array}$ & $\begin{array}{c}\text { O/S } \\
\text { Mass } \\
(\mathrm{TPH})\end{array}$ & $\begin{array}{c}\text { O/S } \\
\text { Mass } \\
(\mathrm{TPH})\end{array}$ & $\begin{array}{c}\text { Feed } \\
\text { Mass } \\
(\mathrm{TPH})\end{array}$ & $\begin{array}{c}\text { Delta } \\
\text { Mass } \\
(\mathrm{TPH})\end{array}$ & $\begin{array}{c}\mathrm{V} \\
\text { Value } \\
(\mathrm{f}-\mathrm{u})\end{array}$ & $\begin{array}{c}\mathrm{X} \\
\text { Value } \\
(\mathrm{0}-\mathrm{u})\end{array}$ & $\begin{array}{c}\text { Partition } \\
\text { Factor } \\
(-)\end{array}$ \\
\hline & 212 & 0.06 & 0.00 & 0.06 & 0.00 & 4.76 & 4.76 & 1.00 \\
\hline 212 & 150 & 0.06 & 0.00 & 0.06 & 0.00 & 5.27 & 5.27 & 1.00 \\
\hline 150 & 75 & 0.17 & 0.01 & 0.18 & 0.00 & 14.91 & 14.44 & 0.97 \\
\hline 75 & 45 & 0.12 & 0.01 & 0.13 & 0.00 & 10.34 & 9.62 & 0.94 \\
\hline 45 & 25 & 0.06 & 0.01 & 0.07 & 0.00 & 5.03 & 4.41 & 0.89 \\
\hline 25 & 13.1 & 0.10 & 0.05 & 0.15 & 0.00 & 8.72 & 4.39 & 0.67 \\
\hline 13.1 & 6.5 & 0.09 & 0.07 & 0.16 & 0.00 & 7.83 & 1.89 & 0.57 \\
\hline 6.5 & 3.3 & 0.08 & 0.07 & 0.16 & 0.00 & 7.23 & 1.06 & 0.54 \\
\hline 3.3 & 1.5 & 0.06 & 0.05 & 0.10 & 0.00 & 4.93 & 0.88 & 0.55 \\
\hline 1.5 & & 0.05 & 0.05 & 0.10 & 0.00 & 4.44 & 0.18 & 0.51 \\
\hline Totals & -- & 0.86 & 0.31 & 1.17 & 0.00 & & & \\
\hline & Slurry (TPH) & 15.48 & 12.81 & 25.43 & 0.90 & & & \\
\hline
\end{tabular}




\section{HYDROCYCLONE EVALUATION (BALANCED)}

\begin{tabular}{|c|c|}
\hline No. 30 & \\
\hline Feed Flow (GPM) & 100.0 \\
\hline Feed Pressure (PSI) & 21.0 \\
\hline Water Flow (GPM) & 15.0 \\
\hline Water Pressure (PSl) & 10.0 \\
\hline Feed Rate (TPH) & 1.15 \\
\hline
\end{tabular}

\begin{tabular}{|r|c|c|c|}
\hline & OS & U/S & Feed \\
\hline Solids Content (\%) & 6.79 & 2.57 & 4.51 \\
\hline Ash Content (\%) & 23.74 & 48.18 & 34.58 \\
\hline Solids Yield (\%) & 55.6 & 44.4 & 100.0 \\
\hline Ash Yield (\%) & 55.6 & 44.4 & 100.0 \\
\hline Size Class Yield (\%) & 55.6 & 44.4 & 100.0 \\
\hline
\end{tabular}

\begin{tabular}{|c|c|c|c|c|c|c|c|c|}
\hline $\begin{array}{c}\text { Pass } \\
\text { Size } \\
(u m)\end{array}$ & $\begin{array}{c}\text { Retain } \\
\text { Size } \\
(u m)\end{array}$ & $\begin{array}{c}\text { O/S } \\
\text { Sample } \\
(\mathrm{gms})\end{array}$ & $\begin{array}{c}\text { U/S } \\
\text { Sample } \\
(\mathrm{gms})\end{array}$ & $\begin{array}{c}\text { O/S } \\
\text { Mass } \\
(\%, \text { strm) }\end{array}$ & $\begin{array}{c}\text { U/S } \\
\text { Mass } \\
(\%, \text { strm) }\end{array}$ & $\begin{array}{c}\text { OSS } \\
\text { Mass } \\
(\%, \text { feed })\end{array}$ & $\begin{array}{c}\text { U/S } \\
\text { Mass } \\
(\%, \text { feed })\end{array}$ & $\begin{array}{c}\text { Feed } \\
\text { Mass } \\
(\%, \text { feed })\end{array}$ \\
\hline & 212 & 1.63 & 0.00 & 7.75 & 0.00 & 4.31 & 0.00 & 4.31 \\
\hline 212 & 150 & 1.89 & 0.00 & 8.99 & 0.00 & 5.00 & 0.00 & 5.00 \\
\hline 150 & 75 & 5.19 & 0.02 & 24.64 & 0.12 & 13.71 & 0.05 & 13.76 \\
\hline 75 & 45 & 3.83 & 0.27 & 18.18 & 1.59 & 10.12 & 0.71 & 10.82 \\
\hline 45 & 25 & 1.45 & 0.40 & 6.89 & 2.37 & 3.83 & 1.05 & 4.89 \\
\hline 25 & 13.1 & 1.98 & 2.94 & 9.42 & 17.60 & 5.24 & 7.81 & 13.05 \\
\hline 13.1 & 6.5 & 1.60 & 3.76 & 7.59 & 22.48 & 4.22 & 9.98 & 14.20 \\
\hline 6.5 & 3.3 & 1.44 & 3.80 & 6.82 & 22.76 & 3.80 & 10.10 & 13.90 \\
\hline 3.3 & 1.5 & 0.95 & 2.55 & 4.50 & 15.27 & 2.50 & 6.77 & 9.28 \\
\hline 1.5 & & 1.10 & 2.97 & 5.22 & 17.80 & 2.90 & 7.90 & 10.80 \\
\hline Totals & -- & 21.07 & 16.70 & 100.00 & 100.00 & 55.63 & 44.37 & 100.00 \\
\hline
\end{tabular}

\begin{tabular}{|c|c|c|c|c|c|c|c|c|}
\hline $\begin{array}{c}\text { Pass } \\
\begin{array}{c}\text { Size } \\
(u m)\end{array}\end{array}$ & $\begin{array}{c}\text { Retain } \\
\text { Size } \\
(u m)\end{array}$ & $\begin{array}{c}\text { O/S } \\
\text { Mass } \\
(\mathrm{TPH})\end{array}$ & $\begin{array}{c}\text { O/S } \\
\text { Mass } \\
(\mathrm{TPH})\end{array}$ & $\begin{array}{c}\text { Feed } \\
\text { Mass } \\
(\mathrm{TPH})\end{array}$ & $\begin{array}{c}\text { Delta } \\
\text { Mass } \\
(\mathrm{TPH})\end{array}$ & $\begin{array}{c}\mathrm{V} \\
\text { Value } \\
(\mathrm{f}-\mathrm{u})\end{array}$ & $\begin{array}{c}\mathrm{X} \\
\text { Value } \\
(\mathbf{0}-\mathrm{u})\end{array}$ & $\begin{array}{c}\text { Partition } \\
\text { Factor } \\
(-)\end{array}$ \\
\hline & 212 & 0.05 & 0.00 & 0.05 & 0.00 & 4.31 & 4.31 & 1.00 \\
\hline 212 & 150 & 0.06 & 0.00 & 0.06 & 0.00 & 5.00 & 5.00 & 1.00 \\
\hline 150 & 75 & 0.16 & 0.00 & 0.16 & 0.00 & 13.71 & 13.65 & 1.00 \\
\hline 75 & 45 & 0.12 & 0.01 & 0.12 & 0.00 & 10.12 & 9.41 & 0.93 \\
\hline 45 & 25 & 0.04 & 0.01 & 0.06 & 0.00 & 3.83 & 2.78 & 0.78 \\
\hline 25 & 13.1 & 0.06 & 0.09 & 0.15 & 0.00 & 5.24 & -2.57 & 0.40 \\
\hline 13.1 & 6.5 & 0.05 & 0.11 & 0.16 & 0.00 & 4.22 & -5.75 & 0.30 \\
\hline 6.5 & 3.3 & 0.04 & 0.12 & 0.16 & 0.00 & 3.80 & -6.30 & 0.27 \\
\hline 3.3 & 1.5 & 0.03 & 0.08 & 0.11 & 0.00 & 2.50 & -4.27 & 0.27 \\
\hline 1.5 & & 0.03 & 0.09 & 0.12 & 0.00 & 2.90 & -5.00 & 0.27 \\
\hline Totals & -- & 0.64 & 0.51 & 1.15 & 0.00 & & & \\
\hline & Slurry (TPH) & 9.39 & 19.79 & 25.43 & 0.00 & & & \\
\hline
\end{tabular}




\begin{tabular}{|c|c|c|c|c|c|c|c|}
\hline \multirow{3}{*}{$\begin{array}{l}\text { Experimental } \\
\text { Partition } F \text { actor }\end{array}$} & \multirow{3}{*}{$\begin{array}{c}\text { Mean } \\
\text { Particle } \\
\text { Size ( } \mu \mathrm{m})\end{array}$} & \multirow{3}{*}{$\begin{array}{c}\text { Predicted } \\
\text { Partition } \\
\text { Factor }\end{array}$} & \multirow{3}{*}{$\begin{array}{c}\text { Predicted } \\
\text { Partition } \\
\text { Factor }\end{array}$} & (Plitt) & Predicted & (Linch) & Predicted \\
\hline & & & & D50 & 24.20 & D50 & 24.11 \\
\hline & & & & $m$ & 1.78 & Alpha & 2.48 \\
\hline$(-)$ & $(-)$ & (Plitt) & (Linch) & Bypass(Rf) & 0.52 & Bypass(Rf) & 0.52 \\
\hline 1.00 & 299.81 & 1.000 & 1.000 & & & & \\
\hline 1.00 & 178.33 & 1.000 & 1.000 & & & & \\
\hline 0.97 & 106.07 & 1.000 & 1.000 & & & & \\
\hline 0.94 & 58.09 & 0.982 & 0.987 & & & & \\
\hline 0.89 & 33.54 & 0.862 & 0.872 & & & & \\
\hline 0.67 & 18.08 & 0.683 & 0.676 & & & & \\
\hline 0.57 & 9.25 & 0.577 & 0.576 & & & & \\
\hline 0.54 & 4.62 & 0.538 & 0.541 & & & & \\
\hline 0.55 & 2.32 & 0.526 & 0.527 & & & & \\
\hline 0.51 & 1.64 & 0.524 & 0.523 & & & & \\
\hline & WSSQ & 0.005 & 0.005 & & & & \\
\hline & TWSSQ & 0.010 & & & & & \\
\hline
\end{tabular}

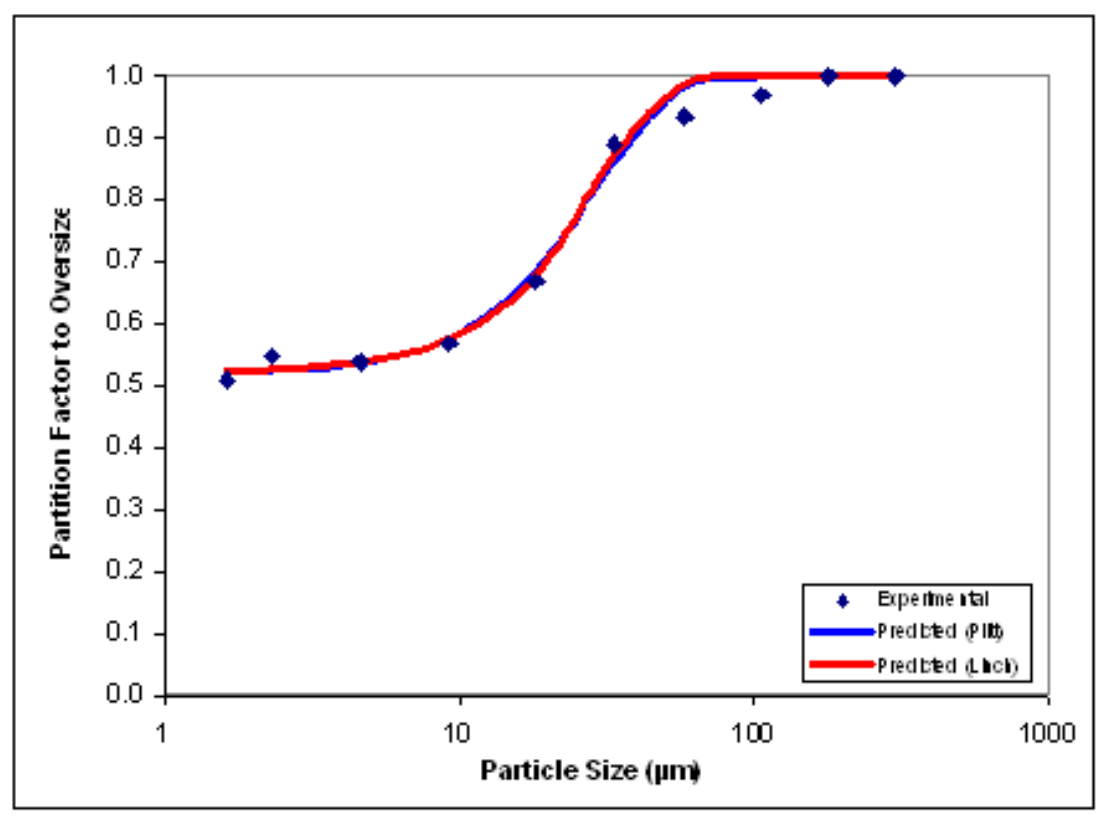




\section{HYDROCYCLONE EVALUATION (UNBALANCED)}

\begin{tabular}{|c|c|}
\hline No. 55 & \\
\hline Feed Flow (GPM) & 100.0 \\
\hline Feed Pressure (PSI) & 21.0 \\
\hline Water Flow (GPM) & 0.0 \\
\hline Water Pressure (PSl) & 0.0 \\
\hline Feed Rate (TPH) & 1.27 \\
\hline
\end{tabular}

\begin{tabular}{|r|c|c|c|}
\hline & OS & U/S & Feed \\
\hline Solids Content (\%) & 9.40 & 2.86 & 5.00 \\
\hline Ash Content (\%) & 25.46 & 48.91 & 34.04 \\
\hline Solids Yield (\%) & 61.5 & 38.5 & 100.0 \\
\hline Ash Yield (\%) & 63.4 & 36.6 & 100.0 \\
\hline Size Class Yield (\%) & 84.4 & 15.6 & 100.0 \\
\hline
\end{tabular}

\begin{tabular}{|c|c|c|c|c|c|c|c|c|}
\hline $\begin{array}{c}\text { Pass } \\
\text { Size } \\
(u m)\end{array}$ & $\begin{array}{c}\text { Retain } \\
\text { Size } \\
(u m)\end{array}$ & $\begin{array}{c}\text { O/S } \\
\text { Sample } \\
(\mathrm{gms})\end{array}$ & $\begin{array}{c}\text { U/S } \\
\text { Sample } \\
(\mathrm{gms})\end{array}$ & $\begin{array}{c}\text { O/S } \\
\text { Mass } \\
(\%, \text { strm) }\end{array}$ & $\begin{array}{c}\text { U/S } \\
\text { Mass } \\
(\%, \text { strm) }\end{array}$ & $\begin{array}{c}\text { OSS } \\
\text { Mass } \\
(\%, \text { feed })\end{array}$ & $\begin{array}{c}\text { U/S } \\
\text { Mass } \\
(\%, \text { feed })\end{array}$ & $\begin{array}{c}\text { Feed } \\
\text { Mass } \\
(\%, \text { feed })\end{array}$ \\
\hline & 212 & 2.04 & 0.00 & 8.30 & 0.00 & 5.26 & 0.00 & 5.26 \\
\hline 212 & 150 & 1.82 & 0.00 & 7.40 & 0.00 & 4.70 & 0.00 & 4.70 \\
\hline 150 & 75 & 5.66 & 0.15 & 23.03 & 0.94 & 14.60 & 0.34 & 14.95 \\
\hline 75 & 45 & 4.21 & 0.37 & 17.13 & 2.32 & 10.86 & 0.85 & 11.71 \\
\hline 45 & 25 & 1.83 & 0.50 & 7.45 & 3.13 & 4.72 & 1.15 & 5.87 \\
\hline 25 & 13.1 & 2.72 & 2.64 & 11.08 & 16.56 & 7.03 & 6.06 & 13.09 \\
\hline 13.1 & 6.5 & 2.09 & 3.48 & 8.50 & 21.83 & 5.39 & 7.99 & 13.38 \\
\hline 6.5 & 3.3 & 1.82 & 3.77 & 7.39 & 23.62 & 4.68 & 8.64 & 13.32 \\
\hline 3.3 & 1.5 & 1.15 & 2.66 & 4.68 & 16.70 & 2.97 & 6.11 & 9.08 \\
\hline 1.5 & & 1.24 & 2.38 & 5.04 & 14.90 & 3.20 & 5.45 & 8.65 \\
\hline Totals & -- & 24.58 & 15.95 & 100.00 & 100.00 & 63.41 & 36.59 & 100.00 \\
\hline
\end{tabular}

\begin{tabular}{|c|c|c|c|c|c|c|c|c|}
\hline $\begin{array}{l}\text { Pass } \\
\text { Size } \\
\text { (um) }\end{array}$ & $\begin{array}{l}\text { Retain } \\
\text { Size } \\
\text { (um) }\end{array}$ & $\begin{array}{c}\text { O/S } \\
\text { Mass } \\
\text { (TPH) }\end{array}$ & $\begin{array}{c}\text { O/S } \\
\text { Mass } \\
\text { (TPH) }\end{array}$ & $\begin{array}{l}\text { Feed } \\
\text { Mass } \\
\text { (TPH) }\end{array}$ & $\begin{array}{l}\text { Delta } \\
\text { Mass } \\
\text { (TPH) }\end{array}$ & $\begin{array}{c}Y \\
\text { Value } \\
(f-u)\end{array}$ & $\begin{array}{c}X \\
\text { Value } \\
(0-u)\end{array}$ & $\begin{array}{l}\text { Partition } \\
\text { Factor } \\
\text { (-) }\end{array}$ \\
\hline & 212 & 0.07 & 0.00 & 0.07 & 0.00 & 5.26 & 5.26 & 1.00 \\
\hline 212 & 150 & 0.06 & 0.00 & 0.06 & 0.00 & 4.70 & 4.70 & 1.00 \\
\hline 150 & 75 & 0.19 & 0.00 & 0.19 & 0.00 & 14.60 & 14.26 & 0.98 \\
\hline 75 & 45 & 0.14 & 0.01 & 0.15 & 0.00 & 10.86 & 10.01 & 0.93 \\
\hline 45 & 25 & 0.06 & 0.01 & 0.07 & 0.00 & 4.72 & 3.57 & 0.80 \\
\hline 25 & 13.1 & 0.09 & 0.08 & 0.17 & 0.00 & 7.03 & 0.97 & 0.54 \\
\hline 13.1 & 6.5 & 0.07 & 0.10 & 0.17 & 0.00 & 5.39 & -2.59 & 0.40 \\
\hline 6.5 & 3.3 & 0.06 & 0.11 & 0.17 & 0.00 & 4.68 & -3.96 & 0.35 \\
\hline 3.3 & 1.5 & 0.04 & 0.08 & 0.12 & 0.00 & 2.97 & -3.14 & 0.33 \\
\hline 1.5 & & 0.04 & 0.07 & 0.11 & 0.00 & 3.20 & -2.25 & 0.37 \\
\hline Totals & -- & 0.81 & 0.47 & 1.27 & 0.00 & & & \\
\hline & Slurry (TPH) & 8.59 & 16.29 & 25.47 & 0.59 & & & \\
\hline
\end{tabular}


HYDROCYCLONE EVALUATION (BALANCED)

\begin{tabular}{|c|c|}
\hline No. 55 & \\
\hline Feed Flow (GPM) & 100.0 \\
\hline Feed Pressure (PSl) & 21.0 \\
\hline Water Flow (GPM) & 0.0 \\
\hline Water Pressure (PSl) & 0.0 \\
\hline Feed Rate (TPH) & 1.15 \\
\hline
\end{tabular}

\begin{tabular}{|r|c|c|c|}
\hline & OS & U/S & Feed \\
\hline Solids Content (\%) & 6.79 & 2.57 & 4.51 \\
\hline Ash Content (\%) & 23.74 & 48.18 & 34.58 \\
\hline Solids Yield (\%) & 69.2 & 30.8 & 100.0 \\
\hline Ash Yield (\%) & 55.6 & 44.4 & 100.0 \\
\hline Size Class Yield (\%) & 55.6 & 44.4 & 100.0 \\
\hline
\end{tabular}

\begin{tabular}{|c|c|c|c|c|c|c|c|c|}
\hline $\begin{array}{c}\text { Pass } \\
\text { Size } \\
(u m)\end{array}$ & $\begin{array}{c}\text { Retain } \\
\text { Size } \\
(u m)\end{array}$ & $\begin{array}{c}\text { O/S } \\
\text { Sample } \\
(\mathrm{gms})\end{array}$ & $\begin{array}{c}\text { U/S } \\
\text { Sample } \\
(\mathrm{gms})\end{array}$ & $\begin{array}{c}\text { O/S } \\
\text { Mass } \\
(\%, \text { strm) }\end{array}$ & $\begin{array}{c}\text { U/S } \\
\text { Mass } \\
(\%, \text { strm) }\end{array}$ & $\begin{array}{c}\text { OS } \\
\text { Mass } \\
(\%, \text { feed })\end{array}$ & $\begin{array}{c}\text { U/S } \\
\text { Mass } \\
(\%, \text { feed })\end{array}$ & $\begin{array}{c}\text { Feed } \\
\text { Mass } \\
(\%, \text { feed })\end{array}$ \\
\hline & 212 & 1.63 & 0.00 & 7.75 & 0.00 & 4.31 & 0.00 & 4.31 \\
\hline 212 & 150 & 1.89 & 0.00 & 8.99 & 0.00 & 5.00 & 0.00 & 5.00 \\
\hline 150 & 75 & 5.19 & 0.02 & 24.64 & 0.12 & 13.71 & 0.05 & 13.76 \\
\hline 75 & 45 & 3.83 & 0.27 & 18.18 & 1.59 & 10.12 & 0.71 & 10.82 \\
\hline 45 & 25 & 1.45 & 0.40 & 6.89 & 2.37 & 3.83 & 1.05 & 4.89 \\
\hline 25 & 13.1 & 1.98 & 2.94 & 9.42 & 17.60 & 5.24 & 7.81 & 13.05 \\
\hline 13.1 & 6.5 & 1.60 & 3.76 & 7.59 & 22.48 & 4.22 & 9.98 & 14.20 \\
\hline 6.5 & 3.3 & 1.44 & 3.80 & 6.82 & 22.76 & 3.80 & 10.10 & 13.90 \\
\hline 3.3 & 1.5 & 0.95 & 2.55 & 4.50 & 15.27 & 2.50 & 6.77 & 9.28 \\
\hline 1.5 & & 1.10 & 2.97 & 5.22 & 17.80 & 2.90 & 7.90 & 10.80 \\
\hline Totals & -- & 21.07 & 16.70 & 100.00 & 100.00 & 55.63 & 44.37 & 100.00 \\
\hline
\end{tabular}

\begin{tabular}{|c|c|c|c|c|c|c|c|c|}
\hline $\begin{array}{c}\text { Pass } \\
\text { Size } \\
(u m)\end{array}$ & $\begin{array}{c}\text { Retain } \\
\text { Size } \\
(u m)\end{array}$ & $\begin{array}{c}\text { O/5 } \\
\text { Mass } \\
(\mathrm{TPH})\end{array}$ & $\begin{array}{c}\text { O/S } \\
\text { Mass } \\
(\mathrm{TPH})\end{array}$ & $\begin{array}{c}\text { Feed } \\
\text { Mass } \\
(\mathrm{TPH})\end{array}$ & $\begin{array}{c}\text { Delta } \\
\text { Mass } \\
(\mathrm{TPH})\end{array}$ & $\begin{array}{c}\mathrm{V} \\
\text { Value } \\
(\mathrm{f}-\mathrm{u})\end{array}$ & $\begin{array}{c}\mathrm{X} \\
\text { Value } \\
(\mathrm{0}-\mathrm{u})\end{array}$ & $\begin{array}{c}\text { Partition } \\
\text { Factor } \\
(-)\end{array}$ \\
\hline & 212 & 0.05 & 0.00 & 0.05 & 0.00 & 4.31 & 4.31 & 1.00 \\
\hline 212 & 150 & 0.06 & 0.00 & 0.06 & 0.00 & 5.00 & 5.00 & 1.00 \\
\hline 150 & 75 & 0.16 & 0.00 & 0.16 & 0.00 & 13.71 & 13.65 & 1.00 \\
\hline 75 & 45 & 0.12 & 0.01 & 0.12 & 0.00 & 10.12 & 9.41 & 0.93 \\
\hline 45 & 25 & 0.04 & 0.01 & 0.06 & 0.00 & 3.83 & 2.78 & 0.78 \\
\hline 25 & 13.1 & 0.06 & 0.09 & 0.15 & 0.00 & 5.24 & -2.57 & 0.40 \\
\hline 13.1 & 6.5 & 0.05 & 0.11 & 0.16 & 0.00 & 4.22 & -5.75 & 0.30 \\
\hline 6.5 & 3.3 & 0.04 & 0.12 & 0.16 & 0.00 & 3.80 & -6.30 & 0.27 \\
\hline 3.3 & 1.5 & 0.03 & 0.08 & 0.11 & 0.00 & 2.50 & -4.27 & 0.27 \\
\hline 1.5 & & 0.03 & 0.09 & 0.12 & 0.00 & 2.90 & -5.00 & 0.27 \\
\hline Totals & -- & 0.64 & 0.51 & 1.15 & 0.00 & & & \\
\hline & Slurry (TPH) & 9.39 & 19.79 & 25.43 & -3.75 & & & \\
\hline
\end{tabular}




\begin{tabular}{|c|c|c|c|}
\hline $\begin{array}{c}\text { Experimental } \\
\text { Partition Factor }\end{array}$ & $\begin{array}{c}\text { Mean } \\
\text { Particle } \\
\text { Size }(\mu \mathrm{m})\end{array}$ & $\begin{array}{c}\text { Predicted } \\
\text { Partition } \\
\text { Factor }\end{array}$ & $\begin{array}{c}\text { Predicted } \\
\text { Partition } \\
\text { Factor }\end{array}$ \\
\hline$(--)$ & $(--)$ & (Plitt) & (Linch) \\
\hline 1.00 & 299.81 & 1.000 & 1.000 \\
\hline 1.00 & 178.33 & 1.000 & 1.000 \\
\hline 0.98 & 106.07 & 1.000 & 0.999 \\
\hline 0.93 & 58.09 & 0.961 & 0.965 \\
\hline 0.80 & 33.54 & 0.779 & 0.784 \\
\hline 0.54 & 18.08 & 0.547 & 0.541 \\
\hline 0.40 & 9.25 & 0.411 & 0.414 \\
\hline 0.35 & 4.62 & 0.359 & 0.363 \\
\hline 0.33 & 2.32 & 0.343 & 0.342 \\
\hline 0.37 & 1.64 & 0.339 & 0.336 \\
\hline & $W S S \mathrm{Q}$ & 0.004 & 0.004 \\
\hline
\end{tabular}

\begin{tabular}{|c|c|c|c|}
\hline (Plitt) & Predicted & (Linch) & Predicteo \\
\hline D50 & 25.58 & D50 & 25.41 \\
\hline$m$ & 1.71 & Alpha & 2.17 \\
\hline Bypass(Rt) & 0.34 & Bypass(Rf) & 0.32 \\
\hline
\end{tabular}

\begin{tabular}{|l|l|}
\hline TWSSQ & 0.008 \\
\hline
\end{tabular}

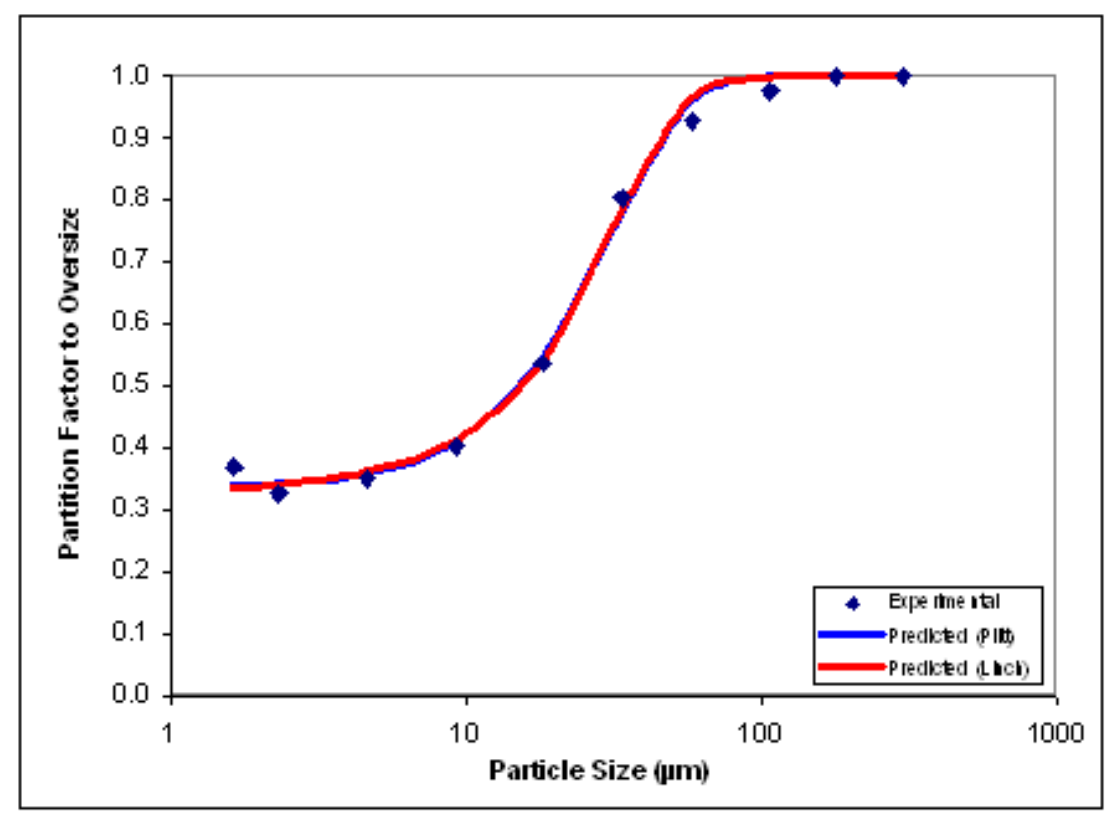


HYDROCYCLONE EVALUATION (UNBALANCED)

\begin{tabular}{|c|c|}
\hline No. 56 & \\
\hline Feed Flow (GPM) & 100.0 \\
\hline Feed Pressure (PSI) & 21.0 \\
\hline Water Flow (GPM) & 10.0 \\
\hline Water Pressure (PSl) & 11.0 \\
\hline Feed Rate (TPH) & 1.23 \\
\hline
\end{tabular}

\begin{tabular}{|r|c|c|c|}
\hline & OS & U/S & Feed \\
\hline Solids Content (\%) & 8.25 & 2.81 & 4.85 \\
\hline Ash Content (\%) & 24.47 & 48.86 & 34.03 \\
\hline Solids Yield (\%) & 55.2 & 44.8 & 100.0 \\
\hline Ash Yield (\%) & 60.8 & 39.2 & 100.0 \\
\hline Size Class Yield (\%) & 75.8 & 24.2 & 100.0 \\
\hline
\end{tabular}

\begin{tabular}{|c|c|c|c|c|c|c|c|c|}
\hline $\begin{array}{l}\text { Pass } \\
\text { Size } \\
\text { (um) }\end{array}$ & $\begin{array}{c}\text { Retain } \\
\text { Size } \\
\text { (um) }\end{array}$ & $\begin{array}{c}\text { O/S } \\
\text { Sample } \\
\text { (gms) }\end{array}$ & $\begin{array}{c}\text { U/S } \\
\text { Sample } \\
\text { (gms) }\end{array}$ & $\begin{array}{c}\text { orS } \\
\text { Mass } \\
(\% \text {, strm })\end{array}$ & $\begin{array}{c}\text { U/S } \\
\text { Mass } \\
(\% \text {, strm) }\end{array}$ & $\begin{array}{c}\text { OS } \\
\text { Mass } \\
(\% \text {, feed })\end{array}$ & $\begin{array}{c}\text { U/S } \\
\text { Mass } \\
(\%, \text { feed })\end{array}$ & $\begin{array}{c}\text { Feed } \\
\text { Mass } \\
(\% \text {, feed })\end{array}$ \\
\hline & 212 & 2.09 & 0.00 & 9.37 & 0.00 & 5.70 & 0.00 & 5.70 \\
\hline 212 & 150 & 1.56 & 0.00 & 7.00 & 0.00 & 4.25 & 0.00 & 4.25 \\
\hline 150 & 75 & 5.46 & 0.19 & 24.48 & 1.05 & 14.89 & 0.41 & 15.30 \\
\hline 75 & 45 & 393 & 0.42 & 17.62 & 2.33 & 10.72 & 0.91 & 11.63 \\
\hline 45 & 25 & 1.85 & 0.52 & 8.30 & 2.88 & 5.04 & 1.13 & 6.17 \\
\hline 25 & 13.1 & 2.53 & 3.04 & 11.34 & 16.84 & 6.90 & 6.60 & 13.50 \\
\hline 13.1 & 6.5 & 1.78 & 3.80 & 7.97 & 21.07 & 4.85 & 8.26 & 13.10 \\
\hline 6.5 & 3.3 & 1.39 & 4.26 & 6.25 & 23.65 & 3.80 & 9.27 & 13.07 \\
\hline 3.3 & 1.5 & 0.83 & 3.01 & 3.72 & 16.70 & 2.26 & 6.54 & 8.81 \\
\hline 1.5 & & 0.88 & 2.79 & 3.94 & 15.48 & 2.40 & 6.07 & 8.46 \\
\hline Totals & -- & 22.30 & 18.03 & 100.00 & 100.00 & 60.80 & 39.20 & 100.00 \\
\hline
\end{tabular}

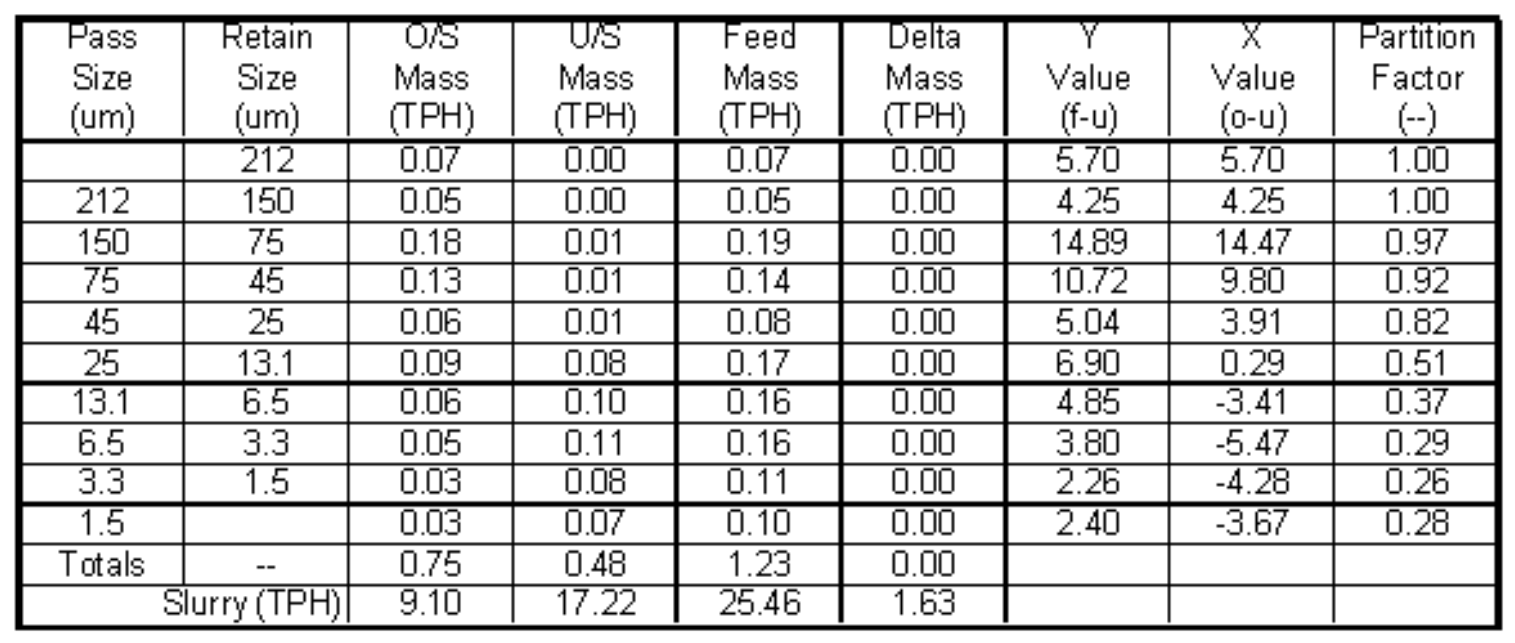


HYDROCYCLONE EVALUATION (BALANCED)

\begin{tabular}{|c|c|}
\hline No. 56 & \\
\hline Feed Flow (GPM) & 100.0 \\
\hline Feed Pressure (PSI) & 21.0 \\
\hline Water Flow (GPM) & 10.0 \\
\hline Water Pressure (PSl) & 11.0 \\
\hline Feed Rate (TPH) & 1.15 \\
\hline
\end{tabular}

\begin{tabular}{|r|c|c|c|}
\hline & OS & U/S & Feed \\
\hline Solids Content (\%) & 6.79 & 2.57 & 4.51 \\
\hline Ash Content (\%) & 23.74 & 48.18 & 34.58 \\
\hline Solids Yield (\%) & 60.1 & 39.9 & 100.0 \\
\hline Ash Yield (\%) & 55.6 & 44.4 & 100.0 \\
\hline Size Class Yield (\%) & 55.6 & 44.4 & 100.0 \\
\hline
\end{tabular}

\begin{tabular}{|c|c|c|c|c|c|c|c|c|}
\hline $\begin{array}{l}\text { Pass } \\
\text { Size } \\
\text { (um) }\end{array}$ & $\begin{array}{c}\text { Retain } \\
\text { Size } \\
\text { (um) }\end{array}$ & $\begin{array}{c}\text { O/S } \\
\text { Sample } \\
\text { (gms) }\end{array}$ & $\begin{array}{c}\text { U/S } \\
\text { Sample } \\
\text { (gms) }\end{array}$ & $\begin{array}{c}\text { orS } \\
\text { Mass } \\
(\% \text {, strm })\end{array}$ & $\begin{array}{c}\text { U/S } \\
\text { Mass } \\
(\% \text {, strm) }\end{array}$ & $\begin{array}{c}\text { OS } \\
\text { Mass } \\
(\% \text {, feed })\end{array}$ & $\begin{array}{c}\text { U/S } \\
\text { Mass } \\
(\%, \text { feed })\end{array}$ & $\begin{array}{c}\text { Feed } \\
\text { Mass } \\
(\% \text {, feed })\end{array}$ \\
\hline & 212 & 1.63 & 0.00 & 7.75 & 0.00 & 4.31 & 0.00 & 4.31 \\
\hline 212 & 150 & 1.89 & 0.00 & 8.99 & 0.00 & 5.00 & 0.00 & 5.00 \\
\hline 150 & 75 & 5.19 & 0.02 & 24.64 & 0.12 & 13.71 & 0.05 & 13.76 \\
\hline 75 & 45 & 3.83 & 0.27 & 18.18 & 1.59 & 10.12 & 0.71 & 10.82 \\
\hline 45 & 25 & 1.45 & 0.40 & 6.89 & 2.37 & 3.83 & 1.05 & 4.89 \\
\hline 25 & 13.1 & 1.98 & 2.94 & 9.42 & 17.60 & 5.24 & 7.81 & 13.05 \\
\hline 13.1 & 6.5 & 1.60 & 3.76 & 7.59 & 22.48 & 4.22 & 9.98 & 14.20 \\
\hline 6.5 & 3.3 & 1.44 & 3.80 & 6.82 & 22.76 & 3.80 & 10.10 & 13.90 \\
\hline 3.3 & 1.5 & 0.95 & 2.55 & 4.50 & 15.27 & 2.50 & 6.77 & 9.28 \\
\hline 1.5 & & 1.10 & 2.97 & 5.22 & 17.80 & 2.90 & 7.90 & 10.80 \\
\hline Totals & -- & 21.07 & 16.70 & 100.00 & 100.00 & 55.63 & 44.37 & 100.00 \\
\hline
\end{tabular}

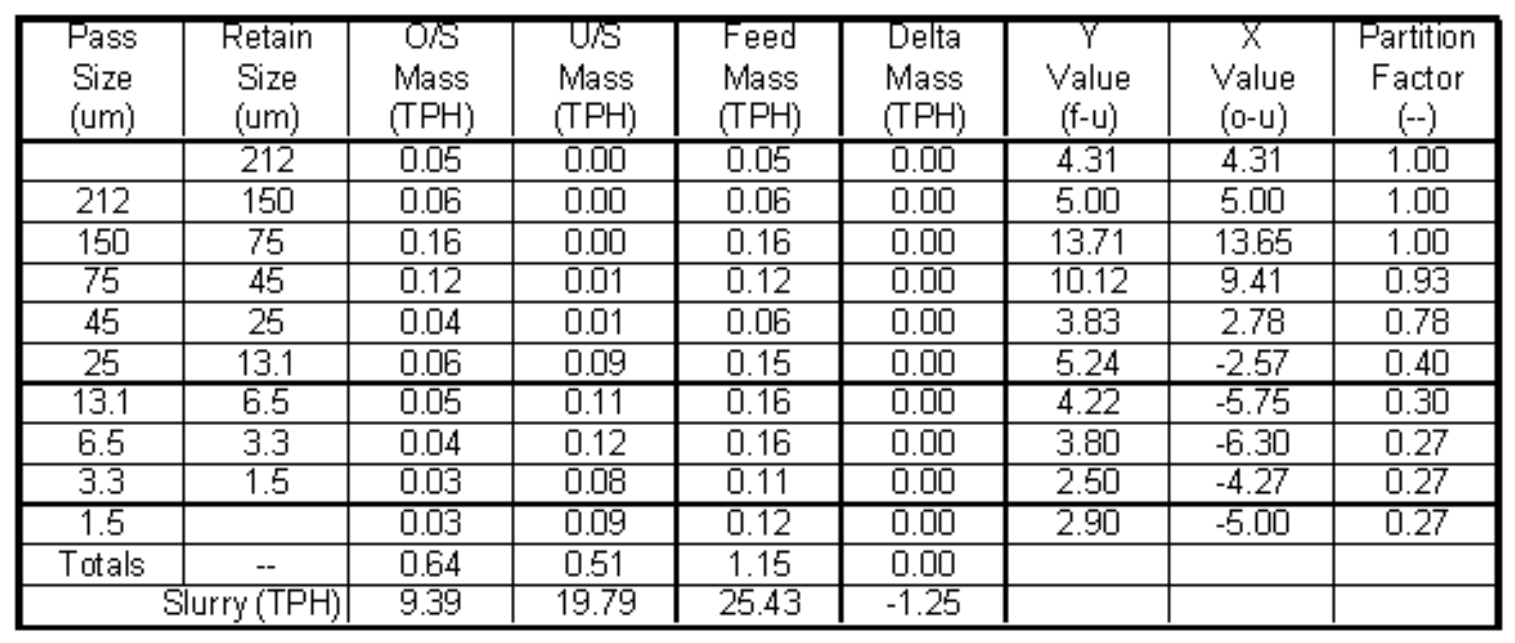




\begin{tabular}{|c|c|c|c|}
\hline $\begin{array}{c}\text { Experimental } \\
\text { Partition F actor }\end{array}$ & $\begin{array}{c}\text { Mean } \\
\text { Particle } \\
\text { Size }(\mu \mathrm{m})\end{array}$ & $\begin{array}{c}\text { Predicted } \\
\text { Partition } \\
\text { Factor }\end{array}$ & $\begin{array}{c}\text { Predicted } \\
\text { Partition } \\
\text { Factor }\end{array}$ \\
\hline$(-)$ & $(--)$ & (Plitt) & (Linch) \\
\hline 1.00 & 299.81 & 1.000 & 1.000 \\
\hline 1.00 & 178.33 & 1.000 & 1.000 \\
\hline 0.97 & 106.07 & 1.000 & 0.999 \\
\hline 0.92 & 58.09 & 0.961 & 0.966 \\
\hline 0.82 & 33.54 & 0.784 & 0.790 \\
\hline 0.51 & 18.08 & 0.532 & 0.525 \\
\hline 0.37 & 9.25 & 0.366 & 0.367 \\
\hline 0.29 & 4.62 & 0.295 & 0.299 \\
\hline 0.26 & 2.32 & 0.270 & 0.270 \\
\hline 0.28 & 1.64 & 0.264 & 0.262 \\
\hline & MSSQ & 0.004 & 0.004 \\
\hline
\end{tabular}

\begin{tabular}{|c|c|c|c|}
\hline (Plitt) & Predicted & (Linch) & Predicted \\
\hline D50 & 23.35 & D50 & 23.20 \\
\hline$m$ & 1.59 & Alpha & 1.94 \\
\hline Bypass(Rt) & 0.26 & Bypass(Rf) & 0.24 \\
\hline
\end{tabular}

\begin{tabular}{|l|l}
\hline TWSSQ & 0.009 \\
\hline
\end{tabular}

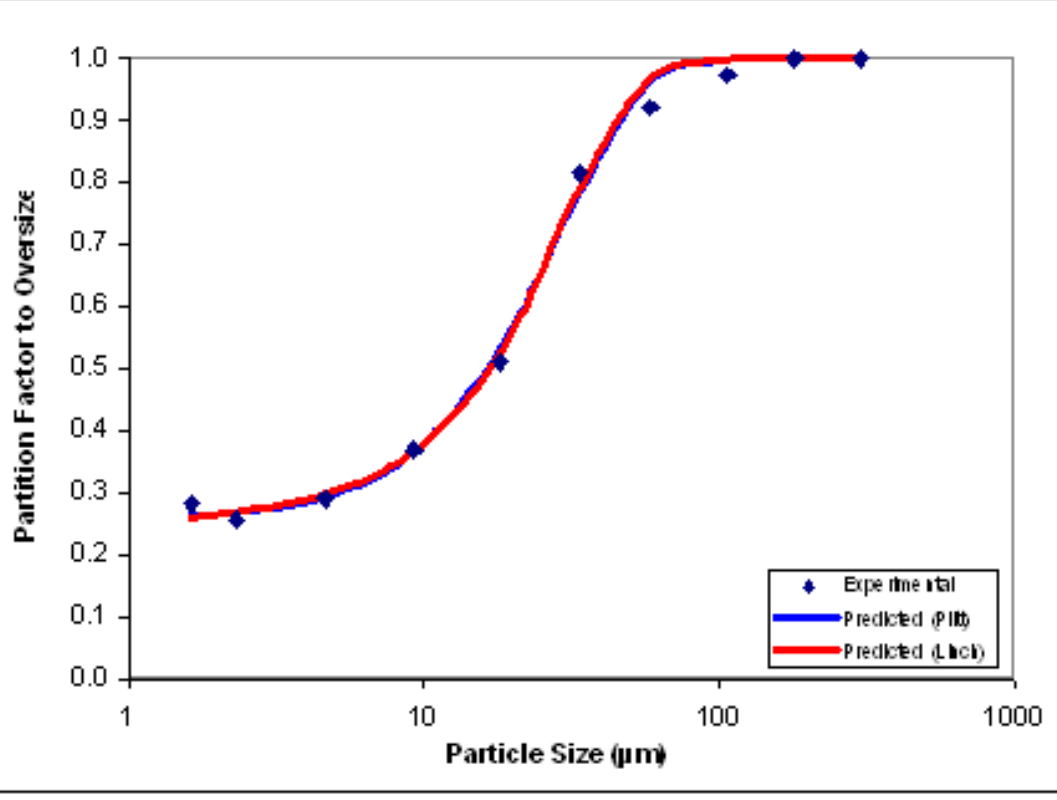


HYDROCYCLONE EVALUATION (UNBALANCED)

\begin{tabular}{|c|c|}
\hline No. 57 & \\
\hline Feed Flow (GPM) & 100.0 \\
\hline Feed Pressure (PSI) & 21.0 \\
\hline Water Flow (GPM) & 20.0 \\
\hline Water Pressure (PSl) & 14.0 \\
\hline Feed Rate (TPH) & 1.15 \\
\hline
\end{tabular}

\begin{tabular}{|r|c|c|c|}
\hline & OS & U/S & Feed \\
\hline Solids Content (\%) & 6.94 & 2.76 & 4.51 \\
\hline Ash Content (\%) & 21.64 & 48.57 & 34.07 \\
\hline Solids Yield (\%) & 44.4 & 55.6 & 100.0 \\
\hline Ash Yield (\%) & 53.8 & 46.2 & 100.0 \\
\hline Size Class Yield (\%) & 53.9 & 46.1 & 100.0 \\
\hline
\end{tabular}

\begin{tabular}{|c|c|c|c|c|c|c|c|c|}
\hline $\begin{array}{c}\text { Pass } \\
\text { Size } \\
(u m)\end{array}$ & $\begin{array}{c}\text { Retain } \\
\text { Size } \\
(u m)\end{array}$ & $\begin{array}{c}\text { O/S } \\
\text { Sample } \\
(\mathrm{gms})\end{array}$ & $\begin{array}{c}\text { U/S } \\
\text { Sample } \\
(\mathrm{gms})\end{array}$ & $\begin{array}{c}\text { O/S } \\
\text { Mass } \\
(\%, \text { strm) }\end{array}$ & $\begin{array}{c}\text { U/S } \\
\text { Mass } \\
(\%, \text { strm) }\end{array}$ & $\begin{array}{c}0 / S \\
\text { Mass } \\
(\%, \text { feed })\end{array}$ & $\begin{array}{c}\text { U/S } \\
\text { Mass } \\
(\%, \text { feed })\end{array}$ & $\begin{array}{c}\text { Feed } \\
\text { Mass } \\
(\%, \text { feed })\end{array}$ \\
\hline & 212 & 2.00 & 0.00 & 9.51 & 0.00 & 5.12 & 0.00 & 5.12 \\
\hline 212 & 150 & 1.72 & 0.00 & 8.18 & 0.00 & 4.40 & 0.00 & 4.40 \\
\hline 150 & 75 & 5.50 & 0.18 & 26.15 & 0.88 & 14.08 & 0.41 & 14.49 \\
\hline 75 & 45 & 3.89 & 0.47 & 18.50 & 2.31 & 9.96 & 1.07 & 11.03 \\
\hline 45 & 25 & 1.52 & 0.81 & 7.23 & 3.98 & 3.89 & 1.84 & 5.73 \\
\hline 25 & 13.1 & 2.18 & 4.07 & 10.37 & 19.99 & 5.58 & 9.23 & 14.81 \\
\hline 13.1 & 6.5 & 1.50 & 4.60 & 7.14 & 22.61 & 3.84 & 10.44 & 14.28 \\
\hline 6.5 & 3.3 & 1.24 & 4.38 & 5.88 & 21.54 & 3.16 & 9.94 & 13.11 \\
\hline 3.3 & 1.5 & 0.73 & 2.76 & 3.46 & 13.56 & 1.86 & 6.26 & 8.12 \\
\hline 1.5 & & 0.76 & 3.08 & 3.59 & 15.12 & 1.93 & 6.98 & 8.91 \\
\hline Totals & -- & 21.03 & 20.35 & 100.00 & 100.00 & 53.84 & 46.16 & 100.00 \\
\hline
\end{tabular}

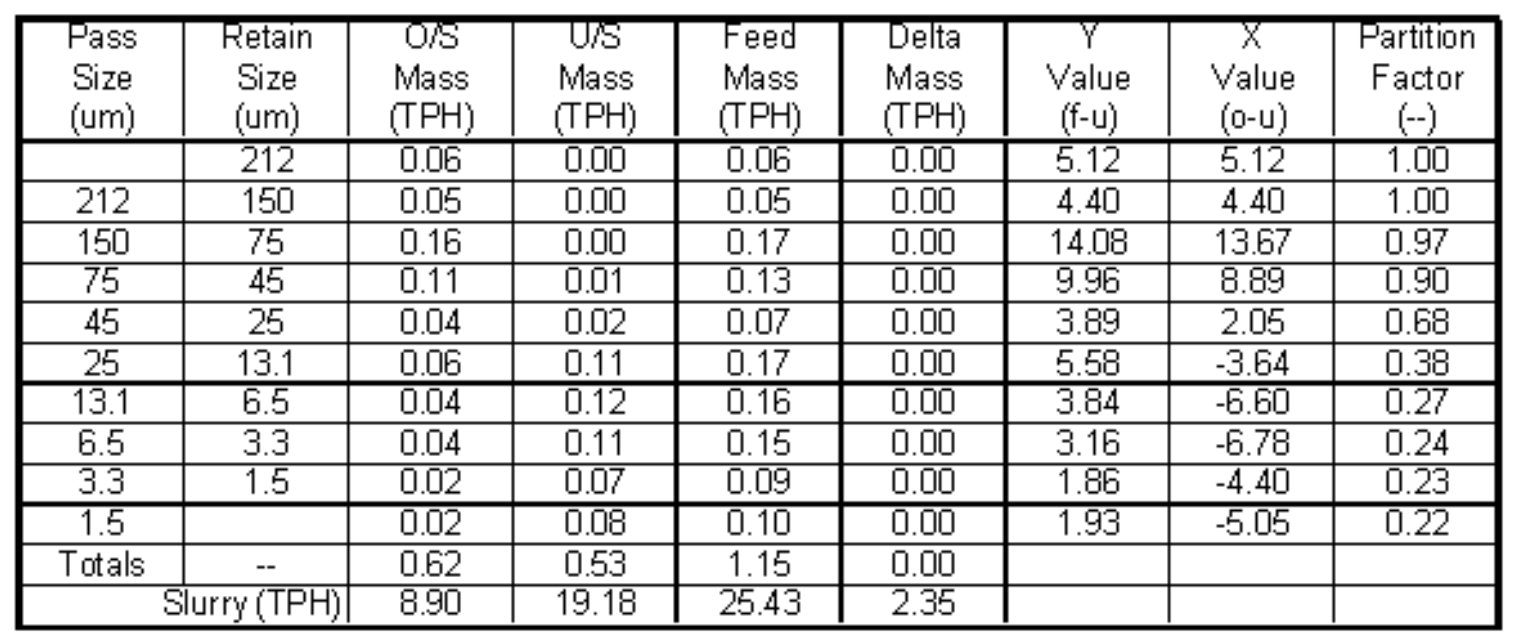


HYDROCYCLONE EVALUATION (BALANCED)

\begin{tabular}{|c|c|}
\hline No. 57 & \\
\hline Feed Flow (GPM) & 100.0 \\
\hline Feed Pressure (PSI) & 21.0 \\
\hline Water Flow (GPM) & 20.0 \\
\hline Water Pressure (PSl) & 14.0 \\
\hline Feed Rate (TPH) & 1.15 \\
\hline
\end{tabular}

\begin{tabular}{|r|c|c|c|}
\hline & OS & U/S & Feed \\
\hline Solids Content (\%) & 6.79 & 2.57 & 4.51 \\
\hline Ash Content (\%) & 23.74 & 48.18 & 34.58 \\
\hline Solids Yield (\%) & 51.1 & 48.9 & 100.0 \\
\hline Ash Yield (\%) & 55.6 & 44.4 & 100.0 \\
\hline Size Class Yield (\%) & 55.6 & 44.4 & 100.0 \\
\hline
\end{tabular}

\begin{tabular}{|c|c|c|c|c|c|c|c|c|}
\hline $\begin{array}{l}\text { Pass } \\
\text { Size } \\
\text { (um) }\end{array}$ & $\begin{array}{c}\text { Retain } \\
\text { Size } \\
\text { (um) }\end{array}$ & $\begin{array}{c}\text { O/S } \\
\text { Sample } \\
\text { (gms) }\end{array}$ & $\begin{array}{c}\text { U/S } \\
\text { Sample } \\
\text { (gms) }\end{array}$ & $\begin{array}{c}\text { orS } \\
\text { Mass } \\
(\% \text {, strm })\end{array}$ & $\begin{array}{c}\text { U/S } \\
\text { Mass } \\
(\% \text {, strm) }\end{array}$ & $\begin{array}{c}\text { OS } \\
\text { Mass } \\
(\% \text {, feed })\end{array}$ & $\begin{array}{c}\text { U/S } \\
\text { Mass } \\
(\%, \text { feed })\end{array}$ & $\begin{array}{c}\text { Feed } \\
\text { Mass } \\
(\% \text {, feed })\end{array}$ \\
\hline & 212 & 1.63 & 0.00 & 7.75 & 0.00 & 4.31 & 0.00 & 4.31 \\
\hline 212 & 150 & 1.89 & 0.00 & 8.99 & 0.00 & 5.00 & 0.00 & 5.00 \\
\hline 150 & 75 & 5.19 & 0.02 & 24.64 & 0.12 & 13.71 & 0.05 & 13.76 \\
\hline 75 & 45 & 3.83 & 0.27 & 18.18 & 1.59 & 10.12 & 0.71 & 10.82 \\
\hline 45 & 25 & 1.45 & 0.40 & 6.89 & 2.37 & 3.83 & 1.05 & 4.89 \\
\hline 25 & 13.1 & 1.98 & 2.94 & 9.42 & 17.60 & 5.24 & 7.81 & 13.05 \\
\hline 13.1 & 6.5 & 1.60 & 3.76 & 7.59 & 22.48 & 4.22 & 9.98 & 14.20 \\
\hline 6.5 & 3.3 & 1.44 & 3.80 & 6.82 & 22.76 & 3.80 & 10.10 & 13.90 \\
\hline 3.3 & 1.5 & 0.95 & 2.55 & 4.50 & 15.27 & 2.50 & 6.77 & 9.28 \\
\hline 1.5 & & 1.10 & 2.97 & 5.22 & 17.80 & 2.90 & 7.90 & 10.80 \\
\hline Totals & -- & 21.07 & 16.70 & 100.00 & 100.00 & 55.63 & 44.37 & 100.00 \\
\hline
\end{tabular}

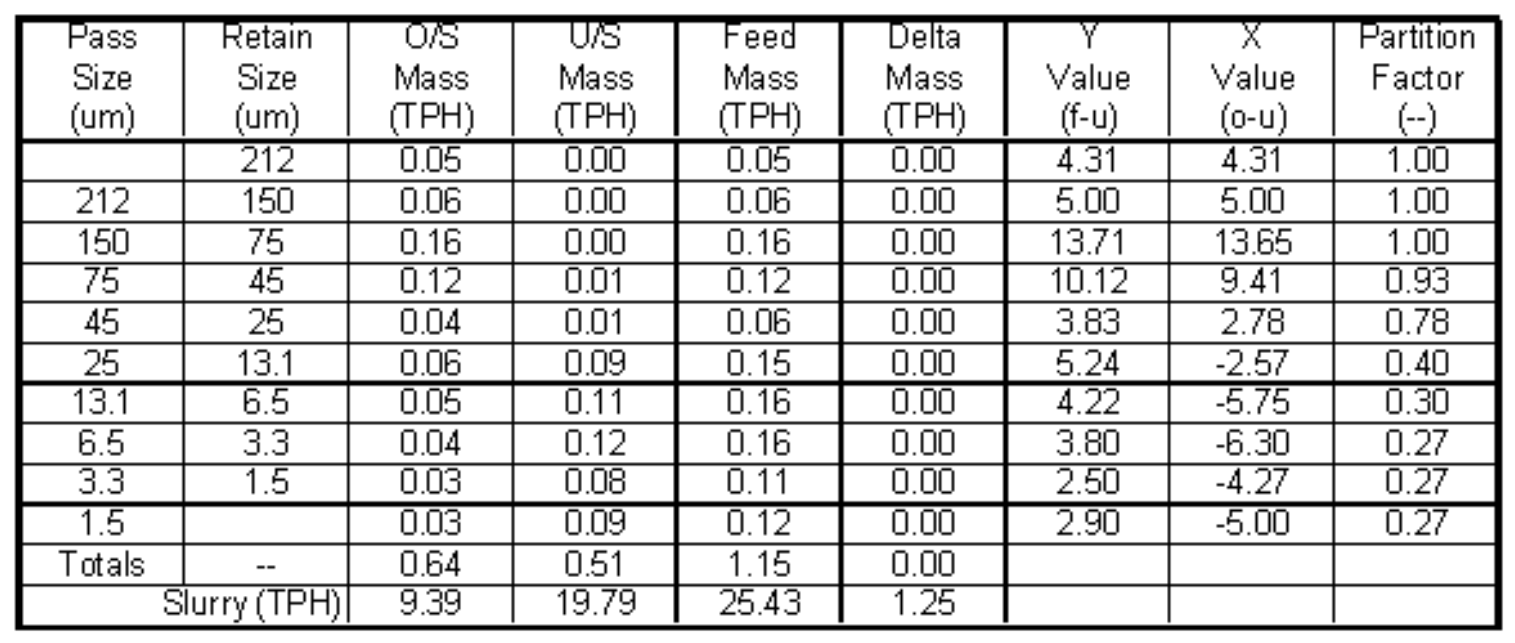




\begin{tabular}{|c|c|c|c|c|c|c|c|}
\hline \multirow{3}{*}{$\begin{array}{l}\text { Experimental } \\
\text { Partition } \mathrm{F} \text { actor }\end{array}$} & \multirow{3}{*}{$\begin{array}{c}\text { Mean } \\
\text { Particle } \\
\text { Size }(\mu \mathrm{m})\end{array}$} & \multirow{3}{*}{$\begin{array}{c}\text { Predicted } \\
\text { Partition } \\
\text { Factor }\end{array}$} & \multirow{3}{*}{$\begin{array}{c}\text { Predicted } \\
\text { Partition } \\
\text { Factor }\end{array}$} & (Plitt) & Predicted & (Linch) & Predicted \\
\hline & & & & D50 & 30.62 & D50 & 30.41 \\
\hline & & & & $m$ & 1.87 & Alpha & 2.43 \\
\hline$(-)$ & $(-)$ & (Plitt) & (Linch) & Bypass(Rf) & 0.22 & Bypass(Rf) & 0.20 \\
\hline 1.00 & 299.81 & 1.000 & 1.000 & & & & \\
\hline 1.00 & 178.33 & 1.000 & 1.000 & & & & \\
\hline 0.97 & 106.07 & 0.999 & 0.998 & & & & \\
\hline 0.90 & 58.09 & 0.921 & 0.927 & & & & \\
\hline 0.68 & 33.54 & 0.656 & 0.656 & & & & \\
\hline 0.38 & 18.08 & 0.396 & 0.394 & & & & \\
\hline 0.27 & 9.25 & 0.274 & 0.280 & & & & \\
\hline 0.24 & 4.62 & 0.234 & 0.237 & & & & \\
\hline 0.23 & 2.32 & 0.222 & 0.220 & & & & \\
\hline 0.22 & 1.64 & 0.220 & 0.215 & & & & \\
\hline & WSSQ & 0.002 & 0.002 & & & & \\
\hline & TWSSQ & 0.004 & & & & & \\
\hline
\end{tabular}

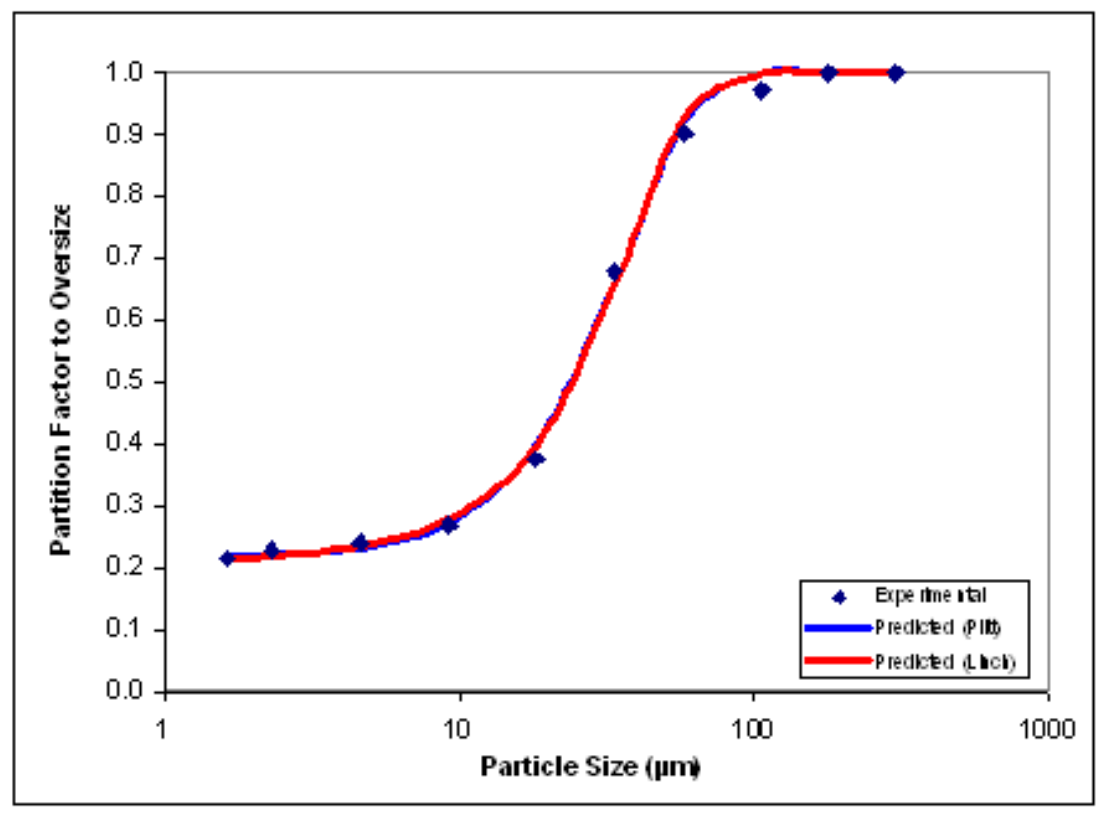


HYDROCYCLONE EVALUATION (UNBALANCED)

\begin{tabular}{l}
\hline Test: No. 58 \\
\begin{tabular}{|l|c|}
\hline \multicolumn{2}{|c|}{} \\
\hline Feed Flow (GPM) & 100.0 \\
\hline Feed Pressure (PSl) & 21.0 \\
\hline Water Flow (GPM) & 30.0 \\
\hline Water Pressure (PSI) & 22.0 \\
\hline Feed Rate (TPH) & 1.14 \\
\hline
\end{tabular}
\end{tabular}

\begin{tabular}{|r|c|c|c|}
\hline & OS & U/S & Feed \\
\hline Solids Content (\%) & 5.45 & 2.80 & 4.50 \\
\hline Ash Content (\%) & 17.20 & 46.85 & 33.88 \\
\hline Solids Yield (\%) & 39.9 & 60.1 & 100.0 \\
\hline Ash Yield (\%) & 43.7 & 56.3 & 100.0 \\
\hline Size Class Yield (\%) & 34.3 & 65.7 & 100.0 \\
\hline
\end{tabular}

\begin{tabular}{|c|c|c|c|c|c|c|c|c|}
\hline $\begin{array}{l}\text { Pass } \\
\text { Size } \\
\text { (um) }\end{array}$ & $\begin{array}{l}\text { Retain } \\
\text { Size } \\
\text { (um) }\end{array}$ & $\begin{array}{c}\text { OS } \\
\text { Sample } \\
\text { (gms) }\end{array}$ & $\begin{array}{c}\text { U/S } \\
\text { Sample } \\
\text { (gms) }\end{array}$ & $\begin{array}{c}\text { O/S } \\
\text { Mass } \\
(\% \text {, strm })\end{array}$ & $\begin{array}{c}\text { USS } \\
\text { Mass } \\
(\% \text {, strm) }\end{array}$ & $\begin{array}{c}\text { OS } \\
\text { Mass } \\
(\% \text {, feed })\end{array}$ & $\begin{array}{c}\text { U/S } \\
\text { Mass } \\
(\%, \text { feed })\end{array}$ & $\begin{array}{c}\text { Feed } \\
\text { Mass } \\
(\% \text {, feed })\end{array}$ \\
\hline & 212 & 1.99 & 0.00 & 11.85 & 0.00 & 5.18 & 0.00 & 5.18 \\
\hline 212 & 150 & 1.52 & 0.00 & 9.05 & 0.00 & 3.96 & 0.00 & 3.96 \\
\hline 150 & 75 & 5.29 & 0.22 & 31.51 & 1.11 & 13.78 & 0.62 & 14.41 \\
\hline 75 & 45 & 3.41 & 0.71 & 20.31 & 3.58 & 8.88 & 2.01 & 10.90 \\
\hline 45 & 25 & 1.16 & 1.20 & 6.91 & 6.05 & 3.02 & 3.40 & 6.43 \\
\hline 25 & 13.1 & 1.30 & 3.54 & 7.76 & 17.84 & 3.40 & 10.04 & 13.43 \\
\hline 13.1 & 6.5 & 0.77 & 4.24 & 4.60 & 21.37 & 2.01 & 12.02 & 14.04 \\
\hline 6.5 & 3.3 & 0.61 & 4.10 & 3.64 & 20.65 & 1.59 & 11.62 & 13.21 \\
\hline 3.3 & 1.5 & 0.36 & 2.66 & 2.15 & 13.43 & 0.94 & 7.56 & 8.50 \\
\hline 1.5 & & 0.37 & 3.16 & 2.23 & 15.96 & 0.97 & 8.98 & 9.95 \\
\hline Totals & -- & 16.79 & 19.83 & 100.00 & 100.00 & 43.74 & 56.26 & 100.00 \\
\hline
\end{tabular}

\begin{tabular}{|c|c|c|c|c|c|c|c|c|}
\hline $\begin{array}{c}\text { Pass } \\
\text { Size } \\
(u m)\end{array}$ & $\begin{array}{c}\text { Retain } \\
\text { Size } \\
(u m)\end{array}$ & $\begin{array}{c}\text { O/5 } \\
\text { Mass } \\
(\mathrm{TPH})\end{array}$ & $\begin{array}{c}\text { O/S } \\
\text { Mass } \\
(\mathrm{TPH})\end{array}$ & $\begin{array}{c}\text { Feed } \\
\text { Mass } \\
(\mathrm{TPH})\end{array}$ & $\begin{array}{c}\text { Delta } \\
\text { Mass } \\
(\mathrm{TPH})\end{array}$ & $\begin{array}{c}\mathrm{V} \\
\text { Value } \\
(\mathrm{f}-\mathrm{u})\end{array}$ & $\begin{array}{c}\mathrm{X} \\
\text { Value } \\
(\mathbf{0}-\mathrm{u})\end{array}$ & $\begin{array}{c}\text { Partition } \\
\text { Factor } \\
(-)\end{array}$ \\
\hline & 212 & 0.06 & 0.00 & 0.06 & 0.00 & 5.18 & 5.18 & 1.00 \\
\hline 212 & 150 & 0.05 & 0.00 & 0.05 & 0.00 & 3.96 & 3.96 & 1.00 \\
\hline 150 & 75 & 0.16 & 0.01 & 0.16 & 0.00 & 13.78 & 13.16 & 0.96 \\
\hline 75 & 45 & 0.10 & 0.02 & 0.12 & 0.00 & 8.88 & 6.87 & 0.82 \\
\hline 45 & 25 & 0.03 & 0.04 & 0.07 & 0.00 & 3.02 & -0.38 & 0.47 \\
\hline 25 & 13.1 & 0.04 & 0.11 & 0.15 & 0.00 & 3.40 & -6.64 & 0.25 \\
\hline 13.1 & 6.5 & 0.02 & 0.14 & 0.16 & 0.00 & 2.01 & -10.01 & 0.14 \\
\hline 6.5 & 3.3 & 0.02 & 0.13 & 0.15 & 0.00 & 1.59 & -10.03 & 0.12 \\
\hline 3.3 & 1.5 & 0.01 & 0.09 & 0.10 & 0.00 & 0.94 & -6.62 & 0.11 \\
\hline 1.5 & & 0.01 & 0.10 & 0.11 & 0.00 & 0.97 & -8.00 & 0.10 \\
\hline Totals & -- & 0.50 & 0.64 & 1.14 & 0.00 & & & \\
\hline & Slurry (TPH) & 9.18 & 22.99 & 25.42 & 0.75 & & & \\
\hline
\end{tabular}


HYDROCYCLONE EVALUATION (BALANCED)

Test: No.58

\begin{tabular}{|l|c|}
\hline Feed Flow (GPM) & 100.0 \\
\hline Feed Pressure (PSl) & 21.0 \\
\hline Water Flow (GPM) & 30.0 \\
\hline Water Pressure (PSl) & 22.0 \\
\hline Feed Rate (TPH) & 1.15 \\
\hline
\end{tabular}

\begin{tabular}{|r|c|c|c|}
\hline & OS & U/S & Feed \\
\hline Solids Content (\%) & 6.79 & 2.57 & 4.51 \\
\hline Ash Content (\%) & 23.74 & 48.18 & 34.58 \\
\hline Solids Yield (\%) & 42.1 & 57.9 & 100.0 \\
\hline Ash Yield (\%) & 55.6 & 44.4 & 100.0 \\
\hline Size Class Yield (\%) & 55.6 & 44.4 & 100.0 \\
\hline
\end{tabular}

\begin{tabular}{|c|c|c|c|c|c|c|c|c|}
\hline $\begin{array}{l}\text { Pass } \\
\text { Size } \\
\text { (um) }\end{array}$ & $\begin{array}{c}\text { Retain } \\
\text { Size } \\
\text { (um) }\end{array}$ & $\begin{array}{c}\text { O/S } \\
\text { Sample } \\
\text { (gms) }\end{array}$ & $\begin{array}{c}\text { U/S } \\
\text { Sample } \\
\text { (gms) }\end{array}$ & $\begin{array}{c}\mathrm{OSS} \\
\text { Mass } \\
(\%, \text { strm) }\end{array}$ & $\begin{array}{c}\text { USS } \\
\text { Mass } \\
(\%, \text { strm) }\end{array}$ & $\begin{array}{c}\text { OSS } \\
\text { Mass } \\
(\%, \text { feed })\end{array}$ & $\begin{array}{c}\text { U/S } \\
\text { Mass } \\
(\%, \text { feed })\end{array}$ & $\begin{array}{c}\text { Feed } \\
\text { Mass } \\
(\%, \text { feed })\end{array}$ \\
\hline & 212 & 1.63 & 0.00 & 7.75 & 0.00 & 4.31 & 0.00 & 4.31 \\
\hline 212 & 150 & 1.89 & 0.00 & 8.99 & 0.00 & 5.00 & 0.00 & 5.00 \\
\hline 150 & 75 & 5.19 & 0.02 & 24.64 & 0.12 & 13.71 & 0.05 & 13.76 \\
\hline 75 & 45 & 3.83 & 0.27 & 18.18 & 1.59 & 10.12 & 0.71 & 10.82 \\
\hline 45 & 25 & 1.45 & 0.40 & 6.89 & 2.37 & 3.83 & 1.05 & 4.89 \\
\hline 25 & 13.1 & 1.98 & 2.94 & 9.42 & 17.60 & 5.24 & 7.81 & 13.05 \\
\hline 13.1 & 6.5 & 1.60 & 3.76 & 7.59 & 22.48 & 4.22 & 9.98 & 14.20 \\
\hline 6.5 & 3.3 & 1.44 & 3.80 & 6.82 & 22.76 & 3.80 & 10.10 & 13.90 \\
\hline 3.3 & 1.5 & 0.95 & 2.55 & 4.50 & 15.27 & 2.50 & 6.77 & 9.28 \\
\hline 1.5 & & 1.10 & 2.97 & 5.22 & 17.80 & 2.90 & 7.90 & 10.80 \\
\hline Totals & -- & 21.07 & 16.70 & 100.00 & 100.00 & 55.63 & 44.37 & 100.00 \\
\hline
\end{tabular}

\begin{tabular}{|c|c|c|c|c|c|c|c|c|}
\hline $\begin{array}{c}\text { Pass } \\
\begin{array}{c}\text { Size } \\
(\mathrm{um})\end{array}\end{array}$ & $\begin{array}{c}\text { Retain } \\
\text { Size } \\
(\mathrm{um})\end{array}$ & $\begin{array}{c}\text { ORS } \\
\text { Mass } \\
(\mathrm{TPH})\end{array}$ & $\begin{array}{c}\text { OSS } \\
\text { Mass } \\
(\mathrm{TPH})\end{array}$ & $\begin{array}{c}\text { Feed } \\
\text { Mass } \\
(\mathrm{TPH})\end{array}$ & $\begin{array}{c}\text { Delta } \\
\text { Mass } \\
(\mathrm{TPH})\end{array}$ & $\begin{array}{c}\text { Value } \\
(\text { f-u) }\end{array}$ & $\begin{array}{c}\text { X } \\
\text { Value } \\
(0-u)\end{array}$ & $\begin{array}{c}\text { Partition } \\
\text { Factor } \\
(--)\end{array}$ \\
\hline & 212 & 0.05 & 0.00 & 0.05 & 0.00 & 4.31 & 4.31 & 1.00 \\
\hline 212 & 150 & 0.06 & 0.00 & 0.06 & 0.00 & 5.00 & 5.00 & 1.00 \\
\hline 150 & 75 & 0.16 & 0.00 & 0.16 & 0.00 & 13.71 & 13.65 & 1.00 \\
\hline 75 & 45 & 0.12 & 0.01 & 0.12 & 0.00 & 10.12 & 9.41 & 0.93 \\
\hline 45 & 25 & 0.04 & 0.01 & 0.06 & 0.00 & 3.83 & 2.78 & 0.78 \\
\hline 25 & 13.1 & 0.06 & 0.09 & 0.15 & 0.00 & 5.24 & -2.57 & 0.40 \\
\hline 13.1 & 6.5 & 0.05 & 0.11 & 0.16 & 0.00 & 4.22 & -5.75 & 0.30 \\
\hline 6.5 & 3.3 & 0.04 & 0.12 & 0.16 & 0.00 & 3.80 & -6.30 & 0.27 \\
\hline 3.3 & 1.5 & 0.03 & 0.08 & 0.11 & 0.00 & 2.50 & -4.27 & 0.27 \\
\hline 1.5 & & 0.03 & 0.09 & 0.12 & 0.00 & 2.90 & -5.00 & 0.27 \\
\hline Totals & -- & 0.64 & 0.51 & 1.15 & 0.00 & & & \\
\hline
\end{tabular}




\begin{tabular}{|c|c|c|c|}
\hline $\begin{array}{c}\text { Experimental } \\
\text { Partition Factor }\end{array}$ & $\begin{array}{c}\text { Mean } \\
\text { Particle } \\
\text { Size }(\mu \mathrm{m})\end{array}$ & $\begin{array}{c}\text { Predicted } \\
\text { Partition } \\
\text { Factor }\end{array}$ & $\begin{array}{c}\text { Predicted } \\
\text { Partition } \\
\text { Factor }\end{array}$ \\
\hline$(-)$ & $(--)$ & (Plitt) & (Linch) \\
\hline 1.00 & 299.81 & 1.000 & 1.000 \\
\hline 1.00 & 178.33 & 1.000 & 1.000 \\
\hline 0.96 & 106.07 & 0.991 & 0.989 \\
\hline 0.82 & 58.09 & 0.803 & 0.809 \\
\hline 0.47 & 33.54 & 0.481 & 0.473 \\
\hline 0.25 & 18.08 & 0.246 & 0.249 \\
\hline 0.14 & 9.25 & 0.147 & 0.156 \\
\hline 0.12 & 4.62 & 0.115 & 0.119 \\
\hline 0.11 & 2.32 & 0.106 & 0.103 \\
\hline 0.10 & 1.64 & 0.105 & 0.099 \\
\hline & WSSQ & 0.002 & 0.001 \\
\hline
\end{tabular}

\begin{tabular}{|c|c|c|c|}
\hline (Plitt) & Predicted & (Linch) & Predicted \\
\hline D50 & 38.10 & $\mathrm{D} 50$ & 38.07 \\
\hline $\mathrm{m}$ & 1.85 & Alpha & 2.39 \\
\hline Bypass(Rt) & 0.10 & Bypass(Rf) & 0.09 \\
\hline
\end{tabular}

\begin{tabular}{l|l}
\hline TWSSQ & 0.003 \\
\hline
\end{tabular}

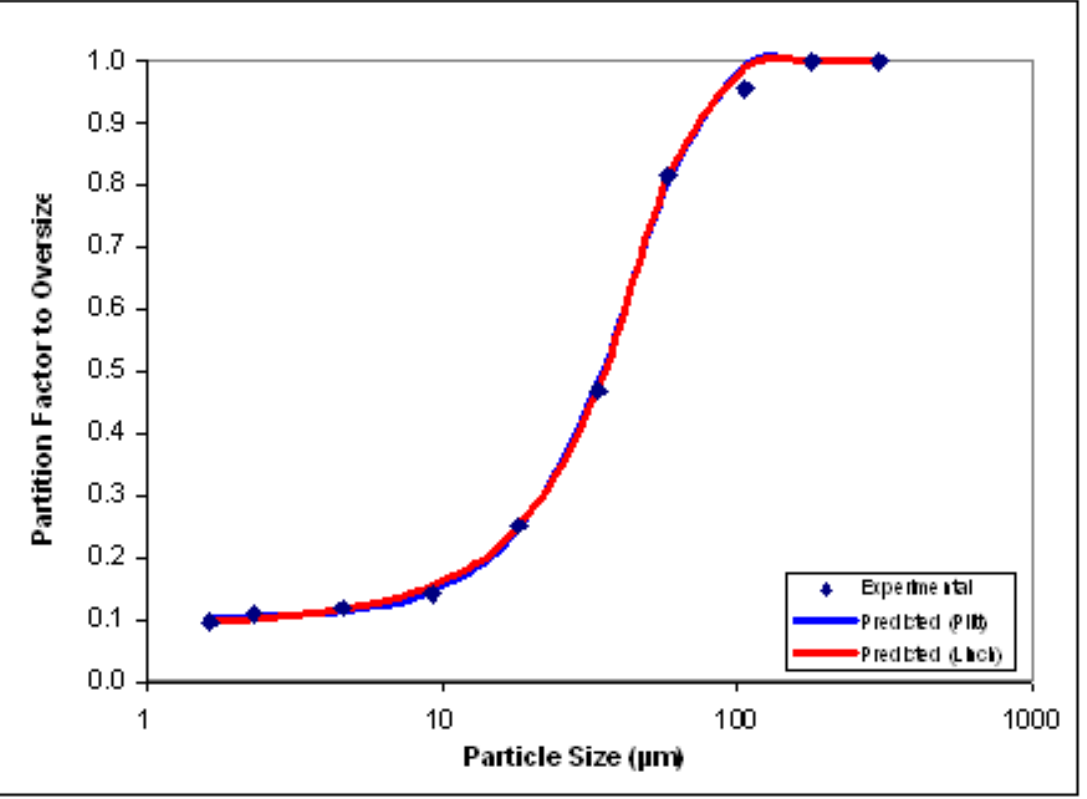


Tabular Summary of Parametric Test Results

\begin{tabular}{|c|c|c|c|c|c|c|c|c|c|c|c|c|c|c|c|c|c|c|c|c|}
\hline \multirow{3}{*}{ std } & \multirow{3}{*}{$\begin{array}{l}\text { Factor A } \\
Q \text { (gpm) }\end{array}$} & \multirow{3}{*}{$\begin{array}{l}\text { Factor B } \\
\boldsymbol{D}_{\text {(inchi) }}\end{array}$} & \multirow{3}{*}{$\left|\begin{array}{l}\text { Fador C } \\
\boldsymbol{D}_{c} \text { (inch) }\end{array}\right|$} & \multirow{3}{*}{$\begin{array}{l}\text { Factor D } \\
\boldsymbol{D}_{3} \text { (inch) }\end{array}$} & \multirow{2}{*}{\multicolumn{2}{|c|}{\begin{tabular}{|c|} 
Response $A$ \\
Cut size $\left(\mathrm{D}_{\mathbf{3 0}, 4 \mathrm{~m})}\right.$
\end{tabular}}} & \multirow{2}{*}{\multicolumn{2}{|c|}{$\begin{array}{l}\text { Response B } \\
\text { Bypass (Rf) }\end{array}$}} & \multirow{2}{*}{\multicolumn{2}{|c|}{$\begin{array}{l}\text { Response C } \\
\text { Sharmess ( } \alpha)\end{array}$}} & \multirow{2}{*}{\multicolumn{2}{|c|}{$\begin{array}{l}\text { Response D } \\
\text { Imperfedion (1) }\end{array}$}} & \multirow{3}{*}{$\begin{array}{l}\text { Sluny Fbwrate } \\
\text { Pressir D DP } \\
\text { coogypm, ps ) }\end{array}$} & \multirow{3}{*}{ 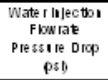 } & \multicolumn{3}{|c|}{ Head Solid Contert } & \multicolumn{3}{|c|}{ Head Ash Content } \\
\hline & & & & & & & & & & & & & & & \multirow{2}{*}{ Feed (s) } & \multirow{2}{*}{ Off $(*)$} & \multirow[b]{2}{*}{ U/F $(*)$} & \multirow{2}{*}{ Feed $(\omega)$} & \multirow{2}{*}{ Of $(*)$} & \multirow{2}{*}{ U/F $*$} \\
\hline & & & & & $\begin{array}{ll}P_{\text {NiU }} \\
\end{array}$ & Lynch & Priu & Linch & 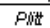 & Linch & Pint & Linch & & & & & & & & \\
\hline 1 & 000 & 0.50 & 4.00 & 100 & 24.02 & 23.94 & 0.37 & 0,36 & 2.87 & 2.70 & 038 & 0.41 & 21.00 & 000 & 4.50 & 2.44 & 8.57 & $\begin{array}{l}34.62 \\
\end{array}$ & 49.01 & 25.88 \\
\hline 2 & 3000 & 0.50 & 4.00 & 100 & 37.86 & 37.42 & 0.17 & 0.16 & 3.41 & 3.61 & 031 & 030 & 21.00 & 2800 & 4.50 & 2.56 & 5.27 & 34.55 & 47.67 & 18.57 \\
\hline 3 & 1500 & 0.50 & 3.50 & 100 & 32.43 & 32.89 & 028 & 027 & 6.92 & 500 & 0.16 & 022 & 21.00 & 1500 & 4.63 & 2.75 & 6.61 & 34.55 & 48.29 & 22.47 \\
\hline 4 & 1500 & 0.50 & 4.50 & 100 & 33.34 & 32.45 & 028 & 029 & 3.53 & 5.19 & 0.30 & 021 & 21.00 & 1500 & 4.63 & 2.80 & 6.73 & 34.97 & 48.10 & 23.87 \\
\hline 5 & 1500 & 0.50 & 4.00 & 0.75 & 34.80 & 34.85 & 0.10 & 009 & 1.84 & 1.76 & 0.51 & 0.83 & 21.00 & 1600 & 4.73 & 2.86 & 8.42 & 34.24 & 47.00 & 18.86 \\
\hline 6 & 1500 & 0.50 & 4.00 & 125 & 23.53 & 23.34 & 0.49 & 0.48 & 192 & 192 & 0.50 & 0.57 & 21.00 & 1300 & 4.59 & 2.43 & 5.69 & 33.96 & 49.25 & 28.07 \\
\hline 7 & 1500 & 0.75 & 3.50 & 0.75 & 40.17 & 39.81 & 0.16 & 0.15 & 3.87 & 386 & 029 & 028 & 21.00 & 1300 & 4.50 & 2.70 & 7.50 & 34.90 & 47.33 & 19.60 \\
\hline 8 & 1500 & 0.75 & 4.50 & 0.75 & 37.18 & 37.00 & 0.16 & 0.15 & 2.84 & 2.88 & 0.36 & 0.38 & 21.00 & 1400 & 4.50 & 2.68 & 7.75 & 34.60 & 47.32 & 20.49 \\
\hline 9 & 000 & 0.75 & 3.50 & 100 & 26.87 & 26.91 & 0.41 & 0.40 & 3.46 & 3.55 & 030 & 031 & 21.00 & 000 & 4.59 & 2.68 & 8.32 & 34.63 & 49.03 & 27.01 \\
\hline 10 & 1500 & 0.75 & 4.50 & 125 & 21.53 & 21.39 & 0.48 & 0.47 & 2.91 & 2.86 & 0.35 & 0.38 & 21.00 & 1200 & 4.50 & 2.31 & 5.18 & 34.98 & 49.41 & 29.20 \\
\hline 11 & 1500 & 0.75 & 4.00 & 100 & 25.55 & 25.33 & 024 & 023 & 1.97 & 1.87 & 0.49 & 0.59 & 21.00 & 1200 & 4.74 & 2.66 & 7.02 & 33.80 & 48.59 & 23.18 \\
\hline 12 & 1500 & 0.75 & 4.00 & 100 & 27.15 & 26.99 & 020 & 0.19 & 1.78 & 1.82 & 0.53 & 0.88 & 21.00 & 1300 & 4.63 & 2.65 & 7.34 & 34.37 & 48.38 & 23.26 \\
\hline 13 & 000 & 0.75 & 4.00 & 0.75 & 26.46 & 26.26 & 0.23 & 021 & 203 & 195 & 0.47 & 0.56 & 21.00 & 000 & 4.65 & 2.73 & 11.23 & 34.28 & 48.60 & 23.84 \\
\hline 14 & 3000 & 0.75 & 4.00 & 0.75 & 47.17 & 47.18 & 003 & 002 & 292 & 308 & 0.35 & 036 & 21.00 & 1800 & 4.63 & 3.00 & 6.05 & 34.11 & 45.61 & 10.52 \\
\hline 15 & 000 & 0.75 & 4.00 & 125 & 22.11 & 22.19 & 0.53 & 0.52 & 2.84 & 2.70 & 0.38 & 0.41 & 21.00 & 000 & 4.55 & 2.49 & 6.52 & 34.42 & 48.70 & 29.38 \\
\hline 16 & 3000 & 0.75 & 4.00 & 125 & 23.99 & 23.94 & 0.37 & 0.36 & 221 & 2.48 & 0.44 & 0.44 & 21.00 & 1400 & 4.57 & 2.40 & 6.41 & 34.56 & 49.30 & 26.16 \\
\hline 17 & 1500 & 0.75 & 4.00 & 100 & 31.62 & 31.44 & 0.30 & 029 & 6.79 & 5.43 & 0.16 & 020 & 21.00 & 1200 & 4.57 & 2.61 & 8.95 & 34.34 & 48.23 & 23.16 \\
\hline 18 & 1500 & 0.75 & 4.00 & 100 & 27.88 & 27.70 & 026 & 025 & 2.33 & 2.37 & 0.42 & 0.46 & 21.00 & 1200 & 4.59 & 2.59 & 6.83 & 33.95 & 48.49 & 23.72 \\
\hline 19 & 3000 & 0.75 & 3.50 & 100 & 33.85 & 33.46 & 0.18 & 0.17 & 2.91 & 3.12 & 0.35 & 035 & 21.00 & 1700 & 4.61 & 2.78 & 5.34 & 33.20 & 47.65 & 18.60 \\
\hline 20 & 1500 & 0.75 & 3.50 & 125 & 24.31 & 24.23 & 0.54 & 0.53 & 3.50 & 3.46 & 030 & 0.32 & 21.00 & 1200 & 4.50 & 2.37 & 5.17 & 34.55 & 49.49 & 29.26 \\
\hline 21 & 000 & 0.75 & 4.50 & 100 & 26.18 & 26.20 & 0.38 & 0.37 & 324 & 3.36 & 0.32 & 0.33 & 21.00 & 000 & 4.50 & 2.48 & 7.87 & 35.04 & 49.16 & 26.99 \\
\hline 22 & 3000 & 0.75 & 4.50 & 100 & 35.69 & 35.23 & 0.19 & 0.18 & 330 & 3.58 & 031 & 0.31 & 21.00 & 1900 & 4.50 & 2.58 & 5.16 & 34.75 & 48.05 & 20.38 \\
\hline 23 & 1500 & 0.75 & 4.00 & 100 & 28.78 & 28.57 & 0.27 & 026 & 251 & 2.70 & 0.40 & 0.41 & 21.00 & 1300 & 4.51 & 2.51 & 6.79 & 34.34 & 48.29 & 23.88 \\
\hline 24 & 1500 & 0.75 & 4.00 & 100 & 29.04 & 28.79 & 024 & 023 & 223 & 223 & 0.44 & 0.49 & 21.00 & 1200 & 4.51 & 2.48 & 6.31 & 34.73 & 48.24 & 23.23 \\
\hline 25 & 000 & 1.00 & 4.00 & 100 & 25.67 & 25.61 & 0.33 & 0.32 & 2.58 & 2.79 & 039 & 039 & 21.00 & 000 & 4.51 & $\frac{2.40}{2.42}$ & 8.10 & 35.36 & 48.96 & 26.33 \\
\hline 26 & 3000 & 1.00 & 4.00 & 100 & 32.94 & 32.77 & 0.18 & 0.17 & 2.34 & 230 & 0.42 & 0.48 & 21.00 & 1400 & 4.51 & 2.51 & 5.25 & 34.50 & 47.88 & 21.17 \\
\hline 27 & 1500 & 1.00 & $\begin{array}{l}.000 \\
.50\end{array}$ & 100 & 29.61 & 29.37 & 0.10 & 0.11 & $\begin{array}{l}2 . N 4 \\
2.14\end{array}$ & $\begin{array}{l}200 \\
208\end{array}$ & 0.45 & 0.53 & 21.00 & 1200 & 4.64 & $\begin{array}{l}2.31 \\
\end{array}$ & 8.200 & 35.15 & 48.000 & 23.68 \\
\hline 28 & 1500 & 1.00 & 4.50 & 100 & 31.22 & 31.04 & 024 & 023 & 232 & 228 & 0.43 & 0.48 & 21.00 & 1200 & 4.63 & 2.80 & 6.53 & 35.30 & 47.80 & 24.36 \\
\hline 29 & 1500 & 1.00 & 4.00 & 0.75 & 37.04 & 37.01 & 0.14 & 0.12 & 2.41 & 2.41 & 0.41 & 0.46 & 21. & 1200 & 4.67 & $\frac{2.00}{2.82}$ & 8.37 & $\begin{array}{l}34.52 \\
\end{array}$ & 47.23 & 20.01 \\
\hline 30 & 1500 & 1.00 & 4.00 & 125 & 24.20 & 24.11 & 0.52 & 0.52 & 228 & 2.48 & 0.43 & 0.44 & 21.00 & 1000 & 4.59 & 2.42 & 5.54 & 34.12 & 49.28 & 28.64 \\
\hline 55 & 000 & 0.75 & 4.00 & 100 & 25.58 & 25.41 & 0,34 & 0,32 & 2.17 & 2.17 & 0.45 & 051 & 21.00 & 000 & 5.00 & 2.86 & 9.40 & 34.04 & 48.91 & 25.46 \\
\hline 56 & 1000 & & 4.00 & 100 & 23.35 & 23.20 & 026 & 024 & 198 & 194 & 0.48 & 0.57 & 21.00 & 1100 & 4.85 & 2.81 & 8.25 & 34.03 & 48.86 & 24.47 \\
\hline 57 & 2000 & 0.75 & 4.00 & 100 & 30.62 & 30.41 & 0.22 & 020 & 2.41 & 2.43 & 0.41 & 0.45 & 21.00 & 1400 & 4.51 & 2.76 & 6.94 & 34.07 & 48.57 & 21.64 \\
\hline 58 & 3000 & 0.75 & 4.00 & 100 & 38.10 & 38.07 & 0.10 & 009 & 238 & 239 & 0.42 & 0.46 & 21.00 & 2200 & 4.50 & 2.80 & 5.45 & 33.88 & 46.85 & 17.20 \\
\hline
\end{tabular}


Appendix 2: Alternative Materials for Dense Medium Separation (KY004) 


\section{FINAL TECHNICAL REPORT}

Contract Title and Number:

Crosscutting Technology Development at the Center for

Advanced Separation Technologies

(DE-FC26-02NT41607)
Period of Performance:

Starting Date: 10/1/03

Ending Date: 10/31/09
Sub-Recipient Project Title:

Alternative Materials for Dense Medium Separation

Principal Investigators:

Rick Q. Honaker

Contact Address:

University of Kentucky

234B Mining and Mineral Resources

Lexington KY 40506

Subcontractor Address:
Report Information:

Type: Final

Number:

Period: 10/01/03-10/31/09

Date: $\quad$ 10/25/09

Code: KY004-FINAL

Contact Information:

Phone: (859) 257-1108

Fax: (858) 323-1962

E-Mail: rhonaker@engr.uky.edu

Subcontractor Information:

Phone:

Fax:

E-Mail:

\section{ABSTRACT}

In response to concerns regarding cost and future supply of magnetite, a study has been performed to evaluate the potential of alternative materials that can be used to generate a dense medium for coal cleaning applications. Alternative materials included waste steel slag, fine sand, high-density material existing in run-of-mine feed and magnetic material from coal combustion bottom ash. Under certain conditions, each of the alternative materials provided separation efficiencies that meet industrial standards including probable error values ranging from 0.03 to 0.05 . Dense medium derived from the non-magnetite sources and magnetic bottom ash particles achieved organic efficiency values exceeding the 95\% level over a medium density range from 1.3RD to $1.6 \mathrm{RD}$ with lower than normal density offsets. Coarse magnetite, which has a lower commercial value than conventional magnetite, provided excellent separation efficiencies when evaluating medium density values greater than 1.6RD. From the assessment of recoverability after the coal cleaning process, the magnetic bottom ash material was easily recovered by a standard low intensity magnetic separator which is the current method used to recover magnetite in operating coal cleaning facilities. 


\section{DISCLAIMER}

This report was prepared as an account of work sponsored by an agency of the United States Government. Neither the United States Government nor any agency thereof, nor any of their employees, make any warranty, express or implied, nor assume any legal liability or responsibility for the accuracy, completeness, or usefulness of any information, apparatus, product, or process disclosed, or represents that its use would not infringe privately owned rights. Reference herein to any specific commercial product, process, or service by trade name, trademark, manufacturer, or otherwise does not necessarily constitute or imply endorsement, recommendation, or favoring by the United States Government or any agency thereof. The views and opinions of authors expressed herein do not necessarily state or reflect those of the United States Government or agency thereof. 


\section{Table of Contents}

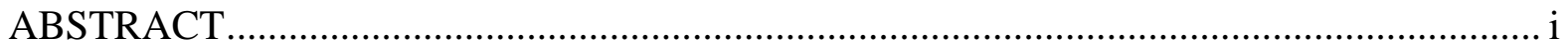

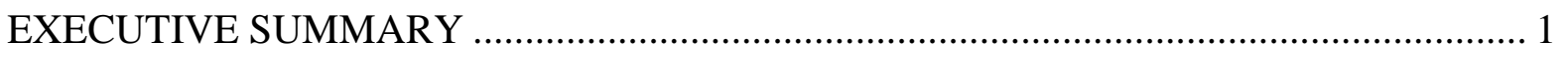

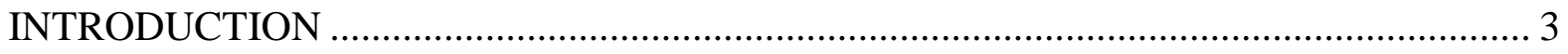

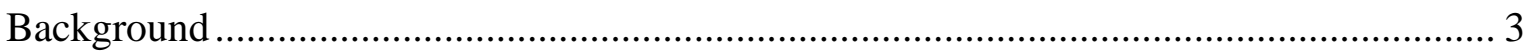

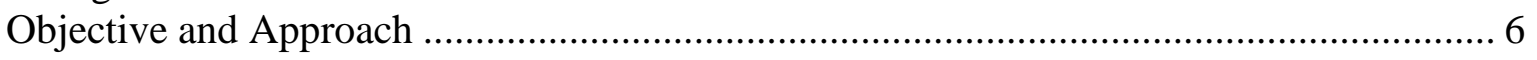

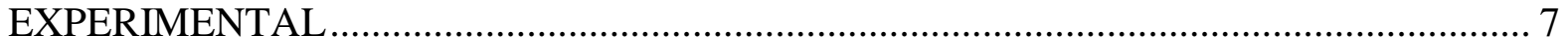

Dense Medium Cyclone Circuit............................................................................. 7

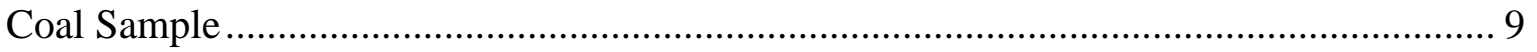

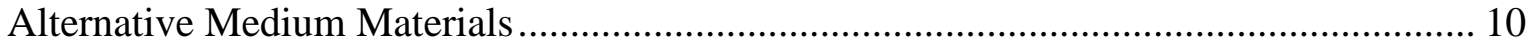

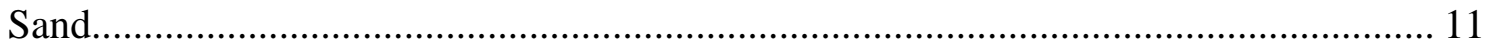

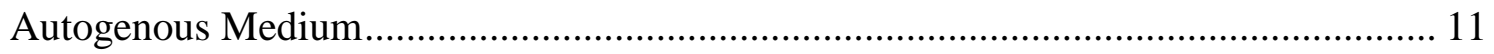

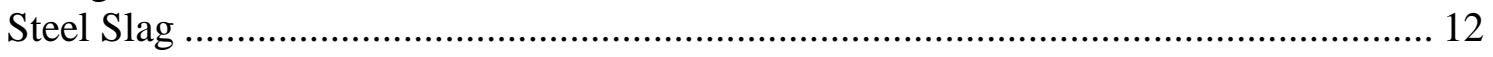

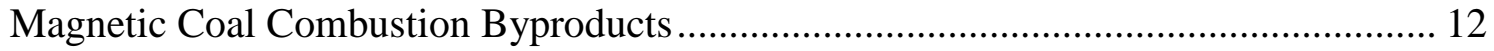

RESULTS AND DISCUSSION ............................................................................ 18

Reference Magnetite Performance............................................................................ 18

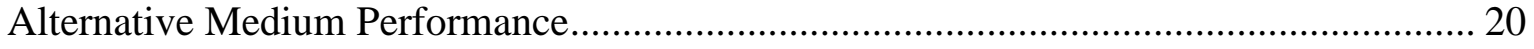

Sand, Autogenous and Coarse Magnetite Medium..................................................... 20

Magnetic Coal Combustion Byproduct .............................................................. 24

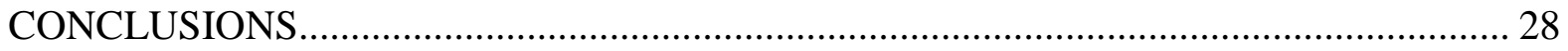

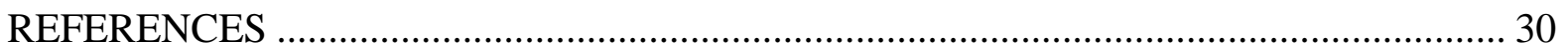

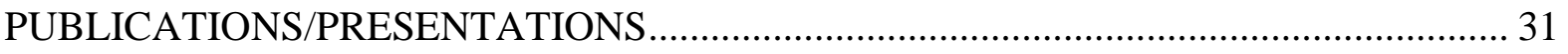




\section{EXECUTIVE SUMMARY}

Dense medium separation processes are currently used to clean over $50 \%$ of the 640 million tons of coal that is processed through preparation plants in the U.S. annually. They provide the most efficient separations for particle sizes greater than $1 \mathrm{~mm}$. To develop the dense medium, magnetite $\left(\mathrm{Fe}_{3} \mathrm{O}_{4}\right)$ particles finer than about 44 micrometer are dispersed in water at a concentration that provides a medium density between the solid densities of coal and rock. After the separation, the medium is passed through a low-intensity magnetic drum separator to recover the magnetite. Magnetite losses are typically within the range of 0.5 to $1.0 \mathrm{lbs} / \mathrm{ton}$ of raw coal processed.

In 2002, most of the magnetite used in the U.S. coal industry was obtained from domestic sources and worldwide production was adequate to meet the needs for coal cleaning and steel production as well as other needs. The cost for a ton of magnetite was $\$ 56$. However, closure of a mine that supplied magnetite to the coal industry containing very low amounts of silica particles resulted in the need to seek international sources of magnetite which resulted in imports mainly from South America and elevated costs. Economic and construction growth in developing countries brought a high demand for iron ore worldwide and, as a result, the concern for adequate magnetite supply for the coal industry. Prices rose to around $\$ 300$ per ton. Concerns over cost and supply resulted in worldwide interest in identifying alternative materials that could potentially replace magnetite is dense medium separation processes.

The project funded through the Center for Advanced Separation Technologies investigated several magnetite alternatives including a source of coarse magnetite that is sold internationally at a lower cost than conventional magnetite. Other materials included magnetic particles recovered from coal combustion bottom ash, fine sand and fine waste generated from steel production. A unique source of alternative material is the high-ash content, high density particles that exist in the fine reject streams in coal preparation plants. The autogenous particles have a density less than half of magnetite which requires the use of twice the amount of autogneous material to achieve a given medium density. The high particle population raises concerns over negative viscosity effects but separations at low medium density values may avoid these concerns.

The characteristics of a medium used to clean coal are that i) the particles forming the medium are relatively stable over a range of medium densities and ii) the medium has low viscosity. Medium stability is achieved by minimizing the settling velocity of the particles forming the medium. Elevating medium density improves stability since the increased volumetric concentration of particles creates a high level of hindered settling which decreases particle settling velocity. However, high particle populations result in viscosity issues that typically reduce the separation efficiency achieved when applied to coal cleaning. Decreasing particle size and sphericity also reduces particle settling rates and thus enhances stability.

The magnetic bottom ash particles collected from the Mill Creek power station, a pulverized coal fired power plant, operated by Louisville Gas and Electric (LG\&E), was comprised of mostly hollow spheres. The Mill Creek plant was selected as a source for the material due to the relatively high pyrite content of the feed coal which results in elevated concentrations of 
iron oxide material in the combustion byproduct streams, particularly in the bottom ash stream due to the high particle density of the iron oxide particles. The iron oxide particles were recovered using a spiral concentrator and a high intensity magnetic separator. The -100 mesh material recovered from the bottom ash stream represented $5.7 \%$ of the total feed. However, the sphericity of the iron oxide particles provided a relatively unstable medium. As such, the material was ground in a ball mill to reduce particle sphericity which resulted in a particle size distribution in which $90 \%$ of the particles were finer than 50 micrometers. Grinding caused liberation of the magnetic material which resulted in only $54 \%$ of the originally recovered magnetic material to be recoverable by a magnetic separator. A positive outcome is that $98 \%$ of the remaining magnetic particles can be recovered by a low intensity magnetic separator with a field strength of 1000 gauss.

The conventional magnetite alternatives were evaluated for their application in dense medium separators using a 15-cm diameter Krebs cyclone operated in a closed-looped circuit. The overflow and underflow streams of the cyclone were returned to a common feed sump from which feed to the cyclone was pumped under pressures ranging from 17.3 to 68.7 $\mathrm{kPa}$ (2.5 to $7.5 \mathrm{psi}$ ). Medium stability was assessed by measuring the difference in the pulp density values of the underflow and overflow streams. Differences less than 0.4 units are indicative of acceptable medium stability. With the exception of the coarse magnetite, each of the alternative materials provided a more stable medium than the conventional magnetite over the entire medium density range evaluated. This outcome was somewhat expected due to the lower solid density values of the alternative materials which means that higher volumetric solid concentrations relative to the conventional magnetite are needed to achieve a given medium density. Medium stability formed from coarse magnetite approached acceptable levels when the relative medium density exceeded 1.6.

The separation performance achievable using medium from each of the alternative sources was assessed using a relatively difficult to clean Coalburg coal having a top particle size of $6 \mathrm{~mm}$ and a bottom size of $0.6 \mathrm{~mm}$. Medium density was varied from 1.4RD to a high of 1.9RD. At medium density values of $1.4 \mathrm{RD}$ and $1.5 \mathrm{RD}$, the separation efficiency values achieved when using steel slag, fine sand, autogenous material, and magnetic bottom ash were nearly equal to the values obtained using the conventional magnetite (e.g., $\mathrm{E}_{\mathrm{p}}=0.03$ and organic efficiency $>95 \%$ ). However, when the medium density was raised to 1.6RD, only the magnetic bottom ash material achieved separation efficiency values near but slighter lower than that realized from conventional magnetite. The inferior performances at 1.6RD are reflective of the elevated medium viscosity caused by particle populations far exceeding that of the conventional magnetite.

On the other hand, the lower cost coarse magnetite provided poor medium stability for all medium density values tested at or below 1.6RD. As a result, the separation efficiencies achieved while cleaning coal were significantly inferior. However, as the medium density was increased above 1.6RD, medium stability and thus separation efficiency improved significantly. In fact, the separation efficiency achieved with the coarse magnetite was superior to conventional magnetite at medium density values of 1.8RD and 1.9RD. The findings support previously reported conclusions that an optimum medium particle size distribution exists for a separation at a given medium density. 


\section{INTRODUCTION}

\section{Background}

Dense-medium separators are the most widely applied gravity concentration units in coal preparation. In the process, the separation of coal from mineral matter is achieved by creating a medium having a density between the solid densities of the two materials being separated. The medium is formed by suspending ultrafine particles in a medium of water. The materials that have been used to form the suspension in commercial applications include sand, clay, quartz, slate, galena, magnetite and ferrosilicon [1]. In the early development of dense medium separators, sand (Chance sand process) was used as medium. However, sand was difficult to recover. When magnetic separators were developed at acceptable capacities, sand was replaced with magnetite [2]. Since then, magnetite has become the predominate material used to form a dense medium when relative separation densities below 2.0 are desired.

Recently, worldwide demand for magnetite has increased significantly due to steel production requirements in developing countries. This has led to a growing interest in seeking alternate materials for dense medium separation. Material that is acceptable for producing a dense medium should provide the following characteristics: good medium stability; capability of achieving a broad range of separation densities, low viscosity and easy recoverability for reuse. Medium stability is typically monitored by the density difference between the overflow and the underflow media streams. Acceptable medium stability is indicated by medium density differences in the discharge streams of less than 0.4 density units. Particle size, shape and density are parameters that play vital roles in the stability and rheological properties of a medium. For example, spherical particles create unstable mediums at low medium densities but may become stable as the medium density is increased.

The control of density, viscosity, and settling rate of a medium is necessary for the efficient separation of coal and impurities. The following equation determines the density of a suspension, $\rho_{m}\left(\mathrm{~g} / \mathrm{cm}^{3}\right)$,

$$
\rho_{m}=\frac{100}{\left(100-\phi_{W}\right)+\frac{\phi_{W}}{\rho_{s}}},
$$

where $\rho_{s}$ is the density of the solid $\left(\mathrm{g} / \mathrm{cm}^{3}\right)$ used to form the medium and $\phi_{W}$ the solid concentration by weight (\%). From Eq. (1), it can be seen that the density of a suspension can be varied by adjusting the proportion of medium solids present in the suspension. However, there is a natural limit to the proportion of solids as imposed by the negative effects of medium viscosity. Relatively low viscosity is needed to permit the free travel of coal and impurities in it. This limiting volumetric concentration is similar for all suspensions with particles of similar size range but can be modified to a limited extent by a change in the size distribution of the medium solids. Eq. (1) also shows that the density of the suspension is a 
function of the relative density (RD) of the medium solids and it follows that media composed of higher specific gravity solids will have a higher density at the limiting volume concentration than suspensions composed of lower specific gravity solids.

Increasing the medium density above the density of pure water by the addition of magnetite or alternative material initially has a positive effect on separation performance due to improved medium stability and the positive impacts on differential density-based particle movement. Medium stabilization is a result of an elevated particle population which decreases the settling rate of the magnetite particles due to hindered settling as quantified by the expression:

$$
v_{H}=\frac{d^{2} g\left(\rho_{s}-\rho_{f}\right)}{18 \mu_{A}}\left(1-\phi_{V}\right)^{3.65}
$$

where $v_{H}$ is the hindered settling velocity $(\mathrm{cm} / \mathrm{s}), d$ particle size $(\mathrm{cm}), g$ acceleration due to gravity $\left(\mathrm{cm} / \mathrm{s}^{2}\right), \rho_{s}$ and $\rho_{f}$ the densities of particle and fluid, respectively, $\phi_{V}$ the solids concentration by volume and $\mu_{A}$ the actual suspension viscosity (poise). Equation (2) is applicable for ultrafine particle suspensions and clearly shows that increasing the solid concentration by volume reduces particle settling rates which leads to stabilization of the medium. However, as many studies have shown, a continued increase in the solids concentration above a critical value leads to a viscous suspension having non-Newtonian fluid characteristics and depressed separation efficiencies when cleaning coarse and fine coal $[3-4]$.

A semi-empirical model was developed by Shi and Napier-Munn [1996] that allows the prediction of slurry viscosity as a function of particle size and volumetric solids concentration of the suspension, i.e.,

$$
\mu_{A}=\mu_{W}\left[C_{1}(H-1)+C_{2} H \Omega+C_{3} \frac{\phi_{V}}{P_{80}} \Omega^{2}+C_{4} \Omega^{3}\right],
$$

in which

$$
H=\exp \left[\frac{\phi_{V}}{1-\phi_{V}\left(P_{20}\right)^{C_{5}} \exp \left(C_{6} T\right)}\right] .
$$


The variable $T$ is the medium temperature $\left({ }^{\circ} \mathrm{C}\right), \Omega$ the bobbin angular velocity of the viscometer (rad/s), $P_{20}$ and $P_{80}$ the $20 \%$ and $80 \%$ passing sizes of the suspended solids (mm), respectively, $\mu_{W}$ the viscosity of water at $20^{\circ} \mathrm{C}\left(0.01\right.$ Poise) and $C_{1}-C_{6}$ model parameters that are determined experimentally for particular suspension type. Predictions using Eqs. (3) and (4) were found to accurately predict the output readings obtained from a rotational viscometer at various bobbin speeds.

An evaluation of three different medium solid materials conducted using Eqs. (2) - (4) shows a window of medium density values in which medium stability is realized and viscosity remains low (Figure 1). One of the materials (MAG) resembles conventional magnetite with a relative solids density of 5.2 and a $P_{80}$ of 36 microns. The characteristics of the other two cases represent those associated with materials that could potentially be used as an alternative to magnetite (FAM: $\rho_{\mathrm{s}}=3.6$ and $P_{80}=45$ microns; SR: $\rho_{\mathrm{s}}=2.45$ and $P_{80}=170$ microns). In all three cases, hindered settling velocity decreases with an elevation in medium density which stabilizes the medium. Simultaneously, medium viscosity increases and the rate of increase becomes significantly greater as medium density is raised above a relative value of 1.60. The case of using a low density solid (SR) requires the use of a relative coarse particle size distribution to reduce particle surface area and minimize particle suspension viscosity. The result is a relatively fast hindered settling velocity at low medium density values and thus medium instability. At a medium density of about 1.5RD, the 'SR' material becomes stable due to the low settling velocity. However, at a medium density of 1.6RD, viscosity increases rapidly and provides undesirable separation conditions. Thus, an operating window exists for each medium type which provides the appropriate medium stability through hindered settling with relatively low viscosity effects.

The results from several recent studies have identified potential low cost materials that could be used as a substitute for magnetite in dense medium separators. Studies with magnetic fly ash particles have shown that the material could be an acceptable substitute for magnetite with some changes in the magnetite recovery circuit [6 - 7]. The research revealed that the raw state of magnetic fly ash has spherical shape of particle sizes between $0.1 \mathrm{~mm}$ and $0.2 \mathrm{~mm}$ which creates unstable suspension conditions. Recent research found that the wet grinding of fly ash liberates the magnetic components and changes the shape of the material. As a result, the medium suspension was found to be relatively stable over a broad range of medium density values. This result was confirmed by pilot scale experiments and in-plant tests conducted at two different coal preparation plants. The recovery of $99.1 \%$ of the magnetic fly ash material was obtained in in-plant test [8]. Another investigation focused on the magnetic fraction from flue dust (MFFD) which achieved satisfactory results in a coal cleaning application [9].

A number of materials have potential for use as a substitute for magnetite in dense medium separations. However, they must provide the medium characteristics that have been previously discussed. A detailed research program has been conducted to evaluate the potential use of a relatively coarse magnetite as well as steel slag waste, fine sand and autogenous medium. The details of the study and the associated results are the subject of this publication. 


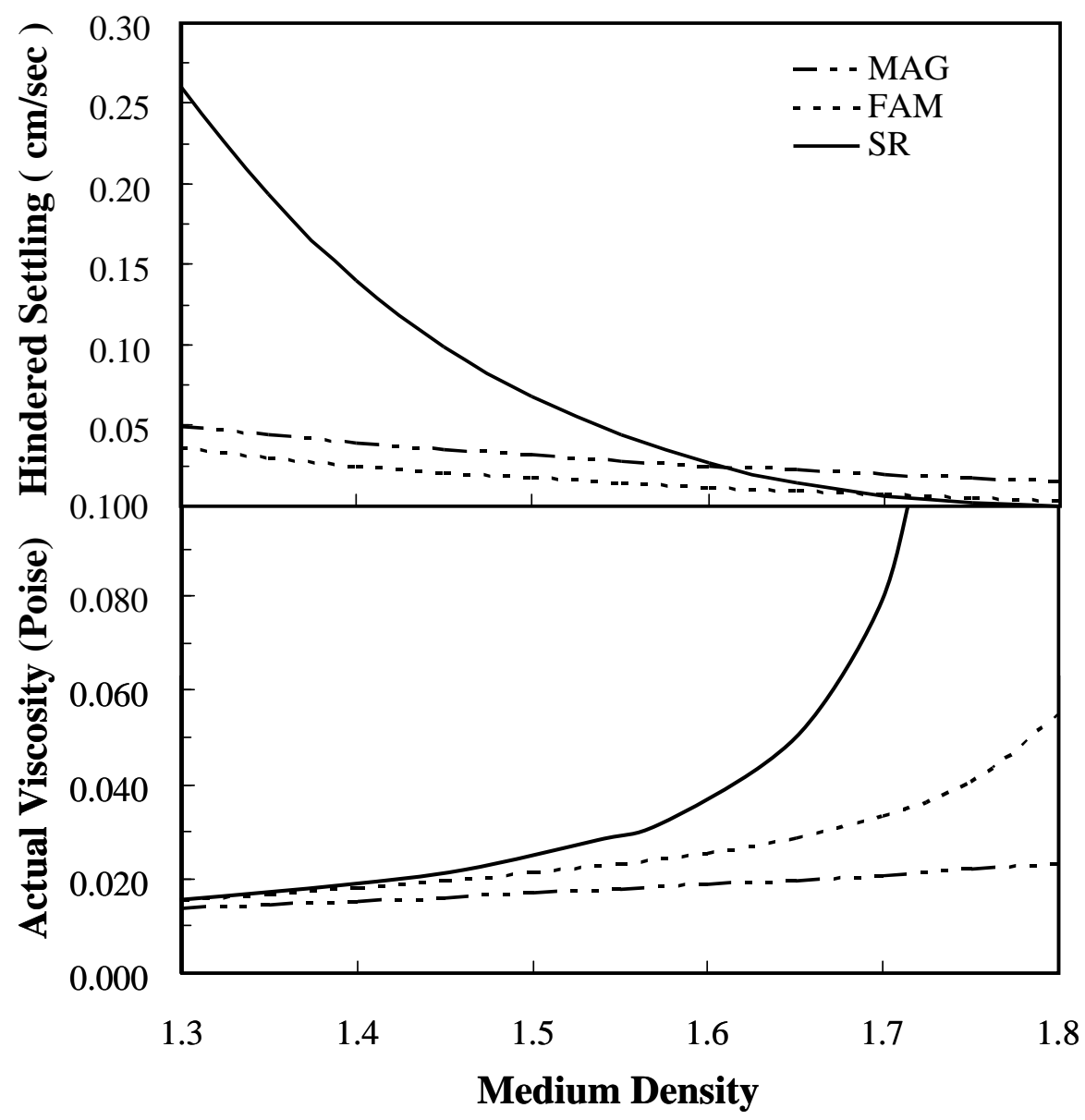

Figure 1. Predicted impact of the type of material used to develop a dense medium on medium stability (hindered settling rate) and medium viscosity; MAG: $\rho_{\mathrm{s}}=5.2$ and $P_{80}=36$ microns; FAM: $\rho_{\mathrm{s}}=3.6$ and $P_{80}=45$ microns; SR: $\rho_{\mathrm{s}}=2.45$ and $P_{80}$ $=170$ microns.

\section{Objective and Approach}

The objectives of the proposal were i) to evaluate alternative materials that could be economically used as a substitute for the traditional magnetite in dense medium processes, ii) to develop and evaluate a process in which fine waste material may be used as whole or part of the material needed for adjusting medium density and iii) to provide an excellent educational experience for a graduate student that can be easily translated to industrial application. 


\section{EXPERIMENTAL}

\section{Dense Medium Cyclone Circuit}

The tests were conducted using a $150 \mathrm{~mm}$ diameter Krebs dense medium cyclone (DMC) with a 63mm diameter vortex and a 20-degree cone angle. The angle of inclination of the set up was 10 degrees from horizontal with inlet pressures operated between 17 to $69 \mathrm{kPa}$ (2.5 to $10 \mathrm{psi}$ ). The apex diameter was $4.5 \mathrm{~cm}$ and was maintained constant throughout the entire study.

The dense medium cyclone was set to operate in closed loop. Figure 2 shows the closed loop circuit set up of the dense medium cyclone. The feed to the cyclone is pumped from the feed sump with the overflow and underflow streams reporting back to the sump. A feed bypass also reported to the sump, which was used to collect samples of feed to cyclone. The feed pump was used to control the inlet feed pressure to the cyclone between 17 and $69 \mathrm{kPa}(2.5$ and 10 psi).

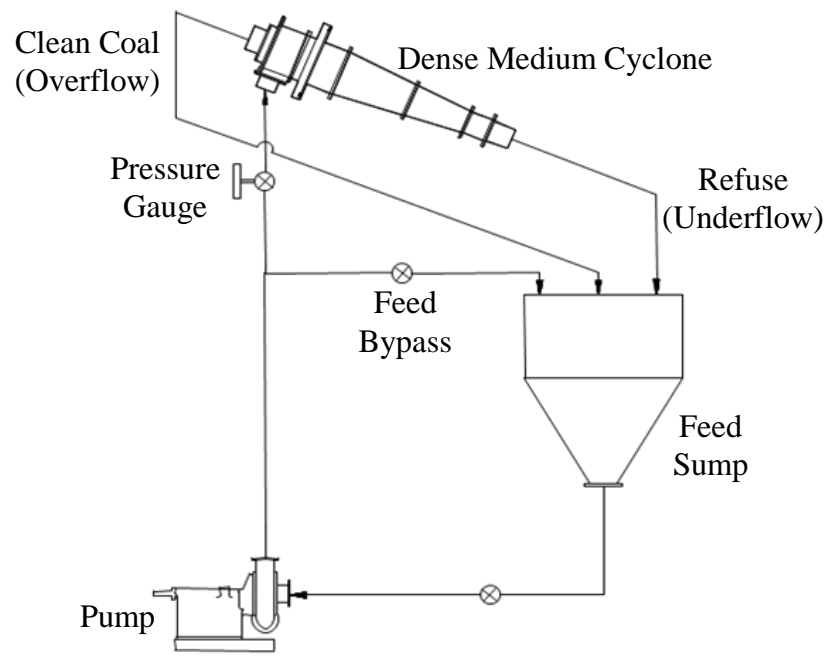

Figure 2. Schematic diagram of the dense medium cyclone circuit.

The evaluation of the material was conducted in two phases. The objective of the first phase was to evaluate the stability of the medium. The second phase evaluated the performances achieved using the medium when treating $6 \times 0.6 \mathrm{~mm}$ Coalburg coal, which has relatively difficult cleaning characteristics.

At the beginning of each stability test, the relative density of medium, which is a suspension of water and the high density magnetic material, was adjusted to the desired value in the circuit feed sump. The density was measured using a Marcy density scale. The medium was pumped to the cyclone at a pre-selected feed inlet pressure. A time interval of 10 minutes was allowed for the medium to stabilize. Samples were taken from the underflow and 
overflow streams to calculate the density differential between the two streams. The stability of the material was tested over a range of relative medium density values from 1.3 to $1.7 \mathrm{RD}$.

In the second phase of the experimental program, the medium was adjusted to the desired value and then the coal sample was added to the medium with the amount equivalent to achieve a medium-to-coal ratio of about 4:1. Again, a time interval of 10 minutes was allowed to ensure adequate mixing and steady state conditions. Samples from the overflow (clean coal and medium) and underflow (reject and medium) streams were taken simultaneously. Also, a feed sample was obtained from the bypass feed stream. The samples collected were wet screened using a $419 \mu \mathrm{m}$ (40 mesh) screen to separate the medium from the coal. Each coal sample was split into two factions for washability analysis and ash content. These results were used for data analysis and performance assessment of the medium

Previous research has shown the significant effect of centrifugal force on the separation performance achieved by DMCs. Near-gravity particles move slowly due to small density differences between the particle and the medium. As the centrifugal force is increased, the velocities of the near-density density particles are enhanced which improves the sharpness of the separation. However, elevated centrifugal forces can destabilize the medium suspension which leads to a deterioration in performance. Thus, an optimum centrifugal force or inlet pressure may exist for a given medium type having a characteristic particle size distribution. According to Bradley (1965) and later Zanker (1977), the centrifugal force applied within a DMC unit having standard dimensions varies with cyclone diameter $\left(d_{c}\right)$ as described by the expression:

$$
N_{g}=2 \alpha^{2} \frac{V_{i}^{2}}{D_{C} g}\left(\frac{D_{C}}{d_{C}}\right)^{(2 n+1)}
$$

where $\alpha$ is a modifying factor for inlet losses approximated by $3.7\left(D_{i} / D_{c}\right), D_{i}$ the cyclone inlet diameter, $D_{c}$ the cyclone diameter, $g$ the gravitational acceleration, $V_{i}$ the inlet velocity and $n$ a constant that normally varies between 0.5 and 0.8. Equation (2) clearly shows that the centrifugal force in the $150 \mathrm{~mm}$ DMC used in this study is significantly greater than the typical DMC units in industry at the same inlet pressure.

To determine the inlet pressure needed to realize a centrifugal force in the $150 \mathrm{~mm}$ DMC unit that is nearly equal to the larger industrial units, a standard inlet pressure equivalent to 9 cyclone diameters was assumed which equates to about $41 \mathrm{kPa}$ for a $700 \mathrm{~mm}$ diameter cyclone. According to data generated from Eq. (5) and those reported by Mengelers (1982) the centrifugal force at this inlet pressure is approximately 36.9g's. Using Eq. (5), the centrifugal force over a range a range of inlet pressures was determined for two common DMC diameters (i.e., $700 \mathrm{~mm}$ and $1000 \mathrm{~mm}$ ) and a $150 \mathrm{~mm}$ diameter DMC (Figure 3). The inlet pressure providing an equivalent centrifugal force in the $150 \mathrm{~mm}$ cyclone is $31 \mathrm{kPa}(4.5$ psi). As such, most of the tests were conducted with an inlet pressure of $34.5 \mathrm{kPa}$ (5 psi) which provides a centrifugal force of $37.5 \mathrm{~g}$ 's while the remaining tests were performed over an inlet pressure range of $17.4 \mathrm{kPa}$ to $68.9 \mathrm{kPa}$. 


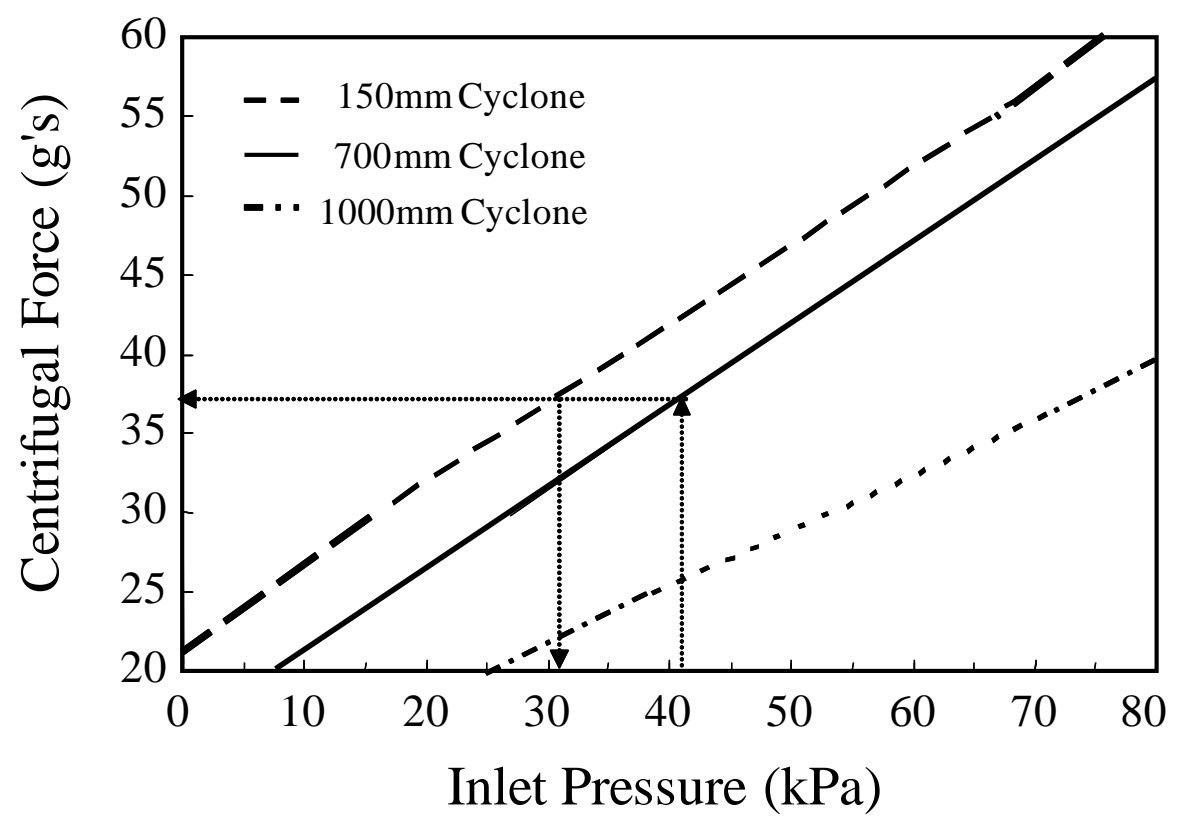

\begin{tabular}{|c|c|}
\hline \multicolumn{2}{|c|}{$\begin{array}{c}\text { Feed Pressure of Cyclone } \\
=9 \times \\
\text { Mengers }(1982)\end{array}$} \\
\hline $\begin{array}{c}\text { Cyclone } \\
\text { Diameter } \\
(\mathrm{mm})\end{array}$ & $\begin{array}{c}\text { Centrifugal } \\
\text { Force }\end{array}$ \\
\hline 250 & $54.9 \times \mathrm{g}$ \\
350 & $50.6 \times \mathrm{g}$ \\
400 & $48.8 \times \mathrm{g}$ \\
500 & $42 . .2 \times \mathrm{g}$ \\
600 & $39.4 \times \mathrm{g}$ \\
700 & $36.2 \times \mathrm{g}$ \\
800 & $33.8 \times \mathrm{g}$ \\
1000 & $31.0 \times \mathrm{xg}$ \\
\hline
\end{tabular}

Figure 3. Centrifugal force as a function of feed inlet pressure and cyclone diameter.

\section{Coal Sample}

The bituminous coal sample was obtained from an operating coal processing plant treating coal extracted from the Coalburg seam located in southern West Virginia, USA. The samples were collected into four 208-liter drums. Upon arrival in the test facility, the samples were dry screened using $6 \mathrm{~mm}(1 / 4-\mathrm{in})$ and $0.6 \mathrm{~mm}$ (28 mesh) screens. The $6 \times 0.6 \mathrm{~mm}$ fraction was retained while the material having a particle size greater than $6 \mathrm{~mm}$ was crushed using a laboratory hammer mill. The mill product was re-screened and the $6 \times 0.6 \mathrm{~mm}$ fraction combined with the previously obtained material. Coal particles that were smaller than $0.6 \mathrm{~mm}$ were discarded. The coal sample was mixed thoroughly and then used as the dense-medium cyclone feed material.

Representative samples were obtained to determine the particle size and density distribution of the $6 \times 0.6 \mathrm{~mm}$ coal. The wet screening results shown in Table 1 indicate that the majority of the total mass existed in the fractions greater than $1 \mathrm{~mm}$ in which the mass is equally distributed. The total ash content of the sample was $33.82 \%$ and the mineral matter was well distributed throughout the size fraction. The Coalburg seam coal typical has a low Hardgrove Index (i.e., 48) which means that the coal is difficult to break. This fact is important since the dense-medium circuit recycled slurry to maintain good suspension. The resistant to break limited particle size reduction as a result of recycling through a pump. 
Table 1. Characteristics of the Coalburg seam coal.

\begin{tabular}{||c|c|c|c|c|c||}
\hline \multicolumn{3}{|c|}{ Particle Size Distribution } & \multicolumn{2}{c|}{ Particle Density Distribution } \\
\hline $\begin{array}{c}\text { Particle Size } \\
(\mathrm{mm})\end{array}$ & $\begin{array}{c}\text { Weight } \\
(\%)\end{array}$ & $\begin{array}{c}\text { Ash } \\
(\%)\end{array}$ & $\begin{array}{c}\text { Specific } \\
\text { Gravity } \\
\text { Fraction }\end{array}$ & $\begin{array}{c}\text { Weight } \\
(\%)\end{array}$ & $\begin{array}{c}\text { Ash } \\
(\%)\end{array}$ \\
\hline+4.8 & 16.42 & 36.74 & 1.3 float & 27.96 & 3.56 \\
$4.8 \times 3.4$ & 18.76 & 34.70 & $1.3 \times 1.40$ & 22.33 & 11.68 \\
$3.4 \times 2.4$ & 22.26 & 34.45 & $1.40 \times 1.50$ & 10.24 & 25.98 \\
$2.4 \times 1.7$ & 18.26 & 30.97 & $1.50 \times 1.60$ & 6.45 & 37.72 \\
$1.7 \times 1.2$ & 15.46 & 27.98 & $1.6 \times 1.75$ & 4.81 & 45.56 \\
$1.2 \times 0.8$ & 4.54 & 34.73 & $1.75 \times 1.90$ & 4.85 & 54.73 \\
$0.8 \times 0.6$ & 1.71 & 36.72 & $1.90 \times 2.0$ & 2.35 & 63.07 \\
-0.6 & 2.26 & 57.84 & 2.0 sink & 21.02 & 84.53 \\
\hline Total & 100.00 & 33.82 & Total & 100.00 & 32.79 \\
\hline \hline
\end{tabular}

The Coalburg coal seam is known to be a moderately difficult coal to clean based on relatively high quantities of mass existing in the $1.5 \times 2.0$ density fractions. Based on the washability data in Table 1 , the cleaning index (C.I. = 1.3 cumulative weight \% float / 1.6 cumulative weight \% float) was determined to be less than 0.5 which indicates a very difficult-to-clean coal using differential densities. Over $15 \%$ of the feed mass is within \pm 0.1 relative density units for density values in the range of 1.4 and 1.9. The benefit to having a large amount of material in the middle density fractions is better data for partition curve development.

\section{$\underline{\text { Alternative Medium Materials }}$}

The tests evaluated five different dense medium materials, i.e., fine sand, steel slag, coarse magnetite, autogenous reject material and magnetic combustion residue. The magnetic combustion residue material was evaluated separately from the other four sources due to the unique recovery processes needed to recover the combustion residue and the time needed in the project to develop the recovery circuit. As such, the characterization and separation performance data and discussions are presented separately in this report.

Conventional grade B magnetite was used as the reference magnetite. The coarse magnetite was explored as an option when density separations greater than 1.6RD are targeted. The particle size distributions and specific gravities of each dense medium material including the conventional source are provided in Table 2. The particle size distributions of the sand, steel slag and autogenous material were made significantly coarser than the conventional magnetite to compensate for the lower solid densities. Low density materials require the addition of a greater volumetric amount of solid to achieve a given medium density, which elevates particle population and thus negative medium viscosity effects. Since viscosity is 
directly related to the amount of exposed particle surface area, increasing the particle size of the medium material can reduce viscosity, thereby allowing a high performance level.

Table 2. Particle size distribution and specific gravity of the alternative dense medium materials.

\begin{tabular}{|c|c|c|c|c|c||}
\hline \multirow{2}{*}{$\begin{array}{c}\text { Particle } \\
\text { Size } \\
\text { (micron) }\end{array}$} & \multicolumn{5}{|c||}{ Weight Distribution (\%) } \\
\cline { 2 - 6 } & Fine Sand & $\begin{array}{c}\text { Coarse } \\
\text { Magnetite }\end{array}$ & $\begin{array}{c}\text { Conventional } \\
\text { Magnetite }\end{array}$ & Steel Slag & $\begin{array}{c}\text { Autogenous } \\
\text { Material }\end{array}$ \\
\hline+150 & 0.26 & 17.91 & 0 & 0.54 & 52.56 \\
150 x 74 & 48.85 & 32.23 & 0.5 & 46.92 & 36.98 \\
$74 \times 44$ & 29.48 & 14.45 & 4.95 & 22.49 & 6.6 \\
44 x 37 & 6.34 & 6.44 & 7.04 & 6.70 & 2.74 \\
37 x 25 & 6.83 & 5.88 & 13.83 & 6.08 & 1.05 \\
-25 & 8.24 & 23.09 & 73.68 & 17.25 & 0.07 \\
\hline Relative & & 5.2 & 4.9 & 3.8 & 2.2 \\
Particle & 2.6 & 5 & & & \\
Density & & &
\end{tabular}

\section{Sand}

Fine sand was obtained from Belleview Sand and Gravel Inc., a local sand producer in Petersburg, Kentucky, U.S. The sand was used to assess the potential of using fine rock existing in the plant feed as an alternative dense medium. It is known in the early development of dense medium separations that sand was a popular dense medium material used for coal cleaning [2]. Upon arriving at the laboratory, the sand was wet screened using $150 \mu \mathrm{m}$ and $44 \mu \mathrm{m}$ Sweco screens. The $150 \times 44 \mu \mathrm{m}$ (100 x 325 mesh) fraction was retained as dense medium material while the $+150 \mu \mathrm{m}$ and $-44 \mu \mathrm{m}$ material from each screening step was discarded.

\section{Autogenous Medium}

Fine spiral reject was obtained from an eastern coal preparation plant located in eastern Kentucky. To obtain this material, the overflow of the high frequency screen currently treating the spiral reject was screened at $210 \mu \mathrm{m}$ (65-mesh) using a Sweco wet screen. Similar to the sand preparation, the underflow material was further screened at $44 \mu \mathrm{m}$. The $210 \mathrm{x}$ $44 \mu \mathrm{m}$ (65 x 325 mesh) fraction was retained while the +210 and $-44 \mu \mathrm{m}$ material from each screening step was discarded. 


\section{Steel Slag}

The steel slag by-product was obtained from a steel production company, the Stein, Inc., Ashland, Kentucky, USA. Steel slag is used for cement manufacturing, concrete aggregates, fill, and glass manufacturing and in agriculture as a soil amendment. Steel slag is hard, dense material and contains significant amounts of free iron which provides its high density, hardness and magnetic susceptibility. Depending on the cooling method, three types of slag are produced, namely air-cooled, expanded, and granulated. When the slag is formed under controlled rapid cooling, the slag tends to be hard and dense, making it suitable for the dense medium application. This type of steel slag is called 'C-type', which has a higher iron content and solid density than the other slag types. Several million tons of C-type steel slag is produced each year in the U.S. and available at a relative low cost. The steel slag material used in the DMC tests was ground in a cage mill and subsequently screened to reach a top size of $150 \mu \mathrm{m}$. According to a Davis Tube test, $64.1 \%$ of the steel slag was magnetic and recoverable using a magnetic field strength of 700 gauss.

\section{Magnetic Coal Combustion Byproducts}

Research conducted by Baird et al. (1998) in Australia suggested the fly ash derived magnetite (FAM) to have potential advantages in terms of lower cost to the end user, greater medium stability at low separation densities, and utilization of waste by-product of coal fired power plant. Similar research by Sripriya et al. (2003) using magnetic fraction of flue dust (MFFD) as a substitute for magnetite showed satisfactory results.

Problems associated with magnetic fly ash are that the particles are unstable in a suspension due to their sphericity and the particles require stronger magnetic strengths to recover. However, Baird et al. (1998) found that grinding the fly ash reduces the sphericity sufficiently to allow a stable medium within a dense medium cyclone. Additionally, the magnetic portions were selectively liberated and thus the magnetic field strength needed for recovery was significantly reduced. However, high-density magnetic particles in fly ash are already extremely fine and grinding creates a significantly high surface area and thus a suspension that may be stable in part due to elevated viscosity.

A potentially better source of magnetic particles is the bottom ash material produced from a pulverized coal boiler. The pneumatic transport of particles through the boiler naturally causes the higher-density particles to concentrate in the boiler ash stream. The composition of bottom ash indicates the presence of Fe-rich magnetic material in the form of spheres mostly fused with Si and Al. The spherical magnetic particles in the bottom ash are coarser than those in the fly ash and thus can be ground to decrease sphericity. Since the initial particle size is coarser than those contained in fly ash, medium viscosity should be lower for the ground bottom ash material.

The initial efforts involved identifying a bottom ash source in which magnetic material can be economically recovered and suitably used in a dense medium suspension .Similar attempts had been made in the past on commercial scale to recover magnetic material but were unsuccessful due to economic constraints and stability issues. There is insufficient amounts 
of magnetic material in the combustion ash to economically justify the recovery of this material as a single product. The economic constraints led to the alternative of incorporating magnetite recovery into a process flowsheet in which several products of combustion ash were recovered. A process flowsheet referred to as FastFloat process was developed at the University of Kentucky Center for Applied Energy Research (CAER), which utilized physical beneficiation for recovering various marketable products from combustion ash.

It has been noted in the past that high iron content in combustion ash is associated with utilities that burn high sulfur coal in turn leading to higher magnetite production. The site selection for magnetite recovery was limited to utilities that burned high sulfur coal. The criteria for site selection was firstly to use a substrate that contained sufficient amounts of magnetic material, and secondly to select a site which either practiced ash beneficiation to recover marketable products in some form or planned to do so in future.

The evaluation suggested the use of material from the Mill Creek utility. The reasons for the selection are the existence of a commercial scale beneficiation process and the high magnetite yield relative to the other test sites. A bottom ash processing facility is in operation at Mill Creek which produces 80,000 tpy of construction grade fill sand from bottom ash and discharges $-49 \mu \mathrm{m}$ (-100 mesh) fines as a reject stream. The addition of magnetite recovery into the process circuitry would enhance the overall economics of the beneficiation facility.

Mill Creek Station, a pulverized coal fired power plant, operated by Louisville Gas and Electric (LG\&E), began its commercial operation in 1972. It burns about 3.7 million tons of coal per year and is rated to produce $1717 \mathrm{MW}$ of power. All the units are fully scrubbed so as to meet the Clean Air Act's Phase II standards for sulfur-dioxide emissions. Bottom ash produced at this facility has historically been stored in an on-site storage pond along with hard coal and pyrite rejected from coal pulverisers. The bottom ash produced at this facility has higher density than that of other power plants, primarily attributed to the exclusive use of high sulfur coal fuel at this site. The high density of material combined with presence of pyrite and coal precluded the use of this ash as a lightweight aggregate, a product that has been produced at several other utility stations.

Market research by Charah Environmental Inc, an ash management company based in Louisville and LG\&E, identified that the material has a significant potential to be utilized as graded backfill for sanitary sewer construction projects in Louisville area. Bottom Ash is currently being processed at Mill Creek by Charah Environmental Inc., utilizing a process which was jointly developed with CAER. This facility has been in operation since 2002 and has successfully marketed about 500,000 tons of graded fill sand produced from the bottom ash at Mill Creek.

The boilers of the plant produced bottom ash which was sluiced into a trench that flowed to the ash pond. The trench was excavated periodically with a track hoe and the excavated material was stockpiled and allowed to drain. The material in the trench near the sluice entry point contained pyrite which is not suitable for beneficiation. So the other material was transported by front end loaders to a feeder hopper and conveyed to the process facility where the oversize (+3/8 inch) material was rejected. The $-3 / 8$ inch ash was dropped into a 
sump containing screw classifier to dewater the ash. The finished fill sand was conveyed to a

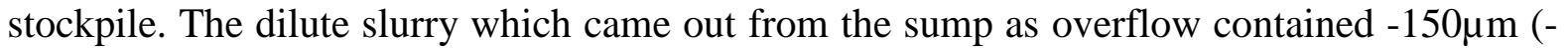
100 mesh) solids, which flow to a separate reject containment area. The material was periodically excavated from this area again using a track hoe and stockpiled so that the fine solids drained out. These stockpiled fines were being used as kiln feed by a nearby cement manufacturing plant named Cemex's Cosmos Plant. This material was used as substrate for magnetite recovery.

The stockpiled fines, which were used as substrate, contained high density magnetic material. The magnetic material was recovered using the process flowsheet as shown in Figure 4. A Bobcat loader was used to excavate the stockpiled fines and dump the material into a feeder hopper. The feeder hopper conveyed it into an agitated mix tank where water was added to make the pulp density $10 \%$ solids by weight. The slurry was fed to a single bank of concentrating spirals at a rate of $35 \mathrm{gpm}$ using a feed pump.

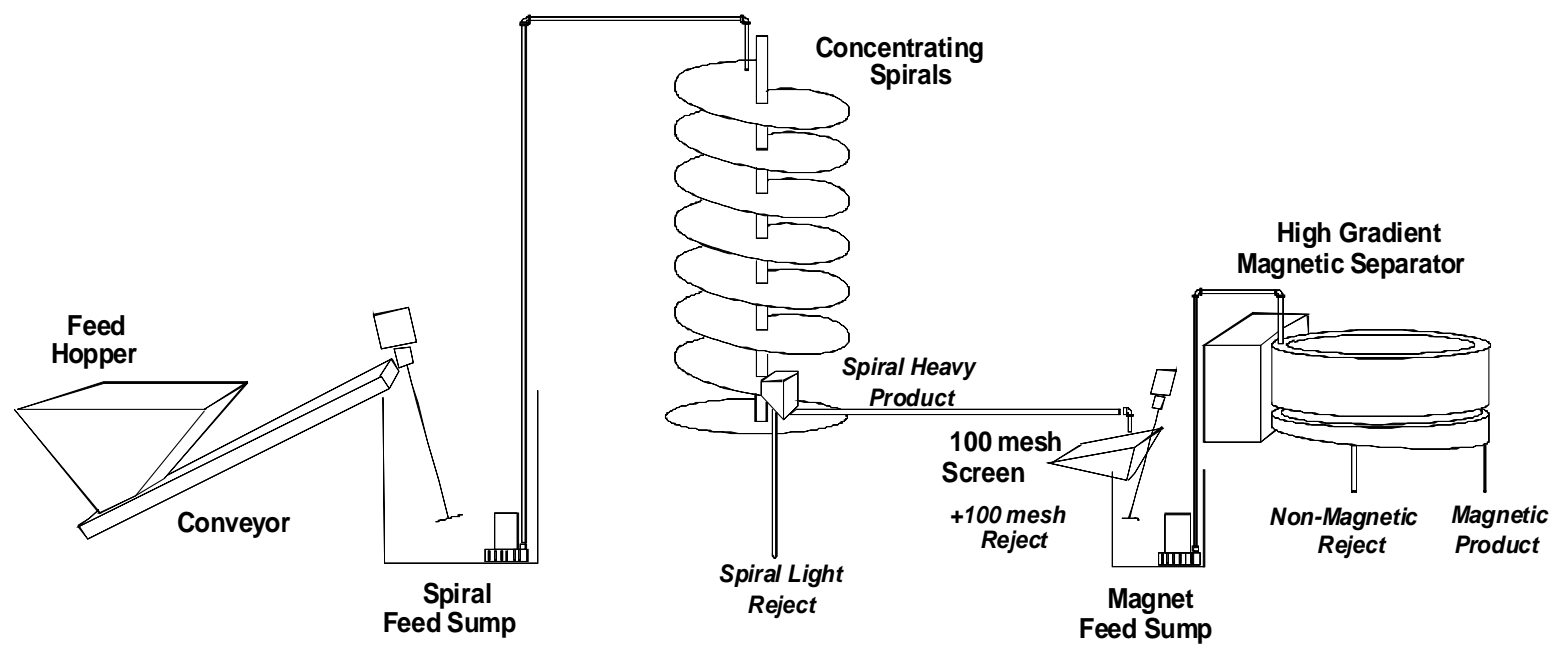

Figure 4. Magnetite recovery process flowsheet.

As the slurry flowed down the spiral, dense magnetite concentrated on the innermost section of race and was collected using a splitter at the bottom of the spiral. The light weight ash and water reported to the outer section of the race which was diverted back to the ash pond as reject. A $150 \mu \mathrm{m}$ (100 mesh) vibrating screen was used to remove the coarser particles from the spiral product. The screened magnetic material was fed to a continuous High Gradient Magnetic Seperator (HGMS) to remove the entrained non-magnetic material.

Samples were collected at each process stage to evaluate the incremental change in the magnetic content of the material. The results obtained during the recovery process are summarized in Figure 5. All the collected samples were dried and weighed, and a representative sample was subjected to Davis Tube Magnetic Separation (DTMS). The weight of material retained in the Davis Tube after two passes was defined to be magnetic. Figure 5 shows the grade of each stream, the incremental yield of each separation process and the overall yield as a weight percent of feed to the spirals. 


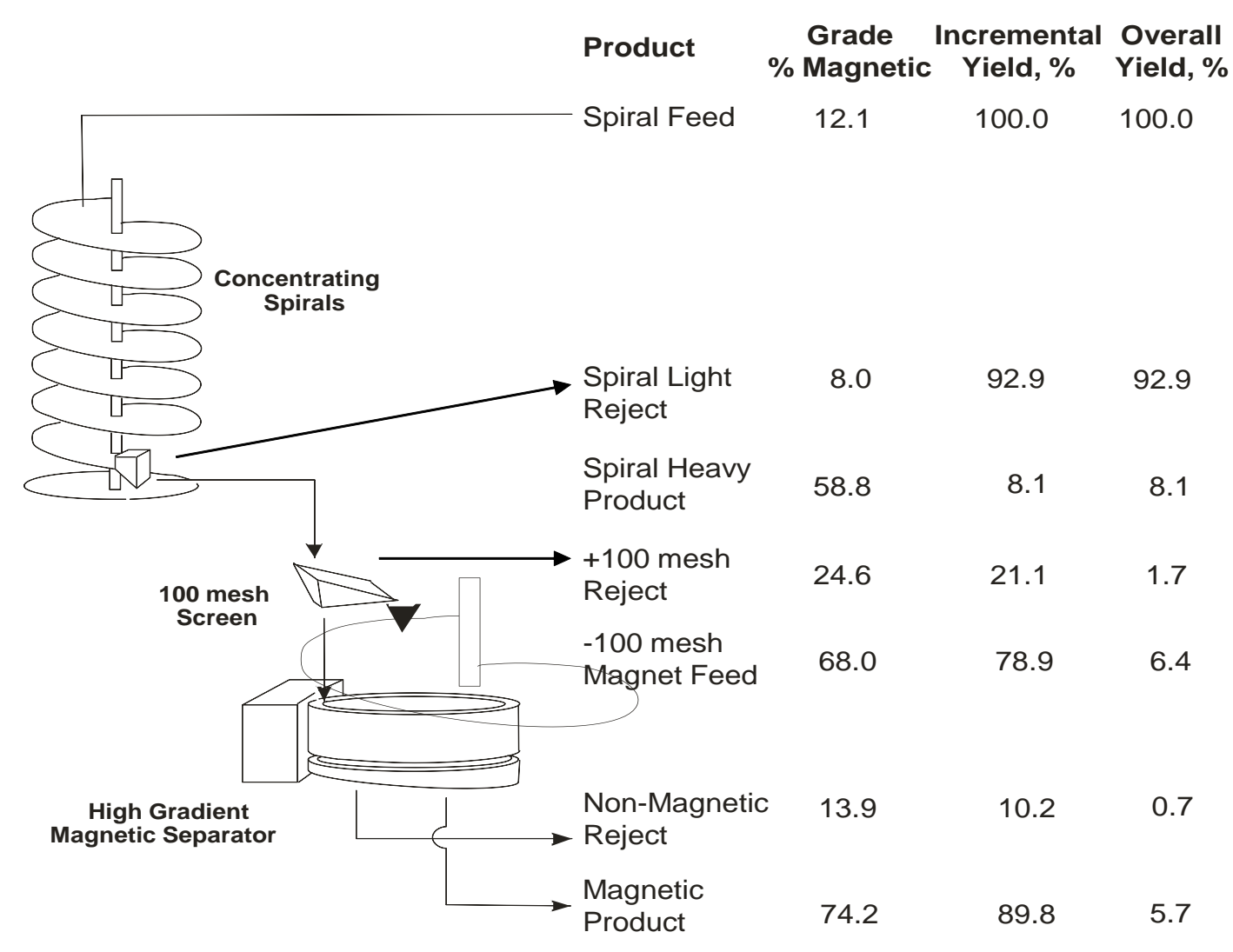

Figure 5. Material balance and magnetic content of the products produced by the magnetic recovery circuit.

The concentrating spiral upgraded the magnetic content from $12.1 \%$ to $58.8 \%$ and rejected a stream of low density ash and water with a magnetic content of about $8 \%$. The overall yield of classification process was $8.1 \%$. Further removal of $+150 \mu \mathrm{m}$ (100 mesh) particles led to the up-gradation of magnetics to $68 \%$ with an incremental yield of $78.9 \%$, resulting in an overall yield of $6.4 \%$. The final stage of magnetic upgrading using a HGMS gave a product which contained $74.2 \%$ magnetic in the recovered material. The overall yield of the magnetic bottom ash recovery process was $5.7 \%$ or 114 lbs per ton of process feed.

Bottom Ash Characterization: Three representative bottom ash samples were used to determine the average solid density. The samples were weighed and mixed into a measured volume of water. The mass of each sample divided by the incremental volume increase when added to the water was used to quantify solid density. The average density from three measurements was $3.44 \mathrm{~g} / \mathrm{ml}$.

Particle size analysis was performed on a representative sample of the HGMS product using a CILAS Particle Size Analyzer. As shown in Table 3, about 87\% of the recovered HGMS product had a particle size less than $74 \mu \mathrm{m}$. This is significantly coarser than conventional magnetite in which $90 \%$ of the particles are less than $44 \mu \mathrm{m}$. As a result of its relatively 
coarse particle size and its spherical shape, particle size reduction was required to obtain a stable medium.

Table 3. Particle size distribution of the magnetic HGMS product.

\begin{tabular}{||c|c|}
\hline $\begin{array}{c}\text { Particle Size } \\
\text { (micron) }\end{array}$ & $\begin{array}{c}\text { Weight } \\
(\%)\end{array}$ \\
\hline+150 & 4.59 \\
$150 \times 74$ & 50.16 \\
$74 \times 44$ & 32.71 \\
$44 \times 37$ & 5.61 \\
$37 \times 25$ & 5.07 \\
-25 & 1.86 \\
\hline
\end{tabular}

Ball Mill Grinding: The preliminary analysis indicated that the particle size distribution of the HGMS product needed to be altered to lower size fractions before its use for evaluation in dense medium separation. A ball mill circuit as shown in the Figure 6 was set up to grind the material. The material was dry fed using a belt feeder and mixed with calculated amounts of water to maintain the pulp density at $33 \%$ solids by weight. The active grind volume in the ball mill was calculated to be $12 \%$ of the total volume. The retention time of material in the ball mill was controlled by controlling the feed rate. A representative sample was collected to analyze the change in the particle size distribution as a result of grinding.

Retention time in the ball mill was defined as the time taken by a particle to travel across the ball mill from feed end to the product end. The ball mill circuit set up was operated in open circuit with no recycle. The retention time was directly controlled by the feed rate to the ball mill. Lower feed rates increased the retention time which in turn increased the chances of the material to be broken, and thus reduce the particle size. 


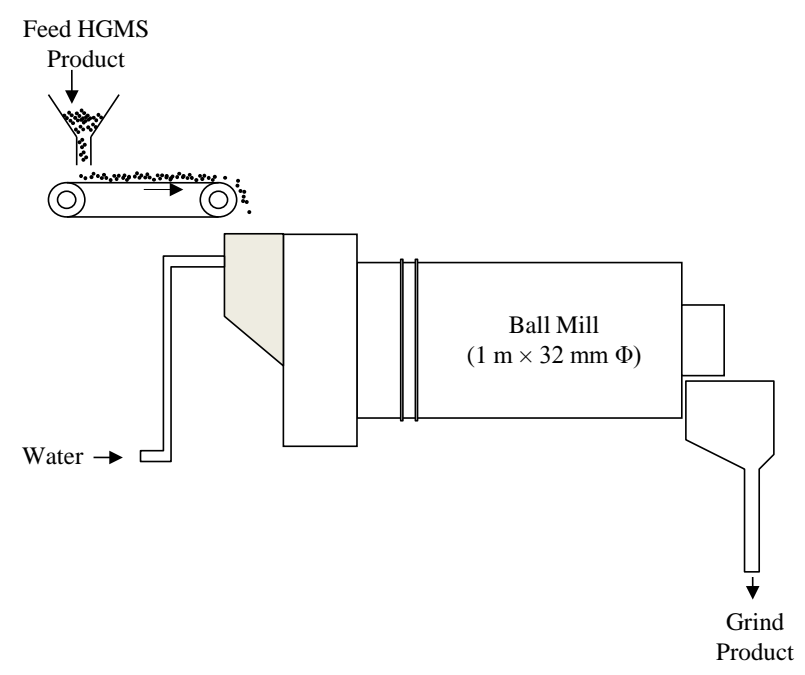

Figure 6. Schematic diagram of the ball mill circuit set up.

Figure 7 shows the cumulative passing of material as a function of particle size fraction for different grind variants produced as a result of grinding in the ball mill. Each grind variant represents a certain cumulative retention time in ball mill. Suspension stability was qualitatively assessed during the grinding trials. A residence time of 52 minutes was required to achieve good medium stability, which provided a mean particle size of about $22 \mu \mathrm{m}$ and a $\mathrm{d}_{90}$ of about $48 \mu \mathrm{m}$.

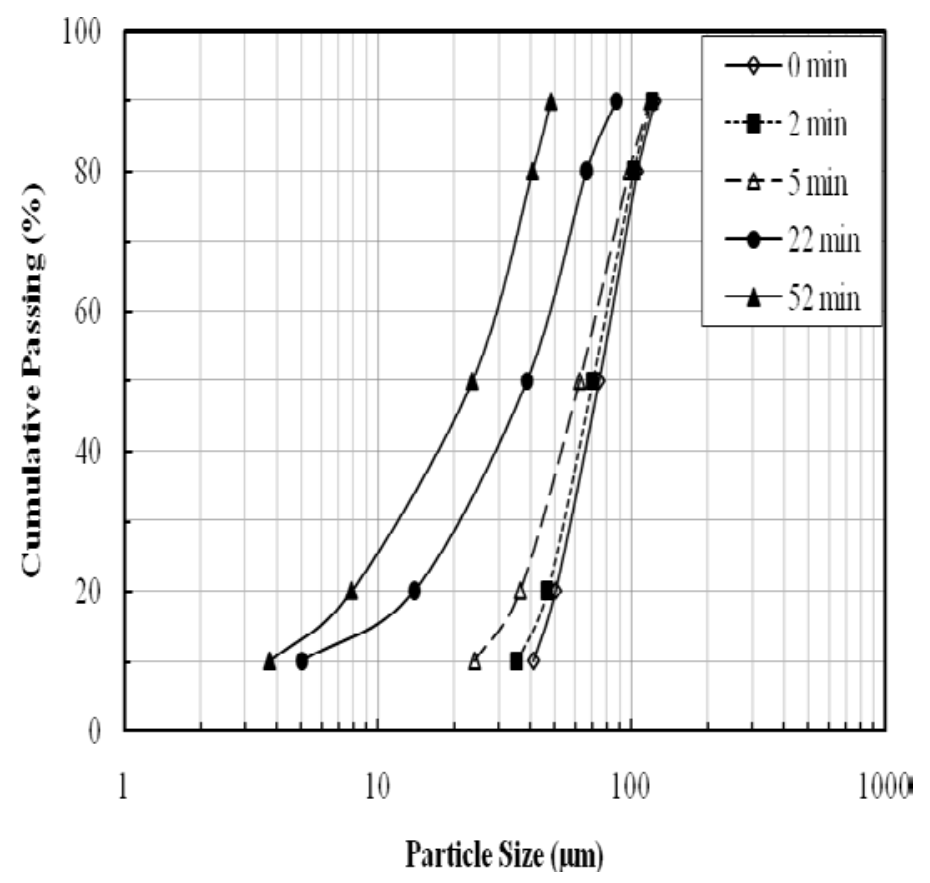

Figure 7. Change in particle size distribution with increased retention time in ball mill. 
The change in particle shape due to grinding is significant as indicated by the micrographs in Figure 8. As shown, the sphericity of the particles was significantly reduced.

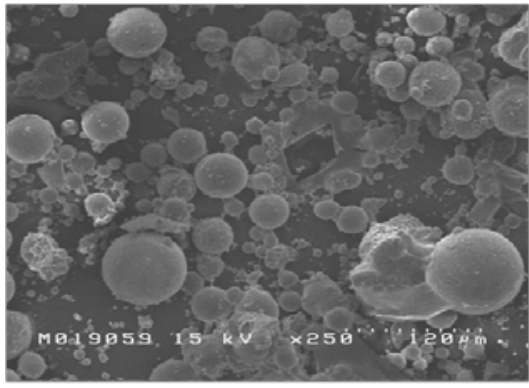

Original

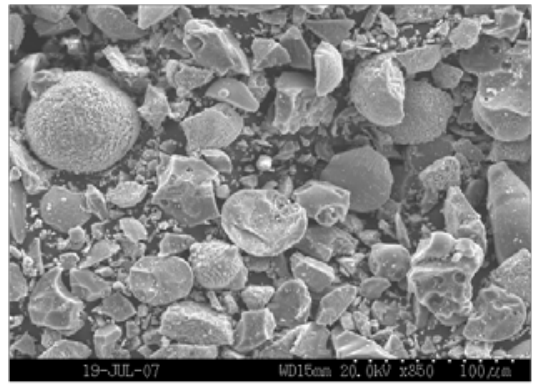

10 min grind

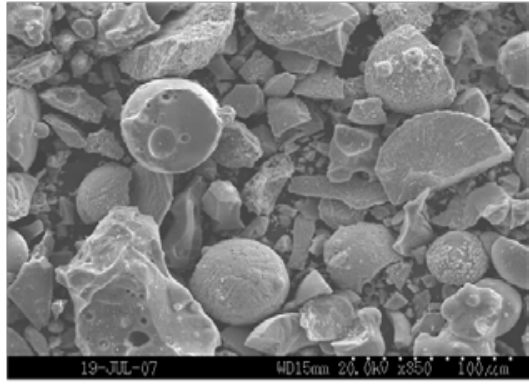

5 min grind

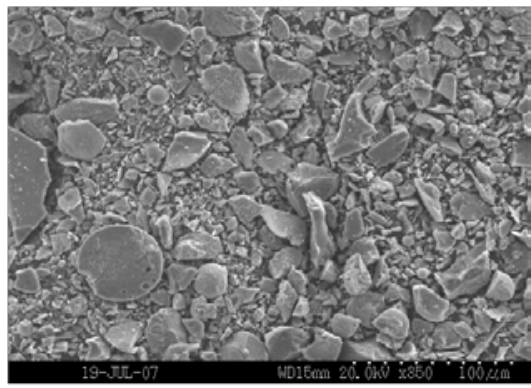

15 min grind

Figure 8. Effect of grinding on particle shape of the magnetic coal combustion byproduct..

\section{RESULTS AND DISCUSSION}

\section{$\underline{\text { Reference Magnetite Performance }}$}

Prior to the evaluation of each alternative material, tests were performed using the grade B magnetite. Medium stability was evaluated in the absence of coal by measuring the difference in the pulp density of the overflow and underflow streams of the DMC under inlet pressures ranging from 34.5 to $68.9 \mathrm{kPa}$ (2.5 to $10.0 \mathrm{psi}$ ). Generally, optimum separation performance is achieved when the relative density difference is maintained at or below 0.40 density units. As shown in Figure 9, stability of the conventional grade B magnetite suspension improved significantly with an increase in medium density and a decrease in inlet pressure. The trend associated with medium density is due to an increase in particle population, which reduces the hindered settling rates of the magnetite particles. Although hindered settling is beneficial for medium stability, another effect realized at high medium densities is an elevation in medium viscosity, which has negative impacts on separation performance. 


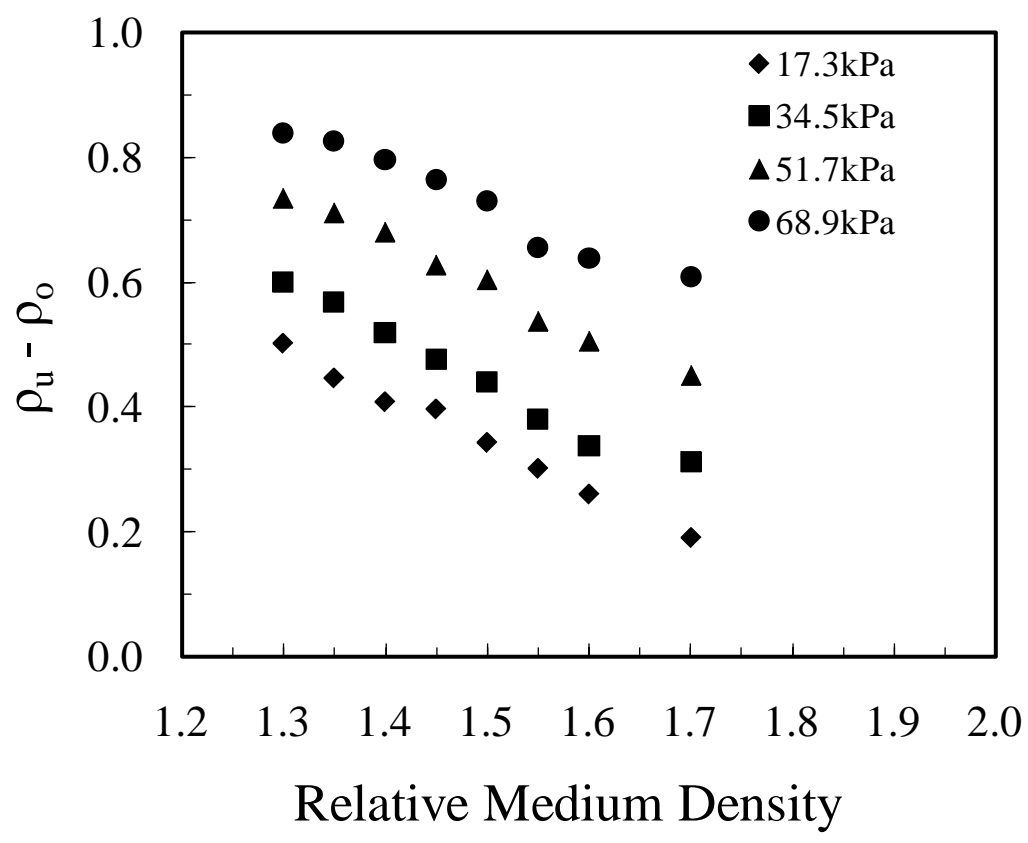

Figure 9. Medium stability achieved by grade B magnetite.

The tests involving the cleaning of the Coalburg coal were conducted over a range of medium density values and inlet pressures. The separation performances achieved using four different medium density values at an inlet pressure of $34.5 \mathrm{kPa}$ are represented by partition curves in Figure 10. The sharpest curves and thus highest separation efficiencies were obtained when using medium densities of 1.5RD and 1.6RD which correspond to improved medium stability as indicated in Figure 9.

The stability of the magnetite suspension when using a medium density of 1.4RD does not meet industrial standards $\left(0.4<\rho_{\mathrm{u}}-\rho_{\mathrm{o}}\right)$ which is likely due to slightly elevated centrifugal forces in the $150 \mathrm{~mm}$ cyclone used in the study. As such, the partition curve is less sharp. Likewise, a lower efficiency is indicated by the $1.7 \mathrm{RD}$ data in Figure 10. In this case, the reduced efficiency is most likely due to elevated medium viscosity which provides a resistance to particle movement. The level of coal cleaning achieved at each medium density is shown in Table 4 . The product ash values achieved when using the reference magnetite ranged from $9.36 \%$ to $14.89 \%$. The large range in mass yield and recovery values is reflective of the difficult cleaning characteristics of the Coalburg coal. 


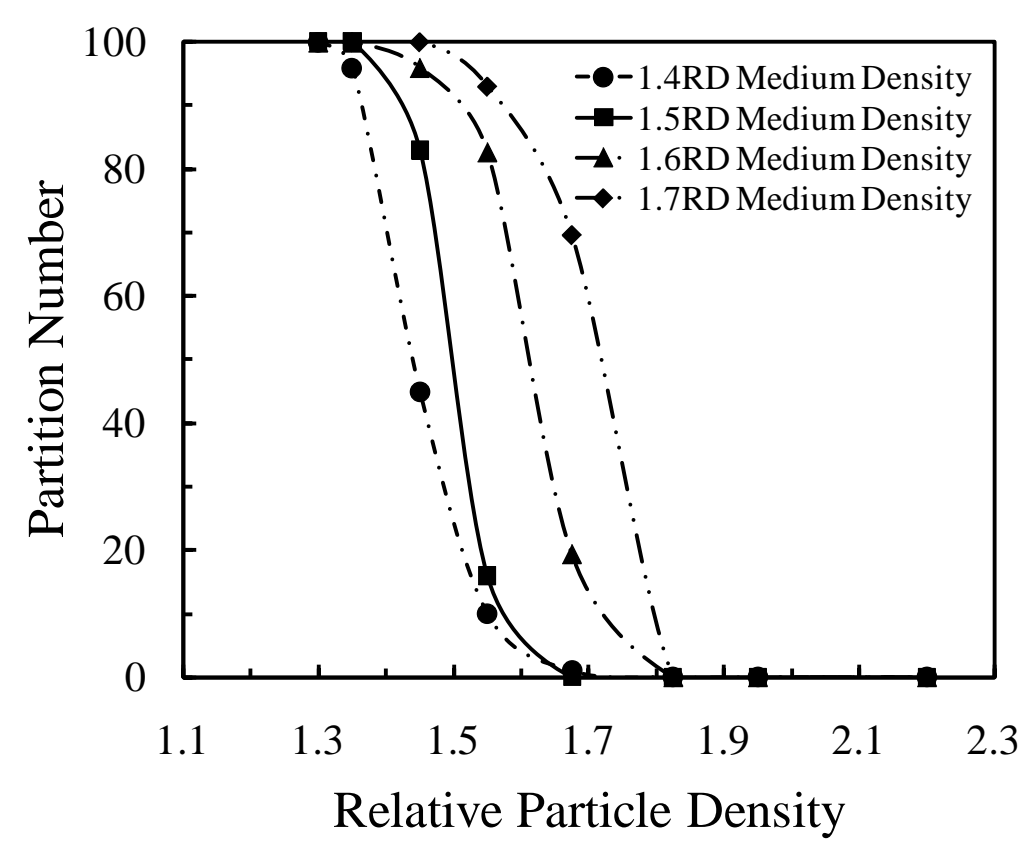

Figure 10. Partition curves achieved using the reference magnetite.

Table 4. Overall separation performances achieved with reference magnetite.

\begin{tabular}{|c|c|c|c|c|}
\hline \multirow{2}{*}{ Variable } & \multicolumn{4}{|c|}{ Relative Medium Density } \\
\cline { 2 - 5 } & 1.4 & 1.5 & 1.6 & 1.7 \\
\hline Feed Ash (\%) & 33.89 & 35.05 & 36.73 & 33.81 \\
Product Ash (\%) & 9.36 & 11.06 & 13.81 & 14.89 \\
Tailings Ash (\%) & 61.41 & 65.71 & 75.29 & 76.41 \\
\hline Yield (\%) & 52.9 & 56.1 & 66.0 & 69.3 \\
Recovery (\%) & 72.5 & 76.9 & 87.5 & 89.0 \\
Organic Efficiency (\%) & 91.3 & 92.9 & 95.7 & 96.7 \\
\hline$\rho_{50}$ & 1.440 & 1.525 & 1.615 & 1.73 \\
$\mathrm{E}_{\mathrm{p}}$ & 0.041 & 0.045 & 0.061 & 0.62 \\
$\rho_{50}-\rho_{\text {medium }}$ & 0.040 & 0.020 & 0.015 & 0.03 \\
\hline
\end{tabular}

\section{Alternative Medium Performance}

\section{Sand, Autogenous and Coarse Magnetite Medium}

Stability and rheology are the two principal medium properties affecting DMC performance and both are strongly dependent on the physical characteristics of the solids comprising the medium. As expected, the coarse magnetite provided very unstable medium with a highly concentrated underflow and a much diluted overflow. However, acceptable stability standards were achieved as the medium density was increased beyond $1.7 \mathrm{RD}$. The 
alternative materials (sand, steel slag and autogenous mediums) provided better medium stability than the reference magnetite. As shown in Figure 11, the density differentials at an inlet pressure of $34.5 \mathrm{kPa}$ ( $5 \mathrm{psi}$ ) were much lower than that of the reference magnetite over the entire medium density range studied. This was mainly due to the lower particle density of the alternative materials which required the addition of a greater volume of material to achieve the same medium density. High volumetric solid concentrations induce hindered settling which aid stability but eventually initiates viscosity effects that negatively impact separation performance.

The higher level of medium stability appeared to be the key in reducing the density offset. Under most conditions, the separation densities provided by the alternative material mediums were nearly equal to the medium density. On the other hand, the density offsets achieved by the reference magnetite-based medium were similar to the typical industrial standards for dense medium cyclones.

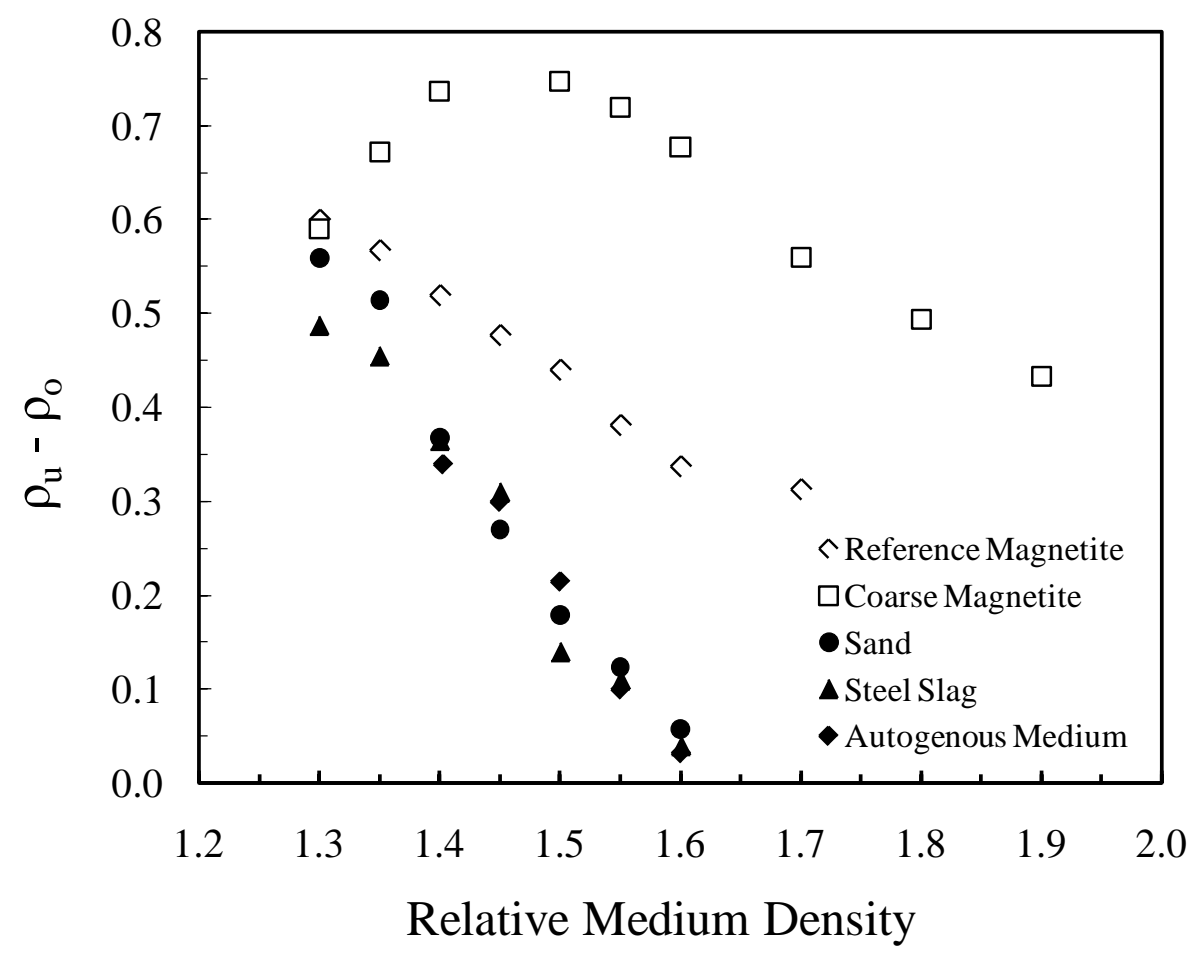

Figure 11. Medium stability as a function of alternative material and medium density at $34.5 \mathrm{kPa}$ (5 psi) feed inlet pressure.

Typical medium densities used in the U.S. coal industry to produce steam coal products are between 1.5 RD and 1.6 RD. As shown in Figures 12 and 13, the separation performances achieved by the alternative materials at 1.5 and 1.6 medium densities were comparable to the reference magnetite with $E_{p}$ values of 0.02 to 0.05 . The partition curves generated by the alternative materials at 1.5 medium density were much sharper than the 1.6 medium density. This suggested the impact of negative viscosity issues at the higher medium densities with the exception of coarse magnetite. 


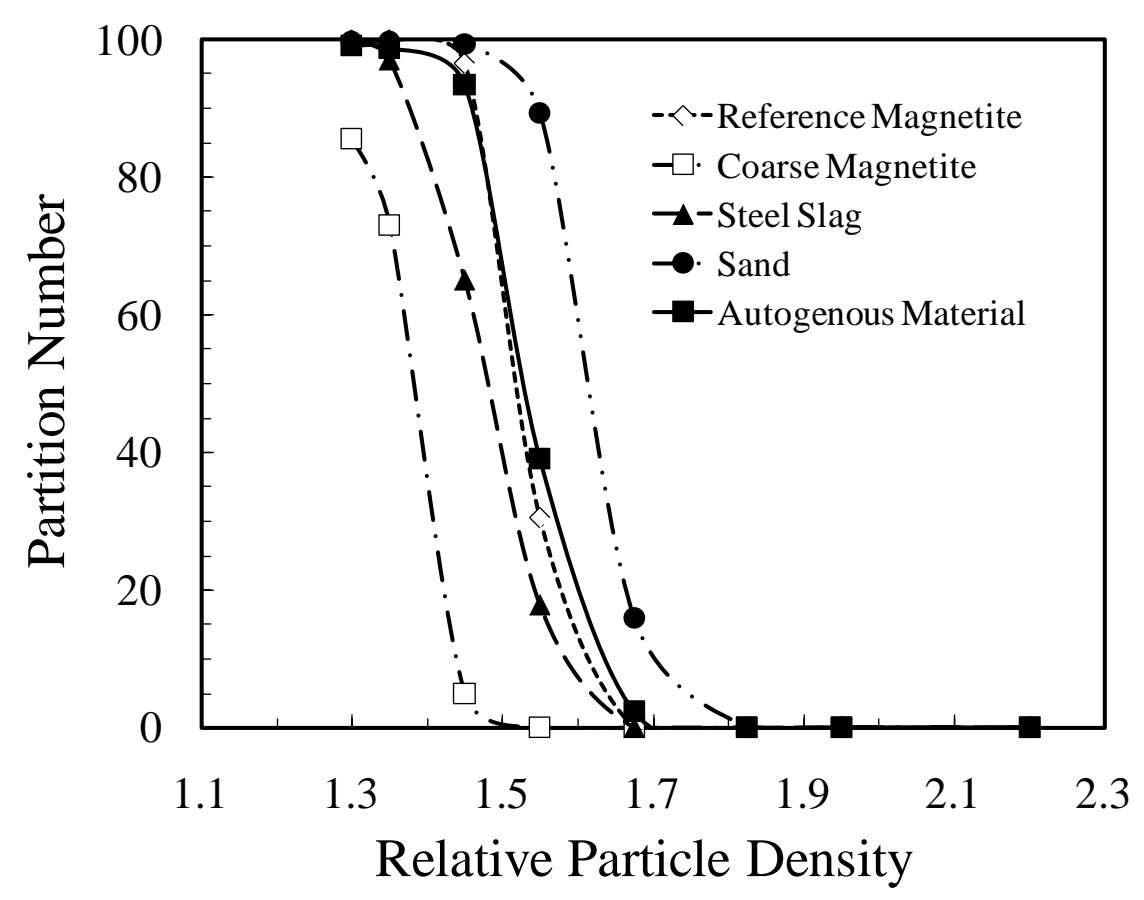

Figure 12. Comparison of separation performance achieved by alternative materials using a medium density of 1.5RD and feed inlet pressure of 34.5kPa (5 psi).

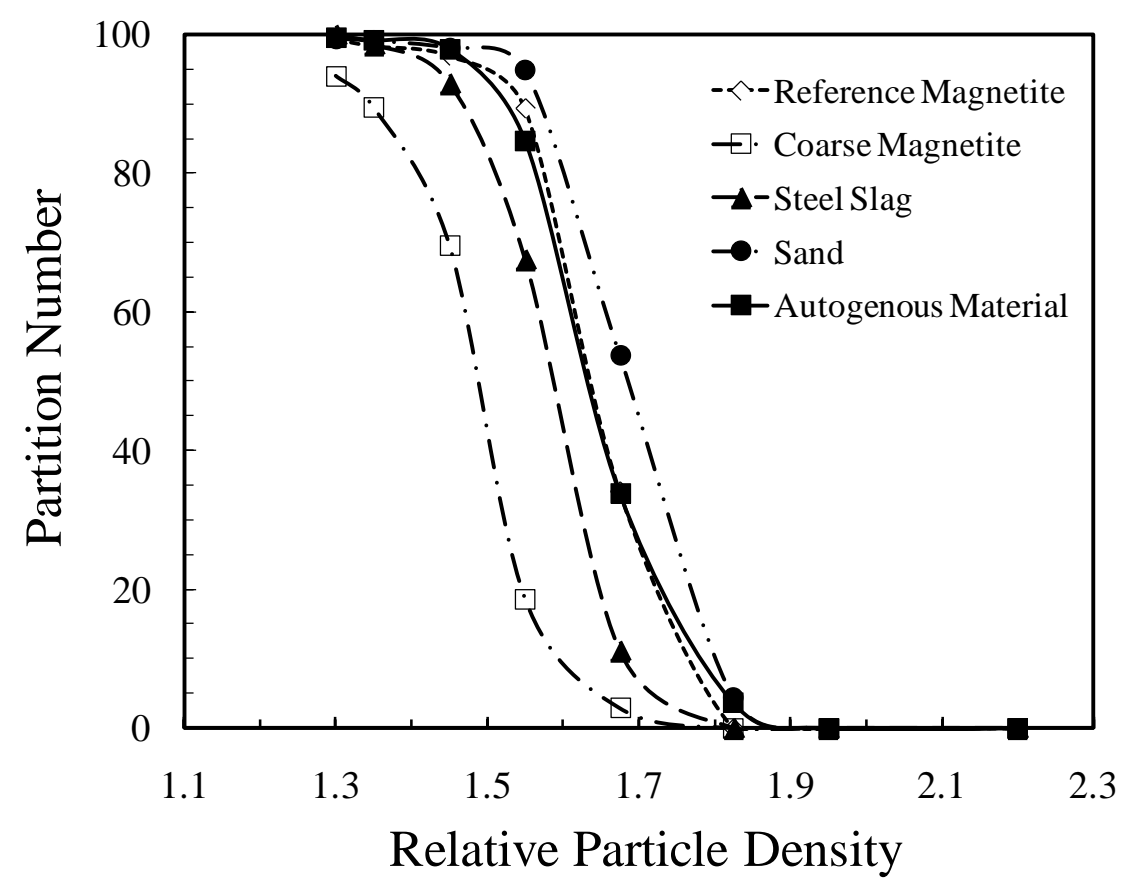

Figure 13. Comparison of separation performance achieved by alternative materials using a medium density of $1.6 \mathrm{RD}$ and an inlet pressure of $34.5 \mathrm{kPa}$ (5 psi). 
With dense media consisting of sand, steel slag, autogenous medium and reference magnetite, the Ep values tend to increase with medium density as expected due to viscosity effects (Figure 14). Surprisingly, the separation efficiency achieved by sand, steel slag and autogenous dense mediums provided $E_{p}$ values similar to the reference magnetite despite their significantly larger particle size distribution. It is believed, that at low medium densities, the relative coarseness of the sand, steel slag and autogenous mediums minimizes the viscosity effects and provides a stable medium due to hindered settling. At high medium density values, separation performance deteriorated due to medium viscosity and yield stress issues. For medium density values greater than 1.6RD, the coarse magnetite seems to become a more attractive option due to a stable, non-viscous medium.

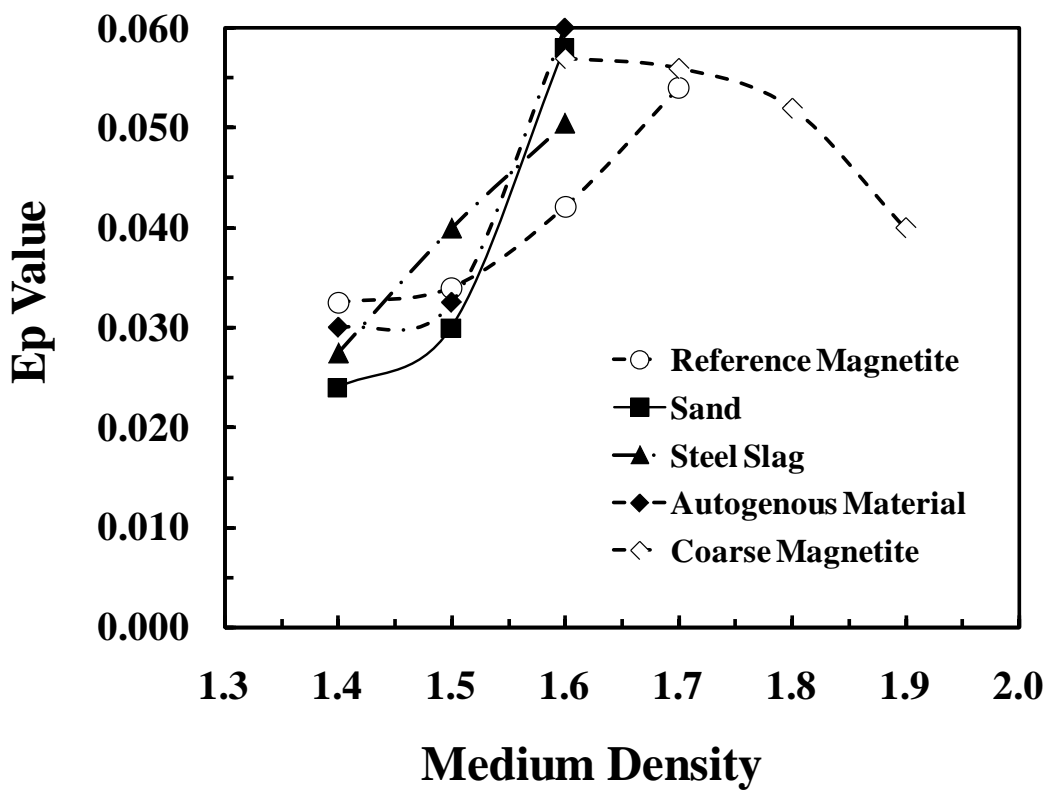

Figure 14. Comparison of the probable error values achieved by the various medium types using an inlet pressure of $34.5 \mathrm{kPa}$ (5 psi).

A summary of selected optimum performances achieved by the alternative materials is provided in Table 4. These performances were near perfect as indicated by the organic efficiency values (= actual yield/theoretical yield*100). One may question the performance achieved by the reference magnetite since the organic efficiency and $E_{p}$ values fall below standards that are commonly achieved in industrial practice. However, the performances obtained using the non-magnetite sources are consistent and would be acceptable in an operating preparation plant. Using coarse magnetite below 1.6RD is not acceptable due to medium stability issues. However, if a plant is often required to vary the medium density from low to high values, there may be a scenario where coarse magnetite provides an optimum solution. As shown in Table 4, the coarse magnetite provides a very good separation performance at a medium density of 1.9RD. 
Table 5. Optimized dense medium separation performances achieved by the alternative materials.

\begin{tabular}{|c|c|c|c|c|c|}
\hline \multirow{2}{*}{ Variable } & \multicolumn{4}{|c|}{$1.5 \mathrm{RD}$} & $1.9 \mathrm{RD}$ \\
\cline { 2 - 6 } & Ref. Mag. & Sand & Steel Slag & $\begin{array}{c}\text { Autogenous } \\
\text { Medium }\end{array}$ & $\begin{array}{c}\text { Coarse } \\
\text { Magnetite }\end{array}$ \\
\hline Feed Ash (\%) & 35.03 & 33.64 & 33.54 & 36.65 & 39.09 \\
Product Ash (\%) & 11.06 & 10.59 & 8.57 & 9.64 & 18.45 \\
Tailings Ash (\%) & 65.71 & 66.32 & 62.35 & 67.88 & 87.15 \\
\hline Yield (\%) & 56.1 & 60.1 & 53.6 & 53.62 & 70.0 \\
Recovery (\%) & 76.9 & 78.2 & 73.7 & 76.49 & 93.7 \\
Organic Efficiency (\%) & 92.9 & 96.3 & 97.4 & 97.2 & 98.5 \\
\hline$\rho_{50}$ & 1.525 & 1.530 & 1.495 & 1.518 & 1.91 \\
$\mathrm{E}_{\mathrm{p}}$ & 0.045 & 0.042 & 0.040 & 0.033 & 0.040 \\
$\rho_{50}-\rho_{\text {medium }}$ & 0.02 & 0.03 & -0.005 & 0.018 & 0.01 \\
\hline
\end{tabular}

\section{Magnetic Coal Combustion Byproduct}

Stability: The medium formed by the original recovered material was unstable with density differential values being over 0.8 density units. As shown in Figure 15, medium stability deteriorated with an increase in medium density. The shape of the bottom ash particles was observed to be spheroidal with a relatively coarse particle size distribution when compared to

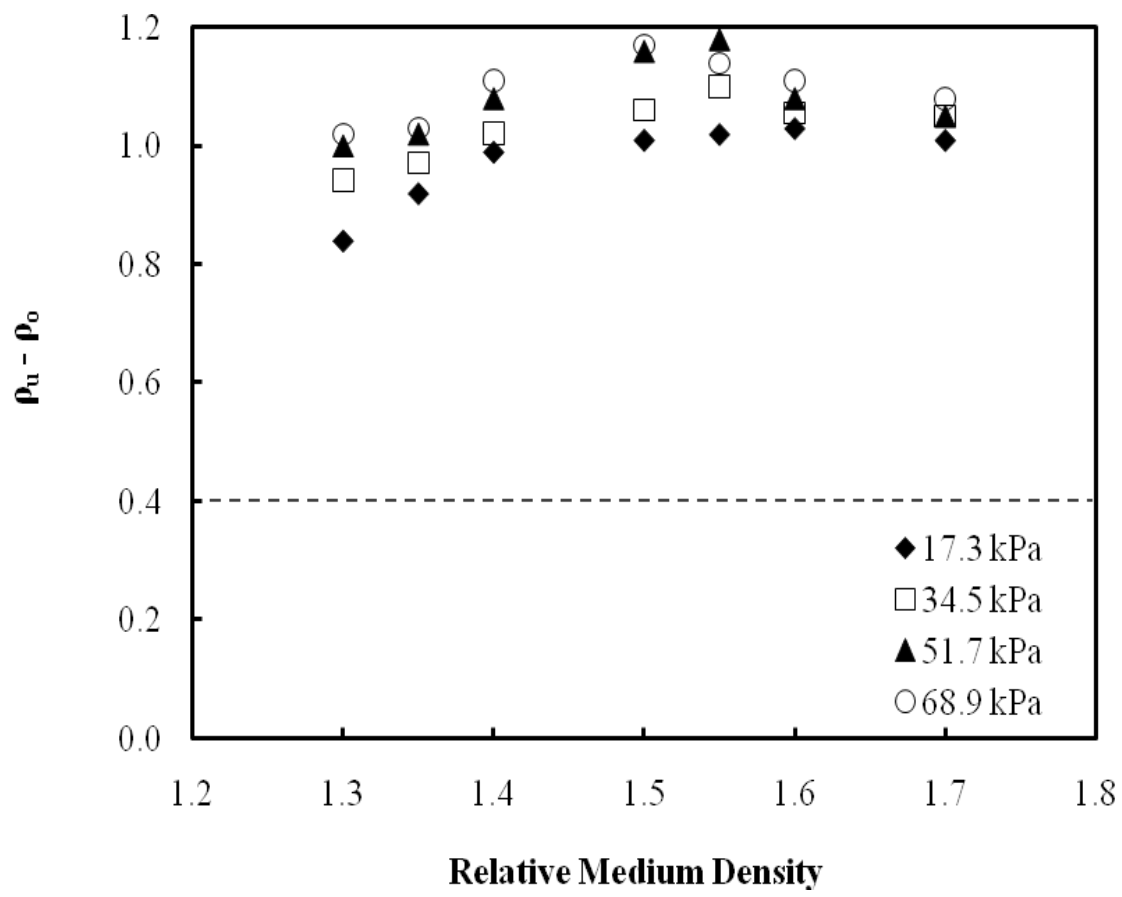

Figure 15. Medium stability achieved using the un-ground magnetic bottom ash. 
conventional magnetite used for coal cleaning. Spheroidal particles tend to exhibit lower drag forces and viscosities for a given particle density and size than irregularly shaped material (Collins et al., 1974). Another effect observed was that an increase in the inlet pressure to the cyclone had a negative impact on the stability at medium density less than $1.6 \mathrm{RD}$. The increase in inlet pressure elevated the centrifugal forces in the cyclone resulting in medium classification. At a relative medium density over $1.6 \mathrm{RD}$, pressure change had almost no effect on stability of the medium. This can be attributed to the combined role of increase in medium viscosity due to the high particle concentration and elevated hindered settling effects.

The medium formed using the ground bottom ash material was observed to be significantly more stable with relative density differences being within the industrial acceptable levels of around 0.4 density units and less (Figure 16). With increase in relative medium density, the suspension provided for improved stability. The particle size distribution of the ground material is nearly equal to that provided by conventional grade B magnetite used for coal cleaning. Enhanced stability with an increase in medium density occurs due to the development of hindered settling conditions as a result of an increased particle population. Also, elevated viscosity also helps to stabilize the suspension.

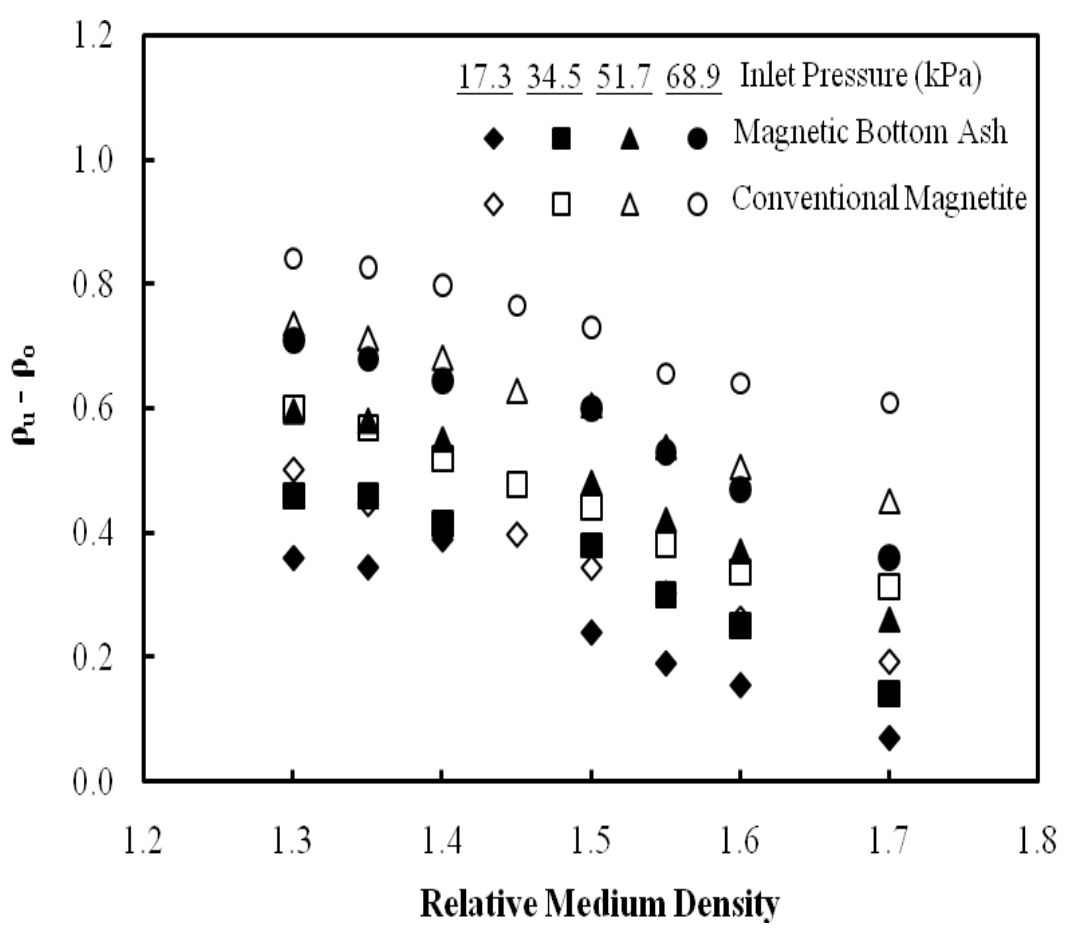

Figure 16. Comparison of medium stability achieved by ground bottom ash and grade 'B' magnetite. 
Dense medium cyclone tests were conducted to assess the separation efficiency achievable when using the magnetic bottom ash. Washability analyses were performed on samples collected from the clean coal, reject and feed process streams. The data was used to develop the partition curves shown in Figure 17 over a range in medium density values from 1.4RD to $1.7 \mathrm{RD}$.

A sign of medium stability is a low density offset, which is the difference between the separation density $\left(\rho_{50}\right)$ and the medium density. At medium density values of 1.4RD and $1.5 \mathrm{RD}$, the density offset was relatively high with values around 0.08 density units. At 1.6RD, the offset drops to 0.04 density units indicating medium stabilization.

Separation efficiency is measured by the slope of the partition curve which is typically quantified by the probable error value (i.e., $\mathrm{Ep}=\left[\rho_{25}-\rho_{75}\right] / 2$ ). Bypass of low-density particles to the reject stream and vice versa are also a sign of inefficiency. A parameter that combines the efficiency associated with the probable error and bypass is the organic efficiency. Organic efficiency is the ratio of the actual energy recovery to the theoretical energy recovery at a given product quality or product ash content. A summary of the separation performances represented in Figure 17 is shown in Table 6.

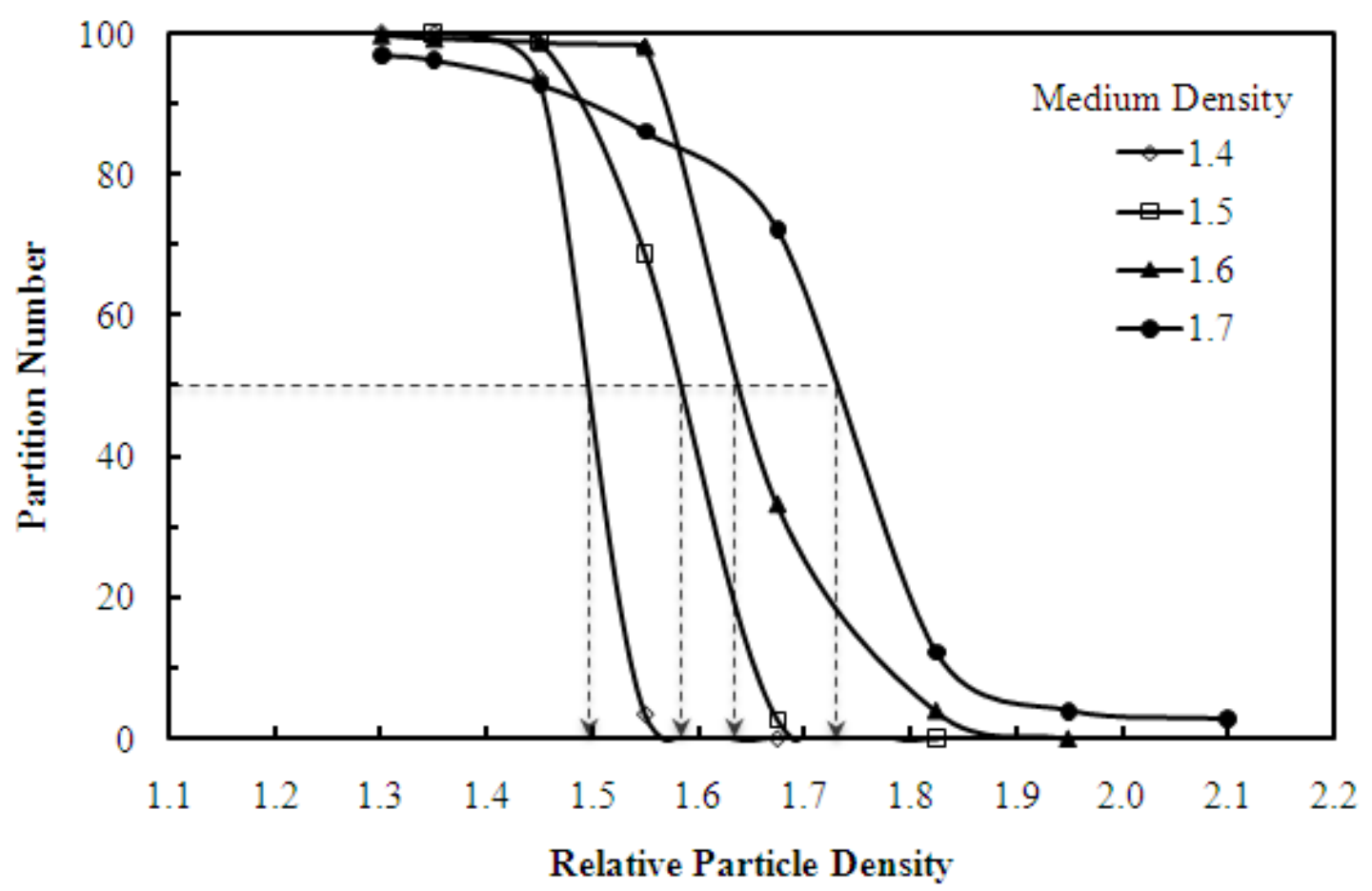

Figure 17 Partition curves generated over a range of medium density values using magnetic bottom ash. 
Table 6. Summary of separation performances achieved using the magnetic bottom ash at an inlet pressure of $34.5 \mathrm{kPa}$.

\begin{tabular}{||c|c|c|c|c||}
\hline \multirow{2}{*}{ Variable } & \multicolumn{4}{|c|}{ Relative Medium Density } \\
\cline { 2 - 5 } & 1.4 & 1.5 & 1.6 & 1.7 \\
\hline Feed Ash (\%) & 40.84 & 40.30 & 33.82 & 32.58 \\
Product Ash (\%) & 6.11 & 8.82 & 11.7 & 15.43 \\
Tailings Ash (\%) & 56.44 & 58.28 & 57.82 & 56.29 \\
\hline Yield (\%) & 31.00 & 36.36 & 52.04 & 58.03 \\
Recovery (\%) & 49.19 & 55.53 & 69.43 & 72.79 \\
Org. Efficiency (\%) & 96.81 & 98.92 & 95.49 & 89.94 \\
\hline$\rho_{50}$ & 1.495 & 1.583 & 1.640 & 1.733 \\
$\mathrm{E}_{\mathrm{p}}$ & 0.023 & 0.044 & 0.051 & 0.063 \\
$\rho_{50} \rho_{\text {medium }}$ & 0.095 & 0.083 & 0.040 & 0.033 \\
\hline \multicolumn{4}{|l|}{}
\end{tabular}

The separation efficiencies measured for the medium densities of $1.4 \mathrm{RD}$ and $1.5 \mathrm{RD}$ are excellent with organic efficiency values around $97 \%$ and probable error values of 0.04 and less. As the case with conventional magnetite, the probable error increases with an increase in medium density which represents a decline in efficiency due to medium viscosity impacts. The probable error values are comparable to those achieved when using conventional magnetite.

A trend that is atypical is the decline in organic efficiency with an elevation in medium density. In most cases, the amount of near gravity material in the feed decreases with an increase in medium density. As a result, organic efficiency generally improves with an increase in medium despite the declining efficiency caused by medium viscosity. For the coal in this study, a peak in middlings content occurs at a density around 1.75 which causes the inefficiencies quantified by the probable error values to by magnified in the determination of organic efficiency.

Magnetic Bottom Ash Recovery: In addition to DMC separation performance, magnetic recovery is another important issue in development of recovered magnetic material as an alternative to magnetite. Magnetic content of the initial recovered material was $74 \%$. After grinding the bottom ash material, the magnetic components were selectively liberated. This action reduced the amount of magnetic particles in the original feed to $54 \%$. However, the magnetic strength needed to recover the material was significantly reduced as shown in Figure 18. The magnetic strength needed is about 1000 gauss to recover $98 \%$ of the material. The typical magnetic field strength provided by the low-intensity wet drum commonly employed in the coal industry is 750 gauss. 


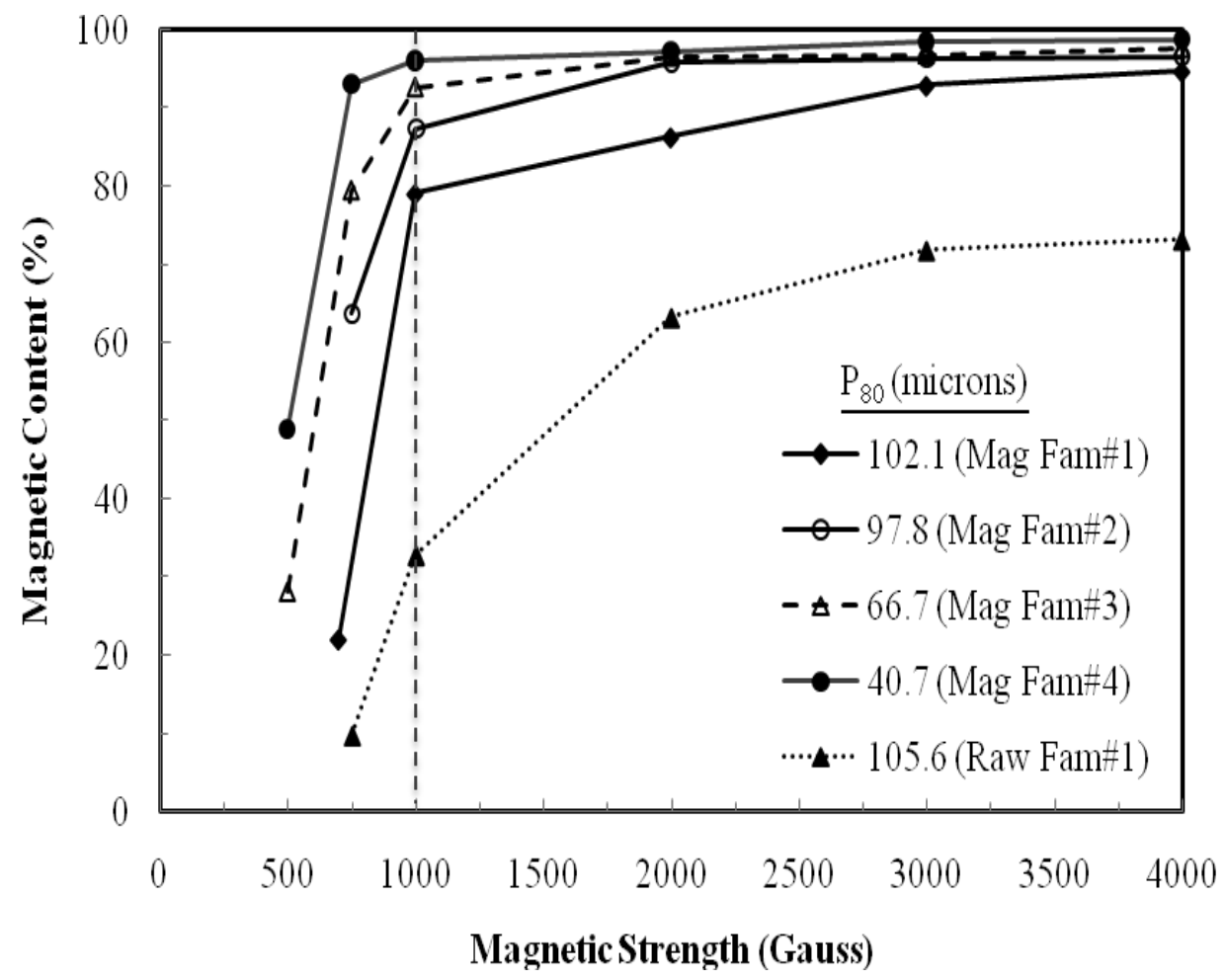

Figure 18. Magnetic intensity needed to recover the magnetic particles over a range of particle sizes.

\section{CONCLUSIONS}

Due to the global demand for iron ore over the last decade, concerns have developed regarding the cost and stable supply of magnetite for use in dense medium separations. In response, a study has been conducted to evaluate the feasibility of using alternative materials to replace magnetite in dense medium processes. Alternative materials included a lower cost, coarse magnetite, steel slag waste, fine sand, autogenous reject material and magnetic bottom ash. The autogenous material was obtained by recovering the finest size fractions from the spiral tailings stream. The evaluations were performed using a $150 \mathrm{~mm}$ diameter dense medium cyclone treating $6 \times 0.6 \mathrm{~mm}$ Coalburg coal over a range of medium densities and feed inlet pressures.

The magnetic bottom ash material was recovered from an electric utility that utilized a feed coal containing a relatively high amount of pyritic sulfur. As a result, the use of a spiral concentrator and a high gradient magnetic separator collected 5.7\% of the total bottom ash having some degree of magnetic susceptibility. However, the sphericity of the -100 mesh material created a very unstable medium that required grinding to alter the particle shape. The result was $90 \%$ of magnetic bottom ash having a particle size less than 50 micrometer. In addition, the magnetic components were selectively liberated which resulted in $54 \%$ of the material being magnetic. The magnetic susceptibility of the magnetic portion was 
significantly improved as indicated by the ability to recover $98 \%$ of the magnetic particles using a relatively weak field strength of 1000 gauss.

With the exception of the coarse magnetite, the dense medium generated using the alternative materials provided superior medium stability when compared to the medium produced from conventional magnetite. This finding was a result of the lower solid density values of the alternative materials which resulted in the need to use greater amounts of the alternative materials to obtain a desired medium density. The higher mass and, more importantly, volume concentrations led to elevated particle populations and thus reduced particle settling velocities due to hindered effects. However, the positive impacts of improved medium stability were negated by a rise in medium viscosity as the medium density is increased. For coarse magnetite, a sufficiently stable medium was achieved only at relative medium density values above 1.7RD due to a need to reach a critical particle population for the development of hindered settling conditions.

The use of fine sand was investigated as an initial assessment of the potential to use the high density reject particles from a spiral concentrator as the material to form a dense medium. As a result of the stable medium formed when using sand, the density offset values (= separation density minus medium density) were positive and comparable to those realized using the conventional grade B magnetite. Organic efficiencies values achieved were greater than $95 \%$ at medium densities of $1.4 \mathrm{RD}$ and $1.5 \mathrm{RD}$ and decreased to $93 \%$ at $1.6 \mathrm{RD}$, which was likely due to the impact of medium viscosity. The performances were slightly better than those achieved by the magnetite-based medium at separation densities of 1.4 RD and 1.5 RD.

The solid density of the spiral reject material was lower than sand density which resulted in a larger amount needed to reach a desired medium density. As result, a coarser particle size distribution was utilized in an effort to minimize the negative impacts of medium viscosity. The autogenous medium provided low density offsets which resulted in lower product ash values than those realized from the magnetite-based medium. The probable error values achieved using the autogenous medium were lower throughout the medium density range of 1.4RD to 1.6RD indicating superior performance. However, a significant elevation in the probable error value occurred at a 1.6RD medium density which is indicative of medium viscosity issues.

Compared to all medium types, steel slag provided the most stable suspension. As a result, the separation density achieved using the steel slag was approximately equal to the medium. The separation efficiency achieved was also superior to all other medium types. The organic efficiency increased from $96.3 \%$ at a medium density of $1.40 \mathrm{RD}$ to $98.9 \%$ at a density of 1.6RD. However, the steel slag medium did experience the typical trend of a rise in the probable error value from 0.025 to 0.051 as the medium density was increased. The partition curves were notably sharp even at the tail ends of the curves. Laboratory Davis Tube tests found that $64 \%$ of the steel slag was magnetic. Of the magnetic portion of the steel slag, nearly $100 \%$ was recovered using a magnetic field strength of around 700 gauss. As such, current magnetite recovery circuits used in the coal industry could be used to recovery the steel slag if applied to dense medium separations. 
The separations achieved using the magnetic bottom ash particles are comparable to those achieved through the use of conventional magnetite. Probable error values ranged from 0.02 at a medium density of $1.4 \mathrm{RD}$ to 0.06 at $1.7 \mathrm{RD}$. For the bottom ash material used in this study, the upper medium density limit appears to be around 1.6RD. Above this value, medium viscosity appears to have a significant impact.

One of the key concerns with the use of coarse magnetite is the ability to maintain a stable suspension. However, an acceptable stable suspension was achieved in a dense medium cyclone at medium densities of 1.7RD and higher at an inlet pressure of $34.5 \mathrm{kPa}$. The stability trend agreed well with data reported by He and Laskowski [1995]. Relatively efficient separations were achieved throughout the medium density range of 1.4RD to 1.9RD despite the instability of the medium at the low density values. Organic efficiencies increased and the probable error values decreased as the medium density was elevated from 1.4RD to 1.9RD, which is the opposite trend observed for the conventional magnetite. This tendency is due to improved stability with little or no viscosity effects. At a $1.9 \mathrm{RD}$, the Ep value was 0.04 and the organic efficiency was $98.5 \%$.

\section{REFERENCES}

Baird, G. A., Hornsby D. T. and Lief, H., 1998, "Development of fly ash derived magnetite for coal cleaning”, 13th Proceedings, International Coal Preparation Conference, Vol. 1, pp. 314-321 (1998).

Birlingmar, D., Murtha, M., and Killmeyer, R., Characterization and Performance of Fly Ash-Derived Heavy Medium Material, DOE Report, IS-4839 (1983).

Bradley, D., The Hydrocyclone, Pergamon Press, Oxford (1965).

Burt, R. O., The Theory of Heavy Medium Separation in Gravity Concentration Technology, Developments in Mineral Processing, Elsevier, Amsterdam. vol. 5, pp.66-68 (1984a).

Davis, J. J., Cleaning Coarse and Small Coal - Dense Medium Processes, in Advanced Coal Preparation Monograph Series Part VIII, Australian Coal Preparation Society, vol. 3, pp. 138 (1994).

He, Y. B. and Laskowski, J. S., Separation of Fine Particles in Dense Medium Cyclone Effect of the Medium Yield Stress", in Proceedings of the 12th International Coal Preparation Congress, Krakow, Poland (1994).

He, Y. B. and Laskowski, J. S., "Dense Medium Cyclone Separation of Fine Particles: Part 2, The Effect of Medium Composition on Dense Medium Cyclone Performance”, International Journal of Coal Preparation, vol. 16, pp. 27-49 (1995).

Leonard, J. W., III and Hardinge, B. C., Coal Preparation, 5th Edition, Society for Mining Metallurgy and Exploration, Inc., Littleton, Colorado, pp 272-300 (1991). 
Luttrell, G.H., Barbee, C.J., Bethell, P.J. and Wood, C.J., “Dense medium optimization”, U.S. Department of Energy Technical Report (2005).

Mengelers, J., "The Influence of Cyclone Diameter on Separating Performance and Economy,” in Proceedings of the 9th International Coal Preparation Congress, New Delhi, India (1982).

Palowitch, R. R., Deurbrock, A. W., Tork, E., and Akers, D. J., 1991, Part 1, Wet Coarse Particle Concentration, Section 1: Dense Media, in Coal Preparation, 5th ed. (J.W. Leonard and B. C. Hardinge) Society for Mining, Metallurgy and Exploration, Littleton, Colorado, pp. 271- 273 (1991).

Shi, F. N. and Napier-Munn, T. J., A Model for Slurry Rheology, International Journal of Mineral Processing, vol. 47, pp. 103-123 (1996).

Sripriya, R., P. Rao, V. T., Bapat, J. P., Singh, N. P. and Das, P., Development of an Alternative to Magnetite for Use as Heavy Media in Coal Washeries, International Journal of Minerals Processing, vol. 71, pp. 55 - 71 (2003).

Zanker, A., Hydrocyclones: Dimensions and Performance, Chemical Engineering Journal, vol. 84, pp. 122-125 (1977).

\section{PUBLICATIONS/PRESENTATIONS}

Honaker, R. Q., Bimpong, C. and Bethell, P., "Alternative Materials for Dense Medium Separations," 136th SME Annual Meeting, Denver, Colorado, February 25 - 28, 2007. (presentation).

Honaker, R. Q. and Bimpong, C., “Alternative Materials for Dense Medium Separations,” Proceedings, $25^{\text {th }}$ International Coal Preparation Conference, Lexington, Kentucky, pp. 19 36, April 28- May 1, 2008, pp. 63 - 79.

Honaker, R. Q., Akram, Z. and Groppo, "Recovery and Utilization of Bottom Ash Magnetics for Coal Cleaning Medium,” Proceedings, Society of Mining, Metallurgy and Exploration Annual Meeting, February 2009, Preprint 09-02.

Honaker, R. Q., and C. Bimpong, “Alternative Materials for Dense Medium Separations,” International Journal of Coal Preparation and Utilization, Vol. 29, No. 4, pp. 173 - 191, 2009. 
Appendix 3: Enhanced Flotation Performance Through Column Froth Enrichment (KY006) 


\section{FINAL REPORT}

Contract Title and Number:

Crosscutting Technology Development at the Center

for Advanced Separation Technologies

(DE-FC26-02NT41607)
Period of Performance:

Starting Date: 10/1/2005

Ending Date: 10/31/10
Sub-Recipient Project Title:

Enhanced Flotation Performance Through Column

Froth Enrichment

Principal Investigators:

Honaker, Tao

Contact Address:

University of Kentucky

234B Mining and Mineral Resources

Lexington KY 40506

Subcontractor Address:

No subcontracts issued.
Report Information:

Type: Final

Number: 6

Period: 10/01/05-10/31/09

Date: $\quad$ 12/15/09

Code: KY006-FINAL

Contact Information:

Phone: (859) 257-1108

Fax: (858) 323-1962

E-Mail: rhonaker@engr.uky.edu

Subcontractor Information:

Phone:

Fax:

E-Mail:

\section{ABSTRACT}

Froth flotation is the most common process used for cleaning coal having a particle size below 150 micrometers. Although the process has been well researched and commercially applied for more than a century, several problems still exist including the flotation of weakly hydrophobic coals, the flotation of coarse (+150 micrometers) and ultrafine (-25 micrometers) coal, and selectivity for difficult-to-clean coals. In this investigation, feed pretreatment using hydrodynamic cavitation, the addition of hydrophobic plastic material and external refluxing a portion of the froth concentrate was evaluated to enhance flotation performance. Cavitation pretreatment nearly doubled the flotation rate and improved the combustion recovery achieved in a flotation column from $71 \%$ to $76 \%$. Using hydrophobic plastic particles in the feed and/or the froth phase improved flotation rates from $1.04 \mathrm{~min}^{-1}$ to $1.31 \mathrm{~min}^{-1}$ which provided an increase in combustible recovery by about 3 absolute percentage points under the same test conditions. Finally, enrichment of the selective detachment mechanism in the froth zone by providing external reflux had a positive impact on the selective detachment process and provided a reduction in the product ash content of nearly 3 absolute percentage points while maintaining the same recovery value. The improvement was realized at low and intermediate feed rates. 


\section{DISCLAIMER}

This report was prepared as an account of work sponsored by an agency of the United States Government. Neither the United States Government nor any agency thereof, nor any of their employees, make any warranty, express or implied, nor assume any legal liability or responsibility for the accuracy, completeness, or usefulness of any information, apparatus, product, or process disclosed, or represents that its use would not infringe privately owned rights. Reference herein to any specific commercial product, process, or service by trade name, trademark, manufacturer, or otherwise does not necessarily constitute or imply endorsement, recommendation, or favoring by the United States Government or any agency thereof. The views and opinions of authors expressed herein do not necessarily state or reflect those of the United States Government or agency thereof. 


\section{TABLE OF CONTENTS}

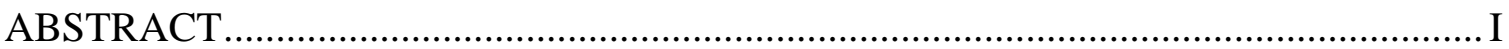

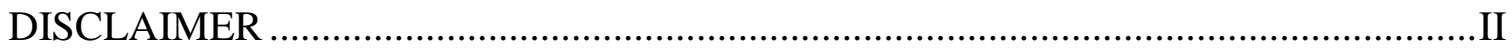

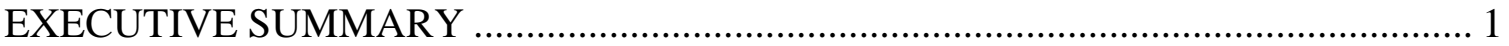

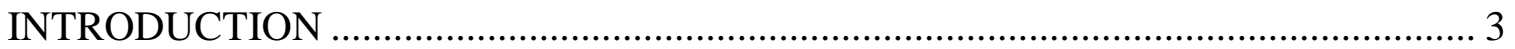

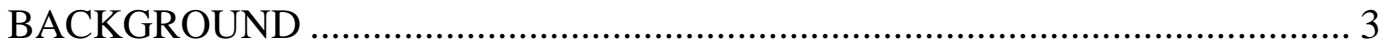

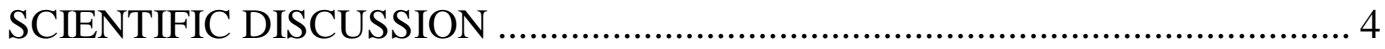

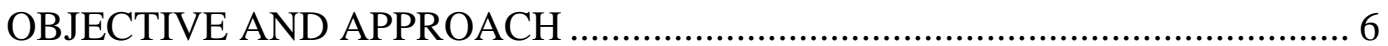

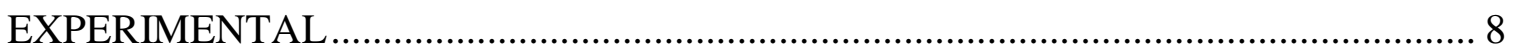

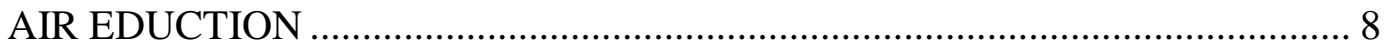

Sample Characterization ......................................................................... 8

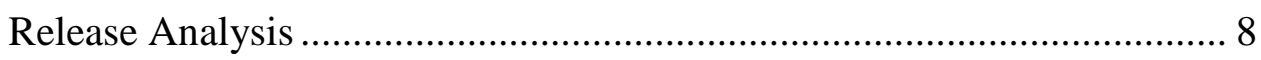

Flotation Rate Test............................................................................. 9

Column Flotation Test ........................................................................... 10

Air Eductor Pretreatment .......................................................................... 11

In-Plant Conventional Flotation Tests ........................................................ 12

MAGNETIC PLASTIC EVALUATION ............................................................... 13

Sample Characterization ........................................................................... 13

Magnetic Plastic Material ..................................................................... 14

Contact Angle Analysis ........................................................................... 16

Zeta Potential Measurements ................................................................. 18

Density Fraction Test ............................................................................ 20

Flotation Rate Test.................................................................................. 20

Column Flotation Test ........................................................................... 20

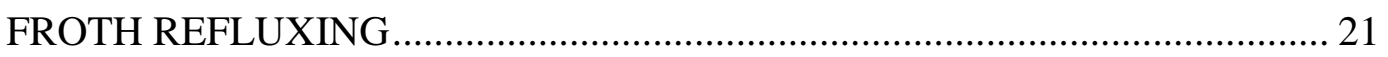

RESULTS AND DISCUSSIONS ....................................................................... 24

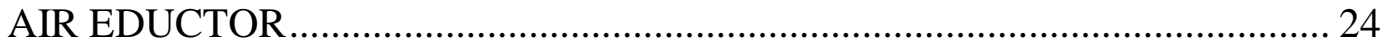

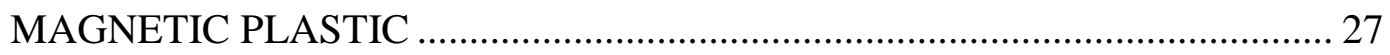

Conventional Flotation Tests ................................................................... 27

Repetitive Performance Evaluation ............................................................ 31 
Effect of Coal Type.............................................................................. 34

Effect of $\mathrm{pH}$................................................................................ 36

Particle Size-by-Size Flotation .................................................................. 40

Column Flotation Tests.......................................................................... 43

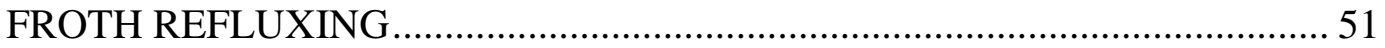

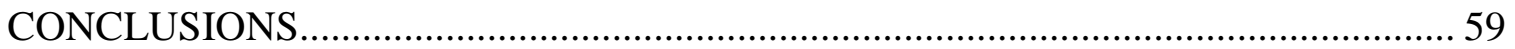

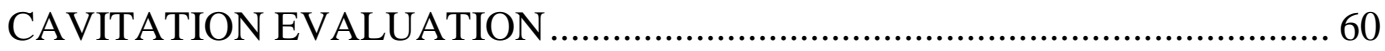

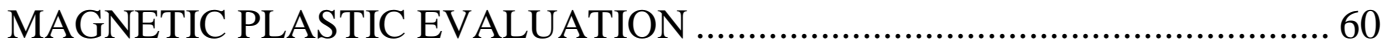

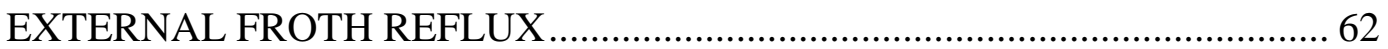

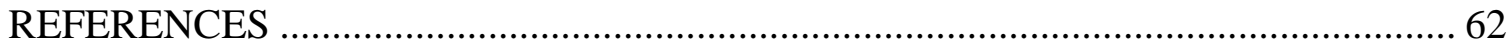

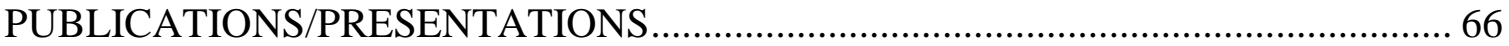




\section{EXECUTIVE SUMMARY}

For over a century, froth flotation has been used as the primary process to recovery coal finer than 150 micrometers. The process exploits differences in surface hydrophobicity between particles in the feed stream to achieve a separation. In contrast to the hydrophilicity of most reject particles, coal particles are naturally hydrophobic but vary in their degree of hydrophobicity within the coal and from coal-to-coal. Although past research has resolved many of the issues that have hampered the widespread use of froth flotation within the coal industry, improvements are still needed including the ability to recover difficult-to-float coals, enhanced selectivity for coals contains a relative large amount of mixed-phase particles and coal pyrite, and increased throughput capacity.

In this investigation, improving selectivity was the primary objective. The proposed methods involved i) the addition of magnetic hydrophobic plastic material directly into the froth zone and ii) enriching the froth zone by refluxing a portion of the froth concentrate back into the froth zone. In both methods, the increased concentration of highly hydrophobic particles crowd the bubble surfaces and cause selective detachment of the more weakly hydrophobic, high ash particles. However, an important finding of the research was that the plastic addition had a greater impact on enhancing coal flotation recovery and this impact became a primary focus of the study. The magnetic characteristic of the plastic allows recovery using a low-intensity magnetic separator. Another method that was discovered to enhance recovery was the pretreatment of the flotation feed through a cavitation tube. As such, all three methods were evaluated to assess and quantify the impact on both flotation recovery and selectivity.

Two coal samples with distinctly different characteristics were used for the flotation experiments. Pittsburgh No. 8 coal is well liberated and thus easy-to-clean in regards to ash reduction. However, the coal contains a significant amount of pyritic sulfur that is weakly-to-moderately hydrophobic. As such, pyrite rejection is typically limited in a froth flotation process. The other flotation feed sample was collected from an active preparation plant that treats coal from the Coalburg seam. Coalburg coal tends to be difficult-to-float and contains a relatively large amount of mixed-phase particles, which results in feed containing particles having a wide range of floatability characteristics.

The impact of adding hydrophobic plastic particles $\left(\theta=84^{\circ}\right)$ directly into the flotation feed slurry of a semi-batch Denver flotation cell was evaluated at concentrations of $2 \%$, $5 \%$ and $10 \%$ weight as related to the amount of dry coal solids in the feed. At a $5 \%$ concentration, combustible recovery increased by 15 absolute percentage points after 1 minute of flotation time when treating the Pittsburgh No. 8 coal. A total recovery improvement of 20 absolute percentage points was realized when using a $10 \%$ plastic concentration. In effort to ensure that the observed improvements were valid, the tests with no plastic material and $10 \%$ plastic addition were repeated five times under identical conditions. By averaging the flotation performances, a flotation rate increase from 1.04 $\min ^{-1}$ to $1.31 \mathrm{~min}^{-1}$ was obtained which corresponds to a $26 \%$ improvement. The corresponding recovery gain was 11 absolute percent points. 
As previously described, the Coalburg seam coal is difficult to float. After 1 minute of flotation time, only $61 \%$ of the coal was recovered from the flotation cell. The flotation rate was a relatively low $0.51 \mathrm{~min}^{-1}$. However, adding the plastic particles directly in the feed resulted in a flotation rate improvement to $0.74 \mathrm{~min}^{-1}$, which resulted in a $77 \%$ recovery of the combustible material.

Using the Pittsburgh No. 8 flotation feed sample, the performance improvement resulting from the plastic particle addition was evaluated as a function of coal particle size. A minimum recovery improvement of 5 absolute percentage points was obtained for particle sizes smaller than 25 micrometer. Recovery improvement increased with particle size to a maximum increase of 35 absolute percentage points for 150 micrometer particles. Additional tests were conducted on individual particle size fractions in separate tests with and without the addition of plastic particles. Very little improvement in the recovery of the coarse particles was observed from the individual size fraction tests. Based on these results, it is hypothesized that the plastic material may be reducing the drainage of the water surrounding the bubble surfaces and thus reducing bubble coalescence and maintaining coarse particle attachment due to high bubble surface area flux rates.

Experiments were also conducted at $\mathrm{pH}$ values of 3.9 and 10.1. Without the addition of plastic particles, flotation recovery was suppressed to values below 35\%. By adding the plastic particles, coal recovery returned to the values achieved under neutral $\mathrm{pH}$ conditions which were around 85\%.

In the continuous column flotation tests, the positive impact on coarse particle recovery was also observed. Feed solid concentrations were varied to assess the carrying-capacity of the column when treating the Pittsburgh No. 8 coal. When plastic particles were continuously added through the wash water distribution ring, the product mass flux rate increased and then became flat at a critical feed mass flux rate which indicates a positive impact on coarse particle recovery. Overall recovery improvements were generally less than the conventional cell results and varied as a function of volumetric feed rate and the amount of plastic particles added. At a 5\% plastic concentration, recovery improvement was from around 4 absolute percentage points at feed rates between $400 \mathrm{ml} / \mathrm{min}$ to 800 $\mathrm{ml} / \mathrm{min}$ for the treatment of Pittsburgh No. 8 coal. For Coalburg coal, the recovery improvement was around 7 absolute percentage points at a feed rate of $600 \mathrm{ml} / \mathrm{min}$.

Further investigations involved the evaluation of the impact of pre-aeration of the feed material through a cavitation tube. Recent investigations found that the bubble-particle attachment rate is increased when small air bubbles pre-exist on the coal particles prior to exposure to the bubbles generated from conventional flotation systems. For the Coalburg coal, pre-aeration using a cavitation tube resulted in an 18 absolute percentage point improvement in recovery after one minute of flotation time in a laboratory Denver cell. Flotation rate was improved from $0.65 \mathrm{~min}^{-1}$ to $1.25 \mathrm{~min}^{-1}$. 


\section{INTRODUCTION}

\section{Background}

The froth flotation process is comprised of two separate and distinctly different zones, i.e., the collection zone and the froth zone. In the collection zone, the separation of the valuable minerals from the non-valuables is achieved based on the bubble-particle attachment process. Due to hydraulic entrainment, a portion of the non-valuable minerals is carried from the collection zone into the froth zone with the mineral-bubble aggregates. Selectivity of the process can be enhanced in the froth zone by providing drainage of the feed pulp and utilizing the selective detachment of the more weak hydrophobic particles as a result of bubble coalescence and the resulting bubble surface area reduction. The importance of the latter sub-process is the subject of recent investigations (van Deventer et al., 2004; Honaker and Ozsever, 2003; Ata et al., 2002; Ralston et al., 1999; Falutsu, 1994; Hewitt et al., 1994; Yianatos et al., 1988). On the other hand, decades of research focused on the froth flotation process have resulted in a clear understanding of the processes and sub-processes involved in the selectivity achieved in the collection zone (Yoon and Mao, 1996; Mao and Yoon, 1997).

Moys (1978) and later Yianatos et al. (1988) confirmed by experimental evidence that the detachment process is selective. From Yianatos et al. (1988), the detachment rate for chalcopyrite under a given set of conditions was $0.30 \mathrm{~min}^{-1}$ whereas pyrite obtained a higher rate at $0.41 \mathrm{~min}^{-1}$. As such, differential detachment rates can be exploited to improve the selectivity between hydrophobic species. Schultz et al. (1991) obtained an improved concentrate grade and recovery in a column by introducing the feed (Alabama shale, $d_{90}=23$ microns) into the froth zone.

Detailed investigations utilizing modified column apparatus designs have provided promising quantifiable evidence of the selective detachment and drop-back processes (Rubio, 1996; Ata et al., 2002; Honaker and Ozsever, 2003). For example, Ata et al. (2002) used a unique cell design to show that highly hydrophobic particles added directly into the froth zone can selectively replace particles of lower hydrophobicity. Specifically, the addition of hydrophobic silica particles in the froth reduced the recovery of hematite particles that were added in the collection zone by 10 percentage points. Based on this finding, it is plausible that the addition of highly hydrophobic particles in the froth zone could assist in the selective rejection of the more weakly hydrophobic material (e.g., low grade particles) reporting from the collection zone, thereby enhancing the overall selectivity between particles of varying hydrophobicity.

The current project exploited the selective detachment process to enhance the selectivity between particles having varying degrees of floatability. External refluxing and the addition of hydrophobic material directly into the froth zone was investigated as a means to improve recovery and selectivity. 


\section{$\underline{\text { Scientific Discussion }}$}

The separation of the desired mineral from the other mineral components in the collection zone is based on differential flotation rates. The collection zone flotation rate of a mineral, $k_{c i}$, can be quantified by the expression:

$k_{c_{i}}=\frac{3}{2} \frac{V_{g}}{D_{b}} P_{c} P_{a} P_{d}$

in which $V_{g}$ is the superficial gas velocity, $D_{b}$ the bubble diameter, $P_{c}$ the probability of collision, $P_{a}$ the probability of attachment and $P_{d}$ the probability of detachment. As shown in Eq. (1), a change in gas velocity or bubble size affects the flotation rate of each mineral species equally and thus does not improve selectivity. Furthermore, bubbleparticle collision is not a selective process and should be maintained at maximum efficiency to ensure high recovery of the floatable mineral. The probability of detachment is a function of both particle size and density and thus may play a minor role in selectivity. However, the bubble-particle attachment process as quantified by $P_{a}$ is the principle mechanism defining the ability to effectively separate minerals in the collection zone of a froth flotation process.

After bubble-particle collision and attachment, the aggregate moves upward through the collection zone and into the froth zone. The transition into the froth zone is characterized by an increase in the air fraction from around $20 \%-30 \%$ to $70 \%-80 \%$ (Finch and Dobby, 1990). In the froth zone, the water filling the voids between the bubbles drain back into the collection zone and the water layer surrounding each bubble thins. Upon approach of the liquid-air interfaces, bubbles coalesce thereby causing a reduction in bubble surface area. If the reduced amount of bubble surface area available in the froth zone is insufficient to carry the solids reporting from the collection zone, particles detach and potentially move with the fluid into the collection zone. As such, froth zone recovery may have a significant influence on overall flotation recovery.

To assess the effect on overall flotation recovery for a given mineral, linear analysis concepts developed by Meloy (1983) can be applied to the mass transport processes illustrated in Figure 1. As proposed by Finch and Dobby (1990), an expression for the overall flotation recovery $R_{O}$ for a mineral component $i$ can be derived as a function of the collection zone recovery $R_{C}$ and froth zone recovery $R_{F}$ (Eq. 2), i.e.,

$$
R_{O}=\frac{R_{C} R_{F}}{R_{C} R_{F}+\left(1-R_{C}\right)}
$$




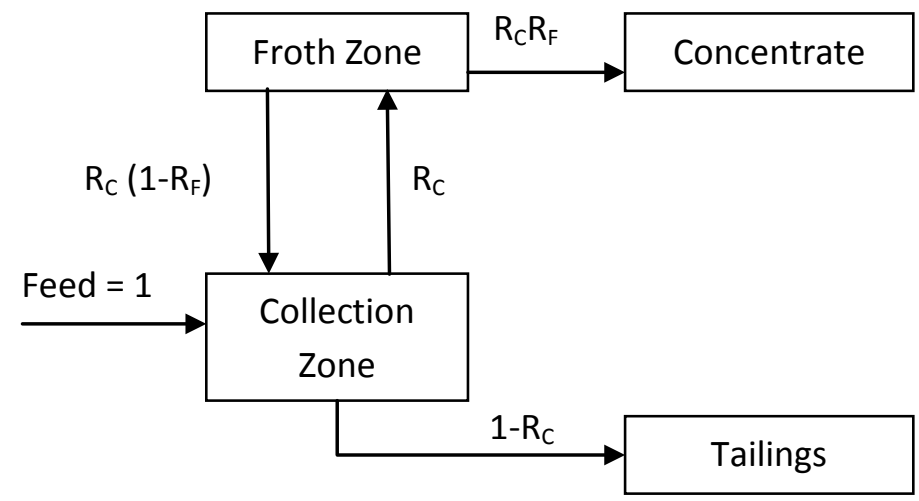

Figure 1. Interaction between zones in a column flotation cell.

One should note that, if $R_{F}$ equals $100 \%, R_{O}$ is equivalent to $R_{C}$.

An analysis of Eq. (2) reveals that froth zone recovery has a relatively small impact on the overall recovery of particles that have a collection zone recovery. Highly floatable particles that are detached in the froth zone are efficiently recovered in the collection zone. However, weakly hydrophobic particles have a low collection zone recovery and thus are less likely to be recovered in the collection zone. As a result, reductions in froth zone recovery have a significant impact on the overall recovery of particles that have moderate-to-weak flotation characteristics. Therefore, the differential effect of froth zone recovery values between particles of varying floatability indicates an additional selectivity mechanism provided by the reflux action between the collection and froth zones.

For systems that contain multiple mineral species or un-liberated particles with varying degrees of hydrophobicity, the selectivity of the detachment process in the froth zone also enhances the overall separation performance achieved by froth flotation as discussed in the previous section. Particles with a low degree of surface hydrophobicity are likely to be weakly bound to the bubble surface and thus will be preferentially detached. By adding particles directly into the froth phase that have a higher degree of hydrophobicity than the low grade particles, selectivity can be enhanced as a result of selective detachment. Figure 2 shows the improvement in ash reduction achieved for an anthracite coal when a more hydrophobic material is added into the froth at concentrations of $5 \%$, $10 \%$ and $15 \%$ of the collection zone feed. The added material was removed from the product and tailing samples by density fractionation. At a given recovery value, the product ash content was reduced from $19 \%$ to nearly $14 \%$ at a concentration equal to $15 \%$ of the feed mass flow rate. 


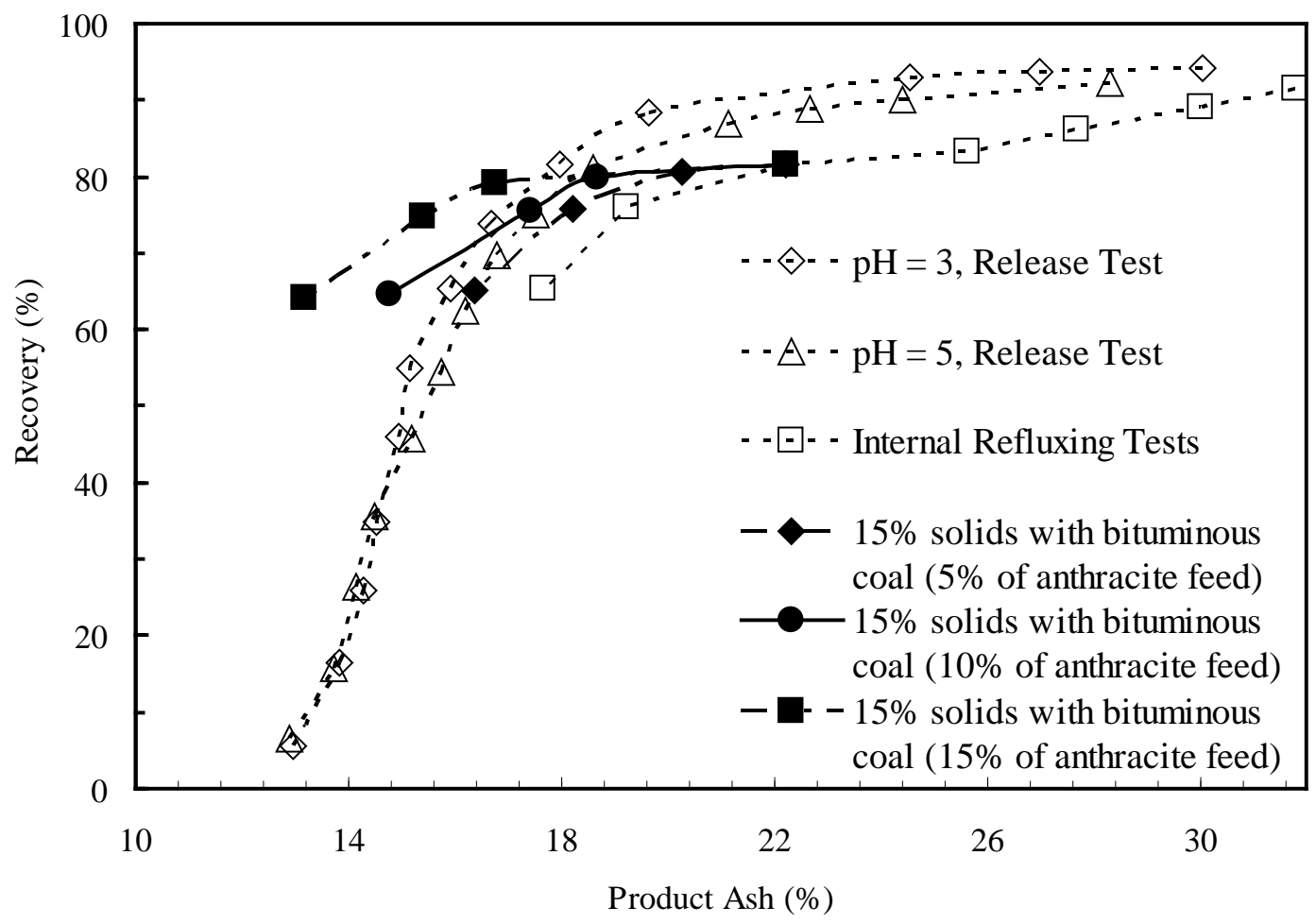

Figure 2. Improvement in the separation performance achieved on anthracite coal.

\section{Objective and Approach}

The main objective of this research focused on the selective detachment process and the hydrodynamic cavitation technology in an effort to enhance the performance of column flotation when cleaning ultrafine coal. Enhanced flotation by the addition of hydrophobic material or external refluxing into the froth zone will promote selective detachment of particles having varying degrees of floatability. To improve recovery, gas nuclei or picobubbles on a particle surface will favor activation of flotation by improving surface hydrophobicity and thus bubble-solid attachment. By using available fundamental relationships, full quantification of the different enhancement alternatives will be performed over a range of operating parameters.

It was hypothesized that the addition of highly hydrophobic particles into the froth zone will increase competition among particles having different degrees of floatability. Thus, the introduction of material with a higher degree of hydrophobicity than the original feed material will result in the detachment of weakly hydrophobic particles, which typically have high ash content. The most hydrophobic particles will be selectively recovered. The selectivity will improve grade without a significant reduction of recovery. A naturally hydrophobic plastic product that contains a significant amount of magnetite and thus recoverable by low intensity magnetic separator was used as froth enrichment material. 
The plastic material is currently provided by Eriez Manufacturing Company. The study involved conventional flotation cell and column flotation experiments with and without the addition of magnetic material in the feed or froth phase.

The same principles were also exploited by refluxing a minor portion of froth concentrate back through the wash water and into the froth phase. As a result, concentration of highly hydrophobic particles occurs which should stimulate the selectivity of the detachment process. Bubble coalescence in the froth phase decreases the amount of bubble surface area needed to recover all of the collected particles. The shock energy of the coalescence event and the lack of bubble surface area cause particle detachment.

In addition, the action of pretreatment feed slurry to promote picobubble formation on the surface of hydrophobic solid will improve flotation rate and thus flotation recovery by increasing the probability of attachment. The advantages will be more prevalent for weakly floatable coals. Effectiveness of this innovative process was evaluated to assess the upgrading potential under different operating conditions. In this particular study, a Coalburg sample obtained from an operating coal preparation plant was used in a column flotation cell, pilot bench-type cells and a continuous column flotation unit.

The specific objectives of the investigation were:

- To develop and demonstrate the potential use of naturally hydrophobic magnetic plastic material to enhance the selectivity achieved when treating coal by froth flotation. Characterization of the plastic material was achieved by evaluating recovery efficiency for a range of magnetic plastic particle sizes and quantifying the surface characteristics. Flotation performance and selectivity detachment was analyzed over a range of conditions (solids concentrations, feed volumetric flow rate, particles size and $\mathrm{pH}$ ).

- To investigate the potential selectivity benefits associated with recycling a portion of the froth product back to the froth phase in a continuous column flotation. The potential separation enhancement will be quantified.

- To demonstrate advantages in flotation performance by treating a weakly floatable and high middling Coalburg feed slurry with a hydrodynamic cavitation technique. The performance enhancement achieved in a continuous column flotation system will be determined over a range of operating conditions. 


\section{EXPERIMENTAL $\underline{\text { Air Eduction }}$}

\section{Sample Characterization}

Three 208-liter drums of coal slurry were collected from a West Virginia coal preparation plant treating coal from the Coalburg seam. Representative flotation feed at the West Virginia preparation plant was obtained on three consecutive days. The slurry contained $5 \%$ solids by weight. The Coalburg slurry sample was used to investigate the impacts of using hydrodynamic cavitation on coal recovery in a froth flotation process. The flotation feed was comprised of about $84 \%$ minus $44 \mu$ m (325 mesh) as shown in Table 1 . The material finer than $44 \mu \mathrm{m}$ contained the largest amount of combustible (floatable) material with $66.6 \%$ of the total amount.

Table 1. Particle size distribution and quality characteristics of a West Virginia preparation plant flotation feed.

\begin{tabular}{|c|c|c|c|c|c|c|}
\hline \multirow{2}{*}{$\begin{array}{c}\text { Particle Size } \\
\begin{array}{c}\text { Fraction } \\
(\mu \mathrm{m})\end{array}\end{array}$} & \multicolumn{3}{|c|}{ Incremental (\%) } & \multicolumn{3}{c|}{ Cumulative (\%) } \\
\cline { 2 - 7 } & Weight & $\begin{array}{c}\text { Ash } \\
\text { Content }\end{array}$ & $\begin{array}{c}\text { Coal } \\
\text { Distribution }\end{array}$ & Weight & $\begin{array}{c}\text { Ash } \\
\text { Content }\end{array}$ & $\begin{array}{c}\text { Coal } \\
\text { Distribution }\end{array}$ \\
\hline+149 & 1.41 & 5.66 & 3.08 & 1.41 & 5.66 & 3.08 \\
$149 \times 44$ & 14.98 & 12.05 & 30.34 & 16.39 & 11.50 & 33.42 \\
-44 & 83.61 & 60.74 & 66.58 & 100.00 & 52.67 & 100.00 \\
\hline Total & 100.00 & 52.67 & 100.00 & & & \\
\hline
\end{tabular}

\section{Release Analysis}

Release analysis is the procedure used to obtain the best possible separation performance achievable by any froth flotation process. As such, the performance of a flotation process can be monitored and improved with the idea to approach the theoretical flotation response curve given by the release analysis curve for a certain feed material. This goal is analogous to the gravity-based washability analysis. Generally, the release analysis test is carried out in conventional laboratory flotation cells and conducted in two-phases with distinctly different goals.

The first stage separates the hydrophobic material away from the hydrophilic material by doing multiple cleaning phases from the initial feed. The sample was introduced in a laboratory Denver flotation cell of 1 liter capacity at a 5\% feed solid content by weight. After mixing collector (Fuel Oil No. 2) and frother (ShurFlot 948) was injected at minimum doses. The fuel oil was added and conditioned for 5 minutes prior to the 
addition of the frother. Flotation was continuously performed in phase I to float all the hydrophobic material from the cell. When needed, frother was added to allow a continuation of flotation. The product was continuously collected in a separate container. When all of the floatable material was collected, the remaining material was placed in a separate container which was labeled as tailings. The floated material was placed back into the flotation cell and refloated. This process was repeated three times to ensure remove of all the hydrophilic particles from the hydrophobic particles.

The second stage has the goal of separating particles into fractions of degrees of surface hydrophobicity. For coal, the more hydrophobic coal contains the least amount of ashforming material with the ash content increasing with a decline in hydrophobicity. Fractionated samples are obtained by controlling air flow rate and rotator revolutions under starvation conditions. Lower air flow rates and rotator revolutions lead to higher floatable particles reporting first until no more particles are able to float (sample 1). Then an incremental increase in air flow rate and/or rotator revolution was allowed to obtain the next most floatable particles, which contained a larger amount of ash (sample 2). A progressive increase in air rate or rotation speed assures the total flotation of the next set of more floatable particles by a fractionation process (sample 3, 4, etc.). The process stops when no more particles can float. The sample were filtered, dried and weighed followed by analysis of the ash content.

\section{Flotation Rate Test}

The flotation rate tests followed the ASTM D5114 procedure with Fuel Oil No.2 as collector and an alcohol-based frother currently used at a West Virginia preparation plant. The collector-to-frother dosage ratio was maintained at 2:1. The coal slurry was treated through an air eductor in an effort to nucleate fine bubbles on the surface of the weakly hydrophobic coal particles prior to flotation tests.

The laboratory semi-batch flotation system was a Denver flotation unit equipped with a 1-liter cell. Flotation was conducted using natural air source. The sample was conditioned with collector for 15 minutes before adding frother, which assured correct adsorption of surfactants without overlapping. The flotation concentrate samples were collected as a function of time. Samples were filtered, dried, weighed and analyzed for ash content using the appropriate ASTM procedures.

In summary, three tests were performed:

Test 1: $\quad$ Rate test using current frother and fuel oil.

Test 2: $\quad$ Rate test on feed slurry using air eductor treatment. Slurry was recycled through the eductor through several cycles.

Test 3: Rate test on feed slurry processed through an air eductor using a single pass. Feed was not recycled back through the eductor. 


\section{Column Flotation Test}

Laboratory flotation tests were performed using a continuously operated flotation column over a range of feed volumetric flow rates which provided a range of retention times. The inlet feed slurry was located at $45 \mathrm{~cm}$ below the overflow lip. The flotation column used the Microcel bubble generator which consisted of a static mixer and a pump configuration to produce the bubbles. The length of the collection and froth zones typically used in the test program were $210 \mathrm{~cm}$ and $30 \mathrm{~cm}$, respectively. With a diameter of $5 \mathrm{~cm}$, the length-to-diameter ratio was around 50:1 which provided near plug-flow conditions. Wash water was added in the froth zone at a depth that was $1 / 3$ of the froth zone height below the overflow lip. A summary of the table operating parameters is provided in Table 2.

Table 2. Operative column flotation conditions use for the entire experimental program test.

Feed rate

Feed solids concentration by weight

Bias factor

Collector/Frother ratio

Froth depth

Superficial gas velocity

Volumetric wash water
$200 \mathrm{ml} / \mathrm{min}$ to $1000 \mathrm{ml} / \mathrm{min}$

$5 \%$

0.7

2:1

$30 \mathrm{~cm}$

$2 \mathrm{~cm} / \mathrm{s}$

$400 \mathrm{ml} / \mathrm{min}$

The tailing flow was adjusted with a control valve attached to the tailing pipe. A microprocessor series 2600 Love Controls received signals from a pressure transducer located at the bottom of the column. The signal adjusts the air pressure that controls the underflow valve whose flow is based on the desired froth level. Conventional air bubbles were generated by processing a portion of the tailing stream through a static mixer at a pressure of $1.36 \mathrm{~atm}$ (20 psi). Frother and air are injected into the stream prior to the static mixer. The output of the static mixer was located inside the cell and near the bottom.

A period of time equivalent to three particle retention times was allowed to achieve steady-state conditions. After reaching steady-state, samples of the feed product and tailing streams were collected simultaneously. The samples were filtered, dried, weighed and analyzed for ash content. 


\section{Air Eductor Pretreatment}

In the study of flotation performance enhancement using hydrodynamic cavitation, feed pretreatment with picobubbles was carried out with the use of a venturi tube. The venture tube consists of a gradual contraction from the pipe diameter $(D)$ to the throat diameter $(d)$ and then a gradual expansion to the original pipe diameter. The high flow velocity leads to hydrodynamic cavitation due to momentarily reduction of vapor pressure in the liquid.

Prior to each test, the feed slurry was conditioned for 15 minutes with Fuel Oil No. 2 which was used to enhance the hydrophobicity of the coal surfaces. Conditioning was conducted in a sump that was equipped with a mixer and four baffles placed vertically and separated by an equal distance along the circumference of the sump.

After conditioning, the slurry was processed through an air eductor to generate micron size bubbles on the coal surfaces. Two different air eductor sizes were used, i.e., $1.27-\mathrm{cm}$ (1/2-in) inner diameter for the laboratory conventional and flotation column tests and 3.8$\mathrm{cm}$ diameter unit for the pilot-scale conventional flotation bank. The slurry was pumped through both air eductors at a volumetric flow rate that provided a pressure of $2.72 \mathrm{~atm}$ (40 psi). Air was injected into the slurry prior to the eductor at a rate of $1 \mathrm{ml} / \mathrm{min}$.

As shown in Figure 3, the process in each experiment involved pretreatment of the conditioned feed through an air eductor. The aerated feed slurry was then pumped to a feed tank which utilized a recirculating line to ensure suspension of all solids. A peristaltic pump was used to draw a pre-determined amount of feed into the flotation column at a point just below the pulp-froth interphase. As a result, the pre-treated coal particles initially move downward in the cell against a rising flow of air bubbles. Upon collision and attachment, the air bubble-particle aggregate rises to the top where they are collected in an overflow launder. The hydrophilic particles remain unattached and flow with the majority of the pulp to the underflow (tailings) stream. 


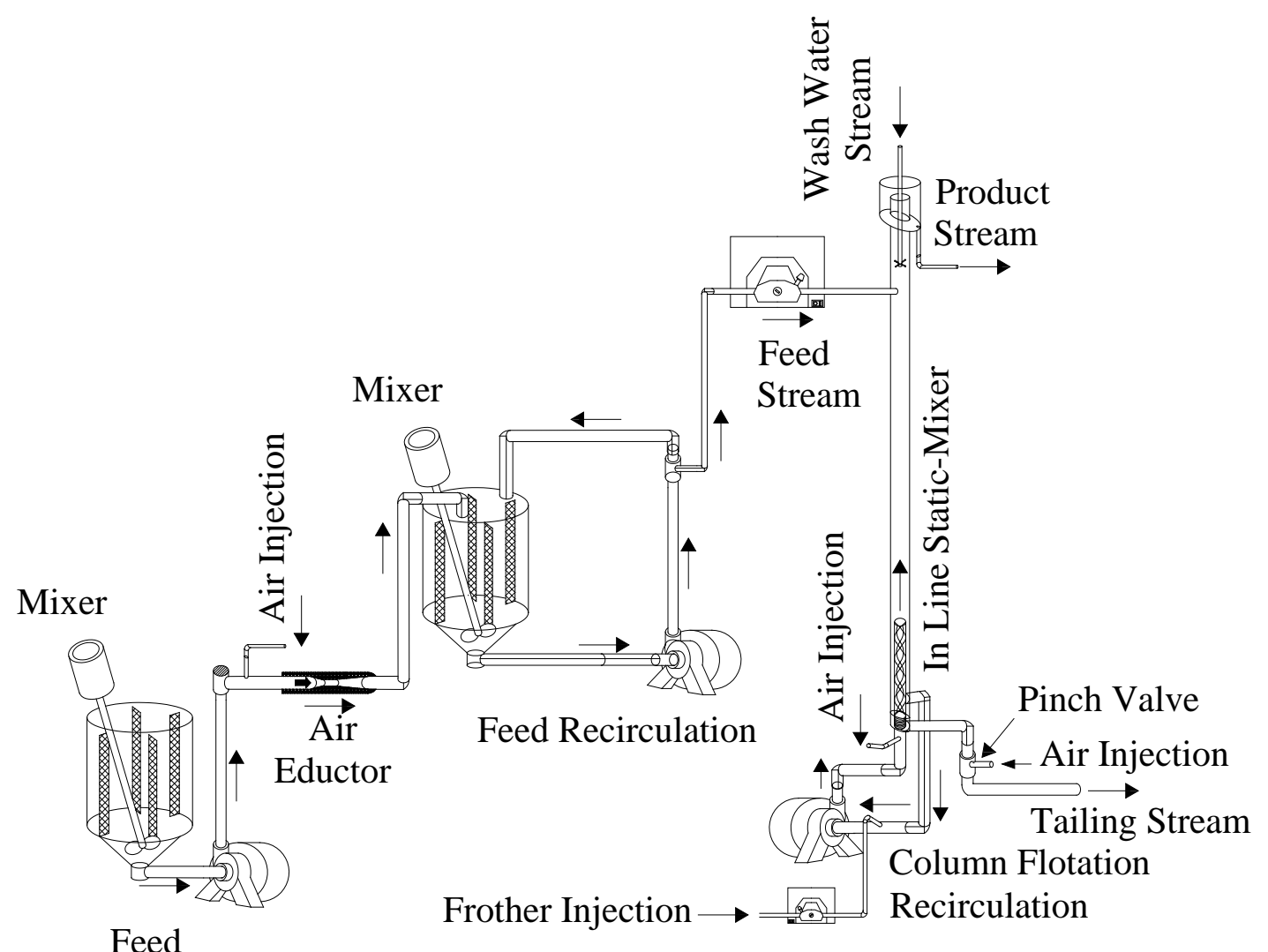

Figure 3. Schematic of the column flotation circuit with the air eductor device.

\section{In-Plant Conventional Flotation Tests}

A 10 liter pilot-scale conventional flotation bank (Figure 4) was used in the West Virginia processing facility to evaluate the performance improvement provided by the use of an air eductor to pretreat the flotation feed. The test was conducted on the fullscale conventional bank currently operating in the West Virginia facility over a period of about 19 hours. A total of 11 tests were performed; 6 air eductor under various air rates, 2 standard tests and 3 tests involving another collector. The frother type was maintained the same and the fuel oil addition was turned off during the period of time that the special collector was being added.

The plant feed during the first three test periods was comprised of Coalburg and Dorothy seam coals at a mass flow rate of $850 \mathrm{tph}$. The solids content in the flotation feed averaged $5.32 \%$ by weight while the mass flow rate to the full-scale flotation bank was determined to be around 67 tph based on a volumetric flow rate of 18,560 liter per minute. 


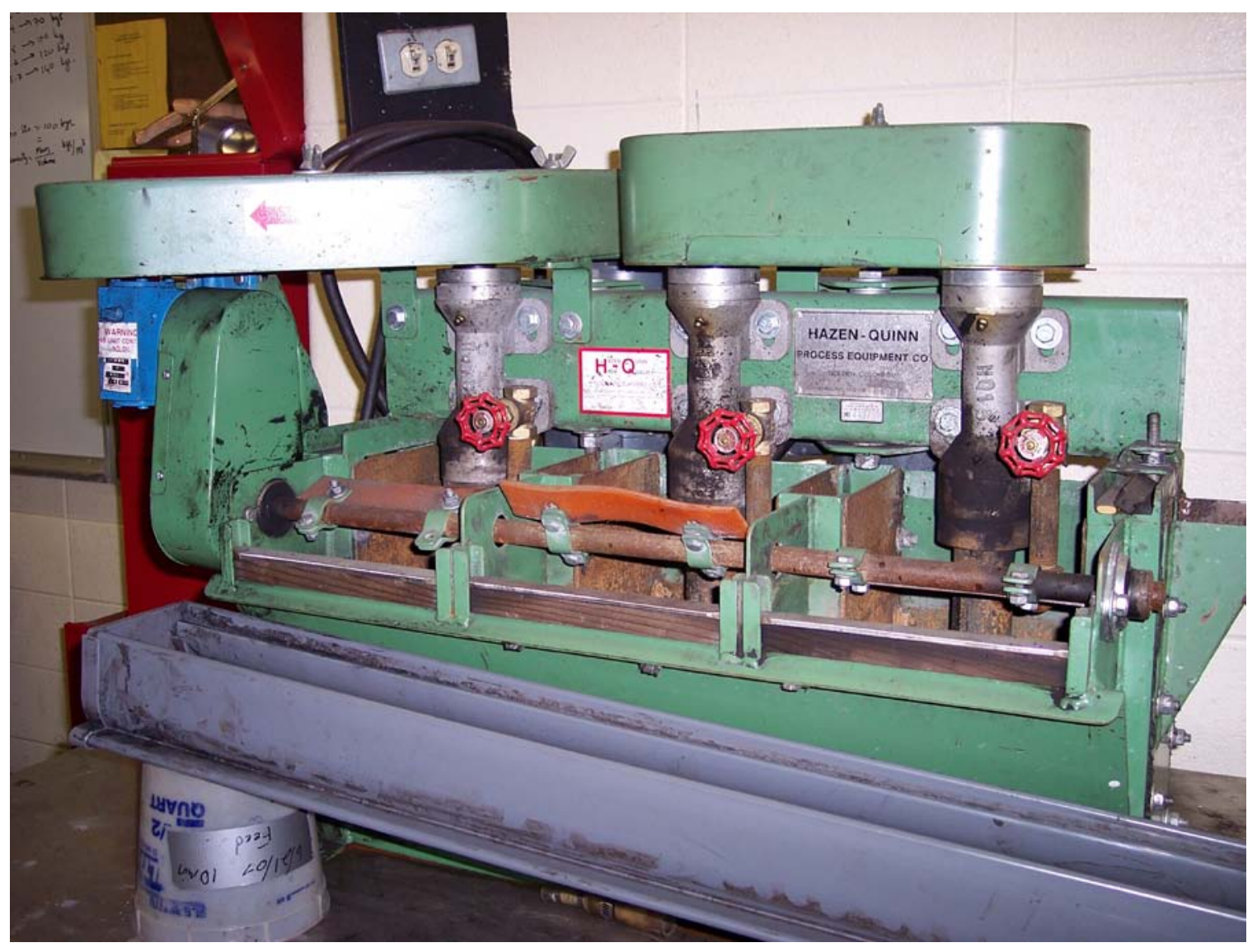

Figure 4. Conventional flotation bank cell used in plant with air eductor.

\section{Magnetic Plastic Evaluation}

\section{Sample Characterization}

As part of the magnetic plastic research, two different bituminous coal samples were independently used to evaluate the impact of selective detachment in a continuous column flotation by the addition of hydrophobic magnetic plastic material. These two samples were Pittsburgh No. 8 and Coalburg seam coal. The Pittsburgh No. 8 coal was very floatable and contained a relatively high amount of coal pyrite while the Coalburg coal had a relatively high content of middling (mixed-phase) particles in a range of $10 \%$ to $20 \%$.

Representative samples of Pittsburgh No.8 and Coalburg coal from operative coal preparation plants in Blacksville and Logan W.V. (USA), respectively, were collected. Both dry samples of minus $8 \mathrm{~cm}$ (3 inch) particle size were stored in drums. Upon arrival to the laboratory, each coal type was crushed with a jaw crusher to obtain $100 \%$ minus 
$1.27 \mathrm{~cm}(1 / 2$-inch) particle size. In a dry room, each coal type was spread out on a clean floor surface for 24 hours to remove excess of moisture and finally combined and quartered to obtain a homogenous representative coal sample that was used during the entire experimental program. In the mean time, the samples were stored into hermetic sealed drums to prevent contamination or prolonged exposure to air. Fresh and intact properties of the coal surface were always considered a high priority. Therefore, the sample was pulverized to minus $177 \mu \mathrm{m}$ ( 80 mesh) just prior to the flotation test.

Characterization of the coal samples was performed by taking representative samples from the storage drum to be prepared and pulverized at minus $177 \mu \mathrm{m}$ and then subjected to analytical analyses for ash content, volatile matter, fixed carbon, total sulfur and caloric value as shown in Table 3.

Table 3. General characterization of Pittsburgh No. 8 and Coalburg sample feed 100\% minus $177 \mu \mathrm{m}$.

\begin{tabular}{l|c|c}
\hline \multicolumn{1}{c|}{ Coal Characterization } & Pittsburgh No. 8 & Coalburg \\
\hline Ash Content (\%) & 16.3 & 36.12 \\
Total Sulfur (\%) & 3.07 & 0.68 \\
Volatile Matter (\%) & 34.11 & 23.21 \\
Calorific Value (Btu/lb) & 12,562 & 9,123 \\
Moisture (\%) & 0.66 & 2.40 \\
\hline
\end{tabular}

\section{Magnetic Plastic Material}

Eriez Equipment Company currently produces a plastic product that contains a significant amount of magnetite. The naturally hydrophobic plastic material is magnetic and thus recoverable by low intensity magnetic separators. The magnetic plastic material arrived as dry sample with a particle size of $100 \%$ minus $0.635 \mathrm{~cm}$ (1/4 inch). A representative sample was collected and ground to obtain material having a particle size less than $177 \mu \mathrm{m}$ which was used in the evaluation of the magnetic strength of the magnetic plastic material by using a Davis tube at full magnetic strength of 4000 Gauss. The results demonstrated that $96 \%$ of sample was reported to the product from a total sample of 28 grams, which indicates the magnetic strength of the plastic material.

In addition, a surface characterization of the magnetic plastic material was performed using a scan electrode image machine (S.E.M) at $500 \mu \mathrm{m}$ from a pulverized sample that was usually utilized during the entire program test. Figure 5 shows a granular, irregular and porous surface with an apparently narrow particle size distribution. It seems like most of the magnetite particles are well coated with the plastic resin after the grinding process. 


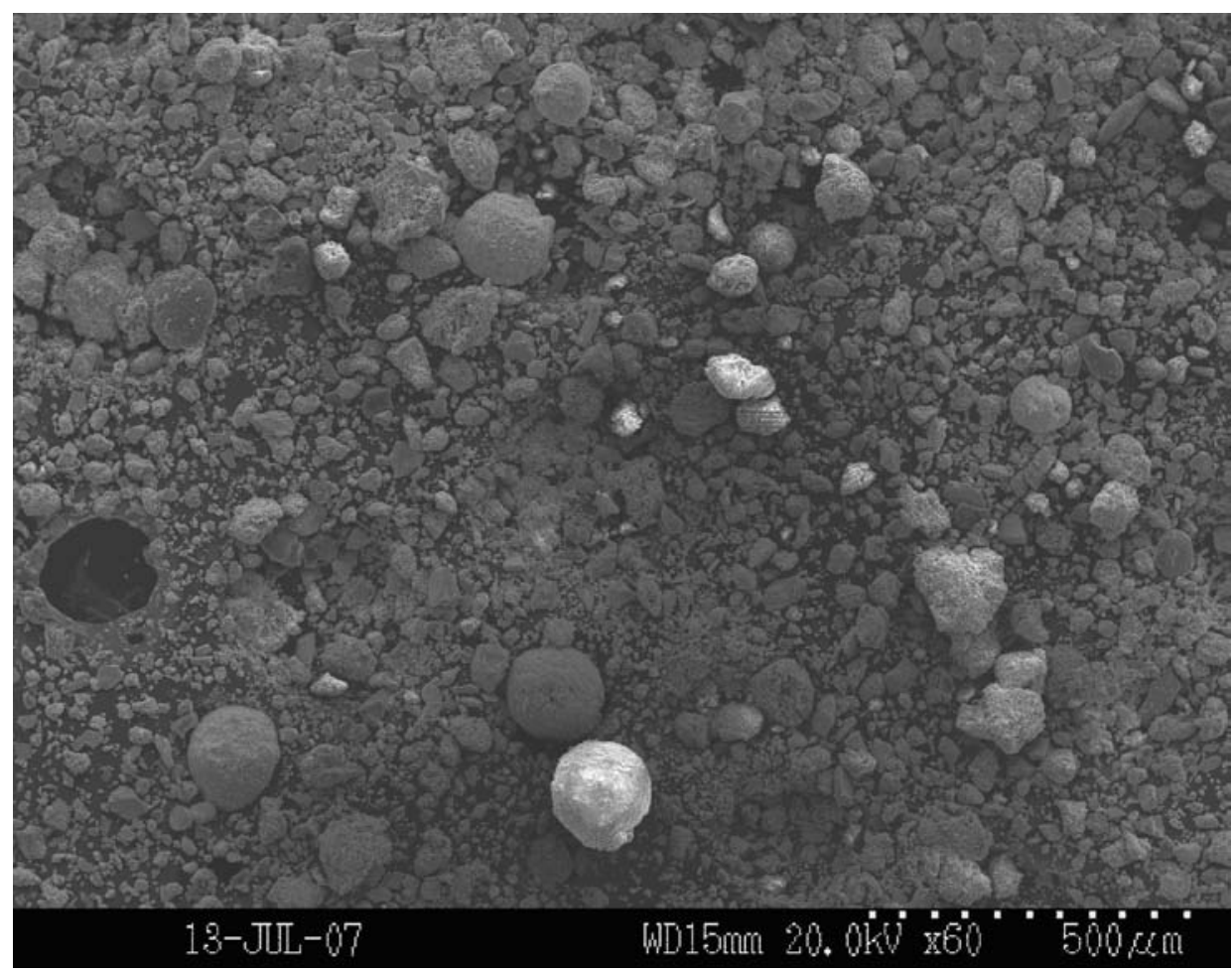

Figure 5. SEM picture of minus $177 \mu$ m particle size of magnetic plastic material at $500 \mu \mathrm{m}$ optical resolution.

An Eriez magnetic separator (Figure 6) was used to recover the magnetic plastic material from the coal slurry. The magnetic separator machine creates a magnetic field in the core of the apparatus where a chamber is located; the chamber is connected with two pieces of pipe line to let the slurry sample pass through the system by gravity action. When the combined slurry of coal and magnetic plastic material pass through a maximum magnetic field focus in the chamber, the magnetic plastic particles are attached by the magnetic gradient fields to the perforated metallic plates locate inside the chamber while the nonmagnetic particles follow water stream path through the chamber. Water is then flushed into the system to assure that all particles have passed through the chamber and only magnetic plastic were attached. Afterward, in another empty and clean container, the adhered magnetic plastic particles are recovered by turning off the magnetic field and flushing water into the chamber to capture the magnetic plastic. The separation process is repeated multiple times to obtain a complete separation. The samples were filtered, dried and weighed to assess magnetic recovery and general performances.

By experimental analysis, it was found that particle size has an important effect on magnetic plastic recovery with an optimum range being minus $177 \mu \mathrm{m}$ particle size material. Lower or higher particle size distributions than the optimum range considerably decrease the magnetic plastic recovery. Thus, lower particles size distribution follows stream water lines while magnetic plastic with higher particles size distribution do not 
attach in the magnetic chamber. Also experimental observation indicates that three passages through the separator are required to ensure maximum recovery of the plastic material. In the first separation process, the magnetic separator was able to recover $87.6 \%$ of the total magnetic sample. A second repetition enhanced recovery to $96.1 \%$ and a final stage resulted in $99.95 \%$ of the magnetic material being recovered.

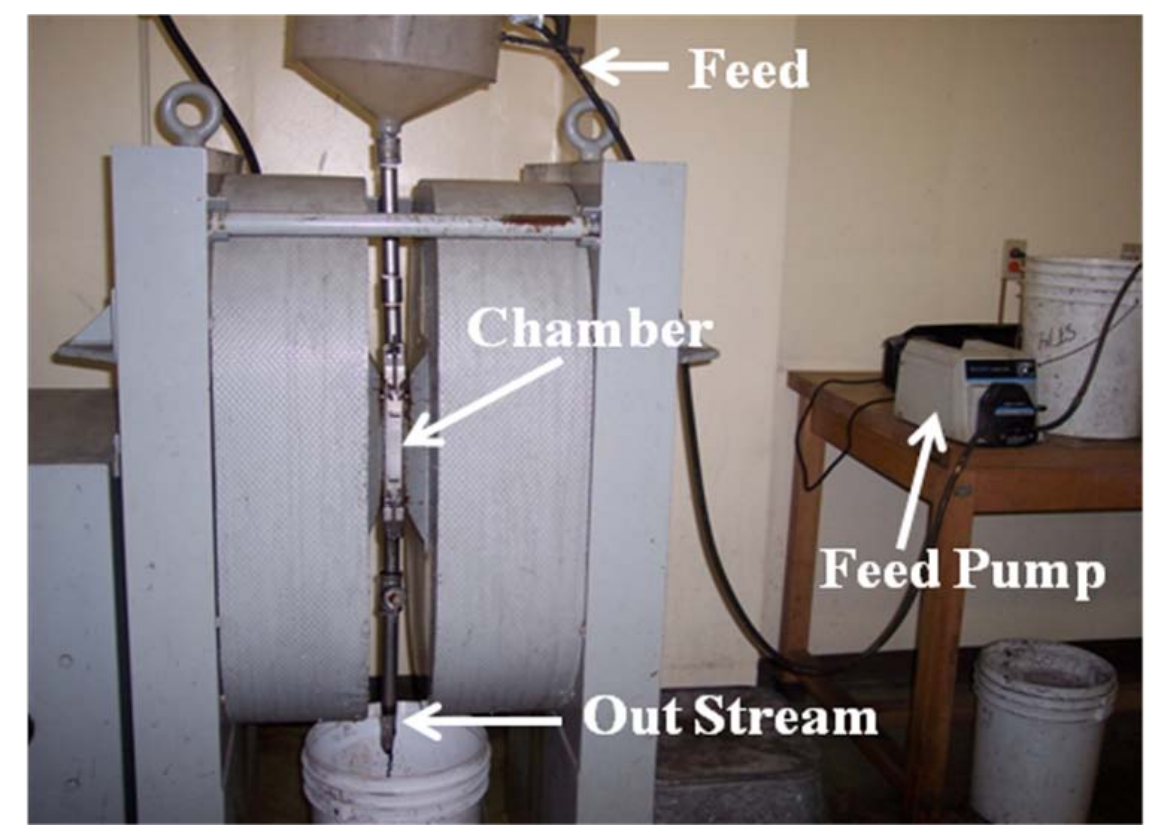

Figure 6. Eriez magnetic plastic separator showing the chamber and machine set up.

\section{Contact Angle Analysis}

The sessile drop method was used to measure the contact angle for the coal and plastic material. Measurements were conducted using a Rame-Hart contact angle goniometer Model 100-00. The model consists of a goniometer with two rotatable cross hairs for measuring contact angle, a specimen stage and a variable intensity illuminator. All the instruments were mounted on an optical bench. The set up for measurement of contact angle is shown in Figure 7. 


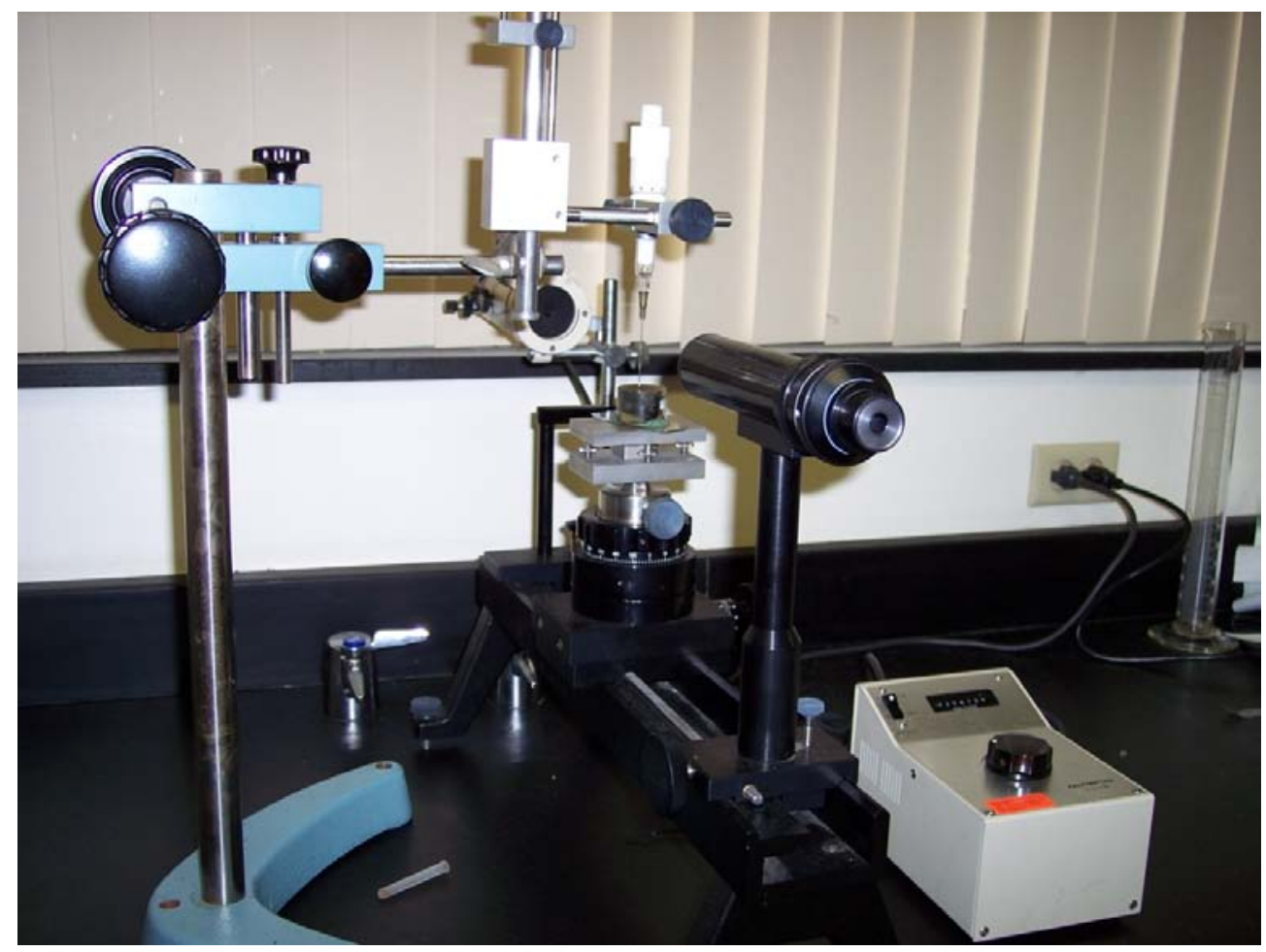

Figure 7. Goniometer used in the determination of contact angles.

The samples of approximately $1 \mathrm{~cm} \times 1.0 \mathrm{~cm}$ x $0.5 \mathrm{~cm}$ size were mounted on an epoxy resin. The mounted specimen was then polished on a Buehler polishing machine using 60, 240, 400 and 600 grit silicon carbide papers. Final polishing was done on a microcloth using an aqueous suspension of 0.05 microns Micropolish Alumina B. As polishing can have a drastic effect on the value of the contact angle, the polishing procedure was kept constant for all measurements. After polishing, the specimen was washed with distilled water and submerged in a microwave bath for a couple of minutes to remove any adhering alumina.

The contact angles of coal and magnetic plastic were measured with distilled water and Methylene Iodide at neutral $\mathrm{pH}$. The equilibrium contact angle on both sides of the water droplet was measured in three different positions. The standard deviation in measuring a single contact angle was $\pm 3^{\circ}$. All measurements were conducted at $25^{\circ} \pm 2^{\circ} \mathrm{C}$. Results in Table 4 clearly indicates that the magnetic plastic surfaces are much more hydrophobic than the surfaces of the Pittsburgh No. 8 coal. 
Table 4. Contact angle measurements with distilled water and methylene iodide.

\begin{tabular}{|c|c|c|c|c|}
\hline \multirow{2}{*}{$\begin{array}{c}\text { Contact Angle } \\
\text { (Degrees) }\end{array}$} & \multicolumn{2}{|c|}{ Distilled Water } & \multicolumn{2}{|c|}{ Methylene Iodide } \\
\cline { 2 - 5 } & Left & Right & Left & Right \\
\hline Mag. Plastic & 81.7 & 84.4 & 61.6 & 62.2 \\
Pittsburgh No. 8 & 54.6 & 55.3 & 41.8 & 42.6 \\
\hline
\end{tabular}

\section{Zeta Potential Measurements}

The surface charge of the Pittsburgh No. 8 coal and magnetic plastic material were studied using a Brookhaven Zeta Plus system (Figure 8). The procedure consisted in prepare enough solution with double distilled water with potassium chloride at $10^{-3} \mathrm{M}$ concentration (4 lt.) for the entire set of experiments. Also, 2 liters of solutions of hydrochloric acid (HCL 25\% concentration) at $3.25 \mathrm{M}$ concentration and sodium hydroxide $(\mathrm{NaOH})$ at $1.73 \mathrm{M}$ concentration were prepared to change the $\mathrm{pH}$ of the potassium chloride solution.

The coal samples and magnetic plastic material were pulverized and wet screened to a particle size below 25 $\mu$ m (500 mesh). For each solid type, six beakers were prepared by the addition of 0.1 grams of solid into a $50 \mathrm{ml}$ potassium chloride solution at $10^{-3} \mathrm{M}$ concentration and agitated until solid dispersion was ensured. Next, the $\mathrm{pH}$ was adjusted by the addition of hydrochloric acid or sodium hydroxide to obtain $\mathrm{pH}$ values within the range of 2 to 12 . The samples were left to reach an equilibrium $\mathrm{pH}$ for approximately 24 hours. 


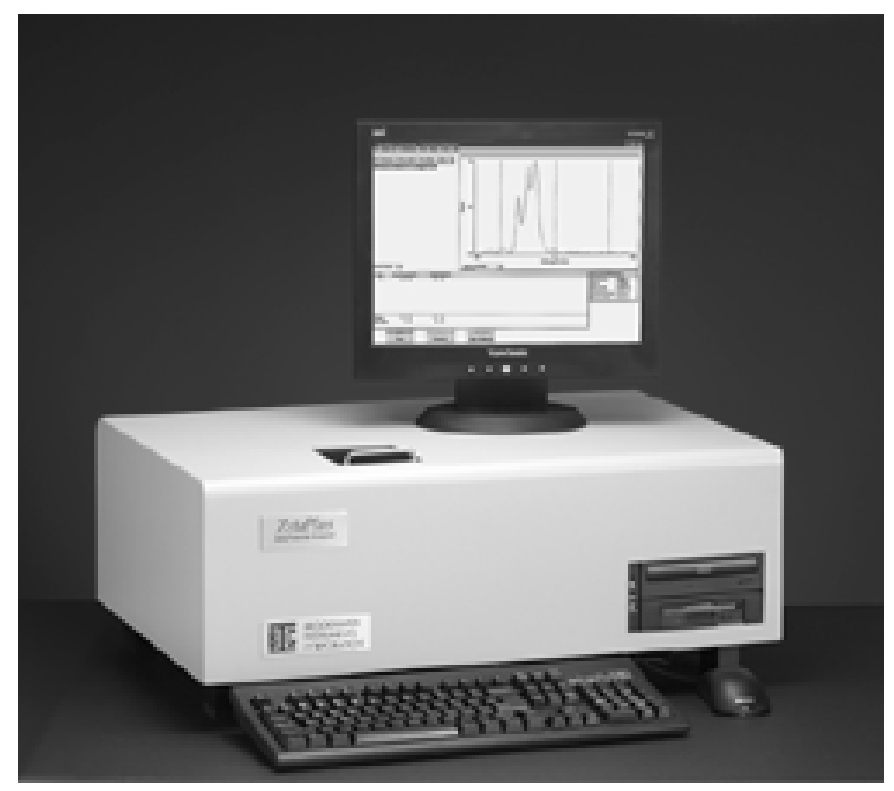

Figure 8. Brookhaven zeta potential analyzer.

Later, representative sample solution of $1.5 \mathrm{ml}$ were taken from each beaker and analyzed by using the Brookhaven ZetaPlus system. Ten (10) measurements were obtained and a final average value determined. The effect of slurry $\mathrm{pH}$ on zeta potential of the coal samples and the magnetic plastic particles is shown in Figure 9. For $\mathrm{pH}$ values greater than about 3, the surface charge is negative for all three solids and the magnitude is significant at $\mathrm{pH}$ values greater than 6 .

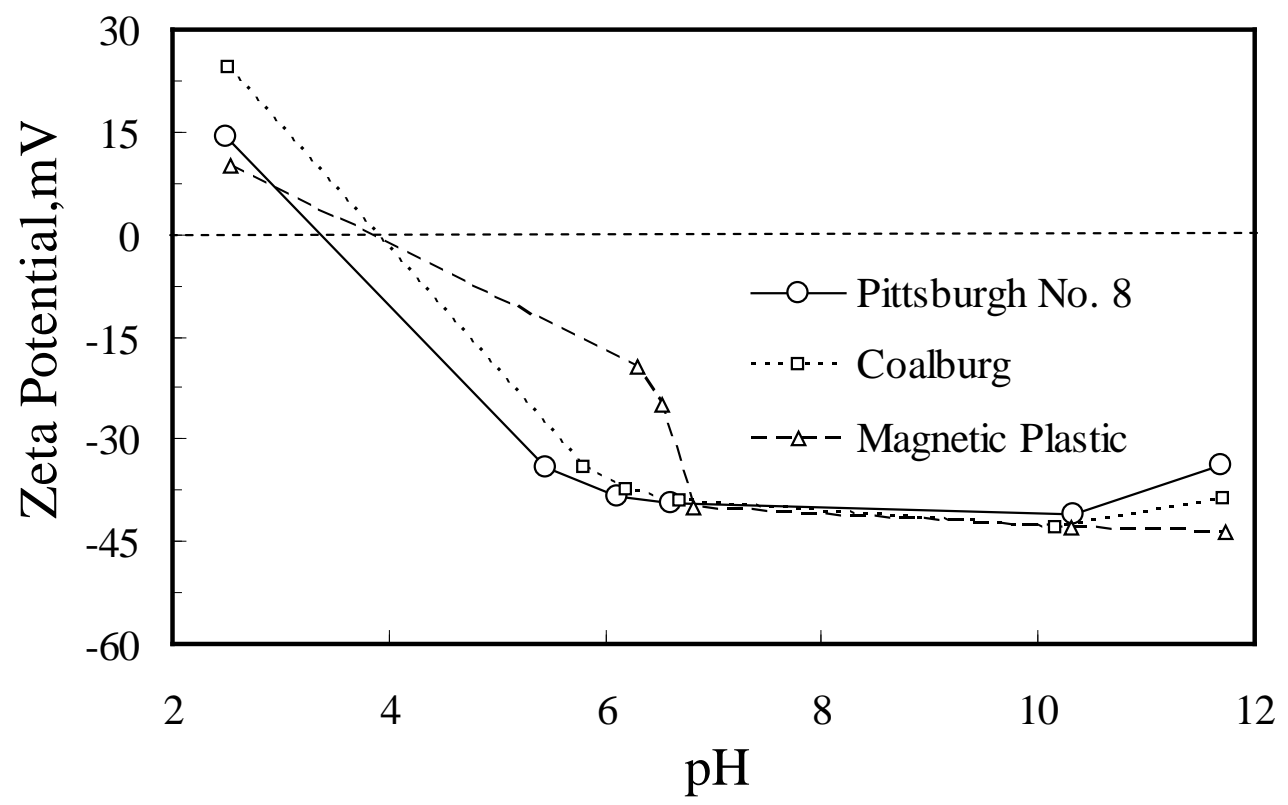

Figure 9. Zeta potential as a function of $\mathrm{pH}$ for the coal and magnetic plastic material. 


\section{Density Fraction Test}

The density fraction test was used to develop a washability curve to estimate the theoretical maximum mass yield and recovery expected while achieving a given product grade for a certain coal. The separation performances predict the theoretical performance, which means the recovery achieved while producing a given product quality can not be greater than the recovery achieved by density fractionation.

The density fraction tests were performed on the Pittsburgh No. 8 and Coalburg sample for particles smaller than $177 \mu \mathrm{m}$. LMT (Lithium Meta-Tungstate) was used as the heavy liquid to increase the density of the solution (specific density of $2.95 \mathrm{RD}$ ) with distilled water. The required medium density (i.e. 1.3, 1.4, 1.5, 1.6, 1.75 and 1.9 RD) was achieved by mixing LMT and distilled water in different beakers. The procedure followed the ASTM D4371 standard. The coal was submerged in the lowest density medium first. After allowing sufficient time for particle separation in the medium, the floated material was removed using a hand held screen. The material that sank to the bottom was recovered by filtration. The sink material was then submerged in the medium with the next highest density. This process was repeated through all of the mediums having a density range from $1.3 \mathrm{RD}$ to $1.9 \mathrm{RD}$. The float products and final tailings were rinsed, filtered, dried and weighed. Each sample was analyzed for ash content using the ASTM D3172 standard procedure.

\section{Flotation Rate Test}

As part of the coal characterization, flotation rate measurements were performed for the Pittsburgh No.8 and Coalburg coal samples with and without the addition of magnetic plastic material. Flotation rate tests were performed in the same manner as described in Section 3.1.3. However, the amount of magnetic plastic material added in the test $(2 \%$, $5 \%$ or $10 \%$ by weight) was subtracted from the total of 215 grams solid to obtain the net amount of coal remaining to be utilized which maintained the solid concentration at $5 \%$ by weight. After collecting the samples, coal was separated from the magnetic plastic material using the Eriez high gradient magnetic separator (Figure 6). After removing the plastic material, the floated coal fractions and final tailings were filtered, dried and weighed and subsequently analyzed for ash content.

\section{Column Flotation Test}

To assess the impact of adding the magnetic plastic material on separation performance, experiments were conducted in a laboratory flotation column. The tests were performed on both the Pittsburgh No. 8 and Coalburg coal samples using the specific parameter settings in Table 5. The ShurFlot 948 was used as the polyglycol frother. 
Table 5. Specific parameter values used for the magnetic plastic addition effects on column flotation performance.

Feed rate

Feed solids concentration by weight

\% of Plastic Added Relative to Feed

Frother concentration (ShurFlot 948)

Collector concentration (Fuel oil No. 2)
$200 \mathrm{ml} / \mathrm{min}$ to $1400 \mathrm{ml} / \mathrm{min}$

$2 \%$ to $12 \%$

$5 \%-10 \%$

15 ppm

$0.45 \mathrm{~kg} / \mathrm{ton}$

Two sets of column flotation tests were performed, the first set established a baseline performance without the use of the magnetic plastic material. These baseline tests were immediately followed by a series of tests that studied the impact of adding various amounts of plastic particles into the froth phase or directly in the feed.

Magnetic plastic material was added into the column flotation in two different ways. The first method was by mixing the external material directly into the feed slurry. In the second method, the injection was in the froth phase. In this case, an auxiliary sump was set up to hold the external material to be pumped into the froth zone. A pump helped to maintain a homogeneous mixture of the plastic particles in water. After obtaining steady state condition in the column flotation, the magnetic plastic material was injected into the froth phase through the wash water as depicted in Figure 10. The injection rate was 100 $\mathrm{ml} / \mathrm{min}$ of magnetic plastic slurry at $5 \%$ or $10 \%$ by weight and $300 \mathrm{ml} / \mathrm{min}$ of water to maintain the same overall volume of wash water that was supplied in the baseline column tests.

\section{Froth Refluxing}

As previously discussed, a method that could induce selective detachment is enrichment of the froth zone by recycling a portion of the froth product back into the froth. The experimental program conducted to investigate the use of external froth refluxing utilized the same fine coal samples as those in the magnetic plastic experiments. A flotation column was used in the circuit shown in Figure 11. Using a Y-connector with a valve on the stream reporting to the final product, the amount of froth concentrate was controlled and material routed to a sump. A pump was used to recycle a controlled amount of the froth product back to the column froth through the wash water system. The rate of injection of slurry into the froth zone was $100 \mathrm{ml} / \mathrm{min}$ plus $300 \mathrm{ml} / \mathrm{min}$ of fresh water to keep the same volume of wash water that was used in the baseline tests. Also, $200 \mathrm{ml} / \mathrm{min}$ of fresh water was required in the launder to flush down the reported concentrate through a pipe line where the Y-tube pipe provides a split of the concentrate stream. The process was totally continuous including the refluxing of the froth product. A period equivalent to three residence times was allowed to reach steady-state prior to the collection of the feed, product and tailing samples. The samples were filtered, dried, weighed and analyzed for ash and total sulfur content. 


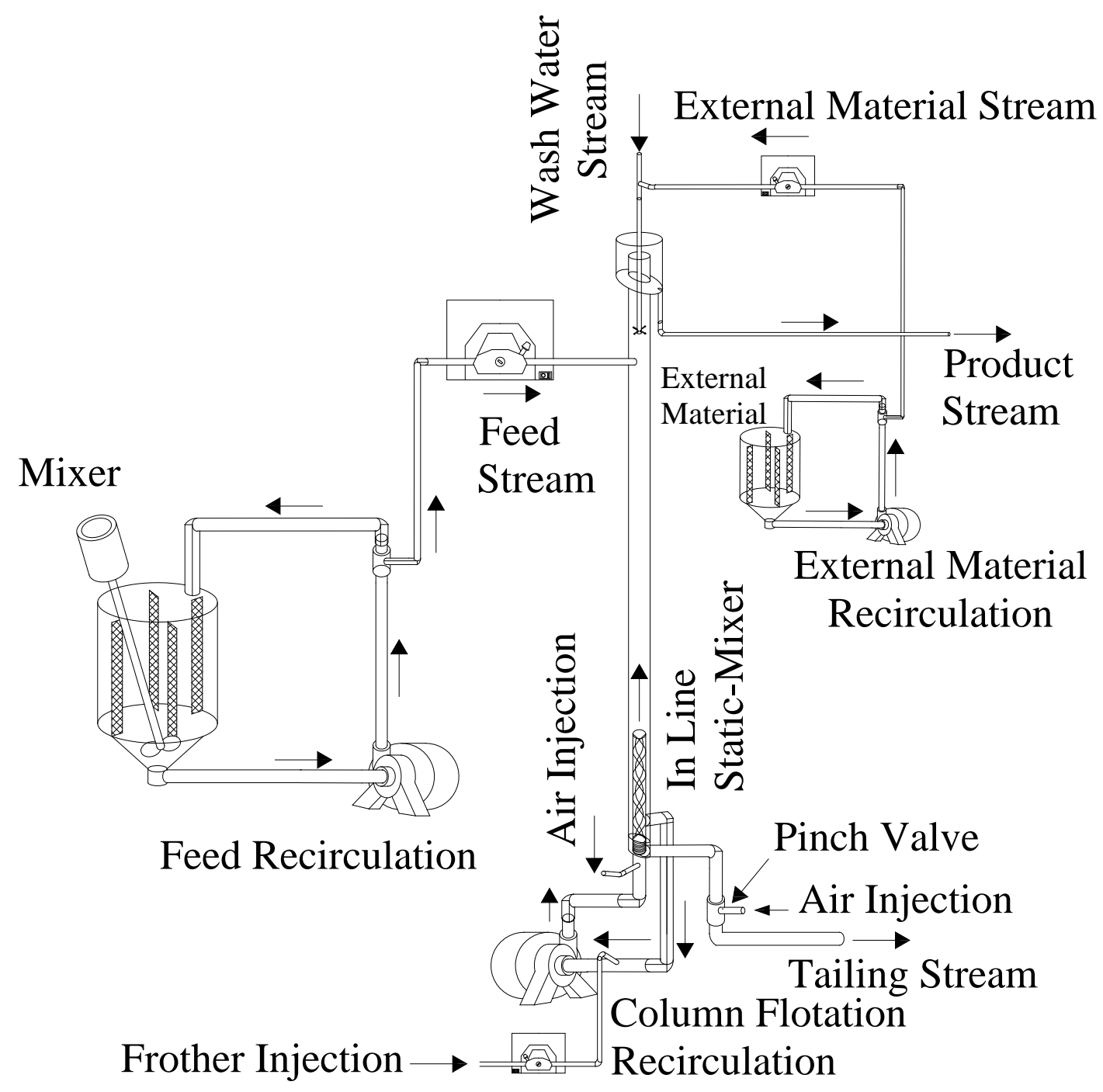

Figure 10. Column flotation circuit used to evaluate the performance benefits of the addition into the froth zone. 


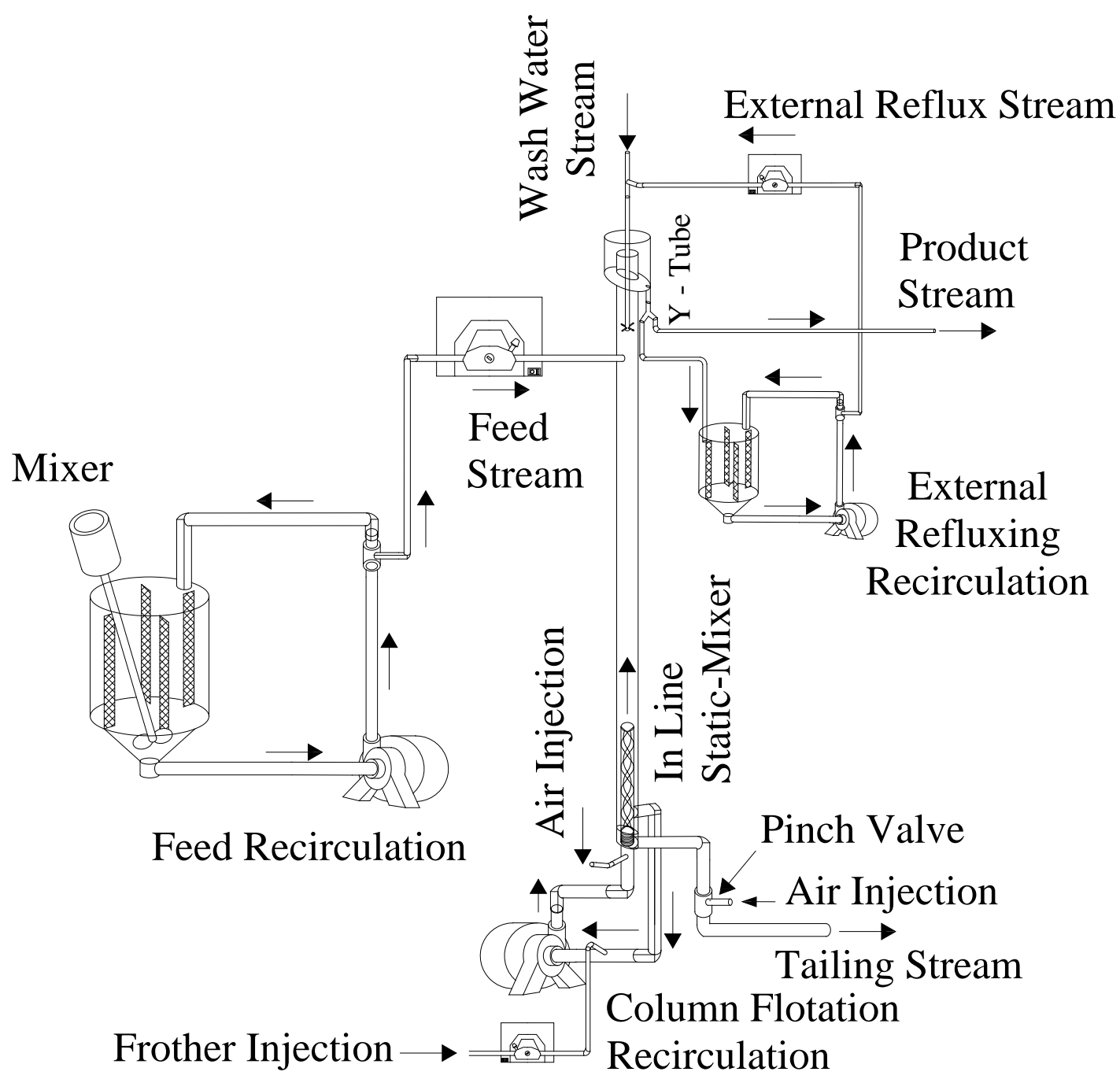

Figure 11. Column flotation used to study the impact of using external froth refluxing to improve separation. 


\section{RESULTS AND DISCUSSIONS}

\section{$\underline{\text { Air Eductor }}$}

Extensive evaluation of the Coalburg feed slurry shows that feed pretreatment through an air eductor has the potential to enhance flotation performance in a conventional cell. In the past, emphasis focused on developing new collectors for low floatability coal. However, a more practical technique that uses an air eductor mechanism has many potential benefits. Figure 12 summarizes the flotation performances achieved in a conventional flotation cell when treating Coalburg coal. After 60 seconds of flotation time, a combustible recovery of about $62 \%$ was achieved. When the feed was treated through a cavitation tube either one or recycled through several times, the recovery after 60 seconds was $80 \%$ which represents an increase of $18 \%$ absolute percentage points.

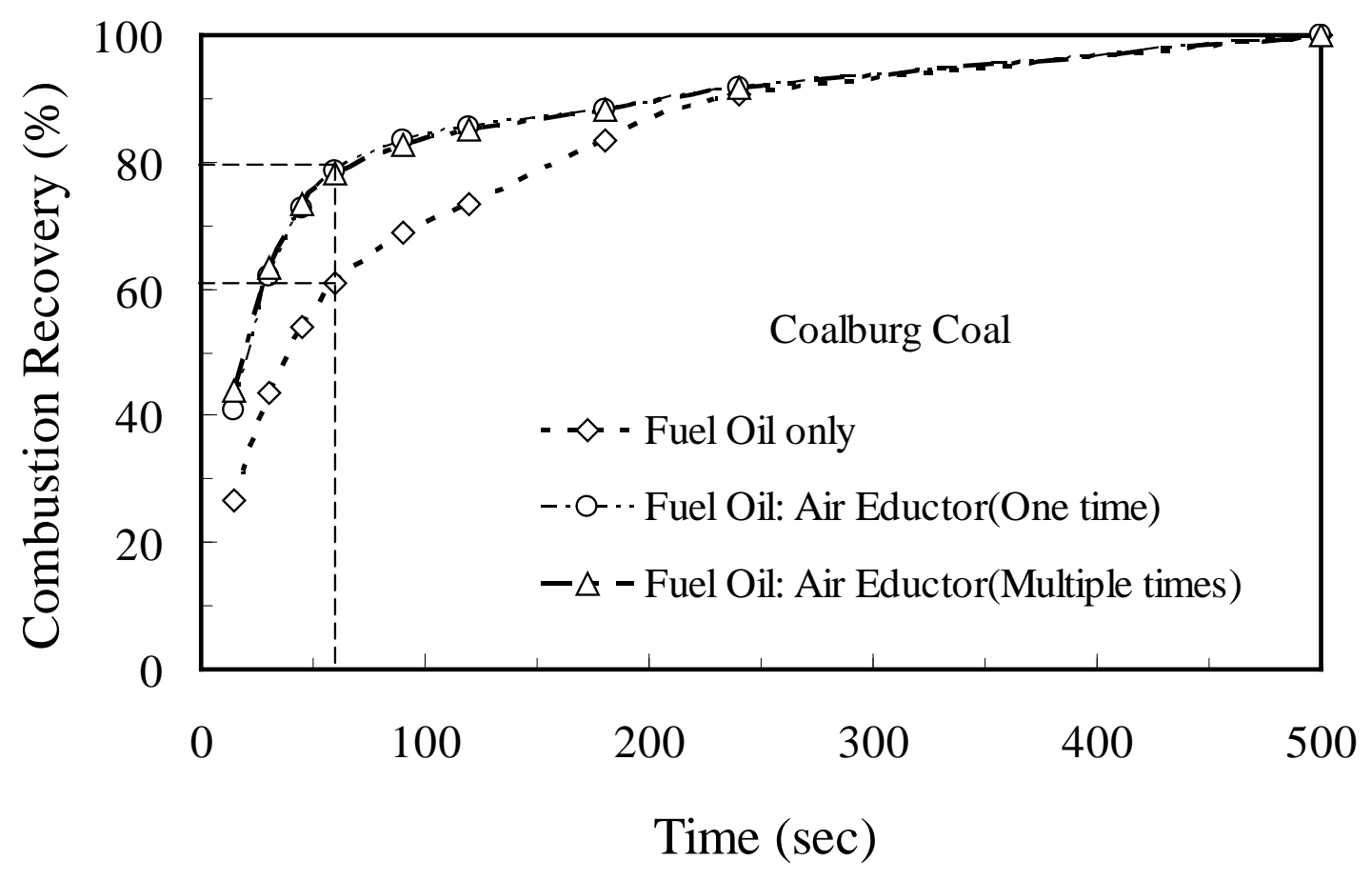

Figure 12. Effects of air eductor performance with the Coalburg seam coal sample in a laboratory conventional flotation cell.

Assuming perfect mixing and first-order kinetics conditions, the flotation rate constants were determined and tabulated for one minute residence time as presented in Table 6 . The flotation rate during the first minute of flotation nearly doubled as a result of using the air eductor. The mechanism for the improvement may be due to the nucleation of the micron-sized air bubbles onto the surface of the weakly hydrophobic coal surface. Evidence provided by Krasocoska and Malysa (2006) indicates that a surface that is 
coated with micron-sized bubbles results in a significant reduction in the induction time upon colliding with a conventional bubble size. As a result, the probability of attachment and thus flotation rate is increased. Similar results in flotation recovery improvement were reported by Attallo et al. (2000) and Zhou et al. (1997).

Table 6. Improvements in flotation rate and recovery using data obtained after one minute of flotation for the Coalburg coal.

\begin{tabular}{|l|c|c|}
\hline \multicolumn{1}{|c|}{$\begin{array}{c}\text { Test } \\
\text { Identification }\end{array}$} & $\begin{array}{c}\text { Flotation } \\
\text { Rate } \\
\left(\mathrm{min}^{-1}\right)\end{array}$ & $\begin{array}{c}\text { Flotation } \\
\text { Recovery } \\
(\%)\end{array}$ \\
\hline Fuel Oil only & 0.65 & 61.0 \\
Air Eductor (Single Pass) & 1.25 & 78.4 \\
Air Eductor (Multiple Pass) & 1.24 & 78.2 \\
\hline
\end{tabular}

Multiple passes of the feed slurry through the air eductor did not further improve the flotation constant rate as indicated in Table 6. However, Figure 13 shows the potential for improving selectivity by passing the slurry through the air eductor multiple times. The improvement is noted by the shift to the left of the recovery-product ash curve. The selectivity improvement may have occurred due to the selective removal of air bubbles from the more weakly hydrophobic particles that contain higher qualities of ash-forming minerals.

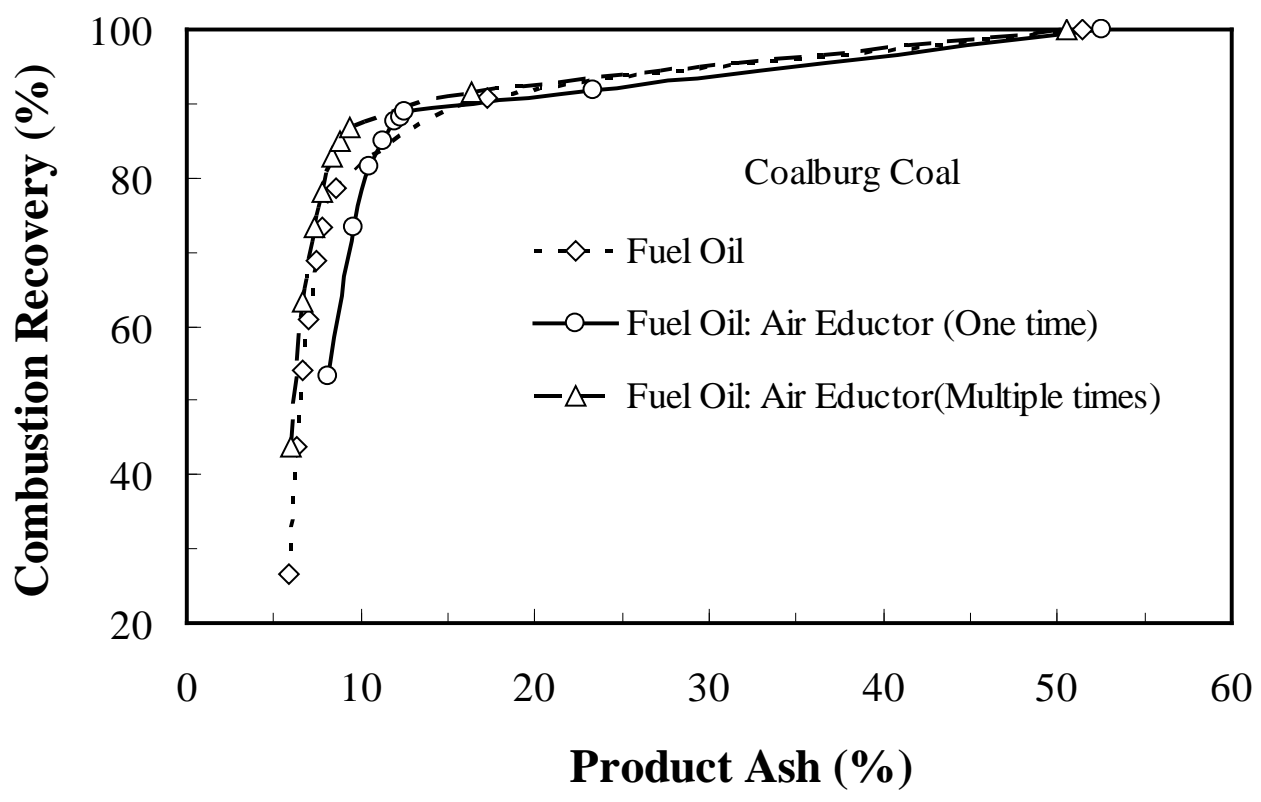

Figure 13. Effect on performance achieves with air eductor for a Coalburg coal sample obtained from flotation rate tests in a conventional flotation cell. 
Considering the notable positive effect observed in the laboratory conventional flotation cells, column flotation tests were performed to evaluate performance on a laboratory flotation column. As indicated in Figure 14, no appreciable difference was obtained from the flotation column tests at the low and high volumetric feed flow rates. However, when moderate volumetric flow rates, the cavitation process improved recovery and reduced the product ash content. A five absolute percentage point improvement in recovery was achieved when the volumetric feed flow rate was between 400 and $600 \mathrm{ml} / \mathrm{min}$.

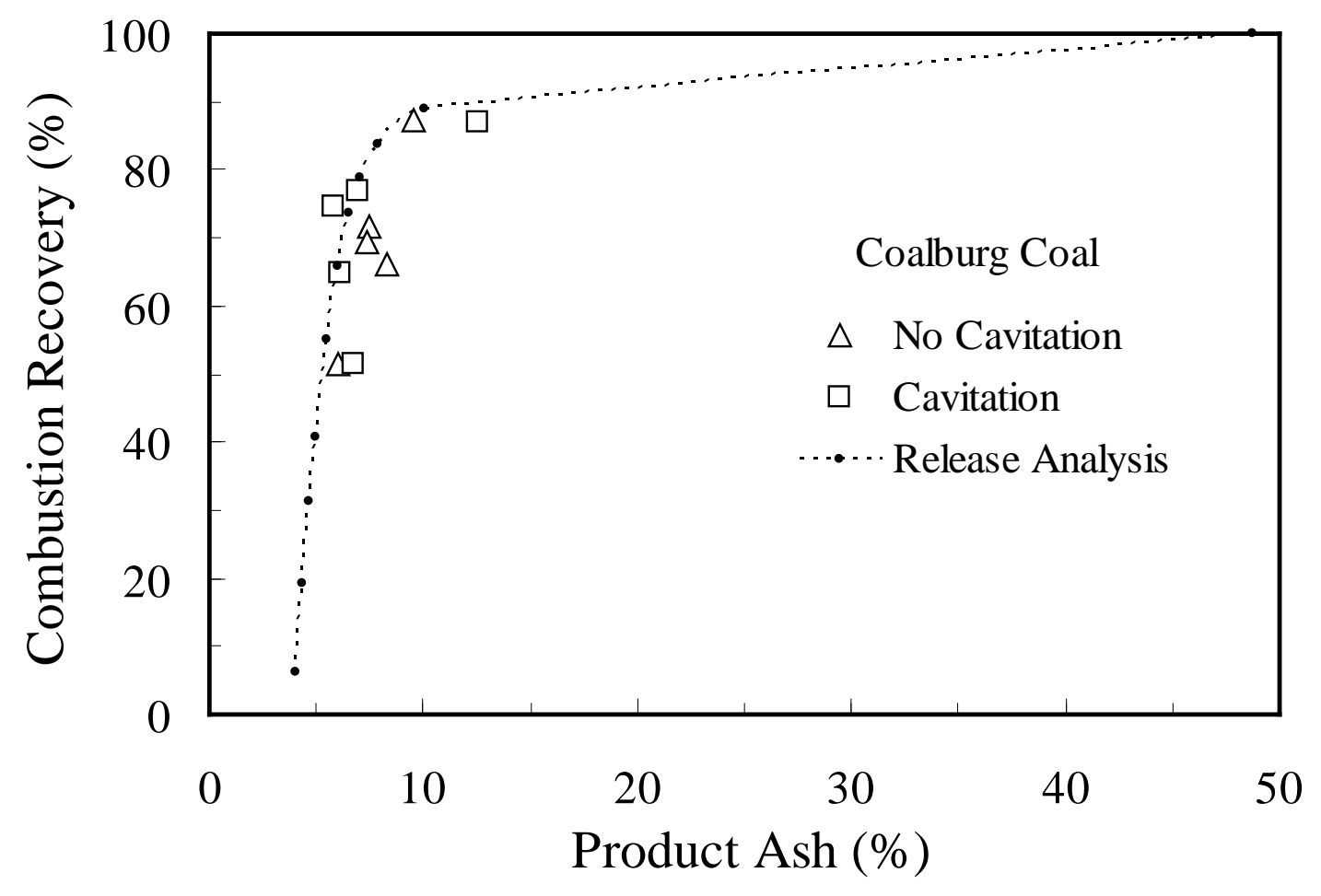

Figure 14. Effects on flotation performance with air eductor utilization for a Coalburg coal preparation plant sample.

In tests investigating the impact of the air eductor feed pretreatment, little-to-no gain in recovery was observed while varying flotation time in a column as shown in Figure 15. This may be due to the experimental set-up which processed the feed stream through a pump and sump after the cavitation tube and before the flotation column. 


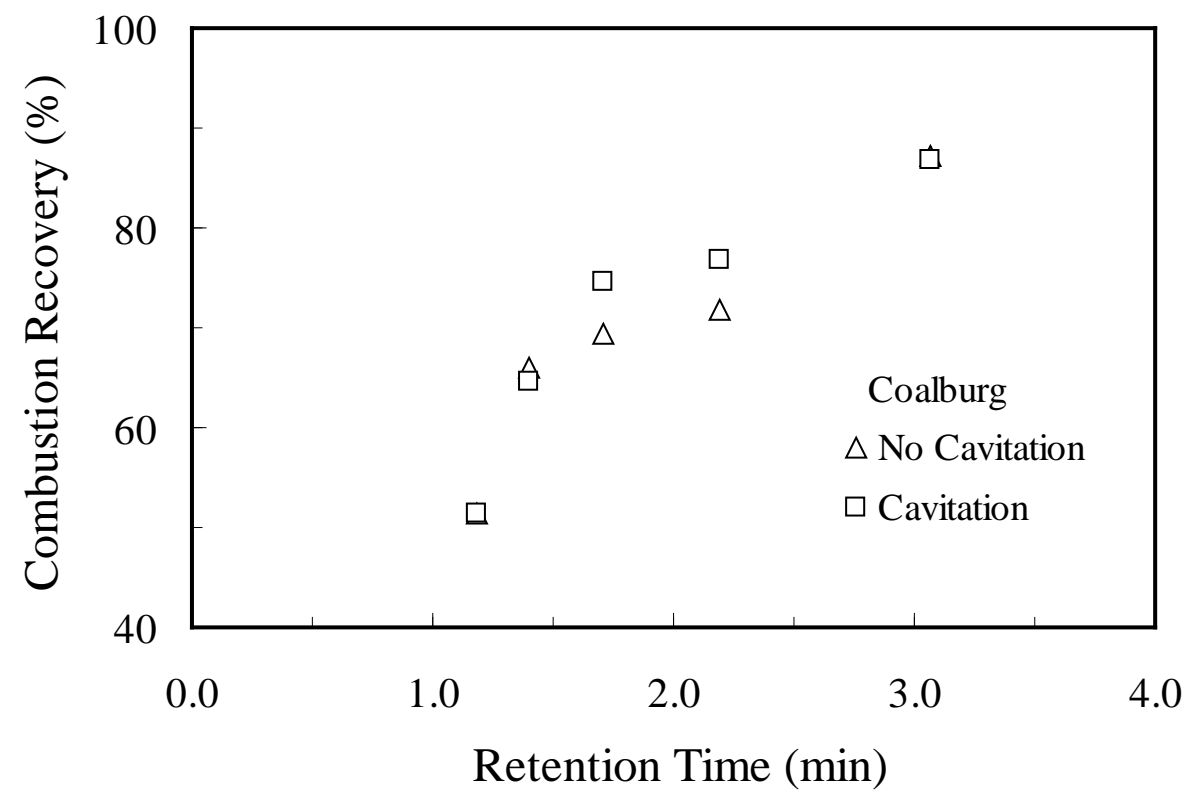

Figure 15. Effects of air eductor on flotation performance under different retention time conditions with a column flotation.

\section{$\underline{\text { Magnetic Plastic }}$}

\section{Conventional Flotation Tests}

The separation performance improvements resulting from the addition of magnetic plastic particles were initially evaluated in a laboratory conventional flotation cell. The plastic particles were mixed into the feed slurry at concentrations expressed as a percentage of the total solid mass. The flotation tests were performed as a function of time on two different coals samples and varying operating conditions.

The impact of magnetic plastic concentration on flotation performance was evaluated over at concentration values of $0 \%, 2 \%, 5 \%$ and $10 \%$ by weight. Figure 16 shows the recovery improvement achieved as a function of time and plastic particle concentration when treating Pittsburgh No. 8 coal. Increasing the plastic concentration from $2 \%$ to 5\% by weight enhanced the combustible recovery by about 15 absolute percentage points. Doubling the concentration to $10 \%$ by weight further increased recovery by another 5 absolute percentage points. Evidence presented in this chapter will point to the high degree of hydrophobicity of the plastic particles $\left(\theta_{\mathrm{w}}=84^{0}\right)$ as the reason for the recovery improvement. 


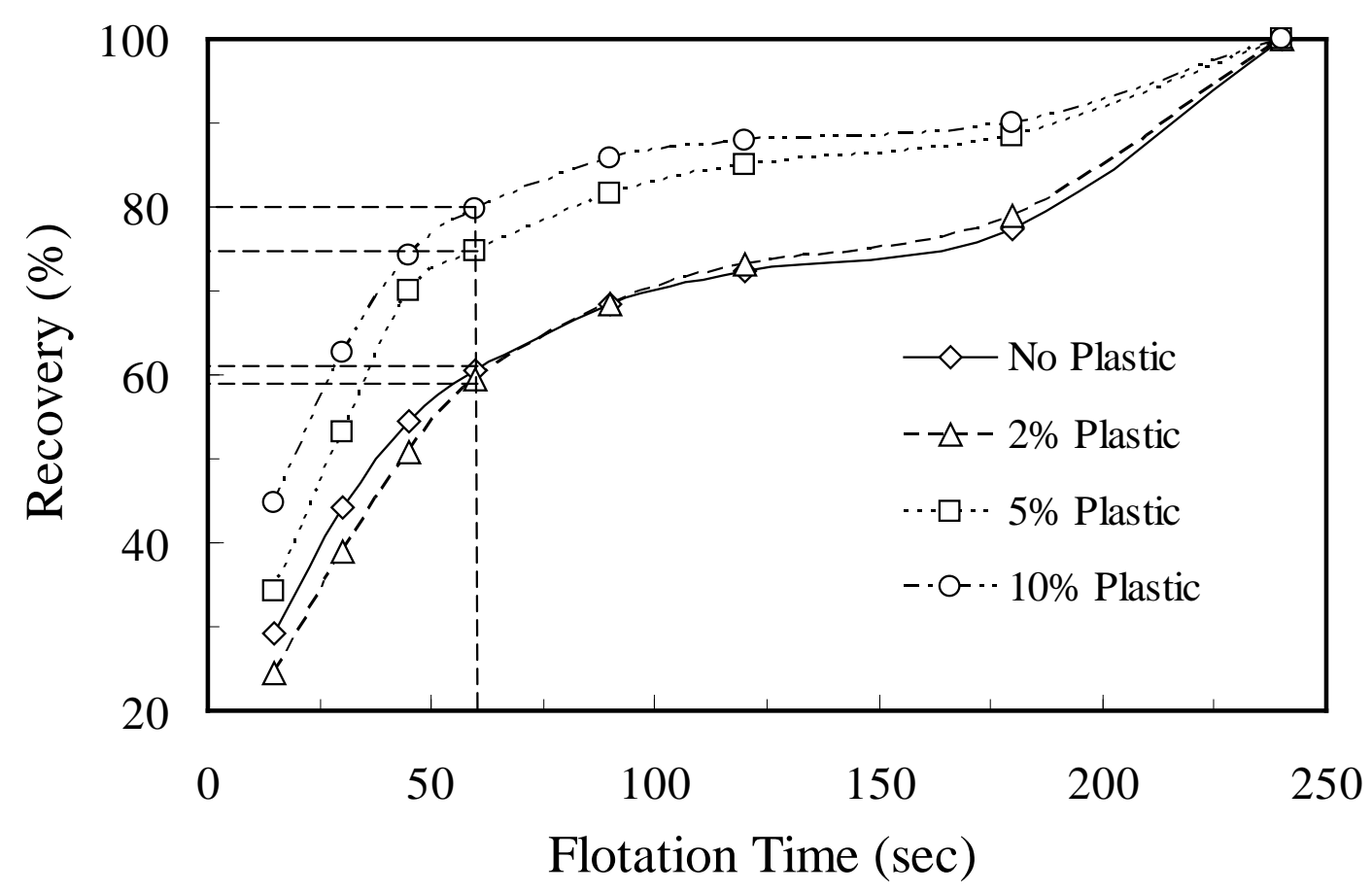

Figure 16. Recovery improvements achieved as a function of flotation time and varying amounts of plastic particles when treating Pittsburgh No. 8 coal in a conventional

flotation cell.

The flotation rates were determined from the data presented in Figure 16. The natural flotation rate of the Pittsburgh No. 8 coal was found to be $0.58 \mathrm{~min}^{-1}$. By adding the plastic particles at a $5 \%$ by weight concentration, the flotation rate was increased to 0.93 $\mathrm{min}^{-1}$, which equates to a $75 \%$ enhancement.

The absolute percentage improvement in recovery as a function of plastic particle concentration is more clearly shown in Figure 17. It is evident that the $10 \%$ plastic concentration has a significant impact on recovery with a 15 absolute percentage point gain after just 15 second of flotation. The maximum recovery improvement occurs after 45 seconds of flotation where a 20 absolute percentage point gain is realized. 


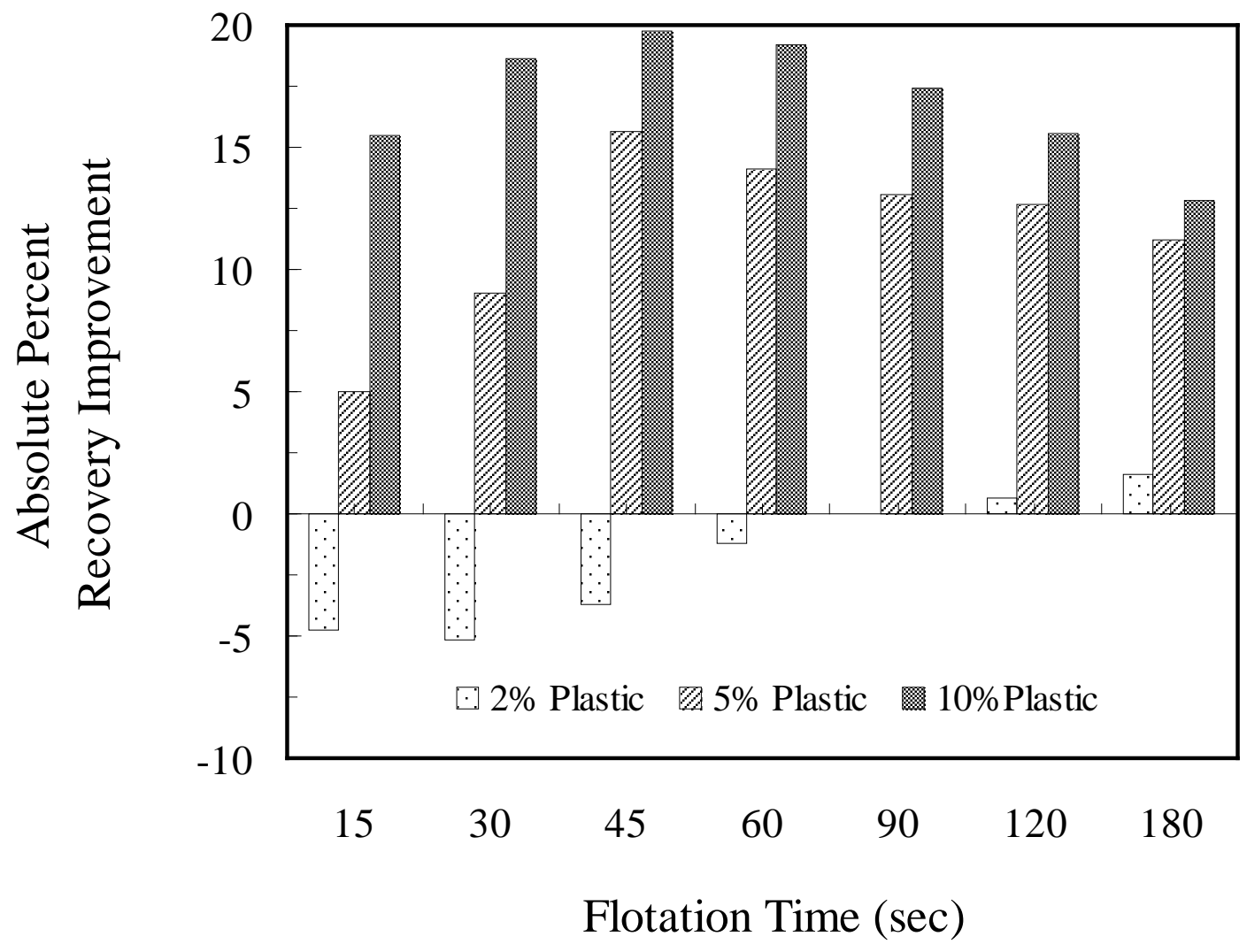

Figure 17. Absolute percent recovery improvement as a function of magnetic plastic concentration in a conventional flotation cell; Pittsburgh No. 8 coal.

The initial tests reveal that no improvement in selectivity was realized when adding the plastic particles. As shown in Figure 18, the ash contents in the froth concentrates were increased with the $5 \%$ and $10 \%$ additions. The finding indicates that the plastic particles may be preferentially enhancing the floatability of the higher ash (middles) particles or reducing drainage of the water containing entrained clay particles surrounding the air bubbles. Also, there is a correlation between the amount of magnetic plastic addition and enhancement in flotation performance as it is described in Table 7. 


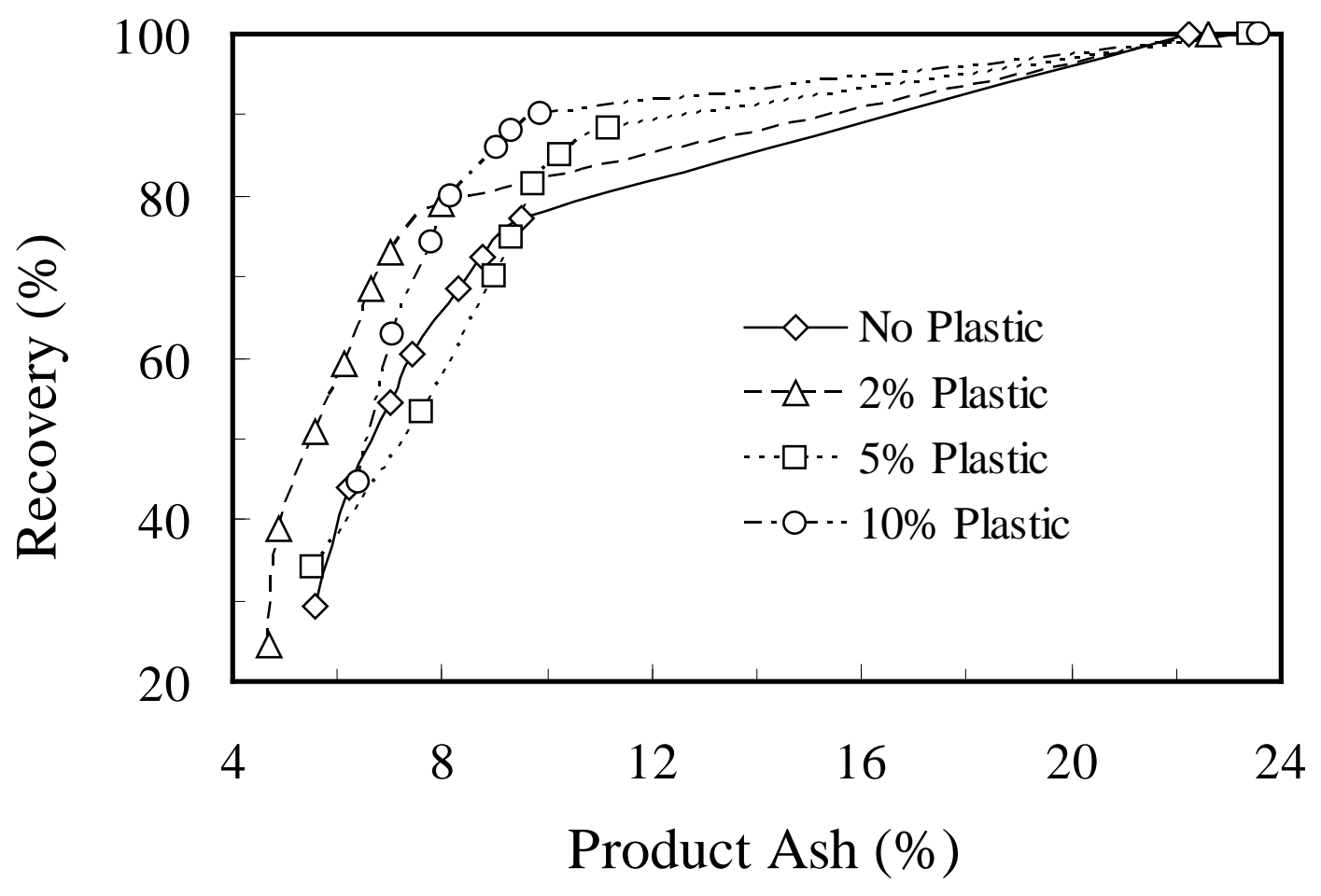

Figure 18. Selectivity performances for varying amounts of magnetic plastic material in a conventional flotation cell treating Pittsburgh No. 8 coal.

Table 7. Flotation performance with addition of $2 \%, 5 \%$ and $10 \%$ magnetic plastic material after one minute of flotation in a conventional cell.

\begin{tabular}{|l|c|c|}
\hline \multicolumn{1}{|c|}{$\begin{array}{c}\text { Test } \\
\text { Identification }\end{array}$} & $\begin{array}{c}\text { Flotation } \\
\text { Rate } \\
\left(\mathrm{min}^{-1}\right)\end{array}$ & $\begin{array}{c}\text { Flotation } \\
\text { Recovery } \\
(\%)\end{array}$ \\
\hline 0\% Plastic Material & 0.58 & 60.6 \\
\hline 2\% Plastic Material & 0.60 & 59.3 \\
\hline 5\% Plastic Material & 0.93 & 74.7 \\
\hline 10\% Plastic Material & 0.95 & 79.8 \\
\hline
\end{tabular}

The recovery of the magnetic particles in the flotation process was also measured as a function of flotation time. The majority of the plastic particles are recovered within the first 15 seconds of flotation and nearly 100\% are recovered in the first minute of flotation (Figure 19). This finding indicates that any benefit in the flotation of coal should occur within a retention time of one minute. 


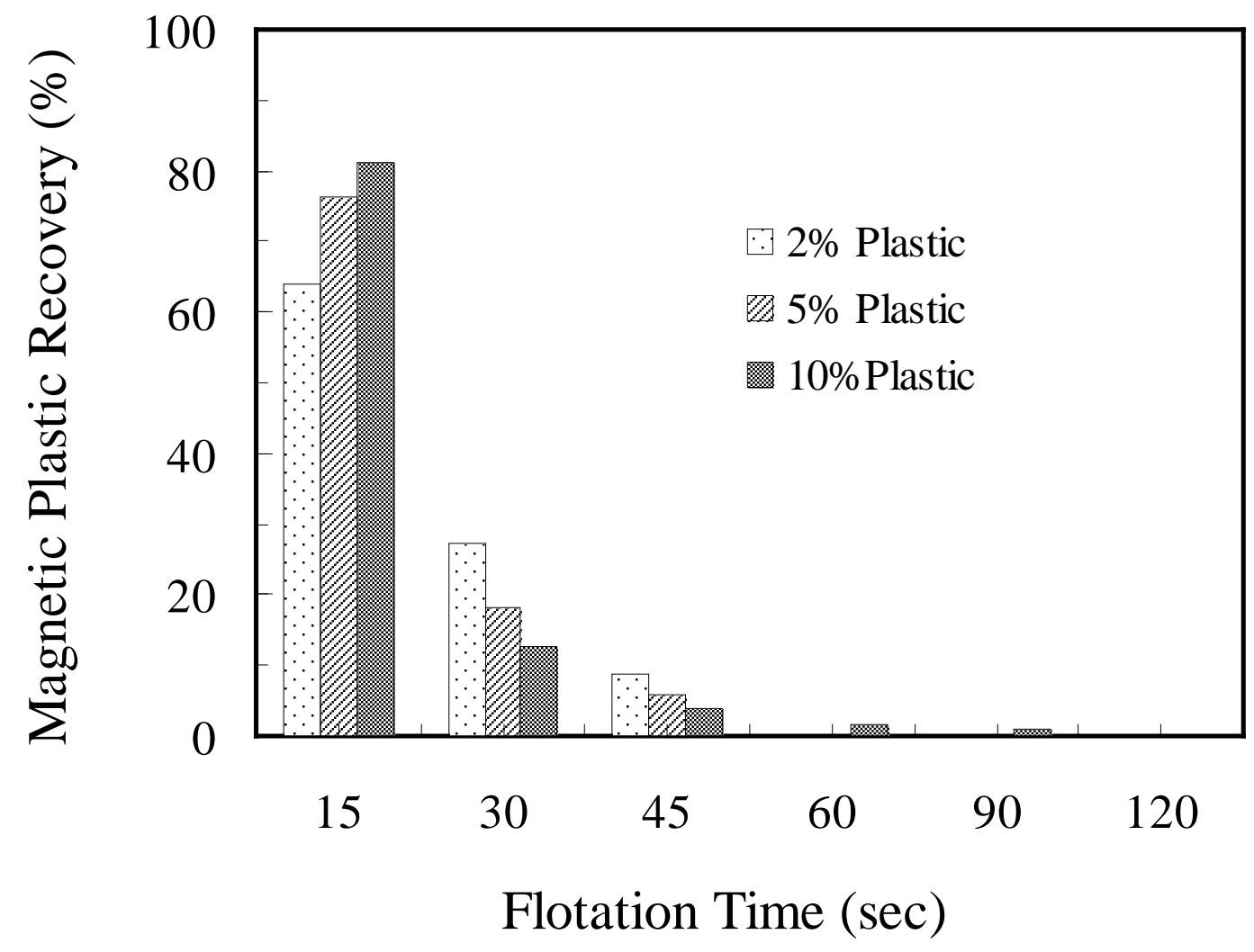

Figure 19. Recovery of magnetic plastic material from flotation kinetic rate test at different magnetic plastic concentration (2\%, 5\% and 10\%).

\section{Repetitive Performance Evaluation}

After the favorable results were obtained, the next evaluation assessed the repeatability of the improved performances at plastic concentrations of $0 \%$ and $10 \%$. The tests were performed under the same conditions five times to obtain statistical evidence of the phenomena. The average results from the analysis are shown in Figure 20. The same positive impact with the use of magnetic plastic material was observed with an improvement of 11 absolute percent point in recovery and almost a 30\% rise in the flotation rate constant as show in Table 8. A small loss in overall recovery was observed but significant improvement in flotation performance was obtained. 


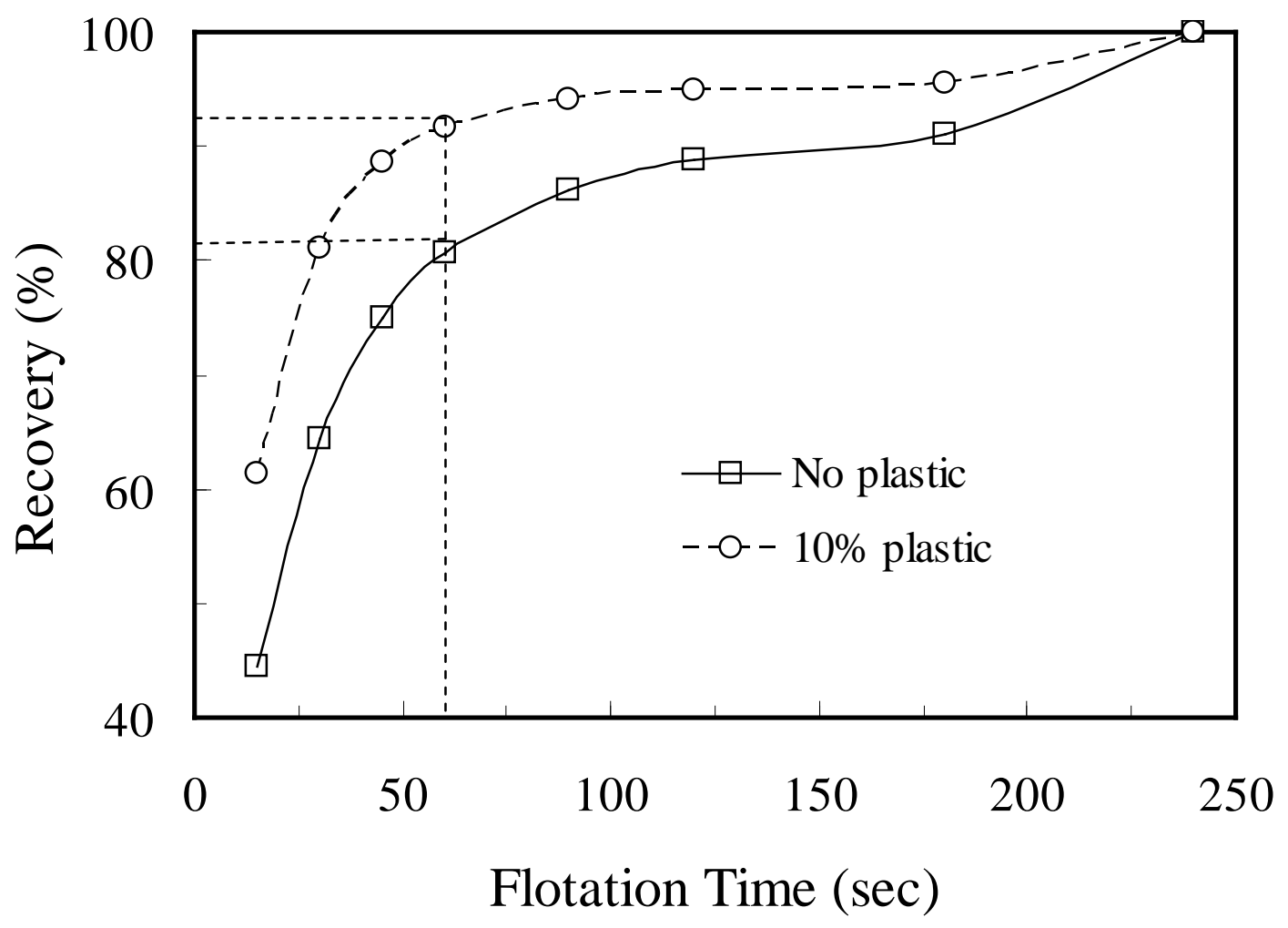

Figure 20. Average recovery improvements achieved after repeating the flotation test under the same conditions with $10 \%$ magnetic plastic and no magnetic plastic for a total of five times; Pittsburgh No. 8 coal.

The recovery data achieved within the first minute of flotation were used to determine the flotation rate. From the average recovery data collected from five test repetitions, the flotation rate of the Pittsburgh No. 8 coal was increased from $1.04 \mathrm{~min}^{-1}$ to $1.31 \mathrm{~min}^{-1}$ by the addition of the magnetic plastic particles at a concentration of $10 \%$ by weight as shown in Table 8 . The rate was increased by $26 \%$.

Table 8. Flotation performances with five times repetition at $0 \%$ and $10 \%$ magnetic plastic material using a residence time of one minute.

\begin{tabular}{|l|c|c|}
\hline \multicolumn{1}{|c|}{$\begin{array}{c}\text { Test } \\
\text { Identification }\end{array}$} & $\begin{array}{c}\text { Flotation } \\
\text { Rate } \\
\left(\mathrm{min}^{-1}\right)\end{array}$ & $\begin{array}{c}\text { Flotation } \\
\text { Recovery } \\
(\%)\end{array}$ \\
\hline No Plastic & 1.04 & 80.7 \\
10\% Plastic & 1.31 & 91.7 \\
\hline
\end{tabular}

Figure 21 addresses the impact on selectivity realized with the addition of $10 \%$ magnetic plastic material compared to the baseline achieved without the addition of the magnetic plastic particles. As typical, higher recovery values are associated with an increase in ash 
content. The results represent the average data obtained from five experimental test repetitions with $0 \%$ and $10 \%$ magnetic plastic material, when treating Pittsburgh No. 8 coal.

The steep portion of the curve was obtained within the first 45 seconds of flotation which is the time period that most of the plastic particles report to the froth concentrates. As shown in Figure 20, this is also the same period of time in which the largest recovery gain is achieved which also leads to elevated product ash content (Figure 21). Considering the individual incremental ash in both cases for each sample at the same period of time, higher incremental ash content is observed with the use of magnetic plastic material. Thus, the sloped curve in the first 45 second of flotation without adding magnetic plastic material is $31 \%(\Delta H / \Delta L)$ against $21 \%\left(\Delta H^{\prime} / \Delta L^{\prime}\right)$ with addition of magnetic plastic material. However, the lower slope curve is corresponds to the higher recovery values observed within the first 15 seconds of flotation when using the magnetic plastic material. The first 45 seconds of regular flotation without magnetic plastic material has the same effect as 30 seconds of flotation with the magnetic plastic material. The majority of the impact is concentrated within the first 15 seconds of flotation. That is the same period during which the majority of the plastic particles report to the flotation concentrates.

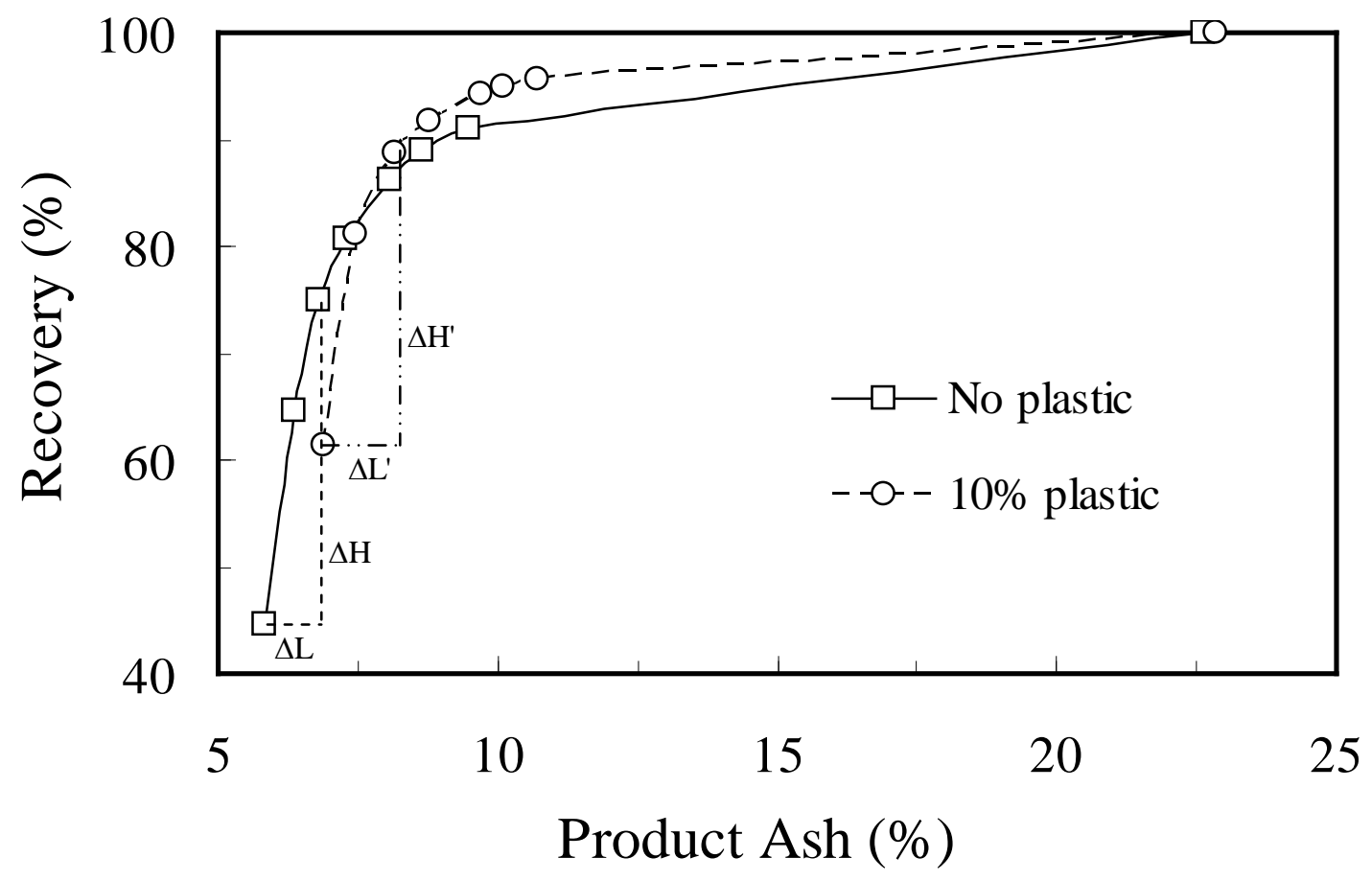

Figure 21. Average improvements in flotation performance achieved by the additional of magnetic plastic material; five experimental repetitions.

The impact of magnetic plastic material on the reduction of pyrite when treating Pittsburgh No. 8 coal samples is minimal based on the data given of Figure 22. The data 
clearly show the improvement in recovery, however, absolute reduction in total sulfur does not indicate a clear impact from the plastic particle addition. It is unclear whether these results reflect the incomplete liberation of the pyrite or the possibility that the plastic particles could adsorb onto the weakly hydrophobic pyrite thereby elevating their surface hydrophobicity. Entrainment could provide a countering effect for any positive selectivity gains (Kawatra and Eisele, 1996).

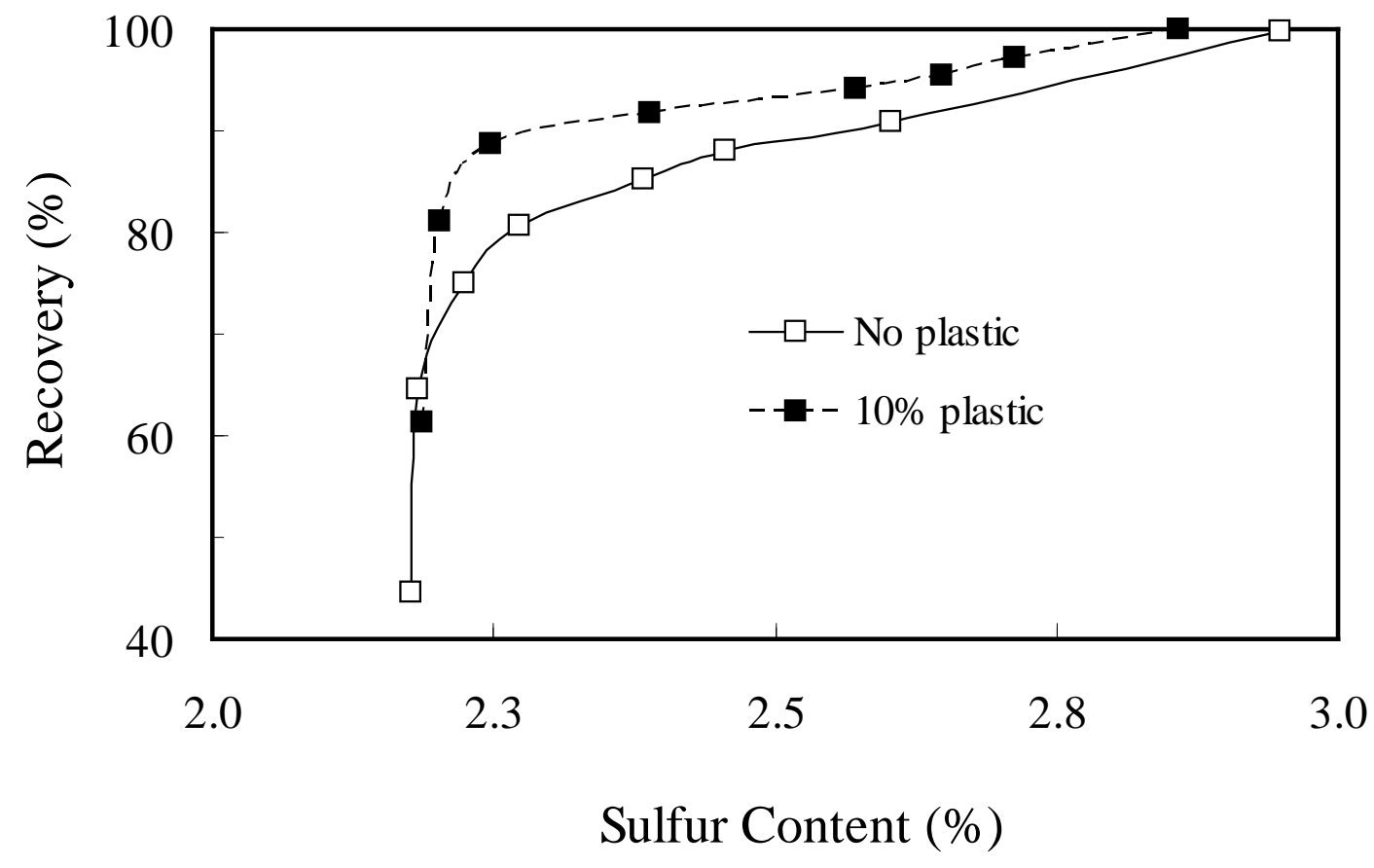

Figure 22. Effect of $10 \%$ magnetic plastic material on sulfur content. Average result of five flotation test.

\section{Effect of Coal Type}

The Coalburg coal seam in the central Appalachia coalfields is well known as a difficultto-clean coal. This characteristic is mainly due to the amount of middling (mixed phase), unliberated particles in the coal, which is in contrast with the Pittsburgh No. 8 coal. The Coalburg coal also has poor floatability characteristics, which is reflected in the low flotation rate value of $0.51 \mathrm{~min}^{-1}$.

The addition of the plastic particles into the Coalburg flotation feed slurry at a $10 \%$ by weight concentration significantly improved the recovery rate. However, the improvement was smaller than that realized for the Pittsburgh No. 8 coal. As shown in Figure 23, coal recovery after one minute of flotation improved from $61.1 \%$ to $77.4 \%$ by the addition of the plastic particles in a conventional cell. As shown in Table 9, the flotation rate increased from $0.51 \mathrm{~min}^{-1}$ to $0.74 \mathrm{~min}^{-1}$ which is a $145 \%$ improvement. 


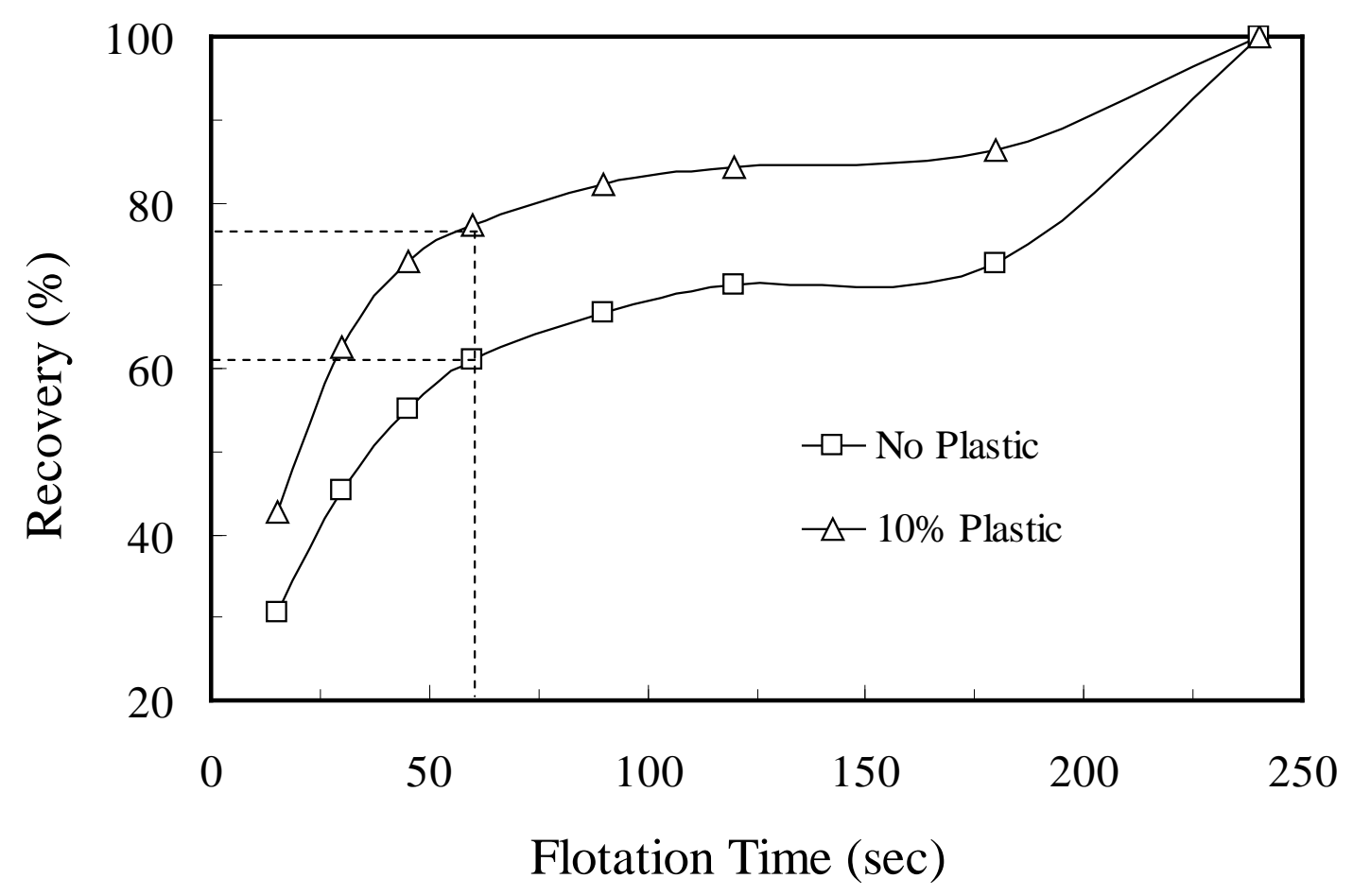

Figure 23. Flotation recovery rate improvement achieved when adding $10 \%$ by weight of plastic particles to the Coalburg flotation feed in a conventional cell.

Table 9. Flotation rate improvements achieved by the addition of plastic particles at a $10 \%$ by weight concentration to Coalburg coal feed slurry.

\begin{tabular}{|c|c|c|}
\hline $\begin{array}{c}\text { Test } \\
\text { Identification }\end{array}$ & $\begin{array}{c}\text { Flotation } \\
\text { Rate } \\
\left(\mathrm{min}^{-1}\right)\end{array}$ & $\begin{array}{c}\text { Flotation } \\
\text { Recovery } \\
(\%)\end{array}$ \\
\hline No Plastic, Coalburg & 0.51 & 61.1 \\
10\% Plastic, Coalburg & 0.74 & 77.4 \\
\hline
\end{tabular}

The selectivity of the flotation process was also enhanced by the plastic particles. As shown in Figure 24, the recovery increase varied as a function of product ash which is indicative of a selectivity improvement. When producing a product containing $15 \%$ ash, coal recovery was increased by 10 absolute percentage points. 


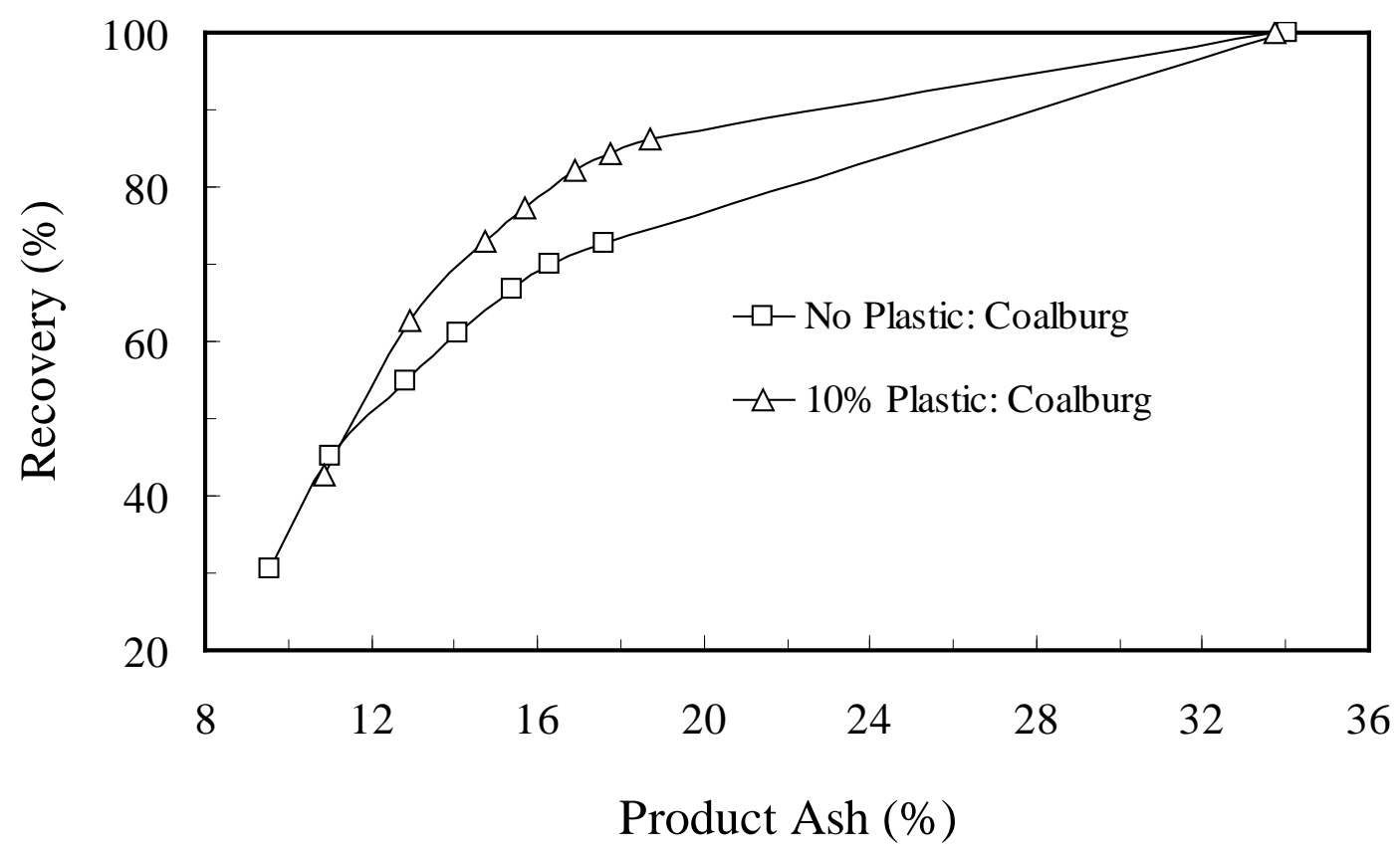

Figure 24. Selectivity performance improvement achieved by the addition of plastic particles at a $10 \%$ by weight concentration to a conventional cell flotation feed containing Coalburg coal.

\section{Effect of $\mathrm{pH}$}

As previously discussed, the electrostatic charge of the particle and bubble surfaces plays a significant role in bubble-particle attachment. The zeta potential, which is an approximation of the surface charge, varies as a function of $\mathrm{pH}$ for both the Pittsburgh No. 8 and plastic particles. In general, the electrostatic charge is negative and increases in magnitude as the $\mathrm{pH}$ is elevated from 3 to 10 . Obviously, higher surface charges lead to greater repulsive forces which could slow or prevent attachment to a bubble. Optimum recovery typically occurs under $\mathrm{pH}$ conditions that provide neutrally charged coal surfaces.

As shown in Figure 25, the best separation performances were achieved under the neutral $\mathrm{pH}$ conditions, which likely corresponded to a $\mathrm{pH}$ value slightly lower than 7.0 for the Pittsburgh No. 8 coal. The flotation recovery obtained under $\mathrm{pH}$ values of 3.9 and 10.1 were significantly depressed as indicated by recovery values of only $32.8 \%$ after 1 minute of flotation, which is about 27 percentage points less than that achieved at the neutral $\mathrm{pH}$ conditions. At $\mathrm{pH} 3.9$, the electrostatic charge on the coal particles was relatively low so the poor recovery was unexpected. However, it is consistent with previously reported coal flotation results. 


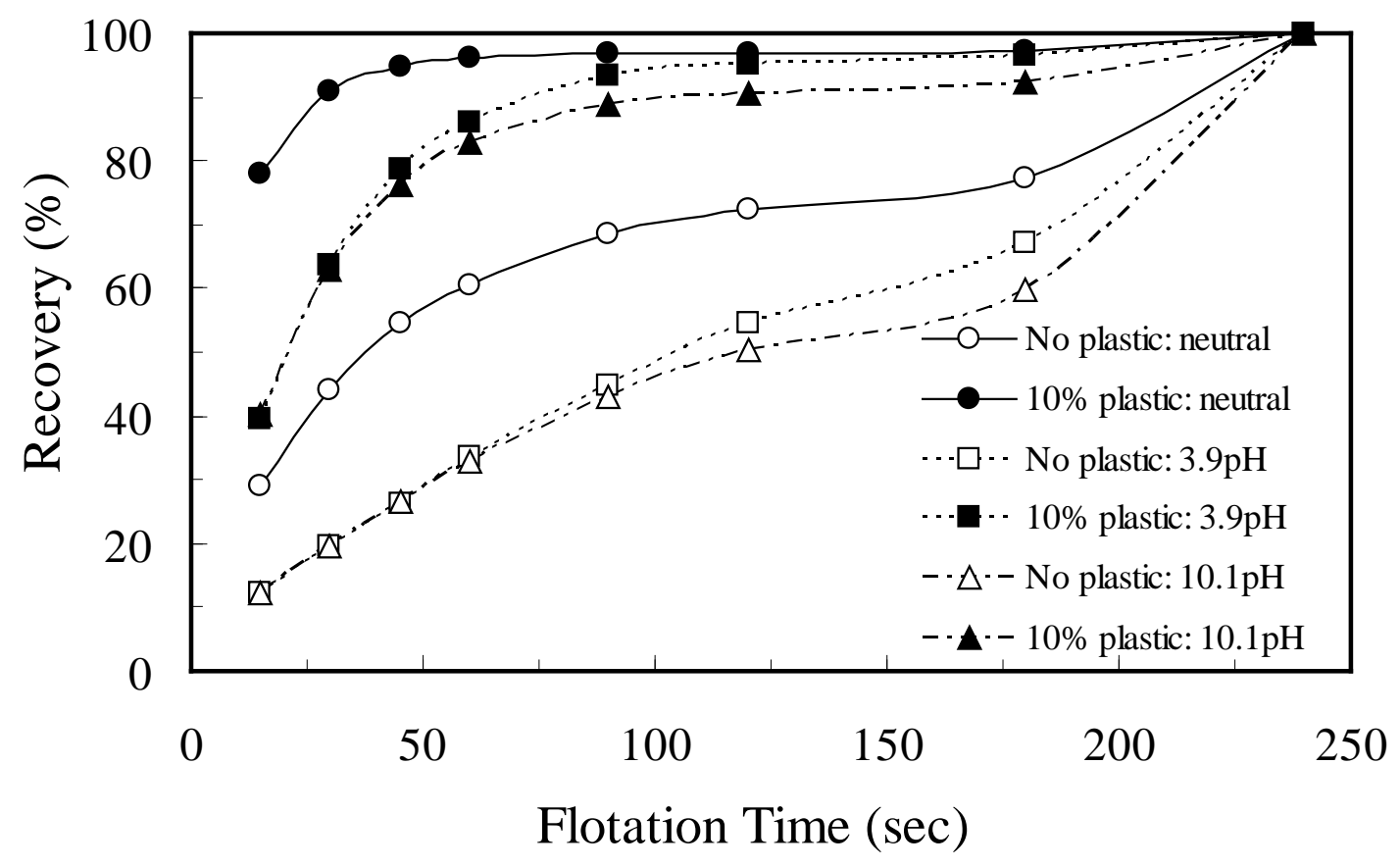

Figure 25. Impact of slurry $\mathrm{pH}$ on the recovery improvements realized when adding plastic particles to a conventional flotation feed containing Pittsburgh No. 8 coal.

By adding the plastic particle at a concentration of $10 \%$ by weight, flotation recovery after one minute increased by nearly 50 absolute percentage points under the $\mathrm{pH}$ conditions of 3.9 and 10.1. The recovery improvement is directly a result of the flotation rate rise from $0.30 \mathrm{~min}^{-1}$ to $1.51 \mathrm{~min}^{-1}$ at $\mathrm{pH} 3.9$ and from $0.30 \mathrm{~min}^{-1}$ to $1.33 \mathrm{~min}^{-1}$ at $\mathrm{pH}$ 10.1 (Table 4.5). The explanation is likely linked to the high hydrophobic plastic particles. A surface with a high degree of surface hydrophobicity produces a highly attractive interactive force with other hydrophobic particles that can overcome the electrostatic repulsive forces. 
Table 10. Flotation rate improvements achieved under varying $\mathrm{pH}$ conditions when adding plastic particles to Pittsburgh No. 8 coal at a concentration of $10 \%$ by weight.

\begin{tabular}{|c|c|c|c|}
\hline \multicolumn{2}{|c|}{ Test Identification } & $\begin{array}{c}\text { Flotation } \\
\text { Rate }\left(\mathrm{min}^{-1}\right)\end{array}$ & $\begin{array}{c}\text { Flotation } \\
\text { Recovery (\%) }\end{array}$ \\
\hline \multirow{2}{*}{ Neutral } & No Plastic & 0.66 & 60.5 \\
& $10 \%$ Plastic & 1.32 & 96.0 \\
\hline \multirow{2}{*}{ pH 3.9 } & No Plastic & 0.30 & 32.8 \\
& $10 \%$ Plastic & 1.51 & 85.8 \\
\hline \multirow{2}{*}{ pH 10.1 } & No Plastic & 0.30 & 32.8 \\
& $10 \%$ Plastic & 1.33 & 82.7 \\
\hline
\end{tabular}

The addition of the plastic material also appears to provide a selectivity improvement at the $\mathrm{pH}$ conditions of 3.9 and 10.1. As shown in Figure 26, the performance improvement appears totally due to recovery gains for the neutral $\mathrm{pH}$ values of 3.9 and 10.1 shift to the left and upward when the plastic particles are added, which indicates a reduction in the product ash content while also elevating coal recovery. The coal recovery gains are more clearly highlighted as a function of flotation time in Figure 27 for the three $\mathrm{pH}$ conditions studied. The neutral $\mathrm{pH}$ condition had the largest gain in the shortest time (15 seconds) while the largest overall improvement was realized under $3.9 \mathrm{pH}$ after 45 seconds of flotation.

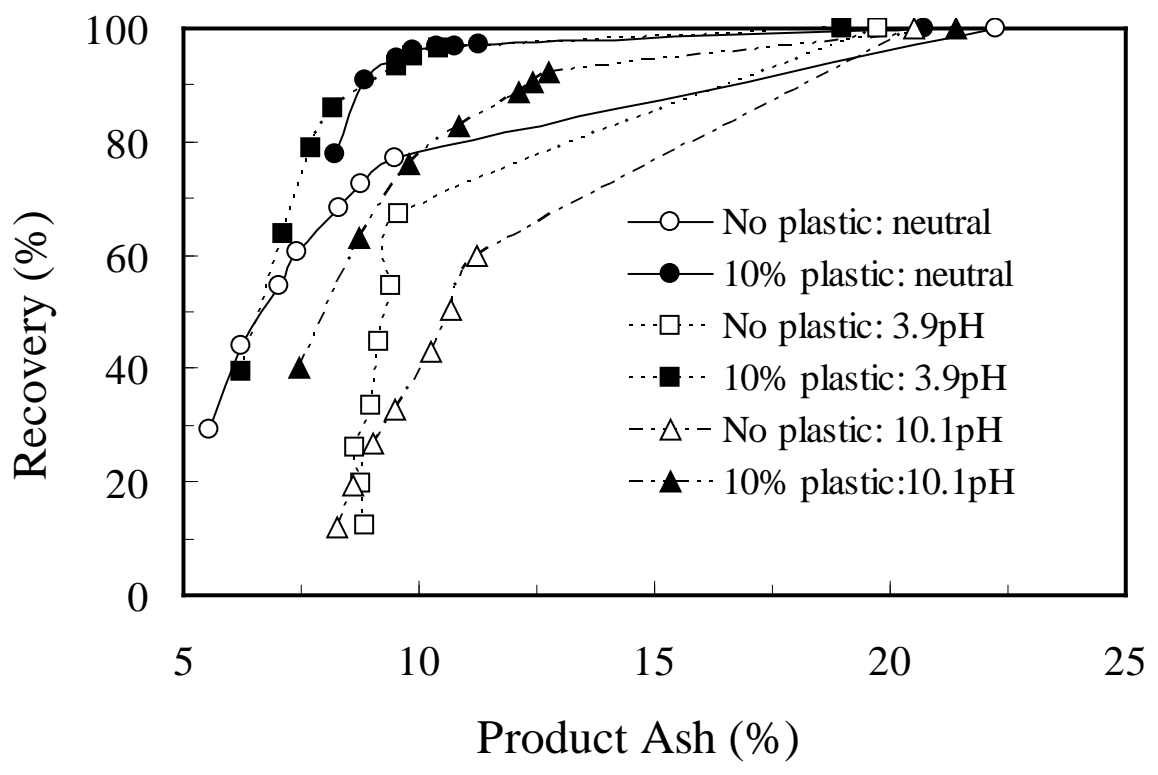

Figure 26. Selectivity performance improvements achieved by the addition of the plastic particles at a concentration of $10 \%$ by weight in a conventional flotation cell; Pittsburgh No. 8 coal. 


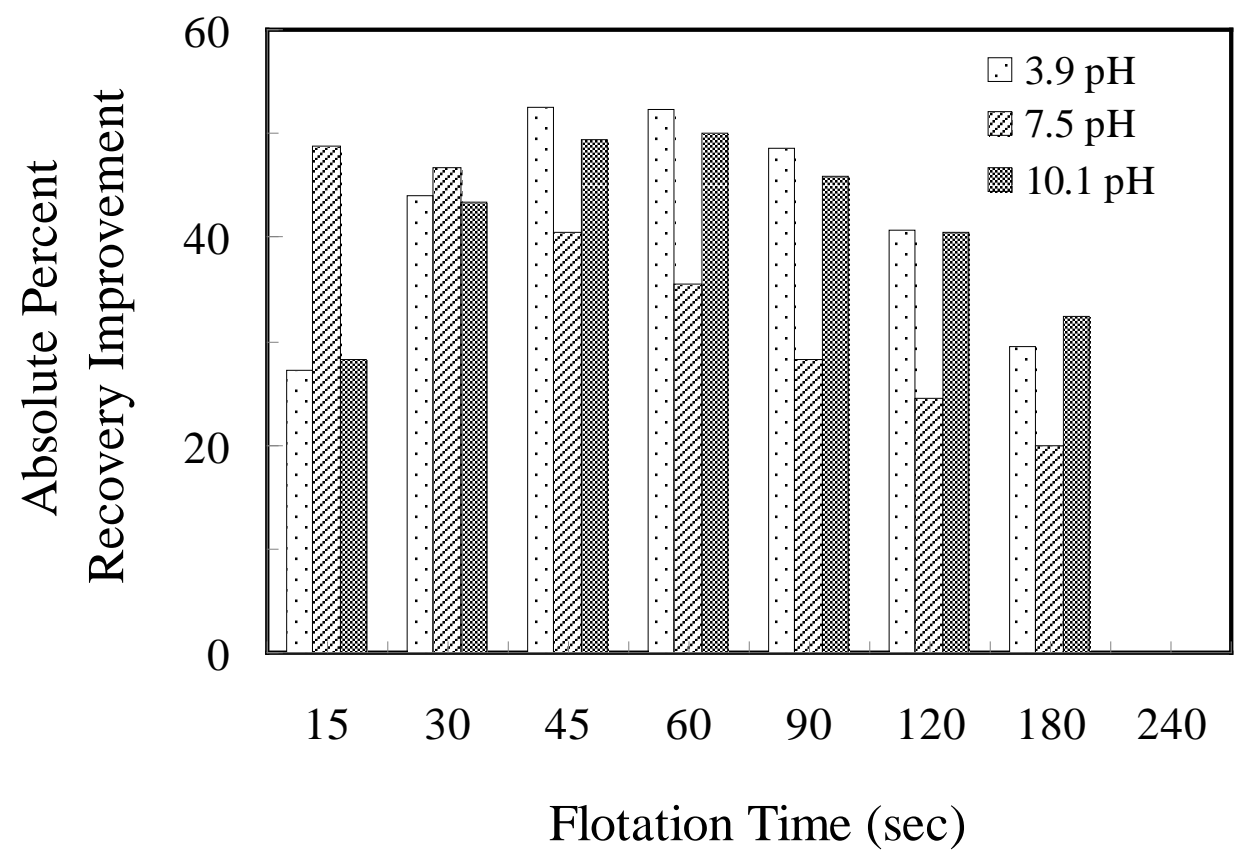

Figure 27. Realized from the addition of plastic particles at a $10 \%$ concentration in a conventional cell when treating Pittsburgh No. 8 coal.

Interestingly, the slurry $\mathrm{pH}$ also had an effect on the recovery time of the plastic particles in the conventional flotation cell. Under neutral $\mathrm{pH}$ conditions, nearly $98 \%$ of the plastic was recovered from the flotation cell within the first 60 seconds of flotation process as shown in Figure 28. After the first 15 seconds, nearly $80 \%$ of the plastic was recovered as compared to only $45 \%$ of the coal. The plastic particle recovery was only slightly depressed under $\mathrm{pH} 10.1$ with about $70 \%$ recovery after 15 seconds of flotation. However, under $\mathrm{pH} 3.9$, plastic recovery was only about $45 \%$ after 15 seconds of flotation time and increments representing $5 \%$ to $10 \%$ absolute percentage units of the total plastic particle concentration reported in all time increments up to 240 seconds ( 4 minutes). The acidity of the slurry could have resulted in chemical changes that negatively affected floatability although significant coal recovery improvements were observed. 


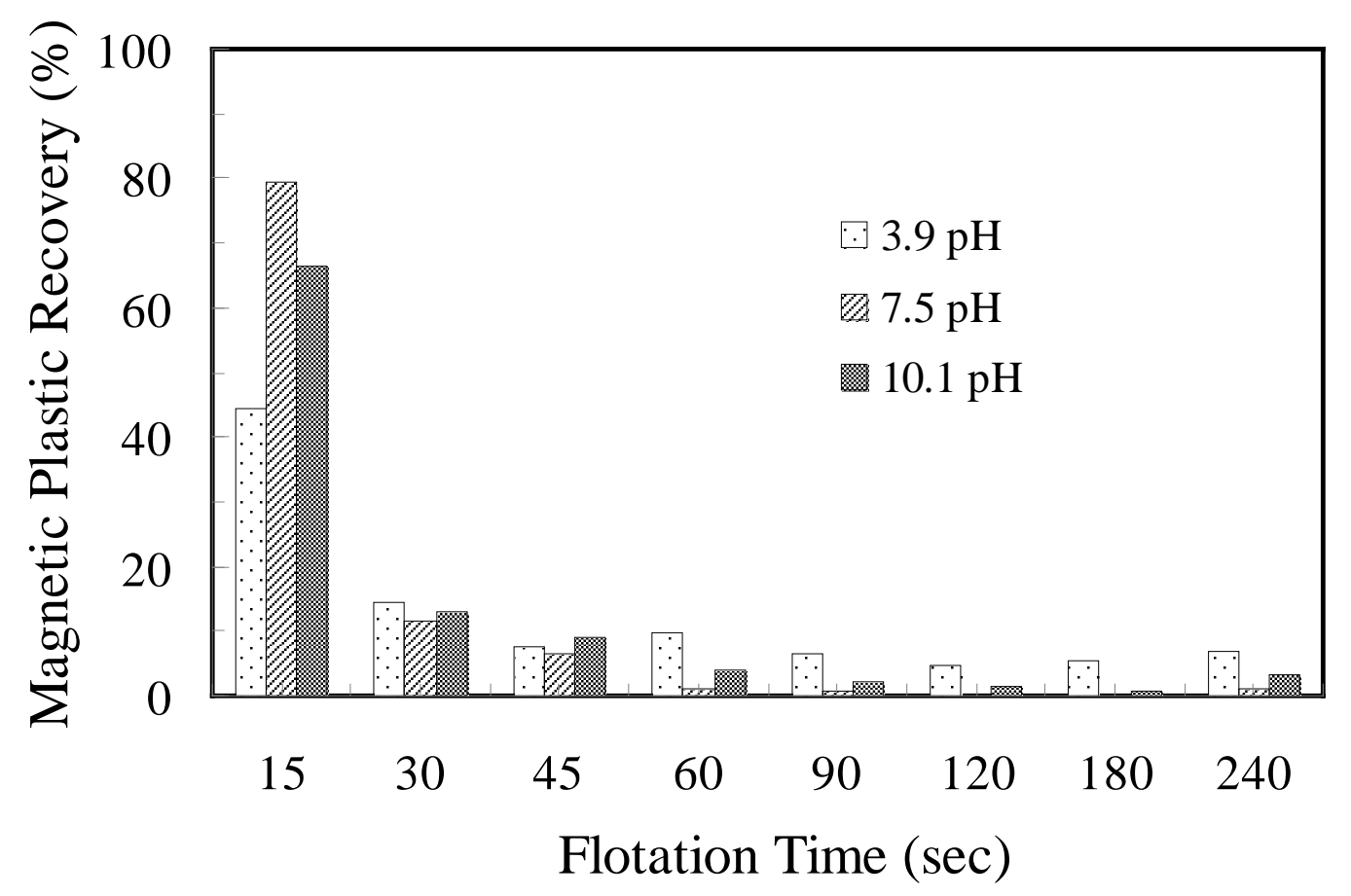

Figure 28. Recovery distribution of magnetic plastic material from the flotation rate tests performed on Pittsburgh No. 8 coal over a range of $\mathrm{pH}$ values.

\section{Particle Size-by-Size Flotation}

Froth flotation performance varies significantly as a function of particle size. In fact, a lower and upper particle size exists and the absolute value of the limits varies from material-to-material. For coal, the typical reported limits are 50 to 500 microns. The lower limit is a result of inefficiencies in the bubble-particle collision process while the upper size limit is associated with the detachment of particles from the bubble surfaces.

To determine the impact of the magnetic plastic material on particle size-by-size performance, a flotation rate test was performed on the Pittsburgh No. 8 coal. Using a $10 \%$ concentration of plastic particles in the feed stream, the weight recovery achieved on the +100 mesh fraction of the total feed was significantly increased as shown in Figure 29. After one minute of flotation, weight recovery was elevated by nearly 30 absolute percentage points. 


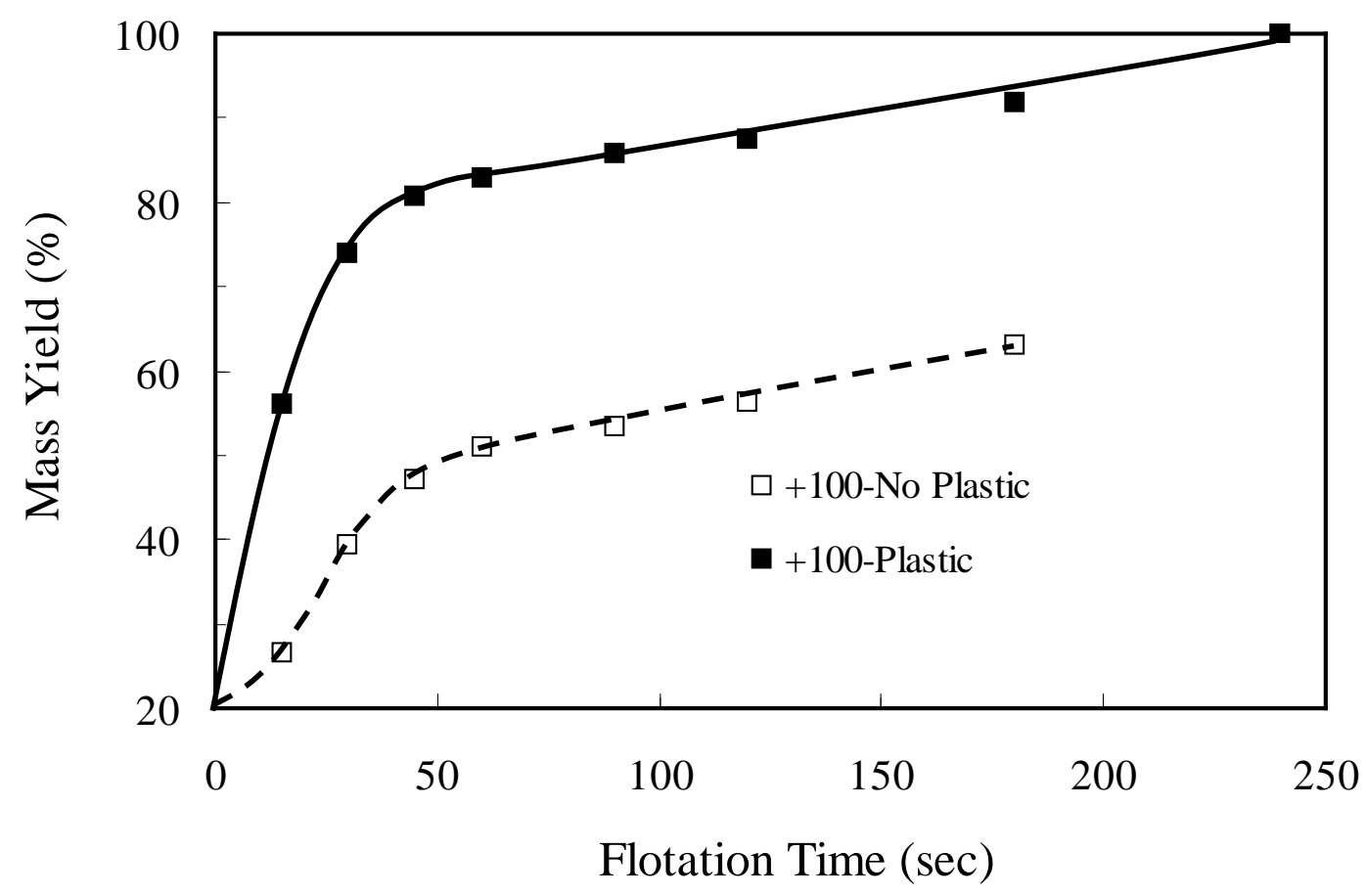

Figure 29. Improvements in the mass recovery rate of the +100 mesh Pittsburgh No. 8 coal by the addition of plastic particles in a conventional flotation cell.

The weight recovery achieved after one minute for each particle size fraction was assessed to quantify the potential change in the lower and upper particle size limits. As shown in Figure 30, mass recovery was increased throughout the particle size range by at least 5 absolute percentage points as a result of the plastic particle addition. However, the impact was much greater for the particles having a size larger than $0.10 \mathrm{~mm}$. A possible explanation is that the presence of more hydrophobic solids on the surface of the air bubbles causes drainage resistance of the water film surrounding an air bubble. As a result, bubble coalescence is reduced thereby maintaining high bubble surface air flux through the froth zone. The availability of bubble surface area reduces preferential detachment of the coarse particles within the froth zone. 


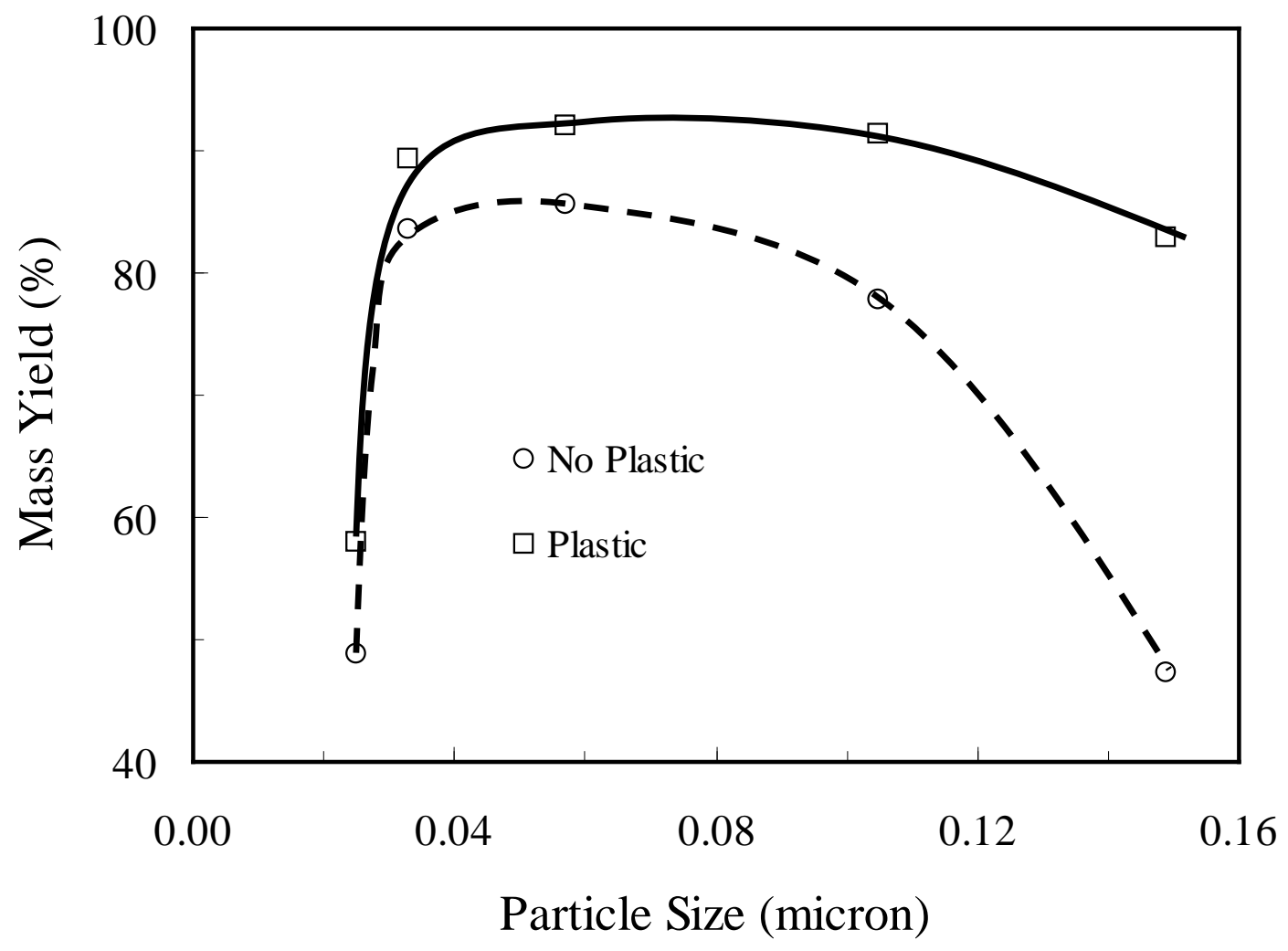

Figure 30. Particle size-by-size mass recovery improvements achieved on Pittsburgh No.

8 coal by the addition of plastic particles at a concentration $10 \%$ by weight in a conventional flotation cell.

Low flotation rates for the coarse particles typically occur due to higher collector adsorption rates of the finer particles and preferential detachment resulting from overloaded surfaces. Tests were performed to determine if the positive impact of the plastic particle addition was maintained if the coarse particles were floated separately from the fine particles. The Pittsburgh No. 8 coal was screened to obtain size fractions of particles greater than 200 mesh, 200 x 500 mesh and finer than 500 mesh. Each particle size fraction was floated separately.

As shown in Figure 31 and Table 11, the flotation rates of the coarser size fractions were much greater when the fractions were floated separately as compared to floating the same size fraction together. Furthermore, the addition of the plastic particles had no positive effect on coarse particle flotation. However, a 5 absolute percentage point increase was obtained for the material finer than 25 microns (500 mesh). In this case, the plastic particles could be either i) serving as a carrier for the fine coal particles and/or ii) the plastic particles act as a seed for hydrophobic agglomeration and the larger coal-plastic agglomerates are more readily collected by the conventionally-sized bubbles. 


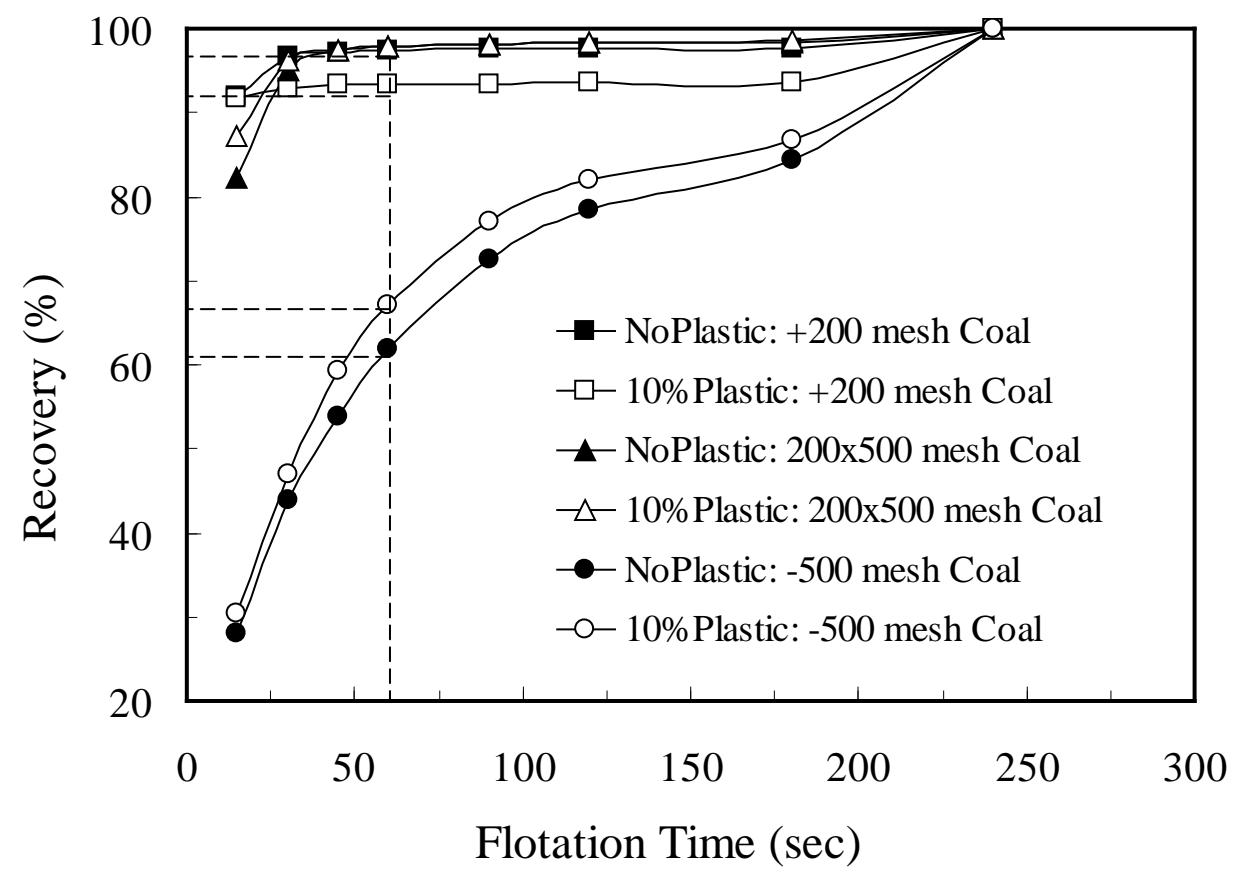

Figure 31. Recovery improvements from the flotation of individual particle size fractions in a conventional flotation cell with and without the addition of plastic particles;

Pittsburgh No. 8 coal.

Table 11. A comparison of the flotation rates and recovery values achieved with and without the addition of plastic particles when floating each size fraction separately; conventional cell, Pittsburgh No. 8 coal.

\begin{tabular}{|c|c|c|c|}
\hline $\begin{array}{c}\text { Particle size } \\
\mu \mathrm{m}\end{array}$ & $\begin{array}{c}\text { External } \\
\text { Material } \\
\text { Addition }\end{array}$ & $\begin{array}{c}\text { Flotation } \\
\text { Rate } \\
\left(\mathrm{min}^{-1}\right)\end{array}$ & $\begin{array}{c}\text { Flotation } \\
\text { Recovery } \\
(\%)\end{array}$ \\
\hline \multirow{2}{*}{+75} & No Plastic & 2.31 & 97.3 \\
& $10 \%$ Plastic & 2.25 & 93.4 \\
\hline $75 \times 25$ & No Plastic & 2.54 & 97.8 \\
& 10\% Plastic & 2.53 & 97.8 \\
\hline \multirow{2}{*}{25} & No Plastic & 0.59 & 61.9 \\
& 10\% Plastic & 0.71 & 67.0 \\
\hline
\end{tabular}

\section{Column Flotation Tests}

An investigation was performed to quantify the overall performance impacts of adding the magnetic plastic particles in a continuously-operated, laboratory flotation column. A major difference between the conventional cell and the flotation column is the 
hydrodynamic conditions which are mostly due to the geometric dimensions of the units. The high length-to-diameter ratio of a laboratory column provides near plug-flow conditions which are optimum for recovery. Therefore, the overall recovery improvements were expected to be lower from the laboratory column unit since the conditions are more ideal than those realized in conventional flotation cells. Since the initial focus of the study was aimed at improving selective detachment in the froth phase, the majority of the column experiments involved injection of the plastic particles through the was water distributer in the froth.

All flotation systems have a limitation on the amount of product that can be conveyed to the collection launder. This limit is typically referred to as the carrying capacity. To determine the carrying capacity, the feed solids concentration is increased while holding the volumetric feed rate constant. As the feed solid content is increased, the product mass rate also rises linearly at a rate that reflects the amount of floatable material entering in the feed stream as shown in Figure 32. When the bubble surfaces reporting to the top of the cell are completely full, the product mass rate reaches a maximum. A further elevation of the feed solids concentration results in a decrease in the product mass flow rate due to selective detachment of the coarse particles, which have more overall mass than the fine particles that remain on the bubble.

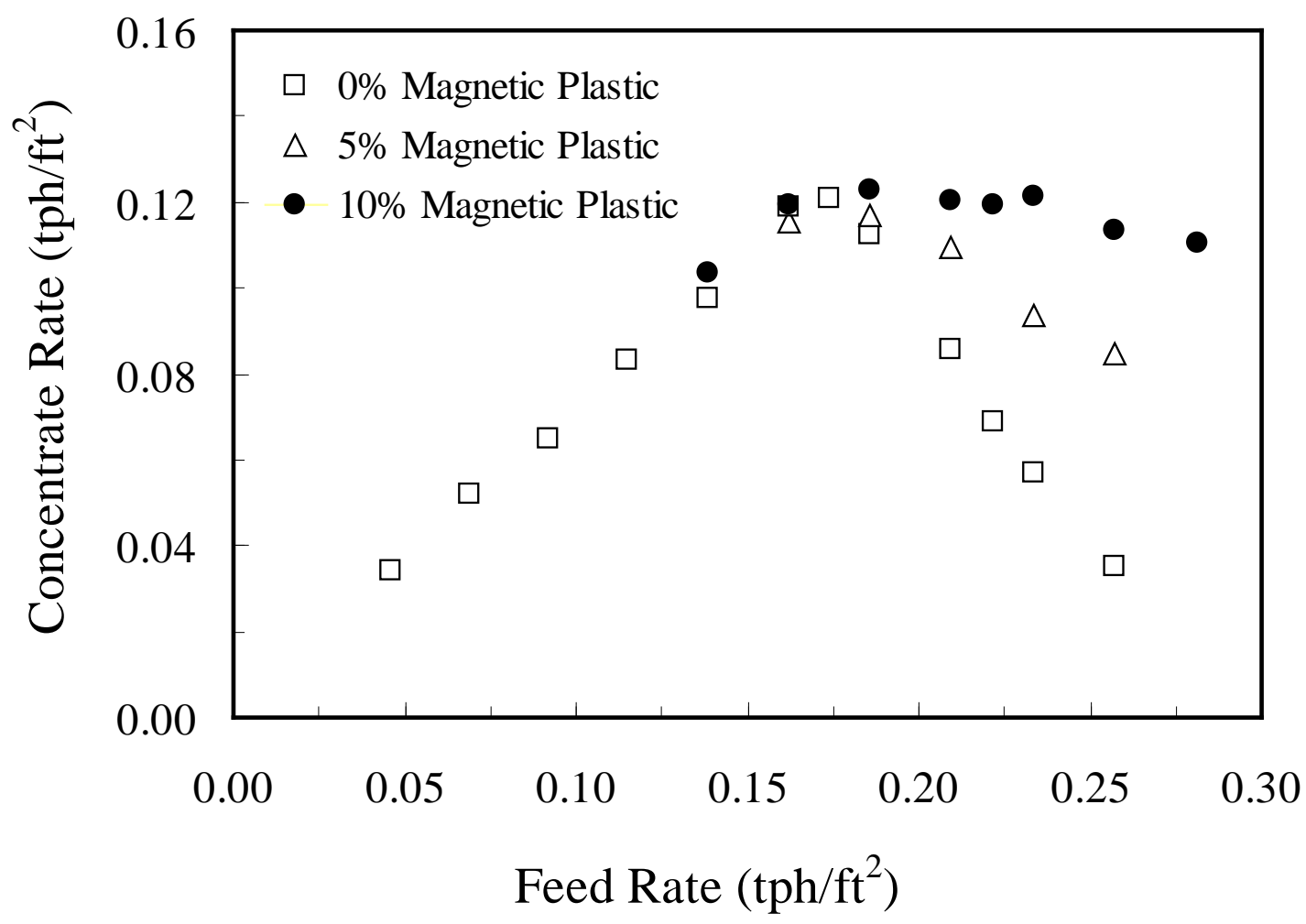

Figure 32. Enhanced carrying capacity characteristics realized by the addition of varying concentrations of magnetic plastic material into the froth phase of a flotation column;

Pittsburgh No. 8 coal. 
As shown in Figure 32, the injection of plastic particles at a concentration of $10 \%$ by weight into the froth phase reduced the detachment of coarse particles as indicated by a near constant product mass rate after reaching carrying capacity. This finding indicates that either the hydrophobic plastic particles are stabilizing the froth and thereby increasing the available surface area or the preferential detachment of coarse particles has been significant reduced. Since the concentrate mass rate reaches a maximum, it is more likely that the selectively detachment of coarse particles is being prevented. A possible explanation is that the plastic particles are coating the coarse coal thereby enhancing the hydrophobicity of the coal surfaces.

The plastic addition had no positive impact on the selectivity achieved on the basis of ash reduction as shown in Figure 33. The range in recovery and product ash values was obtained by varying the volumetric feed flow rate while maintaining the feed solid concentration at $7.5 \%$ by weight. Although the performances are not equal under the same operating conditions, the general recovery versus product ash relationships are nearly equal for the tests not involving plastic addition and those for which plastic was added at $5 \%$ by weight concentration.

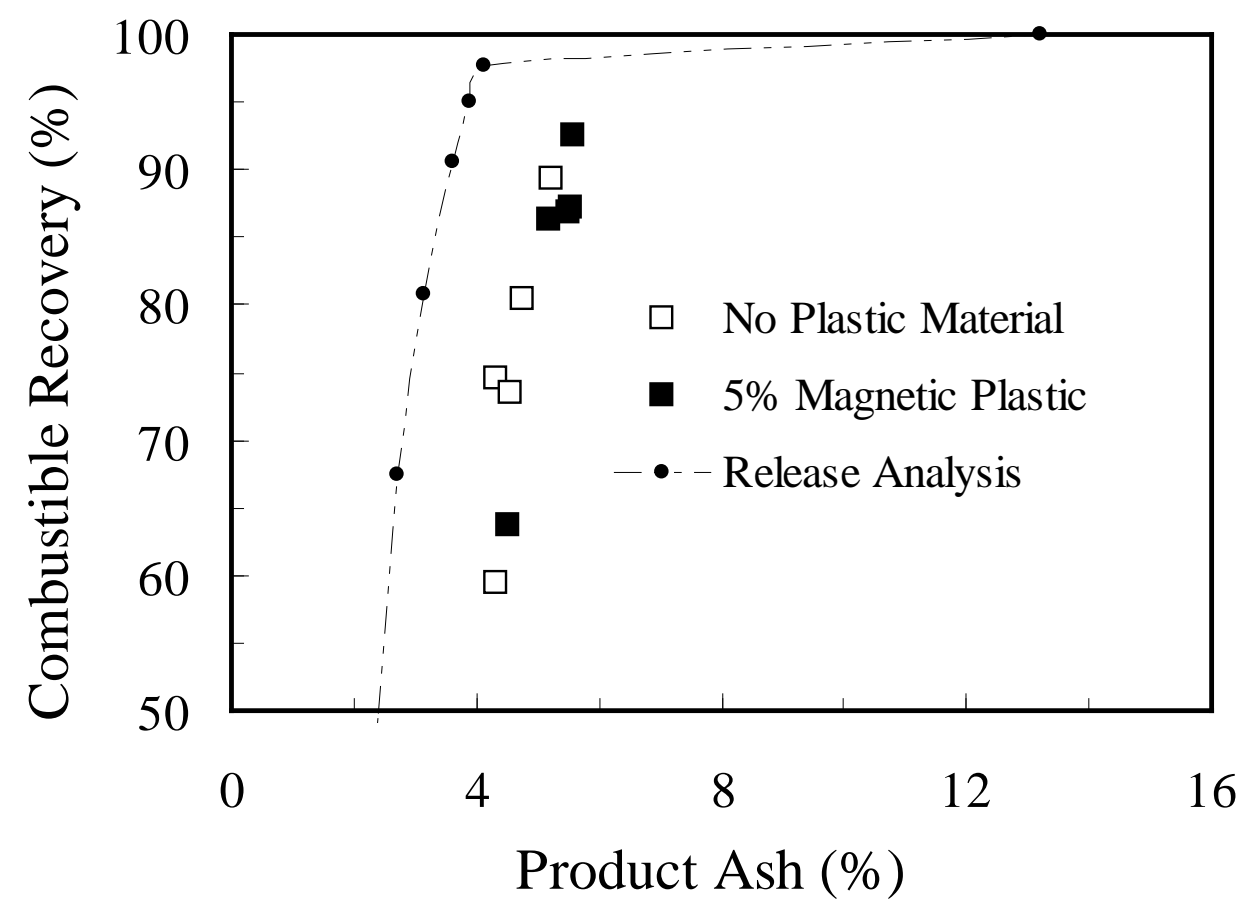

Figure 33. Effect of magnetic plastic material injection on the selectivity of a column flotation froth under elevated solid loading conditions; 7.5\% feed solid concentration; Pittsburgh No. 8 coal.

The performance data in Figure 33 are plotted as a function of volumetric feed rate in Figure 34. At a given feed rate, the combustible recovery is increased by the addition of the plastic material. The recovery gain reaches a maximum of ground 13 absolute 
percentage points in the feed rate range of $600-800 \mathrm{ml} / \mathrm{min}$. This improvement compares well with those obtained from the conventional cell results. However, a negative impact is a slight elevation in ash content in the froth concentrate from around $4.0 \%$ to $5.5 \%$ under equivalent feed rates.

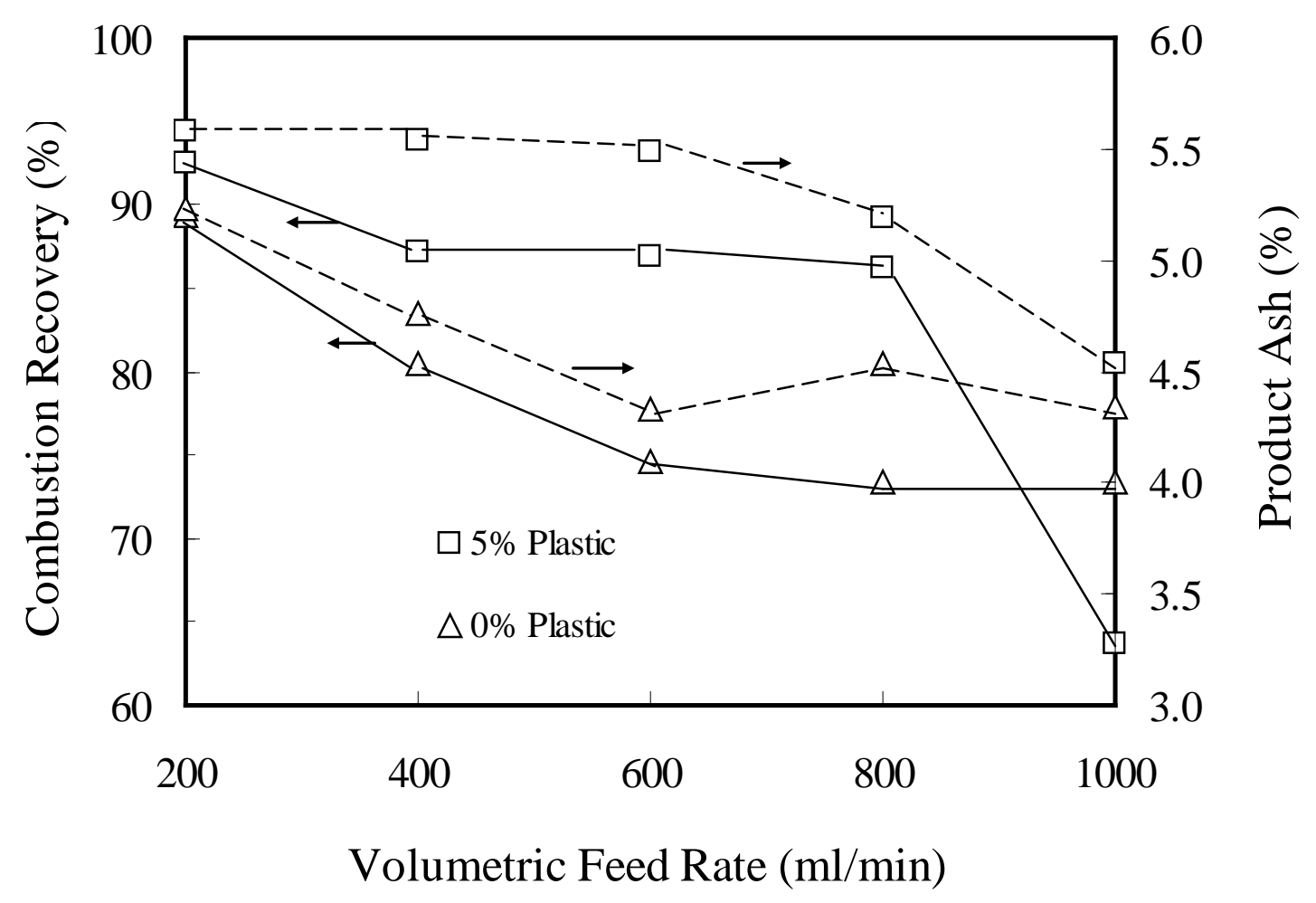

Figure 34. Separation performance improvements achieved as a function of the feed volumetric flows rate when adding $5 \%$ by weight plastic particles into the froth phase of a flotation column treating Pittsburgh No. 8 coal; 7.5\% feed solids concentration.

Two methods of injecting the magnetic plastic particles were studied, i.e., i) adding the particles through the wash water which is distributed in the froth and ii) mixing the plastic particles into the feed coal slurry and injecting into the collection zone of the column. The performances shown in Figure 35 indicate that adding the plastic particles directly into the froth zone provides better selectivity. 


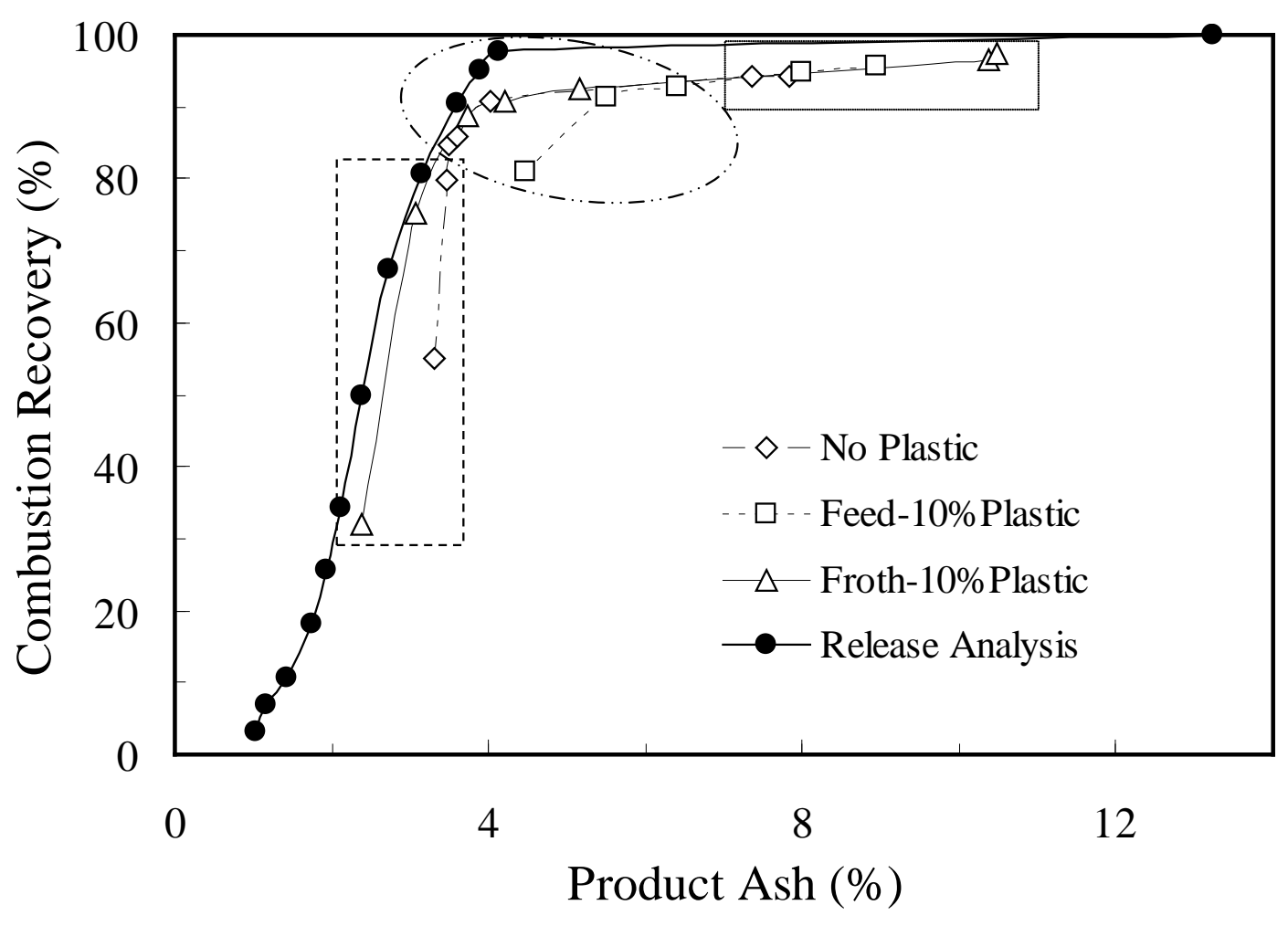

Figure 35. A selectivity improvement comparison between tests in which the plastic particles were added in the froth or in the feed stream of a flotation column at $10 \%$ by weight concentration; Pittsburgh No. 8 coal.

The recovery improvement that was realized from using a $10 \%$ by weight concentration of plastic particles was significantly lower than that achieved with a 5\% concentration. As shown in Figure 36, a recovery gain ranging from 2 to 5 absolute percentage points was obtained for volumetric feed rates below about $1050 \mathrm{ml} / \mathrm{min}$, which interestly corresponds to the feed rates providing the performances near the elbow of the recovery versus product ash curve in Figure 35 . Above the $1050 \mathrm{ml} / \mathrm{min}$ feed rate, recovery drops significantly below the values achieved using no plastic particles. It is also noted that product ash contents were significantly higher when adding plastic particles below the critical feed rate. Overall, the results were inferior to those achieved using a plastic particle concentration of $5 \%$ by weight of the total feed mass flow rate. 


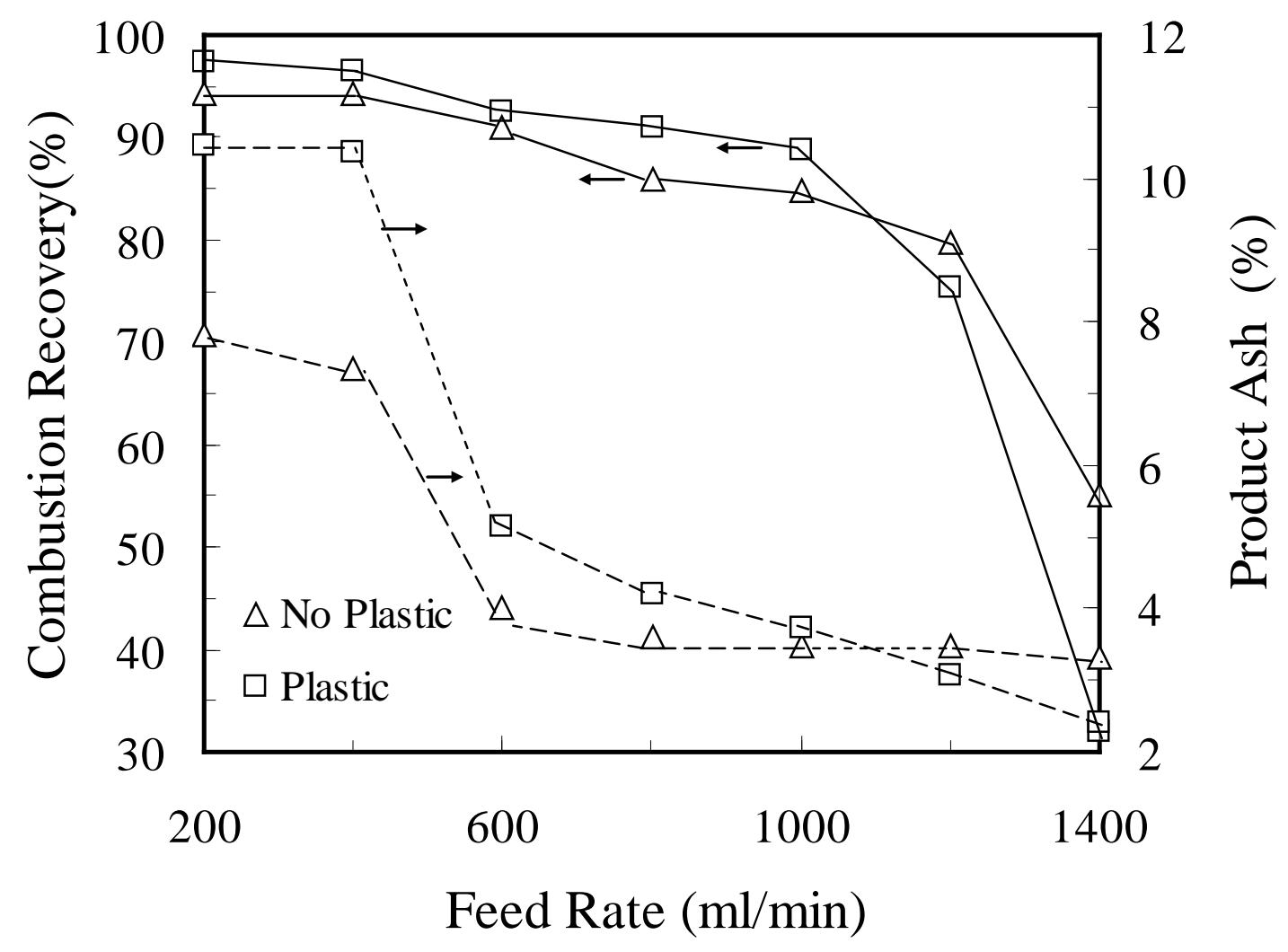

Figure 36. Separation performances as a function of volumetric feed rate to a flotation column when adding plastic particles into the froth phase at a concentration of $10 \%$ of the column feed; Pittsburgh No. 8 coal.

In the conventional cell tests, the plastic material was added directly in the feed and significant recovery improvement was realized. The performances achieved by the flotation column when adding the plastic particles in the column feed stream also indicate an increase in recovery at volumetric feed rate flow rates equal to or lower than 800 $\mathrm{ml} / \mathrm{min}$. As shown in Figure 37, the maximum recovery improvement was about 5 absolute percent points when using a plastic particle concentration of $10 \%$ by weight as compared to recovery values achieved using no plastic particle addition. 


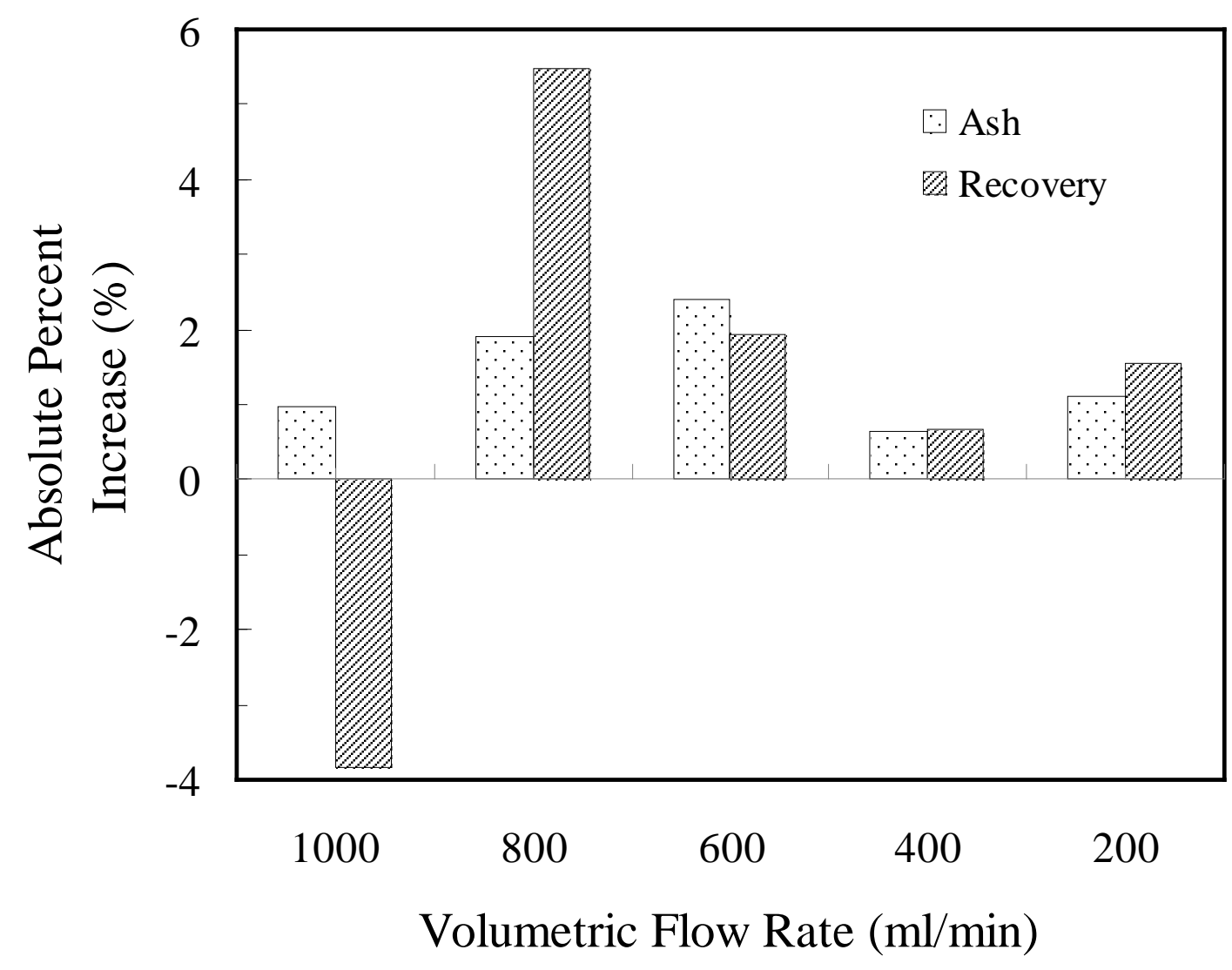

Figure 37. Change in ash content and recovery performance after addition of $10 \%$ magnetic plastic material into the feed slurry in a column flotation respect to baseline performs without addition of external material; Pittsburgh No. 8 coal.

The recovery of the magnetic plastic particles was also somewhat affected by the feed volumetric flow rate. Figure 38 compares the plastic recovery values achieved by adding the plastic particles in the froth phase or the feed stream at a $10 \%$ by weight concentration. As expected, plastic recovery was significantly higher when adding the particles in the froth phase, especially at volumetric feed rates greater than $800 \mathrm{ml} / \mathrm{min}$. The recovery values were nearly $100 \%$ at flow rates below $800 \mathrm{ml} / \mathrm{min}$.

The separation performances achieved on the difficult-to-clean Coalburg coal using the flotation column and plastic particles are shown in Figure 39. Very little if any improvements were realized when using a plastic particle concentration of $10 \%$ by weight of the feed. However, the same results plotted as a function of volumetric feed rate in Figure 40 indicate that a 3 to 5 absolute percentage point improvement in recovery was achieved at feed rates between 600 and $800 \mathrm{ml} / \mathrm{min}$ with minimal effect on product ash content. 


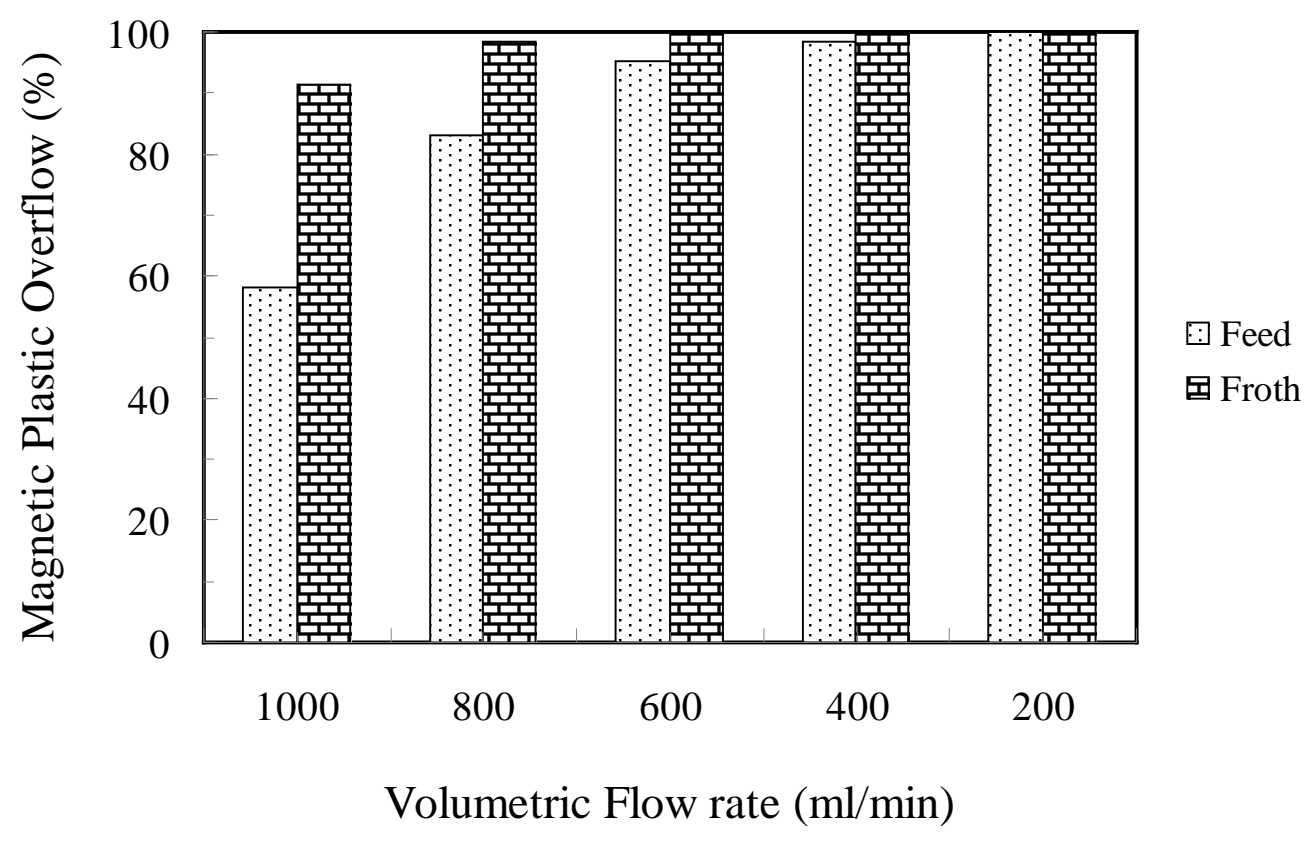

Figure 38. Comparison of the magnet plastic recovery performance achieved when injecting into the froth phase or the feed stream of a flotation column; $10 \%$ plastic particle concentration by weight.

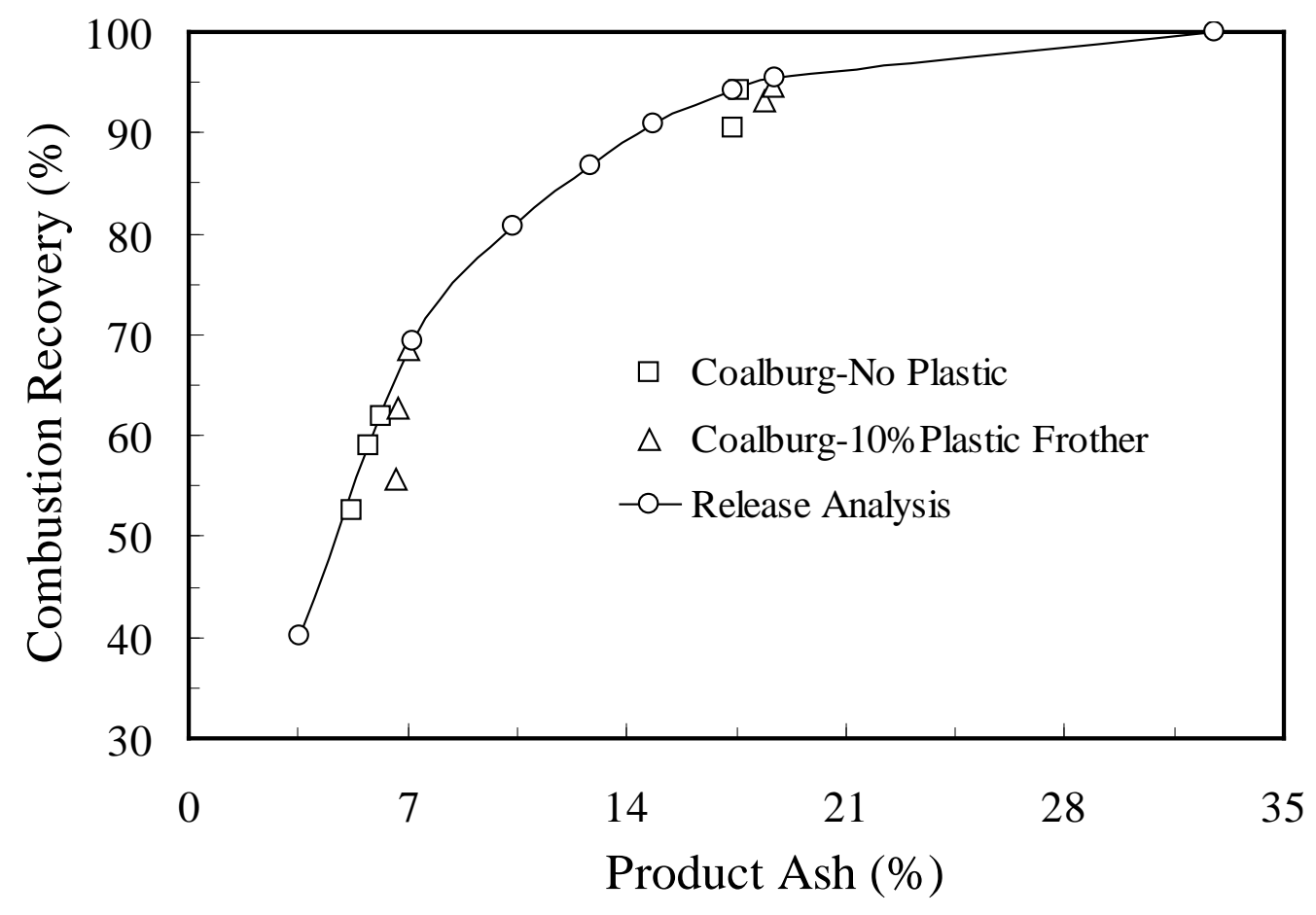

Figure 39. Separation performance achieved when treating Coalburg coal in a flotation column with a $10 \%$ by weight plastic particle injection into the froth phase. 


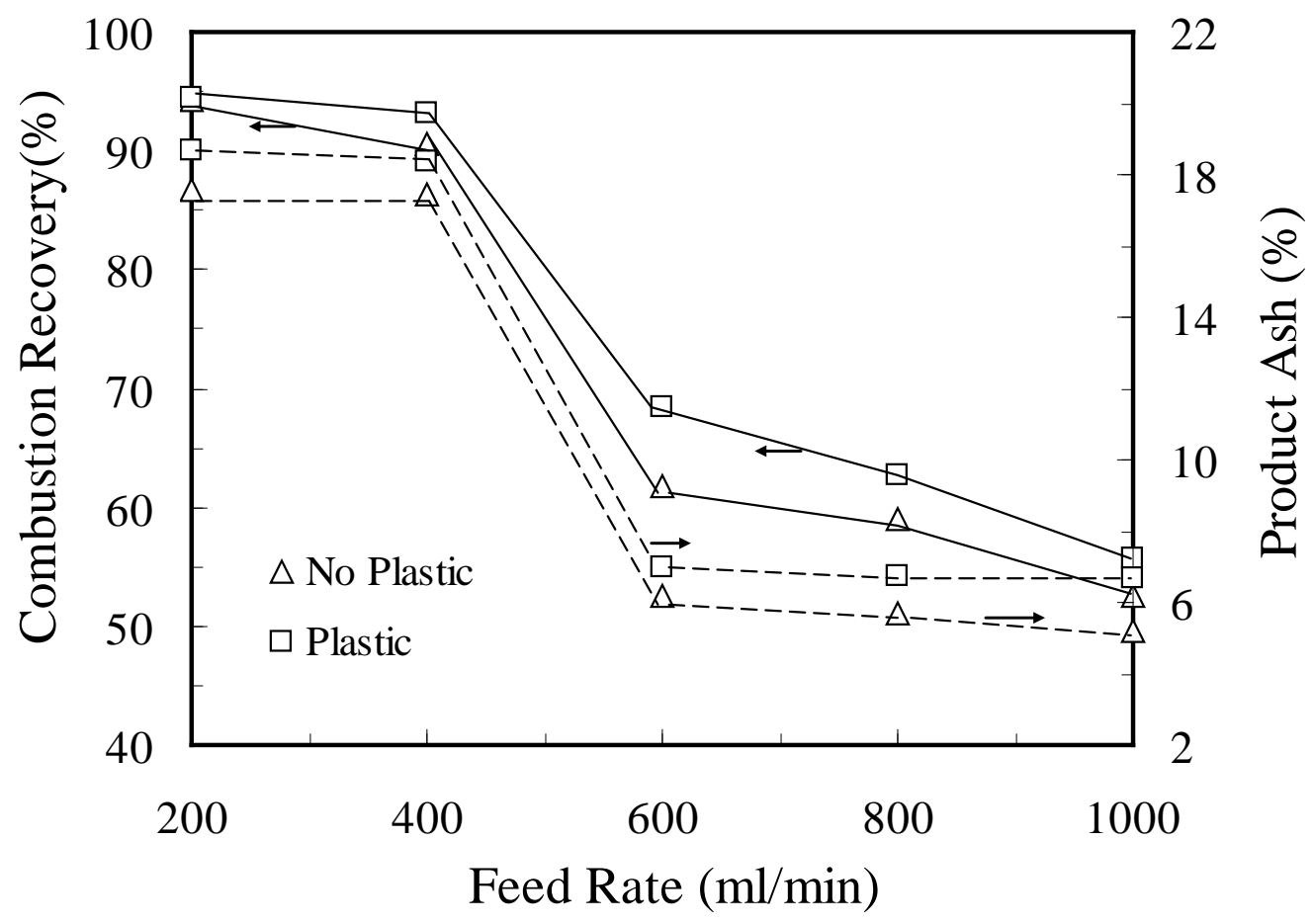

Figure 40. Separation performance achieved as a function of volumetric feed rate when treating Coalburg coal in a flotation column using $10 \%$ by weight plastic particle concentration.

\section{Froth Refluxing}

As previously discussed, selective detachment occurs in the froth zone as a result of bubble coalescence and the subsequent reduction in the amount of surface area per unit of time reporting to the final product. When the amount of particles being conveyed by the bubbles exceed the bubble surface area available, particles are detached. Those particles with a weak binding energy with the bubble surface are preferentially detached. The weak binding energy is likely due to poor surface hydrophobicity which is typically associated with high ash particles. Thus, by promotion of the conditions leading to particle detachment, the selectivity of the process may be improved. Moys (1978) and later Yianatos et al. (1988) have confirmed in previous studies that selective detachment in the froth zone occurs and experiment evidence shows improvements in the product grade.

To activate the selective detachment process, a portion of the froth concentrate stream was continuously collected and recycled through the wash water and into the froth. As a result, the froth was concentrated with high quality, highly hydrophobic coal particles. The flotation column as well as the recycling process was operated continuously. Two test conditions were evaluated in the study: 1 ) low solids loading condition (feed solid content $=2 \%$ by weight) which typically limits selectivity to that achieved on the basis of flotation rate differentials and 2 ) high solids loading (feed solids content $=7.5 \%$ ) which 
achieves selectivity due to both flotation rate differences and selective particle detachment in the froth zone. Froth refluxing was expected to provide the greatest impact under low feed solid conditions since bubble-particle detachment in the froth zone would be minimal due to an abundance of available bubble surface area.

The selectivity achieved by a process is typically indicated by a shift in the recovery versus product grade relationship. As shown in Figure 41, a slight shift to higher recovery and lower product ash values occurred when refluxing a portion of the froth concentrate under the high load condition. The improvements are more easily visualized in Figure 42 as a function of the feed volumetric flow rate. At low feed flow rates which typically provide high recovery values, refluxing reduced the ash content by about 3 absolute percentage points. Since the low flow rates likely provide a low froth loading, detachment is probably limited. Refluxing froth helps to load the froth and encourage the selective detachment of the high ash coal particles. As the feed flow was increased beyond $500 \mathrm{ml} / \mathrm{min}$, the ash reduction benefit was reduced to zero and recovery improvements were realized. The effect on recovery was unexpected and may be due to simper randomness. However, the trend was relatively constant. The increased recovery may be result of the concentration of highly hydrophobic particles and the positive impact on froth stability (more bubble surface area) as well as agglomeration of the coal particles.

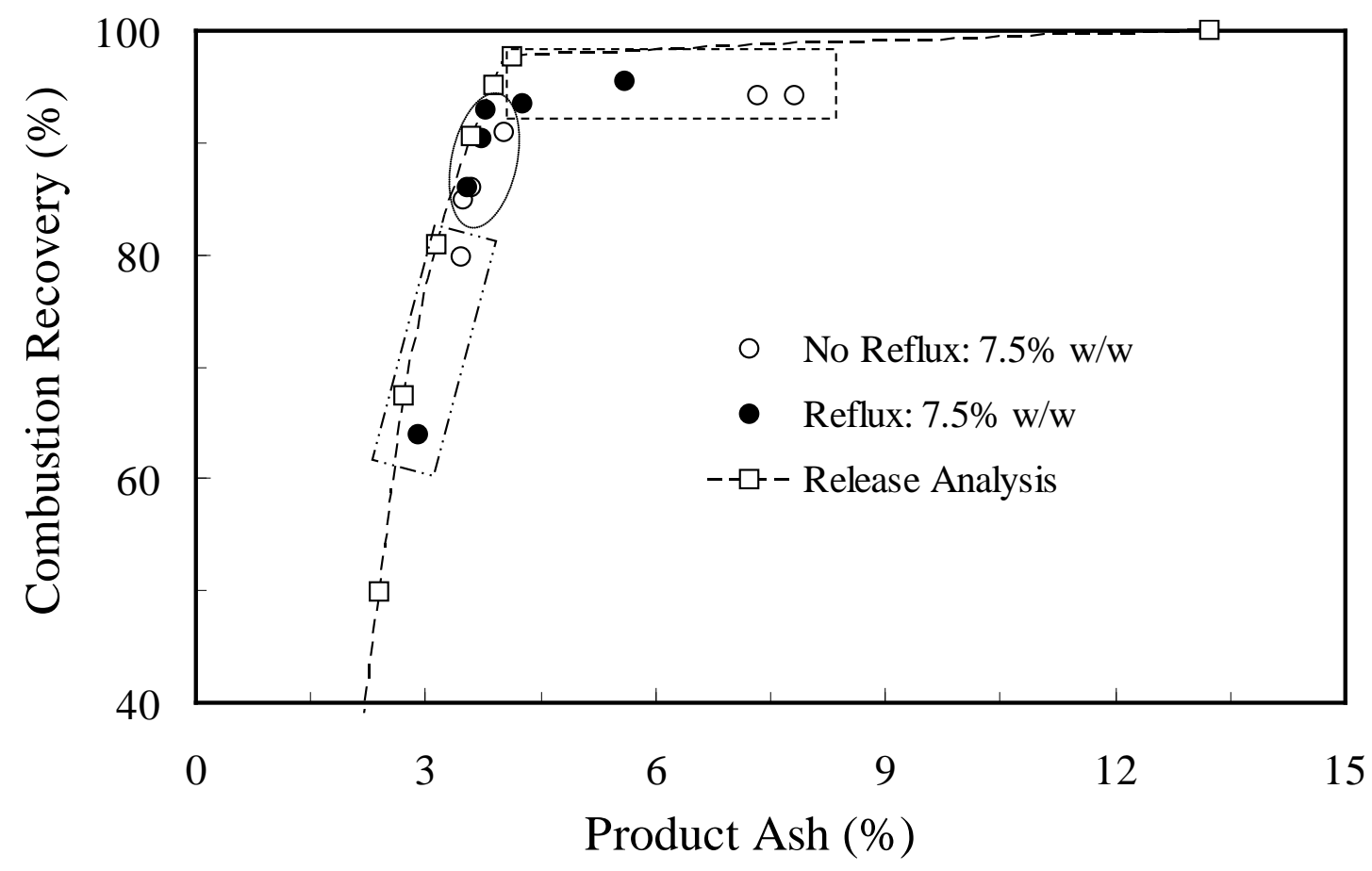

Figure 41. Comparison between continuous column flotation with and without external reflux material at 7.5\% solid content; Pittsburgh No. 8. 


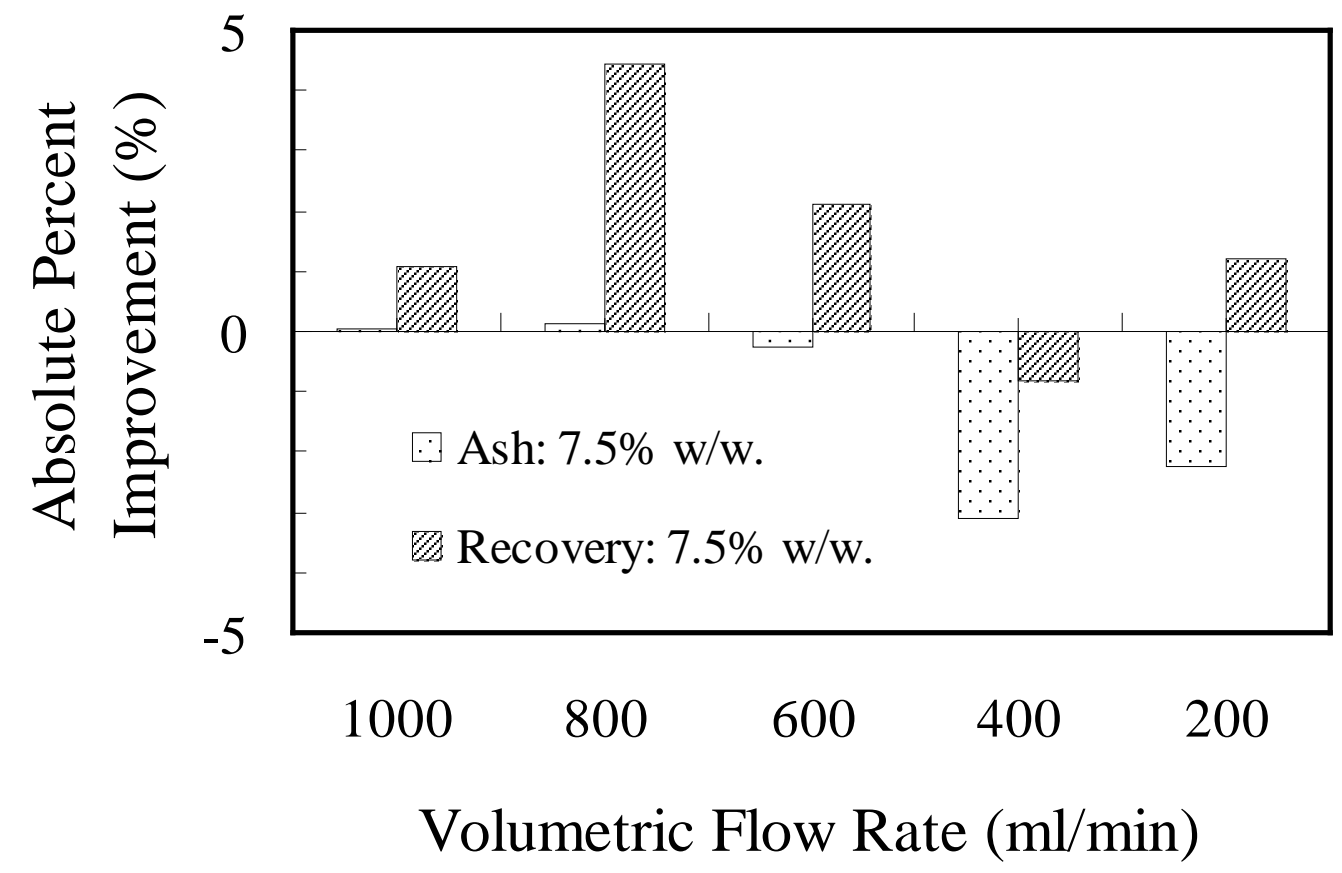

Figure 42. Change in ash content and recovery performance after external reflux into the froth zone in a column flotation respect to baseline performs without refluxing at $7.5 \%$ solid content; Pittsburgh No. 8 coal.

Column flotation tests conducted under low feed solids loading conditions resulted in a similar trend in regards to ash content reduction when employing froth reflux. As shown in Figure 43, the recovery versus product ash curve achieved using refluxing was slightly shifted toward higher recovery and lower ash values as compared to the performance realized when the external froth reflux was not employed. This trend is similar to that described under the high solids conditions in Figure 41. However, the ash content reduction was lower. The ash content was decreased by only 1.5 absolute percentage points at flow rates below $600 \mathrm{ml} / \mathrm{min}$. At higher feed flow rates, a slight ash reduction was realized as shown in Figure 44 but recovery was negatively affected. The overall impact of froth refluxing under low feed solids conditions was minimal which may be due to the availability of bubble surface area even when refluxing a portion of the product. A higher level of refluxing may be needed to realize the impacts on performance under the low solids conditions. 


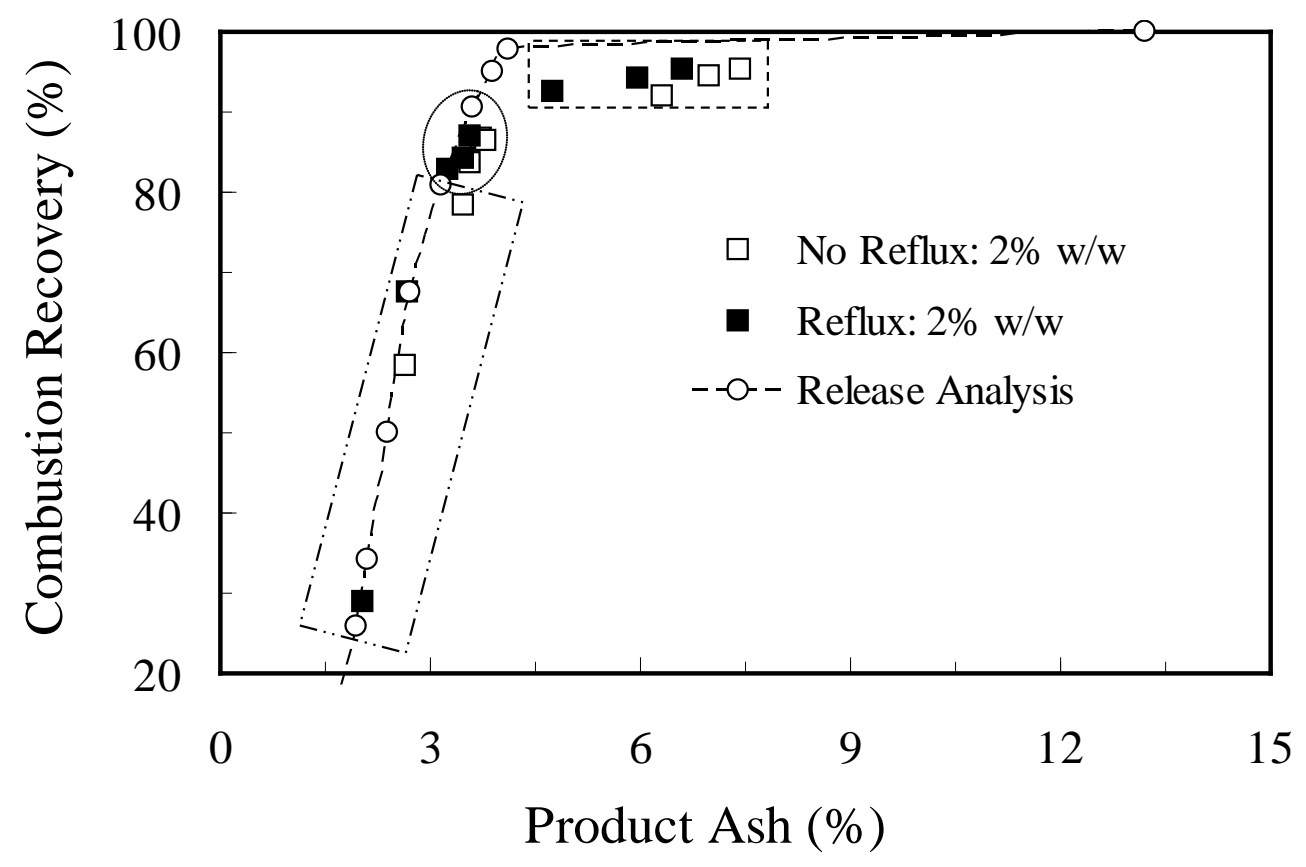

Figure 43. Comparison between continuous column flotation with and without external reflux material at $2 \%$ solid content; Pittsburgh No. 8.

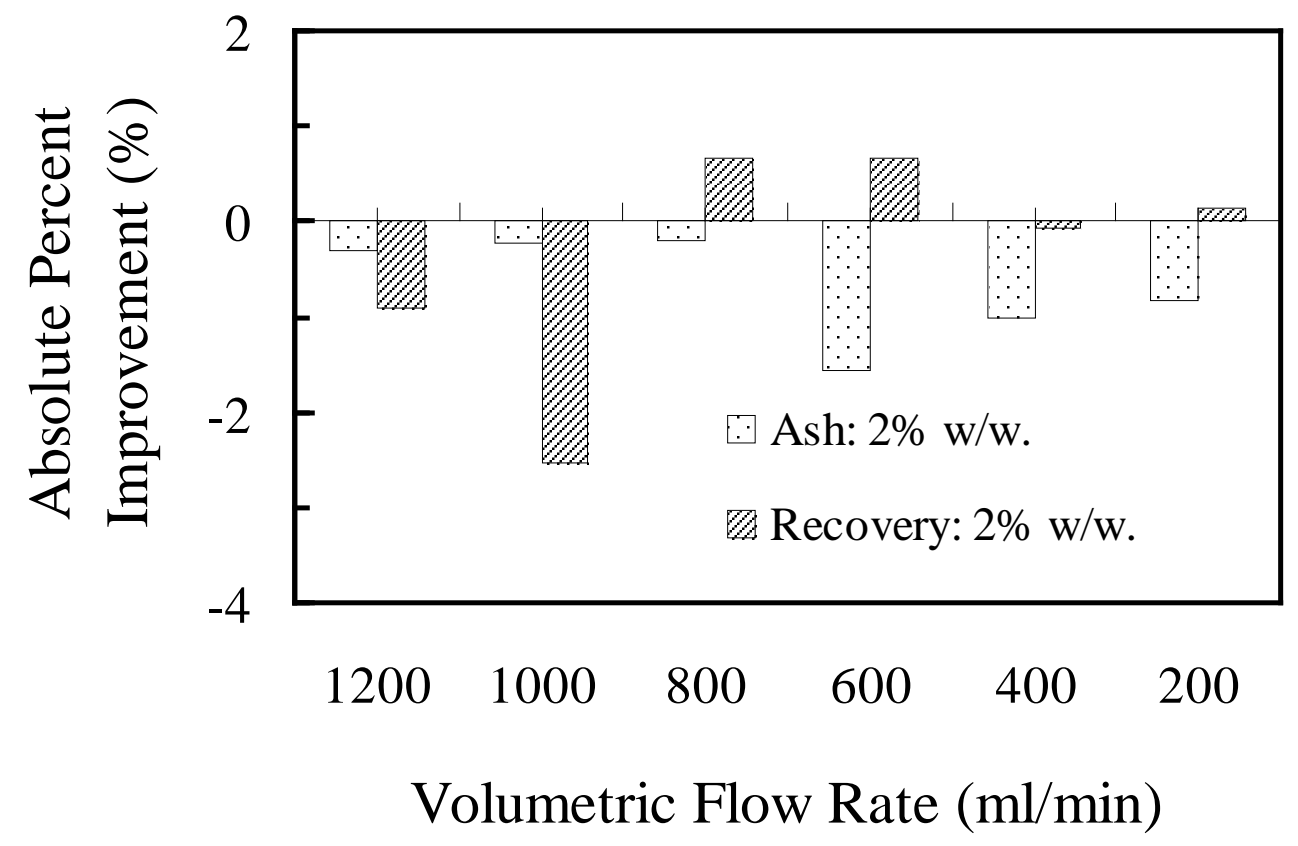

Figure 44. Change in ash content and recovery performance after external reflux into the froth zone in a column flotation respect to baseline performs without refluxing at $2.0 \%$ solid content; Pittsburgh No. 8 coal. 
Coal pyrite is typically weakly-to-moderately hydrophobic naturally. When fuel oil is added, the pyrite floatability increases significantly. The froth reflux action was expected to provide a significant sulfur content reduction benefit for the Pittsburgh No. 8 coal which contains a relatively large amount of pyrite. However, according to the results in Figure 45, no improvement in the sulfur reduction was achieved by froth refluxing in tests conducted under high solids loading conditions. Similar results were also obtained using a $2 \%$ feed solids concentration (Figure 46). The lack of improved selectivity with respect to sulfur reduction may be due to liberation issues and/or the hydrophobic nature of the coal pyrite.

A similar test program was performed on the Coalburg coal with nearly equal results. As described previously, the Coalburg coal contains a significant quantity of middling particles, which are particles comprised of both coal and mineral matter and typically have a low degree of surface hydrophobicity. Thus, selectivity improvements were expected to be greater for the Coalburg coal as compared to that achieved from the treatment of Pittsburgh No. 8 coal.

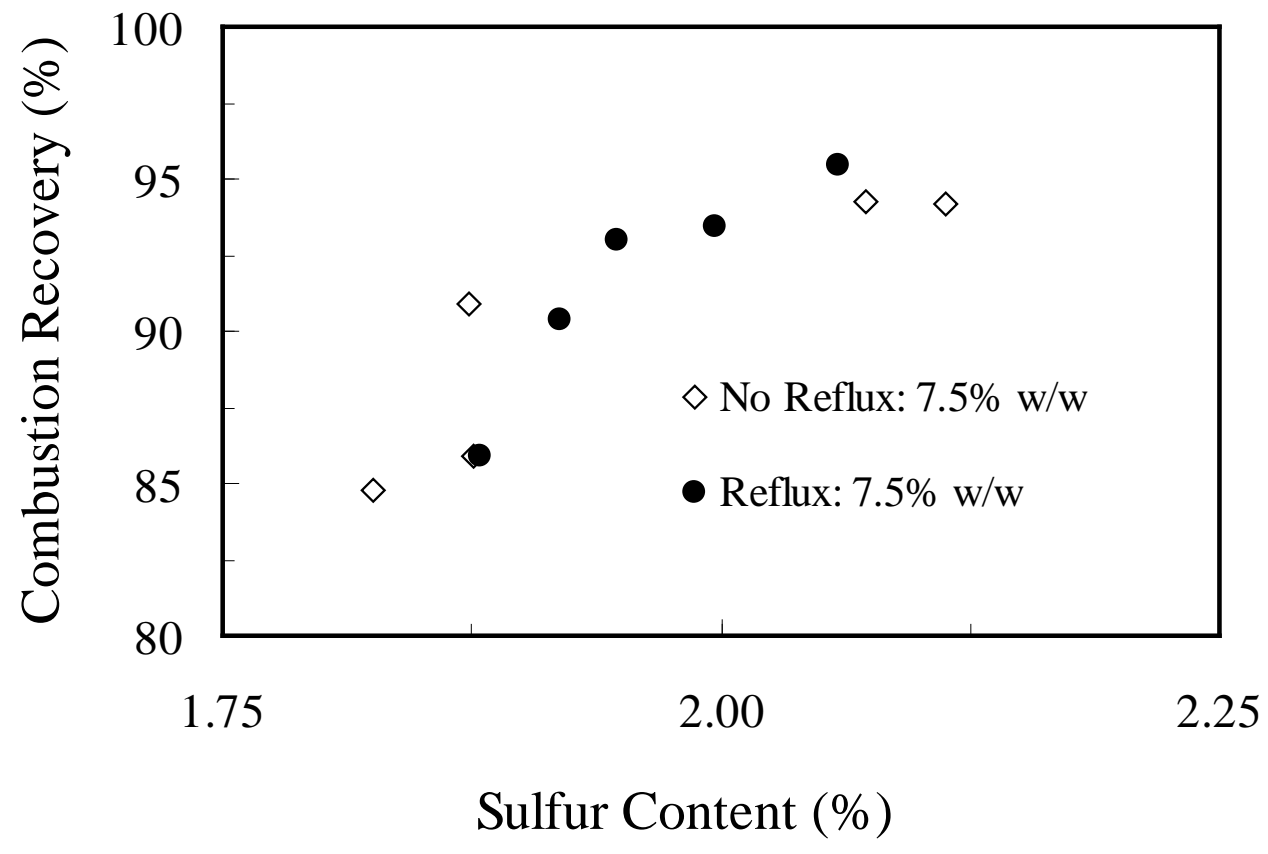

Figure 45. Sulfur reduction comparison from results obtained with and without external froth reflux in a flotation column using a 7.5\% feed solids content; Pittsburgh No. 8 coal. 


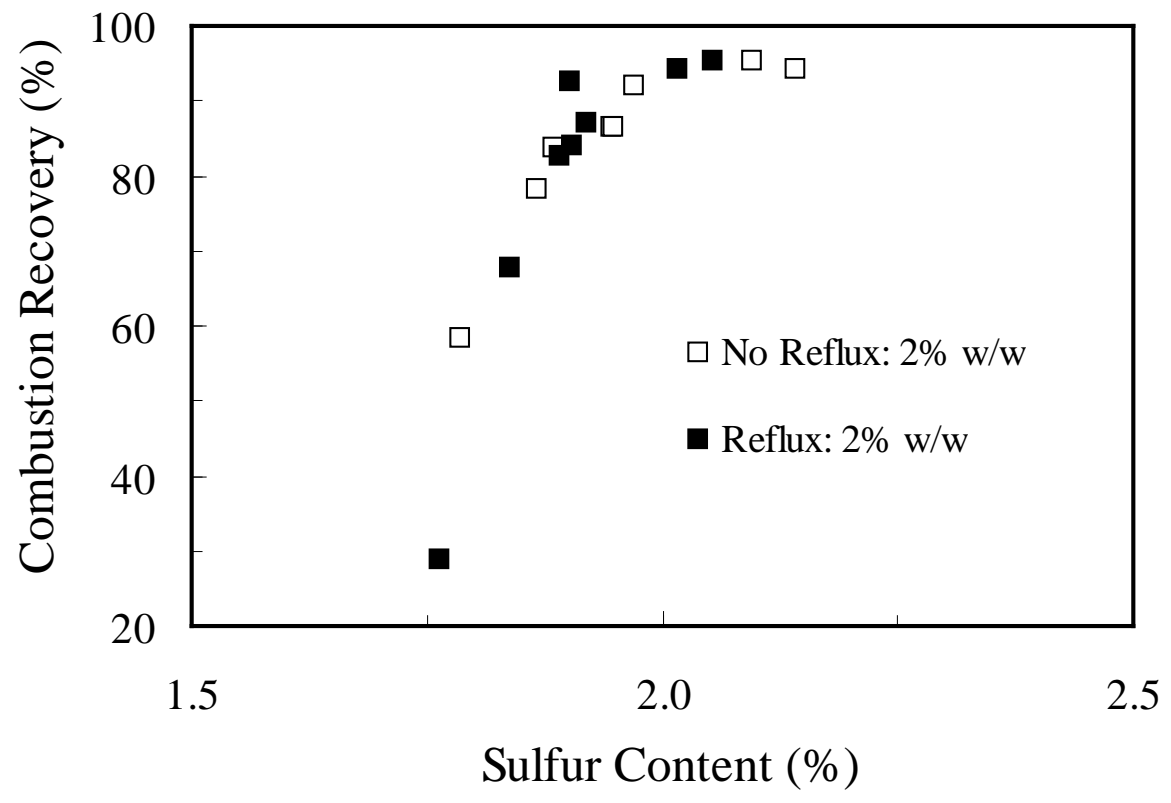

Figure 46. Sulfur reduction performance comparison of results obtained with and without external reflux material in a flotation column being fed at $2 \%$ solids content by weight; Pittsburgh No. 8 coal.

As shown in Figure 47, the recovery versus product ash relationship obtained when refluxing a portion of the froth slightly shifted to lower product ash and recovery values. Figure 48 shows the improvements in both combustible recovery and product ash over the range of volumetric feed rates tested at a feed solids concentration of $7.5 \%$ by weight. The improvement trends were nearly identical to those observed from the data generated when cleaning Pittsburgh No. 8 coal. Ash reductions of 3 to 4 absolute percentage points were achieved at feed rates below $600 \mathrm{ml} / \mathrm{min}$ while little or no improvements were obtained at higher feed flow rates. Positive recovery improvements were obtained at feed rates of $600 \mathrm{ml} / \mathrm{min}$ and higher which was the same finding realized from the Pittsburgh No. 8 coal. 


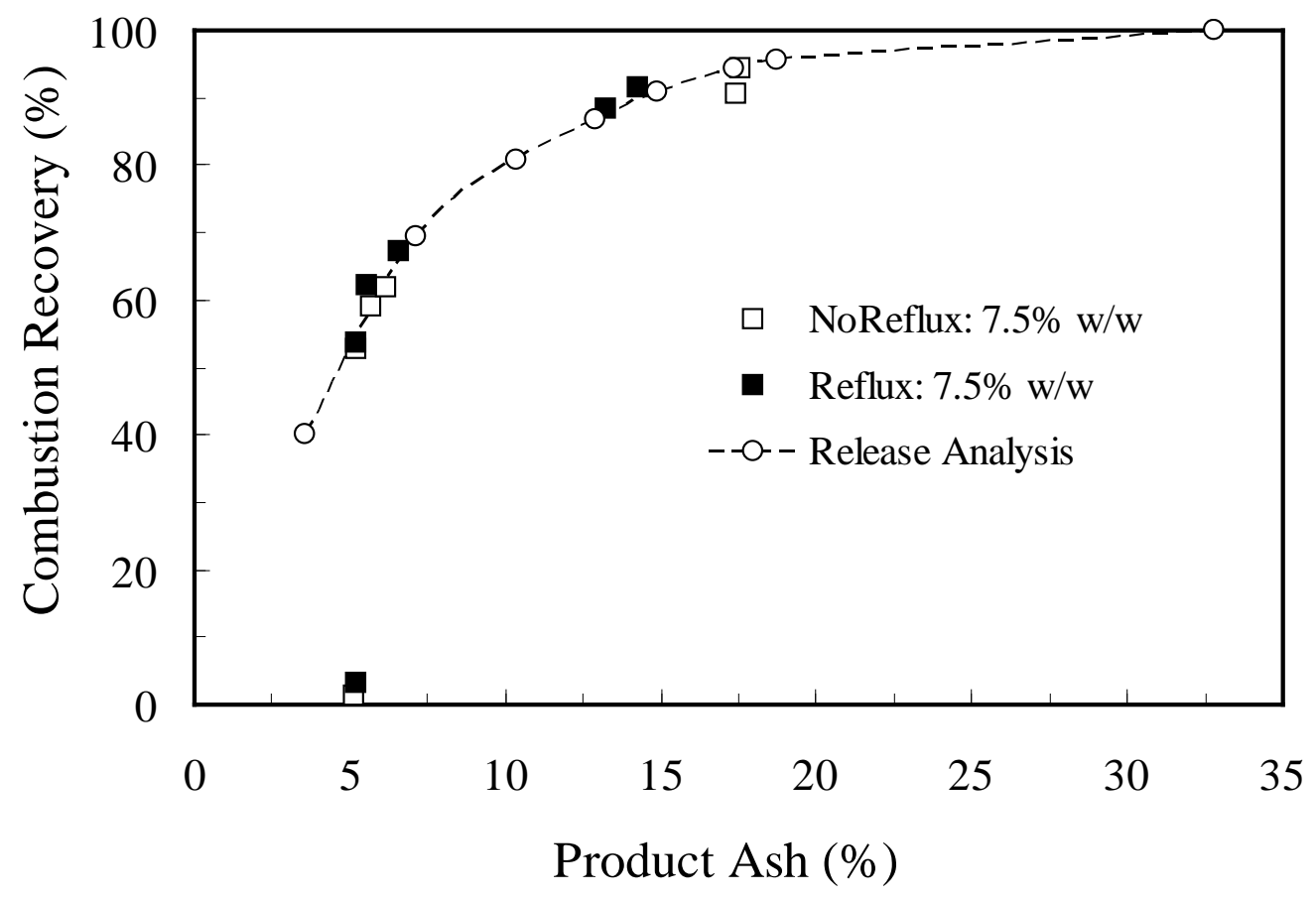

Figure 47. Comparison between separation performances achieved with and without external froth reflux in a flotation column at 7.5\% feed solid content; Coalburg coal.

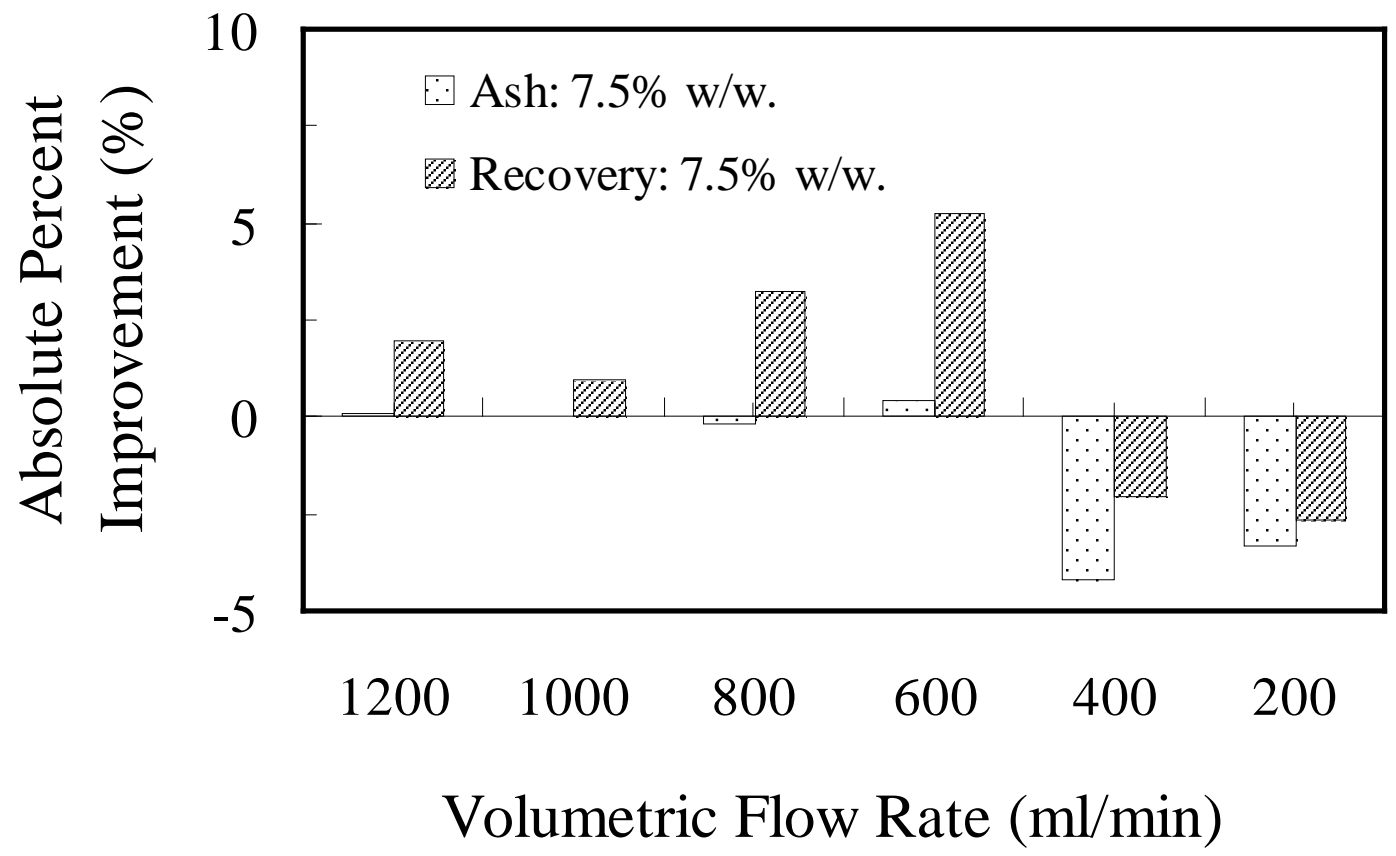

Figure 48. Recovery and product ash content improvements achieved when using external froth refluxing in a flotation column at a feed solids content of $7.5 \%$ by weight; Coalburg coal. 
The results from the $2 \%$ feed solid tests also resemble those achieved on the Pittsburgh No. 8 coal. Figure 49 and 50 clearly indicate ash reductions were achieved up to 4 absolute percentage points at a feed rate of $400 \mathrm{ml} / \mathrm{min}$. The ash reduction was reduced to almost zero as the feed rate was increased above $400 \mathrm{ml} / \mathrm{min}$. No recovery improvement was achieved while a significant reduction was realized at the highest feed rate tested.

Overall, the impact of froth refluxing on separation performance was relatively consistent for the two coal samples and the two solids loading conditions evaluated. The improvement in ash reduction of 3 to 4 absolute percentage points occurred under low feed flow rates which typically are associated with high collection zone recovery values. No recovery improvement was expected; however, elevated recovery values were observed for both coal samples at feed rates above a critical level when using a feed solids concentration of $7.5 \%$ by weight. Surprisingly, no improvement in sulfur reduction resulted from external froth refluxing.

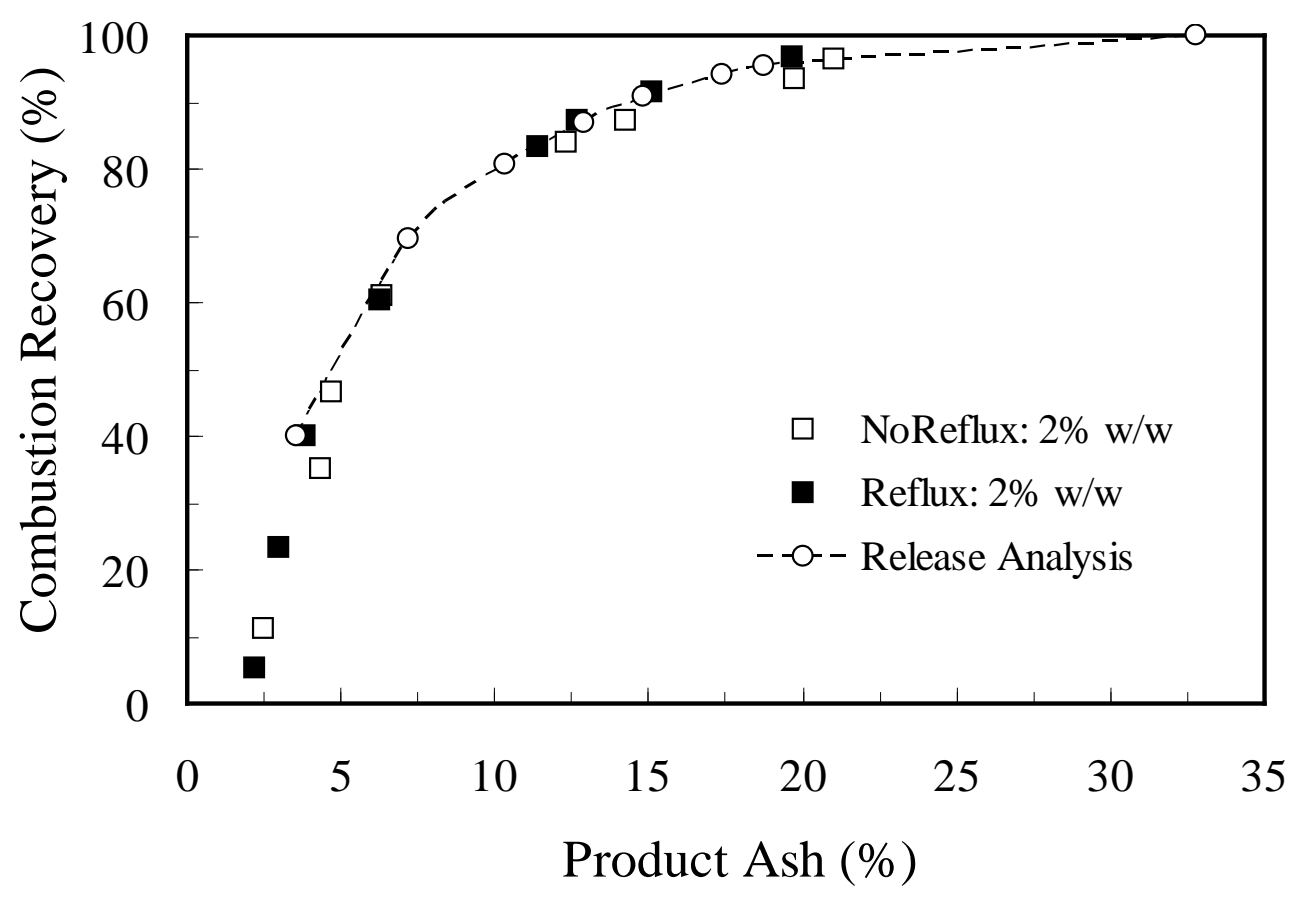

Figure 49. Comparison between separation performances achieved with and without external froth reflux material in a flotation column at $2 \%$ feed solid content; Coalburg coal. 


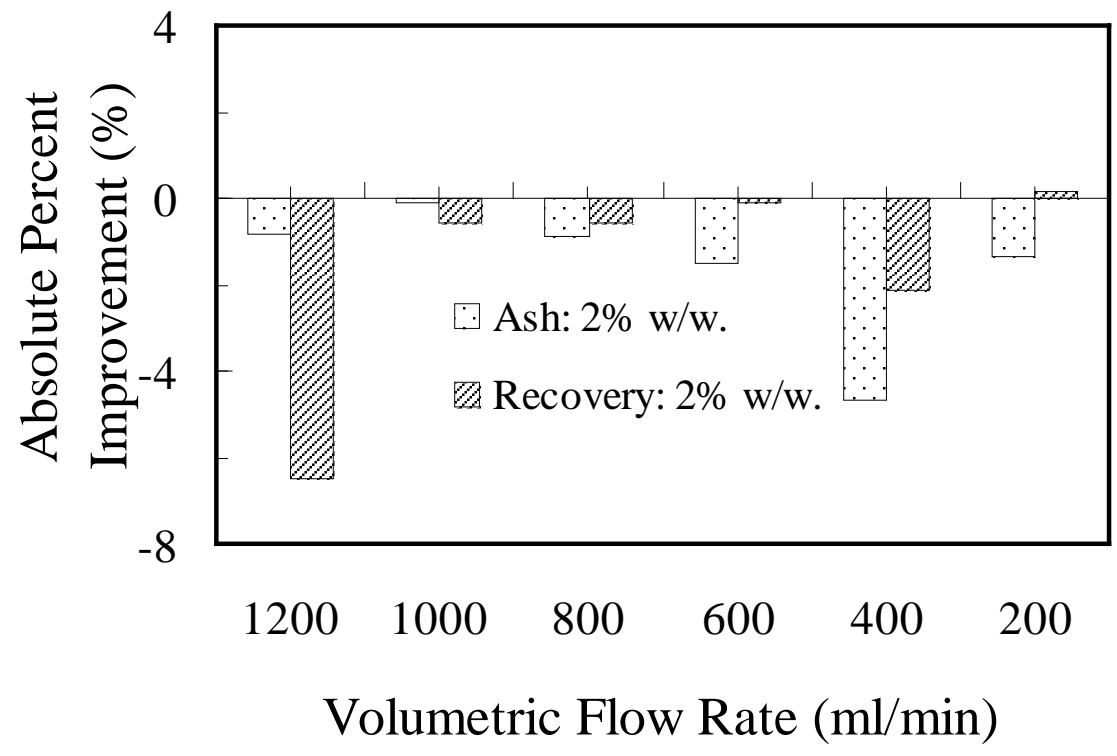

Figure 50. Recovery and product ash content improvements realized from the use of the external froth refluxing with a feed solids content of $2 \%$ by weight; Coalburg coal.

\section{CONCLUSIONS}

In the present study, several techniques to improve flotation performance were explored for the treatment of coal finer than 150 microns, including coal with poor flotation characteristics. Three novel concepts were studied: i) pretreatment of feed coal with hydrodynamic cavitation, ii) injection of magnetic plastic material into the flotation process, and iii) external refluxing to promote selective detachment in the froth zone to improve selectivity. The first technique exploited the picobubble formation phenomena on hydrophobic particles surfaces by hydrodynamic cavitation in an effort to enhance the hydrophobicity of weakly hydrophobic surfaces. The second and third methods used physical and chemical properties of the particles to produce differential flotation rates and selective detachment. Utilization of the selective detachment mechanism and differential flotation rates are the main fundamental principles that determine the overall separation performance.

For the cavitation study, Coalburg seam coal was used for which about $80 \%$ of the material was below 25 microns. The sample was pretreated one or multiple times in the cavitation device before the flotation process. Several flotation rate tests, laboratory column flotation and pilot scale conventional flotation tests in an operating plant were performed.

For the magnetic plastic and froth reflux studies, Pittsburgh No. 8 and Coalburg seam coal samples were utilized. The coal and magnetic plastic material had a particle size that was less than 177 microns. Experiments were carried initially to obtain a baseline 
performance level under standard conditions with no refluxing or plastic additions. For the studies involving the magnetic plastic, flotation rate tests were conducted while varying $\mathrm{pH}$, particle size and the concentration of the added plastic. Subsequently, continuous column flotation tests were performed at optimum parameter levels identified from previous studies. Finally, tests were conducted to evaluate the impact of refluxing a portion of the froth concentrate back to froth zone in a continuous column flotation process. Column flotation tests were performed at different feed solid concentrations and volumetric feed flow rates.

The conclusions obtained from this investigation are provided as follows:

\section{Cavitation Evaluation}

1. The use of an air eductor may be a very inexpensive method to improve coal flotation rate. Micron-sized bubbles are thought to aid in particle attachment to the conventionally-sized bubbles in the flotation cell thereby improving flotation probability. The micron-size bubbles agglomerate fine particles by bridging particles together to the point that the effective size of the agglomerates are able to collide with regular air bubbles more efficiently. Also, the interaction between the bubble coated particle and the conventional bubble is more attractive which reduces the adhesion time and thereby increases flotation rate and recovery, especially for weakly hydrophobic solids.

2. Incorporating a cavitation tube in the feed line to a conventional flotation cell nearly doubled the flotation rate which improved combustible recovery by 18 percentage points. An optimum feed velocity was detected above which recovery decreased. Differences in selectivity were found by using one or multiple repetitions of pretreating the feed slurry. The impact of treatment using the cavitation tube may be greater for particles having a low degree of hydrophobiciy, such as the Coalburg coal. An additional study is recommended to investigate the impact on coal of varying degrees of hydrophobicity.

3. A pilot-scale flotation test was performed side-by-side with a conventional flotation bank in an operating preparation plant. The air eductor test results were superior to those from the full-scale bank. However, the air eductor was not operated properly and thus no real conclusions could be drawn from the pilot-scale study.

\section{Magnetic Plastic Evaluation}

4. The addition of hydrophobic magnetic plastic at a $5 \%$ concentration by weight into feed coal slurry containing moderately hydrophobic Pittsburgh No. 8 coal increased the flotation rate from $0.58 \mathrm{~min}^{-1}$ to $0.93 \mathrm{~min}^{-1}$ under standard conditions. The increase in flotation rate enhanced combustible recovery by about 18 percentage points after one minute of flotation. 
5. For the more weakly hydrophobic Coalburg coal, the flotation rate was improved from $0.51 \mathrm{~min}^{-1}$ to $0.74 \mathrm{~min}^{-1}$ which provided a recovery increase of about 16.3 percentage points after 1 minute of flotation in a conventional cell. The fundamental explanations for the magnetic plastic impact on recovery may include:

a. The hydrophobic plastic particles limit drainage of the water surrounding each air bubble. As a result, bubble coalescence is reduced thereby maximizing the bubble surface area flux rate. The elevation in the amount of surface area available through the froth phase limits preferential detachment of the coarse and weakly hydrophobic particles;

b. Attachment of the plastic material onto the surface of the coal particles thereby yielding a more hydrophobic surface for the bubble-particle attachment process;

c. The hydrophobic plastic material serves as a seed for agglomeration due to strong hydrophobic interaction energies. The large agglomerates are more easily collected in the flotation process;

d. The hydrophobic plastic serves as additional surface area that can be used for the convergence of coal particles into the froth product.

6. It is well known that coal recovery in froth flotation is typically optimum at $\mathrm{pH}$ values around the isoelectric point. Indeed, experiments conducted at $\mathrm{pH}$ values of 3.9 and 10.1 produced flotation rates of around $0.30 \mathrm{~min}^{-1}$ when treating Pittsburgh No. 8 coal. However, after the addition of $10 \%$ magnetic plastic particles, the rate was significantly enhanced to $1.3 \mathrm{~min}^{-1}$ plastic particles at both $\mathrm{pH}$ conditions. Again, this is believed to be due to the strong hydrophobic interactions associated with the plastic particles. The corresponding recovery increase under both conditions was greater than 50 absolute percentage points, which is highly remarkable.

7. The original concept associated with the use of plastic particles involved enrichment of the froth phase to induce selective detachment and, thus improve selectivity. For the Pittsburgh No. 8 coal, very little selectivity improvement was observed from the laboratory conventional cell tests. However, selectivity was significantly improved by the presence of the plastic particle from the treatment of the Coalburg coal. While producing a $15 \%$ ash concentrate, the plastic material increased recovery by nearly 10 absolute percentage points in a conventional cell. The finding can be explained by the large quantity of middling (unliberated) material present in the Coalburg coal.

8. Test results indicate that the coal recovery improvement observed from the addition of the hydrophobic plastic is preferentially associated with the coarse particles, especially those having a particle size equal to $0.10 \mathrm{~mm}$ or greater. This finding 
tends to support the hypothesis that the plastic particles are attaching to the coal surfaces and enhancing their hydrophobicity.

9. This hypothesis was further substantiated by column flotation experiments in which carrying capacity were evaluated. Typically, the product mass flow rate increases and then reaches a maximum at a given feed mass flow rate. Beyond the critical mass flow rate, product mass flow decreases as a result of selective detachment of coarse coal particles. When plastic particles are added directly into the froth, the product mass flow did not decrease but maintained a constant value with an increase in feed flow rate. The only plausible explanation is that the plastic particles reduce the detachment rate of the coarse coal particles. This event occurs due to a stronger binding force between the bubble and the plastic coated coal particles.

10. In the continuous column flotation tests, improvements in recovery were generally less than the conventional cell results and varied as a function of volumetric feed rate and the amount of plastic particles added. At a 5\% concentration, recovery improvement was from around 4 absolute percentage points at feed rates between $400 \mathrm{ml} / \mathrm{min}$ to $800 \mathrm{ml} / \mathrm{min}$ for the treatment of Pittsburgh No. 8 coal. For Coalburg coal, the recovery improvement was around 7 absolute percentage points at a feed rate of $600 \mathrm{ml} / \mathrm{min}$. Lower recovery improvements in the flotation column were expected due to the difference in hydrodynamics conditions between flotation column (i.e. near plug flow) and conventional cells (i.e. near perfectly-mixed). The addition of the magnetic plastic provided no improvement in selectivity.

\section{External Froth Reflux}

11. By refluxing a portion of the froth concentrate back to the froth phase through the wash water, significantly lower product ash contents were achieved under kinetic limiting conditions (low volumetric flow rates). At the same low feed rate, product ash content decreased from around $7 \%$ to nearly $4 \%$ while maintaining recovery at a near constant level for the Pittsburgh No. 8 coal. Similar findings were obtained in tests involving the Coalburg coal.

12. Near the elbow of the recovery-product ash curve, very little to no improvements were observed from froth refluxing. However, at ash content lower than those near the elbow of the curve, the recovery losses due to the froth reflux were evident.

\section{REFERENCES}

Alexander, D.J., Franzidis, J.P. and Manlaping, E.V., 2003, Froth Recovery Measurement in Plant Scale Flotation Cells. Minerals Engineering, 16 (11), 1197-1203.

Ata, S, Ahmed, N. and Jameson, G J, 2002, Collection of Hydrophobic Particles in the Froth Phase, International Journal Of Mineral Processing, 64:101-122. 
Ata, S., Ahmed, N. and Jameson, G.J., 2004, The Effect of Hydrophobicity on The Drainage of Gangue Minerals in Flotation Froths. Mineral Engineering, 17(7-8), 897-901.

Attalla, M., Chao, C. and Nicol, S.K., 2000, The Role of Cavitation in Coal Flotation. Proceedings of the Eight Australian Coal Preparation Conference; Port Stephens, Nov 1216; 337-350.

Bisshop, J.P. and White, M.E., 1976, Study of Particle Entrainment in Flotation Froths. Transactions of The Institution of Mining and Metallurgy, Section C: Mineral Processing and Extractive Metallurgy 85, 191- 194.

Cheng, T. and Holthman, P., 1995, The Particle Detachment Process in Flotation. Minerals Engineering 8 (8), 883-891.

Cilek, E.C. and Yilmazer, B.Z., 2003, Effects of Hydrodynamics Parameters on Entrainment and Flotation, Minerals Engineering, 16 (8), 745-756.

Dippenaar, A., 1982, The Destabilization of Froth by Solids. I. The Mechanism of Film Rupture. International Journal Mineral Processing, 9, 1-14.

Dippenaar, A., 1982, The Destabilization of Froth by Solids. II. The Rate-Determining Step. International Journal Mineral Processing, 9, 15-22.

Falutsu, M., and Dobby, G.S., 1989, Direct Measurement of Froth Drop-Back and Collection Zone Recovery in a Laboratory Flotation Column. Minerals Engineering, 2, 377-386.

Falutsu, M., 1994, Column Flotation Froth Characteristics-Stability of The BubbleParticle System, International Journal of Mineral Processing, 40: 25-243.

Finch, J. A. and Dobby, G. S., 1990, Column Flotation, Pergamon Press: Oxford.

Finch, J.A., Xiao, J., Hardie, C., and Gomez, C.O., 2000, Gas Dispersion Properties: Bubbles Surface Area Flux and Gas Holdup. Minerals Engineering, 13: 365-372.

George, P., Nguyen, A.V., and Jameson, G.J., 2004, Assessment of True Flotation and Entrainment in the Flotation of Submicron Particles by Fine Bubbles. Minerals Engineering, 17 (7-8), 847-853.

Hemmings, C.E., 1981, On the Significance of Flotation Froth Liquid Lamella Thickness Trans. Instn Min. Metall. (Sect. C: Mineral Process. Extr. Metall.) 90, C96.

Honaker, R. Q. and Ozsever, A. V., 2003, Evaluation of the Selective Detachment Process in Flotation Froth, Minerals Engineering, 16(10): 975-982. 
Honaker, R.Q., Ozsever, A.V. and Parekh, B.H., 2006, Selective Detachment Process in Column Flotation Froth, Minerals Engineering, 19(6-8), 687-695.

Johansson, G., and Pugh, R.J., 1992, The Influence of Particle Size and Hydrophobicity on the Stability of Mineralized Froths. International Journal Mineral Processing. 34 (12), $1-21$.

Laplante A.R., Kaya, M., and Smith, H.W., 1989, The Effect of Froth on Flotation Kinetics- A Mass Transfer Approach, In Frothing In Flotation (Laskowski, J.S., Ed.), Gordon and Breach Science Publishers, 77-99.

Malysa, K., 1998, Water Contents and Distribution in Flotation Froths. In: Frothing in Flotation II. Cordon and Breach, Laskowski, J.S., Woodburn, E.T. (Eds.), New York, pp 81-108.

Mathe, Z.T., Harris, M.C. and O’Connor, C.T., and Franzidis, J.P., 1998, Review of Froth Modeling in Steady State Flotation Systems. Minerals Engineering, 11 (5), 397421.

Mathe, Z.T., Harris, M.C., O’Connor, C.T., 2000, A Review of Methods to Model the Froth Phase in Non-Steady Flotation Systems. Minerals Engineering, 13 (2), 127-140.

Moys, M.H., 1978, A Study of a Plug-Flow Model for Flotation Froth Behaviour. International Journal Mineral Processing. 5: 21-38.

Moys, M.H., 1984, Residence Time Distributions and Mass Transport in the Froth Phase of The Flotation Process. International Journal Mineral Processing. 13: 117-142.

Neethling, S.J. and Cilliers, J.J., 1999, Visual Kinematic Model of Flowing Foams Incorporating Coalescence. Powder Technology, 101,249-256.

Neethling, S.J., and Cilliers, J.J., 2002, The Entrainment of Gangue into a Flotation Froth. International Journal of Mineral Processing, 64(2-3), 123-134.

Neethling, S.J., and Cilliers, J.J., 2003, Modelling Flotation Froths. International Journal of Mineral Processing, 72(1-4), 267-287.

Nguyen, A.V., Harvey, P.A., and Jameson, G.J., 2003, Influence of Gas Flow Rate and Frothers on Water Recovery in a Froth Column. Minerals Engineering, 16(11), 11431147.

Ozsever, A. V, 2005, Evaluation of the Selective Detachment Process in Froth Flotation. PhD. Thesis. University of Kentucky. 
Perez, R., and Villar, R.D., 1997. Estimation of Bias and Entrainment in Flotation Columns Using Conductivity Measurements. Canadian Metallurgical Quarterly, 36 (5), 299-307.

Ralston, J., Fornasiero, D and Hayes, R, 1999. Bubble-Particle Attachment and Detachment in Flotation, International Journal of Mineral Processing, 56(1-4),133-164.

Ralston, J. and Dukhin, S.S., 1999. The Interaction between Particles and Bubbles. Colloid Surface, 151, 3-14.

Savassi, O.N., Alexander, D.J., Johnson, N., Franzidis, J.P., and Manlaping, E.V., 1997. Measurement of Froth Recovery of Attached Particles in Industrial Flotation Cells. In: Sixth Mill Operators’ Conference. Madang, Papua New Guinea, pp. 149-156.

Savassi, O.N., Alexander, D.J., Franzidis, J.P., and Manlaping, E.V., 1998. An Empirical Model for Entrainment in Industrial Flotation Plants. Mineral Engineering, 11(3), 243256.

Savassi, O.N., 1998. Direct Estimation of the Degree of Entrainment and Froth Recovery of Attached Particles in Industrial Flotation Cells. Department of Mining, Minerals and Material Engineering, JKMRC. PhD Thesis, University of Queensland, Brisbane, p.393.

Seaman, D., Franzidis, J. and Manlaping E., 2004. Bubble Load Measurement in the Pulp Zone of Industrial Flotation Machines- A New Device For Determining the Froth Recovery of Attached Particles. International Journal of Mineral Processing 74 (1-2), 113.

Subrahmanyam, T.V. and Forssberg, E., 1988. Froth Stability, Particles Entrainment and Drainage in Flotation - A Review. International Journal of Mineral Processing, 23(1-2), 33-53.

Sulman, H.L., Picard H.F.K. and Ballot J., 1905. British patent 7,803, April 12; Duplicated as U.S. Patent 835,120, May 29.

Tao, D., 2004. Role of Bubble Size in Flotation of Coarse and Fine Particles- A review, Separation Science and Technology, 39 (4):741-760.

Van Deventer, J. S. J., Van Dyk, W.A., and Lorenzen, L., 2000. The Separation of Coarse Particles by a Moving Froth Bed. In Proceedings of the $21^{\text {st }}$ International Mineral Processing Congress, Rome. Italy, July 23-28. Amsterdam: Elsevier, C7-1 - C7-8.

Van Deventer, J. S. J., Van Dyk, W.A., Lorenzen, L., and Feng, D., 2000a. The Dynamic Behavior of Particles in Flotation Froths. Part I: Model. Minerals Engineering 15:635645. 
Van Deventer, J. S. J., Van Dyk, W.A., Lorenzen, L., and Feng, D., 2000b. The Dynamic Behavior of Particles in Flotation Froths. Part II: Density Tracer Tests. Minerals Engineering 15:647-657.

Van Deventer, J. S. J., Van Dyk, W.A., Lorenzen, L., and Feng, D., 2000c. The Dynamic Behavior of Particles in Flotation Froths. Part III: Ore particles. Minerals Engineering 15:659-665.

Van Deventer, J. S. J., Feng, D. and Burger, A. J., 2004. Transport phenomena at the Pulp-Froth Interface in a Flotation Column: II Detachment, International Journal of Mineral Processing, 74(1-4): 217-231.

Van Dyk, W.A., van Deventer, J.S.J., and Lorenzen, L., 1995. The Dynamic Behavior of Coarse Particles in Flotation Froths. In Proceeding of the $19^{\text {th }}$ International Minerals Processing Congress. Volume 3. Littleton, CO: SME, 99-103.

Vera, M.A., Franzidis, J.P. and Manlapig, E.V., 1998. Simultaneous Determination of Collection Zone Rate Constant and Froth Zone Recovery Factor. Frothing in Flotation II, Gordon and Breach Science Publications. Amsterdam.

Vera, M.A., Mathe, Z.T., Franzidis, J.P., Harris, M.C., Manlaping, E.V., and O’Connor, C.T., 2002. The Modeling of Froth Zone Recovery in Batch and Continuously Operated Laboratory Flotation Cells. International Journal of Mineral Processing, 64 (2-3), 135151.

Xu, M., Uribe-Salas A. and Finch J.A., 1991. Maximum Gas and Bubble Surface Rates in Column Flotation. International Journal of Mineral Processing, 32, 233-250.

Yianatos, J. B., Finch, J. A. and Laplante, A. R., 1986. Holdup Profile and Bubble Size Distribution of Flotation Column Froths, Can. Met. Q., 25 (1): 23-29.

Yianatos, J. B., Finch, J. A. and Laplante, A. R., 1988. Selectivity in Column Flotation Froths, International Journal of Mineral Processing, 23: 279-292.

Zhou, Z.A., Xu, Z.,. Finch, J.A., Hu, H. and Rao, S.R., 1997. Role of Hydrodynamic Cavitation in Fine Particle Flotation. International Journal Mineral Processing, 51, 139149.

\section{PUBLICATIONS/PRESENTATIONS}

Honaker, R. Q., C. Munoz and M. Mankosa, "Enhancing Coal Flotation Performance Using Recyclable Hydrophobic Particles,” $137^{\text {th }}$ SME Annual Meeting, Salt Lake City, Colorado, February 24 - 27, 2008. 
Appendix 4: Dispersion of Flotation Clays from New Mexico Potash Ores (NM001) 


\section{TECHNICAL REPORT}

Contract Title and Number:

Crosscutting Technology Development at the Center for

Advanced Separation Technologies

(DE-FC26-02NT41607)
Period of Performance:

Starting Date: 04/01/03

Ending Date: 10/31/09
Sub-Recipient Project Title:

Dispersion and Flotation of Clays

from New Mexico Potash Ores

Principal Investigators:

I.H. Gundiler, S.Titkov, M.Yekeler

Contact Address:

New Mexico Tech

Bureau of Geology \& Min. Res.

801 Leroy Place

Socorro, NM 87801

Subcontractor Address:

"No subcontracts issued."
Report Information:

Type: FINAL

Number: R12

Period: $\quad 04 / 01 / 03-03 / 31 / 10$

Date: $\quad 04 / 18 / 2010$

Code: NM001-FINAL

Contact Information:

Phone: (575) 835-5730

Fax: (575) 835-6333

E-Mail: Gundiler@gis.nmt.edu

Subcontractor Information:

Phone:

Fax:

E-Mail:

\section{Disclaimer}

"This report was prepared as an account of work sponsored by an agency of the United States Government. Neither the United States Government nor any agency thereof, nor any of their employees, make any warranty, express or implied, nor assume any legal liability or responsibility for the accuracy, completeness, or usefulness of any information, apparatus, product, or process disclosed, or represents that its use would not infringe privately owned rights. Reference herein to any specific commercial product, process, or service by trade name, trademark, manufacturer, or otherwise does not necessarily constitute or imply endorsement, recommendation, or favoring by the United States Government or any agency thereof. The views and opinions of authors expressed herein do not necessarily state or reflect those of the United States Government or agency thereof." 


\begin{abstract}
Sylvite $(\mathrm{KCl})$ is the most common potash mineral; it is concentrated from sylvinite (KCl$\mathrm{NaCl}$ ) ores by froth flotation using long-chain aliphatic amines in double saturated brines. Clay minerals are the most troublesome impurity in processing potash ores. Because of their strong cation and anion exchange capabilities and very large surface areas, they adsorb flotation reagents and hinder sylvite flotation. Clays therefore are removed by multi-stage mixing and decantation using classifiers, hydrocyclones, and hydroseparators ahead of sylvite flotation. This process, however, is inherently inefficient. Residual clays remaining in the flotation feed rendered innocuous by the use of 'clay blinders', organic polymers such as starch, guar gum, carboxymethyl cellulose (CMC), and recently with urea-formaldehyde resins.
\end{abstract}

New Mexico is the largest potash producer in the US. Potash production in the southeastern part of the state began in the 1940s. There are still large reserves of ores remaining; however, as the low-clay ores are depleted the potash producers have to exploit higher clay ores that are not amenable to processing with the existing clay removal technologies. This study was initiated to investigate increasing mechanical desliming efficiency at existing plants, and explore other means of desliming high-clay ores that heretofore can not be mined.

During the first phase of this study, effects of organic and inorganic dispersants were studied. Sodium or calcium lignin sulfonates, and polyvalent metal salts such as ferric and aluminum sulfate (alum) were found to be effective dispersants that keep the clays in suspension and help increase the efficiency of clay rejection, decrease $\mathrm{KCl}$ loses into slimes and increase recovery in the flotation concentrate. When added into the rod-mill grinding circuit, they also increased the grinding efficiency producing 7-9\% finer product. It appears that dispersants also act as grinding aids that may help reducing the power consumption and/or increase the rod-mill throughput. Conventional slime dispersants, such as sodium silicate or phosphates did not have any effect in this system.

Slime flotation was investigated to augment mechanical desliming. Slimes were first treated with a flocculant and floated using oxyethylated-fatty acids, -fatty amines, or ether amines. In general, slime flotation removes highly surface-active clays from the potash flotation feed, increase recovery, reduce reagent consumption, and produce higher quality concentrates. Low clay ores can be floated with one stage desliming; medium clay ores require coarse-ore desliming followed by grinding and slime flotation, and high-clay ores require two-stage mechanical (coarse and fine ore desliming after grinding) and one stage slime flotation. Ores containing up to $11 \%$ water-insoluble residue were successfully floated with $88 \%$ flotation recovery, $82 \%$ overall recovery. A new flotation plant under consideration in the district will incorporate slime flotation in the process feasibility and design stage.

Finally, urea-formaldehyde resins were investigated as potential substitutes for clay blinders. Although comparable recoveries were obtained with guar and resins in fine ore flotation, resin might have certain advantages such as availability and price, does not require special solution preparation - simply diluted with brine, and reportedly increase filtration rates of the tailings. The use of resins has already been implemented at one of the plants past few years. 


\section{TABLE OF CONTENTS}

TITLE PAGE

DISCLAIMER

$\underline{\text { Page }}$

ABSTRACT

TABLE OF CONTENTS

LIST OF FIGURES

EXECUTIVE SUMMARY vi

INTRODUCTION 1

EXPERIMENTAL METHODS 5

Materials and Methods 5

Slime Dispersion Tests

Slime Flotation Tests $\quad 6$

Potash Flotation Tests $\quad 7$

Urea Formaldehyde Resins $\quad 7$

EXPERIMENTAL RESULTS $\quad 8$

Clay Dispersion Tests $\quad 8$

Slimes Settling Rates $\quad 8$

Zeta Potential Measurements 9

Dispersion and Potash Flotation Tests 10

Low Clay Ores 10

Medium Clay Ores 11

High Clay Ores $\quad 15$

Slime Flotation Tests $\quad 15$

Low Clay Ores 15

Medium Clay Ores $\quad 17$

High Clay Ores 19

Urea-Formaldehyde Resin Depressants 20

Fine Ore Flotation 21

Coarse Ore Flotation (-4 mesh) 22

Coarse Ore Flotation (-5 mesh) 24

Four Factor Screening Tests (-5 mesh) 26

DISCUSSIONS $\quad 29$

SUMMARY AND CONCLUSIONS 31

REFERENCES

$\begin{array}{ll}\text { APPENDIX } & 34\end{array}$ 


\section{LIST OF FIGURES}

Figure 1 - Dispersion of slimes with (left jar) and without dispersants (right jar) after (a) 0.5 minutes, and (b) 5 minutes of settling time.

Figure 2 - Slimes settling rates with Lignin Sulfonate (top) and with Alum as dispersants.

Figure 3 - Comparison of $\mathrm{K}_{2} \mathrm{O}$ and IR recovery into the flotation concentrate after one-stage desliming and flotation tests with low clay ores

Figure 4 - Comparison of low and medium clay ores flotation tests after one-stage desliming with or without Alum dispersant.

Figure 5 - Overall effects of Lignin Sulfonate dispersants on $\mathrm{K}_{2} \mathrm{O}$ recovery into slimes and residual IR in desliming tailings (flotation feed) after coarse grinding and one-stage desliming of medium clay ores (MCO).

Figure 6 - Effect of dispersants on IR and $\mathrm{K}_{2} \mathrm{O}$ recovery into slimes fractions after coarse desliming followed by 3 -minute grinding and 2-stage desliming of medium clay ores

Figure 7 - Overall effects of dispersants on IR and $\mathrm{K}_{2} \mathrm{O}$ recovery into slimes fractions after coarse desliming followed by 5 -minute grinding and 2-stage desliming of medium clay ores

Figure 8 - The influence of dispersants on potash flotation recovery from fine-ground medium-clay ores after 2-stage desliming with or without dispersants.

Figure 9 - Effect of Alum on grinding kinetics in rod-mill grinding of medium clay ore.

Figure 10 - Insoluble residue recovery into slime fraction and slime flotation concentrates.

Figure $11-\mathrm{K}_{2} \mathrm{O}$ recovery into slime concentrates from low-clay ores, based on initial ore.

Figure 12 - IR content in the potash flotation feed after desliming low-clay ores samples.

Figure $13-\mathrm{K}_{2} \mathrm{O}$ recovery in the potash flotation concentrates (at guar 60, amine $30 \mathrm{~g} / \mathrm{t}$ )

Figure 14 -Potash recovery from medium clay ores after 2-stage desliming, followed by sylvite flotation with $60 \mathrm{~g} / \mathrm{t}$ guar and $33 \mathrm{~g} / \mathrm{t}$ amine emulsion.

Figure 15 - Comparison of total insoluble minerals recovery and IR content of the sylvite flotation feed, by siphon decantation and slime flotation methods.

Figure 16 - Overall potash recovery from high clay ore samples by decantation desliming versus slime flotation with $20 \mathrm{~g} / \mathrm{t}$ oxy-ethylated fatty amine collector. 
Figure 17 - Comparison of potash recovery from fine flotation feed with $60 \mathrm{~g} / \mathrm{t}$ amine emulsion containing $23 \mathrm{~g} / \mathrm{t}$ oil and $11 \mathrm{~g} / \mathrm{t}$ frother with guar and UF resin as depressant.

Figure 18 - Effect of amine and resin concentrations on potash recovery in fine ore flotation

Figure 19 - 3D representation of potash recovery from -4 mesh ore as a function of resin and frother concentrations at mid levels of extender oil $(375 \mathrm{~g} / \mathrm{t})$.

Figure 20 - Effect of guar, oil, and resin on potash recovery from minus 5-mesh ore

Figure 21 - Effect of amine and oil on potash recovery from coarse ore.

Figure 22 - Potash recovery in coarse (-5/+28 mesh) ore flotation with $900 \mathrm{~g} / \mathrm{t}$ Resin

Figure 23 - Effect of flotation reagents on potash recovery in coarse ore flotation

Figure 24 - Maximum recovery predicted from 4-factor fractional factorial experiments occur at high resin, low amine, oil, and frother concentrations.

Figure 25 - Typical example of a coarse ore ( -5 mesh) flotation concentrate.

Figure A1- Predicted versus actual recoveries in 4-parameter fractional factorial design tests.

Figure A2 - Normal plot of residuals of the experimental data fitted to a second order model

\section{LIST OF TABLES}

Table 1- Common potash minerals [3]

Table 2 - Zeta potential measurements on grey and mixed clays in dilute brines in the presence of aluminum sulfate as dispersant.

Table 3 - List of slime collectors tested in this study

Table A1 - Analysis of variance (ANOVA) table for selected factorial model

Table A2 - Optimization of Recovery 


\section{EXECUTIVE SUMMARY}

New Mexico (NM) is the largest producer of potash minerals, accounting for over $70 \%$ of the U.S. production. Potash mining and processing in New Mexico began before 1940 in response to the need for domestic production of potassium fertilizer due to World War II. By the 1960's there were six Potash Processing companies in New Mexico. Foreign competition, lower grade ore and higher clay content, thus higher processing costs, have reduced the operating companies to two, Mosaic Potash Carlsbad and Intrepid Potash New Mexico LLC.

Sylvite $(\mathrm{KCl})$ and langbeinite $\left(\mathrm{K}_{2} \mathrm{SO}_{4} \cdot 2 \mathrm{MgSO}_{4}\right)$ are the major ore minerals mined in New Mexico. The main product of the potash industry is MOP, muriate of potash, contains minimum of $95 \% \mathrm{KCl}\left(60 \% \mathrm{~K}_{2} \mathrm{O}\right)$. Major gangue minerals are halite $(\mathrm{NaCl})$, anhydrite $\left(\mathrm{CaSO}_{4}\right)$, silica $\left(\mathrm{SiO}_{2}\right)$, and clay minerals. Sulfates of potassium-magnesium (SOPM or K$\mathrm{Mag})$ are concentrated from the langbeinite $\left(\mathrm{K}_{2} \mathrm{SO}_{4} \cdot 2 \mathrm{MgSO}_{4}\right)$ ores. SOP, sulfate of potash $\left(\mathrm{K}_{2} \mathrm{SO}_{4}\right)$, is produced from langbeinite, and kainite $\left(\mathrm{KCl} \cdot \mathrm{MgSO}_{4} \cdot 3 \mathrm{H}_{2} \mathrm{O}\right)$ concentrates in Utah. Langbeinite is only mined and processed in the world in NM. Low chloride content and lower solubility of the potassium sulfate products make them desirable for certain soil conditions and crops such as citrus fruits, tobacco, and vegetables. Potash products are sold on the basis of their chemical compositions and particle sizes; coarser particles are more desirable.

In general, clay minerals are the most troublesome impurity encountered in processing sylvite ores. Clays are soft and easily hydrated; they swell and disintegrate in water into minute-sized $(<0.002 \mathrm{~mm})$ platelets. Because of their high cation (and anion) exchange capacity, and high surface area, clays preferentially adsorb large amounts of flotation collectors, thus decreasing their effective concentration and hindering sylvite flotation. As low as $0.1 \%$ free clay could reduce the flotation recovery by $20 \%$. Flotation of sylvite virtually ceases in the presence of $1 \%$ clays in the feed. Consequently, clays increase the reagent and energy costs to process potash ores, and increase the losses of fine sized potassium minerals in the separation and densification of the clay slurries.

Slimes, therefore, are removed ahead of sylvite flotation circuit by multi-stage scrubbing and mechanical de-sliming, using screens, hydroseparators, and hydrocyclones. This process is inherently inefficient; slime dispersants and blinding agents, such as starch, (CMC) carboxy methyl cellulose, or guar gum, and recently urea-formaldehyde resins are used as "blinding agents" to prevent adsorption of flotation collectors on clays to alleviate the adverse effects of clays remaining in the flotation feed.

Hydro-mechanical desliming methods, however, are only effective for ores with low clay content, i.e., total insoluble residue (I.R.) content (clay-carbonate admixtures) to about 5\%. Ores with higher I.R. content (or very fine-grained ores) are leached with halite saturated hotbrine in order to dissolve sylvite selectively. After solution purification, the brine is cooled and $\mathrm{KCl}$ is recovered by vacuum crystallization. This process, however, is highly energy and equipment intensive.

Flotation of sylvite in coarse sizes in processing sylvinite $(\mathrm{KCl}-\mathrm{NaCl})$ ores is highly desirable, eliminating the need for compaction, re-crushing, and screening required of the fine flotation 
concentrates. Although it is commonly practiced in most Canadian operations, most New Mexico ores are not amenable to processing in coarse sizes due to fine grain size, hence the necessity for finer grinding to liberate sylvite and halite, and disperse clay minerals.

This study was initiated to address these problems and provide solutions that can easily be implemented in the existing plants. Therefore, the objectives of this study were:

- to investigate the effects of organic and inorganic clay dispersants to increase the efficiency of the existing slime removal technologies in processing NM potash ores

- to investigate flotation separation of clays from sylvinite ores using slime flotation

- to investigate the effects of temperature on the flotation recovery of sylvite

- and to develop and optimize reagent combinations for plant trials.

All experiments were performed in double saturated $(\mathrm{NaCl}-\mathrm{KCl})$ brines. After 5-10 minutes of intense agitation in brines, the coarse samples were deslimed by siphon decantation, ground in a laboratory (10" diameter) rod mill, and deslimed once more for low and medium clay ores, 2-3 times for very high clay ores. Hydrogenated tallow amine (Armeen HT), extender oil (Philflo), and MIBC frother used as flotation reagents. Ore samples used in this study were collected from the operating mines in the Carlsbad Potash District (CPD), NM.

The effect on clay dispersants were investigated in the first phase of this study. Dispersants, such as lignin sulfonate was found to hinder slimes settling rates, hence increase desliming efficiency, resulting 5-7\% increase in potash flotation recoveries in processing low and medium clay ores. Polyvalent metal ion salts, aluminum sulfate $\left(\mathrm{Al}_{2}\left(\mathrm{SO}_{4}\right)_{3}\right)$ (alum) and ferric chloride $\left(\mathrm{FeCl}_{3}\right)$ in particular, similarly effected the settling rates. Common inorganic dispersants, such as sodium silicate $\left(\mathrm{Na}_{2} \mathrm{SiO}_{3}\right)$ and trisodium phosphate $\left(\mathrm{Na}_{3} \mathrm{PO}_{4}\right)$ did not have any effect on slime settling rates. Although polyvalent metal ion salts are generally known as coagulants, neutralizing the surface charge of suspended particles, hence allowing them to settle faster, in saturated brines at high concentrations they cause charge reversal and help disperse clays, and prevent adsorption of positively charged amine molecules on residual clays, thus increasing recovery and purity of the concentrate.

One surprising finding of the dispersant study was that alum and lignin sulfonates behave as grinding aids increasing grinding kinetics and fineness of the product by about $9 \%$. That may suggest that energy consumption for grinding may be reduced, or grinding capacity may be increased by the like amount.

Following the coarse ore desliming, slime flotation tests were performed on rod-mill ground samples instead of, or in conjunction with the siphon decantation. Slimes were flocculated with polyacrylamide (PAA) type flocculant, and floated with oxyethylated fatty acid (OEFAc) or fatty amine (OEFAm) family of collectors, or with ether amines. All three types of reagents yielded comparable recoveries with 10-25 g/t PAA and slime flotation reagent. However, the reagent consumptions should be optimized depending on the nature of clay minerals and the initial clay content of the ore. 
In general, after the first stage coarse ore desliming, low clay ores can be deslimed very efficiently with one-stage slime flotation, and medium clay ores with one-stage decantation desliming followed by slime flotation. Sylvite flotation recoveries have increased with similar decrease of $\mathrm{KCl}$ loses to slimes and tailings, and the concentrate quality increased with lesser clay contamination. Clay depressant (guar) and amine requirements for sylvite flotation also decreased, suggesting surface-active clay minerals were removed during the clay flotation step.

Although low and medium clay ores can be economically processed with the existing plants, those type of ores are being depleted. Incorporation of the slime flotation step into the existing plants will allow processing higher clay ores and provide more uniform feed to the sylvite flotation circuit. For instance, high to very high-clay ores (7-11\%) that heretofore can not be exploited, was successfully floated after two-stage mechanical and one-stage slime flotation desliming with $88 \%$ sylvite flotation recovery ( $82 \%$ overall recovery). Feasibility studies for a new flotation plant under way in the District will incorporate slime flotation in process design.

Urea-formaldehyde resins were also studied as alternative clay depressants. Experiments conducted with fine ore samples yielded high recoveries $(92+\%)$ with moderate quantities of amine, guar or resin depressants. However, resin might have a number of advantages such as cost and availability, does not require special procedures for solution preparation, and reportedly increases filtration and de-brining rates in plant practice. The resins are being used in one potash plant in fine ore flotation circuit at the CDP.

Recoveries with coarse ore samples, however was found to depend on the top size and the fraction of fines contained in the flotation feed. Resin concentrations required for high yields also varied with particle size. Up to $88 \%$ recovery was obtained with minus 6-mesh ore samples using moderate quantities of resin $(600 \mathrm{~g} / \mathrm{t})$, and $87 \%$ recovery was obtained with minus 5-mesh using $1200 \mathrm{~g} / \mathrm{t}$ resin. Without depressants recoveries were very low (33\%), and did not exceed $70 \%$ even with $120-150 \mathrm{~g} / \mathrm{t}$ guar levels.

Four-parameter fractional factorial design experiments helped identify the resin as the most influential factor in coarse ore flotation, followed by amine and oil concentrations. Optimization tests indicated that best recoveries can be obtained at low concentrations of amine $(80 \mathrm{~g} / \mathrm{t})$, oil $(100 \mathrm{~g} / \mathrm{t})$, frother $(11-13) \mathrm{g} / \mathrm{t}$, and high resin concentrations $(1200 \mathrm{~g} / \mathrm{t})$. It is concluded that resin not only depress residual slimes in the flotation feed, but is also adsorbed on sylvite grains rendering them more hydrophobic than amine and oil could. Plant trials are under consideration for the use of resin in the coarse ore flotation circuit at one of the plants. 


\section{INTRODUCTION}

Potash is the common term that denotes the element potassium in a water-soluble form in a variety of chemical combinations. Potassium is an essential element vital for plant and animal life. In combination with fixed nitrogen and phosphorus, over $90 \%$ of the potash produced in the United States is used as agricultural fertilizer; industrial uses account for the rest [1].

United States is the seventh largest producer in the world at 1.1 million metric tons $\mathrm{K}_{2} \mathrm{O}$ equivalent (2.6 Mt concentrates) in 2007 [1]. Canada, Russia, Germany, Belarus, China, and Israel, in that order, are the largest producers. The vast majority of potash ores are mined from underground bedded evaporite deposits. Israel and Jordan harvest potash from the Dead Sea brines. In the U.S., New Mexico (NM) is the largest producer accounting for over 70\% of the U.S. production; Utah and Michigan are the other two potash-producing states. In Utah, production comes from near-surface brines, from solution mining, and from solar evaporation and beneficiation of Great Salt Lake brines, and in Michigan solution mining and mechanical evaporators are used to produce white crystalline $\mathrm{KCl}$ [1].

The most abundant potash ores are sylvinite, which is a mechanical mixture of sylvite $(\mathrm{KCl})$ and halite $(\mathrm{NaCl})$, including minor amounts of clays, silica, anhydrite $\left(\mathrm{CaSO}_{4}\right)$, and potassium complexes such as carnallite, polyhalite, kainite, langbeinite and leonite. The potassium content in potash ores and fertilizer products is reported as $\% \mathrm{~K}_{2} \mathrm{O}$ as a means to compare fairly diverse potash minerals. The chemical composition of principal potash minerals is given in Table 1. Sylvite and langbeinite are the only major ore minerals mined in New Mexico [2].

Table 1. Common potash minerals [3]

\begin{tabular}{llcc}
\hline Mineral & Formula & Specific gravity & $\% \mathrm{~K}_{2} \mathrm{O}$ \\
\hline Sylvite & $\mathrm{KCl}$ & 1.99 & 63.17 \\
Carnallite & $\mathrm{KCl} \cdot \mathrm{MgCl}_{2} \cdot 6 \mathrm{H}_{2} \mathrm{O}$ & 1.60 & 16.95 \\
Kainite & $\mathrm{KCl} \cdot \mathrm{MgSO}_{4} \cdot 3 \mathrm{H}_{2} \mathrm{O}$ & 2.13 & 18.92 \\
Langbeinite & $\mathrm{K}_{2} \mathrm{SO}_{4} \cdot 2 \mathrm{MgSO}_{4}$ & 2.83 & 22.70 \\
Leonite & $\mathrm{K}_{2} \mathrm{SO}_{4} \cdot \mathrm{MgSO}_{4} \cdot 4 \mathrm{H}_{2} \mathrm{O}$ & 2.25 & 25.69 \\
Schoenite & $\mathrm{K}_{2} \mathrm{SO}_{4} \cdot \mathrm{MgSO}_{4} \cdot 6 \mathrm{H}_{2} \mathrm{O}$ & 2.15 & 23.39 \\
Polyhalite & $\mathrm{K}_{2} \mathrm{SO}_{4} \cdot \mathrm{MgSO}_{4} \cdot 2 \mathrm{CaSO}_{4} \cdot 2 \mathrm{H}_{2} \mathrm{O}$ & 2.78 & 15.62 \\
\hline
\end{tabular}

The main product of the potash industry is MOP, muriate of potash, contains minimum of $95 \% \mathrm{KCl}\left(60 \% \mathrm{~K}_{2} \mathrm{O}\right)$. Sulfates of potassium-magnesium (SOPM or K-Mag) are concentrated from the langbeinite $\left(\mathrm{K}_{2} \mathrm{SO}_{4} \cdot 2 \mathrm{MgSO}_{4}\right)$ ores. SOP, sulfate of potash $\left(\mathrm{K}_{2} \mathrm{SO}_{4}\right)$, is produced from kainite and langbeinite concentrates. Low chlorine content and lower solubility of the potassium sulfate products make them desirable for certain soil conditions and crops such as citrus fruits, tobacco, and vegetables. Potash products are sold on the basis of their chemical compositions and particle sizes; coarser particles are more desirable. Pricing is based on the total agricultural nutrient composition. 
Potash producing formations of the Carlsbad potash district in southeastern New Mexico consists of 11 ore zones, generally 1-3 m thick and laterally consistent except when interrupted by barren "salt horses"[2]. The deepest ore horizon (zone 1) accounted for about $80 \%$ of potash production in the past. Potash mining and processing in New Mexico began before 1940 in response to the need for domestic production of potassium fertilizer due to World War II. By the 1960's there were six Potash processing companies in New Mexico. Foreign competition, lower grade ore and higher clay content, thus higher processing costs, have reduced the operating companies to two, Mosaic Potash Carlsbad Inc. and Intrepid Potash New Mexico LLC.

Sylvite ore with low clay content (1-3\%) is now practically depleted. Production is mainly from ore zone 4 (major langbeinite-sylvite), zone 5 (sylvite-langbeinite), and zone 10. Ore zone 10 is the second best (sylvite-sylvinite) zone in the district but with high clay content (6$7 \%$ ). Zones 2, 6, 9, and 11 containing mostly carnallite with kieserite $\left(\mathrm{MgSO}_{4} \cdot \mathrm{H}_{2} \mathrm{O}\right)$ are not mined to date; zone 7 contains moderate reserves of sylvinite with 3-4\% clay [2]. Combined production of the two companies is reported at 922,000 metric tons of $\mathrm{K}_{2} \mathrm{O}$ equivalent of finished products [4]. Potash mining directly employs over 1,200 persons in a sparsely populated region of the state.

Langbeinite ores are mainly beneficiated by gravity concentration (heavy media cyclones) and leaching of the residual halite by water. Sylvite ore processing include a combination of slime separation, flotation, heavy-media separation, leaching, and crystallization circuits designed for a specific ore [5]. In processing New Mexico sylvinite ores, liberation of sylvite from halite is achieved at about 2.4-mm by impact crushing and rod-mill grinding [6]. In contrast, sylvinite ores mined in Saskatchewan, Canada are much coarser grained (liberated at about 9$\mathrm{mm}$ ) and they are amenable to heavy-media separation in coarse sizes. Fine-grained ores are beneficiated by froth flotation in saturated brine where sylvite is preferentially floated using aliphatic amine type collectors with carbon chain lengths of 12-24. Alcohol type frothers and extender oils are also used to aid sylvite flotation.

In general, clay minerals are the most troublesome impurity encountered in processing sylvite ores. Clays are soft and easily hydrated; they swell and disintegrate in water into minute-sized $(<0.002 \mathrm{~mm})$ platelets. Because of their high cation (and anion) exchange capacity, high surface area, and residual charges, clays preferentially adsorb large amounts of flotation collectors, thus decreasing their effective concentration and hindering sylvite flotation [5-9]. It is estimated that the ratio of surface area of residual clays to $\mathrm{KCl}$ a typical flotation feed could be as high as 2,000 to 1 . As low as $0.1 \%$ free clay could reduce the flotation recovery by 20 $\%$. Sylvite flotation virtually ceases in the presence of $1 \%$ clays in the feed. Other deleterious effects of clays may include colloid formation and slime coating of mineral surfaces, hence preventing particle-bubble interaction. Consequently, clays increase the reagent and energy costs to process potash ores, and increase the losses of fine sized potassium minerals associated in the separation and densification of the clays.

Slimes, therefore, are removed ahead of sylvite flotation circuit by multi-stage scrubbing and mechanical de-sliming, using screens, hydroseparators, and hydrocyclones. This process is inherently inefficient; slime dispersants and blinding agents, such as starch, (CMC) carboxy 
methyl cellulose, or guar gum, and recently urea-formaldehyde resins are used as "blinding agents" to prevent adsorption of flotation collectors on clays to alleviate the adverse effects of clays remaining in the flotation feed.

Hydro-mechanical desliming methods, however, are only effective for ores with low clay content, i.e., total water-insoluble residue (I.R.) content (clay-carbonate admixtures) to about $5 \%$. Ores with higher I.R. content (or very fine-grained ores) are leached with halite $(\mathrm{NaCl})$ saturated hot-brine in order to dissolve sylvite selectively. After solution purification, $\mathrm{KCl}$ is recovered by vacuum crystallization. This process, however, is highly energy and equipment intensive. Although the product, $98+\%$ pure $\mathrm{KCl}$, commands higher prices, increasing energy costs in recent years, increasing clay content, and declining ore grades would render this processing route uneconomical. Furthermore, processing mixed (langbeinite-sylvite) ores with the hot-brine leach method makes it technologically very difficult and unattractive.

Flotation of sylvite in coarse sizes in processing sylvinite ores is highly desirable, eliminating the need for compaction, crushing, and screening required of the fine flotation concentrates. However, not many ores are amenable to flotation in coarse sizes because of the liberation size of sylvite from halite grains. New Mexico potash ores are fine-grained, and generally floated after fine grinding. Most Canadian ores, on the other hand, are higher grade and much coarser grained than NM ores. Therefore, in most Canadian operations run of mine ore is crushed to minus 4 to 6 mesh $(4.75-3.36 \mathrm{~mm})$, deslimed and screened through 14-20 mesh $(1.18-0.84 \mathrm{~mm})$. The coarse and fine fractions are then conditioned separately with clay depressants and flotation reagents, combined, and floated together. At some plants rougher flotation tailings are screened, the oversize is re-ground and returned to conditioning stage [10]. At few Canadian and one NM plants coarse and fine fractions are floated separately.

Magnesium bearing minerals, mainly highly-soluble carnallite, most commonly langbeinite, leonite, and kainite present as impurities in sylvite ores, introduce magnesium ions into the $\left(\mathrm{KCl}-\mathrm{NaCl}-\mathrm{H}_{2} \mathrm{O}\right)$ brines. Unlike most potash plants, sylvite flotation at the NM plants was historically performed in brines that contain high concentrations of magnesium ion (1.9$2.2 \%$ ) [12]. Studies showed that magnesium ions in saturated brines aggravate the adverse effect of slimes on $\mathrm{KCl}$ floatability when its concentration exceeds of $0.4 \%$ [11]. Higher brine temperatures and magnesium ion concentrations during summer months both enhances the degree of hydration of $\mathrm{KCl}$ surface and reduce its floatability.

Reduced amine fixation on the surface of $\mathrm{KCl}$ particles impairs the floatability of sylvite particles and requires a greater consumption of a collector (amine). The use of higher amine concentrations to restore $\mathrm{KCl}$ flotation also promotes the conditions suitable for activation and flotation of fine-grained fraction of halite and deteriorates the quality of the sylvite concentrate. The greatest decrease in amine sorption is observed for sylvite fraction larger than $0.25 \mathrm{~mm}$ (60 mesh) in size, whereas, the change in amine sorption on fine-grained sylvite fraction, characterized by considerably larger specific surface $\left(>0.075 \mathrm{~m}^{2} / \mathrm{g}\right)$, is relatively small. Therefore, longer chain amines during the summer months are preferred, particularly for coarse ore processing. 
Some European and Canadian producers have been exploiting high-clay ores, containing 8-12 $\%$ IR [13-17]. Those type of ores are treated with coarse-ore desliming after attrition scrubbing, rod-mill grinding, desliming with hydrocyclones, and finally slime flotation. It has been established that flotation desliming removes highly surface-active slimes from the flotation feed, thus reducing reagent costs and producing cleaner product with less IR contamination. Slime flotation stage also help smooth out variations in the IR content of the run of mine ore, which is generally controlled by selective mining and blending ores underground.

New Mexico's potash industry is facing serious technological and economical difficulties. Thinly bedded deposits $(1-3 \mathrm{~m})$, declining ore grades $(\sim 20 \% \mathrm{KCl})$, increasing clay content in ores (4-7\%), and scarcity of fresh water supplies create problems in mining and processing potash ores. In order to remain viable and competitive, NM producers have to overcome a number of technological challenges. Improving the efficiency of processing high-clay ores is of outmost importance; high losses of potassium minerals coupled with increased processing costs all associated with the clays in the ore are of major concern to the industry.

This study was initiated to address these problems and provide solutions that can easily be implemented in the existing plants. Therefore, the objectives of this study were:

- to investigate the effects of organic and inorganic clay dispersants to increase the efficiency of the existing slime removal technologies in processing NM potash ores

- to investigate flotation separation of clays from sylvinite ores using slime flotation

- to develop and optimize reagent combinations, and make recommendations for plant trials 


\section{EXPERIMENTAL}

\section{Materials and Methods}

Several drums of low-clay ore samples containing 2.2-2.5\% water Insoluble Residue (I.R.) were collected from the impact crusher discharge and another four drums of medium-clay (3.5-3.7\% I.R.) lump ore samples were collected from transfer conveyor at the storage bins. In addition, samples of high-clay (5-7\%) ore from leach-crystallization plant and very high (8$11 \%$ I.R.) gray and brown clay ore samples were collected near the ceiling and the footwall of the ore seams of underground workings of the two operating mines.

One drum of low clay ore sample another drum of medium-clay ore were homogenized and split samples were cut for laboratory tests. Remainder is used to prepare brines. As many as 30 liters of brine was needed for each wet-screening test, and just as much for multiple dispersion and sedimentation tests. Some brines were recycled; however, if a flocculant was used during the test, that brine was discarded.

For coarse ore flotation studies, $-9.5 \mathrm{~mm}\left(-3 / 8^{\prime \prime}\right)$ ore samples collected from the plants were mixed with brine for 15 minutes in a 30 gallon bin with saturated brine under intense agitation, allowed to settle for 1 minute, and the slimes were removed by siphon-decantation. Ore samples were then mixed for another 5 minutes with fresh brine and deslimed. Coarse (+0.6 mm, $28 \mathrm{mesh})$ and fine (-28 mesh) fractions were screened, filtered, washed with methanol until brines were removed, and dried. Coarse fractions were screened through the desired top-size; the oversize was crushed, re-screened, and added into the respective coarse and fine size fractions.

Deslimed fine and coarse flotation feed samples were also collected from two operating plants in the Carlsbad area, de-brined, washed with methanol, dried and stored in sealed containers for side by side flotation tests with plant-deslimed and laboratory deslimed ore samples.

\section{$\underline{\text { Slimes Dispersion Tests }}$}

Slimes dispersion tests were conducted on as received ore (minus $9.5 \mathrm{~mm}$ impact crusher product) and on minus $1.18 \mathrm{~mm}(-14 \mathrm{mesh})$ laboratory rod mill product. The dispersion tests were conducted with $730 \mathrm{~g}$ ore sample and $700 \mathrm{ml}$ saturated brine of specific gravity (SG) 1.248-1.260 at 45\% solids, in a Denver flotation machine in agitator mode. A 50-mm diameter impeller was used at $1000 \mathrm{rpm}$. The agitation was carried out for 15 minutes, which is comparable to total agitation / scrubbing time at the plants. The pulp was then transferred to a 4-L glass beaker, diluted with brine to $16 \%$ solids, and minus 50- $\mu \mathrm{m}$ (270\#), 75 um (200\#), or 100 um (150\#) slime fractions were removed through siphon-decantation by successive mixing and decantation. These tests were repeated in the presence of inorganic dispersant, and with a mixture of organic and inorganic dispersant.

Sylvinite ores processed by flotation at the Carlsbad potash plants generally contains 1.7$4.0 \%$ water-insoluble admixtures, mainly represented by the montmorillonite type clay minerals [12]. Significant tonnages of ore reserves with higher than $4 \%$ clay content exists. Two types of clays were identified; brown clays are readily dispersed, whereas gray clays are poorly dispersed in brine. Therefore, it is impossible to ensure complete recovery of clays in 
the course of slime removal cycle. Gray clays continue to disperse during the subsequent mechanical agitation in the flotation cells causing carry-over of clay slimes into a frothy sylvite product, reduction in sylvite floatability, difficulty in pumping and de-watering of flotation concentrates, and increase in consumption of flotation reagents and energy for concentrate drying.

Inorganic compounds, such as sodium silicate and poly-phosphates, have been utilized as dispersants for clay minerals in a number of flotation systems. However, in high ionic strength brines the surface charge on clay minerals is practically nil due to double layer compression. Inorganic clay dispersants added in the grinding circuit not only augment slime dispersion but also increase the selectivity of liberation of sylvite-halite mixed grains. Dispersant action of inorganic polyvalent salts, such as aluminum sulfate $\left(\mathrm{Al}_{\mathbf{2}}\left(\mathrm{SO}_{4}\right)_{3}\right)$, alum, have been postulated to be due to recharging of the clay particle surfaces in the presence of polyvalent ions.

In this study, aluminum sulfate and sodium and calcium lignin sulfonates have been used either individually or in combination in different proportions. Three different ore samples with I.R. content approximately $2.5 \%, 3.5-4.0 \%$, and 8-11\% were collected from Carlsbad potash operations. Minus $9.5 \mathrm{~mm}(3 / 8$ ") ore samples were scrubbed with or without the addition of dispersant, deslimed, ground in the laboratory rod-mill to minus 20 mesh $(0.85$ $\mathrm{mm}$ ) and again deslimed by siphon decantation. Slimes were filtered, dried, and analyzed for insoluble residue (I.R.) and $\mathrm{K}_{2} \mathrm{O}$ content, and sands fractions were placed in a flotation cell, conditioned with flotation reagents and sylvite was floated. Concentrates and tailings were also filtered, dried, and analyzed for I.R. and $\mathrm{K}_{2} \mathrm{O}$ content.

\section{$\underline{\text { Slime Flotation Tests }}$}

Comparative studies were conducted on slime flotation using different families of clay flotation collectors. Low clay ore samples were dry ground to minus $1.18 \mathrm{~mm}$ (14-mesh), homogenized and split into $670 \mathrm{~g}$ samples. Ore samples were mixed with saturated brine in a 2-L flotation cell, slime flocculant and collector are added simultaneously, and slimes were floated for 2 minutes. Following slime flotation, a measured amount of guar solution as slime depressant, and an emulsion containing amine, extender oil, and frother were added, conditioned and $\mathrm{KCl}$ was floated. The products were filtered, dried, and analyzed for insoluble residue (IR) and $\mathrm{K}_{2} \mathrm{O}$ content.

For high-clay (4-11\% IR) ore samples, minus $9.5 \mathrm{~mm}\left(3 / 8^{\prime \prime}\right)$ ore samples were mixed with brine in attrition scrubber at high pulp density for 10 minutes, transferred into a $4 \mathrm{~L}$ beaker, diluted with brine, mixed and siphon-deslimed in coarse sizes. The process was repeated once or twice depending on the clay content in the ore, then ground in the rod mill to desired particle size and deslimed in fine sizes either by siphon decantation, or transferred into the flotation cell and deslimed by slime flotation before sylvite flotation.

Slime flotation can be performed with the aid of various oxyethylated surface-active agents (SAA) of derivative amines [15-17]. A priory condition of efficient flotation of finely dispersed slime particles is their preliminary flocculation with high-molecular-weight 
polymers. The optimum consumption of a flocculant depends on the hydro-mechanical parameters of operation of the flotation cell and flocculant composition $[15,17]$. The use of column flotation cells for slime flotation from ore fines holds promise as the absence of strong shearing forces on slime flocculi promotes selective slime separation with lower consumption of flotation reagents.

The studies on the application of ether amines as a collecting agent of cationic SAA that were carried out at the Institute of Halurgy in cooperation with Akzo Nobel Surface Chemistry AB showed promise for their application [18]. Comparative tests on slime flotation with amine ethers (Lilaflot 810 and Lilaflot D 817) and oxyethylated fatty acids showed the possibility of a several-fold decrease in the consumption of collectors with the application of amine ethers.

\section{Potash Flotation Tests}

The flotation tests were either conducted with the rod-mill-ground and de-slimed ore samples or deslimed and methanol washed dry samples. The flotation samples were initially conditioned for 2-minutes in saturated brine in the flotation cell for fine ores, or with bottleroll technique for coarse ore samples, 1-3 minutes with clay depressant, either guar gum (Rantec KP-4000) or UF resin (Arclin), primary Tallow amine (Armeen HT, Akzo Nobel or Witco Arosurf MG-140), and extender oil (Philflo Flotation oil), transferred to the Denver D12 flotation cell; frother (methyl isobutyl carbinol - MIBC or Phillips Orfom F2) is added and floated for 2 minutes. The concentrate and tailings were filtered with a vacuum filter, wet weight recorded, dried and weighed. The amount of salts precipitated from the brine on products was calculated from the moisture loss; representative samples were ground and analyzed. These tests were repeated with different dispersant additions.

\section{$\underline{\text { Urea-Formaldehyde Resins }}$}

Adsorption of amines by residual clays in the potash flotation feed is prevented using "clay blinders", such as carboxymethyl cellulose (CMC), starch, or guar gum, depending on the nature of clay minerals, and cost and availability of these depressants. Urea-formaldehyde resins (UFR) as clay depressants were identified and patented by the Potash Company of America (PCA) early in the course of the development of potash flotation in New Mexico [19], however UF resins were not used in flotation plants until recently. A modified UFR formulation, patented and marketed as KS-MF in Russia [20], has been used at Russian potash plants for the past decade. One of the potash companies in NM also has been using UFR, produced by Georgia Pacific (GP) Company [21] for the past few years.

This study was initiated to study the efficacy of one Arclin UF resin, selected based on the results of previous screening tests, as clay depressant in potash flotation and, in particular, as a reagent to aid flotation of coarse ore fractions. 


\section{EXPERIMENTAL RESULTS}

\section{Clay Dispersion Tests}

\section{Slimes Settling Rates}

Effects of organic and inorganic dispersants on clay dispersion during attrition scrubbing and during rod mill grinding were investigated using ores with various clay contents. Appropriate dosage of dispersants was determined for each type of ore using relative settling velocities of salts and clays in jar tests. These tests were also used to screen and verify the effect of dispersants, either individually or in combination in various proportions.

An example of jar test illustrating the effect of dispersants on settling time of clay dispersion after 0.5 and 5 minutes (left jar) for a low-clay ore sample is shown in Fig.-1. Figure-2 shows settling test results with lignin sulfonate (LS) and aluminum sulfate (alum), as compared to settling without dispersant. It is apparent that slimes stay in suspension much longer in the presence of dispersants. Similar tests conducted with sodium silicate $\left(\mathrm{Na}_{2} \mathrm{SiO}_{3}\right)$ and trisodium phosphate $\left(\mathrm{Na}_{3} \mathrm{PO}_{4}\right)$ did not affect settling rates.

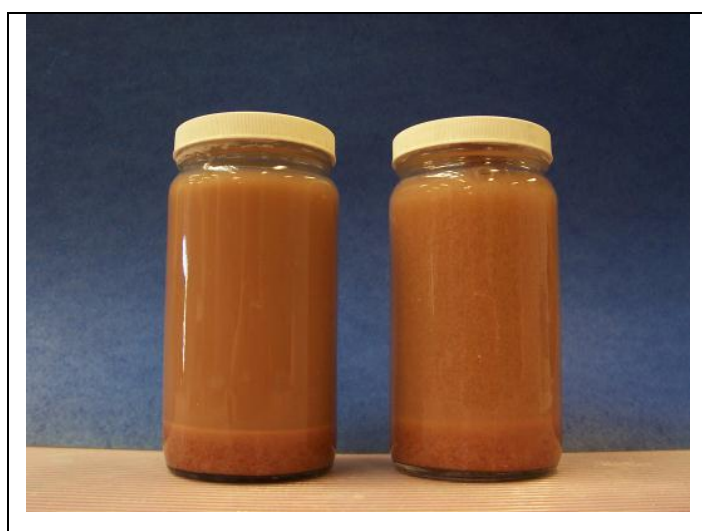

(a)

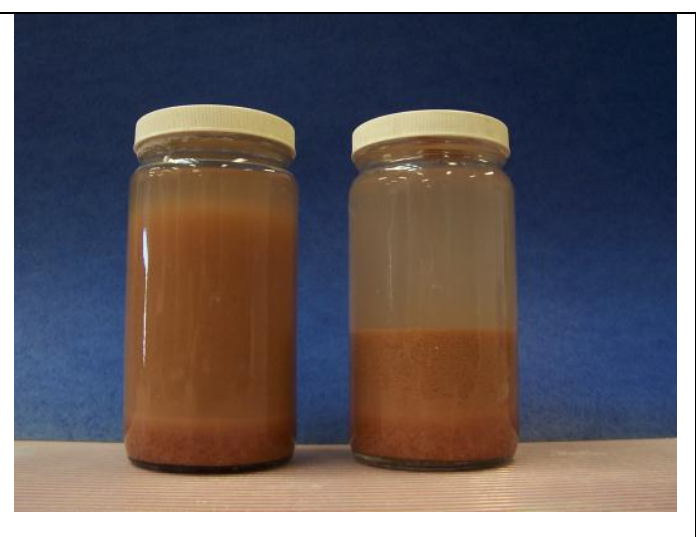

(b)

Figure 1 - Dispersion of slimes with (left jar) and without dispersants (right jar) after (a) 0.5 minutes, and (b) 5 minutes of settling time.

Dispersants, such as calcium (CaLS) or sodium (NaLS) lignin sulfonates, and alum, therefore help keep the slimes (clays) in suspension, rather than being entrained into fast-settling solids, hence help increase the efficiency of desliming process with classifiers, hydrocyclones, and hydro-separators. 

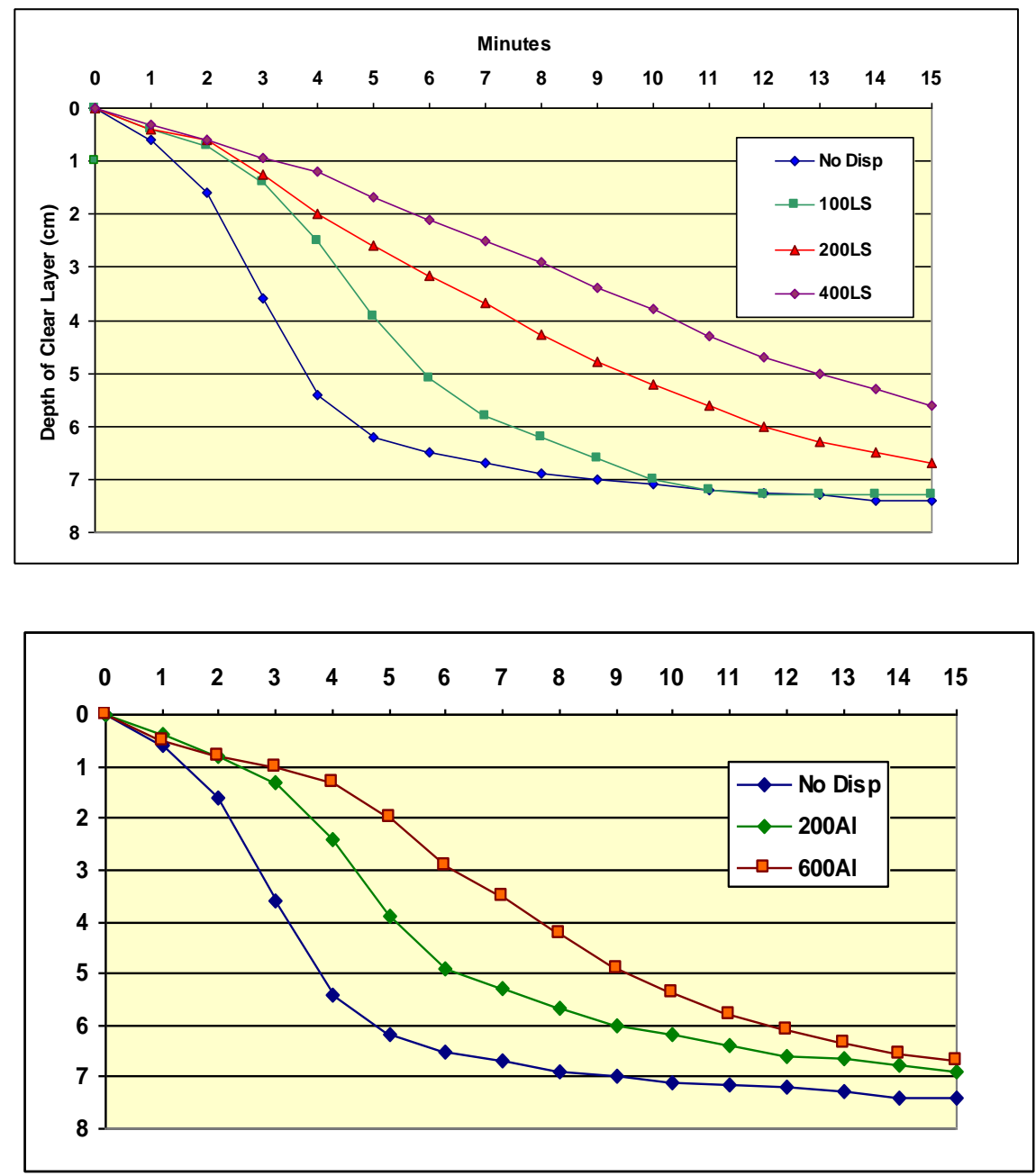

Figure 2 - Slimes settling rates with Lignin Sulfonate (top) and with Alum as dispersants.

\section{$\underline{\text { Zeta Potential Measurement }}$}

Aluminum sulfate is generally used in waste water treatment as coagulating agent. However, it is also known that excess of alum causes charge reversal and dispersion of coagulated solids. Effect of aluminum sulfate concentrations on the zeta potentials of clays in brines were investigated in the laboratory with mixed (brown and grey) and grey clay samples isolated from two different ore samples (Type I \& Type II). Table-2 shows charge reversal is easily observed in dilute $\mathrm{NaCl}$ brine, where as in double saturated $\mathrm{NaCl}-\mathrm{KCl}$ brine, diluted ten times down to $0.66 \mathrm{M}$, much higher alum concentrations were required.

It is also observed that there is about $10 \mathrm{mv}$ difference in the zeta potential measurements of the two types of clays in dilute $\mathrm{NaCl}$ solutions (Table -2). That might be a reason for varying guar and amine requirements experienced in flotation of different types of ores in the plants. 
Table 2. Zeta potential measurements on grey and mixed clays in dilute brines in the presence of aluminum sulfate as dispersant.

\section{Zeta Potential Measurements}

Beckman Coulter Delsa 440SX

$\begin{array}{lccccc}\begin{array}{c}\text { Clay } \\ \text { Type }\end{array} & \text { Matrix } & \begin{array}{c}\mathbf{A l}_{\mathbf{2}}\left(\mathbf{S O}_{\mathbf{4}}\right)_{\mathbf{3}} \\ \mathbf{p p m}\end{array} & \begin{array}{c}\text { Top Layer } \\ (\mathbf{m v})\end{array} & \begin{array}{c}\text { Bottom Layer } \\ (\mathbf{m v})\end{array} & \begin{array}{c}\text { Average } \\ (\mathbf{m v})\end{array} \\ \text { Grey } & 0.001 \mathrm{M} \mathrm{NaCl} & 0 & -26.2 & -29.7 & -28 \\ \text { Grey } & 0.001 \mathrm{M} \mathrm{NaCl} & 500 & 6.65 & 5.89 & 6.3 \\ \text { Grey } & 0.001 \mathrm{M} \mathrm{NaCl} & 1000 & 4.58 & 5.84 & 5.21 \\ & & & & & \\ \text { Grey } & 0.66 \mathrm{M} \text { brine } & 0 & -35.1 & -31.7 & -33.4 \\ \text { Grey } & 0.66 \mathrm{M} \text { brine } & 500 & -19.1 & -16.4 & -16.4 \\ \text { Grey } & 0.66 \mathrm{M} \text { brine } & 1000 & -1.16 & -4.55 & -2.85 \\ & & & & & -36.8 \\ \text { Mixed } & 0.001 \mathrm{M} \mathrm{NaCl} & 0 & -34.2 & -39.5 & -6.52 \\ \text { Mixed } & 0.001 \mathrm{M} \mathrm{NaCl} & 500 & -5.72 & -7.35 & 0.71 \\ \text { Mixed } & 0.001 \mathrm{M} \mathrm{NaCl} & 1000 & 0.51 & 0.92 & -31.5 \\ & & & & & -16.9 \\ \text { Mixed } & 0.66 \mathrm{M} \text { brine } & 0 & -31.5 & -30.2 & -15 \\ \text { Mixed } & 0.66 \mathrm{M} \text { brine } & 500 & -20.8 & -18.8 & \end{array}$

\section{Dispersion and Potash Flotation Tests}

\section{$\underline{\text { Low Clay ores }}$}

Desliming and flotation tests with low-clay ores (I.R. <2.5\%) were conducted with dryground ore samples with one-stage desliming. Figure-3 shows that potash recovery into the flotation concentrate increases in the presence of dispersants, where as overall insoluble residue recovery decreases from 55 to $30 \%$ under the same amine and guar consumptions. 


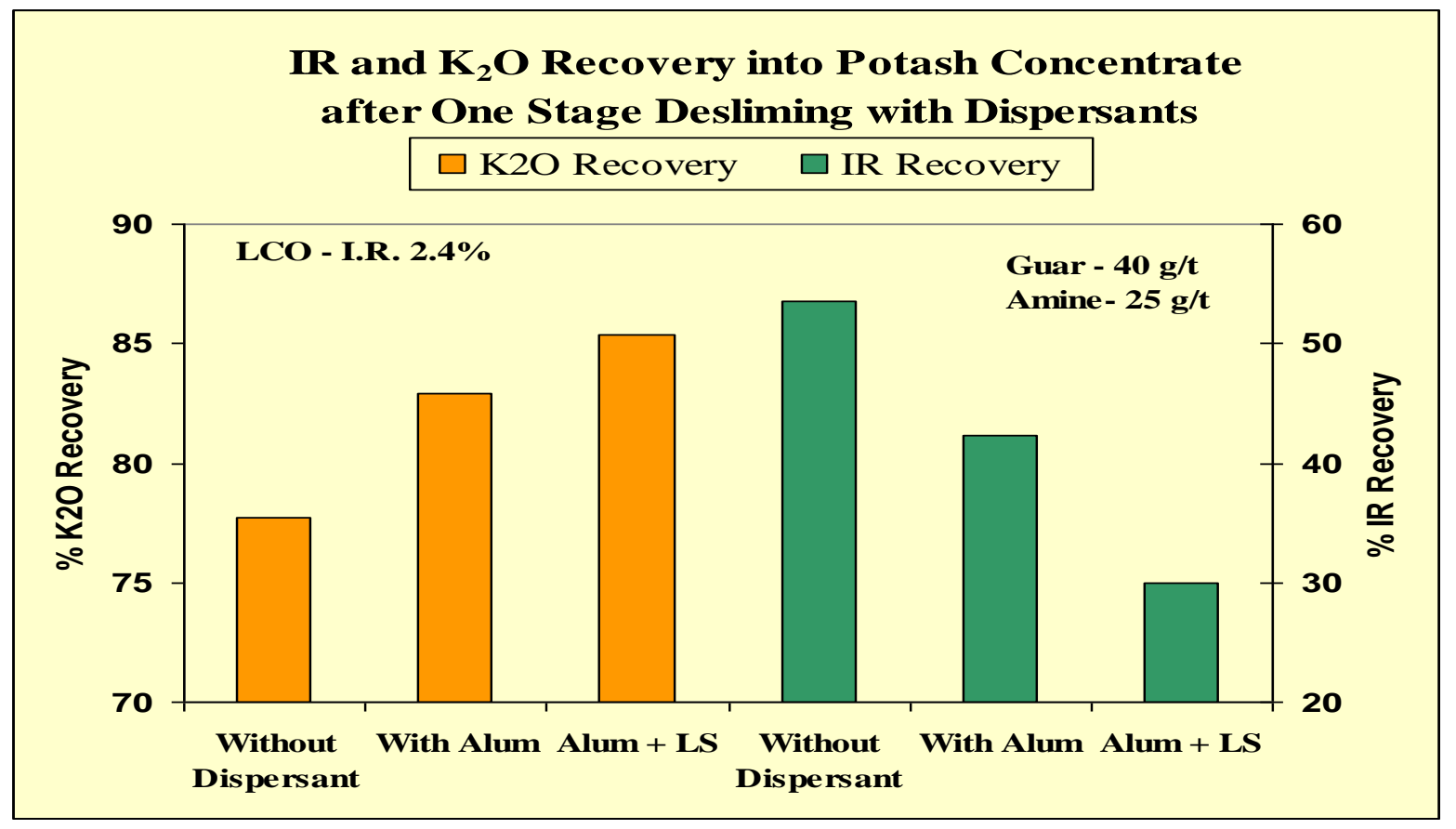

Figure 3 - Comparison of $\mathrm{K}_{2} \mathrm{O}$ and IR recovery into the flotation concentrate after one-stage desliming and flotation tests with low clay ores

Results of these desliming and flotation tests conducted on low-clay ores showed that in the presence dispersants, especially organic and inorganic dispersants combined,

- $\mathrm{K}_{2} \mathrm{O}$ recovery in potash concentrates increases by $5-7 \%$,

- $\mathrm{K}_{2} \mathrm{O}$ losses to slimes decrease

- I.R. recovery in potash concentrates decreases.

- Quality of the concentrate increases due to higher grade and lower I.R. content

\section{Medium Clay Ores}

Medium clay (4-5\% IR) required 2-stage desliming: coarse ore, and after rod-mill grinding. Initial flotation tests conducted after only one-stage desliming (Fig.-4) required much higher amine and guar concentrations.

Figures -5 shows the effects of lignin sulfonate (LS) on $\mathrm{K}_{2} \mathrm{O}$ loses into the slimes fractions and IR remaining in the desliming tailings (flotation feed) after 5-minute grinding and 2-stage desliming. Figures -6 and 7 show the overall effects of dispersants, aluminum sulfate, calcium lignin sulfonate, or combination of both, on the stage-wise IR recovery into slimes fractions and $\mathrm{K}_{2} \mathrm{O}$ loses in 3 minute (7 tests) and 5 minute grinding (9) tests. On the average, the 1st stage (coarse) desliming removes $30 \%$ of IR without dispersants and $37 \%$ with dispersants. $\mathrm{K}_{2} \mathrm{O}$ loses to the $1^{\text {st }}$-stage slimes fraction slightly increases in coarse ore desliming due to increased volume of slimes, however, decreases in 2 nd stage during ground ores desliming in the presence of dispersants. 


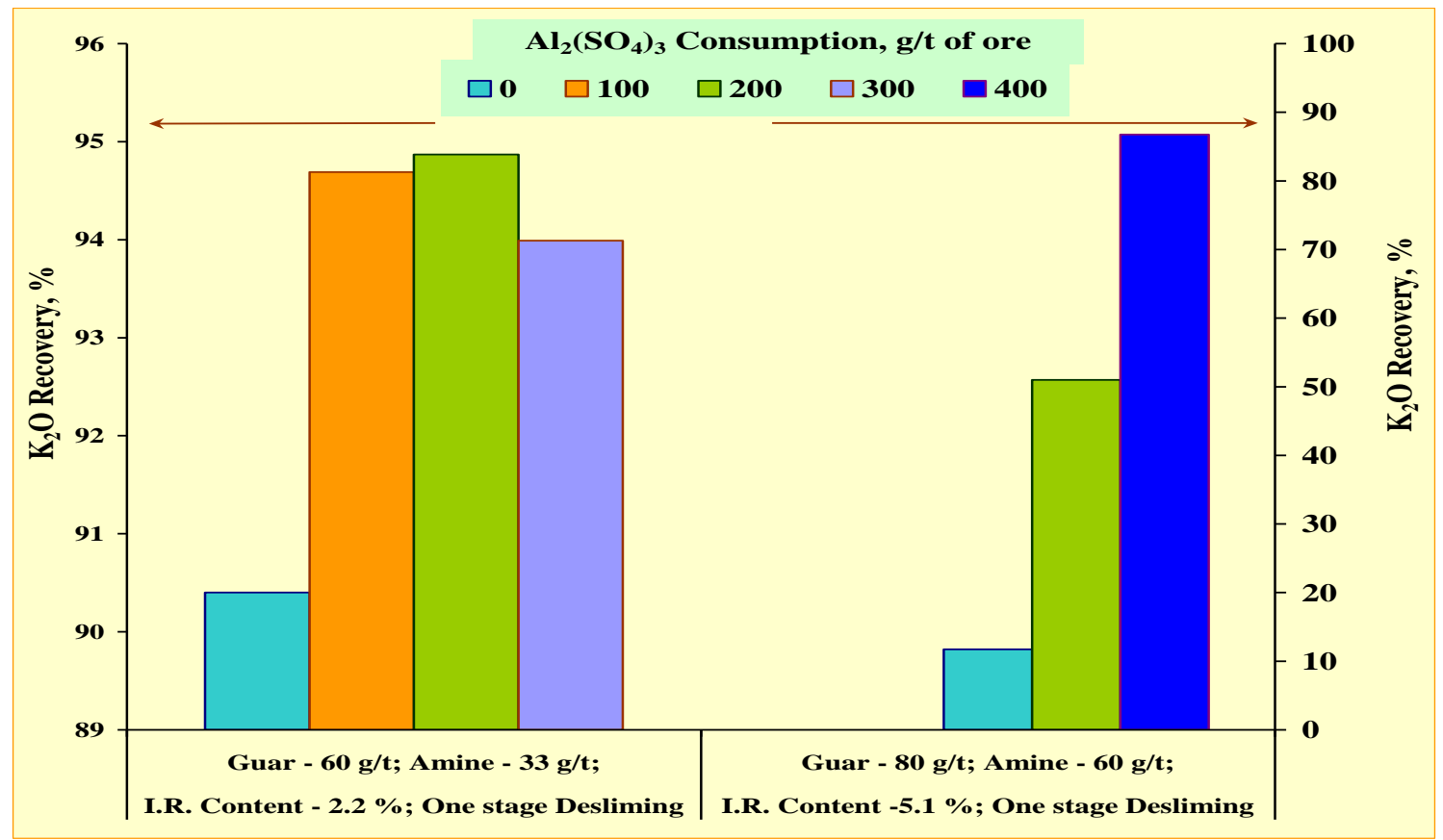

Figure 4 - Comparison of low and medium clay ores flotation tests after one-stage desliming with or without Alum dispersant.

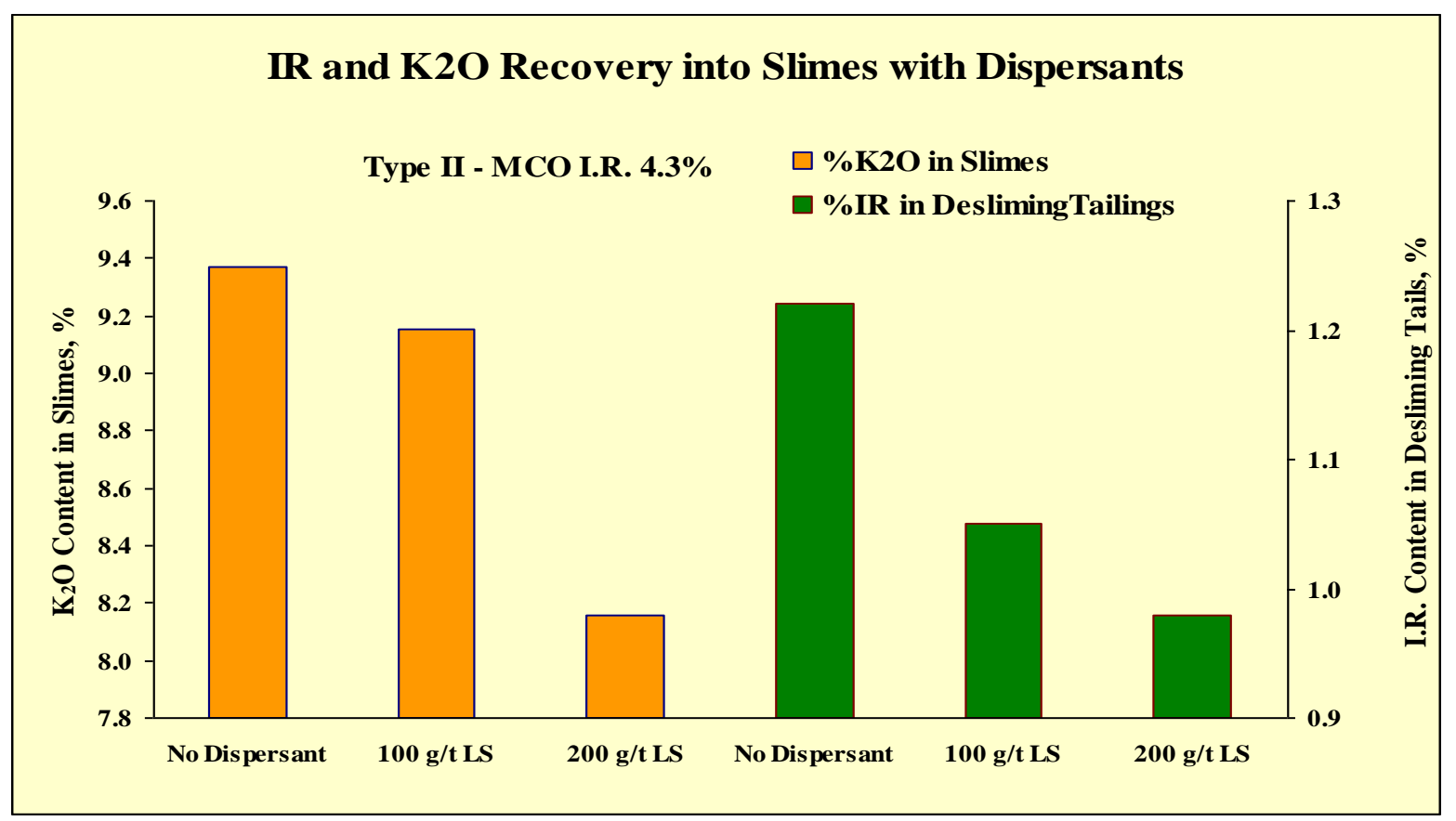

Figure 5 - Overall effects of Lignin Sulfonate dispersants on $\mathrm{K}_{2} \mathrm{O}$ recovery into slimes and residual IR in desliming tailings (flotation feed) after coarse grinding and one-stage desliming of medium clay ores (MCO). 


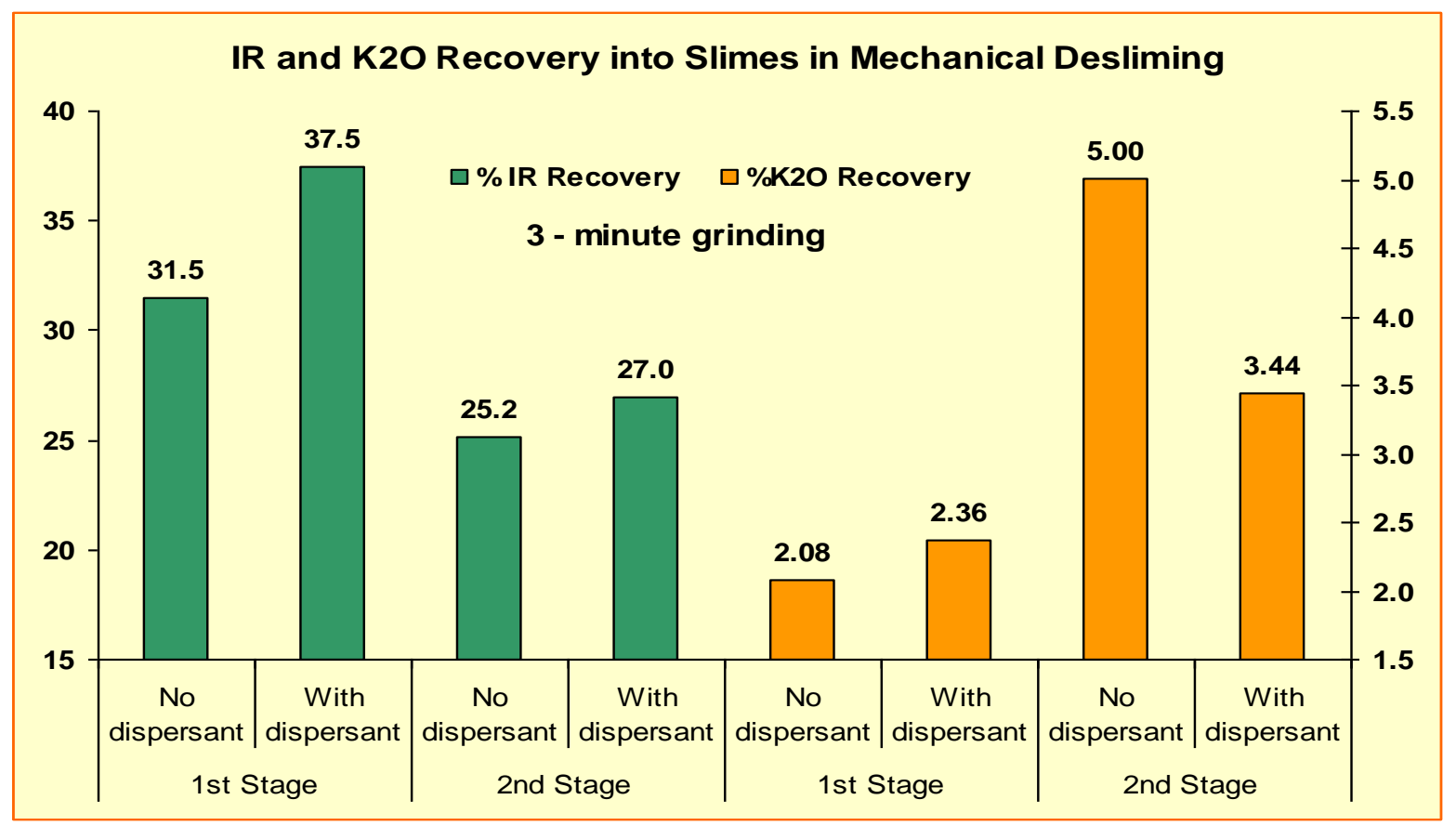

Figure 6 - Effect of dispersants on IR and $\mathrm{K}_{2} \mathrm{O}$ recovery into slimes fractions after coarse desliming followed by 3 -minute grinding and 2-stage desliming of medium clay ores.

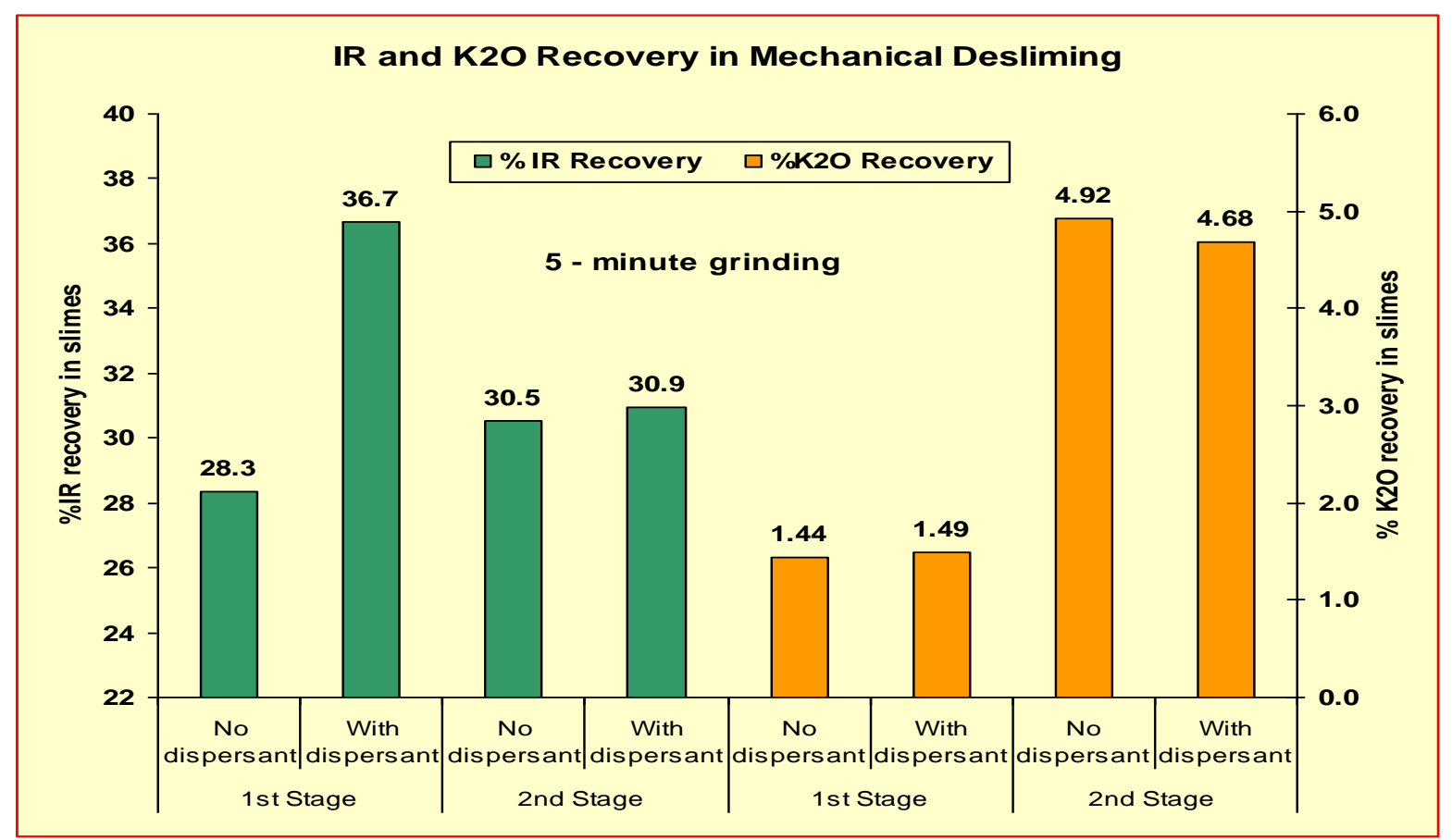

Figure 7 - Overall effects of dispersants on IR and $\mathrm{K}_{2} \mathrm{O}$ recovery into slimes fractions after coarse desliming followed by 5-minute grinding and 2-stage desliming of medium clay ores. 


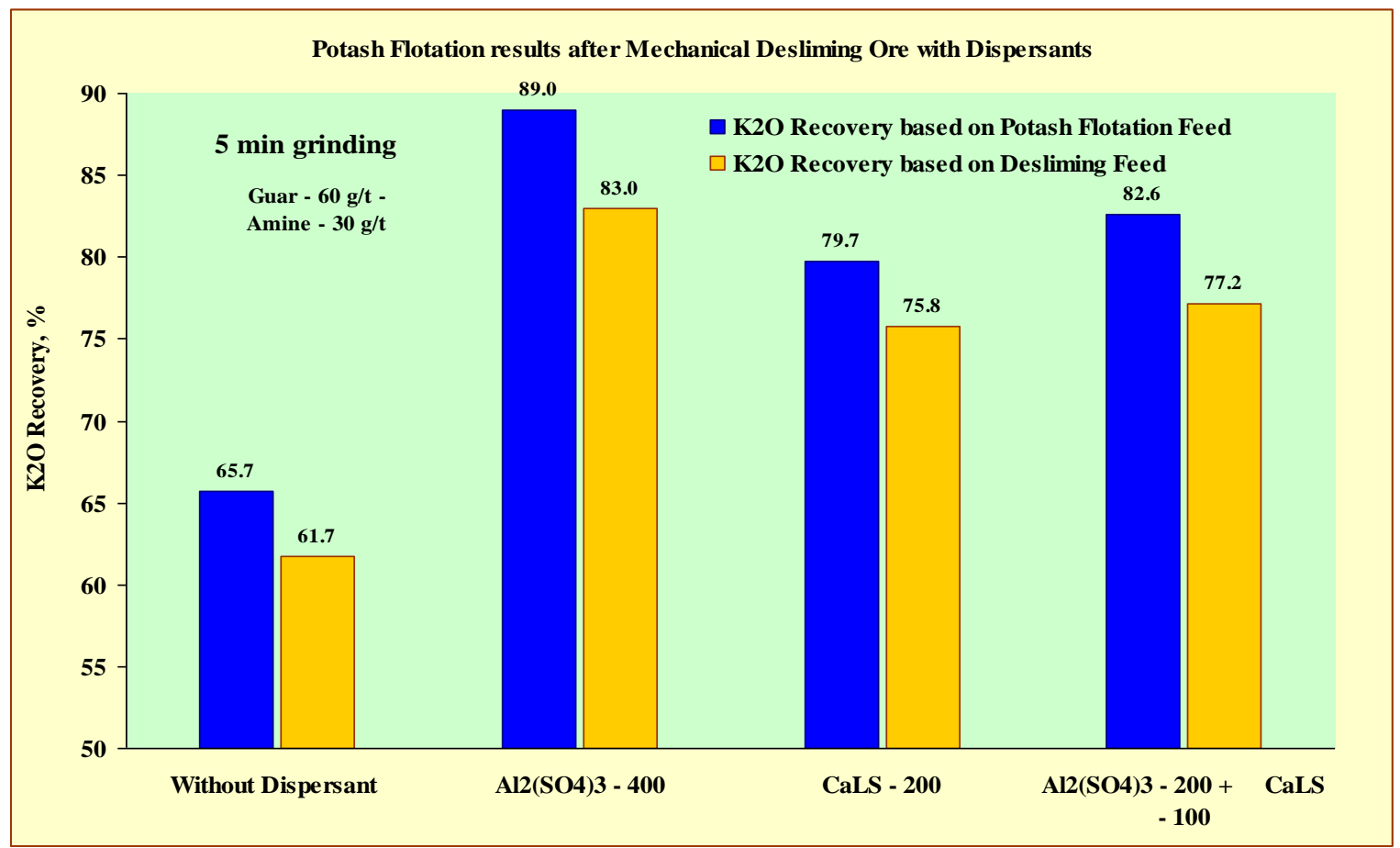

Figure 8 - The influence of dispersants on potash flotation recovery from fine-ground medium-clay ores after 2-stage desliming with or without dispersants.

Aluminum sulfate solution added into the rod mill after first stage desliming, rather than into the rod-mill product and mixed before desliming, revealed that alum also behaves as grinding aid and increases fineness of the product by about $9 \%$, particularly in coarse sizes. Figure- 9 shows the size distribution of the rod mill products after grinding with or without alum.

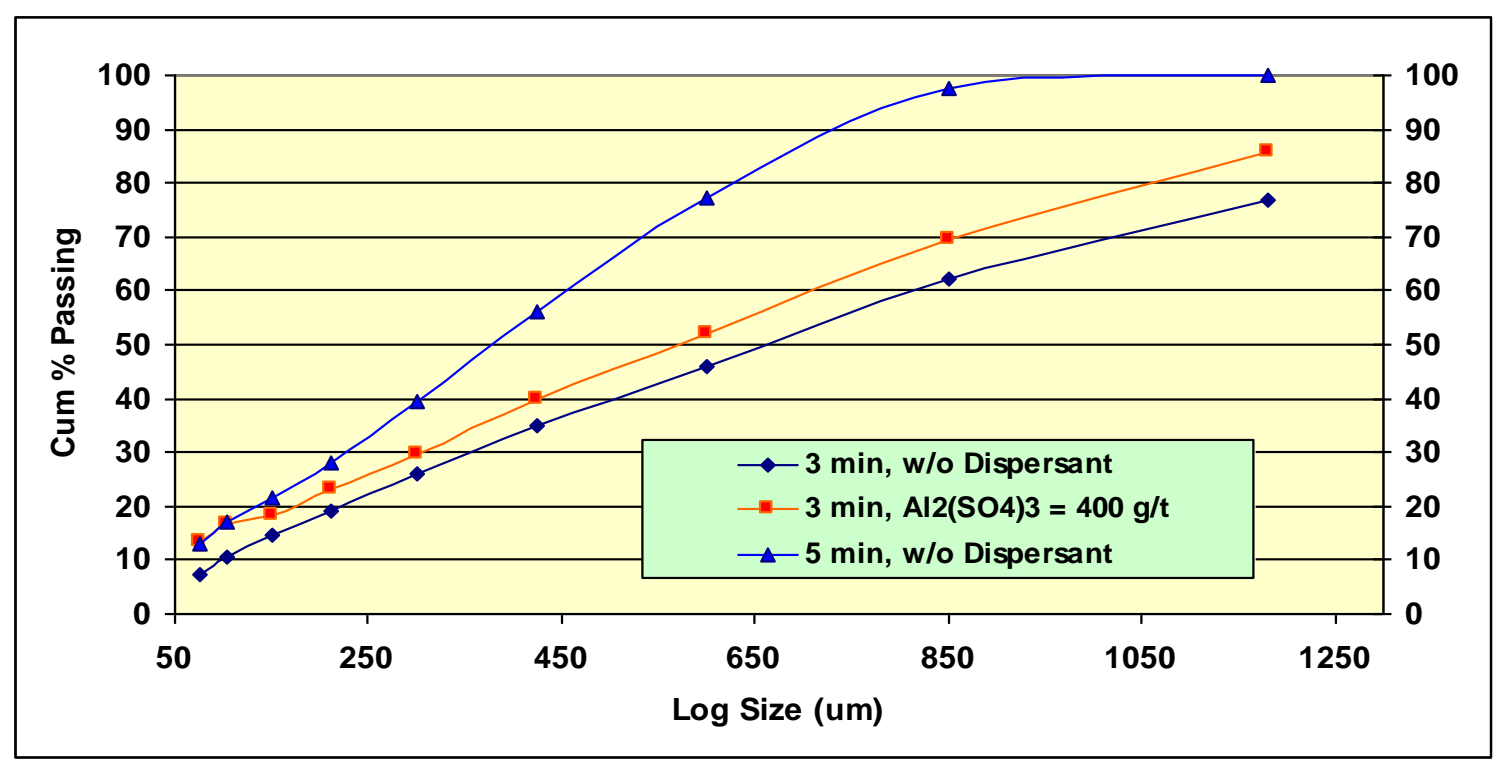

Figure 9 - Effect of Alum on grinding kinetics in rod-mill grinding of medium clay ore. 


\section{$\underline{\text { High Clay Ores }}$}

High-clay ores containing (8-11\%) insoluble residue, required multiple desliming stages depending on the clay content of the ore. Even with the use of various dispersants potash loses to the slimes fraction were high and overall flotation recoveries remained fairly low, 50-60\%. These types of ores could be beneficiated with acceptable recoveries only after slime flotation, as discussed in the following section.

\section{Slime Flotation Tests}

\section{$\underline{\text { Low Clay Ores }}$}

Comparative studies were conducted on slime flotation using different families of clay flotation collectors. Low clay ore samples were dry ground to minus $1.18 \mathrm{~mm}$ (14 mesh), homogenized and split into $670 \mathrm{~g}$ samples. Ore samples were mixed with saturated brine in a 2-L flotation cell, slime flocculant and collector are added simultaneously, and slimes were floated for 2 minutes. Following slime flotation, a measured amount of guar solution as slime depressant, and an emulsion containing amine, extender oil, and frother were added, conditioned and $\mathrm{KCl}$ was floated. The products were filtered, dried, and analyzed for insoluble residue (IR) and $\mathrm{K}_{2} \mathrm{O}$ content.

In conventional slime flotation the fines are first agglomerated with a polymeric flocculant, then slime collectors are added and the fines are floated. A similar procedure was followed in our studies as reported for low and medium clay ores. During the course of this investigation, however, it was observed that slimes floated equally well when the flocculant and collector were added simultaneously. Actually, this procedure resulted in lower reagent consumption and reduced $\mathrm{KCl}$ loses into slimes, thus increasing overall potash recoveries in the concentrates. Further studies are needed to optimize the flocculant and slime collector dosage for different types of ores.

Base-line for potash flotation recovery was established with 1-stage mechanical desliming low-clay ore samples by siphon decantation, followed by flotation tests at constant slime depressant (guar) and amine concentrations. The slime collector reagents tested are tabulated in Table 3.

Table 3 - List of slime collectors tested in this study

\begin{tabular}{|l|l|}
\hline \multicolumn{1}{|c|}{ Type } & \multicolumn{1}{|c|}{ Supplier } \\
\hline \multirow{2}{*}{$\begin{array}{l}\text { Oxyethylated Fatty Acids } \\
(\text { OEFAc })\end{array}$} & Clariant, Germany \\
\cline { 2 - 2 } & Degussa, Goldschmidt Chemical Corporation, USA \\
\cline { 2 - 2 } & Akzo Nobel, USA \\
\hline \multirow{2}{*}{$\begin{array}{l}\text { Oxyethylated Fatty Amines } \\
(\text { OEFAm })\end{array}$} & Akzo Nobel, USA \\
\cline { 2 - 2 } & Degussa, Goldschmidt Chemical Corporation, USA \\
\hline OEFAm proprietary mixture & Cytec, USA \\
\hline Ethermonoamine & Akzo Nobel Surface Chemistry, Sweden \\
\hline Etherdiamine & \\
\hline
\end{tabular}


Figure-10 shows IR recoveries achieved with one-stage mechanical desliming in comparison with slime flotation with different collectors at low flocculant concentrations.

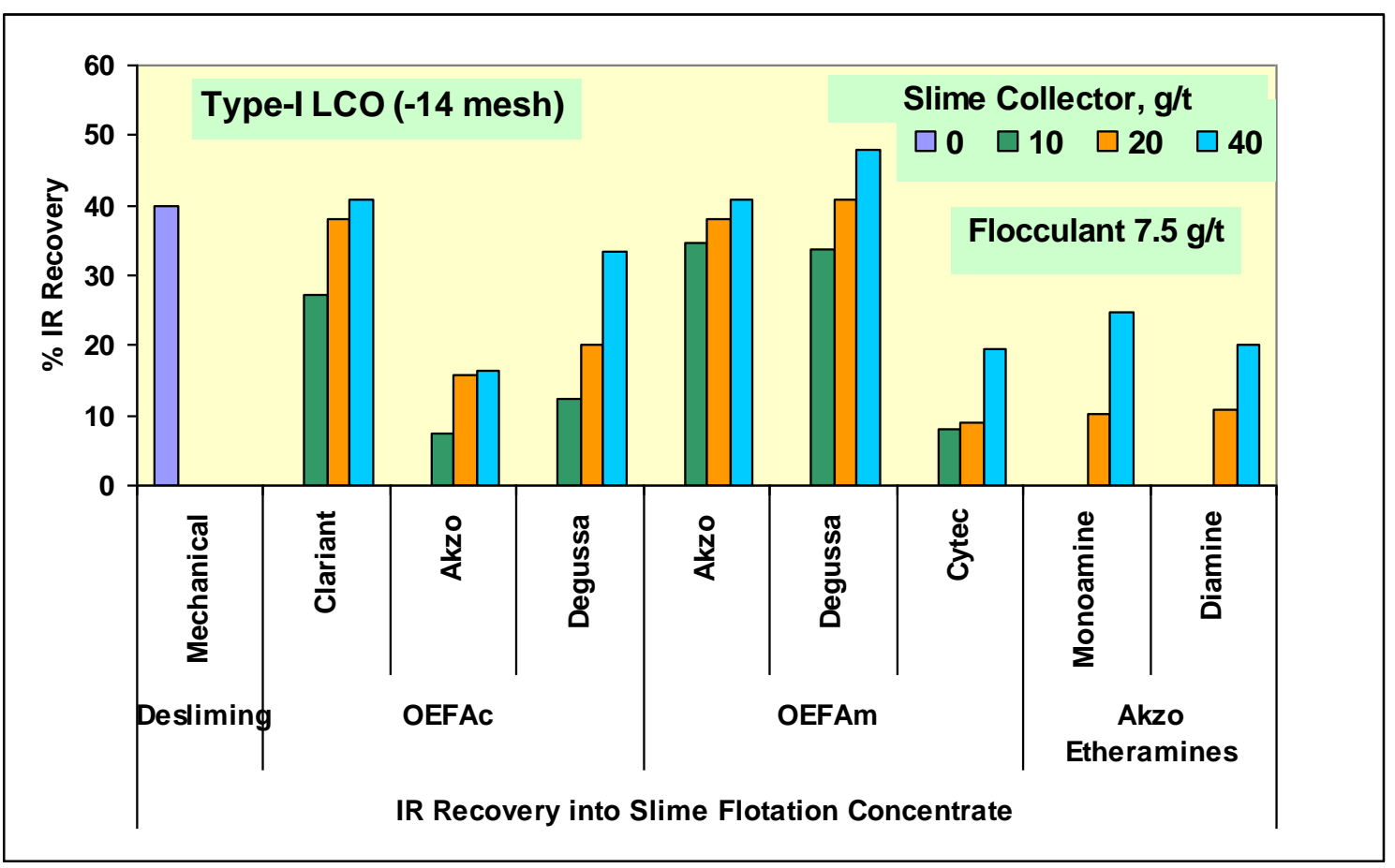

Figure 10 - Insoluble residue recovery into slime fraction and slime flotation concentrates.

Figure-11 shows $\mathrm{K}_{2} \mathrm{O}$ recovery into slime flotation concentrate, and Figure-12 shows the IR content remaining in the potash flotation feed.

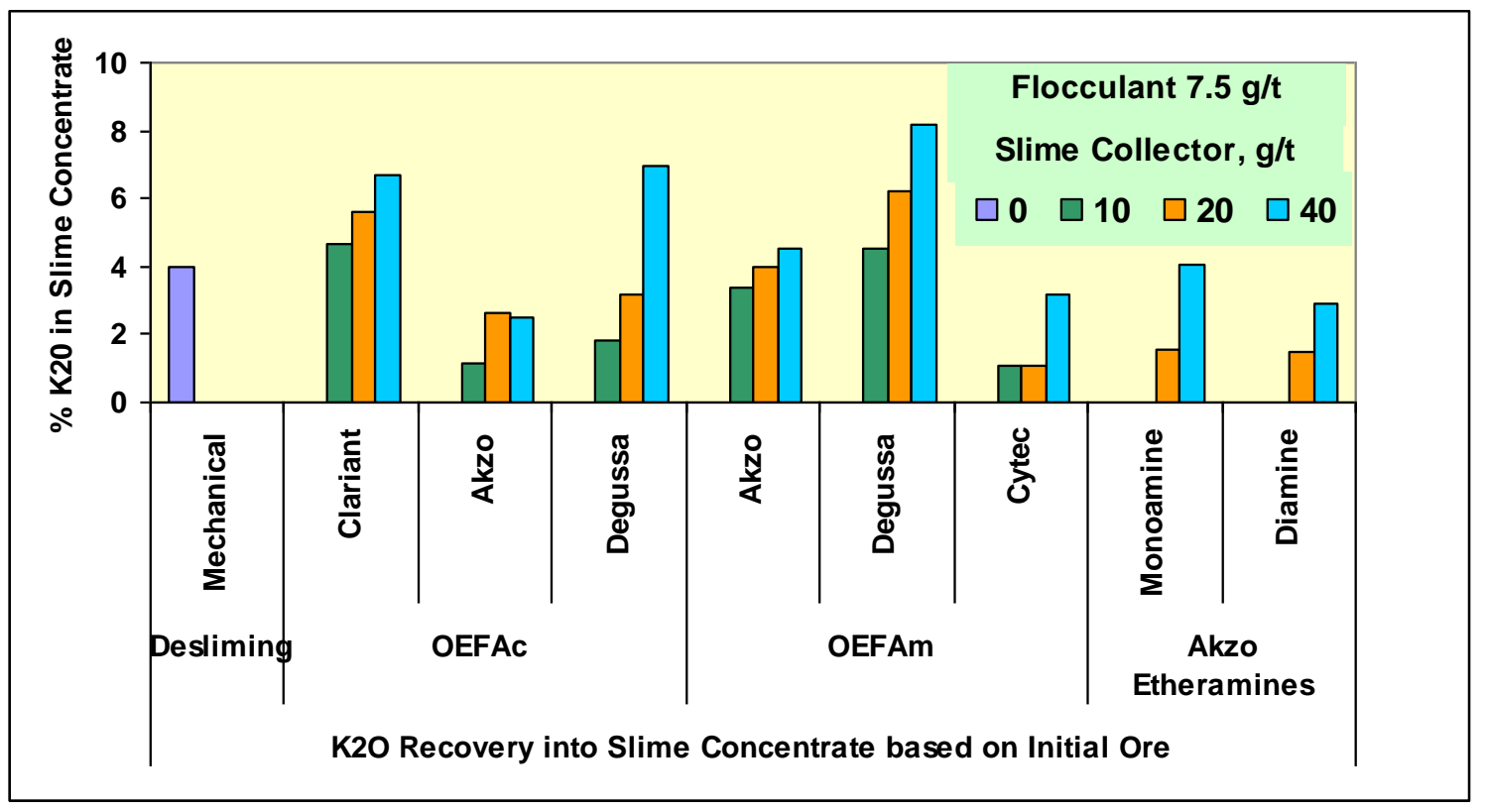

Figure $11-\mathrm{K}_{2} \mathrm{O}$ recovery into slime concentrates from low-clay ores, based on initial ore. 


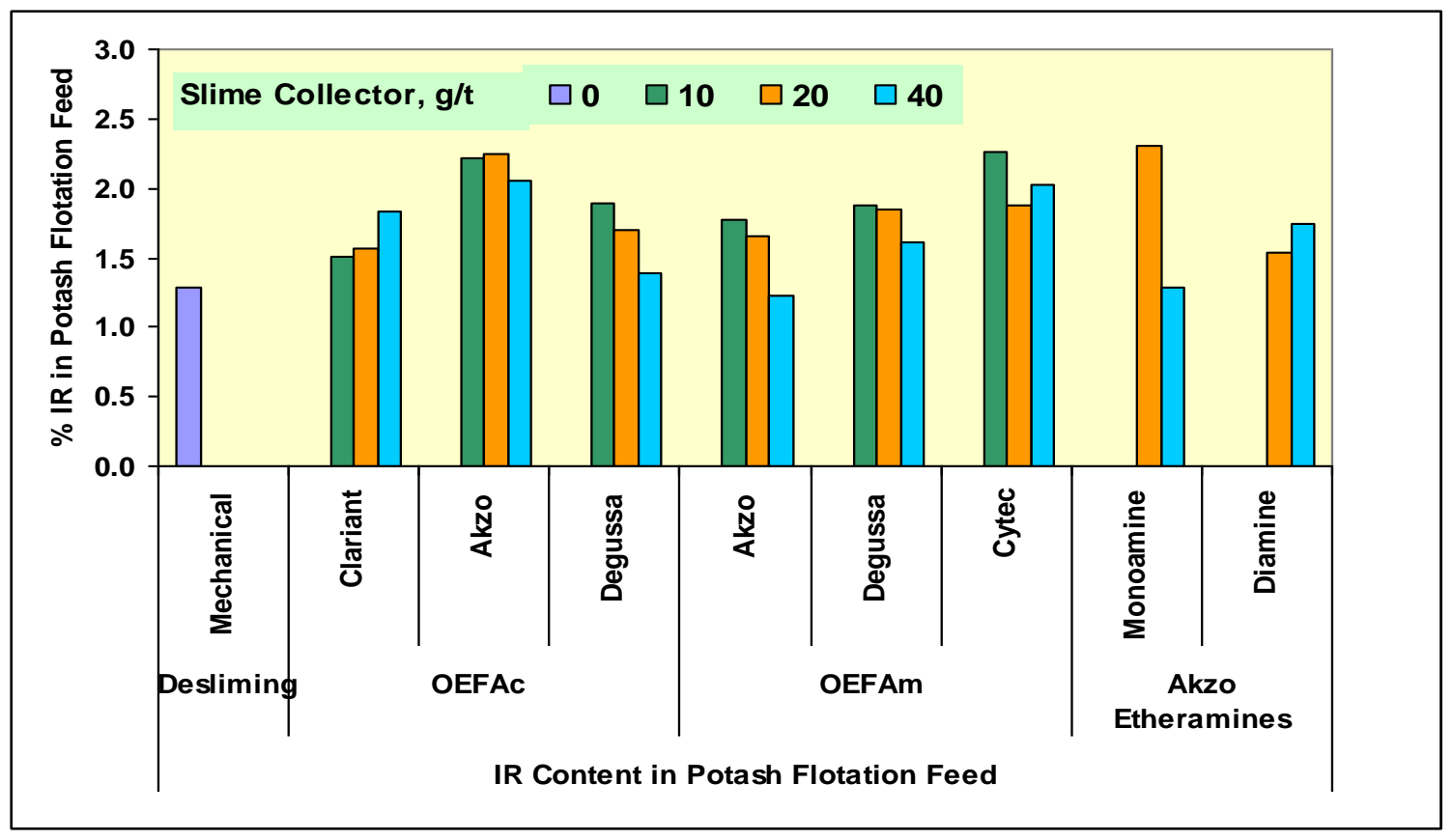

Figure 12 - IR content in the potash flotation feed after desliming low-clay ores samples.

It is noted that those reagents that yield higher IR recovery in the slime concentrate also promote higher $\mathrm{KCl}$ flotation along with slimes (Fig. - 11). Figure-13 shows the $\mathrm{K}_{2} \mathrm{O}$ recovery into the potash flotation concentrate following slime flotation. The potash recoveries closely correlate with the IR content of the flotation feed, i.e., as the IR content increases the potash recovery decreases, since all flotation tests were conducted using the same guar and amine concentrations.

Although there were fairly wide variations in the total IR recoveries, 10-45\% (Figure-10) and the IR content of the flotation feed, 1.1-2.3\% (Figure-11), overall potash recoveries are comparable. Without desliming, potash recovery was only $76 \%$ with the same guar and amine consumption. That indicates, although small in quantity, slime flotation could remove most active clays from feed, preventing slimes from absorbing amine collectors and reporting into the potash concentrate, thus producing cleaner concentrates.

Similarly, potash recoveries (Figure-13) also correlate well with the $\mathrm{KCl}$ loses into the slime flotation concentrate. However, based on plant experience, up to $40 \%$ of the potash fines entrained in the slimes concentrates can be recovered by cleaner flotation in column cells, and added into the potash flotation feed.

\section{Medium Clay Ores}

Medium clay ores floated well after first stage coarse ore desliming, milling, slime flotation followed by sylvite flotation. Comparison of $\mathrm{KCl}$ flotation recoveries after desliming by decantation or slime flotation under different conditions are shown in Figure - 14 . 


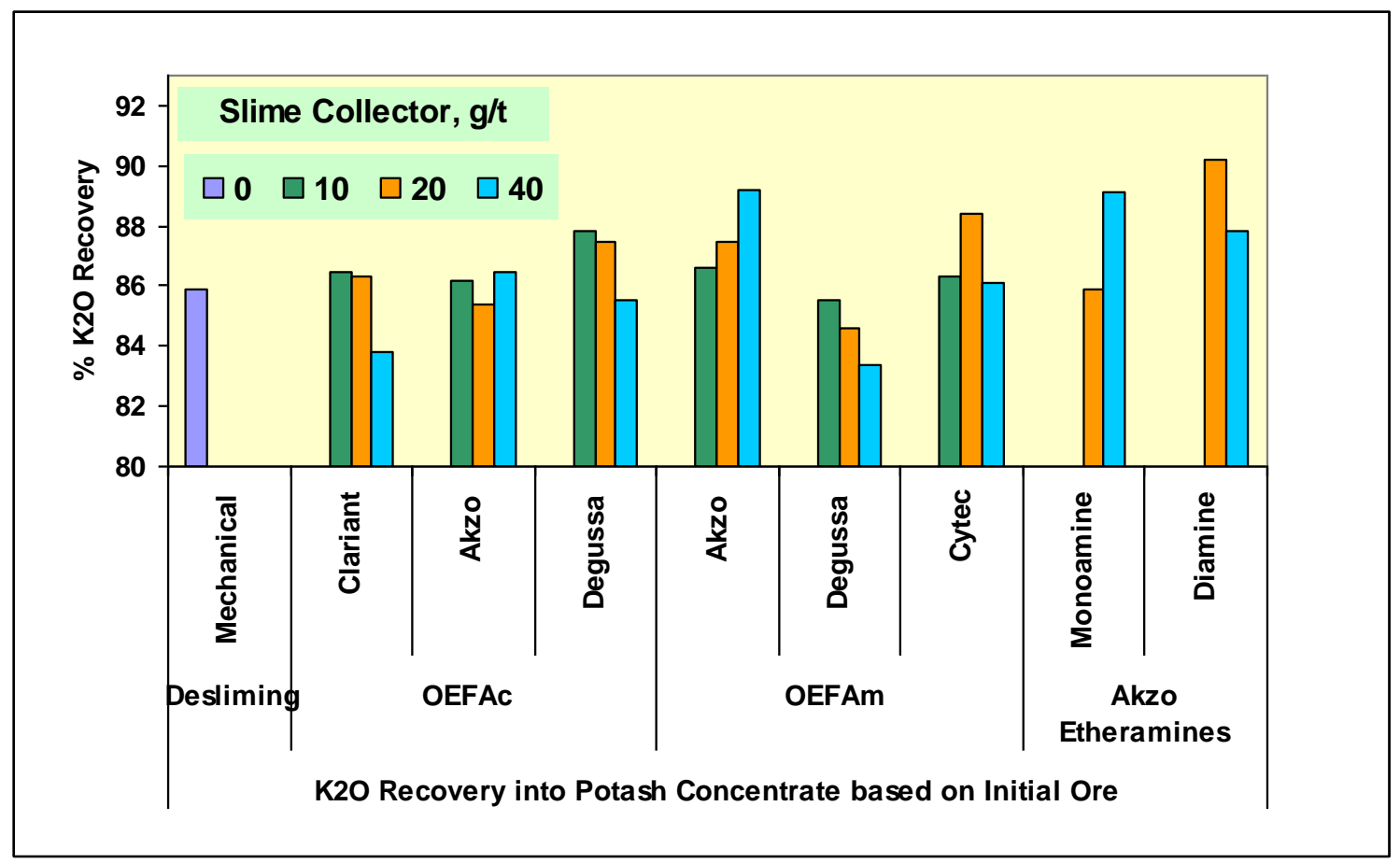

Figure $13-\mathrm{K}_{2} \mathrm{O}$ recovery in the potash flotation concentrates (at guar 60 , amine $30 \mathrm{~g} / \mathrm{t}$ )

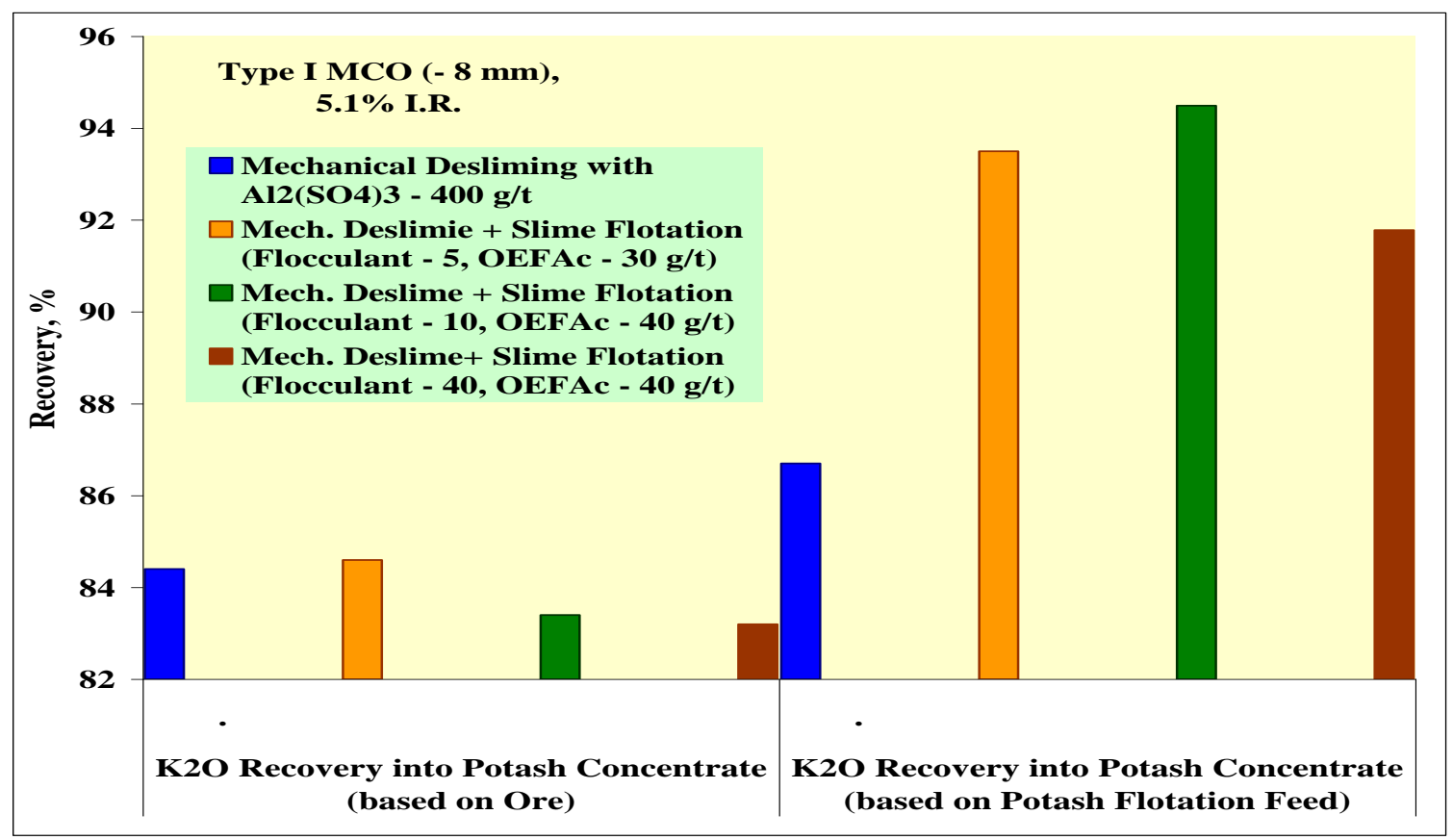

Figure 14 - Potash recovery from medium clay ores after 2-stage desliming, followed by sylvite flotation with $60 \mathrm{~g} / \mathrm{t}$ guar and $33 \mathrm{~g} / \mathrm{t}$ amine emulsion. 


\section{$\underline{\text { High Clay Ores }}$}

High clay ore samples containing as high as $11 \%$ insoluble residue were successfully floated following attrition scrubbing and coarse ore desliming, rod-mill grinding, and two more stages of siphon decantation or slime flotation. Figure - 15 shows that although $80 \%$ of the water-insoluble minerals were removed by decantation, the IR remaining in the flotation feed is higher than run-of-mine low clay ores. Furthermore, sylvite flotation feed after slime flotation has even higher I.R. content, up to $2.9 \%$ vs. $2.5 \%$, than ore deslimed by decantation. Yet the sylvite flotation recoveries are higher with the flotation deslimed ore samples (Figure16), suggesting more of the surface-active clay minerals were removed by slime flotation than that can be removed by decantation.

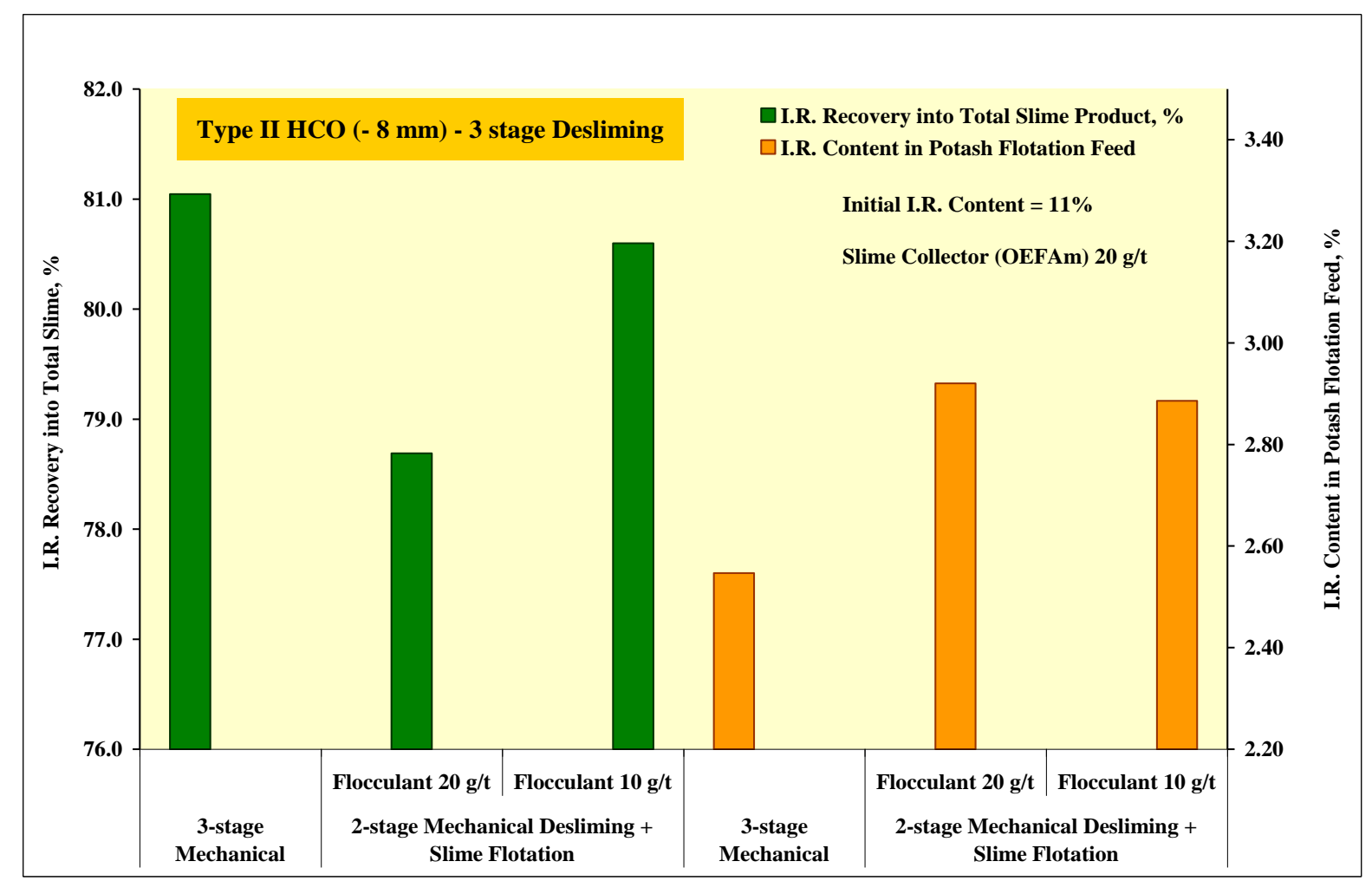

Figure 15 - Comparison of total insoluble minerals recovery and IR content of the sylvite flotation feed, by siphon decantation and slime flotation methods. 


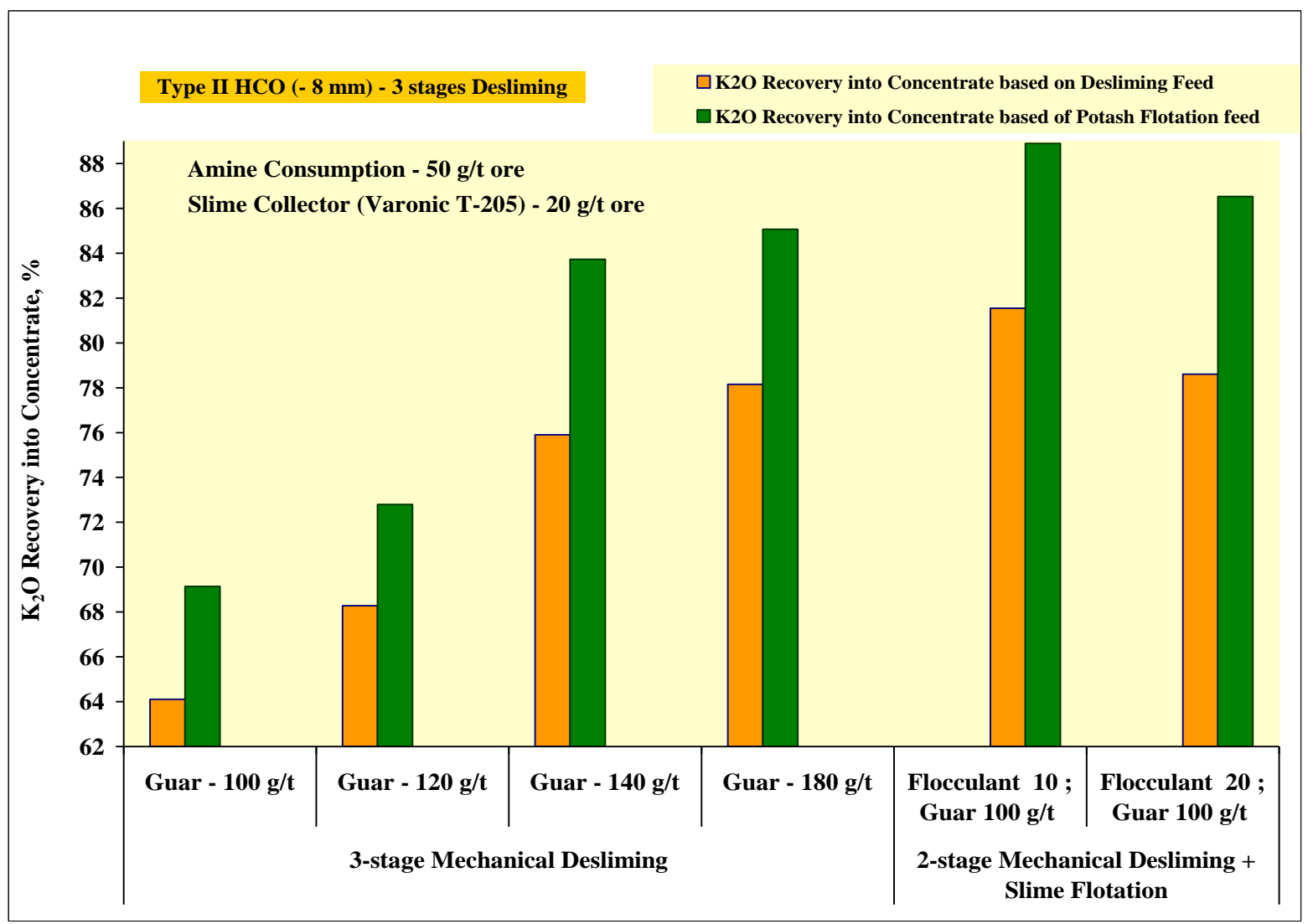

Figure 16 - Overall potash recovery from high clay ore samples by decantation desliming versus slime flotation with $20 \mathrm{~g} / \mathrm{t}$ oxy-ethylated fatty amine collector.

\section{$\underline{\text { Urea-Formaldehyde Resin Depressants }}$}

Flotation tests with UF resin depressants were carried out with previously deslimed, washed and dried ore samples, using 400 grams of fine, or 800 grams of coarse ore fractions in 1.5L and 3L flotation cells, respectively, using Denver D-12 flotation machine. Fine ore samples were conditioned in the flotation cell for one minute with depressant, and one minute with amine emulsion, and floated for 2 minutes. Coarse ore samples were conditioned for 2 minutes with depressant and 3 minutes with amine emulsion, using the bottle-roll technique at $76 \mathrm{rpm}$ in a $2 \mathrm{~L}$ plastic jar fitted with baffles, to simulate likely plant conditions. The coarse ore samples were then transferred into the flotation cell, half-filled with brine while the air was turned on, more brine was gently poured into the cell and the overflowing concentrates were collected. A rubber mixing paddle was also utilized to collect the rest of the concentrate.

Initially a number of preliminary tests were conducted to establish the coarse-ore flotation procedure described above, and screening tests to determine low and high levels of reagent concentrations. Hydrogenated tallow amine (Armeen HT, provided by Akzo Nobel), Philflo flotation oil (Chevron-Phillips), and MIBC frother were used along with guar or Arclin UF resin (170-04-08 239C), which was provided as 50\% water solution, as depressants. 


\section{Fine Ore Flotation}

Preliminary experiments conducted with minus $0.6 \mathrm{~mm}$ ( $28 \mathrm{mesh}$ ) fine flotation feed samples containing $0.62 \%$ water insoluble residue (I.R.) and $13.5 \% \mathrm{~K}_{2} \mathrm{O}$ yielded high recoveries $(92+\%)$ with moderate quantities of amine, guar or resin depressants. Figure-17 shows that comparable recoveries were obtained with both guar and resin in equivalent quantities on dry basis. However, resin might have a number of advantages such as cost and availability; it does not require special solution preparation - simply diluted with brine, and reportedly, increases filtration rates and lowers brine loss into tailings [21]. Tests conducted without depressant yielded only $27 \%$ potash recovery, $33.5 \%$ with $80 \mathrm{~g} / \mathrm{t}$ amine.

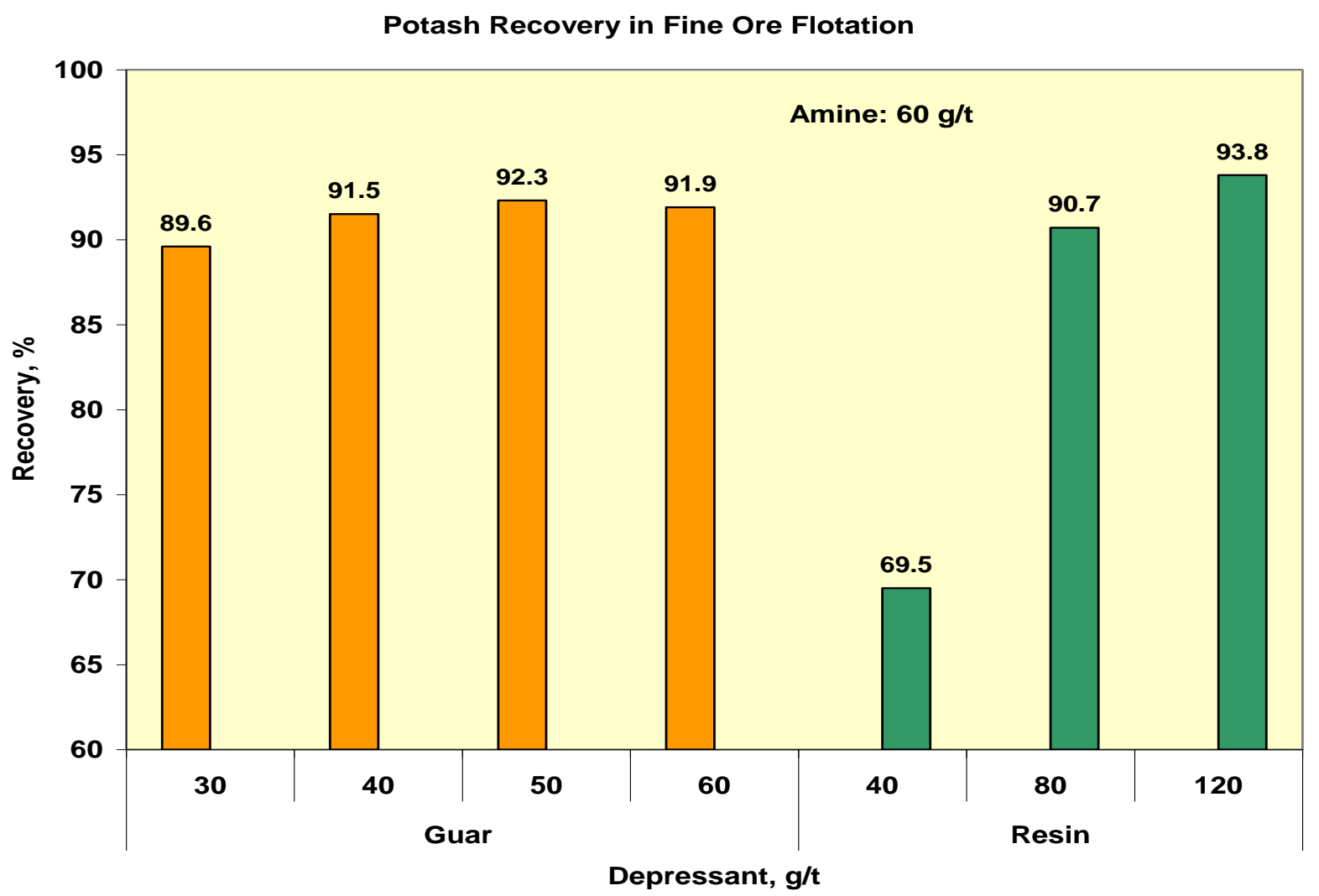

Figure 17 - Comparison of potash recovery from fine flotation feed with $60 \mathrm{~g} / \mathrm{t}$ amine emulsion containing $23 \mathrm{~g} / \mathrm{t}$ oil and $11 \mathrm{~g} / \mathrm{t}$ frother with guar and UF resin as depressant. 


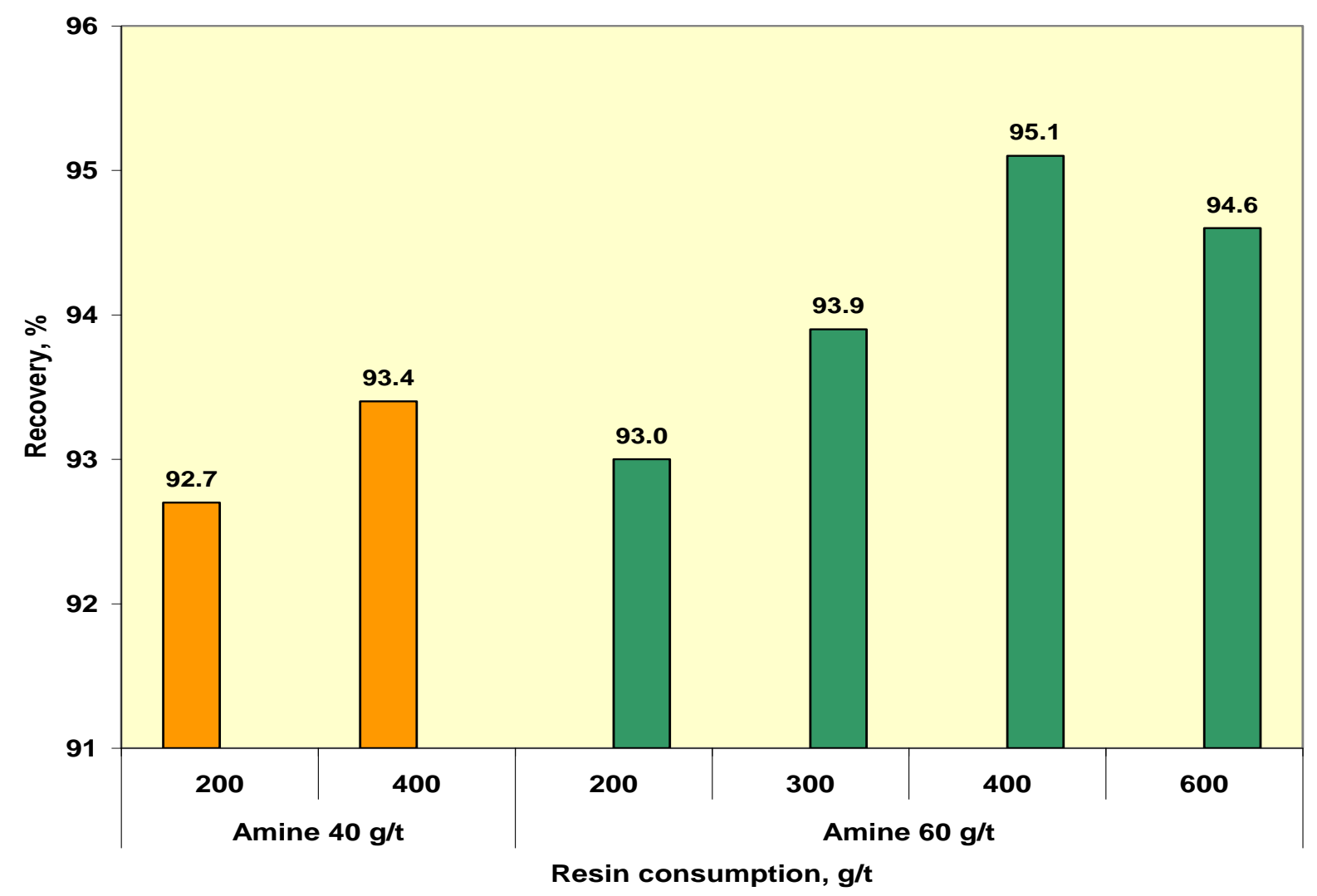

Figure 18 - Effect of amine and resin concentrations on potash recovery in fine ore flotation

Increasing resin concentrations resulted in further increase in potash recovery up to 95\% (Fig.-18). This range of resin concentrations is in agreement with the reported consumptions in aforementioned Russian and US patents. It is also noted that further increase in guar and amine concentrations up to $80 \mathrm{~g} / \mathrm{t}$ levels, did not produce measurable increase in recovery in laboratory tests.

\section{Coarse Ore Flotation (-4 mesh)}

The first batch of coarse ore sample prepared for these tests was screened through 4-mesh and 28 -mesh, contained $10.5 \%+6$ mesh and $12.4 \%$ minus 20 -mesh. On the average, it contained $11.3 \% \mathrm{~K}_{2} \mathrm{O}$ and $0.85 \%$ insolubles. Preliminary screening tests to determine the high and low levels oil, frother, and resin concentrations showed the highest recovery (77\%) was obtained at moderate levels of oil, high levels of resin, and no frother. Concentrates generally assayed about $56 \% \mathrm{~K}_{2} \mathrm{O}$ and $<0.6 \%$ insolubles. These tests also showed that frother helps increase recovery at low levels of resin and oil; it has the opposite effect at high resin levels. However, the UF resin also has frothing properties and excessive frothing was observed in some tests. Addition of small quantity of frother helped mitigate excessive frothing. 
In order to delineate the interactions between reagent concentrations a $2^{3}$ fractional factorial experimental design, including 6 center and 6 orthogonal star points was utilized. Amine was kept at $80 \mathrm{~g} / \mathrm{t}$; resin was varied 300-1100, oil at relatively high levels of 260-550 g/t, and frother from 0 to $54 \mathrm{~g} / \mathrm{t}$ at star points. Again the highest recovery obtained was $77.6 \%$. At very low levels of resin $(95 \mathrm{~g} / \mathrm{t})$ at the star points, recovery was only $60.5 \%$. Although there was considerable scatter in the data, statistical tests showed that the results were acceptable and the model was valid within the design space.

The model also showed that resin and oil have positive contribution to recovery, in that order, whereas resin-frother interaction showed strong negative affect. These results, displayed in a 3D graph in Figure - 19, shows that highest recovery was obtained at high resin and low frother concentrations and at mid levels of oil.

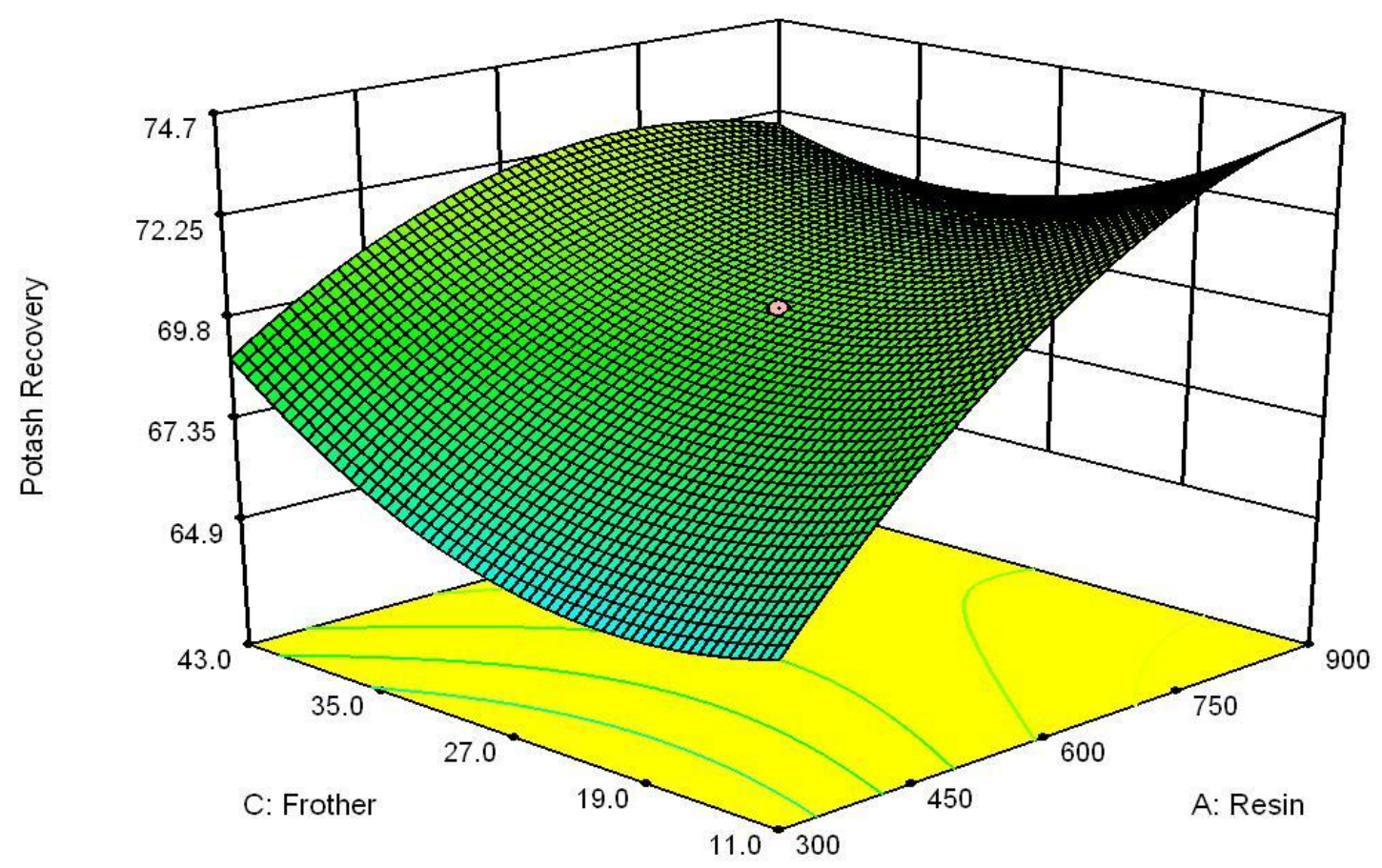

Figure 19 - 3D representation of potash recovery from -4 mesh ore as a function of resin and frother concentrations at mid levels of extender oil $(375 \mathrm{~g} / \mathrm{t})$.

Changing oil concentrations within the design space did not affect the potash recovery appreciably. Furthermore, it was rather messy and difficult to work with oil at concentrations higher than $300 \mathrm{~g} / \mathrm{t}$. In further tests, the oil levels were varied between 100-300 g/t. 
These tests also indicated that the recovery was constrained by the particle size and cell hydrodynamics. It appears that, unless the experiments were carried out in a column cell, no further increase in recovery was possible within this particle size range.

\section{Coarse Ore Flotation (-5 mesh)}

Next batch of test sample was prepared by screening coarse ore through 5 mesh $(4.0 \mathrm{~mm})$ screen. It contained $1.3 \%+6$ mesh and $17.7 \%$ minus 20 mesh fractions. Preliminary potash flotation tests were performed using $100 \mathrm{~g} / \mathrm{t}$ amine and $11 \mathrm{~g} / \mathrm{t}$ frother yielded up to $85 \%$ recovery (Figure - 20).

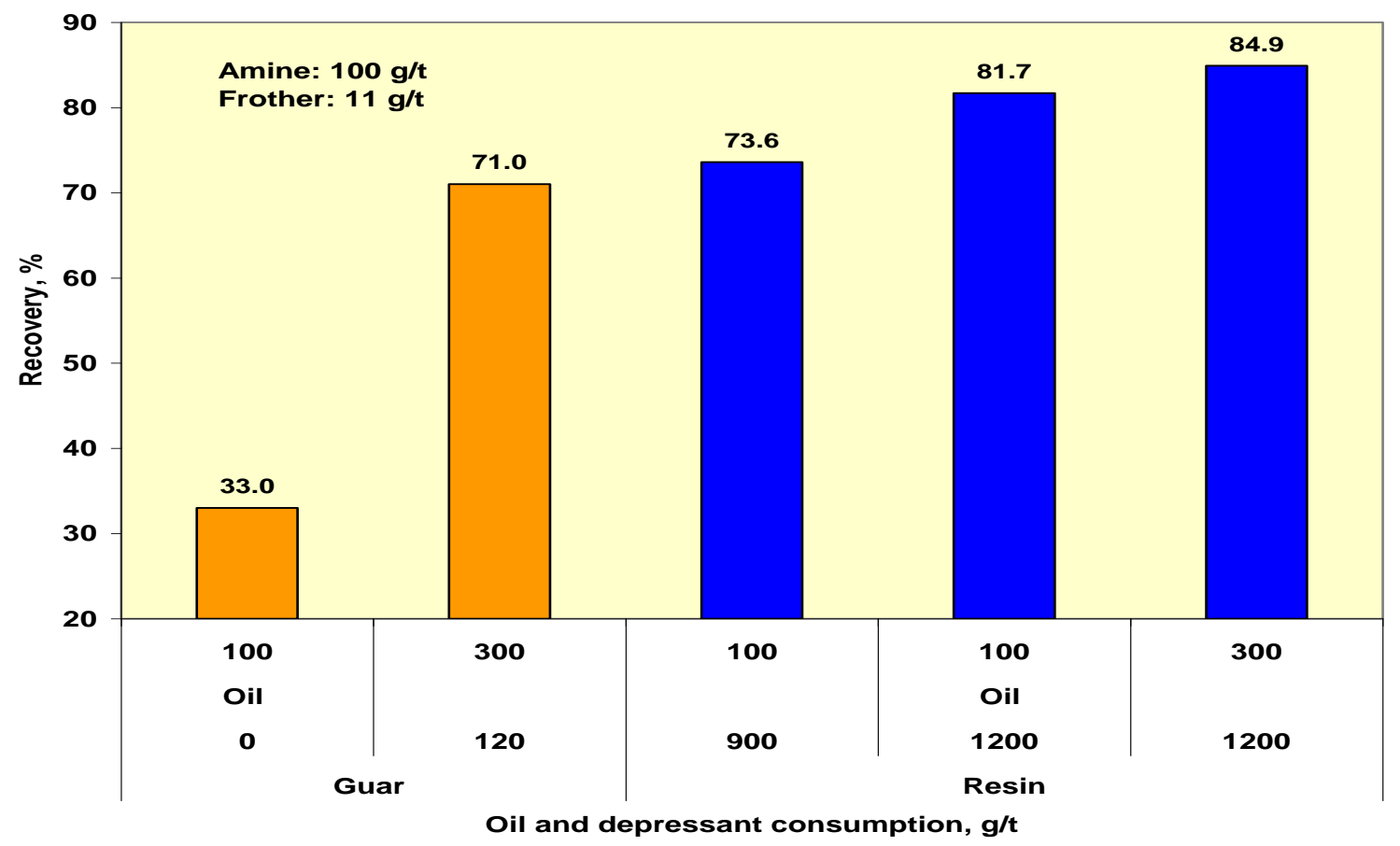

Figure 20 - Effect of guar, oil, and resin on potash recovery from minus 5-mesh ore

The minus 5-mesh batch of ore sample was re-screened through 28 mesh $(0.6 \mathrm{~mm})$ to reduce the fines content in the ore. New size distribution showed $1.6 \%+6$ and $11.3 \%$ minus 20 mesh fractions. Initial tests showed that, at constant resin concentrations $(900 \mathrm{~g} / \mathrm{t})$, increasing the amine-oil-frother emulsion concentrations, recovery also increases (Figure-21).

Tests were also performed to compare the effect of flotation cell volume and samples size on flotation recovery. Figure-22 shows recovery increases with increasing sample size in tests conducted in the $4.5 \mathrm{~L}$ flotation cell. High-low screening tests, conducted at constant amine $(100 \mathrm{~g} / \mathrm{t})$ and frother $(11 \mathrm{~g} / \mathrm{t})$ using 1-kg samples in the large flotation cell also showed that as high as $87 \%$ recovery was achieved with high levels of oil (100-300 g/t) and resin (600-1200 $\mathrm{g} / \mathrm{t})$. Reproducibility was also better with larger samples size and brine volume; however, conducting experiments under these conditions rapidly exhausted deslimed ore samples and brine stocks. 


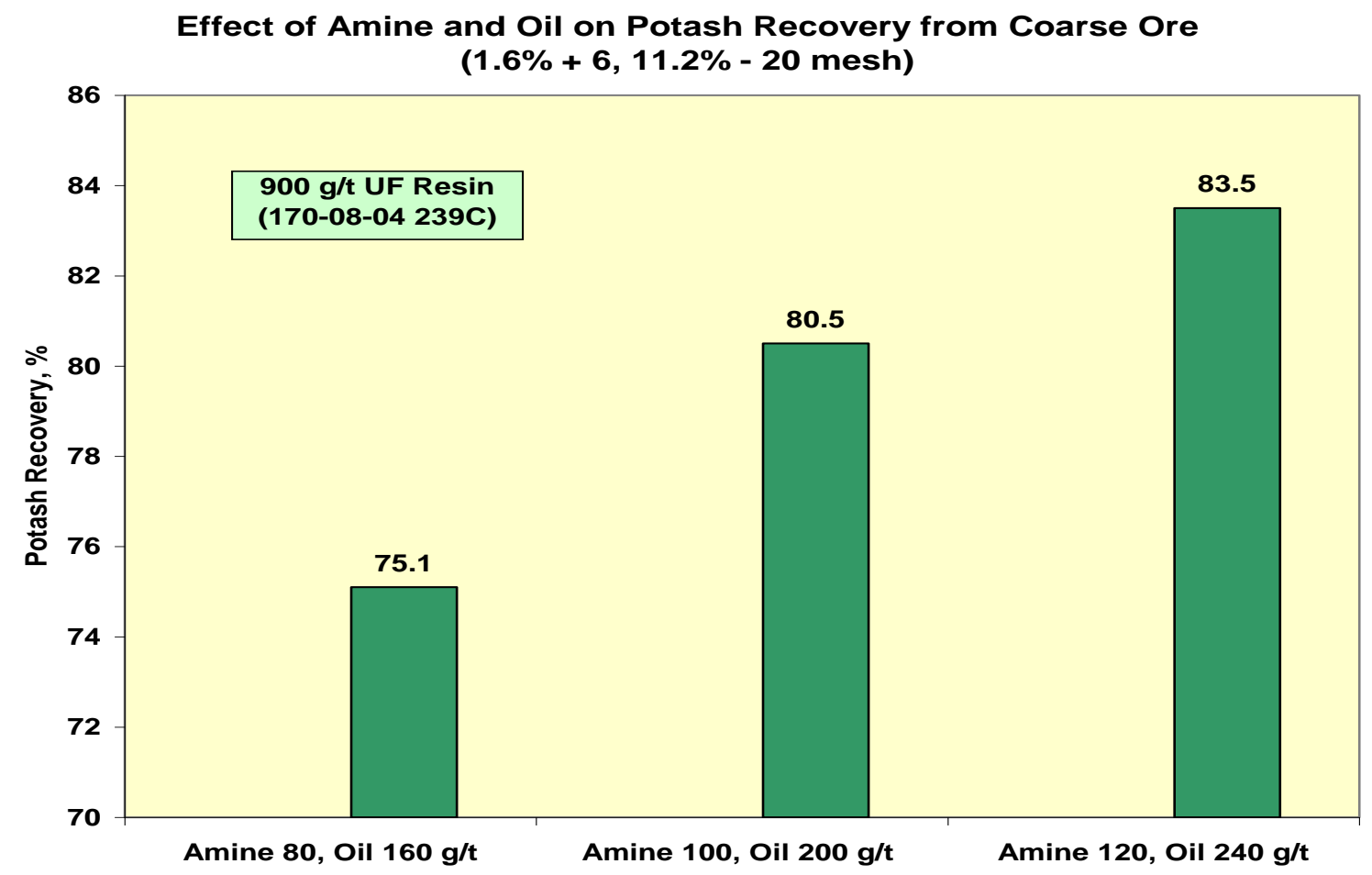

Figure 21 - Effect of amine and oil on potash recovery from coarse ore.

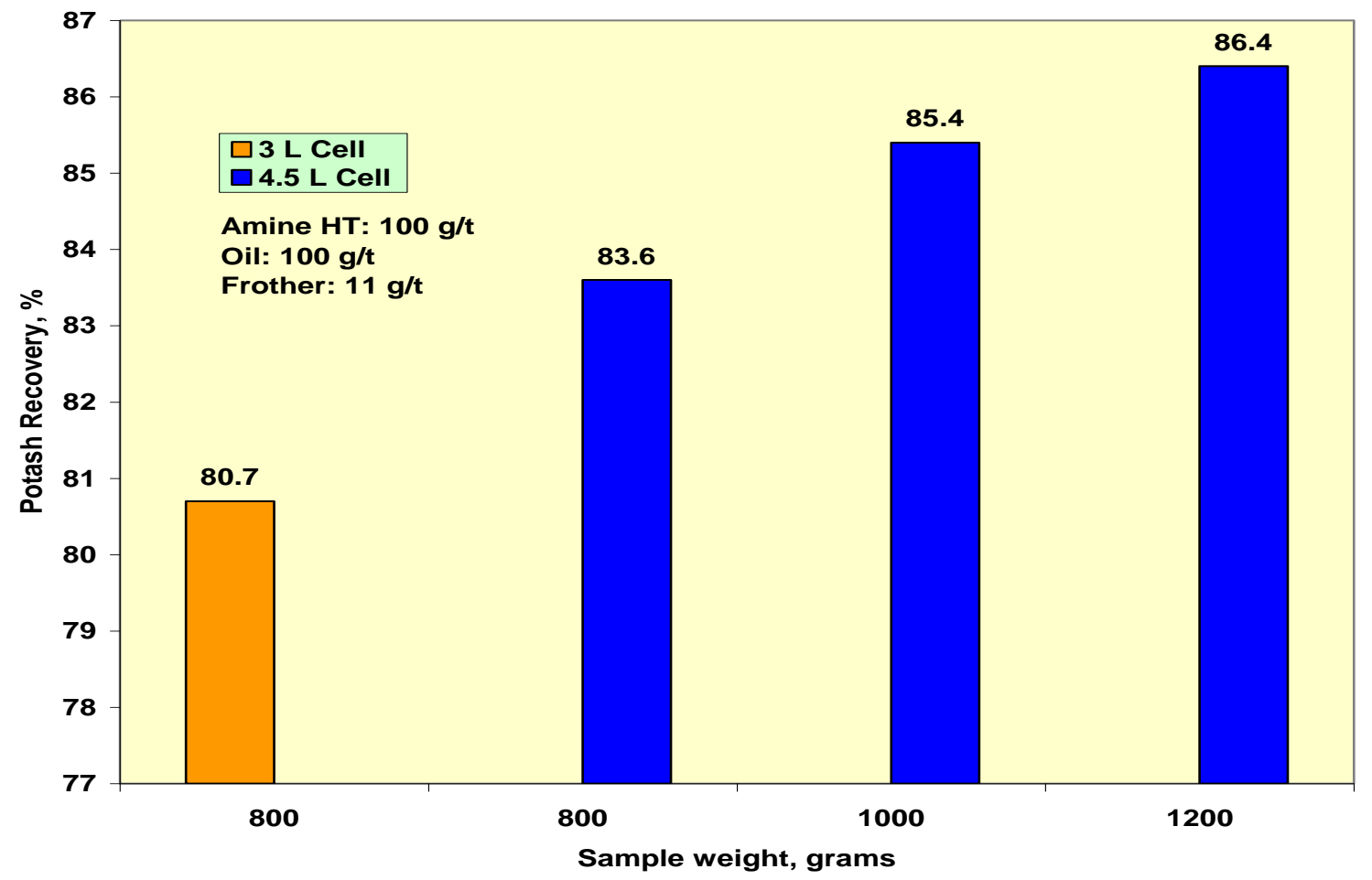

Figure 22 - Potash recovery in coarse (-5/+28 mesh) ore flotation with $900 \mathrm{~g} / \mathrm{t}$ Resin 


\section{Four factor screening tests (-5mesh)}

A new batch of minus 5 mesh ore $(3.5 \%+6$ and $11.2 \%$ minus 20 mesh), containing $12.5 \%$ $\mathrm{K}_{2} \mathrm{O}$ and $0.6 \%$ insoluble, was prepared and $2^{4}$ fractional factorial screening tests, including 6 center points, were carried out using 1-kg ore samples and 80-120 g/t amine, 400-1,200 g/t resin, $100-300 \mathrm{~g} / \mathrm{t}$ oil, and $11-39 \mathrm{~g} / \mathrm{t}$ frother in $4.5 \mathrm{~L}$ cell at $1300 \mathrm{rpm}$. The data, analyzed using Stat-Ease DX.7 software [22], showed little scatter with very good diagnostic statistics (Appendix Table - A1), adjusted $\mathrm{R}^{2}$ and predicted $\mathrm{R}^{2}$ being 0.9913 and 0.9784 , respectively.

There is also good agreement between the predicted and experimental values (App. Fig.1), and the residuals fit the normality criteria (App. Fig.-2). Analysis of variance (ANOVA) also illustrated the complexity of the reagent interactions. The scree chart of the main effects below (Figure-23) show that under the experimental constraints, resin concentration (A) has the most positive effect, followed by amine (B) and oil (C) concentrations. Frother (D) has a strong negative effect, as do resin-amine (AB), resin-frother (AD) interactions; where as oilfrother (CD) and amine-oil (BC) interactions have positive effects.

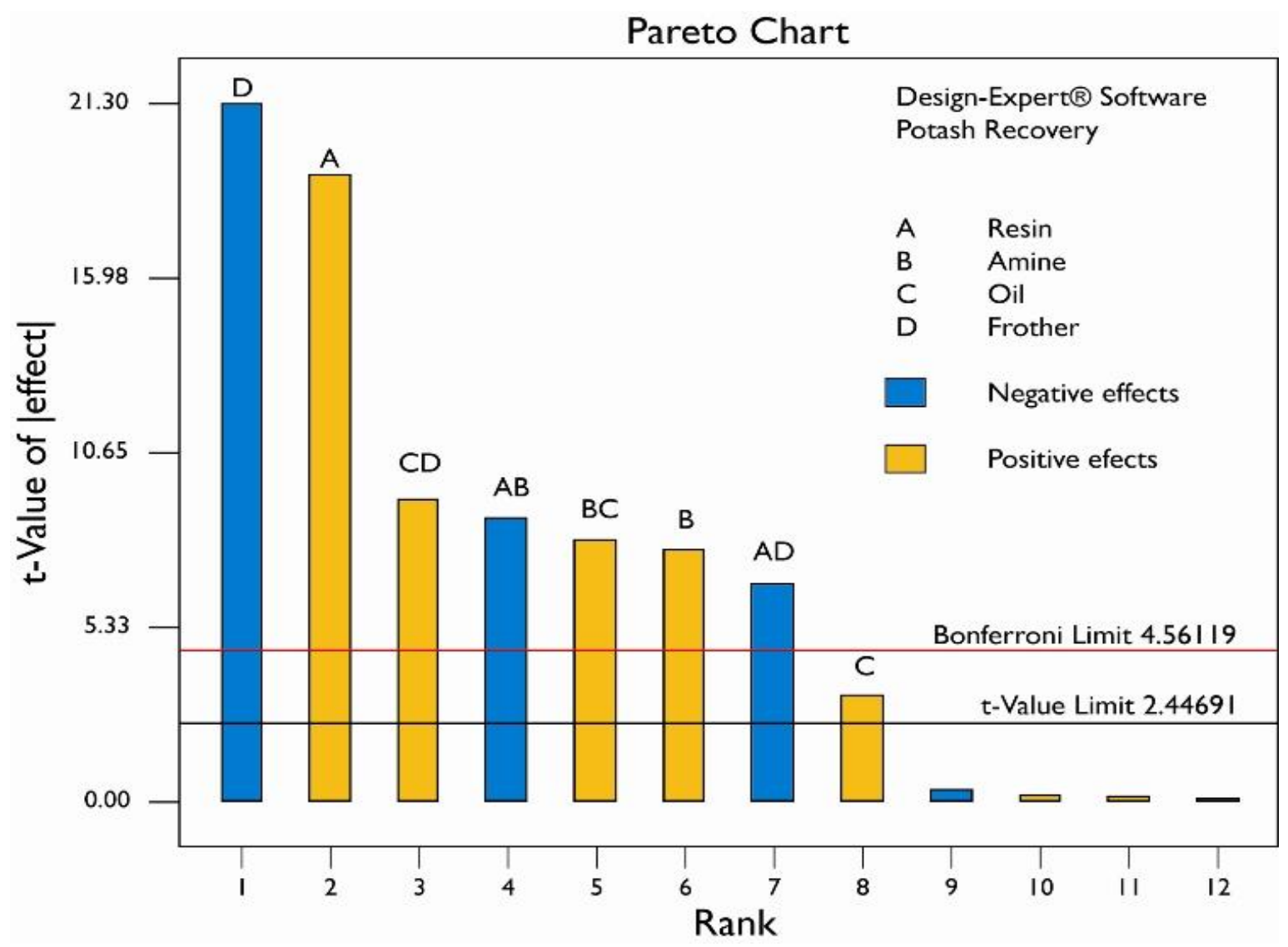

Figure 23 - Effect of flotation reagents on potash recovery in coarse ore flotation 
A two-factor interaction model fitted with regression analysis yielded an equation in terms of coded factors;

$$
\begin{aligned}
\text { Recovery }= & 77.90+1.76^{*} \mathrm{~A}+0.71 * \mathrm{~B}+0.30^{*} \mathrm{C}-1.55^{*} \mathrm{D} \\
& -0.16^{*} \mathrm{~A} * \mathrm{~B}-0.48 * \mathrm{~A}^{*} \mathrm{D}+0.74^{*} \mathrm{~B} * \mathrm{C}+0.67 * \mathrm{C}^{*} \mathrm{D}
\end{aligned}
$$

Final equation in terms of actual factors:

$$
\begin{aligned}
& \text { Recovery }=73.44167 \\
& +0.015604 * \text { Resin }+0.045937 * \text { Amine }-0.042417 * \text { Oil }-0.14776 * \text { Frother } \\
& -8.98438 \mathrm{E}-5 * \text { Resin*Amine }-9.29487 \mathrm{E}-5 * \text { Resin*Frother } \\
& +3.28125 \mathrm{E}-4 * \text { Amine*Oil }+5.16026 \mathrm{E}-4 * \text { Oil } * \text { Frother }
\end{aligned}
$$

Further examinations of the interaction terms help explain the conflicting results observed in during the course of this investigation. In addition to particle size distribution, two-way interaction terms could produce significantly different effects on potash recovery depending on the concentration of other two reagents. For example, recovery reaches a maximum at low amine concentration $(80 \mathrm{~g} / \mathrm{t})$ at low levels of oil and frother with increasing resin concentration, where as at high amine $(120 \mathrm{~g} / \mathrm{t})$ and high levels of oil and frother recovery is not affected by resin concentration and remains below that can be achieved at low amine, oil, and frother levels. The effects of other two-way interaction terms can also be examined graphically by changing the concentrations other two variables.

Further examination of the ANOVA table (App. Table - A1) also shows the presence of significant curvature effects. Curvature effects can best be studied using response surface methodology (RSM) by conducting a set of $2^{\mathbf{3}}$ factorial (resin-amine-oil) experiments, including 6 center and 6 star points, keeping frother concentration at low levels. However, curvature can also be observed in the Design-Expert optimization plots, shown in Figure - 14. Predicted ten best recoveries (App. Table - A2) occur at either low amine - low oil levels (Figure - 24), or high amine - high oil, but all at high resin and low frother concentrations.

Finally, a typical example of the coarse flotation concentrate is shown in Figure -25 . 


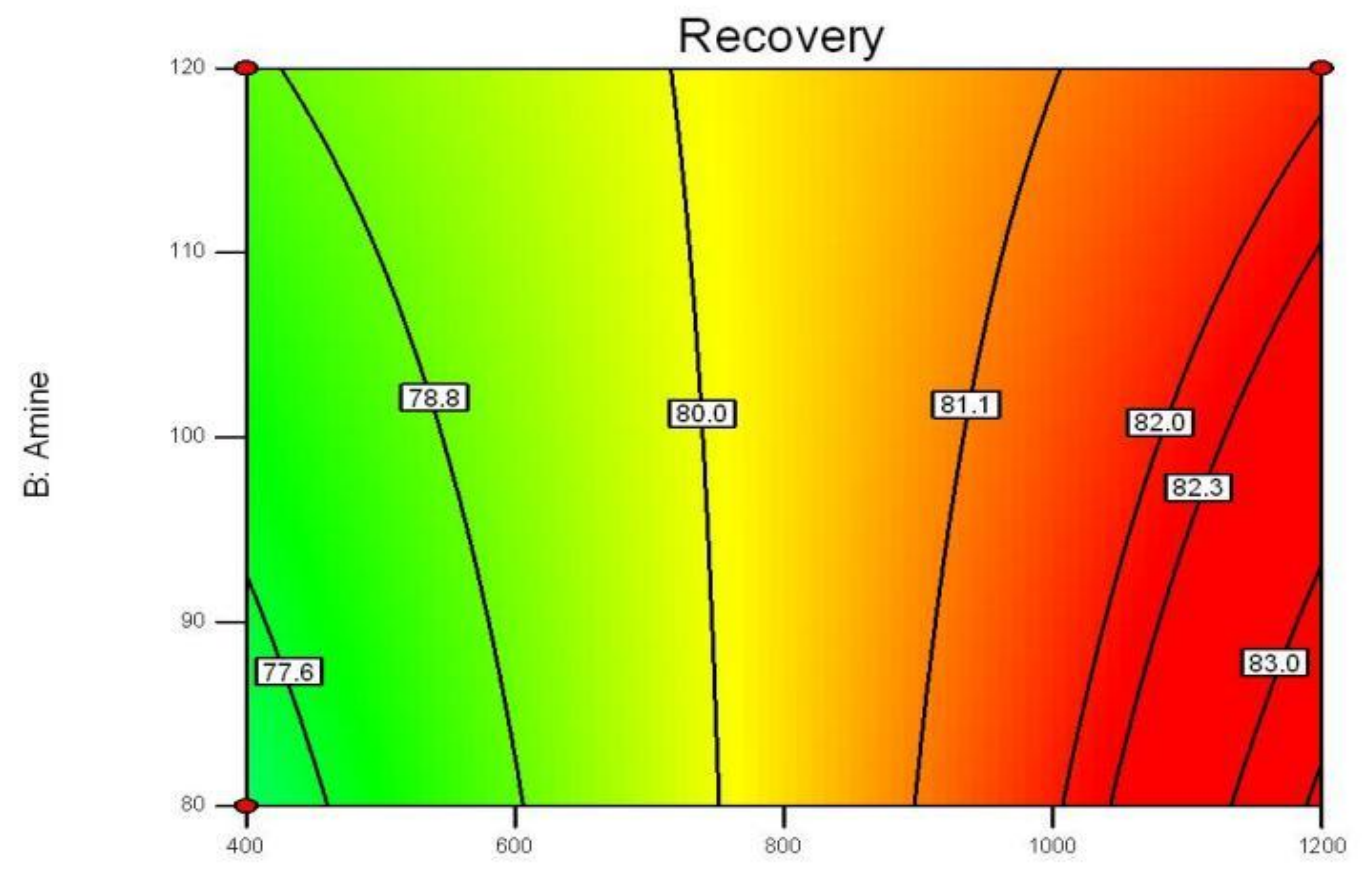

A: Resin

Figure 24 - Maximum recovery predicted from 4-factor fractional factorial experiments occur at high resin, low amine, oil, and frother concentrations.

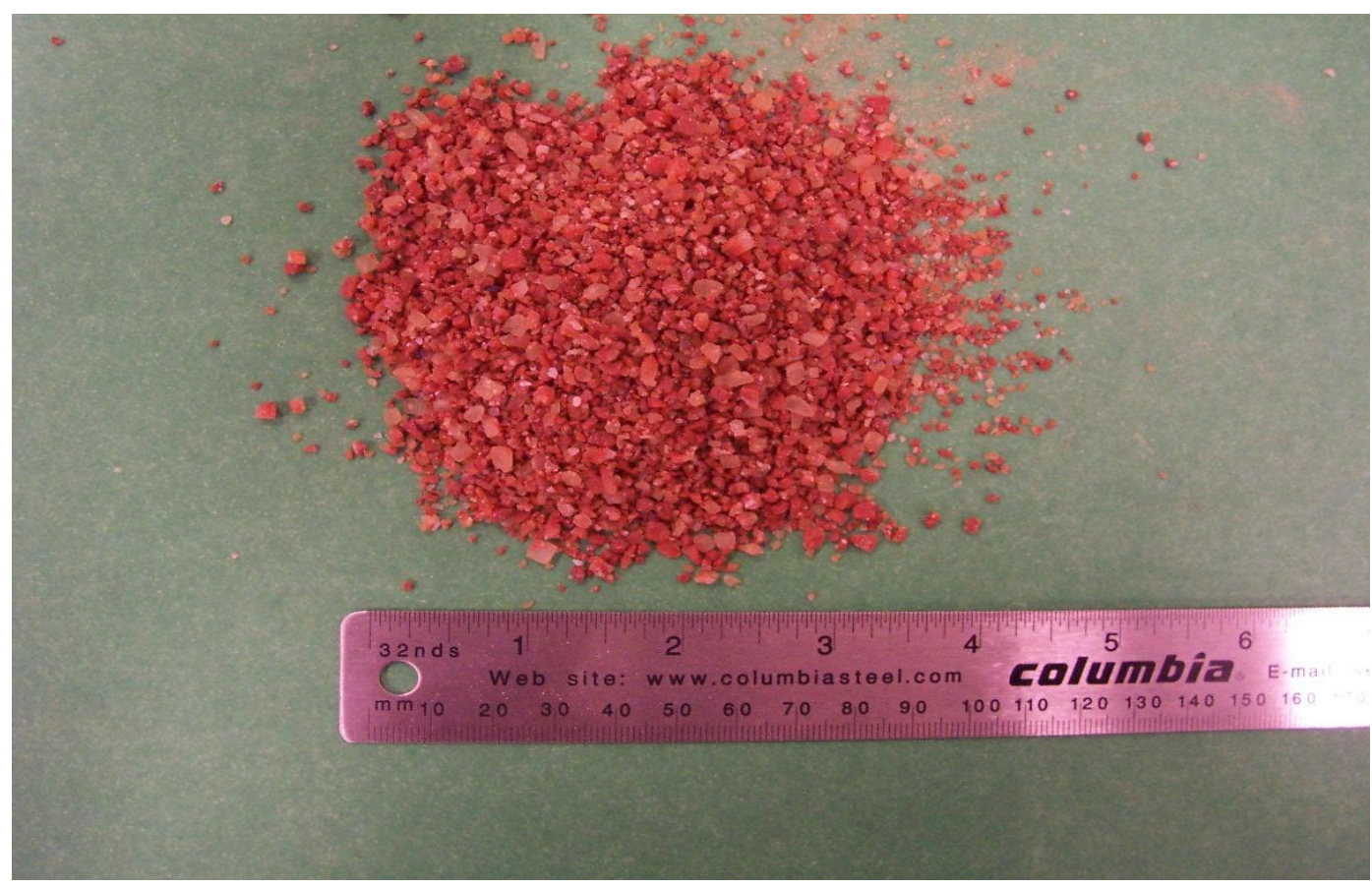

Figure 25 - Typical example of a coarse ore ( -5 mesh) flotation concentrate. 


\section{DISCUSSIONS}

The use of dispersants in non-sulfide minerals flotation is fairly common. Since potash flotation is carried out in high ionic strength saturated brines, however, the surface charge of slimes (mostly clay particles) is practically zero because of double layer compression. Hence clays usually settle along with coarser sylvite and halite particle, and are difficult to remove from the potash flotation feed. In this study it was found that the use of lignin sulfonates and polyvalent metal salts, such as aluminum sulfate (alum) help disperse the clays. Alum most likely causes charge reversal at higher concentrations, rendering clay surfaces positively charged, hence help disperse clay particles and improve mechanical desliming efficiency. Reduced clay content in the flotation feed, in turn, reduces amine consumption, increases potash recovery into flotation concentrate and improves concentrate quality. Conventional dispersants, such as sodium silicate and phosphates did not show any effect in this medium.

Adsorption mechanism of lignin sulfonate and its dispersant effect on clays in brines is not well understood. It is also noteworthy that lignin sulfonates in combination with alum work more effectively and reduce reagent consumptions. Addition of these dispersants into the rod mill also increases grinding kinetics, producing a finer-sized product. This may suggest that dispersants may reduce power consumption and/or increase rod-mill throughput.

Dispersants alone, however, were not effective in desliming very high clay ores that are to be encountered in the near future. Incorporating slime flotation into the process could eliminate process upsets due to fluctuations in clay content of the ores presently mined, and allow highclay ores to be mined and processed economically in the future.

Flotation of sylvite $(\mathrm{KCl})$ in coarse sizes in processing sylvinite ores is highly desirable, eliminating the need for compaction, crushing, and screening required of the fine flotation concentrates. However, not many ores are amenable to flotation in coarse sizes because of the liberation size of sylvite from halite grains. New Mexico potash ores are fine-grained, and generally floated after fine grinding. Most Canadian ores, on the other hand, are higher grade and much coarser grained than NM ores. Therefore, in most Canadian operations run of mine ore is crushed to minus $4 \mathrm{~mm}$, deslimed and screened. The coarse and fine fractions are then conditioned separately with clay depressants and flotation reagents, combined, and floated together. At some plants rougher flotation tailings are screened, the oversize is re-ground and returned to conditioning stage [10]. In few other Canadian and one NM operations, coarse and fine fractions are floated separately.

Although potash flotation with long-chain aliphatic amines has been used in the industry for almost 70 years, underlying principles of soluble salt flotation has not yet been adequately understood. The practice of potash flotation is also highly varied, mostly based on company expertise and characteristics of specific ores. It is generally accepted that extender oils and frothers help improve the flotation of coarse particles. However, fundamental studies on the effects of oil and frother on potash flotation are lacking, particularly in coarse sizes, due to inherent difficulties in experimenting with coarse ore particles in mechanically agitated laboratory flotation cells. 
Studies conducted at the University of British Columbia (UBC) using a short column cell [23] and reagent concentrations as high as $200 \mathrm{~g} / \mathrm{t}$ amine, $1346 \mathrm{~g} / \mathrm{t}$ extender oil and $420 \mathrm{~g} / \mathrm{t}$ methyl isobutyl carbinol frother, showed that very high recoveries (93-99.8\%) can be achieved in flotation of closely sized coarse sylvite ores. UBC researchers were also able to float different ores in a wide range of oil, amine, frother concentrations, but they did not attempt to establish a correlation between reagent concentrations for flotation in coarse sizes [24]. In general, the reagent concentrations were much higher than those reported for Potash Corporation of Saskatchewan (PCS) operations, e.g., 40-90 g/t amine, 9-85 g/t oil, and about $25 \mathrm{~g} / \mathrm{t}$ frother, including the Lanigan operations where coarse and fine ore fractions were floated separately [10].

Since coarse fraction of the flotation feed is removed from the fines by screening, it is generally assumed that clays report to the fines fraction and coarse fractions do not contain high enough clay concentrations to hinder flotation. Yet flotation tests conducted during the course of this study consistently yielded very low recoveries without depressants. Recoveries improved considerably with the use of guar, however, were limited to $70 \%$ levels in coarse ore flotation even with the use of $120-150 \mathrm{~g} / \mathrm{t}$ guar.

Although urea-formaldehyde resins were used in fine ore flotation as clay depressants, it is now recognized that they have other attributes more beneficial to the overall flotation process than clay depressants alone, such as higher filtration rates and lower moisture content for concentrates and tailings that were reported in plant practice. In particular, this study showed that use of UFR increases the coarse ore recovery to levels that could not be achieved with high concentrations of amine, oil, and frother, even with the use of guar depressant. Although similar recoveries were obtained with guar and resin in fine ore flotation at comparable consumption levels on dry basis, up to $88 \%$ recovery was obtained in coarse ore flotation, depending on the fines (-28 mesh) content, albeit at much higher quantities than required for clay depression alone. It is safe to assume that having the unit structure shown below, nitrogen containing resin macro-molecules are also adsorbed on the sylvite grains rendering them much more hydrophobic than amine-oil adsorption alone could.

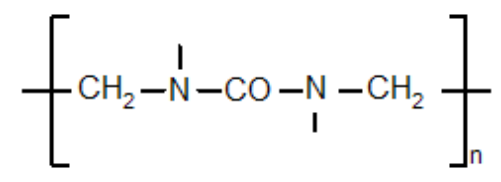

It was also evident that resin renders sylvite particles much more hydrophobic than oil and amine alone, and performs more like a flotation reagent than clay depressant at higher concentrations. Also, resins would likely promote flotation of coarse-size particles in the plant more effectively than that could be observed in the laboratory setting.

Reagent screening tests and $2^{4}$ fractional factorial tests conducted in this study showed that, in addition to top size and the quantity of fine fraction $(-28$ mesh) in the flotation feed, the potash recovery in coarse sizes is affected mostly by resin and frother concentrations at moderate levels of amine and oil. As resin increased recovery at constant amine and oil concentrations, frothers generally had the opposite effect. Although it appears to be counter intuitive, presence of small quantity of frother eliminated excessive frothing generated by the 
resin. The reason for this anomaly is not yet understood; perhaps it can be explained by surface tension measurements in the future.

In addition to hydrodynamic considerations in the laboratory flotation cell, flotation recovery was also hindered by limited degree of liberation of coarse sylvite-halite grains that was easily observed by visual examination. It is also noted that both experimental and predicted recoveries fall within a narrow range, i.e. between 73 and $83 \%$, perhaps because of the range of reagent concentrations selected, based on the preliminary tests, were near optimum. Using longer chain amines with resin might further increase recovery.

Another study conducted with high-grade $(40 \% \mathrm{KCl})$ Canadian ores reported comparable recovery $(85.7 \%)$ using UF resins at $3 \mathrm{lbs} /$ ton levels. However, concentrations of other flotation reagents were not specified [25]. Furthermore, the flotation feed contained $3.8 \%+6$ and $40.0 \%$ minus 28 mesh fractions, as opposed to $<3.5 \%+6$ and $4.6 \%$ minus 28 mesh ore samples used in this study. It should be pointed out that use of resin to augment coarse ore flotation recovery could drastically reduce high extender oil consumptions, which might pose health and safety issues, as suggested in previous studies and applied in some plants.

\section{SUMMARY AND CONCLUSIONS}

Effect of common organic (lignin sulfonate) and inorganic dispersants on desliming potash ores were investigated. It was found that lignin sulfonate and aluminum sulfate, either alone or preferably together, increase mechanical desliming efficiency resulting in cleaner potash flotation feed, reducing reagent consumptions, increasing potash recovery and concentrate grade and purity. Addition of these reagents into the rod mill also increases grinding kinetics, resulting in finer product. Although it has not been tested in the plants yet, this may suggest dispersants may reduce power consumption and/or increase rod-mill throughput.

Flotation of slimes following coarse-ore desliming and grinding have also been investigated with oxyethylated fatty acids, fatty amines, and ether-amine family of collectors. After initial flocculation with high molecular weight poly-acryl amide flocculants, highly surface-active slimes (clays) can be removed from the potash flotation feed by flotation. Further cleaning of slime concentrates in column cells allows additional $\mathrm{KCl}$ recovery, thus reducing loses into slimes and increasing potash recovery in the concentrates.

Slime flotation could eliminate process upsets due to pockets of higher clay ores delivered to the mill and, most importantly, allow exploitation of high-clay ores that heretofore can not be mined or processed with the existing desliming technologies. A new flotation plant planned in the district will incorporate slime flotation in feasibility and process design.

Urea-formaldehyde resins were also investigated as a substitute for organic clay blinder guar. In flotation of fine ore fractions (-28 mesh), comparable recoveries were obtained with the same quantity of reagents on dry basis. However, resin might have certain advantages such as cost and availability, does not require special procedures for solution preparation as guar. In plant trials resins have been found to increase slime filtration and de-brining rates, thus producing drier concentrates and tailings [21]. 
In coarse ore flotation, resins produced much higher recoveries than that could be obtained with guar. It was also evident that resin renders sylvite particles much more hydrophobic than oil and amine alone, and performs more like a flotation reagent than clay depressant at higher concentrations. Plant trial for resin tests in coarse ore flotation is under consideration.

\section{REFERENCES}

1. Jasinski, S.M., Potash, U.S. Geological Survey Minerals Yearbook, 2008.

2. Barker, J.M., and Austin, G.S., Overview of Carlsbad Potash District, New Mexico, in Potash Resources of WIPP Site, New Mexico Bureau of Mines \& Mineral Resources, Circular 207, 1999, pp.7-15.

3. Zandon, V.A., Potash, in SME Min. Proc. Handbook, Vol. 2, SME-AIME, New York, N.Y. 1985, Chapter 22, pp. 1-15.

4. NM Energy, Minerals, and Natural Resources Department, Annual Report 2008.

5. Garret, D.E., Potash: Deposits, Processing, Properties, and Uses, Chapman \& Hall, New York, (1996), 736 pages

6. Dancy, W.B., Potassium Compounds, in Kirk \& Othmer Encyclopedia of Chemical Technology, Vol. 18, John Wiley \& Sons, Inc., 1980, pp. 920-950.

7. Arsantiev, V.A., and Leja J., Problems in Potash Flotation Common to Ores in Canada and Soviet Union, CIM Bulletin, March (1977) pp.154-158.

8. Laskowski, J.S., Flotation of Potash Ores, in Reagents for Better Metallurgy, P.S. Mulukutla (Ed.), SME, 1994, pp. 225-243.

9. Yalamanchili, M.R., Kellar, J.J., and Miller, J.D., Adsorption of Collector Colloids in the Flotation of Alkali Halide Particles, International Journal of Mineral Processing, v39, (1993) pp.137-153.

10. Strathdee, G.G., at al., The Processing of Potash Ore by PCS, Proceed. XIV Int. Mineral Processing Congress (IMPC) Toronto, Canada, (1982), vol.-V, paper 12, 20 p.

11. Titkov, N. Panteleeva, A. Chistyakov, L. Pimkina, I. Mikhaylova, Studies of surface and sorption behavior of saline and clay-carbonate minerals in electrolytes, XXI International Mineral Processing Congress, 2000, Rome, Italy, July, pp. c8b-36-42

12. Gamble, S.A., IMC Potash Carlsbad, Inc., Private communication, Oct. 2002.

13. Titkov S.N., Sokolov I.D., Ryzhova M.M., Struzhkov V.N., and Panteleeva., N.N., Development of New Effective Process for Flotation Concentration of Potash Ores with 
High Clay Content. XV Intern. Mineral Processing Congress, Cannes, (1985) part II, pp.122-133.

14. Yalamanchili, M.R. and Miller, J.D., Removal of Insoluble Slimes from Potash Ore by Air-sparged Hydrocylone Flotation, Minerals Engineering, 8 (1995) pp.169-177.

15. Alonso, E.A., and Laskowski, J.S., in Selection of Polymers used as Slime Modifiers in Potash Ore Flotation, $38^{\text {th }}$ Annual Conference of Metallurgists of CIM, Intern. Symposium on Fundamentals of Mineral Processing "Polymers in Mineral Processing", J.S. Laskowski (Ed.), Quebec (1999) pp. 421-436.

16. Perucca, C.F., and Cormode, D.A., The Use of Polymers in Potash Processing at Agrium Potash Plant, ibid., pp. 393-403.

17. Titkov, S., Pantaleeva, N., and Gurkova, T., Use of Polymer Reagents for Processing Clay Slimes, ibid., pp. 375-392.

18. Titkov, S., Sabirov, R., Panteleeva, N., and Gustafsson, J-O., Flotation of Sliming Silicate and Carbonate Minerals: Technology and Reagents, IX. International Mineral Processing Symposium, Cappadocia, Turkey, 18-20 September, (2002), pp. 122-123.

19. Schoeld, E.A., and Egbom, C.W., Clay Depressant, (1962), U.S. Patent 3,017,028.

20. Urea-Formaldehyde Resin Production Process, Russian Patent, (1999), RU 2169740

21. Navarette, J., Johnson, J., and Gamble, S., Use of Urea-Formaldehyde Resin in Potash Ore Flotation, (2006), U.S. Patent Application, 20060226051 A1.

22. Stat-Ease Statistical Software http://www.statease.com/

23. Laskowski, J.S., and Wang, Q., Flotation Process for the Flotation of Coarse Fractions of Potash Ores, (1995), U.S. Patent 5,456,362

24. Laskowski, J.S., and Wang, Q., Amine Containing Oils as Extenders in the Flotation of Sylvinite Ores, Proceed. XX Int. Mineral Processing Congress (IMPC), Aachen, Germany, (1997), pp. 605-616.

25. Tao, D., Zhou, X., Zhao, C., Aron, M., Wright, J., Clay Binders for Enhanced Potash Mineral Beneficiation Using Froth Flotation, (2008), SME Preprint 08-044, 5 p. 


\section{APPENDIX:}

\section{Analysis of 4-factor experimental design test results}

Table - A1. Analysis of variance (ANOVA) table for selected factorial model

Error term includes BD, ABD, ACD, BCD

Ignored terms are $\mathrm{AC}$,

SS: 3.37

df: 3

\begin{tabular}{|c|c|c|c|c|c|c|}
\hline \multicolumn{7}{|c|}{ [Partial sum of squares - Type III] } \\
\hline \multicolumn{3}{|c|}{ Sum of } & \multirow{2}{*}{$\begin{array}{r}\text { Mean } \\
\text { Square }\end{array}$} & \multicolumn{2}{|c|}{$\mathbf{F}$} & p-value \\
\hline Source & Squares & df & & \multicolumn{2}{|c|}{ Value } & Prob $>$ F \\
\hline Block & 2.65 & 1 & 2.65 & & & \\
\hline Model & 93.91 & 6 & 15.65 & \multicolumn{2}{|c|}{229.99} & $<0.0001$ \\
\hline A-Resin & 24.85 & 1 & 24.85 & \multicolumn{2}{|c|}{365.16} & $<0.0001$ \\
\hline B-Amine & 4.06 & 1 & 4.06 & \multicolumn{2}{|c|}{59.68} & 0.0002 \\
\hline C-Oil & 0.72 & 1 & 0.72 & \multicolumn{2}{|c|}{10.58} & 0.0174 \\
\hline D-Frother & r 30.88 & 1 & 30.88 & \multicolumn{2}{|c|}{453.76} & $<0.0001$ \\
\hline$A B$ & 5.12 & 1 & 5.12 & \multicolumn{2}{|c|}{75.23} & 0.0001 \\
\hline$A D$ & 3.02 & 1 & 3.02 & \multicolumn{2}{|c|}{44.36} & 0.0006 \\
\hline$B C$ & 4.35 & 1 & 4.35 & \multicolumn{2}{|c|}{63.94} & 0.0002 \\
\hline$C D$ & 5.82 & 1 & 5.82 & \multicolumn{2}{|c|}{85.45} & $<0.0001$ \\
\hline & 0.67 & 1 & 0.67 & \multicolumn{2}{|c|}{9.80} & 0.0203 \\
\hline Curvature & 0.67 & 1 & 0.67 & \multicolumn{2}{|c|}{9.80} & 0.0203 \\
\hline Residual & 0.41 & 6 & 0.068 & \multirow{2}{*}{\multicolumn{2}{|c|}{0.10}} & \\
\hline Lack of Fit & 0.068 & 4 & 0.017 & & & 0.9720 \\
\hline Pure Error & 0.34 & 2 & 0.17 & & \\
\hline Cor Total & 97.63 & 14 & & & & \\
\hline \multicolumn{2}{|l|}{ Std. Dev. } & 0.26 & \multicolumn{3}{|c|}{ R-Squared $\quad 0.9957$} & \\
\hline \multicolumn{2}{|l|}{ Mean } & 78.02 & \multicolumn{2}{|r|}{ Adj R-Squared } & 0.9913 & \\
\hline \multicolumn{2}{|l|}{ C.V. \% } & 0.33 & \multicolumn{2}{|r|}{ Pred R-Squared } & 0.9784 & \\
\hline \multicolumn{2}{|l|}{ PRESS } & 2.04 & \multicolumn{2}{|r|}{ Adeq Precision } & 40.571 & \\
\hline
\end{tabular}

The Model F-value of 229.99 implies the model is significant. There is only a $0.01 \%$ chance that a "Model F-Value" this large could occur due to noise.

Values of "Prob > F" less than 0.0500 indicate model terms are significant. In this case $\mathrm{A}, \mathrm{B}, \mathrm{C}, \mathrm{D}, \mathrm{AB}, \mathrm{AD}, \mathrm{BC}, \mathrm{CD}$, are significant model terms. 
The "Curvature F-value" of 9.80 implies there is significant curvature (as measured by difference between the average of the center points and the average of the factorial points) in the design space. There is only a $2.03 \%$ chance that a "Curvature F-value" this large could occur due to noise.

The "Lack of Fit F-value" of 0.10 implies the Lack of Fit is not significant relative to the pure error. There is a $97.20 \%$ chance that a "Lack of Fit F-value" this large could occur due to noise.

The "Pred R-Squared" of 0.9784 is in reasonable agreement with the "Adj R-Squared" of 0.9913 .

"Adeq Precision" measures the signal to noise ratio. A ratio greater than 4 is desirable. A ratio of 40.571 indicates an adequate signal. This model can be used to navigate the design space.

Table-A2. Optimization of Recovery

\section{Constraints}

\begin{tabular}{llllllc}
\hline Name & Goal & $\begin{array}{l}\text { Lower } \\
\text { Limit }\end{array}$ & $\begin{array}{l}\text { Upper } \\
\text { Limit }\end{array}$ & $\begin{array}{l}\text { Lower } \\
\text { Weight }\end{array}$ & $\begin{array}{l}\text { Upper } \\
\text { Weight }\end{array}$ & $\begin{array}{c}\text { Impor- } \\
\text { tance }\end{array}$ \\
Resin & is in range & 400 & 1200 & 1 & 1 & 3 \\
Amine & is in range & 80 & 120 & 1 & 1 & 3 \\
Oil & is in range & 100 & 300 & 1 & 1 & 3 \\
Frother & is in range & 13 & 39 & 1 & 1 & 3 \\
Recovery & maximize & 73.6 & 82.2 & 1 & 1 & 4
\end{tabular}

\section{Solutions:}

\begin{tabular}{|c|c|c|c|c|c|}
\hline Resin & Amine & Oil & Frother & Recovery & Desirability \\
\hline 11183.45 & $\underline{84.38}$ & 142.34 & 14.15 & 82.4758 & $\underline{1.0}$ \\
\hline$2 \longdiv { 1 1 9 3 . 3 1 }$ & $1 \overline{19.31}$ & 282.36 & $\overline{13.74}$ & 82.2796 & $\overline{1.0}$ \\
\hline $3 \overline{1193.03}$ & 82.06 & $\overline{112.73}$ & $\overline{16.96}$ & 82.3246 & $\overline{1.0}$ \\
\hline $4 \overline{1200.00}$ & $\overline{80.00}$ & $\overline{100.00}$ & 13.00 & 83.3875 & $\underline{1.0}$ \\
\hline$5 \longdiv { 1 1 9 1 . 0 2 }$ & $\underline{118.34}$ & 281.50 & 14.00 & 82.2133 & $\underline{1.0}$ \\
\hline$6 \longdiv { 1 1 5 5 . 6 2 }$ & $\underline{84.32}$ & $\underline{100.91}$ & 14.11 & 82.6576 & $\overline{1.0}$ \\
\hline $7 \underline{1190.78}$ & $\underline{103.19}$ & 100.71 & 13.30 & $\underline{82.3193}$ & $\underline{1.0}$ \\
\hline $8 \underline{1199.22}$ & $\overline{91.24}$ & $\overline{197.70}$ & $\overline{13.13}$ & 82.2206 & 1.0 \\
\hline $9 \underline{1200.00}$ & $\underline{120.00}$ & $\underline{300.00}$ & $\underline{13.00}$ & $\underline{82.4708}$ & $\underline{1.0}$ \\
\hline $10 \underline{1188.39}$ & 119.23 & 295.30 & 14.73 & 82.2034 & $\underline{1.0}$ \\
\hline
\end{tabular}




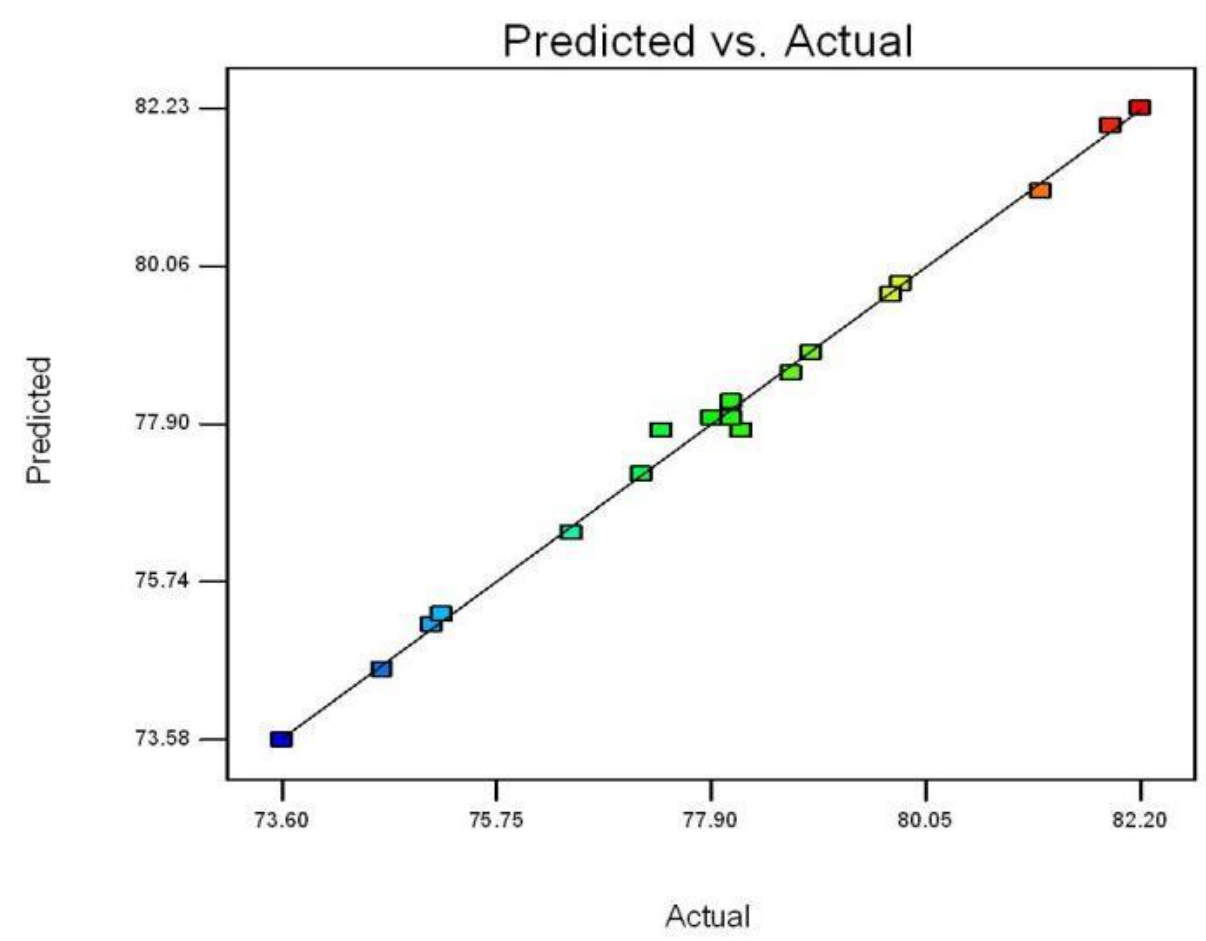

Figure -A1. Predicted versus actual recoveries in 4-parameter fractional factorial design tests.

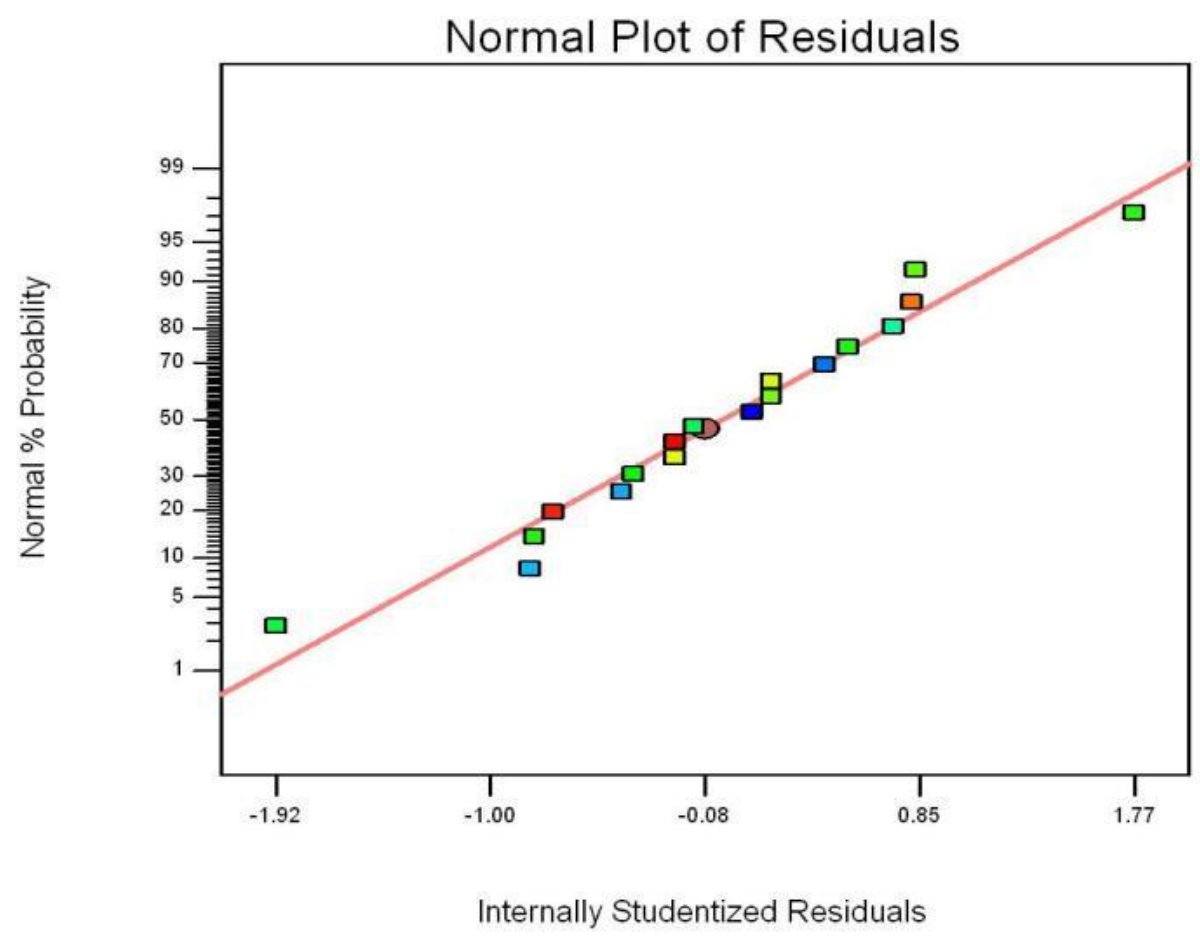

Figure - A2. Normal plot of residuals of the experimental data fitted to a second order model 
Appendix 5: Development of New Reagents for the Flotation of Dolomite from Phosphate Ore (NV002) 


\section{TECHNICAL PROGRESS REPORT}

Contract Title and Number

Establishment of the Center for Advanced Separation

Technologies (DE-FC26-02NT41607)

Sub-Recipient Project Title:

Developments of new Reagents for Flotation

of Dolomite from Phosphate Ores

Principal Investigators

M. C. Fuerstenau

M. Misra

T. W. Bell

Contact Address:

Phone: (775) 784-4310

Chemical and Metallurgical Engineering

University of Nevada, Reno 89557

Subcontractor Address:

University of Nevada, Reno

Reno, NV 89557
Period of Performance

Starting Date: 6/1/2004

Ending Date: 10/31/2008

Report Information:

Type: Final

Number

Date: 3-04-09

Code: NV002-FINAL

Contact Information

Fax: (775) 327-5059

E-mail: mcf@unr.edu

Subcontractor Information:

Phone: (775) 784-4312

Fax: (775) 784-6680

E: stewart@unr.edu 


\section{DISCLAIMER}

"This report was prepared as an account of work sponsored by an agency of the United States Government. Neither the United States Government nor any agency thereof, nor any of their employees, make any warranty, express or implied, nor assume any legal liability or responsibility for the accuracy, completeness, or usefulness of any information, apparatus, product, or process disclosed, or represents that its use would not infringe privately owned rights. Reference herein to any specific commercial product, process, or service by trade name, trademark, manufacturer, or otherwise does not necessarily constitute or imply endorsement, recommendation, or favoring by the United States Government or any agency thereof. The views and opinions of authors expressed herein do not necessarily state or reflect those of the United States Government or agency thereof.” 


\section{ABSTRACT}

The United States is the second largest producer of phosphate rock in the world, which corresponds to $30 \%$ of the world production. Florida accounts for $80 \%$ of the U.S. phosphate production. During the past century, the Florida phosphate industry has produced high quality phosphate with low $\mathrm{MgO}(<0.5 \%)$ content. As low dolomitic phosphate reserves become exhausted, the remaining deposits contain lower amounts of phosphate with significantly higher dolomite (MgO) content. It is generally difficult to obtain a phosphate concentrate from such materials containing less than the desired $\mathrm{MgO}$ content of 1\% MgO. The objective of the research work was to evaluate the effectiveness of new synthesized collectors and some commercially-available collectors for the selective flotation of dolomite from phosphate rock.

The experimentation included evaluating the applicability of synthesized and some commercially-available collectors utilizing adsorption and electrokinetic experiments, microflotation of minerals comprising phosphate ore, and benchtop flotation of dolomitic Florida phosphate rock. 


\section{TABLE OF CONTENTS}

$\underline{\text { Page }}$

Disclaimer $\quad$ i

Abstract ii

Table of Contents iii

List of Figures iv

List of Tables vi

Executive Summary $\quad 1$

Introduction 3

Experimental $\quad 5$

Materials $\quad 5$

Procedures

$\begin{array}{ll}\text { Microflotation Studies } & 7\end{array}$

$\begin{array}{lr}\text { Benchtop Flotation Studies } & 8\end{array}$

Zeta Potential Determinations $\quad 8$

Fourier Transform Infrared Spectroscopy $\quad 8$

Results and Discussion $\quad 8$

Microflotation Studies $\quad 8$

Oleic Acid $\quad 8$

Octadecyl Malonic Acid $\quad 12$

Octadecyl Malonic Acid Ester $\quad 13$

Betaine Analogs $\quad 14$

Hexcadecyl Citrate $\quad 15$

$\begin{array}{lr}\text { Citric Acid Ester } & 17\end{array}$

Sarcosine Analogs $\quad 17$

Electrokinetic Studies $\quad 19$

Fourier Transform Infrared Spectroscopy 21

$\begin{array}{ll}\text { Benchtop Flotation } & 24\end{array}$

$\begin{array}{ll}\text { Conclusions } & 30\end{array}$

References $\quad 31$

Acknowledgment 32 
Figure 1. Crystallographic model of dolomite.................................. 4

Figure 2. Hallimond tube apparatus.......................................... 9

Figure 3. Flotation recovery of apatite and dolomite as a function of oleic acid concentration

Figure 4. Flotation recovery of apatite and dolomite as a function of $\mathrm{pH}$ with $1 \times 10^{-4} \mathrm{M}$ oleic acid

Figure 5. Zeta potential of apatite in distilled water with $5 \times 10^{-3} \mathrm{M}$

Figure 6. Zeta potential of dolomite in distilled water with $5 \times 10^{-3} \mathrm{M}$

Figure 7. The speciation diagram of palmitic acid at various calcium concentrations....

Figure 8. Flotation recovery of dolomite with $1 \times 10^{-4} \mathrm{M}$ octadecyl malonic acid and its methyl ester.

Figure 9. Flotation recovery of apatite and dolomite as a function of $\mathrm{pH}$ with $1 \times 10^{-4} \mathrm{M}$ octadecyl malonic ester concentration....

Figure 10. Flotation recovery of apatite and dolomite as a function of betaine variant concentration.

Figure 11. Flotation recovery of apatite and dolomite as a function of hexadecylcitrate concentration.....

Figure 12. Flotation recovery of apatite and dolomite as a function of $\mathrm{pH}$ with $1 \times 10^{-4} \mathrm{M}$ hexadecylcitrate

Figure 13. Flotation recovery of dolomite with three analogs of sarcosine at $\mathrm{pH}$ 9.5-10.8

Figure 14. Flotation recovery of apatite, dolomite and collophane as a function of myristoyl sarcosine concentration ( $\mathrm{pH}$ 9.4-9.9).

Figure 15. Flotation recovery of apatite and dolomite as a function of $\mathrm{pH}$ with $1 \times 10^{-4} \mathrm{M}$ myristoyl sarcosine as collector. 
Figure 16. Flotation recovery of collophane as a function of $\mathrm{pH}$ with $2 \times 10^{-4} \mathrm{M}$ myristoyl sarcosine as collector.

Figure 17. Zeta potential of collophane as a function of $\mathrm{pH}$ in the absence and presence of $1 \times 10^{-4} \mathrm{M}$ myristoyl sarcosine...

Figure 18. Zeta potential of apatite and dolomite as a function of $\mathrm{pH}$ in the presence of $1 \times 10^{-4} \mathrm{M}$ myristoyl sarcosine

Figure 19. Zeta potential of myristoyl sarcosine precipitate with $2 \times 10^{-3} \mathrm{M} \mathrm{KNO}_{3}$

Figure 20. FTIR spectra of myristoyl sarcosine, calcium precipitate of myristoyl sarcosine, collophane, collophane conditioned with myristoyl sarcosine at $\mathrm{pH} 9$ and $\mathrm{pH} 5$

Figure 21. FTIR spectra of myristoyl sarcosine, calcium precipitate of myristoyl sarcosine, dolomite, and dolomite conditioned with myristoyl sarcosine at $\mathrm{pH} 9$ and $\mathrm{pH} 5$....

Figure 22. FTIR spectra of myristoyl sarcosine, magnesium precipitate of myristoyl sarcosine, dolomite, and dolomite conditioned with myristoyl sarcosine at $\mathrm{pH} 9$ and $\mathrm{pH} 10.9$

Figure 23. MgO removal and content of nonfloat product with oleate as collector as a function of flotation time

Figure 24. MgO removal and content of nonfloat product with lauroyl sarcosine as collector as a function of flotation time 


\section{LIST OF TABLES}

Table 1. Flotation conditions and experimental results obtained with sodium oleate as collector

Table 2. Flotation conditions and experimental results obtained with a phosphorous based collector ..........................................................

Table 3. Flotation conditions and experimental results obtained with a mixture of fatty acids as collector.

Table 4. Flotation conditions and experimental results obtained with lauroyl sarcosine as collector

Table 5. Flotation conditions and experimental results obtained with myristoyl sarcosine as collector.

Table 6. Flotation conditions and experimental results obtained with various additions of oleate as collector

Table 7. Flotation conditions and experimental results obtained with sodium oleate and lauroyl sarcosine as collectors 


\section{EXECUTIVE SUMMARY}

Phosphate rock is the source of phosphate-based chemical fertilizer. The United States is a major producer of this raw material, and most of it is mined and processed in Florida. The principal phosphate producing region is in the state's central and north districts, and the ore underlies some 2,600 square miles of land. Until recently, phosphate production has been confined to the central north district. These deposits are being depleted, however, and future mining will shift to the southern extension of the central district. The southern reserves contain less phosphate minerals and have a serious $\mathrm{MgO}$ contamination, i.e., they contain significant quantities of dolomitic carbonates. The processing of these reserves will require special beneficiation techniques to produce concentrates containing less than the practical limit of about $1 \% \mathrm{MgO}$.

Over the past several years, five flotation processes have been developed to separate dolomite from phosphate or phosphate from dolomite (El Shall 1994). They are the TVA (Tennessee Valley Authority) phosphonic acid process, the UF (University of Florida) two-stage conditioning process, the University of Alabama process, the USBM (United States Bureau of Mines) process, and the IMC (International Minerals and Chemical Corporation) cationic process. For a variety of reasons, none of these processes has been commercialized. The objective of this research was to synthesize new collectors and investigate some commerciallyavailable collectors for possible use in selective flotation of dolomite from phosphate rock prior to processing ore for phosphate recovery.

In the experimental work, flotation response of single minerals was established with a Hallimond flotation cell. One-gram charges of closely sized-mineral were tested; the minerals were apatite, collophane and dolomite. Collector type and concentration, and $\mathrm{pH}$ were the principal variables studied. Some of the collectors were tested on a natural dolomitic phosphate ore. The collectors used in this study were oleate, malonate, betaine, sarcosine, alkyl citrate, and a mixture of fatty acids.

The electrical properties of the mineral surfaces were studied with electrokinetic measurements. The points-of-zero-charge of the minerals and the conditions under which their surfaces are positively or negatively charged were established. The flotation results indicate that different mechanisms are involved in collector adsorption depending on the system $\mathrm{pH}$. In acid medium (under which condition the mineral surface is positively charged), the neutral molecule and precipitate of the collector in the acid form appear to be responsible for flotation. On the basic side, collector anion and a precipitate of the calcium salt of the collector appear responsible for flotation.

Fourier Transform Infrared Analysis (FTIR) analysis was also used to study mechanisms of collector adsorption. These measurements confirmed that the species of collector principally responsible for flotation in basic medium is the bulk precipitate of the calcium salt of the collector. These hydrophobic species probably adsorb on previously adsorbed collector anion or neutral molecule. 
Economics play a key role in the chemical fertilizer industry. The sale price of a concentrate of phosphate rock sells for about $\$ 75 /$ ton. Very inexpensive collectors must be used in concentrating phosphate rock. In this view betaines and their derivatives were examined because their principal source is sugar beet residue, an inexpensive commodity. Unfortunately, reagents developed from this stock did not function effectively as collectors.

Some reagents, synthesized in the laboratory, were shown to float single minerals effectively. In analyzing the cost of the stock and the manufacturing process, in some cases it was deemed that the costs of their use would be excessive. As a result some of the collectors, although effective in flotation in single mineral systems, were not studied on natural ore.

Of the various collectors studied with natural ore, only oleate was shown to yield a concentrate containing less than $1.0 \mathrm{wt} \% \mathrm{MgO}$. Other investigators have also obtained a flotation product from phosphate ore containing less than $1.0 \% \mathrm{MgO}$ using oleate as collector. Using a two stage process (flotation at $\mathrm{pH} 10$ followed by flotation at $\mathrm{pH}$ 3), Moudgil and Ince (1987) obtained a product that contained 0.9\% MgO. Direct flotation at pH 2.7, though, yielded a product that contained $1.5 \% \mathrm{MgO}$. 


\section{INTRODUCTION}

Until 2006, the United States was the largest producer of phosphate rock in the world. About $30 \%$ of the total world production in 1990 was produced in the United States, and Florida accounts for approximately 80\% of U.S. production (Bartels and Gurr 1994; Harbin 1980). During the past 100 years, the Florida phosphate industry has produced high quality products having a MgO content of less than $0.5 \%$. Phosphate reserves and resources in Florida have the potential to continue production at a rate of about 40 - 55 million metric tons/year for hundreds of years (Sandvik 1979).

The Florida phosphate rock deposits are located in the state's central and northern and pebble districts (Moudgil and Ince 1991). The central land pebble district constitutes the major phosphate rock producing region and underlies 2,600 square miles in Polk, Hillborough, Hardee, Manatee, and DeSoto Counties. Until recently, phosphate production has been confined to the Bone Valley of the central district. These deposits, however, are being depleted, and future mining will shift to the southern extension of the central district. The southern reserves contain less phosphate minerals and have a serious $\mathrm{MgO}$ contamination, i.e., they contain significant quantities of dolomitic carbonates (Lawver 1982). The processing of these reserves will require special beneficiation techniques to produce concentrates containing less than the practical limit of about $1 \% \mathrm{MgO}$.

Over the past several years, five flotation processes have been developed to separate dolomite from phosphate or phosphate from dolomite (El Shall 1994). They are the TVA (Tennessee Valley Authority) phosphonic acid process, the UF (University of Florida) two-stage conditioning process, the University of Alabama process, the USBM (United States Bureau of Mines) process, and the IMC (International Minerals and Chemical Corporation) cationic process.

The TVA process involves thorough desliming of the feed (48 x 400 mesh), conditioning with diphosphonic acid at high pulp density, followed by conditioning with oleic acid/pine oil. With this technique phosphate concentrate containing less than $1.0 \%$ MgO cannot be obtained.

The University of Florida technique uses two stages of flotation for dolomite separation from phosphate. The first stage is conducted with deslimed 35 x 150 mesh feed using tall oil and diesel fuel at $\mathrm{pH}$ 10. The rougher concentrate is reconditioned at $\mathrm{pH}$ below 5.5 to float dolomite from collophane. Reproducibility of results appears to be a problem with this technique.

The University of Alabama process is a two-stage process involving conditioning $35 \times 150$ mesh deslimed feed at low solids pulp density with fatty acid as collector at $\mathrm{pH}$ 5.5-7.0. The flotation tailing is further conditioned with sodium silicate and more fatty acid, and phosphate is floated from silica at $\mathrm{pH}$ 6.0-7.0. This process fails to float dolomite in the initial flotation step and phosphate in the second step.

The U.S.B.M. process involves 28 x 150 mesh feed which is vigorously attrition-scrubbed to

reduce the softer dolomite to slimes which are deslimed at 150 mesh. The scrubbed solids are 
conditioned at high pulp density in the presence of oleic acid and fuel oil at $\mathrm{pH}$ 9. This mixture is subjected to rougher/cleaner flotation to separate the phosphate from the quartz and dolomite. High levels of reagent are needed, and, further, MgO contents below $1.0 \%$ cannot be achieved.

The IMC process involves floating deslimed feed (28 x 150 mesh) with amine at low pulp density to remove quartz. The flotation tailing is dewatered to $65-70 \%$ solids, conditioned with amine and floated for phosphate recovery. The rougher tailing in this operation contains the dolomite. Reagent consumption is relatively high, and overall phosphate recovery is low. However, acceptable levels of MgO can be achieved.

None of these processes has been commercialized for a variety of reasons. In order to further exploit Florida dolomitic phosphate pebble resources and to improve phosphate beneficiation, the development of effective collectors for dolomite appears to be essential.

The objective of this investigation was to synthesize collectors that have specificity for magnesium, and, hence, dolomite. Flotation characteristics of apatite, dolomite and phosphate rock were determined with those collectors to establish whether physical separation of dolomite from phosphate rock could be obtained.

A crystallographic model of dolomite, $\mathrm{CaCO}_{3} \cdot \mathrm{MgCO}_{3}$, is shown in Figure 1 . This model was established utilizing Crystal Maker Crystallographic Software (England). The data are from Reeder and Markgraf (1986). The standard free energy of formation of $\mathrm{MgCO}_{3}$ is $-1012.1 \mathrm{~kJ} / \mathrm{mol}$ and that of $\mathrm{CaCO}_{3}$ is $-1129.1 \mathrm{~kJ} / \mathrm{mol}$ (Lide 2003), suggesting that the bond strength of $\mathrm{Ca}-\mathrm{O}$ will, then, be greater than that of $\mathrm{Mg}-\mathrm{O}$, since the primary bonds are mainly between magnesium and oxygen and calcium and oxygen. In addition, the iron that is frequently substituted for magnesium in dolomite may be in the (002) plane where magnesium resides which may further increase the propensity to fracture along the preferred cleavage plane (002) during comminution.

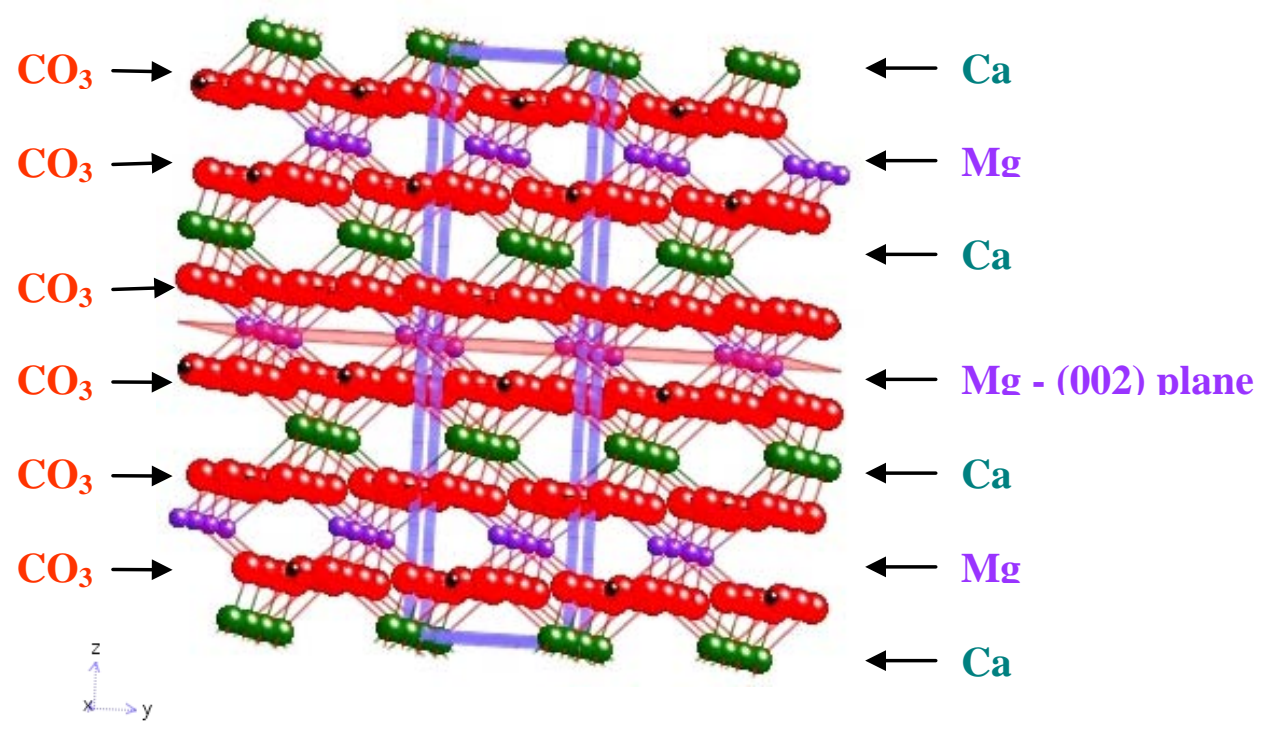

Figure 1. Crystallographic model of dolomite. 
The initial design of collectors for dolomite was, therefore, focused on interaction with $\mathrm{Mg}^{2+}$ It can be noted that each $\mathrm{Mg}^{2+}$ is bound to four $\mathrm{O}^{2-}$. Upon fracture, two magnesium-oxygen bonds will not be satisfied which will lead to bidentate formation upon collector adsorption.

$\mathrm{Ca}^{2}$ ion will be exposed along planes of non-preferential cleavage. Calcium ion is also bonded with four oxide ions. Any fracture between calcium ion and oxide ions will also expose two unsatisfied bonds, and bidentate formation with collector will also occur under these conditions.

Following synthesis of these collectors, preliminary testing of the collectors was conducted in a micro-flotation system to establish if their basic adsorption characteristics would result in selective flotation. Once promising flotation characteristics were seen in a collector, zeta potential determinations were made to clarify the mechanisms of adsorption of the collector as a function of $\mathrm{pH}$. Fourier Transform Infra-Red spectroscopy was also conducted to refine the understanding of the adsorption characteristics under specific conditions. For additional insight, scanning electron microscopy was employed to observe the basic surface characteristics of the dolomite and collophane samples used in the experiments. The final test of the collector potential for industrial application was conducted using benchtop flotation.

\section{EXPERIMENTAL}

\section{$\underline{\text { Materials }}$}

Mineral samples of apatite $(\mathrm{CaF}) \mathrm{Ca}_{4}\left(\mathrm{PO}_{4}\right)_{3}$, dolomite $\left(\mathrm{CaCO}_{3} \cdot \mathrm{MgCO}_{3}\right)$, and collophane (cryptocrystalline apatite) were obtained from Ward's Scientific. Phosphate rock ore was obtained from Florida.

\section{Collectors}

The collectors synthesized were malonate, sarcosine, betaine, and alkyl citrate. Oleate, lauroyl sarcosine, a mixture of fatty acids, and a phosphorous-bearing reagent were obtained from suppliers. Collector synthesis was done in Professor Thomas Bell's laboratory in the Department of Chemistry, University of Nevada, Reno. Structural formulas of these reagents are as follows:

Oleate

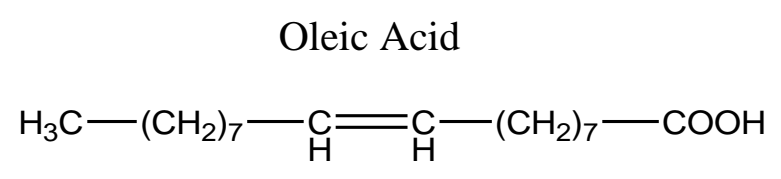


Malonate

Octadecyl Malonic Acid

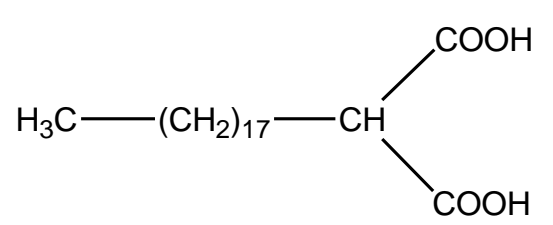

Potassium Salt

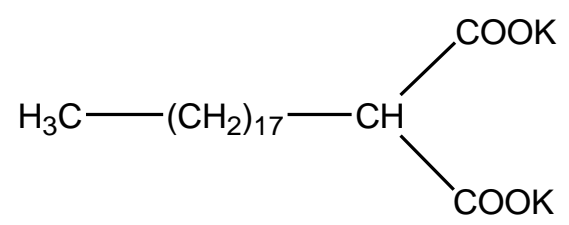

Octadecyl Malonic Acid Ester<smiles>CCCCCC(C(=O)O)C(=O)OC</smiles>

Citrate

Hexadecyl Citrate

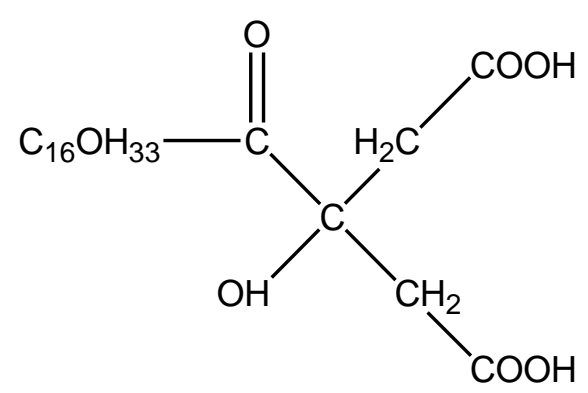

Decyl Betaine<smiles>CC(C)([14CH3])CCCCCCC(=O)O</smiles>

Citric Acid Ester<smiles>CCCCCCCCC(O)(CC(=O)O)C(=O)O</smiles>

Tetradecyl Betaine<smiles>CCCCCCCCCC(=O)O</smiles> 


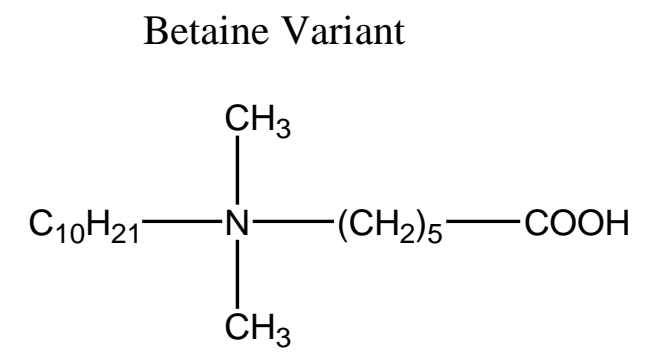

Sarcosine
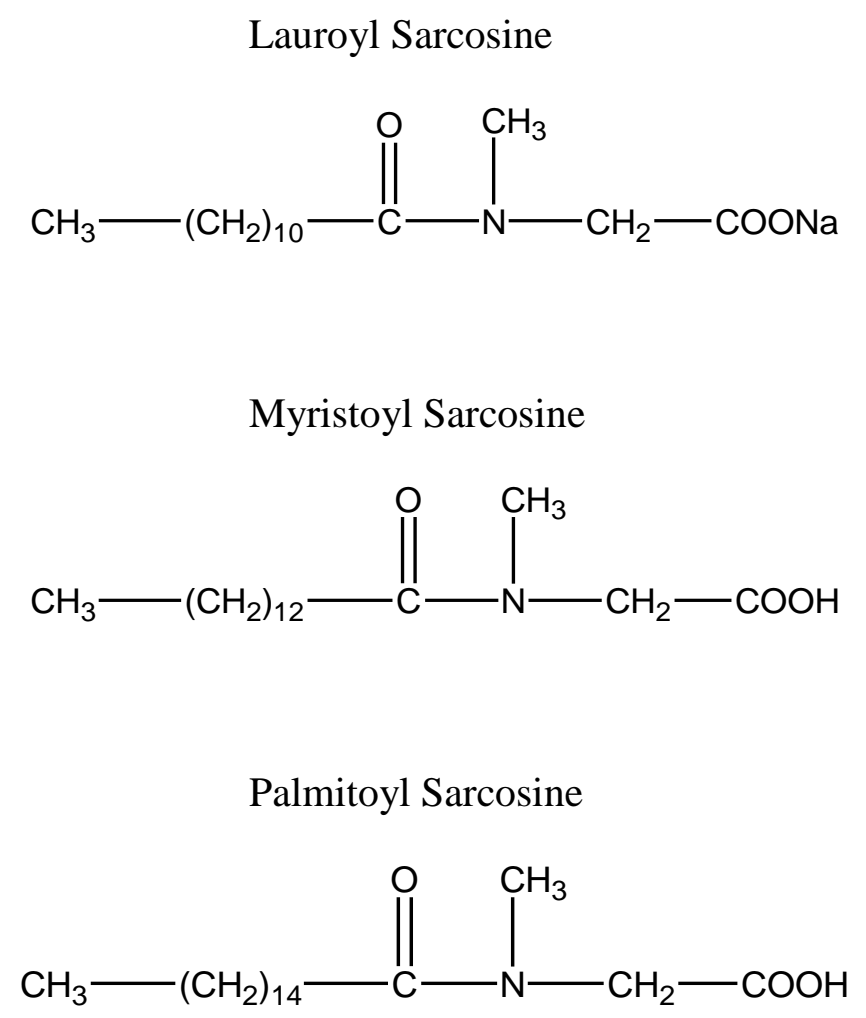

$\underline{\text { Flotation Studies }}$

Micro-flotation

The effect of various collectors on the flotation response of apatite, dolomite, and collophane was established with a Hallimond tube. A schematic of this micro-flotation cell is shown in Figure 2. Pure mineral samples were ground and sized to $104 \mu \mathrm{m} \times 147 \mu \mathrm{m}$. They were conditioned in the presence of known collector concentration and $\mathrm{pH}$ and, then, transferred to the 
Hallimond tube to determine the flotation response. Under continuous stirring with a magnetic stir bar, nitrogen gas from a cylinder was bubbled through the cell to facilitate flotation. Particles attached to the bubbles of nitrogen were carried to the surface of the water and collected in a separate chamber (concentrate tube) without the use of a frother, allowing for the study of only the collector effect. Concentrate and tailings were collected, dried and weighed.

\section{Benchtop Flotation}

To examine the effect of collectors on the flotation of a Florida phosphate ore, a benchtop Denver flotation cell (500-gram) as well as a 100-gram laboratory cell, constructed in our laboratory, were employed. The ore was ground wet in a ball mill and sized to $147 \mathrm{x} 417 \mu \mathrm{m}$. This sized material was then ground to $60 \%-74 \mathrm{~nm}$ for flotation testing. The pulp was conditioned at $30 \mathrm{wt} \%$ solids for 3 minutes in a bottle roller. The conditioned slurry was transferred to the benchtop cell and diluted with 1 liter of tap water for flotation. Frother was added to the slurry, and flotation was conducted for 3 minutes. Flotation products were collected, dried, weighed and sampled. MgO content was then determined analytically.

\section{Zeta Potential Determinations}

The electrical characteristics of the mineral surfaces were established using a zeta meter. A Pen Kem Lazer Zee Meter Model 501 with a fused silica cell was used to determine the zeta potential of the samples at various $\mathrm{pH}$ values in the absence and presence of collector.

\section{Fouier Transform Infrared Spectroscopy (FTIR)}

A Bio-Rad FTS 6000 spectrometer was used to analyze the surface of the minerals prior to and after collector adsorption to establish mechanisms of collector adsorption. Digilab Win-IR Pro software analyzed the data and generated the IR spectra. Spectra of the mineral surfaces were generated in the absence and presence of collector. Mineral samples were hand-ground and screened at $37 \mu \mathrm{m}$. A portion of the minus $37 \mu \mathrm{m}$ sample was combined with a designated concentration of collector at specified $\mathrm{pH}$, and conditioned for $30 \mathrm{~min}$. A final $\mathrm{pH}$ was measured; solids were separated from liquid by centrifugation; the separated mineral was rinsed with deionized water and acetone, and, then, mixed with potassium bromide for measurement.

\section{RESULTS AND DISCUSSION}

\section{Microflotation Studies}

\section{Oleic Acid}

Oleic acid, saponified with sodium hydroxide, was used as a basis to determine the respective effectiveness of the synthesized collectors. Flotation recoveries of both apatite and dolomite were established as a function of oleate concentration and $\mathrm{pH}$ utilizing a Hallimond cell. As can be noted in Figure 3, similar curves of recovery vs. concentration are noted for both minerals, although lower collector concentration was needed for apatite flotation. The $\mathrm{pH}$ profile 
reveals essentially complete flotation of apatite from about pH 5 to 11 (Figure 4). The point-of zero-charge (pzc) of apatite was determined to be $\mathrm{pH} 5.0$ and that of dolomite was determined to

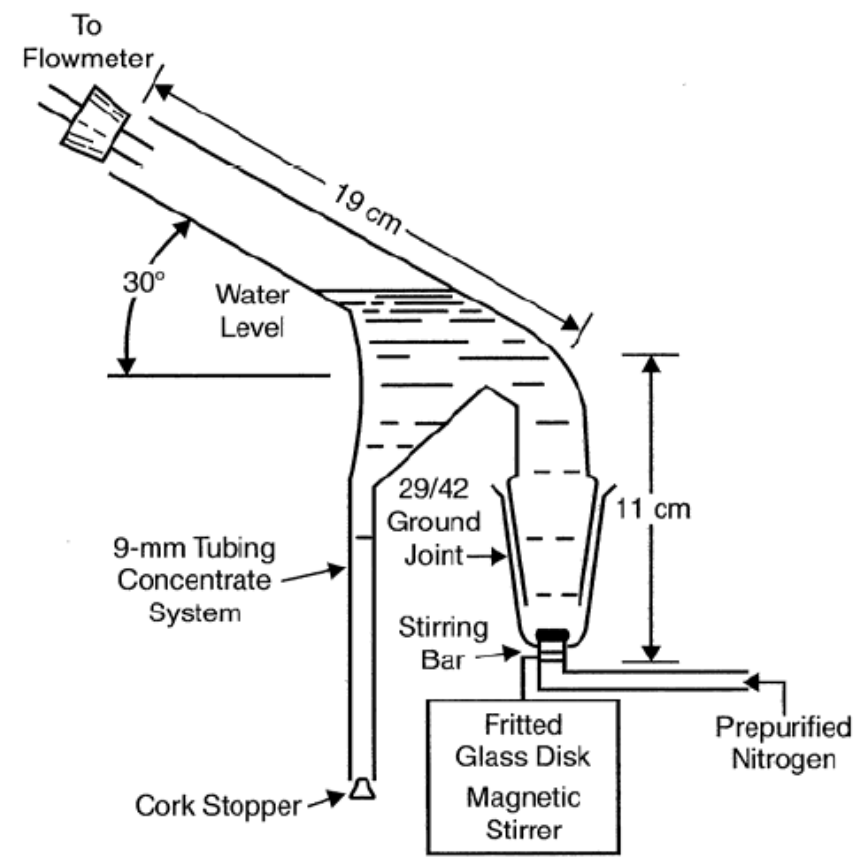

Figure 2. Hallimond tube apparatus (Fuerstenau and Somasundaran 2003).

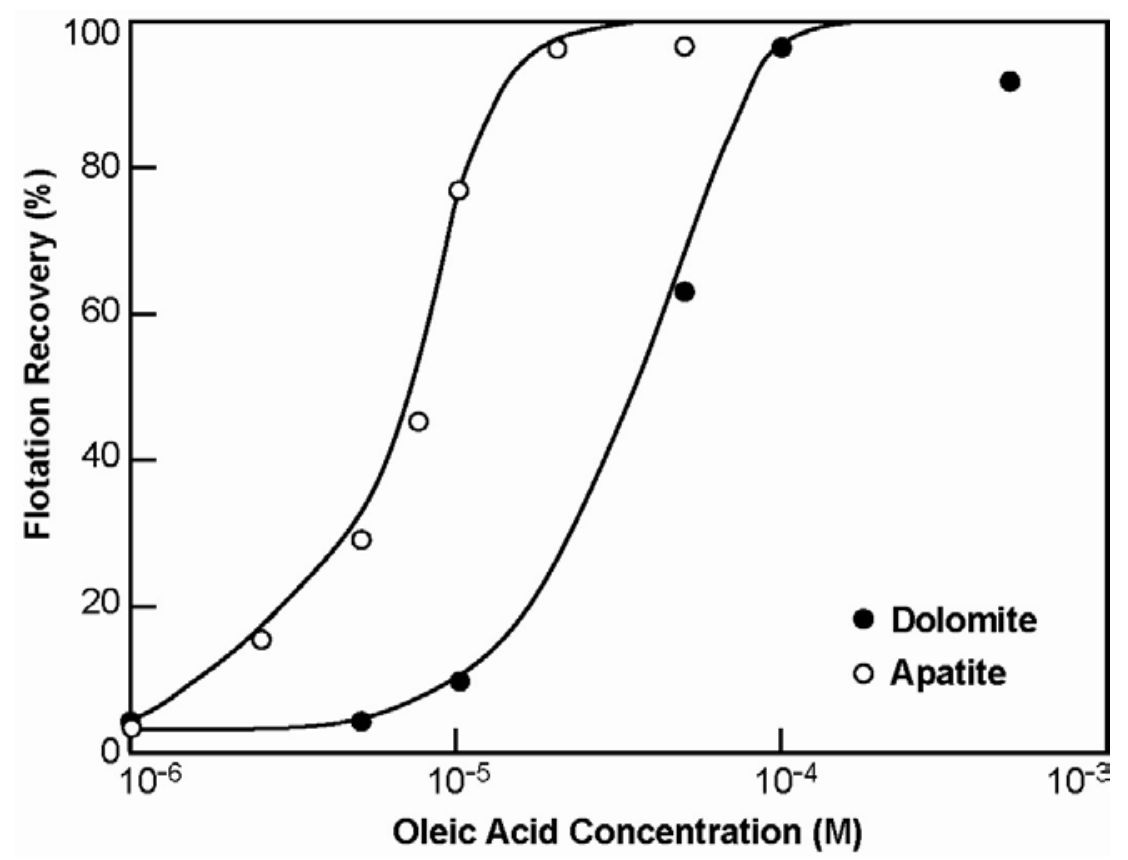

Figure 3. Flotation recovery of apatite and dolomite as a function of oleic acid concentration. 


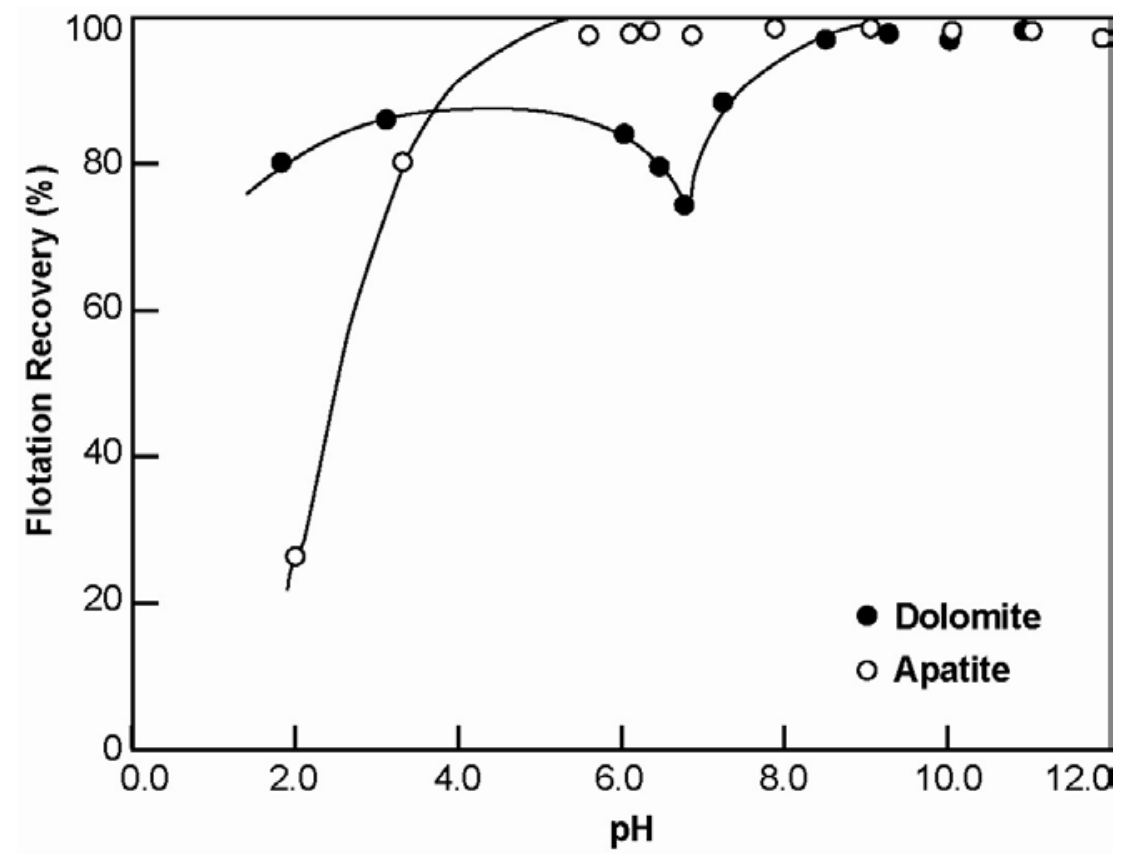

Figure 4. Flotation recovery of apatite and dolomite as a function of $\mathrm{pH}$ with $1 \mathrm{x} 10^{-4} \mathrm{M}$ oleic acid.

be pH 8.0 (Figures 5 and 6). The strength of the metal ion-oleate bonds formed at the surface of these minerals is demonstrated by the strong flotation response much beyond their pzc, under which conditions the surfaces are negatively charged. The flotation of apatite has been shown to be due to the formation and adsorption of precipitated calcium oleate on previously adsorbed oleate ions (Moudgil et al.1987).

In the case of dolomite, a narrow region of flotation depression is noted around $\mathrm{pH}$ 6. Two regions of good flotation separated by a narrow region of depression suggest that different mechanisms of collector adsorption are probably occurring above and below that $\mathrm{pH}$. Flotation in basic medium is the same as with apatite, namely adsorption of calcium oleate on chemisorbed oleate ions. This will be demonstrated in a later section of the report. 


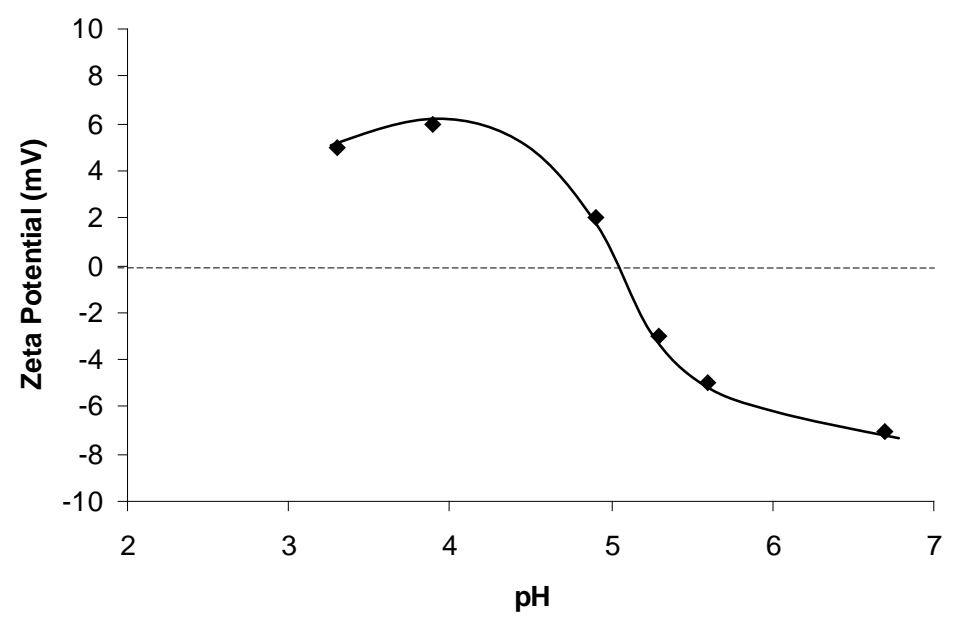

Figure 5: Zeta potential of apatite in distilled water in the presence of $5 \times 10^{-3} \mathrm{M} \mathrm{KNO}_{3}$.

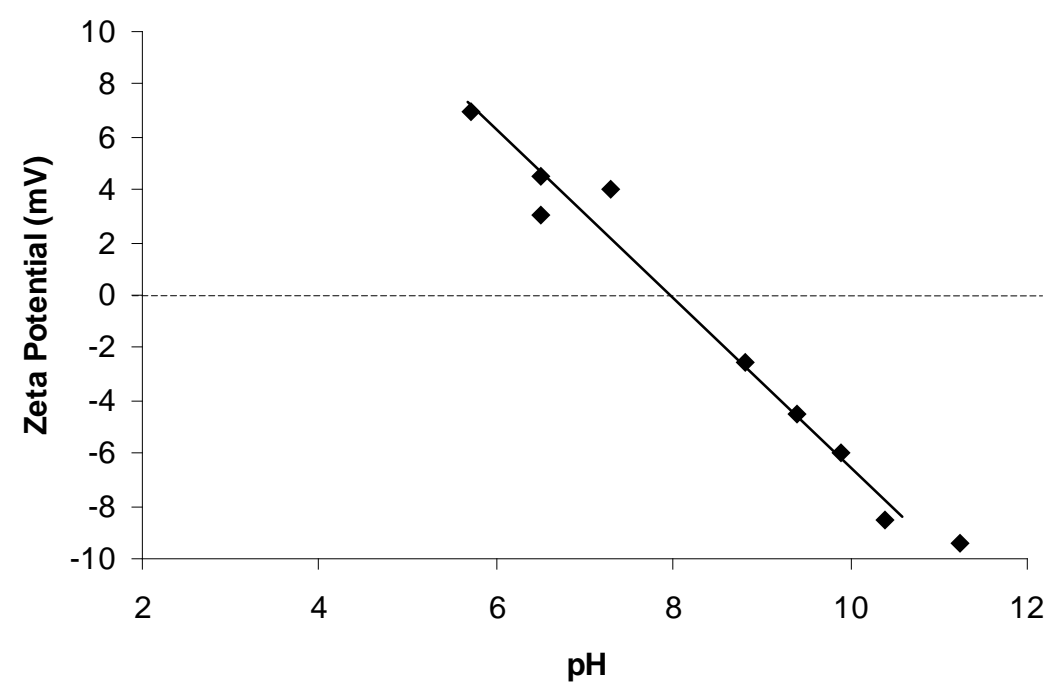

Figure 6. Zeta potential of dolomite in distilled water in the presence of $5 \times 10^{-3} \mathrm{M} \mathrm{KNO}_{3}$.

The speciation of palmitic acid, a long-chained fatty acid $\left(\mathrm{C}_{15} \mathrm{H}_{31} \mathrm{COOH}\right)$, in the presence of various additions of $\mathrm{Ca}^{2+}$ is given in Figure 7. As the calcium addition is increased, the stability region of $\mathrm{HPa}$, precipitated palmitic acid, is reduced while that of calcium palmitate is increased. It can be noted that the predominant species is precipitated palmitic acid (HPa) below about $\mathrm{pH}$ 6. Flotation response in the dolomite/oleic acid system indicates that two mechanisms of collector adsorption may be occurring below and above about $\mathrm{pH}$ 7. Colloidal precipitate of oleic acid could be adsorbing on previously adsorbed oleate ions by hydrophobic bonding, or alternatively, negatively-charged oleic acid precipitate could be adsorbing on the positivelycharged dolomite surface. 


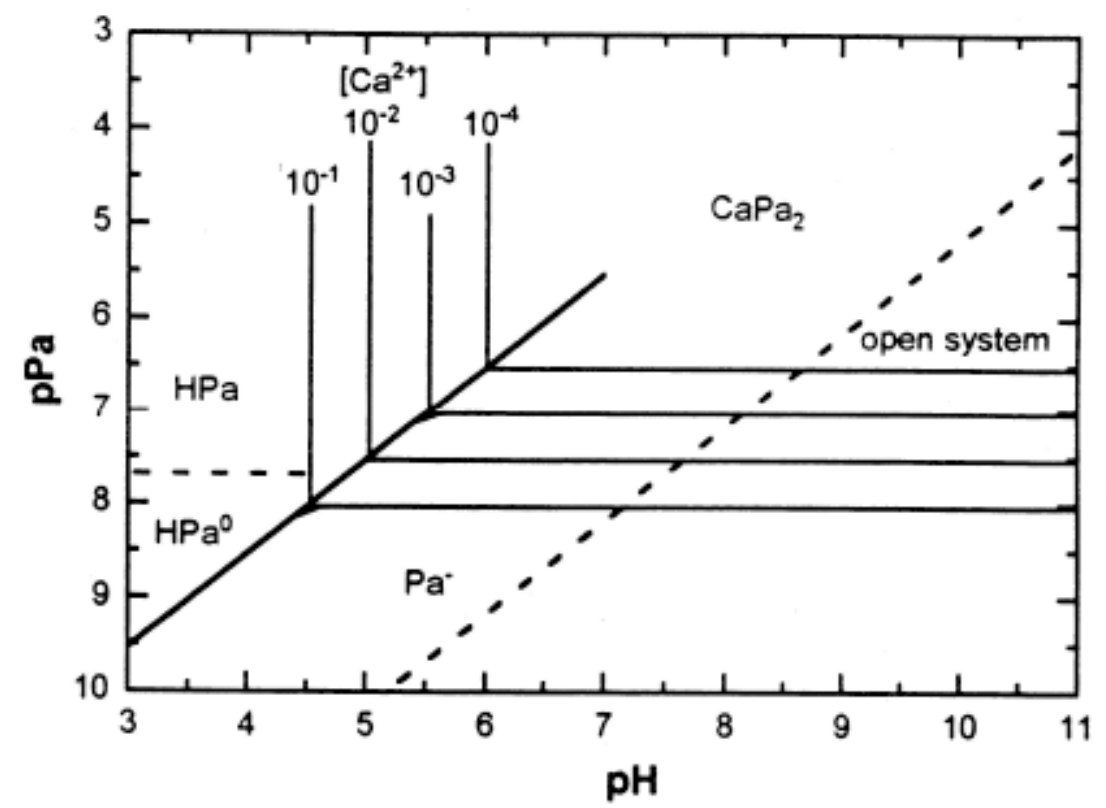

Figure 7. The speciation diagram of palmitic acid at various calcium concentrations. (Bunge and Fuerstenau 2006).

\section{Octadecyl Malonic Acid}

The first alternative collector selected for flotation was octadecyl malonic acid. The flotation response of dolomite as a function of collector concentration is shown in Figure 8. The concentration curve was generated with the potassium salt of the malonic acid to enhance dissolution. Maximal and limited recovery ( 30\%) of dolomite was achieved with $5 \times 10^{-4} \mathrm{M}$ malonic acid. Testing the effect of $\mathrm{pH}$ showed no appreciable flotation between $\mathrm{pH} 3$ and $\mathrm{pH} 11$ with $1 \times 10^{-4} \mathrm{M}$ concentration. Due to the very limited response of dolomite to flotation with this collector, tests were not conducted with apatite. 


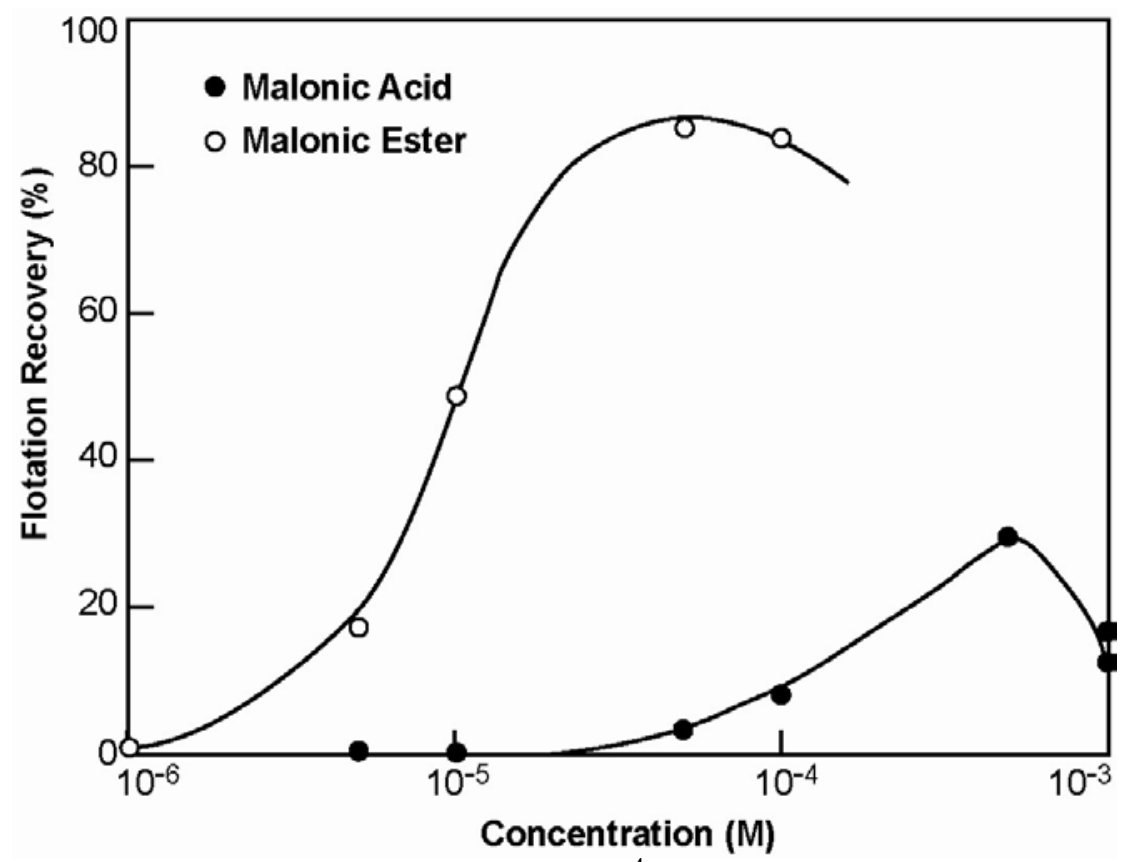

Figure 8. Flotation recovery of dolomite with $1 \times 10^{-4} \mathrm{M}$ octadecyl malonic acid and its methyl ester.

Octadecyl malonic acid exhibits very low solubility in water, and further, the molecule contains two carboxyl groups which could conceivably hinder chemisorption of the collector. In this view a methyl ester of this reagent was prepared and tested.

\section{Octadecyl Malonic Acid Ester}

The flotation characteristics of dolomite as a function of octadecyl malonic acid ester concentration are also shown in Figure 8, and it can be noted that a recovery of about $90 \%$ was achieved with $5 \times 10^{-5} \mathrm{M}$.

Flotation recovery of apatite and dolomite as a function of $\mathrm{pH}$ with the same collector concentration is given in Figure 9. Two maxima in recovery can be noted in both systems but at different $\mathrm{pH}$ values in acid medium. The differences in recovery over the whole $\mathrm{pH}$ spectrum would not enable their selective separation. 


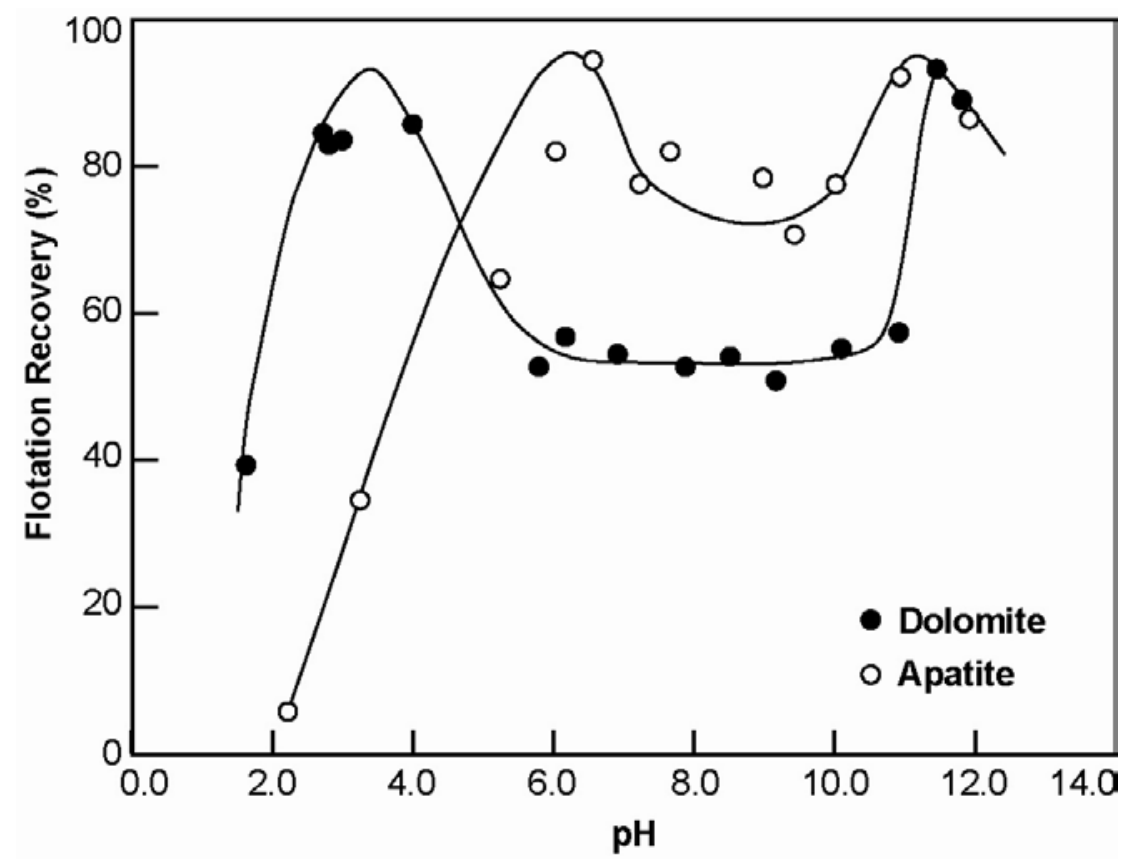

Figure 9. Flotation recovery of apatite and dolomite as a function of $\mathrm{pH}$ with $1 \times 10^{-4} \mathrm{M}$ octadecyl malonic ester concentration.

\section{Betaine Analogs}

Betaine is found in the juice of sugar beets, and the residue from the manufacture of beet sugar is an abundant source. In view of the relatively inexpensive cost of this material, various betaine analogs were synthesized and tested. Three variations of betaine were considered for their potential as selective collectors for apatite and dolomite flotation. These were decyl and tetradecyl betaine and a variant of tetradecyl betaine in which five hydrocarbon groups were placed between the carboxyl group and the nitrogen atom and 10 hydrocarbon groups were placed after the nitrogen atom. With decyl and tetradecyl betaines, maximal recovery of only about 20 percent was obtained at a concentration of $1 \times 10^{-3} \mathrm{M}$ at about $\mathrm{pH} 9$. Very limited flotation response (10-20\%) was obtained with even the high concentration of $1 \times 10^{-3} \mathrm{M}$ of either collector. The response was somewhat higher (40-50\%) at $1 \times 10^{-3} \mathrm{M}$ with the tetradecyl betaine variant (Figure 10). The betaines are not candidates as collectors in this system. 


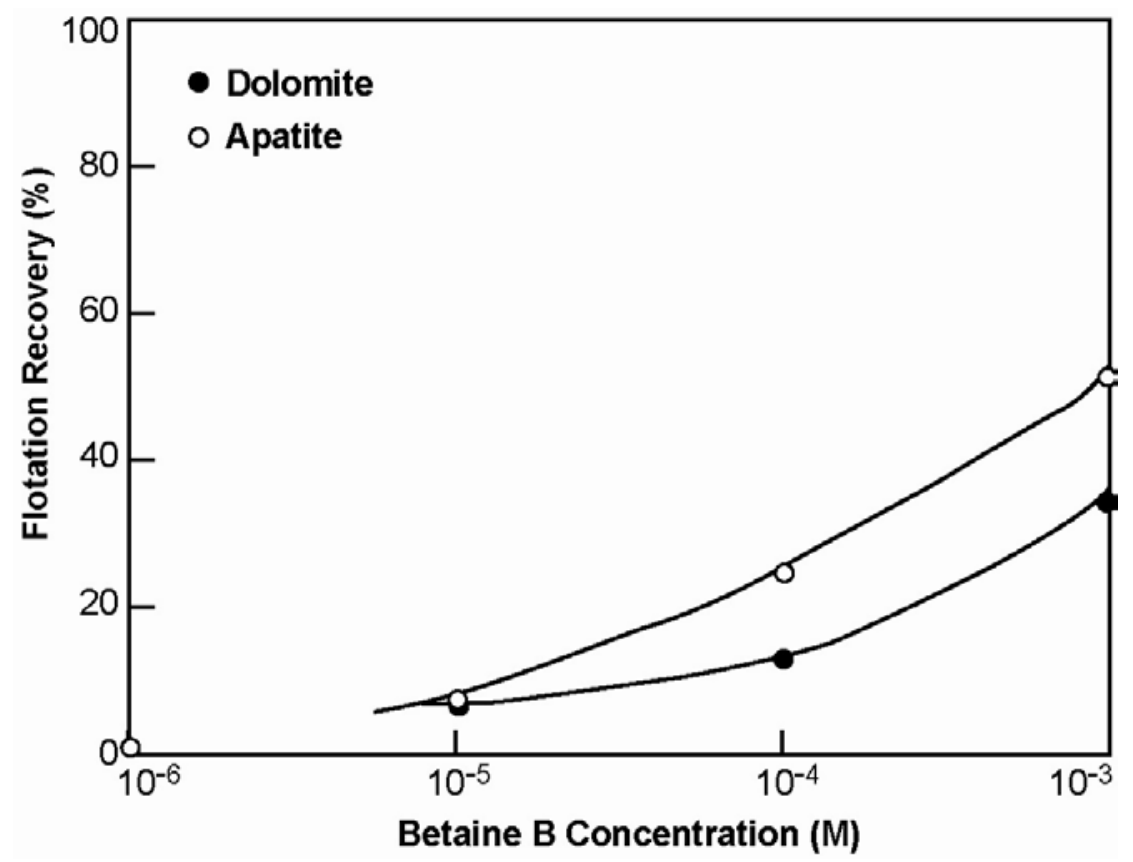

Figure 10. Flotation recovery of apatite and dolomite as a function of betaine variant concentration.

\section{Hexadecyl Citrate}

The next collector examined was hexadecyl citrate. As before, apatite and dolomite were tested with this collector. Figure 11 shows the dependence of recovery in separate systems of apatite and dolomite on collector concentration. It can be noted that apatite responds to flotation at lower concentrations of hexadecyl citrate. The maximum recovery achieved with dolomite was around $80 \%$ at $1 \times 10^{-4} \mathrm{M}$. Increasing the collector concentration above this amount decreased the recovery. This is most likely due to hexadecyl citrate ions adsorbing in reverse fashion (constituting a complete micelle) at the mineral-liquid interface, thereby reducing the hydrophobicity of the surface. 


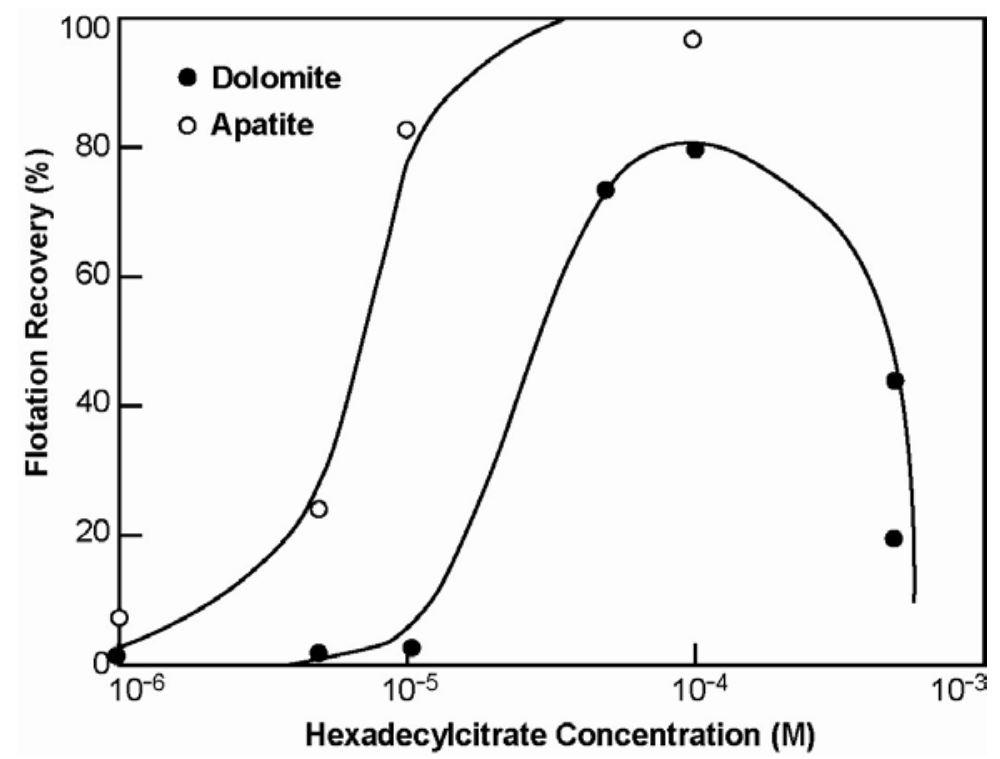

Figure 11. Flotation recovery of apatite and dolomite as a function of hexadecyl citrate concentration..

Flotation recovery as a function of $\mathrm{pH}$ is given in Figure 12. Two maxima are noted for both systems. The dips in recovery, though, are noted to occur at $\sim \mathrm{pH} 5.5$ in the case of dolomite and $\sim \mathrm{pH} 7.5$ for apatite. These data, again, indicate that different mechanisms of collector adsorption occur in these systems, depending on the $\mathrm{pH}$. To the left of the dips in recovery at lower values of $\mathrm{pH}$, electrostatic adsorption of collector ions is probably occurring. Chemisorption of collector ions with possible co-adsorption of precipitated calcium hexadecylsulfate must be occurring to the right of the dips in recovery.

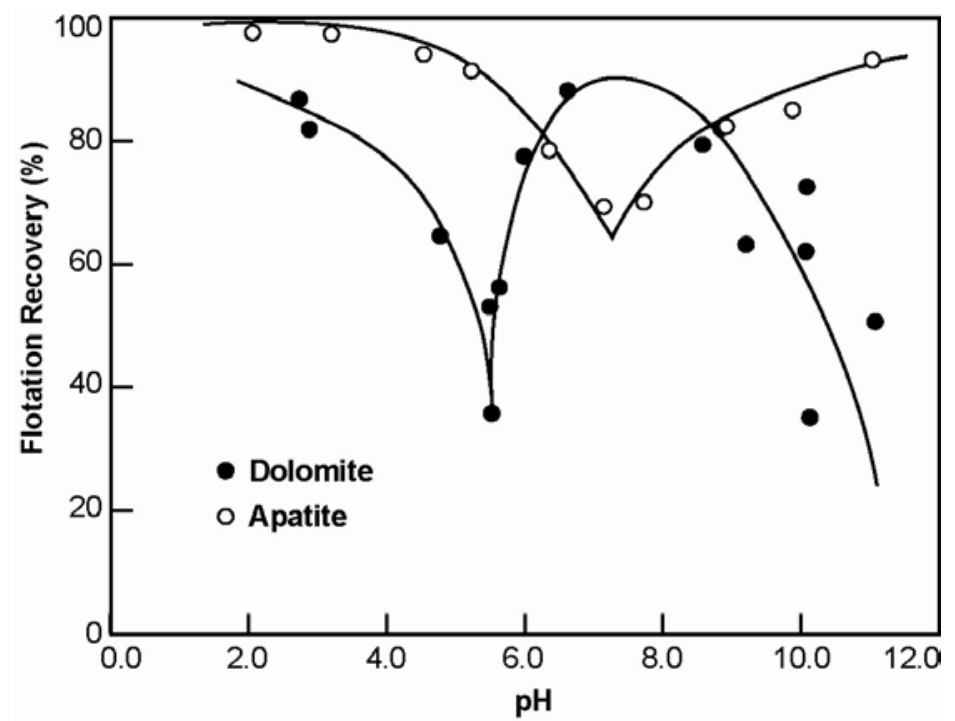

Figure 12. Flotation recovery of apatite and dolomite as a function of $\mathrm{pH}$ with $1 \times 10^{-4} \mathrm{M}$ hexadecyl citrate. 


\section{Citric Acid Ester}

An ester of citric acid was synthesized and tested for selective separation of apatite and dolomite. Solubility of this reagent was limited; and concentrations greater than $1 \times 10^{-4} \mathrm{M}$ could not be used. Flotation recoveries were impacted as a result, especially for dolomite (Kruth 2007).

\section{Sarcosine Analogs}

Sarcosine was the last molecule synthesized as an alternative collector for dolomite. The potential use of this type of collector was examined by Moudgil and Vasudevan (1988) on an ore. They used n-substituted sarcosine and reported favorable phosphate recovery and dolomite rejection. Tthe flotation characteristics of apatite, dolomite and collophane with this molecule were examined in some detail in this study. Three analogs of sarcosine were examined, namely lauroyl, myristoyl, and palmitoyl.

Lauroyl sarcosine was obtained as a sodium salt from a commercial distributor. The lower hydrocarbon chain length of the lauroyl molecule (12 carbon atoms) allowed for easy dissolution for experimentation. Myristoyl (14 carbon atoms) and palmitoyl (16 carbon atoms) sarcosines were synthesized for testing. Dissolution of palmitoyl sarcosine above about $1 \times 10^{-4}$ $\mathrm{M}$ was difficult to achieve. Flotation of dolomite with these three analogs is shown in Figure 13. When the length of the hydrocarbon chain is increased, the hydrophobicity of the mineral surface is increased after collector adsorption. As a result lower concentration of collector is needed with longer chained collectors. This was observed in these systems.

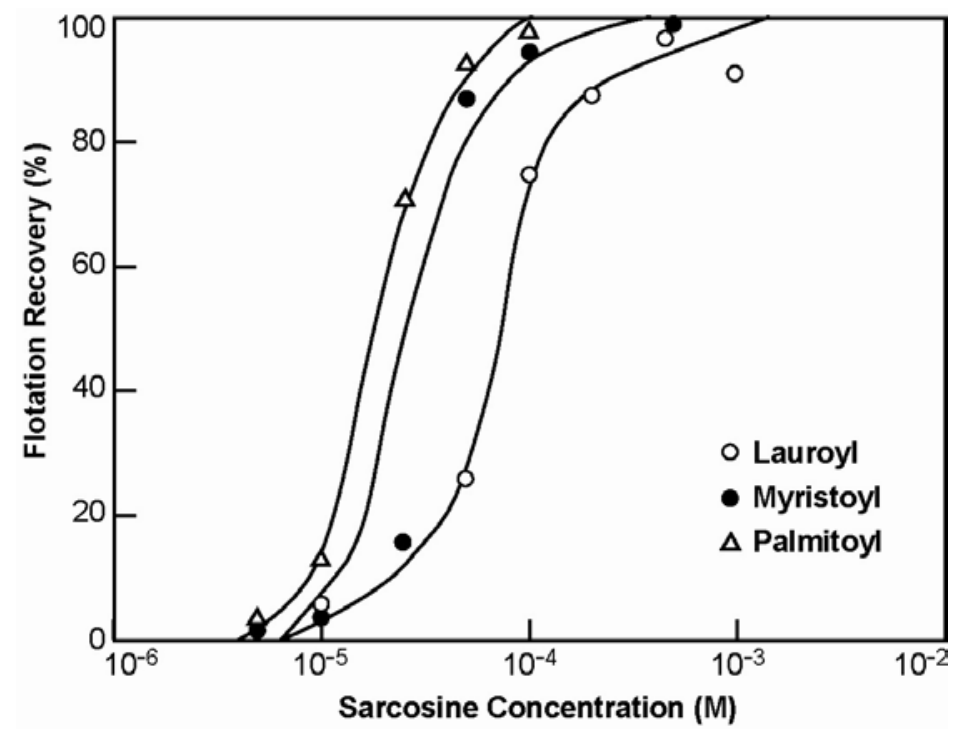

Figure 13. Flotation recovery of dolomite three analogs of sarcosine at pH 9.5-10.8.

The flotation characteristics of the three minerals obtained with myristoyl sarcosine as collector are shown in Figure 14. In these experiments $\mathrm{pH}$ values of pH 9.4 to 9.9 were involved. 


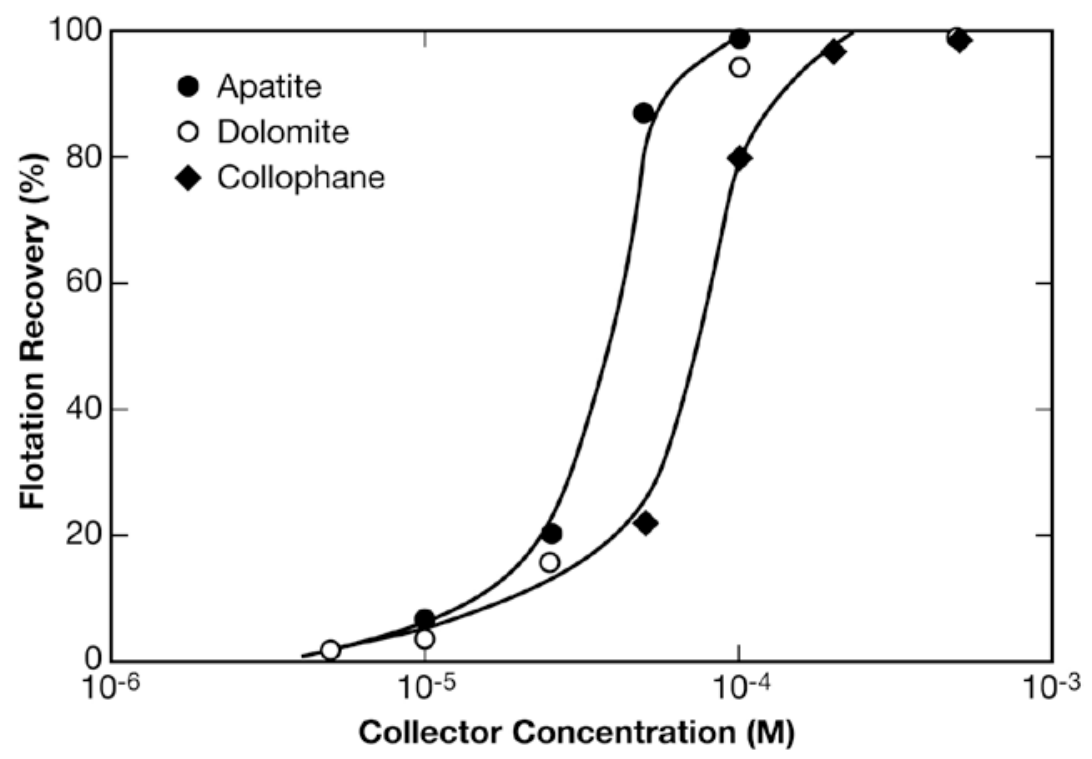

Figure 14. Flotation recovery of apatite, dolomite and collophane as a function of myristoyl sarcosine concentration (pH $9.4-9.9$ ).

The characteristics are similar, but it is noted that of the three, apatite responds at somewhat lower concentration.

The effect of $\mathrm{pH}$ was also examined. As shown in Figures 15 and 16, apatite is floated completely from about $\mathrm{pH} 3$ to 11. A region of flotation depression is noted around $\mathrm{pH}$ 6-7 for both dolomite and collophane and is even greater in extent for collophane.

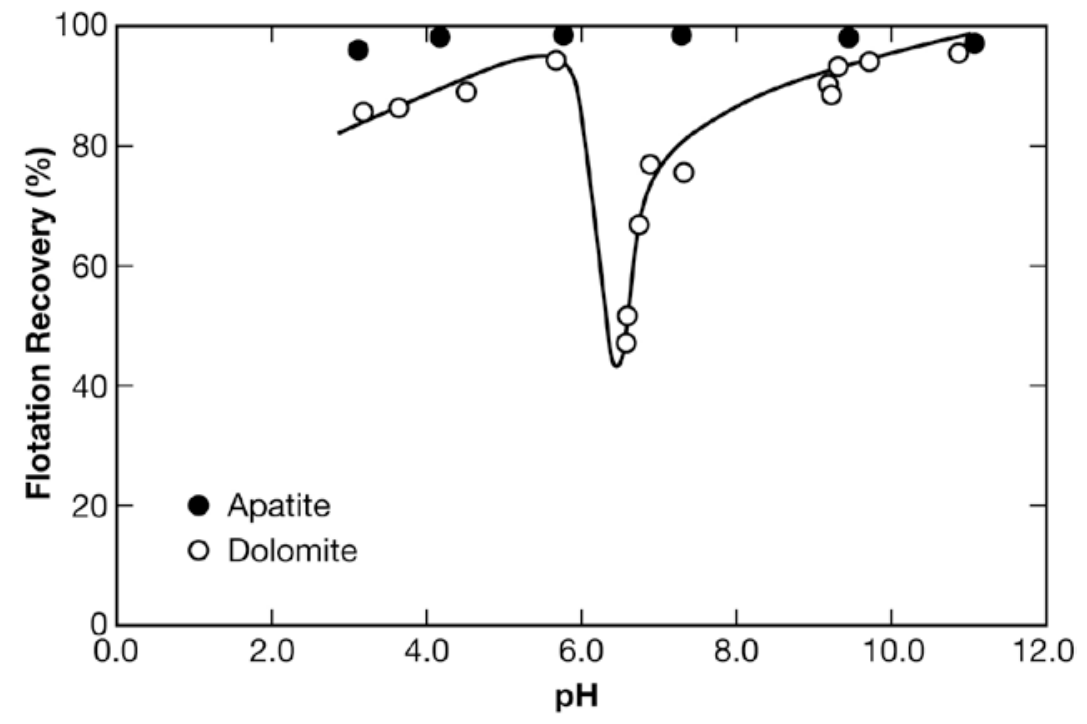

Figure 15. Flotation recovery of apatite and dolomite as a function of $\mathrm{pH}$ with $1 \times 10^{-4} \mathrm{M}$ myristoyl sarcosine as collector. 


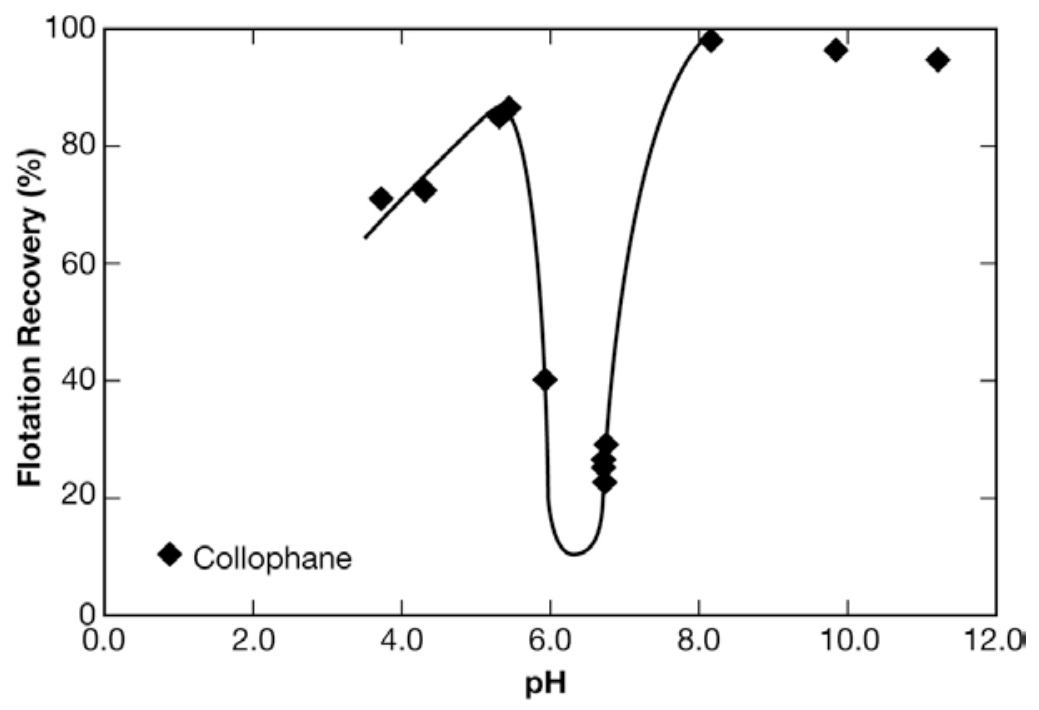

Figure 16. Flotation recovery of collophane as a function of $\mathrm{pH}$ with $2 \times 10^{-4} \mathrm{M}$ myristoyl sarcosine as collector.

\section{Electrokinetic Studies}

To understand the mechanisms involved in collector adsorption on these minerals, electrokinetic and Fourier infrared analysis studies were conducted on the minerals in the presence of myristoyl sarcosine. Electrokinetic tests were conducted on apatite and dolomite in the absence of collector, and the results are found in Figures 5 and 6. The constant addition of 5 $\mathrm{x} 10^{-3} \mathrm{M} \mathrm{KNO}_{3}$ was made to maintain constant ionic strength of the solution. The point-of-zerocharge (pzc) of apatite was measured at $\mathrm{pH}$ 5.0. Other values reported for apatite are $\mathrm{pH} 6.4$ (Fuerstenau et al. 1968) and pH 6.9 (Hanna and Somasundaran 1969). The differences in pzcs can be attributed to differences in impurity contents of the samples.

The pzc of dolomite was measured at $\mathrm{pH}$ 8.0. This agrees with other studies (Pokrovsky et al. 1999). The zeta potential of collophane in water is shown in Figure 17. The zeta potential is negative from $\mathrm{pH} 3-10$. The pzc of francolite has been reported between $\mathrm{pH} 3.8-4.9$ (Hanna and Somasundaran 1976). It was anticipated that a pzc for collophane would be measured in this range, since it is cryptocrystalline francolite. Again, impurity content is probably responsible for this different behavior. 


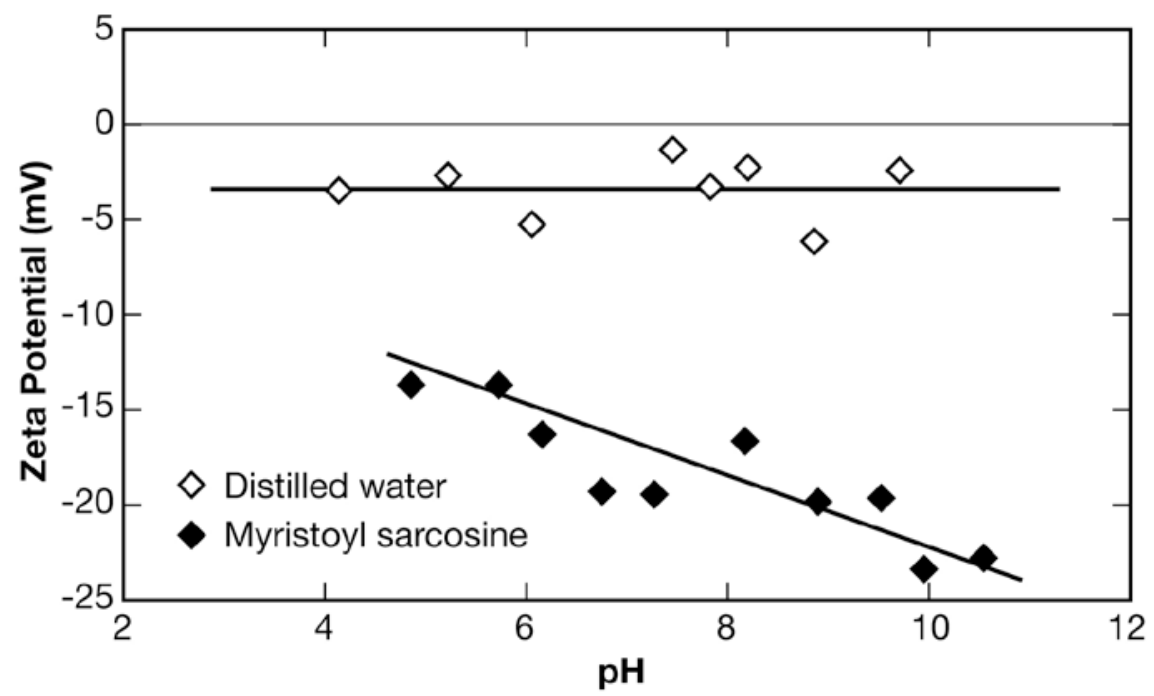

Figure 17. Zeta potential of collophane as a function of $\mathrm{pH}$ in the absence and presence of 1 × $10^{-4} \mathrm{M}$ myristoyl sarcosine

Zeta potentials of apatite, dolomite and collophane in the presence of myristoyl sarcosine are given in Figures 17 and 18. In the case of apatite in the presence of $1 \times 10^{-4} \mathrm{M}$ myristoyl, zeta potential values hovered around $-25 \mathrm{mV}$ between $\mathrm{pH} 4$ and 9.5. At around $\mathrm{pH} 11$, the zeta potential became less negative but did not reverse in electrical sign. This has been shown in other systems to be due to calcium activation from dissolved calcium ion hydrolysis/ adsorption (Fuerstenau and Cummins 1968). With dolomite, zeta potential became increasingly less negative with increased $\mathrm{pH}$ until about $\mathrm{pH} 10$, at which point the zeta potential became very significantly more negative.

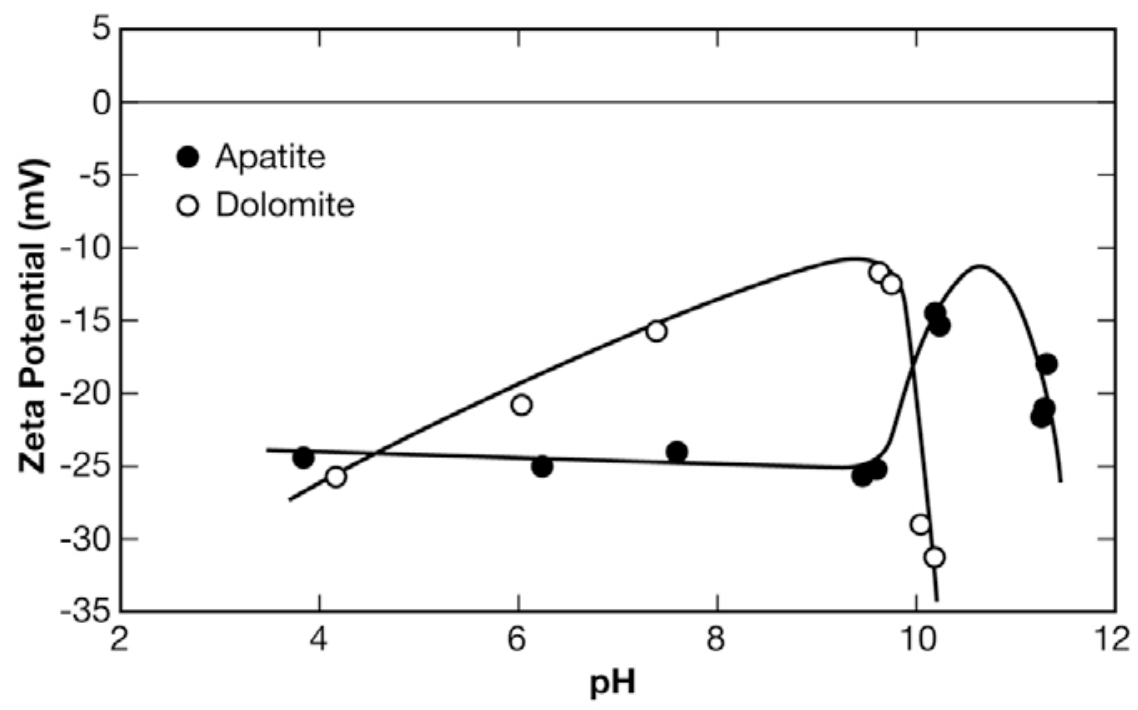

Figure 18. Zeta potential of apatite and dolomite as a function of $\mathrm{pH}$ in the presence of $1 \times 10^{-4} \mathrm{M}$ myristoyl sarcosine. 
Figure 17 shows the zeta potential curve for collophane in the presence of $1 \times 10^{-4} \mathrm{M}$ myristoyl sarcosine. The zeta potential was negative over the $\mathrm{pH}$ range tested, and increased in negative value as the $\mathrm{pH}$ was increased.

The curve of zeta potential of myristoyl sarcosinic acid is shown Figure 19. Myristoyl sarcosinic acid was observed to precipitate below about $\mathrm{pH}$ 7. As can be noted, the surface charge of this precipitate was negative over the $\mathrm{pH}$ range tested but decreased in value with decreasing $\mathrm{pH}$.

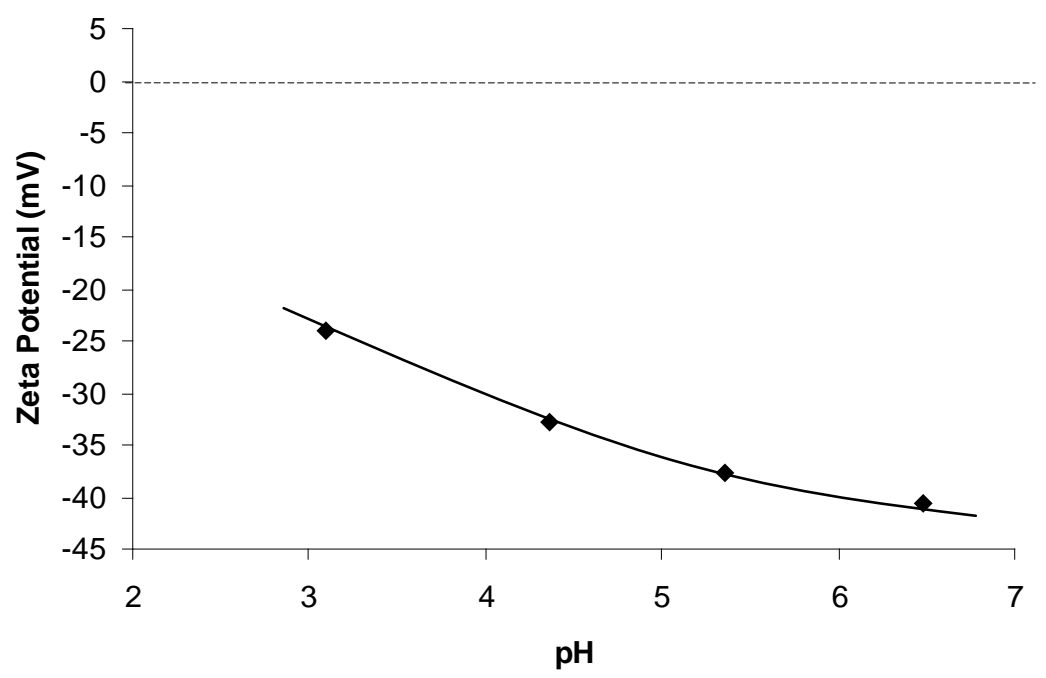

Figure19: Zeta potential of myristoyl sarcosine precipitate with $2 \times 10^{-3} \mathrm{M} \mathrm{KNO}_{3}$.

\section{Fourier Transform Infra-Red Spectroscopy (FTIR)}

To aid in the understanding of the adsorption characteristics of the collector on the mineral surface, FTIR analysis was conducted on dolomite and collophane. The spectra of dolomite, myristoyl sarcosine, calcium and magnesium myristoyl sarcosine, and dolomite after contact with myristoyl sarcosine at $\mathrm{pH} 5$ and $\mathrm{pH} 9$ are shown in Figures 20 and 21. Characteristic peaks of the myristoyl sarcosine occur at 2920 and $2851\left(\mathrm{~cm}^{-1}\right)$ and are visible in the myristoyl sarcosine, the calcium precipitate, and after adsorption on dolomite at $\mathrm{pH} 9$. These peaks are not visible on the dolomite sample at $\mathrm{pH}$ 5. A doublet is visible in the spectrum of the calcium precipitate at 1578 and $1541\left(\mathrm{~cm}^{-1}\right)$. A doublet is also visible on the $\mathrm{pH} 9$ and $\mathrm{pH} 10.9$ samples of dolomite with peaks also at 1578 and $1579\left(\mathrm{~cm}^{-1}\right)$. The peaks for the other doublet in the magnesium precipitate, at 1636 and $1564\left(\mathrm{~cm}^{-1}\right)$, are not visible in any of the dolomite spectra.

The spectra for collophane are shown in Figure 22. The doublet of the calcium precipitate is not visible in the $\mathrm{pH} 5$ spectrum, but is visible in the $\mathrm{pH} 9$ spectrum at the same location, 1580 and $1545\left(\mathrm{~cm}^{-1}\right)$. 


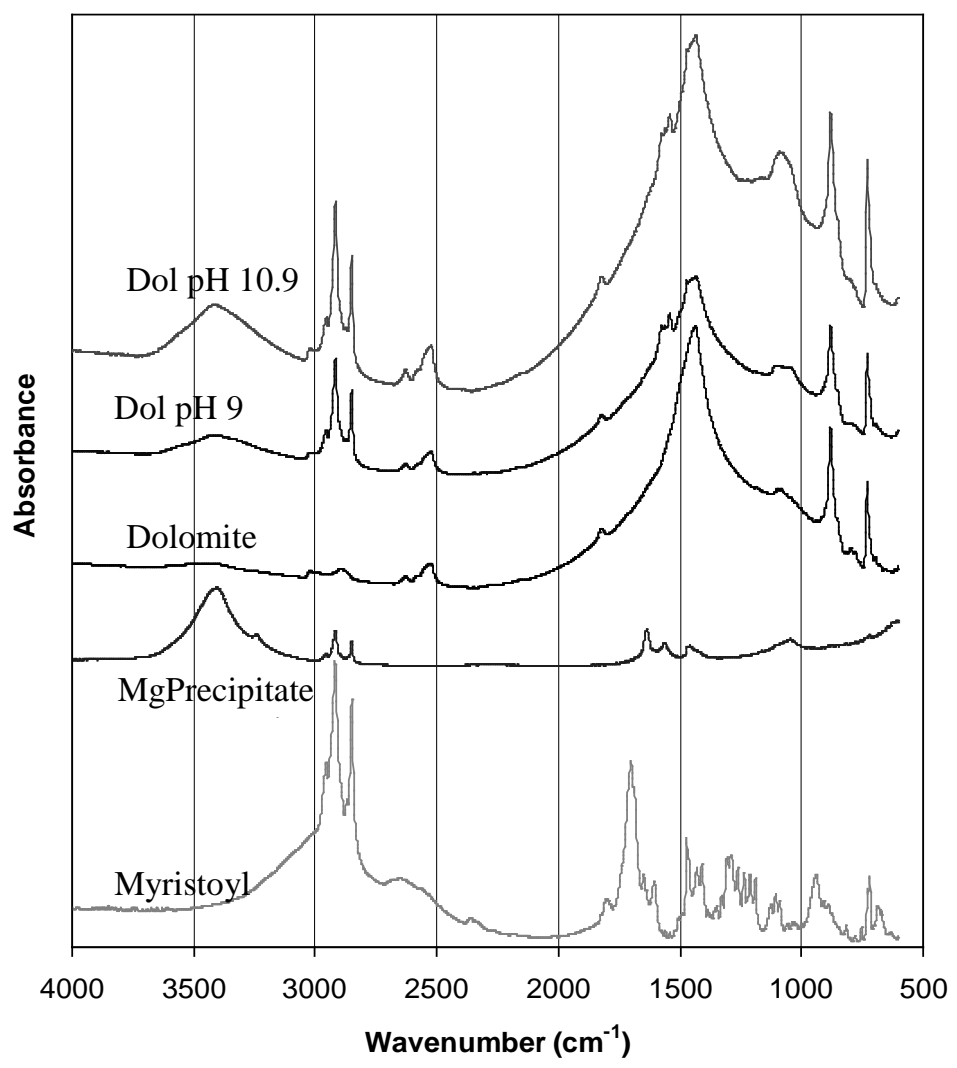

Figure 20: FTIR spectra of myristoyl sarcosine, magnesium precipitate of myristoyl sarcosine, dolomite, dolomite conditioned with myristoyl sarcosine at $\mathrm{pH}$ 9, and dolomite conditioned with myristoyl sarcosine at $\mathrm{pH}$ 10.9. 


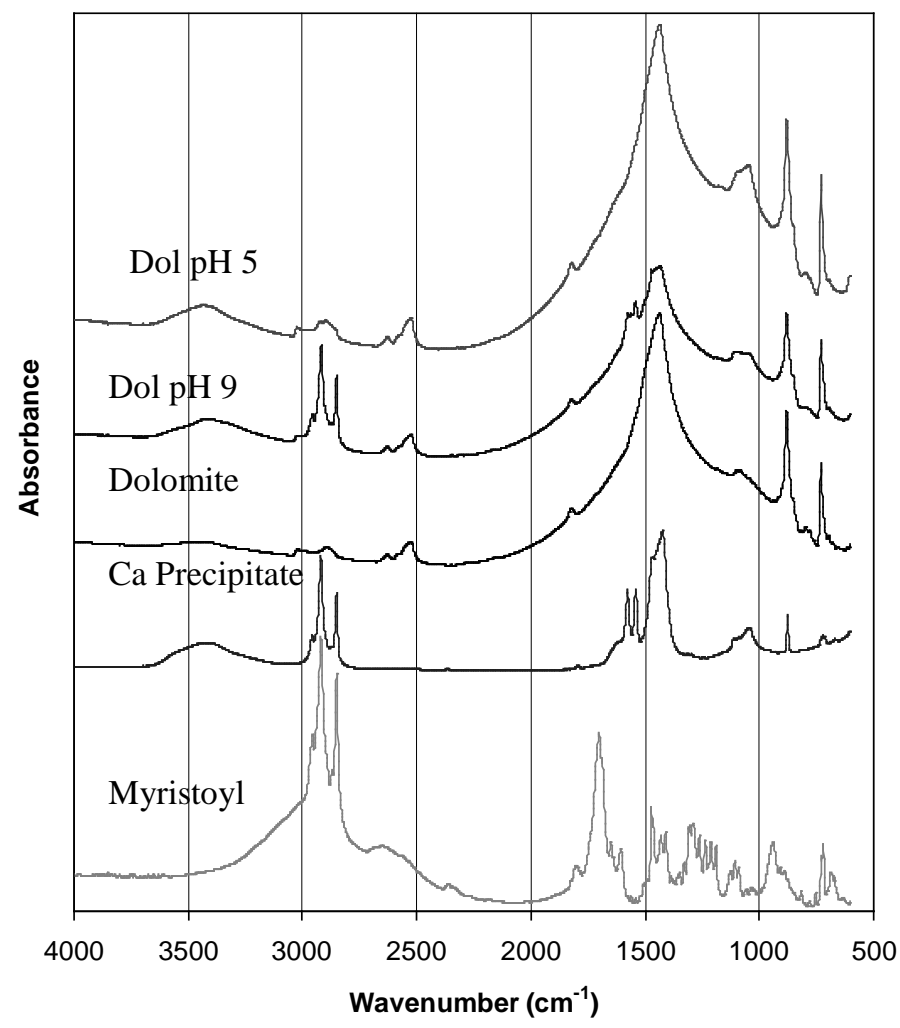

Figure 21: FTIR spectra of myristoyl sarcosine, calcium precipitate of myristoyl sarcosine, dolomite, dolomite conditioned with myristoyl sarcosine at $\mathrm{pH}$ 9, and dolomite conditioned with myristoyl sarcosine at pH 5. 


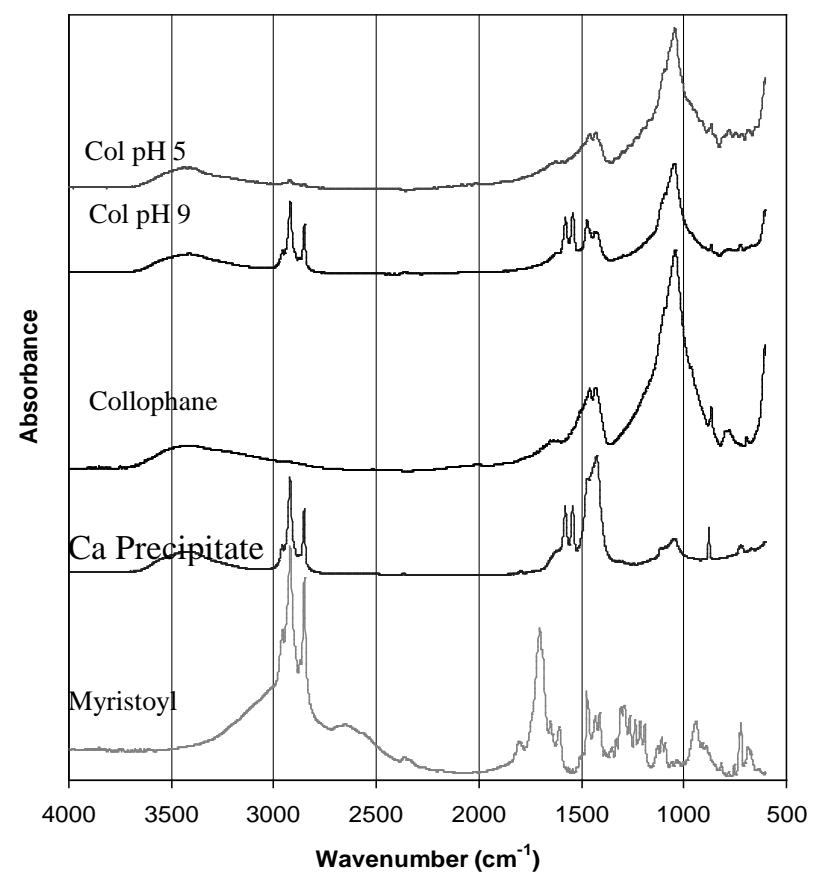

Figure 22: FTIR spectra of myristoyl sarcosine, calcium precipitate of myristoyl sarcosine, collophane , collophane conditioned with myristoyl sarcosine at $\mathrm{pH} 9$, and collophane conditioned with myristoyl sarcosine at pH 5.

In the case of dolomite, at $\mathrm{pH}$ values below 8 , the surface has a positive electrical charge. Either the negatively-charged colloidal precipitate of sarcosinic acid is adsorbed on the mineral surface, or the precipitate is adsorbed on the hydrocarbon chains of previously adsorbed sarcosine ions or molecules. Above $\mathrm{pH} \mathrm{8}$, the presence of bulk precipitated calcium sarcosine on the dolomite surface is evident from the FTIR analysis. Interestingly, precipitated magnesium sarcosine is not present. Sequentially, sarcosine ions must chemisorb on the dolomite surface, calcium sarcosine forms from reaction between dissolved calcium ion and sarcosine, and the precipitate of calcium sarcosine adsorbs on the hydrocarbon chains of the previously chemisorbed sarcosine ions.

These results indicate that if sarcosine is to be used as a collector in these systems, it will have to be used in conjunction with a depressant for the phosphate minerals.

The adsorption of sarcosine (and, hence, flotation) over the entire $\mathrm{pH}$ range under which condition the collophane surface is negatively charged indicates chemisorption of the collector at the mineral surface.

\section{Benchtop Flotation}

To estimate the potential of the collectors in removing dolomite from ore under conditions closer to those observed in industry, flotation testing was conducted with a Florida ore sample in a Denver laboratory cell and also in a cell constructed in our laboratory. Each test was conducted with a 500-g or 125-g sample of ground Florida phosphate ore $(60 \%-74 \mu \mathrm{m})$. To the ground ore, selected quantities of phosphoric acid and sulfuric acid were added with collector and 
conditioned. The conditioned material was subjected to flotation, collected, and dried in an oven. Floated and nonfloated products were analyzed for MgO content.

Sodium oleate was tested first to establish a baseline, since high-molecular weight fatty acids are used in the industry. An oleate addition of $2.2 \mathrm{lb} /$ ton was used in conjunction with 10 $\mathrm{lb} /$ ton phosphoric acid. An addition of $10 \mathrm{lb} /$ ton $\mathrm{H}_{2} \mathrm{SO}_{4}$ was made, and the $\mathrm{pH}$ was 5.2. Frother addition was $0.09 \mathrm{lb} / \mathrm{ton}$. After five minutes of flotation, the tailing contained $1.5 \mathrm{wt} \% \mathrm{MgO}$. About 11 wt \% of the feed solids was floated.

Table 1. Flotation conditions and results obtained with sodium oleate as collector.

\begin{tabular}{|c|c|c|c|c|c|c|c|}
\hline \multicolumn{2}{|c|}{ Reagents } & \multicolumn{6}{c|}{ Results } \\
\hline $\begin{array}{c}\text { Oleate } \\
(\mathrm{lb} / \text { ton) })\end{array}$ & $\begin{array}{c}\mathrm{H}_{3} \mathrm{PO}_{4} \\
(\mathrm{lb} / \text { ton })\end{array}$ & $\begin{array}{c}\text { Float } \\
(\%)\end{array}$ & $\begin{array}{c}\mathrm{MgO} \\
(\%)\end{array}$ & $\begin{array}{c}\text { Nonfloat } \\
(\%)\end{array}$ & $\begin{array}{c}\mathrm{MgO} \\
(\%)\end{array}$ & $\begin{array}{c}\text { MgO\% } \\
\text { Removed }\end{array}$ & $\begin{array}{c}\text { Mass } \\
\text { Balance } \\
\text { MgO\% }\end{array}$ \\
\hline 2.2 & 10 & 11.3 & 12.1 & 88.7 & 1.5 & 51.5 & 2.7 \\
\hline
\end{tabular}

Phosphorous -Based Reagent

A sample of a phosphorous-based collector was obtained and tested at $4 \mathrm{lb} / \mathrm{ton}$. The slurry $\mathrm{pH}$ following conditioning ( 3 minutes at $40 \mathrm{wt} \%$ solids) was about 5.0. Flotation was conducted for 3 minutes on the ore sample. As listed in Table 2, the floated product contained $23.6 \mathrm{wt} \%$ of the feed. An analysis of the float and nonfloat products showed $5.6 \%$ and $2.1 \%$ $\mathrm{MgO}$, respectively.

Table 2. Flotation conditions and results obtained with phosphorous-based collector.

\begin{tabular}{|c|c|c|c|c|c|c|c|}
\hline \multicolumn{2}{|c|}{ Reagents } & \multicolumn{6}{c|}{ Results } \\
\hline $\begin{array}{c}\text { Collector } \\
\text { Conc. }\end{array}$ & $\mathrm{H}_{3} \mathrm{PO}_{4}$ & $\begin{array}{c}\text { Float } \\
(\%)\end{array}$ & $\begin{array}{c}\mathrm{MgO} \\
(\%)\end{array}$ & $\begin{array}{c}\text { Nonfloat } \\
\text { l } \\
(\%)\end{array}$ & $\begin{array}{c}\mathrm{MgO} \\
(\%)\end{array}$ & $\begin{array}{c}\text { MgO\% } \\
\text { Removed }\end{array}$ & $\begin{array}{c}\text { Mass } \\
\text { Balance } \\
\text { MgO\% }\end{array}$ \\
\hline 4 & 8 & 23.6 & 5.6 & 76.4 & 2.1 & 45.2 & 2.9 \\
\hline
\end{tabular}

Mixture of Fatty Acids

A collector containing a mixture of fatty acids was also tested. Phosphoric and sulfuric acids were added as phosphate rock depressant and for $\mathrm{pH}$ adjustment. Addition of the fatty acid was varied, and the (1:1) $\mathrm{H}_{3} \mathrm{PO}_{4} / \mathrm{H}_{2} \mathrm{SO}_{4}$ solution was added until a $\mathrm{pH}$ of 5 was attained. Solids content in the flotation feed was $40 \mathrm{wt} \%$. Conditioning and flotation times were three minutes each. The results obtained with this collector are presented in Table 3. As can be noted, tailings containing less than $1.0 \mathrm{wt} \% \mathrm{MgO}$ were not obtained. This mixture was not effective as a collector for dolomite.

Table 3. Flotation conditions and results obtained with fatty acid mixture as collector.

\begin{tabular}{|c|c|c|c|c|c|c|c|c|c|c|}
\hline \multicolumn{3}{|c|}{ Reagents } & \multicolumn{10}{c|}{ Results } \\
\hline $\begin{array}{c}\text { Collector } \\
\text { (lb/ton) }\end{array}$ & $\begin{array}{c}\text { Promoter } \\
\text { (lb/ton) }\end{array}$ & $\begin{array}{c}\mathrm{pH} \\
\mathrm{H}_{3} \mathrm{PO}_{4} \\
\mathrm{H}_{2} \mathrm{SO}_{4}\end{array}$ & $\begin{array}{c}\text { Flt 1 } \\
(\%)\end{array}$ & $\begin{array}{c}\mathrm{MgO} \\
(\%)\end{array}$ & $\begin{array}{c}\text { Flt 2 } \\
(\%)\end{array}$ & $\begin{array}{c}\text { MgO } \\
(\%)\end{array}$ & $\begin{array}{c}\text { Nonflt } \\
(\%)\end{array}$ & $\begin{array}{c}\text { MgO } \\
(\%)\end{array}$ & $\begin{array}{c}\text { MgO } \\
\text { Removed } \\
(\%)\end{array}$ & $\begin{array}{c}\text { Mass } \\
\text { Balance } \\
\text { MgO\% }\end{array}$ \\
\hline 1.1 & 0 & 5 & 2.9 & 4.4 & & & 97.1 & 2.8 & 4.5 & 2.9 \\
\hline 3 & 2 & 5 & 4.0 & 8.4 & 3.5 & 9.1 & 92.5 & 2.2 & 24.4 & 2.7 \\
\hline
\end{tabular}


Sarcosine

\section{Lauroyl Sarcosine}

The first test conducted with lauroyl sarcosine was with $3 \mathrm{lb} /$ ton collector. Following conditioning, the $\mathrm{pH}$ of the slurry was about 5.2. Flotation was conducted for 2 minutes. Weight of the float product was $22.0 \mathrm{wt} \%$ of the feed. As shown in Table 4, analysis of the float and nonfloat showed $\mathrm{MgO}$ contents of 6.5\%.and 1.8\%, respectively. Another test was conducted at a collector concentration of $4 \mathrm{lb} / \mathrm{ton}$. In this case about $10 \%$ of the feed was floated; the MgO content of the nonfloat was much larger than acceptable levels.

Additional tests were conducted with differing lauroyl sarcosine and phosphoric acid concentrations in the presence of a fatty acid ester promoter, which was added to establish whether its presence would increase the hydrophobicity of the collector-coated surfaces. These results are also shown in Table 4. Two flotation products were obtained in each experiment. The second concentrate was obtained following an additional $1.0 \mathrm{lb} /$ ton collector. Although 13 to 23 wt \% of the ore was floated, the nonfloat products were lowered to only $2.1 \% \mathrm{MgO}$.

Table 4. Flotation conditions and results obtained with lauroyl sarcosine as collector.

\begin{tabular}{|c|c|c|c|c|c|c|c|c|c|c|}
\hline \multicolumn{3}{|c|}{ Reagents } & \multicolumn{9}{c|}{ Results } \\
\hline $\begin{array}{c}\text { Collector } \\
\text { (lb/ton) }\end{array}$ & $\begin{array}{c}\text { Promoter } \\
(\mathrm{lb} / \text { ton) }\end{array}$ & $\begin{array}{c}\mathrm{H}_{3} \mathrm{PO}_{4} \\
(\mathrm{lb} / \text { ton) }\end{array}$ & $\begin{array}{c}\text { Flt 1 } \\
(\%)\end{array}$ & $\begin{array}{c}\text { MgO } \\
(\%)\end{array}$ & $\begin{array}{c}\text { Flt 2 } \\
(\%)\end{array}$ & $\begin{array}{c}\mathrm{MgO} \\
(\%)\end{array}$ & $\begin{array}{c}\text { Nonflt } \\
(\%)\end{array}$ & $\begin{array}{c}\text { MgO } \\
(\%)\end{array}$ & $\begin{array}{c}\text { MgO\% } \\
\text { Removed }\end{array}$ & $\begin{array}{c}\text { Mass } \\
\text { Balance } \\
\text { MgO\% }\end{array}$ \\
\hline 3 & 0 & 8 & 22.6 & 6.5 & -- & -- & 77.4 & 1.8 & 51.3 & 2.9 \\
\hline 4 & 2 & 4 & 7.3 & 5.2 & 15.4 & 3.9 & 77.3 & 2.1 & 37.7 & 2.6 \\
\hline 3 & 2 & 7 & 5.7 & 5.4 & 7.4 & 5.5 & 86.9 & 2.1 & 28.2 & 2.5 \\
\hline
\end{tabular}

\section{Myristoyl Sarcosine}

Myristoyl sarcosine was also used as collector. These results are listed in Table 5. The initial test was conducted with $4 \mathrm{lb} /$ ton myristoyl sarcosine. Solids concentration during conditioning was $40 \mathrm{wt} \%$. The $\mathrm{pH}$ of the slurry following conditioning was about 5.6. Flotation was conducted on the sample for 3 minutes. The float products contained about $4 \mathrm{wt} \%$ of the feed. Flotation was quite selective ( $\mathrm{MgO}$ contents of the concentrates were about 9\%), but the recovery of dolomite was very limited.

Other tests were conducted with constant additions of collector and phosphoric acid but with various additions of the fatty acid ester as promoter. Nonfloat products containing $2 \%$ and greater MgO were obtained. Unfortunately, acceptable products were not obtained with either of lauroyl or myristoyl sarcosine. The industry requires a phosphate product containing less than 1 $\% \mathrm{MgO}$ for phosphoric acid manufacture. 
Table 5. Flotation conditions and results obtained with myristoyl sarcosine as collector.

\begin{tabular}{|c|c|c|c|c|c|c|c|c|c|c|c|}
\hline \multicolumn{3}{|c|}{ Reagents, lb/ton } & \multicolumn{9}{|c|}{ Results } \\
\hline $\begin{array}{c}\text { Collector } \\
\text { Conc. }\end{array}$ & Promoter & $\mathrm{H}_{3} \mathrm{PO}_{4}$ & $\begin{array}{c}\text { Flt 1 } \\
(\%)\end{array}$ & $\begin{array}{c}\mathrm{MgO} \\
(\%)\end{array}$ & $\begin{array}{c}\text { Flt 2 } \\
(\%)\end{array}$ & $\begin{array}{c}\text { MgO } \\
(\%)\end{array}$ & $\begin{array}{c}\text { Flt 3 } \\
(\%)\end{array}$ & $\begin{array}{c}\text { MgO } \\
(\%)\end{array}$ & $\begin{array}{c}\text { Nonf } \\
(\%)\end{array}$ & $\begin{array}{c}\text { MgO } \\
(\%)\end{array}$ & $\begin{array}{c}\text { MgO } \\
\text { Removed } \\
(\%)\end{array}$ \\
\hline 4 & 2 & 8 & 1.4 & 9.1 & 2.4 & 8.7 & -- & -- & 96.2 & 2.1 & 14.1 \\
\hline 3 & 0 & 8 & 5.8 & 6.4 & 4.0 & 5.4 & -- & -- & 90.2 & 2.0 & 24.6 \\
\hline 3 & 1 & 8 & 3.6 & 4.5 & 2.5 & 5.5 & & & 93.9 & 2.3 & 12.3 \\
\hline 3 & 2 & 8 & 1.0 & 5.1 & 1.5 & 8.6 & 2.5 & 5.7 & 95.0 & 2.4 & 12.4 \\
\hline 3 & 1 & 8 & 1.3 & 6.1 & 3.3 & 6.7 & 3.4 & 5.9 & 92.0 & 2.2 & 19.8 \\
\hline
\end{tabular}

In prior experiments, myristoyl sarcosine was found to precipitate out of solution before and during activity in the flotation cell. This has greatly inhibited the effectiveness of the collector. In an attempt to increase the effective amount in solution during flotation, the $\mathrm{pH}$ was raised to a $\mathrm{pH}$ of almost 6 by addition of $\mathrm{NaOH}$. This allowed the myristoyl sarcosine to remain in solution. A counter effect was witnessed as the $\mathrm{pH}$ level inhibited the recovery in flotation.

\section{Flotation Time}

\section{Oleate as Collector}

A usual time for flotation of nonmetallic minerals is three minutes. Physical observation in many of these systems revealed that flotation was essentially complete after 3 to 5 minutes of flotation. This time of flotation was used in the experiments. Tests were also conducted to establish whether longer times of flotation would result in acceptable grades of MgO in the nonfloated material. As shown in Figure 23, with sodium oleate as collector, after 15 minutes of flotation, a final non-float product containing 1.2\% MgO was obtained. Although the nonfloat contained slightly more than $1.0 \% \mathrm{MgO}$, importantly, only about $15 \%$ of the feed was floated under these conditions.

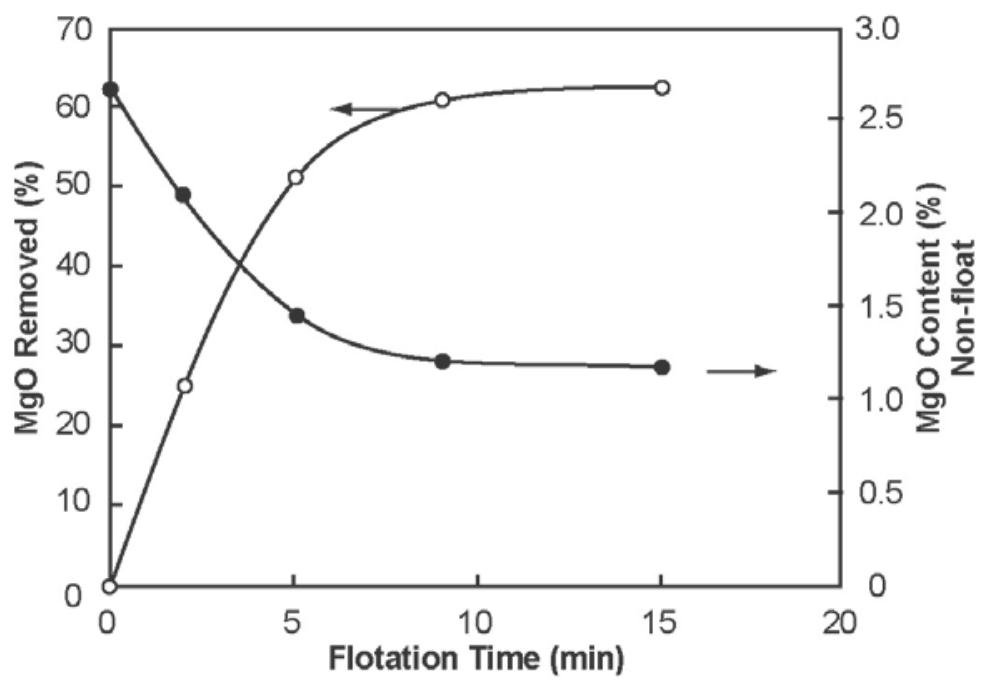

Figure 23. $\mathrm{MgO}$ removed and $\mathrm{MgO}$ content of nonfloat product as a function of flotation time. Conditions: $2.2 \mathrm{lb} /$ ton oleate; $10 \mathrm{lb} /$ ton $\mathrm{H}_{3} \mathrm{PO}_{4} ; 10 \mathrm{lb} /$ ton H2SO4; $0.09 \mathrm{lb} /$ ton frother; flotation feed $75 \%-74 \mu \mathrm{m}$, conditioning $40 \%$ solids for 3 min., $\mathrm{pH}$ 5.1-5.3. 
Additional experiments were run to test the effect of both flotation time and collector addition. Sodium oleate at an initial addition of $3.3 \mathrm{lb} /$ ton was used as collector. Frother addition was $0.35 \mathrm{lb} /$ ton. After 15 minutes of flotation, float 1 was obtained. A second addition of oleate, $0.44 \mathrm{lb} /$ ton in one case was made; the system was conditioned for two minutes and floated again for 6 minutes. The $\mathrm{pH}$ of the system was $\mathrm{pH}$ 5.1-5.5. The solids concentration during conditioning and flotation was about $20 \mathrm{wt} \%$ solids. A second float product was obtained and is designated float 2 . See Table 6 .

Table 6. Flotation conditions and experimental results obtained with sodium oleate as collector.

\begin{tabular}{|c|c|c|c|c|c|c|c|c|c|c|}
\hline \multicolumn{3}{|c|}{ Reagents, lb/ton } & \multicolumn{8}{|c|}{ Results } \\
\hline \multirow[b]{2}{*}{$\mathrm{NaOl}$} & \multirow[b]{2}{*}{$\mathrm{NaOl}$} & \multirow[b]{2}{*}{$\mathrm{H}_{3} \mathrm{PO}_{4}$} & \multicolumn{2}{|c|}{ Float 1} & \multicolumn{2}{|c|}{ Float 2} & \multicolumn{2}{|c|}{ Nonfloat } & \multirow{2}{*}{$\begin{array}{c}\mathrm{MgO} \\
\text { Removed } \\
\%\end{array}$} & \multirow{2}{*}{$\begin{array}{c}\text { Mass } \\
\text { Bal. } \\
\text { MgO\% }\end{array}$} \\
\hline & & & $\begin{array}{c}\mathrm{Wt} \\
\%\end{array}$ & $\begin{array}{c}\mathrm{MgO} \\
\%\end{array}$ & $\begin{array}{c}\mathrm{Wt} \\
\%\end{array}$ & $\begin{array}{c}\mathrm{MgO} \\
\%\end{array}$ & $\begin{array}{c}\mathrm{Wt} \\
\%\end{array}$ & $\underset{\%}{\mathrm{MgO}}$ & & \\
\hline \multirow{3}{*}{3.3} & 0.44 & \multirow{3}{*}{10.6} & 18.4 & 9.4 & 8.1 & 3.5 & 74.5 & 0.93 & 74.7 & 2.7 \\
\hline & 0.70 & & 25.1 & 7.7 & 5.6 & 2.5 & 69.3 & 0.95 & 76.0 & 2.7 \\
\hline & 1.45 & & 26.2 & 8.0 & 5.2 & 2.0 & 68.6 & 0.86 & 78.7 & 2.8 \\
\hline
\end{tabular}

In every case the $\mathrm{MgO}$ content of the valuable product was less than $1.0 \mathrm{wt} \%$; about $75 \mathrm{wt} \%$ of the $\mathrm{MgO}$ was removed which reported to float products containing 25-30 wt \% of the ore. These results are similar to those reported by Moudgil and Ince (1987) who obtained a product containing $0.9 \% \mathrm{MgO}$ from an ore containing $10.3 \% \mathrm{MgO}$ using a two-stage technique. The $\mathrm{pH}$ of their first float was about $\mathrm{pH} 10$ and that of the second was $\mathrm{pH}$ 2.7-3.1.

\section{Lauroyl Sarcosine as Collector}

The effect of flotation time was also investigated with lauroyl sarcosine. Collector addition was $1.1 \mathrm{lb} /$ ton. A lower addition was used as compared with oleate because of the excessive foaming properties of sarcosine. No frother was added. Total flotation time of five minutes was used, since flotation was deemed complete after that time. Analysis of the ore showed that this sample contained 1.8\% MgO. As shown in Figure 24, about 60\% of the MgO was removed after five minutes of flotation. The MgO concentration of the nonfloat was about $1.2 \%$. Although the nonfloat product was close to the acceptable $\mathrm{MgO}$ content, about $35 \%$ of the feed was floated. 


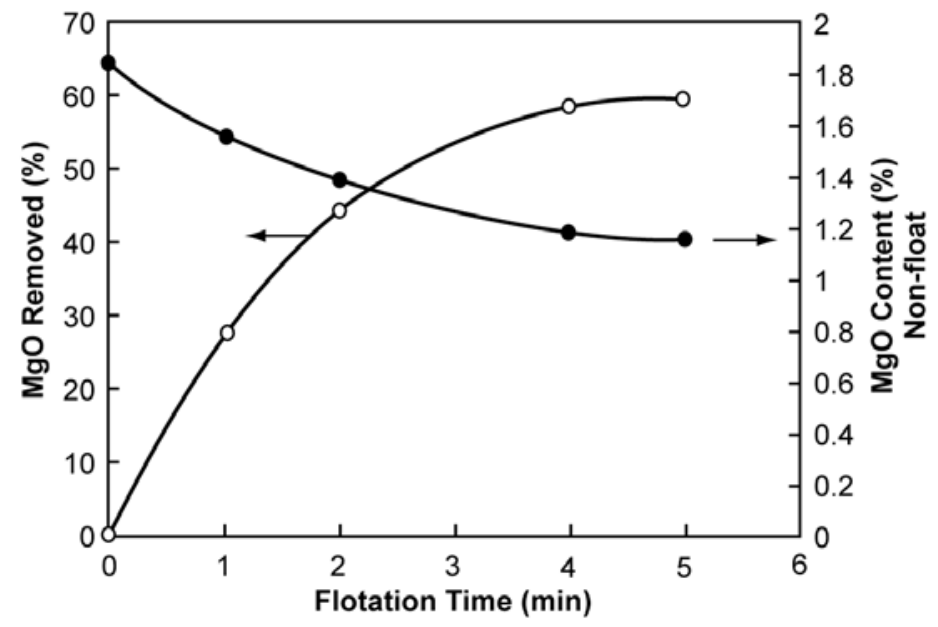

Figure 24. MgO removed and MgO content of nonfloat product as a function of flotation time. Conditions: $1.1 \mathrm{lb} /$ ton lauroyl sarcosine; $10 \mathrm{lb} /$ ton $\mathrm{H}_{3} \mathrm{PO}_{4} ; 20 \mathrm{lb} /$ ton $\mathrm{H}_{\mathrm{s}} \mathrm{SO}_{4}$; , flotation feed: 75\% -74 $\mu \mathrm{m}$; conditioning: 40\% solids for 3 minutes,;pH 5.1-5.3.

\section{Oleate and Lauroyl Sarcosine}

A series of experiments were conducted to establish whether a synergistic effect could be obtained between oleate and lauroyl sarcosine in the flotation system (See Table 7). Initially, 3.3 $\mathrm{lb} /$ ton oleate $(\mathrm{NaOl})$ was added, and flotation was effected at about $\mathrm{pH} 5$ for 15 minutes. Frother addition was $0.35 \mathrm{lb} / \mathrm{ton}$. Following this, various amounts of lauroyl sarcosine were added, and after conditioning for 3 minutes, flotation was conducted for another 6 minutes. When a relatively small quantity of $0.088 \mathrm{lb} /$ ton sarcosine was added, weight to float and $\mathrm{MgO}$ content of the nonfloat were about the same as when oleate only was added (Table 6). Increasing the amount of lauroyl sarcosine increased the weight to the float. With an addition of $0.26 \mathrm{lb} / \mathrm{ton}$ sarcosine, nearly $40 \mathrm{wt} \%$ of the feed was floated.

Table 7. Flotation conditions and experimental results obtained with sodium oleate and lauroyl sarcosine as collectors.

\begin{tabular}{|c|c|c|c|c|c|c|c|c|c|c|}
\hline \multicolumn{3}{|c|}{ Reagents, lb/ton } & \multicolumn{8}{|c|}{ Results } \\
\hline \multirow[b]{2}{*}{$\mathrm{NaOl}$} & \multirow[b]{2}{*}{ Sarcosine } & \multirow[b]{2}{*}{$\mathrm{H}_{3} \mathrm{PO}_{4}$} & \multicolumn{2}{|c|}{ Float 1} & \multicolumn{2}{|c|}{ Float 2} & \multicolumn{2}{|c|}{ Nonfloat } & \multirow{2}{*}{$\begin{array}{c}\text { MgO } \\
\text { Removed } \\
\%\end{array}$} & \multirow{2}{*}{$\begin{array}{c}\text { Mass } \\
\text { Bal. } \\
\text { MgO\% }\end{array}$} \\
\hline & & & $\begin{array}{c}\mathrm{Wt} \\
\%\end{array}$ & $\begin{array}{c}\mathrm{MgO} \\
\%\end{array}$ & $\begin{array}{c}\mathrm{Wt} \\
\%\end{array}$ & $\begin{array}{c}\mathrm{MgO} \\
\%\end{array}$ & $\begin{array}{c}\mathrm{Wt} \\
\%\end{array}$ & $\begin{array}{c}\mathrm{MgO} \\
\%\end{array}$ & & \\
\hline \multirow{3}{*}{3.3} & 0.088 & \multirow{3}{*}{10.6} & 20.5 & 9.1 & 5.9 & 2.9 & 73.6 & 0.92 & 74.9 & 2.7 \\
\hline & 0.14 & & 25.0 & 8.0 & 6.8 & 2.0 & 68.2 & 0.78 & 80.0 & 2.7 \\
\hline & 0.26 & & 23.5 & 8.4 & 14.2 & 1.5 & 62.3 & 0.97 & 78.3 & 2.8 \\
\hline
\end{tabular}




\section{CONCLUSIONS}

The presence of more than 1.0 wt \% MgO in concentrates of phosphate rock is problematic in phosphoric acid production. Combination of ores containing higher quantities of $\mathrm{MgO}$ with those of lower $\mathrm{MgO}$ content has enabled meeting this requirement. Future processing will involve processing only ore that contains relatively high $\mathrm{MgO}$ contents. There is a need, then, to develop technology that will enable selective separation of phosphate rock from dolomite. This study was undertaken to determine whether certain collectors could be synthesized that might exhibit greater specificity for magnesium over calcium and also to investigate conditions under which certain commercially-available collectors might be used this flotation separation.

A variety of collectors were tested. They included: oleate, malonic acid and ester, betaine, alkyl citrate and ester, sarcosine, and two collectors obtained from suppliers. The flotation results indicate that different mechanisms are involved in collector adsorption depending on the system $\mathrm{pH}$. In acid medium the neutral molecule and precipitate of the collector in the acid form are responsible for flotation. On the basic side, collector anion and a precipitate of the calcium salt of the collector are responsible for flotation.

Betaine can be obtained from very inexpensive stock, namely sugar beet processing residue. Unfortunately, the all of the betaines tested did not exhibit favorable collector attributes.

Octadecyl malonic acid did not function effectively as collector due probably to its lack of solublility. Octadecyl malonic acid ester was shown to float both apatite and dolomite. However, the flotation characteristics of both minerals with this collector were quite similar.

Similar phenomena were noted with hexadecyl citrate. Hexadecyl citrate functions effectively as a collector, but the conditions under which flotation is obtained with both apatite and dolomite are so similar that using this reagent in a selective separation is not possible.

Sarcosine was suggested as a flotation collector for selective flotation of dolomite by Moudgil and Vasudevan (1988). Lauroyl, myristoyl and palmitoyl sarcosines were all shown in the present study to function as collectors for apatite, collophane, and dolomite. The flotation characteristics of these minerals were shown to be quite similar, but some selective flotation of dolomite was obtained when phosphoric acid was added as a depressant for phosphate mineral. The dolomite content of the nonfloat product was still too high for its use in phosphoric acid production. Excessive foaming was observed in the ore flotation which would make its commercial use difficult.

Of the various collectors studied with natural ore, only sodium oleate was shown to yield a nonfloat product containing less than $1.0 \mathrm{wt} \% \mathrm{MgO}$. Nonfloat product containing as low as 0.86 $\% \mathrm{MgO}$ was obtained when the ore was floated in single stage in modestly acidic medium $(\mathrm{pH}$ 5.0-5.5). Earlier investigators (Moudgil and Ince 1987) obtained a nonfloat product containing less than 1.0\% MgO, but their technique involved a two stage (pH 10 and $\mathrm{pH}$ 2.7) flotation process. 


\section{REFERENCES}

Bartels, J. J., and Gurr, T. M., 1994, "Phosphate Rock,” Industrial Minerals and Rocks, Sixth Edition, Carr, D. D., ed. SME, Littleton, CO. pp 751-764.

Buchowicz, W., and Mol, J. C., 1999, J. Molecular Catalysis A: Chemical, 148, 97-103.

Bunge, R. C. and Fuerstenau, D. W., 2006, “The Complex Behavior of Fatty Acids in Fluorite Flotation," Proceedings of the XXIII International Mineral Processing Congress. Ankara,Turkey, Vol. 1, pp. 510-515.

El Shall, H., 1994, “Evaluation of Dolomite Separation Techniques, FIPR Contract No. 93-02994, Final Report.

Fuerstenau, M.C. and W. F. Cummins, 1967, “The Role of Basic Aqueous Complexes in Anionic Flotation of Quartz,” Vol. 238, p. 196.

Hamann, J., 1933, Metall. und Erz., vol. 30, p. 455.

Harben, P., 1980, “Where is Florida’s Phosphate Industry Going?” Industrial Minerals, No. 148, pp. 48-55.

Kotick, M. P., 1991, “Method for Regioselective Preparation of 1-or 2-monoalkyl Citrates as Surfactants,” U.S. Patent No. 5049699.

Lawver, J. et al., 1982, “Phosphate Reserves Enhancement by Beneficiation,” Mining Congress Journal, Vol. 68, pp. 27-31.

Lide, D. R., Chief Editor, 2003, CRC Handbook of Chemistry and Physics, $84^{\text {th }}$ Edition, CRC Press.

Marvey, B. B., du Plessis, J. A. K., Vosloo, J. C. M., and Mol, J. C., 2003, J. Molecular Catalysis A: Chemical, Vol. 201, 297-308.

Moudgil, B. M., and Chanchani, R., 1985, "Selective Flotation of Dolomite from Francolite Using Two-Stage Conditioning,” Minerals \& Met. Proc., Vol. 2, pp. 331-341.

Moudgil, b. M., T. W. Vasudevan, and J. Blackmeer, 1987, “Adsorption of Oleate on Apatite,” Trans. AIME, 282:50.

Moudgil, B. M. and Ince, D., (1987). See also Moudgil and Vasudevan (1988).

Moudgil, B. M. and T. V. Vasudevan, 1988, "Beneficiation of Phosphate Ores Containing Carbonate and Silica Gangue,” Minerals \& Met. Proc., Vol. 5, p. 120. 
Moudgil, B. M., and Ince, D., 1991, "Effect of Sodium Chloride on Flotation of Dolomite from Apatite,” Minerals and Met. Proc., Vol, 8, pp.139-143.

Reeder, R. J., and Markgraf, S. A., 1986, American Mineralogist, Vol. 71, pp. 795-804.

Sandvik, P. O., 1979, “U.S. Phosphate-Abundant Resources Will Last for Hundreds of Years," Eng. \& Min. J., Vol. 180, No. 10, pp. 99-101.

Sillen, L. G., and Martell, A. E., 1964, Stability Constants of Metal-Ion Complexes, Chemical Society, London, pp. 385-386.

Stock, D. I., and Davies, C. W., 1949, J. Chem. Soc., pp. 1371-1374.

Zhang, J., 1994, "Phosphate Beneficiation - Challenges and Opportunities," Separation Processes:Heavy Metals, Ions and Minerals," Misra, M., ed., The Metallurgical Society, Warrendale, PA, pp. 167-183.

Zheng, X. P., Misra, M., Smith, R. W., and Raichur, A., 1998, “Anionic Flotation of Apatite from Dolomite Modified by Presence of a Bacterium,” Mineral and Met. Proc., Vol, 15, p. 52.

Zheng, X. P., Smith, R. W., Misra, M., Mehra, R. K., and Raichur, A., 1998, "Effect of a Water Soluble Fraction Derived from Mycobacterium phlei on the Surface Characteristics and Fotation of Apatite and Dolomite,“ Miner. Proc. Ext. Met. Review, Vol, 19, pp. 335341.

\section{ACKNOWLEDGMENT}

The authors wish to acknowledge the experimental work performed by David Kruth and Kangnian Zhong. 
Appendix 6: Flotation Technology for the Trona Industry (UT001) 


\section{TECHNICAL REPORT}

\begin{tabular}{lll}
\hline Contract Title and Number: & \multicolumn{2}{l}{ Period of Performance: } \\
\cline { 2 - 2 } Crosscutting Technology Development at the Center for & Starting Date: & 10/01/2002 \\
Advanced Separation Technologies & Ending Date: & 10/31/2009 \\
(DE-FC26-02NT41607 & &
\end{tabular}

\begin{tabular}{|c|c|c|}
\hline Sub-Recipient Project Title: & Report Information: & \\
\hline & Type: & Final Report \\
\hline Flotation Technology for the Trona Industry & Number: & \\
\hline & Period: & \\
\hline Principal Investigators: & Date: & $4 / 3 / 2007$ \\
\hline J.D. Miller & Code: & UT001-Final \\
\hline Contact Address: & Contact Information: & \\
\hline Department: Metallurgical Engineering & Phone: & $801-581-6386$ \\
\hline University of Utah & Fax: & $801-581-4937$ \\
\hline Salt Lake City & E-Mail: & jdmiller@mines.utah.edu \\
\hline Utah 84112 & & \\
\hline Subcontractor Address: & $\underline{\text { Subcontractor Inform }}$ & ation: \\
\hline No subcontracts issued. & Phone: & \\
\hline & Fax: & \\
\hline & E-Mail: & \\
\hline
\end{tabular}

"This report was prepared as an account of work sponsored by an agency of the United States Government. Neither the United States Government nor any agency thereof, nor any of their employees, make any warranty, express or implied, nor assume any legal liability or responsibility for the accuracy, completeness, or usefulness of any information, apparatus, product, or process disclosed, or represents that its use would not infringe upon privately owned rights. Reference herein to any specific commercial product, process, or service by trade name, trademark, manufacturer, or otherwise does not necessarily constitute or imply endorsement, recommendation, or favoring by the United States Government or any agency thereof. The views and opinions of the authors expressed herein do not necessarily state or reflect those of the United States Government or agency thereof. ” 


\begin{abstract}
$\underline{\text { Abstract }}$
Trona (sodium sesquicarbonate), having the formula, $\mathrm{Na}_{2} \mathrm{CO}_{3} \cdot \mathrm{NaHCO}_{3} \cdot 2 \mathrm{H}_{2} \mathrm{O}$ is typically associated with insoluble gangue minerals such as dolomite, shale, clay and quartz. Removal of these gangue minerals may be desirable prior to dissolution in hot brine when the trona feed contains a significant amount of impurities. The development of new flotation technology for the separation of gangue minerals and production of trona concentrate has been substantiated by bench scale flotation experiments at the University of Utah. Laboratory flotation tests showed that insolubles in the feed can be successfully floated and a trona concentrate of $99 \%$ purity obtained with a recovery of $90 \%$. In order to achieve a high separation efficiency, evaluation of reagents and formulation procedures was undertaken together with an examination of flotation chemistry conditions. In this regard various process variables such as amine dosage, flotation percent solids, amine to oil ratio, $\mathrm{pH}$ of amine solution, collector structure, brine composition, feed particle size, etc. were studied to provide the preferred conditions and operating variables for pilot-plant testing.

Preferred flotation conditions obtained from the bench scale experiments were applied to pilot plant tests $(1 \mathrm{t} / \mathrm{h})$ at the General Chemical plant-site in Wyoming. During plant-site tests, some significant problems (usage of mine water, greater amount of fines, etc.) were identified. Possible solutions to the problems faced at the plant-site are discussed. Due to weather conditions, feed availability, and time considerations, a new trona pilot plant flotation system $(110 \mathrm{lb} / \mathrm{h})$ was assembled and tested at the University of Utah laboratories. The results from the University of Utah pilot plant tests indicated that under normal conditions, a good single stage separation can be achieved with a trona concentrate containing $2-4 \%$ insolubles at $70-80 \%$ recovery.
\end{abstract}


Table of Contents

Page

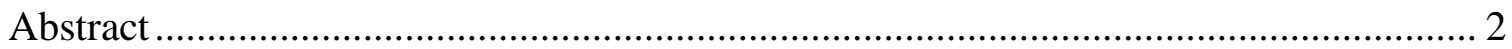

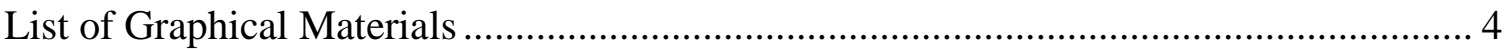

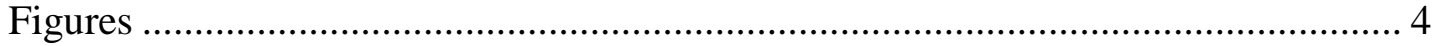

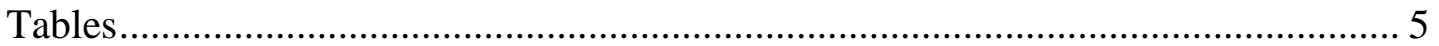

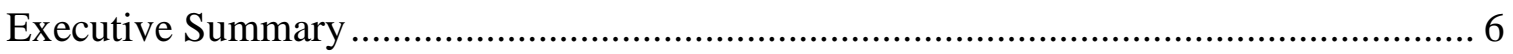

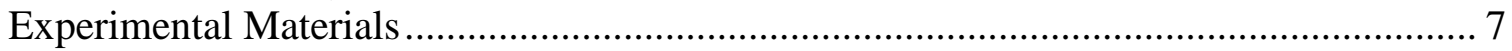

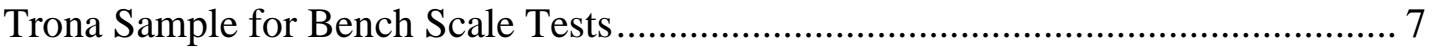

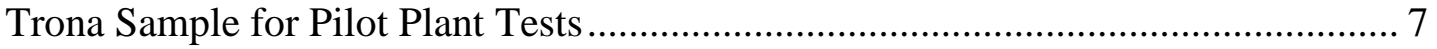

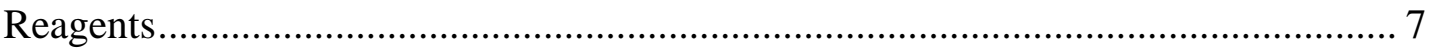

Experimental Procedure, Bench Scale Tests at General Chemical................................... 7

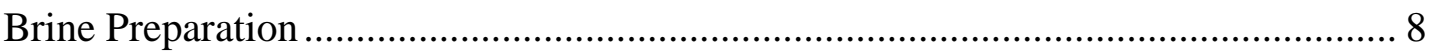

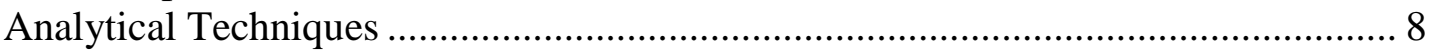

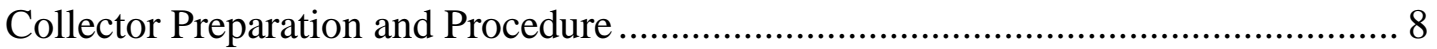

Experimental Procedure, Pilot Scale Tests at General Chemical ................................... 9

Experimental Procedure, Pilot Scale Tests at University of Utah ................................... 9

Collector Characterization Study (Emulsion Droplet Size Distribution) ........................ 10

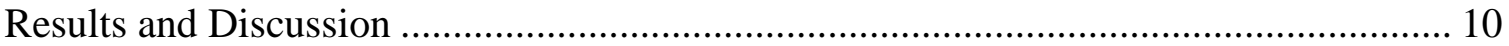

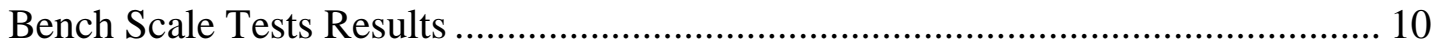

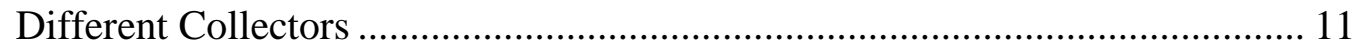

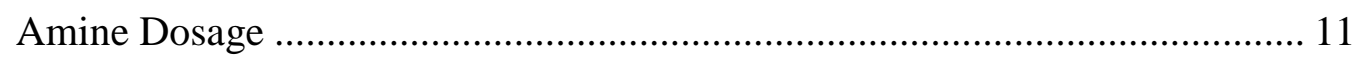

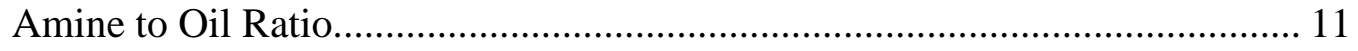

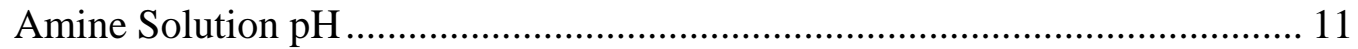

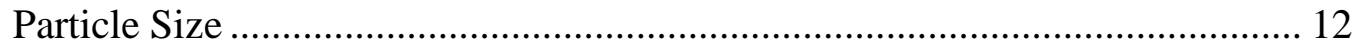

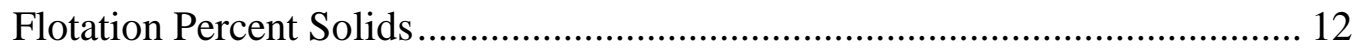

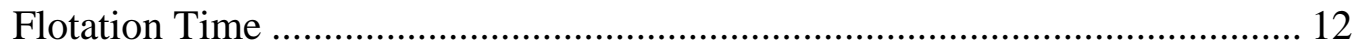

Flotation with Recycled Saturated Solutions............................................... 12

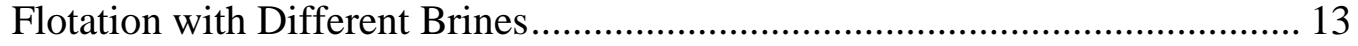

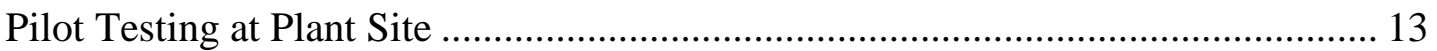

Preparation for Plant-Site Testing .............................................................. 13

First Pilot Plant Results ......................................................................... 14

Large Scale Batch Flotation........................................................................ 14

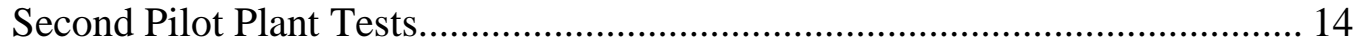

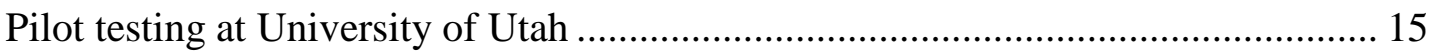

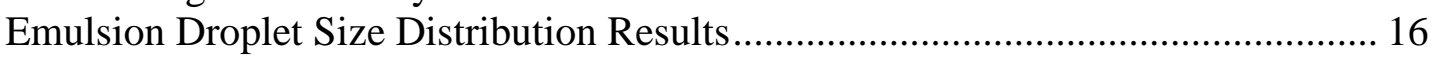

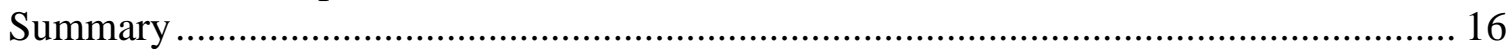

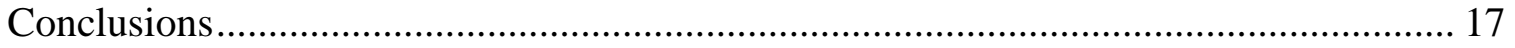

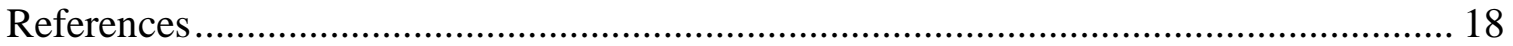

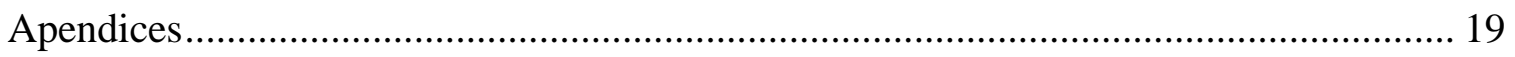




\section{List(s) of graphical materials}

\section{$\underline{\text { Figures }}$}

Figure 1 Total mass, insoluble and trona distributions with respect to particle size for the bench scale flotation feed sample.

Figure 2 Total mass, insoluble and trona distributions with respect to particle size for the pilot plant flotation feed sample.

Figure 3 Denver flotation cell, high solids conditioning and centrifuge for dewatering of flotation products.

Figure 4 Procedure for bench scale flotation experiments.

Figure 5 The flotation equipment (EIMCO) used for first pilot scale tests.

Figure 6 Hazen quinn flotation equipment for second pilot plant study at General Chemical plant site.

Figure 7 Trona flotation pilot plant set-up with high solids conditioning tank and dilution tank.

Figure 8 Effect of different amine collectors on trona flotation.

Figure 9 Effect of amine dosage on trona flotation.

Figure 10 Effect of amine/kerosene ratio on trona flotation.

Figure 11 Effect of $\mathrm{pH}$ of amine solution on trona flotation.

Figure 12 Effect of particle size on trona floation.

Figure 13 Effect of flotation percent solids on trona flotation.

Figure 14 Effect of flotation time on trona flotation.

Figure 15 The modified flowsheet for the pilot plant trona flotation system.

Figure 16 Facilities for the second pilot scale flotation test campaign at General Chemical plant site.

Figure 17 Conditioning process in trona flotation.

Figure 18 Concentrate product.

Figure 19 Flotation of gangue minerals.

Figure 20 Trona flotation performance as a function of collector dosage for flotation feed. Figure 21 Effect of flotation time on continuous trona flotation.

Figure 22 Photomicrograph of the dispersed drops in 1\% and 2\% DAH emulsion.

Figure 23 Photomicrograph of the dispersed drops in 3\% and 4\% DAH emulsion.

Figure 24 Comparison of droplet diameter distributions of kerosene emulsion at different DAH concentrations.

Figure 25 Effect of amine concentration on trona flotation. 


\section{Tables}

Table 1 Comparison of results from flotation with fresh saturated solution and recycled saturated solution.

Table 2 Results from different brines in trona flotation.

Table 3 Plant-site operating conditions for pilot plant tests.

Table 4 Plant-site operating conditions for second pilot plant tests.

Table 5 Results from pilot plant trona flotation system under the condition of $5 \mathrm{~kg} / \mathrm{t}$ amine dosage.

Table 6 Results from pilot plant trona flotation system under the condition of $4 \mathrm{~kg} / \mathrm{t}$ amine dosage.

Table 7 Results from pilot plant trona flotation system under the condition of $3 \mathrm{~kg} / \mathrm{t}$ amine dosage.

Table 8 Results from continuous (30 min.) U of U pilot plant trona flotation system under the condition of $4 \mathrm{~kg} / \mathrm{t}$ amine dosage.

Table 9 Comparison of trona flotation results for bench scale, batch and continuous operation. 


\section{Executive Summary}

Soda ash, an essential raw material for major industries such as the glass, chemical, soap and detergent, pulp and paper, and water treatment industries, is traditionally produced by chemical and thermal treatment of trona ore. The treatment processes for trona ore are not only chemically expensive but also energy intensive. Hence, the production of high purity trona concentrate at lower energy and operating cost is of considerable interest to the trona industry.

Almost all (90\%) of the soda ash production in the U.S. comes from the trona deposits of the Green River Basin in Wyoming. The trona reserves of greater than 120 billion tons (20-25 billion tons economical to mine) are extensive, covering an area of 900 sq. miles, and will be the most significant source of soda ash in the years to come. It is estimated that these reserves of trona could satisfy the world's demand for many centuries (Kostick, 1994).

The trona beds occur with layers of oil shale, marlstone, and sandstone/mudstone. These insoluble contaminants include a number of double carbonate salts in addition to dolomitic shale, quartz, and clays (Garrett, 1991). In the industry, conventional production of soda ash from trona is a complex process with many steps needed to eliminate the insoluble components of the trona ore.

Removal of insoluble gangue minerals from trona by particle separation processes is not practiced by industry. Nevertheless, research and development efforts have been made to find an appropriate particle separation process for the removal of insoluble gangue minerals prior to traditional thermo chemical processing. It was believed that removal of gangue minerals from the trona plant feed prior to dissolution in hot brine could be accomplished by froth flotation in a comparatively simple and inexpensive approach. Such a preprocessing strategy at ambient temperature and pressure has been limited by the lack of satisfactory flotation technology. Some researchers (Brison and Gathje, 1981; Rosar et al., 1974; Datta and Salotti, 1990; Garrett and White, 1970) claimed to be able to float the organic matter, but it is not known whether their reagents were useful in floating insolubles. Nevertheless, an appropriate flotation technique for trona recovery has not yet been commercialized. More recently, a unique flotation procedure was discovered. Based on results from laboratory research at the University of Utah, it was observed that run-of-mine trona can be treated at ambient temperature and pressure using a special reverse flotation procedure to separate the gangue minerals and make a trona concentrate (Wang et al., 2002).

The overall objective of this study was to demonstrate the feasibility of a new flotation technology for the production of a high grade trona (sodium sesquicarbonate) concentrate at a satisfactory level of recovery. Appropriate conditions for trona flotation technology were established from exploratory bench-scale experiments at the University of Utah and subsequently by factorially designed experiments. Preferred conditions obtained from the bench scale experiments were applied to plant-site pilot scale flotation tests. Construction of the pilot plant system was completed and installed at the General Chemical plant site in Wyoming. The tests were carried out under preferred operating conditions. However, this pilot system was not able to yield satisfactory results due to certain problems (usage of mine water, greater amount of fines, etc.). Therefore, another pilot plant campaign 
was carried out at the University of Utah to investigate the problems faced in plant-site testing. The pilot plant flowsheet was modified with some new design features to appropriately conduct reverse flotation of gangue minerals from trona. Under normal conditions a good separation was achieved typically producing a trona concentrate containing $2-4 \%$ insolubles at $70-80 \%$ recovery.

\section{Experimental Materials}

\section{Trona Sample for Bench Scale Tests}

A natural trona sample was obtained from General Chemical, Green River Formation in Wyoming. The sample (bulk density, $0.97 \mathrm{~g} / \mathrm{cm}^{3}$ ) was taken from the crushing circuit before calcination. The sample was then classified into different particle size fractions by laboratory screening. Shown in Figure 1 are the total mass distribution and gangue mineral distribution (insoluble content) with respect to particle size for the minus $2 \mathrm{~mm}$ material taken from the calcination feed. The results in Figure 1 indicate that the feed material is mainly distributed in the minus 200 mesh fraction with only 1 to $2 \%$ insoluble content. The overall grade for this feed material was about $11.3 \%$. In this regard, it was decided that the minus 200 mesh fraction could be removed since the minus 200 mesh material only contains 1 to $2 \%$ insoluble content and can be used directly as a trona concentrate.

\section{Trona Sample for Pilot Plant Tests}

Another trona sample, obtained from General Chemical, Green River, Wyoming, was used for pilot plant tests at the plant-site and at the University of Utah as well. This run of mine trona ore was crushed to a fairly fine size (30x200 mesh) using a hammer mill. The crushed sample was stored in about 36 super sacks (plastic bags with 1 ton capacity). For pilot plant testing, the crushed ore was air dried at ambient temperature of about $15-20^{\circ} \mathrm{C}$. The dry sample was then sieved at 200 mesh to remove fine particles (minus 200 mesh) because the crushed sample had about 50\% fine particles (minus 200 mesh). The insoluble content of the feed was about $18 \%$. As seen from Figure 2, $41 \%$ of the total mass is distributed in the minus 200 mesh size fraction with only $1 \%$ insoluble content.

\section{$\underline{\text { Reagents }}$}

A procedure to float associated gangue minerals (shales, dolomite, clays) from the trona was developed using DAH as collector. Dodecylamine hydrochloride (DAH) is a primary longchain amine $\mathrm{C}_{12} \mathrm{H}_{27} \mathrm{~N}$.HCl (molecular weight 221.81, TCI Chemical Co.), which was purchased from TCI Chemical Co. and had a specified purity of 98\%. Commercial amines were used for further reagent investigation and were obtained from Akzo Nobel Chemical Co. Kerosene, which is a nonpolar oil, was also used to extend the hydrophobic effect of DAH. Kerosene was purchased from a local company, Hi-Valley Chemical, Inc. 


\section{Experimental Procedure, Bench Scale Tests at General Chemical}

Trona flotation was conducted using a bench-scale Denver Flotation machine with a one-liter stainless steel flotation cell. A Denver conditioning machine was used for conditioning the feed with brine and reagents. Figure 3 shows the facility used for trona flotation.

About 150 grams of dry trona feed was pulped by adding 25-30 ml brine (saturated trona solution) followed by addition of the flotation collector (blend of amine and kerosene). The suspension was conditioned for about $2 \mathrm{~min}$. After conditioning, the pulp was transferred to the flotation cell, diluted with brine and then flotation was carried out. Usually the flotation time was about 4-5 min. Flotation products, sink (trona concentrate) and float (tailing), were dewatered by using a centrifuge and then dried in an oven at $40^{\circ} \mathrm{C}$. The water-insoluble material in both float and sink products was determined by gravimetric analysis as described in the section titled Analytical Techniques. In some cases, the solution from centrifugation was taken for use in further flotation experiments as recycled solution. Figure 4 shows the flowsheet for the bench scale flotation testing.

\section{Brine Preparation}

The flotation of naturally occurring, highly soluble salts must be carried out in saturated solutions. In order to prepare the saturated solution for flotation experiments, a sufficient amount of trona was dissolved in water ( $40 \%$ solids $+60 \%$ water) and stirred for over $10 \mathrm{hrs}$ to reach saturation (light yellow colored solution, specific gravity: $1.21 \mathrm{~g} / \mathrm{cm}^{3}$ ). Since the solubility of the sodium carbonate salts is highly sensitive to temperature, the solution was stirred in a constant temperature water bath. Then the saturated solution was stored overnight to ensure that suspended solids had settled completely. The solution was then decanted and transferred to another container. The solution was also filtered to remove fine particles.

\section{$\underline{\text { Analytical Techniques }}$}

The analytical method for insoluble mineral analysis of the flotation products (concentrate and tailing) was as follows: After drying and weighing, a representative sample ( $25-30 \%)$ of product was taken for insoluble mineral analysis. This representative sample was mixed with water and stirred for $1 \mathrm{~min}$. Then it was decanted after $5 \mathrm{~min}$. This process was repeated several times until all soluble salts (light yellow color) were dissolved completely, leaving only insoluble minerals (dark brown color) in the container. These insoluble minerals were then transferred onto a pyrex glass plate and heated at $40-50^{\circ} \mathrm{C}$ for a few minutes followed by cooling at room temperature. The dry sample was then weighed and the insoluble mineral content determined.

\section{$\underline{\text { Collector Preparation and Procedure }}$}

Armeen 12D (Dodecylamine, $\mathrm{C}_{12} \mathrm{H}_{27} \mathrm{~N}$ ), which is a commercial reagent manufactured by Akzo Nobel Surface Chemistry Llc., was chosen for bench scale flotation experiments. A 3\% concentration of amine solution was prepared by dissolving $6 \mathrm{~g}$ of Armeen 12D in $200 \mathrm{~g}$ of distilled water. To this amine solution, $0.022 \mathrm{cc} \mathrm{HCl}$ (3 drops) was added to maintain $\mathrm{pH} 3$, 
and the solution was then stirred using a Cole Parmer Overhead mixer at 1200-1500 rpm for 1 hour to completely dissolve the amine. Since the amine has a low solubility in water, the solution was heated to $70^{\circ} \mathrm{C}$ in order to obtain a clear solution. The solution was then cooled down for an hour in order to use for the preparation of the emulsion in the next step.

The emulsions were prepared by dispersing kerosene in the $3 \%$ aqueous solution of the Armeen 12D (alkaline, $\mathrm{pH} 10$ ) in the ratio of 1:12 (1 gram amine to 12 grams kerosene) by weight. The mixture was mixed using a Cole Parmer mixer at $1500 \mathrm{rpm}$ speed for 2 min to obtain the emulsion. The mixing speed of $1500 \mathrm{rpm}$ was established after using different mixing speeds to see the effect on the emulsion properties. As expected, at high speed (1500 rpm), the average drop size obtained was smaller than at low speed (700 rpm).

\section{Experimental Procedure, Pilot Scale Test at General Chemical}

The pilot plant test series was conducted in order to establish preferred conditions for trona flotation and to determine the influence of operating variables on the separation efficiency. The dry feed was conveyed to high solids conditioning tanks, in which the calibrated amount of reagents and mine water were added to obtain $75 \%$ solids conditioning. The slurry was then conditioned for 2 min in both conditioning tanks and then discharged by gravity into the dilution tank. In the dilution tank, the slurry was diluted to about $15 \%$ solids with the addition of fresh saturated trona solution and pumped to the flotation equipment. The slurry flow rate was adjusted with the control valve. Each test lasted for at least 30 min during which time operating variables were kept constant at desired values. Samples of float and sink products were taken for analysis. Such samples were dried, weighed and analyzed for insoluble mineral content.

For the pilot-plant setup, the flowsheet and equipment list were prepared with the assistance of Roberts \& Schaefer during the summer semester. The trona flotation system includes two high solids ( $>70 \%$ by weight) reagent conditioning tanks with an impeller for stirring, dilution tank with a mixer, vibratory feeder with belt conveyor to transfer feed material to the conditioning tank, pulp circulation pipe, Galigher pump to pump 15\% solids into the flotation cells and a $10 \mathrm{ft}^{2}$ belt filter. In addition, the system includes an amine preparation tank, reagent preparation tank, mine water/trona saturation tank, impellers for the amine and reagent tanks and a reagent pump and concentrate recirculation pump.

Initially, the flotation machine shown in Figure 5 was used for the first pilot plant tests and several flotation tests were performed with this unit under the desired operating conditions. Since some problems occurred during previous continuous pilot scale flotation experiments, several batch flotation experiments were performed in one of the large cells (9.3 gallons) (Figure 6) to find the preferred conditions in the second pilot plant system.

\section{Experimental Procedure, Pilot Scale Test at University of Utah}

A pilot flotation circuit was also installed at the University of Utah. Set-up and installation of the pilot plant was accomplished with some new design features to appropriately conduct reverse flotation of gangue minerals from the trona feed. The trona flotation system included a Hazen-Quinn Process Co. flotation machine (2 cells, 70 liter total), high solids (60-75\% by 
weight) reagent conditioning tank with an impeller for stirring, dilution tank with a mixer, high solids slurry discharge valve, brine storage tank, vibratory feeder with a pipe to transfer feed material to the conditioning tank, peristaltic pump to pump emulsion into the conditioning tank, sump pump to prepare saturated trona solution (brine), mixers for amine and emulsion preparation and filtration equipment.

Shown in Figure 7 is the modified trona flotation pilot plant system. Once constructed and properly calibrated, the pilot plant system followed a simple procedure. The trona sample was screened to remove fines (minus 200 mesh) in order to obtain increased flotation separation efficiency. The overall grade of feed sample was $18 \%$ insolubles. The feed sample was fed into the high solids conditioning tank through the vibratory feeder at a calculated solids flow rate. In the conditioning step emulsion (calculated mixture of amine and oil) and brine were prepared to get $65 \%-70 \%$ solids during conditioning. The slurry was mixed for $3-$ 5 minutes and then discharged by gravity into the dilution tank. In the dilution tank, the slurry was diluted to about $15 \%$ solids with the addition of fresh saturated trona solution and discharged into the 35 liter flotation cell.

\section{Collector Characterization Study (Emulsion Droplet Size Distribution)}

In this part of the research the droplet size distributions of emulsions were measured by optical microscopy. Reagents used (amine and kerosene oil) as well as their concentrations were identical to those used in the bench scale flotation studies. Dodecylamine hydrochloride (DAH) was used as a surfactant, and kerosene was used for the oil phase. In this study, four different concentrations of amine solution were selected, i.e., $1 \%, 2 \%, 3 \%$, and $4 \%\left(4.51 \times 10^{-}\right.$ ${ }^{2} \mathrm{M}, 9.02 \times 10^{-2} \mathrm{M}, 1.35 \times 10^{-1} \mathrm{M}$, and $1.80 \times 10^{-1} \mathrm{M}$, respectively).

The emulsions were stabilized by 20 cc $(0.23 \mathrm{M})$ of DAH solution using a Cole Parmer laboratory mixer (2 min, $1500 \mathrm{rpm}$ ). The ratio of amine to oil was 1:12 by weight for each experiment. In each case, the emulsion was equilibrated for $2 \mathrm{~min}$. A sample of emulsion was collected and immediately submitted to microscopic analysis. An optical microscope, model AXIOPLAN (ZEISS, Germany) and microscopic slide with master scale were used. Ten photos of each sample were taken and the droplet sizes were determined from these photographs. Five hundred to 700 droplets were counted to obtain the size distribution curve (5 photographs were selected for each distribution and every droplet on each photograph was counted).

\section{$\underline{\text { Results and Discussion }}$}

\section{Bench Scale Tests Results}

A series of 1-liter bench scale flotation experiments were conducted in order to explore the influence of flotation variables. The effects of particle size distribution of feed, collector dosage, different collectors, amine to oil ratio, $\mathrm{pH}$ of amine solution, conditioning/flotation time, conditioning pulp density, flotation percent solids and brine composition were studied. With these experiments the aim was to establish appropriate flotation conditions and 
operating variables for plant-site testing. Consequently commercial amines were used for the flotation tests.

\section{Different Collectors}

Three different amine solutions using Armeen 12D, Armeen 16D \& Armeen 18D (12, 16 \&18 carbon atoms respectively) were prepared for the bench scale flotation experiments in the laboratory. In these experiments, $5 \mathrm{~kg} / \mathrm{t}$ of amine dosage was used. Flotation results in Figure 8 indicate that as the chain length of the amine increases, the flotability of solubles and insolubles also increases slightly, which reduces the recovery of trona but improves the quality of the trona concentrate. It was found that Armeen 18D's longer chain and therefore greater collecting ability than Armeen 12D causes more of the trona to be floated and hence reduces the recovery of trona. With Armeen 18D, a high quality trona concentrate (99.1\%) was achieved in comparison to the concentrate quality with Armeen 12D (98.6\%), but the recovery was low, which may be improved by reducing the amine dosage. However, amine dosage reduction might result in a trona concentrate of low quality. Thus, Armeen 12D was chosed for use in further tests because of its consistently good flotation results and improved froth stability.

\section{Amine Dosage}

Standard laboratory flotation experiments were carried out to determine the effect of amine dosage for different feed grades. The results are presented in Figure 9. Amine dosages were varied from $2 \mathrm{~kg} / \mathrm{t}$ to $5 \mathrm{~kg} / \mathrm{t}$. The flotation results for different amine dosages show that the amount of amine needed to achieve a specified grade increases with a poor quality feed. However, there is not a big difference in recovery.

\section{$\underline{\text { Amine to Oil Ratio }}$}

The effect of dry amine to oil ratio on the flotation of trona was investigated. Flotation results are shown in Figure 10. It was found that, with respect to concentrate grade, the best A/O ratio is 1:12 for the emulsion, prepared with the high-speed mixer. At $5 \mathrm{~kg} / \mathrm{t}$ of amine dosage and a $1: 12 \mathrm{~A} / \mathrm{O}$ ratio, a concentrate grade of about $99.5 \%$ was obtained with $91 \%$ recovery of trona. The grade is increased to $99.8 \%$ if the $\mathrm{A} / \mathrm{O}$ ratio is changed to $1: 8$. However, as can be seen from Figure 10, the recovery is clearly low if the A/O ratio is 1:8.

\section{Amine Solution $\mathrm{pH}$}

The $\mathrm{pH}$ of the amine solution is an important factor that may influence floatability. Several experiments were done to investigate the effect of $\mathrm{pH}$ on the amine solution on the insoluble content of the concentrate. The $\mathrm{pH}$ was varied from 4 to 10 and the optimum floatability of insoluble minerals was achieved between $\mathrm{pH} 8.5$ and 10. The results, shown in Figure 11, indicate that as the $\mathrm{pH}$ of the amine solution increases, the floatability of insoluble minerals increases, and therefore the $\mathrm{pH}$ of the amine solution must be greater than $\mathrm{pH} 8.5$ to realize a high purity concentrate. 
Conductivity measurements were also carried out to investigate the relation between the $\mathrm{pH}$ and the conductivity of the amine solution. The conductivity data are also presented in Figure 11 and support the above finding. It was found that when the $\mathrm{pH}$ increases, the floatability of insoluble minerals also increases and these conditions correspond to a low value of conductivity. The experimental results reveal that there is a direct correlation between $\mathrm{pH}$ and conductivity of the amine solution.

\section{$\underline{\text { Particle Size }}$}

Flotation experiments for different particle sizes were carried out and the results are shown in Figure 12. It can be seen that for the fine particle size distributions, especially in the presence of a large amount of slimes, flotation is not as effective. The best separation was achieved for coarse particle size material. In this regard it seems that the particle size for flotation feed material should be minus $1 \mathrm{~mm}$ top size with the minus 200 mesh fraction removed since the minus 200 mesh material contains only 1 to $2 \%$ insoluble and can be used directly as concentrate.

\section{$\underline{\text { Flotation Percent Solids }}$}

The effect of flotation percent solids on reverse flotation performance is presented in Figure 13. These bench scale experiments were performed with the 30x200 mesh size fraction. From the data given in Figure 13 it is evident that the diluted pulps (5\%) do not float insoluble minerals well resulting in a poor grade (3.2\%) of concentrate with about $85 \%$ recovery. When the flotation tests were carried out in suspensions, $15 \%$ solids or more, the flotation response of insoluble minerals changed considerably. At 15\% solids, trona concentrate with a purity of almost $99 \%$ trona at a recovery of more than $75 \%$ was obtained.

\section{$\underline{\text { Flotation Time }}$}

Flotation tests were carried out with the 30x200 mesh trona sample to investigate the effect of flotation time. It was found that $75 \%$ of the gangue minerals were floated in 2 min. However, for successful flotation, $4 \mathrm{~min}$ are required to float most of the gangue minerals. The flotation recovery decreased when the flotation time was lengthened to $4 \mathrm{~min}$ as trona started floating with gangue minerals. The results are shown in Figure 14.

\section{Flotation with Recycled Saturated Solution}

Flotation with recycled saturated solution is of considerable importance in order to reduce consumption of water and brine. Flotation experiments with recycled brine (one time recycled) were conducted in order to study the effect on flotation efficiency. A feed material (16 x 100 mesh) containing about 18\% insolubles was used. Shown in Table 1 is the result for flotation with both fresh saturated solution and recycled saturated solution. The results indicate that when the recycled saturated solution is used, there is no significant effect on the efficiency of the flotation separation. It should be noted that if brine is recycled more than one time then separation efficiency decreases due to the poor quality of the brine. 


\section{$\underline{\text { Flotation with Different Brines }}$}

Four different brines were prepared for the bench scale flotation experiments in the laboratory using a high intensity mixer. These four brines were trona saturated solution, mine water, synthetic sodium carbonate solution, and sodium carbonate solution from the plant. For the preparation of saturated solutions, a special hot bath and a filtration device were employed to maintain constant temperature $\left(40^{\circ} \mathrm{C}\right)$ throughout the experiment, since sodium carbonate has the tendency to crystallize at room temperature. The results from flotation with different saturated solutions are shown in Table 2.

The results presented in Table 2 indicate that the trona saturated solution is the most effective for the trona flotation process. It can also be seen from the results that saturated mine water may be used for flotation instead of trona brine since it also gives a good recovery of trona and reduces the cost of brine preparation. The mine water obtained from the mine may be contaminated and hence affect the adsorption/wetting of the collector at the gangue particle surface. The effect of temperature on the flotation of insoluble minerals was studied and the results indicate that when the temperature increases, the floatability of insoluble minerals decreases. In the case of sodium carbonate solution, the high viscosity ( 3 CP) may contribute to the poor trona flotation.

\section{$\underline{\text { Pilot Testing at Plant Site }}$}

\section{Preparation for Plant-Site Testing}

Pilot plant testing is of considerable importance for the evaluation of the flotation technology by industry. The objectives of designing, constructing and operating the pilot plant were to: (1) evaluate the efficiency and practical applicability of the proposed technology in an industrial setting, (2) ascertain that the flotation parameters and conditions are reliable to apply in continuous tests at the plant site, and (3) obtain comparative and performance data for the flotation processes.

In this regard, the design of the pilot plant for the trona flotation system was accomplished with the assistance of General Chemical, Roberts \& Schaefer and EIMCO. The construction and installation of the pilot plant involved flowsheet development, preparation of an equipment list, and process design and monitoring. The flowsheet was developed using MODSIM software after taking into account all basic input information provided by General Chemical. This flowsheet contains major items of equipment and all relevant flows in and out of each piece of equipment. The detailed flowsheet is shown in Figure 15.

A photo of the facilities (Vibratory Feeder, Belt Conveyor, Conditioning and Dilution Tank) for the pilot scale flotation test campaign at General Chemical is shown in Figure 16. The mass and water flow rates were calculated for 1 tph of feed. The specific gravity of trona feed 
was $2.2 \mathrm{~g} / \mathrm{cm}^{3}$, while the density of the trona saturated solution (brine) was $1.21 \mathrm{~g} / \mathrm{cm}^{3}$. In addition, the density of the amine solution and kerosene were 0.98 and $0.75 \mathrm{~g} / \mathrm{cm}^{3}$, respectively. These values were used for mass and water flow rate calculations. Table 4 shows the equipment list for plant site tests.

\section{$\underline{\text { First Pilot Plant Results }}$}

The first pilot plant tests and several flotation tests were performed under optimum operating conditions obtained from bench-scale flotation experiments as described in Table 3. Experimental observations showed that this flotation unit was not convenient for our study. The equipment was too large and hard to handle since its capacity is about 66 gal, consuming a huge amount of feed material and producing a concentrate of poor quality (about 9\% insolubles) with an exceptionally low trona recovery. The possible reasons for poor flotation separation during pilot plant tests were believed to be inadequate conditioning, overmixing of slurry in the dilution tank, unstable adsorption of reagents on solids, greater amount of fines (50\%) in flotation feed and usage of mine water instead of trona brine. Therefore, another pilot plant was designed taking the above issues into account.

\section{Large Scale Batch Flotation}

Due to the problems mentioned above during previous continuous pilot scale flotation experiments, several batch flotation experiments were performed in a 2-cell flotation machine shown in Figure 6 to find the optimum conditions in the second pilot plant system. The total flotation volume of this second pilot plant system is about 18.6 gal. Tests were first performed in one of the large cells (9.3 gallons) with mine water, which was obtained from the plant. A trona concentrate of $97.43 \%$ purity was obtained with a recovery of $60.79 \%$. Removing the fine particles from the system increased the recovery, but only slightly reduced the insoluble content of the product.

As the insoluble content of the trona concentrates are high $(2.57 \%$ and $2.3 \%)$, it was decided to use trona brine instead of mine water for flotation experiments to see the effect of brine composition on pilot scale flotation tests. The brine also has a significant effect on flotation. By using trona brine, it is possible to reduce the insoluble content of the feed to less than $1 \%$. In addition, the removal of fines from the system not only increased the recovery but also reduced the insoluble content of the product. Hence a trona concentrate of $99.57 \%$ purity was obtained with a recovery of $87.27 \%$ using commercial amine with pilot scale flotation equipment. However, this situation requires the use of considerably more trona for preparation of the brine. As mentioned previously the presence of fines significantly affects the recovery of trona. Further results showed that a trona saturated solution is most effective for the trona flotation process and a trona concentrate of $99.5 \%$ purity can be obtained with a recovery of around $90 \%$. Under these conditions, the trona product meets concentrate grade specifications for use in the glass industry and for other purposes.

$\underline{\text { Second Pilot Plant Tests }}$ 
The results obtained from these large scale experiments were applied in further continuous pilot scale flotation tests. Instead of two conditioning tanks, one tank was used to avoid the long conditioning time. In addition, the dilution tank and Galigher pump were removed from the system and conditioned slurry was discharged into a plastic container (as a dilution tank), which was directly connected to the flotation cell. The trona brine was also added in this container for dilution. This modified system is shown in Figure 16. A couple of continuous tests were carried out with this new system, but the campaign had to be terminated because of weather conditions and time constraints. Second pilot plant tests were carried out under operating conditions as listed in Table 4.

It is quite clear from the results that this trona flotation system works very well for both small and large scale batch experiments. It is important to note that usage of brine on screened feed (after removing minus 200 mesh particles) significantly improved the grade and recovery of trona. The results indicate that a trona concentrate of $99.57 \%$ purity was obtained with a recovery of $87.27 \%$ using the University of Utah's pilot scale flotation equipment. Thus, further study of this flotation technology was continued at the University of Utah pilot plant due to weather conditions, feed availability, and time considerations. It was expected that the above mentioned problems could be avoided by proper adjustment of flotation operating conditions and variables. For example, brine (trona saturated solution) was to be used instead of mine water to obtain better results. Also, the fine particles (minus 200 mesh) from the trona feed were to be screened out in order to reduce the dissolution of fine particles during trona flotation.

\section{$\underline{\text { Pilot Testing at University of Utah }}$}

The trona flotation system was installed in such a way to insure that efficient and reliable flotation experiments could be carried out in a reasonable time interval so that more design and operating variables could be established for each pilot scale experiment, which involves from 3-30 kg feed material depending on the flotation retention time and percentage solids to be examined. The pilot scale flotation experiments were carried out in laboratory at the University of Utah. Figure 17 is the conditioning process. Shown in Figure 18 and 19 are the flotation separation in the flotation cell and the concentrate product, respectively.

In order to determine the proper operating conditions and values for reagent dosages (amine and oil), several flotation experiments were carried out for different amine dosages. The results are presented in Figure 20. For these flotation tests, the results for concentrate (\% gangue) and recovery (\% trona), are $2 \%$ and $75 \%$ respectively as shown from data presented in Tables $5-7$. These results indicate that an increased trona grade and recovery are possible at low levels of collector addition. However, one continuous operation of trona flotation at collector dosage of $4 \mathrm{~kg} / \mathrm{t}$ (shown in Table 8 ) resulted in only a modest decrease in grade; a collector dosage of $3 \mathrm{~kg} / \mathrm{t}$ seems to be appropriate under these conditions.

Another continuous pilot plant test was performed with $4 \mathrm{~kg} / \mathrm{t}$ of amine dosage. This test was carried out to ensure the validity of previous results. The total run-time was $30 \mathrm{~min}$. The results obtained from this test are shown in Table 8. As can be seen from Table 8, a trona concentrate of $96.04 \%$ purity was obtained with a recovery of $72.14 \%$, using commercial 
amine with pilot scale flotation equipment. It was observed that the high-solids conditioning tank takes approximately $15 \mathrm{~kg}$ feed to be able to discharge the slurry (dry solids + reagents + brine) into the dilution tank. In this continuous operation, the concentrate products were collected in 5 gallon buckets after every 5 minutes. The concentrate products were then dewatered, dried and analyzed separately. The results shown in Figure 21 indicate that a uniform product of consistent grade can be achieved for the first $15 \mathrm{~min}$. However, the trona recovery decreased rapidly due to inconsistent feed rate and brine flow rate when the flotation operation was lengthened to more than 15 min. This problem can be avoided by proper adjustment of the vibratory feeder and brine discharge tank.

Table 8 shows the results for pilot plant at the University of Utah operating continuously for $30 \mathrm{~min}$ at optimum operating conditions. The results from pilot plant tests indicate that under optimum operating conditions good separation can be achieved in reverse flotation of trona with a capacity of about $110 \mathrm{lb} / \mathrm{h}$ dry solids and an amine consumption of $4 \mathrm{~kg} / \mathrm{t}$. Again the flotation results from the trona flotation pilot plant system indicate that this trona flotation technology can be used by the trona industry for the production of a high quality trona concentrate.

\section{Emulsion Droplet Size Distribution Results}

Micrographs are shown in Figures 22 to 23. The droplet size distributions for oil-in-water emulsions are shown in Figure 24. It can be seen from Figure 24 that there is, essentially, no change in size distribution for $1 \%$ and $2 \%$ DAH. After $2 \%$, the droplet size distribution by number becomes narrower and shifts to smaller sizes.

The results indicate that the size of droplets decreases with an increase in amine concentration. The data show that $3 \%$ and $4 \%$ solutions are finely dispersed emulsions which affect the trona flotation as shown in Figure 25. The small oil droplets facilitate the fast and selective distribution at gangue surface.

Figure 25 shows that grades with 3\% amine were better than those obtained with 1\%, and $2 \%$ amine, and slightly better than $4 \%$ amine. Diluted amine emulsions (1\% and $2 \%$ ) require a higher amount of collector for flotation than $3 \%$ and $4 \%$ amine emulsions. For instance, at $5 \mathrm{~kg} / \mathrm{t}$ collector dosage, the $1 \%$ amine solution will need 75 cc of collector whereas the $3 \%$ solution requires only 25 cc. The high volume of amine solution causes the dissolution of trona which results in inferior flotation results. In addition, 3\% and $4 \%$ amine emulsions have finer droplets than $1 \%$ and $2 \%$ as shown in Figure 24, and facilitate fast and stable adsorption on solids.

In addition, a few bench-scale flotation experiments were performed with different amine concentrations (1\%, $2 \%, 3 \%$ and $4 \%$ ) to complement the emulsion droplet size distribution study.

\section{Summary}


Soda ash $\left(\mathrm{Na}_{2} \mathrm{CO}_{3}\right)$ produced from the trona deposits of the Green River Basin in Wyoming by direct chemical treatment of the ore is important for major industries such as the glass, chemical, soap and detergent, pulp and paper, and water treatment industries. In order to reduce energy demand and operating costs, the production of a high purity trona concentrate for the soda ash market is of considerable interest.

The overall objective of this research was to demonstrate the feasibility of a new flotation technology for the production of a high grade trona (sodium sesquicarbonate) concentrate at a satisfactory level of recovery. Appropriate conditions for trona flotation technology were established from exploratory bench-scale experiments at University of Utah and subsequently by factorially designed experiments. Preferred conditions obtained from the bench scale experiments were applied to plant-site pilot scale flotation tests. Construction of the pilot plant system was completed and installed at the General Chemical plant site in Wyoming. The tests were carried out under preferred operating conditions. However, this pilot system was not able to yield satisfactory results due to the previously mentioned problems. Therefore, another pilot plant campaign was carried out at the University of Utah to investigate the problems faced in plant-site testing. The pilot plant flowsheet was modified with some new design features to appropriately conduct reverse flotation of gangue minerals from trona. Under normal conditions a good separation was achived typically producing a trona concentrate containing $2-4 \%$ insolubles at $70-80 \%$ recovery.

\section{Conclusions}

From these research results it can be concluded that:

- The new flotation technology for the separation of gangue minerals and production of a trona concentrate of high purity should be further considered for use by industry with appropriate adjustment of flotation operating variables. A comparison of results from bench-scale experiments and pilot plant tests is presented in Table 9.

- The insoluble content of feed is one of the most important variables in the reverse flotation of gangue. Successful flotation of insolubles is possible by proper control of the flotation conditions.

- Laboratory tests showed that even better flotation of gangue minerals from the feed is possible and a trona concentrate of about $99 \%$ purity can be obtained with a recovery of about $90 \%$ at an amine dosage of $5.0 \mathrm{~kg} / \mathrm{t}$.

- The $\mathrm{pH}$ of the amine solution is an important factor that influences floatability. As the $\mathrm{pH}$ of the amine solution increases, the floatability of insolubles increases.

- The effect of amine dosage was identified. The results show that if the feed has a high insoluble content, a higher amine dosage is required.

- Flotation percent solids was found to have significant effect on trona flotation. Diluted pulps were not successful to float insoluble minerals due to inefficient distribution of the insoluble collector at the gangue mineral surface.

- Particle size also influences trona flotation. It was found that the coarse particle size fractions (30x200 mesh) are more easily and efficiently separated by flotation at a lower amount of collector. 
- The effect of amine to oil ratio on trona flotation was investigated experimentally and statistically. It was found that the best $\mathrm{A} / \mathrm{O}$ ratio is $1: 12$, which can produce about a $99.5 \%$ of concentrate grade with $91 \%$ recovery of trona.

- The results from the $U$ of $U$ pilot plant tests indicate that under normal conditions a good separation can be achieved in amine flotation with a capacity of about $110 \mathrm{lb} / \mathrm{h}$ dry solids.

\section{$\underline{\text { References }}$}

Brison, R.J., and Gathje, J.C., 1981. "Beneficiation of Trona by Flotation,” U.S. Patent 4,283,277, Stauffer, 5 pp.

Datta, R.S., and Salotti, C.A. 1990. "Process for Beneficiating Oil Shale Using Froth Flotation,” U.S. Patent 4,968,413.

Garrett, D.E., and White, W.R., 1970. "Method for Separating Choride, Sulfate and Carbonate Salts, and theLlike," U.S. Patent 3,525,434, 3 pp.

Garrett, D.E., 1991. Natural Soda Ash; Occurrences, Processing, and Use, Van Nonstrand Reinhold, NewYork.

Kostick, Dennis, 1994. Soda Ash: Industrial Minerals \& Rocks, Editors D.D. Carr, Sr., $6^{\text {th }}$ Edn. SME, Littleton, CO.

Rosar, E.C., Hobaugh, J.R., and Lepetic, V.M., 1974. "Froth Flotation Method of Separating Nacholite from Ores Containing Nacholite,” U.S. Patent 3,806,044, Ind. Resources, 4 pp.

Wang, X., Li, M., and Miller, J. D., 2002. "Flotation as a Process Alternative for the Treatment ofTrona Resources,” SME Annual Meeting, Phoenix, Arizona. 


\section{$\underline{\text { Appendices }}$}

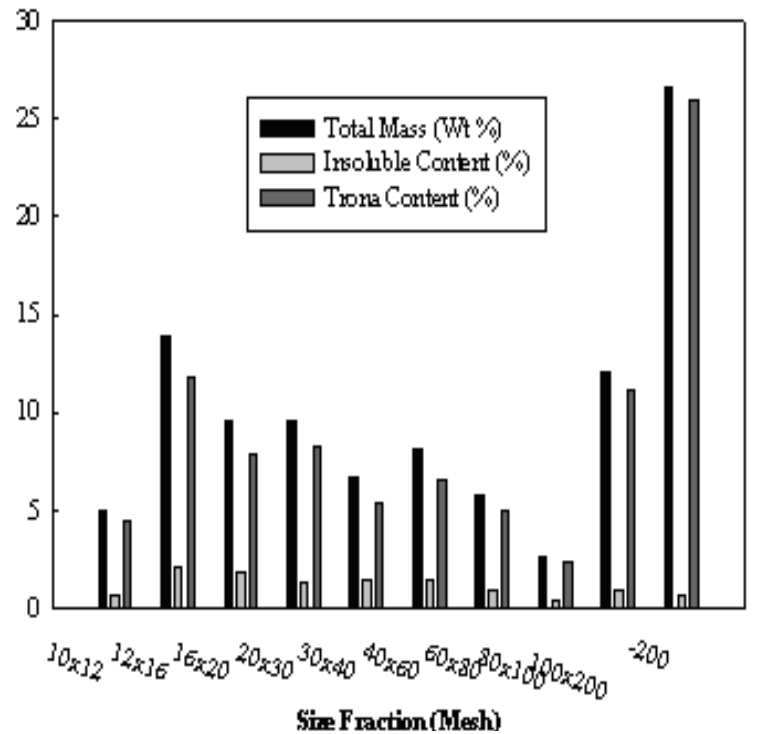

Figure 1. Total mass, insoluble and trona distributions with respect to particle size for the bench scale flotation feed sample

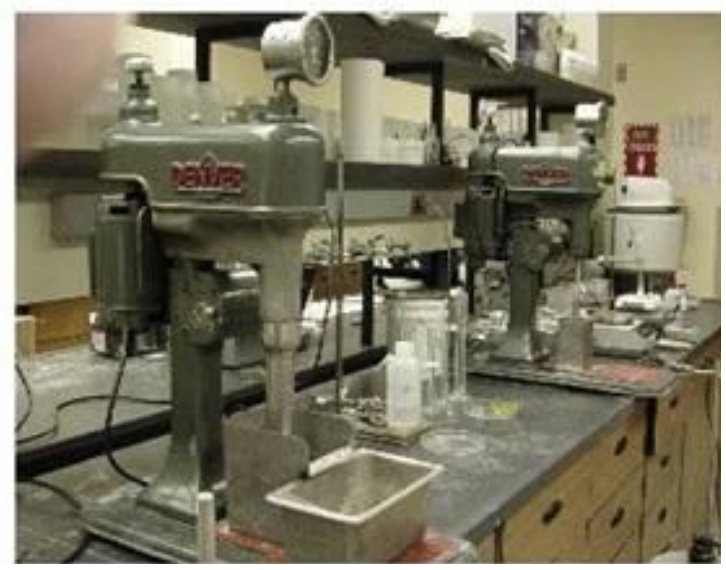

Figure 3. Denver flotation cell, high solids conditioning and centrifuge for dewatering of flotation products

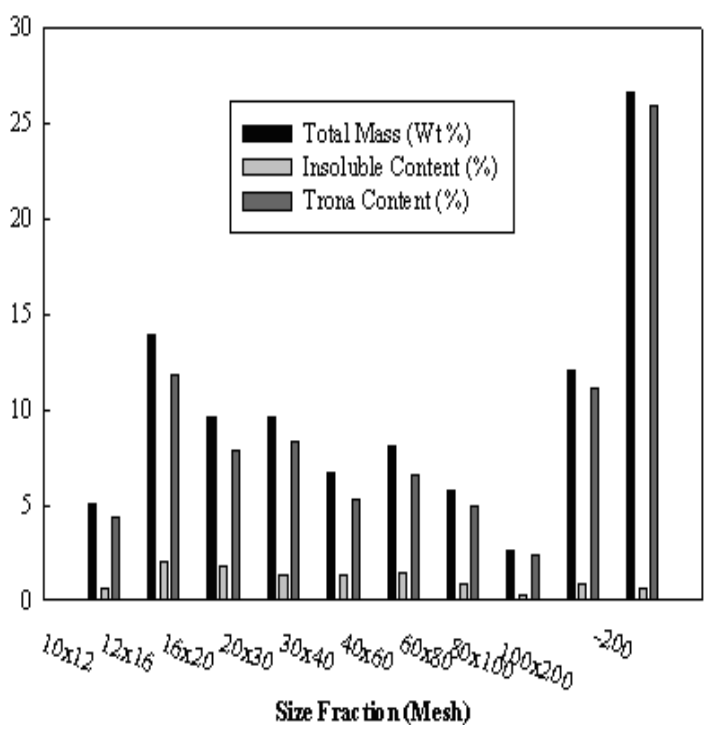

Figure 2. Total mass, insoluble and trona distributions with respect to particle size for the pilot plant flotation feed sample

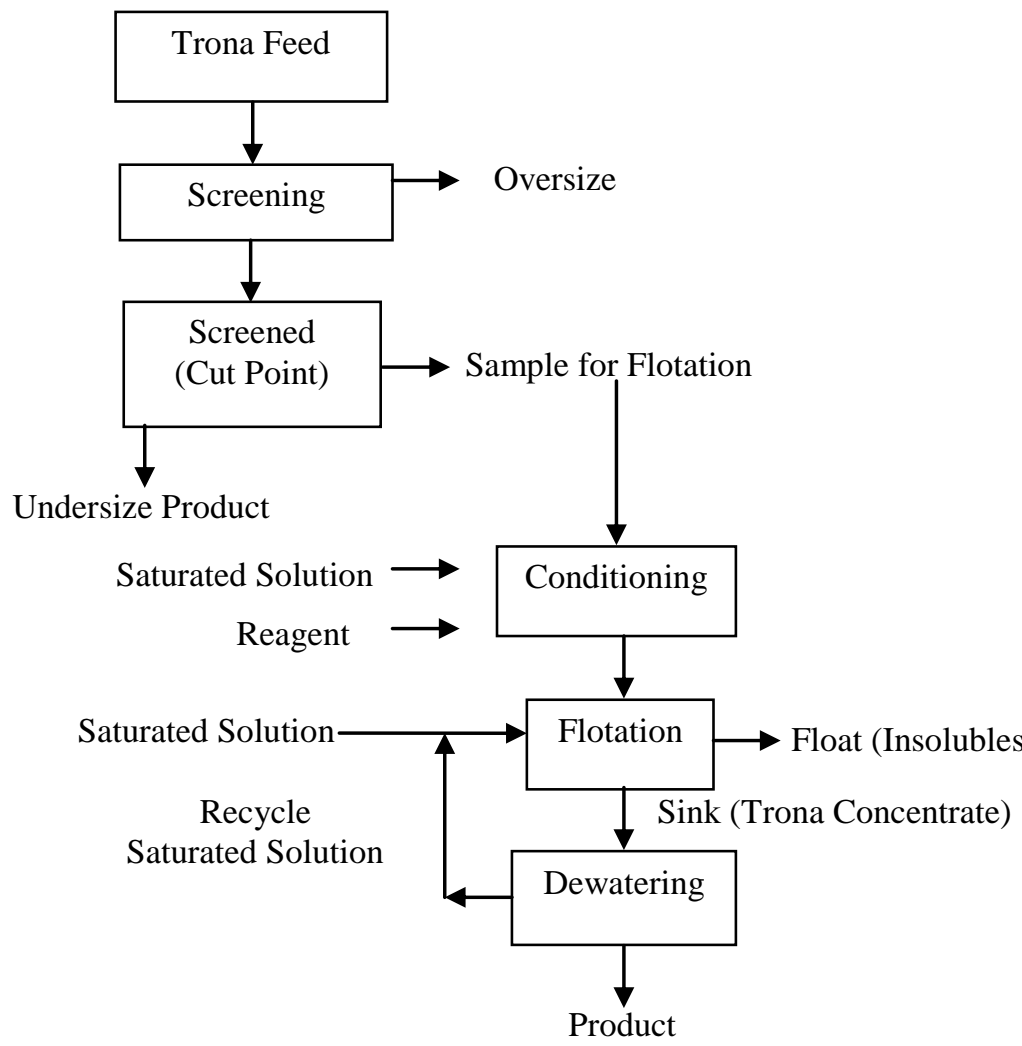

Figure 4. Procedure for bench scale flotation experiments 


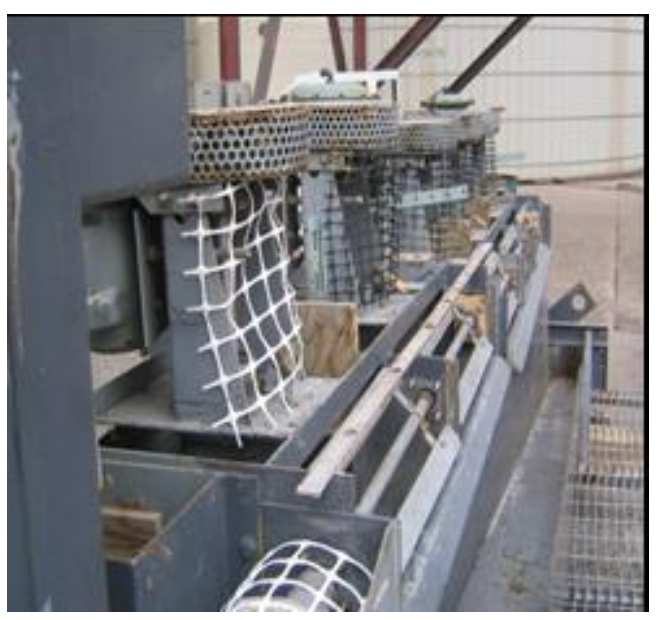

Figure 5. The flotation equipment (EIMCO) used for first pilot scale tests

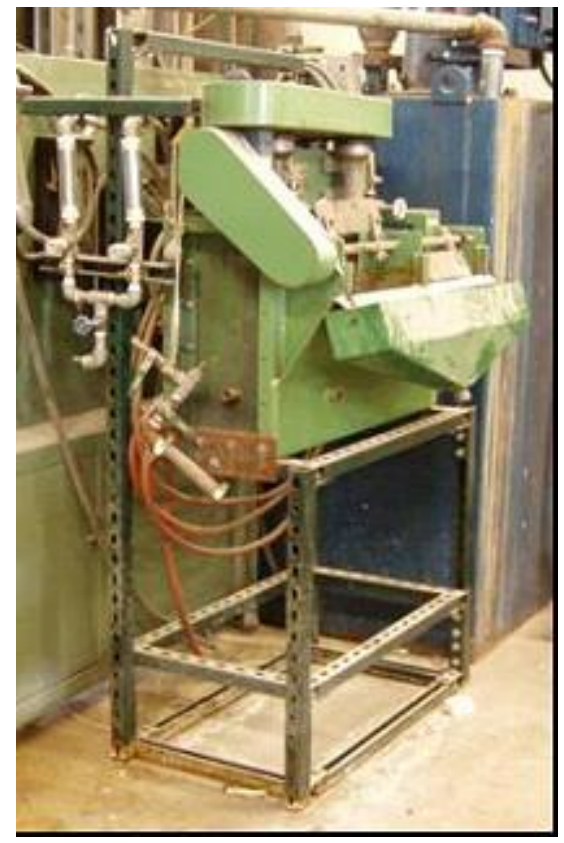

Figure 6. Hazen quinn flotation equipment for second pilot plant study at General Chemical plant site

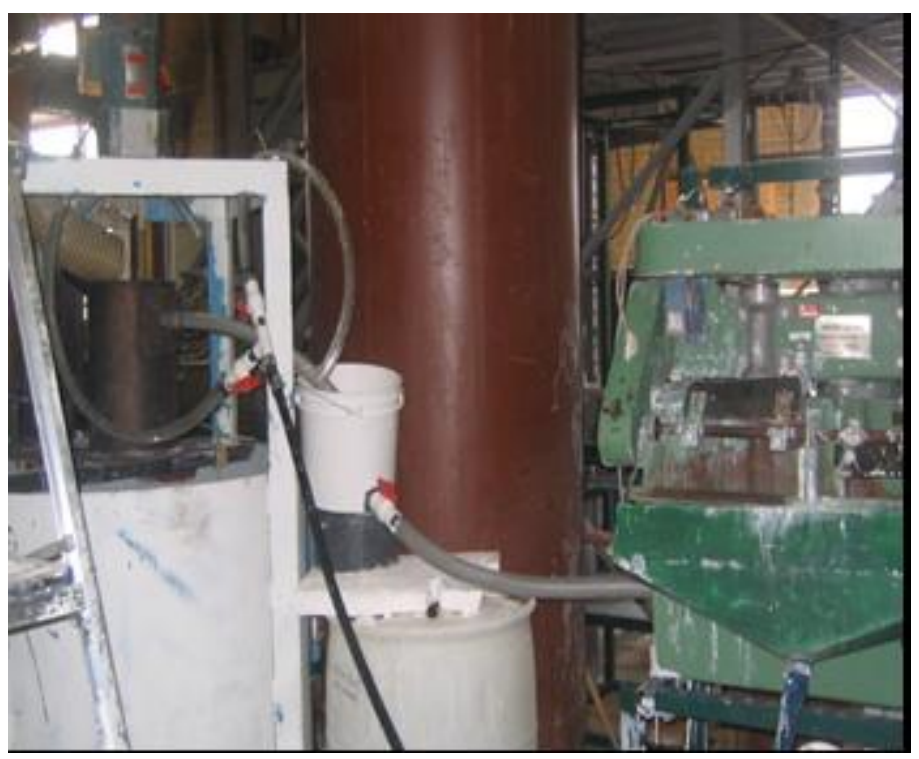

Figure 7. Trona flotation pilot plant set-up with high solids conditioning tank and dilution tank 


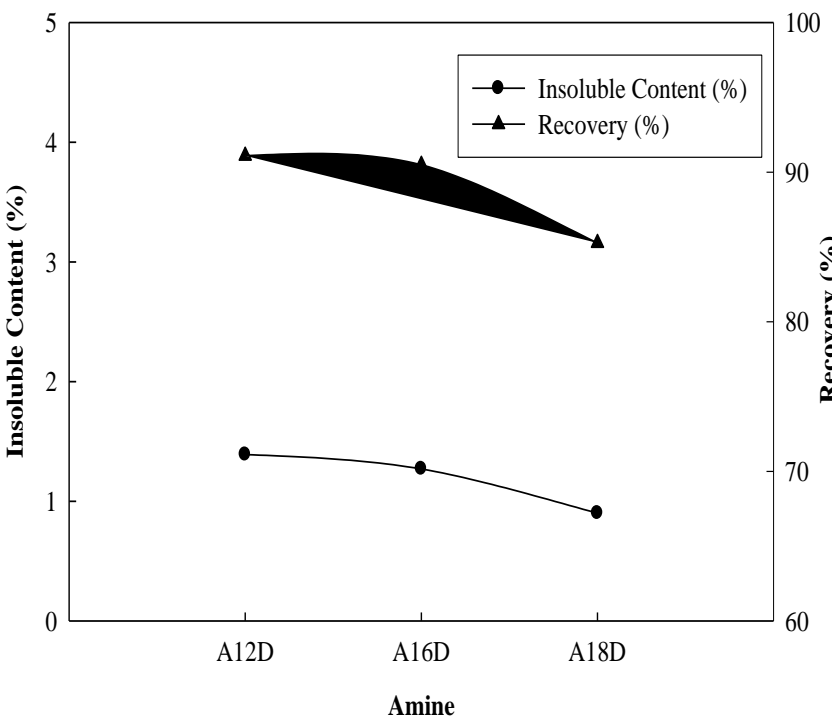

Figure 8. Effect of different amine collectors on trona flotation

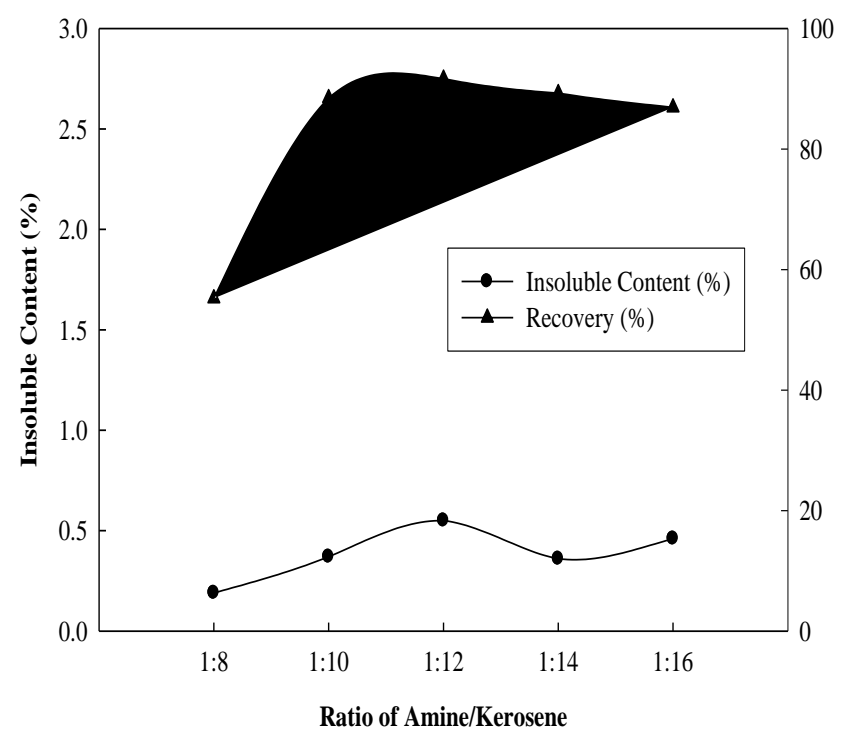

Figure 10. Effect of amine/kerosene ratio on trona flotation

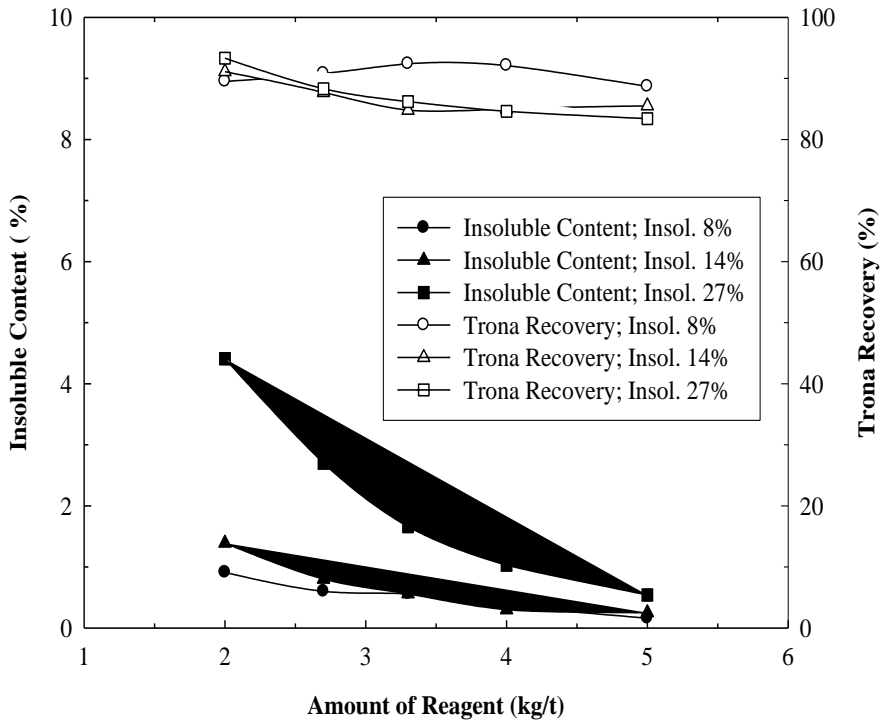

Figure 9. Effect of amine dosage on trona flotation

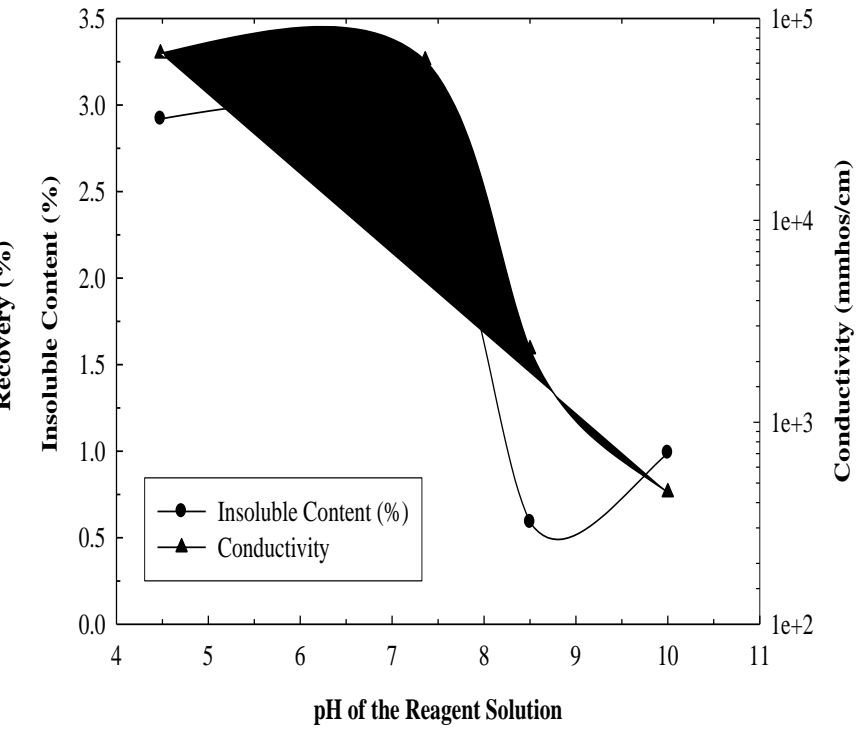

Figure 11. Effect of $\mathrm{pH}$ of amine solution on trona flotation 


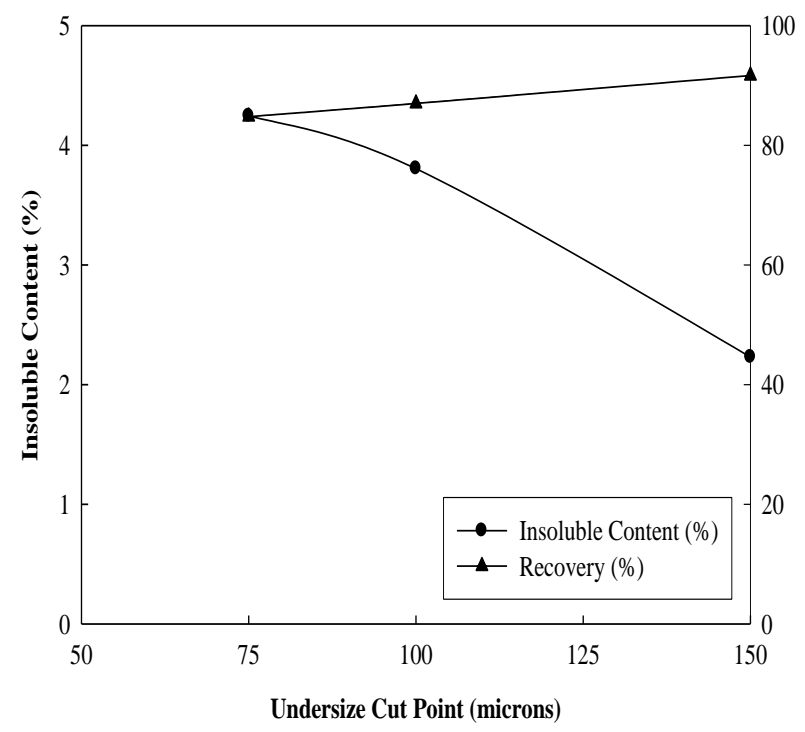

Figure 12. Effect of particle size on trona flotation

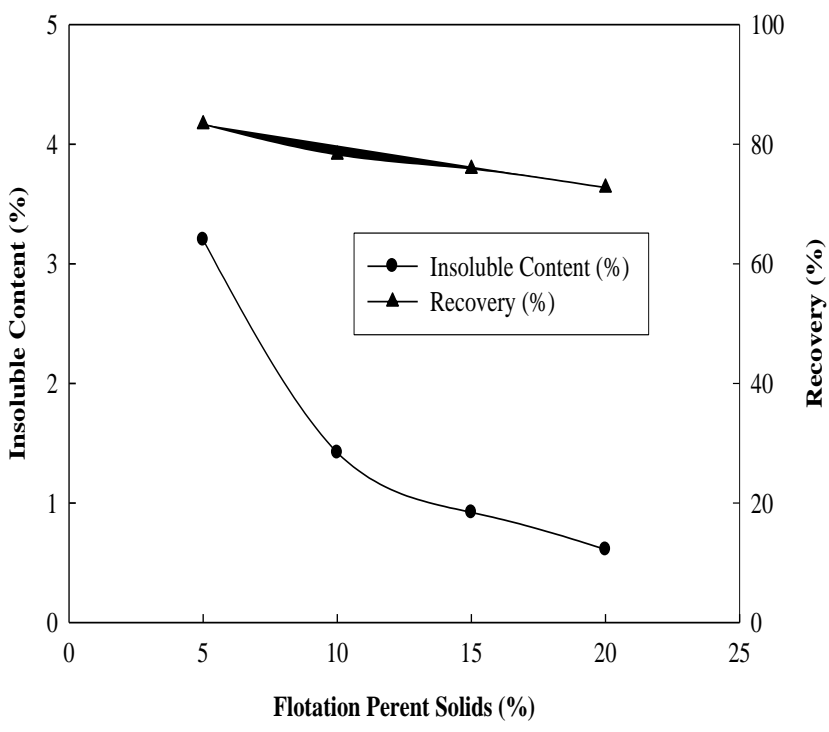

Figure 13. Effect of flotation percent solids on trona flotation

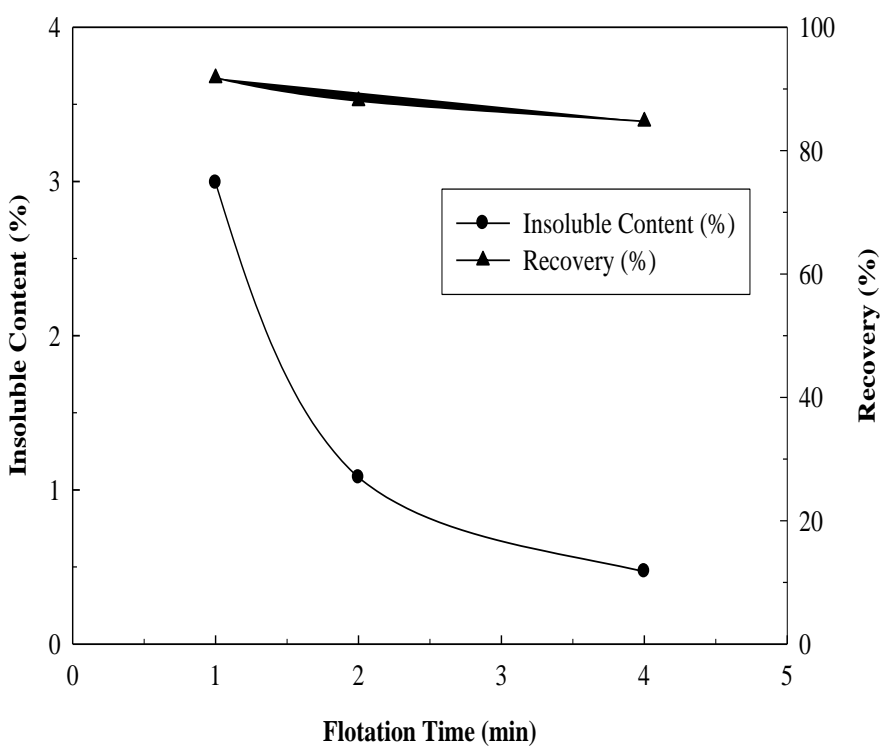

Figure 14. Effect of flotation time on trona flotation 


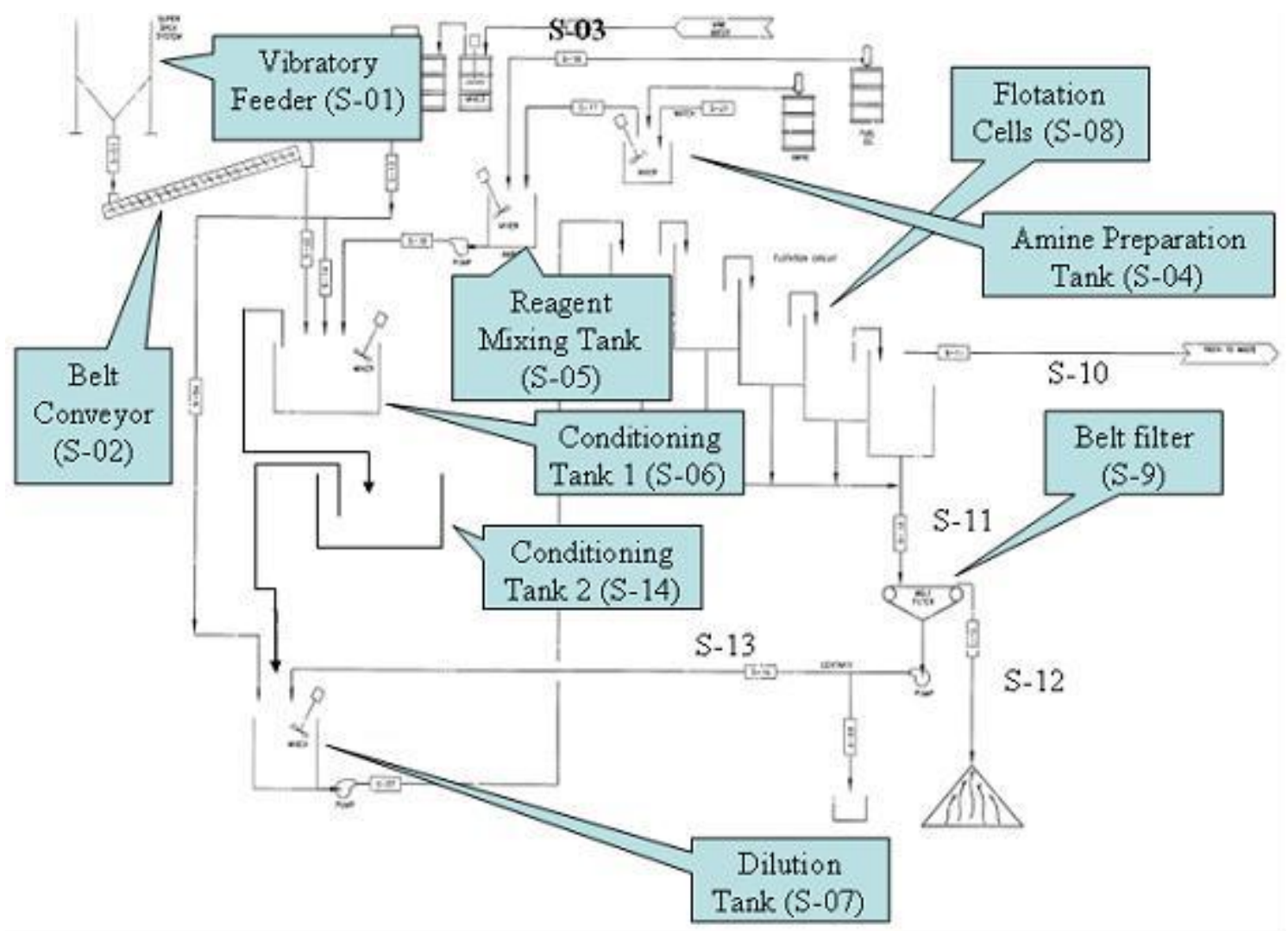

Figure 15. The modified flowsheet for the pilot plant trona flotation system

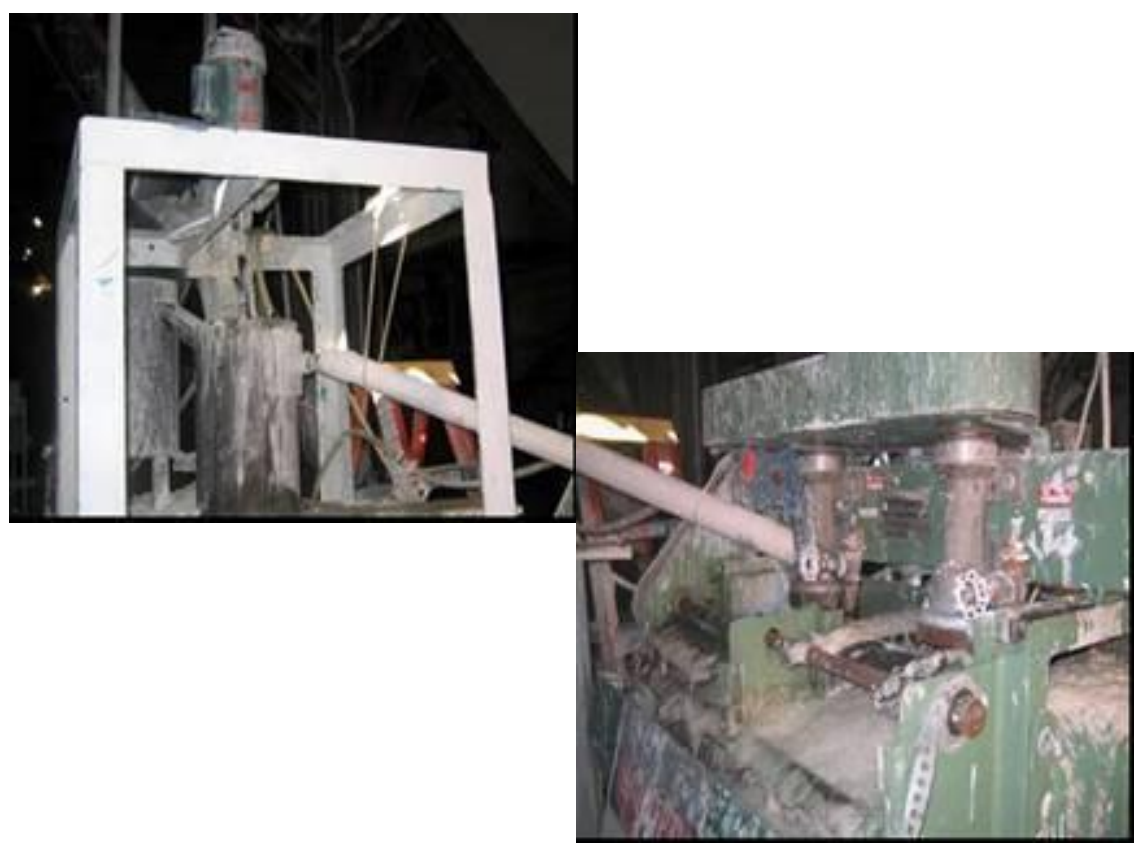

Figure 16. Facilities for the second pilot scale flotation test campaign at

General Chemical plant site 


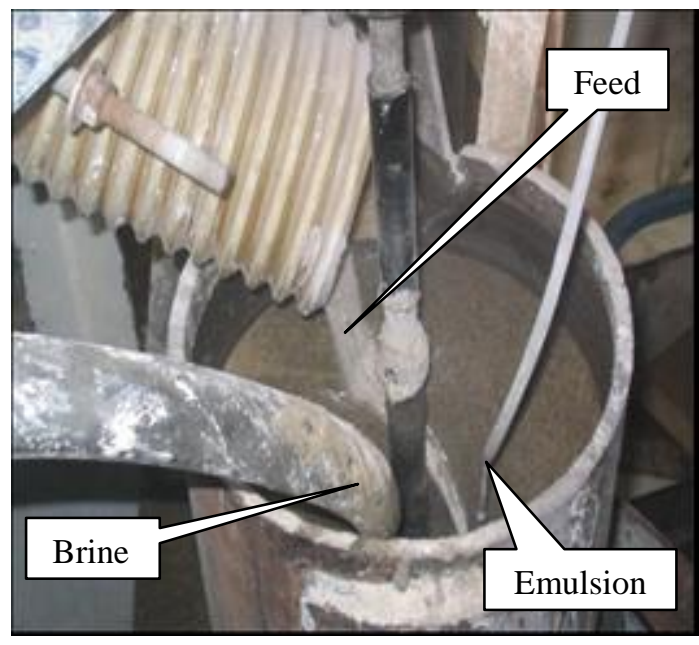

Figure 17. Conditioning process in trona flotation

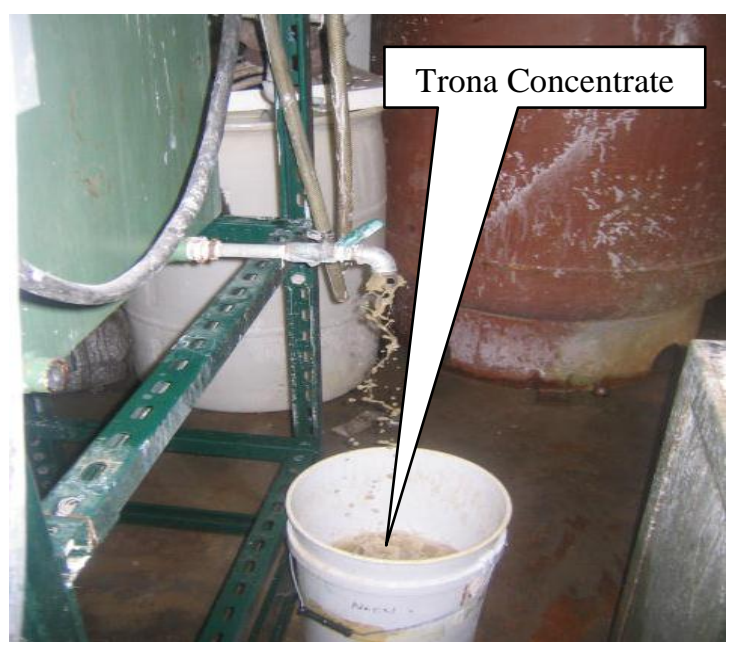

Figure 18. Concentrate product

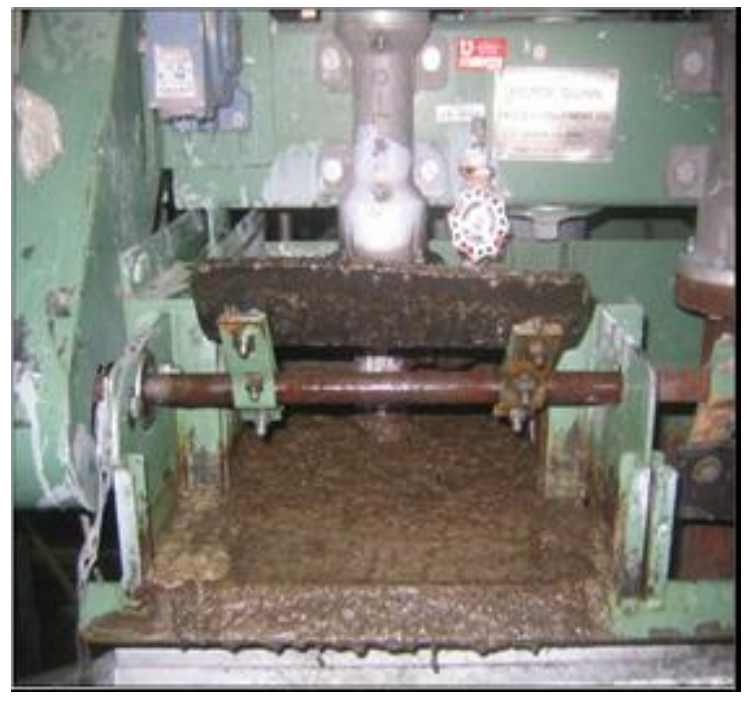

Figure 19. Flotation of gangue minerals 


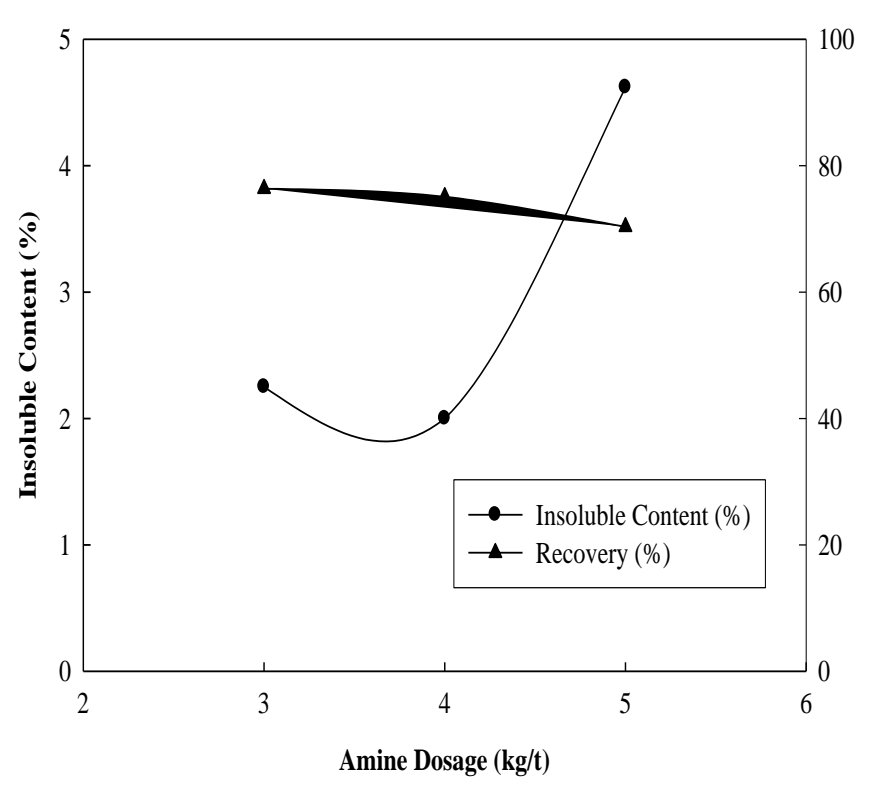

Figure 20. Trona flotation performance as a function of collector dosage for flotation feed

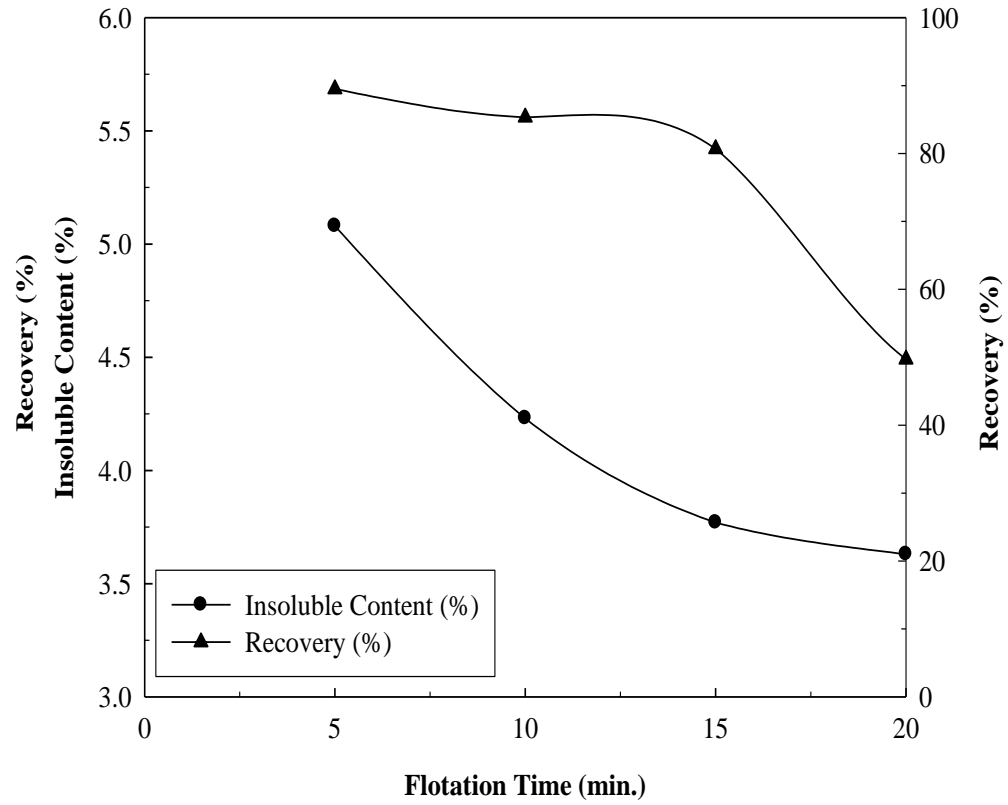

Figure 21. Effect of flotation time on continuous trona flotation
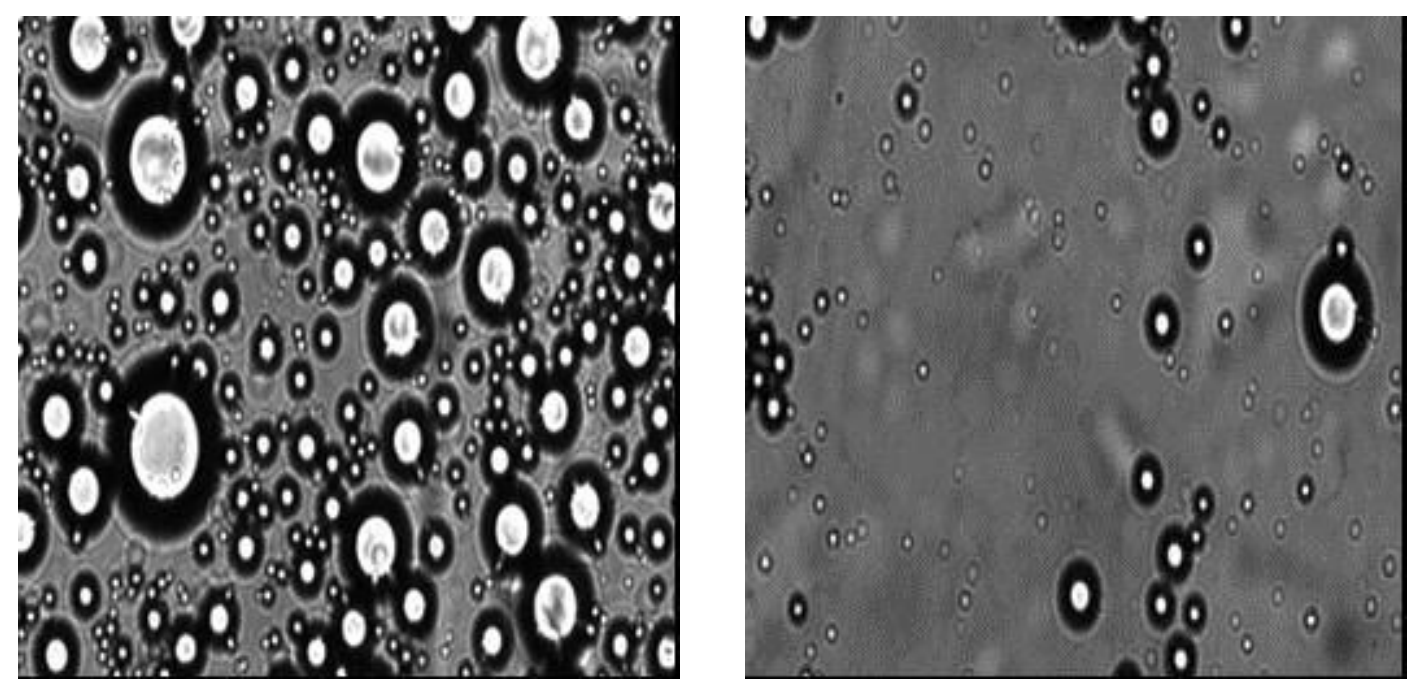

Figure 22. Photomicrograph of the dispersed drops in 1\% and 2\% DAH emulsion 

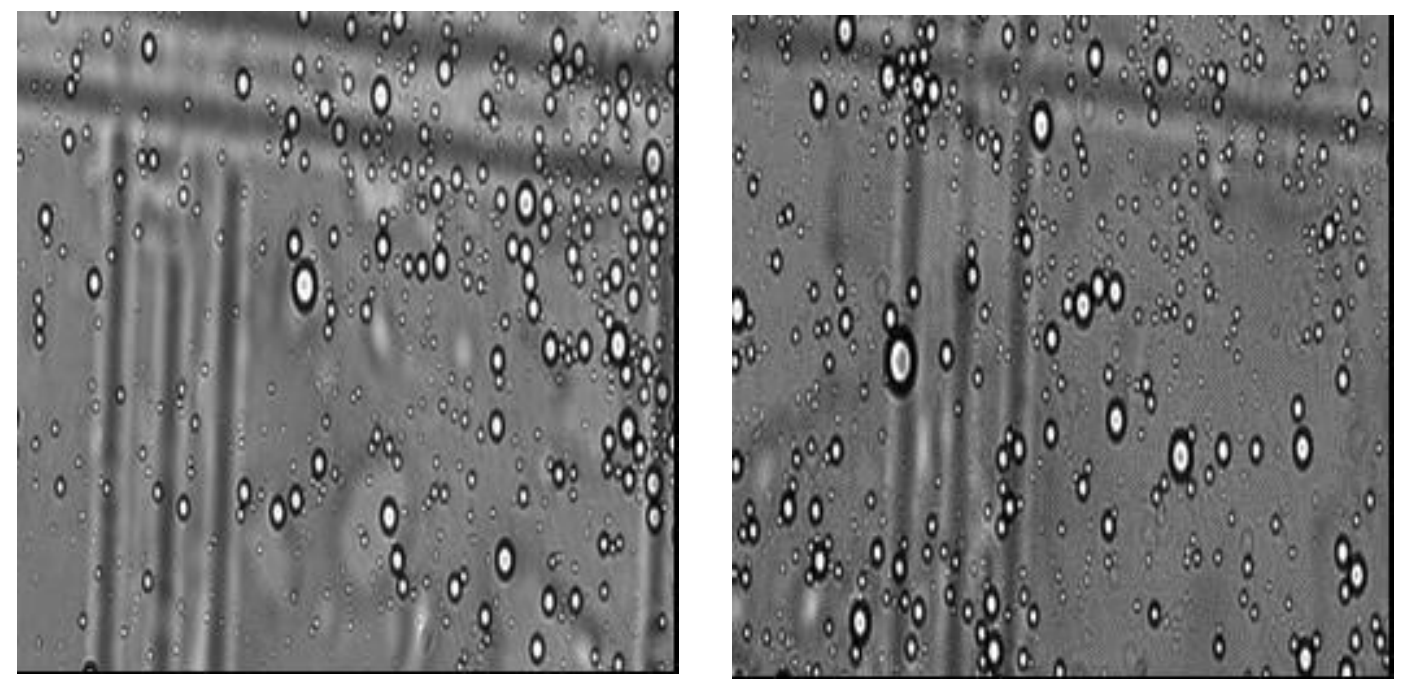

Figure 23. Photomicrograph of the dispersed drops in 3\% and 4\% DAH emulsion

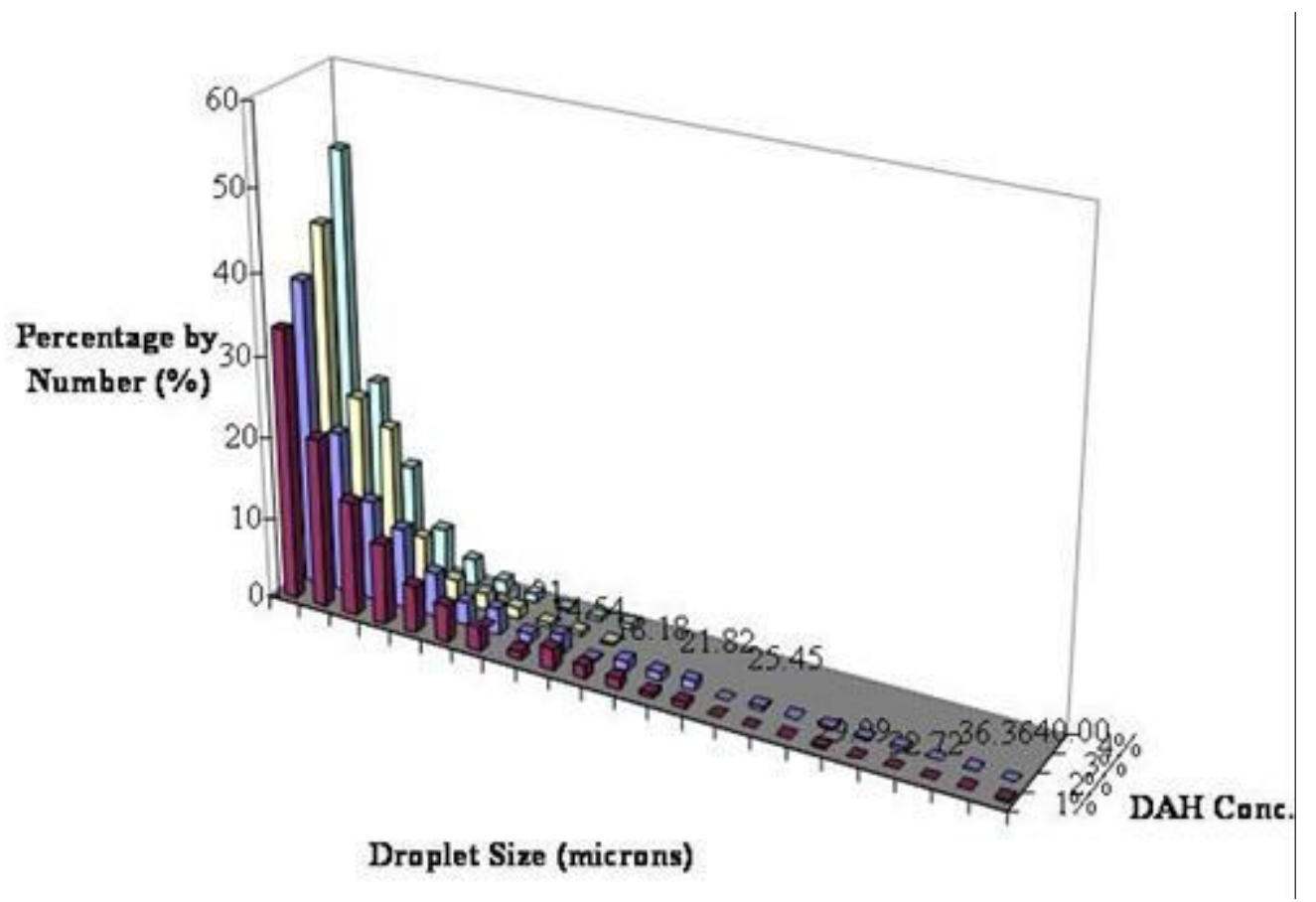

Figure 24. Comparison of droplet diameter distributions for kerosene emulsion at different DAH concentrations 


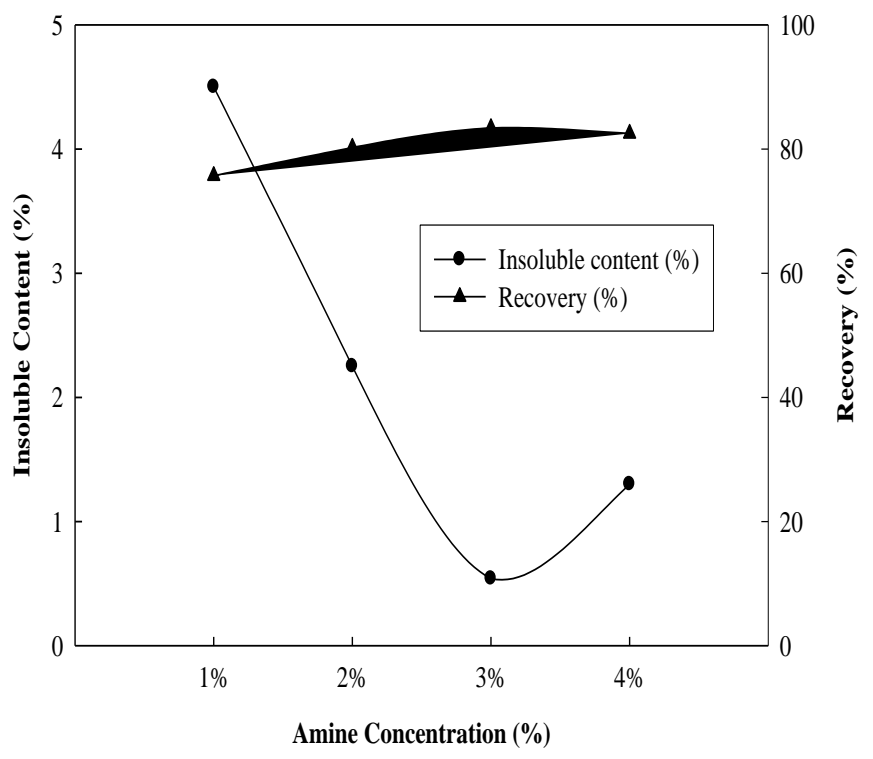

Figure 25. Effect of amine concentration on trona flotation 
Table 1. Comparison of results from flotation with fresh saturated solution and recycled saturated solution

\begin{tabular}{|c|c|c|c|c|c|}
\hline $\begin{array}{c}\text { Saturated } \\
\text { Solution }\end{array}$ & Products & $\begin{array}{c}\text { Wt. } \\
\mathrm{g}\end{array}$ & $\begin{array}{c}\text { Wt. } \\
\%\end{array}$ & $\begin{array}{c}\text { Insol } \\
\%\end{array}$ & $\begin{array}{c}\text { Trona } \\
\text { Recovery } \%\end{array}$ \\
\hline \hline \multirow{2}{*}{$\begin{array}{c}\text { Fresh } \\
\text { Saturated } \\
\text { Solution }\end{array}$} & Float & 33.50 & 23.11 & 70.30 & 8.35 \\
\cline { 2 - 6 } & Sink & 111.44 & 76.89 & 2.24 & 91.65 \\
\hline \hline Recycled & Total & 144.94 & 100.00 & 17.80 & 100.00 \\
\cline { 2 - 6 } Saturated & Float & 32.72 & 23.77 & 70.69 & 8.60 \\
\cline { 2 - 6 } Solution & Sink & 104.96 & 76.23 & 2.98 & 91.40 \\
\cline { 2 - 6 } & Total & 137.68 & 100.00 & 18.98 & 100.00 \\
\hline
\end{tabular}

Table 2. Results from different brines in trona flotation

\begin{tabular}{|c|c|c|c|c|c|c|}
\hline Trona Product & $\begin{array}{l}\text { Trona } \\
\text { Brine } \\
20^{\circ} \mathrm{C}\end{array}$ & $\begin{array}{l}\text { Mine } \\
\text { Water } \\
20^{\circ} \mathrm{C}\end{array}$ & $\begin{array}{c}\text { Trona } \\
\text { Brine } \\
40^{\circ} \mathrm{C}\end{array}$ & $\begin{array}{l}\text { Mine } \\
\text { Water } \\
40^{\circ} \mathrm{C}\end{array}$ & $\begin{array}{c}\mathrm{Na}_{2} \mathrm{CO}_{3} \\
40^{\circ} \mathrm{C} \\
\text { (Synthetic) }\end{array}$ & $\begin{array}{c}\mathrm{Na}_{2} \mathrm{CO}_{3} \\
40^{\circ} \mathrm{C} \\
(\text { Plant })\end{array}$ \\
\hline Insolubles, (\%) & 2.24 & 3.47 & 5.67 & 9.4 & 9.57 & 11.40 \\
\hline Recovery, (\%) & 91.65 & 90.28 & 72.88 & 83.86 & 66.13 & 61.68 \\
\hline
\end{tabular}

Table 3. Plant-site operating conditions for pilot plant tests

\begin{tabular}{cc}
\hline Item & Condition \\
\hline Conditioning Pulp Density & $70-75 \%$ \\
Flotation Percent Solids & $15 \%$ \\
Conditioning Time & $2-4$ minutes \\
Amine Dosage (AR 12D) & $4-5 \mathrm{~kg} / \mathrm{t}$ \\
Reagent Ratio (A/O) by weight & $1 / 12$ \\
Amine Concentration & $3 \%$ \\
Dry Solids Throughput & $1 \mathrm{tph}$ \\
\hline
\end{tabular}

Table 4. Plant-site operating conditions for second pilot plant tests

\begin{tabular}{cc}
\hline Item & Condition \\
\hline Conditioning Pulp Density & $70-75 \%$ \\
Flotation Percent Soilds & $15 \%$ \\
Conditioning Time & $2-4$ minutes \\
Amine Dosage (AR 12D) & $4-5 \mathrm{~kg} / \mathrm{t}$ \\
Reagent Ratio (A/O) by weight & $1 / 12$ \\
Amine Concentration & $3 \%$ \\
Throughput & $150 \mathrm{lb} / \mathrm{h}$ \\
\hline
\end{tabular}


Table 5. Results from pilot plant trona flotation system under the condition of $5 \mathrm{~kg} / \mathrm{t}$ amine dosage

\begin{tabular}{cccccc}
\hline Products & Weight $(\mathbf{k g})$ & Weight $(\boldsymbol{\%})$ & $\begin{array}{c}\text { Insoluble } \\
\text { Weight }(\boldsymbol{\%})\end{array}$ & $\begin{array}{c}\text { Trona } \\
\text { Weight }(\boldsymbol{\%})\end{array}$ & Recovery (\%) \\
\hline Tailing & 0.70 & 41.63 & 43.57 & 56.43 & 29.68 \\
Concentrate & 0.98 & 58.37 & $\mathbf{4 . 6 2}$ & 95.38 & $\mathbf{7 0 . 3 2}$ \\
Total & 1.68 & 100.00 & 20.84 & 79.16 & 100.00 \\
& $\mathbf{2 . 5 0}$ & & & & \\
\hline
\end{tabular}

Table 6. Results from pilot plant trona flotation system under the condition of $4 \mathrm{~kg} / \mathrm{t}$ amine dosage

\begin{tabular}{cccccc}
\hline Products & Weight $(\mathbf{k g})$ & Weight $(\boldsymbol{\%})$ & $\begin{array}{c}\text { Insoluble } \\
\text { Weight }(\boldsymbol{\%})\end{array}$ & $\begin{array}{c}\text { Trona } \\
\text { Weight }(\boldsymbol{\%})\end{array}$ & Recovery (\%) \\
\hline Tailing & 1.16 & 37.04 & 44.72 & 55.28 & 24.92 \\
Concentrate & 1.97 & 62.96 & $\mathbf{2 . 0 0}$ & 98.00 & $\mathbf{7 5 . 0 8}$ \\
Total & 3.13 & 100.00 & 17.82 & 82.18 & 100.00 \\
& $\mathbf{3 . 7 5}$ & & & & \\
\hline
\end{tabular}

Table 7. Results from pilot plant trona flotation system under the condition of $3 \mathrm{~kg} / \mathrm{t}$ amine dosage

\begin{tabular}{cccccc}
\hline Products & Weight $(\mathbf{k g})$ & Weight $(\boldsymbol{\%})$ & $\begin{array}{c}\text { Insoluble } \\
\text { Weight }(\boldsymbol{\%})\end{array}$ & $\begin{array}{c}\text { Trona } \\
\text { Weight }(\boldsymbol{\%})\end{array}$ & Recovery $(\boldsymbol{\%})$ \\
\hline Tailing & 3.87 & 36.19 & 46.64 & 53.36 & 23.65 \\
Concentrate & 6.82 & 63.81 & $\mathbf{2 . 2 5}$ & 97.75 & $\mathbf{7 6 . 3 5}$ \\
Total & 10.69 & 100.00 & 18.31 & 81.69 & 100.00 \\
& $\mathbf{1 4 . 0 0}$ & & & & \\
\hline
\end{tabular}

Table 8. Results from continuous ( $30 \mathrm{~min}$ ) $U$ of $U$ pilot plant trona flotation system under the condition of $4 \mathrm{~kg} / \mathrm{t}$ amine dosage

\begin{tabular}{cccccc}
\hline Products & Weight $\mathbf{( k g )}$ & $\begin{array}{c}\text { Weight } \\
(\boldsymbol{\%})\end{array}$ & $\begin{array}{c}\text { Insoluble } \\
\text { Weight }(\boldsymbol{\%})\end{array}$ & $\begin{array}{c}\text { Trona } \\
\text { Weight }(\boldsymbol{\%})\end{array}$ & $\begin{array}{c}\text { Recovery } \\
(\boldsymbol{\%})\end{array}$ \\
\hline Tailing & 8.27 & 35.65 & 33.03 & 66.97 & 27.86 \\
Concentrate & 14.92 & 64.35 & $\mathbf{3 . 9 3}$ & 96.07 & $\mathbf{7 2 . 1 4}$ \\
Total & 23.19 & 100.00 & 14.30 & 85.70 & 100.00 \\
& $\mathbf{2 6 . 0 0}$ & & & & \\
\hline
\end{tabular}


Table 9. Comparison of trona flotation results for bench scale, batch and continuous operation

\begin{tabular}{cccc}
\hline System & $\begin{array}{c}\text { Feed } \\
\text { Insoluble (\%) }\end{array}$ & $\begin{array}{c}\text { Concentrate } \\
\text { Insoluble (\%) }\end{array}$ & $\begin{array}{c}\text { Trona } \\
\text { Recovery (\%) }\end{array}$ \\
\hline $\begin{array}{c}\text { Bench Scale } \\
\text { (1 liter) }\end{array}$ & 6.07 & 1.25 & 97.37 \\
$\begin{array}{c}\text { Batch Operation } \\
\text { (30 liter) }\end{array}$ & 8.38 & 0.43 & 87.27 \\
$\begin{array}{c}\text { Continuous Operation } \\
\text { (30 liter) }\end{array}$ & 18.31 & 2.25 & 76.35 \\
\hline
\end{tabular}


Appendix 7: High Frequency Eddy Current Separation of Metallic Residue from Slags, Sands, Electronic Scraps and Other Wastes (UT004) 


\section{FINAL TECHNICAL REPORT}

Contract Title and Number: Crosscutting Technology Development at the Center for Advanced Separation Technologies (DE-FC26-02NT41607
Period of Performance:

Starting Date: 10/01/2002

Ending Date: 10/31/2009
Sub-Recipient Project Title:

High Frequency Eddy Current Separation of Metallic Residue from Slags, Sands, Electroni Scrap and Other Wastes

Principal Investigators:

Raj K. Rajamani

Contact Address:

University of Utah

135 South 1460 E. Room 412

Salt Lake City UT 84112

Subcontractor Address:

University of Utah

135 South 1460 E. Room 412

Salt Lake City UT 84112
Report Information:

Type: Final

Number:

Period: 10/1/05-8/31/06

Date: $\quad$ 8/31/06

Code: UT004-Final

Contact Information:

Phone: (801) 581-3107

Fax: $\quad$ (801) 581-8119

E-Mail: rajamani@mines.utah.edu

Subcontractor Information:

Phone: (801) 581-3107

Fax: (801) 581-4937

E-Mail:_Raj.rajamani@mines.utah.edu 


\section{Disclaimer}

This report was prepared as an account of work sponsored by an agency of the United States Government. Neither the United States Government nor any agency thereof, nor any of their employees, make any warranty, express or implied, nor assume any legal liability or responsibility for the accuracy, completeness, or usefulness of any information, apparatus, product, or process disclosed, or represents that its use would not infringe privately owned rights. Reference herein to any specific commercial product, process, or service by trade name, trademark, manufacturer, or otherwise does not necessarily constitute or imply endorsement, recommendation, or favoring by the United States Government or any agency thereof. The views and opinions of authors expressed herein do not necessarily state or reflect those of the United States Government or agency thereof. 


\begin{abstract}
$\underline{\text { Abstract }}$
The basic aim of the experiments carried out was to separate different non-ferrous metallic particles from waste streams as efficiently as possible. The research carried out explored the possibility of using magnetic fields generated between two ends of a dipole at high frequencies using. Towards this end, a high power amplifier was used to feed a LCR-circuit. Experiments were conducted to increase the separation capacity of ferrite core. The objectives were to: 1) increase the efficiency of the circuit, 2) decrease the frequency by changing the capacitance on the given single core and measure the deflection of the particles, 3) design a new optimally shaped cores, 4) design a new core with greater surface area provided for more magnetic lines of force coming out, 5) test the probable outcomes by forming a bigger core by joining multiple cores to provide greater surface area and, 6) optimize the newly joined core-set to get much lower frequency. The effect of the field on the particle was studied with the help of a pendulum, which was placed at various points in the magnetic field. The circuit was optimized by conducting experiments for all possible combinations of resistance, inductance and capacitance. The optimization of single core and a core-set, made up of two joined cores, were carried out and their differences were studied based on the applied magnetic field in a toroid. The frequency of the single core was tested with much high rated capacitors to get the minimum frequency. A new bigger core was designed to further test it on pilot plant scale. Prior to that a core-set was made by joining smaller cores and was tested in order to generate much lower frequency field. Also, particles were suspended in the field to asses the magnitude of force the metallic particles would encounter. For a gap of 14 $\mathrm{mm}$ outer and $7 \mathrm{~mm}$ inner diameter the peak voltage for the dipole was found to be 5219 Volts with current flowing through the dipole at $568 \mathrm{~mA}$ which gives a power of 2964 Watts. The circuit designed by sandwiching multiple cores showed very high magnetic flux and separation was possible between copper and aluminum particles which lead to the conclusion that increasing the number of turns or/and increasing the size of the core would lead to greater separation capacity. The single core with $16 \mathrm{~mm}$ outer and $1 \mathrm{~mm}$ inner opening size gave a minimum frequency of $3.28 \mathrm{kHz}$ at $158 \mathrm{nF}$ capacitance. The bigger core-set made up of two cores produced a frequency of $2.8 \mathrm{KHz}$ and the particles tested were encountering the same magnitude of force. Such force levels would increase the capacity of the eddy current separator.
\end{abstract}




\section{$\underline{\text { Table of contents }}$}

1. $\quad$ LIST OF GRAPHIC MATERIALS ............... 6

2. INTRODUCTION $\quad$...............

3. EXECUTIVE SUMMARY $\quad \ldots \ldots \ldots \ldots \ldots \ldots . \ldots$

4. EXPERIMENTAL $\quad \ldots \ldots \ldots \ldots \ldots \ldots . . . .9$

5. RESULTS AND DISCUSSIONS $\ldots \ldots \ldots \ldots \ldots . . . . .12$

6. CONCLUSIONS $\quad \ldots \ldots \ldots \ldots \ldots \ldots$

7. REFERENCES $\quad$................ 19 


\section{$\underline{\text { List(s) of graphical materials }}$}

\section{List of figures:}

Figure 1 Basic setup of the eddy current separator................................9

Figure 2 Front view of the dual core-set.................................... 11

Figure 3 Plan view of the core set............................................11

Figure 4 Deflections observed at different locations of the core opening............... 14

Figure 5 Proposed shape of the core openings................................. 14

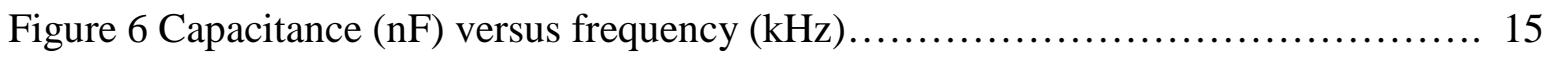

Figure 7: Frequency $(\mathrm{kHz})$ versus output voltage (volts) $\ldots \ldots \ldots \ldots \ldots \ldots \ldots \ldots \ldots \ldots \ldots$

\section{List of tables:}

Table 1 Output potential with change in capacitance at constant \pm 25 volts input at feedback resistance of $4.7 \mathrm{k} \Omega$ of the amplifier.......................................... 12

Table 2 Change in voltage with change in feedback resistor........................ 13

Table 3 Horizontal distance covered by copper particles of different diameters.......... 17

Table 4 Effect of increasing capacitance on frequency on single core.................. 17

Table 5 Effect of increasing capacitance on frequency on core-set.................... 18 


\section{$\underline{\text { Introduction }}$}

Recovery of primary metal from earth and secondary metal from used product economically has become a major challenge nowadays. Recycling of metal reduces landfill disposal and increases the recovery of valuable metals for proper reuse. In US, electronic scraps (escraps), also known as e-scraps, which consist of circuit board of desk top computers, cellular phones, televisions, calculators etc, have been increasing tremendously (Shuey and Taylor, 2005). Such wastes increase the burden on landfills. E-scraps contain plastics and other constituents along with metals. Plastics add 7 to 8 million Btu to each short ton of waste (Shuey and Taylor, 2005). Pyrometallurgical operations have been carried out to recycle these metallic wastes but the energy required in this operation is very high. This increases the operating cost of the process. Magnetic separators have been used widely to separate magnetic materials from wastes but recovery of non-ferrous metals from scraps using least energy source is still a challenge.

Eddy current separation is the best-known technique for separating non-ferrous metallic wastes from waste streams. The product developed in this report is a prototype high frequency eddy current separator, which can separate non-ferrous metallic particles from well-mixed wastes. The wastes have to be dried and ground properly into small size of around 3-10 mm. These particles will then be allowed to fall freely through an alternate magnetic field region. This will produce eddy current on the surface of the particle which produces Lorentz force. This force pushes the particle outwards towards the line of the magnetic field and the particle gets separated (Saviliev et al., 1998). The objective of this study is to increase the capacity of separation in order to separate wastes in larger scale. 
Executive Summary

The main aim of this phase's research was to increase the separation capacity to conduct separation of wastes at a higher rate. To increase the feed rate the core gap had to be increased. The increase in the air gap leads to a decrease in the magnetic field around the opening. So in order to maintain the same separation efficiency higher magnetic field had to be maintained in the gap to enable the same magnitude of force experienced in previous experiments of electrodynamic separation. This was done by characterization of the LCRcircuit, studying the effect of core shape and conducting the separation experiments. Characterization of the circuit was done first on a core of $7 \mathrm{~mm}$ inner and $14 \mathrm{~mm}$ outer opening size. It was found that at $4.2 \mathrm{nF}$, the circuit's performance was highest for separation. Experiments were also conducted by changing feedback resistance to see the effect on resonance frequency and voltage across the coil. Minimum resonance frequency of 11.4 KHz was obtained and it was found that the change in feedback resistance did not affect the resonance frequency. However, it affected the voltage across the coil a lot and the maximum voltage was found to be around $5500 \mathrm{~V}$ across the coil. This voltage was assumed to be better for separation but pendulum experiments did not give good results. This was due to the bigger gap on both sides of the core gap. This opening was so large that it reduced the magnetic lines of force per unit area as a result of which particles could not experience greater force. So, the core shape was studied to find a better shape that would give rise to a greater force at the same circuit characteristics. New core was finally designed (1 mm inner and $16 \mathrm{~mm}$ outer gap) which could separate particles at the same feed rate as that of the previous core shape. This shape was able to transfer more magnetic line of force because of its converging shape. This shape could align more magnetic lines outwards per unit area. Pendulum experiments were first conducted to asses the force. That force was found to be high enough for deflection of particles. Some deflection testes for copper particles were conducted and it was found that the field was enough to deflect the particle when allowed to fall freely through the alternating magnetic field. More characterization experiments were done in order to see the effect of magnetic field at low resonance frequency. That was done by increasing the capacitance of the circuit and consequentially a resonance frequency of 3.5 $\mathrm{kHz}$ was achieved. After performing pendulum experiments it was conclude that the metallic particles were experiencing greater force than before. So, it was concluded that bringing frequency low up to 1 or $2 \mathrm{KHz}$ would serve our purpose better. But from in that single core, the frequency became stagnant at $3.5 \mathrm{KHz}$ i.e., it was the minimum frequency that could be achieved for that core. Next aim was to create a bigger core that could work with the same circuit to conduct pilot scale experiments. Two cores were first joined together in order to test the significance of bigger core in the same circuit and then the circuit was optimized. It was found that the resonance frequency reduced more, to $2.8 \mathrm{kHz}$. The particles were experiencing the same amount of force as before. This concluded that bigger core would work in the same circuit, after circuit optimization. A bigger core has already been designed and is going to be ordered very soon. Within the next two months separation experiments in continuous scale will be performed to strengthen the conclusion. The pilot scale eddy current separators can be scaled for commercial scale production. 


\section{Experimental}

Equipments used:

Ferrite core: OW 48613 TC

Oscilloscope: HP 54645D

Function generator: HP 33120A

Power supply: HP E3631A

Multimeter: HP 34401

The experimental setup used the eddy-current separator shown in Figure 1. The separator consists of electrical circuit for alternating current generation and the hardware for magnetic field generation.

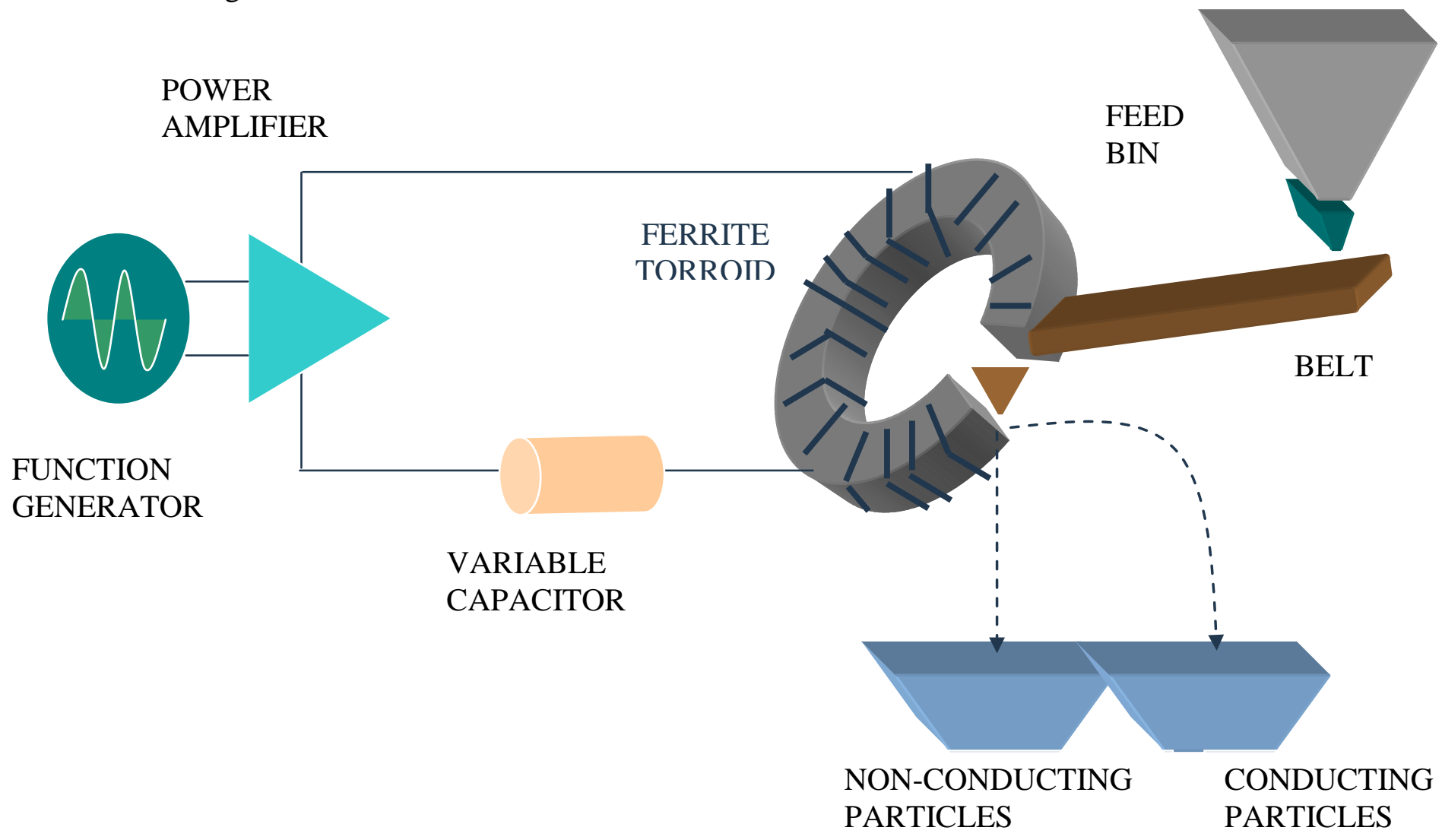

Figure 1: Basic set up of the eddy current separator.

Following steps were followed in conducting the experiments: 
Step 1: Characterization of LCR circuit using ferrite core of $7 \mathrm{~mm}$ and $14 \mathrm{~mm}$ as inner and outer openings respectively: An amplifier was designed with an amplification factor 400 (by changing the feedback resistance this gain can be increased or decreased). The capacitor of the LCR-circuit was optimized to get a maximum value of output potential across the ends of the dipole. This value was found to be $4.2 \mathrm{nF}$. The dipole was fixed with 600 turns with outer edge being $14 \mathrm{~mm}$ apart and the inner $7 \mathrm{~mm}$ apart. The dipole was not varied any further.

Step 2: System setup characterization and particle deflection tests of newly designed core of $1 \mathrm{~mm}$ inner and $16 \mathrm{~mm}$ outer gap: The next task was to study the magnetic field intensity at various points between the openings of the core gap. The main objective was to determine the field strength at various positions which would determine the correct position of the feed stream coming in to the gap. It would also help in determining the proper shape of the core opening. Experiments were also conducted to study and optimize the new electrical circuit by changing the capacitance and feedback resistance along with the effect of magnetic field on particle separation.

Setup

1. System was set up to give about $5400 \mathrm{~V}$ at $11.349 \mathrm{kHz}$. In order to study the magnetic field, copper (6 $\mathrm{mm}$ diameter approx.) and aluminum (3.5mm diameter approx.) particles were suspended from weightless strings. The strings were moved from various positions and the field strength was studied.

2. Voltage across the core was $2300 \mathrm{~V}$ at a frequency of $10.215 \mathrm{kHz}$. The particles were dropped from a height of $13 \mathrm{~cm}$.

Step 3: More system characterization and particle deflection tests after step 2: The effect on the particles was studied on the newly designed core. The experiments were conducted to study the new optimized circuit by changing the capacitance and feedback resistance along with the effect of magnetic field on the particle. Experiments were also conducted to study the deflections of copper and aluminum particles on the generated magnetic field. At first a copper particle of spherical shape (7 mm diameter) was taken and dropped in to the magnetic field generated by the new core. The particles were made to fall up from a height of $13 \mathrm{~cm}$ where it touched a horizontal surface. The horizontal distance up to which the particle traveled was recorded by using a scale. Seven similar experiments were conducted for one particle size and results were noted down. Similar seven experiments were conducted for $5 \mathrm{~mm}$ and $3 \mathrm{~mm}$ particles and the results were recorded.

Step 4: Single core circuit optimization of newly designed core to get minimum frequency: The next task was to decrease the frequency to the lowest possible extent at comparable voltage by increasing the capacitance in the LCR circuit. Experiments were conducted to study and optimize the new electrical circuit by changing the capacitance along with the effect of magnetic field on particle separation. 
System was set up to give about $1600 \mathrm{~V}$ at $6.57 \mathrm{kHz}$. In order to study the magnetic field, copper (6 mm diameter approx.) particle were tested. The horizontal distance traveled by these particles from a height of $13 \mathrm{~cm}$ were recorded.

Step 5: Design and characterization of bigger core made by joining two small cores: A bigger core was designed in this phase in order to provide greater opening surface and consecutively greater magnetic lines of force for greater separation capacity. In order to test the characteristics of bigger core a new core was made by joining two single cores like that in previous setup. This core-set provided greater surface area through which when a particle was made to fall freely under the gravity, it would encounter greater magnetic lines of force for greater deflection.

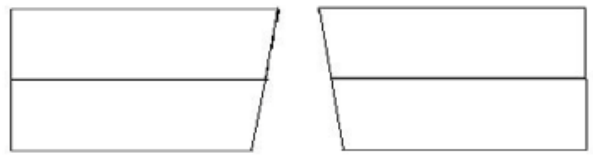

Figure 2: Front view of the dual core-set

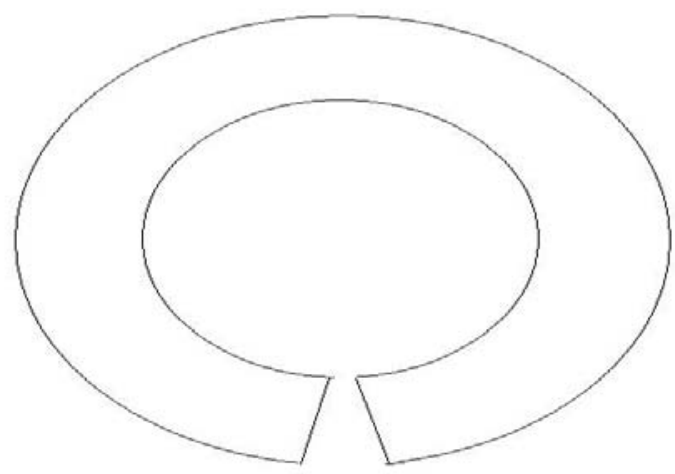

Figure 3: Plan view of the core-set

This setup was similar to the initial set up. The only difference was the use of dual core-set instead of a single core. The circuit was set at $1700 \mathrm{~V}$ and $38 \mathrm{nF}$ capacitance. The initial 
frequency at this set up was found to be $4.8 \mathrm{KHz}$. Then the capacitance was decreased subsequently and the frequency was recorded.

Results and Discussion

The following table shows the output potential (across the dipole) with change in capacitance:

Table 1: Output potential with change in capacitance at constant \pm 25 volts input using a feedback resistance of $4.7 \mathrm{k} \Omega$ on the amplifier.

\begin{tabular}{|c|c|c|c|}
\hline $\begin{array}{c}\text { Capacitor } \\
\text { (nF) }\end{array}$ & $\begin{array}{c}\text { Resonance } \\
\text { Frequency } \\
\text { (kHz) }\end{array}$ & $\begin{array}{c}\text { Current through } \\
\text { dipole } \\
\text { (mA) }\end{array}$ & $\begin{array}{c}\text { Voltage across Dipole } \\
\text { Vp-p } \\
\text { (V) }\end{array}$ \\
\hline 2.1 & 15.787 & 93 & 1219 \\
\hline 4.1 & 11.461 & 104 & 1180 \\
\hline 4.2 & 11.295 & 111 & 1312 \\
\hline 4.5 & 10.979 & 105 & 1156 \\
\hline 4.7 & 10.685 & 108 & 1188 \\
\hline 5.2 & 10.170 & 110 & 1180 \\
\hline 6.2 & 9.2479 & 111 & 1086 \\
\hline
\end{tabular}

Therefore a capacitor value of $4.2 \mathrm{nF}$ produced the maximum output voltage.

The following table shows the change in voltage (across the dipole) with respect to a changing value of the feedback resistance of the amplifier: 
Table 2: Change in voltage with change in feedback resistor

\begin{tabular}{|c|c|c|c|c|c|c|}
\hline SI.No. & $\begin{array}{c}\text { Feedback } \\
\text { resistor } \\
\text { value } \\
(\mathbf{k} \Omega)\end{array}$ & $\begin{array}{c}\text { Signal } \\
\text { Generator } \\
\text { output Vp-p } \\
(\mathrm{mV})\end{array}$ & $\begin{array}{c}\text { Resonance } \\
\text { Frequency } \\
\text { (f0) } \\
(\mathbf{k H z})\end{array}$ & $\begin{array}{c}\text { Power } \\
\text { supply } \\
\text { DC } \\
\text { Current } \\
\text { (mA) }\end{array}$ & $\begin{array}{c}\text { AC } \\
\text { Current } \\
\text { through } \\
\text { coil } \\
\text { (mA) }\end{array}$ & 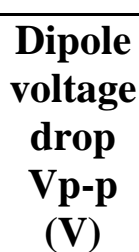 \\
\hline \multirow[t]{4}{*}{1} & 180 & 196.9 & 11.284 & 132 & 192 & 1789 \\
\hline & & 395.4 & 11.284 & 201 & 352 & 3281 \\
\hline & & 595.4 & 11.306 & 278 & 525 & 4844 \\
\hline & & $\begin{array}{l}655.4 \\
\end{array}$ & 11.372 & 297 & 568 & 5219 \\
\hline \multirow[t]{3}{*}{2} & 490 & 99.22 & 11.286 & 155 & 252 & 2344 \\
\hline & & 157.8 & 11.288 & 214 & 393 & 3641 \\
\hline & & 200 & 11.300 & 252 & 491 & 4594 \\
\hline \multirow[t]{3}{*}{3} & 500 & 99.22 & 11.285 & 156 & 250 & 2312 \\
\hline & & 157.8 & 11.283 & 214 & 382 & 3531 \\
\hline & & 200 & 11.300 & 255 & 496 & 4656 \\
\hline \multirow[t]{3}{*}{4} & 510 & 99.22 & 11.285 & 161 & 264 & 2453 \\
\hline & & 157.8 & 11.286 & 219 & 406 & 3794 \\
\hline & & 200 & 11.306 & 261 & 513 & 4766 \\
\hline \multirow{3}{*}{5} & 520 & 99.22 & 11.286 & 161 & 264 & 2453 \\
\hline & & 157.8 & 11.292 & 228 & 428 & 4000 \\
\hline & & 200 & 11.310 & 263 & 519 & 4828 \\
\hline
\end{tabular}

For the gap of $14 \mathrm{~mm}$ outer and $7 \mathrm{~mm}$ inner diameter the peak voltage for the dipole was found to be 5219 Volts with current flowing through the dipole at $568 \mathrm{~mA}$. The power at this setting is 2964 Watts.

Observations from step 2

(a) Aluminum particle: When aluminum particle was suspended, it was found that its interaction with the magnetic field destabilized the field. In order to verify this, the copper particle was suspended next. The aluminum particle weighed $0.0601 \mathrm{gm}$. Its diameter was $3.5 \mathrm{~mm}$. 
(b) Copper particle: Similar result was observed for copper particle. Hence, it was concluded that the voltage across the core had to be reduced in order to sustain the magnetic field. The copper particle weighed $1.3396 \mathrm{gm}$. Its diameter was $6 \mathrm{~mm}$.

Based on the earlier conclusion the voltage was reduced to $4000 \mathrm{~V}$ and similar experiments were reconducted. It was found that at a voltage of $4000 \mathrm{~V}$ or lower across the core, the system was stable. Figure 4 shows the direction of deflection of copper particle as was observed during the experiment:

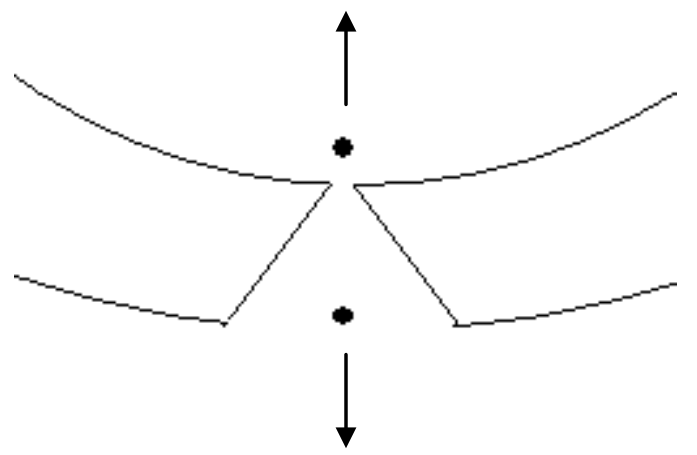

Figure 4: Deflections observed at different locations of the core opening.

It was found that aluminum particles experienced little or no deflection when suspended at any point. The copper particle showed maximum deflection at the inner edge than at the outer edge. From the above, it was concluded that the magnetic field intensity at the inner edge was much stronger than at the outer edge. Hence a new design of the core opening was proposed. The design looks as shown in the Figure 5 below.

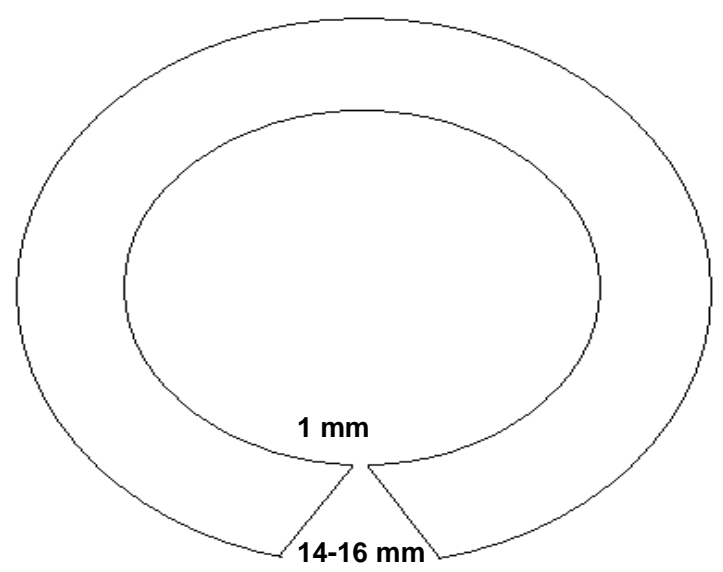

Figure 5: Proposed shape of the core opening. 
Results are shown for the circuit optimization, which was done to get maximum and stable field within the core.

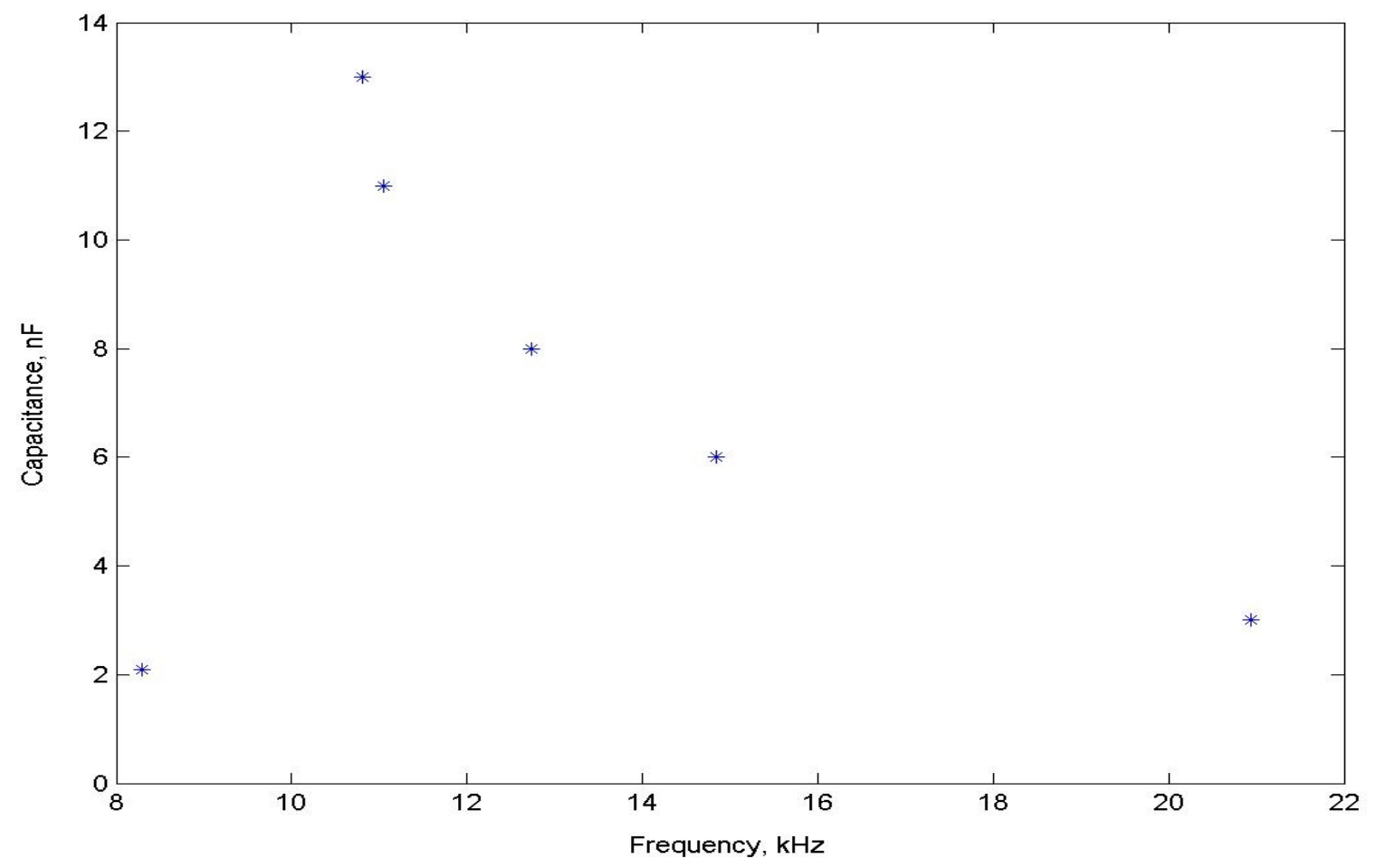

Figure 6: Capacitance $(\mathrm{nF})$ versus frequency $(\mathrm{kHz})$ 


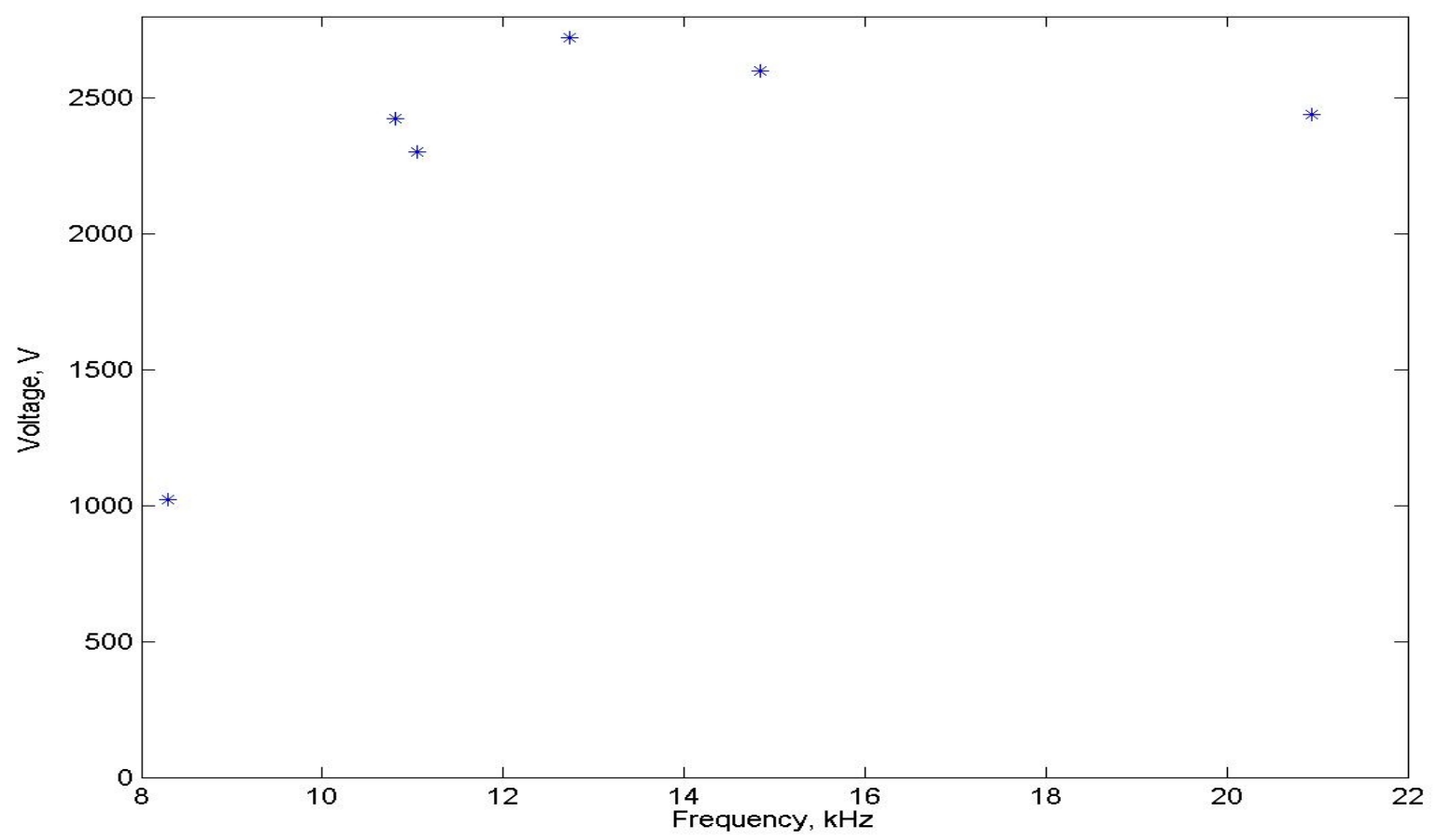

Figure 7: Frequency (kHz) versus output voltage (volts)

From figure 6, it was found that as capacitance increased, the frequency, at which maximum output was obtained, dropped except for the very first reading, which can be neglected because the output voltage was only around 1000 volts. When interpolated, it was presumed that for $20 \mathrm{nF}$ capacitance, we should get about $4 \mathrm{kHz}$. From Figure 7, it was found that the output voltage almost stabilized and did not change much with increase in capacitance or decrease in frequency. The feedback resistance was set as 100 kilo Ohms as it was found to be near optimum for the entire range of capacitance. 
Following readings were recorded when particles were dropped from $13 \mathrm{~cm}$ height:

Table 3: Horizontal distance covered by copper particles of different diameters

\begin{tabular}{|c|c|c|c|}
\hline Diameter & $\mathbf{7 m m}$ & $\mathbf{5} \mathbf{~ m m}$ & $\mathbf{3} \mathbf{~ m m}$ \\
\hline \multirow{4}{*}{ Copper } & \multicolumn{3}{|c|}{ Horizontal distance covered $\mathbf{( c m )}$} \\
\cline { 2 - 4 } & 6 & 6.5 & 5 \\
\cline { 2 - 4 } & 6 & 7.5 & 6 \\
\cline { 2 - 4 } & 4 & 7 & 5 \\
\cline { 2 - 4 } & 4.5 & 7.5 & 5 \\
\cline { 2 - 4 } & 6 & 6.5 & 6 \\
\cline { 2 - 4 } & 4.5 & 7 & 5 \\
\hline Average & 6 & 7 & 5.5 \\
\hline
\end{tabular}

Observations from step 4

Copper particles: When the copper particles were led to drop freely under gravity through this magnetic field, proper horizontal deflection was seen through a height of $13 \mathrm{~cm}$. The copper particle weighed 1.3396 gm. Its diameter was $6 \mathrm{~mm}$.

Based on the experiments conducted, the following table showed the decrease in frequency with the increase of capacitance and deflections recorded at respective frequencies:

Table 4: Effect of increasing capacitance on frequency on single core

\begin{tabular}{|c|c|c|c|c|}
\hline Vpp (V) & Capacitance (nF) & Frequency (KHz) & $\begin{array}{c}\text { Feedback Resistance } \\
(\mathbf{K} \boldsymbol{\Omega})\end{array}$ & Deflection (cm) \\
\hline 1600 & 38 & 6.57 & 100 & 5 \\
\hline 1575 & 49 & 6.03 & 100 & 5.5 \\
\hline 1550 & 60 & 5.71 & 100 & 6 \\
\hline 1514 & 71 & 5.24 & 100 & 6 \\
\hline 1478 & 82 & 5.014 & 100 & 6 \\
\hline 1442 & 93 & 4.83 & 100 & 7 \\
\hline 1416 & 104 & 4.59 & 100 & 6 \\
\hline 1380 & 115 & 4.42 & 100 & 6.5 \\
\hline 1344 & 126 & 4.25 & 100 & 7 \\
\hline 1308 & 137 & 4.09 & 100 & 7 \\
\hline 1272 & 143 & 3.97 & 100 & 7 \\
\hline 1236 & 150 & 3.64 & 100 & \\
\hline 1200 & 158 & 3.28 & 100 & \\
\hline
\end{tabular}


Table 5: Effect of increasing capacitance on frequency on core-set

\begin{tabular}{|c|c|c|c|c|}
\hline Vpp (V) & $\begin{array}{c}\text { Capacitance } \\
(\mathrm{nF})\end{array}$ & Frequency (KHz) & $\begin{array}{c}\text { Feedback } \\
\text { Resistance (K } \Omega \text { ) }\end{array}$ & $\begin{array}{c}\text { Input Voltage } \\
(\mathrm{mV})\end{array}$ \\
\hline 1700 & 38 & 4.8 & 100 & 320 \\
\hline 1650 & 49 & 6.0 & 100 & 320 \\
\hline 1810 & 60 & 4.0 & 100 & 320 \\
\hline 1880 & 71 & 3.2 & 100 & 320 \\
\hline 1930 & 82 & 3.1 & 100 & 320 \\
\hline 2300 & 93 & 2.8 & 100 & 320 \\
\hline
\end{tabular}

Table 4 showed a decrease in frequency with the increase in capacitance. The final frequency from the single core was found to be $3.28 \mathrm{KHz}$ at $158 \mathrm{nF}$. It was also found that there was a decrease in voltage with the increase in capacitance but the deflection encountered on the particle was found to increase with increasing frequency. Capacitance above $158 \mathrm{nF}$ was also tested but the frequency and deflection was found to have become stagnant.

Table 5 showed better results with the core-set. The frequency was found to be $4.8 \mathrm{KHz}$ at $1700 \mathrm{~V}$ and $38 \mathrm{nF}$. The nature of frequency change with increasing capacitance was again found to be monotonous. It was found that with the increase in capacitance the frequency decreased to $2.8 \mathrm{KHz}$ at $93 \mathrm{nF}$. Also, the voltage was found to increase, in this case, with the increase in capacitance. This increase in voltage was unexpected as compared to the results shown in Table 1. The reason for this change in nature was unidentified and hence is under investigation.

\section{Conclusion}

From the above experiments it was concluded that shape changing and circuit optimization head in the right direction for the criteria of eddy current separation. A very high force was experienced by the particles in the magnetic field. The average deflection for copper was found to be much higher than for aluminum particles. This suggests that change in particle size could also affect the deflections. It was concluded that lower frequency can produce better deflection of non-ferrous metallic particles. The results of the core-set showed better results than the single set so, performing experiments on bigger cores is feasible using the same electrical set up.

New bigger core is going to be ordered. The objective is higher separation capacity. The above results show promise in advancing towards pilot scale separation experiments within the next two months. Once pilot separation experiments are conducted, the device would be ready for prototype testing and scale-up. 


\section{$\underline{\text { References }}$}

Naidu, H., Electrodynamic separation of metallic granules from flowing mixed waste stream, M.S. thesis, University of Utah, 2002.

Saviliev, L.V., System and method for separating electrically conducting particles. U.S. Patent 5,772,043, June 30, 1998.

Shuey, S.A., and Taylor, P., "Review of pyrometallurgical treatment of electronic scrap," Mining Engineering, April 2005, pp. 67-70. 
Appendix 8: Direct Measurement of Forces in Flotation (VA004) 


\section{FINAL TECHNICAL REPORT}

Contract Title and Number:

Crosscutting Technology Development at the Center

for Advanced Separation Technologies

(DE-FC26-02NT41607
Period of Performance:

Starting Date: 10/01/2002

Ending Date: 10/31/2009
Sub-Recipient Project Title:

Direct Measurement of Forces in Flotation Systems

Principal Investigators:

Roe-Hoan Yoon and William Ducker

Contact Address:

146 Holden Hall and 304 Davidson Hall

Virginia Polytechnic Institute \& State University

Blacksburg, VA 24061

Subcontractor Address:

No subcontracts issued.
Report Information:

Type: Final

Number:

Period: $\quad$ 9/17/01-5/31/07

Date:

Code: VA004-Final

Contact Information:

Phone: (540)231-4508

Fax: (540)231-3948

E-Mail: cast@vt.edu

Subcontractor Information:

Phone:

Fax:

E-Mail:

\section{$\underline{\text { Disclaimer }}$}

This report was prepared as an account of work sponsored by an agency of the United States Government. Neither the United States Government nor any agency thereof, nor any of their employees, make any warranty, express or implied, nor assume any legal liability or responsibility for the accuracy, completeness, or usefulness of any information, apparatus, product, or process disclosed, or represents that its use would not infringe privately owned rights. Reference herein to any specific commercial product, process, or service by trade name, trademark, manufacturer, or otherwise does not necessarily constitute or imply endorsement, recommendation, or favoring by the United States Government or any agency thereof. The views and opinions of authors expressed herein do not necessarily state or reflect those of the United States Government or agency thereof. 


\begin{abstract}
$\underline{\text { Abstract }}$
The objective of this project is to directly measure the interaction force between a particle and bubbles as a function of separation distance. This force controls the attachment and detachment of particles to bubbles, which is an essential step in determining the efficiency of a flotation process. In the past ten years, one predominant explanation for the long range attraction between hydrophobic surfaces is the nanobubble/gas cavity theory. Firstly, an Atomic Force Microscope was utilized to study the hydrophobic force between solid surfaces to clarify the applicability of nanobubble theory in the solid/surfactant system. The effects of degassing, salt concentration and chain length on the hydrophobic force are systemically studied. The results are beneficial for the understanding of the origin of the hydrophobic force and the particle/bubble interaction in Flotation. Secondly, a specially designed device, wetting film apparatus was fabricated for the direct particle/bubble force measurements. The experiment results show that between hydrophilic substrate/bubble, when the separation decreases, there is a strong repulsive disjoining pressure, which leads to a thick water film between particle/bubble, therefore, a less likely flotation. The wetting film between a hydrophobic substrate and a bubble ruptures in tens of milliseconds drastically, suggesting a possible flotation after the solid particles are hydrophobized. The rupture process is proposed to be studied systemically using a high speed video camera.
\end{abstract}




\section{Table of contents}

Abstract 2

Table of contents 3

List(s) of graphical materials $\quad 4$

Executive Summary $\quad 6$

Introduction $\quad 7$

Experimental $\quad 8$

Materials $\quad 8$

Degassing procedure 9

Air-equilibrated solutions 9

Surface Force Measurement 9

AFM Imaging 10

$\zeta$-Potential Measurement 10

Contact Angle Measurement 11

Film thickness measurement 11

Results and Discussion $\quad 11$

Contact Angle Measurement 11

Forces in Air-Equilibrated Solution 12

Effect of Degassing on AFM Force Measurement 14

Effect of Salt Concentration on the Forces in $C_{18}$ TACl Solutions 19

Force Measurements in $C_{n}$ TACl Surfactant Solutions 20

AFM Images 23

Possible Origin(s) of Hydrophobic Force 28

1) Attractive Double-layer Forces 29

2) Charged Patches Model 30

3) Eriksson et al.'s theory 33

Measurement of wetting film thickness 36

Conclusions $\quad 40$

Bibliography $\quad 41$ 


\section{$\underline{\text { List(s) of graphical materials }}$}

Figure 1. Sessile drop and captive bubble contact angles of the fused silica plates as a function of the time that the plate was exposed to $10^{-5} \mathrm{M} \mathrm{C}_{18} \mathrm{TACl}$ solutions.

Figure 2. Forces (F) normalized by the radius (R) of the sphere in air-equilibrated $\mathrm{C}_{18} \mathrm{TACl}$ solutions.

Figure 3. Forces (F) normalized by the radius (R) of sphere in degassed $\mathrm{C}_{18} \mathrm{TACl}$ solutions.

Figure 4. Comparison of the forces measured in degassed and air-equilibrated $5 \times 10^{-6}$ and $1 \times 10^{-5} \mathrm{M} \mathrm{C}_{18} \mathrm{TACl}$ solutions.

Figure 5. Effect of $\mathrm{pH}$ on forces in air-equilibrated $10^{-5} \mathrm{M} \mathrm{C}_{18} \mathrm{TACl}$ solutions.

Figure 6. Comparison of the $\zeta$-potentials measured in degassed $\mathrm{C}_{18} \mathrm{TACl}$ solutions with those measured in air-equilibrated solutions whose $\mathrm{pH}$ have been adjusted to the same $\mathrm{pH}(=6.6)$ as the degassed solutions.

Figure 7. Comparison of the $\zeta$-potentials measured in air-equilibrated $\mathrm{C}_{18} \mathrm{TACl}$ solutions of $\mathrm{pH} 5.7$ with those measured in solutions whose $\mathrm{pH}$ was adjusted to $\mathrm{pH}$ 6.6.

Figure 8. Effect of $\mathrm{NaCl}$ on the forces measured in air-equilibrated solutions of a) $5 \times 10^{-6} \mathrm{M}$ and b) $8 \times 10^{-6} \mathrm{M} \mathrm{C}_{18} \mathrm{TACl}$ solutions.

Figure 9. The AFM force curves obtained with the glass sphere and silica plate immersed in $\mathrm{C}_{12} \mathrm{TACl}$ solutions at $\mathrm{pH} \approx 6$.

Figure 10. The AFM force curves obtained with the glass sphere and silica plate immersed in $\mathrm{C}_{14} \mathrm{TACl}$ solutions.

Figure 11. The AFM force curves obtained with the glass sphere and silica plate immersed in $\mathrm{C}_{16} \mathrm{TACl}$ solutions.

Figure 12. The AFM force curves measured with the glass sphere/silica plate immersed in $\mathrm{C}_{18} \mathrm{TACl}$ solutions.

Figure 13. The AFM force curves obtained with glass sphere and silica plate at the p.c.n.’s of the $\mathrm{C}_{\mathrm{n}} \mathrm{TACl}$ homologues $(\mathrm{n}=12,14,16,18)$.

Figure 14. A soft contact mode AFM image of the fused silica plate immersed in water.

Figure 15. The soft contact mode AFM images of the silicon wafer surfaces immersed in $\mathrm{C}_{18} \mathrm{TACl}$ solutions at different concentrations and scale.

Figure 16. A relationship between the p.c.n.'s for the silica- $\mathrm{C}_{\mathrm{n}} \mathrm{TACl}$ system and the number of carbons in the hydrocarbon chains.

Figure 17. Force curves for the silica plate-glass sphere interaction in an airequilibrated $5 \times 10^{-6} \mathrm{M} \mathrm{C}_{18}$ TAC solution.

Figure 18. Decay length vs. the Debye length plots. 
Figure 19. A decay length $(D)$ vs. $\left(C_{0} n\right)^{-1 / 2}$ plot for the data obtained in $C_{n} T A C l$ solutions in the absence of electrolyte.

Figure 20. Film Thickness Measured Using a Micro-interference Technique

Figure 21. Theoretical equilibrium film thickness at different salt concentrations.

Figure 22. Kinetic thinning of the wetting film on a hydrophilic silicon wafer surface as a function of thinning time.

Figure 23. Experimental equilibrium film thickness at $1 \times 10^{-3} \mathrm{M} \mathrm{NaCl}$ concentration

Figure 24. Experimental and theoretical equilibrium film thickness at different $\mathrm{NaCl}$ concentration.

\section{$\underline{\text { List(s) of Tables }}$}

Table 1. Effects of $\mathrm{NaCl}$ on Debye Lengths $\left(\kappa^{-1}\right)$ and Decay Lengths $\left(\mathrm{D}_{2}\right)$ in $\mathrm{C}_{18} \mathrm{TACl}$ Solutions.

Table 2. The parameters of Eq. (1) obtained for the force curves measured at the p.c.n's of the silica- $\mathrm{C}_{\mathrm{n}} \mathrm{TACl}$ system.

Table 3. The parameters of Miklavic et al.'s charged-patch model as obtained by fitting the decay lengths obtained at the p.c.n.'s of the silica/ $\mathrm{C}_{\mathrm{n}} \mathrm{TACl}$ system

Table 4. Experimental and theoretical equilibrium film thickness at different $\mathrm{NaCl}$ concentration. 


\section{Executive Summary}

Sakamoto et al. (2002) conducted AFM force measurements between silica sphere and fused-silica plate in aqueous octadecyltrimethylammonium chloride $\left(\mathrm{C}_{18} \mathrm{TACl}\right)$ solutions, and concluded that long-range attractive force is not observed in carefully degassed solutions. In the present work, AFM force measurements were conducted by following the procedures described by Sakamoto et al. The results showed the presence of the long-range attractive force both in air-saturated and degassed solutions. It was most attractive at $5 \times 10^{-6} \mathrm{M} \mathrm{C} 18 \mathrm{TACl}$, where the surface was most hydrophobic. At this concentration, which is close to the point of charge neutralization (p.c.n.) of the glass sphere, the long-range decay lengths $\left(D_{2}\right)$ were 34 and $38 \mathrm{~nm}$ in airsaturated and degassed solutions, respectively. At $10^{-5} \mathrm{M}$, the decay length decreased from 30 to $4 \mathrm{~nm}$ upon degassing, which is explicable by simple $\mathrm{pH}$ change associated with the changes in the concentration of dissolved $\mathrm{CO}_{2}$. The attractive force was screened by an added electrolyte $(\mathrm{NaCl})$, indicating that the attractive force may be of electrostatic origin. Therefore, the very long decay lengths observed in the absence of electrolyte may be ascribed to the fact that the p.c.n. occurs at a very low surfactant concentration.

Surface forces were measured using an AFM with silica surfaces immersed in $\mathrm{C}_{\mathrm{n}} \mathrm{TACl}(\mathrm{n}=12-18)$ solutions in the absence of added salt. The results showed longrange attractive forces that cannot be explained by the DLVO theory. The long-range attractions increased with increasing surfactant concentration, reaching a maximum at the point of charge neutralization (p.c.n.) and then decreased. The long-range forces decayed exponentially, with the decay lengths increasing from 3 to $32 \mathrm{~nm}$ as the chain length of the surfactants increased from C-12 to C-18. The measured forces can be fitted to the charged-patch model of Miklavic et al. (1994) by assuming patch sizes that are much larger than the values reported in the literature.

It was found that the decay length decreases linearly with the effective concentration of the $\mathrm{CH}_{2} / \mathrm{CH}_{3}$ groups of the $\mathrm{C}_{\mathrm{n}} \mathrm{TACl}$ homologues raised to the power of $1 / 2$, which is in line with the Eriksson et al.'s (1989) hydrophobic force model derived using a mean-field approach. It appears, therefore, that the long-range attractions observed in the present work are hydrophobic forces originating from changes in water structure across the thin surfactant solution film between the silica surfaces. It is conceivable that hydrocarbon chains in solution disrupt the surface-induced water structure and cause a decrease in hydrophobic force.

The film thicknesses of a wetting film in different $\mathrm{NaCl}$ solutions on oxidized hydrophilic silicon wafer surface show that equilibrium film thickness decreases with salt concentration increasing, which is the same as predicted by the DLVO theory. The experiment results also suggest that there is an equilibrated thick water film between a hydrophilic solid and a bubble, therefore, a less likely flotation. However, the wetting film between a hydrophobic substrate and a bubble ruptures in tens of milliseconds drastically, suggesting a possible flotation after the solid particles are hydrophobized. The rupture process is proposed to be studied systemically using a high speed video camera. 


\section{Introduction}

Of the many variables affecting flotation, particle hydrophobicity plays the most decisive role. In general, the hydrophobicity determines both selectivity and recovery. Various collectors are used to control the hydrophobicity, and many investigators studied ways to increase the hydrophobicity and hence improve the separation process.

In colloid chemistry, it is recognized that the kinetics of particle coagulation is controlled by the surface forces. In general, coagulation is slow when the surface forces create an energy barrier and the coagulation is fast when the energy barrier becomes zero. Derjaguin and Dukhin (1961) were the first to model flotation (or bubble-particle interaction) using the surface forces that are known to be present in colloidal systems; namely, the electrostatic force due to double-layer interaction and the van der Waals dispersion force. However, both of these forces are repulsive in most of the conditions encountered in flotation, which makes it difficult to model flotation using the DLVO theory. Blake and Kitchener (1972) showed later that the thin film of water (wetting film) between a hydrophobic solid and air bubble ruptures spontaneously and that it does so at much larger separation distances than predicted by the classical DLVO theory. These investigators suggested, therefore, the presence of 'hydrophobic force' in wetting films. In fact, the presence of the third force (i.e., hydrophobic force) had been suggested by Laskowski and Kichener (1969) three years prior to the Blake and Kitchener's work. The former discovered that methylated and pure silica particles exhibited practically the same $\zeta$-potentials and yet the former floated while the latter did not, which led to their suggestion that a long-range 'hydrophobic influence' caused by the instability of the water structure in the vicinity of a hydrophobic surface may be responsible for the flotation.

It was not until 1982 when Israelachvili and Pashley (1982) actually measured the hydrophobic force using the surface force apparatus (SFA). Since then, numerous investigators conducted follow-up experiments, many of which confirmed the existence of the hydrophobic force while others did not.

Tsao and Evans (1993) observed long-range attractive forces with mica surfaces coated with double-chain cationic surfactants. They found that the forces were screened by electrolyte and, therefore, concluded that they are electrostatic in origin. Further, longchain surfactants self-assembled at interfaces/surfaces form domains and the hydrocarbon chains are tilted relative to the interface. The tilted structure generates in-plane dipole moment, which creates electric fields extending large distances. More recently, we developed a mathematical model relating attractive forces to the dipole moments of the domains (or patches) that are formed during self-assembly processes. (Pazhianur and Yoon, 2003) Miklavic et al. (1994) also suggested that net-neutral surfaces consisting of charged patches can create attractive double-layer forces, provided that the charged species are free to migrate.

The strongest opposition came from those who observed nano-bubbles on hydrophobic surfaces during measurement. It was suggested that the so-called 
hydrophobic force is due to the capillary force caused by the coalescence of preexisting nano-bubbles when two hydrophobic surface approach each other. (Sakamoto et al., 2002; Attard, 1996; Ishida et al., 2000a, 2000b; Tyrrell and Attard, 2002) Recent work from the group of Higashitani (Ishida et al., 2000a, 2000b) offered support for the preexisiting adsorbed bubble hypothesis, which may be acceptable for the surfaces whose water angles $(\theta)$ were greater than $90^{\circ}$ due to hydrophobization with silanes Thermodynamically, cavitation should occur at $\theta>90^{\circ}$. However, Sakamoto, et al. (2002) reported that air bubbles are also the cause for the hydrophobic forces observed with surfaces coated with a water-soluble surfactant (octadecyltrimethylammonium chloride, $\left.\mathrm{C}_{18} \mathrm{TACl}\right)$. They observed long-range $(60-250 \mathrm{~nm})$ attractive forces between silica surfaces in $1-3 \times 10^{-5} \mathrm{M} \mathrm{C}_{18} \mathrm{TACl}$ when the solution was equilibrated with air. The force vs. distance curves exhibited discontinuities (or steps), as were also reported by other investigators. (Parker, et al. 1994; Nuyen et al. 2003) When the measurement was conducted in a degassed solution, both the steps and the long-range attraction disappeared, which lead to the conclusion that the long-range attraction is caused by the gas bridges formed between two hydrophobic surfaces interacting with each other. They stated further that long-range attraction never appears in completely air-free $\mathrm{C}_{18} \mathrm{TACl}$ solutions.

In the present work, the AFM force measurements conducted by Sakamoto et al. (2002) in $\mathrm{C}_{18} \mathrm{TACl}$ solutions were repeated by exactly following their procedure in order to help clarify the origin of the long range attraction. What is more, systematic studies on the effect of chain length on hydrophobic force were carried out by measuring the hydrophobic forces between silica surfaces in solutions of alkylammoniumtrimetyl chloride $\left(\mathrm{C}_{\mathrm{n}} \mathrm{TACl}\right)$ homologues. The results are also used to discuss the role of hydrocarbon chain length on the mechanisms of adsorption of the long-chain surfactants and to discuss the possible origins of hydrophobic force

Finally, we performed direct measurements of the interaction between solid substrates of different surface hydrophobicity and an air bubble using a wetting film apparatus. The obtained information is utilized to analyze the effect of surface hydrophobicity of solid on the solid/bubble interaction, which is important in flotation practice.

\section{Experimental}

\section{Materials}

Nanopure water was obtained by using the Nanopure Ш (Barnstead IA) water system. The conductivity of the water was $18.2 \mathrm{M} \Omega / \mathrm{cm}$ at $25^{\circ} \mathrm{C}$ and the surface tension was $72.5 \mathrm{mN} / \mathrm{m}$ at $22^{\circ} \mathrm{C}$. The water and surfactant solutions were used without purging with ultra pure nitrogen to exclude atmospheric $\mathrm{CO}_{2}$. Thus, the $\mathrm{pH}$ of the solutions was in the range of 5.6-5.8. $\mathrm{NaOH}$ (99\%, Mallinckrodt Baker) was used without further purification. Liquid reagents, such as chloroform (99.9\%, Burdick \& Jackson), $\mathrm{H}_{2} \mathrm{SO}_{4}$ (98\%, VMR International) and $\mathrm{H}_{2} \mathrm{O}_{2}$ (30\%, VMR International) were used as received without further purification. The surfactants used in the present work included dodecyltrimetyl-ammonium chloride $\left(\mathrm{C}_{12} \mathrm{TACl}\right)$, tetradecyltrimetylammonium chloride 
( $\left.\mathrm{C}_{14} \mathrm{TACl}\right)$ hexadecyltrimetylammonium chloride $\left(\mathrm{C}_{16} \mathrm{TACl}\right)$ and octadecyltrimetylammonium chloride $\left(\mathrm{C}_{18} \mathrm{TACl}\right)$ all from TCI and of greater than $97 \%$ purity. They were used without further purification.

Quartz plates (Hereaus Amersil, Inc.) and silicon wafers (100 oriented, Polished Wafer, Sumco, Oregon) were boiled in a $\mathrm{H}_{2} \mathrm{SO}_{4} / \mathrm{H}_{2} \mathrm{O}_{2}$ solution ( $7: 3$ by volume) at $120^{\circ} \mathrm{C}$ for 60 minutes. The plates were rinsed thoroughly with a large amount of Nanopure water, equilibrated with the water in a sealed vial for at least 20 minutes, removed and dried by blowing pure nitrogen gas over the surface, and then used for experiments.

\section{Degassing procedure}

To conduct AFM force measurements under air-free conditions, surfactant solutions were degassed by following the procedure described by Sakamoto et al. (2002) Nanopure water was boiled for one hour, frozen in a vessel with liquid nitrogen, evacuated for two hours with a final air pressure at 20mbar, and then melted under reduced pressure. This procedure was repeated three times. During the third degassing step, a known amount of surfactant was placed on the ice and evacuated for two hours. The vessel containing the ice and surfactant powder was then allowed to reach thermal equilibrium with ambient temperature before use. According to Sakamoto et al. (2002), this procedure "guarantees that the solution is completely free from dissolved gas".

When the target surfactant concentration was very low, i.e., $10^{-6} \mathrm{M}$, it was difficult to add accurately a desired amount of dry surfactant to frozen water unless a very large amount solution was boiled, frozen, and degassed. Therefore, we prepared a 1 $\mathrm{mL}$ of $10^{-4} \mathrm{M} \mathrm{C}_{18} \mathrm{TACl}$ solution and $125 \mathrm{~mL}$ of water, degassed them in the manner described above, mixed the two, and boiled the mixture for 1 hour. During the boiling, only about $25 \mathrm{mg}$ of water evaporated so that the surfactant concentration remained close to $10^{-6} \mathrm{M}$. The boiled solution was frozen by liquid nitrogen, degassed, melted under vacuum, and allowed to reach a thermal equilibrium with the ambient before use. For comparison purpose, we prepared higher concentrations of $\mathrm{C}_{18} \mathrm{TACl}$ solutions using this modified procedure and Sakamoto el al.'s, and conducted AFM force measurements. We found no significant differences between the two methods of obtaining air-free solutions.

\section{Air-equilibrated solutions}

Air-equilibrated $\mathrm{C}_{18} \mathrm{TACl}$ solutions were prepared in a vessel such that the amount of air above the liquid was greater than the amount of air in the equilibrated solution. This was easily accomplished because the concentration of gas molecules in air $(\sim 40 \mathrm{mM})$ at STP is about 40 times that in water. In each experiment, a solution vessel was thoroughly shaken to speed equilibration, and then was left sealed for 30 minutes. At the end of this period, the seal was broken to allow mixing with ambient air for a few minutes. This procedure was repeated 4 times.

\section{Surface Force Measurement}

Surface forces were measured using a Digital Instrument Nanoscope Шa AFM at room temperature $\left(\sim 22^{\circ} \mathrm{C}\right)$ using the colloidal probe technique. (Ducker et al., 1991, 1992) The separation distance $(\mathrm{H})$ between the probe (glass sphere) and the substrate 
(slica plate) was measured by monitoring the deflection of the cantilever on which the probe was attached. Silicon-nitride NP-20 cantilevers were obtained from Veeco, CA. Triangular cantilevers with spring constant of $0.58 \mathrm{~N} / \mathrm{m}$ were used. The spring constants were determined using the resonant frequency technique. (Cleveland et al., 1993) The error associated with this technique was $\pm 7 \%$. The glass spheres $(12-20 \mu \mathrm{m}$ in diameter) were obtained from Polysciences Inc. Warrington, PA. They were soaked in chloroform for 30 minutes and UV-irradiated for 30 minutes to remove possible organic contaminants. In each experiment, a glass sphere was glued onto a cantilever with EPON 1004 resin (Shell Chemical Co.). The diameter of the glass sphere was measured using an Olympus BH-2 light microscope with a video caliper with an error of $\pm 0.5 \mu \mathrm{m}$. Combined error between the measurements of the spring constants and the sphere diameters was about $\pm 12 \%$ in force/diameter (F/R) calculations.

Surface forces were measured for a series of surfactant concentrations. For a given series of measurements, the surfactant solutions were injected into an AFM liquid cell starting from the lowest concentration and continuing to the highest one. For each new concentration, the glass sphere and silica plate were exposed to the surfactant solution for a period of 20 minutes before force measurements were commenced. Forces reported in this paper were recorded about 1 hour after exposure of the solids to a new solution. Although the forces measured during the periods of 20 minutes to 1 hour were reproducible, we found that the forces varied slowly with time after 1 hour, presumably because of slow adsorption kinetics and possible rearrangement of the surfactant molecules on the surface. Thus, we have no evidence that the forces reported here were at adsorption equilibrium.

All reported forces, $F$, are normalized by the particle radius, $R$. For measurements at equilibrium, when $R$ is much greater than the range of the force, $F / 2 \pi R$ is equal to the energy of interaction per unit area for an infinite flat plate interacting with another infinite flat plate of the same material. (Derjaguin, 1934)

\section{AFM Imaging}

All AFM images reported in this communication are height (constant force) images captured at $22 \pm 1^{\circ} \mathrm{C}$ with integral and proportional gains of about 2. Silicon nitride cantilevers with nominal $\mathrm{k}=0.12 \mathrm{Nm}^{-1}$ were used. The images are of the same type of silica plate as used in the force measurements. The images were captured after a substrate was soaked in an aqueous solution for about 1 hour using the soft contact-mode imaging technique. Minimum contact forces were used in order not to disturb the adsorbed surfactant layer.

\section{$\zeta$-Potential Measurement}

The silica plates and glass spheres that were used for the AFM force measurements were subjected to $\zeta$-potential measurements. The glass spheres were suspended in water, and the coarser spheres were allowed to settle. The finer spheres $(<3$ $\mu \mathrm{m})$ left in suspension were used for the $\zeta$-potential measurements. For silica plates, the measurements were conducted after pulverizing the plates by means of an agate mortar and pestle and isolating the finer particles by sedimentation. The $\zeta$-potential 
measurements were conducted in both air-equilibrated and degassed $\mathrm{C}_{18} \mathrm{TACl}$ solutions using a Lazer Zee meter, Model 501 (Pen Kem, Inc, NY). The $\zeta$-potentials were calculated from the mobilities using the Smoluchowski equation. Reported results represent arithmetic averages of six measurements.

\section{Contact Angle Measurement}

A silica plate was immersed in a surfactant solution for a desired period of time, removed from the solution, and dried by blowing nitrogen gas over the surface. A drop of the surfactant solution was then placed on the surface and the contact angles were measured by means of a contact angle goniometer. The contact angles were also measured using the captive bubble technique, in which silica plates were kept in solution while air bubbles were brought to contact. A total of six contact angles were measured at a given experimental condition and averaged.

\section{Film thickness measurement}

The thickness of wetting film on a silicon wafer has been obtained in $\mathrm{NaCl}$ solutions at different electrolyte concentrations from the measurement of light intensity. The value was compared to that calculated from the disjoining pressure using classical DLVO theory. The capillary radius is about $0.75 \mathrm{~mm}$. Hydrophilic substrate is obtained by oxidizing the silicon wafer in $\mathrm{H}_{2} \mathrm{SO}_{4} / \mathrm{H}_{2} \mathrm{O}_{2}$ solution (7:3 by volume) at $100^{\circ} \mathrm{C}$ for 60 minutes. Hydrophobic surface is obtained by hydrophobizing silicon wafer using dilute OTS solution with a resulting contact angle about 30 50 degrees.

\section{$\underline{\text { Results and Discussion }}$}

\section{Contact Angle Measurement}

Figure 1 shows the equilibrium contact angles of the fused silica plates immersed in $10^{-5} \mathrm{M} \mathrm{C}_{18} \mathrm{TACl}$ solutions. The contact angles measured with the captive bubble technique reached a maximum after approximately 2 hours, which was shorter than

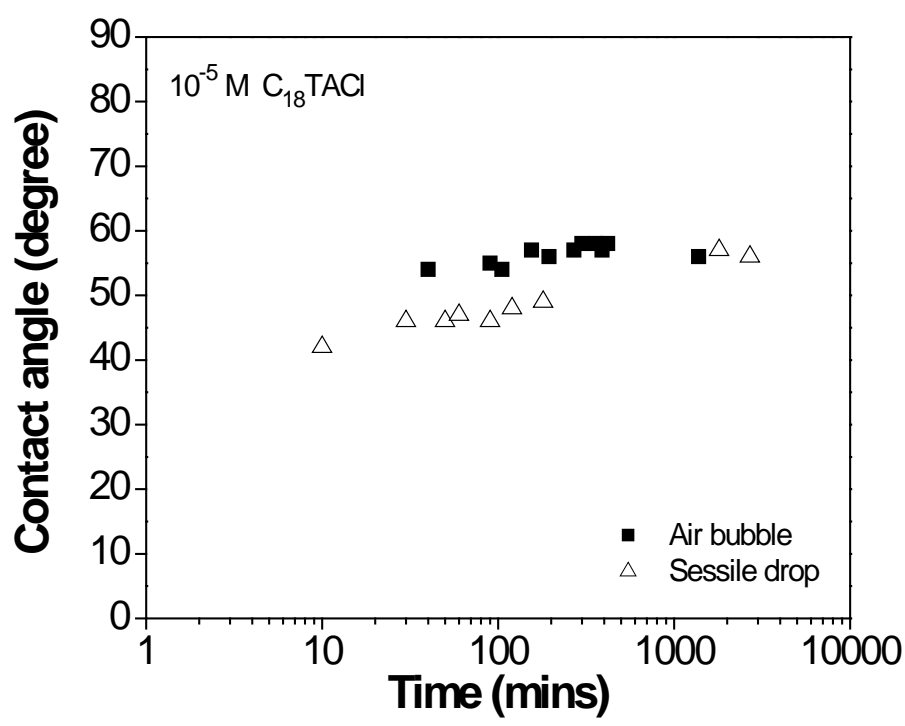

Figure 1. Sessile drop and captive bubble contact angles of the fused silica plates as a function of the time that the plate was exposed to $10^{-5} \mathrm{M} \mathrm{C}_{18} \mathrm{TACl}$ solutions. 
suggested by ellipsometry. The maximum contact angles were less than $60^{\circ}$ despite the long chain length of the surfactant, which may be attributed to the possibility that some of the surfactant molecules adsorb with the head group facing toward the solution, that is, the inverse (or flip-flop) orientation.

\section{Forces in Air-Equilibrated Solution}

Figure 2 shows the results of the AFM force measurements conducted as a function of the $\mathrm{C}_{18} \mathrm{TACl}$ concentration in air-equilibrated solutions. It shows the same general trend as the forces measured previously in cationic surfactant solutions. (Subramanian and Ducker, 2001; Lokar and Ducker, 2002, 2004) Addition of a small amount of the cationic surfactant reduces the repulsive force between the negatively charged surfaces, and a further increase in concentration lead to a repulsive force. In the present work, however, the changes in force were observed at lower concentrations of $\mathrm{C}_{18} \mathrm{TACl}$ than of $\mathrm{C}_{12} \mathrm{TABr}$ or $\mathrm{C}_{16} \mathrm{TABr}$, as expected of a longer chain surfactant. Also, the range of the forces was much greater. This can be attributed to the fact that the changes in forces was brought about at substantially lower surfactant concentrations than with $\mathrm{C}_{12} \mathrm{TABr}$. It is important to note here that a) the most attractive force is much greater

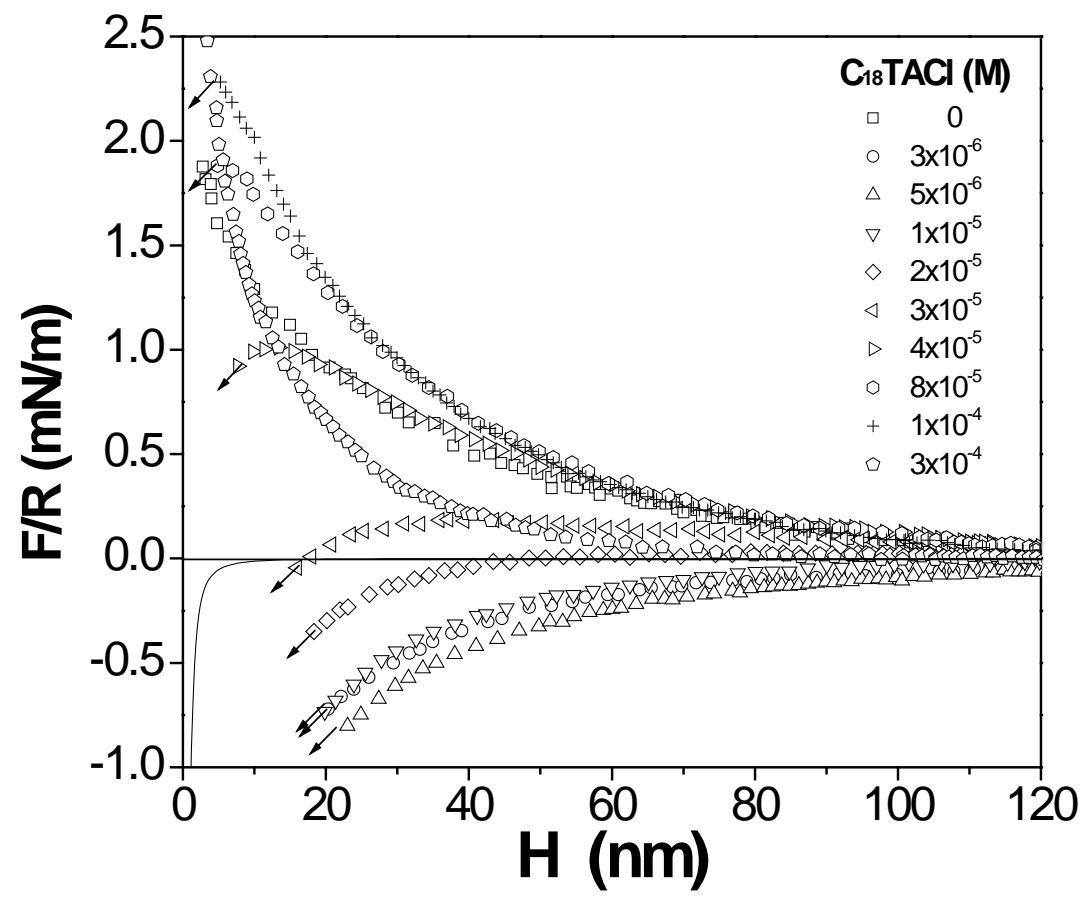

Figure 2. Forces (F) normalized by the radius (R) of the sphere in air-equilibrated $\mathrm{C}_{18} \mathrm{TACl}$ solutions. The solid line represents the theoretical van der Waals force calculated using a Hamaker constant of $8 \times 10^{-21} \mathrm{~J}$. The measured forces were repulsive in $0-3 \times 10^{-4} \mathrm{M} \mathrm{C}_{18} \mathrm{TACl}$ solutions. At $0 \mathrm{M}$, the surface potential obtained from a fit to Poisson-Boltzman theory was $-85 \mathrm{mV}$. The arrows represent the point where the cantilever undergoes a mechanical instability. Forces at smaller separations were measured, but are not reproduced here because the deflection of the spring does not give the surface force when it is not in mechanical equilibrium. Forces were measured in the order of increasing surfactant concentrations with the same sphere-plate combination. The forces were measured $\sim 60$ minutes after injection of each new reagent addition. 
than the van der Waals force between either silica solids or hydrocarbon solids, and that b) there is a very large gradient in the force as a function of concentration near the minimum (most attractive) force. The measured force was most attractive at $5 \times 10^{-6} \mathrm{M}$ and became progressively weaker at higher concentrations.The force became net repulsive above $3 \times 10^{-5} \mathrm{M}$. The concentration at which the measured force became most attractive was close to the p.c.n. of the glass sphere and silica plate, as will be shown later. Other investigators also showed that long-range force is most attractive near p.c.n. (Kerkicheff and Spalla, 1995; Parker et al. 1993)

The decrease in attractive force above the p.c.n. $\left(5 \times 10^{-6} \mathrm{M}\right)$ is due to the adsorption of the cationic surfactant in excess of the negative charge sites available on silica. The ability of the cationic surfactant to adsorb above p.c.n. is due to net attraction between the hydrocarbon chains, which is known as "hydrophobic effect". According to Somasundaran et al. (1964) the standard free energy change $\left(\Delta \mathrm{G}^{\mathrm{o}}\right)$ associated with this process is given by $\Delta G^{\circ}=-0.85 n \mathrm{kcal} / \mathrm{mole}$, where $n$ is the number of $\mathrm{CH}_{2}$ groups of a hydrocarbon chain that are in contact with the $\mathrm{CH}_{2}$ groups of the neighboring hydrocarbon chains. This value is comparable to the free energy of transferring hydrocarbon chain to water, $0.88 \mathrm{kcal}$ per mole of $\mathrm{CH}_{2}$ group. (Tanford, 1980)

It is possible that $\mathrm{C}_{18} \mathrm{TA}^{+}$ions adsorbing above p.c.n. may do so with inverse (or

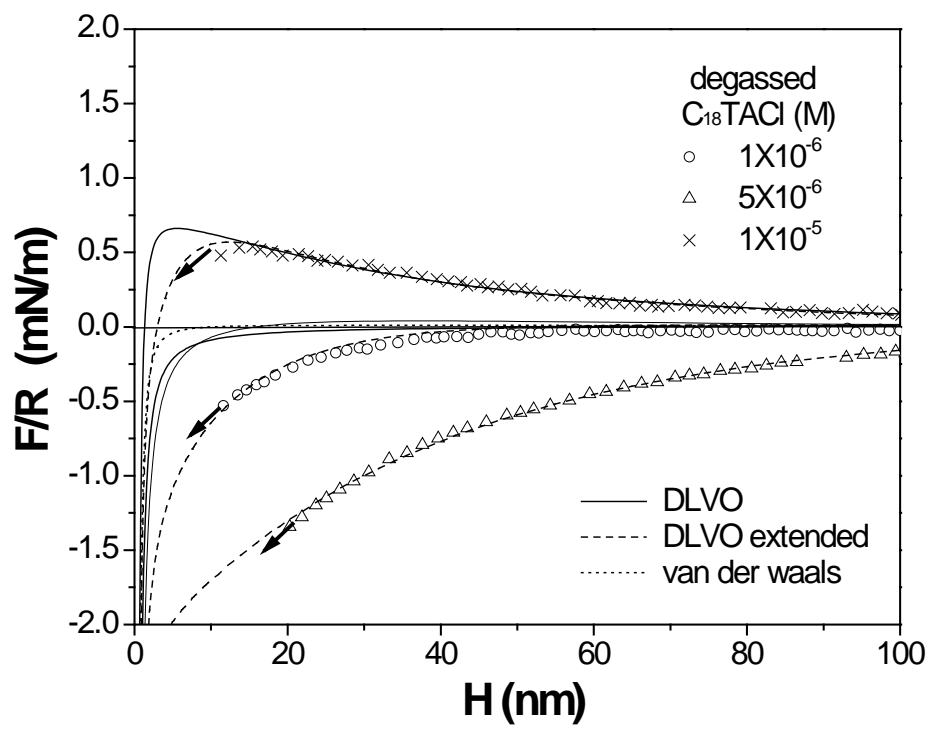

Figure 3. Forces (F) normalized by the radius (R) of sphere in degassed $\mathrm{C}_{18} \mathrm{TACl}$ solutions. The magnitudes of the forces are similar to those measured in airequilibrated solutions shown in Figure 2. The force in $1 \times 10^{-5} \mathrm{M}$ solution fits well to DLVO theory that was calculated using an exact numerical solution of the Poisson Boltzmann equation at separations great than $\sim 15 \mathrm{~nm}$, which is shown as a line though the points. The addition of an exponential force term improves the fit as shown by the dashed line. The forces at $1 \times 10^{-6}$ and $5 \times 10^{-6} \mathrm{M}$ are much more attractive than the van der Waals force alone, which is shown as a dotted line. 
flip-flop) orientation, i.e., with the head groups pointing toward the solution. The inverse orientation may occur after the surface has become hydrophobic by virtue of the normal mode of orientation, i.e., the head group in touch of the surface and the tails pointing to the solution. The possible inverse orientation may provide an explanation for the relatively low contact angles observed in $\mathrm{C}_{18} \mathrm{TACl}$ solutions (Figure 1).

The forces measured by Sakamoto et al. in air-equilibrated $\mathrm{C}_{18} \mathrm{TACl}$ solutions are about 30-100 times larger than shown in Figure 2. Furthermore, we did not observe the steps that were observed by these investigators and attributed to bridging air bubbles. Other investigators also observed the steps and attributed them to bridging bubbles. (Parker, et al. 1994; Nuyen et al. 2003)

\section{Effect of Degassing on AFM Force Measurement}

Figure 3 shows the forces between glass sphere and fused silica plate in solutions that were degassed. Overall, the forces in degassed and air-equilibrated solutions show the same trends. The measured forces in degassed solutions were attractive at a concentration as low as $10^{-6} \mathrm{M}$. The strongest attractive force was observed at $5 \times 10^{-6} \mathrm{M}$, as was the case with the measurements conducted in air-equilibrated solutions (Figure 2). In fact, the force measured in degassed solution was slightly more attractive than in the air-saturated solution (Figure 4a). As the concentration was increased to $10^{-5} \mathrm{M}$, the measured force in degassed solution became repulsive while the force measured in airsaturated solution remained attractive (Figure 4b). Below we explain these differences without using the theory of bridging air bubbles.

The measured forces were fitted to an exponential function,

$$
\frac{F}{R}=C \exp \left(-\frac{H}{D}\right)
$$

where $F$ is the surface force, $R$ the radius of the glass sphere used for the force measurements, $H$ the closest separation distance between the sphere and plate, and $C$, and $D$ are fitting parameters. It was assumed that the electrical potentials of the silica plates and glass spheres were zero and that $C=-1.4 \mathrm{mN} / \mathrm{m}$. Also, the fitting was carried out after subtracting the forces due to van der Waals interaction from the measured forces.

Note here that the glass sphere and the fused silica plate jumped into contact in the degassed $10^{-5} \mathrm{M} \mathrm{C}_{18} \mathrm{TACl}$ solution at a separation distance of $12 \mathrm{~nm}$, suggesting the presence of an attractive force not considered in the DLVO theory. The solid line of Figure 3 represents the DLVO fit of the data obtained in $10^{-5} \mathrm{M}$ degassed solution. The dashed lines represent the extended DLVO theory, which considers the contributions from non-DLVO attractive force whatever its origin is. Details of the fitting procedures will be discussed later.

The results given in Figures 2 and 3 show that, although the effect of degassing was quite dramatic at some individual concentrations of surfactant, degassing the solution 

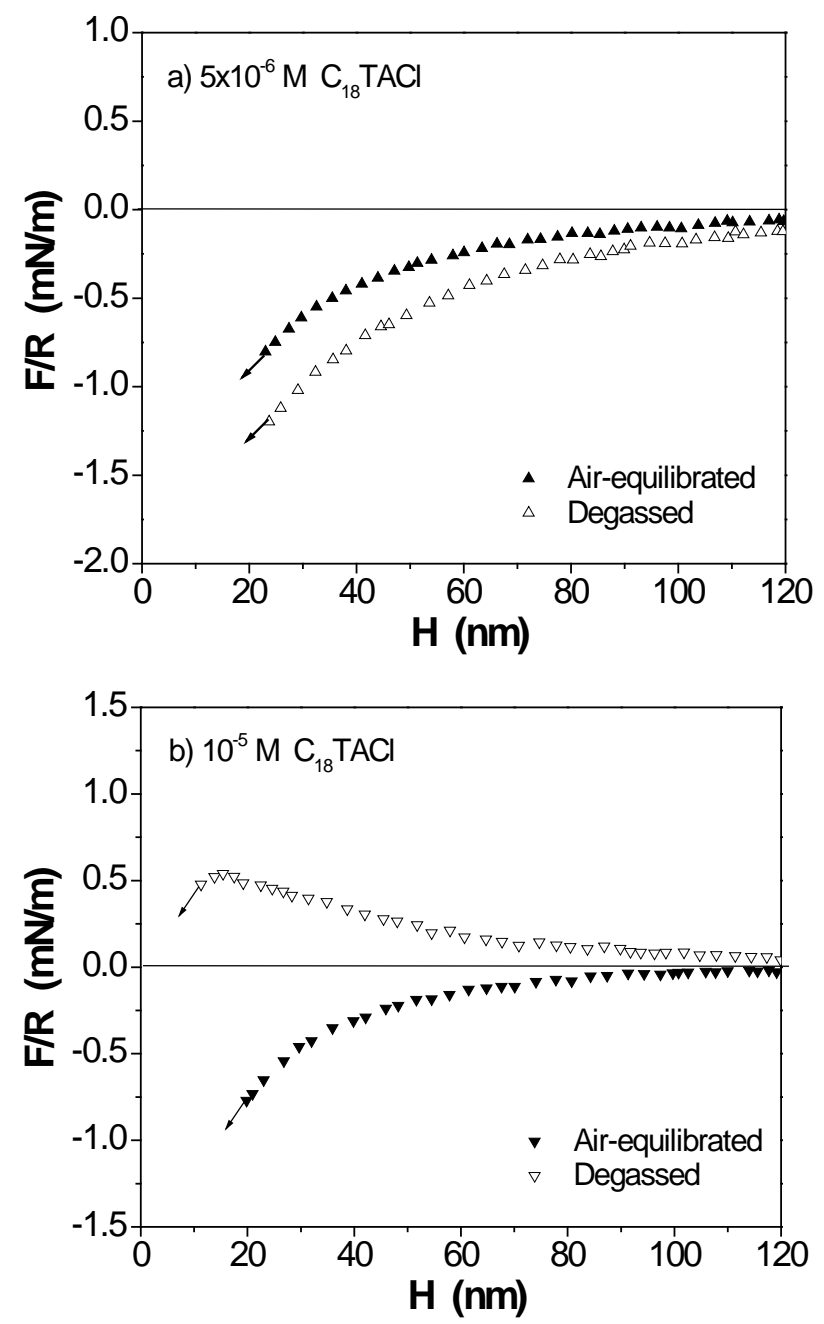

Figure 4. Comparison of the forces measured in degassed and air-equilibrated $5 \times 10^{-}$ ${ }^{6}$ and $1 \times 10^{-5} \mathrm{M} \mathrm{C}_{18} \mathrm{TACl}$ solutions. The data were taken from Figures 2 and 3 . At $5 \times 10^{-6}$ $\mathrm{M}, \mathrm{D}_{2}$ is 38 and $34 \mathrm{~nm}$ in degassed and air-equilibrated solutions, respectively. At $1 \mathrm{x} 10^{-}$ ${ }^{5} \mathrm{M}, \mathrm{D}_{2}$ is 30 and $4 \mathrm{~nm}$ in air-equilibrated and degassed solutions, respectively. The decrease in decay lengths by degassing can be attributed to $\mathrm{pH}$ change upon degassing.

did not change the overall trends, and caused only a small change in magnitude particularly at the concentration of maximum attractive force. In the remainder of this communication, we will discuss 1) our hypothesis for the mechanism of the changes in force upon degassing surfactant solutions, and 2) further experiments to reveal the nature of the attractive force.

Our hypothesis is that the primary effect of degassing the solution in our experiments was to raise the $\mathrm{pH}$. As the $\mathrm{pH}$ increases, the surface charge becomes more negative, which in turn causes an increase in the adsorption of the $\mathrm{C}_{18} \mathrm{TA}^{+}$ions and, hence, a change in surface force. We found that the $\mathrm{pH}$ of the air-equilibrated solutions was 5.7, which increased to 6.6 when the solution was degassed. Degassing removes $\mathrm{CO}_{2}$ from the solution, causing shift in the following set of equilibria to the left: (Stumm and Morgan, 1996) 


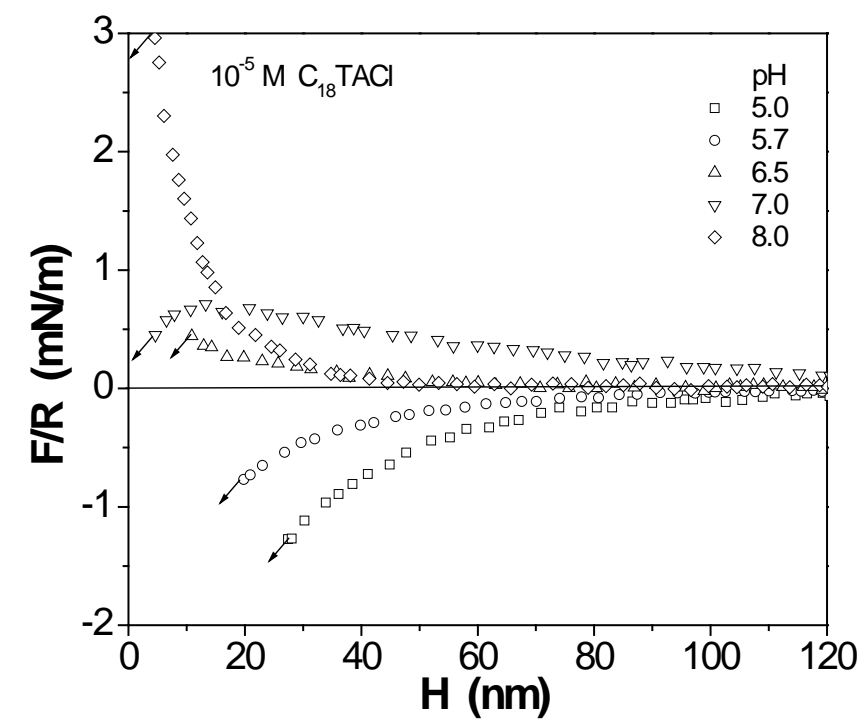

Figure 5. Effect of $\mathrm{pH}$ on forces in air-equilibrated $10^{-5} \mathrm{M} \mathrm{C} 18 \mathrm{TACl}$ solutions. The data for $\mathrm{pH}=5.7$ were taken from Figure 6; all other data were for the same particle/plate combination

$$
\mathrm{H}_{2} \mathrm{O}+\mathrm{CO}_{2}(\mathrm{aq}) \rightleftharpoons \mathrm{H}_{2} \mathrm{CO}_{3}(\mathrm{aq}) \rightleftharpoons \mathrm{H}^{+}(\mathrm{aq})+\mathrm{HCO}_{3}^{-}(\mathrm{aq})
$$

The fact that the solution remained at $\mathrm{pH} 6.6$ rather than 7.0 suggests that we had not completely degassed the solution, or that some $\mathrm{CO}_{2}$ had returned on the timescale of our $\mathrm{pH}$ measurement. Using the known equilibrium constant for the composite reaction above $\left(\mathrm{K}=4.47 \times 10^{-7}\right)$, (Stumm and Morgan, 1996) we can calculate that our degassing

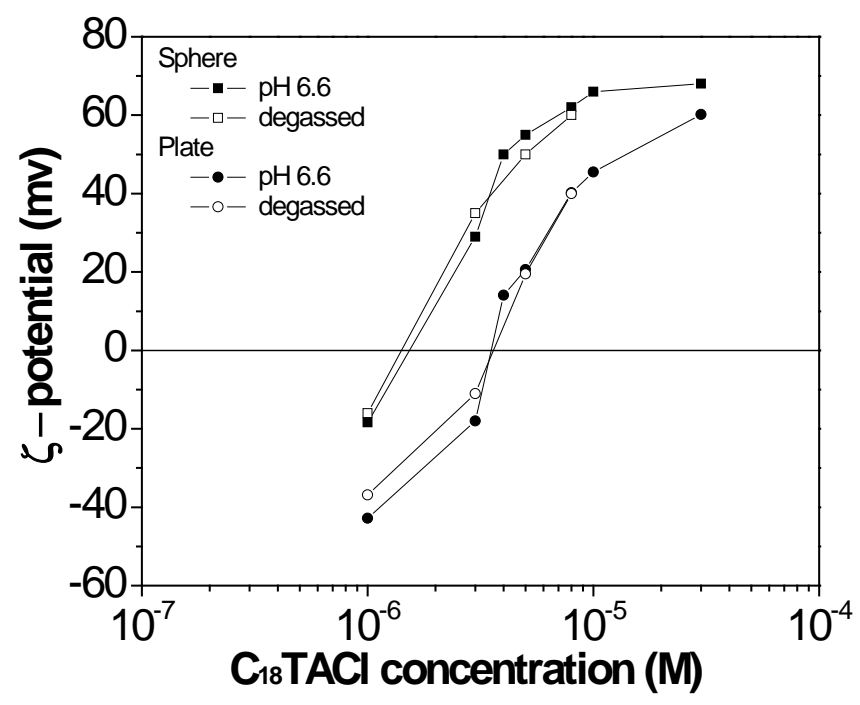

Figure 6. Comparison of the $\zeta$-potentials measured in degassed $\mathrm{C}_{18} \mathrm{TACl}$ solutions with those measured in air-equilibrated solutions whose $\mathrm{pH}$ have been adjusted to the same $\mathrm{pH}(=6.6)$ as the degassed solutions. 
procedure decreased the concentration of dissolved carbon dioxide from $8.9 \times 10^{-6}$ to $1.2 \times 10^{-7} \mathrm{M}$, i.e. we removed about $99 \%$ of the $\mathrm{CO}_{2}$.

Figure 5 shows the forces measured in air-equilibrated $10^{-5} \mathrm{M} \mathrm{C}_{18} \mathrm{TACl}$ solutions by changing the $\mathrm{pH}$ with $\mathrm{NaOH}$ or $\mathrm{HCl}$. The effect of increasing the $\mathrm{pH}$ was very similar to the effect of degassing (compare Figure 5 with Figure 4). As the $\mathrm{pH}$ increases, the surface becomes more negative, which should cause an increase in the adsorption of the $\mathrm{C}_{18} \mathrm{TA}^{+}$ions and render the surface more hydrophobic. As the surface becomes more hydrophobic, additional $\mathrm{C}_{18} \mathrm{TA}^{+}$ions adsorb on the surface, the adsorption mechanism being hydrophobic effect between the hydrocarbon chains. The additional adsorption of the cationic surfactant should lead to an increased surface charge and, hence, an increased repulsive surface force, as shown in Figures 4 and 5.

To examine the above mechanism, we have measured the $\zeta$-potentials of the glass particles and the pulverized plate. Figure 6 shows the $\zeta$-potentials as a function of the $\mathrm{C}_{18} \mathrm{TACl}$ concentration. The measurements were conducted both in degassed and airequilibrated solutions. The $\mathrm{pH}$ of the degassed solutions was 6.6, and the $\mathrm{pH}$ of the airequilibrated solutions was adjusted to 6.6 with $\mathrm{NaOH}$. Clearly, the effect of degassing on the $\zeta$-potential was reproduced by increasing the $\mathrm{pH}$ of the air-saturated solution.

Figure 7 compares the $\zeta$-potentials of the silica plate and glass sphere measured at $\mathrm{pH} 5.7$ and $\mathrm{pH}$ 6.6. As the $\mathrm{pH}$ was increased from 5.7 to that of the degassed solution, i.e., $\mathrm{pH} 6.6$, the $\zeta$-potentials became considerably more positive, indicating that $\mathrm{C}_{18} \mathrm{TA}^{+}$ ions adsorb more readily at the higher $\mathrm{pH}$. The increased adsorption of the cationic surfactant at the higher $\mathrm{pH}$ (and hence the higher surface potential) may be responsible

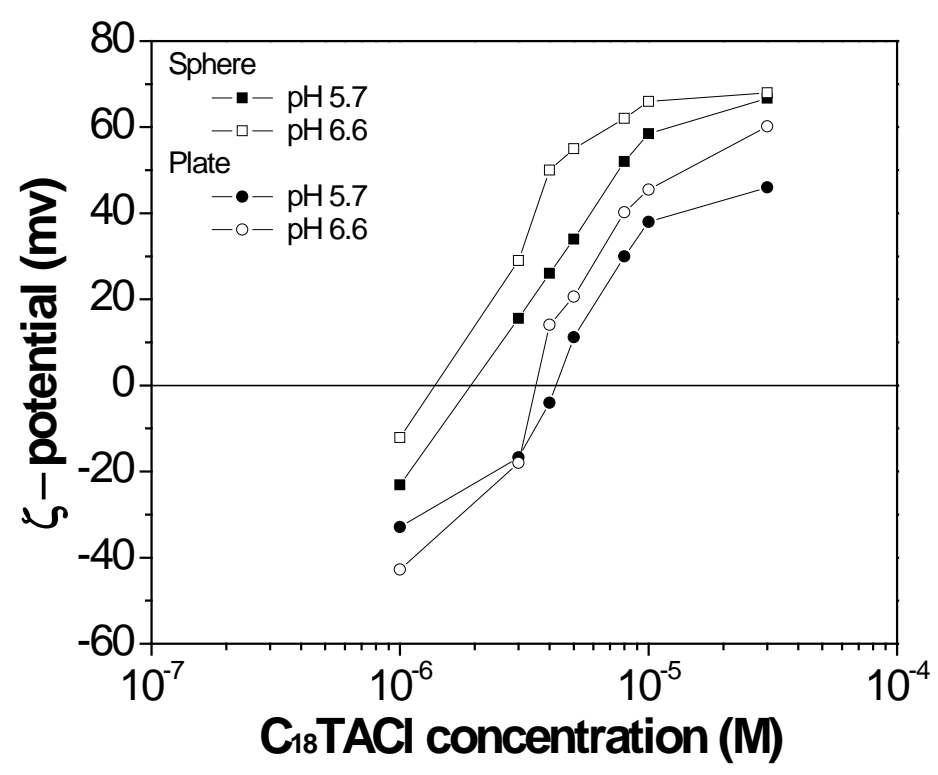

Figure 7. Comparison of the $\zeta$-potentials measured in air-equilibrated $\mathrm{C}_{18} \mathrm{TACl}$ solutions of $\mathrm{pH} 5$.7 with those measured in solutions whose $\mathrm{pH}$ was adjusted to $\mathrm{pH}$ 6.6. 
for the transition of the measured forces from the long-range attraction to long-range repulsion when a $10^{-5} \mathrm{M} \mathrm{C}_{18} \mathrm{TACl}$ solution was degassed (see Figure 4b). That the measured force became net repulsive does not mean that the surface became hydrophilic. It simply indicates that the repulsive force was greater than the attractive force. In the degassed solution, the two surfaces jumped into contact at $12 \mathrm{~nm}$, which was much larger than predicted by the DLVO theory. It can be stated, therefore, that the long-range attractive force still existed in the degassed $10^{-5} \mathrm{M} \mathrm{C}_{18} \mathrm{TACl}$ solution.

The $\zeta$-potential measurements also show that the potentials of the glass sphere
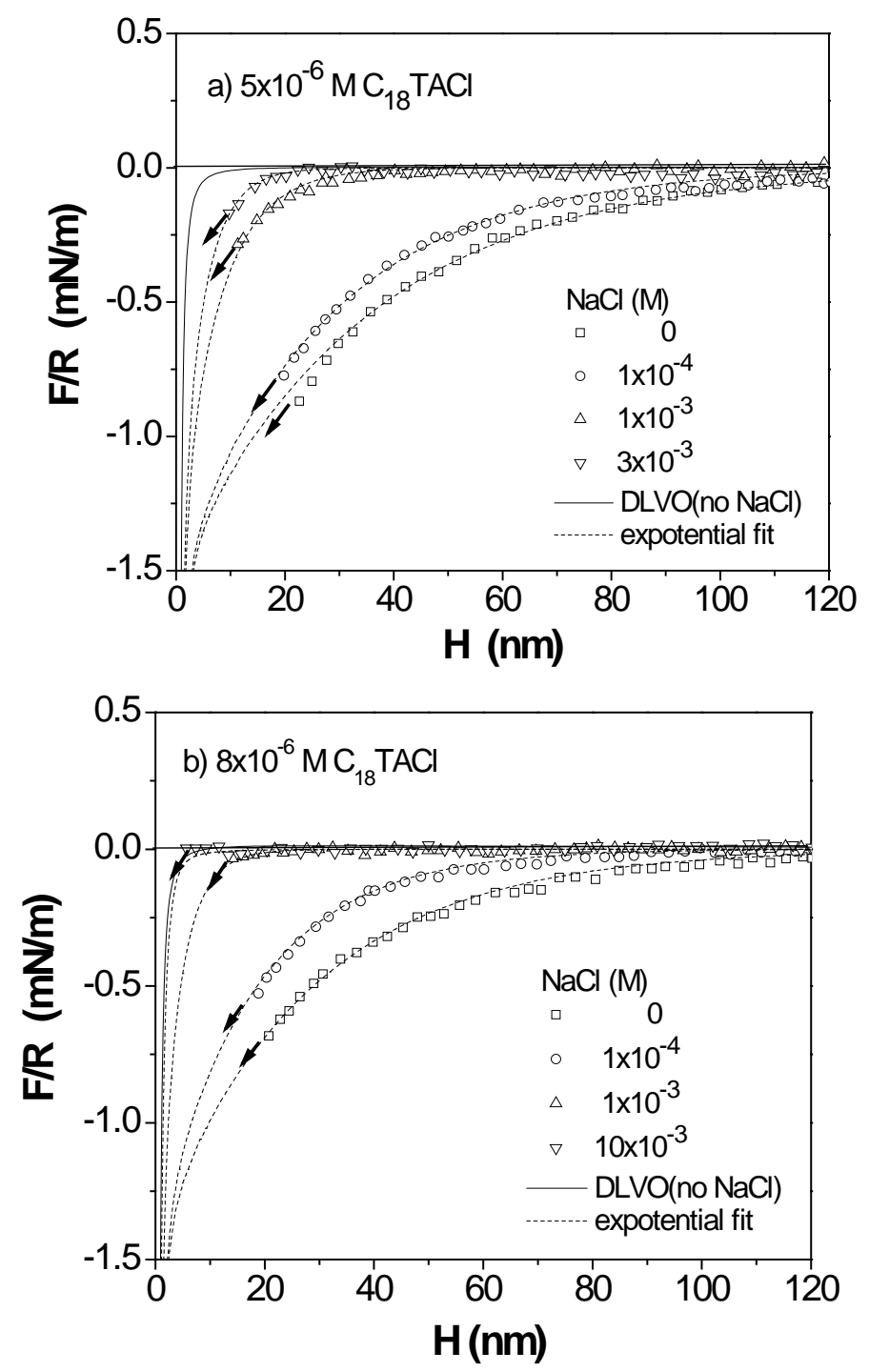

Figure 8. Effect of $\mathrm{NaCl}$ on the forces measured in air-equilibrated solutions of a) $5 \times 10^{-6} \mathrm{M}$ and b) $8 \times 10^{-6} \mathrm{M} \mathrm{C}_{18} \mathrm{TACl}$ solutions. The solid lines represent the DLVO fit, and the dashed lines represent the extended DLVO theory with a double-exponential force term (Eq. [3]). The fitting parameters are given in Table 1. The decrease in the long-range attractive force suggests that it may be of electrostatic origin. 
Table 1. Effects of $\mathrm{NaCl}$ on Debye Lengths $\left(\kappa^{-1}\right)$ and Decay Lengths $\left(\mathrm{D}_{2}\right)$ in $\mathrm{C}_{18} \mathrm{TACl}$ Solutions.

\begin{tabular}{cccc}
\hline \multirow{2}{*}{$\begin{array}{c}\mathrm{NaCl} \\
(\mathrm{mM})\end{array}$} & $\kappa^{-1}$ & \multicolumn{2}{c}{$\mathrm{D}_{2}(\mathrm{~nm})$} \\
\cline { 3 - 4 } & $(\mathrm{nm})$ & 33 & $\mathrm{C}_{18} \mathrm{TAC}$ Concentration $(\mathrm{M})$ \\
\cline { 3 - 4 } 0 & 136 & $5 \times 10^{-6}$ & 28 \\
0.1 & 30 & 26 & 18 \\
1 & 9.6 & 7.5 & 4 \\
3 & 5.6 & 3 & - \\
10 & 3 & - & 1 \\
\hline
\end{tabular}

were different from those of the ground silica plate. This finding suggests that we should consider the possibility that the long-range attractive forces measured in the present work could be attributed to attractive double-layer interactions.

Effect of Salt Concentration on the Forces in $C_{18}$ TACl Solutions

One possible explanation for the attractive forces measured in air-equilibrated and degassed $\mathrm{C}_{18} \mathrm{TACl}$ solutions and given in Figures 2 and 3, respectively, is that the force is of electrical origin. In this case, the magnitude of the force should decrease with an increase in salt concentration. Figure 8 shows the effect of added salt $(\mathrm{NaCl})$ on the forces measured in $5 \times 10^{-6}$ and $8 \times 10^{-6} \mathrm{M} \mathrm{C}_{18} \mathrm{TACl}$ solutions. Attractive forces decreased with increasing $\mathrm{NaCl}$ concentration. In all cases, the forces were approximately exponential. It is not likely that the decrease in force was caused by desorption of surfactant. Goloub, et al. (1997) showed that the addition of salt near a p.c.n. does not change the amount of adsorbed surfactant.

The measured forces were fitted to a double-exponential function,

$$
\frac{F}{R}=C_{1} \exp \left(-\frac{H}{D_{1}}\right)+C_{2} \exp \left(-\frac{H}{D_{2}}\right)
$$

where $F$ is the surface force, $R$ the radius of the glass sphere used for the force measurements, $H$ the closest separation distance between the sphere and plate, and $C_{1}$, $C_{2}, D_{1}$ and $D_{2}$ are fitting parameters. It was assumed that the electrical potentials of the silica plates and glass spheres were zero, and that $C_{1}=-50 \mathrm{mN} / \mathrm{m}, \mathrm{D}_{1}=1.0 \mathrm{~nm}$, and $\mathrm{C}_{2}=-1.4$ $\mathrm{mN} / \mathrm{m}$. Also, the fitting was carried out after subtracting the forces due to van der Waals interaction from the measured forces.

The decay lengths $\left(D_{2}\right)$ obtained from the fitting procedure described above are given in Table 1 . Also shown in the table are the Debye lengths $\left(\kappa^{-1}\right)$ calculated from the $\mathrm{NaCl}$ concentrations used. The values of $\mathrm{D}_{2}$ obtained from the force curves obtained in $5 \times 10^{-6}$ and $8 \times 10^{-6} \mathrm{M} \mathrm{C}_{18} \mathrm{TACl}$ solutions show the same trends although the values are not 


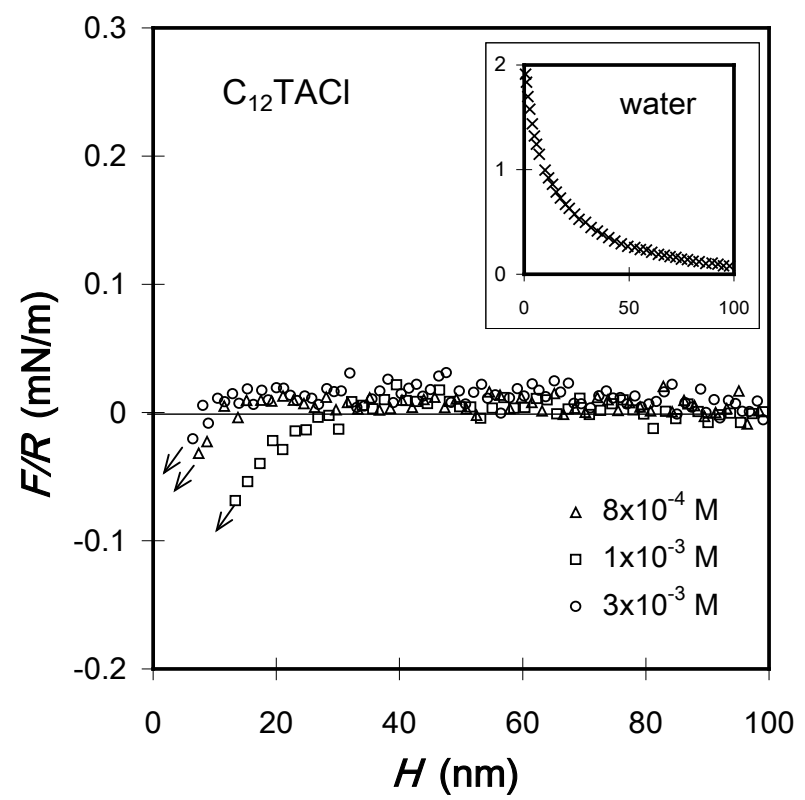

Figure 9. The AFM force curves obtained with the glass sphere and silica plate immersed in $\mathrm{C}_{12} \mathrm{TACl}$ solutions at $\mathrm{pH} \approx 6$. The repulsive forces measured in the presence of the surfactant are much lower than that obtained in pure water (inset). The arrows represent the distance where the two surfaces jumped into contact due to attractive forces. The attraction was strongest at $10^{-3} \mathrm{M} \mathrm{C}_{12} \mathrm{TACl}$.

identical. Unfortunately, the slow equilibration of the solution complicated the analysis of these results. Nevertheless, the decrease in the attractive force with $\mathrm{NaCl}$ suggests that the long-range attractive force may be of electrical origin and, therefore, is screened by the salt.

\section{Force Measurements in $C_{n}$ TACl Surfactant Solutions}

Figure 9 shows the surface forces $(F / R)$ measured between a glass sphere of radius $R$ and a flat silica plate in $\mathrm{C}_{12} \mathrm{TACl}$ solutions as a function of the closest distance $(H)$ separating the two macroscopic surfaces. The inset, which represents the force curve obtained in pure water, shows a strong repulsive force due to the electrostatic repulsion between two negatively charged surfaces. All of the force curves shown in Figures 9-12 were smooth, without showing steps or kinks, indicating that the adsorption systems were relatively free of contamination or air bubbles.

In the presence of the cationic surfactant, the repulsive force disappeared almost completely due to charge neutralization. The force curve obtained at $8 \times 10^{-4} \mathrm{M} \mathrm{C}_{12} \mathrm{TACl}$ showed a weakly attractive force at $\mathrm{H}<10 \mathrm{~nm}$. At $10^{-3} \mathrm{M} \mathrm{C}_{12} \mathrm{TACl}$, the attractive force became stronger and considerably longer ranged, the net attractive force being detectable at $H \approx 25 \mathrm{~nm}$. As the surfactant concentration was further increased to $3 \times 10^{-3} \mathrm{M}$, the attractive force was significantly reduced.

Several investigators (Rutland and Parker, 1994; Christenson and Claesson, 2001) showed that the attractive forces measured between hydrophobic surfaces became the 


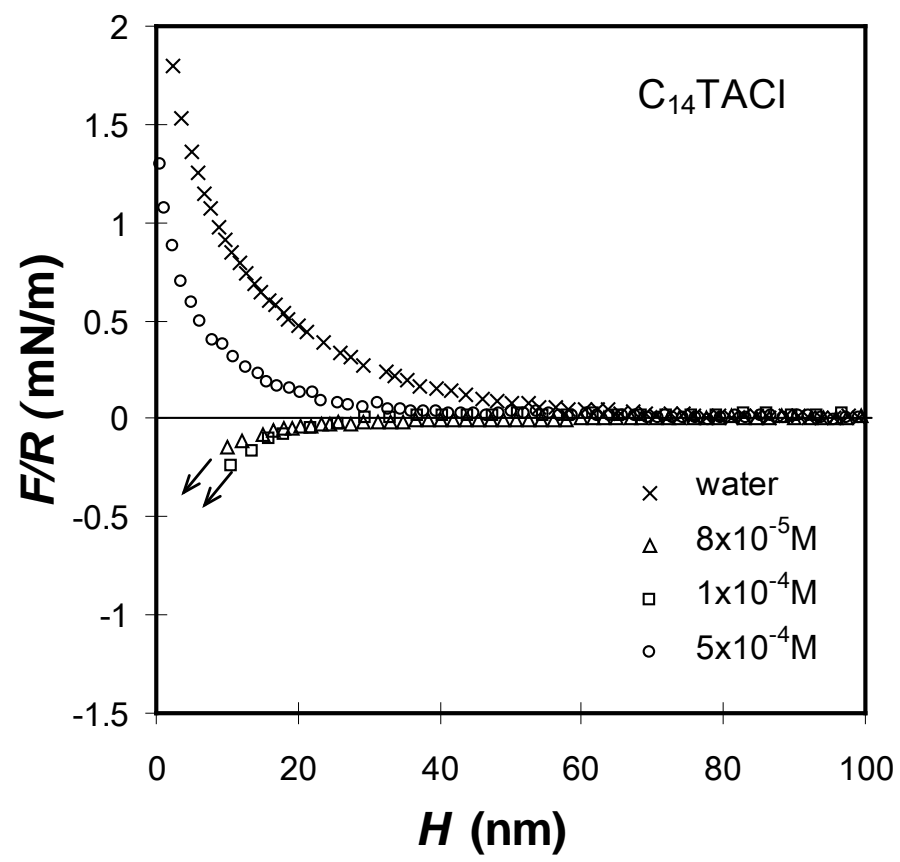

Figure 10. The AFM force curves obtained with the glass sphere and silica plate immersed in $\mathrm{C}_{14} \mathrm{TACl}$ solutions.

strongest at the surfactant concentrations close to the points of charge neutralization (p.c.n.), which is defined as the concentrations where the surface charge of the substrate is neutralized by the adsorption of an ionic surfactant. The $F / R$ vs. $H$ curves given in Figure 9 show the p.c.n. for the silica- $\mathrm{C}_{12}$ TAC system is approximately $10^{-3} \mathrm{M}$, as the attractive force was the strongest at this concentration. At the p.c.n., most of the negative sites are occupied by the $\mathrm{C}_{12} \mathrm{TA}^{+}$ions, forming hemimicelles in which the hydrocarbon tails of the adsorbed surfactant molecules are closely packed. (Somasundaran et al. 1964)

At $3 \times 10^{-3} \mathrm{M} \mathrm{C}_{12} \mathrm{TACl}$, additional $\mathrm{C}_{12} \mathrm{TA}^{+}$ions adsorb on silica, but most probably with inverse (or flip-flop) orientation. Such an orientation would be favored as it would cost less energy to insert additional surfactant molecules into the adsorption layer with their polar heads farthest apart from each other. The inverse orientation exposes the head groups toward the aqueous phase and, hence, causes the surface to be less hydrophobic. Therefore, the decrease in attractive force at the higher concentration can be attributed to the inverse orientation.

Figure 10 shows the surface forces measured between a glass sphere and a silica plate in the presence of a longer-chain $\left(\mathrm{C}_{14} \mathrm{TACl}\right)$ homologue. The trend is the same as observed with $\mathrm{C}_{12} \mathrm{TACl}$. At $8 \times 10^{-5} \mathrm{M} \mathrm{C}_{14} \mathrm{TACl}$, the repulsive double layer force that was observed in pure water was completely suppressed by charge neutralization, and a netattractive force was observed. At $10^{-4} \mathrm{M} \mathrm{C}_{14} \mathrm{TACl}$, the net-attractive force became stronger. As the surfactant concentration was further increased to $5 \times 10^{-4} \mathrm{M} \mathrm{C}_{14} \mathrm{TACl}$, the measured force became totally repulsive. It appears, therefore, that the p.c.n. is close to $10^{-4} \mathrm{M} \mathrm{C}_{14} \mathrm{TACl}$. At concentrations above the p.c.n., additional $\mathrm{C}_{14} \mathrm{TA}^{+}$ions adsorbed, 


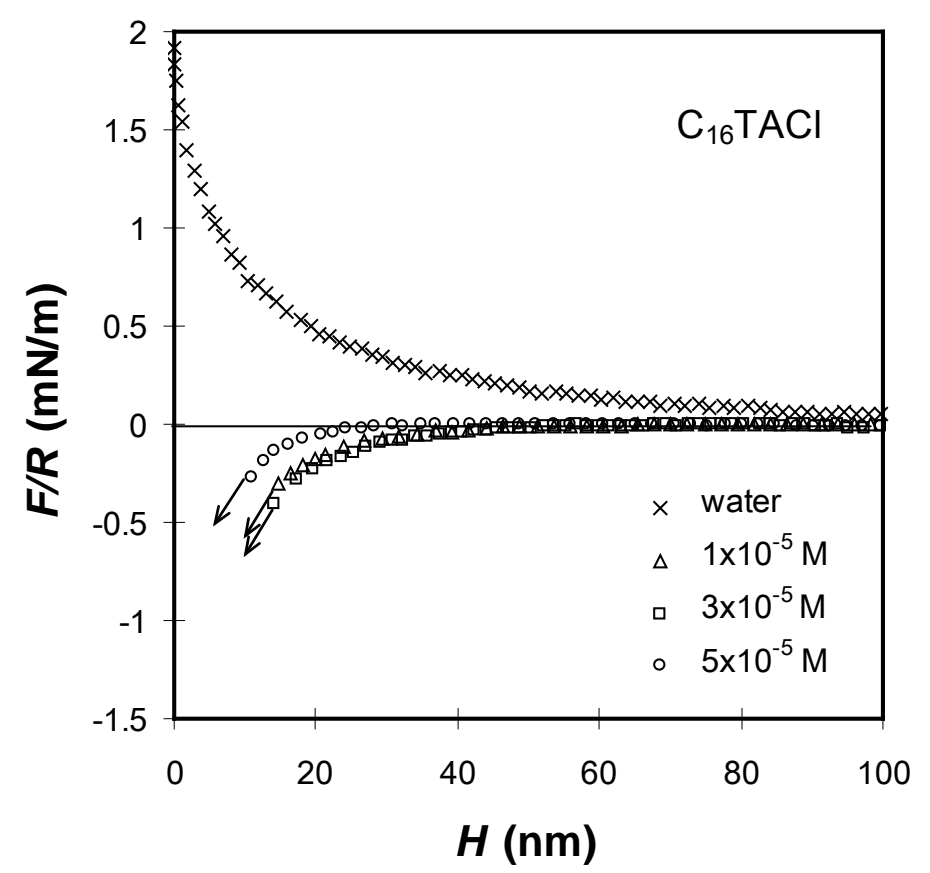

Figure 11. The AFM force curves obtained with the glass sphere and silica plate immersed in $\mathrm{C}_{16} \mathrm{TACl}$ solutions. The measured forces are most attractive at $3 \times 10^{-5} \mathrm{M}$ $\mathrm{C}_{16} \mathrm{TACl}$. The long-range forces become less negative at the higher concentration possibly due to inverse orientation.

causing the observed force totally repulsive. It is likely that additional surfactant ions adsorbed with inverse orientation, rendering the surface hydrophilic. It is interesting to note here that an increase in the number of ethylene groups by two caused a ten-fold decrease in the concentration (p.c.n.) at which the strongest attractive force was observed. This observation is a powerful message that longer-chain surfactants be used to increase the attractive force between hydrophobic surfaces.

Figure 11 shows the results obtained with an even longer-chain $\left(\mathrm{C}_{16} \mathrm{TACl}\right)$ homologue. At $10^{-5} \mathrm{M} \mathrm{C}_{16} \mathrm{TACl}$, the repulsive force disappeared completely and a netattractive force appeared. At $3 \times 10^{-5} \mathrm{M}$, the measured force became most attractive, indicating that the p.c.n. for the silica- $\mathrm{C}_{16} \mathrm{TACl}$ was close to this concentration. At $5 \times 10^{-5}$ $\mathrm{M}$, the attractive force was somewhat reduced, most probably due to the inverse orientation of the $\mathrm{C}_{16} \mathrm{TA}^{+}$ions. It is interesting to note here that the attractive forces observed with the C-16 cationic surfactant were substantially longer ranged than those observed with shorter-chain (i.e., C-12 and C-14) homologues.

The results obtained with $\mathrm{C}_{18} \mathrm{TACl}$ are presented in Figure 12. At $3 \times 10^{-6} \mathrm{M}$ $\mathrm{C}_{18} \mathrm{TACl}$, a very long-ranged and net-attractive force was observed. When the concentration was raised to $5 \times 10^{-6} \mathrm{M}$, the attractive force was strongest. At $10^{-5} \mathrm{M}$, the attractive force was reduced a little. Thus, the p.c.n. of the silica- $\mathrm{C}_{18} \mathrm{TACl}$ system was approximately $3 \times 10^{-5} \mathrm{M}$, which was significantly lower than observed for the shorter chain homologues. The most interesting aspect of the data shown in Figure 4 was that the 


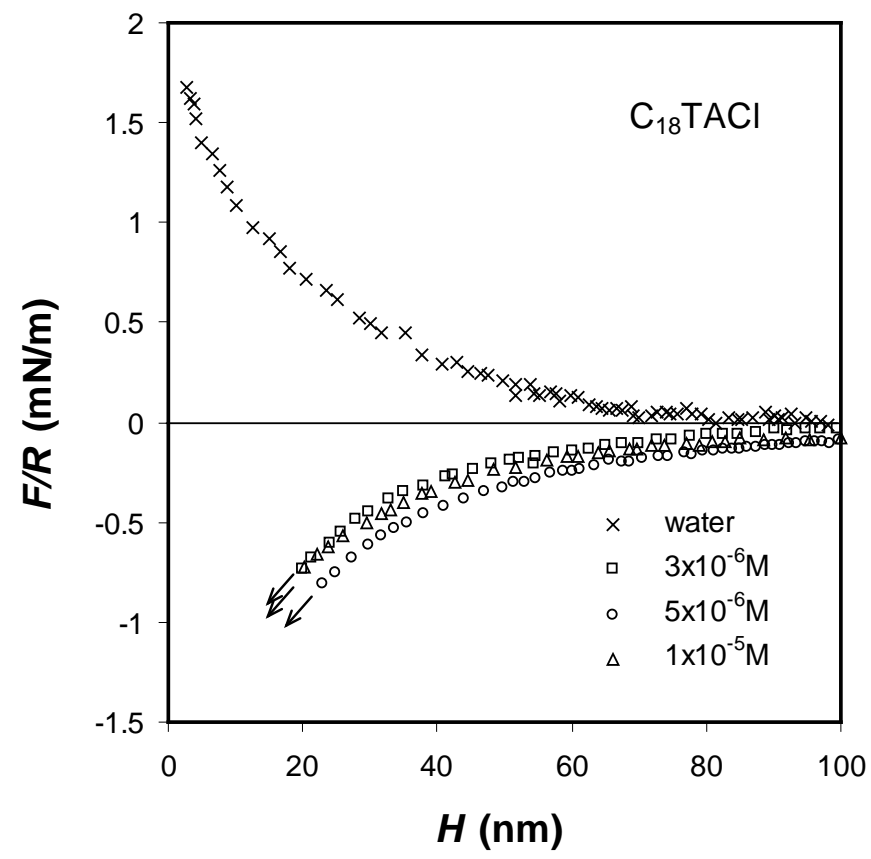

Figure 12. The AFM force curves measured with the glass sphere/silica plate immersed in $\mathrm{C}_{18} \mathrm{TACl}$ solutions. The long-range forces measured are most attractive at $5 \times 10^{-6} \mathrm{M} \mathrm{C}_{18} \mathrm{TACl}$, which is close to the p.c.n.

C-18 surfactant gave much stronger and longer-range attractive forces than observed for the shorter-chain homologues. With the $\mathrm{C}_{18} \mathrm{TACl}$, net attractive forces were observed at separations as large as $100 \mathrm{~nm}$.

The strongest attractive force curves shown in each of Figures 9-12 are redrawn in Figure 13, and were fitted to a single-exponential force law, viz. eq[1]. Table 2 gives the values of these parameters as well as the Debye length $\left(\kappa^{-1}\right)$. From Figure 5, it is clearly seen that the maximum attractive force that can be created by the adsorption of a $\mathrm{C}_{\mathrm{n}} \mathrm{TACl}$ on silica increases with increasing hydrocarbon chain length. As shown in Table 2, the decay length $(D)$ of the attractive force increases with chain length. It was only $3 \mathrm{~nm}$ at $n=12$. As $n$ was increased to 14,16 , and $18, D$ was increased to 6,14 , and $32 \mathrm{~nm}$, respectively. While $D$ was very sensitive to chain length, the values of $C$ stayed practically the same, $1.3-1.4 \mathrm{mN} / \mathrm{m}$. It is important to note here that the surfactant concentration at which surface charge is neutralized and the maximum hydrophobic force observed decreases substantially with increasing chain length.

\section{AFM Images}

Figure 14 shows the contact mode AFM images of a fused quartz plate immersed in water. It shows that the surface is smooth with a roughness of less than $2 \mathrm{~nm}$ on a $100 \times 100 \mathrm{~nm}$ scale. The AFM images show boundaries between fused silica particles of approximately $100 \mathrm{~nm}$.

The presence of the grain boundaries on the fused silica surfaces can make it difficult to see the surfactant molecules adsorbing on the surface. Therefore, the images of surfactant 


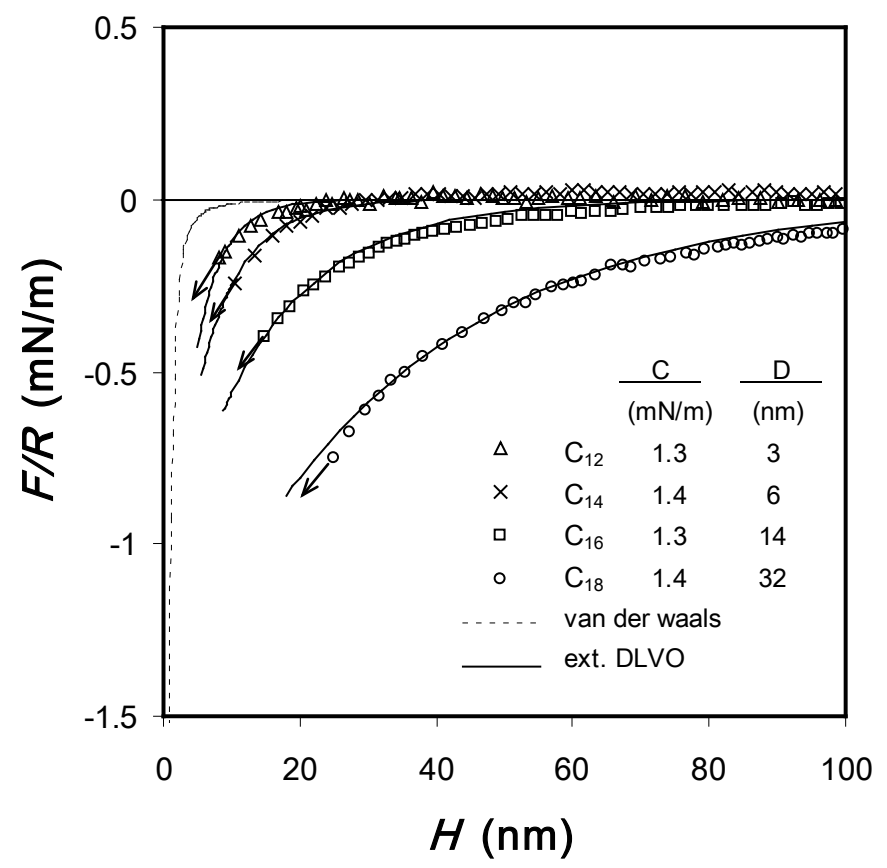

Figure 13. The AFM force curves obtained with glass sphere and silica plate at the p.c.n.'s of the $\mathrm{C}_{\mathrm{n}} \mathrm{TACl}$ homologues $(\mathrm{n}=12,14,16,18)$. The dashed line represents the van der Waals force with the Hamaker constant $A=0.8 \times 10^{-20} \mathrm{~J}$. The solid lines represent the extended DLVO theory which includes contributions from the van der Waals force and the hydrophobic force. (The double layer force is considered to be zero.) The hydrophobic force is represented by the single-exponential force law (Eq. (1)) with a preexponential parameter $\mathrm{C}$ and the decay length $\mathrm{D}$ as fitting parameters. It is shown that the hydrophobic force increases with increasing chain length.

molecules adsorbing on $\mathrm{SiO}_{2}$ surfaces were taken using silicon wafers as substrates. Figure 15a shows an AFM image of a silicon wafer surface immersed in Nanopure water. Before taking the images, the substrate had been boiled in a $\mathrm{H}_{2} \mathrm{SO}_{4} / \mathrm{H}_{2} \mathrm{O}_{2}$ solution (7:3 by volume) at $120^{\circ} \mathrm{C}$ for 1 hour to remove possible organic contaminants and to generate a "clean" oxide surface. The $10 \times 10 \mu \mathrm{m}$ image shows that the substrate surface is smooth with $0.2 \mathrm{~nm}$ roughness. No lumps or scratches were seen, indicating that the surface was clean and free of contaminants. Figure $15 \mathrm{~b}$ shows an image of a silicon wafer surface that had been immersed in $10^{-5} \mathrm{M} \mathrm{C}_{18} \mathrm{TACl}$ solution for 1 hour. It shows that the surfactant molecules cover most of the surface with some areas unoccupied, suggesting the formation of patches or hemi-micelles. The height of the adsorption layer is approximately $2.2 \mathrm{~nm}$, which is a little less than the length $(2.3 \mathrm{~nm})$ of a fully extended $\mathrm{C}_{18}$ hydrocarbon chain.

Figure $15 \mathrm{c}$ and d show the AFM images of a silicon wafer immersed in a $3 \times 10^{-5}$ $\mathrm{M} \mathrm{C}_{18} \mathrm{TACl}$ solution which was above its p.c.n. The images obtained at this concentration show that the adsorption occurs in patches. The heights of some of the patches are higher than $2.3 \mathrm{~nm}$, indicating bilayer formation. Some of the patches are of 20-30 nm in dimension, while others are larger. 


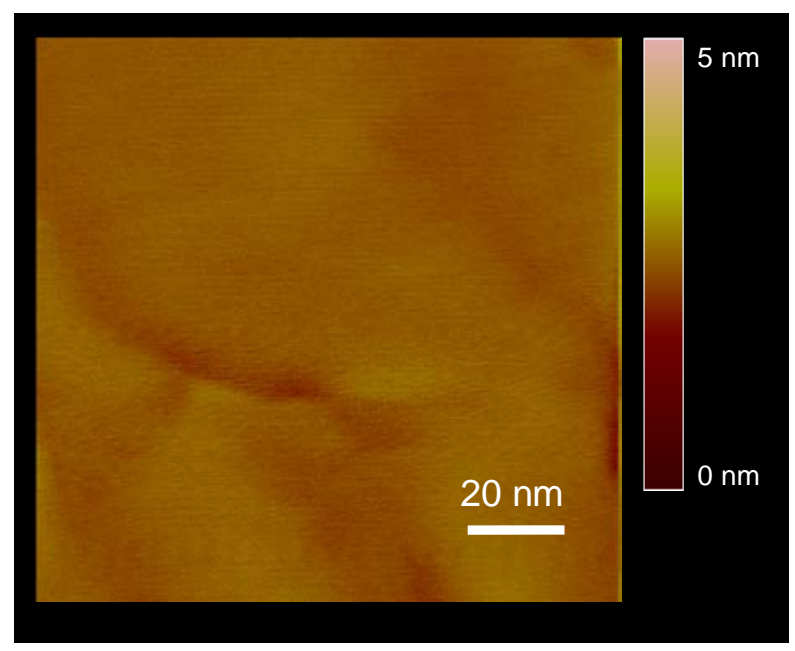

Figure 14. A soft contact mode AFM image of the fused silica plate immersed in water. The dimension of the scanning area is given by a scale bar. Height data for the surface were obtained using the scale bar to the right.

\section{Adsorption Mechanism}

When a glass or silica substrate is immersed in water, its surface becomes eclectically charged by the following mechanism:

$$
-\stackrel{\mathrm{S} i}{\mathrm{i}}-\mathrm{OH}_{2}^{+} \stackrel{\mathrm{H}^{+}}{\Leftrightarrow}-\mathrm{S} \mathrm{Si}-\mathrm{OH} \stackrel{\mathrm{OH}^{-}}{\Leftrightarrow}-\mathrm{S} \mathrm{S}-\mathrm{O}^{-}+\mathrm{H}_{2} \mathrm{O}
$$

in which silanol (Si-OH) groups are protonated to become positive $\left(\mathrm{Si}-\mathrm{OH}_{2}{ }^{+}\right)$sites or are hydroxylated to become negative $\left(\mathrm{Si}-\mathrm{OH}_{2}{ }^{-}\right)$sites depending on $\mathrm{pH}$. The point of zero charge (p.z.c.) of silica is approximately pH 2. (Parks, 1965; Papirer, 2000) Thus, silica surfaces are negatively charged at pHs above 2. It has been shown, however, that the negative charge density is low until the $\mathrm{pH}$ increases above 6 (Zajac et al., 1996; Goloub et al., 1996) In the present work, direct force measurements were conducted at $\mathrm{pH} \approx 5.7$, where surface charge densities are relatively low. Under this condition, the adsorption of cationic surfactants may occur in patches. (Rutland, J.L. Parker, 1994; Wong et al. 1989)

In 1955, Gaudin and Fuerstenau (1955) studied the adsorption of dodecylammonium hydrochloride (DAH) on quartz, and introduced the concept of "hemi-micelle" formation (Fuerstenau, 1970). More detailed studies conducted by Somasundaran and Fuerstenau (1966) and Wakamatsu and Fuerstenau (1968) showed that the onset of the hemi-micelle formation is indicated by a sharp decrease in $\zeta$ potential. Below this concentration, which is referred to as the critical hemi-micelle concentration (c.h.c.), the surfactant molecules adsorb individually by displacing the counter-ions, causing no changes in the $\zeta$-potentials. Above the c.h.c., however, the hydrocarbon chains of the surfactant molecules associate with each other, causing a sharp increase in adsorption density and, hence, a sharp decrease in $\zeta$-potentials. When the surfactant concentration is increased further, the surface charge can be neutralized. Above this concentration, which is referred to as point of charge neutralization (p.c.n.), the surfactant molecules adsorb with inverse (or flip-flop) orientation, causing a decrease 


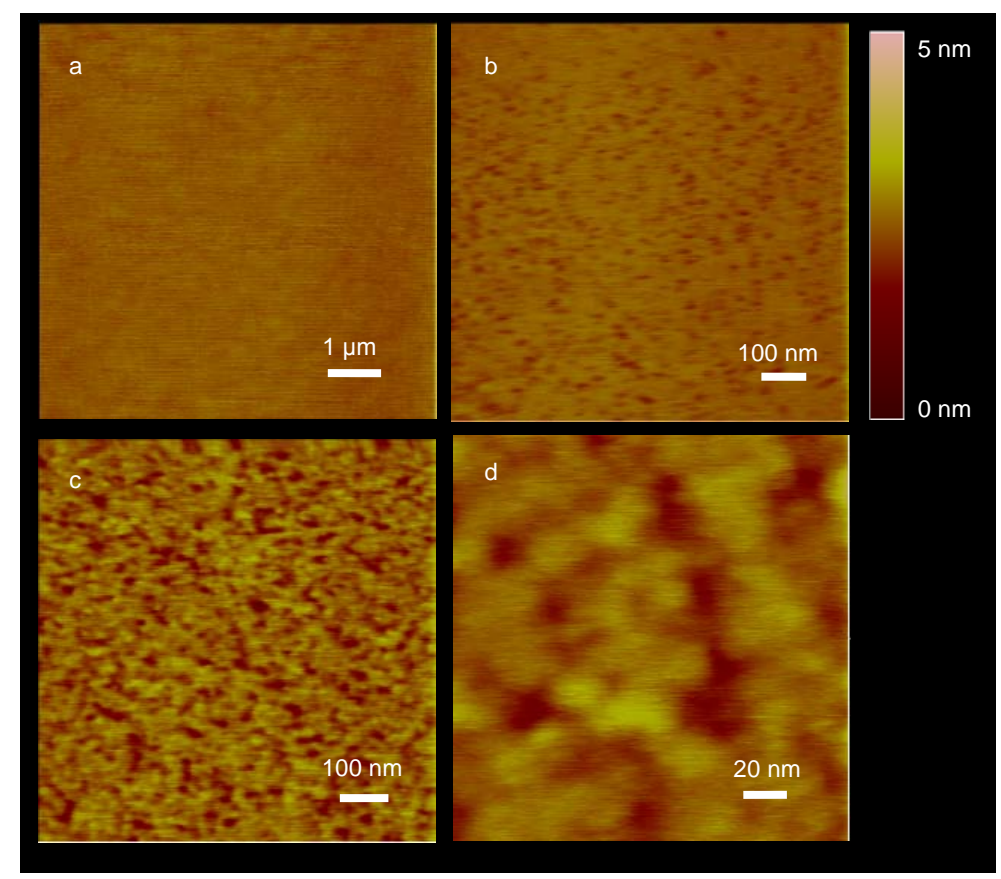

Figure 15. The soft contact mode AFM images of the silicon wafer surfaces immersed in $\mathrm{C}_{18} \mathrm{TACl}$ solutions at different concentrations and scale. The scale of the image is given in each image. The scale bar on the side gives height information. All of the images were taken 1 hour after the solution had been injected into the AFM cell. a) $10 \times 10 \mu \mathrm{m}$ image in water; b) $1 \times 1 \mu \mathrm{m}$ image in a $10^{-5} \mathrm{M} \mathrm{C}_{18}$ TACl solution; c) $1 \times 1 \mu \mathrm{m}$ image in a $3 \times 10^{-5} \mathrm{M} \mathrm{C}_{18} \mathrm{TACl}$ solution; d) $200 \times 200 \mathrm{~nm}$ image in a $3 \times 10^{-5} \mathrm{M} \mathrm{C}_{18} \mathrm{TACl}$ solution.

in hydrophobicity. Thermodynamically, the inverse orientation is favored as it is not necessary to remove some of the hydrated water molecules from polar heads.

It was shown by Herder (1990) that the details concerning the formation of an adsorption layer of an ionic surfactant (DAH) onto an ion-exchanging solid surface (mica) are surprisingly complex. Upon raising the surfactant concentration, a localized (site-wise) adsorption mechanism is gradually being replaced by an adsorption mechanism that in the end involves migrating (mobile) surfactant ions. Simultaneously, hemi-micelles are formed, first as immobile patches at low surfactant concentrations (typically two to three orders of magnitude below the c.m.c.) and possibly also as mobile surfactant aggregates at higher surfactant concentrations (typically one order of magnitude below the c.m.c.) which are more closely related to ordinary surfactant micelles in bulk solution. A full bilayer is as a rule finally formed in the c.m.c. concentration range. Reaching equilibrium for an adsorption layer of this kind is an amazingly slow process (many hours), especially for surfactants with a long hydrocarbon tail.

The AFM images of the silicon wafers immersed in surfactant solutions support the adsorption mechanism discussed above. Figure 15b, which was obtained at $10^{-5} \mathrm{M}$ $\mathrm{C}_{18} \mathrm{TACl}$, which was close to the p.c.n. $\left(=5 \times 10^{-6} \mathrm{M}\right)$, shows patches (or hemi-micelles) of 


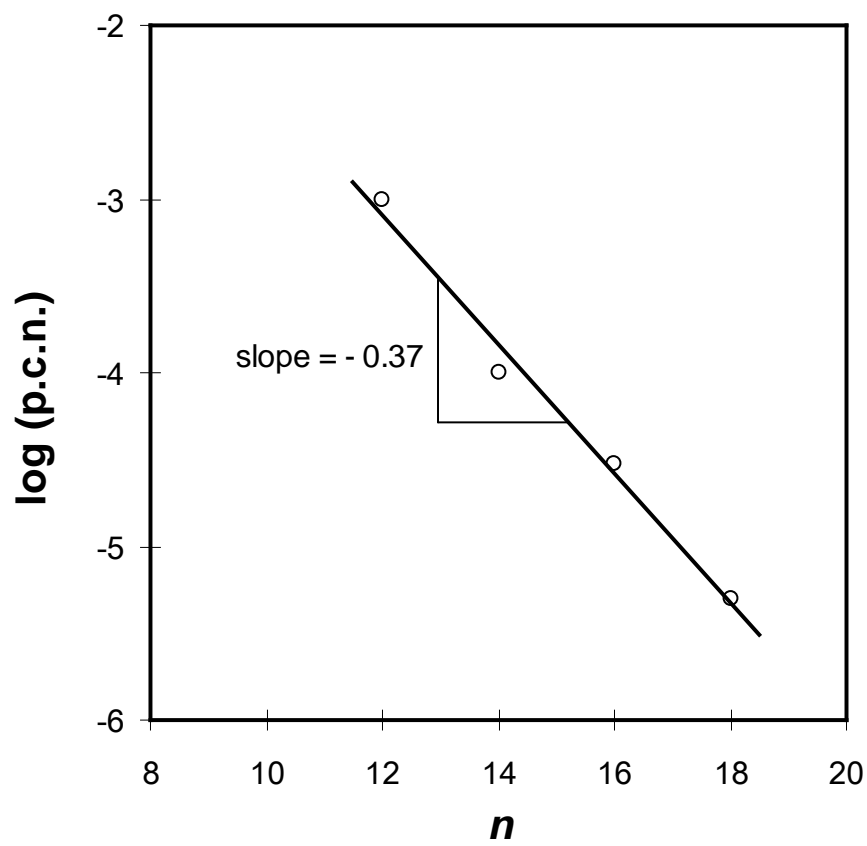

Figure 16. A relationship between the p.c.n.'s for the silica- $\mathrm{C}_{\mathrm{n}} \mathrm{TACl}$ system and the number of carbons in the hydrocarbon chains. According to the Stern-Graham equation (Eq. 5), the slope of -0.37 gives $\phi=-0.85 \mathrm{kT}$ per $\mathrm{CH}_{2}$ group.

the $\mathrm{C}_{18} \mathrm{TA}^{+}$ions adsorbed on the silica substrate. Many of the patches have coalesced to form a self-assembled monolayer with parts of the surface unoccupied. At this concentration, it appears that the surfactants have the normal mode of orientation with their head groups in contact with the surface and the hydrocarbon tail stretching out toward water. An evidence for this is that the thickness of the adsorption layer is close to the length of the fully-extended C-18 hydrocarbon chains. At $3 \times 10^{-5} \mathrm{M}$, which is well above the p.c.n, the adsorption layer was non-uniform and the heights of the clusters are much larger than the hydrocarbon chain length, indicating that additional surfactant molecules adsorb on top of the monolayer most probably with inverse orientation.

It has been suggested that the attractive hydrophobic force becomes the strongest at the p.c.n. of a surfactant. Thus, one can determine the p.c.n. from a set of force curves obtained at different concentrations of a given surfactant. Table 2 gives the values of the p.c.n.'s determined from the data obtained for the $\mathrm{C}_{\mathrm{n}} \mathrm{TACl}$-silica systems studied in the present work. Also shown in the table for comparison are the points of zeta-potential reversal (p.z.r.) obtained by Somasundaran et al. (1964) for the quatz-alkylammonium acetate system. In Figure 16, the p.c.n. values given in Table 2 have been plotted vs. the number of hydrocarbons $(n)$ in the alkyl chains of the $\mathrm{C}_{n} \mathrm{TACl}$ homologues. This plot represents the Stern-Grahame equation (Grahame, 1947):

$$
\ln C_{0}=-n_{c}(\phi / k T)-\ln \left(\Gamma_{\delta}^{+}\right)_{0}-\ln (2 r)
$$


Table 2. The parameters of Eq. (1) obtained for the force curves measured at the p.c.n's of the silica- $\mathrm{C}_{\underline{n}}$ TACl system

\begin{tabular}{cccccc}
\hline Surfactant & $\begin{array}{c}\text { p.c.n. } \\
(\mathrm{M})\end{array}$ & $\begin{array}{c}\text { p.z.r. } \\
(\mathrm{M})\end{array}$ & $\begin{array}{c}\mathrm{C} \\
(\mathrm{mN} / \mathrm{m})\end{array}$ & $\begin{array}{c}\mathrm{D} \\
(\mathrm{nm})\end{array}$ & $\begin{array}{c}\kappa^{-1} \\
(\mathrm{~nm})\end{array}$ \\
\hline $\mathrm{C}_{12} \mathrm{TACl}$ & $1 \times 10^{-3}$ & $2 \times 10^{-3}$ & 1.3 & 3 & 9.5 \\
$\mathrm{C}_{14} \mathrm{TACl}$ & $1 \times 10^{-4}$ & $4 \times 10^{-4}$ & 1.4 & 6 & 30 \\
$\mathrm{C}_{16} \mathrm{TACl}$ & $3 \times 10^{-5}$ & $7 \times 10^{-5}$ & 1.3 & 14 & 54.8 \\
\hline $\mathrm{C}_{18}$ TACl & $5 \times 10^{-6}$ & $8 \times 10^{-6}$ & 1.4 & 32 & 134 \\
\hline
\end{tabular}

*point of zeta-potential reversal, from Somasundaran et al. [30].

in which $C_{0}$ is the., $\phi$ is the standard adsorption free energy per mole of $\mathrm{CH}_{2}$ or $\mathrm{CH}_{3}$ group and $\left(\Gamma_{\delta}^{+}\right)_{0}$ is the adsorption density of the cationic surfactant at $\zeta=0, r$ is the radius of the polar head of the surfactant, and $k$ is the Boltzmann constant. If the product of $\left(\Gamma_{\delta}^{+}\right)_{0}$ and $2 r$ remain relatively constant for the $\mathrm{C}_{\mathrm{n}} \mathrm{TACl}$ homologues studied in the present work, a plot of log (p.c.n) vs. $n$ should give a slope of $-\phi / 2.3 k T$, from which one can obtain the value of $\phi=-0.85 k T$ ( $-0.50 \mathrm{kcal}$ per mole). This value is pretty close to the value of $-0.97 k T$ obtained from a plot of $\log$ (p.z.r.) vs. $n$ for the quartz-primary amine system (Somasundaran et al., 1964; Somasundaran and Huang, 2000). For the ordinary micelles forming in solutions of ionic surfactants, the corresponding figure is about $-0.75 k T$ per $\mathrm{CH}_{2}$ group.

The above discussion shows that a major driving force for adsorption of a longchain surfactant is the hydrocarbon chain association, which is commonly recognized as hydrophobic effect. (Tanford, 1980) Therefore, the longer the hydrocarbon chain length is, the larger the hydrophobic effect becomes, which in turn causes a decrease in the p.c.n.

\section{Possible Origin(s) of Hydrophobic Force}

We see little merit in attributing the long-range attractive forces observed in present work to the effects of adsorbed bubbles because: 1) the effects of degassing are minor, and can be explained by the effect of a small $\mathrm{pH}$ change, 2) the observed changes in AFM images on addition of surfactant could be explained simply by the adsorption of surfactant, rather than by the adsorption of bubbles, and 3) we did not observe steps (small discontinuities) in force curves that could be attributed to bridging bubbles. Therefore, for the remainder of this paper, we will consider three alternate hypotheses for the long-range attractive forces: a) attractive double-layer forces; b) a long-range hydrophobic force, and c) attractive forces due to correlations between charged patches. 
Figure 13 and Table 2 show that the long-range attraction becomes stronger and longer ranged with increasing hydrocarbon chain length. Further, the concentration at which the strongest attractive force was observed increased with increasing carbon number $(n)$ in a chain.

It has been more than twenty years since Israelachvilli and Pashley $(1982,1984)$ observed long-range attractive forces between two mica surfaces hydrophobized with hexadecyltrimethylammonium bromide $\left(\mathrm{C}_{16} \mathrm{TABr}\right)$. Numerous other investigators observed similar results, (Christenson and Claesson, 1988; Rabinovich and Derjaguin, 1988; Wood and Sharma, 1995; Hato, 1996; Yoon and Ravishankar, 1996) and proposed possible causes for the long-range attraction. (Claesson and Christenson, 1988; Eriksson et al., 1989; Miklavic et al., 1994; Sakamoto et al., 2002) However, its origin is not yet fully understood. In the following, we discuss the possible origin(s) of the long-range attraction between hydrophobic surfaces. Discussing the effect of the chain length may shed light from a different angle on this complex and continually perplexing issue.

It was shown that the long-range attractive force deceases in the presence of inorganic electrolyte, which may be taken as evidence that the non-DLVO force is of electrostatic origin. In this regard, it is of interest to analyze the force data obtained in the present work in terms of the charged-patch model developed by Miklavic et al. (1994). Their model suggests that the long-range attraction may originate from the electrostatic correlation attraction between the patches of surfactant molecules formed on surfaces. It was necessary to assume, of course, that the patches are electrically charged. The charged-patch model was based on the recognition that long-chain surfactants adsorb in patches (or hemi-micelles) at a relatively low concentration. According to Fuerstenau and Pradip (2005), hemi-micelles begin to form at concentrations 2-3 orders of magnitude below the c.m.c. The AFM (Zhang et al., 2005) and SEM (Delamarche, et al., 1994) images of the surfactant-coated surfaces indeed showed patches, as is also shown in Figure 15. Further, the aggregation numbers of the hemi-micelles (or 'soloids') determined using the electron spin resonance (ESR) and fluorescence spectroscopic techniques showed the patch-wise adsorption mechanism (Fan et al., 1997; Ström et al., 2000).

\section{1) Attractive Double-layer Forces?}

The possibility of explaining the long-range attractive forces observed in the present work as due to double-layer interaction was considered in an earlier publication of one of us. ${ }^{33}$ An appeal for this approach was that it is a known interaction, and the measured forces have the appropriate functional form (exponential). In the present work, however, we are immediately in trouble with the assumptions of DLVO theory. Not only do we have the usual problems with additivity of the double-layer and van der Waals forces, but also the fact that $\mathrm{C}_{18} \mathrm{TACl}$ ions are not point-sized, that there are significant interactions between them, and that the charge regulation is unknown. Furthermore, we have good evidence that the surfaces were inhomogeneous on the length scale of the forces, and the adsorption was not at equilibrium. Nevertheless, we went ahead and estimated the values of the surface potentials $\left(\psi_{0}\right)$ that are required to fit the measured 


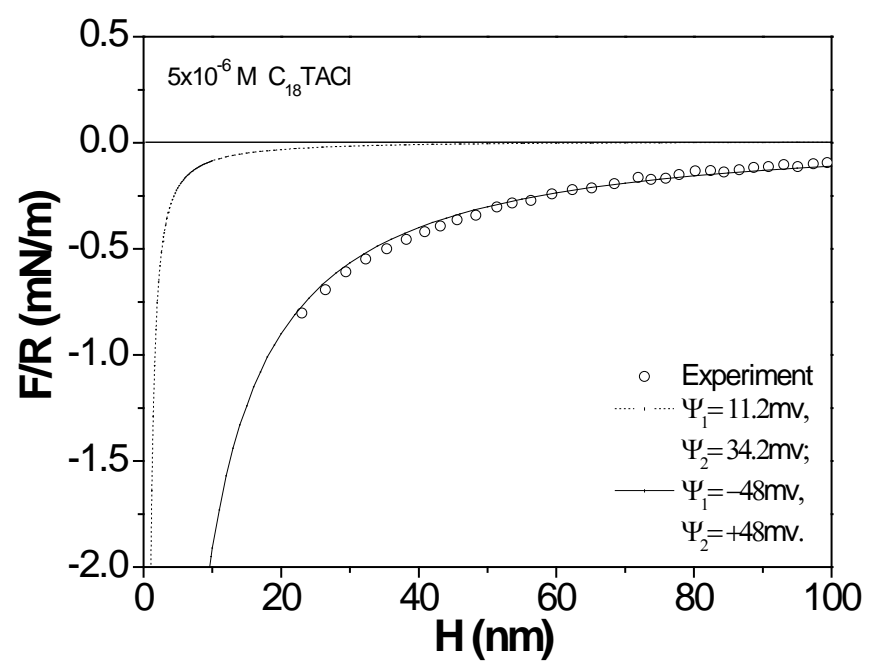

Figure 17. Force curves for the silica plate-glass sphere interaction in an airequilibrated $5 \times 10^{-6} \mathrm{M} \mathrm{C}_{18}$ TAC solution. The dotted line represents the DLVO fit with $\Psi_{1}=+11.2 \mathrm{mV}$ and $\Psi_{2}=+34.2 \mathrm{mV}$, which were taken from the $\zeta$-potential measurements. To fit the long-range attractive force, it was necessary to assume that $\Psi_{1}=+48 \mathrm{mv}$ and $\Psi_{2}=-48 \mathrm{mv}$, which is unreasonable.

forces assuming that that Poisson-Boltzmann theory applies. We used the constant potential boundary condition.

Figure 17 shows the force curve obtained in an air-equilibrated $5 \times 10^{-6} \mathrm{M} \mathrm{C}_{18} \mathrm{TACl}$ solution. The measured force can be reproduced by assuming that one surface has $\psi_{1}=+48 \mathrm{mV}$ and the other has $\psi_{2}=-48 \mathrm{mV}$ with $\kappa^{-1}=130 \mathrm{~nm}$. However, these potentials were very different to the zeta potential measurements. As shown in Figures 6 and 7, the $\zeta$-potentials of the silica plate and glass sphere were +34 and $+11 \mathrm{mV}$, respectively, in air-saturated solutions of $\mathrm{pH}$ 5.7. The force curve obtained with these values as $\psi_{1}$ and $\psi_{2}$, and $\kappa^{-1}=130.4 \mathrm{~nm}$ became weakly attractive. Clearly, the agreement was not good. Although the long-range attractive force observed in the air-saturated $5 \times 10^{-6} \mathrm{M} \mathrm{C}_{18} \mathrm{TACl}$ solution decayed exponentially, it was definitely not due to double-layer force.

\section{2) Charged Patches Model}

Miklavic et al. (1994) considered that the patches are charged but the surface as a whole is neutral. The model implies that the interaction between the two surfaces covered with patches of positive and negative charges is always attractive when the patches are sufficiently mobile and free to correlate with each other. At an infinite separation, the patches may be distributed randomly. As the separation decreases, the patches migrate laterally on the surface in order for the system to minimize the free energy. Assuming that the patches are distributed in a square lattice with a dimension of $a$, the free energy of interaction per unit area $(G(H))$ is given as follows:

$$
G(H)=-\frac{A}{k T} \Omega^{2} \exp \left(-2 q_{\min } H\right)
$$


Table 3. The parameters of Miklavic et al.'s charged-patch model as obtained by fitting the decay lengths obtained at the p.c.n.'s of the silica/ $\mathrm{C}_{n} \underline{\mathrm{TACl}}$ system

\begin{tabular}{cccccccc}
\hline & $\begin{array}{c}\text { p.c.n. } \\
(\mathrm{mM})\end{array}$ & $\begin{array}{c}\mathrm{D} \\
(\mathrm{nm})\end{array}$ & $q_{\min }$ & $\kappa^{2}$ & $(\pi / a)^{2}$ & $\begin{array}{c}a \\
(\mathrm{~nm})\end{array}$ & $\begin{array}{c}b \\
(\mathrm{~nm})\end{array}$ \\
\hline $\mathrm{C}_{12} \mathrm{TACl}$ & 1 & 3 & 0.167 & 0.011 & 0.0168 & 24 & 18 \\
& & & & & & & \\
$\mathrm{C}_{14} \mathrm{TACl}$ & 0.1 & 6 & 0.083 & 0.0011 & 0.0058 & 41 & 31 \\
$\mathrm{C}_{16} \mathrm{TACl}$ & 0.03 & 14 & 0.036 & 0.00033 & 0.00097 & 101 & 76 \\
$\mathrm{C}_{18} \mathrm{TACl}$ & 0.005 & 32 & 0.0156 & 0.000056 & 0.000188 & 229 & 172 \\
\hline
\end{tabular}

$b=0.75 a$

where $A$ is the area of the unit cell, $\Omega$ is a constant which varies with surface property (surface potential or charge), reciprocal lattice vector, and lateral displacement. As shown, the interaction energy decays exponentially, with a decay length of $\left(2 q_{\min }\right)^{-1}$, in which $q_{\min }$ is given as follows:

$$
q_{\min }=\left[(\pi / a)^{2}+\kappa^{2}\right]^{1 / 2}
$$

where $\kappa$ is the reciprocal Debye length. At a high electrolyte concentration, $q_{\min } \approx \kappa$; therefore, the interaction energy should decay as $(2 \kappa)^{-1}$. At a low electrolyte concentration, $\kappa$ is small; therefore, $q_{\min }$ should vary inversely with $a$.

From the decay lengths, $D\left(=\left(2 q_{\min }\right)^{-1}\right)$, measured in the present work, one can obtain the values of $q_{\min }$, which can then be used to obtain the values of $a$ using Eq. (7). In this exercise, the $\kappa$ values have been calculated from the concentrations of the ionic surfactants used in force measurements. As shown in Table 3, the lattice patch size $b$, which may be roughly estimated from the value of $a$ by multiplying it with a factor 0.75 used by Miklavic et al., increases with chain length. This finding is in agreement with the results obtained by Fan et al. (1997) and Strom et al.. (2000), who showed that the aggregation numbers of the hem-micelles increased with increasing chain length. These investigators studied the adsorption of $\mathrm{C}_{12} \mathrm{TABr}, \mathrm{C}_{14} \mathrm{TABr}$, and $\mathrm{C}_{16} \mathrm{TABr}$ on silica and alumina.

Eq. (7) suggests that decay length (or $q_{\min }$ ) is mainly controlled by two parameters, i.e., $a$ and $\kappa$. The data presented in Table 3 show that the contribution from $a$ is more significant than from $\kappa$ in determining the range of attraction, but $\kappa$ still plays an important role, particularly with the shorter chain surfactant, e.g., $\mathrm{C}_{12} \mathrm{TACl}$. These findings may be anticipated from the fact that all of the force data presented in Table 3 
were obtained in the absence of supporting electrolyte. Under this condition, the residual concentrations of the ionic surfactants act as electrolytes that can dampen the hydrophobic force.

It has been shown in the present work that the long-range attraction was strongest at p.c.n., and that the adsorption of the $\mathrm{C}_{n} \mathrm{TACl}$ on silica is likely to occur as patches. Both of these observations provide a basis for discussing the origin of the long-range attraction in view of the model developed by Miklavic et al.. A question remains, however, as to whether it is physically possible for the patches to be positively charged, while unoccupied silica surface remains negatively charged. This would undoubtedly be the case if the adsorption occurs as micelles (Gu and Huang, 1989), or as bilayers (Yeskie and Harwell, 1988). In both of these cases, the number of the surfactant molecules adsorbing in each patch (aggregation number) can exceed the negative charge sites underneath, causing it net positively charged. However, the micellar or bilayer adsorption should render the surface hydrophilic due to the orientation of the polar heads toward the aqueous phase. Therefore, such adsorption mechanisms cannot explain the experimental observations that both the long-range attraction and contact angle reached the maxima at a p.c.n..

Somasundaran and Fuerstenau (1966) showed, on the other hand, that long-chain surfactants adsorb as hemi-micelles, in which the head groups of the surfactant molecules are in touch with substrates and the hydrocarbon tails point toward the aqueous phase.

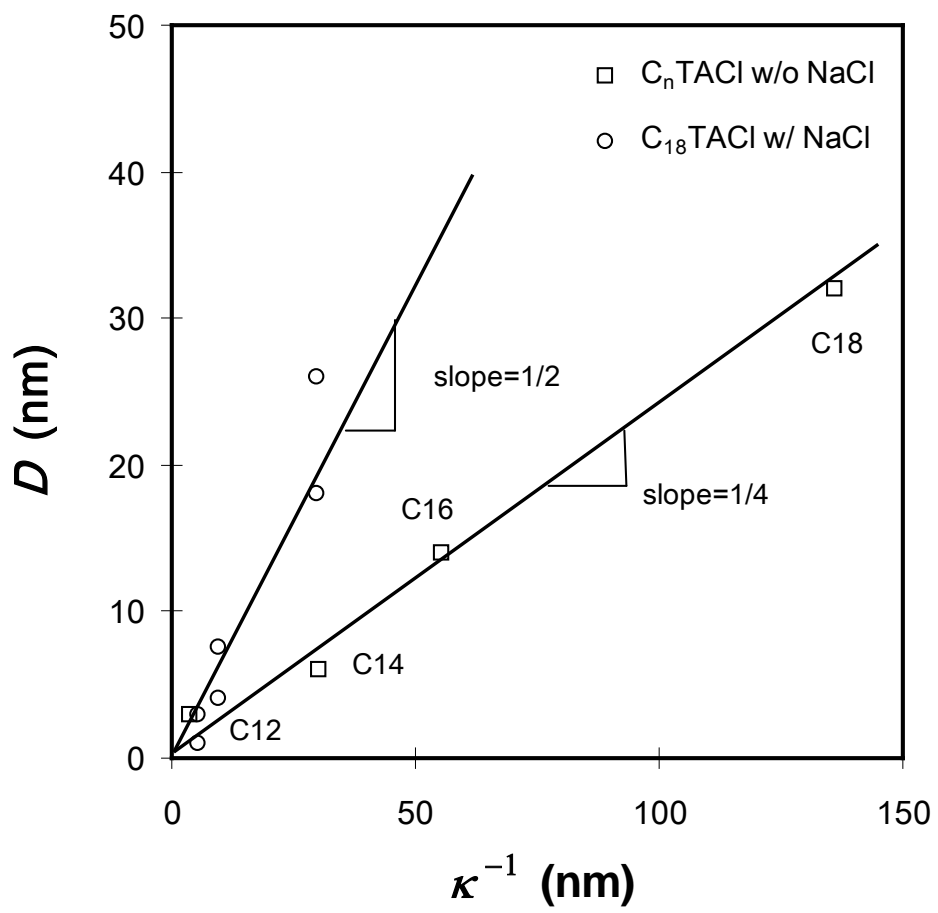

Figure 18. Decay length vs. the Debye length plots for the data obtained with $\mathrm{C}_{18} \mathrm{TACl}$ with $\mathrm{NaCl}$ and for the data obtained in $\mathrm{C}_{n} \mathrm{TACl}$ solutions at the p.c.n.'s in the absence of electrolyte. The former fits the charged patch model of Miklavic et al., while the latter cannot be explained with any available theory. 
Such orientation renders the surface hydrophobic. Once the surface has been neutralized, the surfactant molecules begin to adsorb with inverse orientation, rendering the surface hydrophilic. Thus, the surface should become most hydrophobic at a p.c.n, as has been demonstrated by Fan et al. (1997) with a series of vacuum flotation experiments.

For the case of $\mathrm{C}_{n} \mathrm{TA}^{+}$ions adsorbing on silica, the hemi-micelles formed at a p.c.n. must be positively charged in order for the surface, which includes the patches of unoccupied negative charge sites, to be neutral as a whole. The positive charges associated with the hemi-micelles should come from the surfactant molecules coadsorbing into the hemi-micelles along with those that adsorb on negatively charged sites. Fuerstenau and Pradip (2005) showed that at p.c.r. the co-adsorption occurs with the head groups in contact with the surface which gives rise to optimal flotation. Beyond the p.c.r., the co-adsorption occurs with the head groups oriented toward the water phase.

It appears, therefore, that the charged patch model of Miklavic et al. (1994) requires substantially larger patches than observed experimentally with self-assembled monolayers to predict the long-range attractive forces measured in the present work. Moreover, it seems unlikely that the hemi-micelles (patches) formed at or slightly below the p.c.n. can migrate on the surfaces rapidly enough to actually generate an attractive correlation force.

\section{3) Eriksson et al.'s theory}

Figure 18 shows a plot of $D$ vs. $\kappa^{-1}$ for the data obtained with $C_{n}$ TACl homologues. Also shown is a plot of the $D$ values obtained with $\mathrm{C}_{18} \mathrm{TACl}$ in the presence of varying concentrations of $\mathrm{NaCl}$. The latter has a slope of $1 / 2$ as predicted by Eq. (7). The former, on the other hand, has a slope of $1 / 4$, for which there is so far no adequate theoretical explanation.

Figure 19 shows a plot of $D$ vs. $\left(C_{0} n\right)^{-1 / 2}$, where $C_{0}$ is the concentration of $\mathrm{C}_{\mathrm{n}} \mathrm{TACl}$ in bulk solution and $n$ is the number of carbons in a hydrocarbon chain. The linear relationship suggests that the range of hydrophobic force is simply a function $C_{0} n$, which is the molar concentration of the $\mathrm{CH}_{2}$ and $\mathrm{CH}_{3}$ groups in solution. In effect, the results presented in Figure 18 shows that the long-range attraction decreases with increasing concentration of the species that can disrupt the structure of water, i.e., hydrocarbons. That the long-range attraction decreases in the presence of $\mathrm{NaCl}$ may be given the same explanation, that is, the long-range attraction decreases with increasing concentration of the species, i.e., $\mathrm{Na}^{+}$and $\mathrm{Cl}^{-}$ions in this case, that can disrupt the water structure. Thus, the results presented in Figures 8 and 13 show that the long-range attraction is strongest when water is in its pristine state. Any foreign species added to water causes a decrease in $D$.

According to the Derjaguin approximation (Derjaguin, 1934), the surface force $(F)$ normalized by the diameter of the sphere $(R)$ can be given as follows: 


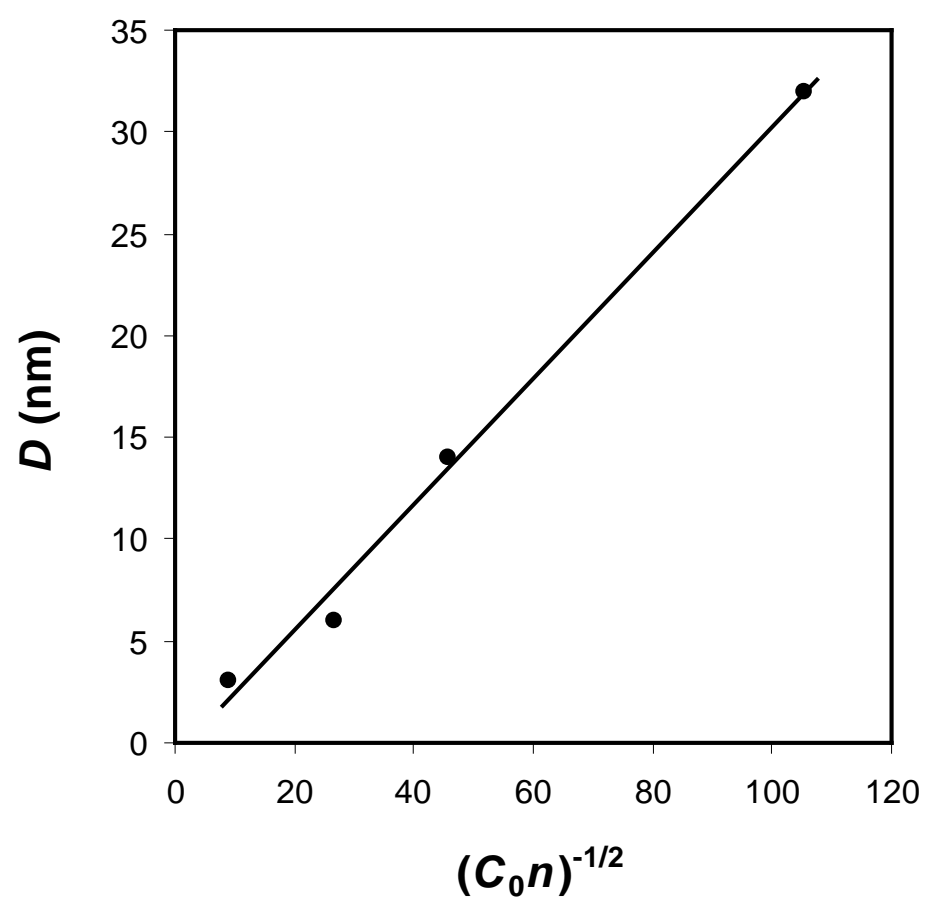

Figure 19. A decay length $(D)$ vs. $\left(C_{0} n\right)^{-1 / 2}$ plot for the data obtained in $C_{n} T A C l$ solutions in the absence of electrolyte. $C_{0}$ represents the surfactant concentration, and $D$ is equivalent to $b^{-1}$ of Eriksson et al.'s hydrophobic force model (Eq. [11]) derived from the mean-field theory. $\mathrm{C}_{0} n$ represents the effective concentration of $\mathrm{CH}_{2} / \mathrm{CH}_{3}$ species in solution.

$$
F / R=2 \pi\left(\gamma^{\mathrm{f}}-\gamma_{\infty}^{\mathrm{f}}\right)
$$

Eriksson et al. (1989) derived theoretical expressions for $\gamma^{\mathrm{f}}$ and $\gamma_{\infty}^{\mathrm{f}}$ using a mean field approach based on the 'square-gradient approximation'. By substituting those into Eq. (8), they obtained the following expression for the hydrophobic force:

$$
F / R=-B[\operatorname{coth}(b H / 2)-1]
$$

which can be simplified to:

$$
F / R=-2 B \exp (-b H)
$$

for large enough separations. Eq. (10) shows that $b^{-1}$ can be identified with $D$ and that $2 B$ is equivalent to $C$ of Eq. (1). Furthermore, according to Eriksson et al.,

$$
b^{-1}=\sqrt{c_{3} / 2 c_{2}}
$$

where $c_{2}$ and $c_{3}$ are constants which account for the free energy costs of structuring the water and forming order parameter gradients, respectively. From Figure 18, it is seen that 
the decay length $D$ (or $b^{-1}$ ) varies as $\left(C_{0} n\right)^{-1 / 2}$. According to Eq. 11 , such a relationship can be generated if we assume that $c_{2}$ is proportional to $\left(C_{0} n\right)^{1 / 2}$ and $c_{3}$ is inversely proportional to $\left(C_{0} n\right)^{1 / 2}$. In other words, the presence of surfactant ions would make it more costly free-energy-wise to obtain a certain ordered water state, whereas it would be less costly with the gradients in the order parameter function in the vicinity of a hydrophobic surface. Although these assumptions seem reasonable, they are nevertheless in need of further justification.

Based on the data obtained in the present work, two possible origins for the longrange attractions have been discussed. The charged-patch model of Miklavic et al. seems to provide an explanation at high electrolyte concentration. At low electrolyte concentrations, however, it is necessary to assume that the patch sizes are much larger than known from experimental work. A basic premise of the model is that the patches have lateral mobility. Otherwise, the model predictions are too small to predict the longrange forces measured between hydrophobic surfaces. A question arises then as to whether the charged patches are indeed mobile enough to give rise to long-range attractions. It is conceivable that physically adsorbing surfactant molecules might possess the necessary molecular and micellar mobility that is a prerequisite for the applicability of the theory of Miklavic et al.. However, ionic surfactants adsorb primarily on oppositely charged surface sites rather than being evenly distributed across the surface. For a surfactant molecule to be mobile, it may have to jump from one site to another, which is likely to be a slow process when the surfactant-site bond is fairly strong. Needless to say, the corresponding aggregate mobility should be exceedingly slow. Thus, correlation attraction might possibly arise in the concentration range well above the p.c.n., where, however, electrostatic repulsion as a rule predominates. Further, the charged-patch model does not explain the long-range forces observed with silylated surfaces and Langmuir-Blodgett-deposited surfaces. The surfactants in neither of these two surfaces are likely to be mobile. It should also be noted here that long-range attractions have been observed with thiolated gold surfaces, which is a well-known chemisorbing system.

The third possibility discussed above is related to water structure. It suggests that surfactant monomers and inorganic electrolytes break the hydrogen bond network present in thin watery films between hydrophobic surfaces, thereby reducing the long-range attraction. A short-chain surfactant requires a higher monomer concentration in solution to obtain a given surface coverage, which would be the reason that it gives a relatively short-range attraction. Chemisorbing surfactants such as thiols would leave very few monomers in solution, which may be conducive to creating long-range attractions. The very long-range attractions observed with silylated and LB-deposited surfaces may be explained in the same vein. The circumstance that the experimental data support Eriksson et al.'s theoretical model (Eriksson et al. 1989), which is based on the changes in water structure across a hydrophobic surface-solution interface, is yet another reason to further explore the possibility of the hydrophobic force actually being a structural force. 


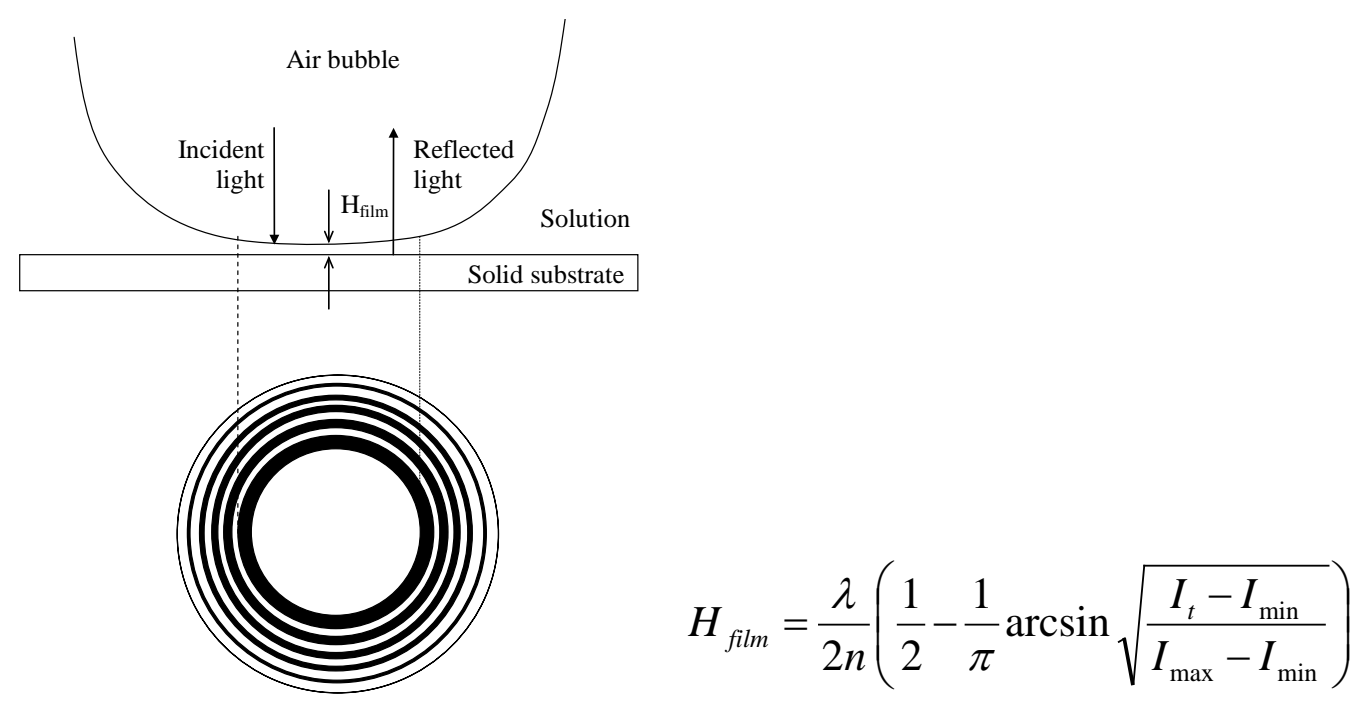

Figure 20. Film Thickness Measured Using a Micro-interference Technique

\section{Measurement of wetting film thickness}

The thickness of wetting film at different $\mathrm{NaCl}$ solution has been carried out on oxidized hydrophilic and hydrophobic Silicon wafer to study the effect of surface hydrophobicity on the wetting film thickness. Also, the kinetics of film thinning in certain salt solution was studied. However, due to the formation of the "dimple" at the time of film thinning, the kinetics of film thinning does not apply to the theoretical Reynold equation. However, on hydrophilic substrate, the equilibrium film thickness was finally obtained after 30 seconds thinning and the values were compared to those calculated from classical DLVO theory. (Schulze et al., 2001)

The theoretical disjoining pressure at different salt concentrations can be calculated using the following equations from Eq. (13) to Eq. (17) and plotted as Fig. 21. The theoretical equilibrium film thickness can be obtained by picking up the point at which the disjoining pressure is $194 \mathrm{~Pa}$, which equals the capillary pressure, $P_{\mathrm{c}}$ in present wetting film system, on the disjoining pressure-distance curves for each salt concentration; thus the obtained distance, $H$ gives the equilibrium film thickness, $H_{\mathrm{e}}$ from the DLVO theory.

$$
\begin{aligned}
& \Pi=\Pi_{e l}+\Pi_{v d w} \\
& \Pi-P_{c}=0 \\
& P_{c}=\frac{2 \gamma}{R_{c}} \\
& \Pi_{v d w}=-\frac{A_{s l v}}{6 \pi h^{3}} \\
& \Pi_{e l}=-\frac{1}{2}\left[\kappa\left(\frac{\sigma_{2}^{2}}{\varepsilon_{0} \varepsilon \kappa}-\varepsilon_{0} \varepsilon \kappa \Psi_{1}^{2}\right) \frac{1}{\cosh ^{2}(\kappa h)}-2 \kappa \Psi_{1} \sigma_{2} \frac{\sinh (\kappa h)}{\cosh ^{2}(\kappa h)}\right]
\end{aligned}
$$

in which, 


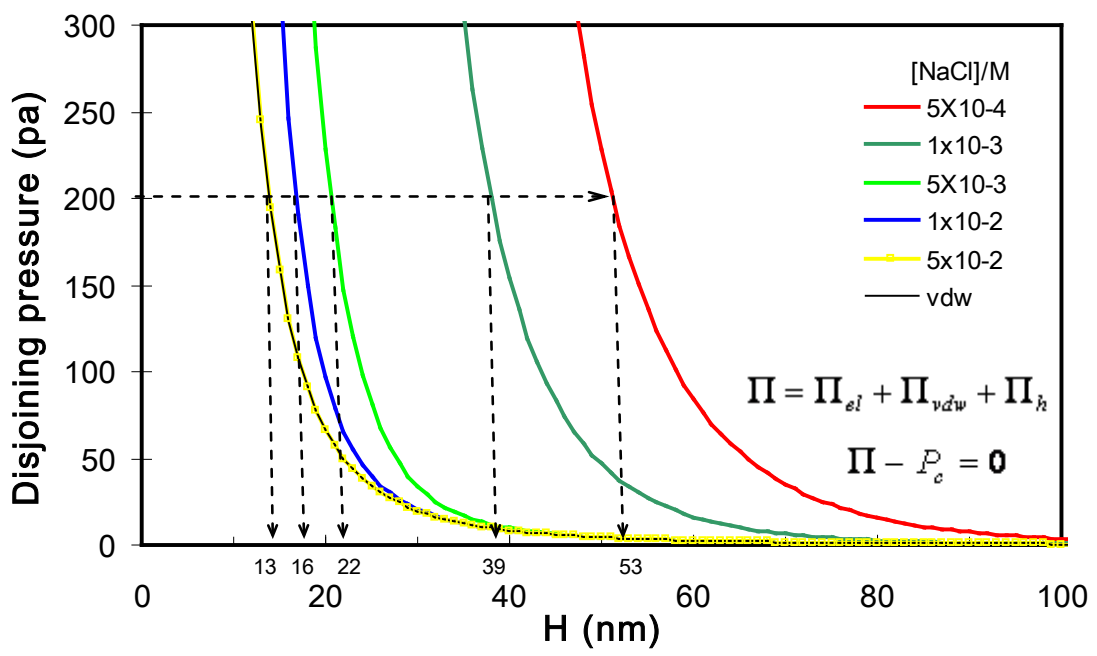

Figure 21 Theoretical equilibrium film thickness at different salt concentrations.

- $\kappa$ : Debye-Huckel Parameter;

$-\varepsilon_{0}$ : absolute dielectric constant;

- $\varepsilon$ : relative dielectric constant;

- $\sigma_{2}$ : charge density of silica surface;

- $\psi_{1}$ : surface potential of gas bubble;

- $\mathrm{h}$ : film thickness;

$-\gamma$ : surface tension of liquid.

- $\mathrm{R}_{\mathrm{c}}$ : radius of capillary tube;

- $\mathrm{A}_{\text {slv }}$ : Hamaker constant for wetting film(silica/water/air system), $1 \times 10^{-20} \mathrm{~J}$.

For example, as shown in Figure 21, the equilibrium film thickness, $H_{\mathrm{e}}$, in $1 \times 10^{-}$ ${ }^{3} \mathrm{M} \mathrm{NaCl}$ solutions should be $39 \mathrm{~nm}$ at a capillary pressure of 194 pa and the film thickness is $22 \mathrm{~nm}$ accordingly in $5 \times 10^{-3} \mathrm{M}$ salt solutions.

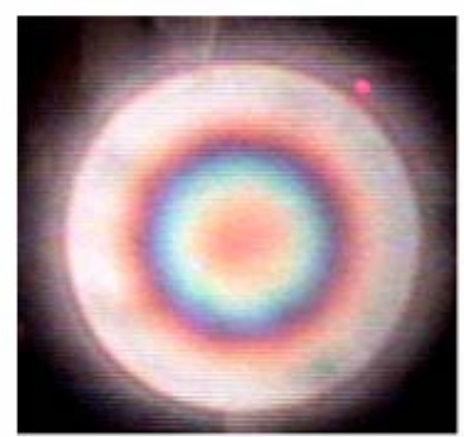

$0 \mathrm{~s}$

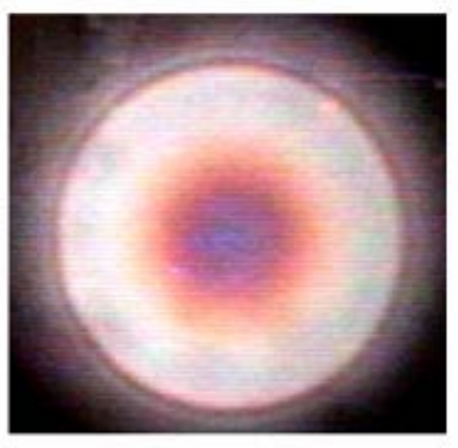

$5 s$

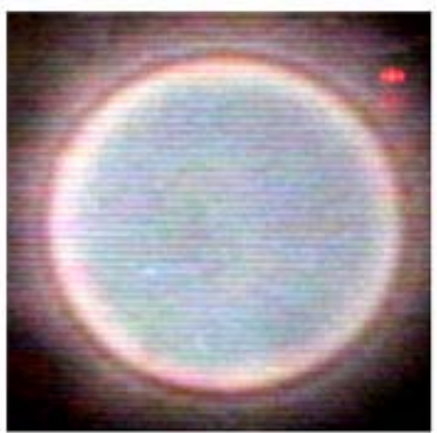

$10 \mathrm{~s}$

Time (s)

Figure 22 Kinetic thinning of wetting film on a hydrophilic silicon wafer surface as a function of thinning time. After 10 seconds, an almost equilibrated wetting film is obtained. 


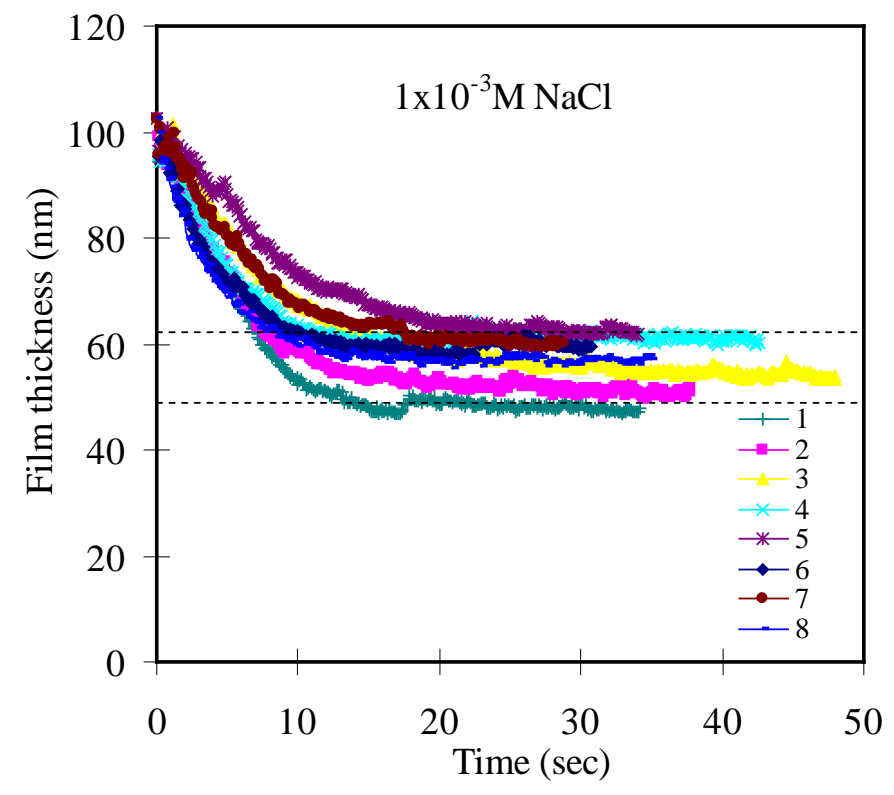

Figure 23. Experimental equilibrium film thickness at $1 \times 10^{-3} \mathrm{M} \mathrm{NaCl}$ concentration.

Figure 22 and 23 shows the kinetic thinning of wetting film on an oxidized silicon wafer in $1 \times 10^{-3} \mathrm{M} \mathrm{NaCl}$ solutions. The initial film thickness was set at $102 \mathrm{~nm}$; then the water film keeps on thinning until the strong repulsive disjoining pressure makes the two interfaces apart, as which is predicted by the classical DLVO theory. After nearly 15 seconds, an equilibrium film thickness is obtained and the value is read directly from the curve shown in Figure 23. Due to the formation of dimple at the time of film forming, the

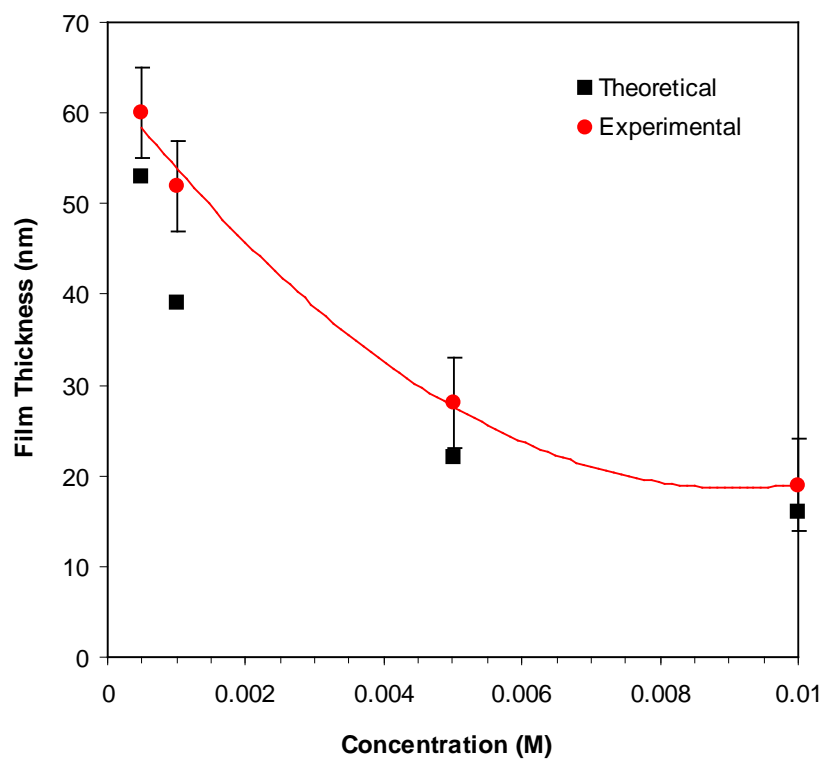

Figure 24. Experimental and theoretical equilibrium film thickness at different $\mathrm{NaCl}$ concentration. 
Table 4. Experimental and Theoretical Equilibrium Film Thickness at different NaCl Concentration.

\begin{tabular}{ccc}
\hline Concentration (M) & $\begin{array}{c}\text { Equilibrium Film } \\
\text { Thickness } \\
\text { Theoretical (nm) }\end{array}$ & $\begin{array}{c}\text { Equilibrium Film Thickness } \\
\text { Experimental(nm) }\end{array}$ \\
\hline 0.0005 & 53 & 60 \\
0.001 & 39 & 52 \\
0.005 & 22 & 28 \\
0.01 & 16 & 20 \\
\hline
\end{tabular}

experiment results show some variations. It is shown in the figure that the equilibrium film thickness varies from $45 \mathrm{~nm}$ to $65 \mathrm{~nm}$. This experiment error in present investigation therefore is much higher than that obtained in foam film thickness measurement because of the less pronounced dimple formation in the latter.

The experimental equilibrium film thicknesses measured in $\mathrm{NaCl}$ solutions at different salt concentrations are listed in Figure 24. In addition, the theoretical values of film thicknesses are also shown in Figure 24 for comparison. The figure shows that, both from theoretical and experimental data, the equilibrium film thickness decreases with salt concentration increasing. This is mainly due to the depression of repulsive double layer disjoining pressure with ionic strength increasing in solution, while the van der waals force doesn't change much at large surface separation. Table 4 also shows that the experiment film thickness generally has larger value than that calculated from theory. This may be partially due to the fact that experimental equilibrium film thickness is obtained within 30 seconds, which is not yet at a full equilibrium. It may origin from the underestimate of the capillary diameter, which accordingly decreases the theoretical equilibrium film thickness. In another possibility, the surface charge density on oxidized silicon wafer may be higher than the one used in theoretical calculation, of which the value is taken from silica substrate

The experiment results of kinetic thinning and equilibrium film thickness show that between hydrophilic substrate/bubble, when the separation decreases, there is a strong repulsive disjoining pressure, which leads to a thick water film between particle/bubble. The water film doesn't rupture even when the film thickness is as thin as $20 \mathrm{~nm}$; therefore, flotation is very less likely to occur.

The wetting film between a hydrophobic substrate, with contact angle varying from 30-50 degree, and a bubble ruptures in tens of milliseconds drastically. No kinetic film thinning data was obtained in the present investigation due to the extremely short film life time. Of course, no equilibrium film thickness was obtained because the film ruptured, which suggested a possible flotation after the solid particles are hydrophobized. This phenomenon is the same as what has been observed by Blake and Kitchener (1972). The wetting film ruptures are a very long surface separation, which is far beyond the 
range of the attractive van der waal force. That is, there must be attractive hydrophobic force acting between hydrophobic solid and a bubble. It is this additional non-DLVO hydrophobic attraction that introduces the rupture of wetting film on hydrophobic substrate. The rupture process is proposed to be studied systemically using a high speed video camera.

\section{Conclusions}

In the present work, AFM force measurements were conducted by following the procedures described by Sakamoto et al. The results showed the presence of the longrange attractive force both in air-saturated and degassed solutions. It was most attractive at $5 \times 10^{-6} \mathrm{M} \mathrm{C}_{18} \mathrm{TACl}$, where the surface was most hydrophobic. At this concentration, which is close to the point of charge neutralization (p.c.n.) of the glass sphere, the longrange decay lengths $\left(D_{2}\right)$ were 34 and $38 \mathrm{~nm}$ in air-saturated and degassed solutions, respectively. At $10^{-5} \mathrm{M}$, the decay length decreased from 30 to $4 \mathrm{~nm}$ upon degassing, which is explicable by simple $\mathrm{pH}$ change associated with the changes in the concentration of dissolved $\mathrm{CO}_{2}$. The attractive force was screened by an added electrolyte $(\mathrm{NaCl})$, indicating that the attractive force may be of electrostatic origin. Therefore, the very long decay lengths observed in the absence of electrolyte may be ascribed to the fact that the p.c.n. occurs at a very low surfactant concentration.

A series of surface force measurements were measured with silica surfaces coated in situ with $\mathrm{C}_{\mathrm{n}} \mathrm{TACl}$ homologues with $\mathrm{n}=12-18$ using an AFM. The measured forces decayed exponentially with separation, with the decay lengths varying in the range of 3$32 \mathrm{~nm}$. With a given surfactant, the strongest attractions were observed at the p.c.n. Both the maximum decay lengths measured at the p.c.n.'s and the adhesion forces increased with increasing chain length. The adhesion forces can be predicted from the solid-liquid interfacial tensions, with appropriate corrections for surface roughness. It was found that the roughness increased with increasing chain length.

The long-range forces measured in the present work were analyzed in terms of the migrating charged-patch model, which might explain the forces measured in high electrolyte concentrations. For the long-range attractions measured in the absence of added salt, however, it was necessary to assume that the patch sizes were substantially larger than those measured experimentally and reported in the literature. Furthermore, there is no evidence that hemimicelles are mobile in the p.c.n. concentration range.

A plot of the maximum decay lengths measured at p.c.n.'s vs. the concentration of the $\mathrm{CH}_{2}$ and $\mathrm{CH}_{3}$ groups in solution shows a linear relationship, which supports the formalism derived by Eriksson et al. from the mean-field theory by considering the structural changes across the thin water solution film between the silica surfaces. It is possible that the residual surfactant monomers in solution break the structure of the water and cause the long-range force to decrease.

The film thicknesses of a wetting film in different $\mathrm{NaCl}$ solutions on oxidized hydrophilic silicon wafer surface show that equilibrium film thickness decreases with salt 
concentration increasing, which is the same as predicted by the DLVO theory. The experiment results also suggest that there is an equilibrated thick water film between a hydrophilic solid and a bubble, therefore, a less likely flotation. However, the wetting film between a hydrophobic substrate and a bubble ruptures in tens of milliseconds drastically, suggesting a possible flotation after the solid particles are hydrophobized. The results also suggest that, between hydrophobic solid and a bubble, there must be an additional non-DLVO attractive hydrophobic force, which introduces the rupture of wetting film on hydrophobic substrate. The rupture process is proposed to be studied systemically using a high speed video camera.

\section{Bibliography}

Attard, P., Langmuir 1996, 12, 1693-1695.

Blake, T. D.; Kitchener, J. A., J. Chem. Soc Faraday Trans. 1972, 68, 1435-1442.

Christenson, H. K.; Claesson, P. M., Adv. Colloid Interface Sci., 2001, 91, 391- 436.

Christenson, H.K.; Claesson, P..M., Science, 1988, 239, 390.

Claesson, P. M.; Christenson, H. K., J. Phys. Chem. 1988, 92, 1650-1655.

Cleveland, J. P.; Manne, S.; Bocek, D.; Hansma, P. K., Rev. Sci. Instrum. 1993, 64, 403406.

Delamarche, E. ; Michel, B.; Gerber, Ch.; Anselmetti, D.; Guntherodt, H.-J.; Wolf, H.; Ringsdorf, H., Langmuir 1994,10, 2869-2871.

Derjaguin, B. V., Duhkin, S. S., Trans. Inst. Min. Metall. 1961, 70, 221.

Derjaguin, B.V., Kolloid Z. 1934, 69, 155.

Ducker, W. A.; Senden, T. J.; Pashley, R. M., Langmuir 1992, 8, 1831-1836.

Ducker, W. A.; Senden, T. J.; Pashley, R. M., Nature 1991, 353, 239-241.

Eriksson, J. C.; Ljunggren, S.; Claesson, P. M., J. Chem. Soc., Faraday Trans. 2, 1989, $85,163-176$.

Fan, P. Somasundaran, N.J. Turro, Langmuir, 1997, 13, 506.

Fuerstenau, D. W., Pure Appl Chem 1970, 24, 135.

Fuerstenau, D.W.; Pradip, T., Adv. Colloid Interface Sci., 2005, 114-115, 9-26.

Gaudin, M.; Fuerstenau, D. W., Trans. AIME, 1955, 202,958.

Goloub, T. P.; Koopal, L. K. Langmuir 1997, 13, 673-681.

Goloub, T. P.; Koopal, L. K.; Bijsterbosch, B. H., Langmuir, 1996, 12, 3188-3194.

Grahame, D. C., Chem. Rev., 1947, 40, 441.

Gu, T.; Huang, Z., Colloids Surf., 40, 1989, 71.

Hato, M., J. Phys. Chem. 1996, 100, 18530-18538.

Herder, P. C., J. Colloid and Interface Sci., 1990, 134, 2, 346-356.

Ishida, N.; Inoue, T.; Miyahara, M.; Higashitani, K., Langmuir, 2000, 16, 6377-6380

Ishida, N.; Sakamoto, M.; Miyahara, M.; Higashitani, K., Langmuir, 2000, 16, 56815687.

Israelachvili, J. N.; Pashley, R. M., J. Colloid Interface Sci., 1984, 98, 500.

Israelachvili, J. N.; Pashley, R. M., Nature, 1982, 300, 341.

Kerkicheff, P. and Spalla, O., 1995, Phys. Rev. Lett. 75, 1851.

Laskowski, J.; Kitchener, J.A., J. Colloid Interface Sci. 1969, 30, 391.

Lokar, W. J.; Ducker, W. A. Langmuir 2002, 18, 3167-3175. 
Lokar, W. J.; Ducker, W. A. Langmuir 2004, 20, 378-388.

Miklavic, S. J.; Chan, D. Y. C.; White, L. R.; Healy, T. W., J. Phys. Chem. 1994, 98, 9022-9032.

Nuyen, A.V.; Nalaskowski, Miller. J.; Butt, H.-J. Int. J. Miner. Process., 2003, 72, 215225.

Papirer, E.(ed), ‘Adsorption on Silica Surface’, Surfactant Science Series, Vol. 90, New York, Marcel Dekker, Inc. 2000.

Parker, J. L.; Claesson, P.; Attar, P. J. Phys. Chem. 1994, 98, 8468

Parker, J.L.;Yaminsky, V.V.; Claesson, P.M., J. Phys. Chem. 1993, 97, 7706.

Parks, G. A., Chem. Rev., 1965, 65(2), 177-198.

Pazhianur, R. and Yoon, R.-H., Minerals \& Metallurgical Processing, 2003, 20, 178-184.

Rabinovich, Ya. I.; Derjaguin, B.V., Colloids Surf., 1988, 30, 243.

Rutland, M.W.; Parker, J.L., Langmuir 10, 1994, 1110-1121.

Sakamoto, M.; Kanda, Y.; Miyahara, M.; Higashitani, K., Langmuir 2002, 18, $5713-$ 5719.

Schulze, H.J.; Stockelhuber, K.W.; Wenger, A., J. Colloids and Surfaces A 192 (2001) 61-74.

Somasundaran, P.; Fuerstenau, D. W., J Phys Chem 1966;70:90.

Somasundaran, P.; Healy, T. W.; Fuerstenau, D. W., J. Phys. Chem. 1964, 68, 35623566.

Somasundaran, P.; Huang, L., Adv. Colloid Interface Sci., 2000, 88, 179-208

Ström, P. Hansson, B. Jönsson, O. Söderman, Langmuir, 2000, 16, 2469.

Stumm, W.; Morgan, J. J. Aquatic Chemistry: Chemical Equilibria and Rates in Natural Waters Environmental Science and Technology; John Wiley \& Sons: New York, 1996.

Subramanian, V.; Ducker, W. J. Phys. Chem. B 2001, 105, 1389-1402.

Tanford, C., “The Hydrophobic Effect,” 1980, 2nd ed. Wiley, New York.

Tanford, C., 1980, "The Hydrophobic Effect," 2nd ed.. Wiley, New York, p.9.

Tsao, Y. H.; Evans, D. F.; Wennerström, H., Science 1993, 262, 547-550.

Tyrrell, J. W. G. ; Attard, P., Langmuir, 2002, 18, 160-167.

Wakamatsu, T.; Fuerstenau, D. W., Adv Chem Ser 1968;79:161.

Wong, K.; Cabane, B.; Duplessix, R.; Somasundaran, P., Langmuir, 1989, 5, 1346-1350.

Wood, J.; Sharma, R., Langmuir 1995, 11, 4797-4802

Yeskie, M. A.; Harwell, J. H., J. Phys. Chem., 1988, 92, 8, 2346-2352.

Yoon, R.-H.; Ravishankar, S. A., J. Colloid and Interface Sci., 1996, 179, 403-411

Zajac, J.; Trompette, J. L.; Partyka, S., Langmuir, 1996, 12, 1357-1367.

Zhang, J.; Yoon, R.-H.; Mao, M.; Ducker, W. A., Langmuir, 2005; 21, 5831-5841.

\section{List of Acronyms and Abbreviations}

None

\section{Appendices (if necessary)}

None 
Appendix 9: Flotation Processes, Experiments and analysis (VA009) 


\section{FINAL TECHNICAL REPORT}

Contract Title and Number:

Crosscutting Technology Development at the Center

for Advanced Separation Technologies

(DE-FC26-02NT41607

Sub-Recipient Project Title:

Flotation Processes/ Experiments and Analysis

Principal Investigators:

Demetri P. Telionis and Pavlos P. Vlachos

Contact Address:

Virginia Tech

Engineering Science and Mechanics Dept.

219 Norris Hall

Blacksburg, VA, 24061

Subcontractor Address:

No subcontracts issued.
Period of Performance:

Starting Date: $\quad 10 / 01 / 2002$

Ending Date: $\quad$ 10/31/2009
Report Information:

Type: Final

Number:

Period: $\quad 4 / 1 / 04$ to $8 / 31 / 06$

Date: $\quad$ August 25, 2006

Code: $\quad$ VA 009-FINAL

Contact Information:

Phone: $\quad 540-231-7492$

Fax: 540-231-3867

E-Mail: Telionis@vt.edu

Subcontractor Information:

Phone:

Fax:

E-Mail:

\section{DISCLAIMER}

This report was prepared as an account of work sponsored by an agency of the United States Government. Neither the United States Government nor any agency thereof, nor any of their employees, make any warranty, express or implied, nor assume any legal liability or responsibility for the accuracy, completeness, or usefulness of any information, apparatus, product, or process disclosed, or represents that its use would not infringe privately owned rights. Reference herein to any specific commercial product, process, or service by trade name, trademark, manufacturer, or otherwise does not necessarily constitute or imply endorsement, recommendation, or favoring by the United States Government or any agency thereof. The views and opinions of authors expressed herein do not necessarily state or reflect those of the United States Government or agency thereof. 


\begin{abstract}
Flotation processes involve complex, three-phase flow interactions between a liquid, bubbles and solid particles. For decades engineers and researchers based their calculations of such interactions on algebraic formulas that model such processes. These formulas were derived from simple models, from experimental data or just from arbitrary assumptions. Considerable progress has been made so far but this approach is far for providing a reliable tool for the design of flotation machines.
\end{abstract}

Most of the experimental data were obtained decades ago with literally primitive methods. Modern experimental tools are employed in this effort to measure with great accuracy the basic features of the motion of all three phases in homogenous isotropic turbulent flow. We carried out measurements in a water tunnel with controlled grid turbulent fluid motions. We have also designed and constructed a Rushton stirring tank and obtained data on the flow patterns and the turbulence thus generated.

We employ a unique in the US Digital Particle Image Velocimeter (DPIV) that can record with great accuracy and $\mathrm{kHz}$ temporal resolution velocity vectors of all three phases, namely the fluid, the solid particles and the air bubbles. In this report we describe our work in estimating experimentally the turbulence dissipation and its relationship to the fluctuations of bubbles and particles. We also employ our experimental data to investigate the validity of models that relate collision rates of bubbles and particles to turbulent dissipation. 


\section{TABLE OF CONTENTS}

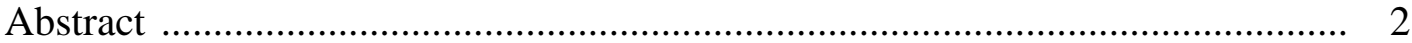

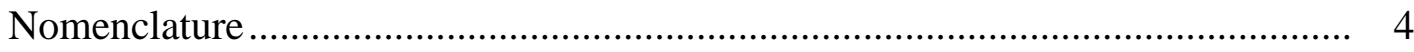

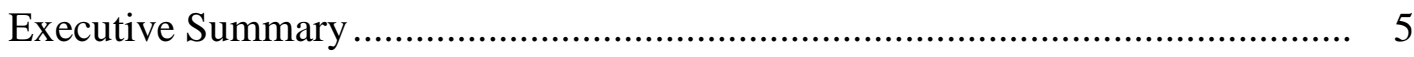

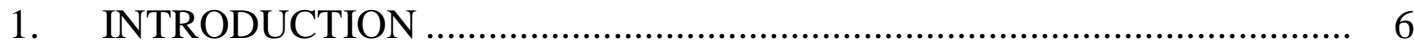

Background ............................................................................................. 6

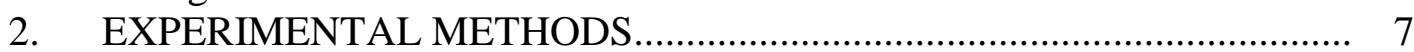

2.1 Analytical Considerations ..................................................... 7

2.2 Turbulent Energy Dissipation ......................................................... 9

2.3 Digital Particle Image Velocimetry .................................................. 11

2.4 Facilities and Instrumentation..................................................... 12

3. RESULTS OBTAINED WITH GRID TURBULENCE .................... 15

4. RESULTS OBTAINED WITH RUSHTON TANK ................................... 22

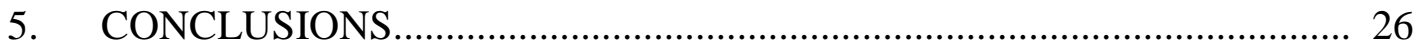

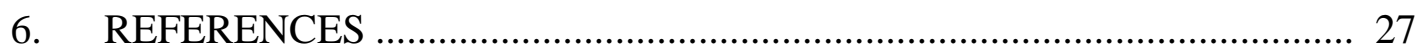

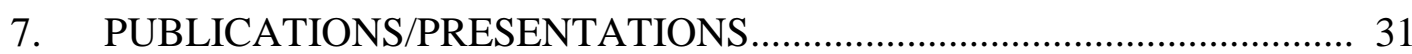




\begin{tabular}{|c|c|}
\hline Nomenclature & \\
\hline$\overline{\text { Subscript } \mathrm{p}}$ & particle \\
\hline Subscript b & bubble \\
\hline Subscript l & liquid \\
\hline Superscript * & nondimentional quantity \\
\hline$<>$ & time average \\
\hline $\mathrm{C}_{12}$ & collision frequency coefficient $\left[\mathrm{m}^{3} \cdot \mathrm{s}^{-1}\right]$ \\
\hline $\mathrm{d}_{\mathrm{i}}$ & diameter of i $[\mathrm{m}]$ \\
\hline $\mathrm{d}_{12}$ & sum of diameters of particle and bubble \\
\hline $\mathrm{N}_{\mathrm{i}}$ & number density of i $\left[\mathrm{m}^{-3}\right]$ \\
\hline $\mathrm{R}_{12}$ & radius of collision $[\mathrm{m}]=\left(\mathrm{d}_{1}+\mathrm{d}_{2}\right) / 2=\mathrm{R}_{1}+\mathrm{R}_{2}$ \\
\hline $\mathrm{u}_{\mathrm{i}}$ & $\mathrm{i}^{\text {th }}$ component of fluctuating velocity \\
\hline${\sqrt{\mathbf{U}_{i}^{2}}}^{2}$ & root-mean-squared velocity of $\mathrm{i}$ with respect to flow $\left[\mathrm{m} \cdot \mathrm{s}^{-1}\right]$ \\
\hline$\overline{\mathbf{U}_{\mathrm{i}}^{2}}$ & variance of i with respect to flow $\left[\mathrm{m}^{2} \cdot \mathrm{s}^{-2}\right]$ \\
\hline $\mathbf{U}^{2}$ & variance of flow velocity \\
\hline $\mathrm{Z}_{12}$ & collision frequency between particle and bubble $\left[\mathrm{m}^{-3} \cdot \mathrm{s}^{-1}\right]$ \\
\hline$\varepsilon$ & Kinetic Energy Dissipation rate $\left[\mathrm{m}^{2} \cdot \mathrm{s}^{-3}\right]$ \\
\hline$v$ & kinematic viscosity $\left[\mathrm{m}^{2} \cdot \mathrm{s}^{-1}\right]$ \\
\hline$\rho_{\mathrm{i}}$ & density of $\mathrm{i}\left[\mathrm{kg} \cdot \mathrm{m}^{-3}\right]$ \\
\hline$\tau_{\mathrm{i}}$ & relaxation time of i $[\mathrm{s}]$ \\
\hline $\mathrm{L}$ & Characteristic length \\
\hline Uo & Free stream velocity \\
\hline $\operatorname{Re}$ & Reynolds number \\
\hline $\mathrm{T}$ & flow time scale \\
\hline
\end{tabular}




\section{EXECUTIVE SUMMARY}

We employ DPIV (Digital Particle Image Velocimetry) to study experimentally the direct interaction of bubbles and particles. The streams are seeded with two types of particles. The first type is the order of $100 \mu$, and simulate coal particles. The second type of particles is sized down to the order of $1 \mu$ and their purpose is to allow accurate measurements of the instantaneous velocity field around the bubbles. These are essentially seed particles. We document the individual velocities and turbulence fluctuations of each individual phase.

We measured the trajectories and velocities of solid particles traveling around bubbles in homogenous isotropic turbulence. From the experimental results we computed the energy dissipation rate, which we will be incorporated into Schubert's formulation. Data were obtained in grid turbulence and in a stirring tank. We then employed established formulas that return the RMS of the fluctuation of bubbles and particles and compared with the actual experimental measurements of such fluctuations to provide information on their validity. 


\section{INTRODUCTION}

\section{Background}

Flotation cells use bubbles to collect solid particles selectively. They are designed to maximize the collision frequencies between particles and bubbles. The flotation process occurs within a highly turbulent three-phase flow, a detailed understanding of which is still limited. Knowledge of the dispersed phase velocities is a basis for understanding of what occurs in a turbulent, multi-phase environment. In many analytical formulations of flotation processes, the rate of collision is modeled in terms of the fluctuations of bubbles and particles. Typical examples of such models are presented in the present conference (Ralston et al. 2005, Sherrell and Yoon 2005). These in turn are related to the character of the turbulent flow that drives both bubbles and particles. These are either empirical models based on experimental data analysis (Liepe and Möckel, 1976), or analytical formulations based on theoretical approximations (Levins and Glastonbury, 1972; Saffman and Turner, 1956; Abrahamson, 1975). The Liepe and Möckel model was developed nearly thirty years ago on the basis of the data obtained using laboratory techniques of limited accuracy, while the analytical models are all approximations. No attempt has been made so far to validate either the empirical or the analytical models using modern experimental tools. This is the purpose of the present communication.

The model which is most frequently cited in the flotation literature is one of Liepe and Möckel (1976), who compiled experimental data from six different earlier publications. Schubert (1999) used the model extensively in his studies on the effect of various hydrodynamic parameters on flotation. One should note here that all of these experiments were carried out with solid particles heavier than water. Therefore, use of the Liepe and Möckel's model to estimate the turbulent root mean square velocities of bubbles in a flotation cell is questionable. Moreover, the predictions from the analytical models have not been compared either with each other or against experimental data. We present experimental results obtained with modern powerful methods and compare the performance of the most popular models against the new experimental data and against each other.

The first set of the present experiments were conducted in a facility where turbulence is generated by a grid of cylindrical rods. This offers a wide range of turbulence scales, beginning with the regions immediately downstream of the rods, where large vortical structures simulating the environment near an impeller reside. Far from the grid, turbulence tends to become homogeneous. The turbulence characteristics are, therefore, similar to those found in flotation cells, but they are well defined and the experiments are repeatable.

Closer to the flotation mechanisms are the three-phase motion generated in stirred tanks. There is a wealth of experimental and numerical results of the turbulent characteristics of the flow in stirred tanks. These are cylindrical tanks in which a stirring device generates turbulence. A typical stirrer is the Rushton turbine, which is essentially a set of flat plates with their plane positioned in the radial direction and parallel to the axis of symmetry of the devise. In the tests reported here, we use an impeller type of a 
stirrer. The aim has always been to measure the basic parameters that characterize turbulence. Recall that each of the models discussed in our previous report, relies on the accurate determination of the kinetic energy dissipation rate. Our measurement technique to determine the dissipation rate is now discussed.

Researchers used a variety of methods to record velocity fluctuations in stirred tanks, as for example hot wire velocimetry, (Rao and Brodkey 1972), laser-Doppler velocimetry (Costes and Couderc 1988, Wu et al. 1989, Okamoto et al. 1981, Wu and Patterson 1989) and more recently particle-image velocimetry (Saarenrinne and Piirto 2000). Wu et al. (1989) performed an energy balance on measured turbulent kinetic energy over a control volume to obtain local values of turbulent dissipation. Rao and Brodkey (1972) and Okamoto et al. (1981) applied power spectrum methods, while others (Rao and Brodkey 1972, Wu and Patterson 1989 and Kresta and Wood 1993) estimated the integral scale by integration of the auto-correlation function.

A well-documented approach of the performance of flotation cells is based on analytical or numerical determination of the rate constant (Moon, et al. (2002). An accepted estimate of the collision rate is given by the Abrahamson (1975) equation, which requires the root-mean square of the velocities of phase 1 and 2, here the coal particles and the bubbles relative to the fluid, respectively. Abrahamson's analysis is valid only if the phase density is much larger than the fluid density. This is the case for coal particles but not for bubbles.

Schubert (1999) proposes instead

$$
\overline{U_{i}^{2}}=0.33 \frac{\varepsilon^{4 / 9} d_{i}}{v^{1 / 3}}\left(\frac{\rho_{i}-\rho_{f}}{\rho_{f}}\right)^{2 / 3}
$$

This formulation is an empirical, curve-fitting procedure carried out by Liepe and Moeckel (1976), based on experimental data collected by many authors. All these data were obtained decades ago, with almost primitive tools. Levins and Glastonbury (1972) for example record visual data in a flotation machine, along two planes and then proceed to measure manually thousands of particle-path segments. From such data they then generate the RMS of flow and particle velocity fluctuations.

\section{EXPERIMENTAL METHODS}

\section{$\underline{2.1 \text { Analytical Considerations }}$}

The knowledge of particle and bubble velocities within a turbulent environment is a basis for understanding what occurs within a flotation cell. This knowledge, along with other relevant parameters, allows the prediction of a rate constant which in turn allows the prediction of recovery and grade of minerals. The flotation process occurs within a highly turbulent three-phase flow, where a detailed understanding of these flows is limited. These velocities are used to model collision frequencies which are in the form

$$
\mathbf{Z}_{12}=\mathbf{C}_{12} \mathbf{N}_{1} \mathbf{N}_{2} \mathbf{R}_{12}^{2}
$$

where $\mathrm{Z}_{12}$ is a collision frequency coefficient and contains the relevant velocities. 
Saffman and Turner (ref) proposed two collision models both assuming Stokes numbers below 1 . The first took into account only the shear mechanism for collision and assumes a Stokes number of zero.

$$
\mathbf{Z}_{12}=\sqrt{\frac{8 \pi}{15}} \mathbf{R}_{12}^{3} \mathbf{N}_{1} \mathbf{N}_{2} \sqrt{\frac{\varepsilon}{v}}
$$

Abrahamson (1975) then came up with a collision frequency where the Stokes number of the colliding particles is infinite; essentially assuming the motion of the bubbles and particles is completely uncorrelated with the flow. This frequency only accounted for the accelerative mechanism for collisions.

$$
\mathbf{Z}_{12}=2^{3 / 2} \pi^{1 / 2} \mathbf{N}_{1} \mathbf{N}_{2} \mathbf{d}_{12}^{2} \sqrt{\left(\overline{\mathbf{U}_{1}^{2}}+\overline{\mathbf{U}_{2}^{2}}\right)}
$$

There are various methods to calculate the RMS of the particle and bubble velocities, $\overline{\mathbf{U}_{\mathbf{i}}^{2}}$. One such method is a derivation from the BBO equation by Levins and Glastonbury (1972) that assumed single frequency oscillations,

$$
\begin{aligned}
\frac{\overline{\mathbf{U}_{\mathbf{i}}^{2}}}{\overline{\mathbf{U}^{2}}}=\frac{(1-\mathbf{b})^{2}}{\mathbf{a T}+1} & \\
\mathbf{a}=\frac{36 \mu}{\left(2 \mathbf{p}_{\mathbf{i}}+\mathbf{p}_{\mathbf{f}}\right) \mathbf{d}_{\mathbf{p}}{ }^{2}} & \mathbf{b}=\frac{3 \mathbf{p}_{\mathbf{f}}}{2 \mathbf{p}_{\mathbf{i}}+\mathbf{p}_{\mathbf{f}}} .
\end{aligned}
$$

Abrahamson also included a simplification to Equation 4 by assuming solid particles in a gas $\left(\mathrm{b}=0, \mathrm{a}=1 / \tau_{\mathrm{p}}\right)$ and calculating the time scale, $\mathbf{T}_{\mathbf{L}}=0 . \overline{\mathbf{U}^{2}} / \varepsilon$, therefore reducing to equation 5 , which is a common formula used in the flotation industry,

$$
{\overline{\left(\mathbf{U}_{\mathbf{i}}^{2}\right.}}_{\mathbf{a b s}}=\frac{\overline{\mathbf{U}^{2}}}{1+1.5 \tau_{\mathbf{i}} \varepsilon / \overline{\mathbf{U}^{2}}} .
$$

The most common form used in the flotation industry is a combination of the collision frequency given by Abrahamson and the particle root-mean-squared velocity with respect to the flow given by Liepe and Mockel (1976).

$$
\sqrt{\overline{\mathbf{U}_{\mathbf{i}}^{2}}}=0.33 \frac{\varepsilon^{4 / 9} \mathbf{d}_{\mathbf{i}}^{7 / 9}}{v^{1 / 3}}\left(\frac{\rho_{\mathbf{i}}-\rho_{\mathbf{l}}}{\rho_{\mathbf{l}}}\right)^{2 / 3}
$$

This velocity was experimentally developed and is valid at intermediate Stokes numbers where the flotation process occurs.

There are limitations to all of these models for flotation. The velocity components of Saffman and Turner's models assume Stokes numbers of 1 or less while Abrahamson assumes Stokes numbers of infinity. Neither of these conditions is valid within the flotation environment over the particle sizes that are significant. Liepe and Mockel's model should be valid for particles at intermediate Stokes numbers. The limitation on this is the assumption that the density of the particle is greater than that of 
the fluid, which is not the case with air bubbles. To determine how greatly these limitations affect their collision frequencies, fluid velocity measurements have been recorded in grid turbulence. The velocity at the Kolmogorov scale, $U=(v \varepsilon)^{1 / 4}$, has been determined from these and compared against the RMS velocities predicted by the three models described above. The nondimentionalized forms of the Levins and Glastonbury, and Shubert models respectively are shown in Equations 6 and 7. These comparisons will show how well each predicts small particle flow.

$$
\begin{gathered}
\frac{\overline{\mathbf{U}^{*^{2}}}}{\overline{\mathbf{U}^{* 2}}}=\frac{(1-\mathbf{b})^{2}}{\mathbf{a} \mathbf{T}^{*} \mathbf{L} / \mathbf{U}_{\mathbf{o}}+1} \\
\sqrt{\overline{\mathbf{U}_{\mathbf{i}}^{* 2}}}=0.33 \mathbf{R e}^{1 / 3} \varepsilon^{* 4 / 9} \mathbf{d}_{\mathbf{i}}^{* 7 / 9}\left(\frac{\rho_{\mathbf{i}}-\rho_{\mathbf{f}}}{\rho_{\mathbf{f}}}\right)^{2 / 3}
\end{gathered}
$$

The main objective of this project is to record the fluctuations of particles in a homogenous turbulent flow field. The RMS of theses velocity fluctuations corresponding to the Kolmogorov micro scale will then be calculated in order to confirm or modify accordingly the Schubert equation given in Equation 7, where $\overline{U_{i}^{2}}$ is the RMS of the velocity fluctuation of species $i$, with respect to the flow. In addition, the measured particle velocity fluctuations will be compared to those presented by Levins and Glastonbury (Equation 6).

\subsection{Turbulent Energy Dissipation}

Each of these models are dependent upon the kinetic energy dissipation rate, which has been shown to determine the degree of droplet and bubble break up and rates of chemical reactions. Investigating the flow physics of turbulent flows requires the accurate measurement of turbulence dissipation rate (TDR). This task becomes intractable when the flow field under investigation presents inhomogeneous anisotropic characteristics which require spatio-temporally resolving the full range of turbulent scales.

There are various methods to estimate TDR, including turbulent kinetic energy balance, Taylor's hypothesis, and dimensional analysis. These methods are based on the stipulation that the flow field is resolved down to the Kolmogorov scales and most of them assume homogeneous isotropic turbulence. Yet, neither of these assumptions is valid for most realistic turbulent flows. In particular, spatial or temporal resolution limitations of conventional experimental flow-diagnostic systems prohibit us from resolving the dissipative Kolmogorov scales. As a result, until recently, computational fluid dynamics methods such as large-eddy simulations (LES) and direct numerical simulation (DNS) provided the only viable solution.

Digital Particle Image Velocimetry (DPIV) presents a new opportunity for TDR estimation by overcoming many of the limitations of existing methods. In particular, it allows the estimation of global space and time derivatives. In one of the first DPIV-based TDR studies, Saarenrinne and Piirto [2000], estimated turbulence dissipation limited to the 2-dimensional isotropic homogeneous relation (obtained from the theoretical definition of viscous dissipation) and calculated the error associated with the TDR values 
as a function of the image area size and the interrogation area size. Piirto et al. [2003] estimated turbulence energy in a backward-facing step flow using a three-component stereo PIV system. In this study, TDR estimation was performed using the direct 3dimensional theoretical definition and found to be a function of the spatial resolution, i.e. TDR values were higher for the higher resolutions; yet, the study also showed that the measurement resolution was not close to the dissipation scale. These studies reveal that although PIV is capable of providing multi-point instantaneous measurements, spatial resolution limitations hamper TDR estimations.

An alternative to resolving the dissipative scales using DPIV was proposed by Sheng et. al. (2000) by employing a large eddy approximation inspired from computational methods for fluid dynamics. The basis for this method is that since turbulent kinetic energy is mainly generated at the integral scale, the same amount of energy is dissipated near the Kolmogorov scales, i.e. production equals dissipation. Sheng et al. [2000] approximated TDR from PIV measurements of a stirred vessel by employing a large eddy estimation inspired from two LES subgrid scale eddy viscosity models (Smagorinsky and gradient) and compared them to the dimensional analysis relation

$$
\varepsilon=(\mathrm{A} / \mathrm{L}) \mathrm{u}^{\prime 3},
$$

where $A=1$ and $L$ is the integral length scale]. This relation requires the assumption of isotropic homogeneous turbulence, which is not actually valid for this specific flow field and most other complex flows. Additionally, a constant value for the Smagorinsky coefficient, $\mathrm{C}_{\mathrm{s}}$, was used, which is not valid for inhomogeneous anisotropic turbulent flows whose local structure dictates $\mathrm{C}_{\mathrm{s}}$. Therefore, it is evident that there is a need for more accurate TDR approximations in anisotropic inhomogeneous turbulent flows.

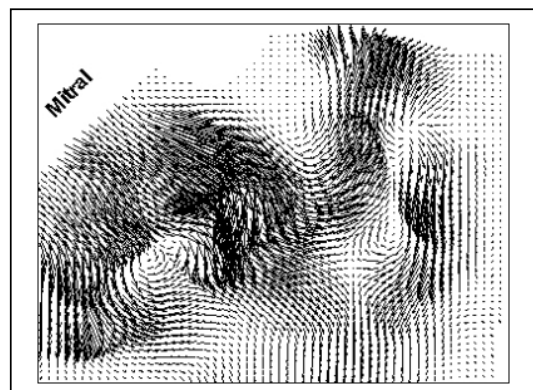

Figure 2.1. Sample DPIV velocity field inside the left

In this effort, we bridge the numerical and experimental models for calculating TDR by using methods from both LES-based subgrid scale models and the direct definition of TDR without assuming homogeneous isotropic turbulence. This is accomplished by using Time-Resolved DPIV (TRDPIV) with high spatio-temporal resolution. The performance and accuracy of the various TDR estimation models is validated.

The LES-based PIV method approximates TDR by computing the Reynolds averaged sub-grid scale (SGS) dissipation rate

$$
\varepsilon \approx\left\langle\varepsilon_{\mathrm{SGS}}\right\rangle=-2\left\langle\tau_{\mathrm{ij}} \overline{\mathrm{S}}_{\mathrm{ij}}\right\rangle
$$

where $\varepsilon$ is the dissipation, $\tau_{\mathrm{ij}}$ is the SGS stress tensor, and $\mathrm{S}_{\mathrm{ij}}$ is the strain rate tensor. In this method, both $\tau_{\mathrm{ij}}$ and $\mathrm{S}_{\mathrm{ij}}$ are computed directly from the TRDPIV resolved velocity measurements (inertial scale). Moreover, the SGS stress tensor $\tau_{\mathrm{ij}}$ can be approximated by various methods, the most common being the Smagorinsky model given by

$$
\tau_{\mathrm{ij}}=-\mathrm{C}_{\mathrm{s}}^{2} \Delta^{2}|\overline{\mathrm{S}}| \bar{S}_{\mathrm{ij}}
$$


where $\mathrm{C}_{\mathrm{s}}$ is the Smagorinsky constant and $\Delta$ is the filter width which is a function of the interrogation window. In order to overcome the shortcomings of the Smagorinsky model, which assumes a constant Smagorinsky coefficient $\mathrm{C}_{\mathrm{s}}$, a dynamically obtained Smagorinsky coefficient, which depends on the local structure of the resolved velocity field, will also be applied. Use of this novel method will allow for estimating the turbulence dissipation over the entire flow field and for any type of flow. This LESbased PIV estimation of the TDR will be compared with the direct theoretical approximation of the viscous dissipation:

$$
\varepsilon=v \overline{\left(\frac{\partial u_{i}}{\partial x_{j}}+\frac{\partial u_{j}}{\partial x_{i}}\right) \frac{\partial u_{j}}{\partial x_{i}}}
$$

Since the planar DPIV measurements will only provide five of the twelve terms of the expanded form of Equation (3), assumptions of local homogeneity will be made so that we can approximate the seven unresolved gradients.

In this effort, three types of flow fields will be investigated. A homogeneous isotropic turbulent flow field (grid turbulence data) is explored and serves as a reference case to compare the accuracy of the various models of estimating TDR. For this flow field, the Kolmogorov length scale is on the order of $56 \mu \mathrm{m}$ while the spatial resolution is on the order of $110 \mu \mathrm{m}$. The velocity measurements were produced by an iterative DPIV procedure with a minimum interrogation window of 8x8 pixels with 3 pixels spacing (62\% overlap) between consecutive vectors. Secondly, stirring tank data and also data of the flow inside a flexible left ventricle (Kolmogorov length $~ 85 \mu \mathrm{m}$ ) in the presence of mechanical heart valves will be investigated for their anisotropic inhomogeneous turbulence properties and to study the performance of the various models. Figure 2.1 shows a sample PIV velocity field (fixed vector-to-vector spacing of $\sim 700 \mu \mathrm{m}$ ) with vortical structures (eddies) inside the LV past a mechanical heart valve prosthesis. Cardiovascular flows are very complex due to the pulsatility of the flow, thus, the dissipation rates of both the mean velocity and the periodic contributions will be considered separately and

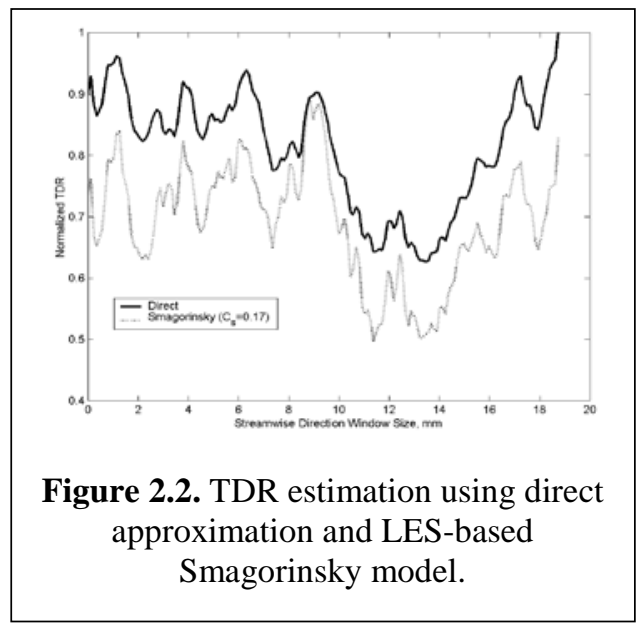
compared. Figure 2.2 shows preliminary results of the mean normalized TDR as a function of the streamwise direction of the grid turbulence data (isotropic, homogeneous). The two curves correspond to the Smagorinsky model (Equations 1 and 2) with $\mathrm{C}_{\mathrm{s}}=0.17$ and the direct theoretical approximation (Equation 3). There is good correlation between the direct approximation and the LES-based Smagorinsky model. The correlation can be further improved by optimizing $\mathrm{C}_{\mathrm{s}}$ and later by employing a dynamic $\mathrm{C}_{\mathrm{s}}$.

\subsection{Digital Particle Image Velocimetry}

The results presented herein were generated using Particle Image Velocimetry (PIV). During the past two decades, numerous publications have appeared presenting improvements on the PIV method. Applications of the method range from low-speed 
liquid and two-phase flows to supersonic gas flows. Review articles by Hasselinc (1988); Adrian (1991) and Grant $(1994,1997)$ provide comprehensive summaries of the principles of the method and its various applications. The work of Willert and Gharib (1991), Westerweel (1993 a,b), Huang and Gharib (1997) and Huang et al. (1997) established the digital implementation of PIV, namely DPIV. The applicability of the method in resolving a variety of length scales in the flow has investigated by Adrian (1997). Despite the advances in improving the spatial resolution and accuracy of the method, conventional DPIV systems provide a limited temporal resolution $(\sim 30 \mathrm{~Hz})$, which is insufficient for resolving the fluctuations present in a turbulent flow field. For moderate Reynolds numbers $\left(10^{3}-10^{5}\right)$ and small length scales, which are common in a laboratory environment, the sampling frequency necessary in order to resolve adequately the turbulent characteristics of the flow is indeed in the order of $10^{3} \mathrm{~Hz}$. The system developed by the present team extends the range of time resolution to this range of frequencies.

Past implementation of time-resolved systems employed digital sensors with limited resolution (Whybrew et al, 1999) or drum cameras that are limited both in recording times as well as frame rate (Lecordier and Trinite, 1999). Recent work by Upatnieks et al (2002) presented an analog based kilohertz frame rate PIV system capable of recording up to $4000 \mathrm{fps}$. By employing analog recording, the system delivers superior spatial resolution $(>1 \mathrm{Kx} 1 \mathrm{~K})$, however due to the fact that such high frame rates require extreme rotation speed for the film, the registration of the images is compromised by film alignment errors that are added on the common digitization errors. Super resolution, iterative, and hybrid DPIV algorithms were developed in order to carry out the task of the velocity evaluation by Scarano and Riethmuller (1999), Guezennec and Kiritsis (1990), Cowen and Monismith (1997) and Wereley and Meinhart (2002). Finally, several implementations of the method demonstrated its applicability in two-phase and multiphase flows Boedec and Simoens (2001) Khalitov and Longmire (2002).

Previous work by the present team (Vlachos 2000) presented a fully digital system with $1 \mathrm{KHz}$ sampling rate, which however was limited to 256x256-pixel resolution. More recently we presented time resolved DPIV (TRDPIV) measurements with $\mathrm{kHz}$ sampling rate in multi-phase flows (Abiven and Vlachos, 2002) and provided detailed error analysis (Abiven et. al. 2002) In the present effort we have improved upon the earlier works to employ a time-resolved DPIV system capable of resolving multiphase flows with $\sim 1 \mathrm{kHz}$ sampling rate and enhanced spatial resolution on the order of $60 \mu \mathrm{m}$.

\subsection{Facilities and Instrumentation}

Experiments to characterize the particle and bubble response to turbulence were performed. A small-scale water tunnel has been constructed in which we generated homogenous isotropic turbulence (Figure 2.3). The design allows quick change of the fluid/particle mixture and flushing of the system for more tests. The facility is appropriate for mixing different sizes of particles with different densities, as well as generating air bubbles of different sizes. Turbulence is generated by a grid of circular cylinders $1 \mathrm{~cm}$ in diameter, spaced by $1 \mathrm{~cm}$ in both the length and width directions. This is the simplest reproducible and repeatable example of homogeneous turbulence. On the other hand, the 
space immediately above the cylinders displays a range of large turbulent structures that simulate the turbulent flow next to a flotation cell impeller.

The Reynolds number based on the grid spacing was on the order of $\mathrm{Re}=1000$.

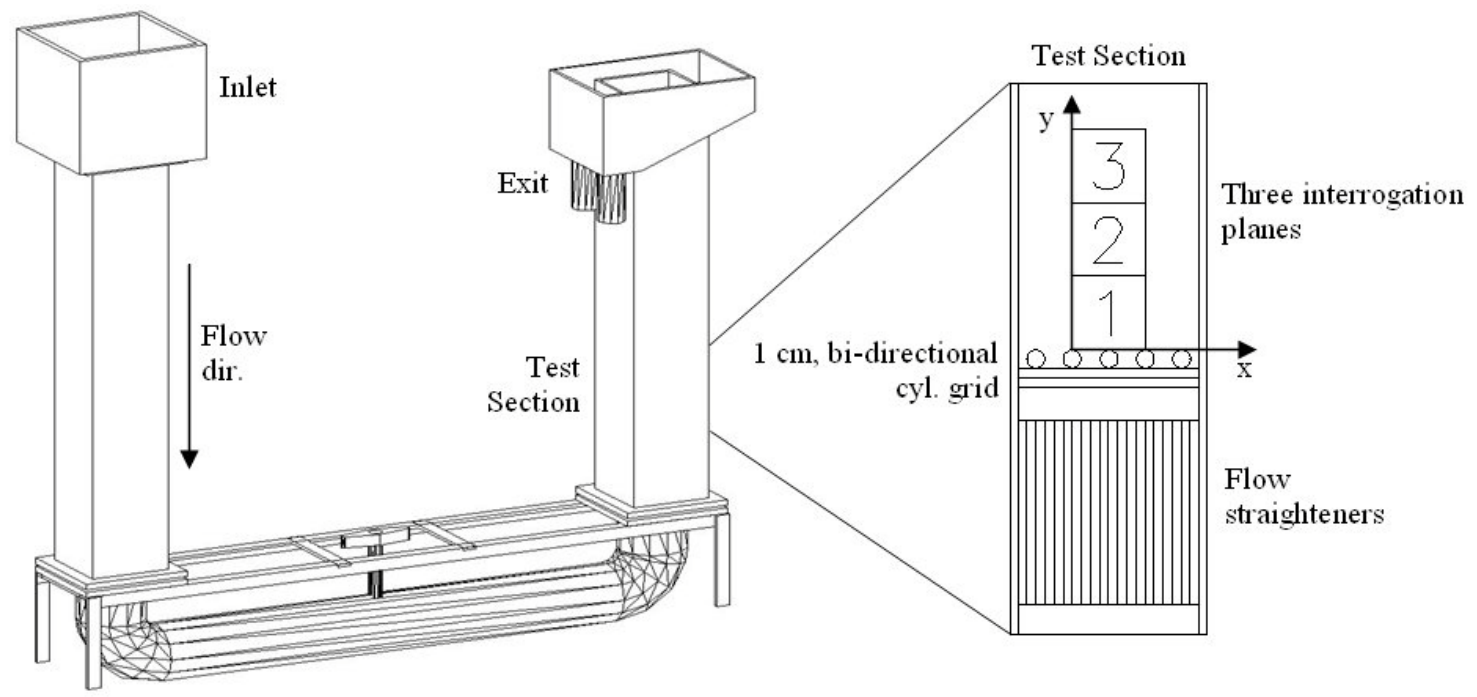

Figure 2.3 - Schematic of water tunnel and expanded view of test section

The Kolmogorov length scale is of the order of $50 \mu \mathrm{m}$ and the corresponding time scale was $100 \mathrm{~ms}$. The experiments were performed with a sampling frequency of $1000 \mathrm{~Hz}$ $(\Delta \mathrm{t}=1 \mathrm{~ms})$ and a spatial resolution of $60 \mu \mathrm{m} /$ pixel. The DPIV data analysis scheme was optimized for the maximum spatial resolution with the minimum spatial averaging effects introduced by the cross-correlation. Therefore the velocity measurements were carried out with a minimum interrogation window of $8 \times 8$ pixels with 3 pixels (62\%) overlap spacing between consecutive vectors. This results in spatial resolution for the flow field of the order of $180 \mu \mathrm{m}$.

Non-invasive optical flow diagnostics measurements were performed in order to characterize the particle and/or bubble interactions within homogeneous isotropic turbulence. DPIV requires a plane sheet of intense laser light. This sheet cuts the domain along planes of interest. The DPIV component arrangement shown in Figure 2.4 records the instantaneous flow field in a plane. In the present case the plane of interest is vertical and parallel to the plane $\mathrm{x}-\mathrm{y}$ plane. Data were collected along three square sections, each with sides equal to $4 \mathrm{~cm}$, or $4 \mathrm{~L}$. The length scale $\mathrm{L}$ was chosen to be equal to one rod diameter. The origin of the coordinate system is at the lower left corner of the domain of measurements, as shown in Fig. 2.3. We employ a digital camera to record the instantaneous position of different objects in the flow field. Recording the position of seed particles that follow the flow allows us to calculate the instantaneous flow velocity field. Over the past few years, we were able to improve this method by increasing the frequency response by two orders of magnitude over commercially available systems. We have also employed this method to record two-phase flows, namely spray flows or bubbly flows.

Digital Particle Image Velocimetry (DPIV) is the most established global flowfield measurement technique. However, time-resolved measurements with simultaneous velocity shape and size characterization of multiple phases remains a great challenge. In the current effort, we integrate the appropriate hardware and software components 


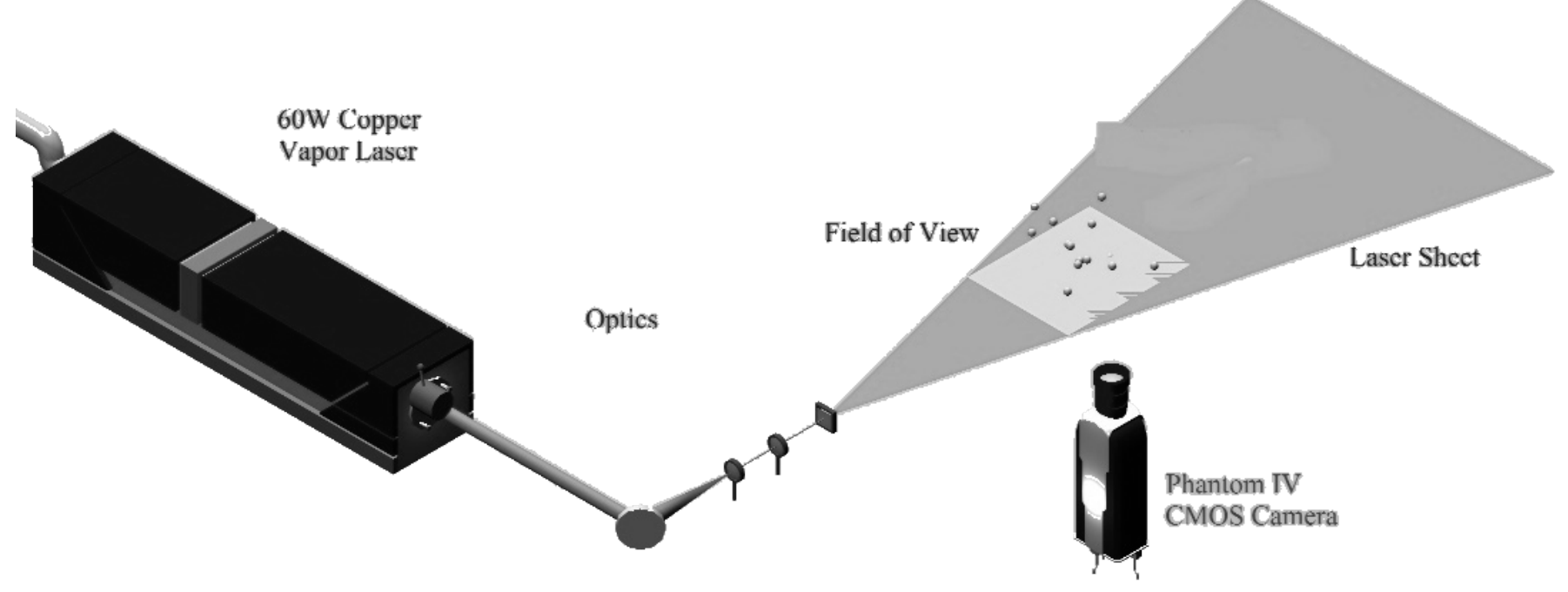

Figure 2.4: View of a typical DPIV experimental setup

necessary to perform such measurements. A Vision Research Phantom-IV camera with frame rate up to 1000 frames per second (fps) and spatial resolution of 512x512 is employed using single laser exposure per frame for the recording of the DPIV images. The camera is synchronized with a high repetition rate Copper-vapor pulsing laser with emission wavelengths at 511nm and 578nm. The pulse duration is of the order of 25nsecs, sufficient for performing measurements for low and moderate speeds.

For multiphase flows, saturation of the image from overexposure of bubbles or droplets is a detrimental parameter for carrying out accurate quantitative measurements of size and position. Conventional DPIV systems employ CCD cameras, which suffer from leakage effects, namely, the excessive charge from overexposed pixels leaks to the neighboring ones saturating the whole area. In contrast, CMOS sensors isolate the individual pixels behaving as a cut-off filter without leaking the energy. Therefore, by employing CMOS technology we eliminate the blooming effect, allowing resolution of a multi-phase flow with direct imaging within a laser sheet. This feature is of great importance, since it simplifies the experimental setup, enhances the signal-to-noise ratio, and more importantly allows accurate shape and size quantification of particles or bubbles present in the flow. In addition, it improves the performance of the position estimator, which is essential for the performance of the particle tracking methodology. Details on the performance characteristics and accuracy of the system can be found in Abiven and Vlachos (2002).

Traditional, cross-correlation-based PIV data-processing software was used to determine the flow velocities. This requires relatively dense seeding, namely at least 5 to 8 seed particles in each interrogation window. Because model particles and bubbles appear more sparsely in the images, they were measured using another processing technique, namely Particle Tracking Velocimetry (PTV). PTV tracks individual particle locations over multiple frames, from which velocities can be determined with any order of accuracy desired. PTV allows us to measure $\overline{\left(\mathbf{U}_{\mathbf{i}}^{2}\right)_{\mathbf{a b s}}}$, and $\overline{\mathbf{U}_{\mathbf{i}}^{2}}$ experimentally, where $\mathbf{i}$ takes the values 1 or 2 , for solid particles or bubbles respectively. The kinetic energy dissipation rate was calculated using traditional, cross-correlation-based PIV, providing a means to calculate $\overline{\left(\mathbf{U}_{\mathbf{i}}^{2}\right)_{\mathbf{a b s}}}$ and $\overline{\mathbf{U}_{\mathbf{i}}^{2}}$ from the models in Equations (10)-(14).

We also designed and constructed a mixing tank. This tank consisted of a smallscale cylindrical, baffled vessel made of Plexiglas with diameter $D=0.1504 \mathrm{~m}$ as it shown in Fig. 2.5. Four axial baffles with width $\left(w_{b}\right)$ one tenth of the tank diameter were 
mounted along the wall. An impeller with four blades was located at a height $0.0762 \mathrm{~m}$
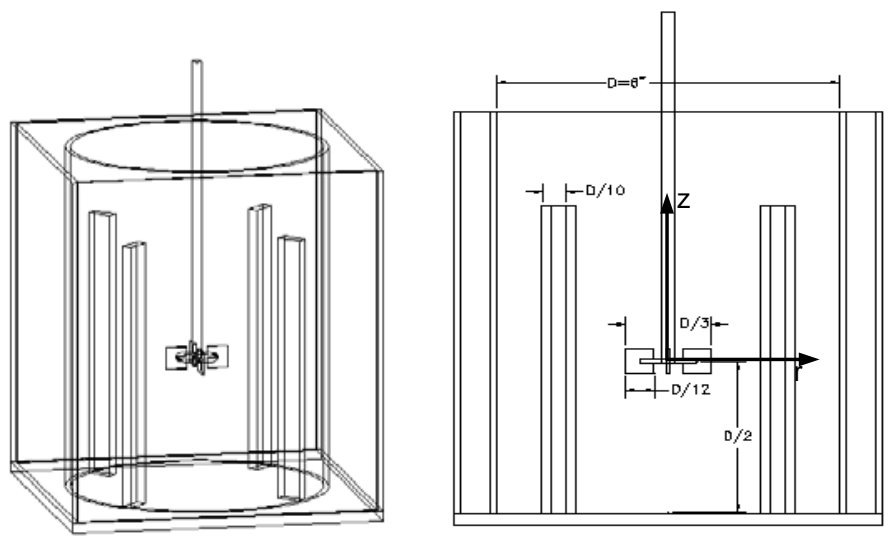

Figure 2.5.Dimentions of the stirring tank

used for the PIV experiment

from the bottom and was driven at $225 \mathrm{rpm}$. The ratio between the vessel and the impeller diameter was $d$ / $D=1 / 3$. The ratio of blade width $\left(w_{i}\right)$ to the impeller diameter $(D)$ was 0.25 . The tank was open at the top and was filled with filtered water as the working fluid. The height of the water was maintained at $0.1504 \mathrm{~m}$, equal to the tank diameter. The cylindrical tank was housed in an outer octagon tank filled with water to eliminate the optical distortion of light beams passing a curved boundary of media with different indices of refraction. The flow was seeded with $6 \mu \mathrm{m}$ diameter spherical fluorescent tracers made of polystyrene. In the present paper we studied two cases. One only with the seed particles so we can examine the flow field of the mixing and one in which we added glass particles (specific gravity 3.6) with diameter of $86 \mu \mathrm{m}$ to simulate coal particles. The aim is to determine the trajectories and slip velocities of the coal particles and relate them to the local flow properties.

The Reynolds number is calculated $\mathrm{Re}=N \cdot d^{2} / v$ based on the impeller diameter and was about 9677 . The Kolmogorov length scale can be calculated from $\eta=d \cdot(\mathrm{Re})^{-3 / 4}$ and was in the order of $50 \mu \mathrm{m}$, and the corresponding time scale was 0.267 .The experiments were performed with sampling frequency $1000 \mathrm{~Hz}$ and a spatial resolution of 49.8 microns/pixel.

\section{RESULTS OBTAINED WITH GRID TURBULENCE}

Very small particles characterized by Stokes numbers less than 1 behave as fluid elements, responding to all the fluctuations of the flow. The $U_{\text {rms }}$ corresponding to the particle motion is defined by the velocity at the level of the Kolmogorov micro-scale for homogeneous isotropic turbulence, namely, $\mathbf{U}=(\varepsilon v)^{\frac{1}{4}}$. Figure 3.1 shows two sample images over the range $y / L=0-4$, just the above turbulence grid and the resulting velocity field produced by cross correlation. Figure 3.2 displays results averaged over 1500 instantaneous frames. The color contours correspond to magnitudes of the quantity plotted. The region where data were obtained consist of the three square frames, centered over two of the two gaps between three of the grid rods as shown in Fig. 2.3. Two jets 

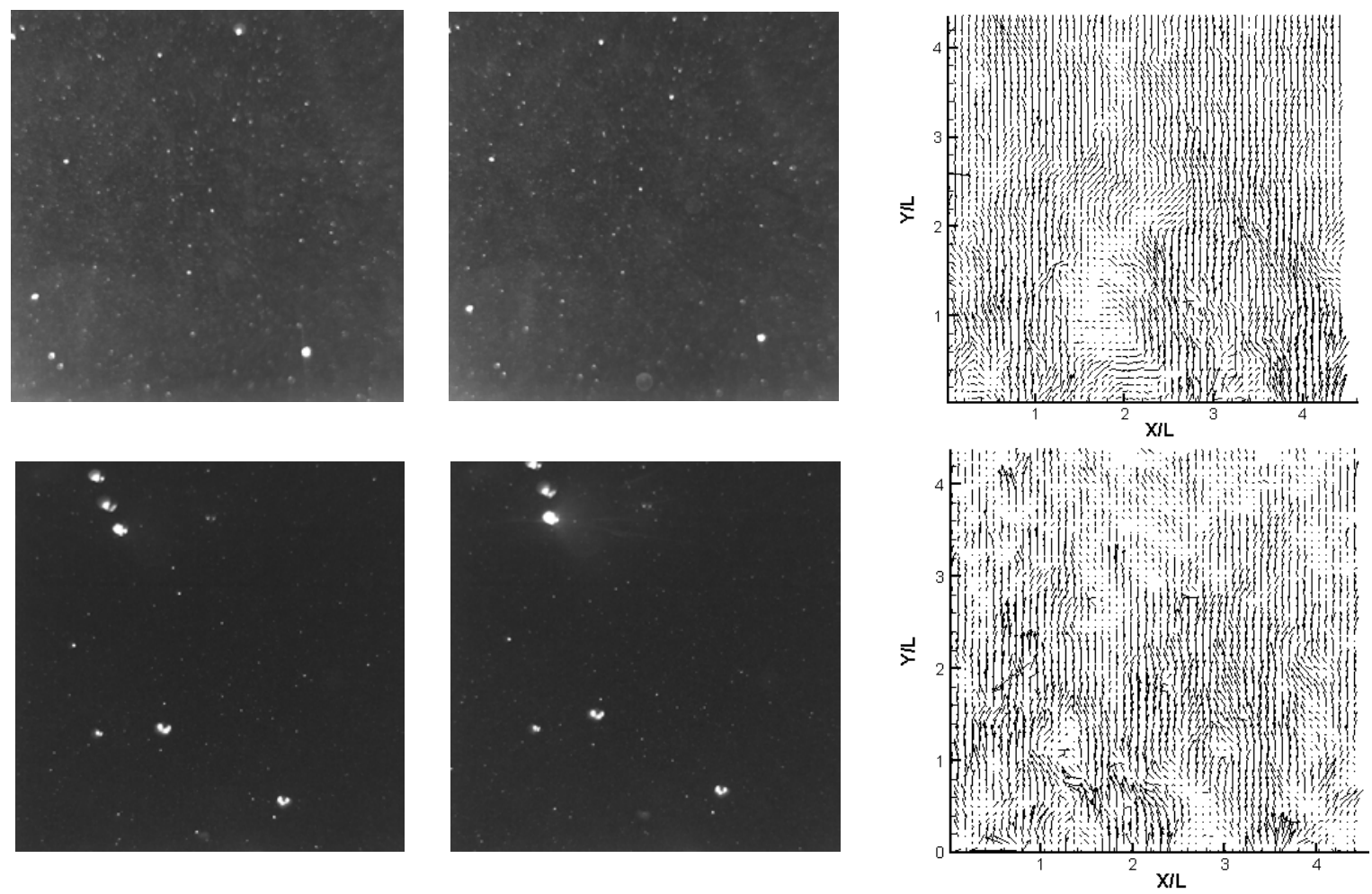

Figure 3.1 - Two sample images and corresponding DPIV vectors with $80 \mu \mathrm{m}$ solid particles and flow tracers (top row), and $1.2 \mathrm{~mm}$ bubbles and flow tracers (bottom row) separated by 0.001 sec and corresponding velocity field from cross correlation 

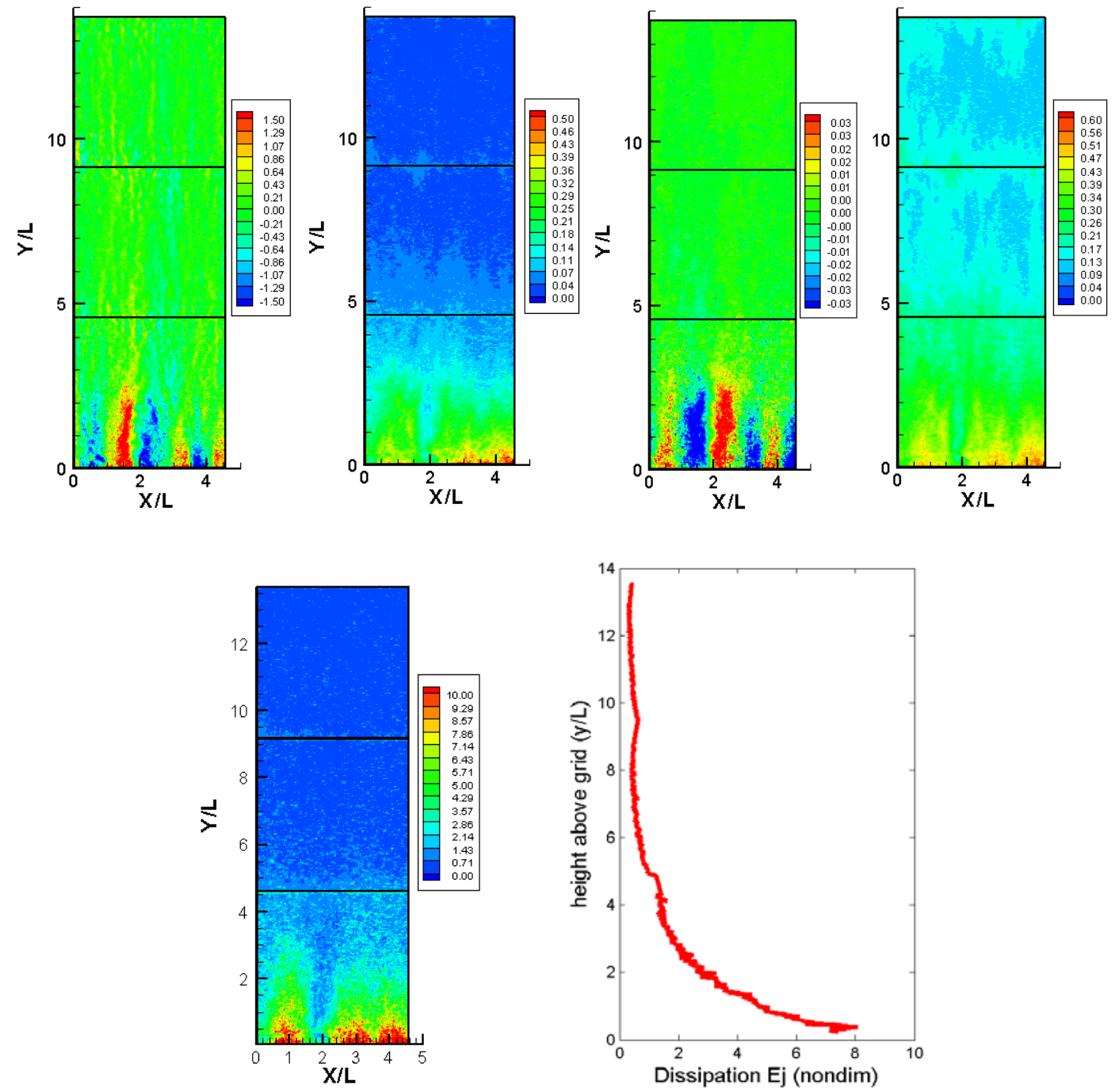

Figure 3.2: Time averaged results of three interrogation planes (all nondimentionalized by Uo and $L$ ) downstream of the turbulence grid over a length of approximately 14 grid spacings. From left to right and top to bottom : vorticity, turbulent kinetic energy, Reynolds stresses, Vrms, dissipation, and dissipation averaged over time and $x$. Dissipation is normalized by dividing by $\mathrm{Uo}^{3} \mathrm{~L}^{-1}$. 
issuing between each pair of cylinders are therefore captured in the lower frame. Immediately downstream of the grid the turbulence is not homogenous. Homogeneity and isotropy evolve as we move up and away from the grid. The time-averaged vorticity contours clearly show two regions of intense vorticity on either side of the jet. The dissipation rate was computed using Taylor's hypothesis of frozen-turbulence, namely Equation (15). Figure 3.2 clearly illustrates a leveling off of the dissipation rate beyond three grid lengths.

Particle Tracking Velocimetry (PTV) was utilized to track the positions of the 80$\mu \mathrm{m}$ glass particles. Figure 3.2 shows particle trajectories at three regions above the turbulence grid tracked for 50 frames. These three frames were obtained by zooming in to the frames shown in Fig. 2.3, and the units on the axes are in number of pixels of the camera. The circles represent the location and diameter of each particle measured by PTV. A small component of the motion is perpendicular to the plane of measurement. The size of the particle appears to be changing with time. This is because the particle may be going through the lighted region. An estimate of the size of the particle is therefore the largest diameter recorded along a trajectory. The region just above the turbulence grid shows a domain of unsteady but coherent motion, especially on the extreme left and right of the image, which are in the immediate downstream region over each rod. Flow reversal in the wake of the rods is evident. Further above the grid at $y / L=4-8$, the particle accelerations are not as severe; however large-scale fluid motion is now evident. Finally, in the region $y / L=812$ above the grid, small scale turbulence is the only noticeable type of particle fluctuation.

\section{Comparison of models}

In Figures 3.4 to 3.6 we present experimental data and analytical predictions along the height of the measuring domain, which covers different levels of the dissipation rates. The analytical predictions were calculated in terms of dimensionless expressions shown in Table 1. Using the experimental dissipation rate estimates, the predicted RMS velocities of the $80-\mu \mathrm{m}$ particles were calculated and compared to the measured RMS velocities of the particles obtained via the Particle Tracking algorithm. Figure 3.4 indicates that the RMS slip velocity measurements agree well with those predicted by the Liepe-and-Möckel model. The RMS velocities do not deviate from the experimental results by more than $12 \%$ over the region of interest. The model of Levins and Glastonbury underestimated the particle RMS by about $50 \%$ in the fully-developed flow region $(y / L>8)$. Figure 3.5 shows the absolute particle RMS velocity and the corresponding predictions from Abrahamson and Williams and Crane. Abrahamson's model provides a reasonable estimate (18\% mean error) in the fully developed region. The Williams and Crane model did not provide a good prediction, generating a $40 \%$ mean error.

The bubble RMS slip velocity, shown in Figure 3.6 is significantly higher than the particle RMS by about $100 \%$ in the homogeneous isotropic region, indicating relatively large fluctuations compared to the flow. Liepe and Möckel's empirical model, although not developed for the case of bubbles, is used in the flotation industry and is shown along with the Levins and Glastonbury model. Both models overestimate the bubble RMS with the Levins and Glastonbury model providing a better prediction with a mean error of 
22\%. Figure 9 shows the results of the other models. Results obtained with the Abrahamson and the Williams and Crane models are in good agreement with the
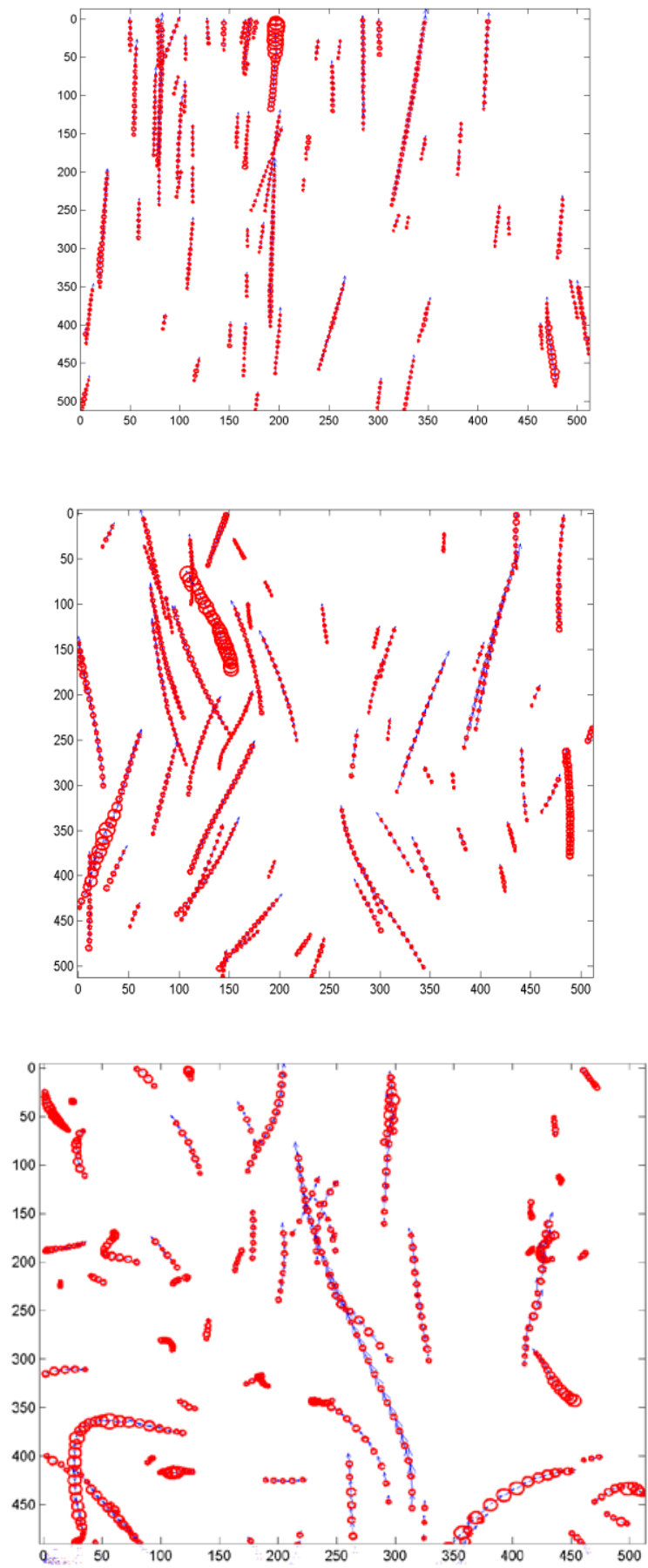

Figure 3.3 - Trajectories of $80 \mu \mathrm{m}$ particles: bottom $y / L=0-2$ grid lengths, middle $y / l=4-6$, top $y / l=10$ 12. Units on the axes are in number of pixels. 


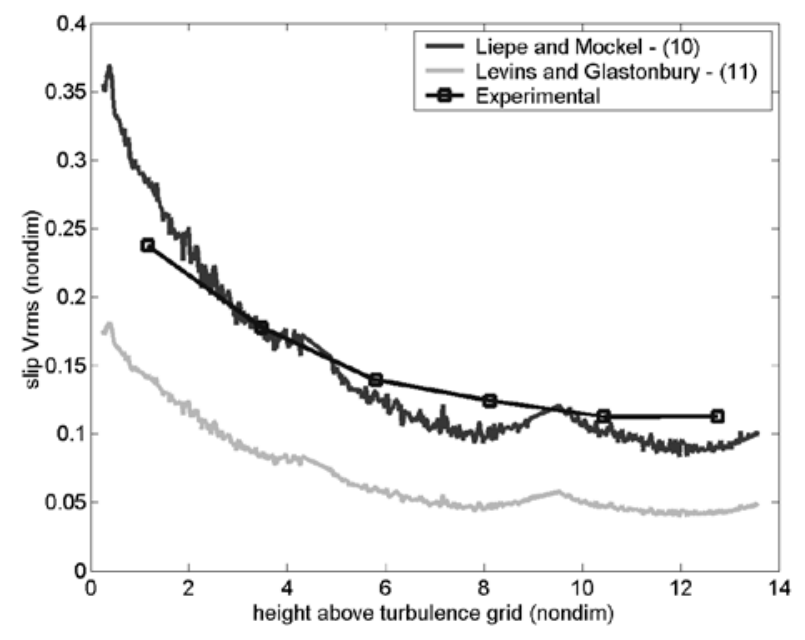

Figure 3.4 - RMS of slip velocity versus height above turbulence grid of $80 \mu \mathrm{m}$ particles and corresponding predictions from models. Equations numbers are shown in Table 1.

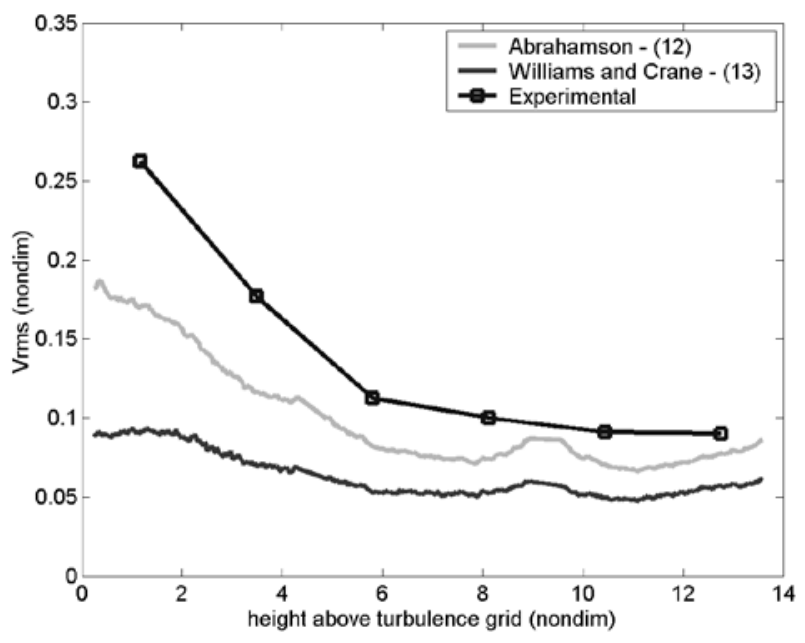

Figure 3.5 - RMS velocity versus height above turbulence grid of $80 \mu \mathrm{m}$ particles and corresponding predictions from models. Equations numbers are shown in Table 1. 


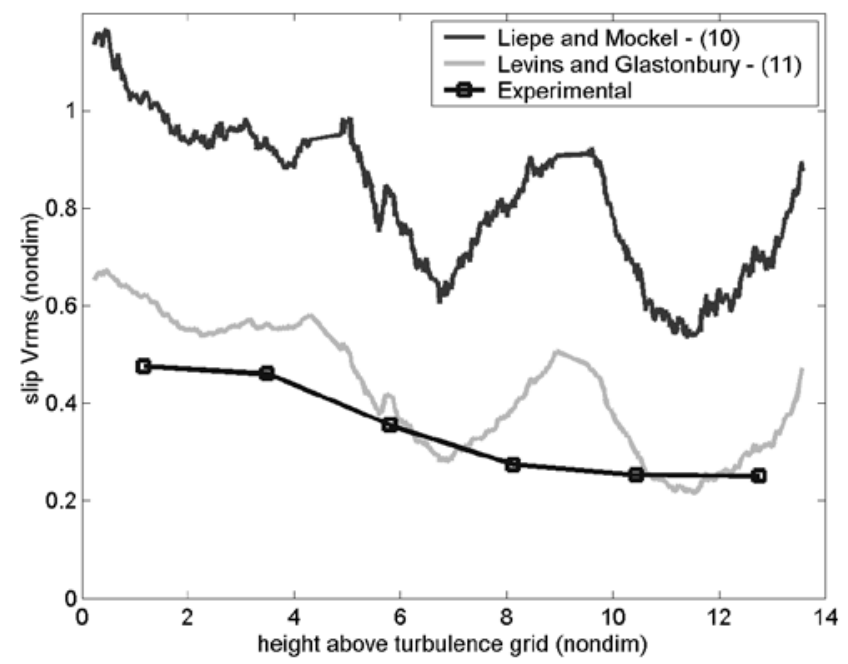

Figure 3.6 - RMS of slip velocity versus height above turbulence grid of $1.2 \mathrm{~mm}$ bubbles and corresponding predictions from models. Equations numbers are shown in Table 1.

Table 1 - Summary of models

\begin{tabular}{|c|c|c|c|}
\hline $\begin{array}{c}\text { Original } \\
\text { Derivation }\end{array}$ & valid for: & Nondimentionalized Equation & Eq. no. \\
\hline Liepe and Möckel & Intermediate St, & $\sqrt{\overline{\mathbf{U}_{\mathbf{i}}^{* 2}}}=0.33 \mathbf{R e}^{1 / 3} \varepsilon^{* 4 / 9} \mathbf{d}_{\mathbf{i}}^{* 7 / 9}\left(\frac{\rho_{\mathbf{i}}-\rho_{\mathbf{f}}}{\rho_{\mathbf{f}}}\right)^{2 / 3}$ & (10) \\
\hline $\begin{array}{l}\text { Levins and } \\
\text { Glastonbury }\end{array}$ & & $\frac{\overline{\mathbf{U}_{\mathbf{i}}^{* 2}}}{\overline{\mathbf{U}^{* 2}}}=\frac{(1-\mathbf{b})^{2}}{\mathbf{a} \mathbf{T}^{*} \mathbf{L} / \mathbf{U}_{\mathbf{0}}+1}$ & (11) \\
\hline Abrahamson & $\begin{array}{c}\text { St }>>1 \text {, } \\
\text { Accelerative } \\
\text { mechanism only }\end{array}$ & $\overline{\left(\mathbf{U}_{\mathbf{i}}^{* 2}\right)_{\mathbf{a b s}}}=\frac{\overline{\mathbf{U}^{* 2}}}{1+1.5 \tau_{\mathbf{i}}^{*} \varepsilon^{*} / \overline{\mathbf{U}^{2}}}$ & (12) \\
\hline $\begin{array}{l}\text { Williams and } \\
\text { Crane }\end{array}$ & $\begin{array}{c}\text { St }>>1 \text {, } \\
\text { Accelerative } \\
\text { mechanism only }\end{array}$ & 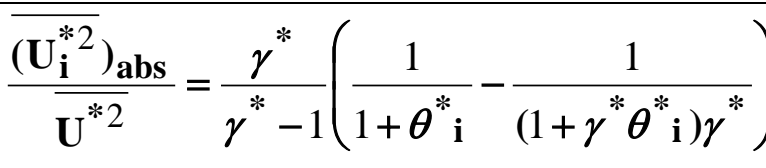 & (13) \\
\hline $\begin{array}{l}\text { Lee and } \\
\text { Erickson. }\end{array}$ & Bubbles & $\left.\overline{\left(\mathbf{U}_{\mathbf{i}}^{* 2}\right.}\right)_{\mathbf{a b s}}=2\left(\mathbf{d}_{12}^{*} \boldsymbol{\varepsilon}^{*}\right)^{2 / 3}$ & (14) \\
\hline
\end{tabular}


measured bubble RMS (13\% and 12\% mean errors, respectively). The model developed by Lee et al. provided the poorest estimates of the bubble RMS with a mean error of at least $350 \%$.

\section{RESULTS OBTAINED WITH RUSHTON TANK}

The same experimental instrumentation was employed to conduct measurements in the Rushton tank. Figure 4.1 (a)-(f) shows instantaneous vorticity contours superimposed with stream traces over one complete blade cycle for $\mathrm{Re}=20000$. The time elapsed between frames is $1 / 250 \mathrm{sec}$, corresponding to an angular displacement of a blade equal to 10.0 degrees. This corresponds to about 36 frames per impeller revolution for the smallest Reynolds number. In these figures the passage of the blade is evident in terms of the large velocity magnitudes that persist for the entire sequence. Figure 4.1 (a) is the start of the cycles when one of the impeller blades is parallel to the laser sheet. The general flow pattern is a radial jet with a pair of tip vortices. In Figure 4.1 (f), a new set of tip vortices are generated, and the old pair that was generated in Figure 4.1 (a) is still convecting outward. Apparently the flow is sustained for a certain period after the blade has cut through the plane of interrogation. The 2-D vortices shown are actually a vortex ring extending outward, most likely with a strong out of plane velocity component.

Turbulence is also evident in the distribution of the time-averaged results shown in Figures 4.2 and 4.3 for the extreme two Reynolds numbers, 20000 and 50000 . The results were averaged over 500 velocity fields, or 1000 frames. The vorticity distribution in Figures 4.2 (a) and 4.3 (a) clearly shows the dominant role the tip vortices have as they convect all the way out, until the end of the interrogation region. The distribution of the diagonal component of the Reynolds stresses, and the $u_{r}$ RMS clearly show unsteady fluctuations in the velocity.

Figures 4.4 through 8 describe the power spectrum of the $\mathrm{u}_{\mathrm{z}}$ velocity for two Reynolds numbers, 20000 and 40000 . Figure 5 shows a line at $r / \mathrm{D}_{\mathrm{imp}}=0.56$ and three points. A series of power spectra were calculated along this line and are shown as contour plots in Figures 4.5 and 4.6. A total of 153 power spectra were performed to generate each of the two plots. The normalized Power Spectral Density is shown as a function of $\mathrm{z}$ in the impeller stream and the normalized (with the blade passing) frequency. The relative maxima at a normalized frequency of 1 in both contour plots indicate a very sharp spectrum. Also, notice Figures 6 and 7 contain two relative maxima in the impeller stream, precisely where the tip vortices pass by. The center of the impeller stream, which corresponds to the $\mathrm{z} / \mathrm{D}_{\text {imp }}=0$, does not contain high power at the blade frequency. Three of the 153 power spectra are taken out of Figure 4.5 and displayed as line plots in Figure 4.7 (a)-(c). These are located at the three points shown in Figure 4.4.

Figures 4.8-4.12 show how some selected turbulence statistics scale with the Reynolds number. We show dissipation contours in the impeller stream for $\mathrm{Re}=30000$ in Figure 4.8, and a corresponding rectangular region where a maximum and a mean value were determined. Turbulent dissipation rate is a very important quantity directly connected with turbulent mixing. A variety of methods are available for the calculation of turbulent dissipation rate. We chose a direct method from the strain rate tensor, based on the assumption of isotropy. 

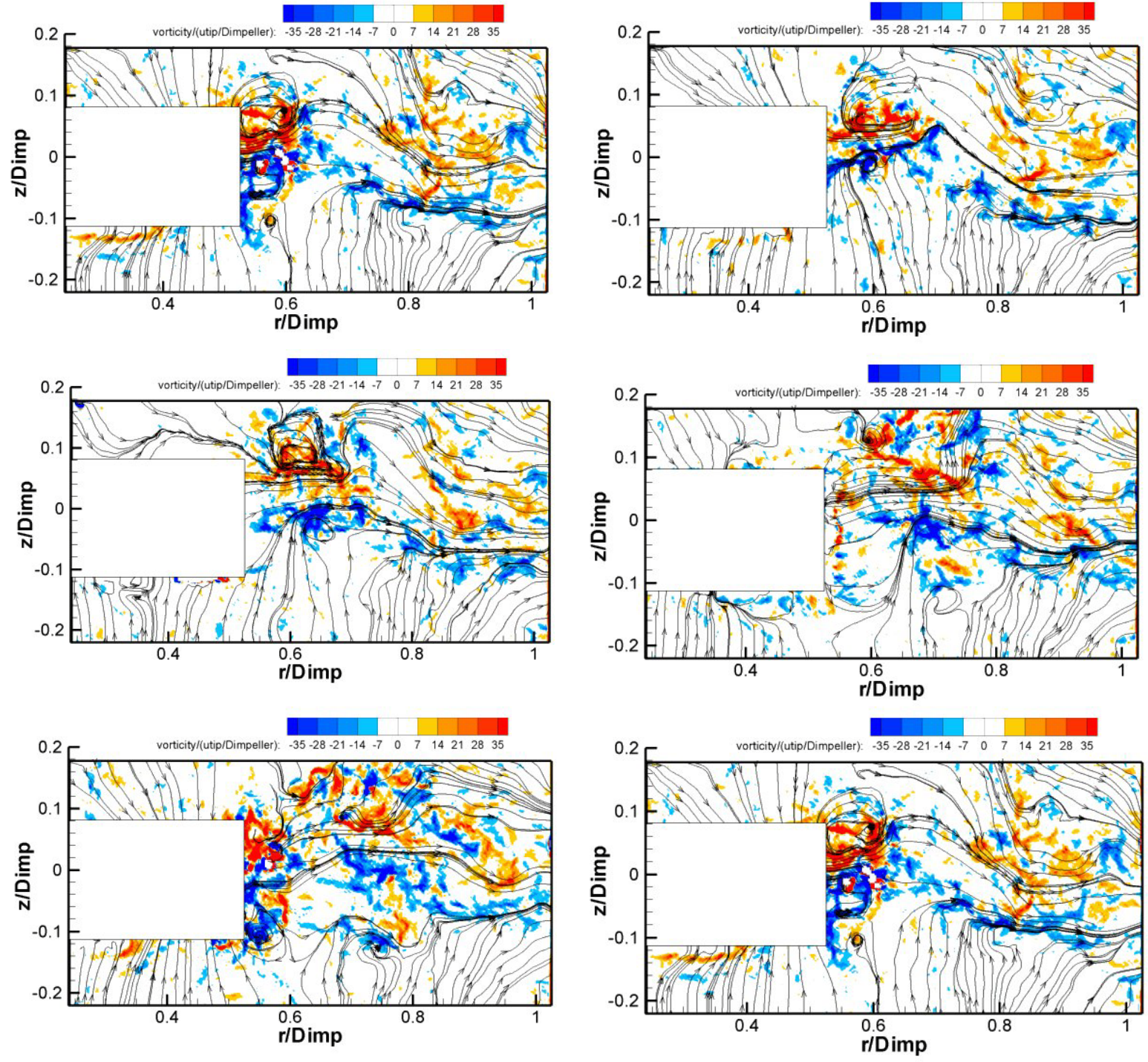

Figure 4.1(a)-(f) left to right, top to bottom. Instantaneous vorticity contours with streamtraces over one blade cycle. $\Delta t=1 / 250 \mathrm{sec}$

$$
\varepsilon=15 v\left(\overline{\left(\frac{\partial \mathrm{u}_{\mathrm{r}}}{\partial \mathrm{x}_{\mathrm{r}}}\right)^{2}}\right)
$$

Figures 4.11 and 4.12 show how maximum and mean dissipation in the impeller stream scale with Reynolds number. The dissipation is normalized with $\mathrm{N}^{3} \mathrm{D}^{2}$, which is consistent with previous publications. A general decreasing trend is observed for the normalized dissipation with increasing Reynolds number. Excellent agreement with a previous study was obtained (Baldi and Yianneskis 2004). Other quantities, namely vorticity magnitude, Urms and Vrms appear to follow constant trends in the range of Reynolds numbers investigated here. 

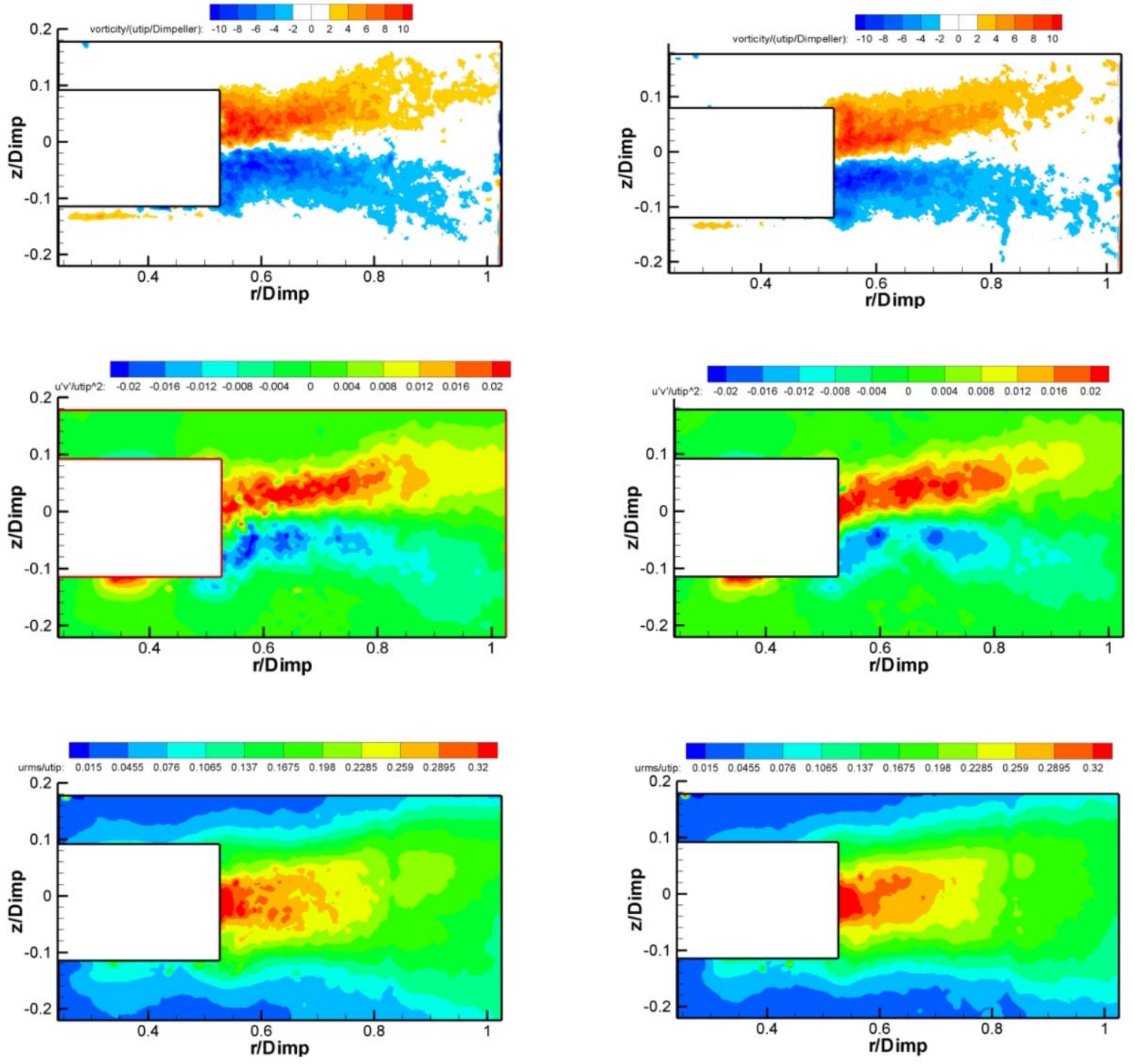

Figure 4.2 (a), (b), (c). $\operatorname{Re}=20000$, time average. top to bottom: vorticity, $u_{r}$ ' $u_{z}$ ' Reynolds stress and $u_{r}$ RMS. all normalized with $u_{t i p}$ and Dimpeller

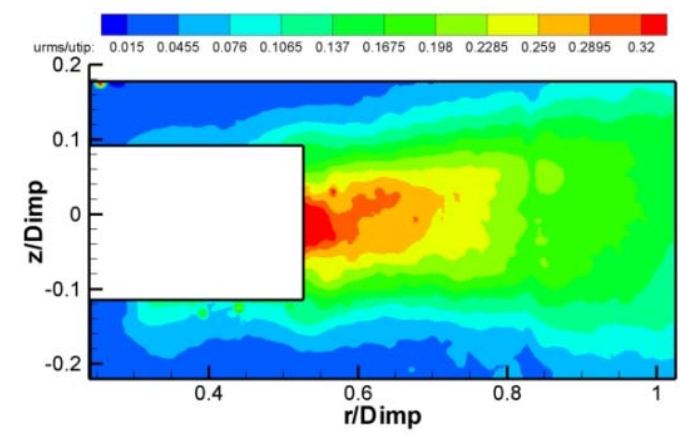

Figure 4.3 (a), (b), (c). $R e=50000$, time average. top to bottom: vorticity, $u_{\mathrm{r}}$ ' $\mathrm{u}_{\mathrm{z}}$ ' Reynolds stress and $u_{r}$ RMS. all normalized with $u_{\text {tip }}$ and Dimpeller 


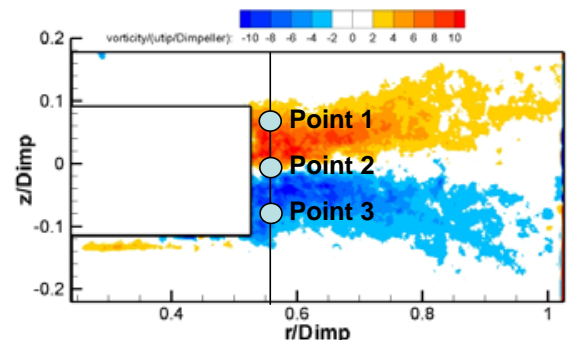

Figure 4.4. Schematic showing locations where Power Spectrums were taken. Line located at $r / D_{\text {imp }}=0.56$

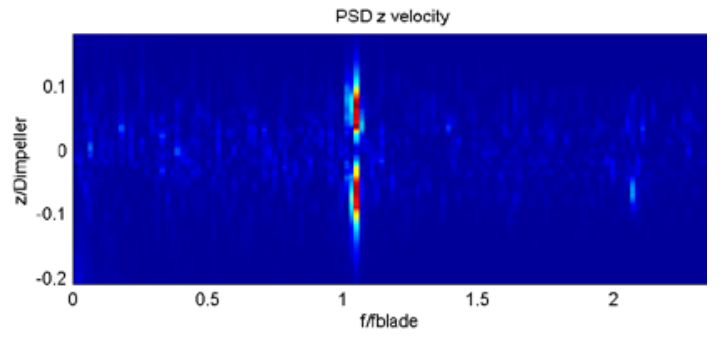

Figure 4.5 Contours of Power Spectrum of $u_{z}, R e=20,000$. frequency normalized with blade frequency

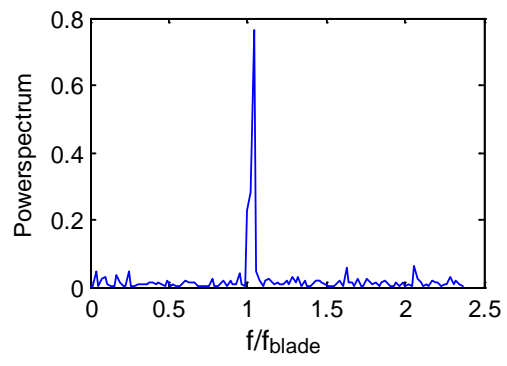

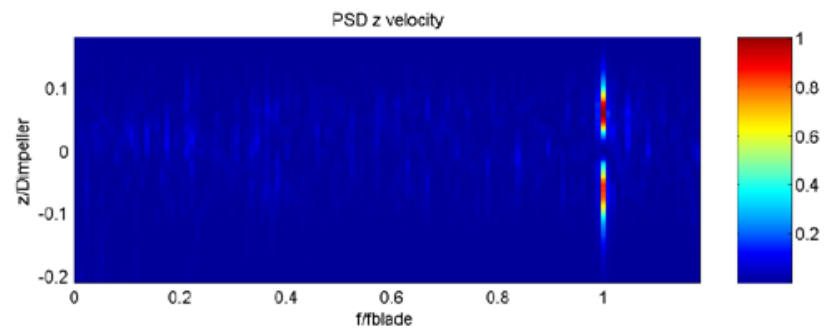

Figure 4.6. Contours of Power Spectrum of $\mathbf{u}_{z}$, $R e=40,000$. frequency normalized with blade frequency
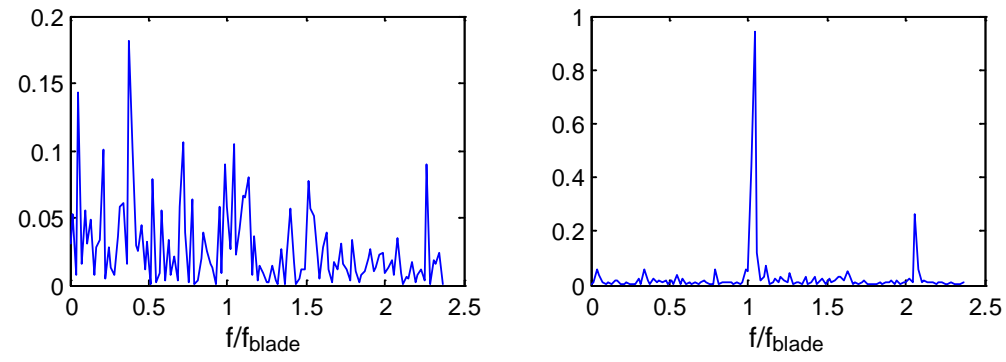

Figure 4.7 (a), (b), (c) left to right. Power Spectrum of $u_{z}$, for $R e=20,000$. (a) corresponds to point 1 in Figure 25, (b) to point 2, and 


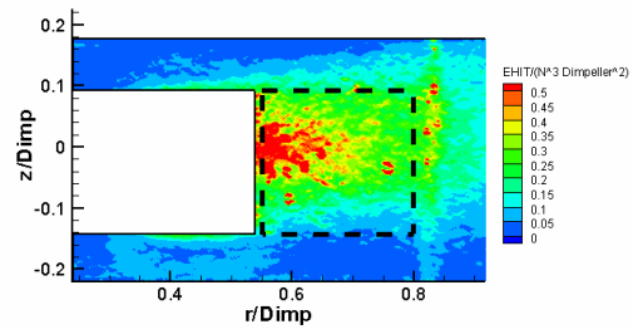

Figure 4.8. Dissipation contours in the impeller stream for $R e=30000$.

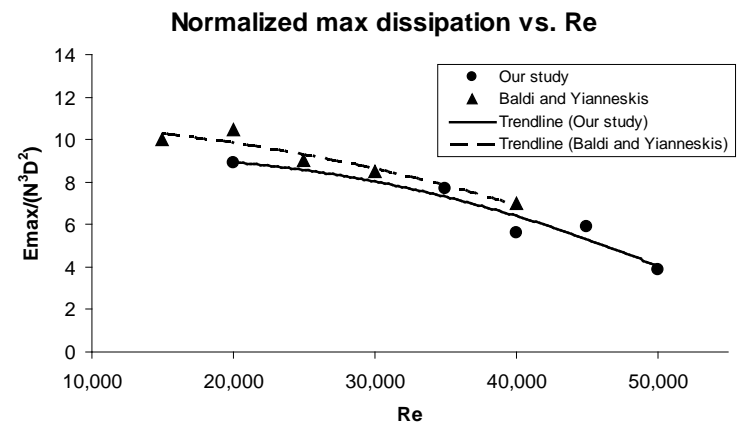

Figure 4.9 Normalized max dissipation vs. Re in dashed region of Figure 28

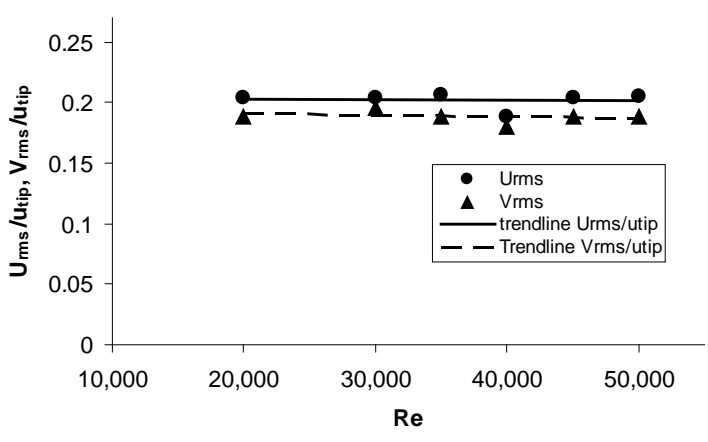

Figure 4.11 Normalized mean Urms and Vrms vs. Re in dashed region of Figure 28

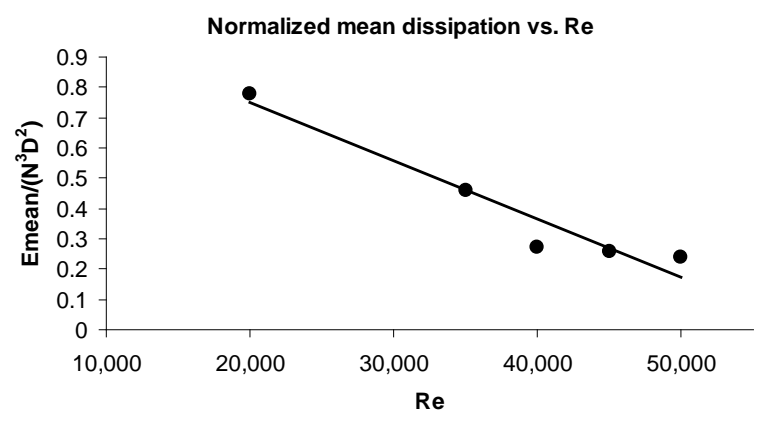

Figure 4.10. Normalized mean dissipation vs. $R e$ in dashed region of figure 28

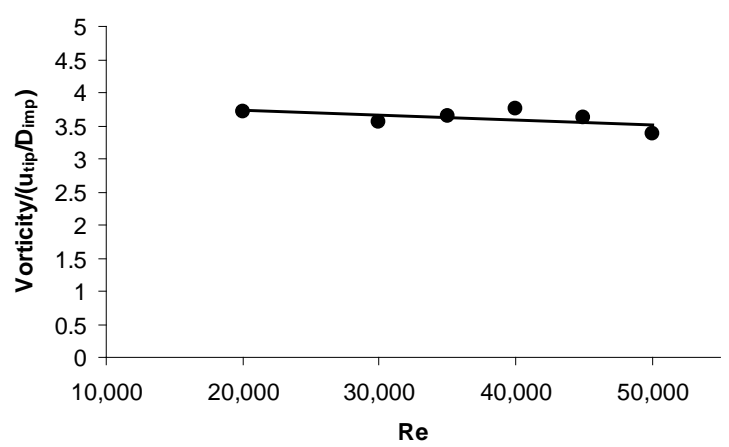

Figure 4.12 Normalized mean Vorticity magnitude vs. Re in dashed region of Figure

\section{CONCLUSIONS}

A range of turbulence characteristics, including isotropic turbulence was created in a model flotation cell through turbulence grid. The turbulent dissipation rate was measured using a cross-correlation-based Particle Image Velocimeter. Particle Tracking Velocimetry was used to determine the velocity fluctuations of the solid particles and the bubbles relative to the fluid phase. RMS velocities of particles and bubbles were calculated and compared to experimental and theoretical models that are based on the turbulent dissipation rate. The particle RMS slip velocity was in good agreement with Liepe and Möckel's empirical model, demonstrating 12\% mean error. The theoretical model, derived by Levins and Glastonbury was found to be in good agreement with the 
bubble RMS, but substantially underpredicted the RMS of the glass particles. In addition, the theoretical models derived by Abrahamson, and Williams and Crane provided good and almost identical agreement in predicting the RMS of the bubble velocity, but did not do as well in predicting particle fluctuations.

It has been known that dissipation is larger in the neighborhood of the agitating mechanism, in our case the Rushton impeller. This is somewhat anti-intuitive, because energy is dissipated at the smallest eddy scales, and the immediate vicinity of the impeller contains large vortical structures and provides little space or time for such structures to break down. We provide further evidence that larger dissipation values in the vicinity of the impeller is consistent with the dynamic motion generated by the blade passage. The flow in the impeller stream of a Rushton impeller can be best summarized as a radial jet with a pair of tip vortices. The maximum and mean normalized dissipation in the impeller stream showed decreasing trends with the Reynolds number. Other normalized turbulence quantities, namely $\mathrm{U}_{\mathrm{rms}}, \mathrm{V}_{\mathrm{rms}}$ and vorticity magnitude scaled as constant values with the Reynolds number. Estimates of turbulence characteristics and in particular distributions of turbulent energy dissipation determined in this study can be used in estimating rates of collisions of bubbles and particles in flotation cells.

\section{REFERENCES}

Abiven, C, Vlachos, P P, Papadopoulos, G, 2002. DPIV Strategies for resolving high shear and vortical flows, ASME Int. Mech. Eng. Congress 2002, Nov. 17-22, 2002, New Orleans, LA.

Abiven, C Vlachos, P P, 2002. Super spatio-temporal resolution, digital PIV system for multi-phase flows with phase differentiation and simultaneous shape and size quantification, Int. Mech. Eng. Congress, Nov. 17-22, 2002, New Orleans, LA

Abiven, C, Vlachos, P P, 2002. Comparative study of established DPIV algorithms for planar velocity measurements ASME IMECE, IMECE2002-33170.

Abrahamson, J, 1975. Collision rates of small particles in a vigorously turbulent fluid, Chem Eng Sci, 30: 1371-1379.

Adrian, R J, 1991. Particle-imaging techniques for experimental fluid mechanics. ARFM, 23: 261-304.

Adrian, R J, 1997. Dynamic ranges of velocity and spatial resolution of particle image velocimetry. Measurement Science and Technology, 8: 1393-1398.

Adrian, R J, 1991: Particle-imaging techniques for experimental fluid mechanics. ARFM, 23, 261-304.

Adrian, R J, 1997: Dynamic ranges of velocity and spatial resolution of particle image velocimetry, Measurement Science and Technology, 8 1393-1398. 
Adrian, R J, Yao, C-S, 1985: Pulsed laser technique application to liquid and gaseous flows and the scattering power of seed materials, App Optics, 24, 44-52.

Baldi, S, Yianneskis, M, 2004, "On the quantification of energy dissipation in the impeller stream of a stirred vessel from fluctuating velocity gradient measurements" Chem. Eng. Sci., 59, pp. 2659-2671.

Brady, M, Telionis, D P, Sherrell, I, Yoon, R H and Vlachos, P P, 2004 Turbulent Bubble-Particle Interactions Measured by Particle Image Velocimetry, Society of Mining Engineer 2004s, no. 04:193, February, 2004

Boedec, T, Simoens, S, 2001: Instantaneous and simultaneous planar velocity field measurements of two phases for turbulent mixing of high pressure sprays. Experiments in Fluids, 31, 506-518.

Costes, J, Couderc, J P, 1988. Study by laser Doppler anemometry of the turbulent flow induced by a Rushton turbine in a stirred tank: influence of the size of the units-1. Mean flow and turbulence, Chemical Engineering Science, 43:2751-2764.

Cowen, B, Monismith, S, 1997: A hybrid digital particle tracking velocimetry technique. Experiments in Fluids, 22, 199-211.

Fan, J, Rao, Q, Wang, Y, Fei, W, 2004. "Spatio-Temporal analysis of macro-instability in a stirred vessel via digital particle image velocimetry (DPIV) Chemical Engineering Science 59 (2004) pp.1863-1873.

Grant, I. 1994. Selected papers on particle image velocimetry. SPIE Milestone Series MS99, SPIE Optical Engineering Press, Bellingham, Washington.

Grant, I. 1997. Particle image velocimetry: A review. Proc., Institute of Mechanical Engineers.

Guezennec, Y G, Kiritsis, N, 1990. Statistical investigation of errors in particle image velocimetry. Experiments in Fluids, 10, 138-146.

Hasselinc, L. (1988): Digital image processing in flow visualization. ARFM 20:421-485.

Hinze, J O. 1994 Turbulence. (McGraw-Hill, Inc: New York:).

Höfken, M, Schäfer, M, Durst, F, 1996. Detaillierte Untersuchung des Strömungsfeldes innerhalb eines Sechs-Blatt-Scheibenrührers, Chemie Ingenieur Technik, 68:803-809.

Huang, H T,Dabiri, D, Gharib, M, 1997. On Errors of digital particle image velocimetry. Meas. Science and Technology, 8: 1427-1440. 
Huang, H T, Gharib, M, 1997: Processing error in digital particle image velocimetry, FEDSM97-3068.

Huang, H T, Dabiri, D, Gharib, M 1997: On Errors of digital particle image velocimetry, Meas. Science and Technology, .8, 1427-1440.

Jenne, M, Reuss, M, 1999. “A critical assessment on the use of $k$-e turbulence models for simulation of the turbulent liquid flow induced by a Rushton-turbine in baffled stirredtank reactors”, Chemical Engineering Science 54 (1999) pp.3921-3941.

Khalitov, D A, Longmire, E K, 2002: Simultaneous two-phase PIV by two-parameter phase discrimination, Experiments in Fluids, 32, 252-268.

Kresta, A M and Wood, P E, 1993. The flow field produced by a pitched blade turbine: characterization of the turbulence and estimation of the dissipation rate, Chemical Engineering Science, 48:1761-1774.

La Fontaine, R F, Shepherd, I C, 1996. "Particle Image Velocimetry Applied to a Stirred Vessel ”, Experimental Thermal and Fluid Science (1996) 12, pp. 256-264.

Lamberto, D J, Muzzio, F J, Swanson, PD, Tonkovich, A L, 1996 “Using TimeDependent RPM to Enhance Mixing in Stirred Vessels”, Chem. Engng. Sci., 51, 1996, 733-741.

Laufhütte, H D, Mersmann, A, 1985. Proceedings of $5^{\text {th }}$ European Conference on Mixing, 331 (BHRA Fluid Eng., Cranfield, England).

Lecordier, B, Trinite, M, 1999: Time resolved PIV measurements for high speed flows, Third International Workshop on Particle Image Velocimetry. (Santa Barbara, 16-18 Sept 1999).

Lee, C A, Erickson, L E. Bubble breakup and coalescence in turbulent gas-liquid dispersions, Chemical eng. comm. 59(1-6), 65-84.

Levins, B E, Glastonbury, J R, 1972. Trans. Inst. Chem. Engr., 50, 32, 132.

Levins, D M, Glastonbury, J R, 1972. Particle-liquid hydrodynamics and mass transfer in a stirred vessel, Part I-particle-liquid vessel, Trans. Inst. Chem. Engrs., 40:32-41.

Liepe, F und Möckel Hans-Otto, 1976, Untersuchungen zum Stoffvereinigen in Flüssiger Phase, Chem. Techn., 28, Jg., Heft 4.

Montantea, G, Leeb, K C, Brucatoa, A, Yianneskis, M, 2001. "Numerical simulations of the dependency of flow pattern on impeller clearance in stirred vessels" Chemical Engineering Science 56 (2001) pp.3751-3770. 
Mununga, L, Hourigan, K, Thompson, M, 2001. "Comparative study of flow in a mixing vessel stirred by a solid disk and a four bladed impeller"14th Australasian Fluid Mechanics Conference Adelaide University, Adelaide, Australia 10-14 December 2001.

Okamoto, Y, Nishikawa, N, Hashimoto, K, 1981. Energy dissipation rate distribution in mixing vessels and its effects on liquid-liquid dispersion and solid-liquid mass transfer, International Chemical Engineering, 21:88-94.

Piirto M., Saarenrinne P., Eloranta H., Karvinen R. 2003 Measuring Turbulence Energy with PIV in a Backward-facing Step Flow. Experiments in Fluids, 35, pp. 219-236.

Ralston, J, Fornasiero, D, Grano, S, Duan, J and Akroyd, T, 2005. Flotation rate constant prediction for metal sulfide particles. Centenary of Flotation 2005 Symposium.

Rao, M A, Brodkey, R S, 1972. Continuous flow stirred tank turbulence parameters in the impeller stream, Chemical Engineering Science, 27:137-156.

Saarenrinne P., Piirto M. 2000. Turbulent Kinetic Energy Dissipation Rate Estimation from PIV Velocity Vector Fields. Experiments in Fluids, [Suppl.], pp. S300-307.

Saffman, PG, Turner, J S, 1956. On the collision of drops in turbulent clouds. Journal of Fluid Mechanics, 1, 16-30.

Scarano, F, Rieuthmuller, M L, 1999. Iterative multigrid approach in PIV image processing with discrete window offset. Experiments in Fluids, 26: 513-523.

Scarano, F, Rieuthmuller, M L, 1999: Iterative multigrid approach in PIV image processing with discrete window offset, Experiments in Fluids, 26, 513-523.

Sheng, J, Meng, H and Fox, R O, 2000. A large eddy PIV method for turbulence dissipation rate estimation. Chemical Engineering Science, 55: 4423-4434.

Sherrell, I, Yoon, R H, 2005. Development of a Turbulent Flotation Model, Centenary of Flotation 2005 Symposium.

Sheng J., Meng H., Fox R.O. 2000 A Large Eddy PIV Method for Turbulence Dissipation Rate Estimation. Chemical Engineering Science, 55, pp. 4423-4434.

Sheng, J, Meng, H, Fox, R O, 2000. A large eddy PIV method for turbulence dissipation rate estimation, Chemical Engineering Science, 55:4423-4434.

Schubert, H., (1999) On the Turbulence-Controlled Microprocesses in Flotation Machines. Int. J. Miner. Process. 56: 257-276. 
Upatnieks, A, Laberteaux, K, Ceccio, S L, 2002:A kilohertz frame rate cinematographic PIV system for laboratory-scale turbulent and unsteady flows, Experiments in Fluids, 32, 87-98.

Vlachos, P, 2000: A spatio-temporal analysis of separated flows over bluff bodies using quantitative flow visualization. PhD. Dissertation, Department of Engineering Science and Mechanics, Virginia Polytechnic Institute and State University, Blacksburg, VA.

Wereley, S T, Meinhart, C D, 2001: Second-order accurate particle image velocimetry. Experiments in Fluids, 31, 258-268.

Westerweel, J, 1993a. Digital Particle Image Velocimetry, Theory and Application, Delft University Press, Delft, the Netherlands.

Westerweel, J, 1993b. Analysis of PIV interrogation with low pixel resolution. In Optical diagnostics in fluid and thermal flow, (ed) Trolinger J. D., Proc SPIE Vol. 2005, pp. 624635.(San Diego July 1993).

Whybrew, A, Reeves, M, Slagle, R L, Boaler, J J, Baughan, A K, Nicholls, T R, Towers, D P, Tavender B and Buckberry, C H,1999. Two techniques for all-digital time-resolved PIV. Third International Workshop on Particle Image Velocimetry. (Santa Barbara, 1618 Sept 1999).

Willert, C E and Gharib, M, 1991. Digital particle image velocimetry. Experiments in Fluids, 10: 181-193.

Williams, J J E and Crane, R I, 1983. Particle collision rate in turbulent flow. Int. J. Multiphase Flow, 9, No. 4: 421-435.

Wu, H and Patterson, G K, 1989. Laser-Doppler measurements of turbulent-flow parameters in a stirred mixer, Chemical Engineering Science, 44:2207-2221.

Yoon, H S, Sharp, K V, Hillc, D F, Adrian, R J, Balachandar, S, Haa, M Y, Kard, K, 2001. "Integrated experimental and computational approach to simulation of flow in a stirred tank” Chemical Engineering Science 56 pp.6635-6649.

\section{PUBLICATIONS/PRESENTATIONS}

1. “Turbulent Particle Bubble Interactions Measured by Particle Image Velocimetry”, by Mike Brady, Demetri Telionis, Pavlos Vlachos, Ian Sherrell and Roe-Hoan Yoon. Presented at the SME Conference. Denver Colorado, February 2004.

2. "Velocities of Particles and Bubbles in Grid Turbulence Measured by Particle Image Velocimetry”. By Mike Brady, Demetri Telionis, Pavlos Vlachos and Roe-Hoan Yoon, presented the Centenary of Flotation 2005 Symposium, Brisbane, Australia, June 2005. 
3. "Stirring Tank Estimates of Turbulent Energy Dissipation”. By Mike Brady, Demetri Telionis, Pavlos Vlachos and Roe-Hoan Yoon. presented at the First IC-EpsMso (First International Conference on Experiments/Process/System Modeling/Simulation/Optimization) Athens, July 2005.

4. "Evaluation of Multiphase Flotation Models in Grid Turbulence via Particle Image Velocimetry”, by Mike Brady, Demetri Telionis, Pavlos Vlachos and Roe-Hoan Yoon, International Journal of Mineral Processing, Vol. 80, pp. 133-143, 2006. 
Appendix 10: Engineering Development of a Fine Particle Heavy Medium Separator (VA012) 


\section{TECHNICAL REPORT}

Contract Title and Number:

Continuation of Crosscutting Technology

Development at CAST

(DE-FC26-02NT42457)
Period of Performance:

Starting Date: $6 / 1 / 05$

Ending Date: $\quad 10 / 31 / 09$ $\underline{\text { Sub-Recipient Project Title: }}$

Engineering Development of a Fine Particle Dense

Medium Separator

Principal Investigators:

R. Bratton, G.H. Luttrell and R.-H. Yoon

Contact Address:

146 Holden Hall

Virginia Tech

Blacksburg, VA 24061

Subcontractor Address:

No subcontracts issued.
Report Information:

Type: Final

Number:

Period:

Date: $\quad$ 23/6/2010

Code: VA012-FINAL

Contact Information:

Phone: (540) 231-4508

Fax: $\quad$ (540) 231-3948

E-Mail:1uttrell@vt.edu

Subcontractor Information:

Phone:

Fax:

E-Mail: 


\section{DISCLAIMER}

"This report was prepared as an account of work sponsored by an agency of the United States Government. Neither the United States Government nor any agency thereof, nor any of their employees, make any warranty, express or implied, nor assume any legal liability or responsibility for the accuracy, completeness, or usefulness of any information, apparatus, product, or process disclosed, or represents that its use would not infringe privately owned rights. Reference herein to any specific commercial product, process, or service by trade name, trademark, manufacturer, or otherwise does not necessarily constitute or imply endorsement, recommendation, or favoring by the United States Government or any agency thereof. The views and opinions of authors expressed herein do not necessarily state or reflect those of the United States Government or agency thereof." 


\begin{abstract}
The objective of this project was to develop an innovative dense medium separator that can reduce the cost and improve the efficiency of fine coal cleaning. The technology is designed to replace inefficient water-based separators such as spirals and water-only cyclones that are currently used by industry to upgrade $1 \times 0.15 \mathrm{~mm}$ run-of-mine coal. The new dense medium separator incorporates novel design features that allow it make sharp separations without the need for costly micronized magnetite. Also, the new technology can share circulating medium from other circuits, thereby avoiding the expense of installing and maintaining an additional medium circuit. These advantages, together with the low headroom design of the module, make it possible to integrate this technology into an existing plant with minimal retrofit costs. The project tasks included (i) design and construction, (ii) pilot-scale testing, (iii) field testing, and (iv) flowsheet development.
\end{abstract}




\section{TABLE OF CONTENTS}

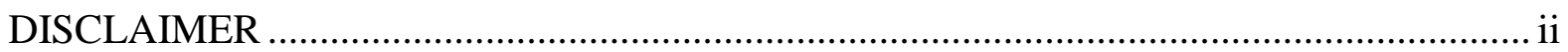

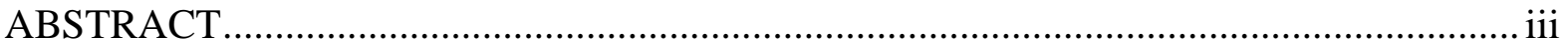

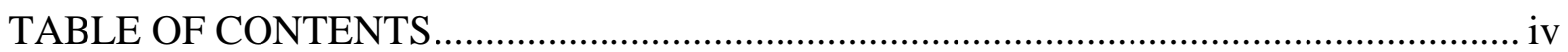

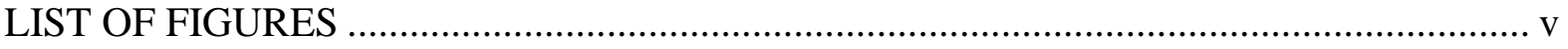

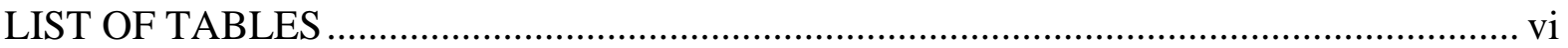

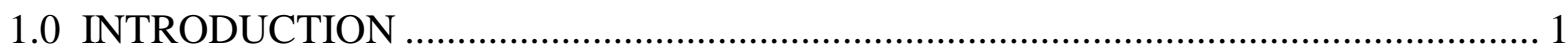

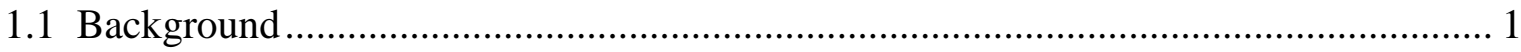

1.2 Objective and Approach ………………………............................................. 3

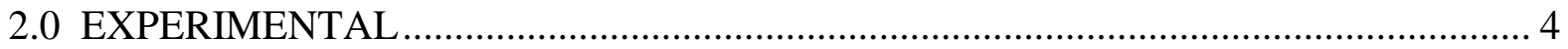

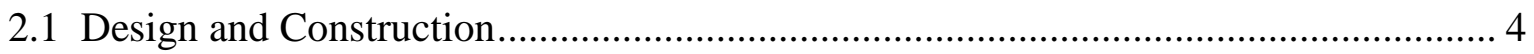

2.2 Pilot-Scale Testing …………………………………...................................... 5

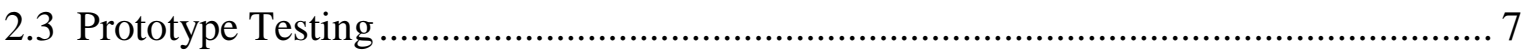

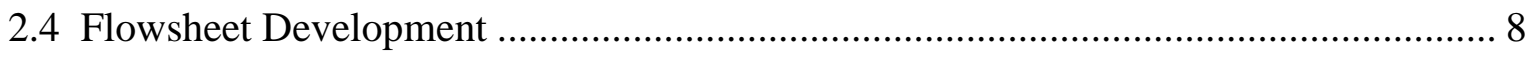

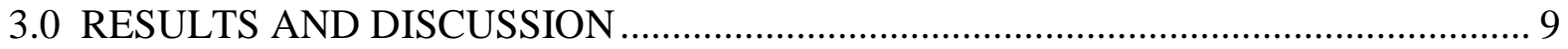

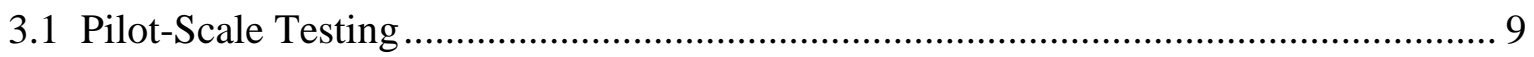

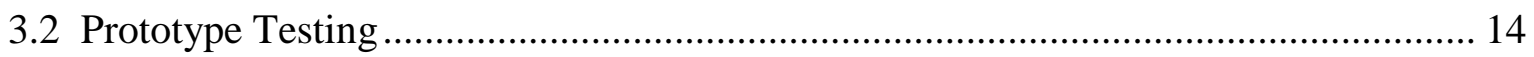

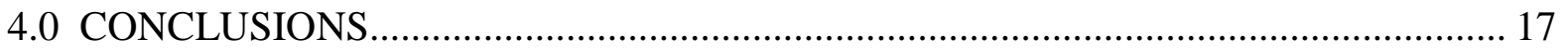

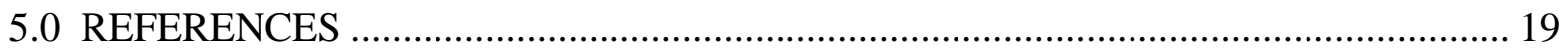




\section{LIST OF FIGURES}

Figure 1. Retrofit flowsheet for the new fine coal dense medium circuit............................ 3

Figure 2. Layout for the pilot-scale test circuit........................................................... 4

Figure 3. Unit operating on water with stable air core. ............................................... 6

Figure 4. Fine coal separator performance. ............................................................. 14 


\section{LIST OF TABLES}

Table 1 - Particle size distribution at 1.60 SG with water/magnetite medium..................... 10

Table 2 - Particle size distribution and ash content for the third experimental work. .......... 11

Table 3 - Particle size distribution and ash content for the fourth experimental work......... 13

Table 4 - Fine coal separator performance............................................................... 13

Table 5. Prototype unit flow characterization tests................................................. 15 


\subsection{INTRODUCTION}

\section{$\underline{1.1 \text { Background }}$}

Dense medium cyclones (DMCs) have been used for more than 50 years by the mining industry to upgrade a wide variety of raw materials including coal, magnesite, dolomite, diamonds, potash, and lead-zinc ores. These inexpensive, high-capacity units utilize centrifugal forces to enhance the separation of smaller particles that cannot be efficiently treated using static density-based separators (e.g., dense medium vessels and jigs). In the U.S. coal industry, dense medium cyclones are used in nearly $80 \%$ of all coal preparation plants and represent a total installed capacity of nearly 80,000 ton/hr of feed coal. Most of these units treat feed coals in the 50 x $0.5 \mathrm{~mm}$ size range (Wood, 1997). More recently, there is renewed interest in using dense medium cyclones to treat finer coal feeds (i.e., down to $0.15 \mathrm{~mm}$ ) to take advantage of yield gains that may be realized by replacing inefficient water-based separators.

Data reported in the literature indicate that a properly configured dense medium cyclone circuit can provide Ep values that are 3-4 times better than can be achieved using water-based separators such as spirals or water-only cyclones (Moorhead, 1999). Unfortunately, the use of dense medium cyclones to upgrade fine coal has failed to be widely accepted by industry since existing designs of dense medium cyclones require the use of ultrafine (micronized) magnetite in order to efficiently separate fine coal. The ultrafine magnetite is needed to prevent coal losses associated with an internal buildup of middlings that occur due to excessive medium segregation (Wood, 1990). The milling infrastructure required to generate sufficient tonnages of ultrafine magnetite at an acceptable cost is also currently unavailable. Production reports also indicate that the consumption of ultrafine 
magnetite is unacceptably high due to the limited effectiveness of conventional magnetic separators in recovering micronized particles. These difficulties have combined to make the use of dense medium cyclones impractical for treating coal feeds finer than $0.5 \mathrm{~mm}$ (Robertson et al., 1997).

Cyclone designers at Krebs Engineers have been working to develop a new dense medium separator that can be used to effectively treat fine coal. The proprietary technology incorporates novel design features that allow it make sharp separations without the need for costly micronized magnetite. As a result, the new technology can share circulating medium from other circuits, thereby avoiding the expense of installing and maintaining an additional medium circuit. These advantages, together with the low headroom design of the module, make it possible to integrate this technology into an existing plant with minimal retrofit costs.

A conceptual illustration of the proposed circuitry for the new technology is shown in Figure 1. During operation, feed medium is injected into the unit along with the fine $(1 \times 0.15$ $\mathrm{mm}$ ) run-of-mine coal. The clean coal product is taken as overflow from the unit, while refuse is taken as underflow. The product streams are then passed through two different magnetic separators to recover the clean coal and refuse solids from the magnetite medium prior to dewatering of the products. The proposed configuration makes the new circuit very cost effective since the magnetite needed for operation can be "robbed" from other dense medium circuits that are already in service within the plant. Preliminary calculations show that only a small portion of the underflow from a typical plus $0.5 \mathrm{~mm}$ dense medium cyclone circuit would be more than adequate to supply the coarse medium necessary to operate the new separator. The sharing of medium avoids the costs of installing and operating an independent medium circuit for the new separator. In addition, the headroom and floor space 


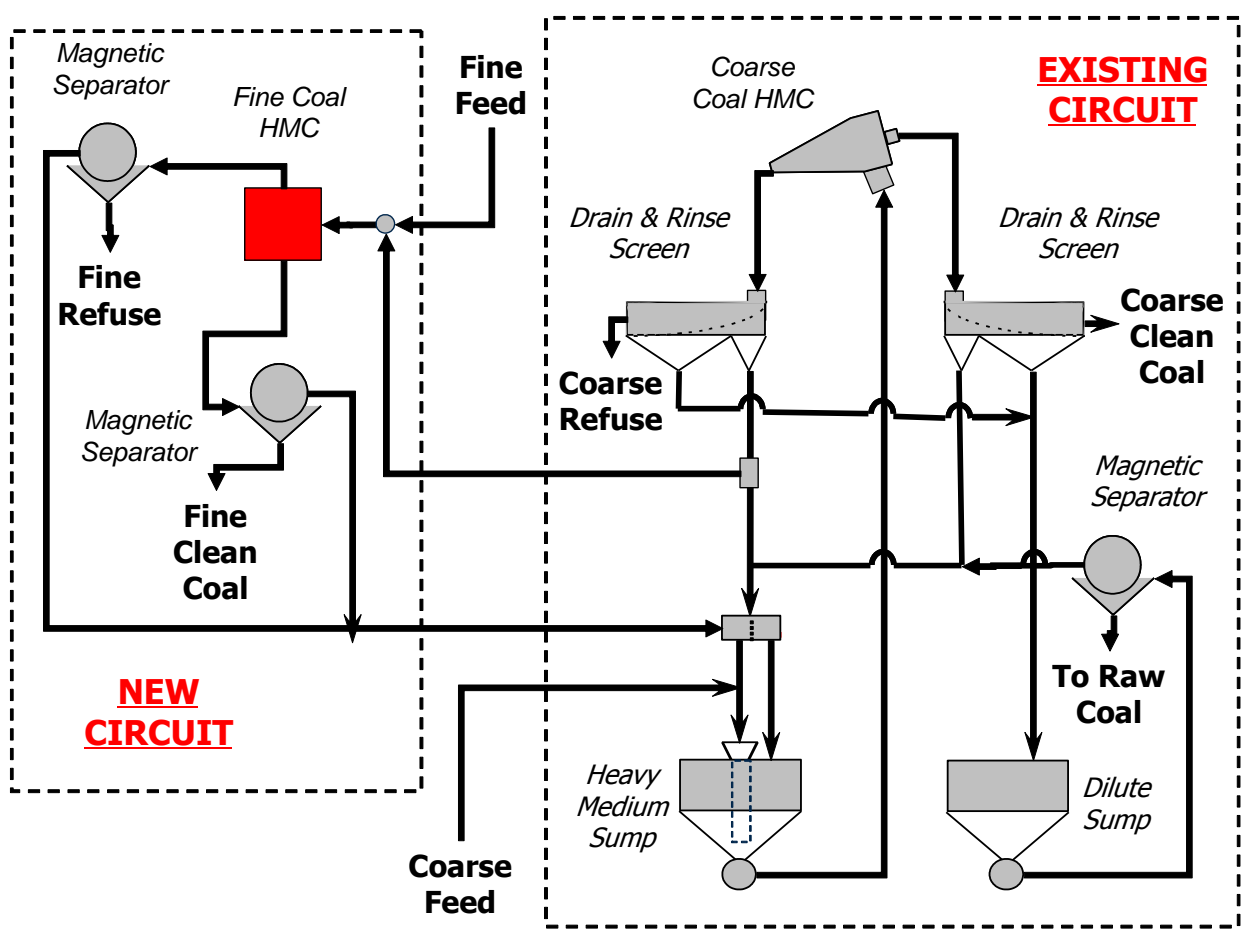

Figure 1. Retrofit flowsheet for the new fine coal dense medium circuit.

requirements for the new module are expected to be similar in size to those required by an existing spiral circuit. Thus, this technology could be easily retrofit into an existing plant flowsheet without major renovations to the facility.

\subsection{Objective and Approach}

The primary objective of this project was to complete the engineering development of an innovative dense medium separator that can reduce the cost and improve the efficiency of fine coal cleaning. The technology is designed to replace inefficient water-based separators such as spirals and water-only cyclones that are currently used by industry to upgrade $1 \mathrm{x}$ $0.15 \mathrm{~mm}$ run-of-mine coal. The proposed work included (i) design and construction, (ii) pilot-scale testing, (iii) field testing, and (iv) flowsheet development. 


\subsection{EXPERIMENTAL}

\section{$\underline{2.1 \text { Design and Construction }}$}

The work conducted under this task accomplished (i) the assembly of the prototype 6inch diameter heavy medium separator using components designed and largely provided by Krebs Engineers and (ii) the construction of the closed-loop test circuitry required to evaluate the separator under controlled pilot-scale conditions. The components to assemble the separator and the slurry pump equipment were donated by Krebs Engineers and Morris Coker Equipment, respectively, as cost-sharing contributions to the project.

A schematic of the pilot-scale test circuit constructed for this project is given in Figure 2. The circuitry includes a 240 gallon feed medium sump, a variable-flow centrifugal feed pump, associated control valves and proportional sample cutters. During operation, feed slurry and appropriate medium is circulated by the pump into the fine particle heavy medium

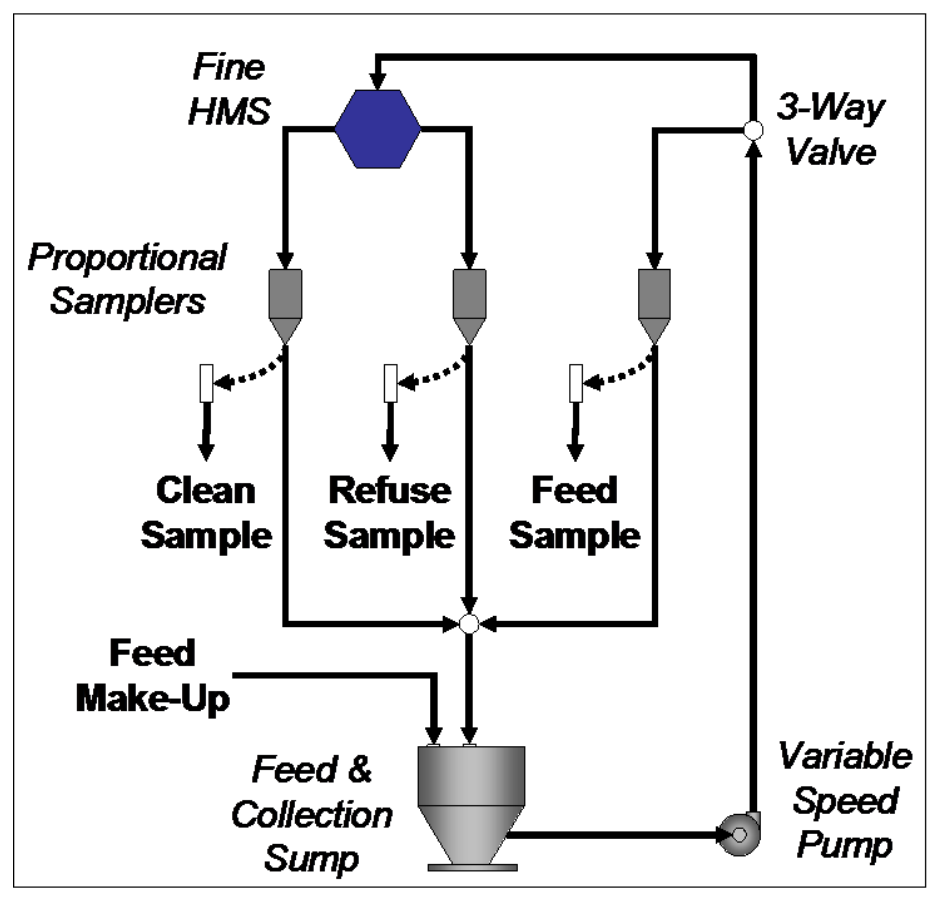

Figure 2. Layout for the pilot-scale test circuit. 
separator. A three-way valve is used to divert the entire feed through a proportional sampler so that a representative sample of the feed stream can be collected at the beginning and end of each test run. The two products from the separator are passed through similar proportional samples so that independent clean coal and refuse samples can be obtained in equal proportions. The slurry flows that bypass the proportional samplers are directed back to the feed/collection sump so they can be circulated back through the circuitry. The circuit is equipped with instruments so that pressure and flow can be monitored on-line. The specific gravity of the medium was determined with a density cup scale.

\subsection{Pilot-Scale Testing}

\subsubsection{Series 1 Testing}

Following the fabrication and assembly of the single-stage separator and the set up of the closed-loop test circuit, the first experimental test work was conducted using water as the medium to evaluate the ability of the unit to accommodate the projected fluid flow and to maintain a stable air core within the unit at the recommended feed flow rate and pressure. The unit operating on water with a stable air core is pictured in Figure 3. The testing with water also indicated the flow split between the overflow and underflow streams could be controlled by varying the back pressure on the underflow stream.

\subsubsection{Series 2 Testing}

To conduct the second experimental test work, the feed/collection sump was charged with water and magnetite to form a medium density at 1.50 specific gravity (SG). The volumetric flow split between the overflow and underflow streams was calculated using the measured feed, overflow, and underflow densities. The same measurements were taken with 


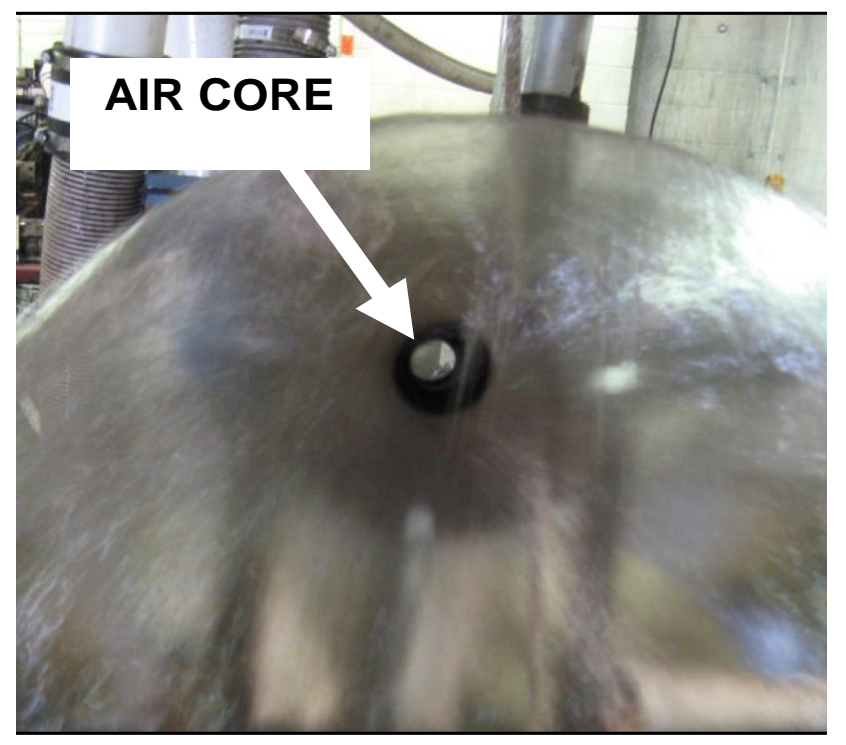

Figure 3. Unit operating on water with stable air core.

the feed density at 1.60 SG and 1.70 SG. Fine particle size analysis was conducted on a set of samples collected during the tests to evaluate the distribution, by size, of the magnetite to the overflow and the underflow streams.

\subsubsection{Series 3 Testing}

Following a review of the favorable and encouraging results of the previous experimental testing, the third experimental work was conducted to evaluate the separating performance and determine the operating characteristics with a raw coal feed to the cyclone. The feed for the experimental work was intended to be a typical spiral separator raw coal feed, $1 \times 0.15 \mathrm{~mm}$, but the actual material received for the feed was a clean coal product with an ash content of $6.9 \%$. The circulating medium density in the closed-loop system was established at 1.63 SG. Before the coal feed was introduced to the unit, the overflow and underflow stream densities were measure at $1.52 \mathrm{SG}$ and $1.92 \mathrm{SG}$, respectively, resulting in a medium flow split of $73 \%$ (volume basis) reporting to the overflow. 


\subsubsection{Series 4 Testing}

The fourth experimental work was conducted to specifically evaluate the unit's capability for a high-density separation. The circulating medium density in the testing system was established at $1.88 \mathrm{SG}$. The medium feed was adjusted to provide an operating inlet pressure equivalent to about nine heads of medium $(258 \mathrm{~cm})$. Before the raw coal feed was introduced to the unit, the overflow and underflow stream densities were measured at 1.76 SG and 2.04 SG, respectively, resulting in a medium flow split of 57\% (volume basis) reporting to the overflow. A typical spiral separator raw coal feed with $58.2 \%$ ash content was obtained for this experimental work.

\subsection{Prototype Testing}

In light of the successful pilot-scale tests conducted at Virginia Tech, a three-stage prototype unit was designed, fabricated and constructed for additional experimental testing of the proposed technology. Unfortunately, the volumetric flow requirements for the prototype unit were beyond the capabilities of the pilot-scale circuitry available at Virginia Tech. Therefore, testing for the three-stage unit was moved to the field where the necessary pumping equipment could be accessed.

The initial testing of the prototype unit focused on the determination of the relationship between back pressure and volumetric flow for each stage in the unit. This data was used to establish flow-capacity curves based on the inlet and outlet pressures for each stage. 


\section{$\underline{2.4 \text { Flowsheet Development }}$}

The development of a preliminary design for a full-scale fine coal dense medium separator depends on established flow-capacity curves for the separating unit. Work to establish the curves for a new separation unit requires a full suite of controlled prototype testing to determine the flow characteristics for the expected range of operating pressure and separator fittings (inlets, vortex finders, apexes) configuration. This test work continues to be conducted by Krebs Engineers. 


\subsection{RESULTS AND DISCUSSION}

\section{$\underline{3.1 \text { Pilot-Scale Testing }}$}

\subsubsection{Series 1 Testing}

The first experimental test work was conducted using water as the medium to evaluate the ability of the unit to accommodate the projected fluid flow and to maintain a stable air core within the unit. The results from the tests using water indicated the unit will accept the expected feed rate at the recommended feed pressure of nine times the diameter of the unit or $137 \mathrm{~cm}$ (54 in) of water head. The first test also verified that (i) a stable air core could be maintained within the unit at the recommended feed flow rate and pressure (Figure 3) and (ii) the flow split between the overflow and underflow streams could be controlled by varying the back pressure on the underflow stream.

\subsubsection{Series 2 Testing}

To conduct the second experimental test work, the feed/collection sump was charged with water and magnetite to form a medium density at $1.50 \mathrm{SG}$. The results of the test with the medium at $1.50 \mathrm{SG}$ and with the recommended feed pressure indicated that the unit would operate under stable conditions. The volumetric flow split between the overflow and underflow streams was calculated using the feed, overflow, and underflow densities. The calculated value correlated very well with the volumetric flows measured for the overflow and underflow streams. The same measurements were taken with the feed density at $1.60 \mathrm{SG}$ and 1.70 SG.

Fine particle size analysis was conducted on a set samples collected during the tests to evaluate the distribution, by size, of the magnetite to the overflow and the underflow. Table 1 presents the data for the size distribution of the streams for the medium density of 1.60 SG. 
Table 1 - Particle size distribution at 1.60 SG with water/magnetite medium.

\begin{tabular}{ccccccc}
\hline $\begin{array}{c}\text { Particle Size } \\
\text { (micron) }\end{array}$ & $\begin{array}{c}\text { Feed (wt \%) } \\
\text { Individual }\end{array}$ & $\begin{array}{c}\text { Overflow (wt \%) } \\
\text { Cumulative }\end{array}$ & $\begin{array}{c}\text { Underflow (wt \%) } \\
\text { Individual }\end{array}$ & $\begin{array}{c}\text { Cumulative } \\
\text { Individual }\end{array}$ & Cumulative \\
\hline+88 & 5.07 & 5.07 & 2.31 & 2.31 & 6.66 & 6.66 \\
$88 \times 44$ & 24.79 & 29.86 & 16.85 & 19.16 & 33.84 & 40.50 \\
$44 \times 22$ & 34.22 & 64.08 & 36.75 & 55.91 & 31.76 & 72.26 \\
$22 \times 11$ & 18.13 & 82.21 & 21.65 & 77.56 & 13.83 & 86.09 \\
$11 \times 5.5$ & 11.75 & 93.96 & 14.80 & 92.36 & 8.81 & 94.90 \\
-5.5 & 6.04 & 100.00 & 7.64 & 100.00 & 5.10 & 100.00 \\
\hline
\end{tabular}

As expected the underflow medium stream exhibited a coarser size distribution than the overflow medium stream.

\subsubsection{Series 3 Testing}

Following a review of the favorable and encouraging results of the previous experimental testing, the third experimental work was conducted to evaluate the separating performance and determine the operating characteristics with a coal feed to the cyclone. The feed for the experimental work was intended to be a typical spiral separator raw coal feed, 1 $\mathrm{x} 0.15 \mathrm{~mm}$, but the actual material received for the feed was a clean coal product with an ash content of $6.9 \%$.

The circulating medium density in the closed-loop system was established at 1.63 SG. Before the coal feed was introduced to the unit, the overflow stream density was $1.52 \mathrm{SG}$ and the underflow stream density was $1.92 \mathrm{SG}$, resulting in a medium flow split of $73 \%$ (volume basis) reporting to the overflow.

Initially, it was feared that the low ash feed material would nullify the test, but subsequent analyses of the data indicated a very favorable indication of a gravity separation 
at the established circulating medium density. Table 2 presents the size distribution and ash content for the products in the overflow and underflow streams, and the calculated reconstituted feed stream. Because the source of the feed was spiral separator clean coal, which had previously been effectively cleaned to a nominal size of 70 mesh, the overflow ash content for all particles larger than 70 mesh was less than $6 \%$ and the underflow ash content for all particles larger than 70 mesh was less than 19\%. This indicates that the separator did in fact concentrate low-density +70 mesh particles in the overflow stream. The underflow ash content for this same size was also low because the spiral separator had effectively removed the high-density refuse in the commercial cleaning process.

One exceedingly important result to note, however, is that the unit effectively produced a $6.7 \%$ ash content in the overflow stream for the 70 mesh x 100 mesh size fraction while simultaneously producing a $42.4 \%$ ash content in the underflow stream for that same size fraction. This indicates that the unit is capable of effectively separating particles as small as 100 mesh. Based on conventional knowledge of dense-medium separation practices, it was anticipated that the unit would merely split the feed for particles smaller than 60-70 mesh due the relatively coarse-sized magnetite used in for the experimental work. This

Table 2 - Particle size distribution and ash content for the third experimental work.

\begin{tabular}{|c|c|c|c|c|c|c|c|c|c|c|c|c|}
\hline \multirow[b]{2}{*}{ Size } & \multicolumn{4}{|c|}{ Cyclone Overflow } & \multicolumn{4}{|c|}{ Cyclone Underflow } & \multicolumn{4}{|c|}{ Reconstituted Feed } \\
\hline & $\begin{array}{r}\text { Indi } \\
\text { Wt }(\%) \\
\end{array}$ & $\begin{array}{l}\text { vidual } \\
\text { Ash (\%) }\end{array}$ & $\begin{array}{r}\text { Cumu } \\
\mathrm{Wt}(\%) \\
\end{array}$ & $\begin{array}{l}\text { ulative } \\
\text { Ash (\%) }\end{array}$ & $\begin{array}{r}\text { Indi } \\
\mathrm{Wt}(\%) \\
\end{array}$ & $\begin{array}{l}\text { ividual } \\
\text { ) Ash (\%) }\end{array}$ & $\begin{array}{r}\text { Cumı } \\
\text { ) Wt (\%) } \\
\end{array}$ & $\begin{array}{l}\text { lulative } \\
\text { Ash (\%) }\end{array}$ & $\begin{array}{r}\text { Indi } \\
\text { Wt }(\%) \\
\end{array}$ & $\begin{array}{l}\text { ividual } \\
\text { ) Ash (\%) }\end{array}$ & $\begin{array}{r}\text { Cum } \\
\mathrm{Wt}(\%) \\
\end{array}$ & $\begin{array}{l}\text { ulative } \\
\text { Ash (\%) }\end{array}$ \\
\hline+16 mesh & 12.64 & 5.15 & 12.64 & 5.15 & 7.29 & 13.84 & 7.29 & 13.84 & 11.62 & 6.20 & 11.62 & 6.20 \\
\hline 16 mesh x 40 mesh & 64.61 & 4.90 & 77.26 & 4.94 & 64.92 & 10.76 & 72.21 & 11.07 & 64.67 & 6.03 & 76.29 & 6.06 \\
\hline 40 mesh x 70 mesh & 22.25 & 5.43 & 99.51 & 5.05 & 26.62 & 18.96 & 98.84 & 13.20 & 23.09 & 8.43 & 99.38 & 6.61 \\
\hline 70 mesh x 100 mesh & 0.32 & 6.74 & 99.82 & 5.06 & 0.53 & 42.40 & 99.37 & 13.35 & 0.36 & 16.93 & 99.73 & 6.65 \\
\hline-100 mesh & 0.18 & 69.78 & 100.00 & 5.17 & 0.63 & 95.73 & 100.00 & 13.87 & 0.27 & 81.68 & 100.00 & 6.85 \\
\hline
\end{tabular}


finding contradicts some presently held concepts regarding dense-medium separations.

\subsubsection{Series 4 Testing}

Although the results from the third experimental work were encouraging, the separating capability of the unit for a typical raw coal feed remained undetermined. Because the concept relies on a series of staged separations, the unit must be capable of performing progressive separations starting with a high-density separation in the first module. For this concept to be successful, the pilot-scale unit must demonstrate that an effective high-density separation can be achieved on a typical raw coal feed, which will produce an underflow of acceptably high ash content.

The fourth experimental test work was conducted to specifically evaluate the unit's capability for a high-density separation. The circulating medium density in the testing system was established at $1.88 \mathrm{SG}$ by adjusting the amounts of water and magnetite in the feed sump. The feed medium was adjusted until an operating inlet pressure was obtained equivalent to about nine heads of medium $(258 \mathrm{~cm})$. Before the raw coal feed was introduced to the unit, the overflow and underflow stream densities were $1.76 \mathrm{SG}$ and $2.04 \mathrm{SG}$, respectively, resulting in a medium flow split of $57 \%$ (volume basis) reporting to the overflow.

A typical spiral separator raw coal feed with $58.2 \%$ ash content was obtained for this experimental work. The size distribution and ash content for the products in the overflow and underflow streams, and the calculated reconstituted feed stream are presented in Table 3 . The results indicate that the unit is capable of producing a high ash content product in the underflow stream where the +70 mesh size fraction had an ash content of $88.5 \%$ and the 70 mesh x 100 mesh size fraction had an ash content of $80.3 \%$. Thus the first stage module in a 
Table 3 - Particle size distribution and ash content for the fourth experimental work.

\begin{tabular}{|c|c|c|c|c|c|c|c|c|c|c|c|c|}
\hline \multirow[b]{2}{*}{ Size } & \multicolumn{4}{|c|}{ Cyclone Overflow } & \multicolumn{4}{|c|}{ Cyclone Underflow } & \multicolumn{4}{|c|}{ Reconstituted Feed } \\
\hline & $\begin{array}{r}\text { Indi } \\
\text { Wt }(\%)\end{array}$ & $\begin{array}{l}\text { vidual } \\
\text { Ash (\%) }\end{array}$ & $\begin{array}{r}\text { Cum } \\
\text { Wt (\%) }\end{array}$ & $\begin{array}{l}\text { ulative } \\
\text { Ash (\%) }\end{array}$ & $\begin{array}{r}\text { Indi } \\
\text { Wt }(\%)\end{array}$ & $\begin{array}{l}\text { vidual } \\
\text { Ash (\%) }\end{array}$ & $\begin{array}{r}\text { Cum } \\
\text { Wt (\%) }\end{array}$ & $\begin{array}{l}\text { ulative } \\
\text { Ash (\%) }\end{array}$ & $\begin{array}{r}\text { Indi } \\
\text { Wt }(\%)\end{array}$ & $\begin{array}{l}\text { vidual } \\
\text { Ash (\%) }\end{array}$ & $\begin{array}{r}\text { Cum } \\
\text { Wt (\%) }\end{array}$ & $\begin{array}{l}\text { ulative } \\
\text { Ash (\%) }\end{array}$ \\
\hline+16 mesh & 17.31 & 42.02 & 17.31 & 42.02 & 7.29 & 88.41 & 7.29 & 88.41 & 14.97 & 47.30 & 14.97 & 47.30 \\
\hline 16 mesh x 40 mesh & 23.85 & 42.62 & 41.16 & 42.37 & 64.90 & 89.33 & 72.19 & 89.24 & 33.45 & 63.81 & 48.41 & 58.70 \\
\hline 40 mesh $\times 70$ mesh & 47.95 & 53.44 & 89.11 & 48.33 & 26.61 & 86.52 & 98.80 & 88.51 & 42.97 & 58.23 & 91.38 & 58.48 \\
\hline 70 mesh x 100 mesh & 9.88 & 53.47 & 99.00 & 48.84 & 0.53 & 80.30 & 99.33 & 88.46 & 7.70 & 53.90 & 99.07 & 58.13 \\
\hline-100 mesh & 1.00 & 59.43 & 100.00 & 48.95 & 0.67 & 84.09 & 100.00 & 88.43 & 0.93 & 63.60 & 100.00 & 58.18 \\
\hline
\end{tabular}

multi-stage unit would be capable of achieving an efficient high-density scalping-type separation which would produce a primary underflow stream with very low carbon loss.

Table 4 shows the size-by-size recovery and ash rejection obtained from the pilotscale test run conducted with the raw spiral feed. The data, which is also plotted in Figure 4 for comparison, show that the unit is capable of producing very high combustible recoveries $(>87 \%)$ for all size fractions treated. However, a high ash rejection of greater than $60 \%$ was only obtained for the 16 mesh x 40 mesh size class. Material outside this critical size range was separated with an extremely high combustible recovery exceeding 95\%, but no ash

Table 4 - Fine coal separator performance.

\begin{tabular}{ccccc}
\hline $\begin{array}{c}\text { Mean } \\
\text { Size } \\
(\mathbf{m m})\end{array}$ & $\begin{array}{c}\text { Mass } \\
\text { Yield } \\
(\boldsymbol{\%})\end{array}$ & $\begin{array}{c}\text { Combust } \\
\text { Recovery } \\
(\boldsymbol{\%})\end{array}$ & $\begin{array}{c}\text { Ash } \\
\text { Rejection } \\
(\boldsymbol{\%})\end{array}$ & $\begin{array}{c}\text { Separation } \\
\text { Efficiency } \\
(\boldsymbol{\%})\end{array}$ \\
\hline 1.414 & 88.62 & 97.50 & 21.27 & 18.77 \\
0.648 & 54.63 & 86.62 & 63.51 & 50.13 \\
0.297 & 85.52 & 95.33 & 21.51 & 16.84 \\
0.177 & 98.40 & 99.32 & 2.39 & 1.70 \\
0.081 & 83.09 & 92.61 & 22.36 & 14.97 \\
\hline Total & 76.62 & 93.53 & 35.53 & 29.07 \\
\hline
\end{tabular}




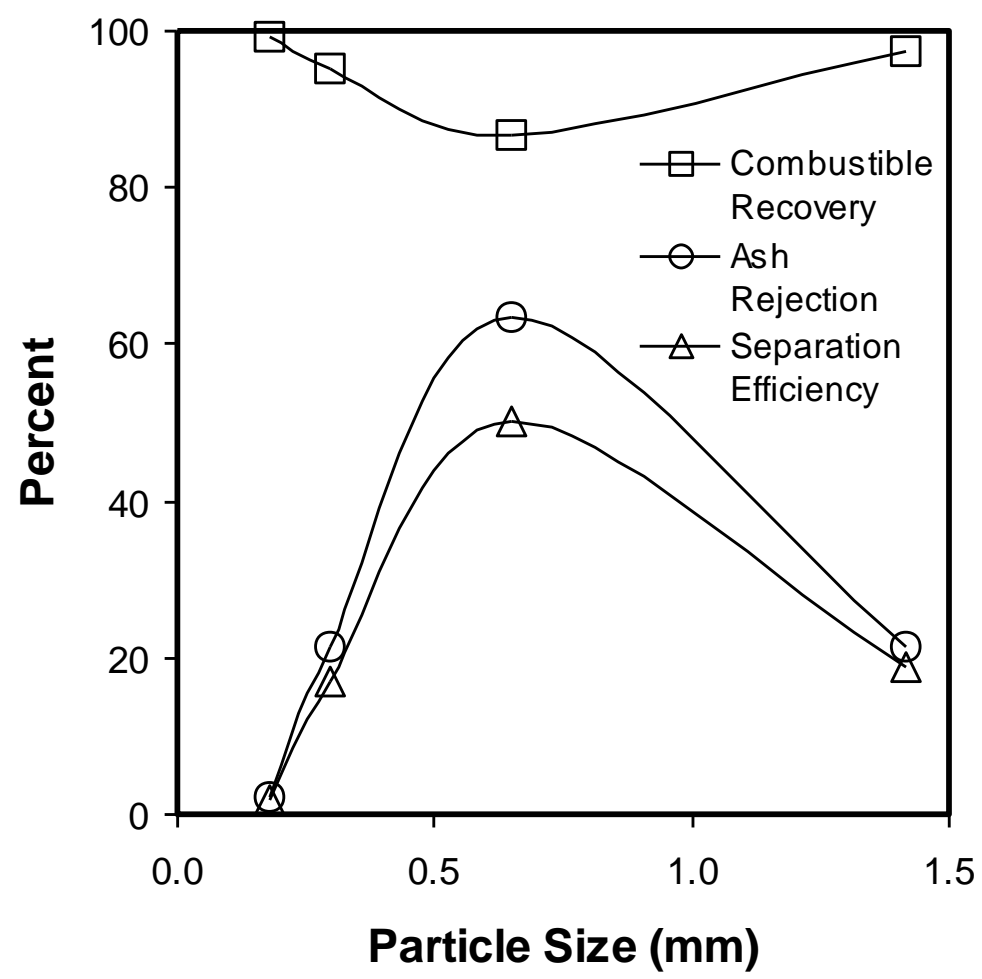

Figure 4. Fine coal separator performance.

rejection values greater than $21 \%$ were obtained. These findings indicate that a commercial unit would incorporate an initial stage of high-recovery "scalping" followed by several stages of high-rejection "cleaning" to improve the product grade.

The results obtained from the third and fourth experimental test work significantly demonstrated that (i) the unit is capable of producing a low-density separation without indications of inducing medium instability despite the use of coarse magnetite and (ii) the unit produced sufficient energy to provide a high-density separation as a scalping stage.

\subsection{Prototype Testing}

The four pilot-scale experimental tests above validated the concept of the design for a single stage unit. The next step required fabrication, assembly, and testing with a multi-stage unit. A three-stage prototype unit was fabricated for the next series of tests. The required 
fluid flows for the three-stage unit testing were beyond the capability of the equipment at the Virginia Tech Pilot Plant. The testing for the three-stage unit was moved to the Krebs Engineers research lab in Tucson, Arizona, which can provide the required pumping equipment and closed circuit capacity.

The primary objectives for the testing of the three-stage unit was to determine the influence of the back pressure at the reject (tails) outlet on the volumetric flow from the outlet and the effect on the flows around the unit as the individual outlet back pressure changes. All the testing to-date with the three-stage unit has used water as the medium.

The initial testing results were used to develop preliminary flow capacity curves and equations based on the standard inlet pressure and at the various reject outlet pressures. The three inlet pressures were set at the standard head pressure of nine times the cyclone diameter. Unfortunately, results from the first round of prototype testing were somewhat inconclusive. Table 5 presents the initial flow characterization measurements conducted at two feed inlet pressures. Tests A and B were conducted at $237 \mathrm{~cm}$ (54 in) water head and Tests C and D were conducted at $152 \mathrm{~cm}$ (60 in) water head. Although the flows were more balanced between the tails ports at the higher inlet pressure, the variability is beyond acceptable limits for stable operation in all three stages. Additional testing is currently

Table 5. Prototype unit flow characterization tests.

\begin{tabular}{ccccccc}
\hline & Inlet Pressure & \multicolumn{5}{c}{ Flow at Port (volume \%) } \\
\cline { 3 - 7 } Test & $(\mathbf{c m}$ water) & Tails 1 & Tails 2 & Tails 3 & Product & Total \\
\hline A & 137 & 23.1 & 14.1 & 15.5 & 47.3 & 100.0 \\
B & 137 & 22.3 & 15.2 & 15.8 & 46.7 & 100.0 \\
C & 152 & 19.8 & 20.5 & 23.4 & 36.3 & 100.0 \\
D & 152 & 19.3 & 19.9 & 22.5 & 38.2 & 100.0 \\
\hline
\end{tabular}


underway with tighter controls on the operating parameters.

Unfortunately, detailed information concerning the multi-stage unit (photos, circuit configuration, dimensions, etc.) and test data are unavailable at this time due to the proprietary nature of the innovative design of the unit and the anticipated application. 


\subsection{CONCLUSIONS}

The primary objective of this project was to complete the engineering development of an innovative dense medium separator that can reduce the cost and improve the efficiency of fine coal cleaning. The technology is designed to replace inefficient water-based separators such as spirals and water-only cyclones that are currently used by industry to upgrade $1 \mathrm{x}$ $0.15 \mathrm{~mm}$ run-of-mine coal. The project included design, fabrication and construction of a single-stage pilot-scale and the assembly of a three-stage prototype unit. The experimental test work included pilot-scale testing and field testing.

The results from the tests with only water indicated the unit will accept the expected feed flow rate at the recommended feed pressure of nine times the diameter of the unit or 137 cm (54 in) of water head. The first test also verified that (i) a stable air core could be maintained within the unit at the recommended feed flow rate and pressure and (ii) the flow split between the overflow and underflow streams could be controlled by varying the back pressure on the underflow stream.

Test work conducted with a magnetite and water medium verified the expected distribution, by size, of the magnetite to the overflow and the underflow with the underflow medium stream exhibiting a coarser size distribution than the overflow stream.

On a low ash content feed $(6.9 \%)$, the pilot-scale unit was capable of reducing the overflow ash content to $5 \%$ for the +70 mesh fraction. One exceedingly important result to note, however, is that the unit effectively produced a $6.7 \%$ ash content in the overflow stream for the 70 mesh x 100 mesh size fraction while simultaneously producing a $42.4 \%$ ash content in the underflow stream for that same size fraction. This indicates that the unit is capable of effectively separating particles as small as 100 mesh. This finding contradicts 
some presently held concepts regarding dense-medium separations. Also, this test work demonstrated that the unit is capable of producing a low-density separation without indications of inducing medium instability despite the use of coarse magnetite.

The results from the test work with typical spiral circuit raw coal feed (58\% ash) significantly demonstrated that the unit produced sufficient energy to provide a high-density separation as a scalping stage.

A three-stage prototype unit was fabricated for the field tests. The initial testing for the three-stage unit was moved to the Krebs Engineers research lab in Tucson, Arizona, to develop the flow-capacity curves required to install and test the unit in an operating processing plant. The development of the flow-capacity curves and other testing is presently being conducted by Krebs. 


\subsection{REFERENCES}

1. Wood, C.J., 1997. "Coal Preparation Expertise in Australia: In-Plant Issues and the Potential Impact of Broader Applications," Proceedings, Coal Prep '97, Lexington, Kentucky, pp. 179-198.

2. Moorhead, R., 1999. "Heavy Media Cyclones \& Classifying Cyclones in the Coal Industry," Unpublished Workshop Report, Workshop sponsored by Powell Construction Company at the Mountaineer Conference Center, Beckley, West Virginia, June 10-11, 1999.

3. Robertson, R., Placha, D., Terry, R., and Watters, L., 1997. "Recent Developments in Dense Medium Cyclone Circuit Design,” Proceedings, SME Annual Meeting, Denver, Colorado, February 24-27, 1997, Preprint No. 97-153, 6 pp. 
Appendix 11: Development of a Turbulent Flotation Model and a Computer Simulator (VA009) 


\section{TECHNICAL PROGRESS REPORT}

Contract Title and Number: $\quad$ Period of Performance:

Crosscutting Technology Development at the Center for Starting Date: 6/1/2005

Advanced Separation Technologies

Ending Date: 10/31/2007

(DE-FC26-02NT41607)

Sub-Recipient Project Title:

Development of a Turbulent Flotation Model and a

Computer Simulator

Principal Investigators:

Yoon, Luttrell

Contact Address:

Subcontractor Address:

No subcontracts issued.
Report Information:

Type: Final

Number: 3

Period: $\quad 10 / 1 / 06-3 / 31 / 07$

Date: $\quad 04 / 12 / 07$

Code: VA015-FINAL

Contact Information:

Phone:

Fax:

E-Mail: ryoon@vt.edu

Subcontractor Information:

Phone:

Fax:

E-Mail: 


\begin{abstract}
Mineral flotation is effective over a relatively narrow particle size range of approximately 10 to $100 \mu \mathrm{m}$. If the upper particle size limit can be increased, however, a run-of-mine ore may be coarse ground, and only the middlings are ground further to achieve required grades. This will result in significant energy savings and increased throughput. In this communication, methods of increasing the upper particle size limit will be discussed on the basis of a flotation model that can predict flotation from both hydrodynamic and surface chemical parameters. An example of increasing flotation recovery by control of a chemistry parameter is given.
\end{abstract}

\title{
Introduction
}

Flotation is an efficient and cost-effective method of upgrading run-of-mine (ROM) ores; therefore, it is widely used for processing various ores and coal. However, the process is efficient only at a relatively narrow particle size range, typically between 10 to $100 \mu \mathrm{m}$ for mineral flotation (Lynch et al., 1981) and 40 to $500 \mu \mathrm{m}$ for coal flotation. In coal industry, coal fines are generated during mining, crushing, and attrition (during agitation and transport), while in base metals industry an ROM ore is purposefully size-reduced to conform to the optimum particle size range noted above. Otherwise, coarse particles would drop off from air bubbles, and recovery would suffer. On the other hand, fine grinding is energy intensive and costly, particularly at a time of high energy prices.

The above situation is particularly the case for the copper industry in the U.S., where much of the ores mined and processed today are of low grades. It would be of interest if a low-grade copper ore, as an example, is floated at a coarser particle size, and only the middlings (or rougher concentrates) are ground further using regrind mills. This will minimize the tonnages of the gangue minerals to be finely ground and, thereby, save significant amounts of energy. Further, by allowing grinding mills to produce coarser products, one can increase the throughput, which by itself represents large energy savings. Further, increasing throughput entails shorter retention times in the mill, which should also help minimize generating ultrafine particles that are difficult to float.

Thus, extending the upper particle size limit of flotation would be of interest to the mining industry, particularly for the base metals industry, where processing low-grade and hard-to-grind ores are common. In this communication, we will describe a flotation model that can predict recoveries as functions of practically all of the process variables, including both the surface chemistry and hydrodynamic parameters. Because the model has been developed mostly from first principles, it has predictive capabilities. Also, the model identifies the key variables that can be controlled to maximize flotation recovery, particularly for coarse particles.

\section{Flotation model Development}

Yoon and Mao (1996) derived a first-order flotation rate equation based on first principles. It can predict flotation recovery from both hydrodynamic and surface chemistry parameters. However, the model can predict flotation under quiescent-flow conditions only and does not consider the events occurring in the froth phase of a flotation cell. Therefore, the model is not useful for predicting the performance of conventional flotation machines that are operating under turbulent flow conditions, with the froth phase playing an important role.

Abrahamson (1975) derived a collision model for turbulent flow conditions. The collision frequency, $\mathrm{Z}_{12}$, between particle $\mathbf{1}$ and $\mathbf{2}$ is given by the following relation:

$$
Z_{12}=-2^{3 / 2} \pi^{1 / 2} N_{1} N_{2}\left(r_{1}+r_{2}\right)^{2} \sqrt{\left(\bar{U}_{1}^{2}+\bar{U}_{2}^{2}\right)}
$$


in which $N_{1}$ the number of particle 1 per unit volume of the suspension, $N_{2}$ the same for particle 2 , and $r_{1}$ and $r_{2}$ the radii of the particles, and $\sqrt{\bar{U}_{1}^{2}}$ and $\sqrt{\bar{U}_{2}^{2}}$ are the RMS velocities of the particles. If $\mathbf{1}$ represents the mineral particles in a flotation cell and 2 represents air bubbles, Eq. [1] can serve as the flotation rate equation for the turbulent flow conditions, provided that all of the collisions result in successful flotation. Of course, this condition is not met in flotation. In fact, if it does, the process will be a failure, that is, all of the particles that collide with air bubbles, regardless whether they are hydrophilic or hydrophobic, will float, resulting in no separation.

Assuming that flotation is a first-order process, one can write the following relation:

$$
\frac{d N_{1}}{d t}=-k N_{1}=-Z_{12} P R_{f}
$$

where $t$ is the flotation time, $k$ the first-order rate constant; $P$ is the probability of bubbles and particles colliding and being attached to each other and thereby entering the froth phase; and $R_{\mathrm{f}}$ is the probability of the bubble-particle aggregates being transported successfully through the froth phase.

From Eqs. [1] and [2], one can readily derive an expression for the first-order flotation rate constant as follows:

$$
k=2^{3 / 2} \pi^{1 / 2} N_{2}\left(r_{1}+r_{2}\right)^{2} \times \sqrt{\left(\bar{U}_{1}^{2}+\bar{U}_{2}^{2}\right)} P_{c} P_{a}\left(1-P_{d}\right) R_{f}
$$

where $P$ is considered as a product of the subprocesses of collision $(P c)$, adhesion $\left(P_{a}\right)$ and detachment $\left(P_{d}\right)$.

In the present work, the RMS velocities were calculated using the following empirical relationships:

$$
\begin{aligned}
& \sqrt{\bar{U}_{1}^{2}}=0.4 \frac{\varepsilon^{4 / 9} d_{1}^{7 / 9}}{v^{1 / 3}}\left(\frac{\rho_{1}-\rho_{3}}{\rho_{3}}\right)^{2 / 3} \\
& \bar{U}_{2}^{2}=C_{0}\left(\varepsilon d_{2}\right)^{2 / 3}
\end{aligned}
$$

for particles (Liepe and Moeckel, 1976) and bubbles (Lee et al., 1987), respectively. In Eqs. [4] and [5], $\varepsilon$ is energy dissipation, $d_{1}$ and $d_{2}$ are particle and bubble diameters, $v$ kinematic viscosity, $\rho_{1}$ particle density, $\rho_{3}$ is liquid density, and $C_{0}$ $(=2.0)$ is a constant.

The probabilities of the three subprocesses introduced in Eq. [3] can be calculated using the following relations:

$$
\begin{aligned}
& P_{c}=\frac{3}{2}\left(\frac{d_{1}}{d_{2}}\right)^{2}\left[1+\frac{\frac{3}{16} \operatorname{Re}}{1+0.249 \operatorname{Re}^{0.56}}\right] \\
& P_{a}=\exp \left(-E_{1} / E_{k}\right) \\
& P_{d}=\exp \left(-\left(W_{a}+E_{1}\right) / E_{k}^{\prime}\right)
\end{aligned}
$$

as suggested previously (Luttrell and Yoon, 1992; Yoon and Mao, 1996). In using Eqs. [7] and [8], the values of the energy barriers $\left(E_{1}\right)$ were calculated using the extended DLVO theory (Yoon, 2000), while the kinetic energies for bubble-particle attachment were obtained by calculating the particle velocity at the critical distance $\left(H_{c}\right)$ at which $E_{1}$ is located. Eq. [7] suggests in effect that bubble-particle adhesion should occur when the kinetic energy of attachment $\left(E_{k}\right)$ exceeds $E_{1}$. In the present work, the values of $E_{k}$ were determined using the following equation:

$$
E_{k}=0.5 m_{1}\left(\sqrt{\bar{U}_{1}^{2}} / \beta\right)^{2}
$$

in which $m_{1}$ is the mass of the particle under consideration and $\beta$ is the parameter representing the hydrodynamic resistance to film thinning, which in turn can be obtained using the lubrication theory of Goren and O’Neil (1971). In calculating the energy barriers for detachment $\left(E_{k}^{\prime}\right)$, we used the velocities of bubble-particle aggregates that can be calculated from a 
momentum balance. It was assumed also that all of the air bubbles in a flotation cell are $50 \%$ covered by particles. In calculating $P_{d}$ using Eq. [8], we calculated the work of adhesion $\left(W_{a}\right)$ from contact angles as follows (Yoon and Mao, 1996):

$$
W_{a}=\gamma_{l v} \pi r_{1}^{2}(1-\cos \theta)^{2}
$$

where $\gamma_{1 v}$ is the surface tension of water and $\theta$ is the water contact angle of the particle of radius $r_{1}$.

In calculating the particle and bubble velocities using Eqs. [4-5], it was necessary to know the values of energy dissipation. We assumed that $80 \%$ of the energy input goes for generating air bubbles and slurry agitation and transport, and only $20 \%$ is available for bubble-particle collision and attachment. We found also that for bubble-particle detachment to occur, the bubble-particle aggregate velocity must be high. It was, therefore, assumed that the detachment occurs in the impeller zone of a flotation cell.

In froth phase, bubble size grows (or coarsens) with time or with increasing froth height, which entails a decrease in bubble surface area and hence particle detachment. Intuitively, the detachment probability should increase with decreasing particle hydrophobic and with increasing particle size. Also, some of the particles are recovered by entrainment rather than bubble-particle adhesion. By considering all of these phenomena, a froth recovery model has been developed to calculate $R_{f}$, (Do et al., 2006; Sherrel, 2004). In the present work, the froth recovery model developed by Do et al. has been employed $R_{f}$.

\section{Simulation Results}

Figure 1 shows the model predictions made for the flotation of chalcopyrite (S.G. $=4.1)$. The simulations were done at 1 $\mathrm{mm}$ bubble size $\left(d_{2}\right), 1.0 \mathrm{~W} / \mathrm{kg}$ energy dissipation, $30 \mathrm{~cm}$ froth height $\left(h_{\mathrm{f}}\right), 2 \mathrm{~cm} / \mathrm{s}$ superficial gas velocity $\left(V_{\mathrm{g}}\right), 60^{\circ}$ water

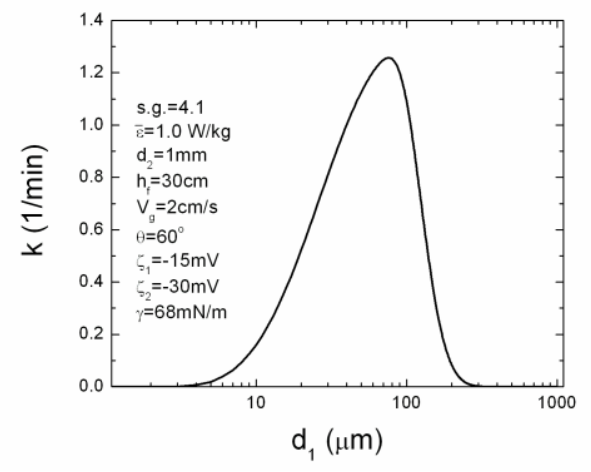

Figure. 1-A. The flotation rate constants $(k)$ vs. particle size $\left(d_{1}\right)$ as predicted from the model. It was assumed that the energy dissipations in the collection zone was $20 \%$ of the mean energy dissipation $(\bar{\varepsilon})$. The fractional recovery at the froth phase $\left(R_{F}\right)$ was obtained by assuming that bubbles grow in size by 20 times as they rise from the bottom to the top of the froth phase.

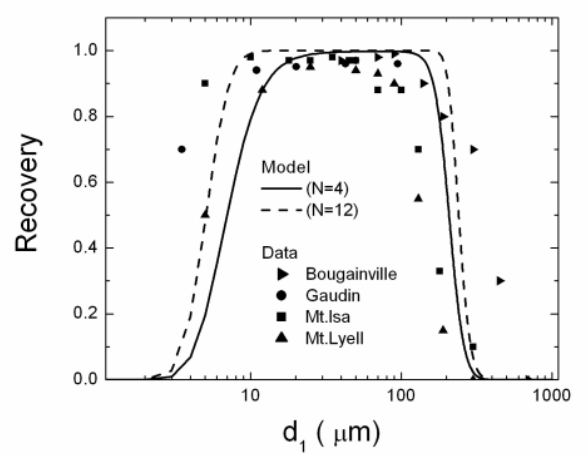

Figure. 1-B. Fractional recovery of chalcopyrite predicted using Eq. [11] from the flotation rate constants given in Figure 2-A. The data points represent the size-by-size recoveries obtained at operating plants as reported by Gaudin (1931) and Lynch et al. (1981). As these references did not specify the operational details, the predictions were made for four-cell bank $(\mathrm{N}=4)$ and twelve-cell banks $(\mathrm{N}=12)$ with 3-minute retention time in each cell.

contact angle $(\theta)$, and $-15 \mathrm{mV}$ particle zeta potential $\left(\zeta_{1}\right),-30 \mathrm{mV}$ bubble zeta-potential $\left(\zeta_{1}\right)$, and $68 \mathrm{mN} / \mathrm{m}$ surface tension of water. These may represent typical hydrodynamic and surface chemistry parameters that are employed in copper flotation. The predicted flotation rate constants are plotted $v s$. particle size in Figure 1-A. One can transform the $k v s$. $d_{1}$ plot into a fractional recovery $(R)$ vs. $d_{1}$ plot shown in Figure 1-B by using the well-known relation:

$$
R=1-(1+k t)^{-N}
$$


where $k$ is the flotation rate constant, $t$ is the retention time of particles in a flotation cell, and $N$ is the number of cells in a bank. The simulations were carried out with $N=4$ (solid line) and 12 (dotted line). Also shown in Figure 1-B are the plant operating data reported in the literature (Lynch et al., 1981, Gaudin et al., 1931. Trahar and Warren, 1976). As shown, the model predictions are in general agreement with the industrial flotation practice. One can see that copper flotation is difficult approximately below $10 \mu \mathrm{m}$ and above $150 \mu \mathrm{m}$.

Figure 2 shows the simulation results obtained by increasing energy dissipation at $1,1.5$ and $2.0 \mathrm{~mm}$ bubble sizes. At a given bubble size $(1 \mathrm{~mm})$, an increase in energy dissipation caused a significant increase in recovery, suggesting that high energy input helps flotation in general. Note that $R v s$. $d_{1}$ curves shift toward the left, indicating that the high-intensity flotation is particularly helpful for fine particle flotation. As bubble size is increased, the curves shifted toward the right, suggesting that large bubbles may help coarse particle flotation. But this is not a good strategy for coarse particle flotation, because the overall flotation rate is decreased significantly.

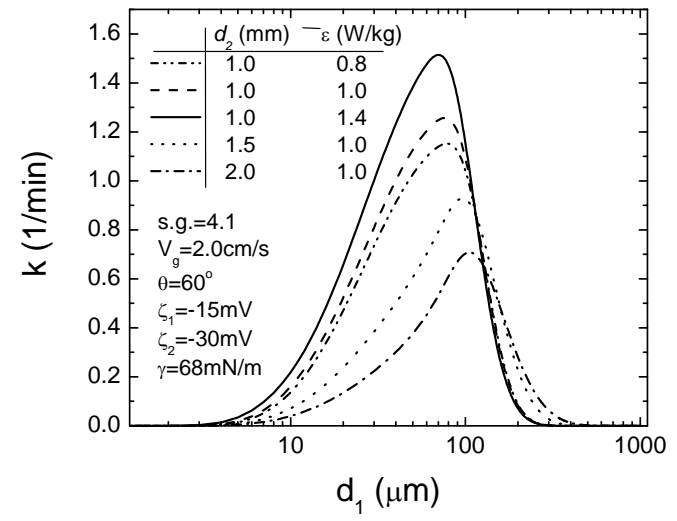

Figure 2. Effect of changing bubble size $\left(d_{2}\right)$ and mean energy input $(\varepsilon)$ on $k$. Smaller bubbles and higher energy inputs are beneficial to flotation, especially for fine particles.

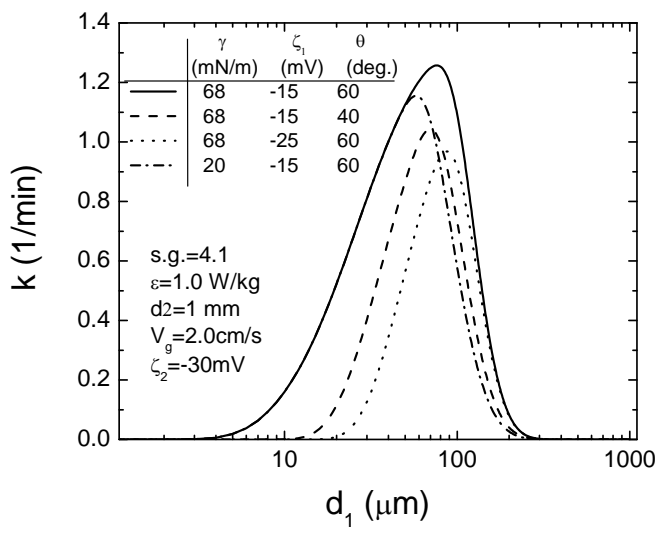

Figure 3. Effect of changing surface tension $(\gamma)$ and particle zeta-potential $\left(\zeta_{1}\right)$ on $k$. Control of the former helps coarse particle flotation, while control of the latter helps the flotation of fine particles. Increase in contact angle $(\theta)$ improves the flotation of both coarse and fine particles.

Figure 3 shows the effects of changing three surface chemistry parameters, i.e., $\theta, \zeta_{1}$, and $\gamma$. As the contact angle was increased from 40 to $60^{\circ}$, with all of the other parameters kept constant, $k$ increased substantially. As the zeta potential was changed from $-15 \mathrm{mV}$ to $-25 \mathrm{mV}$, with all the other variables kept constant, $k$ decreased substantially. As the surface tension was decreased from 68 to $20 \mathrm{mN} / \mathrm{m}$ with the other variables kept constant, the $k$ for coarse particle flotation decreased considerably. This is because the work of adhesion is a function of surface tension (see Eq. [10]). Although flotation is never run at $20 \mathrm{mN} / \mathrm{m}$, the message is clear, that is, over dose of frothers is detrimental to coarse particle flotation.

\section{Experimental Results}

Of the various model parameters investigated by simulation, it appears that contact angle is probably the most important variable for flotation. For this reason, many researchers during the early days of flotation research and technology development focussed on contact angle measurement and new collector development. Therefore, a series of flotation collectors that can increased the contact angles beyond what can be achieved using conventional collectors have been developed. Figure 4 shows the results obtained using novel collectors for the flotation of anthracite coal from Korea. As shown, all of the three new collectors gave substantially high recoveries throughout the particle size range investigated. As shown in model predictions (Figures 1-3), there exists an optimum particle size. It happens that the optimum for coal flotation occurs at $0.5 \mathrm{~mm}$, which is actually the top size that is usually employed in coal industry worldwide. The reagents listed in Figure 4 are currently marketed by Nalco. 
Other examples of controlling various parameters to improve flotation will be given at the presentation. In addition, implications of the model predictions on flotation cell and circuit design will be discussed.

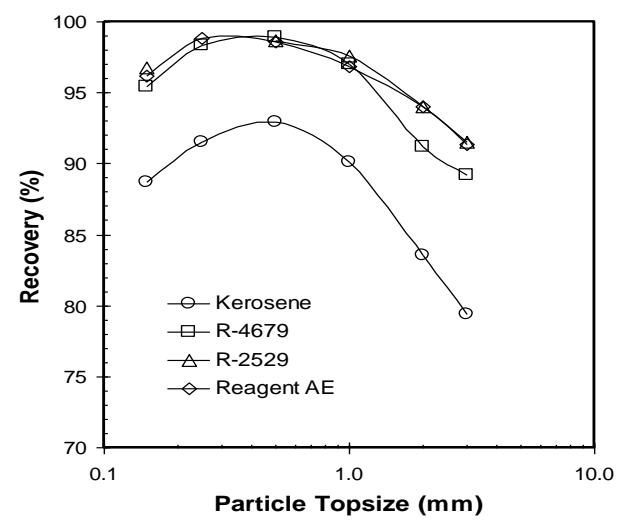

Figure 4. Effect of top particle size on maximum combustible recovery for the anthracite coal sample

\section{Summary and conclusions}

A turbulent flotation model has been developed mostly based on first principles. It can, therefore, predict flotation from both hydrodynamic and surface chemistry parameters. Simulation results showed that an increase in energy dissipation can increase the rate of flotation, particularly that of fine particles. Model also shows that decrease in bubble size is good for the flotation of fine particles. Use of large bubbles tend to shift the recovery $v s$. particle size curves to the direction of larger particles, but the over all recovery is well below those obtainable using smaller air bubbles. Control of surface chemistry parameters such as contact angle, zeta-potentials and surface tension can help the flotation of coarse particles. The benefits of using more powerful collectors for coal flotation are shown.

\section{References}

Abrahamson, J., 1975. Collision rates of small particles in a vigorously turbulent fluid. Chemical Engineering Science, 30(11): 1371-1379.

Brown, D J and Smith, H G, 1954. The flotation of coal as a rate process, Trans Ins. of Mining Eng, 113:1001-1020.

Do, H., Sherrel, I.M. and Yoon, R.H., 2006. Development of a froth recovery model in mineral processing. Minerals Engineering, to be submitted.

Gaudin, A.M., Groh, J.O. and Henderson, H.B., 1931. AIME Eng., Tech. Pub. No. 414 (1931).

Goren, S.L. and O'Neil, M.E., 1971. On the hydrodynamic resistance to a particle of a dilute suspension when in the neighbourhood of a large obstacle Chem. Eng. Sci, 26: 325-338.

Jowett, A., In: P. Somasundaran (Editor), International symposium on fine particles: Fine Particle Processing. SME, Las Vegas, 1980, p.720.

Lee, C.H., Erickson, L.E. and Glasgow, L.A., 1987. Bubble breakup and coalescence in turbulent gas-liquid dispersions. Chemical Engineering Communications, 59(1-6): 65-84.

Liepe, F. and Moeckel, H.O., 1976. Studies of the combination of substances in liquid phase. Part 6: The influence of the turbulence on the mass transfer of suspended particles. Chemische Technik (Leipzig, Germany), 28(4): 205-209.

Lynch, A.J., Johnson, N.W., Manlapig, E.V. and Thorne, C.G. (Editors), 1981. Mineral and coal flotation circuits- their simulation and control. Developments in mineral processing, 3. Elsevier, Amsterdam.

Schimmoller, B.K., Luttrell, G.H. and Yoon, R.-H., 1993. Hydrodynamic surface force model for bubble particle collection, 18th International Mineral Processing Congress, Sydney, Australia, pp. 751-756.

Sherrell, I.M., 2004. Ph.D. thesis, Virginia Tech, Blacksburg. 
Trahar, W.J., 1981. A rational interpretation of the role of particle size in flotation. International Journal of Mineral Processing, 8(4): 289-327.

Yoon, R.-H. and Mao, L., 1996. Application of extended DLVO theory, IV. Derivation of flotation rate equation from first principles. Journal of Colloid and Interface Science, 181(2): 613-626.

Yoon, R. -H., 2000, The role of surface forces in flotation kinetics, 21 th International Mineral Processing Congress, Rome, Italy, B8a1-7. 
Appendix 12: Measurement of Surface Forces Between Hydrophobic Surfaces (VA016) 


\section{TECHNICAL PROGRESS REPORT}

Contract Title and Number:

Crosscutting Technology Development at the Center for

Advanced Separation Technologies

(DE-FC26-02NT41607)
Period of Performance:

Starting Date: 6/1/2005

Ending Date: 10/31/2009 $\underline{\text { Sub-Recipient Project Title: }}$

Measurement of Surface Forces Between Hydrophobic

Surfaces

Principal Investigators:

Yoon

Contact Address:

Subcontractor Address:

Insert address of subcontractor. If none awarded, insert "No subcontracts issued."
Report Information:

Type: Final

Number:

Period: $\quad$ 9/30/07

Date:

Code: VA016-FINAL

Contact Information:

Phone:

Fax:

E-Mail: ryoon@vt.edu

Subcontractor Information:

Phone:

Fax:

E-Mail:

\section{ABSTRACT}

Froth flotation is the most widely used method for separating fine particles in the mining industry. The process is based on rendering a selected mineral hydrophobic, so that it can be separated from other minerals that are hydrophilic. Thus, control of particle hydrophobicity is critically important in flotation. However, the nature of the hydrophobicity is not fully understood, particularly in view of the forces acting between two hydrophobic particles suspended in water. In the current reporting period, surface force measurements have been conducted using an atomic force microscope (AFM). The force measurements were conducted with gold surfaces after coating them with xanthate surfactant. In addition, the adsorption process of xanthate ions onto gold was investigated by cyclic voltammetry (CV) and contact angle measurements, which show that the amyl xanthate can reversely adsorb on gold surfaces. Long range attractive forces in excess of van der Waals force have been detected using AFM for gold in amyl xanthate solution.

\section{INTRODUCTION}

\section{$\underline{\text { Background }}$}

Froth flotation is the most widely used solid-solid separation technique in the mining industry. It is based on the hydrophobicity difference of particles. Thus, rendering an 
interested mineral more hydrophobic than others is the key to the success of a flotation process. For this reason, the early days of flotation research was focused on hydrophobizing minerals and monitoring the changes in hydrophobicity by measuring contact angles. However, contact angle is a thermodynamic property and does not provide kinetic information. On the other hand, flotation is a kinetic process and the industry strives for improving flotation kinetics and, hence, increasing recovery and throughput.

In colloid chemistry, the kinetics of coagulation can be predicted by the DLVO theory, which considers two surface forces, namely, repulsive double-layer force and attractive van der Waals force. The theory is useful for describing interactions between particles. However, it is inadequate for studying bubble-particle interactions, as both of these forces are repulsive under typical flotation conditions. It was not until recently that one could actually measure the surface forces acting between two hydrophobic surfaces, and observed additional attractive force, which is naturally referred to as "hydrophobic force". It has been shown that the use of the hydrophobic force allows one to model coagulation of hydrophobic particles (Xu and Yoon, 1989, 1990) and bubble-particle adhesion (Yoon, 1991; Yoon and Mao, 1996; Mao and Yoon, 1997). However, there has been a great deal of controversy regarding the existence of the hydrophobic force and its possible origins. Furthermore, most of the hydrophobic force measurements conducted in the past was made with mica and silica surfaces using surfactants that are not commonly used for flotation. It is therefore, proposed to conduct direct force measurements using sulfide minerals, gold, and rutile $\left(\mathrm{TiO}_{2}\right)$ in the presence of thiols, cationic surfactants, and anionic surfactants. The results of the proposed work should help alleviate the controversy and collect information that can help further the flotation technology.

\section{Objective and Approach}

The objective in the reporting period is to perform direct measurement of forces acting between gold surfaces in xanthate solutions. Cyclic Voltammetry and contact angle measurements will be employed to investigate the adsorption process of amyl xanthate on gold. The obtained data is helpful for the controlling the hydrophobicity of gold in xanthate solution. The surface force measurement between gold surfaces in the amyl xanthate solutions will be conducted using AFM technique.

\section{PROJECT TASKS}

\section{$\underline{\text { Task } 1 \text { Purification of Xanthates }}$}

Potassium amyl xanthate (KAX) was used in the experiment to hydrophobizing gold surfaces. The purification of xanthates was conducted by using pure acetone and diethyl ether, which is described in the literature (Rao, 1971). The xanthates were recrystallized at least twice prior to use.

\section{$\underline{\text { Task } 2 \text { Electrochemical Measurements }}$}


Cyclic voltammetry (CV) was used to verify the chemisorption of the potassium amyl xanthate on gold surfaces. CV experiments were conducted in a standard electrochemical cell using a 273A potentiostat. The cell was equipped with a saturated calomel electrode (SCE) as reference electrode and a counter electrode made of platinum. Gold coated glass slide was used as working electrode, and $10^{-1} \mathrm{M}$ of $\mathrm{NaClO}_{4}$ was employed as supporting electrolyte. When testing the blocking capability of the xanthate layer, the electrolyte solution containing $10^{-2} \mathrm{M} \mathrm{K}_{3} \mathrm{Fe}(\mathrm{CN})_{6} / \mathrm{K}_{4} \mathrm{Fe}(\mathrm{CN})_{6}$ was used.

Cyclic voltammograms of gold electrode in $10^{-1} \mathrm{M} \mathrm{NaClO}_{4}$ and $10^{-1} \mathrm{M}+10^{-4} \mathrm{M} \mathrm{KAX}$ are shown in Fig. 1. The dotted curve represents the relationship of current density $v s$. potential for gold electrode in $10^{-1} \mathrm{M} \mathrm{NaClO}_{4}$ electrolyte solution, while the cyclic voltammogram of gold in $10^{-1} \mathrm{M} \mathrm{NaClO}_{4}+10^{-4} \mathrm{M} \mathrm{KAX}$ is represented by the solid curve. For the dotted curve, during the anodic scan, the small anodic current at potential around $190 \mathrm{mV}$ is supposed to be caused by the adsorption of perchloride ions on gold. And the anodic

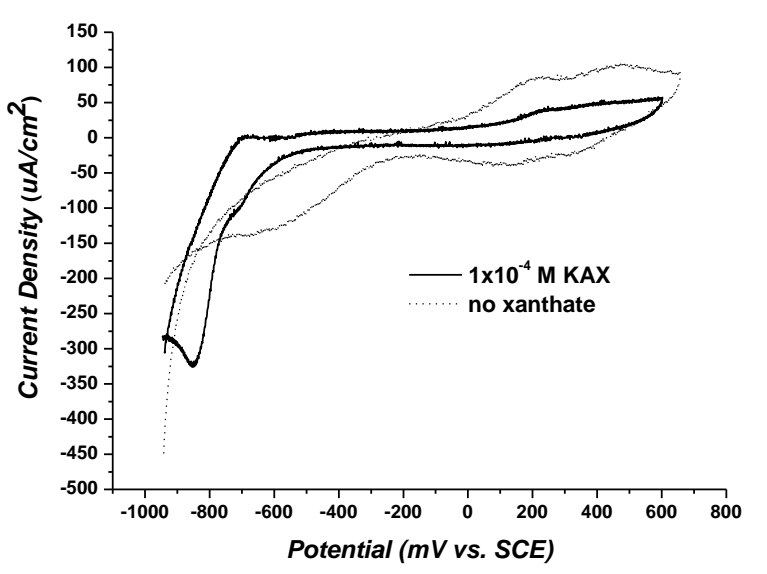

Fig 1. Cyclic voltammograms of gold in $10^{-1} \mathrm{M}$ $\mathrm{NaClO}_{4}$ solution with a scan rate of $250 \mathrm{mV} / \mathrm{s}$.

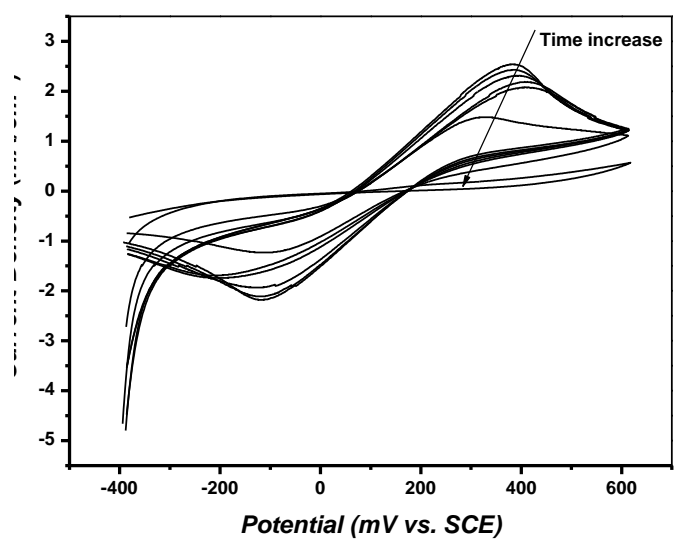

Fig 2. Cyclic voltammograms of gold recorded in $10^{-2}$ $\mathrm{M} \mathrm{K}_{3} \mathrm{Fe}(\mathrm{CN})_{6} / \mathrm{K}_{4} \mathrm{Fe}(\mathrm{CN})_{6}, 10^{-1} \mathrm{M} \mathrm{NaClO}_{4}$ and $10^{-5} \mathrm{M}$ $\mathrm{KAX}$ at different adsorption time, with scan rate of $200 \mathrm{mV} / \mathrm{s}$. current at potential about $500 \mathrm{mV}$ is believed to be due to the oxidation of gold. As the potential is swept in the reverse direction (cathodic scan), the reduction of gold begins at a potential about $150 \mathrm{mV}$. For the solid curve, the anodic current peak at $-700 \mathrm{mV}$ is attributed to the adsorption of xanthate ions, and the current peak at $250 \mathrm{mV}$ is due to the oxidation of ethyl xanthate ions to diamyldixanthogen. The two cathodic current peaks around potential of $-690 \mathrm{mV}$ and $-840 \mathrm{mV}$ are believed to due to the reduction of diamyldixanthogen to amyl xanthate ions, and the desorption of amyl xanthate ions, respectively. The cyclic voltammetry experiment shows that the amyl xanthate ions from water solution spontaneously chemically adsorb onto gold surface and form xanthate layer.

The blocking capability of the xanthate layer formed with different immersion time towards the ferri/ferro redox couples was studied by cyclic voltammetry as shown Fig. 2 . Application of a bare cleaned gold electrode gave the expected $\mathrm{CV}$ of $\mathrm{K}_{3} \mathrm{Fe}(\mathrm{CN})_{6} / \mathrm{K}_{4} \mathrm{Fe}(\mathrm{CN})_{6}$. It clearly appears that the blocking capability of xanthate layer to the access of the redox 
couple to the Au electrode surface increases with time. After 1260 min of immersion time, the xanthate layer gave the highest efficient blocking capability, which indicates that the layers have few pinholes and defects. The layer is thus, from an electrochemical point of view, well packed. It is also indirectly indicates that the adsorption of amyl xanthate onto gold surface is a rather slow process, which requires at least 20 hours.

\section{$\underline{\text { Task } 3 \text { Contact Angles Measurements }}$}

Contact angle measurement is another way to examine the adsorption process of amyl xanthate ions in water adsorb onto gold surface. The result of water contact angle of gold is shown in Fig 3. Generally, the contact angles of gold surfaces reach maximum value with less time for higher concentration of amyl xanthate, which means that the adsorption rate is determined by the concentration of xanthate in the bulk solution. In the xanthate solutions of higher concentrations $\left(5 \times 10^{-6} \mathrm{M}\right.$, $\left.1 \times 10^{-5} \mathrm{M}\right)$, the contact angles of gold decrease after reaching the maximum values. It is supposed that a second layer which reversely orients toward water bulk solution is formed. According to the above CV test, for the concentration of $1 \times 10^{-5} \mathrm{M}$, the blocking capability reaches the highest level after an immersion time of 21 hours. While the contact angle measurement shows that the contact angle value of gold reaches maximum after 1 hour of immersion time in

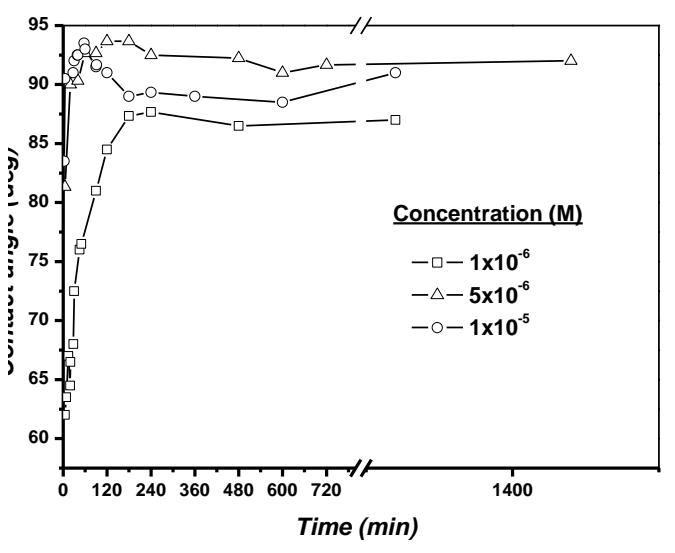

Fig 3. Contact angles of amyl xanthate layers on gold surface formed in different xanthate aqueous solutions with varying immersion time. $1 \times 10^{-5} \mathrm{M}$ xanthate solution. It is suggested that the high contact angle might not determine the closely packed structure of the xanthate layer.

\section{$\underline{\text { Task } 4 \text { Surface Force Measurements }}$}

Gold microspheres and gold coated glass flat plates were used in the surface force experiments during this reporting period. The surface forces were measured at room temperature $\left(\sim 25^{\circ} \mathrm{C}\right)$ using the colloidal probe technique developed by Ducker et al. (1991, 1992). Triangular cantilevers with spring constant of $0.58 \mathrm{~N} / \mathrm{m}$ were used in the current experiment. To remove contamination, the gold coated glass plates were boiled in a $\mathrm{H}_{2} \mathrm{SO}_{4} / \mathrm{H}_{2} \mathrm{O}_{2}$ (Piranha solution) solution for $30 \mathrm{~min}$, rinsed thoroughly with nanopure water and absolute ethanol. Figures 4, 5 and 6 show the results of measurement of the forces between a gold micro particle and a flat gold coated glass plate in aqueous KAX solution with different concentration $\left(1 \times 10^{-6} \mathrm{M}, 5 \times 10^{-6} \mathrm{M}\right.$ and $\left.1 \times 10^{-5} \mathrm{M}\right)$ at varying immersion time. The experimental data show that the most attractive forces are much greater in magnitude than the van der Waals force. In the $1 \times 10^{-6} \mathrm{M} \mathrm{KAX}$ solution, the repulsive force decreases with time, and the contact angle (from $81^{\circ}$ to $87^{\circ}$ ) increases with time. In the $5 \times 10^{-6} \mathrm{M} \mathrm{KAX}$, 
with the extending time, first the repulsive force decreases, and then becomes strongly attractive, then becomes repulsive again. For the contact angle, it increases at first (from $81^{\circ}$

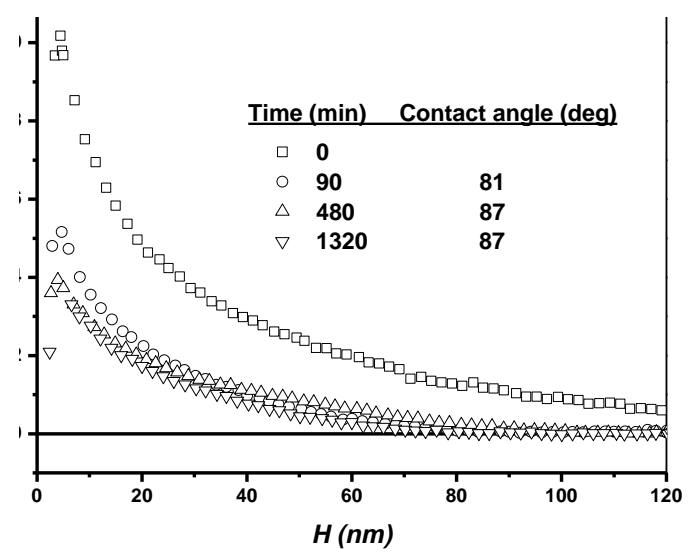

Fig 4. Surface forces in $1 \times 10^{-6} \mathrm{M} \mathrm{KAX}$ aqueous solution.

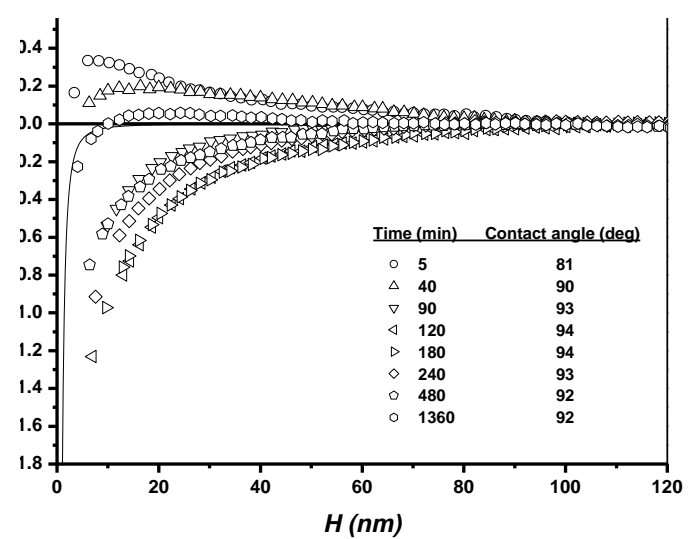

Fig 5. Surface forces in $5 \times 10^{-6} \mathrm{M} \mathrm{KAX}$ aqueous solution.

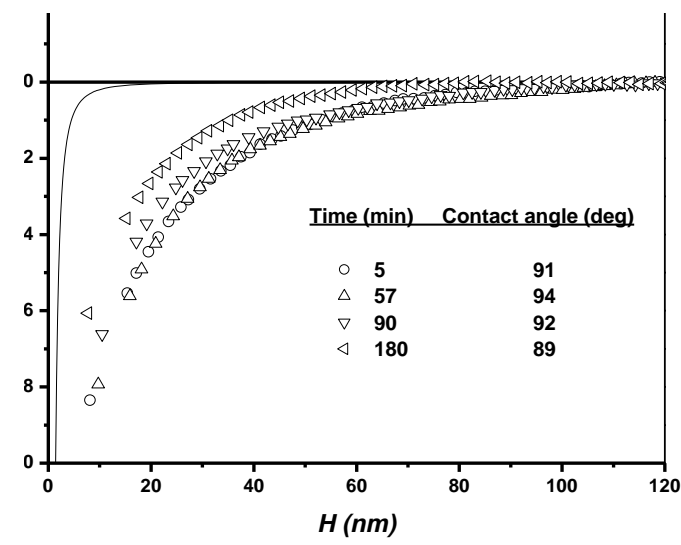

Fig 6. Surface Forces in $1 \times 10^{-5} \mathrm{M}$ KAX aqueous solution. to $94^{\circ}$ ), and reaches maximum, then decreases (from $94^{\circ}$ to $92^{\circ}$ ). In a higher concentration of $1 \times 10^{-5} \mathrm{M}$, the strongly attractive force decreases with time. According to the above surface force data, it is confirmed that in the higher concentration of KAX solution, a second layer reversely adsorbs onto the first monolayer of KAX. The anionic group of $\mathrm{KAX}$ on the surface renders the repulsive double layer force, which counteracts the hydrophobic attractive force.

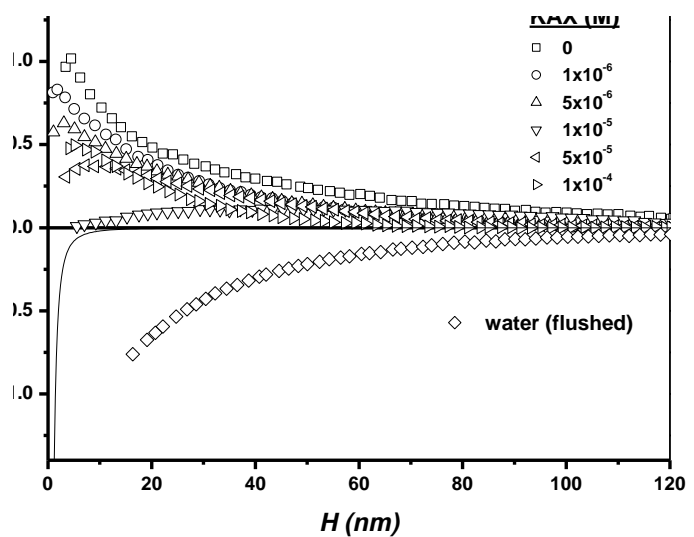

Fig 7. The surface forces as a function of concentration of KAX.

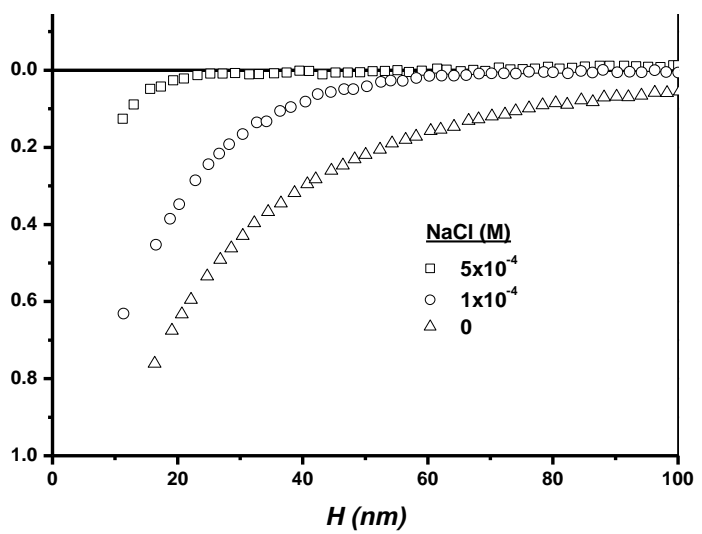

Fig 8. The surface forces between KAX hydrophobic layers in different concentration of $\mathrm{NaCl}$ aqueous solution. 
To further explore the reverse orientation phenomena of KAX on gold, the surface force was measured as a function of concentration of KAX. The Fig 7 shows that, when the concentration of KAX surfactant is increased, first the repulsive force decreases, then becomes attractive, then becomes repulsive again. After adding the KAX solution with concentration of $1 \times 10^{-4} \mathrm{M}$, the liquid cell was flushed with pure water. A strongly attractive hydrophobic force is observed. It is probably that the physically adsorbed top layer was removed by the flushing water.

After flushed with pure water, the liquid cell was filled by $\mathrm{NaCl}$ solution with different concentration $\left(5 \times 10^{-4} \mathrm{M}\right.$ and $\left.1 \times 10^{-4} \mathrm{M}\right)$, and the experimental data is shown in Fig 8. It is shown that the addition of $\mathrm{NaCl}$ decreases the magnitude and range of the attractive hydrophobic force. It is believed that the addition of $\mathrm{NaCl}$ disturbs the water structure between the hydrophobic surfaces.

\section{SUMMARY}

During the current reporting period, CV, contact angle and surface force measurements were used to study the adsorption of amyl xanthate on gold, and the hydrophobic force between hydrophobic xanthate layer surfaces. The CV test verified the chemiadsorption of amyl xanthate on gold. The contact angle and surface force measurements revealed the reverse orientation phenomena of amyl xanthate on gold surface. According to the surface force measurement, the attractive hydrophobic force which is much greater in magnitude and range than expected for the van der waals force between hydrocarbon surfaces has been detected at the high water contact angles.

\section{FUTURE WORK}

Much of the work conducted during the next reporting period will focus on the surface force measurements between the $\mathrm{ZnS}$ surfaces.

\section{REFERENCES}

Rao, S. R., Xanthates and Related Compounds. Marcel Dekker, Inc: 1971.

Xu, Z., Yoon, R. H., J. Colloid Interface Sci 1989, 132, (2), 532-541.

Xu, Z., Yoon, R. H., J. Colloid Interface Sci 1990, 134, (2), 427-434.

Ducker, W. A., Senden, T. J., Pashley, R. M., Nature 1991, 353, 239-241.

Yoon, R. H. In Hydrodynamic and Surface Forces in Bubble-Particle Interactions, XVII International Mineral Processing Congress, Dresden, Germany, September 23-28, 1991, Dresden, Germany.

Ducker, W. A., Senden, T. J., Pashley, R. M., Langmuir 1992, 8, 1831-1836.

Yoon, R. H., Mao, Laiqun, J. Colloid Interface Sci 1996, 181, 613-626.

Mao, L., Yoon, R. H., Int. J. Mineral Process 1997, 50, 171-181.

\section{PUBLICATIONS/PRESENTATIONS}

To date, no major publications have resulted from the current reporting period. 
Appendix 13 Novel Surfactants as Collectors for Froth Flotation (VA017) 


\section{FINAL TECHNICAL REPORT}

Contract Title and Number:

Crosscutting Technology Development at the Center for

Advanced Separation Technologies

(DE-FC26-02NT41607

Sub-Recipient Project Title:

NOVEL SURFACTANTS AS COLLECTORS FOR

FROTH FLOTATION.

Principal Investigators:

Richard D. Gandour

Contact Address:

Department of Chemistry, MC 0212

Virginia Tech

Blacksburg, VA 24061

Subcontractor Address:

Insert address of subcontractor. If none awarded, insert

"No subcontracts issued."
Period of Performance:

Starting Date: 10/01/2002

Ending Date: $\quad$ 10/31/2009
Report Information:

Type: Final

Number:

Period:

Date:

Code: VA017-Final

Contact Information:

Phone: 540-231-3731

Fax: $\quad$ 540-231-3255

E-Mail: gandour@vt.edu

Subcontractor Information:

Phone:

Fax:

E-Mail: 


\section{DISCLAIMER}

The Disclaimer must follow the Title Page and must contain the following paragraph: "This report was prepared as an account of work sponsored by an agency of the United States Government. Neither the United States Government nor any agency thereof, nor any of their employees, make any warranty, express or implied, nor assume any legal liability or responsibility for the accuracy, completeness, or usefulness of any information, apparatus, product, or process disclosed, or represents that its use would not infringe privately owned rights. Reference herein to any specific commercial product, process, or service by trade name, trademark, manufacturer, or otherwise does not necessarily constitute or imply endorsement, recommendation, or favoring by the United States Government or any agency thereof. The views and opinions of authors expressed herein do not necessarily state or reflect those of the United States Government or agency thereof. ”

\section{ABSTRACT}

The abstract should be a brief and concise summary that highlights the important accomplishments of the research for the current reporting period

\section{TABLE OF CONTENTS}

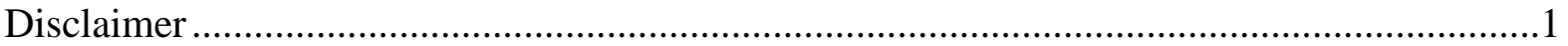

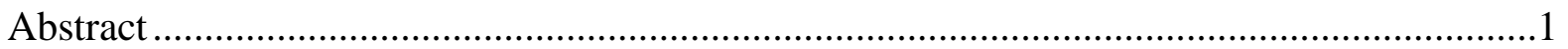

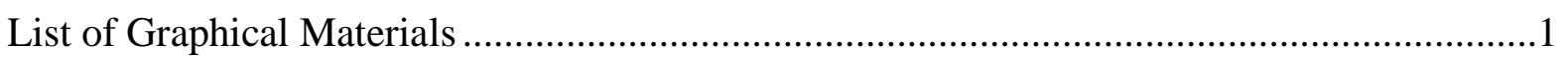

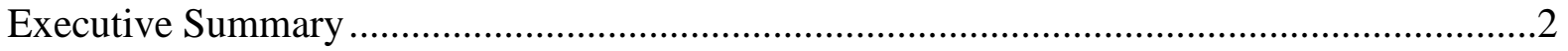

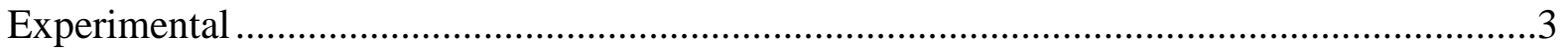

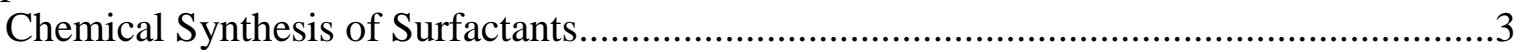

Absorption of Surfactants on Iron Nanoparticles. .....................................................19

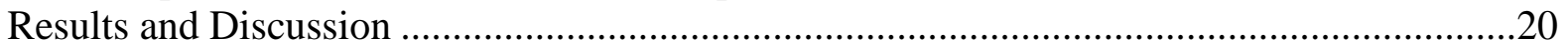

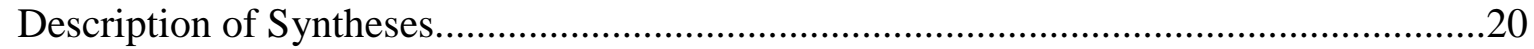

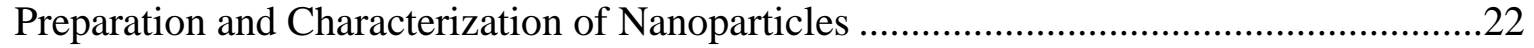

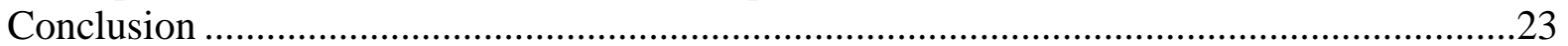

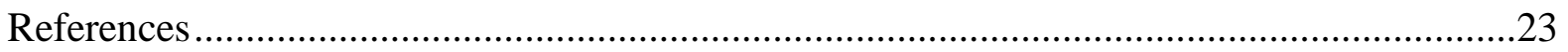

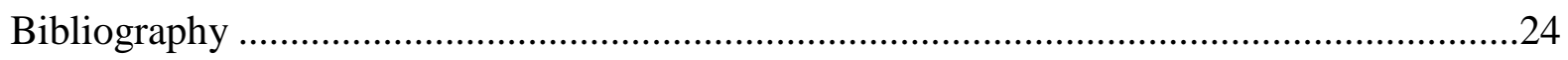

\section{LIST OF GRAPHICAL MATERIALS}

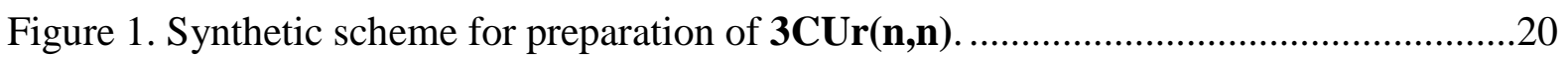

Figure 2. Synthetic scheme for preparation of 3CUr1(n,n)........................................20

Figure 3. Synthetic scheme for preparation of 3CCb1(n,n) .......................................21

Figure 4. Synthetic scheme for preparation of 3CAm1(n,n)...........................................21

Figure 5. Synthetic scheme for the preparation of 3CbPEGmw. .....................................22

Figure 6. Exchanging oleic acid with $\mathrm{HCOOH}$ and 3CCbPEGmw on Iron Nanoparticles. ..22 


\section{EXECUTIVE SUMMARY}

Froth flotation involves separating finely divided solid particulates suspended in water. Many mining companies worldwide employ froth flotation to enhance the concentration of desired mineral and coal fines. Processing occurs on the scale of billions of tons of ore annually and consumes millions of kilograms of reagents-collectors, depressants, activators, modifiers, and frothers.(Fuerstenau and Herrera-Urbina, 1989) The proposed triheaded surfactants with two tails will function as collectors. The goal is to find a collector that will operate at very low concentrations such that the amount of reagent needed is drastically reduced. This reduction will lead to energy savings and lessen the environmental impact of reagent disposal.

We synthesized 25 new surfactants in three different series-3CUr1(n,n), 3CUr(n,n), 3CAm1(n,n), 3CCb1(n,n), and 3CCbPEGmw; the shorthand terms are 3C = three carboxyl groups, $\mathrm{Am}=$ amido linker, $\mathrm{Ur}=$ ureido linker, $\mathrm{Cb}=$ carbamato linker, and $\mathrm{n}=$ the number of carbons in the fatty chain. These surfactants were water soluble in basic aqueous solutions, especially those comprising triethanolamine as the base, up to $20 \mathrm{mM}$ for the longest chains. The 3CCbPEGmw series are water soluble at any $\mathrm{pH}$.
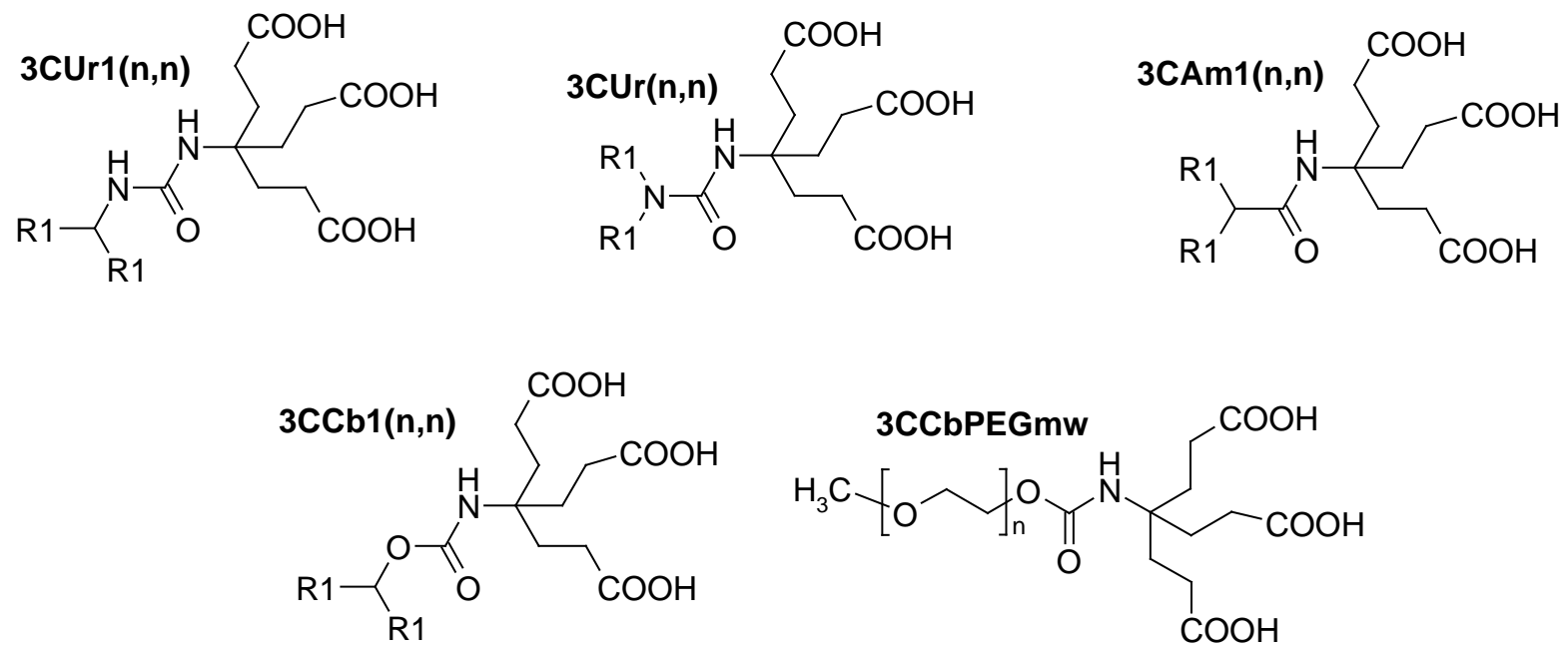<smiles>CC(C)(C)OCCCOC(=O)NC(CCC(=O)O)(CCC(=O)O)CCC(=O)O</smiles>

Although the 3CCbPEGmw series was not included in the original proposal, a collaborator needed a material to change a hydrophobic iron nanoparticle into a hydrophilic nanoparticle. We prepared 3CCbPEG550, 3CCbPEG2000, and 3CCbPEG5000. These modified polymers bind to magnetite nanoparticles, and as a result, the coated nanoparticles become water soluble. These polymers can attach onto nanoparticles through a two-step ligand-exchange process: 1) replace oleic acid with $\mathrm{HCOOH}$ in a concentration-driven process; and 2) exchange $\mathrm{HCOOH}$ with 3CCbPEGmw in an entropically driven process. Preliminary dynamic light scattering (DLS) and Fourier transform infrared spectroscopy (FTIR) studies have shown that Step 1 is feasible and that we can even exchange the 3CCbPEG2000 directly for oleic acid without Step 1. 


\section{EXPERIMENTAL}

Chemical Synthesis of Surfactants.

General Comments. Unless specified, solvents and reagents were used as received. Behera's amine(Newkome et al., 1991a) and Weisocyanate ${ }^{\mathrm{TM}}$ (Newkome et al., 1997) were prepared as described. Analytical thin layer chromatography was performed on silica gel $60 \AA$ and detected by dipping in a solution of $10 \%$ ethanolic phosphomolybdic acid reagent (20 wt. \% solution in ethanol) and then heated with a heat gun. The $\mathrm{R}_{\mathrm{f}}$ for $3 \mathbf{E C b n}$ in hexane:THF (6:1 $\mathrm{v}: \mathrm{v})$ was $0.32-0.41$. Flash column chromatography was carried out on silica gel (60 $\AA$ ). The samples were introduced as concentrated solutions in hexane:THF (6:1 v:v). After eluting the solvent mixture $(100 \mathrm{~mL})$, fractions $(25 \mathrm{~mL})$ were collected. The flow rate $(\sim 64 \mathrm{~mL} / \mathrm{min})$ was controlled by compressed air. The product appeared in fractions 3 to 12. Solutions were concentrated by rotary evaporation. Melting points were determined in open capillary tubes at $1^{\circ} \mathrm{C} / \mathrm{min}$ and uncorrected. NMR spectra of 3CCbn series were recorded at 400 and 100 $\mathrm{MHz}$ for ${ }^{1} \mathrm{H}$ and ${ }^{13} \mathrm{C}$, respectively, and reported in ppm. References in ${ }^{1} \mathrm{H}$ and ${ }^{13} \mathrm{C}$ spectra were TMS and DMSO- $d_{6}$, respectively. NMR spectra of 3CAm14 and 3CAm16 were recorded at 500 and $125 \mathrm{MHz}$ for ${ }^{1} \mathrm{H}$ and ${ }^{13} \mathrm{C}$, respectively, and reported in ppm. References in ${ }^{1} \mathrm{H}$ and ${ }^{13} \mathrm{C}$ spectra were $\mathrm{CD}_{3} \mathrm{OD}$ and DMSO- $d_{6}$, respectively. IR spectra were recorded on neat samples with an FTIR equipped with a diamond ATR system, and reported in $\mathrm{cm}^{-1}$. HRMS data were obtained on a dual-sector mass spectrometer in FAB mode with 2nitrobenzylalcohol as the proton donor. Elemental analyses were performed by a commercial vendor.

General procedures for N-alkylalkanamide, 3a,c,e. An alkan-1-amine 1 (13.0 mmol) and $\mathrm{Et}_{3} \mathrm{~N}$ (15.6 mmol) were combined, dissolved in dry THF (38 mL), and stirred in the ice bath. An acid chloride 2 (14.3 mmol) was added dropwise to the solution, which was maintained at $2.5 \pm 1.0^{\circ} \mathrm{C}$. After the addition, the ice bath was removed, and the reaction was stirred for another $15 \mathrm{~min}$ at rt. The resulting reaction was washed successively with $\mathrm{HCl}(1 \mathrm{M}, 7.5$ $\mathrm{mL})$, saturated aqueous $\mathrm{NaHCO}_{3}(12 \mathrm{~mL})$, and water $(12 \mathrm{~mL})$. The organic layer was dried with $\mathrm{Na}_{2} \mathrm{SO}_{4}$ and concentrated to dryness to give a white solid, which was purified by flash column chromatography to give a pure white solid, which gave a single spot on TLC with $2 \% \mathrm{MeOH}$ in $\mathrm{CH}_{2} \mathrm{Cl}_{2}\left(R_{f}=0.23-0.31\right)$.

$\mathrm{N}$-Heptylheptanamide, 3a. The general procedure described above afforded a white solid (2.50 g, 85\%); mp 45.0-45.7 ${ }^{\circ} \mathrm{C}$ (lit.(Djedovič et al., 2005) mp 44-46 $\left.{ }^{\circ} \mathrm{C}\right) ;{ }^{1} \mathrm{H}$ NMR $\left(\mathrm{CDCl}_{3}\right)$ $\delta 0.88$ (t, 6H), 1.20-1.35 (bm, 14H), 1.50 (m, 2H), 1.62 (m, 2H), 2.17 (t, 2H), 3.24 (m, 2H), 5.69 (bs, 1H) (lit. $250 \mathrm{MHz}$,(Troyanskii et al., 1984) $300 \mathrm{MHz}\left(\right.$ Djedovič et al., 2005)); ${ }^{13} \mathrm{C}$ NMR $\left(\mathrm{CDCl}_{3}\right) \delta 14.19,14.20,22.69,22.75,26.0,27.1,29.15,29.17,29.9,31.7,31.9,37.1$, 39.7, 173.3 (lit.(Djedovič et al., 2005) 75MHz); IR 3287, 2955, 2921, 2851, 1640, 1555, 1467 (lit.(Troyanskii et al., 1984) neat); HRMS: for $\mathrm{C}_{14} \mathrm{H}_{30} \mathrm{NO}[\mathrm{M}+\mathrm{H}]^{+}$calcd 228.2327, found 228.2328.

$\mathrm{N}$-Nonylnonanamide, 3c. The general procedure described above afforded a white solid (3.05 g, 83\%); mp 62.7-63.3.9 ${ }^{\circ} \mathrm{C}$ (lit.(Gall and Bosies, 1988) mp 52-55 ${ }^{\circ} \mathrm{C}$ ); ${ }^{1} \mathrm{H}$ NMR $\left(\mathrm{CDCl}_{3}\right) \delta 0.88(\mathrm{t}, 3 \mathrm{H}), 1.20-1.35(\mathrm{bm}, 22 \mathrm{H}), 1.48(\mathrm{~m}, 2 \mathrm{H}), 1.62(\mathrm{~m}, 2 \mathrm{H}), 2.15(\mathrm{t}, 2 \mathrm{H}), 3.25$ (m, 2H), 5.36 (bs, 1H); ${ }^{13} \mathrm{C} \mathrm{NMR}\left(\mathrm{CDCl}_{3}\right) \delta 14.1,22.66,22.68,25.9,26.9,29.18,29.25$, 29.32, 29.35, 29.5, 29.7, 31.84, 31.87, 37.0, 39.5, 173.03; IR 3287, 2955, 2918, 2849, 1638, 
1551, 1467; HRMS: for $\mathrm{C}_{18} \mathrm{H}_{38} \mathrm{NO}[\mathrm{M}+\mathrm{H}]^{+}$calcd 284.2953, found 284.2940.

$\mathrm{N}$-Undecylundecanamide, 3e. The general procedure described above afforded a white solid (3.12 g, 71\%); mp 73.9-74.7 ${ }^{\circ} \mathrm{C}$ (lit.(Froger and Parisot, 1954) mp $\left.73{ }^{\circ} \mathrm{C}\right) ;{ }^{1} \mathrm{H}$ NMR $\left(\mathrm{CDCl}_{3}\right)$ $\delta 0.88(\mathrm{t}, 6 \mathrm{H}), 1.20-1.35(\mathrm{bm}, 32 \mathrm{H}), 1.49(\mathrm{~m}, 2 \mathrm{H}), 1.62(\mathrm{~m}, 2 \mathrm{H}), 2.15(\mathrm{t}, 2 \mathrm{H}), 3.24(\mathrm{~m}, 2 \mathrm{H})$, 5.36 (bs, $1 \mathrm{H}) ;{ }^{13} \mathrm{C}$ NMR $\left(\mathrm{CDCl}_{3}\right) \delta 14.3,22.9,26.1,27.2,29.53,29.54,29.59,29.73,29.77$, 29.79, 29.80, 29.9, 32.1, 37.2, 39.7, 173.3; IR 3312, 2953, 2914, 2849, 1634, 1544, 1471 (lit.(Yoshioka and Tashiro, 2003) bands); HRMS: for $\mathrm{C}_{22} \mathrm{H}_{46} \mathrm{NO}[\mathrm{M}+\mathrm{H}]^{+}$calcd 340.3579, found 340.3584 .

General procedures for $N$-alkylalkan-1-amine, 4a,c,e. An $N$-alkylalkanamide 3 (9.58 mmol) was added slowly to a stirred suspension of $\mathrm{LiAlH}_{4}(574 \mathrm{mg}, 14.7 \mathrm{mmol})$ in $\mathrm{Et}_{2} \mathrm{O}(50 \mathrm{~mL})$. Addition took place in an ice-salt bath, while the temperature was maintained at $0.0 \pm 1.0^{\circ} \mathrm{C}$. After the addition, the reaction was warmed to rt and then heated to reflux. After being refluxed overnight, the resulting reaction was cooled to rt. Successive addition of water (2 $\mathrm{mL}), \mathrm{NaOH}(10 \mathrm{M}, 2.5 \mathrm{~mL})$, and water $(4 \mathrm{~mL})$ yields a sticky white solid. The organic layer was decanted, and the sticky white solid was washed once with $\mathrm{Et}_{2} \mathrm{O}(10 \mathrm{~mL})$. The organic layers were combined, dried with $\mathrm{Na}_{2} \mathrm{SO}_{4}$, and concentrated to give clear liquids $(\mathrm{n}=7,9)$, and a white solid ( $\mathrm{n}=11)$ in $80-85 \%$ yield. Each resulting $N$-alkylalkan-1-amine (4a,c,e) was used for the next step without further purification.

$N$-Heptylheptan-1-amine, 4a. The general procedure described above afforded a clear liquid (1.72 g, 84\%) (lit.(Djedovič et al., 2005) mp 30-31 $\left.{ }^{\circ} \mathrm{C}\right) ;{ }^{1} \mathrm{H} \mathrm{NMR}\left(\mathrm{CDCl}_{3}\right) \delta 0.88$ (t, 6H), 1.20-1.35 (bm, 16H), 1.48 (m, 4H), 2.58 (t, 4H) (lit. $60 \mathrm{MHz}$,(Rao and Bharathi, 2002) 300 $\mathrm{MHz}\left(\right.$ Djedovič et al., 2005)); ${ }^{13} \mathrm{C}$ NMR $\left(\mathrm{CDCl}_{3}\right) \delta 14.3,22.8,27.6,29.5,30.4,32.0,50.4$ (lit.(Djedovič et al., 2005) 75MHz); IR 2955, 2922, 2853, 1457, 1377, 1129 (lit.(Rao and Bharathi, 2002) neat); HRMS: for $\mathrm{C}_{14} \mathrm{H}_{32} \mathrm{~N}[\mathrm{M}+\mathrm{H}]^{+}$calcd 214.2535, found 214.2538.

$N$-Nonylnonan-1-amine, 4c. The general procedure described above afforded a clear liquid (2.14 g, 83\%) (lit.(Gall and Bosies, 1988) $\left.37-39{ }^{\circ} \mathrm{C}\right) ;{ }^{1} \mathrm{H}$ NMR $\left(\mathrm{CDCl}_{3}\right) \delta 0.88$ (t, 6H), 1.20 1.35 (bm, 24H), 1.47 (m, 4H), $2.58(\mathrm{t}, 4 \mathrm{H}) ;{ }^{13} \mathrm{C} \mathrm{NMR}\left(\mathrm{CDCl}_{3}\right) \delta$ 14.3, 22.9, 27.7, 29.5, 29.79, 29.82, 30.5, 32.1, 50.4; IR 2954, 2921, 2852, 1466, 1377, 1130; HRMS: for $\mathrm{C}_{18} \mathrm{H}_{40} \mathrm{~N}$ [M + $\mathrm{H}]^{+}$calcd 270.3161, found 270.3148 .

$\mathrm{N}$-Undecylundecan-1-amine, 4e. The general procedure described above afforded a white solid (2.50 g, 80\%); mp 47.5-48.3 ${ }^{\circ} \mathrm{C}$ (lit.(Wright and Elderfield, 1946) $51.5-52.5{ }^{\circ} \mathrm{C}$ ); ${ }^{1} \mathrm{H}$ NMR $\left(\mathrm{CDCl}_{3}\right) \delta 0.88(\mathrm{t}, 6 \mathrm{H}), 1.20-1.35(\mathrm{bm}, 32 \mathrm{H}), 1.47(\mathrm{~m}, 4 \mathrm{H}), 2.58(\mathrm{t}, 4 \mathrm{H}) ;{ }^{13} \mathrm{C} \mathrm{NMR}$ $\left(\mathrm{CDCl}_{3}\right) \delta 14.3,22.9,27.7,29.6,29.8,30.5,32.1,50.4$; IR 2955, 2913, 2827, 1469, 1375, 1127; HRMS: for $\mathrm{C}_{22} \mathrm{H}_{48} \mathrm{~N}[\mathrm{M}+\mathrm{H}]^{+}$calcd 326.3787, found 326.3775 .

General procedures for long chain tri-tert-butyl esters, 5(n,n). A dialkylamine 4 (4.95 mmol) was added slowly to a solution of WeNCO $(4.71 \mathrm{mmol})$ in $\mathrm{CH}_{2} \mathrm{Cl}_{2}(20 \mathrm{~mL})$. The resulting transparent solution was stirred at rt. After stirring overnight, the solution was concentrated to leave a crude white solid. The crude solid was purified by flash column chromatography with 5:1 (v:v) hexane:EtOAc to give a white solid (60-85\%), which gave a single spot on TLC (hexane:EtOAc, 5:1, $R_{f}=0.18-0.25$ ). 
Di-tert-butyl 4-(2-tert-butoxycarbonylethyl)-4-(3,3-diheptylureido)heptanedioate, 5(7,7). The general procedure described above afforded a white solid (2.44 g, 79\%); mp 53.5-54.1 ${ }^{\circ} \mathrm{C} ;{ }^{1} \mathrm{H}$ NMR $\left(\mathrm{CDCl}_{3}\right) \delta 0.88$ (t, 6H), 1.20-1.35 (bm, 16H), 1.43 (bs, 27H), 1.51 (m, 4H), $1.96(\mathrm{~m}, 6 \mathrm{H}), 2.22(\mathrm{~m}, 6 \mathrm{H}), 3.12(\mathrm{~m}, 4 \mathrm{H}), 4.56(\mathrm{~s}, 1 \mathrm{H}) ;{ }^{13} \mathrm{C} \mathrm{NMR}\left(\mathrm{CDCl}_{3}\right) \delta$ 14.0, 22.5, 27.0, 28.0, 28.7, 29.1, 29.9, 30.6, 31.8, 47.2, 56.6, 80.3, 156.1, 173.1; IR 3368, 2925, 1733, 1725, 1615, 1365, 1143; HRMS: for $\mathrm{C}_{37} \mathrm{H}_{71} \mathrm{~N}_{2} \mathrm{O}_{7}[\mathrm{M}+\mathrm{H}]^{+}$calcd 655.5261, found 655.5242. Anal. Calcd for $\mathrm{C}_{37} \mathrm{H}_{70} \mathrm{~N}_{2} \mathrm{O}_{7}$ : C, 67.85; H, 10.77; N, 4.28. Found: C, 67.89; H, 10.84; N, 4.30.

Di-tert-butyl 4-(2-tert-butoxycarbonylethyl)-4-(3,3-dioctylureido)heptanedioate, 5(8,8). The general procedure described above afforded a white solid (1.93 g, 60\%); mp 56.8-57.5 ${ }^{\circ} \mathrm{C}$; ${ }^{1} \mathrm{H}$ NMR $\left(\mathrm{CDCl}_{3}\right) \delta 0.89(\mathrm{t}, 6 \mathrm{H}), 1.20-1.35$ (bm, 20H), 1.43 (s, 27H), 1.50 (m, 4H), 1.96 (m, $6 \mathrm{H}), 2.21(\mathrm{~m}, 6 \mathrm{H}), 3.11(\mathrm{~m}, 4 \mathrm{H}), 4.55$ (s, 1H); ${ }^{13} \mathrm{C} \mathrm{NMR}\left(\mathrm{CDCl}_{3}\right) \delta 14.1,22.6,27.0,28.1$, 28.7, 29.27, 29.44, 29.9, 30.6, 31.8, 47.3, 56.6, 80.4, 156.1, 173.2; IR 3366, 2923, 1732, 1724, 1615, 1365, 1144; HRMS: for $\mathrm{C}_{39} \mathrm{H}_{75} \mathrm{~N}_{2} \mathrm{O}_{7}[\mathrm{M}+\mathrm{H}]^{+}$calcd 638.5574, found 638.5540. Anal. Calcd for $\mathrm{C}_{39} \mathrm{H}_{74} \mathrm{~N}_{2} \mathrm{O}_{7}$ : C, 68.58; H, 10.92; N, 4.10. Found: C, 68.55; H, 11.07; N, 4.10.

Di-tert-butyl 4-(2-tert-butoxycarbonylethyl)-4-(3,3-dinonylureido)heptanedioate, $\mathbf{5}(\mathbf{9 , 9})$. The general procedure described above afforded a white solid (2.34 g, 70\%); mp 62.6-63.4 ${ }^{\circ} \mathrm{C}$; ${ }^{1} \mathrm{H}$ NMR $\left(\mathrm{CDCl}_{3}\right) \delta 0.88$ (t, 6H), 1.20-1.35 (bm, 24H), 1.44 (bs, 27H), 1.51 (bm, 4H), 1.97 (m, 6H), $2.22(\mathrm{~m}, 6 \mathrm{H}), 3.12(\mathrm{~m}, 4 \mathrm{H}), 4.57(\mathrm{~s}, 1 \mathrm{H}) ;{ }^{13} \mathrm{C} \mathrm{NMR}\left(\mathrm{CDCl}_{3}\right) \delta 14.1,22.6,27.0,28.1$, 28.7, 29.2, 29.49, 29.58, 29.9, 30.6, 31.8, 47.3, 56.6, 80.4, 156.1, 173.15; IR 3377, 2922, 1732, 1725, 1617, 1365, 1144; HRMS: for $\mathrm{C}_{41} \mathrm{H}_{79} \mathrm{~N}_{2} \mathrm{O}_{7}[\mathrm{M}+\mathrm{H}]^{+}$calcd 711.5887, found 711.5893. Anal. Calcd for $\mathrm{C}_{41} \mathrm{H}_{78} \mathrm{~N}_{2} \mathrm{O}_{7}$ : C, 69.25; H, 11.06; N, 3.94. Found: C, 69.34; $\mathrm{H}$, 11.27; N, 3.96.

Di-tert-butyl 4-(2-tert-butoxycarbonylethyl)-4-(3,3-didecylureido)heptanedioate, 5(10,10). The general procedure described above afforded a white solid (2.96 g, 85\%); mp 49.7-50.4 ${ }^{\circ} \mathrm{C} ;{ }^{1} \mathrm{H}$ NMR $\left(\mathrm{CDCl}_{3}\right) \delta 0.88$ (t, 6H), 1.20-1.35 (bs, 28H), 1.43 (s, 27H), 1.50 (m, 4H), 1.96 (m, 6H), $2.21(\mathrm{~m}, 6 \mathrm{H}), 3.11(\mathrm{~m}, 4 \mathrm{H}), 4.55(\mathrm{~s}, 1 \mathrm{H}) ;{ }^{13} \mathrm{C}$ NMR $\left(\mathrm{CDCl}_{3}\right) \delta 14.1,22.7,27.0,28.1$, 28.7, 29.3, 29.49, 29.55, 29.63, 29.9, 30.6, 31.9, 47.3, 56.6, 80.4, 156.1, 173.2; IR 3389, 2925, 1729, 1719, 1618, 1365, 1143; HRMS: for $\mathrm{C}_{43} \mathrm{H}_{83} \mathrm{~N}_{2} \mathrm{O}_{7}[\mathrm{M}+\mathrm{H}]^{+}$calcd 739.6200, found 739.6213. Anal. Calcd for $\mathrm{C}_{43} \mathrm{H}_{82} \mathrm{~N}_{2} \mathrm{O}_{7}$ : C, 69.88; H, 11.18; N, 3.79. Found: C, 70.02; H, 11.41; N, 3.80.

Di-tert-butyl 4-(2-tert-butoxycarbonylethyl)-4-(3,3-diundecylureido)heptanedioate, 5(11,11). The general procedure described above afforded a white solid (2.64 g, 73\%); mp 42.9-43.5 ${ }^{\circ} \mathrm{C} ;{ }^{1} \mathrm{H}$ NMR $\left(\mathrm{CDCl}_{3}\right) \delta 0.88$ (t, 6H), 1.20-1.35 (bm, 32H), 1.43 (bs, 27H), 1.50 (bm, 4H), $1.96(\mathrm{~m}, 6 \mathrm{H}), 2.21(\mathrm{~m}, 6 \mathrm{H}), 3.11(\mathrm{~m}, 4 \mathrm{H}), 4.56(\mathrm{~s}, 1 \mathrm{H}) ;{ }^{13} \mathrm{C} \mathrm{NMR}\left(\mathrm{CDCl}_{3}\right) \delta 14.1,22.65$, 27.03, 28.1, 28.7, 29.3, 29.49, 29.59, 29.62, 29.9, 30.6, 31.9, 47.3, 56.6, 80.35, 156.1, 173.1; IR 3372, 2923, 1734, 1725, 1616, 1365, 1143; HRMS: for $\mathrm{C}_{45} \mathrm{H}_{87} \mathrm{~N}_{2} \mathrm{O}_{7}[\mathrm{M}+\mathrm{H}]^{+}$calcd 767.6513, found 767.6498. Anal. Calcd for $\mathrm{C}_{45} \mathrm{H}_{86} \mathrm{~N}_{2} \mathrm{O}_{7}$ : C, 70.45; H, 11.30; N, 3.65. Found: C, 70.45; H, 11.46; N, 3.71. 
A tri-tert-butyl ester $\mathbf{5}(\mathbf{n}, \mathbf{n})(3.33 \mathrm{mmol})$ was dissolved in $99 \% \mathrm{HCOOH}$ so that its concentration was $0.1 \mathrm{M}$. The mixture might need to be warmed slightly to get all the $\mathbf{5 ( n , n )}$ to dissolve. Once dissolved to give a clear colorless solution, the solution was stirred at rt. After $9 \mathrm{~h}$, the resulting milky white solution was concentrated. The white solid was recrystallized from EtOAc to yield a white solid (77-90\%).

4-(2-Carboxyethyl)-4-(3,3-diheptylureido)heptanedioic acid, $\mathbf{3 C U r} \mathbf{U}(\mathbf{7}, 7)$. The general procedure described above afforded a white solid (1.46 g, 90\%); mp 125.3-125.9 ${ }^{\circ} \mathrm{C} ;{ }^{1} \mathrm{H}$ NMR (DMSO-d $\left.)_{6}\right) \delta .83(\mathrm{t}, 6 \mathrm{H}), 1.10-1.30$ (bm, 16H), 1.38 (m, 4H), 1.81 (m, 6H), 2.09 (m, 6H), 3.09 (m, 4H), 5.07 (s, 1H), 12.00 (bs, 3H); ${ }^{13} \mathrm{C}$ NMR (DMSO-d $\left.{ }_{6}\right) \delta 14.4,22.5,26.7$, 28.5, 28.7, 29.0, 30.2, 31.7, 46.3, 56.4, 156.3, 175.1; IR 2926, 1735, 1701, 1590, 1527, 1285, 1174; HRMS: for $\mathrm{C}_{25} \mathrm{H}_{47} \mathrm{~N}_{2} \mathrm{O}_{7}[\mathrm{M}+\mathrm{H}]^{+}$calcd 487.3383 found 487.3391. Anal. Calcd for $\mathrm{C}_{25} \mathrm{H}_{46} \mathrm{~N}_{2} \mathrm{O}_{7}$ : C, 61.70; H, 9.53; N, 5.76. Found: C, 61.61; H, 9.50; N, 5.75.

4-(2-Carboxyethyl)-4-(3,3-dioctylureido)heptanedioic acid, $\mathbf{3 C U} \mathbf{U}(\mathbf{8 , 8})$. The general procedure described above afforded a white solid (1.47 g, 86\%); mp $120.4-120.8{ }^{\circ} \mathrm{C} ;{ }^{1} \mathrm{H}$ NMR (DMSO-d $\left.d_{6}\right) \delta 0.80$ (t, 6H), 1.10-1.30 (bm, 20H), 1.38 (m, 4H), 1.81 (m, 6H), 2.08 (m, 6H), 3.09 (m, 4H), 5.06 (s, 1H), 12.00 (bs, 3H); ${ }^{13} \mathrm{C}$ NMR (DMSO-d $\left.d_{6}\right) \delta 13.9,22.0,26.2$, 28.0, 28.2, 28.7, 28.8, 29.7, 31.2, 45.8, 55.9, 155.9, 174.7; IR 2921, 1713, 1694, 1607, 1533, 1293, 1175; HRMS: for $\mathrm{C}_{27} \mathrm{H}_{51} \mathrm{~N}_{2} \mathrm{O}_{7}[\mathrm{M}+\mathrm{H}]^{+}$calcd 515.3702, found 515.3696. Anal. Calcd for $\mathrm{C}_{27} \mathrm{H}_{50} \mathrm{~N}_{2} \mathrm{O}_{7}$ : C, 63.01; H, 9.79; N, 5.40. Found: C, 62.98; H, 9.79; N, 5.40 .

4-(2-Carboxyethyl)-4-(3,3-dinonylureido)heptanedioic acid, $\mathbf{3 C U r} \mathbf{( 9 , 9 ) . ~ T h e ~ g e n e r a l ~}$ procedure described above afforded a white solid (1.59 g, 88\%); mp $121.3-121.7^{\circ} \mathrm{C} ;{ }^{1} \mathrm{H}$ NMR (DMSO-d $\left.)_{6}\right) \delta 0.83(\mathrm{t}, 6 \mathrm{H}), 1.10-1.30(\mathrm{bm}, 24 \mathrm{H}), 1.38(\mathrm{~m}, 4 \mathrm{H}), 1.82(\mathrm{~m}, 6 \mathrm{H}), 2.09(\mathrm{~m}$, 6H), 3.09 (m, 4H), 5.07 (s, 1H), 11.98 (bs, 3H); ${ }^{13} \mathrm{C}$ NMR (DMSO-d ${ }_{6}$ ) $\delta 14.6,22.8,27.0$, 28.7, 29.0, 29.3, 29.58, 29.66, 30.4, 32.0, 46.6, 56.6, 156.6, 175.4; IR 3446, 2919, 1712, 1694, 1608, 1534, 1293, 1175; HRMS: for $\mathrm{C}_{29} \mathrm{H}_{55} \mathrm{~N}_{2} \mathrm{O}_{7}[\mathrm{M}+\mathrm{H}]^{+}$calcd 543.4009, found 543.3997. Anal. Calcd for $\mathrm{C}_{29} \mathrm{H}_{54} \mathrm{~N}_{2} \mathrm{O}_{7}$ : C, 64.18; $\mathrm{H}, 10.03$; N, 5.16. Found: C, 63.93; $\mathrm{H}$, 10.02; N, 5.15 .

4-(2-Carboxyethyl)-4-(3,3-didecylureido)heptanedioic acid, 3CUr(10,10). The general procedure described above afforded a white solid (1.63 g, 88\%); mp 120.9-121.2 ${ }^{\circ} \mathrm{C} .{ }^{1} \mathrm{H}$ NMR (DMSO-d $\left.d_{6}\right) \delta 0.83(\mathrm{t}, 6 \mathrm{H}), 1.10-1.30(\mathrm{bm}, 28 \mathrm{H}), 1.37(\mathrm{~m}, 4 \mathrm{H}), 1.81(\mathrm{~m}, 6 \mathrm{H}), 2.08(\mathrm{~m}$, 6H), 3.09 (m, 4H), 5.08 (s, 1H), 11.99 (bs, 3H); ${ }^{13} \mathrm{C}$ NMR (DMSO-d $\left.{ }_{6}\right) \delta 14.4,22.5,26.7$, 28.5, 28.7, 29.1, 29.32, 29.40, 29.46, 30.2, 31.7, 46.3, 56.4, 156.3, 175.2; IR 2919, 1712, 1694, 1608, 1534, 1293, 1175; HRMS: for $\mathrm{C}_{31} \mathrm{H}_{59} \mathrm{~N}_{2} \mathrm{O}_{7}[\mathrm{M}+\mathrm{H}]^{+}$calcd 571.4322, found 571.4315. Anal. Calcd for $\mathrm{C}_{31} \mathrm{H}_{58} \mathrm{~N}_{2} \mathrm{O}_{7}$ : C, 65.23; H, 10.24; N, 4.91. Found: C, 65.33; $\mathrm{H}$, 10.34; N, 4.93.

4-(2-Carboxyethyl)-4-(3,3-diundecylureido)heptanedioic acid, $\mathbf{3 C U r ( 1 1 , 1 1 ) . ~ T h e ~ g e n e r a l ~}$ procedure described above afforded a white solid (1.53 g, 77\%); mp $121.4-122.1{ }^{\circ} \mathrm{C} ;{ }^{1} \mathrm{H}$ NMR (DMSO- $\left.d_{6}\right) \delta 0.83(\mathrm{t}, 6 \mathrm{H}), 1.10-1.30(\mathrm{bm}, 32 \mathrm{H}), 1.38(\mathrm{~m}, 4 \mathrm{H}), 1.81(\mathrm{~m}, 6 \mathrm{H}), 2.08(\mathrm{~m}$, 6H), 3.09 (m, 4H), 5.07 (s, 1H), 12.00 (bs, 3H); ${ }^{13} \mathrm{C}$ NMR (DMSO- $d_{6}$ ) $\delta 14.4,22.6,26.7$, 28.5, 28.7, 29.17, 29.33, 29.46, 30.2, 31.8, 46.4, 56.4,156.3, 175.2; IR 2917, 1713, 1694, 
1608, 1535, 1295, 1185; HRMS: for $\mathrm{C}_{33} \mathrm{H}_{63} \mathrm{~N}_{2} \mathrm{O}_{7}[\mathrm{M}+\mathrm{H}]^{+}$calcd 599.4635, found 599.4620. Anal. Calcd for $\mathrm{C}_{33} \mathrm{H}_{62} \mathrm{~N}_{2} \mathrm{O}_{7}$ : C, 66.19; H, 10.44; N, 4.68. Found: C, 66.17; H, 10.40; N, 4.69.

General procedure for the preparation of dialkylcarbinols, 7a-f. To a three-neck round bottom flask containing Mg turnings ( $2.34 \mathrm{~g}, 100 \mathrm{mmol}$ ) was added several chips of $\mathrm{I}_{2}$. After the flask was flushed with $\mathrm{N}_{2}$, the mixture was stirred for a couple minutes until $\mathrm{I}_{2}$ sublimed. THF (12 mL) was added, following a solution of a bromoalkane 6 (48.1 mmol) in THF (36 $\mathrm{mL}$ ) in a dropwise (1 drop/3 sec) manner through a dropping funnel. Then, $\mathrm{HCO}_{2} \mathrm{Et}(1.43 \mathrm{~g}$, $19.0 \mathrm{mmol})$ in dry THF (24 mL) was added at the same rate through a dropping funnel. The reaction was stirred and heated $\left(65-70{ }^{\circ} \mathrm{C}\right)$. Monitoring the reaction with TLC showed that 3 $\mathrm{d}$ were needed to achieve the best yields. The reaction mixture was diluted with THF (36 $\mathrm{mL})$, followed by successive addition of $\mathrm{MeOH}(4 \mathrm{~mL})$ and saturated $\mathrm{NH}_{4} \mathrm{Cl}(30 \mathrm{~mL})$. The organic layer was separated and washed with saturated $\mathrm{NaCl}(65 \mathrm{~mL})$. The organic layer was dried and concentrated to give a light yellow solid, which was recrystallized from EtOAc to give a pure white solid (11) in moderate yield (49-56\%); ${ }^{1} \mathrm{H}$ NMR $\left(\mathrm{CDCl}_{3}\right) \delta 0.88(\mathrm{t}, 6 \mathrm{H})$, 1.20-1.50 (bm, 24-44Hs), 3.58 (bm, 1H) (lit.(Boal et al., 2002) $200 \mathrm{MHz}$, 7f); 7a mp 53.5$54.3^{\circ} \mathrm{C}$ (lit.(Breusch and Sokullu, 1953) 52-52.6 ${ }^{\circ} \mathrm{C}$ ); 7b $61.7-62.2^{\circ} \mathrm{C}$ (lit.(Breusch and Sokullu, 1953) 60.8-61.2 ${ }^{\circ} \mathrm{C}$ ); 7c 67.0-67.4 ${ }^{\circ} \mathrm{C}$ (lit.(Meakins and Sack, 1951) 65.9-66.1 ${ }^{\circ} \mathrm{C}$ ); 7d 71.1-72.0 ${ }^{\circ} \mathrm{C}$ (lit.(Overmars et al., 1994) 71.3-72.5 ${ }^{\circ} \mathrm{C}$ ); 7e 76.8-77.6 ${ }^{\circ} \mathrm{C}$ (lit.(Breusch and Sokullu, 1953) $75.5-75.7^{\circ} \mathrm{C}$ ); 7f 80.2-81.0 ${ }^{\circ} \mathrm{C}$ (lit.(Baykut, 1954) 79.5-80.5 ${ }^{\circ} \mathrm{C}$ ).

General procedure for the preparation of bis(alkyl)methyl mesylates, 8a-f. To a solution of 7 (8.96 mmol) and $\mathrm{Et}_{3} \mathrm{~N}(9.86 \mathrm{mmol})$ in THF $(59 \mathrm{~mL})$ was added $\mathrm{MsCl}(25.7 \mathrm{mmol})$ dropwise through a syringe at rt. In the middle of the addition, the clear solution became cloudy. After the addition, the reaction was stirred for another $15 \mathrm{~min}$. The resulting suspension was filtered; the filtrate was washed successively with water $(8 \mathrm{~mL})$ and saturated aqueous $\mathrm{NaCl}(2 \mathrm{~mL})$. The organic layer was dried with $\mathrm{Na}_{2} \mathrm{SO}_{4}$, filtered, and concentrated to a give light yellow liquid (8) in $90-98 \%$ yield. The product was used for the next step without further purification; ${ }^{1} \mathrm{H}$ NMR $\left(\mathrm{CDCl}_{3}\right) \delta 0.88(\mathrm{t}, 6 \mathrm{H}), 1.20-1.50(\mathrm{bm}, 24-44 \mathrm{Hs})$, 1.60-1.75 (m, 4H), 3.00 (s, 3H), 4.70 (quin, 1H) (lit.(Meekel et al., 2000) $200 \mathrm{MHz}, \mathbf{8}, \mathrm{R}=$ $\left.n-\mathrm{C}_{16} \mathrm{H}_{33}\right)$.

General procedures for bis(alkyl)azidomethanes, 9a-f. To a stirred solution of 8 (7.41 $\mathrm{mmol}$ ) in DMF (49 $\mathrm{mL})$ at rt was slowly added $\mathrm{NaN}_{3}(36.3 \mathrm{mmol})$. The suspension was heated to $85^{\circ} \mathrm{C}$ for $4 \mathrm{~h}$. After the resulting yellow solution was cooled to rt, hexane $(98 \mathrm{~mL})$ and water $(16 \mathrm{~mL})$ were added. The organic layer was separated and washed successively with saturated $\mathrm{NaHCO}_{3}(8 \mathrm{~mL})$ and saturated $\mathrm{NaCl}(8 \mathrm{~mL})$. The organic layer was dried with $\mathrm{Na}_{2} \mathrm{SO}_{4}$ and concentrated to give a colorless oil, which was purified by flash column chromatography with hexane to give a pure colorless oil (9a-e) or a white solid (9f), which gave a single spot on TLC with hexane $\left(R_{f}=0.41\right)$, in $78-97 \%$ yield.

8-Azidopentadecane, 9a. The general procedure described above afforded a colorless liquid (1.46 g, 78\%); ${ }^{1} \mathrm{H}$ NMR $\left(\mathrm{CDCl}_{3}\right) \delta 0.89$ (m, 6H), 1.20-1.53 (bm, 24H), 3.22 (quin, $\left.1 \mathrm{H}\right) ;{ }^{13} \mathrm{C}$ NMR $\left(\mathrm{CDCl}_{3}\right) \delta 14.3,22.9,26.4,29.4,29.6,32.0,34.6$, 63.4; IR 2924, 2855, 2093; HRMS: for $\mathrm{C}_{15} \mathrm{H}_{32} \mathrm{~N}_{3}[\mathrm{M}+\mathrm{H}]^{+}$calcd 254.2596, found 254.2597. Anal. Calcd for $\mathrm{C}_{15} \mathrm{H}_{31} \mathrm{~N}_{3}$ : C, 71.09; 
H, 12.33; N, 16.58. Found: C, 71.16; H, 12.44; N, 16.44.

9-Azidoheptadecane, 9b. The general procedure described above afforded a colorless liquid (1.73 g, 83\%); ${ }^{1} \mathrm{H}$ NMR $\left(\mathrm{CDCl}_{3}\right) \delta 0.88(\mathrm{t}, 6 \mathrm{H}), 1.20-1.53(\mathrm{bm}, 28 \mathrm{H}), 3.22$ (quin, $\left.1 \mathrm{H}\right) ;{ }^{13} \mathrm{C}$ NMR $\left(\mathrm{CDCl}_{3}\right) \delta 14.3,22.9,26.4,29.5,29.68,29.70,32.1$, 34.6, 63.4; IR 2923, 2854, 2091; HRMS: for $\mathrm{C}_{17} \mathrm{H}_{36} \mathrm{~N}_{3}[\mathrm{M}+\mathrm{H}]^{+}$calcd 254.2848, found 254.2847. Anal. Calcd for $\mathrm{C}_{17} \mathrm{H}_{35} \mathrm{~N}_{3}$ : C, 72.54; H, 12.53; N, 14.93. Found: C, 72.83; H, 12.68; N, 14.86.

10-Azidononadecane, 9c. The general procedure described above afforded a colorless liquid (2.11 g, 92\%); ${ }^{1} \mathrm{H}$ NMR $\left(\mathrm{CDCl}_{3}\right) \delta 0.88$ (t, 6H), 1.20-1.54 (bm, 32H), 3.22 (quin, $\left.1 \mathrm{H}\right) ;{ }^{13} \mathrm{C}$ NMR $\left(\mathrm{CDCl}_{3}\right) \delta 14.3,22.9,26.4,29.5,29.65,29.72,32.1,34.6,63.3$; IR 2922, 2853, 2093 ; HRMS: for $\mathrm{C}_{19} \mathrm{H}_{40} \mathrm{~N}_{3}[\mathrm{M}+\mathrm{H}]^{+}$calcd 282.3161, found 282.3149. Anal. Calcd for $\mathrm{C}_{19} \mathrm{H}_{39} \mathrm{~N}_{3}$ : C, 73.73; H, 12.70; N, 13.57. Found: C, 73.91; H, 12.77; N, 13.42.

11-Azidohenicosane, 9d. The general procedure described above afforded a colorless liquid (2.47 g, 97\%); ${ }^{1} \mathrm{H}$ NMR $\left(\mathrm{CDCl}_{3}\right) \delta 0.89$ (t, 6H), 1.24-1.55 (bm, 36H), 3.22 (quin, $\left.1 \mathrm{H}\right) ;{ }^{13} \mathrm{C}$ NMR $\left(\mathrm{CDCl}_{3}\right) \delta 14.3,22.9,26.3,29.5,29.65,29.71,29.76,29.78,32.1,34.6,63.4$; IR 2922, 2853, 2093; HRMS: for $\mathrm{C}_{21} \mathrm{H}_{44} \mathrm{~N}_{3}[\mathrm{M}+\mathrm{H}]^{+}$calcd 310.3474, found 310.3467. Anal. Calcd for $\mathrm{C}_{21} \mathrm{H}_{43} \mathrm{~N}_{3}$ : C, 74.71; H, 12.84; N, 12.45. Found: C, 74.86; H, 12.98; N, 12.44.

12-Azidotricosane, 9e. The general procedure described above afforded a colorless liquid (2.28 g, 84\%); ${ }^{1} \mathrm{H}$ NMR $\left(\mathrm{CDCl}_{3}\right) \delta 0.89$ (t, 6H), 1.20-1.54 (bm, 40H), 3.23 (quin, $\left.1 \mathrm{H}\right) ;{ }^{13} \mathrm{C}$ NMR $\left(\mathrm{CDCl}_{3}\right) \delta 14.3,22.9,26.4,29.6,29.7,29.75,29.78,29.85,29.86,32.1$, 34.6, 63.4; IR 2921, 2852, 2094; HRMS: for $\mathrm{C}_{23} \mathrm{H}_{48} \mathrm{~N}_{3}[\mathrm{M}+\mathrm{H}]^{+}$calcd 338.3787, found 338.3794. Anal. Calcd for $\mathrm{C}_{23} \mathrm{H}_{47} \mathrm{~N}_{3}$ : C, 75.55; H, 12.96; N, 11.49. Found: C, 75.78; H, 13.10; N, 11.49.

13-Azidopentacosane, 9f. The general procedure described above afforded a white solid (2.63 g, 90\%); mp 36.8-37.4 ${ }^{\circ} \mathrm{C} ;{ }^{1} \mathrm{H}$ NMR $\left(\mathrm{CDCl}_{3}\right) \delta 0.88$ (t, 6H), 1.26-1.52 (bm, 44H), 3.22 (quin, $1 \mathrm{H}) ;{ }^{13} \mathrm{C} \mathrm{NMR}\left(\mathrm{CDCl}_{3}\right) \delta 14.3,22.9,26.4,29.6,29.67,29.75,29.8,29.86,29.88,32.1$, 34.6, 63.4; IR 2913, 2848, 2085; HRMS: for $\mathrm{C}_{25} \mathrm{H}_{52} \mathrm{~N}_{3}[\mathrm{M}+\mathrm{H}]^{+}$calcd 366.4100, found 366.4090. Anal. Calcd for $\mathrm{C}_{25} \mathrm{H}_{51} \mathrm{~N}_{3}$ : C, 72.67; H, 13.06; N, 10.67. Found: C, 76.35; H, $13.14 ; \mathrm{N}, 10.63$.

General procedures for bis(alkyl)methanamines, 10a-f. To a solution of 9 (7.03 $\mathrm{mmol})$ in hexane (35 mL) was added $\mathrm{Pd} / \mathrm{C}$ ( $3 \%$ weight of azidoalkane). The resulting solution was shaken and hydrogenated under $62 \mathrm{psi}$ at rt for $3 \mathrm{~h}$. After sitting overnight, the resulting solution was filtered. The filtrate was concentrated to give a colorless liquid (10a-d) or a white solid (10e,f) in 75-88\% yield. The product was used in the next step without purification.

Pentadecan-8-amine, 10a. The general procedure described above afforded a colorless liquid (1.22 g, 76\%); ${ }^{1} \mathrm{H}$ NMR $\left(\mathrm{CDCl}_{3}\right) \delta 0.88$ (t, 6H), 1.20-1.45 (bm, 26H; 2Hs exchange with $\left.\mathrm{D}_{2} \mathrm{O}\right), 2.67$ (bm, $\left.1 \mathrm{H}\right) ;{ }^{13} \mathrm{C}$ NMR $\left(\mathrm{CDCl}_{3}\right) \delta 14.3,22.8,26.4,29.5,30.0,32.0,38.4$, 51.40; IR 2955, 2921, 2852, 1464, 767.

Heptadecan-9-amine, 10b. The general procedure described above afforded a colorless 
liquid (1.35 g, 75\%); ${ }^{1} \mathrm{H}$ NMR $\left(\mathrm{CDCl}_{3}\right) \delta 0.88$ (t, 6H), 1.20-1.45 (bm, 30H), 2.67 (bm, 1H);

${ }^{13} \mathrm{C}$ NMR $\left(\mathrm{CDCl}_{3}\right) \delta 14.3,22.9,26.4,29.5,29.8,30.0,32.1,38.2,51.4$; IR 2955, 2921, 2852, $1464,800$.

Nonadecan-10-amine, 10c. The general procedure described above afforded a colorless liquid (1.67 g, 84\%); ${ }^{1} \mathrm{H}$ NMR $\left(\mathrm{CDCl}_{3}\right) \delta 0.88$ (t, 6H), $1.20-1.45$ (bm, 34H; 2Hs exchange with $\mathrm{D}_{2} \mathrm{O}$ ), 2.68 (bm, 1H) (lit.(Wescott and Mattern, 2003) $\left.500 \mathrm{MHz}\right) ;{ }^{13} \mathrm{C} \mathrm{NMR}\left(\mathrm{CDCl}_{3}\right) \delta$ 14.3, 22.9, 26.4, 29.5, 29.8, 29.9, 30.0, 32.1, 38.4, 51.4; IR 2955, 2921, 2852, 1464, 797 (lit.(Wescott and Mattern, 2003) KBr pellet).

Henicosan-11-amine, 10d. The general procedure described above afforded a colorless liquid (1.93 g, 88\%); ${ }^{1} \mathrm{H}$ NMR $\left(\mathrm{CDCl}_{3}\right) \delta 0.88$ (t, 6H), $1.20-1.45$ (bm, 38H; 2Hs exchange with $\left.\mathrm{D}_{2} \mathrm{O}\right), 2.67(\mathrm{bm}, 1 \mathrm{H}) ;{ }^{13} \mathrm{C}$ NMR $\left(\mathrm{CDCl}_{3}\right) \delta 14.3,22.9,26.4,29.5,29.83,29.85,29.87$, 30.0, 32.1, 38.3, 51.4; IR 2955, 2920, 2852, 1464, 792.

Tricosan-12-amine, 10e. The general procedure described above afforded a white solid (1.93 g, 81\%); mp 65.5-66.4 ${ }^{\circ} \mathrm{C} ;{ }^{1} \mathrm{H}$ NMR $\left(\mathrm{CDCl}_{3}\right) \delta 0.88$ (t, 6H), $1.13(\mathrm{~s}, 2 \mathrm{H}), 1.20-1.45$ (bm, $42 \mathrm{H}$; 2Hs exchange with $\left.\mathrm{D}_{2} \mathrm{O}\right), 2.67(\mathrm{bm}, 1 \mathrm{H}) ;{ }^{13} \mathrm{C} \mathrm{NMR}\left(\mathrm{CDCl}_{3}\right) \delta 14.3,22.9,26.4,29.6$, 29.86, 29.89, 30.1, 32.1, 33.3, 51.4; IR 2953, 2913, 2848, 1468, 720.

Pentacosan-13-amine, 10f. The general procedure described above afforded a white solid (2.15 g, 86\%); mp 79.7-80.4 ${ }^{\circ} \mathrm{C}$; ${ }^{1} \mathrm{H}$ NMR $\left(\mathrm{CDCl}_{3}\right) \delta 0.88$ (t, 6H), $1.20-1.45$ (bm, 46H; $2 \mathrm{Hs}$ exchange with $\left.\mathrm{D}_{2} \mathrm{O}\right), 2.67$ (bm, $\left.1 \mathrm{H}\right) ;{ }^{13} \mathrm{C}$ NMR $\left(\mathrm{CDCl}_{3}\right) \delta 14.3,22.9,26.4,29.6,29.87$, 29.89, 29.91, 30.1, 32.1, 38.4, 51.4; IR 2952, 2914, 2848, 1467, 720.

General procedures for long chain tri-tert-butyl esters, 10(n,n) An amine 10 (4.95 mmol) was added slowly to a solution of WeNCO $(4.71 \mathrm{mmol})$ in $\mathrm{CH}_{2} \mathrm{Cl}_{2}(20 \mathrm{~mL})$. The resulting transparent solution was stirred at rt. After stirring overnight, the solution was concentrated to afford a crude white solid. This crude product was purified by flash column chromatography to give a white solid, which gave a single spot on TLC (hexane:EtOAc, 5:1, $\left.R_{f}=0.17-0.30\right)$. The isolated solid was then recrystallized from $\mathrm{CH}_{3} \mathrm{CN}(60-87 \%)$.

Di-tert-butyl 4-(2-tert-butoxycarbonylethyl)-4-[3-(1-heptyloctyl)ureido] heptanedioate, 11(7,7). The general procedure described above afforded a white solid (1.89 g, 60\%); mp 109.0-109.8 ${ }^{\circ} \mathrm{C} ;{ }^{1} \mathrm{H}$ NMR (CDCl $) \delta 0.88$ (t, 6H), 1.20-1.35 (bm, 22H), 1.43 (bs, 29H), 1.94 $(\mathrm{m}, 6 \mathrm{H}), 2.23(\mathrm{~m}, 6 \mathrm{H}), 3.52(\mathrm{bm}, 1 \mathrm{H}), 3.76(\mathrm{~d}, 1 \mathrm{H}), 4.41(\mathrm{~s}, 1 \mathrm{H}) ;{ }^{13} \mathrm{C} \mathrm{NMR}\left(\mathrm{CDCl}_{3}\right) \delta 14.0$, 22.6, 25.8, 28.0, 29.2, 29.6, 29.8, 30.6, 31.8, 35.7, 50.2, 56.4, 80.4, 156.4, 173.0; IR 3329, 2922, 2852, 1726, 1650, 1151; HRMS: for $\mathrm{C}_{38} \mathrm{H}_{73} \mathrm{~N}_{2} \mathrm{O}_{7}[\mathrm{M}+\mathrm{H}]^{+}$calcd 669.5418, found 669.5419. Anal. Calcd for $\mathrm{C}_{38} \mathrm{H}_{72} \mathrm{~N}_{2} \mathrm{O}_{7}$ : C, 68.22; H, 10.85; N, 4.19. Found: C, 67.96; H, $10.91 ;$ N, 4.26 .

Di-tert-butyl 4-(2-tert-butoxycarbonylethyl)-4-[3-(1-octylnonyl)ureido] heptanedioate, 11(8,8). The general procedure described above afforded a white solid (2.30 g, 70\%); mp 108.5-109.3 ${ }^{\circ} \mathrm{C}$; ${ }^{1} \mathrm{H}$ NMR $\left(\mathrm{CDCl}_{3}\right) \delta 0.88$ (t, 6H), 1.20-1.35 (bm, 26H), 1.43 (bs, 29H), 1.94 $(\mathrm{m}, 6 \mathrm{H}), 2.23(\mathrm{~m}, 6 \mathrm{H}), 3.51(\mathrm{bm}, 1 \mathrm{H}), 3.75(\mathrm{~d}, 1 \mathrm{H}), 4.41(\mathrm{~s}, 1 \mathrm{H}) ;{ }^{13} \mathrm{C} \mathrm{NMR}\left(\mathrm{CDCl}_{3}\right) \delta$ 14.0, 
22.6, 25.8, 28.0, 29.2, 29.5, 29.7, 29.8, 30.6, 31.8, 35.7, 50.2, 56.4, 80.4, 156.4, 173.0; IR 3317, 2925, 2855, 1730, 1630, 1147; HRMS: for $\mathrm{C}_{40} \mathrm{H}_{77} \mathrm{~N}_{2} \mathrm{O}_{7}[\mathrm{M}+\mathrm{H}]^{+}$calcd 697.5731, found 697.5731. Anal. Calcd for $\mathrm{C}_{40} \mathrm{H}_{76} \mathrm{~N}_{2} \mathrm{O}_{7}$ : C, 68.92; H, 10.99; N, 4.02. Found: C, 69.06; H, 11.17; N, 4.06.

Di-tert-butyl 4-(2-tert-butoxycarbonylethyl)-4-[3-(1-nonyldecyl)ureido] heptanedioate, 11(9,9). The general procedure described above afforded a white solid (2.90 g, 85\%); mp 89.6-90.3 ${ }^{\circ} \mathrm{C} ;{ }^{1} \mathrm{H}$ NMR $\left(\mathrm{CDCl}_{3}\right) \delta 0.88$ (t, 6H), 1.20-1.40 (bm, 30H), 1.43 (bs, 29H), 1.93 $(\mathrm{m}, 6 \mathrm{H}), 2.23(\mathrm{~m}, 6 \mathrm{H}), 3.51(\mathrm{bm}, 1 \mathrm{H}), 3.76(\mathrm{~d}, 1 \mathrm{H}), 4.41(\mathrm{~s}, 1 \mathrm{H}) ;{ }^{13} \mathrm{C} \mathrm{NMR}\left(\mathrm{CDCl}_{3}\right) \delta 14.0$, 22.6, 25.9, 28.0, 29.3, 29.55, 29.56, 29.7, 29.9, 30.7, 31.8, 35.7, 50.2, 56.4, 80.4, 156.4, 173.0; IR 3328, 2922, 2852, 1726, 1651, 1148; HRMS: for $\mathrm{C}_{42} \mathrm{H}_{81} \mathrm{~N}_{2} \mathrm{O}_{7}[\mathrm{M}+\mathrm{H}]^{+}$calcd 725.6044, found 725.6028. Anal. Calcd for $\mathrm{C}_{42} \mathrm{H}_{80} \mathrm{~N}_{2} \mathrm{O}_{7}$ : C, 69.57; H, 11.12; N, 3.86. Found: C, 69.60; H, 11.22; N, 3.85.

Di-tert-butyl 4-(2-tert-butoxycarbonylethyl)-4-[3-(1-decylundecyl)ureido]heptandioate, 11(10,10). The general procedure described above afforded a white solid (2.73 g, 77\%); mp 87.0-87.6 ${ }^{\circ} \mathrm{C} ;{ }^{1} \mathrm{H}$ NMR $\left(\mathrm{CDCl}_{3}\right) \delta 0.88(\mathrm{t}, 6 \mathrm{H}), 1.20-1.40$ (bm, 34H), 1.44 (bs, 29H), 1.93 $(\mathrm{m}, 6 \mathrm{H}), 2.23(\mathrm{~m}, 6 \mathrm{H}), 3.52(\mathrm{bm}, 1 \mathrm{H}), 3.86(\mathrm{~d}, 1 \mathrm{H}), 4.47(\mathrm{~s}, 1 \mathrm{H}) ;{ }^{13} \mathrm{C} \mathrm{NMR}\left(\mathrm{CDCl}_{3}\right) \delta 14.1$, 22.6, 25.9, 28.02, 28.04, 29.3, 29.57, 29.61, 29.68, 29.9, 30.6, 31.9, 35.7, 50.2, 56.4, 80.4, 156.4, 173.0; IR 3376, 2919, 2850, 1730, 1675, 1146; HRMS: for $\mathrm{C}_{44} \mathrm{H}_{85} \mathrm{~N}_{2} \mathrm{O}_{7}[\mathrm{M}+\mathrm{H}]^{+}$ calcd 753.6357, found 753.6376. Anal. Calcd for $\mathrm{C}_{44} \mathrm{H}_{84} \mathrm{~N}_{2} \mathrm{O}_{7}$ : C, 70.17; H, 11.24; N, 3.72 . Found: C, 69.89; H, 11.33; N, 3.70.

Di-tert-butyl 4-(2-tert-butoxycarbonylethyl)-4-[3-(1-undecyldodecyl)ureido] heptanedioate, 11(11,11). The general procedure described above afforded a white solid (3.16 g, 86\%); mp 92.9-93.8 ${ }^{\circ} \mathrm{C} ;{ }^{1} \mathrm{H}$ NMR $\left(\mathrm{CDCl}_{3}\right) \delta 0.88(\mathrm{t}, 6 \mathrm{H}), 1.20-1.40(\mathrm{bm}, 38 \mathrm{H}), 1.44$ (bs, 29H), 1.94 $(\mathrm{m}, 6 \mathrm{H}), 2.24(\mathrm{~m}, 6 \mathrm{H}), 3.52(\mathrm{bm}, 1 \mathrm{H}), 3.83(\mathrm{~m}, 1 \mathrm{H}), 4.45(\mathrm{~s}, 1 \mathrm{H}) ;{ }^{13} \mathrm{C} \mathrm{NMR}\left(\mathrm{CDCl}_{3}\right) \delta 14.0$, 22.6, 25.9, 28.0, 29.3, 29.58, 29.60, 29.7, 29.9, 30.7, 31.9, 35.7, 50.2, 56.4, 80.4, 156.4, 173.0; IR 3374, 2920, 2850, 1722, 1676, 1148; HRMS: for $\mathrm{C}_{46} \mathrm{H}_{89} \mathrm{~N}_{2} \mathrm{O}_{7}[\mathrm{M}+\mathrm{H}]^{+}$calcd 781.6670, found 781.6657. Anal. Calcd for $\mathrm{C}_{46} \mathrm{H}_{88} \mathrm{~N}_{2} \mathrm{O}_{7}$ : C, 70.72; H, 11.35; N, 3.59. Found: C, 70.49; H, 11.37; N, 3.67.

Di-tert-butyl 4-(2-tert-butoxycarbonylethyl)-4-[3-(1-dodecyltridecyl)ureido] heptanedioate, 11(12,12). The general procedure described above afforded a white solid (3.49 g, 87\%); mp 73.8-74.3 ${ }^{\circ} \mathrm{C} ;{ }^{1} \mathrm{H}$ NMR $\left(\mathrm{CDCl}_{3}\right) \delta 0.89$ (t, 6H), 1.20-1.40 (bm, 42H), 1.44 (bs, 29H), 1.94 $(\mathrm{m}, 6 \mathrm{H}), 2.24(\mathrm{~m}, 6 \mathrm{H}), 3.51(\mathrm{bm}, 1 \mathrm{H}), 3.80(\mathrm{~d}, 1 \mathrm{H}), 4.44(\mathrm{~s}, 1 \mathrm{H}) ;{ }^{13} \mathrm{C} \mathrm{NMR}\left(\mathrm{CDCl}_{3}\right) \delta 14.1$, 22.7, 25.9, 28.0, 29.3, 29.61, 29.63, 29.66, 29.70, 29.85, 30.6, 31.9, 35.7, 50.2, 56.4, 80.4, 156.4, 173.1; IR 3319, 2917, 2851, 1730, 1654, 1147; HRMS: for $\mathrm{C}_{48} \mathrm{H}_{93} \mathrm{~N}_{2} \mathrm{O}_{7}[\mathrm{M}+\mathrm{H}]^{+}$ calcd 809.6983, found 809.7014. Anal. Calcd for $\mathrm{C}_{48} \mathrm{H}_{92} \mathrm{~N}_{2} \mathrm{O}_{7}$ : C, 71.24; H, 11.46; N, 3.46. Found: C, 71.43; H, 11.58; N, 3.50.

General procedures for long chain triacid, 3CUr1(n,n). A tri-tert-butyl ester 11(n,n) (3.00 $\mathrm{mmol}$ ) was dissolved in $99 \% \mathrm{HCOOH}$ so that the concentration was $0.1 \mathrm{M}$. Some mixtures needed warming to completely dissolve 11(n,n). Once dissolved to give a clear colorless solution, the mixture was stirred at rt. After stirring $9 \mathrm{~h}$, the resulting milky white solution was concentrated. The white solid was recrystallized from EtOAc to yield a white solid (77- 
$90 \%)$.

4-(2-Carboxyethyl)-4-[3-(1-heptyloctyl)ureido] heptanedioic acid, 3CUr1(7,7). The general procedure described above afforded a white solid (1.28 g, 85\%); mp $168.5-169.2{ }^{\circ} \mathrm{C} ;{ }^{1} \mathrm{H}$ NMR (CD $\left.{ }_{3} \mathrm{OD}\right) \delta 0.89(\mathrm{t}, 6 \mathrm{H}), 1.20-1.40(\mathrm{bm}, 24 \mathrm{H}), 1.44(\mathrm{~m}, 2 \mathrm{H}), 1.95(\mathrm{~m}, 6 \mathrm{H}), 2.28(\mathrm{~m}$, $6 \mathrm{H}), 3.55$ (bm, $1 \mathrm{H}) ;{ }^{13} \mathrm{C}$ NMR (DMSO-d $\left.{ }_{6}\right) \delta 14.4,22.5$, 25.8, 28.6, 29.2, 29.4, 30.5, 31.7, 35.8, 48.3, 55.4, 157.4, 175.0; IR 3405, 2924, 2855, 1732, 1700 1585; HRMS: for $\mathrm{C}_{26} \mathrm{H}_{49} \mathrm{~N}_{2} \mathrm{O}_{7}[\mathrm{M}+\mathrm{H}]^{+}$calcd 501.3540, found 501.3562. Anal. Calcd for $\mathrm{C}_{26} \mathrm{H}_{48} \mathrm{~N}_{2} \mathrm{O}_{7}$ : C, 62.37; H, 9.66; N, 5.60. Found: C, 62.28; H, 9.57; N 5.52.

4-(2-Carboxyethyl)-4-[3-(1-octylnonyl)ureido] heptanedioic acid, 3CUr1(8,8). The general procedure described above afforded a white solid (1.36g, 86\%); mp $164.3-165.1{ }^{\circ} \mathrm{C} ;{ }^{1} \mathrm{H}$ NMR (CD $\left.{ }_{3} \mathrm{OD}\right) \delta 0.89(\mathrm{t}, 6 \mathrm{H}), 1.20-1.40(\mathrm{bm}, 28 \mathrm{H}), 1.44(\mathrm{~m}, 2 \mathrm{H}), 1.95(\mathrm{~m}, 6 \mathrm{H}), 2.28(\mathrm{~m}$, 6H), 3.54 (bm, 1H); ${ }^{13} \mathrm{C}$ NMR (DMSO-d $)_{6} \delta 14.7,22.8,26.1,28.8,29.4,29.69,29.73,30.8$, 31.9, 36.0, 48.5, 55.6, 157.6, 175.2; IR 3404, 2921, 2852, 1730, 1699, 1556; HRMS: for $\mathrm{C}_{28} \mathrm{H}_{53} \mathrm{~N}_{2} \mathrm{O}_{7}[\mathrm{M}+\mathrm{H}]^{+}$calcd 529.3853, found 529.3837. Anal. Calcd for $\mathrm{C}_{28} \mathrm{H}_{52} \mathrm{~N}_{2} \mathrm{O}_{7}$ : C, 63.61; H, 9.91; N, 5.30. Found: C, 63.65; H, 9.94; N 5.20.

4-(2-Carboxyethyl)-4-[3-(1-nonyldecyl)ureido] heptanedioic acid, 3CUr1(9,9). The general procedure described above afforded a white solid (1.39g 83\%); mp 165.1-165.7 ${ }^{\circ} \mathrm{C} ;{ }^{1} \mathrm{H}$ NMR $\left(\mathrm{CD}_{3} \mathrm{OD}\right) \delta 0.86$ (t, 6H), 1.20-1.35 (bm, 30H), 1.41 (m, 2H), $1.92(\mathrm{~m}, 6 \mathrm{H}), 2.25(\mathrm{~m}, 6 \mathrm{H})$, 3.51 (bm, $1 \mathrm{H}) ;{ }^{13} \mathrm{C}$ NMR (DMSO-d $\left.d_{6}\right) \delta 14.4$, 22.5, 25.8, 28.6, 29.1, 29.36, 29.43, 30.5, 31.7, 35.7, 48.3, 55.4, 157.3, 174.9; IR 3405, 2921, 2852, 1730, 1699, 1554; HRMS: for $\mathrm{C}_{30} \mathrm{H}_{57} \mathrm{~N}_{2} \mathrm{O}_{7}[\mathrm{M}+\mathrm{H}]^{+}$calcd 557.4166, found 557.4172. Anal. Calcd for $\mathrm{C}_{30} \mathrm{H}_{56} \mathrm{~N}_{2} \mathrm{O}_{7}$ : C, 64.72; H, 10.14; N, 5.03. Found: C, 64.73; H, 10.20; N 5.01.

4-(2-Carboxyethyl)-4-[3-(1-decylundecyl)ureido] heptanedioic acid, 3CUr1(10,10). The general procedure described above afforded a white solid $(1.42,81 \%)$; mp $162.8-163.5^{\circ} \mathrm{C}$; ${ }^{1} \mathrm{H}$ NMR $\left(\mathrm{CD}_{3} \mathrm{OD}\right) \delta 0.86(\mathrm{t}, 6 \mathrm{H}), 1.20-1.35$ (bm, 34H), $1.41(\mathrm{~m}, 2 \mathrm{H}), 1.87(\mathrm{t}, 6 \mathrm{H}), 2.25(\mathrm{t}$, $6 \mathrm{H}), 3.51$ (bm, 1H); ${ }^{13} \mathrm{C}$ NMR (DMSO-d $\left.{ }_{6}\right) \delta 14.4,22.5,25.8,28.5,29.1,29.41,29.43,30.5$, 31.7, 35.6, 48.3, 55.4, 157.3, 174.9; IR 3404, 2921, 2852, 1731, 1699, 1557; HRMS: for $\mathrm{C}_{32} \mathrm{H}_{61} \mathrm{~N}_{2} \mathrm{O}_{7}[\mathrm{M}+\mathrm{H}]^{+}$calcd 585.4479, found 585.4501. Anal. Calcd for $\mathrm{C}_{32} \mathrm{H}_{60} \mathrm{~N}_{2} \mathrm{O}_{7}$ : C, 65.72; H, 10.34; N, 4.79. Found: C, 65.52; H, 10.31; N 4.78.

4-(2-Carboxyethyl)-4-[3-(1-undecyldodecyl)ureido] heptanedioic acid, 3CUr1(11,11). The general procedure described above afforded a white solid (1.56 g, 85\%); mp 164.6-165.1 ${ }^{\circ} \mathrm{C}$; ${ }^{1} \mathrm{H}$ NMR (CD $\left.\mathrm{CD}_{3}\right) \delta 0.86$ (t, 6H), 1.20-1.35 (bm, 38H), 1.41 (m, 2H), 1.92 (m, 6H), 2.25 (m, 6H), 3.51 (bm, 1H); ${ }^{13} \mathrm{C}$ NMR (DMSO-d $\left.{ }_{6}\right) \delta 14.4,22.5,25.7,28.5,29.1,29.41,29.44,30.5$, 31.7, 35.6, 48.2, 55.3, 157.3, 174.9; IR 3404, 2921, 2852, 1732, 1699, 1557; HRMS: for $\mathrm{C}_{34} \mathrm{H}_{65} \mathrm{~N}_{2} \mathrm{O}_{7}[\mathrm{M}+\mathrm{H}]^{+}$calcd 613.4792, found 613.4792. Anal. Calcd for $\mathrm{C}_{34} \mathrm{H}_{64} \mathrm{~N}_{2} \mathrm{O}_{7}$ : C, 66.63; H, 10.53; N, 4.57. Found: C, 66.77; H, 10.64; N 4.56.

4-(2-Carboxyethyl)-4-[3-(1-dodecyltridecyl)ureido] heptanedioic acid, 3CUr1(12,12). The general procedure described above afforded a white solid (1.67 g, 87\%); mp 163.3-163.9 ${ }^{\circ} \mathrm{C}$; ${ }^{1} \mathrm{H}$ NMR (CD $\left.{ }_{3} \mathrm{OD}\right) \delta 0.86$ (t, 6H), 1.20-1.35 (bm, 42H), 1.41 (m, 2H), 1.95 (m, 6H), 2.25 (m, 6H), 3.51 (bm, 1H); ${ }^{13} \mathrm{C}$ NMR $\left(\mathrm{DMSO}_{-}\right.$) $\delta 14.6,22.8,26.0,28.8,29.4,29.67,29.71,29.75$, 
30.8, 32.0, 35.9, 48.5, 55.6, 157.6, 175.2; IR 3403, 2920, 2851, 1731, 1700, 1559; HRMS: for $\mathrm{C}_{36} \mathrm{H}_{69} \mathrm{~N}_{2} \mathrm{O}_{7}[\mathrm{M}+\mathrm{H}]^{+}$calcd 641.5105, found 641.5095. Anal. Calcd for $\mathrm{C}_{36} \mathrm{H}_{68} \mathrm{~N}_{2} \mathrm{O}_{7}$ : C, 67.46; H, 10.69; N, 4.37. Found: C, 67.31; H, 10.62, N 4.34.

General procedures for long chain tri-tert-butyl esters, 12(n,n). Dialkylcarbinol 7(a-f) (2.2 mmol), WeNCO (1.70 g, $3.85 \mathrm{mmol})$, and $\mathrm{Et}_{3} \mathrm{~N}(3.9 \mathrm{~mL})$ were combined in a $50-\mathrm{mL}$ round bottom flask and stirred at $95^{\circ} \mathrm{C}$ for $48 \mathrm{~h}$. The mixture was allowed to cool to rt, and diluted with $\mathrm{Et}_{2} \mathrm{O}(40 \mathrm{~mL})$. The resulting solution was washed with $2-\mathrm{M} \mathrm{HCl}(3 \times 6 \mathrm{~mL})$, then satd $\mathrm{NaHCO}_{3}(3 \times 6 \mathrm{~mL})$, and finally satd $\mathrm{NaCl}(1 \times 6 \mathrm{~mL})$. The solution was dried with $\mathrm{MgSO}_{4}$, filtered and concentrated to a yellow solid. The resulting solid was purified via flash chromatography with 9:1 hex/EtOAc to give a white solid (45-55 \% yield).

Di-tert-butyl 4-(2-(tert-butoxycarbonyl)ethyl)-4-(1-heptyloctyloxycarbonylamino)heptanedioate, 12(7,7). The general procedure gave a white solid (50 \% yield). mp 54.5$57.0{ }^{\circ} \mathrm{C} ;{ }^{1} \mathrm{H}$ NMR $\left(\mathrm{CDCl}_{3}\right) \delta 0.88(\mathrm{t}, 3 \mathrm{H}), 1.25$ (m, 22H), 1.42 (s, 27H), 1.57 (broad p, 2H), 1.91 (t, 6H), 2.21 (t, 6H), 3.96 (broad t, $2 \mathrm{H}), 4.69$ (broad s, $1 \mathrm{H}) ;{ }^{13} \mathrm{C} \mathrm{NMR}\left(\mathrm{CDCl}_{3}\right) \delta 14.1$, 22.7, 25.9, 28.08, 28.99, 29.35, 29.37, 29.58, 29.62, 29.67, 29.69, 29.72, 30.2, 31.9, 56.4, 64.6, 80.6, 154.6, 172.6; IR 3371, 2916, 1727, 1703, 1363, 1142, 1075; IR 3354, 2922, 1729, 1713, $1148 \mathrm{~cm}^{-1}$; HRMS (FAB+) calcd for $\mathrm{C}_{38} \mathrm{H}_{71} \mathrm{NO}_{8}[\mathrm{M}+\mathrm{H}]^{+}$669.9915, found 670.5266; anal. calcd for $\mathrm{C}_{38} \mathrm{H}_{71} \mathrm{NO}_{8}$ : C, 68.12; H, 10.68; N, 2.09. Found: C, 68.28; H, 10.63; N, 2.15.

Di-tert-butyl 4-(2-(tert-butoxycarbonyl)ethyl)-4-(1-octylnonyloxycarbonylamino)heptanedioate, 12(8,8). The general procedure gave a white solid $(45 \%)$. mp $74.4-75.6{ }^{\circ} \mathrm{C}$; ${ }^{1} \mathrm{H}$ NMR $\left(\mathrm{CDCl}_{3}\right) \delta 0.89$ (t, 3H), 1.27 (m, 26H), 1.43 (s, 27H), 1.59 (broad p, 2H), 1.91 (t, $3 \mathrm{H}), 2.21$ (t, 3H), 3.98 (broad t, $2 \mathrm{H}), 4.69$ (broad s, $1 \mathrm{H}) ;{ }^{13} \mathrm{C} \mathrm{NMR}\left(\mathrm{CDCl}_{3}\right) \delta 14.13,22.70$, 25.90, 28.08, 29.00, 29.34, 29.37, 29.57, 29.62, 29.67, 29.70, 30.16, 31.93, 56.37, 64.55, 80.57, 154.75, 172.57; IR 3362, 2916, 1727, 1713, $1142 \mathrm{~cm}^{-1}$; HRMS (FAB+) calcd for $\mathrm{C}_{40} \mathrm{H}_{75} \mathrm{NO}_{8}[\mathrm{M}+\mathrm{H}]^{+}$698.0457, found 698.5594; anal. calcd for $\mathrm{C}_{40} \mathrm{H}_{75} \mathrm{NO}_{8}$ : C, 68.83; $\mathrm{H}$, 10.83; N, 2.01. Found: C, 69.05; H, 10.87; N, 1.94.

Di-tert-butyl 4-(2-(tert-butoxycarbonyl)ethyl)-4-(1-nonyldecyloxycarbonylamino)heptanedioate, 12(9,9). The general procedure gave a white solid (55 \%). mp $71.4-72.5{ }^{\circ} \mathrm{C}$; ${ }^{1} \mathrm{H}$ NMR $\left(\mathrm{CDCl}_{3}\right) \delta 0.89$ (t, 3H), 1.27 (m, 26H), 1.43 (s, 27H), 1.59 (broad p, 2H), 1.91 (t, $3 \mathrm{H}), 2.21$ (t, 3H), 3.98 (broad t, $2 \mathrm{H}), 4.69$ (broad s, $1 \mathrm{H}) ;{ }^{13} \mathrm{C} \mathrm{NMR}\left(\mathrm{CDCl}_{3}\right) \delta 14.13,22.70$, 25.90, 28.08, 29.00, 29.34, 29.37, 29.57, 29.62, 29.67, 29.70, 30.16, 31.93, 56.37, 64.55, 80.57, 154.75, 172.57; IR 3357, 2916, 1722, 1708, $1142 \mathrm{~cm}^{-1}$; HRMS (FAB+) calcd for $\mathrm{C}_{42} \mathrm{H}_{79} \mathrm{NO}_{8}[\mathrm{M}+\mathrm{H}]^{+}$726.0998, found 726.5880; anal. calcd for $\mathrm{C}_{42} \mathrm{H}_{79} \mathrm{NO}_{8}$ : C, 69.48; $\mathrm{H}$, 10.97; N, 1.93. Found: C, 69.51; H, 10.87; N, 1.92.

Di-tert-butyl 4-(2-(tert-butoxycarbonyl)ethyl)-4-(1-decylundecyloxycarbonylamino)heptanedioate, 12(10,10). The general procedure gave a white solid (46 \%). $\mathrm{mp}$ 75.6-76.2 ${ }^{\circ} \mathrm{C} ;{ }^{1} \mathrm{H} \mathrm{NMR}\left(\mathrm{CDCl}_{3}\right) \delta 0.89$ (t, 3H), 1.27 (m, 26H), 1.43 (s, 27H), 1.59 (broad p, 2H), 1.91 (t, 3H), 2.21 (t, 3H), 3.98 (broad t, 2H), 4.69 (broad s, $1 \mathrm{H}) ;{ }^{13} \mathrm{C} \mathrm{NMR}\left(\mathrm{CDCl}_{3}\right) \delta 14.13$, 22.70, 25.90, 28.08, 29.00, 29.34, 29.37, 29.57, 29.62, 29.67, 29.70, 30.16, 31.93, 56.37, 64.55, 80.57, 154.75, 172.57; IR 3358, 2918, 1728, 1712, $1146 \mathrm{~cm}^{-1}$; HRMS (FAB+) calcd 
for $\mathrm{C}_{44} \mathrm{H}_{83} \mathrm{NO}_{8}[\mathrm{M}+\mathrm{H}]^{+} 754.1540$, found 754.6234; anal. calcd for $\mathrm{C}_{44} \mathrm{H}_{83} \mathrm{NO}_{8}$ : C, 70.08; $\mathrm{H}$, 11.09; N, 1.86. Found: C, 70.25; H, 11.11; N, 1.90.

Di-tert-butyl 4-(2-(tert-butoxycarbonyl)ethyl)-4-(1-undecyldodecyloxycarbonylamino)heptanedioate, 12(11,11). The general procedure gave a white solid (51\%). mp 76.8-77.4 ${ }^{\circ} \mathrm{C} ;{ }^{1} \mathrm{H} \mathrm{NMR}\left(\mathrm{CDCl}_{3}\right) \delta 0.89$ (t, 3H), 1.27 (m, 26H), 1.43 (s, 27H), 1.59 (broad p, 2H), 1.91 (t, 3H), 2.21 (t, 3H), 3.98 (broad t, $2 \mathrm{H}), 4.69$ (broad s, $1 \mathrm{H}) ;{ }^{13} \mathrm{C}$ NMR $\left(\mathrm{CDCl}_{3}\right) \delta 14.13$, 22.70, 25.90, 28.08, 29.00, 29.34, 29.37, 29.57, 29.62, 29.67, 29.70, 30.16, 31.93, 56.37, 64.55, 80.57, 154.75, 172.57; IR 3353, 2915, 1729, 1713, $1146 \mathrm{~cm}^{-1}$; HRMS (FAB+) calcd for $\mathrm{C}_{46} \mathrm{H}_{87} \mathrm{NO}_{8}[\mathrm{M}+\mathrm{H}]^{+} 782.2082$, found 782.6492; anal. calcd for $\mathrm{C}_{46} \mathrm{H}_{87} \mathrm{NO}_{8}$ : C, 70.64; $\mathrm{H}$, 11.21; N, 1.79. Found: C, 70.77; H, 11.35; N, 1.81.

Di-tert-butyl 4-(2-(tert-butoxycarbonyl)ethyl)-4-(1-dodecyltridecyloxycarbonylamino)heptanedioate, 12(12,12); The general procedure gave a white solid (51 \%). mp 67.5-67.8 ${ }^{\circ} \mathrm{C} ;{ }^{1} \mathrm{H}$ NMR $\left(\mathrm{CDCl}_{3}\right) \delta 0.89$ (t, 3H), 1.27 (m, 26H), 1.43 (s, 27H), 1.59 (broad p, 2H), 1.91 (t, 3H), 2.21 (t, 3H), 3.98 (broad t, 2H), 4.69 (broad s, $1 \mathrm{H}) ;{ }^{13} \mathrm{C} \mathrm{NMR}\left(\mathrm{CDCl}_{3}\right) \delta 14.13$, 22.70, 25.90, 28.08, 29.00, 29.34, 29.37, 29.57, 29.62, 29.67, 29.70, 30.16, 31.93, 56.37, 64.55, 80.57, 154.75, 172.57; IR 3357, 2921, 1727, 1708, 1530, 1363, 1257, $1142 \mathrm{~cm}^{-1}$; HRMS (FAB+) calcd for $\mathrm{C}_{48} \mathrm{H}_{91} \mathrm{NO}_{8}[\mathrm{M}+\mathrm{H}]^{+}$810.2624, found 810.6818; anal. calcd for $\mathrm{C}_{48} \mathrm{H}_{91} \mathrm{NO}_{8}$ : C, 71.15; H, 11.32; N, 1.73. Found: C, 71.37; H, 11.11; N, 1.77.

General procedures for two-tailed triacids, 3CCb1(n,n) Compound 12(n,n) (1 mmol) was combined with THF ( $\mathrm{mL}$ ) in a $50 \mathrm{~mL}$ round bottom flask and all solids were allowed to dissolve. $\mathrm{HCO}_{2} \mathrm{H}(\mathrm{mL})$ was added and the solution stirred at $\mathrm{rt}$ for $48 \mathrm{~h}$. The solution was concentrated to a white solid and then dried under high vacuum for $48 \mathrm{~h}$ to give a white solid. Solid was recrystallized from $\mathrm{HOAc} / \mathrm{H}_{2} \mathrm{O}$ to give white crystals (92-98\%).

4-(2-Carboxyethyl)-4-(1-heptyloctyloxycarbonylamino)heptanedioic acid, 3CCb1(7,7). The general procedure gave white crystals $(92 \%)$. mp 159.5-160.3 ${ }^{\circ} \mathrm{C} ;{ }^{1} \mathrm{H}$ NMR $\left(\mathrm{CDCl}_{3}\right) \delta 0.88$ (t, 3H), 1.25 (m, 22H), 1.42 (s, 27H), 1.57 (broad p, 2H), 1.91 (t, 6H), 2.21 (t, 6H), 3.96 (broad t, 2H), 4.69 (broad s, $1 \mathrm{H}) ;{ }^{13} \mathrm{C} \mathrm{NMR}\left(\mathrm{CDCl}_{3}\right) \delta 14.1,22.7,25.9,28.08,28.99,29.35$, 29.37, 29.58, 29.62, 29.67, 29.69, 29.72, 30.2, 31.9, 56.4, 64.6, 80.6, 154.6, 172.6; IR 3371, 2916, 1727, 1703, 1363, 1142, 1075; IR 3352, 2924, 1740, 1701, 1530, $1243 \mathrm{~cm}^{-1}$; HRMS $\left(\mathrm{FAB}+\right.$ ) calcd for $\mathrm{C}_{26} \mathrm{H}_{47} \mathrm{NO}_{8}[\mathrm{M}+\mathrm{H}]^{+}$501.6664, found 502.3370; anal. calcd for $\mathrm{C}_{26} \mathrm{H}_{47} \mathrm{NO}_{8}$ : C, 62.25; H, 9.44; N, 2.79. Found: C, 62.18; H, 9.37; N, 2.79.

4-(2-Carboxyethyl)-4-(1-octylnonyloxycarbonylamino)heptanedioic acid, 3CCb1(8,8). The general procedure gave white crystals $(93 \%)$. mp $133.0-133.9^{\circ} \mathrm{C} ;{ }^{1} \mathrm{H} \mathrm{NMR}\left(\mathrm{CDCl}_{3}\right) \delta 0.88$ (t, 3H), 1.25 (m, 22H), 1.42 (s, 27H), 1.57 (broad p, 2H), 1.91 (t, 6H), $2.21(\mathrm{t}, 6 \mathrm{H}), 3.96$ (broad t, 2H), 4.69 (broad s, $1 \mathrm{H}) ;{ }^{13} \mathrm{C} \mathrm{NMR}\left(\mathrm{CDCl}_{3}\right) \delta 14.1,22.7,25.9,28.08,28.99,29.35$, 29.37, 29.58, 29.62, 29.67, 29.69, 29.72, 30.2, 31.9, 56.4, 64.6, 80.6, 154.6, 172.6; IR 3371, 2916, 1727, 1703, 1363, 1142, 1075; IR 3352, 2916, 1737, 1698, 1531, $1238 \mathrm{~cm}^{-1}$; HRMS $(\mathrm{FAB}+)$ calcd for $\mathrm{C}_{28} \mathrm{H}_{51} \mathrm{NO}_{8}[\mathrm{M}+\mathrm{H}]^{+}$529.7206, found 530.3716; anal. calcd for $\mathrm{C}_{28} \mathrm{H}_{51} \mathrm{NO}_{8}$ : C, 63.49; H, 9.70; N, 2.64. Found: C, 63.21; H, 9.78; N, 2.67. 
The general procedure gave white crystals (94\%). mp 130.4-130.9 ${ }^{\circ} \mathrm{C} ;{ }^{1} \mathrm{H} \mathrm{NMR}\left(\mathrm{CDCl}_{3}\right) \delta$ 0.88 (t, 3H), 1.25 (m, 22H), 1.42 (s, 27H), 1.57 (broad p, 2H), 1.91 (t, 6H), 2.21 (t, 6H), 3.96 (broad t, 2H), 4.69 (broad s, $1 \mathrm{H}) ;{ }^{13} \mathrm{C} \mathrm{NMR}\left(\mathrm{CDCl}_{3}\right) \delta 14.1,22.7,25.9,28.08,28.99,29.35$, 29.37, 29.58, 29.62, 29.67, 29.69, 29.72, 30.2, 31.9, 56.4, 64.6, 80.6, 154.6, 172.6; IR 3371, 2916, 1727, 1703, 1363, 1142, 1075; IR 3357, 2921, 1737, 1699, 1531, $1244 \mathrm{~cm}^{-1}$; HRMS (FAB+) calcd for $\mathrm{C}_{30} \mathrm{H}_{55} \mathrm{NO}_{8}[\mathrm{M}+\mathrm{H}]^{+}$557.7748, found 558.3959; anal. calcd for $\mathrm{C}_{30} \mathrm{H}_{55} \mathrm{NO}_{8}$ : C, 64.60; H, 9.94; N, 2.51. Found: C, 64.48; H, 10.03; N, 2.52.

4-(2-Carboxyethyl)-4-(1-decylundecyloxycarbonylamino)heptanedioic acid, 3CCb1(10,10). The general procedure gave white crystals $(94 \%)$. mp 132.5-133.3 ${ }^{\circ} \mathrm{C} ;{ }^{1} \mathrm{H} \mathrm{NMR}\left(\mathrm{CDCl}_{3}\right) \delta$ 0.88 (t, 3H), 1.25 (m, 22H), 1.42 (s, 27H), 1.57 (broad p, 2H), 1.91 (t, 6H), 2.21 (t, 6H), 3.96 (broad t, 2H), 4.69 (broad s, $1 \mathrm{H}) ;{ }^{13} \mathrm{C} \mathrm{NMR}\left(\mathrm{CDCl}_{3}\right) \delta 14.1,22.7,25.9,28.08,28.99,29.35$, 29.37, 29.58, 29.62, 29.67, 29.69, 29.72, 30.2, 31.9, 56.4, 64.6, 80.6, 154.6, 172.6; IR 3371, 2916, 1727, 1703, 1363, 1142, 1075; IR 3433, 2914, 1741, 1703, 1686, 1514, 1283, $1234 \mathrm{~cm}^{-}$ 1; HRMS (FAB+) calcd for $\mathrm{C}_{32} \mathrm{H}_{59} \mathrm{NO}_{8}[\mathrm{M}+\mathrm{H}]^{+}$585.8289, found 586.4304; anal. calcd for $\mathrm{C}_{32} \mathrm{H}_{59} \mathrm{NO}_{8}$ : C, 65.61; H, 10.15; N, 2.39. Found: C, 65.53; H, 10.35; N, 2.38.

4-(2-Carboxyethyl)-4-(1-undecyldodecyloxycarbonylamino)heptanedioic acid, 3CCb1(11,11).

The general procedure gave white crystals (96 \%). mp 128.3-129.3 ${ }^{\circ} \mathrm{C} ;{ }^{1} \mathrm{H}$ NMR $\left(\mathrm{CDCl}_{3}\right) \delta$ 0.88 (t, 3H), 1.25 (m, 22H), 1.42 (s, 27H), 1.57 (broad p, 2H), 1.91 (t, 6H), 2.21 (t, 6H), 3.96 (broad t, 2H), 4.69 (broad s, $1 \mathrm{H}) ;{ }^{13} \mathrm{C} \mathrm{NMR}\left(\mathrm{CDCl}_{3}\right) \delta 14.1,22.7,25.9,28.08,28.99,29.35$, 29.37, 29.58, 29.62, 29.67, 29.69, 29.72, 30.2, 31.9, 56.4, 64.6, 80.6, 154.6, 172.6; IR 3371, 2916, 1727, 1703, 1363, 1142, 1075; IR 3358, 2916, 1740, 1698, 1691, 1536, 1294, $1267 \mathrm{~cm}^{-}$ 1; HRMS (FAB+) calcd for $\mathrm{C}_{34} \mathrm{H}_{63} \mathrm{NO}_{8}[\mathrm{M}+\mathrm{H}]^{+}$613.8831, found 614.4680; anal. calcd for $\mathrm{C}_{34} \mathrm{H}_{63} \mathrm{NO}_{8}$ : C, 66.52; H, 10.34; N, 2.28. Found: C, 66.42; H, 10.53; N, 2.16.

\section{4-(2-Carboxyethyl)-4-(1-dodecyltridecyloxycarbonylamino)heptanedioic acid,}

\section{CCb1(12,12)}

The general procedure gave white crystals (98\%). mp 125.0-125.8 ${ }^{\circ} \mathrm{C} ;{ }^{1} \mathrm{H}$ NMR $\left(\mathrm{CDCl}_{3}\right) \delta$ 0.88 (t, 3H), 1.25 (m, 22H), 1.42 (s, 27H), 1.57 (broad p, 2H), 1.91 (t, 6H), 2.21 (t, 6H), 3.96 (broad t, 2H), 4.69 (broad s, $1 \mathrm{H}) ;{ }^{13} \mathrm{C} \mathrm{NMR}\left(\mathrm{CDCl}_{3}\right) \delta 14.1,22.7,25.9,28.08,28.99,29.35$, 29.37, 29.58, 29.62, 29.67, 29.69, 29.72, 30.2, 31.9, 56.4, 64.6, 80.6, 154.6, 172.6; IR 3371, 2916, 1727, 1703, 1363, 1142, 1075; IR 3358, 2918, 1741, 1702, 1514, 1266, $1234 \mathrm{~cm}^{-1}$; HRMS (FAB+) calcd for $\mathrm{C}_{36} \mathrm{H}_{67} \mathrm{NO}_{8}[\mathrm{M}+\mathrm{H}]^{+}$641.9373, found 642.4896; anal. calcd for $\mathrm{C}_{36} \mathrm{H}_{67} \mathrm{NO}_{8}$ : C, 67.36; H, 10.52; N, 2.18. Found: C, 67.46; H, 10.60; N, 2.28.

General procedure of the preparation of dialkylmalonate diesters, 13a-e. Sodium metal (1.886 g, $82 \mathrm{mmol}$ ) was added into a three-neck-round bottom flask containing absolute ethanol (50 mL) with stirring. Alkylmalonate diester $(82 \mathrm{mmol})$ was added when all the sodium as dissolved. The resulting solution was then heated and kept under reflux for about 60 minutes. Alkyl bromide 6 (68 mmol) was added into the reaction mixture dropwise. The reaction mixture was heated under reflux for 18 hours. The solution was cooled to room temperature after reflux. The cool solution was then concentrated by rotatory evaporation. The residue was extracted with ether $(3 \times 50 \mathrm{~mL})$. Filtration separated the white solid from the ether solution. The ether solution was then concentrated by rotatory evaporation to yield light 
yellow oil. The dialkylmalonate diester was vacuumed distilled in a bulb-to-bulb apparatus and collected at $140-180^{\circ} \mathrm{C}$ as colorless oil.

Diethyl dihexylmalonate, 13a. Vacuum distilled to give a colorless oil (36\%): TLC (1:1 Hexane/ $\mathrm{CH}_{3} \mathrm{Cl}$ ) $\mathrm{R}_{\mathrm{f}}=0.5$; bp: $140{ }^{\circ} \mathrm{C}, 0.5$ torr (lit.(Asinger and Bochnia, 1961) bp 136-137 ${ }^{\circ} \mathrm{C}, 0.8$ torr)

Diethyl diheptylmalonate, 13b. Vacuum distilled to give a colorless oil (51\%): TLC (1:1 Hexane $/ \mathrm{CH}_{3} \mathrm{Cl}$ ) $\mathrm{R}_{\mathrm{f}}=0.5$; bp: $150{ }^{\circ} \mathrm{C}, 0.5$ torr (lit.(Stanley et al., 1929) bp $178-180{ }^{\circ} \mathrm{C}, 3$ torr)

Diethyl dioctylmalonate, 13c. Vacuum distilled to give a colorless oil (34\%): TLC (1:1 Hexane/ $\mathrm{CH}_{3} \mathrm{Cl}$ ) $\mathrm{R}_{\mathrm{f}}=0.5$; bp: $160{ }^{\circ} \mathrm{C}, 0.5$ torr (lit.(Stanley et al., 1929) bp $192-195{ }^{\circ} \mathrm{C}, 3$ torr)

Diethyl dinonylmalonate, 13d. Vacuum distilled to give a colorless oil (34\%): TLC (1:1 Hexane $/ \mathrm{CH}_{3} \mathrm{Cl}$ ) $\mathrm{R}_{\mathrm{f}}=0.5$; bp: $170^{\circ} \mathrm{C}, 0.5$ torr (no lit. data)

Diethyl didecylmalonate, 13e. Vacuum distilled to give a colorless oil (34\%): TLC (1:1 Hexane/ $\mathrm{CH}_{3} \mathrm{Cl}$ ) $\mathrm{R}_{\mathrm{f}}=0.5$; bp: $180{ }^{\circ} \mathrm{C}, 0.5$ torr (lit.(Braun and Schattner, 1941) bp $196-198{ }^{\circ} \mathrm{C}$, 0.2 torr)

General procedure of the preparation of dialkylacetic acid, 14a-e. Dialkylmalonate diester 13 (50 mmol) was added into a 250-mL reaction flask containing 50:50 wt KOH: $\mathrm{H}_{2} \mathrm{O}$ solution $(200 \mathrm{~mL})$. The reaction mixture was heated and kept under reflux for 8 hours. Enough water $(\sim 200 \mathrm{~mL})$ was added to dissolve the white solid formed after the reflux. The resulting solution was then washed with ether $(50 \mathrm{~mL})$ once before concentrated $\mathrm{HCl}$ was added dropwise until no more solid formed. Ether $(3 \times 50 \mathrm{~mL})$ was used to extract the reaction solution and concentrated by rotatory evaporation, which yields yellow solid. The yellow solid was transferred into a $250-\mathrm{mL}$ reaction flask and heated to $180^{\circ} \mathrm{C}$ for 3 hours. The cooled down residue was then washed with ether $(3 \times 50 \mathrm{~mL})$. The collected ether solution was concentrated to yield light yellow oil. The yellow oil was vacuumed distilled in a bulbto-bulb apparatus to give colorless oil or a white solid, which solidifies on cooling below rt. The ${ }^{1} \mathrm{H}$ NMR spectrum indicated the presence of impurities, which were removed by washing with acetone $(3 \times 25 \mathrm{~mL})$.

2-Hexyloctanoic acid, 14a. Colorless oil (81\%): $\mathrm{mp} 13.6-14.0^{\circ} \mathrm{C}$; (lit. (Asinger, 1963) mp $\left.13{ }^{\circ} \mathrm{C}\right) ;{ }^{1} \mathrm{H}$ NMR $(500 \mathrm{MHz}) \delta 0.87(6 \mathrm{H}, \mathrm{t}, J=7 \mathrm{~Hz}), 1.26(16 \mathrm{H}, \mathrm{b}), 1.46(2 \mathrm{H}, \mathrm{m}), 1.61(2 \mathrm{H}$, m), 2.33 (1H, m); (lit.(Hwang et al., 1974) $60 \mathrm{MHz}{ }^{1} \mathrm{H}$ NMR)

2-Heptylnonanoic acid, 14b. Colorless oil (81\%): $\mathrm{mp} 26.5-27^{\circ} \mathrm{C}$; (lit.(Stanley et al., 1929) mp 26-27 $\left.{ }^{\circ} \mathrm{C}\right) ;{ }^{1} \mathrm{H}$ NMR $(500 \mathrm{MHz}) \delta 0.87(6 \mathrm{H}, \mathrm{t}, J=7 \mathrm{~Hz}), 1.26(20 \mathrm{H}, \mathrm{b}), 1.46(2 \mathrm{H}, \mathrm{m})$, 1.61 (2H, m), 2.33 (1H, m); (lit.(Hwang et al., 1974) $60 \mathrm{MHz}{ }^{1} \mathrm{H}$ NMR)

2-Octyldecanoic acid, 14c. White solid (81\%): mp 35-35.6 ${ }^{\circ} \mathrm{C}$; (lit. (Stanley et al., 1929) mp 35-36 $\left.{ }^{\circ} \mathrm{C}\right) ;{ }^{1} \mathrm{H}$ NMR $(500 \mathrm{MHz}) \delta 0.87(6 \mathrm{H}, \mathrm{t}, J=7 \mathrm{~Hz}), 1.26(24 \mathrm{H}, \mathrm{b}), 1.46(2 \mathrm{H}, \mathrm{m})$, $1.61(2 \mathrm{H}, \mathrm{m}), 2.34$ (1H, m) (lit.(Hwang et al., 1974) $60 \mathrm{MHz}{ }^{1} \mathrm{H}$ NMR) 
2-Nonylundecanoic acid, 14d. White solid (80\%): mp 47-47.5 ${ }^{\circ} \mathrm{C}$; (lit.(Birch and Robinson, 1942) mp 47-47.5 $\left.{ }^{\circ} \mathrm{C}\right) ;{ }^{1} \mathrm{H}$ NMR $(500 \mathrm{MHz}) \delta 0.87$ (6H, t, $\left.J=7 \mathrm{~Hz}\right), 1.26$ (28H, b), 1.46 (2H, m), 1.61 (2H, m), 2.33 (1H, m) (lit.(Hwang et al., 1974) $60 \mathrm{MHz}{ }^{1} \mathrm{H}$ NMR);

2-Decyldodecanoic acid, 14e. White solid (80\%): mp 54-54.8 ${ }^{\circ} \mathrm{C}$; (lit.(Birch and Robinson, 1942) mp $\left.54{ }^{\circ} \mathrm{C}\right) ;{ }^{1} \mathrm{H}$ NMR $(500 \mathrm{MHz}) \delta 0.87(6 \mathrm{H}, \mathrm{t}, J=7 \mathrm{~Hz}), 1.26(32 \mathrm{H}, \mathrm{b}), 1.46(2 \mathrm{H}, \mathrm{m})$, 1.61 (2H, m), 2.33 (1H, m) (lit.(Hwang et al., 1974) $60 \mathrm{MHz}{ }^{1} \mathrm{H}$ NMR);

General procedure of the preparation of dialkylacetyl chloride 15a-e. Dialkylacetic acid $(7.0 \mathrm{mmol})$ was added into a $250-\mathrm{mL}$ beaker containing distilled water (50 mL) and $\mathrm{NaOH}$ $(0.84 \mathrm{~g}, 21.0 \mathrm{mmol})$. The resulting water solution was heated to boiling. After the water was gone, the white solid left in the beaker was collected and put in an oven for 10 minutes to remove the water. The dried white solid was then added into a $100-\mathrm{mL}$ reaction flask containing thionyl chloride $(50 \mathrm{~mL})$ in small portions over 5 minutes. After stirring for another 30 minutes, the resulting solution was concentrated by rotatory evaporation The residue was dissolved in dry toluene $(50 \mathrm{~mL})$ and filtered. The filtrate was concentrated to yield light yellow solid as the product, which was used without further purification.( 98\% crude yield)

2-Hexyloctanoyl chloride, 15a. ${ }^{1} \mathrm{H}$ NMR (500 MHz) $\delta 0.88(6 \mathrm{H}, \mathrm{t}, J=7 \mathrm{~Hz}), 1.27$ (16H, b), $1.53(2 \mathrm{H}, \mathrm{m}), 1.73(2 \mathrm{H}, \mathrm{m}), 2.74(1 \mathrm{H}, \mathrm{m})$.

2-Heptylnonanoyl chloride, 15b. ${ }^{1} \mathrm{H}$ NMR $(500 \mathrm{MHz}) \delta 0.87(6 \mathrm{H}, \mathrm{t}, J=7 \mathrm{~Hz}), 1.27(20 \mathrm{H}$, b), $1.54(2 \mathrm{H}, \mathrm{m}), 1.72(2 \mathrm{H}, \mathrm{m}), 2.73(1 \mathrm{H}, \mathrm{m})$.

2-Octyldecanoyl chloride, 15c. ${ }^{1} \mathrm{H}$ NMR (500 MHz) $\delta 0.88(6 \mathrm{H}, \mathrm{t}, J=7 \mathrm{~Hz}), 1.27(24 \mathrm{H}, \mathrm{b})$, $1.53(2 \mathrm{H}, \mathrm{m}), 1.73(2 \mathrm{H}, \mathrm{m}), 2.74(1 \mathrm{H}, \mathrm{m})$.

2-Nonylundecanoyl chloride, 15d. ${ }^{1} \mathrm{H}$ NMR $(500 \mathrm{MHz}) \delta 0.88(6 \mathrm{H}, \mathrm{t}, J=7 \mathrm{~Hz}) .1 .26(28 \mathrm{H}$, b), 1.54 (2H, m), 1.72 (2H, m), $2.74(1 \mathrm{H}, \mathrm{m})$.

2-Decyldodecanoyl chloride, 15e. ${ }^{1} \mathrm{H}$ NMR $(500 \mathrm{MHz}) \delta 0.88(6 \mathrm{H}, \mathrm{t}, J=7 \mathrm{~Hz}), 1.27(32 \mathrm{H}$, b), 1.53 (2H, m), $1.73(2 \mathrm{H}, \mathrm{m}), 2.74(1 \mathrm{H}, \mathrm{m})$.

General procedure of preparation of the two-tailed triesters, 16(n,n). Dialkylacetyl chloride 15 (2.78 mmol) was added into a 50-mL reaction flask containing dry $\mathrm{CH}_{2} \mathrm{Cl}_{2}(10 \mathrm{~mL})$.

Behera's amine (1.155 g, $2.78 \mathrm{mmol})$ was added in portions. The reaction mixture was then sonicated for 10 minutes. Triethylamine ( $0.617 \mathrm{~g}, 6.12 \mathrm{mmol}, 2.2 \mathrm{eq})$ was then added dropwise. After sonicated for another $2 \mathrm{~h}$, the resulting solution was stirred for $24 \mathrm{~h}$. The reaction mixture was filtered and, then, the filtrate washed with diluted $\mathrm{HCl}$ solution (50 $\mathrm{mL}$ ). The organic layer was collected and dried with magnesium sulfate. The resulting solution was filtered and concentrated to yield light solid as the product.

Di-tert-butyl 4-(2-tert-butoxycarbonylethyl)-4-(2-hexyloctanoylamino)octanedioate, 16(6,6). ${ }^{1} \mathrm{H}$ NMR $(500 \mathrm{MHz}) \delta 0.89-0.85$ (t, 6H, J=7 Hz), 1.29-1.21 (b, 16H), 1.43 (s, 9H), 1.981.94 (m, 6H), 2.24-2.19 (m, 6H). 
Di-tert-butyl 4-(2-tert-butoxycarbonylethyl)-4-(2-heptylnonanoylamino)heptanedioate, 16(7,7). ${ }^{1} \mathrm{H}$ NMR $\delta \quad 0.89-0.85$ (t, 6H, $J=7$ Hz), 1.29-1.21 (b, 20H), 1.43 (s, 9H), 1.98-1.94 (m, 6H), 2.24-2.19 (m, 6H).

Di-tert-butyl 4-(2-tert-butoxycarbonylethyl)-4-(2-octyldecanoylamino)heptanedioate, 16(8,8). ${ }^{1} \mathrm{H}$ NMR $\delta \quad 0.89-0.85$ (t, 6H, $J=7$ Hz), 1.29-1.21 (b, 24H), 1.43 (s, 9H), 1.98-1.94 (m, 6H), 2.24-2.19 (m, 6H).

Di-tert-butyl 4-(2-tert-butoxycarbonylethyl)-4-(2-nonylundecanoylamino)heptanedioate, 16(9,9) ${ }^{1} \mathrm{H}$ NMR $\delta$ 0.89-0.85 (t, 6H, J=7 Hz), 1.29-1.21 (b, 28H), 1.43 (s, 9H), 1.98-1.94 (m, 6H), 2.24-2.19 (m, 6H).

Di-tert-butyl 4-(2-tert-butoxycarbonylethyl)-4-(2-decyldodecanoylamino)heptanedioate, 16(10,10). ${ }^{1} \mathrm{H}$ NMR $\delta \quad 0.89-0.85$ (t, 6H, $J=7$ Hz), 1.29-1.21 (b, 32H), 1.43 (s, 9H), 1.98$1.94(\mathrm{~m}, 6 \mathrm{H}), 2.24-2.19(\mathrm{~m}, 6 \mathrm{H})$.

General procedure of the preparation of two-tailed triacids, 3CAm1(n,n)

Triester 16(n,n) (3 mmol) was added into a 50-mL reaction flask containing formic acid (20 $\mathrm{mL}$ ). The reaction mixture was stirred for 8 hours under rt and then concentrated to yield crude di-tailed triacids as light yellow solid. The crude product was then chromatographed $\left(\mathrm{SiO}_{2}\right)$, eluting with 1:1:0.1 hexane:EtOAc:AcOH to give the white solids (10-60\%).

4-(2-Carboxyethyl)-4-(2-hexyloctanoylamino)heptanedioic acid, 3CAm1(6,6). The general procedure described above afforded a white solid in 10\% yield; mp $184.8-185.7^{\circ} \mathrm{C} ;{ }^{1} \mathrm{H}$ NMR (500 MHz, $d_{6}$-DMSO) $\delta$ 0.87-0.81 (t, 6H), 1.30-1.12 (b, 16H), 1.43-1.32 (b, 4H), 1.89-1.79 (m, 6H), 2.14-2.08 (m, 6H), 2.21-2.15 (m, 1H).; ${ }^{13} \mathrm{C}$ NMR (125 MHz, $d_{6^{-}}$ Acetone) $\delta 13.52,22.52,27.62,27.82,28.55,28.70,28.85,29.01,29.17,29.32,29.47,29.50$, 31.70, 33.27, 47.27, 57.16, 173.8, 175.1; IR: 1709, 1622, $1554 \mathrm{~cm}^{-1}$. HRMS: calcd for $\mathrm{C}_{24} \mathrm{H}_{44} \mathrm{NO}_{7}[\mathrm{M}+\mathrm{H}]^{+}$calcd 458.3118, found 458.3098 .

4-(2-Carboxyethyl)-4-(2-heptylnonanoylamino)heptanedioic acid, 3CAm1(7,7) The general procedure described above afforded a white solid in 10\% yield; mp $174.6-175.4{ }^{\circ} \mathrm{C} ;{ }^{1} \mathrm{H}$ NMR (500 MHz, $d_{6}$-DMSO) $\delta 0.87-0.81$ (t, 6H), 1.30-1.12 (b, 20H), 1.43-1.32 (b, 4H), $1.89-1.79(\mathrm{~m}, 6 \mathrm{H}), 2.14-2.08(\mathrm{~m}, 6 \mathrm{H}), 2.21-2.15(\mathrm{~m}, 1 \mathrm{H}) ;{ }^{13} \mathrm{C}$ NMR (in $d_{6}$-Acetone) $\delta$ 13.54, 22.5, 27.68, 27.83, 28.56, 28.71, 28.87, 29.02, 29.17, 29.33, 29.48, 29.52, 29.62, 31.79, 33.26, 47.27, 57.18, 173.9, 175.2; IR: 1708, 1622, $1554 \mathrm{~cm}^{-1}$. HRMS: calcd for $\mathrm{C}_{26} \mathrm{H}_{48} \mathrm{NO}_{7}[\mathrm{M}+\mathrm{H}]^{+}$486.3431, found 486.3445 .

4-(2-Carboxyethyl)-4-(2octyl-decanoylamino)heptanedioic acid, 3CAm1(8,8) The general procedure described above afforded a white solid in $~ 40 \%$ yield; mp $164.8-165.3{ }^{\circ} \mathrm{C} ;{ }^{1} \mathrm{H}$ NMR (500 MHz, d $d_{6}$-DMSO) 0.87-0.81 (t, 6H), 1.30-1.12 (b, 24H), 1.43-1.32 (b, 4H), 1.89$1.79(\mathrm{~m}, 6 \mathrm{H}), 2.14-2.08(\mathrm{~m}, 6 \mathrm{H}), 2.21-2.15$ (b, 1H); ${ }^{13} \mathrm{C}$ NMR (in $d_{6}$-Acetone) $\delta 13.56$, 22.50, 27.68, 27.85, 28.56, 28.87, 29.03, 29.18, 29.25, 29.33, 29.47, 29.53, 29.68, 31.82, 33.25, 47.26, 57.19, 173.9, 175.2; IR: 1708, 1623, $1554 \mathrm{~cm}^{-1}$. HRMS: for $\mathrm{C}_{28} \mathrm{H}_{52} \mathrm{NO}_{7}[\mathrm{M}+$ $\mathrm{H}]^{+}$calcd 514.3744, found 514.3761. 
4-(2-Carboxyethyl)-4-(2-nonylundecanoylamino)heptanedioic acid, 3CAm1(9,9) The general procedure described above afforded a white solid in 40\% yield; mp 155.0-155.8 ${ }^{\circ} \mathrm{C} ;{ }^{1} \mathrm{H}$ NMR (500 MHz, $d_{6}$-DMSO) 0.87-0.81 (t, 6H), 1.30-1.12 (b, 28H), 1.43-1.32 (b, $4 \mathrm{H}), 1.89-1.79(\mathrm{~m}, 6 \mathrm{H}), 2.14-2.08(\mathrm{~m}, 6 \mathrm{H}), 2.21-2.15(\mathrm{~m}, 1 \mathrm{H}) ;{ }^{13} \mathrm{C}$ NMR (in $d_{6}$-Acetone) $\delta$ 13.55, 22.50, 27.68, 27.85, 28.57, 28.72, 28.87, 29.03, 29.19, 29.26, 29.34, 29.52, 29.63, 29.68, 31.81, 33.24, 47.26, 57.21, 174.0, 175.3; IR: 1710, 1624, $1560 \mathrm{~cm}^{-1}$. HRMS: for $\mathrm{C}_{30} \mathrm{H}_{56} \mathrm{NO}_{7}[\mathrm{M}+\mathrm{H}]^{+}$calcd 542.4057, found 542.4055.

4-(2-Carboxyethyl)-4-(2-decyldodecanoylamino)heptanedioic acid, 3CAm1(10,10) The general procedure described above afforded a white solid in 60\% yield; mp 144.8-145.7 ${ }^{\circ} \mathrm{C}$; ${ }^{1} \mathrm{H}$ NMR (500 MHz, $d_{6}$-DMSO) 0.87-0.81 (t, 6H), 1.30-1.12 (b, 32H), 1.43-1.32 (b, $4 \mathrm{H}), 1.89-1.79(\mathrm{~m}, 6 \mathrm{H}), 2.14-2.08(\mathrm{~m}, 6 \mathrm{H}), 2.21-2.15(\mathrm{~m}, 1 \mathrm{H}) ;{ }^{13} \mathrm{C}$ NMR (in $d_{6}$-Acetone) $\delta$ 13.55, 22.51, 27.67, 27.85, 28.57, 28.72, 28.87, 29.02, 29.18, 29.26, 29.34, 29.51, 29.55, 29.59, 29.67, 31.84, 33.23, 47.24, 57.19, 173.9, 175.3; IR: 1703, 1645, $1536 \mathrm{~cm}^{-1}$. HRMS: for $\mathrm{C}_{32} \mathrm{H}_{60} \mathrm{NO}_{7}[\mathrm{M}+\mathrm{H}]^{+}$calcd 570.4370, found 570.4374 .

General procedure of preparation of the PEG triesters, 17(PEGmw). WeNCO (1.1 mmol), mPEGmw (0.57 mmol) and $5 \mathrm{ml} \mathrm{TEA} \mathrm{were} \mathrm{added} \mathrm{to} \mathrm{a} \mathrm{round} \mathrm{bottom} \mathrm{flask.} \mathrm{The} \mathrm{solution} \mathrm{was}$ then heated to $100{ }^{\circ} \mathrm{C}$ and refluxed for $24 \mathrm{~h}$. The resulting solid was purified by recrystallization in ethyl acetate $(10 \mathrm{~mL})$, and hexane $(10 \mathrm{~mL})$. The resulting white solids were rinsed with hexane and dried under vacuum overnight ( $75 \%$ yield).

Di-tert-butyl 4-(2-(tert-butoxycarbonyl)ethyl)-4-(550polyethylenoxycarbonylamino)heptanedioate, 17(PEG550): ${ }^{1} \mathrm{H}$ NMR $\left(\mathrm{CDCl}_{3}\right)$ : $\delta=1.39$ (s, 27H), 1.85 (t, 5.19H), $2.16(\mathrm{t}$, $5.66 \mathrm{H}), 3.33(\mathrm{~s}, 2.95 \mathrm{H}), 3.61(\mathrm{~m}, 40.47 \mathrm{H}), 4.10(\mathrm{~s}, 1.55 \mathrm{H}), 4.78(\mathrm{~s}, 0.68 \mathrm{H}) .{ }^{13} \mathrm{C} \mathrm{NMR}$ $\left(\mathrm{CDCl}_{3}\right): \delta=13.1,28.1,29.7,56.5,59.1,61.7,63.5,69.6,70.6,72.0,77.2,80.6,154.2,172.6$. IR: 2868.2, 1725.2, 1458.1, 1366.3, 1300.8, 1245.2, 1144.4, 1101.2, 945.3, 847.2

Di-tert-butyl 4-(2-(tert-butoxycarbonyl)ethyl)-4-(2000polyethylenoxycarbonylamino)heptanedioate, 17(PEG2000) ${ }^{1} \mathrm{H}$ NMR $\left(\mathrm{CDCl}_{3}\right): \delta=1.42(\mathrm{~m}, 27.56 \mathrm{H}), 1.88(\mathrm{~m}, 6.02 \mathrm{H}), 2.20$ (m, 6.24H), 3.55 (s, 3H), $3.64(\mathrm{~m}, 185.64 \mathrm{H}), 4.14(\mathrm{~m}, 1.93 \mathrm{H}), 4.77$ (m, 0.89H). ${ }^{13} \mathrm{C} \mathrm{NMR}$ $\left(\mathrm{CDCl}_{3}\right): \delta=28.16,29.73,30.10,59.13,72.01,76.87,80.65,172.59$. IR: 2888, 172.98, 1533.1, 1465.6, 1342.2, 1278.6, 1240, 1106.9, 960.4, $840.8 \mathrm{~cm}^{-1}$.

Di-tert-butyl 4-(2-(tert-butoxycarbonyl)ethyl)-4-(5000polyethylenoxycarbonylamino)heptanedioate, 17(PEG5000) ( 75\% yield) ${ }^{1} \mathrm{H} \mathrm{NMR}\left(\mathrm{CDCl}_{3}\right): \delta=1.59(\mathrm{~s}, 27.50 \mathrm{H}), 1.88(\mathrm{t}$, $5.88 \mathrm{H}), 2.20$ (t, 6.9H), 3.54 (s, 3H), $5.65(\mathrm{~m}, 466.15 \mathrm{H}), 4.14(\mathrm{~s}, 2.12 \mathrm{H}), 4.77(\mathrm{~s}, 0.85 \mathrm{H}) .{ }^{13} \mathrm{C}$ NMR $\left(\mathrm{CDCl}_{3}\right): \delta=28.16,29.74,30.13,59.12,70.66,72.02,80.64,172.56$. IR: 2882, 1272, 1466, 1454, 1359, 1341, 1279, 1240, 1147, 1098, 1060, 946, 841.

General procedure of preparation of the PEG triacids, 3CCbPEGmw. Formic acid (20 mL) was added to 17(PEGmw) (1.5 g) and stirred for $48 \mathrm{~h}$. The resulting solution was concentrated by rotary evaporation to remove formic acid, and then dried under vacuum overnight. The resulting white powder was recrystallized in ethyl acetate $(10 \mathrm{~mL})$, and hexanes $(10 \mathrm{~mL})$ and rinsed with hexanes two times, then dried under vacuum ( 78\% yield). 
4-(2-Carboxyethyl)-4-(550polyethylenoxycarbonylamino)heptanedioic acid, 3CCbPEG550. ${ }^{1} \mathrm{H} \mathrm{NMR}\left(\mathrm{CDCl}_{3}\right): \delta=1.96(\mathrm{~m}, 6 \mathrm{H}), 2.30(\mathrm{~m}, 5.63 \mathrm{H}), 3.36(\mathrm{~s}, 3),{ }^{13} \mathrm{C} \mathrm{NMR}\left(\mathrm{CDCl}_{3}\right): \delta=$ 28.45, 29.52, 56.14, 59, 70.56, 68.097, 71.89, 163.47. IR: 3000-3300 (broad), 2872, 1718, 1530, 1456, 1344, 1238, 1079, 945, 843.

4-(2-Carboxyethyl)-4-(2000polyethylenoxycarbonylamino)heptanedioic acid, 3CCbPEG2000. ${ }^{1} \mathrm{H}$ NMR $\left(\mathrm{CDCl}_{3}\right): \delta=2.01$ (m, 5.92H), 2.32 (m, 5.90H), 3.54 (s, 1.87H), 4.12(m, 1.89H), $4.88(\mathrm{~m}, 1.03 \mathrm{H}) .{ }^{13} \mathrm{C} \mathrm{NMR}\left(\mathrm{CDCl}_{3}\right): \delta=28.39,29.51,56.94,59.10,70.63$, 70.88, 72.00, 176.23. IR: 2878, 1718, 1454, 1348, 1300.9, 1243.6, 1081, 948, 842, $689 \mathrm{~cm}^{-1}$.

4-(2-Carboxyethyl)-4-(5000polyethylenoxycarbonylamino)heptanedioic acid, 3CCbMPEG5000 ( 80\% yield) ${ }^{1} \mathrm{H}$ NMR $\left(\mathrm{CDCl}_{3}\right): \delta=2.01(\mathrm{t}, 4.28 \mathrm{H}), 2.32(\mathrm{t}, 4.48 \mathrm{H}), 3.37$ (s, 3H), 3.63 (m, 475.63H), 4.16 (s, 1.08H), 4.96 (s, 0.53H). IR: 2882, 1727, 1466, 1454, 1359, 1341, 1279, 1240, 1144, 1098, 1060, 946, 841.

Molar Mass Characterization. The molar mass of starting methoxyPEGs and as-prepared 17(PEGmw) were characterized by GPC. The number average molar masses are calculated by ${ }^{1} \mathrm{H}$ NMR results. The $\mathrm{M}_{\mathrm{n}}$ and polydispersity indices are shown in Table 1 . Molar masses of 17PEGmw are around 440 daltons higher than starting methoxyPEG.

Table 1. Molar Mass Results (g/mol) obtained with GPC and ${ }^{1} \mathrm{H}$ NMR

\begin{tabular}{llll}
\hline Sample & $\mathbf{M}_{\mathbf{n}}$ (GPC) & PDI & $\mathbf{M}_{\mathbf{n}}$ (NMR) \\
\hline mPEG550 & 400 & 1.15 & 560 \\
mPEG2000 & 2220 & 1.02 & 2030 \\
mPEG5000 & 5510 & 1.01 & 5100 \\
17(PEG 550) & 1020 & 1.11 & 1000 \\
17(PEG 2000) & 2650 & 1.07 & 2448 \\
17(PEG 5000) & 5880 & 1.01 & 5540 \\
\hline
\end{tabular}

Absorption of Surfactants on Iron Nanoparticles.

Particle synthesis. Monodisperse $\mathrm{Fe}_{3} \mathrm{O}_{4}$ magnetic nanoparticles (MNPs) are synthesized according to the method reported. ${ }^{4} \mathrm{Fe}(\text { acac })_{3}$ (2 mmol), 1,2-hexadecanediol (10 mmol), oleic acid (6 mmol), oleylamine (6 mmol), and benzyl ether $(20 \mathrm{~mL})$ were mixed and stirred under a flow of nitrogen. The mixture was heated to $200{ }^{\circ} \mathrm{C}$ for 2 hours and then, under a blanket of nitrogen, heated to reflux $\left(300{ }^{\circ} \mathrm{C}\right.$ ) for another 1 hour. Under ambient conditions, ethanol (40 $\mathrm{mL}$ ) was added to the mixture, and a black material precipitated and was separated via centrifugation. The black product was dissolved in hexane in the presence of oleic acid (0.05 $\mathrm{mL}$ ) and oleylamine $(0.05 \mathrm{~mL})$. Centrifugation (6000 rpm, $10 \mathrm{~min})$ was applied to remove any undispersed residue. The product was then precipitated with ethanol, centrifuged (6000 rpm, $10 \mathrm{~min}$ ) to remove the solvent and dried under vacuum overnight.

Ligand Exchange. For a typical ligand exchange reaction, $20 \mathrm{mg}$ MNPs were mixed with 3CCbPEGmw (100 mg) in $5 \mathrm{~g} \mathrm{CHCl}_{3}$. The solution was sonicated for $30 \mathrm{~min}$ and stirred for $24 \mathrm{~h}$. The particles were precipitated by adding hexanes and dried in vacuum. The hydrodynamic radii are characterized by dynamic light scattering (DLS). 


\section{RESULTS AND DISCUSSION}

Description of Syntheses. Our research team of three graduate students and one undergraduate student synthesized four homologous series of three-headed, two-tail amphiphiles-, 3CUr1(n,n), 3CCb1(n,n), and 3CAm1(n,n). In addition, another student synthesized a short series of modified polymers, 3CCbPEGmw.

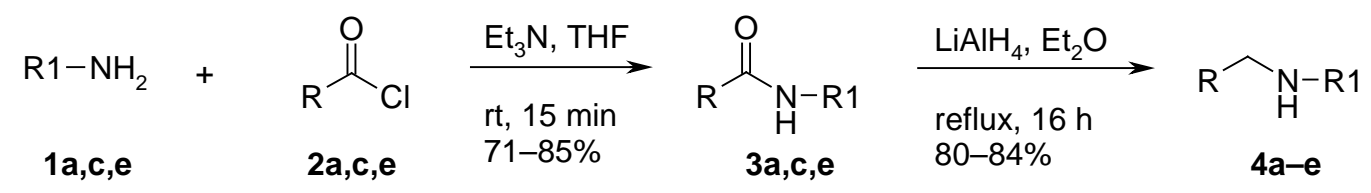

a, R1 = $\mathrm{C}_{7} \mathrm{H}_{15}, \mathrm{R}=\mathrm{C}_{6} \mathrm{H}_{13} ; \mathbf{c}, \mathrm{R} 1=\mathrm{C}_{9} \mathrm{H}_{19}, \mathrm{R}=\mathrm{C}_{8} \mathrm{H}_{17} ; \mathbf{e}, \mathrm{R} 1=\mathrm{C}_{11} \mathrm{H}_{23}, \mathrm{R}=\mathrm{C}_{10} \mathrm{H}_{21}$

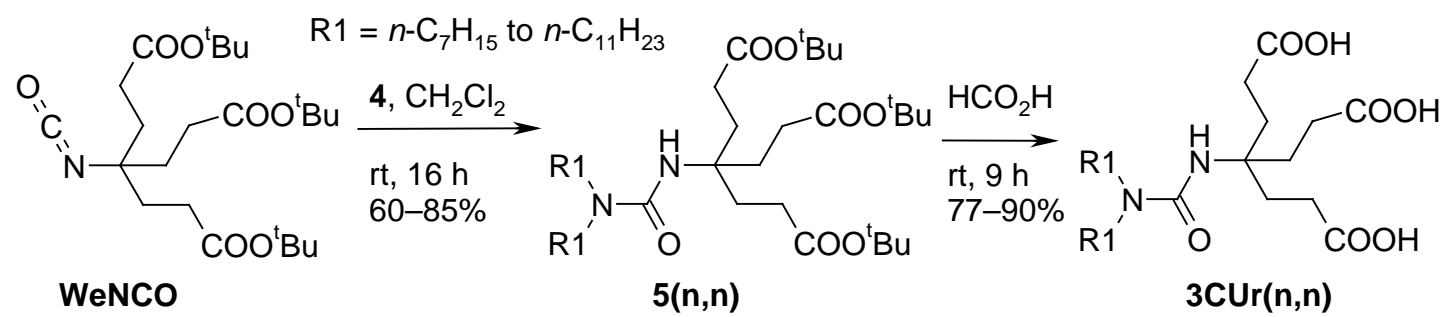

Synthesis of $\mathbf{3 C U}(\boldsymbol{n}, \boldsymbol{n})$. The synthesis (Figure 1) began with the preparation of the odd-chain dialkylamines, 4a,c,e. The reaction of WeNCO (Newkome et al., 1998) and 4 occurred at room temperature to produce triesters $\mathbf{5}(\mathbf{n}, \mathbf{n})$. Formolysis of $\mathbf{5}(\mathbf{n}, \mathbf{n})$ produced the triacids 3CUr(n,n). All compounds were fully characterized by the standards of organic chemistry. The compounds were soluble in water as tristriethanolammonium salts at a concentration of $1 \times 10^{-3} \mathrm{M}$ or greater.

Figure 1. Synthetic scheme for preparation of $3 \mathbf{C U r}(\mathbf{n}, \mathbf{n})$.

Synthesis of $\mathbf{3 C U r 1 ( n , n ) . ~ T h e ~ s y n t h e s i s ~ ( F i g u r e ~ 2 ) ~ b e g a n ~ w i t h ~ t h e ~ p r e p a r a t i o n ~ o f ~}$ dialkylcarbinols, 6, which were converted into alkyl mesylates, 8. Substitution of the mesylate group by azide gave the alkyl azides, $\mathbf{9}$. Reduction of $\mathbf{9}$ by catalytic hydrogentation gave the key intermediate amine, 10. The reaction of WeNCO (Newkome et al., 1998) and $\mathbf{1 0}$ occurred at room temperature to produce triesters 11(n,n). Formolysis of 11(n,n) produced the triacids $\mathbf{3 C U} \mathbf{C} \mathbf{1}(\mathbf{n}, \mathbf{n})$. All compounds were fully characterized by the standards of organic chemistry.
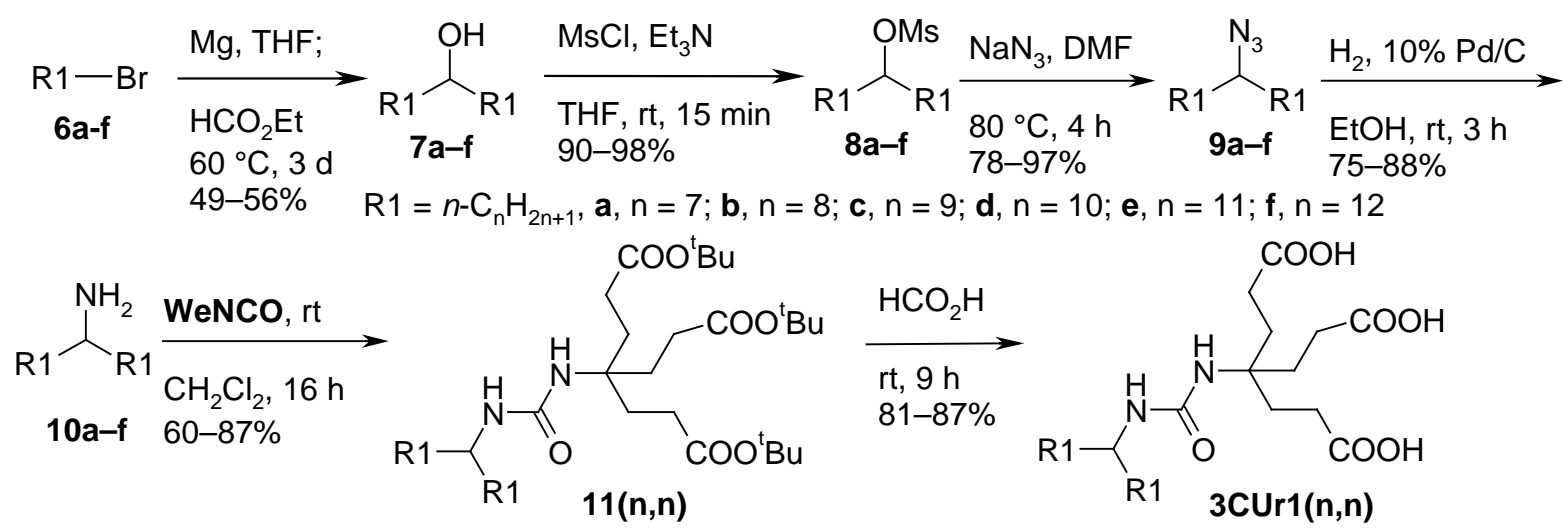

Figure 2. Synthetic scheme for preparation of $\operatorname{3CUr1(n,n).}$<smiles>[R]C([R])NC(=O)NC(CCC(=O)O)(CCC(=O)O)CCC(=O)O</smiles> 
Synthesis of $\mathbf{3 C C b 1}(\mathbf{n}, \boldsymbol{n})$. The synthesis (Figure 3) began with the preparation of dialkylcarbinols, 7 (Figure 2). The reaction of WeNCO (Newkome et al., 1998) and 7 occurred at elevated temperatures in triethylamine as a solvent and catalyst to produce triesters 12(n,n). Formolysis of $\mathbf{1 2 ( n , n ) ~ p r o d u c e d ~ t h e ~ t r i a c i d s ~} \mathbf{3 C U r ( n , n ) . ~ A l l ~ c o m p o u n d s ~}$ were fully characterized by the standards of organic chemistry.

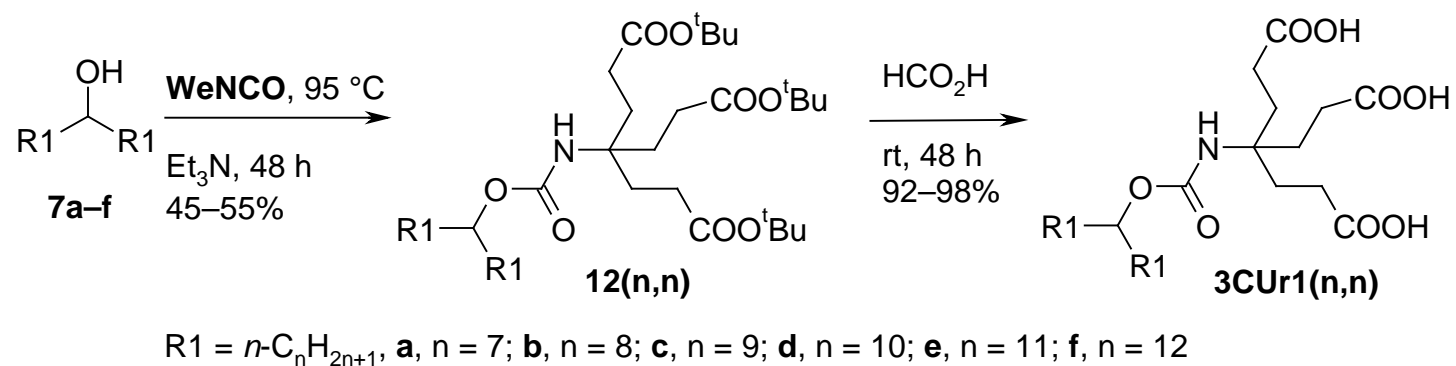

Figure 3. Synthetic scheme for preparation of $3 \mathbf{C C b 1 ( n , n ) . ~}$

Synthesis of $\mathbf{3 C A m 1 ( n , n ) . ~ T h e ~ s y n t h e s i s ~ ( F i g u r e ~ 4 ) ~ b e g a n ~ w i t h ~ t h e ~ p r e p a r a t i o n ~ o f ~}$ dialkylacetic acids, 14, via the malonic ester synthetic route. Bisalkylation of diethyl malonate proceeded smoothly to give 13, which was saponified, acidified, and decarboxylated to dialkylacetic acid 14. Converting 14 into acid chloride 15 occurred via the carboxylate salt. Condensation of 15 with Behera's amine (Newkome et al., 1991b) gave the triester $\mathbf{1 6}(\mathbf{n}, \mathbf{n})$. As before, formolysis of $\mathbf{1 6}(\mathbf{n}, \mathbf{n})$ gave the target triacids $\mathbf{3 C A m 1 ( n , n ) . ~}$
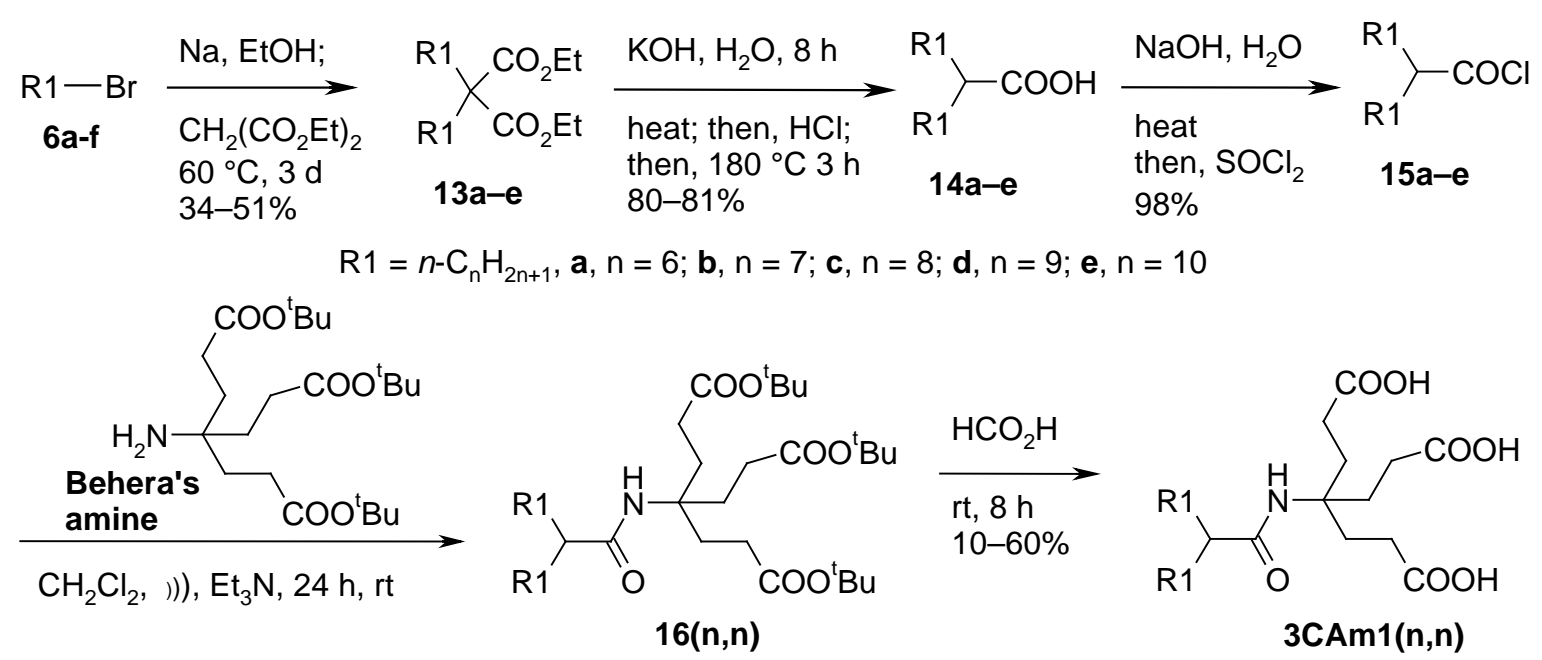

Figure 4. Synthetic scheme for preparation of 3CAm1(n,n).

Synthesis of 3CCbPEGmw. The synthesis (Figure 5) began with the addition of commercial methoxy-terminated polyethylene glycols mPEGmw to WeNCO (Newkome et al., 1998). These reactions proceeded smoothly. The molecular masses (Table 1) of resulting modified polymers were characterized by gel permeation chromatography (GPC) and by integration of the peaks in 1H NMR spectra. Formolysis of the 17(PEGmw) gave the desired triacidterminated PEGs, 3CbPEGmw, with no degradation of the polymer. 


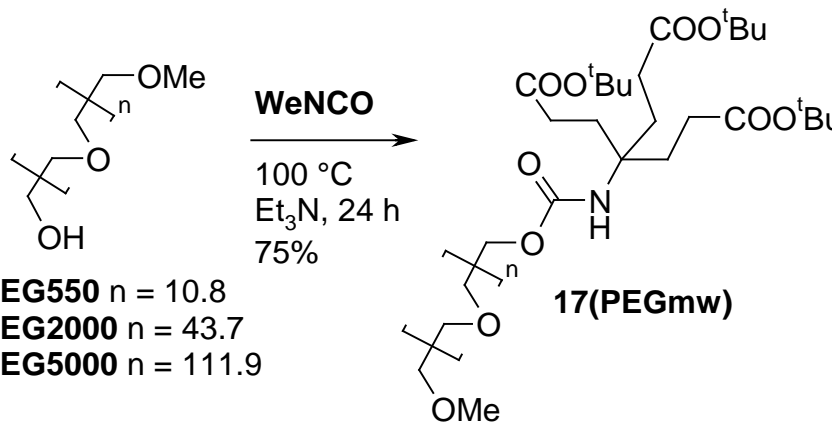

Figure 5. Synthetic scheme for the preparation of 3CbPEGmw.

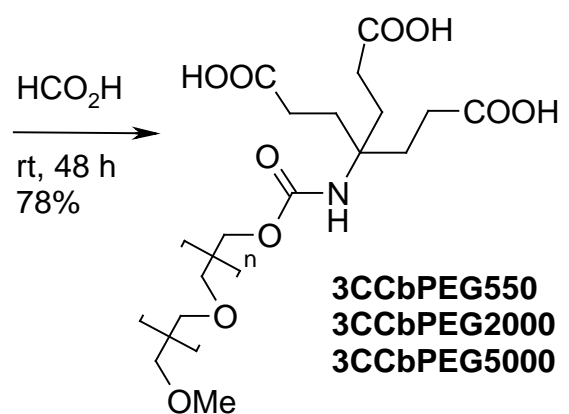

Preparation and Characterization of Nanoparticles. In collaboration with Professor Alan Esker (Virginia Tech, Chemistry), we explored the ability of these polymers to bind to iron nanoparticles, we mixed the polymers and oleate-coated nanoparticles in a solution. These modified polymers bind to magnetite nanoparticles, and as a result, the coated nanoparticles become water soluble (Figure 6). These polymers can attach onto nanoparticles through a twostep ligand exchange process: 1) replace oleic acid with $\mathrm{HCOOH}$ in a concentration-driven process; and 2) exchange $\mathrm{HCOOH}$ with 3CCbPEGmw in an entropically driven process (Figure 6, top). Preliminary dynamic light scattering (DLS) and Fourier transform infrared spectroscopy (FTIR) studies have shown that Step 1 is feasible and that we can even exchange the 3CCbPEG2000 directly for oleic acid without Step 1 (Step 3 in Figure 6, top). Nonetheless, the two-step process minimizes the amount of stabilizer required for the ligand exchange and completely eliminates residual oleic acid from the initial synthesis of the metal-oxide. The final products can be fractionated to narrow size distributions with magnetic separation equipment. More recently, we have found that carrying out the ligand exchange via Step 3 at $100{ }^{\circ} \mathrm{C}$ can completely eliminate the larger aggregates for 3CCbPEG2000 and 3CCbPEG5000, thereby eliminating the need for fractionation. These preliminary results suggest that 3CCbPEGmw can overcome the challenge of producing water-soluble magnetic nanoparticles with a narrow size distribution by direct exchange of oleic acid from the nanoparticle surface. These experiments also confirm that triheads attach to metal-oxide nanoparticles.

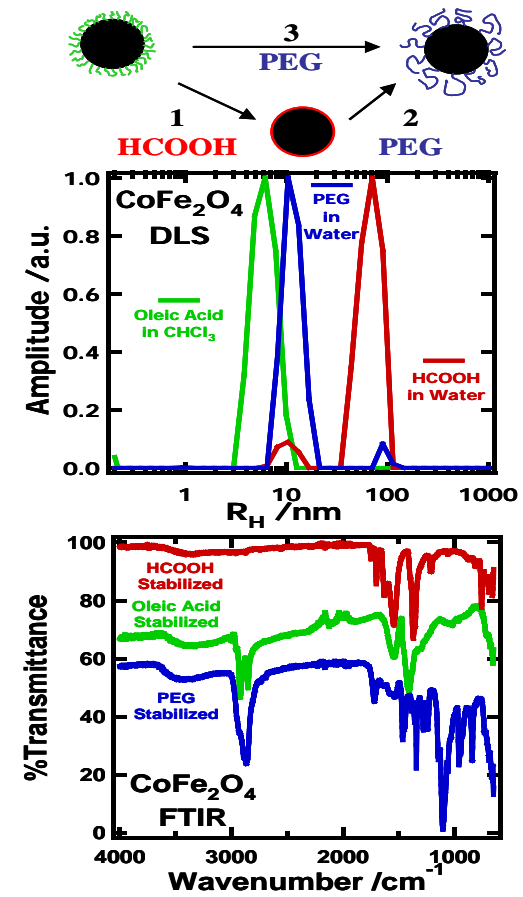

Figure 6. Exchanging (top) oleic acid with $\mathrm{HCOOH}$ and $3 \mathbf{C C b P E G m w}$ on Iron Nanoparticles. DLS (middle) shows oleic acid stabilized magnetic nanoparticles can be converted into water soluble $\mathrm{HCOOH}$ (1) or 3CCbPEGmw (1 \& 2, or 3) coated particles. Second peaks at larger hydrodynamic radii, $\mathrm{R}_{\mathrm{H}}$, indicate aggregates. FTIR (bottom) shows that $\mathrm{HCOOH}$ and 3CCbPEGmw can replace oleic acid. 


\section{CONCLUSION}

We have developed procedures for making two-tailed triheaded surfactants. These versatile procedures can be used for reactions with inexpensive hydrophobic materials and mixtures thereof to create inexpensive surfactants, which could serve as collectors for froth flotation. Measuring contact angles of thin films of these surfactants and comparing the results with thos e of one-tailed surfactants will test our hypothesis about the increased density of chains leading to more hydrophobic surfaces.

The modification of PEGs represents an opportunity to produce the some very inexpensive, high performing surfactants that attach to mineral surfaces. The experiments reported here demonstrate convincingly that the triheads can bind more efficiently than single headed surfactants to iron nanoparticle. Although this example demonstrated the change of hydrophobic surface to a hydrophilic surface, the ready availability of hydrophobic polymers with amino, carboxy, and alcohol end groups bodes well for this technology in the mining industry. In fact, anionic surfactant-PEG mixtures can improve the wettability of coal(Kilau, 1993).

\section{REFERENCES}

Asinger, F., 1963. J. Prakt. Chem., 22: 153-172.

Asinger, F. and Bochnia, D., 1961. J. Prakt. Chem., 13: 1-22.

Baykut, F., 1954. The bihomologous series of the symmetrical dialkyl ketones and dialkylcarbinols, up to C41. Rev. Fac. Sci. Univ. Istanbul, 19: 121-124.

Birch and Robinson, 1942. J. Chem. Soc.: 494-451.

Boal, A.K., Das, K., Gray, M. and Rotello, V.M., 2002. Monolayer exchange chemistry of gamma-Fe2O3 nanoparticles. Chemistry of Materials, 14(6): 2628-2636.

Braun, V. and Schattner, 1941. Chem. Ber., 74: 22-25.

Breusch, F.L. and Sokullu, S., 1953. Isomeric and homologous series. IV. Synthesis of dlhydroxyparaffins with 14-23 carbon atoms. Chem. Ber., 86: 678-684.

Djedovič, N. et al., 2005. The $C$-and $N$-terminal residues of synthetic heptapeptide ion channels influence transport efficacy through phospholipid bilayers. New J. Chem., 29: 291-305.

Froger, C. and Parisot, A., 1954. The melting points of the $N$-monosubstituted amides. Compt. rend., 238: 1589-1591.

Fuerstenau, D.W. and Herrera-Urbina, R., 1989. Flotation Reagents. In: S. Chander and R.R. Klimpel (Editors), Advances in Coal and Mineral Processing Using Flotation. Society for Mining, Metallurgy, and Exploration, Inc., Littleton, Colorado, pp. 3-18.

Gall, R. and Bosies, E., 1988. Preparation of diphosphonic acid derivatives for treating calcium metabolic disorders., Germany, pp. 12pp.

Hwang, Y.S., Mulla, M.S. and Arias, J.R., 1974. J. Agr. Food Chem., 22(6): 1004-1006.

Kilau, H.W., 1993. The Wettability of Coal and Its Relevance to the Control of Dust during Coal-Mining. J. Adhes. Sci. Technol., 7(6): 649-667.

Meakins, R.J. and Sack, R.A., 1951. The dielectric properties of symmetrical long-chain secondary alcohols in the solid state. Australian J. Sci. Research (1951), 4A 21328. Aust. J. Sci. Res., 4A: 213-228. 
Meekel, A.A.P. et al., 2000. Synthesis of Pyridinium Amphiphiles Used for Transfection and Some Characteristics of Amphiphile/DNA Complex Formation. Eur. J. Org. Chem., 2000(4): 665-673.

Newkome, G.R., Behera, R.K., Moorefield, C.N. and Baker, G.R., 1991a. Chemistry of micelles. 18. Cascade polymers: syntheses and characterization of one-directional arborols based on adamantane. J. Org. Chem., 56(25): 7162-7167.

Newkome, G.R., Behera, R.K., Moorefield, C.N. and Baker, G.R., 1991b. Chemistry of micelles. 18. Cascade polymers: syntheses and characterization of one-directional arborols based on adamantane. J. Org. Chem., 56(25): 7162-7.

Newkome, G.R. et al., 1998. Isocyanate-based dendritic building blocks: Combinatorial tier construction and macromolecular-property modification. Angewandte ChemieInternational Edition, 37(3): 307-310.

Newkome, G.R., Weis, C.D., Moorefield, C.N. and Fronczek, F.R., 1997. Chemistry of micelles series .66. A useful dendritic building block: Di-tert-butyl 4-[(2-tertbutoxycarbonyl)ethyl]-4-isocyanato-1,7-heptanedicarboxylate. Tetrahedron Lett., 38(40): 7053-7056.

Overmars, F.J.J., Engberts, J.B.F.N. and Weringa, W.D., 1994. Synthesis of and vesicle formation from phosphorylcholine amphiphiles with one symmetrically branched alkyl chain. Recl. Trav. Chim. Pays-Bas, 113(5): 293-296.

Rao, H.S.P. and Bharathi, B., 2002. Reduction of oximes with sodium borohydride-copper (II) sulfate in methanol. Indian J. Chem. B, 41(5): 1072-1074.

Stanley, Jay and Adams, 1929. J. Amer. Chem. Soc., 51: 1266-1268.

Troyanskii, E.I., Ioffe, V.A., Mizintsev, V.V. and Nikishin, G.I., 1984. Oxidative cyanation of aminyl radicals in the sodium peroxydisulfate-sodium cyanide system. Izv. Akad. Nauk SSSR, Ser. Khim., 8: 1814-1822.

Wescott, L.D. and Mattern, D.L., 2003. Donor- $\alpha$-acceptor molecules incorporating a nonadecyl-swallowtailed perylenediimide acceptor. J. Org. Chem., 68(26): 1005810066.

Wright, J.B. and Elderfield, R.C., 1946. 9-Alkylamino carbinols derived from 9-acyl-1,2,3,4tetrahydrophenanthrene derivatives. J. Org. Chem., 11: 111-122.

Yoshioka, Y. and Tashiro, K., 2003. Structural changes in phase transitions of nylon model compounds. 1. Transition behavior of model compounds of R-NHCO-R ' type. J. Phys. Chem. B, 107(43): 11835-11842.

\section{BIBLIOGRAPHY}

Sugandhi, E. W.; Falkinham, J. O., III; Gandour, R. D. Synthesis and Antimicrobial Activity of Symmetrical Two-tailed Dendritic Tricarboxylato Amphiphiles. Bioorg. Med. Chem. 2007, 14, 0000-0000. (BMC-5561, accepted on March 8, 2007) 
Appendix 14: Continuation of Project-Dry Particle Separation in a CFB Rise System (WV009) 


\section{TECHNICAL REPORT}

\begin{tabular}{|c|c|}
\hline Contract Title and Number: & Period of Performance: \\
\hline Establishment of the Center for Advanced Separation & Starting Date: 6/1/2004 \\
\hline Technologies (DE-FC26-02NT41607) & $\begin{array}{l}10 / 31 / 2006 \text { (no } \\
\text { cost extension to } \\
10 / 31 / 2007 \text { ) }\end{array}$ \\
\hline
\end{tabular}

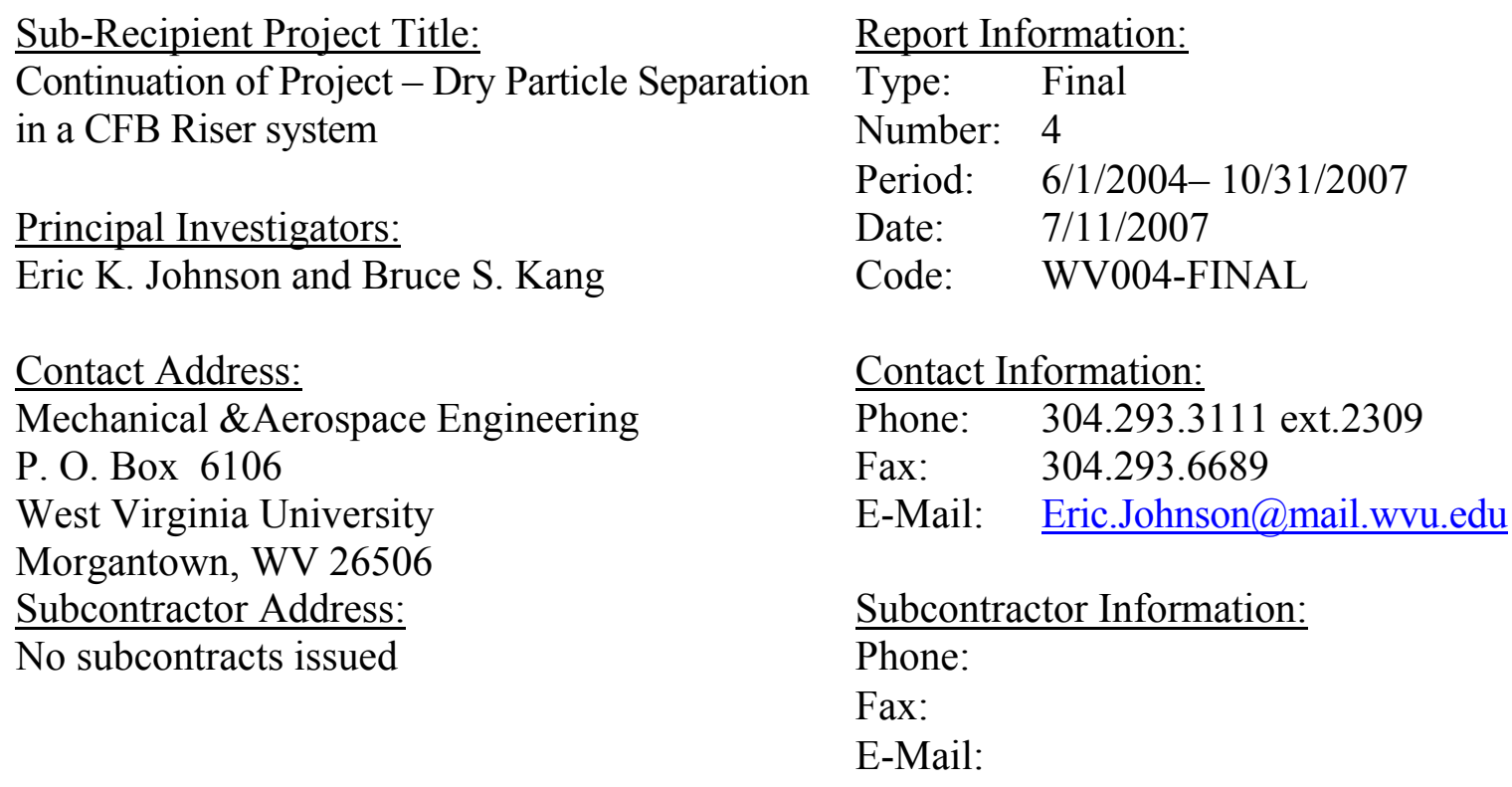

\section{DISCLAIMER}

This report was prepared as an account of work sponsored by an agency of the United States Government. Neither the United States Government nor any agency thereof, nor any of their employees, make any warranty, express or implied, nor assume any legal liability or responsibility for the accuracy, completeness, or usefulness of any information, apparatus, product, or process disclosed, or represents that its use would not infringe privately owned rights. Reference herein to any specific commercial product, process, or service by trade name, trademark, manufacturer, or otherwise does not necessarily constitute or imply endorsement, recommendation, or favoring by the United States Government or any agency thereof. The views and opinions of authors expressed herein do not necessarily state or reflect those of the United States Government or agency thereof. 


\begin{abstract}
The goal of this research activity was to investigate the potential for dry separation of particles in a circulating fluidized bed (CFB) riser system. This specific research project was to continue a previous project, CAST WV002, which demonstrated that steel shot may be efficiently separated from sand in the riser. This research project focuses on refining and expanding our test conditions and to develop a more comprehensive understanding of the separation process. This continuation project consists of three tasks: 1) separation of particles of the same material, due to size differences, 2) separation of particles, with the same size distribution, due to density differences, and 3) separation of clean coal from "dirty" coal. Dirty coal is loosely defined as coal with a significant amount of pyrite. Task 1 consisted of separating sand into particles with diameters smaller or larger than a prescribed diameter. The experimental results indicated excellent separation at appropriate operating conditions. Task 2 consisted of separating steel shot from sand, both with the same size distribution. Again, the experimental results were excellent and they collaborated the results of the initial study in which the size distributions of the two materials were not to different. Task 3 consisted of attempting to separate clean coal from dirty coal. In this case, there is no distinct difference in either size or density. Initially, inconsistent and therefore inconclusive results were obtained. This phase of the research is denoted as Task $3 \mathrm{a}$.
\end{abstract}

Pyrite particles are frequently very small and not uniformly distributed throughout the coal particles. Therefore, it was decided to crush the coal to a finer size in the hope that some particles would contain sufficiently more pyrite globules than others. Consequently, a density difference would then be created between the clean coal and the dirty coal. With the desire to separate finer coal, it was apparent that a small riser system be constructed to investigate the flow of fine powers in the separation process. Task $3 \mathrm{~b}$ consisted of building the small riser and obtaining the desired experience with coal fines. It was determined that the initial separation process had to be modified to obtain good separation of fines. Two modifications were developed. Task $3 \mathrm{c}$ consisted of creating a stronger jet of fine particles into the riser, and task $3 \mathrm{~d}$ consisted of creating pulsating gas flow in the riser. Both modifications produced a coal that had a sulfur content that was $20 \%$ less than that of the feed coal.

This project contains essentially six experimental sub-projects, resulting in four theses. There was significant activity in developing three distinct experimental systems and the associated support equipment to produce the required separation results. This final report only summarizes these activities with respect to the separation results. The interested reader is recommended to read the four theses for the details of this project.

In conclusion, the dry separation has been demonstrated to efficiently separate solid particles according to size and density and has the potential to become an acceptable coal cleaning process for coal fines. 
TABLE OF CONTENTS

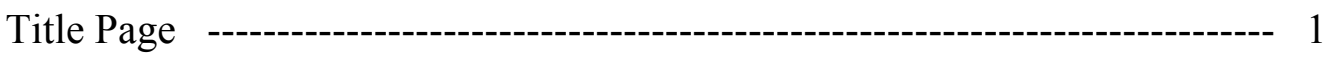

Disclaimer ------ 1

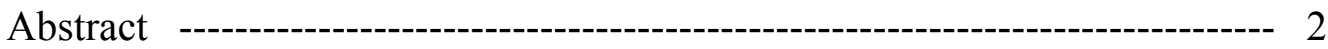

Table of Contents ----o- 3

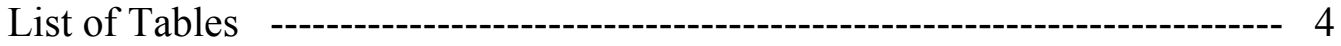

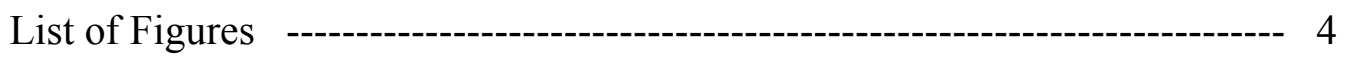

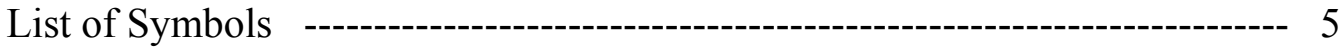

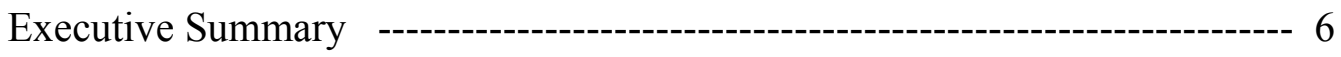

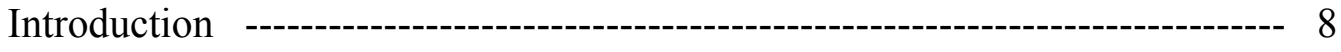

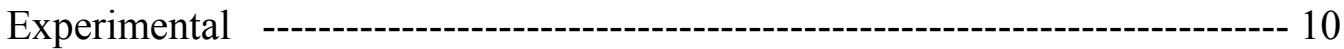

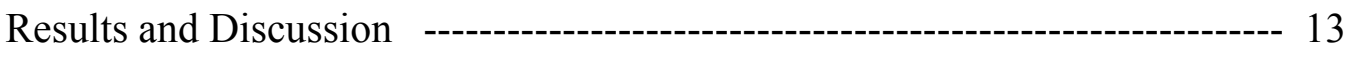

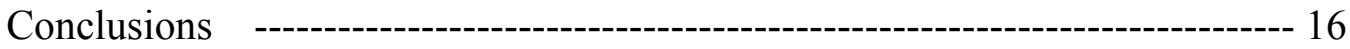

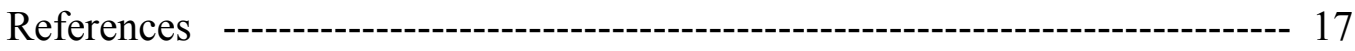




\section{List of Tables}

Table 1 Experimental results for the separation of sand based on size difference, Task1.

Table 2 Experiment results for the separation of shot from sand, both with same size distribution, Task 2

Table 3 Experimental results for the separation of steel shot and sand for evaluation of the system modifications for the Task $3 \mathrm{C}$

Table 4 Test matrix for the separation of fine coal in Task 3C

Table 5 Flow velocities and volumetric flow rates for $0 \mathrm{~Hz}, 1 \mathrm{~Hz}$, and $2 \mathrm{~Hz}$ pulsation tests. The solid mass flow rate was held constant at $2.5 \mathrm{~kg} / \mathrm{hr}$

\section{List of Figures}

Figure 1: Basic terminal velocity-particle diameter curve for sand.

Figure 2: Basic terminal velocity-particle diameter curves for steel shot and sand. Also a superficial gas velocity curve for separating the two materials.

Figure 3: Terminal velocity-particle diameter curves for coal with different specific gravities.

Figure 4: Experimental system used in Task 1, 2, 3a, and 3c, For Task 3c the distributor region was modified, as shown in Figure 6.

Figure 5: Small scale exploratory system for Task $3 \mathrm{~b}$. Riser inside diameter is $5 \mathrm{~cm}$. Note the collection ring is below the solids circuit inlet and the solids injector position was designed to hold several type of injectors.

Figure 6: Modifications to the original system for Task 3c. 
Figure 7: Experimental system for the investigation of particle separation in pulsating flow. This was a small scale system with a $5 \mathrm{~cm}$ ID riser.

Figure 8: Size distributions for Test \#1. The riser height was $4.3 \mathrm{~m}$, bend outlet, $\mathrm{u}_{\mathrm{o}}=2.4 \mathrm{~m} / \mathrm{s}$, and $\mathrm{G}=1.24 \mathrm{~kg} / \mathrm{m}^{2} \mathrm{~s}$, Task 1 .

Figure 9: Multiple phase tests.

Figure 10: Process diagrams to illustrate the production of material (sand) in a selected size range, Task 1.

Figure 11: Distribution of the final product after 2 and 4 passes compared to the size distribution of the initial mixture, Task 1. 26

Figure 12: Percent sulfur change comparison for gas flow rates. 27

Figure 13: Product percent change in sulfur vs. mass flux. 27

Figure 14: Percent sulfur change comparison for repeatability tests. 28

Figure 15: Results from tests with $1 \mathrm{~Hz}$ pulsations, coal particles $\left(105<\mathrm{d}_{\mathrm{p}}<210\right) \mu \mathrm{m}$, and decreasing superficial gas velocity with grouped product bins. ------- 28

Figure 16: Results from tests with $2 \mathrm{~Hz}$ pulsations, coal particles $(105<\mathrm{dp}<210) \mu \mathrm{m}$, and decreasing superficial gas velocity with grouped product bins.

Figure 17: Results from tests with superficial gas velocity $\mathrm{u}_{0}=0.9621 \mathrm{~ms}^{-1}$, coal particles $\left(105<\mathrm{d}_{\mathrm{p}}<210\right) \mu \mathrm{m}$, varying pulsation rates $(0 \mathrm{~Hz}, 1 \mathrm{~Hz}$, and $2 \mathrm{~Hz})$, and grouped product bins.

\section{Liist of Symbols}

$\begin{array}{ll}\text { C } & \text { accumulated mass less than } \\ d_{P} & \text { particle diameter } \\ d_{P}^{*} & \text { particle cut diameter } \\ \mathrm{u}_{\mathrm{O}} & \text { superficial gas velocity } \\ \mathrm{v}_{\mathrm{T}} & \text { terminal particle velocity } \\ \mathrm{CFB} & \text { circulating fluidized bed } \\ \mathrm{NRCCE} & \text { National Research Center for Coal and Energy } \\ \mathrm{Re}_{\mathrm{P}} & \text { particle Reynolds number }\end{array}$




\section{EXECUTIVE SUMMARY}

A initial exploratory project, CAST I WV002, demonstrated that solids may be separated by density differences in a dry process in a circulating fluidized bed, CFB, riser system. The goal of the present project, CAST II WV 008, is to continue the initial project in order to refine and expand the basic understanding of the separation process gained in the initial project. The approach taken to achieve this goal was to conduct this research in three tasks. These tasks were: Task 1, investigation of the separation of particles of the same material, due to size differences, Task 2, an investigation of separation of particles, with the same size distribution, due to density differences, and Task 3 , investigate the potential for the separation of clean coal from dirty coal. Dirty coal is loosely defined as coal containing a relatively large amount of pyrite globules.

The data obtained in the Task 1 effort demonstrated excellent results in dividing sand into two distinct piles, one with a diameter greater than $d_{P}^{*}$ and one with diameters less than $d_{P}^{*}$. These tests were referred to as single cut tests. For a separation diameter of $d_{P}^{*}=$ $340 \mu \mathrm{m}$, the results for the capture of the smaller particles was about $90 \%$ and the mass fraction of the smaller particles in the product bin was also about $90 \%$. In other tests, sand was passed through the system up to four runs, in order to produce a product consisting of a selected range of particle sizes. In one test of four runs, for a desired product range of particles between $500 \mu \mathrm{m}$ and $250 \mu \mathrm{m}$, the final product bin contained $96 \%$ less than $500 \mu \mathrm{m}$ and $4 \%$ less than $250 \mu \mathrm{m}$. The separation of particles by size may be considered an efficient dry process.

Task 2 was a investigation of the separation of steel shot and sand, each having the same size distribution. The selected size distribution was $-500 \mu \mathrm{m} x+250 \mu \mathrm{m}$ with a match at $355 \mu \mathrm{m}$ for $* 80 \%$ less than. For the appropriate operating conditions, the capture of the steel shot was greater than $80 \%$ and the mass fraction of the steel shot in the dense bin was greater than $80 \%$. These results essentially support the results of the initial study in which the size distributions were not to different. The separation of particles due to density differences in a dry process may be considered a success.

Task 3 was the investigation into the potential to separated clean coal from dirty coal. This task represented a significant challenge because coal has a continuous variation in density and size. However, it was hoped that the dirty coal would tend to be heavier or larger in size. The first experimental runs employed coal in the size range $-500 \mu \mathrm{m} x+$ $250 \mu \mathrm{m}$. The results were inconclusive and when a float-sink test on the products of one test run showed separation and the chemical analysis did not, it was decided to re-think what we were trying to do. Successful separation tests were conducted using particles greater than $250 \mu \mathrm{m}$. If the pyrite globules are small and distributed throughout the coal particles in a non-homogeneous fashion, then crushing the coal to a smaller size may lead to particles that are dense dirty coal particles compared to clean coal particles. A separation based on density difference would then be possible. Subsequently, three new subtasks of Task 3 were formulated. Task 3 a denotes the initial work on separating coal. Task $3 \mathrm{~b}$ was the preliminary investigation on the movement of small coal particles, (fines), in the separation system. This would then lead to modifications in the riser 
system. Because of the difficulty in separating fines in the original riser, two alternative methods for separation were devised. Task $3 \mathrm{c}$ consisted in modifying the flow field, within the riser, with a strong gas-solid feed jet flowing upward along the axis of the riser. Tests were conducted with coal in the size range $-210 \mu \mathrm{m} x+105 \mu \mathrm{m}$ and with a $0.95 \%$ sulfur content. The sulfur content of the coal in the product bin was reduced by 10 $\%$ under some conditions. Because the coal had been previously cleaned, these results appear to be very good. Task $3 \mathrm{~d}$ consisted of constructing a smaller riser system and installing a system for creating gas pulsations in the riser. Again, tests were conducted with coal in the size range $-210 \mu \mathrm{m} x+105 \mu \mathrm{m}$ and with a sulfur content of $1.7 \%$. The sulfur content of the coal in the product bin was reduced by up to $20 \%$ for some test conditions. The results from the Tasks $3 \mathrm{c}$ and $3 \mathrm{~d}$ were more conclusive than those from Task $3 \mathrm{a}$ The reasons for this is that the coal samples to the chemistry laboratory were larger and the coal particles were smaller; consequently, the scatter in the data was significant less. This was considered a successful separation of clean coal from dirty coal, especially since the feed coal had been previously cleaned.

This final report is essentially a summary of the activities relating to the investigation of the dry separation of particles in the riser of a CFB system. Initially, the separation was to be based on density difference; however, separation based on size difference was also found to be significant. In this essentially exploratory study, six separate subprojects (Tasks) were completed and reported in four MS theses. The reader is recommended to read these theses for a more comprehensive understanding of what was accomplished.

In conclusion, the dry separation of solids based on size difference or density difference in a CFB riser system has been demonstrated. 


\section{INTRODUCTION}

This project is a continuation of an initial "exploratory" study to determine the potential for the dry separation of particles based on density differences. This exploratory study, CAST I WV002, produced many practical and interesting results ( Register 2004, Johnson 2005). This continuation project, CAST II WV004, focuses on the refinement and expansion of the original concepts in order to develop a more comprehensive understanding of the separation process. This project consists of three tasks. Task 1 investigates the separation of particles of the same material according to sizes. Task2 investigates the separation of particles, with the same size distribution, according to density differences. Task 3 is to investigate the separation of clean coal from dirty coal. In this case, there is no distinct density difference or size difference relating to pyrite in a coal particle.

The analysis of the separation process has produced four dimensionless parameters. They are:

$$
\Pi_{1}=\frac{v_{T}}{u_{0}} \quad \Pi_{2}=\frac{d_{P}}{u_{0}} \frac{\partial u}{\partial r} \quad \Pi_{3}=\frac{(u-v) d_{P}}{v} \quad \Pi_{4}=\frac{G}{\rho u_{0}}
$$

The first parameter, $\Pi_{1}$, determines which particles may remain in the riser and which will be blown out of the riser. The second term, $\Pi_{2}$, is a measure of the potential to push the particle towards the wall. The third term, $\Pi_{3}$, is the familiar Reynolds number, which indicates the characteristics of the flow about the particle. The last term, $\Pi_{4}$, relates the mass flow of solids to the mass flow of the air.

The performance parameters for the separation process in Tasks 1 and 2 were:

1) capture efficiency

$$
\eta_{H}=\frac{\text { mass of desired particles captured }}{\text { mass of desired particles in the original mixture }}
$$

2) mass fraction efficiency

$$
\omega_{H}=\frac{\text { mass of desired particles collected }}{\text { mass of all particles collected }}
$$

The subscript $\mathrm{H}$ denotes either the desired diameter for the split of the mixture or the density of the desired particles (in this report, the heavy particles).

For Task 1, play sand was initially employed in the size range of $-500 \mathrm{x}+100 \mu \mathrm{m}$. Later tests, to verify the results of the initial tests, brown construction sand was employed with a size range of $-840 \times 0 \mu \mathrm{m}$. For Task 2 , sand and steel shot were used to form a mixture with essentially the same size distribution. The size distribution selected was $-500 \mathrm{x}+250$ 
$\mu \mathrm{m}$. The separation results for both tasks were excellent, as reported in the progress reports.

The size range for the coal used in Task 3 initially was $-500 \mathrm{x}+250 \mu \mathrm{m}$. Problems arose relating to mass and sulfur balances. After cleaning and slight modification of the riser system, inconsistent results were still obtained. The problem appeared to be the method of chemical analysis employed. When a float-sink showed better separation than the chemical analysis, it was decided to revise Task 3 . This initial work was designated as Task 3a. Because pyrite globules are frequently small and non-homogenously dispersed in the coal particle, it was decided to crush the coal to a small size than originally employed. The goal was to determine if coal particles containing relatively large number of globules of pyrite could be produced; thereby, creating a density difference between dirty coal and relatively clean coal. This then would create a condition for separation by density difference. However; considering the first three dimensionless parameters, the smaller particles will be more difficult to separate. Consequently, Task $3 \mathrm{~b}$ was designed to investigate the operational problems of coal fines a small scale riser system. This riser had a $5 \mathrm{~cm}$ ID riser and the size distribution investigated was $-150 \mathrm{x}+74 \mu \mathrm{m}$. After gaining operating experience with the coal fines, two new tasks were formulated, which are Tasks $3 \mathrm{c}$ and $3 \mathrm{~d}$. Task $3 \mathrm{c}$ consists of modifying the flow field in the riser to create larger gas velocity gradients, and Task $3 \mathrm{~d}$ consists of creating a pulsating gas flow in the riser to enhance the relative motion between the particle and the gas.

Tasks $3 \mathrm{c}$ and $3 \mathrm{~d}$ employed a different efficiency parameter; the percent change in sulfur content, $\varphi$.

$$
\phi=\frac{\text { Percent of sulfur in the feed - percent of sulfur in the product }}{\text { Percent of sulfur in the feed }}
$$

Note that $\varphi_{C}$ will be less than one for the clean coal and $\varphi_{D}$ greater than one for the dirty coal. With very little material being collected in the filter, the two $\varphi$ 's should add up to one.

For Task $3 c$, the original riser system was modified to create a high velocity gas-particle jet shooting upward along the axis of the riser. This modification was intended to produce large velocity gradients in the flow field. The coal was crushed to a size range of $-210 \mathrm{x}+$ $105 \mu \mathrm{m}$ and the coal had an initial sulfur content of about $0.95 \%$ (previously cleaned coal). The results of Task $3 \mathrm{c}$ were unexpected. The percent change in sulfur was about $10 \%$ for some conditions. However, the clean coal reported to the dense bin instead of the product bin. The only plausible explanation is that during the crushing process, the cleaner coal was at the larger end of the size range and the coal containing the pyrite was at the smaller end of the size range. Nevertheless, the fact that the sulfur content of the previously cleaned coal was reduced by $10 \%$ is impressive.

A new, small, riser system was built for Task 3d, and a pulsating flow circuit was installed. This modification was intended to create periods of large relative velocities between the gas and the fine particles. The coal used was crushed to a size range of -210 
$\mathrm{x}+105 \mu \mathrm{m}$ and the coal had an initial sulfur content of $1.7 \%$ (again previously cleaned). The percent change in sulfur content reached $20 \%$ at certain operating conditions. Considering that the coal was previously cleaned, the reduction of the sulfur content of the product coal in significant.

\section{EXPERIMENTAL}

\section{$\underline{\text { Introduction }}$}

The goal of this project was to improve our understanding of the dry separation process occurring in a CFB riser system. This project was a continuation of the CAST I WV002 project, which indicated that there was efficient separation of steel shot and sand at certain operating conditions. To accomplish this goal, there were six distinguishable experimental studies undertaken. The first task, Task 1, was to investigate the separation process, for the same material, based on size differences. Task 2 was to separate steel shot from sand with each material having the same size distribution. Task 3 was to an attempt to determine if the system could separate clean coal from dirty coal. Task 3 produced several challenging experimental problems which were overcome by modifying the original separation system. The initial tests with coal and the ensuing challenges were then denoted as Task 3a. In order to create a basis for separation, the coal was crushed in the hope that the pyrite globules were not uniformly distributed throughout the coal thus creating a density difference between the clean coal and the dirty coal. Task $3 \mathrm{~b}$ was directed towards obtaining operating experience with smaller particles of coal. Tasks $3 \mathrm{c}$ and $3 \mathrm{~d}$ employed modified riser systems for their experimental work.

\section{$\underline{\text { Selection of Test Conditions }}$}

The research conducted in this project continued to be of an exploratory nature. Therefore, the initial test conditions were selected based on the initial CAST I WV002, project (Regester 2004). As experience was gained, the dimensionless parameters described in the INTRODUCTION section of this report were developed. The first quantity to consider was the size distribution of the particles of interest. A terminal velocity, $\mathrm{v}_{\mathrm{T}}$, was determined as a function of particle size and plotted as shown in Figure 1. If there was only one component in the material, then only one curve would appear on the diagram, Figure 1. If the material contained two components, with one component more dense than the other, then two curves on the $v_{T}-d_{P}$ diagram, Figure 2. A superficial gas velocity was then selected to separate the material by size, as shown in Figure 1, or by density, as shown in Figure 2. This method was used to select a superficial velocity in Task 1 and to select a range of superficial velocities for Task 2. After the selection of the superficial gas velocity and with a constant riser diameter, the gas velocity was fixed.

The experimental work for Task 3 was complicated because there were no specific cut diameters or density difference in the material to be separated. After the initial tests, Task 3a, it became apparent that an attempt must be made to create a useful density difference in the coal. This problem is described in Figure 3. It was now assumed that if the coal is crushed to a smaller size range, a density difference may appear between the clean coal 
and the dirty coal. This was done by crushing the coal to $-210 \mu \mathrm{m} x+105 \mu \mathrm{m}$. Because the particle diameters are smaller, the original separation process is no longer efficient. Task $3 \mathrm{~b}$ involved building a small scale riser system, to investigate these problems. The basic problem to be resolved was the flowability of the fine coal into the riser and to have a more acceptable filter. The results of this work were used to modify the original system, Task $3 \mathrm{c}$, and to build a new small system for Task $3 \mathrm{~d}$. The selection of superficial gas velocities were now again based on $\mathrm{a} \mathrm{v}_{\mathrm{T}}-\mathrm{d}_{\mathrm{P}}$ diagram.

The acceptance of the mass flux of solids for a successful test was based on the stability and operating conditions produced in the riser. By visual inspection, it was determined if the flow in the riser was in the transition region between fast fluidization and entrained flow. This was a necessary condition for good performance. The other condition to satisfy was that the flow rate from the feed hopper was essentially steady. Because this was an exploratory investigation, the necessary equipment to ideally solve the feed hoper problem appeared to be too expensive.

\section{Experimental Systems}

The initial system used in investigating the dry separation of steel shot and sand is shown in Figure 4. This system was used in Tasks 1, 2, and 3a and modified for Task 3c. Two filter systems were employed. A water tank was employed when a material balance was not necessary. A filter bag was employed when a mass balance was required. The filter bag was essentially a vacuum cleaner bag attached to the end of the cycle exit pipe.

Task 1. Initially, play sand with a size distribution of $-500 \mu \mathrm{m} x+250 \mu \mathrm{m}$ was to be used to form two batches of sand, in the size range:

$$
\begin{aligned}
& -420 \mu \mathrm{m} x+292 \mu \mathrm{m} \text {-------------batch \#1 } \\
& -210 \mu \mathrm{m} x+105 \mu \mathrm{m} \text {------------batch \#2 }
\end{aligned}
$$

With a fixed amount of mass from each batch, the two were thoroughly mixed together. The mixture was then passed through the separation system to determine if the initial mass from each batch could be recovered. The results were inconsistent. The problem was traced to the Denver 4x4 Sieving Machine. After an unsuccessful attempt to repair the machine and screens, it was decided to use the smaller Ro-Tap Sieving Machine. During this period, it became apparent that a more productive approach to satisfying the goal of Task1 could be employed. This approach consists of starting with a wide size distribution and attempting to divide the batch into two batches, one less than $d_{P}^{*}$ and one batch greater than that diameter.

The experimental procedure consisted of first determining the size distribution and the mass of sand placed in the feed hopper. The test was then run at selected conditions, depending on the riser flow field. The load cell reading was then inspected to determine if the transient potion of the run was less than $10 \%$ of the total run time. The slowest gage to indicate steady state was the load cell reading. After the test, the mass and size 
distribution in the product and dense bin were determined. Mass closure was achieved in these tests.

A second set of tests were performed to determine if a narrow size batch of particles could be produced in this separation system. A batch of sand was passed through the separator at a velocity $3.6 \mathrm{~m} / \mathrm{s}$ which corresponds to a diameter of $500 \mu \mathrm{m}$. The sand in the product hopper has sand essentially smaller than $500 \mu \mathrm{m}$. The sand from the product hopper was then passed through the separator at a velocity $1.8 \mathrm{~m} / \mathrm{s}$, which corresponds to a diameter of $250 \mu \mathrm{m}$. The material collected in the dense bin now has a diameter which is essentially greater than $250 \mu \mathrm{m}$ and less than $500 \mu \mathrm{m}$. This procedure was denoted as a two pass test. This procedure was repeated with the products from the two-pass test to see if an improvement in results could be obtained. This then was denoted as a four-pass test.

Task 2. Task 2 consisted of investigating the he separation of two materials, each with the same size distribution, but of different densities. A batch of steel shot and a batch of sand each had their size distribution adjusted so that the percent less than were equal at 250 , 355 , and $500 \mu \mathrm{m}$. A know mass was then taken from each batch and thoroughly mixed together forming a known mass fraction for the steel shot in the feed mixture. The mixture was then passed through the separation system and the mass of the material collected in the dense bin and the product bin were recorded. The mass of the steel shot in the dense bin was determined by passing a magnet through the material until no more steel shot was collected by the magnet. The mass of the steel shot collected was then used to determine the capture efficiency and the mass fraction of steel shot associated with the capture.

Task 3 Task 3 was divided into four separate tasks after the initial tests to satisfy the goals of the program

Task 3a. Task 3a represents the initial effort to separate clean coal from dirty coal. Coal was sieved to obtain a size range corresponding to the previous tests, $-500 \mu \mathrm{m} \mathrm{x}+212$ $\mu \mathrm{m}$. A known amount of this coal was then passed through the separation system. The amount of mass reporting to the product bin, the dense bin, and the filter were recorded. Three samples were taken from the initial feed, the material in the product bin, the material in the dense bin, and the material in the filter. These samples were sent to the WVU NRCCE Chemical Analysis Group for analysis. Usually, there was not enough material in the filter for analysis. The results indicated there was no closure for mass, sulfur, or ash. The problem was two-fold, the samples sent for analysis were to small, and that there were locations in which coal was trapped in one test and released in a following test. Since there were no problems with the flow of steel shot and sand through the system, it was concluded that the system had to be modified for coal to eliminate passages with a small slope.

Task $3 \mathrm{~b}$. When it was decided to investigate the separation process with smaller particles, a smaller, simpler, $5 \mathrm{~cm}$ ID riser system was built to a conduct an exploratory study on the flowability of fine coal particles. This smaller system is shown in Figure 5. The specific goals for this task were to investigate the flow of the fine coal through the 
injection system at the bottom of the fed hopper, flow fields in the riser, and flow in the cyclones. The results of this effort produced the necessary background design information for Tasks $3 \mathrm{c}$ and $3 \mathrm{~d}$.

Task $3 \mathrm{c}$. This investigation involved attempting to modify the flow field in the riser by imposing a strong jet flow of gas and solids upward, axially, in the riser. This design is directed towards improving the velocity gradients in the flow field. The modification to the original riser system is shown in Figure 6. The gas-solids feed port in the riser was machined to allow nozzles to be attached. Each nozzle had a different opening to create a different exit velocity. A second modification to the system was to allow for a variable height ring wall.

In order to determine what effect the modifications may have on the previous results, a series of runs was conducted with steel shot and sand in the size range $-500 \mu \mathrm{m} \mathrm{x}$ $+250 \mu \mathrm{m}$. The test procedure was the same as described in Task 2 . These results may then be directly compared to those results. The results indicated that the results were the same and that the nozzle opening or division of flow rates had no effect on the performance of the separation process.

After the initial system tests, the investigation of the potential for the separation of clean coal from dirty coal began. Coal from the Kingwood Coal Company was crushed and sieved to a size range $-210 \mu \mathrm{m} \times 105 \mu \mathrm{m}$. The tests were conducted as described in Task 3a. With the system modifications and a different procedure for chemical analysis, the closure for mass, sulfur, and ash were now acceptable. Samples sent to the NRCCE Chemical Analysis Group were now 3-5 grams and a wet Inductively Coupled Plasma process was employed.

Task 3d. A small CFB riser system was built and instrumented, as shown in Figure 7. This riser system had a $5 \mathrm{~cm}$ ID riser and was operated essentially in the same manner as the other systems. However, a fast acting open-close valve was inserted into the air flow circuit to the distributor plate. This valve created pulsations in the riser flow. The pulsating frequencies selected for these tests were 1 and $2 \mathrm{hz}$. Coal from a CONSOL preparation plant was crushed and sieved to a size range of $-210 \mu \mathrm{m} x+105 \mu \mathrm{m}$. Samples from each solids bin were sent to the NRCCE Chemical Analysis Group for analysis. Acceptable results were obtained for the closure for mass, sulfur, and ash.

\section{RESULTS AND DISCUSSION}

This project consisted of three separate tasks. This section presents the results and conclusions of the project as three independent projects. This effort was conducted in two phases, one is referred to as the single pass tests and the other is referred to as the multiple pass tests.

Task 1-The goal of this task was to investigate the separation of a material by size. This effort consisted of two phases. The initial phase consisted of separating play sand, with a size range of $-500 \mu \mathrm{m} x+250 \mu \mathrm{m}$, into two piles, one pile with particle diameters larger 
than $d_{P}^{*}$ and one pile with smaller diameter particles. This cut occurs in a single pass through the riser system. The results of this phase are presented in Table 1. Figure 8 shows the size distribution curves for the initial mixture, the product bin, and the dense bin of test \#1.

If a single pass size cut is effective, the ability to do a double cut should be possible. The goal of the multiple pass tests was to produce a product where the particles are all within a specified size range. The multiple phase tests consisted of starting with a specified size range and then constructing the velocity-particle size curve for the material, as shown in Figure 9. The sand used in this phase was brown construction sand. The smaller size particle diameter is denoted as $d_{P S}^{*}$ and the larger size particle diameter is denoted as $d_{P L}^{*}$. The two riser operating superficial gas velocities are then $\mathrm{u}_{0 \mathrm{~S}}$ and $\mathrm{u}_{01}$. The first pass through the riser was selected to remove the larger particles from the mixture, sending them to the dense bin. The particles from the product bin were then passed through the riser and particles with a diameter less than $d_{P S}^{*}$ reported to the product bin. The material in the dense bin now contained the particles in the desired size range. The process is shown in Figure 10. To improve the sharpness of the size range, two experimental runs involved four passes, the two-pass process repeated twice. Figure 11, shows the size distribution of the sand after 2 and 4 passes through the riser system. Unfortunately, no efficiencies were determined due to the loss of material during each transfer of particles between bins and the sieving machine.

Task 2 - The goal of this task was to separate particles, with the same size distribution, due to a density difference. Steel shot and sand were sieved separately to a size range of $-500 \mu \mathrm{m} \mathrm{x}+250 \mu \mathrm{m}$ with a percent less than match at $355 \mu \mathrm{m}$. The two materials were then mixed to form a mixture with a mass fraction of 0.2 . The test conditions and the results are shown in Table 2. The results are consistent with the initial separation project, Regester 2005. Test number 12 produced inconsistent results, consequently, test 13 was a was performed at the essentially the same operating conditions, and it now appears that test 12 produced inconsistent results.

Task 3 - This task was divided into four separate phases, all directed towards the original goal of Task 3, that is, separating clean coal from dirty coal. Task 3 a continued to employ the separation techniques of Tasks 1 and 2. However, now there are no distinctive terminal velocity curves for the clean and dirty coals. Waste coal from a Consol coal preparation plant was sieved to a size range of $-500 \mu \mathrm{m} x+250 \mu \mathrm{m}$. Six exploratory tests were conducted. Unfortunately, there was a long wait between the testing and the reports on the sulfur content of each stream. When the reports arrived from the NRCCE Chemistry Analysis Group, it was found that there was no mass closure for the ash and sulfur. The experimental system was then modified to obtain better results, but to no avail. Again, the results were inconclusive. The analysis of the coal consisted of three small samples (about 1 gr) in a dry process in an Inductively Coupled Plasma machine. The small samples probably contributed to the scatter of the data. A float-sink test was conducted on the particles from on test and it was found that some separation had 
occurred. The $50 \%$ mass point was reduced from a $1.3 \mathrm{sg}$ to $1.27 \mathrm{sg}$. The details of this work may be found in (Almond.2006).

At this point in time, it was concluded that there was no significant difference in the densities of clean coal and dirty coal. Also, the size of the particle had no relationship to the cleanliness of a coal particle in this size range. However, since the pyrite globules are frequently reported as very small, (of the order of $30 \mu \mathrm{m}$ ), and are probably not uniformly distributed in the coal particles, crushing the coal to a smaller diameter may lead to a density variation between the clean and dirty coal. Consequently, Task 3 was reorganized into four phases. Task 3 a denotes the initial effort to separate clean from dirty coal. Task $3 \mathrm{~b}$ represents the effort to obtain experience with the movement of coal fines. Task $3 \mathrm{c}$ was to investigate the separation of coal in a modified flow field. The flow field in the riser had to be modified in order to create conditions conducive to separation of fine particles. Task $3 \mathrm{~d}$ represents a second flow modification in the riser, using pulsating gas flow, for separation.

A small riser system, $5 \mathrm{~cm}$ ID, was constructed for Task $3 \mathrm{~b}$. Coal in the size range, -150 $\mu \mathrm{m} x+74 \mu \mathrm{m}$ was employed to develop an improved coal feed injector and evaluate the other components of the system. Also, a brief investigation was undertaken to determine if a more dense substance could be found that would attach itself to the dirty coal particle by means of a van der Waals force. This literature review did not find a suitable material for this purpose. The basic outcome from Task $3 \mathrm{~b}$ was that valuable experience was gained in working with coal fines in the riser system which greatly aided in the design of the system modifications for Tasks $3 \mathrm{c}$ and $3 \mathrm{~d}$. Details of this work may be found in (Aktar, 2006).

The flow field modification in Task $3 \mathrm{c}$ consisted of employing a strong, solid-gas jet, flowing upward along the axis of the riser. The jet velocity was the primary variable. The desired jet velocity was created by the nozzle throat size, which is the listed variable for the gas velocity. The total gas flow was essentially constant at $170 \mathrm{SCMH}$. The percent flow through the nozzle was from $35 \%$ to $72 \%$. This configuration created relatively large velocity gradients near the distributor plate. This approach allowed the superficial gas velocity to be relatively low in the upper part of the riser so as not to prematurely blow the fines out of the system. The first set of runs employed a mixture of steel shot and sand. The purpose of these runs was to compare the separation efficiencies of the modified riser system with those of the original system. The size range of the material was $-210 \mu \mathrm{m} x+105 \mu \mathrm{m}$ and the mass fraction of the steel shot was 0.20 .The specific test conditions and the results for each run are shown in Table 3. The separation efficiencies are similar to those of the original system operating with larger particles.

The final set of tests employed coal from the Kingwood Coal Company. This coal contained about $1 \%$ sulfur and the researchers were informed after the tests that the coal had been previously cleaned. The coal was crushed to $-210 \mu \mathrm{m} x+105 \mu \mathrm{m}$ for these tests. The test matrix for the coal separation tests are presented in Table 4 . The results for the separation are shown in Figures 12, 13 and 14. Note that points above the zero percent change line represent an increase in sulfur content and the points below that line 
represent coal with a reduction in sulfur content. It may also be noted that the dense bin contains the coal with a smaller sulfur content. This is inconsistent with previous results where the heavier material reports to the dense bin. The only plausible explanation is that the pyrite in a coal particle contributes to the crushing process and the pyrite containing particles end up in the smaller end of the size distribution. Consequently, the smaller particles report to the product bin and the larger clean particles report to the dense bin as they would in a size separation process. The coal analysis for each collection of coal particles consisted of 3, 3-5 grams, samples analyzed wet in a Inductance Coupled Plasma machine. The larger coal samples and the smaller particles apparently contributed to a small scatter in the data. Consequently, a high degree of confidence in the results was attained.

Task 3d. A small riser system was built for the study of separation of fine particles in a pulsating flow field. The air flow circuit to the distributor plate was modified to provide for a steady flow component and a pulsating flow component. The flow through the solids entry port was kept constant. Based on the work of Task $3 b$, new and more appropriate instrumentation was installed on this system. Coal, from a CONSOL coal preparation plant, was crushed and sieved to a size range of $-210 \mu \mathrm{m} x+105 \mu \mathrm{m}$. This coal had been previously cleaned and had a sulfur content of $1.7 \%$. The test matrix for the pulsating flow separation tests are presented in Table RR. The results of the separation tests are represented in Figures 15, 16, and 17. Again, note that the points above the zero line represent coal with an increased sulfur content and the points below the zero line represents coal with a reduced sulfur content. The coal fines collected in the filter showed the lowest sulfur content, but these fines passed through essentially two cleaning processes. The coal analysis consisted of obtaining three samples, each $0.3-3.5$ grams, from each bin and employing the wet Inductance-Coupled-Plasma process. The analysis showed consistent results; consequently, a high degree of confidence in the results was attained.

\section{CONCLUSIONS}

The goal of this research activity was to refine and expand the results of a previous project, CAST I WV002. This exploratory project had shown that there was a potential for the efficient separation of small particles, based on density difference, in a dry process. The initial project efficiently separated steel shot from sand in a circulating fluidized bed riser system. This continuation project was conducted in three tasks. Task 1 was to investigate the separation of the same material based on size difference. Task 2 was to investigate the separation of two materials, with the same size distribution, based on density difference. Task 3 was to investigate the potential for the separation clean coal from dirty coal.

Four dimensionless parameters evolved from the initial study and the early activities in this project. These parameters have been useful in explaining the results of Tasks 1 and 2and planning the work in Task 3. These parameters are presented in the INTRODUCTION section of this report. The excellent results in separation in Tasks 1 and 2 were due to the relatively large particles which then lead to values for the four 
parameters. From these parameters, the most useful quantity became the terminal velocity of the particles. For the large particles, a relatively large terminal velocity was calculated, which in turn allowed for a large superficial gas velocity. This then produced effective velocity gradients in the flow and a relatively large Reynolds number. All of these quantities combined to produce an effective lift force.

In order to separate clean coal from dirty coal, a significant difference between the clean coal and the dirty coal had to be established. It was found that crushing the coal to less than $200 \mu \mathrm{m}$, some coal particles contained more pyrite globules than other coal particles. Consequently, a density difference appeared that would be the basis for separation. However, returning to the four dimensionless parameters, the smaller particles will lead to smaller values for these parameters. Consequently, two exploratory investigations were undertaken to determine if favorable conditions for separation could be established. Task $3 \mathrm{c}$ employed a gas-solid jet to increase the particle Reynolds number and the velocity gradients in the flow field. Although separation was achieved, the clean coal reported to the dense bin and the dirty coal reported to the product hopper. This implies that the pyrite contributed to the crushing process and appeared in the smaller coal particles whereas the clean coal particles appeared at the larger end of the size distribution. A second exploratory study, Task $3 \mathrm{~d}$, employed a pulsating gas flow in the riser. The results indicated a potential for good separation of the clean coal from the dirty coal. For this Task, the dirty coal reported to the dense bin and the clean coal reported to the product bin.

The separation principles for large, $d_{\mathrm{P}}>250 \mu \mathrm{m}$, particles are now well established. The exploratory work done to separate clean coal from dirty coal showed that the potential for doing this exists, but more research on this subject is required before definite conclusions may be drawn.

\section{REFERENCES}

Aktar,H. (2006), "Development of a Small Separation System for Fine Coal Particles", MSME Thesis, West Virginia University.

Almond, R. R.(2005), "Small Particle Separation in a Circulating Fluidized Bed Riser System", MSAE Thesis, West Virginia University.

Johnson, E. K., Register, J. L. and Kang, B. S-J.(2005), "Fluidized Bed Riser as a Dry Particle Separation System", Minerals \& Metallurgical Processing, Vol.22, no.3, 130134.

Musser, J. (2007),'Development of a Separation Riser with Flow Pulsations for Small Coal Particles", MSME Thesis, Wesr Virginia University.

Regester, J. L. (2004),"Separation of Small Particles ue to Density Differences in a CFB Riser System", MSME Thesis, West Virginia University.

Wimer, B. M.(2007),"Fine Particle Separation bt Density in a Circulating Fluidized Bed", MSME Thesis, West Virginia University. 
Table 1 Experimental results for the separation of sand based on size difference, Task1.

\begin{tabular}{|c|c|c|c|c|c|c|}
\hline & 1 & 2 & 3 & 4 & 5 & 6 \\
\hline $\mathbf{u}_{\mathbf{o}} \mathbf{m} / \mathbf{s}$ & \multicolumn{3}{|c|}{2.4} & \multicolumn{3}{|c|}{2.0} \\
\hline$G \mathrm{~kg} / \mathrm{m}^{2} \mathrm{~s}$ & 1.24 & 1.48 & 1.90 & 1.22 & 1.45 & 1.70 \\
\hline$D_{p}^{*} \mu m$ & \multicolumn{3}{|c|}{$340 \mu \mathrm{m}$} & \multicolumn{3}{|c|}{$290 \mu \mathrm{m}$} \\
\hline $\mathbf{m}_{\mathbf{p b}}$ & 55.63 & 68.13 & 69.38 & 40.94 & 36.88 & 40.00 \\
\hline$C_{p}\left(d_{p}^{*}\right) \%$ & 92.2 & 88 & 78 & 81 & 79 & 85 \\
\hline$C_{m}\left(d_{p} *\right) \%$ & 61.5 & 62 & 62 & 51 & 52 & 51 \\
\hline$\eta_{s}$ & 83 & 97 & 87 & 65 & 56 & 67 \\
\hline$\omega_{\mathrm{s}}$ & 92.2 & 88 & 78 & 81 & 79 & 85 \\
\hline
\end{tabular}

$\mathrm{m}_{\mathrm{pb}}$ percent mass in the product bin

$C_{p}\left(d_{p} *\right)$ percent mass less than $d_{p} *$ in the product hopper

$\mathrm{C}_{\mathrm{m}}\left(\mathrm{d}_{\mathrm{p}}^{*}\right) *$ ) percent mass less than $\mathrm{d}_{\mathrm{p}} *$ in the initial mixture

Table 2 Experiment results for the separation of shot from sand, both with same size distribution, Task 2.

\begin{tabular}{|c|c|c|c|c|}
\hline Test & $\begin{array}{c}\mathbf{u}_{\mathbf{0}} \\
\mathbf{m} / \mathbf{s}\end{array}$ & $\begin{array}{c}\mathbf{G} \\
\mathrm{kg} / \mathbf{m}^{2} \mathbf{s}\end{array}$ & $\begin{array}{c}\boldsymbol{\eta}_{\mathbf{H}} \\
\%\end{array}$ & $\begin{array}{c}\omega_{\mathbf{H}} \\
\%\end{array}$ \\
\hline 1 & 3.5 & 1.20 & 98 & 78 \\
\hline 2 & 3.5 & 1.92 & 97 & 70 \\
\hline 3 & 3.5 & 2.17 & 98 & 65 \\
\hline 4 & 3.5 & 2.55 & 97 & $\mathbf{6 5}$ \\
\hline 5 & 4.3 & 1.20 & 94 & 86 \\
\hline 6 & 4.3 & 1.95 & 88 & 80 \\
\hline 7 & 4.3 & 2.06 & 85 & 80 \\
\hline 8 & 4.3 & 2.18 & 86 & 87 \\
\hline 9 & 4.8 & 1.32 & 75 & 85 \\
\hline 10 & 4.8 & 1.49 & 70 & 85 \\
\hline 11 & 4.8 & 1.90 & 71 & 82 \\
\hline 12 & 4.8 & 2.29 & 68 & 80 \\
\hline
\end{tabular}

Steel shot mass fraction in the initial mixture, $20 \%$ 
Table 3 Experimental results for the separation of steel shot and sand for evaluation of the system modifications for the Task $3 C$.

\begin{tabular}{|c|c|c|c|c|c|c|c|c|c|}
\hline $\begin{array}{c}\text { Run } \\
\text { Number }\end{array}$ & $\begin{array}{c}\text { Total } \\
(\text { SCMH) }\end{array}$ & $\begin{array}{c}\text { Distributor } \\
\text { Flow } \\
(\text { SCMH) }\end{array}$ & $\begin{array}{c}\text { Transport } \\
\text { Flow } \\
(\text { SCMH) }\end{array}$ & $\begin{array}{c}\text { Nozzle } \\
\text { Inlet Size } \\
(\mathbf{c m})\end{array}$ & $\begin{array}{c}\text { Mass Flux } \\
\left(\mathbf{k g} / \mathbf{m}^{\wedge} 2 \mathrm{~s}\right)\end{array}$ & $\begin{array}{c}\text { Ring } \\
\text { Wall } \\
\text { Height } \\
(\mathbf{m})\end{array}$ & $\begin{array}{c}\text { Dense } \\
\text { Mass } \\
\text { Fraction }\end{array}$ & $\begin{array}{c}\text { Dense } \\
\text { Collection } \\
\text { Efficiency }\end{array}$ & $\begin{array}{c}\% \\
\text { Nozzle } \\
\text { Flow }\end{array}$ \\
\hline 1 & 169.0 & 108.2 & 60.8 & 1.905 & 0.725 & 0.3048 & $99.40 \%$ & $78.00 \%$ & 36.0 \\
\hline 2 & 172.9 & 51.2 & 121.7 & 1.905 & 0.81 & 0.3048 & $99.30 \%$ & $84.80 \%$ & 70.4 \\
\hline 3 & 170.0 & 109.3 & 60.8 & 1.270 & 0.893 & 0.3048 & $97.90 \%$ & $84.40 \%$ & 35.7 \\
\hline 4 & 167.6 & 68.4 & 99.2 & 1.270 & 1.49 & 0.3048 & $93.40 \%$ & $81.50 \%$ & 56.2 \\
\hline 5 & 168.2 & 113.2 & 60.7 & 1.111 & 0.746 & 0.3048 & $92.20 \%$ & $91.60 \%$ & 36.1 \\
\hline 6 & 169.0 & 94.0 & 76.9 & 1.111 & 0.893 & 0.3048 & $96.50 \%$ & $93.30 \%$ & 45.5 \\
\hline 7 & 169.0 & 108.2 & 60.8 & 1.905 & 1.05 & 0.381 & $99.40 \%$ & $89.10 \%$ & 36.0 \\
\hline 8 & 170.0 & 47.5 & 122.5 & 1.905 & 1.27 & 0.381 & $90.30 \%$ & $70.80 \%$ & 72.1 \\
\hline 9 & 170.0 & 109.3 & 60.8 & 1.270 & 0.81 & 0.381 & $97.00 \%$ & $81.70 \%$ & 35.7 \\
\hline 10 & 167.6 & 68.4 & 99.2 & 1.270 & 1.19 & 0.381 & $97.40 \%$ & $85.20 \%$ & 59.2 \\
\hline 11 & 170.5 & 111.4 & 59.2 & 1.111 & 1.05 & 0.381 & $99.70 \%$ & $86.50 \%$ & 34.7 \\
\hline 12 & 168.2 & 93.5 & 74.9 & 1.111 & 1.79 & 0.381 & $98.80 \%$ & $84.70 \%$ & 44.5 \\
\hline
\end{tabular}

Steel shot mass fraction in the initial mixture, $20 \%$ 
Table 4 Test matrix for the separation of fine coal in Task $3 \mathrm{C}$.

\begin{tabular}{|c|c|c|c|c|c|c|}
\hline $\begin{array}{c}\text { Run } \\
\text { Number }\end{array}$ & $\begin{array}{l}\text { Nozzle - } \\
\text { Distributor } \\
\text { Flow }\end{array}$ & $\begin{array}{c}\text { Air Flow } \\
\text { Rate } \\
\text { (SCMH) }\end{array}$ & $\begin{array}{l}\text { Particle } \\
\text { Mass Flux } \\
\left(\mathrm{kg} / \mathrm{m}^{\wedge} 2 \mathrm{~s}\right)\end{array}$ & $\begin{array}{l}\text { Riser } \\
\text { Wall } \\
\text { Height } \\
\text { (m) }\end{array}$ & $\begin{array}{l}\text { Nozzle } \\
\text { Inlet Size } \\
\text { (cm) }\end{array}$ & Comments \\
\hline 1 & $50 / 50$ & 42.25 & 0.399 & 0.3048 & 1.905 & \multirow{5}{*}{$\begin{array}{l}\text { Initial test runs to } \\
\text { check velocity } \\
\text { value and how it } \\
\text { corresponds with } \\
\text { particle inlet size. }\end{array}$} \\
\hline 2 & $50 / 50$ & 42.25 & 0.352 & 0.3048 & 1.27 & \\
\hline 3 & $50 / 50$ & 42.25 & 0.346 & 0.3048 & 1.11 & \\
\hline 4 & $50 / 50$ & 42.25 & 0.388 & 0.3048 & 0.714 & \\
\hline 5 & $50 / 50$ & 42.25 & 0.334 & 0.3048 & 0.556 & \\
\hline 6 & $50 / 50$ & 50.7 & 0.298 & 0.3048 & 1.905 & \multirow{5}{*}{$\begin{array}{l}\text { Test runs to check } \\
\text { higher velocity } \\
\text { value and how it } \\
\text { corresponds with } \\
\text { particle inlet size. }\end{array}$} \\
\hline 7 & $50 / 50$ & 50.7 & 0.328 & 0.3048 & 1.27 & \\
\hline 8 & $50 / 50$ & 50.7 & 0.298 & 0.3048 & 1.11 & \\
\hline 9 & $50 / 50$ & 50.7 & 0.280 & 0.3048 & 0.714 & \\
\hline 10 & $50 / 50$ & 50.7 & 0.334 & 0.3048 & 0.556 & \\
\hline 11 & $50 / 50$ & 50.7 & 1.192 & 0.3048 & 1.905 & \multirow{4}{*}{$\begin{array}{c}\text { High mass flux } \\
\text { values with .3048 } \\
\text { meter ring wall } \\
\text { height. Aimed for } \\
1.2 \mathrm{~kg} / \mathrm{m}^{\wedge} 2 \mathrm{~s} . \\
\end{array}$} \\
\hline 13 & $50 / 50$ & 50.7 & 1.157 & 0.3048 & 1.27 & \\
\hline 15 & $50 / 50$ & 50.7 & 1.246 & 0.3048 & 1.11 & \\
\hline 17 & $50 / 50$ & 50.7 & 1.127 & 0.3048 & 0.714 & \\
\hline 12 & $50 / 50$ & 50.7 & 0.608 & 0.3048 & 1.905 & \multirow{4}{*}{$\begin{array}{c}\text { Low mass flux } \\
\text { values with .3048 } \\
\text { meter ring wall } \\
\text { height. Aimed for } \\
.60 \mathrm{~kg} / \mathrm{m}^{\wedge} 2 \mathrm{~s} \text {. }\end{array}$} \\
\hline 14 & $50 / 50$ & 50.7 & 0.608 & 0.3048 & 1.27 & \\
\hline 16 & $50 / 50$ & 50.7 & 0.614 & 0.3048 & 1.11 & \\
\hline 18 & $50 / 50$ & 50.7 & 0.572 & 0.3048 & 0.714 & \\
\hline 19 & $50 / 50$ & 50.7 & 1.318 & 0.381 & 1.905 & \multirow{4}{*}{$\begin{array}{c}\text { High mass flux } \\
\text { values with .381 } \\
\text { meter ring wall } \\
\text { height. Aimed for } \\
1.2 \mathrm{~kg} / \mathrm{m}^{\wedge} 2 \mathrm{~s} \text {. }\end{array}$} \\
\hline 21 & $50 / 50$ & 50.7 & 1.282 & 0.381 & 1.27 & \\
\hline 23 & $50 / 50$ & 50.7 & 1.127 & 0.381 & 1.11 & \\
\hline 25 & $50 / 50$ & 50.7 & 1.133 & 0.381 & 0.714 & \\
\hline 20 & $50 / 50$ & 50.7 & 0.638 & 0.381 & 1.905 & \multirow{4}{*}{$\begin{array}{c}\text { Low mass flux } \\
\text { values with } .381 \\
\text { meter ring wall } \\
\text { height. Aimed for } \\
.60 \mathrm{~kg} / \mathrm{m}^{\wedge} 2 \mathrm{~s} \text {. } \\
\end{array}$} \\
\hline 22 & $50 / 50$ & 50.7 & 0.620 & 0.381 & 1.27 & \\
\hline 24 & $50 / 50$ & 50.7 & 0.614 & 0.381 & 1.11 & \\
\hline 26 & $50 / 50$ & 50.7 & 0.608 & 0.381 & 0.714 & \\
\hline 27 & $50 / 50$ & 50.7 & 0.306 & 0.3048 & 1.905 & \multirow{6}{*}{$\begin{array}{c}\text { Re-runs of test } \\
\text { numbers } 6,7, \& 8 \text { to } \\
\text { check for } \\
\text { repeatability of } \\
\text { results }\end{array}$} \\
\hline 28 & $50 / 50$ & 50.7 & 0.280 & 0.3048 & 1.905 & \\
\hline 29 & $50 / 50$ & 50.7 & 0.310 & 0.3048 & 1.27 & \\
\hline 30 & $50 / 50$ & 50.7 & 0.317 & 0.3048 & 1.27 & \\
\hline 31 & $50 / 50$ & 50.7 & 0.311 & 0.3048 & 1.11 & \\
\hline 32 & $50 / 50$ & 50.7 & 0.317 & 0.3048 & 1.11 & \\
\hline
\end{tabular}

Size range of coal fine 
Table 5 Flow velocities and volumetric flow rates for $0 \mathrm{~Hz}, 1 \mathrm{~Hz}$, and $2 \mathrm{~Hz}$ pulsation tests. The solid mass flow rate was held constant at $2.5 \mathrm{~kg} / \mathrm{hr}$.

\begin{tabular}{|c|c|c|c|c|c|c|}
\hline & \multicolumn{2}{|c|}{$0 \mathrm{~Hz}$ Tests } & \multicolumn{4}{c|}{ 1Hz and 2Hz Tests } \\
\hline & $\mathrm{u}$ & $\mathrm{V}$ & $\mathrm{u}_{\mathrm{U}}$ & $\mathrm{u}_{\mathrm{L}}$ & $\mathrm{V}_{\mathrm{U}}$ & $\mathrm{V}_{\mathrm{L}}$ \\
\hline & $\mathrm{ms}^{-1}$ & $\mathrm{SCFH}$ & $\mathrm{ms}^{-1}$ & $\mathrm{~ms}^{-1}$ & $\mathrm{SCFH}$ & $\mathrm{SCFH}$ \\
\hline Set 1 & 0.9621 & 248 & 0.9621 & 0.9221 & 248 & 238 \\
\hline Set 2 & 0.9101 & 234 & 0.9101 & 0.8691 & 234 & 224 \\
\hline Set 3 & 0.8581 & 221 & 0.8581 & 0.8181 & 221 & 211 \\
\hline Set 4 & 0.8061 & 208 & 0.8061 & 0.7661 & 208 & 198 \\
\hline
\end{tabular}

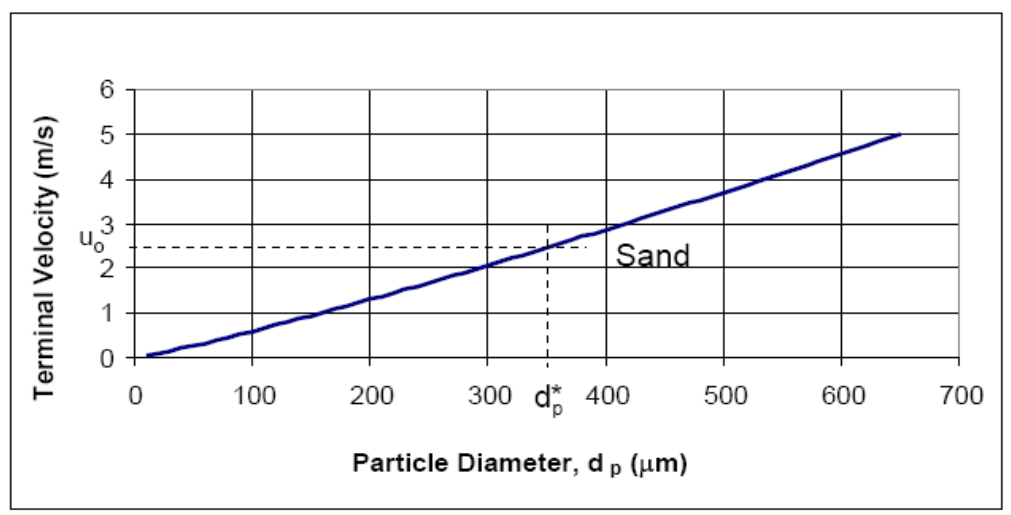

Figure 1: Basic terminal velocity-particle diameter curve for sand.

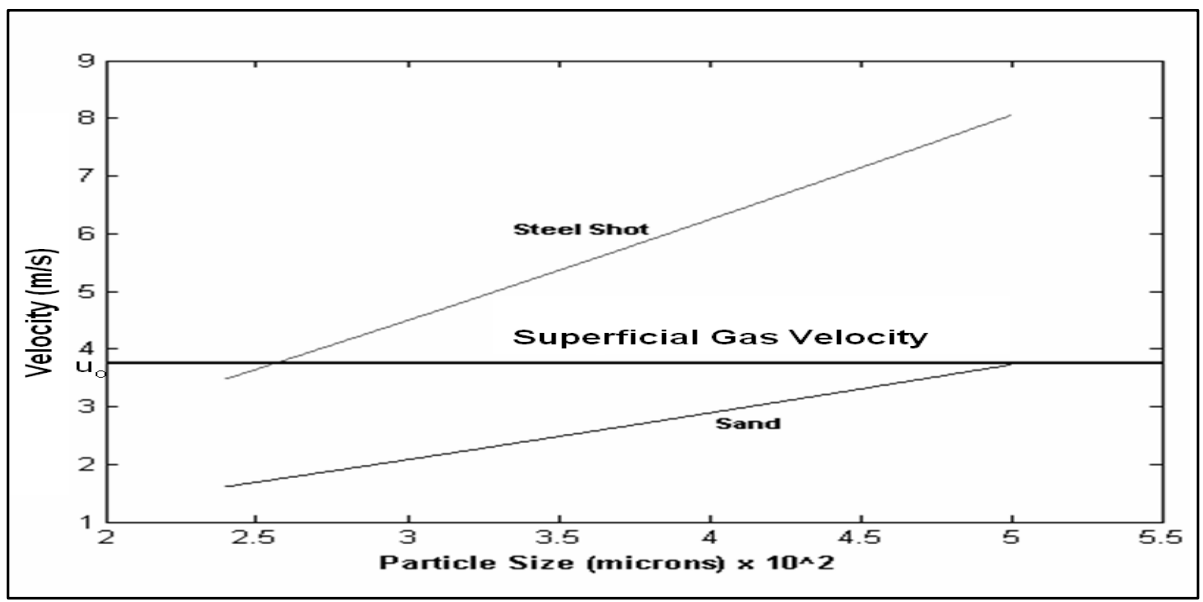

Figure 2: Basic terminal velocity-particle diameter curves for steel shot and sand. Also a superficial gas velocity curve for separating the two materials. 


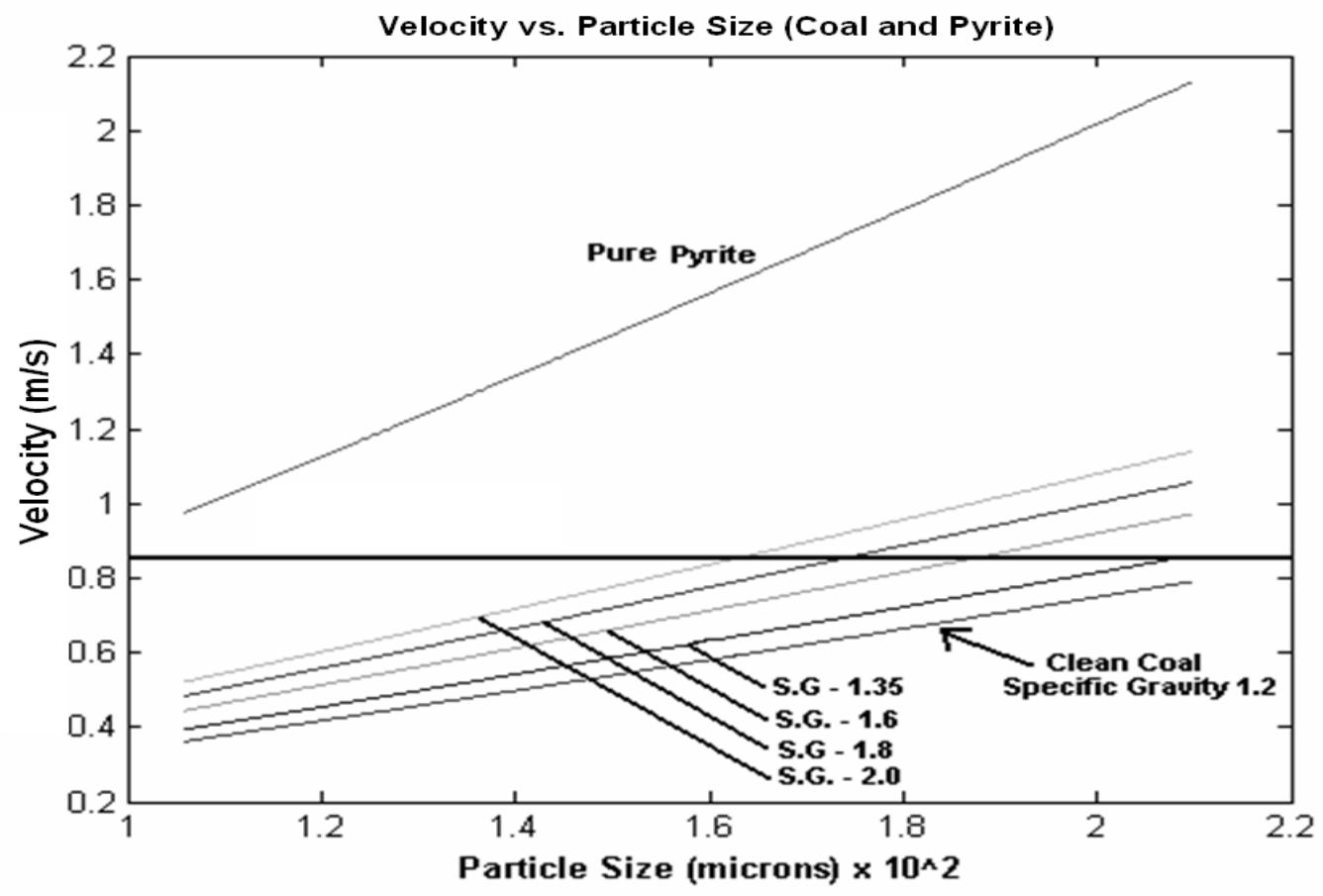

Figure 3: Terminal velocity-particle diameter curves for coal with different specific gravities.

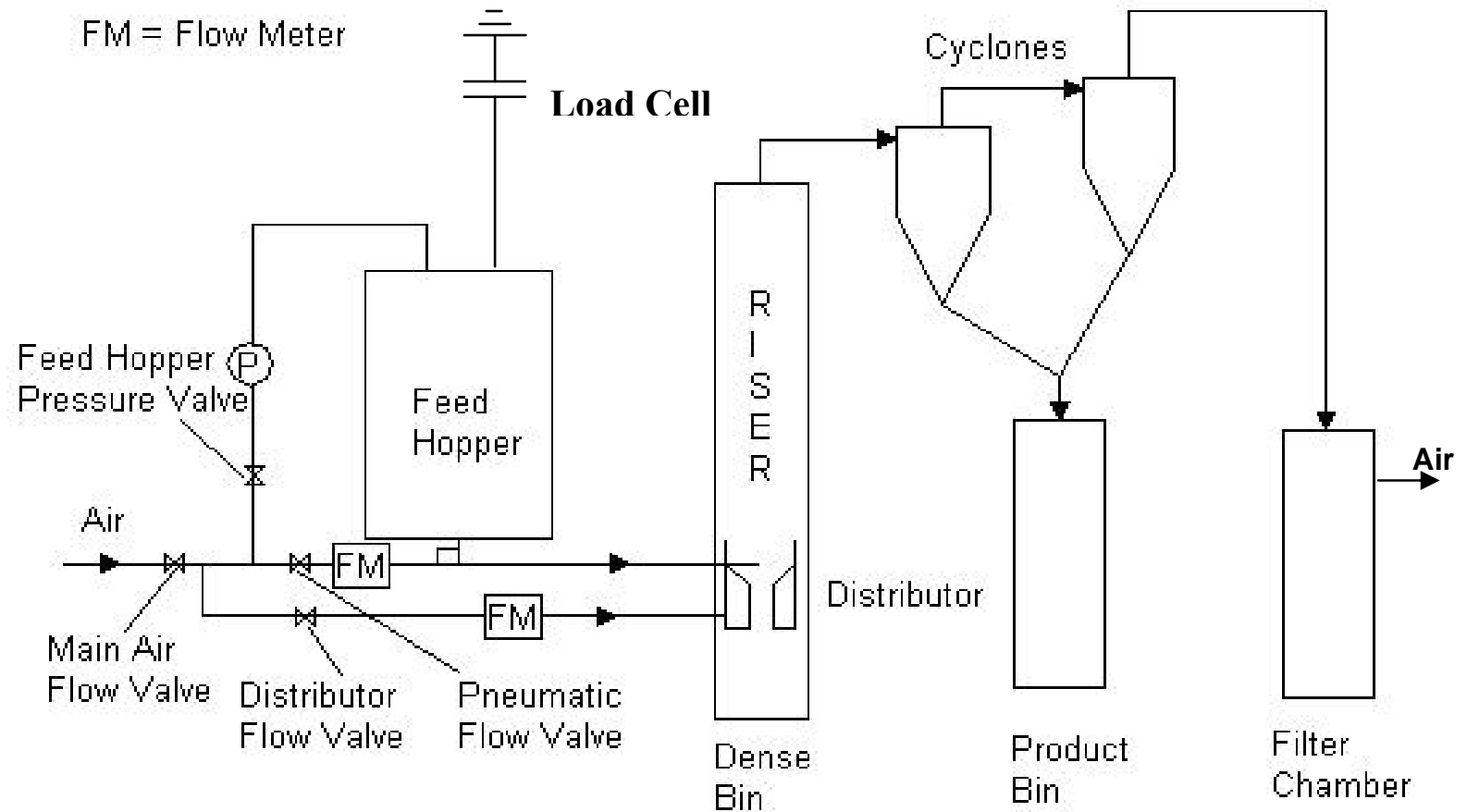

Figure 4: Experimental system used in Task 1, 2, 3a, and 3c, For Task $3 \mathrm{c}$ the distributor region was modified, as shown in Figure 6. 


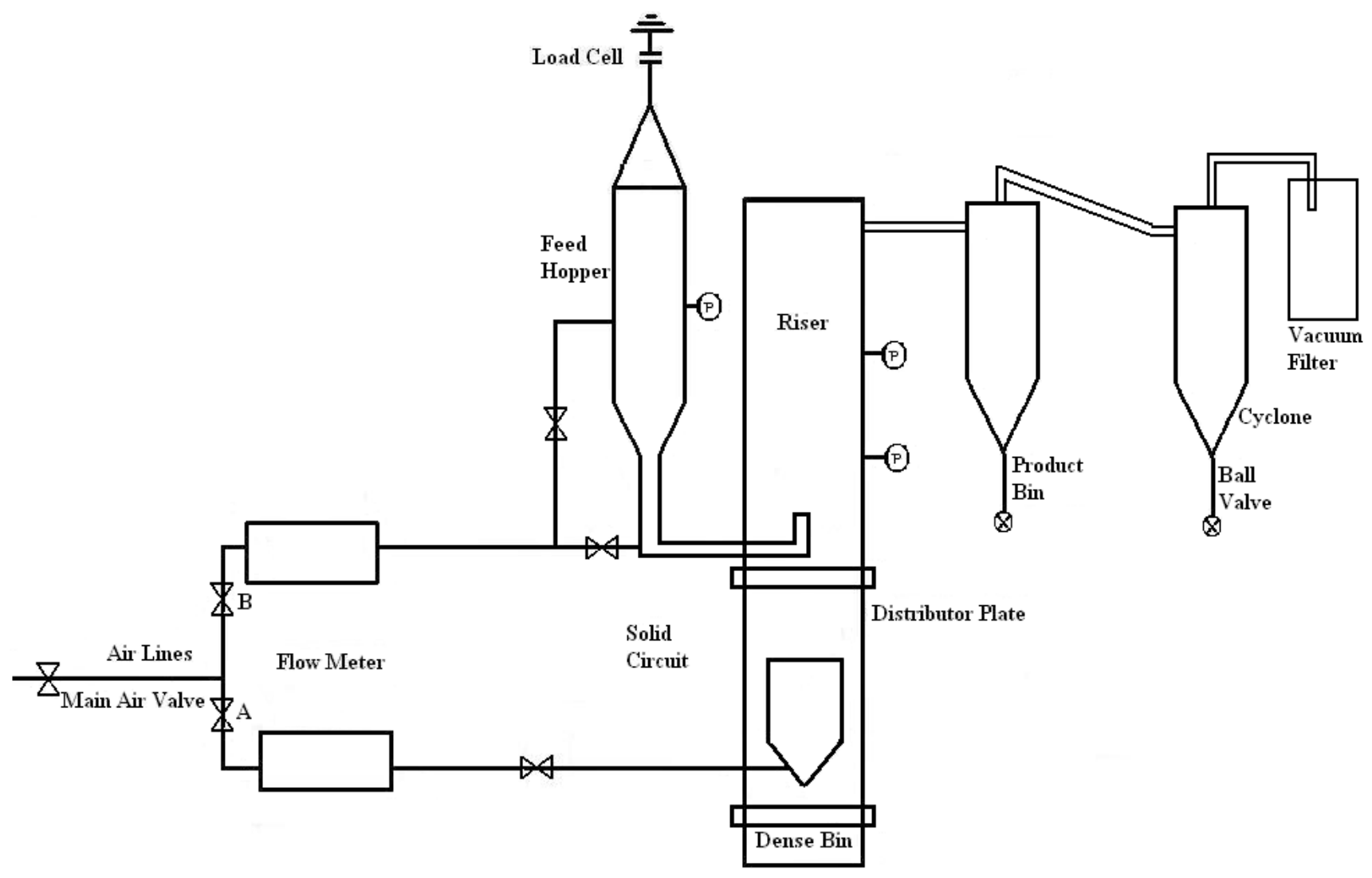

Figure 5: Small scale exploratory system for Task $3 \mathrm{~b}$. Riser inside diameter is $5 \mathrm{~cm}$.

Note the collection ring is below the solids circuit inlet and the solids injector position was designed to hold several type of injectors.

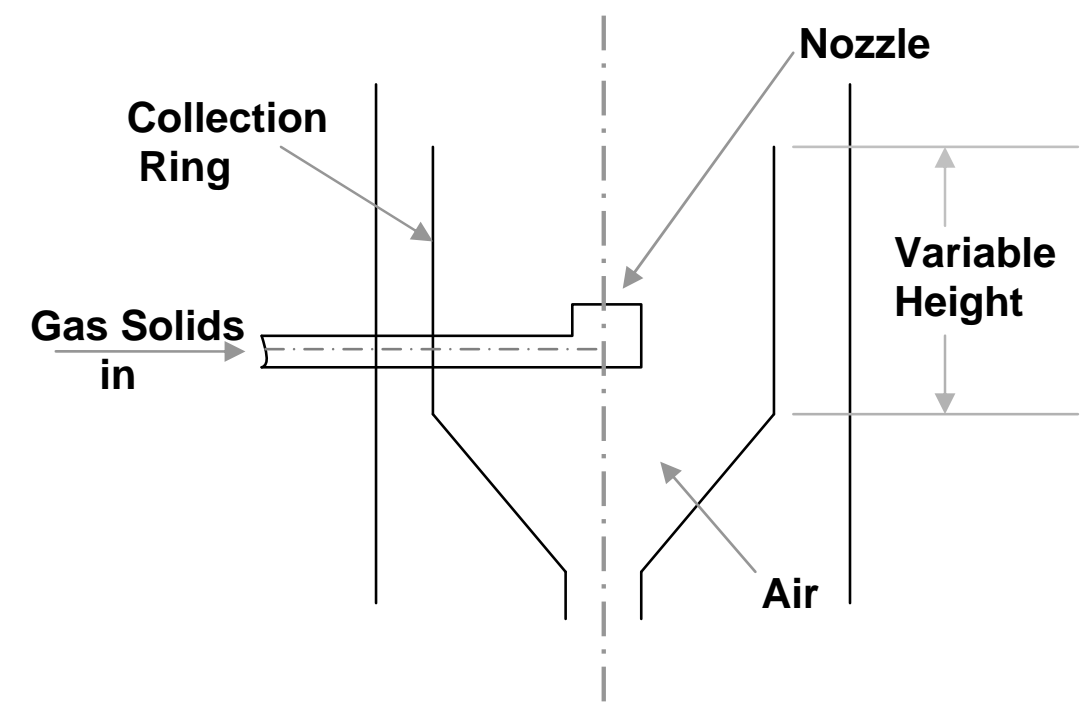

Figure 6: Modifications to the original system for Task 3c. 


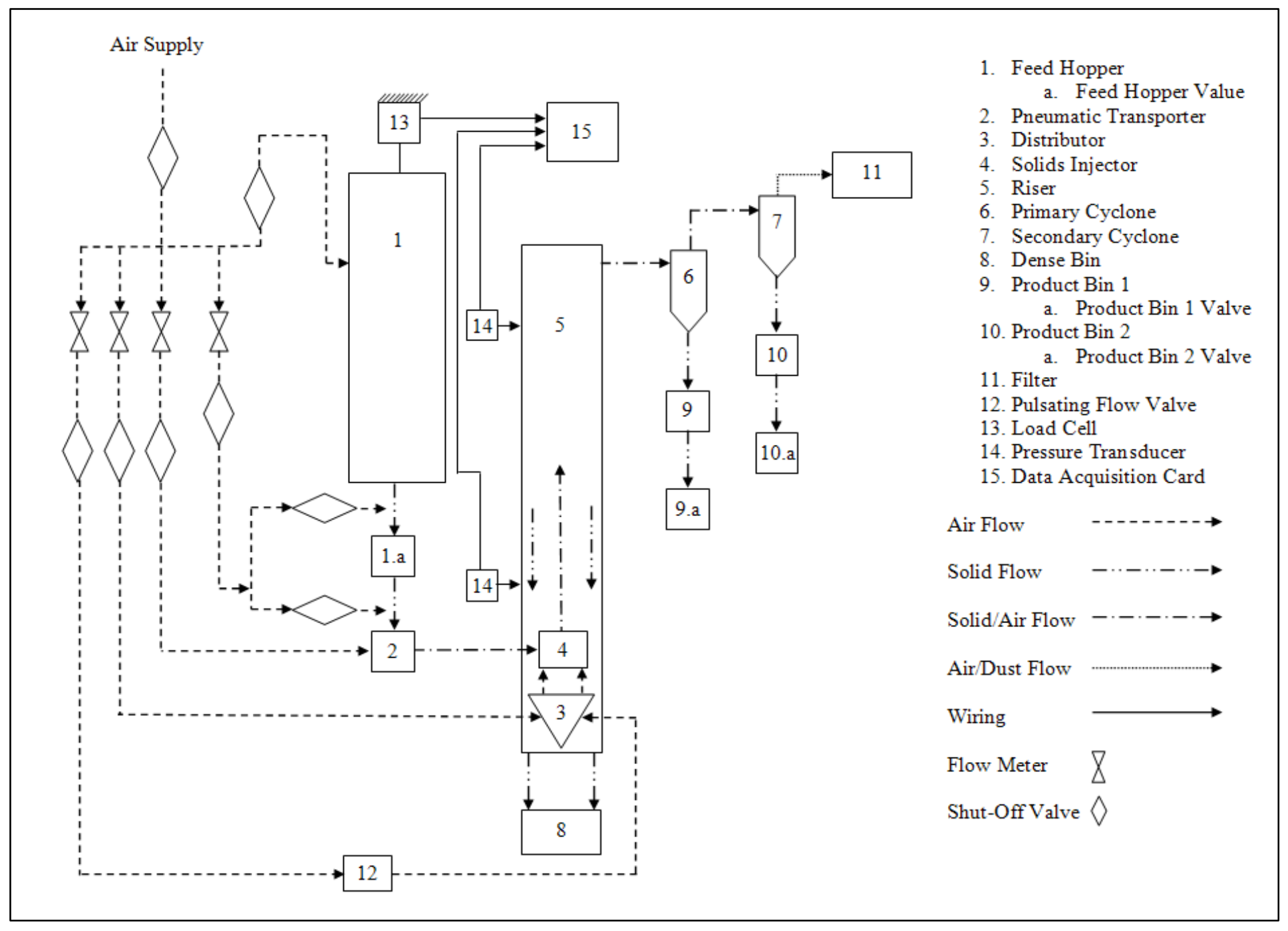

Figure 7: Experimental system for the investigation of particle separation in pulsating flow. This was a small scale system with a $5 \mathrm{~cm}$ ID riser. 


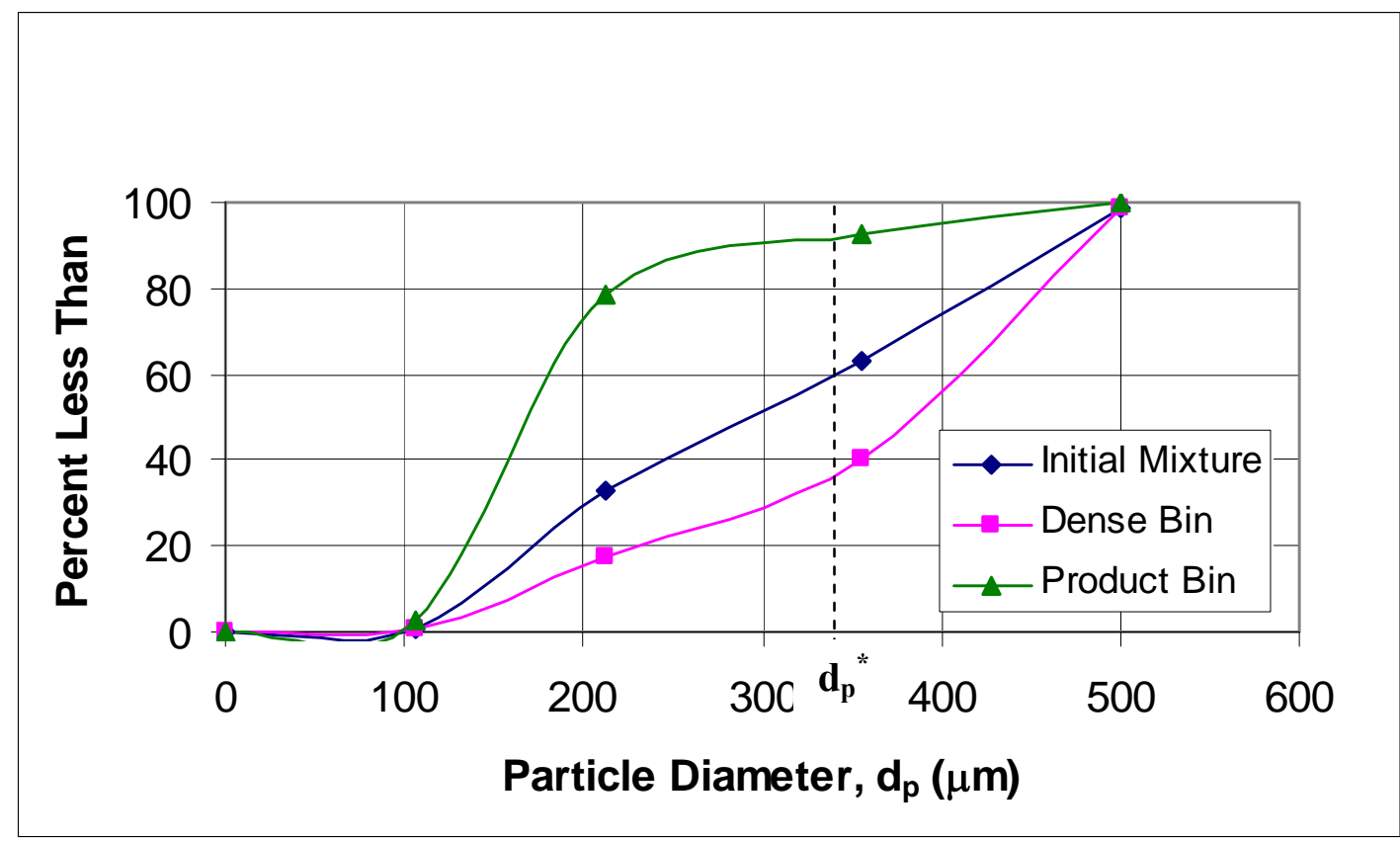

Figure 8: Size distributions for Test \#1. The riser height was $4.3 \mathrm{~m}$, bend outlet, $u_{0}=2.4 \mathrm{~m} / \mathrm{s}$, and $G=1.24 \mathrm{~kg} / \mathrm{m}^{2} \mathrm{~s}$, Task 1 .

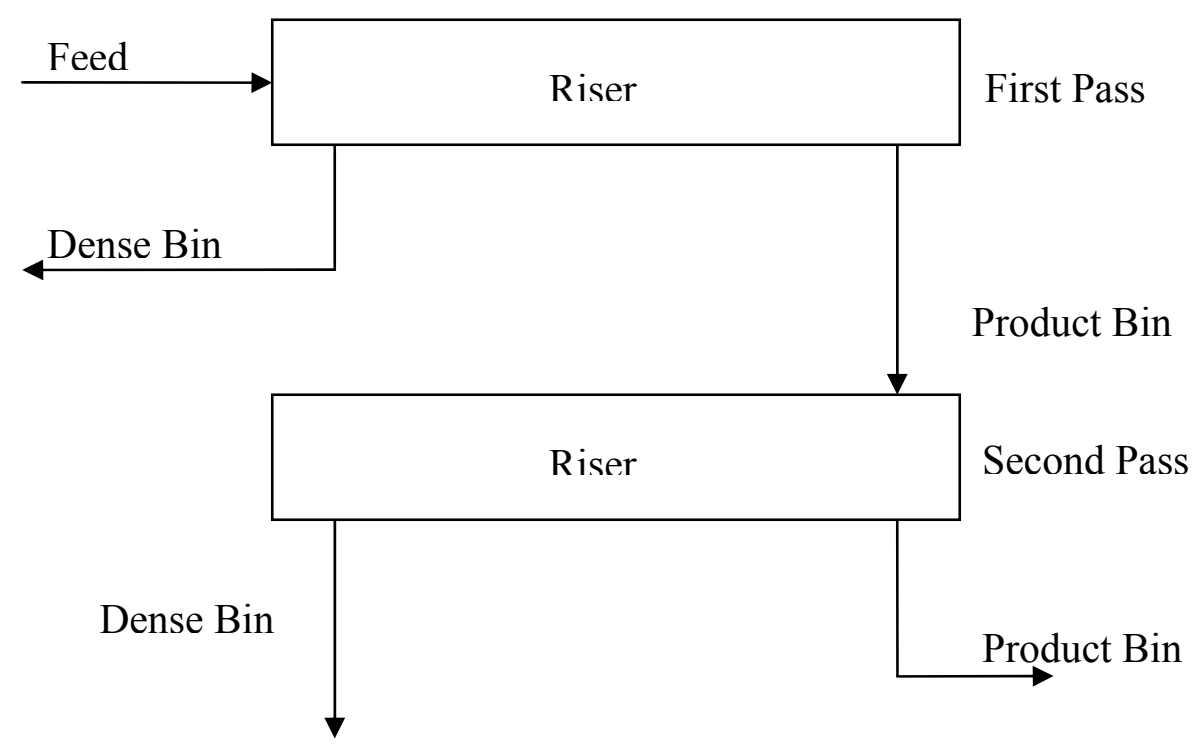

Final Product

Figure 9: Multiple phase tests. 


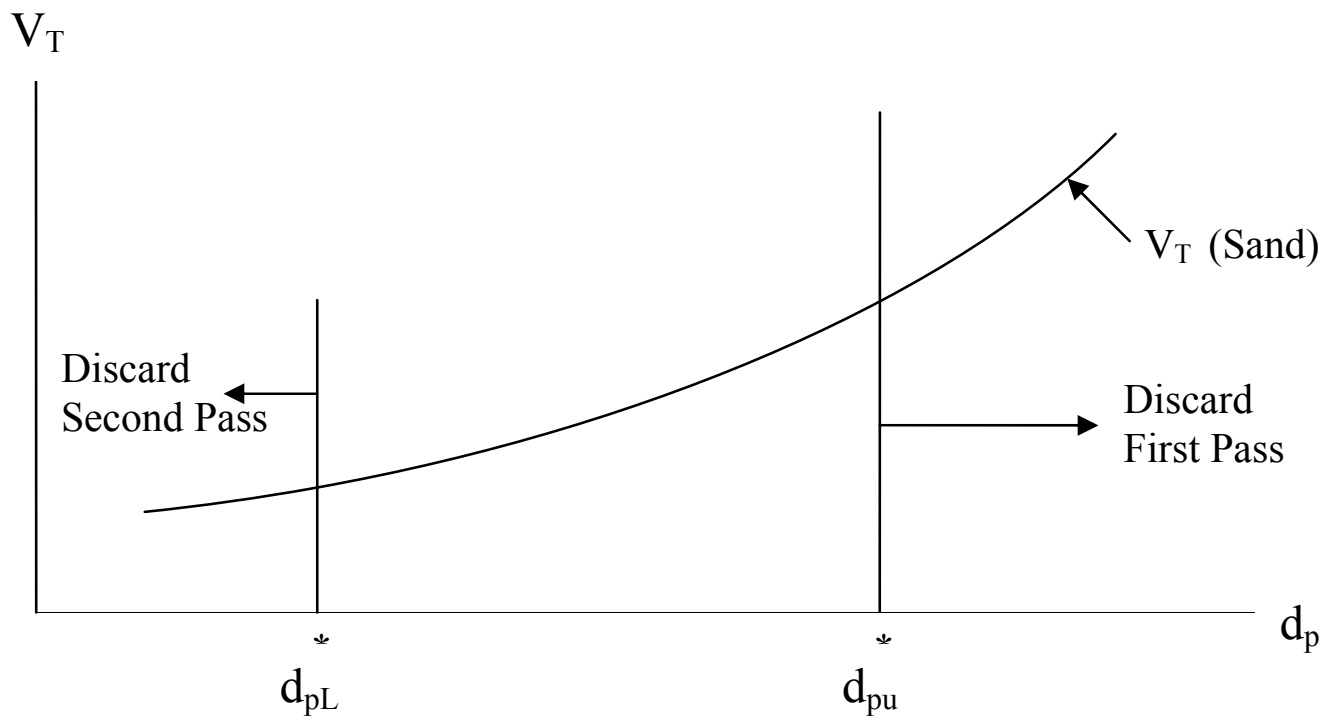

Figure 10: Process diagrams to illustrate the production of material (sand) in a selected size range, Task 1 .

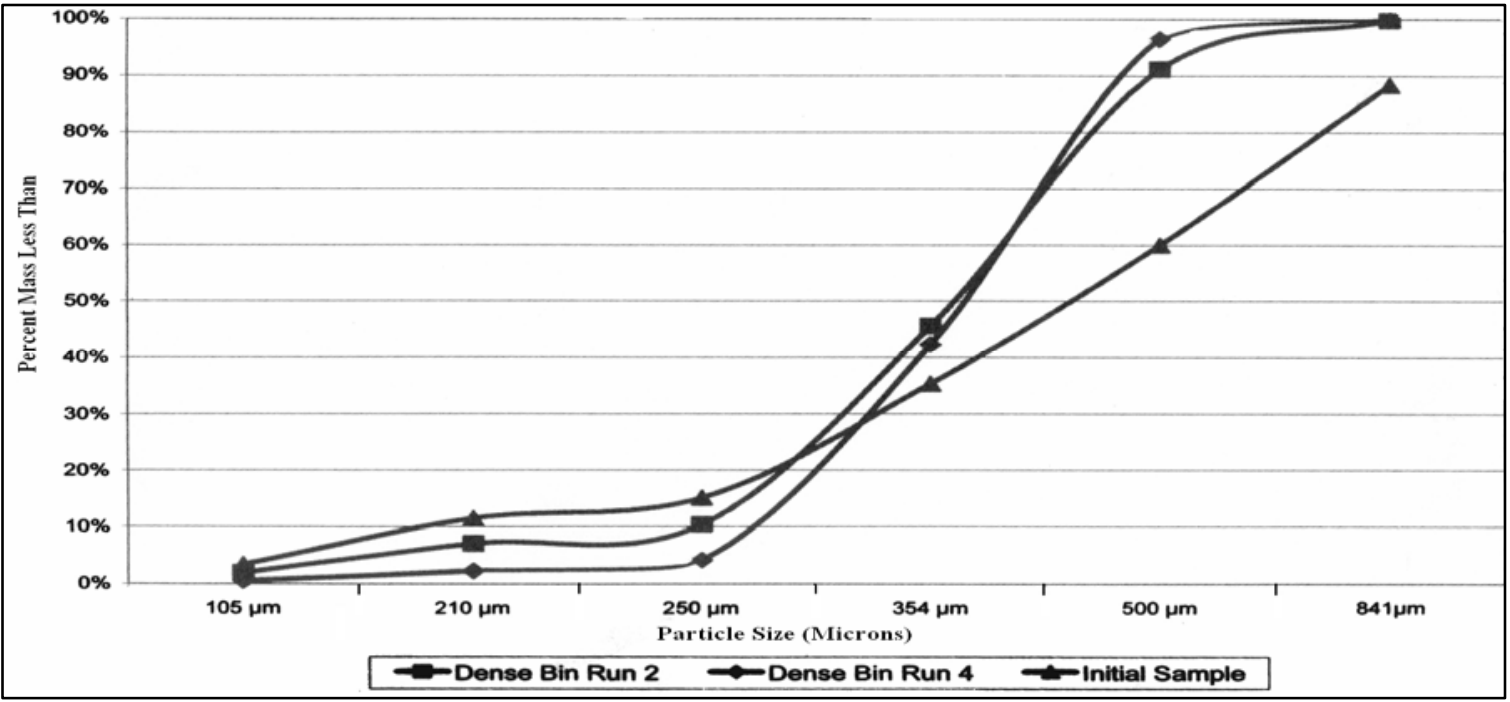

Figure 11: Distribution of the final product after 2 and 4 passes compared to the size distribution of the initial mixture, Task 1. 


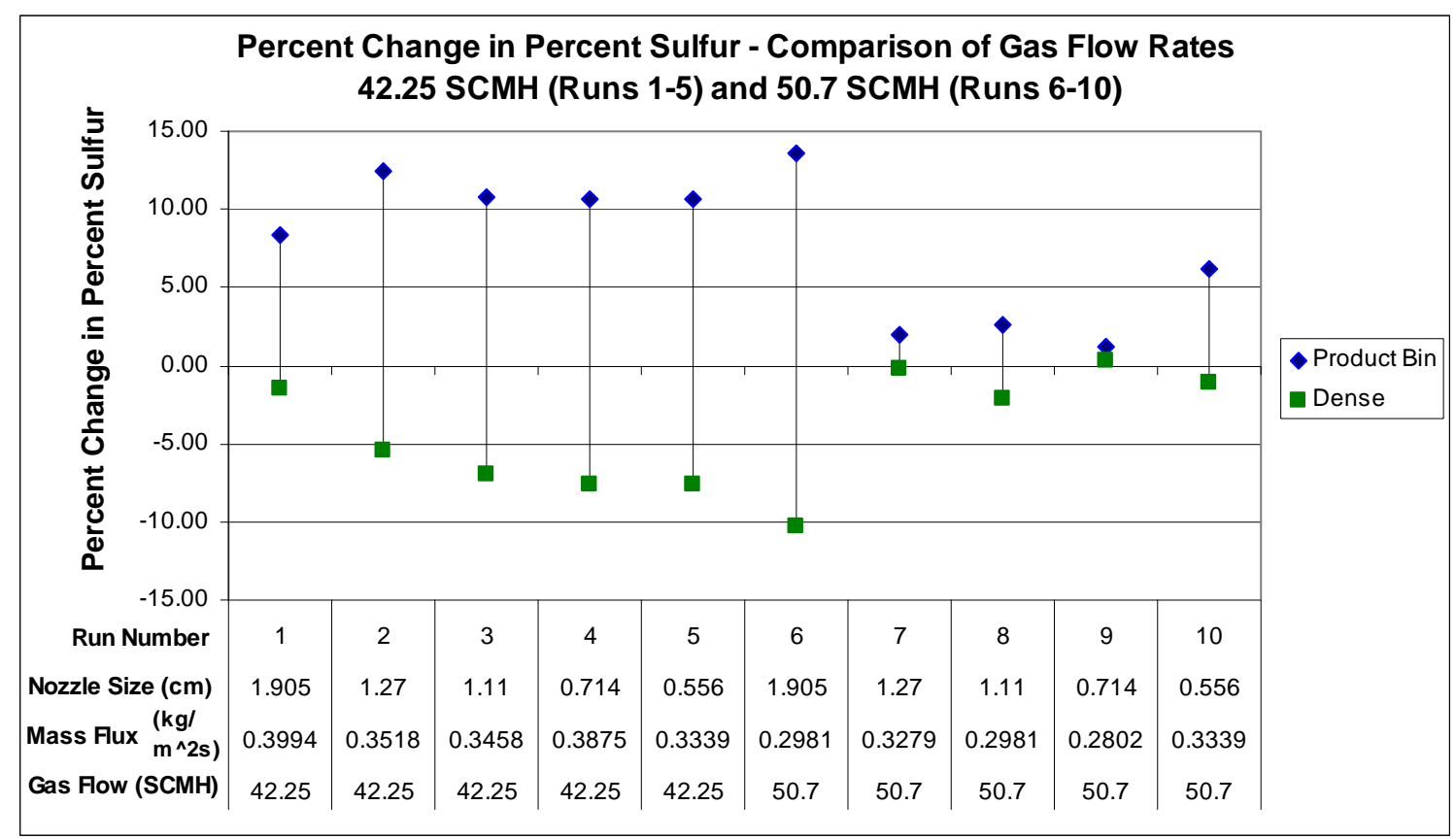

Figure 12: Percent sulfur change comparison for gas flow rates.

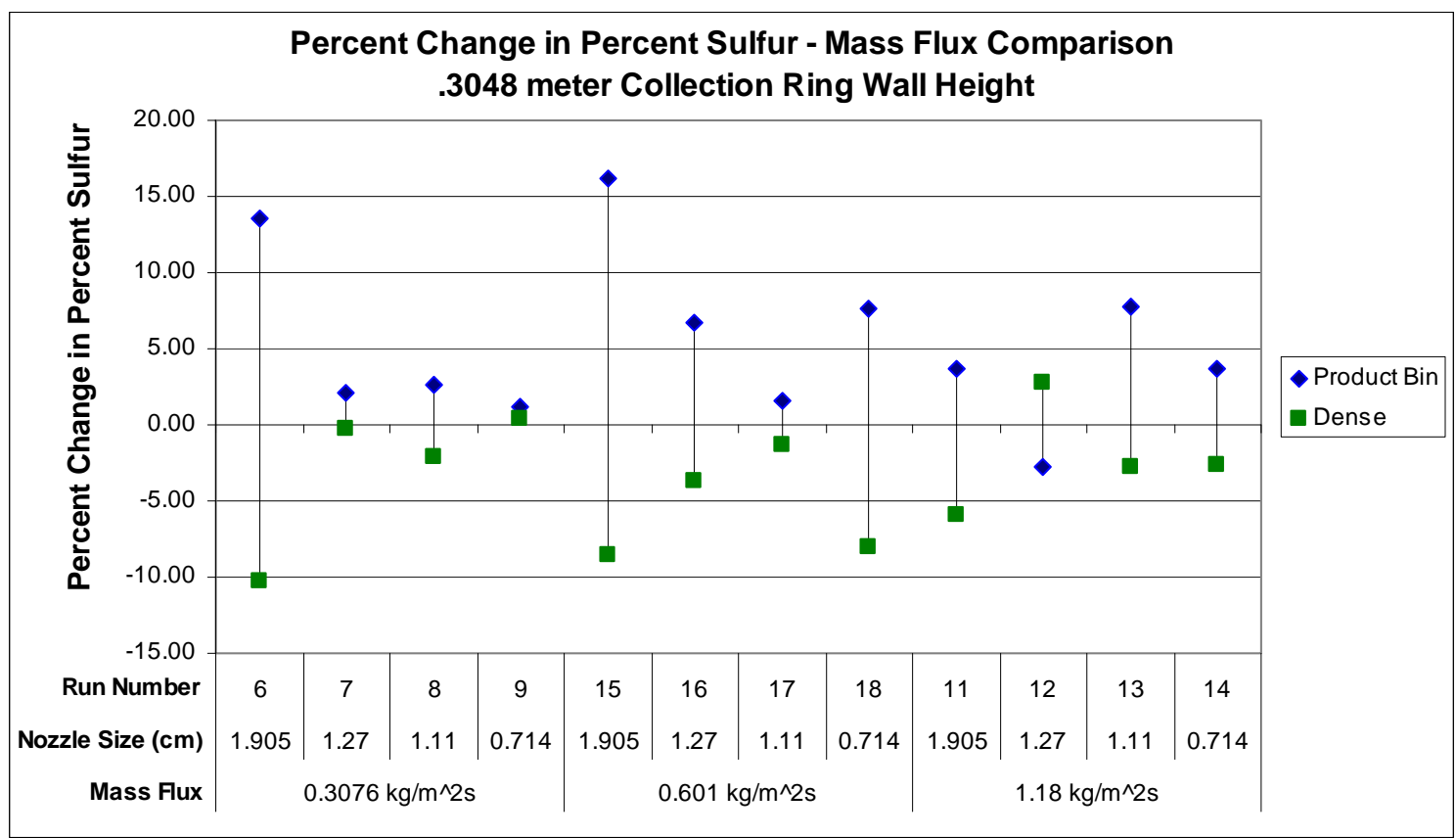

Figure 13: Product percent change in sulfur vs. mass flux. (0.3048 meter Collection Ring Wall Height) 


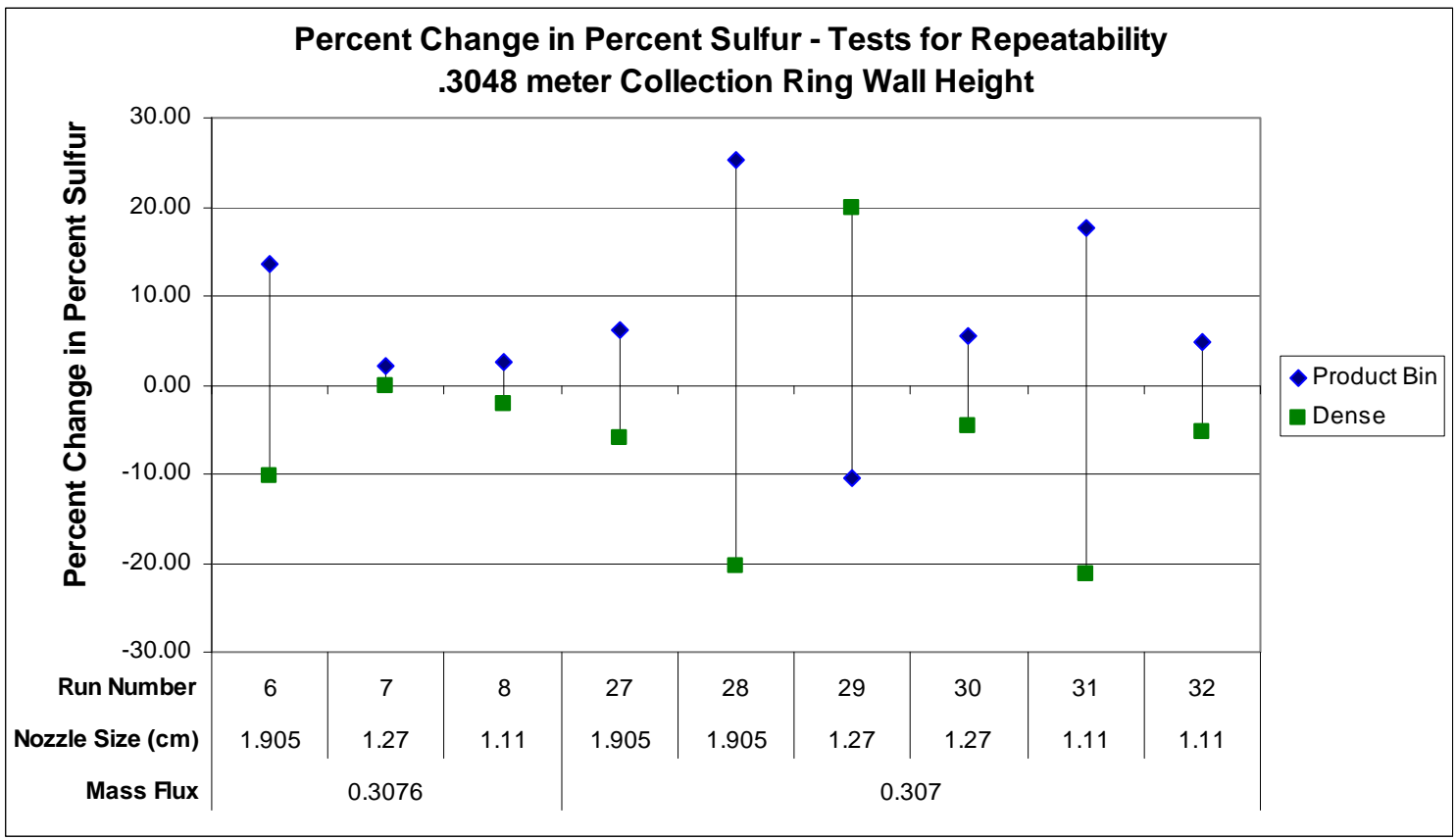

Figure 14: Percent sulfur change comparison for repeatability tests.

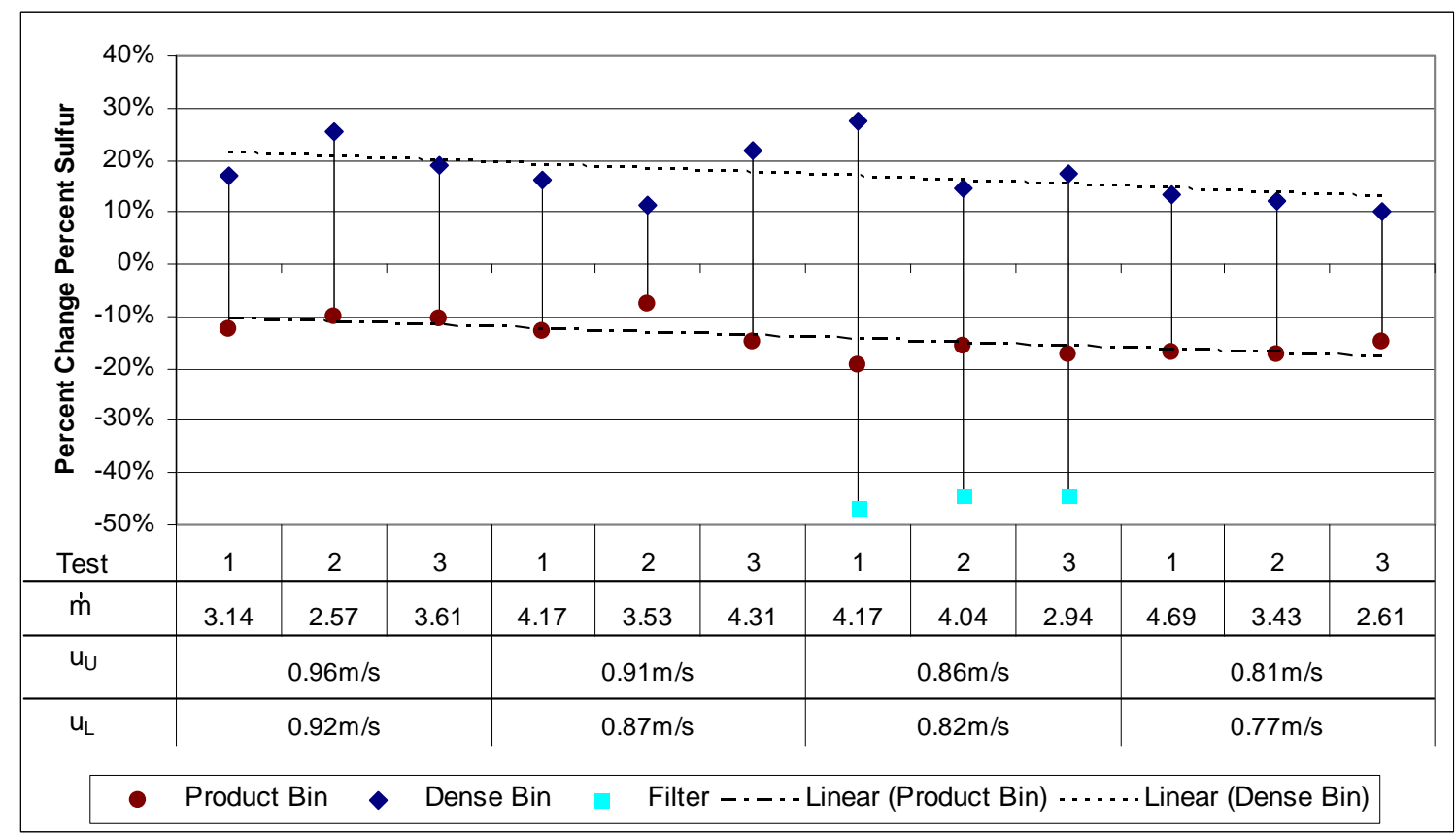

Figure 15: Results from tests with $1 \mathrm{~Hz}$ pulsations, coal particles $\left(105<\mathbf{d}_{\mathrm{p}}<210\right) \mu \mathrm{m}$, and decreasing superficial gas velocity with grouped product bins. 


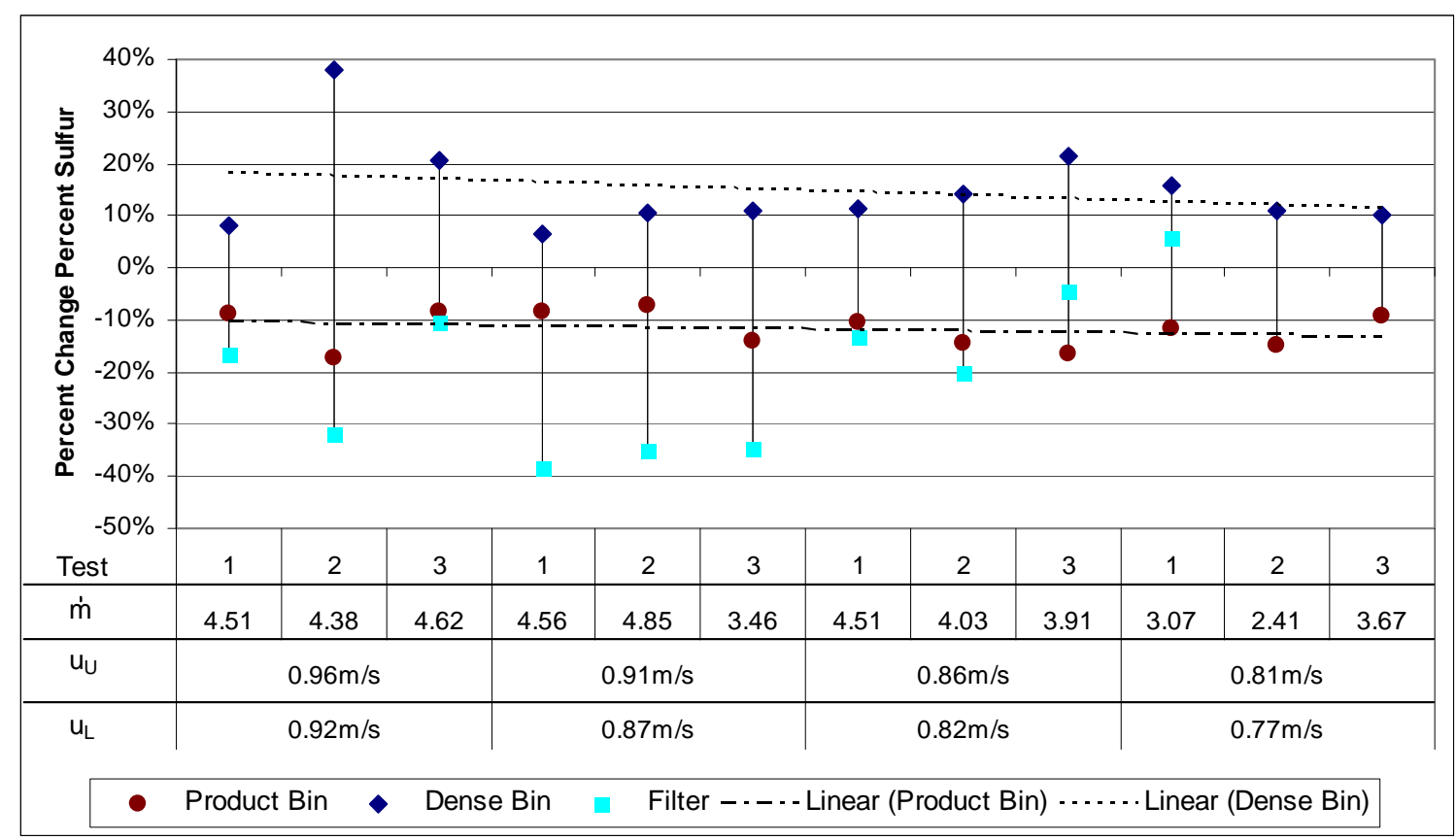

Figure 16: Results from tests with $2 \mathrm{~Hz}$ pulsations, coal particles $(\mathbf{1 0 5}<\mathrm{dp}<210) \mu \mathrm{m}$, and decreasing superficial gas velocity with grouped product bins.

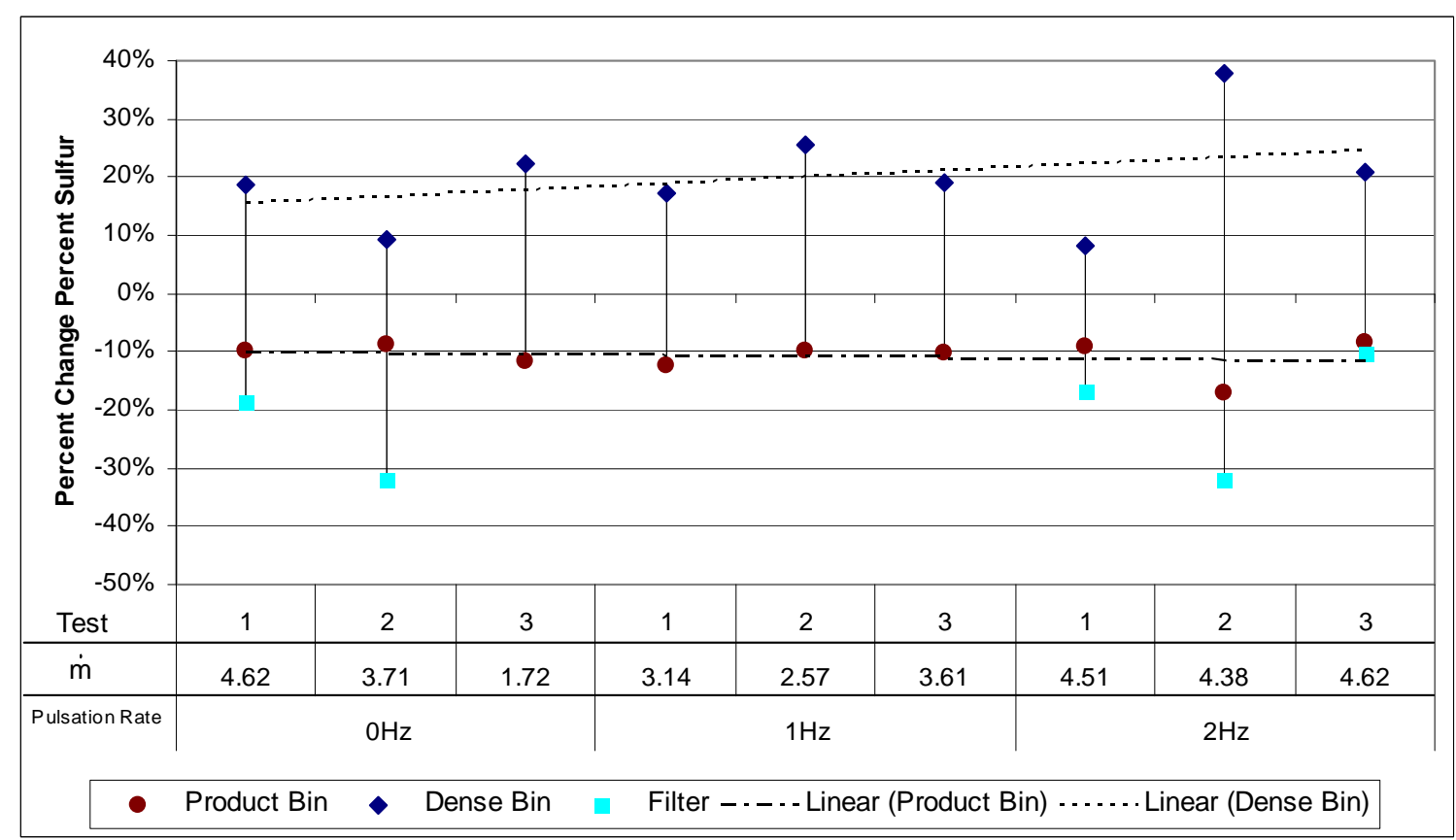

Figure 17: Results from tests with superficial gas velocity $u_{0}=0.9621 \mathrm{~ms}^{-1}$, coal particles $\left(105<d_{p}<210\right) \mu \mathrm{m}$, varying pulsation rates $(0 \mathrm{~Hz}, 1 \mathrm{~Hz}$, and $2 \mathrm{~Hz})$, and grouped product bins. 
Appendix 15: Development of an Optimized Control System for HMC Circuits (VA021) 


\section{FINAL TECHNICAL REPORT}

\begin{tabular}{ll} 
Contract Title and Number: & \multicolumn{2}{l}{ Period of Performance: } \\
$\begin{array}{l}\text { Crosscutting Technology Development at } \\
\text { Center for Advanced Separation Technologies }\end{array}$ & $\begin{array}{l}\text { Starting Date: } \\
\text { (DE-FC26-02NT41607) }\end{array}$
\end{tabular}

Sub-Recipient Project Title:

Development of an Optimized Control System for Heavy Medium Cyclone Circuits

Report Information:

Type: Final

Number: 4

Principal Investigators:

Period: $\quad$ 10/1/08-3/30/09

Gerald H. Luttrell, Robert Bratton, and Roe-Hoan Code: VA021-FINAL Yoon

Contact Address:

Contact Information:

146 Holden Hall

Phone: (540) 231-4508

Virginia Tech

Blacksburg, VA 24061

Fax:

(540) 231-3948

E-Mail: luttrell@vt.edu

Subcontractor Address:

No subcontracts issued.

Subcontractor Information:

Phone:

Fax:

E-Mail:

\section{Disclaimer}

This report was prepared as an account of work sponsored by an agency of the United States Government. Neither the United States Government nor any agency thereof, nor any of their employees, make any warranty, express or implied, nor assume any legal liability or responsibility for the accuracy, completeness, or usefulness of any information, apparatus, product, or process disclosed, or represents that its use would not infringe privately owned rights. Reference herein to any specific commercial product, process, or service by trade name, trademark, manufacturer, or otherwise does not necessarily constitute or imply endorsement, recommendation, or favoring by the United States Government or any agency thereof. The views and opinions of authors expressed herein do not necessarily state or reflect those of the United States Government or agency thereof. 


\title{
Development of a Multi-Stream Monitoring and Control System for Dense Medium Cyclones
}

\author{
Coby Braxton Addison
}

\begin{abstract}
Dense medium cyclones (DMCs) have become the workhorse of the coal preparation industry due to their high efficiency, large capacity, small footprint and low maintenance requirements. Although the advantages of DMCs make them highly desirable, size-by-size partitioning data collected from industrial operations suggest that DMC performance can suffer in response to fluctuations in feed coal quality. In light of this problem, a multi-stream monitoring system that simultaneously measures the densities of the feed, overflow and underflow medium around a DMC circuit was designed, installed and evaluated at an industrial plant site. The data obtained from this real-time data acquisition system indicated that serious shortcomings exist in the methods commonly used by industry to monitor and control DMC circuits. This insight, together with size-by-size partition data obtained from in-plant sampling campaigns, was used to develop an improved control algorithm that optimizes DMC performance over a wide range of feed coal types and operating conditions. This document describes the key features of the multi-stream monitoring system and demonstrates how this approach may be used to potentially improve DMC performance.
\end{abstract}




\section{TABLE OF CONTENTS}

ABSTRACT.

i

ACKNOWLEDGEMENTS

Error! Bookmark not defined.

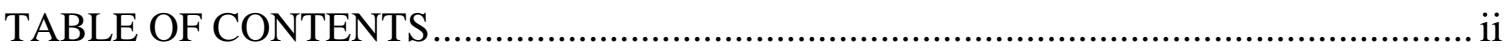

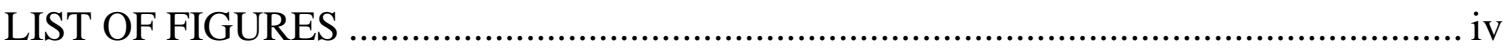

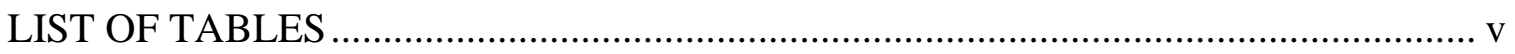

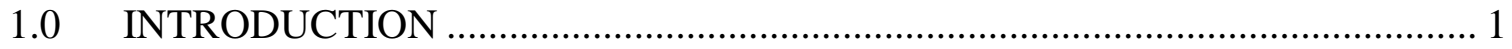

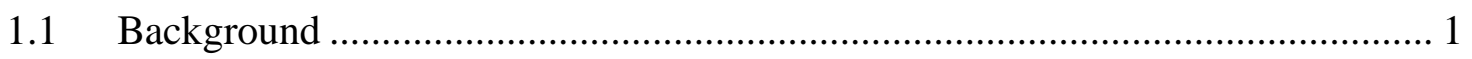

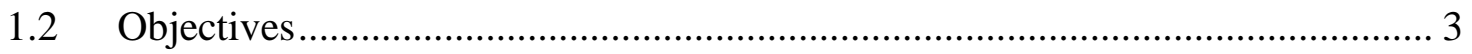

$2.0 \quad$ LITERATURE REVIEW …................................................................... 5

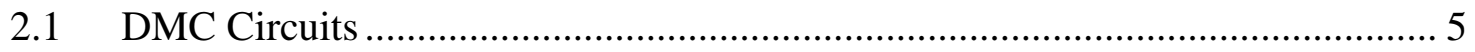

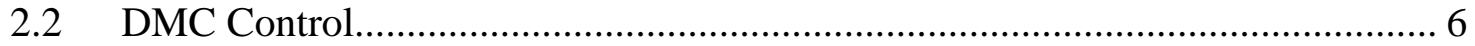

2.3 Specific Gravity Measurement Techniques ................................................ 8

2.4 Specific Gravity Cutpoint..................................................................... 10

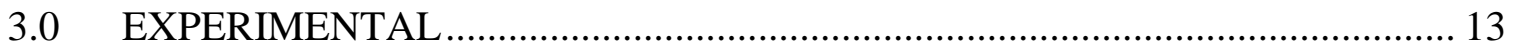

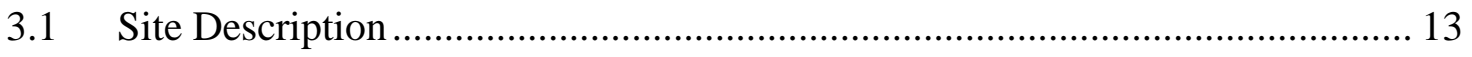

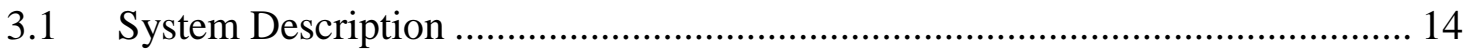

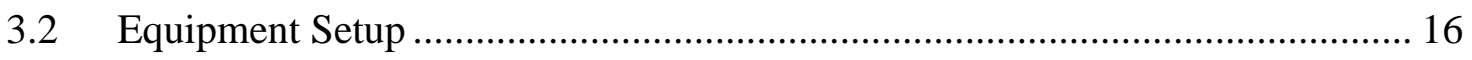

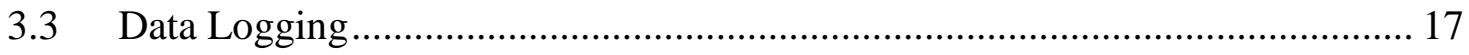

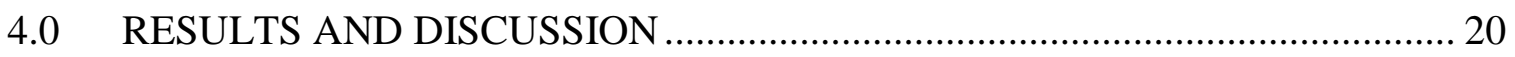

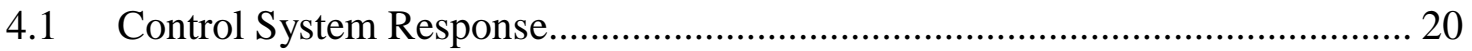

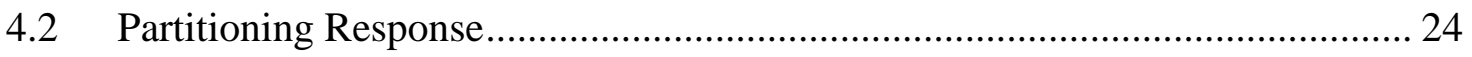

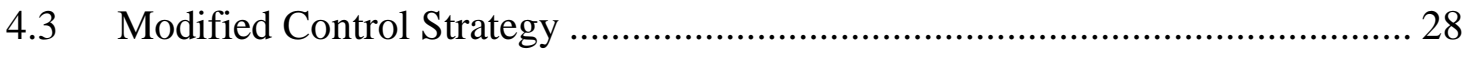




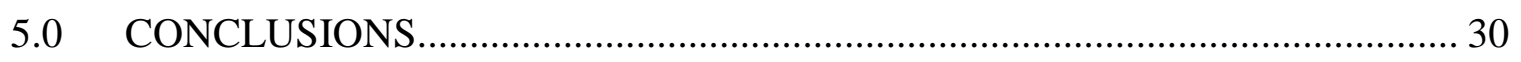

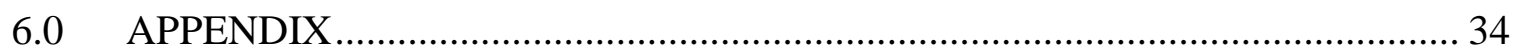

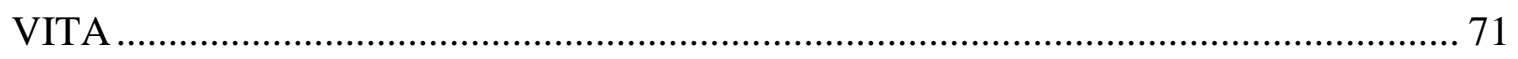




\section{LIST OF FIGURES}

Figure 1. Photograph of an industrial dense medium cyclone (DMC) installation. ........... 1

Figure 2. DMC circuit with feed medium monitored by nuclear density gauge. .............. 7

Figure 3. Photographs of (a-left) density scale and (b-right) nuclear density gauge.......... 9

Figure 4. Photograph of the Tom's Creek preparation plant facility.............................. 13

Figure 5. DMC circuit with all three medium streams monitored by nuclear density

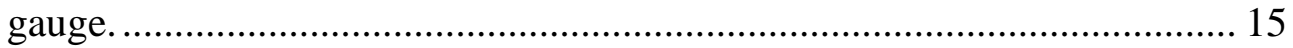

Figure 6. Rack supporting nuclear density gauge and feed pipe medium SGs................ 17

Figure 7. Screen-shot for PLC data monitoring system.............................................. 18

Figure 8. Comparison of plant SG (control SG) with the feed, overflow and underflow medium SGs for low density range.

Figure 9. Comparison of plant SG (control SG) with the feed, overflow and underflow medium SGs for intermediate density range.

Figure 10. Comparison of plant SG (control SG) with the feed, overflow and underflow medium SGs for high density range......................................................... 23

Figure 11. Comparison of plant nuclear gauge SG and experimental cutpoint SG $_{50} \ldots . . .26$

Figure 12. Comparison of plant nuclear gauge SG and experimental cutpoint $\mathrm{SG}_{50} . \ldots . . .27$

Figure 13. Comparison of estimated (model) and experimentally measured $\mathrm{SG}_{50} \ldots \ldots . . . .30$ 


\section{LIST OF TABLES}

Table 1. Effect of SG range and feed coal type on DMC medium behavior ...........25

Table 2. Effect of density range and feed coal type on DMC partitioning performance

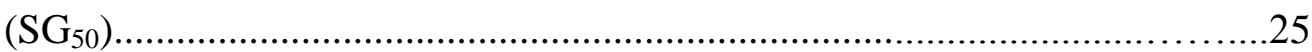




\subsection{INTRODUCTION}

\subsection{Background}

Dense medium cyclones (DMCs) are used in the coal preparation industry to clean particles in the 50-0.5 mm size range. These high-capacity units (Figure 2) use centrifugal forces to enhance the separation of fine particles that cannot be upgraded in static dense medium separators. In the U.S. alone, DMC circuits account for an annual production of about 160 million tons of clean coal (16\% of total U.S. production) and represent an installed capacity approaching 75,000 tons per hour. Due to the high tonnage treated, a small increase in DMC efficiency can have a large impact on plant profitability. Estimates suggest that a modest two percentage point increase in the DMC efficiency

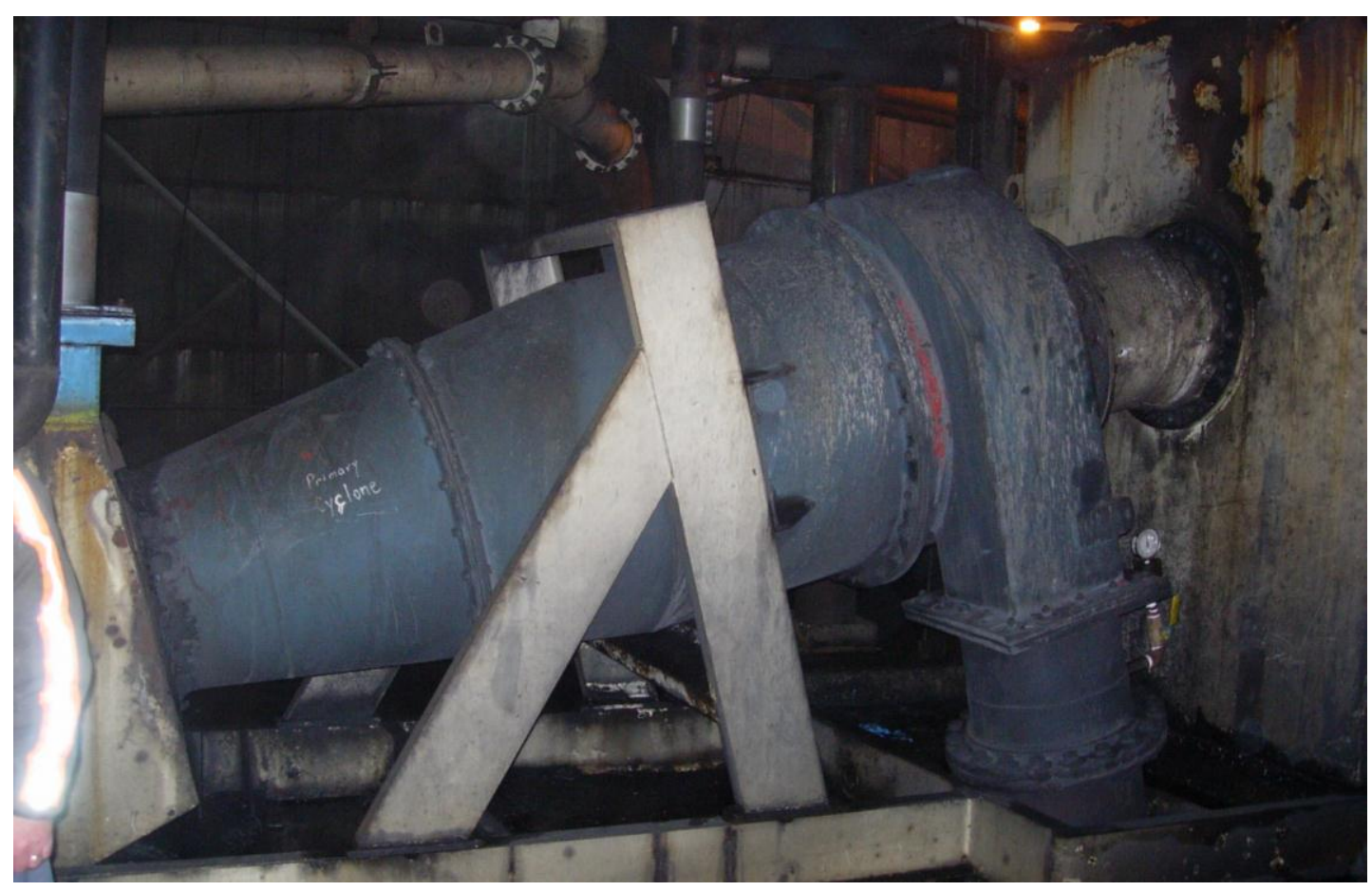

Figure 1. Photograph of an industrial dense medium cyclone (DMC) installation. 
would produce 3.2 million tons of additional clean coal in the U.S. from the same tonnage of mined coal. At a market price of $\$ 50$ per ton, the recovered tonnage represents annual revenues of nearly $\$ 156$ million for the U.S. coal industry or nearly $\$ 660,000$ per year for an average preparation plant.

Dense medium cyclones are frequently installed in banks of two or more parallel units or in parallel with other separators (such as dense medium vessels) in order to meet the production requirements of a given plant. Theoretical analyses show that the clean coal yield from these parallel circuits is maximized when all of the separators are operated at the same specific gravity cutpoint (Abbott, 1982; Clarkson, 1991; Luttrell et al., 2000). This optimization principle is valid regardless of the desired quality of the total clean coal product or the ratios of different coals passed through the circuits.

To illustrate the importance of this optimization concept, consider a 500-tph circuit consisting of two identical DMCs operating in parallel. Both of the DMCs are capable of producing an $8 \%$ total ash product when they operate at the same cutpoint of 1.55 SG. The overall yield from these two DMCs is $68.2 \%$. However, the two units can also produce a combined clean coal ash of $8 \%$ by operating the first DMC at 1.59 SG (which produces an $8.5 \%$ ash) and by operating the second cyclone at 1.51 SG (which produces a $7.5 \%$ ash). Although the combined product is still $8 \%$ ash, operation at a cutpoint difference of $0.08 \mathrm{SG}$ units reduces the overall yield from the combined circuit from $69.6 \%$ to $68.2 \%$ (i.e., a 1.4 percentage reduction). If the cyclones are operated for 6,000 hrs per year, the annual revenue lost due to the cutpoint difference is \$2.1 MM annually (i.e., $1.4 \%$ x $500 \mathrm{ton} / \mathrm{hr} \times 6000 \mathrm{hr} / \mathrm{yr} \times \$ 50 /$ ton $=\$ 2,100,000$ ). Therefore, it is 
important that all dense medium circuits (vessels and DMCs) be operated at the same SG cutpoint to optimize total plant profitability.

The industrial application of cutpoint optimization is relatively straightforward for dense medium vessels. Vessels tend to operate at a density cutpoint that is predictable based on the specific gravity (SG) of the feed medium. On the other hand, the segregation of medium by the centrifugal field within a DMC makes it very difficult to estimate the true SG cutpoint for cyclones. Typically, the underflow medium from a DMC has a substantially higher SG than that of the overflow medium due to preferential classification of the magnetite particles used to create the artificial medium. The thickening of the medium tends to increase the SG cutpoint for the DMC above that of the feed medium SG. Because of this phenomenon, the actual cutpoint of the DMC is about 0.05-0.10 SG units higher than that of the measured SG of the feed medium. This "offset" between true and measured density can vary substantially depending on the feed medium density, extend of cyclone wear, and characteristics of the feed coal. In some cases, negative offset values have even been reported from plant studies due to the utilization of poor grades of magnetite. As a result, the normal practice of on-line monitoring the feed medium SG using nuclear density gauges cannot be used to accurately estimate the true cutpoint for DMCs. As discussed previously, this inability to estimate and maintain the SG cutpoint can result in coal losses that have a tremendous impact on plant profitability.

\section{$1.2 \quad$ Objectives}

The primary objective of this project was to develop an on-line monitoring and control system to optimize the performance of dense medium cyclone (DMC) circuits. 
The system developed from this work utilizes multi-stream on-line monitoring of the feed, overflow and underflow streams using low-cost nuclear density gauges. The realtime data from these sensors is passed through a mathematical algorithm that accurately estimates the specific gravity (SG) cutpoints of the DMC circuitry. This information makes it possible to globally optimize plant performance under conditions of changing coal types and feed blends, which are now common in industrial operations. The specific tasks completed as part of this applied R\&D project included (i) equipment installation, (ii) in-plant sampling, (iii) control algorithm development and (iv) control system testing. All of the experimental work was performed on-site at an industrial plant site in order to promote the rapid deployment of this technology in the coal preparation industry. 


\subsection{LITERATURE REVIEW}

\subsection{DMC Circuits}

There are three major DMC circuits used throughout the coal processing industry. These three circuits are gravity feed circuit, wing tank circuit, and pump feed circuit. In a gravity feed circuit, the DMCs are located below the pulping column that feeds the distributor for the cyclones. The pulping column is a vertical mixing pipe for the circulating medium and feed material to the cyclones. In this type of circuit there is no need for a pump. Since the pulping column must be an adequate length to provide a desired feed inlet pressure, there is always a consistent feed pressure to the cyclones. The specific gravity of the circulating medium can easily be measured prior to entering the column and without the presence of the feed material, i.e. coal and rock, which provides an accurate specific gravity measurement for the feed medium.

In a pump feed circuit, the DMCs are located above the sump and pump that feeds the distributor to the cyclones. Since a pump provides the desired feed inlet pressure, a pump feed circuit requires less building height than a gravity feed circuit. With this circuit the feed inlet pressure dependent on the wear and proper maintenance of the feed pump, as compared to relying solely on gravitational forces for the gravity feed circuit. Unless medium is combined before being introduced to the pulping column within the sump, the measurement of the feed medium is in the presence of the raw feed material to the cyclones. Measuring the specific gravity of the feed medium in the presence of raw feed material with a nuclear density gauge fails to provide an accurate value since the specific gravity of the raw feed material will bias the specific gravity measurement. 
In a wing tank circuit, the circulating medium is returned to a correct medium sump before being introduced to a smaller mixing tank along with the raw feed material. Measuring the medium pumped from the correct medium sump (without the presence of raw feed material) with a nuclear density gauge provides an accurate method of measuring the specific gravity of the feed medium. This circuit requires more building area for the correct medium sump and pump, as compared to the previous two circuits in which there is only one medium sump and pump.

\subsection{DMC Control}

The cutpoint is defined as the specific gravity at which a particle has an equal chance of reporting to the overflow or underflow of the cyclone. Since the medium is subjected to the centrifugal forces inside the DMC, the specific gravity of the medium will increase toward the apex of the cyclone. This tendency always makes the specific gravity of the medium in the overflow of the cyclone lower than the feed medium specific gravity, and, accordingly, the specific gravity of the medium in the underflow higher than the feed medium specific gravity, thus the cutpoint in a DMC is always higher than the circulating feed medium.

There are various control implementations to monitor the specific gravity of the medium entering a DMC. The most common method for the widely used pump feed circuit is the placement of a nuclear density gauge on the feed pipe to the DMC (Figure 2). Since the medium has not been subjected to the cyclone's forces at this point, this implementation may not accurately provide the cutpoint. Also, since the difference between the cutpoint and circulating feed medium specific gravity relies heavily on various parameters (inlet pressure, geometry, fittings, etc.) and the density of the raw feed 


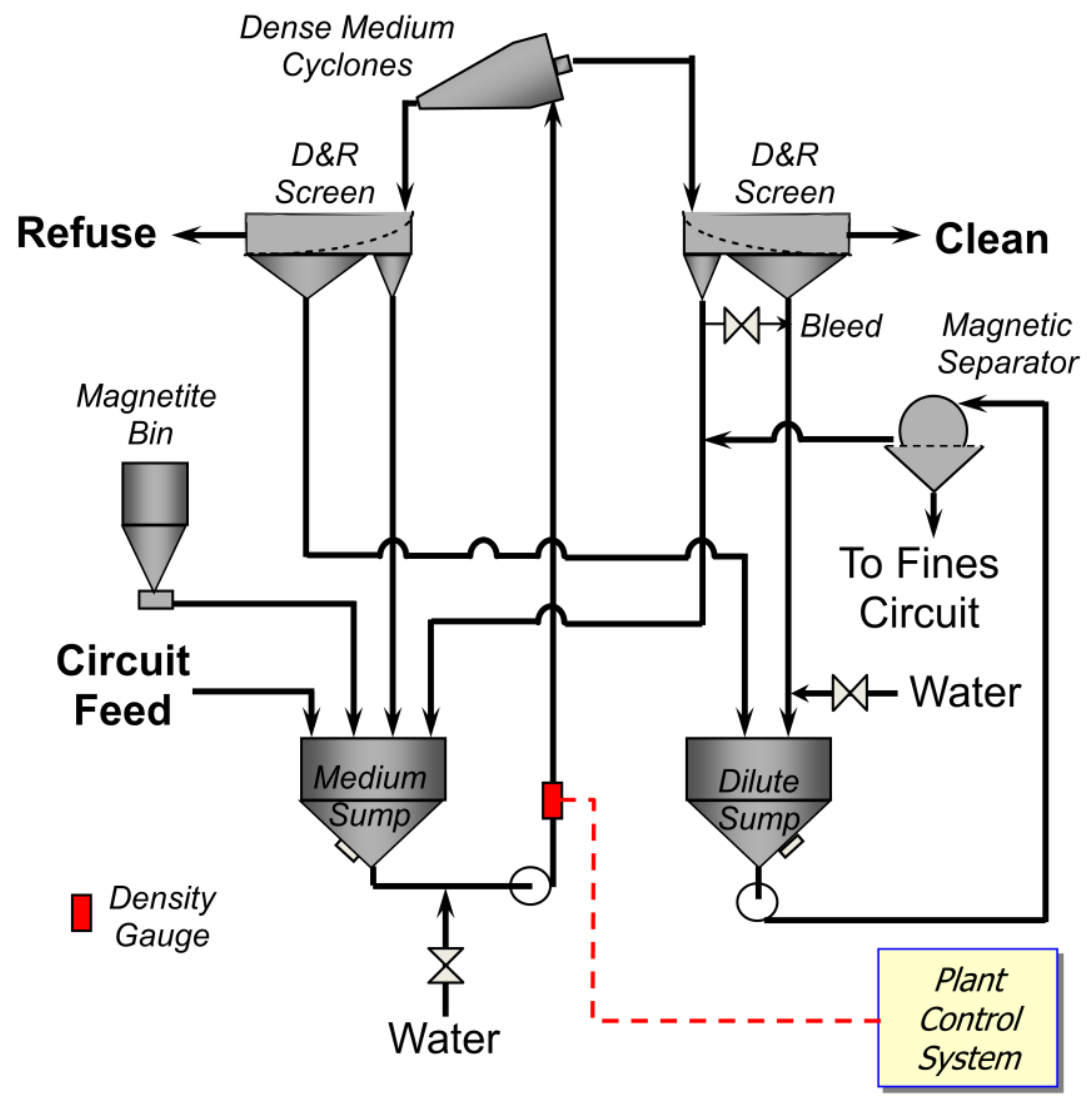

Figure 2. DMC circuit with feed medium monitored by nuclear density gauge.

material, monitoring the feed medium specific gravity provides an unstable method for controlling the cutpoint.

It has been observed that a direct correlation exists between magnetite distribution and the DMC cutpoint. Cyclone geometry is changed in order to improve performance in dense medium cyclones, such as changing the apex and vortex finder sizes, thus affecting the cutpoint. The cutpoint of the cyclone can be accurately obtained by monitoring the distribution of the medium in the cyclone. In this case, the overflow and underflow 
medium streams are monitored in order to obtain a ratio of magnetite distribution for control of the cutpoint (Burgess et al., 1987).

In most scenarios, about two-thirds of the medium that reports to the feed inlet of the cyclone should report to the overflow of the cyclone. This split can be manually calculated by collecting samples of the feed, overflow, and underflow medium streams, and using the formula:

$$
\beta=\frac{S G_{u}-S G_{f}}{S G_{u}-S G_{o}}
$$

where $\beta$ is the medium split to overflow and $\mathrm{SG}_{\mathrm{f}}, \mathrm{SG}_{\mathrm{o}}$ and $\mathrm{SG}_{\mathrm{u}}$ are the specific gravity of the medium of the feed, overflow and underflow streams, respectively. In order to obtain an accurate specific gravity measurement of the medium streams, the measurement must be obtained after the medium has been screened from the processed material. This measurement is very useful since it can help identify problems with a cyclone, i.e., corrective actions can be performed when the medium split to overflow drops below twothirds (Luttrell et al., 2002).

\subsection{Specific Gravity Measurement Techniques}

There are various techniques for measuring the specific gravity of a particular medium. The two most common methods are the manual specific gravity scale and the use of a nuclear density gauge (Figure 3).

The manual method typically consists of using a customized sample collection device with a volume of one-liter and dial-type spring scale. To calibrate the device correctly, water is used to check the 1.00 specific gravity (SG) point and a known weight 

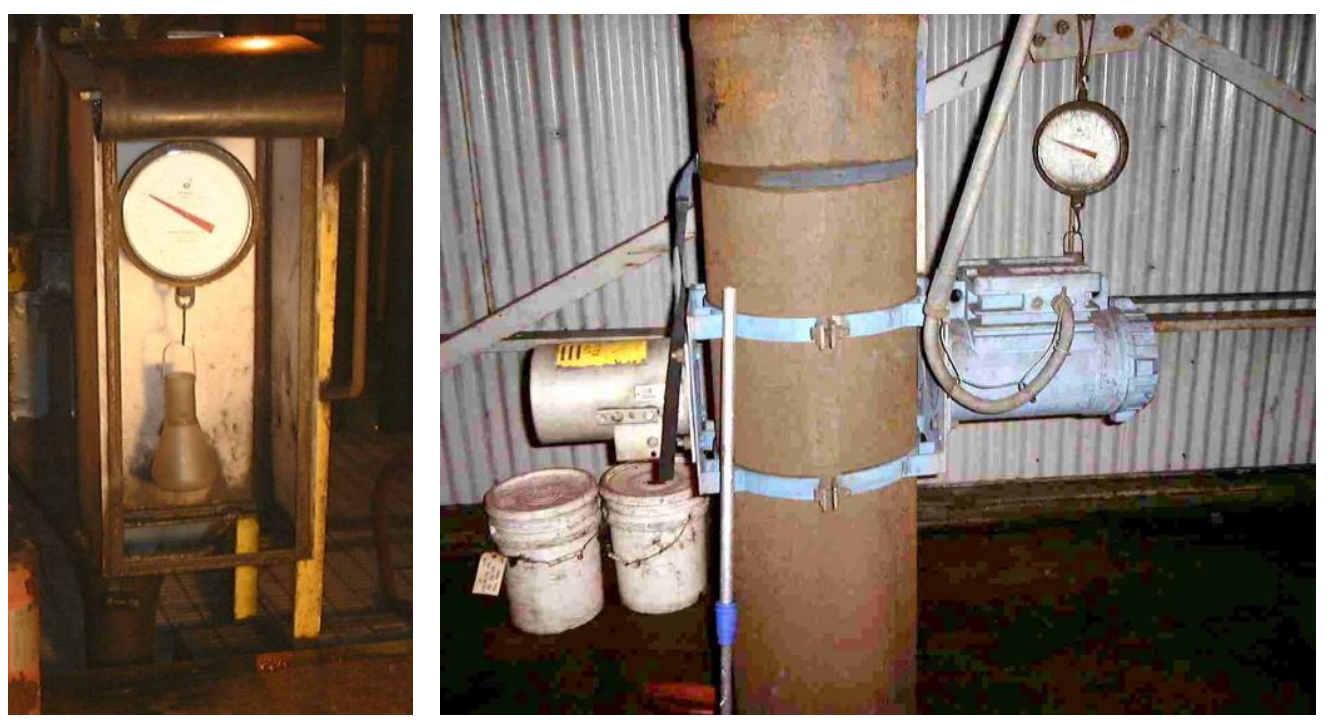

Figure 3. Photographs of (a-left) density scale and (b-right) nuclear density gauge.

is used to check the SG point near the medium specific gravity. The method includes properly collecting a representative sample of the medium with the collection device and weighing the device filled with medium on the calibrated scale.

The nuclear density gauge is a device that is placed on a pipe in which the medium is flowing. The nuclear source, typically an isotope of Cesium (Cs137) irradiates a narrow beam of gamma particles that strike a detector on the opposite side of the pipe after passing through the contents of the pipe. The specific gravity of the contents in the pipe is calculated based on the attenuation of the gamma particles as compared to calibration points set with water (1.0 SG) and a known SG point near the normal operating range of the medium. The more dense the material in the pipe, the more the gamma particles are attenuated and fewer gamma particles reach the detector. Fewer 
gamma particles seen by the detector yields a higher specific gravity, and more gamma particles seen by the detector yields a lower specific gravity.

\subsection{Specific Gravity Cutpoint}

The cutpoint is defined as the specific gravity at which a particle has an equal chance of reporting to the overflow or underflow of the cyclone. Since the medium is subjected to the centrifugal forces inside the dense medium cyclone, the specific gravity of the medium will increase toward the apex of the cyclone. This tendency decreases the specific gravity of the medium in the overflow of the cyclone and increases the specific gravity of the medium in the underflow compared to the feed medium specific gravity. The cutpoint specific gravity in a DMC is typically higher than the circulating feed medium due to enhanced settling created by the centrifugal forces in the cyclone.

Besides sampling the feed, overflow, and underflow streams of the DMCs and obtaining float/sink analysis from a commercial lab, the separation performance or determination of the specific gravity cutpoint of a DMC can be predicted using a partition model which assumes that the partition curve for each particle size class passes through a common pivot point (Scott, 1988). The specific gravity $\left(\mathrm{SG}_{50} *\right.$ ) corresponding to the pivot point can be estimated from an empirical expression given by Wood (1981):

$$
S G_{50}^{*}=0.360 S G_{f i n}+0.274 S G_{u m}+0.532 S G_{o m}-0.205
$$

where $\mathrm{SG}_{\mathrm{fm}}, \mathrm{SG}_{\mathrm{um}}$ and $\mathrm{SG}_{\mathrm{om}}$ are the specific gravities of the feed, underflow and overflow streams, respectively. The $\mathrm{SG}_{50}^{*}$ value represents the effective SG cutpoint of an infinitely large particle separated under a zero medium viscosity. The second defining 
term for the pivot point is obtained at a partition number that is numerically equal to the medium split to underflow (Su) given by (Restarick and Krnic, 1990):

$$
S_{u}=\frac{S G_{f m}-S G_{o m}}{S G_{u m}-S G_{o m}}
$$

Once the pivot point is identified, the specific gravity cutpoint $\left(\mathrm{SG}_{50}\right)$ for each particle size class can be obtained using (Wood, 1990; 1997):

$$
S G_{50}=S G_{50}^{*}+0.910 E p \ln \left[\left(1-S_{u}\right) / S_{u}\right]
$$

To utilize this expression, it is assumed that the unknown Ep value for each particle size class can be estimated using (Barbee et al., 2005):

$$
E p=D_{c}^{0.5} /\left(398 D_{p}\right)
$$

in which $D_{p}$ is the mean particle diameter (in $\mathrm{mm}$ ) of each size class and Dc is the cyclone diameter (in mm). Equations [2]-[5] show that it is possible to predict the SG cutpoints for a DMC provided that the values of $\mathrm{SG}_{\mathrm{fm}}, \mathrm{SG}_{\mathrm{um}}$ and $\mathrm{SG}_{\mathrm{om}}$ are known. Unfortunately, only the feed medium density $\left(\mathrm{SG}_{\mathrm{fm}}\right)$ is typically measured in most industrial DMC circuits.

Since the previous equations calculate a specific gravity cutpoint of a DMC based on all three medium streams, feed, overflow and underflow, specific gravity measurements of these streams in a preparation plant could provide an accurate, real-time specific gravity cutpoint. This cutpoint could be used as an input for the control system 
and optimize the preparation plant performance by operating dense medium circuits at the same specific gravity cutpoint. 


\subsection{EXPERIMENTAL}

\subsection{Site Description}

Seeing the opportunity for optimization, Alpha Natural Resources, LLC, decided for collaboration with Virginia Tech by providing a facility for in-plant testing and sampling. The Tom's Creek Preparation Plant, located in Coeburn, Virginia, was chosen as the host site for the proposed test work. The plant (Figure 2) was commissioned in 1980 and was acquired by Alpha Natural Resources on January 31, 2003, when Coastal Coal Company was purchased by Alpha Natural Resources. By mid-2003, the decision had been made to idle the Ramsey preparation plant and bring the 80,000 tons per month being processed and shipped there to Tom's Creek. Tom's Creek was upgraded in order to increase the throughput capacity and add a middlings recovery circuit starting in October 2003. The preparation plant now has a design capacity of 1,100 raw tons per

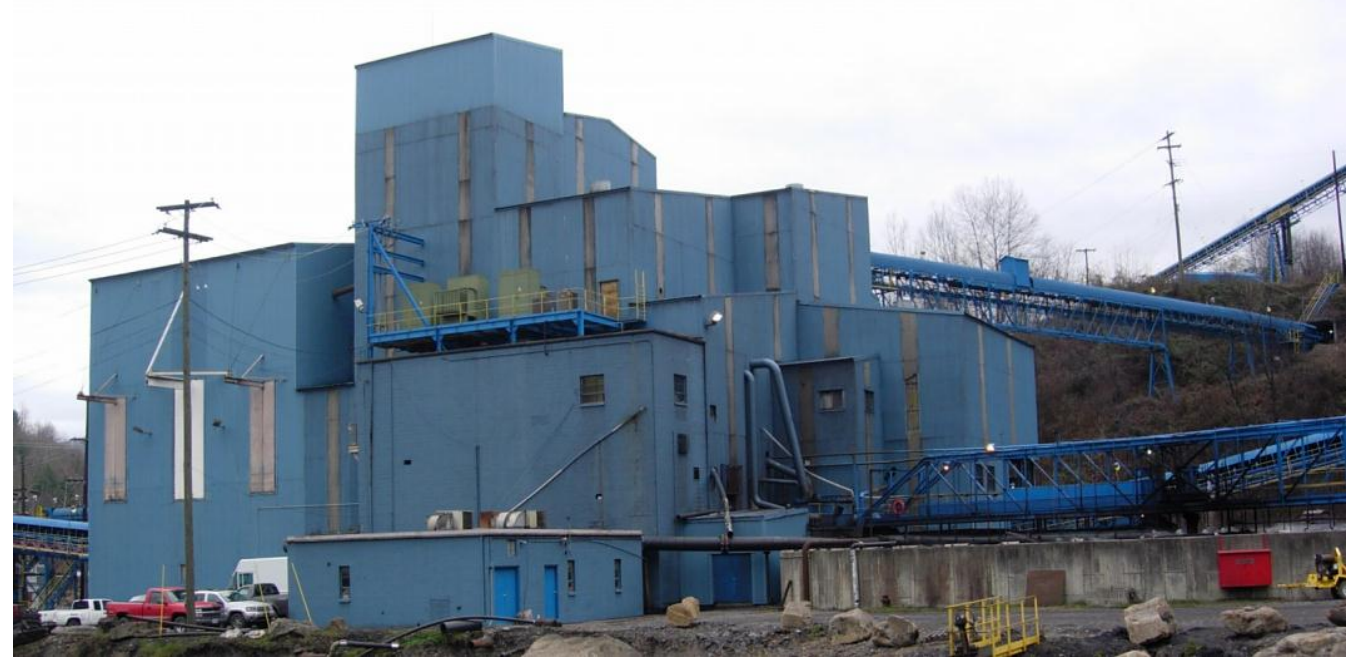

Figure 4. Photograph of the Tom's Creek preparation plant facility. 
hour with a maximum capacity of 1,300 raw tons per hour. Changes were completed to each of the four circuits: coarse, intermediate, fine, and ultra fine, and a middlings recovery circuit was added to the intermediate circuit in order to give the option of a rewash of material. The middlings recovery circuit was designed to minimize the amount of rock being pumped and re-handled in the plant and utilize the smaller 33-inch diameter dense medium cyclones for the lower gravity separation. The 40-inch diameter primary dense medium cyclone is utilized for the high gravity separation. This eliminates the rehandling of rock increasing the wear life of the operating equipment components. The overflow from the primary dense medium cyclone reports to the two secondary dense medium cyclones in order to achieve the product split between the premium product and middlings.

\subsection{System Description}

Equations [2]-[5] suggest that it is possible to predict and properly optimize the SG cutpoints for a DMC provided that the values of $\mathrm{SG}_{\mathrm{fm}}, \mathrm{SG}_{\mathrm{um}}$ and $\mathrm{SG}_{\mathrm{om}}$ are known. Unfortunately, only the feed medium density $\left(\mathrm{SG}_{\mathrm{fm}}\right)$ is typically measured in most industrial DMC circuits. Also, density for the feed medium $\left(\mathrm{SG}_{\mathrm{fm}}\right)$ is often measured with coal present so that the true medium density is not known. To overcome this limitation, an improved monitoring and control system was developed that utilizes multi-stream online measurements of the feed, overflow and underflow medium densities using low-cost nuclear density gauges and pressure transmitters. A schematic of the multi-stream monitoring system is provided in Figure 5.

The multi-stream monitoring system uses four nuclear density gauges to simultaneously monitor medium density throughout the entire circuit. The first density 


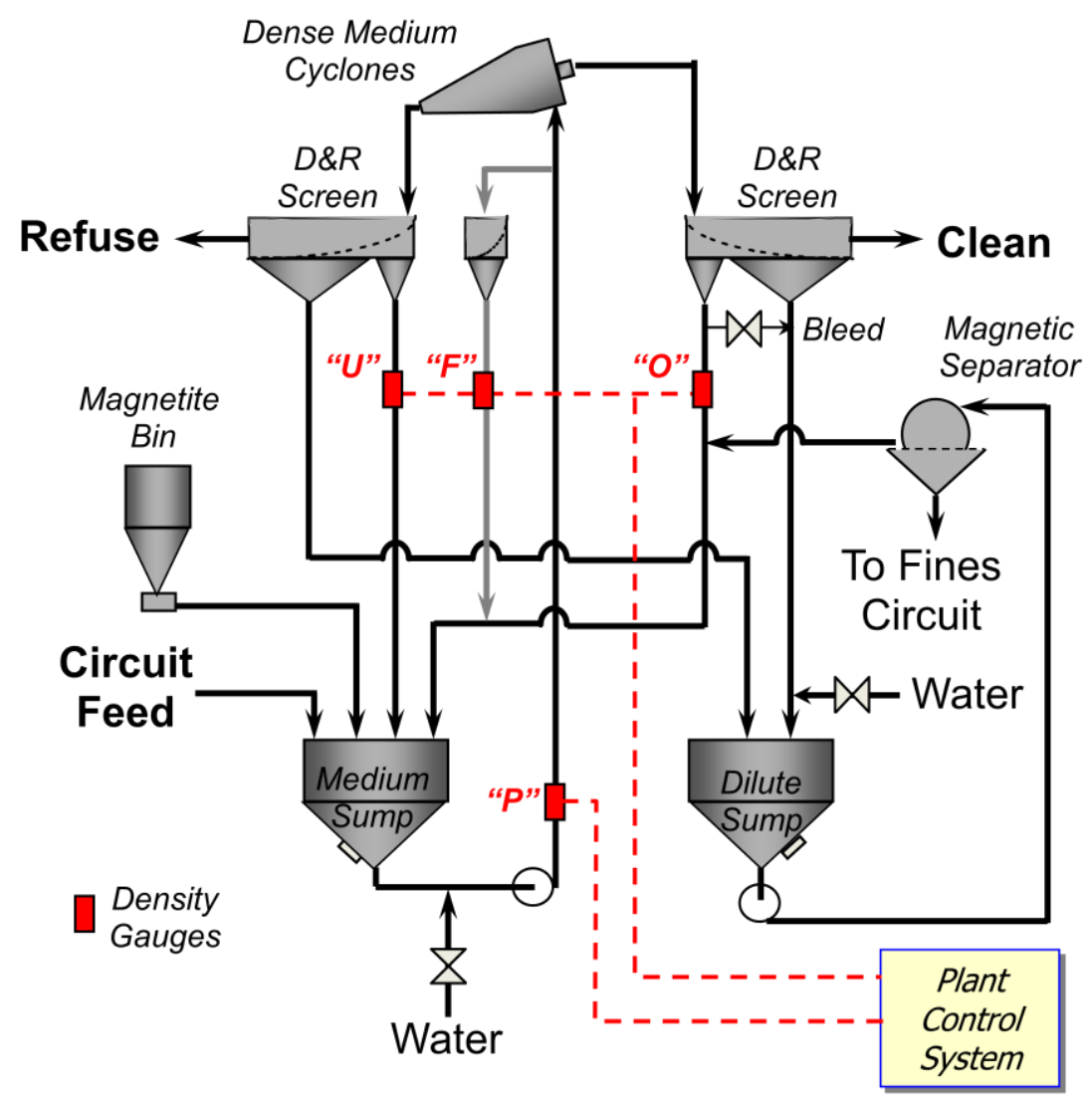

Figure 5. DMC circuit with all three medium streams monitored by nuclear density gauge.

gauge ("P"), which was already installed at the plant, was used to monitor and control the density of the circulating medium that was fed to the DMCs. Unfortunately, since this stream also contains coal particles, the reading from this density gauge does not necessarily represent the true density of the circulating medium. To determine the true medium density, a small slipstream from the DMC feed line was passed across a small sieve screen. The underflow from the sieve screen reported to another nuclear density gauge ("F"). Two additional nuclear density gauges ("U" and "O") were installed in the return medium streams from the drain sections of the overflow and underflow drain-andrinse screens. These four measurement points made it possible to monitor the density of 
the feed medium (with and without coal present), underflow medium and overflow medium. Data from the electronic sensors was continuously logged on-line using a PLC data recorder. In principle, the real-time data from these sensors can be passed through a mathematical algorithm to estimate the "true" SG cutpoint for the DMCs (see Equation [4]). As such, this information makes it possible to fully optimize DMC cutpoints under conditions of changing coal types and feed blends.

\subsection{Equipment Setup}

The nuclear density gauges were mounted in custom fabricated portable racks as illustrated in Figure 6. A vertical feed pipe above the gauge was used to ensure a high velocity flow that prevented any settling of the magnetite through the system. The vertical feed pipe was fitted with an overflow at the top. Flow to the rack and density gauge was set to provide an overflow stream at the top of the vertical feed pipe to ensure a full feed pipe and to eliminate any air bubbles in the medium passing through the gauge. The medium that passed through the nuclear density gauge and from the overflow was routed back to the DMC feed sump. The density gauge rack for the underflow medium sample was installed, along with the associated sampling points and piping, to receive medium flow from either the clean coal or refuse drain-and-rinse screens.

After the installation of the density gauges, manual density (Marcy) cup measurements were taken and flows were established to insure that the flow through the gauges was an accurate representation of the actual medium flows around the DMC. The next step involved energizing the three nuclear density gauges, checking the electrical connections, setting the proper configuration parameters, and then standardizing the gauges with clear water. Circulating medium was then routed through the gauges for the 


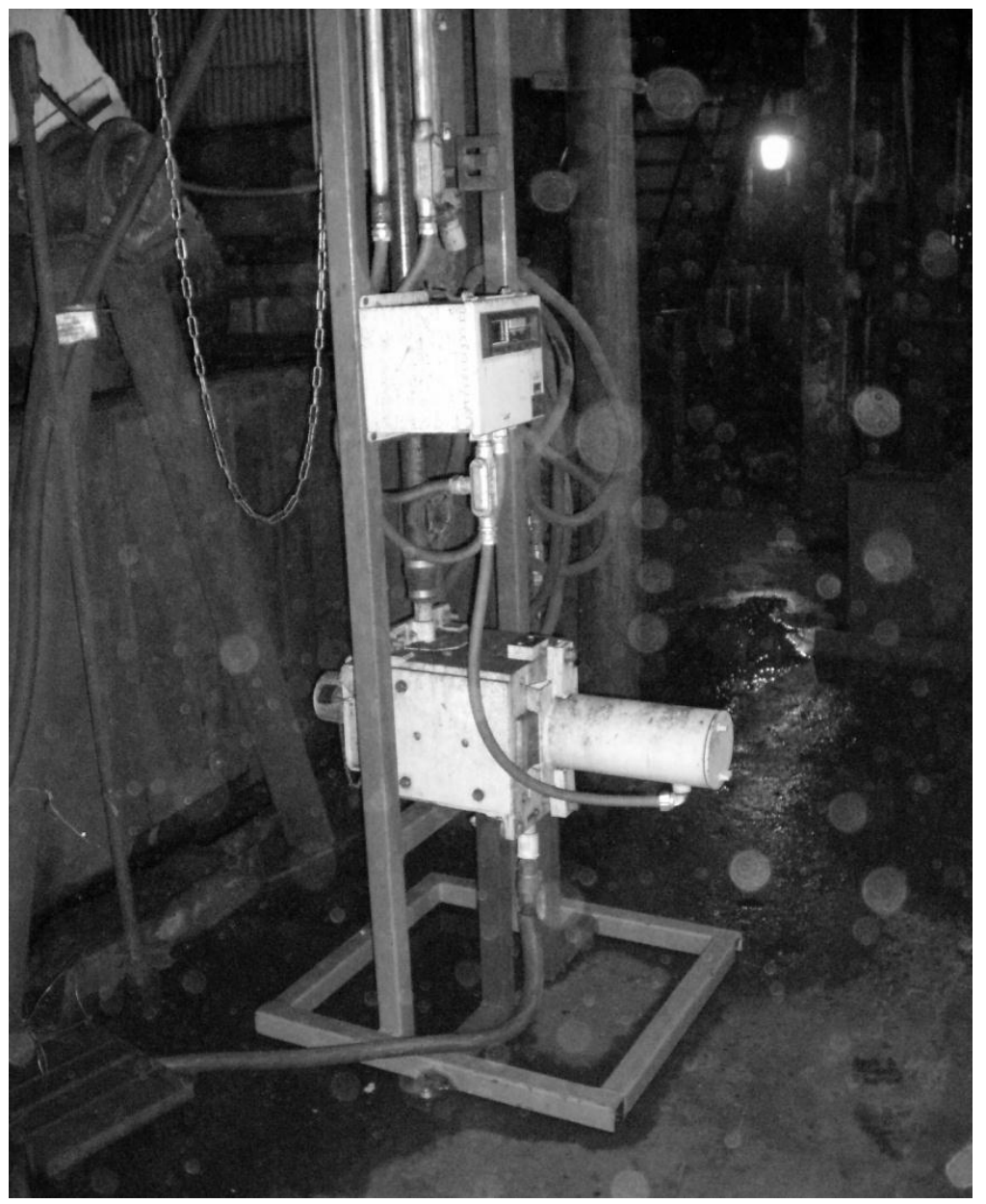

Figure 6. Rack supporting nuclear density gauge and feed pipe medium SGs.

calibration procedure. A pulp density scale, calibrated using a two-point procedure at 1.0 and 1.6 SG, was used to obtain an accurate medium SG for the density gauge calibration. The process signals from the density gauges representing the SG for the streams were connected to the plant PLC control system via 4-20 mA analog inputs.

\subsection{Data Logging}

A dedicated data monitoring and logging system was developed to retrieve the relevant process information from the plant PLC control system during the sampling 


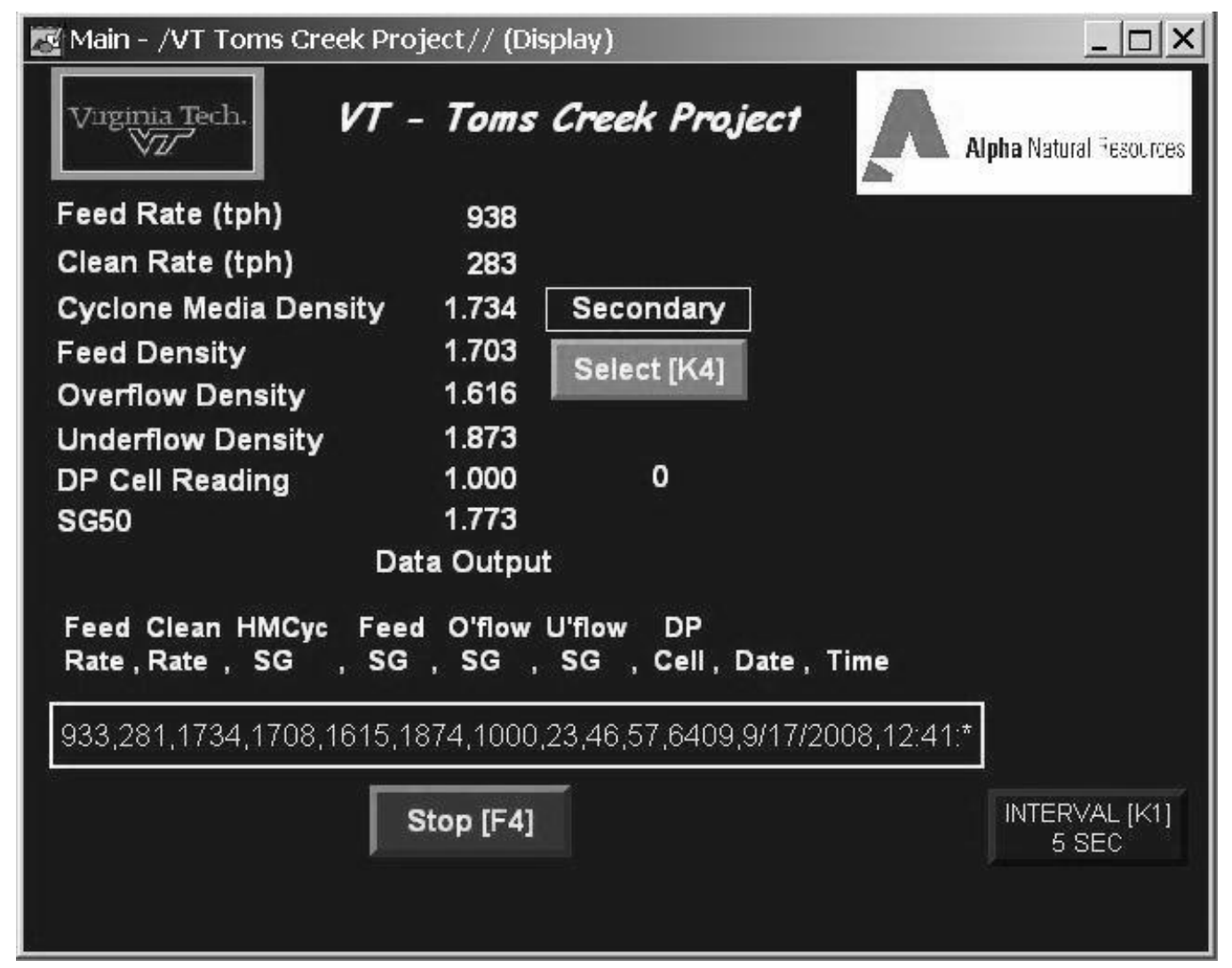

Figure 7. Screen-shot for PLC data monitoring system.

periods. The system provided a means to monitor the process data while collecting samples and logged all the data to a computer text file. A screen shot of the data monitoring system is illustrated in Figure 7. The process data was logged every five seconds, with a time and date stamp, and included:

\footnotetext{
- $\quad$ Plant Feed Rate (tph)

- $\quad$ Clean Coal Rate (tph)

- $\quad$ Plant Secondary DMC Feed Medium Density (used for circuit SG control)

- $\quad$ Project Secondary DMC Feed Medium Density (from sieve screen underflow)

- $\quad$ Project Secondary DMC Overflow Medium Density
} 
- $\quad$ Project Secondary DMC Underflow Medium Density

- $\quad$ Differential Pressure Cell Transmitter

- $\quad$ Calculated SG Cutpoint

The differential pressure $(\mathrm{d} / \mathrm{p})$ cell transmitter, noted in the above list, was installed on the vertical feed pipe for the one of the density gauges and the signal was intended to be logged in an attempt to correlate that data with the density gauge data. Problems were encountered in obtaining reliable data from the $d / p$ cell transmitter, so this data was not reported in this article. The calculated SG cutpoint for particles was based on the mathematical model described by Equation [4]. While the accuracy of this equation was not verified in the present work, it was believed to provide a relative indicator of the expected cutpoint densities for particles passing through the DMC circuit. 


\subsection{RESULTS AND DISCUSSION}

\subsection{Control System Response}

Three series of test runs were conducted at low, medium and high SG setpoints using the four SG monitoring stations. A complete summary of the experimental data and associated partition computations for all three series of tests are provided in the appendix. In each run, the values for the feed, underflow and overflow medium were recorded using density gauges " $F$ ", "U" and "O". The reading from the existing plant density gauge ("P") was also recorded. At the midpoint of each test run, the feed coal to the circuit was intentionally switched from a low-ash feed coal containing a low amount of reject rock to a high-ash feed coal containing a large amount of reject rock. This switch was intentionally initiated so that the effects of feedstock quality on DMC control system response could be fully assessed.

Figures 8-10 summarize the results of the medium measurements conducted around the DMC circuit for various density ranges and feed coal types. The data collected for the lowest setpoint of approximately 1.3 SG is shown in Figure 8. For the low-reject feed, a relatively constant value of 1.33 SG was obtained by both the plant gauge ("P") and the slipstream feed gauge ("F"). The density values for the overflow and underflow streams were found to be about 1.21 and 1.57 SG, respectively. However, when the plant switched to the high-reject feed, the reading from the slipstream feed gauge (" $F$ ") dropped by about 0.02 SG to about 1.31 SG. The reason for the drop is that the plant gauge ("P") misinterpreted the extra rock in the feed as high density medium. In response, the plant control system added more water to drop the true density of the 


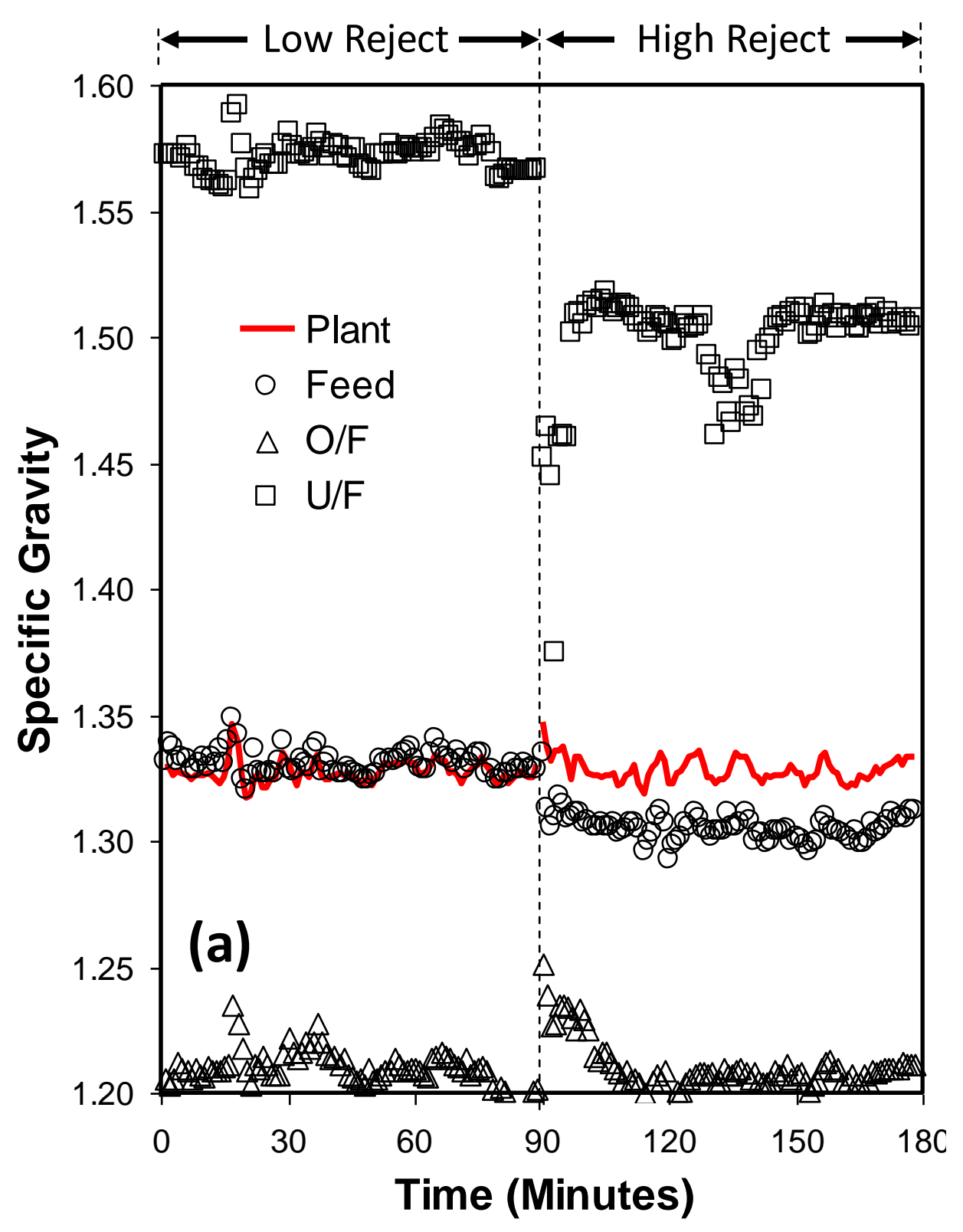

Figure 8. Comparison of plant SG (control SG) with the feed, overflow and underflow medium SGs for low density range. 


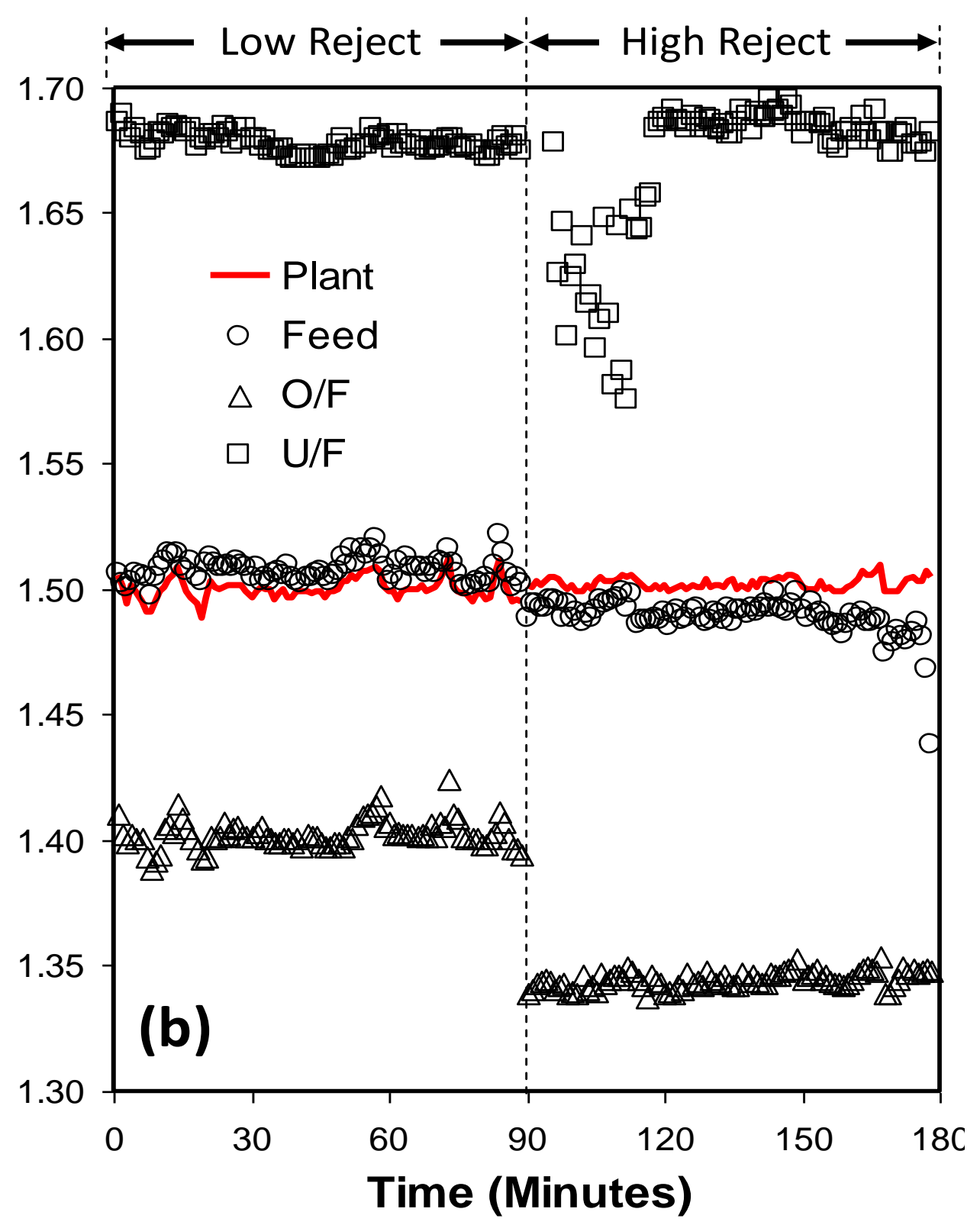

Figure 9. Comparison of plant SG (control SG) with the feed, overflow and underflow medium SGs for intermediate density range. 


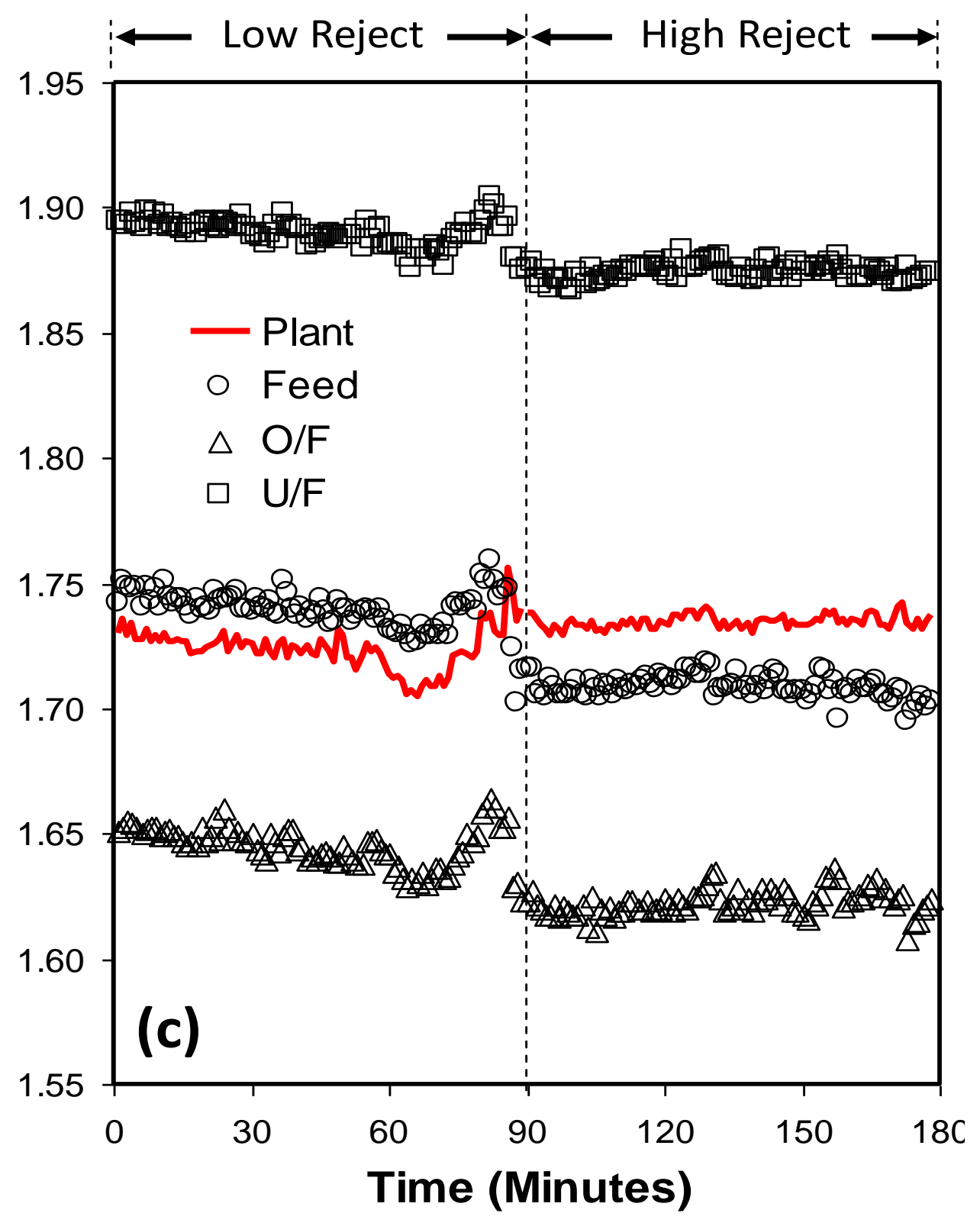

Figure 10. Comparison of plant SG (control SG) with the feed, overflow and underflow medium SGs for high density range. 
circulating medium. Under this new condition, the densities of the overflow and underflow streams changed to 1.21 and 1.50 SG, respectively.

The density data for the test runs conducted using an intermediate SG setpoint is shown in Figure 9. In this case, the plant density gauge ("P") indicated that the circulating medium was $1.50 \mathrm{SG}$. The feed density ("F") measured without coal showed a slightly higher value of 1.51 SG when running the low-reject feed coal. The switch to the high-reject feed coal sharply reduced this value from 1.51 SG down to 1.49 SG. Once again, the existing plant density gauge ("P") and control system misinterpreted the higher rock content in the high-reject feed coal as too much medium and reduced the density. This unexpected change was not apparent in the readings from the plant density gauge ("P") which remained relatively constant at about $1.50 \mathrm{SG}$ during the entire test period.

Finally, Figure 10 shows the density values obtained for the test run performed using a very high SG setpoint. While more variability was observed in the plant density gauge ("P") readings during this particular run, the data still showed a strong dependence between coal type and true medium density. For the low-reject feed coal, the true medium density reported by the feed gauge ("F") was significantly higher than that from the plant gauge ("P"). The trend was exactly opposite when running a high-reject feed, i.e., the true medium density was significantly lower than the plant gauge reading.

\subsection{Partitioning Response}

After completing the medium response tests, three additional series of test runs were conducted to examine the partitioning performance of the DMC circuit. The detailed numerical data and associated partition computations for these tests are provided in the appendix. As before, the test runs were conducted at low, medium and high SG 
setpoints for different quality feeds (i.e., high- and low-reject feedstocks). In each test, representative samples of the feed, clean and reject products were collected and subjected to float-sink analysis. The float-sink analyses were conducted on a size-by-size basis for 12.7x6.35, 6.35x3.18, 3.18x1.41 and $1.41 \times 0.707 \mathrm{~mm}$ size classes. Measurements of the feed, underflow and overflow medium were also obtained using manual sampling and via the on-line medium monitoring stations. The medium response data and partitioning results are summarized in Tables 1 and 2, respectively.

Table 1. Effect of SG range and feed coal type on DMC medium behavior.

\begin{tabular}{ccccccc}
\hline & \multicolumn{2}{c}{ Low SG } & \multicolumn{2}{c}{ Medium SG } & \multicolumn{2}{c}{ High SG } \\
\cline { 2 - 7 } Parameter & $\begin{array}{c}\text { Low } \\
\text { Reject }\end{array}$ & $\begin{array}{c}\text { High } \\
\text { Reject }\end{array}$ & $\begin{array}{c}\text { Low } \\
\text { Reject }\end{array}$ & $\begin{array}{c}\text { High } \\
\text { Reject }\end{array}$ & $\begin{array}{c}\text { Low } \\
\text { Reject }\end{array}$ & $\begin{array}{c}\text { High } \\
\text { Reject }\end{array}$ \\
\hline Gauge SG & 1.330 & 1.350 & 1.500 & 1.501 & 1.699 & 1.713 \\
Feed SG & 1.309 & 1.267 & 1.483 & 1.516 & 1.787 & 1.761 \\
O/F SG & 1.223 & 1.200 & 1.419 & 1.324 & 1.618 & 1.603 \\
U/F SG & 1.546 & 1.453 & 1.687 & 1.683 & 1.813 & 1.796 \\
\hline
\end{tabular}

Table 2. Effect of density range and feed coal type on DMC partitioning performance $\left(\mathrm{SG}_{50}\right)$.

\begin{tabular}{ccccccc}
\hline \multirow{2}{*}{$\begin{array}{c}\text { Size Class } \\
(\mathbf{m m})\end{array}$} & $\begin{array}{c}\text { Low } \\
\text { Reject }\end{array}$ & $\begin{array}{c}\text { High } \\
\text { Reject }\end{array}$ & $\begin{array}{c}\text { Low } \\
\text { Reject }\end{array}$ & $\begin{array}{c}\text { High } \\
\text { Reject }\end{array}$ & $\begin{array}{c}\text { Low } \\
\text { Reject }\end{array}$ & $\begin{array}{c}\text { High } \\
\text { Reject }\end{array}$ \\
\hline $12.7 \times 6.35$ & 1.336 & 1.263 & 1.510 & 1.476 & 1.714 & 1.691 \\
$6.35 \times 3.18$ & 1.342 & 1.272 & 1.511 & 1.481 & 1.715 & 1.687 \\
$3.18 \times 1.41$ & 1.347 & 1.283 & 1.500 & 1.494 & 1.734 & 1.710 \\
$1.41 \times 0.707$ & 1.353 & 1.315 & 1.534 & 1.545 & 1.834 & 1.790 \\
Composite & 1.349 & 1.275 & 1.506 & 1.484 & 1.719 & 1.679 \\
\hline
\end{tabular}


Figure 11 shows the variation in SG readings between the slipstream feed gauge ("F") and plant nuclear density gauge ("P"). At low operating densities, the true SG of the circulating medium was typically lower than indicated by the plant gauge. The reason for the drop is that the plant gauge ("P") misinterpreted rock in the feed as high-density medium. In response, the plant control system added more water and dropped the true density of the circulating medium. The decrease was most noticeable for the high-reject feed since this feedstock contained a larger amount of high density material. Likewise, at high operating densities, the true SG of the circulating medium was typically higher than indicated by the plant gauge. In this case, the plant gauge ("P") misinterpreted coal in the feed as low density medium. In response, the plant control system added less water and

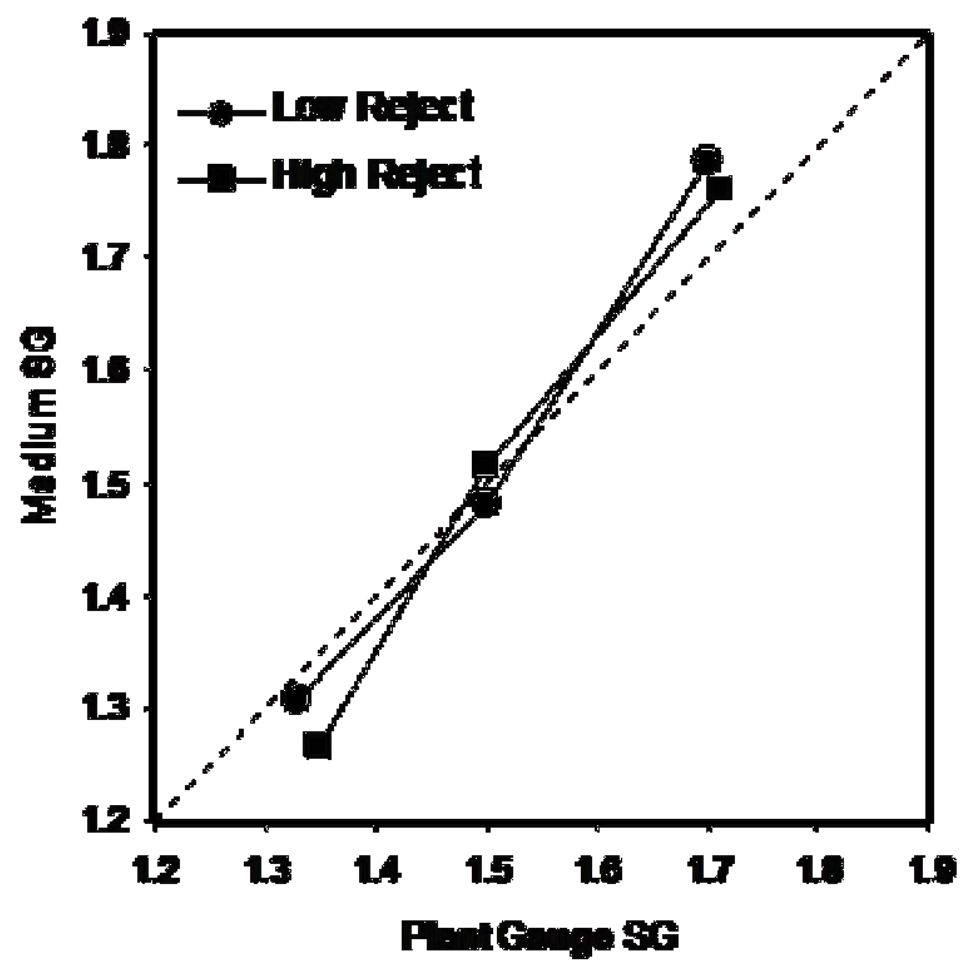

Figure 11. Comparison of plant nuclear gauge SG and experimental cutpoint $\mathbf{S G}_{\mathbf{5 0}}$. 
raised the true density of the circulating medium. The increase was greater for the low reject feed since this feedstock contained the most low-density coal.

Figure 12 shows the relationship between plant gauge reading ("F") and the experimentally measured cutpoint density of relatively coarse $(12.7$ x $6.35 \mathrm{~mm})$ particles. For the case of the low-reject feedstock, the cutpoint densities were surprisingly well matched by the plant gauge (albeit slightly high in all cases). In contrast, the cutpoint SGs were not well matched between the plant gauge and the true circulating medium when operating with a high-reject feed. The discrepancy was particularly large in the lowdensity region where the nuclear gauge suggested a cutpoint of 1.350 SG while particles were separated at a very low density of 1.263 SG. Differences such as these make it

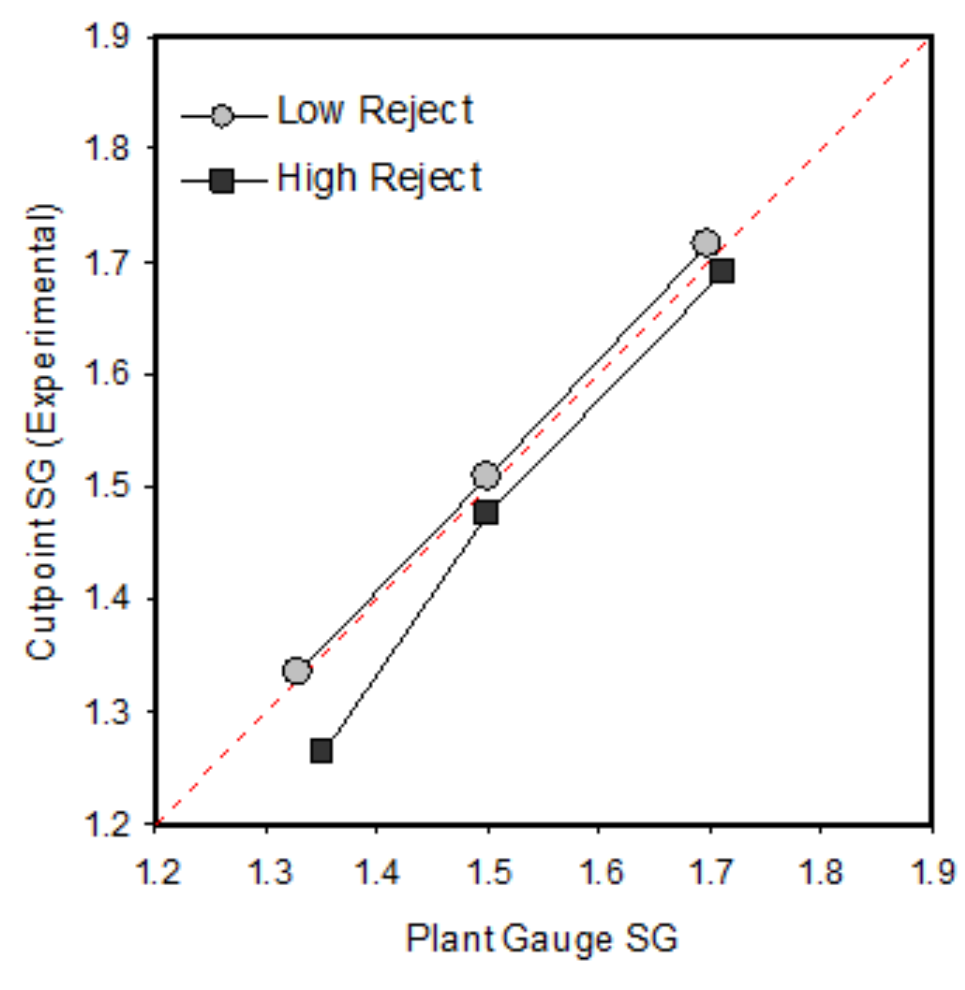

Figure 12. Comparison of plant nuclear gauge SG and experimental cutpoint $\mathrm{SG}_{50}$. 
difficult to optimize DMC circuit performance in cases where the plant feed coal characteristics routinely change throughout the production period. This problem can be particularly severe when operating in the low density range (Chedgy et al., 1986).

\subsection{Modified Control Strategy}

There are numerous expressions available in the technical literature that can be used to model DMC performance (Napier-Munn, 1984; Rao et al., 1986; Davis, 1987; Scott, 1988; Clarkson and Wood, 1991; Barbee et al., 2005). One such model reported by Wood (1990) indicates that the SG cutpoint for a DMC can be estimated using an empirical linear equation of the form:

$$
\mathrm{SG}_{50 \mathrm{c}}=\mathrm{a}_{0}+\mathrm{a}_{1}\left(\mathrm{SG}_{\mathrm{um}}\right)+\mathrm{a}_{2}\left(\mathrm{SG}_{\mathrm{om}}\right)+\mathrm{a}_{3}\left(\mathrm{SG}_{\mathrm{fm}}\right)
$$

where $\mathrm{SG}_{\mathrm{um}}, \mathrm{SG}_{\mathrm{om}}$ and $\mathrm{SG}_{\mathrm{fm}}$ are the specific gravities of the underflow, overflow and feed medium, respectively, and $\mathrm{a}_{0}, \mathrm{a}_{1}, \mathrm{a}_{2}$ and $\mathrm{a}_{3}$ are fitting coefficients. $\mathrm{SG}_{50 \text { c }}$ represents the effective SG cutpoint of relatively large $(>4 \mathrm{~mm})$ particles that are efficiently separated. Once known, the density cutpoint $\left(\mathrm{SG}_{50 \mathrm{p}}\right)$ for other particle size classes can be estimated from:

$$
\mathrm{SG}_{50 \mathrm{p}}=\mathrm{SG}_{50 \mathrm{c}}+0.0674\left(1 / \mathrm{D}_{\mathrm{p}}-0.10\right)
$$

where $D_{p}$ is the particle diameter (mm) of the size class of interest (Wood et al., 1987). These equations indicate that it is possible to predict and properly optimize the SG cutpoints for a DMC provided that the values of $\mathrm{SG}_{\mathrm{fm}}, \mathrm{SG}_{\mathrm{um}}$ and $\mathrm{SG}_{\mathrm{om}}$ are known.

The results of the in-plant DMC tests demonstrate the importance of designing plants with layouts that allow for the proper monitoring of circulating medium. Ideally, 
dense medium circuits should be configured with sufficient headroom to allow return medium to be recombined, homogenized and monitored prior to the addition of feed coal. However, this preferred option is not available in many existing coal preparation facilities operating in the U.S. Therefore, another option is needed for this type of existing situation. One promising alternative is to utilize information from only the overflow and underflow medium streams for controlling the DMC cutpoint. While not "ideal", this approach is believed to offer improved monitoring and control in cases where feedstock quality and cutpoint values change frequently and dramatically. This scheme assumes that the cutpoint density $\left(\mathrm{SG}_{50 c}\right.$ ) can be estimated using a simplified form of Eq. [6], i.e.:

$$
\mathrm{SG}_{50 \mathrm{c}}=\mathrm{a}_{0}+\mathrm{a}_{1}\left(\mathrm{SG}_{\mathrm{um}}\right)+\mathrm{a}_{2}\left(\mathrm{SG}_{\mathrm{om}}\right)
$$

where $\mathrm{SG}_{\mathrm{um}}$ and $\mathrm{SG}_{\mathrm{om}}$ are the specific gravities of the underflow and overflow medium, respectively, and $\mathrm{a}_{0}, \mathrm{a}_{1}$ and $\mathrm{a}_{2}$ are fitting coefficients. In this case, the constant $\mathrm{a}_{3}$ shown previously in Eq. [6] is assumed to be zero. For the data collected in the present work, the fitting coefficients were found to be $\mathrm{a}_{0}=0.640, \mathrm{a}_{1}=0.518$ and $\mathrm{a}_{2}=-0.290$.

Figure 13 shows the correlation between the cutpoint values $\left(\mathrm{SG}_{50 c}\right)$ predicted from Eq. [8] and experimentally measured cutpoint $\left(\mathrm{SG}_{50}\right)$ values from float-sink analysis of the $12.7 \times 6.35 \mathrm{~mm}$ size class. As shown, this simple mathematical model provides a very good estimate of the particle cutpoint density for this particular operation. The model can be used by the plant control system to adjust the medium SG up or down to maintain a constant SG cutpoint so that DMC performance can be optimized. By avoiding the use of feed medium density in the control algorithm, problems associated with changing feedstock quality can be somewhat mitigated by this approach. In practice, 


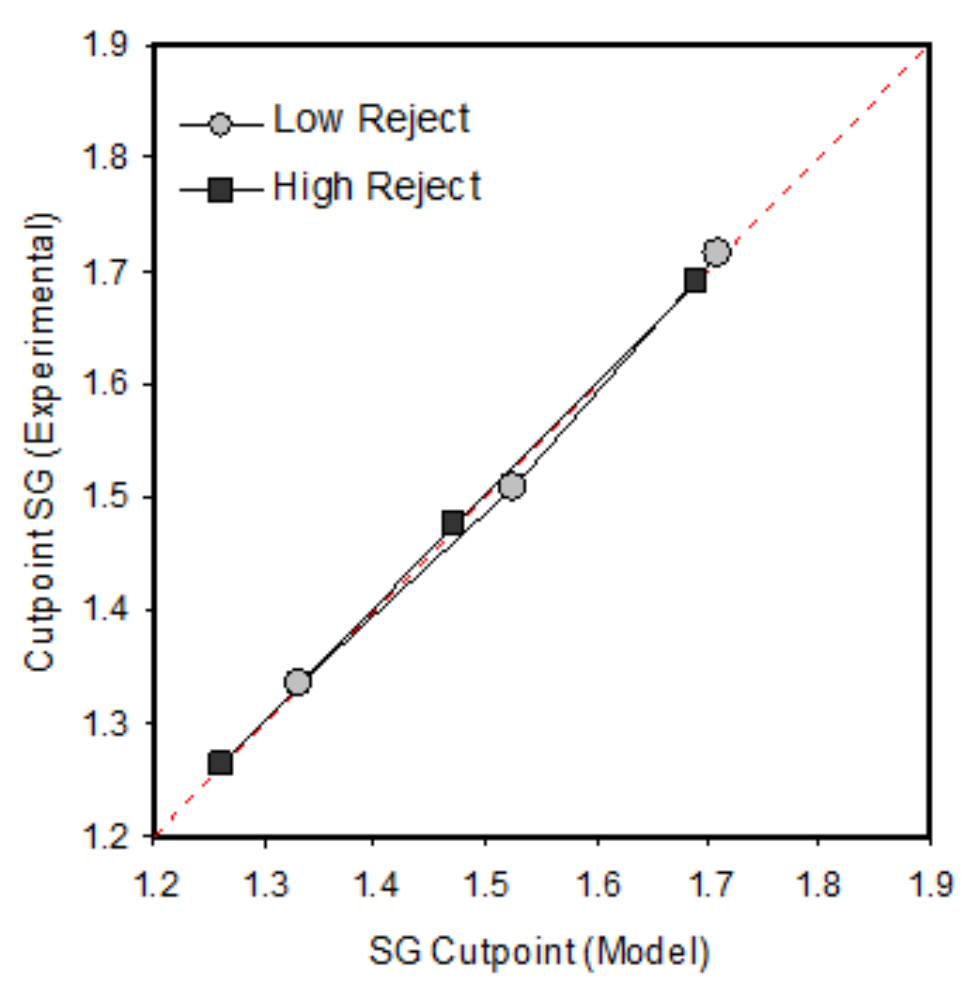

Figure 13. Comparison of estimated (model) and experimentally measured $\mathbf{S G}_{50}$.

all three streams, i.e., feed, overflow and underflow, should still be monitored on-line to ensure that the predicted cutpoint from the model makes logical sense when compared to the plant gauge density. In this manner, the plant density gauge would provide a secondary indication as to whether the plant feedstock was high in reject or low in reject. For example, a plant gauge reading lower than the model SG cutpoint set by the control algorithm would serve as an indication that the plant feedstock is high in reject, which could then be confirmed by the plant operator. 


\subsection{CONCLUSIONS}

Test data collected in the current study indicate that optimization of dense medium cyclone (DMC) performance cannot be realistically achieved for cases in which only the feed medium density is monitored in the presence of coal. This problem appears to be created by incorrect density readings which interpret the presence of large amounts of high-density rock as overdense medium. To avoid errors in density readings, it is recommended that plant circuits be designed with a means to monitor the true density of circulating medium in the absence of feed coal. Ideally, representative streams of return overflow and underflow medium from the drain-and-rinse screens should be recombined, homogenized and then passed through the density gauge. This layout requires that the plant be designed with sufficient headroom for a monitoring station between the drainand-rinse screens and the DMC feed sump. In existing facilities where combined return medium cannot be realistically obtained, a control system that makes use of only the medium SGs of the overflow and underflow SG values is suggested as a possible approach for dealing with feedstocks that are highly variable. This multi-stream monitoring system makes use of a simple mathematical model to estimate the DMC cutpoint density using only the returned overflow and underflow medium streams. In this approach, the feed medium density would still be monitored, but only as a secondary check on whether the circuit is behaving logically in response to perceived changes in feed quality. 


\subsection{REFERENCES}

1. Abbott, J., 1982. The Optimisation of Process Parameters to Maximise the Profitability from a Three-Component Blend, 1st Australian Coal Preparation Conference, April 6-10, Newcastle, Australia, 87-105.

2. Abbot and Miles......

3. Barbee, C.J., Luttrell, G.H., Wood, C.J., and Bethell, P.J., 2005. "Simulation of Heavy Medium Cyclone Performance," Minerals \& Metallurgical Processing, Vol. 22, No. 1, pp. 38-42.

4. Burgess, C.L., McGuire, W.J., Perez, V.M., Thompson, R.S., 1987. "System for Controlling Separation Gravity in a Dense-Media Cyclone Circuit”, 4th International Coal Preparation Conference, Lexington, Kentucky, pp. 181-193.

5. Chedgy, D.G., Watters, L.A., and Higgins, S.T., 1986. Heavy Medium Cyclone Separations at Ultra-Low Specific Gravity, 10th International Coal Preparation Congress, Edmonton, 60-79.

6. Clarkson, C.J. \& Wood, C.J., 1991. A Model of Dense Medium Cyclone Performance, Proceedings of the 5th Australian Coal Preparation Conference, Australia, 65-79.

7. Davis, J.J., 1987. A Study of Coal Washing Dense Medium Cyclones, PhD Thesis, University of Queensland, Australia.

8. Gottfried, B.S. \& Jacobsen, P.S., 1977. A Generalized Distribution Curve for Characterizing the Performance of Coal Cleaning Equipment, USBM. Report 8238.

9. King, R.P. \& Juckes, A.H., 1988. Performance of Dense Medium Cyclone when Beneficiating Fine Coal, Coal Preparation: An International Journal, 5: 188-210. 
10. Luttrell, G.H., Catarious, D.M., Miller, J.D., \& Stanley, F.L., 2000. An Evaluation of Plantwide Control Strategies for Coal Preparation Plants, Control 2000, J.A. Herbst (Ed.), SME, Littleton, CO, 175-184.

11. Luttrell, G.H., Barbee, C.J., Wood, C.J., and Bethell, P.J., 2003. "Operating Guidelines for Heavy-Media Cyclone Circuits," Coal Age, Vol. 108, No. 4, April 2003, pp. 30-34.

12. Napier-Munn, T.J., 1984. The Mechanism of Separation in Dense Medium Cyclones, Ph.D. Thesis, University of Lon-don, England.

13. Rao, T.C., Vanagamudi, M., \& Sufiyan, S.A., 1986. Modelling of Dense Medium Cyclones Treating Coal, International Journal of Mineral Processing, 17: 287-301.

14. Restarick, C.J. \& Krnic, Z., 1990. Effect of Underflow/Overflow Ratio on Dense Medium Cyclone Operation, 4th Samancor Symposium on Dense Medium Separations, Cairns.

15. Scott, I., 1988. A Dense Medium Cyclone Model Based on the Pivot Phenomenon, Ph.D. Thesis, University of Queensland, Australia.

16. Wood, C.J., Davis, J.J. \& Lyman, G.J., 1987. Towards a Medium Behavior Based Performance Model for Coal Wash-ing Dense Medium Cyclones, Australian IMM, Brisbane, 247-256.

17. Wood, C.J. 1990. A Performance Model for Coal-Washing Dense Medium Cyclones. PhD Thesis (unpublished), University of Queensland, Australia.

18. Wood, C.J., 1997. Coal Preparation Expertise in Australia: In-Plant Issues and the Potential Impact of Broader Applications, Proceedings of Coal Prep '97, Lexington, Kentucky, 179-198. 
6.0 APPENDIX 
APPENDIX 6.1 - INTERMEDIATE SG \& LOW REJECT FEED 
Circuit: TOMS CREEK - SECONDARY DMC CIRCUIT TEST 1

\begin{tabular}{|c|c|c|c|}
\hline Clean Rate (t/hr): & 410.4 & Clean Yield (\%): & 82.08 \\
\hline Refuse Rate (t/hr): & 89.6 & Refuse Yield (\%): & 17.92 \\
\hline Feed Rate (t/hr): & 500.0 & Feed Ash (\%): & 12.09 \\
\hline
\end{tabular}

\begin{tabular}{|c|c|c|c|c|c|c|c|c|}
\hline Pass & Retain & Mean & Clean & Clean & Refuse & Refuse & Feed & Feed \\
\hline Size & Size & Size & Mass & Ash & Mass & Ash & Mass & Ash \\
\hline$(\mathrm{mm})$ & $(\mathrm{mm})$ & $(\mathrm{mm})$ & $(\%)$ & $(\%)$ & $(\%)$ & $(\%)$ & $(\%)$ & $(\%)$ \\
\hline 12.7 & 6.35 & 8.98 & 14.50 & 6.54 & 35.94 & 40.63 & 18.34 & 18.51 \\
\hline 6.35 & 3.175 & 4.49 & 28.51 & 5.85 & 19.24 & 39.11 & 26.85 & 10.12 \\
\hline 3.175 & 1.41 & 2.12 & 44.28 & 5.28 & 32.24 & 39.23 & 42.12 & 9.94 \\
\hline 1.41 & 0.707 & 1.00 & 11.10 & 5.41 & 11.53 & 52.44 & 11.18 & 14.11 \\
\hline \multirow[t]{2}{*}{0.707} & 0 & 0.00 & 1.61 & 8.66 & 1.05 & 52.40 & 1.51 & 14.11 \\
\hline & & Totals & 100.00 & 5.69 & 100.00 & 41.37 & 100.00 & 12.09 \\
\hline Pass & Retain & Mean & Clean & Refuse & Feed & Clean & Refuse & Feed \\
\hline Size & Size & Size & Yield & Yield & Yield & Mass & Mass & Mass \\
\hline$(\mathrm{mm})$ & $(\mathrm{mm})$ & $(\mathrm{mm})$ & $(\%)$ & $(\%)$ & $(\%)$ & (Cum\%) & (Cum\%) & (Cum\%) \\
\hline 12.7 & 6.35 & 8.98 & 64.88 & 35.12 & 100.00 & 14.50 & 35.94 & 18.34 \\
\hline 6.35 & 3.175 & 4.49 & 87.16 & 12.84 & 100.00 & 43.01 & 55.18 & 45.19 \\
\hline 3.175 & 1.41 & 2.12 & 86.28 & 13.72 & 100.00 & 87.29 & 87.42 & 87.31 \\
\hline 1.41 & 0.707 & 1.00 & 81.51 & 18.49 & 100.00 & 98.39 & 98.95 & 98.49 \\
\hline \multirow[t]{2}{*}{0.707} & 0 & 0.00 & 87.53 & 12.47 & 100.00 & 100.00 & 100.00 & 100.00 \\
\hline & & Totals & 82.08 & 17.92 & 100.00 & & & \\
\hline
\end{tabular}

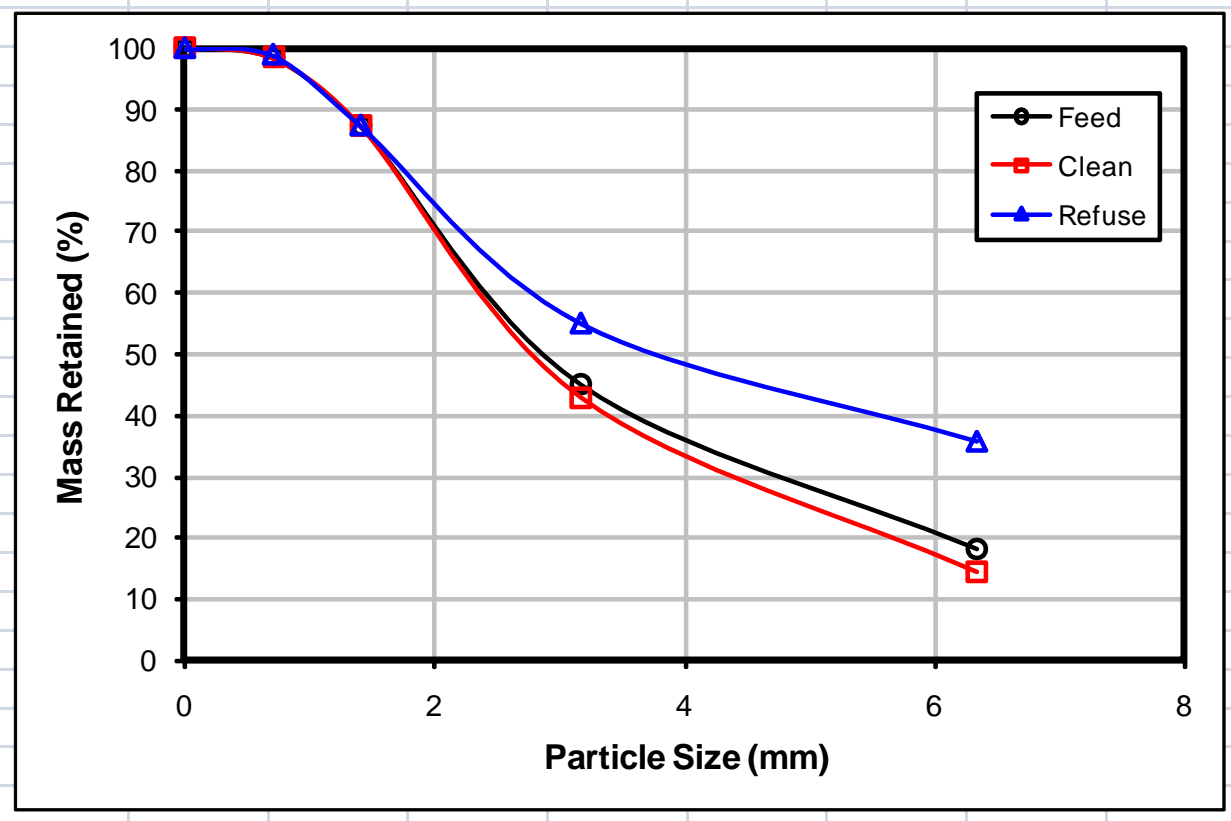


Circuit: TOMS CREEK - SECONDARY DMC CIRCUIT TEST 1

Size: $12.7 \times 6.35 \mathrm{~mm}$

Clean Yield (\%)

Refuse Yield (\%)

\begin{tabular}{|l|}
\hline 64.88 \\
\hline 35.12 \\
\hline
\end{tabular}

SG Cutpoint (SG50)

Probable Error (Ep):
Weighting $(\mathrm{Y} / \mathrm{N})$ ? Low SG Offset:

\begin{tabular}{|c|}
\hline $\mathrm{N}$ \\
\hline 0.00 \\
\hline
\end{tabular}

\begin{tabular}{|c|c|c|c|c|c|c|c|c|c|}
\hline & & & Clean & Refuse & Feed & Measured & Fitted & Fitting & Weighted \\
\hline Sink & Float & Mean & Mass & Mass & Mass & Refuse & Refuse & Weight & Squared \\
\hline$S G$ & $S G$ & $S G$ & (\% Strm) & (\% Strm) & (\% Strm) & Partition & Partition & Factor & Error \\
\hline 1.260 & 1.300 & 1.280 & 51.63 & 0.04 & 33.51 & 0.00 & 0.00 & 0.10 & 0.00 \\
\hline 1.300 & 1.400 & 1.350 & 42.94 & 0.18 & 27.92 & 0.00 & 0.00 & 0.10 & 0.00 \\
\hline 1.400 & 1.450 & 1.425 & 3.10 & 0.22 & 2.09 & 0.04 & 0.01 & 0.10 & 0.00 \\
\hline 1.450 & 1.500 & 1.475 & 1.34 & 0.53 & 1.06 & 0.18 & 0.15 & 0.18 & 0.00 \\
\hline 1.500 & 1.525 & 1.513 & 0.54 & 1.03 & 0.71 & 0.51 & 0.53 & 0.49 & 0.00 \\
\hline 1.525 & 1.550 & 1.538 & 0.43 & 2.99 & 1.33 & 0.79 & 0.80 & 0.21 & 0.00 \\
\hline 1.550 & 1.575 & 1.563 & 0.00 & 5.55 & 1.95 & 1.00 & 0.94 & 0.10 & 0.00 \\
\hline 1.575 & 1.600 & 1.588 & 0.00 & 8.21 & 2.88 & 1.00 & 0.98 & 0.10 & 0.00 \\
\hline 1.600 & 1.650 & 1.625 & 0.00 & 19.38 & 6.81 & 1.00 & 1.00 & 0.10 & 0.00 \\
\hline 1.650 & 1.750 & 1.700 & 0.00 & 43.19 & 15.17 & 1.00 & 1.00 & 0.10 & 0.00 \\
\hline 1.750 & 2.000 & 1.875 & 0.00 & 17.38 & 6.10 & 1.00 & 1.00 & 0.10 & 0.00 \\
\hline 2.000 & 2.400 & 2.200 & 0.02 & 1.30 & 0.47 & 0.97 & 1.00 & 0.10 & 0.00 \\
\hline & & Totals & 100.00 & 100.00 & 100.00 & & & WSSQ: & 0.01 \\
\hline
\end{tabular}
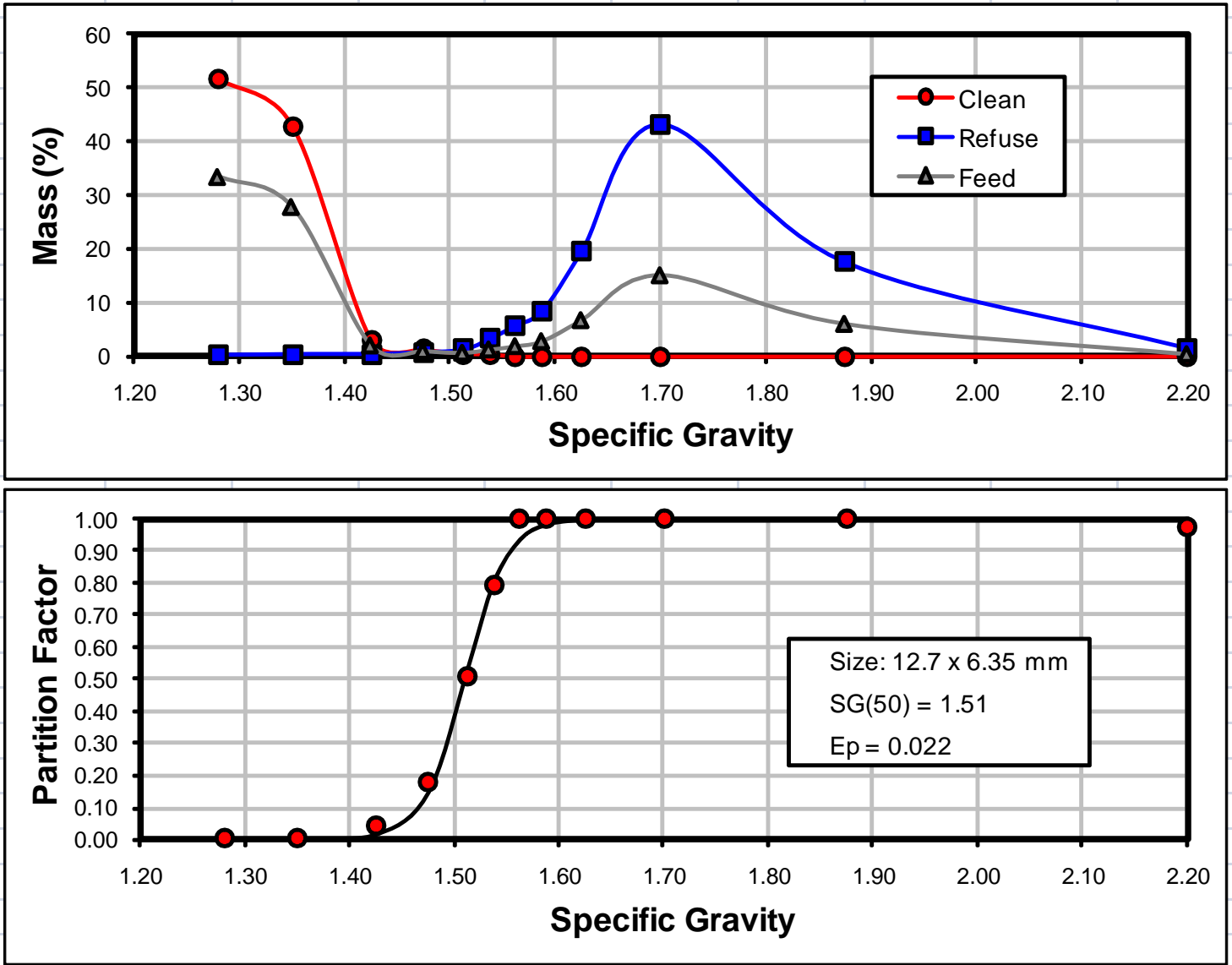
Circuit: TOMS CREEK - SECONDARY DMC CIRCUIT TEST 1

Size: $\quad 6.35 \times 3.175 \mathrm{~mm}$

Clean Yield (\%)

Refuse Yield (\%)

\begin{tabular}{|l|}
\hline 87.16 \\
\hline 12.84 \\
\hline
\end{tabular}

\begin{tabular}{|c|c|c|c|c|c|c|c|c|c|}
\hline & & & Clean & Refuse & Feed & Measured & Fitted & Fitting & Weighted \\
\hline Sink & Float & Mean & Mass & Mass & Mass & Refuse & Refuse & Weight & Squared \\
\hline$S G$ & SG & SG & (\% Strm) & (\% Strm) & (\% Strm) & Partition & Partition & Factor & Error \\
\hline 1.260 & 1.300 & 1.280 & 62.71 & 0.67 & 54.74 & 0.00 & 0.00 & 0.10 & 0.00 \\
\hline 1.300 & 1.400 & 1.350 & 33.09 & 1.70 & 29.06 & 0.01 & 0.00 & 0.10 & 0.00 \\
\hline 1.400 & 1.450 & 1.425 & 2.17 & 1.11 & 2.03 & 0.07 & 0.02 & 0.10 & 0.00 \\
\hline 1.450 & 1.500 & 1.475 & 1.27 & 2.15 & 1.38 & 0.20 & 0.16 & 0.20 & 0.00 \\
\hline 1.500 & 1.525 & 1.513 & 0.41 & 2.57 & 0.69 & 0.48 & 0.52 & 0.48 & 0.00 \\
\hline 1.525 & 1.550 & 1.538 & 0.27 & 6.00 & 1.01 & 0.77 & 0.78 & 0.23 & 0.00 \\
\hline 1.550 & 1.575 & 1.563 & 0.00 & 8.64 & 1.11 & 1.00 & 0.92 & 0.10 & 0.01 \\
\hline 1.575 & 1.600 & 1.588 & 0.00 & 12.16 & 1.56 & 1.00 & 0.97 & 0.10 & 0.00 \\
\hline 1.600 & 1.650 & 1.625 & 0.00 & 17.51 & 2.25 & 1.00 & 1.00 & 0.10 & 0.00 \\
\hline 1.650 & 1.750 & 1.700 & 0.00 & 29.04 & 3.73 & 1.00 & 1.00 & 0.10 & 0.00 \\
\hline 1.750 & 2.000 & 1.875 & 0.01 & 14.40 & 1.86 & 1.00 & 1.00 & 0.10 & 0.00 \\
\hline 2.000 & 2.400 & 2.200 & 0.07 & 4.05 & 0.58 & 0.90 & 1.00 & 0.10 & 0.01 \\
\hline & & Totals & 100.00 & 100.00 & 100.00 & & & WSSQ: & 0.02 \\
\hline
\end{tabular}
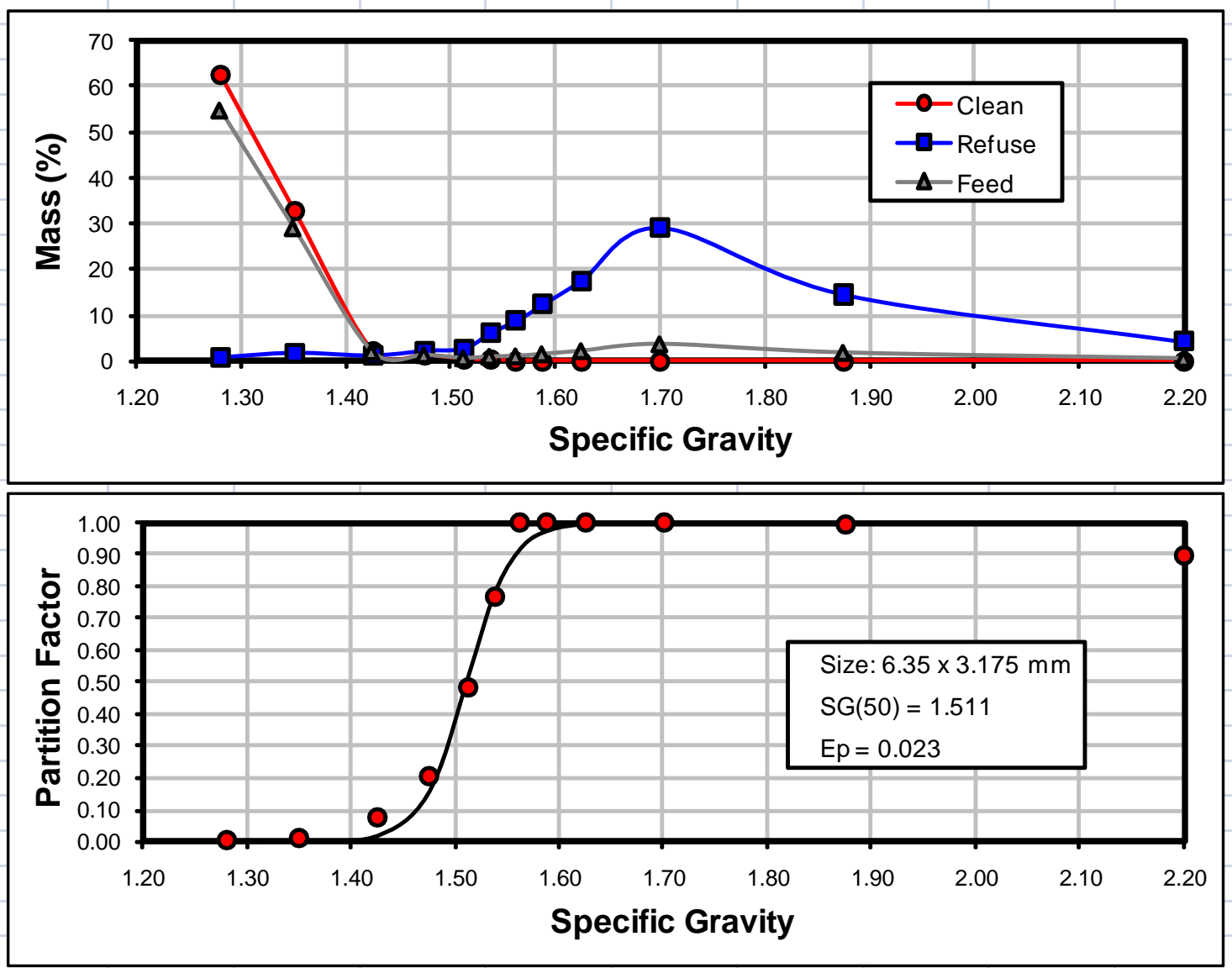
Circuit: TOMS CREEK - SECONDARY DMC CIRCUIT TEST 1

Size: $\quad 3.175 \times 1.41 \mathrm{~mm}$

Clean Yield (\%)

Refuse Yield (\%)

\begin{tabular}{|l|}
\hline 86.28 \\
\hline 13.72 \\
\hline
\end{tabular}

\begin{tabular}{|c|c|c|c|c|c|c|c|c|c|}
\hline & & & Clean & Refuse & Feed & Measured & Fitted & Fitting & Weighted \\
\hline Sink & Float & Mean & Mass & Mass & Mass & Refuse & Refuse & Weight & Squared \\
\hline $\mathrm{SG}$ & $S G$ & SG & (\% Strm) & (\% Strm) & (\% Strm) & Partition & Partition & Factor & Error \\
\hline 1.260 & 1.300 & 1.280 & 69.59 & 8.32 & 61.18 & 0.02 & 0.00 & 0.10 & 0.00 \\
\hline 1.300 & 1.400 & 1.350 & 25.99 & 6.65 & 23.34 & 0.04 & 0.01 & 0.10 & 0.00 \\
\hline 1.400 & 1.500 & 1.450 & 3.37 & 4.84 & 3.57 & 0.19 & 0.19 & 0.19 & 0.00 \\
\hline 1.500 & 1.600 & 1.550 & 0.70 & 18.69 & 3.17 & 0.81 & 0.81 & 0.19 & 0.00 \\
\hline 1.600 & 1.700 & 1.650 & 0.05 & 22.74 & 3.16 & 0.99 & 0.99 & 0.10 & 0.00 \\
\hline 1.700 & 1.800 & 1.750 & 0.01 & 14.55 & 2.00 & 1.00 & 1.00 & 0.10 & 0.00 \\
\hline 1.800 & 1.900 & 1.850 & 0.01 & 6.61 & 0.92 & 0.99 & 1.00 & 0.10 & 0.00 \\
\hline 1.900 & 2.000 & 1.950 & 0.01 & 3.01 & 0.42 & 0.98 & 1.00 & 0.10 & 0.00 \\
\hline 2.000 & 2.400 & 2.200 & 0.27 & 14.59 & 2.23 & 0.90 & 1.00 & 0.10 & 0.01 \\
\hline & & & & & & & & & \\
\hline & & & & & & & & & \\
\hline & & Totals & 100.00 & 100.00 & 100.00 & & & WSSQ: & 0.01 \\
\hline
\end{tabular}

\begin{tabular}{|l|l|}
\hline SG Cutpoint (SG50): & 1.500 \\
\hline Probable Error (Ep): & 0.038 \\
\hline
\end{tabular}

Low SG Offset:

\begin{tabular}{|c|}
\hline $\mathrm{N}$ \\
\hline 0.00 \\
\hline
\end{tabular}

Weighting $(\mathrm{Y} / \mathrm{N})$ ?
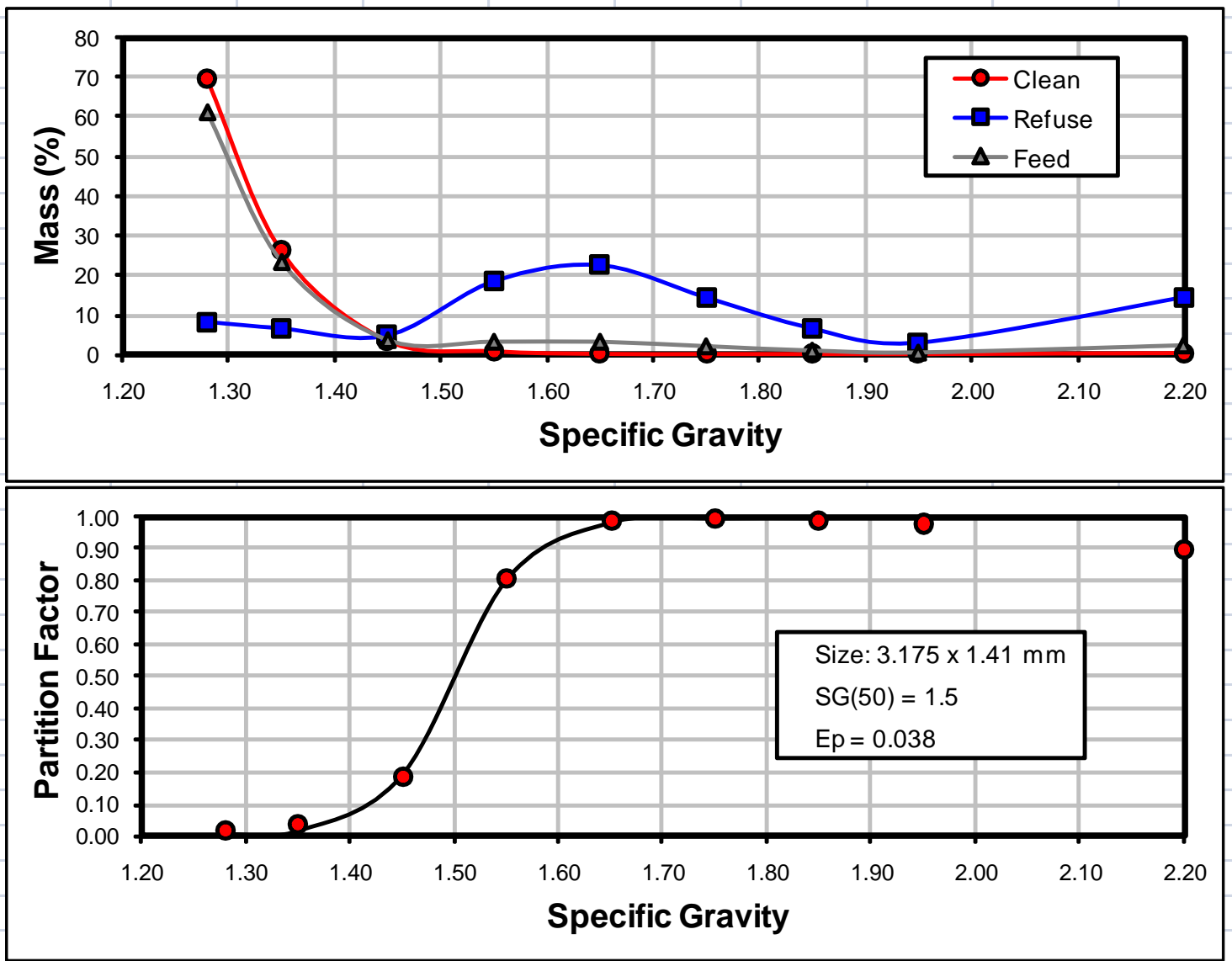
Circuit: TOMS CREEK - SECONDARY DMC CIRCUIT TEST 1

Size: $\quad 1.41 \times 0.707 \mathrm{~mm}$

Clean Yield (\%)

Refuse Yield (\%)

\begin{tabular}{|l|}
\hline 81.51 \\
\hline 18.49 \\
\hline
\end{tabular}

SG Cutpoint (SG50)

Probable Error (Ep):
Weighting $(\mathrm{Y} / \mathrm{N})$ ? Low SG Offset:

\begin{tabular}{|c|}
\hline $\mathrm{N}$ \\
\hline 0.00 \\
\hline
\end{tabular}

\begin{tabular}{|c|c|c|c|c|c|c|c|c|c|}
\hline & & & Clean & Refuse & Feed & Measured & Fitted & Fitting & Weighted \\
\hline Sink & Float & Mean & Mass & Mass & Mass & Refuse & Refuse & Weight & Squared \\
\hline$S G$ & $S G$ & $S G$ & (\% Strm) & (\% Strm) & (\% Strm) & Partition & Partition & Factor & Error \\
\hline 1.260 & 1.300 & 1.280 & 70.92 & 12.04 & 60.03 & 0.04 & 0.01 & 0.10 & 0.00 \\
\hline 1.300 & 1.400 & 1.350 & 23.62 & 7.43 & 20.63 & 0.07 & 0.04 & 0.10 & 0.00 \\
\hline 1.400 & 1.500 & 1.450 & 3.33 & 3.16 & 3.30 & 0.18 & 0.18 & 0.18 & 0.00 \\
\hline 1.500 & 1.600 & 1.550 & 1.10 & 6.10 & 2.02 & 0.56 & 0.57 & 0.44 & 0.00 \\
\hline 1.600 & 1.700 & 1.650 & 0.22 & 9.86 & 2.00 & 0.91 & 0.89 & 0.10 & 0.00 \\
\hline 1.700 & 1.800 & 1.750 & 0.06 & 8.90 & 1.69 & 0.97 & 0.98 & 0.10 & 0.00 \\
\hline 1.800 & 1.900 & 1.850 & 0.03 & 6.52 & 1.23 & 0.98 & 1.00 & 0.10 & 0.00 \\
\hline 1.900 & 2.000 & 1.950 & 0.02 & 4.27 & 0.81 & 0.98 & 1.00 & 0.10 & 0.00 \\
\hline 2.000 & 2.400 & 2.200 & 0.70 & 41.72 & 8.28 & 0.93 & 1.00 & 0.10 & 0.00 \\
\hline & & & & & & & & & \\
\hline & & Totals & 100.00 & 100.00 & 100.00 & & & WSSQ: & 0.01 \\
\hline
\end{tabular}
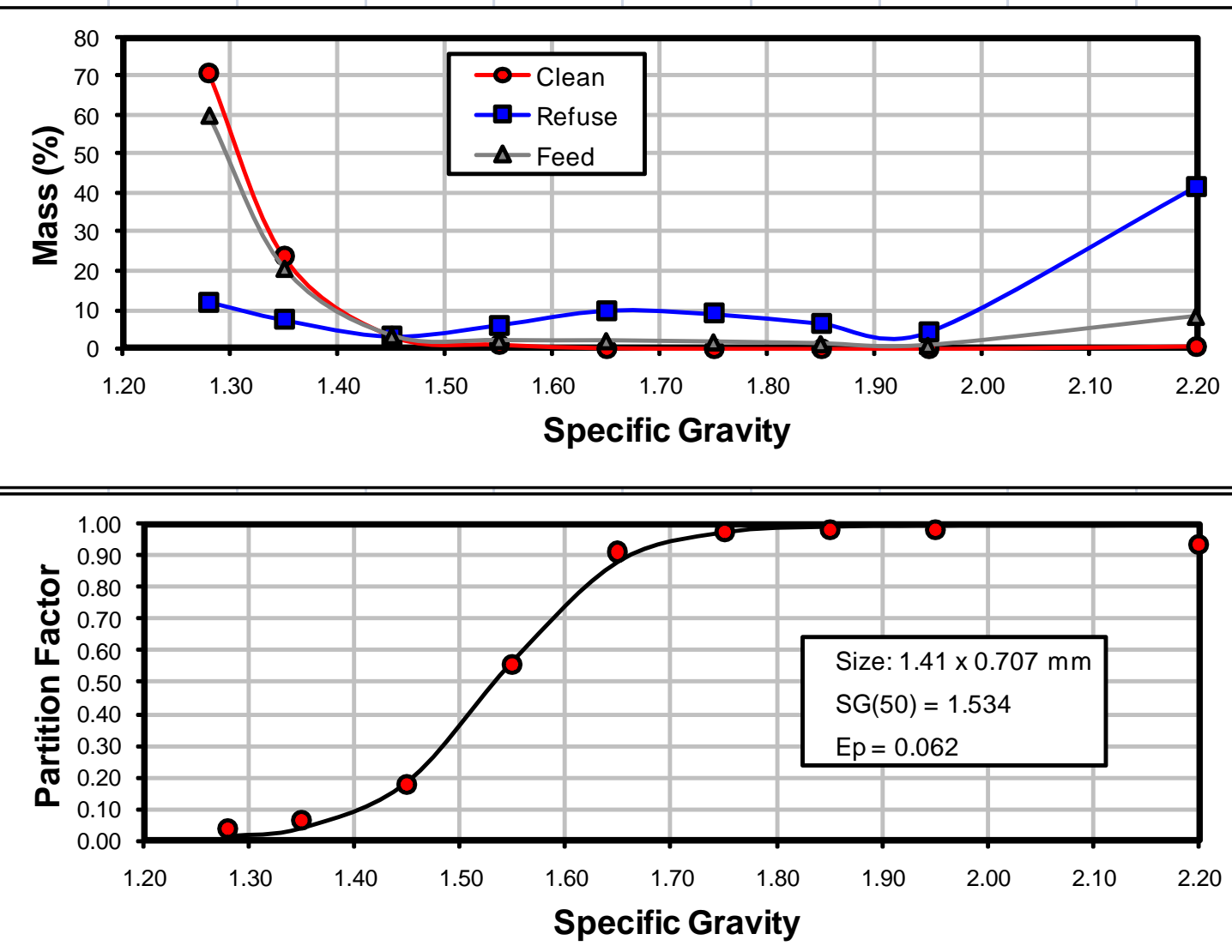
APPENDIX 6.2 - LOW SG RANGE AND LOW REJECT FEED 
Circuit: TOMS CREEK - SECONDARY DMC CIRCUIT TEST 2

\begin{tabular}{|c|c|c|c|c|c|c|c|c|}
\hline \multicolumn{2}{|c|}{ Clean Rate (t/hr): } & 295.1 & & \multicolumn{2}{|c|}{ Clean Yield (\%): } & 59.01 & & \\
\hline \multicolumn{2}{|c|}{ Refuse Rate (t/hr): } & 204.9 & & \multicolumn{2}{|c|}{ Refuse Yield (\%): } & 40.99 & & \\
\hline \multicolumn{2}{|c|}{ Feed Rate $(\mathrm{t} / \mathrm{hr})$ : } & 500.0 & & \multicolumn{2}{|c|}{ Feed Ash (\%): } & 12.49 & & \\
\hline Pass & Retain & Mean & Clean & Clean & Refuse & Refuse & Feed & Feed \\
\hline Size & Size & Size & Mass & Ash & Mass & Ash & Mass & Ash \\
\hline$(\mathrm{mm})$ & $(\mathrm{mm})$ & $(\mathrm{mm})$ & $(\%)$ & $(\%)$ & $(\%)$ & $(\%)$ & $(\%)$ & $(\%)$ \\
\hline 12.7 & 6.35 & 8.98 & 12.96 & 5.15 & 30.97 & 28.24 & 20.34 & 19.56 \\
\hline 6.35 & 3.175 & 4.49 & 40.33 & 4.76 & 27.89 & 22.18 & 35.23 & 10.41 \\
\hline 3.175 & 1.41 & 2.12 & 32.48 & 4.53 & 27.65 & 19.40 & 30.50 & 10.05 \\
\hline 1.41 & 0.707 & 1.00 & 12.57 & 4.47 & 11.81 & 24.83 & 12.26 & 12.51 \\
\hline \multirow[t]{3}{*}{0.707} & 0 & 0.00 & 1.66 & 6.26 & 1.68 & 26.40 & 1.67 & 14.57 \\
\hline & & & & & & & & \\
\hline & & Totals & 100.00 & 4.72 & 100.00 & 23.67 & 100.00 & 12.49 \\
\hline Pass & Retain & Mean & Clean & Refuse & Feed & Clean & Refue & Feed \\
\hline Size & Size & Size & Yield & Yield & Yield & Mass & Mass & Mass \\
\hline$(\mathrm{mm})$ & $(\mathrm{mm})$ & $(\mathrm{mm})$ & $(\%)$ & $(\%)$ & $(\%)$ & (Cum\%) & (Cum\%) & (Cum\%) \\
\hline 12.7 & 6.35 & 8.98 & 37.60 & 62.40 & 100.00 & 12.96 & 30.97 & 20.34 \\
\hline 6.35 & 3.175 & 4.49 & 67.56 & 32.44 & 100.00 & 53.29 & 58.86 & 55.57 \\
\hline 3.175 & 1.41 & 2.12 & 62.85 & 37.15 & 100.00 & 85.77 & 86.51 & 86.07 \\
\hline 1.41 & 0.707 & 1.00 & 60.51 & 39.49 & 100.00 & 98.34 & 98.32 & 98.33 \\
\hline 0.707 & 0 & 0.00 & 58.72 & 41.28 & 100.00 & 100.00 & 100.00 & 100.00 \\
\hline & & & & & & & & \\
\hline & & Totals & 59.01 & 40.99 & 100.00 & & & \\
\hline
\end{tabular}

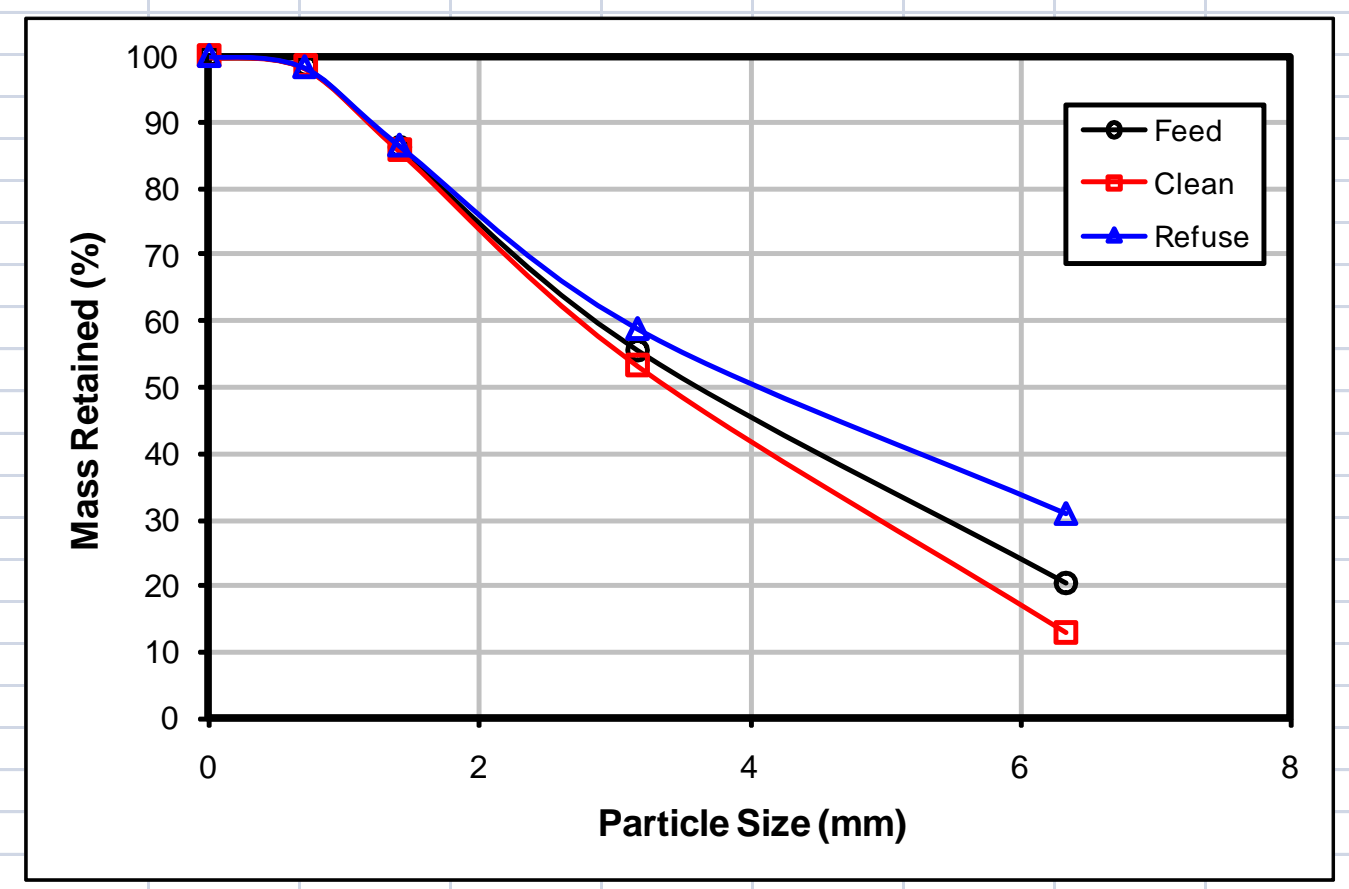


Circuit: TOMS CREEK - SECONDARY DMC CIRCUIT TEST 2

Size: $12.7 \times 6.35 \mathrm{~mm}$

Clean Yield (\%)

Refuse Yield (\%)

\begin{tabular}{|l|}
\hline 37.60 \\
\hline 62.40 \\
\hline
\end{tabular}

SG Cutpoint (SG50)

Probable Error (Ep):
Weighting $(\mathrm{Y} / \mathrm{N})$ ? Low SG Offset:

\begin{tabular}{|c|}
\hline $\mathrm{N}$ \\
\hline 0.00 \\
\hline
\end{tabular}

\begin{tabular}{|c|c|c|c|c|c|c|c|c|c|}
\hline & & & Clean & Refuse & Feed & Measured & Fitted & Fitting & Weighted \\
\hline Sink & Float & Mean & Mass & Mass & Mass & Refuse & Refuse & Weight & Squared \\
\hline$S G$ & $S G$ & $S G$ & (\% Strm) & (\% Strm) & (\% Strm) & Partition & Partition & Factor & Error \\
\hline 1.210 & 1.250 & 1.230 & 2.20 & 0.02 & 0.84 & 0.01 & 0.00 & 0.10 & 0.00 \\
\hline 1.250 & 1.300 & 1.275 & 64.50 & 2.46 & 25.79 & 0.06 & 0.01 & 0.10 & 0.00 \\
\hline 1.300 & 1.350 & 1.325 & 30.77 & 7.81 & 16.44 & 0.30 & 0.30 & 0.30 & 0.00 \\
\hline 1.350 & 1.375 & 1.363 & 2.10 & 9.97 & 7.01 & 0.89 & 0.88 & 0.11 & 0.00 \\
\hline 1.375 & 1.400 & 1.388 & 0.42 & 7.83 & 5.04 & 0.97 & 0.98 & 0.10 & 0.00 \\
\hline 1.400 & 1.425 & 1.413 & 0.00 & 5.28 & 3.29 & 1.00 & 1.00 & 0.10 & 0.00 \\
\hline 1.425 & 1.450 & 1.438 & 0.00 & 5.94 & 3.71 & 1.00 & 1.00 & 0.10 & 0.00 \\
\hline 1.450 & 1.500 & 1.475 & 0.00 & 10.51 & 6.56 & 1.00 & 1.00 & 0.10 & 0.00 \\
\hline 1.500 & 1.600 & 1.550 & 0.00 & 18.42 & 11.49 & 1.00 & 1.00 & 0.10 & 0.00 \\
\hline 1.600 & 1.800 & 1.700 & 0.00 & 25.46 & 15.89 & 1.00 & 1.00 & 0.10 & 0.00 \\
\hline 1.800 & 2.000 & 1.900 & 0.00 & 1.49 & 0.93 & 1.00 & 1.00 & 0.10 & 0.00 \\
\hline 2.000 & 2.400 & 2.200 & 0.01 & 4.81 & 3.01 & 1.00 & 1.00 & 0.10 & \\
\hline & & Totals & 100.00 & 100.00 & 100.00 & & & WSSQ: & 0.00 \\
\hline
\end{tabular}
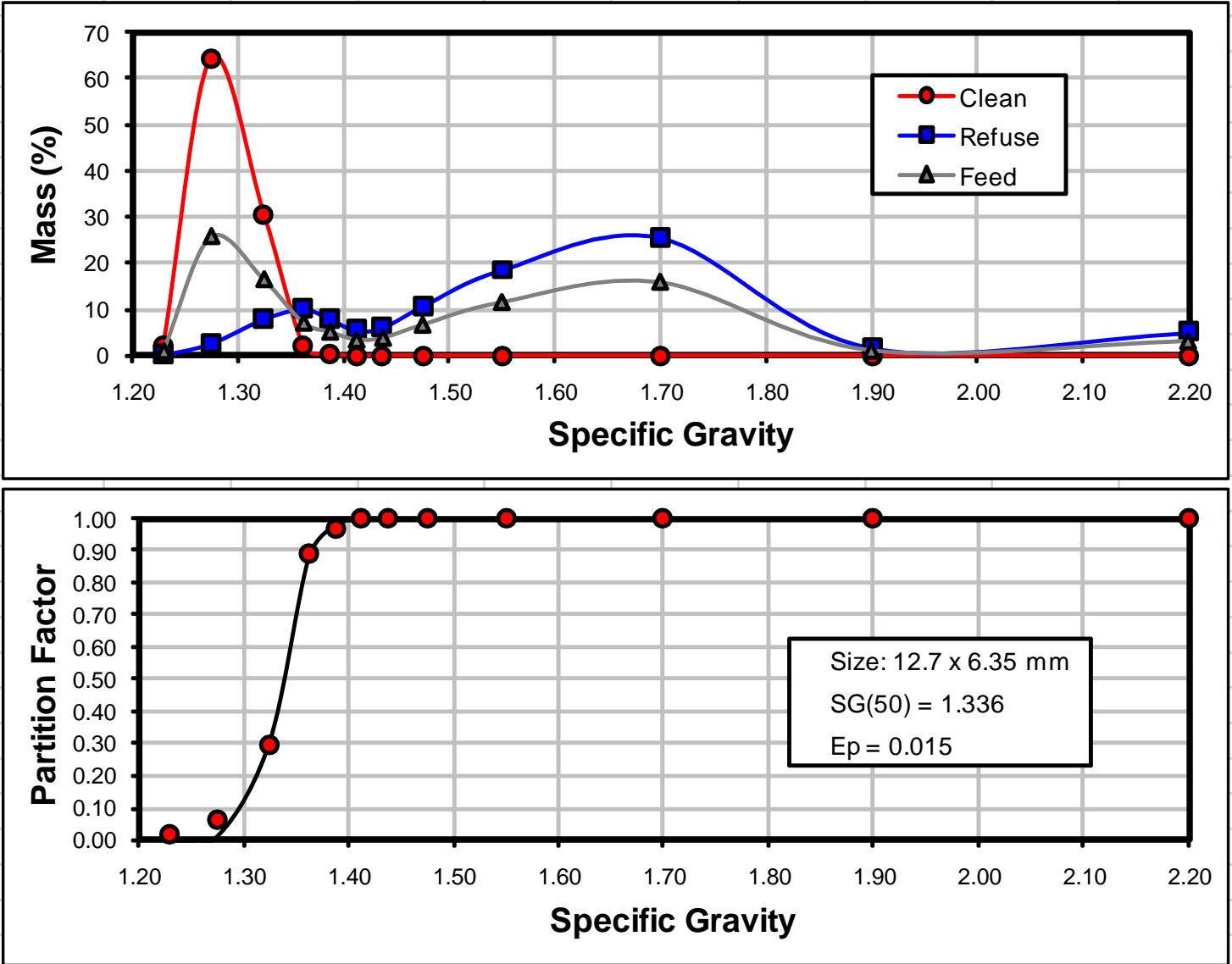
Circuit: TOMS CREEK - SECONDARY DMC CIRCUIT TEST 2 Size: $\quad 6.35 \times 3.175 \mathrm{~mm}$

Clean Yield (\%)

Refuse Yield (\%)

\begin{tabular}{|l|}
\hline 67.56 \\
\hline 32.44 \\
\hline
\end{tabular}

\begin{tabular}{|c|c|c|c|c|c|c|c|c|c|}
\hline & & & Clean & Refuse & Feed & Measured & Fitted & Fitting & Weighted \\
\hline Sink & Float & Mean & Mass & Mass & Mass & Refuse & Refuse & Weight & Squared \\
\hline SG & SG & SG & (\% Strm) & (\% Strm) & (\% Strm) & Partition & Partition & Factor & Error \\
\hline 1.210 & 1.250 & 1.230 & 3.74 & 0.56 & 2.71 & 0.07 & 0.00 & 0.10 & 0.00 \\
\hline 1.250 & 1.300 & 1.275 & 71.32 & 14.31 & 52.82 & 0.09 & 0.03 & 0.10 & 0.00 \\
\hline 1.300 & 1.350 & 1.325 & 22.18 & 16.13 & 20.22 & 0.26 & 0.29 & 0.26 & 0.00 \\
\hline 1.350 & 1.375 & 1.363 & 1.98 & 14.46 & 6.03 & 0.78 & 0.75 & 0.22 & 0.00 \\
\hline 1.375 & 1.400 & 1.388 & 0.55 & 8.52 & 3.14 & 0.88 & 0.92 & 0.12 & 0.00 \\
\hline 1.400 & 1.425 & 1.413 & 0.00 & 7.75 & 2.51 & 1.00 & 0.98 & 0.10 & 0.00 \\
\hline 1.425 & 1.450 & 1.438 & 0.00 & 4.54 & 1.47 & 1.00 & 0.99 & 0.10 & 0.00 \\
\hline 1.450 & 1.500 & 1.475 & 0.00 & 5.29 & 1.72 & 1.00 & 1.00 & 0.10 & 0.00 \\
\hline 1.500 & 1.600 & 1.550 & 0.01 & 10.74 & 3.49 & 1.00 & 1.00 & 0.10 & 0.00 \\
\hline 1.600 & 1.800 & 1.700 & 0.01 & 9.68 & 3.15 & 1.00 & 1.00 & 0.10 & 0.00 \\
\hline 1.800 & 2.000 & 1.900 & 0.00 & 1.43 & 0.46 & 1.00 & 1.00 & 0.10 & 0.00 \\
\hline 2.000 & 2.400 & 2.200 & 0.21 & 6.59 & 2.28 & 0.94 & 1.00 & 0.10 & 0.00 \\
\hline & & Totals & 100.00 & 100.00 & 100.00 & & & WSSQ: & 0.02 \\
\hline
\end{tabular}
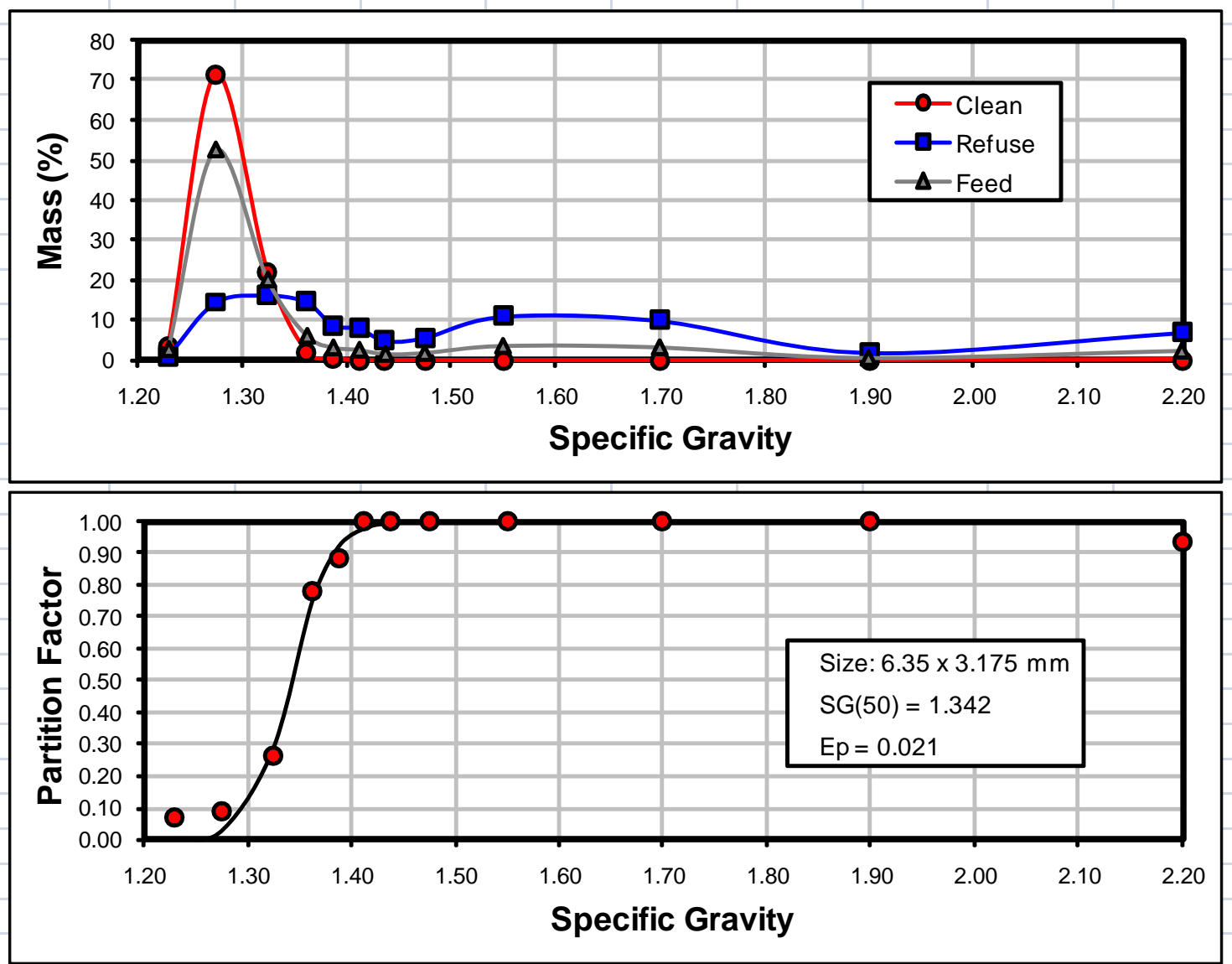
Circuit: TOMS CREEK - SECONDARY DMC CIRCUIT TEST 2

Size: $\quad 3.175 \times 1.41 \mathrm{~mm}$

Clean Yield (\%)

Refuse Yield (\%)

\begin{tabular}{|l|}
\hline 62.85 \\
\hline 37.15 \\
\hline
\end{tabular}

\begin{tabular}{|c|c|c|c|c|c|c|c|c|c|}
\hline & & & Clean & Refuse & Feed & Measured & Fitted & Fitting & Weighted \\
\hline Sink & Float & Mean & Mass & Mass & Mass & Refuse & Refuse & Weight & Squared \\
\hline SG & $S G$ & SG & (\% Strm) & (\% Strm) & (\% Strm) & Partition & Partition & Factor & Error \\
\hline 1.260 & 1.300 & 1.280 & 77.55 & 23.01 & 57.29 & 0.15 & 0.14 & 0.15 & 0.00 \\
\hline 1.300 & 1.400 & 1.350 & 21.48 & 38.38 & 27.76 & 0.51 & 0.52 & 0.49 & 0.00 \\
\hline 1.400 & 1.500 & 1.450 & 0.34 & 15.37 & 5.92 & 0.96 & 0.95 & 0.10 & 0.00 \\
\hline 1.500 & 1.600 & 1.550 & 0.02 & 7.30 & 2.72 & 1.00 & 1.00 & 0.10 & 0.00 \\
\hline 1.600 & 1.700 & 1.650 & 0.01 & 3.66 & 1.37 & 1.00 & 1.00 & 0.10 & 0.00 \\
\hline 1.700 & 1.800 & 1.750 & 0.01 & 3.05 & 1.14 & 0.99 & 1.00 & 0.10 & 0.00 \\
\hline 1.800 & 1.900 & 1.850 & 0.01 & 1.46 & 0.55 & 0.99 & 1.00 & 0.10 & 0.00 \\
\hline 1.900 & 2.000 & 1.950 & 0.01 & 0.71 & 0.27 & 0.98 & 1.00 & 0.10 & 0.00 \\
\hline 2.000 & 2.400 & 2.200 & 0.57 & 7.06 & 2.98 & 0.88 & 1.00 & 0.12 & 0.01 \\
\hline & & & & & & & & & \\
\hline & & & & & & & & & \\
\hline & & Totals & 100.00 & 100.00 & 100.00 & & & WSSQ: & 0.02 \\
\hline
\end{tabular}

SG Cutpoint (SG50): 1.347

Probable Error (Ep): 0.040 \begin{tabular}{r|c|} 
Weighting $(\mathrm{Y} / \mathrm{N}) ?$ & $\mathrm{~N}$ \\
\hline Low SG Offset: & 0.00 \\
\hline
\end{tabular}

Low SG Offset: 0.00
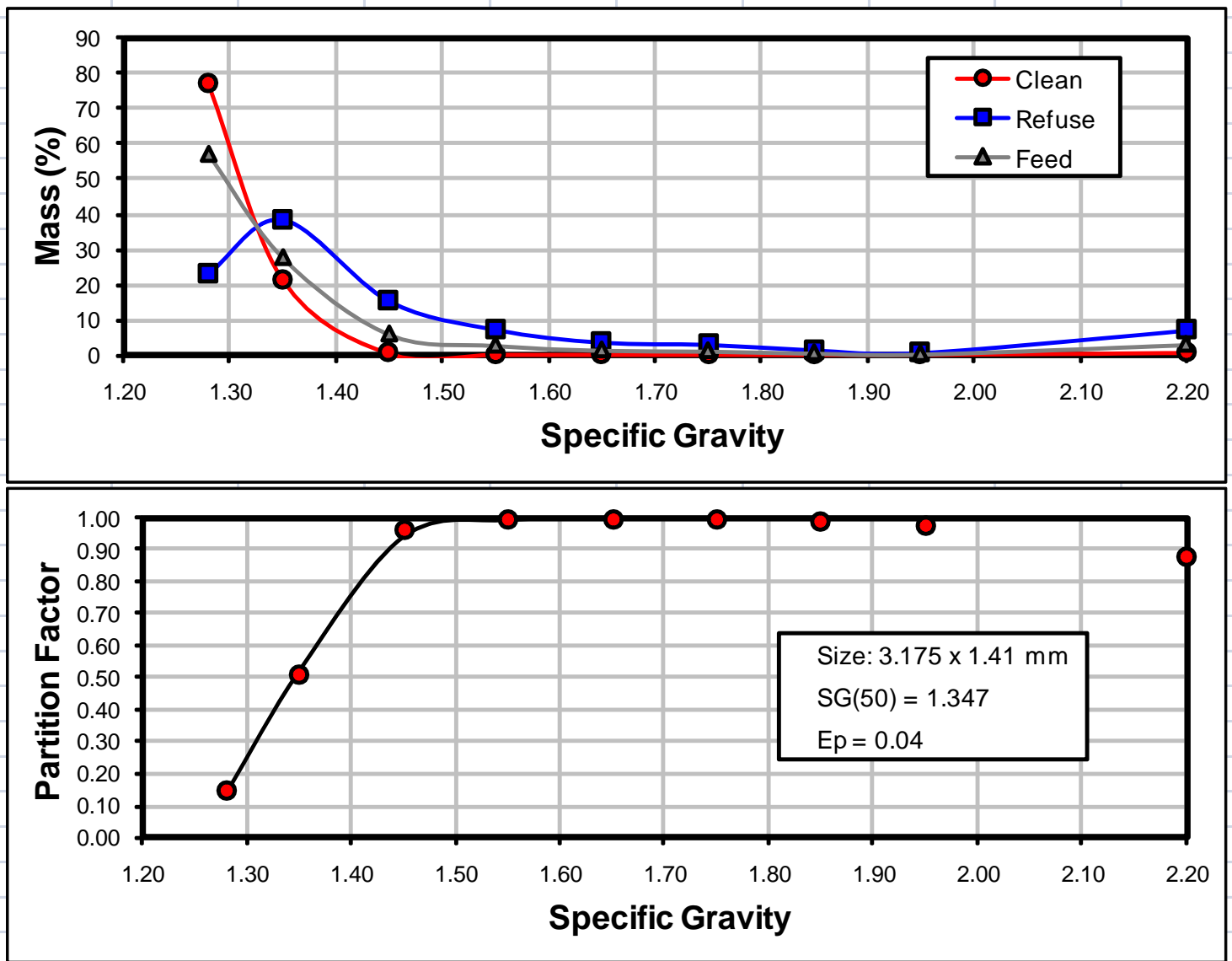
Circuit: TOMS CREEK - SECONDARY DMC CIRCUIT TEST 2

Size: $\quad 1.41 \times 0.707 \mathrm{~mm}$

Clean Yield (\%)

Refuse Yield (\%)

\begin{tabular}{|l|}
\hline 60.51 \\
\hline 39.49 \\
\hline
\end{tabular}

\begin{tabular}{|c|c|c|c|c|c|c|c|c|c|}
\hline & & & Clean & Refuse & Feed & Measured & Fitted & Fitting & Weighted \\
\hline Sink & Float & Mean & Mass & Mass & Mass & Refuse & Refuse & Weight & Squared \\
\hline SG & $\mathrm{SG}$ & SG & (\% Strm) & (\% Strm) & (\% Strm) & Partition & Partition & Factor & Error \\
\hline 1.260 & 1.300 & 1.280 & 78.15 & 30.97 & 59.52 & 0.21 & 0.18 & 0.21 & 0.00 \\
\hline 1.300 & 1.400 & 1.350 & 20.22 & 26.01 & 22.51 & 0.46 & 0.48 & 0.46 & 0.00 \\
\hline 1.400 & 1.500 & 1.450 & 0.79 & 11.73 & 5.11 & 0.91 & 0.88 & 0.10 & 0.00 \\
\hline 1.500 & 1.600 & 1.550 & 0.08 & 6.16 & 2.48 & 0.98 & 0.98 & 0.10 & 0.00 \\
\hline 1.600 & 1.700 & 1.650 & 0.03 & 4.00 & 1.60 & 0.99 & 1.00 & 0.10 & 0.00 \\
\hline 1.700 & 1.800 & 1.750 & 0.02 & 2.64 & 1.05 & 0.99 & 1.00 & 0.10 & 0.00 \\
\hline 1.800 & 1.900 & 1.850 & 0.01 & 1.90 & 0.76 & 0.99 & 1.00 & 0.10 & 0.00 \\
\hline 1.900 & 2.000 & 1.950 & 0.01 & 1.33 & 0.53 & 0.99 & 1.00 & 0.10 & 0.00 \\
\hline 2.000 & 2.400 & 2.200 & 0.69 & 15.26 & 6.44 & 0.94 & 1.00 & 0.10 & 0.00 \\
\hline & & & & & & & & & \\
\hline & & & & & & & & & \\
\hline & & Totals & 100.00 & 100.00 & 100.00 & & & WSSQ: & 0.01 \\
\hline
\end{tabular}
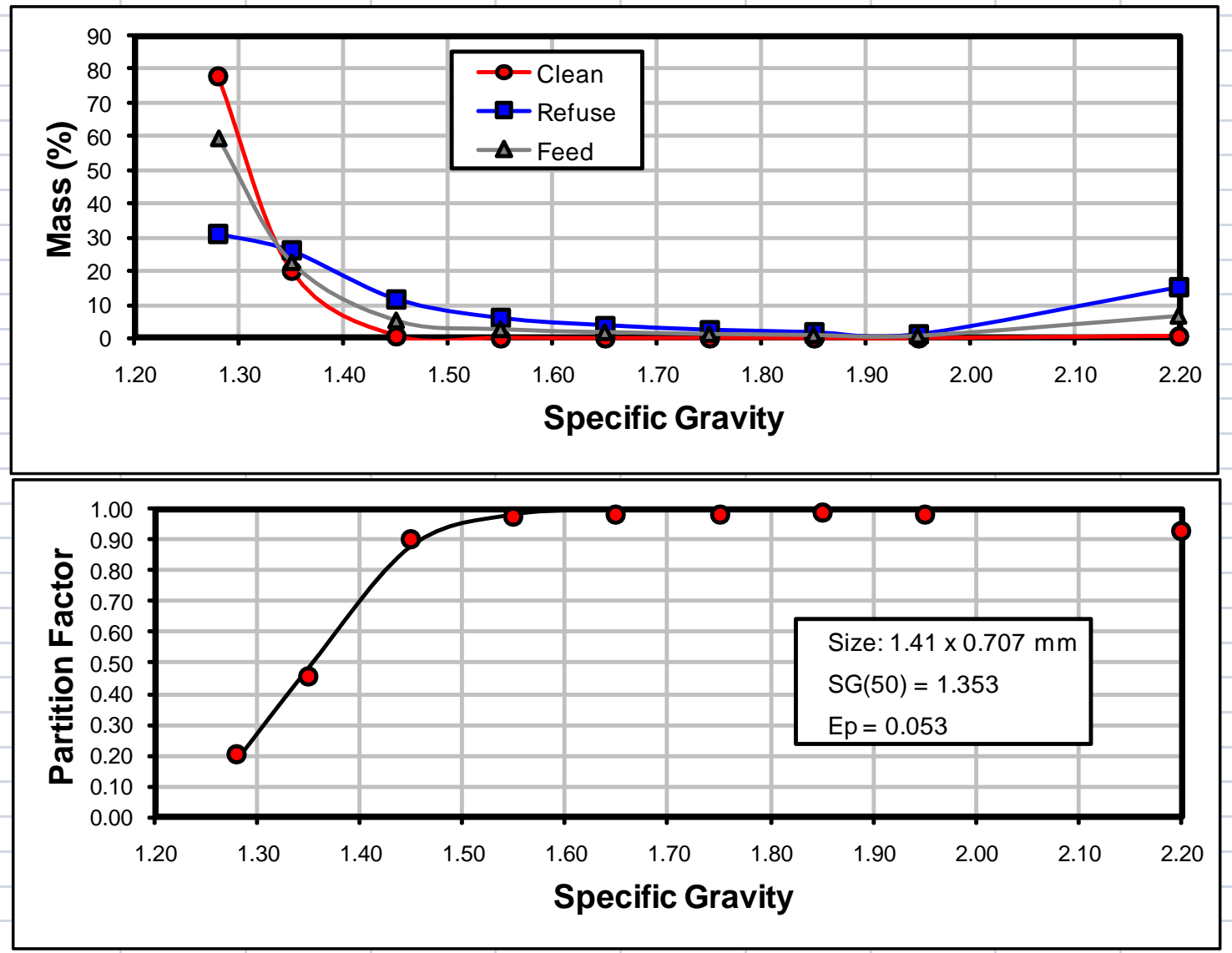
APPENDIX 6.3 - LOW SG RANGE AND HIGH REJECT FEED 
Circuit: TOMS CREEK - SECONDARY DMC CIRCUIT TEST 3

\begin{tabular}{|c|c|c|c|c|c|c|c|c|}
\hline \multirow{3}{*}{\multicolumn{2}{|c|}{$\begin{array}{l}\text { Clean Rate }(\mathrm{t} / \mathrm{hr}) \text { : } \\
\text { Refuse Rate }(\mathrm{t} / \mathrm{hr}) \text { : } \\
\text { Feed Rate }(\mathrm{t} / \mathrm{hr}) \text { : }\end{array}$}} & 96.0 & & \multicolumn{2}{|c|}{ Clean Yield (\%): } & 19.19 & & \\
\hline & & 404.0 & & \multicolumn{2}{|c|}{ Refuse Yield (\%): } & 80.81 & & \\
\hline & & 500.0 & & \multicolumn{2}{|c|}{ Feed Ash (\%): } & 38.45 & & \\
\hline Pass & Retain & Mean & Clean & Clean & Refuse & Refuse & Feed & Feed \\
\hline Size & Size & Size & Mass & Ash & Mass & Ash & Mass & Ash \\
\hline$(\mathrm{mm})$ & $(\mathrm{mm})$ & $(\mathrm{mm})$ & $(\%)$ & $(\%)$ & $(\%)$ & $(\%)$ & $(\%)$ & $(\%)$ \\
\hline 12.7 & 6.35 & 8.98 & 10.57 & 3.72 & 33.62 & 52.82 & 29.20 & 49.41 \\
\hline 6.35 & 3.175 & 4.49 & 37.51 & 3.57 & 32.79 & 43.42 & 33.70 & 34.91 \\
\hline 3.175 & 1.41 & 2.12 & 34.64 & 3.83 & 25.95 & 42.77 & 27.62 & 33.40 \\
\hline 1.41 & 0.707 & 1.00 & 15.49 & 4.14 & 6.86 & 47.42 & 8.52 & 32.31 \\
\hline \multirow[t]{2}{*}{0.707} & 0 & 0.00 & 1.79 & 5.83 & 0.78 & 42.53 & 0.97 & 29.58 \\
\hline & & Totals & 100.00 & 3.80 & 100.00 & 46.68 & 100.00 & 38.45 \\
\hline Pass & Retain & Mean & Clean & Refuse & Feed & Clean & Refuse & Feed \\
\hline Size & Size & Size & Yield & Yield & Yield & Mass & Mass & Mass \\
\hline$(\mathrm{mm})$ & $(\mathrm{mm})$ & $(\mathrm{mm})$ & $(\%)$ & $(\%)$ & $(\%)$ & (Cum\%) & (Cum\%) & (Cum\%) \\
\hline 12.7 & 6.35 & 8.98 & 6.95 & 93.05 & 100.00 & 10.57 & 33.62 & 29.20 \\
\hline 6.35 & 3.175 & 4.49 & 21.37 & 78.63 & 100.00 & 48.08 & 66.41 & 62.89 \\
\hline 3.175 & 1.41 & 2.12 & 24.07 & 75.93 & 100.00 & 82.72 & 92.36 & 90.51 \\
\hline 1.41 & 0.707 & 1.00 & 34.91 & 65.09 & 100.00 & 98.21 & 99.22 & 99.03 \\
\hline \multirow[t]{2}{*}{0.707} & 0 & 0.00 & 35.28 & 64.72 & 100.00 & 100.00 & 100.00 & 100.00 \\
\hline & & Totals & 19.19 & 80.81 & 100.00 & & & \\
\hline
\end{tabular}

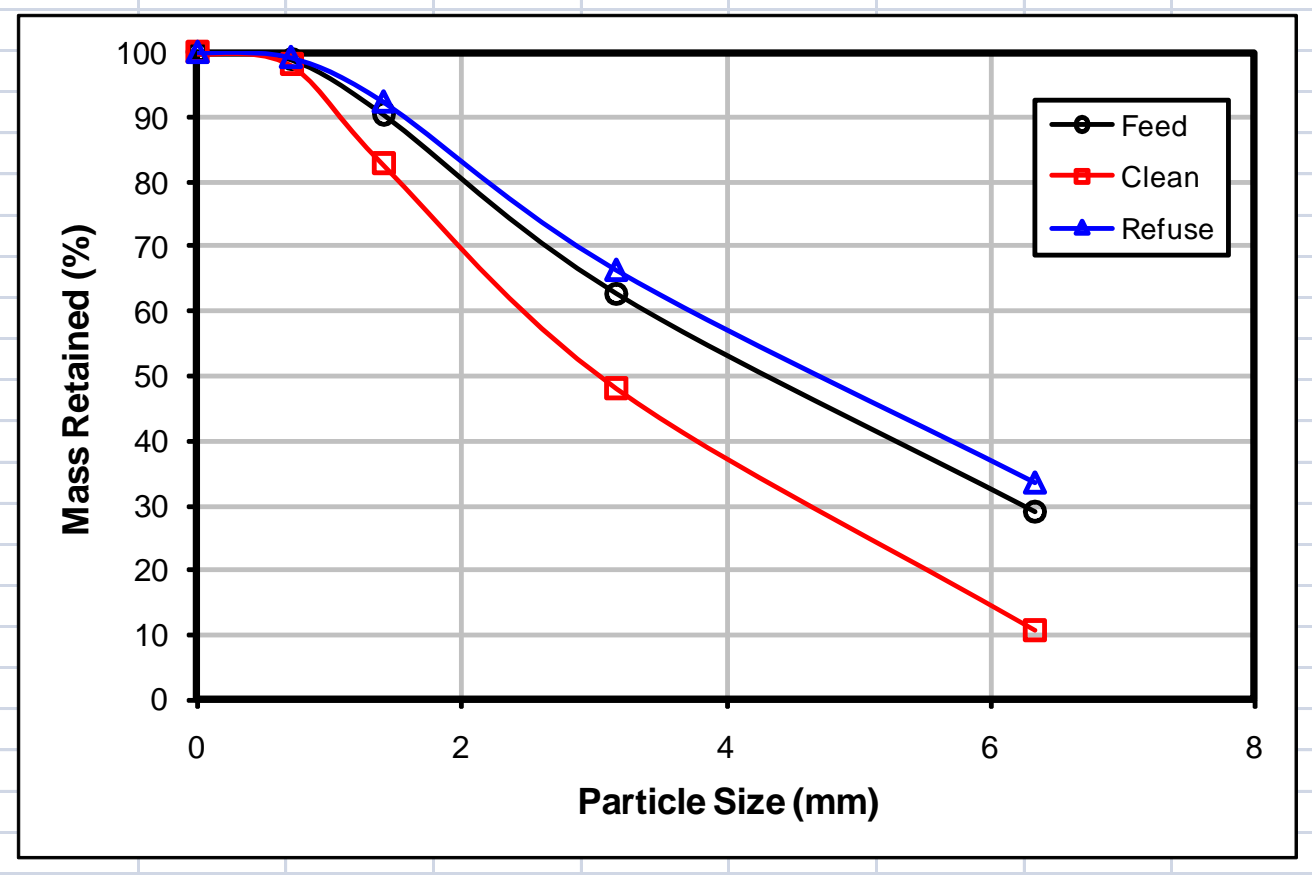


Circuit: TOMS CREEK - SECONDARY DMC CIRCUIT TEST 3

Size: $12.7 \times 6.35 \mathrm{~mm}$

Clean Yield (\%)

Refuse Yield (\%)

\begin{tabular}{|c|}
\hline 6.95 \\
\hline 93.05 \\
\hline
\end{tabular}

\begin{tabular}{|c|c|c|c|c|c|c|c|c|c|}
\hline & & & Clean & Refuse & Feed & Measured & Fitted & Fitting & Weighted \\
\hline Sink & Float & Mean & Mass & Mass & Mass & Refuse & Refuse & Weight & Squared \\
\hline SG & $S G$ & SG & (\% Strm) & (\% Strm) & (\% Strm) & Partition & Partition & Factor & Error \\
\hline 1.210 & 1.250 & 1.230 & 5.21 & 0.07 & 0.43 & 0.15 & 0.15 & 0.15 & 0.00 \\
\hline 1.250 & 1.300 & 1.275 & 88.07 & 11.95 & 17.24 & 0.65 & 0.65 & 0.35 & 0.00 \\
\hline 1.300 & 1.350 & 1.325 & 6.58 & 18.38 & 17.56 & 0.97 & 0.96 & 0.10 & 0.00 \\
\hline 1.350 & 1.375 & 1.363 & 0.06 & 3.37 & 3.14 & 1.00 & 0.99 & 0.10 & 0.00 \\
\hline 1.375 & 1.400 & 1.388 & 0.02 & 2.93 & 2.73 & 1.00 & 1.00 & 0.10 & 0.00 \\
\hline 1.400 & 1.425 & 1.413 & 0.02 & 1.51 & 1.41 & 1.00 & 1.00 & 0.10 & 0.00 \\
\hline 1.425 & 1.450 & 1.438 & 0.00 & 1.24 & 1.15 & 1.00 & 1.00 & 0.10 & 0.00 \\
\hline 1.450 & 1.500 & 1.475 & 0.00 & 1.99 & 1.85 & 1.00 & 1.00 & 0.10 & 0.00 \\
\hline 1.500 & 1.600 & 1.550 & 0.01 & 2.29 & 2.13 & 1.00 & 1.00 & 0.10 & 0.00 \\
\hline 1.600 & 1.800 & 1.700 & 0.00 & 3.19 & 2.97 & 1.00 & 1.00 & 0.10 & 0.00 \\
\hline 1.800 & 2.000 & 1.900 & 0.00 & 1.67 & 1.55 & 1.00 & 1.00 & 0.10 & 0.00 \\
\hline 2.000 & 2.400 & 2.200 & 0.03 & 51.41 & 47.84 & 1.00 & 1.00 & 0.10 & \\
\hline & & Totals & 100.00 & 100.00 & 100.00 & & & WSSQ: & 0.00 \\
\hline
\end{tabular}
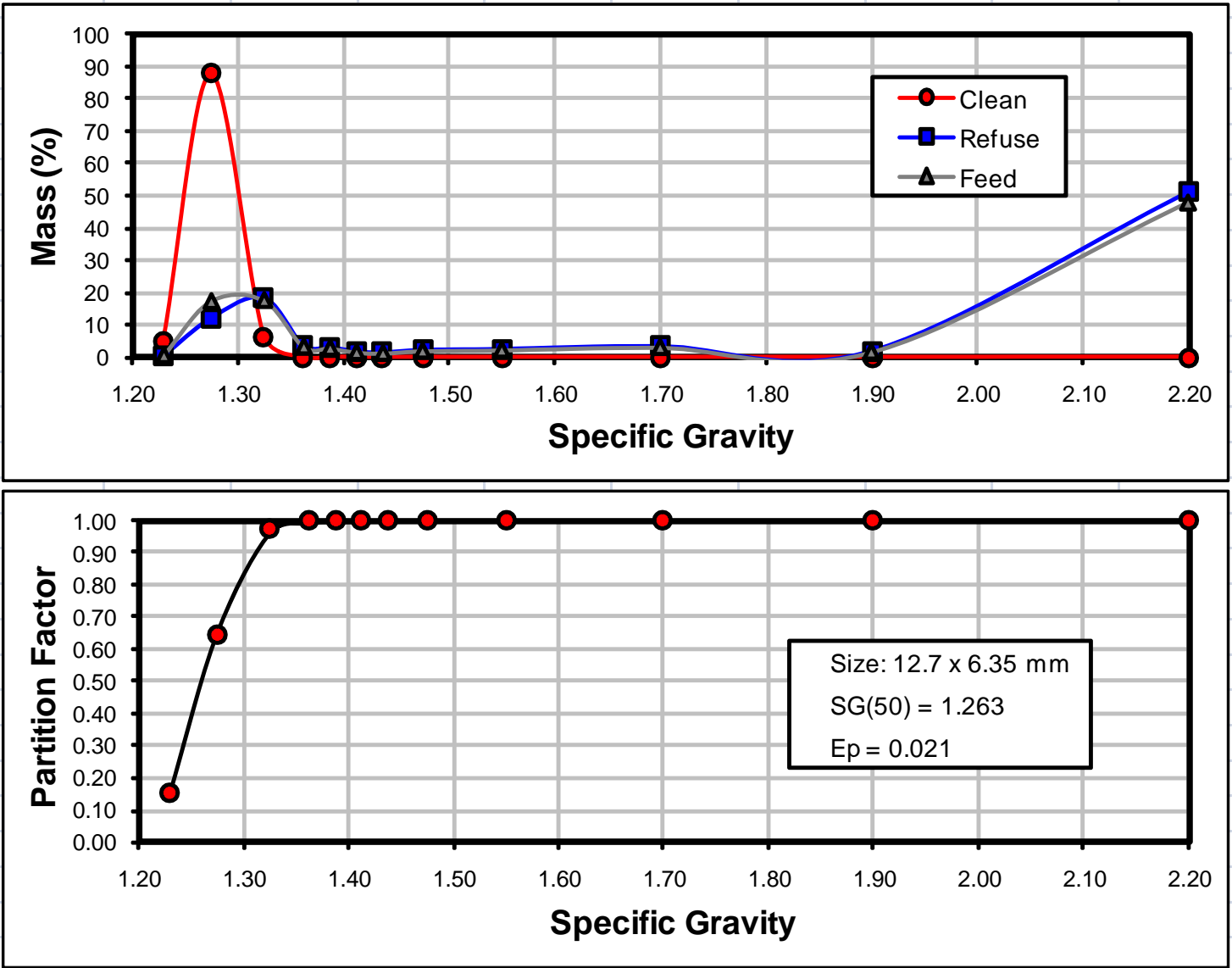
Circuit: TOMS CREEK - SECONDARY DMC CIRCUIT TEST 3

Size: $\quad 6.35 \times 3.175 \mathrm{~mm}$

Clean Yield (\%)

Refuse Yield (\%)

\begin{tabular}{|l|}
\hline 21.37 \\
\hline 78.63 \\
\hline
\end{tabular}

\begin{tabular}{|l|l|}
\hline SG Cutpoint (SG50): & 1.272 \\
\hline Probable Error (Ep): & 0.030 \\
\hline
\end{tabular}

\begin{tabular}{r|c|} 
Weighting $(\mathrm{Y} / \mathrm{N}) ?$ & $\mathrm{~N}$ \\
\hline Low SG Offset: & 0.00 \\
\hline
\end{tabular}

\begin{tabular}{|c|c|c|c|c|c|c|c|c|c|}
\hline & & & Clean & Refuse & Feed & Measured & Fitted & Fitting & Weighted \\
\hline Sink & Float & Mean & Mass & Mass & Mass & Refuse & Refuse & Weight & Squared \\
\hline$S G$ & $S G$ & SG & (\% Strm) & (\% Strm) & (\% Strm) & Partition & Partition & Factor & Error \\
\hline 1.210 & 1.250 & 1.230 & 2.80 & 0.14 & 0.71 & 0.16 & 0.18 & 0.16 & 0.00 \\
\hline 1.250 & 1.300 & 1.275 & 89.06 & 30.85 & 43.29 & 0.56 & 0.53 & 0.44 & 0.00 \\
\hline 1.300 & 1.350 & 1.325 & 7.67 & 9.77 & 9.32 & 0.82 & 0.87 & 0.18 & 0.00 \\
\hline 1.350 & 1.375 & 1.363 & 0.10 & 4.70 & 3.72 & 0.99 & 0.96 & 0.10 & 0.00 \\
\hline 1.375 & 1.400 & 1.388 & 0.05 & 2.76 & 2.18 & 1.00 & 0.99 & 0.10 & 0.00 \\
\hline 1.400 & 1.425 & 1.413 & 0.02 & 1.99 & 1.57 & 1.00 & 0.99 & 0.10 & 0.00 \\
\hline 1.425 & 1.450 & 1.438 & 0.00 & 1.16 & 0.91 & 1.00 & 1.00 & 0.10 & 0.00 \\
\hline 1.450 & 1.500 & 1.475 & 0.01 & 1.58 & 1.24 & 1.00 & 1.00 & 0.10 & 0.00 \\
\hline 1.500 & 1.600 & 1.550 & 0.00 & 2.13 & 1.67 & 1.00 & 1.00 & 0.10 & 0.00 \\
\hline 1.600 & 1.800 & 1.700 & 0.01 & 2.20 & 1.73 & 1.00 & 1.00 & 0.10 & 0.00 \\
\hline 1.800 & 2.000 & 1.900 & 0.00 & 1.36 & 1.07 & 1.00 & 1.00 & 0.10 & 0.00 \\
\hline 2.000 & 2.400 & 2.200 & 0.28 & 41.36 & 32.58 & 1.00 & 1.00 & 0.10 & 0.00 \\
\hline & & Totals & 100.00 & 100.00 & 100.00 & & & WSSQ: & 0.01 \\
\hline
\end{tabular}
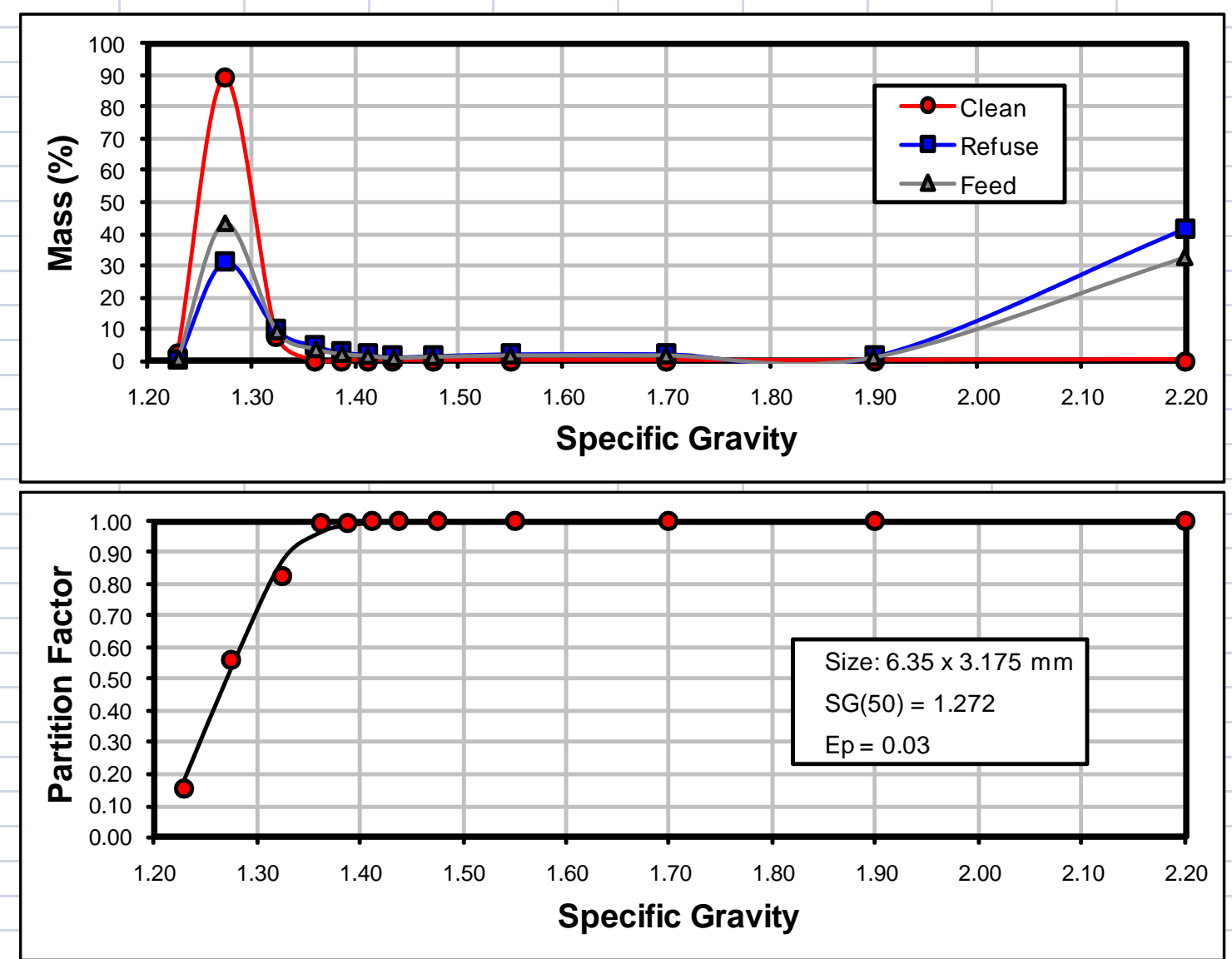
Circuit: TOMS CREEK - SECONDARY DMC CIRCUIT TEST 3

Size: $\quad 3.175 \times 1.41 \mathrm{~mm}$

Clean Yield (\%)

Refuse Yield (\%)

\begin{tabular}{|l|}
\hline 24.07 \\
\hline 75.93 \\
\hline
\end{tabular}

\begin{tabular}{|c|c|c|c|c|c|c|c|c|c|}
\hline & & & Clean & Refuse & Feed & Measured & Fitted & Fitting & Weighted \\
\hline Sink & Float & Mean & Mass & Mass & Mass & Refuse & Refuse & Weight & Squared \\
\hline SG & $S G$ & SG & (\% Strm) & (\% Strm) & (\% Strm) & Partition & Partition & Factor & Error \\
\hline 1.260 & 1.300 & 1.280 & 85.06 & 25.29 & 39.68 & 0.48 & 0.48 & 0.48 & 0.00 \\
\hline 1.300 & 1.400 & 1.350 & 14.16 & 23.43 & 21.20 & 0.84 & 0.84 & 0.16 & 0.00 \\
\hline 1.400 & 1.500 & 1.450 & 0.13 & 4.95 & 3.79 & 0.99 & 0.98 & 0.10 & 0.00 \\
\hline 1.500 & 1.600 & 1.550 & 0.02 & 2.18 & 1.66 & 1.00 & 1.00 & 0.10 & 0.00 \\
\hline 1.600 & 1.700 & 1.650 & 0.01 & 1.24 & 0.94 & 1.00 & 1.00 & 0.10 & 0.00 \\
\hline 1.700 & 1.800 & 1.750 & 0.01 & 0.90 & 0.69 & 1.00 & 1.00 & 0.10 & 0.00 \\
\hline 1.800 & 1.900 & 1.850 & 0.01 & 0.74 & 0.56 & 1.00 & 1.00 & 0.10 & 0.00 \\
\hline 1.900 & 2.000 & 1.950 & 0.01 & 0.68 & 0.52 & 1.00 & 1.00 & 0.10 & 0.00 \\
\hline 2.000 & 2.400 & 2.200 & 0.59 & 40.59 & 30.96 & 1.00 & 1.00 & 0.10 & 0.00 \\
\hline & & & & & & & & & \\
\hline & & & & & & & & & \\
\hline & & Totals & 100.00 & 100.00 & 100.00 & & & WSSQ: & 0.00 \\
\hline
\end{tabular}

\begin{tabular}{|l|l|}
\hline SG Cutpoint (SG50): & 1.283 \\
\hline Probable Error (Ep): & 0.044 \\
\hline
\end{tabular}

Weighting $(\mathrm{Y} / \mathrm{N})$ ? Low SG Offset:

$\frac{N}{0.00}$
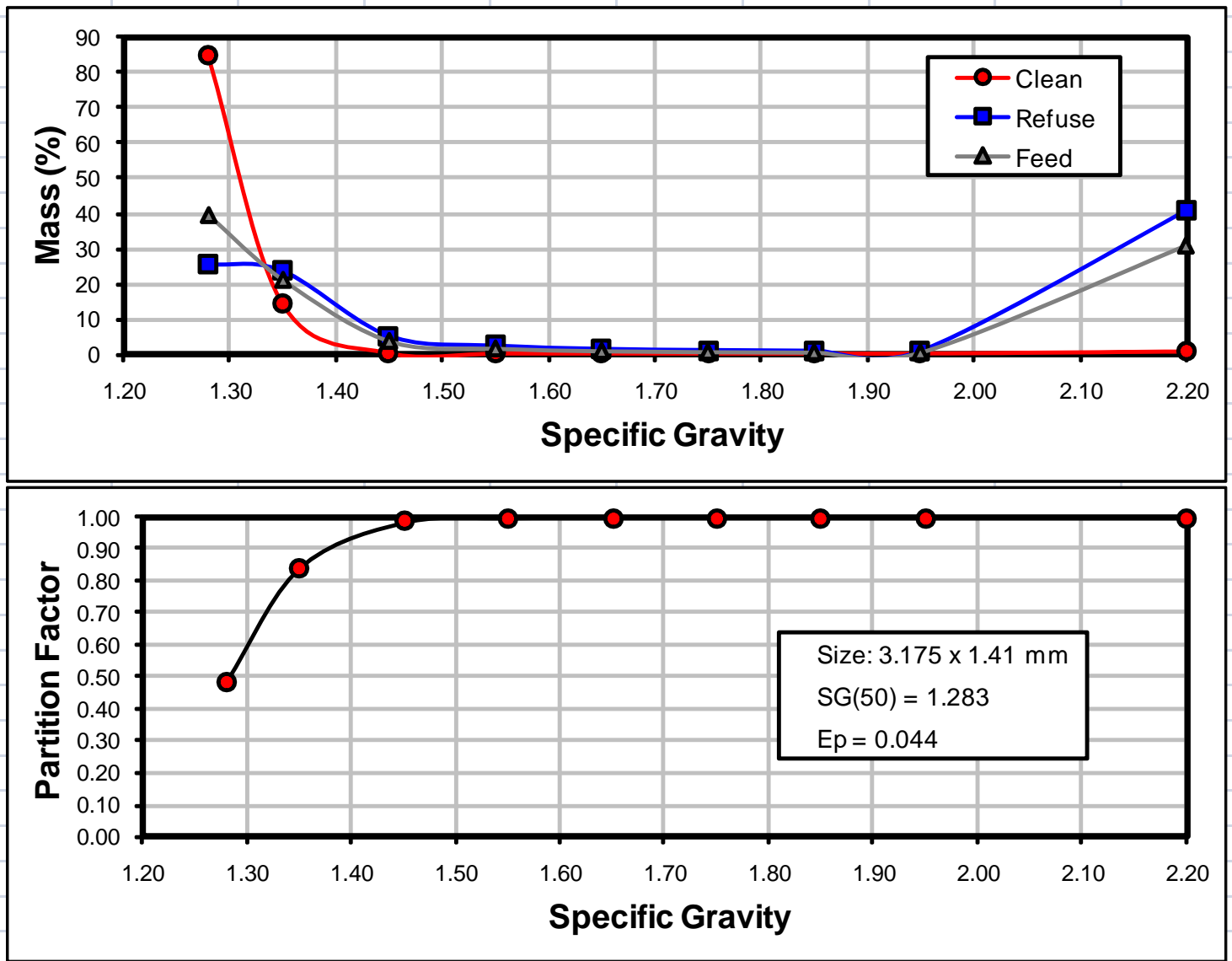
Circuit: TOMS CREEK - SECONDARY DMC CIRCUIT TEST 3

Size: $\quad 1.41 \times 0.707 \mathrm{~mm}$

Clean Yield (\%)

Refuse Yield (\%)

\begin{tabular}{|l|}
\hline 34.91 \\
\hline 65.09 \\
\hline
\end{tabular}

\begin{tabular}{|c|c|c|c|c|c|c|c|c|c|}
\hline & & & Clean & Refuse & Feed & Measured & Fitted & Fitting & Weighted \\
\hline Sink & Float & Mean & Mass & Mass & Mass & Refuse & Refuse & Weight & Squared \\
\hline$S G$ & $S G$ & SG & (\% Strm) & (\% Strm) & (\% Strm) & Partition & Partition & Factor & Error \\
\hline 1.260 & 1.300 & 1.280 & 80.25 & 22.19 & 42.46 & 0.34 & 0.33 & 0.34 & 0.00 \\
\hline 1.300 & 1.400 & 1.350 & 18.64 & 18.40 & 18.48 & 0.65 & 0.67 & 0.35 & 0.00 \\
\hline 1.400 & 1.500 & 1.450 & 0.35 & 6.26 & 4.20 & 0.97 & 0.94 & 0.10 & 0.00 \\
\hline 1.500 & 1.600 & 1.550 & 0.08 & 2.71 & 1.79 & 0.98 & 0.99 & 0.10 & 0.00 \\
\hline 1.600 & 1.700 & 1.650 & 0.03 & 1.59 & 1.05 & 0.99 & 1.00 & 0.10 & 0.00 \\
\hline 1.700 & 1.800 & 1.750 & 0.02 & 1.13 & 0.74 & 0.99 & 1.00 & 0.10 & 0.00 \\
\hline 1.800 & 1.900 & 1.850 & 0.02 & 0.95 & 0.63 & 0.99 & 1.00 & 0.10 & 0.00 \\
\hline 1.900 & 2.000 & 1.950 & 0.02 & 1.19 & 0.78 & 0.99 & 1.00 & 0.10 & 0.00 \\
\hline 2.000 & 2.400 & 2.200 & 0.59 & 45.58 & 29.87 & 0.99 & 1.00 & 0.10 & 0.00 \\
\hline & & & & & & & & & \\
\hline & & & & & & & & & \\
\hline & & Totals & 100.00 & 100.00 & 100.00 & & & WSSQ: & 0.00 \\
\hline
\end{tabular}

\begin{tabular}{|l|l|}
\hline SG Cutpoint (SG50): & 1.315 \\
\hline Probable Error (Ep): & 0.054 \\
\hline
\end{tabular}

Weighting $(\mathrm{Y} / \mathrm{N})$ ?

Low SG Offset:

\begin{tabular}{|c|}
\hline $\mathrm{N}$ \\
\hline 0.00 \\
\hline
\end{tabular}
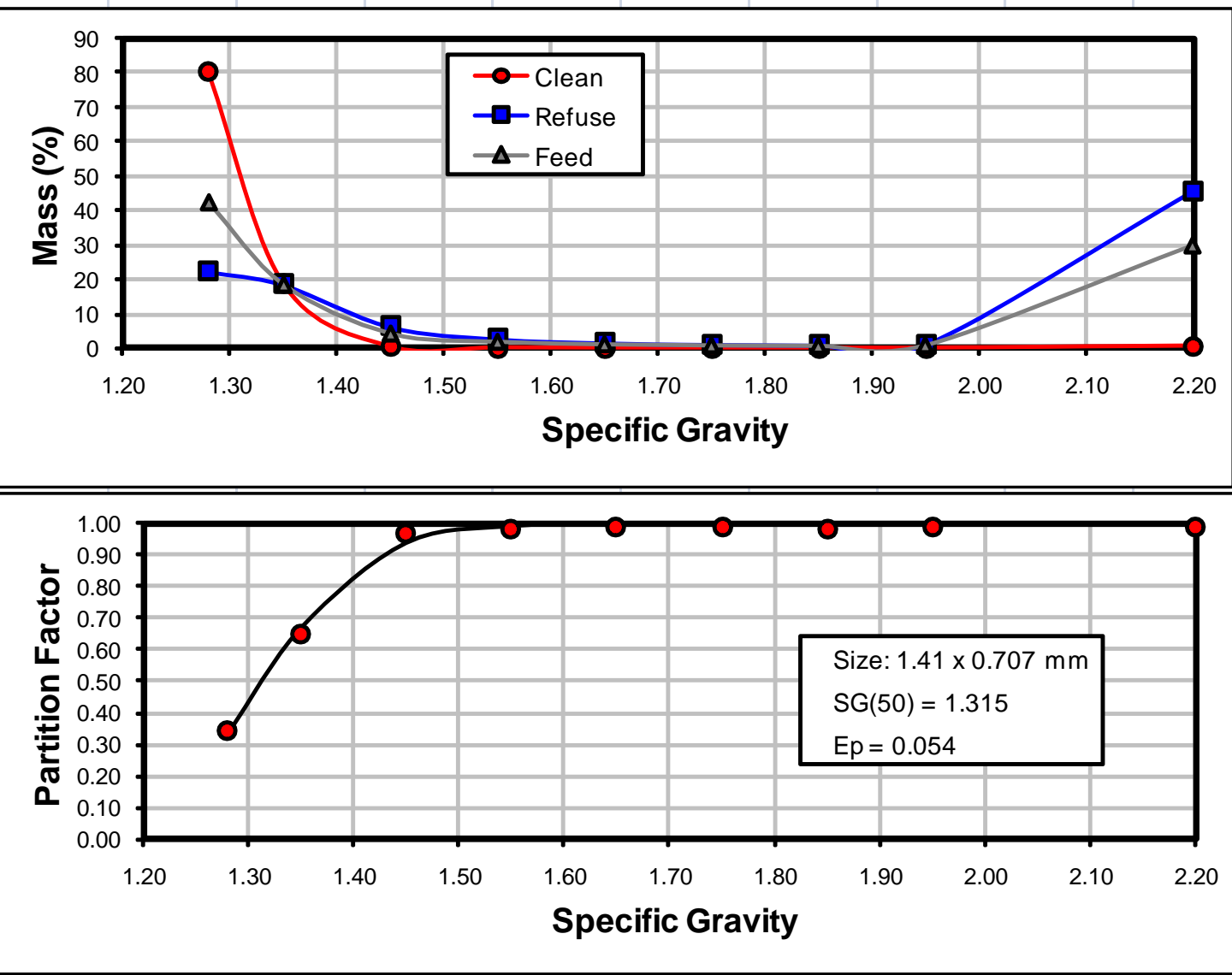
APPENDIX 6.4 - INTERMEDIATE SG RANGE AND HIGH REJECT FEED 
Circuit: $\quad$ TOMS CREEK - SECONDARY DMC CIRCUIT TEST 4

\begin{tabular}{|c|c|c|c|c|c|c|c|c|}
\hline \multirow{3}{*}{\multicolumn{2}{|c|}{$\begin{array}{l}\text { Clean Rate }(\mathrm{t} / \mathrm{hr}) \text { : } \\
\text { Refuse Rate }(\mathrm{t} / \mathrm{hr}) \text { : } \\
\text { Feed Rate }(\mathrm{t} / \mathrm{hr}) \text { : }\end{array}$}} & 250.6 & & \multicolumn{2}{|c|}{ Clean Yield (\%): } & \multicolumn{3}{|l|}{50.11} \\
\hline & & 249.4 & & \multicolumn{2}{|c|}{ Refuse Yield (\%): } & 49.89 & & \\
\hline & & 500.0 & & \multicolumn{2}{|c|}{ Feed Ash (\%): } & 45.50 & & \\
\hline Pass & Retain & Mean & Clean & Clean & Refuse & Refuse & Feed & Feed \\
\hline Size & Size & Size & Mass & Ash & Mass & Ash & Mass & Ash \\
\hline$(\mathrm{mm})$ & $(\mathrm{mm})$ & $(\mathrm{mm})$ & $(\%)$ & $(\%)$ & $(\%)$ & $(\%)$ & $(\%)$ & $(\%)$ \\
\hline 12.7 & 6.35 & 8.98 & 24.36 & 5.17 & 40.13 & 87.16 & 32.23 & 56.10 \\
\hline 6.35 & 3.175 & 4.49 & 35.88 & 5.00 & 29.81 & 86.43 & 32.85 & 41.86 \\
\hline 3.175 & 1.41 & 2.12 & 30.13 & 4.72 & 24.18 & 84.78 & 27.16 & 40.28 \\
\hline 1.41 & 0.707 & 1.00 & 8.70 & 4.86 & 5.41 & 85.11 & 7.06 & 35.55 \\
\hline \multirow[t]{2}{*}{0.707} & 0 & 0.00 & 0.93 & 6.87 & 0.47 & 79.04 & 0.70 & 31.03 \\
\hline & & Totals & 100.00 & 4.96 & 100.00 & 86.22 & 100.00 & 45.50 \\
\hline Pass & Retain & Mean & Clean & Refuse & Feed & Clean & Refuse & Feed \\
\hline Size & Size & Size & Yield & Yield & Yield & Mass & Mass & Mass \\
\hline$(\mathrm{mm})$ & $(\mathrm{mm})$ & $(\mathrm{mm})$ & $(\%)$ & $(\%)$ & $(\%)$ & (Cum\%) & (Cum\%) & (Cum\%) \\
\hline 12.7 & 6.35 & 8.98 & 37.88 & 62.12 & 100.00 & 24.36 & 40.13 & 32.23 \\
\hline 6.35 & 3.175 & 4.49 & 54.73 & 45.27 & 100.00 & 60.24 & 69.94 & 65.08 \\
\hline 3.175 & 1.41 & 2.12 & 55.59 & 44.41 & 100.00 & 90.37 & 94.12 & 92.24 \\
\hline 1.41 & 0.707 & 1.00 & 61.76 & 38.24 & 100.00 & 99.07 & 99.53 & 99.30 \\
\hline 0.707 & 0 & 0.00 & 66.53 & 33.47 & 100.00 & 100.00 & 100.00 & 100.00 \\
\hline & & & & & & & & \\
\hline & & Totals & 50.11 & 49.89 & 100.00 & & & \\
\hline
\end{tabular}

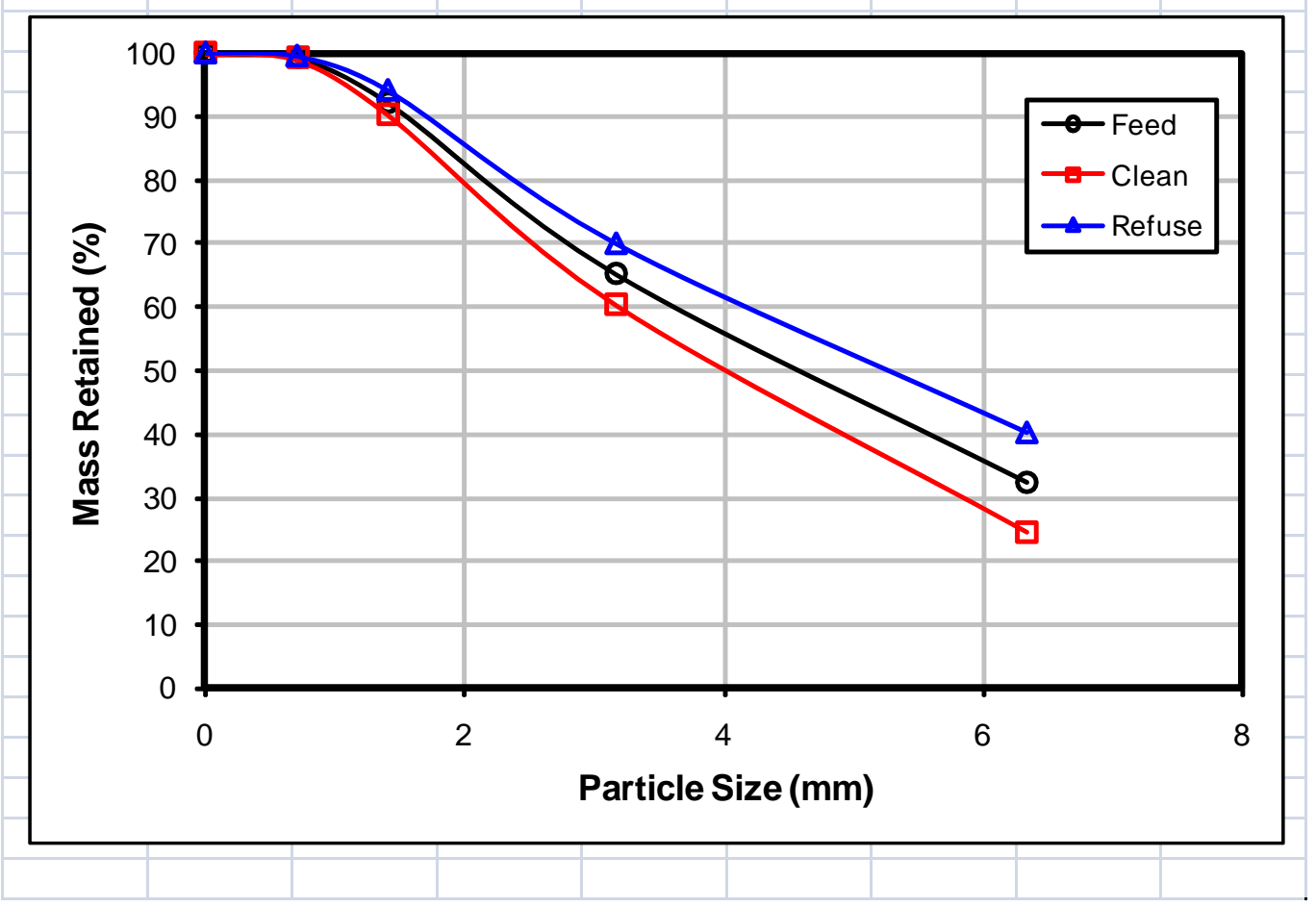


Circuit: TOMS CREEK - SECONDARY DMC CIRCUIT TEST 4

Size: $12.7 \times 6.35 \mathrm{~mm}$

Clean Yield (\%)

Refuse Yield (\%)

\begin{tabular}{|l|}
\hline 37.88 \\
\hline 62.12 \\
\hline
\end{tabular}

SG Cutpoint (SG50)

Probable Error (Ep)
Weighting $(\mathrm{Y} / \mathrm{N})$ ? Low SG Offset:

\begin{tabular}{|c|}
\hline $\mathrm{N}$ \\
\hline 0.00 \\
\hline
\end{tabular}

\begin{tabular}{|c|c|c|c|c|c|c|c|c|c|}
\hline & & & Clean & Refuse & Feed & Measured & Fitted & Fitting & Weighted \\
\hline Sink & Float & Mean & Mass & Mass & Mass & Refuse & Refuse & Weight & Squared \\
\hline$S G$ & $S G$ & $S G$ & (\% Strm) & (\% Strm) & (\% Strm) & Partition & Partition & Factor & Error \\
\hline 1.260 & 1.300 & 1.280 & 76.14 & 0.02 & 28.85 & 0.00 & 0.00 & 0.10 & 0.00 \\
\hline 1.300 & 1.400 & 1.350 & 20.05 & 0.07 & 7.64 & 0.01 & 0.00 & 0.10 & 0.00 \\
\hline 1.400 & 1.450 & 1.425 & 2.81 & 0.12 & 1.14 & 0.07 & 0.03 & 0.10 & 0.00 \\
\hline 1.450 & 1.500 & 1.475 & 0.90 & 0.48 & 0.64 & 0.47 & 0.48 & 0.47 & 0.00 \\
\hline 1.500 & 1.525 & 1.513 & 0.05 & 0.40 & 0.27 & 0.93 & 0.91 & 0.10 & 0.00 \\
\hline 1.525 & 1.550 & 1.538 & 0.01 & 0.36 & 0.23 & 0.98 & 0.98 & 0.10 & 0.00 \\
\hline 1.550 & 1.575 & 1.563 & 0.00 & 0.37 & 0.23 & 1.00 & 1.00 & 0.10 & 0.00 \\
\hline 1.575 & 1.600 & 1.588 & 0.00 & 0.34 & 0.21 & 1.00 & 1.00 & 0.10 & 0.00 \\
\hline 1.600 & 1.650 & 1.625 & 0.00 & 0.52 & 0.32 & 1.00 & 1.00 & 0.10 & 0.00 \\
\hline 1.650 & 1.700 & 1.675 & 0.00 & 0.57 & 0.35 & 1.00 & 1.00 & 0.10 & 0.00 \\
\hline 1.700 & 1.800 & 1.750 & 0.00 & 0.88 & 0.55 & 1.00 & 1.00 & 0.10 & 0.00 \\
\hline 1.800 & 2.000 & 1.900 & 0.00 & 1.83 & 1.14 & 1.00 & 1.00 & 0.10 & 0.00 \\
\hline 2.000 & 2.400 & 2.200 & 0.04 & 94.04 & 58.44 & 1.00 & 1.00 & 0.10 & 0.00 \\
\hline & & Totals & 100.00 & 100.00 & 100.00 & & & WSSQ: & 0.00 \\
\hline
\end{tabular}
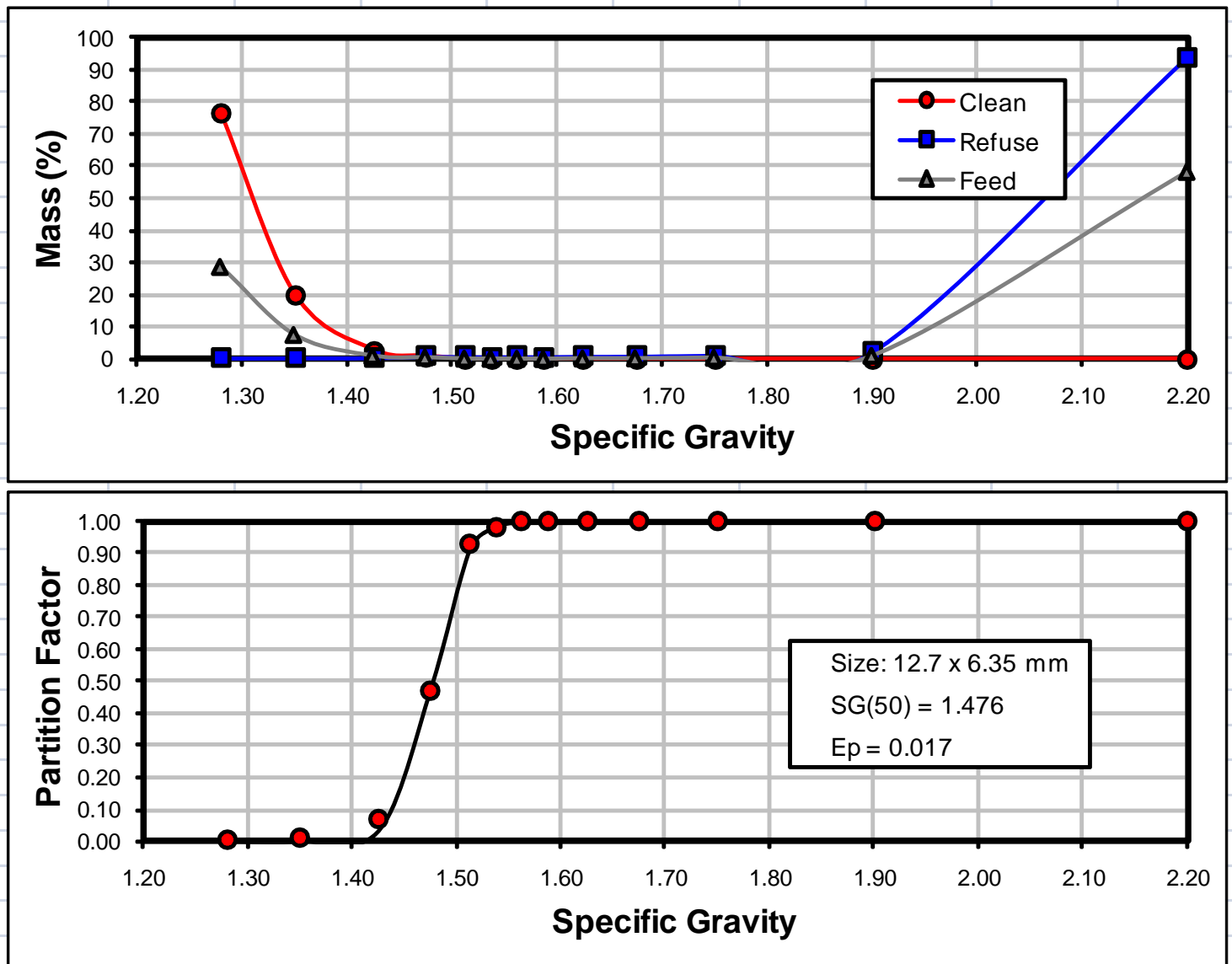
Circuit: TOMS CREEK - SECONDARY DMC CIRCUIT TEST 4 Size: $\quad 6.35 \times 3.175 \mathrm{~mm}$

Clean Yield (\%)

Refuse Yield (\%)

\begin{tabular}{|l|}
\hline 54.73 \\
\hline 45.27 \\
\hline
\end{tabular}

SG Cutpoint (SG50)

Probable Error (Ep):
Weighting $(\mathrm{Y} / \mathrm{N})$ ? Low SG Offset:

\begin{tabular}{|c|}
\hline$N$ \\
\hline 0.00 \\
\hline
\end{tabular}

\begin{tabular}{|c|c|c|c|c|c|c|c|c|c|}
\hline & & & Clean & Refuse & Feed & Measured & Fitted & Fitting & Weighted \\
\hline Sink & Float & Mean & Mass & Mass & Mass & Refuse & Refuse & Weight & Squared \\
\hline$S G$ & SG & SG & (\% Strm) & (\% Strm) & (\% Strm) & Partition & Partition & Factor & Error \\
\hline 1.260 & 1.300 & 1.280 & 81.23 & 0.18 & 44.54 & 0.00 & 0.00 & 0.10 & 0.00 \\
\hline 1.300 & 1.400 & 1.350 & 15.82 & 0.26 & 8.78 & 0.01 & 0.00 & 0.10 & 0.00 \\
\hline 1.400 & 1.450 & 1.425 & 1.78 & 0.25 & 1.09 & 0.10 & 0.06 & 0.10 & 0.00 \\
\hline 1.450 & 1.500 & 1.475 & 0.87 & 0.73 & 0.81 & 0.41 & 0.42 & 0.41 & 0.00 \\
\hline 1.500 & 1.525 & 1.513 & 0.11 & 0.52 & 0.30 & 0.80 & 0.82 & 0.20 & 0.00 \\
\hline 1.525 & 1.550 & 1.538 & 0.00 & 0.54 & 0.24 & 1.00 & 0.94 & 0.10 & 0.00 \\
\hline 1.550 & 1.575 & 1.563 & 0.00 & 0.56 & 0.25 & 1.00 & 0.98 & 0.10 & 0.00 \\
\hline 1.575 & 1.600 & 1.588 & 0.00 & 0.47 & 0.21 & 1.00 & 0.99 & 0.10 & 0.00 \\
\hline 1.600 & 1.650 & 1.625 & 0.00 & 0.84 & 0.38 & 1.00 & 1.00 & 0.10 & 0.00 \\
\hline 1.650 & 1.700 & 1.675 & 0.00 & 0.59 & 0.27 & 1.00 & 1.00 & 0.10 & 0.00 \\
\hline 1.700 & 1.800 & 1.750 & 0.00 & 1.16 & 0.53 & 1.00 & 1.00 & 0.10 & 0.00 \\
\hline 1.800 & 2.000 & 1.900 & 0.00 & 2.17 & 0.98 & 1.00 & 1.00 & 0.10 & 0.00 \\
\hline 2.000 & 2.400 & 2.200 & 0.19 & 91.73 & 41.63 & 1.00 & 1.00 & 0.10 & 0.00 \\
\hline & & Totals & 100.00 & 100.00 & 100.00 & & & WSSQ: & 0.01 \\
\hline
\end{tabular}
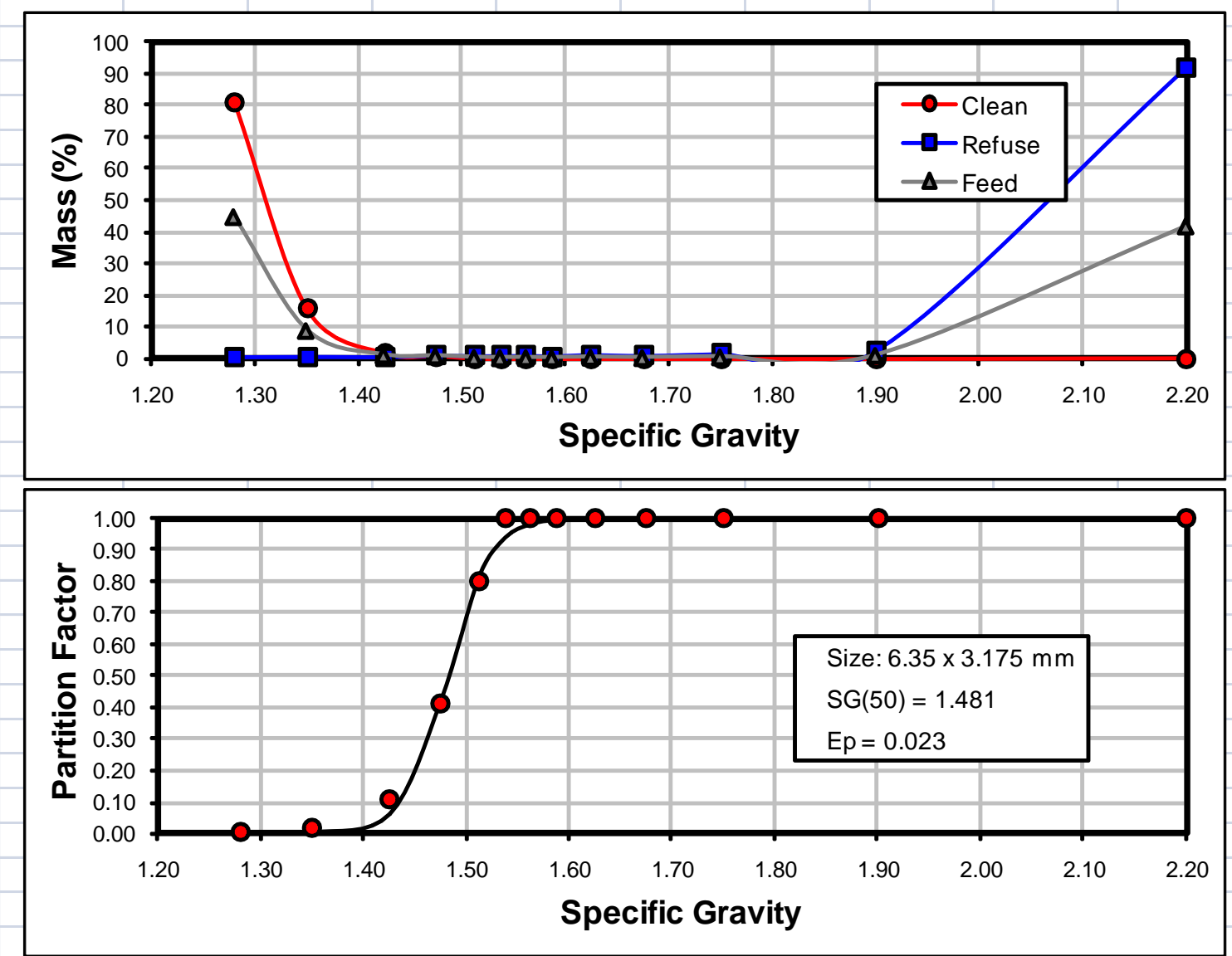
Circuit: $\quad$ TOMS CREEK - SECONDARY DMC CIRCUIT TEST 4

Size: $\quad 3.175 \times 1.41 \mathrm{~mm}$

Clean Yield (\%)

Refuse Yield (\%)
SG Cutpoint (SG50): 1.494

Probable Error (Ep): 0.036 \begin{tabular}{r|c|} 
Weighting $(\mathrm{Y} / \mathrm{N}) ?$ & $\mathrm{~N}$ \\
& $\mathbf{L}$ \\
\cline { 2 - 2 } &
\end{tabular}

\begin{tabular}{|c|c|c|c|c|c|c|c|c|c|}
\hline & & & Clean & Refuse & Feed & Measured & Fitted & Fitting & Weighted \\
\hline Sink & Float & Mean & Mass & Mass & Mass & Refuse & Refuse & Weight & Squared \\
\hline SG & $S G$ & SG & (\% Strm) & (\% Strm) & (\% Strm) & Partition & Partition & Factor & Error \\
\hline 1.260 & 1.300 & 1.280 & 80.64 & 0.81 & 45.19 & 0.01 & 0.00 & 0.10 & 0.00 \\
\hline 1.300 & 1.400 & 1.350 & 15.61 & 0.70 & 8.99 & 0.03 & 0.01 & 0.10 & 0.00 \\
\hline 1.400 & 1.500 & 1.450 & 2.99 & 0.96 & 2.09 & 0.20 & 0.21 & 0.20 & 0.00 \\
\hline 1.500 & 1.600 & 1.550 & 0.28 & 1.99 & 1.04 & 0.85 & 0.85 & 0.15 & 0.00 \\
\hline 1.600 & 1.700 & 1.650 & 0.00 & 1.48 & 0.66 & 1.00 & 0.99 & 0.10 & 0.00 \\
\hline 1.700 & 1.800 & 1.750 & 0.00 & 1.17 & 0.52 & 1.00 & 1.00 & 0.10 & 0.00 \\
\hline 1.800 & 1.900 & 1.850 & 0.00 & 1.16 & 0.52 & 1.00 & 1.00 & 0.10 & 0.00 \\
\hline 1.900 & 2.000 & 1.950 & 0.01 & 1.17 & 0.53 & 0.99 & 1.00 & 0.10 & 0.00 \\
\hline 2.000 & 2.400 & 2.200 & 0.47 & 90.56 & 40.48 & 0.99 & 1.00 & 0.10 & 0.00 \\
\hline & & & & & & & & & \\
\hline & & & & & & & & & \\
\hline & & & & & & & & & \\
\hline & & Totals & 100.00 & 100.00 & 10000 & & & WSSQ: & 0.00 \\
\hline
\end{tabular}
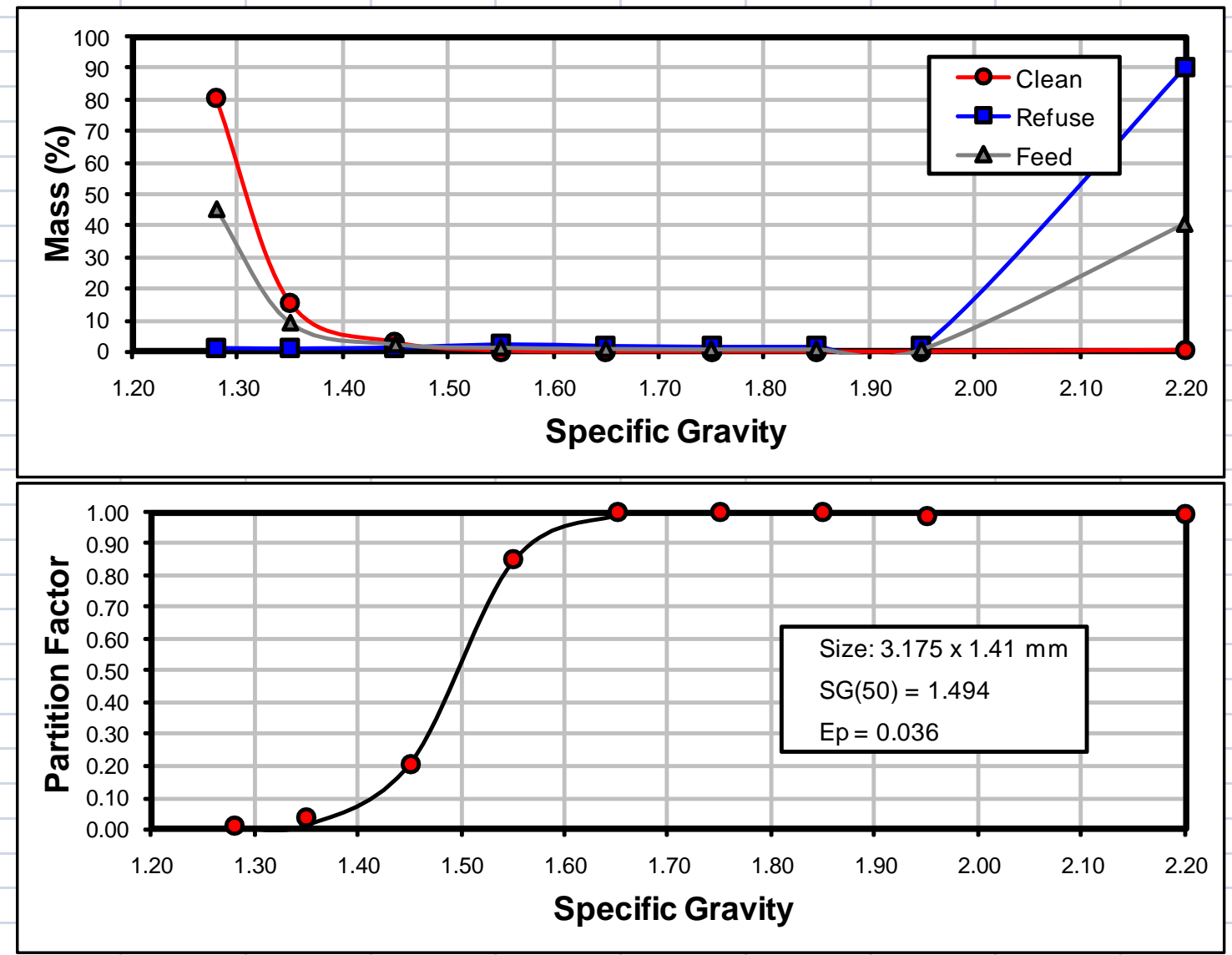
Circuit: TOMS CREEK - SECONDARY DMC CIRCUIT TEST 4

Size: $\quad 1.41 \times 0.707 \mathrm{~mm}$

Clean Yield (\%)

Refuse Yield (\%)

\begin{tabular}{|l|}
\hline 61.76 \\
\hline 38.24 \\
\hline
\end{tabular}

SG Cutpoint (SG50)

Probable Error (Ep):
Weighting $(\mathrm{Y} / \mathrm{N})$ ?

Low SG Offset:

\begin{tabular}{|c|}
\hline $\mathrm{N}$ \\
\hline 0.00 \\
\hline
\end{tabular}

\begin{tabular}{|c|c|c|c|c|c|c|c|c|c|}
\hline & & & Clean & Refuse & Feed & Measured & Fitted & Fitting & Weighted \\
\hline Sink & Float & Mean & Mass & Mass & Mass & Refuse & Refuse & Weight & Squared \\
\hline SG & SG & $S G$ & (\% Strm) & (\% Strm) & (\% Strm) & Partition & Partition & Factor & Error \\
\hline 1.260 & 1.300 & 1.280 & 76.59 & 1.17 & 47.75 & 0.01 & 0.00 & 0.10 & 0.00 \\
\hline 1.300 & 1.400 & 1.350 & 18.07 & 0.73 & 11.44 & 0.02 & 0.02 & 0.10 & 0.00 \\
\hline 1.400 & 1.500 & 1.450 & 3.71 & 0.58 & 2.51 & 0.09 & 0.12 & 0.10 & 0.00 \\
\hline 1.500 & 1.600 & 1.550 & 0.71 & 1.38 & 0.97 & 0.55 & 0.53 & 0.45 & 0.00 \\
\hline 1.600 & 1.700 & 1.650 & 0.12 & 1.44 & 0.62 & 0.88 & 0.90 & 0.12 & 0.00 \\
\hline 1.700 & 1.800 & 1.750 & 0.03 & 1.21 & 0.48 & 0.96 & 0.99 & 0.10 & 0.00 \\
\hline 1.800 & 1.900 & 1.850 & 0.02 & 1.15 & 0.45 & 0.97 & 1.00 & 0.10 & 0.00 \\
\hline 1.900 & 2.000 & 1.950 & 0.00 & 1.10 & 0.42 & 1.00 & 1.00 & 0.10 & 0.00 \\
\hline 2.000 & 2.400 & 2.200 & 0.75 & 91.24 & 35.35 & 0.99 & 1.00 & 0.10 & 0.00 \\
\hline & & & & & & & & & \\
\hline & & & & & & & & & \\
\hline & & Totals & 100.00 & 100.00 & 100.00 & & & WSSQ: & 0.00 \\
\hline
\end{tabular}
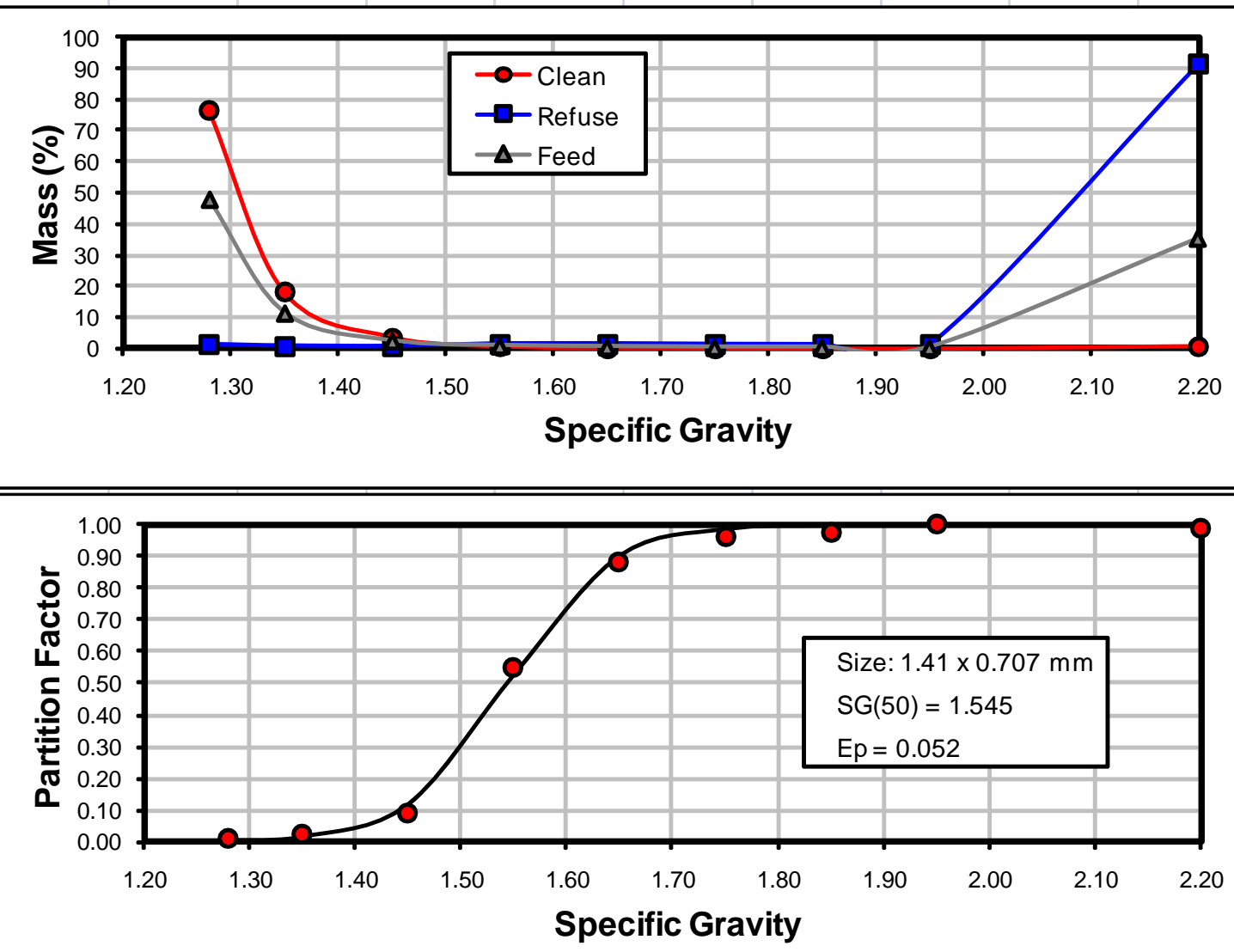
APPENDIX 6.5 - HIGH SG RANGE AND LOW REJECT FEED 
Circuit: TOMS CREEK - SECONDARY DMC CIRCUIT TEST 5

\begin{tabular}{|c|c|c|c|c|c|c|c|c|}
\hline \multirow{3}{*}{\multicolumn{2}{|c|}{$\begin{array}{l}\text { Clean Rate }(\mathrm{t} / \mathrm{hr}): \\
\text { Refuse Rate }(\mathrm{t} / \mathrm{hr}) \\
\text { Feed Rate }(\mathrm{t} / \mathrm{hr}) \text { : }\end{array}$}} & 334.0 & & \multicolumn{2}{|c|}{ Clean Yield (\%): } & \multirow{2}{*}{$\begin{array}{l}66.79 \\
33.21\end{array}$} & & \\
\hline & & 166.0 & & \multicolumn{2}{|c|}{ Refuse Yield (\%): } & & & \\
\hline & & 500.0 & & \multicolumn{2}{|c|}{ Feed Ash (\%): } & 33.16 & & \\
\hline Pass & Retain & Mean & Clean & Clean & Refuse & Refuse & Feed & Feed \\
\hline Size & Size & Size & Mass & Ash & Mass & Ash & Mass & Ash \\
\hline$(\mathrm{mm})$ & $(\mathrm{mm})$ & $(\mathrm{mm})$ & $(\%)$ & (\%) & $(\%)$ & (\%) & $(\%)$ & $(\%)$ \\
\hline 12.7 & 6.35 & 8.98 & 20.84 & 8.27 & 40.28 & 85.95 & 27.30 & 46.34 \\
\hline 6.35 & 3.175 & 4.49 & 33.17 & 7.08 & 28.97 & 86.71 & 31.78 & 31.19 \\
\hline 3.175 & 1.41 & 2.12 & 31.94 & 6.45 & 24.38 & 84.19 & 29.43 & 27.84 \\
\hline 1.41 & 0.707 & 1.00 & 11.89 & 6.70 & 5.83 & 83.52 & 9.88 & 21.76 \\
\hline \multirow[t]{2}{*}{0.707} & 0 & 0.00 & 2.16 & 8.50 & 0.54 & 76.66 & 1.62 & 16.04 \\
\hline & & Totals & 100.00 & 7.11 & 100.00 & 85.55 & 100.00 & 33.16 \\
\hline Pass & Retain & Mean & Clean & Refuse & Feed & Clean & Refuse & Feed \\
\hline Size & Size & Size & Yield & Yield & Yield & Mass & Mass & Mass \\
\hline$(\mathrm{mm})$ & $(\mathrm{mm})$ & $(\mathrm{mm})$ & $(\%)$ & $(\%)$ & $(\%)$ & (Cum\%) & (Cum\%) & (Cum\%) \\
\hline 12.7 & 6.35 & 8.98 & 50.99 & 49.01 & 100.00 & 20.84 & 40.28 & 27.30 \\
\hline 6.35 & 3.175 & 4.49 & 69.72 & 30.28 & 100.00 & 54.01 & 69.25 & 59.07 \\
\hline 3.175 & 1.41 & 2.12 & 72.49 & 27.51 & 100.00 & 85.95 & 93.63 & 88.50 \\
\hline 1.41 & 0.707 & 1.00 & 80.40 & 19.60 & 100.00 & 97.84 & 99.46 & 98.38 \\
\hline \multirow[t]{2}{*}{0.707} & 0 & 0.00 & 88.94 & 11.06 & 100.00 & 100.00 & 100.00 & 100.00 \\
\hline & & Totals & 66.79 & 33.21 & 100.00 & & & \\
\hline
\end{tabular}

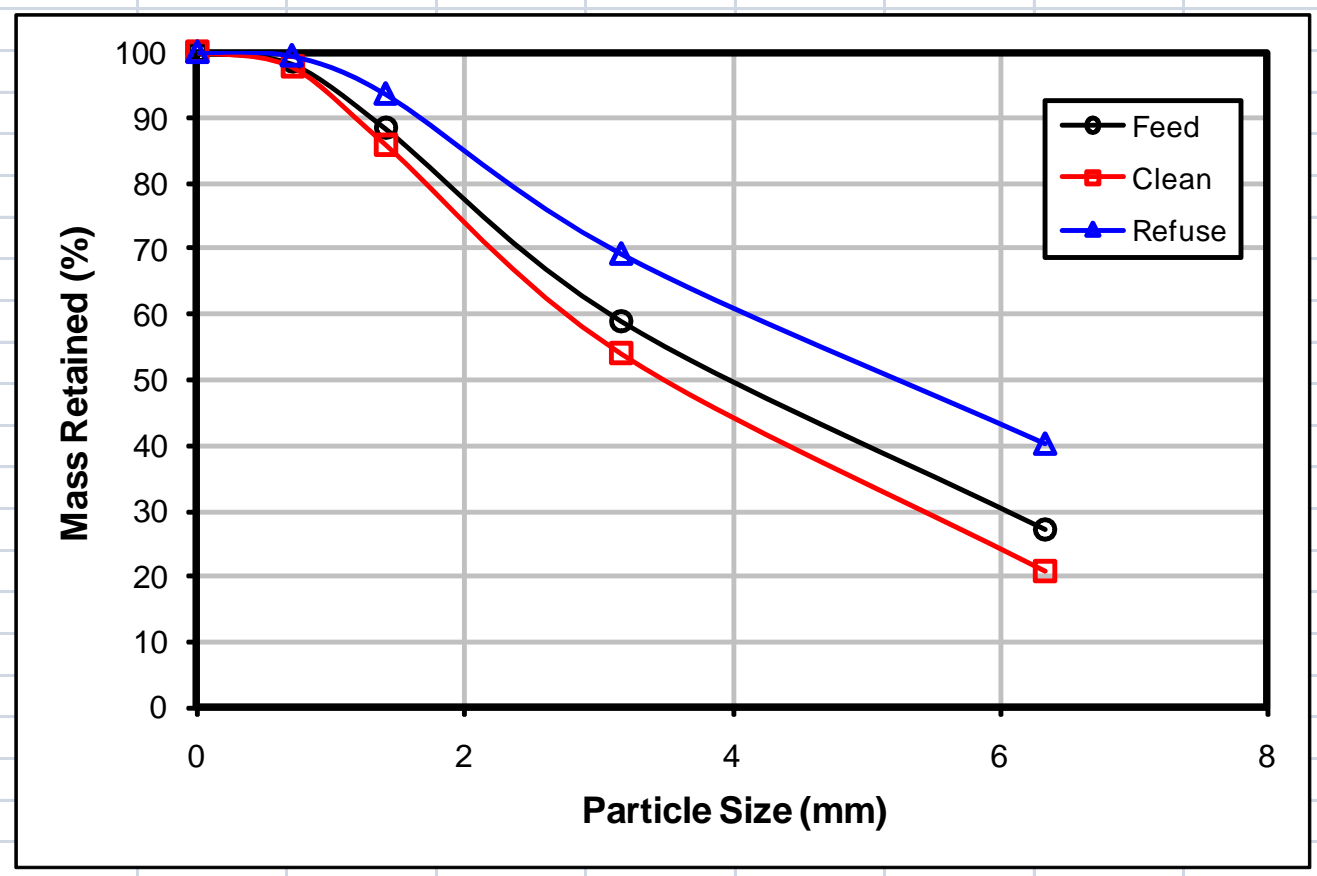


Circuit: TOMS CREEK - SECONDARY DMC CIRCUIT TEST 5

Size: $12.7 \times 6.35 \mathrm{~mm}$

Clean Yield (\%)

Refuse Yield (\%)

\begin{tabular}{|l|}
\hline 50.99 \\
\hline 49.01 \\
\hline
\end{tabular}

SG Cutpoint (SG50)

Probable Error (Ep):
Weighting $(\mathrm{Y} / \mathrm{N})$ ? Low SG Offset:

\begin{tabular}{|c|}
\hline $\mathrm{N}$ \\
\hline 0.00 \\
\hline
\end{tabular}

\begin{tabular}{|c|c|c|c|c|c|c|c|c|c|}
\hline & & & Clean & Refuse & Feed & Measured & Fitted & Fitting & Weighted \\
\hline Sink & Float & Mean & Mass & Mass & Mass & Refuse & Refuse & Weight & Squared \\
\hline$S G$ & $S G$ & $S G$ & (\% Strm) & (\% Strm) & (\% Strm) & Partition & Partition & Factor & Error \\
\hline 1.260 & 1.300 & 1.280 & 48.26 & 0.03 & 24.62 & 0.00 & 0.00 & 0.10 & 0.00 \\
\hline 1.300 & 1.500 & 1.400 & 47.36 & 0.02 & 24.16 & 0.00 & 0.00 & 0.10 & 0.00 \\
\hline 1.500 & 1.600 & 1.550 & 2.51 & 0.02 & 1.29 & 0.01 & 0.00 & 0.10 & 0.00 \\
\hline 1.600 & 1.650 & 1.625 & 0.83 & 0.04 & 0.44 & 0.04 & 0.00 & 0.10 & 0.00 \\
\hline 1.650 & 1.700 & 1.675 & 0.69 & 0.18 & 0.44 & 0.20 & 0.00 & 0.20 & 0.04 \\
\hline 1.700 & 1.725 & 1.713 & 0.34 & 0.25 & 0.30 & 0.41 & 0.42 & 0.41 & 0.00 \\
\hline 1.725 & 1.750 & 1.738 & 0.00 & 0.45 & 0.22 & 1.00 & 0.98 & 0.10 & 0.00 \\
\hline 1.750 & 1.775 & 1.763 & 0.00 & 0.60 & 0.29 & 1.00 & 1.00 & 0.10 & 0.00 \\
\hline 1.775 & 1.800 & 1.788 & 0.00 & 0.58 & 0.28 & 1.00 & 1.00 & 0.10 & 0.00 \\
\hline 1.800 & 1.850 & 1.825 & 0.00 & 1.19 & 0.58 & 1.00 & 1.00 & 0.10 & 0.00 \\
\hline 1.850 & 1.900 & 1.875 & 0.00 & 1.30 & 0.64 & 1.00 & 1.00 & 0.10 & 0.00 \\
\hline 1.900 & 2.000 & 1.950 & 0.00 & 2.25 & 1.10 & 1.00 & 1.00 & 0.10 & 0.00 \\
\hline 2.000 & 2.400 & 2.200 & 0.01 & 93.09 & 45.62 & 1.00 & 1.00 & 0.10 & 0.00 \\
\hline & & Totals & 100.00 & 100.00 & 100.00 & & & WSSQ: & 0.04 \\
\hline
\end{tabular}
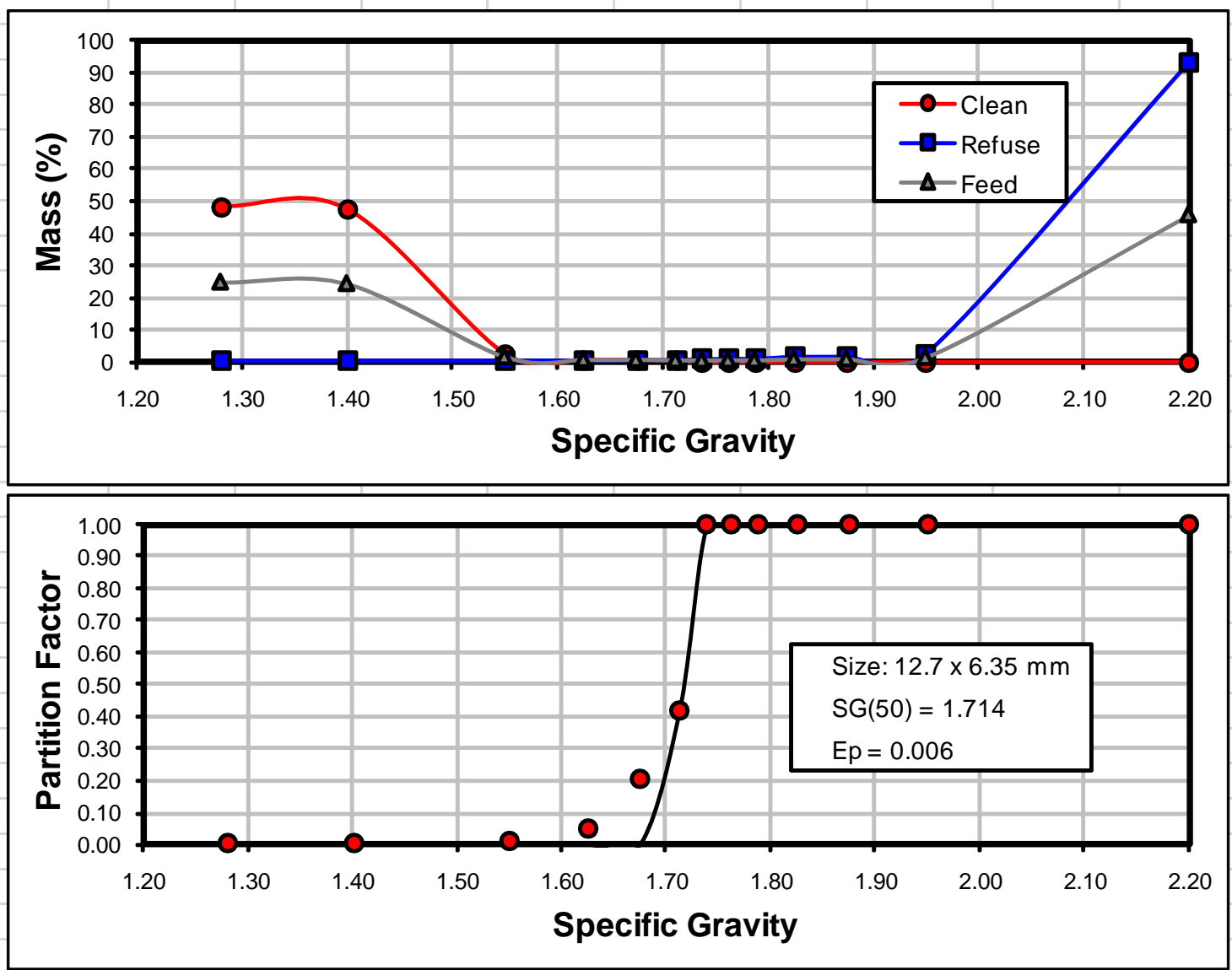
Circuit: TOMS CREEK - SECONDARY DMC CIRCUIT TEST 5 Size: $\quad 6.35 \times 3.175 \mathrm{~mm}$

Clean Yield (\%)

Refuse Yield (\%)

\begin{tabular}{|l|}
\hline 69.72 \\
\hline 30.28 \\
\hline
\end{tabular}

\begin{tabular}{|c|c|c|c|c|c|c|c|c|c|}
\hline & & & Clean & Refuse & Feed & Measured & Fitted & Fitting & Weighted \\
\hline Sink & Float & Mean & Mass & Mass & Mass & Refuse & Refuse & Weight & Squared \\
\hline SG & SG & SG & (\% Strm) & (\% Strm) & (\% Strm) & Partition & Partition & Factor & Error \\
\hline 1.260 & 1.300 & 1.280 & 57.61 & 0.20 & 40.23 & 0.00 & 0.00 & 0.10 & 0.00 \\
\hline 1.300 & 1.500 & 1.400 & 39.11 & 0.20 & 27.33 & 0.00 & 0.00 & 0.10 & 0.00 \\
\hline 1.500 & 1.600 & 1.550 & 1.80 & 0.07 & 1.28 & 0.02 & 0.00 & 0.10 & 0.00 \\
\hline 1.600 & 1.650 & 1.625 & 0.61 & 0.09 & 0.45 & 0.06 & 0.00 & 0.10 & 0.00 \\
\hline 1.650 & 1.700 & 1.675 & 0.48 & 0.28 & 0.42 & 0.20 & 0.00 & 0.20 & 0.04 \\
\hline 1.700 & 1.725 & 1.713 & 0.27 & 0.20 & 0.25 & 0.24 & 0.17 & 0.24 & 0.01 \\
\hline 1.725 & 1.750 & 1.738 & 0.00 & 0.43 & 0.13 & 1.00 & 0.76 & 0.10 & 0.06 \\
\hline 1.750 & 1.775 & 1.763 & 0.00 & 0.61 & 0.18 & 1.00 & 0.98 & 0.10 & 0.00 \\
\hline 1.775 & 1.800 & 1.788 & 0.00 & 0.60 & 0.18 & 1.00 & 1.00 & 0.10 & 0.00 \\
\hline 1.800 & 1.850 & 1.825 & 0.00 & 1.15 & 0.35 & 1.00 & 1.00 & 0.10 & 0.00 \\
\hline 1.850 & 1.900 & 1.875 & 0.00 & 1.37 & 0.41 & 1.00 & 1.00 & 0.10 & 0.00 \\
\hline 1.900 & 2.000 & 1.950 & 0.00 & 2.54 & 0.77 & 1.00 & 1.00 & 0.10 & 0.00 \\
\hline 2.000 & 2.400 & 2.200 & 0.12 & 92.26 & 28.02 & 1.00 & 1.00 & 0.10 & 0.00 \\
\hline & & Totals & 100.00 & 100.00 & 100.00 & & & WSSQ: & 0.11 \\
\hline
\end{tabular}
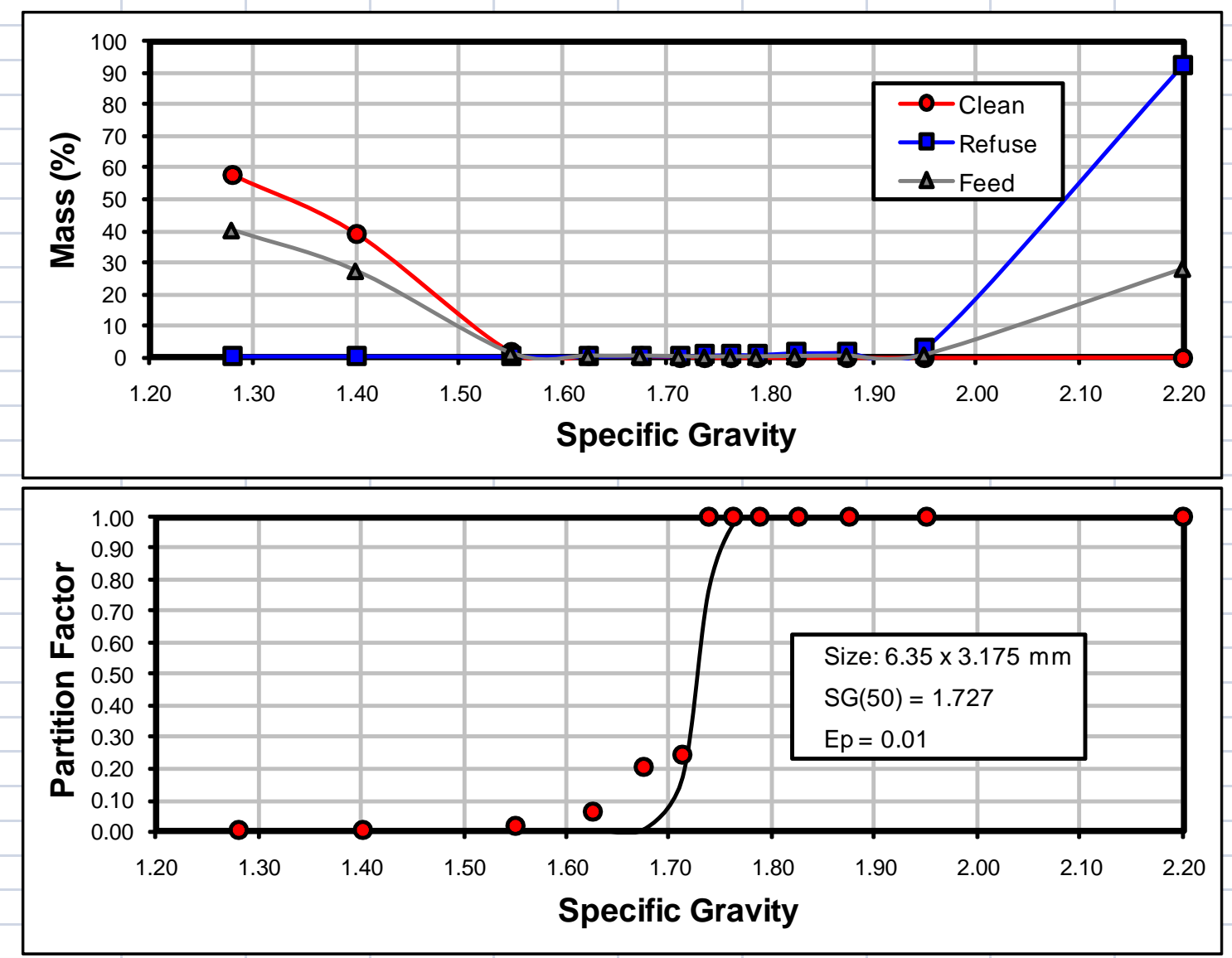

62 
Circuit: TOMS CREEK - SECONDARY DMC CIRCUIT TEST 5

Size: $\quad 3.175 \times 1.41 \mathrm{~mm}$

Clean Yield (\%)

Refuse Yield (\%)

\begin{tabular}{|l|}
\hline 72.49 \\
\hline 27.51 \\
\hline
\end{tabular}

\begin{tabular}{|c|c|c|c|c|c|c|c|c|c|}
\hline & & & Clean & Refuse & Feed & Measured & Fitted & Fitting & Weighted \\
\hline Sink & Float & Mean & Mass & Mass & Mass & Refuse & Refuse & Weight & Squared \\
\hline SG & $S G$ & SG & (\% Strm) & (\% Strm) & (\% Strm) & Partition & Partition & Factor & Error \\
\hline 1.260 & 1.300 & 1.280 & 64.98 & 1.76 & 47.59 & 0.01 & 0.00 & 0.10 & 0.00 \\
\hline 1.300 & 1.400 & 1.350 & 27.46 & 1.32 & 20.27 & 0.02 & 0.00 & 0.10 & 0.00 \\
\hline 1.400 & 1.500 & 1.450 & 4.17 & 0.19 & 3.08 & 0.02 & 0.00 & 0.10 & 0.00 \\
\hline 1.500 & 1.600 & 1.550 & 1.74 & 0.17 & 1.31 & 0.04 & 0.02 & 0.10 & 0.00 \\
\hline 1.600 & 1.700 & 1.650 & 0.94 & 0.39 & 0.79 & 0.14 & 0.14 & 0.14 & 0.00 \\
\hline 1.700 & 1.800 & 1.750 & 0.38 & 1.40 & 0.66 & 0.58 & 0.59 & 0.10 & 0.00 \\
\hline 1.800 & 1.900 & 1.850 & 0.05 & 2.29 & 0.67 & 0.95 & 0.93 & 0.10 & 0.00 \\
\hline 1.900 & 2.000 & 1.950 & 0.02 & 2.36 & 0.66 & 0.98 & 0.99 & 0.10 & 0.00 \\
\hline 2.000 & 2.400 & 2.200 & 0.26 & 90.12 & 24.98 & 0.99 & 1.00 & 0.10 & 0.00 \\
\hline & & & & & & & & & \\
\hline & & & & & & & & & \\
\hline & & & & & & & & & \\
\hline & & Totals & 100.00 & 100.00 & 100.00 & & & WSSQ: & 0.00 \\
\hline
\end{tabular}
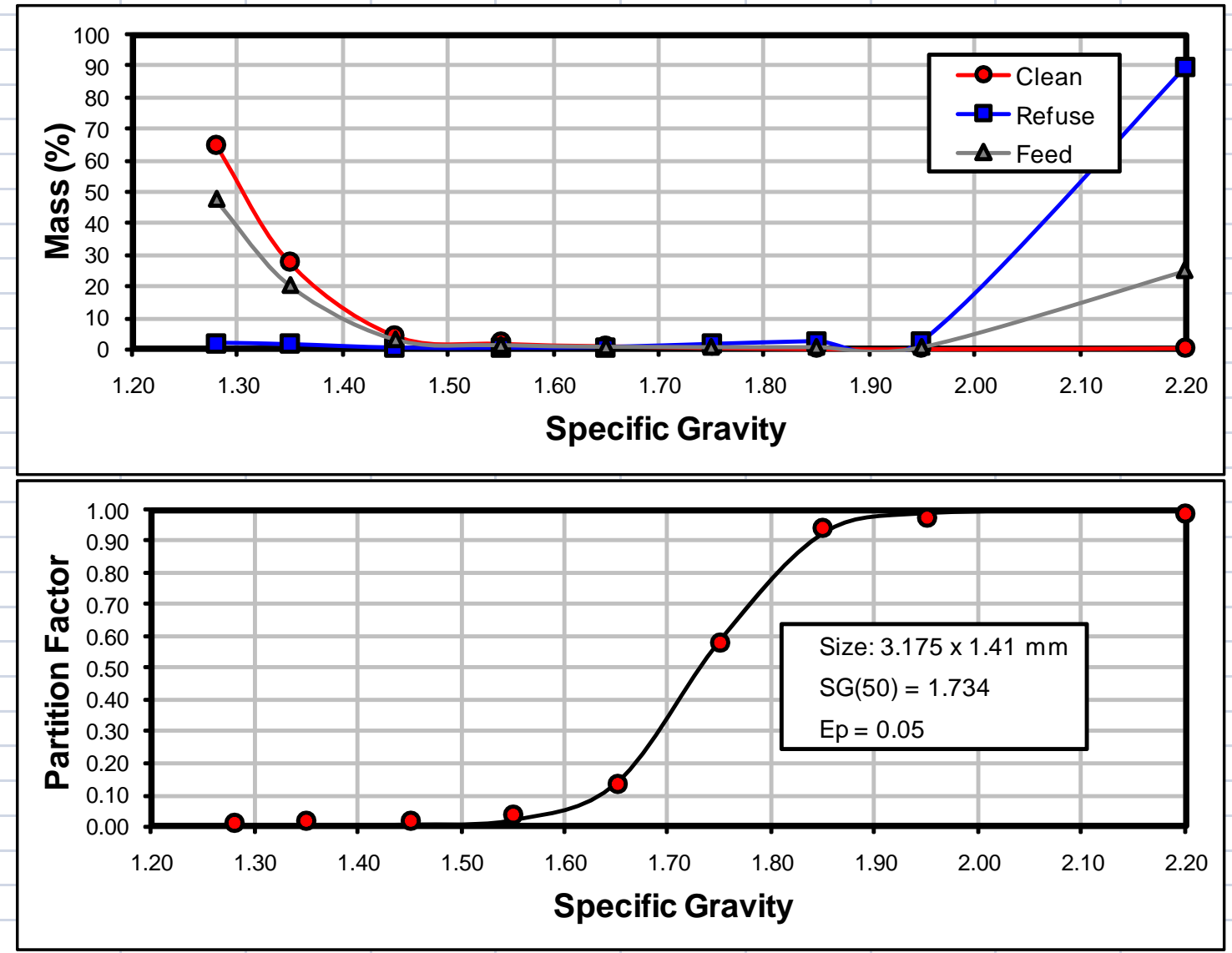
Circuit: TOMS CREEK - SECONDARY DMC CIRCUIT TEST 5

Size: $\quad 1.41 \times 0.707 \mathrm{~mm}$

Clean Yield (\%)

Refuse Yield (\%)

\begin{tabular}{|l|}
\hline 80.40 \\
\hline 19.60 \\
\hline
\end{tabular}

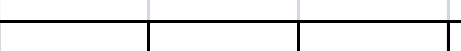

\begin{tabular}{|c|c|c|c|c|c|c|c|c|c|}
\hline & & & Clean & Refuse & Feed & Measured & Fitted & Fitting & Weighted \\
\hline Sink & Float & Mean & Mass & Mass & Mass & Refuse & Refuse & Weight & Squared \\
\hline$S G$ & SG & SG & (\% Strm) & (\% Strm) & (\% Strm) & Partition & Partition & Factor & Error \\
\hline 1.260 & 1.300 & 1.280 & 64.52 & 3.13 & 52.49 & 0.01 & 0.00 & 0.10 & 0.00 \\
\hline 1.300 & 1.400 & 1.350 & 26.67 & 1.92 & 21.82 & 0.02 & 0.00 & 0.10 & 0.00 \\
\hline 1.400 & 1.500 & 1.450 & 4.42 & 0.31 & 3.61 & 0.02 & 0.00 & 0.10 & 0.00 \\
\hline 1.500 & 1.600 & 1.550 & 1.74 & 0.18 & 1.43 & 0.02 & 0.01 & 0.10 & 0.00 \\
\hline 1.600 & 1.700 & 1.650 & 0.95 & 0.26 & 0.81 & 0.06 & 0.06 & 0.10 & 0.00 \\
\hline 1.700 & 1.800 & 1.750 & 0.58 & 0.59 & 0.58 & 0.20 & 0.22 & 0.20 & 0.00 \\
\hline 1.800 & 1.900 & 1.850 & 0.28 & 1.58 & 0.53 & 0.58 & 0.56 & 0.42 & 0.00 \\
\hline 1.900 & 2.000 & 1.950 & 0.09 & 2.01 & 0.47 & 0.84 & 0.86 & 0.16 & 0.00 \\
\hline 2.000 & 2.400 & 2.200 & 0.75 & 90.02 & 18.25 & 0.97 & 1.00 & 0.10 & 0.00 \\
\hline & & & & & & & & & \\
\hline & & & & & & & & & \\
\hline & & & & & & & & & \\
\hline & & Totals & 100.00 & 100.00 & 100.00 & & & WSSQ: & 0.00 \\
\hline
\end{tabular}
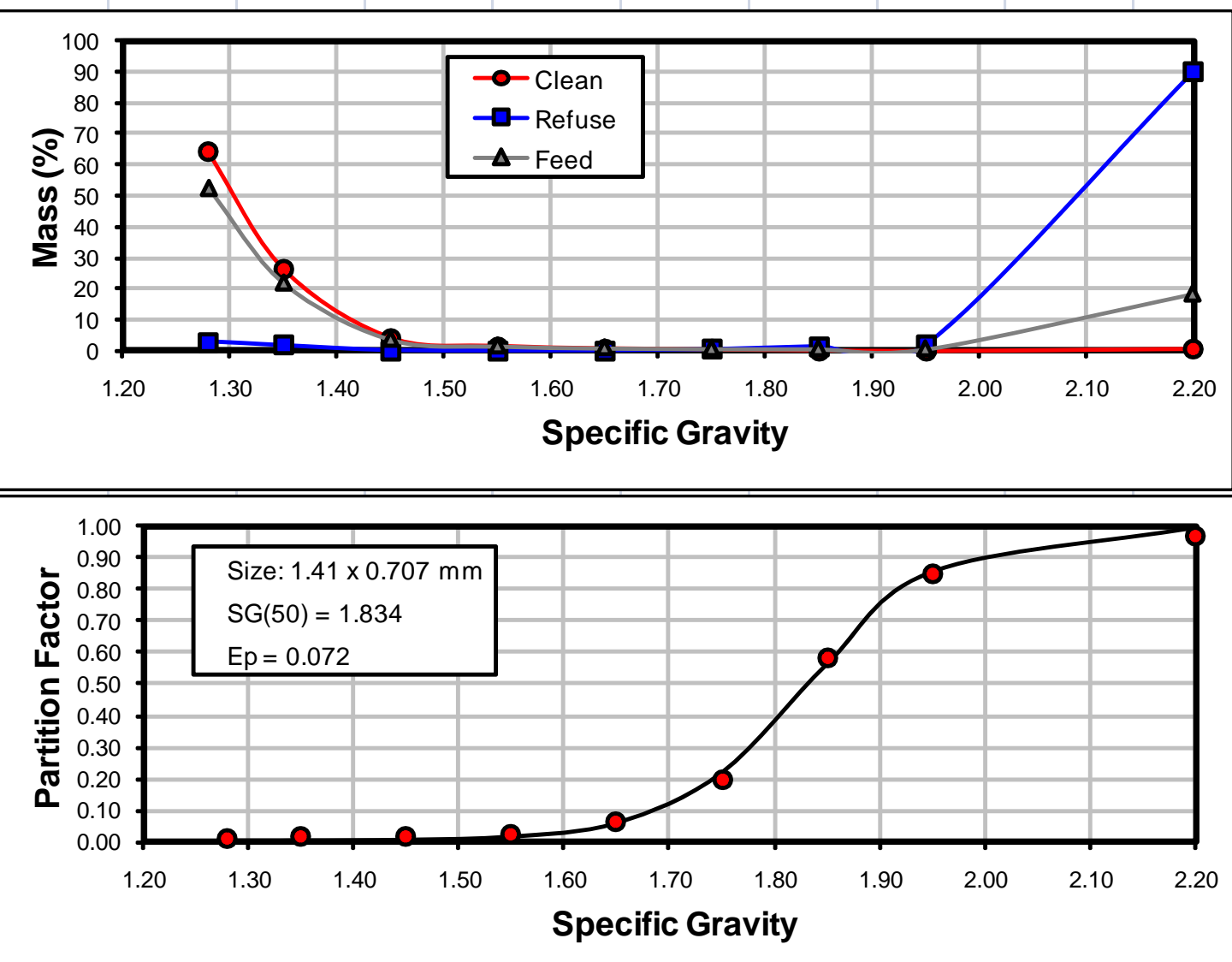
APPENDIX 6.6 - HIGH SG RANGE AND HIGH REJECT FEED 
Circuit: TOMS CREEK - SECONDARY DMC CIRCUIT TEST 6

\begin{tabular}{|c|c|c|c|c|c|c|c|c|}
\hline \multirow{3}{*}{\multicolumn{2}{|c|}{$\begin{array}{l}\text { Clean Rate }(\mathrm{t} / \mathrm{hr}) \text { : } \\
\text { Refuse Rate }(\mathrm{t} / \mathrm{hr}) \text { : } \\
\text { Feed Rate }(\mathrm{t} / \mathrm{hr}):\end{array}$}} & 273.7 & & \multicolumn{2}{|c|}{ Clean Yield (\%): } & \multicolumn{3}{|l|}{54.74} \\
\hline & & 226.3 & & \multicolumn{2}{|c|}{ Refuse Yield (\%): } & 45.26 & & \\
\hline & & 500.0 & & \multicolumn{2}{|c|}{ Feed Ash (\%): } & 43.61 & & \\
\hline Pass & Retain & Mean & Clean & Clean & Refuse & Refuse & Feed & Feed \\
\hline Size & Size & Size & Mass & Ash & Mass & Ash & Mass & Ash \\
\hline$(\mathrm{mm})$ & $(\mathrm{mm})$ & $(\mathrm{mm})$ & $(\%)$ & $(\%)$ & $(\%)$ & $(\%)$ & $(\%)$ & $(\%)$ \\
\hline 12.7 & 6.35 & 8.98 & 27.16 & 6.33 & 35.15 & 89.58 & 30.78 & 49.36 \\
\hline 6.35 & 3.175 & 4.49 & 36.45 & 5.86 & 34.90 & 88.86 & 35.75 & 42.53 \\
\hline 3.175 & 1.41 & 2.12 & 27.95 & 5.80 & 23.98 & 88.81 & 26.15 & 40.24 \\
\hline 1.41 & 0.707 & 1.00 & 7.59 & 6.46 & 5.43 & 88.62 & 6.61 & 36.99 \\
\hline \multirow[t]{2}{*}{0.707} & 0 & 0.00 & 0.85 & 8.35 & 0.54 & 84.29 & 0.71 & 34.50 \\
\hline & & Totals & 100.00 & 6.04 & 100.00 & 89.06 & 100.00 & 43.61 \\
\hline Pass & Retain & Mean & Clean & Refuse & Feed & Clean & Refuse & Feed \\
\hline Size & Size & Size & Yield & Yield & Yield & Mass & Mass & Mass \\
\hline$(\mathrm{mm})$ & $(\mathrm{mm})$ & $(\mathrm{mm})$ & $(\%)$ & $(\%)$ & $(\%)$ & (Cum\%) & (Cum\%) & (Cum\%) \\
\hline 12.7 & 6.35 & 8.98 & 48.31 & 51.69 & 100.00 & 27.16 & 35.15 & 30.78 \\
\hline 6.35 & 3.175 & 4.49 & 55.82 & 44.18 & 100.00 & 63.61 & 70.05 & 66.52 \\
\hline 3.175 & 1.41 & 2.12 & 58.51 & 41.49 & 100.00 & 91.56 & 94.03 & 92.68 \\
\hline 1.41 & 0.707 & 1.00 & 62.84 & 37.16 & 100.00 & 99.15 & 99.46 & 99.29 \\
\hline 0.707 & 0 & 0.00 & 65.57 & 34.43 & 100.00 & 100.00 & 100.00 & 100.00 \\
\hline & & & & & & & & \\
\hline & & Totals & 54.74 & 45.26 & 100.00 & & & \\
\hline
\end{tabular}

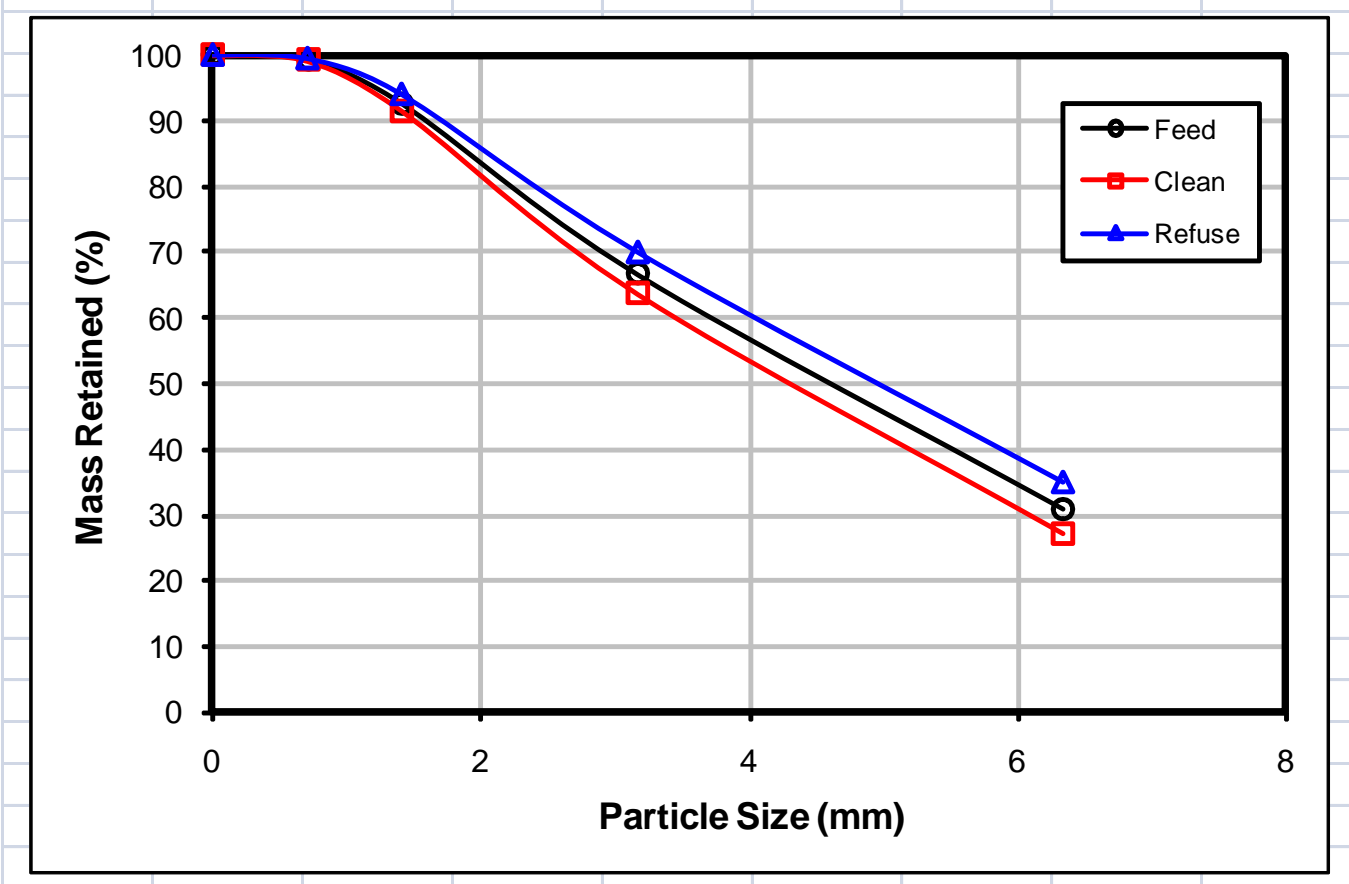


Circuit: TOMS CREEK - SECONDARY DMC CIRCUIT TEST 6

Size:

$12.7 \times 6.35 \mathrm{~mm}$

Clean Yield (\%)

Refuse Yield (\%)

\begin{tabular}{|l|}
\hline 48.31 \\
\hline 51.69 \\
\hline
\end{tabular}

\begin{tabular}{|c|c|c|c|c|c|c|c|c|c|}
\hline & & & Clean & Refuse & Feed & Measured & Fitted & Fitting & Weighted \\
\hline Sink & Float & Mean & Mass & Mass & Mass & Refuse & Refuse & Weight & Squared \\
\hline SG & SG & SG & (\% Strm) & (\% Strm) & (\% Strm) & Partition & Partition & Factor & Error \\
\hline 1.260 & 1.300 & 1.280 & 70.80 & 0.00 & 34.21 & 0.00 & 0.00 & 0.10 & 0.00 \\
\hline 1.300 & 1.500 & 1.400 & 26.22 & 0.01 & 12.67 & 0.00 & 0.00 & 0.10 & 0.00 \\
\hline 1.500 & 1.600 & 1.550 & 1.95 & 0.02 & 0.95 & 0.01 & 0.00 & 0.10 & 0.00 \\
\hline 1.600 & 1.650 & 1.625 & 0.53 & 0.04 & 0.28 & 0.07 & 0.03 & 0.10 & 0.00 \\
\hline 1.650 & 1.700 & 1.675 & 0.38 & 0.16 & 0.27 & 0.31 & 0.31 & 0.31 & 0.00 \\
\hline 1.700 & 1.725 & 1.713 & 0.07 & 0.17 & 0.12 & 0.72 & 0.75 & 0.28 & 0.00 \\
\hline 1.725 & 1.750 & 1.738 & 0.01 & 0.25 & 0.13 & 0.96 & 0.92 & 0.10 & 0.00 \\
\hline 1.750 & 1.775 & 1.763 & 0.00 & 0.22 & 0.11 & 1.00 & 0.98 & 0.10 & 0.00 \\
\hline 1.775 & 1.800 & 1.788 & 0.00 & 0.20 & 0.10 & 1.00 & 0.99 & 0.10 & 0.00 \\
\hline 1.800 & 1.850 & 1.825 & 0.00 & 0.48 & 0.25 & 1.00 & 1.00 & 0.10 & 0.00 \\
\hline 1.850 & 1.900 & 1.875 & 0.00 & 0.58 & 0.30 & 1.00 & 1.00 & 0.10 & 0.00 \\
\hline 1.900 & 2.000 & 1.950 & 0.00 & 0.87 & 0.45 & 1.00 & 1.00 & 0.10 & 0.00 \\
\hline 2.000 & 2.400 & 2.200 & 0.04 & 97.00 & 50.15 & 1.00 & 1.00 & 0.10 & 0.00 \\
\hline & & Totals & 100.00 & 100.00 & 100.00 & & & WSSQ: & 0.01 \\
\hline
\end{tabular}
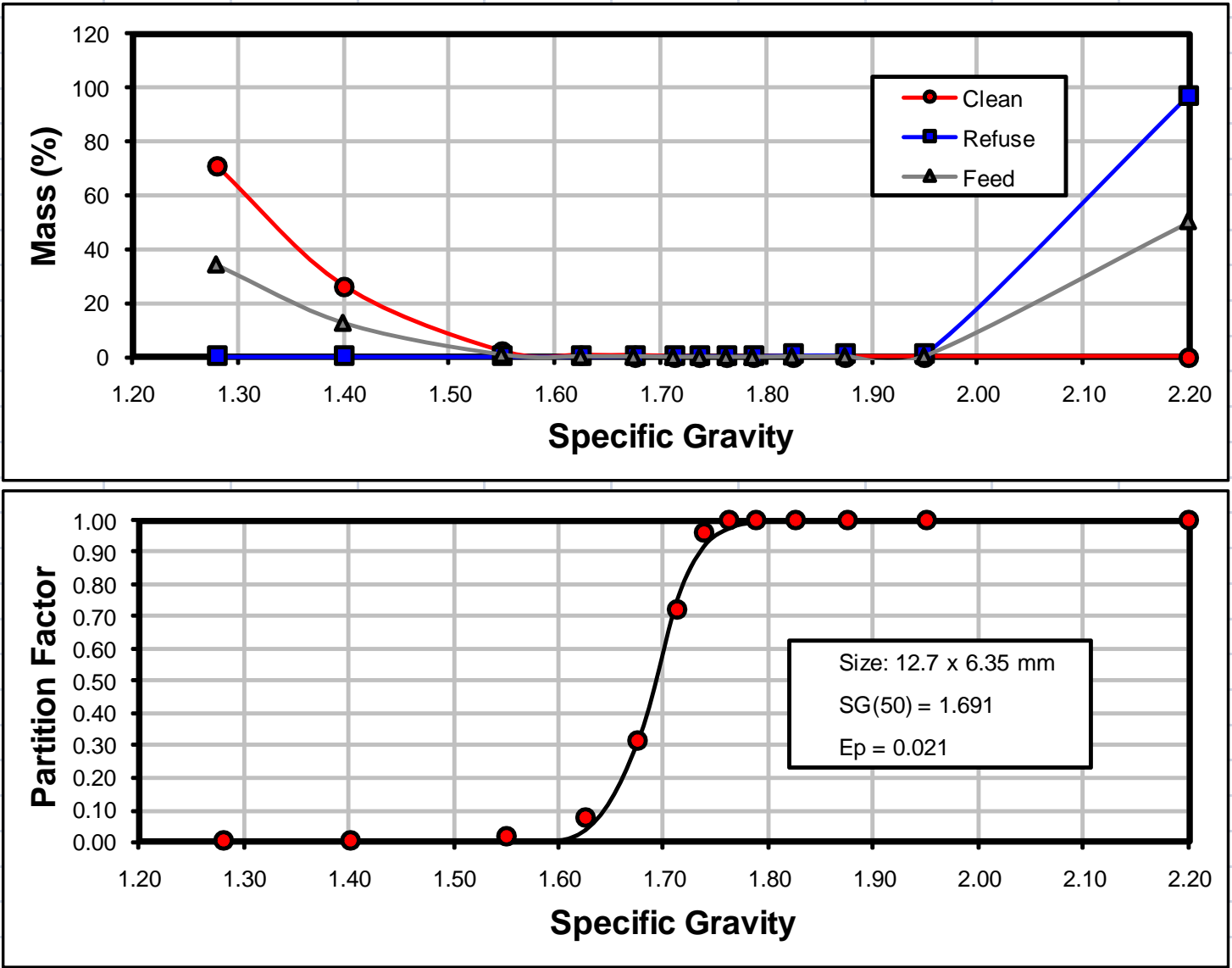
Circuit: TOMS CREEK - SECONDARY DMC CIRCUIT TEST 6

Size: $\quad 6.35 \times 3.175 \mathrm{~mm}$

Clean Yield (\%)

Refuse Yield (\%)

\begin{tabular}{|l|}
\hline 55.82 \\
\hline 44.18 \\
\hline
\end{tabular}

\begin{tabular}{|c|c|c|c|c|c|c|c|c|c|}
\hline & & & Clean & Refuse & Feed & Measured & Fitted & Fitting & Weighted \\
\hline Sink & Float & Mean & Mass & Mass & Mass & Refuse & Refuse & Weight & Squared \\
\hline SG & SG & SG & (\% Strm) & (\% Strm) & (\% Strm) & Partition & Partition & Factor & Error \\
\hline 1.260 & 1.300 & 1.280 & 74.12 & 0.04 & 41.39 & 0.00 & 0.00 & 0.10 & 0.00 \\
\hline 1.300 & 1.500 & 1.400 & 23.14 & 0.04 & 12.93 & 0.00 & 0.00 & 0.10 & 0.00 \\
\hline 1.500 & 1.600 & 1.550 & 1.59 & 0.06 & 0.91 & 0.03 & 0.00 & 0.10 & 0.00 \\
\hline 1.600 & 1.650 & 1.625 & 0.54 & 0.06 & 0.33 & 0.08 & 0.08 & 0.10 & 0.00 \\
\hline 1.650 & 1.700 & 1.675 & 0.32 & 0.24 & 0.28 & 0.37 & 0.38 & 0.37 & 0.00 \\
\hline 1.700 & 1.725 & 1.713 & 0.05 & 0.20 & 0.12 & 0.76 & 0.73 & 0.24 & 0.00 \\
\hline 1.725 & 1.750 & 1.738 & 0.05 & 0.30 & 0.16 & 0.83 & 0.88 & 0.17 & 0.00 \\
\hline 1.750 & 1.775 & 1.763 & 0.00 & 0.20 & 0.09 & 1.00 & 0.95 & 0.10 & 0.00 \\
\hline 1.775 & 1.800 & 1.788 & 0.00 & 0.23 & 0.10 & 1.00 & 0.98 & 0.10 & 0.00 \\
\hline 1.800 & 1.850 & 1.825 & 0.00 & 0.56 & 0.25 & 1.00 & 1.00 & 0.10 & 0.00 \\
\hline 1.850 & 1.900 & 1.875 & 0.00 & 0.69 & 0.30 & 1.00 & 1.00 & 0.10 & 0.00 \\
\hline 1.900 & 2.000 & 1.950 & 0.00 & 1.22 & 0.54 & 1.00 & 1.00 & 0.10 & 0.00 \\
\hline 2.000 & 2.400 & 2.200 & 0.19 & 96.16 & 42.59 & 1.00 & 1.00 & 0.10 & 0.00 \\
\hline & & Totals & 100.00 & 100.00 & 100.00 & & & WSSQ: & 0.01 \\
\hline
\end{tabular}
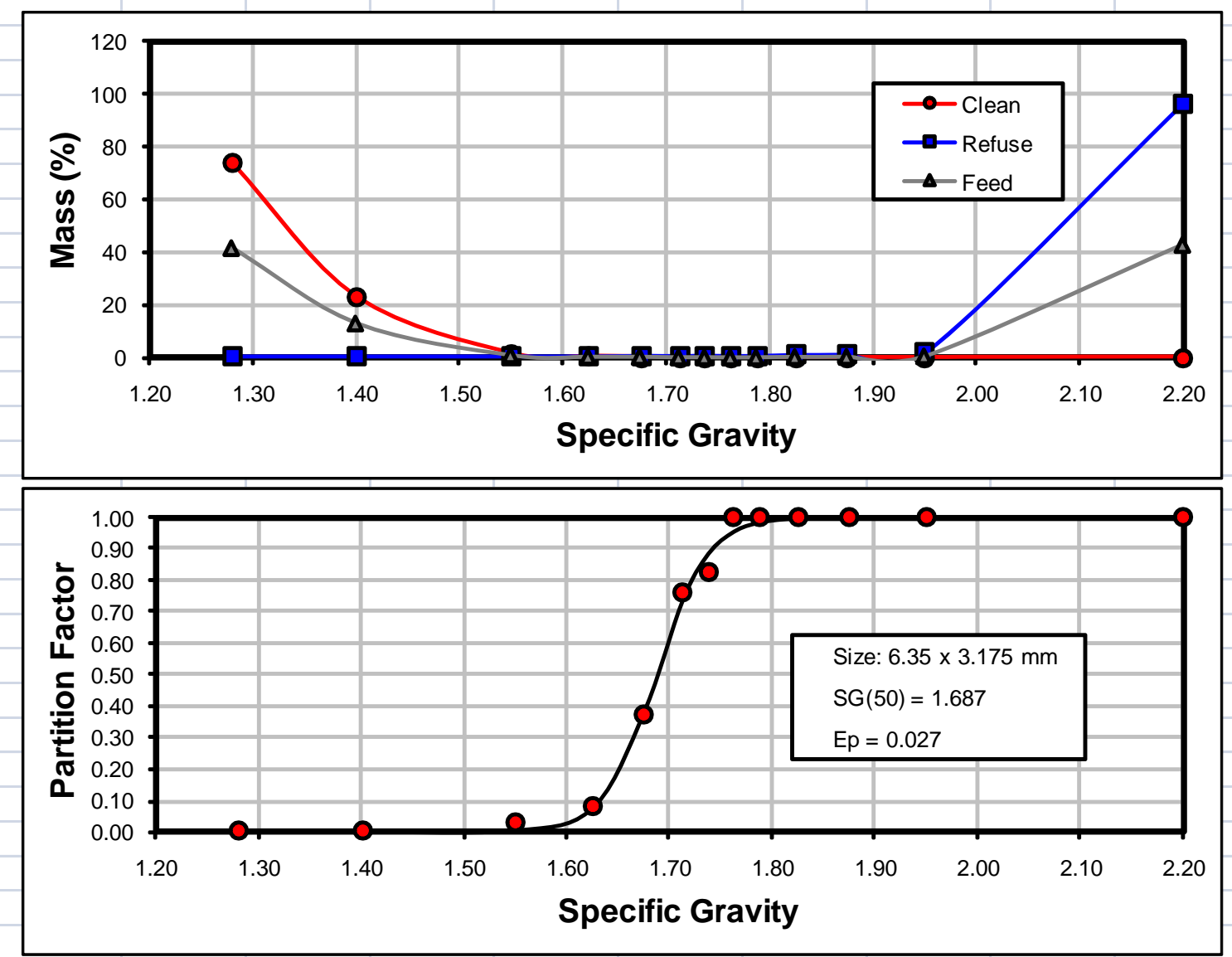
Circuit: TOMS CREEK - SECONDARY DMC CIRCUIT TEST 6

Size: $\quad 3.175 \times 1.41 \mathrm{~mm}$

Clean Yield (\%)

Refuse Yield (\%)

\begin{tabular}{|l|}
\hline 58.51 \\
\hline 41.49 \\
\hline
\end{tabular}

\begin{tabular}{|c|c|c|c|c|c|c|c|c|c|}
\hline & & & Clean & Refuse & Feed & Measured & Fitted & Fitting & Weighted \\
\hline Sink & Float & Mean & Mass & Mass & Mass & Refuse & Refuse & Weight & Squared \\
\hline SG & $S G$ & $S G$ & (\% Strm) & (\% Strm) & (\% Strm) & Partition & Partition & Factor & Error \\
\hline 1.260 & 1.300 & 1.280 & 76.12 & 0.55 & 44.76 & 0.01 & 0.00 & 0.10 & 0.00 \\
\hline 1.300 & 1.400 & 1.350 & 16.49 & 0.17 & 9.72 & 0.01 & 0.00 & 0.10 & 0.00 \\
\hline 1.400 & 1.500 & 1.450 & 3.98 & 0.06 & 2.35 & 0.01 & 0.00 & 0.10 & 0.00 \\
\hline 1.500 & 1.600 & 1.550 & 1.68 & 0.10 & 1.02 & 0.04 & 0.02 & 0.10 & 0.00 \\
\hline 1.600 & 1.700 & 1.650 & 0.92 & 0.27 & 0.65 & 0.17 & 0.18 & 0.17 & 0.00 \\
\hline 1.700 & 1.800 & 1.750 & 0.23 & 0.90 & 0.51 & 0.74 & 0.73 & 0.10 & 0.00 \\
\hline 1.800 & 1.900 & 1.850 & 0.03 & 1.16 & 0.50 & 0.96 & 0.97 & 0.10 & 0.00 \\
\hline 1.900 & 2.000 & 1.950 & 0.01 & 1.37 & 0.57 & 0.99 & 1.00 & 0.10 & 0.00 \\
\hline 2.000 & 2.400 & 2.200 & 0.54 & 95.42 & 39.91 & 0.99 & 1.00 & 0.10 & 0.00 \\
\hline & & & & & & & & & \\
\hline & & & & & & & & & \\
\hline & & Totals & 100.00 & 100.00 & 10000 & & & WSSO. & 000 \\
\hline
\end{tabular}
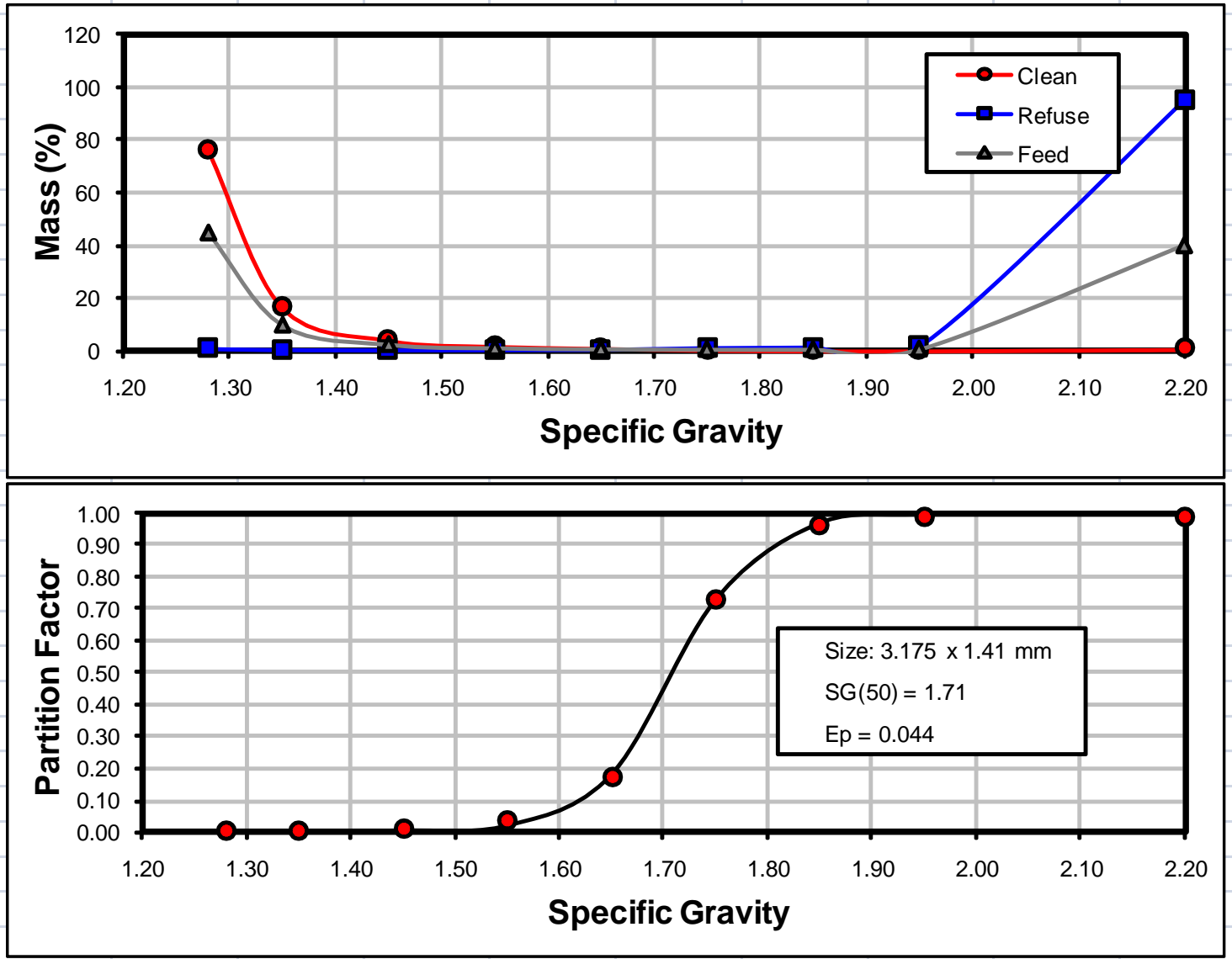
Circuit: TOMS CREEK - SECONDARY DMC CIRCUIT TEST 6

Size: $\quad 1.41 \times 0.707 \mathrm{~mm}$

Clean Yield (\%)

Refuse Yield (\%)

\begin{tabular}{|l|}
\hline 62.84 \\
\hline 37.16 \\
\hline
\end{tabular}

\begin{tabular}{|l|l|l|l|}
\hline & & & Cl \\
\hline
\end{tabular}

\begin{tabular}{|c|c|c|c|}
\hline Sink & Float & Mean & SG \\
SG & SG & SG & (\% \\
\hline & 1260 & 1.300 & 1.280
\end{tabular}

1.260

1.300

1.400

1.500

1.600

1.700

1.800

1.900

2.000

1.300

1.400

1.500

1.600

1.700

1.800

1.900

2.000

2.400
1.350

1.450

1.550

1.650

1.750

1.850

1.950

2.200

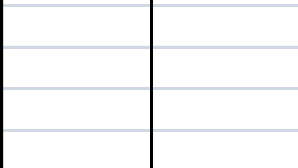

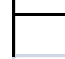
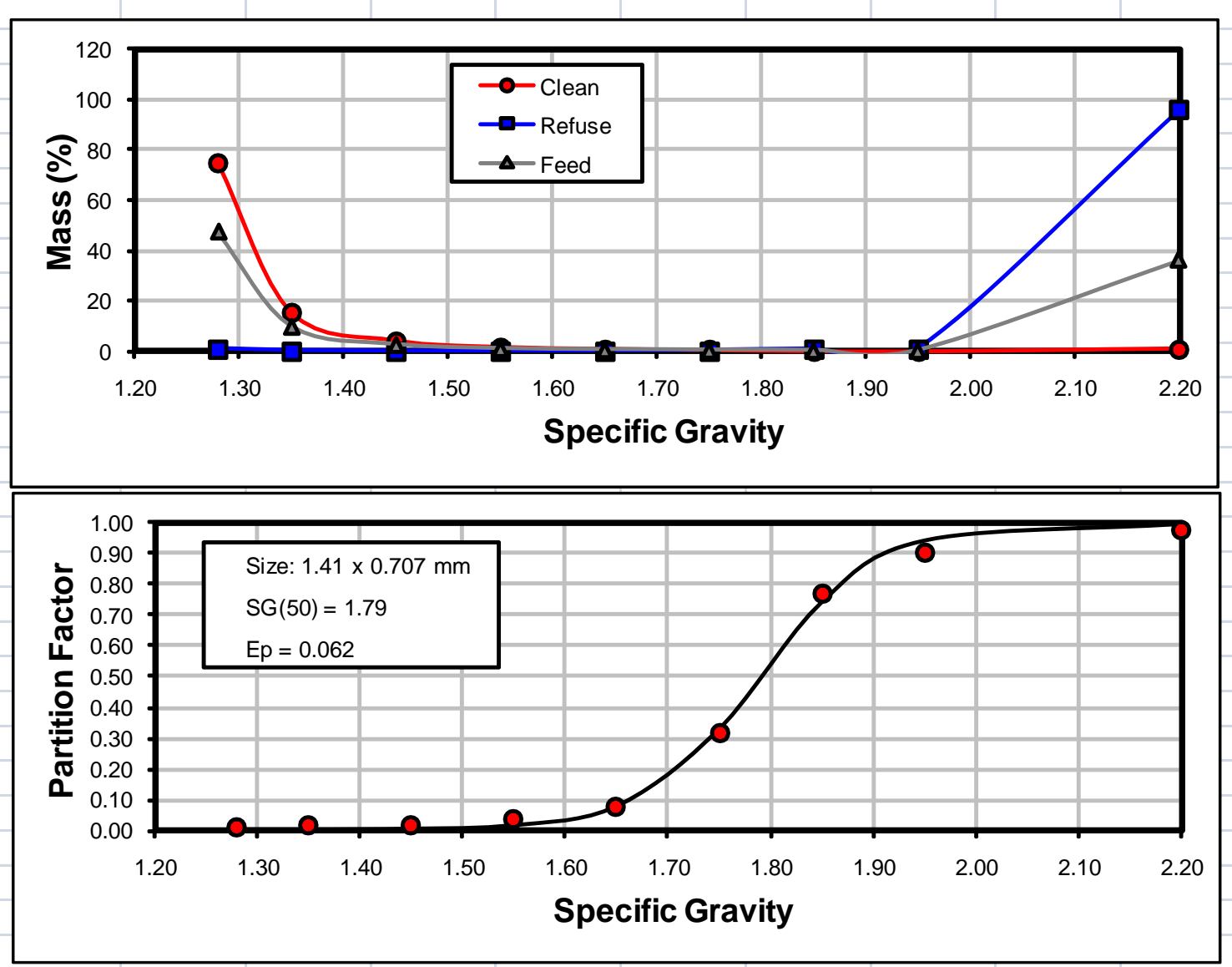
Appendix 16: Densification of Fine Coal Refuse Slurries to Eliminate Slurry Ponds (KY002) 


\section{FINAL TECHNICAL REPORT}

Contract Title and Number: Crosscutting Technology Development at the Center for Advanced Separation Technologies (DE-FC26-02NT41607)

Sub-Recipient Project Title: Improving Densification of Fine Coal Refuse Tailings to Eliminate Slurry Ponds

Principal Investigators:

B.K.Parekh (P.I.)

R.Honaker, D.P.Patil

Contact Address:

Center for Applied Energy Research 2540 Research Park Dr. Lexington, KY 40511

Subcontractor Address:

No subcontracts issued
Period of Performance:

Starting Date: 10/01/2002

Ending Date: 10/31/2009
Report Information:

Type: Final

Number: 7

Period: $\quad$ 4/01/03 - 08/31/2006

Date: $\quad 08 / 25 / 2006$

Code: KY002-Final

Contact Information:

Phone: 859-257-0239

Fax: $\quad$ 859-257-0302

E-Mail: Parekh@caer.uky.edu

Subcontractor Information:

Phone:

Fax:

E-Mail: 


\section{DISCLAIMER}

This report was prepared as an account of work sponsored by an agency of the United States Government. Neither the United States Government nor any agency thereof, nor any of their employees, make any warranty, express or implied, nor assume any legal liability or responsibility for the accuracy, completeness, or usefulness of any information, apparatus, product, or process disclosed, or represents that its use would not infringe privately owned rights. Reference herein to any specific commercial product, process, or service by trade name, trademark, manufacturer, or otherwise does not necessarily constitute or imply endorsement, recommendation, or favoring by the United States Government or any agency thereof. The views and opinions of authors expressed herein do not necessarily state or reflect those of the United States Government or agency thereof. 


\section{ABSTRACT}

A coal preparation plant washing coal discharges fine size (minus 100 mesh) refuse-coal slurry in an impoundment. The coal industry is required to monitor the impoundment regularly. Impoundment breakage has become a serious problem for the industry. In this project an advanced thickening technique known as "Deep Cone Thickener" was tested at three different coal preparation plants to produce a paste type material which could be pumped and stored on a slightly elevated surface, thus eliminating the impoundment and the damn breakage problem. The results of the present study showed that the conventional thickener discharge from a coal preparation plant containing about 25 to 30 percent solids could be thickened to about 50 to 55 percent solids, which is similar to the dewatered refuse tailings produced by a belt filter press. A couple of coal preparation plants have decided to install the technique. 


\section{Table of Contents}

Page

Disclaimer

2

Abstract 3

List of Graphical Materials

Executive Summary 6

$\begin{array}{ll}\text { Introduction } & 7\end{array}$

$\begin{array}{ll}\text { Experimental } & 13\end{array}$

$\begin{array}{ll}\text { Results and Discussions } & 15\end{array}$

Summary and Conclusions $\quad 29$

$\begin{array}{ll}\text { References } & 30\end{array}$ 


\section{Lists of Graphical Materials}

Page

Figure 1 Schematic diagram of a gravity thickener $\quad 8$

Figure 2 The EIMCO E-CAT clarifier thickener $\quad 9$

Figure 3. Schematic diagram of deep cone thickener 11

Figure 4. Zeta Potential of the fine refuse slurry solids with respect to $\mathrm{pH}$. 14

Figure 5. Effect of dosages of different anionic flocculants on $\begin{array}{ll}\text { suspension supernatant quality. } & 15\end{array}$

Figure 6. Settling flux of flocculated solids with respect to percent solids in suspension. 15

Figure 7. Yield stress of flocculated thickener underflow $\begin{array}{ll}\text { with respect to percent solids. } & 16\end{array}$

Figure 8. Pilot-scale Deep Cone Thickener unit installed at the Clean Coal Corp.

Figure 9. Deep Cone Thickener underflow containing about 40 percent solids.

Figure 10. Deep Cone Thickener underflow containing about 53 percent solids.

Figure 11.Effect of flocculant AD609XX dosage on settling flux and settled percent solids.

Figure 12. Effect of mode of addition of flocculants on settling flux using the Nalco flocculants

Figure 13. Effectof mode of flocculant addition on settled percent using the Nalco flocculants

Figure 14. T-Floc apparatus set-up showing setteled solids and clear supernatant

Figure 15. Flowsheet of the pilot-scale set-up at the Arch Coal Co.

Figure 16. Deep Cone Thickener underflow containing about 45 percent solids after shear thinning.

Figure 17. Deep Cone thickener underflow containing about 52 percent solids after shear thinning

Figure 18. Yield stress determination of the thickener underflow using the slump test

Figure 19. Particle size analysis of the Lone Mountain thickener underflow

Figure 20. Intitial settling flux vs. solids concentration of the Lone Mountain slurry 


\section{EXECUTIVE SUMMARY}

Increased mechanization in the underground coal mining industry has decreased selectivity and increased the volume of refuse. Coal preparation separates noncombustibles material from coal. Thus, a coal preparation plant separates the material it receives into a product stream and a reject stream, which may be further divided into coarse and fine refuse streams. Depending on the source, 20 to 50 percent of the run-ofmine material ends up in reject stream. One of the reject streams is slurry, which is a blend of water, coal fines, silt, sand, and clay particles, and is commonly disposed of in an impoundment. As of August 2001, Mining Safety and Health Administration (MSHA) oversee 713 active fresh-water and slurry impoundments in the United States. The coal industry is required to monitor the impoundment constantly and maintain the dikes holding the slurry. In the past, there have been several incidents of impoundment breakthrough, of these, the Buffalo Creek in W.Va in1972 and the Martin County Coal in Kentucky in the year 2000 have drawn attention of Federal and State Governments and local people due to heavy loses of life and property.

Impoundment maintenance is an on going problem for the coal industry and its breakage creates environmental as well as financial problems. The U.S. coal industry is seeking help in eliminating the refuse ponds. The Deep Cone ${ }^{\mathrm{TM}}$ thickener technology which has been successfully applied in the alumina industry to dewater red mud into a paste was evaluated for the fine coal refuse slurry. In this project a laboratory as well as a pilotscale Deep Cone Thickener tests were conducted at three different coal preparation plants. Samples of thickener underflow slurries were collected from the preparation plants for laboratories studies to determine the feasibility of producing a paste from both the slurries. Laboratory results identified that mode of addition of flocculants (anionic followed by cationic flocculant) is an important step in producing desired types of floccs which should be large and when hold in hand should be squeezable. Pilot-scale studies identified that about 6 to 8 hours retention time of the slurry in the Deep Cone thickener is sufficient to produce a paste material containing about 52 weight percent solids. The rheology (yield stress) of the paste was in the range of 150 to $250 \mathrm{~Pa}$ indicating its pump ability for easy disposal. The study recommends testing of the technology at a preparation plant to study disposal at the site and also test the technology for the clean coal slurry with very low ash content. 


\section{INTRODUCTION}

Increased mechanization in the underground coal mining industry has decreased selectivity and increased the amount of refuse created. Coal preparation separates noncombustible material from coal. Thus, a coal preparation plant separates the material it receives into a product stream and a reject stream, which may be further divided into coarse and fine refuse streams. Depending on the source, 20 to $50 \%$ of the run-of-mine material ends up in reject streams. One of the reject streams is a slurry stream. This is a blend of water, coal fines, silt, sand, and clay particles, which is commonly disposed of in an impoundment. As of August 2001, MSHA has overseen 713 active fresh-water and slurry impoundments in the United States. The coal industry is required to monitor the impoundment constantly and maintain the dikes holding the slurry. There have been several incidents of impoundment breakthrough. Of these, Buffalo Creek in West Virginia in1972 and recently, Martin County Coal in Kentucky, have drawn the attention of federal and state governments and local people due to heavy losses of life and property.

In this project an advanced thickening technique known as "Paste Thickening Technology" marketed by the Dorr-Oliver EIMCO, which has been successfully applied in the alumina processing industry for thickening of the red mud, was evaluated at three different coal preparation plants for the dewatering of fine coal tailings. The DEEP CONE Thickener ${ }^{\mathrm{TM}}$ is specially designed to concentrate tailings into a high percent solid, so it can discharge as a paste. The thickened paste material could be stacked at a low angle of repose rather than stored in a pond. Thus, with utilization of this technique it is anticipated that the fine refuse slurry ponds could be completely eliminated.

The main objective of the program is to evaluate the application of the DEEP CONETM Thickener technology for the disposal of fine coal refuse slurry as a paste. It was also the objective of the program to obtain basic information on the flocculation of the refuse slurries and the rheological properties of the thickened sludge. The laboratory and the pilot-scale testing conducted at three different coal preparation plants showed that the Deep Cone Thickener was able to dewater the conventional thickener underflow material and produce a paste containing about 50 to 55 . wt. \% solids. The dewatered solids had the same moisture contents as obtained by a belt filter machine.

\section{Background}

The development of thickeners started in the early $20^{\text {th }}$ century. The modern era of thickening started in the year 1905 with the invention of Dorr thickener. It made possible continuous dewatering giving a clear overflow and thick underflow. Rakes were driven using a rake mechanism and the primary reason of the rakes was to move the solids to the discharge end. The rakes moved very slowly with speeds less than $1 \mathrm{rpm}$ so as not to cause agitation and not interfere with settling of solids (Parekh and Matoney 1991). 
From that period there has been a tremendous improvement in the design of thickeners and various designs and technologies are currently being used. Thickeners may be generally classified as follows according to the percent solids they produce or the type of equipment they have.

- Conventional thickeners

- High Rate thickeners

- High Rate Rakeless thickeners

- Ultra high density or Paste thickener

\section{Conventional Thickeners}

Conventional thickeners have served the coal and minerals industry for the last hundred years and are continuing to do so all over the world. Their major advantage lies in the fact that the design is simple and can handle large volumes of material. Most of the conventional thickeners have the same major components as listed below and shown in Figure 1.

- tank

- feedwell

- overflow and underflow withdrawal systems

- rake systems which includes rake, drive and support structure.

The design of each of these components is based on specific requirements of that particular operation. The rake system helps in keeping the solids in motion continuously and sends them to the discharge point. Its second function is to provide channels through which the water can be released to the overflow thus thickening the underflow. The thickeners are usually made up of steel or concrete. The diameter of the thickener ranges from $20 \mathrm{~m}$ to $45 \mathrm{~m}$. The slope of the thickener bottom varies from 1:12 to 1:3. The design of rake mechanism is very critical to the performance of the thickener.

The conventional thickeners have been a back bone for the coal and minerals industry for a long period of time. Their major advantage lies in the simplicity of their design and high capacity. But later in the twentieth century the industry felt the need for developing more advanced thickeners to produce higher percentage solids in the underflow. This is so because the conventional thickeners can produce an underflow with 35 percent solids which was sufficient before but with stringent environmental requirements and high cost of maintaining impoundments, there was an urgent need for a drastic improvement of the thickener design to produce paste like underflow.

For a thickener the main objective is to obtain high solids concentration in the underflow while maintaining as clear an overflow as possible but clarity in the overflow is not a main priority for a thickener. There are several designs available for choosing a suitable thickener and rapid advances have been made for making the thickeners perform as better as possible. In these days where economical and environmental problems share equal importance, the mining industry is striving to produce higher density underflow solids; the thickeners are evolving to meet those demands. The new and scientific designs of 
thickeners require advanced feed and flocculation systems, higher torque drives and controls which did not exist in the thickeners before.

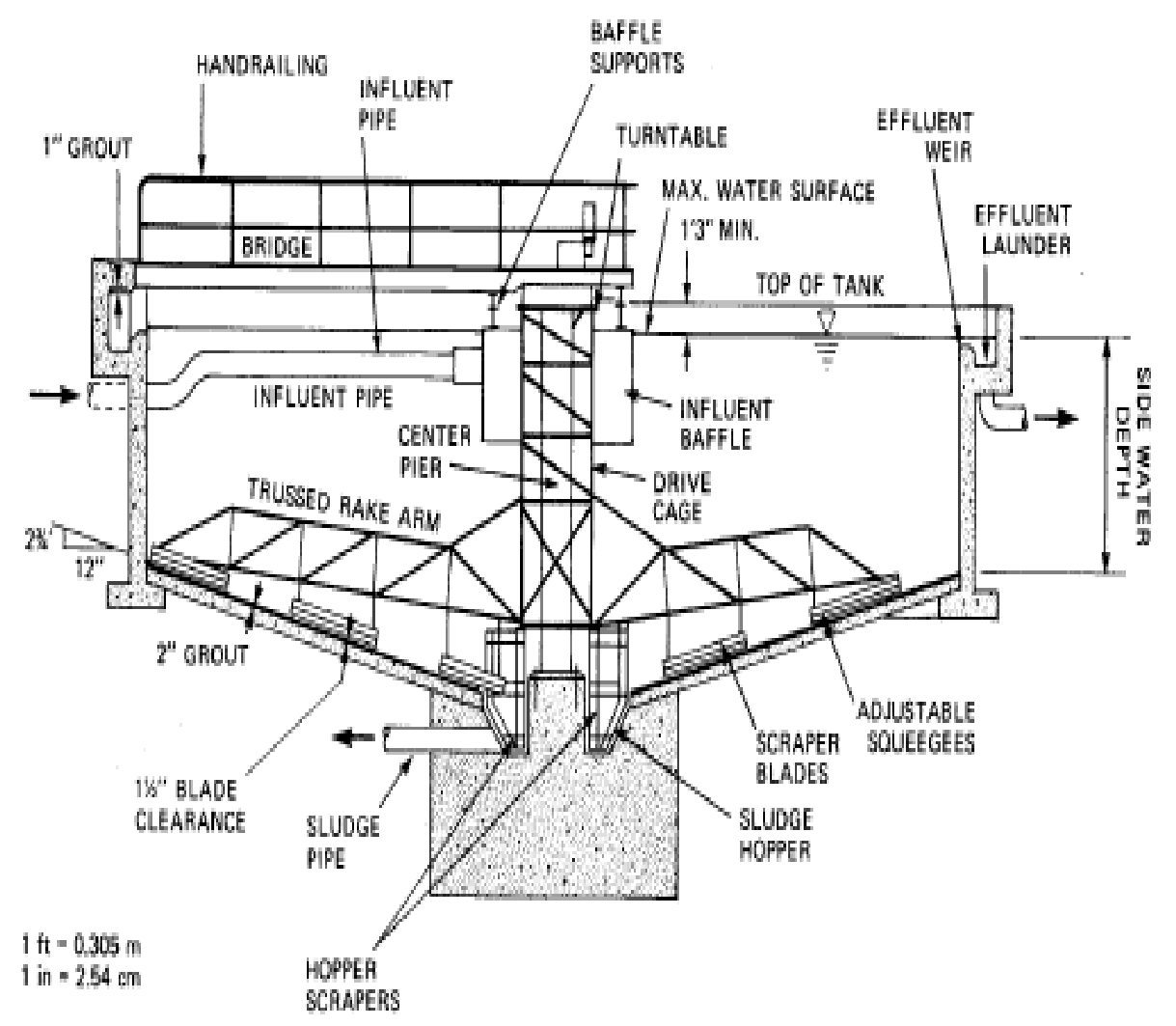

Figure 1: Schematic diagram of a gravity thickener

\section{High Rate Thickeners}

High rate thickeners can produce more tonnage per unit area and have a faster settling rate than conventional thickeners. The two pioneers in designing high rate thickeners are Eimco Process Company and Enviroclear. The success of high rate thickeners was because of optimum use of flocculants and special feed well systems and controls. The relationship between settling flux and feed percent solids was well understood and it was determined that there exists an optimum feed percent solids at which the settling flux and underflow percent solids is maximum. The optimum feed percent solids are determined in the lab using bench scale testing and by conducting pilot scale studies. Special feed wells were designed with and without bottom plates and dilution features to reach the optimum flux concentration. Once this was achieved the throughput capacity of the thickener improved to a great extent. The two most widely used dilution system are EDUC $^{\mathrm{R}}$ feed dilution system (EIMCO) and Supaflo Autodil ${ }^{\mathrm{TM}}$ system (Outokumpu).

\section{Ultra-High Rate Rakeless Thickener}


The design of the thickener was continuously improved due to persistent research efforts and a new design of thickener without rakes was developed called the rake less thickener, which consists of a deep thickener tank with specially designed dewatering cones at its center. This feature plus the cylinder ringing outside the feed well produces a clear overflow. Figure 2 shows the Rakeless thickener called the E-Cat Clarifier Thickener patented by EIMCO.

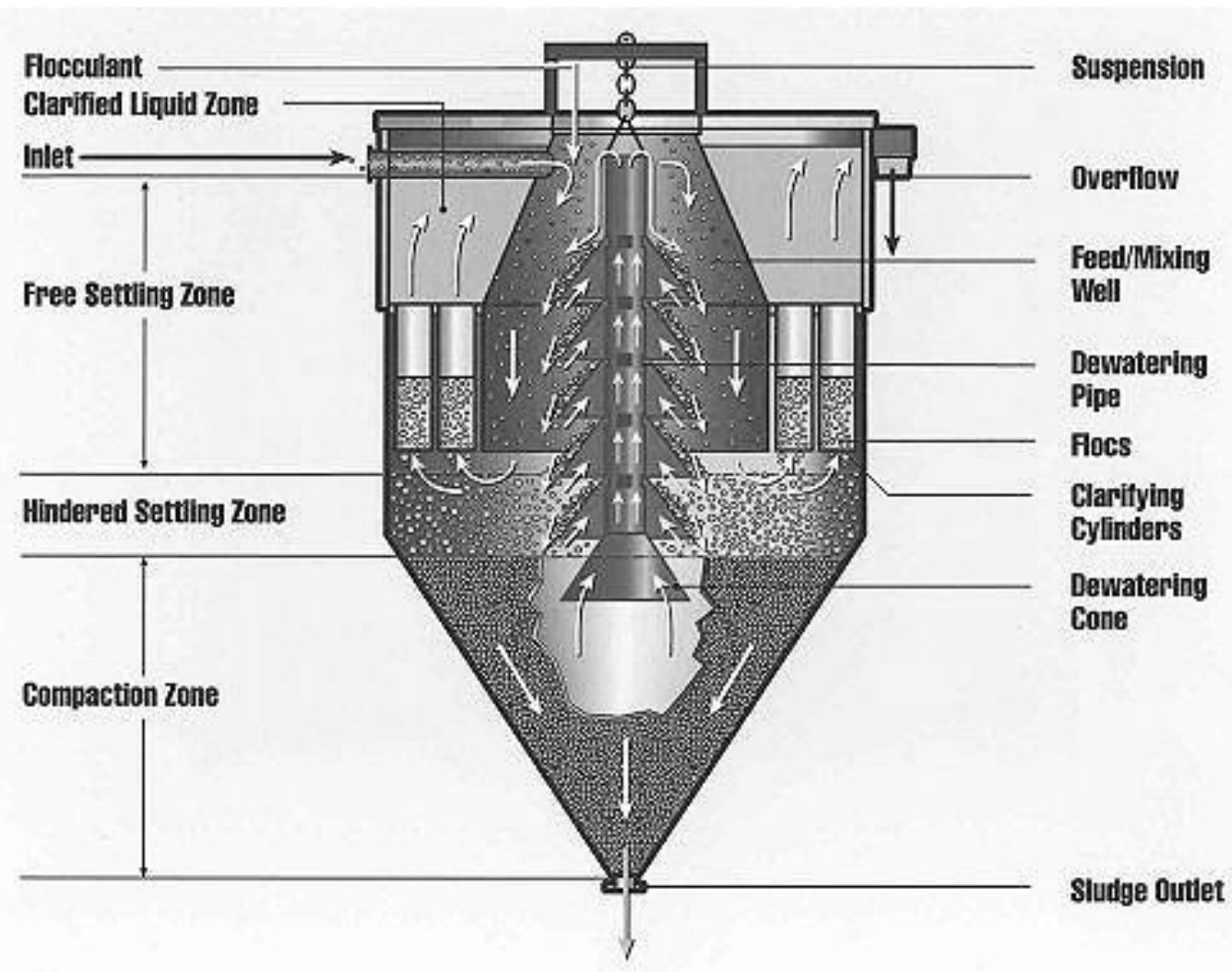

Figure 2: The EIMCO E-CAT clarifier thickener

In the rake less thickener the rapid removal of water from the feed collapses the hindered settling zone of the thickener taking solids from the free settling zone into the compaction zone. The deep tank and high cone angles up to 60 degrees provides a deep compression that results in high density underflow solids. The Rakeless thickener requires a high degree of instrumentation. Changes in sedimentation are very rapid and cannot be controlled manually. 


\section{Deep Cone Thickener}

Most of the thickeners in use today are conventional thickeners. Initially, when thickeners were first introduced in the industry, no chemicals or settling aids were used. As knowledge regarding flocculants increased, it was determined that by using proper chemicals settling can be enhanced rapidly.

During the last twenty years because of environmental and economical reasons there was enormous effort to improve the design of the thickeners and use proper conditioning to achieve higher percent solids. This was achieved using special feedwell systems, controls and by optimizing flocculants usage. As mentioned earlier flocculants perform at their best at a particular feed concentration. This optimum feed concentration or feed percent solids must be determined in the laboratory using bench scale tests and pilot operations.

The Deep Cone Thickener was initially designed by the British coal industry. Alcan bought the rights to the technology and improved the design to handle thickening of red mud. After 1996 the paste technology was successfully applied to other minerals as well such as copper, lead and zinc tailings. The thickened tailings were either disposed of on the surface or used for back filling operations underground.

Deep cone paste thickeners handle 20 times the solids mass flow and 10 times the hydraulic loading of conventional thickeners (EIMCO, 2003). This is accomplished by optimizing solids concentration for feed dilution, chemical addition and floc formation The deep cone thickener produces underflow with very high percent solids because of the following factors

- increasing the L: $\mathrm{D}$ ratio by to obtain a deep bed of compression

- by using steeper cone angles, generally from 30 to 45 degree

- selection of best flocculants at optimum dosage, by conducting lab scale studies.

- by adopting a feed dilution system such as E -Duc ${ }^{\mathrm{TM}}$ (Dorr Oliver Eimco)

- designing a new rake system to handle high torques

- high degree of precision and instrumentation

Rake drives are key components of conventional thickeners but in deep cone thickener, they are absolutely critical. These drives can be powered with either hydraulic or electric motors. Drives must be designed to meet unusually high continuous torque requirements because of high viscosities encountered when the slurry reaches a paste consistency and also because of high mud depths. The rake mechanism is designed to keep the solids in continuous motion and to keep the solids flowing towards the discharge and to assist with releasing water from the paste bed. The schematic diagram of the Deep cone thickener is shown in Figure 3. 


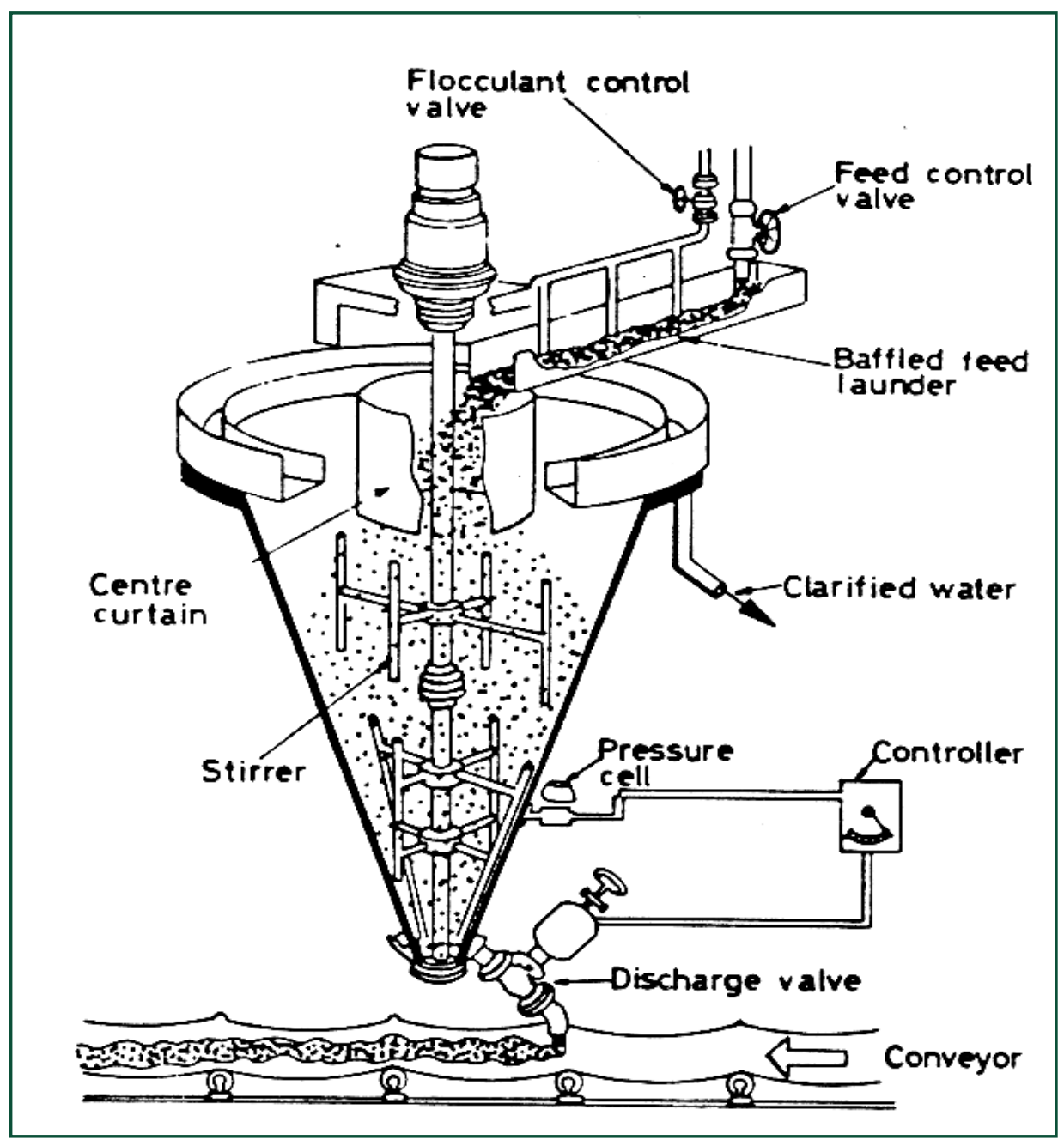

Figure 3. Schematic diagram of deep cone thickener

Advantages of Deep Cone Thickener over Conventional Thickener

The Deep Cone Thickener by the virtue of its design and handling capacities offers numerous advantages over conventional thickeners. They are as follows

- the area required for installation of a deep cone thickener is much less when compared to that of conventional thickener. This is so because of higher L/D ratio.

- the higher water recovery as the underflow of the deep cone thickener contains higher percent solids.

- the area required to dispose of the thickened paste will be much less when compared to a conventional thickener

- the tailing ponds are not necessary as disposal is by stacking, not ponding. 
- the tailings even though they contain high percent solids can be transported using pumps.

- the optimum use of flocculants there by reducing chemical costs.

Paste Disposal Techniques

In conventional disposal methods the slurry is thickened to about $35 \%$ solids but using paste thickening techniques the underflow can be thickened to about $60 \%$ solids, the resulting waste can be disposed of by high density residue stacking.

High density stacking was developed more than 25 years ago for handling red mud tailings and it has been used for disposing gold and other base metals for the last 10 years. Deep cone thickeners produce a homogenous non segregating paste. The paste can be disposed of in thin layers over the disposal site at an angle of 2 to 5 percent and an impoundment structure is not required. This method is most suitable for homogenous residues of fine gradation where the thickening process prevents segregation of particles. Perimeter drains are required to catch the runoff from the waste.

The current research program evaluates the use of Deep Cone Thickener paste technology for thickening fine coal waste.

\section{EXPERIMENTAL}

For the present study three coal preparation plants namely, Coal Clean Corp. Dry Branch, W.V., the Arch Coal of West Virginia located at Yoln, W.V. and Arch Coal located at Logan, VA were selected for the study. A representative sample of the slurry was characterized for $\mathrm{pH}$, particle size distributions and ash contents. Table 1 lists the characterization data for the Coal clean Corporation, and two of Arch Coal Co. slurries. Note, that the average particle size $\left(D_{50}\right)$ varies from 9.1 to 29.5 microns. The large $D_{50}$ at the Arch coal companies is attributed to the absence of fine coal recovery circuit at the plants.

Table 1. Characterization data of the Thickener underflow slurries

\begin{tabular}{|l|l|l|c|c|c|}
\hline Plant Name & $\begin{array}{l}\text { Solids } \\
\text { Concentration } \\
\text { weight \% }\end{array}$ & $\begin{array}{l}\mathbf{+ 3 2 5} \text { mesh } \\
\text { material }\end{array}$ & $\begin{array}{c}\mathbf{D}_{\mathbf{5 0}} \\
\text { ( microns) }\end{array}$ & pH & \% Ash \\
\hline $\begin{array}{l}\text { Coal Clean } \\
\text { Corp. }\end{array}$ & 28.70 & 27.9 & 9.1 & 7.00 & 62.55 \\
\hline $\begin{array}{l}\text { Arch Coal } \\
\text { of WV. }\end{array}$ & 29.85 & 48.6 & 29.5 & 7.2 & 47.63 \\
\hline $\begin{array}{l}\text { Arch Coal, } \\
\text { Logan, VA }\end{array}$ & 29.66 & -------- & 25.0 & 8.5 & 55.65 \\
\hline
\end{tabular}




\section{Selection of Flocculants:}

To identify the best combination of flocculants to provide a high rate of settling of the flocculated particles, tests were conducted using $1000 \mathrm{ml}$ of the refuse slurry in a graduated cylinder. A known amount of flocculant solution was added to the slurry in the cylinder and was mixed applying 10 moderate up-and-down strokes using a plunger. The plunger technique closely approximates the ideal situation in which dispersion of the flocculant throughout the slurry is almost instantaneous. The settling rate (height of the sediment with respect to time) of the flocculated solids was monitored using a stop watch. For the Deep Cone Thickener, the settling flux is an important parameter was calculated using the following equation.

Settling Flux $\left(\right.$ tonnes $\left./ \mathrm{hr} / \mathrm{m}^{2}\right)=\left(\frac{\text { Settling Dis } \tan c e(\text { meters }) \bullet \text { Slurry Concentration }(\mathrm{Kg} / \mathrm{l}) \bullet 3600}{\text { Settling Time }(\mathrm{sec})}\right)$

The percent solids in the settled solids was determined by removing the supernatant and filtering the settled solids, weighing them wet and then drying it overnight in an oven and weighing it again.. The percent solids were calculated using the following equation,

Percent Solids $=\frac{\text { Weight of dry solids }}{\text { Weight of slurry }} \times 100$

\section{Yield Stress Measurement:}

Rheology of the thickened slurry is an important parameter, as it determines the amount of force necessary for the thickened slurry to pump and also amount of torque required in the thickener. Yield Stress is the force (units of pressure) that must be applied to the slurry to initiate the flow. Yield stress could be measured using a Viscometer and reported in Pascals $(\mathrm{Pa})$ units. However, in the field the slump test is a quick way to obtain the yield stress. A slump test basically involves filling a cylinder with a representative sample of the thickened slurry, then lifting the cylinder, allowing the sample to slump (Pashias and Boger, 1996 ). The measured amount of slump is a measure of yield stress, which is calculated using the following equation.

$$
\text { Yield Stress }=(0.5-0.5 \sqrt{(s / h)}) \rho g h
$$

Where $s$ is the slump in the slurry, $h$ is the height of the cylinder, $\rho$ the specific gravity of the slurry and g acceleration due to gravity in $\mathrm{m} / \mathrm{sec}^{2}$. 


\section{RESULTS AND DISCUSSIONS}

- Most of the fine refuse tailings contain coal, quartz, alumina, and clays. The surface charge on the particles plays an important role in flocculation Figure 4 shows the Zeta Potential values of the solids present in the slurry with respect to $\mathrm{pH}$. Note, that the particles remains negatively charged for all the $\mathrm{pH}$ range.

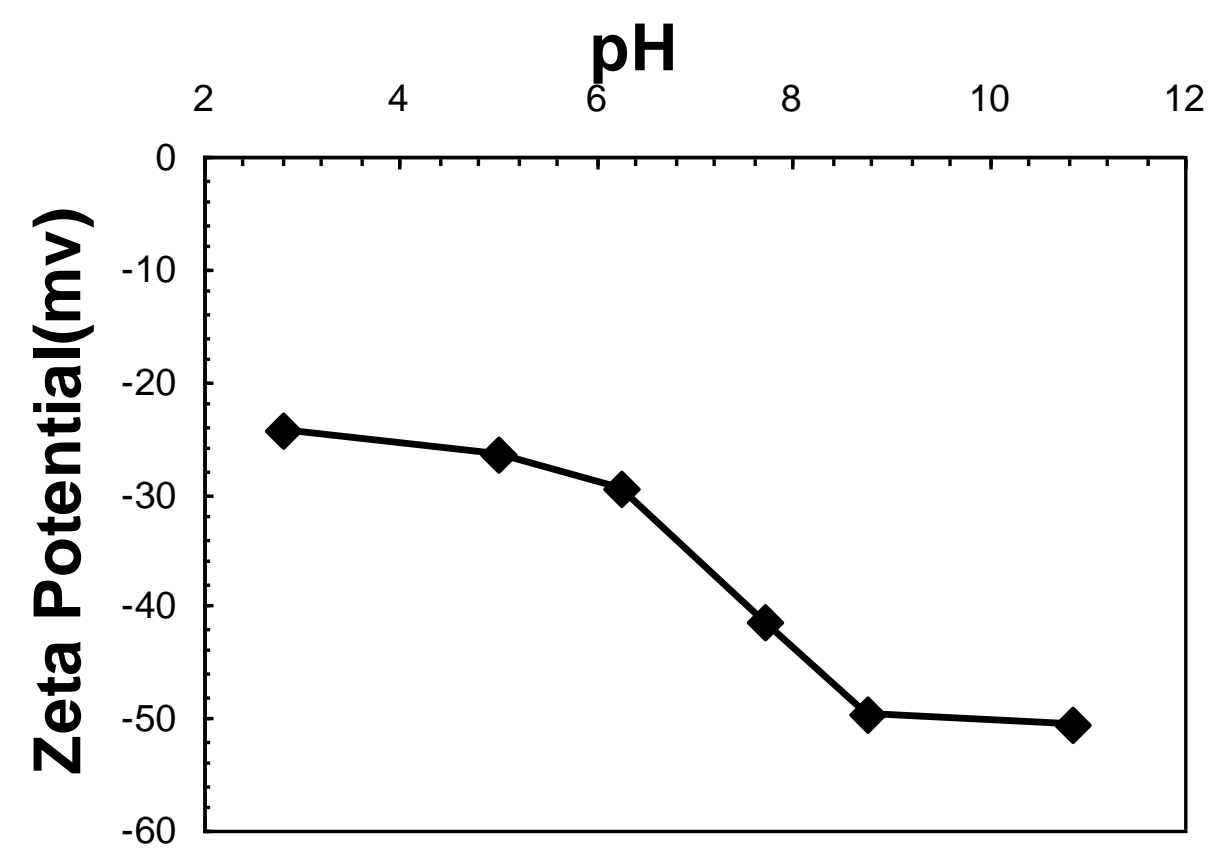

Figure 4. Zeta Potential of the fine refuse slurry solids with respect to $\mathrm{pH}$. (Coal Clean Corp. thickener underflow)

The results of the study are described below for all the three coal preparation plants refuse slurries.

\section{(a) Coal Clean Corporation:}

A series of flocculants obtained were evaluated on the thickener underflow to determine their effectiveness in settling and providing a clear supernatant. Figure 5 shows the supernatant quality with respect to the flocculant dosage. To avoid any preferential treatment or favoritism the flocculants were identified by the letters. The figure shows that flocculant $\mathrm{A}$ was the most effective in providing lowest solids content in the supernatant, compared to the other three flocculants.

Figure 6 shows the settling flux rate for the thickener underflow. For the thickener underflow, the highest settling flux of $0.4 \mathrm{t} / \mathrm{hr} / \mathrm{m}^{2}$ is obtained at $5 \mathrm{wt} \%$ diluted slurry. 
These results indicate that the slurry will have to be diluted to obtain a high settling flux rate in the Deep Cone Thickener.

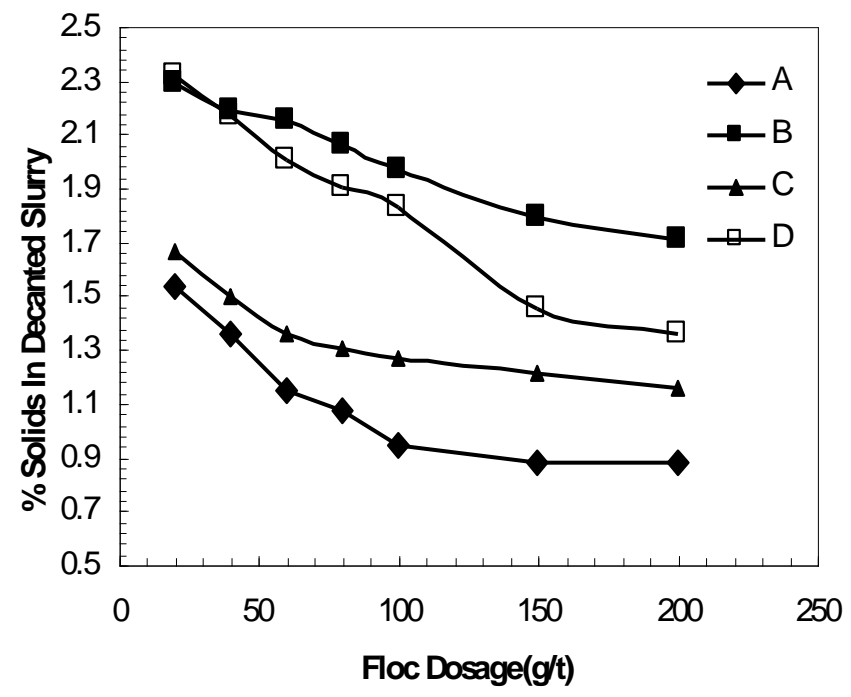

Figure 5. Effect of different anionic flocculants dosages of on suspension supernatant quality.

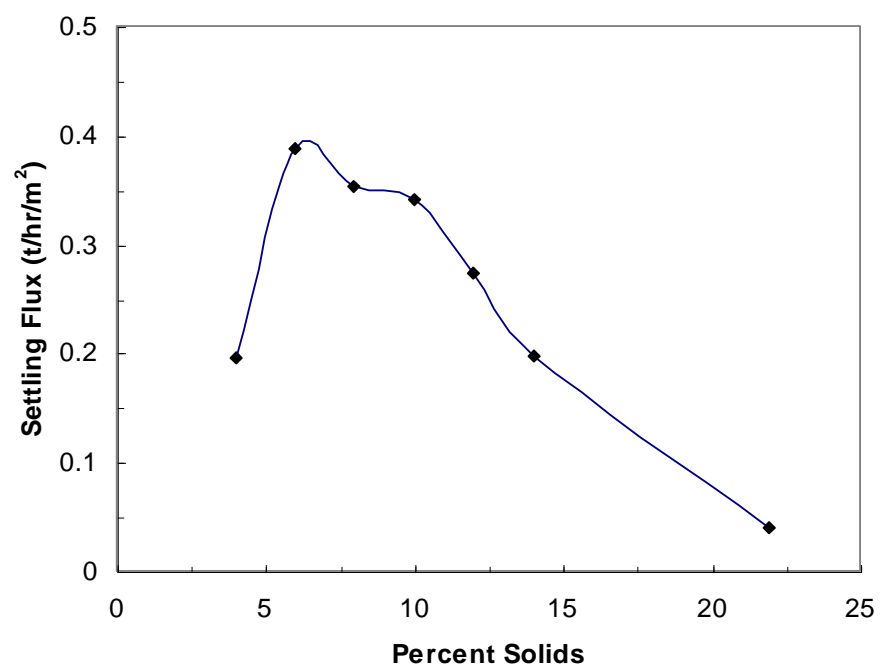

Figure 6. Settling flux of flocculated solids with respect to percent solids in suspension.

Another important criterion for the successful application of the Deep Cone Thickener is the yield stress value of the flocculated settled solids. Figure 7 shows the yield stress of 
thickener underflow diluted to various concentrations. A yield stress of close to $150 \mathrm{~Pa}$ is preferred for easy pumping of the paste. Thus, for the thickener underflow slurry paste with 60 wt $\%$ could be obtained at about 150 Pa yield stress.

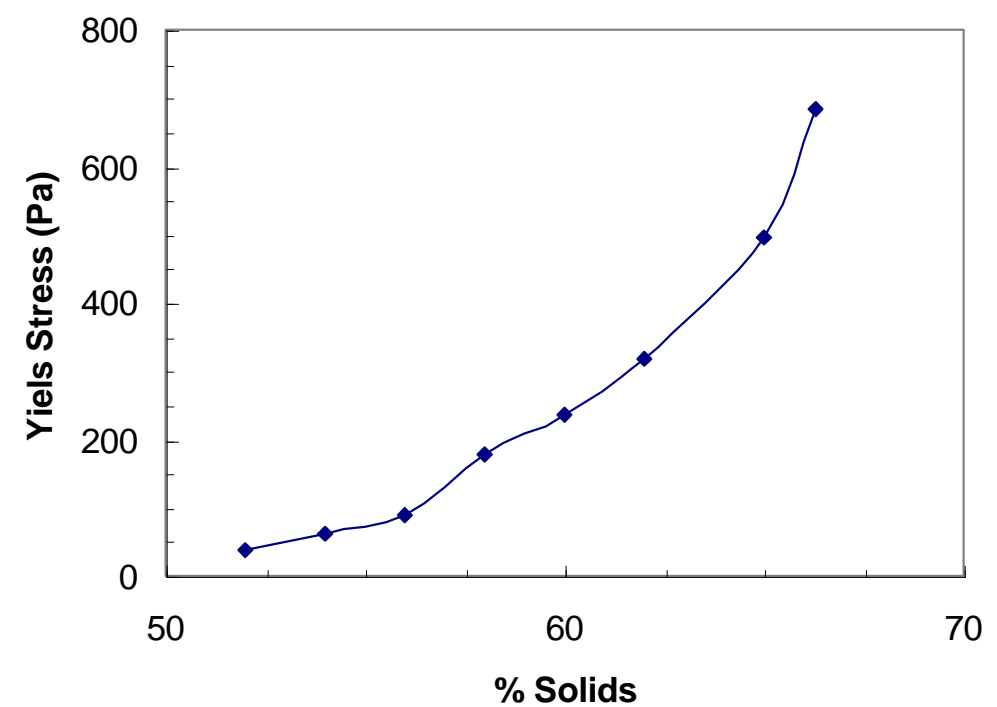

Figure 7. Yield stress of flocculated thickener underflow with respect to percent solids.

Summary of the laboratory tests is given in Table. 2. It shows that for the thickener underflow a paste with about 50 percent solids could be achieved.

Table 2. Summary of the Laboratory Test Data

\begin{tabular}{|l|c|c|c|c|c|}
\hline Sample & $\begin{array}{c}\text { Initial } \\
\text { Solid } \\
(\text { Wt \%) }\end{array}$ & $\begin{array}{c}\text { Settling } \\
\text { Velocity } \\
\mathbf{m} / \text { day }\end{array}$ & $\begin{array}{c}\text { Mag Floc } \\
\mathbf{3 5 8} \\
(\mathbf{p p m})\end{array}$ & $\begin{array}{c}\text { Percol } \\
\mathbf{3 6 8} \\
(\mathbf{p p m})\end{array}$ & $\begin{array}{c}\text { Ultimate solids } \\
\text { concentration } \\
(\mathbf{W t} \%)\end{array}$ \\
\hline $\begin{array}{l}\text { Thickener } \\
\mathrm{U} / \mathrm{F}\end{array}$ & 5.0 & 628 & 30 & 30 & 49.0 \\
\hline
\end{tabular}

$\underline{\text { Pilot-Scale Test Results }}$ 
For the pilot-scale test a Deep Cone Thickener of $1.5 \mathrm{~m}$ diameter and $7.5 \mathrm{~m}$ height, supplied by the Dorr Oliver Eimco, was installed at the Coal Clean Corp. Figure 8 shows the pilot-plant unit in operation. A stream of the thickener underflow was diverted to the unit. Initially the flocculation of the thickener underflow was conducted in the tank installed at the top of the thickener, using only the anionic flocculant. After conducting tests for a week it was found that using the only the anionic flocculant alone a paste with about $40 \%$ solids could be obtained (Figure 9 ).

Later on, it was decided to utilize the thickener underflow feed that was flocculated by anionic and cationic flocculants and being fed to the filter press. The flocculated material appeared to have squeezability, that is one can hold the flocs in hand and squeeze water out to produce a paste type material. For the pilot-plant testing a small stream of the flocculated slurry was fed to the Deep Cone thickener.

Table 3 shows the data of the tests conducted by varying the bed depth of the flocculated solids. It shows that by varying the bed depth from $2.4 \mathrm{~m}(8 \mathrm{ft})$ to $3.35 \mathrm{~m}(11 \mathrm{ft})$ the solids contents of the paste varies from $51.5 \mathrm{wt} . \%$ to $52.7 \mathrm{wt} \%$. The table also shows the solids content of the filter press material which was $52.5 \mathrm{wt} \%$. Figure 16 shows the paste product obtained for these tests. These data shows that the Deep Cone thickener is able to produce a material similar to that produced by filter press.

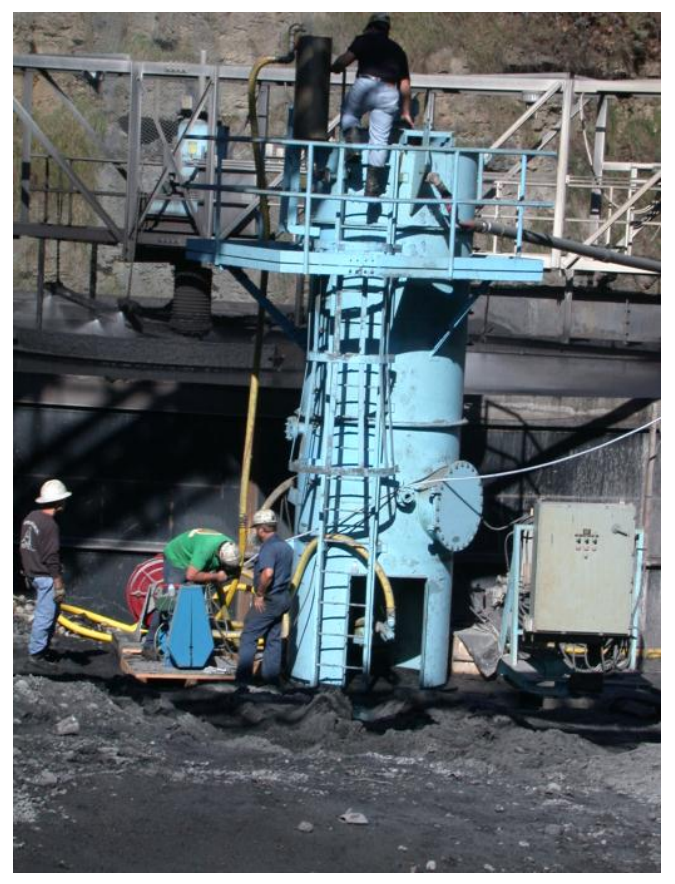

Figure 8. Pilot-scale Deep Cone Thickener unit installed at the Clean Coal Corp 


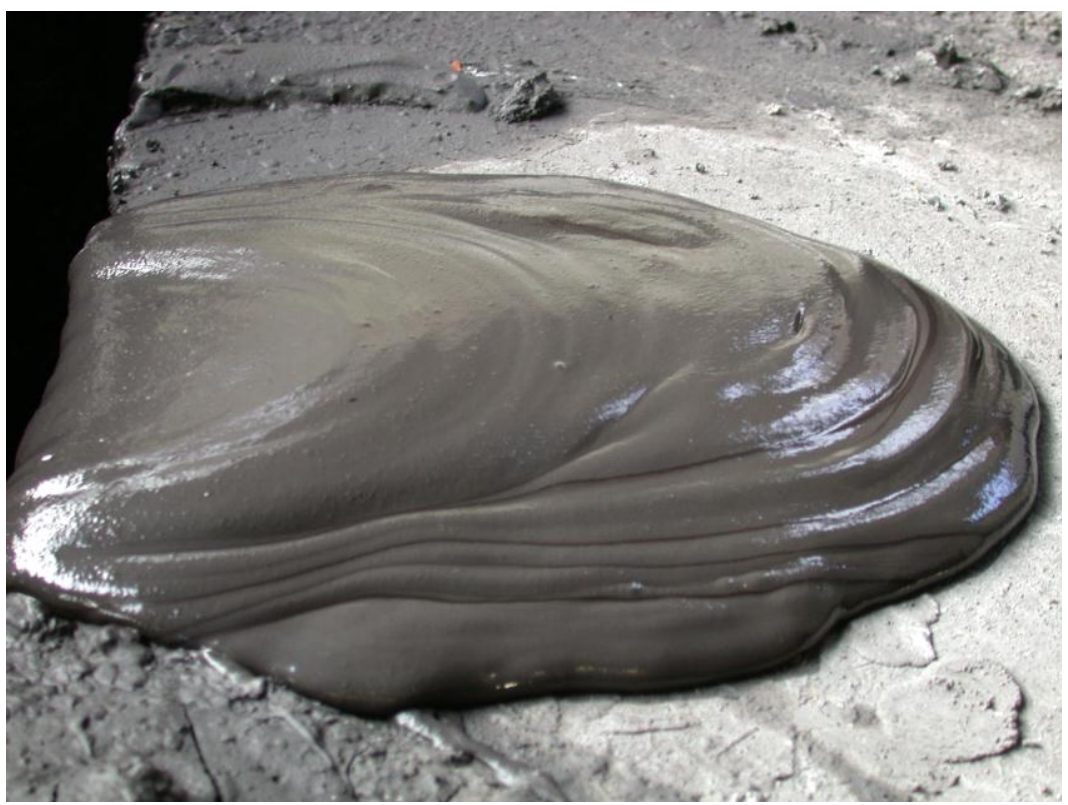

Figure 9. Deep Cone Thickener underflow containing about 40 percent solids.

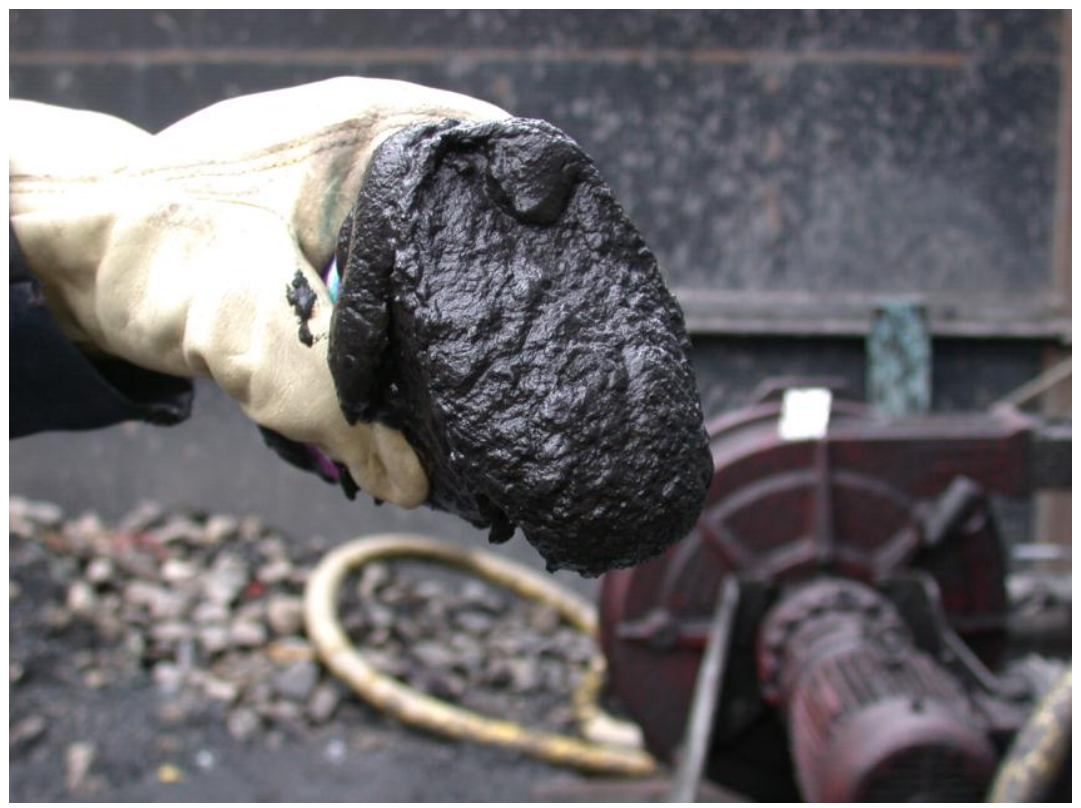

Figure 10. Deep Cone Thickener underflow containing about 53 percent solids. 
Table 3 - Pilot scale data of Deep Cone thickener (Feed rate $\approx 15 \mathrm{gpm}$ at $11 \%$ solids).

\begin{tabular}{|c|c|}
\hline Bed Height & \% Solids in UF \\
\hline $2.43 \mathrm{~m}(8 \mathrm{ft})$ & 51.5 \\
\hline $3.04 \mathrm{~m}(10 \mathrm{ft})$. & 52.9 \\
\hline $3.35 \mathrm{~m}(11 \mathrm{ft})$. & 52.7 \\
\hline $\begin{array}{l}\text { Filter cake from the } \\
\text { existing filter press }\end{array}$ & 52.5 \\
\hline
\end{tabular}

\section{(b) Arch Coal of West Virginia:}

Flocculation and settling tests were conducted with flocculants having different properties (Table 4) using a 2 liter cylinder and the procedure developed by the DorrOliver Eimco Company. During the settling tests, a rake running at a speed of $5 \mathrm{~min} / \mathrm{rev}$ was utilized to simulate thickener mechanism and maximized solids content in the settled solids. In order to determine the optimum flocculant dosage for providing higher settling flux and percent solids, flocculation tests were conducted with the thickener underflow slurry diluted from $35 \%$ to $10 \%$ solids to facilitate the observation of the settling of flocculated solids.

The tests with the anionic flocculants can be summarized as follows;

- Flocculants having lower charge densities (15-20\%) provided higher settling flux and settled percent solids.

- The settling flux and settled percent solids increased with increased in molecular weight of the polymer flocculant.

- A flocculant (AD609XX )dosage of $400 \mathrm{~g} / \mathrm{t}$ (dry basis) provided a settling flux of 3.21 (tonne dry solids $/ \mathrm{hr} \mathrm{m}^{2}$ ) with settled percent solids of $42 \%$ (Figure 11)

Table 4: Properties of anionic flocculants evaluated in the laboratory Study

(Supplied by Zinkan Enterprises Inc.)

\begin{tabular}{|c|c|c|}
\hline Flocculant & $\begin{array}{c}\text { Molecular weight } \\
\text { (million) }\end{array}$ & $\begin{array}{c}\text { Charge Density } \\
(\%)\end{array}$ \\
\hline AD 206 & \multirow{2}{*}{$10-12$} & 30 \\
\cline { 1 - 1 } & & 40 \\
\hline AD 207 & & \\
\hline & \multirow{2}{*}{$14-16$} & 10 \\
\cline { 1 - 1 } AD 608 & & 30 \\
\hline AD 603 & & 40 \\
\hline AD 605 & & 15 \\
\cline { 1 - 1 } & & 20 \\
\hline AD 609XX & \multirow{2}{*}{$18-20$} & 40 \\
\hline AD 604XX & & \\
\cline { 1 - 1 } AD 605XX & & \\
\cline { 1 - 1 } & &
\end{tabular}




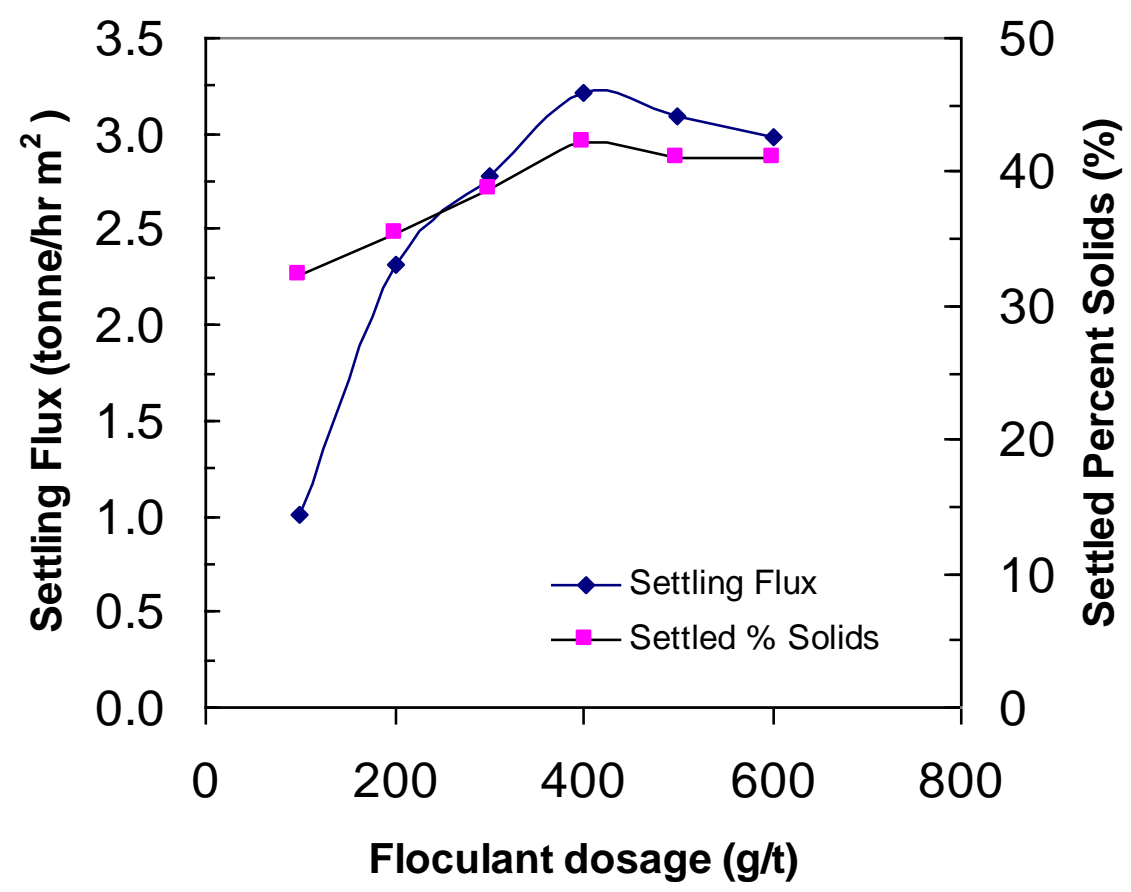

Figure 11 : Effect of flocculant AD609XX dosage on settling flux and settled percent solids.

Effect of slurry concentration

Experiments were carried out to evaluate the effect of percent solids in the feed on the initial settling velocity. The objective was to find out optimum solids concentration at which solids settling velocity and flocculation rate are maximum. The settling flux was calculated by multiplying the initial settling velocity (meter/hr) and the slurry concentration (tonne dry solids $/ \mathrm{m}^{3}$ ) . It was observed that the settling flux was higher at a slurry concentration of $8-10 \%$ solids.

\section{Mode of addition of anionic and cationic flocculants}

Usually, polymers of high molecular weight are employed for flocculating oppositely charged suspensions. However, improved effect is generally observed using cationic and anionic flocculants. The preferred method of addition of these flocculants to the negatively charged suspensions is to add cationic flocculant first, then followed by anionic flocculant. In some cases the cationic flocculant is added after conditioning the slurry with anionic flocculant. Therefore, tests were carried out to evaluate the effects of mode of addition of flocculants on settling flux and settled percent solids. 


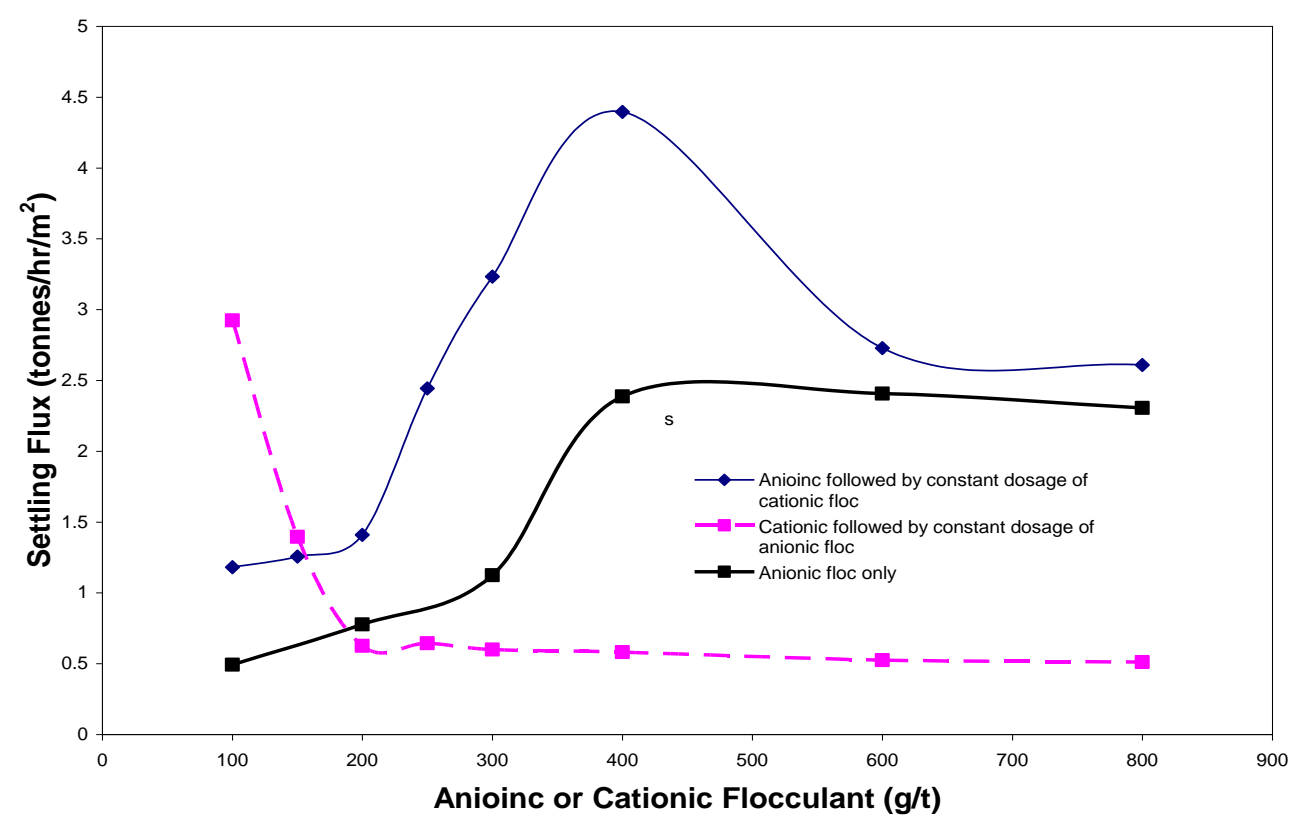

Figure 12: Effect of mode of addition of flocculants on settling flux using the Nalco flocculants

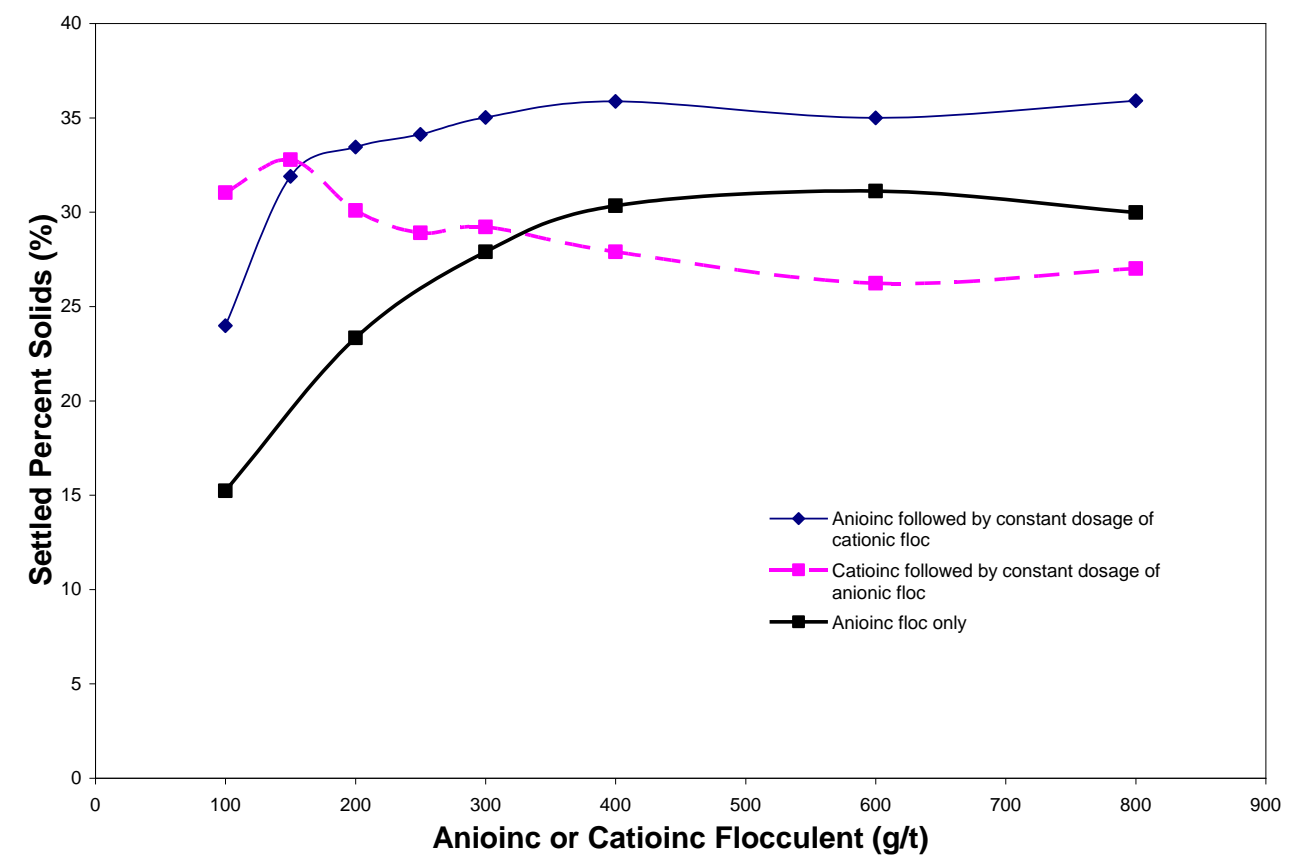

Figure 13: Effect of mode of addition of flocculants on settled percent solids using the Nalco flocculants 
Figures 12 and 13 show that pretreatment of slurry with anionic flocculant before the addition of cationic flocculant, improves the settling flux and percent solids. At $400 \mathrm{~g} / \mathrm{t}$ anionic flocculant dosage, the settling flux and percent solids increased from 2.38 to 4.39 tonnes $/ \mathrm{hr} / \mathrm{m}^{2}$ and $30 \%$ to $35 \%$ respectively.

\section{Tests with T-Floc Apparatus}

The test conditions described earlier were utilized while conducting tests with the DorrOliver Eimco's designed T-floc apparatus (Figure 14). A flocculation tank was designed and arrangements were made to add anionic and cationic flocuulent to the slurry in the required mode. Tests were conducted using the as received slurry, in a semi continuous mode, using anionic (400 g/t) and cationic (100 g/t) flocculent. After 4 hours residence time, the underflow solids concentration reached $57 \%$ solids, which clearly indicated that the refuse slurry could be thickened as a paste. A sample of the thickened (paste) slurry is shown as inset in Figure 14.

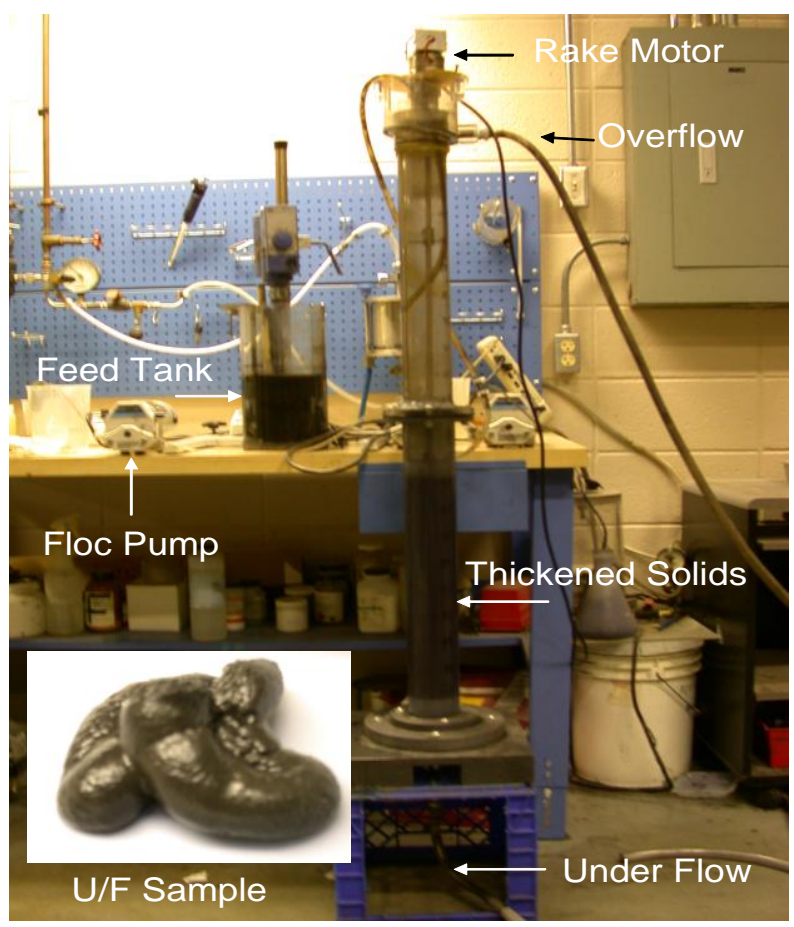

Figure 14: T-Floc apparatus setup showing settled solids and clear supernatant 


\section{$\underline{\text { Pilot-Scale Test Results }}$}

For the pilot-scale testing slurry was obtained from just above the thickener underflow pump using a 1-inch valve, which provided slurry under a high pressure. About $100-\mathrm{ft}$ long high pressure rubber tube was used to transport the slurry to the tank which reduced the pressure considerably before the slurry entered the Deep Cone thickener. The pilotplant set-up schematic is shown in Figure 15. For the test, Nalco's anionic and cationic flocculants about $0.45 \mathrm{~kg} / \mathrm{t}(1 \mathrm{lb} / \mathrm{ton})$ and $0.11 \mathrm{~kg} / \mathrm{t}$ ( $0.25 \mathrm{lb} / \mathrm{ton})$, respectively, were used for flocculating the slurry. The anionic flocculant was injected inline, before the entrance of the slurry into a flocculation tank, which provided enough time to condition the slurry. The cationic flocculant was added to the flocculation tank in such a way to give enough time for the slurry interact with anionic flocculant first, then with cationic flocculant. Both flocculants were diluted before mixing with the slurry. Deep cone thickener set-up at the Arch Coal Co.
preparation plant

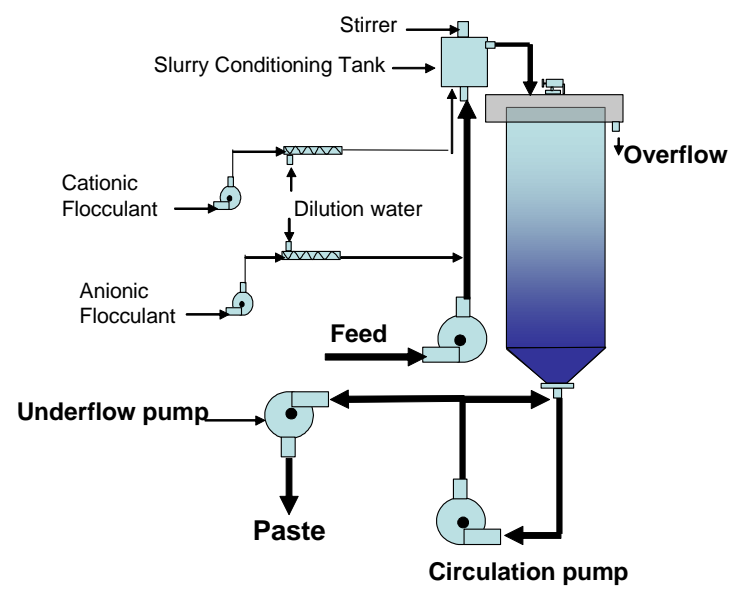

Figure 15. Flowsheet of the pilot-scale set-up at the Arch Coal Co.

The pilot-scale test conditions are listed below:

Feed rate

Anionic flocculant

Cationic flocculant

Rake speed
20-25 gpm

$300-400 \mathrm{~g} / \mathrm{t}$

$100-200 \mathrm{~g} / \mathrm{t}$

$5 \mathrm{~min} / \mathrm{rev}$

Using these conditions after a 4 hours residence time the solids thickened to about 45\% (Figure 16 )solids and after 6 hours residence time solids thickened to about 50\% solids 
(Figure17 ). The yield stress of the paste containing about 50\% solids as determined using the slump test (Figure 18)was about $207 \mathrm{~Pa}$, which indicates that the paste will be pump-able using the conventional positive discharge pump.

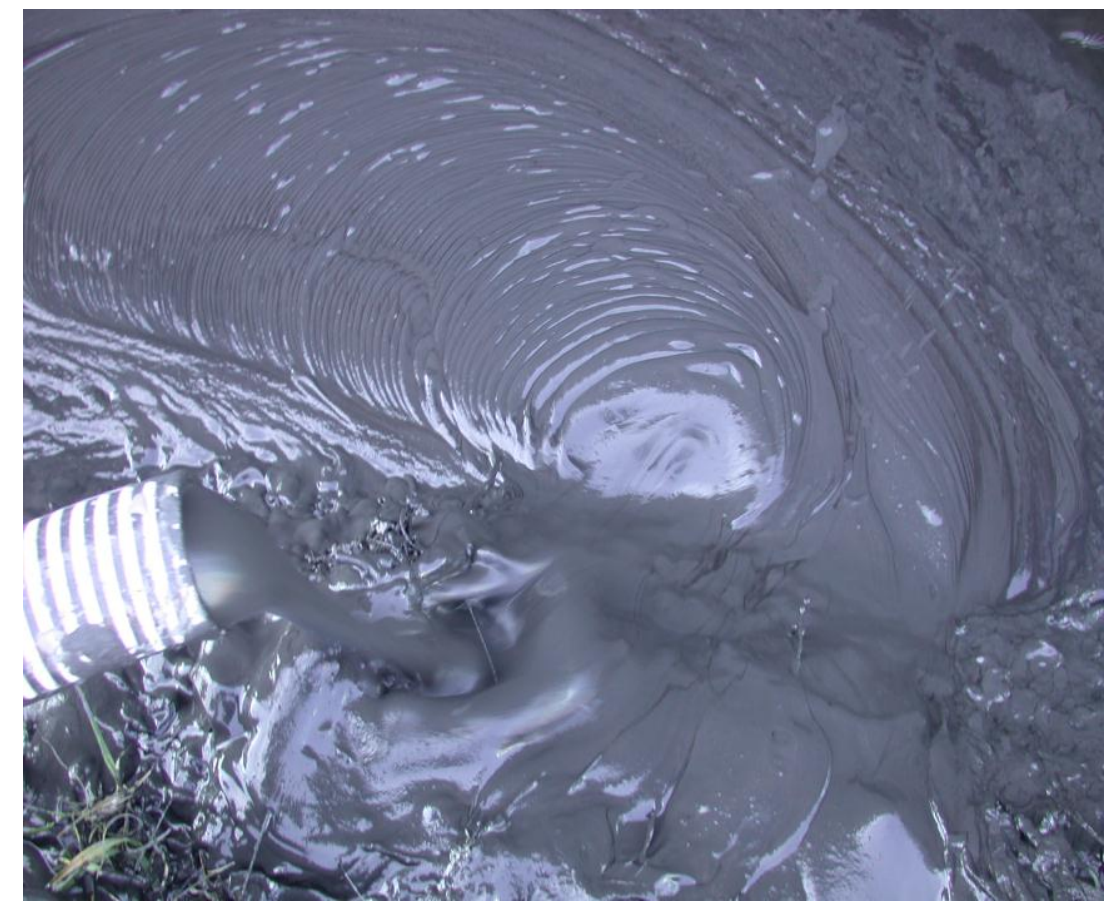

Figure 16. Deep cone thickener under flow containing about $45 \%$ solids after shear thinning 


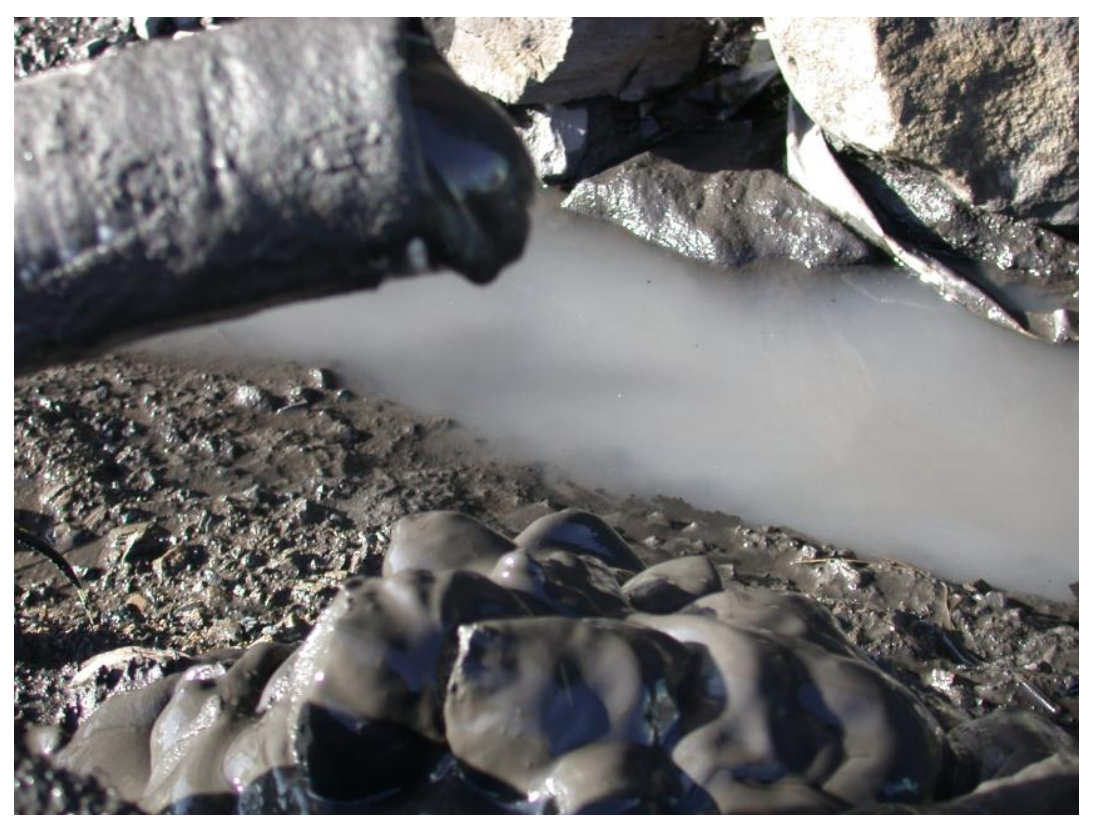

Figure 17. Deep cone thickener under flow containing about $50-52 \%$ solids after shear thinning

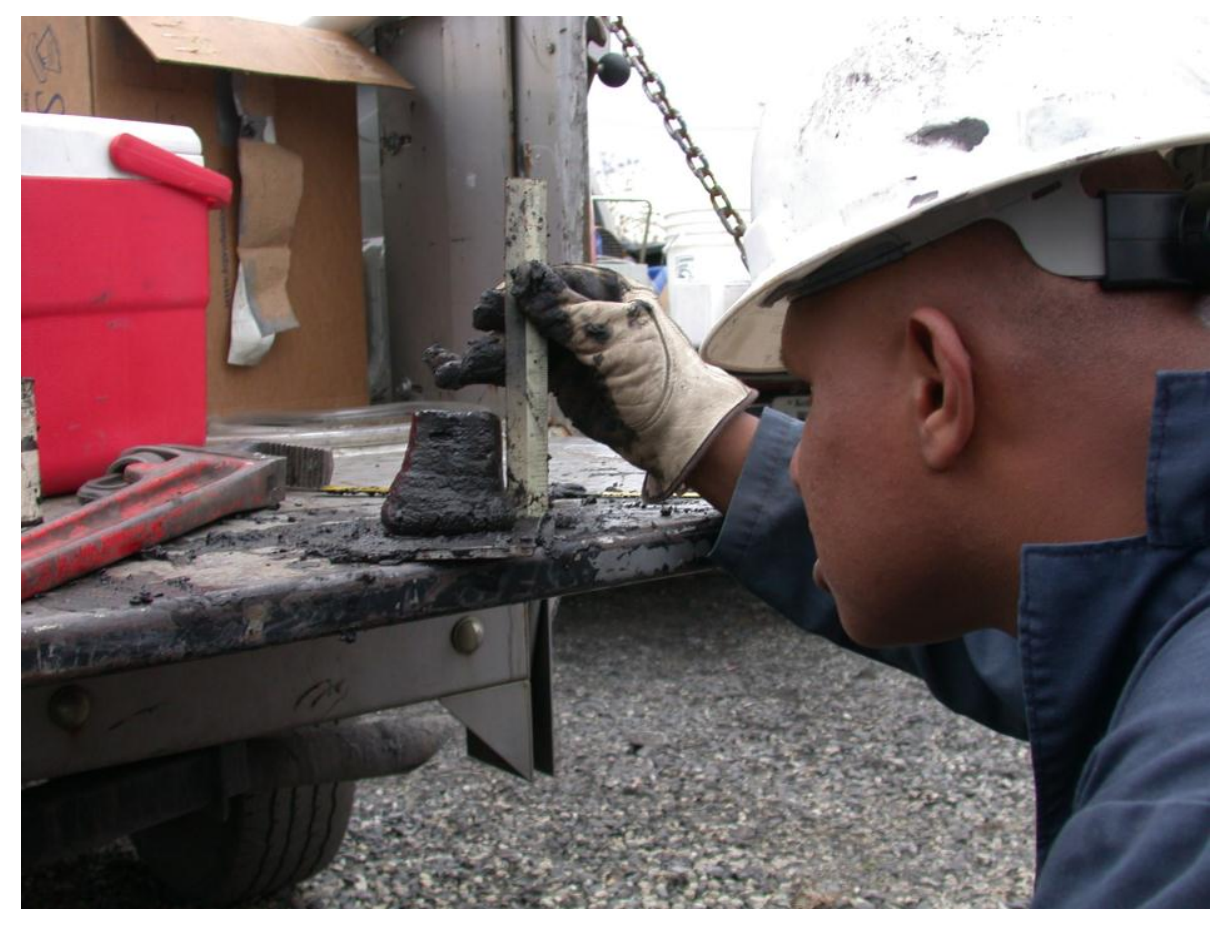

Figure 18. Determination of yield stress of the Deep cone thickener under flow using the slump test. 


\section{$\underline{\text { Arch Coal Co., Lone Mountain }}$}

Two five gallons buckets of thickener underflow slurry were collected from the Arch Coal Lone Mountain plant. A representative sample of the slurry obtained using the Carpco Rotary Slurry Sampler was characterized for $\mathrm{pH}$, particle size distribution, solid and ash contents and mineralogy. The solids content of the slurry was $29.62 \%$. The pH of the slurry was 7.72 with ash and sulfur contents of $47.69 \%$ and $0.44 \%$ respectively.

The particle size distribution of the thickener underflow slurry is shown in Figure 19. At this plant there are two thickeners, thickener 1 little coarser particles than the thickener No.2..

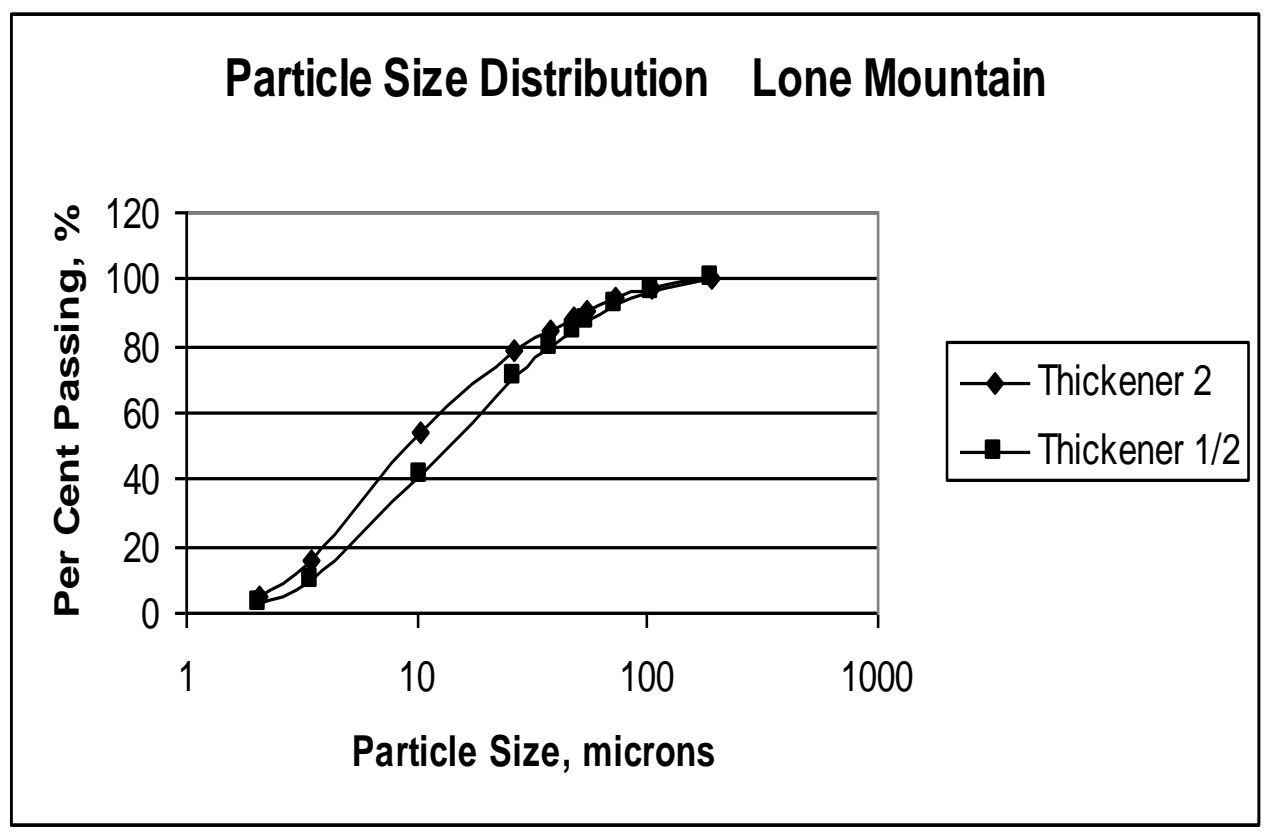

Figure 19. Particle size analysis of the Lone Mountain thickener underflow

\section{Laboratory Studies:}

Settling flux tests were conducted using cationic followed by anionic at doses of $35 \mathrm{~g} / \mathrm{t}$ and $30 \mathrm{~g} / \mathrm{t}$, respectively. Solids concentration was varied through the range of 20 to 5 wt\%. Figure 2 shows the effect of diluting the feed to optimize flocculation and settling velocity. 
The results shown in Figure 20, indicate that diluting the feed to $7 \mathrm{wt} \%$ will result in the highest solids settling flux rate. Designing at this feed solids point gives the smallest diameter unit recommended. According to Figure 20 and using a 50\% scale up factor, a design settling flux of $33 \mathrm{tpd} / \mathrm{m}^{2}\left(0.03 \mathrm{~m}^{2} / \mathrm{tpd}\right)$ would be chosen for scale up at feed solids concentrations of $7 \mathrm{wt} \%$ and $35 \mathrm{~g} / \mathrm{t}$ cationic and $30 \mathrm{~g} / \mathrm{t}$ anionic.

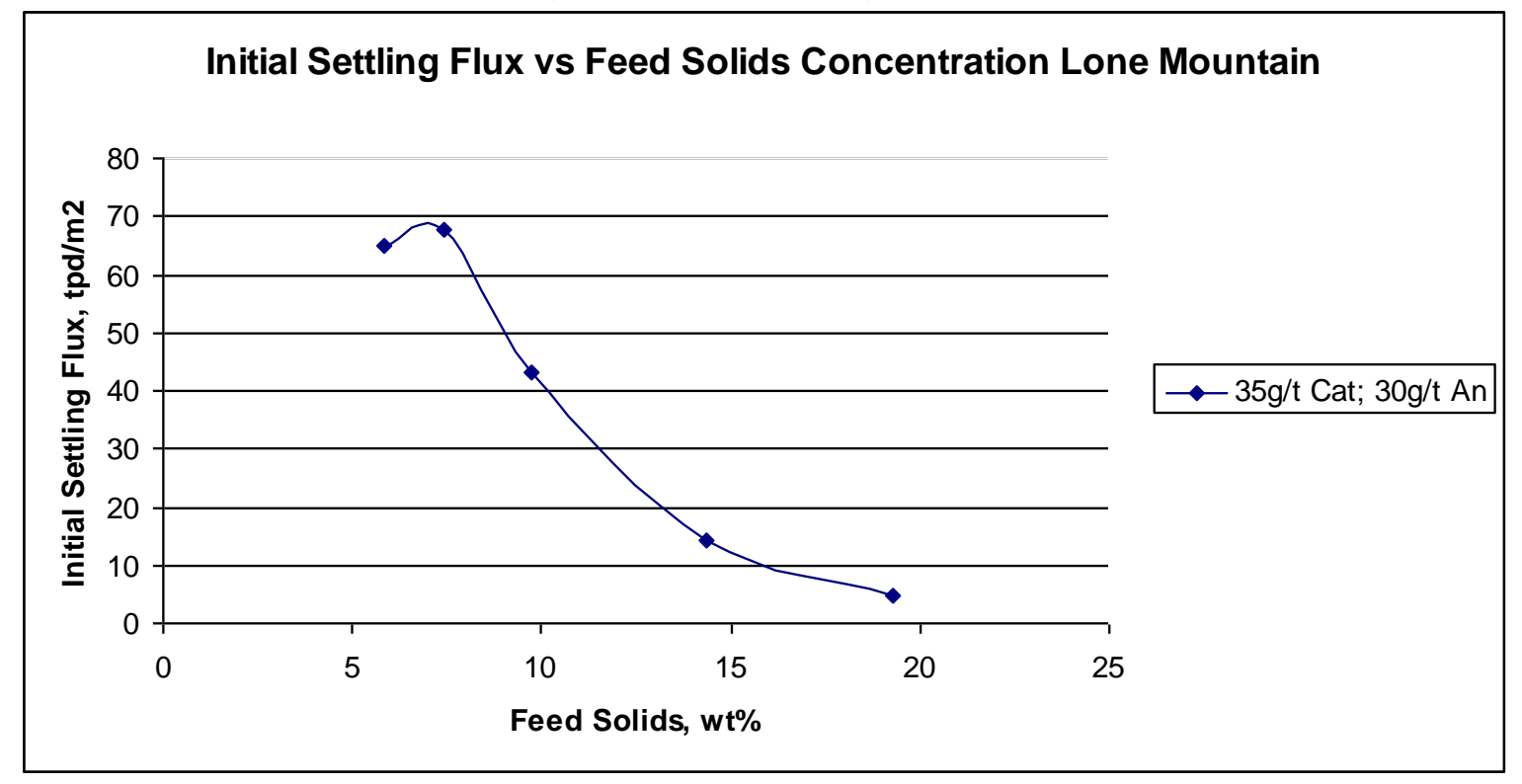

Figure 20. Initial Settling Flux vs Solids Concentration. for the Lone Mountain thickener underflow

\section{$\underline{\text { Pilot-Scale Test Results }}$}

The goal of the test was to confirm stable operation at the minimum diameter (design unit loading) originally predicted from the initial tests. This was confirmed at $12.2 \mathrm{tpd} / \mathrm{m}^{2}$ provided the feedwell flocculation solids were at $7 \mathrm{wt} \%$, and flocculant solutions were added to provide at least $30 \mathrm{~g} / \mathrm{t}$ each of cationic and anionic. The No. 1 thickener overflow was at $898 \mathrm{mg} / \mathrm{l}$. Due to a shut down for plant maintenance during operation, it was not possible to run continuously beyond the initial fill period used to confirm maximum loading. After obtaining a bed up to bottom of the feedwell, the underflow was turned off and the bed was allowed to consolidate. Samples were taken through a 14 hour residence time to note the rate of compression and the thickened slurry solids concentration. With a $50 \mathrm{wt} \%$ underflow as the minimum target, it appears that a residence time of at least 9 hours will be required. Design of the full scale unit will therefore have at least 9 hours minimum residence time, and design will be based on 12 hours using an additional $30 \%$ for safety. Underflow density reached $52-53 \mathrm{wt} \%$ at the 12 hour mark, which was slightly better than the earlier pilot run in January.

The pilot-scale test conditions are listed below: 
Feed rate

20-25 gpm

Anionic flocculant

$300-400 \mathrm{~g} / \mathrm{t}$

Cationic flocculant

$100-200 \mathrm{~g} / \mathrm{t}$

Rake speed

$5 \mathrm{~min} / \mathrm{rev}$

Using these conditions after a 2 hours residence time the solids thickened to about 45\% solids and after 9 hours residence time solids thickened to about 50\% solids.The yield stress of the paste containing about $50 \%$ solids is about $207 \mathrm{~Pa}$ which indicates that the paste will be pump-able using the conventional positive discharge pump. The thickener overflow was better than currently discharged with the conventional thickener.

\section{SUMMARUY AND CONCLUSIONS}

Based on the laboratory and pilot-plant results it can be concluded that

- Efficient flocculation of the fine coal refuse slurry is necessary to produce squeezable flocs for paste thickening.

- The flocculant molecular weight and charge density affect the settling velocity of the flocs and settled percent solids.

- It was observed that a high molecular weight (18 -20 million) anionic flocculant having a lower charge density (10\%- 20\%) provided better flocculation and settling flux.

- Mode of addition of flocculant is important in obtaining a higher settling flux. For the thickener underflow, the addition of anionic flocculant followed by cationic flocculant provided a higher settling flux and settled percent solids.

- The pilot scale Deep Cone thickener was able to produce a paste type material from the thickener underflow containing about $52 \mathrm{wt} \%$ solids by maintaining a bed height of about $2.4 \mathrm{~m}(8 \mathrm{ft})$. The paste product was similar to the dewatered product obtained using the filter press.

- The paste produced by the Deep Cone thickener had a suitable yield stress of 150 Pa to $250 \mathrm{~Pa}$ for pumping.

\section{Recommendations}

- It is recommended that paste pumping studies as well as monitoring of the paste disposal site should be conducted to obtain more detailed information on the possibility of using the technology in the U.S. Coal industry at a couple of coal preparation plants where Deep Cone Thickeners will be installed.

- It is also recommended that the Deep Cone Thickener technology should be tested for dewatering of fine clean coal slurry produced in the fine coal circuit at a coal preparation pant. 


\section{Acknowledgement}

Financial support for the project was provided by the USDOE through the Center for Advanced Separation Technology. Dorr Oliver Eimco support for providing the pilotscale equipment as well as technical and financial support for the project is acknowledged. Support also provided by the Clean Soil and Energy, Coal Clean Corp., Arch Coal Company and assistance provided by Roger Carter of the Nalco is great fully acknowledged.

\section{REFERENCES}

(1) "Coal Waste Impoundments", Risk, Response, and Alternatives, National Research Council, National Academy Press, 2002.

(2) Dorr Oliver Eimco, Personal Communication, 2003

(3) Parekh, B.K. and .P.Matoney, "Mechanical Dewatering" Chapter 8, Coal Preparation, $5^{\text {th }}$ Edition, J.W.Leonard, Ed. Society for Mining, Metallurgy, and Exploration, Inc, 1991

(4) Pashias, N. and D.V. Boger, A fifty cent rheometer for yield stress measurement' Journal of Rheology,40(6), page 1179, 1996 
Appendix 17: Development of a Fine Particle centrifuge (VA006) 


\section{FINAL TECHNICAL REPORT}

Contract Title and Number:

Crosscutting Technology Development at the Center for

Advanced Separation Technologies

(DE-FC26-02NT41607)

Sub-Recipient Project Title:

Development of a Fine Particle Centrifuge

Principal Investigators:

S. Keles, G.H. Luttrell and R.-H. Yoon

Contact Address:

100 Holden Hall

Virginia Tech

Blacksburg, Virginia 24061

Subcontractor Address:

No subcontracts issued.
Period of Performance:

Starting Date: 10/1/02

Ending Date: 10/31/09
Report Information:

Type: Final

Number: VA006

Period:

Date: $\quad 6 / 30 / 10$

Code: VA006-FINAL

Contact Information:

Phone: 540-231-6314

Fax: $\quad$ 540-231-4070

E-Mail: luttrell@vt.edu

Subcontractor Information:

Phone:

Fax:

E-Mail: 


\section{DISCLAIMER}

"This report was prepared as an account of work sponsored by an agency of the United States Government. Neither the United States Government nor any agency thereof, nor any of their employees, make any warranty, express or implied, nor assume any legal liability or responsibility for the accuracy, completeness, or usefulness of any information, apparatus, product, or process disclosed, or represents that its use would not infringe privately owned rights. Reference herein to any specific commercial product, process, or service by trade name, trademark, manufacturer, or otherwise does not necessarily constitute or imply endorsement, recommendation, or favoring by the United States Government or any agency thereof. The views and opinions of authors expressed herein do not necessarily state or reflect those of the United States Government or agency thereof." 


\begin{abstract}
The solid-solid separation processes employed by modern coal preparation plants require large amounts of process water that must be removed from the surfaces of particles using mechanical dewatering equipment. Unfortunately, the existing processes that are used to dewater fine particles are inefficient in terms of moisture reduction and/or solids recovery. Many coal preparation plants are forced to discard fine coal particles because of the inability of existing technologies to reduce the moisture content of this product to an acceptable level. In light of this problem, a new ultrafine dewatering process called hyperbaric filter centrifugation (HFC) has been developed. This novel method combines centrifugation and pressure filtration within a single process to substantially reduce moistures over what can be achieved using conventional dewatering systems.
\end{abstract}

In the current study, empirical and theoretical dewatering models were developed in order to be able to simulate the behavior of the HFC technology. The empirical model was based on grain-size properties. On the other hand, the theoretical model was based on fundamental theories of filtration and centrifugation. Although the theoretical model provided a better understanding of the working principles of the process, the empirical model produced more accurate equilibrium moisture predictions. Therefore, the empirical model was used to further investigate the effects of several parameters on cake moistures. As such, the empirical model was useful for scale up and design purposes.

The empirical dewatering model was also used to perform an economical analysis of potential applications of the HFC technology. The model was used to investigate a variety of new circuit designs that have the potential to be commercially applied in the coal industry. The results clearly showed that this new technology would allow coal companies to process difficult- 
to-dewater ultrafines using the HFC process, while coarser solids would be more appropriately dewatered using conventional technologies such as vacuum filters or screenbowl centrifuges. This "split dewatering" concept would provide substantially higher profitability due to lower moistures and higher recoveries of ultrafine solids than could be achieved using a single dewatering process.

Laboratory- and pilot-scale versions of this technology has been constructed and tested at the facilities of Mining \& Minerals Engineering Department of Virginia Tech. Results of this testing program showed that $30-50 \%$ lower moisture values than the ones obtained using conventional mechanical dewatering processes could be achieved with the HFC technology. Based on these promising results, a pilot-scale prototype unit, which was tested successfully at several commercial U.S. coal plants, was also constructed by Decanter Machine, Inc. Finally, the process of developing of this novel technology was successfully completed with the sale of the first full-scale commercial unit by Decanter Machine, Inc. to a major U.S. coal producer. 


\section{TABLE OF CONTENTS}

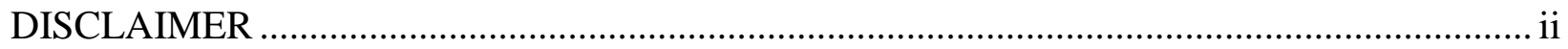

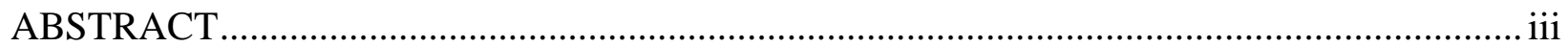

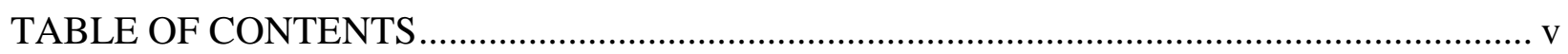

LIST OF FIGURES ………………………………........................................................ viii

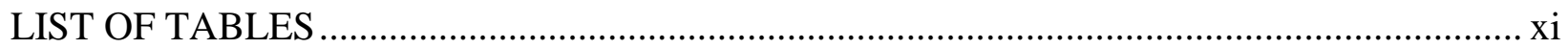

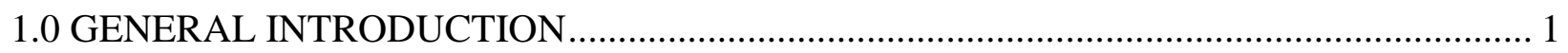

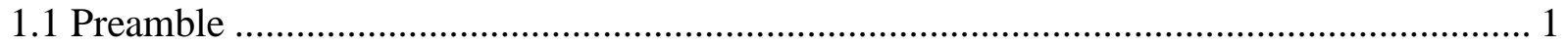

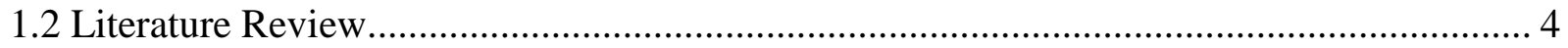

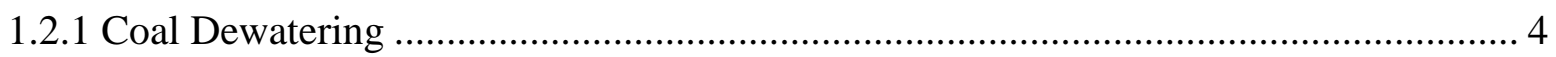

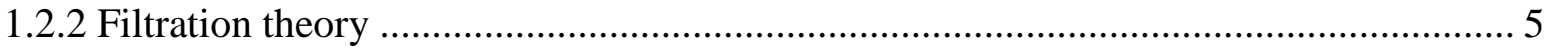

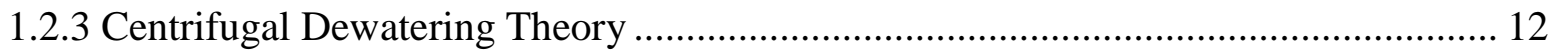

1.2.4 Centrifugal Dewatering Equipment ................................................................. 14

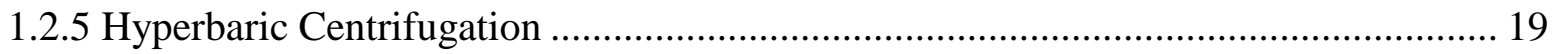

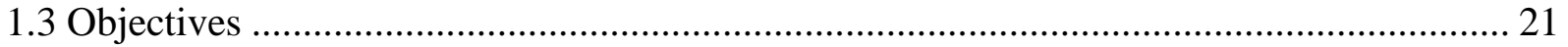

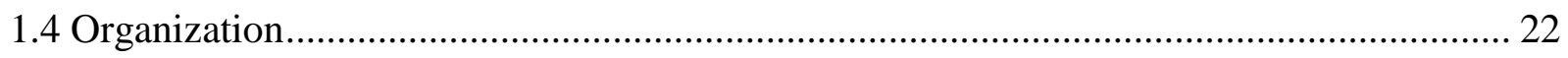

2.0 A LABORATORY STUDY OF HYPERBARIC CENTRIFUGATION ........................... 23

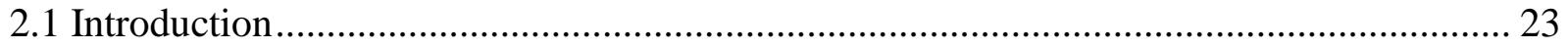

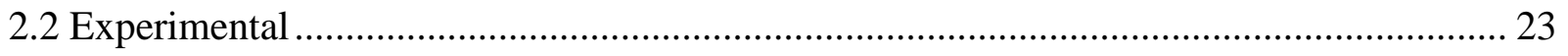

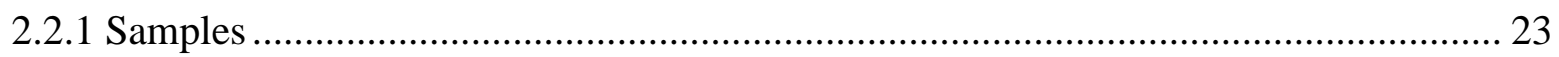

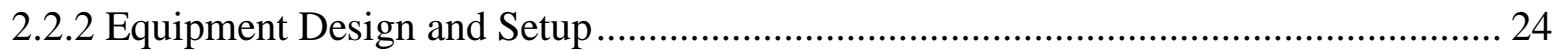

2.2.3 Hyperbaric Centrifuge Apparatus with Screen Bars............................................. 28

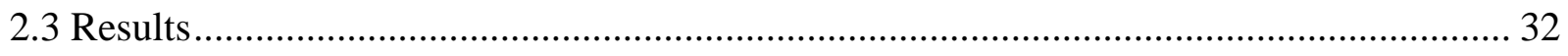

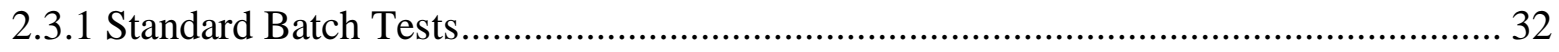

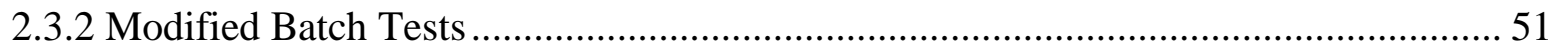

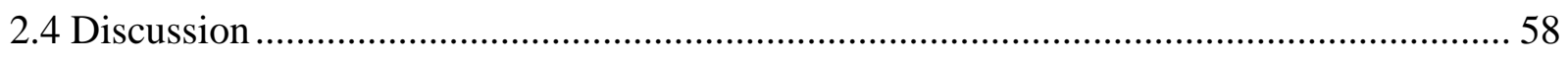

2.4.1 Solids Recovery and Product Moisture............................................................. 58

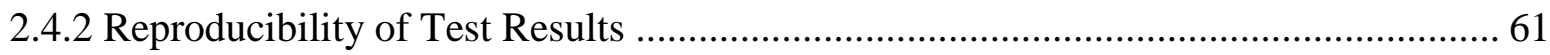

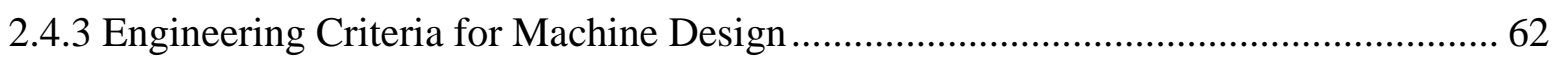

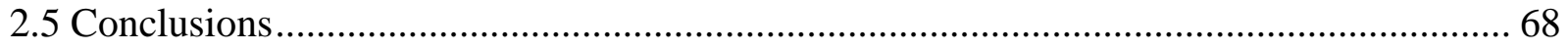


3.0 A PILOT-SCALE STUDY OF HYPERBARIC CENTRIFUGATION ….............................69

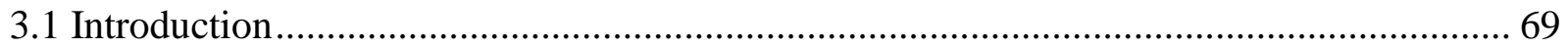

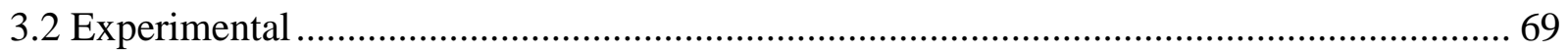

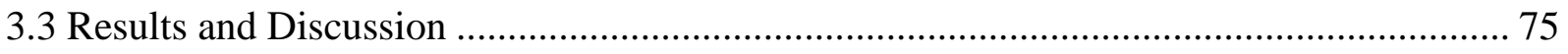

3.3.1 Effect of Rotation Speed and Rotation Time ............................................................. 75

3.3.2 Effect of Rotation Time and Air Injection ................................................................ 76

3.3.3 Effect of Thickening ............................................................................................... 77

3.3.4 Effect of Feed Size Distribution............................................................................. 79

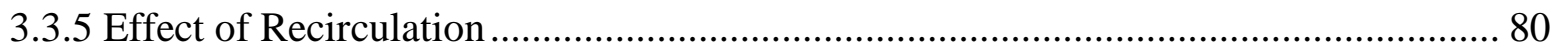

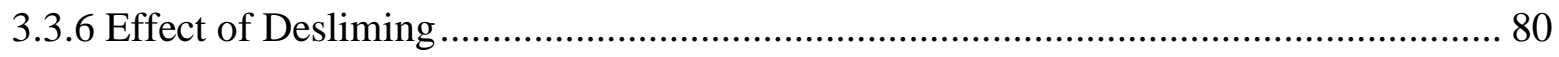

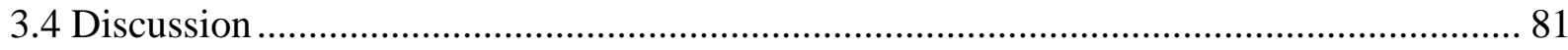

3.4.1 Solids Recovery and Product Moisture...................................................................................... 81

3.4.2 Engineering Criteria for Machine Design ........................................................................ 82

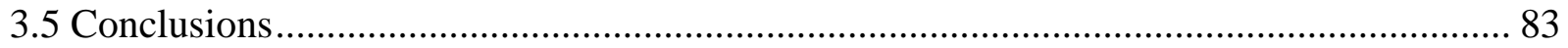

4.0 FIELD TESTING OF A PROTOTYPE HYPERBARIC CENTRIFUGE .............................. 84

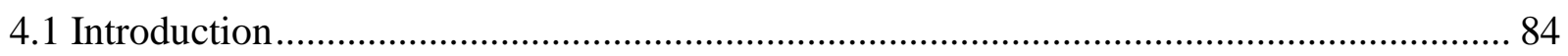

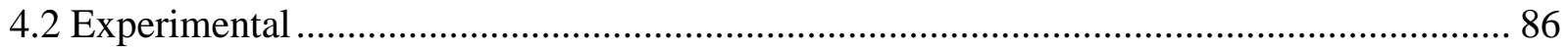

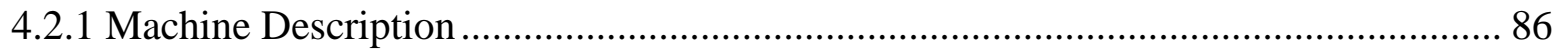

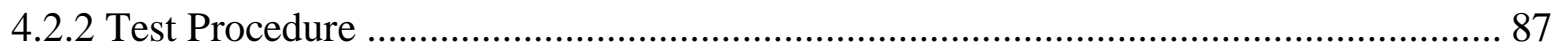

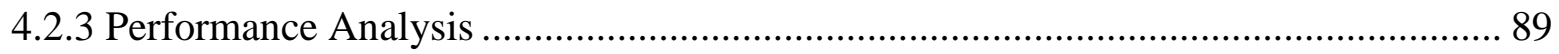

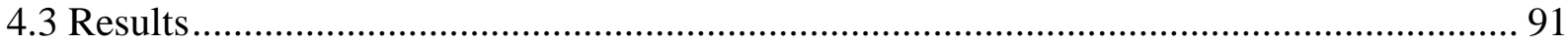

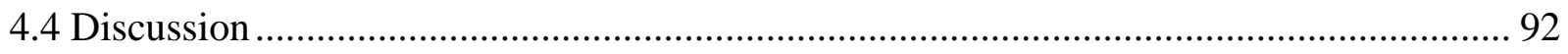

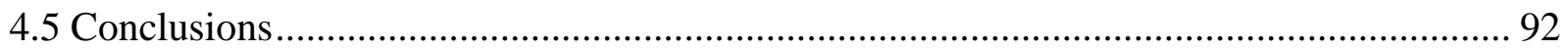

5.0 MODELING OF A HYPERBARIC FILTER CENTRIFUGE ………………….................. 94

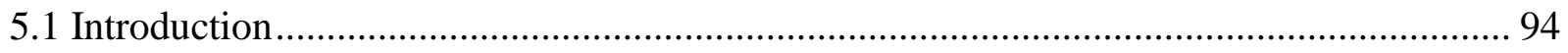

5.2 Pressure Distribution Model .............................................................................................. 95

5.3 Cake Moisture Models .................................................................................................... 98

5.3.1 Empirical Dewatering Model....................................................................................... 98

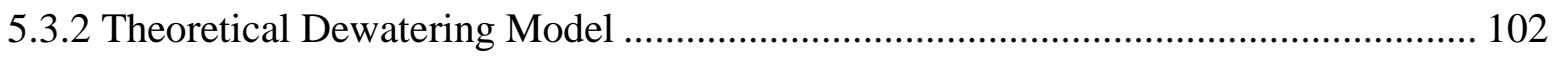

5.4 Model Integration and Simulations.............................................................................. 107

5.4.1 Empirical Dewatering Model Simulations...................................................................... 107 
5.4.2 Theoretical Dewatering Model Simulations .............................................................. 117

5.5 Mechanical Degradation ............................................................................................. 121

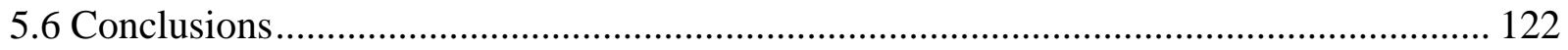

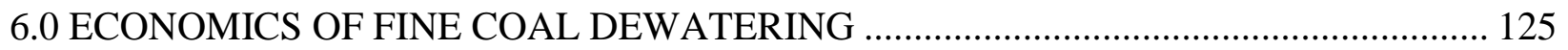

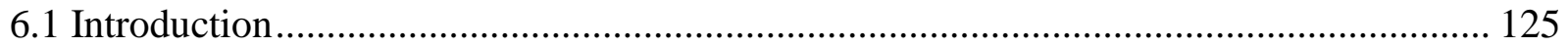

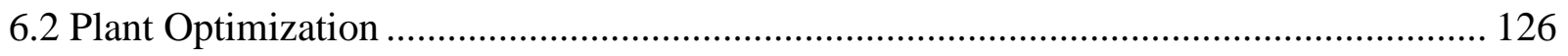

6.2.1 Optimization by Constant Incremental Ash............................................................... 126

6.2.2 Incremental Ash and Specific Gravity ………….................................................. 128

6.2.3 Effect of Moisture on Optimization .............................................................................. 130

6.2.4 Incremental Quality and Coal Value........................................................................ 131

6.3 Case Study …………………………………………….............................................. 134

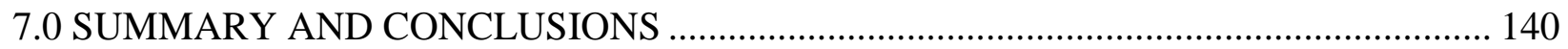

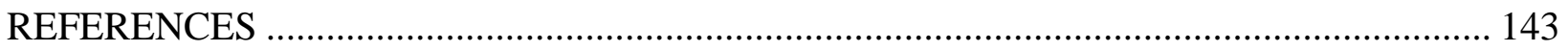

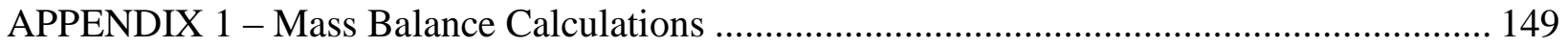

APPENDIX 2 - Economic Calculations …………….............................................................. 150 


\section{LIST OF FIGURES}

Figure 1.1 Electric power generation by energy source in the U.S. in 2008 (EIA, 2010) .............. 1

Figure 1.2 Coal production and consumption in the United States (EIA, 2010) ............................. 3

Figure 1.3 Stages of filtration ............................................................................................. 7

Figure 1.4 Plots of (a) $t / V$ versus filtrate volume $V$ for constant-pressure filtration and (b) $\Delta P$

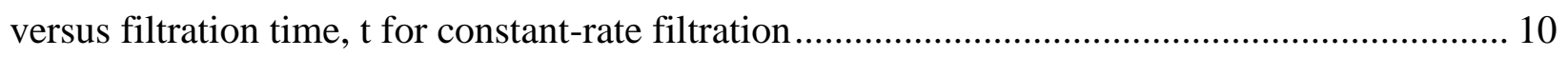

Figure 1.5 Representation of contact angles between solid, liquid and vapor phases ................... 11

Figure 1.6 States of a liquid in particulate bed ....................................................................... 13

Figure 1.7 Relationship between capillary pressure and saturation of a particulate cake ............ 14

Figure 1.8 Components of a solid-bowl centrifuge (Decanter Machine, 2010) …....................... 17

Figure 1.9 Components of a screenbowl centrifuge (Decanter Machine, 2010) ........................... 18

Figure 1.10 Pressure in a centrifuge filter cake under ambient pressure (Zeitsch, 1990)............. 20

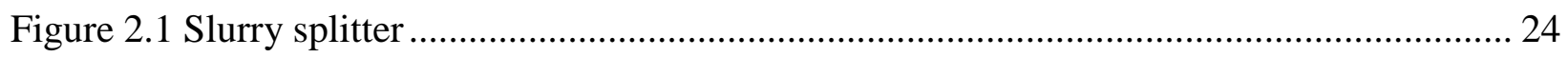

Figure 2.2 Schematic of the hyperbaric centrifuge unit used in standard batch tests................... 25

Figure 2.3 Beckman centrifuge machine ....................................................................................... 26

Figure 2.4 Sealed compartment of the Beckman centrifuge machine ......................................... 26

Figure 2.5 The test setup used for modified batch tests................................................................ 27

Figure 2.6 Schematic of the hyperbaric centrifuge unit used in modified batch tests ................... 27

Figure 2.7 Hyperbaric centrifuge constructed with screen bars .................................................... 29

Figure 2.8 Different sides of tungsten carbide bars used to build the screen section .................... 29

Figure 2.9 Schematic of the batch bench-scale centrifuge unit used in standard batch tests ....... 29

Figure 2.10 Schematic of the solid-bowl centrifuge unit used in standard batch tests.................. 31

Figure 2.11 Specially designed lid of the solid-bowl unit ............................................................ 31

Figure 2.12 The effect of size distribution on moisture at $500 \mathrm{~g}$ 's................................................. 49

Figure 2.13 The effect of size distribution on moisture at 2700 g's.............................................. 50

Figure 2.14 The first set of kinetic tests with filter cloth and closer looks to air injection parts of the curves ((d) and (f)) .............................................................................................................. 53

Figure 2.15 The second set of kinetic tests with filter cloth ......................................................... 54

Figure 2.16 The third set of kinetic tests with $1.5 \mathrm{~mm}$ screen mesh............................................... 55

Figure 2.17 Effect of feed charge on product solid content......................................................... 57

Figure 2.18 Effect of feed charge on solids loss to effluent ........................................................ 57 
Figure 2.19 Effect of centrifugal force on product solid content........................................... 57

Figure 2.20 Effect of centrifugal force on solids loss to effluent ......................................... 57

Figure 2.21 Effect of feed rate on product solids content.................................................. 57

Figure 2.22 Effect of feed rate on solids loss to effluent .................................................... 57

Figure 2.23 Effect of feed solid content on product solid content .......................................... 58

Figure 2.24 Effect of feed solid content on solids loss to effluent ........................................ 58

Figure 2.25 Effect of amount of fines on moisture reduction.............................................. 59

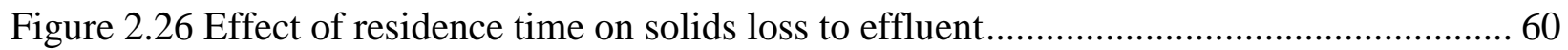

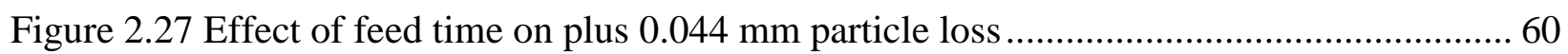

Figure 2.28 Charts showing reproducibility of test results at (a) 2700 g's and (b) 500 g's.......... 61

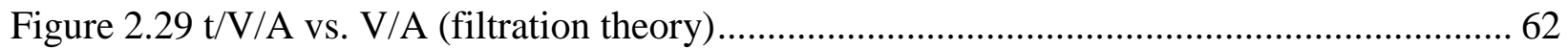

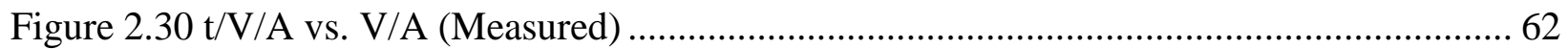

Figure 2.31 Rate curve fit for the first set of kinetic tests at 300 g's ..................................... 64

Figure 2.32 Rate curve fits for the second set of kinetic tests at (a) 300 g's and (b) 800g's ........ 65

Figure 2.33 Rate curve fits for the third set of kinetic tests at (a) 300 g's and (b) 800g's............ 66

Figure 2.34 Rate curve fit for the air injection part of the kinetic tests at 300 g's ..................... 67

Figure 3.1 Simplified schematic of the hyperbaric filter centrifuge ..................................... 71

Figure 3.2 HFC with identification labels for key components ........................................... 71

Figure 3.3 Process steps utilized by the hyperbaric filter centrifuge ...................................... 72

Figure 3.4 Rotating drum assembly for the pilot-scale centrifuge......................................... 73

Figure 3.5 Motor, drive belt and shaft assembly for the pilot-scale centrifuge ......................... 73

Figure 3.6 Valve system for the introduction of feed slurry and air...................................... 73

Figure 3.7 Electronic control panel for the pilot-scale centrifuge .......................................... 73

Figure 3.8 Effect of Rotation Speed and Rotation Time ................................................... 76

Figure 3.9 Effect of rotation time and air injection on product moisture ................................ 77

Figure 3.10 Effect of thickening on product moisture ....................................................... 79

Figure 4.1 Prototype hyperbaric centrifuge of Decanter Machine ......................................... 84

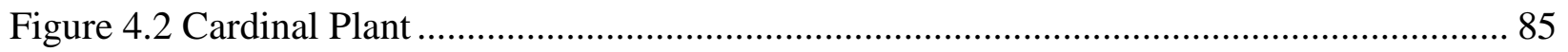

Figure 4.3 Simplified flowsheet of Cardinal Plant (Single Module) ....................................... 86

Figure 4.4 Diagram of the prototype hyperbaric centrifuge ............................................... 87

Figure 4.5 Effect of minus 0.025 mm particle amount on cake moisture................................ 90 
Figure 5.1 Geometry of centrifugal dewatering. 96

Figure 5.2 Changes in pressure across the filter cake formed on a centrifugal filter: $\mathrm{r}_{\mathrm{B}}=0.8 \mathrm{~m}$ and $\mathrm{r}_{\mathrm{S}}=0.6 \mathrm{~m}$. Calculations were made at $\mathrm{r}_{0}=0.50,0.55$ and $0.60 \mathrm{~m}$. At $\mathrm{r}_{0}=0.60 \mathrm{~m}$, the thickness of the water film on the cake become zero. In case (a), pressure in the cake becomes lower than the ambient, as shown by the dotted line. Whereas, in case (b), pressure inside the cake is positive. 97

Figure 5.3 Empirical centrifugal dewatering model setup in Excel.... 108

Figure 5.4 Comparison of empirical simulation results and measured moistures using three different methods of permeability calculation at 500 g's and 2700 g's 110

Figure 5.5 Effect of porosity on moisture with empirical model. 115

Figure 5.6 Effect of cake thickness on moisture with empirical model. 115

Figure 5.7 Effect of permeability on moisture with empirical model. 115

Figure 5.8 Effect of rotation time on moisture with empirical model 115

Figure 5.9 Effect of viscosity on moisture with empirical model. 116

Figure 5.10 Effect of air pressure on moisture with empirical model 116

Figure 5.11 Effect of centrifugal acceleration on moisture with empirical model 116

Figure 5.12 Comparison of predicted moistures to measured moistures at (a) 500 g's and (b) 2700 g's using the theoretical dewatering model ..... 118

Figure 5.13 Effect of (a) contact angle, (b) cake thickness, (c) centrifugal acceleration, (d) air pressure, (e) cake permeability and (f) liquid surface tension on moisture with theoretical dewatering model. 120

Figure 6.1 Clean coal products obtained by (a) treating parallel streams at constant cumulative ash and (b) treating parallel streams at constant incremental ash. 127 Figure 6.2 Relationship between specific gravity and individual ash content of different size classes 129

Figure 6.3 Different circuit configurations evaluated for economic analysis 136

Figure 6.4 Size distributions of coals A and B used for the case study 137 


\section{LIST OF TABLES}

Table 1.1 Recoverable coal reserves at the end of 2008 (BP, 2009) ....................................... 2

Table 1.2 Leading coal producers of the world and their production numbers in 2008 (Thousand

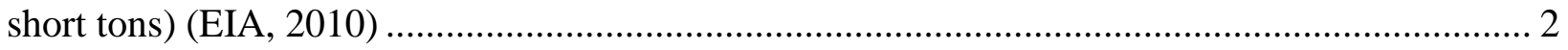

Table 2.1 Size distributions of the screenbowl feed samples obtained from different plants. ..... 32 Table 2.2 Test results obtained with Tom's Creek coal plant screenbowl feed ( $1 \mathrm{~mm}$ x 0$)$......... 33 Table 2.3 Test results obtained with Coal Clean coal plant screenbowl feed (1 mm x 0, Eagle Seam). 34

Table 2.4 Test results obtained with Weatherby coal plant screenbowl feed (1 mm x 0, Deslimed,

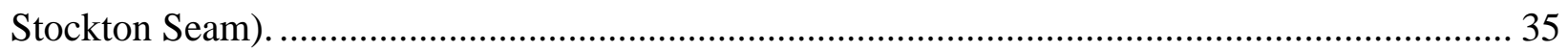

Table 2.5 Test results obtained with Moss No. 3 coal plant screenbowl feed ( $1 \mathrm{~mm}$ x 0$)$ ).......... 36 Table 2.6 Size distributions of the flotation concentrate samples obtained from two plants for

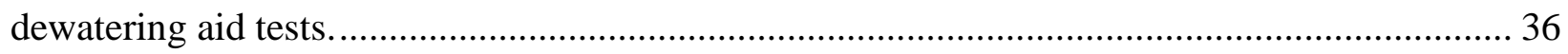

Table 2.7 Effect of dewatering aids on moisture (East Gulf Coal Plant flotation concentrate sample). 37

Table 2.8 Vacuum filtration test results (East Gulf Plant flotation concentrate sample) 39

Table 2.9 Effect of dewatering aids on moisture (Cardinal Coal Plant flotation concentrate

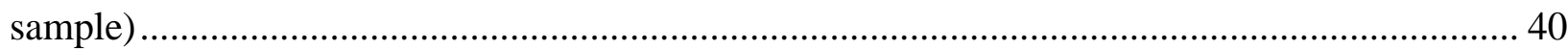

Table 2.10 Effect of rotation time with 1 lbs/t Reagent $U$ addition....................................... 41

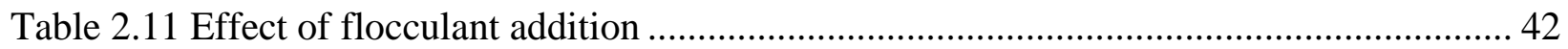

Table 2.12 Vacuum filtration test results at $120 \mathrm{~s}$ filtration time (Cardinal Plant flotation concentrate sample). .44

Table 2.13 Vacuum filtration test results at $60 \mathrm{~s}$ filtration time (Cardinal Plant flotation

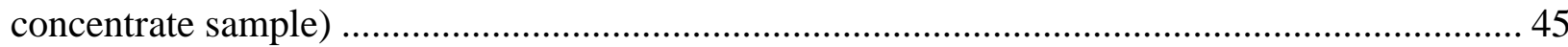

Table 2.14 Vacuum filtration test results with flocculants (Cardinal Plant flotation concentrate

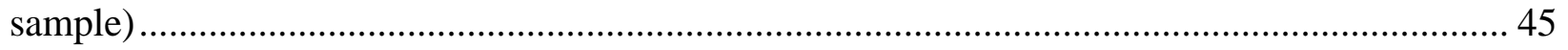

Table 2.15 Effect of air pressure on cake moisture ............................................................. 46

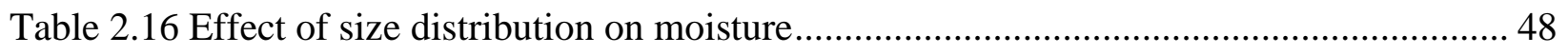

Table 2.17 Equilibrium moistures obtained at (a) the first and (b) the second sets of kinetic tests with filter cloth, and (c) the third set of kinetic tests with screen mesh ................................... 52

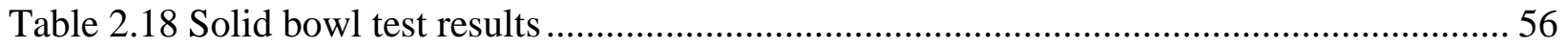


Table 3.1 Size distributions of samples tested with the pilot-scale unit . 74

Table 3.2 Effect of Rotation Speed and Rotation Time.......................................................... 75

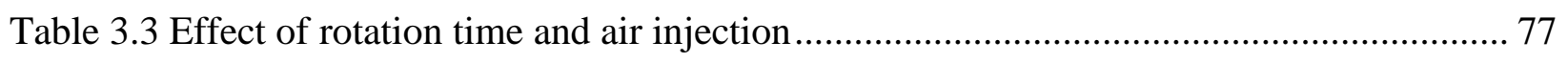

Table 3.4 Equilibrium moistures and solids recoveries obtained when samples were thickened 78

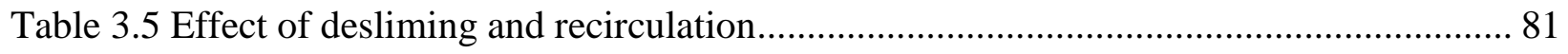

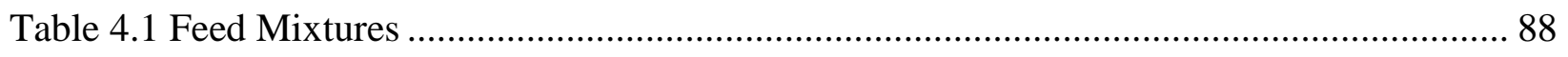

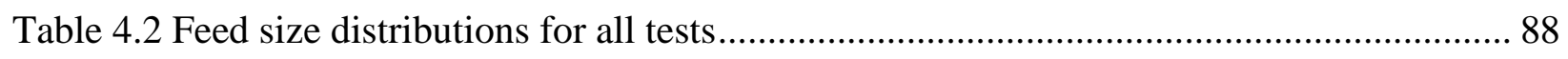

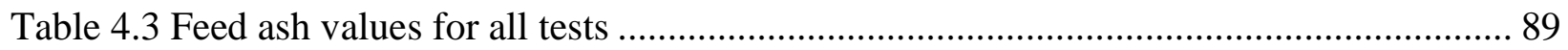

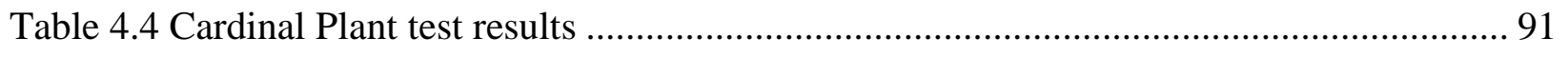

Table 5.1 Parameters used in empirical dewatering simulations ......................................... 107

Table 5.2 Measured and predicted moisture values using three different permeability estimation

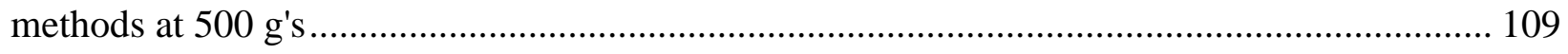

Table 5.3 Measured and predicted moisture values using three different permeability estimation

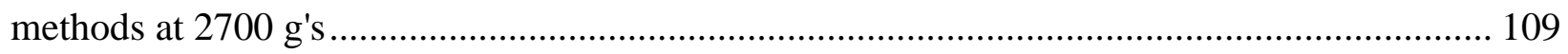

Table 5.4 Size distributions used in empirical dewatering simulations to study the synergistic effect of air pressure and centrifugal acceleration ............................................................. 111

Table 5.5 Parameters used in empirical dewatering simulations to study the synergistic effect of air pressure and centrifugal acceleration........................................................................... 111 Table 5.6 Moisture values obtained with coal A using empirical dewatering simulations at different air pressures and centrifugal accelerations........................................................... 112 Table 5.7 Moisture values obtained with coal B using empirical dewatering simulations at different air pressures and centrifugal accelerations....................................................... 112

Table 5.8 Magnitude of change in moisture caused by each parameter ................................. 113

Table 5.9 Parameters used in dynamic dewatering simulations .......................................... 117 Table 5.10 Measured and predicted moisture values using the theoretical dewatering model at 500 g's

Table 5.11 Measured and predicted moisture values using three different permeability estimation methods at $2700 \mathrm{~g}$ 's.....

Table 6.1 Simplified value calculations for particles contained in run-of-mine coal conducted (a) without any moisture and (b) with $16.67 \%$ moisture. 132 
Table 6.2 Moisture values and coal worth for (a) the coarse feed and (b) the fine feed with

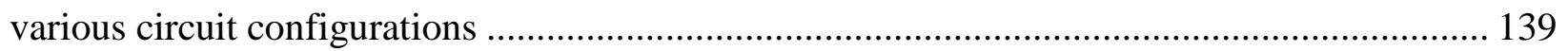




\subsection{GENERAL INTRODUCTION}

\subsection{Preamble}

The United States has the world's largest reported coal reserve with approximately 263 billion tons of coal, which represents about $29 \%$ of the total proved global coal reserves (see Figure 1.1). In 2008, the U.S. coal production reached a record level of 1,171.5 million tons (see Table 1.2) and 96\% of the coal produced was used domestically (BP, 2009; Freme, 2009). Therefore, if the consumption continues at the same rate, there is enough coal to meet the domestic consumption for more than 200 years. In 2008, more than $90 \%$ of the coal produced was used for electric power generation. Moreover, coal was the largest source of electrical power generation, with a share of almost 50\% (Figure 1.1).

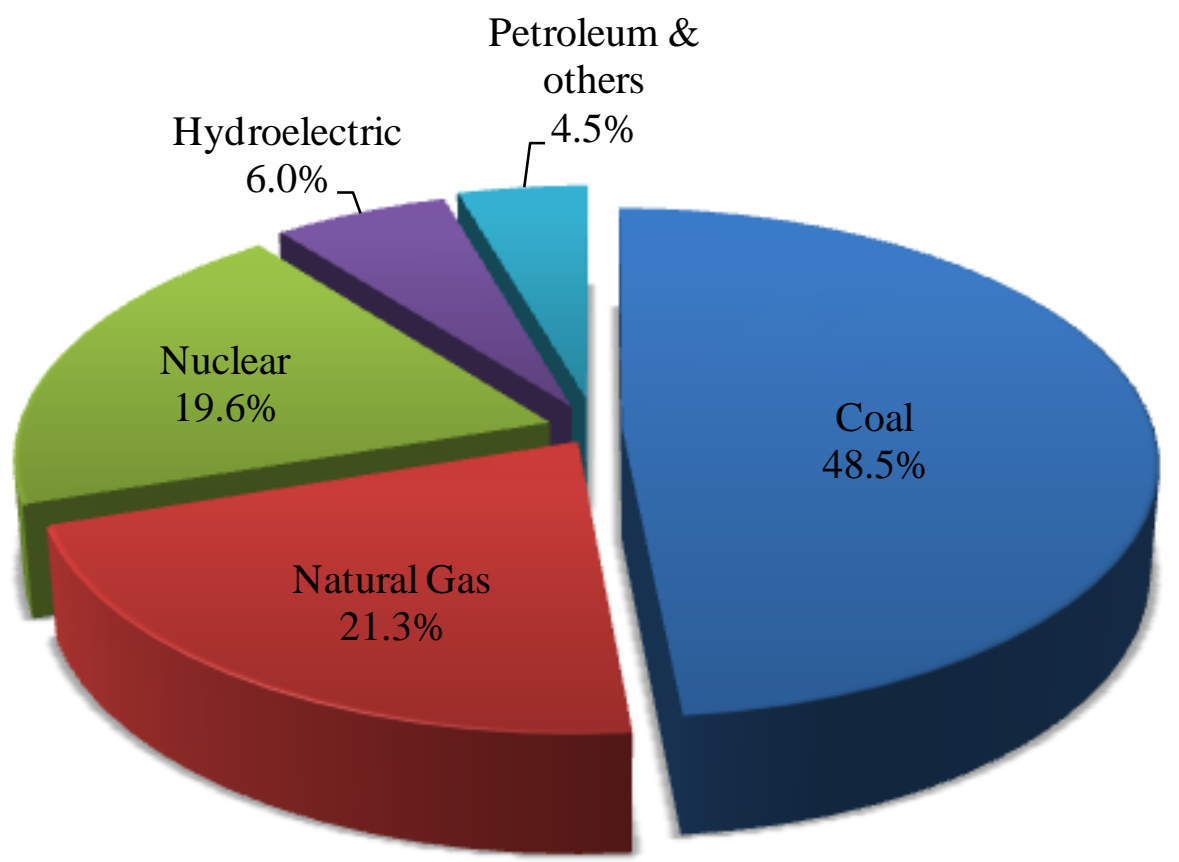

Figure 1.1 Electric power generation by energy source in the U.S. in 2008 (EIA, 2010) 
Table 1.1 Recoverable coal reserves at the end of 2008 (BP, 2009)

\begin{tabular}{cc}
\hline Country & $\begin{array}{c}\text { Recoverable Reserve } \\
\text { (Million short tons) }\end{array}$ \\
\hline United States & 262,689 \\
Russia & 173,073 \\
China & 126,214 \\
Australia & 83,996 \\
India & 64,595 \\
Ukraine & 37,339 \\
Kazakhstan & 34,502 \\
South Africa & 33,519 \\
Poland & 8,269 \\
Brazil & 7,781 \\
\hline
\end{tabular}

Table 1.2 Leading coal producers of the world and their production numbers in 2008 (Thousand short tons) (EIA, 2010)

\begin{tabular}{lccc}
\hline & Anthracite & Bituminous & Lignite \\
\cline { 2 - 4 } China & 539,454 & $2,197,069$ & 111,461 \\
United States & 1,701 & $1,094,098$ & 75,684 \\
India & 0 & 532,782 & 35,541 \\
Australia & 218 & 358,480 & 79,807 \\
Indonesia & 0 & 304,997 & 8,234 \\
Africa & 1,434 & 264,601 & 0 \\
Russia & 10,828 & 261,533 & 83,824 \\
South Africa & 1,414 & 258,182 & 0 \\
Kazakhstan & 0 & 115,100 & 4,708 \\
Poland & 0 & 92,217 & 65,665 \\
\hline
\end{tabular}

Even though the global coal consumption increase slowed in 2008, coal still was the fastest growing fossil fuel with $3.1 \%$ growth among the other fuels. While the domestic coal consumption decreased as a consequence of the slowing domestic economy and poor weather in 2008, production and exports increased to levels not seen in 30 years as a result of the increased international demand (Figure 1.2). Furthermore, coal prices also increased because of rising international demand and the increased cost of transportation due to elevated oil prices (Freme, 
2009). Therefore, coal is likely to remain as one of the most important energy sources in the U.S. and in the world.

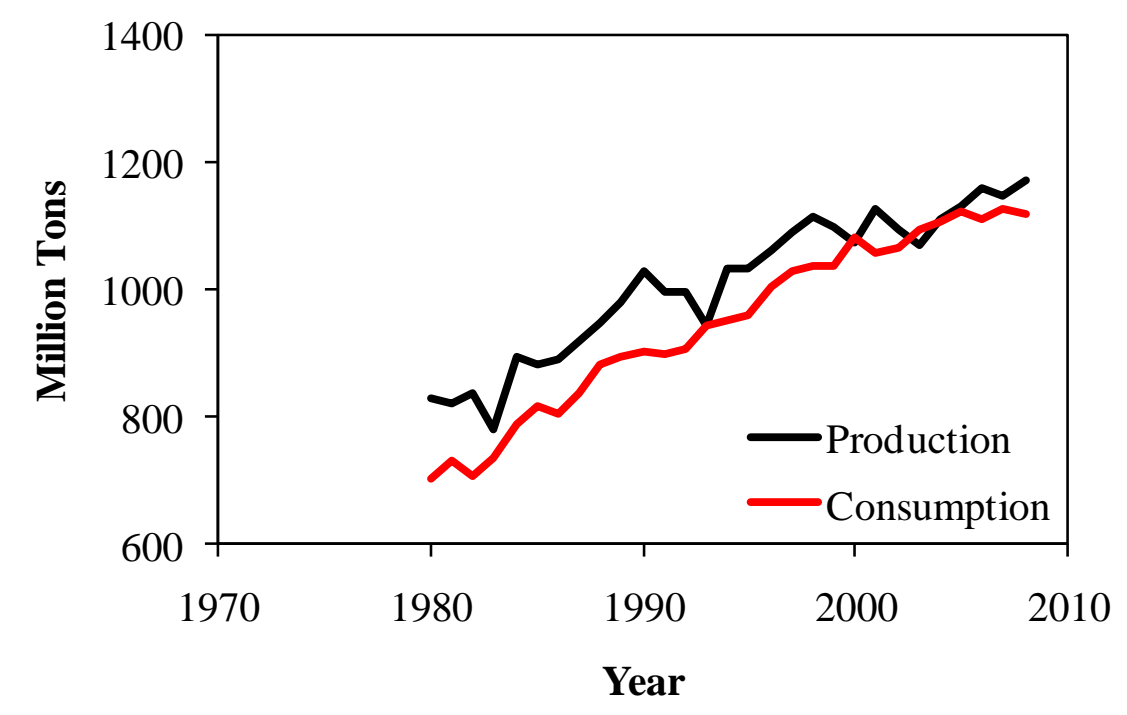

Figure 1.2 Coal production and consumption in the United States (EIA, 2010)

Much of the coal consumed in the U.S. is pretreated using coal preparation operations. These facilities are used to remove unwanted mineral matter that otherwise would decrease coal heating value, increase transportation costs, and adversely impact environmental emissions. Unfortunately, most of the cleaning processes used in modern coal preparation facilities are performed in an aqueous medium. As such, a variety of dewatering processes are used in coal preparation plants to remove excess surface moisture from clean coal products. Coarser particles can be readily dewatered using simple screening systems, while finer particles require costly unit operations such as centrifuges and filters (Osborne, 1988). Moisture that is not removed by these processes reduces the heating value and increases the cost of transporting the clean coal. Excess moisture can also create unacceptable handling problems for both the coal producer and downstream consumers by plugging chutes, bins, and rail cars. In colder regions, coal handling 
can be particularly severe during winter months because of freezing (Kaytaz, Acarkan, \& Halvorsen, 1994; L. R. Lyons, 1950).

The dewatering of fine (minus $0.150 \mathrm{~mm}$ ) coal is particularly difficult due to the large surface area of particles in this size fraction (Sandy, Matoney, \& Dahlstrom, 1991). At present, there are no mechanical dewatering processes that can inexpensively reduce the surface moisture of fine coal to a level that is acceptable for most industrial consumers. This shortcoming often forces U.S. coal producers to deslime their fine coal streams and to discard the ultrafine (minus $0.044 \mathrm{~mm}$ ) fraction into refuse impoundments. Recent estimates for the U.S. coal industry suggest that approximately 2 billion tons of fine coal has been discarded in abandoned ponds and 500-800 million tons are in active ponds (Orr, 2002). In addition, U.S. preparation plants discard 30-40 million tons of additional fresh fine coal to ponds each year. This activity represents a loss of valuable natural resources, loss of profit for coal producers, and the creation of a potential environmental liability.

\subsection{Literature Review}

\subsubsection{Coal Dewatering}

The solid-solid separation processes employed by modern coal preparation plants require large amounts of process water. After cleaning, the unwanted water must be removed from the surfaces of the particles. Only about less than $10 \%$ of the existing U.S. coal plants still utilizes thermal dryers for moisture control (Bratton, 2010) because obtaining new permits for new thermal dryers is very difficult. Therefore, dewatering operations are mostly conducted by mechanical dewatering devices in modern coal preparation plants. Depending on the size of the coal, several types of mechanical devices are employed for coal dewatering. Coal particles larger than $5 \mathrm{~mm}$ are dewatered using screens. Shaking and vibrating screens are commonly used for 
this purpose. Moreover, sieve bends are generally used for preliminary dewatering of coal prior to vibrating screens and centrifuges. $5 \mathrm{~mm} \times 0.5 \mathrm{~mm}$ size range is sent to basket type centrifuges for dewatering. Screenbowl centrifuges are widely used in coal industry to dewater $1 \mathrm{~mm} \times 0$ size range of clean coal coming from froth flotation and spirals. However, the biggest disadvantage of screenbowl centrifuges is that they discard approximately $50 \%$ of the minus $0.044 \mathrm{~mm}$ material. If high coal recovery is desirable, then the fine coal $(0.5 \mathrm{~mm} \times 0)$ can be dewatered using vacuum filters. Two types of vacuum filters traditionally used are drum filters and disc filters. Vacuum disc filters are the most commonly employed type in coal preparation due to their large filter areas and small footprint (Dahlstrom \& Klepper, 1982; Kaytaz, et al., 1994; Sandy, et al., 1991).

While clean coal is dewatered using the above mentioned units, coal tailings also have to be dewatered. The first step in fines tailing dewatering is sedimentation, which is done by thickeners. In the second step, thickened refuse sludge is sent to a dewatering device for further dewatering. Among all the available units, belt filter presses are the most commonly used devices to dewater fine coal refuse due to their good performance with very fine clay, which is often the main component of fine coal refuse. While the solid refuse material obtained is discarded to a refuse pond, clarified water recovered from thickeners and filters is recycled back to the plant to meet the water requirements of different processes (Dahlstrom \& Klepper, 1982; Osborne, 1988).

\subsubsection{Filtration theory}

Filtration is used to separate liquids from solids by passing the solid-liquid mixture through a permeable medium, which accumulates most of the solid particles while permeating the liquid. There are two types of filters: 
- Surface filters accumulate solids in the form of a cake atop a thin filter medium. Filtration conducted using surface filters is also called cake filtration.

- Depth filters accumulate the solids inside a thicker filter medium.

Surface filters are the most commonly used type of filters in coal preparation. Although, the filter medium is very thin, the cake formed on top of the filter medium becomes an extension to the original medium. Consequently, the cake acts as a depth filter and continues to accumulate particles inside and on the top as the slurry flow continues (Osborne, 1988; Svarovsky, 1990).

Cake filtration consists of several stages. First, initial bridging of particles occurs across the filter medium (Figure 1.3(a)). Then, cake formation starts and the cake gradually grows, while single-phase flow of water passes through the filter medium (Figure 1.3(b)). After the cake is formed (Figure 1.3(c)), cake compression occurs if the material is compressible (Figure 1.3(d)). This stage is often not important for coal applications because fine (i.e., $0.5 \mathrm{~mm} \times 0$ ) coal is not considered compressible (Condie, Hinkel, \& Veal, 1996). Up to this point, the process is called filtration, as there is only single-phase flow of water. After that, the dewatering part of the process begins. Two-phase flow of air and water empties the largest pores (Figure 1.3(e)) and finally air breakthrough occurs (Figure 1.3(f)). In this stage, small pores still contain water. If there is a significant amount of cake cracking as result of the air breakthrough, dewatering stops because the vacuum pressure is lost (Condie, et al., 1996; Gala, Kakwani, Chiang, Tierney, \& Klinzing, 1981). 


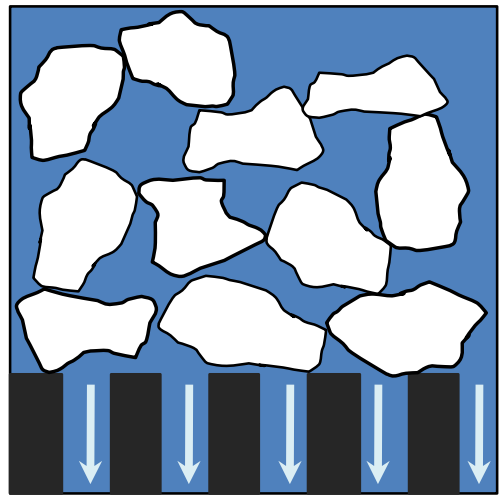

(a)

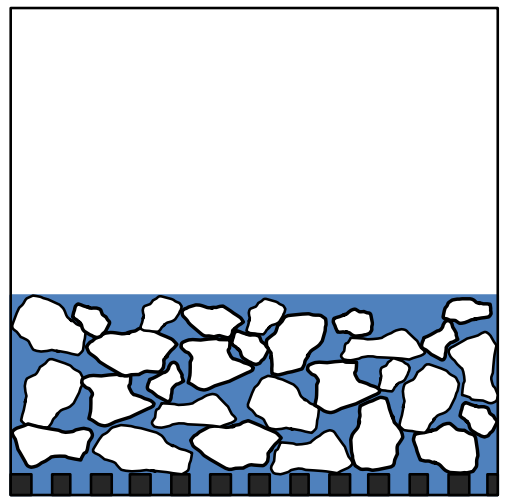

(d)

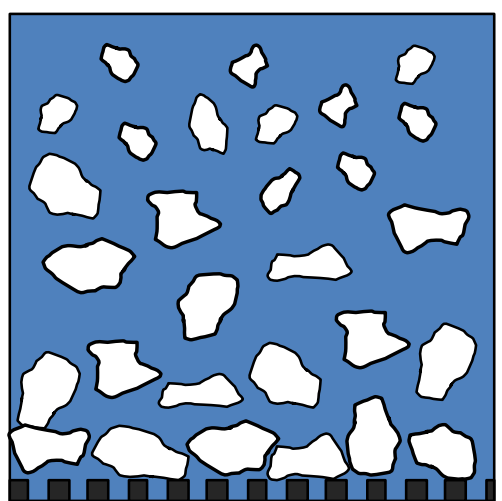

(b)

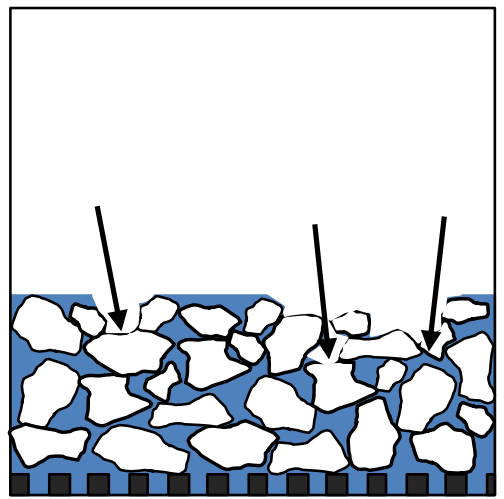

(e)

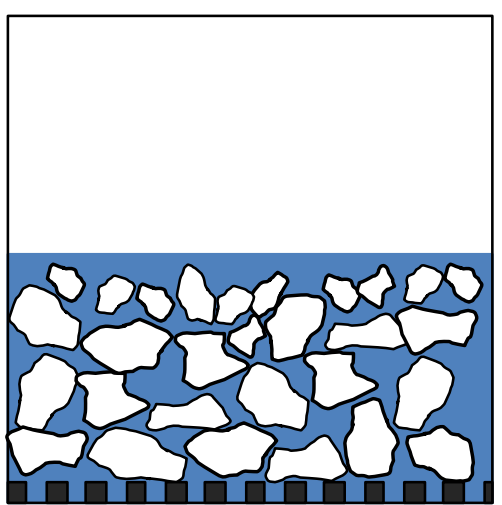

I

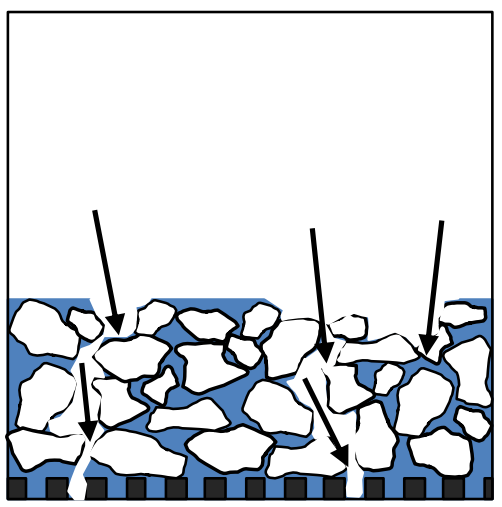

(f)

Figure 1.3 Stages of filtration

\section{a) Kinetics of Filtration}

Darcy (1856) defined filtration with the following basic equation, which relates the liquid flow rate through a filter cake to several parameters:

$$
\frac{d V}{d t}=\frac{K \Delta P A}{\eta L}
$$

where $V$ is the volume of liquid passing through the cake, $t$ is the filtration time, $K$ is the permeability of the bed, $\Delta P$ is the pressure drop across the cake, $A$ is the cross-sectional area of the cake, $\eta$ is the viscosity of the fluid and $H$ is the cake thickness. In this equation $\Delta \mathrm{P}$ is the most important parameter, as it is the driving force for liquid flow and dewatering. 
Eq. [1.1] contains the permeability term $K$, which was defined by Kozeny (1927) using the following equation:

$$
K=\frac{\varepsilon^{3}}{k(1-\varepsilon)^{2} S_{v}{ }^{2}}
$$

where $S_{v}$ is the specific surface area of the cake $\left(\mathrm{m}^{2} / \mathrm{m}^{3}\right), \varepsilon$ is the porosity of the cake and $k$ is the Kozeny constant, which normally takes value of 5 in fixed or slowly moving beds. For many industrial filter cakes formed in the presence of flocculants, $k$ is often greater than 5 and can be as large as several thousands (Gray, 1958). Substituting Eq. [1.2] into Eq. [1.1] gives the Kozeny-Carman equation (Carman, 1956):

$$
\frac{d V}{d t}=\frac{\Delta P A}{\eta L} \frac{\varepsilon^{3}}{k(1-\varepsilon)^{2} S_{\nu}{ }^{2}}
$$

In vacuum filtration and pressure filtration, the liquid filtered is subjected to medium resistance, $R_{m}$, and cake resistance, $R_{c}$. The medium resistance is assumed to stay more or less constant, while the cake resistance changes with time. The $L / K$ term in Eq. [1.1] can be expressed in terms of these two resistance values, and the equation becomes:

$$
\frac{d V}{d t}=\frac{\Delta P A}{\eta\left(R_{c}+R_{m}\right)}
$$

If the cake formed is assumed to be incompressible, then $R_{c}$ can be expressed as:

$$
R_{c}=\alpha W
$$

where $\alpha$ is the specific cake resistance $(\mathrm{m} / \mathrm{kg})$ and $W$ is the cake mass accumulated per unit area $\left(\mathrm{kg} / \mathrm{m}^{2}\right) . W$ can also be expressed as: 


$$
W=c \frac{V}{A}
$$

where $\mathrm{c}$ is the concentration of solids in slurry $\left(\mathrm{kg} / \mathrm{m}^{3}\right)$. Therefore, substituting Eq. [1.5] and Eq. [1.6] into Eq. [1.4] results in:

$$
\begin{gathered}
\frac{d V}{d t}=\frac{\Delta P A}{\eta\left(\alpha c \frac{V}{A}+R_{m}\right)} \\
\frac{d t}{d V}=\frac{\alpha \eta c V}{\Delta P A^{2}}+\frac{\eta R_{m}}{\Delta P A}
\end{gathered}
$$

Integration of Eq. [1.8] for constant-pressure filtration condition gives:

$$
\frac{t}{V}=\left(\frac{\alpha \eta c}{2 \Delta P A^{2}}\right) V+\left(\frac{\eta R_{m}}{\Delta P A}\right)
$$

which produces a straight line when $t / V$ is plotted against $V$, as shown in Figure 1.4(a). The parameter $a$ is the slope of the line and $b$ is the y-axis intercept of the line. Moreover, for constant-rate filtration, $V / t$ is constant and $\Delta \mathrm{P}$ varies as follows:

$$
\Delta P=\left(\frac{\alpha \eta c V}{A^{2} t}\right) V+\left(\frac{\eta R_{m}}{A} \frac{V}{t}\right)
$$

Similarly, plotting volume of liquid collected, V, versus filtration time, t, results in a straight line relationship such as that shown in Figure 1.4(b) (Dahlstrom, 1985; Osborne, 1988; Ranjan \& Hogg, 1996; Rushton, Ward, \& Holdich, 2000b; Wakeman \& Tarleton, 1999). 


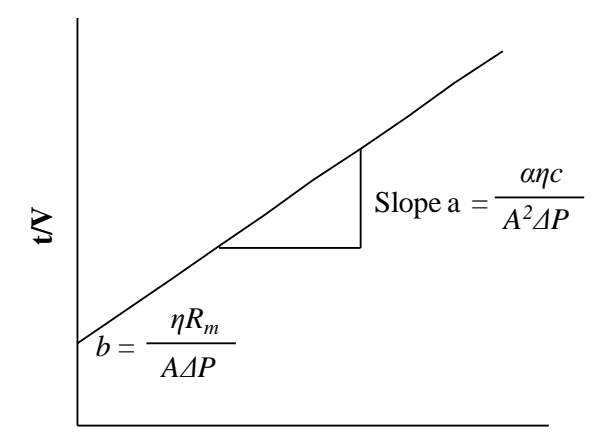

(a)
Volume of liquid collected, $\mathrm{V}$

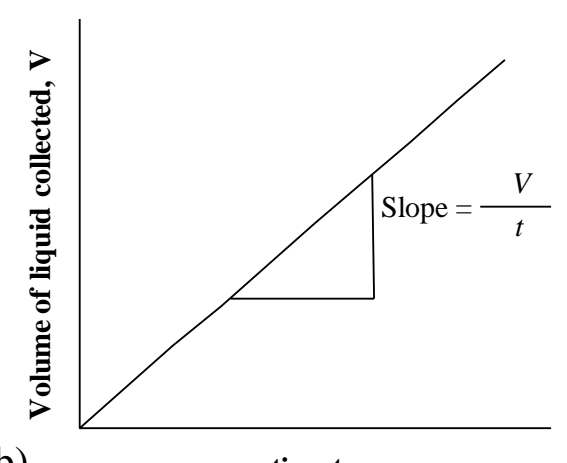

(b)

time,t

Figure 1.4 Plots of (a) $t / V$ versus filtrate volume $V$ for constant-pressure filtration and (b) $\Delta P$ versus filtration time, $t$ for constant-rate filtration

Another fundamental equation of filtration was developed by Poiseuille (1841), which defines the flow of a liquid through a capillary tube with circular cross-section:

$$
\frac{d V}{d t}=\frac{\Delta P \pi r^{4}}{8 \eta L}
$$

where $\mathrm{r}$ is the capillary radius (Akers \& Ward, 1977).

\section{b) Thermodynamics of Filtration}

In order to move a liquid contained in a capillary tube, the capillary pressure, $P$, defined by the Laplace equation (1806) given below has to be overcome (Ralston \& Newcombe, 1992).

$$
P=\frac{2 \gamma_{L V} \cos \theta}{r}
$$

where $\gamma_{L V}$ is the surface tension of the liquid, $\theta$ is the water contact angle and $r$ is the radius of the capillary. Eq. [1.12] indicates that $P$ can be reduced by decreasing $\gamma_{L V}$, increasing $r$ or increasing $\theta$. Therefore, the Laplace equation suggests that a cake with coarser size distribution would be easier to dewater than a finer cake, as the coarser cake would have larger capillaries. 
Surface tension and contact angle values can be changed in favor of dewatering by addition of appropriate surface-active dewatering aids to the feed slurry. Figure 1.5 shows a liquid droplet attached to a solid surface. Surface tension vectors for solid-liquid $\left(\gamma_{\mathrm{SL}}\right)$, solidvapor $\left(\gamma_{\mathrm{SV}}\right)$ and liquid-vapor $\left(\gamma_{\mathrm{LV}}\right)$ interfaces and the contact angle which is measured between the solid and the liquid interfaces are shown in the figure. The relationship between surface tensions and the contact angle can be quantified using Young's equation (Erbil, 2006; Ralston \& Newcombe, 1992; Young, 1805):

$$
\gamma_{S L}+\gamma_{L V} \cos \theta=\gamma_{S V}
$$

The work of adhesion, which can be defined as the energy required to separate the liquid droplet from the solid surface, is defined by the following equation:

$$
W_{a d h}=\gamma_{S V}+\gamma_{L V}-\gamma_{S L}
$$

Combining Eq. [1.13] and Eq. [1.14] gives the Young-Dupré equation (Dupré, 1869; Erbil, 2006; Ralston \& Newcombe, 1992):

$$
W_{a d h}=\gamma_{L V}(1+\cos \theta)
$$

According to Eq. [1.15], the work of adhesion reaches its maximum value when $\theta$ is $0^{\circ}$, while at

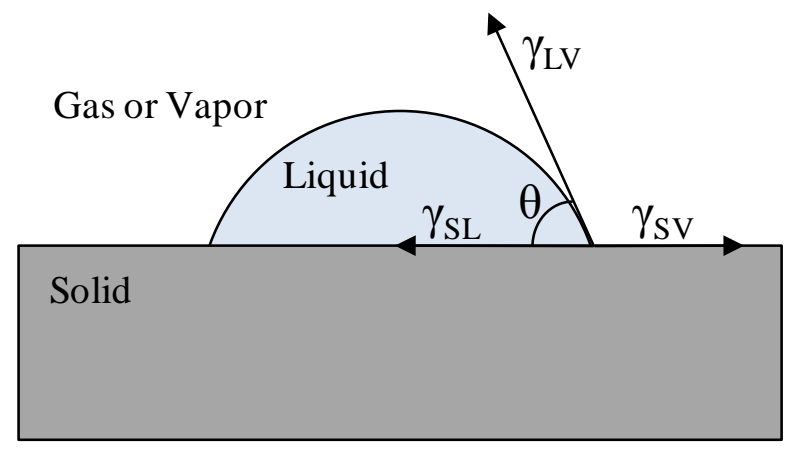

Figure 1.5 Representation of contact angles between solid, liquid and vapor phases 
a $180^{\circ}$, the work of adhesion would be minimum. Therefore, the addition of proper surfaceactive dewatering aids which increase the contact angle and reduce the surface tension can help to reduce the work of adhesion, resulting in better dewatering performance.

\subsubsection{Centrifugal Dewatering Theory}

Centrifugal dewatering is a solid-liquid separation technique in which solid particles are separated from a liquid by means of a combination of sedimentation and filtration mechanisms driven by centrifugal force. Although gravitational sedimentation and centrifugation employs the same basic principle, which is density separation, centrifugation is a much faster process because of the magnitude of the centrifugal force applied. Most of the centrifugal dewatering devices used in coal industry operate at 50-3000 times of gravitational force (Osborne, 1988).

Under the effect of centrifugal force, particles in suspension move away tangentially from the rotation axis until they are stopped by the inner surface of the centrifuge, thus forming an annular cake. If the centrifuge has a perforated wall, the cake acts as filter medium and allows liquid to flow out; thus sedimentation and filtration are combined in this case. However, if the centrifuge has a solid wall, then only sedimentation occurs (Osborne, 1988; Rushton, Ward, \& Holdich, 2000a).

Generally, the centrifugal force created by a centrifuge is expressed in terms of the number of times this force exceeds the gravitational force, or "the number of g's", and it is formulated as follows:

$$
F_{C}=\frac{4 \pi^{2} r N^{2}}{g}
$$

Where $F_{c}$ is the centrifugal force, $r$ is the centrifuge radius, $N$ is the number of rotations per second and $g$ is the gravitational acceleration. 
The particulate bed, or the cake, formed inside a centrifuge contains three types of water: surface water, structure water and chemical water (Lowry, 1963). Chemical water is held in the chemical structure of the solids and cannot be removed. Surface water that lies on the particle surfaces can be removed easily by centrifugation, while the structure water that is found inside the capillary structure of the cake is more difficult to remove (Wakeman, 1984).

The liquid content of a cake is the most important factor among the factors that induce the capillary pressure. The liquid can be contained in a particulate bed in three states, i.e. pendular, funicular and capillary states as shown in Figure 1.6 States of a liquid in particulate bed. The relationship given in Figure 1.7 shows the capillary pressure for different states. As seen from the figure, capillary pressure is the smallest at the capillary state. As some of the liquid is drained from the cake, the capillary pressure increases and becomes far larger in magnitude at the funicular state than at the capillary state. If liquid drainage is continued, the capillary pressure increases rapidly and when the saturation of the particulate bed decreases to a certain level. Under this condition, the centrifugal force will not be large enough to overcome the capillary pressure; therefore, drainage of the liquid will stop (Hwang, Chu, \& Lu, 2001).

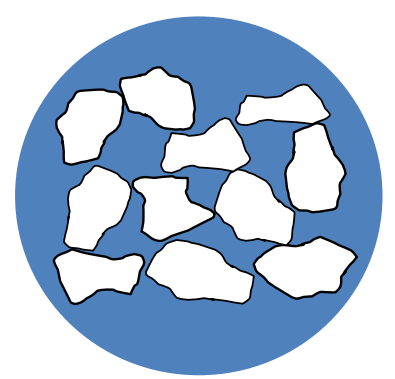

(a) Capillary State

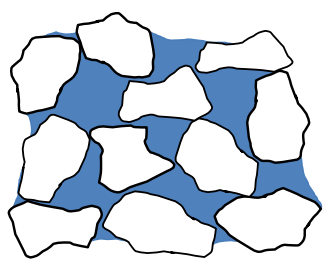

(b) Funicular state

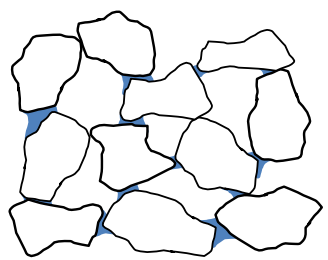

(c) Pendular State

Figure 1.6 States of a liquid in particulate bed 


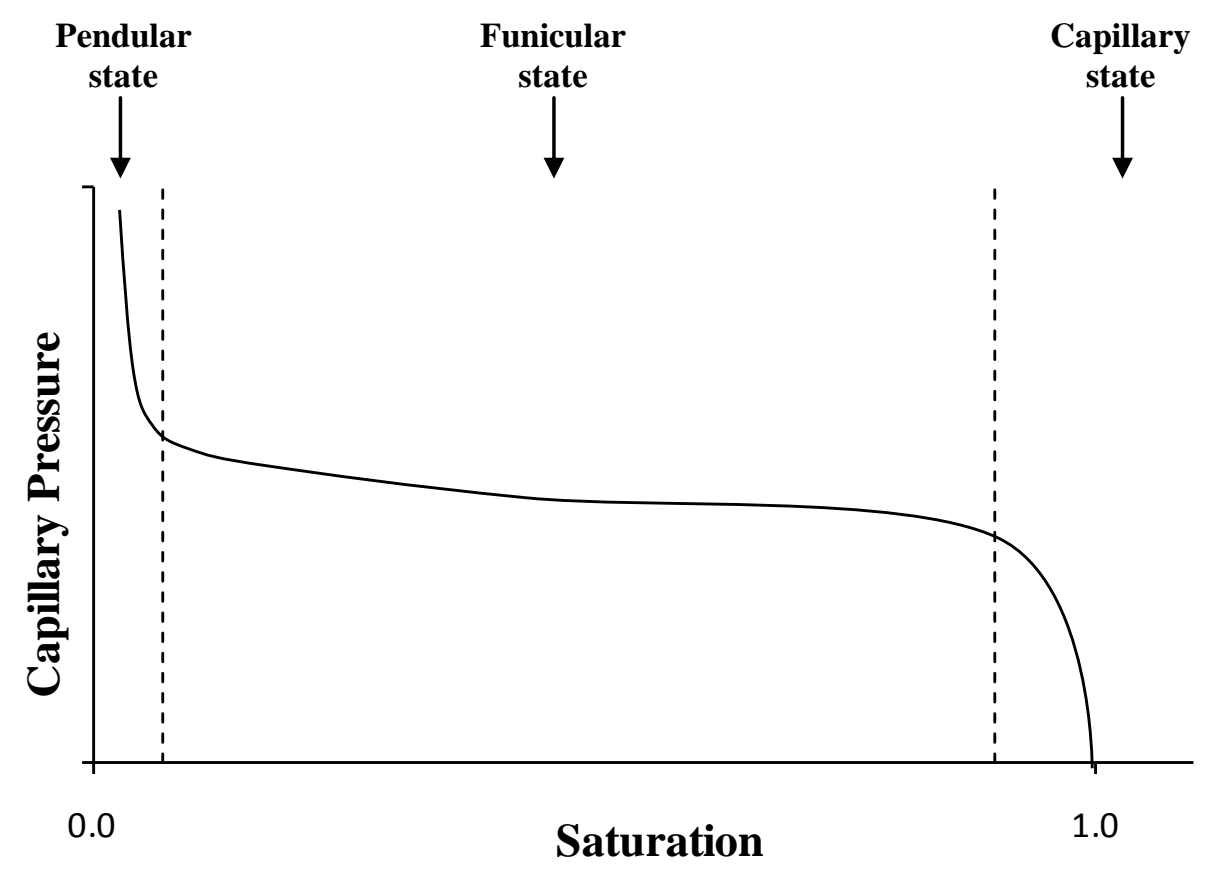

Figure 1.7 Relationship between capillary pressure and saturation of a particulate cake

\subsubsection{Centrifugal Dewatering Equipment}

Industrial centrifuges can be divided into two main categories, namely, continuously fed machines and batch machines. Centrifuges employed in coal industry are all continuously fed type machines, which can treat particle sizes from $50 \mathrm{~mm}$ to a few micrometers. Available feed capacities range from 0.25 to 100 tons of dry solids per hour (tph). However, no single machine can handle this entire particle size range.

In general, continuously fed centrifuges can be divided into two types (Osborne, 1988; Rushton, et al., 2000a; Wills, 2006; Zeitsch, 1990)

1) Perforate basket centrifuges (horizontal or vertical axis of rotation);

2) Bowl type centrifuges (horizontal axis of rotation); 
Each main group has many types of machines as follows:

- Perforate basket type

○ Without a transport device

○ With a transport device

- With positive discharge system

○ Vibrating basket

- Bowl type

○ Solid-bowl

○ Screen-bowl

\section{a) Perforate Basket Centrifuges}

Perforate basket centrifuges without transport devices are the simplest centrifuge designs. They consist of a conical perforated basket with an upward pointed apex of the cone. The coal is accelerated by the rotating bowl and the water is drained through the perforations of the basket. Coal slides down the inclined wall and is discharged at the base of the bowl. These centrifuges can handle $10 \mathrm{~mm}$ to $1 \mathrm{~mm}$ size range at capacities up to 50 tph, producing 6-8\% moistures by weight (Osborne, 1988; Sandy, et al., 1991).

Perforate basket centrifuges with transport devices are commonly used in coal industry. They consist of a positive discharge mechanism, which is a solid cone with helical flights, inside a conical perforated bowl. Wet coal enters the apex of the bowl and is transported by the rotating flights towards the base of the bowl where it is discharged. These centrifuges can handle $10 \mathrm{~mm}$ to $0.5 \mathrm{~mm}$ size range at 80 tph capacity, producing $6-7 \%$ moisture by weight. Another design with a transport device, which is a horizontally rotated machine, can handle finer coal feeds of 
minus $0.5 \mathrm{~mm}$ at 35 tph capacity and can produce a cake containing 10-12\% moisture (Osborne, 1988; Sandy, et al., 1991).

Vibrating basket centrifuges, which can have either a vertical or a horizontal cone shaped basket, are also very commonly employed in coal industry. Perforated basket of the centrifuge vibrates in such a way that causes the coal particles move towards the discharge point. These machines do not rotate at as high speeds as the previously mentioned centrifuges. In the vertical axis type, the coal particles enter the centrifuge from the top through a hopper, and travel upwards on the perforated side walls by the vibrating action. Feed size for this type of centrifuge can be as coarse as $75 \times 6.7 \mathrm{~mm}$, which can be handled at 200 tph to produce $2 \%$ moisture by weight. A finer feed of 6.7 x $0.5 \mathrm{~mm}$ can also be handled at up to 110 tph capacity to produce

about $7.5 \%$ moisture by weight. In the horizontal axis type, the coal particles enter the apex of the basket and the water is drained through the perforations on the walls. The vibrating action of the basket distributes and transports the coal through the machine. The horizontal axis type machines have the similar capabilities to vertical types (Osborne, 1988; Sandy, et al., 1991).

\section{b) Bowl Type Centrifuges}

Bowl type centrifuges, which are also called as decanter centrifuges, were started being used in the coal industry in mid 1960s with the introduction of solid-bowl centrifuge (Figure 1.8) for treating coal refuse. These centrifuges contain two rotating elements, namely the conveyor and the bowl. The bowl consists of a long cylindrical shaped region and a shorter cone shaped region. The conveyor with one or more helical flights that follow the contour of the bowl rotates at a slightly slower or faster speed than the bowl to transport the material. The transport speed is controlled by a mechanical gear reducer that operates at a fixed gear ratio. Clearance between the flights and the bowl is kept to a minimum. Solid particles are transported by the conveyor up the 
slope of the cone above the liquid level, which is controlled by weirs at the other end of the centrifuge. Since solid particles can be very abrasive, conveyor flights are usually lined with a wear resistant material (Osborne, 1988; Sanders \& Sutherland, 2001; Sandy, et al., 1991).

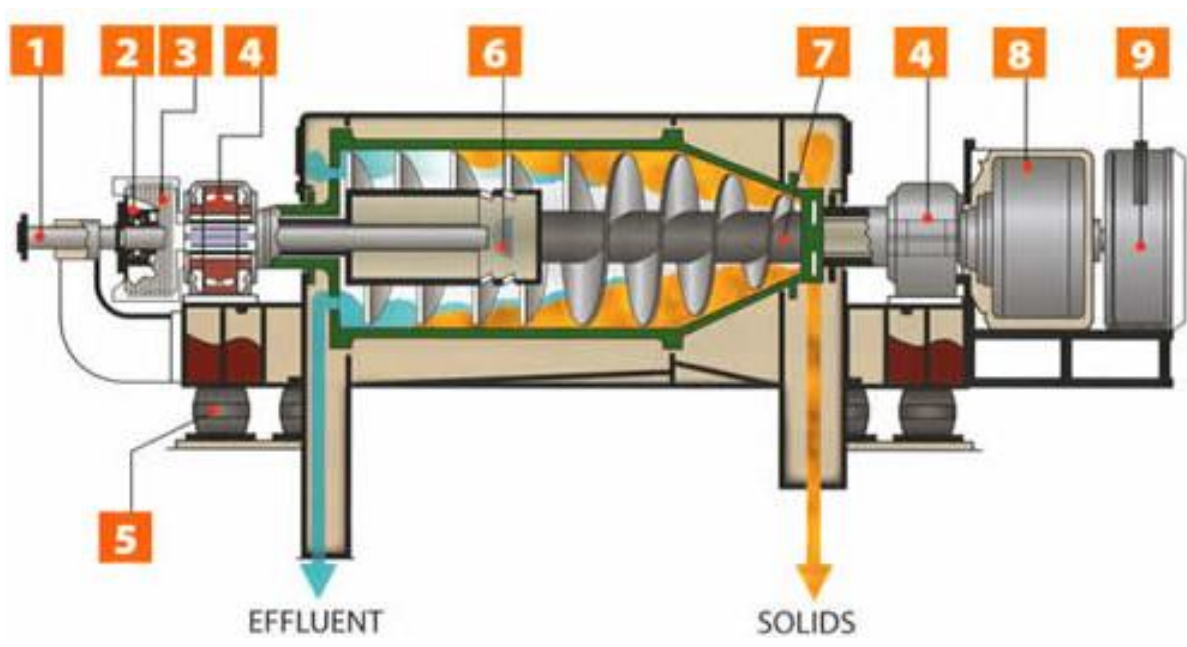

1. Feed Pipe

2. Thrust Bearings

3. Driven Sheave

4. Main Bearings

5. Vibration

Isolators

6. Feed

Compartment

7. Screw Conveyor

8. Planetary

Gearbox

9. Back drive

Figure 1.8 Components of a solid-bowl centrifuge (Decanter Machine, 2010)

A bowl centrifuge can have either a concurrent or a countercurrent feed arrangement. In the concurrent feed design, the material enters the centrifuge at the larger cylindrical section of the bowl and the cake moves in the same direction as the centrate towards the conical end. Concurrent machines operate at slower speeds than countercurrent machines, therefore they are found to be attractive in disposal of coal tailings where higher product moistures (35-45\%) can be accepted. In the countercurrent design, the material enters the centrifuge near where the conical section starts and the cake moves in the opposite direction to centrate flow. Higher wear and more power consumption are unavoidable with countercurrent centrifuges due to the higher speeds at which they operate. However, lower moistures can be achieved as a result of the much higher speeds they operate. 
A screenbowl centrifuge (Figure 1.9) has an added cylindrical screen section to the solid bowl that allows for further water drainage. The screen is made up of tungsten carbide bars that have wedge profiles to prevent near size solid particles from getting stuck between the bars. By design, a screenbowl centrifuge is a countercurrent machine. Feed slurry enters the acceleration chamber, where it is brought up to speed, through a stationary pipe. Then, it is distributed inside the bowl through the feed ports. Solids settled under the acceleration force are carried up the slope of the cone by the helical conveyor, as in solid-bowl centrifuges. However, unlike solidbowl centrifuges, thickened cake of solids pass over a screen section where the remaining excess water is filtered through the cake and discarded by the screen openings. Since the liquid drained through the screen section has such a small volume compared to the feed slurry and it can contain useful material, this liquid is usually recycled back to the feed (Osborne, 1988; Policow \& Orphanos, 1983).

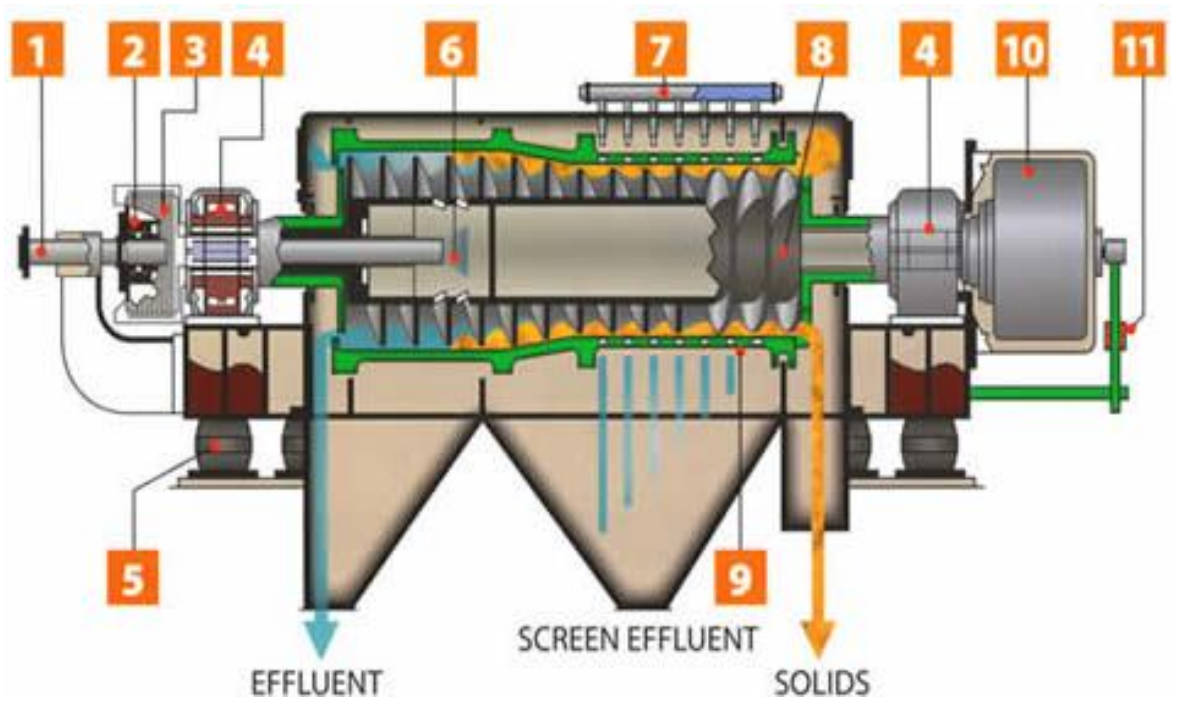

1. Feed Pipe 2. Thrust Bearings

3. Driven Sheave

4. Main Bearings

5. Vibration Isolators

6. Feed

Compartments

7. Screen Wash

Header

8. Screw Conveyor

9. Dewatering Screen

10. Planetary

Gearbox

11. Torque Sensor

Figure 1.9 Components of a screenbowl centrifuge (Decanter Machine, 2010) 


\subsubsection{Hyperbaric Centrifugation}

Injection of air into a centrifuge to improve dewatering performance was first described in the literature by Veal et al. (1998, 1999). This technology consists of the injection of a turbulent stream of high velocity air through the coal bed formed inside a vibrating basket centrifuge. The turbulent flow strips water from the surface of the particles. This technique can be effective when used with a particle size range of 0.5 to $50 \mathrm{~mm}$. However, it is not applicable for fine coal.

Friedmann et al. (2004) conducted hyperbaric centrifugation experiments with a beaker centrifuge using EPDM elastomer particles (ethylene-propylene-diene-polymethylene elastomers), which form a highly compressible filter cake. They concluded that the hyperbaric centrifugation can provide better results in solid-liquid separation for highly compressible materials. They obtained the best results with respect to dewatering rate when a superimposed static pressure was used at lower centrifugal accelerations.

According to Darcy's law (Eq. [1.1]) the driving force for the flow is the pressure differential. In vacuum filtration, the pressure differential is less than $1 \mathrm{~atm}$, while in pressure filtration it can be as high as $10 \mathrm{~atm}$ or more. In centrifugal filtration, the layer of water atop the filter cake creates the pressure differential needed to dewater the particle bed. This pressure can be calculated using:

$$
\Delta P=\frac{1}{2} \rho \omega^{2} h
$$

in which $\rho$ is the density of water, $\omega$ the angular velocity, and $h$ is the thickness of the water above the cake surface. One can see that $\Delta \mathrm{P}$ becomes zero when $h$ becomes zero (i.e., when the water disappears from the cake surface). In fact, the pressure inside the filter cake becomes lower than the ambient pressure when the water level drops below the surface of the cake as 
shown in the example given in Figure 1.10 (Zeitsch, 1990). In the figure, the horizontal axis represents the distance from the axis of rotation and vertical axis shows the pressure differential across the filter cake. The cake surface is $0.6 \mathrm{~m}$ away from the axis of rotation. The water level first forms close to the center of rotation and proceeds towards the cake surface with time. Pressure differentials for three cases are provided in the figure. The top line represents the case when the water level is $0.5 \mathrm{~m}$ away from the center of rotation. The middle and the bottom lines show the pressures when the water level is $0.55 \mathrm{~m}$ and $0.60 \mathrm{~m}$ away from the center of rotation, respectively. As can be seen, the pressure profile across the cake decreases as the water level moves towards the cake surface. Eventually, the pressure drops below ambient, essentially creating a negative pressure, when the water level reaches the cake surface (at $0.60 \mathrm{~m}$ ). This phenomenon greatly reduces the rate of dewatering during the drainage period of the centrifugal filtration process. In order to compensate the loss of the pressure, a higher centrifugal acceleration is typically recommended. Unfortunately, this action has practical limitations and

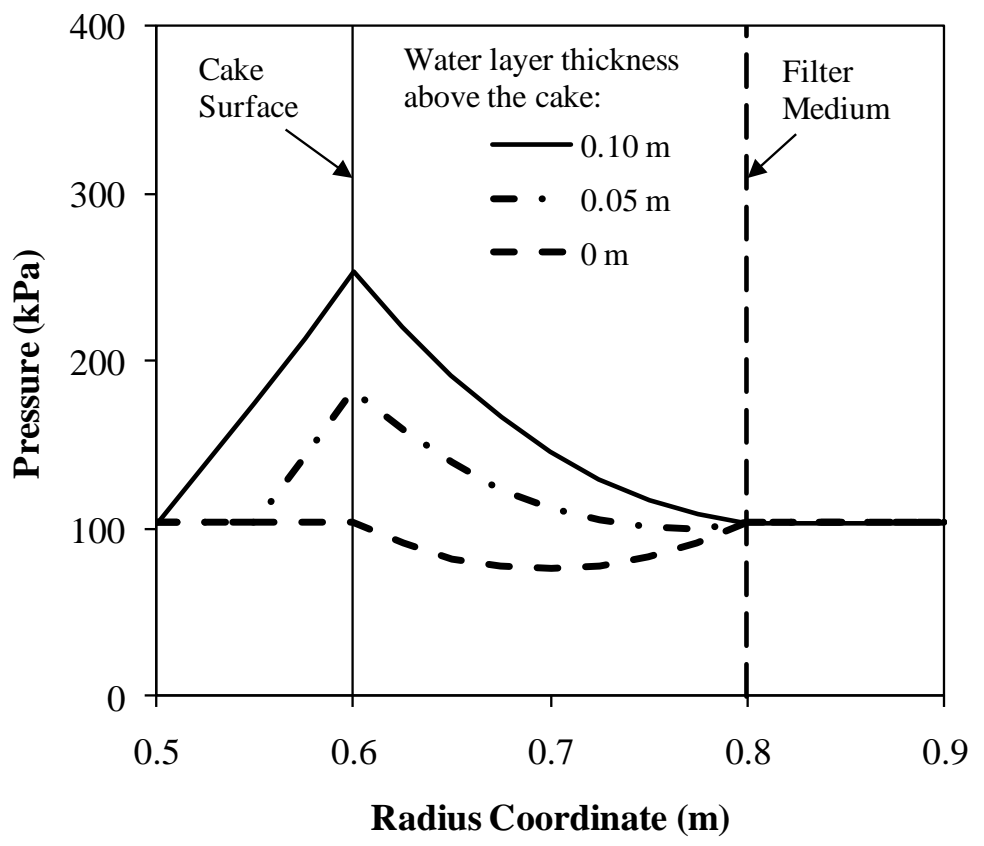

Figure 1.10 Pressure in a centrifuge filter cake under ambient pressure (Zeitsch, 1990) 
typically leads to higher machine wear and maintenance requirements.

Another method of increasing the driving force for dewatering is to introduce compressed gas into the centrifuge chamber. The compressed gas increases the pressure differential across the filter cake and increases the rate of dewatering in accordance with Darcy's law (Eq. [1.1]). A larger pressure differential is useful for increasing the rate of dewatering during the drainage period, which is critical in achieving lower cake moistures.

Using the concepts introduced in the preceding paragraphs, Yoon and Asmatulu (2002) developed a novel centrifugal dewatering device for fine coal dewatering. The novel feature of this device is the use of air pressure as an additional driving force to the centrifugal force for removing water from a fine coal cake. With this technology, the rate of dewatering increases due to the increased driving force. Consequently, laboratory test results showed that hyperbaric centrifugation could provide substantially better moisture reductions than with air pressure or centrifugation alone (Asmatulu, Luttrell, \& Yoon, 2005).

\subsection{Objectives}

The objectives of this study are:

- To fully develop a novel fine coal dewatering technology which can be employed commercially to recover the finest coal particles that were once discarded due to their high moisture content and lack of economic value. This goal has been achieved by:

○ conducting laboratory-scale parametric studies with batch test units so that an improved understanding of the parameters affecting hyperbaric centrifugation could be fully investigated,

○ conducting semi-continuous bench-scale and fully-continuous pilot-scale tests to establish engineering criteria for the design and operation of a prototype unit, and 
- conducting a demonstration of the technology at an industrial site using a full-scale continuous prototype constructed by a commercial partner.

- To successfully develop a dewatering model that can be used to accurately simulate the hyperbaric centrifugation process by extending and improving existing centrifugal dewatering models, and

- To demonstrate the capability of this novel technology for reducing environmental impacts associated with coal production while simultaneously creating a potential source of new revenue and profit for coal producers.

\subsection{Organization}

This dissertation consists of seven chapters. The first chapter gives an introduction to the research and provides detailed background information obtained from various literature sources. While the second chapter represents and discusses all the laboratory-scale test results obtained, the third chapter gives details of the pilot-scale test program. The fourth chapter provides the details and results of field tests conducted with a prototype unit. The fifth chapter discusses the theoretical basis of this research project and provides two models developed in order to simulate this novel dewatering process. The sixth chapter provides an economical evaluation of this novel process via a case study. Finally, the seventh chapter summarizes all the findings of this research and development project. 


\subsection{A LABORATORY STUDY OF HYPERBARIC CENTRIFUGATION}

\subsection{Introduction}

Yoon et al. (2001; 2005) performed several series of laboratory tests to demonstrate the effectiveness of hyperbaric centrifugation for fine coal dewatering. Based on the results obtained, a pilot-scale test unit was constructed to study this novel technology furthermore. However, as it will be explained in Chapter 3, the originally planned complete testing program could not be finished because of the many technical problems faced with the pilot-scale unit. To overcome this problem, Decanter Machine, Inc., which is the largest producer of screenbowl centrifuges in the U.S., was chosen and licensed as a partner for developing a pilot-scale prototype hyperbaric centrifuge. While construction of the pilot-scale prototype was being completed, it was decided to conduct a more detailed laboratory-scale testing program to fully understand the hyperbaric centrifugation process. For this purpose, several laboratory-scale test units were constructed and tested using various coal samples obtained from different coal preparation plants.

\subsection{Experimental}

\subsubsection{Samples}

All slurry samples used for laboratory studies were clean coal samples (i.e. screenbowl feed or flotation product) obtained from different commercial coal plants. These samples typically contained $8-12 \%$ ash. A low-ash $(<10 \%)$ unprocessed dry coal sample from Cucumber Mine in West Virginia was also obtained for testing. All slurry samples were split upon receipt by a custom-made slurry splitter (Figure 2.1) down to the amount required. This step was found to be essential to have a representative sample of the slurry so that reproducible results could be obtained. Past experience showed that when dealing with a coarser sample (i.e. $1 \mathrm{~mm}$ topsize), 
sampling from a mixed bucket was not a trustworthy method and often resulted in biased results. The use of a hand-held scoop to collect feed samples was found to produce erroneous and non-reproducible results. The dry coal sample was also split using a riffle sample divider. After splitting, all samples were screened to determine size distributions. Slurry samples were tested within 2-3 weeks upon receipt to avoid changing of the sample due to surface oxidization.

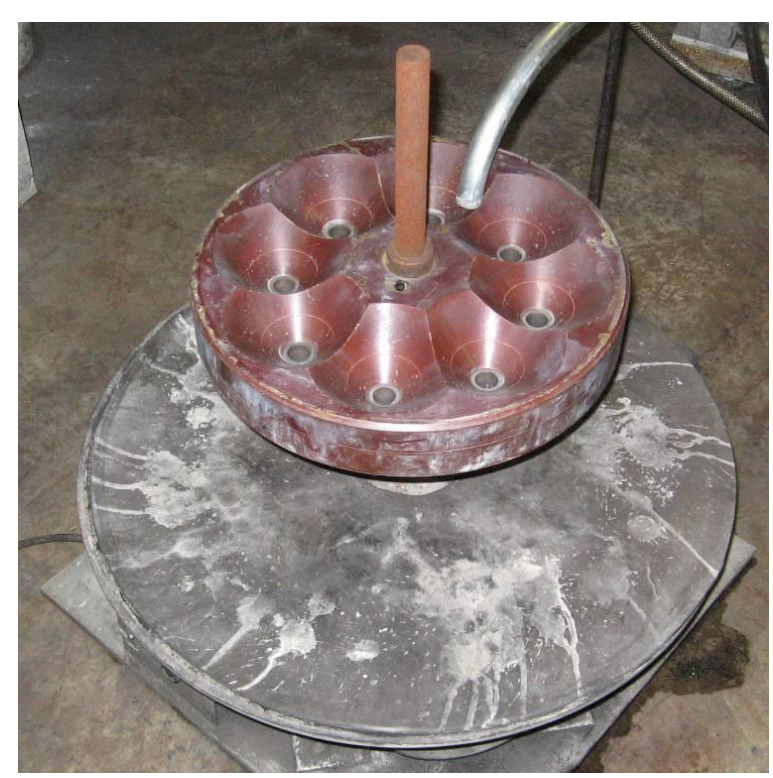

Figure 2.1 Slurry splitter

\subsubsection{Equipment Design and Setup}

\section{a) Hyperbaric Centrifuge Apparatus}

Figure 2.2 shows a schematic of the batch test unit used in the laboratory-scale test program. The unit was constructed with a 4-inch diameter and 3-inch height using stainless steel. Evenly spaced holes with 3.0, 2.4 and $1.6 \mathrm{~mm}$ diameters were drilled on the sidewalls for water drainage from the cake. The unit was used in two different setups with two different pressure lids for a variety of tests. 


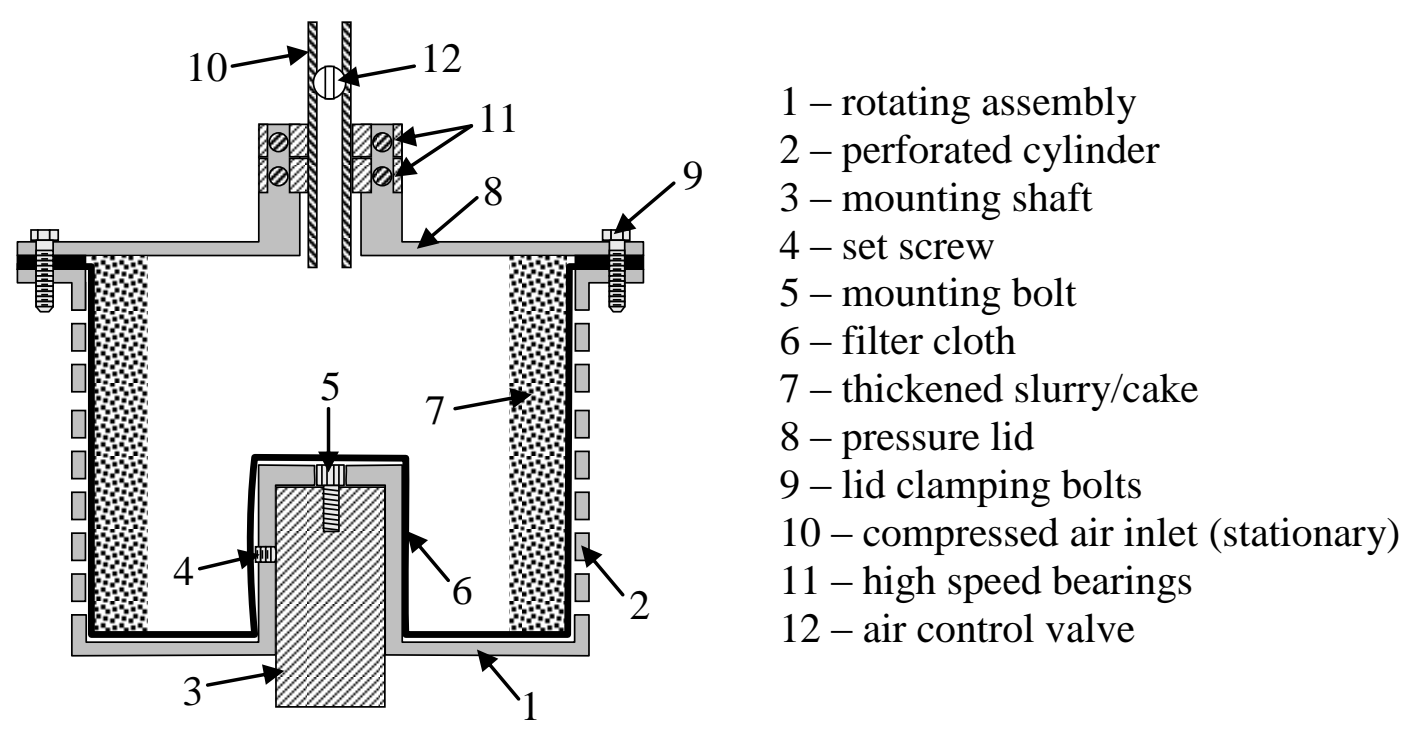

Figure 2.2 Schematic of the hyperbaric centrifuge unit used in standard batch tests

The first setup was used for the standard batch tests described in Section 2.3.1. For this setup, the centrifuge basket was placed inside in a Beckman variable-speed centrifuge machine (Figure 2.3). This "ultracentrifuge" is capable of high rotational speeds of up to approximately 20,000 rpm. The basket was attached to the rotating shaft by means of a mounting bolt. Also, a set screw was used to secure the basket in place to avoid any balancing issues that might arise at high rotation speeds. Since the basket was sealed inside the centrifuge machine (Figure 2.4), very high rotation speeds (and high-g levels) were possible without any safety concerns. Two highspeed ball bearings were attached to the lid in order to insert and support a pressurized air line. The air line was equipped with an on/off valve and a pressure gauge for monitoring and controlling air pressure. Since, this test setup did not also allow for collecting the effluent, coal recovery values could not be determined from these batch experiments. 


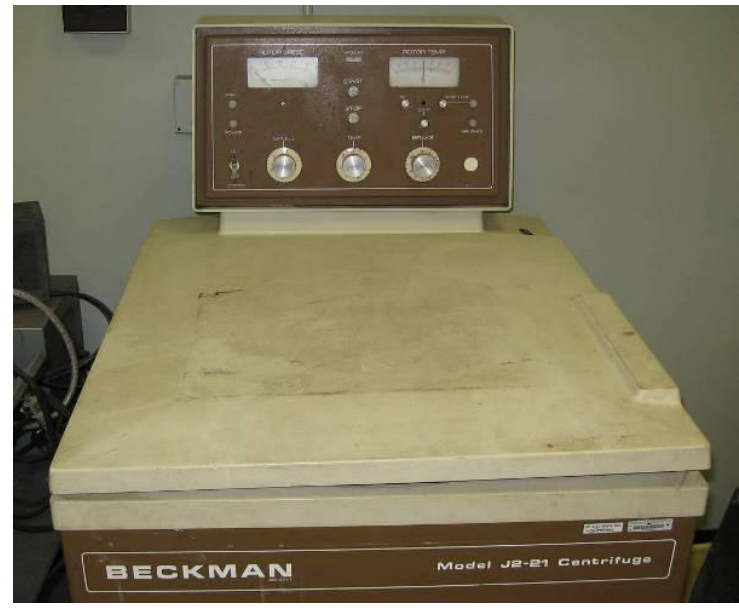

Figure 2.3 Beckman centrifuge machine

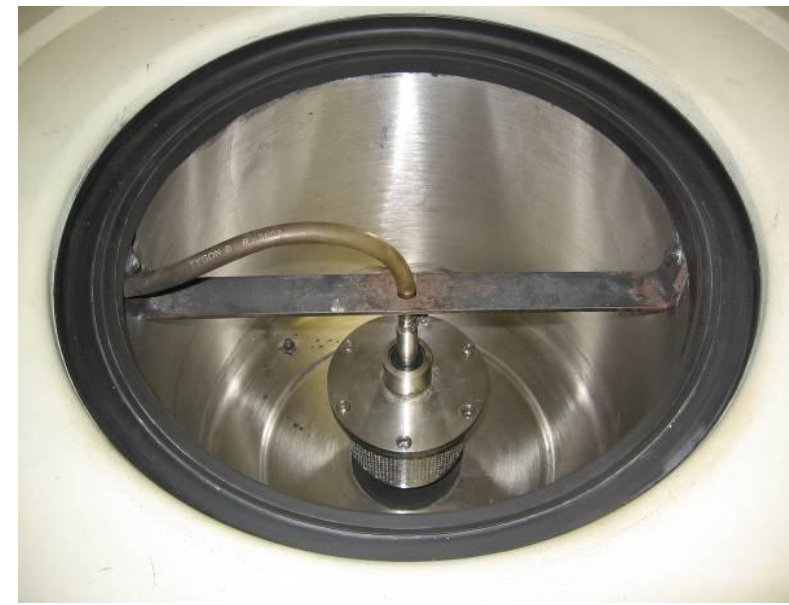

Figure 2.4 Sealed compartment of the Beckman centrifuge machine

The second setup (see Figure 2.5) was used for the modified batch tests described in Section 2.3.2. In this setup, the unit was mounted on a vertical rotor shaft and fixed in place using a set screw. A variable speed motor was used to control the rotational speed of the centrifuge. In order to pressurize the unit, a bearing and a sealed connector were used to attach a compressed air line to the center of the lid. The air-line was equipped with an on/off valve and a pressure gauge so that the air pressure can be monitored and controlled. A container suspended from a digital balance was used to collect water removed during kinetic testing. The balance continuously recorded the mass of water accumulated via a data acquisition computer. As such, this setup made it possible to monitor the kinetics of dewatering. 


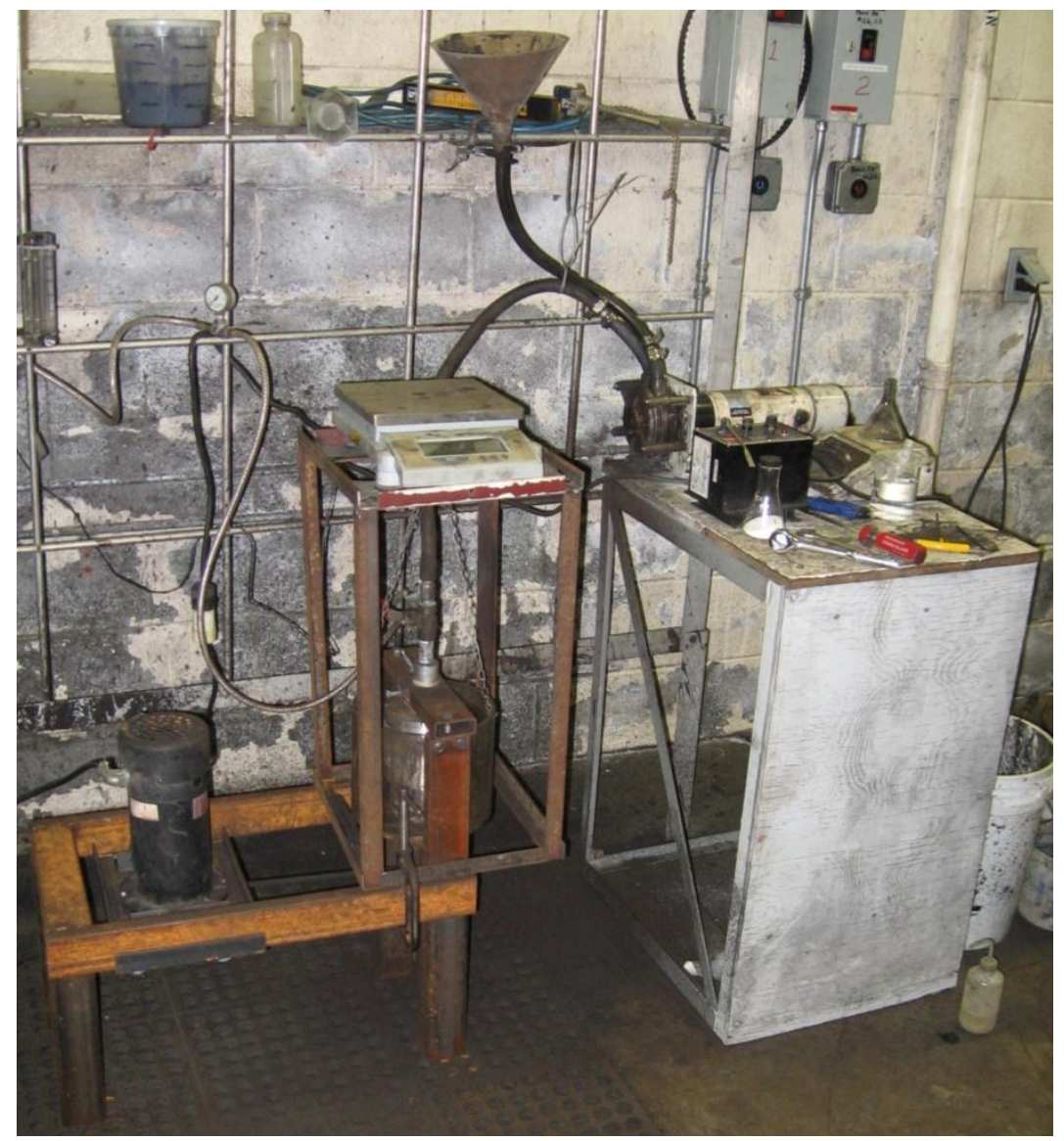

Figure 2.5 The test setup used for modified batch tests

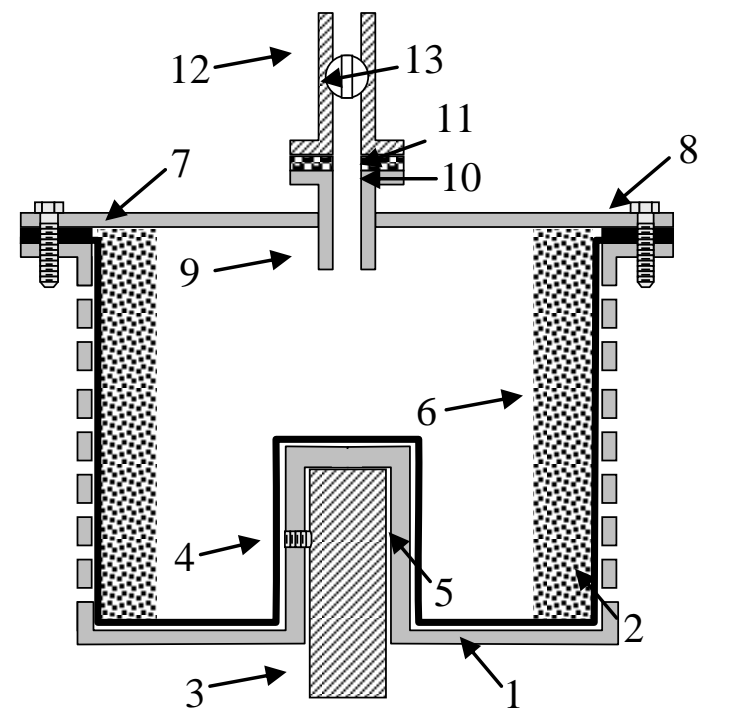

1 - rotating assembly

2 - perforated cylinder

3 - mounting shaft

4 - set screw

5 - filter cloth (or mesh)

6 - cake

7 - pressure lid

8 - lid clamping bolts

9 - compressed air inlet

10 - rotating seal face

11 - rotating pressure seal

12 - stationary inlet

13 - air control valve

Figure 2.6 Schematic of the hyperbaric centrifuge unit used in modified batch tests 
In most of the tests, the centrifuge basket was lined with a filter cloth that had a 0.015 mm aperture size. However, a somewhat coarser filter cloth (unknown aperture size) and a 1.5 mm screen mesh were also used to study the effect of filter medium on product moisture and solid recovery. The first test setup described above did not allow for feeding the unit while the basket was rotating. Therefore, the basket was filled with coal slurry before the lid was securely closed in every test. Since only a limited amount of slurry could fit inside the basket, all the excess water contained in slurries had to be removed before testing. This was accomplished by allowing solid particles to settle followed by removing the excess water using a syringe. On the other hand, in the second test setup, the coal slurry could be fed to the unit while the basket was rotating. A peristaltic pump was used for this purpose so as to minimize degradation of particle size.

\subsubsection{Hyperbaric Centrifuge Apparatus with Screen Bars}

\section{a) Screen Section}

After experimenting with the hyperbaric batch filter centrifuge, another small scale batch unit was built using tungsten carbide bars in the screen section. These bars were the same as those employed in commercial screenbowl centrifuges. The aim of this study was to replace the screen mesh or the filter cloth, which cannot be used commercially, with a wear resistant part that is widely used in the industry. The test unit, which is shown in Figure 2.7, was constructed from aluminum with 4.25-inch height, 5-inch outside diameter and 3.9-inch inside diameter. Slots of approximately 1/8-inch wide were cut on the sidewalls of the basket for water removal. T-shaped tungsten carbide bars (Figure 2.8) were glued inside the basket. These bars were designed by the manufacturer in such a way that when two of them are put together, the gap between them expands towards the outside (i.e., similar to the configuration used in a wedge- 
wire sieve). This arrangement reduces the probability of near-size particles getting trapped between the bars.

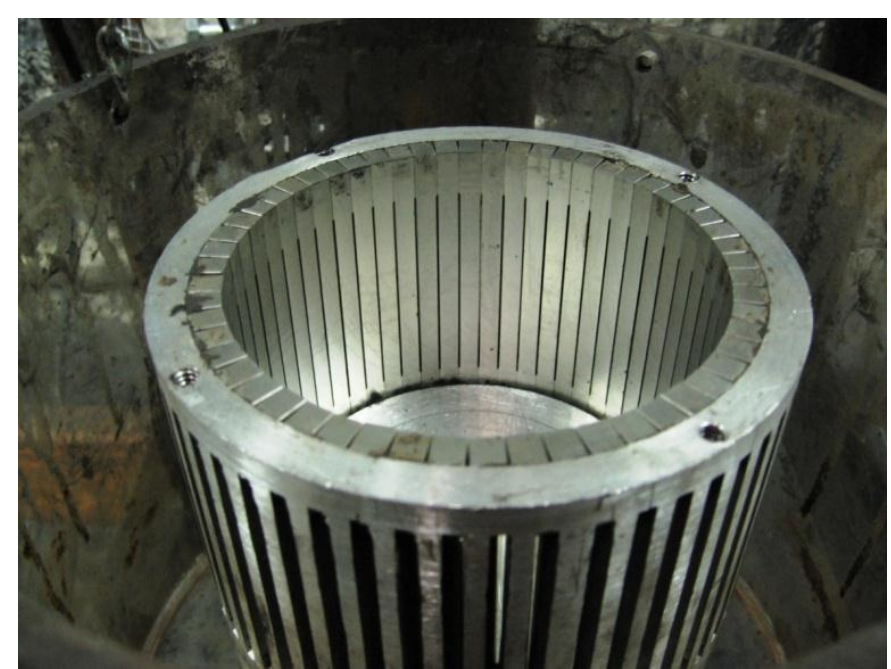

Figure 2.7 Hyperbaric centrifuge constructed with screen bars

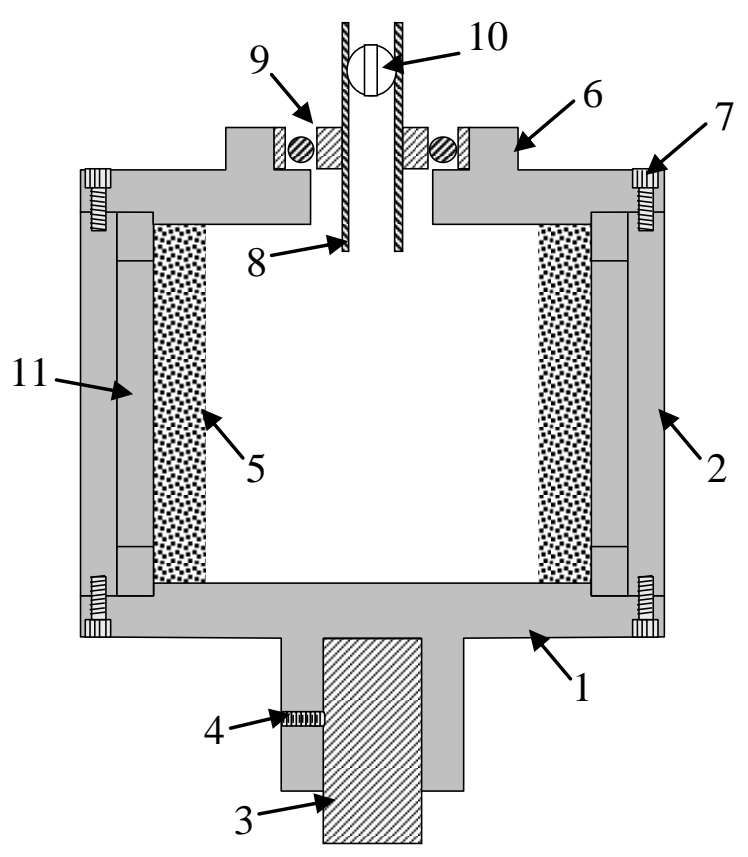

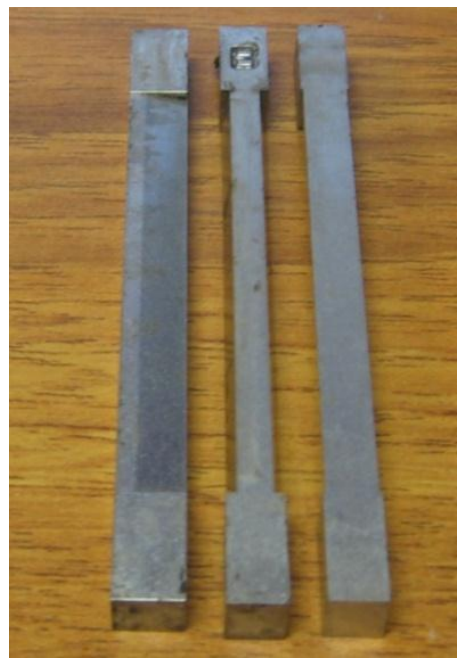

Figure 2.8 Different sides of tungsten carbide bars used to build the screen section
1 - rotating assembly

2 - cylinder with slots

3 - mounting shaft

4 - set screw

5 - thickened slurry/cake

6 - pressure lid

7 - lid clamping bolts

8- compressed air inlet (stationary)

9 - high speed bearing

10 - air control valve

11 - tungsten carbide bars

Figure 2.9 Schematic of the batch bench-scale centrifuge unit used in standard batch tests 
The test unit was mounted on a vertical rotor shaft and fixed in place using a set screw (Figure 2.9). A variable speed motor was used to control the rotational speed of the centrifuge. A high-speed bearing was attached to the lid, in order to connect a pressurized air line. The air-line was equipped with an on/off valve and a pressure gauge so that the air pressure could be accurately monitored and controlled.

\section{b) Solid-Bowl Section}

One of the aims of this project was to use a feed finer than what is normally used with screenbowl centrifuges. Some preliminary tests were run with the batch bench-scale unit with screen bars using minus $0.15 \mathrm{~mm}$ flotation product, but all the particles passed through the openings. Trials with flocculant addition also failed, because the agglomerates formed were not large enough to be retained in the centrifuge. Commercially available screenbowl centrifuges contain a solid-bowl section before the screen-section so that the feed can be thickened and excessive water and ultrafine particles can be discarded for more efficient dewatering. Based on this fact, a separate solid-bowl centrifuge was constructed to produce a thickened-feed for the batch bench-scale centrifuge with screen bars.

The solid-bowl unit (Figure 2.10) was constructed from aluminum with 4.25-inch height, 5-inch outside diameter and 4.4-inch inside diameter. The unit was mounted on a vertical shaft and fixed in place using a set screw. A variable-speed motor was used to control the rotational speed of the centrifuge. A high-speed bearing was attached to the lid, in order to connect a slurry

feed line. Slurry was gravity fed into the unit. The lid of the unit (Figure 2.11) was designed in such a way that excess water could escape the unit easily while solid particles form a cake under the lid. Also, four small threaded-holes were drilled on the sides near the top of the centrifuge, in 
order to discard the water that could not be discarded through the lid. These holes were covered with small pieces of filter cloth to prevent solid particles from escaping the unit.

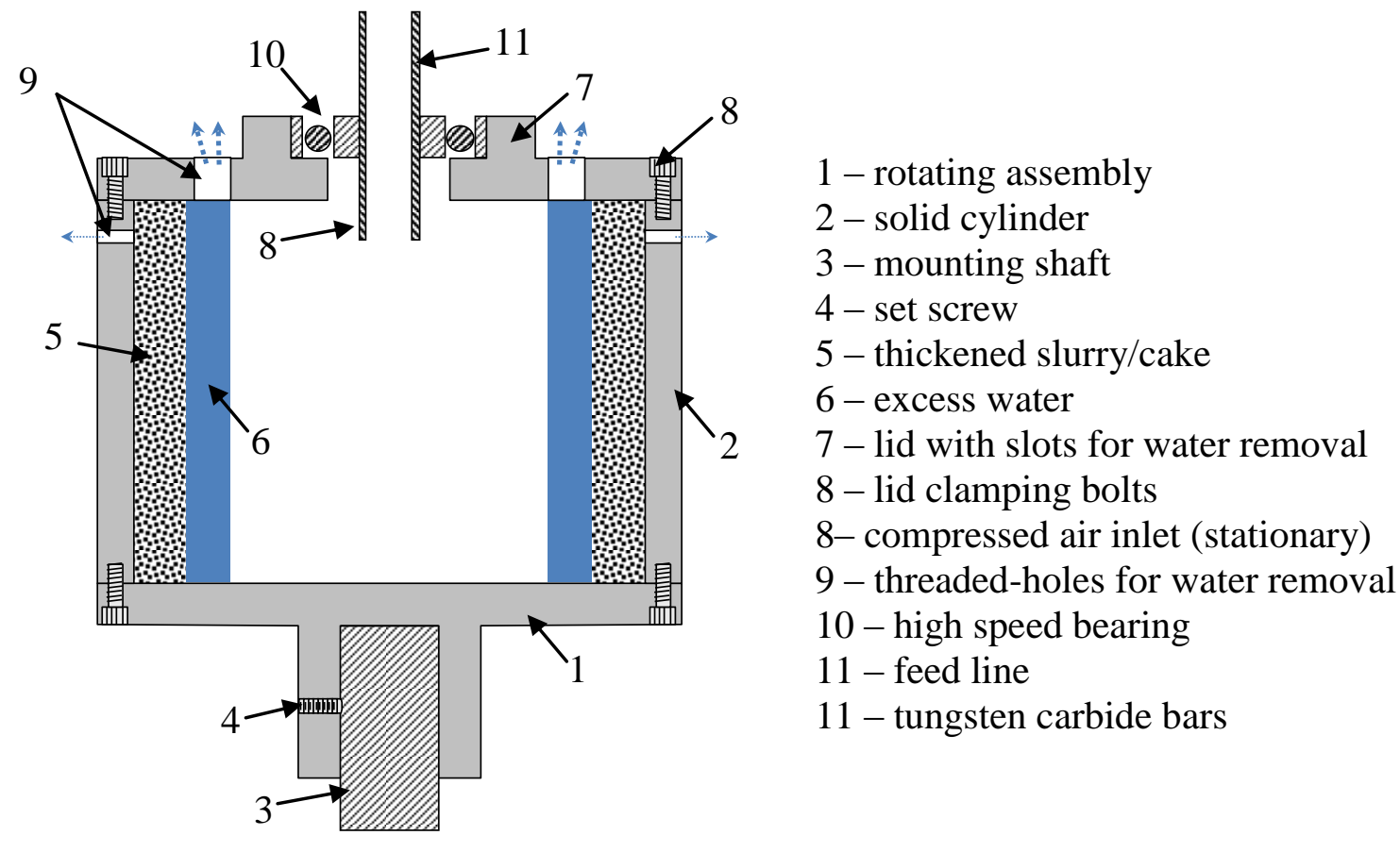

Figure 2.10 Schematic of the solid-bowl centrifuge unit used in standard batch tests.

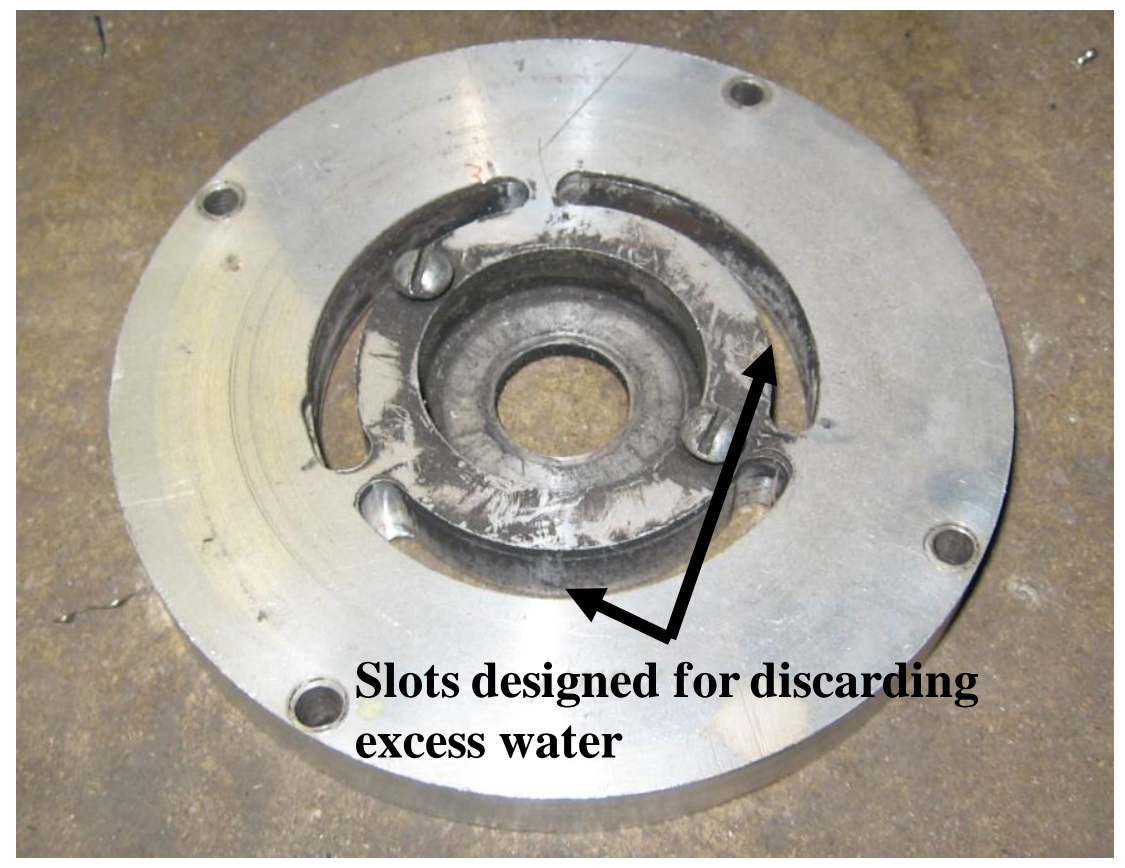

Figure 2.11 Specially designed lid of the solid-bowl unit 
All tests that were conducted with this unit consisted of two steps. In the first step, threaded holes on the sides of the bowl were sealed using bolts, so that the water could only go out of the holes on the top, and the centrifuge was run until there was no water leaving. Then, in the second step, bolts on the sides were unscrewed and the centrifuge was run again to let the remaining free water leave the unit.

\subsection{Results}

\subsubsection{Standard Batch Tests}

\section{a) Effect of Coal Type}

This parametric study of hyperbaric centrifugation was carried out using coal samples from various coal preparation plants. Screenbowl feed samples taken from Tom's Creek, Moss No. 3, Coal Clean and Weatherby coal plants, all of which process different coal seams, were used for these tests. Tests were run as function of centrifugal acceleration (i.e. 1500, 2000 and 2700 g), rotation time (i.e. 30, 60 and 120 second) and air pressure (with and without air pressure of $200 \mathrm{kPa}$ ). Air pressure was on for the whole duration of the tests with air. The particle size distributions of all the different coal samples were determined by wet screening (see Table 2.1). After sizing, an anionic flocculant was added to all samples for the purpose of thickening. The

Table 2.1 Size distributions of the screenbowl feed samples obtained from different plants.

\begin{tabular}{ccccc}
\hline Size & $\begin{array}{c}\text { Tom's Creek } \\
\text { Coal Plant } \\
\text { (Mass, \%) }\end{array}$ & $\begin{array}{c}\text { Coal Clean } \\
\text { Coal Plant } \\
\text { (Mass, \%) }\end{array}$ & $\begin{array}{c}\text { Weatherby } \\
\text { Coal Plant } \\
\text { (Mass, \%) }\end{array}$ & $\begin{array}{c}\text { Moss \#3 } \\
\text { Coal Plant } \\
\text { (Mass, \%) }\end{array}$ \\
\hline$+0.5 \mathrm{~mm}$ & 39.05 & 45.40 & 53.83 & 1.80 \\
$0.5 \times 0.25 \mathrm{~mm}$ & 21.43 & 17.22 & 18.43 & 7.81 \\
$0.25 \times 0.15 \mathrm{~mm}$ & 6.21 & 5.44 & 5.76 & 6.04 \\
$0.15 \times 0.075 \mathrm{~mm}$ & 10.40 & 8.58 & 8.62 & 13.24 \\
$0.075 \times 0.044 \mathrm{~mm}$ & 5.99 & 5.63 & 4.69 & 13.91 \\
$-0.044 \mathrm{~mm}$ & 16.92 & 17.74 & 8.67 & 57.20 \\
\hline
\end{tabular}


excess water was decanted and discarded.

Test results obtained with the Tom's Creek coal sample are given in Table 2.2. The data showed that single-digit moistures were easily achievable when 1500 g's or more of centrifugal acceleration was used, even without applying air pressure. The baseline product moisture without air was $11.5 \%$ for the lowest g level and shortest rotation time. This value was reduced down to 7.3\% moisture at the highest g level and the longest rotation time. These values were further reduced from $11.5 \%$ to $8.3 \%$ and from $7.3 \%$ down to $6.2 \%$, respectively, when air pressure was applied. The results also showed that the effect of air pressure on moisture reduction was more significant at lower rotation speeds (lower g levels). For example, at 1500 g's and 60 seconds of rotation time, the moisture was reduced from $9.9 \%$ down to $8.1 \%$ with air, corresponding to a 1.8 percentage point reduction. However, at $2700 \mathrm{~g}$ 's, the moisture was reduced from $8.5 \%$ down to $7.0 \%$ with air, corresponding to a lower moisture reduction of 1.5 percentage points. Also, the moisture reduction dropped for all g levels as the rotation time increased from 30 to 120 seconds.

Table 2.2 Test results obtained with Tom's Creek coal plant screenbowl feed (1 mm $x$ 0).

\begin{tabular}{|c|c|c|c|c|c|c|}
\hline \multirow{5}{*}{$\begin{array}{c}\text { Rotation } \\
\text { Time } \\
\text { (s) }\end{array}$} & \multicolumn{6}{|c|}{ Cake Moisture } \\
\hline & \multicolumn{3}{|c|}{ Without Air } & \multicolumn{3}{|c|}{ With Air } \\
\hline & 1500 & 2000 & 2700 & 1500 & 2000 & 2700 \\
\hline & g's & g's & g's & g's & g's & $g^{\prime} \mathbf{s}$ \\
\hline & $(\%)$ & $(\%)$ & $(\%)$ & $(\%)$ & $(\%)$ & $(\%)$ \\
\hline 0 & 47.7 & 47.7 & 47.7 & 47.7 & 47.7 & 47.7 \\
\hline 30 & 11.5 & 10.3 & 9.6 & 8.3 & 8.1 & 8.1 \\
\hline 60 & 9.9 & 9.4 & 8.5 & 8.1 & 7.7 & 7.0 \\
\hline 120 & 8.9 & 7.8 & 7.3 & 7.0 & 7.1 & 6.2 \\
\hline
\end{tabular}

The Coal Clean sample had a very similar size distribution to that of the Tom's Creek sample. Even though the samples were taken from coal plants that were processing different coal 
seams, the test results were very similar owning to the similar size distributions. As shown in Table 2.3, baseline moistures obtained ranged from $11.5 \%$ for the lowest g level and shortest rotation time down to $8.1 \%$ for highest g level with the longest rotation time. Moreover, similar to the results obtained with Tom's Creek sample, the moisture reduction obtained by applying air pressure dropped with increased g levels. For example, the moisture at $1500 \mathrm{~g}$ 's and 60 seconds of rotation time was reduced from $11.0 \%$ down to $8.5 \%$ with air, corresponding to a 2.5 percentage point moisture reduction. Whereas, at $2700 \mathrm{~g}$ 's, the moisture was reduced from $8.9 \%$ down to $7.4 \%$, corresponding to a lower moisture reduction of 1.5 percentage points.

\section{Table 2.3 Test results obtained with Coal Clean coal plant screenbowl feed $(1 \mathrm{~mm} x \mathbf{0}$, Eagle Seam).}

\begin{tabular}{|c|c|c|c|c|c|c|}
\hline \multirow{5}{*}{$\begin{array}{c}\text { Rotation } \\
\text { Time } \\
\text { (s) }\end{array}$} & \multicolumn{6}{|c|}{ Cake Moisture } \\
\hline & \multicolumn{3}{|c|}{ Without Air } & \multicolumn{3}{|c|}{ With Air } \\
\hline & 1500 & 2000 & 2700 & 1500 & 2000 & 2700 \\
\hline & $g^{\prime} s$ & g's & $g^{\prime} s$ & g's & $g^{\prime} \mathbf{s}$ & $g^{\prime} \mathbf{s}$ \\
\hline & $(\%)$ & $(\%)$ & $(\%)$ & $(\%)$ & $(\%)$ & $(\%)$ \\
\hline 0 & 50.0 & 50.0 & 50.0 & 50.0 & 50.0 & 50.0 \\
\hline 30 & 11.5 & 10.8 & 10.0 & 9.3 & 8.8 & 8.0 \\
\hline 60 & 11.0 & 9.9 & 8.9 & 8.5 & 7.8 & 7.4 \\
\hline 120 & 9.9 & 9.1 & 8.1 & 7.4 & 7.1 & 6.8 \\
\hline
\end{tabular}

The coal sample from the Weatherby plant was partially deslimed at $0.044 \mathrm{~mm}$ as a result of the use of fine classifying cyclones utilized in the plant circuitry. Because the sample was deslimed, all the moisture values obtained with this coal product were less than $10 \%$. While the baseline moisture for the lowest centrifugal field of 1500 g's and the shortest rotation time of 30 seconds was 9.4\%, for the highest centrifugal field of 2700 g's and the longest rotation time of 120, it was $7.4 \%$. When air pressure was introduced, moistures decreased down to only $8.7 \%$ and $6.5 \%$, respectively, for the same conditions. Therefore, the lack of fines reduced the moisture 
drop and severely limited the impact of air injection on moisture reduction. The smallest moisture drop, which was 0.3 percentage points, was obtained with 2700 g's and 30 seconds rotation time. The largest moisture drop was only about 1.0 percentage point at 2000 g's and 120 seconds rotation time.

Table 2.4 Test results obtained with Weatherby coal plant screenbowl feed $(1 \mathrm{~mm} x$, Deslimed, Stockton Seam).

\begin{tabular}{|c|c|c|c|c|c|c|}
\hline \multirow[b]{3}{*}{$\begin{array}{c}\text { Rotation } \\
\text { Time } \\
\text { (s) }\end{array}$} & \multicolumn{6}{|c|}{ Cake Moisture } \\
\hline & \multicolumn{3}{|c|}{ Without Air } & \multicolumn{3}{|c|}{ With Air } \\
\hline & $\begin{array}{c}1500 \\
g^{\prime} s \\
(\%)\end{array}$ & $\begin{array}{c}2000 \\
g^{\prime} s \\
(\%)\end{array}$ & $\begin{array}{c}2700 \\
g^{\prime} s \\
(\%)\end{array}$ & $\begin{array}{c}1500 \\
\text { g's } \\
(\%)\end{array}$ & $\begin{array}{c}2000 \\
\text { g's } \\
(\%)\end{array}$ & $\begin{array}{c}2700 \\
\text { g's } \\
(\%)\end{array}$ \\
\hline 0 & 42.0 & 42.4 & 42.4 & 42.4 & 42.4 & 42.4 \\
\hline 30 & 9.4 & 9.1 & 8.5 & 8.7 & 8.1 & 8.2 \\
\hline 60 & 9.0 & 8.4 & 8.2 & 8.2 & 7.9 & 7.2 \\
\hline 120 & 8.4 & 8.1 & 7.4 & 7.4 & 7.1 & 6.5 \\
\hline
\end{tabular}

The Moss No. 3 sample was a finer sample compared to the other samples with $57.2 \%$ minus $0.044 \mathrm{~mm}$ particles. As a result of the increased surface area, product moistures also increased to values more than two times the values obtained with the other samples (see Table 2.5). For the lowest $g$ level and the shortest rotation time, the baseline cake moisture was $26.5 \%$. This value was reduced down to $20.2 \%$ for the highest g level and the longest rotation time. When air pressure was introduced, these values decreased further from $26.5 \%$ down to $21.0 \%$ and from $20.2 \%$ down to $15.5 \%$, respectively. Greater moisture reductions compared to the other samples were also observed as a consequence of the increased surface area. For example, at 1500 g's and 60 seconds of rotation time, the moisture was reduced from $25.3 \%$ to $19.0 \%$ with air, corresponding to a 6.3 percentage point reduction. Similar to the tests with other samples, moisture reductions with air were dropped at higher g levels. For instance, at 2700 g's and 60 
seconds of rotation time, the moisture was reduced from $21.7 \%$ to $16.7 \%$ with air, corresponding to a lower moisture reduction of 5.0 percentage points.

Table 2.5 Test results obtained with Moss No. 3 coal plant screenbowl feed (1 mm $x$ 0).

\begin{tabular}{|c|c|c|c|c|c|c|}
\hline \multirow{5}{*}{$\begin{array}{c}\text { Rotation } \\
\text { Time } \\
\text { (s) }\end{array}$} & \multicolumn{6}{|c|}{ Cake Moisture } \\
\hline & \multicolumn{3}{|c|}{ Without Air } & \multicolumn{3}{|c|}{ With Air } \\
\hline & 1500 & 2000 & 2700 & 1500 & 2000 & 2700 \\
\hline & g's & g's & g's & g's & g's & g's \\
\hline & $(\%)$ & $(\%)$ & $(\%)$ & $(\%)$ & $(\%)$ & $(\%)$ \\
\hline 0 & 67.3 & 67.3 & 67.3 & 67.3 & 67.3 & 67.3 \\
\hline 30 & 26.5 & 24.3 & 22.6 & 21.0 & 19.6 & 18.0 \\
\hline 60 & 25.3 & 23.4 & 21.7 & 19.0 & 18.4 & 16.7 \\
\hline 120 & 24.0 & 22.1 & 20.2 & 17.6 & 16.1 & 15.5 \\
\hline
\end{tabular}

\section{b) Effect of Dewatering Aids}

Two sets of flotation concentrate samples (nominally $0.15 \times 0 \mathrm{~mm}$ ) obtained from the East Gulf Plant of United Coal Company and the Cardinal Plant of Arch Coal Company, both located in West Virginia, were used to test the effect of dewatering aids on cake moistures. The main difference between the samples was the size distribution, as the East Gulf sample was much finer than the Cardinal sample (see Table 2.6). While the East Gulf sample contained 43.24\%

Table 2.6 Size distributions of the flotation concentrate samples obtained from two plants for dewatering aid tests.

\begin{tabular}{ccc}
\hline Size & $\begin{array}{c}\text { East Gulf } \\
\text { Coal Plant } \\
\text { (Mass, \%) }\end{array}$ & $\begin{array}{c}\text { Cardinal } \\
\text { Coal Plant } \\
\text { (Mass, \%) }\end{array}$ \\
\hline$+0.3 \mathrm{~mm}$ & 0.34 & 5.04 \\
$0.3 \times 0.15 \mathrm{~mm}$ & 1.61 & 30.57 \\
$0.15 \times 0.075 \mathrm{~mm}$ & 17.47 & 30.43 \\
$0.075 \times 0.044 \mathrm{~mm}$ & 17.33 & 11.93 \\
$0.044 \times 0.025 \mathrm{~mm}$ & 20.01 & 9.74 \\
$-0.025 \mathrm{~mm}$ & 43.24 & 12.29 \\
\hline
\end{tabular}


minus $0.025 \mathrm{~mm}$ material, the Cardinal sample's minus $0.025 \mathrm{~mm}$ material content was only 12.29\%. All the centrifuge tests were run at a relatively lower centrifugal acceleration level of 500 g's to show the effect of dewatering aids, because higher centrifugal accelerations tend to reduce the effect of other parameters. Also, rotation time was kept at 60 seconds and the air pressure was kept at $200 \mathrm{kPa}$ for the tests with air. For comparison, vacuum filtration tests were conducted using the same samples. Several dewatering aids (i.e., reagents U, V and W) and proprietary shear resistant flocculants (i.e., Nalco 71300, 71303, 71306 and 71321) were used in several of the experiments.

Table 2.7 Effect of dewatering aids on moisture (East Gulf Coal Plant flotation concentrate sample).

\begin{tabular}{|c|c|c|c|c|}
\hline $\begin{array}{c}\text { Air } \\
\text { Pressure } \\
\text { (kPa) }\end{array}$ & $\begin{array}{c}\text { Chemical } \\
\text { Type }\end{array}$ & $\begin{array}{c}\text { Chemical } \\
\text { Dosage* } \\
\text { (lbs/t) }\end{array}$ & $\begin{array}{c}\text { Moisture } \\
(\%)\end{array}$ & $\begin{array}{c}\text { Moisture } \\
\text { Reduction } \\
(\%)\end{array}$ \\
\hline 0 & - & 0 & 40.8 & 0.0 \\
\hline 200 & - & 0 & 25.1 & 38.5 \\
\hline 0 & RU & 1 & 40.2 & 1.3 \\
\hline 0 & RU & 2 & 38.6 & 5.4 \\
\hline 0 & RU & 4 & 29.7 & 27.3 \\
\hline 200 & RU & 1 & 24.0 & 41.0 \\
\hline 200 & RU & 2 & 20.1 & 50.8 \\
\hline 200 & RU & 4 & 18.4 & 54.8 \\
\hline 0 & $\mathrm{RV}$ & 1 & 41.7 & -2.4 \\
\hline 0 & $\mathrm{RV}$ & 2 & 41.7 & -2.4 \\
\hline 0 & RV & 4 & 39.8 & 2.4 \\
\hline 200 & $\mathrm{RV}$ & 1 & 24.7 & 39.3 \\
\hline 200 & RV & 2 & 24.8 & 39.1 \\
\hline 200 & RV & 4 & 23.6 & 42.1 \\
\hline 0 & RW & 1 & 39.6 & 2.9 \\
\hline 0 & RW & 2 & 39.7 & 2.6 \\
\hline 0 & RW & 4 & 37.7 & 7.5 \\
\hline 200 & RW & 1 & 22.8 & 44.0 \\
\hline 200 & RW & 2 & 20.0 & 50.9 \\
\hline 200 & RW & 4 & 17.5 & 56.9 \\
\hline
\end{tabular}

* Dosage of active chemical 
Centrifugation test results obtained with the East Gulf sample are given in Table 2.7. All the tests were conducted using 1, 2 and $4 \mathrm{lbs} / \mathrm{t}$ dosages of reagents $\mathrm{U}, \mathrm{V}$ and $\mathrm{W}$. Tests without any chemical addition were also performed. Moisture reductions compared to the baseline test, which was run without dewatering aids and without air pressure, are shown in the same table, as well. While the baseline test produced $40.8 \%$ moisture, the lowest moisture value of $27.9 \%$ without air injection was obtained using $4 \mathrm{lbs} / \mathrm{t}$ of reagent $\mathrm{U}$ corresponding to $27.3 \%$ moisture reduction. While reagent $\mathrm{W}$ slightly reduced the moisture down to $37.7 \%$, reagent $\mathrm{V}$ had almost no effect and it produced 39.8\% moisture at $4 \mathrm{lbs} / \mathrm{t}$ dosage. In fact, at the lower dosages of reagent $\mathrm{V}$, moisture slightly increased compared to the baseline. When air pressure was applied, $25.1 \%$ cake moisture, which corresponds to $38.5 \%$ moisture reduction, was obtained without any dewatering aids. Reagents $\mathrm{U}$ and $\mathrm{W}$ had similar impacts on water removal and produced $18.4 \%$ and $17.5 \%$ moistures, respectively. This level of performance corresponds to moisture reductions of about 55-56\%. Reagent $\mathrm{V}$, on the other hand, caused only a slight moisture reduction compared to the no chemical case and produced $23.6 \%$ cake moisture at the highest dosage.

Vacuum filtration test results obtained with the East Gulf Plant sample are shown in Table 2.8. Since reagent V did not have any effect in centrifuge tests, it was not included in filtration tests. Vacuum pressure was kept at $133 \mathrm{kPa}$ for all the tests. Two cake thicknesses of 12 $\mathrm{mm}$ and 22-24 $\mathrm{mm}$ were tested. Since reagent $\mathrm{U}$ gave the best results in centrifuge tests and since there was a limited amount of coal sample left, only reagent $U$ was tested with both cake thicknesses. Test results given in Table 2.8 show that when the cake thickness was $12 \mathrm{~mm}$, moisture was reduced to about $25 \%$ with both reagents $\mathrm{U}$ and $\mathrm{W}$ compared to the baseline moisture of $30.3 \%$. Also, when a $22-24$ mm cake thickness was produced, the baseline moisture of $36.5 \%$ was reduced to $31.5 \%$ with reagent $\mathrm{U}$. 
Table 2.8 Vacuum filtration test results (East Gulf Plant flotation concentrate sample)

\begin{tabular}{cccccc}
\hline $\begin{array}{c}\text { Vacuum } \\
\text { Pressure } \\
(\mathbf{k P a})\end{array}$ & $\begin{array}{c}\text { Chemical } \\
\text { Type }\end{array}$ & $\begin{array}{c}\text { Chemical } \\
\text { Dosage } \\
(\mathbf{l b s} / \mathbf{t})\end{array}$ & $\begin{array}{c}\text { Cake } \\
\text { Thickness } \\
(\mathbf{m m})\end{array}$ & $\begin{array}{c}\text { Filtration } \\
\text { Time } \\
(\mathbf{s})\end{array}$ & $\begin{array}{c}\text { Moisture } \\
(\mathbf{\%})\end{array}$ \\
\hline 133 & - & 0 & 11 & 120 & 30.3 \\
\hline 133 & RU & 2 & 12 & 120 & 26.3 \\
133 & RU & 4 & 12 & 120 & 24.9 \\
\hline 133 & RW & 2 & 12 & 120 & 25.7 \\
133 & RW & 4 & 12 & 120 & 25.0 \\
\hline 133 & - & 0 & $22-24$ & 300 & 36.5 \\
\hline 133 & RU & 2 & $22-24$ & 240 & 32.8 \\
133 & RU & 4 & $22-24$ & 240 & 31.5 \\
\hline
\end{tabular}

Several series of centrifugation tests were conducted using samples from the Cardinal Plant. The first set was performed to study the effect of reagents $\mathrm{U}, \mathrm{V}$ and $\mathrm{W}$ on cake moisture. Table 2.9 shows the test results obtained. Since this sample was coarser than the East Gulf sample, the need for the chemical dosage reduced due to the smaller specific surface area (surface area per unit weight) of the sample. Therefore, 0.3, 1.0 and $1.7 \mathrm{lbs} / \mathrm{t}$ dosages were used for these experiments. While the baseline test produced $20.0 \%$ moisture, similar moisture values of approximately $15-16 \%$ and $11.5 \%$ were obtained when no air pressure was applied with all dewatering aids at the lowest and the highest dosages, respectively. When air pressure was applied, reagents $\mathrm{U}$ and $\mathrm{W}$ produced similar moisture values at all dosages. However, the lowest moisture value of $7.8 \%$ was obtained using reagent $\mathrm{W}$ at the highest dosage of $1.7 \mathrm{lbs} / \mathrm{t}$. Moistures produced using reagent $\mathrm{V}$ were slightly higher than the ones with reagents $\mathrm{U}$ and $\mathrm{W}$, but still lower than the moisture of $13.4 \%$, which was produced without chemicals. 

Table 2.9 Effect of dewatering aids on moisture (Cardinal Coal Plant flotation concentrate
sample)

\begin{tabular}{ccccc}
\hline $\begin{array}{c}\text { Air } \\
\text { Pressure } \\
(\mathbf{k P a})\end{array}$ & $\begin{array}{c}\text { Chemical } \\
\text { Type }\end{array}$ & $\begin{array}{c}\text { Chemical } \\
\text { Dosage* } \\
\text { (lbs/t) }\end{array}$ & $\begin{array}{c}\text { Moisture } \\
(\boldsymbol{\%})\end{array}$ & $\begin{array}{c}\text { Moisture } \\
\text { Reduction } \\
(\boldsymbol{\%})\end{array}$ \\
\hline 0 & - & 0 & 20.0 & 0.00 \\
200 & - & 0 & 13.4 & 33.19 \\
\hline 0 & RU & 0.3 & 14.9 & 25.7 \\
0 & RU & 1.0 & 12.2 & 38.9 \\
0 & RU & 1.7 & 11.7 & 41.5 \\
\hline 200 & RU & 0.3 & 10.4 & 47.9 \\
200 & RU & 1.0 & 8.4 & 58.2 \\
200 & RU & 1.7 & 8.3 & 58.6 \\
\hline 0 & RV & 0.3 & 14.9 & 25.8 \\
0 & RV & 1.0 & 12.9 & 35.6 \\
0 & RV & 1.7 & 11.4 & 43.1 \\
\hline 200 & RV & 0.3 & 11.1 & 44.4 \\
200 & RV & 1.0 & 10.4 & 48.0 \\
200 & RV & 1.7 & 8.9 & 55.7 \\
\hline 0 & RW & 0.3 & 16.1 & 19.5 \\
0 & RW & 1.0 & 11.3 & 43.3 \\
0 & RW & 1.7 & 11.6 & 42.0 \\
\hline 200 & RW & 0.3 & 10.5 & 47.4 \\
200 & RW & 1.0 & 8.8 & 55.9 \\
200 & RW & 1.7 & 7.8 & 60.8 \\
\hline
\end{tabular}

* Dosage of active chemical

Another set of tests was run with the Cardinal sample to study the effect of different rotation times when a dewatering aid was used. Since reagents $\mathrm{U}, \mathrm{V}$ and $\mathrm{W}$ had similar effects in the previous set of tests, only reagent $U$ at a mid-dosage of $1 \mathrm{lbs} / \mathrm{t}$ was used. Moreover, all the tests were run without air. Test results given in Table 2.10 showed that when no chemical was used, the moisture content was highly correlated to rotation time, as the moisture decreased from $35.9 \%$ at 15 seconds rotation time down to $21.7 \%$ at 60 seconds rotation time. However, when reagent $U$ was added to the feed slurry, even at the shortest rotation time, moisture was reduced down to $14.8 \%$, which corresponds to about 21 percentage points difference compared to no chemical case. Even though the difference was less at the highest rotation time, reagent $U$ still 
produced a lower moisture value of $12.7 \%$ compared to the no chemical case. Therefore, while the product moistures were reduced by using a dewatering aid, the amount of time required for dewatering was also lowered.

Table 2.10 Effect of rotation time with 1 lbs/t Reagent $U$ addition

\begin{tabular}{cccc}
\hline $\begin{array}{c}\text { Rotation } \\
\text { Time } \\
\text { (s) }\end{array}$ & $\begin{array}{c}\text { Chemical } \\
\text { Type }\end{array}$ & $\begin{array}{c}\text { Chemical } \\
\text { Dosage* } \\
\text { (lbs/t) }\end{array}$ & $\begin{array}{c}\text { Moisture } \\
\text { (\% by weight) }\end{array}$ \\
\hline 15 & - & 0 & 35.9 \\
30 & - & 0 & 28.1 \\
60 & - & 0 & 21.7 \\
\hline 15 & RU & 1 & 14.8 \\
30 & RU & 1 & 12.6 \\
60 & RU & 1 & 12.7 \\
\hline *
\end{tabular}

* Dosage of active chemical

The last set of centrifugation tests with the Cardinal Plant sample were conducted using proprietary shear resistant flocculants 71300, 713003, 71306 and 71321 obtained from Nalco Company. Test results given in Table 2.11 showed that using a proper dosage is of utmost importance when dealing with flocculants. When no air was used, employing flocculants reduced the moisture compared to the no chemical case at even the lowest dosage. However, while an additional moisture reduction was obtained with all flocculants when the dosage increased from 0.00033 to $0.00055 \mathrm{lbs} / \mathrm{t}$, increasing the dosage further to $0.0011 \mathrm{lbs} / \mathrm{t}$ resulted in elevated moistures. For example, when 71321 was used, the baseline moisture of $22.2 \%$ was reduced to $17.1 \%$ and $16.8 \%$, at $0.00033 \mathrm{lbs} / \mathrm{t}$ and $0.00055 \mathrm{lbs} / \mathrm{t}$ dosages, respectively. However, the moisture value increased to $17.3 \%$ when $0.0011 \mathrm{lbs} / \mathrm{t}$ dosage employed. The same trend was also observed when air pressure was used, only lower overall moistures were obtained due to the elevated air pressure. 
Similar moisture values ranging from $16.8 \%$ to $20.9 \%$ were obtained with flocculants when no air was used. However, the best results were achieved using the flocculant 71321 . The lowest moisture obtained was $16.8 \%$ at $0.00055 \mathrm{lbs} / \mathrm{t}$ dosage, which corresponds to about 5.4 percentage points moisture reduction compared to the baseline case. When air pressure was applied, moistures produced with flocculants were still in a narrow range of $10.9 \%-14.6 \%$. The flocculant 71321 once more produced the lowest moisture value of $10.9 \%$ at $0.00033 \mathrm{lbs} / \mathrm{t}$ dosage, which corresponds to 11.3 percentage points or $51 \%$ moisture reduction.

Table 2.11 Effect of flocculant addition

\begin{tabular}{cccc}
\hline $\begin{array}{c}\text { Air } \\
\text { Pressure } \\
\text { (kPa) }\end{array}$ & $\begin{array}{c}\text { Chemical } \\
\text { Type }\end{array}$ & $\begin{array}{c}\text { Chemical } \\
\text { Dosage } \\
\text { (lbs/t) }\end{array}$ & $\begin{array}{c}\text { Moisture } \\
\text { (\% by weight) }\end{array}$ \\
\hline 0 & - & 0 & 22.2 \\
\hline 200 & - & 0 & 13.6 \\
\hline 0 & 71300 & 0.00033 & 18.6 \\
0 & 71300 & 0.00055 & 18.1 \\
0 & 71300 & 0.00110 & 19.1 \\
\hline 200 & 71300 & 0.00033 & 13.1 \\
200 & 71300 & 0.00055 & 12.8 \\
200 & 71300 & 0.00110 & 14.6 \\
\hline 0 & 71303 & 0.00033 & 18.7 \\
0 & 71303 & 0.00055 & 17.7 \\
0 & 71303 & 0.00110 & 18.7 \\
\hline 200 & 71303 & 0.00033 & 12.9 \\
200 & 71303 & 0.00055 & 12.9 \\
200 & 71303 & 0.00110 & 13.0 \\
\hline 0 & 71306 & 0.00033 & 20.9 \\
0 & 71306 & 0.00055 & 18.6 \\
0 & 71306 & 0.00110 & 18.7 \\
\hline 200 & 71306 & 0.00033 & 13.8 \\
200 & 71306 & 0.00055 & 12.4 \\
200 & 71306 & 0.00110 & 13.5 \\
\hline 0 & 71321 & 0.00033 & 17.1 \\
0 & 71321 & 0.00055 & 16.8 \\
0 & 71321 & 0.00110 & 17.3 \\
\hline 200 & 71321 & 0.00033 & 10.9 \\
200 & 71321 & 0.00055 & 11.3 \\
200 & 71321 & 0.00110 & 11.4 \\
\hline & & & \\
\hline
\end{tabular}


Several series of vacuum filtration tests were conducted with the Cardinal Plant sample. The first and second sets were performed to provide a comparison to the results given in Table 2.9. While reagents $\mathrm{U}, \mathrm{V}$ and $\mathrm{W}$ were used in the first set of tests, reagent $\mathrm{U}$ was not used in the second set of tests because of the limited amount of sample available for testing. Also, only 0.3 and $1 \mathrm{lbs} / \mathrm{t}$ dosages were used in the second series of tests. The first set of tests was conducted at 120 seconds of filtration time, whereas the second set was performed at 60 seconds of filtration time. As expected, the former produced lower moisture values due to the longer filtration time. At 120 seconds of filtration time, reagent $\mathrm{W}$ produced the lowest moistures, ranging from $11.2 \%$ at the highest chemical dosage to $12.7 \%$ at the lowest chemical dosage. Generally, moistures decreased as the dosage increased from 0.3 to $1.7 \mathrm{lbs} / \mathrm{t}$ for all the chemicals tested. In fact, all moistures produced with chemicals were lower than the baseline moisture of $15.0 \%$. At 60 seconds filtration time, moistures decreased as the chemical dosage increased, resembling the trends observed in the first set of tests. While reagent $\mathrm{W}$ produced the lowest moisture of $14.2 \%$ at $1 \mathrm{lbs} / \mathrm{t}$ dosage, the moisture obtained with reagent $\mathrm{V}$ was $16.7 \%$ at the same dosage. The baseline moisture of $18.7 \%$ was the highest value obtained.

Results showed that when 120 seconds filtration time was used, vacuum filtration produced lower moistures than centrifugation alone at the lowest chemical dosage. For instance, at the lowest dosage of reagent $\mathrm{W}$, centrifugation alone produced $16.1 \%$ moisture. Whereas, a lower moisture value of $12.7 \%$ was produced with vacuum filtration at the same conditions. However, moisture values obtained at higher dosages with centrifugation were either similar to or lower than the ones obtained with vacuum filtration. For example, for the same conditions given above, centrifugation with air pressure produced 10.5\% moisture. When 60 seconds 
filtration time was employed, even the moistures produced with only centrifugation were lower than vacuum filtration.

The last set of vacuum filtration tests were performed with flocculants described earlier. Again, because of the limited amount of available samples, only flocculants 71303 and 71321, which performed the best at centrifugation tests, were evaluated at 0.00033 and $0.00055 \mathrm{lbs} / \mathrm{t}$ dosages. As in the case with the centrifugation tests, flocculant 71321 produced the lowest moistures. However, this time, the lowest moistures were obtained at $0.00033 \mathrm{lbs} / \mathrm{t}$ dosage, rather than $0.00055 \mathrm{lbs} / \mathrm{t}$, which was the optimum dosage in the centrifuge tests. The application of flocculants made an improvement over the baseline moisture of $18.3 \%$. For this particular sample, moistures of $15.3 \%$ and $17.2 \%$ were produced using flocculants 71321 and 71303 , respectively, at a dosage of $0.00033 \mathrm{lbs} / \mathrm{t}$. While the moistures obtained with vacuum filtration were generally lower than centrifugation only results, the application of air pressure together with centrifugation improved moistures and resulted in 25-30\% moisture reductions compared to vacuum filtration.

Table 2.12 Vacuum filtration test results at 120 s filtration time (Cardinal Plant flotation concentrate sample)

\begin{tabular}{cccccc}
\hline $\begin{array}{c}\text { Vacuum } \\
\text { Pressure } \\
(\mathbf{k P a})\end{array}$ & $\begin{array}{c}\text { Chemical } \\
\text { Type }\end{array}$ & $\begin{array}{c}\text { Chemical } \\
\text { Dosage } \\
(\mathbf{l b s} / \mathbf{t})\end{array}$ & $\begin{array}{c}\text { Cake } \\
\text { Thickness } \\
(\mathbf{m m})\end{array}$ & $\begin{array}{c}\text { Filtration } \\
\text { Time } \\
(\mathbf{s})\end{array}$ & $\begin{array}{c}\text { Moisture } \\
(\boldsymbol{\%})\end{array}$ \\
\hline 133 & - & 0 & 12 & 120 & 15.0 \\
\hline 133 & RU & 0.3 & 12 & 120 & 13.4 \\
133 & RU & 1.0 & 12 & 120 & 12.1 \\
133 & RU & 1.7 & 12 & 120 & 12.7 \\
\hline 133 & RV & 0.3 & 12 & 120 & 13.8 \\
133 & RV & 1.0 & 12 & 120 & 13.3 \\
133 & RV & 1.7 & 12 & 120 & 13.4 \\
\hline 133 & RW & 0.3 & 12 & 120 & 12.7 \\
133 & RW & 1.0 & 12 & 120 & 12.1 \\
133 & RW & 1.7 & 12 & 120 & 11.2 \\
\hline
\end{tabular}


Table 2.13 Vacuum filtration test results at 60 s filtration time (Cardinal Plant flotation concentrate sample)

\begin{tabular}{cccccc}
\hline $\begin{array}{c}\text { Vacuum } \\
\text { Pressure } \\
(\mathbf{k P a})\end{array}$ & $\begin{array}{c}\text { Chemical } \\
\text { Type }\end{array}$ & $\begin{array}{c}\text { Chemical } \\
\text { Dosage } \\
\text { (lbs/t) }\end{array}$ & $\begin{array}{c}\text { Cake } \\
\text { Thickness } \\
(\mathbf{m m})\end{array}$ & $\begin{array}{c}\text { Filtration } \\
\text { Time } \\
(\mathbf{s})\end{array}$ & $\begin{array}{c}\text { Moisture } \\
(\mathbf{\%})\end{array}$ \\
\hline 133 & - & 0 & 12 & 60 & 18.7 \\
\hline 133 & RV & 0.3 & 13 & 60 & 17.2 \\
133 & RV & 1.0 & 13 & 60 & 16.7 \\
\hline 133 & RW & 0.3 & 13 & 60 & 14.5 \\
133 & RW & 1.0 & 13 & 60 & 14.2 \\
\hline
\end{tabular}

Table 2.14 Vacuum filtration test results with flocculants (Cardinal Plant flotation concentrate sample)

\begin{tabular}{cccccc}
\hline $\begin{array}{c}\text { Vacuum } \\
\text { Pressure } \\
(\mathbf{k P a})\end{array}$ & $\begin{array}{c}\text { Chemical } \\
\text { Type }\end{array}$ & $\begin{array}{c}\text { Chemical } \\
\text { Dosage } \\
(\mathbf{l b s} / \mathbf{t})\end{array}$ & $\begin{array}{c}\text { Cake } \\
\text { Thickness } \\
(\mathbf{m m})\end{array}$ & $\begin{array}{c}\text { Filtration } \\
\text { Time } \\
(\mathbf{s})\end{array}$ & $\begin{array}{c}\text { Moisture } \\
(\boldsymbol{\%})\end{array}$ \\
\hline 133 & - & 0 & 12 & 60 & 18.3 \\
\hline 133 & 71321 & 0.00033 & 13 & 60 & 15.4 \\
133 & 71321 & 0.00055 & 13 & 60 & 15.9 \\
\hline 133 & 71303 & 0.00033 & 13 & 60 & 17.8 \\
133 & 71303 & 0.00055 & 13 & 60 & 18.4 \\
\hline
\end{tabular}

\section{c) Effect of Air Pressure}

To study the effect of air pressure on cake moisture, an additional set of dewatering tests was conducted using the East Gulf Plant sample. The tests were performed as a function of centrifugal acceleration and air pressure. Rotation time was held constant at 60 seconds for all tests. The results given in Table 2.15 showed that increasing air pressure beyond $100-200 \mathrm{kPa}$ at 500 g's decreased the product moisture from $26.1 \%$ to $23.4 \%$. Since this improvement corresponds to only about 2.7 percentage point moisture reduction, the use of high air pressures may not be necessary for effective dewatering. Moreover, at 3000 g's, moistures obtained at 
different air pressures were practically identical. Therefore, 100-200 kPa of air pressure appears to be adequate for hyperbaric centrifugation.

Table 2.15 Effect of air pressure on cake moisture

\begin{tabular}{ccc}
\hline $\begin{array}{c}\text { Centrifugal } \\
\text { Acceleration } \\
(\mathbf{g} \text { s) }\end{array}$ & $\begin{array}{c}\text { Air } \\
\text { Pressure } \\
(\mathbf{k P a})\end{array}$ & $\begin{array}{c}\text { Moisture } \\
\text { (\% by weight) }\end{array}$ \\
\hline 500 & 0 & 40.8 \\
\hline 500 & 100 & 26.1 \\
500 & 200 & 25.8 \\
500 & 400 & 23.4 \\
\hline 3000 & 100 & 15.4 \\
3000 & 200 & 14.2 \\
3000 & 400 & 15.8 \\
\hline
\end{tabular}

\section{d) Effect of Particle Size Distribution}

Another set of laboratory tests was performed to investigate the effect of feed particle size distribution on cake moisture. To minimize the impacts of heterogeneities, a low-ash unprocessed coal sample taken from Cucumber Mine in West Virginia was used in these experiments. The sample was screened at $1.2 \mathrm{~mm}$ to eliminate large chunks of coal. After screening, the dry sample was split down to about 400 grams. Each split was then ground in a laboratory ball mill for different periods of time, varying from 30 seconds to 2 hours, to obtain progressively smaller size distributions. Using these samples, hyperbaric centrifuge tests were run at 500 and 2700 g's. These centrifugal accelerations were purposely chosen to imitate the accelerations applied by the standard screenbowl centrifuges and the new Decanter Centribaric ${ }^{\mathrm{TM}}$ centrifuge (described later in this document). An air pressure of about $200 \mathrm{kPa}$ was applied during the tests. The air injection and rotation time was held constant at 120 seconds for all the tests. 
The data given in Table 2.16 showed that moisture correlated very well with particle size. For instance, when the centrifugal acceleration was 500 g's, the coarsest product had 9.8\% moisture with air and $13.8 \%$ moisture without air, while the moisture went up to $37.7 \%$ with air and $44.9 \%$ without air with the finest cake. The effect of different size fractions on final moisture is more clearly illustrated in Figure 2.12 and Figure 2.13. These plots show the total moisture content obtained as a function of the dry mass percentage below a given particle top-size. As seen from the plots, the correlation between final product moisture and mass percent passing was the poorest for the coarsest (i.e., minus $0.6 \mathrm{~mm}$ ) size fraction (Figure 2.12(a) and Figure 2.13(a)). The best correlation was obtained with the finest (minus $0.025 \mathrm{~mm}$ ) size fraction (Figure 2.12(f) and Figure 2.13(f)). Therefore, this fraction was chosen to further investigate the impact of size distribution on cake moisture.

Figure 2.13(f) shows that at 2700 g's, when no air pressure was used, the moisture content increased linearly with an increase in the amount of minus $0.025 \mathrm{~mm}$ particles. When air pressure was applied, there was a constant 2 percentage-point moisture improvement until the minus $0.025 \mathrm{~mm}$ solids accounted for about $60 \%$ of the total sample mass. After this point, the moisture remained steady around 25\% with air injection, while it kept increasing without air injection. The absolute difference between adding air and not adding air was about 12 absolute percentage points when the amount of minus $0.025 \mathrm{~mm}$ size fraction reached $99 \%$.

Figure 2.12(f) shows that, at 500 g's, all the moisture values increased due to the decrease in centrifugal acceleration. The moisture difference between with and without air cases started at about 4 percentage points for the coarsest cake and increased up to about 17 percentage points when the minus $0.025 \mathrm{~mm}$ solids accounted for about $70 \%$ of the total sample mass. After this point, the difference started to decrease, because of the rapid change in moisture when air 
Table 2.16 Effect of size distribution on moisture

\begin{tabular}{|c|c|c|c|c|c|c|c|c|}
\hline \multirow[b]{2}{*}{$\begin{array}{c}\text { Air } \\
\text { Pressure } \\
\text { (kPa) }\end{array}$} & \multirow[b]{2}{*}{$\begin{array}{c}\text { Centrifugal } \\
\text { Force } \\
\text { (g's) }\end{array}$} & \multicolumn{6}{|c|}{ Size Distribution (\% by weight) } & \multirow[b]{2}{*}{$\begin{array}{c}\text { Moisture } \\
(\%)\end{array}$} \\
\hline & & $\begin{array}{c}-0.6 \\
\mathbf{m m} \\
(\%) \\
\end{array}$ & $\begin{array}{l}-0.3 \\
\mathbf{m m} \\
(\%) \\
\end{array}$ & $\begin{array}{c}-0.15 \\
\mathrm{~mm} \\
(\%) \\
\end{array}$ & $\begin{array}{c}-0.074 \\
\mathbf{m m} \\
(\%)\end{array}$ & $\begin{array}{c}-0.044 \\
\text { mm } \\
(\%)\end{array}$ & $\begin{array}{c}-\mathbf{- 0 . 0 2 5} \\
\mathbf{m m} \\
(\%) \\
\end{array}$ & \\
\hline 0 & 500 & 66.83 & 48.62 & 33.04 & 23.29 & 18.88 & 13.84 & 13.8 \\
\hline 0 & 500 & 66.83 & 48.62 & 33.04 & 23.29 & 18.88 & 13.84 & 13.7 \\
\hline 0 & 500 & 100.00 & 100.00 & 95.52 & 71.98 & 58.00 & 43.62 & 31.4 \\
\hline 0 & 500 & 100.00 & 100.00 & 99.89 & 96.93 & 88.60 & 70.04 & 40.2 \\
\hline 0 & 500 & 100.00 & 100.00 & 99.87 & 99.06 & 96.98 & 85.00 & 42.1 \\
\hline 0 & 500 & 100.00 & 100.00 & 99.87 & 99.06 & 96.98 & 85.00 & 42.4 \\
\hline 0 & 500 & 100.00 & 100.00 & 100.00 & 99.93 & 99.59 & 97.82 & 44.7 \\
\hline 0 & 500 & 100.00 & 100.00 & 100.00 & 99.93 & 99.59 & 97.82 & 44.9 \\
\hline 200 & 500 & 66.83 & 48.62 & 33.04 & 23.29 & 18.88 & 13.84 & 9.8 \\
\hline 200 & 500 & 100.00 & 100.00 & 95.52 & 71.98 & 58.00 & 43.62 & 20.3 \\
\hline 200 & 500 & 100.00 & 100.00 & 99.89 & 96.93 & 88.60 & 70.04 & 23.6 \\
\hline 200 & 500 & 100.00 & 100.00 & 99.87 & 99.06 & 96.98 & 85.00 & 30.1 \\
\hline 200 & 500 & 100.00 & 100.00 & 99.87 & 99.06 & 96.98 & 85.00 & 30.5 \\
\hline 200 & 500 & 100.00 & 100.00 & 100.00 & 99.93 & 99.59 & 97.82 & 38.3 \\
\hline 200 & 500 & 100.00 & 100.00 & 100.00 & 99.93 & 99.59 & 97.82 & 37.7 \\
\hline 0 & 2700 & 69.28 & 48.67 & 33.45 & 24.44 & 20.61 & 15.76 & 9.2 \\
\hline 0 & 2700 & 88.74 & 65.43 & 44.72 & 31.48 & 26.28 & 19.99 & 11.0 \\
\hline 0 & 2700 & 97.19 & 81.59 & 57.79 & 43.42 & 35.62 & 27.69 & 14.8 \\
\hline 0 & 2700 & 99.86 & 97.93 & 83.72 & 62.60 & 52.69 & 39.99 & 18.8 \\
\hline 0 & 2700 & 99.94 & 99.30 & 90.70 & 71.63 & 60.14 & 46.03 & 20.4 \\
\hline 0 & 2700 & 100.00 & 99.50 & 96.15 & 86.38 & 77.58 & 63.26 & 28.3 \\
\hline 0 & 2700 & 99.98 & 99.46 & 92.79 & 76.60 & 66.31 & 52.36 & 21.7 \\
\hline 0 & 2700 & 99.96 & 99.65 & 98.26 & 86.28 & 74.75 & 55.46 & 24.5 \\
\hline 0 & 2700 & 100.00 & 99.92 & 99.64 & 98.70 & 95.62 & 85.35 & 32.1 \\
\hline 0 & 2700 & 100.00 & 99.92 & 99.64 & 98.70 & 95.62 & 85.35 & 32.0 \\
\hline 0 & 2700 & 100.00 & 99.74 & 99.55 & 99.52 & 99.13 & 97.24 & 40.0 \\
\hline 200 & 2700 & 68.38 & 49.19 & 34.08 & 24.25 & 20.54 & 15.85 & 7.9 \\
\hline 200 & 2700 & 88.74 & 65.43 & 44.72 & 31.48 & 26.28 & 19.99 & 9.6 \\
\hline 200 & 2700 & 97.19 & 81.59 & 57.79 & 43.42 & 35.62 & 27.69 & 11.9 \\
\hline 200 & 2700 & 99.86 & 97.93 & 83.72 & 62.60 & 52.69 & 39.99 & 16.1 \\
\hline 200 & 2700 & 99.94 & 99.30 & 90.70 & 71.63 & 60.14 & 46.03 & 17.2 \\
\hline 200 & 2700 & 100.00 & 99.50 & 96.15 & 86.38 & 77.58 & 63.26 & 23.7 \\
\hline 200 & 2700 & 99.98 & 99.46 & 92.79 & 76.60 & 66.31 & 52.36 & 19.8 \\
\hline 200 & 2700 & 99.96 & 99.65 & 98.26 & 86.28 & 74.75 & 55.46 & 22.1 \\
\hline 200 & 2700 & 100.00 & 99.92 & 99.64 & 98.70 & 95.62 & 85.35 & 21.4 \\
\hline 200 & 2700 & 100.00 & 99.92 & 99.64 & 98.70 & 95.62 & 85.35 & 22.5 \\
\hline 200 & 2700 & 100.00 & 99.74 & 99.55 & 99.52 & 99.13 & 97.24 & 28.0 \\
\hline 200 & 2700 & 100.00 & 99.74 & 99.55 & 99.52 & 99.13 & 97.24 & 27.2 \\
\hline
\end{tabular}




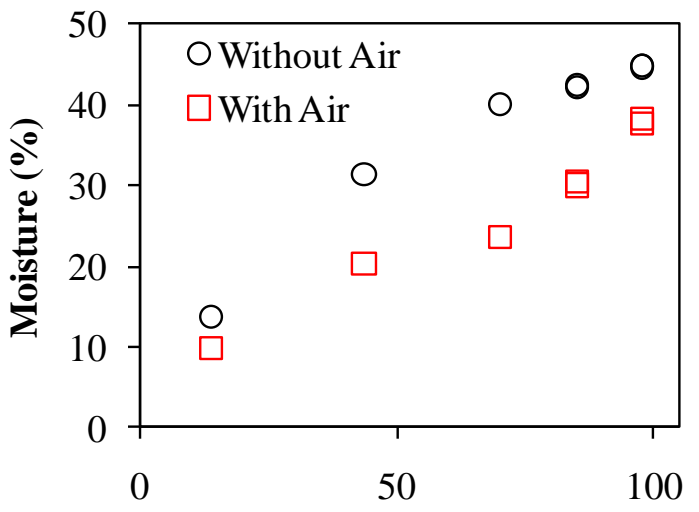

(a) minus $0.025 \mathrm{~mm}(\%)$

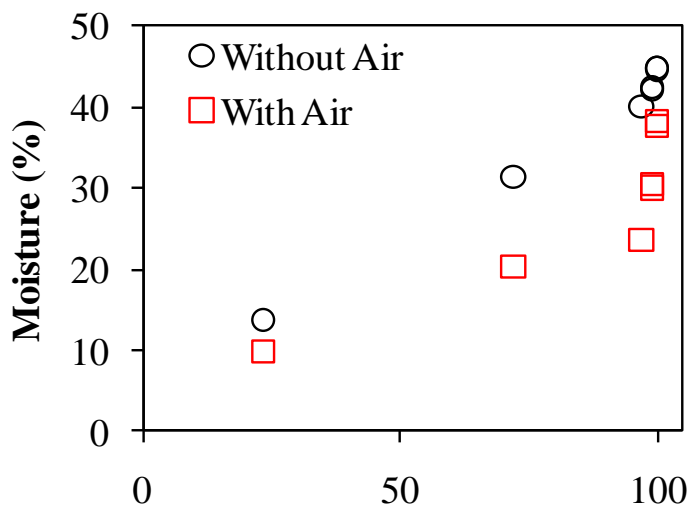

(c) minus $0.074 \mathrm{~mm}(\%)$

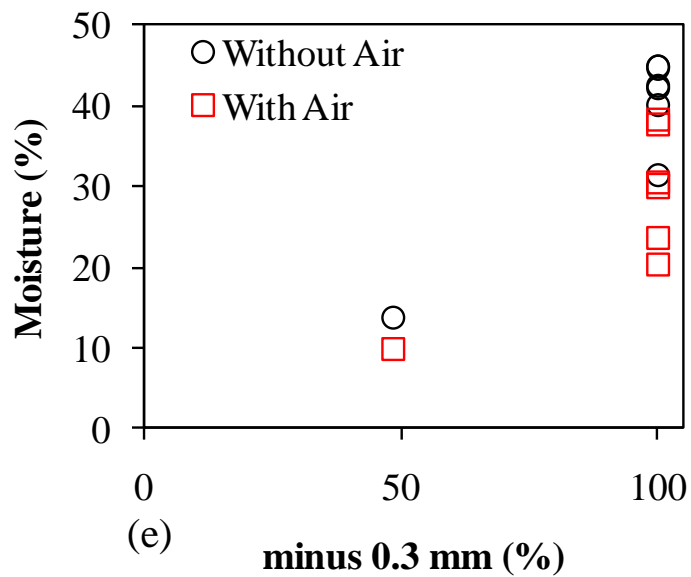

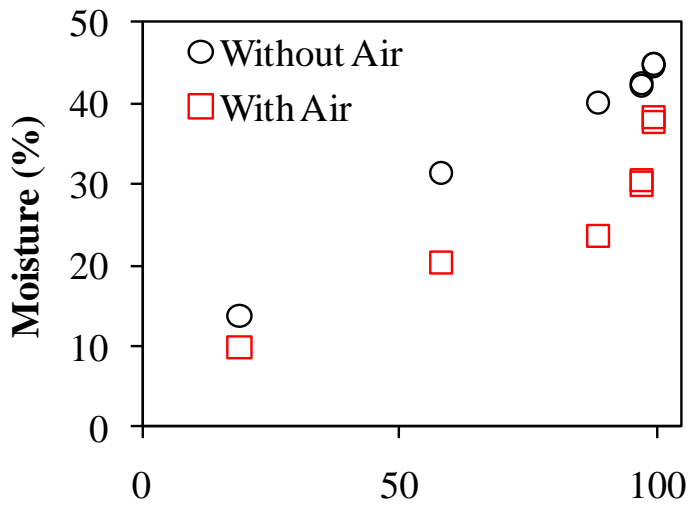

(b) minus $0.044 \mathrm{~mm}(\%)$
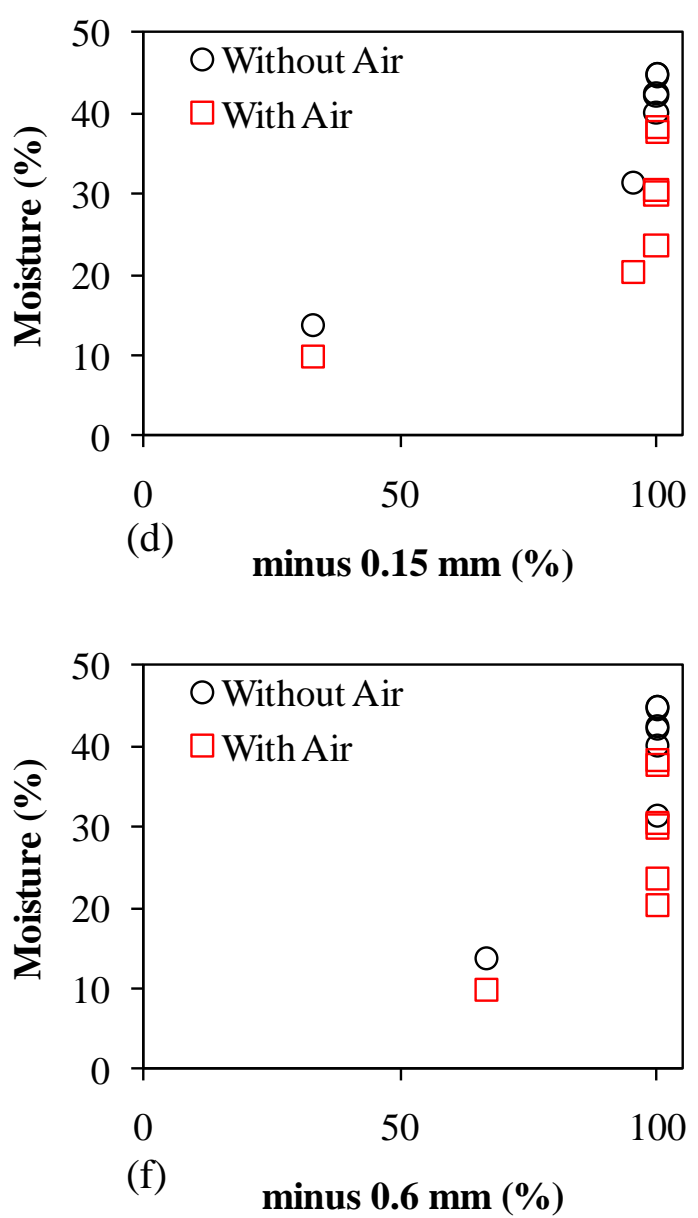

Figure 2.12 The effect of size distribution on moisture at $500 \mathrm{~g}$ 's 

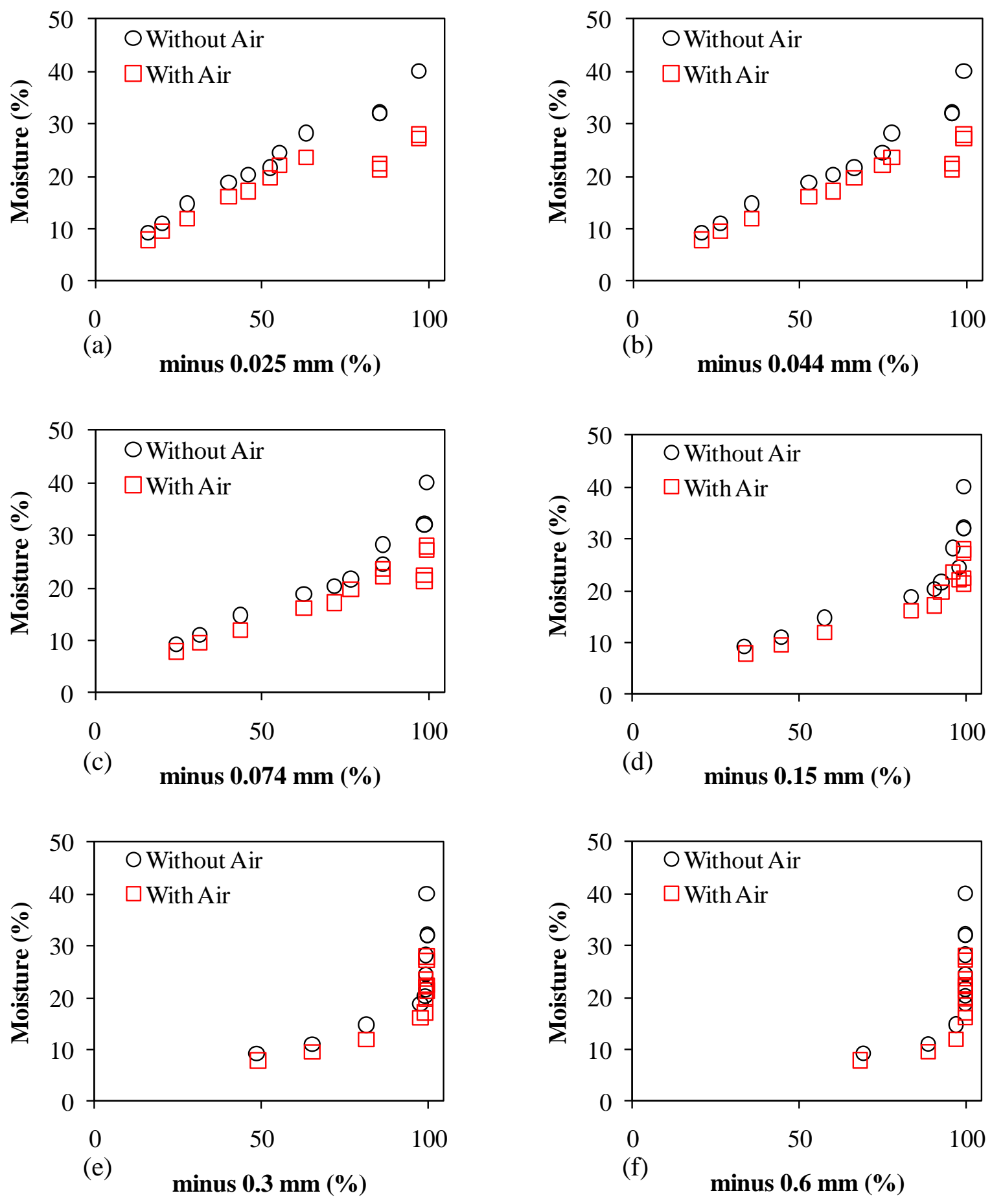

Figure 2.13 The effect of size distribution on moisture at $2700 \mathrm{~g}$ 's 
pressure was applied. As a result, decreasing the centrifugal acceleration from 2700 to 500 g's rendered air less effective when the cake was too fine, thus causing the two performance curves to merge.

\subsubsection{Modified Batch Tests}

\section{a) Effect of Media Type and Dewatering Kinetics}

Several series of tests were run to examine the kinetics of hyperbaric centrifugation. The coal used for these tests was the screenbowl feed sample $(1 \mathrm{~mm} \times 0 \mathrm{~mm})$ taken from Tom's Creek Coal Plant, which was described in section 2.2.1. The first two sets were run with either a standard filter cloth, while a screen mesh with $1.5 \mathrm{~mm}$ aperture was used as the filter media for the last set of runs. The feed charge was held relatively constant at 485-495 gm using either 29\% or $35 \%$ solids contents. Two different levels of centrifugal acceleration (i.e., 300 and $800 \mathrm{~g}$ 's) were used during the tests. Flocculant and coagulant were added to all test samples, in order to increase the solids recovery. Air pressure was also varied from 0 to $200 \mathrm{kPa}$.

After a few trial tests, it was seen that the test setup was not precise enough to record the small amount of water weight removed from the cake by air injection. Therefore, in the first set of tests, instead of recording the weight continuously, the drum was removed and weighed every 10 to 20 seconds after the air injection started until the end of the test run. Since this procedure was time consuming and the curves needed were obtained using the first set of tests, only the final weight of the drum was measured for the rest of the tests.

The test results given in Tables 2.17-2.19 compare the cake moisture and solid recovery values under different conditions. The biggest impact of air injection was achieved when filter cloth was used, due to higher solid recoveries. While the maximum moisture drop was 6 
percentage points when filter cloth was used as the filter media, it was about 1 percentage point with the larger-aperture screen mesh.

Table 2.17 Equilibrium moistures obtained at (a) the first and (b) the second sets of kinetic tests with filter cloth, and (c) the third set of kinetic tests with screen mesh

(a)

\begin{tabular}{ccccccc}
\hline $\begin{array}{c}\text { Run } \\
\text { Number }\end{array}$ & $\begin{array}{c}\text { Feed } \\
\text { Solids } \\
(\boldsymbol{\%})\end{array}$ & $\begin{array}{c}\text { Feed } \\
\text { Charge } \\
(\mathbf{g})\end{array}$ & $\begin{array}{c}\text { Air } \\
\text { Pressure } \\
(\mathbf{k P a})\end{array}$ & $\begin{array}{c}\text { Centrifugal } \\
\text { Force } \\
(\mathbf{g} \mathbf{s})\end{array}$ & $\begin{array}{c}\text { Moisture } \\
(\boldsymbol{\%})\end{array}$ & $\begin{array}{c}\text { Recovery } \\
(\mathbf{\%})\end{array}$ \\
\hline K1-1 & 34.70 & 494.5 & 0 & 300 & 26.8 & 90.7 \\
K1-2 & 34.70 & 494.5 & 0 & 800 & 22.5 & 92.4 \\
K1-3 & 34.70 & 494.5 & 100 & 300 & 22.8 & 86.9 \\
K1-4 & 34.70 & 494.5 & 200 & 300 & 20.8 & 92.9 \\
\hline
\end{tabular}

(b)

\begin{tabular}{ccccccc}
\hline $\begin{array}{c}\text { Run } \\
\text { Number }\end{array}$ & $\begin{array}{c}\text { Feed } \\
\text { Solids } \\
(\boldsymbol{\%})\end{array}$ & $\begin{array}{c}\text { Feed } \\
\text { Charge } \\
(\mathbf{g})\end{array}$ & $\begin{array}{c}\text { Air } \\
\text { Pressure } \\
(\mathbf{k P a})\end{array}$ & $\begin{array}{c}\text { Centrifugal } \\
\text { Force } \\
(\mathbf{g} \mathbf{s})\end{array}$ & $\begin{array}{c}\text { Moisture } \\
(\boldsymbol{\%})\end{array}$ & $\begin{array}{c}\text { Recovery } \\
(\boldsymbol{\%})\end{array}$ \\
\hline K2-1 & 28.76 & 486.3 & 0 & 300 & 27.6 & 86.3 \\
K2-2 & 28.76 & 486.3 & 0 & 800 & 22.6 & 90.2 \\
K2-3 & 28.76 & 486.3 & 100 & 300 & 25.4 & 82.7 \\
K2-4 & 28.76 & 486.3 & 100 & 800 & 18.9 & 92.2 \\
\hline
\end{tabular}

(c)

\begin{tabular}{ccccccc}
\hline $\begin{array}{c}\text { Run } \\
\text { Number }\end{array}$ & $\begin{array}{c}\text { Feed } \\
\text { Solids } \\
(\boldsymbol{\%})\end{array}$ & $\begin{array}{c}\text { Feed } \\
\text { Charge } \\
(\mathbf{g})\end{array}$ & $\begin{array}{c}\text { Air } \\
\text { Pressure } \\
(\mathbf{k P a})\end{array}$ & $\begin{array}{c}\text { Centrifugal } \\
\text { Force } \\
(\mathbf{g} \mathbf{s})\end{array}$ & $\begin{array}{c}\text { Moisture } \\
(\boldsymbol{\%})\end{array}$ & $\begin{array}{c}\text { Recovery } \\
(\boldsymbol{\%})\end{array}$ \\
\hline K3-1 & 28.76 & 486.3 & 0 & 300 & 21.87 & 64.0 \\
K3-2 & 28.76 & 486.3 & 0 & 800 & 17.38 & 56.3 \\
K3-3 & 28.76 & 486.3 & 100 & 300 & 19.94 & 59.2 \\
K3-4 & 28.76 & 486.3 & 100 & 800 & 16.75 & 68.5 \\
K3-5 & 28.76 & 486.3 & 166 & 300 & 22.63 & 71.1 \\
K3-6 & 28.76 & 486.3 & 166 & 800 & 16.61 & 66.4 \\
\hline
\end{tabular}

Figures 2.14-2.16 indicate that the greatest moisture drop took place in the first 50-60 seconds of centrifugation. After 60 seconds, the rate of dewatering decreased dramatically. Also, Figure 2.14(d) and Figure 2.14(f) show that only the first 10 seconds of air injection was effective in reducing moisture. This finding is encouraging since it shows that cakes need not be 
subjected to long periods of air pressure in a production machine in order to obtain low cake moistures during hyperbaric centrifugation.

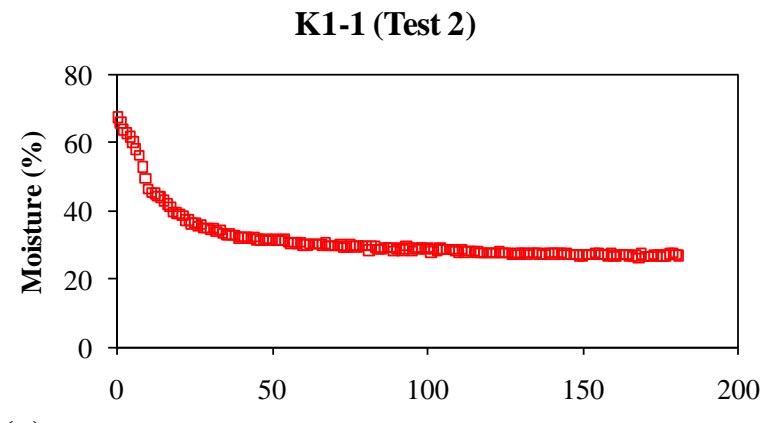

(a)

Time (sec)
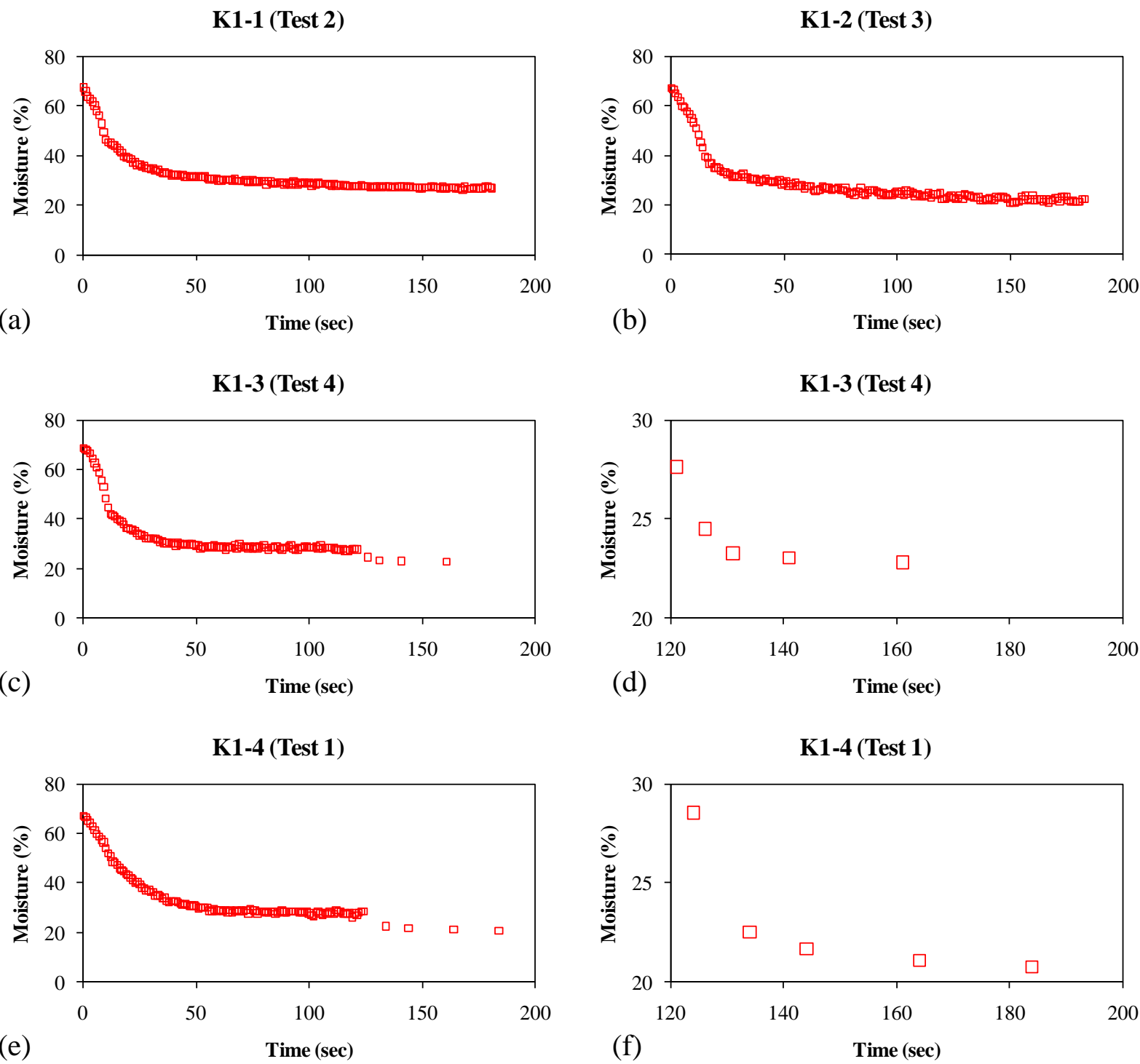

Figure 2.14 The first set of kinetic tests with filter cloth and closer looks to air injection parts of the curves $((d)$ and $(f))$ 
K2-1 (Test 2)

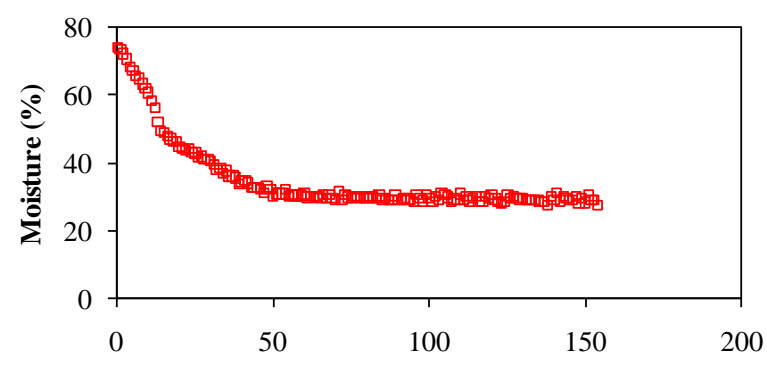

(a)

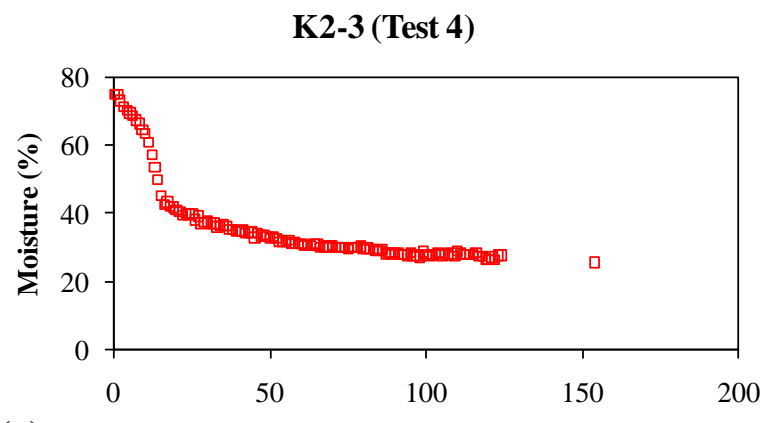

(c)

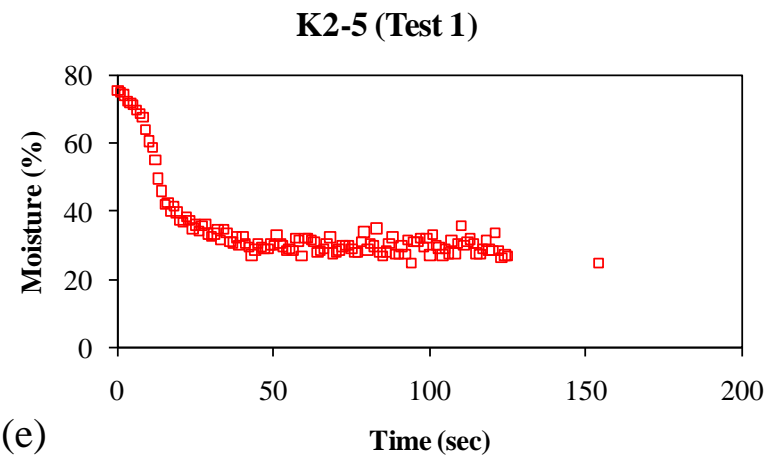

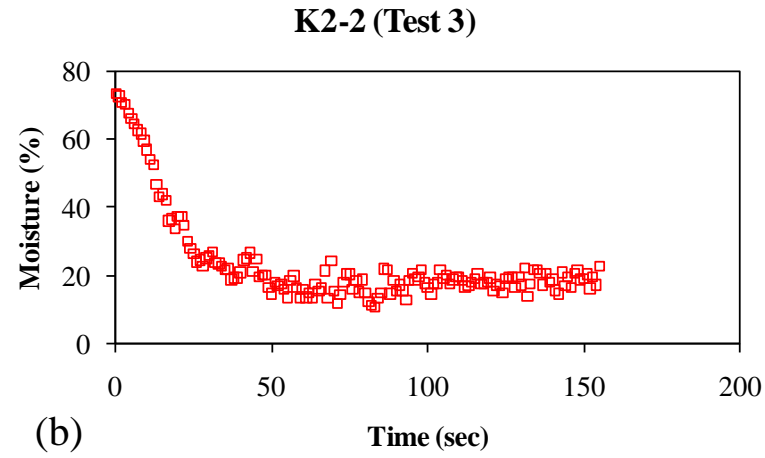

K2-4 (Test 6)

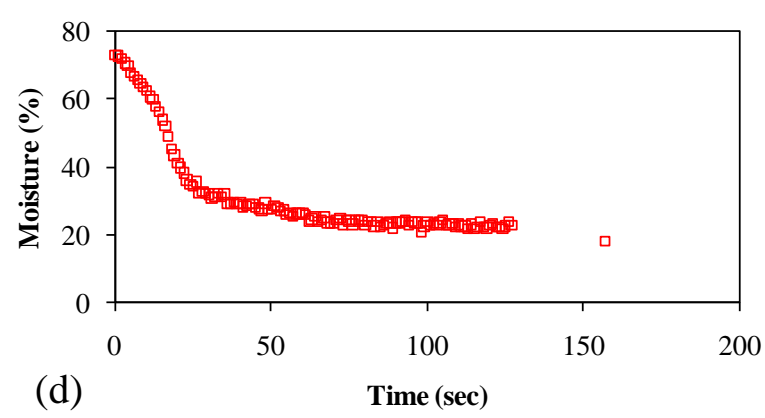

K2-6 (Test 5)

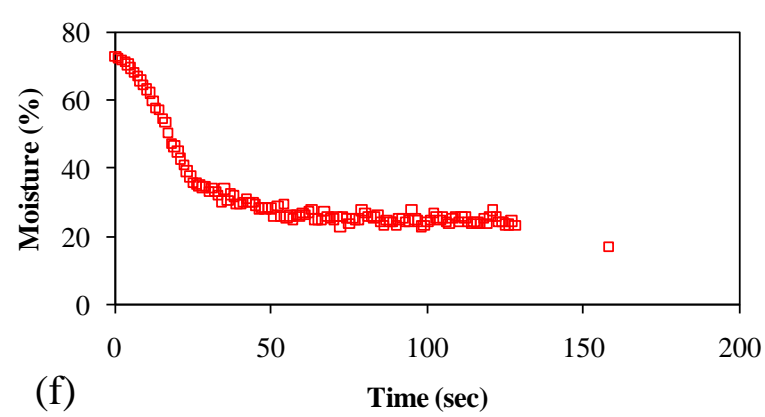

Figure 2.15 The second set of kinetic tests with filter cloth 
K3-1 (Test 2)

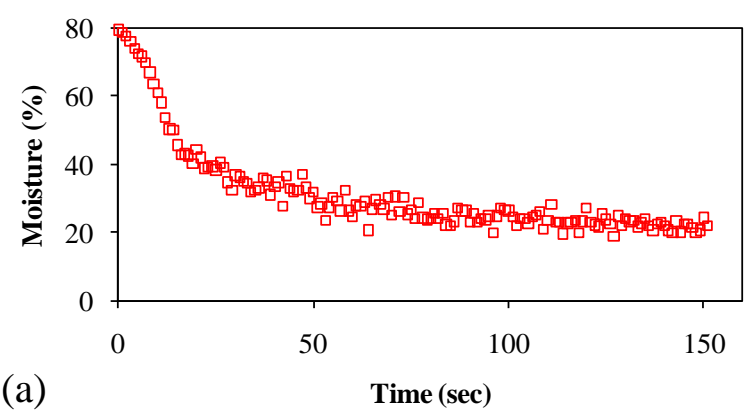

K3-3 (Test 4)

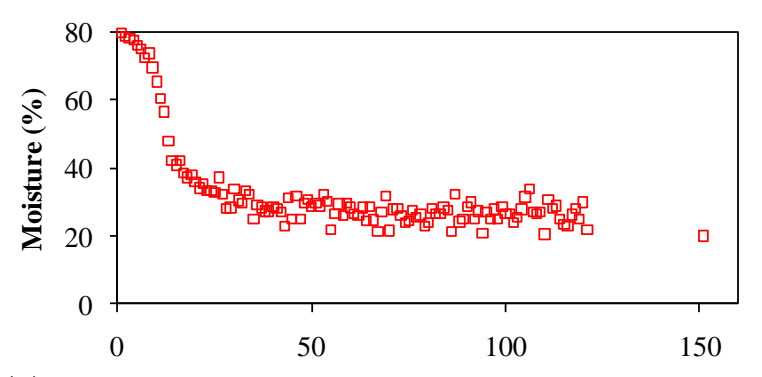

(c)

Time (sec)

K3-5 (Test 1)

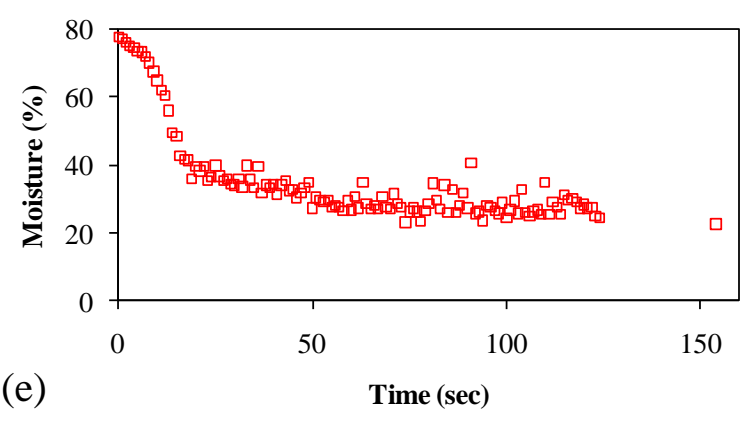

K3-2 (Test 3)

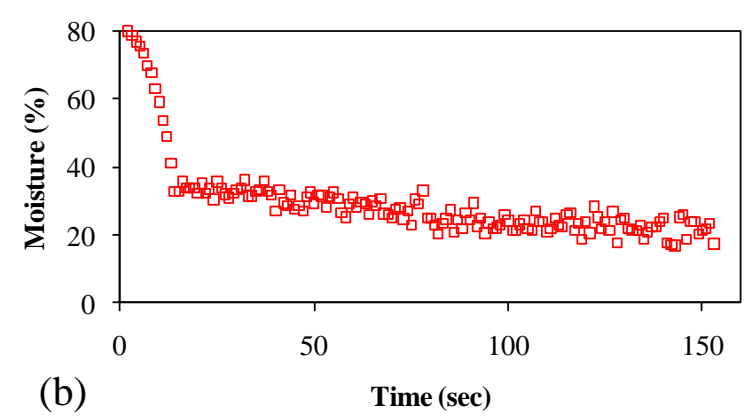

K3-4 (Test 6)

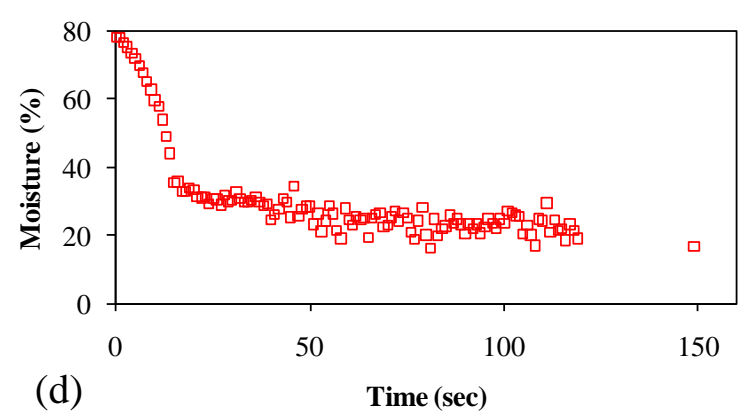

K3-6 (Test 5)

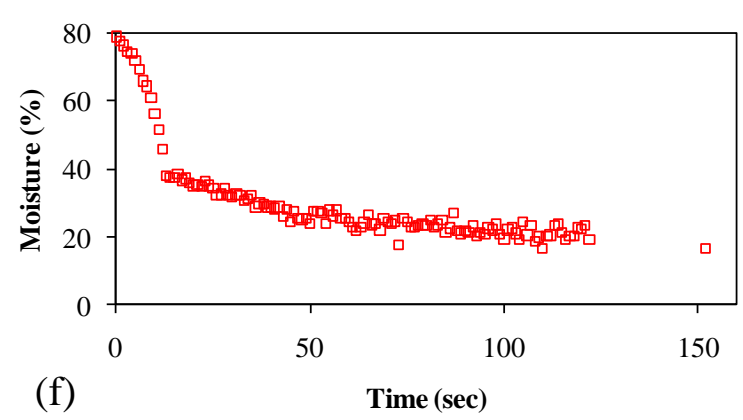

Figure 2.16 The third set of kinetic tests with $1.5 \mathrm{~mm}$ screen mesh 


\section{b) Solid Bowl Tests}

This set of tests was run to identify optimum conditions for producing a thickened feed in a solid-bowl centrifuge. Ideally, this material would be passed to a filter-screen section at a high solids content to ensure that a high recovery of solids could be maintained. The test unit described previously in section 2.2.3.2 was used for this portion of the experimentation. Parameters examined in the study included feed charge, feed rate, feed percent solids and centrifugal acceleration. A flotation product sample $(0.15 \times 0 \mathrm{~mm})$ taken from Tom's Creek coal plant was used for the tests.

The results given in Table 2.18 and Figures 2.17-2.24 showed that changing the amount of feed charge had almost no effect on either the percent solids of the product or the solids loss to

Table 2.18 Solid bowl test results

\begin{tabular}{ccccccc}
\hline $\begin{array}{c}\text { Feed } \\
\text { Charge } \\
(\mathbf{l})\end{array}$ & $\begin{array}{c}\text { Feed } \\
\text { Charge } \\
\text { (dry g) }\end{array}$ & $\begin{array}{c}\text { Centrifugal } \\
\text { Force } \\
(\mathbf{g} \text { 's) }\end{array}$ & $\begin{array}{c}\text { Feed } \\
\text { Rate } \\
(\boldsymbol{\%})\end{array}$ & $\begin{array}{c}\text { Feed } \\
\text { Solid Content } \\
(\boldsymbol{\%})\end{array}$ & $\begin{array}{c}\text { Product } \\
\text { Solid Content } \\
(\boldsymbol{\%})\end{array}$ & $\begin{array}{c}\text { Solids loss } \\
\text { to Effluent } \\
(\boldsymbol{\%})\end{array}$ \\
\hline 0.50 & 78.03 & 760 & 10 & 15.0 & 64.3 & 3.0 \\
0.70 & 109.25 & 760 & 10 & 15.0 & 64.1 & 2.8 \\
1.00 & 156.07 & 760 & 10 & 15.0 & 64.3 & 2.8 \\
1.25 & 195.09 & 760 & 10 & 15.0 & 65.9 & 2.8 \\
1.50 & 234.10 & 760 & 10 & 15.0 & 62.9 & 2.9 \\
\hline 1.00 & 156.07 & 100 & 10 & 15.0 & N/A & 100.0 \\
1.00 & 156.07 & 365 & 10 & 15.0 & 64.7 & 3.6 \\
1.00 & 156.07 & 760 & 10 & 15.0 & 67.0 & 2.7 \\
1.00 & 156.07 & 1045 & 10 & 15.0 & 66.0 & 2.0 \\
1.00 & 156.07 & 1080 & 10 & 15.0 & 67.3 & 1.5 \\
\hline 1.00 & 156.07 & 760 & 10 & 15.0 & 65.3 & 2.6 \\
1.00 & 156.07 & 760 & 30 & 15.0 & 67.4 & 4.0 \\
1.00 & 156.07 & 760 & 50 & 15.0 & 64.2 & 4.0 \\
1.00 & 156.07 & 760 & 70 & 15.0 & 64.7 & 2.9 \\
1.00 & 156.07 & 760 & 100 & 15.0 & 74.4 & 5.5 \\
\hline 3.13 & 156.07 & 760 & 10 & 5.0 & 66.8 & 3.8 \\
1.52 & 156.07 & 760 & 10 & 10.0 & 65.4 & 2.8 \\
1.00 & 156.07 & 760 & 10 & 15.0 & 64.7 & 2.6 \\
0.58 & 156.07 & 760 & 10 & 25.0 & 62.6 & 0.7 \\
0.35 & 156.07 & 760 & 10 & 40.0 & 66.0 & 0.4 \\
\hline
\end{tabular}




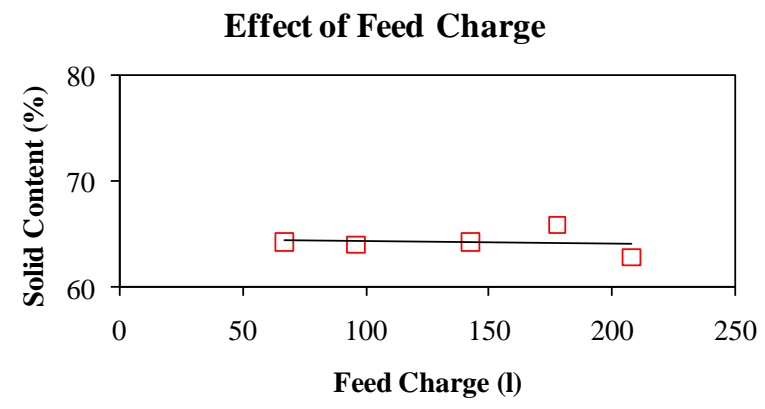

Figure 2.17 Effect of feed charge on product solid content

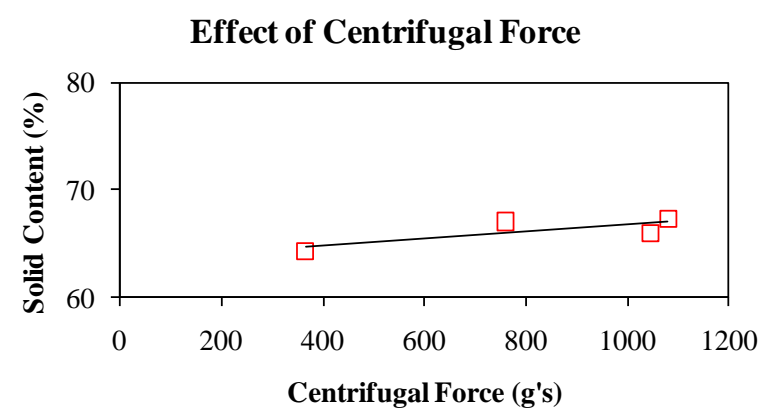

Figure 2.19 Effect of centrifugal force on product solid content

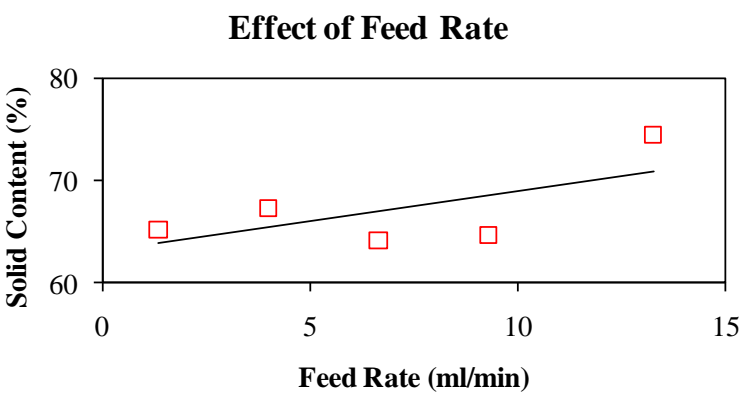

Figure 2.21 Effect of feed rate on product solids content
Effect of Feed Charge

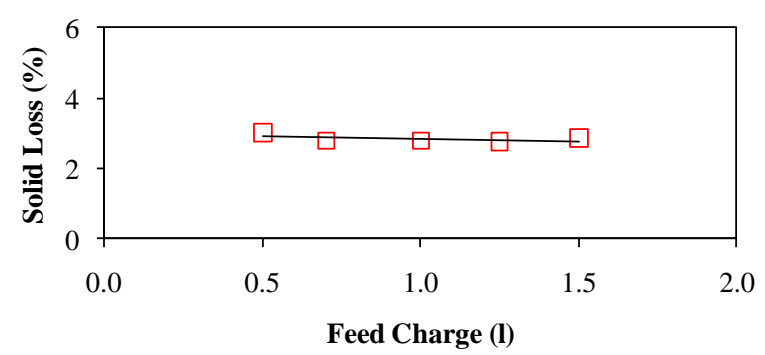

Figure 2.18 Effect of feed charge on solids loss to effluent

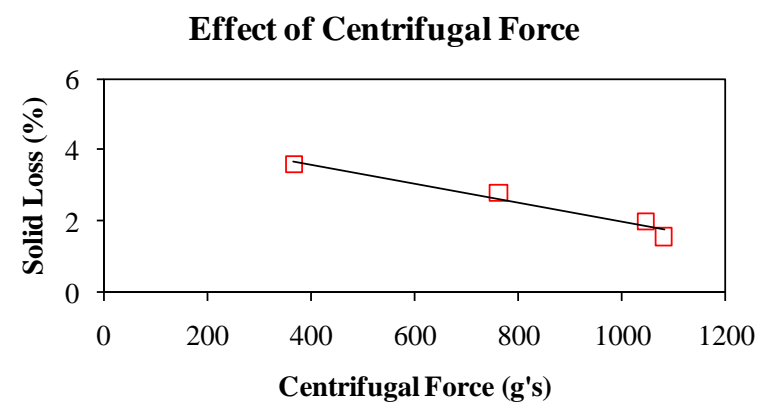

Figure 2.20 Effect of centrifugal force on solids loss to effluent

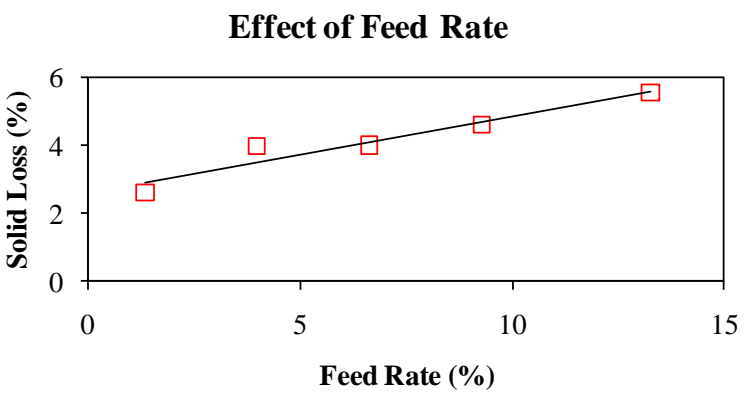

Figure 2.22 Effect of feed rate on solids loss to effluent 
Effect of Solid \%

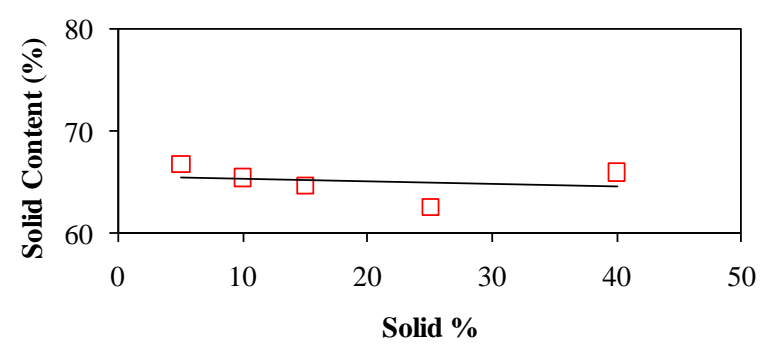

Figure 2.23 Effect of feed solid content on product solid content

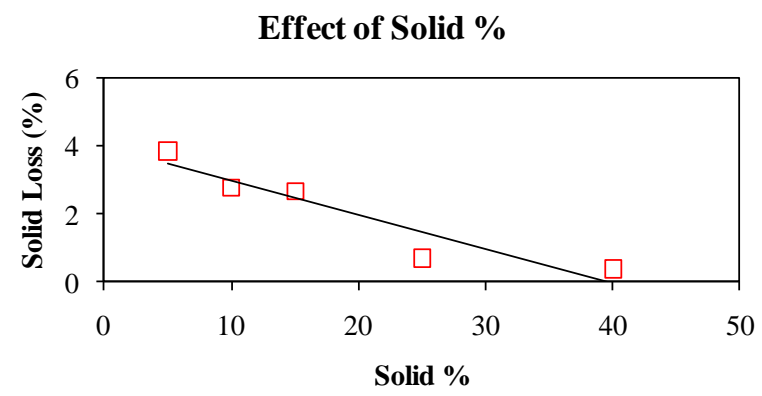

Figure 2.24 Effect of feed solid content on solids loss to effluent

the effluent. In contrast, the loss of solids to effluent significantly decreased from about $4 \%$ down to $1.5 \%$ when the centrifugal acceleration was increased. At the same time, the solids content of the product slightly increased from about $64 \%$ to $67 \%$, while the loss of solids increased significantly from about $2.5 \%$ to $5.5 \%$, due to the increasing feed rate. Finally, increasing the feed solids content decreased the solids loss from about $4 \%$ down to nearly zero, while the product solids content held constant at about $65 \%$.

\subsection{Discussion}

\subsubsection{Solids Recovery and Product Moisture}

Standard batch tests conducted with different coal types showed that the product moisture greatly depended on particle size distribution of the feed slurry. Employing finer feed samples resulted in higher product moistures as a result of the increased specific surface area. Moreover, the application of air pressure was more effective when the feed was finer, which suggests that the higher capillary pressure created by smaller capillaries due to finer particle sizes could only be overcome by applying pressurized air in addition to the centrifugal acceleration.

Moisture reductions obtained using slurries with different size distributions at various levels of centrifugal accelerations are given in Figure 2.25. According to these plots, similar 
moisture reductions were obtained using 500 and 2700 g's when the product contained less than 40\% minus $0.025 \mathrm{~mm}$ material (compared to the baseline moisture obtained at 500 g's without using air pressure). Therefore, it may be unnecessary to use a costly high-g machine when the same level of performance can be obtained using a cost effective lower-g machine for products containing less than $60 \%$ minus $0.025 \mathrm{~mm}$ material. However, when the product contained more than $60 \%$ minus $0.025 \mathrm{~mm}$ material, applying 2700 g's with air was more effective than the other options. These results indicate that the hyperbaric centrifuge is best suited for the dewatering of coals containing substantial amounts of ultrafine solids.

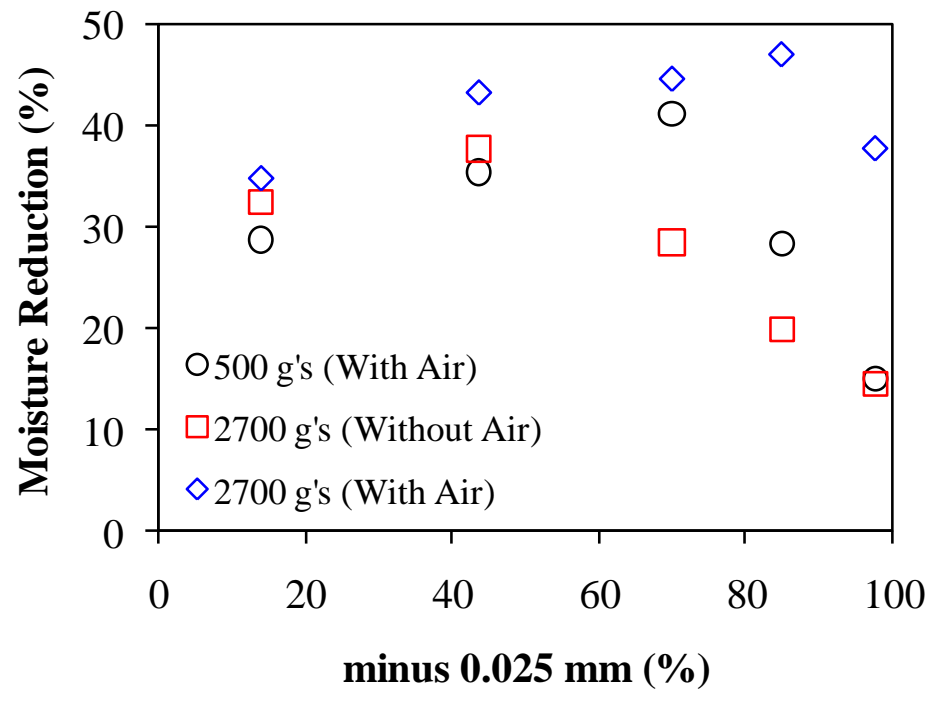

Figure 2.25 Effect of amount of fines on moisture reduction

Tests conducted with various filter media showed that solid recovery can drop dramatically (down to 50-60\%) when a conventional screenbowl feed is used with a coarse screen mesh. Fortunately, solids recoveries were improved by using filter cloth without greatly impacting moisture. However, since it is not possible to use filter cloth in industrial applications, 
employing a method such as pre-thickening appears to be the best option for increasing solids recoveries.

Pre-thickening is usually performed in the solid bowl section of a screenbowl centrifuge. Hence, a separate lab scale solid bowl centrifuge was built and tested to optimize the feed to the hyperbaric centrifuge. Figure 2.26 shows the effect of residence time on the amount of solids

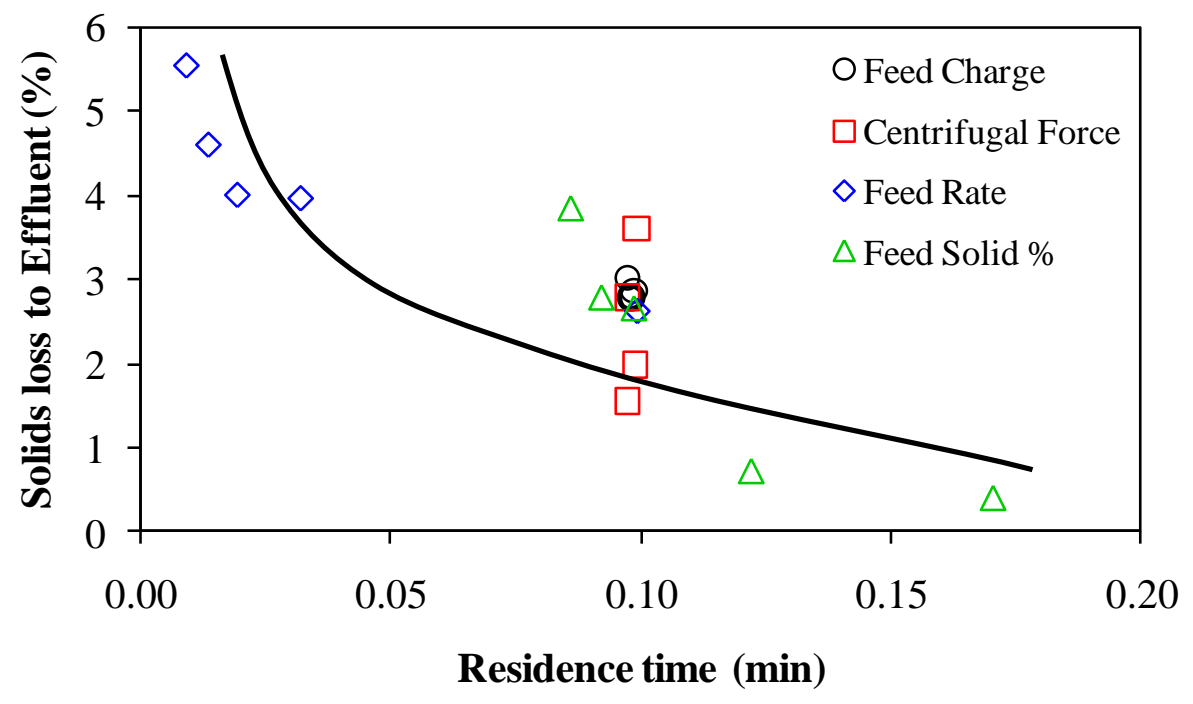

Figure 2.26 Effect of residence time on solids loss to effluent

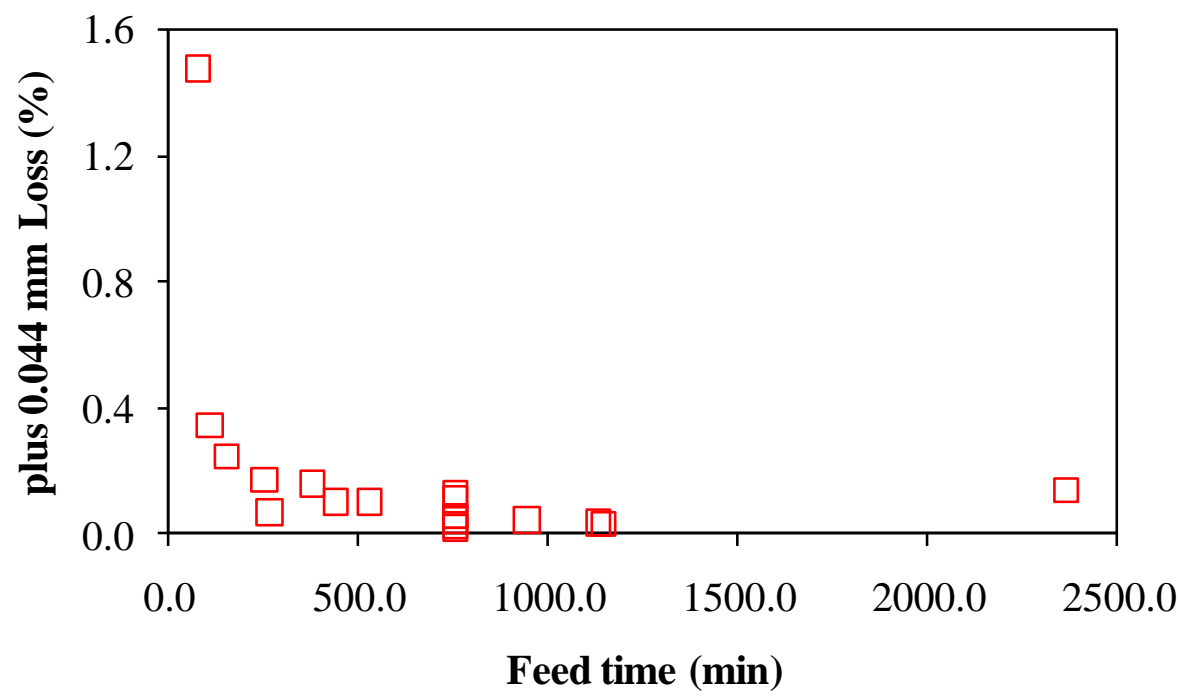

Figure 2.27 Effect of feed time on plus $0.044 \mathrm{~mm}$ particle loss 
reported to effluent at all the conditions tested. Generally, solids loss to the effluent increased as the residence time decreased. Also, as shown in Figure 2.27, the loss of plus $0.044 \mathrm{~mm}$ particles to effluent was found to be correlated to the feed time and, therefore, the feed rate. While the loss was lower than $0.2 \%$ at feed times longer than 0.25 minutes, it increased up to $1.5 \%$ as the feed time became shorter.

\subsubsection{Reproducibility of Test Results}

Although a full experimental design was not performed for a complete statistical analysis, some of the tests described in section 2.3.1d) were repeated to determine the reproducibility of the data. Average moisture values were calculated for each repeated test and these values were used in the charts given in Figure 2.28. Error bars for each repeated test were then determined by adding and subtracting three times the standard deviation value to this average value, which gave a 99.7\% confidence interval. As can be seen, the reproducibility of results without air pressure was high. The calculated relative errors approximately 0.3 percentage points for 2700 g's and 0.7 percentage points for 500 g's when no air was used. Although the addition of air pressure

(a)

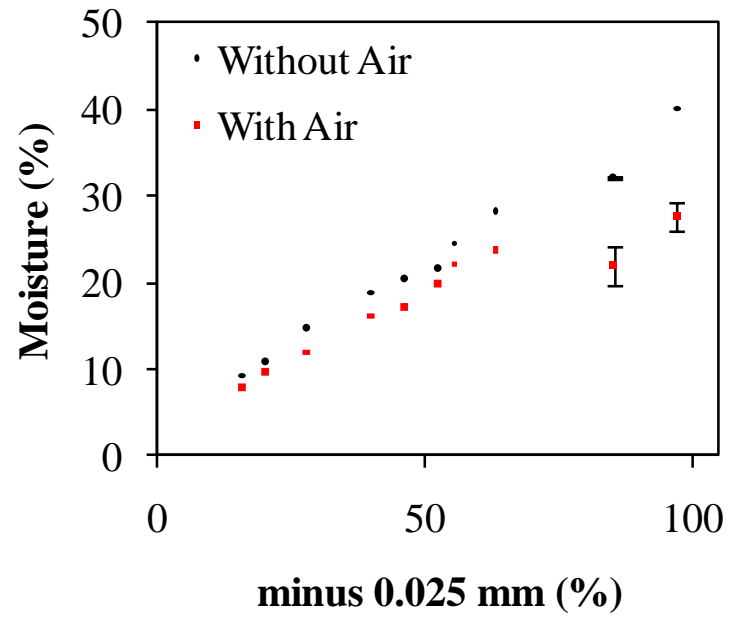

(b)

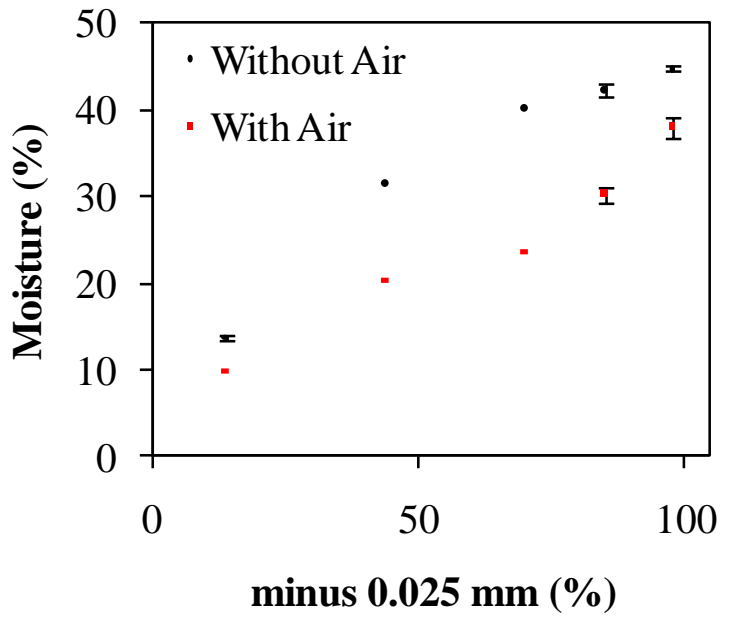

Figure 2.28 Charts showing reproducibility of test results at (a) $2700 \mathrm{~g}$ 's and (b) $500 \mathrm{~g}$ 's 
increased the relative error values to about 0.7 and 1.2 percentage points for 2700 g's and 500 g's, respectively, these variations were still far from being very significant when compared to variations resulting from manual changes to air pressure or centrifugal acceleration.

\subsubsection{Engineering Criteria for Machine Design}

\section{a) Rate Constants}

The kinetic tests run with different filter media showed that the kinetics of centrifugal dewatering is quite different than filtering. The following filtration equation was derived by Dahlstrom from the commonly employed Poiseuille equation (Dahlstrom, 1985):

$$
\frac{t}{V / A}=\frac{\mu a w V}{2 \Delta P A}+\frac{\mu r}{\Delta P}
$$

where $V$ is the volume of filtrate, $A$ is the area of filtration, $t$ is the time, $\Delta P$ is the pressure drop across the filter, $\mu$ is the liquid viscosity, $a$ is the specific cake resistance, $w$ is the weight of dry cake solids per unit volume of filtrate and $r$ is the resistance of filter cloth and drainage network.

Eq. [2.1] yields a straight line plot of t/V/A vs. V/A as given in Figure 2.29. However, when the same plot was created using the data obtained from centrifugal kinetics tests (Figure 2.30), it was not possible to obtain a straight line relationship, because centrifugal acceleration

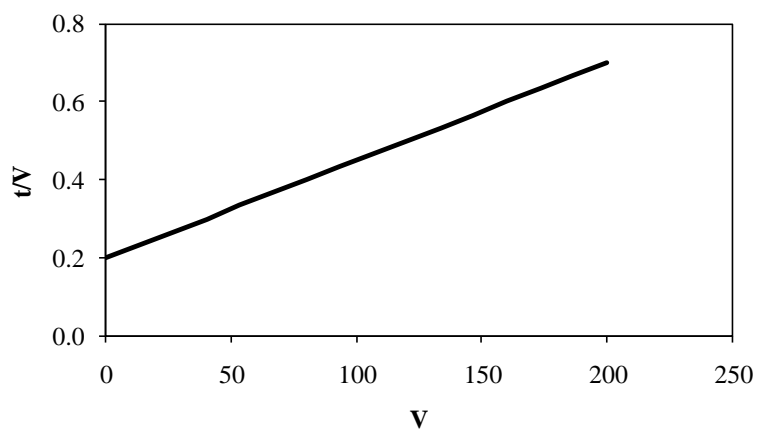

Figure 2.29 t/V/A vs. V/A (filtration theory)

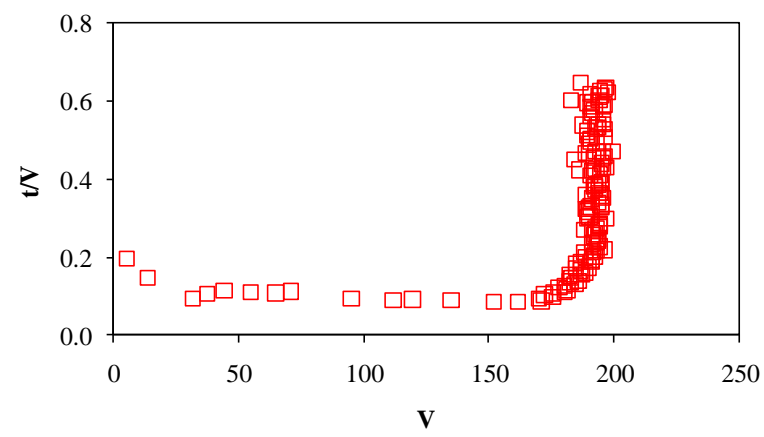

Figure 2.30 t/V/A vs. V/A (Measured) 
changes with time as opposed to the constant pressure used in conventional filtering. Therefore, the Dahlstrom filtration equation cannot be used directly to describe hyperbaric centrifugation.

In the current study, a multiple-term rate equation was used to describe the centrifugal dewatering process. The model assumes that a portion of water was removed quickly from the cake, while another portion was removed slower. Also, another portion of water was assumed to be non-removable by mechanical means, thus staying inside the cake as final product moisture. The resultant dewatering equation can be expressed as:

$$
W / W_{0}=\emptyset_{s} \cdot \exp \left(-K_{s} \cdot t\right)+\emptyset_{f} \cdot \exp \left(-K_{f} \cdot t\right)+\emptyset_{n}
$$

where $W$ is the weight of water remaining in cake, $W_{0}$ is the initial weight of water in feed, $K_{s}$ and $K_{t}$ are the rate constants for slow removed and fast removed water from the cake, respectively; $\varphi_{s}, \varphi_{f}$ and $\varphi_{n}$, are the proportions of slow removed, fast removed and not removed water and $t$ is the time. In general, this expression provided a good correlation between the weight of water remaining in the cake and the dewatering time.

During all of the kinetic tests, the centrifuge was run for a certain amount of time to remove most of the water that could be removed by only centrifugation. Then, air pressure was applied to remove an additional amount of residual water. Therefore, curve fitting was done for every centrifugal acceleration applied using all available data in the relevant set by simply eliminating the values obtained with air. The results of curve fitting based on Eq. [2.2] are given in Figures 2.30-2.32. Even though the moisture and recovery values were reasonable for Tests 3 of the first and second sets, kinetic data recorded were not in agreement with rest of the tests run at the same speed. Also, data for Test 3 of the second set of tests contained an excess amount of scatter. This problem can attributed to the difficulty of recording data with a sensitive digital balance connected to centrifuge running at thousands of rpm. Therefore, the data for Test 3 from 
both sets were not included in the plots, since, possibly, for these particular tests, the weights recorded were not accurate.

The kinetic test results showed that the first and second sets of tests that were run with filter cloth had only one dewatering rate, while the third set run with the screen mesh had two rates. More solids and more fines were recovered during the tests with filter cloth, because filter cloth had a much smaller opening size than screen mesh. After a certain amount of time, fines blinded the filter cloth and dewatering rate diminished; thus, there was no slow rate. On the other hand, the screen mesh was coarse enough for dewatering not to stop, but continue at a slower rate. Therefore, the tests with screen mesh had two different dewatering rates.

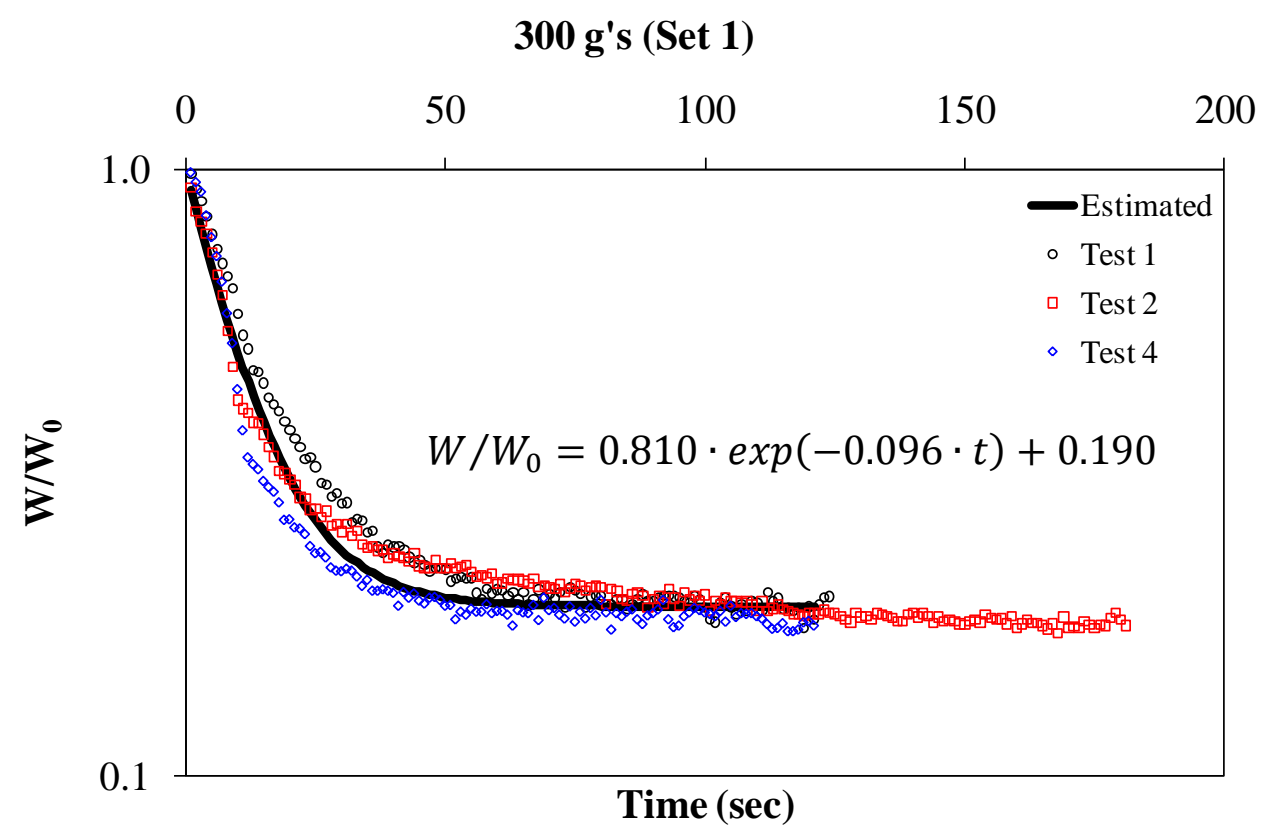

Figure 2.31 Rate curve fit for the first set of kinetic tests at $300 \mathrm{~g}$ 's 

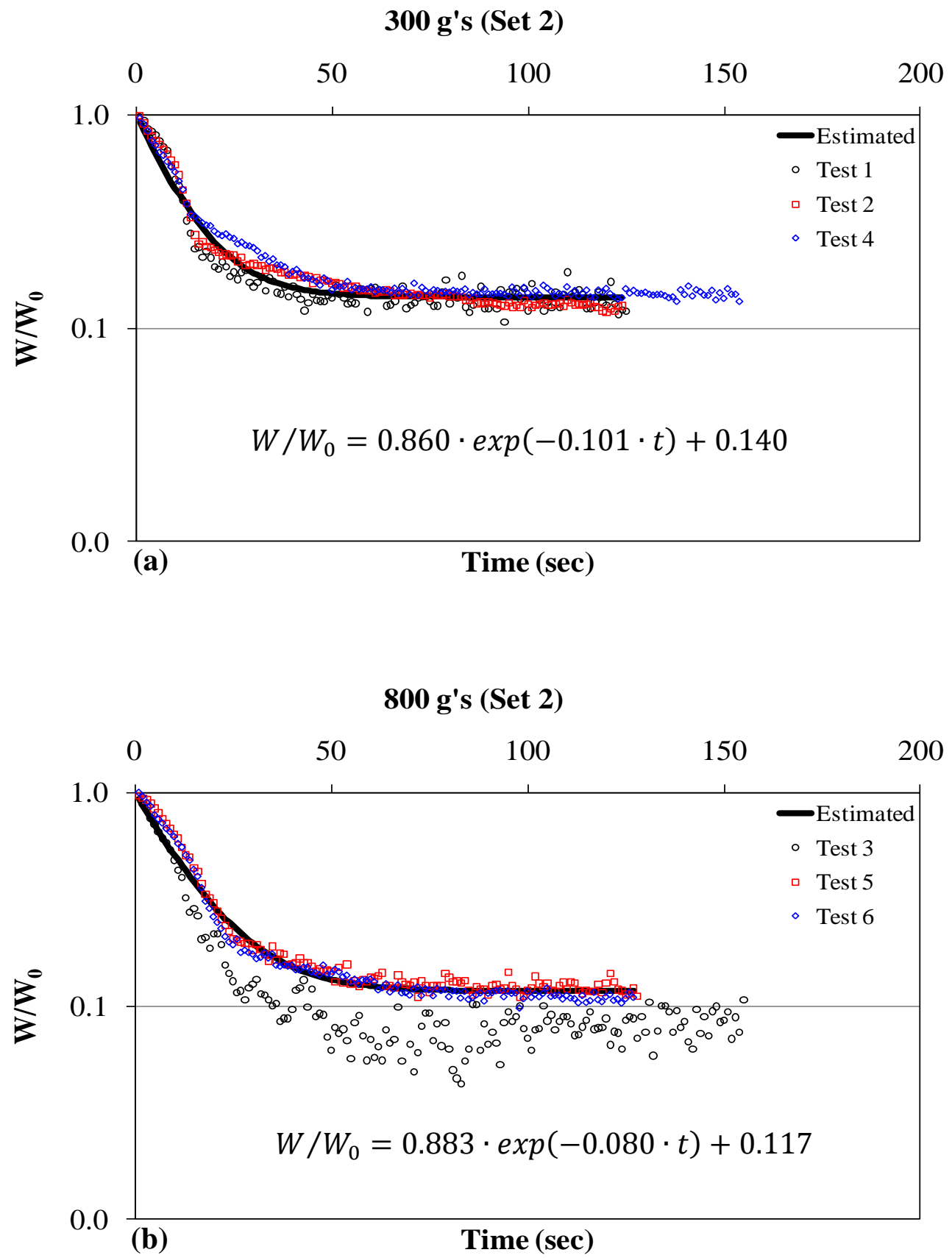

Figure 2.32 Rate curve fits for the second set of kinetic tests at (a) $300 \mathrm{~g}$ 's and (b) $800 \mathrm{~g}$ 's 

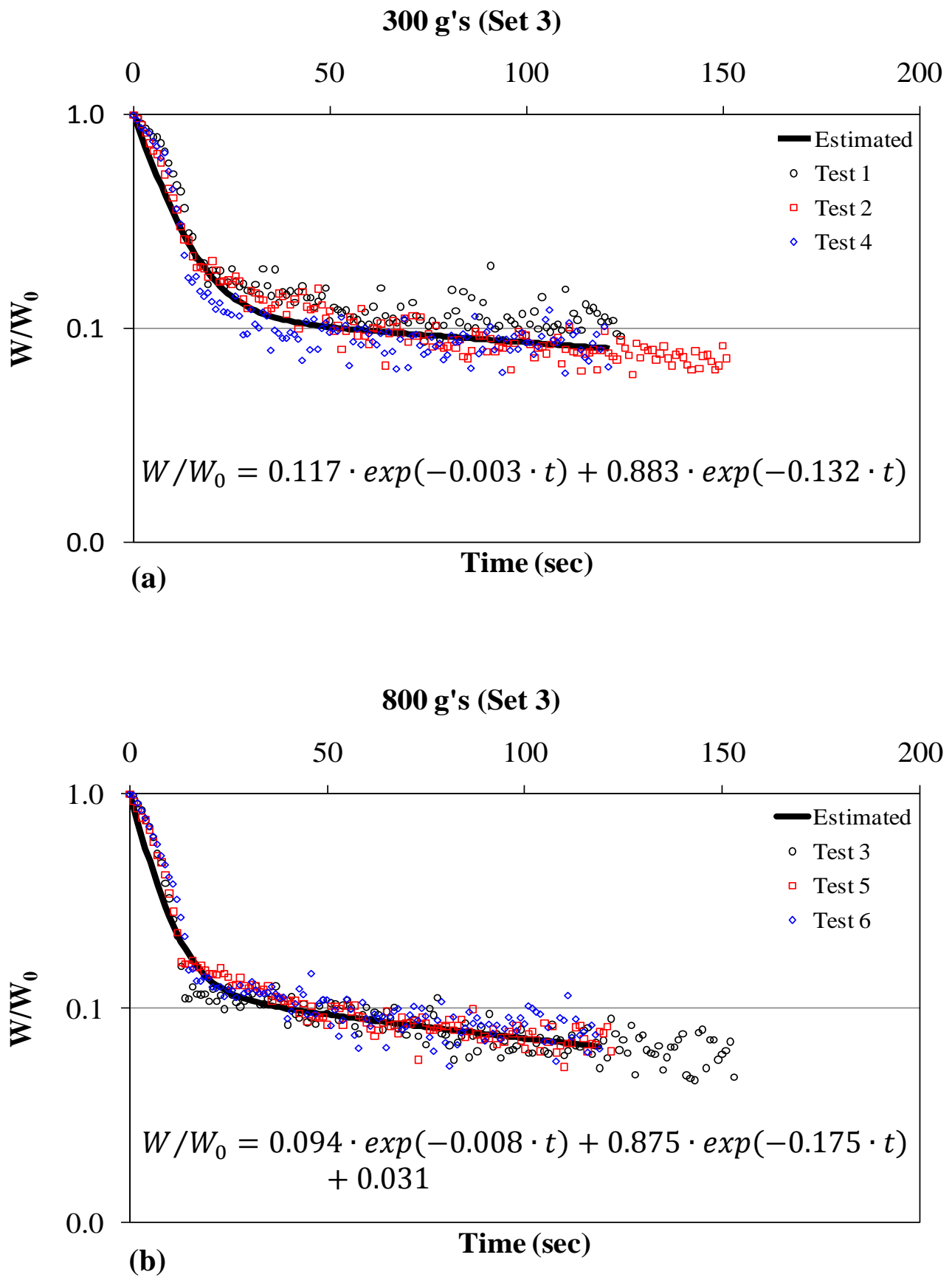

Figure 2.33 Rate curve fits for the third set of kinetic tests at (a) $300 \mathrm{~g}$ 's and (b) $800 \mathrm{~g}$ 's 
As explained in Section 2.3.2.1, the effect of pressurized air injection was studied by measuring cake weight at every 10-20 seconds (Figure 2.14(d) and (f)) at 300 g's. These tests readily demonstrated that air injection provided a further decrease in product moisture. Eq. [2.2] was applied to these data to determine the kinetics of water removal by pressurized air injection during centrifugation. The results given in Figure 2.34 suggest that this process was also a tworate process. The bulk of the water was removed quickly during the first 10 seconds of air injection with a very high rate constant of $9.0 \mathrm{sec}^{-1}$. After 10 seconds, air lost its effectiveness and the rate constant declined dramatically down to about $0.2 \mathrm{sec}^{-1}$. Therefore, these results indicate that injecting air for a short amount of time can be just as efficient as applying air pressure during the entire centrifugation time.

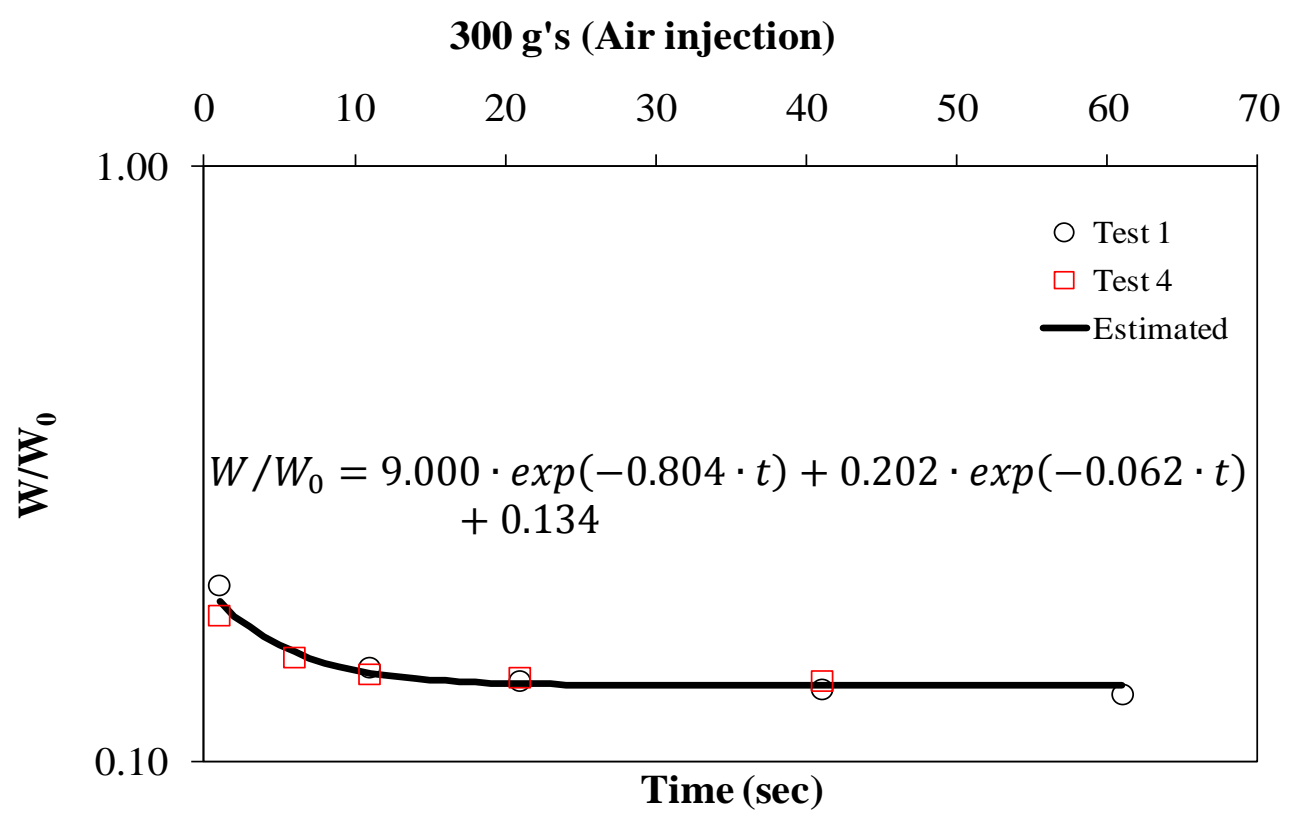

Figure 2.34 Rate curve fit for the air injection part of the kinetic tests at $300 \mathrm{~g}$ 's 


\subsection{Conclusions}

A detailed parametric study of hyperbaric centrifugation was conducted using several laboratory-scale test units. Tests performed with different coal types showed that it was possible to achieve very low moistures using hyperbaric centrifugation. Moreover, tests performed using different size distributions showed that as long as the product contained less than about $60 \%$ minus $0.025 \mathrm{~mm}$ particles, a lower centrifugal acceleration of about $500 \mathrm{~g}$ 's with air pressure was as effective as employing a high centrifugal acceleration of 2700 g's. Therefore, it was concluded that when the fines content of a product was below a certain amount, the use of a high-g centrifuge is probably not necessary. However, when the minus $0.025 \mathrm{~mm}$ particle content was above $60 \%$, the use of a high centrifugal acceleration together with air pressure was found to be essential to achieve good moisture values.

Tests conducted using several surface active dewatering aids and flocculants showed that moisture reductions as much as about 60\% (compared to no-chemical and no-air cases) were possible when dewatering aids were used along with air pressure. Although it was not possible to measure solid recoveries because of the limitations of the test setups, it was visually observed that the use of chemical additives (especially flocculants) vastly improved solids recoveries.

Test results obtained with the modified batch test setup showed that centrifugal dewatering process could be described as a kinetic process. While the bulk of the water was removed quickly from the cake during centrifugation, a smaller amount was removed at a slower rate and a certain amount was not removed at all, which represented the final moisture content of the product. Therefore, it was necessary to employ a rate equation with multiple rate constants to represent the different stages of cake formation and moisture removal. 


\subsection{A PILOT-SCALE STUDY OF HYPERBARIC CENTRIFUGATION}

\subsection{Introduction}

In the light of previous studies done on hyperbaric centrifugation at Virginia Tech (Asmatulu et. al., 2005), a two-stage pilot-scale unit was designed and built. The design of the pilot-scale unit incorporated two integrated processes: (i) filtration in a pressurized rotating filter basket (pressure chamber) with scraper discharge and (ii) slurry pre-thickening and retreatment of recycled fines in an intermittent feed tank. The rotating pressure chamber is mounted along the central axis of a hollow shaft. This shaft is driven by a variable speed motor. A second hollow shaft is located within the drive shaft to allow feed and air to enter the pressure chamber. The second shaft is also connected to two sets of end plates that serve to seal the pressure chamber on one end and to discharge the cake on the other. A conceptual drawing of the rotating filter basket is shown in Figure 3.1. For comparison, a photograph of the assembled pilot-scale unit is provided in Figure 3.2.

\subsection{Experimental}

During a typical production cycle, the basket (see Figure 3.4) is rotated at speeds up to 319 g's using a variable speed motor and drive belt (see Figure 3.5). Slurry from the feed tank is pumped into a distributor pipe located within the center of the shaft of the rotating basket. The basket is constructed using wedge-shaped tungsten carbide bars spaced at $0.1 \mathrm{~mm}$ intervals. All particles larger than the bar spacing are retained along with most of the fines (via entrapment in the cake). The feeding cycle is continued until a desired cake thickness is achieved (Figure 3.3(a)). The feed flow is then diverted back to the feed tank via an automated three-way valve 
(see Figure 3.6). Compressed air is then introduced into the filter chamber to create a differential pressure across the cake. The combined force of the centrifugal field and air pressure drives out nearly all of the surface moisture and some ultrafine solids through the spacing between the basket bars (Figure 3.3(b)). This effluent stream is passed back to the feed tank where the solids are concentrated by flocculation and recycled back to centrifuge to maintain a high recovery of solids. After this pressure drying cycle, the air pressure is released and the cake is discharged by a pneumatically driven scraper (Figure 3.3(c)). The edges of the scraper are sealed by rubber ORings to provide an excellent seal and to serve as a replaceable wear element. After discharge is completed, the scraper returns to its original position and the entire cycle of dewatering is repeated. The entire operation requires 30-120 seconds, depending on the range of operating conditions selected, feed particle size distribution, and target moisture level. The entire sequencing of the feeding, drying and discharge cycles is fully automated and controlled using a programmable logic controller (see Figure 3.7). 


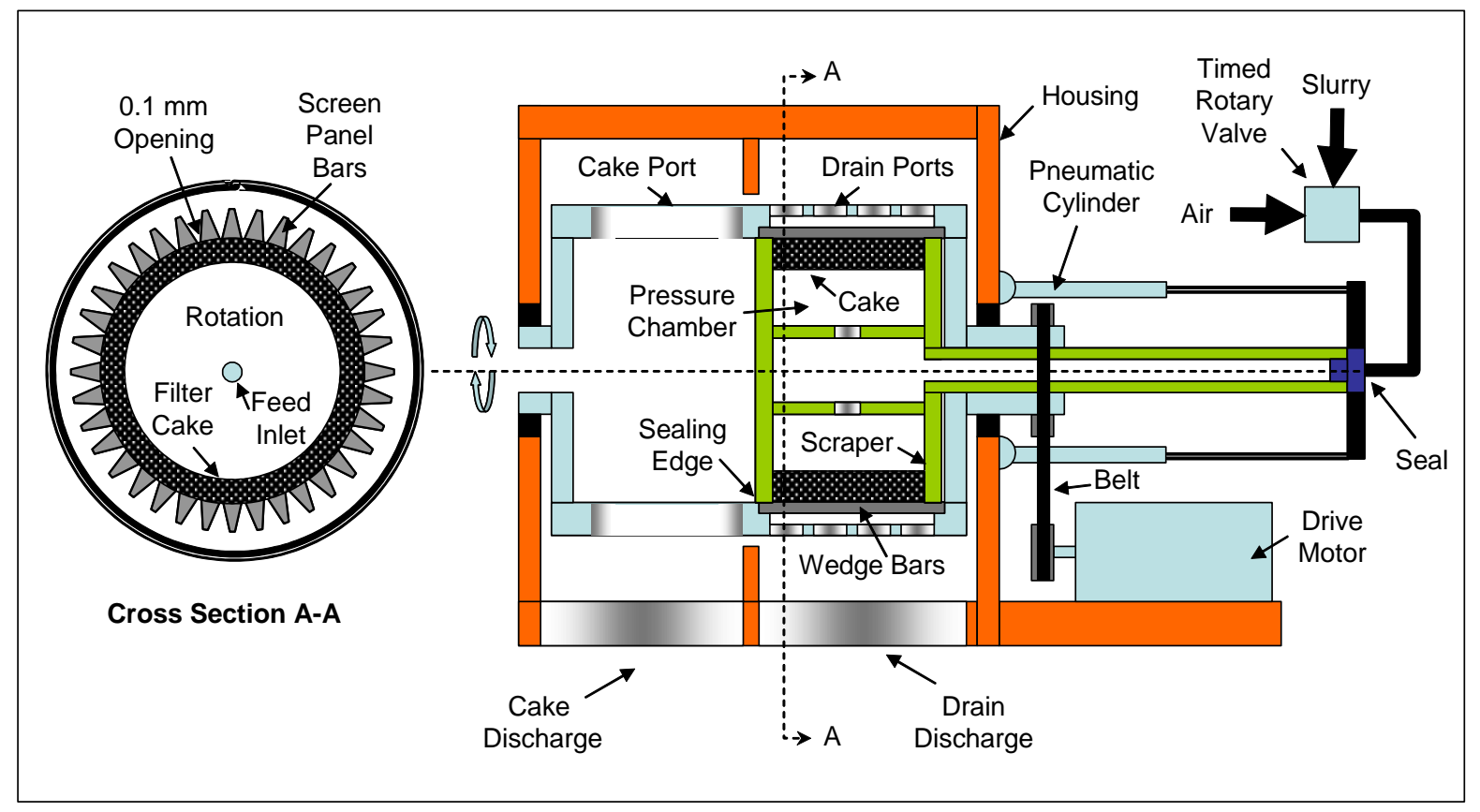

Figure 3.1 Simplified schematic of the hyperbaric filter centrifuge

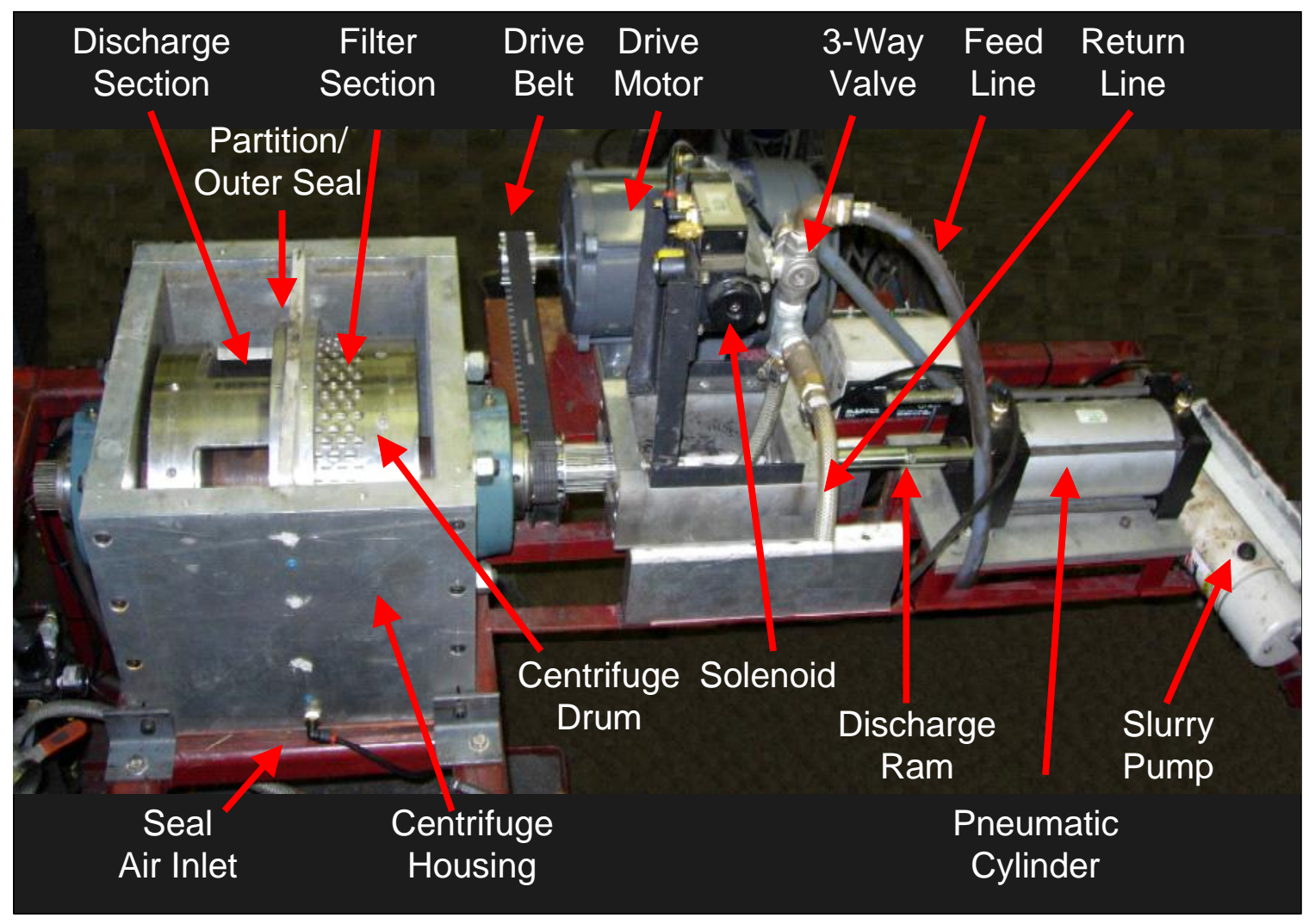

Figure 3.2 HFC with identification labels for key components 

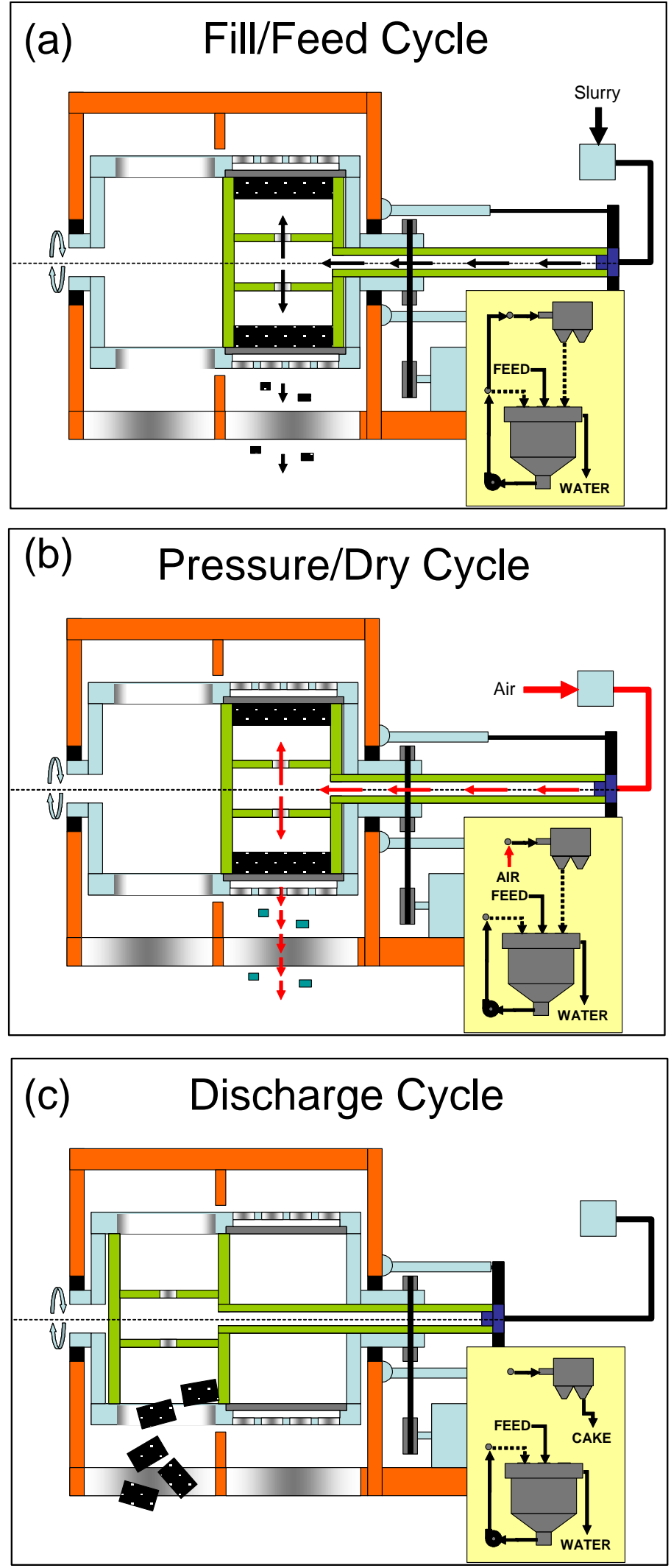

Figure 3.3 Process steps utilized by the hyperbaric filter centrifuge 


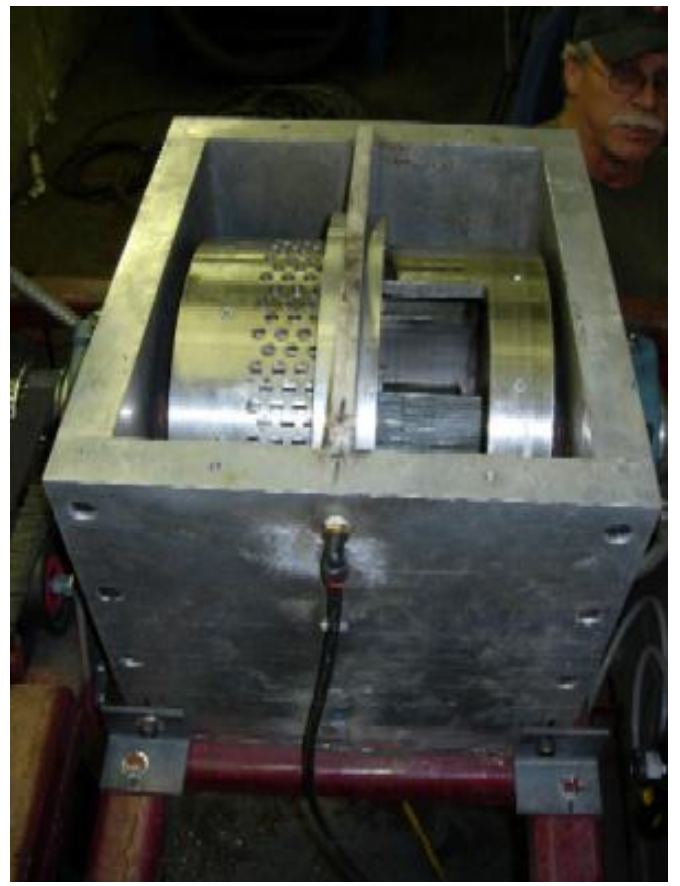

Figure 3.4 Rotating drum assembly for the pilot-scale centrifuge

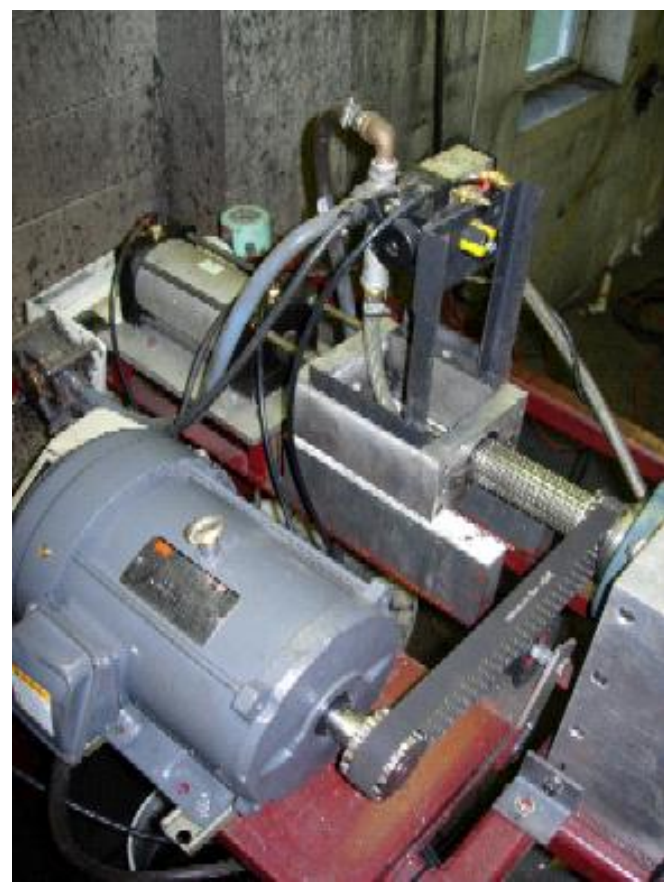

Figure 3.6 Valve system for the introduction of feed slurry and air

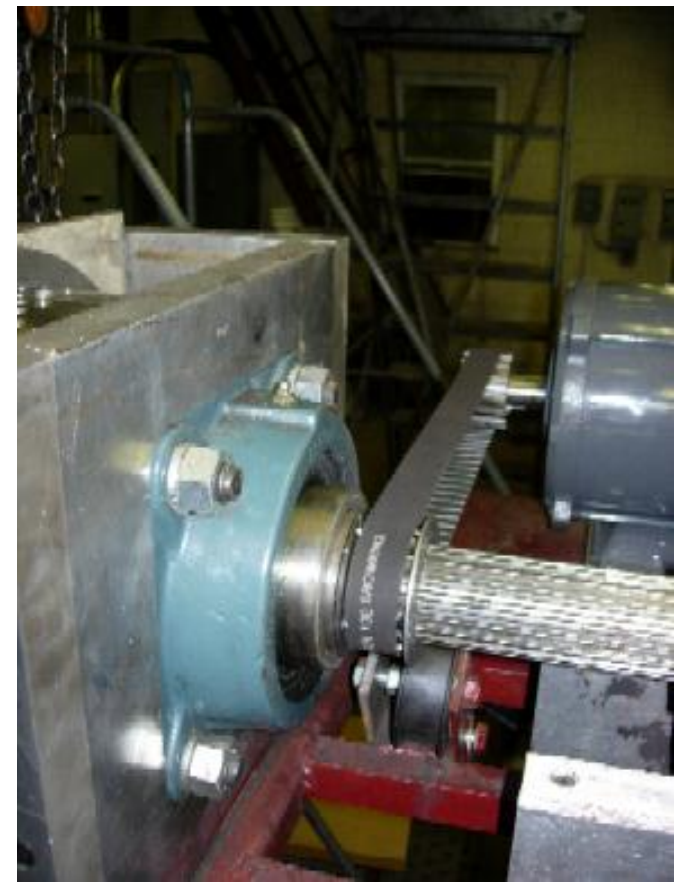

Figure 3.5 Motor, drive belt and shaft assembly for the pilot-scale centrifuge

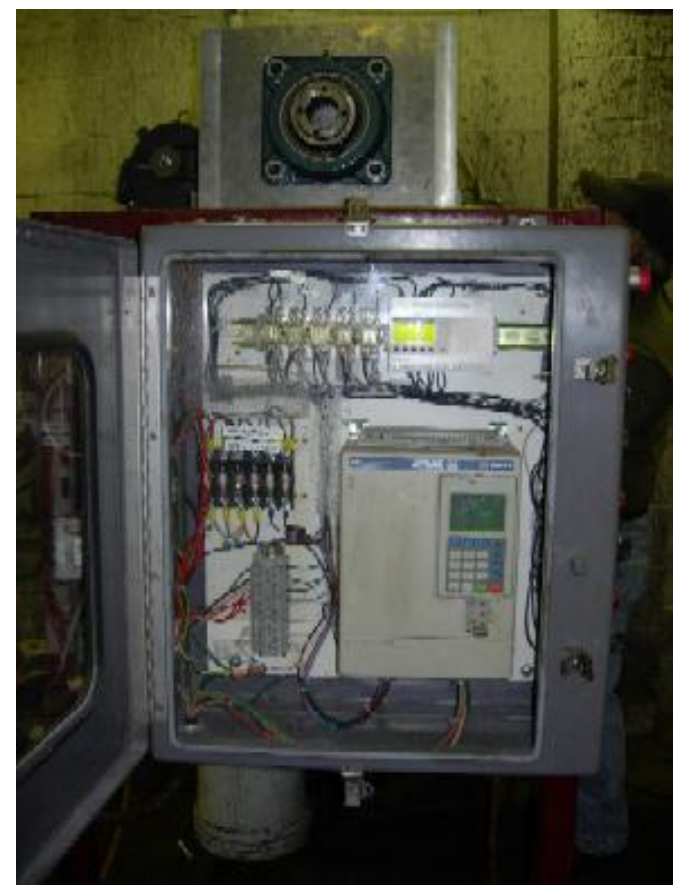

Figure 3.7 Electronic control panel for the pilot-scale centrifuge 
After conducting the tests that are given in sections 3.3.1 through 3.3.3 with the setup mentioned above, the feeding mechanism was modified to allow recycling of the effluent slurry that drains through the screen section back to the feed inlet of the pilot-scale unit. The recycling of the effluent for a certain amount of time was found to be necessary in order to increase the capture of fine solids by the cake. With this system, a known amount of coal slurry was pumped from a 6-inch diameter by 17-inch tall feed tank to the centrifuge unit. Effluent was collected in the same tank and was pumped back to the centrifuge. After the effluent became clear, the valve at the bottom of the tank was closed and pumping stopped.

Coal samples from two different coal plants were used to test the pilot-scale unit. The first sample, which was used with tests described in sections 3.3.1 through 3.3.4, was a nondeslimed screenbowl centrifuge feed (nominally $1 \mathrm{~mm}$ x 0) obtained from Tom's Creek Coal Preparation Plant. The second sample, which was used with the tests mentioned in section 3.3.5, was a deslimed screenbowl centrifuge feed (nominally $1 \mathrm{~mm}$ x $0.15 \mathrm{~mm}$ ) from Pinnacle Pond Reclamation Plant. The size distributions of the samples are given in Table 3.1.

Table 3.1 Size distributions of samples tested with the pilot-scale unit

\begin{tabular}{ccc}
\hline Size & $\begin{array}{c}\text { Tom's Creek } \\
(\boldsymbol{\%})\end{array}$ & $\begin{array}{c}\text { Pinnacle } \\
(\boldsymbol{\%})\end{array}$ \\
\hline$+0.6 \mathrm{~mm}$ & 26.54 & 16.08 \\
$0.6 \times 0.15 \mathrm{~mm}$ & 33.53 & 72.38 \\
$0.15 \times 0.044 \mathrm{~mm}$ & 17.76 & 5.48 \\
$-0.044 \mathrm{~mm}$ & 22.17 & 6.06 \\
\hline
\end{tabular}




\subsection{Results and Discussion}

\subsubsection{Effect of Rotation Speed and Rotation Time}

Filling time, which controls the amount of feed slurry entering the test unit, was kept constant at 15 seconds for all test runs. Unfortunately, the baseline tests (without air injection) could not be run due to plugging of the feed line that occurred when no air was introduced. Therefore, air was on for the whole duration of each test. Tests were performed as a function of centrifugal acceleration (i.e. 142 and 319 g's) and rotation time (i.e. 10, 60 and 120 s).

The results from the pilot-scale tests are summarized in Table 3.2. The data show that the moisture dropped from just over $20 \%$ at the lowest air injection time and rotation speed to below $11 \%$ at the largest air injection time and rotation speed. The data are plotted in Figure 3.8 for comparison. This plot shows that an increase in rotation time from 10 to $120 \mathrm{~s}$ incrementally improved the moisture removal by about 5 percentage points for the lowest centrifugal acceleration of 142 g's. The incremental improvement was somewhat smaller (i.e., about 3 percentage points) for the higher centrifugal acceleration of 319 g's. Moreover, increasing centrifugal acceleration from $142 \mathrm{~g}$ 's to $319 \mathrm{~g}$ 's caused the moisture to decrease about 7 percentage points for the lowest rotation time and 4 percentage points for the highest rotation time.

Table 3.2 Effect of Rotation Speed and Rotation Time

\begin{tabular}{cccc}
\hline $\begin{array}{c}\text { Rotation } \\
\text { Time } \\
(\mathbf{s})\end{array}$ & $\begin{array}{c}\text { Rotation } \\
\text { Speed } \\
(\mathbf{r p m})\end{array}$ & $\begin{array}{c}\text { Centrifugal } \\
\text { Acceleration } \\
\left(\mathbf{g}^{\prime} \mathbf{s}\right)\end{array}$ & $\begin{array}{c}\text { Moisture } \\
\text { Content } \\
(\%)\end{array}$ \\
\hline 10 & 1000 & 142 & 20.2 \\
60 & 1000 & 142 & 18.3 \\
120 & 1000 & 142 & 15.0 \\
\hline 10 & 1500 & 319 & 13.9 \\
60 & 1500 & 319 & 13.2 \\
120 & 1500 & 319 & 10.9 \\
\hline
\end{tabular}




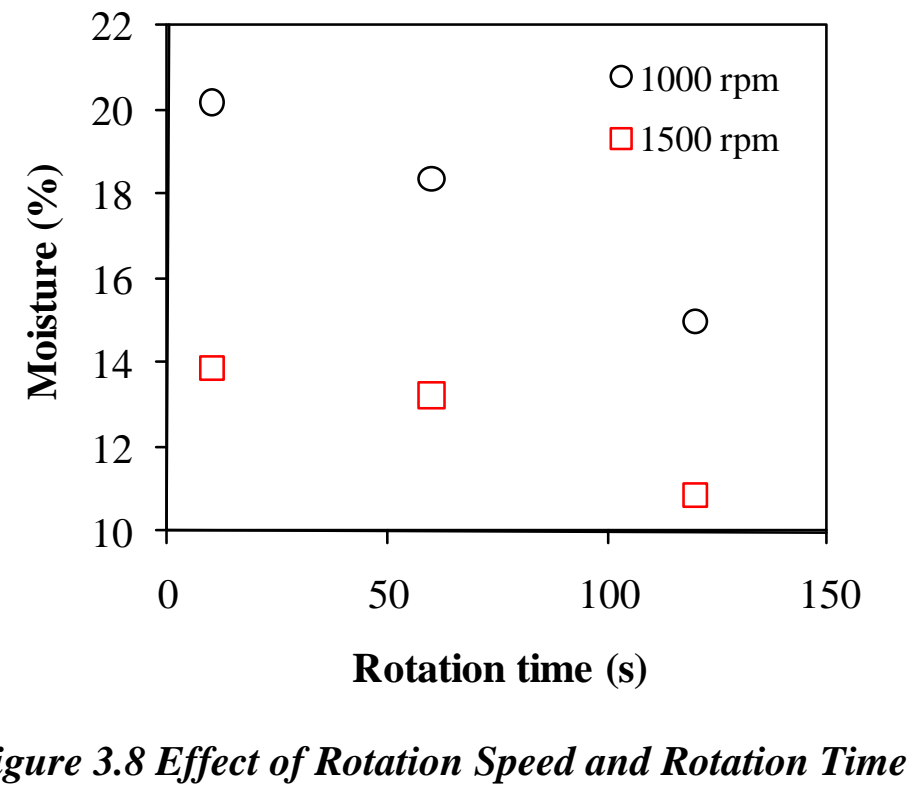

\subsubsection{Effect of Rotation Time and Air Injection}

Several test runs were also conducted to investigate the effects of rotation time (i.e. 5, 10, 30, and $120 \mathrm{~s}$ ) and air pressure (i.e. with and without air) on the performance of the pilot-scale test unit. The centrifugal acceleration was kept at $319 \mathrm{~g}$ 's during these tests. The results are summarized in Table 3.3. A comparison of the data is also provided in Figure 3.9. The test data indicate that the injection of air provided approximately 2 percentage points reduction in moisture with rotation times of 5, 10 and 30 seconds. However, all of the moisture values obtained in these tests were already very low (around $12 \%$ or less). In fact, the values obtained with air injection at centrifugation drying times of $30 \mathrm{~s}$ or greater were all single-digit values. This can be explained by low solid recovery values obtained. Because the coal slurry was not thickened before it was fed to the unit, most of the fine solids were lost during centrifugation. Therefore, only $55-60 \%$ of solids were recovered during all the tests. The absence of fines drove moisture values down to below $12 \%$. Moreover, since moisture values obtained at $120 \mathrm{~s}$ rotation 
time with and without air were similar (i.e. 9.7\%, 9.6\%), the effect of air injection on moisture may have diminished due to long rotation time.

Table 3.3 Effect of rotation time and air injection

\begin{tabular}{cccc}
\hline $\begin{array}{c}\text { Rotation } \\
\text { Time } \\
(\mathbf{s})\end{array}$ & $\begin{array}{c}\text { Air } \\
\text { Injection }\end{array}$ & $\begin{array}{c}\text { Moisture } \\
\text { Content } \\
(\boldsymbol{\%})\end{array}$ & $\begin{array}{c}\text { Solid } \\
\text { Recovery } \\
(\boldsymbol{\%})\end{array}$ \\
\hline 5 & No & 12.6 & 56.37 \\
10 & No & 11.2 & 58.34 \\
30 & No & 10.8 & 57.15 \\
120 & No & 9.6 & 55.79 \\
\hline 5 & Yes & 10.6 & 60.76 \\
10 & Yes & 10.3 & 60.61 \\
30 & Yes & 9.6 & 60.46 \\
120 & Yes & 9.7 & 59.17 \\
\hline
\end{tabular}

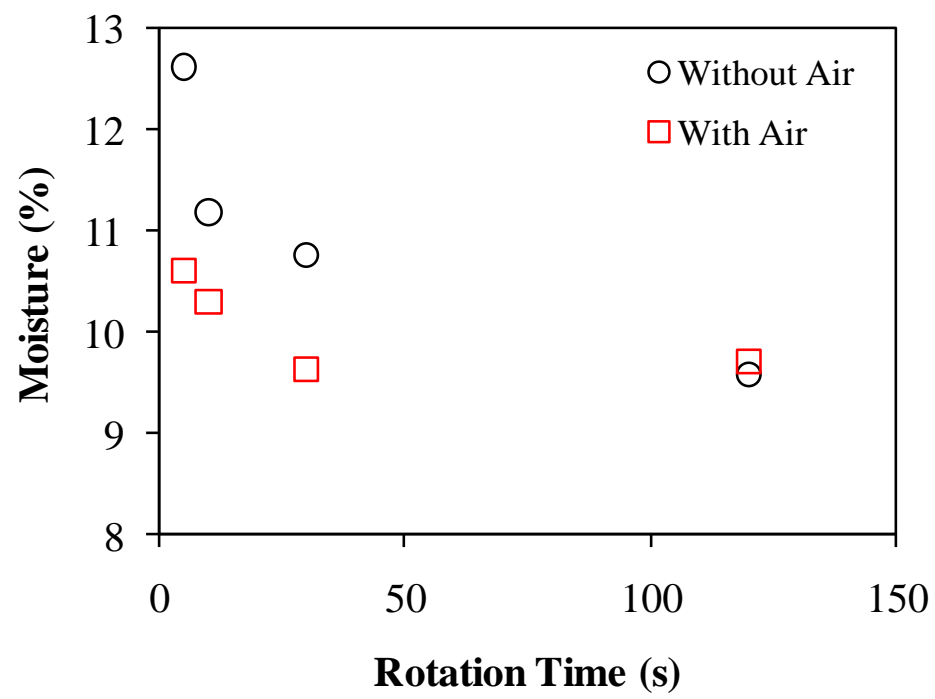

Figure 3.9 Effect of rotation time and air injection on product moisture

\subsubsection{Effect of Thickening}

Test results given in the previous section showed that only low solid recoveries could be achieved when dilute coal slurry was directly fed to the centrifuge. Therefore, for the next set of tests, the coal slurry was thickened by addition of an anionic flocculant before it was fed to the 
centrifuge. Although, a more extensive test program with several cake thicknesses and with and without air injection was planned for this section, only a fraction of these tests could actually be conducted because of several mechanical failures occurred during testing. Tests were conducted as a function of centrifugal acceleration (i.e. $142 \mathrm{~g}$ 's and $319 \mathrm{~g}$ 's), rotation time (i.e. 15, 30, 60 and 120 s) and air pressure (i.e. with and without air).

The test results given in Table 3.4 and Figure 3.10 show that the moisture of $18.7 \%$ obtained at the shortest rotation time of 15 seconds with 142 g's reduced to $16.1 \%$ when the centrifugal acceleration increased to 319 g's. A further moisture reduction down to $13.2 \%$ was achieved when air was injected. While the same trend continued at the other rotation speeds, all moisture values improved as the rotation time increased. At the longest rotation time of 120 seconds, $14.1 \%$ moisture value obtained with $142 \mathrm{~g}$ 's reduced down to $10.0 \%$ at $319 \mathrm{~g}$ 's with air injection.

As a result of thickening the feed slurry, higher solid recoveries of more than $90 \%$ were obtained. Since no effluent sample was collected during testing, solid recovery values were

Table 3.4 Equilibrium moistures and solids recoveries obtained when samples were thickened

\begin{tabular}{cccccc}
\hline $\begin{array}{c}\text { Rotation } \\
\text { Time } \\
(\mathbf{s})\end{array}$ & $\begin{array}{c}\text { Rotation } \\
\text { Speed } \\
(\mathbf{r p m})\end{array}$ & $\begin{array}{c}\text { Centrifugal } \\
\text { Acceleration } \\
(\mathbf{g} \mathbf{s})\end{array}$ & $\begin{array}{c}\text { Air } \\
\text { Injection }\end{array}$ & $\begin{array}{c}\text { Moisture } \\
\text { Content } \\
(\boldsymbol{\%})\end{array}$ & $\begin{array}{c}\text { Solid } \\
\text { Recovery } \\
(\boldsymbol{\%})\end{array}$ \\
\hline 15 & 1000 & 142 & No & 18.7 & 92.9 \\
30 & 1000 & 142 & No & 16.3 & 97.5 \\
60 & 1000 & 142 & No & 15.1 & 96.0 \\
120 & 1000 & 142 & No & 14.1 & 96.2 \\
\hline 15 & 1500 & 319 & No & 16.1 & 68.6 \\
30 & 1500 & 319 & No & 14.6 & 89.4 \\
60 & 1500 & 319 & No & 13.0 & 88.4 \\
120 & 1500 & 319 & No & 12.3 & 73.7 \\
\hline 15 & 1500 & 319 & Yes & 13.2 & 98.6 \\
30 & 1500 & 319 & Yes & 11.9 & 72.5 \\
60 & 1500 & 319 & Yes & 11.2 & 91.4 \\
120 & 1500 & 319 & Yes & 10.0 & 96.8 \\
\hline
\end{tabular}


calculated by dividing the dry cake weight by dry feed weight. However, this approach created potentially inaccurate recovery values, because feed samples were not taken for all the tests. Therefore, although the solid recoveries seem to be generally improved by thickening, the accuracy of these values is arguable.

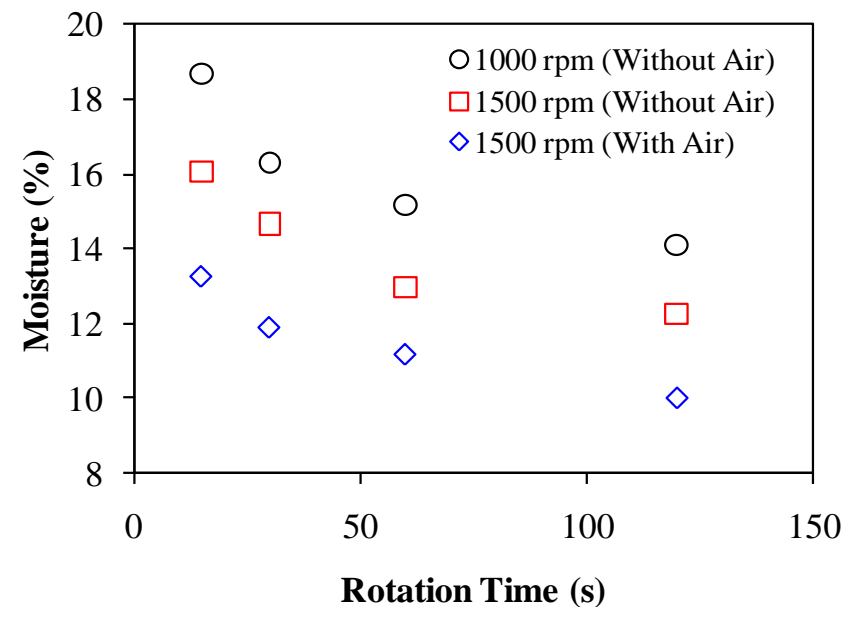

Figure 3.10 Effect of thickening on product moisture

\subsubsection{Effect of Feed Size Distribution}

Screenbowl centrifuges are widely used in coal industry to dewater fine coal feeds containing nominally $1 \mathrm{~mm}$ x 0 size particles. However, a screenbowl centrifuge can only recover about $50 \%$ of the minus $0.044 \mathrm{~mm}$ size fraction. Therefore, one of the expectations from the hyperbaric centrifuge technology was the ability of dewatering the minus $0.044 \mathrm{~mm}$ size fraction effectively while keeping solid recoveries high. To examine this possibility, the effect of feed size distribution was one of the major parameters examined in the pilot-scale tests. Unfortunately, all the test attempts with finer size feeds failed due to several factors. First of all, when a feed finer than $0.5 \mathrm{~mm}$ was used, all the particles passed through the screen openings and a cake could not be formed. Secondly, although flocculant addition seemed to be effective with 
the coarser feed as explained in the previous section, the sizes of the particle aggregates formed with finer feeds were not sufficiently big to facilitate a cake formation. Even though a shearresistant flocculant was used, the high shear forces introduced by the feed pump caused these particle aggregates to break, thus they were not able reach the size needed for cake formation. Therefore, the recirculation of the screen effluent appeared to be the only viable option for improving solids recoveries.

\subsubsection{Effect of Recirculation}

After running the tests that were given in previous sections, the test setup was changed to allow recirculation of screen effluent back to the feed port. This was done using the feeding arrangement explained in section 3.2. Recirculation was considered to be necessary to maximize the solid recovery. First, the non-deslimed Tom's Creek sample (Table 3.1) was tested. However, none of the tests could be completed, because recirculation caused fine particles to form a slime coating atop the cake. This coating prevented the passage of pressurized air through the cake, thus causing the airline to plug and burst several times. Moreover, using a flocculant to form larger agglomerates did not work either, since these weak agglomerates broke after passing through the feed pump several times. Consequently, it was decided to use a deslimed sample to see the effect of recirculation. The results are described in the next section.

\subsubsection{Effect of Desliming}

These tests were conducted using the deslimed screenbowl feed obtained from the Pinnacle Pond Reclamation plant. The size distribution of the sample is given in Table 3.1. The centrifugal acceleration was maintained at 319 g's for all tests. After 30 seconds of recirculation, the cake was centrifuged for an additional 30 seconds before ending the test run. The test results 
given in Table 3.5 indicate that the use of the deslimed sample, which contained very few particles finer than $0.044 \mathrm{~mm}$, resulted in very low values of cake moisture (i.e., 5-7\%). Also, by recycling the effluent, very high solid recoveries were achieved (i.e., 97-99\%). Moreover, injecting air at different pressures lowered the moistures by 1-2 percentage points compared to the baseline values. Therefore, single-digit moistures could be achieved by desliming ahead of the filter centrifuge.

Table 3.5 Effect of desliming and recirculation

\begin{tabular}{ccc}
\hline $\begin{array}{c}\text { Air } \\
\text { Pressure } \\
(\mathbf{k P a})\end{array}$ & $\begin{array}{c}\text { Moisture } \\
\text { Content } \\
(\boldsymbol{\%})\end{array}$ & $\begin{array}{c}\text { Solid } \\
\text { Recovery } \\
(\boldsymbol{\%})\end{array}$ \\
\hline 0 & 6.9 & 97.7 \\
93 & 5.9 & 97.2 \\
107 & 5.6 & 99.1 \\
133 & 5.1 & 97.4 \\
\hline
\end{tabular}

\subsection{Discussion}

\subsubsection{Solids Recovery and Product Moisture}

The tests conducted with the pilot-scale unit showed that thickening the feed slurry is a necessity for achieving high solid recoveries. Also, recirculation of the effluent stream back to the feed stream gave a chance to fine particles to be captured by the cake, thus increasing the recovery of solids. However, increased solid recovery caused product moisture to escalate about 2.7 to 5.4 percentage points depending on centrifugation period (see Table 3.3 and Table 3.4). Considering the increased moisture values were still below $20 \%$ and higher recoveries will help with the capacity requirements, the somewhat higher moisture values were found to be acceptable. 


\subsubsection{Engineering Criteria for Machine Design}

Ability of running a continuous operation is essential for maximizing the capacity in industrial applications. However, the design of the pilot-scale unit did not allow for a truly continuous operation. First of all, it was not possible to feed the unit continuously because of the unit's cyclic operation procedure that was explained in section 3.2. Secondly, coal slurry had to be thickened outside and pumped into the unit, which caused problems with flocculation since the agglomerates broke due to the high shear forces inside the feed pump.

The pilot-scale unit consisted of two sections, a screen section, which was also used as a pressure chamber, and a discharge section. The need for thickening the slurry outside the unit could be eliminated by adding a third "solid" section to the rotating unit, which is similar to the solid-bowl part of a screenbowl centrifuge. Therefore, coal slurry would be fed directly into the unit and be thickened inside. Integrating the thickening operation to the centrifuge would have many advantages over using a conventional thickener. First of all, the space required for a thickening tank would be eliminated. Also, thickening can be done much faster and more efficiently with centrifugation. Furthermore, feeding slurry directly to the unit can effectively reduce the amount of chemicals needed for thickening. Also, since the cake would already be formed in the solid section, the problems encountered with cake formation using fine particles can be overcome. Addition of a third section to the centrifuge unit would not eliminate the need for a feed tank because of the semi-continuous nature of the unit. However, a smaller buffer tank can be used instead of a wide thickening tank. The only downside to using a solid bowl section as part of the hyperbaric centrifuge is that it would need to operate at a very high speed to provide the centrifugal forces necessary to capture ultrafine solids. Calculations suggest that the 
required centrifugal field is likely to be in the order to 2,000 to $3,000 \mathrm{~g}$ 's for the effective capture of minus $0.044 \mathrm{~mm}$ solids now lost in standard screenbowl machines operating at $500 \mathrm{~g}$.

\subsection{Conclusions}

A pilot-scale centrifuge that utilizes pressurized air was built and tested under several conditions. The results showed that higher rotational speeds and longer centrifugation times were needed for producing lower moistures. Also, injecting pressurized air through the cake further improved the moisture.

It was also concluded that thickening was an essential step for centrifugal dewatering, as it increased the recovery of solids. Also, it was found that solids recovery could be further increased when effluent stream was re-circulated back to the feed stream. However, this exercise caused a slime coating to form atop the cake when a non-deslimed feed was used due to the breakage of flocculated agglomerates inside the feed pump. Integration of the thickening process via the addition of a solid bowl section during centrifugation can help with this problem by eliminating the need for this step.

Originally, a more detailed and a complete parametric study of the pilot-scale unit was planned. However, all the attempts for running complete series of tests were unsuccessful due to the fundamental operational problems. For example, the hollow shaft that was used for material feeding and other feed lines and valves could not be designed properly for such a small machine to allow an easy passage of the typical feed material $(1 \mathrm{~mm} \times 0)$. As a result, the lines were plugged several times during testing and led to some failures. Due to the mechanical and design problems faced, it was decided pursue the development of a prototype machine that would include both solid- and screen-bowl sections. 


\subsection{FIELD TESTING OF A PROTOTYPE HYPERBARIC CENTRIFUGE}

\subsection{Introduction}

The laboratory and pilot-scale test programs provided sufficient promising data to warrant the construction of a commercial prototype of the Centribaric ${ }^{\mathrm{TM}}$ technology. The one-ofa-kind machine was designed and fabricated by Decanter Machine, Inc., a well-known manufacturer of solid- and screen-bowl centrifuges. The prototype unit was tested at several industrial sites by Decanter Machine. Virginia Tech participated in one of these test programs at Cardinal Plant of Arch Coal.

Cardinal Plant (Figure 4.2) is located near the town of Sharples in Logan County, West Virginia. Plant production is intended mainly for steam coal market. The plant has three 700 tph capacity modules. Each module employs single unit operations. Multiple modules provide

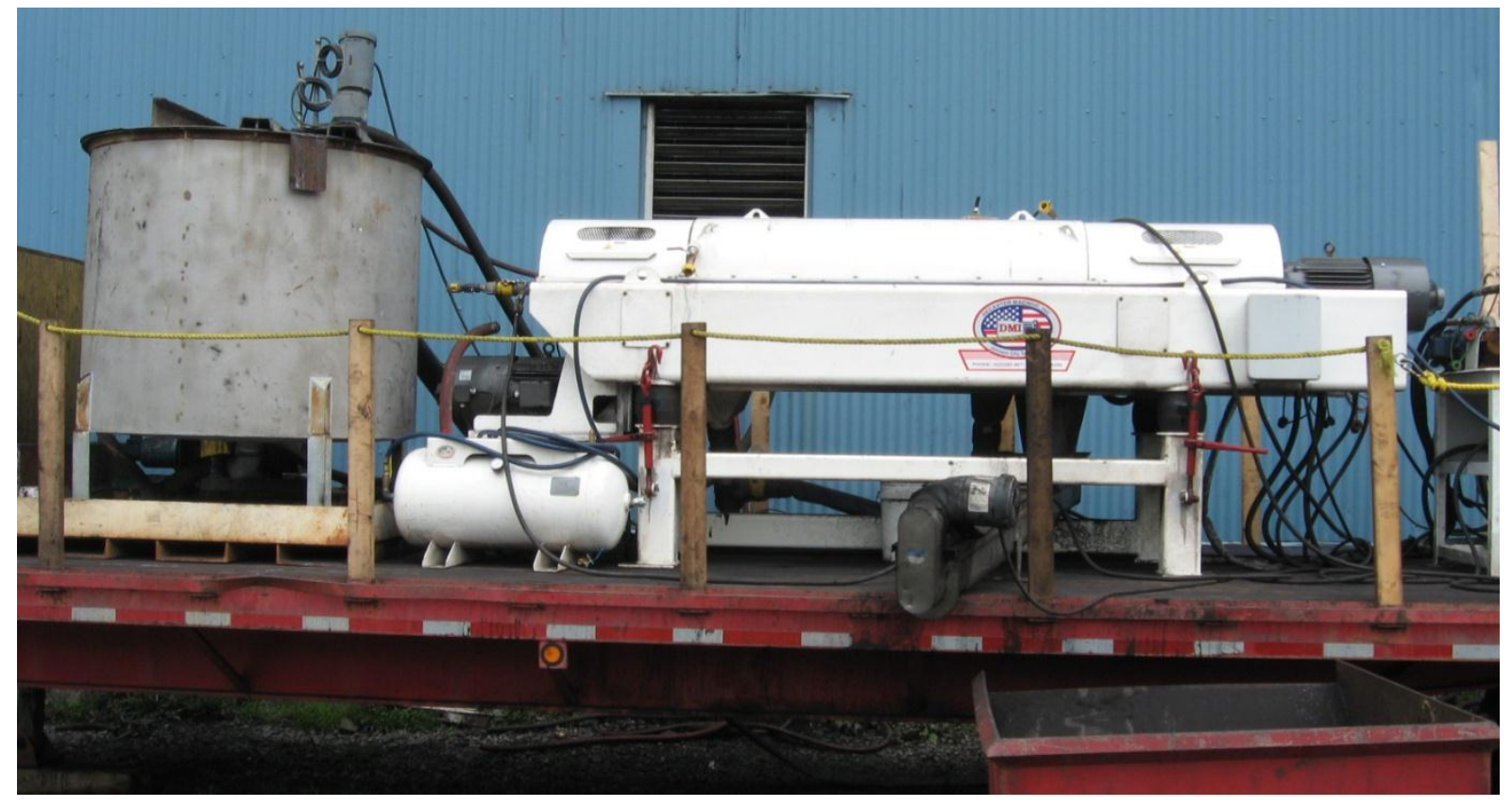

Figure 4.1 Prototype hyperbaric centrifuge of Decanter Machine 


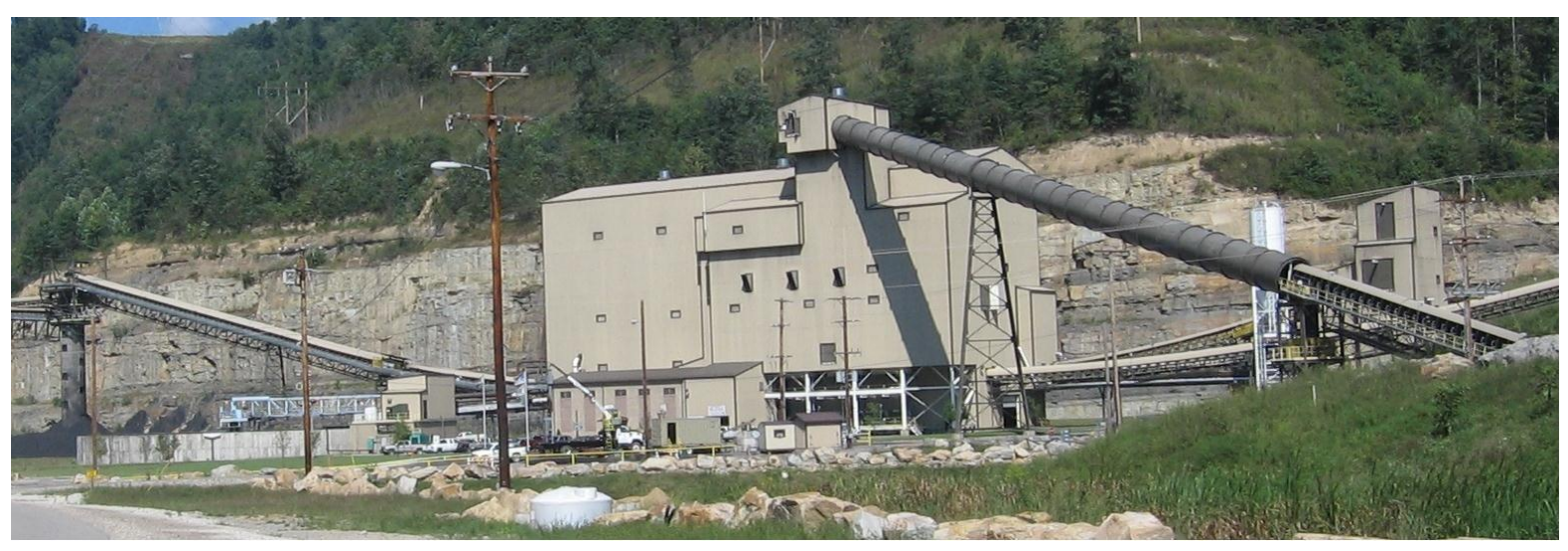

Figure 4.2 Cardinal Plant

flexibility of running with one or more modules being idled for maintenance. As shown in Figure 4.3, each module has a 10x20-ft double deck banana screen, which is used as a combined raw coal/deslime screen, cutting at 0.5 -inch on the top deck and $1 \mathrm{~mm}$ on the bottom deck. The coarse material then goes to a dense medium vessel for density separation. Compound spirals are incorporated for treating 1 x $0.2 \mathrm{~mm}$ size fraction, while column flotation is used to clean deslimed fine coal (0.25 x $0.044 \mathrm{~mm})$. Finally, combined deslimed spiral/column concentrate is dewatered by $42 x 144$-inch screenbowl centrifuges. A single thickener is used to treat plant tailings. Thickener underflow is pumped to an established impoundment, while the coarse refuse is hauled to a separate coarse refuse area. (Bethell and DeHart, 2006) 


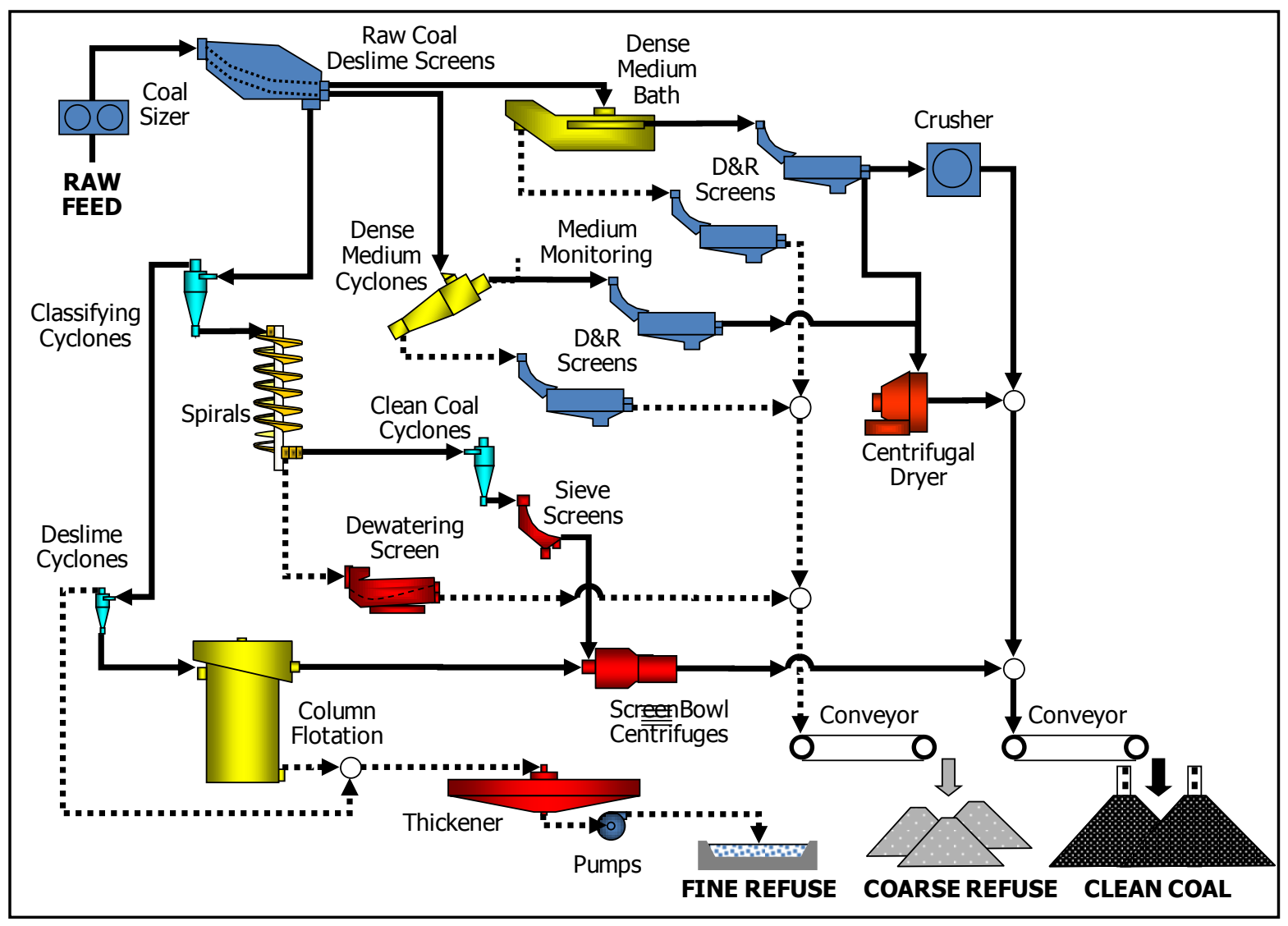

Figure 4.3 Simplified flowsheet of Cardinal Plant (Single Module)

\subsection{Experimental}

\subsubsection{Machine Description}

The prototype was designed to handle a volumetric capacity of 120 liter/minute of feed slurry. The prototype unit and all ancillary components were trailer-mounted so the equipment could be easily relocated to different industrial sites (Figure 4.1). The mobile platform included space for a 1,750 liter feed storage tank, tank mixer, slurry pump, gas compressor, discharge collection bin, effluent pump/sump and electrical control systems. While a detailed description of the feed/discharge system, sealing mechanisms and solids transport assemblies cannot be discussed due to the proprietary nature of this patent-pending technology, the basic concept is 
similar to other centrifugal dewatering processes in terms of applicability, operation and maintenance. (Figure 4.4).

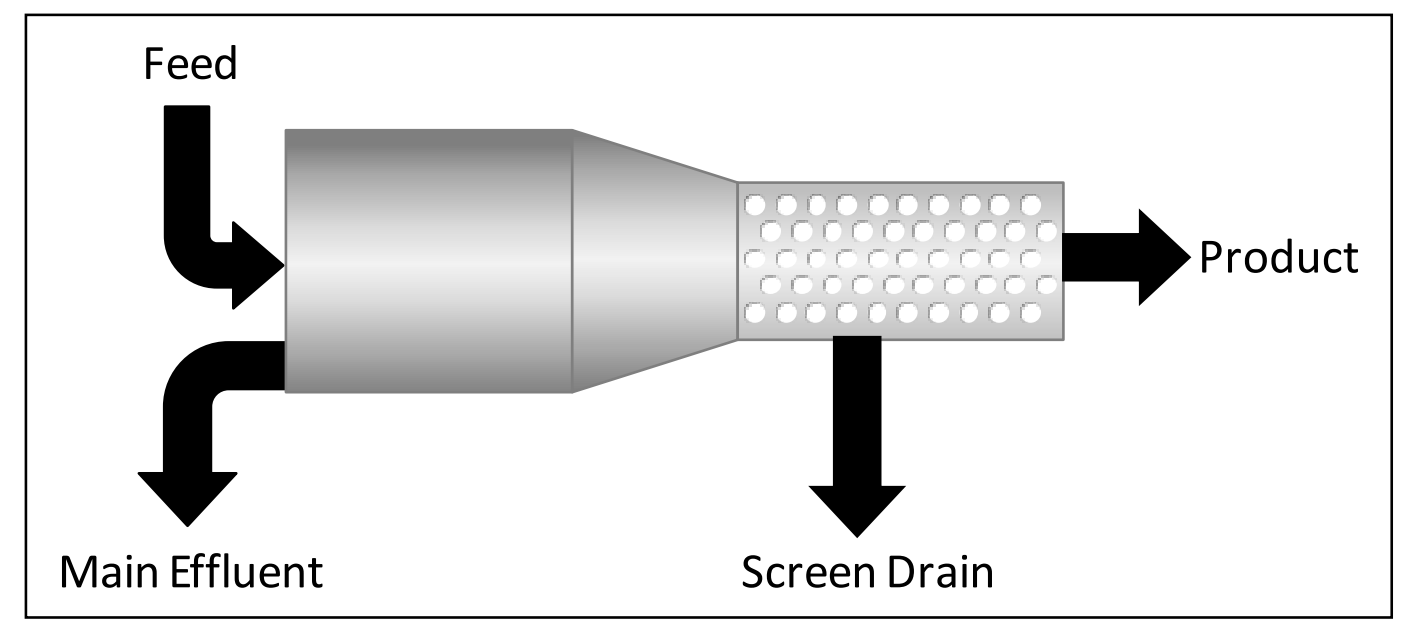

Figure 4.4 Diagram of the prototype hyperbaric centrifuge

\subsubsection{Test Procedure}

In total, seven different feed mixture combinations were evaluated at this particular test site. The coal used as the feed to the test unit was Alma A coal from the Mountaineer II Mine. The 6-inch cyclone overflow stream (minus $0.044 \mathrm{~mm}$ ) of the plant was floated with a 20inchx10-ft pilot scale flotation column, and it was mixed in various proportions, which are given in Table 4.1, with the plant's screenbowl centrifuge screen drain to prepare the feed for every batch. Note that a progressively finer feed stream was used in each subsequent test run. This feeding arrangement made it possible to investigate the effects of a wide range of particle size distributions on dewatering performance and solid/slurry throughput capacity. The mixture was kept agitated in a holding tank during the tests. For the last test, instead of screenbowl screen drain, plant's column flotation concentrate (nominal 0.25 x $0.044 \mathrm{~mm}$ ) was mixed with leftover slurry from the previous test. 
Table 4.1 Feed Mixtures

\begin{tabular}{cc}
\hline Test & Mixing Ratio \\
\hline 1 & $50 \%$ Column concentrate / 50\% Screenbowl screen drain \\
2 & $60 \%$ Column concentrate / 40\% Screenbowl screen drain \\
3 & $70 \%$ Column concentrate / 30\% Screenbowl screen drain \\
4 & $80 \%$ Column concentrate / 20\% Screenbowl screen drain \\
5 & $90 \%$ Column concentrate / 10\% Screenbowl screen drain \\
6 & $100 \%$ Column concentrate / 0\% Screenbowl screen drain \\
7 & NA\% Column concentrate / NA\% Plant column concentrate \\
\hline
\end{tabular}

Timed samples from feed, product, screen effluent and main effluent streams were taken during test runs. While the entire product and screen effluent streams were collected for each test, only 2-4 cuts were taken from feed and main effluent streams to fill up two 5 gallon buckets for each stream. After cutting the sample from the main effluent, a full stream sample of the feed was taken by diverting the feed stream from the centrifuge to a bucket. Feed stream was redirected back to the centrifuge and sufficient time was given to reach steady-state before cutting the next set of samples. The holding tank was filled up before every test at the desired mixing ratio. All the samples were sealed after each test to prevent any evaporation. Analyses of the samples were done by a commercial laboratory. Size distributions and ash data for all the feeds are given in Table 4.2 and Table 4.3, respectively.

Table 4.2 Feed size distributions for all tests

\begin{tabular}{cccccc}
\hline \multicolumn{5}{c}{ Weight $(\mathbf{\%})$} \\
\hline & $\mathbf{+ 0 . 5}$ & $\begin{array}{c}\mathbf{0 . 5} \mathbf{~ 0 . 1 5} \\
\mathbf{m m}\end{array}$ & $\begin{array}{c}\mathbf{0 . 1 5} \mathbf{x ~ 0 . 0 4 4} \\
\mathbf{m m}\end{array}$ & $\begin{array}{c}\mathbf{0 . 0 4 4} \mathbf{x} \mathbf{0 . 0 2 5} \\
\mathbf{m m}\end{array}$ & $\begin{array}{c}\mathbf{- 0 . 0 2 5} \\
\mathbf{m m}\end{array}$ \\
\hline 1 & $\mathbf{m m}$ & 26.08 & 20.47 & 0.58 & 36.24 \\
2 & 16.63 & 17.56 & 23.28 & 7.35 & 41.30 \\
3 & 10.51 & 14.63 & 22.53 & 8.78 & 45.99 \\
4 & 8.07 & 9.97 & 21.45 & 9.20 & 53.86 \\
5 & 5.52 & 7.37 & 16.58 & 11.13 & 62.15 \\
6 & 2.77 & 1.40 & 20.42 & 3.70 & 74.30 \\
7 & 0.18 & 0.44 & 3.20 & 1.89 & 94.46 \\
\hline
\end{tabular}


Table 4.3 Feed ash values for all tests

\begin{tabular}{cccccc}
\hline \multicolumn{6}{c}{ Ash (\%) } \\
\hline Test & $\mathbf{+ 0 . 5}$ & $\begin{array}{c}\mathbf{0 . 5} \mathbf{x ~ 0 . 1 5} \\
\mathbf{~ m m}\end{array}$ & $\begin{array}{c}\mathbf{0 . 1 5} \mathbf{x ~ 0 . 0 4 4} \\
\mathbf{~ m m}\end{array}$ & $\begin{array}{c}\mathbf{0 . 0 4 4} \mathbf{x ~ 0 . 0 2 5} \\
\mathbf{~ m m ~}\end{array}$ & $\begin{array}{c}\mathbf{- 0 . 0 2 5} \\
\mathbf{~ m m}\end{array}$ \\
\hline 1 & 7.65 & 5.43 & 6.68 & 20.50 & 10.21 \\
2 & 10.59 & 5.88 & 5.41 & 7.68 & 7.83 \\
3 & 8.60 & 6.27 & 6.47 & 8.97 & 8.12 \\
4 & 10.83 & 6.80 & 5.29 & 6.55 & 7.94 \\
5 & 12.90 & 7.30 & 4.28 & 5.03 & 6.86 \\
6 & 29.09 & 7.54 & 2.33 & 3.25 & 6.67 \\
7 & 28.65 & 4.61 & 3.60 & 3.85 & 7.19 \\
\hline
\end{tabular}

\subsubsection{Performance Analysis}

To create a reliable set of data out of the raw data obtained from the laboratory, a steadystate mass balance routine that was set up in an Excel spreadsheet was used. This routine makes the least possible change to the measured values, so that the mass of components (i.e. solid, water, ash, etc.) entering the unit is equal to the mass of components leaving the unit. Different amounts of confidence were given to measured values, using weighted sum-of-squares, to be able to let the routine selectively adjust these values.

It was observed that the feed flow rates calculated from the timed feed samples were higher than the combination of product and effluent stream flow rates. Even though the feed pump was a positive displacement type, it was assumed that there was enough wear on the rotor of the pump that allowed surging while it was operating under pressure. Whereas, when the full feed stream was diverted from the centrifuge to a bucket during sampling, resulting pressure drop on the pump caused the flow rate to increase. Therefore, very little dependence was put on the measured feed flow rate numbers when mass balancing. Also, according to the laboratory analysis, $0.044 \times 0.025 \mathrm{~mm}$ size fractions of every sample had unrealistically small mass values compared to the upper and lower size fractions. When mass balancing was tried, the routine 
made large changes to these values. The coarsest size fraction values were also substantially changed by the mass balance routine. Therefore, these size fractions were combined with other size fractions to obtain a more reliable size distribution. As a result, the input parameters used for mass balances are given below:

- Size distribution (plus $0.15 \mathrm{~mm}, 0.15 \times 0.025 \mathrm{~mm}$ and minus $0.025 \mathrm{~mm}$ )

- Ash contents (\%) of plus $0.15 \mathrm{~mm}, 0.15$ x $0.025 \mathrm{~mm}$ and minus $0.025 \mathrm{~mm}$ size fractions

- Solids content (\%, by weight)

- Slurry rate $(\mathrm{kg} / \mathrm{hr})$

Since screen drain stream was not re-circulated back to the feed stream during testing, there was no experimental data representing the effect of circulating load. To simulate recirculation, combinations of screen drain and feed for every test were calculated by iteration in Excel. New moisture values were predicted using the polynomial curve that was fit to the experimental data (Figure 4.5). Details of the calculations are given in Appendix 1.

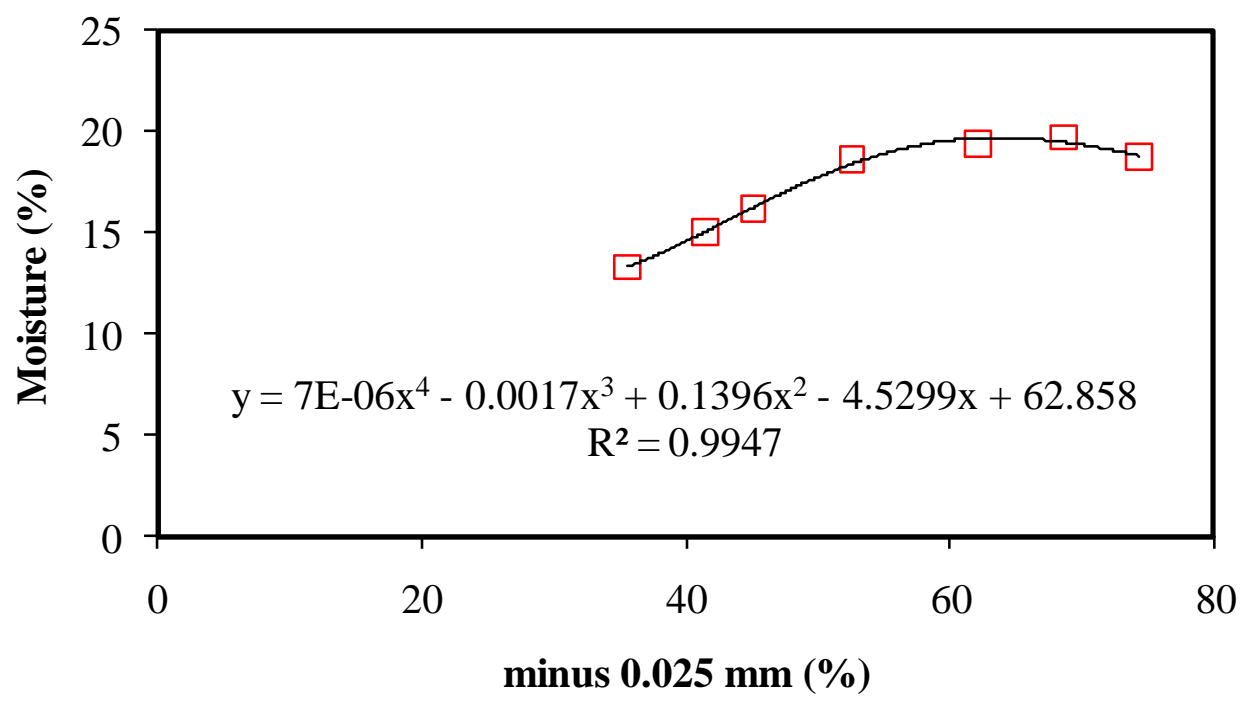

Figure 4.5 Effect of minus $0.025 \mathrm{~mm}$ particle amount on cake moisture 


\subsection{Results}

Summary of test results is given in Table 4.4. As can be seen, a low moisture value of $13.7 \%$ was achieved at the first test run. This particular run employed a relatively coarse feedstock prepared using a 50/50 mixture of screenbowl screen drain (minus $0.5 \mathrm{~mm}$ ) and column flotation concentrate (minus $0.044 \mathrm{~mm}$ ). From this baseline test run, the moisture climbed up to $19.4 \%$ as the mass percent of ultra-fines (minus $0.025 \mathrm{~mm}$ ) increased to its maximum value of $94.5 \%$ by weight. The fact that the moisture in the final run was still below $20 \%$ was considered to be very impressive given the extreme fineness of the feed material used in the final test run. Conventional dewatering processes (e.g., screenbowl centrifuges) typically generate moistures $>50 \%$ for this ultrafine size class.

The prototype test data also demonstrated that very high recoveries of ultrafine solids could be achieved while maintaining low moisture contents. The highest recovery of dry solids (i.e., 99.9\%) was obtained for the coarsest feed mixture containing only $36.2 \%$ minus $0.025 \mathrm{~mm}$, while a slightly lower recovery (i.e., 97.9\%) was obtained for the finest feed mixture containing $94.5 \%$ minus $0.025 \mathrm{~mm}$.

\section{Table 4.4 Cardinal Plant test results}

\begin{tabular}{|c|c|c|c|c|c|}
\hline \multirow[b]{2}{*}{ Test } & \multirow[b]{2}{*}{ Condition } & \multicolumn{2}{|c|}{$\begin{array}{l}\text { No Recirculation } \\
\text { (Measured) }\end{array}$} & \multicolumn{2}{|c|}{$\begin{array}{l}\text { With Recirculation } \\
\text { (Predicted) }\end{array}$} \\
\hline & & $\begin{array}{c}\text { Moisture } \\
(\%)\end{array}$ & $\begin{array}{c}\text { Solid } \\
\text { Recovery } \\
(\%)\end{array}$ & $\begin{array}{c}\text { Moisture } \\
(\%)\end{array}$ & $\begin{array}{c}\text { Solid } \\
\text { Recovery } \\
(\%)\end{array}$ \\
\hline 1 & 50 / 50 : Col Conc / Scrn Drain & 13.3 & 96.1 & 13.7 & 99.9 \\
\hline 2 & 60 / 40 : Col Conc / Scrn Drain & 15.0 & 95.5 & 15.1 & 98.9 \\
\hline 3 & 70 / 30 : Col Conc / Scrn Drain & 16.2 & 93.4 & 17.0 & 99.9 \\
\hline 4 & 80 / 20 : Col Conc / Scrn Drain & 18.6 & 93.8 & 18.6 & 99.2 \\
\hline 5 & 90 / 10 : Col Conc / Scrn Drain & 19.4 & 94.5 & 19.7 & 99.4 \\
\hline 6 & 100 / 0 : Col Conc / Scrn Drain & 18.7 & 83.2 & 18.3 & 97.3 \\
\hline 7 & $\begin{array}{c}\text { NA/NA : Col Conc / Plant Col } \\
\text { Conc }\end{array}$ & 19.7 & 89.4 & 19.4 & 97.9 \\
\hline
\end{tabular}




\subsection{Discussion}

It was originally anticipated that a linear correlation would be observed between the moisture values and the amount of ultrafine solids in the product. This correlation has been observed for most of conventional dewatering processes used in the coal industry (Arnold, 1999). However, as shown in Figure 4.5 , the moisture obtained using the Centribaric ${ }^{\mathrm{TM}}$ technology increased linearly until the mass percent of minus $0.025 \mathrm{~mm}$ solids reached $60 \%$. The moisture then reached a plateau at about $19.4 \%$ and did not increase further as the amount of minus $0.025 \mathrm{~mm}$ solids increased. This surprising trend was also found to be reproducible in laboratory tests conducted with air injection. No such plateau was observed in the laboratory tests where no air injection was employed (i.e., the moisture continued to increase in a linear fashion as the amount of ultrafines increased). As such, the Centribaric ${ }^{\mathrm{TM}}$ technology appears to be ideally suited for dewatering of ultrafine feeds to low moisture values.

\subsection{Conclusions}

The prototype hyperbaric centrifuge unit that was built by Decanter Machine Inc. was tested with various feed conditions at Arch Coal's Cardinal Plant. Test results showed that moistures of 13.3-19.7\%, which are very low numbers compared to conventional filtration, with high recoveries of $83.2-96.1 \%$ were easily achievable when screen drain of the test unit was discarded. Whereas, slightly higher but still low moistures from $13.7 \%$ to $19.4 \%$ with $97.3-$ 99.9\% recoveries were predicted to be achievable if the screen drain was re-circulated. Also, mixing fine (minus $0.044 \mathrm{~mm}$ ) column concentrate with a coarser stream was found to be effective on reducing moisture and increasing solid recovery. To avoid a substantially higher capacity requirement, a smaller ratio of coarse/fine can be used as the test results show that even 
a small amount of coarse material was enough to keep the moisture content low and the recovery high. 


\subsection{MODELING OF A HYPERBARIC FILTER CENTRIFUGE}

\subsection{Introduction}

Coal cleaning is a water based process after which a product with certain amount of moisture is produced. Excess process water is removed by dewatering equipment at the end of the cleaning process. Dewatering is an important step of coal cleaning, because coal producers can only ship a limited amount of moisture due to contractual requirements. Therefore, successfully simulating dewatering equipment using a reliable and accurate model would greatly benefit coal producers, as the value of coal can be estimated under different conditions even before actually running the dewatering equipment.

The final moisture content of a cake depends on many variables such as the physical properties of particles (i.e. size, shape, surface properties, etc.) and operating parameters of the dewatering equipment. Because of the large number of particles involved with randomly distributed sizes and shapes, it is impossible to completely describe this complex system. Instead, the best estimates of process variables can be used to make an accurate prediction.

Dewatering models commonly involve a balance of de-saturating forces that push the water out of the cake and capillary forces that retain water inside the cake. The applied desaturating forces can be either mechanical as in conventional centrifuge machines or can be hydrodynamic as in pressurized air injection. These forces can also be used in conjunction with each other. Even though there are several empirical and theoretical models developed by researchers to predict moisture content of centrifuge and filter products, there is no model currently available in the technical literature for a hyperbaric centrifuge. Therefore, the purpose of this chapter was to develop a model for hyperbaric centrifugation. For this purpose, two types 
of models were developed. The first type of model, which is explained in section 5.2, was developed to explain the pressure distribution inside an HFC cake. This model made it possible to understand why injection of pressurized air helped with dewatering. The second type, which is explained in section 5.3, was intended for prediction of moisture content of HFC products and it involved development of two different models, one of which was a theoretical model and the other one was an empirical model.

\subsection{Pressure Distribution Model}

The centrifugal dewatering process can be characterized by a filter cake submerged in an annulus of clear liquid (Figure 5.1). Zeitsch (1990) developed a model to predict centrifugal filtration rate based on the first principle considerations. According to Zeitsch, the fundamental law of filtration as formulated by Darcy (1856) is not applicable to centrifugal filtration as it fails to take mass forces into account. Darcy's law suggests that there has to be a pressure difference in order to move a liquid through a porous medium, as follows:

$$
Q=\frac{K \Delta P A}{\eta H}
$$

where $Q$ is the flow rate of liquid passing through the cake, $K$ is the permeability of the bed, $\Delta P$ is the pressure drop across the cake, $A$ is the filter area, $\eta$ is the viscosity of the fluid and $H$ is the cake thickness. With reference to Figure 5.1, during centrifugation when the liquid layer reaches the cake surface $\left(r_{0}=r_{S}\right)$, pressures at $r_{S}$ and $r_{B}$ will be equal to the ambient pressure. Therefore, according to Eq. [5.1], the liquid should not be flowing at this point because the pressure difference across the cake is zero. However, the liquid will keep flowing because of the acting centrifugal force. 


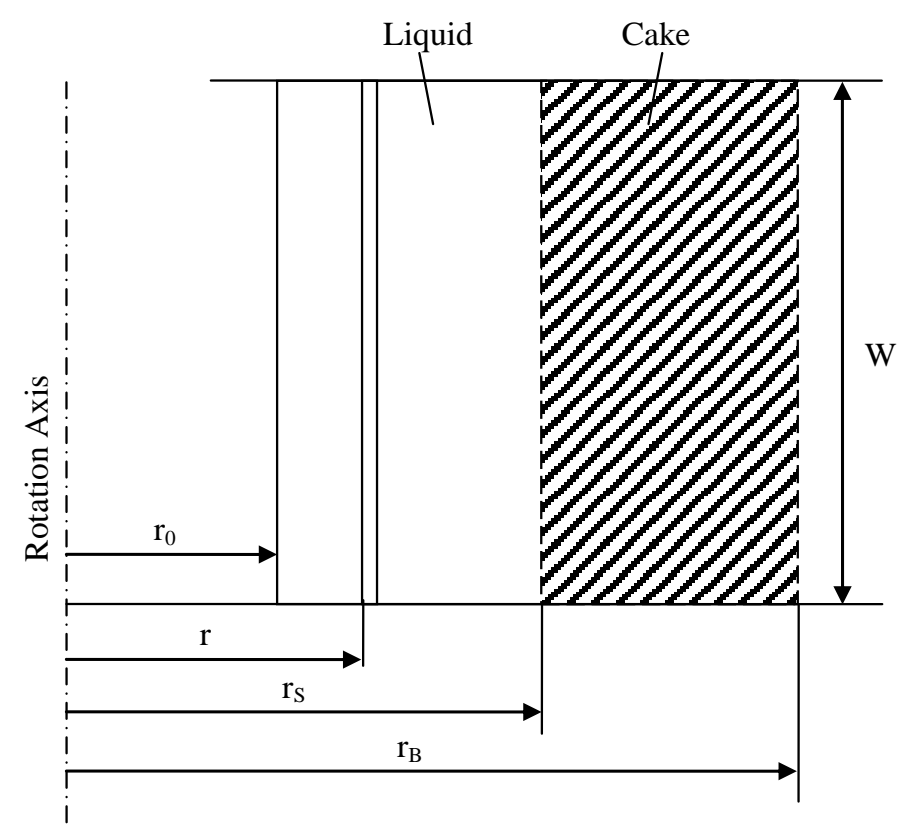

Figure 5.1 Geometry of centrifugal dewatering

Zeitsch (1990) developed the following relationship, which gives the pressure distribution inside a cake during centrifugation, based on the situation given in Figure 5.1:

$$
P(r)=\frac{\ln \left(r_{B} / r\right)}{\ln \left(r_{B} / r_{S}\right)} P_{S}+\frac{\rho \omega^{2} r_{B}^{2}}{2}\left[\frac{\ln \left(r_{B} / r\right)}{\ln \left(r_{B} / r_{S}\right)}\left(1-\frac{r_{S}^{2}}{r_{B}^{2}}\right)-\left(1-\frac{r^{2}}{r_{B}^{2}}\right)\right]
$$

where $P_{s}$ is the pressure on cake surface, $\rho$ is the liquid density and $\omega$ is the angular velocity.

In the current study, this equation was revised to reflect the effect of application of air pressure, as follows:

$$
P(r)=\frac{\ln \left(r_{B} / r\right)}{\ln \left(r_{B} / r_{S}\right)}\left(P_{S}+P_{\text {air }}\right)+\frac{\rho \omega^{2} r_{B}^{2}}{2}\left[\frac{\ln \left(r_{B} / r\right)}{\ln \left(r_{B} / r_{S}\right)}\left(1-\frac{r_{S}^{2}}{r_{B}^{2}}\right)-\left(1-\frac{r^{2}}{r_{B}^{2}}\right)\right]
$$

A numerical example of Eq. [5.3] is given in Figure 5.2(a). The plot shows that the pressure inside a centrifuge cake drops below ambient pressure when the liquid layer reaches the cake surface. However, the pressure may be increased above the ambient pressure by injecting 
pressurized air into the centrifuge (Figure 5.2(b)), thus increasing dewatering kinetics as the total de-saturating force increases.
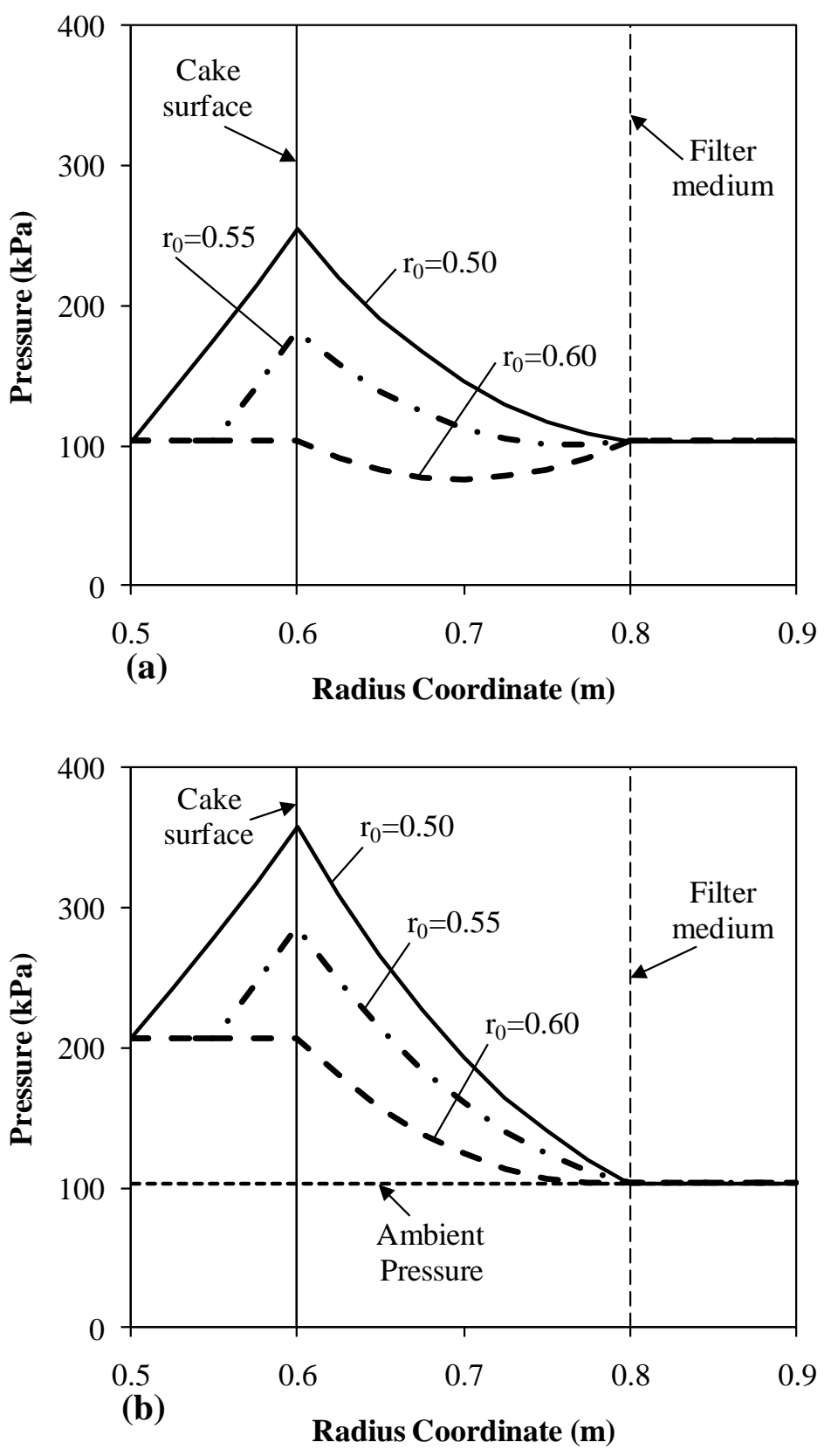

Figure 5.2 Changes in pressure across the filter cake formed on a centrifugal filter: $r_{B}=$ $0.8 \mathrm{~m}$ and $r_{S}=0.6 \mathrm{~m}$. Calculations were made at $r_{0}=0.50,0.55$ and $0.60 \mathrm{~m}$. At $r_{0}=0.60 \mathrm{~m}$, the thickness of the water film on the cake become zero. In case (a), pressure in the cake becomes lower than the ambient, as shown by the dotted line. Whereas. in case (b). pressure inside the cake is positive 


\subsection{Cake Moisture Models}

\subsubsection{Empirical Dewatering Model}

The moisture content of a centrifuge product is calculated in two steps. First, the minimum water content, which is achievable when a de-saturating force acts for a very long time, is calculated and then this value is adjusted upwards to allow for the fact that there is only a finite amount of time available for dewatering. Moreover, when carrying out moisture content calculations, saturation $(S)$ values are used instead of moisture values for ease of computation.

The saturation is estimated by using a dimensionless number called capillary number $\left(N_{c}\right)$. The capillary number represents a ratio of de-saturating forces to water retaining forces. Several researchers such as Brownell and Katz (1947), Haruni and Storrow (1953), Dombrowski and Brownell (1954) defined capillary number using the permeability factor $K$, but Wakeman et al. (1976) showed that the capillary number and the previously used permeability expressions to correlate residual saturation data were inadequate because of the inability of Brown (1950) method to calculate $K$ for centrifugally spun beds. However, Swindells (1979) replaced permeability term, $K$, with the squared mean particle size term, $d_{p}^{2}$, which resulted in a better correlation:

$$
N_{c}=\frac{\rho_{w} N_{g} g d_{p}^{2}}{\sigma \cos \theta}
$$

where $\rho_{w}$ is the density of water, $N_{g}$ is the number of g's, $d_{p}$ is the mean particle diameter, $\sigma$ is surface tension of water and $\theta$ is the contact angle. In the current study, this equation was modified to allow for pressurized air injection as follows:

$$
N_{c}=\frac{\left(\rho_{w} N_{g} g+P_{a i r} / H\right) d_{p}^{2}}{\sigma \cos \theta}
$$


where $P_{\text {air }}$ is the air pressure and $H$ is the cake thickness. $d_{p}$ can be calculated using the following equation:

$$
d_{p}=\frac{1}{\sum_{i=1}^{n}\left(\left(\sum_{j=1}^{m} F_{i j}^{*}\right) / A_{i}\right)}
$$

Above equation gives the diameter of a particle with the same surface area per unit volume as the total surface are per unit volume of collection of all particles.

It is important to determine the mean particle size accurately, since it greatly affects the simulation results. Size distribution of a particulate system is commonly determined by wet screening. However, because of the limitations of this method the size distribution below 0.025 mm cannot be measured. Moreover, a practical method for determining the size distribution of this size fraction does not exist. Therefore, when conducting simulations, the well known GatesGaudin-Schumann (GGS) particle size distribution equation was fitted to size distributions obtained for different feed samples to determine the distribution below $0.025 \mathrm{~mm}$. This allowed for calculating a more realistic mean particle size, especially for the finer size distributions. While fits obtained using GGS equation were not the perfect, it was deemed to be sufficiently accurate for the current study.

Using the data he obtained during his tests and the data available in the literature, Ledger (1999) suggested an empirical relationship that yielded a good correlation between the capillary number and the residual saturation, which is the minimum saturation achievable:

$$
S_{r}=0.051 e^{-N_{c} / 91.3}+0.0045
$$

However, due to the limited amount of time available for dewatering, a higher saturation, which is called effective saturation, $S_{e}$, is normally seen in commercial centrifuges. Brown (1950) derived a relationship that defines saturation, $S_{e}$, in terms of $S_{r}$ and $S$ : 


$$
S_{e}=\frac{S-S_{r}}{1-2 S_{r}+S S_{r}}
$$

Assuming that the cake is fully saturated at time $0, S_{e}$ at any time can be determined by the following equation.

$$
S_{e}^{1-y}=1+\frac{2 t(y-1)}{C_{t}\left(\left(1-S_{r}\right)^{2}+1\right)}
$$

where $\mathrm{t}$ is time and $C_{t}$ is a parameter given by:

$$
C_{t}=\frac{\eta \varepsilon H}{K p}
$$

where $\eta$ is viscosity of water, $\varepsilon$ is porosity of the cake, $K$ is permeability of the cake and $p$ is the pressure differential across the cake. $p$ value can be calculated using the following equation:

$$
p=\rho_{w} N_{g} g
$$

Permeability, $K$, is another important parameter for modeling of the dewatering process. The use of an accurate value of $K$ is crucial for determining the correct moisture content. The best way of determining $K$ is to measure it using one of the available methods in the literature (Zeitsch, 1990). However, when measurement is not possible, the permeability, $K$, can be estimated using one of the many equations that employ porosity and mean particle size values available in the literature. Brown (1950) proposed the following simple equation for estimating $K$ :

$$
K=\frac{\varepsilon^{3} d_{p}^{2}}{180(1-\varepsilon)^{2}}
$$

Panda et al. (1994) also developed the following equation:

$$
K=\frac{\varepsilon^{3} d_{p}^{2}}{72 \tau(1-\varepsilon)^{2}}\left(\frac{S_{k} C_{D_{p}}^{3}+3 C_{D_{p}}^{2}+1}{1+C_{D_{p}}^{2}}\right)^{2}
$$


where $\tau$ is the tortuosity, $S_{k}$ is the skewness of the particle size distribution and $C_{D p}$ is the coefficient of variation of the particle size distribution, which is the standard deviation divided by the mean size. An average value of 3 can be used as the tortuosity. Another equation for predicting permeability was developed by Vidal et al. (2009) as follows:

$$
K=\frac{1}{S_{0}^{2}\left(c_{1} S_{k}+c_{2}\right)} \frac{\varepsilon^{3}}{(1-\varepsilon)^{5 / 3}}
$$

where $c_{1}$ and $c_{2}$ are fitting parameters, which were found to be 0.22 and 4.00 , respectively, and $S_{0}$ is the specific surface area that can be estimated using:

$$
S_{0}=\frac{6}{d_{p}}
$$

Eq. [5.10] was also modified to allow for pressurized air injection, as follows:

$$
C_{t}=\frac{\eta \varepsilon H}{K\left(\rho_{w} N_{g} g+P_{\text {air }} / H\right)}
$$

The dimensionless parameter $y$ in Eq. [5.9] is a function of mean particle size, $d_{p}$, and can be approximated by:

$$
y=1.4355+0.17213 \log d_{p}+0.1401\left(\log d_{p}\right)^{2}
$$

Residual and effective saturation values obtained using previous equations can be combined using the following equation to determine the total saturation value:

$$
S=\frac{S_{e}-2 S_{e} S_{r}+S_{r}}{1-S_{e} S_{r}}
$$

Finally, the moisture content of the product is given by:

$$
M=\frac{S \varepsilon \rho_{w}}{S \varepsilon \rho_{w}+(1-\varepsilon)^{2}}
$$




\subsubsection{Theoretical Dewatering Model}

Zeitsch (1990) developed a theoretical model of centrifugal dewatering using fundamental concepts. For modeling purposes, structure of a filter cake is far too complicated to take size, shape and mutual arrangements of particles into account. However, a filter cake can be characterized as a whole using parameters that are readily measurable, such as the permeability, porosity and volumetric degree of saturation. For his model, Zeitsch assumed that a filter cake would consist of a solid body perforated with cylindrical pores parallel to acting acceleration.

In the current work, modifications were made to Zeitsch's model to allow for pressurized air injection. The velocity of a liquid flowing in a single pore of diameter $s=2 b$ and height $H$ is given by the following differential equation:

$$
\pi r^{2} P_{a i r}+\pi r^{2} y(t, s) \rho_{w} N_{g} g=\pi r^{2} \frac{2 \sigma \cos \theta}{b}-2 \pi r y(t, s) \eta \frac{\mathrm{d} w}{\mathrm{~d} r}
$$

where $r$ is the radius coordinate starting at the pore axis and $y(t, s)$ is the height of the liquid at time $t$. Integrating both sides of this equation yields the radial velocity distribution:

$$
w(r)=\frac{b^{2}-r^{2}}{4 y(t, s) \eta}\left(P_{\text {air }}+\rho_{w} N_{g} g y(t, s)-\frac{2 \sigma \cos \theta}{b}\right)
$$

The volume flow between radii $r$ and $(r+\mathrm{d} r)$ during the time interval $t$ is given by:

$$
\mathrm{d} V_{r / r+\mathrm{d} r}=2 \pi r \mathrm{~d} r w(r) \mathrm{d} t
$$

Substituting Eq. [5.21] into Eq. [5.22] results in:

$$
\mathrm{d} V_{r / r+\mathrm{d} r}=\frac{\pi \mathrm{d} t}{2 y(t, s) \eta}\left(P_{a i r}+\rho_{w} N_{g} g y(t, s)-\frac{2 \sigma \cos \theta}{b}\right)\left(b^{2} r \mathrm{~d} r-r^{3} \mathrm{~d} r\right)
$$

and integration between $r=0$ and $r=b$ leads to:

$$
\mathrm{d} V=\frac{\pi b^{4} \mathrm{~d} t}{8 y(t, s) \eta}\left(P_{\text {air }}+\rho_{w} N_{g} g y(t, s)-\frac{2 \sigma \cos \theta}{b}\right)
$$


When this volume element leaves the pore, the height of the liquid diminishes, which can expressed using:

$$
\mathrm{d} V=-b^{2} \pi \mathrm{d} y
$$

Substituting Eq. [5.24] into Eq. [5.25] yields:

$$
\mathrm{d} y=\frac{b^{2}}{8 y(t, s) \eta}\left(\frac{2 \sigma \cos \theta}{b}-\rho_{w} N_{g} g y(t, s)-P_{\text {air }}\right) d t
$$

Moreover, the introduction of the following three terms:

$$
\begin{aligned}
& \zeta=\frac{2 \sigma \cos \theta}{b \rho_{w} N_{g} g H} \\
& \beta=\frac{b^{2} \rho_{w} N_{g} g}{8 \eta} \\
& X=\frac{P_{\text {air }}}{\rho_{w} N_{g} g H}
\end{aligned}
$$

leads to a simplified expression given by:

$$
\mathrm{d} t=\frac{d y}{\left[\left(\zeta-X-\frac{y(t, s)}{H}\right) \beta \frac{H}{y(t, s)}\right]}
$$

Eq. [5.30] can be integrated to give:

$$
t=-\frac{\frac{y(t, s)}{H}+(\zeta-X) \ln \left[\left(X+\frac{y(t, s)}{H}-\zeta\right) H\right]}{\frac{\beta}{H}}+C
$$

Since the drainage process starts with $y(t, s)=H$ at $t=0$, it is possible to show that:

$$
C=\frac{1+(\zeta-X) \ln [(X+1-\zeta) H]}{\frac{\beta}{H}}
$$

Substituting Eq. [5.32] into Eq. [5.31] yields 


$$
\frac{\beta}{H} t=1-\frac{y(t, s)}{H}+(\zeta-X) \ln \left[\frac{1+X-\zeta}{\frac{y(t, s)}{H}+X-\zeta}\right]
$$

The well known Laplace equation (1806) was modified to calculate the equilibrium height, $h_{0}$, of a liquid in a pore of diameter $s$ when air pressure was present, as follows:

$$
h_{0}=\frac{4 \sigma \cos \theta}{\rho_{w} g N_{g} s}-\frac{P_{a i r}}{\rho_{w} g N_{g}}
$$

Therefore, if the pore diameter is small enough to yield:

$$
h_{0}=\frac{4 \sigma \cos \theta}{\rho_{w} g N_{g} s}-\frac{P_{a i r}}{\rho_{w} g N_{g}}>H
$$

where $H$ is the cake height, the liquid cannot be drained. So, there is a critical pore diameter, $s^{*}$

$$
s^{*}=\frac{4 \sigma \cos \theta}{\rho_{w} g N_{g} H+P_{\text {air }}}
$$

below which the pores remain filled with the liquid. Comparing equations [5.27] and [5.34] shows that:

$$
\zeta=\frac{4 \sigma \cos \theta}{\rho_{w} g N_{g} s H}=\left(\frac{h_{0}}{H}+\frac{P_{\text {air }}}{\rho_{w} g N_{g} H}\right)
$$

Finally, substituting equations [5.27], [5.28], [5.29] and [5.37] into Eq. [5.33] yields:

$$
\frac{\beta}{H} t=1-\frac{y(t, s)}{H}+\frac{h_{0}}{H} \ln \left[\frac{1+\frac{h_{0}}{H}}{\frac{y(t, s)}{H}-\frac{h_{0}}{H}}\right]
$$

This equation gives the relationship between the momentary height, $y$, of the liquid column in a single pore with time $t$. Eq. [5.38] does not permit expressing $y$ as an explicit function of $t$. Therefore, this equation was replaced by the following exponential equation that has the same functional form: 


$$
\frac{y(t, s)}{H}=\left(1-\frac{h_{0}}{H}\right) e^{-\frac{\beta}{H} t}+\frac{h_{0}}{H}
$$

Until this point, dewatering of a single pore has been considered. However, it is now possible to model dewatering of a total cake.

The model assumes that a centrifuge cake consists of two types of pores. The first type comprises the pores that cannot be drained at all, while the second type comprises all the other pores. The separation between these two types of pores is defined by the critical pore diameter, $s^{*}$, given by Eq. [5.36]. Assuming that pore diameters have a Boltzmann-type distribution, the following equation gives the size distribution of pores in a filter cake:

$$
g(s)=\frac{2 \varepsilon S}{\pi \hat{\mathrm{S}}^{4}} e^{-\frac{1}{2}\left(\frac{S}{\hat{\mathrm{s}}}\right)^{2}}
$$

where:

$$
\hat{\mathrm{s}}=\sqrt{\frac{8 K}{\varepsilon}}
$$

The volume of the liquid contained in the first type pores for a cake with height $H$ and area $A$ can be expressed as:

$$
V_{R}=\int_{0}^{s^{*}} g(s) \frac{s^{2} \pi}{4} y(t, s) A d s
$$

Substituting Eq. [5.40] into Eq. [5.42] yields:

$$
V_{R}=\frac{\varepsilon A H}{2 \hat{\mathrm{s}}^{4}} \int_{0}^{s^{*}} s^{3} e^{-\frac{1}{2}\left(\frac{s}{\hat{s}}\right)^{2}} d s
$$

After integration and substituting Eq. [5.41] into Eq. [5.43], it can be shown that:

$$
\frac{V_{R}}{\varepsilon A H}=1-\left[\frac{\varepsilon}{16 K} s^{* 2}+1\right] e^{-\frac{\varepsilon}{16 K} s^{* 2}}
$$

where $V_{R} / \varepsilon A H$ represents the residual saturation of the cake that cannot be drained. 
Similarly, the volume of liquid contained in the second type of pores is expressed as:

$$
V_{E}=\int_{s^{*}}^{\infty} g(s) \frac{s^{2} \pi}{4} \frac{y(t, s)}{H} A d s
$$

Substituting Eq. [5.39] and Eq. [5.40] into Eq. [5.45] yields:

$$
V_{E}=\frac{\varepsilon A H}{2 \hat{s}^{4}} \int_{s^{*}}^{\infty} s^{3} e^{-\frac{1}{2}\left(\frac{S}{\hat{s}}\right)^{2}}\left[\left(1-\frac{h_{0}}{H}\right) e^{-\frac{\beta}{H} t}+\frac{h_{0}}{H}\right] d s
$$

Integration of this expression gives:

$$
\frac{V_{E}}{\varepsilon A H}=\frac{I_{1}-I_{2}+I_{3}-I_{4}}{2 \hat{s}^{4}}
$$

where $V_{E} / \varepsilon A H$ represents the additional saturation that is not removed due to the limited amount of time available for drainage. The terms $\mathrm{I}_{1}$ through $\mathrm{I}_{4}$ are given by:

$$
\begin{aligned}
& I_{1}=\frac{1+\left(\frac{\rho_{w} g N_{g} t}{32 \eta H}+\frac{1}{2 \hat{s}^{2}}\right) s^{* 2}}{2\left(\frac{\rho_{w} g N_{g} t}{32 \eta H}+\frac{1}{2 \hat{s}^{2}}\right)^{2}}\left(1+\frac{P_{\text {air }}}{\rho_{w} N_{g} g H}\right) e^{-\left(\frac{\rho_{w} g N_{g} t}{32 \eta H}+\frac{1}{2 \hat{s}^{2}}\right) s^{* 2}} \\
& I_{2}=\left(\frac{s^{*}}{2\left(\frac{\rho_{w} g N_{g} t}{32 \eta H}+\frac{1}{2 \hat{\mathrm{s}}^{2}}\right)^{2}} e^{-\left(\frac{\rho_{w} g N_{g} t}{32 \eta H}+\frac{1}{2 \hat{\mathrm{s}}^{2}}\right) s^{* 2}}+\frac{\sqrt{\pi} \operatorname{Erfc}\left(\sqrt{\frac{\rho_{w} g N_{g} t}{32 \eta H}+\frac{1}{2 \hat{\mathrm{s}}^{2}} s^{*}}\right)}{4\left(\frac{\rho_{w} g N_{g} t}{32 \eta H}+\frac{1}{2 \hat{\mathrm{s}}^{2}}\right)^{\frac{3}{2}}}\right) \frac{4 \sigma \cos \theta}{\rho_{w} g N_{g} H} \\
& I_{3}=\left(s^{*} \hat{\mathrm{s}}^{2} e^{-\frac{1}{2}\left(\frac{s}{\hat{\mathrm{s}}}\right)^{2}}+\frac{\sqrt{\pi} \operatorname{Erfc}\left(\sqrt{\frac{1}{2 \hat{\mathrm{s}}^{2}} s^{*}}\right)}{4\left(\frac{1}{2 \hat{\mathrm{s}}^{2}}\right)^{\frac{3}{2}}}\right) \frac{4 \sigma \cos \theta}{\rho_{w} g N_{g} H} \\
& I_{4}=\frac{1+\frac{1}{2}\left(\frac{s^{*}}{\hat{s}}\right)^{2}}{2\left(\frac{1}{2 \hat{s}^{2}}\right)^{2}}\left(\frac{P_{\text {air }}}{\rho_{w} N_{g} g H}\right) e^{-\frac{1}{2}\left(\frac{s^{*}}{\hat{s}}\right)^{2}}
\end{aligned}
$$


Finally, the total saturation of the cake can be calculated by adding residual and additional saturation values as follows:

$$
\frac{V_{T}}{\varepsilon A H}=\frac{V_{E}}{\varepsilon A H}+\frac{V_{R}}{\varepsilon A H}
$$

This total saturation value can be converted to a moisture value using Eq. [5.19].

\subsection{Model Integration and Simulations}

\subsubsection{Empirical Dewatering Model Simulations}

A simulation routine was setup in Microsoft Excel for the empirical model (see Figure 5.3) and was run using different size distributions of coal given in section d) (see Table 5.2 and Table 5.3 for detailed size distributions). The routine was setup so that cake permeability could be calculated using different methods defined in the previous sections. Simulations were conducted with and without $200 \mathrm{kPa}$ air injection at 500 and 2700 g's. Results obtained with the simulation routine were then compared to experimental results. All the input parameters used in these simulations are summarized in Table 5.1.

Table 5.1 Parameters used in empirical dewatering simulations

\begin{tabular}{ccc}
\hline Parameter & Unit & Value \\
\hline$k_{d}$ & $\mathrm{~m}^{-1}$ & 400 \\
$\rho_{s}$ & $\mathrm{~kg} / \mathrm{m}^{3}$ & 1400 \\
$\rho_{w}$ & $\mathrm{~kg} / \mathrm{m}^{3}$ & 1000 \\
$\varepsilon$ & -- & $0.45-0.55$ \\
$\sigma$ & $\mathrm{N} / \mathrm{m}$ & 0.072 \\
$\mu$ & $\mathrm{Pa} . \mathrm{s}$ & 0.001 \\
$H$ & $\mathrm{~m}$ & 0.0085 \\
$t$ & $\mathrm{~s}$ & 120 \\
$N_{g}$ & -- & $500-2700$ \\
$P_{\text {air }}$ & $\mathrm{kPa}$ & $0-200$ \\
& $(\mathrm{psi})$ & $(0-30)$ \\
$g$ & $\mathrm{~m} / \mathrm{s}^{2}$ & 9.81 \\
$\theta$ & $\circ$ & 60 \\
\hline
\end{tabular}




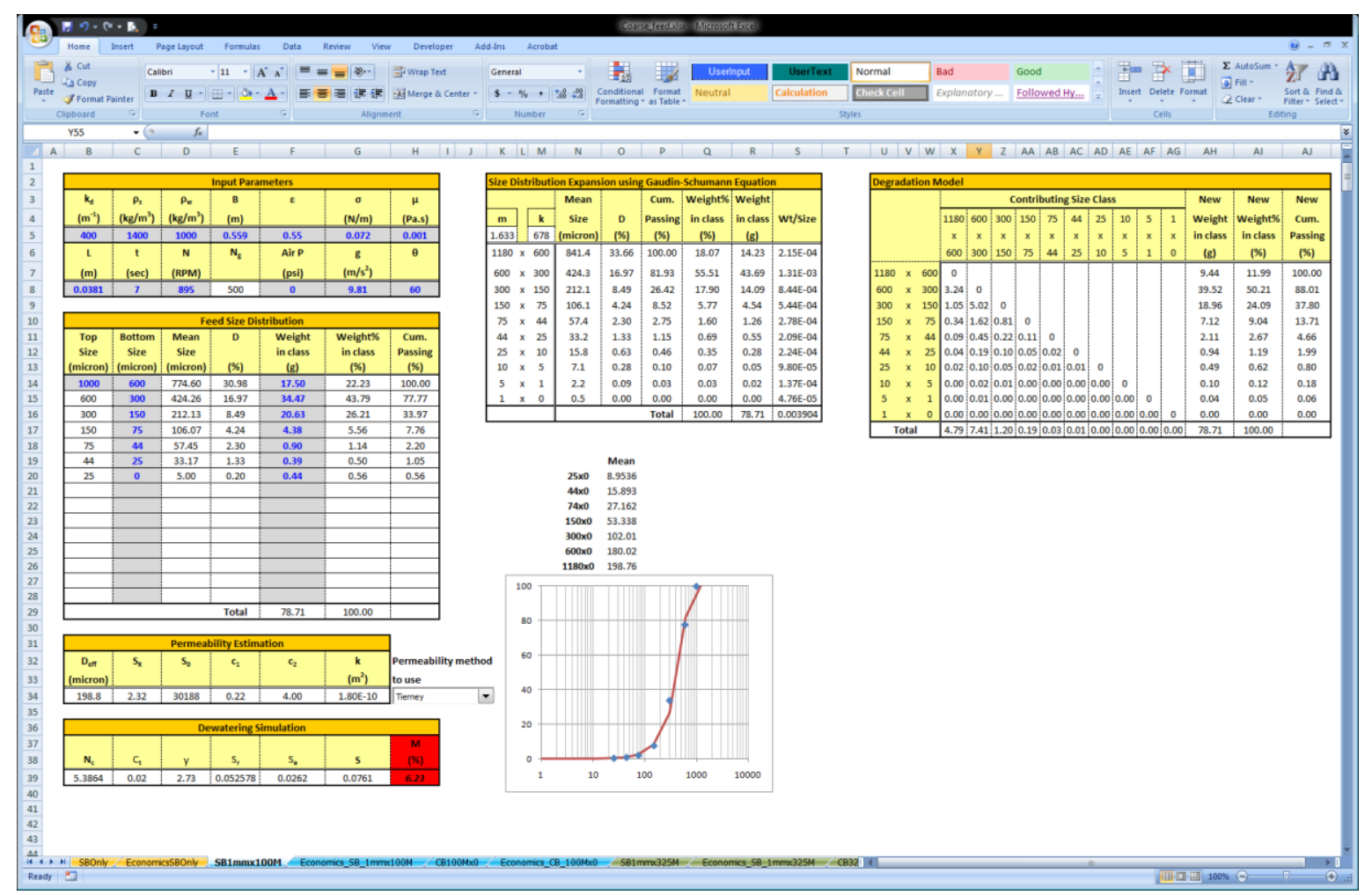

Figure 5.3 Empirical centrifugal dewatering model setup in Excel

Comparisons of simulation results to experimental results obtained at 500 and 2700 g's are given in Figure 5.4 and Tables 5.2 and 5.3. As can be seen, using permeability values calculated by different methods resulted in very similar results at both centrifugal acceleration levels. At 500 g's, very high coefficient of variation $\left(R^{2}\right)$ values, which ranged from 0.996 to 0.998 without air injection and from 0.980 to 0.985 with air injection, obtained using different permeability value calculation methods. At 2700 g's, $R^{2}$ values obtained without air injection using permeability values calculated using different methods were identical (i.e. 0.926). However, these values ranged from 0.937 to 0.947 when air was used. 
Table 5.2 Measured and predicted moisture values using three different permeability estimation methods at $500 \mathrm{~g}$ 's

\begin{tabular}{|c|c|c|c|c|c|c|c|c|c|c|}
\hline \multicolumn{7}{|c|}{ Mass (\%) } & \multirow{2}{*}{$\begin{array}{c}\text { Measured } \\
\text { Moisture } \\
(\%)\end{array}$} & \multicolumn{3}{|c|}{ Predicted Moistures (\%) } \\
\hline $\begin{array}{c}-1.18 \\
\mathrm{~mm}\end{array}$ & $\begin{array}{l}-0.6 \\
\mathrm{~mm}\end{array}$ & $\begin{array}{l}-0.3 \\
\mathrm{~mm}\end{array}$ & $\begin{array}{c}-0.15 \\
\mathrm{~mm}\end{array}$ & $\begin{array}{c}-0.075 \\
\mathrm{~mm}\end{array}$ & $\begin{array}{c}-0.044 \\
\mathrm{~mm}\end{array}$ & $\begin{array}{c}-0.025 \\
\mathrm{~mm}\end{array}$ & & $\begin{array}{l}\text { Panda } \\
\text { et al. }\end{array}$ & Brown & $\begin{array}{l}\text { Vidal } \\
\text { et al. }\end{array}$ \\
\hline \multicolumn{11}{|c|}{ (a) Without Air } \\
\hline 100 & 100 & 100 & 100 & 99.9 & 99.6 & 97.8 & 44.7 & 43.8 & 43.5 & 43.9 \\
\hline 100 & 100 & 100 & 99.9 & 99.1 & 97.0 & 84.4 & 42.1 & 41.2 & 40.6 & 41.2 \\
\hline 100 & 100 & 100 & 99.9 & 96.9 & 88.6 & 70.0 & 40.2 & 39.3 & 38.6 & 39.2 \\
\hline 100 & 100 & 100 & 95.5 & 72.0 & 58.0 & 43.6 & 31.4 & 31.1 & 30.3 & 30.3 \\
\hline 100 & 66.8 & 48.6 & 33.0 & 23.3 & 18.9 & 13.8 & 13.7 & 14.8 & 14.3 & 14.7 \\
\hline \multicolumn{11}{|c|}{ (b) With Air } \\
\hline 100 & 100 & 100 & 100 & 99.9 & 99.6 & 97.8 & 37.8 & 38.3 & 37.8 & 38.5 \\
\hline 100 & 100 & 100 & 99.9 & 99.1 & 97.0 & 84.4 & 30.5 & 34.2 & 33.5 & 34.2 \\
\hline 100 & 100 & 100 & 99.9 & 96.9 & 88.6 & 70.0 & 23.6 & 25.5 & 24.8 & 25.0 \\
\hline 100 & 100 & 100 & 95.5 & 72.0 & 58.0 & 43.6 & 20.3 & 23.3 & 22.6 & 22.6 \\
\hline 100 & 66.8 & 48.6 & 33.0 & 23.3 & 18.9 & 13.8 & 9.8 & 10.5 & 10.2 & 10.4 \\
\hline
\end{tabular}

Table 5.3 Measured and predicted moisture values using three different permeability estimation methods at $2700 \mathrm{~g}$ 's

\begin{tabular}{|c|c|c|c|c|c|c|c|c|c|c|}
\hline \multicolumn{7}{|c|}{ Mass (\%) } & \multirow{2}{*}{$\begin{array}{c}\text { Measured } \\
\text { Moisture } \\
(\%)\end{array}$} & \multicolumn{3}{|c|}{ Predicted Moistures (\%) } \\
\hline $\begin{array}{c}-1.18 \\
\mathrm{~mm}\end{array}$ & $\begin{array}{l}-0.6 \\
\mathrm{~mm}\end{array}$ & $\begin{array}{l}-0.3 \\
\mathrm{~mm}\end{array}$ & $\begin{array}{c}-0.15 \\
\mathrm{~mm}\end{array}$ & $\begin{array}{c}-0.075 \\
\mathrm{~mm}\end{array}$ & $\begin{array}{c}-0.044 \\
\mathrm{~mm}\end{array}$ & $\begin{array}{c}-0.025 \\
\mathrm{~mm}\end{array}$ & & $\begin{array}{c}\text { Panda } \\
\text { et al. }\end{array}$ & Brown & $\begin{array}{l}\text { Vidal } \\
\text { et al. }\end{array}$ \\
\hline \multicolumn{11}{|c|}{ (a) Without Air } \\
\hline 100 & 100 & 99.7 & 99.6 & 99.5 & 99.1 & 97.2 & 40.0 & 35.5 & 34.9 & 35.4 \\
\hline 100 & 100 & 99.9 & 99.6 & 98.7 & 95.6 & 85.3 & 32.1 & 31.5 & 31.5 & 31.5 \\
\hline 100 & 100 & 99.5 & 96.1 & 86.4 & 77.6 & 63.3 & 28.3 & 26.8 & 26.8 & 26.8 \\
\hline 100 & 100 & 99.7 & 98.3 & 86.3 & 74.7 & 55.5 & 24.5 & 21.5 & 21.5 & 21.5 \\
\hline 100 & 100 & 99.5 & 92.8 & 76.6 & 66.3 & 52.4 & 21.7 & 23.0 & 23.0 & 23.0 \\
\hline 100 & 99.9 & 97.9 & 83.7 & 62.6 & 52.7 & 40.0 & 18.8 & 18.6 & 18.6 & 18.6 \\
\hline 100 & 97.2 & 81.6 & 57.8 & 43.4 & 35.6 & 27.7 & 14.8 & 15.5 & 15.5 & 15.5 \\
\hline 100 & 88.7 & 65.4 & 44.7 & 31.5 & 26.3 & 20.0 & 11.0 & 12.5 & 12.5 & 12.5 \\
\hline \multicolumn{11}{|c|}{ (b) With Air } \\
\hline 100 & 100 & 99.7 & 99.6 & 99.5 & 99.1 & 97.2 & 27.2 & 32.5 & 32.5 & 32.5 \\
\hline 100 & 100 & 99.5 & 96.1 & 86.4 & 77.6 & 63.3 & 23.7 & 24.3 & 24.3 & 24.3 \\
\hline 100 & 100 & 99.7 & 98.3 & 86.3 & 74.7 & 55.5 & 22.1 & 21.4 & 21.4 & 21.4 \\
\hline 100 & 100 & 99.9 & 99.6 & 98.7 & 95.6 & 85.3 & 21.4 & 27.3 & 27.3 & 27.3 \\
\hline 100 & 100 & 99.5 & 92.8 & 76.6 & 66.3 & 52.4 & 19.8 & 20.6 & 20.6 & 20.6 \\
\hline 100 & 100 & 97.9 & 83.7 & 62.6 & 52.7 & 40.0 & 16.1 & 16.5 & 16.5 & 16.5 \\
\hline 100 & 97.2 & 81.6 & 57.8 & 43.4 & 35.6 & 27.7 & 12.0 & 13.7 & 13.7 & 13.7 \\
\hline 100 & 88.7 & 65.4 & 44.7 & 31.5 & 26.3 & 20.0 & 9.6 & 11.0 & 11.0 & 11.0 \\
\hline 100 & 68.4 & 49.2 & 34.1 & 24.2 & 20.5 & 15.9 & 8.0 & 9.0 & 9.0 & 9.0 \\
\hline
\end{tabular}


(a) Panda et. al

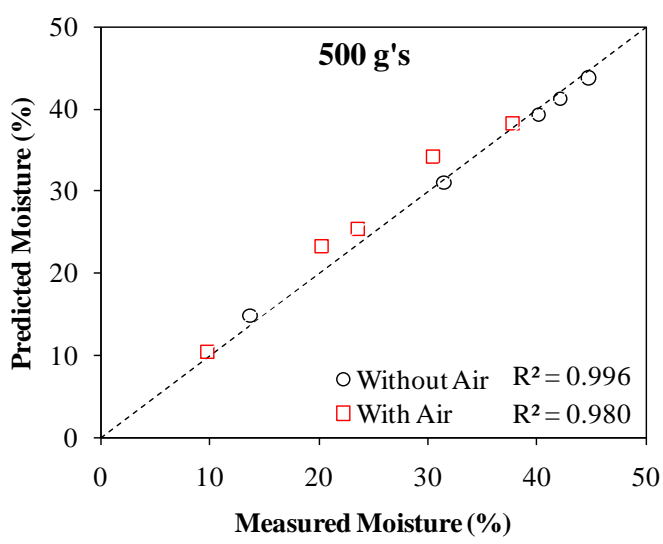

(d) Panda et. al

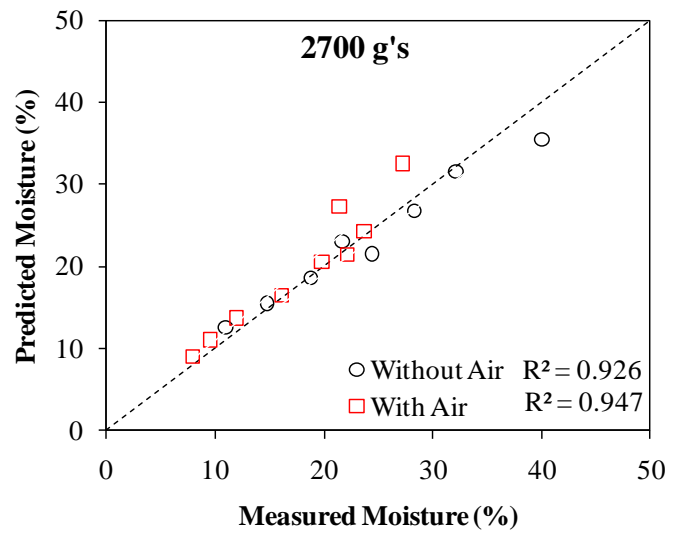

(b) Brown

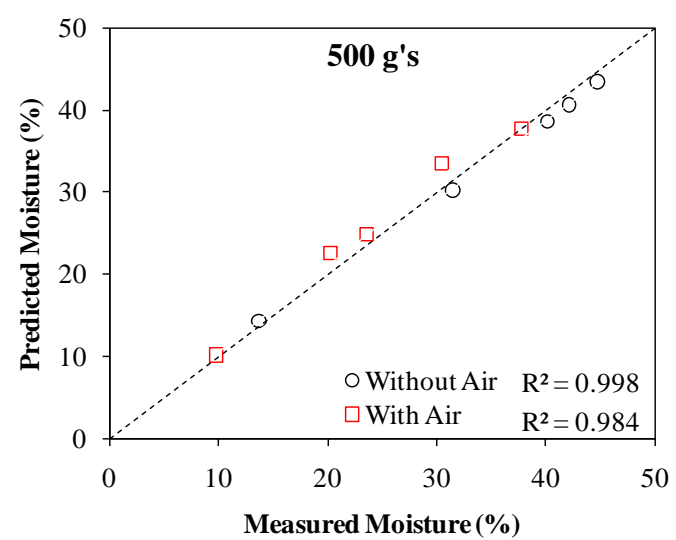

(e) Brown

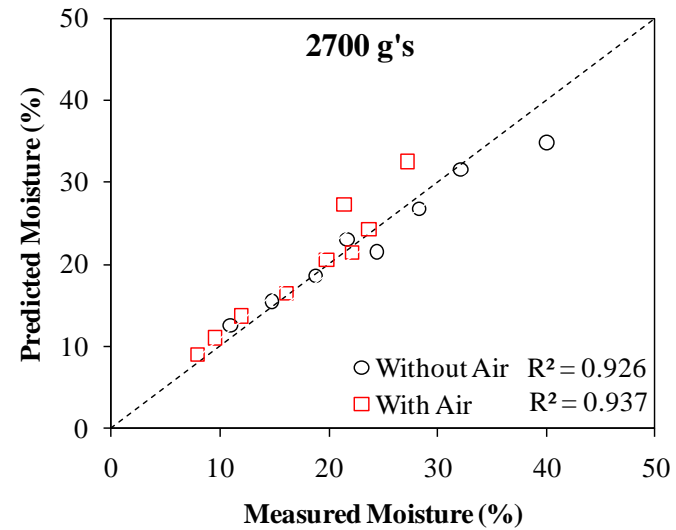

(c) Vidal et. al

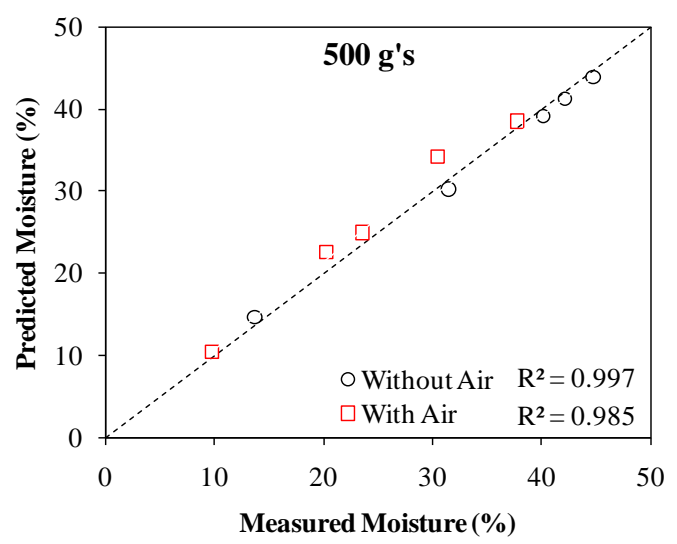

(f) Vidal et. al

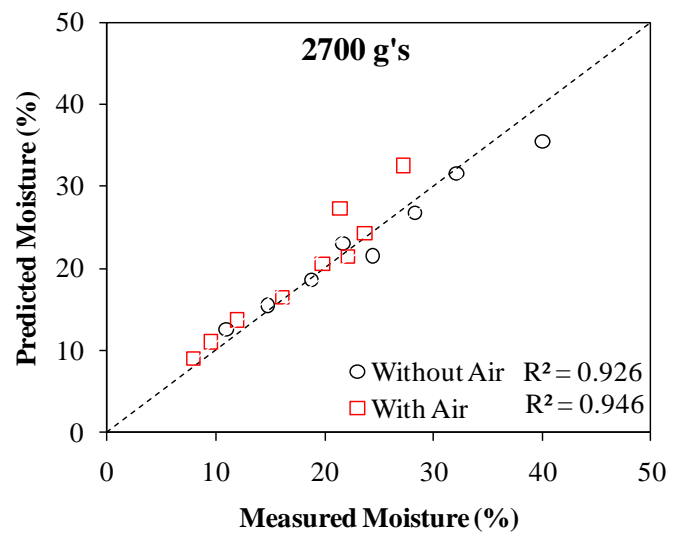

Figure 5.4 Comparison of empirical simulation results and measured moistures using three different methods of permeability calculation at $500 \mathrm{~g}$ 's and $2700 \mathrm{~g}$ 's 
Another set of simulation runs were conducted to study the synergistic effect of air pressure and centrifugal acceleration on cake moistures. For this set of runs, a fine particle size distribution and a relatively coarse particle size distribution were chosen (see Table 5.4). Air pressure and centrifugal acceleration were varied between 0-400 kPa and 100-3000 g's, respectively, while all the other parameters were kept constant (see Table 5.5).

Table 5.4 Size distributions used in empirical dewatering simulations to study the synergistic effect of air pressure and centrifugal acceleration

\begin{tabular}{ccc}
\hline $\begin{array}{c}\text { Size } \\
(\mathbf{m m})\end{array}$ & $\begin{array}{c}\text { Coal } \\
\mathbf{A} \\
\text { (Mass, \%) }\end{array}$ & $\begin{array}{c}\text { Coal } \\
\mathbf{B} \\
(\text { Mass, \%) }\end{array}$ \\
\hline $1.2 \times 0.6$ & 0.14 & 0.00 \\
$0.6 \times 0.3$ & 1.88 & 0.06 \\
$0.3 \times 0.15$ & 13.88 & 0.20 \\
$0.15 \times 0.075$ & 20.62 & 0.69 \\
$0.075 \times 0.044$ & 9.68 & 2.25 \\
$0.044 \times 0.025$ & 12.41 & 7.50 \\
$0.025 \times 0$ & 39.05 & 62.33 \\
\hline
\end{tabular}

Table 5.5 Parameters used in empirical dewatering simulations to study the synergistic effect of air pressure and centrifugal acceleration

\begin{tabular}{ccc}
\hline Parameter & Unit & Value \\
\hline$\rho_{s}$ & $\mathrm{~kg} / \mathrm{m}^{3}$ & 1400 \\
$\rho_{w}$ & $\mathrm{~kg} / \mathrm{m}^{3}$ & 1000 \\
$\varepsilon$ & - & 0.5 \\
$\sigma$ & $\mathrm{N} / \mathrm{m}$ & 0.072 \\
$\mu$ & $\mathrm{Pa} . \mathrm{s}$ & 0.001 \\
$H$ & $\mathrm{~m}$ & 0.0085 \\
$t$ & $\mathrm{~s}$ & 120 \\
$N_{g}$ & - & $100-3000$ \\
$P_{\text {air }}$ & $\mathrm{kPa}$ & $0-400$ \\
& $(\mathrm{psi})$ & $(0-60)$ \\
$g$ & $\mathrm{~m} / \mathrm{s}^{2}$ & 9.81 \\
$\theta$ & $\circ$ & 60 \\
\hline
\end{tabular}


Simulation results given in Table 5.6 and Table 5.7 showed that, as expected, for both coarse and fine coals, the highest moisture value would be obtained at the lowest g level without air injection and the lowest moisture value would be obtained at the highest g level with air injection. Moreover, it would be possible to avoid using higher g levels to achieve lower moisture values by application of more air pressure. For example, using $200 \mathrm{kPa}$ of air pressure at 500 g's would produce the same moisture content as 3000 g's would produce without air pressure for both coarse and fine cakes (values highlighted in Table 5.6 and Table 5.7). Experimental results discussed in section d) that were run at 500 g's and 2700 g's also confirmed this conclusion. In those tests, using $200 \mathrm{kPa}$ air pressure at $500 \mathrm{~g}$ 's had produced similar moistures to the ones obtained at 2700 g's without air pressure.

Table 5.6 Moisture values obtained with coal A using empirical dewatering simulations at different air pressures and centrifugal accelerations

\begin{tabular}{ccccccc}
\hline $\mathbf{P}_{\text {air }}(\mathbf{k P a}) \mathbf{| g}$ 's & $\mathbf{1 0 0}$ & $\mathbf{3 0 0}$ & $\mathbf{5 0 0}$ & $\mathbf{1 0 0 0}$ & $\mathbf{2 0 0 0}$ & $\mathbf{3 0 0 0}$ \\
\hline $\mathbf{0}$ & 32.4 & 27.4 & 25.1 & 22.2 & 19.6 & 18.2 \\
$\mathbf{6 7}$ & 22.5 & 21.8 & 21.1 & 19.9 & 18.4 & 17.4 \\
$\mathbf{1 3 3}$ & 20.1 & 19.7 & 19.3 & 18.6 & 17.6 & 16.8 \\
$\mathbf{2 0 0}$ & 18.7 & 18.4 & 18.2 & 17.7 & 16.9 & 16.3 \\
$\mathbf{2 6 7}$ & 17.8 & 17.6 & 17.4 & 17.0 & 16.4 & 15.9 \\
$\mathbf{3 3 3}$ & 17.1 & 16.9 & 16.8 & 16.5 & 16.0 & 15.5 \\
$\mathbf{4 0 0}$ & 16.5 & 16.4 & 16.3 & 16.1 & 15.6 & 15.2 \\
\hline
\end{tabular}

Table 5.7 Moisture values obtained with coal B using empirical dewatering simulations at different air pressures and centrifugal accelerations

\begin{tabular}{ccccccc|}
\hline $\mathbf{P}_{\text {air }}(\mathbf{k P a}) \backslash \mathbf{g}$ 's & $\mathbf{1 0 0}$ & $\mathbf{3 0 0}$ & $\mathbf{5 0 0}$ & $\mathbf{1 0 0 0}$ & $\mathbf{2 0 0 0}$ & $\mathbf{3 0 0 0}$ \\
\hline $\mathbf{0}$ & 40.5 & 38.7 & 37.4 & 35.2 & 32.7 & 31.1 \\
$\mathbf{6 7}$ & 35.5 & 34.8 & 34.2 & 33.0 & 31.3 & 30.1 \\
$\mathbf{1 3 3}$ & 33.2 & 32.7 & 32.4 & 31.6 & 30.3 & 29.4 \\
$\mathbf{2 0 0}$ & 31.7 & 31.4 & 31.1 & 30.5 & 29.5 & 28.7 \\
$\mathbf{2 6 7}$ & 30.6 & 30.4 & 30.2 & 29.7 & 28.9 & 28.2 \\
$\mathbf{3 3 3}$ & 29.7 & 29.6 & 29.4 & 29.0 & 28.3 & 27.7 \\
$\mathbf{4 0 0}$ & 16.5 & 16.4 & 16.3 & 16.1 & 15.6 & 15.2 \\
\hline
\end{tabular}


The next set of simulation runs were conducted to do a sensitivity analysis of different parameters that were used in the empirical model. The first step of the sensitivity analysis was to establish a baseline moisture value by running the empirical simulation routine using average values of all the input parameters. New moisture values were then predicted by changing each input parameter value by $\pm 10 \%$. Finally, the percent change of the baseline moisture was calculated for each parameter using the new moisture values. Therefore, this study was conducted to determine the magnitude of the influence of different input parameters on moisture prediction of the empirical model.

The results of the sensitivity analysis given in Table 5.8 showed that the most influential parameter was the cake porosity, as a $10 \%$ increase in the porosity caused a $16.9 \%$ increase in the moisture and a $10 \%$ decrease caused a $15.4 \%$ decrease in moisture. Moreover, increasing cake thickness by $10 \%$ increased the moisture content by $2.7 \%$, while the moisture decreased by $3.0 \%$ with a $10 \%$ decrease in cake thickness. While the rest of the parameters had similar magnitudes of effects, changing surface tension and contact angle had very small effect on moisture. However, as it was explained in section b), it is a known fact that lower moisture values can be achieved by adjusting surface tension and contact angle values using dewatering

Table 5.8 Magnitude of change in moisture caused by each parameter

\begin{tabular}{|c|c|c|c|}
\hline Inputs & $\begin{array}{l}\text { Baseline values } \\
\text { of parameters }\end{array}$ & $\begin{array}{c}\% \Delta \text { Moisture with } \\
+10 \% \text { change }\end{array}$ & $\begin{array}{c}\% \Delta \text { Moisture with } \\
-10 \% \text { change }\end{array}$ \\
\hline$\varepsilon$ & 0.5 & 16.9 & -15.4 \\
\hline$\sigma$ & 0.072 & 0.0 & 0.0 \\
\hline$\mu$ & 0.001 & 1.6 & -1.7 \\
\hline $\mathrm{L}$ & 0.0127 & 2.7 & -3.0 \\
\hline $\mathrm{t}$ & 60 & -1.5 & 1.7 \\
\hline $\mathrm{N}_{\mathrm{g}}$ & 500 & -0.4 & 0.4 \\
\hline Pair & 30 & -1.2 & 1.3 \\
\hline$\theta$ & 60 & 0.0 & 0.0 \\
\hline $\mathrm{K}$ & $10^{-14}$ & -1.5 & 1.7 \\
\hline
\end{tabular}


chemicals in accordance with filtration laws. Even though surface tension and contact angle values are included in Eq. [5.4], which is used to calculate the capillary number, these values are rendered ineffective when the capillary number is divided by the value of 91.3 in Eq. [5.7], since the value of the capillary number was usually much lower than 91.3. Therefore, this was found to be one of the shortcomings of the empirical model and further investigation of this shortcoming is suggested for future work.

The final set of simulation runs were conducted to provide a more detailed look at the effects of different parameters on moisture. The results of these runs are shown in Figures 5.5 5.11. Since the sensitivity analysis showed that contact angle and surface tension values had very small effects on moisture when the empirical model was used, these parameters were not included in the figures.

It was observed from these plots that the porosity and the moisture content had a straight line relationship, while the correlation of the moisture with other parameters was more in a logarithmic fashion. It was also observed that an increase in porosity, cake thickness and viscosity values caused the moisture content to increase, while an increase in centrifugal acceleration, air pressure, permeability and time values caused the moisture content to decrease. Moreover, as it was observed with the sensitivity analysis, Figure 5.10 and Figure 5.11 showed that increasing air pressure caused a bigger drop of moisture than it was caused by increasing centrifugal acceleration. 


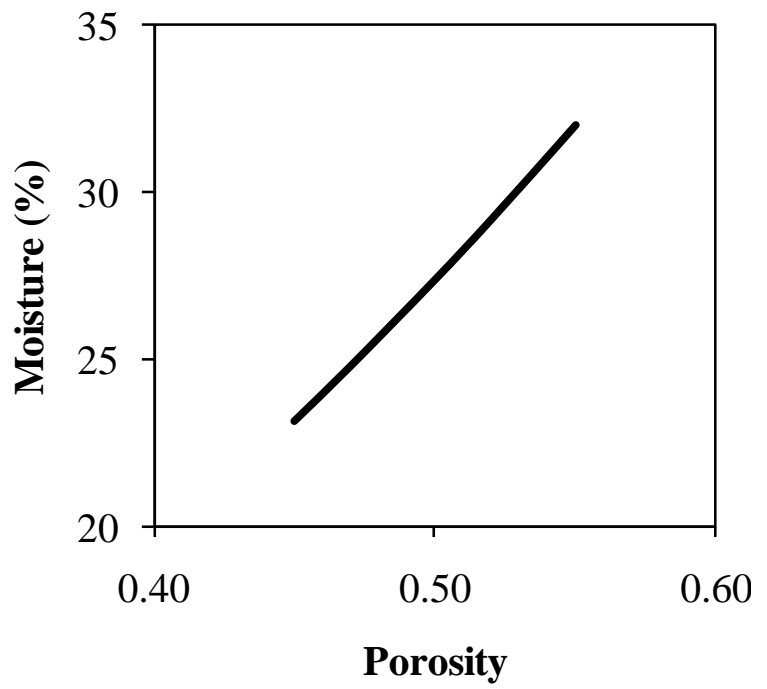

Figure 5.5 Effect of porosity on moisture with empirical model

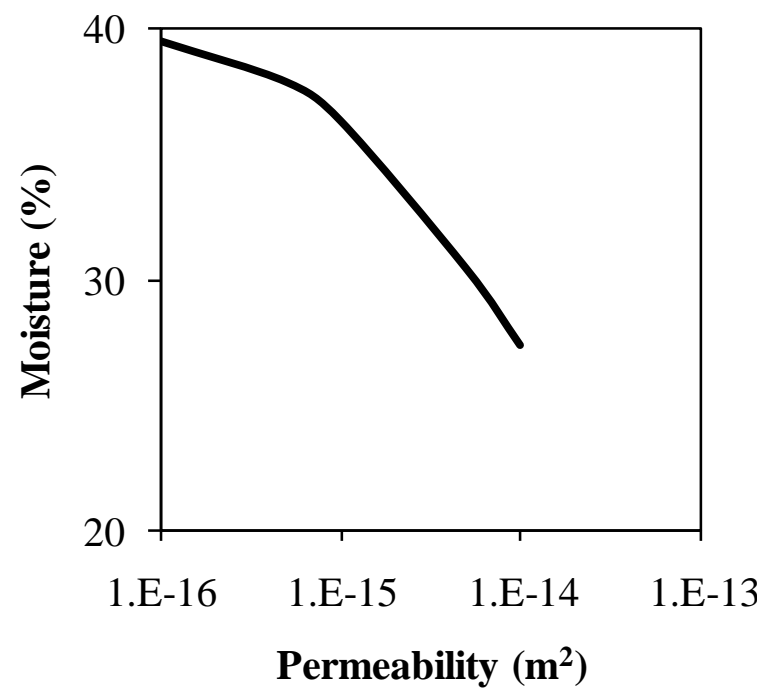

Figure 5.7 Effect of permeability on moisture with empirical model

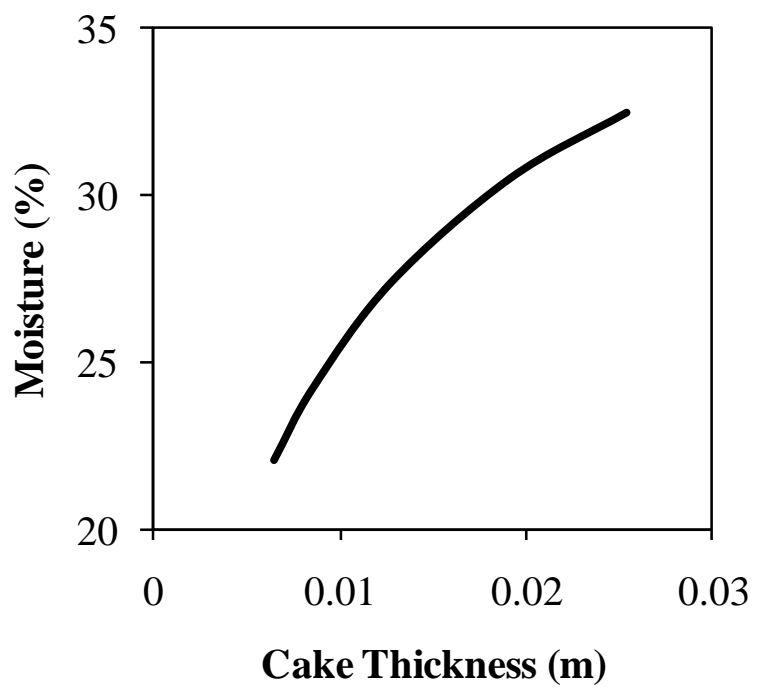

Figure 5.6 Effect of cake thickness on moisture with empirical model

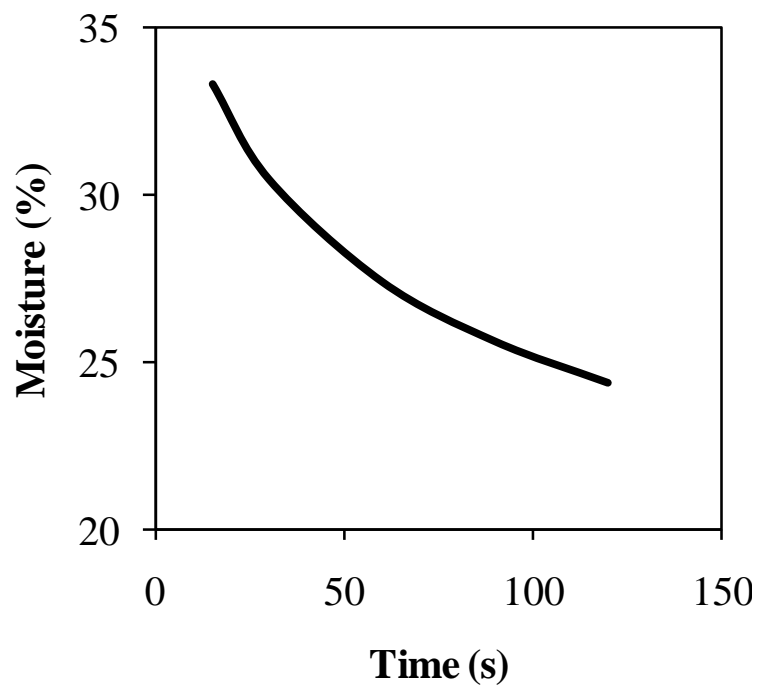

Figure 5.8 Effect of rotation time on moisture with empirical model 


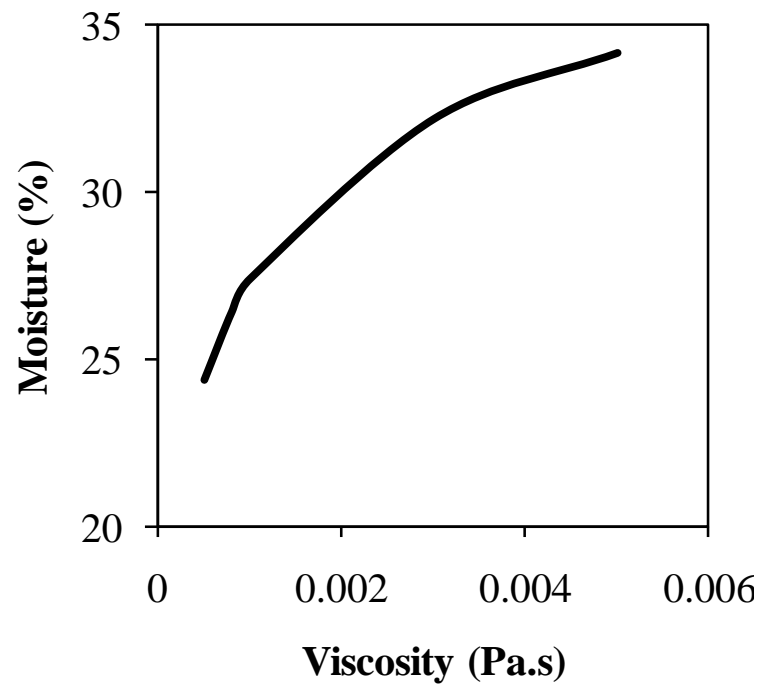

Figure 5.9 Effect of viscosity on moisture with empirical model

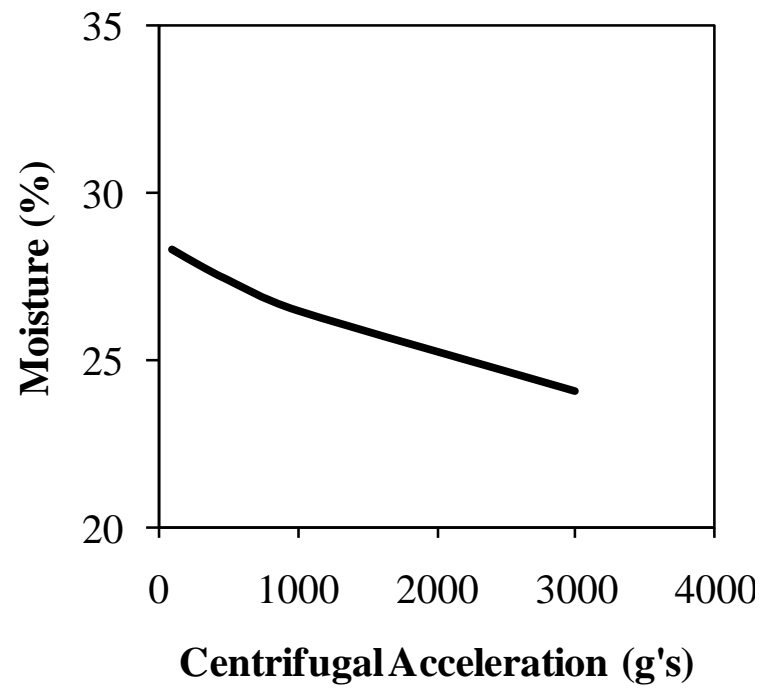

Figure 5.11 Effect of centrifugal acceleration on moisture with empirical model

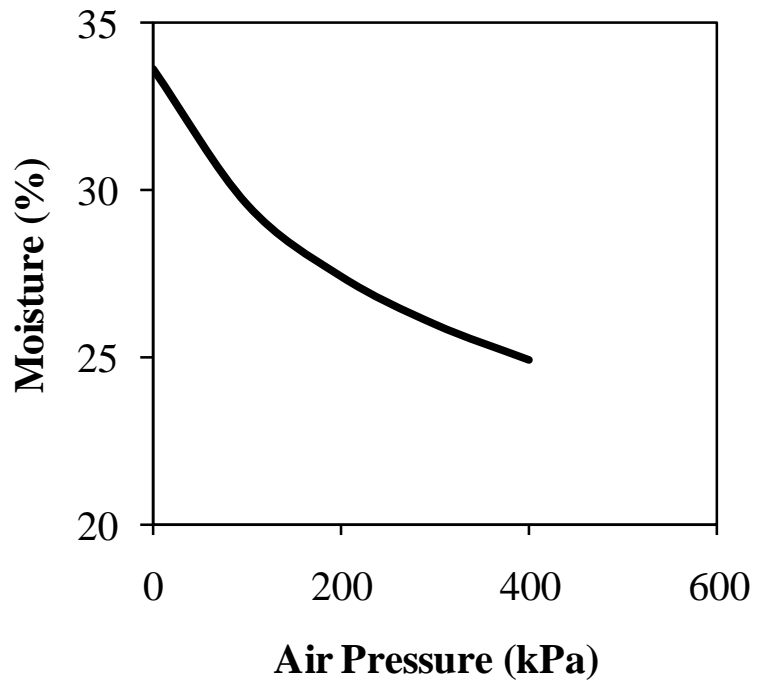

Figure 5.10 Effect of air pressure on moisture with empirical model 


\subsubsection{Theoretical Dewatering Model Simulations}

A simulation routine for the theoretical dewatering model was setup in Microsoft Excel using equations described in section 5.4 and was run using the same set of coals and parameters that were used to run the empirical dewatering model. Simulations were conducted with and without $200 \mathrm{kPa}$ air injection at 500 and 2700 g's. Results obtained with simulation routine were then compared to experimental results. All the input parameters used in these simulations are given in Table 5.9.

Table 5.9 Parameters used in dynamic dewatering simulations

\begin{tabular}{ccc}
\hline Parameter & Unit & Value \\
\hline$\rho_{s}$ & $\mathrm{~kg} / \mathrm{m}^{3}$ & 1400 \\
$\rho_{w}$ & $\mathrm{~kg} / \mathrm{m}^{3}$ & 1000 \\
$\varepsilon$ & -- & $0.45-0.55$ \\
$\sigma$ & $\mathrm{N} / \mathrm{m}$ & 0.072 \\
$\mu$ & $\mathrm{Pa.s}$ & 0.001 \\
$H$ & $\mathrm{~m}$ & 0.0085 \\
$t$ & $\mathrm{~s}$ & 120 \\
$N_{g}$ & -- & $500-2700$ \\
$P_{\text {air }}$ & Pascal & $0-206,843$ \\
& $(\mathrm{psi})$ & $(0-30)$ \\
$g$ & $\mathrm{~m} / \mathrm{s}^{2}$ & 9.81 \\
$\theta$ & $\circ$ & 60 \\
\hline
\end{tabular}

Conducting simulations using this theoretical model revealed serious issues related to the model. As can be seen from the charts given in Figure 5.12, predicted values were mostly off and did not match the experimental results. The model was able to predict relatively accurate moistures at 2700 g's without air injection with a low $\mathrm{R}^{2}$ value of 0.794 . However, all the other simulations conducted at different conditions (i.e. 500 g's with and without air and 2700 g's with air), produced negative $\mathrm{R}^{2}$ values, which showed that there was no correlation between predicted and measured values. The model had difficulty with producing decent results, especially with 
coarser cakes when air pressure was applied. As can be seen in Tables 5.10 and 5.11, it was not even possible to make any predictions for some of the cakes. The best estimations were obtained at 2700 g's without air injection.

(a)

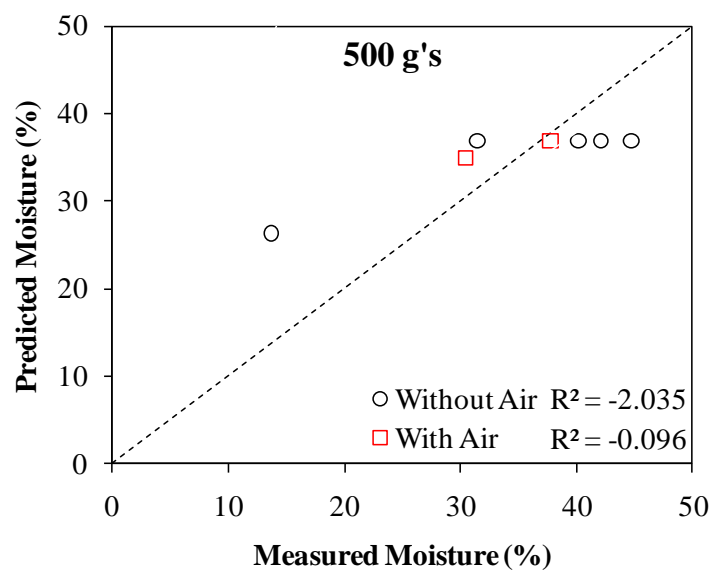

(b)

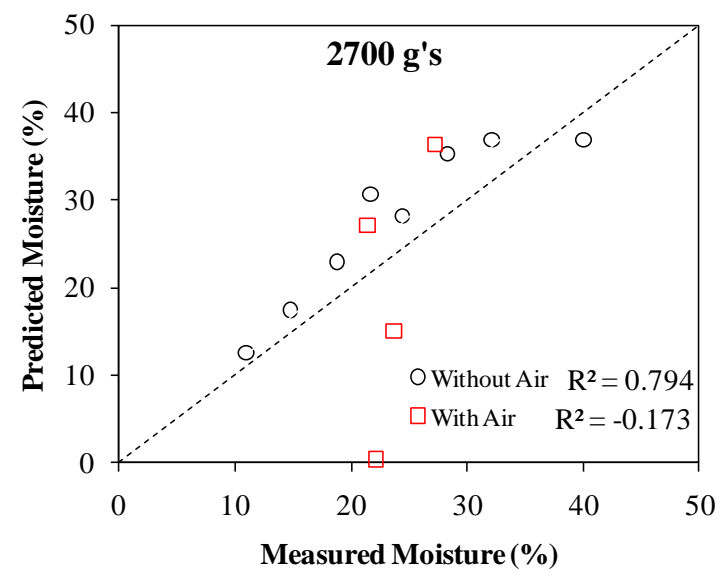

Figure 5.12 Comparison of predicted moistures to measured moistures at (a) $500 \mathrm{~g}$ 's and (b) $2700 \mathrm{~g}$ 's using the theoretical dewatering model

Table 5.10 Measured and predicted moisture values using the theoretical dewatering model at $500 \mathrm{~g}$ 's

\begin{tabular}{|c|c|c|c|c|c|c|c|c|}
\hline \multicolumn{7}{|c|}{ Mass (\%) } & \multirow{2}{*}{$\begin{array}{c}\text { Measured } \\
\text { Moisture } \\
(\%)\end{array}$} & \multirow{2}{*}{$\begin{array}{c}\text { Predicted } \\
\text { Moisture } \\
(\%)\end{array}$} \\
\hline $\begin{array}{c}-1.18 \\
\mathrm{~mm}\end{array}$ & $\begin{array}{l}-\mathbf{0 . 6} \\
\mathrm{mm}\end{array}$ & $\begin{array}{l}-0.3 \\
\mathbf{m m}\end{array}$ & $\begin{array}{c}-0.15 \\
\mathbf{m m}\end{array}$ & $\begin{array}{c}-\mathbf{- 0 . 0 7 5} \\
\mathrm{mm}\end{array}$ & $\begin{array}{c}-0.044 \\
\mathrm{~mm}\end{array}$ & $\begin{array}{c}-\mathbf{0 . 0 2 5} \\
\mathrm{mm}\end{array}$ & & \\
\hline \multicolumn{9}{|c|}{ (a) Without Air } \\
\hline 100 & 100 & 100 & 100 & 99.9 & 99.6 & 97.8 & 44.7 & 36.9 \\
\hline 100 & 100 & 100 & 99.9 & 99.1 & 97.0 & 84.4 & 42.1 & 36.9 \\
\hline 100 & 100 & 100 & 99.9 & 96.9 & 88.6 & 70.0 & 40.2 & 36.9 \\
\hline 100 & 100 & 100 & 95.5 & 72.0 & 58.0 & 43.6 & 31.4 & 36.9 \\
\hline 100 & 66.8 & 48.6 & 33.0 & 23.3 & 18.9 & 13.8 & 13.7 & 26.3 \\
\hline \multicolumn{9}{|c|}{ (b) With Air } \\
\hline 100 & 100 & 100 & 100 & 99.9 & 99.6 & 97.8 & 37.8 & 36.9 \\
\hline 100 & 100 & 100 & 99.9 & 99.1 & 97.0 & 84.4 & 30.5 & 34.9 \\
\hline 100 & 100 & 100 & 99.9 & 96.9 & 88.6 & 70.0 & 23.6 & N/A \\
\hline 100 & 100 & 100 & 95.5 & 72.0 & 58.0 & 43.6 & 20.3 & N/A \\
\hline 100 & 66.8 & 48.6 & 33.0 & 23.3 & 18.9 & 13.8 & 9.8 & N/A \\
\hline
\end{tabular}


Table 5.11 Measured and predicted moisture values using three different permeability estimation methods at $2700 \mathrm{~g}$ 's

\begin{tabular}{|c|c|c|c|c|c|c|c|c|}
\hline \multicolumn{7}{|c|}{ Mass (\%) } & \multirow{2}{*}{$\begin{array}{c}\text { Measured } \\
\text { Moisture } \\
(\%)\end{array}$} & \multirow{2}{*}{$\begin{array}{c}\text { Predicted } \\
\text { Moisture } \\
(\%)\end{array}$} \\
\hline $\begin{array}{c}-1.18 \\
\mathrm{~mm}\end{array}$ & $\begin{array}{l}-0.6 \\
\mathrm{~mm}\end{array}$ & $\begin{array}{l}-0.3 \\
\mathrm{~mm}\end{array}$ & $\begin{array}{c}-0.15 \\
\mathrm{~mm}\end{array}$ & $\begin{array}{c}-0.075 \\
\mathrm{~mm}\end{array}$ & $\begin{array}{c}-0.044 \\
\mathrm{~mm}\end{array}$ & $\begin{array}{c}-0.025 \\
\text { mm }\end{array}$ & & \\
\hline \multicolumn{9}{|c|}{ (a) Without Air } \\
\hline 100 & 100 & 99.7 & 99.6 & 99.5 & 99.1 & 97.2 & 40.0 & 36.9 \\
\hline 100 & 100 & 99.9 & 99.6 & 98.7 & 95.6 & 85.3 & 32.1 & 36.9 \\
\hline 100 & 100 & 99.5 & 96.1 & 86.4 & 77.6 & 63.3 & 28.3 & 35.2 \\
\hline 100 & 100 & 99.7 & 98.3 & 86.3 & 74.7 & 55.5 & 24.5 & 28.2 \\
\hline 100 & 100 & 99.5 & 92.8 & 76.6 & 66.3 & 52.4 & 21.7 & 30.6 \\
\hline 100 & 99.9 & 97.9 & 83.7 & 62.6 & 52.7 & 40.0 & 18.8 & 22.9 \\
\hline 100 & 97.2 & 81.6 & 57.8 & 43.4 & 35.6 & 27.7 & 14.8 & 17.4 \\
\hline 100 & 88.7 & 65.4 & 44.7 & 31.5 & 26.3 & 20.0 & 11.0 & 12.5 \\
\hline \multicolumn{9}{|c|}{ (b) With Air } \\
\hline 100 & 100 & 99.7 & 99.6 & 99.5 & 99.1 & 97.2 & 27.2 & 36.4 \\
\hline 100 & 100 & 99.5 & 96.1 & 86.4 & 77.6 & 63.3 & 23.7 & 15.1 \\
\hline 100 & 100 & 99.7 & 98.3 & 86.3 & 74.7 & 55.5 & 22.1 & 0.39 \\
\hline 100 & 100 & 99.9 & 99.6 & 98.7 & 95.6 & 85.3 & 21.4 & 27.1 \\
\hline 100 & 100 & 99.5 & 92.8 & 76.6 & 66.3 & 52.4 & 19.8 & N/A \\
\hline 100 & 100 & 97.9 & 83.7 & 62.6 & 52.7 & 40.0 & 16.1 & N/A \\
\hline 100 & 97.2 & 81.6 & 57.8 & 43.4 & 35.6 & 27.7 & 12.0 & N/A \\
\hline 100 & 88.7 & 65.4 & 44.7 & 31.5 & 26.3 & 20.0 & 9.6 & N/A \\
\hline 100 & 68.4 & 49.2 & 34.1 & 24.2 & 20.5 & 15.9 & 8.0 & N/A \\
\hline
\end{tabular}

Another set of simulation runs were conducted to study the effect of different parameters on cake moistures with the theoretical dewatering model. For this purpose, the case that produced the best correlation before (i.e. 2700 g's without air injection) was chosen as the baseline case. While keeping all the other parameters constant, value of only one parameter was changed at a time to demonstrate the effect of that parameter on the cake moisture.

The simulation results given in Figure 5.13 showed that although most of the parameters used in the theoretical dewatering model would affect the kinetics of centrifugal dewatering in an expected way, the predicted equilibrium moistures were unreasonable. Model predictions showed that changing the cake permeability would affect dewatering kinetics immensely. 
However, when significantly lower permeability values were used, the model's moisture predictions were as low as $0 \%$. Moreover, increasing contact angle, centrifugal acceleration and air pressure values and decreasing surface tension of the liquid also produced faster dewatering kinetics and lower equilibrium moistures, but again some of the moisture predictions were as low

(a)

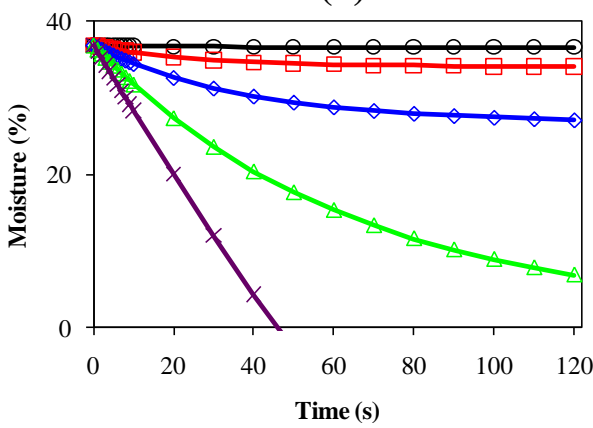

(c)

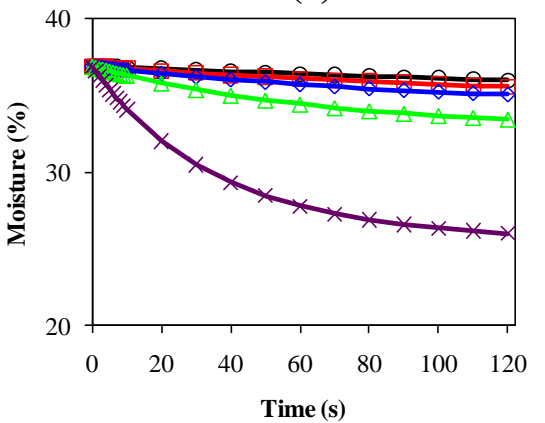

(e)

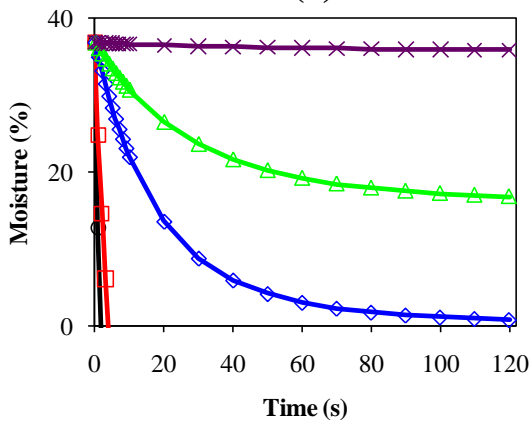

(b)

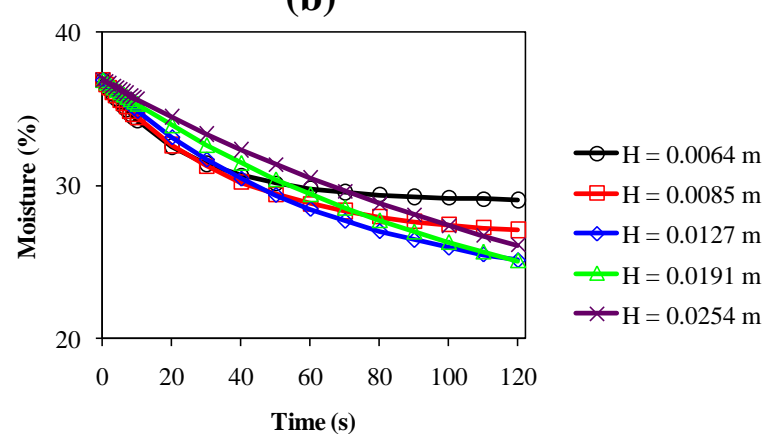

(d)

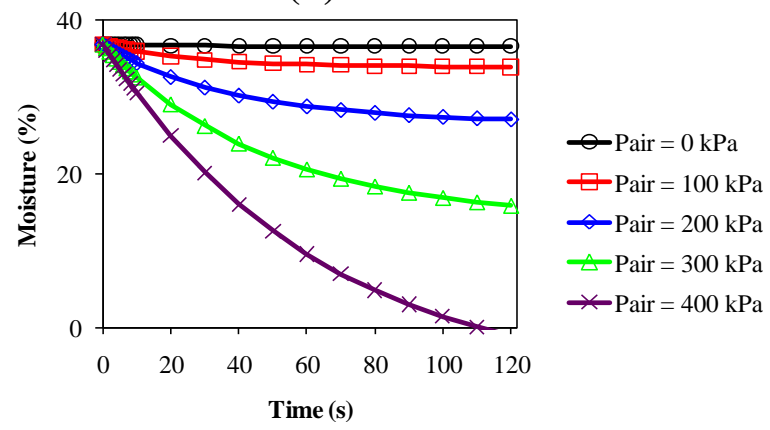

(f)

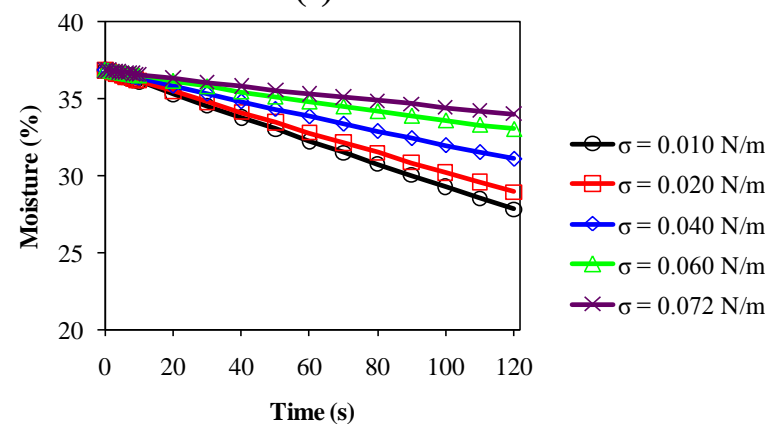

Figure 5.13 Effect of (a) contact angle, (b) cake thickness, (c) centrifugal acceleration, (d) air pressure, $(e)$ cake permeability and $(f)$ liquid surface tension on moisture with theoretical dewatering model 
as $0 \%$. Furthermore, according to the model, the equilibrium moisture content first decreased while the cake thickness increased from $0.0064 \mathrm{~m}$ to $0.0127 \mathrm{~m}$. However, after this point the moisture increased with increased cake thickness.

As a result, it was concluded that there was a fundamental problem with this model, which could not be solved. While the effects of different parameters on cake moistures were generally reasonable, the equilibrium moisture predictions were unreasonable. An investigation directed at improving the theoretical model is suggested as a topic for future research.

\subsection{Mechanical Degradation}

Mechanical degradation of particles is unavoidable in centrifugation due to the forces involved in this process. This effect was not taken into consideration when the empirical and theoretical model simulations given in previous sections were conducted, because those size distributions were obtained from products of real HFC tests, which were already degraded. However, it is essential to use a degradation model when dealing with feed size distributions, i.e. size reduction of the feed inside a centrifuge has to be taken into account before any attempt to model centrifugal dewatering process.

Lyons (1951) did a systematic study of mechanical degradation and he found out that larger particles were subject to size reduction more than finer particles. He also found out that the size distribution of newly created fine particles was similar to the ones already existed in the feed.

Particles existing in the feed stream of a centrifuge can be divided into $n$ size ranges, where size 1 is the coarsest size fraction and size $n$ is the finest. It is assumed that the fraction of size class $i$ that can be degraded is linearly related to the average particle size by the following relation: 


$$
D_{i}=k_{d} A_{i} ; 0<D_{i}<0.9
$$

where $D_{i}$ is the fraction of size class $i$ degraded, $A_{i}$ is the average particle size of the size class $i$ and $k_{d}$ is a parameter unique to the equipment. Tierney and Gottfried (1983) reported that an average $k_{d}$ value of $98.4 \mathrm{~m}^{-1}$ can be used for most centrifuges. However, when simulations given in the following sections were conducted, it was observed that a $k_{d}$ value of $400 \mathrm{~m}^{-1}$ was required to match data from commercially available centrifuges. Therefore, a value of $400 \mathrm{~m}^{-1}$ was used for all the empirical simulations conducted in the present work.

The degraded fraction of size class $i$, given by $D_{i}$, is distributed among all size classes that are finer than the size class $i$, in proportion to the fractions of these size classes in the feed. Moreover, the distribution will be different for each gravity class $j$. Therefore, the contribution to size class $p(p>i)$ due to the degradation of size class $i$ in gravity class $j$ is given by:

$$
G_{p, i, j}=F_{i, j} D_{i} F_{p, j} / \sum_{k=i+1}^{n}\left(F_{k, j}\right)
$$

where $F_{i, j}$ is the weight fraction of feed in size class $i$ and gravity class $j$ before degradation. The total contribution to size $p$ from each size class coarser than $p$ can be obtained by summing all $G_{p, i, j}$ values from $i=1$ to $p-1$. The corrected fraction of feed in size class $p$ and gravity class $j$ after degradation can be determined by adding the summation of $G_{p, i, j}$ values to amount that was not degraded in size class $p$.

$$
F_{p, j}^{*}=\sum_{i=1}^{p-1}\left(G_{p, i, j}\right)+\left(1-D_{p}\right) F_{p, j}
$$

\subsection{Conclusions}

An empirical dewatering model and a theoretical dewatering model were developed to describe the process of dewatering during hyperbaric centrifugation. While very accurate results 
were obtained using the empirical model, the theoretical model had difficulty in predicting accurate product moistures, which suggested that attempts to precisely model a complicated system such as a filter cake using theoretical equations might lead to inaccurate results due to the vast number of unknowns and assumptions. Consequently, only the empirical model is recommended for predicting the equilibrium moistures obtained during hyperbaric centrifugation. 


\section{NOMENCLATURE}

\begin{tabular}{|c|c|c|c|}
\hline A: & Surface area of filter cake, $\mathrm{m}^{2}$ & $S_{0}:$ & $\begin{array}{l}\text { Specific surface area defined by Eq. } \\
\text { [5.15] }\end{array}$ \\
\hline$A_{i}:$ & $\begin{array}{l}\text { Average size, mean of upper and } \\
\text { lower mesh sizes, } m\end{array}$ & $S_{K}$ & $\begin{array}{l}\text { Skewness of the particle size } \\
\text { distribution }\end{array}$ \\
\hline$b:$ & Radius of a capillary, m & $S_{r}:$ & Residual Saturation \\
\hline$c_{1}, c_{2}$ & Fitting parameters used in Eq. [5.14] & $S_{e}:$ & Effective Saturation \\
\hline$C_{D P}:$ & Coefficient of variation & $s:$ & Diameter of a capillary, m \\
\hline$C_{t}:$ & Defined by Eq. [5.10] & $s^{*}:$ & Critical pore diameter, m \\
\hline$D_{i}:$ & $\begin{array}{l}\text { Fraction of particles of size i which } \\
\text { is degraded }\end{array}$ & $\hat{s}:$ & Predominant pore diameter, m \\
\hline$D_{p}:$ & Particle diameter, m & $t:$ & Time, s \\
\hline$F_{i, j} F_{i, j}^{*}$ & $\begin{array}{l}\text { Weight fraction of feed in size range } \\
\mathrm{i} \text { and gravity range } \mathrm{j} \text { before and after } \\
\text { degradation }\end{array}$ & $V_{R}:$ & $\begin{array}{l}\text { Volume of water that cannot be } \\
\text { removed from the filter cake, } \mathrm{m}^{3}\end{array}$ \\
\hline$g(s):$ & Pore size distribution function & $V_{E}:$ & $\begin{array}{l}\text { Volume of water that is not removed } \\
\text { from the filter cake due to time } \\
\text { limitation, } \mathrm{m}^{3}\end{array}$ \\
\hline$g:$ & Gravitational acceleration, $\mathrm{m} / \mathrm{s}^{2}$ & $y:$ & $\begin{array}{l}\text { Dimensionless parameter defined by } \\
\text { Eq. [5.17] }\end{array}$ \\
\hline$G_{p, i, j}:$ & $\begin{array}{l}\text { Fraction of feed size } \mathrm{i} \text { and gravity } \mathrm{j} \\
\text { which appears in size } \mathrm{p} \text { after } \\
\text { degradation }\end{array}$ & $y(t, s):$ & $\begin{array}{l}\text { Momentary height of liquid in a } \\
\text { capillary with diameter } s \text { at time } t, \mathrm{~m}\end{array}$ \\
\hline$h_{0}:$ & $\begin{array}{l}\text { Equilibrium height of a liquid in a } \\
\text { pore, } m\end{array}$ & $\eta:$ & Viscosity of the liquid, Pa.s \\
\hline$H:$ & Cake thickness, m & $\rho_{w}, \rho_{s}:$ & Density o water and solids, $\mathrm{kg} / \mathrm{m}^{3}$ \\
\hline$k_{d}:$ & Degradation constant, dimensionless & $\sigma:$ & Surface tension of liquid, N/m \\
\hline$K:$ & Permeability of filter cake, $\mathrm{m}^{2}$ & $\theta:$ & Contact angle \\
\hline$N_{c}:$ & Capillary number, dimensionless & $\varepsilon:$ & Porosity or the void fraction \\
\hline$N_{g}:$ & Number of g's & $\tau:$ & Tortuosity of particles \\
\hline$P_{\text {air }}$ : & Air pressure, $\mathrm{kPa}$ & $\zeta$ & Parameter defined by Eq. [5.27] \\
\hline$P:$ & Pressure, $\mathrm{Pa}$ & $\beta$ : & Parameter defined by Eq. [5.28] \\
\hline$r:$ & $\begin{array}{l}\text { Radius coordinate starting at the } \\
\text { pore axis, } m\end{array}$ & $X:$ & Parameter defined by Eq. [5.29] \\
\hline$S:$ & Total Saturation & & \\
\hline
\end{tabular}




\subsection{ECONOMICS OF FINE COAL DEWATERING}

\subsection{Introduction}

Modern coal preparation plants typically incorporate a series of sequential unit operations for sizing, cleaning, and dewatering. This sequence of steps is repeated for each size fraction. This subdivision is necessary since the cleaning processes used in modern plants have a limited range of applicability in terms of particle size. As a result, modern plants may include as many as four separate processing circuits for treating the coarse (plus $50 \mathrm{~mm}$ ), small (50 x $1 \mathrm{~mm}$ ), fine (1 x $0.15 \mathrm{~mm}$ ), and ultrafine (minus $0.15 \mathrm{~mm}$ ) material.

In the U.S., coarse and small size coal is typically cleaned using dense medium processes such as vessels and cyclones. The coarser products are dewatered using screens, while smaller coal is dewatered using centrifugal dryers. Fine coal is usually upgraded using water-based density separators such as spirals and water-only cyclones. Ultrafine coal is either cleaned using froth flotation or discarded as waste. The fine and ultrafine coal products are dewatered using various combinations of centrifugal dryers, screenbowl centrifuges and/or filters. After dewatering, the clean coal products from the parallel circuits are usually blended together prior to shipment to market.

An important task for plant managers is to select operating points for each plant process to ensure that the coal products from the plant meet quality criteria dictated by sales contracts. These specifications may include limits on ash, sulfur, moisture, heat content, or other quality indicators. Unfortunately, plant operators often choose set points that provide an identical clean coal quality in every plant circuit. As discussed later, operation of all circuits at a constant product quality typically does not optimize plant yield. Moreover, managers are often forced to 
select set points that are less than ideal for a given coal due to contractual limitations that may or may not be justified from economic considerations. A mismatch between the inherent cleanability of a given coal (including its tendency to retain moisture) and the specifications in a sales contract can substantially lower overall plant yield and overall profitability.

\subsection{Plant Optimization}

\subsubsection{Optimization by Constant Incremental Ash}

Many articles in the technical literature advocate the use of simulation routines to identify optimum set points for processes used in coal preparation plants (King, 1999; Peng \& Luckie, 1991). Such data are used to determine the total plant yield and clean coal quality that may be obtained for any combination of operating points. The optimum points are those that provide the highest total plant yield at the desired target quality. However, the simulations require detailed data describing the washability of the feed coals and empirical and/or theoretical partition data describing the separating capabilities of the cleaning equipment. Unfortunately, the lack of such detailed data makes this approach impractical to implement in modern facilities. This trial-anderror approach also provides little insight regarding the underlying principles that control the optimization process.

A more attractive method for plant optimization is to employ constant incremental ash. This concept, which has long been recognized in coal preparation (Abbott, 1981; Dell, 1956; Mayer, 1950; Rayner, 1987), states that the clean coal yield for parallel operations is highest when all circuits are operated at the same incremental ash. This statement is true for any number of parallel circuits and is independent of the size and washability characteristics of the feed coal. Mathematical proof of the incremental ash concept has been done by Luttrell et al. (2003). However, the fundamental basis for this concept can be easily demonstrated using the simple 
illustrations provided in Figure 6.1(a) and Figure 6.1(b). In each diagram, the same two feed streams (" $\mathrm{A}$ " and "B") are to be treated. The two feeds may represent coal fed to two different cleaning operations in the same plant, the streams fed to two different plants that both service the same customer, or the same cleaning unit at two different points in time. The feed coals are comprised of various amounts of carbonaceous matter (black) and ash-bearing matter (gray). In this example, the washability of Feed "A" makes it relatively easy to upgrade since most of the particles are well liberated. In contrast, Feed "B" has a difficult washability due to the presence of a large percentage of middlings (composite) particles.

Figure 6.1(a) shows the result that is obtained when the separation is conducted to provide a target ash content of $25 \%$ for both streams. For Feed "A", the operating point is raised
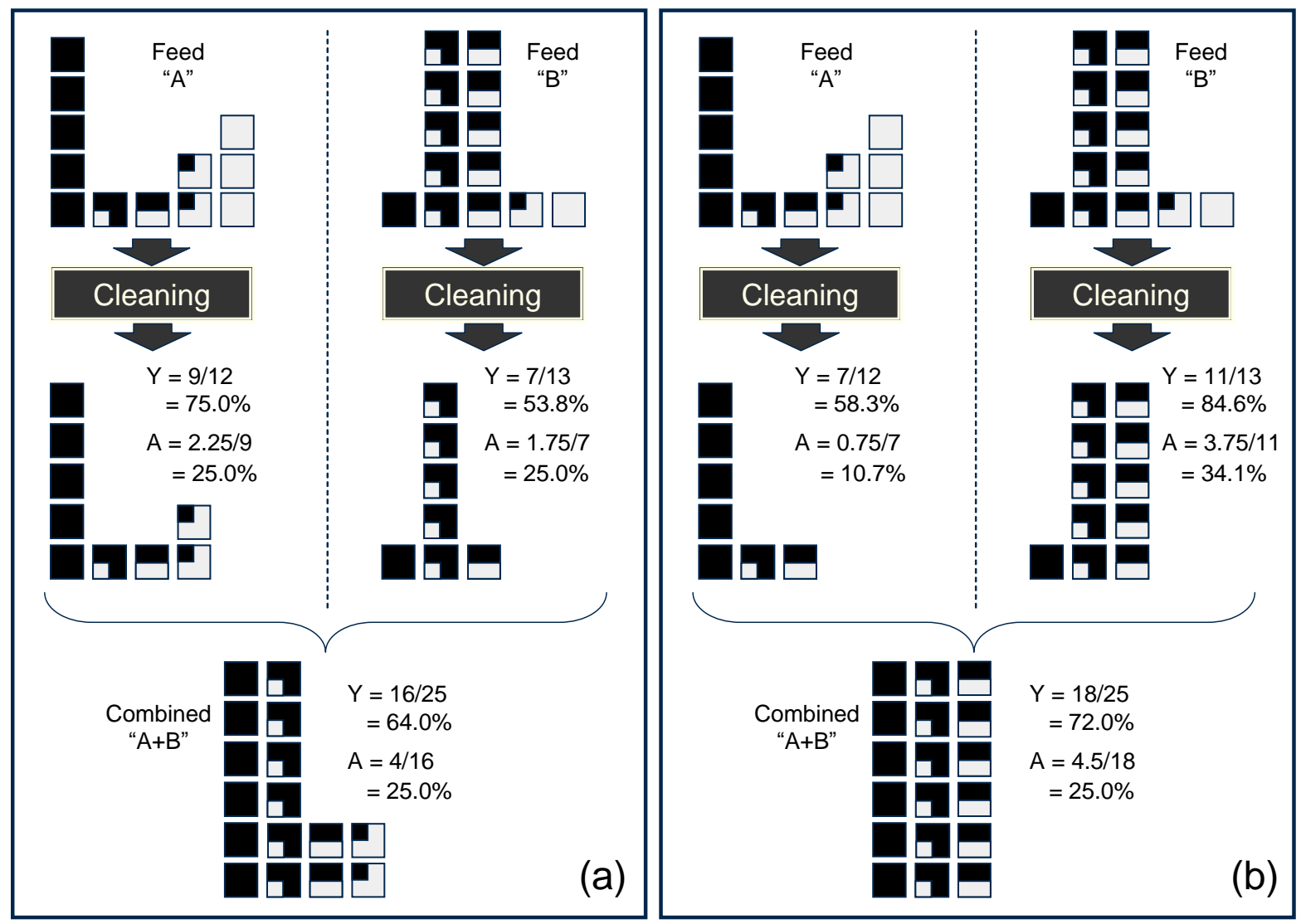

Figure 6.1 Clean coal products obtained by (a) treating parallel streams at constant cumulative ash and (b) treating parallel streams at constant incremental ash 
from left to right until a total of 9 units (blocks) are recovered out of a total of 12 units. This operating point produces a yield of $75 \%$ (i.e., $9 / 12=75 \%$ ) at the desired target ash of $25 \%$ (i.e., $2.25 / 9=25 \%$ ). For Feed "B", a yield of only $53.8 \%$ (i.e., $7 / 13=53.8 \%$ ) can be realized before the target ash of $25 \%$ (i.e., $1.75 / 7=25 \%$ ) is exceeded. The lower yield is due to the poorer liberation of Feed "B". The clean products from these two streams are blended together to produce a combined yield of $64 \%$ at the desired target ash of $25 \%$. At first inspection, this appears to be an acceptable result since the target grade is met. However, a closer examination shows that particles containing $75 \%$ ash reported to clean coal when treating Feed " $\mathrm{A}$ ", while at the same time many particles containing just 50\% ash were discarded when treating Feed "B". This combination is obviously not optimum since higher quality particles were discarded so that poorer quality particles could be taken.

The incremental ash concept dictates that maximum yield can only be obtained when the ash content of the last unit recovered from each feed stream is identical. To test this principle, the feed streams shown in Figure 6.1(b) are each separated so that the last unit recovered in both cases contained $50 \%$ ash. This combination of operating points decreased the ash content of Feed "A" to $10.7 \%$ and increased the ash content of Feed "B" to $34.1 \%$. However, when the two streams are blended together, the combined clean coal product still meets the required target ash of $25 \%$. More importantly, this new set of operating points provides a higher combined yield (i.e., $72 \%$ versus $64 \%$ ). In fact, separation at a constant incremental ash will always provide the maximum yield of coal at a given ash content.

\subsubsection{Incremental Ash and Specific Gravity}

Incremental ash is a mathematical term that cannot be directly monitored in operating industrial plants. However, this value can be estimated for ideal separations. This approximation 
assumes that the particles in run-of-mine coals contain only two components, i.e., a low-density ash-free carbonaceous component and a high-density pure-ash mineral component. Under this assumption, the ash content of an individual particle must increase linearly with the reciprocal of the composite particle density $(\rho)$ according to the expression:

$$
\operatorname{Ash}(\%)=\frac{100}{\rho_{c}-\rho_{a}}\left[\frac{\rho_{c}+\rho_{a}}{\rho}+\rho_{a}\right]
$$

where $\rho_{\mathrm{c}}$ and $\rho_{\mathrm{a}}$ are the densities of the light (carbonaceous) and dense (ash bearing) components, respectively (Abbott \& Miles, 1990). The utility of this expression can be demonstrated by the data shown in Figure 6.2. This illustration shows the ash contents of narrowly partitioned density fractions obtained from experimental float-sink tests conducted on six different size fractions of a run-of-mine coal. For particles coarser than $0.6 \mathrm{~mm}$, the data show that the same individual ash is obtained for any specific gravity regardless of the size fraction treated. The deviation noted for the fractions finer than $0.6 \mathrm{~mm}$ can normally be attributed to inefficiencies in the experimental float-sink procedures at finer particle sizes. In any case, plant optimization requires that all parallel circuits be operated at the same incremental ash. Since Eq. [6.1] shows that incremental ash is fixed by specific gravity, this implies that total plant yield is maximized when all parallel circuits are operated at the same specific gravity cutpoint (Clarkson, 1992). This statement is true regardless of the size distribution or

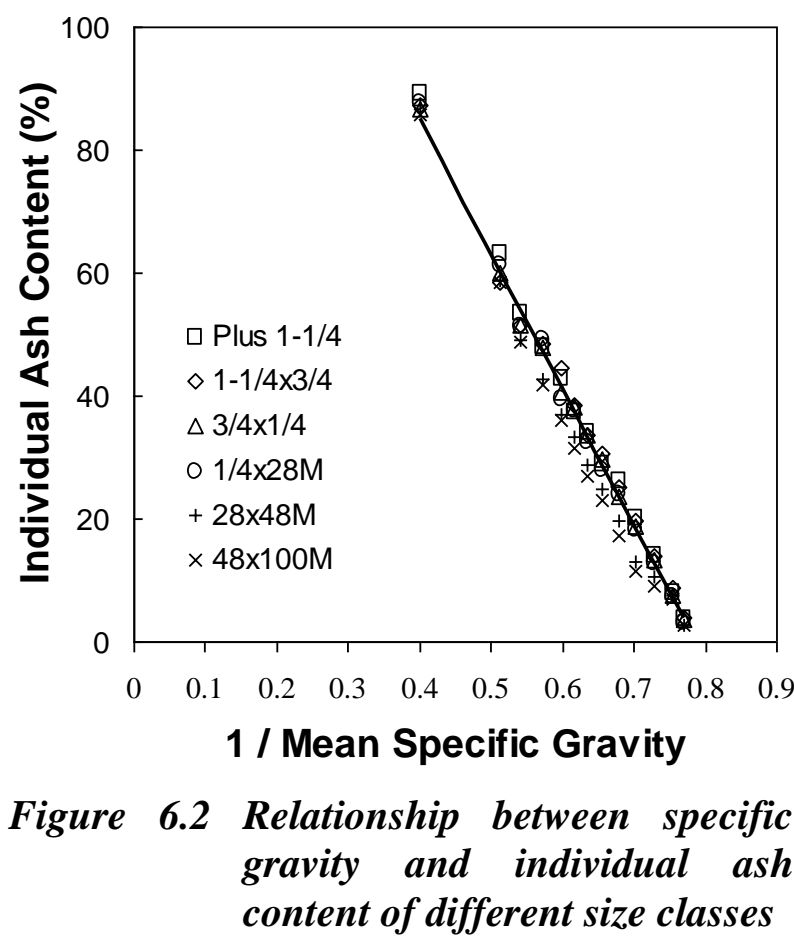


washability characteristics of the feed coal, provided that very efficient separations are maintained in each circuit. If the separation is less efficient (which is typical of fine coal separators), a correction must be made to account for the impacts of misplaced material on the incremental quality. This correction generally requires that less efficient units be operated at a slightly higher specific gravity cutpoint (Luttrell, et al., 2003).

\subsubsection{Effect of Moisture on Optimization}

The incremental ash approach to plant optimization works well for processing metallurgical coals where ash is the primary quality specification. Unfortunately, most coal is sold in the steam (utility) market where other quality specifications are also important (e.g. moisture, heat content, etc.). In particular, the combined ash and moisture content of a coal is very important in this market since this sum dictates the heat content of the coal. Therefore, in the case of steam market, optimization requires that the "incremental value" of the coal be held constant. In other words, the market value of the next particle of coal recovered should be the identical for all circuits. As such, the incremental value concept includes not only incremental ash, but also incremental moisture and, if appropriate, other incremental values that impact the monetary value of the coal product (e.g., sulfur). The inclusion of moisture in the incremental value approach typically dictates that density cutpoints for operations treating coarser particles be set higher than the density cutpoints used to treat finer particles. This difference is due to the fact that moisture content increases (and coal value decreases) as the particle becomes finer. As can be seen from the following example, the increased moisture can have a dramatic impact on the operating points needed for plant yield optimization. 


\subsubsection{Incremental Quality and Coal Value}

Any discussion of plant optimization must address the issue of coal value. Although coal payments in the steam (utility) market are typically reported on a per ton basis, price adjustments are nearly always applied to compensate for variations in the heat content of the as-received product. For example, consider the tabular analysis illustrated in Table 6.1(a) and Table 6.1(b). In this simplified example, two scenarios for the same cleaning operation are shown. In the first scenario, it is assumed that the product is dry; therefore, moisture has no effect on the coal value. In the second case, the product contains $16.67 \%$ moisture, which drives down both the heat content and the market value of the coal. In this example, pure particles of carbonaceous matter ( $\mathrm{SG}=1.3$ ) were assigned a heat value of 15,000 BTU/lb (dry ash-free basis), while pure particles of rock ( $\mathrm{SG}=2.5)$ were assigned no heat value. The sales agreement for this example dictates a market price of $\$ 30.00$ per ton of as-received clean coal with quality specifications of 12,500 BTU/lb and 10\% ash (as-received). The coal payment schedule is prorated at \$0.20 per 100 BTU above/below 12,500 BTU/lb and \$0.50 per 1\% ash above/below $10 \%$ ash. A $\$ 10.00$ per ton sales related cost has also been applied to account for miscellaneous fees, taxes and other sales expenses associated with the coal production.

The calculations for the first case indicate that pure coal particles carry a $\$ 5.00$ per ton BTU premium (i.e., $[15,000-12,500]$ x $\$ 0.20 / 100=\$ 5.00$ ) and $\$ 5.00$ per ton ash premium (i.e., [10-0] x \$0.50/1 = \$5.00). After subtracting a sales related cost of $\$ 10.00$ per ton, the net value of these high grade particles is $\$ 30.00$ per ton (i.e., $\$ 30.00$ base $+\$ 10.00$ premium $-\$ 10.00$ sales

$=\$ 30.00)$. In contrast, the pure rock particles carry a $\$ 25.00$ per ton BTU penalty (i.e., [0$12,500] / 100 \times \$ 0.20=\$ 25.00)$ and $\$ 45.00$ per ton ash penalty (i.e., [10-100]/1 x $\$ 0.50=-$ $\$ 45.00)$. Thus, after subtracting a sales related cost of $\$ 10.00$ per ton, these low-grade particles 
have a net negative value of minus $\$ 50.00$ per ton (i.e., $\$ 30.00$ base $-\$ 70.00$ penalty $-\$ 10.00$ sales $=-\$ 50.00)$. In other words, the producer gains $\$ 30.00$ for every ton of pure carbonaceous material shipped to the customer and loses $\$ 50.00$ per ton of pure rock shipped. Composite particles containing mixed amounts of coal and rock have market values between these two limits. In order to maximize profitability, the coal producer for this example should obviously ship only those particles that have a positive net value and should never ship particles that have a

Table 6.1 Simplified value calculations for particles contained in run-of-mine coal conducted (a) without any moisture and (b) with $16.67 \%$ moisture

Scenario (a)

\begin{tabular}{cccccccc}
\hline & & & & & & \\
& & & & & & & \\
& & & & & & & \\
& & & & & & & \\
\hline Particle Type & 1.30 & 1.55 & 1.60 & $\mathbf{1 . 6 5}$ & 1.70 & 2.50 \\
Ash Content (\%) & 0.0 & 29.2 & 33.3 & $\mathbf{3 7 . 5}$ & 41.7 & 100.0 \\
Moisture Content (\%) & 0 & 0 & 0 & $\mathbf{0}$ & 0 & 0 \\
Heat Content (BTU/lb) & 15000 & 10625 & 10000 & $\mathbf{9 3 7 5}$ & 8750 & 0 \\
\hline BTU Value Adjustment & $\$ 5.00$ & $-\$ 3.75$ & $-\$ 5.00$ & $\mathbf{- \$ 6 . 2 5}$ & $-\$ 7.50$ & $-\$ 25.00$ \\
Ash Value Adjustment & $\$ 5.00$ & $-\$ 9.58$ & $-\$ 11.67$ & $\mathbf{- \$ 1 3 . 7 5}$ & $-\$ 15.83$ & $-\$ 45.00$ \\
Sales Cost & $-\$ 10.00$ & $-\$ 10.00$ & $-\$ 10.00$ & $\mathbf{- \$ 1 0 . 0 0}$ & $-\$ 10.00$ & $-\$ 10.00$ \\
\hline Net Value & $\$ 30.00$ & $\$ 6.67$ & $\$ 3.33$ & $\mathbf{\$ 0 . 0 0}$ & $-\$ 3.33$ & $-\$ 50.00$ \\
\hline
\end{tabular}

Scenario (b)

\begin{tabular}{cccccccc}
\hline & & & & & & \\
& & & & & & & \\
& & & & & & & \\
& & & & & & & \\
\hline Particle Type & 1.30 & 1.55 & $\mathbf{1 . 6 0}$ & 1.65 & 1.70 & 2.50 \\
Ash Content (\%) & 0.0 & 29.2 & $\mathbf{3 3 . 3}$ & 37.5 & 41.7 & 100.0 \\
Moisture Content (\%) & 16.67 & 16.67 & $\mathbf{1 6 . 6 7}$ & 16.67 & 16.67 & 16.67 \\
Heat Content (BTU/lb) & 12500 & 8854 & $\mathbf{8 3 3 3}$ & 7813 & 7292 & 0 \\
\hline BTU Value Adjustment & $\$ 0.00$ & $-\$ 7.29$ & $\mathbf{- \$ 8 . 3 3}$ & $-\$ 9.37$ & $-\$ 10.42$ & $-\$ 25.00$ \\
Ash Value Adjustment & $\$ 5.00$ & $-\$ 9.58$ & $\mathbf{- \$ 1 1 . 6 7}$ & $-\$ 13.75$ & $-\$ 15.83$ & $-\$ 45.00$ \\
Sales Cost & $-\$ 10.00$ & $-\$ 10.00$ & $\mathbf{- \$ 1 0 . 0 0}$ & $-\$ 10.00$ & $-\$ 10.00$ & $-\$ 10.00$ \\
\hline Net Value & $\$ 25.00$ & $\$ 3.13$ & $\mathbf{\$ 0 . 0 0}$ & $-\$ 3.13$ & $-\$ 6.25$ & $-\$ 50.00$ \\
\hline
\end{tabular}


negative net value. In this example, the "zero value" particle, which contains just enough heat content to justify its shipment to market, occurs at a density of 1.75 SG (i.e., an incremental ash of 37.5\%). Therefore, for this simplistic example, which does not include the effect of moisture on coal value, the plant operator should set and maintain an effective specific gravity cutpoint of 1.75 SG to ensure that profitability is maximized.

The second scenario is identical to the first except the impact of moisture has been included in the value calculation. In this scenario, the heat value of particles drops with the presence of moisture, thus driving down the net value of each coal particle. The pure particles of carbonaceous matter (SG=1.3) now have a heat value of 12,500 BTU/lb (i.e., 15,000 x [10016.67] $/ 100=12,500 \mathrm{BTU} / \mathrm{lb})$. Therefore, these particles no longer carry a BTU premium. They still carry the same ash premium of $\$ 5.00$ per ton, since the ash content did not change. (In reality, the presence of moisture would reduce the as-received ash content slightly). Thus, after subtracting the sales cost of $\$ 10$ per ton, these particles now have a lower net value of $\$ 25$ per ton (i.e., $\$ 30$ base $+\$ 5$ premium $-\$ 10$ sales $=\$ 25.00)$. The pure rock particles $(\mathrm{SG}=2.5)$ still have the same net value of $-\$ 50$ since moisture does not further reduce the heat value of 0 BTU/lb material. When all these factors are considered, the presence of moisture lowered the optimum density cutpoint for the "zero value" particle from 1.75 to $1.70 \mathrm{SG}$. In other words, the presence of moisture reduces the optimum density for plant optimization for plants providing coals for the steam market. In fact, very fine particles may contain so much moisture that they have negative value even if they contain no ash. In such cases, the use of desliming circuits to remove and discard ultrafine coal without further processing would be well justified (Bethell \& Luttrell, 2005). 
This above example is important because it shows that the specific gravity for optimum plant operation is controlled by the coal sales agreement and is largely independent of the coal washability. Moreover, penalties attributable to other factors that influence the monetary value of coal such as moisture, sulfur, etc., can shift the optimum density for separations to values that are lower than those predicted by the well-known incremental ash concept. Although this example has been oversimplified, the underlying optimization principle illustrated by this analysis can conceptually be applied to any generalized case if the contractual details are available. Such an example is examined in the following case study.

\subsection{Case Study}

The optimization of plant yield via proper management of incremental value and the effect of moisture on incremental value can be best illustrated by means of a case study. The study was conducted to evaluate three different circuit designs that treated a minus $1 \mathrm{~mm}$ coal stream (see Figure 6.3). The first circuit design was a standard fine coal dewatering circuit used at U.S. coal plants with desliming. In this circuit design, underflow stream of a 15-inch classifying hydrocyclone (nominal $1 \times 0.150 \mathrm{~mm}$ ) was sent to a spiral for cleaning, while the overflow stream (nominal $0.150 \times 0 \mathrm{~mm}$ ) was sent to a 6-inch desliming hydrocyclone. The underflow stream (nominal $0.150 \times 0.044 \mathrm{~mm}$ ) of this hydrocyclone was cleaned with flotation and the clean product was then dewatered using a screenbowl centrifuge along with the spiral product. Moreover, the overflow stream (nominal 0.044 x $0 \mathrm{~mm}$ ) of the desliming hydrocyclone and the main effluent stream of the screenbowl centrifuge were discarded. In the second circuit configuration, the desliming hydrocyclone was removed and the whole overflow stream of the 15-inch hydrocyclone was cleaned using flotation. Again, the underflow stream of this hydrocyclone was cleaned using a spiral. However, in this circuit design, while the spiral product 
(nominal $1 \times 0.150 \mathrm{~mm}$ ) was dewatered using a screenbowl centrifuge, the flotation product and the main effluent of the screenbowl centrifuge (nominal $0.150 \times 0 \mathrm{~mm}$ ) were sent to a Centribaric $^{\mathrm{TM}}$ centrifuge for dewatering. Finally, the third circuit design consisted of a 6-inch hydrocyclone similar to the first circuit configuration. However, the overflow stream (nominal $0.044 \times 0 \mathrm{~mm}$ ) of this hydrocyclone was not discarded, but was cleaned with an ultrafine flotation column. While the combined products of the spiral and the deslime flotation (nominal 1 x $0.044 \mathrm{~mm}$ ) were dewatered using a screenbowl centrifuge, ultrafine flotation product along with the main effluent of the screenbowl centrifuge (nominal $0.044 \times 0 \mathrm{~mm}$ ) were dewatered using a Centribaric ${ }^{\mathrm{TM}}$ centrifuge. 

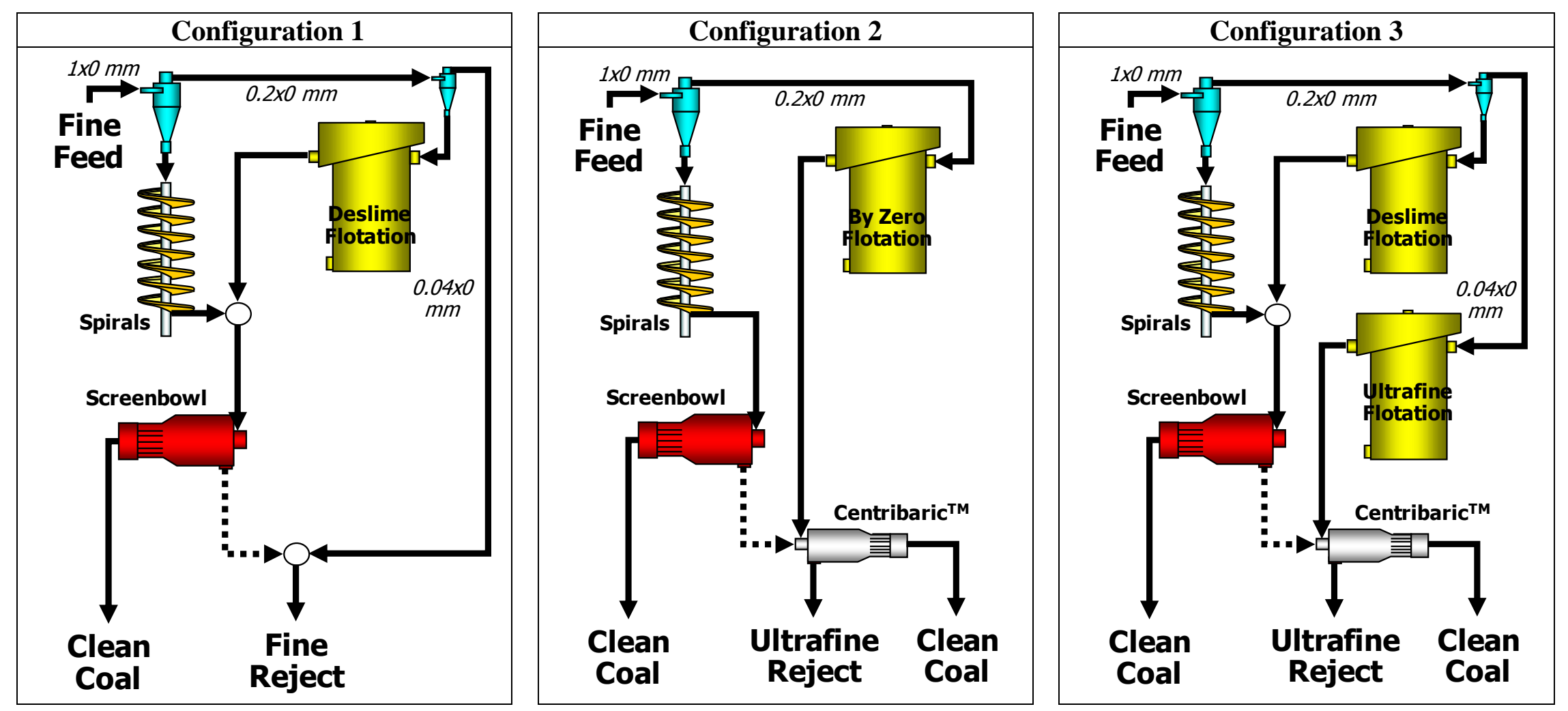

Figure 6.3 Different circuit configurations evaluated for economic analysis 
All three circuit configurations were tested with two different coals with size distributions given in Figure 6.4, where coals A and B represent two extreme cases with Hardgrove indexes of 47 and 116, respectively. Assumptions in the analyses include freight charges of \$20/ton coal, evaporation costs of $\$ 3 /$ ton of water, ash handling expenses of $\$ 20 /$ ton of ash, emission penalties of $\$ 200 /$ ton of $\mathrm{SO}_{2}$ emitted, and miscellaneous charges of $\$ 1.00 /$ ton of clean coal handled. A constant fuel cost to the boiler of \$2.44/MM BTU was maintained for all calculations. Also, the feed stream was assumed to contain clean coal particles with an average of 1.4 SG, which corresponds to about $14.5 \%$ dry ash content. All calculations were conducted based on 100 dry tph of feed coal and were performed in a similar fashion to the ones that are given in Table 6.1 (see Appendix 2 for detailed calculations).

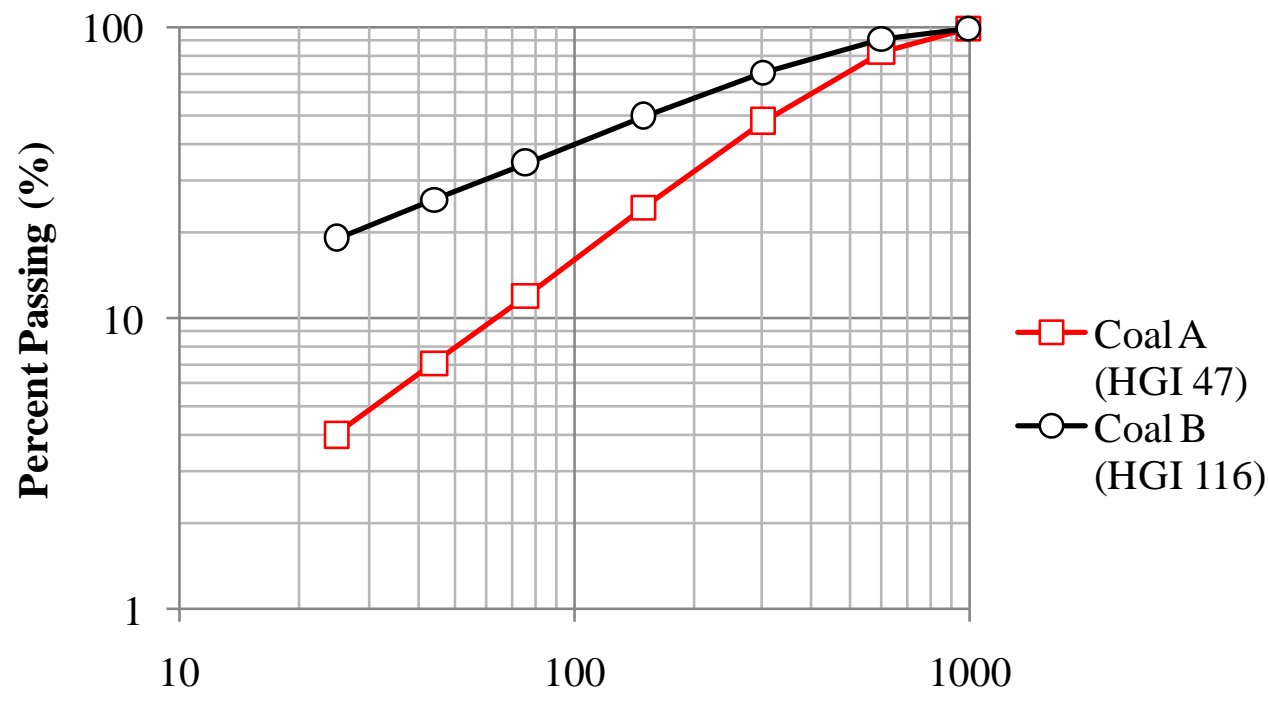

Particle Size (micron)

Figure 6.4 Size distributions of coals $A$ and $B$ used for the case study

Since it was assumed that no coal particles would be lost to effluent streams due to recirculating effluent when the hyperbaric centrifuge was used, both the second and the third 
circuit configurations yielded $100 \%$ coal to product; whereas some coal particles were lost in the first configuration due to discarding the effluent. Results of the analyses given in Table 6.2 showed that for both coals A and B, the lowest moisture value would be produced when the second circuit configuration is used. While $\$ 32.7 /$ ton was the highest product value obtained for coal A, it was a lower value of $\$ 29.4$ for the coal B because of its higher moisture content due to its finer size distribution. Calculations showed that, if the second circuit configuration is used for coal A, an additional $\$ 1,541,735$ would be gained per year compared to the first circuit configuration. Similarly, a lower but still a positive gain of $\$ 1,081,748$ can be achieved with the second configuration for coal B. 
Table 6.2 Moisture values and coal worth for (a) the coarse feed and (b) the fine feed with various circuit configurations

\begin{tabular}{|c|c|c|c|c|c|c|c|c|c|}
\hline Circuit Config. & $\begin{array}{c}\text { Moisture } \\
(\%)\end{array}$ & $\begin{array}{c}\text { Coal } \\
\text { Treated } \\
\text { With SB } \\
\text { (dry tph) }\end{array}$ & $\begin{array}{c}\text { Coal } \\
\text { Treated } \\
\text { With HFC } \\
\text { (dry tph) }\end{array}$ & $\begin{array}{c}\text { Total } \\
\text { Product } \\
\text { (dry tph) }\end{array}$ & $\begin{array}{c}\text { Total } \\
\text { Product } \\
\text { (tph) }\end{array}$ & $\begin{array}{c}\text { Value } \\
\text { (\$/ton) }\end{array}$ & $\begin{array}{l}\text { Total } \\
\text { Value } \\
(\$ / h) \\
\end{array}$ & $\begin{array}{l}\text { Net } \\
\text { Gain } \\
(\$ / h)\end{array}$ & $\begin{array}{c}\text { Net } \\
\text { Gain } \\
\text { (\$/year) }\end{array}$ \\
\hline \multicolumn{10}{|l|}{ (a) Coal $\mathrm{A}$} \\
\hline 1 & 11.1 & 95.0 & -- & 95.0 & 106.9 & $\$ 30.9$ & $\$ 3,304$ & -- & -- \\
\hline 2 & 8.2 & 92.6 & 7.4 & 100.0 & 109.0 & $\$ 32.7$ & $\$ 3,561$ & $\$ 257$ & $\$ 1,541,735$ \\
\hline 3 & 9.0 & 94.1 & 5.9 & 100.0 & 109.9 & $\$ 32.2$ & $\$ 3,539$ & $\$ 236$ & $\$ 1,413,281$ \\
\hline \multicolumn{10}{|l|}{ (b) Coal B } \\
\hline 1 & 18.8 & 85.2 & -- & 85.2 & 104.9 & $\$ 26.2$ & $\$ 3,221$ & -- & -- \\
\hline 2 & 13.5 & 75.8 & 24.2 & 100.0 & 115.6 & $\$ 29.4$ & $\$ 3,401$ & $\$ 180$ & $\$ 1,081,748$ \\
\hline 3 & 14.6 & 79.5 & 20.5 & 100.0 & 117.1 & $\$ 28.8$ & $\$ 3,367$ & $\$ 146$ & $\$ 873,832$ \\
\hline
\end{tabular}




\subsection{SUMMARY AND CONCLUSIONS}

Several series of laboratory-scale, pilot-scale and field tests were conducted using a novel fine-particle-dewatering technology called hyperbaric filter centrifugation. Based on the results obtained from this work, Decanter Machine Inc. constructed and sold a full-scale prototype that has now been successfully demonstrated at an industrial plant site. The technology is now marketed under the trade name Centribaric ${ }^{\mathrm{TM}}$ centrifuge.

Several laboratory-scale test units were constructed for the purpose of conducting a detailed parametric study of hyperbaric filter centrifugation. Test results obtained with samples containing different size distributions showed that as long as the dewatered product contained less than about $60 \%$ minus $0.025 \mathrm{~mm}$ particles, centrifugation could be improved through the injection of pressurized air without having to resort to very high centrifugal fields. For example, a centrifugal acceleration of 500 g's with air pressure was found to be as effective for dewatering as a high centrifugal acceleration of 2700 g's without air pressure. Therefore, it was concluded that the use of a costly high-g centrifuge could be avoided when the fines content of a product was below a certain amount. However, when the minus $0.025 \mathrm{~mm}$ particle content was above $60 \%$, the use of a high centrifugal acceleration with air pressure was found to be essential to achieve low moisture values. Moreover, tests performed using different coal samples obtained from commercial coal plants showed that it was possible to achieve single-digit moisture values when desliming was performed ahead of hyperbaric centrifugation. Furthermore, tests conducted to study the effects of several surface active dewatering aids and flocculants showed that moisture reductions as large as 60\% (compared to no-chemical, and no-air cases) were possible by using chemicals in conjunction with air injection. 
Dewatering kinetics tests performed at the laboratory-scale showed that centrifugal dewatering process could be described as a rate process. While the bulk of the water was removed quickly from the cake during centrifugation, a smaller amount was removed at a slower rate. Moreover, a certain amount of water was not removed at all, which represented the final moisture content of the product. The application of air pressure assisted in the removal of water at both fast and slow rates from the cake and lowered the final equilibrium cake moisture.

Pilot-scale test results showed that higher rotational speeds and longer centrifugation times were beneficial for producing lower moistures. Also, injecting pressurized air through the cake further improved the moisture. It was also concluded that thickening was an essential step for centrifugal dewatering, as it increased the recovery of solids. The experiments also showed that solids recovery could be further increased when the effluent stream was re-circulated back to the feed stream. Although a more detailed and a complete parametric study of the pilot-scale unit was originally intended, all attempts to run a complete series of tests were unsuccessful due to the frequent operational issues associated with the use of a small pilot-scale machine.

In light of the problems associated with the pilot-scale tests, a continuous prototype hyperbaric centrifuge was built by Decanter Machine Inc. for use in field testing. The prototype unit successfully demonstrated that low moisture values could be obtained using hyperbaric centrifugation. Low moistures values in the range of 13 to $20 \%$ were obtainable with high solid recoveries of $83-96 \%$. Slightly higher, but still low, moistures ranging from $14 \%$ to $19 \%$ with 97-99\% recoveries were predicted to be realistically achievable by circulating the screen drain back to the feed stream. Also, mixing fine (minus $0.044 \mathrm{~mm}$ ) column concentrate with a coarser stream was found to be effective in reducing moisture and increasing solids recovery. It was concluded that a smaller ratio of coarse-to-fine particles could be used to avoid a substantial 
capacity de-rating, as the test results showed that even a very small amount of coarse material was enough to keep the moisture content low and the recovery high.

An empirical dewatering model and a theoretical dewatering model were developed for the hyperbaric centrifugation. While very accurate results were obtained using the empirical model, the theoretical model had difficulty in predicting reliable product moistures. Different centrifugal dewatering circuit configurations employing the hyperbaric centrifuge were simulated using the empirical model and an economic analysis was conducted. Based on the simulation results, it was determined that utilization of hyperbaric centrifuges in a coal plant would likely produce an attractive economic gain compared to utilizing only screenbowl centrifuges. 


\section{REFERENCES}

1. Abbott, J. (1981). The Optimization of Process Parameters to Maximize the Profitability from a Three-Component Blend. Paper presented at the First Australian Coal Preparation Conference, Newcastle, Australia.

2. Abbott, J., \& Miles, N. J. (1990). Smoothing and Interpolation of Float-Sink Data for Coals. Paper presented at the Inter. Symp. on Gravity Separation, Sept. 12-14, Cornwall, England.

3. Akers, R. J., \& Ward, A. S. (1977). Filtration: Principles and Practices Part 1 (Vol. 10). New York and Basel: Marcel Dekker, Inc.

4. Asmatulu, R. (2001). Advanced Chemical-Mechanical Dewatering of Fine Particles. Doctor of Philosophy, Virginia Polytechnic Institute and State University, Blacksburg.

5. Asmatulu, R., Luttrell, G., \& Yoon, R.-H. (2005). Dewatering of Fine Coal Using Hyperbaric Centrifugation. Coal Preparation, 25, 117-127.

6. Bethell, P. J., \& Luttrell, G. H. (2005). Effects of Ultrafine Desliming on Coal Flotation Circuits. Paper presented at the Centenary of Flotation Symposium, June 2005, Brisbane, Australia.

7. BP. (2009). Statistical Review of World Energy, from http://www.bp.com/statisticalreview

8. Bratton, R. (2010). [Personal Communication].

9. Brown, G. G., \& Associates. (1950). Unit Operations: Wiley.

10. Brownell, L. E., \& Katz, D. L. (1947). Flow of Fluids Through Porous Media - Part II. Chemical Engineering Progress, 43(11), 601-612.

11. Carman, P. C. (1956). Flow of Gases Through Porous Media. London: Butterworths. 
12. Clarkson, C. J. (1992). Optimisation of Coal Production from Mine Face to Customer. Paper presented at the 3rd Large Open Pit Mining Conference, Aug. 30 - Sept. 3, Makcay, Australia.

13. Condie, D. J., Hinkel, M., \& Veal, C. J. (1996). Modelling the vacuum filtration of fine coal. Filtration \& Separation, 33(9), 825-834.

14. Dahlstrom, D. A. (1985). Filtration. In N. L. Weiss (Ed.), SME Mineral Processing Handbook. New York, N. Y.: Society of Mining Engineers of the American Institute of Mining, Metallurgical, and Petroleum Engineers.

15. Dahlstrom, D. A., \& Klepper, R. P. (1982). Practical Aspects of Filtration and Dewatering in Physical Cleaning of Fine Coal. In Y. A. Liu (Ed.), Pyhsical Cleaning of Coal: Present and Developing Methods. New York: M. Dekker.

16. Darcy, H. (1856). Les fontaines publiques de la ville de Dijon. Paris.

17. Decanter Machine, I. (2010), from http://www.decantermachine.com

18. Dell, C. C. (1956). The Mayer Curve. Colliery Guardian, 33, 412-414.

19. Dombrowski, H. S., \& Brownell, L. E. (1954). Residual Equilibrium Saturation of Porous Media. Industrial Engineering Chemistry, 46(6), 1207-1219.

20. Dupré, A. (1869). Theorie Mecanique de la Chaleur. Paris: Gauthier-Villars.

21. EIA. (2010). International Energy Statistics, from http://www.eia.doe.gov

22. Erbil, H. Y. (2006). Contact Angle of Liquid Drops on Solids. In H. Y. Erbil (Ed.), Surface Chemistry of Solid and Liquid Interfaces (pp. 308-334). India: Blackwell Publishing.

23. Freme, F. (2009). U. S. Coal Supply and Demand: 2008 Review, from http://eia.doe.gov 
24. Friedmann, T., Lustenberger, C., \& Windhab, E. J. (2004). Filtration experiments with compressible filter cakes in centrifugal fields with superimposed static pressure. International Journal of Mineral Processing, 73(2-4), 261-267.

25. Gala, H. B., Kakwani, R., Chiang, S. H., Tierney, J. W., \& Klinzing, G. E. (1981). Filtration and Dewatering of Fine Coal. Separation Science and Technology, 16(10), 1611 - 1632.

26. Gray, V. R. (1958). The Dewatering of Fine Coal. Journal of the Energy Institute, 31, 96108.

27. Haruni, M. M., \& Storrow, J. A. (1953). Hydroextraction VII: Residual Moisture in Whizzed Cakes. Chemical Engineering Science, 2, 203-212.

28. Hwang, K.-J., Chu, W.-T., \& Lu, W.-M. (2001). A Method To Determine The Cake Properties In Centrifugal Dewatering. Separation Science and Technology, 36(12), 2693 2706.

29. Kaytaz, Y., Acarkan, N., \& Halvorsen, W. J. (1994). Dewatering of Coal. In O. Kural (Ed.), Coal: Resources, Properties, Utilization, Pollution (pp. 239-245).

30. King, R. P. (1999). Practical Optimization Strategies for Coal-Washing Plants. Coal Preparation, 20, 13-34.

31. Kozeny, J. (1927). Uber Kapillare Leitung Des Wassers in Boden (Vol. 136(2a), pp. 271306): Wien Akad. Wiss.

32. Laplace, P. S. (1806). Théorie de l'Action Capillaire Supplement au livre dixième de la Mechanique Celeste. Paris.

33. Ledger, B. (1999). Ultimate Saturation of Packed Beds. Master's Thesis, The University of Queensland.

34. Lowry, H. H. (1963). Chemistry of Coal Utilization. New York: Wiley. 
35. Luttrell, G. H., Barbee, C. J., \& Stanley, F. L. (2003). Optimum Cutpoints for Heavy Medium Separations. In R. Q. Honaker \& W. R. Forrest (Eds.), Advances in Gravity Concentration (pp. 81-91). Littleton, Colorado: Society for Mining, Metallurgy, and Exploration, Inc.

36. Lyons, L. R. (1950). Dewatering and Thermal Drying. In D. R. Mitchell (Ed.), Coal Preparation (2nd ed.). New York: American Institute of Mining and Metallurgical Engineers.

37. Lyons, O. R. (1951). An Approximate Method of Predicting and Comparing Expected Results When Dewatering Coal by Centrifuge. Transactions AIME, Mining Engineering, $191,417-425$.

38. Mayer, F. W. (1950). A New Washing Curve. Gluckauf, 86, 498-509.

39. Orr, F. M. (2002). Coal Waste Impoundments: Risks, Responses and Alternatives. Washington, D. C.: National Research Council.

40. Osborne, D. G. (1988). Solid-Liquid Separation Coal Preparation Technology (Vol. 1, pp. 478-542). London; Boston: Graham \& Trotman.

41. Panda, M. N., \& Lake, L. W. (1994). Estimation of single-phase permeability from parameters of particle-size distribution. American Association of Petroleum Geologists Bulletin, 78(7), 1028-1039.

42. Peng, F. F., \& Luckie, P. T. (1991). Process Control, Part 1 - Separation Evaluation. In J. Leonard \& B. Hardinge (Eds.), Coal Preparation (pp. 659-716). Baltimore, Maryland: Society of Mining Engineers, Port City Press, Inc.

43. Poiseuille, J. L. M. (1841). Comptes Rendus, 12, 1041. 
44. Policow, N. D., \& Orphanos, J. S. (1983). Development of the Screen Bowl Centrifuge for Dewatering Fine Coals. Mining Engineering, 35(4), 333-336.

45. Ralston, J., \& Newcombe, G. (1992). Static and Dynamic Contact Angles. In J. S. Laskowski \& J. Ralston (Eds.), Colloid Chemistry in Mineral Processing. Amsterdam: Elsevier.

46. Ranjan, S., \& Hogg, R. (1996). The Role of Cake Structure in the Dewatering of Fine Coal by Filtration. Coal Preparation, 17(1), 71 - 87.

47. Rayner, J. G. (1987). Direct Determination of Washing Parameters to Maximize Yield at a Given Ash. Bull. Proc. Australia Inst. Mining and Metallurgy, 292(8), 67-70.

48. Rushton, A., Ward, A. S., \& Holdich, R. G. (2000a). Centrifugal Separation Solid-Liquid Filtration and Separation Technology (pp. 295-365). Weinhheim: Wiley-VCH.

49. Rushton, A., Ward, A. S., \& Holdich, R. G. (2000b). Filtration Fundamentals Solid-Liquid Filtration and Separation Technology (pp. 295-365). Weinhheim: Wiley-VCH.

50. Sanders, A., \& Sutherland, K. (2001). Decanter Centrifuge Handbook: Elsevier.

51. Sandy, E. J., Matoney, J. P., \& Dahlstrom, D. A. (1991). Dewatering. In J. W. Leonard (Ed.), Coal Preparation (5th ed.). Littleton, Colorado: Society for Mining, Metallurgy, and Exploration.

52. Svarovsky, L. (1990). Filtration Fundamentals. In L. Svarovsky (Ed.), Solid-Liquid Separation (3rd ed.). London; Boston: Butterworths.

53. Swindells, R. J., \& White, E. T. (1979). Drainage of Molasses from Sugar Crystals in a Continuous Centrifuge. Paper presented at the Seventh Australian Conference on Chemical Engineering.

54. Tierney, J. W., \& Gottfried, B. S. (1983). A Simulator for Dewatering of Coal Fines. Proceedings of the First Conference on Use of Computers in the Coal Industry, 230-236. 
55. Veal, C. J., Nicol, S. k., \& Johnston, K. (1998). USA Patent No. 5771601.

56. Veal, C. J., Nicol, S. k., \& Johnston, K. (1999). USA Patent No. 5956858.

57. Vidal, D., Ridgway, C., Pianet, G., Schoelkopf, J., Roy, R., \& Bertrand, F. (2009). Effect of particle size distribution and packing compression on fluid permeability as predicted by lattice-Boltzmann simulations. Computers and Chemical Engineering, 33, 256-266.

58. Wakeman, R. J. (1984). Residual saturation and dewatering of fine coals and filter cakes. Powder Technology, 40(1-3), 53-63.

59. Wakeman, R. J., Rushton, A., \& Brewis, L. N. (1976). Residual Saturation Of Dewatered Filter Cakes. Chemical Engineer-London(314), 668-670.

60. Wakeman, R. J., \& Tarleton, E. S. (1999). Filtration: Equipment Selection Modelling and Process Simulation (1st ed.): Elsevier.

61. Wills, B. A. (2006). Wills' Mineral Processing Technology: An Introduction to the Practical Aspects of Ore Treatment and Mineral Recovery (7th ed.). Amsterdam, London: BH.

62. Yoon, R.-H., \& Asmatulu, R. (2002). USA Patent No. 6440316 B1.

63. Young, T. (1805). Philosophical Transactions of the Royal Society (London), 95(1805), 65.

64. Zeitsch, K. (1990). Centrifugal Filtration. In L. Svarovsky (Ed.), Solid-Liquid Separation (3 ed.). London; Boston: Butterworths. 


\section{APPENDIX 1 - Mass Balance Calculations}




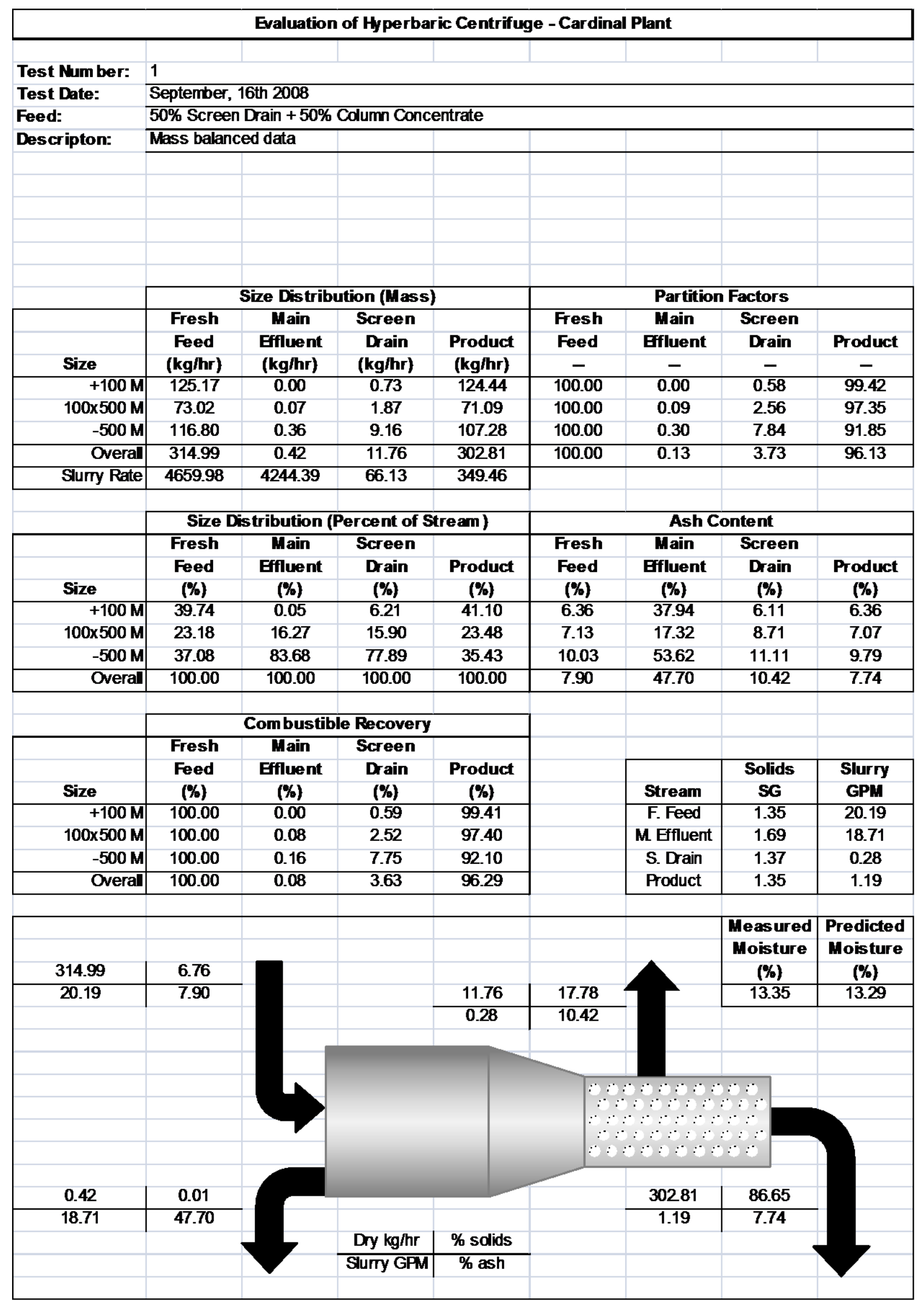




\begin{tabular}{|c|c|c|c|c|c|c|c|c|}
\hline \multicolumn{9}{|c|}{ Evaluation of Hyperbaric Centrifuge - Cardinal Plant } \\
\hline Test Num ber: & \multicolumn{8}{|c|}{1} \\
\hline Test Date: & \multicolumn{8}{|c|}{ September, 16th 2008} \\
\hline Feed: & \multicolumn{8}{|c|}{$50 \%$ Screen Drain + 50\% Column Concentrate } \\
\hline Descripton: & \multicolumn{8}{|c|}{ Simulated data for screen drain recirculation } \\
\hline & & & & & & & & \\
\hline & & & & & & & & \\
\hline & & & & & & & & \\
\hline & & & & & & & & \\
\hline & & & & & & & & \\
\hline & & & & & & & & \\
\hline & \multicolumn{4}{|c|}{ Size Distribution (Mass) } & \multicolumn{4}{|c|}{ Partition Factors } \\
\hline & Com bined & Main & Screen & & Fresh & Main & Screen & \\
\hline & Feed & Effluent & Drain & Product & Feed & Effluent & Drain & Product \\
\hline Size & (kg/hr) & (kg/hr) & (kg/hr) & (kg/hr) & - & - & - & - \\
\hline$+100 \mathrm{M}$ & 125.91 & 0.00 & 0.73 & 125.17 & 100.00 & 0.00 & 0.59 & 100.00 \\
\hline $100 \times 500 \mathrm{M}$ & 74.94 & 0.07 & 1.92 & 72.95 & 100.00 & 0.10 & 2.63 & 99.90 \\
\hline$-500 \mathrm{M}$ & 126.73 & 0.39 & 9.94 & 116.41 & 100.00 & 0.33 & 8.51 & 99.67 \\
\hline Overal & 327.59 & 0.46 & 12.59 & 314.54 & 100.00 & 0.14 & 4.00 & 99.86 \\
\hline \multirow[t]{5}{*}{ Slurry Rate } & 4727.06 & 4295.53 & 67.08 & 364.45 & & & & \\
\hline & & & & & & & & \\
\hline & \multicolumn{4}{|c|}{ Size Distribution (Percent of Stream) } & \multicolumn{4}{|c|}{ Ash Content } \\
\hline & Com bined & Main & Screen & & Com bined & Main & Screen & \\
\hline & Feed & Effluent & Drain & Product & Feed & Effluent & Drain & Product \\
\hline Size & (\%) & (\%) & (\%) & (\%) & $(\%)$ & (\%) & $(\%)$ & (\%) \\
\hline$+100 \mathrm{M}$ & 38.44 & 0.05 & 5.84 & 39.80 & 6.36 & 37.94 & 6.11 & 6.36 \\
\hline $100 \times 500 \mathrm{M}$ & 22.88 & 15.52 & 15.24 & 23.19 & 7.13 & 17.32 & 8.71 & 7.07 \\
\hline$-500 \mathrm{M}$ & 38.69 & 84.43 & 78.92 & 37.01 & 10.03 & 53.62 & 11.11 & 9.79 \\
\hline \multirow[t]{4}{*}{ Overal } & 100.00 & 100.00 & 100.00 & 100.00 & 7.95 & 47.97 & 10.45 & 7.80 \\
\hline & \multicolumn{4}{|c|}{ Combustible Recovery } & & & & \\
\hline & Com bined & Main & Screen & & & & & \\
\hline & Feed & Effluent & Drain & Product & & & Solids & Slurry \\
\hline Size & $(\%)$ & (\%) & $(\%)$ & $(\%)$ & & Stream & SG & GPM \\
\hline$+100 \mathrm{M}$ & 100.00 & 0.00 & 0.59 & 100.00 & & C. Feed & 1.35 & 20.47 \\
\hline $100 \times 500 \mathrm{M}$ & 100.00 & 0.08 & 2.52 & 99.96 & & M. Effluent & 1.69 & 18.94 \\
\hline$-500 \mathrm{M}$ & 100.00 & 0.16 & 7.75 & 99.93 & & S. Drain & 1.37 & 0.28 \\
\hline \multirow[t]{4}{*}{ Overal } & 100.00 & 0.08 & 3.74 & 99.96 & & Product & 1.35 & 1.25 \\
\hline & & & & & & & & \\
\hline & & & & & & & Measured & Predicted \\
\hline & & & & & & & Moisture & Moisture \\
\hline 314.99 & 6.76 & & & 12.59 & 18.77 & & (\%) & (\%) \\
\hline 20.19 & 7.90 & & & 0.28 & 10.45 & & - & 13.70 \\
\hline & & & $---\cdots$ & - & ---- & -2 & & \\
\hline & & & & & & Li & & \\
\hline 327.59 & 6.93 & & & & & $\because: \because: \because$ & $\because \because$ & \\
\hline 20.47 & 7.95 & & & & & $\because \because \because \because \because$ & $\because:$ & \\
\hline & & & & & $\because \because$ & $\because \because \because \because \because: \because$ & $\because \because$ & \\
\hline 0.46 & 0.01 & & & & & 314.54 & 86.30 & \\
\hline 18.94 & 47.97 & & & & & 1.25 & 7.80 & \\
\hline & & & Dry kg/hr & \% solids & & & & \\
\hline & & & Surry GPM & \% ash & & & & \\
\hline & & & & & & & & \\
\hline
\end{tabular}




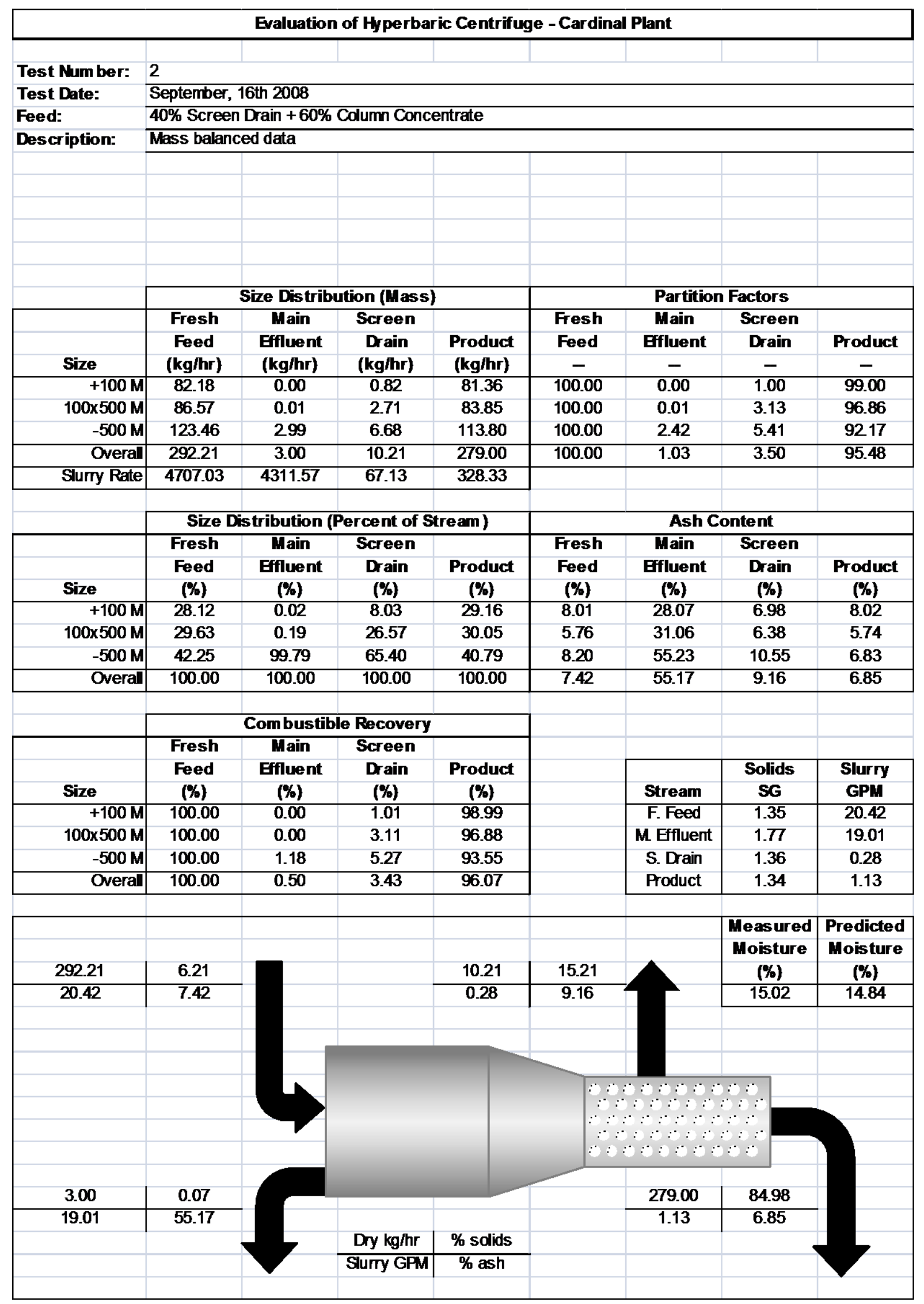




\begin{tabular}{|c|c|c|c|c|c|c|c|c|}
\hline \multicolumn{9}{|c|}{ Evaluation of Hyperbaric Centrifuge - Cardinal Plant } \\
\hline Test Num ber: & \multicolumn{8}{|c|}{2} \\
\hline Test Date: & \multicolumn{8}{|c|}{ September, 16th 2008} \\
\hline Feed: & \multicolumn{8}{|c|}{ 40\% Screen Drain + 60\% Column Concentrate } \\
\hline Description: & \multicolumn{8}{|c|}{ Simulated data for screen drain recirculation } \\
\hline & & & & & & & & \\
\hline & & & & & & & & \\
\hline & & & & & & & & \\
\hline & & & & & & & & \\
\hline & & & & & & & & \\
\hline & & & & & & & & \\
\hline & \multicolumn{4}{|c|}{ Size Distribution (Mass) } & \multicolumn{4}{|c|}{ Partition Factors } \\
\hline & Com bined & Main & Screen & & Fresh & Main & Screen & \\
\hline & Feed & Effluent & Drain & Product & Feed & Effluent & Drain & Product \\
\hline Size & (kg/hr) & (kg/hr) & (kg/hr) & (kg/hr) & - & - & - & - \\
\hline$+100 \mathrm{M}$ & 83.00 & 0.00 & 0.83 & 82.18 & 100.00 & 0.00 & 1.01 & 100.00 \\
\hline $100 \times 500 \mathrm{M}$ & 89.37 & 0.01 & 2.80 & 86.57 & 100.00 & 0.01 & 3.24 & 99.99 \\
\hline$-500 \mathrm{M}$ & 130.52 & 3.16 & 7.06 & 120.30 & 100.00 & 2.56 & 5.72 & 97.44 \\
\hline Overal & 302.90 & 3.17 & 10.69 & 289.05 & 100.00 & 1.08 & 3.66 & 98.92 \\
\hline \multirow[t]{5}{*}{ Slurry Rate } & 4775.14 & 4366.53 & 68.10 & 340.51 & & & & \\
\hline & \multirow{2}{*}{\multicolumn{4}{|c|}{ Size Distribution (Percent of Stream) }} & \multirow{2}{*}{\multicolumn{4}{|c|}{ Ash Content }} \\
\hline & & & & & & & & \\
\hline & Com bined & Main & Screen & & Com bined & Main & Screen & \\
\hline & Feed & Effluent & Drain & Product & Feed & Effluent & Drain & Product \\
\hline Size & (\%) & (\%) & (\%) & (\%) & $(\%)$ & (\%) & $(\%)$ & (\%) \\
\hline$+100 \mathrm{M}$ & 27.40 & 0.02 & 7.75 & 28.43 & 8.01 & 28.07 & 6.98 & 8.02 \\
\hline $100 \times 500 \mathrm{M}$ & 29.51 & 0.19 & 26.20 & 29.95 & 5.76 & 31.06 & 6.38 & 5.74 \\
\hline$-500 \mathrm{M}$ & 43.09 & 99.80 & 66.05 & 41.62 & 8.20 & 55.23 & 10.55 & 6.83 \\
\hline \multirow[t]{4}{*}{ Overal } & 100.00 & 100.00 & 100.00 & 100.00 & 7.43 & 55.18 & 9.18 & 6.84 \\
\hline & \multicolumn{4}{|c|}{ Combustible Recovery } & & & & \\
\hline & Com bined & Main & Screen & & & & & \\
\hline & Feed & Effluent & Drain & Product & & & Solids & Slurry \\
\hline Size & $(\%)$ & (\%) & $(\%)$ & $(\%)$ & & Stream & SG & GPM \\
\hline$+100 \mathrm{M}$ & 100.00 & 0.00 & 1.01 & 99.99 & & C. Feed & 1.35 & 20.71 \\
\hline $100 \times 500 \mathrm{M}$ & 100.00 & 0.00 & 3.11 & 100.02 & & M. Effluent & 1.77 & 19.25 \\
\hline$-500 \mathrm{M}$ & 100.00 & 1.18 & 5.27 & 98.90 & & S. Drain & 1.36 & 0.29 \\
\hline \multirow[t]{4}{*}{ Overal } & 100.00 & 0.51 & 3.46 & 99.54 & & Product & 1.34 & 1.18 \\
\hline & & & & & & & & \\
\hline & & & & & & & Measured & Predicted \\
\hline & & & & & & & Moisture & Moisture \\
\hline 292.21 & 6.21 & & & 10.69 & 15.70 & & (\%) & (\%) \\
\hline 20.42 & 7.42 & & & 0.29 & 9.18 & & - & 15.11 \\
\hline & & & $---\cdots$ & 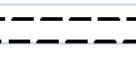 & $-\cdots--$ & - & & \\
\hline & & & & & & Li & & \\
\hline 302.90 & 6.34 & & & & & $: \because: \div:$ & $\because:-$ & \\
\hline 20.71 & 7.43 & & & & & $\because \because \because \because \because$ & $\because:$ & \\
\hline & & & & & $\because \because$ & $\because \because \because \because \because$ & $\because \because$ & \\
\hline 3.17 & 0.07 & & & & & 289.05 & 84.89 & \\
\hline 19.25 & 55.18 & & & & & 1.18 & 6.84 & \\
\hline & & & Dry kg/hr & \% solids & & & & \\
\hline & & & Surry GPM & \% ash & & & & \\
\hline & & & & & & & & \\
\hline
\end{tabular}




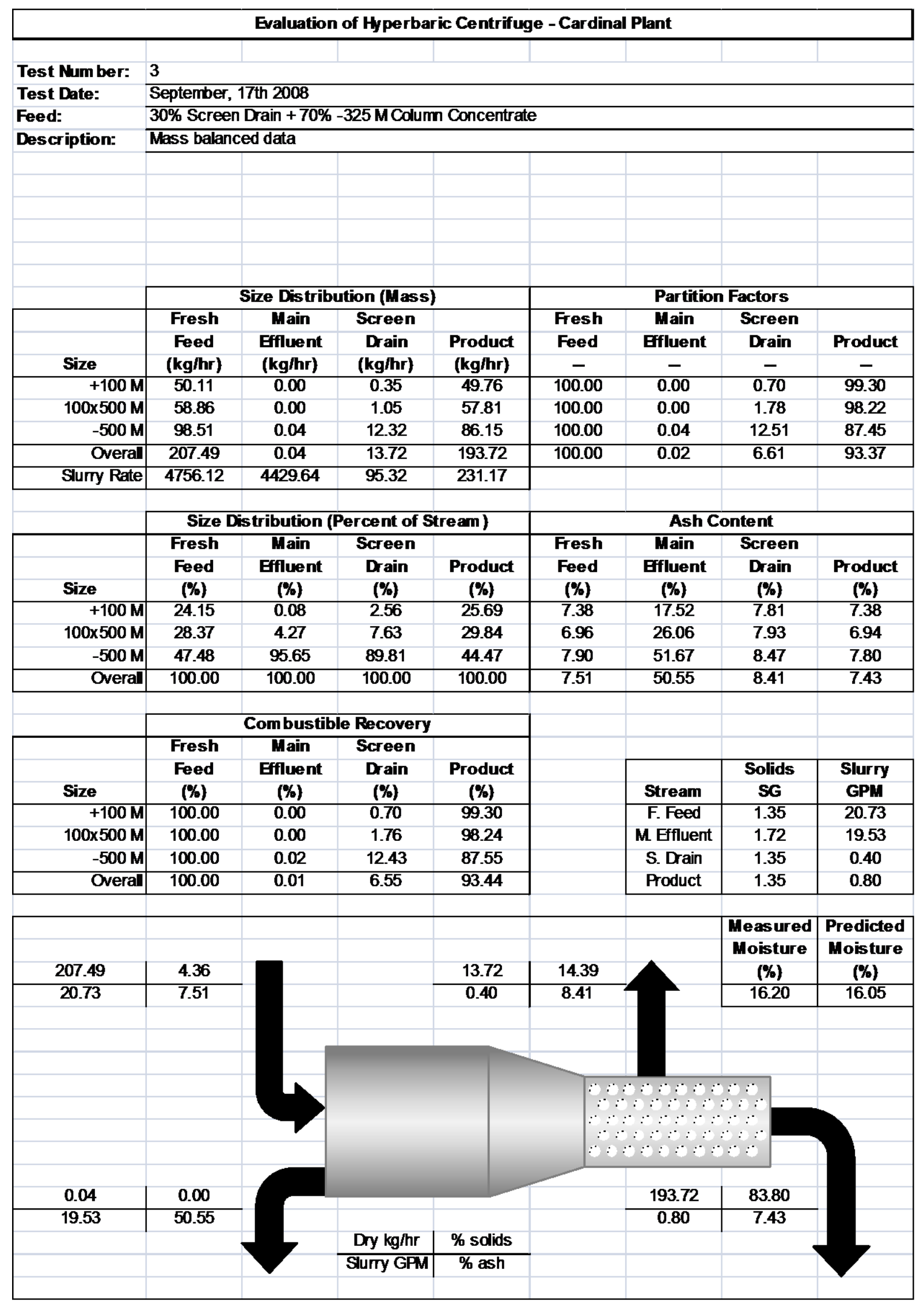




\begin{tabular}{|c|c|c|c|c|c|c|c|c|}
\hline \multicolumn{9}{|c|}{ Evaluation of Hyperbaric Centrifuge - Cardinal Plant } \\
\hline Test Num ber: & \multicolumn{8}{|l|}{3} \\
\hline Test Date: & \multicolumn{8}{|c|}{ September, 17th 2008} \\
\hline Feed: & \multicolumn{8}{|c|}{$30 \%$ Screen Drain + 70\% -325 M Column Concentrate } \\
\hline Description: & \multicolumn{8}{|c|}{ Simulated data for screen drain recirculation } \\
\hline & & & & & & & & \\
\hline & & & & & & & & \\
\hline & & & & & & & & \\
\hline & & & & & & & & \\
\hline & & & & & & & & \\
\hline & & & & & & & & \\
\hline & \multicolumn{4}{|c|}{ Size Distribution (Mass) } & \multicolumn{4}{|c|}{ Partition Factors } \\
\hline & Com bined & Main & Screen & & Fresh & Main & Screen & \\
\hline & Feed & Effluent & Drain & Product & Feed & Effluent & Drain & Product \\
\hline Size & (kg/hr) & (kg/hr) & (kg/hr) & (kg/hr) & - & - & - & - \\
\hline$+100 \mathrm{M}$ & 50.46 & 0.00 & 0.35 & 50.11 & 100.00 & 0.00 & 0.71 & 100.00 \\
\hline $100 \times 500 \mathrm{M}$ & 59.93 & 0.00 & 1.07 & 58.86 & 100.00 & 0.00 & 1.81 & 100.00 \\
\hline$-500 \mathrm{M}$ & 112.60 & 0.05 & 14.08 & 98.47 & 100.00 & 0.05 & 14.30 & 99.95 \\
\hline Overal & 222.99 & 0.05 & 15.50 & 207.44 & 100.00 & 0.02 & 7.47 & 99.98 \\
\hline \multirow[t]{5}{*}{ Slurry Rate } & 4853.39 & 4506.16 & 97.27 & 249.97 & & & & \\
\hline & & & & & & & & \\
\hline & \multicolumn{4}{|c|}{ Size Distribution (Percent of Stream) } & \multicolumn{4}{|c|}{ Ash Content } \\
\hline & Com bined & Main & Screen & & Com bined & Main & Screen & \\
\hline & Feed & Effluent & Drain & Product & Feed & Effluent & Drain & Product \\
\hline Size & (\%) & (\%) & (\%) & (\%) & $(\%)$ & (\%) & $(\%)$ & (\%) \\
\hline$+100 \mathrm{M}$ & 22.63 & 0.07 & 2.28 & 24.16 & 7.38 & 17.52 & 7.81 & 7.38 \\
\hline $100 \times 500 \mathrm{M}$ & 26.88 & 3.82 & 6.88 & 28.38 & 6.96 & 26.06 & 7.93 & 6.94 \\
\hline$-500 \mathrm{M}$ & 50.49 & 96.11 & 90.84 & 47.47 & 7.90 & 51.67 & 8.47 & 7.80 \\
\hline \multirow[t]{4}{*}{ Overal } & 100.00 & 100.00 & 100.00 & 100.00 & 7.53 & 50.67 & 8.42 & 7.45 \\
\hline & \multicolumn{4}{|c|}{ Combustible Recovery } & & & & \\
\hline & Com bined & Main & Screen & & & & & \\
\hline & Feed & Effluent & Drain & Product & & & Solids & Slurry \\
\hline Size & $(\%)$ & (\%) & $(\%)$ & $(\%)$ & & Stream & SG & GPM \\
\hline$+100 \mathrm{M}$ & 100.00 & 0.00 & 0.70 & 100.00 & & C. Feed & 1.35 & 21.15 \\
\hline $100 \times 500 \mathrm{M}$ & 100.00 & 0.00 & 1.76 & 100.02 & & M. Effluent & 1.72 & 19.87 \\
\hline$-500 \mathrm{M}$ & 100.00 & 0.02 & 12.43 & 100.06 & & S. Drain & 1.35 & 0.41 \\
\hline \multirow[t]{4}{*}{ Overal } & 100.00 & 0.01 & 6.89 & 100.03 & & Product & 1.35 & 0.87 \\
\hline & & & & & & & & \\
\hline & & & & & & & Measured & Predicted \\
\hline & & & & & & & Moisture & Moisture \\
\hline 207.49 & 4.36 & & & 15.50 & 15.94 & & (\%) & (\%) \\
\hline 20.73 & 7.51 & & & 0.41 & 8.42 & & - & 17.01 \\
\hline & & & -- - & $-3+2+3$ & -ーー- & 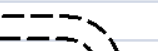 & & \\
\hline & & & & & & LI & & \\
\hline 222.99 & 4.59 & & & & & $: \because: \div:$ & $\because:$ & \\
\hline 21.15 & 7.53 & & & & & $\because \because \because \because \because$ & $\because:$ & \\
\hline & & & & & $\because \because$ & $\because \because \because \because \because$ & $\because \because$ & \\
\hline 0.05 & 0.00 & & & & & 207.44 & 8299 & \\
\hline 19.87 & 50.67 & & & & & 0.87 & 7.45 & \\
\hline & & & Dry kg/hr & \% solids & & & & \\
\hline & & & Surry GPM & $\%$ ash & & & & \\
\hline & & & & & & & & \\
\hline
\end{tabular}




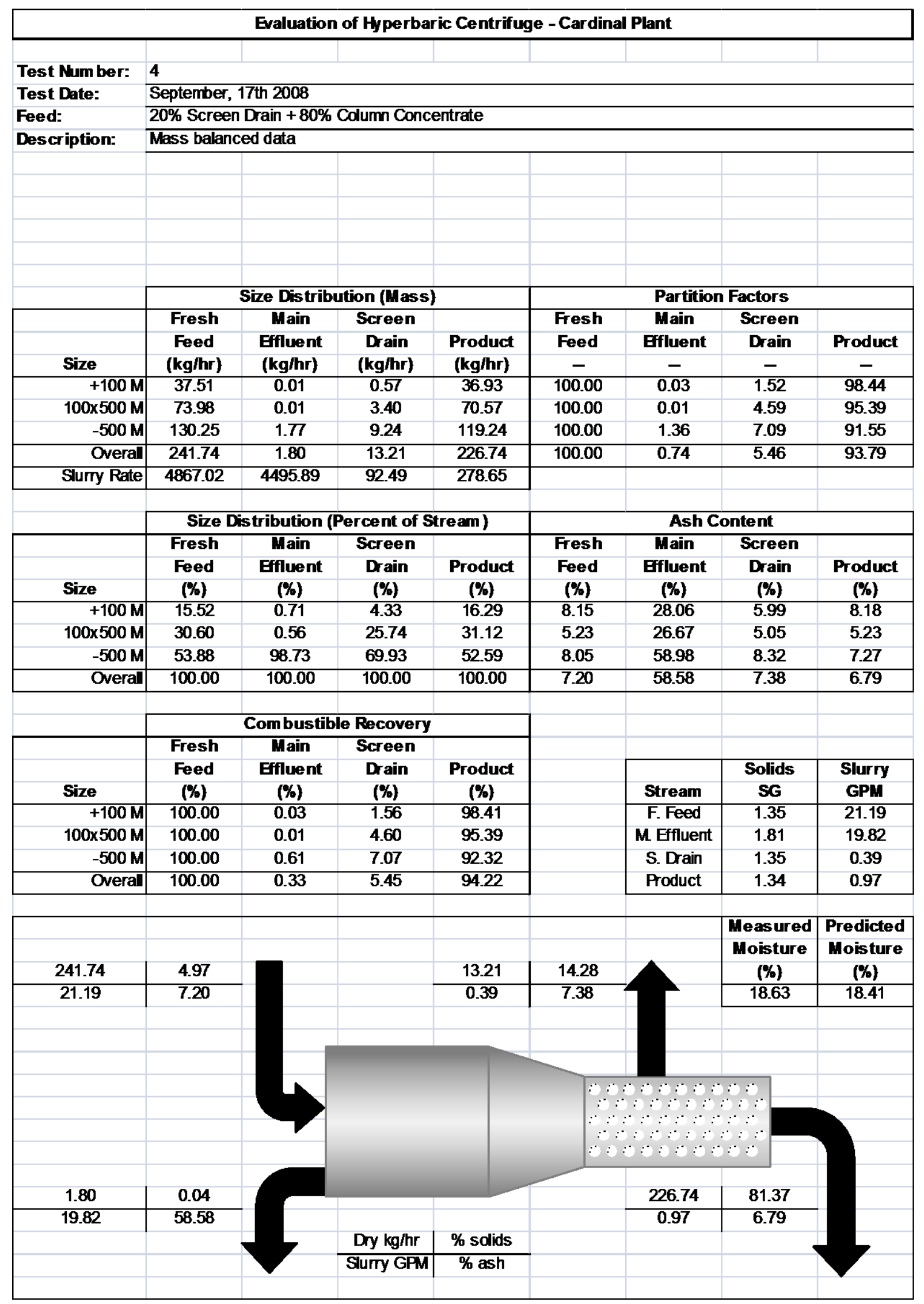




\begin{tabular}{|c|c|c|c|c|c|c|c|c|}
\hline \multicolumn{9}{|c|}{ Evaluation of Hyperbaric Centrifuge - Cardinal Plant } \\
\hline Test Num ber: & \multicolumn{8}{|c|}{4} \\
\hline Test Date: & \multicolumn{8}{|c|}{ September, 16th 2008} \\
\hline Feed: & \multicolumn{8}{|c|}{$20 \%$ Screen Drain + 80\% Column Concentrate } \\
\hline Description: & \multicolumn{8}{|c|}{ Simulated data for screen drain recirculation } \\
\hline & & & & & & & & \\
\hline & & & & & & & & \\
\hline & & & & & & & & \\
\hline & & & & & & & & \\
\hline & & & & & & & & \\
\hline & & & & & & & & \\
\hline & \multicolumn{4}{|c|}{ Size Distribution (Mass) } & \multicolumn{4}{|c|}{ Partition Factors } \\
\hline & Com bined & Main & Screen & & Fresh & Main & Screen & \\
\hline & Feed & Effluent & Drain & Product & Feed & Effluent & Drain & Product \\
\hline Size & (kg/hr) & (kg/hr) & (kg/hr) & (kg/hr) & - & - & - & - \\
\hline$+100 \mathrm{M}$ & 38.09 & 0.01 & 0.58 & 37.50 & 100.00 & 0.03 & 1.55 & 99.97 \\
\hline $100 \times 500 \mathrm{M}$ & 77.54 & 0.01 & 3.56 & 73.96 & 100.00 & 0.01 & 4.82 & 99.99 \\
\hline$-500 \mathrm{M}$ & 140.19 & 1.91 & 9.94 & 128.34 & 100.00 & 1.47 & 7.63 & 98.53 \\
\hline Overal & 255.82 & 1.93 & 14.08 & 239.81 & 100.00 & 0.80 & 5.83 & 99.20 \\
\hline \multirow[t]{5}{*}{ Slurry Rate } & 4961.30 & 457234 & 94.28 & 294.68 & & & & \\
\hline & & & & & & & & \\
\hline & \multicolumn{4}{|c|}{ Size Distribution (Percent of Stream) } & \multicolumn{4}{|c|}{ Ash Content } \\
\hline & Com bined & Main & Screen & & Com bined & Main & Screen & \\
\hline & Feed & Effluent & Drain & Product & Feed & Effluent & Drain & Product \\
\hline Size & (\%) & (\%) & (\%) & (\%) & $(\%)$ & (\%) & $(\%)$ & (\%) \\
\hline$+100 \mathrm{M}$ & 14.89 & 0.67 & 4.12 & 15.64 & 8.15 & 28.06 & 5.99 & 8.18 \\
\hline $100 \times 500 \mathrm{M}$ & 30.31 & 0.55 & 25.30 & 30.84 & 5.23 & 26.67 & 5.05 & 5.23 \\
\hline$-500 \mathrm{M}$ & 54.80 & 98.78 & 70.58 & 53.52 & 8.05 & 58.98 & 8.32 & 7.27 \\
\hline \multirow[t]{4}{*}{ Overal } & 100.00 & 100.00 & 100.00 & 100.00 & 7.21 & 58.60 & 7.40 & 6.79 \\
\hline & \multicolumn{4}{|c|}{ Combustible Recovery } & & & & \\
\hline & Com bined & Main & Screen & & & & & \\
\hline & Feed & Effluent & Drain & Product & & & Solids & Slurry \\
\hline Size & $(\%)$ & (\%) & $(\%)$ & $(\%)$ & & Stream & SG & GPM \\
\hline$+100 \mathrm{M}$ & 100.00 & 0.03 & 1.56 & 99.94 & & C. Feed & 1.35 & 21.59 \\
\hline $100 \times 500 \mathrm{M}$ & 100.00 & 0.01 & 4.60 & 99.98 & & M. Effluent & 1.81 & 20.16 \\
\hline$-500 \mathrm{M}$ & 100.00 & 0.61 & 7.07 & 99.37 & & S. Drain & 1.35 & 0.40 \\
\hline \multirow[t]{4}{*}{ Overal } & 100.00 & 0.34 & 5.49 & 99.65 & & Product & 1.34 & 1.03 \\
\hline & & & & & & & & \\
\hline & & & & & & & Measured & Predicted \\
\hline & & & & & & & Moisture & Moisture \\
\hline 241.74 & 4.97 & & & 14.08 & 14.94 & & (\%) & (\%) \\
\hline 21.19 & 7.20 & & & 0.40 & 7.40 & & - & 18.62 \\
\hline & & & --- & - & $-\cdots-$ & -1 & & \\
\hline & & & & & & LI & & \\
\hline 255.82 & 5.16 & & & & & $: \because: \because:$ & $\because \because$ & \\
\hline 21.59 & 7.21 & & & & & $\because \because \because \because$ & $\because:$ & \\
\hline & & & & & $\because \because$ & $\because \because \because \because \because$ & $\because \because$ & \\
\hline 1.93 & 0.04 & & & & & 239.81 & 81.38 & \\
\hline 20.16 & 58.60 & & & & & 1.03 & 6.79 & \\
\hline & & & Dry kg/hr & \% solids & & & & \\
\hline & & & Surry GPM & \% ash & & & & \\
\hline & & & & & & & & \\
\hline
\end{tabular}




\begin{tabular}{|c|c|c|c|c|c|c|c|c|}
\hline \multicolumn{9}{|c|}{ Evaluation of Hyperbaric Centrifuge - Cardinal Plant } \\
\hline Test Num ber: & \multicolumn{8}{|l|}{5} \\
\hline Test Date: & \multicolumn{8}{|c|}{ September, 17th 2008} \\
\hline Feed: & \multicolumn{8}{|c|}{ 10\% Screen Drain + 90\% Column Concentrate } \\
\hline Description: & \multicolumn{8}{|c|}{ Mass balanced data } \\
\hline & & & & & & & & \\
\hline & & & & & & & & \\
\hline & & & & & & & & \\
\hline & & & & & & & & \\
\hline & & & & & & & & \\
\hline & & & & & & & & \\
\hline & \multicolumn{4}{|c|}{ Size Distribution (Mass) } & \multicolumn{4}{|c|}{ Partition Factors } \\
\hline & Fresh & Main & Screen & & Fresh & Main & Screen & \\
\hline & Feed & Effluent & Drain & Product & Feed & Effluent & Drain & Product \\
\hline Size & (kg/hr) & (kg/hr) & (kg/hr) & (kg/hr) & - & - & - & - \\
\hline$+100 \mathrm{M}$ & 18.08 & 0.01 & 0.02 & 18.05 & 100.00 & 0.05 & 0.11 & 99.84 \\
\hline $100 \times 500 \mathrm{M}$ & 49.72 & 0.01 & 0.17 & 49.54 & 100.00 & 0.03 & 0.34 & 99.63 \\
\hline$-500 \mathrm{M}$ & 108.13 & 0.88 & 8.63 & 98.62 & 100.00 & 0.82 & 7.98 & 91.20 \\
\hline Overal & 175.93 & 0.91 & $\mathbf{8 . 8 1}$ & 166.21 & 100.00 & 0.52 & 5.01 & 94.47 \\
\hline \multirow[t]{5}{*}{ Slurry Rate } & 4805.43 & 4533.53 & 65.70 & 206.20 & & & & \\
\hline & & & & & & & & \\
\hline & \multicolumn{4}{|c|}{ Size Distribution (Percent of Stream) } & \multicolumn{4}{|c|}{ Ash Content } \\
\hline & Fresh & Main & Screen & & Fresh & Main & Screen & \\
\hline & Feed & Effluent & Drain & Product & Feed & Effluent & Drain & Product \\
\hline Size & (\%) & (\%) & (\%) & (\%) & $(\%)$ & (\%) & $(\%)$ & (\%) \\
\hline$+100 \mathrm{M}$ & 10.28 & 0.97 & 0.23 & 10.86 & 8.85 & 18.19 & 7.37 & 8.85 \\
\hline $100 \times 500 \mathrm{M}$ & 28.26 & 1.65 & 1.91 & 29.80 & 4.26 & 15.56 & 4.29 & 4.26 \\
\hline$-500 \mathrm{M}$ & 61.46 & 97.38 & 97.86 & 59.33 & 6.49 & 45.63 & 6.34 & 6.15 \\
\hline \multirow[t]{4}{*}{ Overal } & 100.00 & 100.00 & 100.00 & 100.00 & 6.10 & 44.86 & 6.30 & 5.88 \\
\hline & \multicolumn{4}{|c|}{ Combustible Recovery } & & & & \\
\hline & Fresh & Main & Screen & & & & & \\
\hline & Feed & Effluent & Drain & Product & & & Solids & Slurry \\
\hline Size & $(\%)$ & (\%) & $(\%)$ & (\%) & & Stream & SG & GPM \\
\hline$+100 \mathrm{M}$ & 100.00 & 0.04 & 0.11 & 99.84 & & F. Feed & 1.34 & 20.99 \\
\hline $100 \times 500 \mathrm{M}$ & 100.00 & 0.03 & 0.34 & 99.63 & & M. Effluent & 1.66 & 19.99 \\
\hline$-500 \mathrm{M}$ & 100.00 & 0.48 & 7.99 & 91.53 & & S. Drain & 1.34 & 0.28 \\
\hline \multirow[t]{4}{*}{ Overal } & 100.00 & 0.30 & 5.00 & 94.70 & & Product & 1.34 & 0.72 \\
\hline & & & & & & & & \\
\hline & & & & & & & Measured & Predicted \\
\hline & & & & & & & Moisture & Moisture \\
\hline 175.93 & 3.66 & & & 8.81 & 13.42 & & (\%) & (\%) \\
\hline \multirow[t]{5}{*}{20.99} & 6.10 & & & 0.28 & 6.30 & & 19.39 & 19.52 \\
\hline & & & & & & & & \\
\hline & & & & & & & & \\
\hline & & & & & \multirow{2}{*}{\multicolumn{3}{|c|}{ 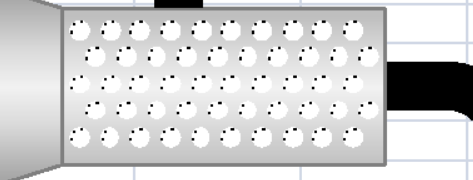 }} & \\
\hline & & & & & & & & \\
\hline 0.91 & 0.02 & & & & & 166.21 & 80.61 & \\
\hline \multirow[t]{3}{*}{19.99} & 44.86 & & & & & 0.72 & 5.88 & \\
\hline & & & Dry kg/hr & \% solids & & & & \\
\hline & & & Slumy GPM & \% ash & & & & \\
\hline & & & & & & & & \\
\hline
\end{tabular}




\begin{tabular}{|c|c|c|c|c|c|c|c|c|}
\hline \multicolumn{9}{|c|}{ Evaluation of Hyperbaric Centrifuge - Cardinal Plant } \\
\hline Test Num ber: & \multicolumn{8}{|c|}{5} \\
\hline Test Date: & \multicolumn{8}{|c|}{ September, 17th 2008} \\
\hline Feed: & \multicolumn{8}{|c|}{ 10\% Screen Drain + 90\% Column Concentrate } \\
\hline Description: & \multicolumn{8}{|c|}{ Simulated data for screen drain recirculation } \\
\hline & & & & & & & & \\
\hline & & & & & & & & \\
\hline & & & & & & & & \\
\hline & & & & & & & & \\
\hline & & & & & & & & \\
\hline & & & & & & & & \\
\hline & \multicolumn{4}{|c|}{ Size Distribution (Mass) } & \multicolumn{4}{|c|}{ Partition Factors } \\
\hline & Com bined & Main & Screen & & Fresh & Main & Screen & \\
\hline & Feed & Effluent & Drain & Product & Feed & Effluent & Drain & Product \\
\hline Size & (kg/hr) & (kg/hr) & (kg/hr) & (kg/hr) & - & - & - & - \\
\hline$+100 \mathrm{M}$ & 18.10 & 0.01 & 0.02 & 18.07 & 100.00 & 0.05 & 0.11 & 99.95 \\
\hline $100 \times 500 \mathrm{M}$ & 49.89 & 0.02 & 0.17 & 49.71 & 100.00 & 0.03 & 0.34 & 99.97 \\
\hline$-500 \mathrm{M}$ & 117.50 & 0.96 & 9.37 & 107.17 & 100.00 & 0.89 & 8.67 & 99.11 \\
\hline Overal & 185.49 & 0.99 & 9.56 & 174.95 & 100.00 & 0.56 & 5.44 & 99.44 \\
\hline \multirow[t]{5}{*}{ Slurry Rate } & 487204 & 4587.69 & 66.61 & 217.74 & & & & \\
\hline & & & & & & & & \\
\hline & \multicolumn{4}{|c|}{ Size Distribution (Percent of Stream) } & \multicolumn{4}{|c|}{ Ash Content } \\
\hline & Com bined & Main & Screen & & Com bined & Main & Screen & \\
\hline & Feed & Effluent & Drain & Product & Feed & Effluent & Drain & Product \\
\hline Size & (\%) & (\%) & (\%) & (\%) & $(\%)$ & (\%) & $(\%)$ & (\%) \\
\hline$+100 \mathrm{M}$ & 9.76 & 0.90 & 0.21 & 10.33 & 8.85 & 18.19 & 7.37 & 8.85 \\
\hline $100 \times 500 \mathrm{M}$ & 26.90 & 1.53 & 1.77 & 28.41 & 4.26 & 15.56 & 4.29 & 4.26 \\
\hline$-500 \mathrm{M}$ & 63.35 & 97.58 & 98.02 & 61.26 & 6.49 & 45.63 & 6.34 & 6.15 \\
\hline \multirow[t]{4}{*}{ Overal } & 100.00 & 100.00 & 100.00 & 100.00 & 6.12 & 44.92 & 6.30 & 5.89 \\
\hline & \multicolumn{4}{|c|}{ Combustible Recovery } & & & & \\
\hline & Com bined & Main & Screen & & & & & \\
\hline & Feed & Effluent & Drain & Product & & & Solids & Slurry \\
\hline Size & $(\%)$ & (\%) & $(\%)$ & $(\%)$ & & Stream & SG & GPM \\
\hline$+100 \mathrm{M}$ & 100.00 & 0.04 & 0.11 & 99.95 & & C. Feed & 1.34 & 21.27 \\
\hline $100 \times 500 \mathrm{M}$ & 100.00 & 0.03 & 0.34 & 99.97 & & M. Effluent & 1.66 & 20.23 \\
\hline$-500 \mathrm{M}$ & 100.00 & 0.48 & 7.99 & 99.47 & & S. Drain & 1.34 & 0.28 \\
\hline \multirow[t]{4}{*}{ Overal } & 100.00 & 0.31 & 5.15 & 99.66 & & Product & 1.34 & 0.77 \\
\hline & & & & & & & & \\
\hline & & & & & & & Measured & Predicted \\
\hline & & & & & & & Moisture & Moisture \\
\hline 175.93 & 3.66 & & & 9.56 & 14.36 & & (\%) & (\%) \\
\hline 20.99 & 6.10 & & & 0.28 & 6.30 & & - & 19.66 \\
\hline & & & --- & - & $-\cdots-$ & -1 & & \\
\hline & & & & & & Li & & \\
\hline 185.49 & 3.81 & & & & & $: \because: \div:$ & $\because:-$ & \\
\hline 21.27 & 6.12 & & & & & $\because \because \because \because \because$ & $\because:$ & \\
\hline & & & & & $\because \because$ & $\because \because \because \because \because$ & $\because \because$ & \\
\hline 0.99 & 0.02 & & & & & 174.95 & 80.34 & \\
\hline 20.23 & 44.92 & & & & & 0.77 & 5.89 & \\
\hline & & & Dry kg/hr & \% solids & & & & \\
\hline & & & Surry GPM & \% ash & & & & \\
\hline & & & & & & & & \\
\hline
\end{tabular}




\begin{tabular}{|c|c|c|c|c|c|c|c|c|}
\hline \multicolumn{9}{|c|}{ Evaluation of Hyperbaric Centrifuge - Cardinal Plant } \\
\hline Test Num ber: & \multicolumn{8}{|c|}{6} \\
\hline Test Date: & \multicolumn{8}{|c|}{ September, 17th 2008} \\
\hline Feed: & \multicolumn{8}{|c|}{$100 \%-325$ M Column Concentrate } \\
\hline Description: & \multicolumn{8}{|c|}{ Mass balanced data } \\
\hline & & & & & & & & \\
\hline & & & & & & & & \\
\hline & & & & & & & & \\
\hline & & & & & & & & \\
\hline & & & & & & & & \\
\hline & & & & & & & & \\
\hline & \multicolumn{4}{|c|}{ Size Distribution (Mass) } & \multicolumn{4}{|c|}{ Partition Factors } \\
\hline & Fresh & Main & Screen & & Fresh & Main & Screen & \\
\hline & Feed & Effluent & Drain & Product & Feed & Effluent & Drain & Product \\
\hline Size & (kg/hr) & (kg/hr) & (kg/hr) & (kg/hr) & - & - & - & - \\
\hline$+100 \mathrm{M}$ & 211 & 0.02 & 0.00 & 2.09 & 100.00 & 0.84 & 0.06 & 99.10 \\
\hline $100 \times 500 \mathrm{M}$ & 35.01 & 0.01 & 0.03 & 34.96 & 100.00 & 0.02 & 0.10 & 99.88 \\
\hline$-500 \mathrm{M}$ & 139.91 & 3.90 & 25.70 & 110.31 & 100.00 & 2.79 & 18.37 & 78.84 \\
\hline Overal & 177.02 & 3.92 & 25.74 & 147.36 & 100.00 & 2.22 & 14.54 & 83.24 \\
\hline \multirow[t]{5}{*}{ Slurry Rate } & 4704.28 & 4426.59 & 96.34 & 181.35 & & & & \\
\hline & & & & & & & & \\
\hline & \multicolumn{4}{|c|}{ Size Distribution (Percent of Stream) } & \multicolumn{4}{|c|}{ Ash Content } \\
\hline & Fresh & Main & Screen & & Fresh & Main & Screen & \\
\hline & Feed & Effluent & Drain & Product & Feed & Effluent & Drain & Product \\
\hline Size & (\%) & (\%) & (\%) & (\%) & $(\%)$ & (\%) & $(\%)$ & (\%) \\
\hline$+100 \mathrm{M}$ & 1.19 & 0.45 & 0.01 & 1.42 & 9.77 & 4237 & 17.51 & 9.49 \\
\hline $100 \times 500 \mathrm{M}$ & 19.77 & 0.20 & 0.13 & 23.73 & 2.48 & 5201 & $\mathbf{3 . 3 4}$ & 246 \\
\hline$-500 \mathrm{M}$ & 79.03 & 99.35 & 99.87 & 74.86 & 7.36 & 60.70 & 5.89 & 5.82 \\
\hline \multirow[t]{4}{*}{ Overal } & 100.00 & 100.00 & 100.00 & 100.00 & 6.42 & 60.60 & 5.89 & 5.07 \\
\hline & \multicolumn{4}{|c|}{ Combustible Recovery } & & & & \\
\hline & Fresh & Main & Screen & & & & & \\
\hline & Feed & Effluent & Drain & Product & & & Solids & Slurry \\
\hline Size & $(\%)$ & (\%) & $(\%)$ & (\%) & & Stream & SG & GPM \\
\hline$+100 \mathrm{M}$ & 100.00 & 0.54 & 0.06 & 99.41 & & F. Feed & 1.34 & 20.54 \\
\hline $100 \times 500 \mathrm{M}$ & 100.00 & 0.01 & 0.09 & 99.89 & & M. Effluent & 1.83 & 19.51 \\
\hline$-500 \mathrm{M}$ & 100.00 & 1.18 & 18.66 & 80.16 & & S. Drain & 1.34 & 0.40 \\
\hline \multirow[t]{4}{*}{ Overal } & 100.00 & 0.93 & 14.62 & 84.44 & & Product & 1.33 & 0.64 \\
\hline & & & & & & & & \\
\hline & & & & & & & Measured & Predicted \\
\hline & & & & & & & Moisture & Moisture \\
\hline 177.02 & 3.76 & & & 25.74 & 26.71 & & (\%) & (\%) \\
\hline \multirow[t]{5}{*}{20.54} & 6.42 & & & 0.40 & 5.89 & & 18.74 & 18.76 \\
\hline & & & & & & & & \\
\hline & & & & & & & & \\
\hline & & & & & \multirow{2}{*}{\multicolumn{3}{|c|}{ 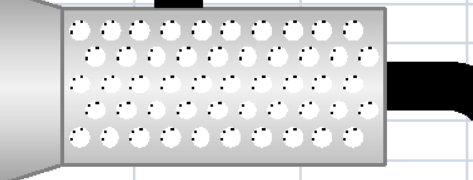 }} & \\
\hline & & & & & & & & \\
\hline 3.92 & 0.09 & & & & & 147.36 & 81.26 & \\
\hline \multirow[t]{3}{*}{19.51} & 60.60 & & & & & 0.64 & 5.07 & \\
\hline & & & Dry kg/hr & \% solids & & & & \\
\hline & & & Slumy GPM & \% ash & & & & \\
\hline & & & & & & & & \\
\hline
\end{tabular}




\begin{tabular}{|c|c|c|c|c|c|c|c|c|}
\hline \multicolumn{9}{|c|}{ Evaluation of Hyperbaric Centrifuge - Cardinal Plant } \\
\hline Test Num ber: & \multicolumn{8}{|c|}{6} \\
\hline Test Date: & \multicolumn{8}{|c|}{ September, 17th 2008} \\
\hline Feed: & \multicolumn{8}{|c|}{$100 \%-325$ M Column Concentrate } \\
\hline Description: & \multicolumn{8}{|c|}{ Simulated data for screen drain recirculation } \\
\hline & & & & & & & & \\
\hline & & & & & & & & \\
\hline & & & & & & & & \\
\hline & & & & & & & & \\
\hline & & & & & & & & \\
\hline & & & & & & & & \\
\hline & \multicolumn{4}{|c|}{ Size Distribution (Mass) } & \multicolumn{4}{|c|}{ Partition Factors } \\
\hline & Com bined & Main & Screen & & Fresh & Main & Screen & \\
\hline & Feed & Effluent & Drain & Product & Feed & Effluent & Drain & Product \\
\hline Size & $(\mathbf{k g} / \mathbf{h r})$ & (kg/hr) & (kg/hr) & (kg/hr) & - & - & - & - \\
\hline$+100 \mathrm{M}$ & 211 & 0.02 & 0.00 & 2.09 & 100.00 & 0.84 & 0.06 & 99.16 \\
\hline $100 \times 500 \mathrm{M}$ & 35.04 & 0.01 & 0.03 & 35.00 & 100.00 & 0.02 & 0.10 & 99.98 \\
\hline$-500 \mathrm{M}$ & 171.39 & 4.77 & 31.49 & 135.13 & 100.00 & 3.41 & 2251 & 96.59 \\
\hline Overal & 208.54 & 4.80 & 31.52 & 17222 & 100.00 & 2.71 & 17.81 & 97.29 \\
\hline \multirow[t]{5}{*}{ Slurry Rate } & 480264 & 4493.50 & 98.36 & 210.78 & & & & \\
\hline & & & & & & & & \\
\hline & \multicolumn{4}{|c|}{ Size Distribution (Percent of Stream) } & \multicolumn{4}{|c|}{ Ash Content } \\
\hline & Com bined & Main & Screen & & Com bined & Main & Screen & \\
\hline & Feed & Effluent & Drain & Product & Feed & Effluent & Drain & Product \\
\hline Size & (\%) & (\%) & (\%) & (\%) & $(\%)$ & (\%) & $(\%)$ & (\%) \\
\hline$+100 \mathrm{M}$ & 1.01 & 0.37 & 0.00 & 1.21 & 9.77 & 4237 & 17.51 & 9.49 \\
\hline $100 \times 500 \mathrm{M}$ & 16.80 & 0.16 & 0.11 & 20.32 & 2.48 & 5201 & $\mathbf{3 . 3 4}$ & 246 \\
\hline$-500 \mathrm{M}$ & 82.19 & 99.47 & 99.89 & 78.46 & 7.36 & 60.70 & 5.89 & 5.82 \\
\hline \multirow[t]{4}{*}{ Overal } & 100.00 & 100.00 & 100.00 & 100.00 & 6.56 & 60.62 & 5.89 & 5.18 \\
\hline & \multicolumn{4}{|c|}{ Combustible Recovery } & & & & \\
\hline & Com bined & Main & Screen & & & & & \\
\hline & Feed & Effluent & Drain & Product & & & Solids & Slurry \\
\hline Size & $(\%)$ & (\%) & $(\%)$ & $(\%)$ & & Stream & SG & GPM \\
\hline$+100 \mathrm{M}$ & 100.00 & 0.54 & 0.06 & 99.47 & & C. Feed & 1.34 & 20.94 \\
\hline $100 \times 500 \mathrm{M}$ & 100.00 & 0.01 & 0.09 & 99.99 & & M. Effluent & 1.83 & 19.80 \\
\hline$-500 \mathrm{M}$ & 100.00 & 1.18 & 18.66 & 98.20 & & S. Drain & 1.34 & 0.40 \\
\hline \multirow[t]{4}{*}{ Overal } & 100.00 & 0.97 & 15.23 & 98.58 & & Product & 1.33 & 0.74 \\
\hline & & & & & & & & \\
\hline & & & & & & & Measured & Predicted \\
\hline & & & & & & & Moisture & Moisture \\
\hline 177.02 & 3.76 & & & 31.52 & 3205 & & (\%) & (\%) \\
\hline 20.54 & 6.42 & & & 0.40 & 5.89 & & - & 18.29 \\
\hline & & & ---- & --1 & ---1 & - & & \\
\hline & & & & & & i i & & \\
\hline 208.54 & 4.34 & & & & & $: \because: \div:$ & $\because:-$ & \\
\hline 20.94 & 6.56 & & & & & $\because \because \because \because \because$ & $\because:$ & \\
\hline & & & & & $\because \because$ & $\because \because \because \because \because: \because$ & $\because \because$ & \\
\hline 4.80 & 0.11 & & & & & 17222 & 81.71 & \\
\hline 19.80 & 60.62 & & & & & 0.74 & 5.18 & \\
\hline & & & Dry kg/hr & \% solids & & & & \\
\hline & & & Surry GPM & \% ash & & & & \\
\hline & & & & & & & & \\
\hline
\end{tabular}




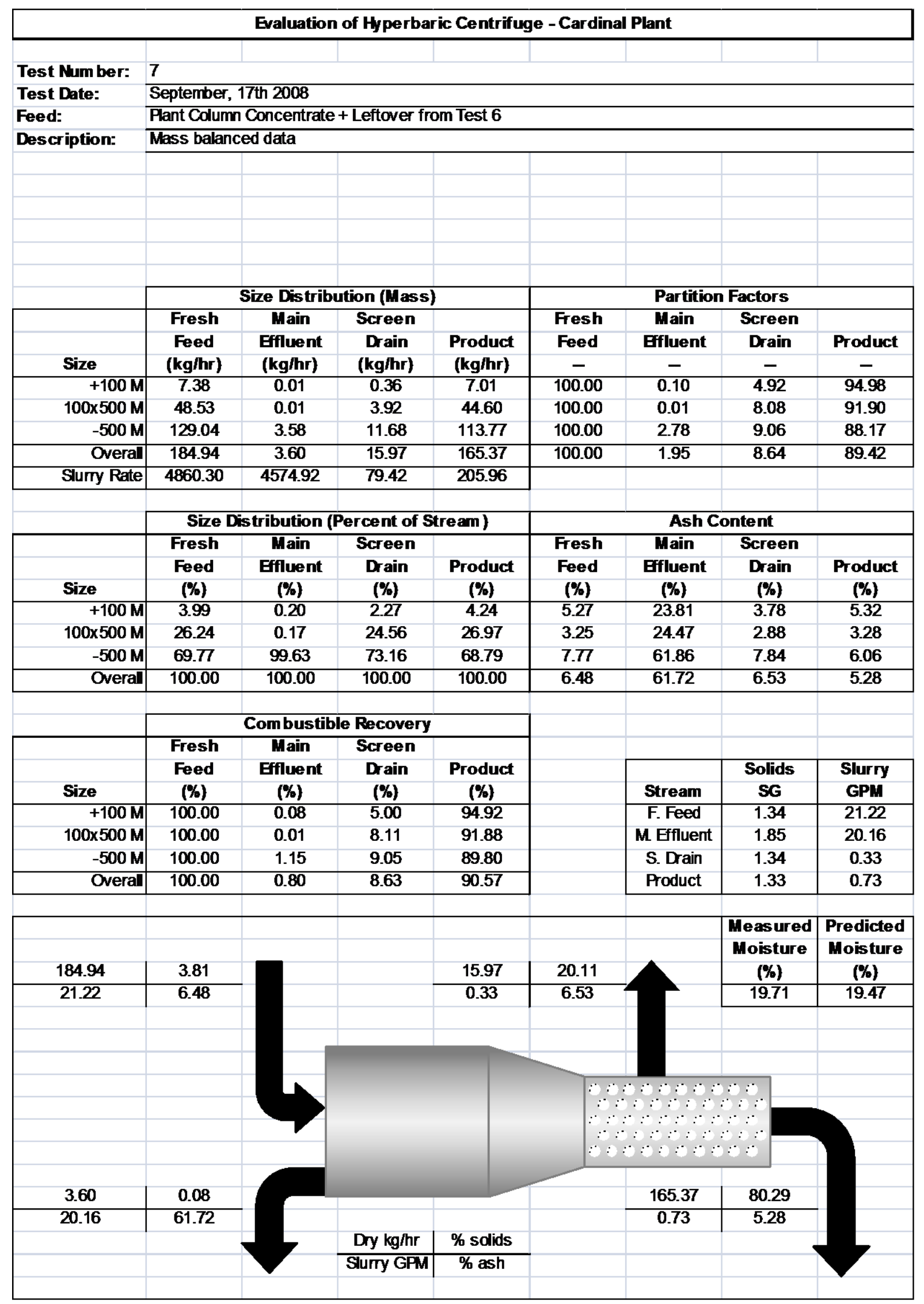




\begin{tabular}{|c|c|c|c|c|c|c|c|c|}
\hline \multicolumn{9}{|c|}{ Evaluation of Hyperbaric Centrifuge - Cardinal Plant } \\
\hline Test Num ber: & \multicolumn{8}{|c|}{7} \\
\hline Test Date: & \multicolumn{8}{|c|}{ September, 17th 2008} \\
\hline Feed: & \multicolumn{8}{|c|}{ Plant Column Concentrate + Leftover from Test 6} \\
\hline Description: & \multicolumn{8}{|c|}{ Simulated data for screen drain recirculation } \\
\hline & & & & & & & & \\
\hline & & & & & & & & \\
\hline & & & & & & & & \\
\hline & & & & & & & & \\
\hline & & & & & & & & \\
\hline & & & & & & & & \\
\hline & \multicolumn{4}{|c|}{\begin{tabular}{|l} 
Size Distribution (Mass) \\
\end{tabular}} & \multicolumn{4}{|c|}{ Partition Factors } \\
\hline & Com bined & Main & Screen & & Fresh & Main & Screen & \\
\hline & Feed & Effluent & Drain & Product & Feed & Effluent & Drain & Product \\
\hline Size & (kg/hr) & (kg/hr) & (kg/hr) & (kg/hr) & - & - & - & - \\
\hline$+100 \mathrm{M}$ & 7.76 & 0.01 & 0.38 & 7.37 & 100.00 & 0.10 & 5.18 & 99.90 \\
\hline $100 \times 500 \mathrm{M}$ & 52.80 & 0.01 & 4.27 & 48.52 & 100.00 & 0.01 & 8.80 & 99.99 \\
\hline$-500 \mathrm{M}$ & 141.88 & 3.94 & 12.85 & 125.10 & 100.00 & 3.05 & 9.96 & 96.95 \\
\hline Overal & 202.44 & 3.96 & 17.50 & 180.99 & 100.00 & 2.14 & 9.46 & 97.86 \\
\hline \multirow[t]{5}{*}{ Slurry Rate } & 4941.04 & 4635.63 & 80.74 & 224.67 & & & & \\
\hline & \multirow{2}{*}{\multicolumn{4}{|c|}{ Size Distribution (Percent of Stream) }} & \multirow{2}{*}{\multicolumn{4}{|c|}{ Ash Content }} \\
\hline & & & & & & & & \\
\hline & Com bined & Main & Screen & & Com bined & Main & Screen & \\
\hline & Feed & Effluent & Drain & Product & Feed & Effluent & Drain & Product \\
\hline Size & (\%) & (\%) & (\%) & (\%) & $(\%)$ & (\%) & $(\%)$ & (\%) \\
\hline$+100 \mathrm{M}$ & 3.83 & 0.19 & 2.18 & 4.07 & 5.27 & 23.81 & 3.78 & 5.32 \\
\hline $100 \times 500 \mathrm{M}$ & 26.08 & 0.17 & 24.39 & 26.81 & 3.25 & 24.47 & 2.88 & 3.28 \\
\hline$-500 \mathrm{M}$ & 70.09 & 99.64 & 73.43 & 69.12 & 7.77 & 61.86 & 7.84 & 6.06 \\
\hline \multirow[t]{4}{*}{ Overal } & 100.00 & 100.00 & 100.00 & 100.00 & 6.50 & 61.72 & 6.54 & 5.28 \\
\hline & \multicolumn{4}{|c|}{ Combustible Recovery } & & & & \\
\hline & Com bined & Main & Screen & & & & & \\
\hline & Feed & Effluent & Drain & Product & & & Solids & Slurry \\
\hline Size & $(\%)$ & (\%) & $(\%)$ & $(\%)$ & & Stream & SG & GPM \\
\hline$+100 \mathrm{M}$ & 100.00 & 0.08 & 5.00 & 99.84 & & C. Feed & 1.34 & 21.56 \\
\hline $100 \times 500 \mathrm{M}$ & 100.00 & 0.01 & 8.11 & 99.96 & & M. Effluent & 1.85 & 20.43 \\
\hline$-500 \mathrm{M}$ & 100.00 & 1.15 & 9.05 & 98.74 & & S. Drain & 1.34 & 0.34 \\
\hline \multirow[t]{4}{*}{ Overal } & 100.00 & 0.80 & 8.64 & 99.12 & & Product & 1.33 & 0.79 \\
\hline & & & & & & & & \\
\hline & & & & & & & Measured & Predicted \\
\hline & & & & & & & Moisture & Moisture \\
\hline 184.94 & 3.81 & & & 17.50 & 21.67 & & (\%) & (\%) \\
\hline 21.22 & 6.48 & & & 0.34 & 6.54 & & - & 19.44 \\
\hline & & & --- & 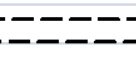 & $-\cdots--$ & - & & \\
\hline & & & & & & Li & & \\
\hline 202.44 & 4.10 & & & & & $: \because: \div:$ & $\because \because$ & \\
\hline 21.56 & 6.50 & & & & & $\because \because \because \because \because$ & $\because:$ & \\
\hline & & & & & $\because \because$ & $\because \because \because \because \because$ & $\because \because$ & \\
\hline 3.96 & 0.09 & & & & & 180.99 & 80.56 & \\
\hline 20.43 & 61.72 & & & & & 0.79 & 5.28 & \\
\hline & & & Dry kg/hr & \% solids & & & & \\
\hline & & & Surry GPM & \% ash & & & & \\
\hline & & & & & & & & \\
\hline
\end{tabular}


APPENDIX 2 - Economic Calculations 
Coal A - Circuit Configuration 1 - Screenbowl Centrifuge Simulation

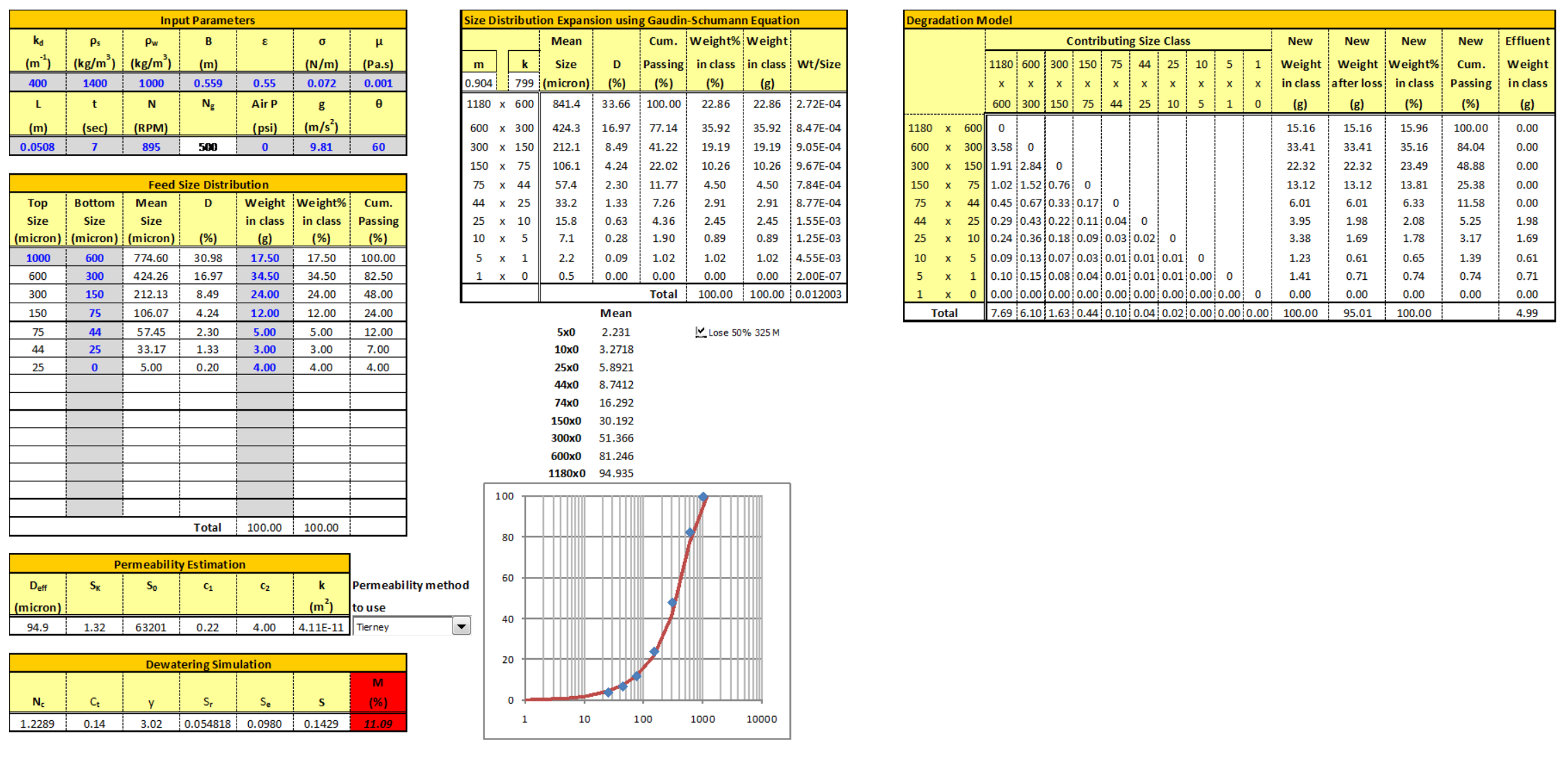


Coal A - Circuit Configuration 1 - Screenbowl Centrifuge Economics

\section{CALCULATION OF OPTIMUM SIZE CUT}

\begin{tabular}{|c|c|c|c|c|c|c|c|c|c|c|c|c|c|}
\hline \multirow{2}{*}{\multicolumn{2}{|c|}{\begin{tabular}{|l|l|} 
FINANCIAL DATA: & \\
& \\
\end{tabular}}} & \multicolumn{2}{|c|}{ Total Boiler Cost $=$} & \multirow{2}{*}{$\begin{array}{r}\$ 2.44 \\
\$ 20.00\end{array}$} & /MM BTU & & \multicolumn{2}{|c|}{ Moisture Evap. = } & \multirow{2}{*}{$\begin{array}{r}\$ 3.00 \\
\$ 20.00\end{array}$} & /ton $\mathrm{H}_{2} \mathrm{O}$ & \multirow{2}{*}{$\begin{array}{l}\mathrm{SO}_{2} \text { Emission }= \\
\text { Other Cost }=\end{array}$} & \multirow{2}{*}{$\begin{array}{r}\$ 200 \\
\$ 1.00\end{array}$} & $0 /$ ton $\mathrm{SO}_{2}$ \\
\hline & & Freight $\mathrm{Co}$ & & & /ton coal & & Ash Handli & $\mathrm{ng}=$ & & /ton ash & & & /ton coal \\
\hline \begin{tabular}{|l|} 
Size Class \\
\end{tabular} & & 1 & 2 & 3 & 4 & 5 & 6 & 7 & Totals & & & & \\
\hline Top Size & $\mu$ & 1180 & 600 & 300 & 150 & 74 & 44 & 25 & $-\cdots$ & & & & \\
\hline Bottom Size & $\mu$ & 600 & 300 & 150 & 74 & 44 & 25 & 0 & $-\ldots$ & & & & \\
\hline SG & $\mu$ & 1.45 & 1.45 & 1.45 & 1.45 & 1.45 & 1.45 & 1.45 & --- & & & & \\
\hline $1 / \mathrm{SG}$ & --- & 0.69 & 0.69 & 0.69 & 0.69 & 0.69 & 0.69 & 0.69 & --- & & & & \\
\hline Mass & $\%$, dry & 15.96 & 35.16 & 23.49 & 13.81 & 6.33 & 2.08 & 3.17 & 100.00 & & & & \\
\hline Solid SG & & 1.40 & 1.40 & 1.40 & 1.40 & 1.40 & 1.40 & 1.40 & & & & & \\
\hline Ash & $\%$, dry & 14.50 & 14.50 & 14.50 & 14.50 & 14.50 & 14.50 & 14.50 & 14.50 & & & & \\
\hline Sulfur & $\%$, dry & 1.00 & 1.00 & 1.00 & 1.00 & 1.00 & 1.00 & 1.00 & 1.00 & & & & \\
\hline$B T U / \mathrm{lb}$ & dry & 12825 & 12825 & 12825 & 12825 & 12825 & 12825 & 12825 & 12825 & & & & \\
\hline BTU/Ib & maf & 15000 & 15000 & 15000 & 15000 & 15000 & 15000 & 15000 & 15000 & & & & \\
\hline Mass & $\%$, ar & 14.63 & 32.74 & 22.77 & 14.53 & 7.58 & 2.83 & 4.92 & 100.00 & & & & \\
\hline Moisture & $\%$, ar & 3.02 & 4.51 & 8.26 & 15.50 & 25.76 & 34.71 & 42.78 & 11.09 & & & & \\
\hline Cum. Moisture & $\%$, ar & 11.09 & 12.47 & 17.43 & 24.42 & 32.88 & 39.83 & 42.78 & --- & & & & \\
\hline Ash & $\%$, ar & 14.06 & 13.85 & 13.30 & 12.25 & 10.76 & 9.47 & 8.30 & 12.89 & & & & \\
\hline Inerts & $\%$, ar & 17.08 & 18.35 & 21.56 & 27.75 & 36.52 & 44.17 & 51.08 & 23.98 & & & & \\
\hline Sulfur & $\%$, ar & 0.97 & 0.95 & 0.92 & 0.85 & 0.74 & 0.65 & 0.57 & 0.89 & & & & \\
\hline BTU/Ib & ar & 12438 & 12247 & 11766 & 10837 & 9522 & 8374 & 7338 & 11403 & & & & \\
\hline Ib SO2/MM BTU & --- & 1.56 & 1.56 & 1.56 & 1.56 & 1.56 & 1.56 & 1.56 & 1.56 & & & & \\
\hline Cost to Boiler & \$/MM BTU & $\$ 2.44$ & $\$ 2.44$ & $\$ 2.44$ & $\$ 2.44$ & $\$ 2.44$ & $\$ 2.44$ & $\$ 2.44$ & $\$ 2.44$ & & & & \\
\hline Freight Cost & $\$ / \mathrm{MM} \mathrm{BTU}$ & $\$ 0.80$ & $\$ 0.82$ & $\$ 0.85$ & $\$ 0.92$ & $\$ 1.05$ & $\$ 1.19$ & $\$ 1.36$ & $\$ 0.88$ & & & & \\
\hline Evap. Cost & $\$ / M M B T U$ & $\$ 0.00$ & $\$ 0.01$ & $\$ 0.01$ & $\$ 0.02$ & $\$ 0.04$ & $\$ 0.06$ & $\$ 0.09$ & $\$ 0.01$ & & & & \\
\hline Ash Cost & $\$ / \mathrm{MM} \mathrm{BTU}$ & $\$ 0.11$ & $\$ 0.11$ & $\$ 0.11$ & $\$ 0.11$ & $\$ 0.11$ & $\$ 0.11$ & $\$ 0.11$ & $\$ 0.11$ & & & & \\
\hline SO2 Cost & $\$ / \mathrm{MMBTU}$ & $\$ 0.04$ & $\$ 0.04$ & $\$ 0.04$ & $\$ 0.04$ & $\$ 0.04$ & $\$ 0.04$ & $\$ 0.04$ & $\$ 0.04$ & & & & \\
\hline \begin{tabular}{|l|} 
Other Cost \\
\end{tabular} & $\$ / \mathrm{MM} \mathrm{BTU}$ & $\$ 0.04$ & $\$ 0.04$ & $\$ 0.04$ & $\$ 0.05$ & $\$ 0.05$ & $\$ 0.06$ & $\$ 0.07$ & $\$ 0.04$ & & & & \\
\hline Coal Worth & $\$ / \mathrm{MM} \mathrm{BTU}$ & $\$ 1.44$ & $\$ 1.43$ & $\$ 1.39$ & $\$ 1.30$ & $\$ 1.15$ & $\$ 0.97$ & $\$ 0.77$ & $\$ 1.36$ & & & & \\
\hline Cost to Boiler & \$/ton & $\$ 60.70$ & $\$ 59.77$ & $\$ 57.42$ & $\$ 52.89$ & $\$ 46.47$ & $\$ 40.86$ & $\$ 35.81$ & $\$ 55.65$ & & & & \\
\hline Freight Cost & $\$ /$ ton & $\$ 20.00$ & $\$ 20.00$ & $\$ 20.00$ & $\$ 20.00$ & $\$ 20.00$ & $\$ 20.00$ & $\$ 20.00$ & $\$ 20.00$ & & & & \\
\hline Evap. Cost & $\$ /$ ton & $\$ 0.09$ & $\$ 0.14$ & $\$ 0.25$ & $\$ 0.46$ & $\$ 0.77$ & $\$ 1.04$ & $\$ 1.28$ & $\$ 0.33$ & & & & \\
\hline Ash Cost & $\$ /$ ton & $\$ 2.81$ & $\$ 2.77$ & $\$ 2.66$ & $\$ 2.45$ & $\$ 2.15$ & $\$ 1.89$ & $\$ 1.66$ & $\$ 2.58$ & & & & \\
\hline SO2 Cost & $\$ /$ ton & $\$ 0.89$ & $\$ 0.88$ & $\$ 0.85$ & $\$ 0.78$ & $\$ 0.68$ & $\$ 0.60$ & $\$ 0.53$ & $\$ 0.82$ & & & & \\
\hline Other Cost & $\$ /$ ton & $\$ 1.00$ & $\$ 1.00$ & $\$ 1.00$ & $\$ 1.00$ & $\$ 1.00$ & $\$ 1.00$ & $\$ 1.00$ & $\$ 1.00$ & & & & \\
\hline Coal Worth & $\$ /$ ton & $\$ 35.90$ & $\$ 34.98$ & $\$ 32.67$ & $\$ 28.19$ & $\$ 21.86$ & $\$ 16.33$ & $\$ 11.34$ & $\$ 30.92$ & & & & \\
\hline Individual Tons & dry & 15.16 & 33.41 & 22.32 & 13.12 & 6.01 & 1.98 & 3.01 & 95.01 & & & & \\
\hline Cum. Tons & dry & 95.01 & 79.85 & 46.44 & 24.12 & 11.00 & 4.99 & 3.01 & & & & & \\
\hline Individual Tons & $\mathrm{ar}$ & 15.64 & 34.99 & 24.33 & 15.53 & 8.10 & 3.03 & 5.26 & 106.86 & & & & \\
\hline Cum. Tons & ar & 106.86 & 91.23 & 56.24 & 31.91 & 16.39 & 8.29 & 5.26 & --- & & & & \\
\hline Cum. Mass & $\%$, ar & 100.00 & 85.37 & 52.63 & 29.86 & 15.33 & 7.76 & 4.92 & $-\cdots$ & & & & \\
\hline Cum. Ash & $\%$, ar & 14.06 & 13.91 & 13.71 & 13.46 & 13.24 & 13.13 & 12.89 & $-\cdots$ & & & & \\
\hline Cum. Sulfur & $\%$, ar & 0.97 & 0.96 & 0.95 & 0.93 & 0.91 & 0.91 & 0.89 & --- & & & & \\
\hline Cum. BTU/lb & ar & 12438 & 12350 & 12221 & 12067 & 11929 & 11834 & 11759 & $-\ldots$ & & & & \\
\hline Individual Worth & $\$$ & $\$ 561.39$ & $\$ 1,223.92$ & $\$ 794.70$ & $\$ 437.69$ & $\$ 177.01$ & $\$ 49.43$ & $\$ 59.66$ & $\$ 3,303.79$ & & & & \\
\hline Cum. Worth & $\$$ & $\$ 561.39$ & $\$ 1,785.30$ & $\$ 2,580.00$ & $\$ 3,017.69$ & $\$ 3,194.70$ & $\$ 3,244.13$ & $\$ 3,303.79$ & --- & & & & \\
\hline
\end{tabular}


Coal A - Circuit Configuration 1 - Coarse Cut - Screenbowl Centrifuge Simulation

\begin{tabular}{|c|c|c|c|c|c|c|}
\hline \multicolumn{7}{|c|}{ Input Parameters } \\
\hline $\begin{array}{c}k_{d} \\
\left(m^{-1}\right)\end{array}$ & $\begin{array}{c}\rho_{\mathrm{s}} \\
\left(\mathrm{kg} / \mathrm{m}^{3}\right)\end{array}$ & $\underset{\left(\mathrm{kg} / \mathrm{m}^{3}\right)}{\rho_{w}}$ & B & $\bar{\varepsilon}$ & $\begin{array}{c}\sigma \\
(\mathrm{N} / \mathrm{m})\end{array}$ & $\stackrel{\mu}{\mu}$ \\
\hline 400 & 1400 & 1000 & 0.559 & 0.55 & 0.072 & 0.001 \\
\hline $\mathrm{L}$ & $t$ & $\mathrm{~N}$ & $\mathrm{~N}_{\mathrm{g}}$ & Air $\mathrm{P}$ & g & $\theta$ \\
\hline (m) & (sec) & (RPM) & & (psi) & $\left(\mathrm{m} / \mathrm{s}^{2}\right)$ & \\
\hline 0.0381 & 7 & 895 & 500 & 0 & 9.81 & 60 \\
\hline
\end{tabular}
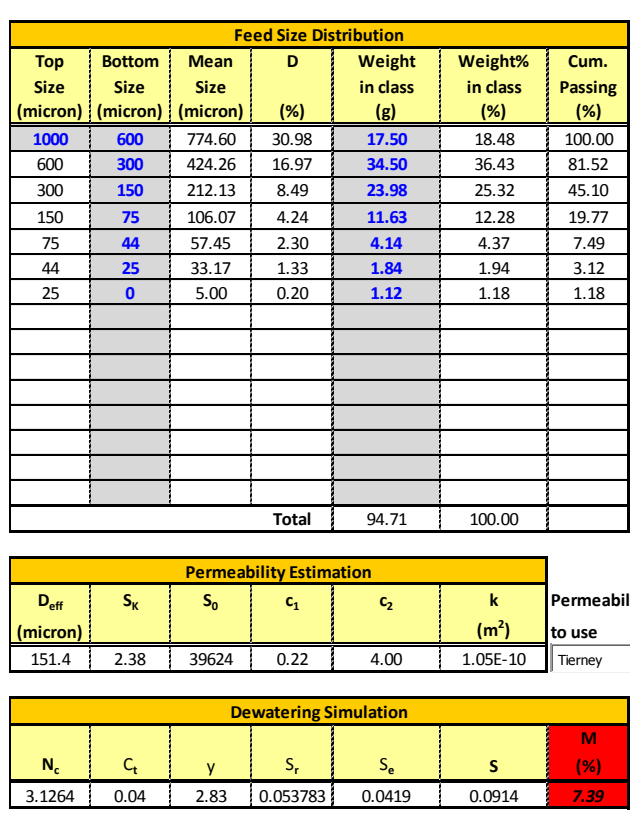
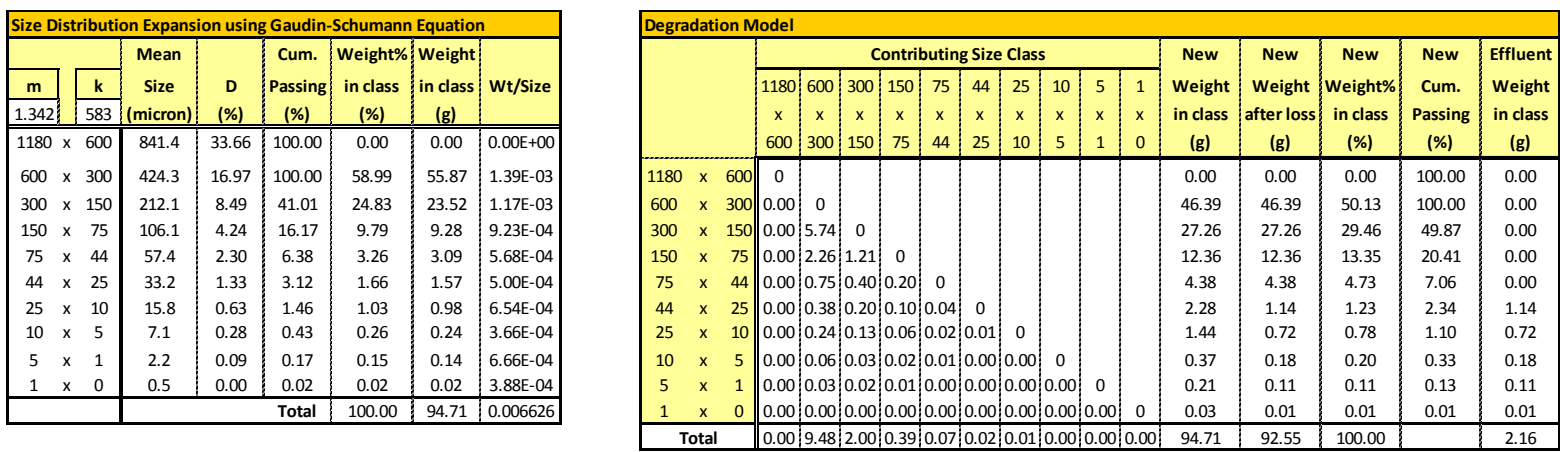

\begin{tabular}{ll} 
& Mean \\
$25 \times 0$ & 6.9885 \\
\hline
\end{tabular}

$\begin{array}{ll}25 \times 0 \\ 44 \times 0 & 11.964 \\ 14 \times 0 & 25.52\end{array}$

$74 \times 0 \quad 25.452$

$\begin{array}{cc}150 \times 0 & 50.608 \\ 300 \times 0 & 91.974\end{array}$

$3000 \times 01.51 .42$

$1180 \times 0$

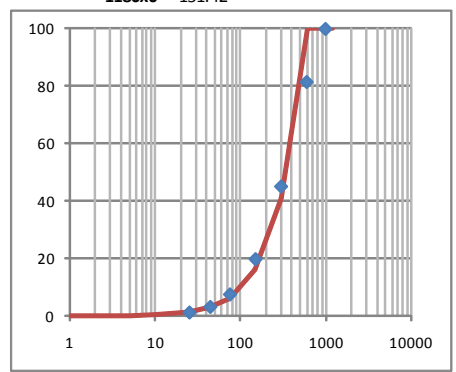


Coal A - Circuit Configuration 1 - Coarse Cut - Screenbowl Centrifuge Economics

CALCULATION OF OPTIMUM SIZE CUT

\begin{tabular}{|c|c|c|c|c|c|c|c|c|c|c|c|c|c|}
\hline \multirow{2}{*}{ FINANCIAL DATA: } & & \multicolumn{2}{|c|}{ Total Boiler Cost $=$} & $\$ 2.44$ & /MM BTU & & Moisture $\mathrm{E}$ & vap. = & $\$ 3.00$ & /ton $\mathrm{H}_{2} \mathrm{O}$ & $\mathrm{SO}_{2}$ Emission $=$ & $\$ 200$ & $/$ ton $\mathrm{SO}_{2}$ \\
\hline & & Freight $\mathrm{Co}$ & & $\$ 20.00$ & /ton coal & & Ash Handli & $\mathrm{ng}=$ & $\$ 20.00$ & /ton ash & Other Cost $=$ & $\$ 1.00$ & /ton coal \\
\hline Size Class & & 1 & 2 & 3 & 4 & 5 & 6 & 7 & Totals & & & & \\
\hline Top Size & $\mu$ & 1180 & 600 & 300 & 150 & 74 & 44 & 25 & --- & & & & \\
\hline Bottom Size & $\mu$ & 600 & 300 & 150 & 74 & 44 & 25 & 0 & $-\cdots$ & & & & \\
\hline$S G$ & $\mu$ & 1.45 & 1.45 & 1.45 & 1.45 & 1.45 & 1.45 & 1.45 & --- & & & & \\
\hline $1 / \mathrm{SG}$ & --- & 0.69 & 0.69 & 0.69 & 0.69 & 0.69 & 0.69 & 0.69 & --- & & & & \\
\hline Mass & $\%$, dry & 0.00 & 50.13 & 29.46 & 13.35 & 4.73 & 1.23 & 1.10 & 100.00 & & & & \\
\hline Solid SG & & 1.40 & 1.40 & 1.40 & 1.40 & 1.40 & 1.40 & 1.40 & & & & & \\
\hline Ash & $\%$, dry & 14.50 & 14.50 & 14.50 & 14.50 & 14.50 & 14.50 & 14.50 & 14.50 & & & & \\
\hline Sulfur & $\%$, dry & 1.00 & 1.00 & 1.00 & 1.00 & 1.00 & 1.00 & 1.00 & 1.00 & & & & \\
\hline BTU/Ib & dry & 12825 & 12825 & 12825 & 12825 & 12825 & 12825 & 12825 & 12825 & & & & \\
\hline BTU/lb & maf & 15000 & 15000 & 15000 & 15000 & 15000 & 15000 & 15000 & 15000 & & & & \\
\hline Mass & $\%$, ar & 0.00 & 48.38 & 29.04 & 13.85 & 5.40 & 1.61 & 1.72 & 100.00 & & & & \\
\hline Moisture & $\%$, ar & 7.39 & 4.05 & 6.04 & 10.73 & 18.93 & 29.20 & 40.57 & 7.39 & & & & \\
\hline Cum. Moisture & $\%$, ar & 7.39 & 7.39 & 10.52 & 16.28 & 25.09 & 35.08 & 40.57 & --- & & & & \\
\hline Ash & $\%$, ar & 13.43 & 13.91 & 13.62 & 12.94 & 11.75 & 10.26 & 8.62 & 13.43 & & & & \\
\hline Inerts & $\%$, ar & 20.82 & 17.96 & 19.66 & 23.67 & 30.68 & 39.47 & 49.19 & 20.82 & & & & \\
\hline Sulfur & $\%$, ar & 0.93 & 0.96 & 0.94 & 0.89 & 0.81 & 0.71 & 0.59 & 0.93 & & & & \\
\hline BTU/Ib & ar & 11877 & 12306 & 12050 & 11449 & 10398 & 9080 & 7621 & 11877 & & & & \\
\hline Ib SO2/MM BTU & $-\ldots$ & 1.56 & 1.56 & 1.56 & 1.56 & 1.56 & 1.56 & 1.56 & 1.56 & & & & \\
\hline Cost to Boiler & $\$ / M M$ BTU & $\$ 2.44$ & $\$ 2.44$ & $\$ 2.44$ & $\$ 2.44$ & $\$ 2.44$ & $\$ 2.44$ & $\$ 2.44$ & $\$ 2.44$ & & & & \\
\hline Freight Cost & $\$ / M M B T U$ & $\$ 0.84$ & $\$ 0.81$ & $\$ 0.83$ & $\$ 0.87$ & $\$ 0.96$ & $\$ 1.10$ & $\$ 1.31$ & $\$ 0.84$ & & & & \\
\hline Evap. Cost & $\$ / \mathrm{MM} \mathrm{BTU}$ & $\$ 0.01$ & $\$ 0.00$ & $\$ 0.01$ & $\$ 0.01$ & $\$ 0.03$ & $\$ 0.05$ & $\$ 0.08$ & $\$ 0.01$ & & & & \\
\hline Ash Cost & $\$ / M M B T U$ & $\$ 0.11$ & $\$ 0.11$ & $\$ 0.11$ & $\$ 0.11$ & $\$ 0.11$ & $\$ 0.11$ & $\$ 0.11$ & $\$ 0.11$ & & & & \\
\hline SO2 Cost & $\$ / M M B T U$ & $\$ 0.04$ & $\$ 0.04$ & $\$ 0.04$ & $\$ 0.04$ & $\$ 0.04$ & $\$ 0.04$ & $\$ 0.04$ & $\$ 0.04$ & & & & \\
\hline Other Cost & $\$ / M M B T U$ & $\$ 0.04$ & $\$ 0.04$ & $\$ 0.04$ & $\$ 0.04$ & $\$ 0.05$ & $\$ 0.06$ & $\$ 0.07$ & $\$ 0.04$ & & & & \\
\hline Coal Worth & $\$ / \mathrm{MM} \mathrm{BTU}$ & $\$ 1.40$ & $\$ 1.43$ & $\$ 1.41$ & $\$ 1.36$ & $\$ 1.25$ & $\$ 1.09$ & $\$ 0.83$ & $\$ 1.40$ & & & & \\
\hline Cost to Boiler & $\$ /$ ton & $\$ 57.96$ & $\$ 60.05$ & $\$ 58.81$ & $\$ 55.87$ & $\$ 50.74$ & $\$ 44.31$ & $\$ 37.19$ & $\$ 57.96$ & & & & \\
\hline Freight Cost & $\$ /$ ton & $\$ 20.00$ & $\$ 20.00$ & $\$ 20.00$ & $\$ 20.00$ & $\$ 20.00$ & $\$ 20.00$ & $\$ 20.00$ & $\$ 20.00$ & & & & \\
\hline Evap. Cost & $\$ /$ ton & $\$ 0.22$ & $\$ 0.12$ & $\$ 0.18$ & $\$ 0.32$ & $\$ 0.57$ & $\$ 0.88$ & $\$ 1.22$ & $\$ 0.22$ & & & & \\
\hline Ash Cost & $\$ /$ ton & $\$ 2.69$ & $\$ 2.78$ & $\$ 2.72$ & $\$ 2.59$ & $\$ 2.35$ & $\$ 2.05$ & $\$ 1.72$ & $\$ 2.69$ & & & & \\
\hline SO2 Cost & $\$ /$ ton & $\$ 0.85$ & $\$ 0.88$ & $\$ 0.87$ & $\$ 0.82$ & $\$ 0.75$ & $\$ 0.65$ & $\$ 0.55$ & $\$ 0.85$ & & & & \\
\hline Other Cost & $\$ /$ ton & $\$ 1.00$ & $\$ 1.00$ & $\$ 1.00$ & $\$ 1.00$ & $\$ 1.00$ & $\$ 1.00$ & $\$ 1.00$ & $\$ 1.00$ & & & & \\
\hline Coal Worth & $\$ /$ ton & $\$ 33.20$ & $\$ 35.26$ & $\$ 34.03$ & $\$ 31.14$ & $\$ 26.07$ & $\$ 19.73$ & $\$ 12.70$ & $\$ 33.20$ & & & & \\
\hline Individual Tons & dry & 0.00 & 46.39 & 27.26 & 12.36 & 4.38 & 1.14 & 1.02 & 92.55 & & & & \\
\hline Cum. Tons & dry & 92.55 & 92.55 & 46.16 & 18.89 & 6.54 & 2.16 & 1.02 & & & & & \\
\hline Individual Tons & $\mathrm{ar}$ & 0.00 & 48.35 & 29.02 & 13.84 & 5.40 & 1.61 & 1.72 & 99.94 & & & & \\
\hline Cum. Tons & $\mathrm{ar}$ & 99.94 & 99.94 & 51.59 & 22.57 & 8.73 & 3.33 & 1.72 & -- & & & & \\
\hline Cum. Mass & $\%$, ar & 100.00 & 100.00 & 51.62 & 22.58 & 8.73 & 3.33 & 1.72 & --- & & & & \\
\hline Cum. Ash & $\%$, ar & 13.43 & 13.91 & 13.80 & 13.67 & 13.57 & 13.51 & 13.43 & --- & & & & \\
\hline Cum. Sulfur & $\%$, ar & 0.93 & 0.96 & 0.95 & 0.94 & 0.94 & 0.93 & 0.93 & -- & & & & \\
\hline Cum. BTU/lb & ar & 11877 & 12091 & 12083 & 12031 & 11980 & 11947 & 11921 & --- & & & & \\
\hline \begin{tabular}{|l} 
Individual Worth \\
\end{tabular} & $\$$ & $\$ 0.00$ & $\$ 1,705.01$ & $\$ 987.59$ & $\$ 431.01$ & $\$ 140.72$ & $\$ 31.74$ & $\$ 21.86$ & $\$ 3,317.94$ & & & & \\
\hline Cum. Worth & $\$$ & $\$ 0.00$ & $\$ 1,705.01$ & $\$ 2,692.60$ & $\$ 3,123.61$ & $\$ 3,264.34$ & $\$ 3,296.08$ & $\$ 3,317.94$ & --- & & & & \\
\hline
\end{tabular}


Coal A - Circuit Configuration 1 - Coarse Cut - Hyperbaric Centrifuge Simulation

\begin{tabular}{|c|c|c|c|c|c|c|}
\hline \multicolumn{7}{|c|}{ Input Parameters } \\
\hline $\begin{array}{c}k_{d} \\
\left(m^{-1}\right)\end{array}$ & $\begin{array}{c}\rho_{\mathrm{s}} \\
\left(\mathrm{kg} / \mathrm{m}^{3}\right)\end{array}$ & $\underset{\left(\mathrm{kg} / \mathrm{m}^{3}\right)}{\rho_{\mathrm{w}}}$ & $\begin{array}{c}\text { B } \\
(\mathrm{m})\end{array}$ & $\varepsilon$ & $\begin{array}{c}\sigma \\
(\mathrm{N} / \mathrm{m})\end{array}$ & $\begin{array}{c}\mu \\
\text { (Pa.s) }\end{array}$ \\
\hline 400 & 1400 & 1000 & 0.381 & 0.52 & 0.072 & 0.001 \\
\hline $\mathrm{L}$ & ${ }^{t}$ & $\mathrm{~N}$ & $\mathrm{~N}_{\mathrm{g}}$ & Air $P$ & $\mathbf{g}$ & $\theta$ \\
\hline (m) & (sec) & (RPM) & & (psi) & $\left(\mathrm{m} / \mathrm{s}^{2}\right)$ & \\
\hline 0.0127 & 7 & 2650 & 2991 & 0 & 9.81 & 60 \\
\hline
\end{tabular}

\begin{tabular}{|c|c|c|c|c|c|c|}
\hline \multicolumn{7}{|c|}{ Feed Size Distribution } \\
\hline $\begin{array}{c}\text { Top } \\
\text { Size } \\
\text { (micron) }\end{array}$ & $\begin{array}{c}\text { Bottom } \\
\text { Size } \\
\text { (micron) }\end{array}$ & $\begin{array}{c}\text { Mean } \\
\text { Size } \\
\text { (micron) }\end{array}$ & $\begin{array}{l}\text { D } \\
(\%)\end{array}$ & $\begin{array}{l}\text { Weight } \\
\text { in class }\end{array}$ & $\begin{array}{l}\text { Weight\% } \\
\text { in class } \\
\text { (\%) }\end{array}$ & $\begin{array}{l}\text { Cum. } \\
\text { Passing } \\
\text { (\%) }\end{array}$ \\
\hline 1000 & 600 & 774.60 & 30.98 & 0.00 & 0.00 & 100.00 \\
\hline 600 & 300 & 424.26 & 16.97 & 0.00 & 0.00 & 100.00 \\
\hline 300 & 150 & 212.13 & 8.49 & 0.02 & 0.22 & 100.00 \\
\hline 150 & 75 & 106.07 & 4.24 & 0.37 & 4.91 & 99.80 \\
\hline 75 & 44 & 57.45 & 2.30 & 0.86 & 11.57 & 94.88 \\
\hline 44 & 25 & 33.17 & 1.33 & 2.30 & 30.89 & 83.32 \\
\hline 25 & 0 & 5.00 & 0.20 & 3.91 & 52.43 & 52.43 \\
\hline & & & & & & \\
\hline & & & & & & \\
\hline & & & & & & \\
\hline & & & & & & \\
\hline & & & & & & \\
\hline & & & Tot? & 705 & 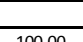 & \\
\hline & & & & & & \\
\hline
\end{tabular}
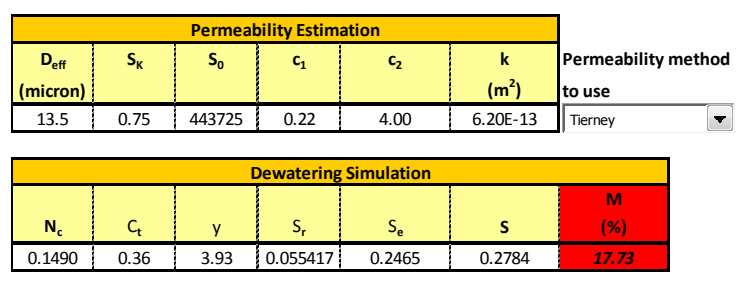

\begin{tabular}{|c|c|c|c|c|c|c|c|}
\hline \multicolumn{8}{|c|}{ Size Distribution Expansion using Gaudin-Schumann Equation } \\
\hline \multicolumn{2}{|c|}{ size vistribut } & \multirow{3}{*}{\begin{tabular}{|c|} 
Mean \\
Size \\
(micron)
\end{tabular}} & \multirow{3}{*}{$\begin{array}{c}D \\
(\%)\end{array}$} & \multirow{3}{*}{$\begin{array}{c}\text { Cum. } \\
\text { Passing } \\
(\%)\end{array}$} & \multirow{3}{*}{\begin{tabular}{|c|} 
Weight $\%$ \\
in class \\
$(\%)$
\end{tabular}} & \multirow{3}{*}{$\begin{array}{c}\text { Weight } \\
\text { in class } \\
\text { (g) }\end{array}$} & \multirow{3}{*}{ Wt/Size } \\
\hline $\mathrm{m}$ & $\mathrm{k}$ & & & & & & \\
\hline 0.543 & 75 & & & & & & \\
\hline 1180 & 600 & 841.4 & 33.66 & 100.00 & 0.00 & 0.00 & $0.00 E+00$ \\
\hline 600 & 300 & 424.3 & 16.97 & 100.00 & 0.00 & 0.00 & $0.00 E+0$ \\
\hline & 150 & & & & & & \\
\hline 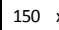 & 75 & 106.1 & 4.24 & & & & 0.00 \\
\hline 75 & 44 & & 2.30 & & & & \\
\hline 44 & 25 & & & & & & \\
\hline 25 & 10 & 15. & & & & & \\
\hline 10 & 5 & & 0.2 & & & & \\
\hline 5 & 1 & & 0.09 & & & & \\
\hline 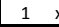 & 0 & 0.5 & 0.00 & & & & \\
\hline & & & & & 100.00 & 7.45 & \\
\hline
\end{tabular}

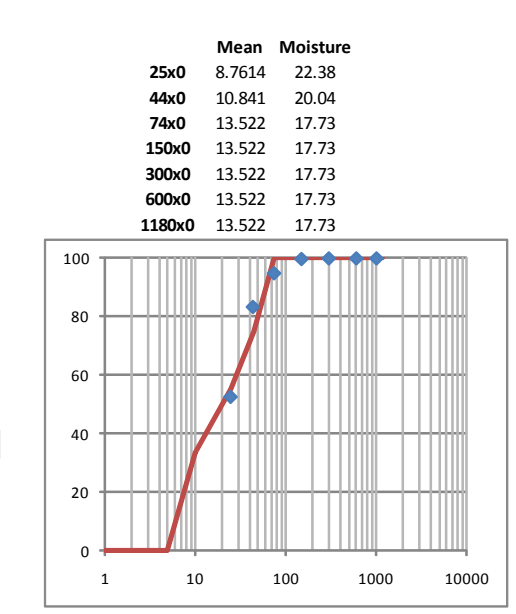

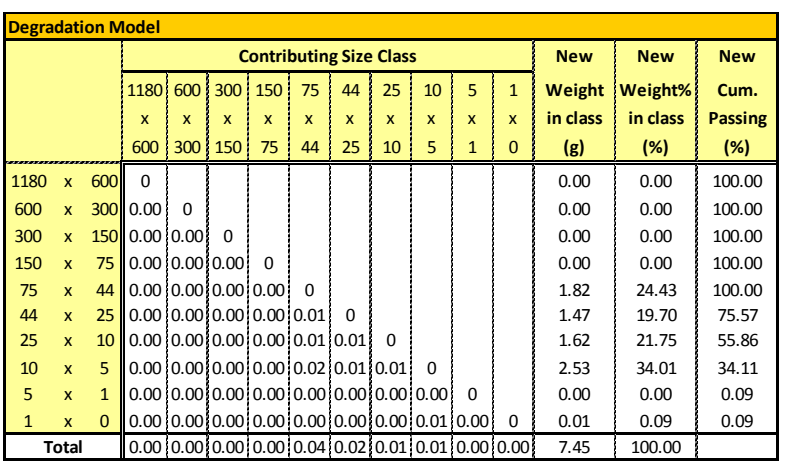

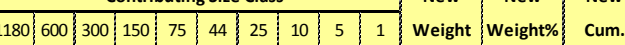
\begin{tabular}{|l|l|l|l|l|l|l|l|l|l|l|l|l|l}
$x$ & $x$ & $x$ & $x$ & $x$ & $x$ & $x$ & in class & in class & Passin \\
\hline
\end{tabular} 100.00

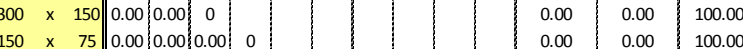

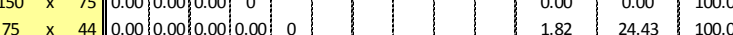

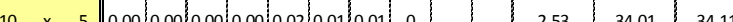
\begin{tabular}{|l|l|l|l|l|l|l|l|l|l|l|}
\hline 0.00 & 0.000 & 0.00 & 0.00 & 0.00 & 0.00 & 0.00 & 0 & & 0.09 \\
\hline
\end{tabular}

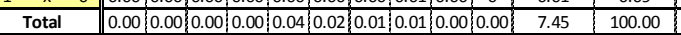


Coal A - Circuit Configuration 1 - Coarse Cut - Hyperbaric Centrifuge Economics

CALCULATION OF OPTIMUM SIZE CUT

\begin{tabular}{|c|c|c|c|c|c|c|c|c|c|c|c|c|c|}
\hline \multicolumn{2}{|l|}{ FINANCIAL DATA: } & \multicolumn{2}{|c|}{ Total Boiler Cost $=$} & \multirow{2}{*}{$\begin{array}{r}\$ 2.44 \\
\$ 20.00\end{array}$} & & \multicolumn{2}{|c|}{ Moisture Evap. = } & & & \multirow{2}{*}{$\begin{array}{l}\mathrm{SO}_{2} \text { Emission }= \\
\text { Other Cost }=\end{array}$} & \multirow{2}{*}{$\begin{array}{r}\$ 200 \\
\$ 1.00\end{array}$} & \\
\hline & & Freight Cos & & & $\begin{array}{l}\text { /MM BTU } \\
\text { /ton coal }\end{array}$ & & Ash Handli & & $\begin{array}{r}\$ 3.00 \\
\$ 20.00\end{array}$ & /ton $\mathrm{H}_{2} \mathrm{U}$ & & & /ton coal \\
\hline Size Class & & 1 & 2 & 3 & 4 & 5 & 6 & 7 & Totals & & & & \\
\hline Top Size & $\mu$ & 1180 & 600 & 300 & 150 & 74 & 44 & 25 & $-\cdots$ & & & & \\
\hline Bottom Size & $\mu$ & 600 & 300 & 150 & 74 & 44 & 25 & 0 & $-\cdots$ & & & & \\
\hline SG & $\mu$ & 1.45 & 1.45 & 1.45 & 1.45 & 1.45 & 1.45 & 1.45 & --- & & & & \\
\hline $1 / \mathrm{SG}$ & --- & 0.69 & 0.69 & 0.69 & 0.69 & 0.69 & 0.69 & 0.69 & --- & & & & \\
\hline Mass & $\%$, dry & 0.00 & 0.00 & 0.00 & 0.00 & 24.43 & 19.70 & 55.86 & 100.00 & & & & \\
\hline Solid SG & & 1.40 & 1.40 & 1.40 & 1.40 & 1.40 & 1.40 & 1.40 & & & & & \\
\hline Ash & $\%$, dry & 14.50 & 14.50 & 14.50 & 14.50 & 14.50 & 14.50 & 14.50 & 14.50 & & & & \\
\hline Sulfur & $\%$, dry & 1.00 & 1.00 & 1.00 & 1.00 & 1.00 & 1.00 & 1.00 & 1.00 & & & & \\
\hline $\mathrm{BTU} / \mathrm{Ib}$ & dry & 12825 & 12825 & 12825 & 12825 & 12825 & 12825 & 12825 & 12825 & & & & \\
\hline BTU/Ib & maf & 15000 & 15000 & 15000 & 15000 & 15000 & 15000 & 15000 & 15000 & & & & \\
\hline Mass & $\%$, ar & 0.00 & 0.00 & 0.00 & 0.00 & 22.24 & 18.55 & 59.21 & 100.00 & & & & \\
\hline Moisture & $\%$, ar & 17.73 & 17.73 & 17.73 & 17.73 & 9.63 & 12.59 & 22.38 & 17.73 & & & & \\
\hline Cum. Moisture & $\%$, ar & 17.73 & 17.73 & 17.73 & 17.73 & 17.73 & 20.04 & 22.38 & --- & & & & \\
\hline Ash & $\%$, ar & 11.93 & 11.93 & 11.93 & 11.93 & 13.10 & 12.67 & 11.25 & 11.93 & & & & \\
\hline Inerts & $\%$, ar & 29.65 & 29.65 & 29.65 & 29.65 & 22.73 & 25.26 & 33.63 & 29.65 & & & & \\
\hline Sulfur & $\%$, ar & 0.82 & 0.82 & 0.82 & 0.82 & 0.90 & 0.87 & 0.78 & 0.82 & & & & \\
\hline BTU/lb & ar & 10552 & 10552 & 10552 & 10552 & 11590 & 11211 & 9955 & 10552 & & & & \\
\hline Ib SO2/MM BTU & \begin{tabular}{|c|}
--- \\
\end{tabular} & 1.56 & 1.56 & 1.56 & 1.56 & 1.56 & 1.56 & 1.56 & 1.56 & & & & \\
\hline Cost to Boiler & $\$ / \mathrm{MM} \mathrm{BTU}$ & $\$ 2.44$ & $\$ 2.44$ & $\$ 2.44$ & $\$ 2.44$ & $\$ 2.44$ & $\$ 2.44$ & $\$ 2.44$ & $\$ 2.44$ & & & & \\
\hline Freight Cost & $\$ / \mathrm{MM} \mathrm{BTU}$ & $\$ 0.95$ & $\$ 0.95$ & $\$ 0.95$ & $\$ 0.95$ & $\$ 0.86$ & $\$ 0.89$ & $\$ 1.00$ & $\$ 0.95$ & & & & \\
\hline Evap. Cost & \$/MM BTU & $\$ 0.03$ & $\$ 0.03$ & $\$ 0.03$ & $\$ 0.03$ & $\$ 0.01$ & $\$ 0.02$ & $\$ 0.03$ & $\$ 0.03$ & & & & \\
\hline Ash Cost & $\$ / \mathrm{MM} \mathrm{BTU}$ & $\$ 0.11$ & $\$ 0.11$ & $\$ 0.11$ & $\$ 0.11$ & $\$ 0.11$ & $\$ 0.11$ & $\$ 0.11$ & $\$ 0.11$ & & & & \\
\hline SO2 Cost & $\$ / \mathrm{MM} \mathrm{BTU}$ & $\$ 0.04$ & $\$ 0.04$ & $\$ 0.04$ & $\$ 0.04$ & $\$ 0.04$ & $\$ 0.04$ & $\$ 0.04$ & $\$ 0.04$ & & & & \\
\hline \begin{tabular}{|l|} 
Other Cost \\
\end{tabular} & $\$ / \mathrm{MM} \mathrm{BTU}$ & $\$ 0.05$ & $\$ 0.05$ & $\$ 0.05$ & $\$ 0.05$ & $\$ 0.04$ & $\$ 0.04$ & $\$ 0.05$ & $\$ 0.05$ & & & & \\
\hline Coal Worth & $\$ / \mathrm{MM} \mathrm{BTU}$ & $\$ 1.27$ & $\$ 1.27$ & $\$ 1.27$ & $\$ 1.27$ & $\$ 1.37$ & $\$ 1.34$ & $\$ 1.20$ & $\$ 1.27$ & & & & \\
\hline Cost to Boiler & \$/ton & $\$ 51.49$ & $\$ 51.49$ & $\$ 51.49$ & $\$ 51.49$ & $\$ 56.56$ & $\$ 54.71$ & $\$ 48.58$ & $\$ 51.49$ & & & & \\
\hline Freight Cost & \$/ton & $\$ 20.00$ & $\$ 20.00$ & $\$ 20.00$ & $\$ 20.00$ & $\$ 20.00$ & $\$ 20.00$ & $\$ 20.00$ & $\$ 20.00$ & & & & \\
\hline Evap. Cost & $\$ /$ ton & $\$ 0.53$ & $\$ 0.53$ & $\$ 0.53$ & $\$ 0.53$ & $\$ 0.29$ & $\$ 0.38$ & $\$ 0.67$ & $\$ 0.53$ & & & & \\
\hline Ash Cost & $\$ /$ ton & $\$ 2.39$ & $\$ 2.39$ & $\$ 2.39$ & $\$ 2.39$ & $\$ 2.62$ & $\$ 2.53$ & $\$ 2.25$ & $\$ 2.39$ & & & & \\
\hline SO2 Cost & $\$ /$ ton & $\$ 0.76$ & $\$ 0.76$ & $\$ 0.76$ & $\$ 0.76$ & $\$ 0.83$ & $\$ 0.81$ & $\$ 0.72$ & $\$ 0.76$ & & & & \\
\hline Other Cost & $\$ /$ ton & $\$ 1.00$ & $\$ 1.00$ & $\$ 1.00$ & $\$ 1.00$ & $\$ 1.00$ & $\$ 1.00$ & $\$ 1.00$ & $\$ 1.00$ & & & & \\
\hline Coal Worth & $\$ /$ ton & $\$ 26.82$ & $\$ 26.82$ & $\$ 26.82$ & $\$ 26.82$ & $\$ 31.82$ & $\$ 29.99$ & $\$ 23.94$ & $\$ 26.82$ & & & & \\
\hline Individual Tons & dry & 0.00 & 0.00 & 0.00 & 0.00 & 1.82 & 1.47 & 4.16 & 7.45 & & & & \\
\hline Cum. Tons & dry & 7.45 & 7.45 & 7.45 & 7.45 & 7.45 & 5.63 & 4.16 & & & & & \\
\hline Individual Tons & ar & 0.00 & 0.00 & 0.00 & 0.00 & 2.01 & 1.68 & 5.36 & 9.05 & & & & \\
\hline \begin{tabular}{|l|} 
Cum. Tons \\
\end{tabular} & ar & 9.05 & 9.05 & 9.05 & 9.05 & 9.05 & 7.04 & 5.36 & --- & & & & \\
\hline Cum. Mass & $\%$, ar & 100.00 & 100.00 & 100.00 & 100.00 & 100.00 & 77.76 & 59.21 & --- & & & & \\
\hline Cum. Ash & $\%$, ar & 11.93 & 11.93 & 11.93 & 11.93 & 13.10 & 12.91 & 11.93 & --- & & & & \\
\hline Cum. Sulfur & $\%$, ar & 0.82 & 0.82 & 0.82 & 0.82 & 0.90 & 0.89 & 0.82 & $-\cdots$ & & & & \\
\hline Cum. BTU/lb & ar & 10552 & 10552 & 10552 & 10552 & 10760 & 10820 & 10740 & --- & & & & \\
\hline Individual Worth & $\$$ & $\$ 0.00$ & $\$ 0.00$ & $\$ 0.00$ & $\$ 0.00$ & $\$ 64.09$ & $\$ 50.36$ & $\$ 128.36$ & $\$ 242.81$ & & & & \\
\hline Cum. Worth & $\$$ & $\$ 0.00$ & $\$ 0.00$ & $\$ 0.00$ & $\$ 0.00$ & $\$ 64.09$ & $\$ 114.44$ & $\$ 242.81$ & --- & & & & \\
\hline
\end{tabular}


Coal A - Circuit Configuration 1 - Fine Cut - Screenbowl Centrifuge Simulation

\begin{tabular}{|c|c|c|c|c|c|c|}
\hline \multicolumn{7}{|c|}{ Input Parameters } \\
\hline $\begin{array}{c}k_{d} \\
\left(m^{-1}\right)\end{array}$ & $\begin{array}{c}\rho_{\mathrm{s}} \\
\left(\mathrm{kg} / \mathrm{m}^{3}\right)\end{array}$ & $\begin{array}{c}\rho_{\mathrm{w}} \\
\left(\mathrm{kg} / \mathrm{m}^{3}\right)\end{array}$ & $\begin{array}{c}\text { B } \\
\text { (m) }\end{array}$ & $\varepsilon$ & $\begin{array}{c}\sigma \\
(\mathrm{N} / \mathrm{m})\end{array}$ & $\begin{array}{c}\mu \\
\text { (Pa.s) }\end{array}$ \\
\hline 400 & 1400 & 1000 & 0.559 & 0.55 & 0.072 & 0.001 \\
\hline $\mathrm{L}$ & $t$ & $\mathrm{~N}$ & $\mathrm{~N}_{\mathrm{g}}$ & Air $\mathrm{P}$ & $\mathbf{g}$ & $\theta$ \\
\hline (m) & (sec) & (RPM) & & (psi) & $\left(\mathrm{m} / \mathrm{s}^{2}\right)$ & \\
\hline 0.0381 & 7 & 895 & 500 & 0 & $\begin{array}{l}9.81 \\
\end{array}$ & 60 \\
\hline
\end{tabular}
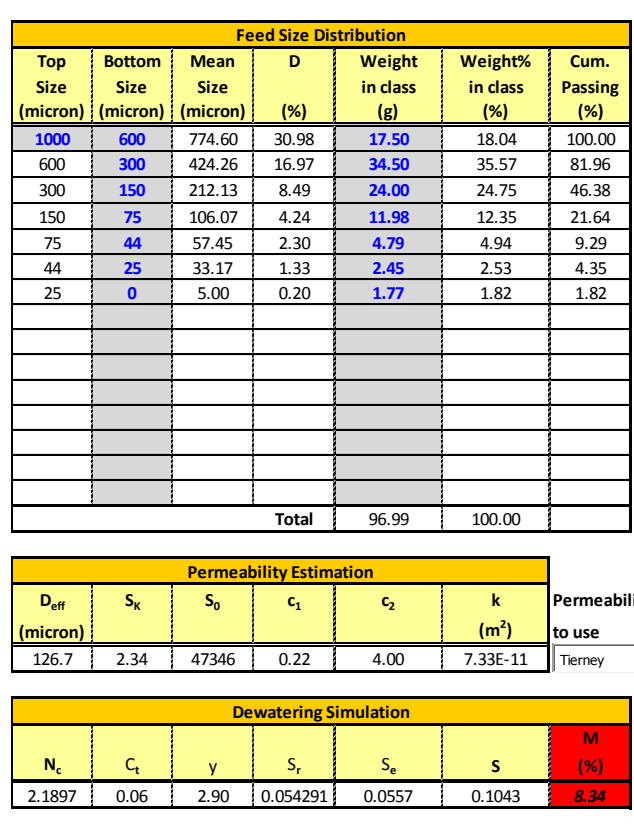

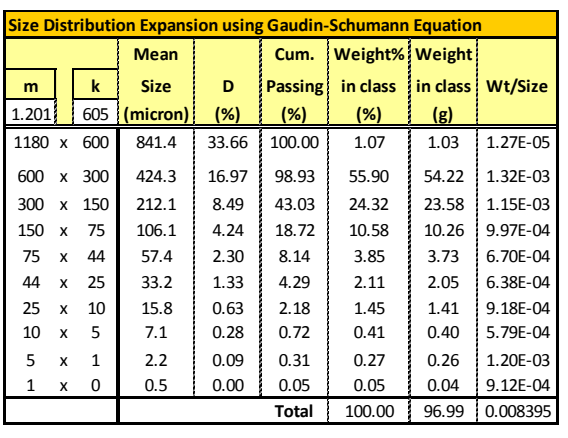

\begin{tabular}{|c|c|c|c|c|c|c|c|c|c|c|c|c|c|c|c|}
\hline \multicolumn{16}{|c|}{ egradation Model } \\
\hline & \multicolumn{10}{|c|}{ Contributing Size Class } & \multirow{2}{*}{$\begin{array}{c}\text { New } \\
\text { Weight } \\
\text { in class } \\
\text { (g) }\end{array}$} & \multirow{2}{*}{\begin{tabular}{|c|} 
New \\
Weight \\
after loss \\
(g) \\
\end{tabular}} & \multirow{2}{*}{\begin{tabular}{|c|} 
New \\
Weight\% \\
in class \\
$(\%)$
\end{tabular}} & \multirow{2}{*}{\begin{tabular}{|c|} 
New \\
cum. \\
Passing \\
$(\%)$
\end{tabular}} & \multirow{2}{*}{$\begin{array}{l}\text { Effluent } \\
\text { Weight } \\
\text { in class } \\
\text { (g) }\end{array}$} \\
\hline & \begin{tabular}{|c|}
1180 \\
$x$ \\
600
\end{tabular} & \begin{tabular}{|c|c|}
600 \\
$x$ \\
300
\end{tabular} & \begin{tabular}{c|c}
300 & $x$ \\
150 & \\
\end{tabular} & $\begin{array}{c}150 \\
x \\
75\end{array}$ & $\begin{array}{l}75 \\
x \\
44\end{array}$ & $\begin{array}{c}44 \\
\times \\
25 \\
\end{array}$ & \begin{tabular}{|l}
25 \\
$x$ \\
10 \\
\end{tabular} & $\begin{array}{l}10 \\
x \\
5\end{array}$ & $\begin{array}{l}5 \\
x \\
1\end{array}$ & $\begin{array}{l}1 \\
x \\
0\end{array}$ & & & & & \\
\hline & \begin{tabular}{|l|l|}
0 & 1
\end{tabular} & & & & & & & & & & & & & 100.00 & \\
\hline & 0.20 & 0 & & & & & & & & & & & & & \\
\hline & \begin{tabular}{|c|c|}
0.09 & \\
\end{tabular} & 5.20 & 0 & & & & & & & & & & & & \\
\hline & & 2.26 & 1.13 & 0 & & & & & & & & & & & \\
\hline & & $\mid$\begin{tabular}{l|}
0.82 \\
\end{tabular} & & & & & & & & & & & & & \\
\hline$x$ & & & & & 0.04 & & & & & & & & & & \\
\hline$x$ & & & & & & & & & & & & & & & \\
\hline$x$ & & & & & & & & & & & & & & & \\
\hline$x$ & & & & & & & & & 0 & & & & & & \\
\hline $\begin{array}{rr}\times & 0 \\
\end{array}$ & & & & & & & & & & & & & & & \\
\hline & & & & & & & & & & & & & 0.00 & & 9 \\
\hline
\end{tabular}

$25 \times 0 \quad 5.978$

$44 \times 0 \quad 9.9675$

$74 \times 0 \quad 20.974$

$\begin{array}{ll}150 \times 0 & 41.896 \\ 300 \times 0 & 75859\end{array}$

$30 \times 075.859$

$1180 \times 0.126 .73$

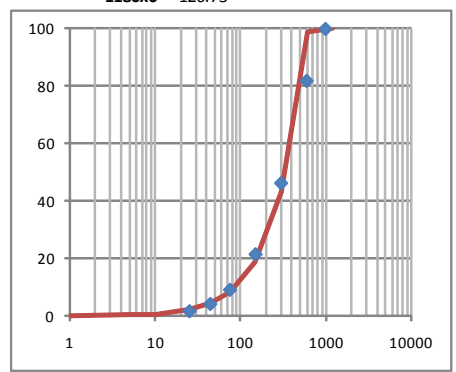


Coal A - Circuit Configuration 1 - Fine Cut - Screenbowl Centrifuge Economics

CALCULATION OF OPTIMUM SIZE CUT

\begin{tabular}{|c|c|c|c|c|c|c|c|c|c|c|c|c|c|}
\hline \multirow{2}{*}{ FINANCIAL DATA: } & & \multicolumn{2}{|c|}{ Total Boiler Cost $=$} & $\$ 2.44$ & /MM BTU & & Moisture $\mathrm{E}$ & vap. = & $\$ 3.00$ & /ton $\mathrm{H}_{2} \mathrm{O}$ & $\mathrm{SO}_{2}$ Emission $=$ & $\$ 200$ & $/$ ton $\mathrm{SO}_{2}$ \\
\hline & & Freight $\mathrm{Co}$ & & $\$ 20.00$ & /ton coal & & Ash Handli & $\mathrm{ng}=$ & $\$ 20.00$ & /ton ash & Other Cost $=$ & $\$ 1.00$ & /ton coal \\
\hline Size Class & & 1 & 2 & 3 & 4 & 5 & 6 & 7 & Totals & & & & \\
\hline Top Size & $\mu$ & 1180 & 600 & 300 & 150 & 74 & 44 & 25 & --- & & & & \\
\hline Bottom Size & $\mu$ & 600 & 300 & 150 & 74 & 44 & 25 & 0 & $-\cdots$ & & & & \\
\hline$S G$ & $\mu$ & 1.45 & 1.45 & 1.45 & 1.45 & 1.45 & 1.45 & 1.45 & --- & & & & \\
\hline $1 / \mathrm{SG}$ & --- & 0.69 & 0.69 & 0.69 & 0.69 & 0.69 & 0.69 & 0.69 & --- & & & & \\
\hline Mass & $\%$, dry & 0.73 & 48.07 & 28.56 & 14.09 & 5.43 & 1.52 & 1.60 & 100.00 & & & & \\
\hline Solid SG & & 1.40 & 1.40 & 1.40 & 1.40 & 1.40 & 1.40 & 1.40 & & & & & \\
\hline Ash & $\%$, dry & 14.50 & 14.50 & 14.50 & 14.50 & 14.50 & 14.50 & 14.50 & 14.50 & & & & \\
\hline Sulfur & $\%$, dry & 1.00 & 1.00 & 1.00 & 1.00 & 1.00 & 1.00 & 1.00 & 1.00 & & & & \\
\hline BTU/Ib & dry & 12825 & 12825 & 12825 & 12825 & 12825 & 12825 & 12825 & 12825 & & & & \\
\hline BTU/lb & maf & 15000 & 15000 & 15000 & 15000 & 15000 & 15000 & 15000 & 15000 & & & & \\
\hline Mass & $\%$, ar & 0.69 & 45.90 & 27.95 & 14.62 & 6.29 & 2.04 & 2.51 & 100.00 & & & & \\
\hline Moisture & $\%$, ar & 3.10 & 4.01 & 6.31 & 11.69 & 20.94 & 31.48 & 41.77 & 8.34 & & & & \\
\hline Cum. Moisture & $\%$, ar & 8.34 & 8.38 & 12.14 & 18.53 & 27.75 & 37.17 & 41.77 & --- & & & & \\
\hline Ash & $\%$, ar & 14.05 & 13.92 & 13.58 & 12.80 & 11.46 & 9.93 & 8.44 & 13.29 & & & & \\
\hline Inerts & $\%$, ar & 17.15 & 17.93 & 19.90 & 24.49 & 32.40 & 41.41 & 50.22 & 21.63 & & & & \\
\hline Sulfur & $\%$, ar & 0.97 & 0.96 & 0.94 & 0.88 & 0.79 & 0.69 & 0.58 & 0.92 & & & & \\
\hline BTU/Ib & ar & 12428 & 12311 & 12015 & 11326 & 10140 & 8788 & 7468 & 11755 & & & & \\
\hline Ib SO2/MM BTU & $-\ldots$ & 1.56 & 1.56 & 1.56 & 1.56 & 1.56 & 1.56 & 1.56 & 1.56 & & & & \\
\hline Cost to Boiler & $\$ / M M$ BTU & $\$ 2.44$ & $\$ 2.44$ & $\$ 2.44$ & $\$ 2.44$ & $\$ 2.44$ & $\$ 2.44$ & $\$ 2.44$ & $\$ 2.44$ & & & & \\
\hline Freight Cost & $\$ / M M B T U$ & $\$ 0.80$ & $\$ 0.81$ & $\$ 0.83$ & $\$ 0.88$ & $\$ 0.99$ & $\$ 1.14$ & $\$ 1.34$ & $\$ 0.85$ & & & & \\
\hline Evap. Cost & $\$ / \mathrm{MM} \mathrm{BTU}$ & $\$ 0.00$ & $\$ 0.00$ & $\$ 0.01$ & $\$ 0.02$ & $\$ 0.03$ & $\$ 0.05$ & $\$ 0.08$ & $\$ 0.01$ & & & & \\
\hline Ash Cost & $\$ / M M B T U$ & $\$ 0.11$ & $\$ 0.11$ & $\$ 0.11$ & $\$ 0.11$ & $\$ 0.11$ & $\$ 0.11$ & $\$ 0.11$ & $\$ 0.11$ & & & & \\
\hline SO2 Cost & $\$ / M M B T U$ & $\$ 0.04$ & $\$ 0.04$ & $\$ 0.04$ & $\$ 0.04$ & $\$ 0.04$ & $\$ 0.04$ & $\$ 0.04$ & $\$ 0.04$ & & & & \\
\hline Other Cost & $\$ / M M B T U$ & $\$ 0.04$ & $\$ 0.04$ & $\$ 0.04$ & $\$ 0.04$ & $\$ 0.05$ & $\$ 0.06$ & $\$ 0.07$ & $\$ 0.04$ & & & & \\
\hline Coal Worth & $\$ / \mathrm{MM} \mathrm{BTU}$ & $\$ 1.44$ & $\$ 1.43$ & $\$ 1.41$ & $\$ 1.35$ & $\$ 1.22$ & $\$ 1.04$ & $\$ 0.80$ & $\$ 1.39$ & & & & \\
\hline Cost to Boiler & $\$ /$ ton & $\$ 60.65$ & $\$ 60.08$ & $\$ 58.64$ & $\$ 55.27$ & $\$ 49.48$ & $\$ 42.88$ & $\$ 36.44$ & $\$ 57.36$ & & & & \\
\hline Freight Cost & $\$ /$ ton & $\$ 20.00$ & $\$ 20.00$ & $\$ 20.00$ & $\$ 20.00$ & $\$ 20.00$ & $\$ 20.00$ & $\$ 20.00$ & $\$ 20.00$ & & & & \\
\hline Evap. Cost & $\$ /$ ton & $\$ 0.09$ & $\$ 0.12$ & $\$ 0.19$ & $\$ 0.35$ & $\$ 0.63$ & $\$ 0.94$ & $\$ 1.25$ & $\$ 0.25$ & & & & \\
\hline Ash Cost & $\$ /$ ton & $\$ 2.81$ & $\$ 2.78$ & $\$ 2.72$ & $\$ 2.56$ & $\$ 2.29$ & $\$ 1.99$ & $\$ 1.69$ & $\$ 2.66$ & & & & \\
\hline SO2 Cost & $\$ /$ ton & $\$ 0.89$ & $\$ 0.88$ & $\$ 0.86$ & $\$ 0.81$ & $\$ 0.73$ & $\$ 0.63$ & $\$ 0.54$ & $\$ 0.85$ & & & & \\
\hline Other Cost & $\$ /$ ton & $\$ 1.00$ & $\$ 1.00$ & $\$ 1.00$ & $\$ 1.00$ & $\$ 1.00$ & $\$ 1.00$ & $\$ 1.00$ & $\$ 1.00$ & & & & \\
\hline Coal Worth & $\$ /$ ton & $\$ 35.85$ & $\$ 35.29$ & $\$ 33.87$ & $\$ 30.55$ & $\$ 24.83$ & $\$ 18.32$ & $\$ 11.96$ & $\$ 32.61$ & & & & \\
\hline Individual Tons & dry & 0.69 & 45.21 & 26.87 & 13.25 & 5.10 & 1.43 & 1.50 & 94.05 & & & & \\
\hline Cum. Tons & dry & 94.05 & 93.37 & 48.15 & 21.29 & 8.04 & 2.93 & 1.50 & & & & & \\
\hline Individual Tons & $\mathrm{ar}$ & 0.71 & 47.10 & 28.68 & 15.01 & 6.46 & 2.09 & 2.58 & 102.62 & & & & \\
\hline Cum. Tons & $\mathrm{ar}$ & 102.62 & 101.91 & 54.81 & 26.13 & 11.12 & 4.67 & 2.58 & $\cdots$ & & & & \\
\hline Cum. Mass & $\%$, ar & 100.00 & 99.31 & 53.41 & 25.46 & 10.84 & 4.55 & 2.51 & --- & & & & \\
\hline Cum. Ash & $\%$, ar & 14.05 & 13.92 & 13.79 & 13.63 & 13.49 & 13.41 & 13.29 & --- & & & & \\
\hline Cum. Sulfur & $\%$, ar & 0.97 & 0.96 & 0.95 & 0.94 & 0.93 & 0.93 & 0.92 & -- & & & & \\
\hline Cum. BTU/lb & ar & 12428 & 12369 & 12295 & 12206 & 12128 & 12077 & 12038 & --- & & & & \\
\hline \begin{tabular}{|l} 
Individual Worth \\
\end{tabular} & $\$$ & $\$ 25.39$ & $\$ 1,662.09$ & $\$ 971.13$ & $\$ 458.35$ & $\$ 160.29$ & $\$ 38.30$ & $\$ 30.85$ & $\$ 3,346.40$ & & & & \\
\hline Cum. Worth & $\$$ & $\$ 25.39$ & $\$ 1,687.48$ & $\$ 2,658.60$ & $\$ 3,116.95$ & $\$ 3,277.25$ & $\$ 3,315.55$ & $\$ 3,346.40$ & --- & & & & \\
\hline
\end{tabular}


Coal A - Circuit Configuration 1 - Fine Cut - Hyperbaric Centrifuge Simulation

\begin{tabular}{|c|c|c|c|c|c|c|}
\hline \multicolumn{7}{|c|}{ Input Parameters } \\
\hline $\begin{array}{c}k_{d} \\
\left(m^{-1}\right)\end{array}$ & $\begin{array}{c}\rho_{\mathrm{s}} \\
\left(\mathrm{kg} / \mathrm{m}^{3}\right)\end{array}$ & $\underset{\left(\mathrm{kg} / \mathrm{m}^{3}\right)}{\rho_{\mathrm{w}}}$ & $\begin{array}{c}\text { B } \\
(\mathrm{m})\end{array}$ & $\varepsilon$ & $\begin{array}{c}\sigma \\
(\mathrm{N} / \mathrm{m})\end{array}$ & $\begin{array}{c}\mu \\
\text { (Pa.s) }\end{array}$ \\
\hline 400 & 1400 & 1000 & 0.381 & 0.52 & 0.072 & 0.001 \\
\hline $\mathrm{L}$ & ${ }^{t}$ & $\mathrm{~N}$ & $\mathrm{~N}_{\mathrm{g}}$ & Air $P$ & $\mathbf{g}$ & $\theta$ \\
\hline (m) & (sec) & (RPM) & & (psi) & $\left(\mathrm{m} / \mathrm{s}^{2}\right)$ & \\
\hline 0.0127 & 7 & 2650 & 2991 & 0 & 9.81 & 60 \\
\hline
\end{tabular}

\begin{tabular}{|c|c|c|c|c|c|c|}
\hline \multicolumn{7}{|c|}{ Feed Size Distribution } \\
\hline $\begin{array}{c}\text { Top } \\
\text { Size } \\
\text { (micron) }\end{array}$ & $\begin{array}{c}\text { Bottom } \\
\text { Size } \\
\text { (micron) }\end{array}$ & $\begin{array}{c}\text { Mean } \\
\text { Size } \\
\text { (micron) }\end{array}$ & $\begin{array}{l}\mathrm{D} \\
(\%)\end{array}$ & $\begin{array}{l}\text { Weight } \\
\text { in class }\end{array}$ & $\begin{array}{l}\text { Weight\% } \\
\text { in class } \\
\text { (\%) }\end{array}$ & $\begin{array}{l}\text { Cum. } \\
\text { Passing } \\
\text { (\%) }\end{array}$ \\
\hline 1000 & 600 & 774.60 & 30.98 & 0.00 & 0.00 & 100.00 \\
\hline 600 & 300 & 424.26 & 16.97 & 0.00 & 0.00 & 100.00 \\
\hline 300 & 150 & 212.13 & 8.49 & 0.00 & 0.00 & 100.00 \\
\hline 150 & 75 & 106.07 & 4.24 & 0.02 & 0.35 & 100.00 \\
\hline 75 & 44 & 57.45 & 2.30 & 0.21 & 3.55 & 99.64 \\
\hline 44 & 25 & 33.17 & 1.33 & 1.98 & 33.33 & 96.09 \\
\hline 25 & 0 & 5.00 & 0.20 & 3.73 & 62.76 & 62.76 \\
\hline & & & & & & \\
\hline & & & & & & \\
\hline & & & & & & \\
\hline & & & & & & \\
\hline & & & & & & \\
\hline & & & Tot? & 509 & 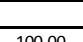 & \\
\hline & & & & & & \\
\hline
\end{tabular}
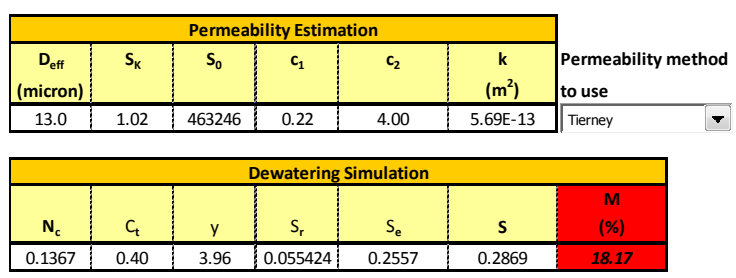
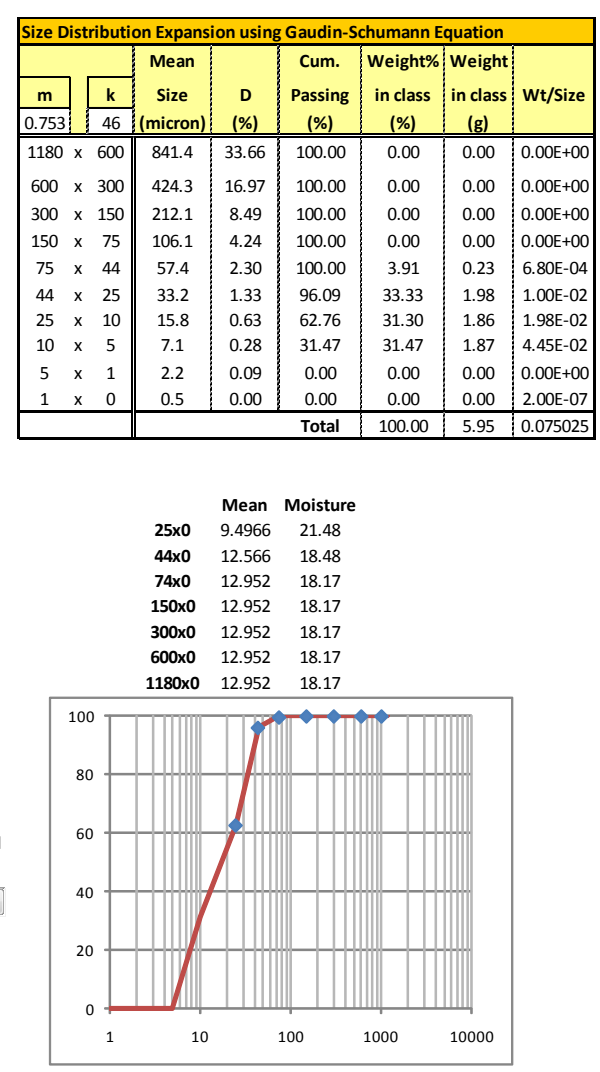

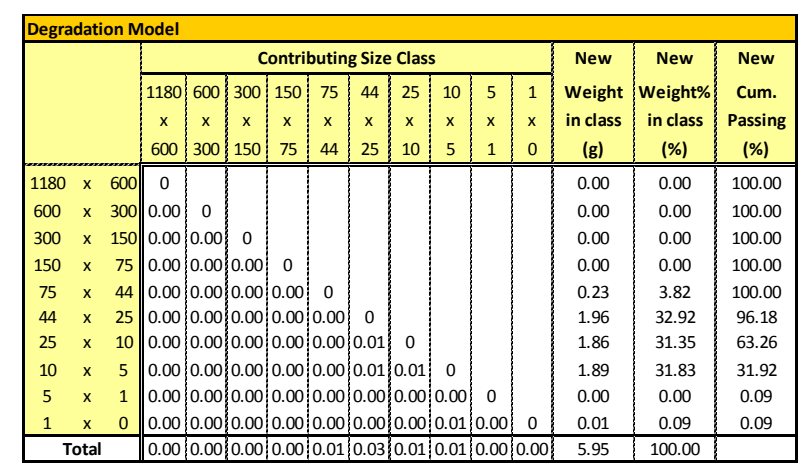

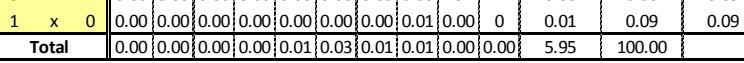


Coal A - Circuit Configuration 1 - Fine Cut - Hyperbaric Centrifuge Economics

CALCULATION OF OPTIMUM SIZE CUT

\begin{tabular}{|c|c|c|c|c|c|c|c|c|c|c|c|c|c|}
\hline \multirow{2}{*}{ FINANCIAL DATA: } & & \multicolumn{2}{|c|}{ Total Boiler Cost $=$} & $\$ 2.44$ & /MMBTU & & Moisture & ap. $=$ & $\$ 3.00$ & /ton $\mathrm{H}_{2} \mathrm{O}$ & $\mathrm{SO}_{2}$ Emission $=$ & $\$ 200$ & /ton $\mathrm{SO}_{2}$ \\
\hline & & Freight $\mathrm{Co}$ & & $\$ 20.00$ & /ton coal & & Ash Handl & & $\$ 20.00$ & /ton ash & Other Cost $=$ & $\$ 1.00$ & /ton coal \\
\hline Size Class & & 1 & 2 & 3 & 4 & 5 & 6 & 7 & Totals & & & & \\
\hline Top Size & $\mu$ & 1180 & 600 & 300 & 150 & 74 & 44 & 25 & --- & & & & \\
\hline Bottom Size & $\mu$ & 600 & 300 & 150 & 74 & 44 & 25 & 0 & $-\cdots$ & & & & \\
\hline$S G$ & $\mu$ & 1.45 & 1.45 & 1.45 & 1.45 & 1.45 & 1.45 & 1.45 & --- & & & & \\
\hline $1 / \mathrm{SG}$ & --- & 0.69 & 0.69 & 0.69 & 0.69 & 0.69 & 0.69 & 0.69 & --- & & & & \\
\hline Mass & $\%$, dry & 0.00 & 0.00 & 0.00 & 0.00 & 3.82 & 32.92 & 63.26 & 100.00 & & & & \\
\hline Solid SG & & 1.40 & 1.40 & 1.40 & 1.40 & 1.40 & 1.40 & 1.40 & & & & & \\
\hline Ash & $\%$, dry & 14.50 & 14.50 & 14.50 & 14.50 & 14.50 & 14.50 & 14.50 & 14.50 & & & & \\
\hline Sulfur & $\%$, dry & 1.00 & 1.00 & 1.00 & 1.00 & 1.00 & 1.00 & 1.00 & 1.00 & & & & \\
\hline BTU/Ib & dry & 12825 & 12825 & 12825 & 12825 & 12825 & 12825 & 12825 & 12825 & & & & \\
\hline BTU/lb & maf & 15000 & 15000 & 15000 & 15000 & 15000 & 15000 & 15000 & 15000 & & & & \\
\hline Mass & $\%$, ar & 0.00 & 0.00 & 0.00 & 0.00 & 3.45 & 30.62 & 65.94 & 100.00 & & & & \\
\hline Moisture & $\%$, ar & 18.17 & 18.17 & 18.17 & 18.17 & 9.39 & 12.01 & 21.48 & 18.17 & & & & \\
\hline Cum. Moisture & $\%$, ar & 18.17 & 18.17 & 18.17 & 18.17 & 18.17 & 18.48 & 21.48 & --- & & & & \\
\hline Ash & $\%$, ar & 11.86 & 11.86 & 11.86 & 11.86 & 13.14 & 12.76 & 11.38 & 11.86 & & & & \\
\hline Inerts & $\%$, ar & 30.03 & 30.03 & 30.03 & 30.03 & 22.53 & 24.77 & 32.87 & 30.03 & & & & \\
\hline Sulfur & $\%$, ar & 0.82 & 0.82 & 0.82 & 0.82 & 0.91 & 0.88 & 0.79 & 0.82 & & & & \\
\hline BTU/Ib & ar & 10495 & 10495 & 10495 & 10495 & 11621 & 11285 & 10070 & 10495 & & & & \\
\hline Ib SO2/MM BTU & $-\ldots$ & 1.56 & 1.56 & 1.56 & 1.56 & 1.56 & 1.56 & 1.56 & 1.56 & & & & \\
\hline Cost to Boiler & $\$ / M M$ BTU & $\$ 2.44$ & $\$ 2.44$ & $\$ 2.44$ & $\$ 2.44$ & $\$ 2.44$ & $\$ 2.44$ & $\$ 2.44$ & $\$ 2.44$ & & & & \\
\hline Freight Cost & $\$ / M M B T U$ & $\$ 0.95$ & $\$ 0.95$ & $\$ 0.95$ & $\$ 0.95$ & $\$ 0.86$ & $\$ 0.89$ & $\$ 0.99$ & $\$ 0.95$ & & & & \\
\hline Evap. Cost & $\$ / \mathrm{MM} \mathrm{BTU}$ & $\$ 0.03$ & $\$ 0.03$ & $\$ 0.03$ & $\$ 0.03$ & $\$ 0.01$ & $\$ 0.02$ & $\$ 0.03$ & $\$ 0.03$ & & & & \\
\hline Ash Cost & $\$ / M M B T U$ & $\$ 0.11$ & $\$ 0.11$ & $\$ 0.11$ & $\$ 0.11$ & $\$ 0.11$ & $\$ 0.11$ & $\$ 0.11$ & $\$ 0.11$ & & & & \\
\hline SO2 Cost & $\$ / M M B T U$ & $\$ 0.04$ & $\$ 0.04$ & $\$ 0.04$ & $\$ 0.04$ & $\$ 0.04$ & $\$ 0.04$ & $\$ 0.04$ & $\$ 0.04$ & & & & \\
\hline Other Cost & $\$ / M M B T U$ & $\$ 0.05$ & $\$ 0.05$ & $\$ 0.05$ & $\$ 0.05$ & $\$ 0.04$ & $\$ 0.04$ & $\$ 0.05$ & $\$ 0.05$ & & & & \\
\hline Coal Worth & $\$ / \mathrm{MM} \mathrm{BTU}$ & $\$ 1.26$ & $\$ 1.26$ & $\$ 1.26$ & $\$ 1.26$ & $\$ 1.38$ & $\$ 1.34$ & $\$ 1.22$ & $\$ 1.26$ & & & & \\
\hline Cost to Boiler & $\$ /$ ton & $\$ 51.22$ & $\$ 51.22$ & $\$ 51.22$ & $\$ 51.22$ & $\$ 56.71$ & $\$ 55.07$ & $\$ 49.14$ & $\$ 51.22$ & & & & \\
\hline Freight Cost & $\$ /$ ton & $\$ 20.00$ & $\$ 20.00$ & $\$ 20.00$ & $\$ 20.00$ & $\$ 20.00$ & $\$ 20.00$ & $\$ 20.00$ & $\$ 20.00$ & & & & \\
\hline Evap. Cost & $\$ /$ ton & $\$ 0.55$ & $\$ 0.55$ & $\$ 0.55$ & $\$ 0.55$ & $\$ 0.28$ & $\$ 0.36$ & $\$ 0.64$ & $\$ 0.55$ & & & & \\
\hline Ash Cost & $\$ /$ ton & $\$ 2.37$ & $\$ 2.37$ & $\$ 2.37$ & $\$ 2.37$ & $\$ 2.63$ & $\$ 2.55$ & $\$ 2.28$ & $\$ 2.37$ & & & & \\
\hline SO2 Cost & $\$ /$ ton & $\$ 0.75$ & $\$ 0.75$ & $\$ 0.75$ & $\$ 0.75$ & $\$ 0.84$ & $\$ 0.81$ & $\$ 0.72$ & $\$ 0.75$ & & & & \\
\hline Other Cost & $\$ /$ ton & $\$ 1.00$ & $\$ 1.00$ & $\$ 1.00$ & $\$ 1.00$ & $\$ 1.00$ & $\$ 1.00$ & $\$ 1.00$ & $\$ 1.00$ & & & & \\
\hline Coal Worth & $\$ /$ ton & $\$ 26.54$ & $\$ 26.54$ & $\$ 26.54$ & $\$ 26.54$ & $\$ 31.97$ & $\$ 30.35$ & $\$ 24.50$ & $\$ 26.54$ & & & & \\
\hline Individual Tons & dry & 0.00 & 0.00 & 0.00 & 0.00 & 0.23 & 1.96 & 3.76 & 5.95 & & & & \\
\hline Cum. Tons & dry & 5.95 & 5.95 & 5.95 & 5.95 & 5.95 & 5.72 & 3.76 & & & & & \\
\hline Individual Tons & $\mathrm{ar}$ & 0.00 & 0.00 & 0.00 & 0.00 & 0.25 & 2.23 & 4.79 & 7.27 & & & & \\
\hline Cum. Tons & ar & 7.27 & 7.27 & 7.27 & 7.27 & 7.27 & 7.02 & 4.79 & $\ldots$ & & & & \\
\hline Cum. Mass & $\%$, ar & 100.00 & 100.00 & 100.00 & 100.00 & 100.00 & 96.55 & 65.94 & --- & & & & \\
\hline Cum. Ash & $\%$, ar & 11.86 & 11.86 & 11.86 & 11.86 & 13.14 & 12.80 & 11.86 & --- & & & & \\
\hline Cum. Sulfur & $\%$, ar & 0.82 & 0.82 & 0.82 & 0.82 & 0.91 & 0.88 & 0.82 & -- & & & & \\
\hline Cum. BTU/lb & ar & 10495 & 10495 & 10495 & 10495 & 10720 & 10812 & 10738 & --- & & & & \\
\hline \begin{tabular}{|l|} 
Individual Worth \\
\end{tabular} & $\$$ & $\$ 0.00$ & $\$ 0.00$ & $\$ 0.00$ & $\$ 0.00$ & $\$ 8.01$ & $\$ 67.53$ & $\$ 117.40$ & $\$ 192.93$ & & & & \\
\hline Cum. Worth & $\$$ & $\$ 0.00$ & $\$ 0.00$ & $\$ 0.00$ & $\$ 0.00$ & $\$ 8.01$ & $\$ 75.54$ & $\$ 192.93$ & --- & & & & \\
\hline
\end{tabular}


Coal A - Circuit Configuration 1 - Summary

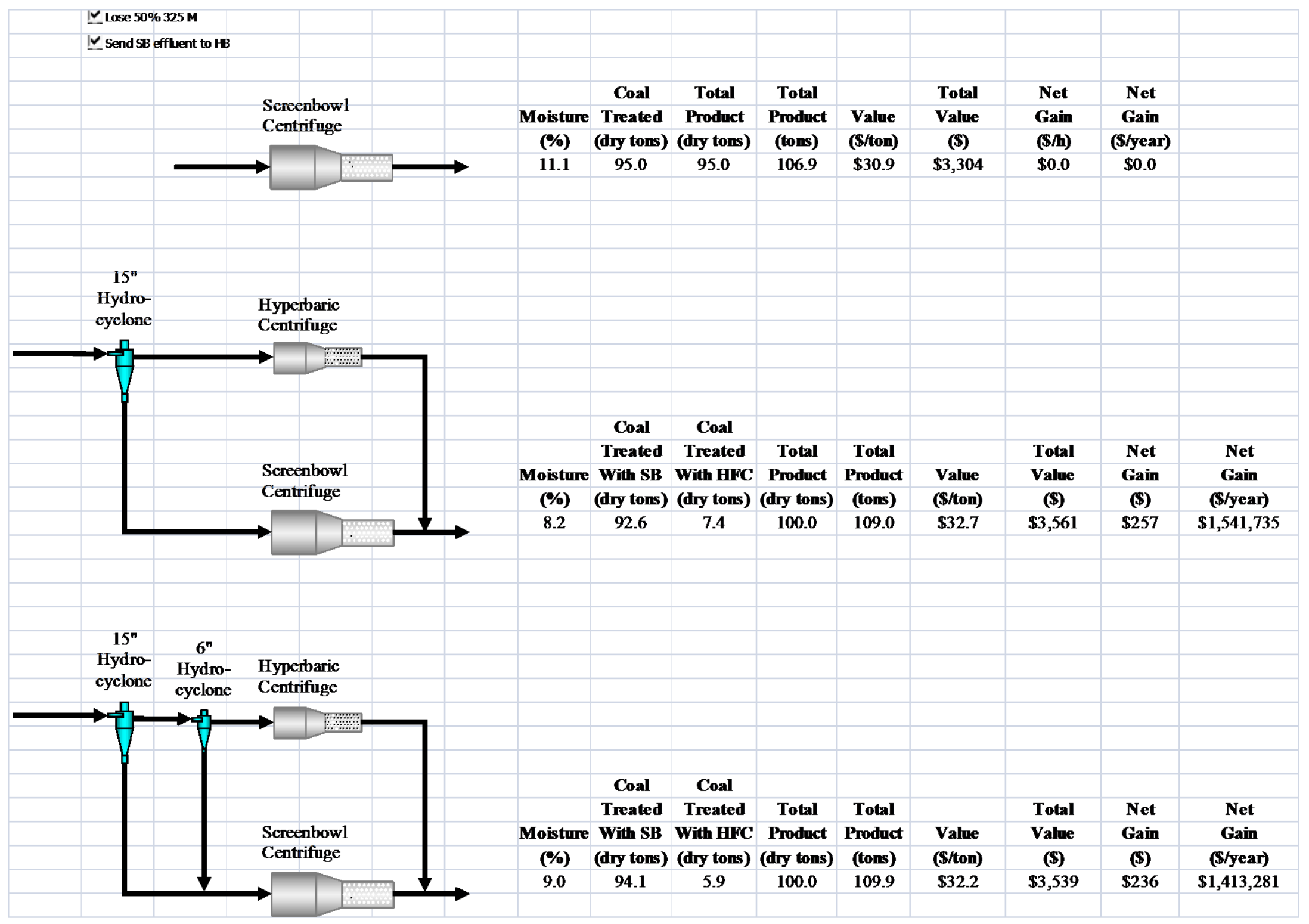


Coal B - Circuit Configuration 1 - Screenbowl Centrifuge Simulation

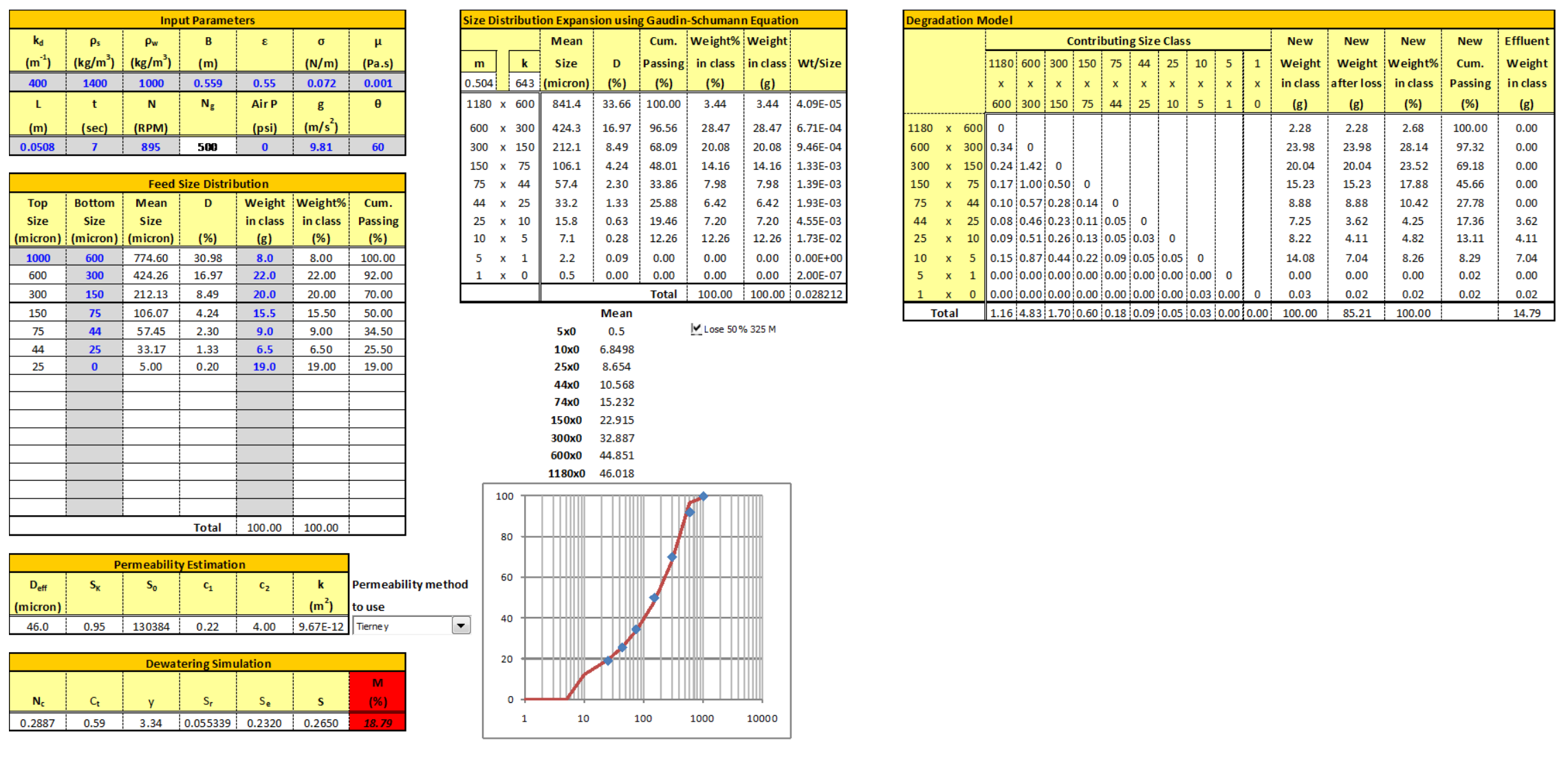


Coal B - Circuit Configuration 1 - Screenbowl Centrifuge Economics

\section{CALCULATION OF OPTIMUM SIZE CUT}

\begin{tabular}{|c|c|c|c|c|c|c|c|c|c|c|c|c|c|}
\hline \multirow{2}{*}{\multicolumn{2}{|c|}{ 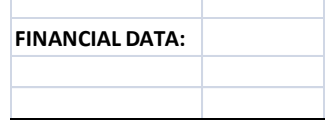 }} & \multicolumn{2}{|c|}{ Total Boiler Cost $=$} & \multirow{2}{*}{$\begin{array}{r}\$ 2.44 \\
\$ 20.00\end{array}$} & /MM BTU & & \multicolumn{2}{|c|}{ Moisture Evap. = } & \multirow{2}{*}{$\begin{array}{r}\$ 3.00 \\
\$ 20.00\end{array}$} & /ton $\mathrm{H}_{2} \mathrm{O}$ & \multirow{2}{*}{$\begin{array}{l}\mathrm{SO}_{2} \text { Emission }= \\
\text { Other Cost }=\end{array}$} & \multirow{2}{*}{$\begin{array}{r}\$ 200 \\
\$ 1.00\end{array}$} & $0 /$ ton $\mathrm{SO}_{2}$ \\
\hline & & Freight $\mathrm{Co}$ & & & /ton coal & & Ash Handli & $\mathrm{ng}=$ & & /ton ash & & & /ton coal \\
\hline \begin{tabular}{|l|} 
Size Class \\
\end{tabular} & & 1 & 2 & 3 & 4 & 5 & 6 & 7 & Totals & & & & \\
\hline Top Size & $\mu$ & 1180 & 600 & 300 & 150 & 74 & 44 & 25 & $-\cdots$ & & & & \\
\hline Bottom Size & $\mu$ & 600 & 300 & 150 & 74 & 44 & 25 & 0 & $-\ldots$ & & & & \\
\hline SG & $\mu$ & 1.45 & 1.45 & 1.45 & 1.45 & 1.45 & 1.45 & 1.45 & --- & & & & \\
\hline $1 / \mathrm{SG}$ & --- & 0.69 & 0.69 & 0.69 & 0.69 & 0.69 & 0.69 & 0.69 & --- & & & & \\
\hline Mass & $\%$, dry & 2.68 & 28.14 & 23.52 & 17.88 & 10.42 & 4.25 & 13.11 & 100.00 & & & & \\
\hline Solid SG & & 1.40 & 1.40 & 1.40 & 1.40 & 1.40 & 1.40 & 1.40 & & & & & \\
\hline Ash & $\%$, dry & 14.50 & 14.50 & 14.50 & 14.50 & 14.50 & 14.50 & 14.50 & 14.50 & & & & \\
\hline Sulfur & $\%$, dry & 1.00 & 1.00 & 1.00 & 1.00 & 1.00 & 1.00 & 1.00 & 1.00 & & & & \\
\hline$B T U / \mathrm{lb}$ & dry & 12825 & 12825 & 12825 & 12825 & 12825 & 12825 & 12825 & 12825 & & & & \\
\hline BTU/Ib & maf & 15000 & 15000 & 15000 & 15000 & 15000 & 15000 & 15000 & 15000 & & & & \\
\hline Mass & $\%$, ar & 2.29 & 24.52 & 21.50 & 17.64 & 11.32 & 5.02 & 17.72 & 100.00 & & & & \\
\hline Moisture & $\%$, ar & 4.86 & 6.80 & 11.16 & 17.70 & 25.21 & 31.15 & 39.92 & 18.79 & & & & \\
\hline Cum. Moisture & $\%$, ar & 18.79 & 19.12 & 23.24 & 28.27 & 33.74 & 37.99 & 39.92 & --- & & & & \\
\hline Ash & $\%$, ar & 13.79 & 13.51 & 12.88 & 11.93 & 10.84 & 9.98 & 8.71 & 11.77 & & & & \\
\hline Inerts & $\%$, ar & 18.65 & 20.31 & 24.04 & 29.64 & 36.05 & 41.13 & 48.63 & 30.56 & & & & \\
\hline Sulfur & $\%$, ar & 0.95 & 0.93 & 0.89 & 0.82 & 0.75 & 0.69 & 0.60 & 0.81 & & & & \\
\hline BTU/Ib & ar & 12202 & 11953 & 11394 & 10555 & 9592 & 8830 & 7705 & 10415 & & & & \\
\hline Ib SO2/MM BTU & --- & 1.56 & 1.56 & 1.56 & 1.56 & 1.56 & 1.56 & 1.56 & 1.56 & & & & \\
\hline Cost to Boiler & \$/MM BTU & $\$ 2.44$ & $\$ 2.44$ & $\$ 2.44$ & $\$ 2.44$ & $\$ 2.44$ & $\$ 2.44$ & $\$ 2.44$ & $\$ 2.44$ & & & & \\
\hline Freight Cost & $\$ / \mathrm{MM} \mathrm{BTU}$ & $\$ 0.82$ & $\$ 0.84$ & $\$ 0.88$ & $\$ 0.95$ & $\$ 1.04$ & $\$ 1.13$ & $\$ 1.30$ & $\$ 0.96$ & & & & \\
\hline Evap. Cost & $\$ / M M B T U$ & $\$ 0.01$ & $\$ 0.01$ & $\$ 0.01$ & $\$ 0.03$ & $\$ 0.04$ & $\$ 0.05$ & $\$ 0.08$ & $\$ 0.03$ & & & & \\
\hline Ash Cost & $\$ / \mathrm{MM} \mathrm{BTU}$ & $\$ 0.11$ & $\$ 0.11$ & $\$ 0.11$ & $\$ 0.11$ & $\$ 0.11$ & $\$ 0.11$ & $\$ 0.11$ & $\$ 0.11$ & & & & \\
\hline SO2 Cost & $\$ / \mathrm{MMBTU}$ & $\$ 0.04$ & $\$ 0.04$ & $\$ 0.04$ & $\$ 0.04$ & $\$ 0.04$ & $\$ 0.04$ & $\$ 0.04$ & $\$ 0.04$ & & & & \\
\hline \begin{tabular}{|l|} 
Other Cost \\
\end{tabular} & $\$ / \mathrm{MM} \mathrm{BTU}$ & $\$ 0.04$ & $\$ 0.04$ & $\$ 0.04$ & $\$ 0.05$ & $\$ 0.05$ & $\$ 0.06$ & $\$ 0.06$ & $\$ 0.05$ & & & & \\
\hline Coal Worth & $\$ / \mathrm{MM} \mathrm{BTU}$ & $\$ 1.42$ & $\$ 1.40$ & $\$ 1.35$ & $\$ 1.27$ & $\$ 1.16$ & $\$ 1.05$ & $\$ 0.85$ & $\$ 1.26$ & & & & \\
\hline Cost to Boiler & \$/ton & $\$ 59.55$ & $\$ 58.33$ & $\$ 55.60$ & $\$ 51.51$ & $\$ 46.81$ & $\$ 43.09$ & $\$ 37.60$ & $\$ 50.83$ & & & & \\
\hline Freight Cost & $\$ /$ ton & $\$ 20.00$ & $\$ 20.00$ & $\$ 20.00$ & $\$ 20.00$ & $\$ 20.00$ & $\$ 20.00$ & $\$ 20.00$ & $\$ 20.00$ & & & & \\
\hline Evap. Cost & $\$ /$ ton & $\$ 0.15$ & $\$ 0.20$ & $\$ 0.33$ & $\$ 0.53$ & $\$ 0.76$ & $\$ 0.93$ & $\$ 1.20$ & $\$ 0.56$ & & & & \\
\hline Ash Cost & $\$ /$ ton & $\$ 2.76$ & $\$ 2.70$ & $\$ 2.58$ & $\$ 2.39$ & $\$ 2.17$ & $\$ 2.00$ & $\$ 1.74$ & $\$ 2.35$ & & & & \\
\hline SO2 Cost & $\$ /$ ton & $\$ 0.88$ & $\$ 0.86$ & $\$ 0.82$ & $\$ 0.76$ & $\$ 0.69$ & $\$ 0.63$ & $\$ 0.55$ & $\$ 0.75$ & & & & \\
\hline Other Cost & $\$ /$ ton & $\$ 1.00$ & $\$ 1.00$ & $\$ 1.00$ & $\$ 1.00$ & $\$ 1.00$ & $\$ 1.00$ & $\$ 1.00$ & $\$ 1.00$ & & & & \\
\hline Coal Worth & $\$ /$ ton & $\$ 34.76$ & $\$ 33.56$ & $\$ 30.87$ & $\$ 26.83$ & $\$ 22.19$ & $\$ 18.53$ & $\$ 13.11$ & $\$ 26.16$ & & & & \\
\hline Individual Tons & dry & 2.68 & 28.14 & 23.52 & 17.88 & 10.42 & 4.25 & 13.11 & 100.00 & & & & \\
\hline Cum. Tons & dry & 100.00 & 97.32 & 69.18 & 45.66 & 27.78 & 17.36 & 13.11 & & & & & \\
\hline Individual Tons & $\mathrm{ar}$ & 2.81 & 30.20 & 26.47 & 21.72 & 13.94 & 6.18 & 21.82 & 123.14 & & & & \\
\hline Cum. Tons & ar & 123.14 & 120.32 & 90.13 & 63.66 & 41.93 & 27.99 & 21.82 & --- & & & & \\
\hline Cum. Mass & $\%$, ar & 100.00 & 97.71 & 73.19 & 51.69 & 34.05 & 22.73 & 17.72 & $-\cdots$ & & & & \\
\hline Cum. Ash & $\%$, ar & 13.79 & 13.54 & 13.24 & 12.89 & 12.59 & 12.43 & 11.77 & --- & & & & \\
\hline Cum. Sulfur & $\%$, ar & 0.95 & 0.93 & 0.91 & 0.89 & 0.87 & 0.86 & 0.81 & --- & & & & \\
\hline Cum. BTU/lb & ar & 12202 & 12079 & 11894 & 11679 & 11480 & 11321 & 11160 & $-\ldots$ & & & & \\
\hline Individual Worth & $\$$ & $\$ 97.83$ & $\$ 1,013.57$ & $\$ 817.23$ & $\$ 582.84$ & $\$ 309.37$ & $\$ 114.47$ & $\$ 285.91$ & $\$ 3,221.21$ & & & & \\
\hline Cum. Worth & $\$$ & $\$ 97.83$ & $\$ 1,111.40$ & $\$ 1,928.63$ & $\$ 2,511.46$ & $\$ 2,820.83$ & $\$ 2,935.30$ & $\$ 3,221.21$ & --- & & & & \\
\hline
\end{tabular}


Coal B - Circuit Configuration 1 - Coarse Cut - Screenbowl Centrifuge Simulation

\begin{tabular}{|c|c|c|c|c|c|c|}
\hline \multicolumn{7}{|c|}{ Input Parameters } \\
\hline $\begin{array}{c}k_{d} \\
\left(m^{-1}\right)\end{array}$ & $\begin{array}{c}\rho_{\mathrm{s}} \\
\left(\mathrm{kg} / \mathrm{m}^{3}\right)\end{array}$ & $\begin{array}{c}\rho_{\mathrm{w}} \\
\left(\mathrm{kg} / \mathrm{m}^{3}\right)\end{array}$ & $\begin{array}{c}\text { B } \\
\text { (m) }\end{array}$ & $\varepsilon$ & $\begin{array}{c}\sigma \\
(\mathrm{N} / \mathrm{m})\end{array}$ & $\begin{array}{c}\mu \\
\text { (Pa.s) }\end{array}$ \\
\hline 400 & 1400 & 1000 & 0.559 & 0.55 & 0.072 & 0.001 \\
\hline $\mathrm{L}$ & $t$ & $\mathrm{~N}$ & $\mathrm{~N}_{\mathrm{g}}$ & Air $\mathrm{P}$ & $\mathbf{g}$ & $\theta$ \\
\hline (m) & (sec) & (RPM) & & (psi) & $\left(\mathrm{m} / \mathrm{s}^{2}\right)$ & \\
\hline 0.0381 & 7 & 895 & 500 & 0 & $\begin{array}{l}9.81 \\
\end{array}$ & 60 \\
\hline
\end{tabular}
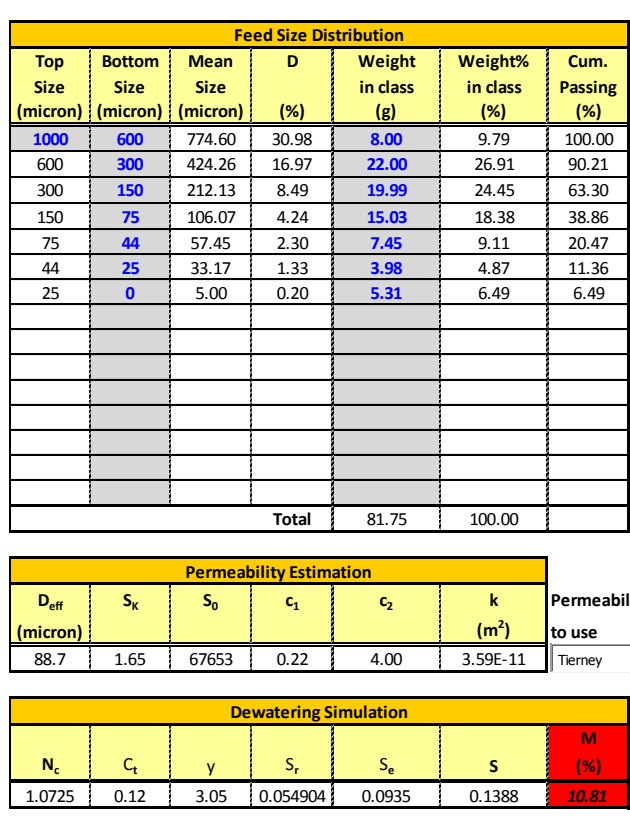
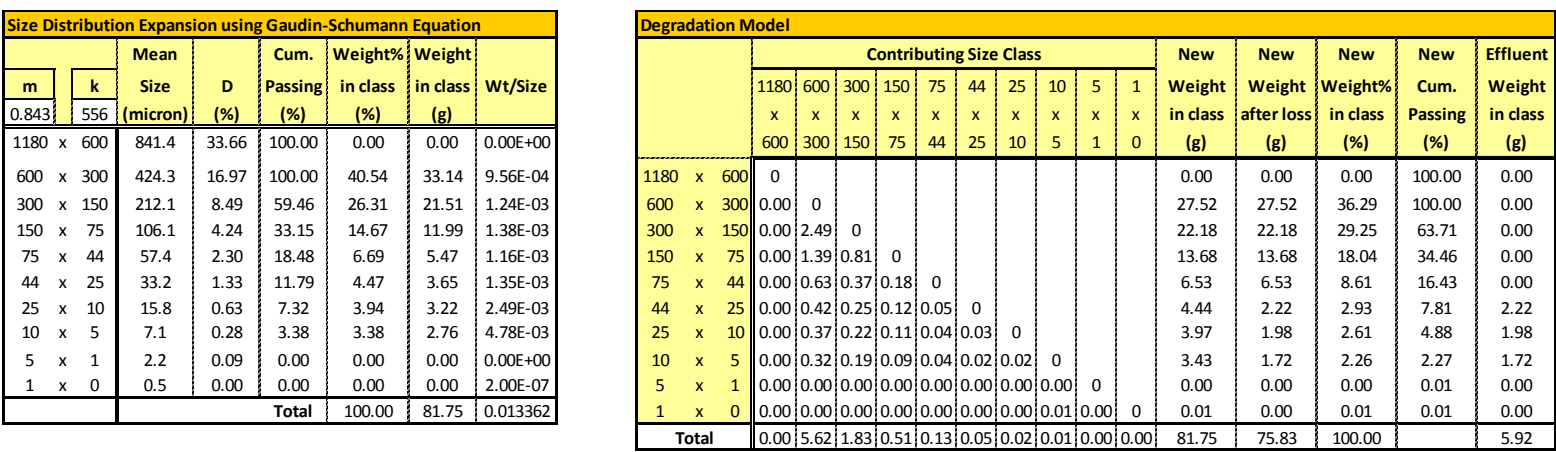

\begin{tabular}{cc} 
& Mean \\
$25 \times 0$ & 9.8493 \\
\hline & 1396
\end{tabular}

\begin{tabular}{ll}
$25 \times 0 \times 0$ & 13.376 \\
\hline $14 \times 0$ & 22376
\end{tabular}

$\begin{array}{ll}74 \times 0 & 22.376 \\ 15000 & 38118\end{array}$

$\begin{array}{ll}150 \times 0 & 38.118 \\ 300 \times 0 & 61.141\end{array}$

$\begin{array}{ll}30000 \times 0 & 81.141 \\ 68.688\end{array}$

$1180 \times 0 \quad 88.688$

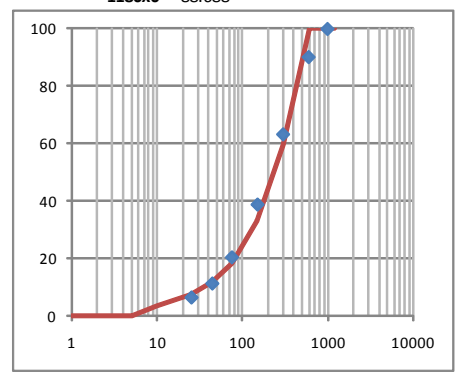


Coal B - Circuit Configuration 1 - Coarse Cut - Screenbowl Centrifuge Economics

CALCULATION OF OPTIMUM SIZE CUT

\begin{tabular}{|c|c|c|c|c|c|c|c|c|c|c|c|c|c|}
\hline \multirow{2}{*}{ FINANCIAL DATA: } & & \multicolumn{2}{|c|}{ Total Boiler Cost $=$} & $\$ 2.44$ & /MM BTU & & Moisture $\mathrm{E}$ & vap. = & $\$ 3.00$ & /ton $\mathrm{H}_{2} \mathrm{O}$ & $\mathrm{SO}_{2}$ Emission $=$ & $\$ 200$ & $/$ ton $\mathrm{SO}_{2}$ \\
\hline & & Freight $\mathrm{Co}$ & & $\$ 20.00$ & /ton coal & & Ash Handli & $\mathrm{ng}=$ & $\$ 20.00$ & /ton ash & Other Cost $=$ & $\$ 1.00$ & /ton coal \\
\hline Size Class & & 1 & 2 & 3 & 4 & 5 & 6 & 7 & Totals & & & & \\
\hline Top Size & $\mu$ & 1180 & 600 & 300 & 150 & 74 & 44 & 25 & --- & & & & \\
\hline Bottom Size & $\mu$ & 600 & 300 & 150 & 74 & 44 & 25 & 0 & $-\cdots$ & & & & \\
\hline$S G$ & $\mu$ & 1.45 & 1.45 & 1.45 & 1.45 & 1.45 & 1.45 & 1.45 & --- & & & & \\
\hline $1 / \mathrm{SG}$ & --- & 0.69 & 0.69 & 0.69 & 0.69 & 0.69 & 0.69 & 0.69 & --- & & & & \\
\hline Mass & $\%$, dry & 0.00 & 36.29 & 29.25 & 18.04 & 8.61 & 2.93 & 4.88 & 100.00 & & & & \\
\hline Solid SG & & 1.40 & 1.40 & 1.40 & 1.40 & 1.40 & 1.40 & 1.40 & & & & & \\
\hline Ash & $\%$, dry & 14.50 & 14.50 & 14.50 & 14.50 & 14.50 & 14.50 & 14.50 & 14.50 & & & & \\
\hline Sulfur & $\%$, dry & 1.00 & 1.00 & 1.00 & 1.00 & 1.00 & 1.00 & 1.00 & 1.00 & & & & \\
\hline BTU/Ib & dry & 12825 & 12825 & 12825 & 12825 & 12825 & 12825 & 12825 & 12825 & & & & \\
\hline BTU/lb & maf & 15000 & 15000 & 15000 & 15000 & 15000 & 15000 & 15000 & 15000 & & & & \\
\hline Mass & $\%$, ar & 0.00 & 33.75 & 27.96 & 18.26 & 9.52 & 3.57 & 6.95 & 100.00 & & & & \\
\hline Moisture & $\%$, ar & 10.81 & 4.11 & 6.71 & 11.87 & 19.29 & 26.75 & 37.29 & 10.81 & & & & \\
\hline Cum. Moisture & $\%$, ar & 10.81 & 10.81 & 14.22 & 19.71 & 26.86 & 33.72 & 37.29 & --- & & & & \\
\hline Ash & $\%$, ar & 12.93 & 13.90 & 13.53 & 12.78 & 11.70 & 10.62 & 9.09 & 12.93 & & & & \\
\hline Inerts & $\%$, ar & 23.74 & 18.01 & 20.23 & 24.65 & 30.99 & 37.37 & 46.39 & 23.74 & & & & \\
\hline Sulfur & $\%$, ar & 0.89 & 0.96 & 0.93 & 0.88 & 0.81 & 0.73 & 0.63 & 0.89 & & & & \\
\hline BTU/Ib & ar & 11439 & 12299 & 11965 & 11303 & 10351 & 9395 & 8042 & 11439 & & & & \\
\hline Ib SO2/MM BTU & $-\ldots$ & 1.56 & 1.56 & 1.56 & 1.56 & 1.56 & 1.56 & 1.56 & 1.56 & & & & \\
\hline Cost to Boiler & $\$ / M M$ BTU & $\$ 2.44$ & $\$ 2.44$ & $\$ 2.44$ & $\$ 2.44$ & $\$ 2.44$ & $\$ 2.44$ & $\$ 2.44$ & $\$ 2.44$ & & & & \\
\hline Freight Cost & $\$ / M M B T U$ & $\$ 0.87$ & $\$ 0.81$ & $\$ 0.84$ & $\$ 0.88$ & $\$ 0.97$ & $\$ 1.06$ & $\$ 1.24$ & $\$ 0.87$ & & & & \\
\hline Evap. Cost & $\$ / \mathrm{MM} \mathrm{BTU}$ & $\$ 0.01$ & $\$ 0.01$ & $\$ 0.01$ & $\$ 0.02$ & $\$ 0.03$ & $\$ 0.04$ & $\$ 0.07$ & $\$ 0.01$ & & & & \\
\hline Ash Cost & $\$ / M M B T U$ & $\$ 0.11$ & $\$ 0.11$ & $\$ 0.11$ & $\$ 0.11$ & $\$ 0.11$ & $\$ 0.11$ & $\$ 0.11$ & $\$ 0.11$ & & & & \\
\hline SO2 Cost & $\$ / M M B T U$ & $\$ 0.04$ & $\$ 0.04$ & $\$ 0.04$ & $\$ 0.04$ & $\$ 0.04$ & $\$ 0.04$ & $\$ 0.04$ & $\$ 0.04$ & & & & \\
\hline Other Cost & $\$ / M M B T U$ & $\$ 0.04$ & $\$ 0.04$ & $\$ 0.04$ & $\$ 0.04$ & $\$ 0.05$ & $\$ 0.05$ & $\$ 0.06$ & $\$ 0.04$ & & & & \\
\hline Coal Worth & $\$ / \mathrm{MM} \mathrm{BTU}$ & $\$ 1.36$ & $\$ 1.43$ & $\$ 1.41$ & $\$ 1.35$ & $\$ 1.25$ & $\$ 1.13$ & $\$ 0.92$ & $\$ 1.36$ & & & & \\
\hline Cost to Boiler & $\$ /$ ton & $\$ 55.82$ & $\$ 60.02$ & $\$ 58.39$ & $\$ 55.16$ & $\$ 50.51$ & $\$ 45.85$ & $\$ 39.25$ & $\$ 55.82$ & & & & \\
\hline Freight Cost & $\$ /$ ton & $\$ 20.00$ & $\$ 20.00$ & $\$ 20.00$ & $\$ 20.00$ & $\$ 20.00$ & $\$ 20.00$ & $\$ 20.00$ & $\$ 20.00$ & & & & \\
\hline Evap. Cost & $\$ /$ ton & $\$ 0.32$ & $\$ 0.12$ & $\$ 0.20$ & $\$ 0.36$ & $\$ 0.58$ & $\$ 0.80$ & $\$ 1.12$ & $\$ 0.32$ & & & & \\
\hline Ash Cost & $\$ /$ ton & $\$ 2.59$ & $\$ 2.78$ & $\$ 2.71$ & $\$ 2.56$ & $\$ 2.34$ & $\$ 2.12$ & $\$ 1.82$ & $\$ 2.59$ & & & & \\
\hline SO2 Cost & $\$ /$ ton & $\$ 0.82$ & $\$ 0.88$ & $\$ 0.86$ & $\$ 0.81$ & $\$ 0.74$ & $\$ 0.68$ & $\$ 0.58$ & $\$ 0.82$ & & & & \\
\hline Other Cost & $\$ /$ ton & $\$ 1.00$ & $\$ 1.00$ & $\$ 1.00$ & $\$ 1.00$ & $\$ 1.00$ & $\$ 1.00$ & $\$ 1.00$ & $\$ 1.00$ & & & & \\
\hline Coal Worth & $\$ /$ ton & $\$ 31.09$ & $\$ 35.23$ & $\$ 33.62$ & $\$ 30.44$ & $\$ 25.85$ & $\$ 21.25$ & $\$ 14.73$ & $\$ 31.09$ & & & & \\
\hline Individual Tons & dry & 0.00 & 27.52 & 22.18 & 13.68 & 6.53 & 2.22 & 3.70 & 75.83 & & & & \\
\hline Cum. Tons & dry & 75.83 & 75.83 & 48.31 & 26.13 & 12.46 & 5.92 & 3.70 & & & & & \\
\hline Individual Tons & $\mathrm{ar}$ & 0.00 & 28.70 & 23.77 & 15.52 & 8.09 & 3.03 & 5.91 & 85.02 & & & & \\
\hline Cum. Tons & $\mathrm{ar}$ & 85.02 & 85.02 & 56.32 & 32.55 & 17.03 & 8.94 & 5.91 & -- & & & & \\
\hline Cum. Mass & $\%$, ar & 100.00 & 100.00 & 66.25 & 38.29 & 20.03 & 10.51 & 6.95 & --- & & & & \\
\hline Cum. Ash & $\%$, ar & 12.93 & 13.90 & 13.73 & 13.51 & 13.32 & 13.22 & 12.93 & --- & & & & \\
\hline Cum. Sulfur & $\%$, ar & 0.89 & 0.96 & 0.95 & 0.93 & 0.92 & 0.91 & 0.89 & -- & & & & \\
\hline Cum. BTU/lb & ar & 11439 & 11869 & 11893 & 11819 & 11728 & 11655 & 11581 & --- & & & & \\
\hline \begin{tabular}{|l|} 
Individual Worth \\
\end{tabular} & $\$$ & $\$ 0.00$ & $\$ 1,010.94$ & $\$ 799.21$ & $\$ 472.38$ & $\$ 209.16$ & $\$ 64.44$ & $\$ 86.99$ & $\$ 2,643.12$ & & & & \\
\hline Cum. Worth & $\$$ & $\$ 0.00$ & $\$ 1,010.94$ & $\$ 1,810.16$ & $\$ 2,282.54$ & $\$ 2,491.69$ & $\$ 2,556.13$ & $\$ 2,643.12$ & --- & & & & \\
\hline
\end{tabular}


Coal B - Circuit Configuration 1 - Coarse Cut - Hyperbaric Centrifuge Simulation

\begin{tabular}{|c|c|c|c|c|c|c|}
\hline \multicolumn{7}{|c|}{ Input Parameters } \\
\hline $\begin{array}{c}k_{d} \\
\left(m^{-1}\right)\end{array}$ & $\begin{array}{c}\rho_{\mathrm{s}} \\
\left(\mathrm{kg} / \mathrm{m}^{3}\right)\end{array}$ & $\underset{\left(\mathrm{kg} / \mathrm{m}^{3}\right)}{\rho_{\mathrm{w}}}$ & $\begin{array}{c}\text { B } \\
(\mathrm{m})\end{array}$ & $\varepsilon$ & $\begin{array}{c}\sigma \\
(\mathrm{N} / \mathrm{m})\end{array}$ & $\begin{array}{c}\mu \\
\text { (Pa.s) }\end{array}$ \\
\hline 400 & 1400 & 1000 & 0.381 & 0.52 & 0.072 & 0.001 \\
\hline $\mathrm{L}$ & ${ }^{t}$ & $\mathrm{~N}$ & $\mathrm{~N}_{\mathrm{g}}$ & Air $P$ & $\mathbf{g}$ & $\theta$ \\
\hline (m) & (sec) & (RPM) & & (psi) & $\left(\mathrm{m} / \mathrm{s}^{2}\right)$ & \\
\hline 0.0127 & 7 & 2650 & 2991 & 0 & 9.81 & 60 \\
\hline
\end{tabular}

\begin{tabular}{|c|c|c|c|c|c|c|}
\hline \multicolumn{7}{|c|}{ Feed Size Distribution } \\
\hline $\begin{array}{c}\text { Top } \\
\text { Size } \\
\text { (micron) }\end{array}$ & $\begin{array}{c}\text { Bottom } \\
\text { Size } \\
\text { (micron) }\end{array}$ & $\begin{array}{c}\text { Mean } \\
\text { Size } \\
\text { (micron) }\end{array}$ & $\begin{array}{l}\mathrm{D} \\
(\%)\end{array}$ & $\begin{array}{l}\text { Weight } \\
\text { in class }\end{array}$ & $\begin{array}{l}\text { Weight\% } \\
\text { in class } \\
\text { (\%) }\end{array}$ & $\begin{array}{l}\text { Cum. } \\
\text { Passing } \\
\text { (\%) }\end{array}$ \\
\hline 1000 & 600 & 774.60 & 30.98 & 0.00 & 0.00 & 100.00 \\
\hline 600 & 300 & 424.26 & 16.97 & 0.00 & 0.00 & 100.00 \\
\hline 300 & 150 & 212.13 & 8.49 & 0.01 & 0.05 & 100.00 \\
\hline 150 & 75 & 106.07 & 4.24 & 0.47 & 1.96 & 99.95 \\
\hline 75 & 44 & 57.45 & 2.30 & 1.55 & 6.42 & 97.99 \\
\hline 44 & 25 & 33.17 & 1.33 & 4.74 & 19.61 & 91.58 \\
\hline 25 & 0 & 5.00 & 0.20 & 17.40 & 71.97 & 71.97 \\
\hline & & & & & & \\
\hline & & & & & & \\
\hline & & & & & & \\
\hline & & & & & & \\
\hline & & & & & & \\
\hline & & & Tot? & 2417 & 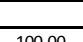 & \\
\hline & & & & & & \\
\hline
\end{tabular}
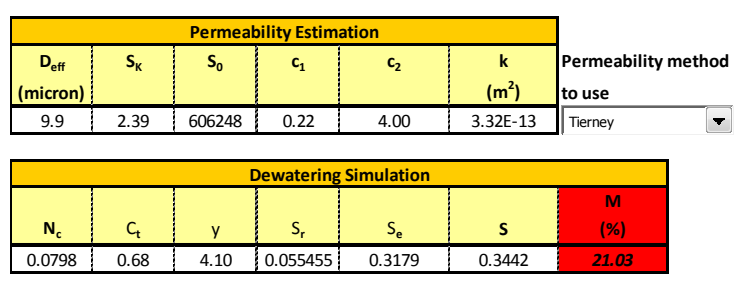

\begin{tabular}{|c|c|c|c|c|c|c|c|}
\hline \multirow{2}{*}{\multicolumn{2}{|c|}{ Size Distributi }} & \multirow{3}{*}{$\begin{array}{l}\text { On Expan } \\
\text { Mean } \\
\text { Size } \\
\text { (micron) }\end{array}$} & \multirow[b]{3}{*}{$\begin{array}{l}\text { D } \\
\text { (\%) }\end{array}$} & \multirow{3}{*}{ 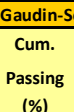 } & \multirow{3}{*}{\begin{tabular}{|l} 
Chumann \\
Weight\% \\
in class \\
(\%)
\end{tabular}} & \multirow{3}{*}{$\begin{array}{l}\text { Equation } \\
\text { Weight } \\
\text { in class } \\
\text { (g) }\end{array}$} & \multirow{3}{*}{ wt/Size } \\
\hline & & & & & & & \\
\hline$\frac{m}{0.282}$ & \begin{tabular}{|l|}
$k$ \\
73 \\
\end{tabular} & & & & & & \\
\hline $1180 \times$ & 2600 & 841.4 & 33.66 & 100.00 & \begin{tabular}{|l|l} 
\\
\end{tabular} & 0.00 & $0.00 E+00$ \\
\hline 600 & 300 & 424.3 & 16.97 & 100.00 & קחת & 00 & 200E \\
\hline 300 & 150 & 212.1 & 8.49 & 100.00 & 0.00 & 0.00 & $0.00 \mathrm{E}+\mathrm{C}$ \\
\hline 150 & 75 & 106. & & & & & 0.00 \\
\hline 75 & 44 & 57. & 2.3 & & & & $2.32 E-03$ \\
\hline 44 & 25 & 33.2 & 1.33 & 86.70 & 12.79 & 3.09 & $3.86 \mathrm{E}-\mathrm{C}$ \\
\hline 25 & 10 & 15.8 & 0.63 & & & & $1.07 E-02$ \\
\hline 10 & 5 & & & & & & $8.07 E-02$ \\
\hline$x$ & 1 & & & & & & .00 \\
\hline & & & 0.00 & & & & \\
\hline & & & & Total & 100.00 & 24.17 & $\mid$\begin{tabular}{|l}
0.09751 \\
\end{tabular} \\
\hline
\end{tabular}

$25 \times 0 \quad 7.8282 \quad 23.64$

$\begin{array}{lll}44 \times 0 & 8.808 & 22.32 \\ 74 \times 0 & 98969 & 21.33\end{array}$

$\begin{array}{lll}74 \times 0 & 9.8969 & 21.03 \\ 150 \times 0 & 9.8969 & 21.03\end{array}$

$300 \times 0 \quad 9.8969 \quad 21.03$

$\begin{array}{lll}1180 \times 0 & 9.8969 & 21.03\end{array}$

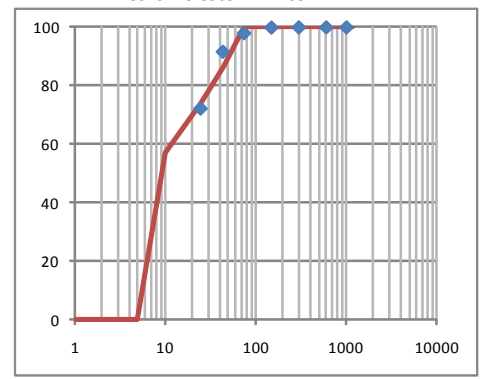

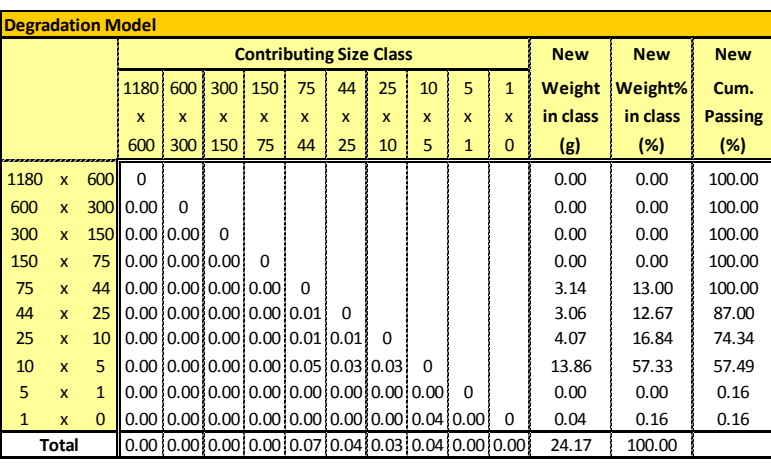

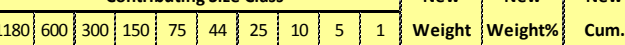

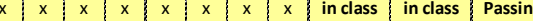

\begin{tabular}{l|l|l|} 
(g) & $(\%)$ & $(\%)$ \\
\hline 0.00 & 0.00 & 100.00 \\
\hline
\end{tabular}

\begin{tabular}{|l|l|l|}
\hline .00 & 100.00 \\
\hline & 0.00 \\
\hline & 10000
\end{tabular}

100.00

100.00
87.00

0.16

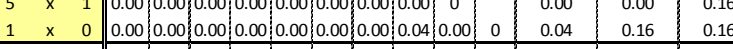

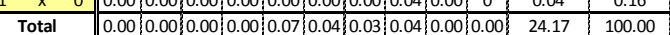


Coal B - Circuit Configuration 1 - Coarse Cut - Hyperbaric Centrifuge Economics

\section{CALCULATION OF OPTIMUM SIZE CUT}

\begin{tabular}{|c|c|c|c|c|c|c|c|c|c|c|c|c|c|}
\hline \multirow{2}{*}{\multicolumn{2}{|c|}{\begin{tabular}{|l|l|} 
FINANCIAL DATA: & \\
& \\
\end{tabular}}} & \multicolumn{2}{|c|}{ Total Boiler Cost $=$} & \multirow{2}{*}{$\begin{array}{r}\$ 2.44 \\
\$ 20.00\end{array}$} & /MM BTU & & \multicolumn{2}{|c|}{ Moisture Evap. = } & \multirow{2}{*}{$\begin{array}{r}\$ 3.00 \\
\$ 20.00\end{array}$} & \multirow{2}{*}{$\begin{array}{l}\text { /ton } \mathrm{H}_{2} \mathrm{O} \\
\text { /ton ash }\end{array}$} & \multirow{2}{*}{$\begin{array}{l}\mathrm{SO}_{2} \text { Emission }= \\
\text { Other Cost = }\end{array}$} & \multirow{2}{*}{$\begin{array}{r}\$ 200 \\
\$ 1.00\end{array}$} & \\
\hline & & Freight $\mathrm{Co}$ & & & /ton coal & & Ash Hand & $\mathrm{ng}=$ & & & & & $\begin{array}{l}\text { /ton } \mathrm{SO}_{2} \\
\text { ton coal }\end{array}$ \\
\hline \begin{tabular}{|l|} 
Size Class \\
\end{tabular} & & 1 & 2 & 3 & 4 & 5 & 6 & 7 & Totals & & & & \\
\hline Top Size & $\mu$ & 1180 & 600 & 300 & 150 & 74 & 44 & 25 & $-\cdots$ & & & & \\
\hline Bottom Size & $\mu$ & 600 & 300 & 150 & 74 & 44 & 25 & 0 & $-\ldots$ & & & & \\
\hline SG & $\mu$ & 1.45 & 1.45 & 1.45 & 1.45 & 1.45 & 1.45 & 1.45 & --- & & & & \\
\hline $1 / \mathrm{SG}$ & --- & 0.69 & 0.69 & 0.69 & 0.69 & 0.69 & 0.69 & 0.69 & --- & & & & \\
\hline Mass & $\%$, dry & 0.00 & 0.00 & 0.00 & 0.00 & 13.00 & 12.67 & 74.34 & 100.00 & & & & \\
\hline Solid SG & & 1.40 & 1.40 & 1.40 & 1.40 & 1.40 & 1.40 & 1.40 & & & & & \\
\hline Ash & $\%$, dry & 14.50 & 14.50 & 14.50 & 14.50 & 14.50 & 14.50 & 14.50 & 14.50 & & & & \\
\hline Sulfur & $\%$, dry & 1.00 & 1.00 & 1.00 & 1.00 & 1.00 & 1.00 & 1.00 & 1.00 & & & & \\
\hline$B T U / \mathrm{lb}$ & dry & 12825 & 12825 & 12825 & 12825 & 12825 & 12825 & 12825 & 12825 & & & & \\
\hline BTU/Ib & maf & 15000 & 15000 & 15000 & 15000 & 15000 & 15000 & 15000 & 15000 & & & & \\
\hline Mass & $\%$, ar & 0.00 & 0.00 & 0.00 & 0.00 & 11.55 & 11.57 & 76.87 & 100.00 & & & & \\
\hline Moisture & $\%$, ar & 21.03 & 21.03 & 21.03 & 21.03 & 11.18 & 13.55 & 23.64 & 21.03 & & & & \\
\hline Cum. Moisture & $\%$, ar & 21.03 & 21.03 & 21.03 & 21.03 & 21.03 & 22.32 & 23.64 & --- & & & & \\
\hline Ash & $\%$, ar & 11.45 & 11.45 & 11.45 & 11.45 & 12.88 & 12.53 & 11.07 & 11.45 & & & & \\
\hline Inerts & $\%$, ar & 32.48 & 32.48 & 32.48 & 32.48 & 24.06 & 26.08 & 34.71 & 32.48 & & & & \\
\hline Sulfur & $\%$, ar & 0.79 & 0.79 & 0.79 & 0.79 & 0.89 & 0.86 & 0.76 & 0.79 & & & & \\
\hline BTU/Ib & ar & 10128 & 10128 & 10128 & 10128 & 11391 & 11087 & 9794 & 10128 & & & & \\
\hline Ib SO2/MM BTU & --- & 1.56 & 1.56 & 1.56 & 1.56 & 1.56 & 1.56 & 1.56 & 1.56 & & & & \\
\hline Cost to Boiler & \$/MM BTU & $\$ 2.44$ & $\$ 2.44$ & $\$ 2.44$ & $\$ 2.44$ & $\$ 2.44$ & $\$ 2.44$ & $\$ 2.44$ & $\$ 2.44$ & & & & \\
\hline Freight Cost & $\$ / \mathrm{MM} \mathrm{BTU}$ & $\$ 0.99$ & $\$ 0.99$ & $\$ 0.99$ & $\$ 0.99$ & $\$ 0.88$ & $\$ 0.90$ & $\$ 1.02$ & $\$ 0.99$ & & & & \\
\hline Evap. Cost & $\$ / M M B T U$ & $\$ 0.03$ & $\$ 0.03$ & $\$ 0.03$ & $\$ 0.03$ & $\$ 0.01$ & $\$ 0.02$ & $\$ 0.04$ & $\$ 0.03$ & & & & \\
\hline Ash Cost & $\$ / M M B T U$ & $\$ 0.11$ & $\$ 0.11$ & $\$ 0.11$ & $\$ 0.11$ & $\$ 0.11$ & $\$ 0.11$ & $\$ 0.11$ & $\$ 0.11$ & & & & \\
\hline SO2 Cost & $\$ / \mathrm{MMBTU}$ & $\$ 0.04$ & $\$ 0.04$ & $\$ 0.04$ & $\$ 0.04$ & $\$ 0.04$ & $\$ 0.04$ & $\$ 0.04$ & $\$ 0.04$ & & & & \\
\hline \begin{tabular}{|l|} 
Other Cost \\
\end{tabular} & $\$ / \mathrm{MM} \mathrm{BTU}$ & $\$ 0.05$ & $\$ 0.05$ & $\$ 0.05$ & $\$ 0.05$ & $\$ 0.04$ & $\$ 0.05$ & $\$ 0.05$ & $\$ 0.05$ & & & & \\
\hline Coal Worth & $\$ / \mathrm{MM} \mathrm{BTU}$ & $\$ 1.22$ & $\$ 1.22$ & $\$ 1.22$ & $\$ 1.22$ & $\$ 1.35$ & $\$ 1.33$ & $\$ 1.18$ & $\$ 1.22$ & & & & \\
\hline Cost to Boiler & \$/ton & $\$ 49.42$ & $\$ 49.42$ & $\$ 49.42$ & $\$ 49.42$ & $\$ 55.59$ & $\$ 54.11$ & $\$ 47.79$ & $\$ 49.42$ & & & & \\
\hline Freight Cost & $\$ /$ ton & $\$ 20.00$ & $\$ 20.00$ & $\$ 20.00$ & $\$ 20.00$ & $\$ 20.00$ & $\$ 20.00$ & $\$ 20.00$ & $\$ 20.00$ & & & & \\
\hline Evap. Cost & $\$ /$ ton & $\$ 0.63$ & $\$ 0.63$ & $\$ 0.63$ & $\$ 0.63$ & $\$ 0.34$ & $\$ 0.41$ & $\$ 0.71$ & $\$ 0.63$ & & & & \\
\hline Ash Cost & $\$ /$ ton & $\$ 2.29$ & $\$ 2.29$ & $\$ 2.29$ & $\$ 2.29$ & $\$ 2.58$ & $\$ 2.51$ & $\$ 2.21$ & $\$ 2.29$ & & & & \\
\hline SO2 Cost & $\$ /$ ton & $\$ 0.73$ & $\$ 0.73$ & $\$ 0.73$ & $\$ 0.73$ & $\$ 0.82$ & $\$ 0.80$ & $\$ 0.70$ & $\$ 0.73$ & & & & \\
\hline Other Cost & $\$ /$ ton & $\$ 1.00$ & $\$ 1.00$ & $\$ 1.00$ & $\$ 1.00$ & $\$ 1.00$ & $\$ 1.00$ & $\$ 1.00$ & $\$ 1.00$ & & & & \\
\hline Coal Worth & $\$ /$ ton & $\$ 24.78$ & $\$ 24.78$ & $\$ 24.78$ & $\$ 24.78$ & $\$ 30.86$ & $\$ 29.40$ & $\$ 23.17$ & $\$ 24.78$ & & & & \\
\hline \begin{tabular}{|l|} 
Individual Tons \\
\end{tabular} & dry & 0.00 & 0.00 & 0.00 & 0.00 & 3.14 & 3.06 & 17.97 & 24.17 & & & & \\
\hline Cum. Tons & dry & 24.17 & 24.17 & 24.17 & 24.17 & 24.17 & 21.03 & 17.97 & & & & & \\
\hline Individual Tons & $\mathrm{ar}$ & 0.00 & 0.00 & 0.00 & 0.00 & 3.54 & 3.54 & 23.53 & 30.61 & & & & \\
\hline Cum. Tons & ar & 30.61 & 30.61 & 30.61 & 30.61 & 30.61 & 27.07 & 23.53 & --- & & & & \\
\hline Cum. Mass & $\%$, ar & 100.00 & 100.00 & 100.00 & 100.00 & 100.00 & 88.45 & 76.87 & $-\cdots$ & & & & \\
\hline Cum. Ash & $\%$, ar & 11.45 & 11.45 & 11.45 & 11.45 & 12.88 & 12.71 & 11.45 & $-\cdots$ & & & & \\
\hline Cum. Sulfur & $\%$, ar & 0.79 & 0.79 & 0.79 & 0.79 & 0.89 & 0.88 & 0.79 & --- & & & & \\
\hline Cum. BTU/lb & ar & 10128 & 10128 & 10128 & 10128 & 10381 & 10487 & 10407 & $-\ldots$ & & & & \\
\hline Individual Worth & $\$$ & $\$ 0.00$ & $\$ 0.00$ & $\$ 0.00$ & $\$ 0.00$ & $\$ 109.14$ & $\$ 104.13$ & $\$ 545.11$ & $\$ 758.38$ & & & & \\
\hline Cum. Worth & $\$$ & $\$ 0.00$ & $\$ 0.00$ & $\$ 0.00$ & $\$ 0.00$ & $\$ 109.14$ & $\$ 213.27$ & $\$ 758.38$ & --- & & & & \\
\hline
\end{tabular}


Coal B - Circuit Configuration 1 - Fine Cut - Screenbowl Centrifuge Simulation

\begin{tabular}{|c|c|c|c|c|c|c|}
\hline \multicolumn{7}{|c|}{ Input Parameters } \\
\hline $\begin{array}{c}k_{d} \\
\left(m^{-1}\right)\end{array}$ & $\begin{array}{c}\rho_{\mathrm{s}} \\
\left(\mathrm{kg} / \mathrm{m}^{3}\right)\end{array}$ & $\begin{array}{c}\rho_{\mathrm{w}} \\
\left(\mathrm{kg} / \mathrm{m}^{3}\right)\end{array}$ & $\begin{array}{c}\text { B } \\
\text { (m) }\end{array}$ & $\varepsilon$ & $\begin{array}{c}\sigma \\
(\mathrm{N} / \mathrm{m})\end{array}$ & $\begin{array}{c}\mu \\
\text { (Pa.s) }\end{array}$ \\
\hline 400 & 1400 & 1000 & 0.559 & 0.55 & 0.072 & 0.001 \\
\hline $\mathrm{L}$ & $t$ & $\mathrm{~N}$ & $\mathrm{~N}_{\mathrm{g}}$ & Air $\mathrm{P}$ & $\mathbf{g}$ & $\theta$ \\
\hline (m) & (sec) & (RPM) & & (psi) & $\left(\mathrm{m} / \mathrm{s}^{2}\right)$ & \\
\hline 0.0381 & 7 & 895 & 500 & 0 & $\begin{array}{l}9.81 \\
\end{array}$ & 60 \\
\hline
\end{tabular}
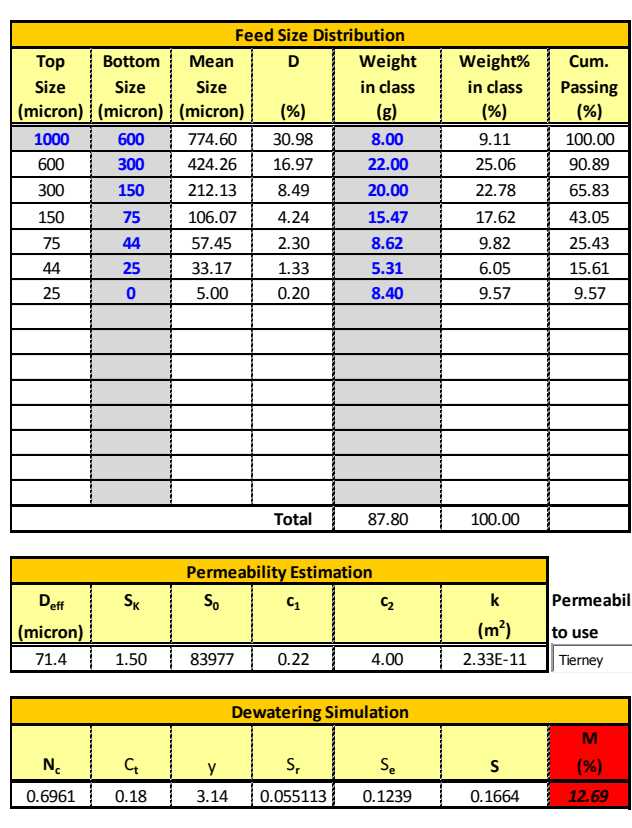

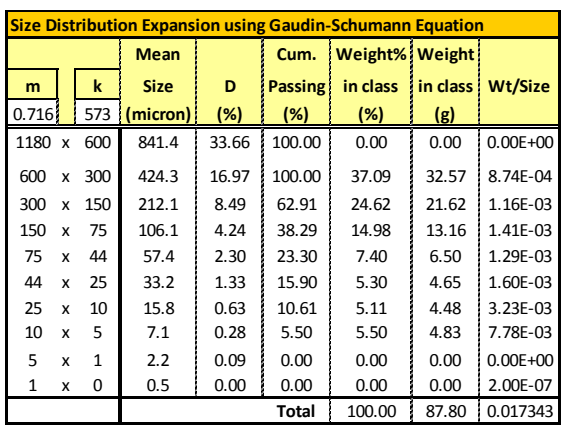

\begin{tabular}{|c|c|c|c|c|c|c|c|c|c|c|c|c|c|c|c|}
\hline \multicolumn{16}{|l|}{ gradation } \\
\hline & \multicolumn{10}{|c|}{ Contributing Size Class } & \multirow{2}{*}{$\begin{array}{c}\text { New } \\
\text { Weight } \\
\text { in class } \\
\text { (g) } \\
\end{array}$} & \multirow{2}{*}{\begin{tabular}{|c|} 
New \\
Weight \\
after loss \\
(g)
\end{tabular}} & \multirow{2}{*}{\begin{tabular}{|c|} 
New \\
Weight\% \\
in class \\
$(\%)$
\end{tabular}} & \multirow{2}{*}{$\begin{array}{c}\text { New } \\
\text { Cum. } \\
\text { Passing } \\
(\%)\end{array}$} & \multirow{2}{*}{$\begin{array}{l}\text { Effluen } \\
\text { Weigh } \\
\text { in class } \\
\text { (g) }\end{array}$} \\
\hline & \begin{tabular}{|c|}
1180 \\
$x$ \\
600
\end{tabular} & \begin{tabular}{|c|c|}
600 \\
$x$ \\
300
\end{tabular} & $\begin{array}{c:c}300 & x \\
150 & \end{array}$ & \begin{tabular}{c|c}
150 \\
75
\end{tabular} & $\begin{array}{l}75 \\
x \\
44\end{array}$ & \begin{tabular}{|c|}
44 \\
\\
25 \\
\end{tabular} & \begin{tabular}{|c|}
25 \\
$x$ \\
10
\end{tabular} & $\begin{array}{l}10 \\
x \\
5\end{array}$ & $\begin{array}{l}5 \\
x \\
1\end{array}$ & $\begin{array}{l}1 \\
\mathrm{x} \\
0\end{array}$ & & & & & \\
\hline & 0 & & & & & & & & & & & & & & \\
\hline & 0.00 & 0 & & & & & & & & & & & & & \\
\hline & \begin{tabular}{|c|c|}
0.00 & \\
\end{tabular} & 2.16 & & & & & & & & & & & & & \\
\hline & \begin{tabular}{|c|c|}
0.00 & \\
\end{tabular} & 1.32 & & 0 & & & & & & & & & & & \\
\hline & & 0.65 & & & & & & & & & & & & & \\
\hline$x$ & & & 0.25 & 0.13 & & & & & & & & & & & \\
\hline 10 & & & & & & & & & & & & & & & \\
\hline$x$ & & & & & & & & & & & & & & & \\
\hline $\mathrm{x}$ & & & & & & & & & 0 & & & & & & \\
\hline$x=$ & & & & & & & & & & & & & & & \\
\hline & & & & & & & & & & & & .4 & 0.00 & & 3 \\
\hline
\end{tabular}

$25 \times 0 \quad 9.41$

$44 \times 0 \quad 12.315$

$74 \times 0 \quad 19.643$

$150 \times 0 \quad 32.258$

$3000 \times 070.004$

$1180 \times 0.71 .448$

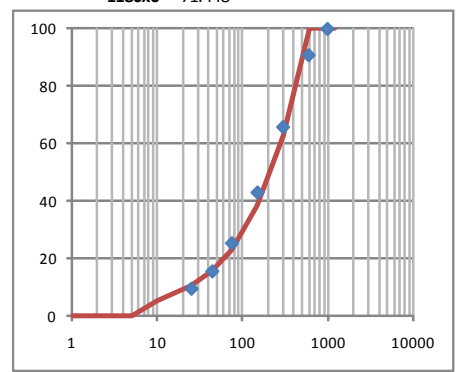


Coal B - Circuit Configuration 1 - Fine Cut - Screenbowl Centrifuge Economics

CALCULATION OF OPTIMUM SIZE CUT

\begin{tabular}{|c|c|c|c|c|c|c|c|c|c|c|c|c|c|}
\hline \multirow{2}{*}{ FINANCIAL DATA: } & & \multicolumn{2}{|c|}{ Total Boiler Cost $=$} & $\$ 2.44$ & /MM BTU & & Moisture $\mathrm{E}$ & vap. = & $\$ 3.00$ & /ton $\mathrm{H}_{2} \mathrm{O}$ & $\mathrm{SO}_{2}$ Emission $=$ & $\$ 200$ & $/$ ton $\mathrm{SO}_{2}$ \\
\hline & & Freight $\mathrm{Co}$ & & $\$ 20.00$ & /ton coal & & Ash Handli & $\mathrm{ng}=$ & $\$ 20.00$ & /ton ash & Other Cost $=$ & $\$ 1.00$ & /ton coal \\
\hline Size Class & & 1 & 2 & 3 & 4 & 5 & 6 & 7 & Totals & & & & \\
\hline Top Size & $\mu$ & 1180 & 600 & 300 & 150 & 74 & 44 & 25 & --- & & & & \\
\hline Bottom Size & $\mu$ & 600 & 300 & 150 & 74 & 44 & 25 & 0 & $-\cdots$ & & & & \\
\hline SG & $\mu$ & 1.45 & 1.45 & 1.45 & 1.45 & 1.45 & 1.45 & 1.45 & --- & & & & \\
\hline $1 / \mathrm{SG}$ & --- & 0.69 & 0.69 & 0.69 & 0.69 & 0.69 & 0.69 & 0.69 & --- & & & & \\
\hline Mass & $\%$, dry & 0.00 & 34.02 & 27.61 & 18.41 & 9.47 & 3.45 & 7.03 & 100.00 & & & & \\
\hline Solid SG & & 1.40 & 1.40 & 1.40 & 1.40 & 1.40 & 1.40 & 1.40 & & & & & \\
\hline Ash & $\%$, dry & 14.50 & 14.50 & 14.50 & 14.50 & 14.50 & 14.50 & 14.50 & 14.50 & & & & \\
\hline Sulfur & $\%$, dry & 1.00 & 1.00 & 1.00 & 1.00 & 1.00 & 1.00 & 1.00 & 1.00 & & & & \\
\hline BTU/Ib & dry & 12825 & 12825 & 12825 & 12825 & 12825 & 12825 & 12825 & 12825 & & & & \\
\hline BTU/lb & maf & 15000 & 15000 & 15000 & 15000 & 15000 & 15000 & 15000 & 15000 & & & & \\
\hline Mass & $\%$, ar & 0.00 & 31.08 & 26.04 & 18.47 & 10.40 & 4.16 & 9.86 & 100.00 & & & & \\
\hline Moisture & $\%$, ar & 12.69 & 4.41 & 7.42 & 12.94 & 20.45 & 27.50 & 37.78 & 12.69 & & & & \\
\hline Cum. Moisture & $\%$, ar & 12.69 & 12.69 & 16.42 & 21.89 & 28.65 & 34.73 & 37.78 & --- & & & & \\
\hline Ash & $\%$, ar & 12.66 & 13.86 & 13.42 & 12.62 & 11.53 & 10.51 & 9.02 & 12.66 & & & & \\
\hline Inerts & $\%$, ar & 25.35 & 18.27 & 20.84 & 25.57 & 31.98 & 38.01 & 46.80 & 25.35 & & & & \\
\hline Sulfur & $\%$, ar & 0.87 & 0.96 & 0.93 & 0.87 & 0.80 & 0.72 & 0.62 & 0.87 & & & & \\
\hline BTU/Ib & ar & 11198 & 12259 & 11874 & 11165 & 10203 & 9298 & 7980 & 11198 & & & & \\
\hline Ib SO2/MM BTU & $-\ldots$ & 1.56 & 1.56 & 1.56 & 1.56 & 1.56 & 1.56 & 1.56 & 1.56 & & & & \\
\hline Cost to Boiler & $\$ / M M$ BTU & $\$ 2.44$ & $\$ 2.44$ & $\$ 2.44$ & $\$ 2.44$ & $\$ 2.44$ & $\$ 2.44$ & $\$ 2.44$ & $\$ 2.44$ & & & & \\
\hline Freight Cost & $\$ / M M B T U$ & $\$ 0.89$ & $\$ 0.82$ & $\$ 0.84$ & $\$ 0.90$ & $\$ 0.98$ & $\$ 1.08$ & $\$ 1.25$ & $\$ 0.89$ & & & & \\
\hline Evap. Cost & $\$ / \mathrm{MM} \mathrm{BTU}$ & $\$ 0.02$ & $\$ 0.01$ & $\$ 0.01$ & $\$ 0.02$ & $\$ 0.03$ & $\$ 0.04$ & $\$ 0.07$ & $\$ 0.02$ & & & & \\
\hline Ash Cost & $\$ / M M B T U$ & $\$ 0.11$ & $\$ 0.11$ & $\$ 0.11$ & $\$ 0.11$ & $\$ 0.11$ & $\$ 0.11$ & $\$ 0.11$ & $\$ 0.11$ & & & & \\
\hline SO2 Cost & $\$ / M M B T U$ & $\$ 0.04$ & $\$ 0.04$ & $\$ 0.04$ & $\$ 0.04$ & $\$ 0.04$ & $\$ 0.04$ & $\$ 0.04$ & $\$ 0.04$ & & & & \\
\hline Other Cost & $\$ / M M B T U$ & $\$ 0.04$ & $\$ 0.04$ & $\$ 0.04$ & $\$ 0.04$ & $\$ 0.05$ & $\$ 0.05$ & $\$ 0.06$ & $\$ 0.04$ & & & & \\
\hline Coal Worth & $\$ / \mathrm{MM} \mathrm{BTU}$ & $\$ 1.34$ & $\$ 1.43$ & $\$ 1.40$ & $\$ 1.33$ & $\$ 1.23$ & $\$ 1.12$ & $\$ 0.90$ & $\$ 1.34$ & & & & \\
\hline Cost to Boiler & $\$ /$ ton & $\$ 54.65$ & $\$ 59.83$ & $\$ 57.94$ & $\$ 54.49$ & $\$ 49.79$ & $\$ 45.37$ & $\$ 38.94$ & $\$ 54.65$ & & & & \\
\hline Freight Cost & $\$ /$ ton & $\$ 20.00$ & $\$ 20.00$ & $\$ 20.00$ & $\$ 20.00$ & $\$ 20.00$ & $\$ 20.00$ & $\$ 20.00$ & $\$ 20.00$ & & & & \\
\hline Evap. Cost & $\$ /$ ton & $\$ 0.38$ & $\$ 0.13$ & $\$ 0.22$ & $\$ 0.39$ & $\$ 0.61$ & $\$ 0.83$ & $\$ 1.13$ & $\$ 0.38$ & & & & \\
\hline Ash Cost & $\$ /$ ton & $\$ 2.53$ & $\$ 2.77$ & $\$ 2.68$ & $\$ 2.52$ & $\$ 2.31$ & $\$ 2.10$ & $\$ 1.80$ & $\$ 2.53$ & & & & \\
\hline SO2 Cost & $\$ /$ ton & $\$ 0.80$ & $\$ 0.88$ & $\$ 0.85$ & $\$ 0.80$ & $\$ 0.73$ & $\$ 0.67$ & $\$ 0.57$ & $\$ 0.80$ & & & & \\
\hline Other Cost & $\$ /$ ton & $\$ 1.00$ & $\$ 1.00$ & $\$ 1.00$ & $\$ 1.00$ & $\$ 1.00$ & $\$ 1.00$ & $\$ 1.00$ & $\$ 1.00$ & & & & \\
\hline Coal Worth & $\$ /$ ton & $\$ 29.93$ & $\$ 35.04$ & $\$ 33.18$ & $\$ 29.77$ & $\$ 25.14$ & $\$ 20.78$ & $\$ 14.43$ & $\$ 29.93$ & & & & \\
\hline Individual Tons & dry & 0.00 & 27.04 & 21.95 & 14.63 & 7.53 & 2.74 & 5.58 & 79.48 & & & & \\
\hline Cum. Tons & dry & 79.48 & 79.48 & 52.44 & 30.49 & 15.86 & 8.33 & 5.58 & & & & & \\
\hline Individual Tons & $\mathrm{ar}$ & 0.00 & 28.29 & 23.70 & 16.81 & 9.46 & 3.78 & 8.98 & 91.02 & & & & \\
\hline Cum. Tons & ar & 91.02 & 91.02 & 62.74 & 39.03 & 22.22 & 12.76 & 8.98 & -- & & & & \\
\hline Cum. Mass & $\%$, ar & 100.00 & 100.00 & 68.92 & 42.88 & 24.41 & 14.02 & 9.86 & --- & & & & \\
\hline Cum. Ash & $\%$, ar & 12.66 & 13.86 & 13.66 & 13.41 & 13.18 & 13.06 & 12.66 & --- & & & & \\
\hline Cum. Sulfur & $\%$, ar & 0.87 & 0.96 & 0.94 & 0.92 & 0.91 & 0.90 & 0.87 & -- & & & & \\
\hline Cum. BTU/lb & ar & 11198 & 11729 & 11766 & 11683 & 11576 & 11485 & 11389 & --- & & & & \\
\hline \begin{tabular}{|l} 
Individual Worth \\
\end{tabular} & $\$$ & $\$ 0.00$ & $\$ 991.22$ & $\$ 786.61$ & $\$ 500.41$ & $\$ 237.90$ & $\$ 78.58$ & $\$ 129.52$ & $\$ 2,724.24$ & & & & \\
\hline Cum. Worth & $\$$ & $\$ 0.00$ & $\$ 991.22$ & $\$ 1,777.83$ & $\$ 2,278.24$ & $\$ 2,516.14$ & $\$ 2,594.73$ & $\$ 2,724.24$ & --- & & & & \\
\hline
\end{tabular}


Coal B - Circuit Configuration 1 - Fine Cut - Hyperbaric Centrifuge Simulation

\begin{tabular}{|c|c|c|c|c|c|c|}
\hline \multicolumn{7}{|c|}{ Input Parameters } \\
\hline $\begin{array}{c}k_{d} \\
\left(m^{-1}\right)\end{array}$ & $\begin{array}{c}\rho_{\mathrm{s}} \\
\left(\mathrm{kg} / \mathrm{m}^{3}\right)\end{array}$ & $\underset{\left(\mathrm{kg} / \mathrm{m}^{3}\right)}{\rho_{\mathrm{w}}}$ & $\begin{array}{c}\text { B } \\
(\mathrm{m})\end{array}$ & $\varepsilon$ & $\begin{array}{c}\sigma \\
(\mathrm{N} / \mathrm{m})\end{array}$ & $\begin{array}{c}\mu \\
\text { (Pa.s) }\end{array}$ \\
\hline 400 & 1400 & 1000 & 0.381 & 0.52 & 0.072 & 0.001 \\
\hline $\mathrm{L}$ & ${ }^{t}$ & $\mathrm{~N}$ & $\mathrm{~N}_{\mathrm{g}}$ & Air $P$ & $\mathbf{g}$ & $\theta$ \\
\hline (m) & (sec) & (RPM) & & (psi) & $\left(\mathrm{m} / \mathrm{s}^{2}\right)$ & \\
\hline 0.0127 & 7 & 2650 & 2991 & 0 & 9.81 & 60 \\
\hline
\end{tabular}

\begin{tabular}{|c|c|c|c|c|c|c|}
\hline \multicolumn{7}{|c|}{ Feed Size Distribution } \\
\hline $\begin{array}{c}\text { Top } \\
\text { Size } \\
\text { (micron) }\end{array}$ & $\begin{array}{c}\text { Bottom } \\
\text { Size } \\
\text { (micron) }\end{array}$ & $\begin{array}{c}\text { Mean } \\
\text { Size } \\
\text { (micron) }\end{array}$ & $\begin{array}{l}\mathrm{D} \\
(\%)\end{array}$ & $\begin{array}{l}\text { Weight } \\
\text { in class }\end{array}$ & $\begin{array}{l}\text { Weight\% } \\
\text { in class } \\
\text { (\%) }\end{array}$ & $\begin{array}{l}\text { Cum. } \\
\text { Passing } \\
\text { (\%) }\end{array}$ \\
\hline 1000 & 600 & 774.60 & 30.98 & 0.00 & 0.00 & 100.00 \\
\hline 600 & 300 & 424.26 & 16.97 & 0.00 & 0.00 & 100.00 \\
\hline 300 & 150 & 212.13 & 8.49 & 0.00 & 0.00 & 100.00 \\
\hline 150 & 75 & 106.07 & 4.24 & 0.03 & 0.13 & 100.00 \\
\hline 75 & 44 & 57.45 & 2.30 & 0.38 & 1.85 & 99.87 \\
\hline 44 & 25 & 33.17 & 1.33 & 3.93 & 19.16 & 98.02 \\
\hline 25 & 0 & 5.00 & 0.20 & 16.18 & 78.85 & 78.85 \\
\hline & & & & & & \\
\hline & & & & & & \\
\hline & & & & & & \\
\hline & & & & & & \\
\hline & & & & & & \\
\hline & & & Tot? & 2052 & 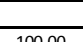 & \\
\hline & & & & & & \\
\hline
\end{tabular}
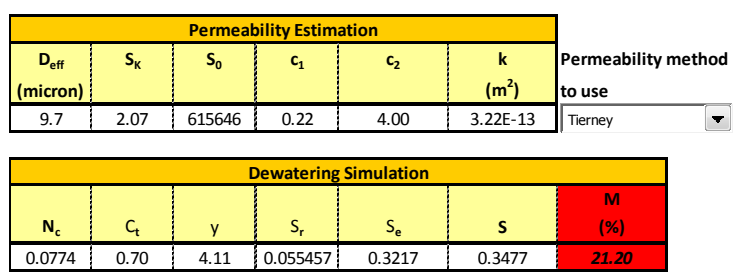

\begin{tabular}{|c|c|c|c|c|c|c|c|}
\hline \multicolumn{8}{|c|}{ Size Distribution Expansion using Gaudin-Schumann Equation } \\
\hline \multicolumn{2}{|c|}{ Size Distribut } & \multirow{3}{*}{$\begin{array}{l}\text { Mean } \\
\text { Size } \\
\text { (micron) }\end{array}$} & \multirow{3}{*}{$\begin{array}{l}D \\
(\%)\end{array}$} & \multirow{3}{*}{$\begin{array}{l}\text { Cum. } \\
\text { Passing } \\
\text { (\%) }\end{array}$} & \multirow{3}{*}{\begin{tabular}{|c|} 
Weight $\%$ \\
in class \\
$(\%)$
\end{tabular}} & \multirow{3}{*}{$\begin{array}{c}\text { Weight } \\
\text { in class } \\
\text { (g) }\end{array}$} & \multirow{3}{*}{ Wt/Size } \\
\hline $\mathrm{m}$ & $\mathrm{k}$ & & & & & & \\
\hline 0.385 . & 46 & & & & & & \\
\hline $1180 \times$ & 600 & 841.4 & 33.66 & 100.00 & 0.00 & 0.00 & $0.00 \mathrm{E}+00$ \\
\hline 600 & 300 & 424.3 & 16.97 & 100.00 & 0.00 & 0.00 & $0.00 E+0$ \\
\hline & 150 & & & & & & \\
\hline 150 & 75 & 106.1 & 4.24 & & & & \\
\hline 75 & 44 & & 2.30 & & & & \\
\hline 44 & 25 & & & & & & \\
\hline 25 & 10 & 15 & & & & & \\
\hline 10 & 5 & 7.1 & 0.2 & & & & \\
\hline 5 & 1 & & 0.09 & & & & \\
\hline $1 \mathrm{x}$ & 0 & 0.5 & 0.00 & & & & \\
\hline & & & & & 100.00 & 20.5 & \\
\hline
\end{tabular}

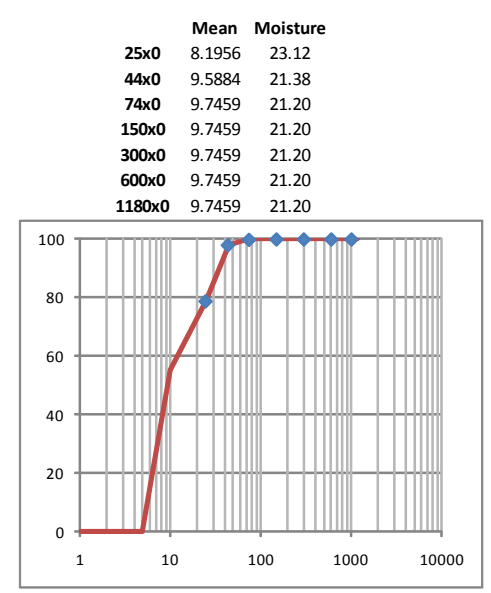

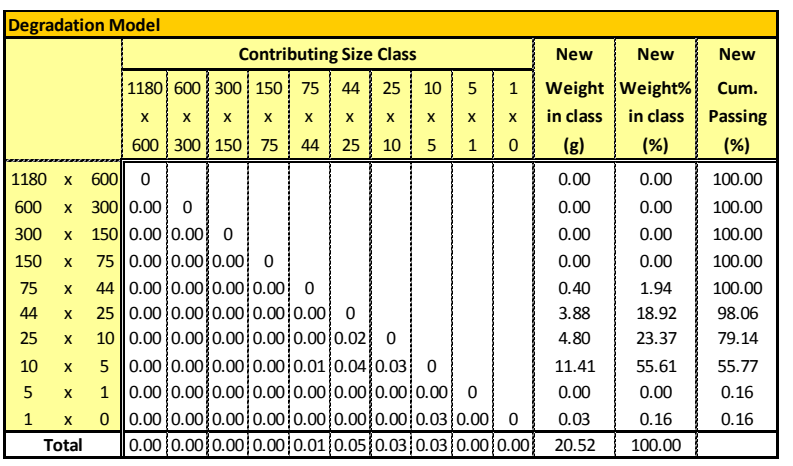

\begin{tabular}{|l|l|l|l|l|l|l|l|l|l|l|l|l|}
\hline 1180 & 600 & 300 & 150 & 75 & 44 & 25 & 10 & 5 & 1 & Weight & Weight\% & Cum. \\
\hline & $x$ & & &
\end{tabular} \begin{tabular}{|l|l|l|l|l|l|l|l|l|l|l|} 
& $x$ & $x$ & $x$ & $x$ & $x$ & $x$ & $x$ & in class & in class & Passin \\
\hline
\end{tabular} \begin{tabular}{c|c|c|}
\hline (g) & $(\%)$ & $(\%)$ \\
\hline 0.00 & 0.00 & 100.00 \\
\hline & 0.00 & 10000 \\
\hline
\end{tabular}

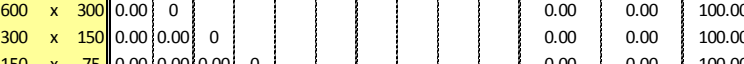

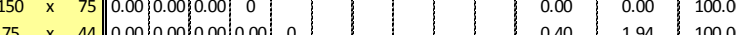

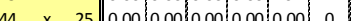
$25 \times 10 \quad 0.00$

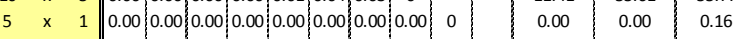

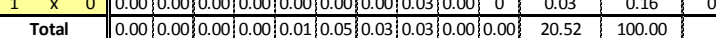


Coal B - Circuit Configuration 1 - Fine Cut - Hyperbaric Centrifuge Economics

CALCULATION OF OPTIMUM SIZE CUT

\begin{tabular}{|c|c|c|c|c|c|c|c|c|c|c|c|c|c|}
\hline \multirow{2}{*}{ FINANCIAL DATA: } & & \multicolumn{2}{|c|}{ Total Boiler Cost $=$} & $\$ 2.44$ & /MMBTU & & Moisture & ap. = & $\$ 3.00$ & /ton $\mathrm{H}_{2} \mathrm{O}$ & $\mathrm{SO}_{2}$ Emission $=$ & $\$ 200$ & $/$ ton $\mathrm{SO}_{2}$ \\
\hline & & \multicolumn{2}{|c|}{ Freight Cost $=$} & $\$ 20.00$ & /ton coal & & \multicolumn{2}{|c|}{ Ash Handling = } & $\$ 20.00$ & /ton ash & Other Cost $=$ & $\$ 1.00$ & /ton coal \\
\hline Size Class & & 1 & 2 & 3 & 4 & 5 & 6 & 7 & Totals & & & & \\
\hline Top Size & $\mu$ & 1180 & 600 & 300 & 150 & 74 & 44 & 25 & --- & & & & \\
\hline Bottom Size & $\mu$ & 600 & 300 & 150 & 74 & 44 & 25 & 0 & $-\cdots$ & & & & \\
\hline$S G$ & $\mu$ & 1.45 & 1.45 & 1.45 & 1.45 & 1.45 & 1.45 & 1.45 & --- & & & & \\
\hline $1 / \mathrm{SG}$ & --- & 0.69 & 0.69 & 0.69 & 0.69 & 0.69 & 0.69 & 0.69 & --- & & & & \\
\hline Mass & $\%$, dry & 0.00 & 0.00 & 0.00 & 0.00 & 1.94 & 18.92 & 79.14 & 100.00 & & & & \\
\hline Solid SG & & 1.40 & 1.40 & 1.40 & 1.40 & 1.40 & 1.40 & 1.40 & & & & & \\
\hline Ash & $\%$, dry & 14.50 & 14.50 & 14.50 & 14.50 & 14.50 & 14.50 & 14.50 & 14.50 & & & & \\
\hline Sulfur & $\%$, dry & 1.00 & 1.00 & 1.00 & 1.00 & 1.00 & 1.00 & 1.00 & 1.00 & & & & \\
\hline BTU/Ib & dry & 12825 & 12825 & 12825 & 12825 & 12825 & 12825 & 12825 & 12825 & & & & \\
\hline BTU/lb & maf & 15000 & 15000 & 15000 & 15000 & 15000 & 15000 & 15000 & 15000 & & & & \\
\hline Mass & $\%$, ar & 0.00 & 0.00 & 0.00 & 0.00 & 1.72 & 17.16 & 81.12 & 100.00 & & & & \\
\hline Moisture & $\%$, ar & 21.20 & 21.20 & 21.20 & 21.20 & 10.96 & 13.14 & 23.12 & 21.20 & & & & \\
\hline Cum. Moisture & $\%$, ar & 21.20 & 21.20 & 21.20 & 21.20 & 21.20 & 21.38 & 23.12 & --- & & & & \\
\hline Ash & $\%$, ar & 11.42 & 11.42 & 11.42 & 11.42 & 12.91 & 12.59 & 11.15 & 11.42 & & & & \\
\hline Inerts & $\%$, ar & 32.62 & 32.62 & 32.62 & 32.62 & 23.87 & 25.73 & 34.27 & 32.62 & & & & \\
\hline Sulfur & $\%$, ar & 0.79 & 0.79 & 0.79 & 0.79 & 0.89 & 0.87 & 0.77 & 0.79 & & & & \\
\hline BTU/Ib & ar & 10106 & 10106 & 10106 & 10106 & 11420 & 11141 & 9860 & 10106 & & & & \\
\hline Ib SO2/MM BTU & $-\ldots$ & 1.56 & 1.56 & 1.56 & 1.56 & 1.56 & 1.56 & 1.56 & 1.56 & & & & \\
\hline Cost to Boiler & $\$ / M M$ BTU & $\$ 2.44$ & $\$ 2.44$ & $\$ 2.44$ & $\$ 2.44$ & $\$ 2.44$ & $\$ 2.44$ & $\$ 2.44$ & $\$ 2.44$ & & & & \\
\hline Freight Cost & $\$ / M M B T U$ & $\$ 0.99$ & $\$ 0.99$ & $\$ 0.99$ & $\$ 0.99$ & $\$ 0.88$ & $\$ 0.90$ & $\$ 1.01$ & $\$ 0.99$ & & & & \\
\hline Evap. Cost & $\$ / \mathrm{MM} \mathrm{BTU}$ & $\$ 0.03$ & $\$ 0.03$ & $\$ 0.03$ & $\$ 0.03$ & $\$ 0.01$ & $\$ 0.02$ & $\$ 0.04$ & $\$ 0.03$ & & & & \\
\hline Ash Cost & $\$ / M M B T U$ & $\$ 0.11$ & $\$ 0.11$ & $\$ 0.11$ & $\$ 0.11$ & $\$ 0.11$ & $\$ 0.11$ & $\$ 0.11$ & $\$ 0.11$ & & & & \\
\hline SO2 Cost & $\$ / M M B T U$ & $\$ 0.04$ & $\$ 0.04$ & $\$ 0.04$ & $\$ 0.04$ & $\$ 0.04$ & $\$ 0.04$ & $\$ 0.04$ & $\$ 0.04$ & & & & \\
\hline Other Cost & $\$ / M M B T U$ & $\$ 0.05$ & $\$ 0.05$ & $\$ 0.05$ & $\$ 0.05$ & $\$ 0.04$ & $\$ 0.04$ & $\$ 0.05$ & $\$ 0.05$ & & & & \\
\hline Coal Worth & $\$ / \mathrm{MM} \mathrm{BTU}$ & $\$ 1.22$ & $\$ 1.22$ & $\$ 1.22$ & $\$ 1.22$ & $\$ 1.36$ & $\$ 1.33$ & $\$ 1.19$ & $\$ 1.22$ & & & & \\
\hline Cost to Boiler & $\$ /$ ton & $\$ 49.32$ & $\$ 49.32$ & $\$ 49.32$ & $\$ 49.32$ & $\$ 55.73$ & $\$ 54.37$ & $\$ 48.12$ & $\$ 49.32$ & & & & \\
\hline Freight Cost & $\$ /$ ton & $\$ 20.00$ & $\$ 20.00$ & $\$ 20.00$ & $\$ 20.00$ & $\$ 20.00$ & $\$ 20.00$ & $\$ 20.00$ & $\$ 20.00$ & & & & \\
\hline Evap. Cost & $\$ /$ ton & $\$ 0.64$ & $\$ 0.64$ & $\$ 0.64$ & $\$ 0.64$ & $\$ 0.33$ & $\$ 0.39$ & $\$ 0.69$ & $\$ 0.64$ & & & & \\
\hline Ash Cost & $\$ /$ ton & $\$ 2.28$ & $\$ 2.28$ & $\$ 2.28$ & $\$ 2.28$ & $\$ 2.58$ & $\$ 2.52$ & $\$ 2.23$ & $\$ 2.28$ & & & & \\
\hline SO2 Cost & $\$ /$ ton & $\$ 0.73$ & $\$ 0.73$ & $\$ 0.73$ & $\$ 0.73$ & $\$ 0.82$ & $\$ 0.80$ & $\$ 0.71$ & $\$ 0.73$ & & & & \\
\hline Other Cost & $\$ /$ ton & $\$ 1.00$ & $\$ 1.00$ & $\$ 1.00$ & $\$ 1.00$ & $\$ 1.00$ & $\$ 1.00$ & $\$ 1.00$ & $\$ 1.00$ & & & & \\
\hline Coal Worth & $\$ /$ ton & $\$ 24.67$ & $\$ 24.67$ & $\$ 24.67$ & $\$ 24.67$ & $\$ 31.00$ & $\$ 29.65$ & $\$ 23.48$ & $\$ 24.67$ & & & & \\
\hline Individual Tons & dry & 0.00 & 0.00 & 0.00 & 0.00 & 0.40 & 3.88 & 16.24 & 20.52 & & & & \\
\hline Cum. Tons & dry & 20.52 & 20.52 & 20.52 & 20.52 & 20.52 & 20.13 & 16.24 & & & & & \\
\hline Individual Tons & $\mathrm{ar}$ & 0.00 & 0.00 & 0.00 & 0.00 & 0.45 & 4.47 & 21.13 & 26.05 & & & & \\
\hline Cum. Tons & $\mathrm{ar}$ & 26.05 & 26.05 & 26.05 & 26.05 & 26.05 & 25.60 & 21.13 & -- & & & & \\
\hline Cum. Mass & $\%$, ar & 100.00 & 100.00 & 100.00 & 100.00 & 100.00 & 98.28 & 81.12 & --- & & & & \\
\hline Cum. Ash & $\%$, ar & 11.42 & 11.42 & 11.42 & 11.42 & 12.91 & 12.62 & 11.42 & --- & & & & \\
\hline Cum. Sulfur & $\%$, ar & 0.79 & 0.79 & 0.79 & 0.79 & 0.89 & 0.87 & 0.79 & -- & & & & \\
\hline Cum. BTU/lb & ar & 10106 & 10106 & 10106 & 10106 & 10369 & 10496 & 10420 & --- & & & & \\
\hline \begin{tabular}{|l|} 
Individual Worth \\
\end{tabular} & $\$$ & $\$ 0.00$ & $\$ 0.00$ & $\$ 0.00$ & $\$ 0.00$ & $\$ 13.86$ & $\$ 132.56$ & $\$ 496.19$ & $\$ 642.60$ & & & & \\
\hline Cum. Worth & $\$$ & $\$ 0.00$ & $\$ 0.00$ & $\$ 0.00$ & $\$ 0.00$ & $\$ 13.86$ & $\$ 146.41$ & $\$ 642.60$ & +--- & & & & \\
\hline
\end{tabular}


Coal B - Circuit Configuration 1 - Summary

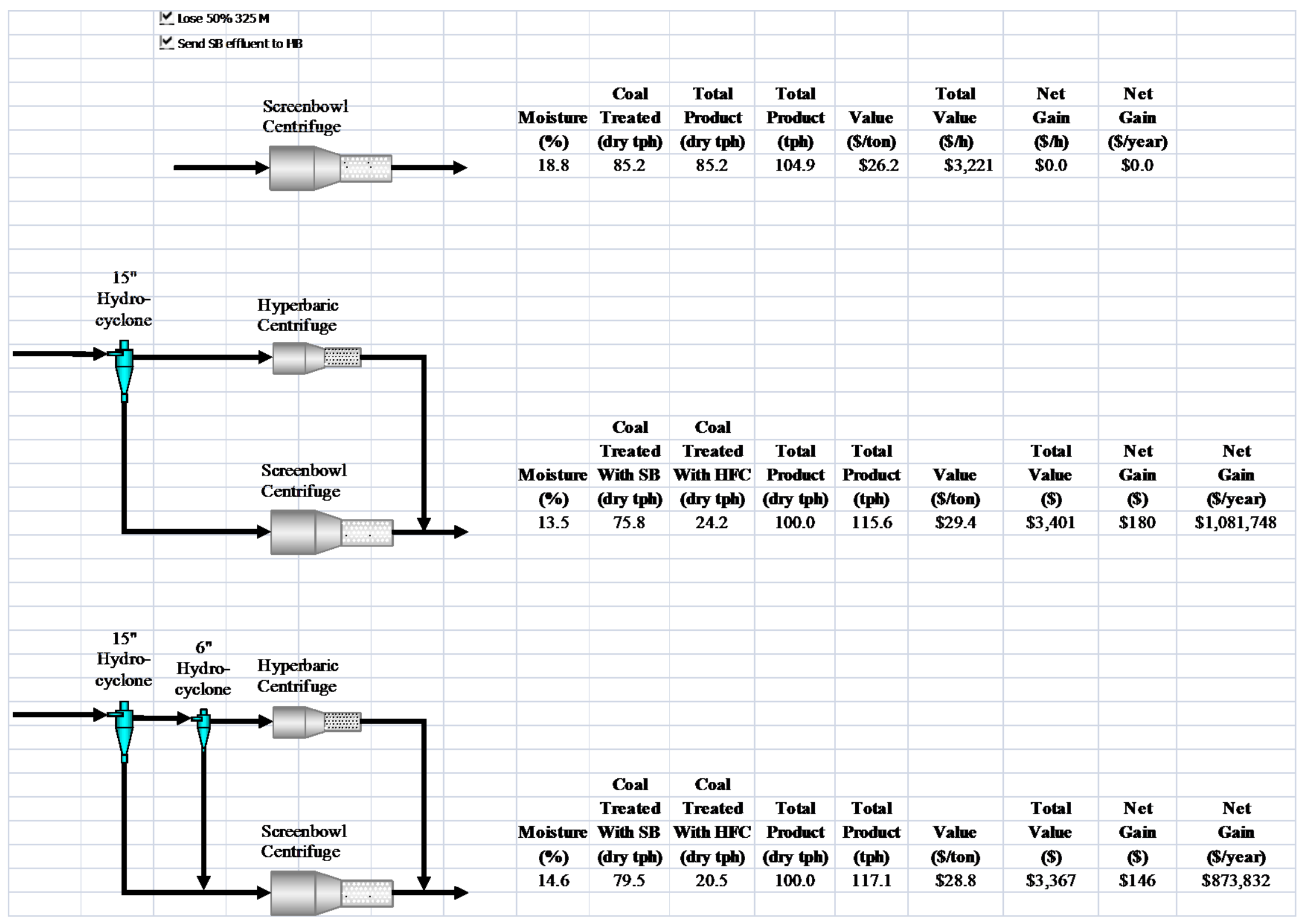


Appendix 18: Development and Testing of a Horizantal Belt Filter (VA010) 


\section{TECHNICAL REPORT}

Contract Title and Number:

Crosscutting Technology Development at the Center for

Advanced Separation Technologies

(DE-FC26-02NT41607)

Sub-Recipient Project Title:

Development and Testing of a Horizontal

Pressure Belt Filter

Principal Investigators:

J. A. Salomon, G.H. Luttrell and R.-H. Yoon

Contact Address:

100 Holden Hall

Virginia Tech

Blacksburg, Virginia 24061

Subcontractor Address:

No subcontracts issued.
Period of Performance:

Starting Date: 10/1/02

Ending Date: 10/31/2009
Report Information:

Type: Final

Number: VA010

Period:

Date: $\quad$ 6/30/2010

Code: VA010-FINAL

Contact Information:

Phone: 540-231-6314

Fax: $\quad$ 540-231-4070

E-Mail: luttrell@vt.edu

Subcontractor Information:

Phone:

Fax:

E-Mail: 


\section{DISCLAIMER}

“This report was prepared as an account of work sponsored by an agency of the United States Government. Neither the United States Government nor any agency thereof, nor any of their employees, make any warranty, express or implied, nor assume any legal liability or responsibility for the accuracy, completeness, or usefulness of any information, apparatus, product, or process disclosed, or represents that its use would not infringe privately owned rights. Reference herein to any specific commercial product, process, or service by trade name, trademark, manufacturer, or otherwise does not necessarily constitute or imply endorsement, recommendation, or favoring by the United States Government or any agency thereof. The views and opinions of authors expressed herein do not necessarily state or reflect those of the United States Government or agency thereof." 


\begin{abstract}
This objective of this project was to develop a new dewatering device that could produce a lower moisture content and better fine particle recovery than current technology. To meet this goal, a hyperbaric horizontal belt filter was designed and constructed over the course of 18 months. Once built, the filter was then thoroughly tested to determine operational capabilities. The test data showed that the lowest moisture content that could be achieved with a coarse feed (minus $1 \mathrm{~mm}$ screen-bowl centrifuge feed) was $8.8 \%$. This value could be further reduced to 8.2\% and capacity increased with the use of dewatering aids. When testing with a fine feed (minus $0.15 \mathrm{~mm}$ column product feed), the lowest moisture content was 35\% without chemicals and $29 \%$ with chemicals. A 50/50 mixture by volume of coarse and fine feeds was artificially created and provided a moisture of $10.8 \%$, which was reduced using reagents to $8.4 \%$. The machine provided a very high recovery rate for all feed materials. Of the coal input, no less than $94 \%$ of it reported to the dry product. The pressure used to dewater the coal was the controlling factor for the air consumption of the unit. The data from these tests suggest that a full size production unit is feasible, although the power requirements for gas compression would be high.
\end{abstract}




\section{TABLE OF CONTENTS}

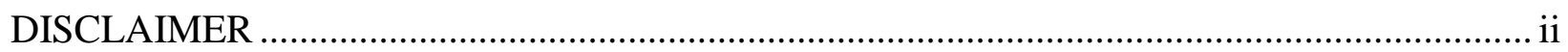

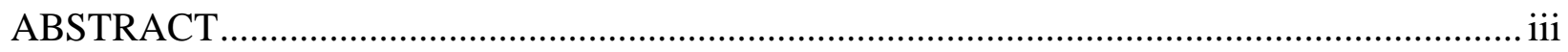

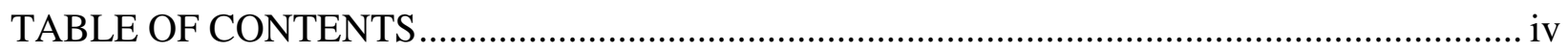

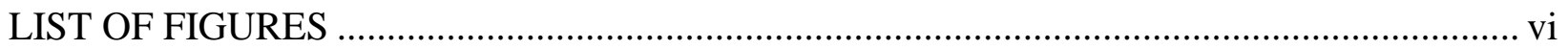

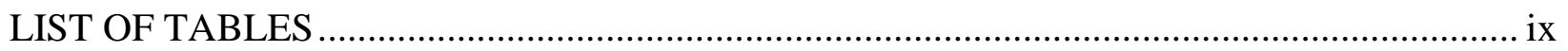

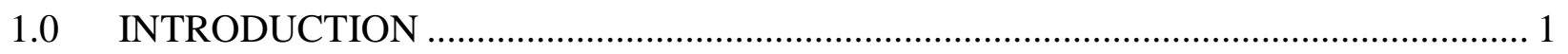

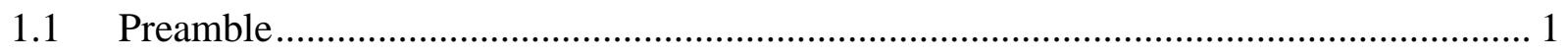

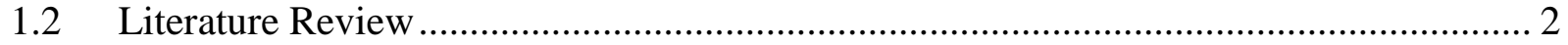

1.2.1 Definition of Filtration ............................................................................... 2

1.2.2 Filtration Methods....................................................................................... 2

1.2.3 Filtration Theory ………………………………................................................. 3

1.2.4 Cake Filtration Using Displacement ...................................................................... 5

1.2.5 Filtration Equipment ………………………................................................ 5

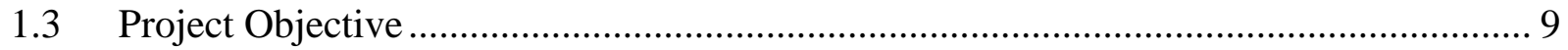

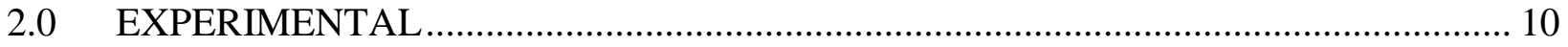

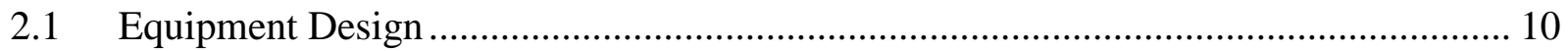

2.1.1 General Design.................................................................................................. 10

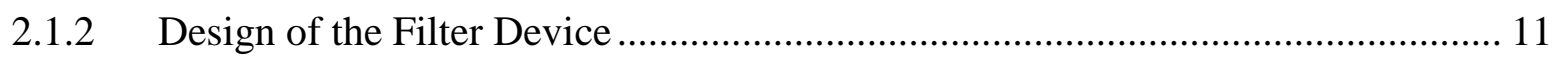

2.1.3 Design of the Pressure Vessel.............................................................................. 19

2.2 Equipment Construction and Assembly ……………………........................................ 27

2.2.1 Filter Device Construction .............................................................................. 27

2.2.2 Pressure Vessel Construction.......................................................................... 28

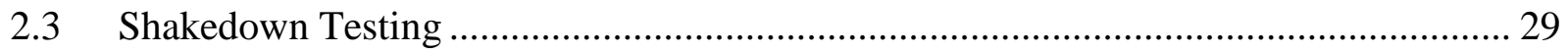

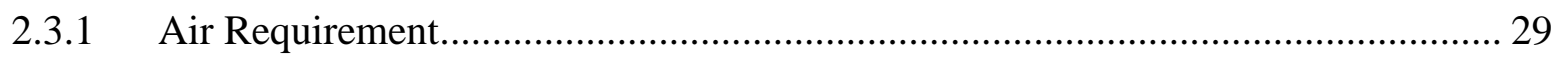

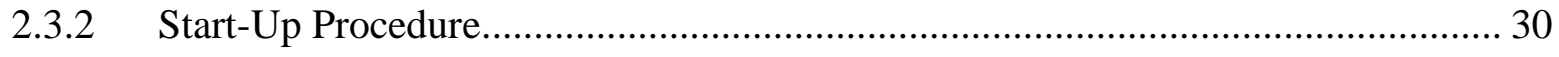

2.4 Detailed Testing ……………………………………............................................... 30

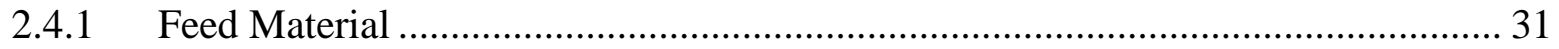

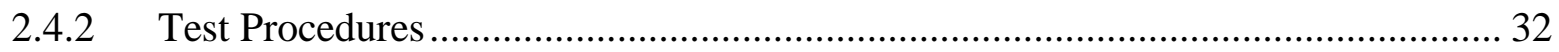




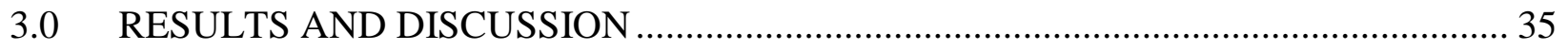

3.1 Laboratory-Scale Batch Testing................................................................................. 35

3.2 Coarse Feed Testing ............................................................................................ 38

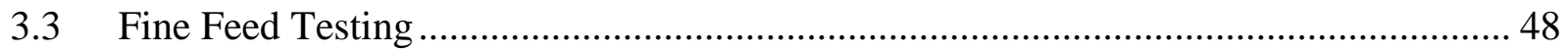

3.4 Mixed Feed Testing.................................................................................................... 55

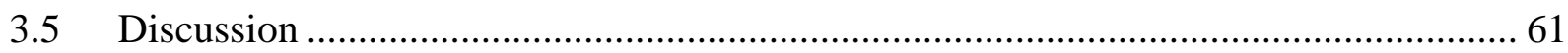

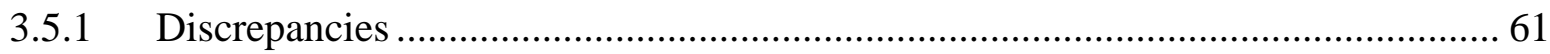

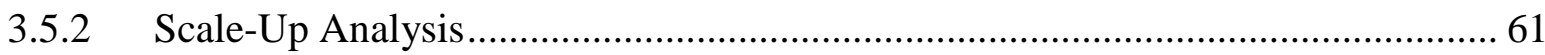

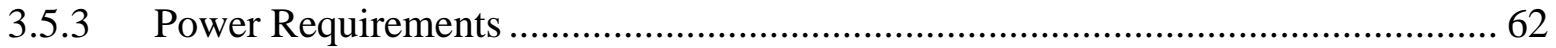

3.5.4 Suggested Design Modifications for a Production Unit ............................................ 63

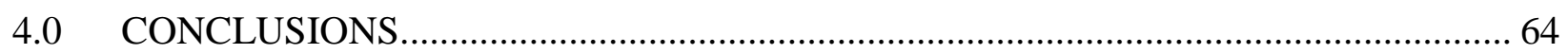

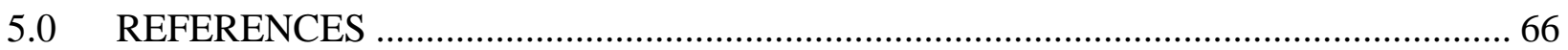

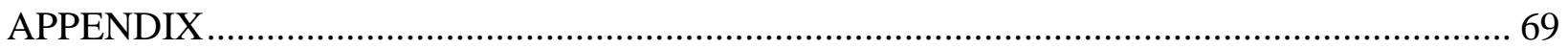

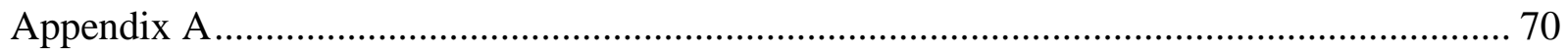

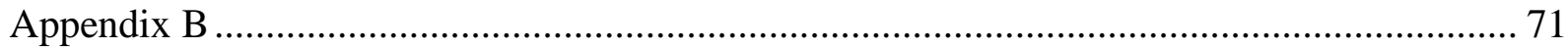

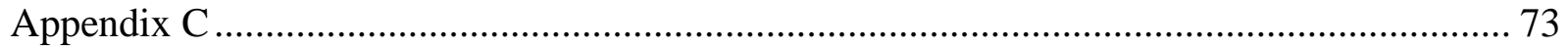

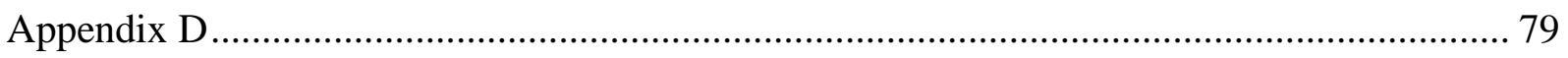

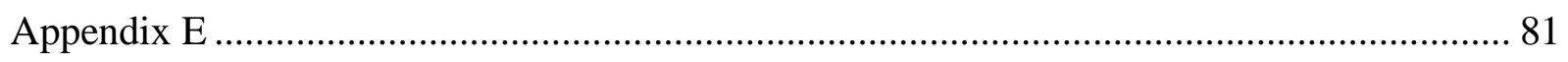




\section{LIST OF FIGURES}

Figure 1.1. Schematic of a vacuum disk filter (Ruthven 1997).......................................... 6

Figure 1.2. Schematic of a drum filter (Ruthven 1997).......................................................... 7

Figure 1.3. Schematic of a vacuum horizontal belt filter (Ruthven 1997). ............................... 8

Figure 2.1. Pressure differential across a horizontal belt filter................................................ 11

Figure 2.2. Design of the filter device frame................................................................... 12

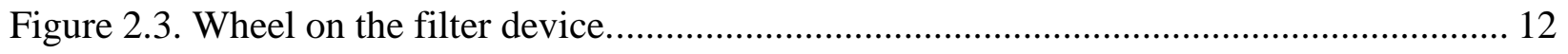

Figure 2.4, A-frame attachment for the filter device. .......................................................... 13

Figure 2.5. Tensioning roller and water spray on the filtering device................................... 14

Figure 2.6. Trough apparatus used to hold slurry on the filter cloth. ...................................... 15

Figure 2.7. Design used for the pressure plate............................................................... 16

Figure 2.8. Plastic strips used to seal the underside of the belt. ........................................... 17

Figure 2.9. Bolts attaching frame to the pressure plate..................................................... 17

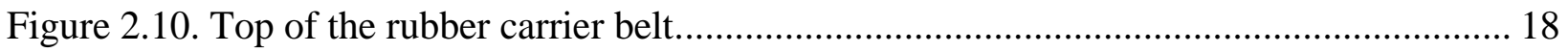

Figure 2.11. Bottom of the rubber carrier belt. ...................................................................... 18

Figure 2.12. Pillow block bearing for the primary drive. .................................................. 19

Figure 2.13. Scrapers used to remove fines from the filter cloth............................................ 19

Figure 2.14. Pipe used to construct the pressure chamber (as received). ................................ 20

Figure 2.15. Steel used to construct the main frame......................................................... 21

Figure 2.16. Schematic of the primary support frame. ........................................................ 21

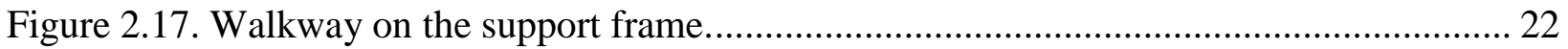

Figure 2.18. Flange attached to the ends of the pressure vessel. ......................................... 23

Figure 2.19. Progressive cavity pump used to feed the pressure filter. .................................... 24

Figure 2.20. Knife gate value used for the air-lock pressure seal........................................... 25 
Figure 2.21. Twin values used for the airlock system (as installed)...................................... 26

Figure 2.22. Rails and support system for the belt filter mechanism. ................................... 27

Figure 2.23. Completely assembled belt filter mechanism............................................... 28

Figure 2.24. Completely assembled pressure vessel....................................................... 29

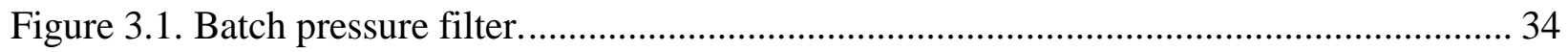

Figure 3.2. Batch test data for $20 \mathrm{ml}$ of coarse feed (60 PSIG)........................................... 36

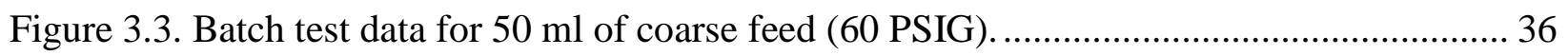

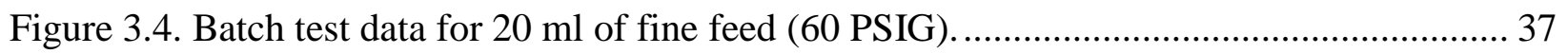

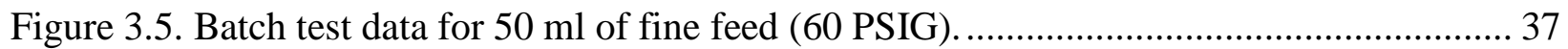

Figure 3.6. Variability of the coarse data (30\% feed pump speed) ......................................... 39

Figure 3.7. Response surface with coarse feed and no chemicals. ...................................... 41

Figure 3.8. Response surface with coarse feed and 3 lb/ton reagent RV (1:10)...................... 43

Figure 3.9. Cake thickness vs. air flow rate for coarse feed.............................................. 45

Figure 3.10. Pressure vs. air flow rate for coarse feed..................................................... 46

Figure 3.11. Pressure vs. air flow rate for coarse feed (base vs. reagent RV)........................ 46

Figure 3.12. Material input vs. losses for coarse feed. .................................................. 47

Figure 3.13. Mass rate vs. recovery for coarse feed. ........................................................... 48

Figure 3.14. Variability of the fine feed data (25\% feed pump speed)................................... 49

Figure 3.15. Response surface for fine feed and no chemicals.......................................... 50

Figure 3.16. Response surface of fine feed with $3 \mathrm{lb} /$ ton reagent RV (1:10). ........................... 51

Figure 3.17. Cake thickness vs. air flow rate for fine feed.................................................. 52

Figure 3.18. Pressure vs. air flow rate for fine feed (base vs. reagent RV)............................. 53

Figure 3.19. Pressure vs. air flow rate for coarse and fine feeds with no chemicals................... 54

Figure 3.20. Pressure vs. air flow rate for coarse and fine feed with reagent RV................... 54 


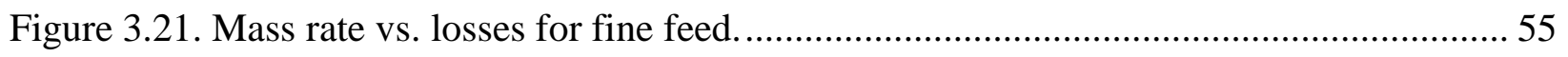

Figure 3.22. Mass flow rate vs. recovery for fine feed. ..................................................... 55

Figure 3.23. Variability of the mixed feed data (30\% feed pump speed)................................. 56

Figure 3.24. Response surface for mixed without chemicals. ............................................. 57

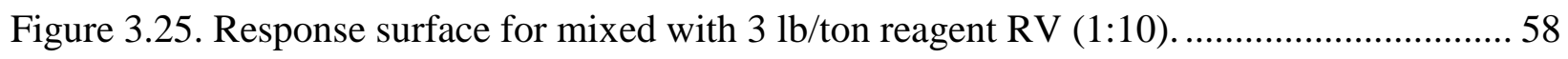

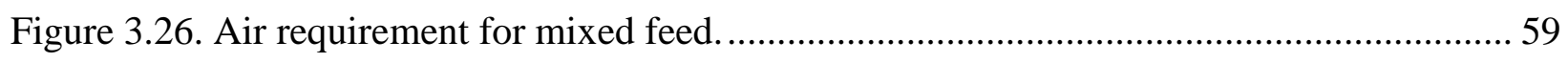

Figure 3.27. Base air flow requirements for mixed feed. .................................................... 59

Figure 3.28. Air flow comparisons for chemically treated feeds.......................................... 60

Figure 3.29. Mass rate vs. recovery for mixed feed.......................................................... 60 


\section{LIST OF TABLES}

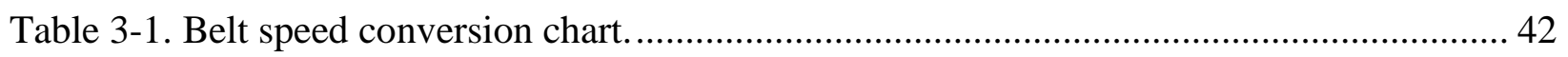

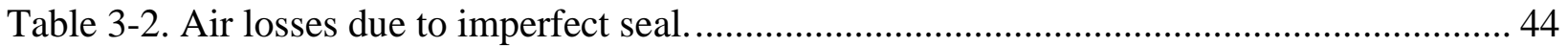




\subsection{INTRODUCTION}

\subsection{Preamble}

Coal is a major energy resource in both the United States and worldwide. The extraction of this resource from the earth requires methods that often mix impurities that do not contain heating value in with the valuable coal. Removal of this excess material requires that the coal be washed in a preparation plant to separate the clean coal from the refuse material. Most known methods for accomplishing this for the fine particle size range (less than $1 \mathrm{~mm}$ ) are wet processes. Water, when mixed with coal, also reduces its BTU (British Thermal Unit) value, thus creating a need to remove it from the final product.

Traditionally, coal producers have handled fine waste slurries in two different ways. The first is to pump the slurry to an impoundment pond where the valuable coal is wasted and creates an environmental hazard. This is done because it becomes increasingly difficult and costly to dewater coal as particle size decreases. The other option is to install thermal dryers. Thermal dryers, however, have high capital and operating costs. It is also difficult to get a permit to install thermal dryers due to air pollution problems.

Thus, there is a need for a third option. An attractive alternative would involve the use of a filtration unit that greatly reduces the cost and improves the effectiveness of fine coal dewatering. This cost is reduced because the energy input needed to evaporate water is high compared to the cost of mechanically removing water. The use of displacement filters combined with surface tension reducing reagents can create a high volume filtration unit that would allow for the economical recovery of these fine coal slurries. Unfortunately, a piece of equipment that takes advantage of all of these factors in a cost effective way does not currently exist. 
The impact of such a unit, if developed, should not be underestimated. It would help reduce the size of hazardous impoundments, reduce the air pollution currently caused by thermal drying, and increase the available energy supply in the world by creating more usable BTUs with less energy input.

\subsection{Literature Review}

\subsubsection{Definition of Filtration}

Filtration is the separation of two forms of matter by passing one of them through a porous medium. This study is limited to the principles relating to the separation between solid particles and liquids. In addition, the primary liquid that is being separated in this case is water, allowing the distinction to be made that this is a dewatering process.

\subsubsection{Filtration Methods}

Dewatering is primarily concerned with removing water that is bound in the capillaries between solid particles. This can be achieved using three basic known methods. The first is to compact the slurry by adding stress to the perimeter of the slurry with a roller, pistons, screw presses, or other mechanical means. The second method is to displace the water using a gas that is applied through either vacuum or pressure. The third method can be achieved by adding an

electrical field to a slurry of charged particles. Each of the methods has its advantages and limitations.

Dewatering that uses mechanical compression is limited to the compressibility of the filter cake. Once the cake has been compressed to the point of virtual incompressibility, no further dewatering is possible since the water has been fully compacted into the capillaries and pores. 
In dewatering by gas displacement, the important factors are the pressure difference across the filter cake and the kinetic dewatering characteristics. This process results in an exchange between water volume and gas volume in the filter cake.

Dewatering using electrical fields creates charged ions to generate the pressure required to force the water out of the capillaries. This process is called electro-osmosis. The primary difference in this method of dewatering is that rather than the driving force being generated from outside the capillaries, it is formed from the electrical forces within the capillaries.

\subsubsection{Filtration Theory}

Scientists and engineers have been working to develop the fundamental theory behind filtration for over 150 years. Despite this large time frame and much attention, the theory regarding filtration is not complete. Most of the work that has been done is in regard to cake formation rates. The ability to predict the moisture content of a cake is beyond the current knowledge of filtration theory. Most work that has been done on this topic is empirical in nature.

The filtration theory that is known is an extension of fluid mechanics. The rate of filtration is directly proportional to the pressure drop across the cake and inversely proportional to the resistance. It is known that the pore sizes in the filter cake are small and the liquid's velocity through the cake is also slow, the filtrate flow maybe considered laminar. Since these assumptions can be made Poiseuille's law is applicable. From this it can be seen that the variable rate of filtration may be expressed as:

$$
u=\frac{d V}{A d t}
$$

where $\quad \mathrm{V}=$ volume of filtrate

$$
A=\text { area of filtration }
$$




$$
\begin{aligned}
& \mathrm{t}=\text { time } \\
& \mathrm{u}=\text { filtration rate }
\end{aligned}
$$

By making the laminar flow assumption and by applying Poiseuille's Law the basic filtration equation, it is possible to show that:

$$
\frac{d V}{A d t}=\frac{\Delta P}{\frac{\mu(a w V+r)}{A}}
$$

where $\Delta \mathrm{P}=$ pressure drop across the filter (including the cloth and drainage system)

$$
\begin{aligned}
& \mu=\text { liquid viscosity } \\
& \mathrm{a}=\text { specific cake resistance } \\
& \mathrm{w}=\text { weight of the dry cake solids per unit volume of filtrate } \\
& \mathrm{r}=\text { resistance of the filter cloth and drainage system }
\end{aligned}
$$

Rearranging Equation (2) yields,

$$
\mu a w V \frac{d V}{A^{2}}+r \frac{d V}{A}=\Delta P d t
$$

It can then be assumed for a thick cake that $\mathrm{r} \approx 0$ since the resistance of the cake is much greater than that of the filter cloth and drainage system. This expression can be integrated between the limits of 0 and $t_{f}$ (where $t_{f}$ is the cake formation time per cycle) and 0 and $V_{f}$ where $V_{f}$ is volume of filtrate in time $t_{f}$. From these limits, it is possible to show that:

$$
\mu a w \int_{0}^{V_{f}} \frac{V d V}{A^{2}}=\Delta P \int_{0}^{t_{f}} d t
$$

The result of the integration gives:

$$
\frac{\mu a w}{2 A^{2}} V_{f}^{2}=\Delta P t_{f}
$$


When both sides are divided by $\mathrm{t}_{\mathrm{f}}{ }^{2}$, rearranged, and square root taken, it can be shown that:

$$
\frac{V_{f}}{A t_{f}}=\sqrt{\frac{2 \Delta P}{\mu a w t_{f}}}
$$

The final step is to get the equation in the form $Z_{s}$. $Z_{s}$ is measured in weight of dry solids per unit area per unit time of cake formation. If $\mathrm{t}_{\mathrm{f}}$ is the time involved in the cake formation, this variation of the equation is realized by multiplying Equation (6) by w, i.e.:

$$
Z_{s}=\frac{w V_{f}}{A t_{f}}=\sqrt{\frac{2 w \Delta P}{\mu a t_{f}}}
$$

This expression shows that the cake production rate is proportional to the square root of the pressure and inversely proportional to the square root of time.

\subsubsection{Cake Filtration Using Displacement}

The most critical aspect of any filtration process is the source of the driving force. The importance of this force is shown in Equation (2), the fundamental relationship for filtration. In that equation, $\Delta \mathrm{P}$ is a critical term and this pressure difference is often referred to as the driving force. In all of the equipment that was studied and analyzed, the driving force is provided by air pressure applied to the filter cake. The filtration theory states that the higher the $\Delta \mathrm{P}$ the greater will be the cake formation rate. Other empirical evidence also shows that the higher the $\Delta \mathrm{P}$ the lower will be the final cake retained moisture (Cheremisinoff, 1995).

\subsubsection{Filtration Equipment}

There are several types of equipment that are commercially available for fine particle dewatering. The most prolific of these are disc filters, drum filters, and belt filters. All of these units are currently used in a vacuum mode and some are used in a pressure mode. Each of these 
units has some advantages over the others. There are other novel pieces of dewatering equipment that are made in various places, but these three are the most prevalent.

a) Disc Filters

Disc filters are used in both a vacuum mode and a pressure mode. Figure 1.1 shows a diagram of a vacuum operated disc filter. Pressurized disc filters work on the same principal only the disks are contained within a pressure vessel. Disc filters use a multitude of rotary discs that are vertically mounted on a horizontal shaft, which are suspended in a slurry reservoir. This has the advantage of having greater floor space utilization than other filters. Each disc is divided into separate sectors often ranging from 12 to 30 per disc. The disc must be mostly submerged because cake formation occurs while the sector is still submerged in the slurry.

Disc filters do have some major disadvantages due to the vertical nature of the disks this

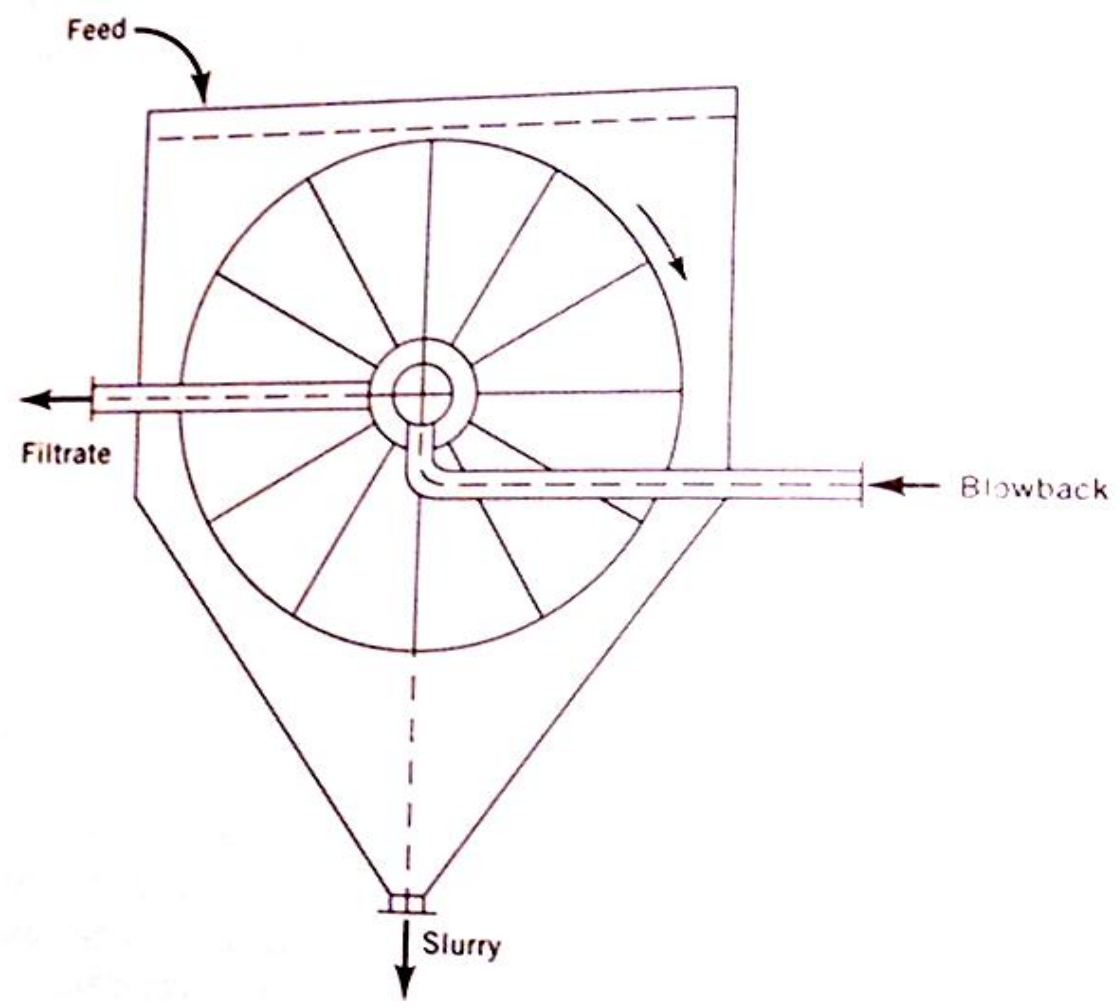

Figure 1.1. Schematic of a vacuum disk filter (Ruthven 1997). 
limits the thickness of the cake since too thick of a cake will not stick to the filter. This limits the capacity for the filters. Also, it is difficult to wash the filter media thus plugging can be a major problem. Since the cake is submerged through much of the rotating cycle of the filter it can result in too high moisture content due to too short of dewatering time.

\section{b) Drum Filters}

Drum filters are the most commonly used air displacement filter. This is a device that has a large drum that rotates slowly about the horizontal axis. The drum is similar to the disc filter in that part of the drum is submerged in the slurry. The drum has multiple sections, each containing a separate vacuum chamber. A diagram of the basic design of a drum filter is shown on Figure 1.2. This filter like the disc filters can be enclosed in a pressure vessel and operated under pressure. The disadvantage of the drum filter is the relatively poor use of space. The entire

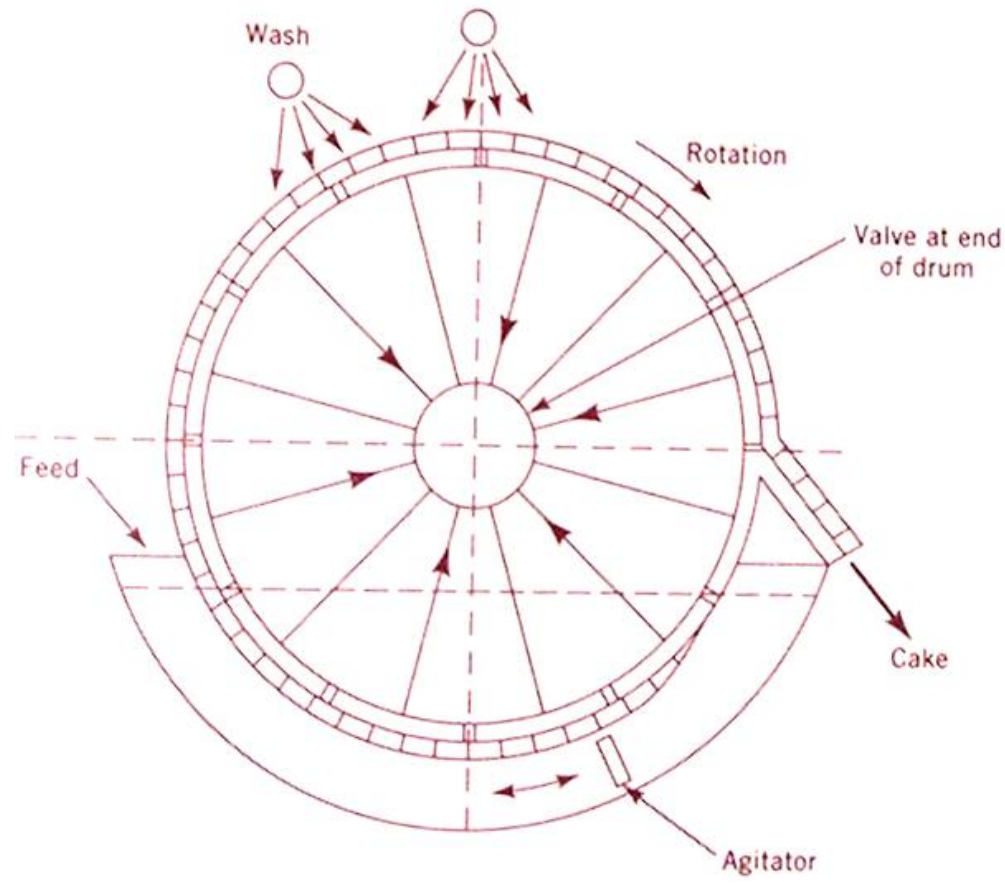

Figure 1.2. Schematic of a drum filter (Ruthven 1997). 
volume of the drum contributes little to the dewatering process and yet consumes much space. Otherwise, the operating principal of the drum filter is similar to the disc filter.

\section{c) Belt Filters}

Horizontal belt filters are the third prevalent type of displacement filtration equipment. The operation of the horizontal belt filter is quite different from that of the disc filter or drum filter. A diagram of the filter's basic operating principal is shown on Figure 1.3. In this unit, a filter belt is continuously rolled across two rollers much like a conveyor belt. The slurry is feed onto one end and then slowly turned across the length of the belt. While traversing the length of the belt, a vacuum is applied to the underside of the belt. This type of unit has an advantage of lower capitol costs than the other filters and it has a high area available for dewatering. Also, the speed and feed rate of the belt can be easily controlled. The primary disadvantage is that the unit has a poor dewatering rate per square footage of floor space. These units are not made in a pressurized version at this time.

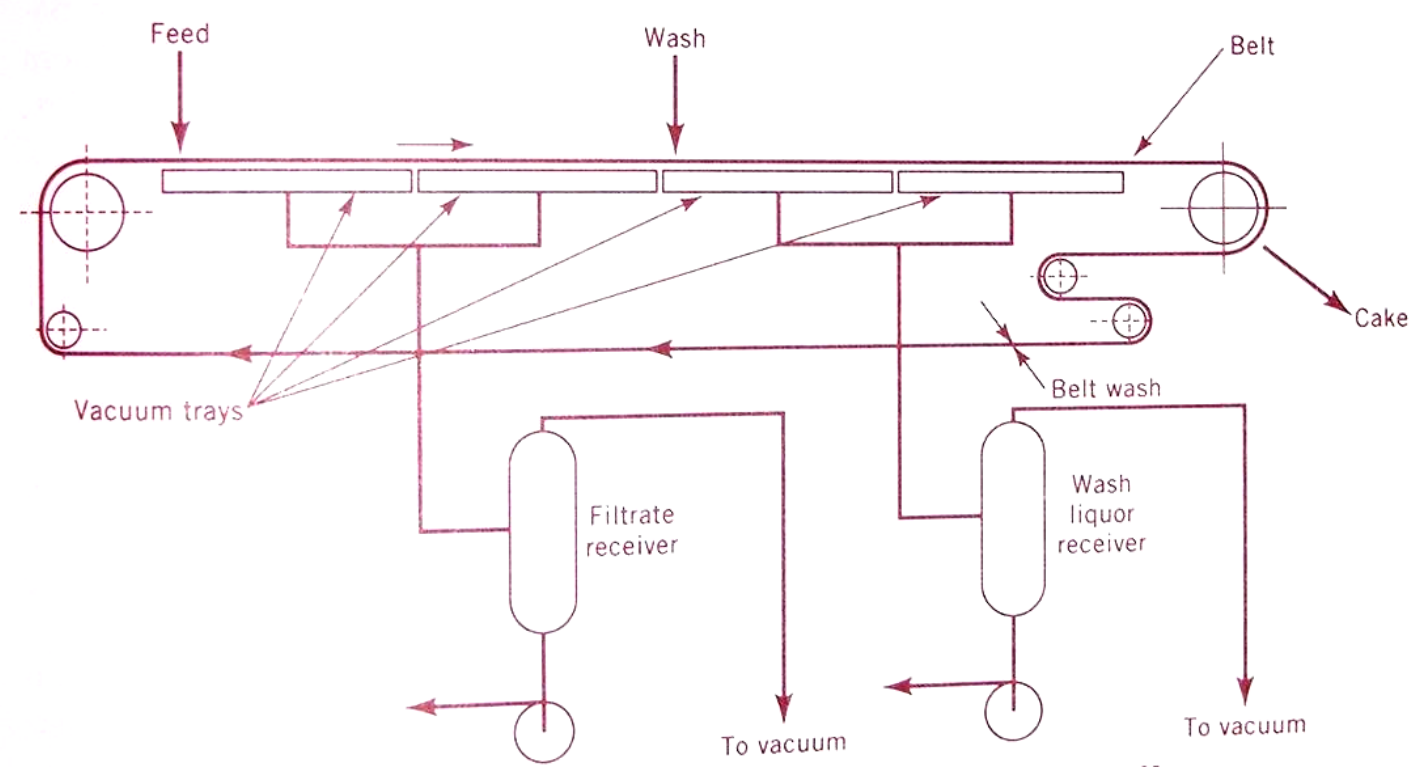

Figure 1.3. Schematic of a vacuum horizontal belt filter (Ruthven 1997). 


\subsection{Project Objective}

A variety of mechanical processes are available for dewatering fine particles in the coal and mineral processing industries. Most of these units have major problems associated with them. All of the units currently available suffer from one or more of a number of difficulties, such as poor dewatering performance, low throughput capacity, high capital costs, and high operating costs. Primarily, the vacuum units suffer from the poor performance and low capacity, while the pressurized units suffer from high capital and operating costs.

The first aspect of this research was to develop a new type of dewatering process that combines the operational flexibility of a continuous horizontal belt filter with the dewatering efficiency of a batch pressure filter. As such, the project involved the design, construction, testing, and evaluation of a pilot-scale prototype unit. The prototype hyperbaric belt filter can be used as a model to assist in the design of a full-size commercial unit. The project demonstrated that the unit is physically feasible and provided critical operating information such as throughput capacity, moisture capabilities, power requirements, etc. 


\subsection{EXPERIMENTAL}

\subsection{Equipment Design}

\subsubsection{General Design}

The first step in the design process was to determine what type of filter was going to be developed to dewater coal. It was determined, that currently, a pressurized belt filter does not exist. This unit has unique advantages over other types of pressure filters. The primary advantage is that it has the potential to have high capacity since the length of the belt can be increased and yet run at a relatively high velocity. Once the decision was made to design a hyperbaric filter, the specific details of the design needed to be worked out. One of the objectives was to make a low cost unit. To this end, the basic components were considered. The lab tests showed that for the unit to physically do what was necessary, the top of the belt would need to be exposed to positive pressure while the bottom of the belt would need be open to atmospheric pressure. This pressure difference across the belt is the key item to a pressure filter. All of the parts of the unit are designed to allow this to occur. Figure 2.1 shows what a pressure difference across the belt looks like.

The most feasible approach to achieve a large pressure differential across a moving belt was one that had the entire filter device enclosed in a pressure vessel. That would create the large positive pressure on the top of the belt. To support the pressure differential across the belt, a rigid pressure plate was devised that was capable of handling a large force load. The other major requirement that this project set out to overcome is that the pressure filter had to be a continuously operating unit. This is something that virtually all of the existing pressure filters are not. Most of them are batch processes that simulate continuous operation by having multiple units operating side by side. This creates numerous difficulties by presenting a situation where 


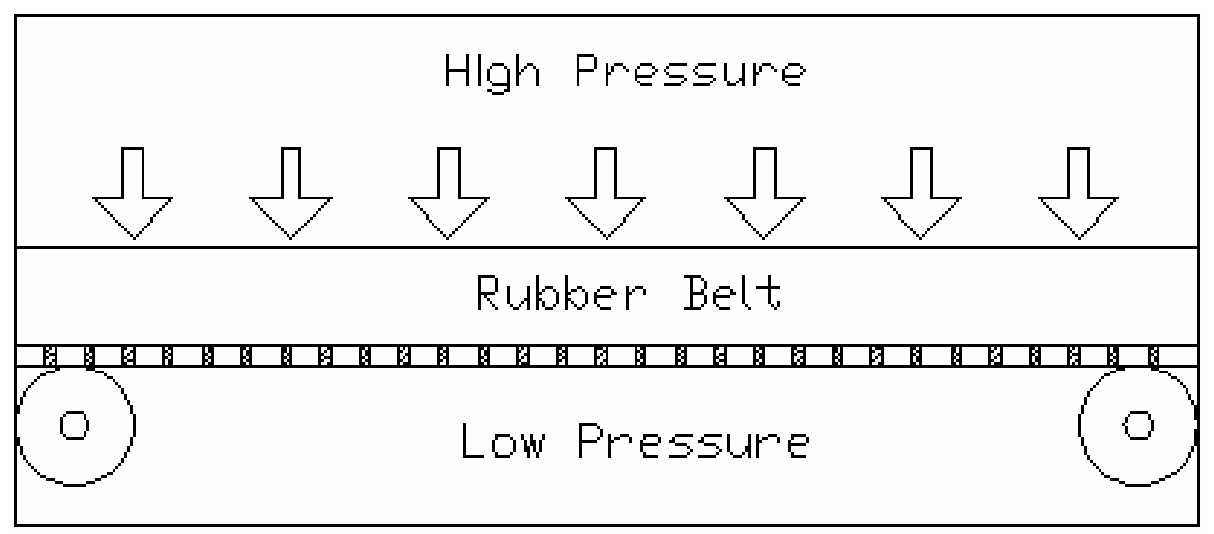

Figure 2.1. Pressure differential across a horizontal belt filter.

material has to both enter and leave a pressurized chamber while maintaining a pressurized environment.

From the general concept of having a belt filter contained in a pressure vessel, it is clear that the project should be separated into two major designs, the filter device and the pressure vessel. Each of the parts of the filter device is discussed in detail in the following sections.

\subsubsection{Design of the Filter Device}

a) Frame

The purpose of the frame is to support all of the other components that are required to make the unit operate. This was designed to be as inexpensive as possible while still minimizing weight and still having the strength characteristics that would be required. The frame was constructed out of carbon steel tubing. Figure 2.2 provides a scale technical drawing of the filter device frame. Additionally, the frame had to be conducive to material handling for maintenance purposes. For this purpose, hooks were added to allow the unit to be handled using an overhead crane. The unit also has wheels that can roll along a track to allow easy access to the unit inside the pressure filter. Figure 2.3 shows the track wheel mounted to the filter device frame. 


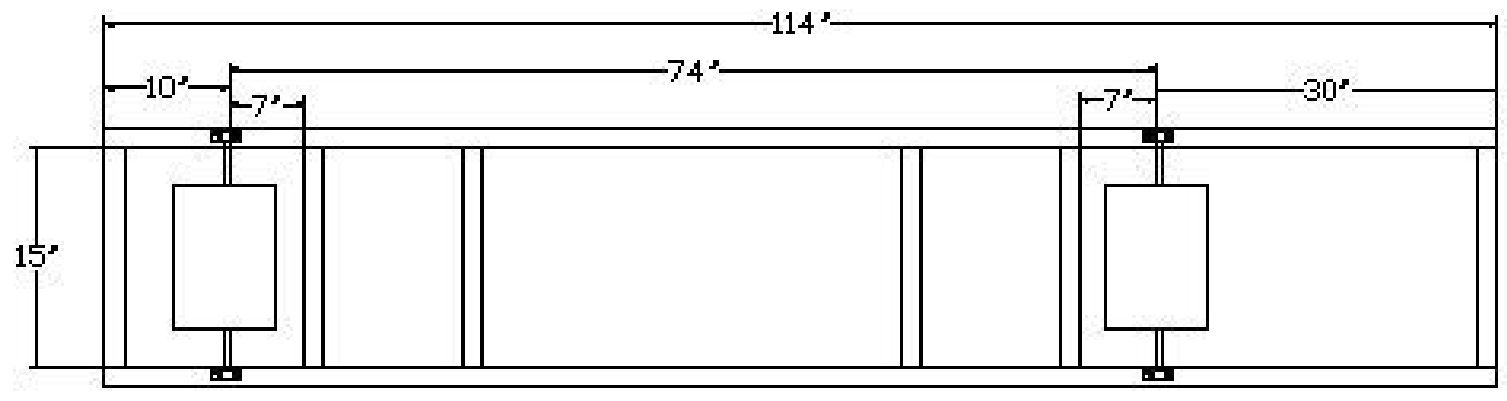

Figure 2.2. Design of the filter device frame.

To assist in the handling of the unit an a-frame attachment was made to fit into the end of the support structure to assist in removing the filter from the pressure vessel. Figure 2.4 is a picture of the A-frame structure inserted into the end of the filter device to support the structure when removing it from the pressure vessel.

\section{b) Filter Cloth}

The filter cloth is the medium that allows the water to pass while holding most of the solids on the top of the belt. The filter cloth that is being used on the unit has openings of 80 microns. Thus, any material that is larger than 80 microns is retained on the filter belt as is a large percentage of finer material. The pressure differential across this medium provides the driving force that allows for the filtration to occur.

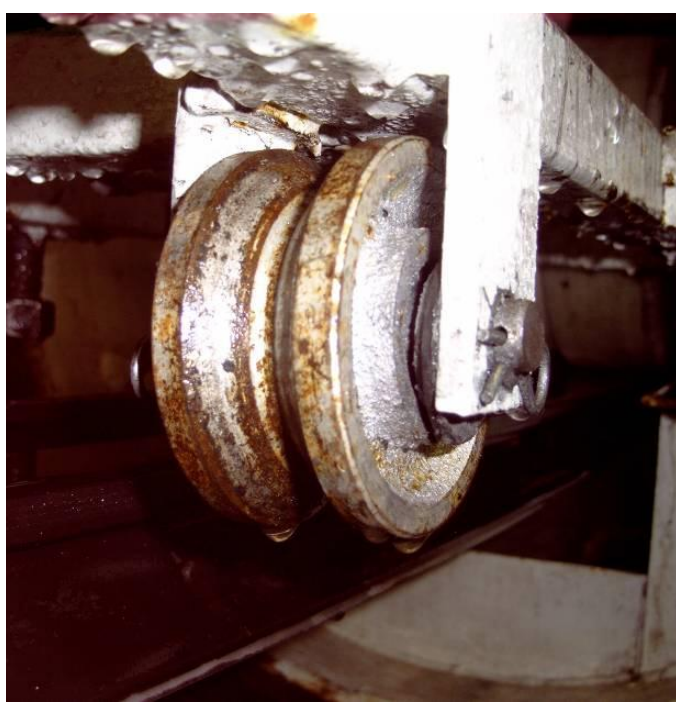

Figure 2.3. Wheel on the filter device. 


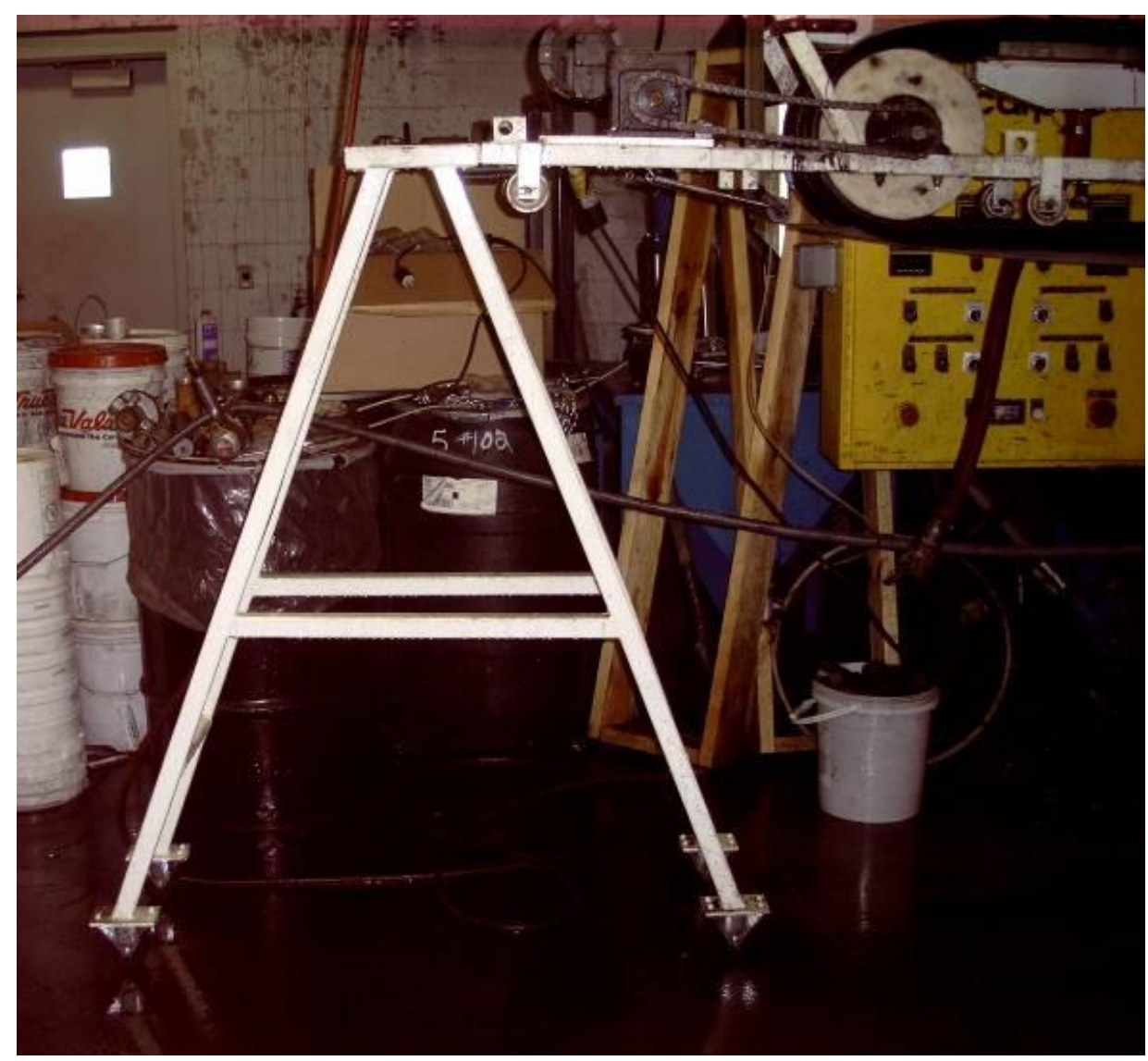

Figure 2.4, A-frame attachment for the filter device.

One of the major concerns of the filter cloth is that over time the cloth would become blinded (plugged) with near size particles. This would cause a situation where water would not be allowed to pass through the cloth and no more filtration would be possible. To prevent this, a high pressure water spray was installed to force water through the back side of the filter cloth to push out any trapped particles. The water spray is located just before the fresh feed is put on the belt to ensure that each run of slurry has a clean cloth to filter through.

Another design concern that had to be addressed in regard to the filter cloth is the tensioning of the filter cloth. This tensioning had to be done separate of the rubber belt so that the water spray would be able to spray the back side of the belt to effectively remove any trapped 


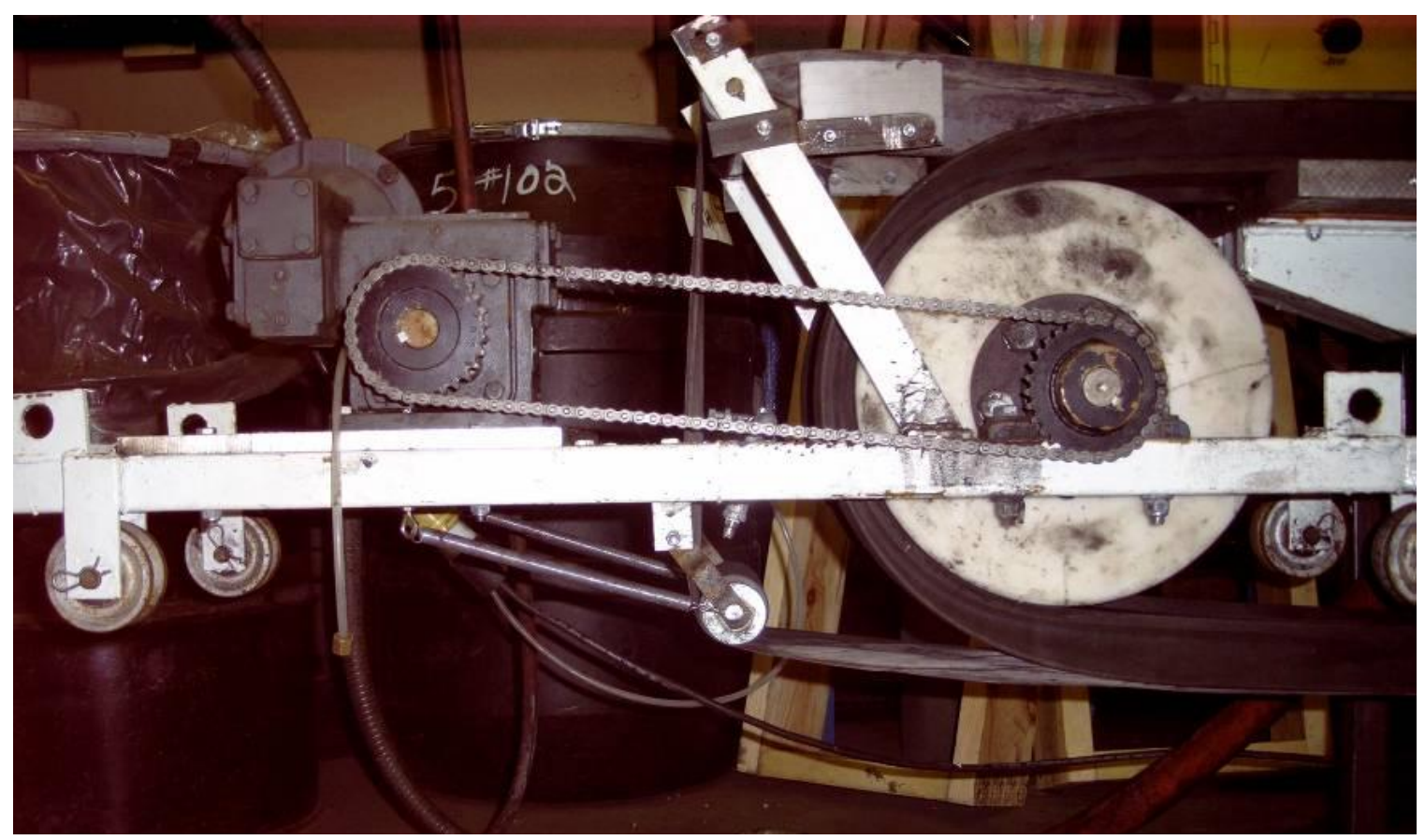

Figure 2.5. Tensioning roller and water spray on the filtering device.

particles. This tensioning is done just behind the drive roller. The roller in the center of Figure 2.5 is the tensioning roller.

The tension is provided by a pair of springs that pull an idler roller back to create the tension on the filter cloth. The filter cloth is in contact with the rubber belt during its entire travel around the non-drive primary roller and the length of the dewatering stage as well as the trip to the drive roller. This means that to tension the filter cloth and allow washing, the amount of distance the filter cloth is apart from the rubber belt is minimized.

To keep the slurry from running off the feed end of the filter, a trough was built to provide a reservoir of slurry to ensure continuous feed to the dewatering section of the belt. This was accomplished using two Teflon guiders for the filter cloth and a roller. A picture of this apparatus is shown in Figure 2.6. 


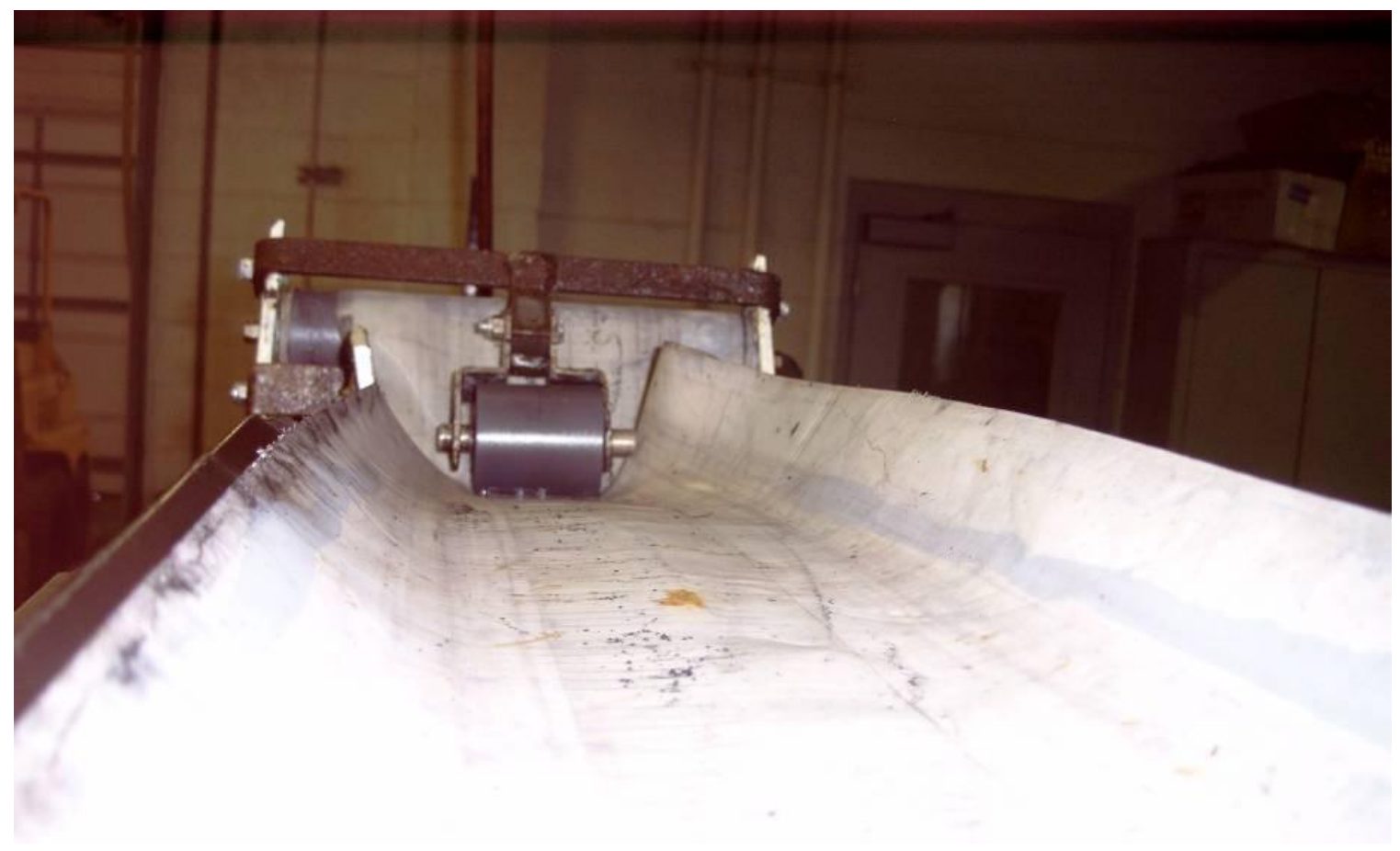

Figure 2.6. Trough apparatus used to hold slurry on the filter cloth.

\section{c) Pressure Plate}

The pressure plate is the part of the filter device that creates the seal necessary to separate the high pressure region from the low (atmospheric) pressure region. The pressure plate consists of an aluminum plate, which has had some machine work done to it, along with a steel box that collects the water. Figure 2.7 shows an isometric view of the pressure plate.

The plate has a slot machined down the center that allows the notch in the rubber belt to fit inside of it. Also, there are holes drilled in a slightly lower slot to allow the water and air to pass through both the pressure plate and the rubber belt. At the ends of the pressure plate, two $3 / 4$ inch rubber strips are mounted. The rubber strips also have a slot machined in them to help seal the ends of the belt and pressure plate as the belt travels across the plate. Also, mounted to the pressure plate are two V-shaped plastic strips that help the length of the belt to seal. Commercial 


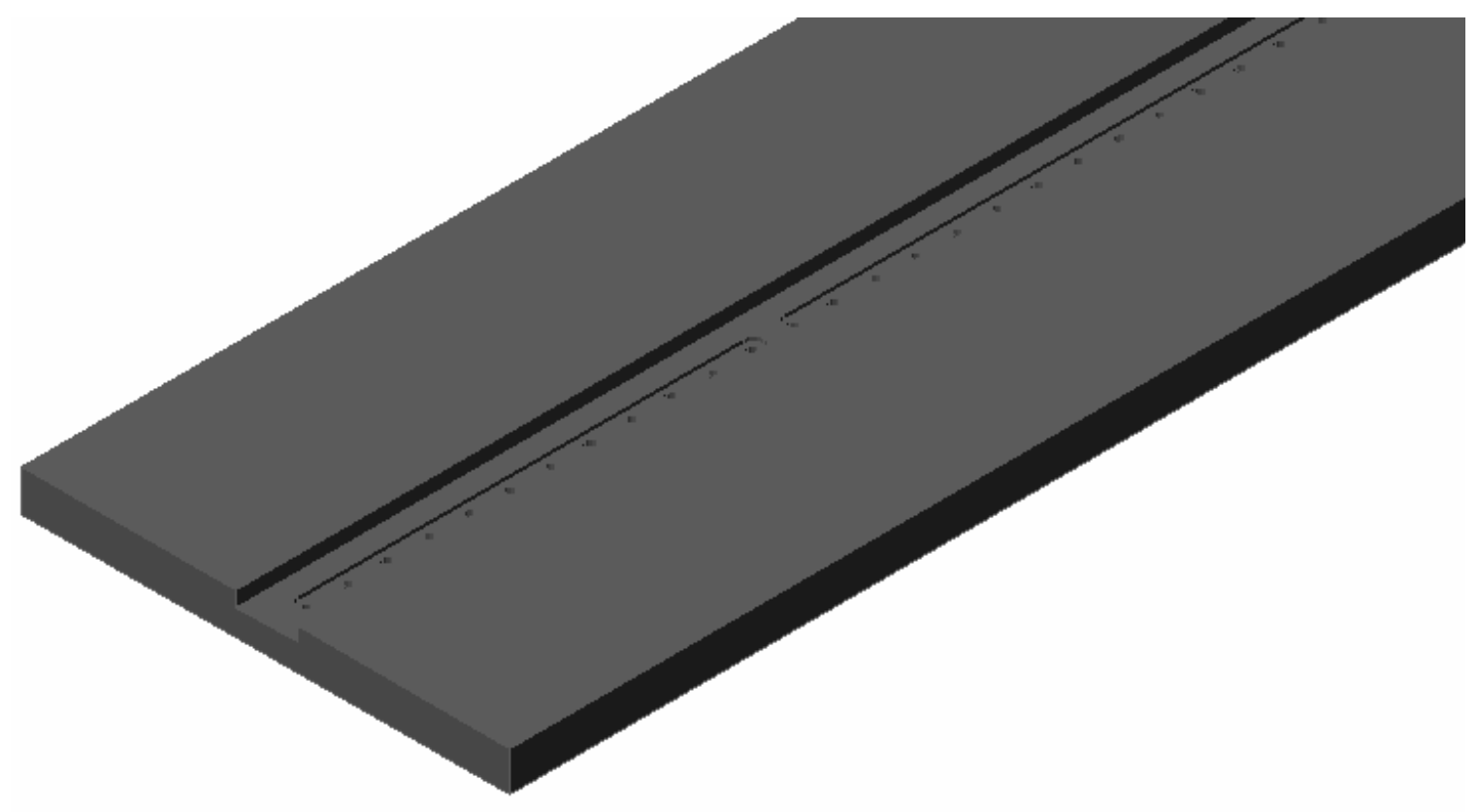

Figure 2.7. Design used for the pressure plate.

weather stripping attached with silicone was found to be ideally suited for sealing the underside of the belt (see Figure 2.8).

On the bottom of the pressure plate is the discharge box that collects the air and water in a central location and sends it to a drain pipe to leave the pressure vessel. The pressure plate is attached to the frame by four one-inch diameter bolts that have a ten-inch adjustment on them. This allows the pressure plate to be pushed against the belt with varying degrees of force. Figure 2.9 shows how the bolts are screwed through four steel blocks that are welded to the frame.

\section{d) Belt and Drive System}

One of the key aspects of the filtration device is the rubber belt that carries the filter cloth over the pressure plate. The rubber belt provides a material with the strength properties needed to take the filter cloth across the pressure plate. The rubber belt supplies the drive to overcome the force that the pressure differential creates to hold the filter cloth in place. The belt has a notch in the center that contains holes for the air and water to pass through. Also, the belt has slots across 


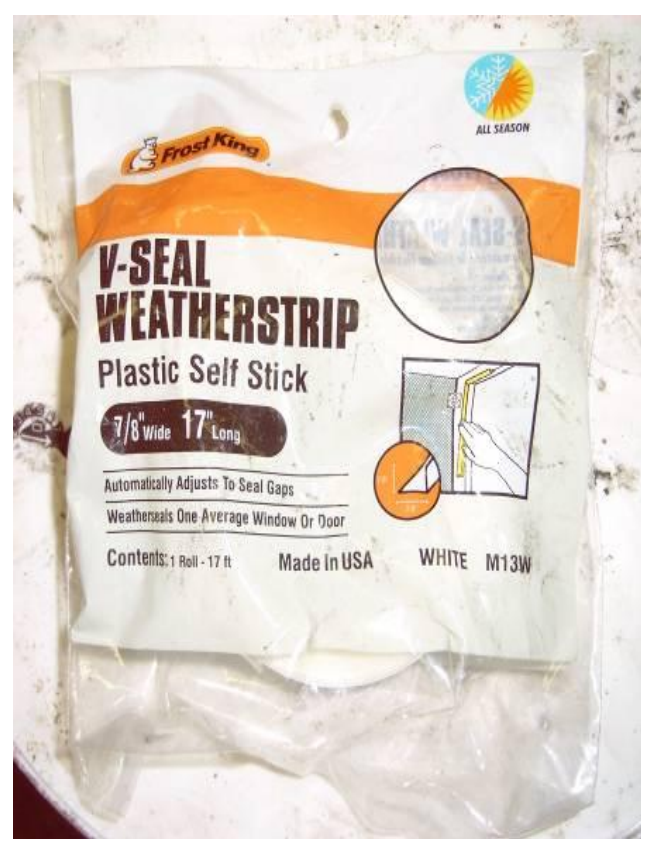

Figure 2.8. Plastic strips used to seal the underside of the belt.

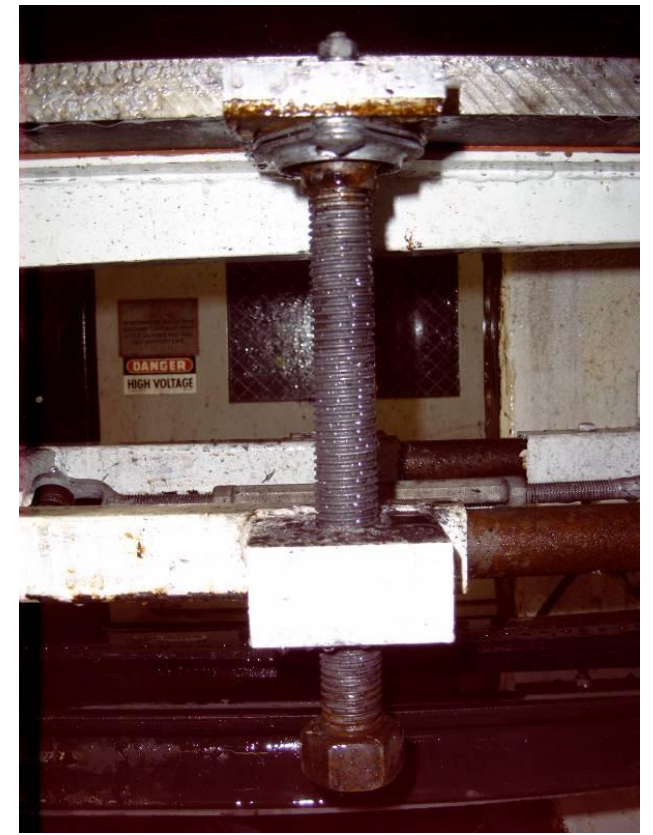

Figure 2.9. Bolts attaching frame to the pressure plate.

it that allow for the pressure to be applied across the entire width of the belt. These attributes are shown in Figures 2.10 and 2.11. The belt has two raised angular rubber pieces on each side to prevent the slurry and cake from falling off the sides of the belt.

The rubber belt is mounted on two nylon rollers. The drive roller is on the same side as the feed is put on the belt. This roller is chain driven by a DC electric motor that has been speed reduced to increase the torque needed to pull the belt across the pressure plate. Both of the nylon rollers are mounted on the frame by two pillow block ball bearings. A diagram of one of the pillow block bearings used to support the two nylon rollers is shown in Figure 2.12.

\section{e) Filter Cloth Scraper}

After studying a lab batch unit that was developed at Virginia Tech, it was determined that to remove the filter cake from the filter cloth after all of the dewatering has been completed would require some sort of scraper. The best location for the placement of the scraper was 


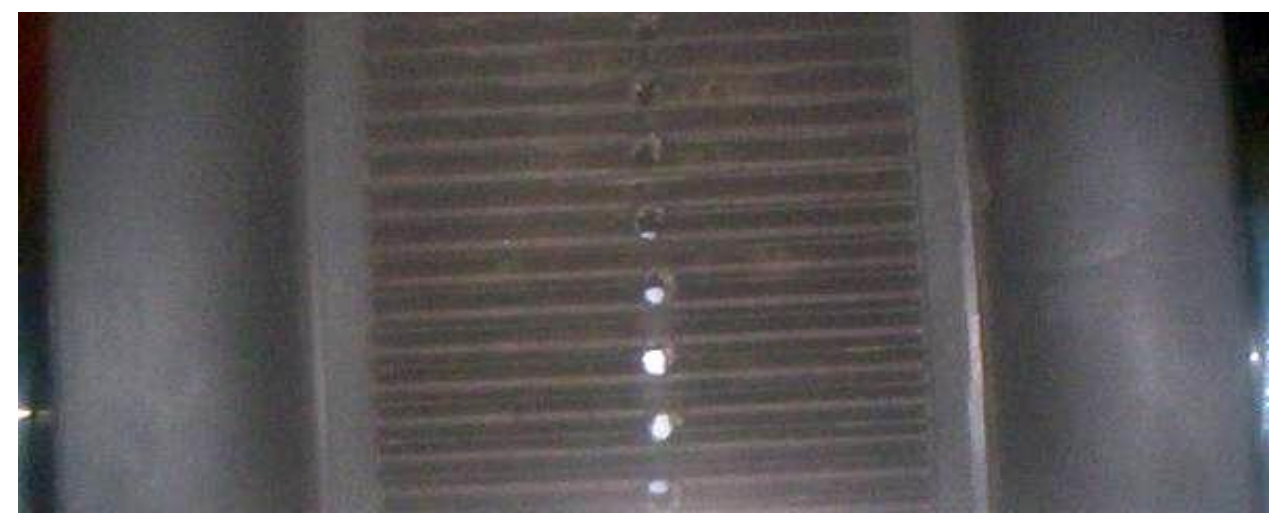

Figure 2.10. Top of the rubber carrier belt.

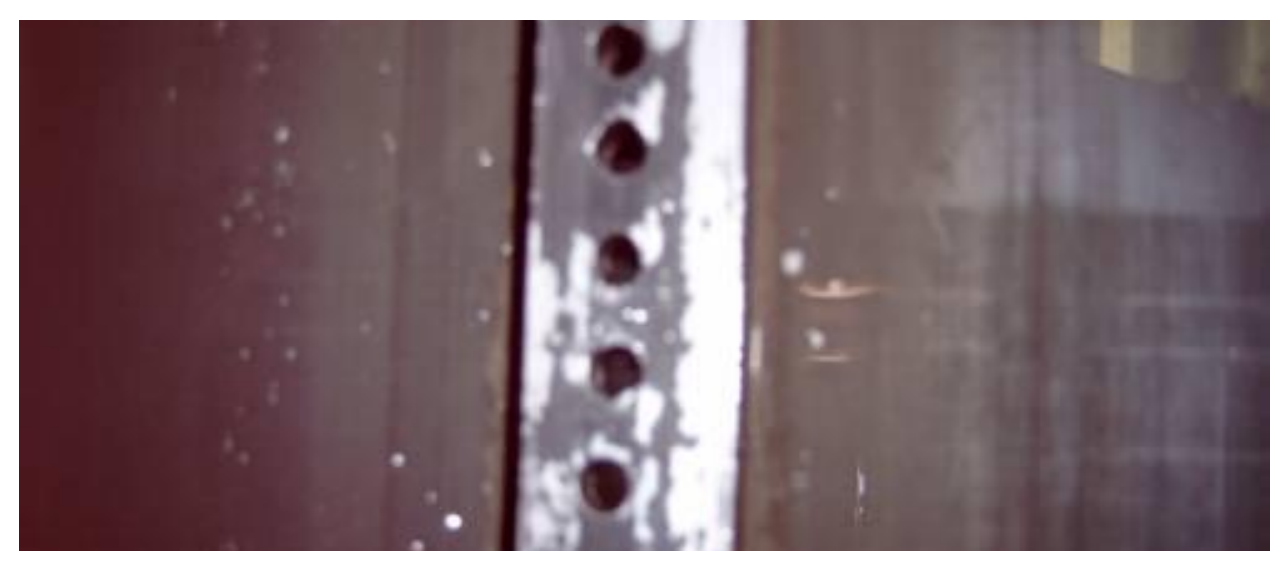

Figure 2.11. Bottom of the rubber carrier belt.

determined to be just over the hopper so that the bulk of the cake would fall into the product discharge chamber. This means that the scraper would have to scrape over a portion of the filter cloth while it is traveling across the nylon roller. Since the scraping site is not flat the scraper needed to be constructed of a material that is soft enough that it would not damage the filter cloth. To meet this requirement, it was determined that a soft rubber would be stiff enough to effectively scrape the cloth and not damage it. 
The other issue that had to be overcome was how to position the scraper in an orientation that the cake would not excessively build up on the scraper. This was accomplished by scraping at an angle with two separate scrapers to cover the entire width of the belt. Figure 2.13 shows the scrapers used to remove fine material coming off of the filter device. Each of the scrapers is adjustable to change the amount of force with which each of the scrapers presses against the belt. The angle of scrape can be adjusted also.

\subsubsection{Design of the Pressure Vessel}

\section{a) Pressure Chamber}

The pressure chamber was designed with two primary concerns in mind. The first was to construct a structure that could safely hold 60 PSI of air pressure for a long period of time. The second concern that had to be addressed was it must be sufficiently large to contain the filtration apparatus. Both of these conditions had to be addressed before the

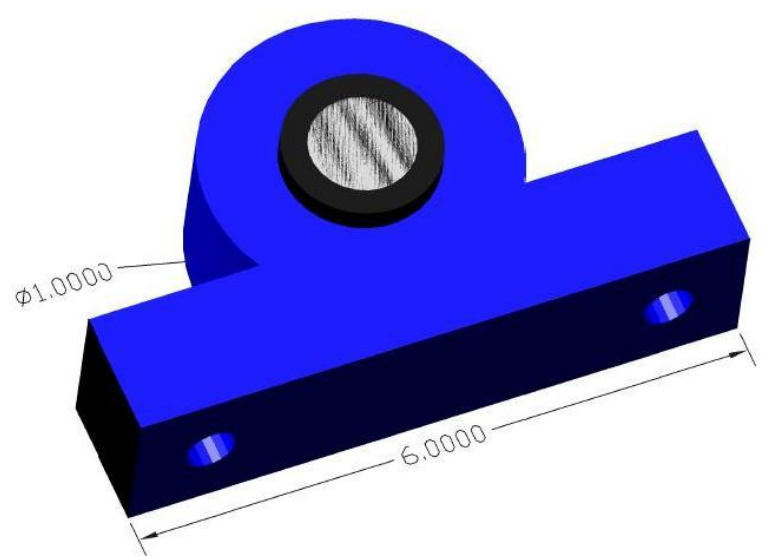

Figure 2.12. Pillow block bearing for the primary drive.

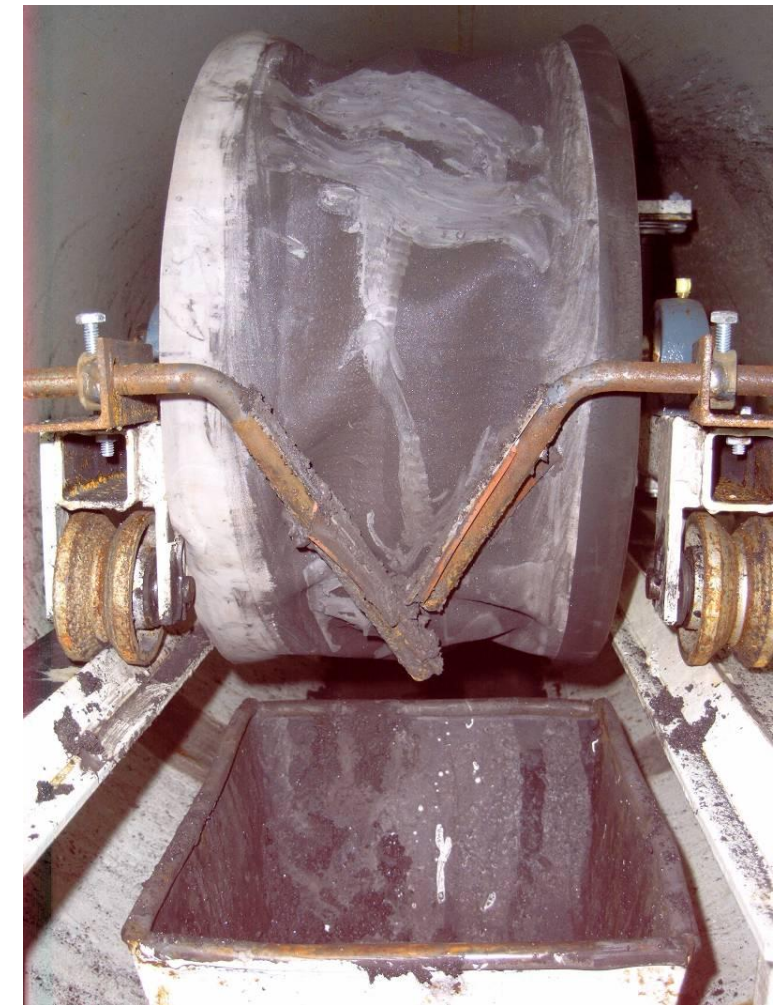

Figure 2.13. Scrapers used to remove fines from the filter cloth. 
smaller technical issues could be resolved.

To form the body of the pressure vessel, a cylindrical steel pipe made the most sense. First, it would be easy to find a pipe that was rated to hold more than 60 PSI of pressure. The second reason for using a steel pipe would be that it is easy to attach accessories to it since it can be welded. The final motivation for using steel is that it is relatively inexpensive. The only major drawbacks to using steel for the pressure vessel is that it is heavy and that there is the potential for corrosion due to the wet environment that exists on the inside of the unit. The other type of material that was seriously considered to form the pressure filter was polyvinyl chloride (PVC). This type of pipe is less accessible but is still widely manufactured. PVC is lighter than steel and has better corrosion resistance. It is more expensive and it would be difficult to cap off the ends. Also, adding a structure to the inside of the pipe to hold the filtration apparatus would present a challenge. After much consideration, the decision was made to use steel pipe. The steel pipe that was used is a schedule 40 pipe that is rated to hold 150 PSI. Figure 2.14 shows the steel pipe in

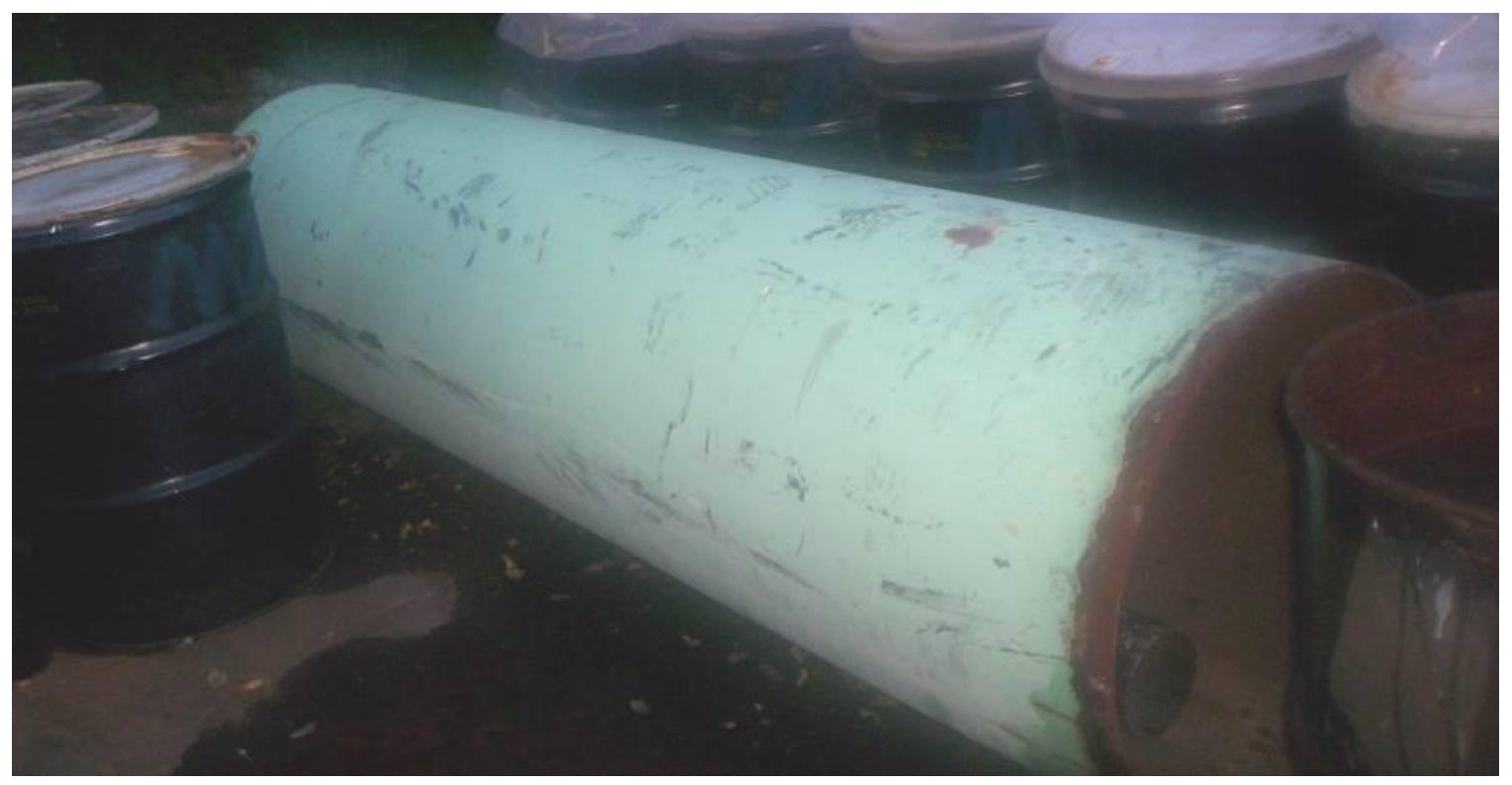

Figure 2.14. Pipe used to construct the pressure chamber (as received). 
the condition it was in when it was received.

The second major concern was to get a vessel that would be large enough to hold the filtration apparatus. From the design specifications it was determined that the smallest circle that could practically hold the entire unit would have to be 30 inches in diameter. From this plan the pipe that was acquired is 30 inches in diameter and 10 feet long.

\section{b) Supporting Frame}

Before the pressure vessel could be assembled, a frame had to be built to hold the vessel and filtration apparatus. This frame was built out of tubular steel. Figure 2.15 shows the dimensions on the tubular steel that was used to construct the frame shown in Figure 2.16. To support the weight of the pressure vessel and all of its components, it was decided that a simple

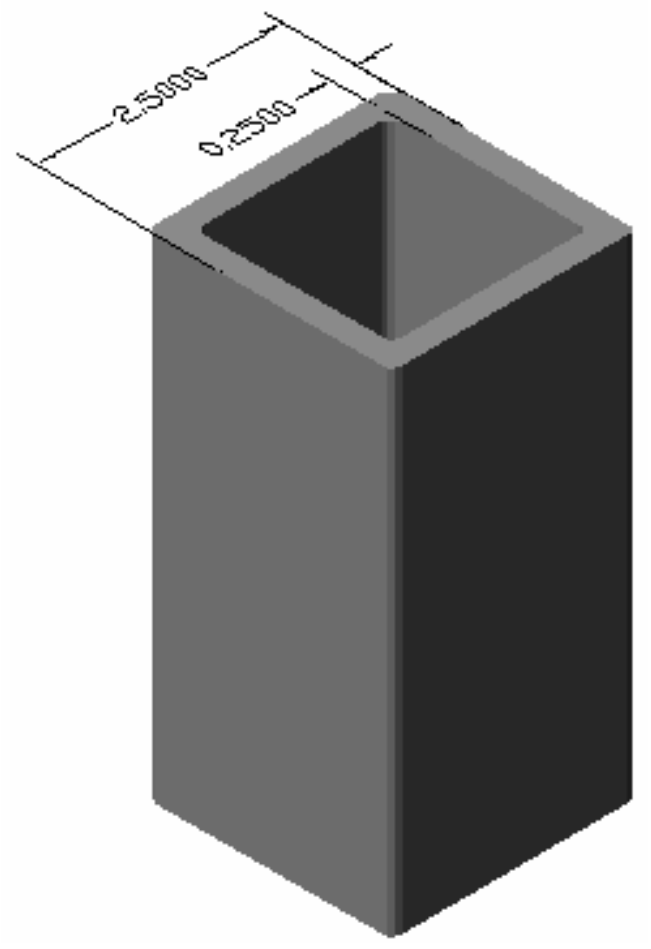

Figure 2.15. Steel used to construct the main frame.

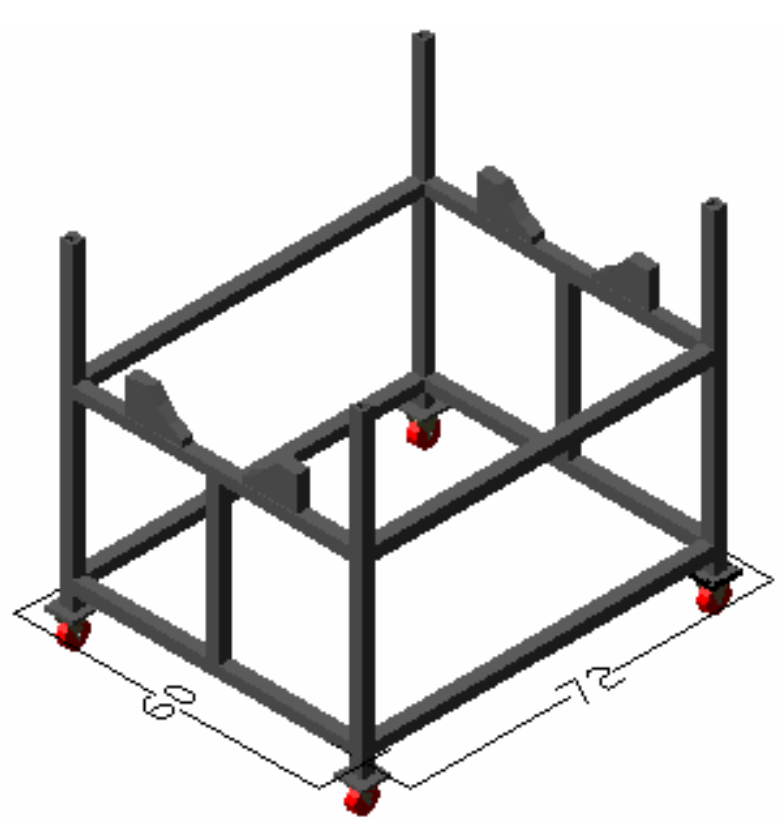

Figure 2.16. Schematic of the primary support frame. 
box frame would be sufficient to hold the pressure vessel. This frame was welded together and then painted. The primary frame was then placed on wheels so that entire hyperbaric belt filter could be easily moved. In addition to holding the pressure vessel, the frame also supports a grate walkway that allows access to the sight glasses that were put into the pressure vessel. Figure 2.17 shows the walkway that was built on the frame and the safety handrails.

\section{c) End Caps}

To seal the ends of the steel pipe, some sort of capping device had to be employed. To create the end caps two possibilities were considered. The first was to make the end caps out of Plexiglas. This would have the great advantage of being transparent. The other possibility was to make the ends out of steel in either a flat cap or a domed structure. The Plexiglas was the first

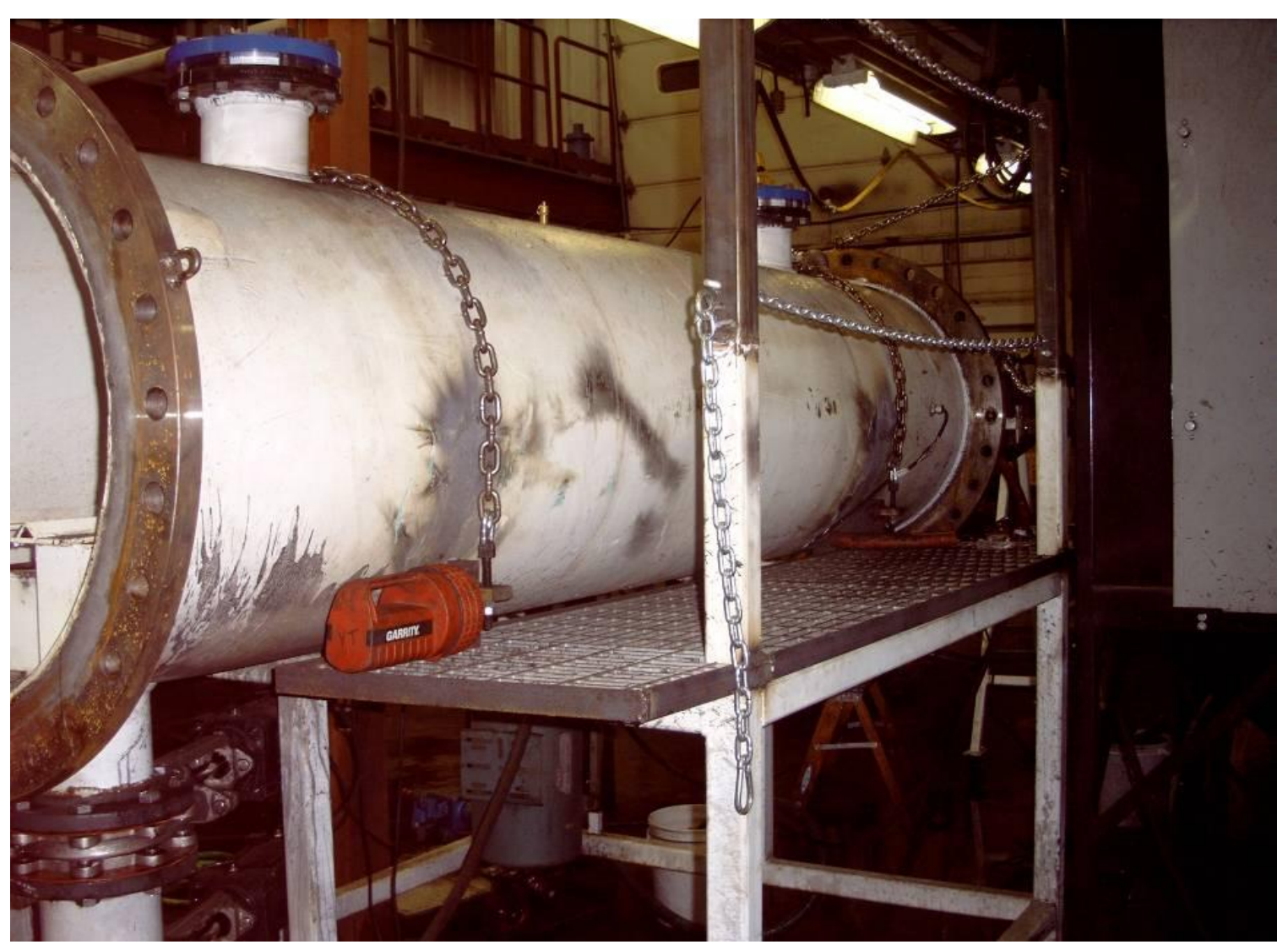

Figure 2.17. Walkway on the support frame. 
choice because of its great visibility. This option was tried until it was determined that to seal a 30 inch flat plate against the pipe holding back 60 PSI of pressure would result in over 42,000 lbs of force. To get a piece of Plexiglas that could withstand these forces would require a 12 inch thick plate. This was determined to be impractical.

That left the steel plate or dome option. Since a blank flanged plate option is simple and these items are fairly commonly produced that was determined to be the best option. Though to have used a dome structure would have allowed the caps to use less steel and thus weight less. The blank flanges would be attached to the pipe using 28 hole flanges that were welded to the pipe. Figure 2.18 shows one of the flanges attached to the pressure vessel.

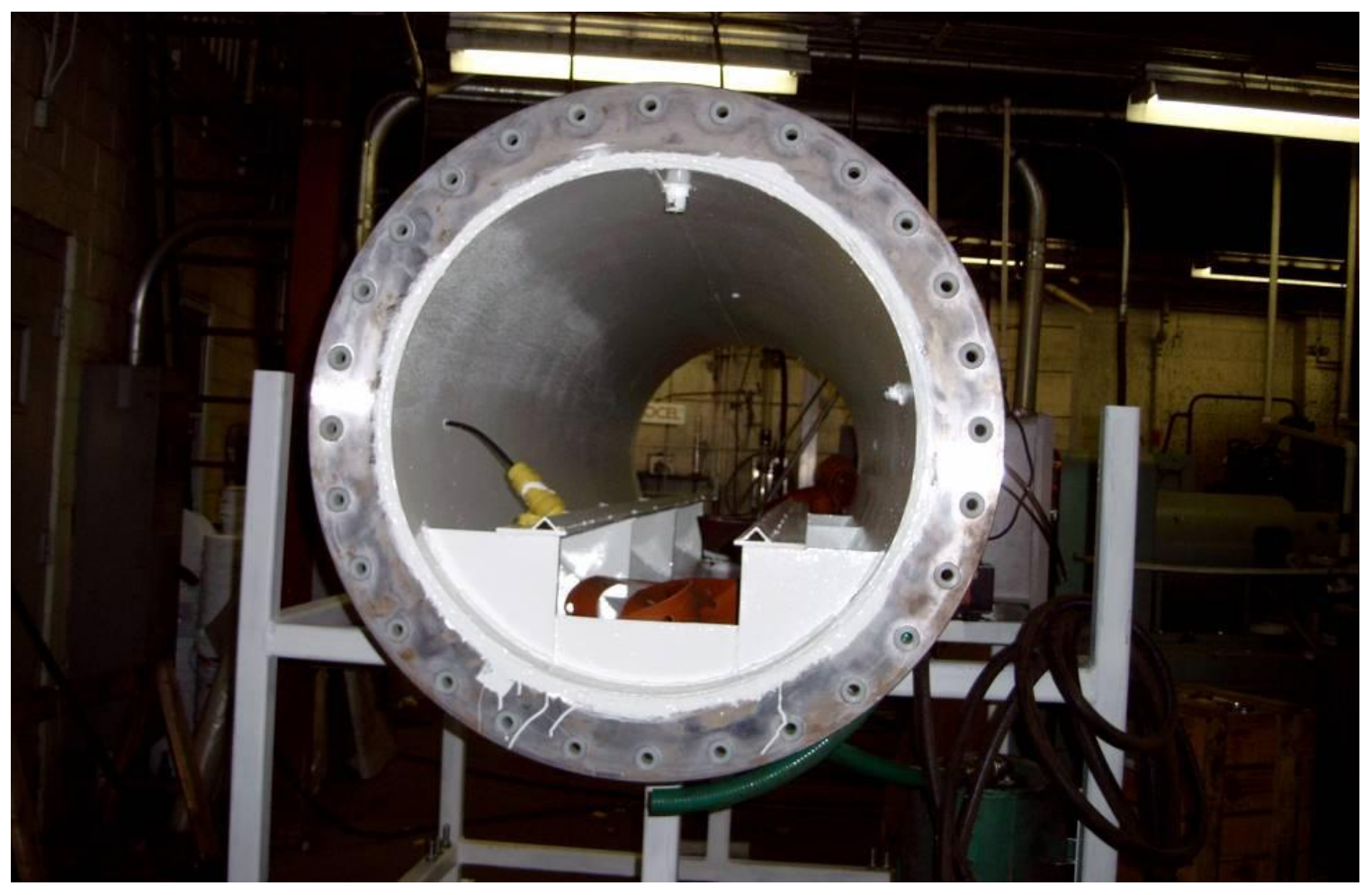

Figure 2.18. Flange attached to the ends of the pressure vessel. 


\section{d) Feed System}

The pressure vessel had to allow for material to be continuously fed to the filter device. Since the input stream to the filter is slurry the only option was to pipe in a line and to pump the slurry onto the filter belt. The main challenge that this presented was that the filter device and pressure vessel where designed to allow operation at various pressures. This means that a normal centrifugal pump could be optimized to create flow at a given pressure but it would be hard to operate the same pump under different conditions. Also, most centrifugal pumps could not produce the pressure required to overcome the back pressure caused by the pressurized tank at a low feed rate. These deficiencies lead to the search for some form of positive displacement pump that could suit the requirements. Since a positive displacement pump could function and get various feed rates with little reaction to the changing back pressure caused by the air. A progressive cavity pump seemed to be the best option for the combination of factors that this situation required. It could generate a low flow rate pulse less flow against high pressure and could handle abrasive slurry. Figure 2.19 shows a picture of the progressive cavity pump that was used with this hyperbaric belt filter unit.

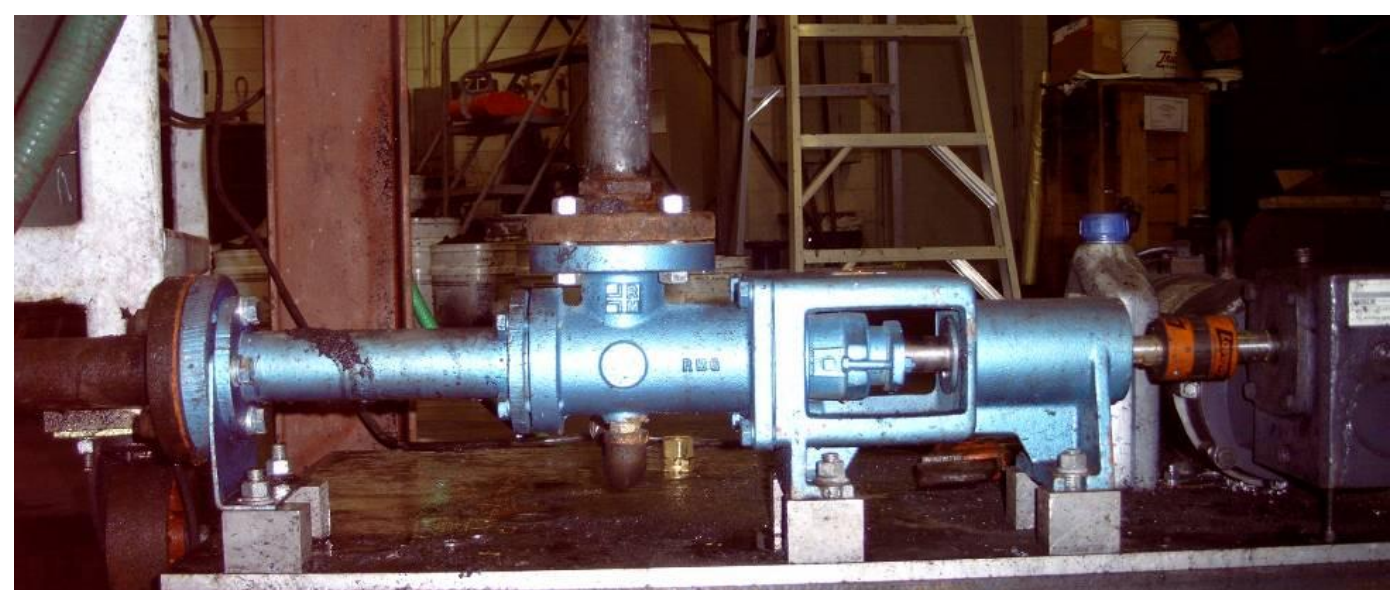

Figure 2.19. Progressive cavity pump used to feed the pressure filter. 


\section{e) Discharge System}

The discharge system is required to allow a large amount of solids to exit the pressure vessel and to not lose air pressure. For this system, two basic concepts were considered. The first was to use a rotary star valve. This is a valve that has several compartments in it that rotate around an axis. While some of the compartments are exposed to the inside of the pressure vessel, others are facing the outside allowing the material to pass but not depressurizing the vessel. This system has the advantage of only requiring one valve. The disadvantage is that these valves are known to have maintenance problems from the solids getting stuck in the rotary portion of the valve preventing it from sealing.

The alternative to this is to use a system of two valves that would act as an air lock. This mean that at no time would both valves be open at the same time. But, one could be opened at a time to allow material to pass through the system. For the pressure vessel, it was decided to use knife gate valves that are pneumatically actuated. These valves are operated on two timers that allow the unit to be fully automated. Figure 2.20 shows one of the knife gate valves prior to being installed. Figure 2.21 shows the entire airlock system after it has been assembled.

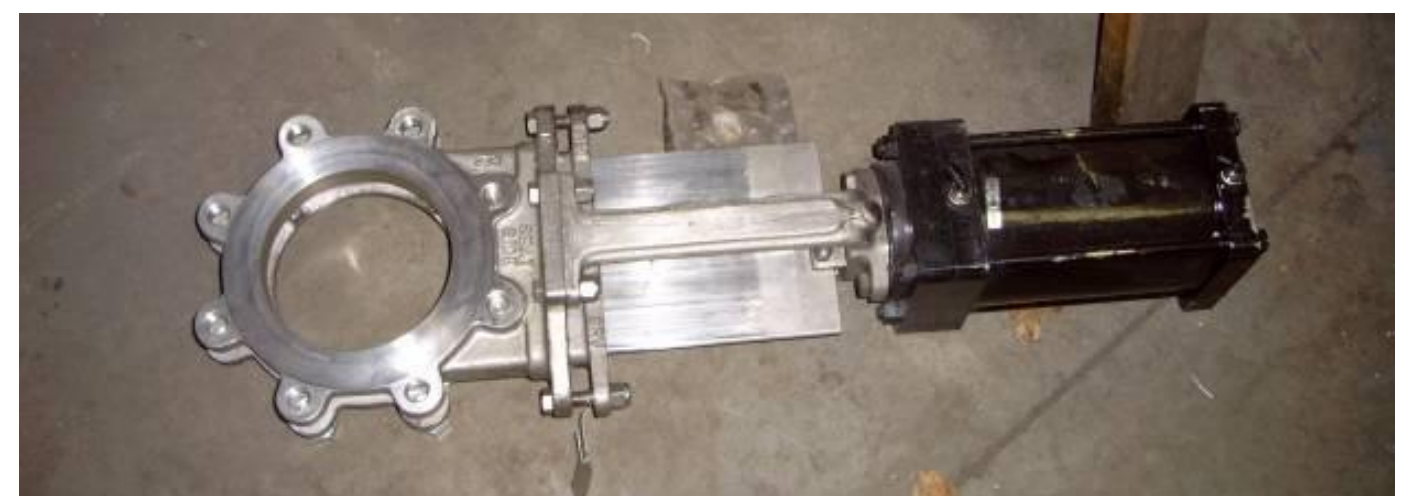

Figure 2.20. Knife gate value used for the air-lock pressure seal. 


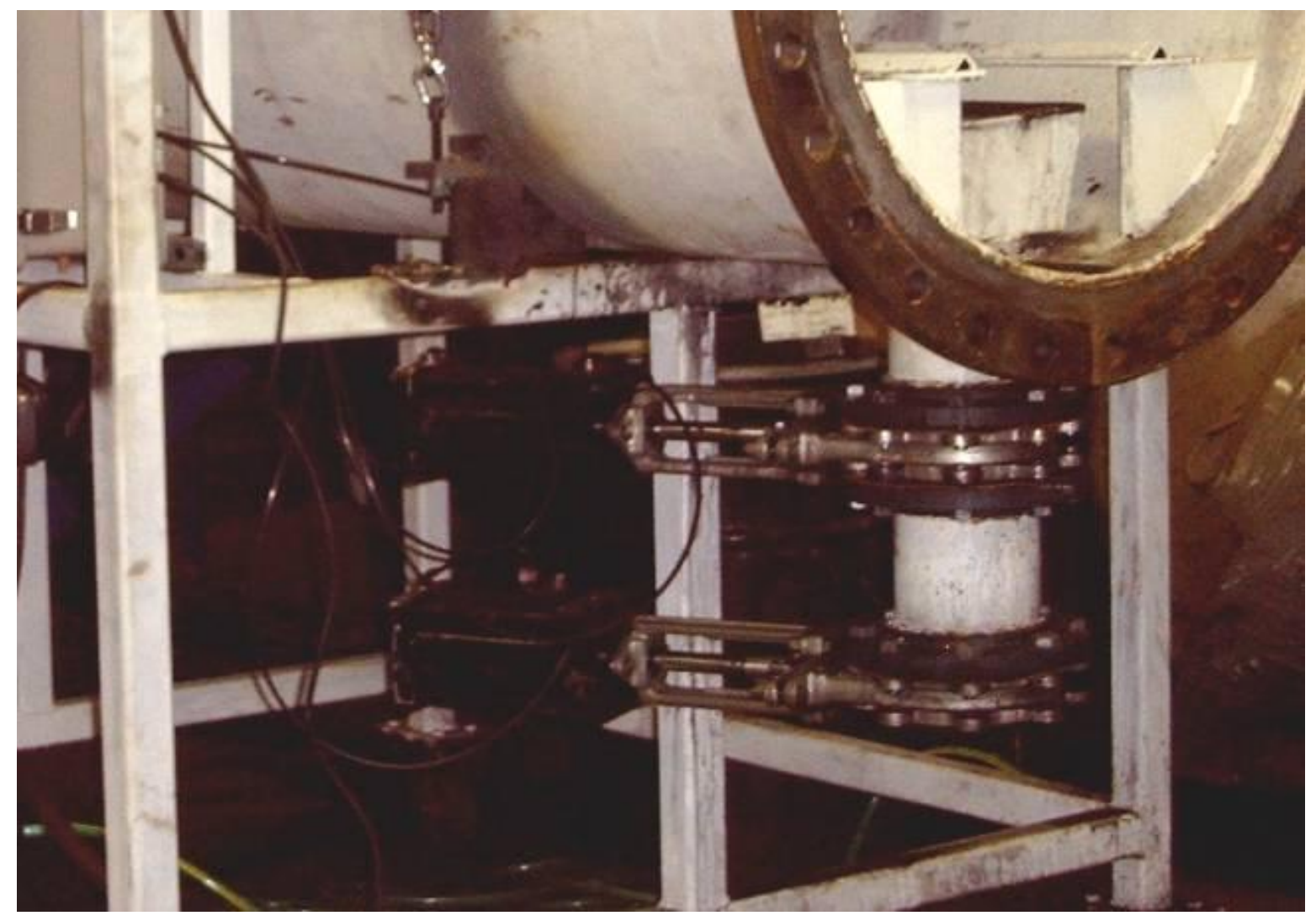

Figure 2.21. Twin values used for the airlock system (as installed).

\section{f) Rail System}

The filter device is supported in the pressure vessel by a rail system. The rail system is a set of angle iron tracks that is supported by steel supports that have been precisely cut to fit into the radius of the pressure vessel. Then the steel supports were welded into place inside the vessel. The tracks were built to fit the wheels on the filter device. Inside the pressure vessel the filter device is free rolling with only its inertia to keep it in place. Although a locking device has not yet been deemed necessary to keep the pressure vessel stationary several have been considered. Figure 2.22 contains a picture of the rail system that was built to allow access to the filter device and to support it inside the pressure vessel. 


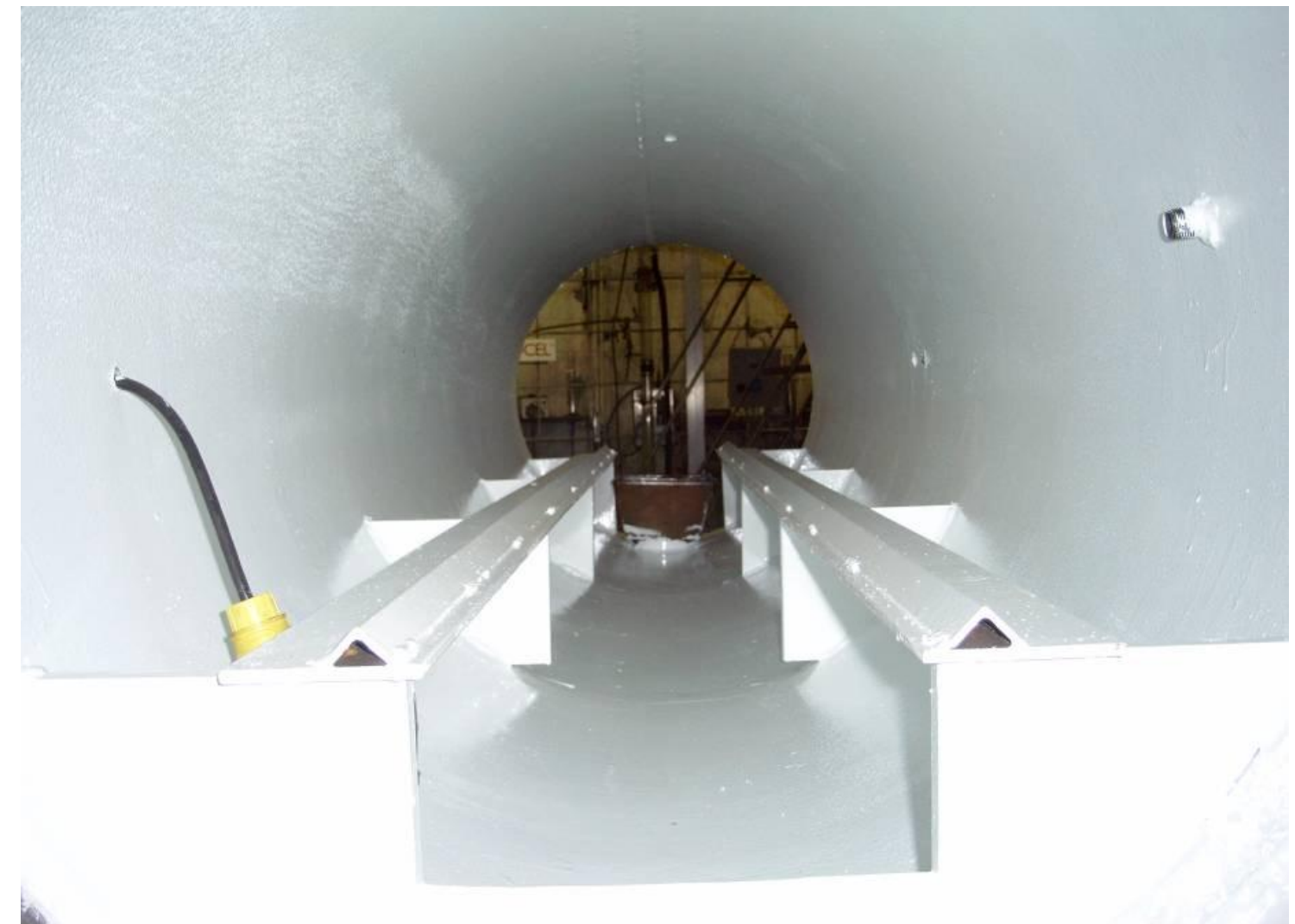

Figure 2.22. Rails and support system for the belt filter mechanism.

\subsection{Equipment Construction and Assembly}

Once the designs for the filtration device and pressure vessel were completed, it took almost one year to complete the construction and assembly of both units. A vast array of equipment was used to construct the hyperbaric belt filter. Appendix A provides a list of equipment that was used.

\subsubsection{Filter Device Construction}

Construction on the filter device started with the tubular steel frame construction. It was then followed by adding the rollers and the tensioning apparatus for the rubber belt. Once that was completed the wheels to slide along the track were added. Then, the screws holding the 
pressure plate were machined and added. While the screws were being made the discharge box was constructed from sheet steel. Next, the hooks were attached to the frame to allow easier handling of the unit. After that, the chain and sprockets were installed to attach the drive motor to the rollers. The roller system for the filter cloth was then made and the filter cloth was added to the unit. The final additions to the filter device were the feed trough, water spray, and scrapers. Once all of the components were added, the unit was painted with epoxy paint. Figure 2.23 shows a picture of the completely assembled filter device.

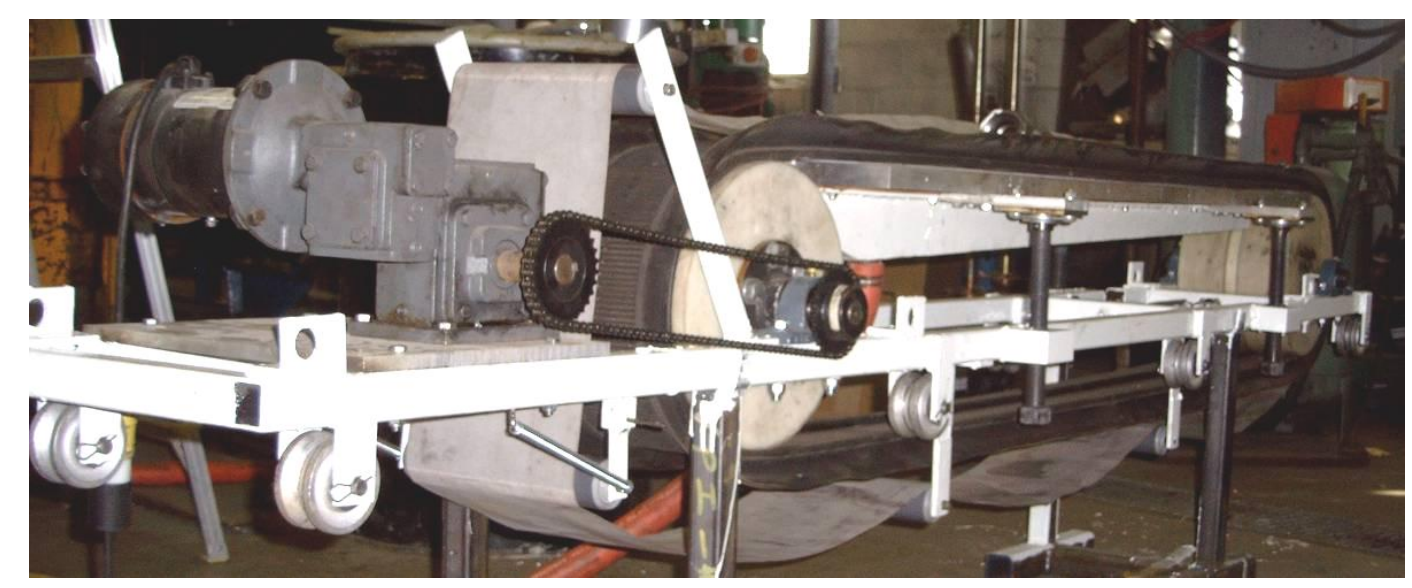

Figure 2.23. Completely assembled belt filter mechanism.

\subsubsection{Pressure Vessel Construction}

The pressure vessel portion of the unit took longer to construct. The construction began with making the frame to hold the pressure vessel. Then, the pipe was placed on the frame. Once on the frame the rail system was added to the inside of the pressure vessel. Then, there was a long break in construction while bids were taken for some work to be done by outside contractors. This work included welding on the pipe fittings for all of the input and output system on the vessel. Also, welded on were the flanges for the end caps and knife gate valves. Once this was done, the end caps were put on and the unit was hydrostatically tested at 100 PSI 


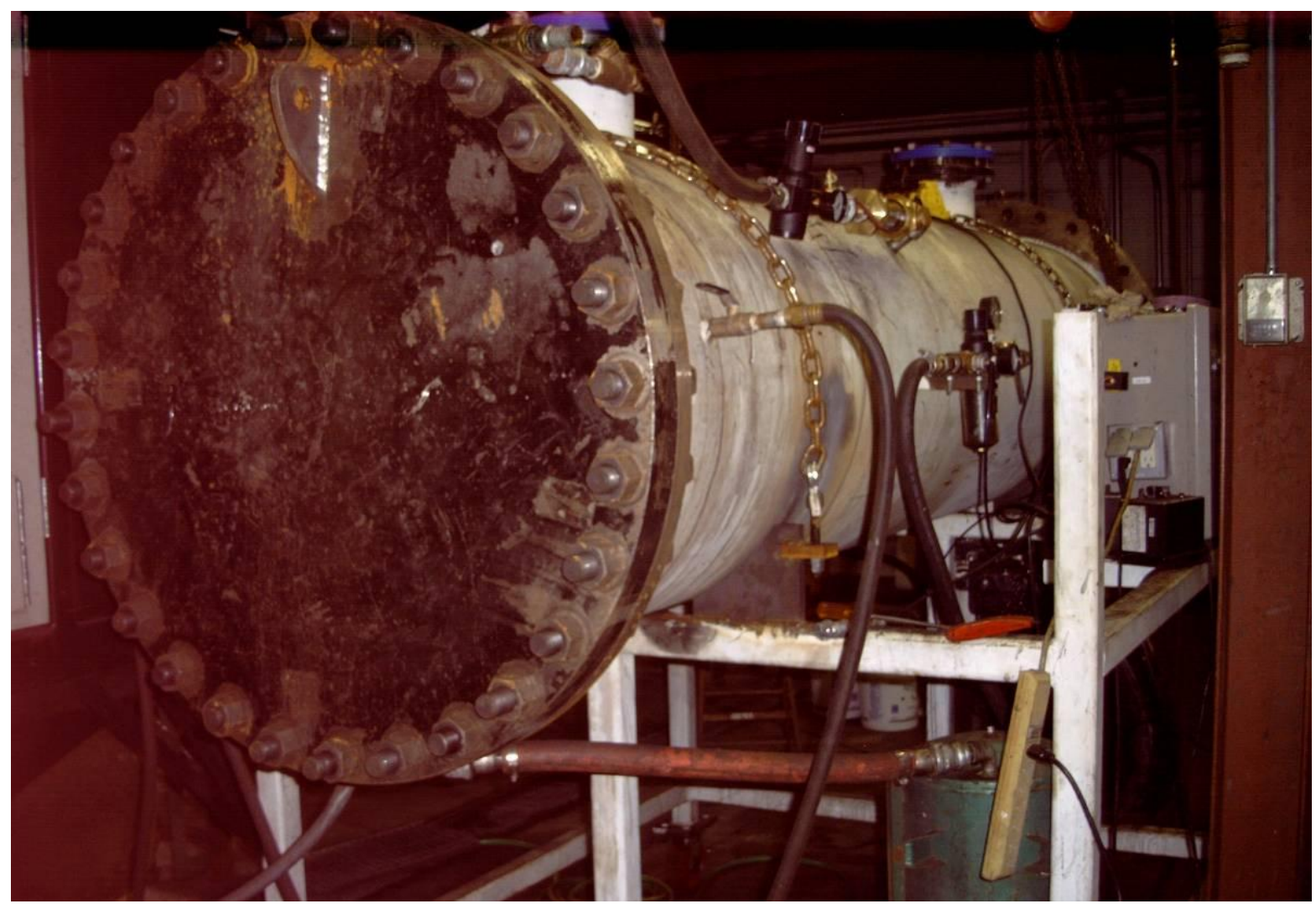

Figure 2.24. Completely assembled pressure vessel.

for one hour. The unit was then returned to the plantation road facilities and painted with water resistant epoxy paint. Figure 2.24 shows a picture of the fully assembled pressure vessel.

\subsection{Shakedown Testing}

When construction of the unit was completed it was necessary to test the hyperbaric belt filter for its mechanical operation. These tests lead to two primary discoveries.

\subsubsection{Air Requirement}

The first operational discovery was that for the unit to operate effectively it would need more volume of air than could be provided by the air compressor at plantation road. The lack of an appropriate air compressor, lead to renting a 185 CFM unit from RSC Rental in 
Christiansburg, Virginia. Once the air requirement for the unit was met the unit began to perform as expected.

\subsubsection{Start-Up Procedure}

The second important discovery during shake-down testing was that the unit like most complex machinery has a start up procedure that must be followed to ensure that the unit will perform as expected. The steps to properly start up the unit are listed below.

1. Check to see that all of the valves on the unit are closed.

2. Start the water spray.

3. Start the belt at the desired speed.

4. Pressurize the vessel to the desired pressure (not to exceed 60 PSI).

5. Check the pressure vessel to ensure that there are no leaks.

6. Start the pump to allow slurry to flow on the belt.

7. Open the valve to allow the dewatering to begin.

8. Open the water discharge valve to ensure that water level in the waste tank is brought to zero.

If these steps are followed the unit has operated effectively with little maintenance required. Once the optimal start up procedure was determined, the unit was ready to be tested to determine the operating parameters.

\section{$2.4 \quad$ Detailed Testing}

The experimental testing of the hyperbaric belt filter was initiated after the design and construction activities were completed. This work is the final phase of the process for developing the hyperbaric belt filter. During this phase, the performance of the prototype unit was tested, analyzed and calculated. The performance was measured on several different aspects. Some of 
these measures focused on the functionality of the unit, while others focused on the dewatering capabilities.

The test program included both batch laboratory tests as well and continuous tests conducted with the new prototype. Because of the importance of particle size in determining filter performance, the tests were conducted using coal feeds that were coarse, fine and intermediate (mixed coarse and fine). Several sets of tests were also performed with dewatering chemicals in order to further enhance the dewatering performance of the unit. Once these tests were completed, the optimal operating conditions for the new hyperbaric belt filter were established. This data was used to develop empirical correlations that could be used to predict the performance of the filter under other conditions not tested in the current study.

\subsubsection{Feed Material}

To test the operation of the filter, feed coal material is needed. It was decided to perform the tests under three primary conditions. The first is to have the filter tested under good filtration conditions. For these tests, the size distribution of the particles in the filter was $1 \mathrm{~mm} \times 0$. This material was obtained as screen-bowl feed for the Alpha Natural Resources Tom's Creek Mine in southwestern Virginia. From filtration theory (Gregory, 1984), it is known that larger particles

are easier to dewater than smaller ones. This higher moisture is primarily due to the increased surface area associated with fine and ultrafine particles.

The second set of tests was also run using fine material that was obtained from Tom's Creek Mine. This material is column flotation overflow material; it is much finer and has low percent solids (less than 10\%). This material was nominally minus $0.15 \mathrm{~mm}$ (minus $100 \mathrm{mesh}$ ).

The third series of tests was done with a mixture of the two feeds. The mixture is $50 \%$ coarse and 50\% fine material by volume. This results in a feed that is $1 \mathrm{~mm} \mathrm{x} 0$, but has a much 
more even size distribution due to the greatly increased amount of fine particles compared to the coarse tests.

Due to the dramatic difference that has been reported in filter performance due to particle size, the unit was tested with both extremes of materials to see how the unit would perform under difficult conditions (Dickenson, 1997). From these tests it should be possible to predict the performance of the filter under a wide range of operating conditions.

\subsubsection{Test Procedures}

All of the procedures used in testing the unit were considered and used for the purposes of functionality and repeatability. In the following sections, each step in the testing process is described in detail to show how the testing was done.

\section{a) Sample Preparation}

The samples were obtained in 55 gallon drums and hauled on a trailer to the Plantation Road processing facilities at Virginia Tech. When it was ready to be used, it was dumped from the drum into a sump. While in the sump, the sample was mixed and aerated by a two horsepower mixer/aerator. Also, it was recirculated from the bottom of the sump back to the top by a pneumatic powered double diaphragm pump to minimize settling. After each had pumped and mixed for size hours, it was not used again to avoid fines generate via attrition. The pumping and mixing was done to help ensure that the sample was as uniform as possible during testing. The sample is meant to be representative of what conditions exist in the processing plant.

\section{b) Percent Solids}

The measurement of percent solids was done by pumping the entire sample through a sample splitter numerous times until it was reduced to approximately $100 \mathrm{ml}$ of sample. Then, the $500 \mathrm{ml}$ beaker was weighed while dry. The $100 \mathrm{ml}$ of sample slurry was placed into it and 
weighed wet. It was then placed in an oven at $80^{\circ} \mathrm{C}$ overnight and weighed dry. From these numbers, it is a simple calculation to determine the percent solids in the feed.

\section{c) Air Flow}

The air flow input into the pressure filter was determined using a spring loaded flow meter. This meter was located after the pressure regulator in the air input line. This allowed it to measure all of the air that went into the unit so that the air requirements at each operating condition would be known.

\section{d) Moisture Content}

The moisture content of the discharge material is of prime importance. Each of the tests was conducted over a five minute period. After five minutes, all of the material that the filter produced was collected by discharging it through the knife gate valve system. Since some of the tests produce a large amount of filter cake it was necessary to take a sample of the discharge. This was done by cutting the sample using a Jones Riffler to reduce to sample to a manageable amount that could be weighed, dried, and reweighed. The sample was reduced in size to less than 50 grams. It was then weighed and placed in an oven at $80^{\circ} \mathrm{C}$ for five hours. This was to ensure that all of the moisture had been removed. Once dried, it was weighed again to determine how much of the original weight could be attributed to moisture.

\section{e) Effluent Characterization}

The effluent from the filtration device was also analyzed. This was to determine how much material was being lost through the filter cloth. This material was collected at the bottom of a stainless steel tank. Once the test was completed, the tank was drained into a container and the contents weighed. This material was also dried to see the weight of the solid material that 
was being lost during the separation process. From this process, it is not possible to determine how much water is being released since much of it is carried with the air to the atmosphere.

\section{f) Batch Test Comparison}

To conduct a batch test each sample is prepared separately. The sample is cut from a five gallon bucket to produce an accurate sample of the bucket's contents. Then, it is placed inside the unit shown in Figure 3.1. This is a pressure vessel that has a filter cloth in the bottom of it. Under the filter cloth there are a series of grooves that lead to a hole which lets air and water escape through the filter media. The process is timed, which varies depending on the conditions of the test. Once the allotted time has elapsed, the bottom is removed from the batch unit and the cake is discharged. It is then carefully placed into an aluminum pan, weighed, and dried. From this process, the cake formation time, total drying time, cake thickness, air consumption, and total moisture

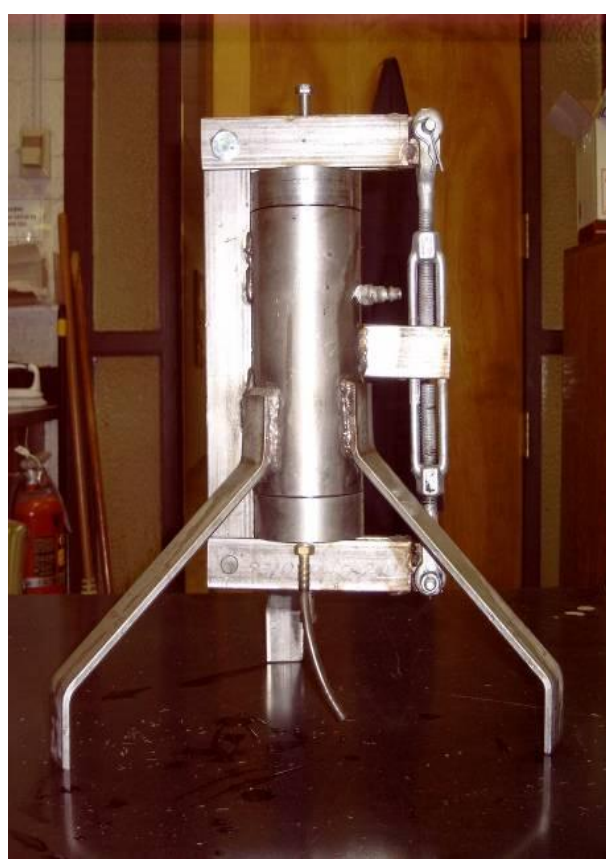

Figure 3.25. Batch pressure filter. are all determined and recorded. 


\subsection{RESULTS AND DISCUSSION}

The results that are described below were arrived at using the methods explained in the experimental section of this report. The results are primarily divided into three sections coarse feed, fine feed, and mixed feed. This segregation is due to the different behavior caused by the different feed material.

\subsection{Laboratory-Scale Batch Testing}

The batch testing was done for two primary reasons. The first was to set a baseline for what types of moisture percentages are possible if conditions in the batch tests could be duplicated in a continuous filter. The second reason for the batch testing was to determine the best possible reagents and dosages for the coal that was to be used for filtration. These optimal reagents would then be tested on the prototype unit.

From the filtration theory described by Equation (2), it is known that one of the controlling factors in dewatering is the driving force created by a $\Delta \mathrm{P}$ across the filter cake. For this reason, all of the tests that conducted were done at 60 PSI. Over fifty tests were done to create each of the charts in this section. The data obtained from the batch tests are plotted in Figures 3.2 and 3.3. The difference between these two charts is the amount of material that was used for each. The later chart shows data from tests run with more slurry, thus increasing the dewatering time and increasing the cake thickness. The tests were conducted using proprietary dewatering reagents provided by Nalco Chemical. In this work, these proprietary reagents have been designated as Reagent RU and RV.

From these charts, it is clear that for this coal sample, which was cut from the larger sample being used to test the continuous unit, that the best reagent combination is $\mathrm{RV}$ at an active ingredient ratio of $1: 10$. It can also be seen that the effect of the reagents in greatly 


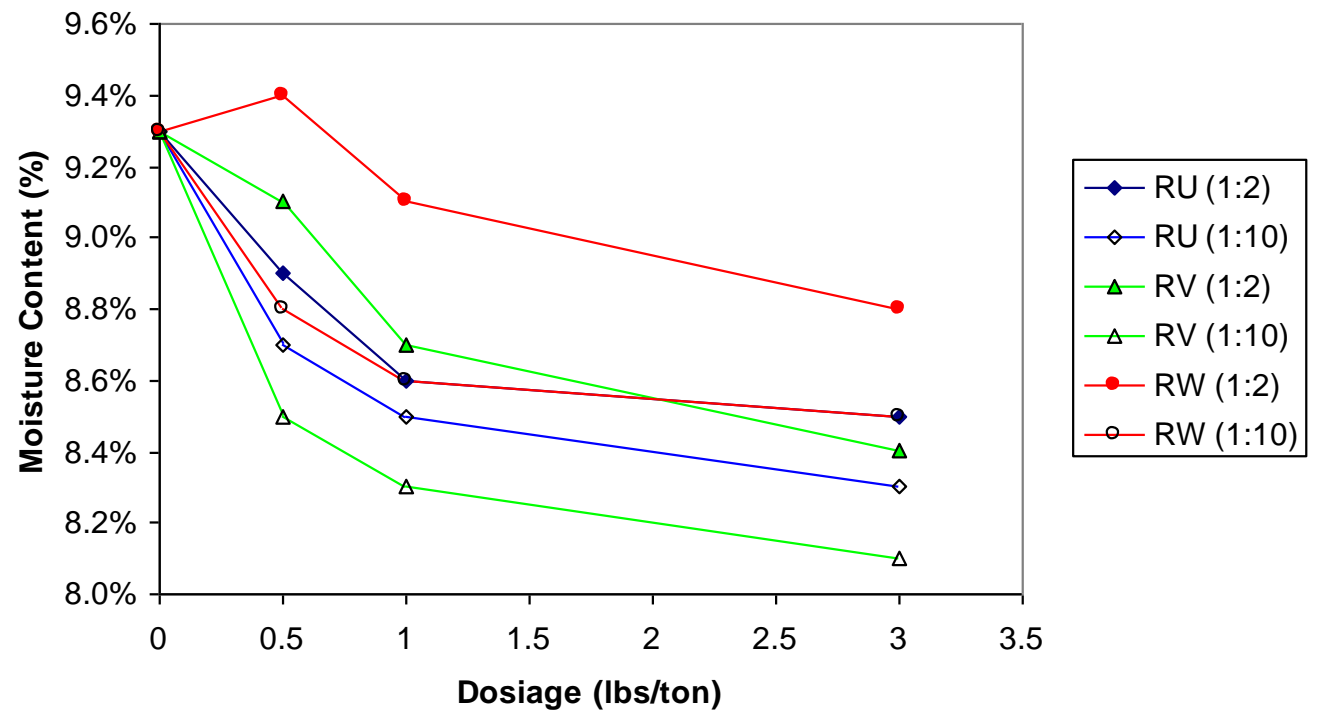

Figure 3.1. Batch test data for $20 \mathrm{ml}$ of coarse feed (60 PSIG).

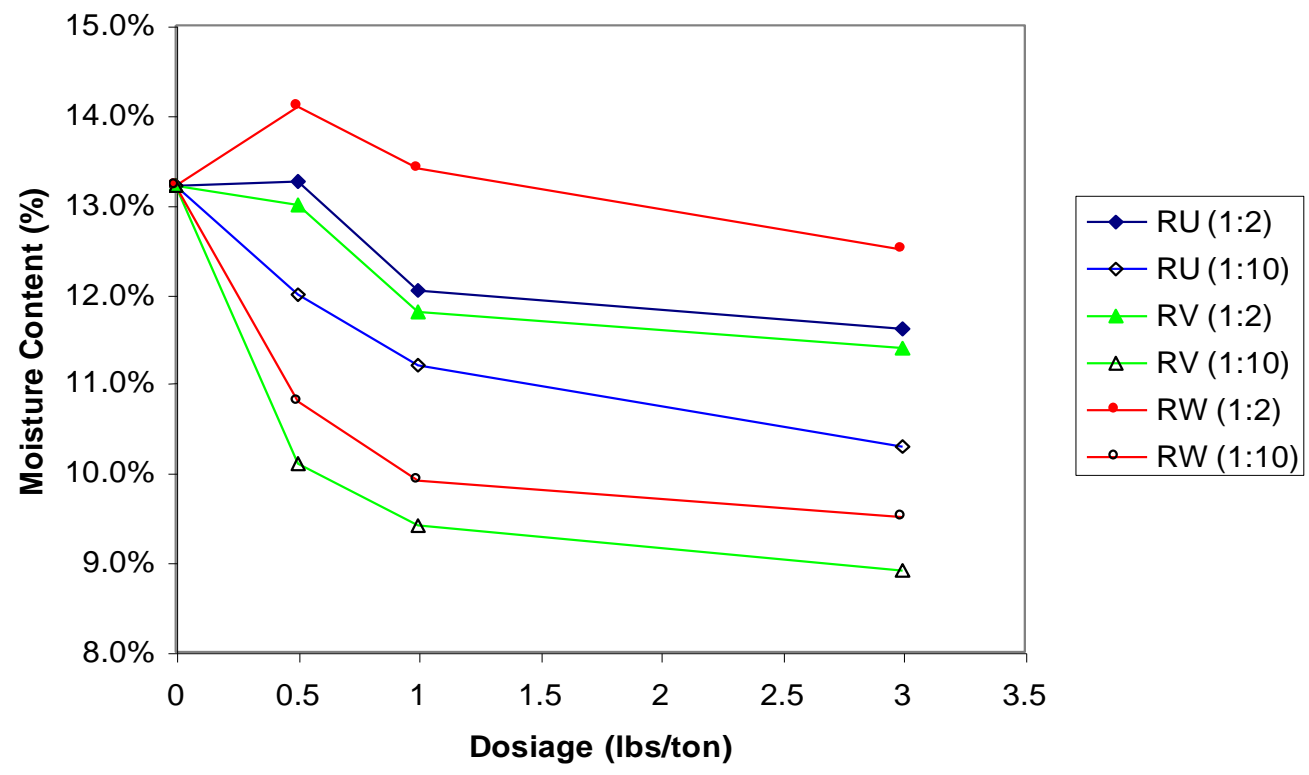

Figure 3.2. Batch test data for $50 \mathrm{ml}$ of coarse feed (60 PSIG).

increased for the thicker filter cake. Thus, if the continuous unit is run with a thick cake, the reagents should provide a substantial benefit compared to that of a very thin cake. Also, from these tests, it is clearly possible to achieve a moisture content less than $9 \%$ if the conditions in the batch test can be replicated in a continuous filter. 


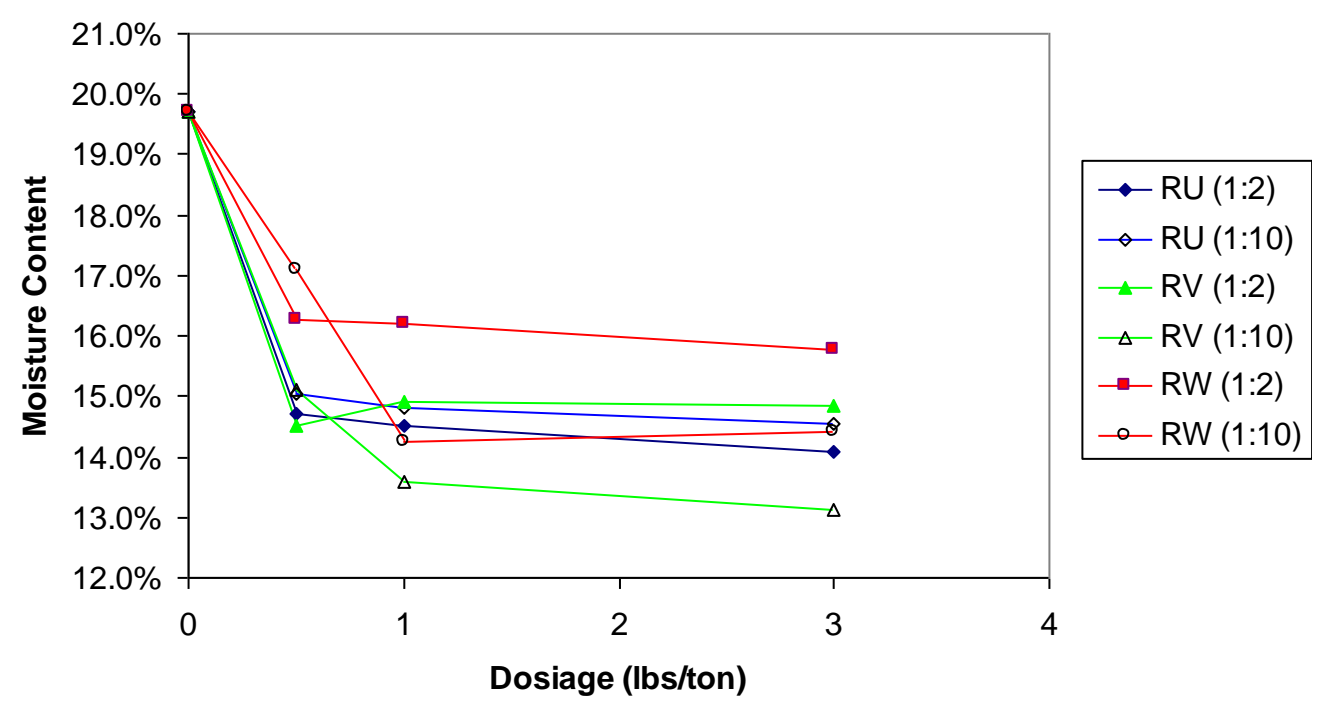

Figure 3.3. Batch test data for $20 \mathrm{ml}$ of fine feed (60 PSIG).

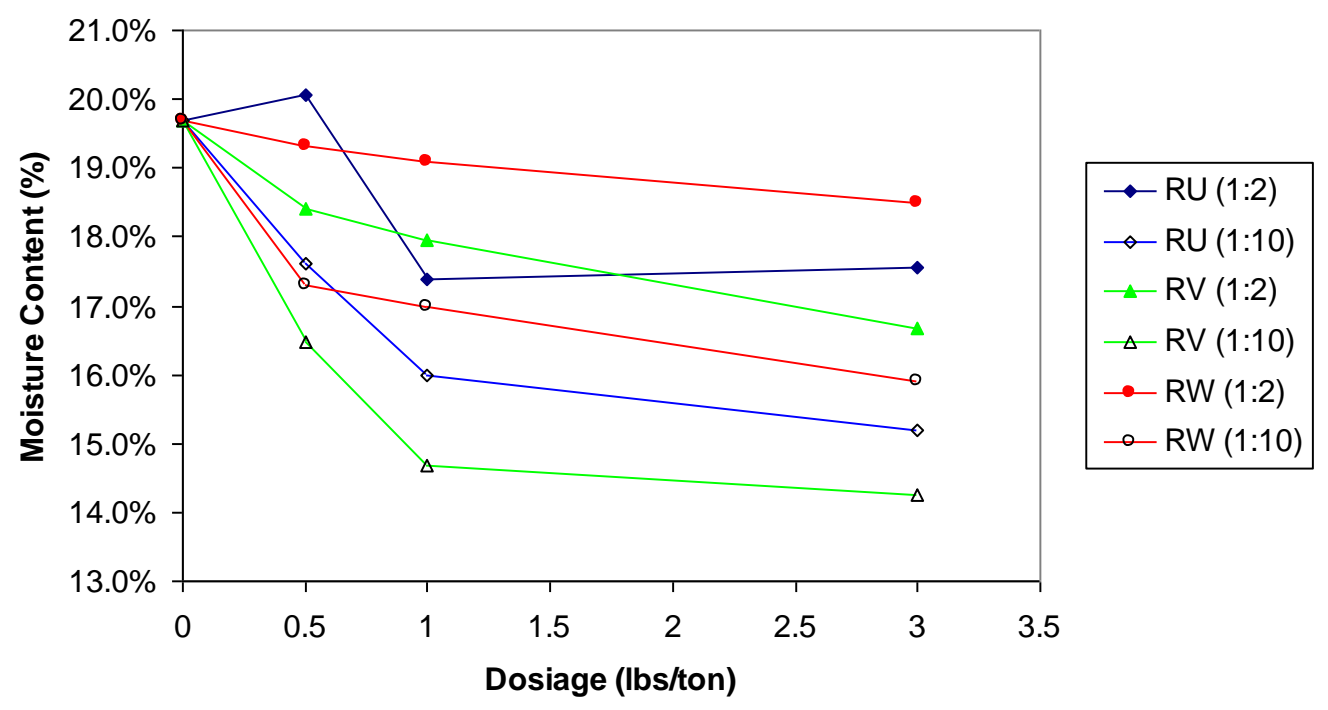

Figure 3.4. Batch test data for $50 \mathrm{ml}$ of fine feed (60 PSIG).

Also tested to determine performance was a fine feed from the same plant. The results of these tests are shown in Figure 3-4 and Figure 3-5. Just as in the coarse feed tests, all of these tests were conducted at 60 PSI. This fine material is $61 \%$ minus 325 mesh. The high percentage of slimes in the feed necessitates the need to use flocculants on all tests. All of the tests have a dosage equivalent to 1 gallon of flocculant per 70 tons of material. This is similar to the amount of flocculants that is used in the preparation plant. 
From the charts, it can be observed that the best reagent is RV with a dosage of active ingredient of 1:10. Also, it can be seen that once again as in the coarse material the less slurry that is on the filter the lower the moisture content of the filtrate. For this fine material, the batch unit was able to produce a moisture content of approximately $14 \%$.

\subsection{Coarse Feed Testing}

On the continuous filter there were four primary controllable parameters that were considered to affect the moisture content of the product. The first has already been mentioned and that is the size of the feed particles. The second is the pressure across the filtrate. Third is the speed that the belt moves and the fourth is the speed at which the material is added to the filter or the mass flow rate. Using these four parameters, it is possible to affect the air flow rate, cake thickness, production rate, and the moisture content of the product. Each of these responses will be addressed separately as they each have their own unique impact on the overall performance of the filter device. The analysis has also been broken down by size class.

This section concerns feed material which is screen-bowl feed from the Tom's Creek Mine. The size of the material is $1 \mathrm{~mm} \times 0$. This material has some spiral material mixed in with it. About $15 \%$ of this material is minus 325 mesh. Thus, it contains a wide range of sizes of particles.

\subsubsection{Moisture Content}

There are several factors that affect the moisture content of the product. First is the size of the material as mentioned before, second the cake thickness, third the $\Delta \mathrm{P}$ across the cake, fourth is the dewatering time, and fifth is the water's affinity to remain attached to the water. With this filter it is possible to affect all of these primary factors but not all of them directly. 
Since some of these factors cannot be controlled directly it makes sense to focus on inputs that can be controlled. These are feed rate, belt speed, pressure, and chemical reagents which affect surface tension.

The first thing that was examined was how consistent the filter's product is when all of the external factors are held constant. The results of these tests are shown in Figure 3.6. The average value of these tests is $11.4 \%$. These tests were conducted at an operating pressure of 60 PSIG. The percent solids for all of the coarse material tests were approximately $24 \%$ solids by weight.

The standard deviation of this data is 0.0074 . This is showing that while the range of the data is $1.1 \%$ by moisture percentage the actual difference between the numbers is small. There are some likely causes for this variation. One unlikely possibility is that the feed material is changing. As described in the procedure section the feed it highly mixed and is also recirculated in the sump. The feed pump is a progressive cavity pump that even with high solids contents and

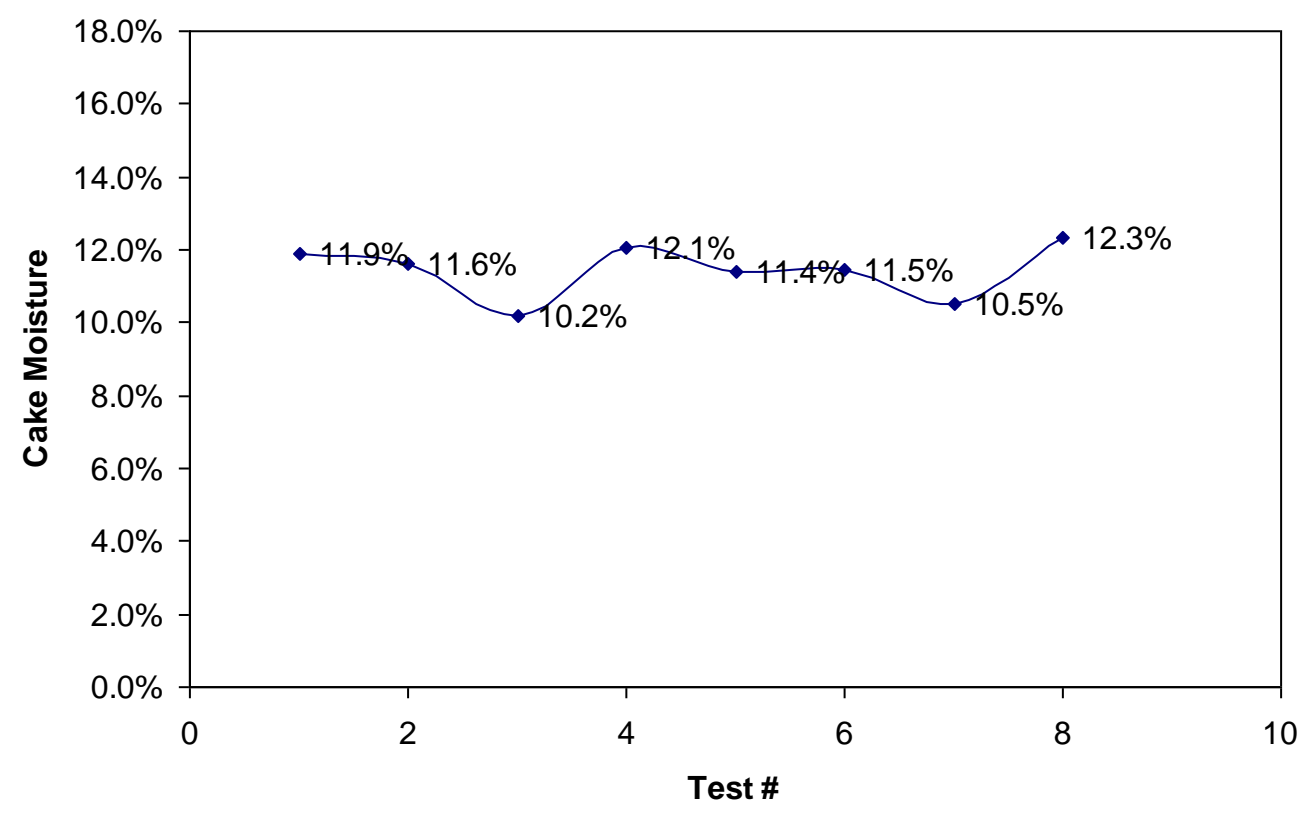

Figure 3.5. Variability of the coarse data (30\% feed pump speed). 
large particles delivers a steady pulseless flow.

Some likely causes of this variation have to do with the moisture in the compressed air itself. The compressor while compressing the air heats it to a temperature of approximately $60^{\circ} \mathrm{C}$. The air is not conditioned in any way - it is straight from the atmosphere in Blacksburg, Virginia. This means that there is a significant amount of moisture in the air. This hot air rises to the top of the pressure vessel and condenses when it meets the cooler exterior wall of the pressure vessel. This causes random droplets of water to periodically fall onto the filtered material. If a few of these drops fall on the filtered material before it is discharged for the pressure vessel it would account for some of the variability. Also, there is the potential for random periodic cracking of the filter cake. When this happens the pressure drop locally across the filtrate material is reduced, which retards the effectiveness of the filtration equipment. Due to this noticeable variability, all of the subsequent data points are actually an average value of three individual data points. By taking an average value, the confidence of each point is significantly improved.

After the variance of the data was determined, Design Expert was used to create a testing matrix of conditions that should be tested to determine the effect of each of the input variables on moisture content. The Design Expert tool analysis method chosen was a Box-Behnken response surface. Given the three parameters that could be changed for each test scenario, the program created a testing schedule that was used. This schedule is shown if Appendix B. Four different response surfaces were created to accommodate the five parameters that were discussed. Since only three are truly controllable by the filter device itself those where the variables in each of the different scenarios.

Two of the response surfaces are related to the coarse feed material. The first is the material with no additives and the second is one where the feed material has been conditioned 
Design-Expert® Sof tware

Moisture

- Design points above predicted value

- Desian points below predicted value

$\prod_{0.088}^{0.653}$

$\mathrm{X} 1=\mathrm{A}$ : Pressure

$\mathrm{X} 2$ = B: Belt

Actual Factor

C: Feed $=25.00$

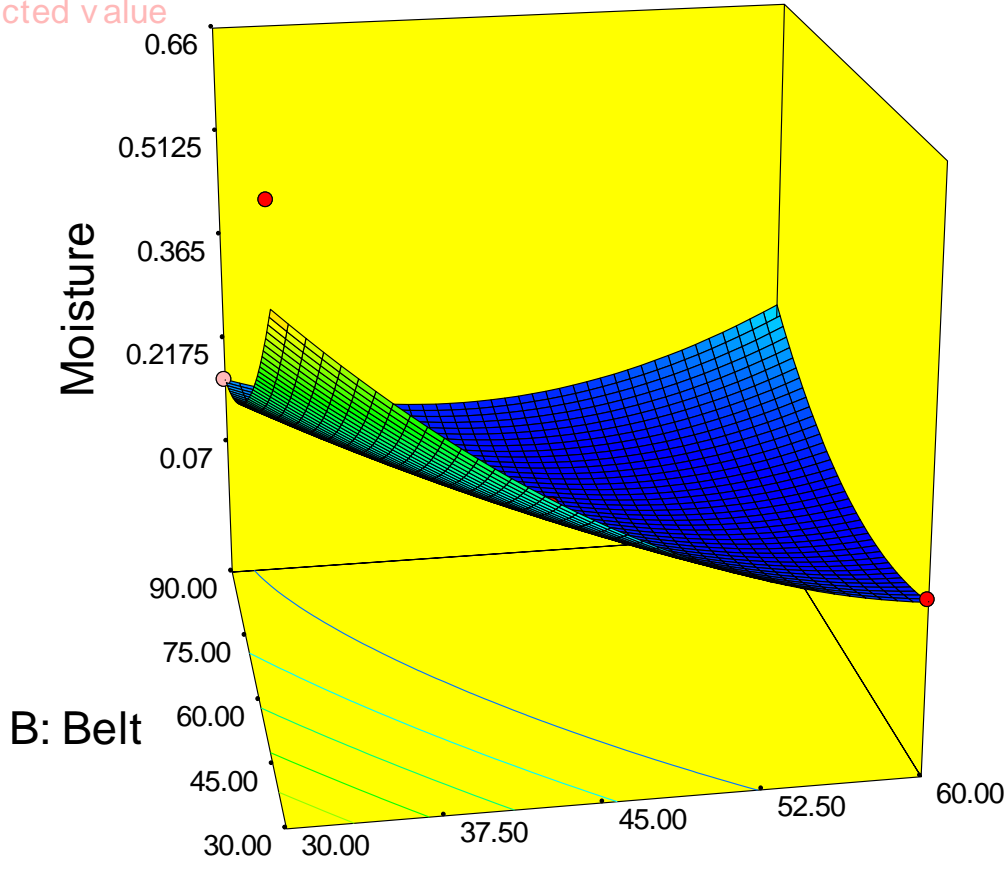

A: Pressure

Figure 3.6. Response surface with coarse feed and no chemicals.

with RV (1:10) based on the results from the batch testing. Figure 3.7 shows the response surface from the base case test with no additives.

The chart shows the pressure on the $\mathrm{x}$-axis and it is measured in PSI. The y-axis is the belt speed and it is shown as a percentage of the maximum speed of the belt, and the z-axis displays the moisture content at a given operating condition expressed as a decimal. Table 3.1 shows the conversion factors between the belt speed as a percentage and feet per minute.

Figure 3.7 shows how the filter device performs at every operating point in the given set. For each input of pressure and belt speed, it shows how the filter will respond. In this case, it is showing that at a low pressure and slow belt speed the moisture content is very high. This is 
Table 3-1. Belt speed conversion chart.

\begin{tabular}{cc}
\hline $\begin{array}{c}\text { Dial Setting } \\
(\%)\end{array}$ & $\begin{array}{c}\text { Belt Speed } \\
(\mathrm{ft} / \mathrm{min})\end{array}$ \\
\hline 20 & 0.2 \\
30 & 0.8 \\
40 & 1.5 \\
50 & 2.1 \\
60 & 2.7 \\
70 & 3.4 \\
80 & 3.9 \\
90 & 4.4 \\
100 & 4.7 \\
\hline
\end{tabular}

because of the slurry being too deep and not enough dewatering time is available due to the short length of the filter belt. With thick slurry, the cake formation time is greatly increased (see cake formation times of batch tests in Appendix E). Also, from the surface it can be seen that the best operating point is a 60 PSI and lies between a belt speed of $45 \%$ and $75 \%$. Finally, it is interesting to note that while moisture content typically drops as cake thickness drops, that is not the case for the $90 \%$ belt speed and the 60 PSI operating point. The reason for this is simply that the belt is moving to rapidly for the dry cake to form in this situation.

That situation changes, however, once the dewatering reagent is added. This chemical, in addition to helping produce lower moisture contents overall, also has the effect of increasing the rate of dewatering. Figure 3.8 shows the response surface generated by the data from the continuous filter using chemical additives. This response surface is for the material that has been conditioned for five minutes with RV (1:10) at a dosage of $3 \mathrm{lbs} / \mathrm{ton}$. This surface shows even more clearly how strong the effect of pressure is on the moisture content of the product. What is different about this surface is that the edges do not flare up in the same manner that they did with 
Design-Expert $\circledast$ Sof tware
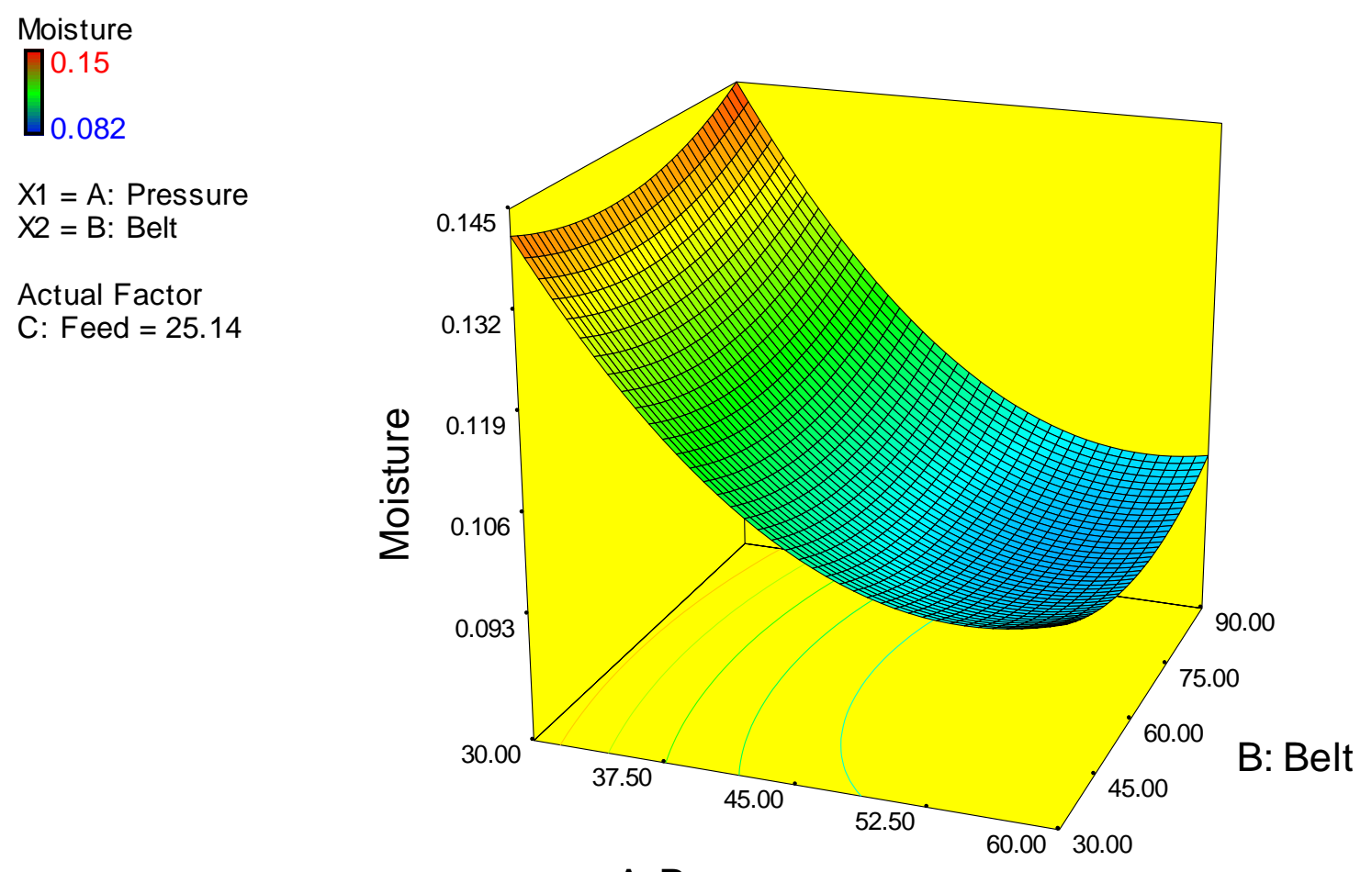

A: Pressure

Figure 3.7. Response surface with coarse feed and $3 \mathrm{lb} / \mathrm{ton}$ reagent RV (1:10).

the chemicals. This is due to the increased rate of filtration associated with the reagents. The thicker cake at the low belt speed can now be dewatered as well as the thin cake that moves quickly across the belt at high speed. It is a little difficult to see, but also the lowest point on the curve is slightly lower than it was without the reagents. Since the scales are different, it is hard to see but the low pressure tests performed much better with the reagents than they did without them. This is also for the same reason. With the lowered surface tension, the filtrate did not require as great a $\Delta \mathrm{P}$ to achieve the same moisture. 


\subsubsection{Air Requirements}

The air requirements for the filter device are important to understanding the cost of operating a production unit. This is without a doubt the most expensive part of the filtration process since it is here that the energy to do the dewatering is applied.

There were several factors that were investigated to see if they had a significant effect on the air requirements of the filter device. These were cake thickness, pressure, feed size, and chemical additions. The other factor that affects the air required is the amount of losses in the air. These losses are the result of air leaking from the pressure vessel into to the discharge without passing through the filtrate.

To approximate how much of the air was being lost, the filter belt was completely sealed off with plastic and then pressurized at the operating pressures to determine how much of the air was being lost. The results of this study are shown in Table 3.2. This loss is a result of an imperfect seal that exists between the rubber belt and the pressure plate. All of the airflow measurements that are shown below have been corrected based on this table.

Table 3-2. Air losses due to imperfect seal.

\begin{tabular}{cccc}
\hline $\begin{array}{c}\text { Pressure } \\
(\text { PSI })\end{array}$ & $\begin{array}{c}\text { Consumption } \\
(\text { CFM })\end{array}$ & $\begin{array}{c}\text { Losses } \\
(\text { CFM })\end{array}$ & $\begin{array}{c}\text { Percentage } \\
\text { Loss }\end{array}$ \\
\hline 30 & 88 & 4 & $4.5 \%$ \\
45 & 103 & 8 & $7.7 \%$ \\
60 & 109 & 12 & $11.0 \%$ \\
\hline
\end{tabular}




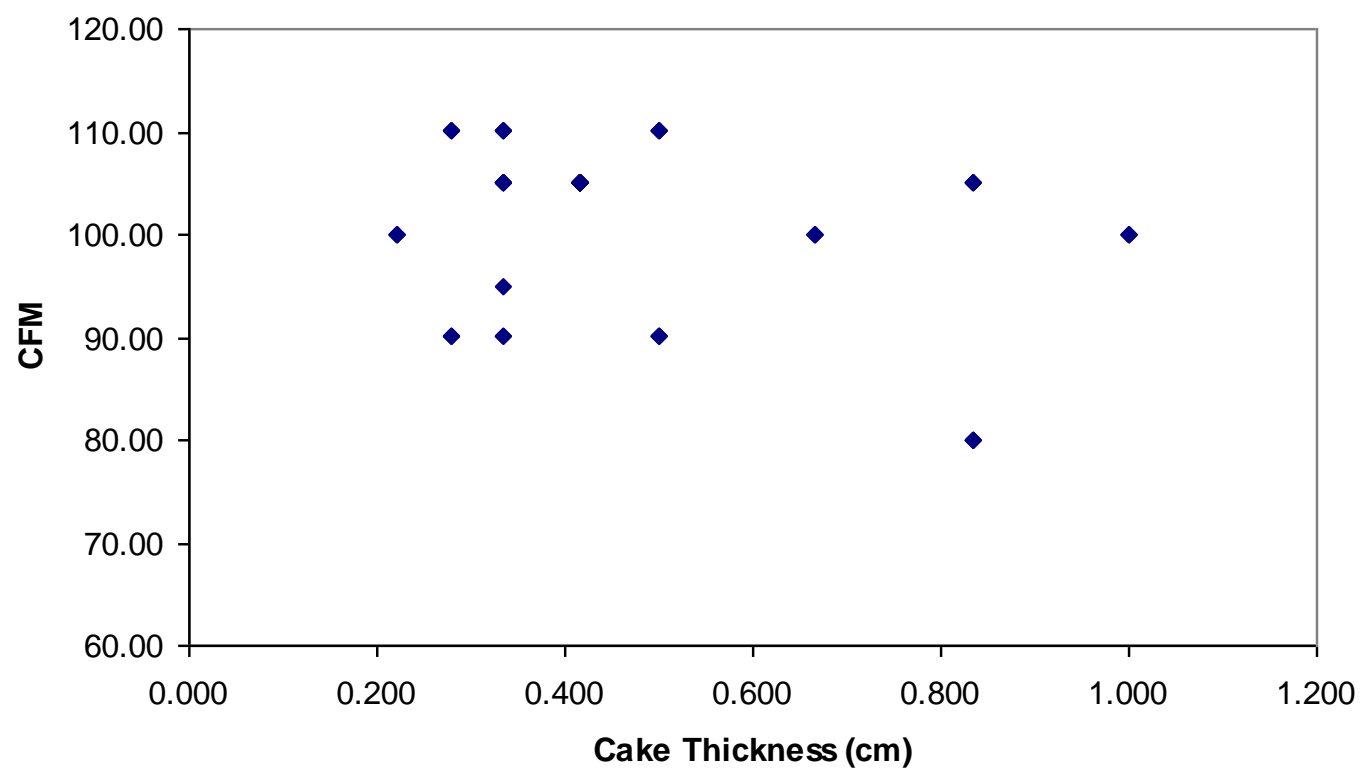

Figure 3.8. Cake thickness vs. air flow rate for coarse feed.

Based on the speed of the belt with the corresponding pumping speed, it is possible to construct an estimate of the cake thickness. After comparing the cake thickness vs. CFM requirements graph, it has been determined that there is only a slight correlation between them. This chart is shown on Figure 3.9.

The pressure vs. CFM chart is shown in Figure 3.10. This chart shows a strong correlation between the two factors. There is clearly a direct relationship between these two. It isn't clear on the chart, there are many more points than it appears, but they are often overlapping.

The last factor that was thought to control the air requirements was reagents. The plot of pressure vs. CFM of both the base tests and the RV tests are shown on Figure 3-11. From this chart it is clear to see that both are directly related and that the coal conditioned with the reagents required more air than the corresponding base test conditions. 


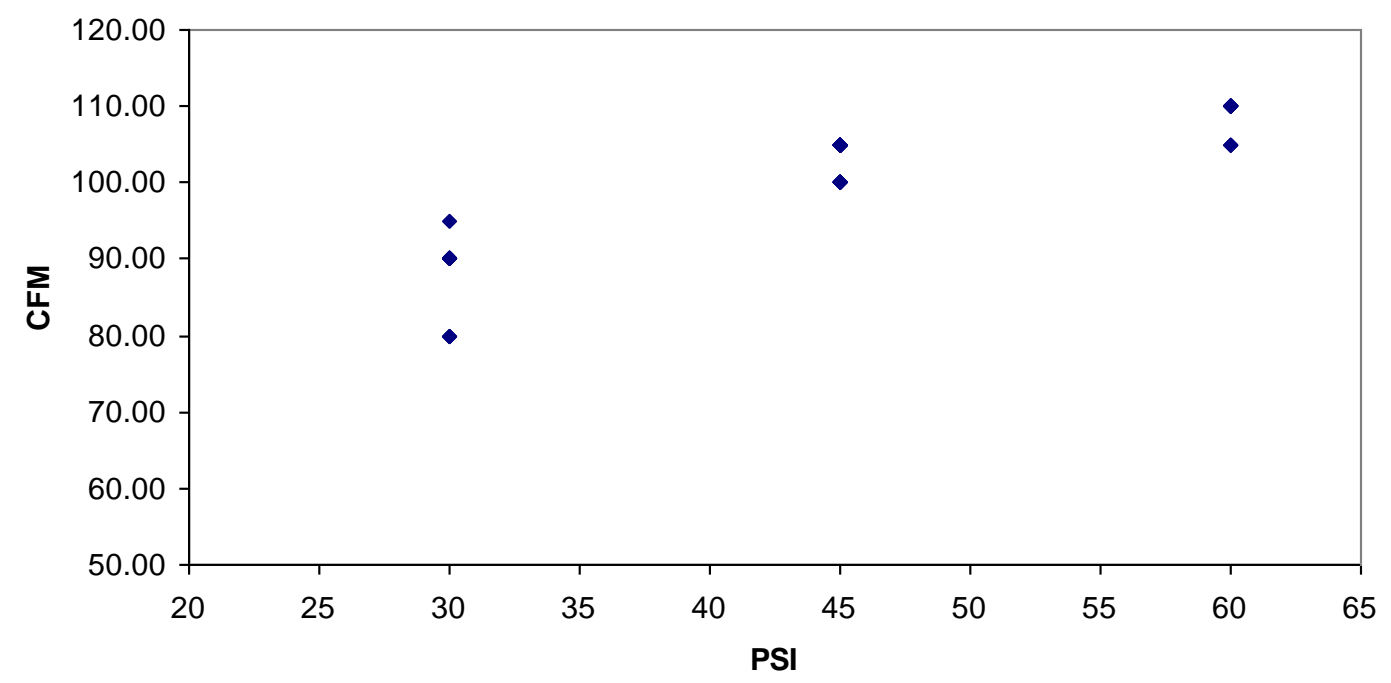

Figure 3.9. Pressure vs. air flow rate for coarse feed.

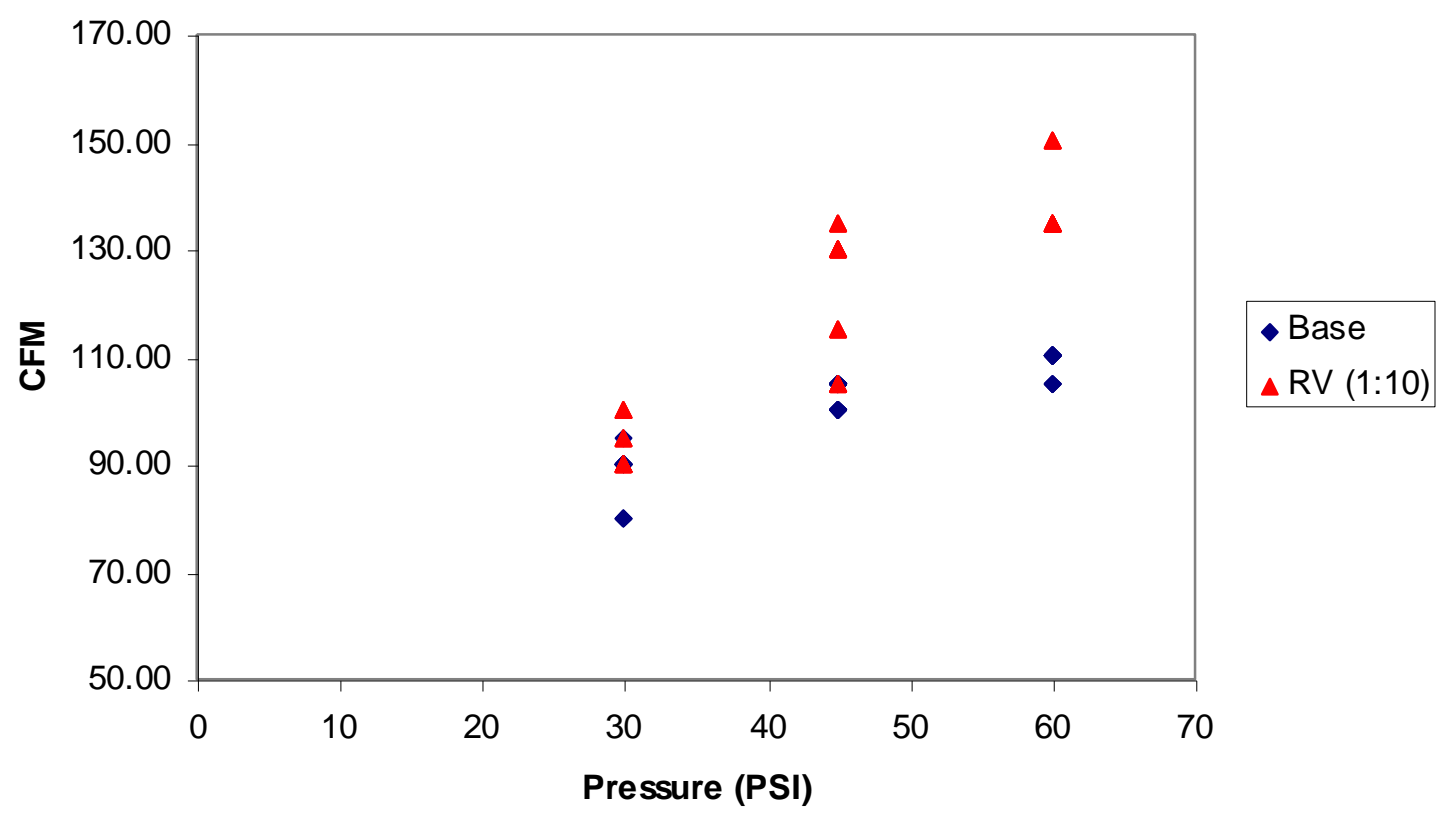

Figure 3.10. Pressure vs. air flow rate for coarse feed (base vs. reagent RV).

From these tests, it is clear that the controlling factor is the pressure in the pressure vessel. The chemical additions also have an effect on the process that causes a wider spread in the air flow and a shift upward in the requirements. This greater variability is caused primary by cake cracking and the increased air requirements are caused by an increase in cake porosity.

\subsubsection{Mass Flow Rate}


The mass flow rate is important to know since it will show what the production capacity of a full size unit would be. This factor is clearly controlled by two factors. That is the percent solids in the feed and the pump speed. This is due to the fact that the amount of material put on the filter directly controls how much comes out as product. The amount of material lost through the filter cloth is important to know. Figure 3.12 shows the mass flow rate into the filter on the horizontal axis and the amount of losses on the vertical axis. It has been found that although losses do increase with more material they increase at a rate slower than the input. This is due to the formation of a bed at the bottom of the filter. This bed once formed greatly reduces the amount of material lost. It is formed at both low and high feed rates.

Figure 3.13 is informative with regard to how the filter is truly performing. It shows the mass cake rate vs. recovery of the filter as an average of all of the values at each mass rate for

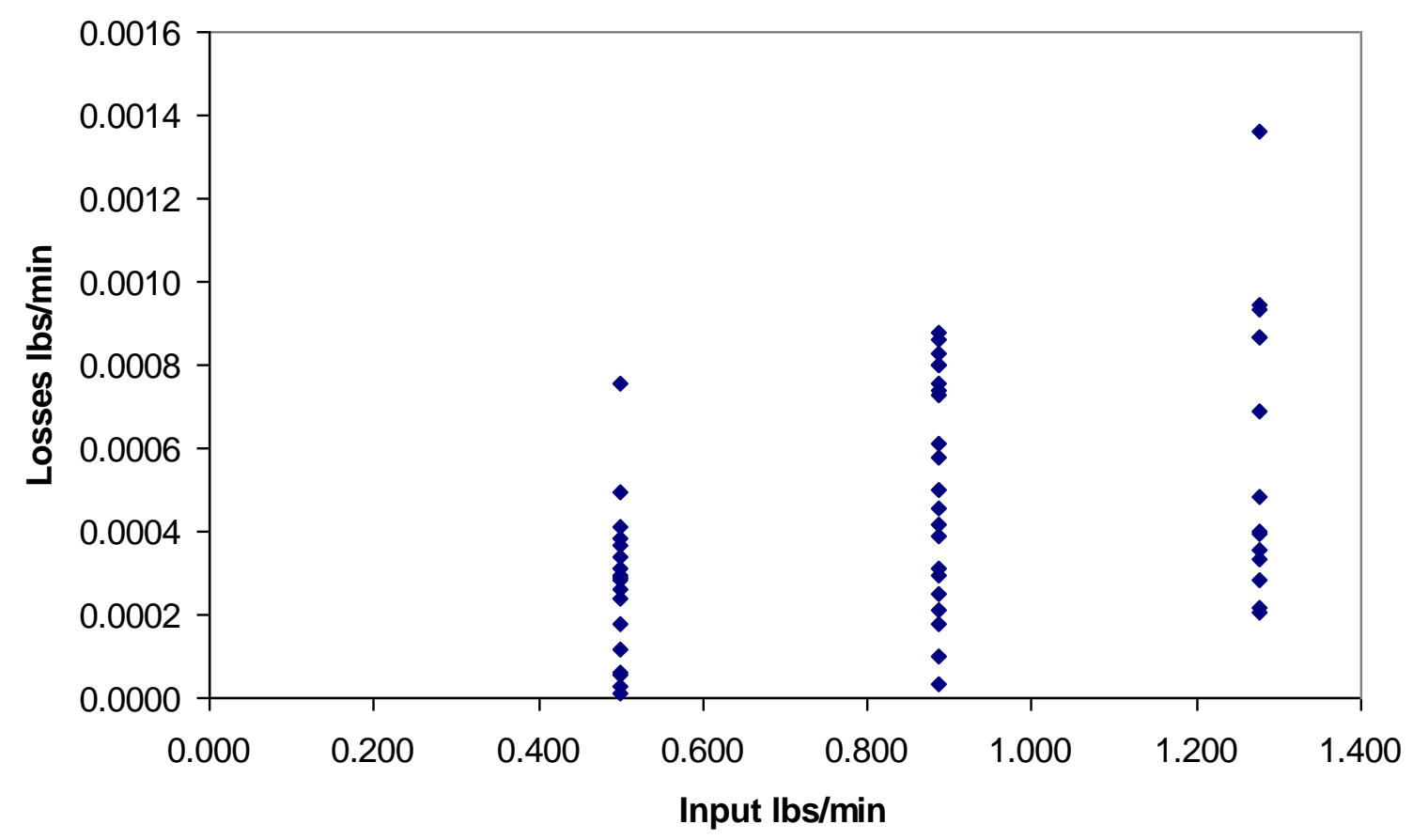

Figure 3.11. Material input vs. losses for coarse feed. 


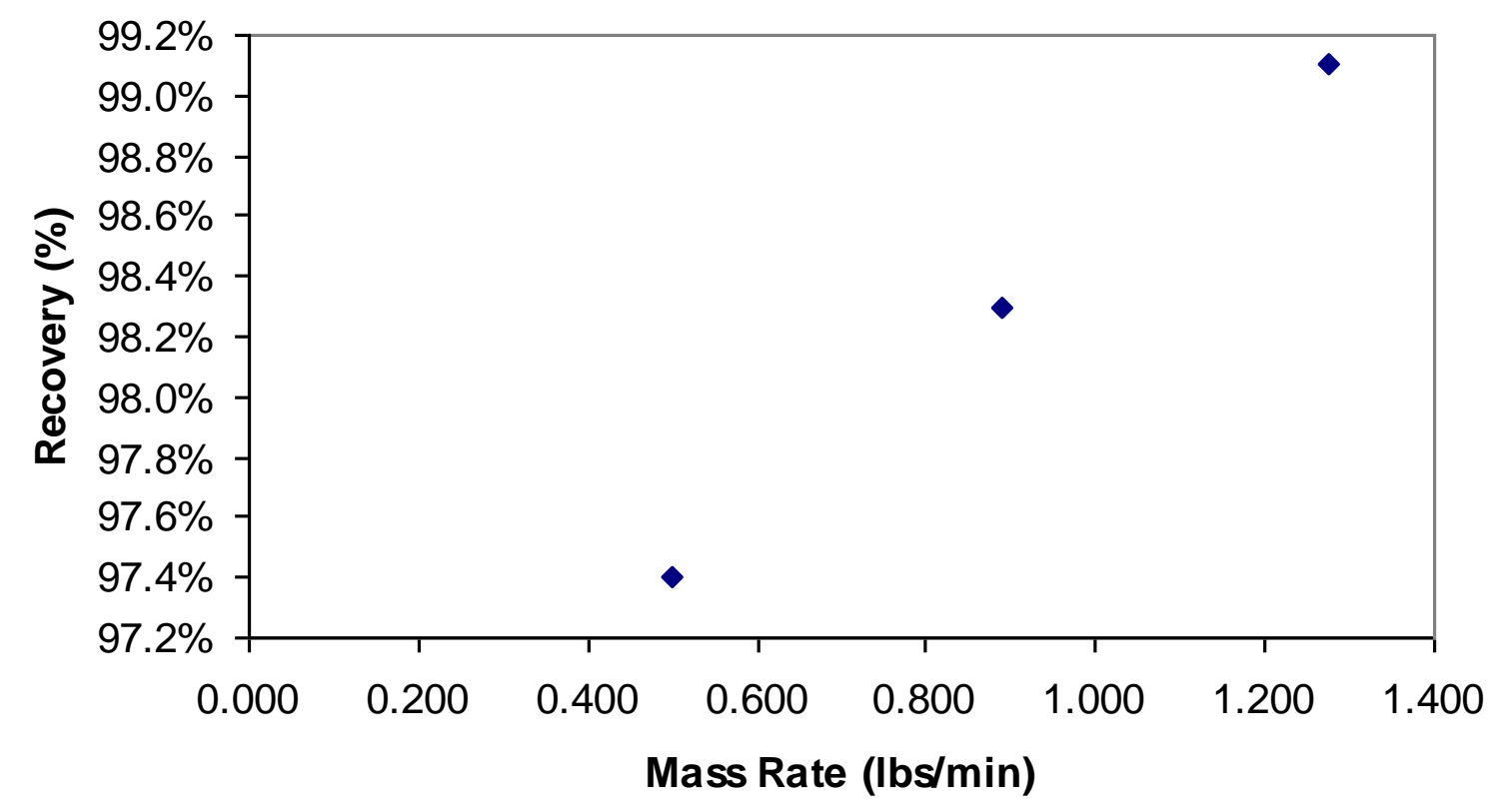

Figure 3.12. Miass rate vs. recovery tor coarse teed.

clarity. The data shows a very slight increase in the recovery of the filter with more material on the filter. If it were possible to put more material on the belt, it is expected that the recovery would asymptotically approach $100 \%$.

\section{$\underline{3.3 \quad \text { Fine Feed Testing }}$}

All of the tests and analysis that was done for the coarse material was also duplicated for the fine material. This material is column product (minus $0.15 \mathrm{~mm}$ ) from Tom's Creek mine. It was tested to see how the belt filter would perform under poor conditions for dewatering. The feed material contains $8 \%$ solids by weight. This material contains $61 \%$ minus 325 mesh. For this reason, all of the tests were conducted with flocculant at a concentration of 1 gallon per 70 tons of coal. The flocculant addition was done by a small pump used to produce very small drops at a slow speed. 


\subsubsection{Moisture Content}

The first tests conducted were designed to see how much variability exists in the filter using the same feed and settings on the pressure filter. The results of these tests are shown on Figure 3.14. It can be seen that the variability is less than that of the coarse feed material on Figure 3.6. The standard deviation of this data is 0.0044 . To keep the testing procedures consistent, it was decided that each data point for the fine feed would also be an average of three test points.

Then same testing schedule was used which is shown in Appendix B. The data from these tests was entered into Design Expert to produce a Box-Behnken response surface. This is a model of how the filter behaves given a set of input conditions. The generated response surface is shown below in Figure 3.15.

The filter in this case performs in much the same manner as it did for the coarse material.

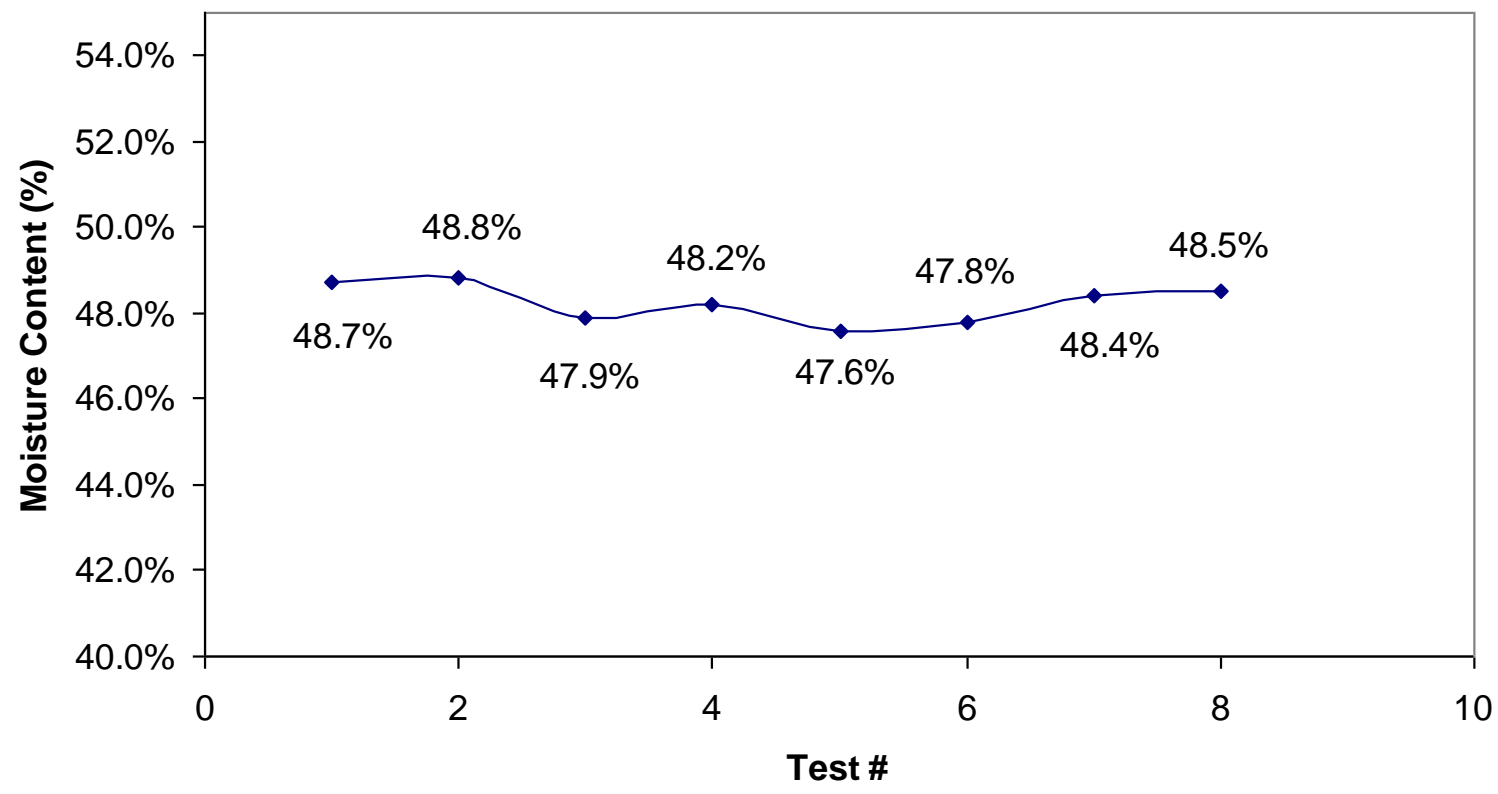

Figure 3.13. Variability of the fine feed data ( $25 \%$ feed pump speed). 
Design-Expert $\circledast$ Sof tware

Moisture

- Design points above predicted value

- Design points below predicted value

0.902

0.35

$\mathrm{X} 1=\mathrm{A}:$ Pressure

$\mathrm{X} 2=\mathrm{B}:$ Belt

Actual Factor

C: Feed $=25.00$

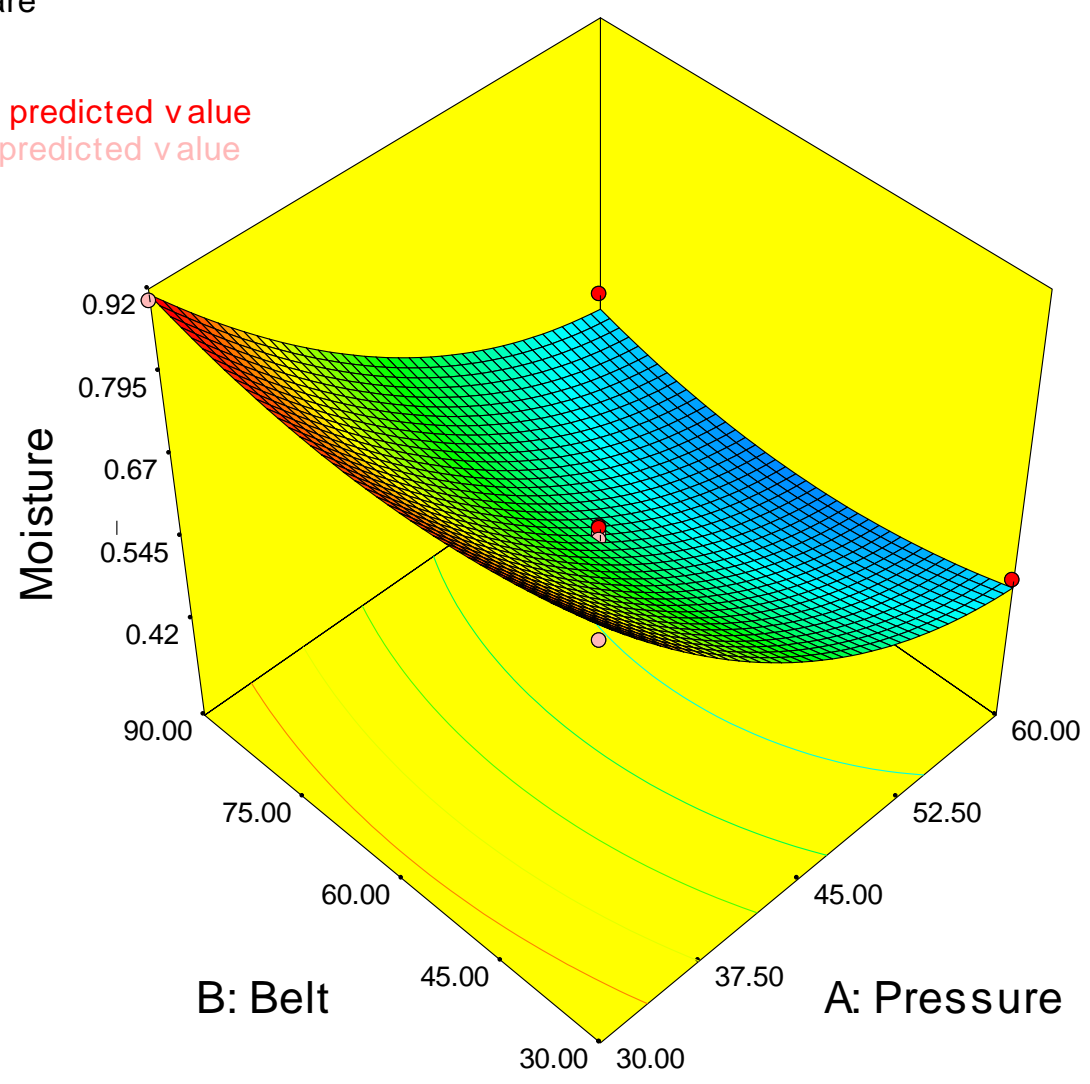

Figure 3.14. Response surface for fine feed and no chemicals.

The most noticeable difference is that the moisture content is much higher than for the fine material. This is due to the fact that it takes more force over a longer period of time to dewater this material. The belt filter was not long enough to give this material sufficient time to fully dewater. From the response surface it is easy to see that the best operating point for moisture was at 60 PSI of pressure and a belt speed of 60 percent.

Based on the results found in the batch testing of this material it was determined that the best reagent to use is the RV (1:10) at $3 \mathrm{lbs} / \mathrm{ton}$. This was then added to the sample and tested. 
The results were input into Design Expert which created the response surface shown in Figure 3.16 .

The response surface shows how the filter behaved in this situation. This material has a lower moisture at every operating point compared to the results without the dewatering chemicals. Most of this gain was realized because of the greatly improved rate of dewatering that was realized. The worst point occurred when the pressure was low and the belt ran slow. This created a situation where the driving force was low and the cake thickness was high. This situation simply did not produce cake formation rates that were fast enough. The best operating point was at high pressure and high belt speed. This was because this had the thinnest cake which realized the greatest moisture reduction. The other corners of the design also exhibit the same

Design-Expert® Sof tware

Moisture

$\prod_{0.291}^{0.793}$

$\mathrm{X} 1=\mathrm{A}$ : Pressure

$\mathrm{X} 2=\mathrm{B}:$ Belt

Actual Factor

C: Feed $=25.01$

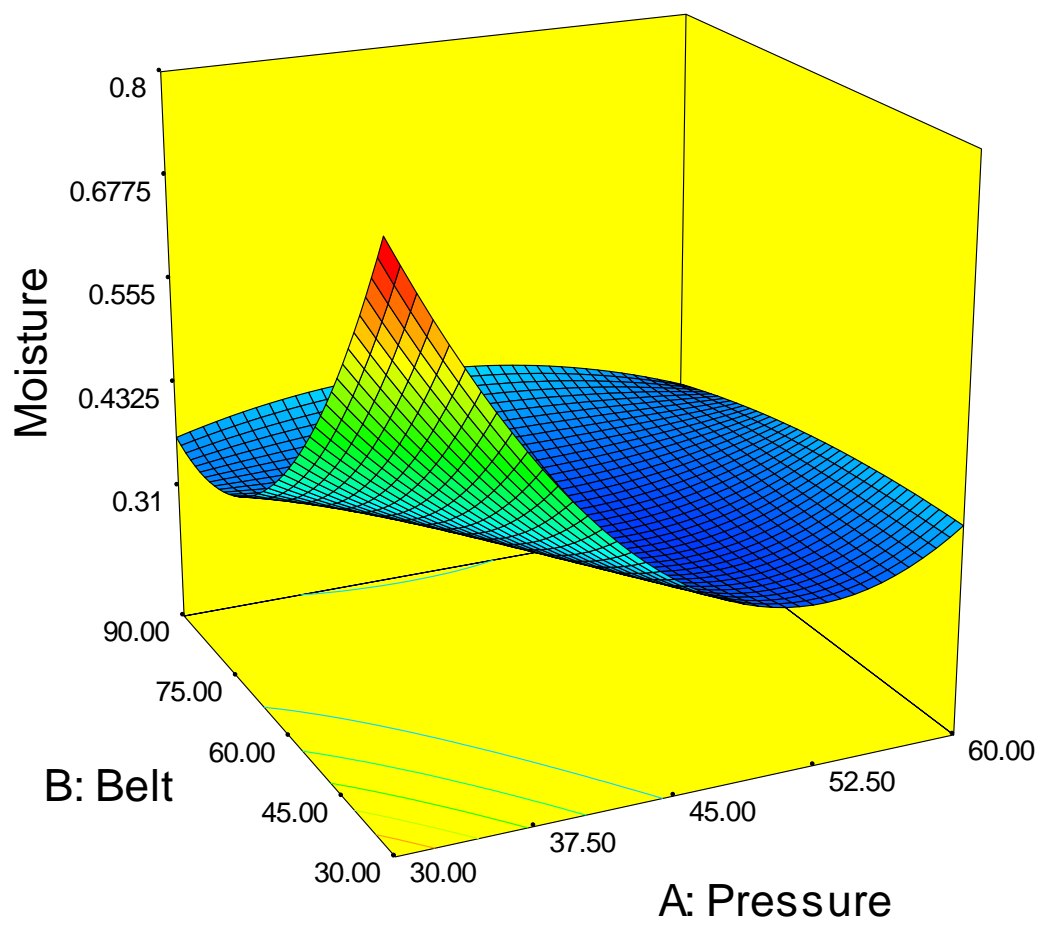

Figure 3.15. Response surface of fine feed with $3 \mathrm{lb} /$ ton reagent RV (1:10). 
behavior only with more moderate results.

\subsubsection{Air Requirements}

The air requirements for the fine feed are important to understand the cost of operating the belt filter. Just as in the coarse feed there are several possible factors that affect the air requirement these are, cake thickness, pressure, chemical additions, and size of the feed.

Figure 3.17 shows the plot of cake thickness vs. air requirements. This chart, like the one for coarse feed, shows that that cake thickness in the filter is not a major determining factor for air consumption.

Pressure was a major controlling factor in the air consumption in the coarse feed material. The addition of chemical reagents also had a shifting effect on the air consumption of the unit. Figure 3.18 shows both the feed with no reagent and that with reagent. From Figure 3.18 it is seen that pressure is a controlling factor for air requirements and that the use of the reagent also

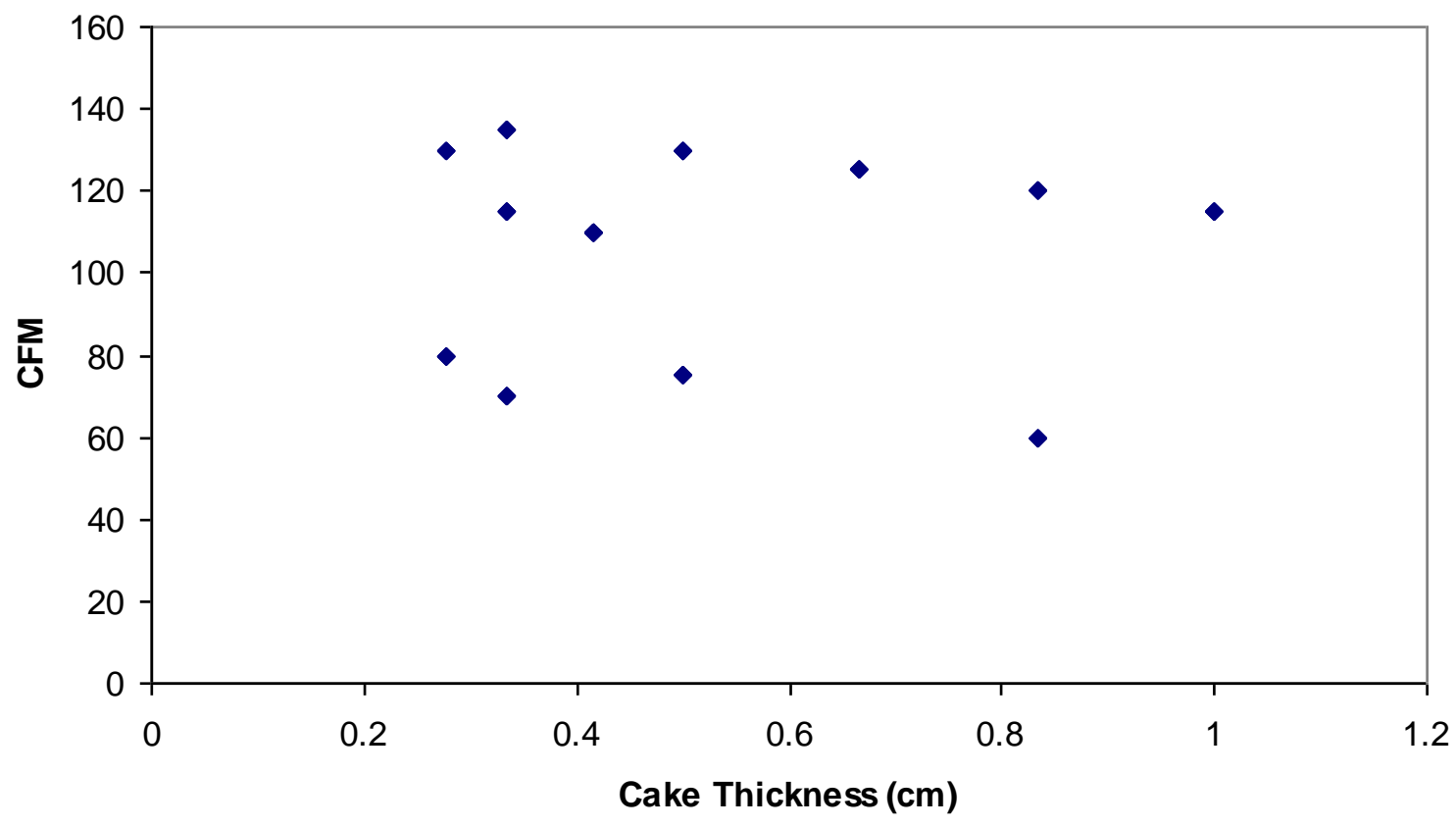

Figure 3.16. Cake thickness vs. air flow rate for fine feed. 


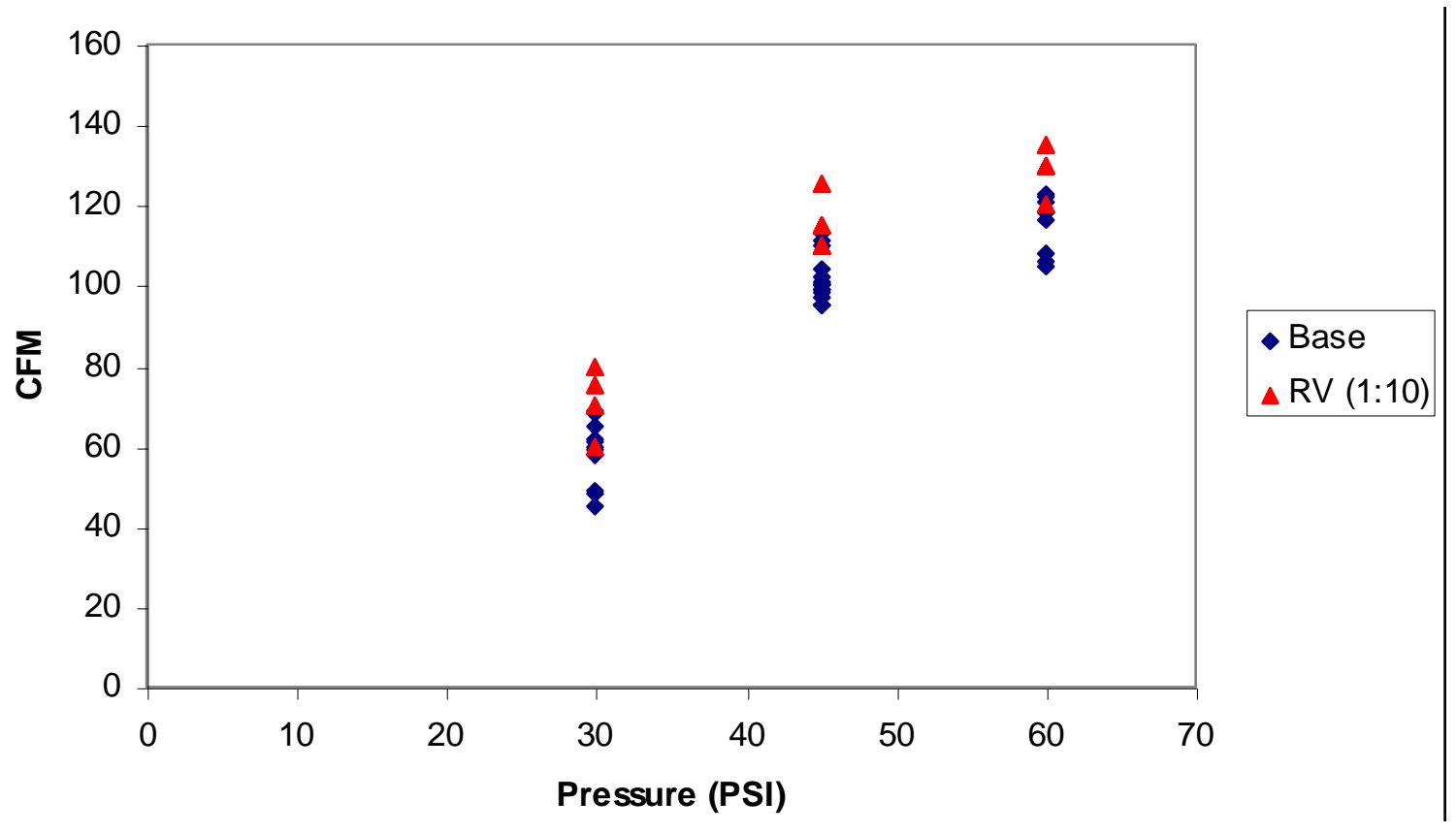

Figure 3.17. Pressure vs. air flow rate for fine feed (base vs. reagent RV).

increases the requirement.

The comparison between the air requirements for the two different feed types is shown on Figures 3.19 and 3.20. On the chart with no reagent it is difficult to discern any meaningful difference in air flow from the two types of feed. This is not true on the material with RV. On this diagram, it shows that the coarse material has a higher airflow than the fine material. This is most due to the greater size in the cracks in the and due to the higher porosity that is created in the cake that contains large particles compared to the small ones.

\subsubsection{Mass Flow Rate}

The mass flow rates are essential for developing a scale up model for this pressure filter. Figure 3.21 shows the mass flow rate vs. losses rate. From this plot, it is shown that the loss rate 


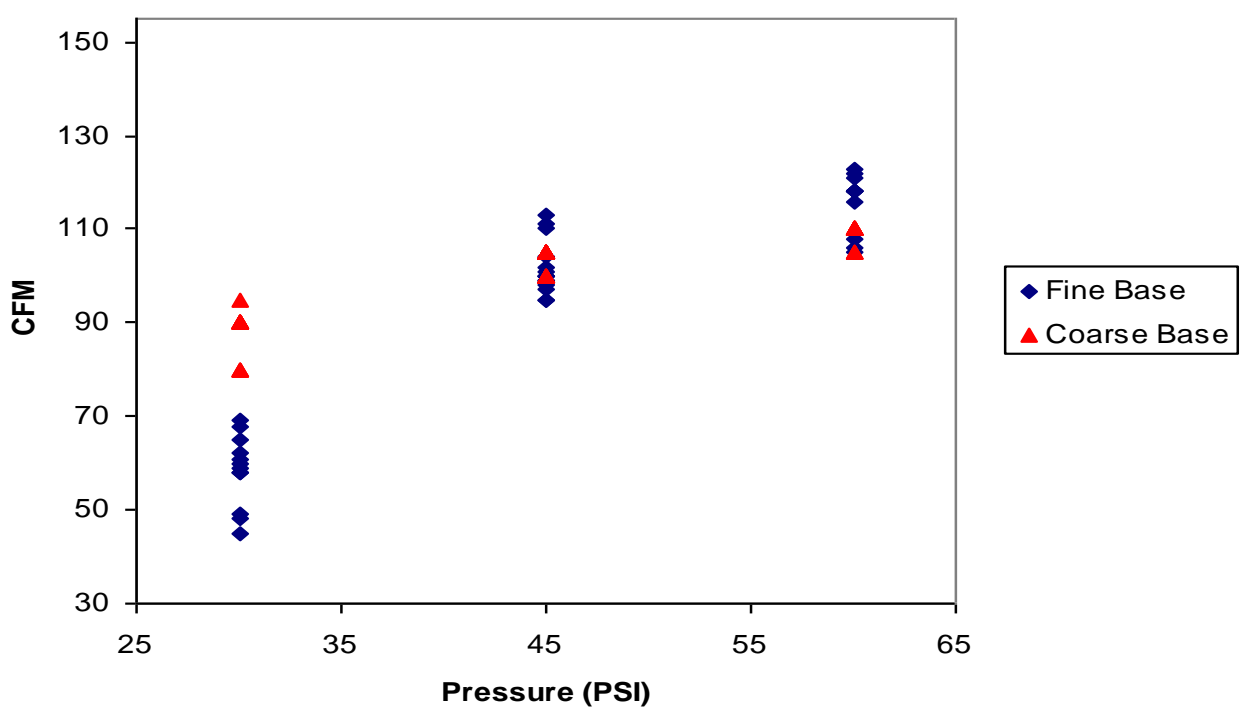

Figure 3.18. Pressure vs. air flow rate for coarse and fine feeds with no chemicals.

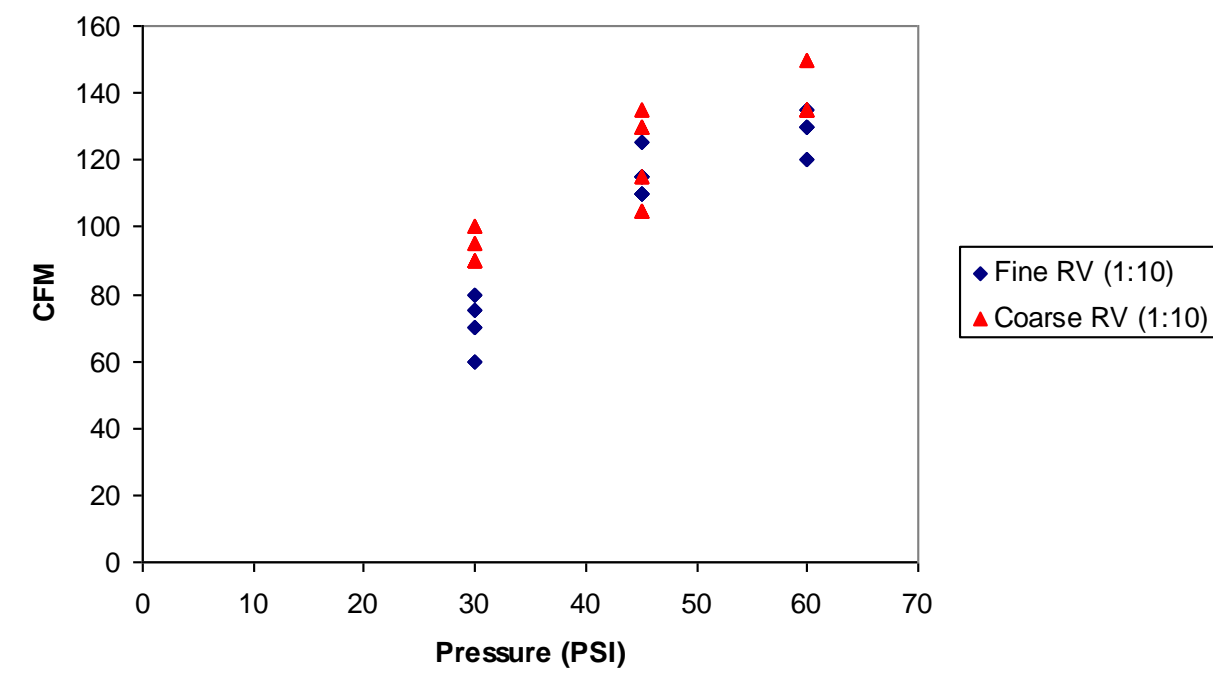

Figure 3.19. Pressure vs. air flow rate for coarse and fine feed with reagent RV.

increases when the feed rate is increased. This is not unexpected since there is more ultrafine material in this feed.

Figure 3.22 shown below is of the input mass rate vs. recovery. This chart shows that there is little change in the recovery of the filter as the mass rate is increased. This is due to the large amount of ultra fine material in that belt that helps slow the rate of dewatering. The time 


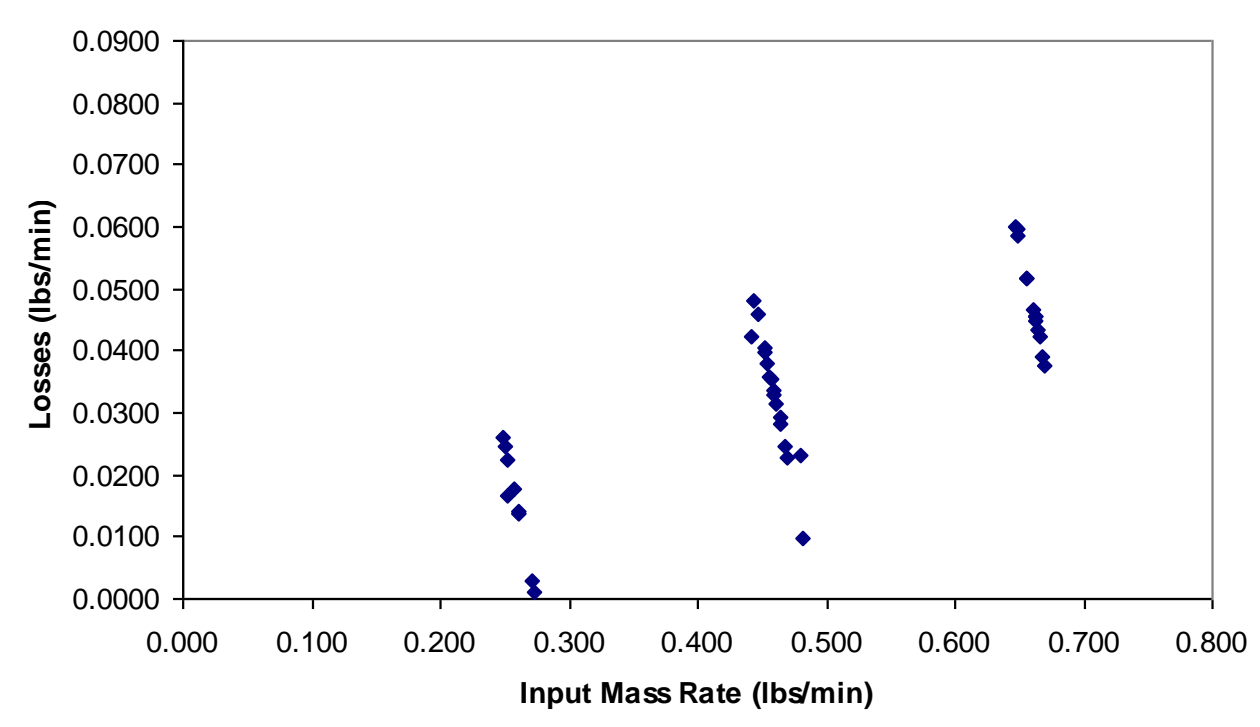

Figure 3.20. Mass rate vs. losses for fine feed.

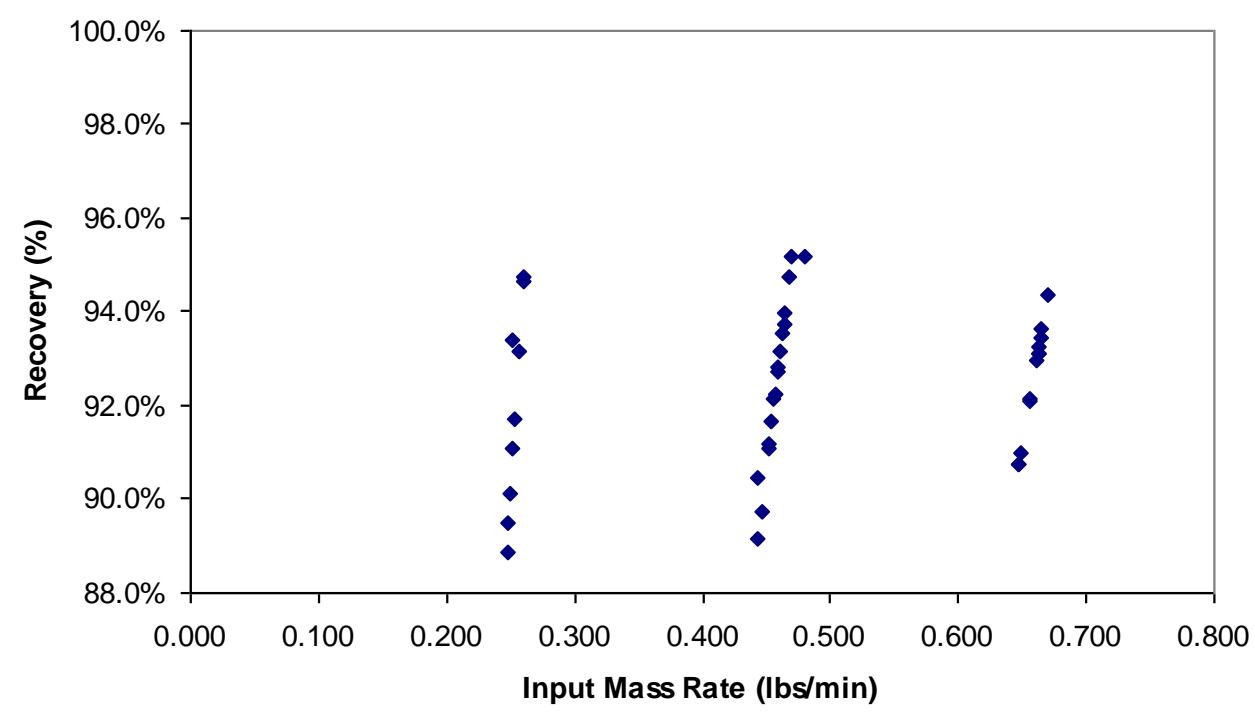

Figure 3.21. Mass flow rate vs. recovery for fine feed.

for the cake to form is increased by a large amount which gives much of the ultra fine material time to escape through the filter cloth before the cake forms to provide a barrier.

\subsection{Mixed Feed Testing}

A mixed feed was the final type of feed that was researched. In this group, the coarse material is mixed with the fine material to produce a feed that has a more evenly dispersed size 
distribution. The size range is still $1 \mathrm{~mm}$ x 0 as with the coarse feed but it has more minus 325 mesh material. This material is $38 \%$ minus 325 mesh, compared to the coarse feed which was $16 \%$ and the fine feed which was $61 \%$. Figure 3.23 show the consistency of the product obtained using the mixed feed. For this group of tests, the standard deviation is 0.0048 .

\subsubsection{Moisture Content}

Once the variability of the data was established, the same series of tests was once again run for mixed sample. The results were then put into Design Expert and analyzed. From this analysis, it was possible to generate a response surface. Figure 3.24 shows the response surface for the mixed data without any chemical additions.

From the surface, the optimal operating point for this material is with a high belt speed and high pressure. This would result in shorter drying times and thinner cake. Also, for this particular feed, the thin cake helps prevent a layer of slimes from coating the surface and

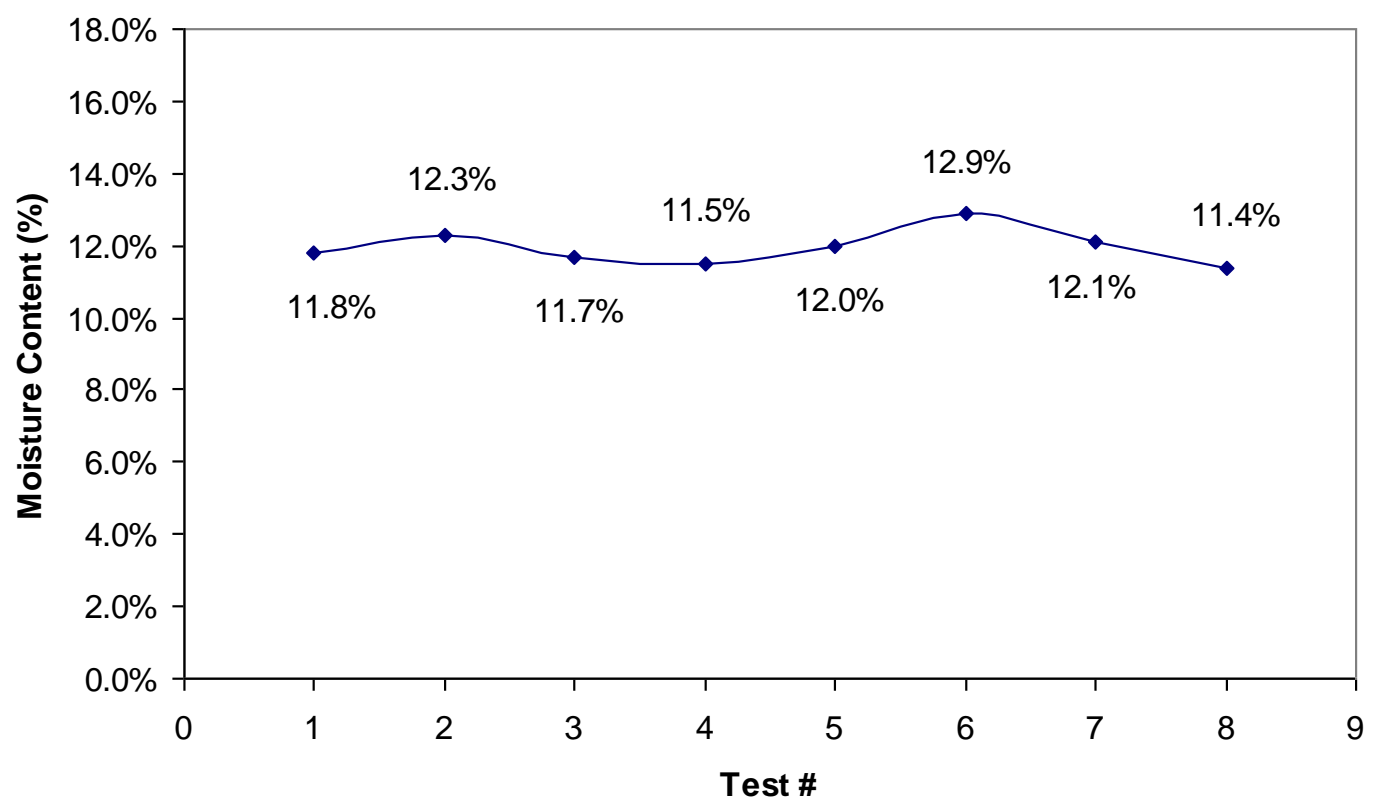

Figure 3.22. Variability of the mixed feed data ( $30 \%$ feed pump speed). 
Design-Expert@ Sof tware
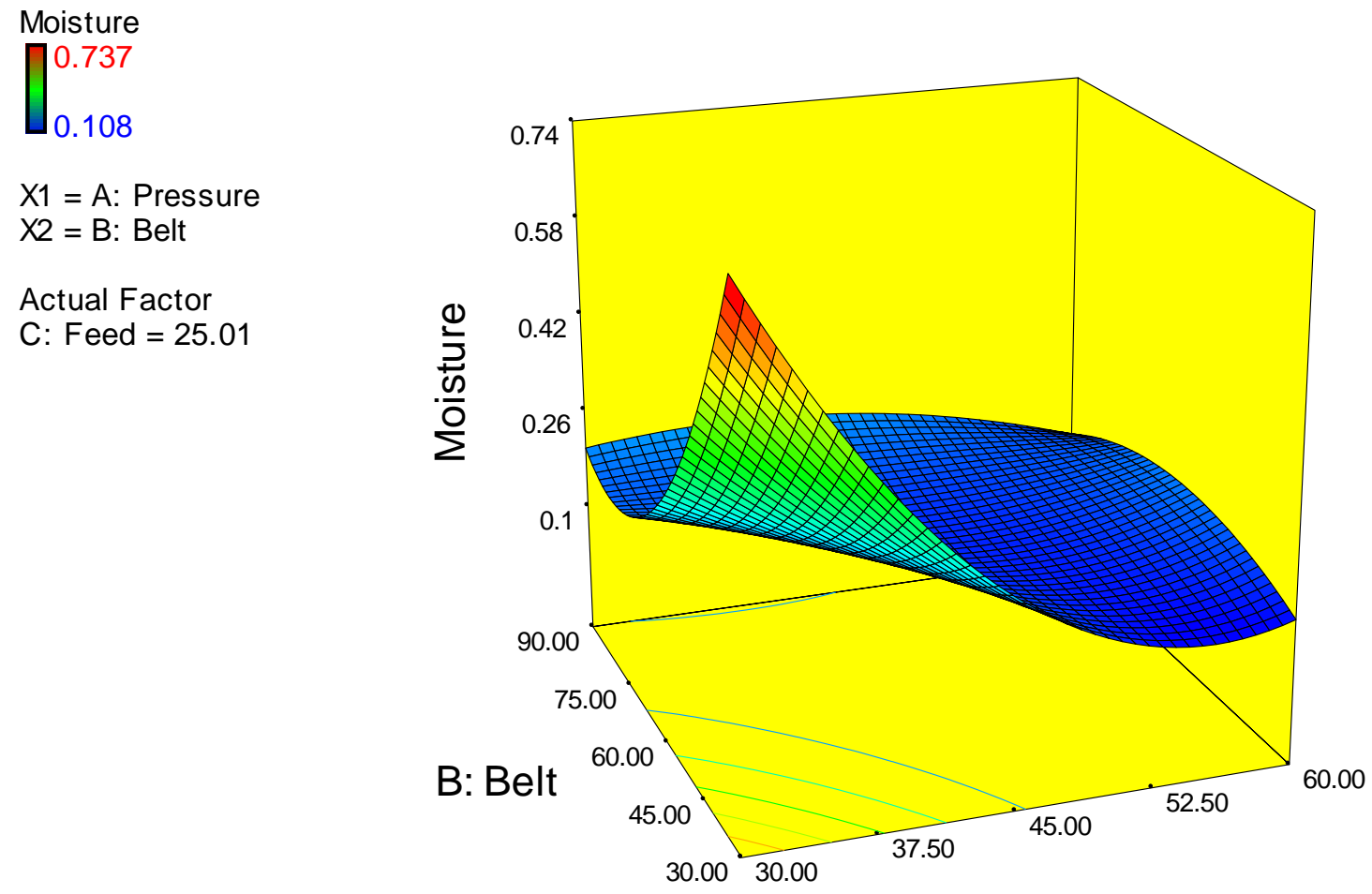

A: Pressure

Figure 3.23. Response surface for mixed without chemicals.

blocking the air from penetrating the cake. The very high moisture that is shown at a slow belt speed and low pressure is the result of the belt flooding due to a cake thickness that is too great and not a long enough time for it to dewater.

Also done were tests on the unit with chemical additions that had been made. These were also completed with RV (1:10) at 3lbs/ton. The resulting response surface is shown in Figure 3.25. This chart indicates that the moisture content can be lowered by a significant amount from those done without the reagents. The moisture reduction is approximately 2-3 percentage points. The best operating conditions for the material under these conditions is with a high pressure and a moderate ranging belt speed. This is a normal optimal condition which is expected. 
Design-Expert® Sof tware
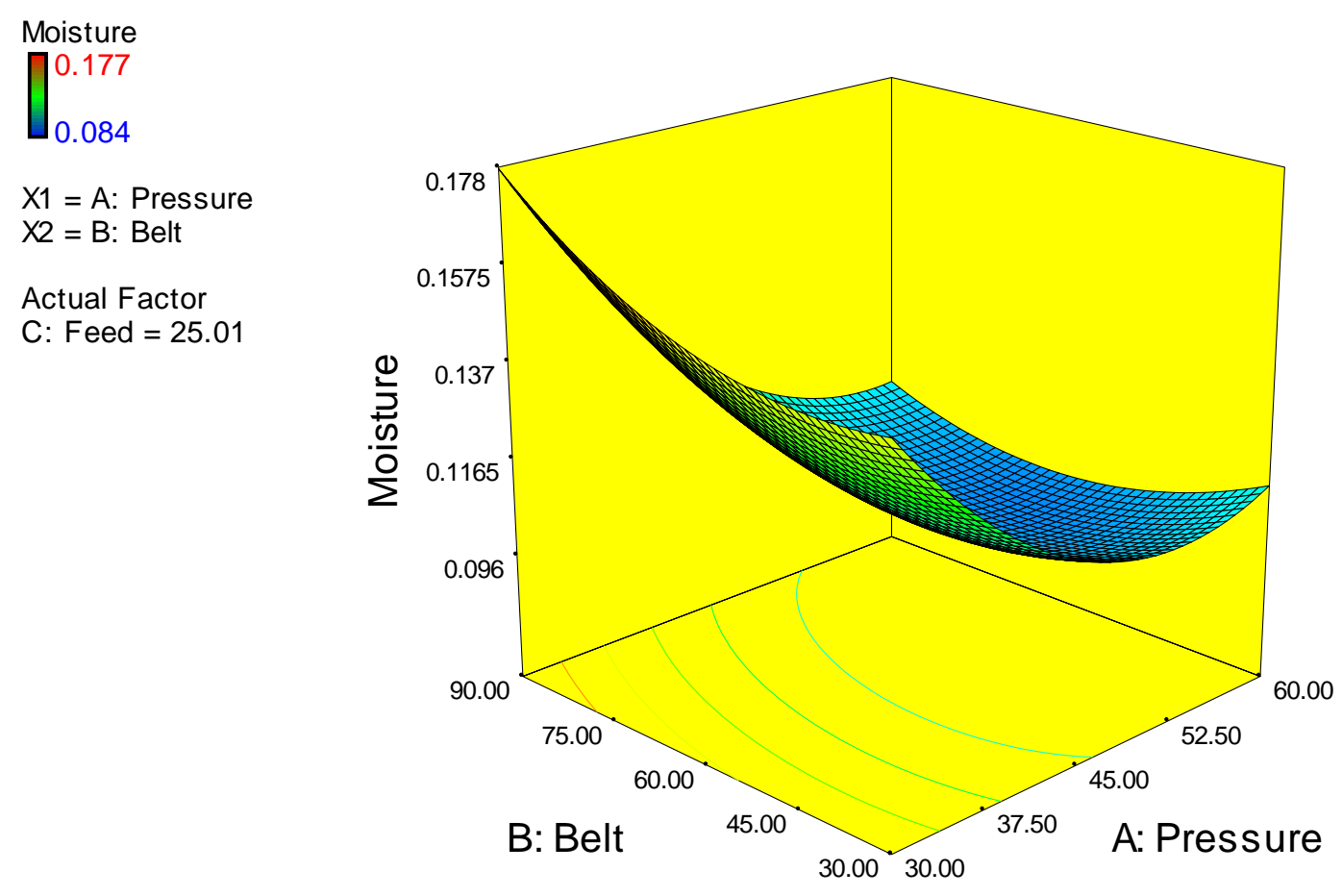

Figure 3.24. Response surface for mixed with $3 \mathrm{lb} /$ ton reagent $\mathrm{RV}(1: 10)$.

The amount of air consumed by the pressure filter during the tests changes depending on the conditions of the test. The amount of air consumption varies primarily with the amount of pressure in the pressure vessel. This can be seen on Figure 3.26.

The amount of air consumed is similar to that of both the fine feed material and the coarse feed material. It largely fits between the two as is seen on Figure 3.27 and Figure 3.28. One of the plots is of the reagent treated material, while the other is with no chemicals material. This further indicates that as the size of the material to be filtered increases, so does the amount of air required for effective filtration. 


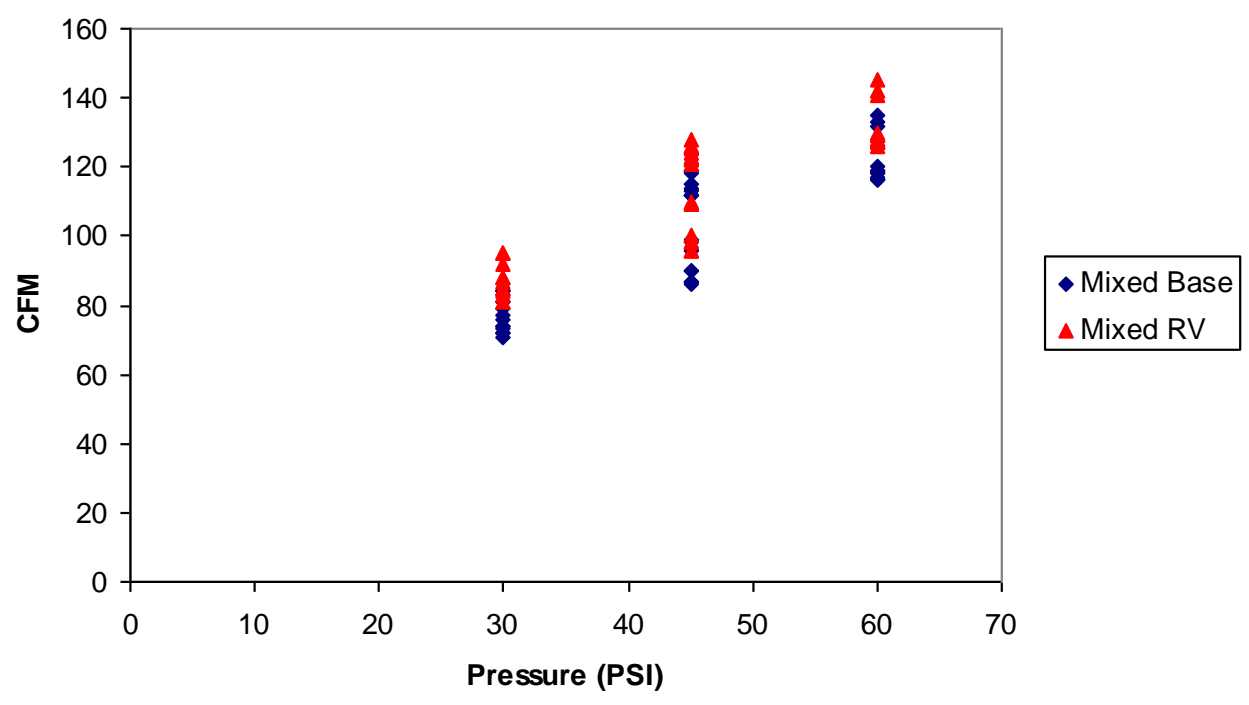

Figure 3.25. Air requirement for mixed feed.

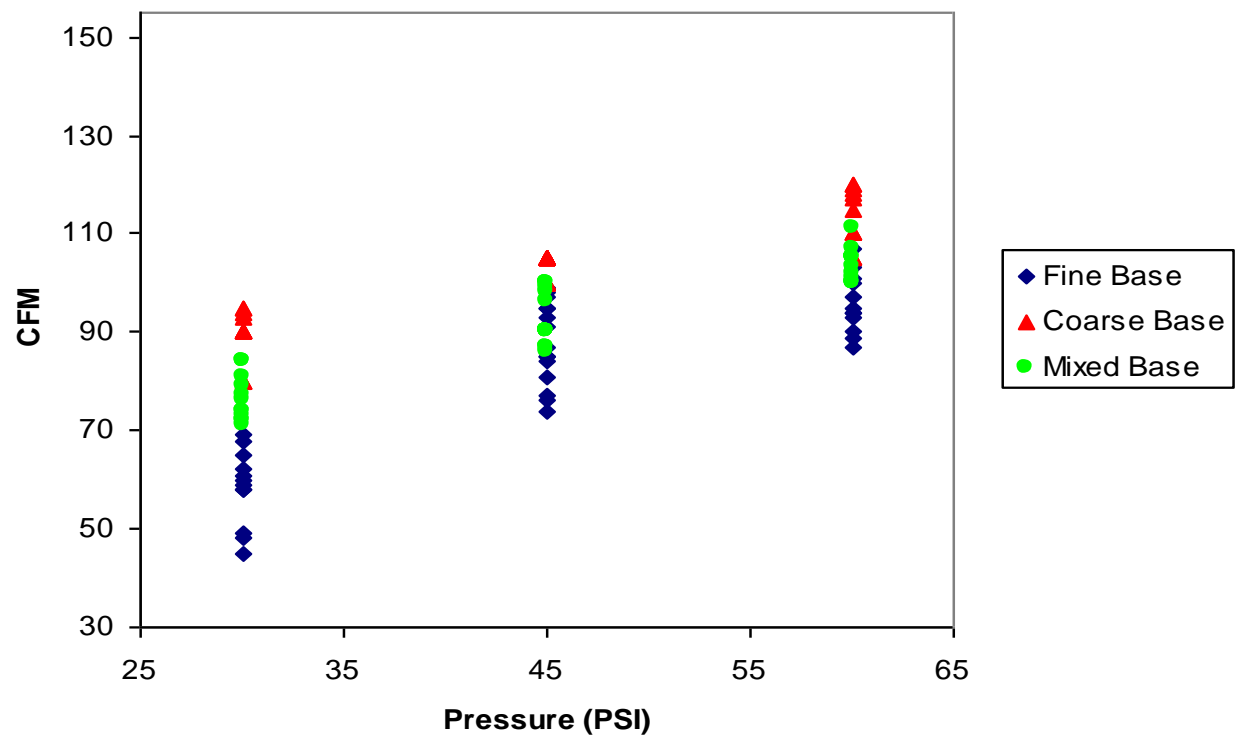

Figure 3.26. Base air flow requirements for mixed feed.

\subsubsection{Mass Flow Rate}

The mass flow rate of this material is of importance in scaling up the equipment to determine how large, a production capacity unit would have to be. The mass flow rate vs. recovery chart is shown on Figure 3.29. This shows that as the amount of material on the belt 
increases so does the recovery of the filter. For this reason there are advantages to creating a filter with high capacity since it will also result in a higher recovery.

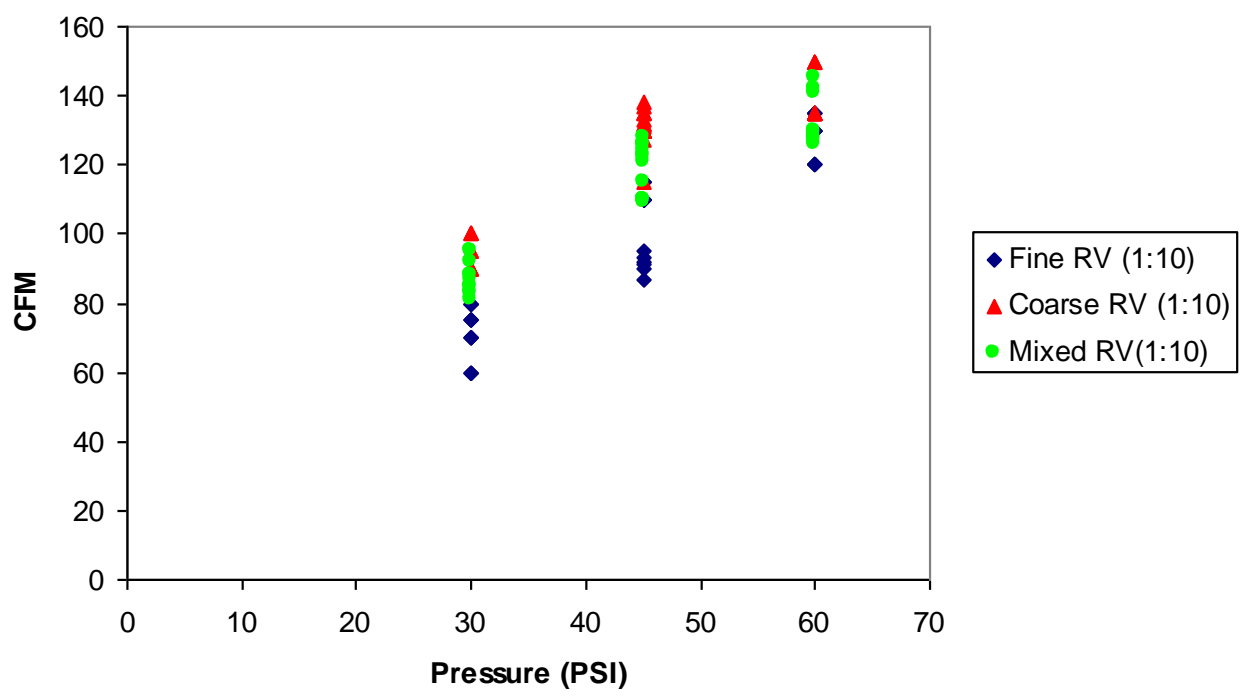

Figure 3.27. Air flow comparisons for chemically treated feeds.

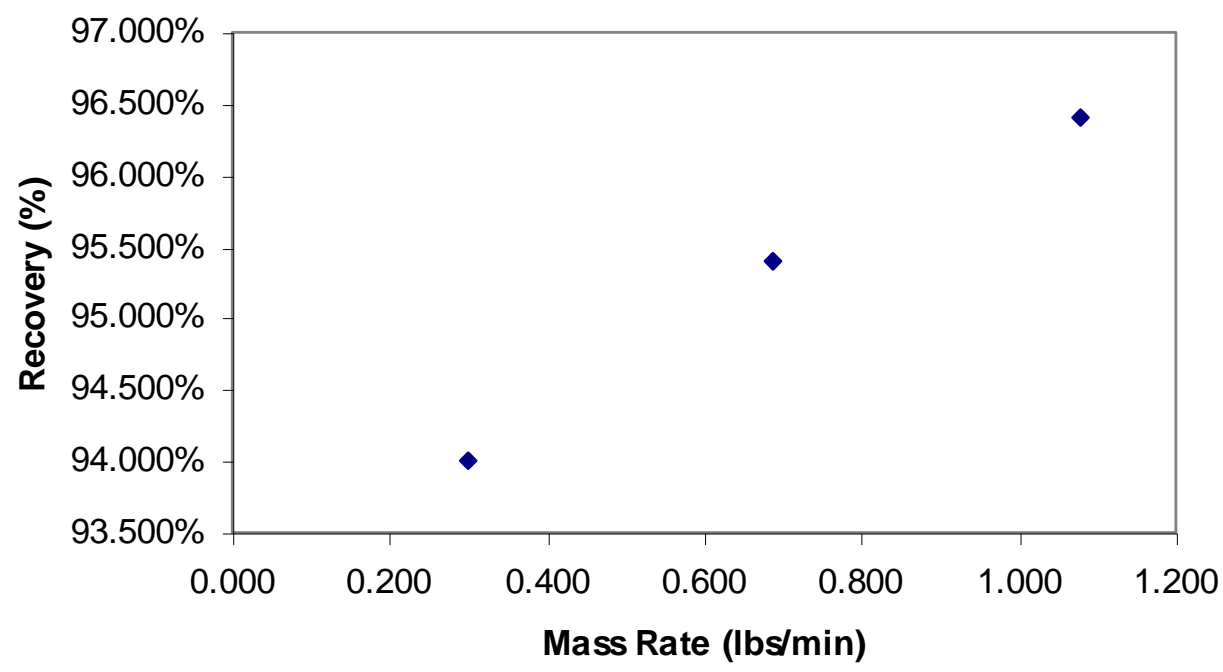

Figure 3.28. Mass rate vs. recovery for mixed feed. 


\subsection{Discussion}

From these results it is possible to scale up this unit and to predict the behavior and requirements of a larger full production capacity unit. This process has several assumptions that must be made and can therefore only provide an estimate as to the performance of such a unit.

\subsubsection{Discrepancies}

In terms of scale up, most of the discrepancies exist in the fine feed batch testing compared to that of the continuous filter. The lowest moisture numbers for the batch test are approximately 14\%, while the lowest moisture numbers for the continuous unit are approximately 29\%. The primary reason for this difference is the dewatering time. In the batch unit, the total time can at times be almost 3.5 minutes. Across the belt the longest time that is has is only 2.5 minutes. Also, the slower the belt moves the greater the cake thickness thus making dewatering even more difficult. If the belt were longer, it would be possible to maintain a thin cake and allow the proper amount of dewatering time. If this occurred, then it is possible that it would produce similar moistures to the batch unit.

The coarse material did not respond in the same manner on the continuous unit as it did on the batch unit. It was not successful at significantly reducing the moisture content of the product. It did, however, increase the rate of dewatering thus allowing greater throughput on the machine. This rate change can be seen from comparing Figure 3.7 and Figure 3.8, where the great improvement was made when the pressure was 30 PSI. The value of this addition will be further explored in the scale up section.

\subsubsection{Scale-Up Analysis}

To do a meaningful scale up the results of two different size machines should so a relationship. The most important factor in this design is the moisture content of the product. For 
this reason, the scale up analysis will be done primarily based on the coarse feed material since its findings can be confirmed more definitely with those of the batch unit. The computer models also suggest that if the dimensions of the filter belt were altered to increase the dewatering time that the fine material would more closely correspond to the lab results.

The scale up analysis will look into determining approximately how much energy would be required to operate a unit with a capacity similar to that of other dewatering equipment. Finally, there are some major design modifications that are suggested for a full time operating unit.

\subsubsection{Power Requirements}

To build a full size capacity unit, the optimal operating point will be used as the design criteria to design for. This is the best that a unit built just like this one could achieve. There are two processes that occur in this unit that require power. The first is turning the rubber belt. This requirement is essential but its overall power requirements are dwarfed by the second requirement. Second is the air compressor that will need to produce large amounts of compressed air continuously.

The optimal operating point produces a cake with $10.7 \%$ moisture required 110 CFM of air, while producing $1 \frac{1 / 2}{2}$ pounds of coal per minute. Assuming that the air consumption has a linear relationship with capacity as it scales up, a unit capable of handling 50 tons per hour would require approximately 120,000 CFM. To produce this much air would require approximately18,360 horsepower. Calculations of this value are shown in Appendix D. The cost associated with load can be calculated as:

$$
\text { Cost }=[18,360 \mathrm{HP} \times 0.747 \mathrm{~kW} / \mathrm{HP} \times \$ 0.04 / \mathrm{kW}-\mathrm{hr}] /[50 \mathrm{ton} / \mathrm{hr}]=\$ 10.97 / \mathrm{ton}
$$


Using the dewatering reagent, it is possible to obtain the same moisture content but with the same amount of air to produce $2 \frac{1}{2}$ pounds of coal per minute. By making the same assumptions, it would require 73,000 CFM of air. That would require 11,169 horsepower to operate. This would reduce the gas compression cost to only $\$ 6.67 /$ ton (i.e., $\$ 10.97$ x 11,169/18360 $=\$ 6.67$ ).

\subsubsection{Suggested Design Modifications for a Production Unit}

A full size production unit will make some design changes to increase reliability and maintenance. The most significant change will be to move the belt drive motor outside of the pressure vessel. This is because inside the pressure vessel is a wet and corrosive environment that would cause the motor to decay at a rapid pace. This new design would most likely involve a sealed bearing connecting a shaft to the outside of the vessel to allow the motor to sit on the outside.A full production unit would also include a way to easily access the water sprays used to clean the belt. This would be done because these water sprays are notorious for getting plugged.

Also, a production unit would design a more robust tracking control system for the filter cloth. At present the filter cloth does not move more than a few inches in either direction. If the tracking did get off it would require the machine to be shut down and disassembled to fix it. A unit designed to be run for more than 100 hours a week would include a way to shift the tracking on the filter cloth without shutting down the equipment.

Finally, for a unit that is built to dewater the fine material, the dimensions of the unit need to be modified. This modification will include an increase in the length of the dewatering section of the belt relative to all the other dimensions. This will allow for longer dewatering times and not increase the thickness of the cake. If this design modification were employed the lab data from the coarse material suggests that it should be possible to produce cake moistures that are similar in value to those of the batch unit. 


\subsection{CONCLUSIONS}

This research set out with two objectives. The first was to design and build and operating pilot-scale hyperbaric belt filter. The first objective was successfully completed when the unit successfully demonstrated a significant moisture reduction with a coal slurry feed. Once this was accomplished and shown that it could be achieved repeatedly, the shakedown tested period came to a close and the unit was declared operational. When this happened, the first goal of constructing an operating device was completed.

The second objective was to determine how low of a moisture content could be achieved via parametric testing of the prototype under various operating conditions. The results obtained from these parametric tests showed that the moisture content of the product depends on many factors including feed particle size, dewatering aid addition, belt speed and vessel pressure. Not surprisingly, the unit was also much more effective in dewatering coarse particles than fine particles. For the coarsest feed material containing $76 \%$ moisture, the unit provided a product moisture of $9 \%$ by weight. This value was even further reduced by adding dewatering aids, which brought the final moisture down to $8 \%$. While the finest feed material provided a relatively high moisture of 35\%, the presence of dewatering aids reduced the product moisture to $29 \%$. As such, the chemical additions had the ability to reduce the moisture content of the fine material in a significant way.

For the case of belt speed, a mid-range value between $40 \%$ and $75 \%$ of full speed was found to provide the best results. Likewise, pressure across the filtrate was also found to have a large impact, particularly for finer feed material. However, it was also determined that the operating pressure of the belt filter was by far the dominate factor in controlling the air consumption of the unit. This is an important consideration in the planning of a production 
capacity unit. Finally, the test results suggest that if the belt's length were increased it would possible to realize lower moisture contents from the fine material than what was recorded with these tests. 


\subsection{REFERENCES}

1. Atak Sunna and Celik Mehmet, Innovations in Mineral and Coal Processing, Rotterdam: A. A. Balkema, 1998.

2. Canbazoglu Mehmet, Changing Scopes in Mineral Processing, Rotterdam: A. A. Balkema, 1996.

3. Cheremisinoff Nicholas P. and Azbel David, Liquid Filtration, Woburn: Ann Arbor Science Publishers, 1983.

4. Cheremisinoff Nicholas P., Pocket Handbook for Solid-Liquid Separations. Houston: Gulf Publishing Company, 1984.

5. Cheremisinoff Paul N., Solid-Liquid Separations. Lancaster: Technomic Publishing Company, 1995.

6. Chuse Robert and Carson Bryce, Pressure Vessels The ASME Code Simplified $7^{\text {th }}$ Edition, New York: McGraw-Hill, 1993.

7. Cummins Arthur and Givens Ivan, SME Mining Engineering Handbook. New York: Port City Press, 1973. p. 27-1 - 27-94

8. Dahlstrom D. A., Challenges in Mineral Processing, Littleton: Society of Mining Engineers, 1989. p. 467-475.

9. Demirel Halim and Ersayin Salih, Progress in Mineral Processing Technology, Rotterdam:
A. A. Balkema Publishers, 1994.

10. Dickenson T. C., Filters and Filtration Handbook $4^{\text {th }}$ Edition, New York: Elsevier Science Ltd, 1997.

11. Ellenberger Phillip and Chuse Robert, Pressure Vessels $8^{\text {th }}$ Edition, New York: McGrawHill, 2004. 
12. Filtration Dictionary, Croydon: Uplands Press Ltd., 1975.

13. Green Robert E., Machinery's Handbook 25 th Edition. New York: Industrial Press Inc, 1996.

14. Gregory J. Solid-Liquid Separation, New York: Ellis Horwood Limited, 1984.

15. Harvey John, Theory and Design of Pressure Vessels $2^{\text {nd }}$ Edition, New York: Van Nostrand Reinhold, 1991.

16. Hicks Tyler G., Handbook of Mechanical Engineering Calculations $2^{\text {nd }}$ Edition. New York: McGraw-Hill, 2006.

17. Hudson Clayton, Filter Cloth Resistance in Constant Pressure Filtration, Thesis. Virginia Tech, 1941.

18. Kutz Myer, Mechanical Engineers’ Handbook. New York: John Wiley \& Sons, 1986.

19. Loving James, The Design, Constriction, \& Operation of a Pressure Continuous Filter, Thesis. Virginia Tech, 1947.

20. Malek Mohammad, Pressure Relief Devices, New York: McGraw-Hill, 2006.

21. Merrit Paul, Book of Flowsheets, New York: McGraw Hill Inc., 1984.

22. Mohanty M.K., Performance Evaluation of a New Fine Coal Dewatering Technology. 2003: p. 1-3.

23. Moss Dennis, Pressure Vessel Design Manual $3^{\text {rd }}$ Edition, Boston: Gulf Professional Publishing, 2004.

24. Myers Drew, Surfactant Science and Technology. Hoboken: John Wiley \& Sons, 2006.

25. Osborne, D.G., Rotary-vacuum filtration of coal flotation concentrates. International Journal of Mineral Processing, 1976. 3(2): p. 175-191.

26. Purchas Derek, Industrial Filtration of Liquids $2^{\text {nd }}$ Edition, London: Leonard Hill Books, 1971. 
27. Ritcey Gordon, Tailings Management, Amsterdam: Elsevier Science Publishing Company, 1989.

28. Ruthven D.M., Encyclopedia of Separation Technology Vol. 1. New York: John Wiley \& Sons, 1997. p.487-513

29. Ruthven D.M., Encyclopedia of Separation Technology Vol. 2. New York: John Wiley \& Sons, 1997. p.829-893

30. Svarovsky L., Solid-Liquid Separation $2^{\text {nd }}$ Edition. London: Butterworth \& Co, 1981.

31. Wade David, The Design, Construction, and Operation of a High Pressure Filter, Thesis. Virginia Tech, 1943.

32. Wang Ching, A Study of the Fine Coal Dewatering by High Temperature and Pressure Filtration, Thesis. Virginia Tech, 1966.

33. Weiss Norman, SME Mineral Processing Handbook. New York: Society of Mining Engineers, 1985. 
APPENDIX 


\section{Appendix A}

A list of tools used in the construction of the hyperbaric pressure filter.

Table A-1: List of tools used in the construction of the hyperbaric belt filer.

\begin{tabular}{cl}
\hline Number & \\
\hline 1 & Tools \\
2 & Ach Inch Chuck Power Drill \\
3 & Ball Pin Hammer \\
4 & Band Saw \\
5 & Belt Sander \\
6 & Bottle Jack \\
7 & Box End Wrenches \\
8 & Calipers \\
9 & C-Clamps \\
10 & Chain Pullers \\
11 & Channel Locks \\
12 & Compound Square \\
13 & Cordless Drill \\
14 & Crescent Wrench \\
15 & Dies \\
16 & Drill Press \\
17 & Flat Head Screw Driver \\
18 & Grinder \\
19 & Hack Saw \\
20 & Impact Wrench 1/2 inch Drive \\
21 & Impact Wrench 3/4 inch Drive \\
22 & Lathe \\
23 & Leather Gloves \\
24 & Mig Welder \\
25 & Mill \\
26 & Miter Saw \\
27 & Paint Brush \\
28 & Paint Roller \\
29 & Phillips Head Screw Driver \\
30 & Pipe Wrench \\
31 & Plainer \\
32 & Sand Blasters \\
33 & Shearer \\
34 & Socket Set and Driver \\
35 & Square \\
36 & Tape Measure \\
37 & Taps \\
38 & Tube Clamps \\
39 & Vice \\
\hline &
\end{tabular}




\section{Appendix B}

This is a table showing the inputs to the Design Expert analysis program.

Table B-1: Input data for Design Expert test runs.

\begin{tabular}{ccccccccc}
\hline STD & Run & Belt $\%$ & $\begin{array}{c}\text { Feed } \\
\%\end{array}$ & Pressure & $\begin{array}{c}\text { Coarse } \\
\text { Moisture }\end{array}$ & $\begin{array}{c}\text { Coarse } \\
\text { Moisture w/ } \\
\text { RV }(1: 10)\end{array}$ & $\begin{array}{c}\text { Fine } \\
\text { Moisture } \\
\text { w/ Floc }\end{array}$ & $\begin{array}{c}\text { Fine Moisture } \\
\text { w/ Floc \& RV } \\
(1: 10)\end{array}$ \\
\hline \hline 1 & 15 & 30 & 20 & 45 & $10.8 \%$ & $11.0 \%$ & $60.4 \%$ & $32.7 \%$ \\
2 & 7 & 30 & 30 & 45 & $11.2 \%$ & $10.6 \%$ & $61.1 \%$ & $31.8 \%$ \\
3 & 8 & 90 & 20 & 45 & $11.4 \%$ & $11.1 \%$ & $58.9 \%$ & $33.0 \%$ \\
4 & 3 & 90 & 30 & 45 & $11.8 \%$ & $11.3 \%$ & $60.7 \%$ & $36.3 \%$ \\
5 & 16 & 60 & 20 & 30 & $14.1 \%$ & $15.0 \%$ & $86.3 \%$ & $38.9 \%$ \\
6 & 14 & 60 & 30 & 30 & $16.6 \%$ & $14.2 \%$ & $88.0 \%$ & $35.3 \%$ \\
7 & 10 & 60 & 20 & 60 & $8.8 \%$ & $8.2 \%$ & $45.0 \%$ & $35.2 \%$ \\
8 & 4 & 60 & 30 & 60 & $11.3 \%$ & $10.7 \%$ & $35.0 \%$ & $29.1 \%$ \\
9 & 17 & 30 & 25 & 30 & $65.3 \%$ & $14.0 \%$ & $89.1 \%$ & $79.3 \%$ \\
10 & 9 & 90 & 25 & 30 & $16.1 \%$ & $13.9 \%$ & $90.2 \%$ & $37.0 \%$ \\
11 & 11 & 30 & 25 & 60 & $10.9 \%$ & $10.8 \%$ & $48.2 \%$ & $39.0 \%$ \\
12 & 5 & 90 & 25 & 60 & $9.9 \%$ & $10.0 \%$ & $50.1 \%$ & $33.6 \%$ \\
13 & 12 & 60 & 25 & 45 & $10.0 \%$ & $9.7 \%$ & $55.7 \%$ & $31.7 \%$ \\
14 & 2 & 60 & 25 & 45 & $10.3 \%$ & $10.5 \%$ & $54.7 \%$ & $33.9 \%$ \\
15 & 6 & 60 & 25 & 45 & $10.2 \%$ & $10.1 \%$ & $54.8 \%$ & $32.5 \%$ \\
16 & 13 & 60 & 25 & 45 & $10.3 \%$ & $10.4 \%$ & $56.6 \%$ & $32.9 \%$ \\
17 & 1 & 60 & 25 & 45 & $9.8 \%$ & $9.9 \%$ & $56.8 \%$ & $31.0 \%$ \\
\hline
\end{tabular}


Table B-1: Input data for Design Expert test runs (continued).

\begin{tabular}{ccccccc}
\hline STD & Run & Belt $\%$ & Feed \% & Pressure & Mixed & $\begin{array}{c}\text { Mixed } \mathbf{w} / \mathrm{RV} \\
(1: 10)\end{array}$ \\
\hline \hline 1 & 15 & 30 & 20 & 45 & $13.9 \%$ & $10.3 \%$ \\
2 & 7 & 30 & 30 & 45 & $13.2 \%$ & $11.6 \%$ \\
3 & 8 & 90 & 20 & 45 & $13.6 \%$ & $12.4 \%$ \\
4 & 3 & 90 & 30 & 45 & $14.1 \%$ & $12.6 \%$ \\
5 & 16 & 60 & 20 & 30 & $16.8 \%$ & $14.9 \%$ \\
6 & 14 & 60 & 30 & 30 & $18.9 \%$ & $15.7 \%$ \\
7 & 10 & 60 & 20 & 60 & $10.8 \%$ & $8.4 \%$ \\
8 & 4 & 60 & 30 & 60 & $12.1 \%$ & $11.7 \%$ \\
9 & 17 & 30 & 25 & 30 & $73.7 \%$ & $15.3 \%$ \\
10 & 9 & 90 & 25 & 30 & $20.0 \%$ & $17.7 \%$ \\
11 & 11 & 30 & 25 & 60 & $12.1 \%$ & $11.2 \%$ \\
12 & 5 & 90 & 25 & 60 & $11.8 \%$ & $10.4 \%$ \\
13 & 12 & 60 & 25 & 45 & $13.4 \%$ & $10.2 \%$ \\
14 & 2 & 60 & 25 & 45 & $13.7 \%$ & $10.8 \%$ \\
15 & 6 & 60 & 25 & 45 & $14.2 \%$ & $10.4 \%$ \\
16 & 13 & 60 & 25 & 45 & $13.7 \%$ & $11.4 \%$ \\
17 & 1 & 60 & 25 & 45 & $11.8 \%$ & $10.2 \%$ \\
\hline
\end{tabular}




\section{Appendix C}

This section shows the results of the hyperbaric belt filter in graphical form showing some of the input factors graphed versus moisture content.

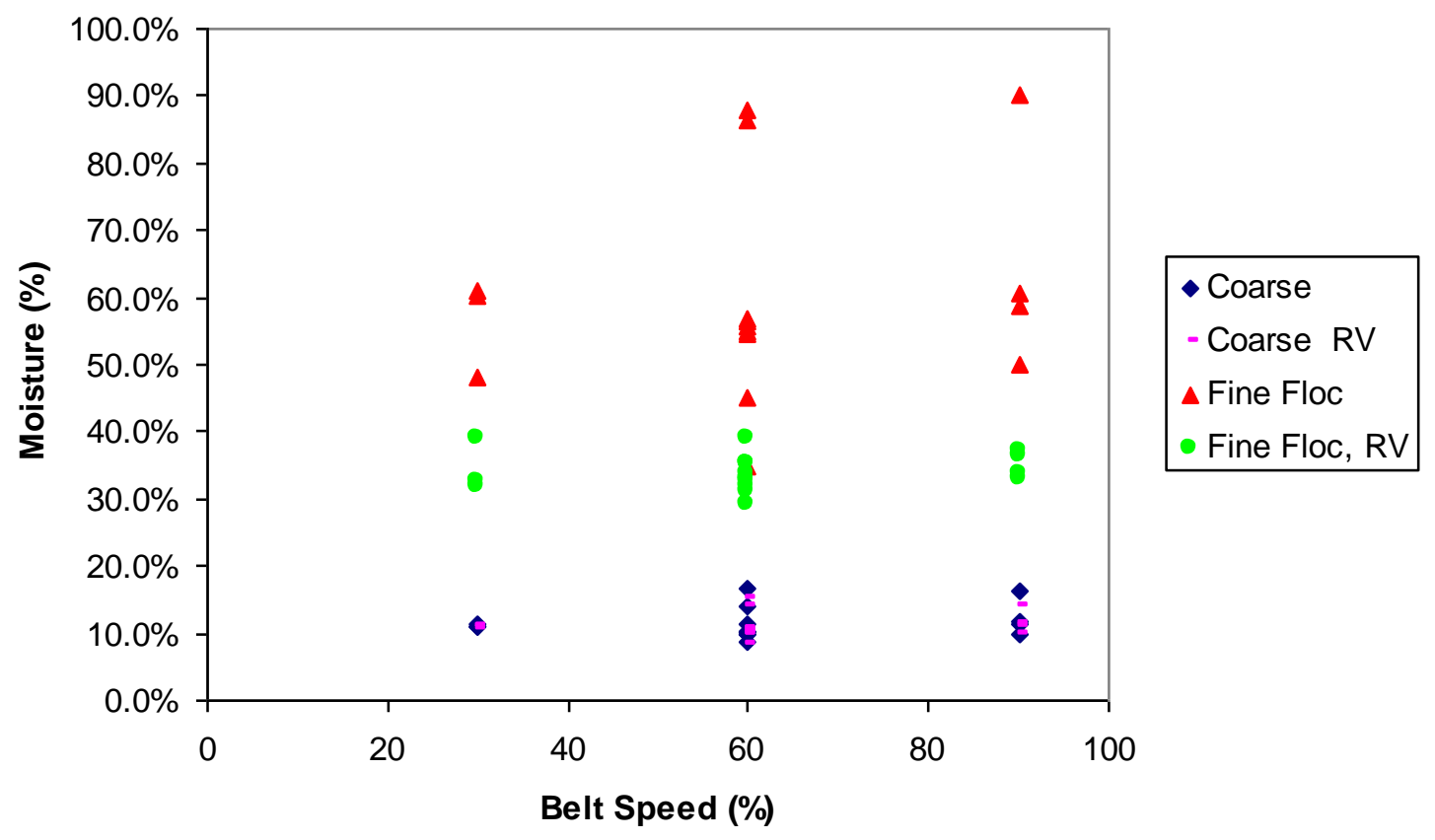

Figure C-1: Belt speed vs. moisture content. 


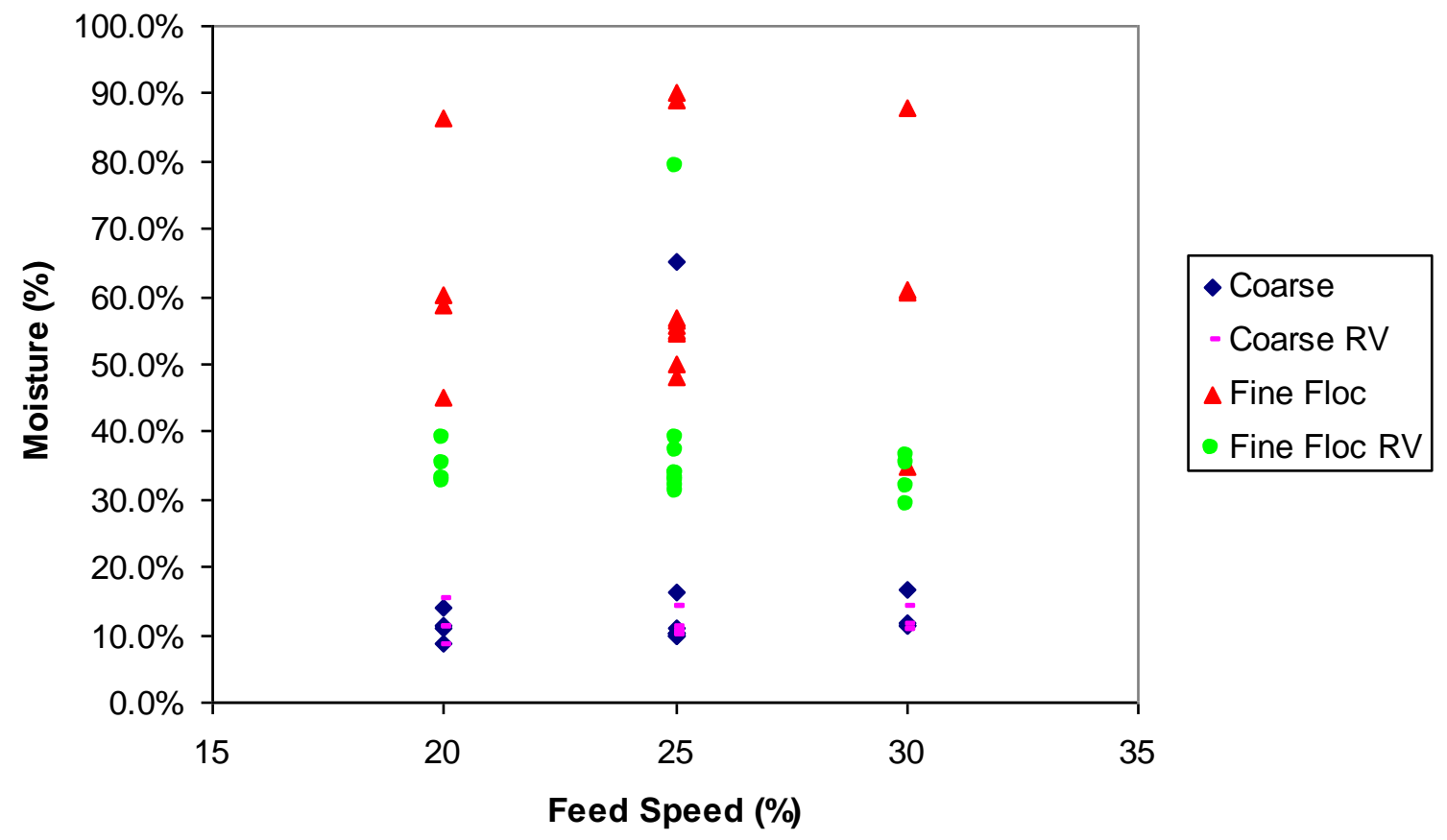

Figure C-2: Feed rate vs. moisture content. 


\section{Coarse No Chemicals}

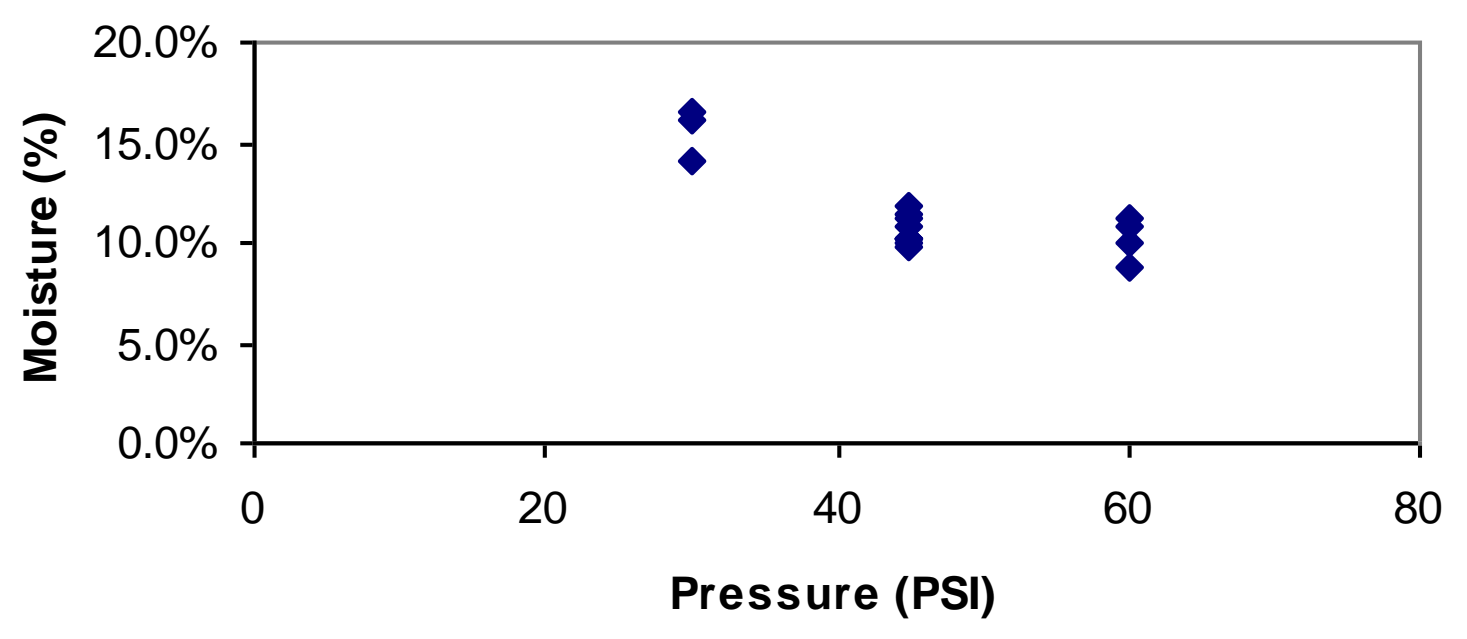

Figure C-3: Pressure vs. moisture content (coarse feed with no chemicals). 


\section{Coarse With RV}

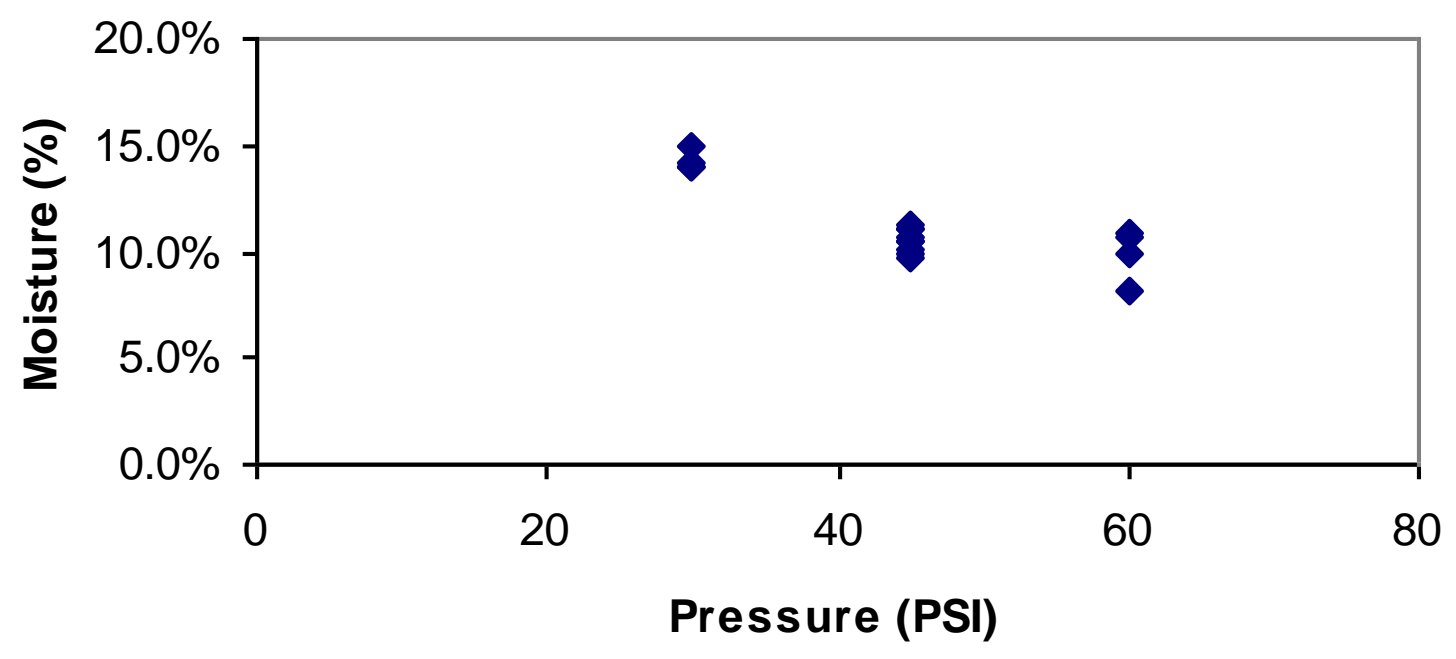

Figure C-4: Pressure vs. moisture content (coarse feed with reagent RV). 


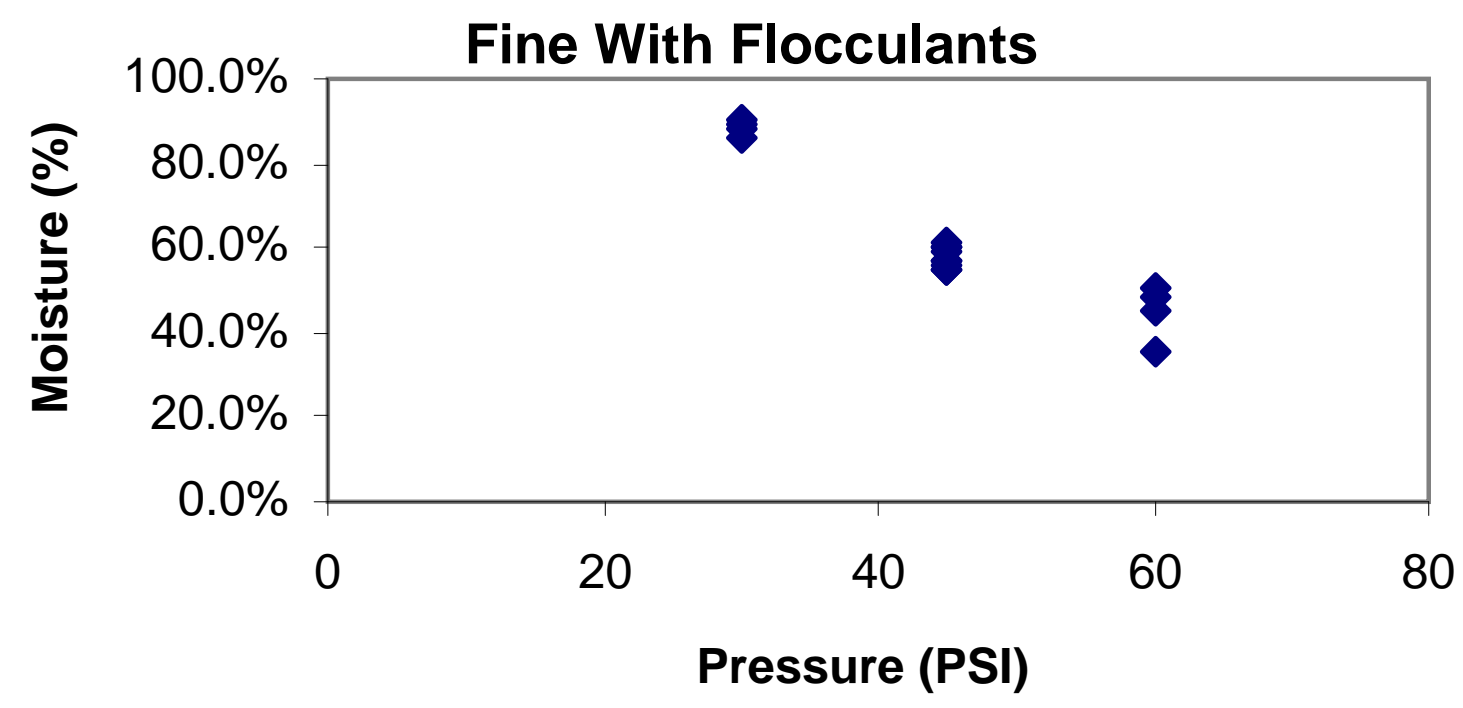

Figure C-5: Pressure vs. moisture content (fine feed with with flocculant). 
Fine With Floc and RV

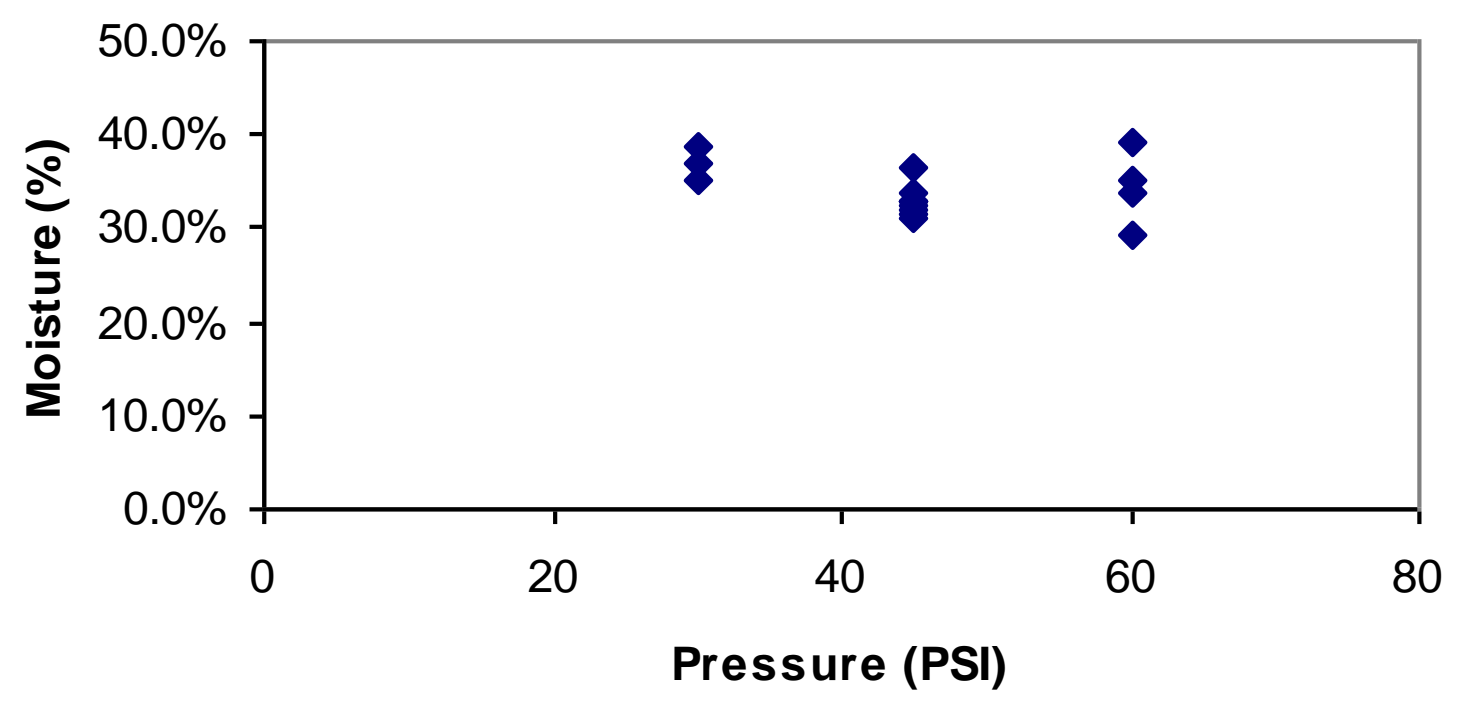

Figure C-6: Pressure vs. moisture content (fine feed with flocculant and reagent RV). 


\section{Appendix D}

This section shows the calculations made to determine the horsepower requirements of the production capacity hyperbaric belt filter. The equation listed below is used of determining the horsepower required to compress atmospheric air to various gauge pressures (Green, 1996). This compression can be done in one, two, three, or four stages. The formula will allow any amount of volume of air that is required to be compressed, adiabatically. Adiabatically is an assumption, but one that holds relatively well for most air compression operations (Hicks, 2006).

$$
H . P .=\frac{144 N P V n}{33000(n-1)}\left[\left(\frac{P_{2}}{P}\right)^{\frac{n-1}{N n}}-1\right]
$$

H.P. = Horsepower

$\mathrm{N}=$ Number of stages of compression

$\mathrm{P}=$ Atmospheric pressure in PSI

$\mathrm{P}_{2}=$ Absolute terminal pressure in PSI

$\mathrm{V}=$ Volume of air to be compressed expressed in CFM

$\mathrm{n}=$ Exponent of the compression curve $=1.41$ for adiabatic compression

To calculate the horsepower required to compress the amount of air that is required for a production capacity unit it will be assumed that the number of stages of compression will be one. From the equation above it would require 15,960 horsepower to compress 120,000 CFM of air at 60 PSIG. However, if the friction and other losses are considered it results in a $15 \%$ loss thus the 
horsepower required is 18,360 . Similarly to generate 73,000 CFM of air at 60 PSIG with the losses included is 11,169 horsepower. 


\section{Appendix E}

In Figure E-1, the cake thickness vs. cake formation times is plotted for the batch tests.

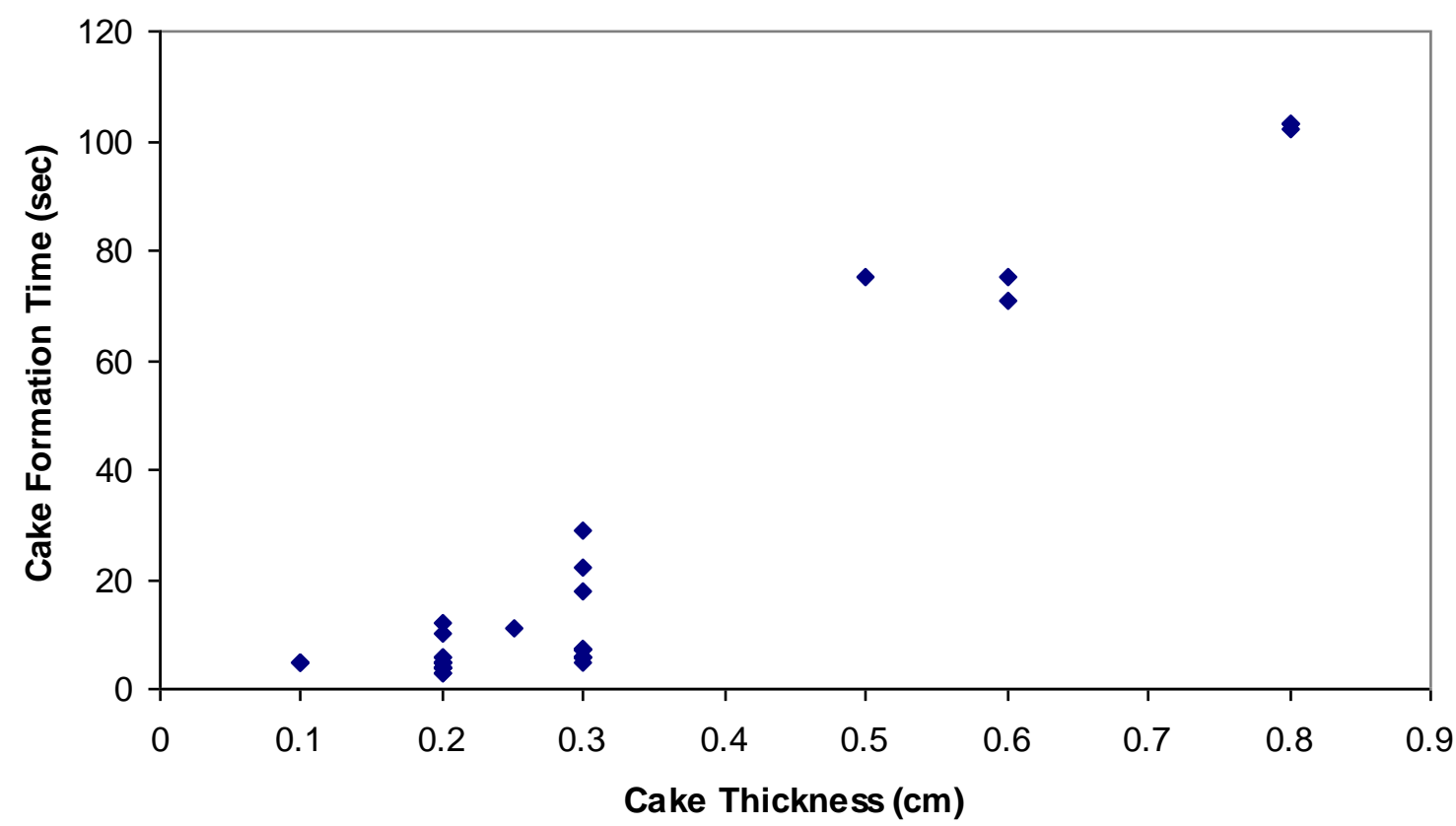

Figure E-1: Batch tests with fine feed. 
Appendix 19: Improvements in Screen Bowl Centrifuge Performance (VA013) 


\section{FINAL TECHNICAL REPORT}

Contract Title and Number:

Crosscutting Technology Development at the Center

for Advanced Separation Technologies

(DE-FC26-02NT41607)

Sub-Recipient Project Title:

Improvements in Screenbowl Centrifuge

Performance

Principal Investigators:

R.T. Burchett, G.H. Luttrell and R.-H. Yoon

Contact Address:

100 Holden Hall

Virginia Tech

Blacksburg, Virginia 24061

Subcontractor Address:

No subcontracts issued.
Period of Performance:

Starting Date: 10/1/02

Ending Date: 10/31/09
Report Information:

Type: Final

Number:

Period:

Date: $\quad$ 6/21/10

Code: VA013-FINAL

Contact Information:

Phone: 540-231-6314

Fax: 540-231-4070

E-Mail: luttrell@vt.edu

Subcontractor Information:

Phone:

Fax:

E-Mail: 


\section{DISCLAIMER}

"This report was prepared as an account of work sponsored by an agency of the United States Government. Neither the United States Government nor any agency thereof, nor any of their employees, make any warranty, express or implied, nor assume any legal liability or responsibility for the accuracy, completeness, or usefulness of any information, apparatus, product, or process disclosed, or represents that its use would not infringe privately owned rights. Reference herein to any specific commercial product, process, or service by trade name, trademark, manufacturer, or otherwise does not necessarily constitute or imply endorsement, recommendation, or favoring by the United States Government or any agency thereof. The views and opinions of authors expressed herein do not necessarily state or reflect those of the United States Government or agency thereof." 


\begin{abstract}
Screenbowl centrifuges are the most commonly used method for dewatering fine coal in the United States. Unfortunately, this process is the least efficient and most costly dewatering operation in the preparation plant. In light of these difficulties, a broad-based research project has been undertaken to develop technological enhancements that improve moisture reduction and increase fine coal recovery for screenbowl centrifuges. The first part of this study involved the field testing of flocculant injection into the lowsolids pool within an industrial machine. The data obtained from these in-plant tests showed that the pool injection technology substantially improved coal recovery by reducing the solids content in the main effluent by nearly an order of magnitude (i.e., from $3.5 \%$ to below $0.33 \%$ ). The second part of this study involved the pilot-scale evaluation of an injection system that added surface tension modifiers directly to the dewatered solids as they passed over the screen section of the centrifuge. The data obtained from these pilot-scale tests indicated that this approach can further reduce the product moisture by up to 2 absolute percentage points.
\end{abstract}




\section{TABLE OF CONTENTS}

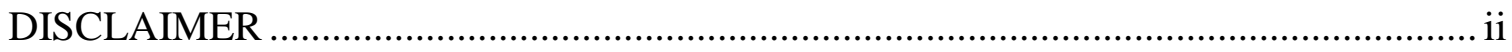

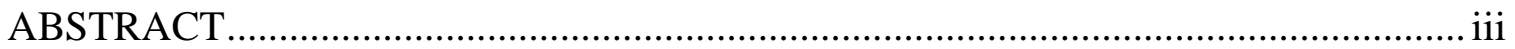

TABLE OF CONTENTS ........................................................................................ iv

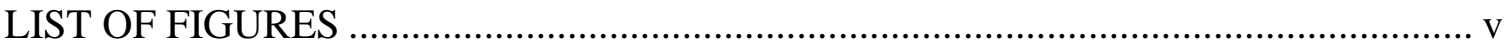

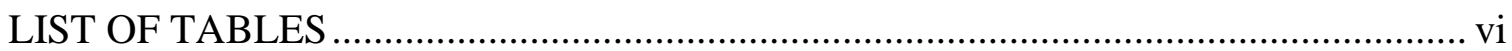

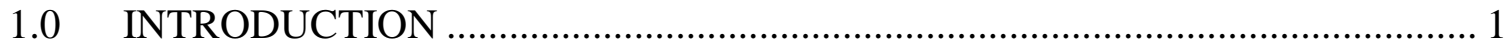

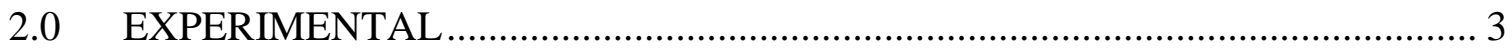

2.1 Improving Solids Recovery ....................................................................... 3

2.2 Improving Moisture Removal ....................................................................... 4

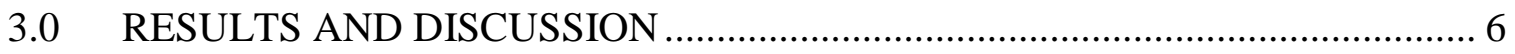

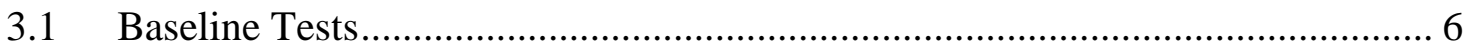

3.2 Field Testing of Polymer Injection................................................................... 11

3.3 Pilot-Scale Testing of Surfactant Injection ....................................................... 15

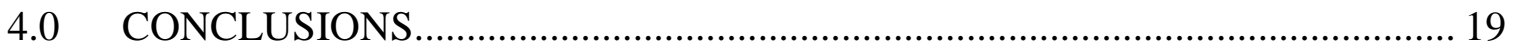

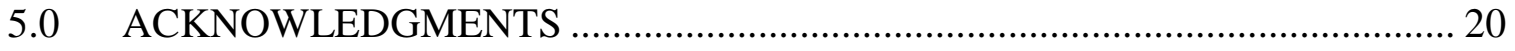

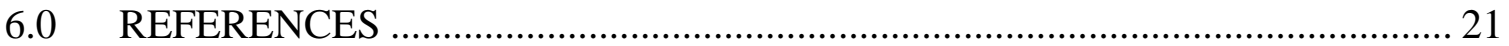




\section{LIST OF FIGURES}

Figure 1. Photograph of an industrial screenbowl centrifuge. ........................................ 2

Figure 2. Schematic showing the injection tube for adding flocculant directly into the dilute low-solids pool within the bowl section.

Figure 3. Schematic showing the injection tube for adding dewatering aides and surface tension modifiers to the partially dewatered cake on the screen section.......... 5

Figure 4. Pilot-scale screenbowl centrifuge used in the reagent injection tests............... 7

Figure 5. Product moisture versus solids recovery for the pilot-scale centrifuge. ............ 9

Figure 6. Mass recovery rates of water and solids for the pilot-scale centrifuge............ 10

Figure 7. Photograph of the modified feed pipe for injecting flocculant into the pool section of an industrial screenbowl centrifuge................................................ 11

Figure 8. Results obtained from field tests conducted to evaluate the effect of polymer injection on screenbowl centrifuge performance.

Figure 9. Pilot-scale screenbowl centrifuge used in the evaluation of dewatering aids. 16

Figure 10. Data obtained from pilot-scale tests conducted to evaluate the effects of dewatering aids on screenbowl centrifuge performance.

Figure 11. Dewatering data obtained after the injection of $2 \mathrm{lb} /$ ton and $8 \mathrm{lb} / \mathrm{ton}$ of surfactant (surface tension reducer) to a screenbowl cake. 


\section{LIST OF TABLES}

Table 1. Typical size-by-size mass balance data for a screenbowl centrifuge........... 6

Table 2. Results of injection tests using various flocculants and polymers ............. 14 


\subsection{INTRODUCTION}

The solid-solid separation processes employed by modern coal preparation plants require large amounts of process water. After cleaning, the unwanted water must be removed from the surfaces of the particles using mechanical dewatering equipment. Coarse particles can be readily dewatered using simple screening systems, while finer particles require more complicated unit operations such as centrifuges and filters for effective dewatering. In select cases, thermal drying may even be required to remove additional moisture so that the final product can meet customer specifications. Moisture that is not removed by these processes reduces the heating value of the final coal product and increases the transportation costs for the solid fuel. In addition, excess moisture can create significant handling problems for both the coal producer and downstream consumers by plugging chutes, bins, rail cars, etc. The problem can be particularly severe during winter months because of freezing.

The most popular process for dewatering fine clean coal in the U.S. is the screenbowl centrifuge (Figure 1). A screenbowl centrifuge is a continuous two-stage solid-liquid separator that combines a centrifugal solid clarifier (bowl) together with a centrifugal filter (screen). The feed slurry treated by a screenbowl is typically introduced through a stationary feed pipe. The feed slurry is brought up to rotational speed in an acceleration chamber. The feed slurry is evenly distributed through feed ports into the solid bowl section where the solids settle under the influence of the centrifugal field. The clarified water from the bowl section is discharged as a waste effluent via an adjustable overflow weir located at the end of the machine. The settled solids are conveyed in the opposite direction to the main effluent using a helical scroll that rotates at a slightly 


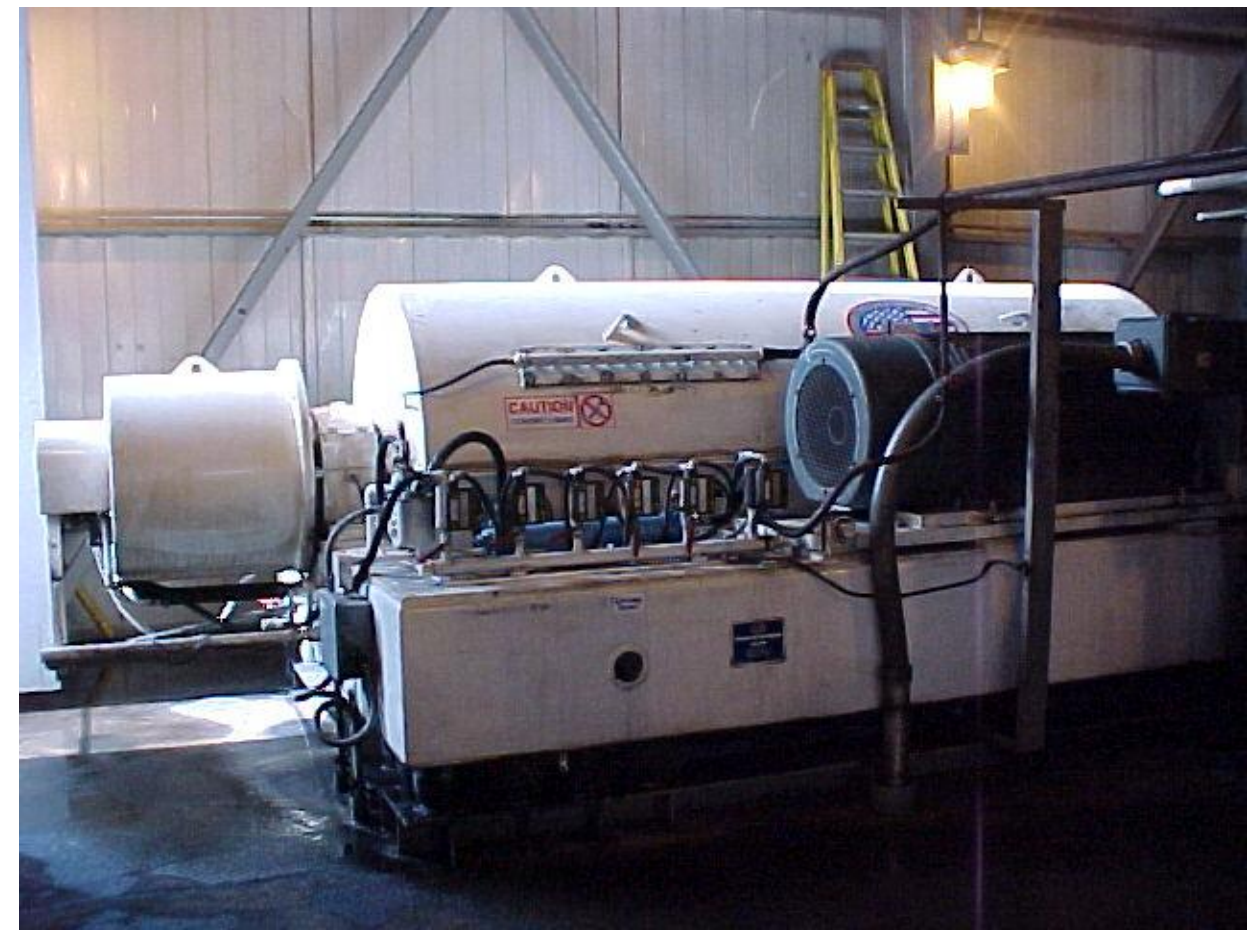

Figure 1. Photograph of an industrial screenbowl centrifuge.

slower speed than the bowl. The scroll carries the solids up a beach and onto a screen section where additional moisture is removed prior to discharge of the final cake. In most cases, the material that drains through the screen section contains valuable coal and is recycled back to the feed inlet so that it can be recovered. 


\subsection{EXPERIMENTAL}

\subsection{Improving Solids Recovery}

Screenbowl centrifuges typically provide lower product moistures compared to other dewatering methods such as vacuum filters. The lower moisture is attributed to the rejection of ultrafine particles that are too small to settle during the relatively short time that they reside within the bowl. This classification step eliminates a large portion of the high surface area solids from the dewatered product. As a result, screenbowl centrifuges can achieve moisture contents 4 to 6 percentage points lower than filters (Gallagher et al., 1981). The ash content of the final cake is also often lower because high-ash ultrafine clays are preferentially rejected with the main effluent stream. Unfortunately, screenbowls also typically achieve a lower overall recovery of coal fines (80-90\%) compared to filters (95-99\%). A standard rule-of-thumb used by industry is that screenbowls recover only about 50\% of particles that are finer than 325 mesh in the feed. In cases where high recoveries of ultrafine solids are desirable (e.g., metallurgical coal plants), screenbowls typically do not perform as well as vacuum filters in recovering fine solids.

Past attempts to improve the recovery of fines by adding flocculants to the screenbowl feed have been largely unsuccessful (Miller and Wen, 1984). However, recent field studies performed by researchers at Virginia Tech indicate that the solids recovery can be dramatically improved using a modified feed tube to introduce a flocculant directly into the low-solids clarified pool within the rotating bowl (Figure 2). The injection pipe used to add the flocculant runs along the inside of the slurry feed pipe and exits just prior to end where the feed slurry is discharged. The flocculant works when 


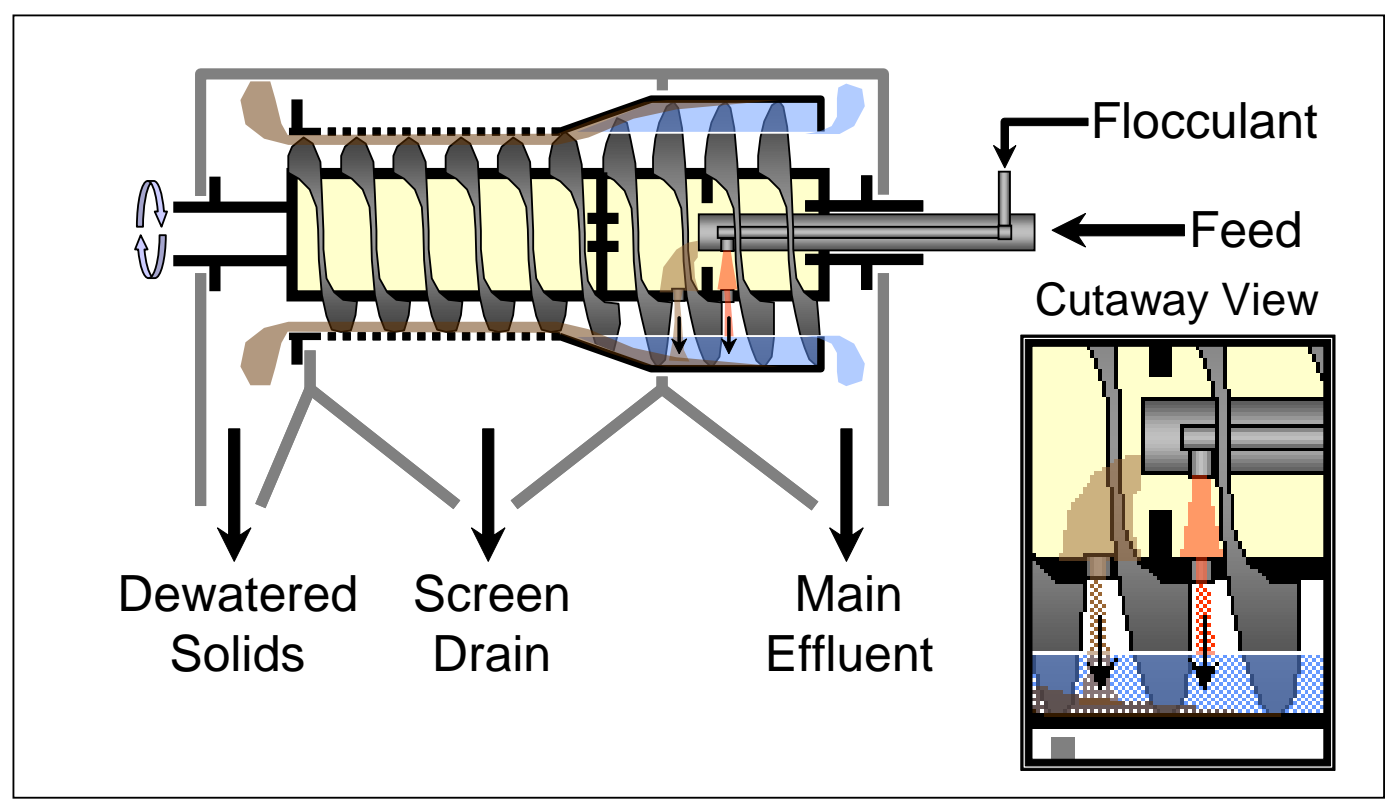

Figure 2. Schematic showing the injection tube for adding flocculant directly into the dilute lowsolids pool within the bowl section.

added directly to the bowl since this approach (i) avoids premature contact between the polymer and vast majority of the feed solids, (ii) prevents shearing of the polymer as it enters the dilute pool, and (iii) provides an ideal low-solids environment for full chemical activation. Although this approach has been used successfully with conventional flocculant applied for water clarification in solid-bowl centrifuges (Records and Sutherland, 2001), no reports have been made in the literature regarding the use of this approach to improve solids recovery in screenbowl centrifuges.

\subsection{Improving Moisture Removal}

A variety of theoretical and experimental studies have shown that the removal of moisture from fine coal can be improved by adding dewatering aids during vacuum filtration (Silverblatt and Dahlstrom, 1954; Baker, 1976; Nicol, 1976; Gala et al., 1982; Baillie and Lumsden, 1988, Yoon et al., 2001). Although fewer studies have been done to 


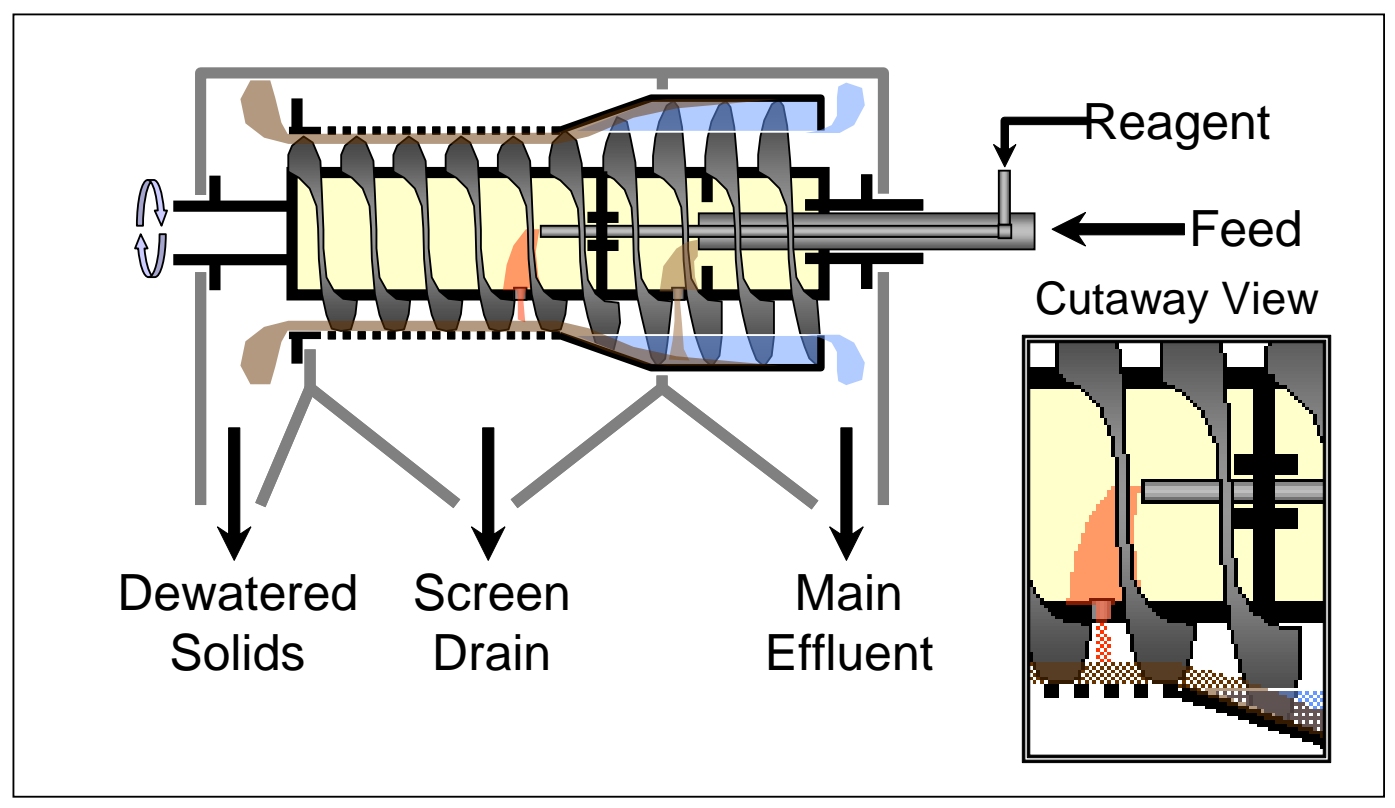

Figure 3. Schematic showing the injection tube for adding dewatering aides and surface tension modifiers to the partially dewatered cake on the screen section.

evaluate the effects of chemical dewatering additives on screenbowl centrifuges, most of the data reported in the literature suggest that these chemical additives are often not effective when used in this application. However, more recent data obtained by researchers at Virginia Tech suggest that specific types of dewatering aides can be successfully used to reduce moisture provided the reagent is properly added to the cake on the screen section of the screenbowl centrifuge. In practice, this is achieved using an injection pipe to introduce the dewatering aid directly onto the solids passing across the screen section of the centrifuge (Figure $2 \mathrm{~b}$ ). This setup allows the chemicals to be added to the partially dewatered coal at a much higher effective concentration than would be possible if the same amount of reagent were added to the dilute feed slurry. 


\subsection{RESULTS AND DISCUSSION}

\subsection{Baseline Tests}

Prior to evaluating the chemical injection technology, baseline tests were conducted (i) to develop engineering expressions that describe the performance of a screenbowl centrifuge and (ii) to calculate the incremental moistures of the products generated. Several series of field tests were completed at an industrial plant site in an attempt to collect the data required to successfully complete this task. A representative set of mass-balanced data from this field testing program is provided in Table 1.

Table 1. Typical size-by-size mass balance data for a screenbowl centrifuge.

\begin{tabular}{|c|c|c|c|c|c|c|c|c|}
\hline & \multicolumn{4}{|c|}{ Experimental Data } & \multicolumn{4}{|c|}{ Balanced Data } \\
\hline & Feed & Product & Drain & Effluent & Feed & Product & Drain & Effluent \\
\hline \multicolumn{9}{|l|}{ Total Mass } \\
\hline Plus $100 \mathrm{M}$ & 63.62 & 69.81 & 53.48 & 0.16 & 63.33 & 70.38 & 53.31 & 0.16 \\
\hline $100 \times 325$ & 13.82 & 14.71 & 23.69 & 0.05 & 12.99 & 14.44 & 24.04 & 0.05 \\
\hline $325 \mathrm{M}$ x 0 & 22.57 & 15.48 & 22.83 & 99.78 & 23.68 & 15.18 & 22.65 & 99.79 \\
\hline Overall & 100.01 & 100.00 & 100.00 & 99.99 & 100.00 & 100.00 & 100.00 & 100.00 \\
\hline \multicolumn{9}{|l|}{ Total Mass } \\
\hline Plus $100 \mathrm{M}$ & 63.62 & 62.92 & 3.21 & 0.02 & 63.33 & 63.31 & 7.78 & 0.02 \\
\hline $100 \times 325$ & 13.82 & 13.26 & 1.42 & 0.00 & 12.99 & 12.99 & 3.51 & 0.01 \\
\hline $325 \mathrm{M}$ x 0 & 22.57 & 13.95 & 1.37 & 9.84 & 23.68 & 13.65 & 3.31 & 10.03 \\
\hline Overall & 100.00 & 90.13 & 6.00 & 9.87 & 100.00 & 89.95 & 14.60 & 10.05 \\
\hline \multicolumn{9}{|l|}{$\overline{\text { Ash (\% }}$} \\
\hline Plus $100 \mathrm{M}$ & 4.44 & 5.87 & 5.35 & 59.84 & 5.01 & 5.00 & 5.30 & 59.82 \\
\hline $100 \times 325$ & 10.97 & 7.48 & 15.40 & 26.11 & 8.08 & 8.07 & 16.03 & 26.11 \\
\hline $325 \mathrm{M}$ x 0 & 16.65 & 12.71 & 19.69 & 23.85 & 17.13 & 12.53 & 19.59 & 23.39 \\
\hline Overall & 8.09 & 7.17 & 11.00 & 23.91 & 8.28 & 6.58 & 11.12 & 23.45 \\
\hline \multicolumn{9}{|l|}{ Total Ash } \\
\hline Plus 100 & 282.24 & 369.36 & 17.17 & 0.94 & 317.23 & 316.26 & 41.26 & 0.96 \\
\hline $100 x$ & 151.57 & 99.18 & 21.89 & 0.13 & 104.99 & 104.86 & 56.27 & 0.13 \\
\hline $325 \mathrm{M} \mathrm{x}$ & 375.61 & 177.34 & 26.97 & 234.79 & 405.76 & 171.10 & 64.79 & 234.66 \\
\hline Overall & 809.34 & 645.87 & 66.03 & 235.88 & 827.97 & 592.22 & 162.31 & 235.75 \\
\hline \multicolumn{9}{|l|}{ Slurry } \\
\hline Solids & 24.51 & 83.37 & 29.57 & 3.29 & 24.18 & 83.36 & 29.57 & 3.29 \\
\hline Rate & 407.99 & 108.11 & 20.29 & 299.88 & 413.49 & 107.90 & 49.36 & 305.59 \\
\hline
\end{tabular}


Unfortunately, the experimental data obtained from the in-plant studies showed large random variations due to fluctuations in feed characteristics and process flow rates. Due to the large degree of data scatter, no discernable trend could be observed in the quality of the centrifuge products (i.e., moisture or solids contents) in response to changes in equipment operating conditions (i.e., volumetric feed slurry rate or feed percent solids).

In light of the difficulties associated with the collection of reliable data in an industrial environment, several series of pilot-scale tests were conducted under controlled laboratory conditions to obtain the information needed to complete this task. The pilotscale tests were conducted using a 6-inch diameter solid-bowl centrifuge that had been reconfigured by Decanter Machine into a screenbowl design. A photograph of the pilot-

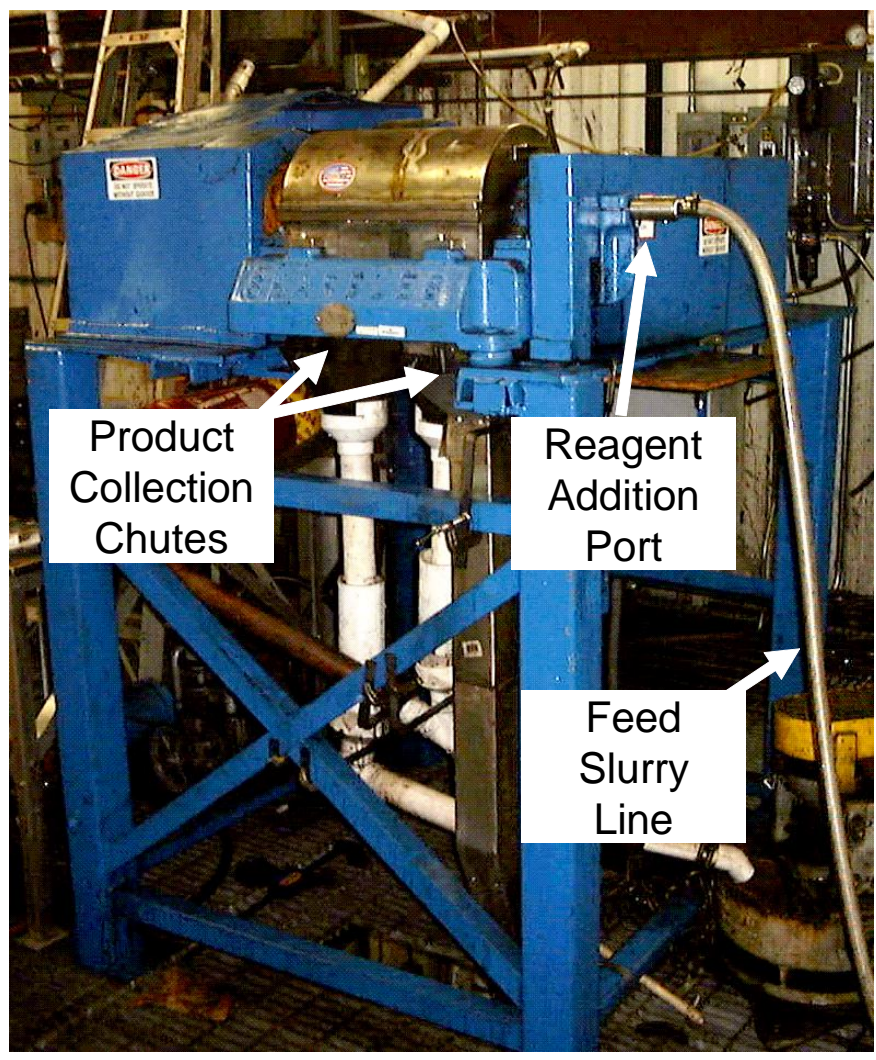

Figure 4. Pilot-scale screenbowl centrifuge used in the reagent injection tests. 
scale test unit is provided in Figure 4. Six different coal samples were used in the pilotscale test work. The volumetric feed flow rate and solids content of the feed slurry were varied in each set of tests.

The results of the pilot-scale tests are summarized in Figure 5. As shown, the pilot-scale test unit provided moisture values in the range of $20-25 \%$ moisture for first four samples and in the range of 30-35\% moisture for the last two samples. In all cases, an increase in solids recovery tended to produce a corresponding increase in moisture. In other words, changes to the operating characteristics of the machine that improved the capture of very fine solids also increased moisture due to the larger surface area associated with the very fine particles.

In order to calculate the "incremental" moisture for these tests, the data have been replotted in Figure 6 to correlate the mass rate of moisture recovered per unit of dry product recovered. The slope of the regression line in each plot represents the ratio of water-to-solids recovered. Most of the slope values for the regression lines fell in the range of $0.34-0.45$, although one test provided a value as large as 0.58 . These values suggest that the incremental moisture for these particular coal samples fall in the range of 25-30\% moisture. However, the worst case slope of 0.58 corresponds to an incremental moisture value of about $37 \%$ by weight. Since incremental moistures up to $40-45 \%$ can sometimes be justified for industrial contracts, these results suggest that the development of technologies to further improve solids recovery and moisture rejection is certainly warranted. 

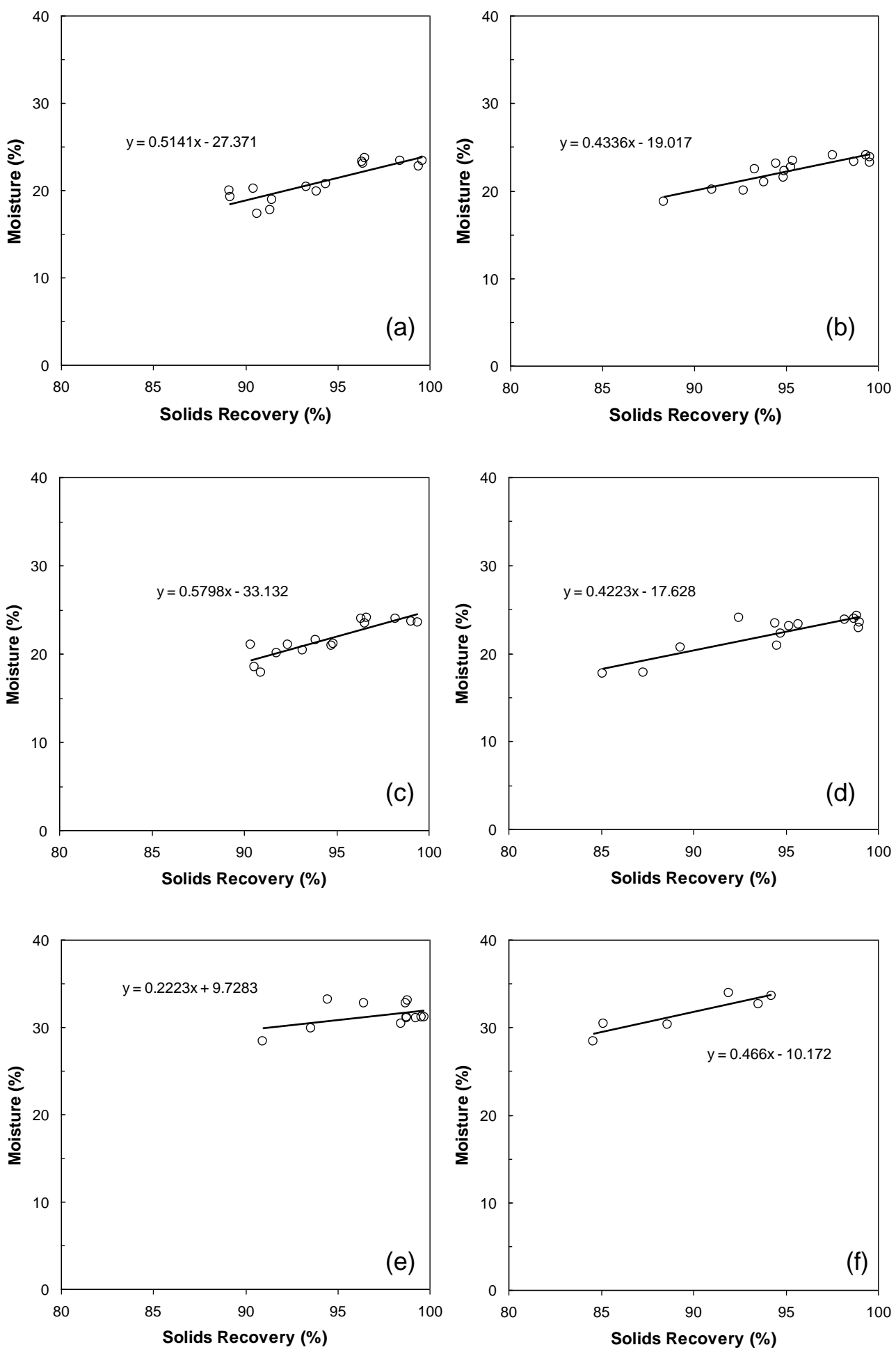

Figure 5. Product moisture versus solids recovery for the pilot-scale centrifuge. 

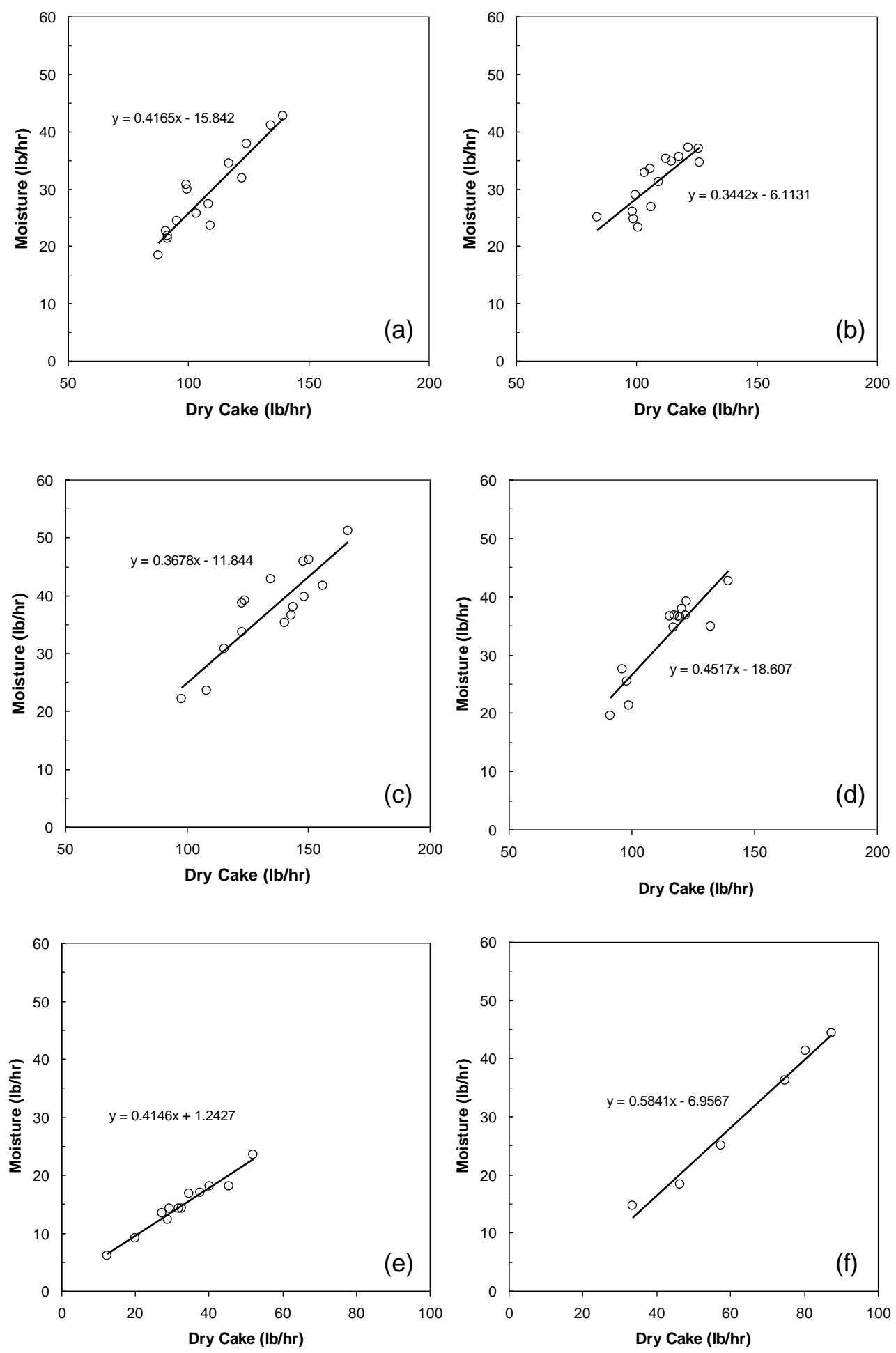

Figure 6. Mass recovery rates of water and solids for the pilot-scale centrifuge. 


\subsection{Field Testing of Polymer Injection}

Several series of industrial field tests were performed to verify that the injection of flocculant into the dilute pool section of a screenbowl centrifuge would improve solids recovery for a production-scale machine. Figure 7 shows a photograph of the modified feed pipe incorporating the flocculant injection pipe. The piping was configured so that different types of chemical additives could be injected at different dosing rates. A structurally-modified synthetic polymer developed by the Nalco Company was used in place of traditional flocculants for these injection tests. The polymer was found to be superior to traditional flocculants in the high stress environment within the centrifuge.

The results obtained the preliminary field tests are summarized in Figure 8. The first test was performed without using the injection tube or any reagents. The baseline test showed that the main effluent, which was being discarded to the waste impoundment, contained 3.54\% solids (Figure 8a). This represented a loss of 2.46 tph of fine solids to

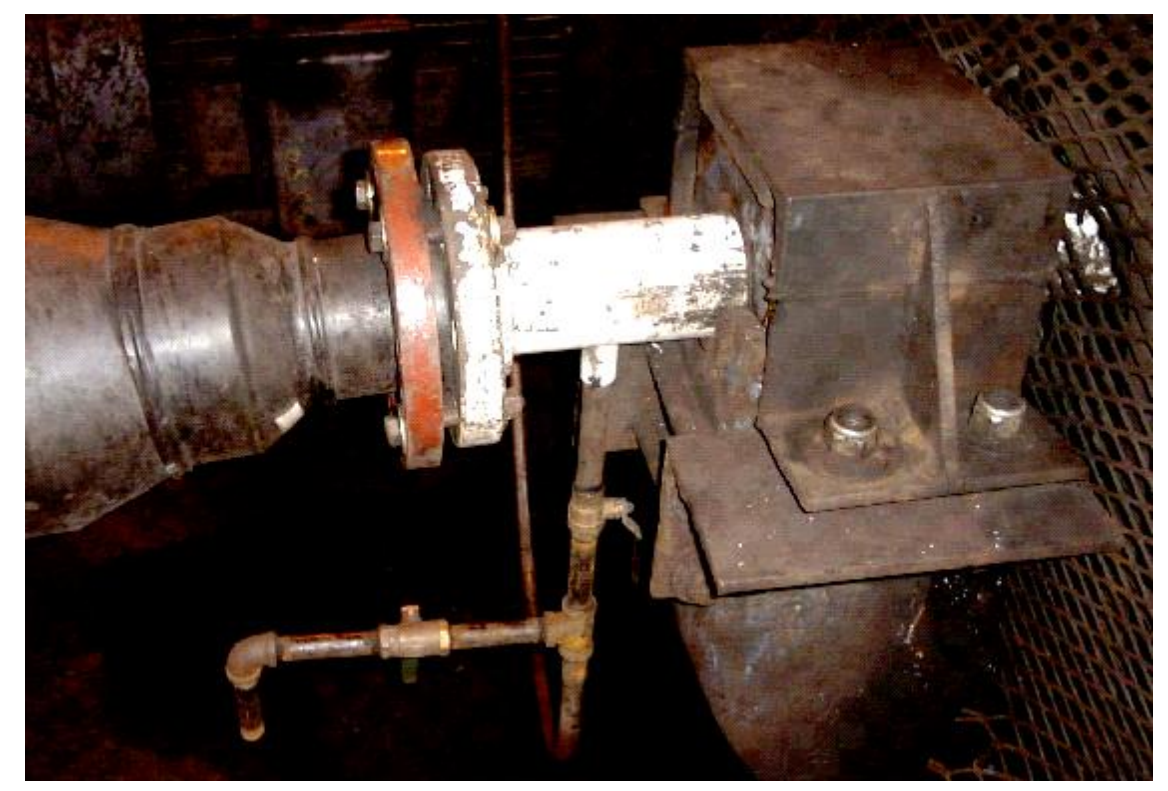

Figure 7. Photograph of the modified feed pipe for injecting flocculant into the pool section of an industrial screenbowl centrifuge 
the main effluent. The corresponding dewatered coal product from the centrifuge contained $\quad 12.84 \%$ moisture (Figure 8b) and 7.06\% ash (Figure 8c). Attempts to improve the solids recovery (i.e., reduce the solids content in the main effluent) by adding conventional flocculant directly to the feed slurry were unsuccessful. As shown in Figure 8a, this approach reduced the solids content in the main effluent by a negligible amount (3.54\% down to $3.45 \%)$. In contrast, the addition of the synthetic polymer through the injection tube substantially reduced the loss of solids to the main effluent. When using polymer "A", the solids content dropped from $3.54 \%$ to a very low value of just 0.33\%. Likewise, when using polymer "B", the solids content dropped to $0.25 \%$. As such, the

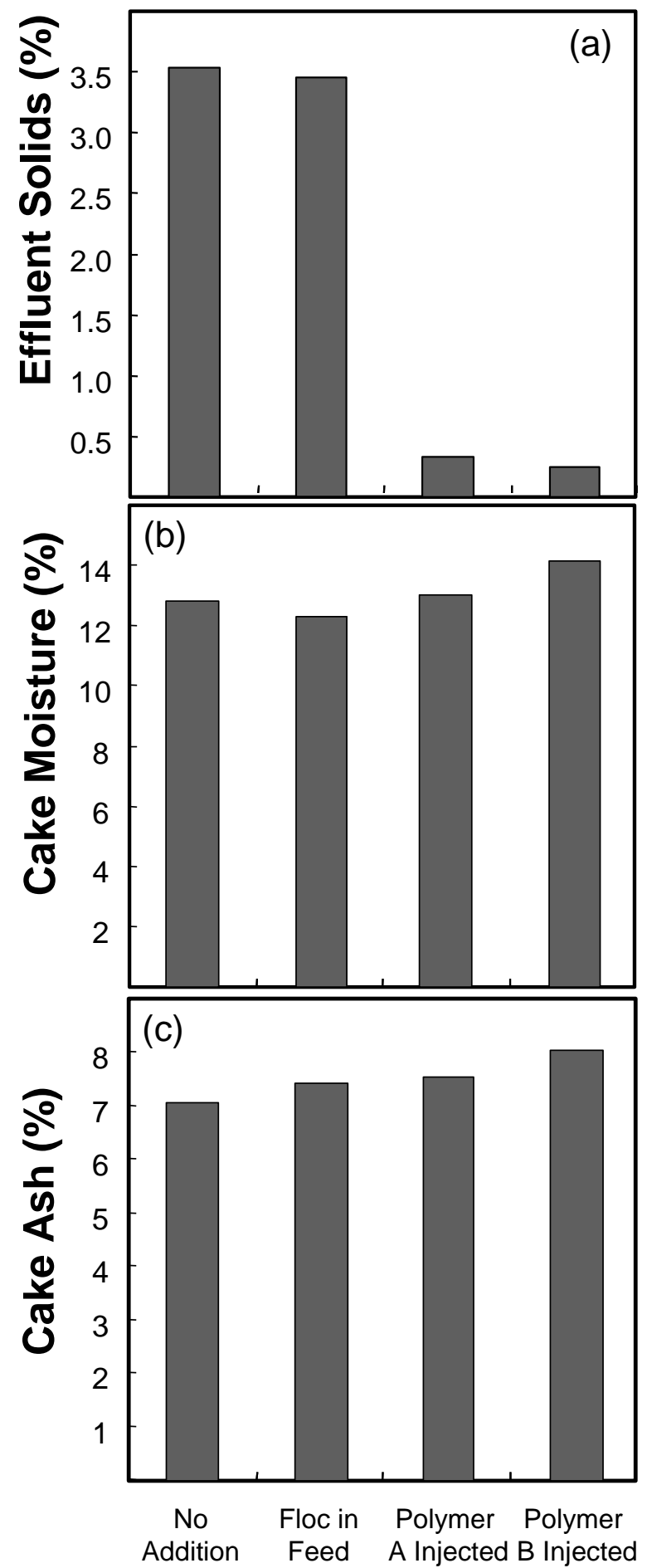

Figure 8. Results obtained from field tests conducted to evaluate the effect of polymer injection on screenbowl centrifuge performance. 
polymer injection reduced the tonnage of lost solids from 2.46 tph down to 0.23 and 0.17 tph using products "A" and "B", respectively. These improvements represent an increase in revenue of more than $\$ 2.0$ million annually for this particular site (2.23 ton/hr/unit x 5 units $\times 6,000 \mathrm{hr} / \mathrm{yr} \times \$ 30 /$ ton $=\$ 2,007,000 / \mathrm{yr})$. In addition, the reduction in the effluent solids content decreased the load on the water clarification circuit and reduced the amount of waste solids that must be permanently deposited in increasingly scarce impoundment space.

The primary downside associated with the use of the injection tube was that it tended to result increase in moisture content of the final dewatered product. The increased moisture was primarily due to the improved recovery of fine particles having a correspondingly high surface area. As shown in Figure 8b, the product moisture increased in this particular case from the baseline value of $12.84 \%$ up to $13.03 \%$ for polymer "A" and up to $14.13 \%$ for polymer "B". The addition of polymer also tended to increase the ash content of the dewatered clean coal product (Figure 8c). The increase was attributed to the improved capture of clay particles that reported to screenbowl product after the polymer injection. Fortunately, the increase in product ash content was not significant enough to negatively impact the sales contract specifications for this particular plant site. The use of the injection tube also marginally increased the operating cost of the dewatering circuit due to the addition of the polymer. However, no attempts have yet been made to determine what impact this addition may have on the overall consumption of chemicals throughout the plant. Preliminary data suggest that the use of polymer in the centrifuge circuit may be partly offset by a decrease in the total flocculant demand for the total plant thickener. 
In light of the promising results obtained from the preliminary tests, several series of additional field tests were performed at another plant site to verify that the injection of polymer flocculant into the dilute pool section of a screenbowl centrifuge would improve solids recovery. In this particular series of tests, another specially formulated polymer (SR polymer) prepared by Nalco Chemical was compared with traditional cationic and anionic flocculants. The results obtained these in-plant tests are summarized in Table 2. In each experimental run, the as-received reagents (polymer or flocculant) were diluted with fresh water down to a $1 \%$ solution before being injected into the screenbowl. The test data from the first series of tests (day 1) show that the solids content in the main effluent was reduced as the polymer dosage was increased up to 4.5 GPM. The highest dosage rate of 4.5 GPM reduced the solids content from about 3.9\% to $0.6 \%$ (2.30 to 0.36

Table 2. Results of screenbowl injection tests using various flocculants and polymers.

\begin{tabular}{|c|c|c|c|c|c|c|c|}
\hline \multirow[b]{2}{*}{ Test Description } & \multirow{2}{*}{$\begin{array}{l}\text { Reagent } \\
\text { Addition } \\
(\mathrm{GPM})\end{array}$} & \multicolumn{3}{|c|}{ Dewatered Product } & \multicolumn{3}{|c|}{ Effluent Discard } \\
\hline & & $\begin{array}{l}\text { Ash } \\
\text { (\%) }\end{array}$ & $\begin{array}{c}\text { Moist. } \\
\text { (\%) }\end{array}$ & $\begin{array}{l}\text { Rate } \\
\text { (TPH) }\end{array}$ & $\begin{array}{l}\text { Ash } \\
(\%)\end{array}$ & $\begin{array}{c}\text { Solids } \\
\text { (\%) }\end{array}$ & $\begin{array}{c}\text { Rate } \\
\text { (TPH) }\end{array}$ \\
\hline No Reagent & -- & 8.70 & 12.84 & 25.72 & 9.58 & 3.94 & 2.30 \\
\hline SR Polymer & 1.25 & 8.63 & 12.26 & 26.45 & 9.44 & 2.69 & 1.57 \\
\hline SR Polymer & 2.50 & 8.48 & 15.77 & 26.47 & 8.61 & 2.66 & 1.55 \\
\hline SR Polymer & 4.50 & 8.97 & 16.54 & 27.66 & 8.32 & 0.62 & 0.36 \\
\hline No Reagent & -- & 8.94 & 11.44 & 25.69 & 9.10 & 3.99 & 2.33 \\
\hline No Reagent & & 8.17 & 11.62 & 25.57 & 11.99 & 4.19 & 2.45 \\
\hline SR Polymer & 4.50 & 9.23 & 16.96 & 27.41 & 9.74 & 1.84 & 1.07 \\
\hline Cationic Floc & 4.50 & 8.65 & 15.31 & 25.93 & 10.07 & 1.05 & 0.61 \\
\hline Anionic Floc & 4.50 & 8.74 & 11.66 & 25.36 & 9.68 & 3.58 & 2.09 \\
\hline
\end{tabular}


TPH). Poorer solids contents of about 2.7\% were obtained at lower dosages of 1.25 and 2.50 GPM of polymer. In addition, test results obtained from a second series of tests (day 2) conducted at a fixed reagent dosage of 4.5 GPM showed that the polymer was superior to traditional anionic flocculant. The cationic flocculant also appeared to work well in the injection tube and reduced the solids content from $4.2 \%$ down to just over $1 \%$. The moisture and ash contents of the dewatered products from these tests increased slightly when adding polymer due to the capture of ultrafine particles.

\subsection{Pilot-Scale Testing of Surfactant Injection}

Several series of preliminary tests were performed to evaluate the potential benefits of adding surface tension modifiers to the screen section of the centrifuge. Due to the exploratory nature of these tests, this work was conducted using a modified $15-\mathrm{cm}$ diameter pilot-scale test unit (Figure 9). The pilot-scale unit was a modified Sharples solid-bowl centrifuge that had been rebuilt by Decanter Machine Inc. as a screenbowl centrifuge. This unit was configured with an internal injection piped that was designed to allow surfactant to be added to the partially dewatered coal at a much higher effective concentration than would be possible if the same amount of reagent were added to the dilute feed slurry. During testing, the test unit was operated at a dry solids capacity of 2.5 $\mathrm{lb} / \mathrm{min}$. Because of the extended length of the bowl section, the pilot-scale unit tended to operate with a longer slurry residence time than was typical for full-scale commercial screenbowl centrifuges. As such, the pilot-scale unit was capable of $>80 \%$ weight recovery of minus 325 mesh solids. The high recovery of ultrafine solids, and correspondingly high moisture content of the dewatered product, made this test unit ideally suited for the evaluation of dewatering aids. 


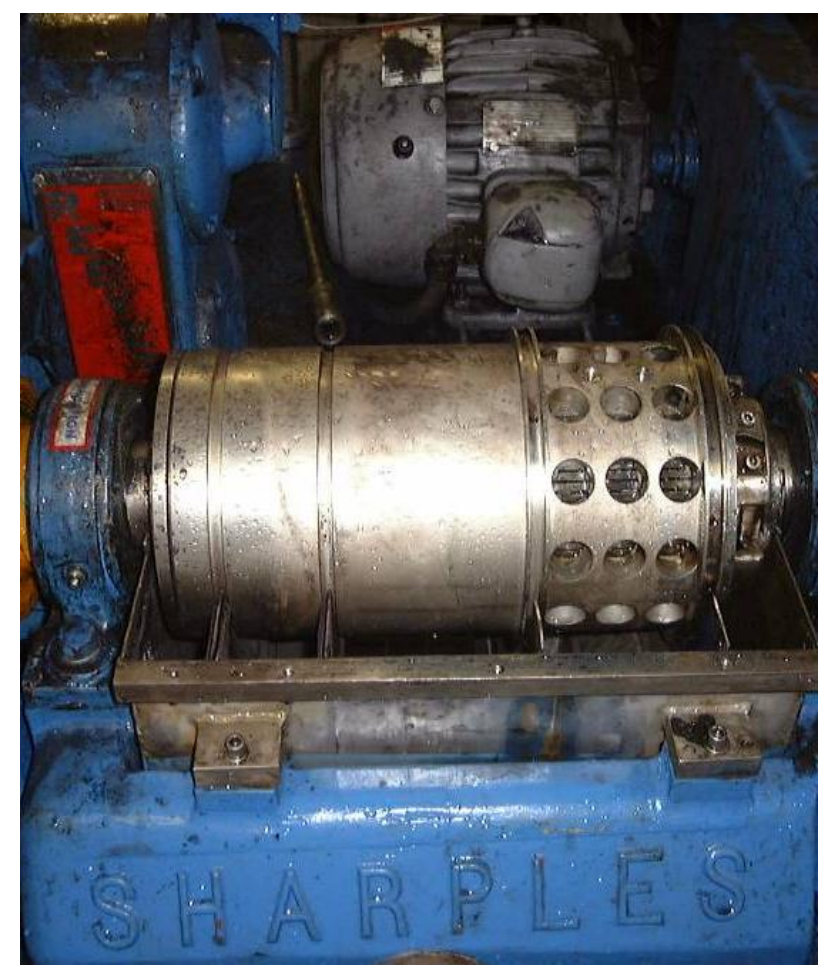

Figure 9. Pilot-scale screenbowl centrifuge used in the evaluation of dewatering aids.

The pilot-scale centrifuge tests were conducted using two different types of dewatering aids. Both of these reagents were generally classified as surface tension modifiers (i.e., Reagent "X" and "Z”). Surface tension measurements conducted using a laboratory tensiometer showed that Reagent "Z" provided a greater drop in surface tension than Reagent " $X$ " at an equivalent dosage level. As such, Reagent "Z" was expected to provide a better level of performance at a lower overall dosage than Reagent "X". For comparison, the effective concentration of each reagent was back-calculated for each test run based on the addition rate of the dewatering aid and the total amount of water that reported to the non-effluent products (i.e., combined sum of water reporting with the dewatered product and screen-drain product). 
The results obtained from the testing of the dewatering aids are summarized in Figure 10. As shown, four separate test runs were performed. The first test run was carried out without the addition of any surface tension modifier. As shown in Figure 10, this baseline test produced a dewatered product containing 16.9\% moisture. The addition of a low dosage (estimated to be $<8 \mathrm{ppm}$ ) of Reagent "X" reduced the moisture by about one percentage point (i.e., from $16.9 \%$ to $15.9 \%$ ). An increase in the amount of Reagent " $\mathrm{X}$ " added to the screen-section of the centrifuge further reduced the moisture content of the dewatered product down to $12.3 \%$. However, the dosage (which was estimated to be more than $25 \mathrm{ppm}$ ) required to achieve this level of dewatering performance was relatively high. Fortunately, the same level of dewatering performance was possible using a much smaller dosage of Reagent "Z". As shown in Figure 10, this stronger surface tension modifier reduced the moisture of the clean coal product down to $12.6 \%$ at a

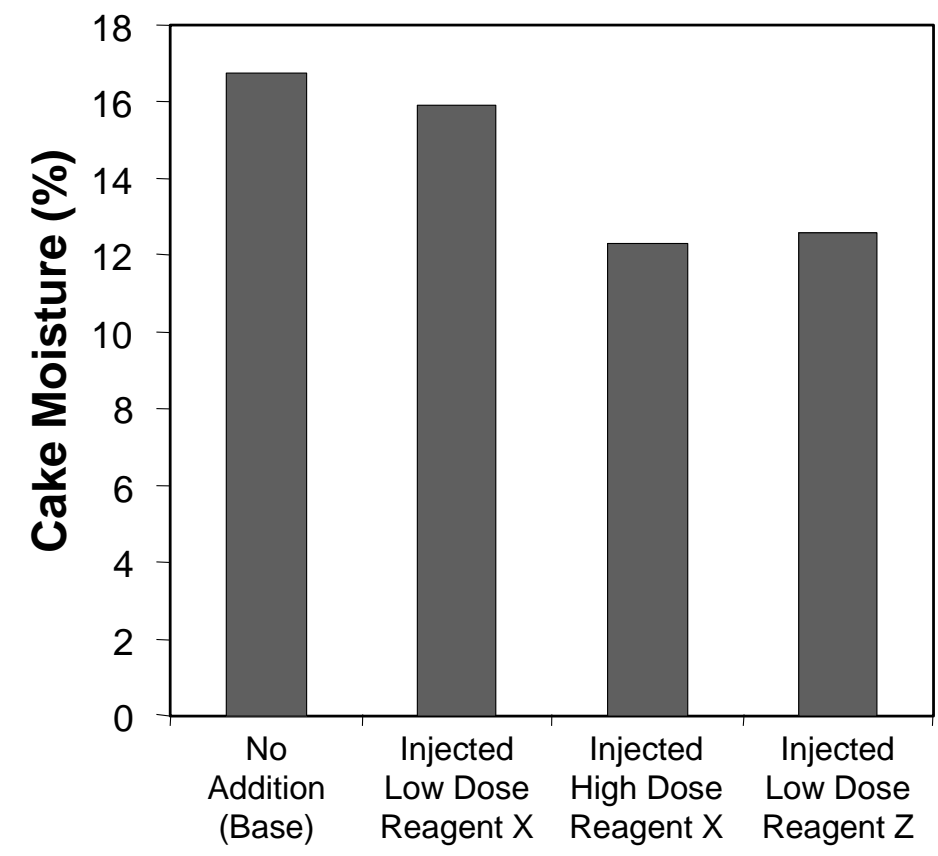

Figure 10. Data obtained from pilot-scale tests conducted to evaluate the effects of dewatering aids on screenbowl centrifuge performance. 
relatively low dosage (estimated to be $12 \mathrm{ppm}$ ). It should be noted that these levels of moisture reduction could only be achieved by adding these reagents directly to the partially dewatered cake on the screen section of the centrifuge. A dosage nearly an order of magnitude higher would be necessary to achieve the same effective concentration if the dewatering aids had been added directly to the dilute feed slurry to the centrifuge.

To investigate the effects of surfactant dosage in greater detail, a second set of pilot-scale tests was conducted at low (2 lb/ton) and high ( $8 \mathrm{lb} / \mathrm{ton})$ dosages. The results obtained from these tests are plotted in Figure 11. In this case, the moisture content of the dewatered product was monitored as a function of time after the injection of reagent was initiated. As shown, the moisture of the final dewatered product decreased steadily from about $21.5 \%$ down to $19.5 \%$ during the testing period of 8 minutes. Surprisingly, both

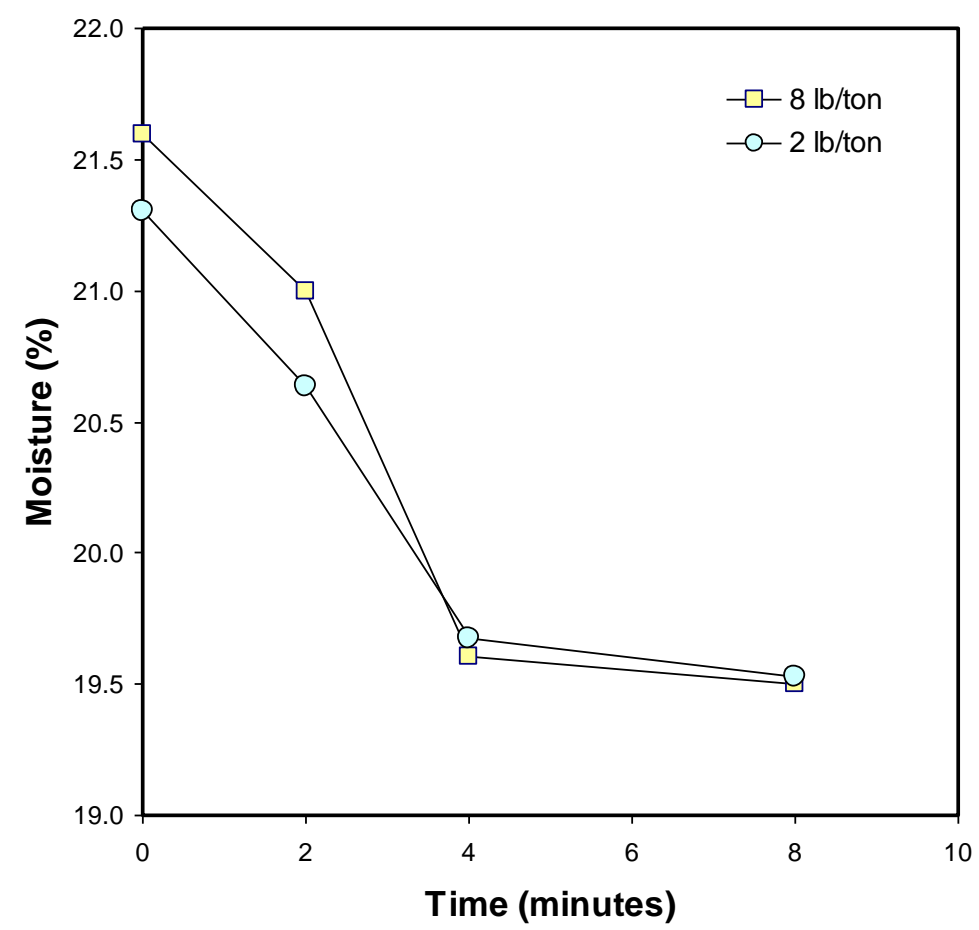

Figure 11. Dewatering data obtained after the injection of $2 \mathrm{lb} /$ ton and $8 \mathrm{lb} /$ ton of surfactant (surface tension reducer) to a screenbowl cake. 
sets of dewatering test runs produced essentially the same level of moisture reduction. This finding suggests that the lower dosage of $2 \mathrm{lb} / \mathrm{ton}$ was adequate to achieve the desired reduction in surface tension and that further additions of chemical would be wasteful. It should be noted that these levels of moisture reduction could only be achieved by adding these reagents directly to the partially dewatered cake on the screen section of the centrifuge. A dosage nearly an order of magnitude higher would be necessary to achieve the same effective concentration if the surfactant had been added directly to the dilute feed slurry to the centrifuge.

\subsection{CONCLUSIONS}

Test data obtained in this investigation have shown that the overall performance of screenbowl centrifuges can be improved through the use of reagent injection tubes. For example, field trials conducted at an industrial site have demonstrated that the recovery of fine solids normally lost to the main effluent can be dramatically improved by injecting a synthetic polymer directly into the low-solids clarified pool within the rotating bowl of the centrifuge. The polymer works optimally when added in this manner since this approach avoids premature contact between the polymer and vast majority of the feed solids. In addition, this type of direct injection prevents shearing of the polymer as it enters the dilute pool and provides an ideal low-solids environment for full chemical activation. The polymer injection system has already been adopted by several coal preparation plants in Virginia, West Virginia, Pennsylvania and Alabama. An economic study conducted at one of the sites showed that this low-cost modification provided a payback period of just a few days. 
Pilot-scale tests conducted in the present work also indicate that surface tension modifiers can be used to effectively reduce the total moisture content of the dewatered product from a screenbowl centrifuge. In this case, an injection tube is used to introduce the surface tension modifier directly onto the partially dewatered solids passing across the screen section of the centrifuge. This method of addition allows the chemicals to be added to the coal particles at a much higher effective concentration than would be possible if the same amount of reagent were added to the dilute feed slurry.

\subsection{ACKNOWLEDGMENTS}

The authors would like to acknowledge the financial support provided by the U.S. Department of Energy (DE-FC26-01NT4109) under a subaward from the Center for Advanced Separation Technologies (CAST Initiative). The technical assistance provided by personnel at the Coal Clean and Moss No. 3 preparation plants is also deeply appreciated. Finally, the technical guidance and fabrication expertise provided by Decanter Machine, Inc. is gratefully acknowledged. 


\subsection{REFERENCES}

1. Baillie, P.F. and Lumsden, B.G., 1988. "Chemical Dewatering of Coal," Proceedings, Fourth Australian Coal Preparation Conference, P. Holtham (Ed.), Gladstone, Australia, May 23-26, 1988, pp. 309-319.

2. Baker, A.F., 1987. "Hot Surfactant Solution as a Dewatering Aid During Filtration," Proceedings of the NCA/BCR Coal Conference and Exposition III, Kentucky Fair and Exposition Center, Louisville, Kentucky, October 1876, pp. 175-183.

3. Gallagher, E. Lewis, J.E., Post, J.J., Swanson, A.R., and Armstrong, L.W., 1981. "Dewatering of Fine Coal by Screenbowl Centrifuges," Proceedings of the First Australian Coal Preparation Conference, Newcastle, Australia, April 6-10, 1981, Swanson. A.R. (Ed.), pp. 135-154.

4. Miller, K.J. and Wen, W.-W., 1984. "Effect of Operating Parameters and Reagent Addition of Fine Coal Dewatering in a Screen Bowl Centrifuge," Pittsburgh Energy Technology Center Report, DOE/PETC/TR-85/1 (DE85002885), 19 pp.

5. Nicol, S.K., 1976. "The Effects of Surfactants on the Dewatering of Fine Coal," Proceedings, Institute of Mining and Metallurgy, No. 260, December 1976, pp. 37-44.

6. Records, A. and Sutherland, K., 2001. "Decanter Centrifuge Handbook," 1st Ed., Elsevier Science Inc., New York, N.Y., 2001, 421 pp.

7. Silverblatt, C.E., and Dahlstrom, D.A., 1954. "Moisture Content of Fine Coal Filter Cake: Effect of Viscosity and Surface Tension," Industrial and Engineering Chemistry, Vol. 46, No. 6, June 1954, pp. 1201-1207. 
8. Yoon, R.-H., Asmatulu, R., and Luttrell, G.H., 2001. "Development of Novel Fine Coal Dewatering Aids," Proceeding fo the 18th International Coal Preparation Conference, May 1-3, 2001, Lexington, Kentucky, pp. 115-122. 
Appendix 20: Development of Alkaline Sulphide Leaching and Recovery of Gold (MT001) 


\section{FINAL TECHNICAL REPORT}

\begin{tabular}{|ll|}
\hline Contract Title and Number: & \multicolumn{2}{l|}{ Period of Performance: } \\
\cline { 2 - 2 } $\begin{array}{l}\text { Crosscutting Technology Development at the Center for Advanced } \\
\text { Separation Technologies }\end{array}$ & Starting Date: Apr. 1, 2003 \\
(DE-FC26-02NT41607) & Ending Date: $\quad$ Oct 31, 2008 \\
\hline
\end{tabular}

Sub-Recipient Project Title:

DEVELOPMENT OF ALKALINE SULFIDE LEACHING AND RECOVERY OF GOLD

Principal Investigators:

Corby G. Anderson

Larry G. Twidwell

Contact Address:

Room 214, ELC Building

1300 West Park Street

Butte, MT 59701-8997

Subcontractor Address:

"No subcontracts issued."
Report Information:

Type: Final

Number:

Period:

Date: $\quad$ April 21, 2008

Code: MT001

Contact Information:

Phone: (406) 496-4794

Fax: (406) 496-4512

E-Mail: canderson@mtech.edu

Subcontractor Information:

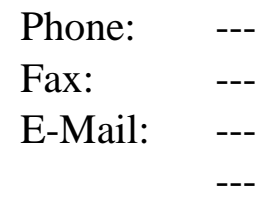

This report was prepared as an account of work sponsored by an agency of the United States Government. Neither the United States Government nor any agency thereof, nor any of their employees, make any warranty, express or implied, nor assume any legal liability or responsibility for the accuracy, completeness, or usefulness of any information, apparatus, product, or process disclosed, or represents that its use would not infringe privately owned rights. Reference herein to any specific commercial product, process, or service by trade name, trademark, manufacturer, or otherwise does not necessarily constitute or imply endorsement, recommendation, or favoring by the United States Government or any agency thereof. The views and opinions of authors expressed herein do not necessarily state or reflect those of the United States Government or agency thereof." 


\begin{abstract}
$\underline{\text { ABSTRACT }}$
The latter part of the $20^{\text {th }}$ century saw great advances in the treatment of refractory gold ores coupled with increased reliance on the use of cyanide for gold processing. Now, in many parts of the world, there is social pressure to limit or eliminate the use of cyanide. As well, treatment of some refractory ores or concentrates which have excessive cyanide consumption, gold cyanide pregrobbing or significant sulfide content remain difficult. This research focused development of alkaline sulfide leaching as an ancillary process to nitrogen species catalyzed (NSC) pressure leaching. Studies on electrochemical fundamentals and the applicable thermodynamics of the alkaline sulfide hydrometallurgical system are included. Flowsheets including functional waste treatment were developed and implemented. As well a successful demonstration plant was built at CAMP and operated to treat several Montana gold ores.
\end{abstract}

\title{
TABLE OF CONTENTS
}

Section

$\underline{\text { Page }}$

Abstract

CAMP Title

Executive Summary

4

Experimental

Results and Discussion

Conclusion

References

Appendices 


\section{EXECUTIVE SUMMARY}

This project was initially funded by Department of Energy through the Center for Advanced Separation Technologies (CAST). CAST funds research studies that have industrial implications. The CAST funds were used as $25 \%$ match for MBRCT funding. From the CAST work and knowledge from a previous fifty years of industrial facility experience and data, there was a basic understanding of alkaline sulfide leaching for antimony, arsenic, mercury, tin and gold. However, it hadn't been applied specifically for industrial gold leaching. The specific goals of the CAST and MBRCT program that were successfully met were;

Task 1: Determine the fundamentals aspects of alkaline sulfide hydrometallurgical system. Optimize conditions for leaching gold from various Montana ore bodies. This was successfully completed.

Task 2: Develop flowsheets and conditions for recovering gold from various Montana ore bodies. This was successfully completed for both Golden Sunlight yretic ores and Yellow Band free milling ores.

Task 3: Integrate gold leaching process with gold recovery and lixiviant regeneration process. This was successfully done.

Task 4: Integrate with proprietary partial oxidation process. This was successfully done with Golden Sunlight ores and Green Creek gravity concentrates.

Task 5: Build demonstration plant. This was successfully done and witnessed.

Overall Goal: Utilize the alkaline sulfide leaching process for more effectively producing gold in Montana. Based on the previous achieved goals, this is being pursued. Given the nature of the business, a larger scale demonstration plant is needed for confirmation for several currently interested entities. 


\section{EXPERIMENTAL}

Experimental procedures for all work are contained in the Darby Stacey and Karel Pramono thesis documents along with the MBRCT report located in the appendices.

\section{RESULTS AND DISCUSSION}

The results and discussion are covered in detail in the appendices. They would be found in the Stacey thesis, the Pramono thesis, the MBRCT report and the CIM paper. Through these combined comprehensive efforts along with fifty years of applied industrial knowledge from the Sunshine alkaline sulfide plant, a sound and in depth understanding of the technology is now available for further applications.

\section{CONCLUSION}

The alkaline sulfide hydrometallurgical system has been evaluated in this research from a fundamental standpoint as well as in application. For troublesome gold and silver ores and concentrates or in areas where traditional cyanidation is restricted, the system stands as a viable technical alternative.

\section{REFERENCES}

All pertinent references are included in the Darby Stacey and Karel Pramono as well as the CIM Proceedings paper. These are contained in the appendices. 
APPENDICES 


\section{MBRCT PROJECT REPORT SUMMARY}

\section{Project Objectives Work Performed and Project Results and Performance Benchmarks, Progress and Achievements}

I. OVERALL PROJECT PERFORMANCE AND ACHIEVEMENT OF OBJECTIVES AS STATED IN THE GRANT AGREEMENT. Project performance and achievement was excellent. Actual funding was received from MBRCT in October 2004 rather than July of 2004. The project was completed in March of 2007. Per our previous discussions with MBCRT personnel, Dr Eric Dahlgren left CAMP in September of 2004 for a more lucrative industrial position with INTEL. This left a huge gap in CAMP manpower. Right now the materials, metals, and mining business is booming and qualified people are scarce. Even new graduates are hard to attract, particularly, for low paying graduate program research. We did replace Dr. Dahlgren with Dr. Paul Miranda and Mr. Darby Stacey at the beginning of March 2004. Dr. Miranda has finished his Ph.D. thesis at the U of Montana and he works fulltime for CAMP. Mr. Darby Stacey graduated with his M.Sc. degree in May 2005. We also recruited Mr. Karel Pramono as a new MS graduate student on this project and he has started working on this project in May, 2005 after his BS Metallurgical Engineering graduation from Montana Tech. As such, we have been expeditious with the budget and we have graduated two M.Sc. Metallurgical Engineering students instead of just one with this project along with achieving our stated goals. We also worked with Dr. Bill Gleason who did part of his Doctoral Research on this project. Dr. Gleason is now a fulltime employee of the Department of Metallurgical and Materials Engineering here at Montana Tech. Lastly, we asked for a no cost project extension from MBRCT which was granted through March 2007. This allowed us to construct and successfully operate the demonstration plant on two different Montana gold ores. Again CAMP, like other service entities in this unprecedented demand boom in mining and energy, was short staffed. This was effectively communicated to the MBRCT personnel who agreed to allow this late final report.

II. ACHIEVEMENT OF PERFORMANCE BENCHMARKS: (1) State the performance benchmarks as presented in the grant agreement; (2) Provide a concise narrative of progress and achievements for each performance benchmark. This will be done and where tasks have been completed illustrative examples will be cited.

\section{Task \#1}

\section{Optimize conditions for leaching various gold ores using statistically designed experiments. Completed.}

Optimum conditions for leaching gold with the alkaline sulfide leaching process were developed using statistically designed experiments based on Stat Ease Design Expert software. Initially, a factorial design was used to identify the important components of the alkaline sulfide leaching lixiviant. A two-level four factor one-half matrix model was 
used to help identify the components that had a significant effect on the alkaline sulfide lixiviants ability to leach gold. The factors included in the design were the free hydroxide concentration, sulfur concentration, sulfide concentration, and thiosulfate concentration. By utilizing factorial design, it was found that thiosulfate additions to the alkaline sulfide lixiviant had a negligible effect on the amount of gold leached. However, it was found that the sulfur, sulfide, and free hydroxide concentrations were all important. A typical surface response model generated in the work showing the effect of sulfide and free hydroxide concentrations and the amount of gold leached is shown in Figure 1. It can be seen from the figure that more gold was leached when the lixiviant contained $0 \mathrm{~g} / \mathrm{L}$ free hydroxide and $50 \mathrm{~g} / \mathrm{L}$ sulfide. The surface response model in Figure 2 shows that a solution containing $50 \mathrm{~g} / \mathrm{L}$ sulfide and $25 \mathrm{~g} / \mathrm{L}$ sulfur resulted in a lixiviant that leached the most gold. (Please bear in mind that while the indicated gold recoveries are relatively low, this is an artificial lab result by design.) These tests were run with excess solid gold to saturate the solutions for ease in ICP and gravimetric analysis. The actual data represents solution gold concentrations which range up to $100 \mathrm{ppm}$ or 50 times that found in real industrial applications). Confirmation of these optimum leaching conditions to real ores and concentrates are better illustrated by reviewing the results of Table 1. Illustrative Scoping Alkaline Sulfide Leach Tests on Ores and Concentrates.

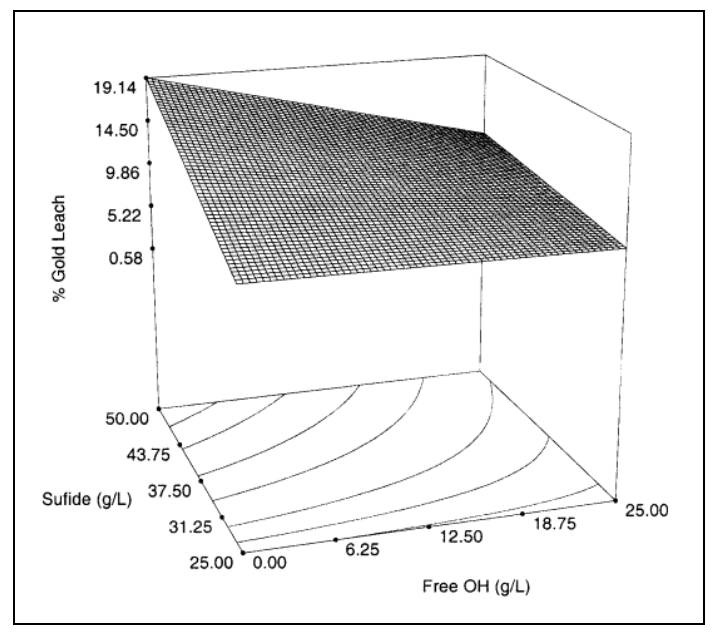

Figure 1. Example surface response model illustrating the percent of gold leached as a function of sulfide and free hydroxide concentrations 


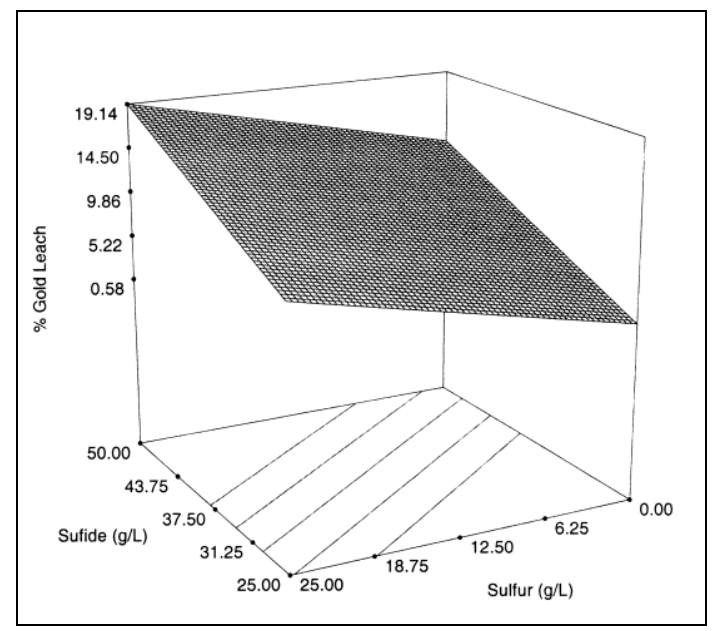

Figure 2. Example surface response model illustrating the percent of gold leached as a function of sulfur and free hydroxide concentrations

\section{Develop a testing procedure for characterizing effectiveness of leach process on various orebodies. Completed.}

Both the metastable characteristics of the ASGLS and the physical properties of the solids tested made it initially difficult to achieve reproducibility with tests conducted in the lab. To combat these phenomena, a strict Standard Operating Procedure (SOP) was created through laboratory experiences. All of the final tests were conducted under the same SOP. To set up tests reproducible with the ones found in Mr. Darby Stacey's M.Sc. thesis, follow the following twelve steps:

1. The final leach volume will be $750 \mathrm{~mL}$; however, reagent additions need to be calculated for a final volume of $1000 \mathrm{~mL}$. Solutions will be made larger than the final volume for two reasons: 1) there will be about a $50 \mathrm{~mL}$ volume loss after filtering the solution, and 2) there was only a $1000 \mathrm{~mL}$ volumetric flask available.

2. Obtain a $2000 \mathrm{~mL}$ flask and fill to about $600 \mathrm{~mL}$ with distilled water.

3. Weigh out sodium hydroxide to the nearest 0.01 grams and add directly to the flask under a fume hood. Gently shake the flask so that the hydroxide does not clog at the bottom of the flask. Let the flask sit while weighing out the sodium sulfide.

4. Weigh sodium sulfide out to the nearest 0.01 grams and add to the flask under the fume hood. By the time the sodium sulfide is added to the flask, all of the sodium hydroxide should be dissolved. Gently shake the flask to wet all of the sodium sulfide flakes.

5. While the sodium sulfide is dissolving, weigh out sulfur to the nearest 0.01 grams. Add the sulfur to the flask under the hood, and gently shake the flask to wet the sulfur flakes. Not all of the sodium sulfide flakes will be dissolved yet. Total solution volume should be closer to $750-800 \mathrm{~mL}$ now, depending on the amount of solid added to the solution. 
6. Place the flask on a hot plate at medium temperature. Because of the dissolution of sodium hydroxide, the solution will have a temperature greater than room temperature. An elevated temperature will aid in the dissolution kinetics; however, it is important that the temperature be held similar to the other tests during dissolution of the sulfur and sodium sulfide. Below is a picture of partially dissolved sulfur and sodium sulfide flakes.

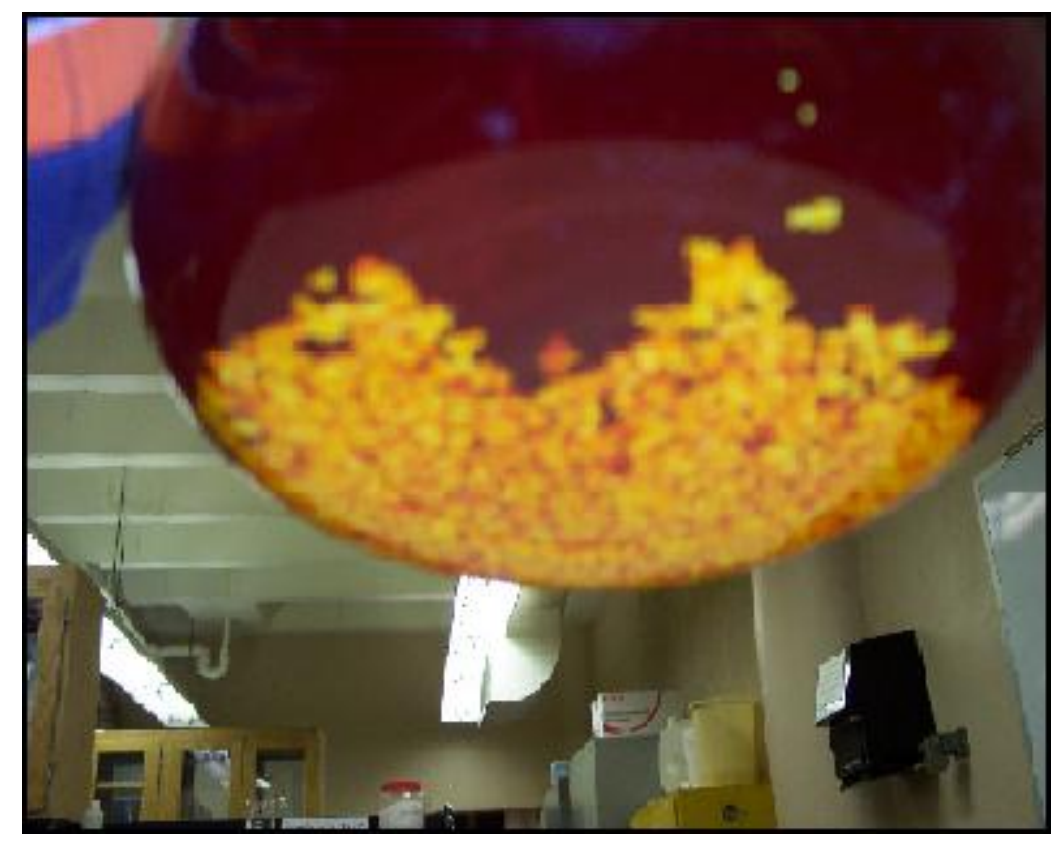

Figure 3. Partially Dissolved Sulfur \& Sulfide Flakes.

7. Keep the solution temperature between $50-60^{\circ} \mathrm{C}$ and shake often during the dissolution process. Total dissolution time should not take longer than 30 minutes. If the heated solutions are kept longer on the hot plate open to the atmosphere it is suspected that the pick up of oxygen will change that chemistry of the solution.

8. As soon as the last solid has dissolved, add to the $1000 \mathrm{~mL}$ volumetric flask. With cold distilled water, wash any remaining solution from the walls of the empty flask and pour it into the volumetric flask until it is near the full line. Place the filled volumetric flask in a bucket of chilled water to bring the temperature back to room temperature. Once the solution has cooled, add distilled water until the proper volume is obtained. Pour the solution from the volumetric flask into a clean, dry flask so that the solution can be agitated to equilibrium.

9. Now the solution must be filtered to remove any solids that did not dissolve. It is suspected that most of the impurities come from the sodium sulfide. A Whatman $185 \mathrm{~mm} \# 3$ filter paper should be placed onto a porcelain filter in the fume hood. To aid in the filtering process the porcelain filter is placed onto a $4000 \mathrm{~mL}$ Buchner flask and sealed with a rubber plug. A vacuum is pulled on the Buchner flask via flowing water passing quickly past an empty hose connected to the Buchner flask. The solution is now poured quickly onto the filter. While wearing a rubber glove, the experimenter may need to adjust the edges of the filter to avoid any short 
circuiting of solution. After filtering is done, pour the solution from the bunker flask into a clean, dry flask. Note the clarity of the solution; if it still looks cloudy there was a short circuit in the filtering, and it needs to be re-filtered.

10. Obtain a $1000 \mathrm{~mL}$ kettle with a lid. Weigh out gold to the nearest 0.001 grams. Unless otherwise noted, all tests done had 0.250 grams of gold added. Add a 2 inch Teflon coated magnet to the kettle with the gold.

11. It is optional to take an ORP of the solution now before gold is added. Into a 250 $\mathrm{mL}$ graduated cylinder, add clean leaching solution. Fill to $250 \mathrm{~mL}$ and add to the kettle with the magnet and gold. Repeat twice to obtain $750 \mathrm{~mL}$ total leach solution in the kettle.

12. Place the lid onto the kettle and plug the probe holes in the lid with rubber stoppers. Again, it is now optional to take an ORP before the solution is brought up to temperature. (See Figure 4.)

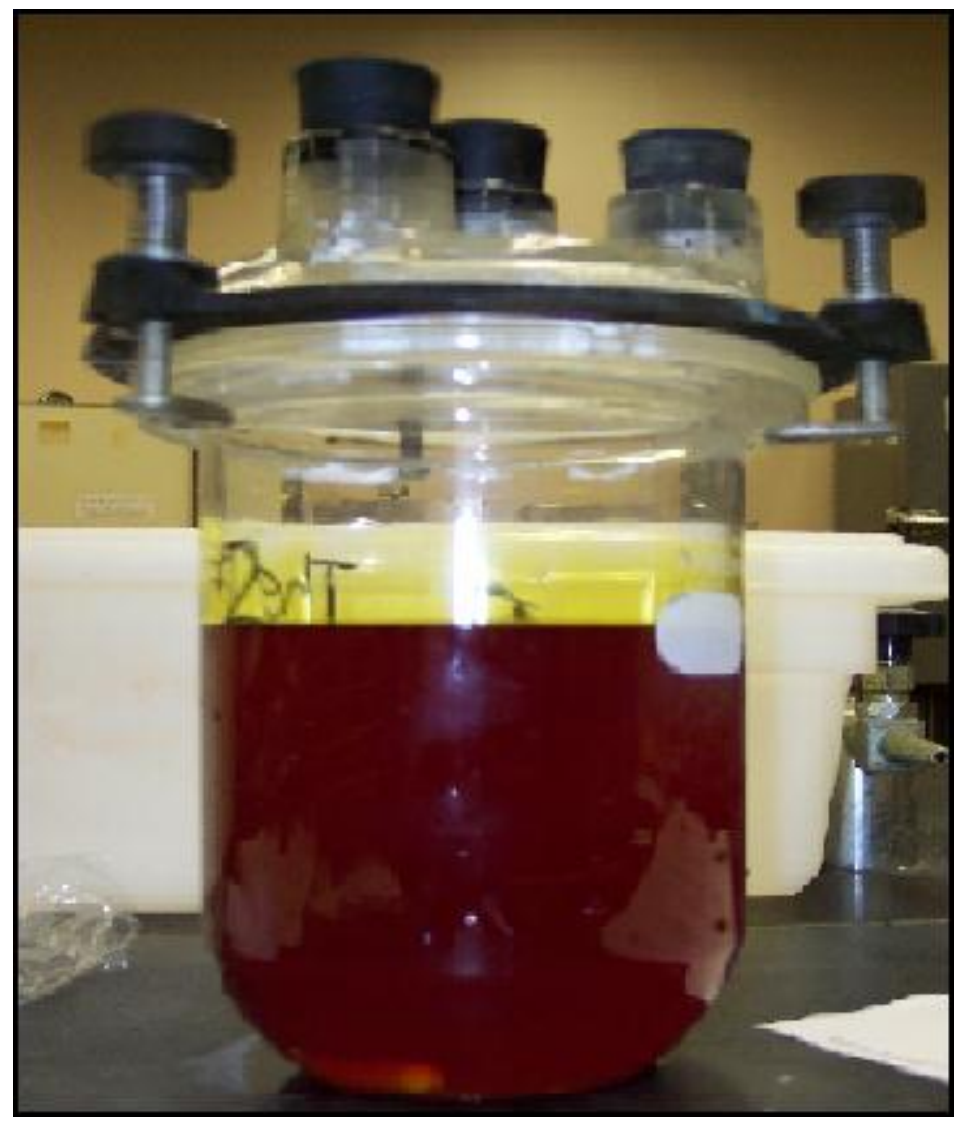

Figure 4. Kettle Test Outside of the Heat Bath

1. Place the filled kettle into a warm water bath at a specified temperature. A magnetic stirrer plate should be place underneath the warm water bath to provide agitation for the system. Start the stirrer 5 minutes after submerging the kettle so that it can be close to leach temperature before the agitation begins. Record to time that agitation starts as the start time for the leach test. Agitation should be held constant for each test at 330 RPM. 
A typical test set up is seen below in Figure 5. In this particular test, three leach tests are being run at $60^{\circ} \mathrm{C}$.

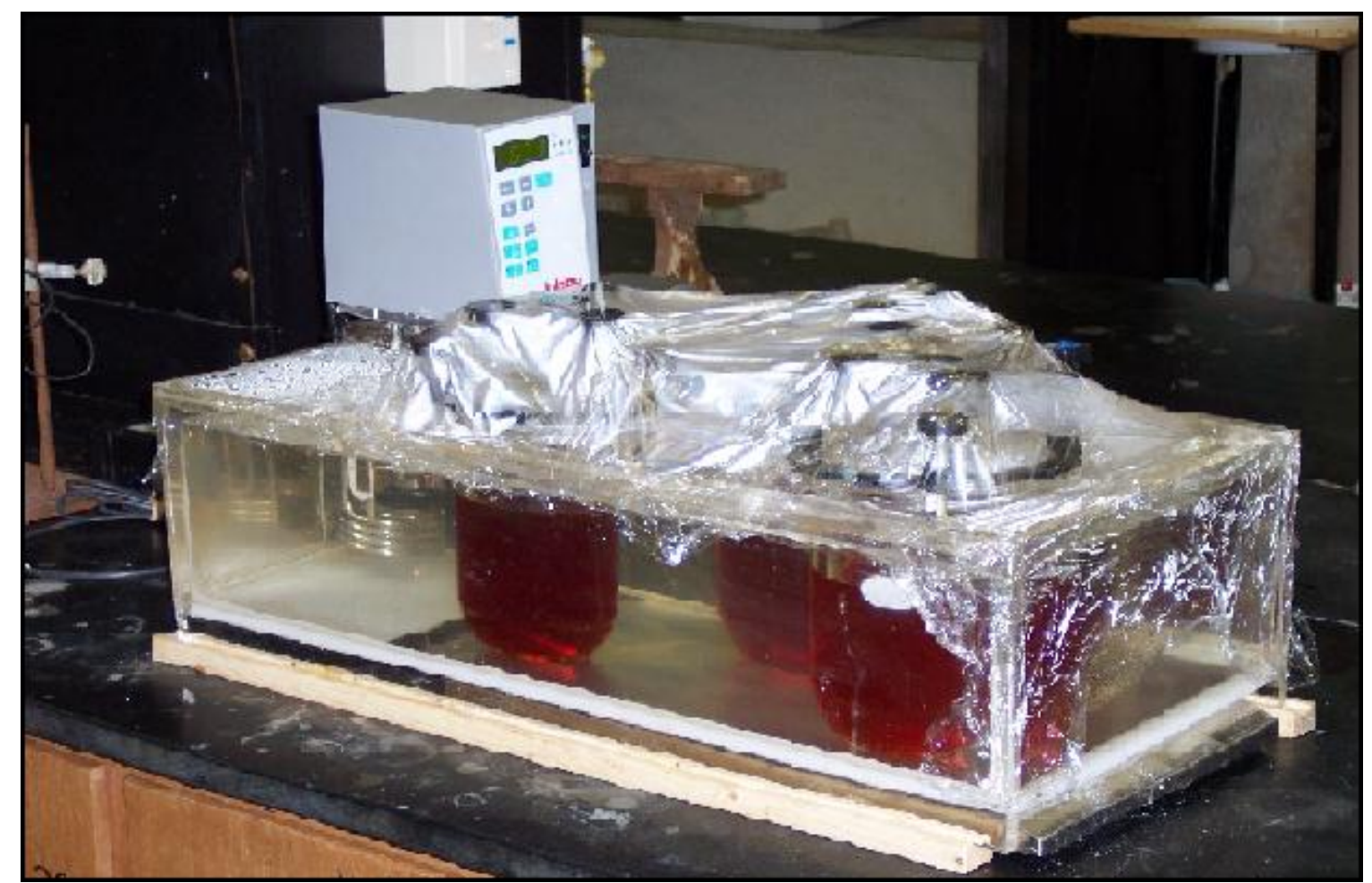

Figure 5. Typical Leach Test.

Note the cellophane draped across the top of the heat bath; it is there because of the fast evaporation kinetics of water at high temperatures. Most of the evaporated water can be caught and drained back into the water bath. Without cellophane the bath would need to be watched hourly so that it did not reach critically low levels.

\section{Demonstrate effectiveness of complete alkaline sulfide leaching process on a gold concentrate obtained from a Montana orebody. Completed.}

Leaching results to demonstrate effectiveness on ores and concentrates including some from Montana is illustrated in Table 1 below. 


\section{Leaching: Industrial Ores and Concentrates}

Placer Dome Golden Sunlight Mine Pyrite Concentrate NSC Partial Oxidation with Alkaline Sulfide Leach 83\% Au Recovery.

Profile Resources Gravity Concentrate Direct Alkaline Sulfide Leach 92\% Au Recovery

Profile Resources Flotation Pyrite Concentrate NSC Partial Oxidation and Alkaline Sulfide Leach $93 \%$ Au Recovery

Cia Minera Ponderosa Peruvian Mine Pyrite Tailings NSC Partial Oxidation with Alkaline Sulfide Leach 97 \% Au Recovery

Elkhorn Carbonaceous Preg Robbing Ore Direct Alkaline Sulfide Leaching $\mathbf{9 1 \%}$ Au Recovery

Elkhorn Gold Chalcopyrite Ore NSC Partial Oxidation and Alkaline Sulfide Leaching 94\% Au Recovery

Table 1. Illustrative Scoping Alkaline Sulfide Leach Tests on Ores and Concentrates

\section{Task \#2}

1. Characterize ore from Montana orebodies. Completed as noted before.

2. Develop thermodynamic models of different ores in the alkaline sulfide system. Completed.

Using StabCal software the alkaline sulfide stable and metastable system was modeled. An illustrative example is shown below in Figure 6. 


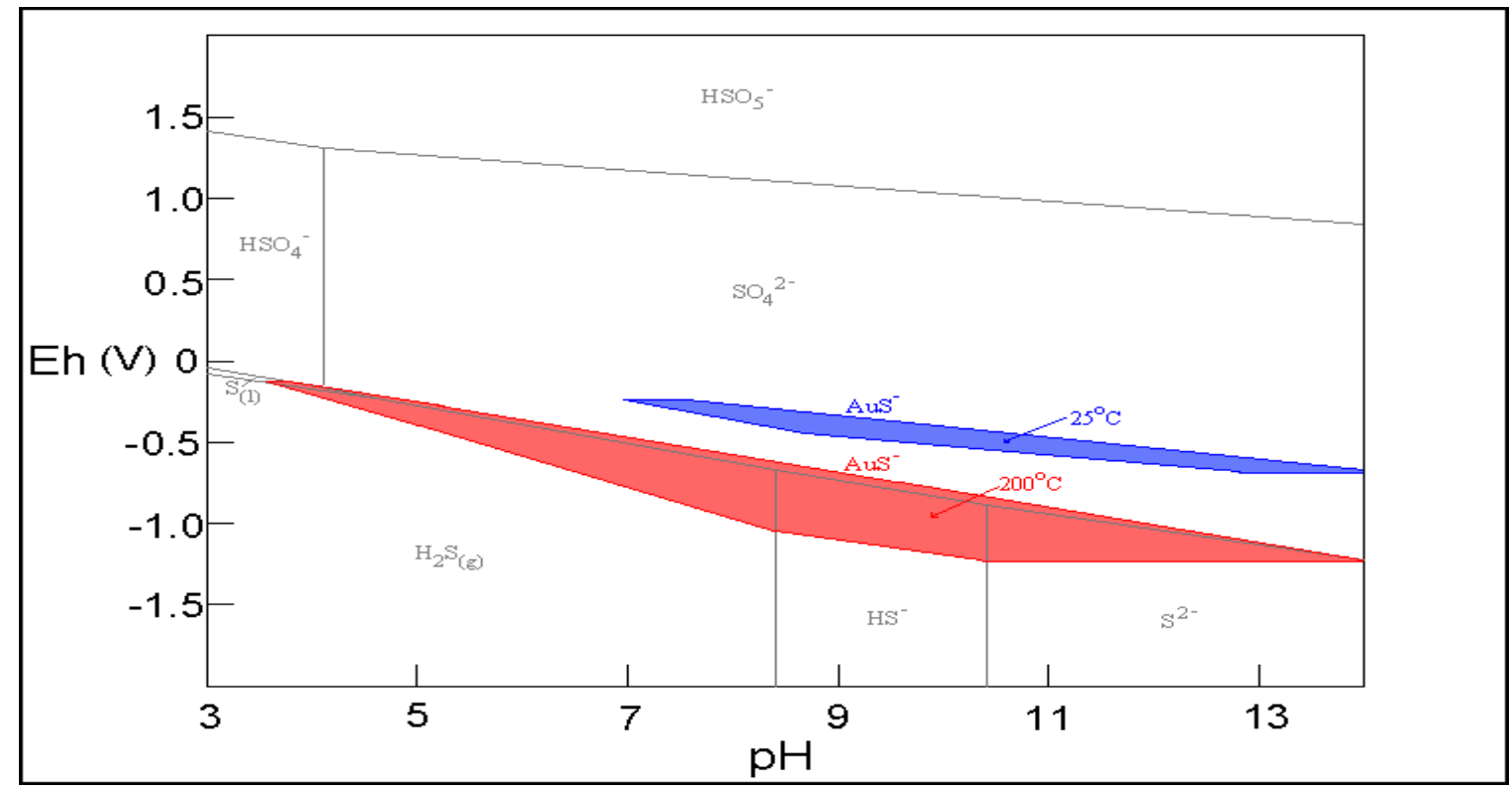

Figure 6. Example alkaline sulfide gold stability diagram with effect of temperature illustrated.

\section{Develop potential flowsheets for different ore bodies located in Montana.}

As an alternative to the currently banned cyanide system, it was thought to be essential to develop similar flowsheet for the alkaline sulfide system. As with any mineral extraction processes, a comminution circuit will precede any extraction unit processes. Furthermore, Figure 7 shows the developed unit processes in a simple flowsheet format. Further research will have to investigate the ideal size reduction before leaching and stripping of gold from pregnant alkaline sulfide solution. 


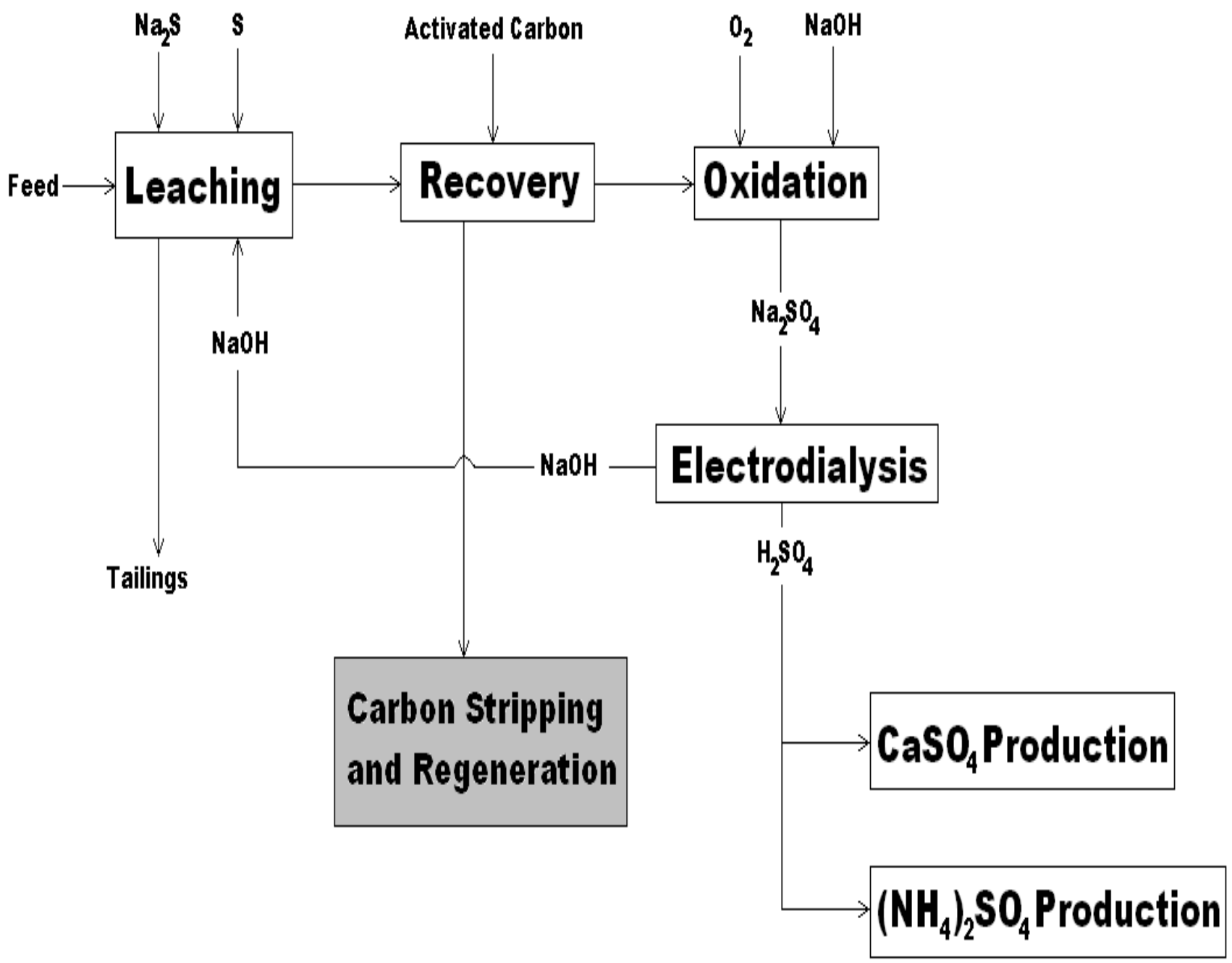

Figure 7. Possible Flowsheet of The Alkaline Sulfide Gold Leaching System for Montana Ores and Concentrates.

Task \#3

1. Optimize gold recovery processes for removing the complexed gold contained in the alkaline sulfide lixiviant. 
Gold was successfully removed from solution with a carbon substrate. (See Table II.) A few ionexchange membranes were also tested as collaboration with PSI Inc. of The University of Montana in Missoula. Inquiries have indicated the possibility of using silica based ion-exchange resins with different active ingredients of quartenary amine and iminodiacetic acid to strip gold out of the alkaline sulfide solution. However, concerns related to the stability of the silica resins in the high $\mathrm{pH}$ system of the alkaline sulfide solution did exist. Thus, styrene based ion-exchange resins with equivalent active ingredients were tested. All tests were done in an alkaline sulfide solution of $80 \mathrm{gr} / \mathrm{L}$ total sulfur concentration, $50 \mathrm{gr} / \mathrm{L} \mathrm{NaOH}$ concentration, and 18.77pm gold.

TABLE II. Description of Substrate Used in the $1^{\text {st }}$ Recovery Experimental Campaign

\begin{tabular}{|l|l|l|l|}
\hline Test & Substrate & Active Ingredient & Base Material \\
\hline RT1-T1 & PSI WP2 & Quartenary Amine & Silica \\
\hline RT1-T2 & Amberlite IRC748 & Iminodiacetic Acid & Styrene-divinylbenzene \\
\hline RT1-T3 & Purolite S930 & Iminodiacetic Acid & Styrene-divinylbenzene \\
\hline RT1-T4 & Sybron Lewatit@ TP207 & Iminodiacetic Acid & Styrene-divinylbenzene \\
\hline RT1-T5 & IRA400 & Quartenary Amine & Styrene-divinylbenzene \\
\hline RT1-T6 & IRA68 & Quartenary Amine & Styrene-divinylbenzene \\
\hline RT1-T7 & Activated Carbon & -- & -- \\
\hline
\end{tabular}

TABLE III. Results of $1^{\text {st }}$ Recovery Experimental Campaign

\begin{tabular}{|l|l|l|l|}
\hline Test & Substrate & $\begin{array}{l}\text { Weight Post-Rec. } \\
\text { (initially 20gr.) }\end{array}$ & \% Rec Fire-Assay \\
\hline RT1-T1 & PSI's WP2 & N/A & N/A \\
\hline RT1-T2 & IRC748 & 9.34 & 3.02 \\
\hline RT1-T3 & S930 & 11.22 & 1.67 \\
\hline RT1-T4 & TP207 & 10.46 & 0.16 \\
\hline RT1-T5 & IRA400 & 21.08 & 53.43 \\
\hline RT1-T6 & IRA68 & 10.34 & 0.16 \\
\hline RT1-T7 & Act. Carbon & 20.83 & 70.06 \\
\hline
\end{tabular}

Results shown in Table III indicate the instability of PSI's WP2 ion-exchange membrane in the high $\mathrm{pH}$ of the alkaline sulfide solution. Only after 4-5hours of shaking, all of the silica-based resins were dissolved in the solution. Additionally, activated carbon was also proven to be the most effective substrate for gold recovery from the alkaline sulfide solution.

Additional experimental campaign was conducted to test the effectiveness of activated carbon and IRA400 ion exchange resin, two of the best performing substrate from previous campaign, in a lower concentration of gold in alkaline sulfide solution. An alkaline sulfide with $80 \mathrm{gr} / \mathrm{L}$ total sulfur concentration, 50gr/L NaOH, and 0.4502ppm gold was used. (See Table IV.) It can be 
concluded that activated carbon is the most effective substrate to remove gold from low concentration alkaline sulfide solution.

TABLE IV. Result of $2^{\text {nd }}$ Recovery Experimental Campaign

\begin{tabular}{|l|l|l|l|}
\hline Test & Substrate & $\begin{array}{l}\text { Weight Post- } \\
\text { Rec. } \\
\text { (initially } \\
\text { 20gr.) }\end{array}$ & $\begin{array}{l}\text { \% Rec Fire- } \\
\text { Assay }\end{array}$ \\
\hline RT2-T1 & IRA400 & 19.3 & 63.79 \\
\hline RT2-T2 & Carbon & 20.14 & 91.62 \\
\hline RT2-T3 & Carbon & 21.32 & 90.17 \\
\hline
\end{tabular}

\section{Optimize alkaline sulfide lixiviant regeneration techniques.}

The focus of this task was to develop a process to treat waste alkaline sulfide lixiviant solution, as it waste-solution treatment is a crucial part of any mineral extraction systems. Since the system deals with a meta-stable sulfide and polysulfide solution, it is desirable to transform the system to sulfate, the highest oxidation state of sulfur. With the excess existence of sodium in the system from the reagent added $\left(\mathrm{Na}_{2} \mathrm{~S}\right.$ and $\left.\mathrm{NaOH}\right)$ in the solution preparation, sodium sulfate $\left(\mathrm{Na}_{2} \mathrm{SO}_{4}\right)$ will be formed during the oxidation of the solution. $\mathrm{Na}_{2} \mathrm{SO}_{4}$ is non hazardous and relatively stable in its solid phase.

Moreover, $\mathrm{Na}_{2} \mathrm{SO}_{4}$ produced from the oxidation process was treated for "salt-splitting" using the newly purchased electrodialysis unit (EDU). The EDU utilizes bipolar, ion-permeable membranes, and the appliance of electrical potential to: split the sodium $\left(\mathrm{Na}^{+}\right)$and sulfate $\left(\mathrm{SO}_{4}{ }^{{ }}\right)$, split water into $\mathrm{H}^{+}$and $\mathrm{OH}^{-}$, and produce separate flows of $\mathrm{NaOH}$ and $\mathrm{H}_{2} \mathrm{SO}_{4}$. Additionally, the EDU is planned to be able to produce $\mathrm{NaOH}$ and ammonium sulfate $\left[\left(\mathrm{NH}_{4}\right)_{2} \mathrm{SO}_{4}\right]$, a bi-product that can be sold as a fertilizer, by feeding in a weak ammonia solution into the system.

Experimental campaigns have been conducted in regards to the lixiviant solution's oxidation. The experiments were done in a pressure vessel (autoclave) at Montana Tech where temperature, oxygen overpressure, and agitation can be controlled accurately. The first experimental campaign was designed to specifically investigate the effects of temperature and oxygen overpressure. Shown in Figure 8 is the 3D surface model of those variables to the sulfur to sulfate conversion: the combination of high temperature and oxygen overpressure produced the most $\mathrm{SO}_{4}{ }^{=}$. 


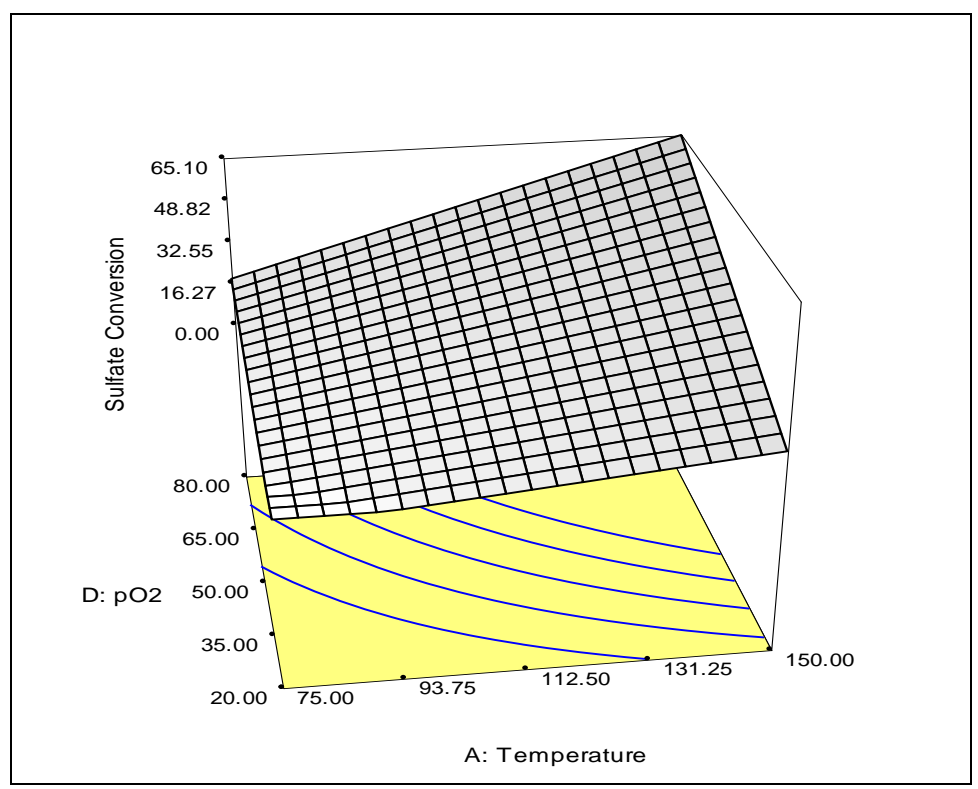

Figure 8. Effects of Temperature and $\mathrm{O}_{2}$ Overpressure on Sulfur to $\mathrm{SO}_{4}{ }^{=}$Conversion

Shown in Figure 9 is the 3D model of the possible retention of thiosulfate $\left(\mathrm{S}_{2} \mathrm{O}_{3}{ }^{\overline{ }}\right)$ and sulfite $\left(\mathrm{SO}_{3}{ }{ }\right)$ anions in solution when the time allowed is insufficient. $\mathrm{S}_{2} \mathrm{O}_{3}{ }{ }$ and $\mathrm{SO}_{3}{ }{ }^{2}$ are products of incomplete oxidation of sulfur; in other words, sulfur are not in their highest oxidation state. Clearly, as Figure 8 also shows, the higher initial sulfur concentration, longer time is needed for conversion. In general, $\mathrm{S}_{2} \mathrm{O}_{3}{ }^{=}$and $\mathrm{SO}_{3}{ }^{=}$are less stable, thus undesirable; and will interfere with the salt splitting process using the electrodialysis unit (EDU).

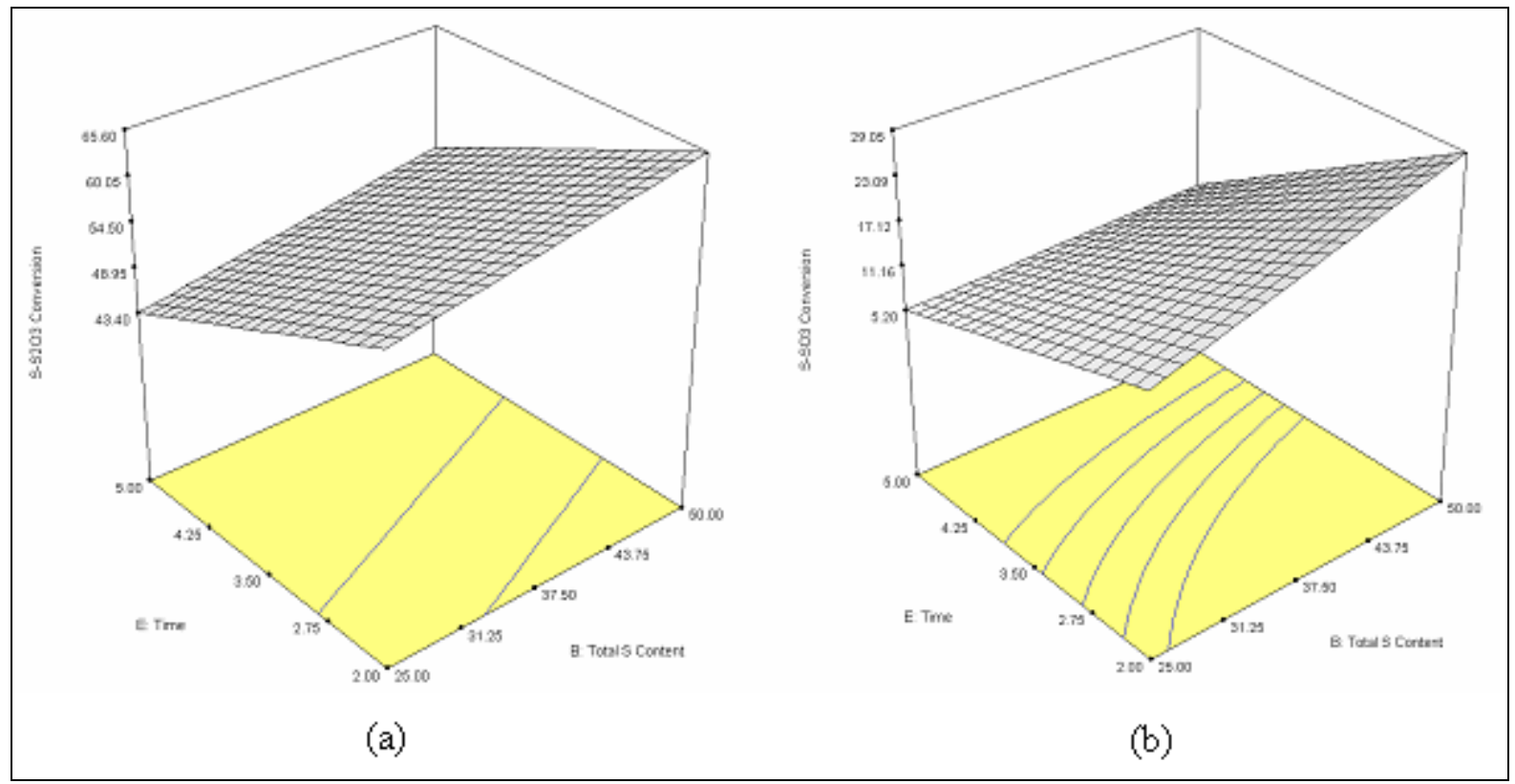

Figure 9. The Effects of Time and Total Sulfur Content on the Formation of (a) $\mathrm{S}_{2} \mathrm{O}_{3}{ }^{=}$and (b) $\mathrm{SO}_{3}{ }^{=}$ 
A $100 \%$ sulfur conversion to sulfate was achieved when time is increased to seven hours and excess $\mathrm{NaOH}$ is provided. Excess $\mathrm{NaOH}$ is needed to supply the system with enough $\mathrm{OH}$ - anions to maintain a high $\mathrm{pH}$. At lower $\mathrm{pH}$, undesirable sulfur solid will be produced.

Experimental campaign was also completed started for the electrodialysis unit (EDU). A standard solution of $\mathrm{NaOH}$ and $\mathrm{Na}_{2} \mathrm{SO}_{4}$ is still being used, instead of the autoclave-oxidized solution. However, results have been very encouraging and indicating that the process will be successful with oxidized lixiviant solution. Figure 10 shows the EDU in operation and Figure 11 shows the basic concept of the electrodialysis process.

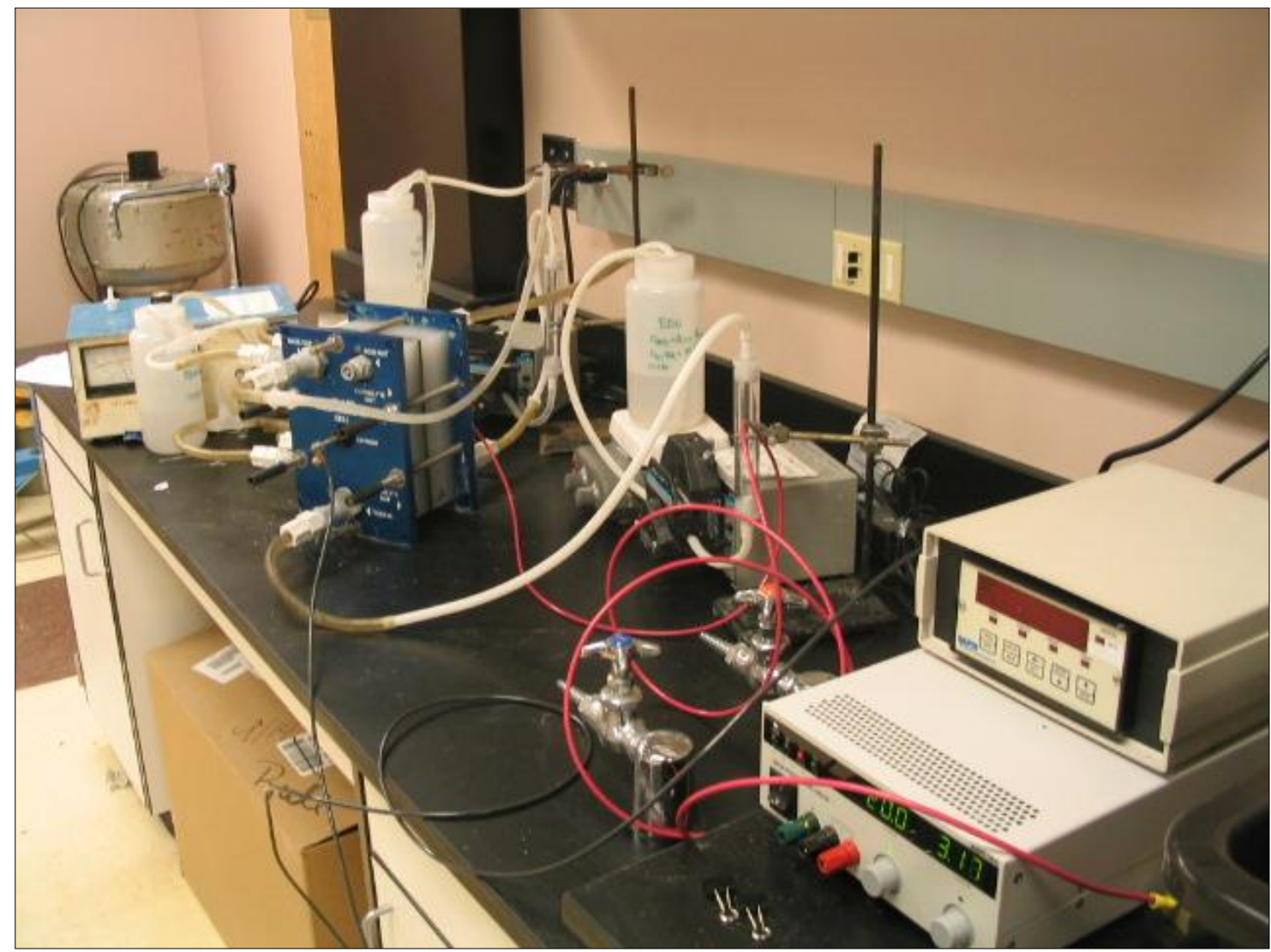

Figure 10. Electrodialysis Unit (EDU) in Operation 


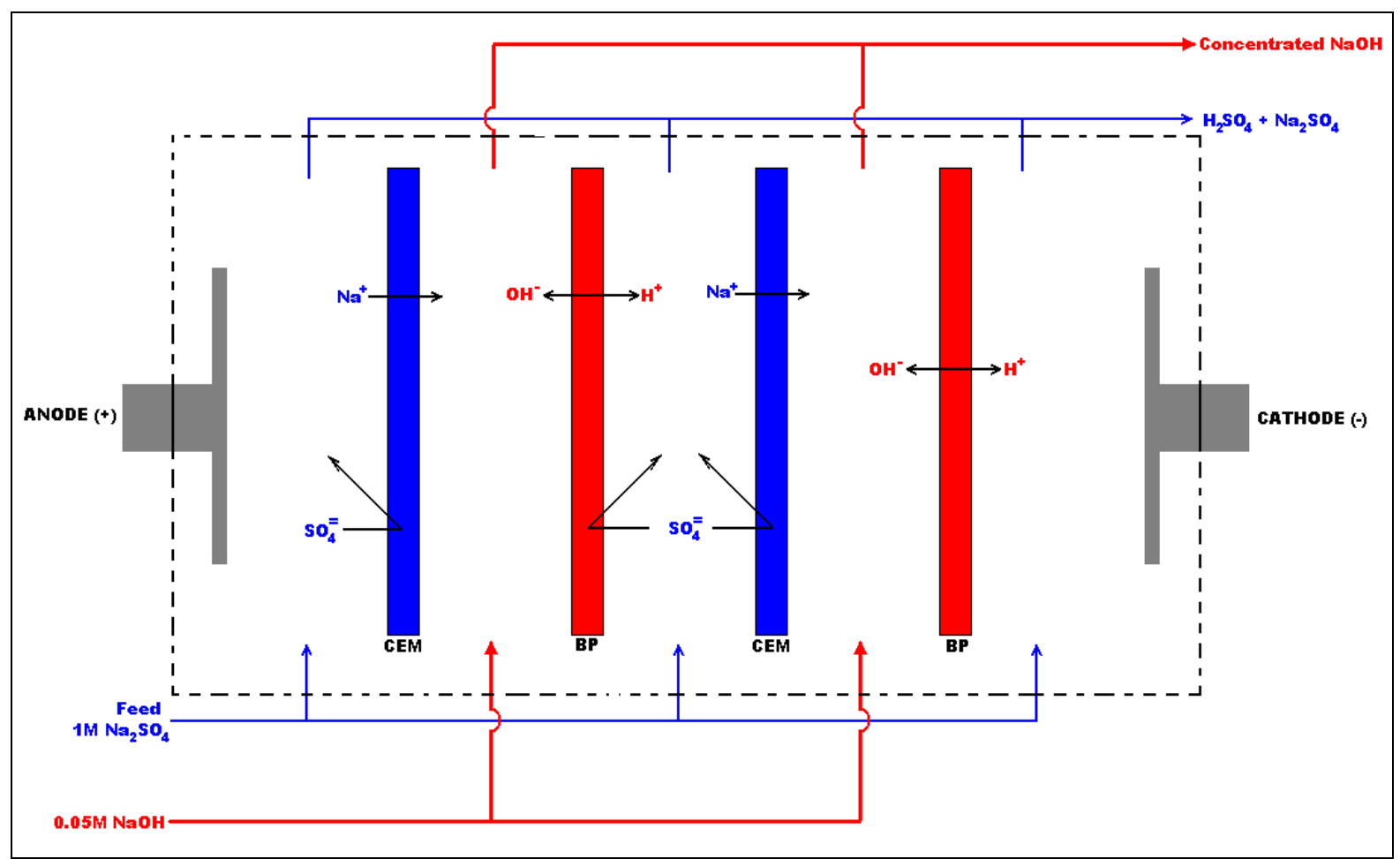

Figure 11. Electrodialysis Unit Ion Migrations using Cationic Membrane (CEM) and Bipolar Membrane (BP) Arrangements

Figure 12 presents the acid/base concentration levels of each flow at different time (with the effect of current density investigated). The experiment was done with constant flow rates $(200 \mathrm{~mL} / \mathrm{min})$ and constant feed and base flow concentrations of $1 \mathrm{M} \mathrm{Na}_{2} \mathrm{SO}_{4}$ and $0.00 \mathrm{NaOH}$, respectively. The effects of current density were investigated in this particular experiment (varied at $400 \mathrm{~A} / \mathrm{m}^{2}$ and $800 \mathrm{~A} / \mathrm{m}^{2}$ ). As shown in Figure 12, final concentrations of $70 \mathrm{gr} / \mathrm{L} \mathrm{NaOH}$ and $130 \mathrm{gr} / \mathrm{L} \mathrm{H}_{2} \mathrm{SO}_{4}$ can be achieved with current density of $800 \mathrm{~A} / \mathrm{m}^{2}$. 


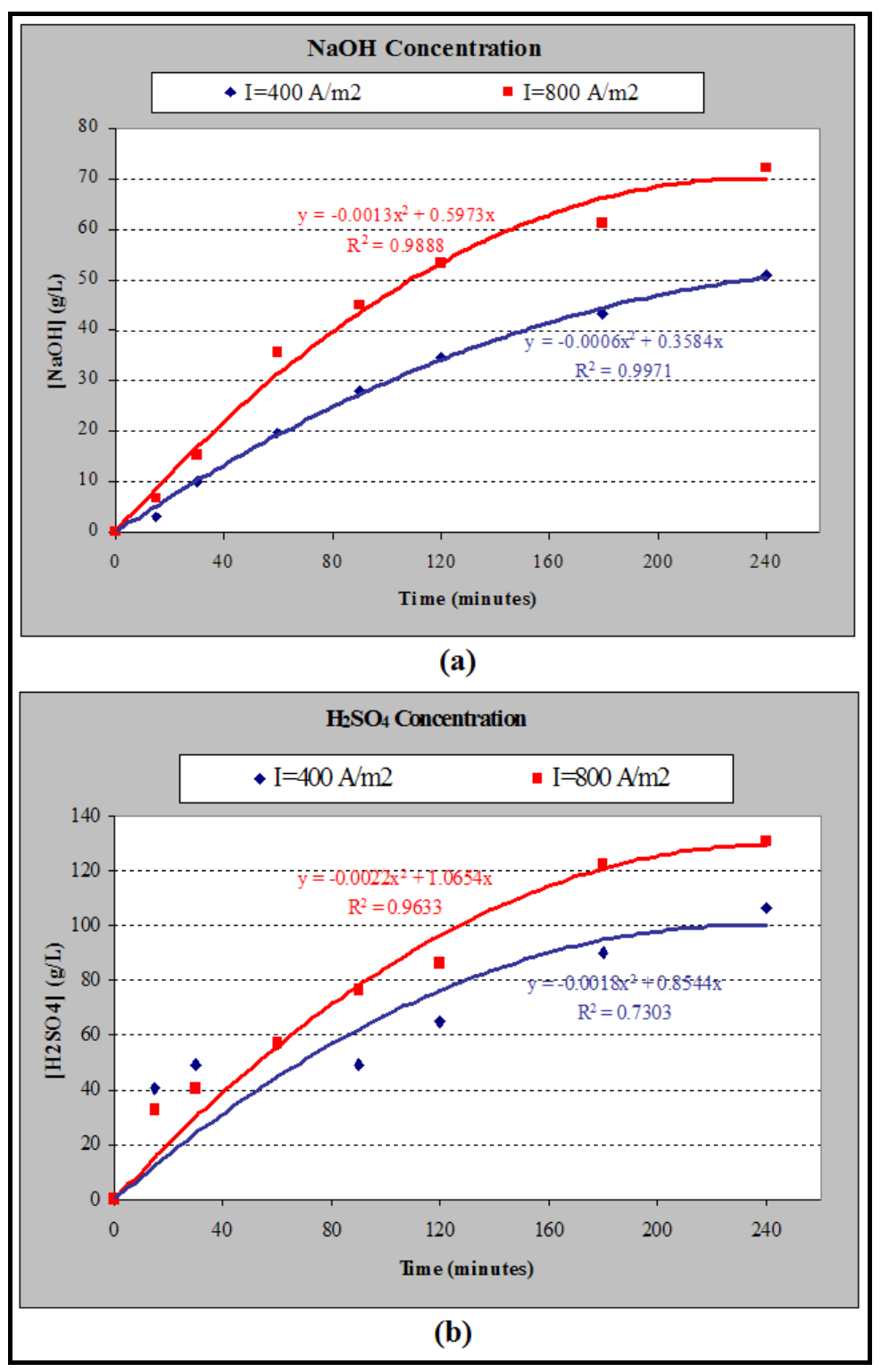

Figure 12. EDU Preliminary Experiment Results Showing Successful Production of (a)NaOH and (b) $\mathrm{H}_{2} \mathrm{SO}_{4}$.

Preliminary work has already been commenced in investigating the treatment of $\mathrm{H}_{2} \mathrm{SO}_{4}$ solution produced by the EDU. Proposed methods are the addition of hydrated lime $\left[\mathrm{Ca}(\mathrm{OH})_{2}\right]$ and ammonium hydroxide $\left[\mathrm{NH}_{4} \mathrm{OH}\right]$ to respectively produce gypsum $\left[\mathrm{CaSO}_{4} \cdot 2 \mathrm{H}_{2} \mathrm{O}\right]$ and ammonium sulfate $\left[\left(\mathrm{NH}_{4}\right)_{2} \mathrm{SO}_{4}\right]$. Both products have minimal environmental risk considerations and potentially can be sold as by-products. Gypsum can be sold as a feed to plaster manufacturing or as fertilizer, while ammonium sulfate can be sold as a nitrogen supplying fertilizer. It has been confirmed that the formation of $\left(\mathrm{NH}_{4}\right)_{2} \mathrm{SO}_{4}$ can be achieved just by adding $\mathrm{NH}_{4} \mathrm{OH}$ solution into the produced $\mathrm{H}_{2} \mathrm{SO}_{4}$ in room temperature. However, since hydrated lime that has to be added to the $\mathrm{H}_{2} \mathrm{SO}_{4}$ solution is solid, a further study of reaction kinetics needs to be done for this alternative. 
3. Test complete gold recovery and lixiviant regeneration process. Completed, as noted above.

\section{Task \#4}

1. Process several different types of ore/concentrates from Montana mining operations with the partial oxidation process.

Partial oxidation experiments were done to the Golden Sunlight pyrite concentrate. Both roasting and nitrogen species catalyzed oxidation was used. Roasting showed a reasonable gain in gold leaching. NSC pre-oxidation tremendously assisted the alkaline sulfide leaching system since NSC exposed all of enclosed gold particles in the pyrite-silica matrix. Additionally, NSC provided more sulfur needed in the leaching system, which decreased the reagent consumption.

2. Process the partially oxidized ore/concentrates with the alkaline sulfide leaching process.

Previous thesis project had successfully investigated the performance of the alkaline sulfide system to several industrial ores:

1. Gravity concentrate of Kennecott Greens Creek Mine (KGCMC);

2. $\quad$ Roaster calcine of Newmont Mining Company; and

3. Montana Elkhorn sulfide concentrate.

These industrial samples were chosen to demonstrate the specific gold leaching capabilities. Leaching tests at elevated temperature $\left(50-60^{\circ} \mathrm{C}\right)$ and high concentrations of total sulfur $(2-3.14 \mathrm{M})$ have achieved leaching performances of $93.5 \%, 84.0 \%$, and $55.59 \%$ for the respective samples. Additionally, the high concentrations of sulfide and cyanicides in $\mathrm{KGCMC}(\mathrm{Pb}, \mathrm{Zn}$, and $\mathrm{Cu})$ and Elkhorn $(\mathrm{Cu})$ samples show the performance of ASGLS in feed materials where cyanide has traditionally been proven to be less effective.

Current research has focused on two additional feed materials: KGCMC Sulfide Composite Concentrate and Barrick's Golden Sunlight Mine (BGSM) Pyrite Concentrate. Through extensive analytical procedures, both of these sample materials have been mineralogically specified. KGCMC sulfide composite concentrate is a combination of galena $(\mathrm{PbS})$ and sphalerite $(\mathrm{ZnS})$ concentrate produced from a flotation circuit and contains 0.690 opt gold and 89.8opt silver. These KGCMC concentrates are currently being sold straight to a smelter with gold credit less than $100 \%$. In the other hand, PDGS concentrate is a pyrite (FeS2) concentrate produced by spiral gravity concentration, which currently being treated by concentrated cyanide solutions to leach the contained $0.104 \mathrm{opt}$ of gold. 
Shown in Table IV are experimental results of leaching tests investigating the effects of total sulfur and hydroxide concentrations to the KGCMC composite concentrate. It was proven that a higher concentration of total sulfur and hydroxide improved the leaching performance of ASGLS on the composite concentrate. Leaching performances up to $79.0 \%$ were achieved. 
TABLE IV. KGCMC Composite Concentrate Leaching Experiment Results

\begin{tabular}{|l|l|l|l|l|l|l|l|l|}
\hline Test \# & $\begin{array}{l}\mathbf{N a} \text { S } \\
\text { (gr/L) }\end{array}$ & $\begin{array}{l}\mathbf{S} \\
(\mathbf{g r} / \mathbf{L})\end{array}$ & $\begin{array}{l}\mathbf{N a O H} \\
(\mathbf{g r} / \mathbf{L})\end{array}$ & $\begin{array}{l}\text { Temperature } \\
\left({ }^{\circ} \mathbf{C}\right)\end{array}$ & $\begin{array}{l}\text { Au TLS } \\
\text { (opt) }\end{array}$ & $\begin{array}{l}\text { Ag TLS } \\
\text { (opt) }\end{array}$ & $\begin{array}{l}\text { Ag } \\
\text { Leache } \\
\text { (\%) }\end{array}$ & $\begin{array}{l}\text { Leached } \\
\text { (\%) }\end{array}$ \\
\hline LGC1-T1 & 40 & 10 & 24 & 50 & 0.524 & 79.5 & $19.0 \%$ & $11.5 \%$ \\
\hline LGC1-T2 & 80 & 20 & 24 & 50 & 0.503 & 77.9 & $22.3 \%$ & $13.3 \%$ \\
\hline LGC1-T3 & 160 & 40 & 24 & 50 & 0.496 & 78.8 & $23.3 \%$ & $12.2 \%$ \\
\hline LGC2-T1 & 160 & 40 & 50 & 50 & 0.235 & 78.5 & $63.7 \%$ & $12.6 \%$ \\
\hline LGC2-T2 & 160 & 40 & 50 & 50 & 0.218 & 82.1 & $66.3 \%$ & $8.6 \%$ \\
\hline LGC2-T3 & 160 & 40 & 50 & 50 & 0.136 & 74.5 & $79.0 \%$ & $17.0 \%$ \\
\hline
\end{tabular}

Shown in Table V are results of leaching tests conducted to the Golden Sunlight pyrite concentrate. Similar to the KGCMC composite concentrate results, higher total sulfur concentration was proven to be more effective in extracting gold. It should also be noted that feed samples of tests LGS2-T1 and LGS2-T2 were roasted at $300^{\circ} \mathrm{C}$ and $400^{\circ} \mathrm{C}$, respectively, for 12 hours prior to leaching. It can be noticed that, similar to the cyanide system, oxidation of sulfide system to sulfur will improve leaching performance.

TABLE V. Golden Sunlight Pyrite Concentrate Leaching Experiment Results

\begin{tabular}{|l|l|l|l|l|l|l|l|l|}
\hline Test \# & $\begin{array}{l}\mathbf{N a}_{2} \mathbf{S} \\
(\mathbf{g r} / \mathbf{L})\end{array}$ & $\begin{array}{l}\mathbf{S} \\
(\mathbf{g r} / \mathbf{L})\end{array}$ & $\begin{array}{l}\mathbf{N a O H} \\
(\mathbf{g r} / \mathbf{L})\end{array}$ & $\begin{array}{l}\text { Temperature } \\
\left({ }^{\circ} \mathbf{C}\right)\end{array}$ & $\begin{array}{l}\text { Au Tails } \\
\text { (opt) }\end{array}$ & $\begin{array}{l}\text { Ag Tails } \\
(\mathbf{o p t})\end{array}$ & $\begin{array}{l}\text { Au Leached } \\
(\%)\end{array}$ & $\begin{array}{l}\text { Ag Leached } \\
(\%)\end{array}$ \\
\hline LGS1-T1 & 80 & 20 & 25 & 50 & 0.042 & 0.4 & $59.6 \%$ & $55.6 \%$ \\
\hline LGS1-T2 & 160 & 40 & 50 & 50 & 0.024 & 0.4 & $76.9 \%$ & $55.6 \%$ \\
\hline LGS1-T3 & 160 & 40 & 50 & 50 & 0.029 & 0.4 & $72.1 \%$ & $55.6 \%$ \\
\hline LGS2-T1 & 160 & 40 & 50 & 50 & 0.016 & 0.4 & $84.6 \%$ & $55.6 \%$ \\
\hline LGS2-T2 & 160 & 40 & 50 & 50 & 0.024 & 0.3 & $76.9 \%$ & $66.7 \%$ \\
\hline
\end{tabular}

\section{Recover gold from the resulting alkaline sulfide solution. Completed as noted before.}

\section{Task \#5}

\section{Testing of pilot plant using a gold concentrate obtained from a Montana ore body.}

A bench-scale batch simulation procedure and schedule have been developed to further test these developed unit processes together (leaching, recovery, and waste treatment). The objective of such simulation is to ultimately test the consistency of the system itself. Additionally, various parameters were monitored before and after each unit processes $(\mathrm{Eh}, \mathrm{pH}$, sulfide concentration, polysulfide concentration, etc), which couldn't be measured before. The effect of solution recirculation, and how much reagent addition in each recirculation needed, could also be investigated. A bench-scale batch simulation has been conducted with KGCMC composite concentrate as feed and has shown an encouraging data where leaching performance was consistent. Additionally, a successful recirculation of $\mathrm{NaOH}$ solution produced using the EDU was achieved, producing a fresh alkaline sulfide solution to be added to the leaching stage. 
As well, demonstration plant testing of the Golden Sunlight's pyrite concentrate was undertaken and successfully completed. In addition, a sample of another Montana gold ore was obtained from Gene and Dirk Nelson who own the Yellow Band deposit.

\section{Demonstration of the pilot plant to interested in industrial entities.}

During the pilot plant demonstration of Golden Sunlight's pyrite concentrate, representatives of the mine were invited and attended observe all of the developed unit processes of this ASGLS system. Mr. Nelson has also witnessed the pilot plant operation of his Montana Yellow Band ores. Newmont has also seen the pilot plant. Shown below is a picture of Mr. Dave Desch of the MBRCT witnessing the demonstration plant operation on 8/23/06. Based on this visit and our judicious use of funds, the MBRCT agreed to allow us to continue our work through 3/31/07.

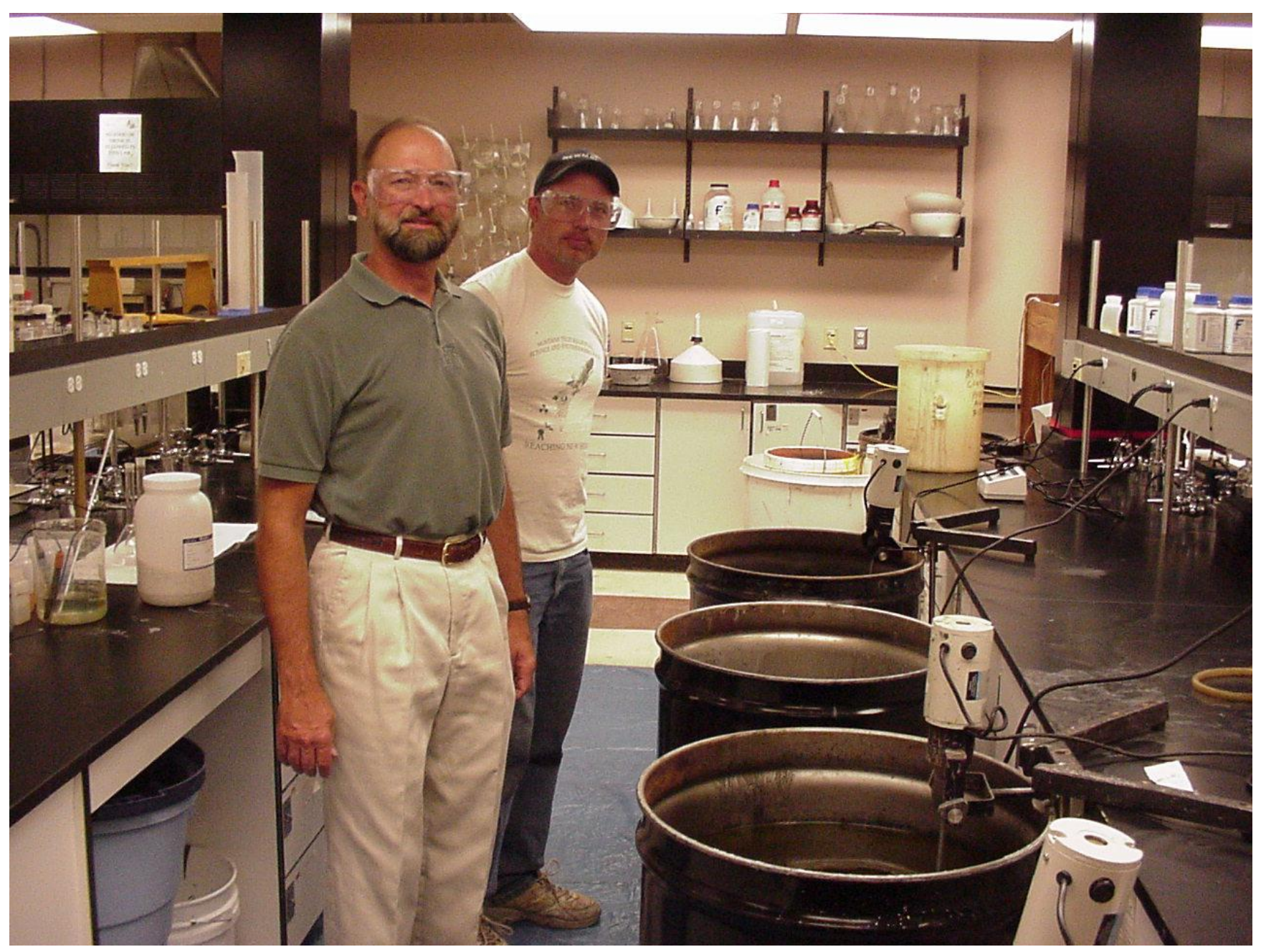




\section{MONTANA BOARD OF RESEARCH AND COMMERCIALIZATION TECHNOLOGY}

\section{FINAL FINANCIAL REPORT}

Grant Agreement: \#05-26 Nontoxic, Alkaline Sulfide Lixiviants for Recovering Gold from Montana Ore Bodies

Principal Investigator: Corby Anderson Phone: 406-496-4794

Email: canderson@mtech.edu

I. FINANCIAL STATUS - Please identify the Banner organizational name and number used to compile this data. Montana Tech B24120

\section{Commercialization Plan.}

Commercialization continues as planned. We currently have several interested global industrial parties who would like to see a larger and more modern demonstration plant capable of treating their gold ores. Our focus is now to design and build such a facility for testing and confirmation.

\section{E. Economic Impacts.}

The current economic impacts have been considerable. As CAMP is a University entity which is entirely financially self sufficient, we were able to graduate two badly needed Master's level engineers and assist in the education of a $\mathrm{Ph}$. $\mathrm{D}$. level engineer who has recently joined the Department of Metallurgical and Materials Engineering at Montana Tech. Further as we progress to the design and operation of our larger demonstration plant for confirmation it is anticipated that our revenue streams will increase allowing sustainability for three engineers at CAMP. Finally, after confirmation at the larger scale, if suitable terms can be arranged with interested industrial entities, the employment and revenue impacts will be significant for CAMP and the mining company. 


\title{
Karel Pramono Thesis
}

\begin{abstract}
As a continuation of the development of the alkaline sulfide gold leaching system (ASGLS), this thesis investigated the performance of the system on two different sulfide concentrates. One concentrate contained high content of $\mathrm{PbS}$ and $\mathrm{ZnS}$ while the other contained high concentrations of $\mathrm{FeS}_{2}$ and $\mathrm{SiO}_{2}$. Leaching experiments on both concentrates showed promising results with precious metal dissolution of up to $80 \%$. Additionally, the method of precious metal recovery from pregnant ASGLS solution was developed. Activated carbon proved to be superior in extracting precious metal compared to ion-exchange resins containing quaternary amine and iminodiacetic acid active ingredients. Gold recovery of $70.0 \%$ and silver extraction of $89.2 \%$ was achieved. A successful investigation of the waste treatment of the ASGLS solution was also conducted. Two developed processes were pressure oxidation for $\mathrm{Na}_{2} \mathrm{SO}_{4}$ production and $\mathrm{NaOH}$ regeneration using electrodialysis. Complete oxidation of sulfide-polysulfide to sulfate was achieved when the proper combination of temperature, oxygen overpressure, time, and $\mathrm{NaOH}$ were supplied. Electrodialysis was also proven to be effective in $\mathrm{NaOH}$ regeneration where a stream of approximately $70-\mathrm{g} / \mathrm{L}$ was achieved after four hours at $800-\mathrm{A} / \mathrm{m}^{2}$.

Ultimately, all developed unit processes were tested simultaneously through a closely controlled Bench-Top Batch Simulation. These simulations were an ultimate test of consistency for the proposed ASGLS process flowsheet. Additionally, monitoring of solution parameters $\left(\mathrm{E}_{\mathrm{H}}, \mathrm{pH}\right.$, sulfide-polysulfide concentration, and free $\mathrm{OH}^{-}$concentration) was conducted. Additional data in regards to the dissolution of other elements in the sulfide concentrates ( $\mathrm{As}, \mathrm{Sb}, \mathrm{Pb}, \mathrm{Zn}, \mathrm{Cu}$, and $\mathrm{Fe}$ ) were also measured. In the end, a constant gold dissolution of approximately $90 \%$ and silver dissolution of $70 \%$ was achieved after three solution circulations through the whole process. Constant precious metal recoveries from pregnant ASGLS solution of approximately $90 \%$ were also achieved through a lab-scale Carbon-In-Column setup.
\end{abstract}

\section{Acknowledgements}

I would like to recognize the Montana Board of Research and Commercialization Technology (MBRCT), the Center for Advanced Separation Technology (CAST), and the Center for Advanced Mineral and Metallurgical Processing (CAMP) for providing the funding and management for this project. Equally, Kennecott Greens Creek Mining Company and Barrick's Golden Sunlight Mining operation have been extremely supportive in providing concentrate samples and valuable information for better understanding the Alkaline Sulfide Gold Leaching System.

My thanks go to the entire staff at Metallurgical and Materials Engineering Department at Montana Tech; their support for the last five years of my college education has been critical and invaluable. I also would like to send special acknowledgements for Dr. Corby Anderson and Dr. Paul Miranda of CAMP for the patience in guiding me through all the challenges and details of this research project. 
Last but not least, I dedicate this thesis to my family, Eddy, Yustina, Stefano and Christopher Pramono, for the constant flow of love and motivation. Equally special thanks go to all of my close friends of Malang, Butte, and Tembagapura.

This thesis wouldn't have been possible without the help and support of many individuals; and for that my extreme gratitude goes to those who have played a role in the progress of the completion of this thesis. 


\section{Table of Contents}

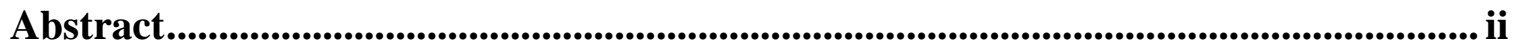

Acknowledgements ........................................................................................................................ ii

Table of Contents .......................................................................................................................... ii

List of Tables .................................................................................................................................. ii

List of Figures.......................................................................................................................... ii

1.0 Introduction ................................................................................................................................ i

$1.1 \quad$ Research Purpose and Objectives .................................................................. i

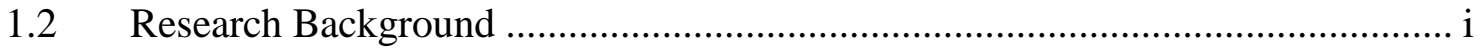

1.3 ASGLS Chemistry .......................................................................................... ii

1.3.1 Solution Chemistry of ASGLS .................................................................ii

1.3.2 Gold Leaching Mechanism in ASGLS .................................................. iv

$1.4 \quad$ Review of Previous Research ...............................................................................

1.4.1 Electrochemistry \& Electrokinetic of ASGLS ........................................... vi

1.4.2 Laboratory Leaching Tests of 99.99\% Gold Powder ................................... vi

1.4.3 Industrial Ore Leaching.............................................................................. vii

1.4.4 Gold Recovery from Pregnant Solution ........................................................ vii

1.4.5 Waste Treatment of Waste ASGLS Solution............................................ vii

1.5 Experimental Nomenclature and Thesis Organization ....................................... viii

2.0 Concentrates Characterization and Leaching Experiments.............................. ix

2.1 Introduction to Concentrates Characterization and Leaching Experiments........ ix

2.2 Kennecott Greens Creek Mining Co.'s (KGCMC) Composite Concentrate ....... x

2.2.1 Introduction \& Background ..................................................................... X

2.2.2 Characterization of KGCMC Sulfide Concentrates..................................... $\mathrm{x}$

2.2.3 Effect of Sulfide-Polysulfide and Hydroxide Concentrations (LGC-1)... xiii

2.2.3.1 LGC-1 Experimental Design .................................................................... xiii

2.2.3.2 LGC-1 Experimental Results \& Analysis ................................................ xiii

2.3 Golden Sunlight Pyrite Concentrate ................................................................. xiv

2.3.1 Introduction \& Background ................................................................... xiv

2.3.2 Characterization of Golden Sunlight's Pyrite Concentrate ...................... xiv

2.3.3 Effect of Sulfide-Polysulfide and Hydroxide Concentration (LGS-1).... xvii

2.3.3.1 LGS-1 Experimental Design \& Setup................................................... xvii

2.3.3.2 LGS-1 Experimental Results \& Analysis ..................................................... i

2.3.4 Effect of Concentrate Pre-Oxidation (LGS-2) ............................................. i

2.3.4.1 LGS-2 Experimental Design ................................................................... i

2.3.4.2 LGS-2 Experimental Results \& Analysis ................................................. i

2.4 Summary of Feed Characterization and Preliminary Leaching Experiments ...... ii

3.0 Precious Metal Recovery from Pregnant ASGLS Solution.................................. ii

3.1 Introduction to Precious Metal Recovery from Pregnant ASGLS Solution ........ ii

3.2 Performance of Activated Carbon and Ion-Exchange Resins (RT-1).................... i

3.2.1 RT-1 Background and Experimental Design ................................................ i

3.2.2 RT-1 Experimental Results and Analysis ................................................... ii

3.3 Effect of Lower Precious Metals Concentration in ASGLS Solution (RT-2) .... iii 
3.3.1 RT-2 Background and Experimental Design .............................................iii

3.3.2 RT-2 Experimental Results and Analysis ..................................................... i

3.4 Summary of Precious Metal Recovery from Pregnant ASGLS Solution .............. i

4.0 Treatment of Waste ASGLS Solution .................................................................. i

4.1 Introduction to Waste Treatment of ASGLS ........................................................ i

4.2 Pressure Oxidation of Waste Alkaline Sulfide Solution.......................................... ii

4.2.1 Sulfide/Polysulfide Oxidation Principles...................................................... ii

4.2.2 Pressure Oxidation Campaign 1 (WO-1): Oxidation Kinetics ........................ v

4.2.2.1 WO1 Background and Experimental Design .............................................. $\mathrm{v}$

4.2.2.2 WO1 Results and Data Analysis .............................................................. vii

4.2.3 Pressure Oxidation Campaign 2 (WO-2): Elemental Sulfur Formation........ ix

4.2.3.1 WO2 Background and Experimental Design........................................... ix

4.2.3.2 WO2 Results and Data Analysis .............................................................. $\mathrm{x}$

4.3 NaOH Regeneration through Electrodialysis with Bipolar Membranes .............. xi

4.3.1 Introduction............................................................................................

4.3.2 The Electrolytica ED-1-BP Unit and It's Troubleshooting.......................... xiv

4.3.3 Effects of Current Density (WED-1) ............................................................iii

4.3.3.1 WED1 Background and Experimental Design ........................................iii

4.3.3.2 WED1 Results and Data Analysis ...........................................................iii

4.3.4 Electrodialysis of Sulfide/Polysulfide Oxidation Produced $\quad \mathrm{Na}_{2} \mathrm{SO}_{4}$ (WED-2) ........................................................................................ vi

4.3.4.1 WED-2 Experimental Design and Setup ……………………………....... vi

4.3.4.2 WED-2 Experimental Results and Discussion ........................................ vii

$4.4 \quad$ Treatment of $\mathrm{H}_{2} \mathrm{SO}_{4}$ Produced from Electrodialysis......................................... viii

4.4.1 Gypsum Production...................................................................................... i

4.4.1.1 Gypsum Production Experimental Campaign 1 (WGP1) ......................... ii

4.4.1.2 Gypsum Production Experimental Campaign 2 (WGP-2)Error! Bookmark not defined.

4.4.2 Ammonium Sulfate Production .............................................................. iv

4.4.2.1 Ammonium Sulfate Production Experimental Campaign 1 (WAS-1) .... v

5.0 ASGLS Bench-Top Batch Simulation......................................................................... $\mathrm{v}$

5.1 Introduction to ASGLS Bench-Top Batch Simulation ..........................................

5.2 Bench-Top Batch Simulation of KGCMC Composite Concentrate (Batch

Simulation 1).......................................................................................... vii

5.2.1 Introduction and Background..................................................................... vii

5.2.3 Experimental Results and Discussion ............................................................ i

5.2.3.2 Leaching Performance and Consistency .................................................... ii

5.2.3.3 Leaching of Other Metals............................................................................. i

5.2.3.4 Precious Metal Recovery from Pregnant ASGLS..................................... ii

5.2.3.4 Waste Treatment of ASGLS Solution and $\mathrm{NaOH}$ Recirculation ................ i

5.3 Bench-Top Batch Simulation of Golden Sunlight's Pyrite Concentrate (Batch

Simulation 2)............................................................................................. ii

5.3.1 Introduction and Background to Batch Simulation 2................................. ii

5.3.2 Experimental Setup, Design, and Daily Operational Schedule....................iii 
5.3.3 Experimental Results and Discussion ........................................................ i

5.3.3.1 Stability of Solution Parameters ………………....................................... i

5.3.3.2 Leaching Performance and Consistency ................................................... ii

5.3.3.3 Leaching of Other Metals.........................................................................iii

5.3.3.4 Precious Metal Recovery Performance and Consistency...........................iii

5.3.3.5 Waste Treatment of ASGLS Solution and $\mathrm{NaOH}$ Recirculation............. iv

5.4 Batch Simulation of Pre-Oxidized Golden Sunlight's Pyrite Concentrate (Batch

Simulation 3) ............................................................................................ i

5.4.1 Introduction and Background...................................................................... i

5.4.2 Experimental Setup, Design, and Daily Operational Schedule...................... i

5.4.3 Experimental Results and Discussion ........................................................ i

5.4.3.1 Stability of Solution Parameters ................................................................. ii

5.4.3.2 Precious Metal Leaching Performance and Consistency .......................... ii

5.4.3.4 Gold Recovery from Pregnant Solution Performance and

Consistency ……....................................................................................ii

5.5 Summary of Bench-Top Batch Simulations ....................................................... iv

6.0 Recommendations of Future Work ............................................................................ iv

7.0 Thesis Conclusion ..................................................................................................................... v

7.1 Sulfide Concentrates Characterization and Leaching Experiments

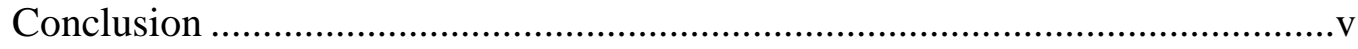

7.2 Precious Metal Recovery from Pregnant ASGLS Solution Conclusion ................ i

7.3 Treatment of Waste ASGLS Solution Conclusion .................................................. i

7.4 Bench-Top Batch Simulations Conclusion ..................................................... i

8.0 Bibliography ........................................................................................................................... ii

Appendix A. Process Flowsheets: Kennecott Greens Creek ........................................ i

Appendix B. Process Flowsheet: Barrick's Golden Sunlight Mine............................... i

Appendix C. ANOVA Analysis of Experimental Campaign WO-1 .......................... i

Appendix D. SOP: ASGLS Solution Preparation............................................................... i

Appendix E. SOP: Batch Leaching................................................................................. i

Appendix F. SOP: Batch Setup of Precious Metal Recovery ....................................... i

Appendix G. CIC Setup of Precious Metal Recovery..................................................... i

Appendix H. SOP: Pressure Oxidation of ASGLS Solution .......................................... i

Appendix I. SOP: Salt Splitting using Electrodialysis....................................................... i

Appendix J. James River Titration ................................................................................ viii

Appendix K. ABC Titration............................................Error! Bookmark not defined.

Appendix L. Free $\mathrm{H}_{2} \mathrm{SO}_{4}$ Titration................................Error! Bookmark not defined.

Appendix M. List of Chemicals and Analytical Equipments ....................................... i 


\section{List of Tables}

Table 1. Selected Oxidation States of Sulfur. iii

Table 2. Solution Parameters for Different Addition Ratios and Total Sulfur Concentrationsiv

Table 3. Experimental Campaign Identifications and Descriptions...................................viii

Table 4. Fire-Assay Results of KGCMC Composite Concentrate........................................ X

Table 5. Carbon-Sulfur Analysis Result of Composite Concentrate ................................. xii

Table 6. Content of Selected Elements in KGCMC Composite Concenctrate................... xii

Table 7. Selected Minerals Existing in KGCMC Ore Body ............................................... xii

Table 8. LGC 1 Experimental Design............................................................................... xiii

Table 9. Experimental Results of Campaign LGC-1 ………............................................ xiv

Table 10. Head Assay of Golden Sunlight's Pyrite Concentrate ......................................... Xv

Table 11. Carbon-Sulfur Analysis Results of Golden Sunlight's Pyrite Concentrate ..... xvi

Table 12. Concentration of Selected Elements in Pyrite Concentrate ............................... xvii

Table 13. Experimental Design of LGS-1 …………….................................................. xvii

Table 14. Experimental Results of LGS-1 ………......................................................... i

Table 15. Fire-Assay Results of Pre-Oxidized Pyrite Concentrate........................................ i

Table 16. Experimental Design of LGS-2 ……………..................................................... i

Table 17. Experimental Results of Experimental Campaign LGS-2 …................................ i

Table 18. Substrates Used in Campaign RT-1 ................................................................ i

Table 19. Experimental Results of RT-1 .................................................................... ii

Table 20. RT-2 Experimental Design ………………….............................................. i

Table 21. Experimental Results of RT-2 ……………................................................ i

Table 22. WO-1's Experimental Design Parameters ...................................................... v

Table 23. WO1 Experimental Design Matrix ................................................................... vi

Table 24. WO1 Product Solution Results ....................................................................... vii

Table 25. Experimental Design of WO-2 ..................................................................... $\mathrm{x}$

Table 26. Experimental Results of Campaign WO-2 …………………………….......... xi

Table 27. ED-1-BP General Specifications ................................................................... i

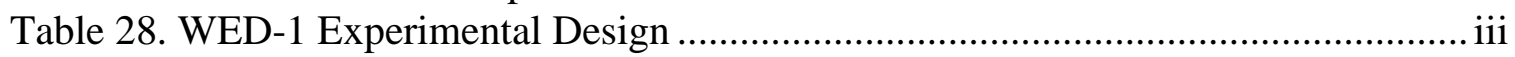

Table 29. WED-2 Experimental Design .......................................................................... vi

Table 30. WGP-1 Experimental Design ..........................................................................iii

Table 31. WGP-1 Analysis Results..................................................................................iii

Table 32. Experimental Results of WGP-2 2.................................................................... iv

Table 33. Process Labels of KGCMC Composite Concentrate Batch Simulation ............. ix

Table 34. Simulation Details Specific to Batch Simulation 1 ............................................. i

Table 35. Solution Parameters Monitoring Results of Batch Simulation 1 1.......................... i

Table 36. Leaching Performance of ASGLS in Batch Simulation 1 ................................... i

Table 37. Results of Leaching of Other Metals in Batch Simulation 1 .............................. ii

Table 38. Gold Recovery Results in Batch Simulation 1 .................................................. i

Table 39. Waste Treatment Stage Results of Batch Simulation 1 ...................................... ii

Table 40. Process Labels of Batch Simulation 2 .............................................................iii

Table 41. Experimental Details of Batch Simulation 2 .................................................... i

Table 42. Solution Parameters of Batch Simulation 2 ……............................................. i 
Table 43. Leaching Performance of ASGLS in Batch Simulation 2 ................................ii

Table 44. Dissolution of Other Metals in Batch Simulation 2 ........................................ii

Table 45. Fire-Assay Results of Batch Simulation 2 Carbon Samples ............................. iii

Table 46. Gradual Loading of Precious Metal in Batch Simulation 2 .............................. iii

Table 47. Total Precious Metal Recovery in Batch Simulation ...................................... iv

Table 48. Waste Treatment Stages of Batch Simulation 2 .............................................. i

Table 49. Monitoring of Solution Parameters in batch Simulation 3 ................................ ii

Table 50. Leaching Performance of ASGLS in Batch Simulation 3 ................................ ii

Table 51. Fire-Assay Results of Carbon Batch of Batch Simulation 3............................iii

Table 52. Gradual Loading of Activated Carbon in Batch Simulation 3..........................iii

Table 53. Total Recovery of Leached Precious Metal in Batch Simulation 3 .................. iv

Table C.1 ANOVA Analysis Table of WO-1 Sulfide-Polysulfide Conversion to

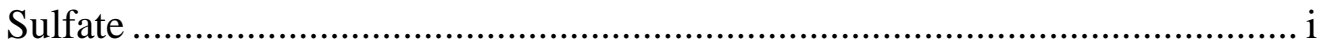

Table M.1 List of Chemicals................................................................................ i 


\section{List of Figures}

Figure 1. Proposed Flowsheet of the ASGLS ............................................................... ii

Figure 2. Dissolution of Sulfur Flakes in ASGLS Solution Preparation .......................... iv

Figure 3. Typical Leaching Test Setup ................................................................ v

Figure 4. XRD Spectrum of KGCMC Composite Concentrate....................................... xi

Figure 5. XRD Spectrum of Golden Sunlight's Pyrite Concentrate ................................ xvi

Figure 6. Proposed Flowsheet of the ASGLS ................................................................. i

Figure 7. Typical Setup of Batch Recovery Experiments .............................................. ii

Figure 8. Dissolved WP2 Ion Exchange Resin in Alkaline Sulfide Solution ..................... iii

Figure 9. Proposed Flowsheet of ASGLS Waste Solution Treatment .............................. ii

Figure 10. Eh-pH Diagram of Sulfur System at Higher Oxidation State............................iii

Figure 11. Pourbaix Diagram of Intermediate Oxidation States of Sulfur ....................... iv

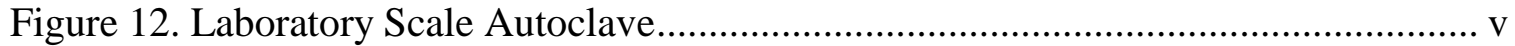

Figure 13. 3D Surface Model Graphs of Sulfide-Polysulfide Conversion to Sulfate (Temp vs. Oxygen Overpressure) ..........................................................................viii

Figure 14. 3D Surface Model Graphs of Sulfide-Polysulfide Conversion to Sulfate (Initial $[\mathrm{NaOH}]$ vs. [Sulfide-Polysulfide])............................................................. viii

Figure 15. Partially Oxidized ASGLS Solution............................................................ ix

Figure 16. Sulfur Solids Produced in ASGLS Oxidation ............................................... $\mathrm{x}$

Figure 17. Product Solution of Pressure Oxidation and Na2SO4 Powder Produced ........ xi

Figure 18. Three Compartment Design of Electrodialysis with BPM, CEM, and AEM.. xii

Figure 19. Two Compartment Design of Electrodialysis with BPM and CEM...............xiii

Figure 20. Two Compartment Design of Electrodialysis with BPM and AEM ..............xiii

Figure 21. Tokuyama Neosepta ${ }^{\circledR}$ a)CMB, b)BP-1, and c) ACM ................................ xiv

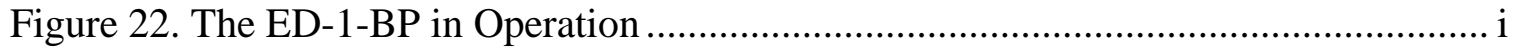

Figure 23. Un-maximized NaOH Regeneration ......................................................... ii

Figure 24. WED-1 NaOH Concentration vs. Time......................................................... iv

Figure 25. Sodium Transfer per Unit Time of WED-1 Tests ....................................... v

Figure 26. $\mathrm{H}_{2} \mathrm{SO}_{4}$ Build Up in WED-1 Tests........................................................... vi

Figure 27. $\mathrm{NaOH}$ Concentration vs. Time in WED-2 .................................................. vii

Figure 28. $\mathrm{H}_{2} \mathrm{SO}_{4}$ Concentration vs. Time in WED-2 ............................................. viii

Figure 29. Pourbaix Diagram of Ca-S System.............................................................. ii

Figure 30. Confirmation of the Formation of (NH4)2SO4 in Campaign WAS-1 …......... v

Figure 31. Process Flowsheet of The ASGLS Batch Simulations ................................... vii

Figure 32. Process Flowsheet of KGCMC Composite Concentrate Batch Simulation ... viii

Figure C.1. Predicted vs. Actual Diagnostic Graph of S to $\mathrm{SO}_{4}{ }^{2-}$ Conversion .................... ii

Figure C.2. Box-Cox Plot Diagnostic Graph of $\mathrm{S}$ to $\mathrm{SO}_{4}{ }^{2-}$ Conversion ............................. ii

Figure C.3. 3D Surface Model Graphs of Sulfide-Polysulfide Conversion to Sulfate (Temp vs. Oxygen Overpressure) ............................................................................... iii

Figure C.4 3D Surface Model Graphs of Sulfide-Polysulfide Conversion to Sulfate (Initial $[\mathrm{NaOH}]$ vs. [Sulfide-Polysulfide]) ...................................................... iv

Figure G.1 Schematic of Laboratory CIC Model ....................................................... i

Figure G.2 Actual CIC Laboratory Setup ................................................................... ii 


\subsection{Introduction}

\subsection{Research Purpose and Objectives}

Currently, cyanidation is the most popular leaching system used in gold extraction for its low reagent consumption and effectiveness. Unfortunately, cyanidation has recently come under heavy criticism, even scrutiny, for its perceived environmental effects. Thus, there is greater interest in the development of alternative gold leaching reagents. Additionally, cyanidation also has recovery limitations in specific ore bodies: refractory, carbonaceous, cyanicides, and sulfide ore bodies. Alternative leaching systems may provide the following benefits:

- $\quad$ Easier processing for refractory, carbonaceous, cyanicides, and sulfide ore bodies (Louw \& Gussmann, 1977);

- $\quad$ Cheaper reagent and operating cost (Anderson, 2004); and

- $\quad$ Opening mine properties in places where cyanide use has been limited by law (Stacey, 2005).

Other lixiviants that recently have been of great interest include: halogens, thiourea, thiosulfate, and the alkaline sulfide gold leaching system (ASGLS). The general purpose of this research project was specifically focused on continued development of the ASGLS. Specifically, this research was designed to validate the applied ASGLS' capability in leaching sulfide containing feed materials. With the recent hike in gold price, it is beneficial to be able to investigate an alternative gold leaching system, especially if it can be implemented in a region like Montana where cyanide use has been limited by law.

Extensive research has previously been conducted in understanding the leaching mechanism of the ASGLS and its performance with pure gold. In regard to waste treatment and use in industrial situations, there has been minimal research conducted. More specifically, the thesis goals can be described as follows:

1. Obtain and characterize sulfide concentrates containing precious metals and their behavior under the ASGLS;

2. Further investigate gold recovery from gold bearing alkaline sulfide pregnant solution using various substrates;

3. Investigate a proposed waste treatment process that includes: pressure oxidation, caustic soda $(\mathrm{NaOH})$ regeneration through electrodialysis, and treatment of sulfuric acid $\left(\mathrm{H}_{2} \mathrm{SO}_{4}\right)$ produced by electrodialysis;

4. Successfully demonstrate performance consistency for all developed unit processes of the ASGLS and solution behavior through a bench-top batch simulation using the characterized sulfide concentrates.

\subsection{Research Background}

Hiskey \& Atluri (1988) have investigated the dissolution of gold in an alkaline sulfide solution. The application of alkaline sulfide solution in metal extraction itself has been known since the 
early ' 80 s. Antimony leaching using alkaline sulfide solution was used in two different industrial locations: Equity Silver Mines Limited in British Columbia and Sunshine Mine in Idaho, in which gold dissolution was observed. Consequently, a research project was proposed in the development and investigation of the ASGLS. Through the project management of the Center for Advanced Metallurgical and Mineral Processing (CAMP) and funding from the Center for Advanced Separations Technology (CAST), a research campaign was initiated on ASGLS in 2003. The Montana Board of Research and Commercialization Technology (MBRCT) have also provided additional funding.

This thesis research was directed to demonstrate the application of the ASGLS in precious metals extraction from actual concentrates. Previous research has characterized the leaching mechanism of ASGLS and this work successfully demonstrated the application of the system to pure gold powder. This research also defined the application of other unit processes required: precious metal recovery from pregnant ASGLS solution and treatment of waste ASGLS solution. Ultimately, these three unit processes were used in a bench-top batch simulation where the application of the system was tested on actual ores and each unit processes was closely monitored. Presented in Figure 1 is the proposed process flowsheet investigated in this research.

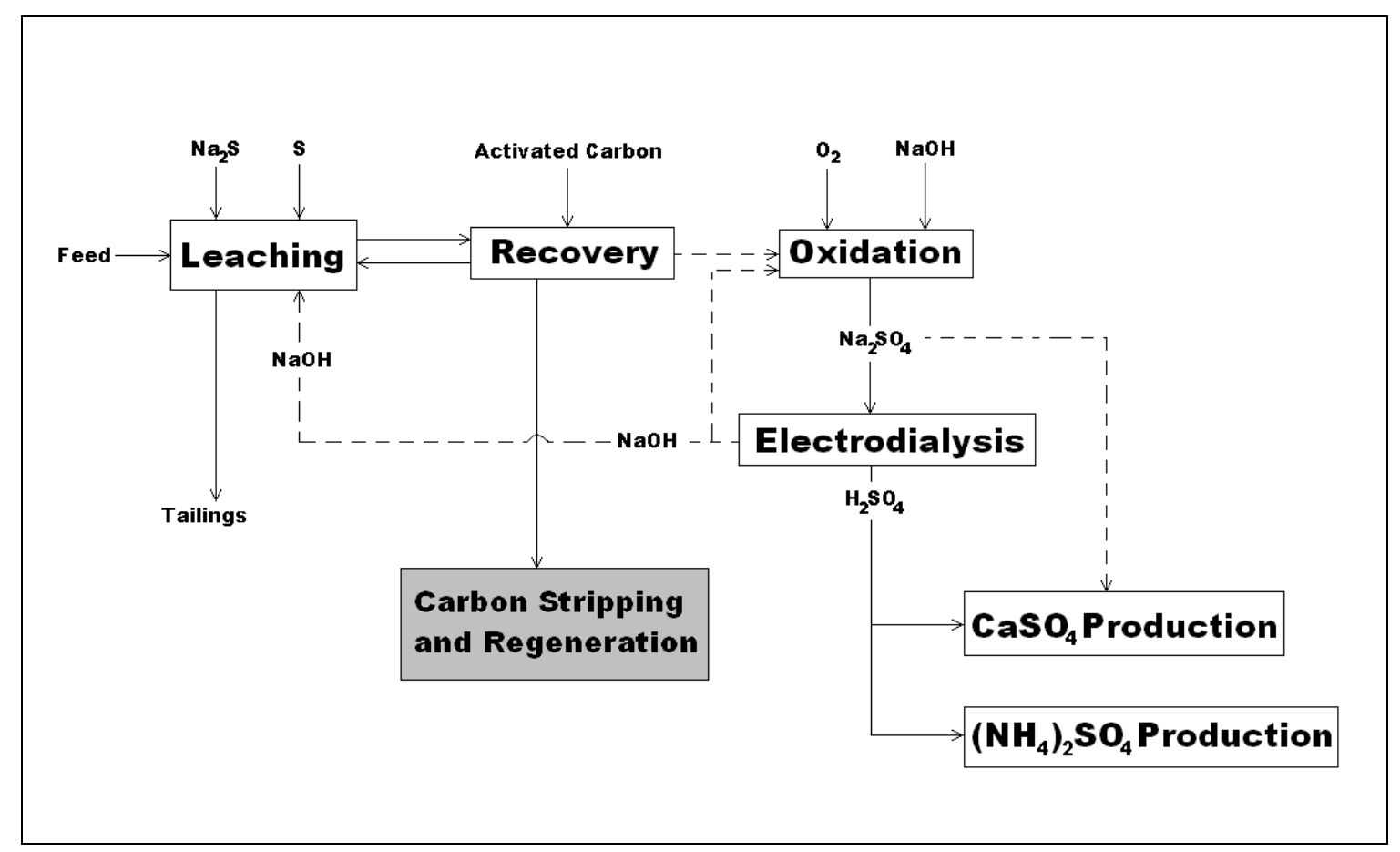

Figure 1. Proposed ASGLS Flowsheet

The following sub-sections describe the leaching chemistry and will review the findings of previous research (Stacey, 2005). Literature research and the background for other processes covered in this thesis (solution recovery and waste treatment) will be discussed separately in their respective chapters. 


\subsection{ASGLS Chemistry}

\subsubsection{Solution Chemistry of ASGLS}

Fundamental research on the chemistry and leaching mechanism of the ASGLS has been conducted collaboratively between Montana Tech and Monash University of Perth, Australia. Jeffrey (2002) has studied the importance of polysulfide and sulfide co-existing in the leaching solution: polysulfide as the oxidant and sulfide as the lixiviant.

Furthermore, Stacey (2005) has developed a standard operating procedure (SOP) for the preparation of the ASGLS solution: sulfide $\left(\mathrm{S}^{2-}\right)$ is provided by the addition of sodium sulfide flakes $\left(\mathrm{Na}_{2} \mathrm{~S}\right)$ and polysulfide $\left(\mathrm{S}_{\mathrm{x}}{ }^{2-}\right)$ is provided by the dissolution of elemental sulfur flakes (S):

$$
\mathrm{S}+(\mathrm{x}-1) \mathrm{S}^{2-} \rightarrow \mathrm{S}_{\mathrm{X}}^{2-}+(2 \mathrm{x}-4) \mathrm{e}^{-} \quad(\mathrm{x}=2,3,4, \text { or } 5)(1)
$$

As seen in Table 1, the ASGLS is metastable in the sulfur system with various oxidation states. The solution preparation SOP developed is crucial: to produce a consistent amount of polysulfide relative to sulfide in the solution. The alkaline sulfide solution preparation standard operating procedure (SOP) is presented in Appendix D.

Table 1. Selected Oxidation States of Sulfur

\begin{tabular}{|l|l|l|}
\hline Specie & Oxidation State of Sulfur & Name \\
\hline $\mathrm{SO}_{4}{ }^{2-}$ & +6 & Sulfate \\
\hline $\mathrm{SO}_{3}{ }^{2-}$ & +4 & Sulfite \\
\hline $\mathrm{S}_{2} \mathrm{O}_{3}{ }^{2-}$ & +2 & Thiosulfate \\
\hline $\mathrm{S}$ & 0 & Elemental Sulfur \\
\hline $\mathrm{S}_{5}{ }^{2-}$ & -0.4 & Polysulfide \\
\hline $\mathrm{S}_{4}{ }^{2-}$ & -0.5 & Polysulfide \\
\hline $\mathrm{S}_{3}{ }^{2-}$ & -0.67 & Polysulfide \\
\hline $\mathrm{S}_{2}{ }^{2-}$ & -1 & Polysulfide \\
\hline $\mathrm{S}^{2-}$ & -2 & Sulfide \\
\hline
\end{tabular}

Shown in Figure 2 is a picture of a typical preparation of an ASGLS solution. Here, Sodium sulfide flakes $\left(\mathrm{Na}_{2} \mathrm{~S}\right)$ is dissolved with elemental sulfur flakes ( $\left.\mathrm{S}\right)$ consequently follows. In Figure 2, $\mathrm{Na}_{2} \mathrm{~S}$ flakes has already been in solution (thus the yellow solution) while the sulfur flakes began to dissolve at elevated temperature, creating a deep red colored solution, confirming the formation of polysulfide in solution. 


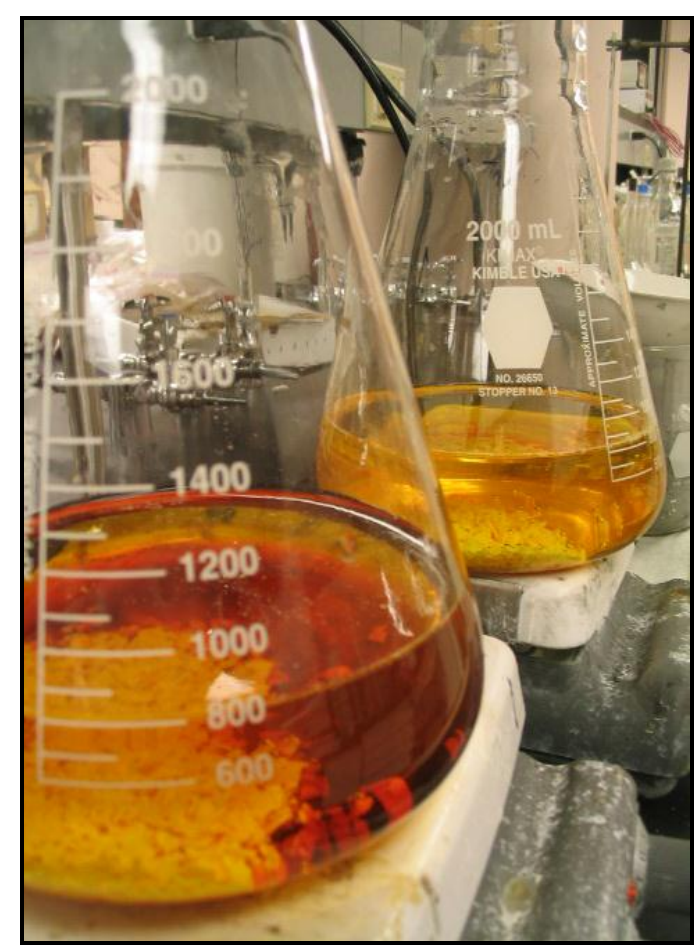

Figure 2. Dissolution of Sulfur Flakes in ASGLS Solution Preparation

A simple campaign was conducted to make ASGLS solutions at different total sulfide-polysulfide concentration and different addition ratio of $S^{2-}$ to $S$. Shown in Table 2 is a simple observation of $\mathrm{E}_{\mathrm{H}}, \mathrm{pH}$, concentration of polysulfide, and concentration of sulfide at different addition methods. All $\mathrm{E}_{\mathrm{H}}$ measurements in this thesis were measured using a standard Ag/AgCl electrode; a correction number of $+220 \mathrm{mV}$ was used to achieve the Standard Hydrogen Electrode (SHE) numbers shown in Table 2.

Table 2. Solution Parameters for Different Addition Ratios and Total Sulfur Concentrations

\begin{tabular}{|c|c|c|c|c|c|c|c|c|}
\hline \multirow{2}{*}{$\begin{array}{c}S^{2-} \text { to } S \\
\text { Ratio }\end{array}$} & \multicolumn{4}{|c|}{ 25-g/L Total Sulfide-Polysulfide } & \multicolumn{4}{|c|}{ 50-g/L Total Sulfide-Polysulfide } \\
\hline & $\begin{array}{c}\mathbf{E}_{\mathbf{H}} \\
(\mathrm{SHE})\end{array}$ & $\mathbf{p H}$ & $\begin{array}{l}{\left[\mathbf{S}_{\mathbf{x}}{ }^{2-}\right]} \\
(\mathrm{g} / \mathrm{L})\end{array}$ & $\begin{array}{l}{\left[\mathrm{S}^{2-}\right]} \\
(\mathrm{g} / \mathrm{L})\end{array}$ & $\begin{array}{c}\mathbf{E}_{\mathbf{H}} \\
(\mathrm{SHE})\end{array}$ & pH & $\begin{array}{l}{\left[\mathbf{S}_{\mathbf{x}}{ }^{2-}\right]} \\
(\mathrm{g} / \mathrm{L})\end{array}$ & $\begin{array}{l}\left.\mathrm{S}^{2-}\right] \\
(\mathrm{g} / \mathrm{L}) \\
\end{array}$ \\
\hline $1: 0$ & -525 & 12.52 & 0.00 & 25.00 & -543 & 12.57 & 0.00 & 25.00 \\
\hline $1: 1$ & -475 & 12.88 & 9.07 & 15.93 & -487 & 12.88 & 8.57 & 16.43 \\
\hline $1: 2$ & -442 & 12.89 & 9.82 & 15.18 & -466 & 12.94 & 10.52 & 14.48 \\
\hline $1: 3$ & -416 & 12.84 & 11.27 & 13.73 & -453 & 12.92 & 11.60 & 13.40 \\
\hline $1: 4$ & -411 & 12.83 & 11.99 & 13.01 & -444 & 12.95 & 12.20 & 12.80 \\
\hline
\end{tabular}

\subsubsection{Gold Leaching Mechanism in ASGLS}

As mentioned before, the ASGLS relies on the co-existence of $\mathrm{S}_{\mathrm{x}}{ }^{2-}$ and $\mathrm{S}^{2-}$ in the leach solution. Jeffrey (2002) has demonstrated the role of these species in the leaching mechanism: $\mathrm{S}_{\mathrm{x}}{ }^{2-}$ acts as the oxidant and $\mathrm{S}^{2-}$ acts as the lixiviant. In this regard, the ASGLS is similar with any other leaching systems: an oxidant is needed to oxidize gold and a lixiviant is needed to form a stable 
gold specie in solution.

The polysulfide reduction and gold lixiviation reactions occurring in the ASGLS are presented in Equation 2 and 3 (Stacey, 2005). Illustrated in Equation 4 is the overall gold dissolution reaction for ASGLS.

$$
\begin{array}{r}
\mathrm{S}_{\mathrm{x}}{ }^{2-}+2(\mathrm{x}-1) \mathrm{e}^{-} \rightarrow \mathrm{xS}^{2-}(\mathrm{x}=2,3,4, \text { or } 5) \\
\mathrm{Au}^{+3}+\mathrm{S}^{2-} \rightarrow \mathrm{AuS}^{-}+\mathrm{e}^{-} \\
2 \mathrm{Au}+\mathrm{S}_{\mathrm{x}}{ }^{2-}+2 \mathrm{~S}^{2-}+(2 \mathrm{x}-4) \mathrm{e}^{-} \rightarrow 2 \mathrm{AuS}^{-}+\mathrm{x} \mathrm{S}^{2-}
\end{array}
$$

A typical leaching test setup is presented in Figure 3. Agitation is provided using a magnetic stir-bar while temperature control is provided using a water bath. The heating recirculator proved to provide a very stable temperature. The cellophane used on top of the leaching vessels was only provided to better control water loss due to evaporation.

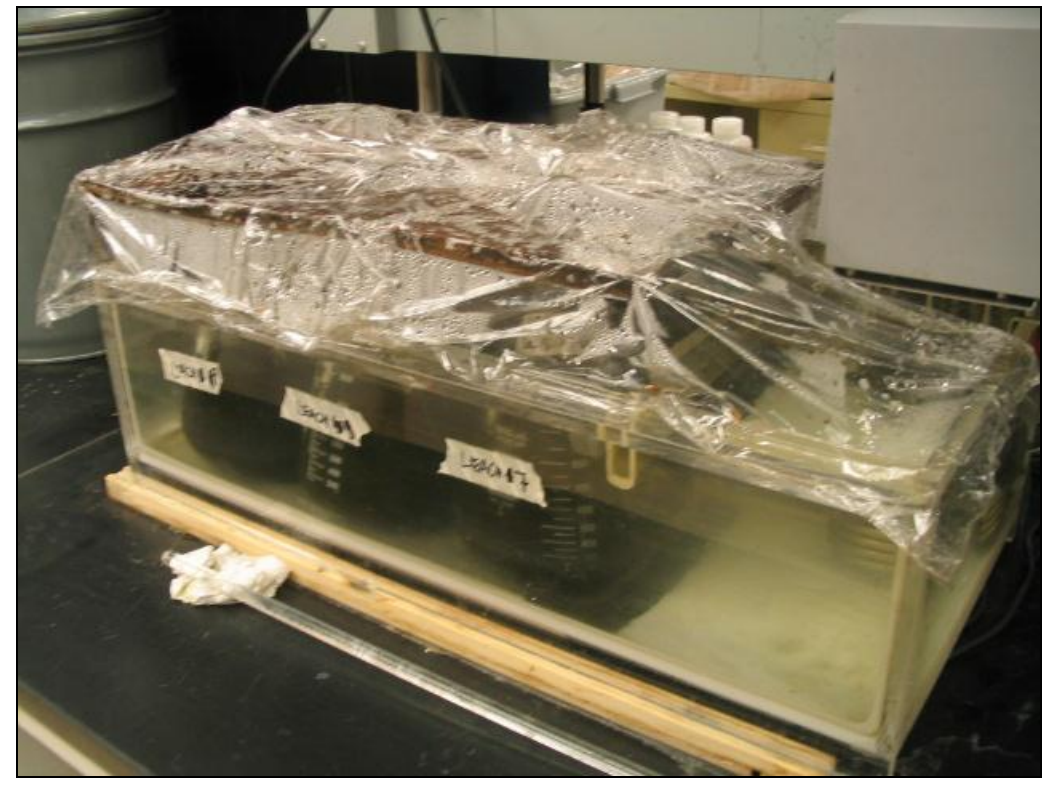

Figure 3. Typical Leaching Test Setup

\subsection{Review of Previous Research}

This section of the thesis will review and compile the findings of the previous ASGLS thesis research accomplished in 2005 by Darby Stacey, a former graduate student at Montana Tech. In depth work was performed on: 1) electrochemistry and electrokinetics of ASGLS and 2) leaching chemistry with gold powder. Additionally, scoping research was conducted in: 1) industrial ore leaching, 2) gold recovery from pregnant solution, and 3) waste treatment of waste ASGLS solution.

The findings, conclusion, and recommendations of this previous research were the starting point 
of this current research. The current research is a continued research; and hence, it is critical to review these important findings of the previous research.

\subsubsection{Electrochemistry \& Electrokinetic of ASGLS}

Most of the electrochemistry and electrokinetic research of the ASGLS were conducted at Monash University, utilizing the availability of the Rotating Electrochemical Quartz Crystal Microbalance

(REQCM). With its ability to measure very minute mass change, accurate measurements of leaching at different solution conditions could be conducted. Variables that were investigated in the system included (Stacey, 2005):

1. Sulfide concentration $\left[\mathrm{S}^{2-}\right]$;

2. Hydrosulfide concentration [HS $\left.{ }^{-}\right]$;

3. Polysulfide concentration $\left[\mathrm{S}_{\mathrm{x}}{ }^{2-}\right]$;

4. $\mathrm{NaOH}$ concentration;

5. Temperature; and

6. Gold purity.

Conclusions reached were:

1. An increase in sulfide concentration increased the dissolution rate of gold;

2. Hydrosulfide had a negative effect on the gold leaching kinetics;

3. The addition of sulfur increased the polysulfide concentration and potential of the system;

4. Higher polysulfide concentration increased leach rate until the potential of the solution becomes so high that leach rate is decreased;

5. Hydroxide additions increased leach kinetics at lower potentials;

6. Temperature had a positive effect on leaching kinetics; and

7. Gold purity affected leach kinetics positively.

\subsubsection{Laboratory Leaching Tests of 99.99\% Gold Powder}

Laboratory leach tests were performed to confirm the findings of the REQCM experiments. At the same time, the laboratory leaching procedure was finalized to produce consistent data. The following conclusions were reached:

1. An increase in total sulfide-polysulfide concentration increases the total amount of gold leaching;

2. $\quad$ An increase in temperature positively affected gold leaching; and

3. Mole ratio of sulfur to sulfide added to the solution should be kept at 1.25:1 to 1:1 (Stacey, 2005). 


\subsubsection{Industrial Ore Leaching}

Two industrial ore and concentrates were successfully leached on a laboratory scale: Kennecott Greens Creek's gravity middlings and Newmont's roaster calcine (pre- and post-cyanide leached). These materials were high in sulfide/sulfur, believed to be beneficial in the ASGLS system (both feed and solution are in the sulfur system).

Kennecott Greens Creek's gravity middlings had $1.11 \%$ total precious metals ( $\mathrm{Au}$ and $\mathrm{Ag}$ ), mostly in the form of electrum. The sample matrix was very high in galena $(\mathrm{PbS})$ with trace amounts of pyrite $\left(\mathrm{FeS}_{2}\right)$ (Stacey, 2005). With a total sulfide-polysulfide concentration of $3.12 \mathrm{M}$ and at $60^{\circ} \mathrm{C}$, leaching performance of $85.7 \%$ and $93.7 \%$ for gold and silver, respectively, was achieved.

Newmont's roaster calcine samples were pre-oxidized feed materials to be leached with cyanide. Both pre- and post-cyanide-leached materials were leached with the ASGLS in the laboratory. Pre-oxidized materials are believed to be higher in elemental sulfur-resulting from oxidation of sulfide to sulfur in the ore. At total sulfide-polysulfide of $3.12 \mathrm{M}$ and $60^{\circ} \mathrm{C}$, these results were obtained:

1. Pre-Cyanide-Leached: $69.1 \%$ Ag and 95.0\%Au leached; and

2. Post-Cyanide-Leached: 33.7\%Ag and 40.0\%Au leached.

\subsubsection{Gold Recovery from Pregnant Solution}

Two experimental matrices were developed by Stacey (2005) to investigate the effects of the following parameters to the performance of activated carbon in recovery of gold from pregnant ASGLS solution:

1. Amount of carbon per liter of pregnant solution; and

2. Sulfide, polysulfide, and hydroxide concentration of pregnant solution.

Additionally, the mechanism of gold extraction from solution was investigated by adding another substrate simultaneously (glass spheres) to some of the experiments. If gold extraction were to be increased by the addition of glass spheres, it might be an indication that gold is precipitating onto the substrate instead of adsorbing onto the carbon. The results of these experiments were inconclusive.

\subsubsection{Waste Treatment of Waste ASGLS Solution}

The waste treatment of ASGLS experiments performed in the previous research only covered the pressure oxidation stage. Using an autoclave-to provide temperature, pressure, and agitation control-the previous experiments were performed mainly to obtain a general range of parameters to obtain sulfide-polysulfide oxidation.

With parameters of oxygen overpressure of 40-80psig, agitation of 700-rpm, temperature of $170^{\circ} \mathrm{C}$, and after 14-hours, sodium sulfate $\left(\mathrm{Na}_{2} \mathrm{SO}_{4}\right)$ crystals were obtained from the resulting oxidized solution. Without any quantitative analysis of the amount of sulfate produced, or if any 
other oxidation state of sulfur existed, it was confirmed that there were zero sulfide $\left(\mathrm{S}^{2-}\right)$ left in the solution.

\section{Experimental Nomenclature and Thesis Organization}

The labeling and naming of each experimental campaign in this research were performed to ease the sample storing management and identification. Shown in Table 3 are all of the experimental campaigns conducted in this research and their respective name coding/nomenclature. These campaign identifications will be used throughout this thesis.

Table 3. Experimental Campaign Identifications and Descriptions

\begin{tabular}{|c|c|}
\hline Campaign ID & Description \\
\hline LGC-1 & $\begin{array}{l}\text { 1st Leaching Campaign of KGCMC } \\
\text { Composite Concentrate }\end{array}$ \\
\hline LGS-1 & $\begin{array}{l}\text { 1st Leaching Campaign of Golden } \\
\text { Sunlight's Pyrite Concentrate }\end{array}$ \\
\hline LGS-2 & $\begin{array}{l}\text { 2nd Leaching Campaign of Golden } \\
\text { Sunlight's Pyrite Concentrate }\end{array}$ \\
\hline RT-1 & $\begin{array}{l}\text { 1st Precious Metal Recovery Campaign } \\
\text { from Pregnant ASGLS Solution }\end{array}$ \\
\hline RT-2 & $\begin{array}{l}\text { 2nd Precious Metal Recovery Campaign } \\
\text { from Pregnant ASGLS Solution }\end{array}$ \\
\hline RT-3 & $\begin{array}{l}\text { 3rd Precious Metal Recovery Campaign } \\
\text { from Pregnant ASGLS Solution }\end{array}$ \\
\hline WO-1 & $\begin{array}{c}\text { 1st Campaign of Pressure Oxidation of } \\
\text { Waste ASGLS Solution }\end{array}$ \\
\hline WO-2 & $\begin{array}{c}\text { 2nd Campaign of Pressure Oxidation of } \\
\text { Waste ASGLS Solution }\end{array}$ \\
\hline WED-1 & $\begin{array}{c}\text { 1st Campaign of } \mathrm{NaOH} \text { Regeneration using } \\
\text { Electrodialysis }\end{array}$ \\
\hline WED-2 & $\begin{array}{l}\text { 2nd Campaign of } \mathrm{NaOH} \text { Regeneration using } \\
\text { Electrodialysis }\end{array}$ \\
\hline WGP-1 & $\begin{array}{l}\text { 1st Campaign of Gypsum Production from } \\
\qquad \mathrm{H}_{2} \mathrm{SO}_{4}\end{array}$ \\
\hline
\end{tabular}




\begin{tabular}{|c|c|}
\hline WAS-1 & $\begin{array}{c}\text { 1st Campaign of Ammonium Sulfate } \\
\text { Production from } \mathrm{H}_{2} \mathrm{SO}_{4}\end{array}$ \\
\hline Batch Simulation 1 & $\begin{array}{c}\text { Bench-Top Batch Simulation with KGCMC } \\
\text { Composite Concentrate }\end{array}$ \\
\hline Batch Simulation 2 & $\begin{array}{c}\text { Bench-Top Batch Simulation with Golden } \\
\text { Sunlight's Pyrite Conc. }\end{array}$ \\
\hline Batch Simulation 3 & $\begin{array}{c}\text { Bench-Top Batch Simulation with Pre- } \\
\text { Oxidized Golden Sunlight Pyrite Conc. }\end{array}$ \\
\hline
\end{tabular}

The organization of this thesis is not based chronologically. Instead, the organization is based on the process flowsheet shown previously in Figure 1. Each of the following chapters will discuss one unit processes covered in this research project. Introduction, background, and literature research of each unit processes will be presented at the beginning of the respective chapters and not in this introduction chapter.

The details of chemicals and analytical equipments used in this thesis are described in Appendix M. The step-by-step procedures of the experimental campaigns and batch simulations conducted in this research are also presented in the Appendices of this thesis.

\subsection{Concentrates Characterization and Leaching Experiments}

\subsection{Introduction to Concentrates Characterization and Leaching Experiments}

The objective of this section of the study is to characterize two different sulfide concentrate materials and their behavior subjected to the ASGLS. Later on, these concentrates were subjected to the Bench Scale Batch Simulation (Batch Simulation). To ensure a good leaching performance in the batch simulations, it was important to conduct characterization studies and preliminary leaching experiments.

The two sulfide concentrates samples were obtained from operating mines:

1. Kennecott Greens Creek Mining Company's (KGCMC) composite concentrate; and 2. Barrick Gold Corp's (Barrick) Golden Sunlight Mining Company pyrite concentrate.

In general, these concentrates are sulfide concentrates containing appreciable amount of precious metals. Sulfide minerals are an ideal feed to the ASGLS and can be troublesome to a cyanide system; it was advantageous to use these feed materials to provide a preliminary idea of the behavior of the ASGLS in a real system.

Detailed discussions of the nature of these sulfide concentrates and their behavior under the treatment of ASGLS will be discussed in the following sections of this chapter. 


\subsection{Kennecott Greens Creek Mining Co.'s (KGCMC) Composite Concentrate}

\subsubsection{Introduction \& Background}

Kennecott Greens Creek Mining Company (KGCMC) is a poly-metallic mine located in Admiralty Island near Juneau, Alaska. KGCMC's concentrator extracts galena (PbS) concentrate, sphalerite $(\mathrm{ZnS})$ concentrate, and bulk concentrate from a flotation circuit; furthermore, gold bullion is produced through gravity concentration--consisting of spirals and shaking tables--and high temperature extraction. A process flowsheet of KGCMC's concentrator is presented in Appendix A.

In fall of 2005, Darby Stacey of KGCMC sent four different concentrate samples to Montana Tech. Samples included: zinc sulfide concentrate, lead concentrate, bulk concentrate, and gravity middling. Although not all of these samples used in the research, a combination of lead and zinc concentrates (composite concentrate) were used in the leaching optimization and batch simulation study. The high gold containing gravity middlings were used to create a high concentration pregnant ASGLS solution used in the gold recovery from pregnant solution study.

\subsubsection{Characterization of KGCMC Sulfide Concentrates}

Prior to any extraction processes, it is essential to characterize the feed materials subjected to the process. In this case, the KGCMC composite concentrate was analyzed using several different methods and analytical methods:

1. Precious Metal (Au and Ag) content with Fire-assay;

2. X-Ray Diffraction (XRD);

3. Carbon-Sulfur Analysis; and

4. Induced Coupled Plasma (ICP) Elemental Sweep.

Each analytical equipment provides unique data to better characterize the concentrate. Fire-assay was performed by Chris Christopherson Inc. (CCI) laboratory in Kellogg, Idaho and determined the gold and silver content of the composite concentrate. Shown in Table 4 are results of the three samples sent. The average content was 0.772-opt Au and 89.25-opt Ag.

Table 4. Fire-Assay Results of KGCMC Composite Concentrate

\begin{tabular}{|c|c|c|}
\hline Sample & Au (opt) & Ag (opt) \\
\hline 1 & 0.856 & 89.8 \\
\hline 2 & 0.647 & 86.5 \\
\hline 3 & 0.812 & 91.45 \\
\hline $\begin{array}{c}\text { Averag } \\
\text { e }\end{array}$ & $\mathbf{0 . 7 7 2}$ & $\mathbf{8 9 . 2 5}$ \\
\hline Std.Dev. & 0.1101 & 2.5204 \\
\hline
\end{tabular}


Most of the gold in the concentrate was electrum, a gold-silver alloy commonly with a ratio of 7:3 of gold to silver. With a gold content of 0.772 -opt, only 0.330 -opt (only $0.37 \%$ of total silver in concentrate) of silver would be in the electrum. While the silver content was analyzed at 89.25opt, it is likely that silver is associated with other sulfide minerals. Some of the minerals shown in Table 5 shows silver bearing minerals that are known to exist in KGCMC's ore body.

XRD provided a qualitative analysis of possible compounds in the concentrate. Shown in Figure 4 is a XRD spectrum produced from the composite concentrate. As expected, the spectrum shows that the concentrate is dominant in galena $(\mathrm{PbS})$ and sphalerite $(\mathrm{ZnS})$. Also, pyrite $\left(\mathrm{FeS}_{2}\right)$ was detected if only in minor amounts. These sulfide compounds were not expected to interfere with the leaching performance of the ASGLS since they are in the same sulfur system. In particular, the ASGLS system is non-reactive with sphalerite whereas cyanide will be consumed by this known cyanicide.

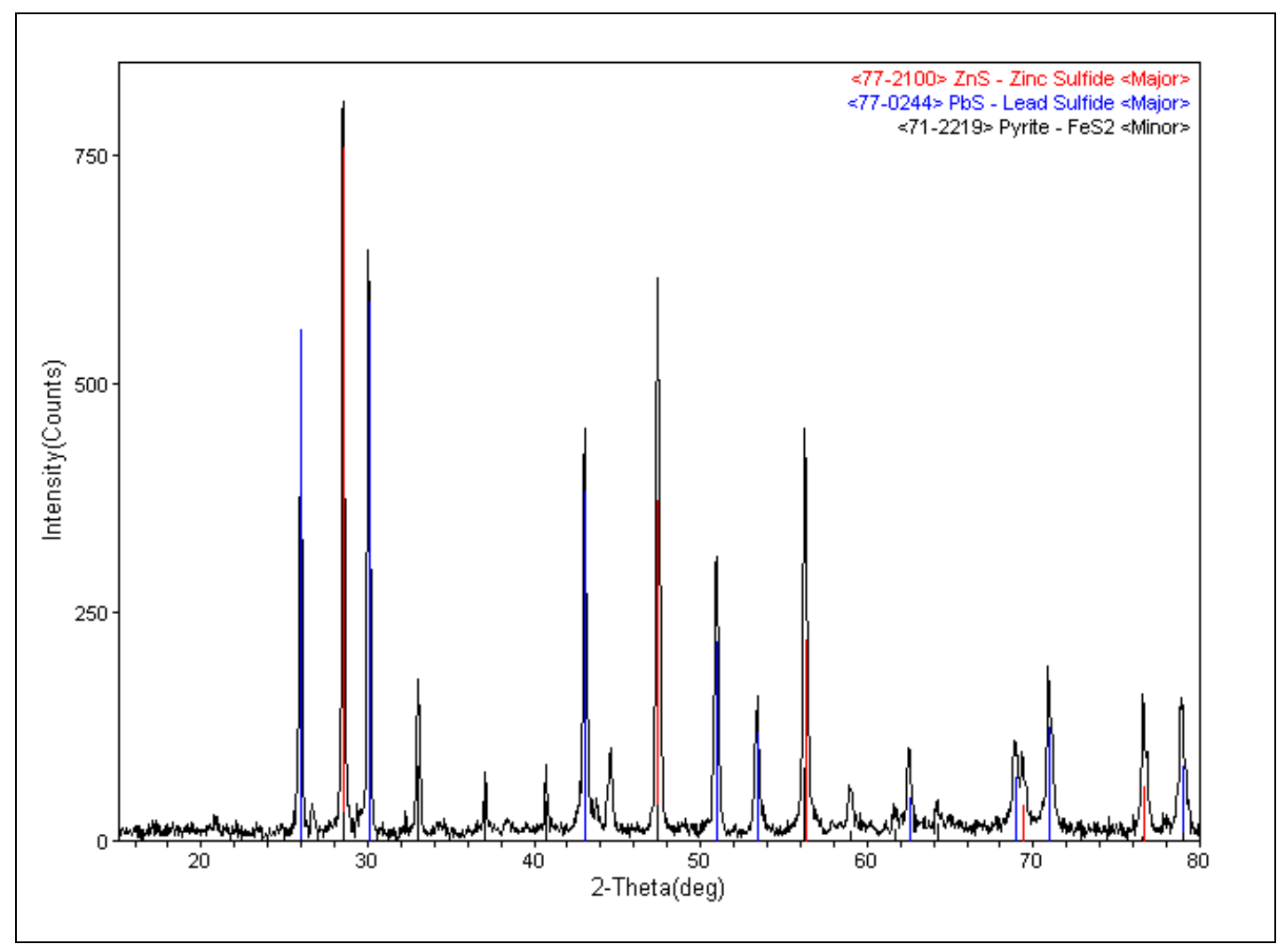

Figure 4. XRD Spectrum of KGCMC Composite Concentrate

Carbon-sulfur analysis was conducted on the sample. This analysis was focused to quantify carbon in the sample: a substantial carbon existing in the concentrate would indicate a carbonaceous (preg-robbing) material. A carbon content of $>5 \%$ results in a significant reduction in gold extraction using cyanide (Mardsen et.al, 1969). Previous success in leaching carbonaceous materials using the ASGLS has demonstrated an advantage to the cyanide system. Shown in Table 5 is the results of carbon-sulfur analysis of the composite concentrate. 
Table 5. Carbon-Sulfur Analysis Result of Composite Concentrate

\begin{tabular}{|c|c|c|}
\hline Sample & \%Carbon & \%Sulfur \\
\hline 1 & 0.942 & 30.06 \\
\hline 2 & 0.878 & 29.47 \\
\hline
\end{tabular}

As expected, since this is essentially a sulfide concentrate, sulfur content is fairly high. Carbon content in the sample, however, is relatively low.

Finally, an analysis of various chemical elements in the sample was conducted using an ICP by the Montana Bureau of Mines and Geology (MBMG) (Olmstead, 2005). Shown in Table 6 are the elements analyzed and their respective concentrations.

Table 6. Content of Selected Elements in KGCMC Composite Concentrate

\begin{tabular}{|c|c|}
\hline Element & Content \\
\hline $\mathbf{A s}(\mathbf{p p m})$ & 4.40 \\
\hline $\mathbf{C u}(\boldsymbol{\%})$ & $1.26 \%$ \\
\hline $\mathbf{F e}(\%)$ & $9.30 \%$ \\
\hline $\mathbf{P b}(\boldsymbol{\%})$ & $20.1 \%$ \\
\hline $\mathbf{S b}(\mathbf{p p m})$ & 6.02 \\
\hline $\mathbf{S i O}_{\mathbf{2}}$ & $3.10 \%$ \\
\hline $\mathbf{Z n}$ & $25.0 \%$ \\
\hline
\end{tabular}

The findings of arsenic (As) and antimony ( $\mathrm{Sb}$ ) in the concentrated prompted an investigation of minerals that these elements exist in the concentrate. Through a personal interview with Stacey (2006), the following minerals shown in Table 7 have been identified in the ore body.

Table 7. Selected Minerals Existing in KGCMC Ore Body

\begin{tabular}{|c|c|}
\hline Mineral & Formula \\
\hline tennantite & {$[\mathrm{CuFe}]_{13} \mathrm{As}_{4} \mathrm{~S}_{13}$} \\
\hline tetrahedrite & {$[\mathrm{CuFe}]_{13} \mathrm{Sb}_{4} \mathrm{~S}_{13}$} \\
\hline covellite & $\mathrm{CuS}$ \\
\hline bornite & $\mathrm{Cu}_{5} \mathrm{FeS}_{4}$ \\
\hline chalcopyrite & $\mathrm{CuFeS}_{4}$ \\
\hline acanthite & $\mathrm{Ag}_{2} \mathrm{~S}$ \\
\hline stromeyerite & $\mathrm{AgCuS}$ \\
\hline jalpaite & $\mathrm{Ag}_{3} \mathrm{CuS}_{2}$ \\
\hline proustite & $\mathrm{AgAsS}_{3}$ \\
\hline pyrargyrite & $\mathrm{AgSbS}_{3}$ \\
\hline polybasite & {$\left[\mathrm{Ag}, \mathrm{Cu}_{16} \mathrm{Sb}_{2} \mathrm{~S}_{11}\right.$} \\
\hline
\end{tabular}


An ICP analysis of the material discharged from the leaching stage of the batch simulations was analyzed to confirm if the ASGLS has the capability to dissolve these elements.

\subsubsection{Effect of Sulfide-Polysulfide and Hydroxide Concentrations (LGC-1)}

\subsubsection{LGC-1 Experimental Design}

Six leaching tests were performed to examine the effect of total sulfide-polysulfide and sodium hydroxide concentrations on the dissolution of precious metals contained in the concentrate composite. Stacey (2005) has demonstrated that with an increase of sulfide-sulfur addition will assist gold dissolution. Table 8 presents the experimental design of this study. Triplicates (labeled T4, T5, and T6) were performed as a simple quality control.

Table 8. LGC 1 Experimental Design

\begin{tabular}{|c|c|c|c|c|}
\hline Test \# & $\mathrm{Na}_{2} \mathbf{S}(\mathbf{g} / \mathbf{L})$ & $\mathbf{S}(\mathbf{g} / \mathbf{L})$ & $\mathbf{N a O H}(\mathbf{g} / \mathbf{L})$ & Temperature $\left({ }^{\circ} \mathbf{C}\right)$ \\
\hline LGC1-T1 & 40.0 & 10.0 & 24.0 & 50.0 \\
\hline LGC1-T2 & 80.0 & 20.0 & 24.0 & 50.0 \\
\hline LGC1-T3 & 160.0 & 40.0 & 24.0 & 50.0 \\
\hline LGC1-T4 & 160.0 & 40.0 & 50.0 & 50.0 \\
\hline LGC1-T5 & 160.0 & 40.0 & 50.0 & 50.0 \\
\hline LGC1-T6 & 160.0 & 40.0 & 50.0 & 50.0 \\
\hline
\end{tabular}

The following parameters were held constant for all the test work:

1. Concentrate weight: 50-grams;

2. Agitation: 170-rpm;

3. Solution volume: $750-\mathrm{mL}$; and

4. Leaching time: 48-hours.

Solution preparation and leaching procedure of this test for these tests are described in the SOPs presented in Appendix D and E. Only 50.0-grams of composite concentrate can be added to each leaching container because of an ineffective agitation using magnetic stir-bars above 50.0-grams.

\subsubsection{LGC-1 Experimental Results \& Analysis}

Shown in Table 9 are fire-assay results and their related leaching performance measured in percent metal leached. It can be seen that increasing total sulfide-polysulfide concentration had positive effect on gold leaching. A maximum of $82.4 \%$ gold dissolution was achieved with a total sulfide-polysulfide solution concentration of 80 grams/L. However, silver leaching did not seem to have any distinct trend in regards to total sulfide-polysulfide concentration in solution. This lack of trend is probably because most of the silver in the concentrate is associated in complex sulfide minerals (noted in Table 7). 
Table 9. Experimental Results of Campaign LGC-1

\begin{tabular}{|c|c|c|c|c|}
\hline Test & $\begin{array}{c}\text { Au Tails } \\
\text { Assay (opt) }\end{array}$ & $\begin{array}{c}\text { Ag Tails } \\
\text { Assay (opt) }\end{array}$ & Au Leached (\%) & Ag Leached (\%) \\
\hline LGC1-T1 & 0.524 & 79.5 & 32.1 & 11.0 \\
\hline LGC1-T2 & 0.503 & 77.9 & 34.8 & 12.8 \\
\hline LGC1-T3 & 0.496 & 78.8 & 35.8 & 11.8 \\
\hline LGC1-T4 & 0.235 & 78.5 & 69.6 & 12.1 \\
\hline LGC1-T5 & 0.218 & 82.1 & 71.8 & 8.1 \\
\hline LGC1-T6 & 0.136 & 74.5 & 82.4 & 16.6 \\
\hline
\end{tabular}

Because of the above results, a total sulfide-polysulfide concentration of 80.0 -g/L was used for the composite concentrate's bench-scale batch simulation (Batch Simulation 1). Batch Simulation monitored the solution parameters (Eh, $\mathrm{pH}$, total sulfide-polysulfide concentration, $\mathrm{NaOH}$ concentration, etc) before and after leaching. Please refer to Chapter 5.0 for detailed discussions of this simulation campaign.

\subsection{Golden Sunlight Pyrite Concentrate}

\subsubsection{Introduction \& Background}

Golden Sunlight Mining Company is a gold mining operation now wholly owned by Barrick Gold Corporation. It is an open-pit mine located in Jefferson County of southwestern Montana and is the only remaining open-pit mining operation in Montana using cyanide extraction technique. Golden Sunlight's concentrator receives 350tph of feed, with cutoff grade of $0.035 \mathrm{opt}$ gold. Presented in Appendix B is the overall process flowsheet of Golden Sunlight's concentrator.

The concentrator utilizes a gravity circuit - consisting of spirals - to further treat the primary leaching circuit discharge; in other words, un-leached precious metals are concentrated through gravity concentration. Since plenty of pyrite is contained in the ore, a pyrite concentrate with elevated content of precious metals is produced. This pyrite concentrate is then ground to $80 \%$ passing 400mesh and further treated with a heavier dose of cyanide (4 lbs/ton.concentrate). A sample of this material, before cyanide treatment, was obtained from the concentrator.

\subsubsection{Characterization of Golden Sunlight's Pyrite Concentrate}

Similar to the characterization study performed on the KGCMC's composite concentrate, extensive analyses using various analytical equipments was performed on Golden Sunlight's pyrite concentrate. Analyses included:

1. Precious Metals Content Analysis through Fire-Assay;

2. XRD; 
3. Carbon-Sulfur Analysis; and

4. ICP Elemental Sweep.

Fire-assay analysis was performed by sending properly mixed-split samples to CCI Laboratory in Kellogg, Idaho. Two samples were sent for each original and pre-oxidized pyrite concentrate as a quality control measure of the fire-assay analysis. As shown in Table 10, all results are consistent to their respective duplicate. Averages of these results were used for all experiments concerning this pyrite concentrate.

Table 10. Head Assay of Golden Sunlight's Pyrite Concentrate

\begin{tabular}{|c|c|c|}
\hline \multirow{2}{*}{ Sample } & \multicolumn{2}{|c|}{ Head Assay (opt) } \\
\cline { 2 - 3 } & $\mathrm{Au}$ & $\mathbf{A g}$ \\
\hline Pyrite & 0.107 & 0.9 \\
\cline { 2 - 3 } Concentrate & 0.101 & 0.9 \\
\hline Average & $\mathbf{0 . 1 0 4}$ & $\mathbf{0 . 9}$ \\
\hline Std.Deviation & 0.004243 & 0 \\
\hline
\end{tabular}

X-Ray Diffraction spectrum was produced for the pyrite concentrate to identify, qualitatively, the major and minor components of the pyrite concentrate. Seen in Figure 5 is the X-Ray spectra produced. It can obviously be seen that pyrite $\left(\mathrm{FeS}_{2}\right)$ and silica $\left(\mathrm{SiO}_{2}\right)$ are the dominant compounds. Gold peaks were not observed, most likely due to its insignificant content in the concentrate, compared to pyrite. 


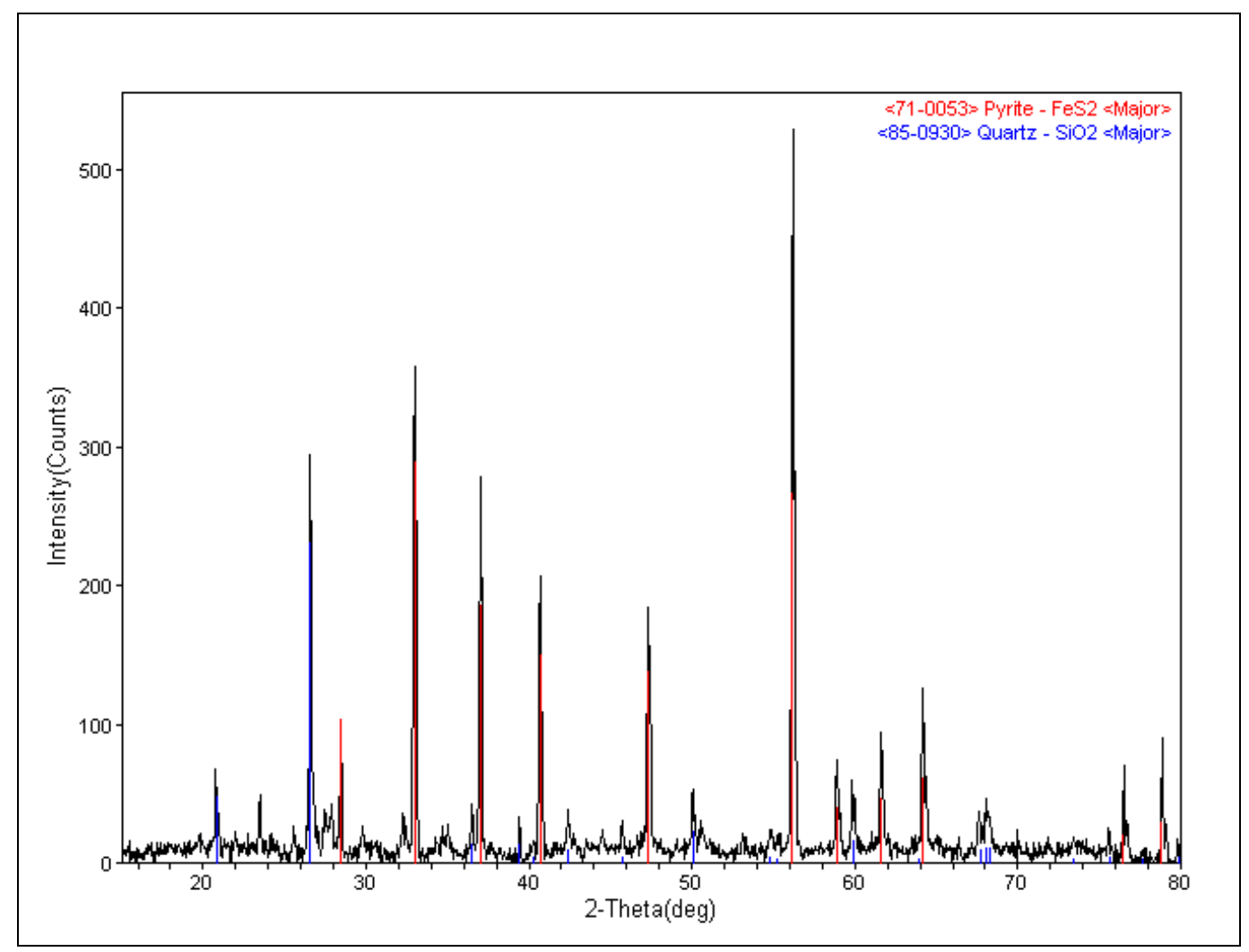

Figure 5. XRD Spectrum of Golden Sunlight's Pyrite Concentrate.

Carbon-sulfur analysis was conducted to provide the amount of carbon present in the pyrite concentrate. Since there have been successful experiments in gold recovery from pregnant ASGLS solutions using activated carbon, a high carbon content on a feed material may lead to a poor ASGLS leaching performance. Shown in Table 11 is the result of this carbon-sulfur analysis. Carbon content was minimal and the pyrite concentrate was determined to be noncarbonaceous.

Table 11. Carbon-Sulfur Analysis Results of Golden Sunlight's Pyrite Concentrate

\begin{tabular}{|c|c|c|}
\hline Sample & \%Carbon & \%Sulfur \\
\hline 1 & 0.450 & 23.89 \\
\hline 2 & 0.354 & 27.76 \\
\hline
\end{tabular}

Finally, an ICP elemental sweep analysis of the sample was conducted using an ICP. This analysis was performed by Wayne Olmstead of the Montana Bureau of Mines and Geology (MBMG). Mainly, the ICP analysis was to define if any other significant elements were present in the composite concentrate. Shown in Table 12 are the elements analyzed and their respective concentrations. It can be seen that the concentrate is fairly simple in composition with only minor amounts of aluminum (Al), chromium (Cr), copper ( $\mathrm{Cu})$, nickel (Ni), and zinc ( $\mathrm{Zn})$. 
Table 12. Concentration of Selected Elements in Pyrite Concentrate

\begin{tabular}{|c|c|}
\hline Element & Content \\
\hline $\mathbf{A l}(\%)$ & $4.02 \%$ \\
\hline $\mathbf{C r}(\mathbf{p p m})$ & 1.40 \\
\hline $\mathbf{C u}(\mathbf{p p m})$ & 0.39 \\
\hline $\mathbf{F e}(\%)$ & $26.00 \%$ \\
\hline $\mathbf{N i}(\mathbf{p p m})$ & 0.19 \\
\hline $\mathbf{S i O}_{\mathbf{2}}(\mathbf{\%})$ & $37.75 \%$ \\
\hline $\mathbf{Z n}(\mathbf{p p m})$ & 0.55 \\
\hline
\end{tabular}

\subsubsection{Effect of Sulfide-Polysulfide and Hydroxide Concentration (LGS-1)}

\subsubsection{LGS-1 Experimental Design \& Setup}

Similar to KGCMC composite concentrate's preliminary leaching tests, the objective of the preliminary leaching experiments on Golden Sunlight's pyrite concentrate was to examine the effect of leaching parameters to maximize gold dissolution. In campaign LGS-1, the effect of initial reagent concentration: total sulfide-polysulfide and sodium hydroxide, was investigated. Shown in Table 13 is a simple experimental design of LGS-1. Test 3 is a duplicate of Test 2 to define reproducibility.

Table 13. Experimental Design of LGS-1

\begin{tabular}{|c|c|c|c|c|c|}
\hline \multirow{2}{*}{ Test \# } & Total Sulfide-Polysulfide & $\mathrm{Na}_{2} \mathbf{S}$ & $\mathbf{S}$ & $\mathbf{N a O H}$ & Temperature \\
\cline { 2 - 6 } & $\mathbf{( g / L )}$ & $(\mathbf{g} / \mathbf{L})$ & $(\mathbf{g} / \mathbf{L})$ & $(\mathbf{g} / \mathbf{L})$ & $\left({ }^{\circ} \mathbf{C}\right)$ \\
\hline LGS1-T1 & 40.0 & 80.0 & 20.0 & 25.0 & 50.0 \\
\hline LGS1-T2 & 80.0 & 160.0 & 40.0 & 50.0 & 50.0 \\
\hline LGS1-T3 & 80.0 & 160.0 & 40.0 & 50.0 & 50.0 \\
\hline
\end{tabular}

All tests were performed using the following constants:

1. Concentrate weight: 50 -grams;

2. Agitation: $170-$ rpm;

3. Solution volume: $750-\mathrm{mL}$; and

4. Leaching time: 48-hours.

All solution preparation and leaching procedures were performed according to the developed SOPs presented in Appendix D and E. 


\subsubsection{LGS-1 Experimental Results \& Analysis}

Shown in Table 14 are fire-assay results of leaching discharge obtained from experimental campaign LGS-1. The reproducibility of the experiments was good with gold assay of tails only varied by 0.005-opt between Test 2 and Test 3 (both having the same experimental parameters). It was concluded that a higher concentration of total sulfide-polysulfide--providing a lower solution potential (as shown in Table 2)--allowed better leaching performance. A gold dissolution of up to $76.9 \%$ was achieved while silver dissolution was constant at 55.6\%.

Table 14. Experimental Results of LGS-1

\begin{tabular}{|c|c|c|c|c|}
\hline \multirow{2}{*}{ Test \# } & Tails Au Assay & Tails Ag Assay & Au Leached & Ag Leached \\
\cline { 2 - 5 } & opt & opt & $\%$ & $\%$ \\
\hline LGS1-T1 & 0.042 & 0.4 & 59.6 & 55.6 \\
\hline LGS1-T2 & 0.024 & 0.4 & 76.9 & 55.6 \\
\hline LGS1-T3 & 0.029 & 0.4 & 72.1 & 55.6 \\
\hline
\end{tabular}

These results were considered promising, proving the ability of the ASGLS to leach precious metals from Montana ore and concentrate now being treated with the cyanide system. Nonetheless, a pre-oxidized pyrite concentrate were also tested. Liberation of any encapsulated precious metal particles and the partial oxidation of sulfide to sulfur are expected to aid ASGLS leaching performance. Experimental campaign LGS-2 was conducted to investigate this.

\subsubsection{Effect of Concentrate Pre-Oxidation (LGS-2)}

\subsubsection{LGS-2 Experimental Design}

Another 2000-gram sample of Golden Sunlight's pyrite concentrate was pre-oxidized using a NSC simulation setup. The NSC is a partial oxidation process utilizing the role of nitrogen species $\left(\mathrm{NO}^{+}\right)$as intermediates and as an expedient means to transport oxygen to the surface of the solid particles (Anderson, 2004). (See Equation 5)

$$
2 \mathrm{MeS}_{(\mathrm{s})}+4 \mathrm{NO}^{+} \rightarrow 2 \mathrm{Me}^{2+}{ }_{(\mathrm{aq})}+2 \mathrm{~S}^{0}+4 \mathrm{NO}_{(\mathrm{g})}
$$

A room temperature solution treatment using $10 \% \mathrm{HNO}_{3}$ solution for 24-hours was used to partially oxidize the pyrite concentrate. It was thought that: 1) partial oxidation of sulfide provides an alternative source of sulfur needed in the ASGLS and 2) partial oxidation liberates any precious metal encapsulation within the pyrite particles.

The intended liberation was indicated by the loss of the $20 \%$ of the original mass (400-grams) at the end of the process; the resulting brown solution produced indicated the dissolution of some iron $(\mathrm{Fe})$ as a cause of this mass loss, which may result in the liberation of encapsulated precious metal particles. 
Table 15. Fire-Assay Results of Pre-Oxidized Pyrite Concentrate

\begin{tabular}{|r|c|c|}
\hline \multirow{2}{*}{ Sample } & \multicolumn{2}{|c|}{ Head Assay (opt) } \\
\cline { 2 - 3 } & $\mathrm{Au}$ & $\mathrm{Ag}$ \\
\hline Pre-Oxidized Pyrite & 0.0937 & 0.9 \\
\cline { 2 - 3 } Concentrate & 0.0973 & 0.9 \\
\hline Average & $\mathbf{0 . 0 9 5 5}$ & $\mathbf{0 . 9}$ \\
\hline Std.Deviation & 0.002546 & 0 \\
\hline
\end{tabular}

Two tests were performed in this experimental campaign. Basically, the best performing solution of LGS-1 was used in this campaign. The two tests were duplicates of each other to ensure experimental consistency. The experimental matrix of LGS-2 is presented in Table 16.

Table 16. Experimental Design of LGS-2

\begin{tabular}{|c|c|c|c|c|c|}
\hline \multirow{2}{*}{ Test \# } & Total Sulfide-Polysulfide & $\mathrm{Na}_{2} \mathrm{~S}$ & $\mathbf{S}$ & $\mathrm{NaOH}$ & Temperature \\
\hline & (grams/L) & (grams/L) & (grams/L) & (grams/L) & (C) \\
\hline LGS2-T1 & 80.0 & 160.0 & 40.0 & 50.0 & 50.0 \\
\hline LGS2-T2 & 80.0 & 160.0 & 40.0 & 50.0 & 50.0 \\
\hline
\end{tabular}

\subsubsection{LGS-2 Experimental Results \& Analysis}

The simple experiments of LGS-2 produced results as shown in Table 17. As expected, leaching of gold was increased after NSC partial oxidation. As seen, average increase of $6.75 \%$ was experienced. Also, silver leaching showed some improvement after NSC partial oxidation with an increase of up to $10 \%$.

Table 17. Experimental Results of Experimental Campaign LGS-2

\begin{tabular}{|c|c|c|c|c|}
\hline \multirow{2}{*}{ Test \# } & Tails Au Assay & Tails Ag Assay & Au Leached & Ag Leached \\
\cline { 2 - 5 } & opt & Opt & $\%$ & $\%$ \\
\hline LGS2-T1 & 0.016 & 0.4 & 83.3 & 55.6 \\
\hline LGS2-T2 & 0.014 & 0.3 & 79.2 & 66.6 \\
\hline
\end{tabular}

With these encouraging results, two different bench-scale batch simulations were conducted for Golden Sunlight's pyrite concentrate: one with pre-oxidation and one without. Discussions and results regarding these batch simulation campaigns are presented in Chapter 5.0. 


\subsection{Summary of Feed Characterization and Preliminary Leaching Experiments}

The composite concentrate blended from KGCMC's galena and sphalerite concentrate has been identified with $\mathrm{PbS}$ and $\mathrm{ZnS}$ as major minerals. Although no diagnostic leaching tests were performed, a separate report performed by KGCMC (Stacey, 2006) was reviewed and correlated to the characterization study in this thesis. Predominantly, gold in the concentrate exists as electrum. Silver, however, was indicated to exist in complex sulfide minerals. Preliminary leaching experiments of the composite concentrate using the ASGLS showed promising results with a gold dissolution of up to 82.4\%. However, silver leaching was only minimal (maximum of $16.6 \%$ ), which was attributed to the association of silver with complex minerals (tennantite, tetrahedrite, stromeyerite, jalpaite, proustite, etc).

The characterization study of Golden Sunlight's pyrite concentrate confirmed the dominance of pyrite $\left(\mathrm{FeS}_{2}\right)$ and minor amounts of silica. Preliminary leaching experiments using the ASGLS showed promising gold-silver dissolution of up to $76.9 \%$ and $55.6 \%(80-\mathrm{g} / \mathrm{L}$ total sulfidepolysulfide in solution). Additionally, pre-oxidation of this pyrite concentrate was conducted using the simulated NSC partial oxidation technique. This pre-oxidation proved to improve gold and silver dissolution of up to $83.3 \%$ and $66.6 \%$, respectively.

\subsection{Precious Metal Recovery from Pregnant ASGLS Solution}

\subsection{Introduction to Precious Metal Recovery from Pregnant ASGLS Solution}

A method of extracting gold out of precious-metal containing ASGLS solution is crucial to the success of the proposed ASGLS process flowsheet. Referring back to the proposed process flowsheet (Figure 6), an effective method of precious metal recovery from pregnant ASGLS solution is crucial to the whole process flowsheet. An effective gold leaching by the ASGLS from the feed will be of no value if a technically and economically effective method of recovery from pregnant solution cannot be performed. 


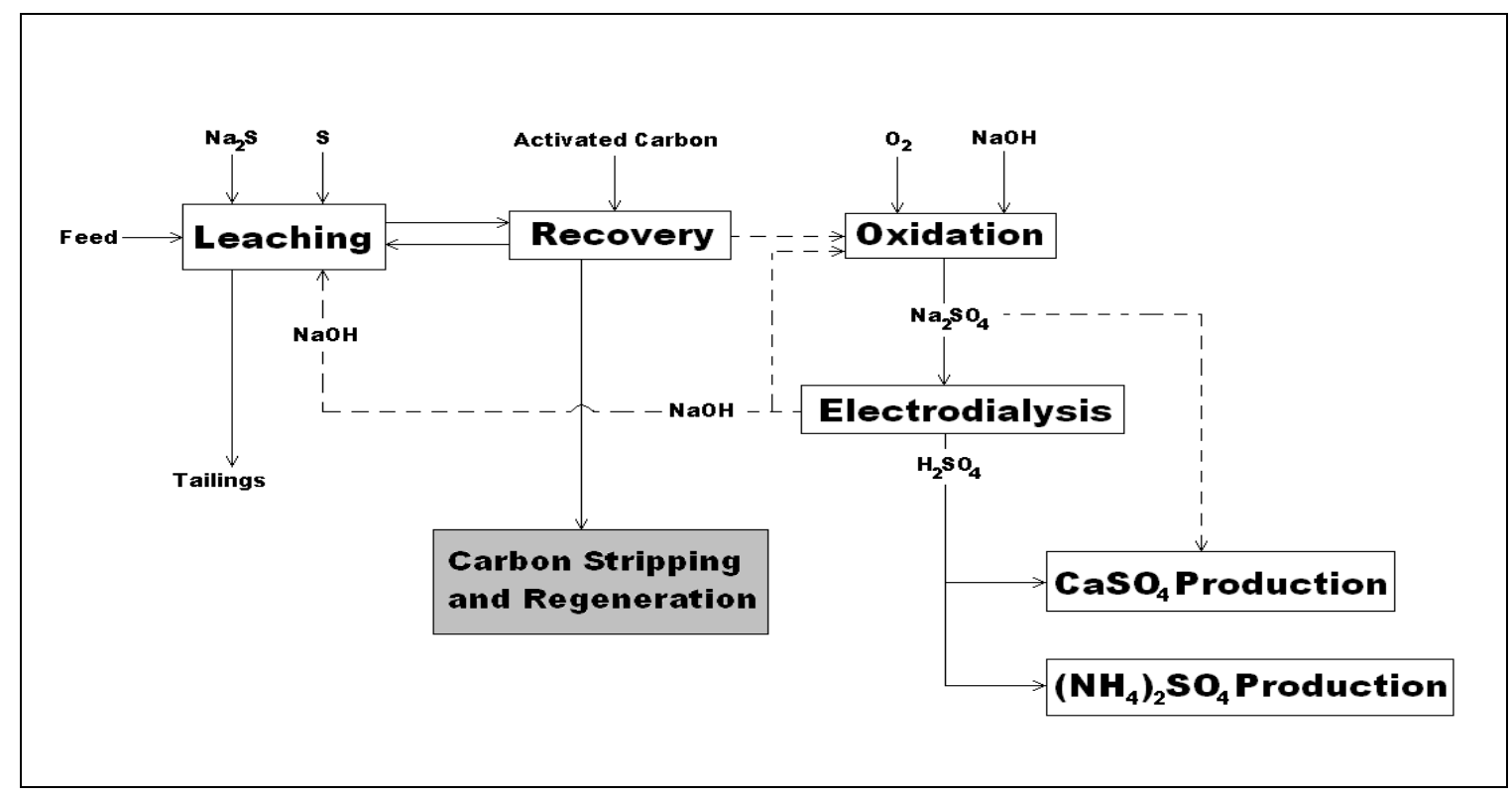

Figure 6. Proposed Flowsheet of the ASGLS

Traditionally, the cyanide system uses activated carbon, mostly produced from coconut shells and charcoal, as a substrate for the adsorption of the gold cyanide specie $\left(\mathrm{Au}(\mathrm{CN})^{2-}\right)$. Adsorption means the species in the liquid phase preferentially partitions onto a solid surface, creating a molecular or atomic film accumulation. Other substrates have also been shown to be effective in gold adsorption in the cyanide system.

Stacey (2005) has shown the possibility of activated carbon as an effective substrate in gold recovery from pregnant solution where a recovery of up to $92.6 \%$ was reported.

Several preliminary experimental campaigns were conducted first to prove the effectiveness of gold loading onto substrates. The first and second campaign dealt with the possibility of ionexchange resins and activated carbon as substrates. A third and fourth campaign were conducted in concurrence with the Batch Simulation campaigns, which provided data of the reliability and the performance of activated carbon using a carbon in columns setup. Additionally, a theory of gold loading mechanism onto the activated carbon has been developed.

\subsection{Performance of Activated Carbon and Ion-Exchange Resins (RT-1)}

\subsubsection{RT-1 Background and Experimental Design}

The first experimental campaign of gold recovery from pregnant solution was aimed to compare the gold recovery capabilities of activated carbon and ion-exchange resins from alkaline sulfide pregnant solution. Table 18 presents the experimental design and substrates used for each test in the

campaign. 
Table 18. Substrates Used in Campaign RT-1

\begin{tabular}{|c|c|c|c|}
\hline Test & Substrate & Active Ingredient & Base Material \\
\hline RT1-T1 & PSI WP2 & Iminoacetic Acid & Amorphous Silica Gel \\
\hline RT1-T2 & Amberlite $^{\circledR}$ IRC748 & Iminodiacetic Acid & Styrene-divinylbenzene \\
\hline RT1-T3 & Purolite $^{\circledR}$ S930 & Iminodiacetic Acid & Styrene-divinylbenzene \\
\hline RT1-T4 & Sybron Lewatit $^{\circledR}$ TP207 & Iminodiacetic Acid & Styrene-divinylbenzene \\
\hline RT1-T5 & Amberlite $^{\circledR}$ IRA400 & Quatenary Amine & Styrene-divinylbenzene \\
\hline RT1-T6 & Amberlite $^{\circledR}$ IRA68 & Quatenary Amine & Styrene-divinylbenzene \\
\hline RT1-T7 & Activated Carbon & -- & \\
\hline
\end{tabular}

This experimental campaign was run as a follow up to the collaboration work between CAMP and Pure System Inc. (PSI) of Missoula, Montana. PSI has developed polyamine-silica composite materials designed to remove transition metal ions from aqueous solutions with collaboration with The University of Montana (Miranda, 2005). Two of PSI's resins have been proposed to be tested under the ASGLS: WP1 ${ }^{\mathrm{TM}}$ and WP2 ${ }^{\mathrm{TM}}$. Both of these resins are silica based resins with a rated operational $\mathrm{pH}$ range of 3-11 (PSI, 2006). Obviously, stability of these resins is of concern because of the ASGLS' $\mathrm{pH}$ of above 12. In experimental campaign RT-1, WP2 was the only silica base resin tested.

Additionally, five different styrene based ion-exchange resins were also tested. Three resins (Amberlite ${ }^{\circledR}$ IRC748, Purolite ${ }^{\circledR}$ S930, and Sybron ${ }^{\circledR}$ TP207) contain the same active ingredient as PSI's WP1: iminodiacetic acid. Two additional resins (IRA400 and IRA68) are styrene-based resins with similar active ingredient as PSI's WP2: quatenary amine.

A standard gold containing alkaline sulfide solution was prepared in one batch from an actual industrial concentrate to provide a precious metal stock solution. The KGCMC gravity middlings were leached with a 40 -g/L total sulfide-polysulfide solution at $45^{\circ} \mathrm{C}$ for 36 -hours. The result was a solution containing 18.8-ppm of gold.

Recovery tests were performed in closed 500-mL flasks with each containing $200 \mathrm{~mL}$ of goldbearing (pregnant) alkaline sulfide solution and 20-grams of substrate. All tests were mixed using a horizontal shaker at 250-rpm for 48-hours. A more detailed description of the experimental setup of this campaign is presented in Appendix F. Shown in Figure 7 is the batch recovery experimental setup used in this campaign. 


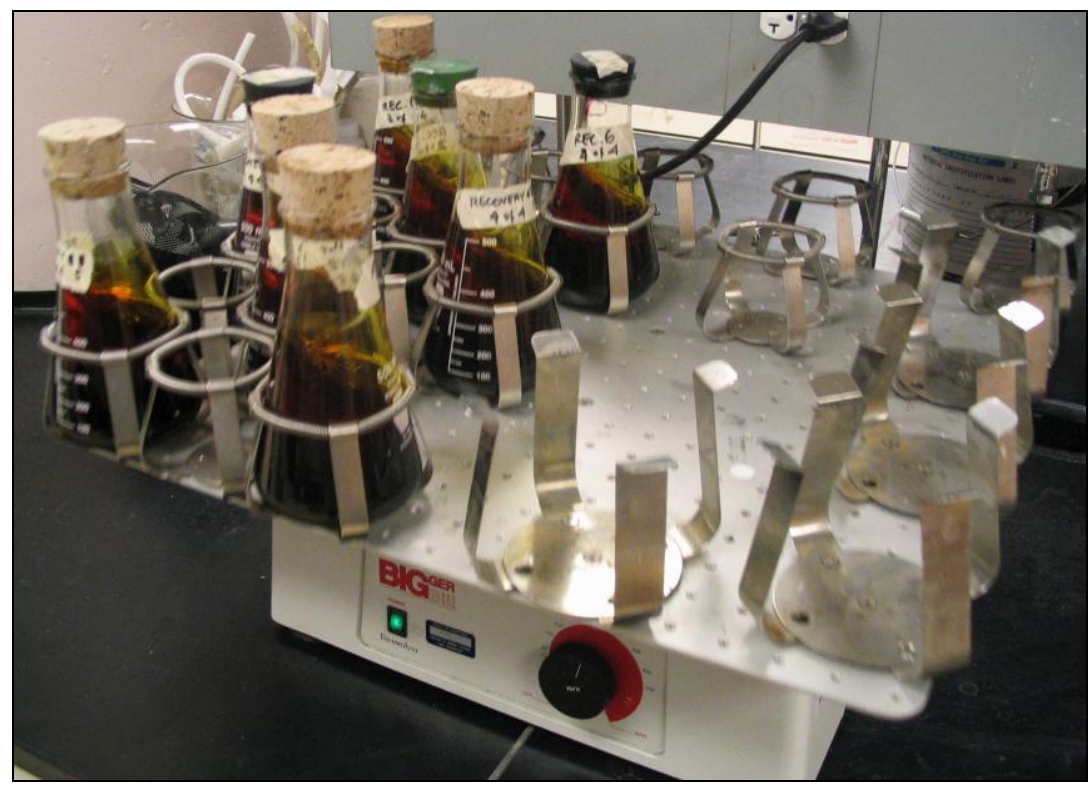

Figure 7. Typical Setup of Batch Recovery Experiments

\subsubsection{RT-1 Experimental Results and Analysis}

To obtain accurate data in recovery performance for each test, fire-assay analyses were performed on the washed, filtered, and then air-dried substrates. With a known amount of initial gold in solution and final gold content within the substrates, simple calculations of percent recovery were easily obtained. Additionally, the weights of the substrates were recorded after each tests to investigate any deterioration of the substrates. The experimental result of campaign RT-1 is presented in Table 19.

Table 19. Experimental Results of RT-1

\begin{tabular}{|c|c|c|c|c|c|c|}
\hline \multirow{2}{*}{ Test } & \multirow{2}{*}{ Substrate } & \multicolumn{2}{|c|}{ Post-Recovery } & \multicolumn{2}{c|}{ Carbon Assay (opt) } & \multicolumn{2}{c|}{ \% Metal Recovered } \\
\cline { 3 - 7 } & & Weight (grams) & Au & Ag & Au & Ag \\
\hline RT1-T1 & WP2 & N/A (Dissolved) & N/A & N/A & N/A & N/A \\
\hline RT1-T2 & IRC748 & 9.34 & 0.379 & 1.2 & 3.02 & 21.43 \\
\hline RT1-T3 & S930 & 11.22 & 0.175 & 0.7 & 1.67 & 15.01 \\
\hline RT1-T4 & TP207 & 10.46 & 0.018 & 0.3 & 0.16 & 6.00 \\
\hline RT1-T5 & IRA400 & 21.08 & 2.936 & 1.1 & 53.43 & 44.33 \\
\hline RT1-T6 & IRA68 & 10.34 & 0.018 & 0.2 & 0.16 & 3.95 \\
\hline RT1-T7 & Act. Carbon & 20.83 & 3.896 & 0.7 & 70.06 & 27.87 \\
\hline
\end{tabular}

It was concluded from Table 19 that activated carbon performed superior to the ion exchange resins. Amberlite ${ }^{\circledR}$ IRA400, a styrene based resin with amine active ingredient, performed reasonably well with a gold recovery of $53.4 \%$ and silver recovery of $70.9 \%$. However, activated carbon is certainly the most suitable substrate for gold recovery (70.1\% recovery). 
The significant superiority of silver recovery of IRA400 over activated carbon suggests a possible two-stage recovery process whereby two separate unit processes could be used to separately recover gold and silver from a pregnant alkaline sulfide solution. The economical consideration of this possibility was not reviewed and is beyond the scope of the thesis.

In addition, the loss of mass on most resins were not caused by the disintegration of the resins; rather, a loss in original moisture content was experienced during drying before analysis.

Equally as important was that PSI's WP2 silica based ion exchange resin did not stand up to the high operating $\mathrm{pH}$ of the alkaline sulfide solution. Just after approximately 3 hours into the experiment, all of the WP2 resin were disintegrated. This disintegration created a lighter polymer like liquid, which floated above the heavier alkaline sulfide solution. (See Figure 8)

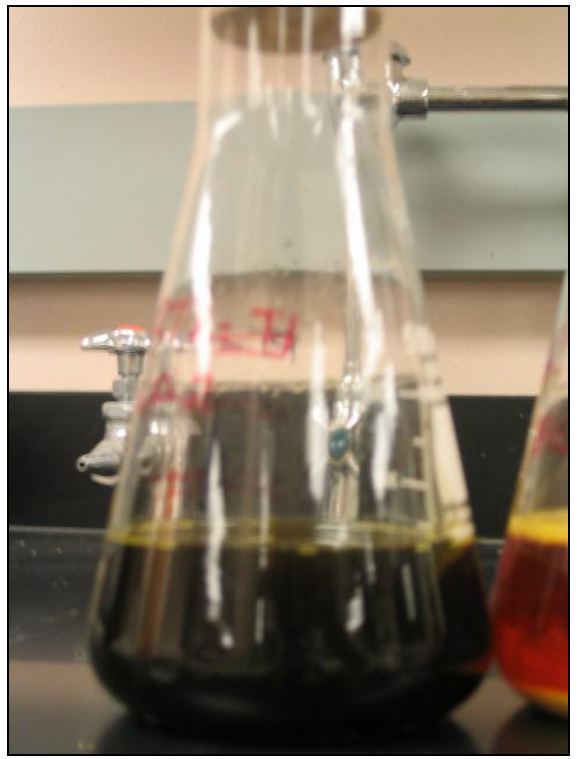

Figure 8. Isintegrated WP2 Ion Exchange Resin in Alkaline Sulfide Solution

\subsection{Effect of Lower Precious Metals Concentration in ASGLS Solution (RT- 2)}

\subsubsection{T-2 Background and Experimental Design}

Experimental campaign RT-2 was designed to investigate the performance of the two most effective substrates found in RT-1 in an alkaline sulfide solution with low gold concentration. In this case, a solution containing 0.45-ppm gold was used, prepared from a method similar to that of RT-1's pregnant solution. A solution with $40-\mathrm{g} / \mathrm{L}$ of total sulfide-polysulfide was used to leach KGCMC gravity middlings for 12 -hours at $50^{\circ} \mathrm{C}$. Shown in Table 20 are the test descriptions of RT-2. 
Table 20. RT-2 Experimental Design

\begin{tabular}{|c|c|c|c|}
\hline Test \# & Substrate & Base & Active Ingredient \\
\hline RT2-T1 & Amberlite ${ }^{\circledR}$ IRA400 & Styrene-divinylbenzene & Quarternary Amine \\
\hline RT2-T2 & Activated Carbon & -- & -- \\
\hline RT2-T3 & Activated Carbon & -- & -- \\
\hline
\end{tabular}

The experimental setup of RT-2 was a batch test similar to the one used in the previous campaign of RT-1 (See Figure 7). 200-mL of solution was added to a 500-mL flask containing 20-grams of desired substrate. Once the flask was tightly closed with a wooden cork, a horizontal shaker set at 250-rpm was used to ensure proper mixing. The experiment was then run for 48-hours. For a more detailed description of the whole procedure, an SOP for batch-recovery experiments is presented in Appendix E.

\subsection{2 $\quad$ RT-2 Experimental Results and Analysis}

A washed, filtered, and then air-dried 15-gram sample was sent for each test of the campaign to an independent laboratory (CCI of Kellogg, Idaho) for assay. The weight of each substrate after the test was recorded to better serve the mass balance calculation and to investigate any substrate disintegration. RT-2's experimental results are presented in Table 21.

Table 21. Experimental Results of RT-2

\begin{tabular}{|c|c|c|c|c|c|c|}
\hline \multirow{2}{*}{ Test \# } & \multirow{2}{*}{ Substrate } & Post-Recovery & \multicolumn{2}{|c|}{ Carbon Assay (opt) } & \multicolumn{2}{c|}{ \% Recovery } \\
\cline { 3 - 7 } & & Weight (grams) & $\mathbf{A u}$ & $\mathbf{A g}$ & $\mathbf{A u}$ & $\mathbf{A g}$ \\
\hline RT2-T1 & IRA400 & 19.30 & 0.093 & 0.8 & 47.31 & 45.41 \\
\hline RT2-T2 & Act. Carbon & 20.14 & 0.128 & 1.4 & 67.95 & 82.93 \\
\hline RT2-T3 & Act. Carbon & 20.32 & 0.119 & 1.5 & 63.74 & 89.65 \\
\hline
\end{tabular}

In general, RT-2 confirmed the result of campaign RT-1: activated carbon performed better for recovering gold from alkaline sulfide solution. As seen in Table 21, activated carbon recovers, in average, $25.0 \%$ more gold from solution. Additionally, a preliminary conclusion of the activated carbon performance consistency was proven with Test 2 and Test 3 having similar results (5.7\% difference in gold recovery).

Again, although important, solution potential and $\mathrm{pH}$ was not measured or altered during experimentation. Further recovery work will optimize these factors. 


\subsection{Summary of Precious Metal Recovery from Pregnant ASGLS Solution}

The objective of these preliminary recovery experiments was to better define the performance of adsorption of precious metal specie in ASGLS solution onto substrates. Previous research (Darby, 2005) has indicated the possibility of activated carbon as an effective adsorption substrate. Silica and styrene based ion exchange resins containing amine and iminodiacetic functional ingredient were also tested. Also, effectively proven substrates were tested in a much lower precious metal containing solution to better simulate a real system. In summary, activated carbon performed superiorly against ion exchange resins containing iminodiacetic acid and polyamine functional groups.

\subsection{Treatment of Waste ASGLS Solution}

\subsection{Introduction to Waste Treatment of ASGLS}

In the development of a new process, particularly with today's environmental awareness, it is crucial to investigate the waste treatment of waste materials generated. Consequently, the mining industry is fully aware of the responsibility of having a sustainable and responsible mining operation. Corporate campaigns promoting sustainability and mine-waste technology research are more active than ever. In accordance, ASGLS waste treatment was investigated in this thesis. In case of reduced leaching performance caused by deterioration of sulfide-polysulfide and/or high level of impurities, a treatment process is needed to stabilize the alkaline sulfide solution. Shown in Figure 9 is the proposed flowsheet for the treatment of waste solution potentially produced by an ASGLS circuit. 


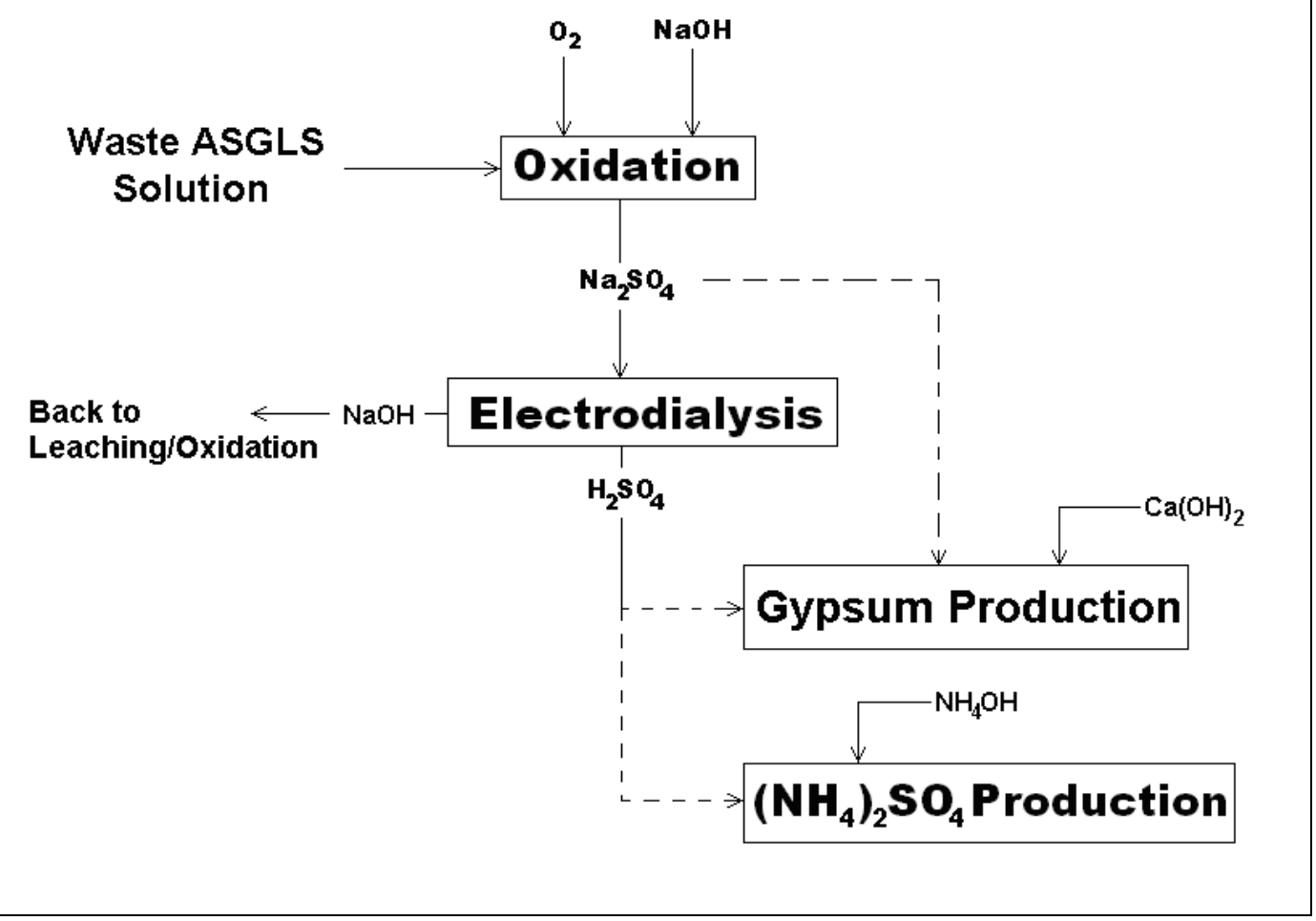

Figure 9. Proposed Flowsheet of ASGLS Waste Solution Treatment

The possible flowsheet includes: pressure oxidation, gypsum production from produced $\mathrm{Na}_{2} \mathrm{SO}_{4}$, salt splitting using electrodialysis, and $\mathrm{H}_{2} \mathrm{SO}_{4}$ treatment using gypsum and/or ammonium sulfate production. In overview, the pressure oxidation stage will bring the metastable alkaline sulfide solution to its highest oxidation state, producing $\mathrm{Na}_{2} \mathrm{SO}_{4}$ powder. $\mathrm{Na}_{2} \mathrm{SO}_{4}$ powders may be treated using gypsum production or using the electrodialysis process, where the $\mathrm{Na}^{+}$and $\mathrm{SO}_{4}{ }^{2-}$ ions will be split to produce $\mathrm{NaOH}$ and $\mathrm{H}_{2} \mathrm{SO}_{4}$. Afterwards, regenerated $\mathrm{NaOH}$ will be recirculated back to leaching or oxidation, while the produced $\mathrm{H}_{2} \mathrm{SO}_{4}$ will be further treated to produce gypsum $\left(\mathrm{Ca}_{2} \mathrm{SO}_{4} \cdot 2 \mathrm{H}_{2} \mathrm{O}\right)$ or ammonium sulfate $\left(\left(\mathrm{NH}_{4}\right)_{2} \mathrm{SO}_{4}\right)$. The use of the electrodialysis process itself will highly depend on the needed current density, which affects the energy consumption of the unit. As current density increases, the cost will be significantly higher. Further discussions of these proposed unit processes will be discussed in the individual sections of this chapter.

\subsection{Pressure Oxidation of Waste Alkaline Sulfide Solution}

\subsubsection{Sulfide/Polysulfide Oxidation Principles}

The initial stage of the proposed waste treatment process is the accelerated oxidation of sulfidepolysulfide, producing sulfate $\left(\mathrm{SO}_{4}{ }^{2-}\right)$. Since ASGLS is a metastable system, it is best to bring the system to a fully stable (oxidized) stage for easier handling, disposal, and/or marketing. With the 
existence of sodium ions $\left(\mathrm{Na}^{+}\right)$added through the initial reagents $\left(\mathrm{Na}_{2} \mathrm{~S}\right.$ and $\left.\mathrm{NaOH}\right)$, it was possible to produce $\mathrm{Na}_{2} \mathrm{SO}_{4}$; and with its relatively low solubility at room temperature, $\mathrm{Na}_{2} \mathrm{SO}_{4}$ crystals was easily formed by a simple crystallization procedure as practiced in industry.

Sulfide oxidation is a fairly simple reaction, as shown in Equation (4.1). Polysulfide oxidation, in the other hand, is a more involved reaction that consumes electrons/hydroxide ions (See Equation 4.2). The rate of oxygen consumption for polysulfide solutions is approximately four times higher than for sulfide solutions (Kleinjan et.al., 2005).

$$
\begin{array}{r}
2 \mathrm{Na}^{+}+\mathrm{S}^{2-}+2 \mathrm{O}_{2}=\mathrm{Na}_{2} \mathrm{SO}_{4} \\
2 \mathrm{x} \mathrm{Na}{ }^{+}+\mathrm{S}_{\mathrm{x}}{ }^{2-}+(\mathrm{x}+1) \mathrm{O}_{2}+(2 \mathrm{x}-2) \mathrm{OH}^{-}=\mathrm{x} \mathrm{Na}_{2} \mathrm{SO}_{4}+(2 \mathrm{x}-2) \mathrm{H}^{+}(\mathrm{x}=2,3,4, \text { or } 5)
\end{array}
$$

Shown in Figure 10 is a Pourbaix diagram of the fully stable sulfur system generated using STABCAL ${ }^{\circledR}$. In theory, the $\mathrm{SO}_{4}{ }^{2-}$ region is substantially large and could be easily achieved.

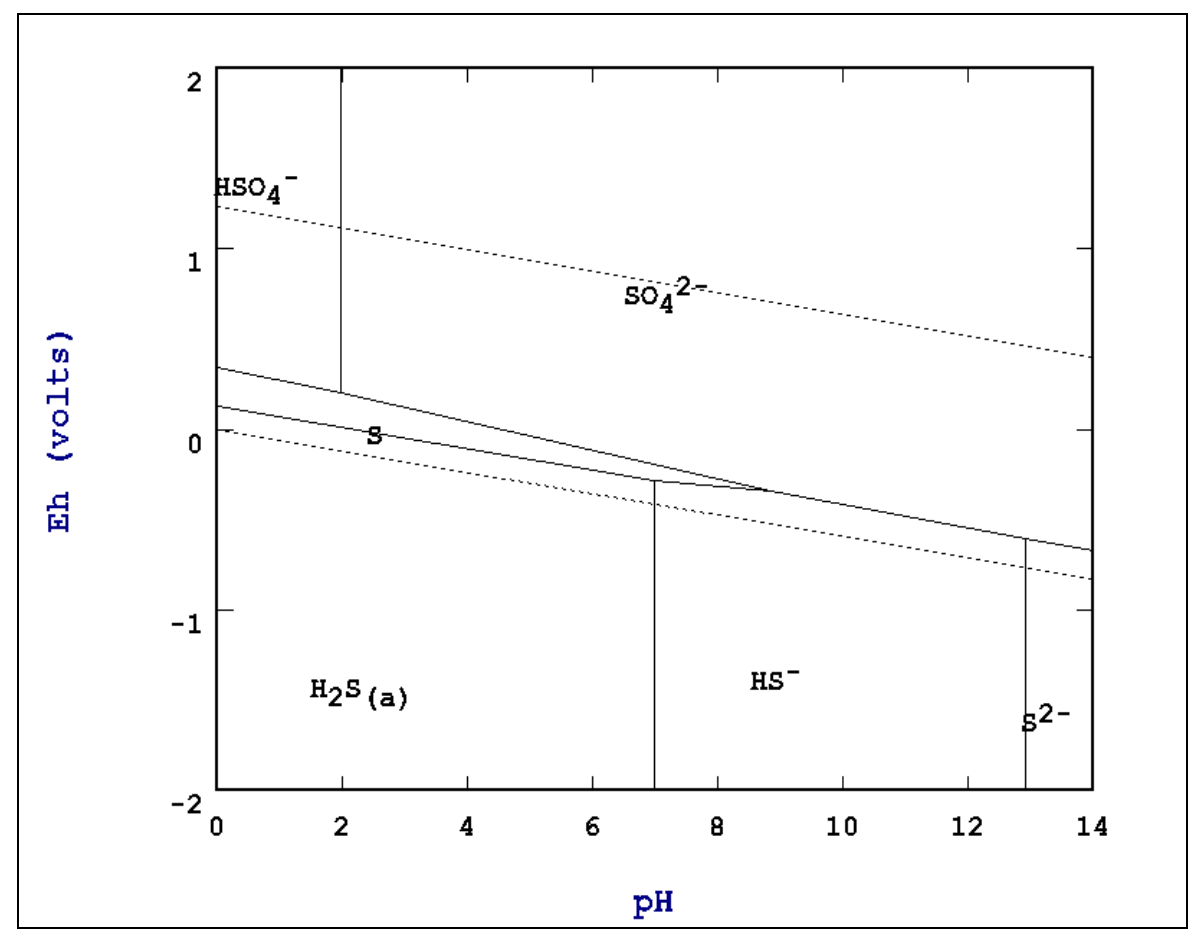

Figure 10. Eh-pH Diagram of Sulfur System at Higher Oxidation State

However, the oxidation process was harder to model than just with one fully stable system as in Figure 11. In ASGLS, oxidation of waste solution will involve the conversion of sulfide to sulfate through all oxidation states of sulfur shown in Table $1\left(\mathrm{~S}^{2-}>\mathrm{S}_{\mathrm{x}}{ }^{2-}>\mathrm{S}>\mathrm{S}_{2} \mathrm{O}_{3}{ }^{2-}>\mathrm{SO}_{3}{ }^{2-}>\mathrm{SO}_{4}{ }^{2-}\right)$. One of the intermediate systems is presented in Figure 11 where partial oxidation has produced thiosulfate. Notice that a sudden decrease in $\mathrm{pH}$, caused by the $\mathrm{OH}^{-}$consumption of polysulfide oxidation, may cause the formation of elemental sulfur in the system. 


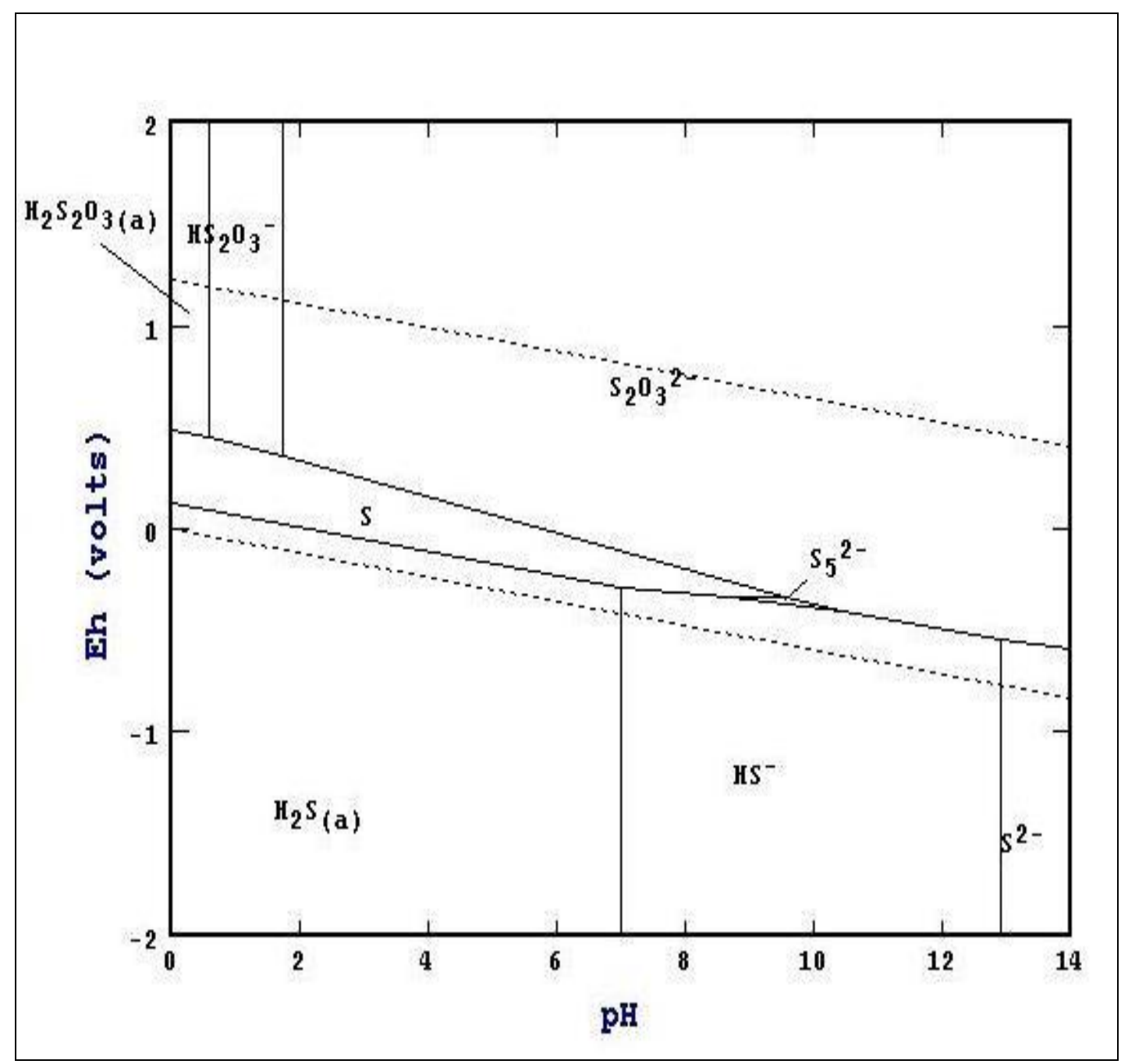

Figure 11. Pourbaix Diagram of Intermediate Oxidation States of Sulfur

There are certainly opportunities to market the produced $\mathrm{Na}_{2} \mathrm{SO}_{4}$ solid powder as a by-product. It is estimated that most sodium sulfate is consumed in the following industries: soap and detergents, textiles, pulp and paper, and glass (Kostick, 1997). Additionally, $\mathrm{Na}_{2} \mathrm{SO}_{4}$ price has been steady at $\$ 114-\$ 134$ per short ton in the last five years. The consideration whether to market $\mathrm{Na}_{2} \mathrm{SO}_{4}$ as a byproduct or not will depend on the economics of the electrodialysis process and relative demand of $\mathrm{Na}_{2} \mathrm{SO}_{4}$. If the electrodialysis process is proven to be more expensive than purchasing fresh $\mathrm{NaOH}$, then it certainly will be most beneficial to eliminate the electrodialysis stage from the flowsheet and finalize $\mathrm{Na}_{2} \mathrm{SO}_{4}$ as a by-product.

The objective of the following experiments was to achieve the optimum autoclave operational settings to achieve a $100 \%$ oxidation of sulfide/polysulfide to sulfate. Shown in Figure 12 is the laboratory scale pressure vessel (autoclave) used in this thesis research. 


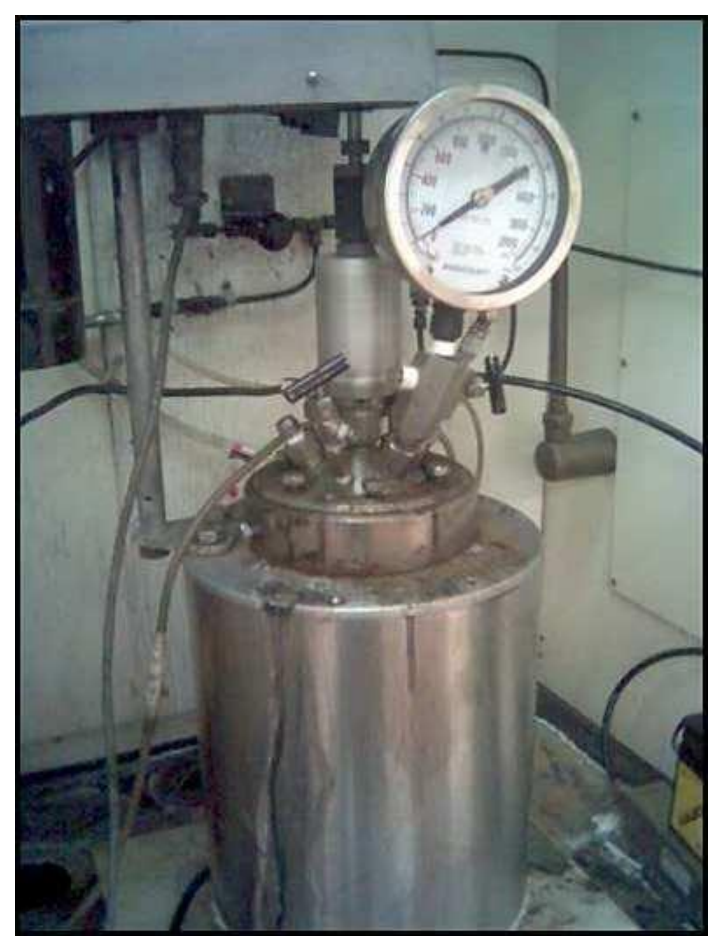

Figure 12. Laboratory Scale Autoclave

The laboratory Standard Operating Procedure (SOP) of this oxidation process is described in Appendix H. Additionally, the analytical procedure used to measure initial and product solutions, using an Ion Chromatograph and ABC-James River titrations, are presented in Appendix J and Appendix K.

\subsubsection{Pressure Oxidation Campaign 1 (WO-1): Oxidation Kinetics}

\subsubsection{WO1 Background and Experimental Design}

The objective of this first autoclave oxidation campaign WO-1 was to investigate the effects of controllable parameters and initial solution concentration to the kinetics of the oxidation reaction. Shown in Table 22 are the range chosen for each varied factor in WO-1.

Table 22. WO-1's Experimental Design Parameters

\begin{tabular}{|c|c|}
\hline Factor & Range of Value \\
\hline Temperature & $75-150^{\circ} \mathrm{C}$ \\
\hline Oxygen Overpressure & 20 to 80 -psi \\
\hline Time & 2 to 5 -hours \\
\hline Initial $\mathrm{S}^{2-}-\mathrm{S}_{\mathrm{x}}^{2-}$ Concentration & 25 to $50-\mathrm{g} / \mathrm{L}$ \\
\hline Initial $\mathrm{NaOH}$ Concentration & 18 to $24-\mathrm{g} / \mathrm{L}$ \\
\hline
\end{tabular}


There were three operational parameters that can be closely controlled using the autoclave:
1. Temperature;
2. Oxygen overpressure; and
3. Time of reaction.

As with any first-order reactive system, increased temperature and overpressure was expected to improve the reaction's kinetics, i.e. shorter reaction time. Simultaneously, it was beneficial at this stage to test the effect of those parameters at a given initial solution concentration. Consequently, these three reagent concentration were also varied:
1. Initial $\mathrm{Na}_{2} \mathrm{~S}$ addition;
2. Initial S addition; and
3. Initial $\mathrm{NaOH}$ addition.

Table 23. WO1 Experimental Design Matrix

\begin{tabular}{|c|c|c|c|c|c|}
\hline Test & Temperature & {$\left[\mathbf{S}^{\mathbf{2}}+\mathbf{S}\right]$} & {$[\mathbf{N a O H}]$} & $\mathbf{O}_{\mathbf{2}}$ Overpressure & Time \\
\hline$\#$ & $\left({ }^{\mathbf{C}}\right)$ & $(\mathbf{g} / \mathbf{L})$ & $(\mathbf{g} / \mathbf{L})$ & $(\mathbf{p s i})$ & $(\mathbf{h r s})$ \\
\hline WO1-T1 & 112.5 & 37.5 & 18 & 50 & 3.5 \\
\hline WO1-T2 & 75.0 & 50.0 & 24 & 20 & 5.0 \\
\hline WO1-T3 & 150.0 & 25.0 & 12 & 80 & 5.0 \\
\hline WO1-T4 & 150.0 & 25.0 & 24 & 80 & 2.0 \\
\hline WO1-T5 & 75.0 & 25.0 & 12 & 80 & 2.0 \\
\hline WO1-T6 & 150.0 & 50.0 & 12 & 80 & 2.0 \\
\hline WO1-T7 & 112.5 & 37.5 & 18 & 50 & 3.5 \\
\hline WO1-T8 & 75.0 & 50.0 & 12 & 20 & 2.0 \\
\hline WO1-T9 & 150.0 & 50.0 & 24 & 80 & 5.0 \\
\hline WO1-T10 & 75.0 & 25.0 & 12 & 20 & 5.0 \\
\hline WO1-T11 & 150.0 & 50.0 & 12 & 20 & 5.0 \\
\hline WO1-T12 & 75.0 & 50.0 & 12 & 80 & 5.0 \\
\hline WO1-T13 & 150.0 & 25.0 & 12 & 20 & 2.0 \\
\hline WO1-T14 & 75.0 & 50.0 & 24 & 80 & 2.0 \\
\hline WO1-T15 & 150.0 & 25.0 & 24 & 20 & 5.0 \\
\hline WO1-T16 & 75.0 & 25.0 & 24 & 20 & 2.0 \\
\hline WO1-T17 & 75.0 & 25.0 & 24 & 80 & 5.0 \\
\hline WO1-T18 & 150.0 & 50.0 & 24 & 20 & 2.0 \\
\hline
\end{tabular}

As seen in Table 23, a half-factorial design was developed, resulting in 16 actual experiments with two additional center points for statistical assurance. The final design only investigated five factors because initial $\mathrm{Na}_{2} \mathrm{~S}$ and $\mathrm{S}$ concentration is fixed to each other at a ratio of 1:1. All tests were performed according to the pressure oxidation SOP shown in Appendix H. Please note that in WO-1, pure (no prior leaching) alkaline sulfide solution was prepared from reagent grade chemicals listed in Appendix M using the solution preparation SOP presented in Appendix D. 
Analysis of the final solution produced by each tests were performed using an Ion Chromatograph (IC) for $\mathrm{SO}_{4}{ }^{=}$and $\mathrm{S}_{2} \mathrm{O}_{3}{ }$; and James River titration for sulfide/polysulfide. SOP for the James River titration can be found in Appendix $\mathrm{J}$.

\subsubsection{WO1 Results and Data Analysis}

Generally, no WO-1 tests were able to achieve $100 \%$ conversion of sulfide/polysulfide to sulfate, as shown in Table 24. Maximum conversion was achieved in WO1-T3 where 71.0\% of sulfide/polysulfide was oxidized thoroughly to sulfate. Additionally, it is also interesting to note that thiosulfate $\left(\mathrm{S}_{2} \mathrm{O}_{3}{ }^{=}\right)$was detected by the IC, indicating that the oxidation process goes through the oxidation steps of the sulfur system $\left(\mathrm{S}^{ }<\mathrm{S}<\mathrm{S}_{2} \mathrm{O}_{3}{ }^{\circ}<\mathrm{SO}_{3}{ }^{=}<\mathrm{SO}_{4}{ }{ }\right)$.

Even though 100\% sulfate conversion was not achieved; these results were effective in optimizing the pathway needed to fulfill this goal. All data shown in Table 24 were entered to STATEASE's Design-Expert software for ANOVA analysis and process modeling.

Table 24. WO1 Product Solution Results

\begin{tabular}{|c|c|c|c|c|c|}
\hline \multirow{2}{*}{ Test \# } & Retained & {$\left[\mathrm{S}_{2} \mathrm{O}_{3}{ }^{=}\right]$} & {$\left[\mathrm{SO}_{3}{ }^{=}\right]$} & {$\left[\mathrm{SO}_{4}{ }^{\circ}\right]$} & Yield Sulfate \\
\hline & $S^{2-} / S_{x}^{2-}(g / L)$ & $(g / L)$ & $(g / L)$ & $(g / L)$ & Conversion (\%) \\
\hline W01-T1 & 1.97 & 34.12 & 0.92 & 0.49 & 1.32 \\
\hline W01-T2 & 2.33 & 43.11 & 3.04 & 1.53 & 3.06 \\
\hline WO1-T3 & 0.00 & 0.06 & 7.19 & 17.75 & 71.00 \\
\hline WO1-T4 & 0.30 & 18.18 & 1.73 & 4.79 & 19.15 \\
\hline WO1-T5 & 0.00 & 22.44 & 1.97 & 0.59 & 2.36 \\
\hline WO1-T6 & 0.00 & 45.96 & 1.78 & 4.26 & 8.51 \\
\hline WO1-T7 & 1.95 & 30.97 & 3.98 & 0.60 & 1.59 \\
\hline W01-T8 & 5.39 & 35.65 & 8.94 & 0.01 & 0.03 \\
\hline WO1-T9 & 0.00 & 0.29 & 15.04 & 34.68 & 69.36 \\
\hline W01-T10 & 0.00 & 22.58 & 2.38 & 0.03 & 0.13 \\
\hline W01-T11 & 3.52 & 47.98 & 0.47 & 0.03 & 0.06 \\
\hline W01-T12 & 0.00 & 45.73 & 4.26 & 0.01 & 0.03 \\
\hline W01-T13 & 3.91 & 16.89 & 3.93 & 0.27 & 1.09 \\
\hline W01-T14 & 1.48 & 45.29 & 2.50 & 0.73 & 1.47 \\
\hline W01-T15 & 0.73 & 18.57 & 2.17 & 3.53 & 14.11 \\
\hline W01-T16 & 2.13 & 19.75 & 2.53 & 0.58 & 2.33 \\
\hline W01-T17 & 0.00 & 16.50 & 1.90 & 6.60 & 26.40 \\
\hline W01-T18 & 0.52 & 23.45 & 24.31 & 1.72 & 3.44 \\
\hline
\end{tabular}

Shown in Figure 13 and 14 are 3D-surface model graphs produced using STATEASE Design Expert from WO-1 data. All ANOVA analysis, significant graph models, and diagnostics of this model are presented in complete in Appendix C. 


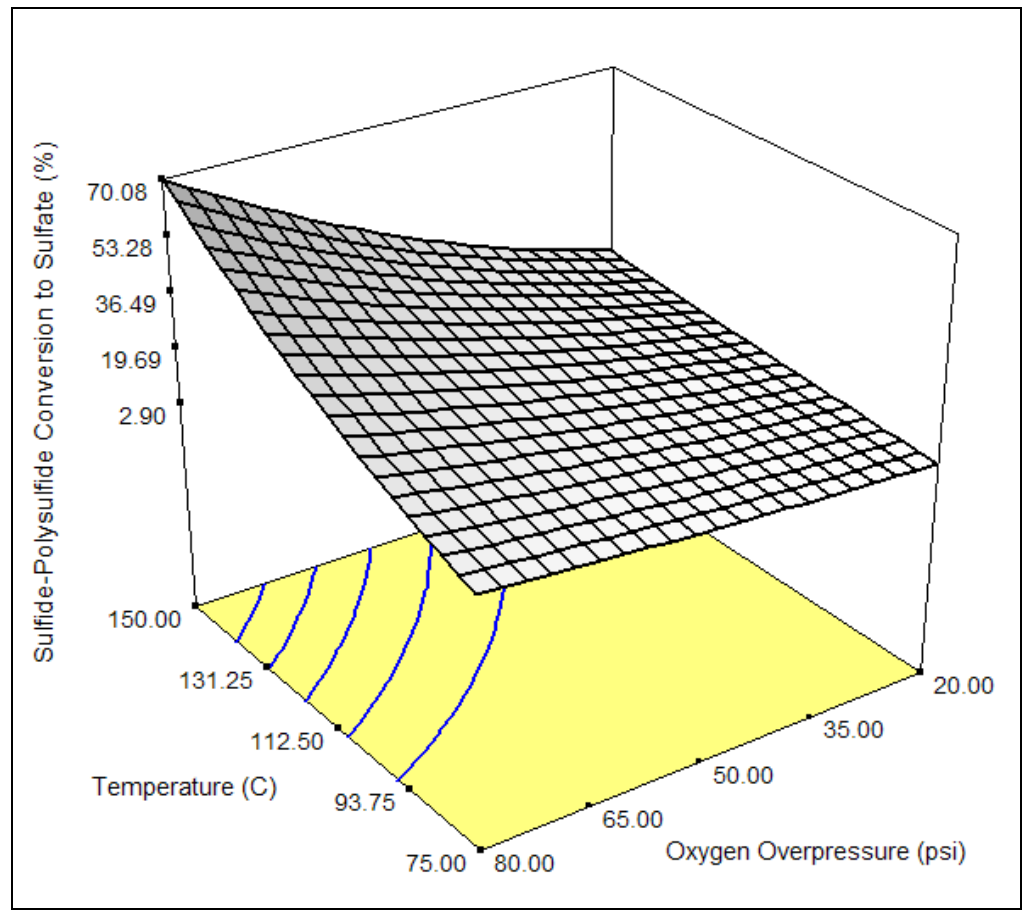

Figure 13. 3D Surface Model Graphs of Sulfide-Polysulfide Conversion to Sulfate (Temp vs. Oxygen Overpressure)

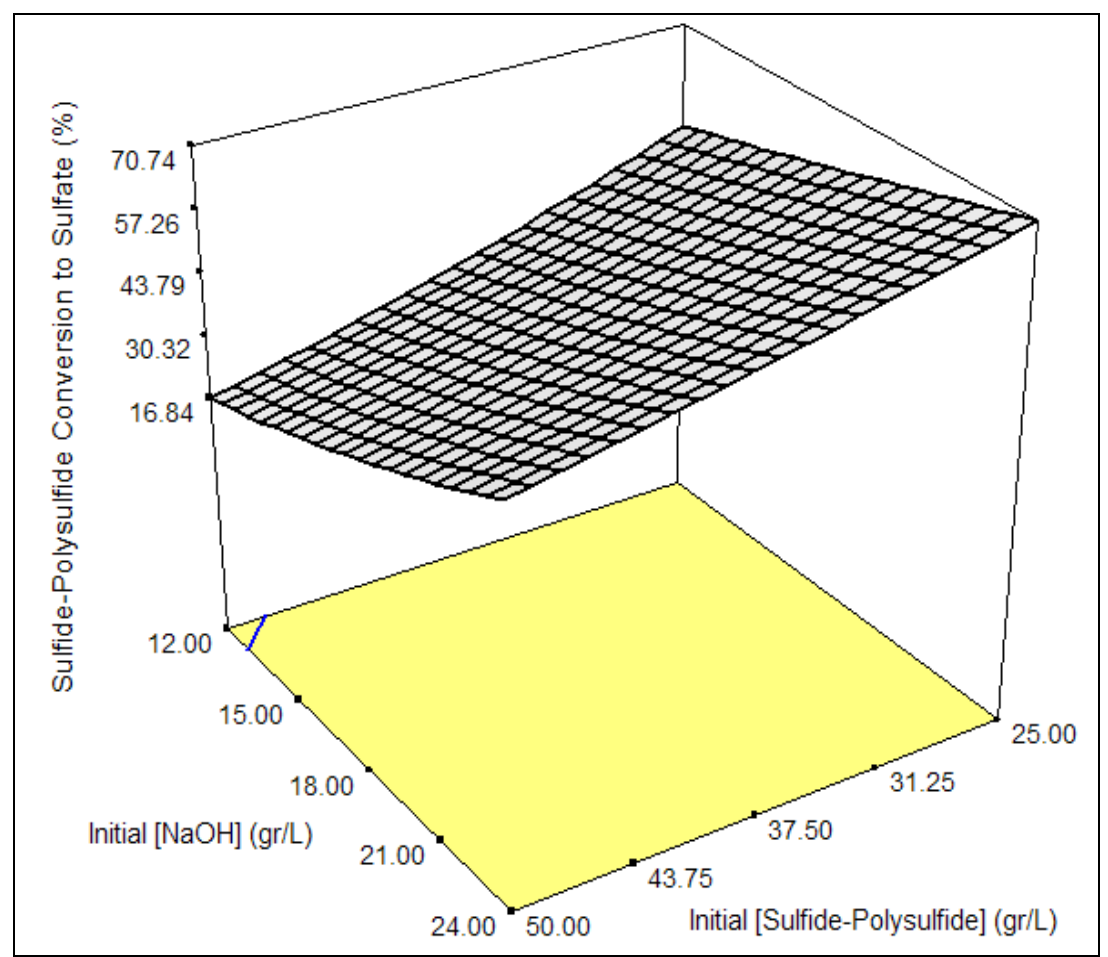

Figure 14. 3D Surface Model Graphs of Sulfide-Polysulfide Conversion to Sulfate (Initial $[\mathrm{NaOH}]$ vs. [Sulfide-Polysulfide]) 
In general, as seen in Figure 13, increasing temperature and oxygen overpressure provides a larger sulfate conversion for a given process time. More important, however, is the interaction effects of temperature and oxygen. As both are increased simultaneously, a larger effect to sulfate conversion was experienced. Another analysis was performed to the effect of initial reagent concentrations on the sulfate conversion at a given time, temperature, and oxygen overpressure (Figure 14). Although it is intuitive that a larger initial concentration of sulfide-polysulfide in the feed solution will yield the need of longer time, higher temperature, and higher overpressure; it was surprising to see the large effect of initial $\mathrm{NaOH}$ concentration. This finding can be attributed to the stoichiometric need of $\mathrm{OH}^{-}$ions in the oxidation of polysulfide.

Shown in Figure 15 is one of the solution produced by WO-1 where partial oxidation of sulfidepolysulfide is achieved. A pale green solution is produced, still containing sulfide/polysulfide and thiosulfate.

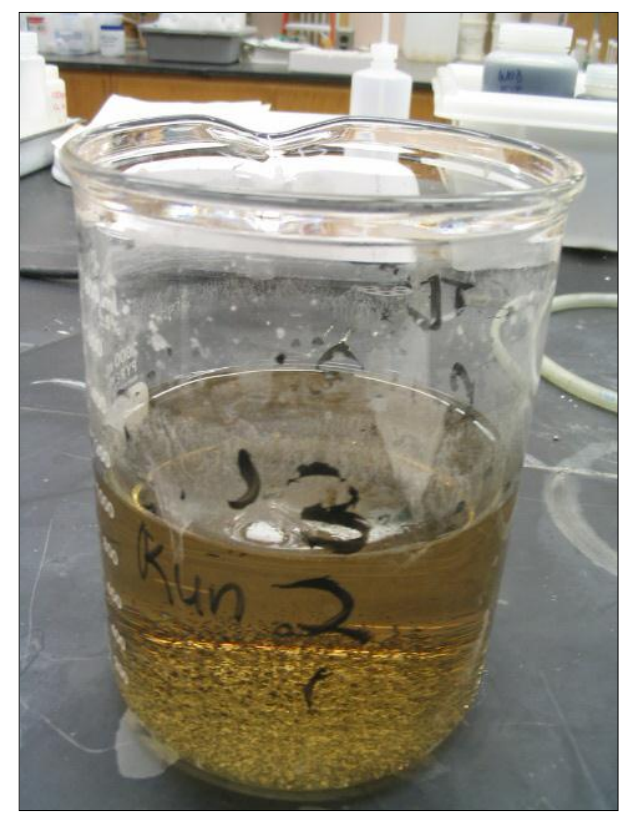

Figure 15. Partially Oxidized ASGLS Solution

\subsubsection{Pressure Oxidation Campaign 2 (WO-2): Elemental Sulfur Formation}

\subsubsection{WO2 Background and Experimental Design}

Campaign WO-2 was originally intended to improve process parameters of WO-1 to achieve the $100 \%$ sulfide/polysulfide conversion to sulfate. Accordingly, an experimental design matrix was developed. However, during the first test, sulfur solids were found after the test was cooled and taken off. The particular test was for 7-hours, with 50-g/L total sulfide-polysulfide, 24-g/L $\mathrm{NaOH}, 80-p s i$, and $150^{\circ} \mathrm{C}$. (See Figure 16) 
This phenomenon was observed because of $\mathrm{pH}$ decrease and $\mathrm{OH}^{-}$deficiency in the initial feed solution. In the case where a partial oxidation of sulfide-polysulfide to thiosulfate $\left(\mathrm{S}_{2} \mathrm{O}_{3}{ }^{2-}\right)$ and a large decrease in $\mathrm{pH}$ is experienced from $\mathrm{OH}^{-}$consumption during polysulfide oxidation, elemental sulfur will be formed. (As previously modeled in Figure 11).

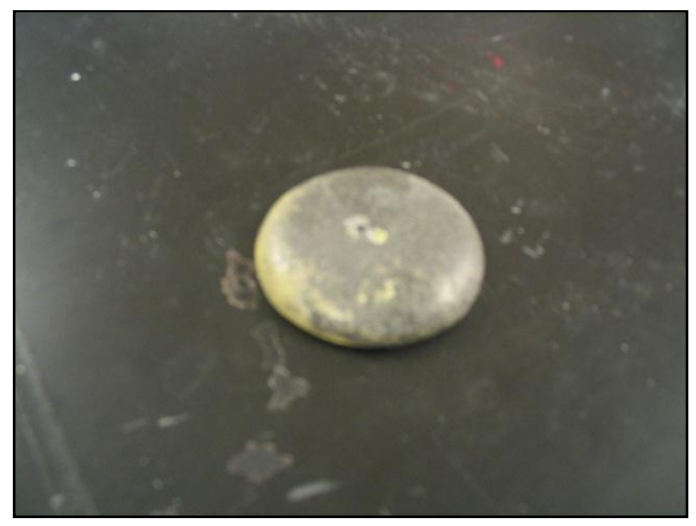

Figure 16. Sulfur Solids Produced in ASGLS Oxidation

To overcome this elemental sulfur formation, a new set of experimental design matrix was made to resolve the decrease of $\mathrm{pH}$ and $\mathrm{OH}^{-}$deficiency by excess addition of $\mathrm{NaOH}$. An experimental design matrix was made to investigate how much $\mathrm{NaOH}$ is needed to avoid sulfur formation (See Table 25). All experimental procedure was identical to that of WO-1: solution preparation SOP is presented in Appendix D and autoclave pressure oxidation SOP is presented in Appendix H.

Table 25. Experimental Design of WO-2

\begin{tabular}{|c|c|c|}
\hline Test & $\begin{array}{c}\text { Time } \\
\text { (hrs) }\end{array}$ & $\begin{array}{c}\text { NaOH } \\
\text { (g/L) }\end{array}$ \\
\hline WO2-T1 & 10 & 45 \\
\hline WO2-T2 & 10 & 50 \\
\hline WO2-T3 & 10 & 55 \\
\hline WO2-T4 & 10 & 60 \\
\hline WO2-T5 & 10 & 65 \\
\hline
\end{tabular}

All tests above were performed at 50-g/L total sulfide-polysulfide, 80 -psi overpressure, $170^{\circ} \mathrm{C}$, and for 10 -hours. Essentially, only $\mathrm{NaOH}$ initial concentration was varied incrementally from 45 to $65-\mathrm{g} / \mathrm{L}$.

\subsubsection{WO2 Results and Data Analysis}

Shown in Table 26 are the results of WO2. No sulfur was formed at 65grams/L of total initial $\mathrm{NaOH}$. This finding confirms that as long as enough $\mathrm{OH}$ - is supplied into the system for polysulfide oxidation, $\mathrm{pH}$ can be maintained above acidic region and no sulfur is formed. 
Table 26. Experimental Results of Campaign WO-2

\begin{tabular}{|c|c|c|c|}
\hline Analysis & ${\text { S converted to } \text { SO }_{4}{ }^{~}{ }^{(\mathbf{g} / \mathbf{L})}}$ & $\mathbf{P H}$ & $\begin{array}{c}\mathbf{S} \\
\text { (grams) }\end{array}$ \\
\hline WO2-T1 & 25.61 & 1.20 & 33.26 \\
\hline WO2-T2 & 31.26 & 2.20 & 23.16 \\
\hline WO2-T3 & 41.98 & 1.60 & 11.98 \\
\hline WO2-T4 & 44.33 & 3.50 & 5.47 \\
\hline WO2-T5 & 49.87 & 7.06 & 0 \\
\hline
\end{tabular}

Thus, a complete oxidation of 50-g/L total sulfide-polysulfide to sulfate required a temperature of $170^{\circ} \mathrm{C}$, oxygen overpressure of $80-\mathrm{psi}$, time of 7-hours, and $\mathrm{NaOH}$ concentration of $65-\mathrm{g} / \mathrm{L}$. Shown in Figure 17 is the final solution where 100\% (WO2-T5) oxidation to sulfate was achieved. Complete oxidation will yield a clear solution containing high $\mathrm{Na}_{2} \mathrm{SO}_{4}$. Also, $\mathrm{Na}_{2} \mathrm{SO}_{4}$ powder was produced by evaporating the product solution, providing crystallization.

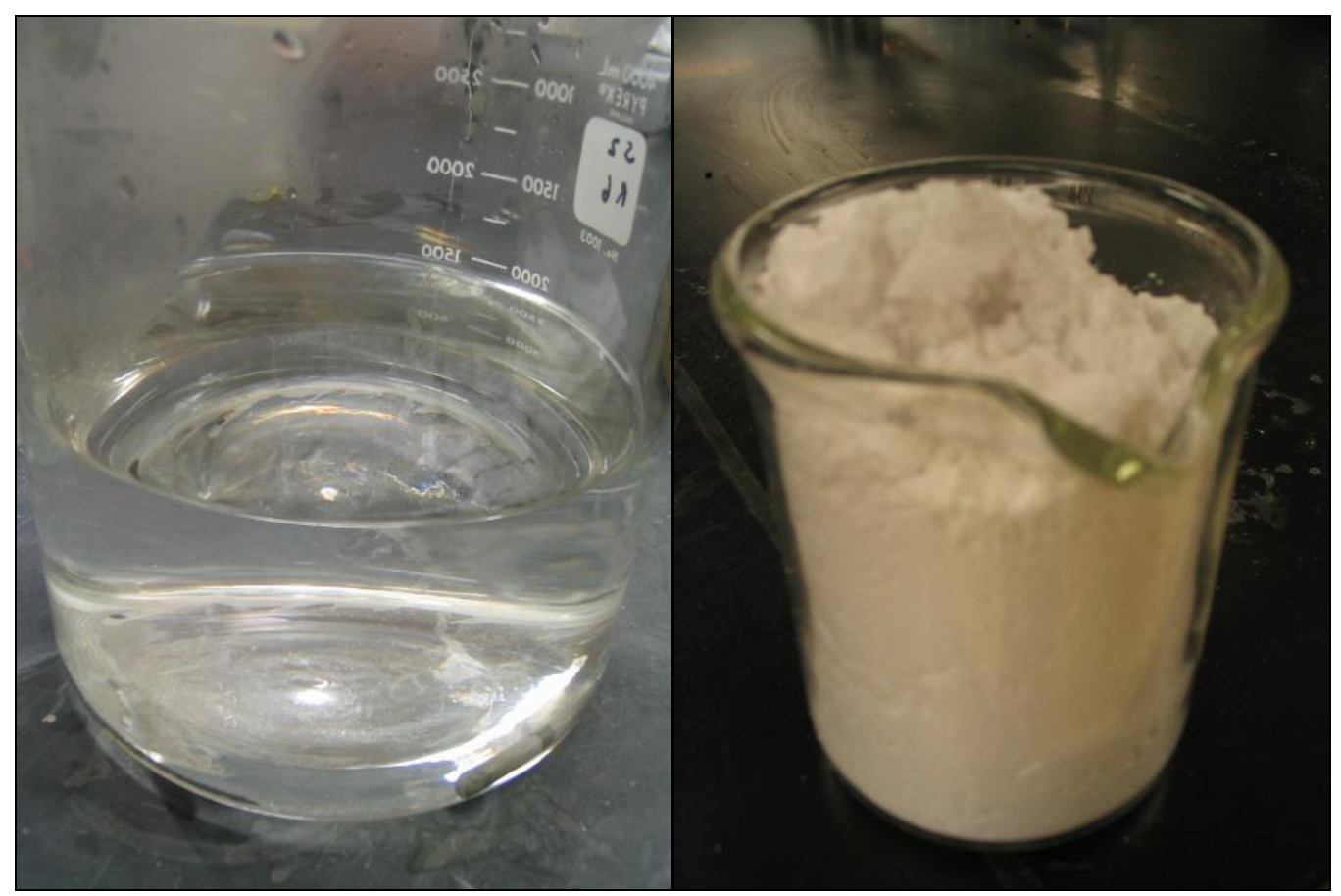

Figure 17. Product Solution of Pressure Oxidation and $\mathrm{Na}_{2} \mathrm{SO}_{4}$ Powder Produced

\subsection{NaOH Regeneration through Electrodialysis with Bipolar Membranes}

\subsubsection{Introduction}

Electrodialysis is a separation process utilizing both applied electrical current and ionexchange/bipolar membranes to produce a mass transport and separation, i.e. cations and anions transport. More simply put, electrodialysis is a general-purpose unit operation for converting water-soluble salts into their corresponding acids and bases (Mani, 1989). Electrodialysis has 
many waste treatment applications since its introduction in the early 1960's as an efficient and economic technique for the desalination of brackish water (Wilson, 1960). In the other hand, bipolar membrane (BPM) has recently gained popularity for its water splitting/dissociation capability, which is considered superior to conventional water electrolysis: no $\mathrm{H}_{2}$ gas is generated, no oxidation and/or reduction of any species, and low initial cost (Xu, 2002). The basic principle of electrodialysis can be seen in Figure 18 below.

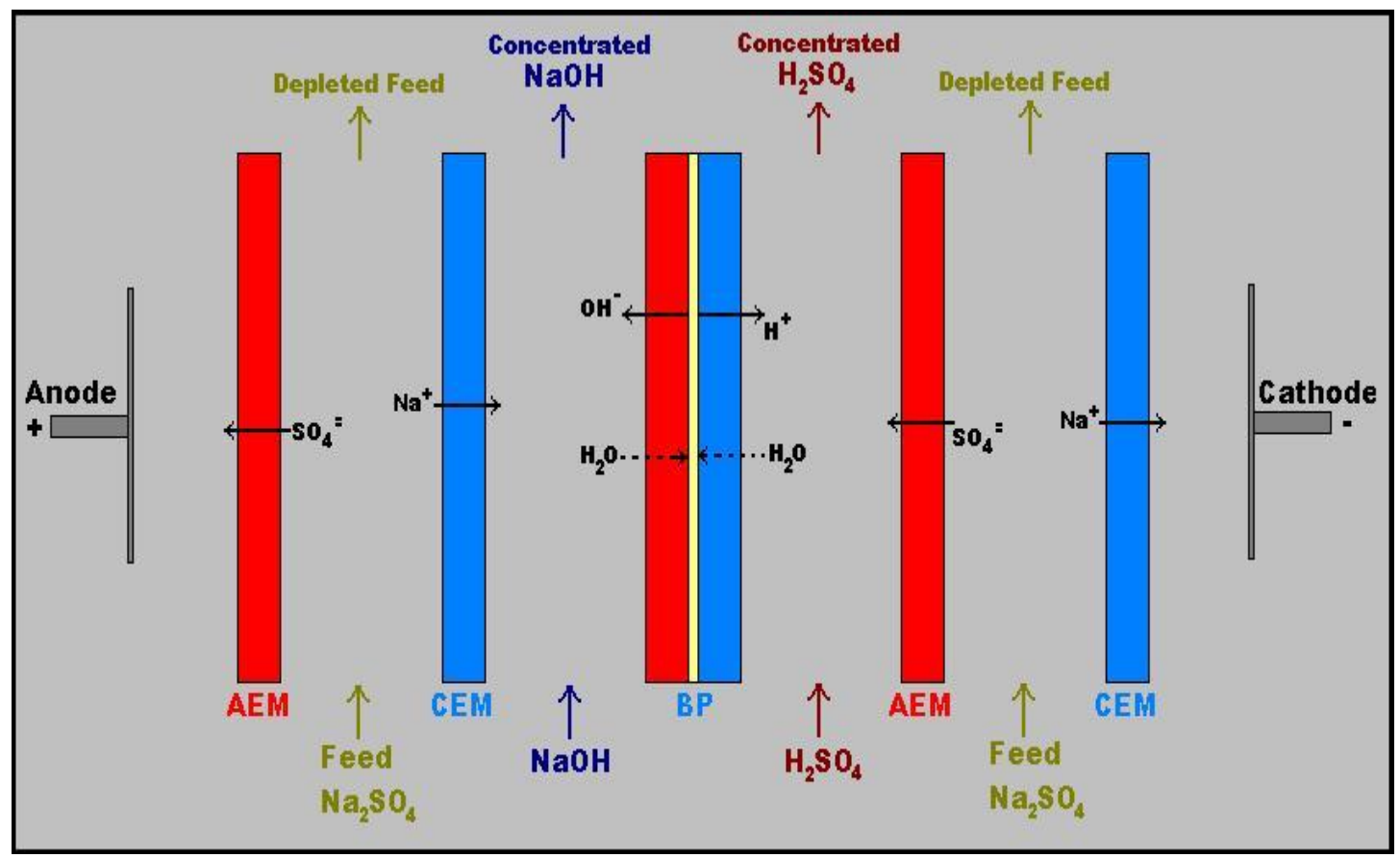

Figure 18. Three Compartment Design of Electrodialysis with BPM, CEM, and AEM

When electrical potential is applied across a membrane stack where ionic solution is driven through, the negatively charged ions naturally move towards the anode $(+)$ and the positively charged ions move towards the cathode (-). The alternatively arranged ion-exchange membranes will retain these ions into the specific compartments and concentration build up is produced.

In the ASGLS, electrodialysis using bipolar membranes was hypothesized to be able to regenerate $\mathrm{NaOH}$ by splitting $\mathrm{Na}^{+}$and $\mathrm{SO}_{4}{ }^{2-}$ ions from produced $\mathrm{Na}_{2} \mathrm{SO}_{4}$ through ion-exchange membranes; simultaneously, Bipolar Membranes (BPM) are used to produce $\mathrm{H}^{+}$and $\mathrm{OH}^{-}$from water splitting. $\mathrm{Na}_{2} \mathrm{SO}_{4}$ is the solid product of an earlier stage waste treatment pressure oxidation process. Additional arrangement, other than the three-compartment design, can be used and are shown in Figure 19 and 20. 


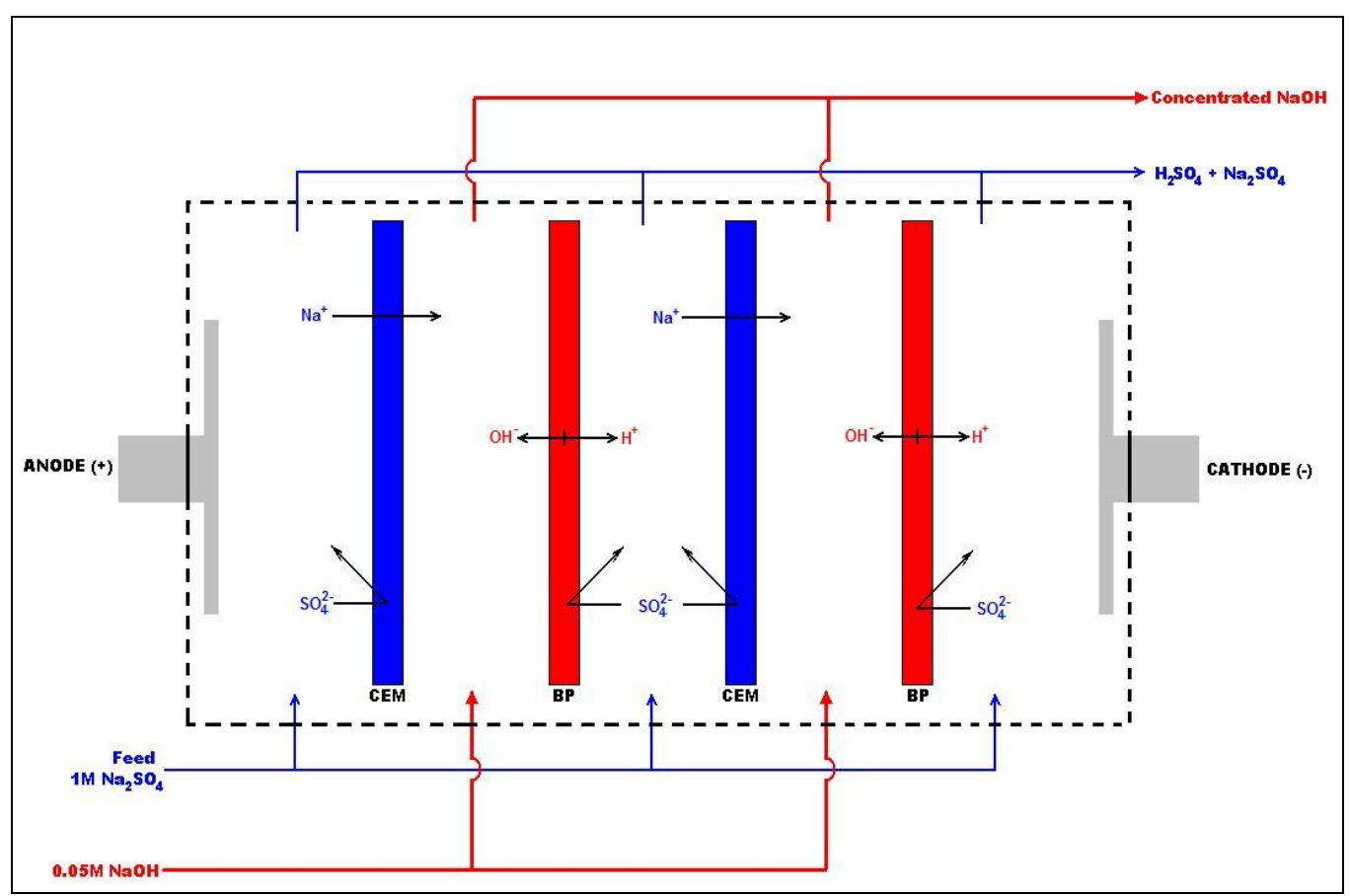

Figure 19. Two Compartment Design of Electrodialysis with BPM and CEM

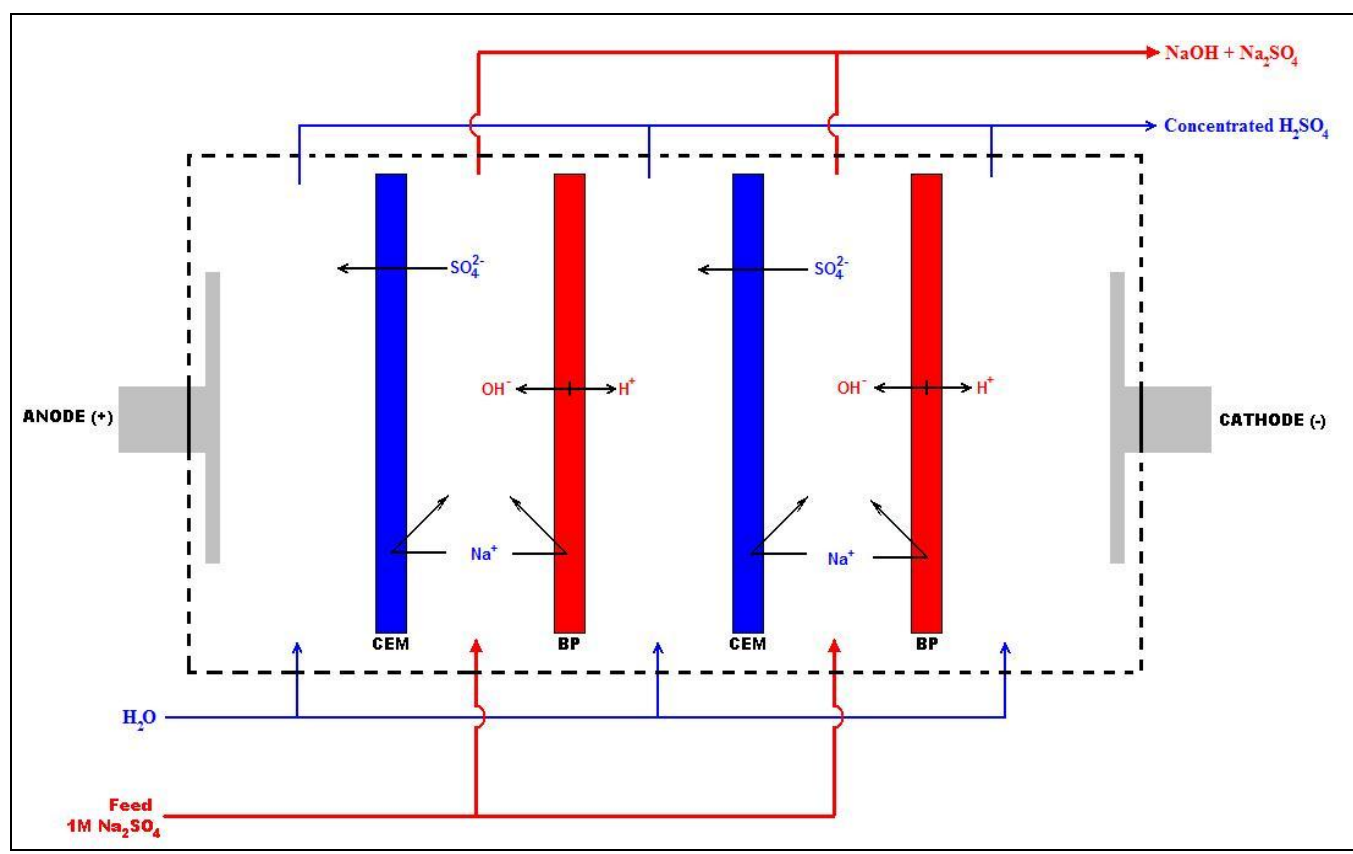

Figure 20. Two Compartment Design of Electrodialysis with BPM and AEM

Ion-exchange membranes used in this research were Tokuyama Neosepta® ACM anion exchange membrane and Tokuyama Neosepta ${ }^{\circledR}$ CMP cation exchange membrane. The bipolar membrane used was a Tokuyama Neosepta ${ }^{\circledR}$ BP-1 bipolar membrane. The ACM was chosen because it is specifically designed in an application where acid concentration takes place as it is optimized for preventing proton back-migration. However, instability in the ACM was observed where a cross- 
flow between feed and acid reservoir was experienced when using the three-compartment design. Shown in Figure 21 is a picture of all membranes used in this research.

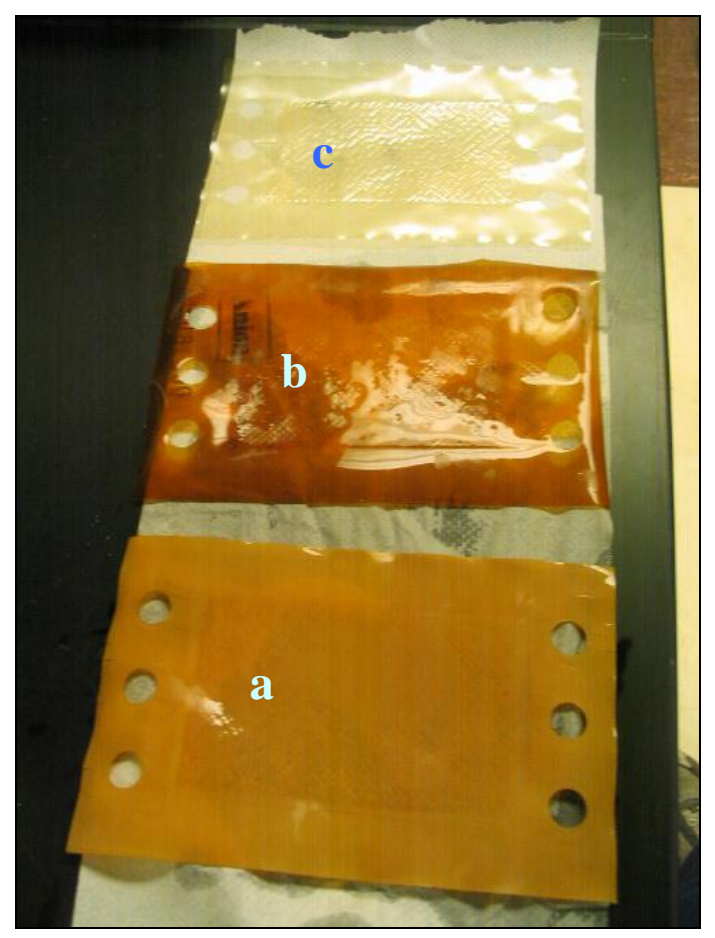

Figure 21. Tokuyama Neosepta® a)CMB, b)BP-1, and c) ACM

\subsubsection{The Electrolytica ED-1-BP Unit and It's Troubleshooting}

A laboratory scale electrodialysis unit (EDU) was acquired in the beginning of summer 2005 from Electrolytica Inc. of Amherst, New York. The ED-1-BP unit is designed specifically with three flow paths to accommodate a traditional electrodialysis process with bipolar membranes. The unit proved to be effective although a complexity in the stack assembly was faced in early experiments. Shown in Table 27 are ED-1-BP's specifications. 
Table 27. ED-1-BP General Specifications

\begin{tabular}{|c|c|}
\hline \multicolumn{2}{|c|}{ Specifications } \\
\hline Cell Configuration & Bipolar \\
\hline Individual Membrane Area $\left(\mathrm{m}^{2}\right)$ & 0.01 \\
\hline Size of Flow Paths (in.) & $3 / 8$ \\
\hline Flow Rate per Chamber (mL/min) & 100 \\
\hline $\begin{array}{c}\text { Electrode Rinse Flow Rates per } \\
\text { Chamber (mL/min) }\end{array}$ & $250-500$ \\
\hline Intermembrane Gap (mm) & 0.75 \\
\hline Anode Electrode & Pt plated Ti \\
\hline Cathode Electrode & Pt plated Ti \\
\hline Cell Body & Polypropylene \\
\hline Turbulence Promoting Mesh & Polypropylene \\
\hline Gasket Materials & EPDM or Viton Rubber \\
\hline Clamping Frame & Anodized Al \\
\hline
\end{tabular}

The construction of the ED-1-BP itself is fairly simple: membrane stack is placed between two electrode-containing polypropylene cell body and clamped together using a anodized aluminum clamping frame. Three flow paths are provided through the cell body in which flow will be propagated through each compartments in between membranes using specially fitted rubber gasket and turbulence promoting mesh made out of polypropylene. A picture of the ED-1-BP in operation is presented in Figure 22.

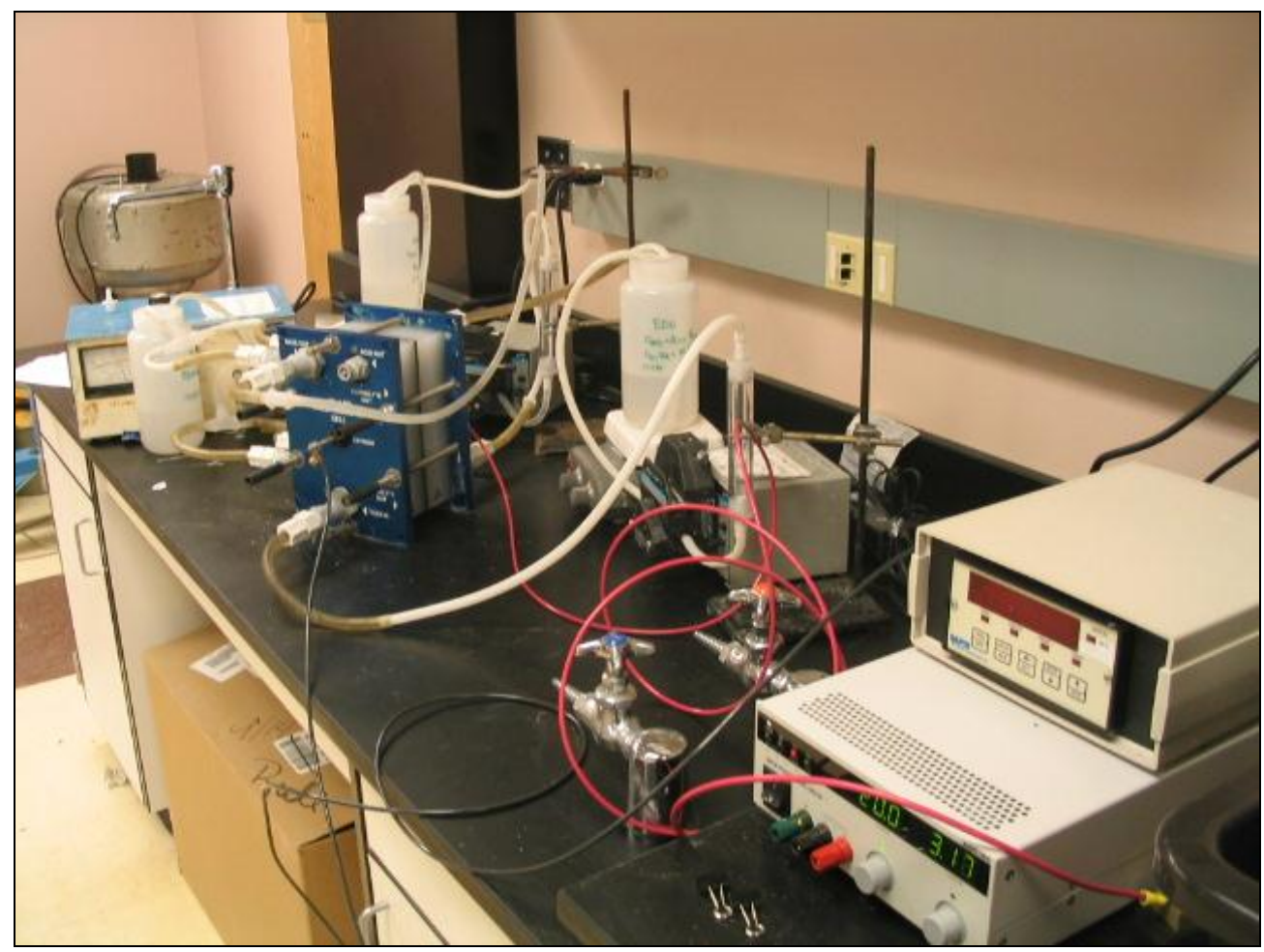

Figure 22. The ED-1-BP in Operation 
Many trials and errors were conducted to produce a legitimate Standard Operating Procedure (SOP) for the laboratory scale electrodialysis experiments. Due to the design of the unit, consistent placement and arrangement of the membrane stack can be very difficult. Moreover, it was important to develop pre- and post-experimental steps to provide consistency and repeatability between consequent experiments without having to disassemble the whole membrane stack. The final SOP is presented in Appendix I.

Early problems encountered include the instability of the AEM. The AEM showed instability in high acid concentration; thus, cross-flow between feed and acid flow was observed. Intermembrane cross-flow is highly undesirable since $\mathrm{Na}^{+}$cations, which should only be in the base flow, existed in the acid flow and creates extra resistance to the applied current.

With lack of time and practical solution to this problem, the two-compartment design using only the BPM and CEM was adopted. This arrangement was considered practical since it produces a pure $\mathrm{NaOH}$ flow and builds up $\mathrm{H}^{+}$cations concentration in the feed flow, creating $\mathrm{H}_{2} \mathrm{SO}_{4}$. Since the main objective of the electrodialysis process in the ASGLS is to regenerate $\mathrm{NaOH}$, as long as the desired concentration of $\mathrm{NaOH}$ is achieved, the two-compartment design is perfectly rational to be used.

Another problem of low $\mathrm{Na}^{+}$cations yield transfer was found after the adoption of the twocompartment design. As can be seen in Figure 23, only a total $\mathrm{NaOH}$ concentration of approximately $50-\mathrm{g} / \mathrm{L}$ was reached after only 75 -minutes at $800-\mathrm{A} / \mathrm{m}^{2}$, in which the concentration curve reached a plateau. Later experiments showed that a $\mathrm{NaOH}$ concentration of $70-\mathrm{g} / \mathrm{L}$ was achievable after 240-minutes.

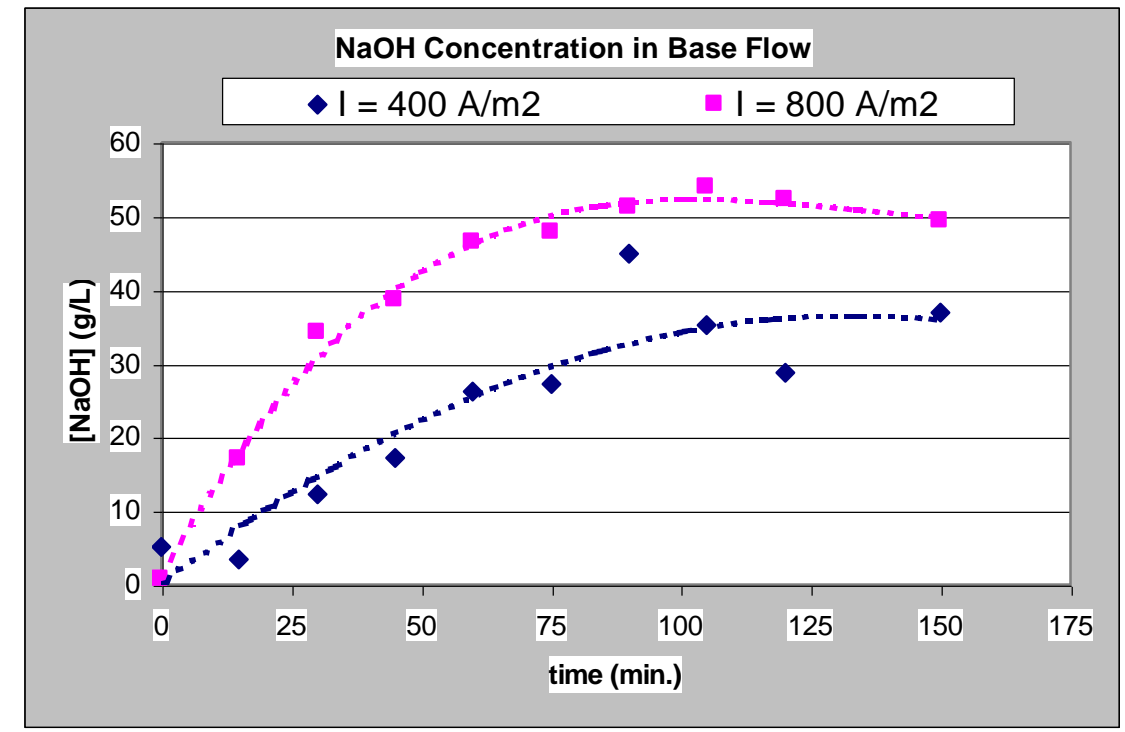

Figure 23. Un-optimized NaOH Regeneration

One possible explanation is the lack of flowrate control employed in the early experiments. Since no practical flow meter was immediately available on campus, the ED-1-BP unit was operated 
without any flow meter. An uneven flowrate created a pressure differential between the compartments and provided means for undesired transfer of ions through the membranes; thus, a poor base concentration was achieved.

Flow-meters were subsequently purchased and greatly improved the performance of the unit, as presented in the experimental campaigns below.

\subsubsection{Effects of Current Density (WED-1)}

\subsubsection{WED1 Background and Experimental Design}

In the first experimental campaign of the use of electrodialysis, the effects of current density (i) was investigated through a simple experimental design. Shown in Table 28 is WED1's experimental design matrix. To achieve better control of the feed, a standard solution prepared from a reagent grade $\mathrm{Na}_{2} \mathrm{SO}_{4}$ crystals was used. All tests and analysis were conducted according to the Standard Operating Procedure (SOP) presented in Appendix I.

Table 28. WED-1 Experimental Design

\begin{tabular}{|c|c|c|c|c|c|c|}
\multirow{2}{*}{ Test \# } & Initial $\mathbf{~ N a O H}$ & Initial $\mathbf{~ N a}_{2} \mathbf{S O}_{4}$ & Temp. & \multirow{2}{*}{ \# of Cells } & Flowrate & Current Density (i) \\
\cline { 2 - 6 } & $\mathbf{M}$ & $\mathbf{M}$ & oC & & $\mathbf{m L} / \mathbf{m i n}$ & $\mathbf{A} / \mathbf{m}^{2}$ \\
\hline WED1-T1 & 0 & 1.5 & 30 & 2 & 200 & 400 \\
\hline WED1-T2 & 0 & 1.5 & 30 & 2 & 200 & 800 \\
\hline
\end{tabular}

It was expected that increasing applied current density will increase the amount of transfer across the CEM at a given time as they will provide more electrical driving force for the $\mathrm{Na}^{+}$cations.

Isolation of other parameters were performed to as follow:

- $\quad$ Total Time of Test = 4-hours;

- Initial Base Flow $(\mathrm{NaOH})$ Concentration = 0M;

- Initial Feed Flow $\left(\mathrm{Na}_{2} \mathrm{SO}_{4}\right)$ Concentration = 1.5M; and

- $\quad$ Temperature of Solution $=30^{\circ} \mathrm{C}$.

For an analysis of each flow throughout the four-hour tests, 5-mL samples were obtained for each flow at the 15, 30, 60,90,120,180, and 240-minutes. The base flow was analyzed for $\mathrm{Na}^{+}$ concentration using ICP, $\mathrm{SO}_{4}{ }^{*}$ contamination through Ion Chromatograph (IC), and $\mathrm{OH}^{-}$ concentration through $\mathrm{ABC}$ titration. Meanwhile, the acid flow was analyzed for $\mathrm{H}_{2} \mathrm{SO}_{4}$ concentration through titration with sodium carbonate and methyl orange indicator. For more specific descriptions of the SOP, please refer to Appendix L.

\subsubsection{WED1 Results and Data Analysis}

Figure 24 shows the significant effect that current density has on the initial and final transfer of sodium ions. At 120 -minutes, a current density of $800-\mathrm{A} / \mathrm{m}^{2}$ achieved the same $\mathrm{NaOH}$ 
concentration that a $400-\mathrm{A} / \mathrm{m}^{2}$ current density achieved in 240-minutes. The final concentration of the $\mathrm{NaOH}$ also shows a significant difference.

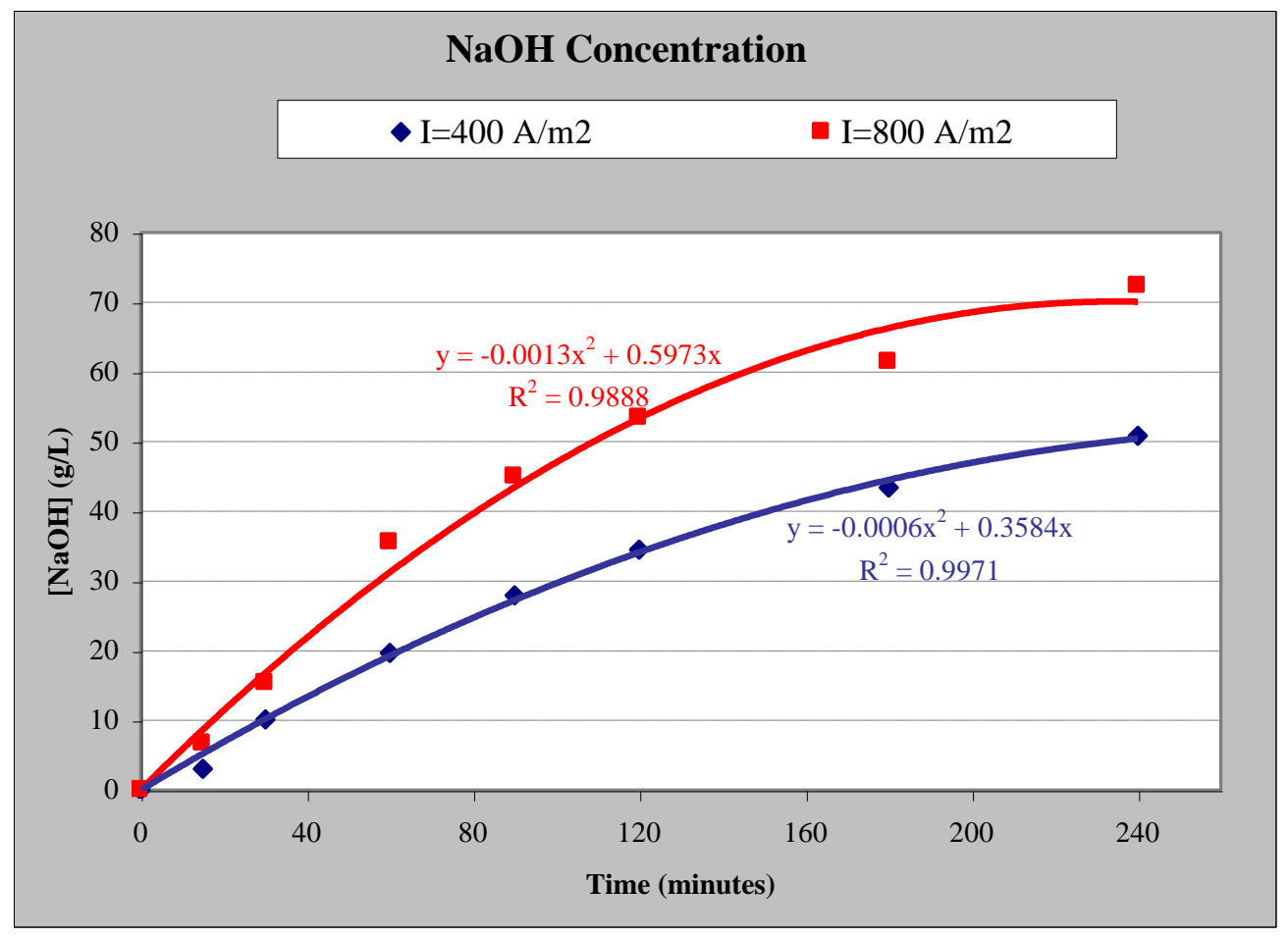

Figure 24. WED-1 NaOH Concentration vs. Time

As equally important is the efficiency of sodium ion transfer at different time of the tests. Seen in Figure 25 is diagram showing the sodium transfer per unit time at the different sampling times. As time is increased and the concentration of sodium in the feed is depleted, the transfer of sodium through the CEM also decreases. This indicates the equal importance of concentration differential between the feed/acid and base flow. In a continuous system where a steady sodium concentration in the feed may be maintained, the curves shown in Figure 25 will be flatter and more stable. 


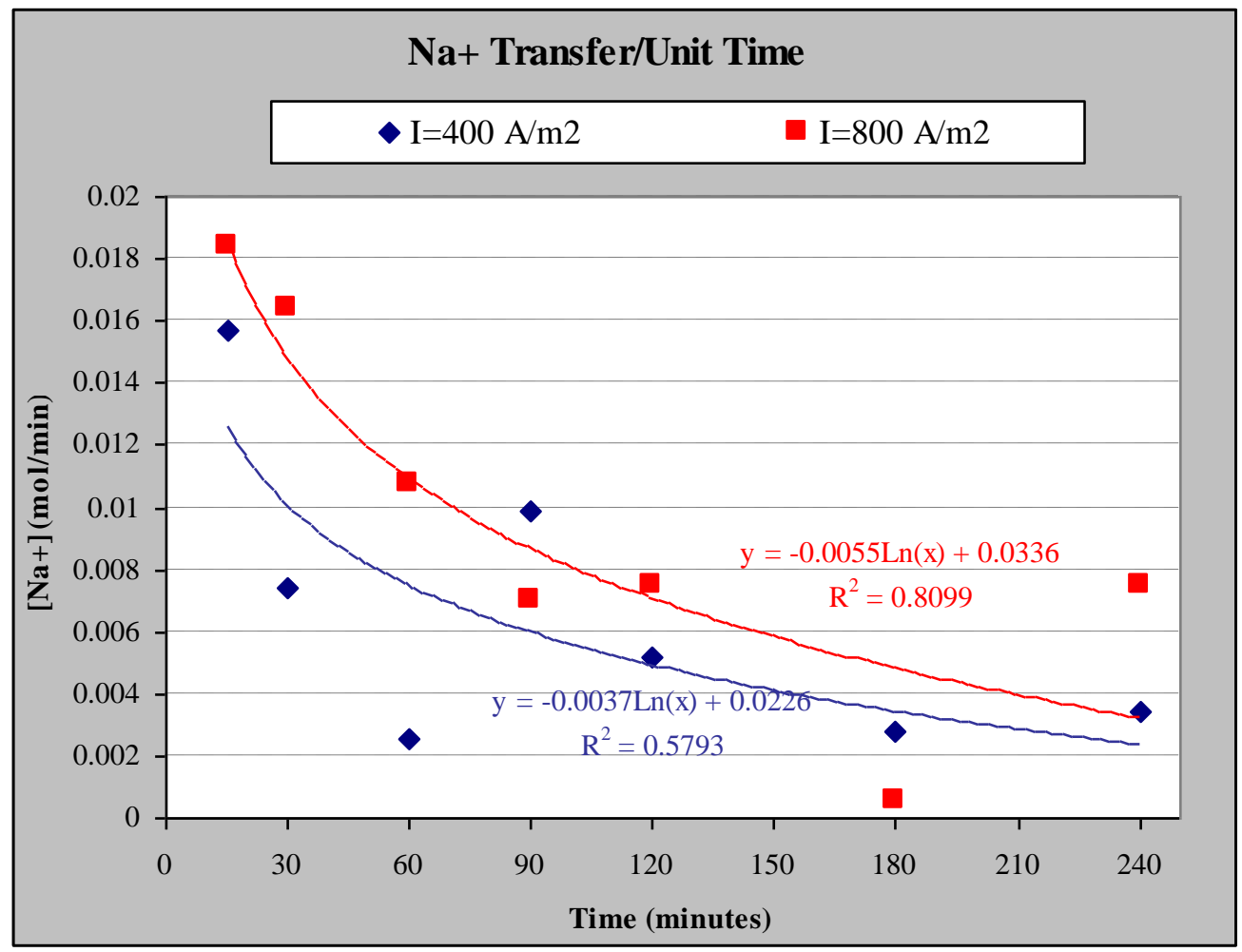

Figure 25. Sodium Transfer per Unit Time of WED-1 Tests

The other product of the electrodialysis, as discussed before, is a acid flow. Shown in Figure 26 is the gradual build up of $\mathrm{H}_{2} \mathrm{SO}_{4}$ in the feed flow. At higher current density, it can be easily concluded that a higher final acid concentration was achieved. Comparable to the build up of the $\mathrm{NaOH}$ in the base flow, a plateau seems to be reached after 240minutes. This plateau might indicate where the unit has reached equilibrium between the two flows so that efficient ion transfer is reduced. 


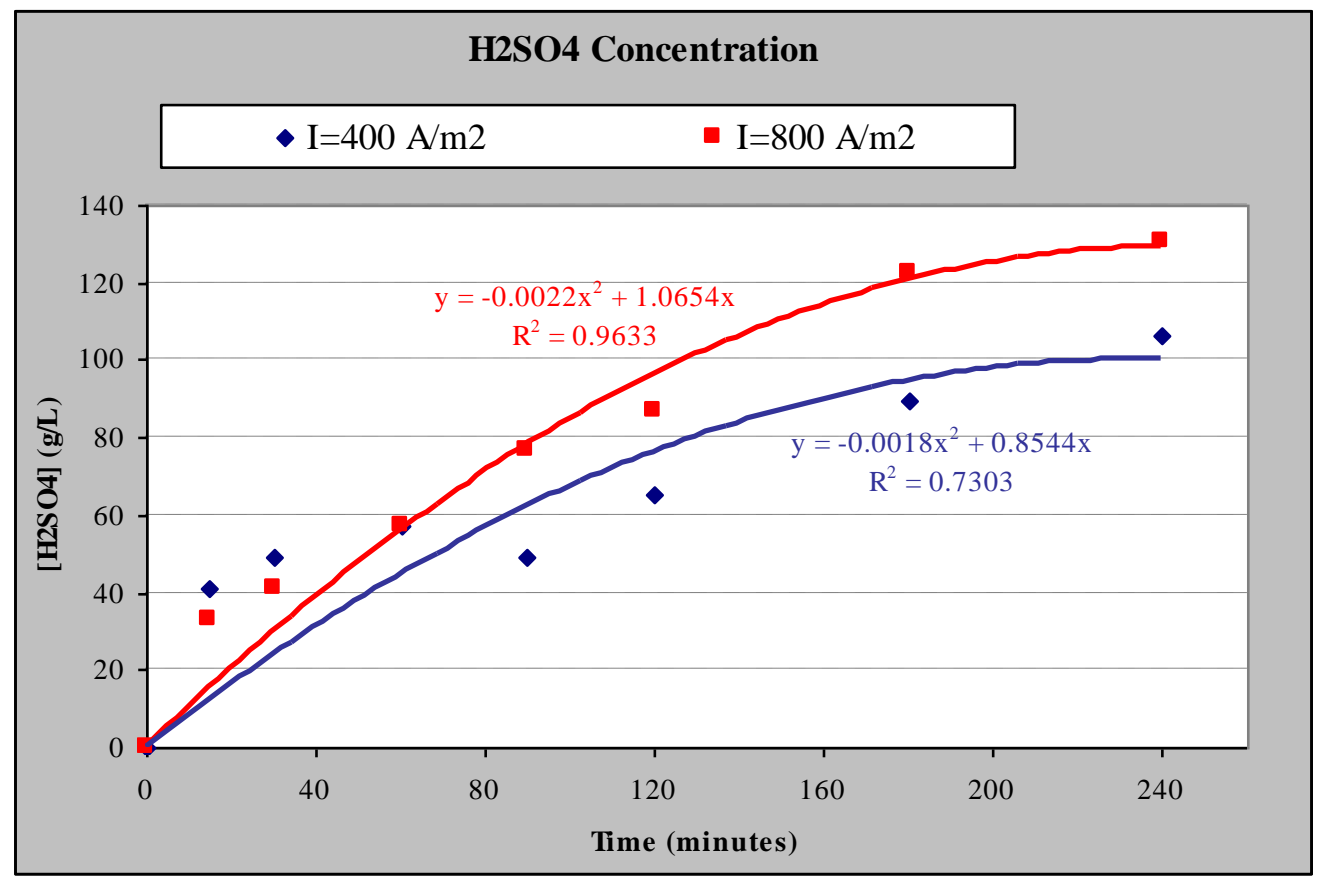

Figure 26. $\mathrm{H}_{2} \mathrm{SO}_{4}$ Build Up in WED-1 Tests

\subsubsection{Electrodialysis of Sulfide/Polysulfide Oxidation Produced $\mathrm{Na}_{2} \mathrm{SO}_{4}$ (WED-2)}

\subsubsection{WED-2 Experimental Design and Setup}

As it has been proven that the EDU process is able to regenerate high concentration of $\mathrm{NaOH}$ from a pure $\mathrm{Na}_{2} \mathrm{SO}_{4}$ solution, it was important to test the unit using feed solution from a $\mathrm{Na}_{2} \mathrm{SO}_{4}$ powder produced from the ASGLS. The $\mathrm{Na}_{2} \mathrm{SO}_{4}$ powder produced from campaign WO-2 was used in these tests. The $\mathrm{Na}_{2} \mathrm{SO}_{4}$ powder was redissolved into DI water producing approximately 1.43M of $\mathrm{Na}_{2} \mathrm{SO}_{4}$.

Consistent with the results of WED-1, $800-\mathrm{A} / \mathrm{m}^{2}$ was used in these tests. Hopefully, the same result may be achieved using a feed provided from the oxidation of ASGLS. Theoretically, the EDU should be as effective unless there are undesirable content of elements that might induce scaling on the membranes. These elements include calcium and magnesium. Shown in Table 29 is the experimental design of WED-2.

Table 29. WED-2 Experimental Design

\begin{tabular}{|c|c|c|c|c|c|c|}
\hline \multirow{2}{*}{ Test \# } & Initial $\mathbf{~ N a O H}$ & Initial $\mathbf{~ N a}_{2} \mathbf{S O}_{4}$ & Temp. & \multirow{2}{*}{ \# of Cells } & Flowrate & Current Density (i) \\
\cline { 2 - 7 } & $\mathbf{M}$ & $\mathbf{M}$ & $\stackrel{\circ}{ } \mathbf{C}$ & & $\mathbf{m L} / \mathbf{m i n}$ & $\mathbf{A} / \mathbf{m}^{2}$ \\
\hline WED1-T1 & 0 & 1.43 & 30 & 2 & 200 & 800 \\
\hline WED1-T2 & 0 & 1.43 & 30 & 2 & 200 & 800 \\
\hline
\end{tabular}




\subsubsection{WED-2 Experimental Results and Discussion}

Seen in Figure 27 is the confirmation of the capabilities of the EDU and also the consistency of the process. The tests showed very similar trend-lines. Again, a final $\mathrm{NaOH}$ concentration of approximately $70-\mathrm{g} / \mathrm{L}$ was achieved.

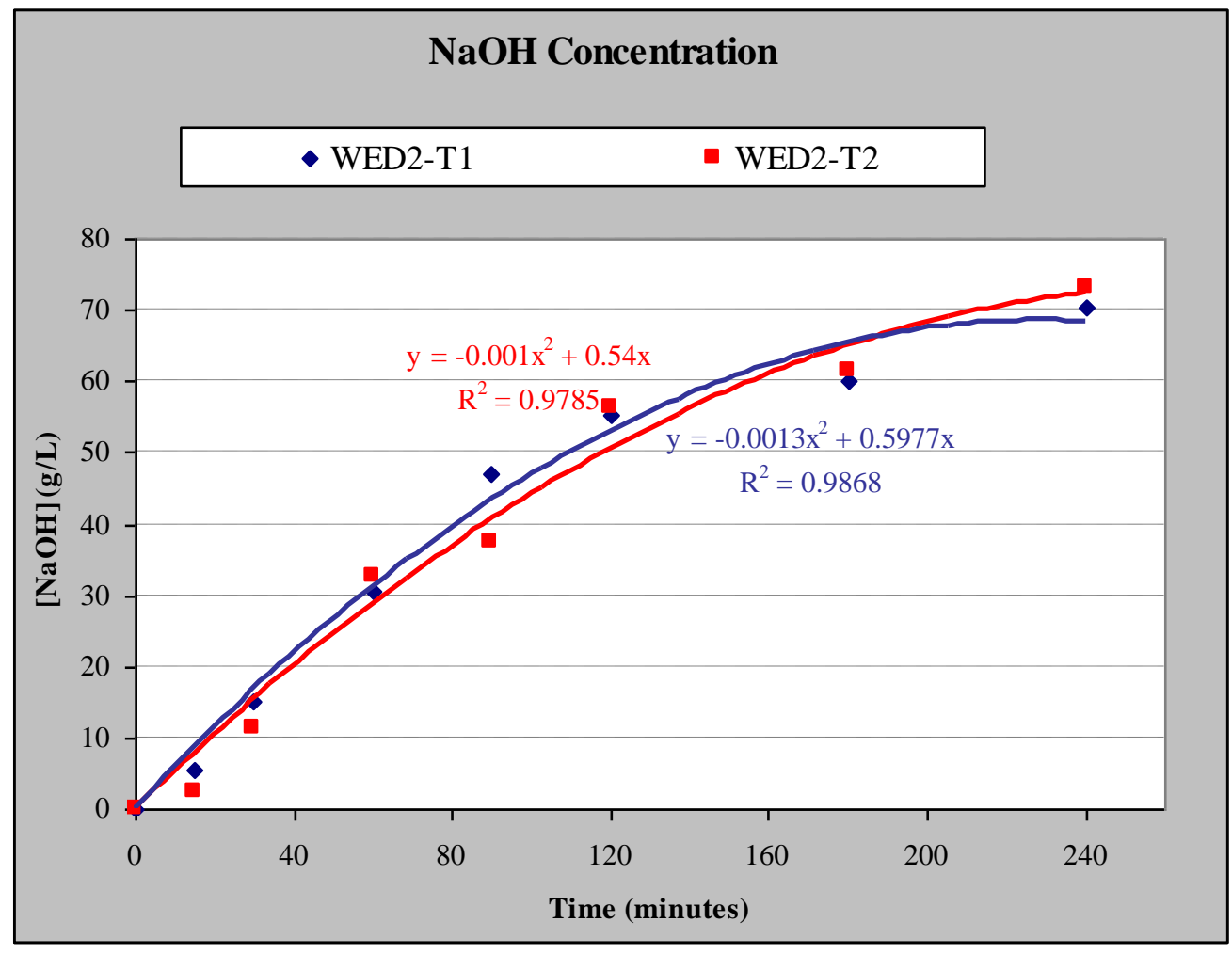

Figure 27. $\mathrm{NaOH}$ Concentration vs. Time in WED-2

Similar consistency was also observed in the acid flow, as shown in Figure 28 below. A final acid concentration of approximately 120-g/L was achieved after 240minutes in both tests of WED-2. 


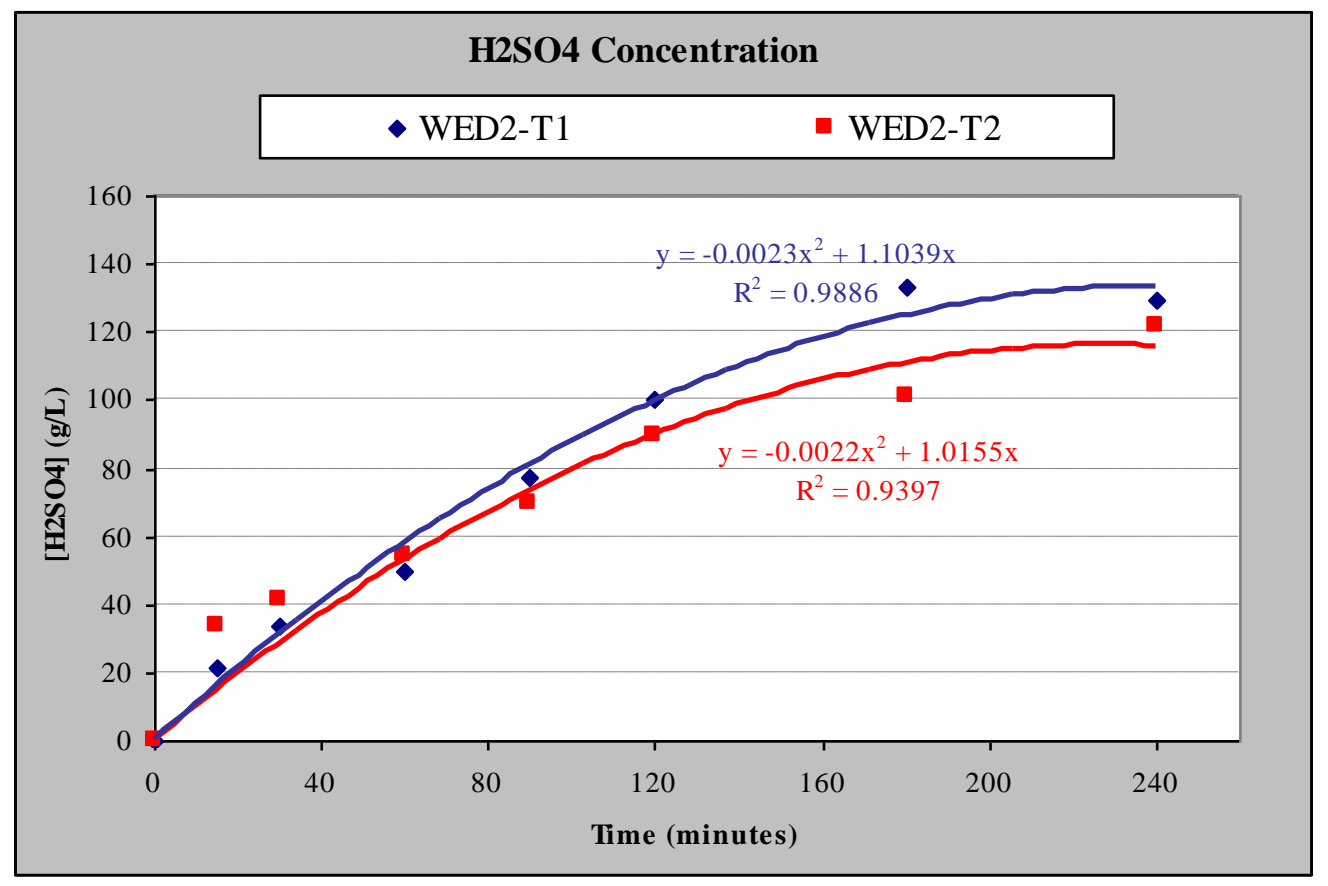

Figure 28. $\mathrm{H}_{2} \mathrm{SO}_{4}$ Concentration vs. Time in WED-2

These test results confirmed the applicability of the EDU to treat $\mathrm{Na}_{2} \mathrm{SO}_{4}$ produced from ASGLS waste treatment.

\subsection{Treatment of $\mathrm{H}_{2} \underline{\mathrm{SO}}_{4} \underline{\text { Produced from Electrodialysis }}$}

Two processes are proposed to treat the $\mathrm{H}_{2} \mathrm{SO}_{4}$ solution produced by electrodialysis process: gypsum production and ammonium sulfate production. Certainly, $\mathrm{H}_{2} \mathrm{SO}_{4}$ can be considered a byproduct of the ASGLS process. However, these two processes are proposed as an alternative to produce possibly more valuable products. Moreover, ammonium sulfate may be used as a fertilizer and may provide a better "approval" of the process.

This section of the thesis will discuss the baseline experiments that have been performed for these processes. Unfortunately, at the time of conducting the Batch Simulations, these processes were not well yet developed and were not included in the simulations. Nonetheless, these studies should give a basic understanding of these processes and motivate future projects. 


\subsubsection{Gypsum Production}

Gypsum production was originally proposed to be an alternative of the electrodialysis unit in $\mathrm{NaOH}$ regeneration. Gypsum production was projected to be significantly cheaper compared to electrodialysis since it does not require any applied voltage; hydrated lime is also abundant and relatively low in cost. Gypsum is a common name to calcium sulfate dihydrate $\left(\mathrm{CaSO}_{4} \cdot 2 \mathrm{H}_{2} \mathrm{O}\right)$; anhydrite $\left(\mathrm{CaSO}_{4}\right)$ and hemihydrite $\left(\mathrm{CaSO}_{4} 1 / 2 \mathrm{H}_{2} \mathrm{O}\right)$ also exist. Chemical reaction of gypsum production is shown in Equation 4.3.

$$
\mathrm{Ca}(\mathrm{OH})_{2}+\mathrm{Na}_{2} \mathrm{SO}_{4}+2 \mathrm{H}_{2} \mathrm{O}=\mathrm{CaSO}_{4} \cdot 2 \mathrm{H}_{2} \mathrm{O}+2 \mathrm{NaOH}
$$

Synthetic gypsum is largely produced from $\mathrm{SO}_{2}$ removal systems used in electrical power plants and in any systems producing $\mathrm{SO}_{2}$ as waste gas. Technologies such as Limestone Forced Oxidation and Dual Alkali utilizes the oxidation of $\mathrm{SO} 2$ to $\mathrm{SO}_{3}{ }^{2}$ and $\mathrm{SO}_{4}{ }^{2}$, to form $\mathrm{CaSO}_{4}$. In 2004, synthetic gypsum accounts for $24.8 \%$ of total crude gypsum sales in the United States at 9.04 millions metric tonnes (Fourier, 2004).

One concern related to this system is the inability of calcium sulfate crystal formation above $\mathrm{pH}$ of 12.5. As shown in Figure 29, calcium sulfate is not stable above 12.5. Furthermore, Greg Roset, Smelter Manager of Stillwater Mining Co., also referred to the operational parameters of $\mathrm{pH}$ below 12.5 to avoid any lime contamination in the final gypsum product (Roset, 2006). 


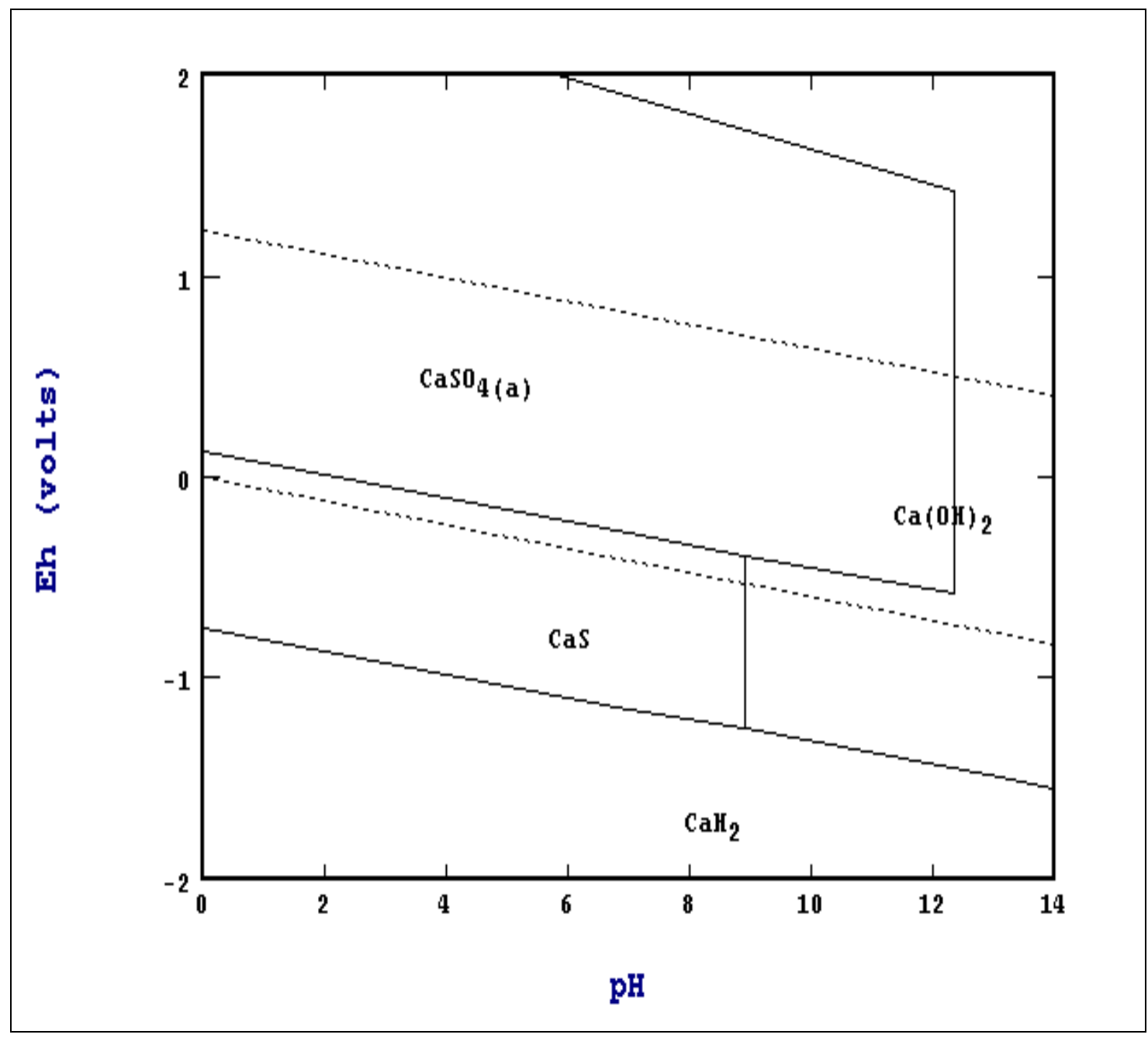

Figure 29. Pourbaix Diagram of Ca-S System

The primary objective of the following experimental campaigns was to optimize the gypsum production in regenerating caustic soda from produced $\mathrm{Na}_{2} \mathrm{SO}_{4}$ precipitates.

\subsubsection{Gypsum Production Experimental Campaign 1 (WGP1)}

WGP1's objective was to develop a strict and practical Standard Operating Procedure (SOP); and at the same time, to investigate possibilities of $\mathrm{NaOH}$ regeneration through a laboratory batch gypsum production experiment.

In review, the procedure used in this thesis was a simple batch test where sodium sulfate solution was prepared from a reagent grade solids and slurry of hydrated lime was added right before the 12-hour agitation was started.

Although most literature and current research in this area uses a continuous system for this reaction (Gominšek et.al. and Abdel-Aal et.al.), it was impossible to be replicated at Montana Tech, due to lack of resources and time. 
Table 30. WGP-1 Experimental Design

\begin{tabular}{|l|l|l|c|}
\hline Test \# & Starting pH & {$\left[\mathrm{Na}_{2} \mathbf{S O}_{4}\right](\mathrm{M})$} & \%solids $\mathrm{Ca}(\mathrm{OH})_{2}$ \\
\hline WGP1-T1 & 7.0 & 1 & 20 \\
\hline WGP1-T2 & 12.5 & 1.5 & 10 \\
\hline WGP1-T3 & 9.75 & 1.25 & 15 \\
\hline WGP1-T4 & 12.5 & 1 & 10 \\
\hline WGP1-T5 & 7.0 & 1 & 10 \\
\hline WGP1-T6 & 7.0 & 1.5 & 10 \\
\hline WGP1-T7 & 12.5 & 1.5 & 20 \\
\hline WGP1-T8 & 12.5 & 1 & 20 \\
\hline WGP1-T9 & 7.0 & 1.5 & 20 \\
\hline
\end{tabular}

As a measure of test performance, two analytical procedures were conducted on the final solution of WGP1 experiments: IC analysis for $\mathrm{SO}_{4}{ }^{2-}$ concentration and $\mathrm{ABC}$ titration for $\mathrm{OH}^{-}$ concentration. Ideally, sulfur analysis would have been performed to the final solids to investigate how much $\mathrm{SO}_{4}{ }^{2-}$ is precipitated to form gypsum; however, within the practical time of the experiments, the LECO X Carbon-Sulfur analyzer was broken and could not be used to obtain accurate numbers. Since the amount of gypsum formed is directly related to the amount of $\mathrm{SO}_{4}{ }^{2-}$ consumed from the solution and the amount of hydroxide produced, IC analysis and ABC titration were sufficient to measure the performance of the experiments.

In general, as shown in Table 31, WGP1 experiments cannot be labeled successful in producing gypsum. Concentration of $\mathrm{NaOH}$ can be generalized to be equal to each other; $\mathrm{pH}$ measurements confirmed this fact. At the same time, only a maximum of $32.80 \%$ of the available $\mathrm{SO}_{4}{ }^{2-}$ was consumed by the reaction.

Table 31. WGP-1 Analysis Results

\begin{tabular}{|c|c|c|c|c|}
\hline Test \# & $\mathbf{p H}$ & {$\left[\mathbf{S O}_{4}{ }^{\circ}\right] \mathbf{~ ( g / L )}$} & Consumed $\mathrm{Na}_{\mathbf{2}} \mathbf{S O}_{\mathbf{4}}(\%)$ & {$[\mathrm{NaOH} \mathbf{~ ( g / L )}$} \\
\hline WGP1-T1 & 13.08 & 45.06 & 24.90 & 2.86 \\
\hline WGP1-T2 & 13.10 & 69.31 & 22.99 & 4.52 \\
\hline WGP1-T3 & 12.84 & 72.81 & 2.92 & 4.10 \\
\hline WGP1-T4 & 13.04 & 46.17 & 23.05 & 4.21 \\
\hline WGP1-T5 & 13.06 & 42.31 & 29.49 & 3.79 \\
\hline WGP1-T6 & 12.95 & 66.07 & 26.59 & 4.35 \\
\hline WGP1-T7 & 13.10 & 67.77 & 24.70 & 4.44 \\
\hline WGP1-T8 & 13.09 & 46.79 & 22.01 & 3.66 \\
\hline WGP1-T9 & 12.96 & 60.48 & 32.80 & 3.96 \\
\hline
\end{tabular}

Interesting results to be considered are final $\mathrm{pH}$ levels on the solution. An average $\mathrm{pH}$ of 13.02 is found in all tests; with previously presented $\mathrm{E}_{\mathrm{H}} \mathrm{pH}$ diagram (Figure 29), it can be concluded that WGP1 has proven that a $\mathrm{pH}$ lower than 12.5-13.0 is needed to obtain complete gypsum 
production. All tests were producing $\mathrm{CaSO}_{4} \cdot 2 \mathrm{H}_{2} \mathrm{O}$ and producing $\mathrm{NaOH}$ until it reaches the maximum $\mathrm{pH}$ where gypsum is no longer stable; thus, $\mathrm{Ca}(\mathrm{OH})_{2}$ will be predominant and formation of more gypsum is unfavorable.

With the WGP1 conclusion, without any $\mathrm{pH}$ control, gypsum production possibly will not be feasible in treating the $\mathrm{Na}_{2} \mathrm{SO}_{4}$ solution straight off the autoclave oxidation. However, it is possible to treat the $\mathrm{H}_{2} \mathrm{SO}_{4}-\mathrm{Na}_{2} \mathrm{SO}_{4}$ solution produced from the electrodialysis unit with this gypsum production process. The acid/feed flow produced by the EDU provides a $\mathrm{pH}$ of 1.5, which can be low enough to accommodate gypsum production. Additionally, it will provide an alternative to the ammonium sulfate production also briefly investigated in this thesis.

Experimental campaign WGP-2 was conducted using the $\mathrm{H}_{2} \mathrm{SO}_{4}$ solutions produced from the previous electrodialysis campaign of WED-2. The produced solutions were of approximately 120 -g/ $\mathrm{L} \mathrm{H}_{2} \mathrm{SO}_{4}$. Unlike WGP-1 where the $\mathrm{Na}_{2} \mathrm{SO}_{4}$ solutions weren't able to provide a $\mathrm{pH}$ low enough to maintain the predominance of gypsum, the $\mathrm{H}_{2} \mathrm{SO}_{4}$ solution with a $\mathrm{pH}$ of approximately 1.50 provided enough room for gypsum formation.

With a simple mass balance using chemical stoichiometry, two identical tests of 10.58-grams of $\mathrm{Ca}(\mathrm{OH})_{2}$ plus $100-\mathrm{mL} \mathrm{H}_{2} \mathrm{SO}_{4}$ solutions were conducted. The reaction was agitated using a horizontal shaker for 12-hours, similar to that of the precious metal recovery batch setup.

Analysis was performed to the filtered solution by measuring $\mathrm{SO}_{4}{ }^{=}$levels using the Ion Chromatograph. It was important to measure how much $\mathrm{SO}_{4}{ }^{2-}$ was extracted out of the solution. Shown in Table 32 are the results of campaign WGP-2.

Table 32. Experimental Results of WGP-2

\begin{tabular}{|c|c|c|c|}
\hline \multirow{2}{*}{ Test \# } & \multicolumn{2}{|c|}{$\mathbf{S O}{ }^{{ }^{*}}{ }^{(\mathbf{g} / \mathbf{L})}$} & \multirow{2}{*}{$\%$ Consumption } \\
\cline { 2 - 3 } & Initial & Final & \\
\hline WGP2-T1 & 137.28 & 33.46 & $75.63 \%$ \\
\hline WGP2-T2 & 137.28 & 20.88 & $84.79 \%$ \\
\hline
\end{tabular}

A maximum sulfate consumption of $84.8 \%$ was achieved by the second test. This is certainly encouraging results, confirming the ability to form gypsum at the lower $\mathrm{pH}$ that the $\mathrm{H}_{2} \mathrm{SO}_{4}$ provided.

\subsubsection{Ammonium Sulfate Production}

Ammonium Sulfate Production is another alternative that can be used in treating produced $\mathrm{H}_{2} \mathrm{SO}_{4}$ from the electrodialysis. Similar to that reaction of gypsum production, a low $\mathrm{pH}$ is needed to be able to produce the product.

$$
2 \mathrm{NH}_{4} \mathrm{OH}+\mathrm{H}_{2} \mathrm{SO}_{4}=\left(\mathrm{NH}_{4}\right)_{2} \mathrm{SO}_{4}+2 \mathrm{H}_{2} \mathrm{O}
$$


Ammonium sulfate has long been known as a nitrogen-based fertilizer. In 2004, it accounted for almost $5 \%$ of the total nitrogen species fertilizer in the US (Kramer, 2004). Prices of the compound have also been quite steady at $\$ 135$ to $\$ 190 /$ short ton.

Again, only simple confirmatory research was performed in this subject of the thesis. Further investigation of the process should certainly be in future planning.

\subsubsection{Ammonium Sulfate Production Experimental Campaign 1 (WAS-1)}

A simple experiment using the WED-2 produced $\mathrm{H}_{2} \mathrm{SO}_{4}$ was performed by adding $30 \%$ Ammonium Hydroxide solution into solution of $120-\mathrm{g} / \mathrm{L} \mathrm{H}_{2} \mathrm{SO}_{4}$. This amount added was calculated by a simple stoichiometric balance. Although there were no quantitative data recorded, an XRD pattern of the white solid product was taken. Shown in Figure 30 is the resultant pattern that confirms the formation of ammonium sulfate crystals. Further work will have to be done to increase the yield and purity of this product.

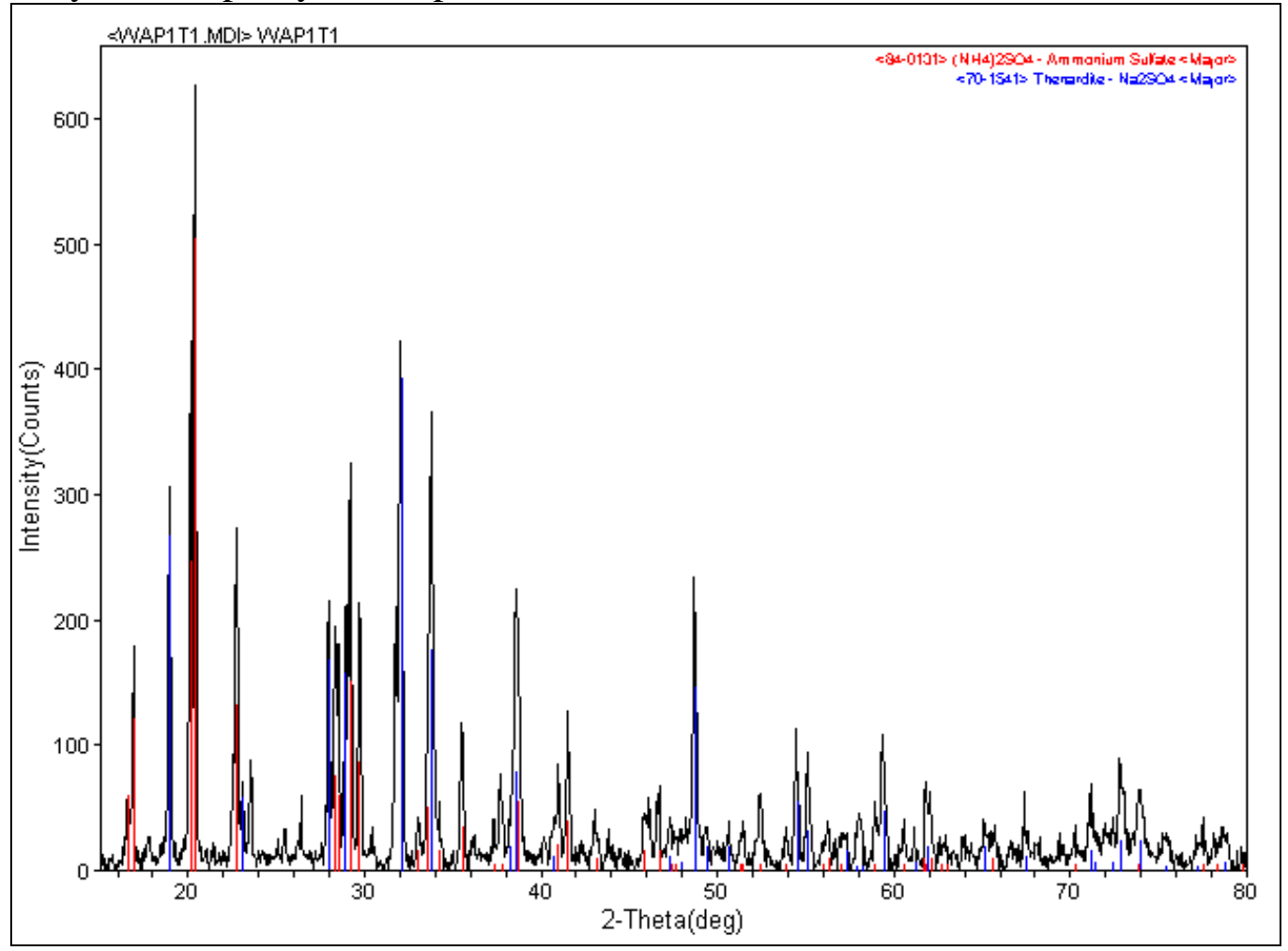

Figure 30. Confirmation of the Formation of $\left(\mathrm{NH}_{4}\right)_{2} \mathrm{SO}_{4}$ in Campaign WAS-1

\subsection{ASGLS Bench-Top Batch Simulation}

\subsection{Introduction to ASGLS Bench-Top Batch Simulation}

The ultimate goal of this research was to show and prove that the ASGLS works consistently on actual ores and concentrates; thus showing the capability of this system as an alternative to the currently used cyanide system. 
To fulfill this goal, a bench-top batch simulation of all the developed unit processes (leaching, recovery, and solution waste treatment) was conducted on three different sulfide feed materials:

1. KGCMC's composite concentrate (Batch Simulation 1);

2. Golden Sunlight's pyrite concentrate (Batch Simulation 2); and

3. Golden Sunlight's pre-oxidized pyrite concentrate (Batch Simulation 3).

A batch circuit was used for the sake of simplicity and because of the lack of time and immediate resources (space, equipments, process controls, etc). With a carefully developed operational schedule, all procedures were kept consistent and timed relatively close. This tight operational control produced highly consistent performances.

The three concentrates were chosen to prove the effective performance of ASGLS to feed materials containing high content of sulfides minerals. KGCMC's composite concentrate is produced from a flotation process where galena $(\mathrm{PbS})$ and sphalerite $(\mathrm{ZnS})$ concentrates are produced. These two concentrates were sampled for this study and than combined, producing the so-called composite concentrate. Some other complex sulfide minerals (tennantite, tetrahedrite, proustite, pyrartyrite, etc) have also been recognized to exist in the composite concentrate. Golden Sunlight's pyrite concentrate is the product of a gravity concentrated cyanide leaching discharge. Detailed descriptions of these concentrates were presented in Chapter 2.

Apart from proving the performance consistency of all developed unit processes, other tremendous benefits were gained from these batch simulations:

1. The ability to monitor solution parameters $\left(\mathrm{E}_{\mathrm{H}}, \mathrm{pH}\right.$, free $\mathrm{OH}^{-}$concentration, sulfide, and polysulfide concentration) before and after each unit processes;

2. The ability to accumulate a great amount of data on the leaching of other elements in the feed materials into the alkaline sulfide solution;

3. The ability to test for the best method of reagent addition to partially deteriorated solutions before recirculation;

4. The ability to test a carbon-in-column (CIC) setup of precious metal recovery from pregnant ASGLS solution;

5. The ability to test the proposed waste treatment processes (pressure oxidation and electrodialysis) in a real ASGLS circuit.

In the experimental setup, all three individual unit processes (leaching, recovery, and waste treatment) followed the same setup as the confirmatory studies in this thesis. Their developed SOPs are presented in the appendices of this thesis. Shown in Figure 31 is a general process flowsheet used in all of the simulations. Specific changes were made in each simulation and will be discussed in their respective sections. 


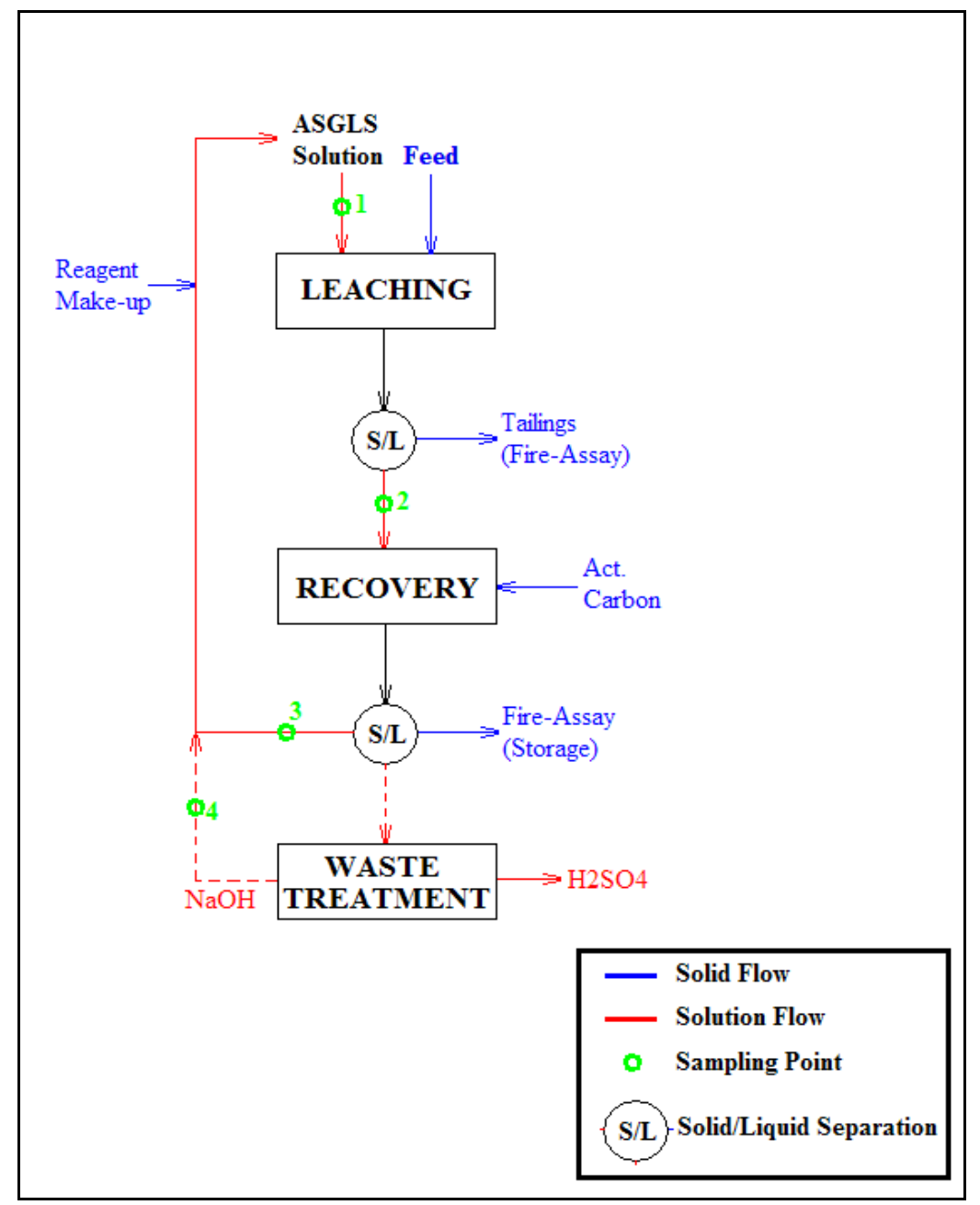

Figure 31. Process Flowsheet of The ASGLS Batch Simulations

\subsection{Bench-Top Batch Simulation of KGCMC Composite Concentrate (Batch Simulation 1)}

\subsection{Introduction and Background}

The KGCMC composite concentrate bench-top batch simulation campaign was conducted in 14 days. An operational schedule was developed to accommodate a day-by-day activity. This daily schedule was important to ensure a time-effective operation and consistent solution treatment. More importantly, the daily schedule ensured a consistent each unit processes' procedure and a consistent sampling of the solution at each sampling point. 


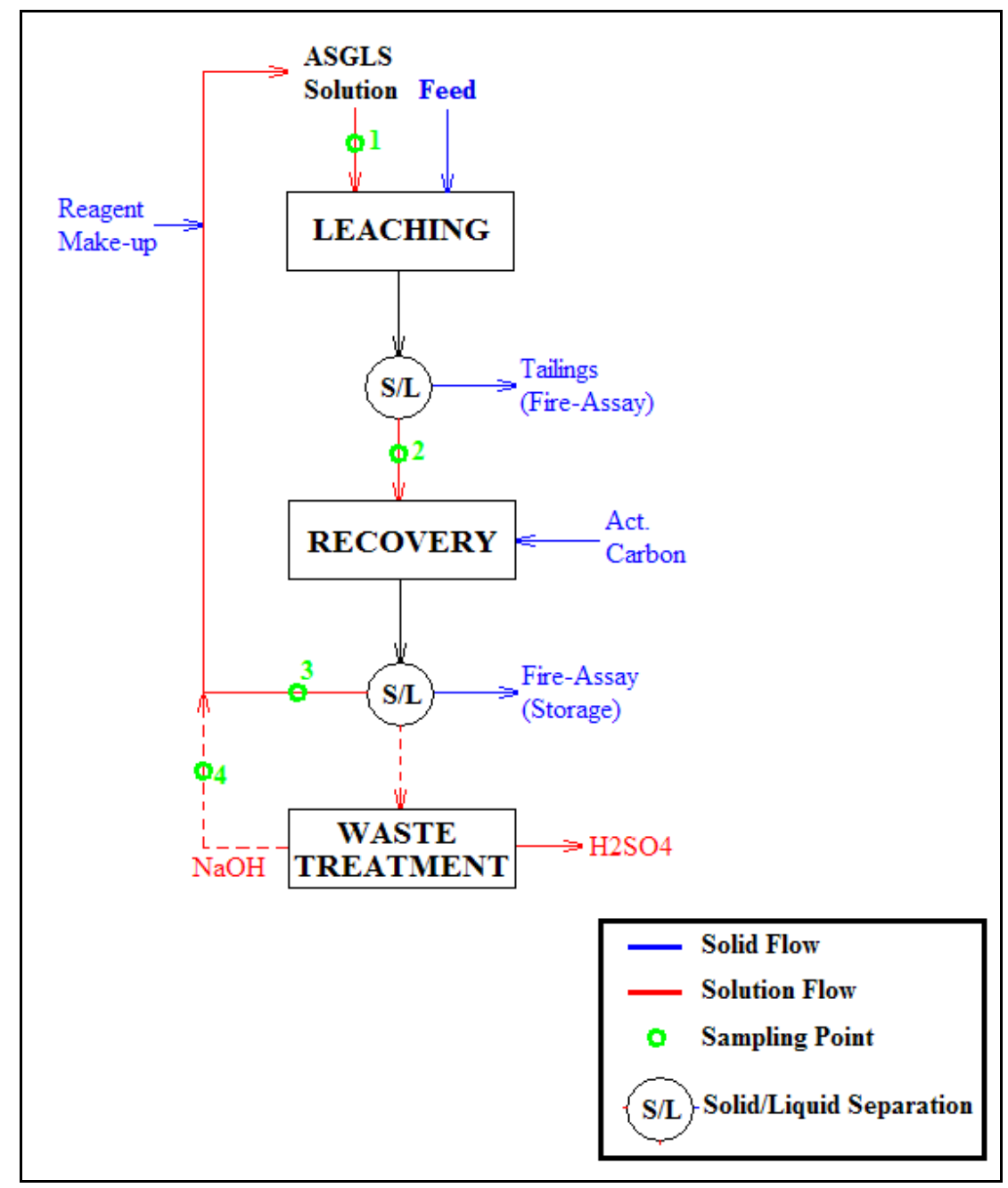

Figure 32. Process Flowsheet of KGCMC Composite Concentrate Batch Simulation

Shown in Figure 32 is a process flowsheet describing the flow experienced by each of the five solutions in this campaign. The dotted lines going into the waste treatment unit process represents a partial bleed-off of the solution. After each circulation $300-\mathrm{mL}$ ( $20 \%$ of original volume) of the solution was taken out of the circuit after recovery to be fed into the waste treatment unit process consisting of pressure oxidation and $\mathrm{NaOH}$ regeneration using the EDU. Due to the nature of the simulation, this loss of 300-mL was made up in Leaching 6-8 by adding fresh stock ASGLS solution and in Leaching 9-10 by adding recirculated ASGLS solution created from produced $\mathrm{NaOH}$ stream from the electrodialysis stage of the waste treatment stage. (See Table 33).

Important are the sampling points within the circuit. At before and after each unit processes, the ASGLS solution is sampled:

1. Sampling point 1 is pre-leaching;

2. Sampling point 2 is post-leaching/pre-recovery;

3. Sampling point 3 is post-recovery/pre-waste treatment; and

4. Sampling point 4 is post-waste treatment. 
Labels dedicated to each stages of the KGCMC composite concentrate and some details of the recirculation methods are presented in Table 33.

Table 33. Process Labels of KGCMC Composite Concentrate Batch Simulation

\begin{tabular}{|l|c|c|c|c|c|c|}
\hline Leaching \# & Solution \# & Feed \# & Recovery \# & $\begin{array}{c}\text { Carbon } \\
\text { Batch \# }\end{array}$ & $\begin{array}{c}\text { Oxidation } \\
\text { Test \# }\end{array}$ & $\begin{array}{c}\text { Origin of Make- } \\
\text { Up Solution }\end{array}$ \\
\hline Leaching 1 & Solution 1 & Feed 1 & Recovery 1 & Carbon 1 & Oxidation 1 & -- \\
\hline Leaching 2 & Solution 2 & Feed 2 & Recovery 2 & Carbon 2 & Oxidation 1 & -- \\
\hline Leaching 3 & Solution 3 & Feed 3 & Recovery 3 & Carbon 3 & Oxidation 1 & -- \\
\hline Leaching 4 & Solution 4 & Feed 4 & Recovery 4 & Carbon 4 & Oxidation 2 & -- \\
\hline Leaching 5 & Solution 5 & Feed 5 & Recovery 5 & Carbon 1 & Oxidation 2 & -- \\
\hline Leaching 6 & Solution 1 & Feed 6 & Recovery 6 & Carbon 2 & Oxidation 2 & Fresh \\
\hline Leaching 7 & Solution 2 & Feed 7 & Recovery 7 & Carbon 3 & Oxidation 3 & Fresh \\
\hline Leaching 8 & Solution 3 & Feed 8 & Recovery 8 & Carbon 4 & Oxidation 3 & Fresh \\
\hline Leaching 9 & Solution 4 & Feed 9 & Recovery 9 & Carbon 1 & Oxidation 3 & Off of Oxidation 1 \\
\hline Leaching 10 & Solution 5 & Feed 10 & Recovery 10 & Carbon 2 & -- & Off of Oxidation 1 \\
\hline
\end{tabular}

As can be seen from Table 33, there were ten leaching stages with Leaching 1-5 were the first circulation for the five solutions; and Leaching 6 through 10 were the second circulation through the circuit. The composite concentrate feed was appropriately labeled 1 through 10, according to their respective leaching batch number. The same labeling was used for the recovery stages.

However, a slightly different activated carbon addition sequence was adopted. Since there were only approximately 600-grams of activated carbon immediately available during the running of the campaign, only four batches of 150-gram activated carbon were created. Thus, a third recirculation of Carbon 1 and Carbon 2 was performed, which was proved to be beneficial since it provided additional data in the effectiveness of $\mathrm{AuS}^{-}$loading onto the activated carbon after multiple recirculations.

Other details of Batch Simulation are presented in Table 34 below. 
Table 34. Simulation Details Specific to Batch Simulation

\begin{tabular}{|c|c|}
\hline \multicolumn{2}{|c|}{ Simulation Variable } \\
\hline \# of Solutions & 5 \\
\hline Volume of Each Solution Batch & $1500-\mathrm{mL}$ \\
\hline Initial Sulfide-Polysulfide Concentration & $80-\mathrm{g} / \mathrm{L}$ \\
\hline Initial NaOH Concentration & $50-\mathrm{g} / \mathrm{L}$ \\
\hline Total Flowsheet Circulations per Solution & 2 \\
\hline Feed Treated per Leach & $100.0-\mathrm{grams}$ \\
\hline Leaching Temperature & $50-\mathrm{C}$ \\
\hline Leaching Time & $72-\mathrm{hours}$ \\
\hline Leaching Agitation & $170-\mathrm{rpm}$ \\
\hline Recovery Stage Setup & Batch \\
\hline Recovery Time & $48-\mathrm{hours}$ \\
\hline Vol. of Solution to Waste Treatment per Circulation & $300-\mathrm{mL}$ \\
\hline Vol. Of Make Up Solution (Fresh or Recirculated) & $300-\mathrm{mL}$ \\
\hline
\end{tabular}

\subsubsection{Experimental Results and Discussion}

As shown in Figure 32, a sample of each solution at different sampling points were taken throughout the simulation campaign. These samples were tested for $\mathrm{E}_{\mathrm{H}}, \mathrm{pH}$, total sulfidepolysulfide concentration, sulfide concentration, and free hydroxide concentration. These parameters are believed to be critical in the ASGLS and a correlation between these parameters and the performance of the ASGLS in each unit process was made. Shown in Table 35 are the average parameters of all solutions at the different sampling points of each circulation.

Table 35. Solution Parameters Monitoring Results of Batch Simulation

\begin{tabular}{|c|c|c|c|c|c|c|c|}
\hline \multicolumn{2}{|c|}{$\begin{array}{c}\text { Circulation/Sampling Point } \\
\text { 1st }\end{array}$} & $\mathbf{E}_{\mathbf{H}}(\mathbf{S H E})$ & $\mathbf{p H}$ & $\begin{array}{c}{\left[\mathbf{S}^{2-}\right]} \\
(\mathbf{g} / \mathbf{L})\end{array}$ & $\begin{array}{c}{\left[\mathbf{S}_{\mathbf{x}}{ }^{2-}\right]} \\
(\mathbf{g} / \mathbf{L})\end{array}$ & $\begin{array}{c}\text { Total } \\
\mathbf{S}^{2-}-\mathbf{S}_{\mathbf{x}}{ }^{2-}\end{array}$ & $\begin{array}{c}\text { Free } \mathbf{O H}^{-} \\
(\mathbf{g} / \mathbf{L})\end{array}$ \\
\cline { 2 - 8 } & Pre-Leach & -478 & 13.22 & 51.64 & 29.46 & 81.10 & 0.14 \\
\cline { 2 - 8 } & Post-Leach/Pre-Recovery & -460 & 13.19 & 54.30 & 26.69 & 80.99 & -0.09 \\
\hline \multirow{3}{*}{ 2nd } & Post-Recovery & -454 & 13.30 & 50.48 & 25.67 & 76.14 & 0.04 \\
\cline { 2 - 8 } & Pre-Leach & -453 & 13.60 & 51.05 & 24.47 & 75.52 & 1.93 \\
\cline { 2 - 8 } & Post-Leach/Pre-Recovery & -412 & 13.56 & 52.17 & 22.68 & 74.85 & -0.08 \\
\cline { 2 - 8 } & Post-Recovery & -412 & 13.56 & 49.66 & 25.18 & 74.85 & -0.02 \\
\hline
\end{tabular}

General trends can be deducted from the first circulation of the solutions:

1. After leaching, sulfide concentration increased and polysulfide concentration decreases;

2. The total concentration of sulfide-polysulfide in the ASGLS solution showed a negative effect on recovery;

3. $\mathrm{pH}$ was stable after leaching and recovery. Free $\mathrm{OH}^{-}$also shows little movement with measurements hovering around zero; and 
4. A increase in $E_{H}$ correlated with a decrease in total sulfide-polysulfide concentration decrease after recovery.

The increase in sulfide concentration after leaching and the decrease polysulfide concentration was expected due to the basic gold oxidation-lixiviation reaction. The decrease in total sulfidepolysulfide concentration after recovery may have been caused by simple natural oxidation during the horizontal shaking of the stage. Although a closed vessel was used in the recovery stage, the system was not in vacuum and air was still available in the vessel for natural oxidation to occur.

During the second circulation of the solutions, no reagent addition of $\mathrm{Na}_{2} \mathrm{~S}$ or $\mathrm{S}$ other than that of the make-up solution was added to the bulk solution before leaching. Since there was no clear method developed for this re-addition of reagents, no action during this campaign was taken. However, the adjustment in free $\mathrm{OH}^{-}$concentration was made by adding sufficient amount into the solution before the second leaching stage. The addition was based on the post-recovery measurement of the available free $\mathrm{OH}^{-}$in solution. A negative free $\mathrm{OH}^{-}$would allow the existence of HS $^{-}$in the solution, which has been proven to have negative effects to leaching.

The average decrease of the total sulfide-polysulfide in solution after the first circulation was $5.58-\mathrm{g} / \mathrm{L}$ from the initial fresh solution (first pre-leach). Most likely, these sulfide-polysulfide experienced a natural oxidation and exist in the solution as thiosulfate $\left(\mathrm{S}_{2} \mathrm{O}_{3}{ }^{2-}\right)$. This accounts to $6.87 \%$ of the initial sulfide-polysulfide in solution oxidized.

The same trends were observed within the second circulation: an increase in sulfide concentration after leaching stage and partial oxidation of sulfide specie in the solution after recovery. These observations will be correlated to the leaching performance of the simulation in the next section below.

\subsubsection{Leaching Performance and Consistency}

In Batch Simulation 1, there were no feed recirculation conducted. The discharge of each leaching batch were considered tailings; two 15-gram samples were taken from each leaching discharge batches and sent to CCI laboratory in Kellogg, Idaho for precious metals analysis by fire-assay techniques. Shown in Table 36 are averages of the fire-assay result of the two samples sent to the laboratory. With a known head grade of 0.772-opt gold and 89.8-opt silver, \% Metal Leached was calculated. 
Table 36. Leaching Performance of ASGLS in Batch Simulation

\begin{tabular}{|c|c|c|c|c|}
\hline \multirow{2}{*}{ Leaching \# } & \multicolumn{2}{|c|}{ Tails Assay (opt) } & \multicolumn{2}{c|}{ \% Metal Leached } \\
\cline { 2 - 5 } & $\mathbf{A u}$ & $\mathbf{A g}$ & $\mathbf{A u}$ & $\mathbf{A g}$ \\
\hline Leaching 1 & 0.345 & 85.3 & 59.87 & 4.48 \\
\hline Leaching 2 & 0.256 & 85.1 & 70.18 & 4.65 \\
\hline Leaching 3 & 0.256 & 84.0 & 70.18 & 5.88 \\
\hline Leaching 4 & 0.262 & 86.5 & 69.48 & 3.14 \\
\hline Leaching 5 & 0.262 & 88.0 & 69.48 & 1.40 \\
\hline & & Average & 67.84 & 3.91 \\
\hline & & Standard Dev. & 4.47 & 1.71 \\
\hline Leaching 6 & 0.406 & 87.4 & 52.71 & 2.07 \\
\hline Leaching 7 & 0.330 & 87.6 & 61.56 & 1.85 \\
\hline Leaching 8 & 0.340 & 87.8 & 60.40 & 1.62 \\
\hline Leaching 9 & 0.308 & 85.1 & 64.12 & 4.65 \\
\hline Leaching 10 & 0.382 & 85.2 & 55.50 & 4.54 \\
\hline & & Average & 58.86 & 2.95 \\
\hline & & Standard Dev. & 4.65 & 1.51 \\
\hline
\end{tabular}

First of all, it should be noted that all leaching batches showed a fairly similar results with reasonable standard deviations relative to the actual results. The leaching performances of the first circulations were similar to those achieved in preliminary leaching test (experimental campaign RT-1), which proves the reproducibility of the system and the experimental procedures.

In average, the solutions had a slightly better gold and silver leaching performance during their first circulations: $67.84 \%$ and $3.91 \%$, versus $58.86 \%$ and $2.95 \%$ of the second circulation. Again, silver leaching was not significant; indicating that minimal attack of the complex silver containing sulfide minerals was performed by the ASGLS. Technically, this ability of the ASGLS to selectively leach free gold-silver (electrum) may be beneficial.

The significantly lower leaching performance of Solution 1 in the first and second circulation (Leaching 1 and 6, respectively) may have caused by an experimental error in analysis and can intuitively be considered as an outlier.

The nearly $10 \%$ decrease in average leaching performance during the second solution circulation was certainly significant. Several correlations can be made in account to this decrease in leaching performance. Firstly, the decrease in total sulfide-polysulfide in solution (likely indicating some thiosulfate in solution) after the first circulation might have impeded leaching. Secondly, the elevated amount of antimony and arsenic leached into solution may have caused extra reagent consumption. (See Table 37)

\subsubsection{Leaching of Other Metals}

From the earlier characterization study of the composite concentrate, it was observed that appreciable amounts of arsenic, antimony, iron, and copper exists in the concentrate. A $2 \mathrm{~mL}$ 
sample of each solution after all leaching stages were obtained and fused with aqua-regia solution to be sent to an outside laboratory for the ICP analysis of those elements. Shown in Table 37 is the result of this analysis.

Table 37. Results of Leaching of Other Metals in Batch Simulation

\begin{tabular}{|l|c|c|c|c|c|c|c|} 
& \multicolumn{7}{|c|}{ Solution Assay (all in ppm, otherwise noted) } \\
\cline { 2 - 9 } & Al & As (ppb) & Cu & Fe & Pb & Sb (ppb) & Zn \\
\hline Post-Leach 1 & 33.37 & 48.91 & 14.51 & 1.18 & 0.70 & 69.00 & 1.09 \\
\hline Post-Leach 2 & 37.59 & 49.23 & 5.23 & 1.40 & -- & 68.48 & 1.68 \\
\hline Post-Leach 3 & -- & 48.16 & 15.17 & 3.93 & -- & 67.58 & 1.36 \\
\hline Post-Leach 4 & -- & 48.40 & 15.59 & 1.14 & -- & 72.82 & 0.97 \\
\hline Post-Leach 5 & -- & 50.16 & 14.44 & 1.04 & -- & 73.19 & 0.54 \\
\hline Post-Leach 6 & -- & 84.31 & 16.36 & 1.06 & -- & 115.46 & 0.64 \\
\hline Post-Leach 7 & 54.99 & 84.71 & 15.80 & 1.05 & -- & 113.80 & 0.50 \\
\hline Post-Leach 8 & 50.22 & 86.84 & 17.21 & 1.08 & -- & 117.68 & 0.62 \\
\hline Post-Leach 9 & -- & 84.78 & 14.91 & 1.20 & -- & 113.14 & 0.55 \\
\hline Post-Leach 10 & -- & 77.12 & 12.50 & 1.25 & -- & 104.06 & 0.56 \\
\hline
\end{tabular}

Appreciable dissolution was only observed in arsenic and antimony where a final concentration of up to 86.84-ppb and 117.68-ppb where found. Although the ASGLS performed exceptionally well against the cyanicides of iron, copper, lead, and zinc in this simulation, it has the known ability to leach arsenic and antimony. This finding may induce future study of the overall effects of these elements in solution to the performance of the ASGLS in all unit processes.

\subsubsection{Precious Metal Recovery from Pregnant ASGLS}

The gold recovery from pregnant solution stage of Batch Simulation 1 was conducted in the same manner of experimental campaign RC-1 and RC-2. Since the available horizontal shaker was only able to accommodate a maximum of 500-mL flasks, the 1500 -mL solution batches were split into four 375-mL tests with 37.5grams of activated carbon as gold loading substrate. At the end of the process, the solution and activated carbon from each flask were again combined. Two 15gram samples of the activated carbon were then taken and sent to CCI laboratory for precious analysis using fire-assay. Shown in Table 38 are the results of the gold recovery stage of Batch Simulation 1. 
Table 38. Gold Recovery Results in Batch Simulation

\begin{tabular}{|c|c|c|c|c|c|c|c|}
\hline \multirow{2}{*}{$\begin{array}{c}\text { Loading } \\
\text { Circulation }\end{array}$} & \multirow{2}{*}{ Carbon Batch \# } & Au in Solution & $\begin{array}{c}\text { Ag in } \\
\text { Solution }\end{array}$ & \multicolumn{2}{|c|}{ Carbon Assay (opt) } & \multicolumn{2}{|c|}{$\%$ Recovery } \\
\cline { 3 - 8 } & CARBON 1 & 1.172 & 9.12 & 0.158 & 0.7 & $\mathbf{4 6 . 1 1 \%}$ & $\mathbf{2 4 . 4 \%}$ \\
\hline \multirow{3}{*}{ 1st Loading } & CARBON 2 & 1.374 & 9.46 & 0.085 & 0.9 & $\mathbf{2 1 . 1 6 \%}$ & $\mathbf{3 2 . 5 \%}$ \\
\cline { 2 - 8 } & CARBON 3 & 1.374 & 11.97 & 0.082 & 0.6 & $\mathbf{2 0 . 4 1 \%}$ & $\mathbf{1 7 . 1 \%}$ \\
\cline { 2 - 8 } & CARBON 4 & 1.360 & 6.39 & 0.064 & 0.7 & $\mathbf{1 6 . 0 9 \%}$ & $\mathbf{3 4 . 8 \%}$ \\
\hline \multirow{3}{*}{ 2nd Loading } & CARBON 1 & 1.360 & 2.85 & 0.184 & 1.5 & $\mathbf{6 . 5 4 \%}$ & $\mathbf{9 6 . 0 0 \%}$ \\
\cline { 2 - 8 } & CARBON 2 & 1.032 & 4.22 & 0.125 & 1.2 & $\mathbf{1 3 . 2 6 \%}$ & $\mathbf{2 4 . 3 2 \%}$ \\
\cline { 2 - 8 } & CARBON 3 & 1.205 & 3.76 & 0.143 & 1.0 & $\mathbf{1 7 . 3 1 \%}$ & $\mathbf{3 1 . 8 2 \%}$ \\
\cline { 2 - 8 } & CARBON 4 & 1.183 & 3.31 & 0.108 & 1.0 & $\mathbf{1 2 . 7 3 \%}$ & $\mathbf{3 6 . 2 1 \%}$ \\
\hline \multirow{3}{*}{ 3rd Loading } & CARBON 1 & 1.256 & 9.46 & 0.210 & 1.4 & $\mathbf{7 . 0 8 \%}$ & $\mathbf{- 1 . 8 1 \%}$ \\
\cline { 2 - 8 } & CARBON 2 & 1.087 & 9.24 & 0.152 & 1.2 & $\mathbf{8 . 3 4 \%}$ & $\mathbf{- 1 . 8 5 \%}$ \\
\hline
\end{tabular}

Referring to Table 38, it can be seen that poor gold recoveries from pregnant solutions were observed. During the first circulation, only Carbon 1 achieved the highest number of only 46.1\%; Carbon 2-4 only achieved an average of 19.2\% gold recovery. These performances are significantly lower than that of previous recovery experimental campaigns; a recovery rate of up to $70.1 \%$ was achieved on experimental campaign RT-2. This significant decrease may possibly be due to the experimental error of higher content of total sulfide-polysulfide of $80.0-\mathrm{g} / \mathrm{L}$ used in this simulation campaign.

As comparison, experimental campaign RT-1 and RT-2 used a solution of 40.0-g/L total sulfidepolysulfide in solution; a gold and silver recovery of $70.1 \%$ and $89.7 \%$ was achieved. Stacey (2005) has shown the sharp decline in gold loading onto activated carbon between a 2.12M and 3.12M total sulfide-polysulfide in solution. This fact was overlooked during this campaign; thus creating lower recovery performances. Nonetheless, further research still is needed to confirm the effect of higher total sulfide-polysulfide concentration on the effectiveness of activated carbon in precious metal recovery from pregnant solution. However, a consistent trend was observed: a further decline of percent metal recovery in the second and third circulation.

\subsubsection{Waste Treatment of ASGLS Solution and NaOH Recirculation}

As described before, there were three waste treatment stages being performed in Batch Simulation 1. From each solution circulation, 300-mL was bled off after recovery. When a cumulative sample of $900-\mathrm{mL}$ was achieved, the volume of the solution was readjusted using DI water to 1500-mL (producing a solution concentration of 50-g/L total sulfide-polysulfide). $\mathrm{Na}_{2} \mathrm{SO}_{4}$ 
produced from the saturation of the oxidation product was then redissolved into a $1.5 \mathrm{M} \mathrm{Na}_{2} \mathrm{SO}_{4}$ solution and was treated using the electrodialysis. Accordingly, $\mathrm{NaOH}$ addition was made to accommodate $65-\mathrm{g} / \mathrm{L}$ concentration needed. Shown in Table 39 is the observations made during each waste treatment stages.

Table 39. Waste Treatment Stage Results of Batch Simulation

\begin{tabular}{|c|c|c|c|}
\hline & Waste Treatment 1 & Waste Treatment 2 & Waste Treatment 3 \\
\hline $\mathrm{Na}_{2} \mathrm{SO}_{4}$ Produced (grams) & 209.23 & 202.17 & 197.56 \\
\hline $\mathrm{NaOH}(\mathrm{g} / \mathrm{L})$ & 68.33 & 65.21 & 62.33 \\
\hline $\mathrm{H}_{2} \mathrm{SO}_{4}(\mathrm{~g} / \mathrm{L})$ & 110.49 & 108.76 & 108.23 \\
\hline \multicolumn{4}{|l|}{ Make up Solution } \\
\hline Total Sulfide-Polysulfide (g/L) & 78.29 & 79.35 & 76.89 \\
\hline Sulfide (g/L) & 51.45 & 54.09 & 49.67 \\
\hline Polysulfide (g/L) & 26.84 & 25.26 & 27.22 \\
\hline
\end{tabular}

As seen above, the performance of the waste treatment stages were certainly comparable to that of earlier studies in this thesis. A NaOH final concentration of up to 68.33-g/L was achieved. More importantly, the electrodialysis stage did not experienced any membrane fouling or any other complications.

In Leaching 9 and 10, 300-mL of these regenerated $\mathrm{NaOH}$ solution was used as make-up solutions before re-circulation. It was found that the dissolution of $\mathrm{Na}_{2} \mathrm{~S}$ and $\mathrm{S}$ in the solution is very good. As seen also in Table 39, great final concentrations of total sulfide-polysulfide, sulfide, and polysulfide were achieved.

\subsection{Bench-Top Batch Simulation of Golden Sunlight's Pyrite Concentrate (Batch Simulation 2)}

\subsubsection{Introduction and Background to Batch Simulation 2}

The second batch simulation treated Golden Sunlight's pyrite concentrate. Actually, two batch simulations of the pyrite concentrate were conducted at the same time: one with original pyrite concentrate and one with pre-oxidized pyrite concentrate. Since each of the provided unique data and analysis, they will be discussed separately in this thesis. Batch Simulation 2 is designated for the batch simulation with original pyrite concentrate; and Batch simulation 3 was designated for the batch simulation with pre-oxidized pyrite concentrate (Section 5.4).

The pyrite concentrate is produced in Golden Sunlight's mill by means of gravity concentration using spirals. A cyanide leach discharge is subject to this gravity concentration, producing pyrite concentrate with elevated amounts of precious metals (Au and Ag). XRD analysis shows the superior existence of pyrite $\left(\mathrm{FeS}_{2}\right)$ and silica $\left(\mathrm{SiO}_{2}\right)$ in the concentrate. Fire-assay analysis provided precious metal content of the concentrate: 0.104-opt Au and 0.9-opt Ag. 
This concentrate was chosen in this study because it provided the study with a feed material currently being treated in Montana less effectively using cyanide.

In Batch Simulation 2, only two solutions, instead of five as in Batch Simulation 1, were being circulated through the unit processes. This change in experimental design was performed since Batch Simulation 2 and 3 were conducted at the same time; the space arrangement of the leaching vessel only allowed two solutions of each simulation to be ran at the same time. Nonetheless, this arrangement provided a monitoring of reproducibility.

\subsubsection{Experimental Setup, Design, and Daily Operational Schedule}

Shown in Figure 32 is a process flowsheet showing all circulations of the two ASGLS solutions of Batch Simulation 2. The same stages were used in this simulation with some improvements applied in experimental procedure:

1. Three circulations (instead of two) were conducted to each solution;

2. Total sulfide-polysulfide concentration of only $60-\mathrm{g} / \mathrm{L}$ was used in this simulation since the Golden Sunlight pyrite concentrate contains less precious metal compared to the KGCMC composite concentrate;

3. A Carbon-In-Column (CIC) setup was used in the recovery stages (rather than batch setup); andWaste treatment was performed continuously within each circulation (rather than every three solution circulations).

These differences in experimental setup provided the research with more valuable applied results. First of all, a third circulation provided a look at the effects of further recirculation to the performance of the developed unit processes. Secondly, a more realistic setup of precious metal recovery from pregnant solution using a CIC (commonly used in industry) was adopted. Finally, more $\mathrm{NaOH}$ recirculation was performed because of the continuous waste treatment unit procedure. Shown in Table 40 is the simulation procedure.

Table 40. Process Labels of Batch Simulation 2

\begin{tabular}{|l|c|c|c|c|c|}
\hline Leaching \# & Solution \# & Feed \# & Recovery \# & Oxidation Test \# & Make-Up Solution \\
\hline Leaching 1 & Solution 1 & Feed 1 & Recovery 1 & Oxidation 1 & -- \\
\hline Leaching 2 & Solution 2 & Feed 2 & Recovery 2 & Oxidation 2 & -- \\
\hline Leaching 3 & Solution 1 & Feed 3 & Recovery 3 & Oxidation 3 & -- \\
\hline Leaching 4 & Solution 2 & Feed 4 & Recovery 4 & Oxidation 4 & From Oxidation 1 \\
\hline Leaching 5 & Solution 1 & Feed 5 & Recovery 5 & Oxidation 5 & From Oxidation 2 \\
\hline
\end{tabular}

The simulation details are presented in Table 41. It is shown that almost all experimental details were similar compared to Batch Simulation 1. 
Table 41. Experimental Details of Batch Simulation 2

5.3 .3

\begin{tabular}{|c|c|}
\hline $\begin{array}{c}\text { Simulation Variable } \\
\text { \# of Solutions }\end{array}$ & 2 \\
\hline Volume of Each Solution Batch & $1500-\mathrm{mL}$ \\
\hline Initial Sulfide-Polysulfide Concentration & $60-\mathrm{g} / \mathrm{L}$ \\
\hline Initial NaOH Concentration & $37.5-\mathrm{g} / \mathrm{L}$ \\
\hline Total Flowsheet Circulations per Solution & 3 \\
\hline Feed Treated per Leach & $100.0-\mathrm{grams}$ \\
\hline Leaching Temperature & $50^{\circ} \mathrm{C}$ \\
\hline Leaching Time & $72-\mathrm{hours}$ \\
\hline Leaching Agitation & $170-\mathrm{rpm}$ \\
\hline Recovery Stage Setup & $\mathrm{ClC}$ \\
\hline Recovery Time & $48-\mathrm{hours}$ \\
\hline vol. of Solution to Waste Treatment per Circulation & $20 \%$ \\
\hline
\end{tabular}

\section{Experimental Results and Discussion}

\subsubsection{Stability of Solution Parameters}

Batch Simulation 2 provided an extra circulation for every solution in the simulation. This extra circulation provided additional information of the leaching performance of the solution. Shown in Table 42 are the monitoring results of the solution parameters of Batch Simulation 2.

Table 42. Solution Parameters of Batch Simulation 2

\begin{tabular}{|c|c|c|c|c|c|c|c|}
\hline \multicolumn{2}{|c|}{ Circulation/Sampling Point } & $\begin{array}{c}\mathbf{E}_{\mathbf{H}} \\
(\mathbf{S H E})\end{array}$ & $\mathbf{p H}$ & $\begin{array}{c}{\left[\mathbf{S}^{2-}\right]} \\
(\mathrm{g} / \mathbf{L})\end{array}$ & $\begin{array}{c}{\left[\mathbf{S}_{\mathbf{x}}^{{ }^{2-}}\right.} \\
(\mathrm{g} / \mathbf{L})\end{array}$ & $\begin{array}{c}\text { Total } \\
\mathbf{S}^{2-}-\mathbf{S}_{\mathbf{x}}^{2-}\end{array}$ & $\begin{array}{c}\text { Free } \mathbf{O H}^{-} \\
(\mathrm{g} / \mathbf{L})\end{array}$ \\
\hline \multirow{3}{*}{ 1st } & Pre-Leach & -500 & 12.94 & 40.89 & 18.69 & 59.44 & 1.349 \\
\cline { 2 - 8 } & Post-Leach/Pre-Recovery & -482 & 13.19 & 39.99 & 15.51 & 55.50 & 0.010 \\
\cline { 2 - 8 } & Post-Recovery & -463 & 12.95 & 39.46 & 10.58 & 50.04 & -0.103 \\
\hline \multirow{3}{*}{ 2nd } & Pre-Leach & -442 & 13.07 & 40.21 & 4.50 & 44.71 & 0.687 \\
\cline { 2 - 8 } & Post-Leach/Pre-Recovery & -442 & 12.67 & 40.43 & 3.66 & 44.09 & -0.190 \\
\cline { 2 - 8 } & Post-Recovery & -427 & 13.20 & 38.14 & 2.51 & 40.65 & -0.330 \\
\hline \multirow{3}{*}{ 3rd } & Pre-Leach & -421 & 12.56 & 33.60 & 4.29 & 37.89 & 0.790 \\
\cline { 2 - 8 } & Post-Leach/Pre-Recovery & -425 & 12.66 & 30.60 & 2.95 & 33.55 & -0.116 \\
\cline { 2 - 8 } & Post-Recovery & -411 & 12.43 & 26.90 & 3.70 & 30.60 & -0.233 \\
\hline
\end{tabular}

As can be seen in the post-recovery parameters in all circulation, a deterioration of up to $4.00-\mathrm{g} / \mathrm{L}$ of sulfide-polysulfide was experienced. This increased rate may be caused by the nature of the CIC setup itself. With a constantly moving solution, more turbulence was experienced by the solution and created a more favorable condition for natural oxidation. Future research will further investigate this effect.

A general trend within each circulation similar to that of Batch Simulation 1 can be observed: 
1. A significant decrease of total sulfide-polysulfide was experienced after each leaching stage. This decrease, however, was not followed by equal deterioration of each sulfide or polysulfide, indicating a simultaneous reduction of polysulfide to sulfide is occurring;

2. $\quad$ Free $\mathrm{OH}$ - in the solution decreases after both leaching and recovery stages;

3. An increase in $\mathrm{E}_{\mathrm{H}}$ is an indication of sulfide-polysulfide content; and

4. $\mathrm{pH}$ of solutions, although minute, can be correlated to the amount of free $\mathrm{OH}-$ in the solution.

In this batch simulation, an alternative method was developed to rejuvinate the solution after each circulation in an effort to maintain a constant polysulfide to sulfide ratio in the solution. Simply put, elemental sulfur was added according to how much polysulfide had decreased and $\mathrm{Na}_{2} \mathrm{~S}$ was added according to how much sulfide has deteriorated. Thus unlike before, there was no set addition ratio set for these reagents.

This method proved to be detrimental to the solution. Shown in the pre-leach parameters of the $2^{\text {nd }}$ circulation that the method did not provide an increase in polysulfide in the solution. Instead, a significant decrease in polysulfide concentration was experienced. This decrease is certainly not in the interest of the ASGLS performance. It can be seen later in the next section that leaching performance is affected by this decrease in polysulfide concentration.

\subsubsection{Leaching Performance and Consistency}

Shown in Table 43. are fire assay results of the leaching stage discharge (tails) and their respective \%Metal Leached of Batch Simulation 2.

Table 43. Leaching Performance of ASGLS in Batch Simulation 2

\begin{tabular}{|c|c|c|c|c|}
\hline \multirow{2}{*}{ Leaching Batch } & \multicolumn{2}{|c|}{ Tails Assay (opt) } & \multicolumn{2}{c|}{ \% Metal Leached } \\
\cline { 2 - 5 } & Au & Ag & Au & Ag \\
\hline Leaching 1 & 0.020 & 0.22 & 79.06 & 75.56 \\
\hline Leaching 2 & 0.023 & 0.20 & 75.92 & 77.78 \\
\hline Leaching 3 & 0.041 & 0.25 & 57.59 & 72.22 \\
\hline Leaching 4 & 0.041 & 0.27 & 57.59 & 70.00 \\
\hline Leaching 5 & 0.035 & 0.23 & 63.35 & 74.44 \\
\hline
\end{tabular}

During the first circulations (Feed $1 \& 2$ ), both leaching stages provided excellent leaching of $79.06 \%$ and $75.92 \%$ for gold, and $75.56 \%$ and $77.78 \%$ for silver. These results are comparable to the preliminary leaching tests (LGS-1) where a maximum gold and silver leaching of $76.90 \%$ and $55.60 \%$ was achieved. It can be concluded that with a fresh ASGLS solution a good recovery of gold and silver in a high containing pyrite concentrate was comparable to or better than that of cyanide systems.

In the second and third circulations, however, a significant drop in gold leaching was observed. This significant drop goes hand in hand with the unsuccessful procedure of sulfide-polysulfide 
regeneration procedure adopted in this campaign. A drop in performance, however, was not observed in silver leaching.

\subsubsection{Leaching of Other Metals}

The Golden Sunlight pyrite concentrate did not contain as much arsenic or antimony as the KGCMC composite concentrate. Accordingly, less dissolution of these elements was experienced in Batch Simulation 2. However, the pyrite concentrate contains a higher content of iron (Fe). As seen in Table 44 below, iron dissolution in the ASGLS is insignificant.

Table 44. Dissolution of Other Metals in Batch Simulation 2

\begin{tabular}{|l|c|c|c|c|c|c|}
\hline \multirow{2}{*}{} & \multicolumn{6}{|c|}{ Solution Assay (all in ppm, otherwise noted) } \\
\cline { 2 - 7 } & Al & As (ppb) & Cu & Fe & Pb & Zn \\
\hline Post-Leach 1 & 173.36 & -- & 0.84 & 1.52 & -- & 0.55 \\
\hline Post-Leach 2 & 211.70 & -- & -- & 1.86 & -- & 0.51 \\
\hline Post-Leach 3 & 187.32 & 3.85 & 9.84 & 1.95 & -- & 0.71 \\
\hline Post-Leach 4 & 222.34 & 1.31 & 1.04 & 3.70 & -- & 0.64 \\
\hline Post-Leach 5 & 225.00 & 1.99 & 1.22 & 3.36 & -- & 0.87 \\
\hline
\end{tabular}

\subsubsection{Precious Metal Recovery Performance and Consistency}

Since the recovery stage setup of Batch Simulation 2 did not use the horizontal shaked flasks setup as in Batch Simulation 1, an analysis of performance consistency cannot be assessed. However, the new CIC setup allowed a more realistic experiment. The nature of the setup (explained in Appendix G) allowed the observance of gradual gold loading onto the activated carbon. Table 45 illustrates the fire-assay results of activated carbon samples sent to CCI laboratory. More importantly, shown in Table 46 is the average metal content of activated carbon batches according to the number of pregnant leach solution they have been subject to.

Table 45. Fire-Assay Results of Batch Simulation 2 Carbon Samples

\begin{tabular}{|c|c|c|}
\hline Sample & Au TLS (opt) & Ag TLS (opt) \\
\hline CARBON 1 & 0.015 & 0.190 \\
\hline CARBON 2 & 0.030 & 0.280 \\
\hline CARBON 3 & 0.040 & 0.320 \\
\hline CARBON 4 & 0.058 & 0.400 \\
\hline CARBON 5 & 0.069 & 0.660 \\
\hline CARBON 6 & 0.040 & 0.340 \\
\hline CARBON 7 & 0.047 & 0.420 \\
\hline CARBON 8 & 0.039 & 0.320 \\
\hline CARBON 9 & 0.016 & 0.22 \\
\hline
\end{tabular}

Table 46. Gradual Loading of Precious Metal in Batch Simulation 2 


\begin{tabular}{|c|c|c|}
\hline \multirow{2}{*}{$\begin{array}{c}\text { \# of Loading } \\
\text { Circulation }\end{array}$} & \multicolumn{2}{|c|}{ Metal in Carbon (opt) } \\
\cline { 2 - 3 } & Au & Ag \\
\hline 1 & 0.016 & 0.205 \\
\hline 2 & 0.035 & 0.300 \\
\hline 3 & 0.044 & 0.370 \\
\hline 4 & 0.049 & 0.370 \\
\hline 5 & 0.069 & 0.660 \\
\hline
\end{tabular}

It can be seen clearly that gradual loading of precious metal occurs in the ASGLS system. This recovery performance, however, is below the maximum performance that can be achieved from activated carbon. As seen in Table 47 below, the total precious metal recovered from the whole batch simulation only reached $62.76 \%$ for gold and $60.54 \%$ for silver. Un-maximized recovery performance may again be attributed to the unsuccessful method of sulfide-polysulfide regeneration techniques employed in this Batch Simulation 2. In retrospect, the regeneration method may have produced elevated concentration of $\mathrm{S}_{2} \mathrm{O}_{3}{ }^{2-}$ that may have hindered the adsorption process onto activated carbon.

Table 47. Total Precious Metal Recovery in Batch Simulation

\begin{tabular}{|c|c|c|c|}
\hline Metal & In Solution $(\mathbf{m g})$ & Recovered $(\mathbf{m g})$ & Total Recovery (\%) \\
\hline $\mathbf{A u}$ & 6.175 & 3.875 & $\mathbf{6 2 . 7 6}$ \\
\hline $\mathbf{A g}$ & 56.96 & 34.48 & $\mathbf{6 0 . 5 4}$ \\
\hline
\end{tabular}

As confirmation learning from these observations, a more effective way of sulfide-polysulfide regeneration was employed in Batch Simulation 3; and total precious metal recovery far superior to that of Batch Simulation 2 was achieved. Batch Simulation 3 produced a recovery rate of 91.12\% for gold and 93.14\% for silver (see Section 5.4.3.3).

\subsubsection{Waste Treatment of ASGLS Solution and NaOH Recirculation}

There were five total waste treatment batches conducted in the Batch Simulation 2. This was possible since a continuous method was adapted. In each circulation, $150 \mathrm{~mL}$ of the initial solution was taken to be fed to the waste treatment process consisting of pressure oxidation and electrodialysis. Nevertheless, Table 48 shows that consistency in the waste treatment process developed is exceptional. Almost $100 \%$ of $\mathrm{Na}_{2} \mathrm{SO}_{4}$ was readily achieved (i.e. a $100 \%$ yield conversion would produce 33.28-grams of $\mathrm{Na}_{2} \mathrm{SO}_{4}$ powder). 
Table 48. Waste Treatment Stages of Batch Simulation 2

\begin{tabular}{|l|c|c|c|c|c|}
\hline \multirow{2}{*}{} & \multicolumn{5}{|c|}{ Waste Treatment Batch } \\
\cline { 2 - 6 } & $\mathbf{1}$ & $\mathbf{2}$ & $\mathbf{3}$ & $\mathbf{4}$ & $\mathbf{5}$ \\
\hline $\mathrm{Na}_{2} \mathrm{SO}_{4}$ Produced (grams) & 31.06 & 30.17 & 29.94 & 32.43 & 28.80 \\
\hline $\mathrm{NaOH}_{(\mathrm{g} / \mathrm{L})}$ & 66.822 & 58.415 & 68.362 & 66.073 & 66.843 \\
\hline $\mathrm{H}_{2} \mathrm{SO}_{4}(\mathrm{~g} / \mathrm{L})$ & 105.18 & 119.736 & 112.14 & 114.744 & 107.988 \\
\hline Make up Solution & & & & & \\
\hline Total Sulfide-Polysulfide $(\mathrm{g} / \mathrm{L})$ & 78.29 & 79.35 & 76.89 & 76.78 & 76.08 \\
\hline Sulfide $(\mathrm{g} / \mathrm{L})$ & 51.45 & 54.09 & 49.67 & 49.96 & 49.07 \\
\hline Polysulfide $(\mathrm{g} / \mathrm{L})$ & 26.84 & 25.26 & 27.22 & 26.82 & 27.01 \\
\hline
\end{tabular}

\subsection{Batch Simulation of Pre-Oxidized Golden Sunlight's Pyrite Concentrate (Batch Simulation 3)}

\subsubsection{Introduction and Background}

A third and final batch simulation campaign was conducted with pre-oxidized Golden Sunlight's pyrite concentrate as feed (Batch Simulation 3). The same pyrite concentrate sample used in Batch Simulation 2 was partially pre-oxidized using a NSC simulation method. The NSC is a partial oxidation process utilizing the role of nitrogen species $\left(\mathrm{NO}^{+}\right)$as intermediates and as an expedient means to transport oxygen to the surface of the solid particles (Anderson, 2004). A room temperature solution treatment using 4000-mL $10 \% \mathrm{HNO}_{3}$ solution for 24 hours was used to partially oxidize 2000-grams of pyrite concentrate.

The result was a further liberation of gold particles in the concentrate and partial oxidation of sulfides to sulfur. As mentioned before, the existence of elemental sulfur in the solution may provide a natural source of reagent and may reduce reagent consumption.

All procedures and experimental setup were identical to that of Batch Simulation 2. However, a better method of sulfide-polysulfide regeneration technique was employed in Batch Simulation 3, resulting in a better maintenance of total sulfide-polysulfide ratio in the solution after recirculation. In the end, a far more consistent leaching performance was experienced even after three circulations. These results will be discussed more in depth in the next sections of this Batch Simulation.

\subsubsection{Experimental Setup, Design, and Daily Operational Schedule}

All experimental setup of Batch Simulation 3 is identical to that of Batch Simulation 2. The only difference is the feed material. Batch Simulation 3 used the pre-oxidized feed material that in preliminary leaching tests proved to improve precious metal leaching significantly. Please refer back to section 5.3.2 for a detailed discussion of Batch Simulation 2's experimental details.

\subsubsection{Experimental Results and Discussion}




\subsubsection{Stability of Solution Parameters}

Shown in Table 49 are the results of the average solution parameters monitoring of Batch Simulation 3 on each sampling points. Similar to Batch Simulation 2, initial concentration of all initial solution batches were $60-\mathrm{g} / \mathrm{L}$.

Table 49. Monitoring of Solution Parameters in batch Simulation 3

\begin{tabular}{|c|c|c|c|c|c|c|c|}
\hline \multicolumn{2}{|c|}{ Circluation/Sampling Point } & $\begin{array}{c}\text { Eh } \\
(\mathbf{S H E})\end{array}$ & $\mathbf{p H}$ & $\begin{array}{c}{\left[\mathbf{S}^{2-}\right]} \\
(\mathrm{g} / \mathrm{L})\end{array}$ & $\begin{array}{c}{\left[\mathbf{S}_{\mathbf{x}}{ }^{2-}\right]} \\
(\mathrm{g} / \mathrm{L})\end{array}$ & $\begin{array}{c}\text { Total } \\
\mathbf{S}^{2-}-\mathbf{S}_{\mathbf{x}}{ }^{2-}\end{array}$ & $\begin{array}{c}\text { Free OH- } \\
(\mathrm{g} / \mathbf{L})\end{array}$ \\
\hline \multirow{3}{*}{ 1st } & Pre-Leach & -491 & 12.87 & 40.27 & 19.42 & 59.69 & 1.165 \\
\cline { 2 - 8 } & Post-Leach/Pre-Recovery & -485 & 12.89 & 41.74 & 14.04 & 55.78 & 1.551 \\
\cline { 2 - 8 } & Post-Recovery & -461 & 12.67 & 40.86 & 11.59 & 52.45 & -0.760 \\
\hline \multirow{3}{*}{ 2nd } & Pre-Leach & -488 & 13.43 & 42.51 & 15.41 & 57.91 & 2.005 \\
\cline { 2 - 8 } & Post-Leach/Pre-Recovery & -475 & 13.02 & 43.72 & 13.74 & 57.46 & 0.240 \\
\cline { 2 - 8 } & Post-Recovery & -466 & 13.04 & 39.93 & 11.05 & 50.98 & -0.225 \\
\hline \multirow{3}{*}{ 3rd } & Pre-Leach & -464 & 13.66 & 41.62 & 13.76 & 55.38 & 1.170 \\
\cline { 2 - 8 } & Post-Leach/Pre-Recovery & -461 & 13.14 & 40.79 & 12.64 & 53.43 & -0.016 \\
\cline { 2 - 8 } & Post-Recovery & -447 & 13.13 & 39.18 & 9.93 & 49.11 & -0.212 \\
\hline
\end{tabular}

However, a different method of sulfide and sulfur addition was employed in this simulation. Instead of using that of in Batch Simulation, a set ratio was kept in adding sulfide and sulfur into the bound to be recirculated solution. The total addition depends on the total deterioration of sulfide-polysulfide shown in the post-recovery measurements. It can be seen that a far greater yield addition of polysulfide concentration increase were achieved in this simulation. This better maintenance of polysulfide concentration in solution proved to be significant. Far greater leaching consistency were achieved. In Batch Simulation 2, the addition method did not provide any polysulfide concentration increase.

\subsubsection{Precious Metal Leaching Performance and Consistency}

As seen in Table 50 presented below, a more steady and consistent leaching performance was achieved in Batch Simulation 3. This consistency in performance may be attributed to the successful method of sulfide-polysulfide concentration maintenance. Leaching performance was maximized after recirculation when the sulfide-polysulfide concentration was also rejuvenated. Compared to the decrease of gold leaching of 39.57\% and 21.47\% in Batch Simulation 1 and 2 after recirculation, the leaching performance of Batch Simulation 3 was more consistent with only a maximum decrease of $7.86 \%$ in gold leaching was experienced.

Table 50. Leaching Performance of ASGLS in Batch Simulation 3

\begin{tabular}{|c|c|c|c|c|}
\hline \multirow{2}{*}{ Leaching Batch } & \multicolumn{2}{|c|}{ Tails Assay (opt) } & \multicolumn{2}{c|}{ \% Metal Leached } \\
\cline { 2 - 5 } & $\mathbf{A u}$ & $\mathbf{A g}$ & $\mathbf{A u}$ & $\mathbf{A g}$ \\
\hline Leaching 1 & 0.011 & 0.20 & 88.48 & 77.78 \\
\hline Leaching 2 & 0.011 & 0.20 & 89.01 & 77.78 \\
\hline Leaching 3 & 0.013 & 0.24 & 86.39 & 73.33 \\
\hline Leaching 4 & 0.018 & 0.22 & 81.15 & 75.56 \\
\hline
\end{tabular}




\begin{tabular}{|l|l|l|l|l|}
\hline Leaching 5 & 0.013 & 0.21 & 86.39 & 76.67 \\
\hline Leaching 6 & 0.010 & 0.20 & 89.53 & 77.78 \\
\hline
\end{tabular}

These results firmly conclude that a consistent leaching performance of the ASGLS can be maintained after multiple solution recirculations. This was a basic objective of this thesis research. Without any solution recirculation, however cheap reagent costs are, it would be impossible to treat fresh feed material continuously with only fresh solution. The cost of storage, handling, and waste treatment would be enormous. With this performance consistency, the ASGLS can certainly compete with cyanide. In Montana, where cyanide use has been limited by law, ASGLS can certainly be a viable alternative.

\subsubsection{Gold Recovery from Pregnant Solution Performance and Consistency}

More encouraging results were achieved in the precious metal recovery stage of Batch Simulation 3. As in Batch Simulation 2, the CIC setup was employed in this campaign. As shown in Table 51 and 52, it can be clearly seen that gradual loading of gold and silver occurs in the CIC setup, according to how many pregnant solution has been treated through each carbon batch.

Table 51 . Fire-Assay Results of Carbon Batch of Batch Simulation 3

\begin{tabular}{|c|c|c|}
\hline Sample & Au (opt) & Ag (opt) \\
\hline CARBON 1 & 0.015 & 0.26 \\
\hline CARBON 2 & 0.023 & 0.30 \\
\hline CARBON 3 & 0.058 & 0.48 \\
\hline CARBON 4 & 0.060 & 0.52 \\
\hline CARBON 5 & 0.117 & 0.74 \\
\hline CARBON 6 & 0.146 & 0.90 \\
\hline CARBON 7 & 0.108 & 0.84 \\
\hline CARBON 8 & 0.082 & 0.70 \\
\hline CARBON 9 & 0.035 & 0.66 \\
\hline CARBON 10 & 0.030 & 0.37 \\
\hline
\end{tabular}

Table 52. Gradual Loading of Activated Carbon in Batch Simulation 3

\begin{tabular}{|c|c|c|}
\hline \multirow{2}{*}{ \# of Treatment } & \multicolumn{2}{|c|}{ Metal in Carbon (opt) } \\
\cline { 2 - 3 } & $\mathrm{Au}$ & $\mathbf{A g}$ \\
\hline 1 & 0.022 & 0.315 \\
\hline 2 & 0.029 & 0.480 \\
\hline 3 & 0.070 & 0.590 \\
\hline 4 & 0.084 & 0.680 \\
\hline 5 & 0.131 & 0.820 \\
\hline
\end{tabular}

More importantly, however, the total recovery of precious metal that Batch Simulation 3 achieved. Seen in Table 53 below is a total \% recovery of both gold and silver throughout campaign. It can be seen that $91.12 \%$ of leached gold into solution was recovered and $93.14 \%$ of 
leached silver into solution was recovered. Compared to results obtained in Batch Simulation where only $62.76 \%$ of gold in solution and $60.54 \%$ of silver in solution is recovered, these results are certainly superior, more realistic, and very promising.

Table 53. Total Recovery of Leached Precious Metal in Batch Simulation 3

\begin{tabular}{|c|c|c|c|}
\hline Metal & In Solution $(\mathbf{m g})$ & Recovered $(\mathbf{m g})$ & Total Recovery (\%) \\
\hline $\mathbf{A u}$ & 10.099 & 9.202 & $\mathbf{9 1 . 1 2}$ \\
\hline $\mathbf{A g}$ & 84.77 & 78.96 & $\mathbf{9 3 . 1 4}$ \\
\hline
\end{tabular}

This significant increase in total recovery of precious metal can certainly again be attributed to the well maintenance of total sulfide-polysulfide in solution.

\subsection{Summary of Bench-Top Batch Simulations}

In general, the three batch simulation studies have provided:

1. A confirmatory data indicating reproducibility of the ASGLS performance on all developed unit processes;

2. The ability to incorporate all unit processes developed to treat actual industrial precious metal containing concentrates at a laboratory bench scale;

3. A developed method of additional reagent addition to better maintain polysulfide and sulfide concentration in the ASGLS solution;

4. An opportunity to test realistic model of a Carbon in Column setup to a recovery circuit using activated carbon. More importantly, the CIC setup itself was proven to be very effective as it provided a better solution distribution within the carbon itself.

\subsection{Recommendations of Future Work}

Future work, immediate and long term, can be suggested from the completion of this thesis:

1. Leaching tests of carbonaceous (cyanide preg-robbing) materials should be performed to assess the effectiveness of the ASGLS. The extremely successful method of precious metal loading onto activated carbon may suggest the inability of ASGLS to perform well in carbonaceous materials. However, a concrete study is needed to investigate this;

2. The mechanism of precious metal loading onto activated carbon needs to be determined and thoroughly tested;

3. The stripping of precious metal from activated carbon was not discussed nor investigated at all in this thesis. An effective method of stripping is critical to the whole developed ASGLS process flowsheet. However, scoping work has indicated viable methodology which will be confirmed;

4. An extensive study of gypsum and/or ammonium production as treatment alternatives for the produced $\mathrm{Na}_{2} \mathrm{SO}_{4}$ by pressure oxidation and/or $\mathrm{H}_{2} \mathrm{SO}_{4}$ by the 
electrodialysis is needed. Only baseline experiments of these processes were performed in this thesis;

5. To test the ASGLS in a larger scale. A pilot scale process will provide a more realistic data in which the ASGLS solution parameters can be monitored more closely and realistically.

\subsection{Thesis Conclusion}

In conclusion, this research has achieved the following:

1. Characterized and successfully leached two gold-silver containing sulfide concentrates, including a Montana gold ore currently processed with cyanide;

2. Tested the ability of activated carbon, and other ion-exchange substrates, in recovering precious metal from pregnant ASGLS solution;

3. Successfully developed the treatment of waste ASGLS solution with pressure oxidation, crystallization, and electrodialysis;

4. Tested and incorporated all developed unit processes on industrial sulfide concentrates using laboratory scale bench-top batch simulations.

\subsection{Sulfide Concentrates Characterization and Leaching Experiments Conclusion}

Successful characterization studies were conducted to two different sulfide concentrates. The KGCMC composite concentrate was a blend of galena and sphalerite concentrate containing mostly $\mathrm{PbS}$ and $\mathrm{ZnS}$. The precious metal content of the composite concentrate was determined to be 0.772-opt gold and 89.8-opt silver. Most of the gold exists in the concentrate as electrum. Additionally, it has been recognized that complex sulfide minerals exists in the concentrate. Most of the silver is bound within these complex compounds and yielded a poor silver recovery in the leaching tests. Gold leaching, however, was very successful with a leaching rate of up to $82.4 \%$ was achieved.

The Golden Sunlight pyrite concentrate was characterized to mostly consist of pyrite $\left(\mathrm{FeS}_{2}\right)$ with appreciable amount of silica in the system. Fire-assay provided a head grade of 0.104-opt gold and 0.9-opt silver. Leaching experiments were performed in which a higher total sulfidepolysulfide concentration yielded a leaching performance of up to $77.25 \%$ of gold and $55.6 \%$ of silver. Additionally, a sample of the pyrite concentrate was partially pre-oxidized using the widely known NSC process. A leaching test of the pre-oxidized concentrate yielded a better performance of up to $83.33 \%$ leaching of gold and $66.6 \%$ leaching of silver. 


\subsection{Precious Metal Recovery from Pregnant ASGLS Solution Conclusion}

Initially, several different substrates were tested through a simple batch setup. Silica based ionexchange resins were determined to be unstable in the ASGLS system as immediate disintegration was observed. Styrene based resins were also tested with great stability in the solution but low recovery of precious metals from pregnant ASGLS solution. Activated carbon proved to be the best substrate for recovery, both in high and low concentration of precious metal in solution. For example, recoveries of up to $70.1 \%$ of gold and $89.3 \%$ of silver were achieved.

\subsection{Treatment of Waste ASGLS Solution Conclusion}

The proposed treatment of waste ASGLS solution included pressure oxidation, crystallization, electrodialysis, and gypsum or ammonium sulfate production for acid stream treatment produced by the electrodialysis. Extensive research was performed in both the pressure oxidation and electrodialysis stage of the proposed process.

Pressure oxidation of the ASGLS solution was successfully investigated so $100 \%$ oxidation of sulfide-polysulfide to sulfate readily achieved. Additionally, Pourbaix diagrams were developed to better investigate the oxidation reaction of the whole sulfur system. Temperature and oxygen overpressure was proven through a factorial experimental design to be very important in the yield of the oxidation reaction. Additionally, sufficient amount of $\mathrm{NaOH}$ needed to be provided to the polysulfide oxidation reaction. An insufficient amount of $\mathrm{OH}$ - in the solution will provide a significant drop in $\mathrm{pH}$ where elemental sulfur may be formed. Temperature of $150^{\circ} \mathrm{C}$, reaction time of 10-hours, overpressure of 80-psi, agitation of 700-rpm, and $\mathrm{NaOH}$ concentration of 65-g/L provided a $100 \%$ oxidation to sulfate of a 50 -g/L solution of total sulfide-polysulfide.

A precise standard operating procedure was developed for the operation of the ED-1-BP unit acquired for the electrodialysis process. In principle, the electrodialysis was able to perform splitting of $\mathrm{Na}_{2} \mathrm{SO}_{4}$ produced from pressure oxidation into two different streams of $\mathrm{NaOH}$ and $\mathrm{H}_{2} \mathrm{SO}_{4}$. Additionally, current density applied to the unit was determined to be significant in producing higher concentrated streams. After 4 hours and at $800-\mathrm{A} / \mathrm{m}^{2}$, streams of $70-\mathrm{g} / \mathrm{L} \mathrm{NaOH}$ and $120-\mathrm{g} / \mathrm{L}$ of $\mathrm{H}_{2} \mathrm{SO}_{4}$ was achieved from a $1.5 \mathrm{M} \mathrm{Na}_{2} \mathrm{SO}_{4}$ solution.

\subsection{Bench-Top Batch Simulations Conclusion}

The developed bench-top simulations provided the ASGLS with an ultimate test for performance consistency in all unit processes developed. Additionally, solution monitoring and effects of recirculation were also to be investigated. The Carbon-In-Column setup was proven to be most effective in extracting precious metal from pregnant ASGLS solution using activated carbon. Waste treatment of the waste ASGLS solution also was proven to be consistently excellent and regeneration of ASGLS solution from the regenerated $\mathrm{NaOH}$ solution was proven to be effective.

A general trend on the solution parameters was observed and a steady decrease of total sulfidepolysulfide in solution was experienced. A method in rejuvenating these oxidized sulfidepolysulfide was developed. This maintenance of sulfide-polysulfide concentration provided consistent leaching and recovery performance during solution recirculation. Consistent gold 
leaching of approximately $90 \%$ and silver leaching of $78 \%$ was achieved from an industrial Montana gold ore when this method was applied in Batch Simulation 3.

Post-leaching solution analysis showed that some leaching of other metals occurs in the ASGLS. However, ASGLS showed tremendous selectivity to the traditional cyanicides of copper, zinc, lead, and iron.

\subsection{Bibliography}

Anderson, C.G. “Applications of NSC Pressure Leaching.” Pressure Hydrometallurgy 34th Annual Hydrometallurgy Meeting of CIM 2004, Banff, Alberta, Canada.

Edwards, C.R. "Engineering The Equity Concentrate Leach Process."

Environmental Protection Agency (EPA).http://www.epa.gov/safewater/mcl.html\#mcls. (Accessed June 01, 2006).

Founie,A. "Minerals Yearbook: Gypsum.” United States Geological Services (2004): page numbers.

Huang, H.H. "Stability Calculation for Aqueous Systems (STABCAL)." Version 1.0. Montana Tech Department of Metallurgical \& Materials Engineering. Butte, Montana. 2006.

Hiskey, J.B., and Atluri, V.P. "Dissolution Chemistry of Gold and Silver in Different Lixiviants." Mineral Processing and Extractive Metallurgy Review (vol.4): 95-134.

Jeffrey, M.I., Chapman, N., Wall, J. "Sulfide/Polysulfide Leaching of Gold." Unpublished paper (2002).

Kostick, D.S. "Minerals Yearbook: Sodium Sulfate.” United States Geological Services (1997): 72.1-72.8.

Kostick, D.S. "Minerals Commodities Summary: Sodium Sulfate.” United States Geological Services (2006): 156-157.

Kleinjan, W.E., de Keizer, A., \& Janssen, A.J.H. "Kinetics of the Chemical Oxidation of Polysulfide Anions in Aqueous Solution.” Water Research 39 (2005): 4093-4100.

Kramer, D.A. "Minerals Yearbook: Nitrogen.” United States Geological Services (2004): 53.153.9.

Louw, N.J., Edwards, A.M., and Gussmann, H.W. “A New Process to Extract Gold and Stibnite from Arsenical Concentrates.” Chemical Engineering Conference (CHEMSA). 1977.

Mani, K.N. "Electrodialysis Water Splitting Technology.” Journal of Membrane Science 58 (1991): 117-138 
Marsden, J., House, I. The Chemistry of Gold Extraction. New York: Ellis Horwood, 1969.

Stacey, D.L. "The Development of The Alkaline Sulfide Gold Leaching System." Master's Thesis: Montana Tech Department of Metallurgical \& Materials Engineering. Butte, MT. 2005.

Stacey, D.L. Associate Metallurgist: Kennecott Greens Creek Mining Company. Personal Interview. 05 Jun. 2006.

STAT-EASE, Inc. Design Expert. Version 5.0.7. Minneapolis, MN. 1996.

Roset, G. Smelter Manager: Stillwater Mining Company. Personal Interview. 07 Nov. 2005

$\mathrm{Xu}, \mathrm{T}$. "Electrodialysis Processes with Bipolar Membranes (EDBM) in Environmental Protection-A Review." Resources, Conservation, and Recycling (2002). [Electronic Version]. 
Appendix A. Process Flowsheets: Kennecott Greens Creek 
Appendix B. Process Flowsheet: Barrick's Golden Sunlight Mine 


\section{Appendix C. ANOVA Analysis of Experimental Campaign WO-1}

\begin{tabular}{|c|l|}
\hline \multicolumn{2}{|c|}{ Factors } \\
\hline A & Temperature $\left({ }^{\circ} \mathrm{C}\right)$ \\
\hline B & Total Sulfide-Polysulfide Concentration $(\mathrm{g} / \mathrm{L})$ \\
\hline C & Initial NaOH Concentration $(\mathrm{g} / \mathrm{L})$ \\
\hline D & Oxygen Overpressure $(\mathrm{psi})$ \\
\hline E & Time (hrs.) \\
\hline
\end{tabular}

Table C.1 ANOVA Analysis Table of WO-1 Sulfide-Polysulfide Conversion to Sulfate

\begin{tabular}{|c|c|c|c|c|c|c|}
\hline \multicolumn{7}{|c|}{ Response: $\mathrm{S}$ to $\mathrm{SO}_{4}{ }^{2-}$ Conversion $($ Square-Root; Lambda $=0.5$ ) } \\
\hline \multirow[b]{2}{*}{ Source } & \multirow{2}{*}{$\begin{array}{c}\text { Sum of } \\
\text { Squares }\end{array}$} & \multirow[b]{2}{*}{ DF } & \multirow{2}{*}{$\begin{array}{c}\text { Mean } \\
\text { Square } \\
\end{array}$} & \multirow{2}{*}{$\begin{array}{c}\text { F } \\
\text { Value }\end{array}$} & \multirow[b]{2}{*}{ Prob $>$ F } & \multirow[b]{2}{*}{ Significance } \\
\hline & & & & & & \\
\hline Model & $\begin{array}{c}8114.60 \\
9\end{array}$ & 12 & 676.217 & $\begin{array}{c}215.93 \\
9 \\
\end{array}$ & $<0.0001$ & significant \\
\hline A & $\begin{array}{c}1423.14 \\
6\end{array}$ & 1 & 1423.146 & $\begin{array}{c}454.45 \\
8 \\
\end{array}$ & $<0.0001$ & \\
\hline $\mathbf{B}$ & 160.239 & 1 & 160.239 & 51.17 & 0.002 & \\
\hline $\mathbf{C}$ & 196.696 & 1 & 196.696 & 62.812 & 0.0014 & \\
\hline D & $\begin{array}{c}1892.86 \\
8\end{array}$ & 1 & 1892.868 & $\begin{array}{l}604.45 \\
6\end{array}$ & $<0.0001$ & \\
\hline $\mathbf{E}$ & $\begin{array}{c}1328.17 \\
1\end{array}$ & 1 & 1328.171 & $\begin{array}{c}424.12 \\
9 \\
\end{array}$ & $<0.0001$ & \\
\hline AD & 970.331 & 1 & 970.331 & $\begin{array}{c}309.85 \\
9 \\
\end{array}$ & $<0.0001$ & \\
\hline $\mathbf{A E}$ & 611.342 & 1 & 611.342 & $\begin{array}{c}195.22 \\
2 \\
\end{array}$ & 0.0002 & \\
\hline BC & 413.013 & 1 & 413.013 & $\begin{array}{c}131.88 \\
9\end{array}$ & 0.0003 & \\
\hline BD & 50.612 & 1 & 50.612 & 16.162 & 0.0159 & \\
\hline BE & 47.848 & 1 & 47.848 & 15.28 & 0.0174 & \\
\hline $\mathbf{C E}$ & 46.66 & 1 & 46.66 & 14.9 & 0.0181 & \\
\hline DE & 973.682 & 1 & 973.682 & $\begin{array}{c}310.92 \\
9 \\
\end{array}$ & $<0.0001$ & \\
\hline Curvature & 275.732 & 1 & 275.732 & 88.05 & 0.0007 & significant \\
\hline Residual & 12.526 & 4 & 3.132 & & & \\
\hline Lack of Fit & 12.49 & 3 & 4.163 & $\begin{array}{c}115.46 \\
9\end{array}$ & 0.0683 & not significant \\
\hline Pure Error & 0.036 & 1 & 0.036 & & & \\
\hline Cor Total & $\begin{array}{c}8402.86 \\
7\end{array}$ & 17 & & & & \\
\hline Std. Dev. & 0.172 & & R-Squared & 0.999 & & \\
\hline Mean & 2.720 & & Adj R-Squared & 0.995 & & \\
\hline C.V. & 6.334 & & Pred R-Squared & 0.968 & & \\
\hline PRESS & 3.264 & & Adeq Precision & 51.071 & & \\
\hline
\end{tabular}




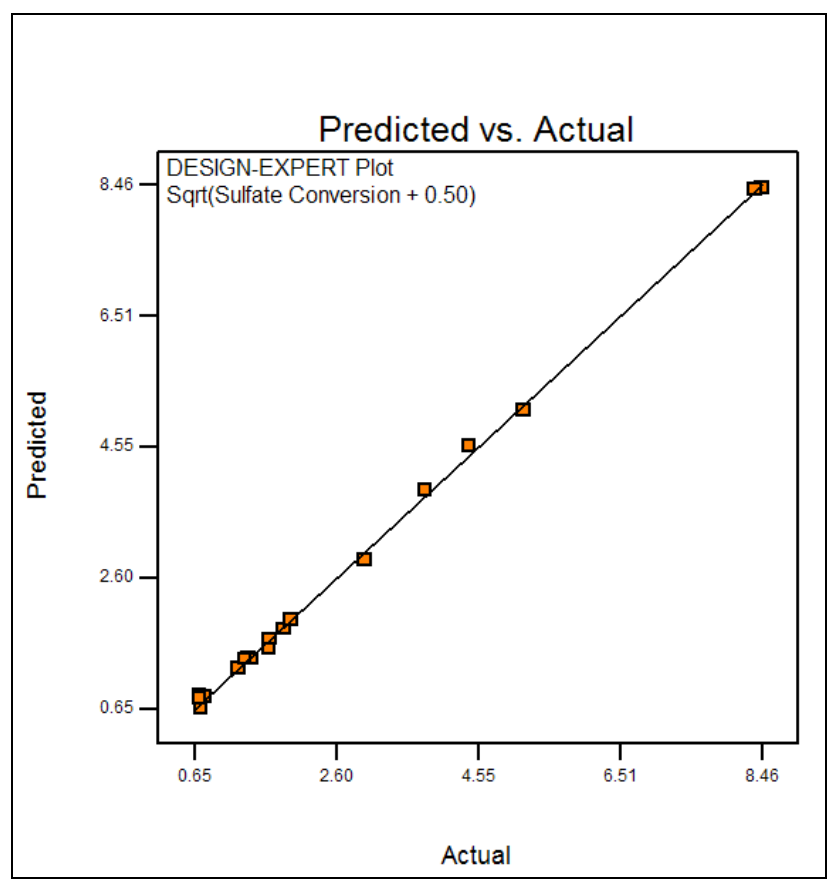

Figure C.1 Predicted vs. Actual Diagnostic Graph of S to $\mathrm{SO}_{4}{ }^{2-}$ Conversion

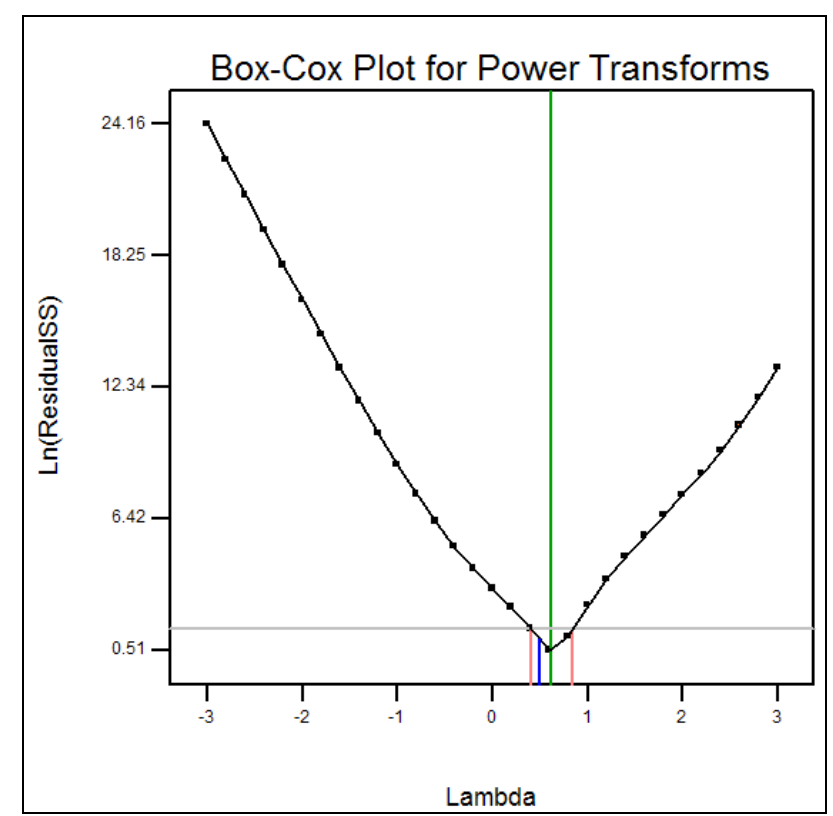

Figure C.2 Box-Cox Plot Diagnostic Graph of S to $\mathrm{SO}_{4}{ }^{2-}$ Conversion 


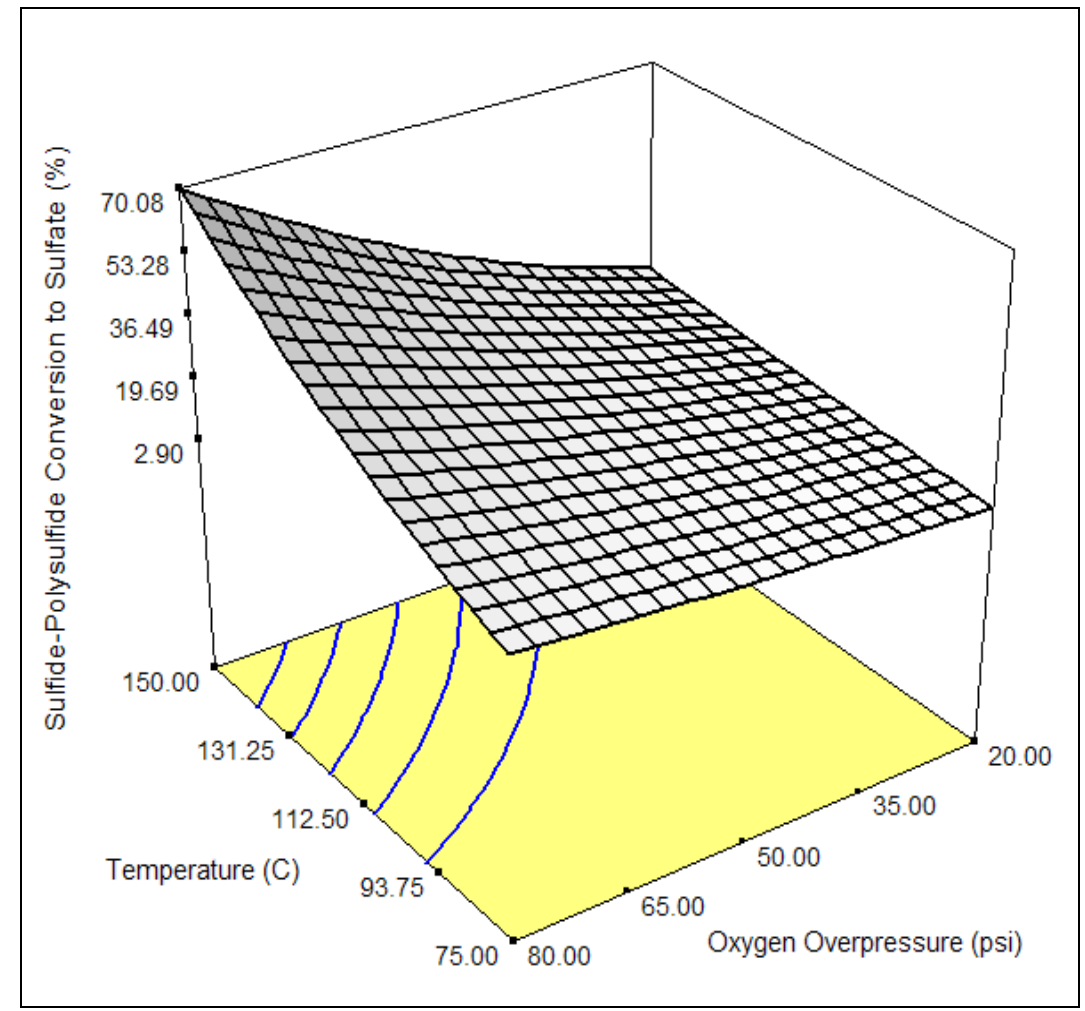

Figure C.3 3D Surface Model Graph of Sulfide-Polysulfide Conversion to Sulfate (Temp vs. Oxygen Overpressure) 


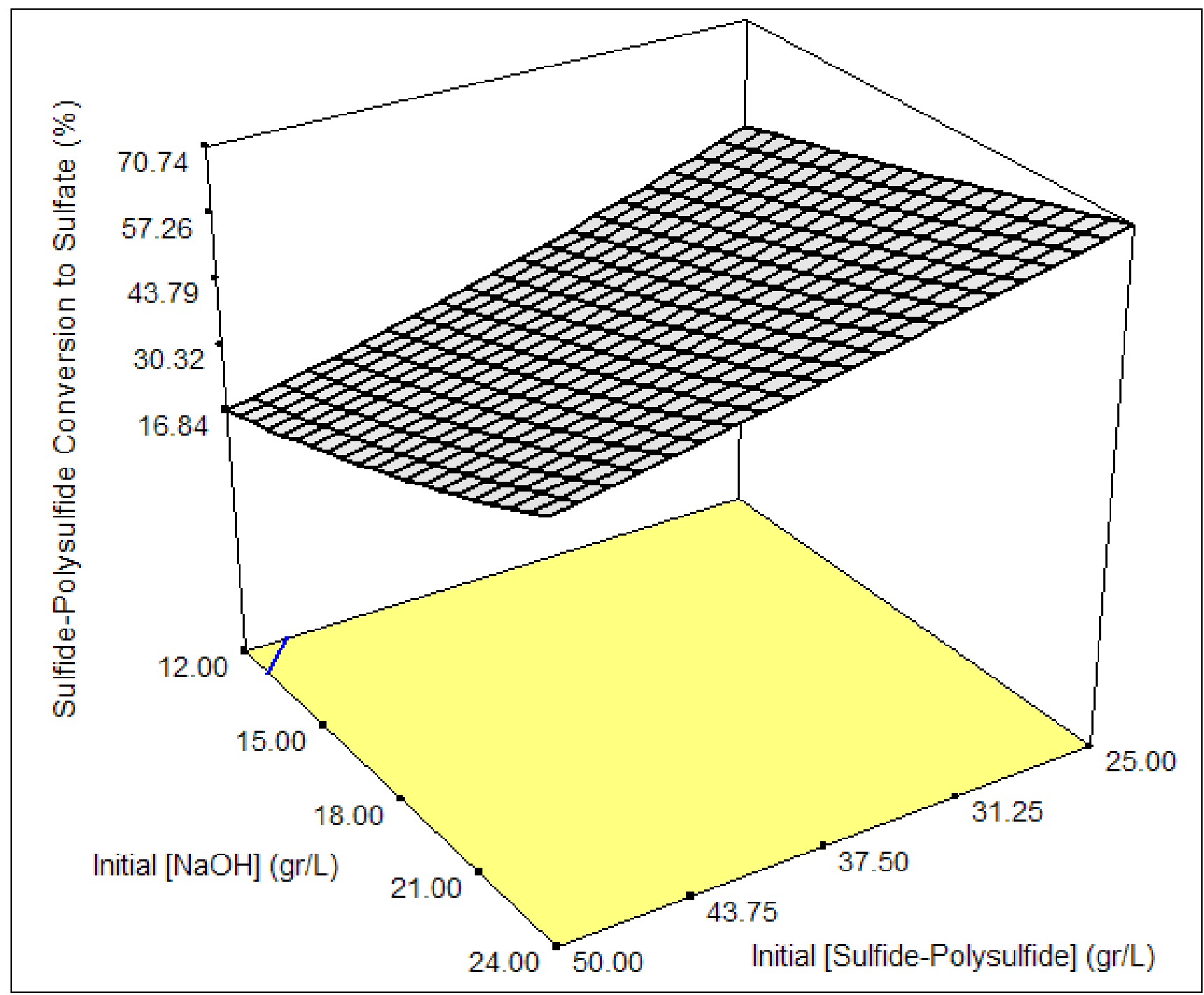

Figure C.4 3D Surface Model Graphs of Sulfide-Polysulfide Conversion to Sulfate (Initial [NaOH] vs. [Sulfide-Polysulfide]) 


\section{Appendix D. SOP: ASGLS Solution Preparation}

A strict Standard Operating Procedure (SOP) has been developed to minimize experimental errors in preparation ASGLS solution. Since ASGLS is a metastable system, exact and accurate solution preparation is critical to achieve the same metastable components in the solution.

Please refer to the previous thesis (Stacey, 2005) for detailed step-by-step description. 


\section{Appendix E. SOP: Batch Leaching}

A strict Standard Operating Procedure (SOP) has been developed to minimize experimental errors in batch leaching using the ASGLS solution. The procedure developed below was used in all preliminary leaching experiments of this thesis and the leaching stage of the batch simulations.

\section{A. Leaching}

1. Into a 1000-mL flask, add the feed material aimed to be leached after being weighed out to the nearest 0.01-grams. Also, add a cleaned and dried magnetic stirrer bar into the flask.

2. Add 750-mL of the prepared ASGLS solution is then added into the feedcontaining flask. Ensure the wetting of all of the sample's particles by gently shaking the flask.

3. After putting the appropriate lid on, insert flask into the pre-heated water bath. Please ensure uniform agitation. Set stirring speed at desired speed (170-rpm was found best for 50.00-grams of feed).

4. Let leaching to occur for the desired time (48-hours) with occasional check-up.

\section{B. Post-Leach Materials Treatment}

5. Separate solids and liquid in the leaching system with a simple vacuum filtering system. Use \#3-185mm Whatman filter. Add minimum amount of DI water in the process of trying to recover all solution/solids. (In case of batch simulations, no DI water was used at all. Lost solution within the filter paper was considered to be a consistent error.)

6. Directly after filtering, store pregnant alkaline sulfide solution in a plastic bottle container and send to analysis/further processing.

7. Agitate solids in a beaker for approximately 10-minutes using 750-mL of DI water as a washing mechanism. It is highly important to wash any remaining alkaline sulfide solution before sending solids to fire-assay analysis. Repeat this washing procedure once.

8. Filter the new mixture using the same vacuum-filtering set-up. Afterwards, put solids in the oven overnight. After dry, weigh and send for fire-assay analysis. 


\section{Appendix F. SOP: Batch Setup of Precious Metal Recovery}

Described below is the developed Standard Operating Procedure (SOP) for the batch setup of precious metal recovery experiments using ion-exchange resins and activated carbon conducted during this research project. The main purpose is to recovery gold from gold-pregnant alkaline sulfide solution; afterwards, alkaline sulfide solution will be circulated back to and reused at the leaching step. Critical steps during the recovery tests include the pregnant-solution preparation and post-leach materials handling.

\section{A.Pregnant-solution Preparation}

1. See Appendix D for alkaline sulfide solution preparation. Adjust volume accordingly as required.

2. To provide more volume to contain a maximum of $1500-\mathrm{mL}$ of solution, the autoclave was used as the leaching vessel, instead of flasks \& water-bath system.

3. Set temperature at $50-55^{\circ} \mathrm{C}$ and agitation at 400 -rpm. Let leaching occur for five days to ensure maximum gold dissolution. Oxygen overpressure feature of the autoclave was not used in this thesis; keep all air intake passage closed.

\section{B. Post-Leach Materials Treatment}

1. Separate solids and liquid in the leaching discharge with a simple vacuum filtering system. Use \#3-185mm Whatmann filter. Add minimum amount of DI water in the process of trying to recover all solution/solids. (In case of batch simulations, no DI water was used at all. Lost solution within the filter paper was considered to be a consistent error.)

2. Directly after filtering, store pregnant alkaline sulfide solution in a plastic bottle container and send to analysis/further processing.

3. Agitate solids in a beaker for approximately 10 -minutes using $750-\mathrm{mL}$ of DI water as a washing mechanism. It is highly important to wash any remaining alkaline sulfide solution before sending solids to fire-assay analysis. Repeat this washing procedure once.

4. Filter the new mixture using the vacuum-filtering set-up. Afterwards, put solids in the oven overnight. After dry, weigh and send for fire-assay analysis.

\section{Recovery Tests}

1. Weigh out the desired amount of ion-exchange resin/activated carbon to the nearest 0.01-grams.

2. $\quad$ Measure desired volume of pregnant alkaline sulfide solution.

3. Mix resin/carbon in a 500-mL flask. Close the flask-top using a rubber or wood cock.

4. Place flask on shaking table; set agitation at 250-rpm. Let recovery test occur according to desired time. Check occasionally for observation and maintenance. 


\section{Post-Recovery Materials Handling}

1. Follow part B of this SOP.

\section{E. Solution/Solids Analysis}

1. Submit all dry solids (resin, carbon, and leaching head/tails) to an outside laboratory for gold and silver fire-assay analysis. 


\section{Appendix G. CIC Setup of Precious Metal Recovery}

The Carbon in Column (CIC) setup was used in the precious metal recovery stage of Batch Simulation 2 and 3. This setup allowed for a more realistic model of a real recovery circuit where solution is introduced from the bottom of the vessel and allowing a more even distribution of flow between the substrate.

Shown in Figure G.1 is a simple schematic representing the laboratory setup. Activated carbon was packed into the vessels, which possessed simple steel-screening wires to prevent any movement of activated carbon between the vessels. Solution was introduced in the opposite direction of gravity so that a packed-bed mechanism can be achieved. However, a maximum flow rate of $150 \mathrm{~mL}$ can only be used since rubber corks on top of the vessels does not have enough friction to handle excessive pressure.

At every change of solution in the batch simulations, the very bottom vessel is removed and the other four vessels were transferred down one step. Another activated carbon filled vessel is then added at the very top of the setup. So, the first solution will be treated with vessel 1 through 5 while the second solution coming through will be treated with vessel 2 through 6l; and so on. There were a total of five recovery stages in Batch Simulation 3; thus, 10 different vessels were used.

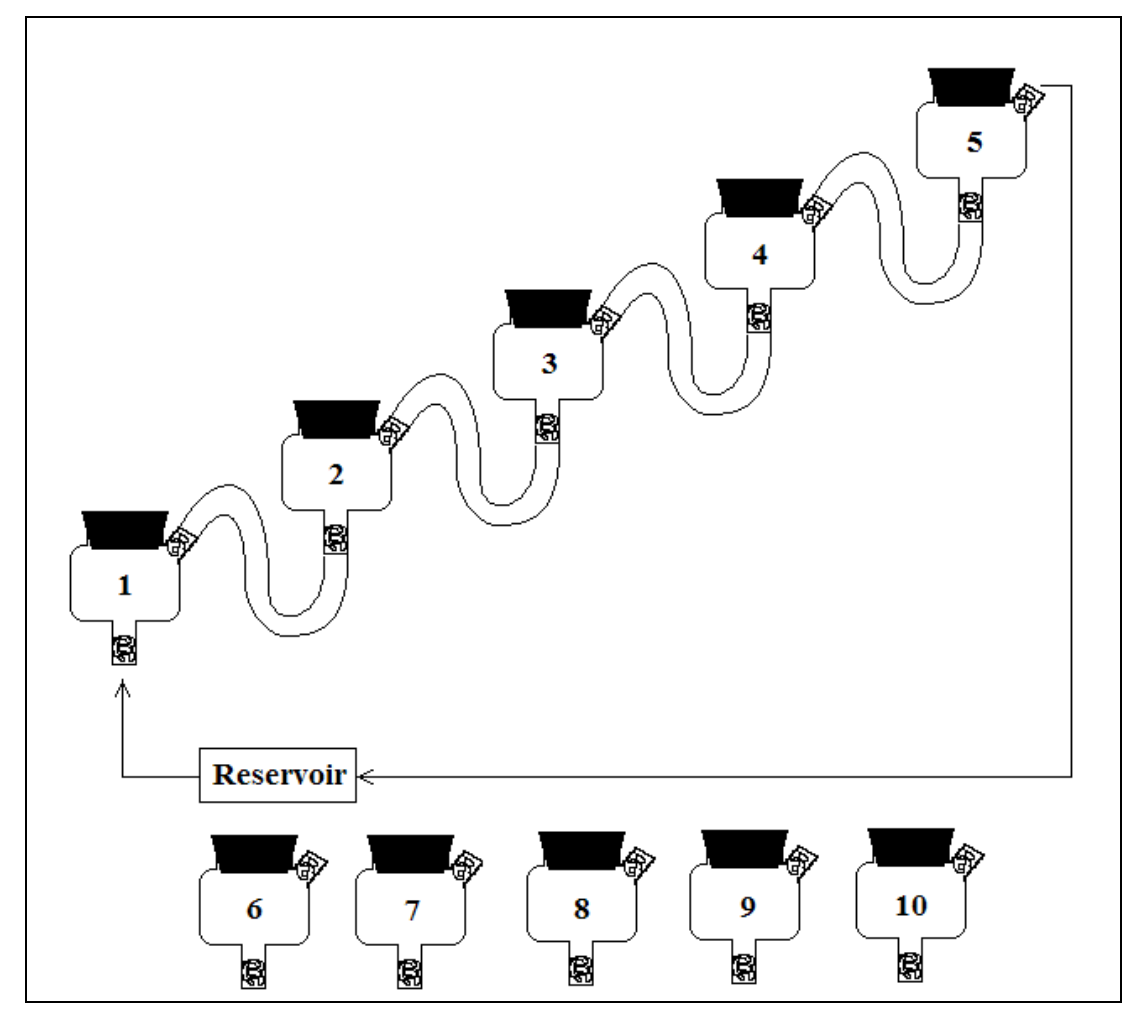

Figure G.1 Schematic of Laboratory CIC Model 


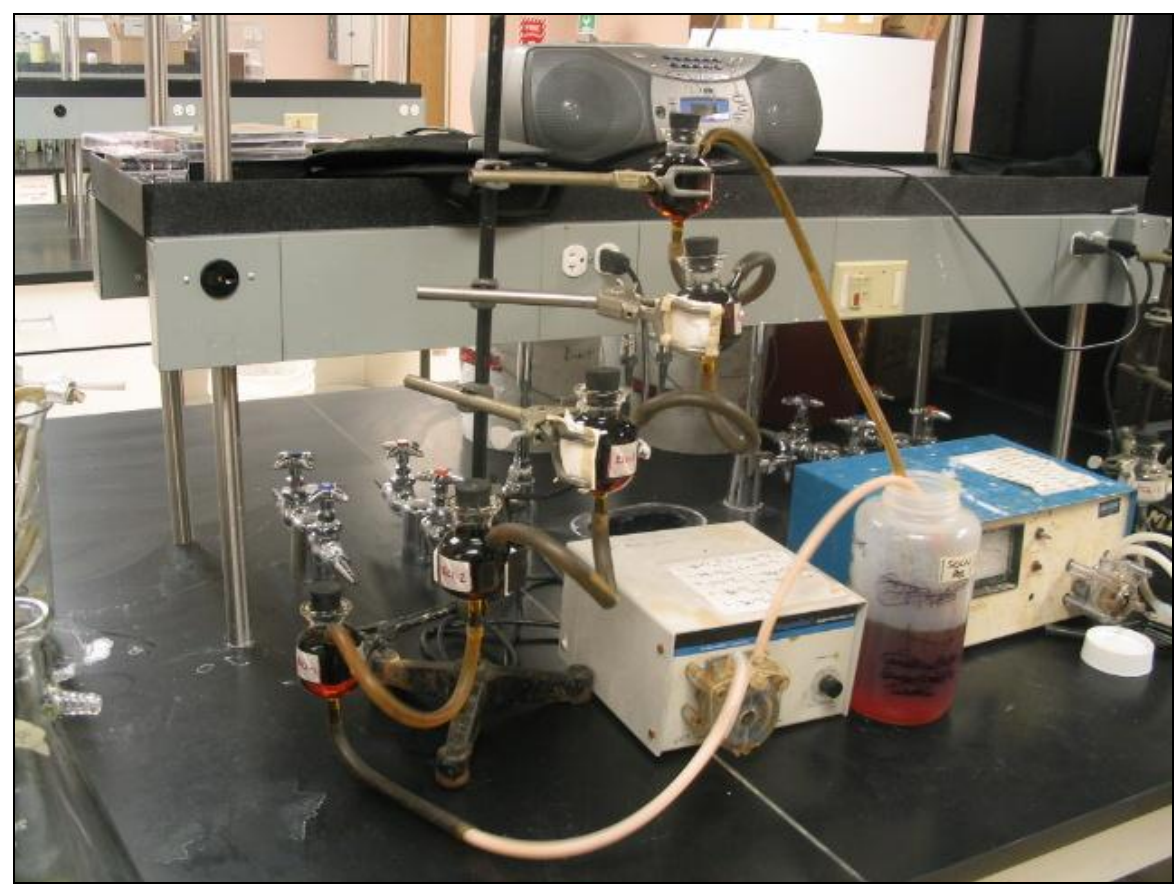

Figure G.2 Actual CIC Laboratory Setup 


\section{Appendix H. SOP: Pressure Oxidation of ASGLS Solution}

As the first stage of solution waste treatment, sulfide/polysulfide oxidation was achieved by utilizing temperature, agitation, and oxygen overpressure. These parameters were able to be controlled automatically and accurately using the autoclave, located in the ELC Building at Montana Tech. Basically, the autoclave is a closed pressure vessel. Listed below is an experimental procedure developed used throughout the waste-treatment campaign of this thesis.

\section{A. Solution Preparation}

1. Solution preparation regarding preliminary work, where "clean" alkaline sulfide solution was used, is very similar to steps described in Appendix D.

\section{B. Autoclave Oxidation}

1. Pour solution into the autoclave. Don't treat more than $1500-\mathrm{mL}$ of autoclave in the heating jacket.

2. Install auxiliaries needed: bearing-cooling water tubes, chamber-cooling air tubes, thermometer, and oxygen supply line.

3. Set temperature solution using the auxiliary control station.

4. After putting on agitator top and all safety clamps securely, put the as desired and turn on heat to high. Start agitation at 50 to 100-rpm, whatever considered sufficient to provide uniform solution heating.

5. As actual temperature approaches the desired temperature, within $5^{\circ} \mathrm{C}$, heat should be switch on low.

6. When desired temperature is reached, change agitation to the desired value (maximum of 700rpm was used in this research). Then, the start time of the experiment can be recorded.

\section{Post-oxidation Solution Handling}

1. At end-time of the experiment, turn off heat and set temperature to $25^{\circ} \mathrm{C}$. Wait and let autoclave to cool down (approximately 30 to 45-minutes).

2. Remove all auxiliary lines and carefully remove safety clamps and agitator top. Pour out solution into an appropriately sized beaker.

3. Insert a magnetic stirrer and put solution under agitation (200 to 300-rpm). If $\mathrm{Na}_{2} \mathrm{SO}_{4}$ concentration is high enough, sulfate crystals will form immediately after saturation.

4. After maximum amount of precipitate is achieved, use a vacuum filter system to achieve a solid-liquid separation.

5. Measure solution volume and solid weight; afterwards, store both samples in a closed container. 


\section{Analysis}

1. Analyze $\mathrm{SO}_{4}{ }^{2-}$ concentration in solution using an Ion Chromatograph; and measure both $\mathrm{pH}$ and Eh.

2. Analyze solids produced using X-Ray Diffractometer (XRD) to confirm $\mathrm{Na}_{2} \mathrm{SO}_{4}$ formation. 


\section{Appendix I. SOP: Salt Splitting using Electrodialysis}

During the progress of this thesis, a Standard Operating Procedure (SOP) was developed on the Electrodialysis Unit (EDU) experiments. Due to the delicate structure and sensitive stackarrangement of the ion-exchange/bipolar membranes, it is necessary to strictly follow this SOP to ensure quality control and reproducibility.

\section{Safety Considerations:}

1. Chemical Handling: Please use protective gloves and glasses when handling chemicals produced in the process $\left(\mathrm{NaOH}\right.$ and $\left.\mathrm{H}_{2} \mathrm{SO}_{4}\right)$.

2. Flammable Gas: Hydrogen is produced in the electrodialysis process. Run the experiment far from any source of combustion to avoid explosion.

3. Electrical Safety: Avoid any direct/conductive contact with electrode connection during power application to the cell.

\section{A. Solution Preparation}

NOTE: Procedure below is to prepare a 2000-mL of 1.5 $\mathrm{M} \mathrm{Na}_{2} \mathrm{SO}_{4}$ solution. Please adjust accordingly to prepare different concentrations and amount.

1. Either using reagent grade or experimentally produced, weigh desired amount of $\mathrm{Na}_{2} \mathrm{SO}_{4}$ crystals to the nearest 0.01 -grams in a 500 -mL beaker.

2. $\quad$ Agitate 2000-mL of DI water in a 2000-mL industrial-grade flask.

3. Slowly pour $\mathrm{Na}_{2} \mathrm{SO}_{4}$ crystals into the agitated DI water. It is important to introduce crystals slowly to avoid any agglomeration, which will cause in a significant increase in dissolution time. For the same reason, liberate any agglomerated crystals as pouring is conducted.

4. Agitate continuously; all crystals should be in solution in 15 to 30-minutes.

\section{B. Unit Preparation and Membrane Stacking}

NOTE: Procedure below was used to prepare the two-cell stack arrangement using the cathodic exchange membrane (CEM) and bipolar membrane (BPM).

1. Place aluminum Clamping Plate marked "CATHODE" on a flat, clean surface. Place this plate with the long threaded studs pointing up. Always make sure that all studs are level.

2. Place one of the polypropylene electrode-cell-body accordingly to the "CATHODE" Clamping Plate. 
3. The membrane now can start to be arranged and stacked. To produce the two-cell stack arrangement using the CEM and BPM, the stacking steps are presented through diagram at the end of this SOP.

4. Place the other polypropylene electrode-cell-body on top of the membrane stack. Make sure all flow-holes are aligned by looking through them.

5. Place clamping plate marked "ANODE" on top of the arrangement.

6. Hand tighten washers and nuts to the six threaded studs with fixed step pattern shown in the unit's manual. Finally, tighten all nuts using the same step pattern to 7-N.m (62-in/lbs) using a micrometer torque wrench.

\section{Experimental Run}

1. Connect all $1 / 2$ " polypropylene connections to the EDU. Check for any imperfect connection immediately.

2. Measure $500-\mathrm{mL}$ of the prepared $1.5 \mathrm{M} \mathrm{Na} \mathrm{SO}_{4}$ solution into a 1-L plastic bottle marked "Feed/Acid Flow". Add a magnetic stirrer into the bottle. A special cap with two drilled holes was used to provide solution access to the $3 / 8$ " tubing.

3. Measure $500-\mathrm{mL}$ of the prepared $1.5 \mathrm{M} \mathrm{Na} \mathrm{SO}_{4}$ solution into a 1-L plastic bottle marked "Electrolyte". This solution will serve as both the anolyte and catholyte (respectively, anode and cathode rinsing solution).

4. Measure 500-mL of DI water serving as the starting solution to the base flow into another bottle marked "Base Flow". Add a magnetic stirrer into the bottle.

5. Agitate each bottle reasonably without creating a vortex.

6. $\quad$ Insert all necessary tubing into bottles.

7. Start pumps individually with the order of: electrolyte, feed, and base. Allow each flow to equilibrate before turning on the next pump. Check all connections for any possible leak.

8. Connect appropriate cathode/anode wires. Make sure that all flows are steady and no leak is present in the system.

9. Turn on the power supply with both voltage and current control knobs turned to the left (zero). Slowly increase both knobs until desired current is achieved (current control knob as the limiting parameter).

10. Let experiment run to the desired length of time.

11. When solution samples are desired during the experiment, use a volumetric pipette (5-mL) to extract solution from the bottles.

\section{Post-run Analysis}

1. Analyze Base solution samples for $\mathrm{SO}_{4}{ }^{2-}$ contamination using the Ion Chromatograph (IC), $\mathrm{pH}$ using a $\mathrm{pH}$ probe, $\mathrm{Na}^{+}$concentration using the ICP, and $\mathrm{OH}^{-}$concentration using the ABC titration.

2. Analyze Feed/Acid solution samples for $\mathrm{SO}_{4}{ }^{2-}$ concentration using the $\mathrm{IC}, \mathrm{pH}$ using a $\mathrm{pH}$ probe, $\mathrm{Na}^{+}$concentration using the $\mathrm{ICP}$, and $\mathrm{H}_{2} \mathrm{SO}_{4}$ concentration using manual titration (sodium carbonate titrate and methyl orange indicator). 
E. Sequence of Membrane Stacking:

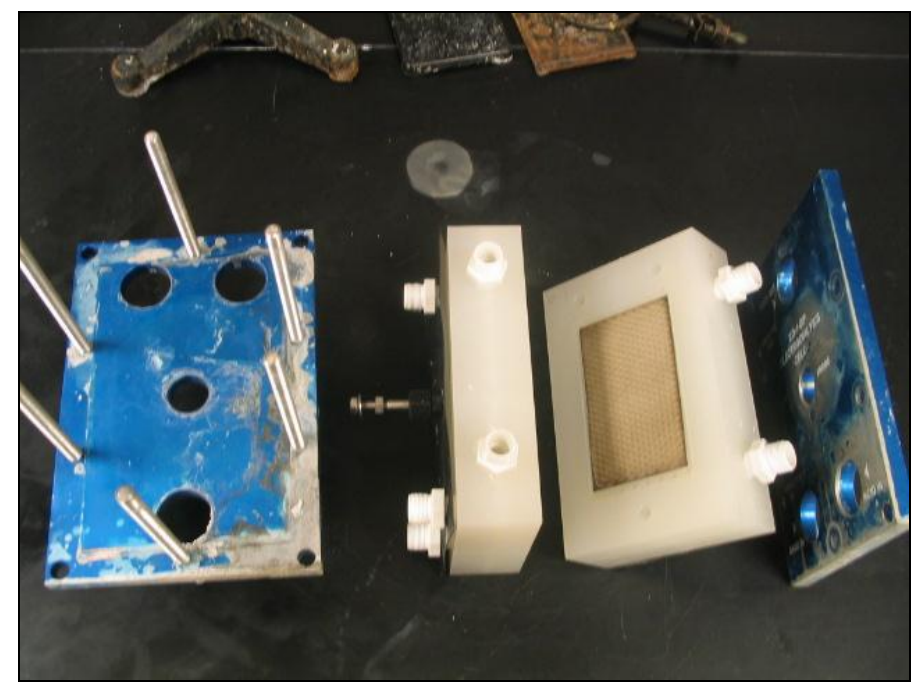

(1)

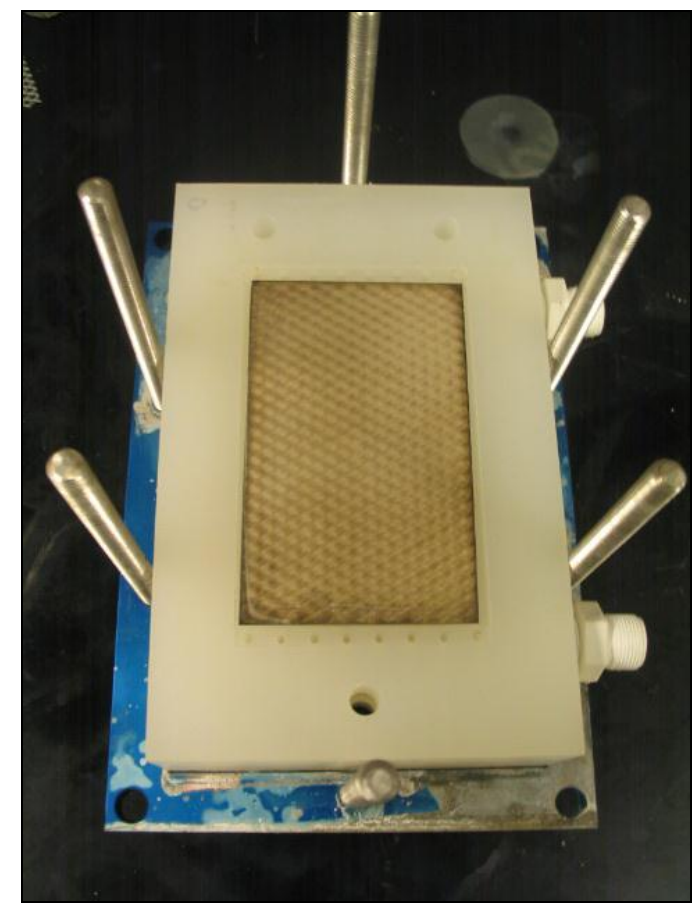

(2) 


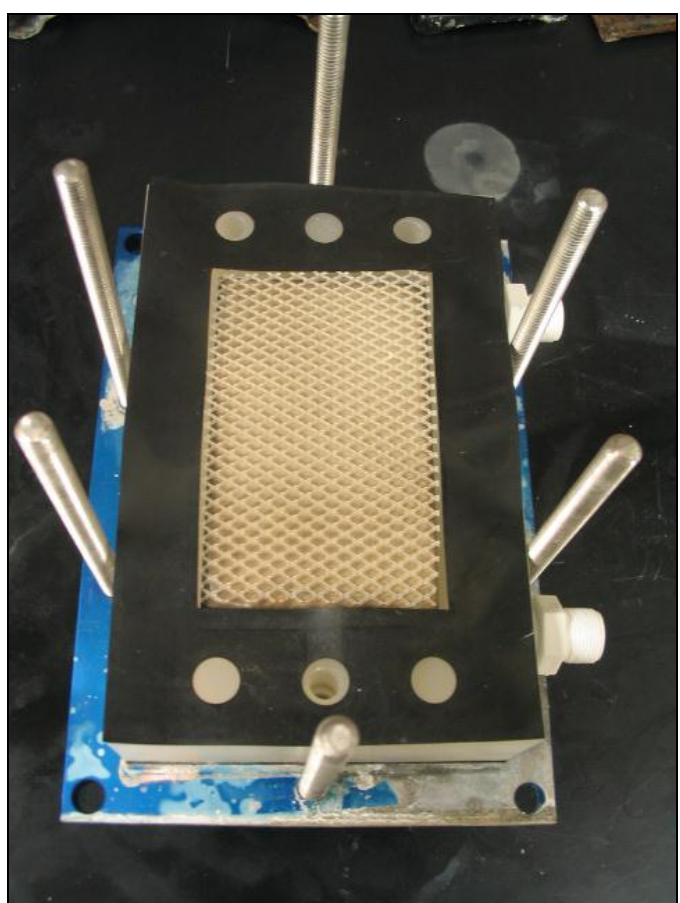

(3)

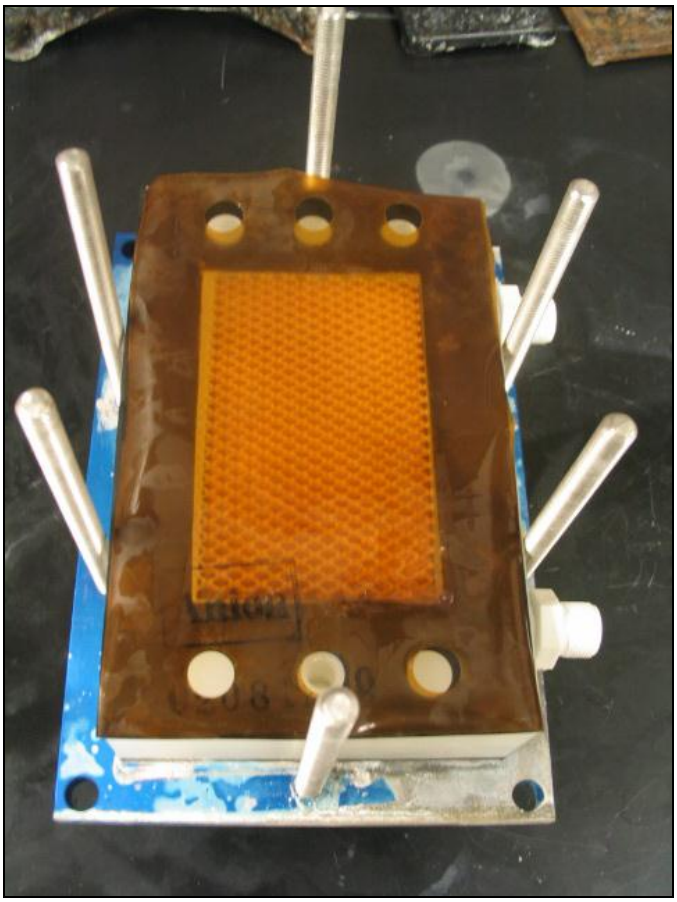

(4) 


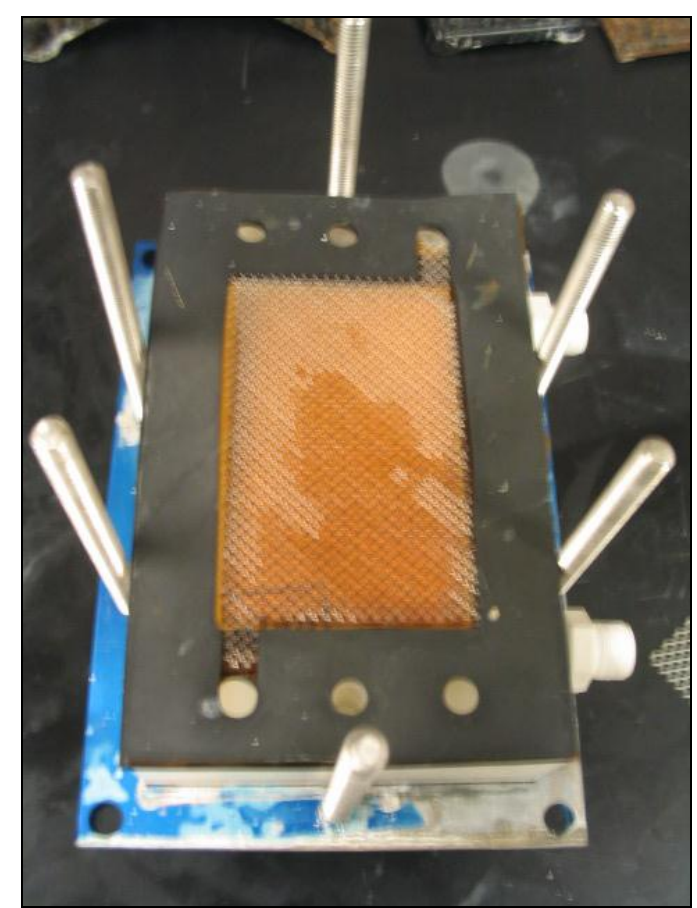

(5)

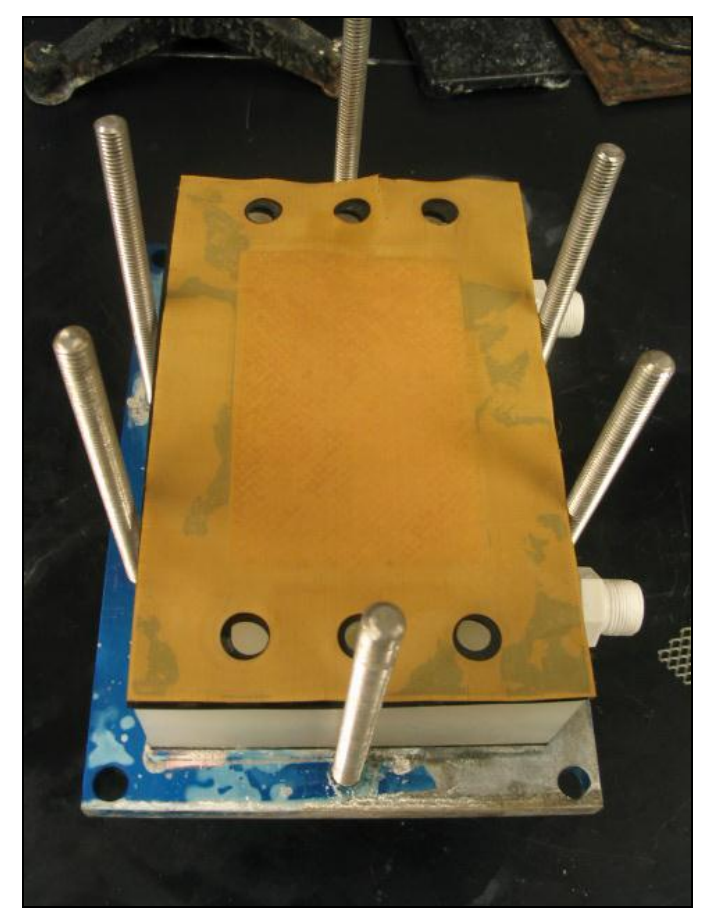

(6) 


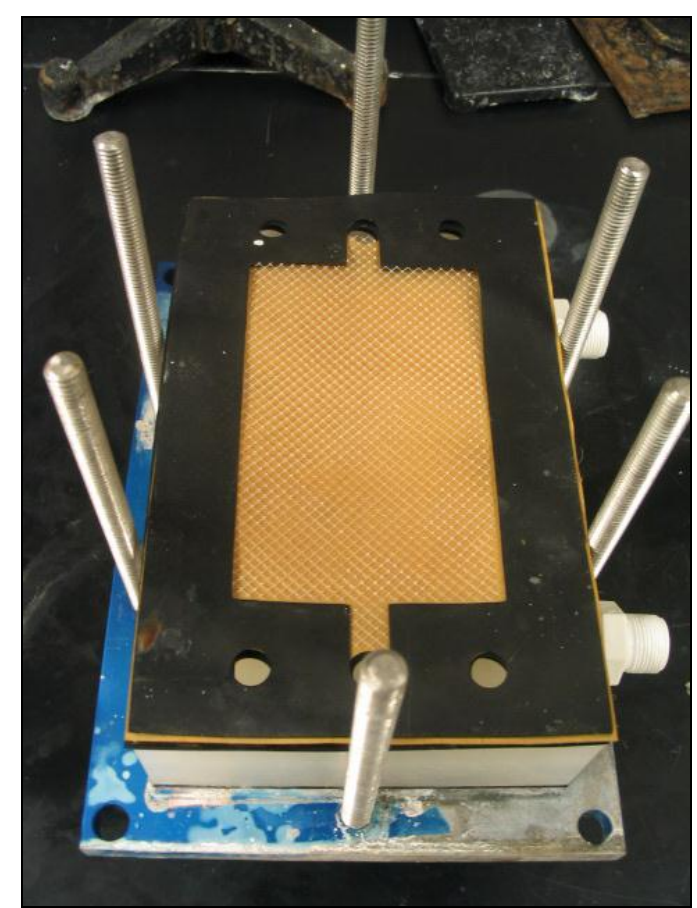

(7)

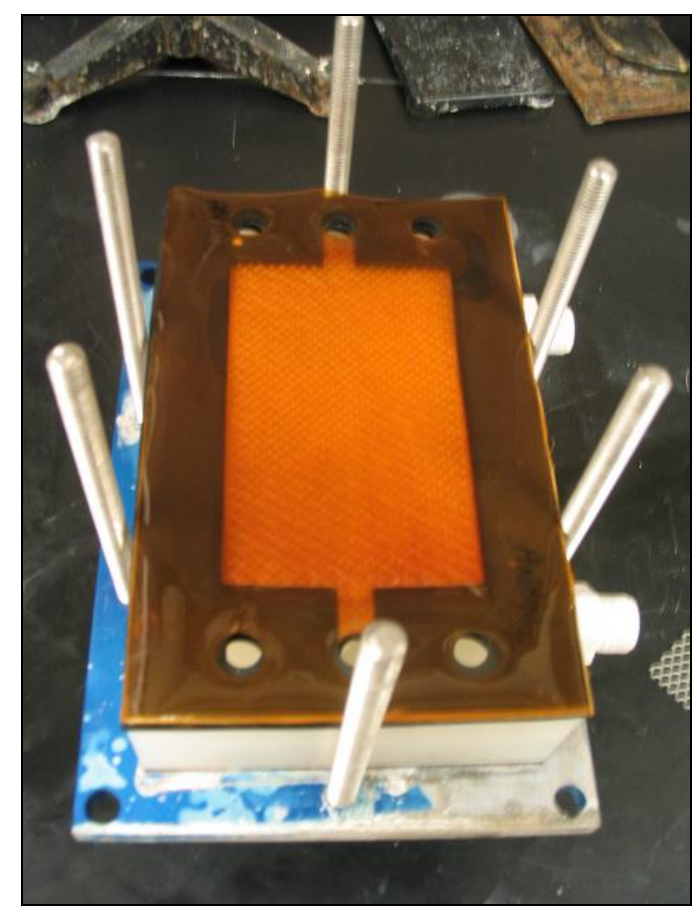

(8) 


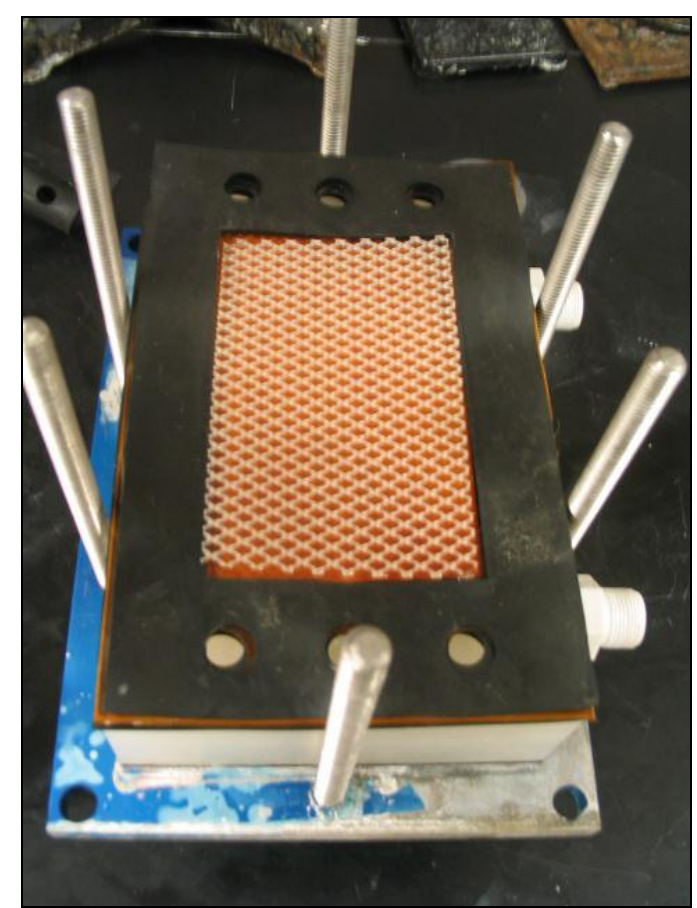

(9)

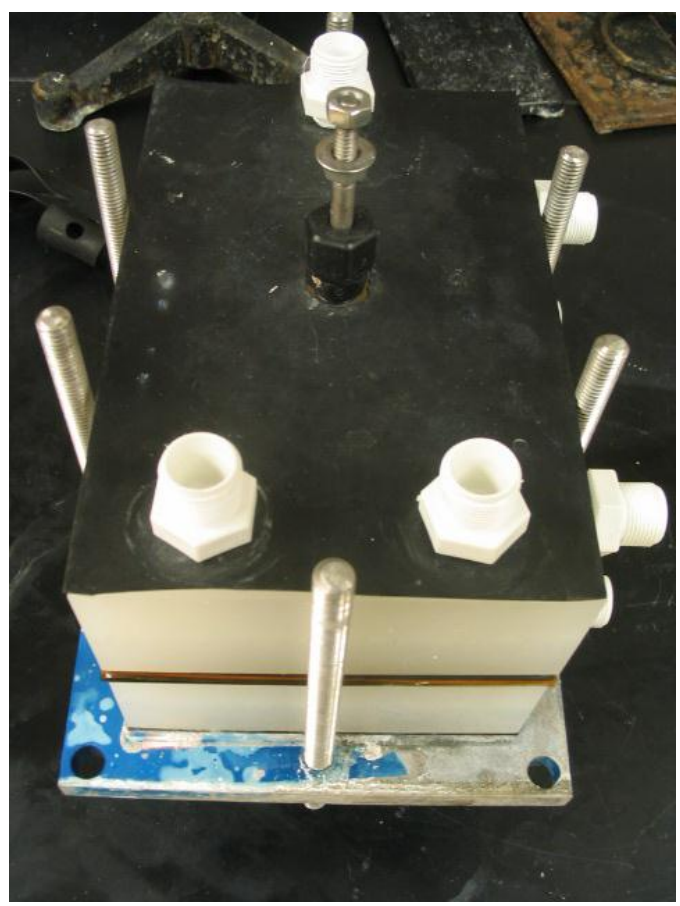

(10) 


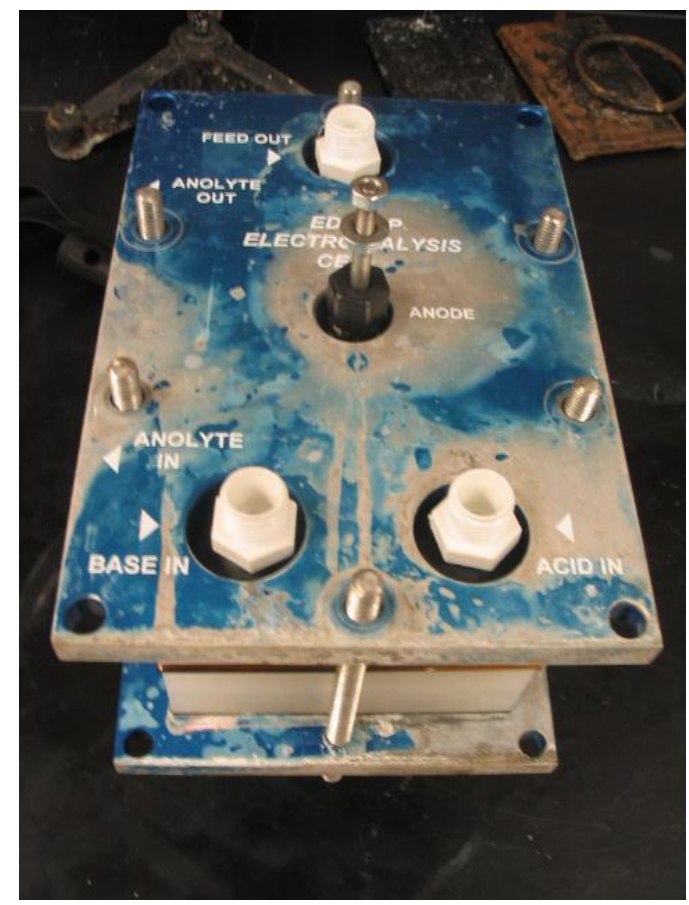

(11) 


\section{Appendix M. List of Chemicals and Analytical Equipments}

Table M.1 List of Chemicals

\begin{tabular}{|c|c|c|c|c|}
\hline Chemical & Purity & Form & Grade & Manufacturer \\
\hline $\mathrm{Na}_{2} \mathrm{~S}$ & Hydrated, $60 \%$ & $\begin{array}{l}\text { Fused } \\
\text { Flakes }\end{array}$ & Technical & $\begin{array}{c}\text { Fisher Chemicals, } \\
\text { S426-212 }\end{array}$ \\
\hline S & $99.9 \%$ & Flakes & Technical & $\begin{array}{c}\text { Montana Sulfur and } \\
\text { Chemical Co. }\end{array}$ \\
\hline $\mathrm{NaOH}$ & $98.7 \%$ & Powder & Technical & $\begin{array}{c}\text { Fisher Chemicals, } \\
\text { S318-5 }\end{array}$ \\
\hline $\mathrm{Na}_{2} \mathrm{SO}_{4}$ & Anhydrous & Powder & USP/FCC & $\begin{array}{c}\text { Fisher Chemicals, } \\
\text { S429-12 }\end{array}$ \\
\hline $\mathrm{Ca}(\mathrm{OH})_{2}$ & $95 \% \min$. & Powder & ACS Certified & Alfa Aesar, \#14662 \\
\hline $\mathrm{NH}_{4} \mathrm{OH}$ & 29.5\% Assay & Liquid & ACS Certified & $\begin{array}{c}\text { Fisher Chemicals, } \\
\text { A669-212 } \\
\end{array}$ \\
\hline $\mathrm{AgNO}_{3}$ & $0.995-0.1005 \mathrm{~N}$ & Liquid & ACS Certified & $\begin{array}{c}\text { Fisher Chemicals, } \\
\text { SS72-500 }\end{array}$ \\
\hline $\mathrm{HCl}$ & $0.995-0.1005 \mathrm{~N}$ & Liquid & ACS Certified & $\begin{array}{c}\text { Fisher Chemicals, } \\
\text { SA48-4 }\end{array}$ \\
\hline
\end{tabular}




\title{
The Development of the Alkaline Sulfide Gold Leaching Process
}

\author{
Completed by \\ Darby Stacey
}

\begin{abstract}
A Thesis Submitted to the Metallurgical Engineering Department of Montana Tech of The University of Montana
\end{abstract}

In Partial Fulfillment of the Requirements for a Master of Science Degree in Metallurgical Engineering

April, 2005 


\begin{abstract}
The Alkaline Sulfide Gold Leaching System (ASGLS) was studied to understand the following: electrochemistry; electrokinetics; a laboratory leaching procedure; optimal leaching parameters; a method for gold removal from solution; industrial ore leaching; and, treating solutions for waste removal.
\end{abstract}

In the ASGLS both sulfide $\left(\mathrm{S}^{2-}\right)$ and polysulfide $\left(\mathrm{S}_{\mathrm{x}}{ }^{2-}\right)$ are required in strong alkaline conditions for gold to leach in the $\mathrm{AuS}^{-}$form. Polysulfide is the system oxidant and sulfide is the lixiviant for gold. As temperatures are increased the Eh-pH stability range for AuS ${ }^{-}$becomes thicker and longer as it shifts to more reducing conditions and more acidic $\mathrm{pH}$.

The optimal ASGLS leach parameters are created when total sulfur and hydroxide are added to saturation. Elemental sulfur should be added with sodium hydroxide at a sulfur ratio of 1:1. Higher temperatures aid in leach kinetics.

Carbon was shown to remove dissolved gold from solution. Gold removal was near $100 \%$ for weak concentrations of total sulfur and hydroxide and almost $0 \%$ for strong solutions. Although tested, stripping carbon with strong solutions was not produced in the laboratory.

Industrial ores were leached successfully with the ASGLS. For a high grade gravity concentrate, 93.5\% of gold was leached. A roaster calcine ore prior to cyanide leaching and after cyanide leaching were also tested with the ASGLS. In the sample prior to cyanide $84 \%$ of gold was leached; in the sample after cyanide an additional $10 \%$ of the gold was leached from the ore.

By oxidizing the sulfides to sulfates a suitable waste solution was created. Solutions were treated for waste removal in an autoclave at $170^{\circ} \mathrm{C}$ and $40 \mathrm{psi}$ of oxygen overpressure. 


\section{Table of Contents}

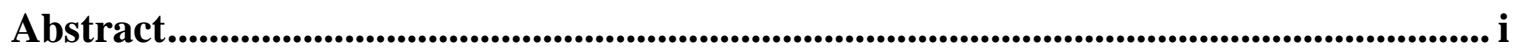
Acknowledgements .....................................................................Error! Bookmark not defined.

Table of Contents ........................................................................................................................... i

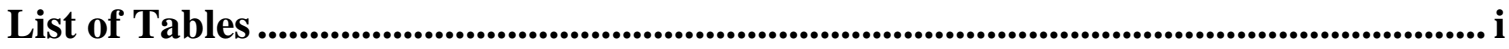

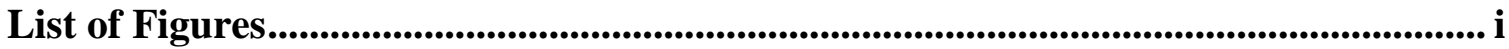

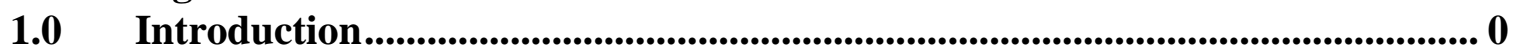

$1.1 \quad$ Research Purpose .................................................................................

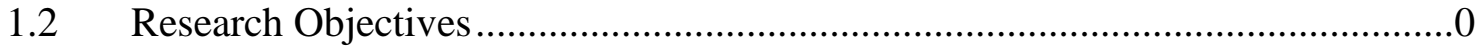

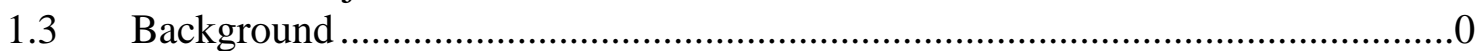

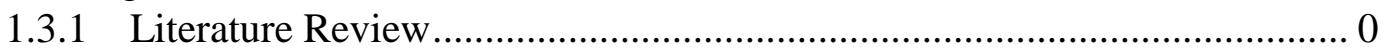

1.3.1.1 Monash University Fundamental Research .............................................. 0

1.1.3.2 Research Associated with the NSC Process ................................................ 0

1.1.3.3 Xian Institute of Metallurgy and Construction Engineering Research .... 0

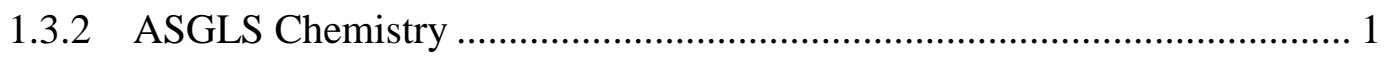

1.3.2.1 Sulfur Chemistry ……………………………….............................. 1

1.3.2.2 Leaching Chemistry .......................................................................... 2

2.0 Electrochemistry and Electrokinetics of the ASGLS.............................................. 4

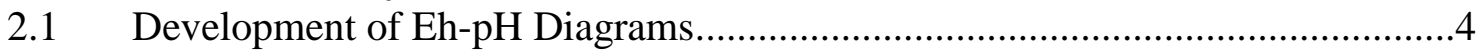

2.1.1 Free Energy Calculations ........................................................................ 4

2.1.2 Stable and Metastable Eh-pH Diagram Production ................................................

2.1.2.3 Stable Eh-pH Diagrams ......................................................................... 4

2.1.2.2 Metastable Eh-pH Diagrams .................................................................... 5

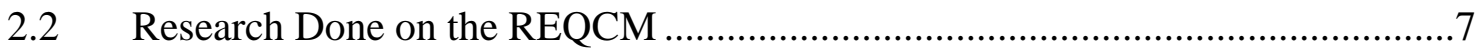

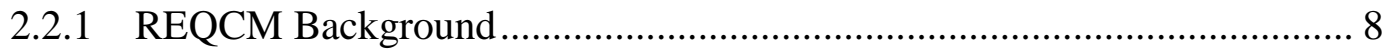

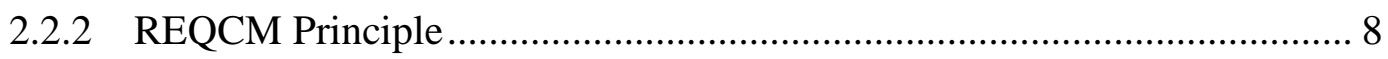

2.2.3 REQCM Critical Components and Pre-test Procedures ............................... 9

2.2.3.1 Platinum Sputter Coating .................................................................... 9

2.2.3.2 MOI Deposition ............................................................................. 9 
2.2.4 Experimental Procedure............................................................................ 11

2.2.4.1 MOI Deposition and Post Experimental Stripping ................................. 11

2.2.5 REQCM Experimental Set Up............................................................ 12

2.2.5.1 Solution Preparation................................................................................ 13

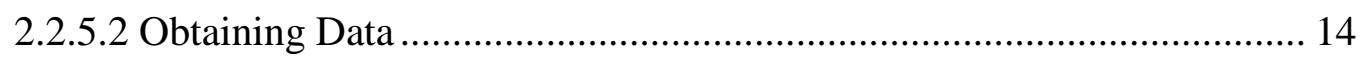

2.2.6 REQCM Experimental Results ............................................................ 15

2.2.6.1 Effect of Sulfide $\left(\mathrm{S}^{2-}\right)$ Concentration ................................................. 15

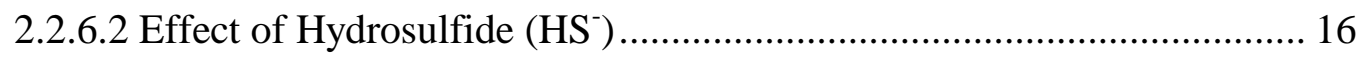

2.2.6.3 Effect of Polysulfide ............................................................................ 16

2.2.6.4 Effect of Hydroxide Addition ................................................................. 19

2.2.6.5 Effect of Temperature .......................................................................... 21

2.2.6.6 Effect of Gold Purity ........................................................................... 23

2.2.7 Conclusions to REQCM Experiments ........................................................... 24

$2.3 \quad$ Laboratory Leach Tests ..................................................................................25

2.3.1 Reagents Used..................................................................................... 25

2.3.2 Laboratory Leach Tests ........................................................................ 25

2.3.2.1 First Year Leach Tests ......................................................................... 25

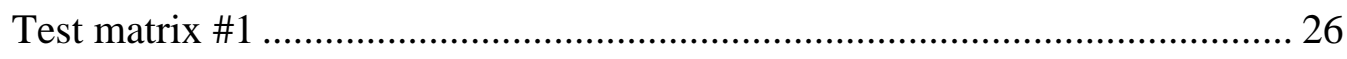

Redo test for Test Matrix \#1 ............................................................................ 27

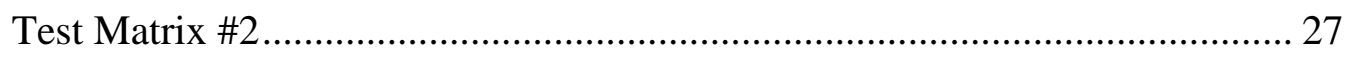

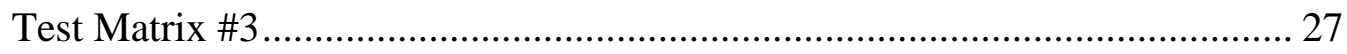

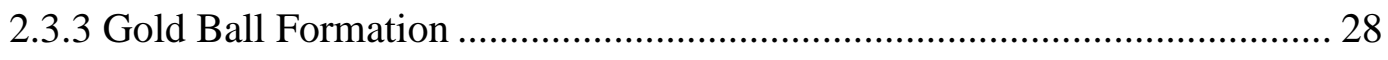

2.2.3.1 Possible Reasons for Gold Ball Formation ................................................ 0 
2.3.4 High Temperature Leach Tests ....................................................................... 0

2.3.4.1 High Temperature Autoclave Test ............................................................. 1

2.3.4.2 High Temperature Kettle Tests ................................................................. 1

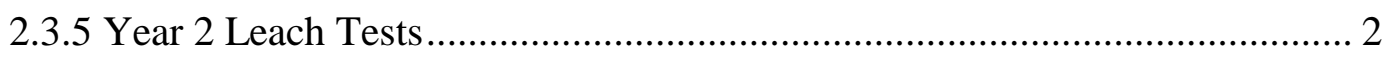

2.3.5.1 Development of a Standard Operating Procedure for Leach Tests ........... 2

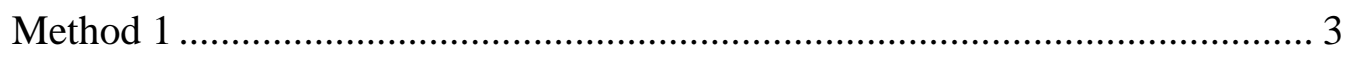

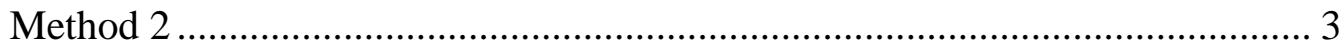

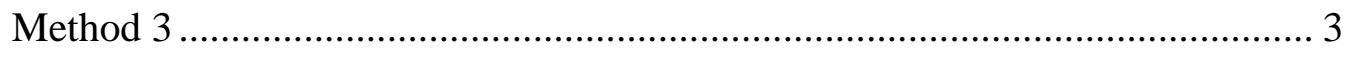

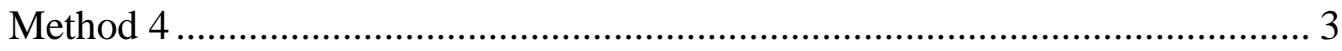

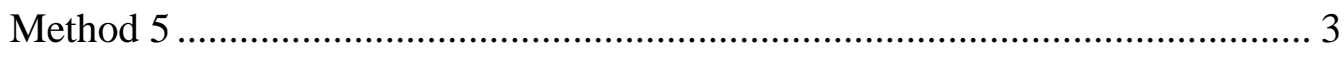

2.3.5.2 Standard Operating Procedure for Leach Tests............................................ 4

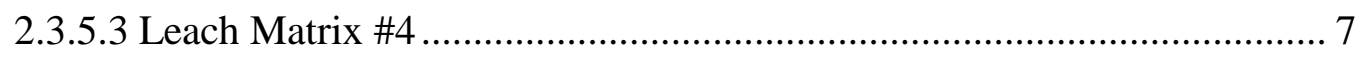

2.3.5.4 Leach Matrix \#4 Experimental Analysis...................................................... 0

2.3.5.5 Measuring Solution Potential.................................................................... 1

2.3.5.6 Analyzing for Dissolved Gold Concentration........................................... 1

2.3.5.7 ICP Standard Analysis Procedure ………………………......................... 1

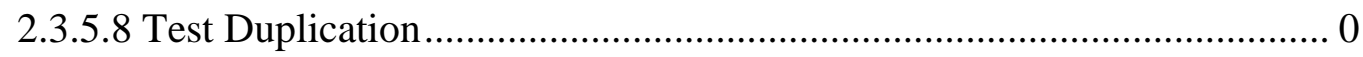

2.3.6 Matrix \#4 Statistical Analysis ................................................................. 0

2.3.6 Matrix \#4 ORP Analysis ..................................................................... 0

2.3.6 Matrix \#4 Statistical Analysis ................................................................ 0

2.3.7 Leach Matrix \#4 Extra Tests ........................................................................... 2

2.3.7.1 Matrix \#4 Extra Test Results ..................................................................... 3

2.3.7.2 Matrix \#4 Extra Test Analysis .................................................................. 3 
3.0 Gold Recovery Tests .............................................................................................................. 4

3.1 Standard Operating Procedure for Recovery Tests ..................................................

3.2 Standard Operating Procedure for Analyzing Recovery Tests ...............................5

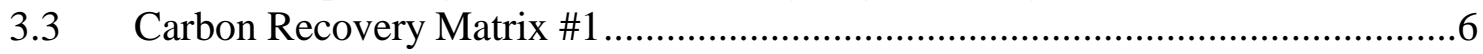

3.3.1 Experimental Set-Up............................................................................. 7

3.3.2 Results of Carbon Recovery Matrix \#1................................................. 7

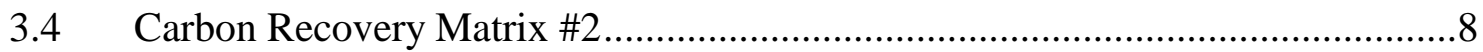

3.4.1 Carbon Recovery Matrix \#2 Results .......................................................... 8

4.0 Industrial Ore Leaching .............................................................................................. 9

4.1 Greens Creek Gravity Middlings …………....................................................

4.1.1 Chemistry of Greens Creek Middlings ........................................................ 9

4.1.2 Greens Creek Middling Leach Test ........................................................... 10

4.2 Newmont Roaster Calcine ………………...................................................11

4.2.1 Newmont Roaster Calcine Leach Tests ..................................................... 11

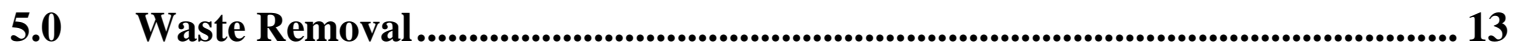

5.1 Standard Laboratory Procedure for Waste Removal Tests ....................................13

6.0 Future Work..................................................................................................... 17

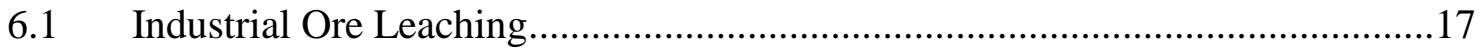

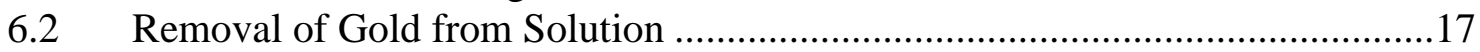

6.3 Stripping Gold from the Gold Removal Substrate...........................................18

6.4 Cleaning of Waste Solutions...........................................................................18

6.5 Processing of Waste Solutions....................................................................18

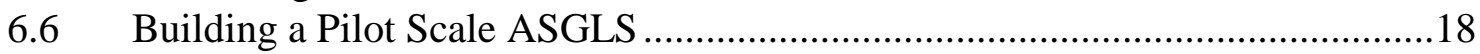

7.0 Thesis Conclusions............................................................................................................... 18

7.1 Electrochemical \& Electrokinetic Conclusions ………......................................18

7.2 Laboratory Leach Test \& Optimization Conclusions .............................................19

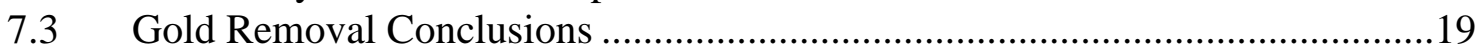

7.4 Industrial Ore Leaching Conclusions .................................................................19

$7.5 \quad$ Waste Removal Conclusions …………………….......................................19

8.0 Bibliography ...................................................................................................................... 0 


\section{List of Tables}

Table I. Oxidation States of Sulfur Species .................................................................. 2

Table II. Dominant Gold Reaction Stability Constants ........................................................ 3

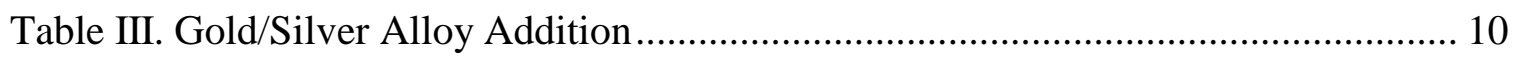

Table IV. Chemicals Used ..................................................................................... 25

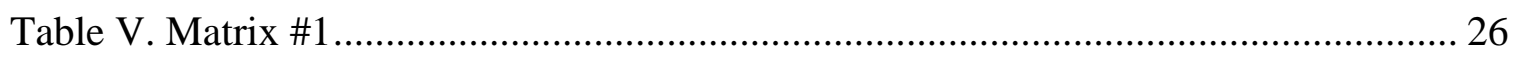

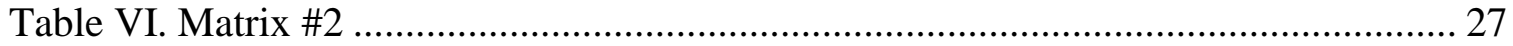

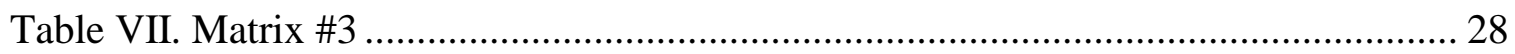

Table VIII. Quantification of Gold Ball Formation ......................................................... 0

Table IX. Autoclave Leach Test Parameters................................................................... 1

Table X. High Temperature Leach Test Parameters ............................................................ 1

Table XI. Leach Matrix \#4 Reagent Additions ................................................................ 0

Table XII. Actual Chemical Additions per Liter.................................................................. 0

Table XIII. ICP Analysis Standard Solutions ……………........................................... 0

Table XIV. Matrix \#4 18hrs. Leaching Results ................................................................ 0

Table XV. Solution Potentials After 18hrs. Leaching ......................................................... 0

Table XVI. Leach Matrix \#4 Extra Tests Parameters ........................................................... 3

Table XVII. Matrix \#4 Extra Tests After 18 hrs. Leaching .................................................. 3

Table XVIII. Carbon Recovery Matrix \#1 …………….............................................. 7

Table XIX. Carbon Recovery Matrix \#1 Results .................................................................. 7

Table XX. Carbon Recovery Matrix \#2 Test Description .................................................. 8

Table XXI. Effect of Concentration on Gold Recovery to Carbon..................................... 8 
Table XXII. Results of Recovery Tests with Glass Added ........................................... 9

Table XXIII. Greens Creek Middling Carbon \& Sulfur Analysis................................... 10

Table XXIV. Greens Creek Middling Leach Test ................................................... 11

Table XXV. Greens Creek Middling Leach Test Results.......................................... 11

Table XXVI. Newmont Roaster Calcine Leach Tests ................................................. 11

Table XXVII. Newmont Calcine Leach Test Results .................................................. 12 


\section{List of Figures}

Figure 1. Final Stability Temperature Comparison Eh-pH Diagram............................... 5

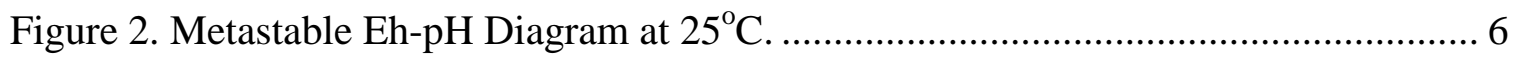

Figure 3. Metastable Eh-pH Temperature Comparison Diagram. .................................. 7

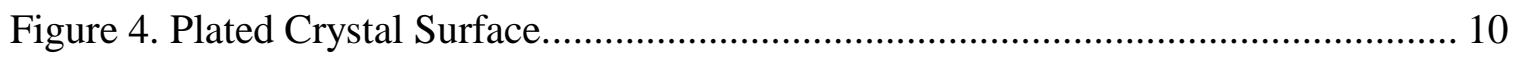

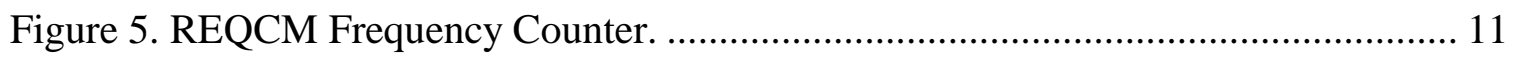

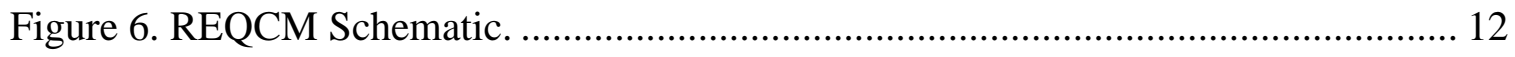

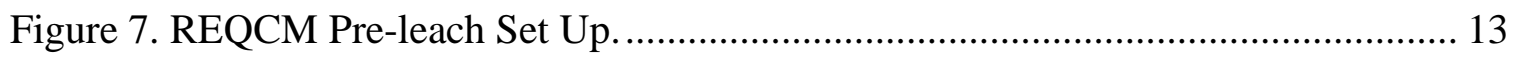

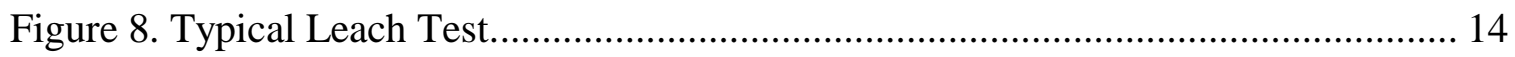

Figure 9. Effect of Sulfide Concentration on Pure Gold Potential Scans. ....................... 15

Figure 10. Effect of Hydrosulfide on Gold Potential Scans.......................................... 16

Figure 11. Effect of Increasing Sulfur Concentration on Gold Potential Scans. .............. 17

Figure 12. Corresponding Leach Tests to Solutions From Figure 11........................... 18

Figure 13. 2M Total Sulfur Pure Gold Potential Scans. ............................................ 19

Figure 14. Effect of Increasing Hydroxide Concentration in $2 \mathrm{M} \mathrm{S}^{2-}$ Solution. ................ 20

Figure 15. Effect of Hydroxide Addition on Gold Oxidation in Polysulfide.................... 20

Figure 16. Effect of Hydroxide Addition on Gold Leach Kinetics................................ 21

Figure 17. Temperature Effects on Gold Oxidation Potential Scans............................. 22

Figure 18. Effect of Temperature on Gold Oxidation in Polysulfide Solutions. .............. 22

Figure 19. Leach Test Varying Temperature in Polysulfide Solutions........................... 23

Figure 20. Effect of Gold Purity on Leach Rate. ..................................................... 24

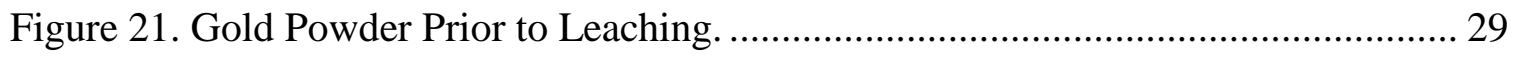

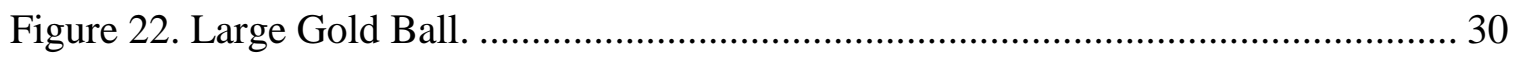


Figure 23. Identical $75^{\circ} \mathrm{C}$ Leach Tests Done with 999.9 Fine Gold. ............................... 2

Figure 24. Partially Dissolved Sulfur \& Sulfide Flakes............................................... 5

Figure 25. Kettle Test Outside of the Heat Bath...................................................... 6

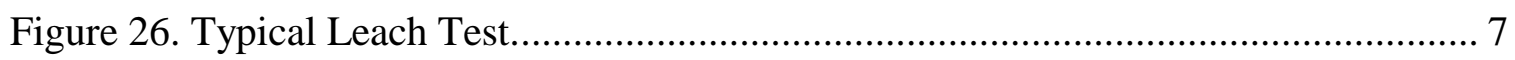

Figure 27. Solution Potential analysis at 18 Hours....................................................... 0

Figure 28. Effect of Sulfur Concentration and Temperature on Gold Leachability............ 1

Figure 29. 3-D Model of Leaching Effects of Hydroxide and Sulfur Concentration. ........ 2

Figure 30. Laboratory ASGLS Recovery Test. ....................................................... 4

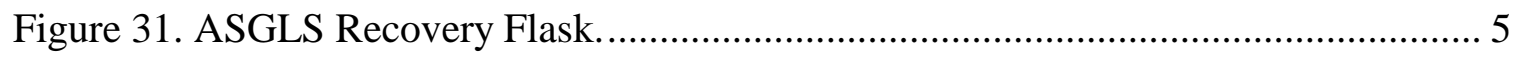

Figure 32. ASGLS Recovery Test Sample Collection. ............................................ 6

Figure 33. Greens Creek Middlings Prior to Leaching. .............................................. 11

Figure 34. Newmont Calcine Heads Before and After Leaching................................... 12

Figure 35. Top View of an Empty Autoclave......................................................... 13

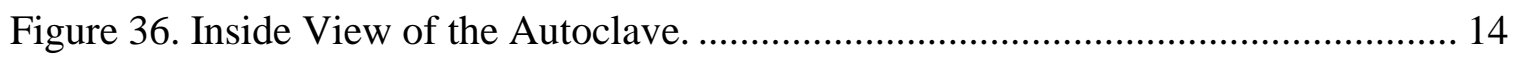

Figure 37. Autoclave Filled With Waste ASGLS Solution. ........................................ 15

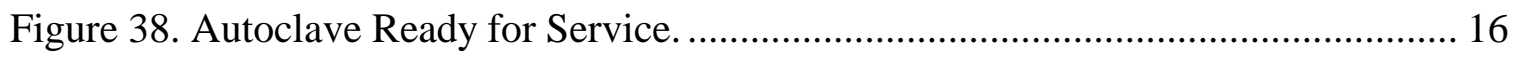

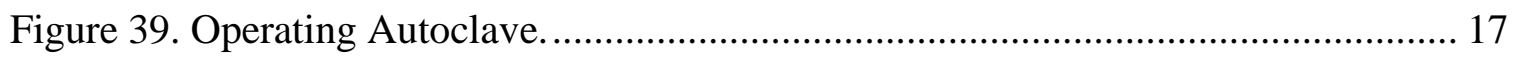




\subsection{Introduction}

\section{1 $\underline{\text { Research Purpose }}$}

Recently, the popular method for leaching gold with cyanide has come under scrutiny for its possible detrimental effects to the environment surrounding the mine site. Also, in cases of some ore bodies, conventional cyanide leaching is not the best method for gold extraction. Currently there is much interest in developing an alternative way to extract gold from ore. Possible benefits of leaching gold with something other than cyanide include the following:

- $\quad$ Easier processing of refractory ore bodies including: carbonaceous, cyanicides, and sulfide. Louw \& Gussmann

- $\quad$ Cheaper reagent and operating costs; ${ }^{\text {Anderson }}$ and,

- $\quad$ Opening of mine properties in places where cyanide use has been limited by the law.

Research for this thesis is focused on the development of the Alkaline Sulfide Gold Leaching System (ASGLS). The purpose of this thesis is to study the fundamentals of the ASGLS, and to apply these fundamentals to an industrial setting to see if it could be a suitable alternative to cyanide.

\section{2 $\quad$ Research Objectives}

To fully develop a method for leaching gold with the ASGLS it is critical to investigate all facets of the leach procedure. Research was focused on the following seven categories:

1. Understand ASGLS electrochemical properties;

2. $\quad$ Understand ASGLS electrokinetic properties;

3. Develop a standard laboratory procedure for leach tests;

4. Optimize the laboratory leaching environment;

5. Develop a method for gold removal from solution;

6. Leach industrial ores with optimized leach solution; and,

7. Develop a method for waste solution treatment.

\subsection{Background}

Gold has been known to readily dissolve in alkaline sulfide systems for some time; Hiskey \& Atluri however, the system has received little attention because of the complex electrochemistry occurring. Jeffery, Chapman, \& Wall . Research specific to the ASGLS has been conducted to account for the formation of elemental sulfur during the partial oxidation of sulfide ores. Anderson Sulfur formation makes it difficult for cyanide to penetrate and lixiviate the gold. Jeffrey \& Anderson The ASGLS is a proposed method to effectively leach gold after partial sulfide oxidation. 


\subsubsection{Literature Review}

\subsubsection{Monash University Fundamental Research}

Most of the electrochemical research done on the ASGLS has been done at Monash University in Melbourne, Australia. Jeffrey \& Anderson Some of the data retrieved from these fundamental studies was re-evaluated and is included in this thesis.

In their fundamental study it was determined that polysulfide is the oxidant and sulfide acts as the system lixiviant. To achieve the best leaching rates a ratio of sulfur : sulfide ratio of 1-1.5: 1 should be used. When hydrosulfide rather than sulfide was added to sulfur leach rates were slowed; it can be implied that leaching in caustic solutions, where sulfide is more stable than hydrosulfide, is beneficial to leaching. Also, silver impurities in the gold increased leach rates.

By measuring the leach rate as a function of temperature it was found that activation energy for leaching was $75 \mathrm{~kJ} / \mathrm{mole}$. From this value it is assumed that the reaction is chemically controlled since diffusion controlled reactions typically have activation energies less than $25 \mathrm{~kJ} / \mathrm{mole}$. Power and Richie

\subsubsection{Research Associated with the NSC Process}

A Nitrogen Species Catalyzed (NSC) method for partial oxidation of sulfide ores has been developed with positive conclusions. Anderson. In the NSC system, $\mathrm{NO}^{+}$is formed from nitrous acid and acts as the sulfide mineral oxidant according to the following equation:

$$
2 \mathrm{MeS}_{(\mathrm{s})}+4 \mathrm{NO}_{(\mathrm{aq})}^{+} \rightarrow 2 \mathrm{Me}^{2+}{ }_{(\mathrm{aq})}+2 \mathrm{~S}+4 \mathrm{NO}_{(\mathrm{g})}
$$

High temperatures and pressures will result in the sulfur being fully oxidized to sulfate.

By adding caustic to the solution critical ASGLS chemicals are formed according to the following equations:

$$
\begin{aligned}
& 4 \mathrm{~S}+6 \mathrm{NaOH} \rightarrow 2 \mathrm{Na}_{2} \mathrm{~S}+\mathrm{Na}_{2} \mathrm{~S}_{2} \mathrm{O}_{3}+3 \mathrm{H}_{2} \mathrm{O} \\
&(\mathrm{X}-1) \mathrm{S}+\mathrm{Na}_{2} \mathrm{~S} \rightarrow \mathrm{Na}_{2} \mathrm{~S}_{\mathrm{x}}
\end{aligned}
$$

The importance of these chemicals in the oxidation and lixiviation of gold will be discussed in the ASGLS Chemistry' section of this thesis.

\subsubsection{Xian Institute of Metallurgy and Construction Engineering Research}

In the two papers (Part I and II) found from this institute a system called Lime-Sulfur-SyntheticSolution (LSSS) was tested for its ability to leach gold. In Part I, gold is described to be oxidized with polysulfide and lixiviated by either polysulfide or thiosulfate. During the experimental work different oxidizing chemicals were added to the LSSS solution to test their effectiveness. For a high sulfur and gold/silver bearing ore precious metal dissolutions of over $90 \%$ were measured after just ten hours in solution at room temperature. Jian, Xinzhe, Feng, \& Zhichang 
In part II, more ores were leached with $+90 \%$ gold dissolution is achieved in less than three hours for a number of ores. The different chemical additions are analyzed on effectiveness per amount added basis. According to the paper to maximize gold dissolution kinetics: sodium sulfite should be added at 0.2 molar; oxidizer ' $\mathrm{G}$ ' should be added at least to 0.10 molar; $\mathrm{NH}_{3} \mathrm{H}_{2} \mathrm{O}$ should be added at 0.60 molar; and $\mathrm{Cu}^{2+}$ should be added at 0.045 molar.

Although the results found in this paper are very positive, the exact methods for achieving such high gold dissolution at room temperatures were poorly explained. Some of the tests were attempted to be recreated in the Montana Tech laboratory with 999.9 fine gold; each test had very poor results (see Matrix \#2). However, the results in these papers are compelling enough that more work should be done in the area of applying oxidizers to assist gold leaching.

\subsubsection{Associated Works}

There has been a lot of research done in areas associated with the ASGLS. A substantial amount of information was found on the hydrosulfide $\left(\mathrm{H}_{2} \mathrm{~S} \& \mathrm{HS}^{-}\right)$leaching of gold at near-natural $\mathrm{pH}$. Although these papers will not be discussed directly in this thesis an overview will be provided below and they are present in the bibliography section. Major hydrosulfide areas covered include the following: high temperature thermodynamic research; high pressure leaching; effect of $\mathrm{pH}$ and solution potential; and the effect of sulfur concentration.

Also, much of the research has been done to find a way of processing ores that are high in arsenic minerals that consume cyanide. Most of these papers pertain to the pressure oxidation of the sulfide minerals and possible ways to treat the ores. The NSC paper described above offers a good method; each of the other papers offer different methods.

In addition to these papers there were two found that pertain to the production of polysulfide for the paper \& pulp industries. These papers offer a good background to polysulfide chemistry while not pertaining directly to gold.

\subsubsection{ASGLSChemistry}

The main leaching component in the ASGLS is sulfur. Sulfur varies widely in oxidation state; a combination of sulfide $\left(\mathrm{S}^{2-}\right)$ and elemental sulfur $\left(\mathrm{S}^{0}\right)$ are needed to make for sufficient leaching kinetics in the ASGLS. Both sulfur chemistry and leaching chemistry will be discussed in further detail below.

\subsubsection{Sulfur Chemistry}

For the ASGLS, the critical solution ingredient is sulfur. Dissolved sulfur can have an oxidation state ranging from ${ }^{-2}$ (sulfide) to ${ }^{+} 6$ (sulfate) depending on potential of the system. As sulfide begins to oxidize towards sulfur it will pass through the metastable polysulfide range. A polysulfide molecule is formed when a chain of sulfur atoms is formed. Each chain can be up to 5 atoms in length; however, for each polysulfide molecule the charge is constant at ${ }^{-2}$. (See Table I). 
Table I. Oxidation States of Sulfur Species

\begin{tabular}{|c|c|c|c|}
\hline Specie & Charge & Name & Oxidation State of Sulfur \\
\hline $\mathrm{SO}_{4}$ & -2 & Sulfate & $(+6)$ \\
\hline $\mathrm{SO}_{3}$ & -2 & Sulfite & $(+4)$ \\
\hline $\mathrm{S}_{2} \mathrm{O}_{3}$ & -2 & Thiosulfate & $(+2)$ \\
\hline $\mathrm{S}$ & 0 & Sulfur & 0 \\
\hline $\mathrm{S}_{5}$ & -2 & Polysulfide & $(-0.4)$ \\
\hline $\mathrm{S}_{4}$ & -2 & Polysulfide & $(-0.5)$ \\
\hline $\mathrm{S}_{3}$ & -2 & Polysulfide & $(-2 / 3)$ \\
\hline $\mathrm{S}_{2}$ & -2 & Polysulfide & $(-1)$ \\
\hline $\mathrm{S}$ & -2 & Sulfide & $(-2)$ \\
\hline
\end{tabular}

Polysulfides can be generated by the additions of sulfur and sulfide to a solution according to the following equation. Relative molar additions of each ingredient will determine the average polysulfide chain length. Also, sulfide can be generated by adding hydroxide to elemental sulfur. Anderson, Nordwick, \& Krys

$$
\begin{aligned}
& \mathrm{S}+(\mathrm{x}-1) \mathrm{S}^{-2} \rightarrow \mathrm{S}_{\mathrm{x}}{ }^{-2} \\
\mathrm{~S}+6 \mathrm{OH}^{-} \rightarrow & 2 \mathrm{~S}^{2-}+\mathrm{S}_{2} \mathrm{O}_{3}{ }^{2-}+3 \mathrm{H}_{2} \mathrm{O}
\end{aligned}
$$

Once the polysulfide solution has been used it will need to be treated for waste. By oxidizing sulfur in solution to the ${ }^{+} 6$ state, or to sulfate, the solution can be disposed of easily. The equations below represent the primary anodic reactions; they provide the cathodic reaction with sufficient electrons. Anderson

$$
\begin{array}{r}
4 \mathrm{OH}^{-} \rightarrow 4 \mathrm{e}^{-}+2 \mathrm{H}_{2} \mathrm{O}+\mathrm{O}_{2} \\
\mathrm{~S}^{-2} \rightarrow 2 \mathrm{e}^{-}+\mathrm{S}
\end{array}
$$

The equations below represents the reduction of polysulfides to sulfate. Note that oxygen is the oxidant in the reaction; during laboratory test work oxygen is provided to help drive the equation to the right.

$$
\mathrm{S}_{\mathrm{x}}{ }^{2-}+(2 \mathrm{x}) \mathrm{O}_{2}+2(\mathrm{x}-1) \mathrm{e}^{-} \rightarrow \mathrm{xSO}_{4}{ }^{2-}
$$

\subsubsection{Leaching Chemistry}

Although there are two gold-sulfur complexes that are responsible for gold dissolution, the dominant lixiviant has been determined to be sulfide. In the table below, stability constants are 
shown for the two prominent ligands; sulfide and bisulfide. Note that the stability constant for the formation of $\mathrm{AuS}^{-}$has a much larger magnitude than $\mathrm{Au}(\mathrm{HS})_{2}{ }^{-}$; this is why it is determined to be the dominant gold-sulfur form. Hiskey \& Atluri

Table II. Dominant Gold Reaction Stability Constants

\begin{tabular}{|c|c|c|c|}
\hline Ligand & Specie & Reaction & Stability Constant $(\beta)$ \\
\hline Sulfide & $\mathrm{AuS}^{-}$ & $\mathrm{AuS}^{-}+\mathrm{e}^{-} \rightarrow \mathrm{AuS}^{2-}$ & $2 * 10^{36}$ \\
\hline Bisulfide & $\mathrm{Au}(\mathrm{HS})_{2}^{-}$ & $\mathrm{Au}(\mathrm{HS})_{2}^{-}+\mathrm{e}^{-} \rightarrow \mathrm{Au}+2 \mathrm{HS}^{-}$ & $1.3 * 10^{30}$ \\
\hline
\end{tabular}

According to research done at Monash University suggests that a combination of sulfides and polysulfides are required for leaching to proceed. Jeffery, Chapman, \& Wall When solutions of sulfide $\left(\mathrm{S}_{2}{ }^{2-}, 50 \mathrm{~g} / \mathrm{L}\right)$, bisulfide (HS, $\left.50 \mathrm{~g} / \mathrm{L}\right)$, and polysulfide $\left(\mathrm{S}_{\mathrm{x}}{ }^{2-}, 50 \mathrm{~g} / \mathrm{L}\right)$ were tested for gold leaching capabilities, neither solution showed any measurable gold dissolution. However, leaching does occur when sulfide and polysulfide are combined. Since polysulfides exist at a higher oxidation state than sulfide, polysulfide is theorized to be the oxidizer in the system. In other words, gold is oxidized $\left(\mathrm{Au} \rightarrow \mathrm{Au}^{+}\right)$as a result of polysulfide reduction $\left(\mathrm{S}_{3}{ }^{2-} \rightarrow \mathrm{S}_{2}{ }^{2-}\right)$.

Experiments were completed at Monash University in an effort to understand what the dominant lixiviant in the system is. Potential scans of solutions containing just polysulfide showed relatively small calculated currents at leaching potentials. Purely sulfide solutions, on the other hand, exhibited high calculated currents at leaching potentials. The conclusion is that sulfide is the active lixiviant in the ASGLS. Jeffery, Chapman, \& Wall.

The gold lixiviation chemical equation can be seen below:

$$
\mathrm{Au}+\mathrm{S}^{2-} \rightarrow \mathrm{AuS}^{-}+\mathrm{e}^{-}
$$

Remember that gold leaching did not occur in solutions of just sulfide. Leaching can occur when there is a simultaneous polysulfide reduction to sulfide reaction:

$$
\mathrm{S}_{\mathrm{x}}^{2-}+2(\mathrm{x}-1) \mathrm{e}^{-} \rightarrow \mathrm{xS}^{2-}
$$

Variable ' $\mathrm{x}$ ' can range from $2-5$. Anderson In the case where $\mathrm{x}=2$ the polysulfide reaction is as follows:

$$
\mathrm{S}_{2}{ }^{2-}+2 \mathrm{e}^{-} \rightarrow 2 \mathrm{~S}^{2-}
$$

Combining the polysulfide reduction (gold oxidation) reaction with the sulfide lixiviant reaction yields the overall reaction. The final ASGLS reaction with the polysulfide molecule as a two atom chain is:

$$
2 \mathrm{Au}+\mathrm{S}_{2}^{2-}+2 \mathrm{~S}^{2-} \rightarrow 2 \mathrm{AuS}^{-}+2 \mathrm{~S}^{2-}
$$

The important aspect of the ASGLS chemistry is to remember that the system oxidant and the system lixiviant are not the same chemicals. Both sulfide and polysulfide must be present in the system if leaching is going to occur. 


\subsection{Electrochemistry and Electrokinetics of the ASGLS}

\subsection{Development of Eh-pH Diagrams}

To better understand the electrochemistry of the ASGLS, Eh-pH diagrams were constructed to determine the conditions where gold is stable as aqueous specie in a sulfur environment. Also, from the work done at Monash University, it was found that the system is highly dependent on temperature. With the computer program Stabcal, Eh-pH stability diagrams were created varying sulfur concentration ( 1 and 5 molar) and temperature $\left(25-200^{\circ} \mathrm{C}\right)$.

\subsubsection{Free Energy Calculations}

All of the Eh-pH diagrams were constructed with Stabcals 'Russia' internal database. Under typical leaching conditions, the most stable aqueous gold specie has been shown to be AuS- ${ }^{-}$effery, Chapman, \& Wall Unfortunately, the Russia database does not contain the free energy data for the AuS $^{-}$specie. The free energy of formation for AuS- is described below: Hiskey \& Atluri.

$$
\mathrm{Au}^{+}+\mathrm{S}^{2-} \rightarrow \mathrm{AuS}^{-} \Delta \mathrm{G}_{\mathrm{f}}^{\mathrm{o}}=11.57 \mathrm{kcal} / \mathrm{mol}
$$

Data for $\mathrm{Au}^{+}$and $\mathrm{S}^{2-}$ was extracted from the database and the free energy for $\mathrm{AuS}^{-}$was calculated to be $9.98 \mathrm{kcal} / \mathrm{mol}$. Free energies for all species were extracted via Stabcal for the following four temperatures: $25,50,100$, and $200^{\circ} \mathrm{C}$.

\subsubsection{Stable and Metastable Eh-pH Diagram Production}

All of the Eh-pH diagrams were produced with an aqueous gold concentration of $100 \mathrm{ppm}$ in 1 atmosphere of pressure. After the diagrams were produced in Stabcal, data for the lines in the diagram is exported as a text file to Excel where the diagrams can be reproduced. Once all of the diagrams are in Excel they are redrawn in AutoCAD so that varying temperatures or concentrations can be superimposed onto one another. By superimposing the diagrams onto one another it becomes easier to see the effect of changing variable in the ASGLS. All of the diagrams created can be seen in Appendix A1-A16.

\subsubsection{Stable Eh-pH Diagrams}

To model the stable ASGLS the Russia database is imported fully and the AuS- is added to the database at a free energy of $9.98 \mathrm{kcal} / \mathrm{mol}$. It is likely that a stable Eh-pH diagram does not represent the leaching system very well; the diagrams were generated to understand how temperature and time affect the ASGLS.

Below is a temperature $\left(25-200^{\circ} \mathrm{C}\right)$ comparison diagram for 5 molar sulfur. The stable sulfur species for $200^{\circ} \mathrm{C}$ are outlined and labeled in gray. The blue region represents final AuS stability for $25^{\circ} \mathrm{C}$ and the red region for $200^{\circ} \mathrm{C}$. 


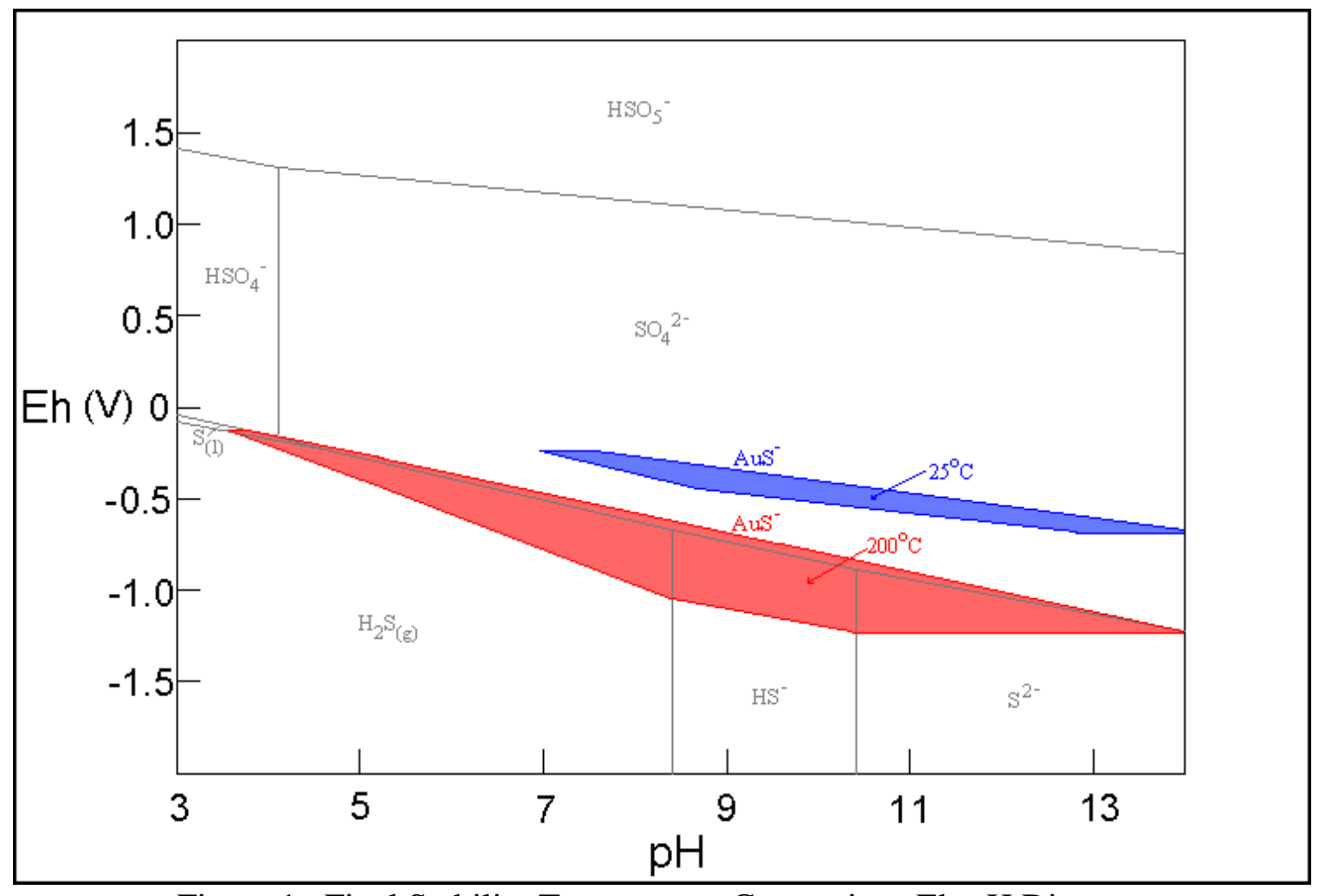

Figure 1. Final Stability Temperature Comparison Eh-pH Diagram

From the figure above it is observed that increasing temperature both widens and thickens the region where gold is stable in solution. Also, the region has been dropped on the order of 500 $\mathrm{mV}$.

Interestingly, by changing the sulfur concentration from 1-5 molar the stability diagrams do not alter very much (see Appendix A4 vs. A8). The biggest effect is the $\mathrm{HS}^{-}$field is widened, which does little to change the AuS ${ }^{-}$region.

\subsubsection{Metastable Eh-pH Diagrams}

In the ASGLS, there are many aqueous sulfur-oxygen-hydrogen species that form; some of the species are more stable than others. When Stabcal produces a diagram it is calculating for the final stability at infinite time of all species involved. In the ASGLS case, gold leaching takes place before the system has reached its final stability. To try and model this metastable period in the leach process some of the final stable products are deleted from the database. These stable products consist of all species that would form if the solution was in contact with oxygen. The species that are left for the calculation are: $\mathrm{S}^{2-} ; \mathrm{S}_{2}{ }^{2-} ; \mathrm{S}_{3}{ }^{2-} ; \mathrm{S}_{4}{ }^{2-} ; \mathrm{S}_{5}{ }^{2-} ; \mathrm{S}_{(\mathrm{l})} ; \mathrm{S}_{(\mathrm{s})} ; \mathrm{H}_{2} \mathrm{~S}_{(\mathrm{g})}$; and, HS .

A metastable Eh-pH diagram (5 molar sulfur, $25^{\circ} \mathrm{C}, 1 \mathrm{ATM}, 100 \mathrm{ppm}$ Au) can be seen below. Gold is stable in solution as AuS- at $100 \mathrm{ppm}$ in the shaded region on the graph. Note that the stable regions for the sulfur species are outlined and labeled in gray. 


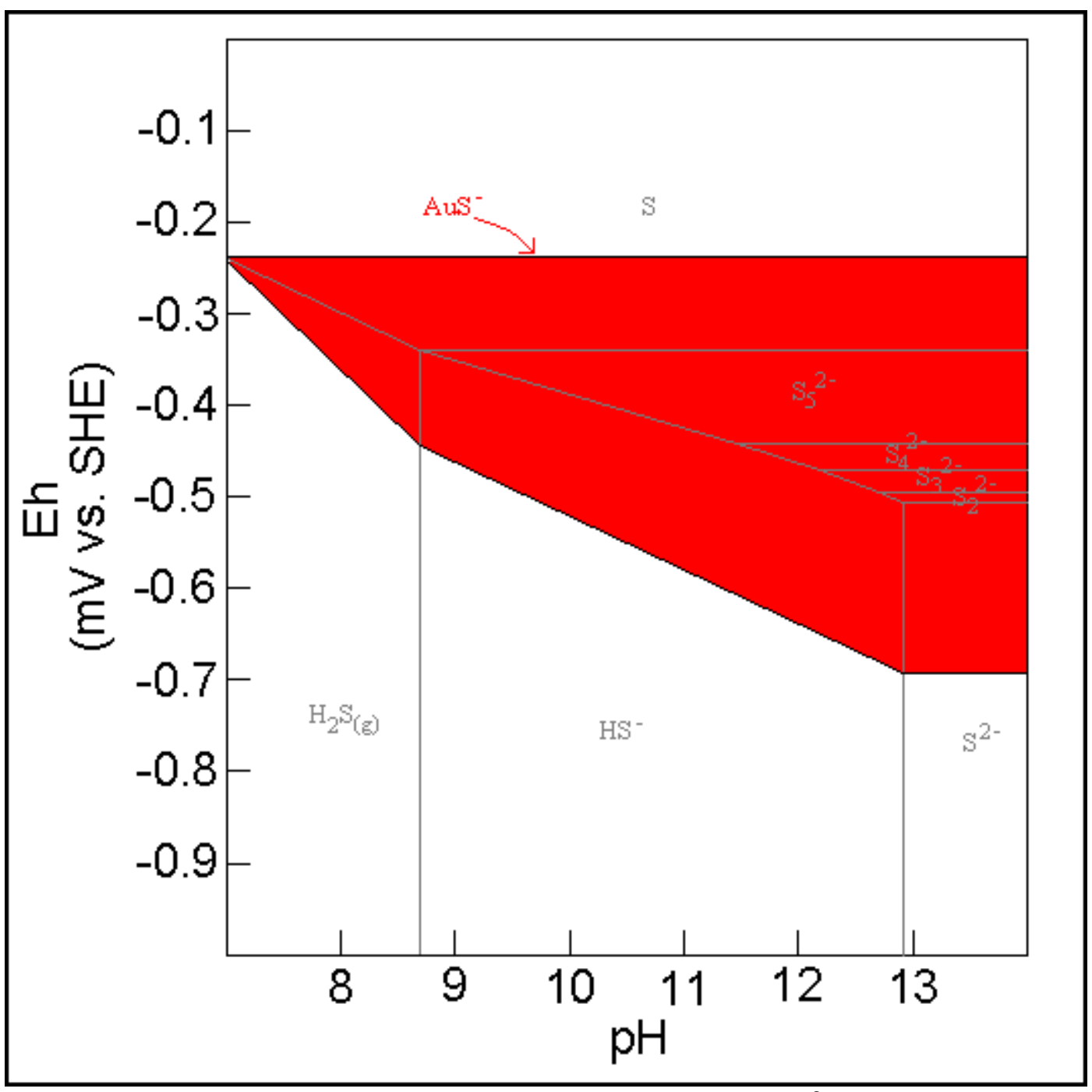

Figure 2. Metastable Eh-pH Diagram at $25^{\circ} \mathrm{C}$

Metastable diagrams were created for temperatures of $25,50,100$, and $200^{\circ} \mathrm{C}$. A comparison diagram for 25 and $200^{\circ} \mathrm{C}$ can be seen below. Gold stability regions are shaded in blue for $25^{\circ} \mathrm{C}$ and red for $200^{\circ} \mathrm{C}$. The area where the AuS stability overlaps is shaded in purple. The sulfur species that are shown on the diagram are representative of the $200^{\circ} \mathrm{C}$ system. Note that in the strong alkaline regions (where the ASGLS is being studied) sulfide is gradually oxidized through the polysulfide regions and eventually to sulfur. 


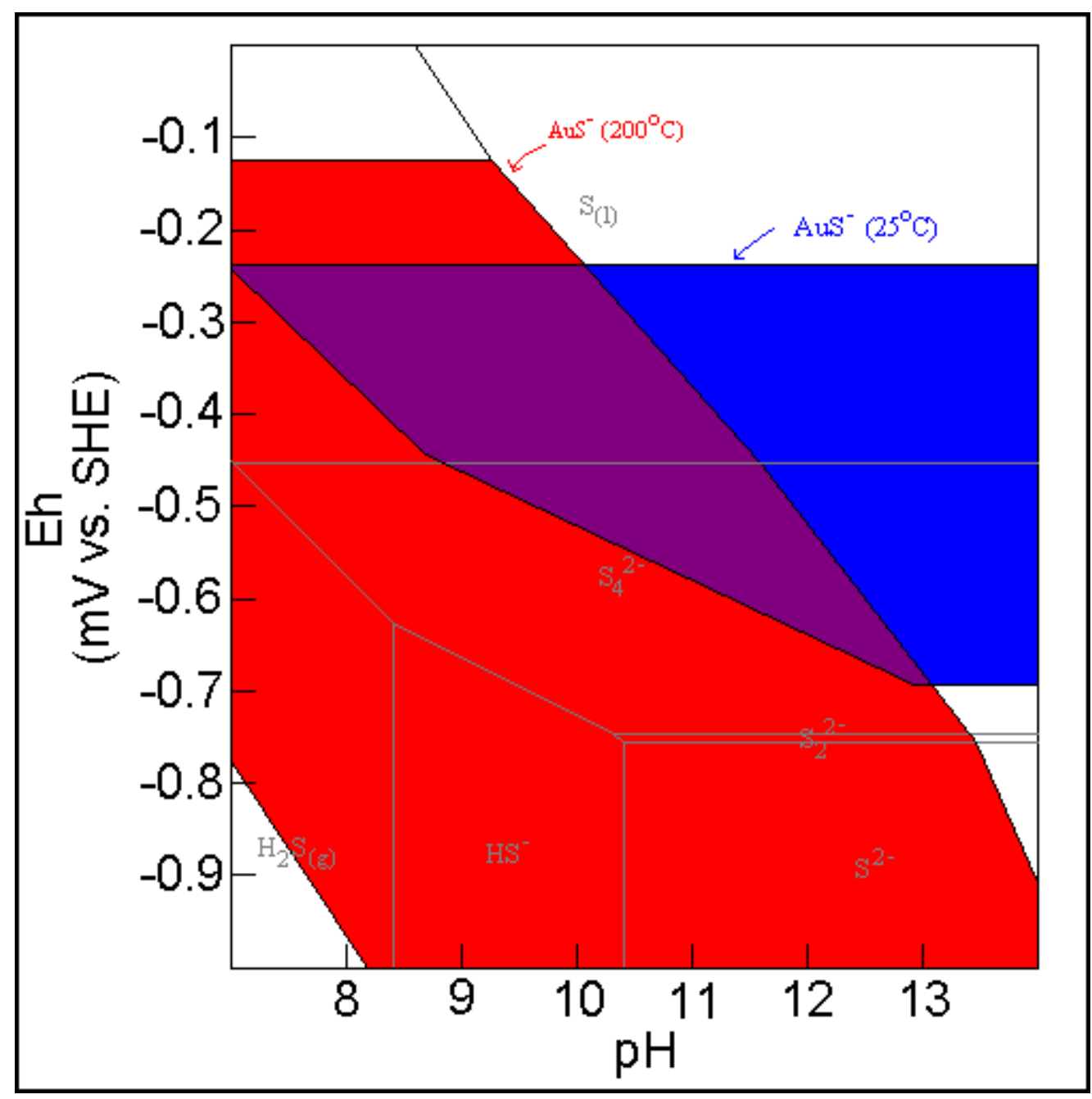

Figure 3. Metastable Eh-pH Temperature Comparison Diagram

As the temperature is increased the $\mathrm{AuS}^{-}$region is shifted to a more reducing potential and a more acidic $\mathrm{pH}$ range. Because the potential range has been extended for the high temperature setting, it should be much easier to get the system into a leaching potential. However, it is important to remember that stability diagrams cannot show anything pertaining to kinetics. Electrokinetics should be studied alongside electrochemistry.

As seen in the stable diagrams, increasing sulfur concentration does not affect the Eh-pH diagrams very much. This can be seen in the Appendix: A12 vs. A16. Apparently adding sulfur only affects the realm of kinetics.

\subsection{Research Done on the REQCM}

During February 2002 and June-August 2004 a Rotating Electrochemical Quartz Crystal Microbalance (REQCM) was used to better understand the fundamental electrochemical and electrokinetic principals in the alkaline sulfide gold leaching system. All of the experiments were done on the REQCM located in the Chemical Engineering Department of Monash 
University, just southeast of Melbourne, Australia. Variables that were investigated in the system include:

- $\quad$ Effect of sulfide concentration $\left[\mathrm{S}^{2-}\right]$;

- $\quad$ Effect of hydrosulfide [HS ${ }^{-}$;

- $\quad$ Effect of polysulfide concentration $\left[\mathrm{S}_{\mathrm{x}}{ }^{2-}\right]$;

- $\quad$ Effect of $\mathrm{NaOH}$ concentration;

- $\quad$ Effect of temperature; and,

- $\quad$ Effect of gold purity.

\subsubsection{REQCM Background}

Under the guidance of Dr. Matthew Jeffery in the Chemical Engineering department at Monash University, there is an abundance of electrochemical research being done on the REQCM. A tool as versatile as the REQCM is required to effectively research complex chemistry like the ASGLS because there are other electrochemical reactions occurring simultaneously to the gold dissolution reaction. Sulfide/Polysulfide leaching of gold What makes the REQCM so versatile is its ability to accurately measure extremely small changes in gold mass while recording the electrical reactions taking place.

The quartz crystal is critical to the REQCM because it makes reading incremental mass changes possible. As current passes through the crystal it will resonate due to the converse piezoelectric effect. Jeffery, Zheng, Richie The frequency of the resonance is related to the mass of the crystal according to the Sauerbary equation: Ward.

$\Delta \mathrm{m}=\left\{-\mathrm{A}_{\mathrm{p}}\left(\mu_{\mathrm{q}} * \rho_{\mathrm{q}}\right)^{1 / 2} * \Delta \mathrm{f}\right\} / 2 \mathrm{f}_{\mathrm{o}}^{2}$

Where:

$\Delta \mathrm{m}=$ change in metal mass;

$A_{p}=$ piezoelectrically active area;

$\mu_{\mathrm{q}}=$ shear modulus of quartz;

$\rho_{\mathrm{q}}=$ quartz density;

$\Delta \mathrm{f}=$ change in frequency; and,

$\mathrm{f}_{\mathrm{o}}=$ resonant frequency.

As a crystal loses weight -- from metal being leached off the surface -- the resonant frequency will increase. As metal is deposited onto the surface the resonant frequency decreases because the total mass of the crystal has increased. Mass changes in the range of \pm 10 nanograms $\left(10^{-9}\right.$ grams) can be accurately measured. Jeffery, Chapman, \& Wall.

\subsubsection{REQCM Principle}

Once the electrode is submerged into the experimental solution the REQCM is able to account for both mass and electrical changes taking place on the Metal of Interest (MOI) surface. Leach 
tests can be run to determine the leachability of certain metals in different solutions. Also, potentioscans can be run to determine the current flow through the MOI at different potentials.

Typically during a potentioscan only the total amount of electrons flowing (total current) can be measured. Again, total current flow in the ASGLS is due to the following two equations:

$$
\begin{aligned}
& \mathrm{Au}+\mathrm{S}^{2-} \rightarrow \mathrm{AuS}^{-}+\mathrm{e}^{-} \\
& \mathrm{S}_{\mathrm{x}}{ }^{2-}+2(\mathrm{x}-1) \mathrm{e}^{-} \rightarrow \mathrm{xS}^{2-}
\end{aligned}
$$

During the course of a potentioscan, some of the MOI will leach into solution. Because the exact quantity of the MOI leached is known, the total occurrence of lixiviation is known. A calculation can be made via Faradays law below to calculate the total amount of electrons produced from only the oxidation of gold.

$$
\mathrm{I}_{\mathrm{calc}}=(\mathrm{nF} / \mathrm{AM}) *(\Delta \mathrm{m} / \Delta \mathrm{t})
$$

The calculated current, or $I_{\text {calc }}$, provides valuable information to the researcher because for any given potential during a potentioscan, the rate of oxidation for the MOI is known.

\subsubsection{REQCM Critical Components and Pre-test Procedures}

The most important piece of equipment on the REQCM is the electrode. During the experiment it will be the working electrode. A MOI is plated onto the end of the electrode prior to being submerged in the leach solution during an experiment. Electrodes are made on site at Monash University. Each electrode consists of five major parts: 1) a thin quartz crystal disk; 2) a small circular deposit of platinum; 3) an electroplated mass of the MOI; 4) two wires connected to the quartz crystal; and, 5) a PVC shell.

\section{Quartz Crystal}

\subsubsection{Platinum Sputter Coating}

On both sides of the quartz crystal, a thin circular layer of platinum is sputtered on the surface. Both sides of the crystal are sputtered so that a potential difference can be measured between the side that is submerged in the leach solution and the side that is not. Platinum is chosen to be sputtered on the crystal surface because of its inert properties; no platinum should react with the solution. A MOI can be electroplated onto the platinum. Therefore the platinum provides both a surface for metal to be electroplated and a surface that electrons can flow through.

\subsubsection{MOI Deposition}

During an experiment $t$ on the REQCM, the solid to be leached is electrically deposited onto the platinum surface until a desired mass has been plated, usually about $150 \mu \mathrm{g}$. All electrodes were plated with the same procedure; however, depending on the MOI to be studied, different plating 
solutions are required. Plating solutions for $\mathrm{Au} / \mathrm{Ag}$ will have the concentrations found in Table III.

\begin{tabular}{|c|c|c|}
\hline \multicolumn{3}{|c|}{ Table III. Gold/Silver Alloy Addition } \\
\hline Chemical & Name & Concentration \\
\hline $\mathrm{KAuCN}$ & Potassium Dicyanoaurate & $0.02 \mathrm{M}$ \\
\hline $\mathrm{K}_{2} \mathrm{CO}_{3}$ & Potassium Carbonate & $0.086 \mathrm{M}$ \\
\hline $\mathrm{KCN}^{\mid 3}$ & Potassium Cyanide & $0.23 \mathrm{M}$ \\
\hline $\mathrm{AgNO}_{3}$ & Silver Nitrate & $*$ \\
\hline \multicolumn{3}{|c|}{} \\
$* \begin{array}{c}\text { (Silver nitrate is added depending on \%-Ag desired in alloy. For } \\
\text { example, a 5\% silver alloy will need 0.5mM silver nitrate.) }\end{array}$ \\
\hline
\end{tabular}

Naturally, a pure Au solution will have no silver nitrate added. A fully plated crystal surface can be seen below.

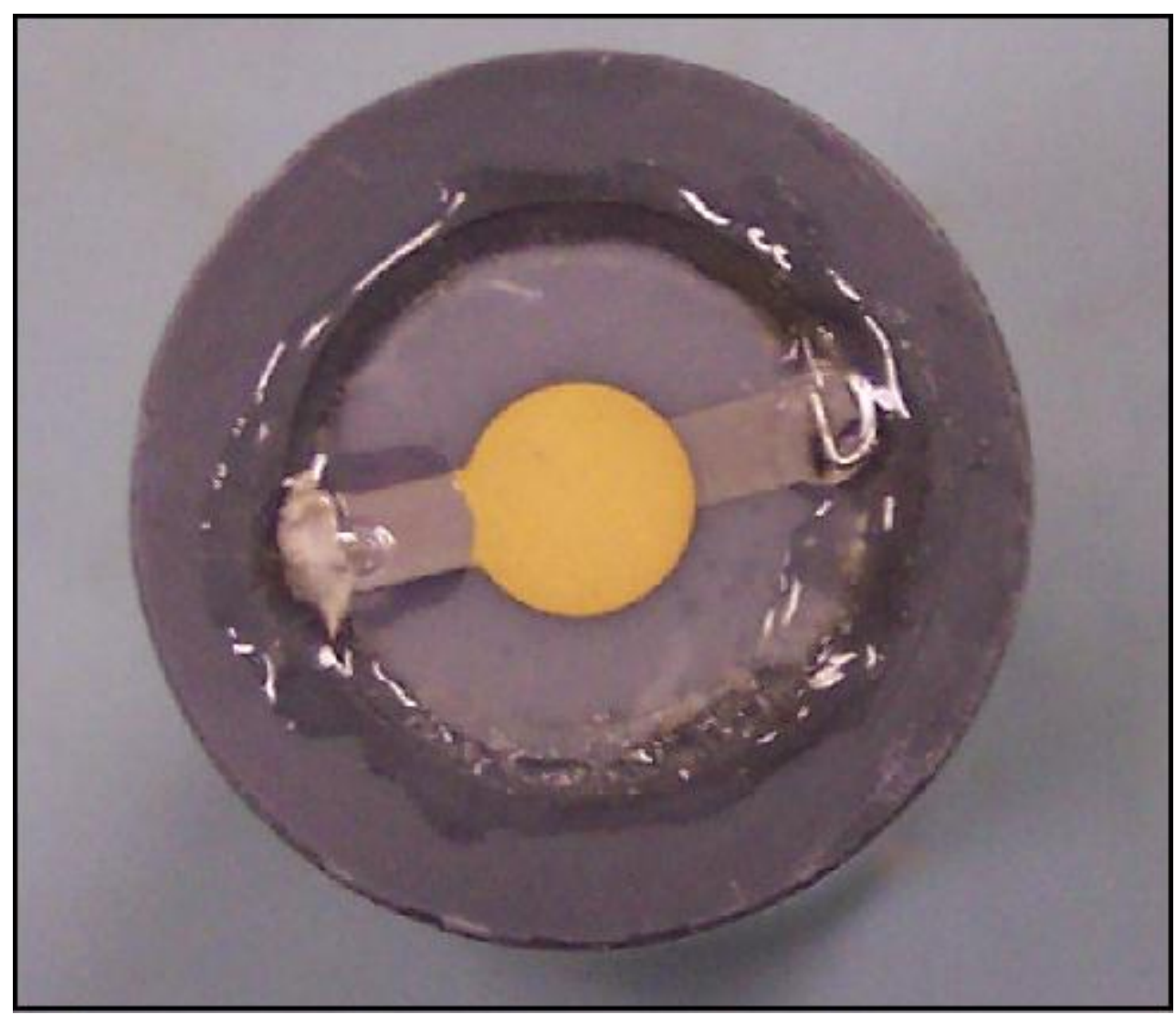

Figure 4. Plated Crystal Surface

After a leach test, there will be some of the MOI that did not fully leach off of the platinum. Rather than disposing of the electrode, the remaining MOI is 'cleaned' off of the platinum 
surface in a weak sodium cyanide solution. A new MOI can be electroplated onto the platinum surface and it can be submitted to a new leaching solution.

To find electrical changes, a voltmeter and ammeter are connected to the circuit. To find the mass changes, current is passed through a quartz crystal, inducing a resonant frequency. Based on frequency changes during the course of an experiment, mass changes can be calculated. Although mass and electrical changes are measured differently, data from the two can be combined to create very useful electrochemical trends.

\subsubsection{Experimental Procedure}

The REQCM was set up in a fume hood in the Monash Chemical Engineering Laboratory so that any dangerous fumes would be exhausted properly. The safety procedure for the ASGLS at Monash University can be found in Appendix B1 - B8.

\subsubsection{MOI Deposition and Post Experimental Stripping}

Once the electrode is plugged in to the holder, the frequency counter will start to transfer resonant frequencies to a computer that calculates the relative mass on the electrode. Below shows the frequency counter (reading about $9.85 \mathrm{MHz}$ ) sitting on the REQCM.

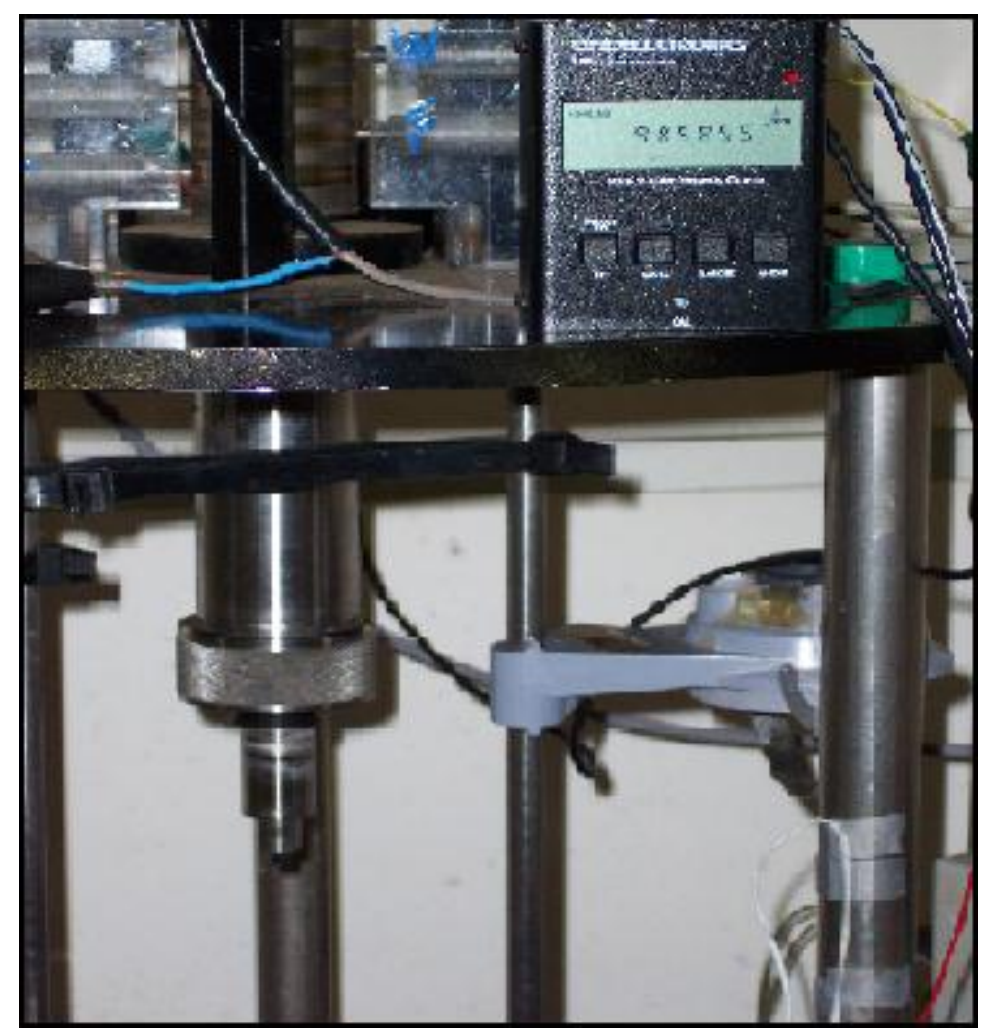

Figure 5. REQCM Frequency Counter

Because each crystal has a unique mass, it is important to note the starting mass without any MOI plated. Electroplating of MOI was turned off once there had been about $150 \mu \mathrm{g}$ of the MOI plated. 
To achieve good MOI surface, all plating is done while the electrode is spinning. The resulting MOI surface is uniform and smooth. After about $150 \mu \mathrm{g}$ has been plated, the current is turned off and the electrode can be removed from the holder.

The freshly MOI plated electrode will have plating solution on it after it is removed. All of the solution is washed off and the electrode and it is then dried off with compressed air. The dry, MOI plated electrode was then plugged back into the holder for experiments.

Each electrode can be reused after a test has been completed. First the crystal surface with remaining MOI is cleaned of any remaining ASGLS solution. Once clean, the electrode is plugged back into the REQCM and the MOI is leached off of the crystal in a weak sodium cyanide solution. After all of the MOI is gone (mass changes will begin to level off to zero change), it can be used again by plating a new MOI onto the crystal.

\subsubsection{REQCM Experimental Set Up}

Once a MOI was plated, experiments could be run to test the electrochemistry of different ASGLS solutions. A schematic of the final set up can be seen below.

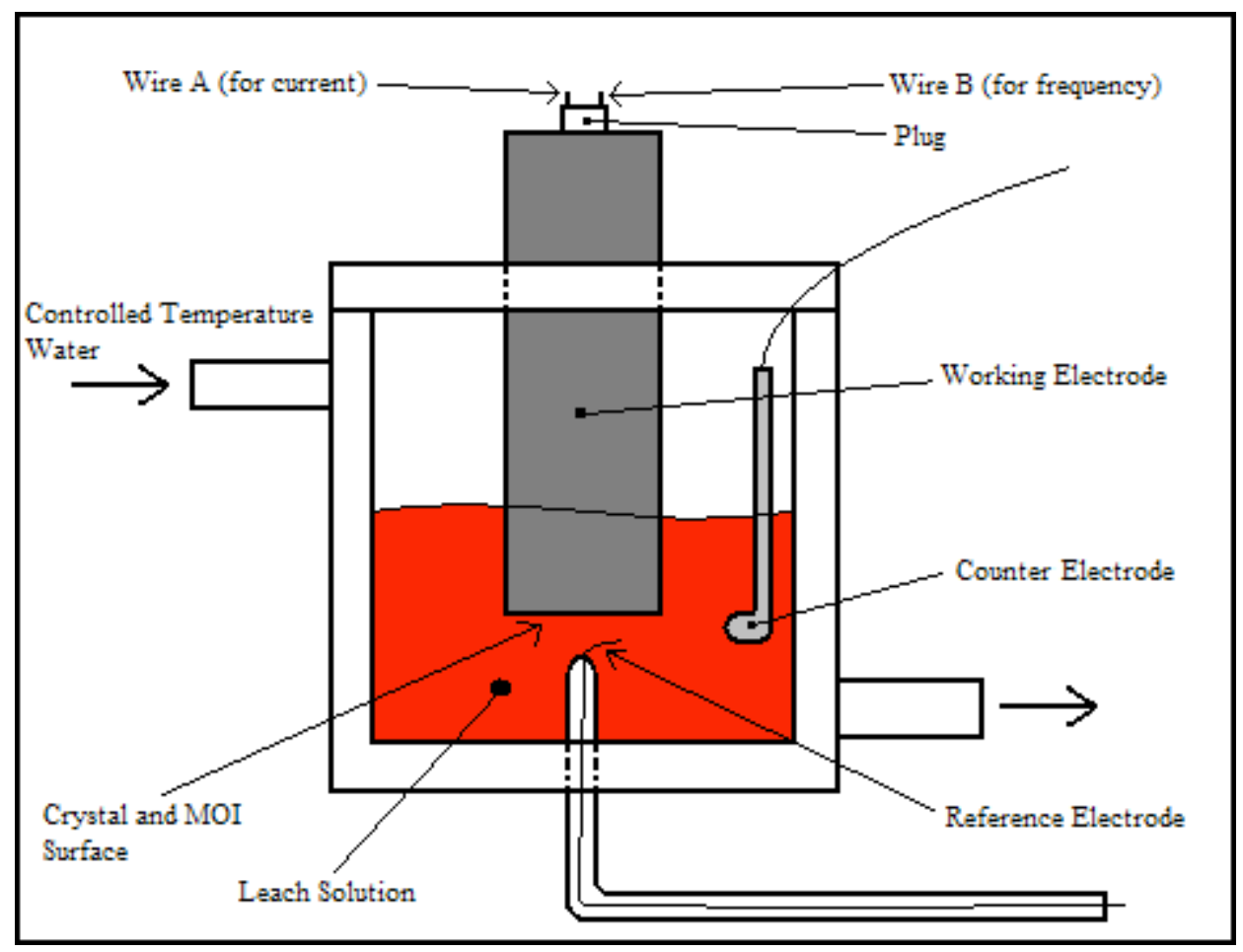

Figure 6. REQCM Schematic

While the crystal surface is being plated and during the course of a potential scan, the counter electrode is providing the electron flow from the DC power output; working electrode is registering the total electron flow at the MOI surface. During a leach test the counter electrode has no current flowing to it, but working electrode is reading the electrical activity at the surface. 
The reference electrode in both the potential scans and leach tests is reading the solution potential against the Standard Calomel Electrode (SCE). A picture of the experimental set up can be seen below.

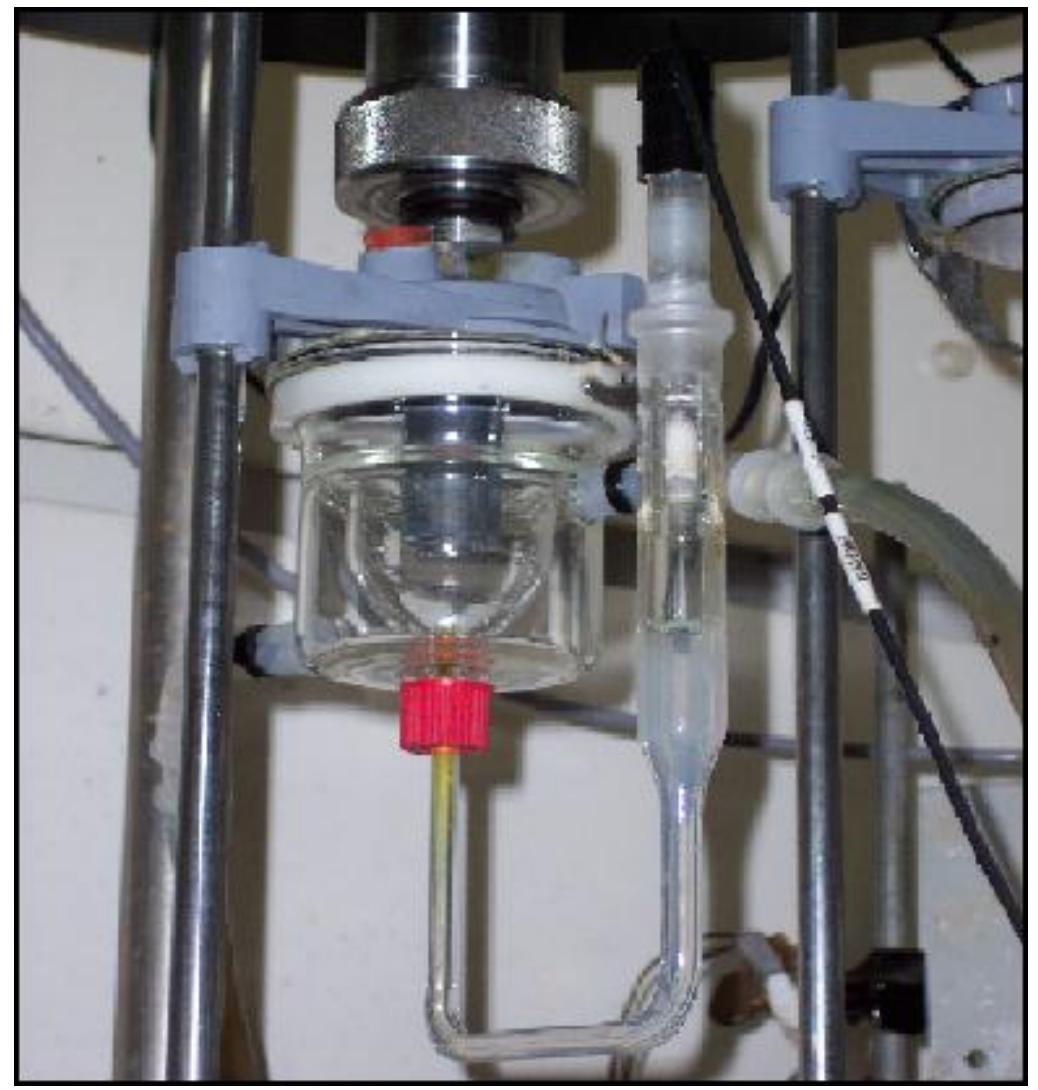

Figure 7. REQCM Pre-leach Set Up

Each test was done with $25 \mathrm{~mL}$ of solution. If the test required the solution to be done at an elevated temperature the controlled temperature water lines were hooked up and the heater was turned on. Experiments could be run at temperatures as high as $60^{\circ} \mathrm{C}$; at higher temperatures the working electrode would lose stability and experimental results were poor.

\subsubsection{Solution Preparation}

All solutions were prepared with solid reagent and de-ionized water. The solids used over the course of work were: sodium sulfide, sulfur, sodium hydroxide, and sodium hydrosulfide. Experimental solutions were prepared in $25 \mathrm{~mL}$ samples. Solids were weighed to $1 / 1000$ of a gram; once all solids were weighed, about $15 \mathrm{~mL}$ of water was added. A magnetic stir bar was dropped and the solution was placed on a hot plate to help in dissolution of solids. As soon as the last solid dissolved, the solution was removed from the heat and allowed to cool. Once cool, the solution was added to a volumetric flask, filled to volume, and added to the REQCM vessel. 


\subsubsection{Obtaining Data}

A computer hooked up to the REQCM automatically calculated the mass on the crystal with inputs of the resonant frequency. Also, a continuous feed of electrical data coming from both the reference electrode and crystal surface is fed into the computer. Two different types of plots can be obtained with the software: leach tests and potential scans.

During a leach test the electrode is simply submerged into the solution and the rate at which metal leaves the surface is recorded along with the Mixed Potential $\left(E_{m}\right)$, the potential at which leaching occurs. A typical leach test can be seen below.

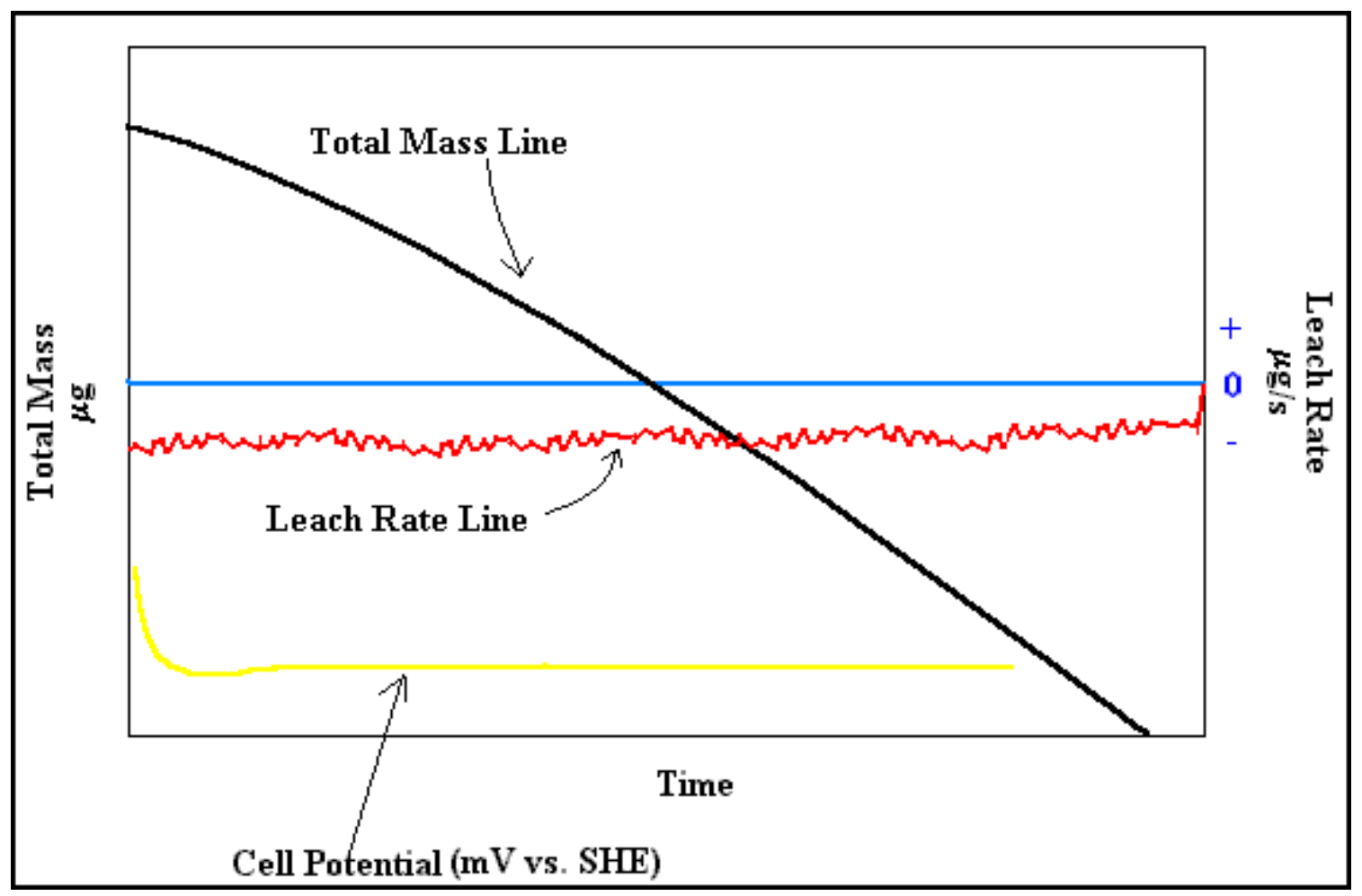

Figure 8. Typical Leach Test

Note that after the system reaches equilibrium the electromotive potential $\left(E_{\mathrm{m}}\right)$ will level off. Also note the noise in the leach rate line. Even though it is relatively noisy, (typically \pm 5 nanograms of the average leach rate), the overall mass loss line is smooth because with a properly working electrode the noise will average out to almost zero over time. Over a short time the total mass line will fall into a constant slope. The leach rate will be a negative value because mass is being lost off of the crystal.

During a potential scan, the cell potential is adjusted during the entire length of the experiment. Total current, leach rate, and cell potential are measured continuously during the scan. Before a scan is started the cell potential should be set at about $10 \mathrm{mV}$ below the $\mathrm{E}_{\mathrm{m}}$ so that the exact point where the total current goes from negative (reducing) to positive (oxidizing) can be seen on the graph. 
Once the potential (forward scan) has risen high enough for the experiment, the scan is reversed and potentials are allowed to drop (reverse scan) to the starting point of the scan. Typically during the forward scan the anodic reaction (gold oxidation) is favored, while in a reverse scan the cathodic reaction (sulfide reduction) is favored. However, tests completed with the REQCM at Monash University showed more gold oxidation during the reverse scan. In the case where the reverse scan has a higher leach rate can be referred to as a hysteresis. Although it is hard to get the reaction started, it proceeds readily once initiated. Jeffery, Chapman, \& Walll

For the ASGLS, if the reverse scan has a lower $\mathrm{I}_{\text {calc, }}$ the surface of the MOI may have been passivated during the forward scan by sulfide oxidizing to sulfur. After tests had been run some of the crystals had an observable dark sulfide tarnish. Passivity on the reverse scan can be avoided by reversing the scan before sulfide can be oxidized to sulfur on the electrode surface.

To avoid other surface phenomena on the forward scan, a leach test should be run first. If no leach test needs to be done, the electrode should sit in solution for about 5 minutes before starting the scan, so that the solution can reach equilibrium with the MOI surface.

\subsubsection{REQCM Experimental Results}

\subsubsection{Effect of Sulfide $\left(S^{2-}\right)$ Concentration}

Four potential scans were run on varying sulfide concentration solutions. No leach tests were run with pure sulfide solutions because leach rates are too slow to measure, even at the test temperature of $50^{\circ} \mathrm{C}$. From the graph below, increases in sulfide concentration enhance gold oxidation. Also, gold oxidation occurs at lower potentials as sulfide concentration increases.

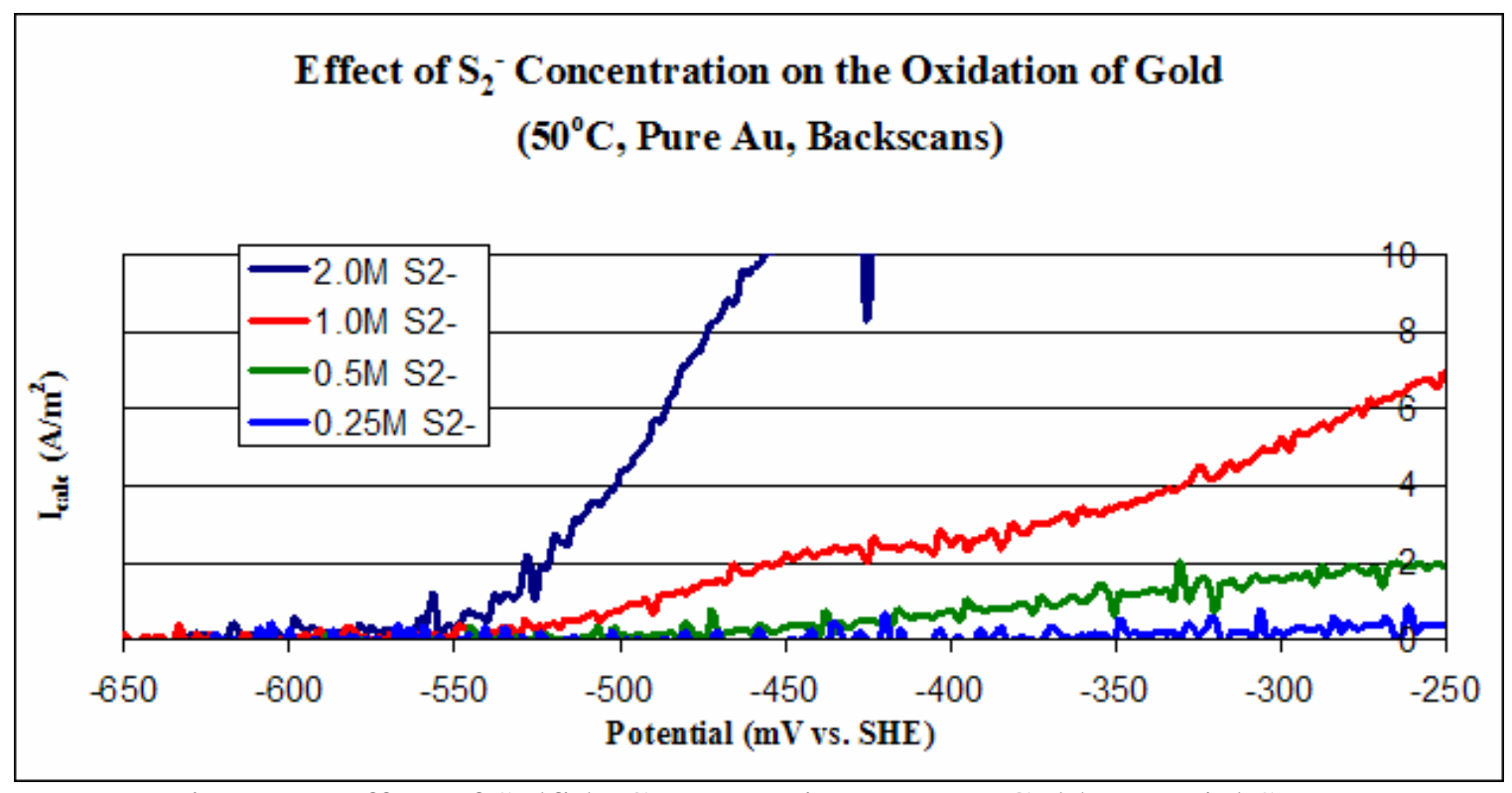

Figure 9. Effect of Sulfide Concentration on Pure Gold Potential Scans 


\subsubsection{Effect of Hydrosulfide (HS')}

Tests were done to see if adding hydrosulfide rather than sulfide had an effect on the oxidation of gold. Below it is shown that hydrosulfide has a negative impact on gold oxidation at higher potentials when compared to sulfide. However, it is important to note that by adding hydrosulfide, the $\mathrm{E}_{\mathrm{m}}$ is raised.
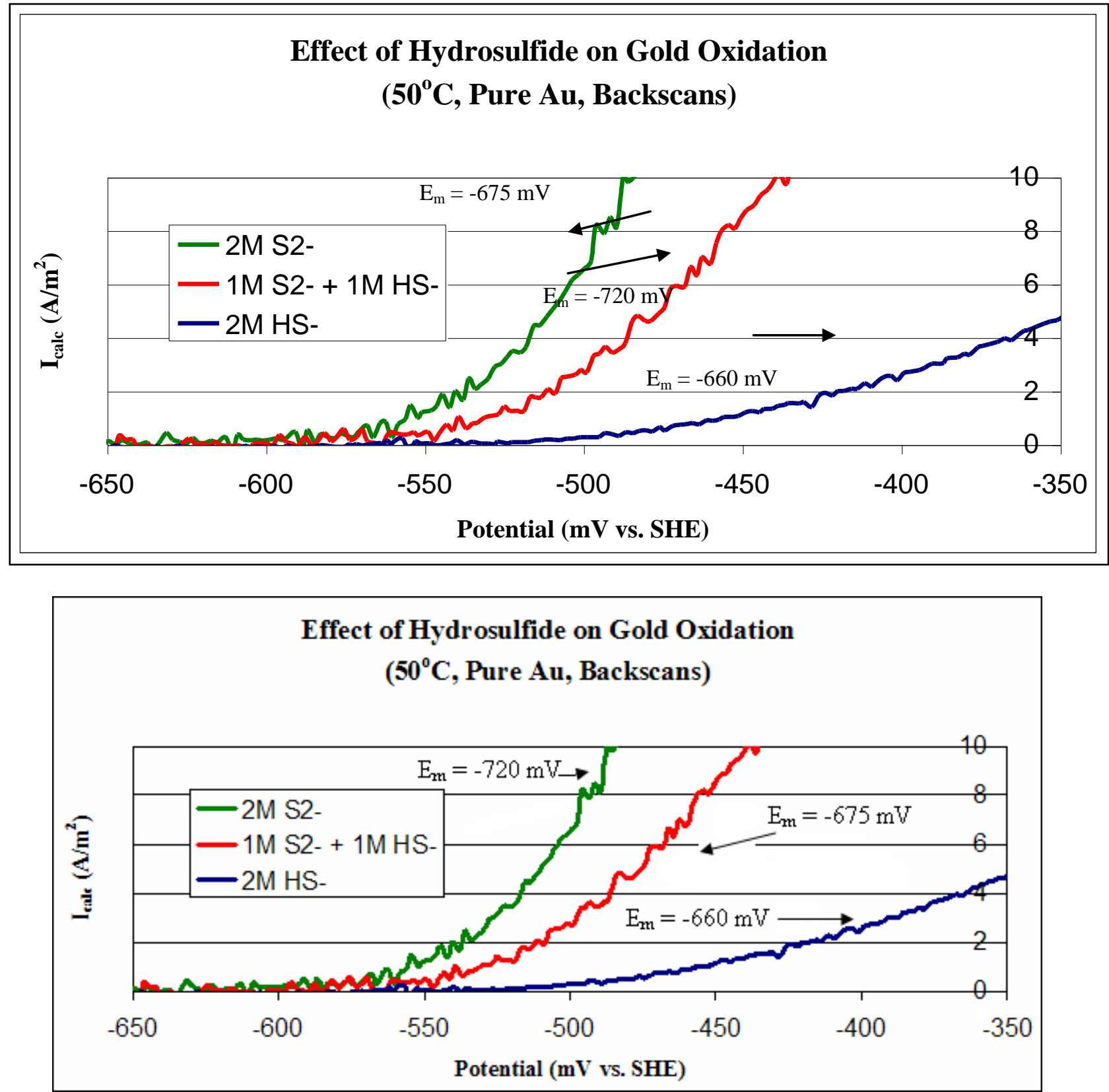

Figure 10. Effect of Hydrosulfide on Gold Potential Scans

\subsubsection{Effect of Polysulfide}


To better understand the effects of polysulfide on gold oxidation two different tests were completed. In the first test the total sulfide concentration was held at 2 molar while the sulfur concentration was increased from 0-3 molar. During the second test the total sulfur (sulfide + sulfur) concentration was held at 2 molar and the mole-\% sulfur in the 2 molar solution was varied from 0-30 mole-\%.

In the figure below a display of what happens with increasing sulfur concentration when sulfide concentration is held at 2 molar. Notice there is little difference when potentials are lower than $400 \mathrm{mV}$ vs. SHE; however, $\mathrm{E}_{\mathrm{m}}$ continues to get higher as sulfur concentration is increased. The effect of $E_{m}$ increasing can be seen in the leach tests in Figure 11.

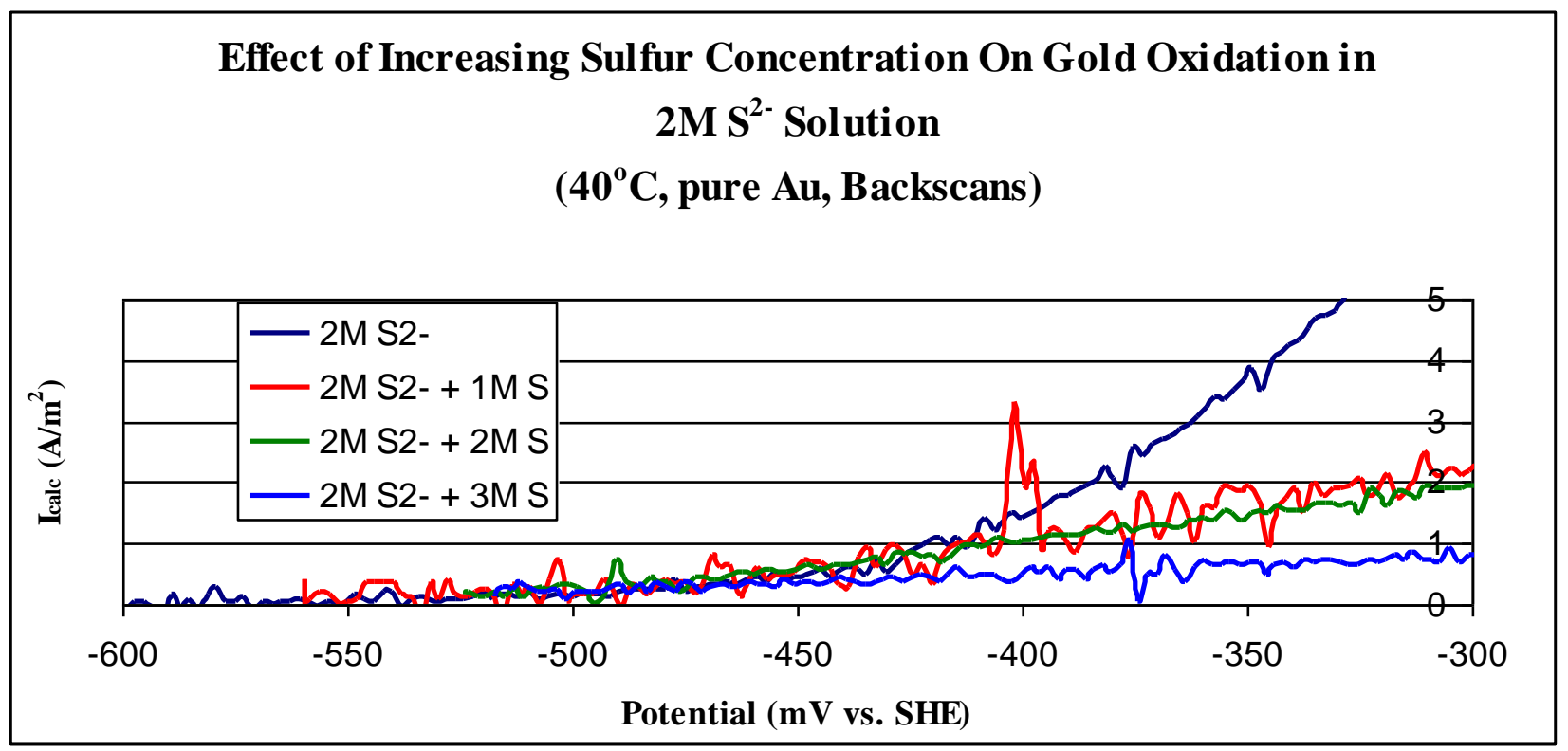

Figure 11. Effect of Increasing Sulfur Concentration on Gold Potential Scans

Solutions of $2 \mathrm{M} \mathrm{S}^{2-}$ essentially did not leach; in other words, the current flow due to the oxidation of gold is essentially zero at the solution potential $\left(E_{m}\right)$. As sulfur concentration increases, the leach rate also increases. Leach rates increased from 0.2 to $6.5,7.2$, and 8.3 nanograms/second respectively for $0,1,2$, and 3 molar sulfur additions to $2 \mathrm{M} \mathrm{S}^{2-}$. 


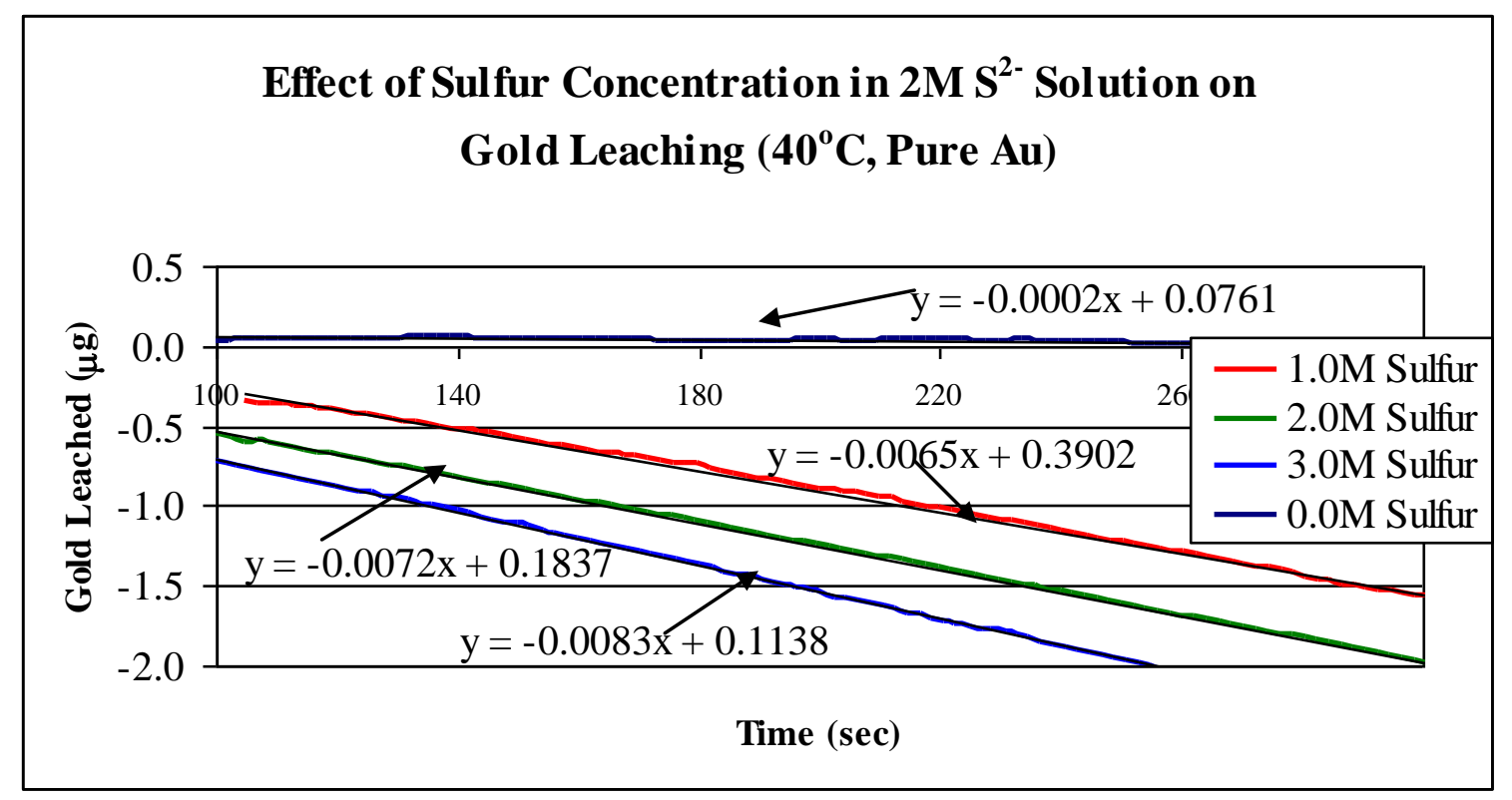

Figure 12. Corresponding Leach Tests to Solutions From Figure 11

Holding the total sulfur concentration at 2 molar produced some informative results as to why leaching is difficult under these conditions. Even though the sulfur and sulfide concentrations are changing, there is still no real leaching occurring because $\mathrm{E}_{\mathrm{m}}$ potentials are occurring where gold oxidation is essentially zero (See Figure 13.) A small amount of leaching can be expected as sulfur concentration goes toward 100 mole-\%( $E_{m}$ increases to gold oxidation regions); however, the leach rates would most likely be insignificant. 


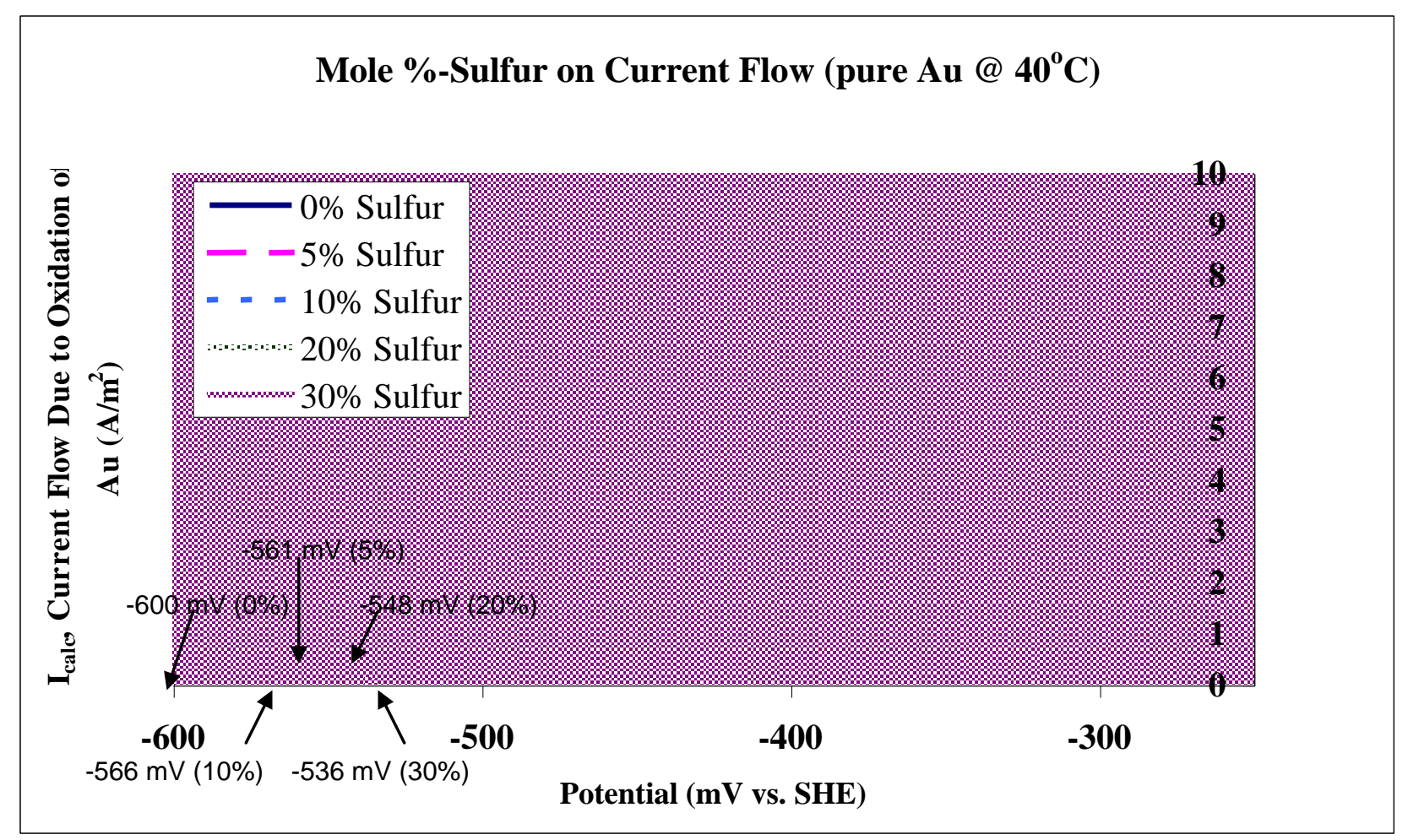

Figure 13. 2M Total Sulfur Pure Gold Potential Scans

Unfortunately the first year of laboratory leach tests done at Montana Tech were done where the total sulfur additions were held constant at 2 molar. Leaching that was observed in these tests is relatively small when compared to tests that were completed in the second year when total sulfur concentrations were increased. Any leaching phenomenon observed in the first year of tests was likely due to experimental error.

\subsubsection{Effect of Hydroxide Addition}

Adding $\mathrm{NaOH}$ to the leaching system was tested to see the effect it had on gold oxidation. The first test that was run was done with $2 \mathrm{M} \mathrm{S}^{2-}$ with concentrations of hydroxide ranging from 0-3 molar. From Figure 7 below it is shown that the addition of hydroxide increases the oxidation of gold to lower potentials. 


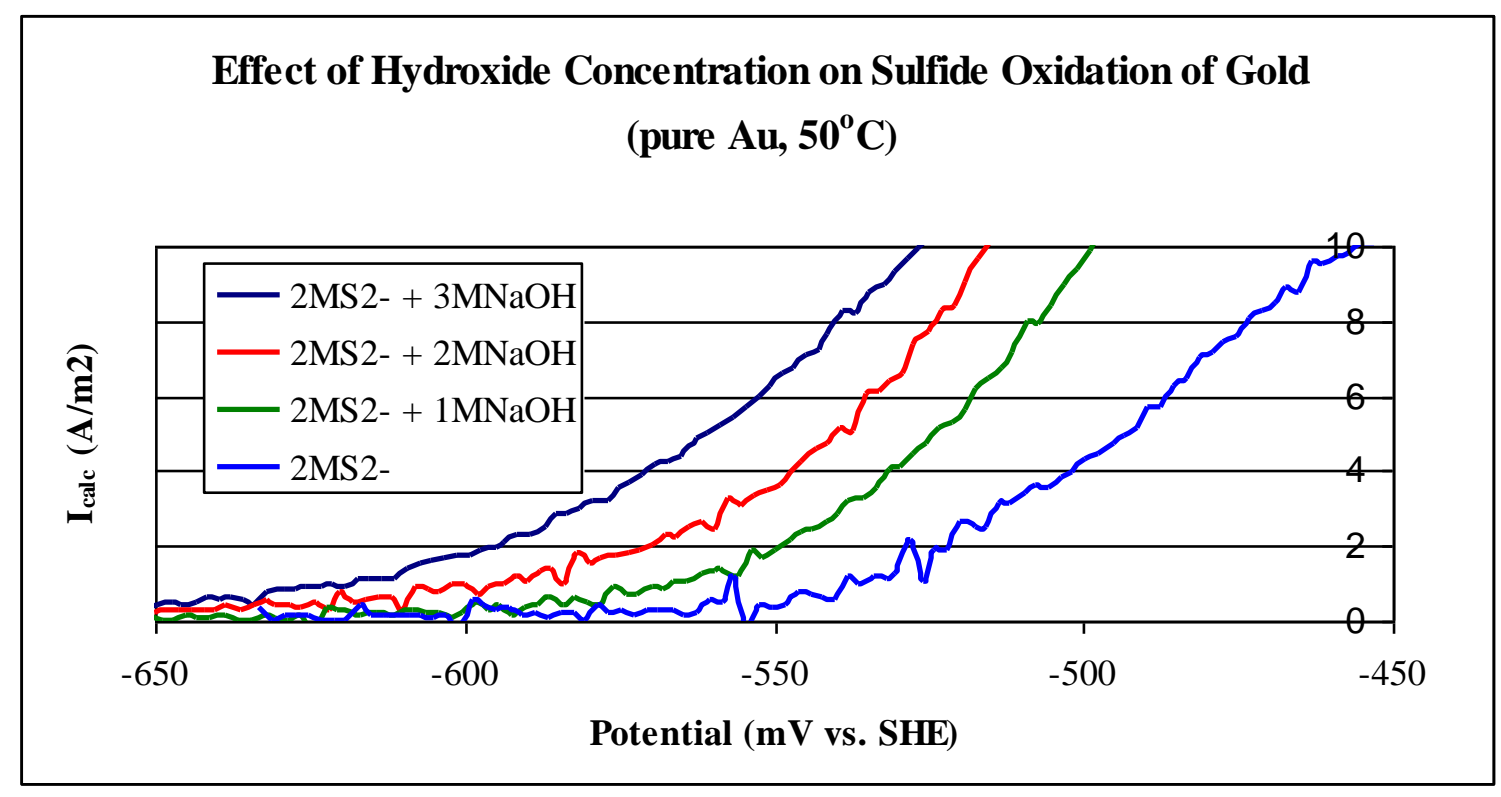

Figure 14. Effect of Increasing Hydroxide Concentration in $2 \mathrm{M} \mathrm{S}^{2-}$ Solution

Tests were also run to see effects of adding hydroxide to a polysulfide solution. From experience, Dr. Corby Anderson suggested adding 1.2 grams of $\mathrm{NaOH}$ for every 1 gram of sulfur in solution will get the solution to around $0 \mathrm{~g} / \mathrm{l}$ free hydroxide. During the polysulfide test work, the 1.2 gram rule was used on the solutions. Two different polysulfide solutions were prepared, one had $2 \mathrm{M} \mathrm{S}^{2-}$ with $2 \mathrm{M} \mathrm{S}$ while the second had $2 \mathrm{M} \mathrm{S}^{2-}$ with $3 \mathrm{M} \mathrm{S}$. To each of these solutions, one had hydroxide added and one did not (See Figure 15.)

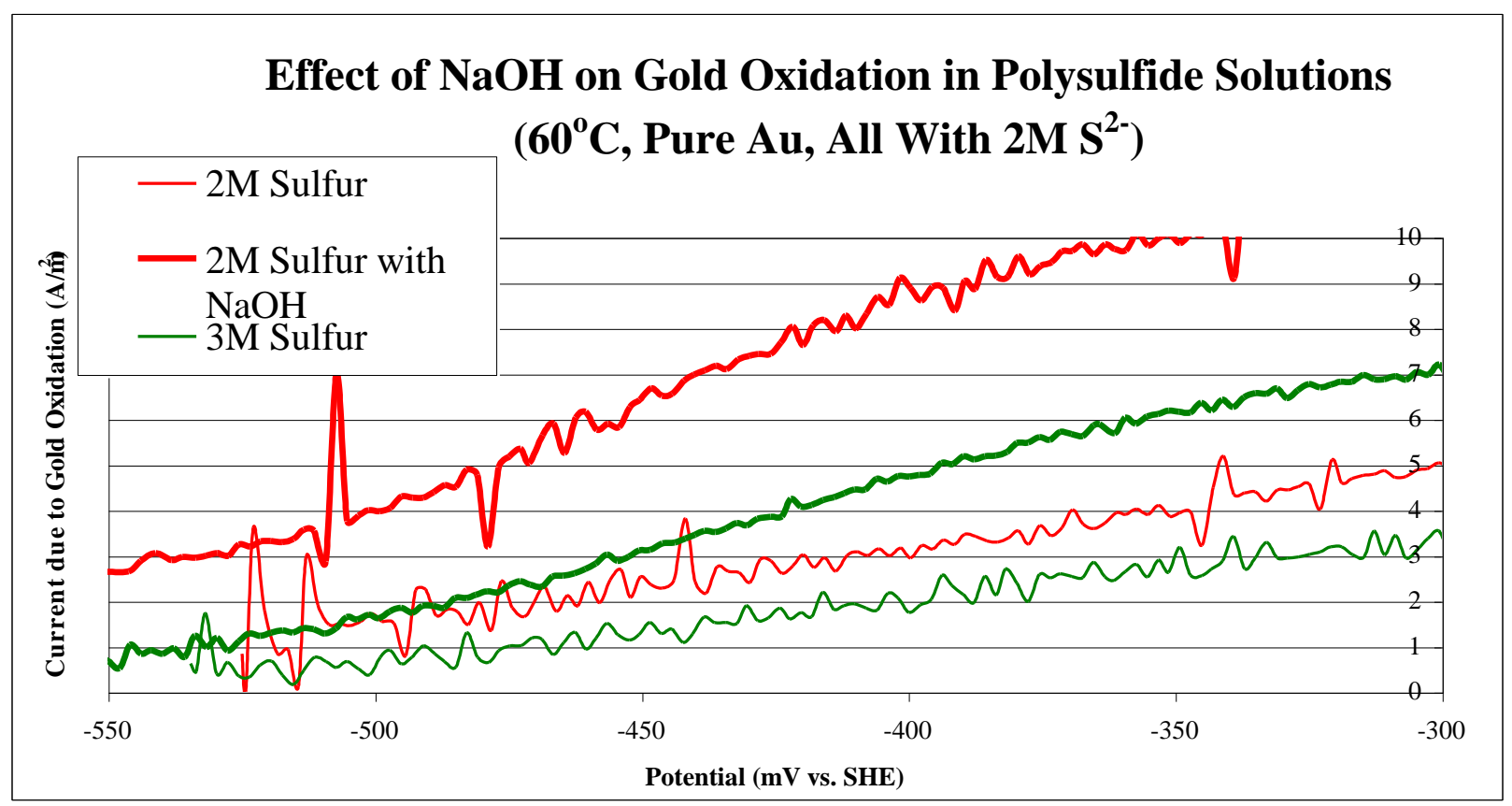

Figure 15. Effect of Hydroxide Addition on Gold Oxidation in Polysulfide 
From Figure 15, gold oxidizes more readily at lower potentials in solutions where hydroxide is added. To see the effect on gold oxidation at $\mathrm{E}_{\mathrm{m}}$, see the figure below. For both solutions, by adding hydroxide the leach rate at $60^{\circ} \mathrm{C}$ is increased $20-25 \mathrm{ng} / \mathrm{s}$.

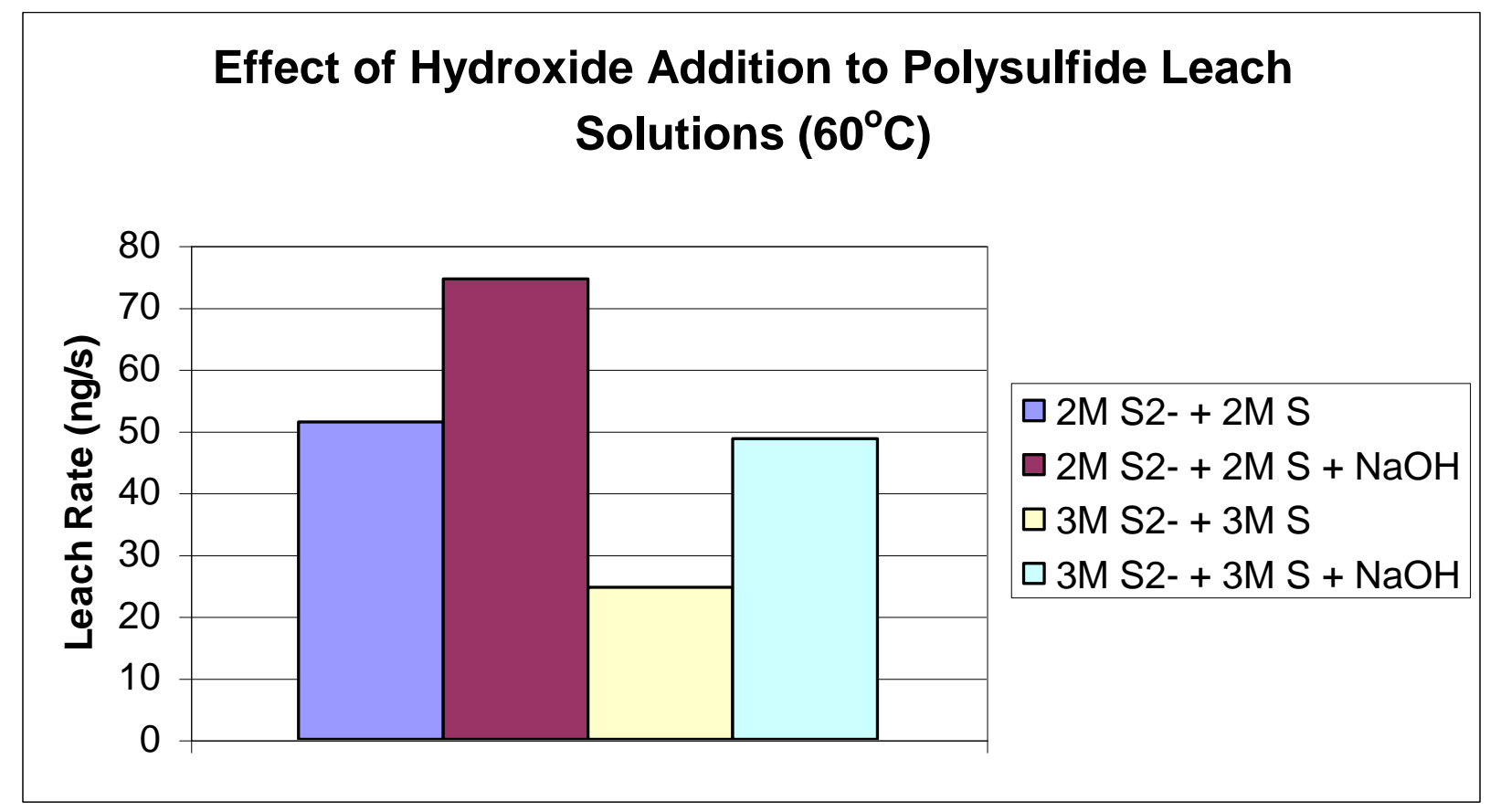

Figure 16 . Effect of Hydroxide Addition on Gold Leach Kinetics

\subsubsection{Effect of Temperature}

In most cases, increasing the temperature of the solution will increase the reaction kinetics. Tests were run to understand how $2 \mathrm{M} \mathrm{S}^{2-}$ solutions and $2 \mathrm{M} \mathrm{S}^{2-}+1 \mathrm{M} \mathrm{S}$ polysulfide solutions were effected by temperature

Figure 17 shows the results for potential scans in $2 \mathrm{M} \mathrm{S}^{2-}$ solutions at temperatures from $30-60^{\circ} \mathrm{C}$. As temperature increases, gold oxidizes more readily at lower potentials. Notice the large jump in gold oxidation as the temperature goes from $50^{\circ} \mathrm{C}$ to $60^{\circ} \mathrm{C}$. 


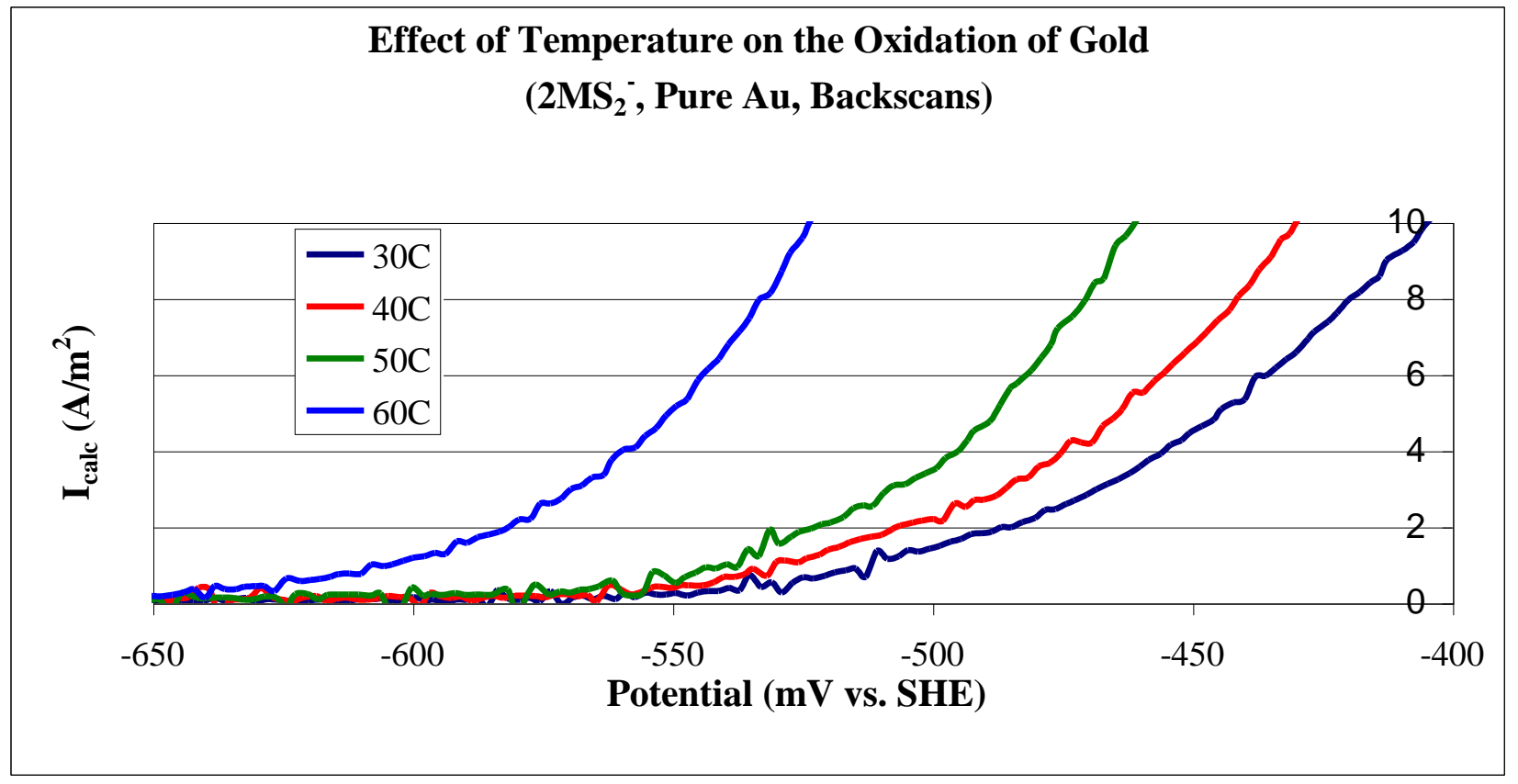

Figure 17. Temperature Effects on Gold Oxidation Potential Scans

In Figure 18, the effect of temperature on gold oxidation in polysulfide solutions is shown. Again, as temperature increases, gold will oxidize more readily at lower potentials. As with the $2 \mathrm{M} \mathrm{S}^{2-}$ solution above, there is a large jump as the temperature goes from $40^{\circ} \mathrm{C}$ to $50^{\circ} \mathrm{C}$.

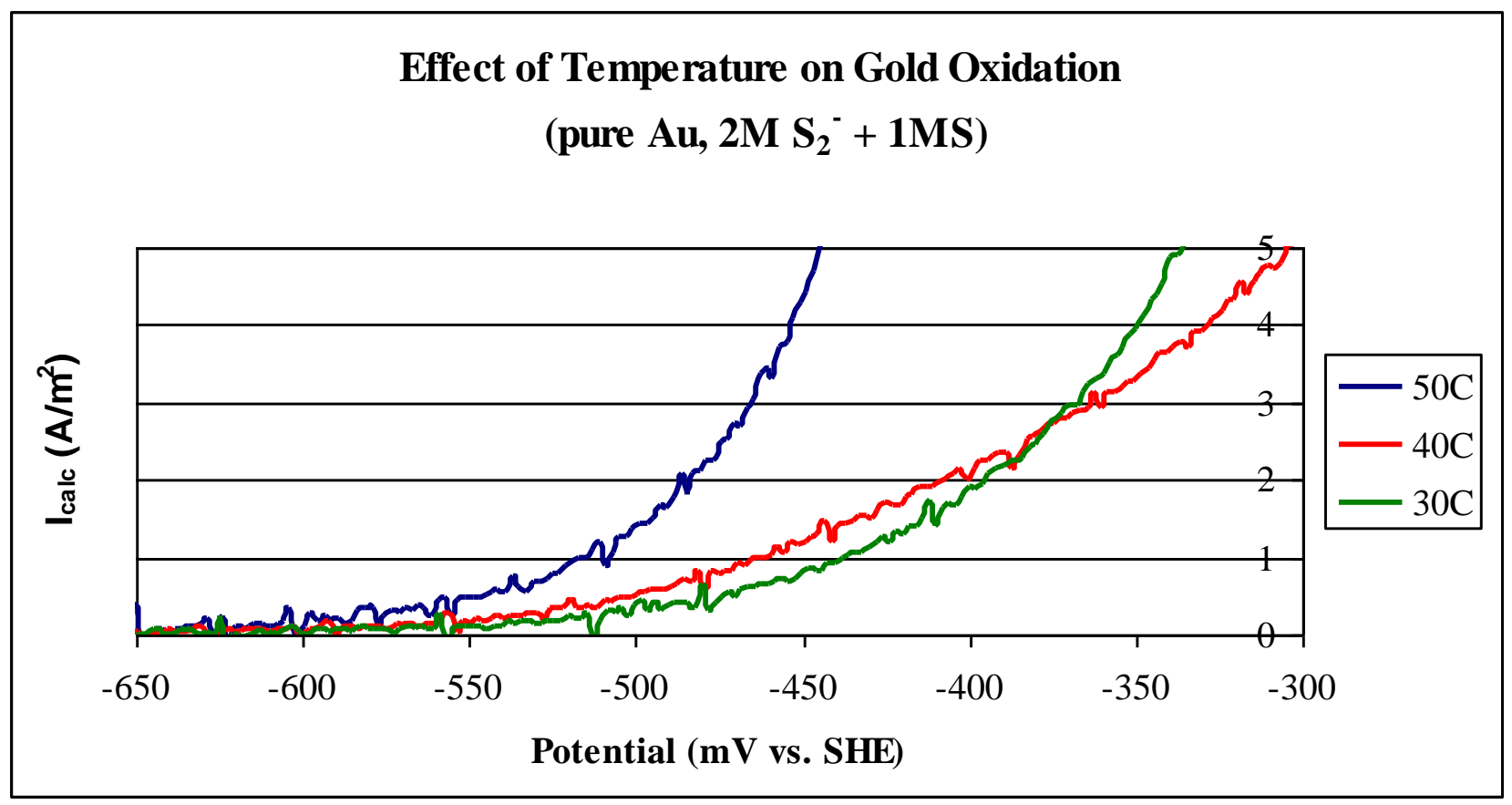

Figure 18. Effect of Temperature on Gold Oxidation in Polysulfide Solutions 
Leach tests were performed on the solutions found in Figure 11; they can be found in Figure 19. The same jump in oxidation kinetics from $40^{\circ} \mathrm{C}$ to $50^{\circ} \mathrm{C}$ can be seen in these leach tests. From $30^{\circ} \mathrm{C}$ to $40^{\circ} \mathrm{C}$ there is only a $0.5 \mathrm{ng} / \mathrm{s}$ rate increase while from $40^{\circ} \mathrm{C}$ to $50^{\circ} \mathrm{C}$ there is a $5.7 \mathrm{ng} / \mathrm{s}$ rate increase.

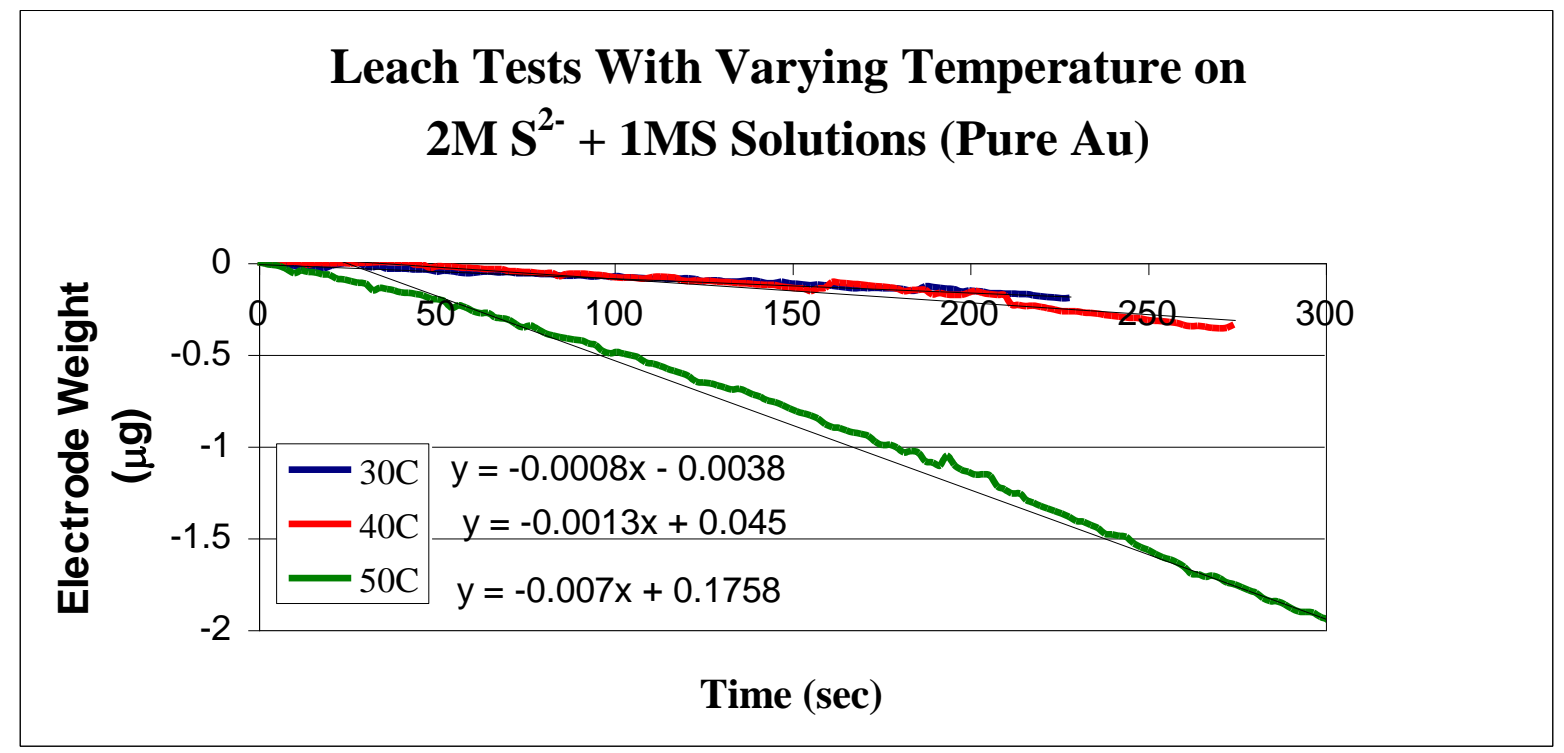

Figure 19. Leach Test Varying Temperature in Polysulfide Solutions

\subsubsection{Effect of Gold Purity}

Gold purity is a variable that needs to be studied because most natural gold deposits have at least 5\% Ag in them (Hurlbut and Klein, 1977). Two identical leach tests were run for three different solutions. The two tests contained one pure gold plate and one 5\% silver plate (see Figure 20.) In the $2 \mathrm{M} \mathrm{S}^{2-}+1 \mathrm{M} \mathrm{S}$ solution, the $5 \%$ silver alloy leached slightly slower than its pure gold counterpart. However, for the more concentrated solutions (2 and $3 \mathrm{MS}$ ), the $5 \%$ enhanced the leach rate almost $100 \%$. 


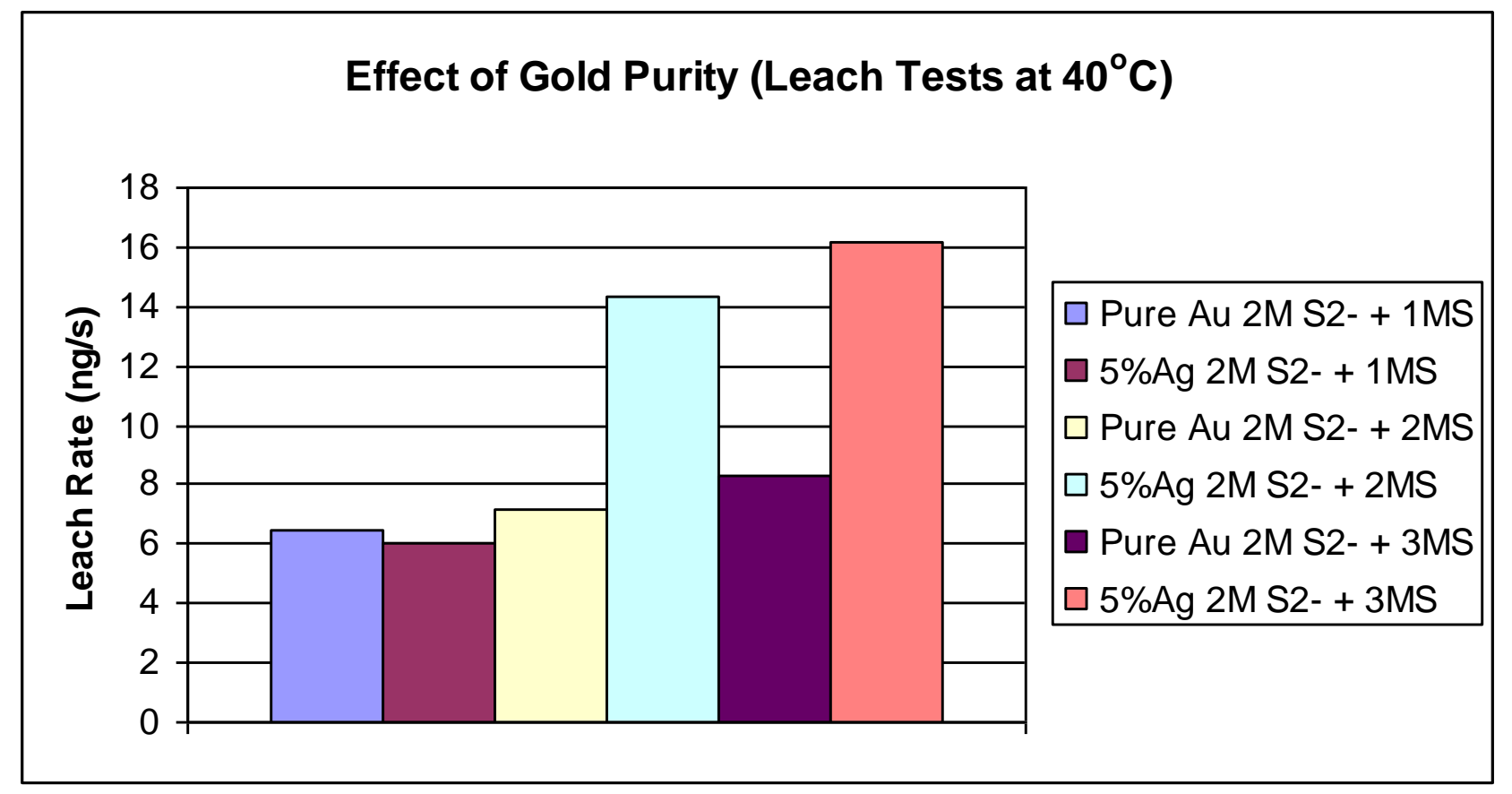

Figure 20. Effect of Gold Purity on Leach Rate

\subsubsection{Conclusions to REQCM Experiments}

Experimental conclusions for the research completed at Monash University are as follows:

- $\quad$ The first variable studied, effect of sulfide concentration, showed that increases in sulfide concentration help to speed the oxidation of gold at lower potentials.

- Hydrosulfide had two major effects on the system: 1) adding hydrosulfide slows gold leaching kinetics at a given potentials; and, 2) hydrosulfide additions raise the solution $\mathrm{E}_{\mathrm{m}}$.

- $\quad$ By adding sulfur to the system, the polysulfide concentration was increased. Higher polysulfide concentrations generally increased leach rates; however, more sulfur both slowed leach kinetics at given potentials and raised the $\mathrm{E}_{\mathrm{m}}$. Eventually the addition of more sulfur will slow the leach reaction.

- $\quad$ Hydroxide additions up to 3 molar were shown to increase leach kinetics at lower potentials in sulfide solutions. Also, hydroxide additions increased leach kinetics in polysulfide solutions.

- $\quad$ As expected, temperature increased leach kinetics; more importantly, there looks to be a large jump in leach kinetics around the $50-60^{\circ} \mathrm{C}$ temperature range.

- $\quad$ The purity of the gold effected leach kinetics positively for concentrated polysulfide solutions above $2 \mathrm{M} \mathrm{S}^{2-}+1 \mathrm{M} \mathrm{S}$. 


\subsection{Laboratory Leach Tests}

\subsubsection{Reagents Used}

Each laboratory leach test consisted of various concentrations of the chemicals found in Table IV. Water used for the leach tests was taken from the distilled water reservoir found in Montana Tech's wet chemistry lab, room 211. Other chemicals used for experimental research during this thesis can be found in Table IV below.

Table IV. Chemicals Used

\begin{tabular}{|c|c|c|c|}
\hline Chemical & Brand and Product Tracking & Purity & Major Impurities \\
\hline $\begin{array}{c}\text { Sodium } \\
\text { Hydroxide }\end{array}$ & $\begin{array}{c}\text { Fisher Chemical } \\
\text { S318-5 }\end{array}$ & $\begin{array}{c}\text { Water, Sodium } \\
\text { Carbonate }\end{array}$ \\
\hline $\begin{array}{c}\text { Sodium } \\
\text { Sulfide }\end{array}$ & $\begin{array}{c}\text { Fisher Chemical } \\
\text { S426-212 }\end{array}$ & Water \\
\hline Sulfur & $\begin{array}{c}\text { Montana Sulfur and Chemical Co. } \\
\text { Hi-Purity SugarHouse Sulfur Flakes }\end{array}$ & $90.0 \%$ & Water \\
\hline Gold & $\begin{array}{c}\text { METALOR } \\
\text { PUAU133; Batch 040900 }\end{array}$ & $\begin{array}{c}999.9 \\
\text { fine }\end{array}$ & Silver \\
\hline
\end{tabular}

\subsubsection{Laboratory Leach Tests}

Leach tests in the laboratory were started at the very beginning of research for this thesis, August, 2003. Prior to the electrochemical and electrokinetic work done at Monash University, the leach tests were done with a large degree of trial and error. Although each trial and error was recorded, numerical data for the first three leach matrices cannot be taken as legitimate experimental data. The major benefit to this thesis that the first three leach matrices is the standard operating procedure for completing a laboratory leach test.

After returning with the valuable electrochemical data from Monash the some of the leach test parameters were deemed to have less importance on the overall leach kinetics than previously thought. Total sulfur concentrations were held at $75 \mathrm{~g} / \mathrm{L}$-- about 2 molar -- total sulfur. As seen in figure 6 earlier, tests that were run at 2 molar total sulfur exhibited electromotive potentials that were too low for significant leach rates. Numerical results from the first year of leach tests are deemed to be insignificant because the leach rates are so slow that experimental error begins to have too big of an influence on results.

\subsubsection{First Year Leach Tests}

Although numerical results from year 1 leach tests are insignificant, the experimental design and laboratory procedures will be detailed below. 


\section{Test Matrix \#1}

Test matrix \#1 consisted of ten leach tests that varied the following four chemical concentrations: free hydroxide; thiosulfate; sulfur; and, sulfide. Free hydroxide, thiosulfate, and sulfur concentrations were varied between 0 and $25 \mathrm{~g} / \mathrm{L}$. Sulfide concentrations ranged between 0 and $50 \mathrm{~g} / \mathrm{L}$. Parameters that were held constant for the first test matrix include: chemical addition order; vessel type; temperature; magnet rotation speed; leach duration; filtering method; and, gold concentration analysis. Since the water bath could only contain a maximum of six leach vessels, the entire matrix was broken into two series of five leach tests. Chemical parameters for test matrix \#1 can be seen in the following table. (See Table V).

\begin{tabular}{|c|c|c|c|c|c|}
\hline \multicolumn{6}{|c|}{ Table V. Matrix \#1 } \\
\hline \multirow[b]{2}{*}{ Test } & \multirow[b]{2}{*}{ Series } & \multicolumn{4}{|c|}{ Goal Reagent Concentrations } \\
\hline & & Free OH- $(\mathrm{g} / \mathrm{L})$ & Sulfide $(\mathrm{g} / \mathrm{L})$ & Sulfur $(g / L)$ & Thiosulfate $(\mathrm{g} / \mathrm{L})$ \\
\hline 1 & 1 & 25 & 25 & 25 & 0 \\
\hline 2 & 1 & 25 & 25 & 0 & 25 \\
\hline 3 & 1 & 0 & 50 & 25 & 0 \\
\hline 4 & 1 & 12.5 & 37.5 & 12.5 & 12.5 \\
\hline 5 & 1 & 0 & 50 & 0 & 25 \\
\hline 6 & 2 & 0 & 25 & 0 & 0 \\
\hline 7 & 2 & 25 & 50 & 0 & 0 \\
\hline 8 & 2 & 0 & 25 & 25 & 25 \\
\hline 9 & 2 & 25 & 50 & 25 & 25 \\
\hline 10 & 2 & 12.5 & 37.5 & 12.5 & 12.5 \\
\hline
\end{tabular}

Chemical addition was carried out by first adding about $400 \mathrm{~mL}$ of distilled water into a $1 \mathrm{~L}$ beaker. Once the hydroxide was added, a magnet was placed into the beaker as it was placed on a hot plate in the fume hood. Next, sodium hydroxide was poured into the solution, followed by sulfur and the thiosulfate. The solution was allowed to heat until the last solids were dissolved into solution.

Once all solids were dissolved in solution it was removed from the heat, allowed to cool, and poured into a $1 \mathrm{~L}$ volumetric flask. The flask was filled with distilled water to the proper volume. From the solution, a sample of about $20 \mathrm{~mL}$ was taken to measure the free hydroxide in the system for the tests that had some target free hydroxide value greater than zero (See Titration Methods). Sodium hydroxide was added to the solution according to how much free hydroxide the titration yielded. No titrations were done on tests that had a goal hydroxide level of zero.

After the hydroxide had been dissolved, $750 \mathrm{~mL}$ of solution were poured into the $1000 \mathrm{~mL}$ flask. At this point about 0.250 grams of 999.9 fine gold powder was added to the solution. A Teflon coated magnet was placed into the solution with the gold. Finally, a glass stopper was placed onto the flask to avoid oxygen getting into the system and the flask was placed into the $40^{\circ} \mathrm{C}$ water bath. Leach time was started after the flasks were in the water bath.

For each test in test matrix \#1, the leach duration was 72 hours. After leaching was complete, three $20 \mathrm{~mL}$ samples were taken for solution analysis. Every solution was measured for gold 
concentration via ICP, as well as sulfide concentration via the James River titration method (see titration methods).

The remaining solution was filtered to trap any remaining gold that had not leached during the test. Filtered solids were placed into a drying oven and weighed after they were completely dry. Remaining solution was bottled, labeled, and stored in a secondary container in the lab.

\section{Redo Test for Test Matrix \#1}

During the first run of tests for matrix \#1, any solution that was to have zero free hydroxide had no hydroxide added to it or a titration done to measure the concentration of free hydroxide. After the first run it became clear that some hydroxide must be added to get the solution to the $0 \mathrm{~g} / \mathrm{L}$ free hydroxide level. The five tests in matrix \#1 that had $0 \mathrm{~g} / \mathrm{L}$ hydroxide were retested, this time with hydroxide additions and titrations prior to leaching. All other test methods were kept exactly the same.

\section{Test Matrix \#2}

The second test matrix was set up to look at the effect of small concentrations of additives that may help the oxidation of gold. All tests were pulled from the same 1 liter of $75 \mathrm{~g} / \mathrm{L} \mathrm{sulfur,} 0 \mathrm{~g} / \mathrm{L}$ free hydroxide solution. Each test was done in a $250 \mathrm{~mL}$ flask with a total solution volume of $100 \mathrm{~mL}$. About 0.100 grams of gold was added to the flasks before all flasks were corked and placed on an orbital shaker and leached for 22 hours at room temperature. Results for Test Matrix \#2 are insignificant because leaching potentials $\left(E_{m}\right)$ are much too low for significant leaching at room temperature. Potentials are provided on the table to give an idea of the effect of hydrogen peroxide on system potentials. The parameters and results for Test matrix \#2 can be seen in Table VI.

Table VI. Matrix \#2

\begin{tabular}{|c|c|c|c|c|c|c|}
\hline Test & \multicolumn{2}{|c|}{$\mathrm{H}_{2} \mathrm{O}_{2}$ Addition } & \multicolumn{2}{|c|}{ Solid Additions (g) } & \multicolumn{2}{|c|}{ Results } \\
\hline & Strength & Volume (mL) & Sodium Sulfite & Sodium thiosulfate & ORP vs. SCE & $\mathrm{ppm} \mathrm{Au}$ \\
\hline 1 & $0 \%$ & 0 & 0 & 0 & -654 & 4.269 \\
\hline 2 & $10 \%$ & 3 & 0.1 & 0 & -637 & 2.573 \\
\hline 3 & $3 \%$ & 3 & 0.1 & 4 & -651 & 5.164 \\
\hline 4 & $3 \%$ & 3 & 1 & 0 & -655 & 1.294 \\
\hline 5 & $10 \%$ & 3 & 1 & 4 & -641 & 3.738 \\
\hline 6 & $\begin{array}{c}10 \% \\
3 \%\end{array}$ & $\begin{array}{l}1.5 \\
1.5\end{array}$ & 0.55 & 2 & -648 & 2.236 \\
\hline 7 & $0 \%$ & 0 & 0 & 0 & -655 & 4.237 \\
\hline
\end{tabular}

\section{Test Matrix \#3}


Test matrix \#3 totaled 18 leach tests. Tests were broken down so that there were three series of six tests each. After Test matrix \#1 showed low significance in regards to thiosulfate concentration, another test matrix was built in order to pinpoint what $\mathrm{S}^{2-} / \mathrm{S}$ ratio was best for leaching in a $75 \mathrm{~g} / \mathrm{L}$ total sulfur solution. For each of the tests the goal free hydroxide concentration was $0 \mathrm{~g} / \mathrm{L}$. Due to an error in hydroxide addition calculation all tests were leached with varying free hydroxide concentrations; all concentration for free hydroxide were greater than zero. Chemical parameters for Test Matrix \#3 can be seen in Table VII below.

Table VII. Matrix \#3

\begin{tabular}{|c|c|c|c|c|c|c|c|}
\hline Test \# & Series & $\begin{array}{c}\text { Goal OH- } \\
(\mathrm{g} / \mathrm{L})\end{array}$ & $\begin{array}{c}\text { Sulfide } \\
(\mathrm{g} / \mathrm{L})\end{array}$ & $\begin{array}{c}\text { Sulfur } \\
(\mathrm{g} / \mathrm{L})\end{array}$ & $\begin{array}{c}\text { OH- by } \\
\text { titration }\end{array}$ & $\begin{array}{c}\text { Actual free } \\
\text { OH- }\end{array}$ \\
\hline 1 & 1 & 0 & 5.00 & 70.00 & 11.87 & 0.00 & 11.87 \\
\hline 2 & 1 & 0 & 5.00 & 70.00 & 10.32 & 0.00 & 10.32 \\
\hline 3 & 1 & 0 & 70.00 & 5.00 & -4.59 & 28.31 & 7.44 \\
\hline 4 & 1 & 0 & 37.50 & 37.50 & 6.71 & 0.00 & 6.71 \\
\hline 5 & 1 & 0 & 21.25 & 53.75 & 14.67 & 0.00 & 14.67 \\
\hline 6 & 1 & 0 & 53.75 & 21.25 & -1.97 & 16.59 & 5.08 \\
\hline 7 & 2 & 0 & 75.00 & 0.00 & -10.08 & 41.86 & 7.71 \\
\hline 8 & 2 & 0 & 56.25 & 18.75 & -4.00 & 16.59 & 3.05 \\
\hline 9 & 2 & 0 & 65.62 & 9.38 & -3.93 & 16.31 & 3.00 \\
\hline 10 & 2 & 0 & 37.50 & 37.50 & 2.96 & 0.00 & 2.96 \\
\hline 11 & 2 & 0 & 46.88 & 28.12 & 2.89 & 0.00 & 2.89 \\
\hline 12 & 2 & 0 & 37.50 & 37.50 & 3.04 & 0.00 & 3.04 \\
\hline 13 & 3 & 0 & 65.75 & 9.25 & -7.34 & 30.49 & 5.62 \\
\hline 14 & 3 & 0 & 70.12 & 4.88 & -7.04 & 29.22 & 5.38 \\
\hline 15 & 3 & 0 & 57.00 & 18.00 & -7.68 & 31.91 & 5.88 \\
\hline 16 & 3 & 0 & 61.38 & 13.62 & -7.09 & 29.44 & 5.42 \\
\hline 17 & 3 & 0 & 74.50 & 0.50 & -8.19 & 34.02 & 6.27 \\
\hline 18 & 3 & 0 & 57.00 & 18.00 & -7.02 & 29.15 & 5.37 \\
\hline
\end{tabular}

Results for Test Matrix \#3 were insignificant. Many of the chemical affects that were explored in Matrix \#3 were found during the electrochemical experiments conducted at Monash University.

\subsubsection{Gold Ball Formation}

During the time where the proper laboratory leaching procedure was being developed a strange phenomenon was observed. While leaching in the $1000 \mathrm{~mL}$ flask with magnetic agitation some of the gold powder would agglomerate in to sizable balls. Gold ball formation was not observed in all tests done in the flasks. Large gold balls were observed in the flasks after as little as three days. Each gold ball was compacted very tightly; balls did not deform when they were pressed upon the laboratory bench top.

To investigate the phenomenon more closely samples were observed under Montana Tech's Scanning Electron Microscope (SEM). In Figure 21 below is some of the gold powder prior to leaching. Note the uniform size from grain to grain; the average size is assumed to be $1.5 \mu \mathrm{m}$ in diameter. 


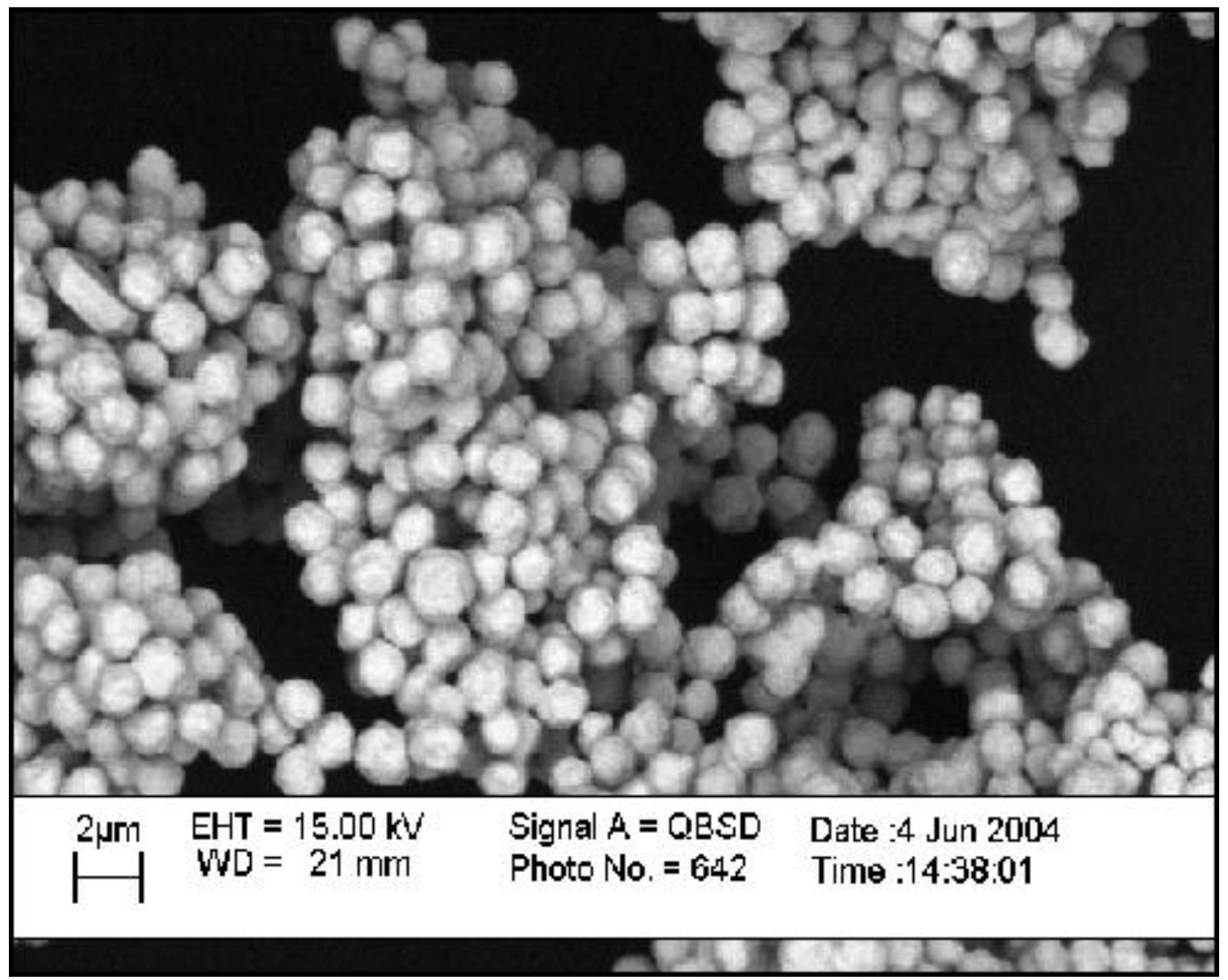

Figure 21. Gold Powder Prior to Leaching

Below is an SEM picture of one of the gold balls that formed during a leach test in the flask. The ball is assumed to be a perfect sphere with a diameter of $650 \mu \mathrm{m}$. Notice the textured surface on the ball; each texture is one of the small gold grains. The discoloration on the surface of the sphere is likely sulfur that has been oxidized from sulfide on the surface of the gold. 


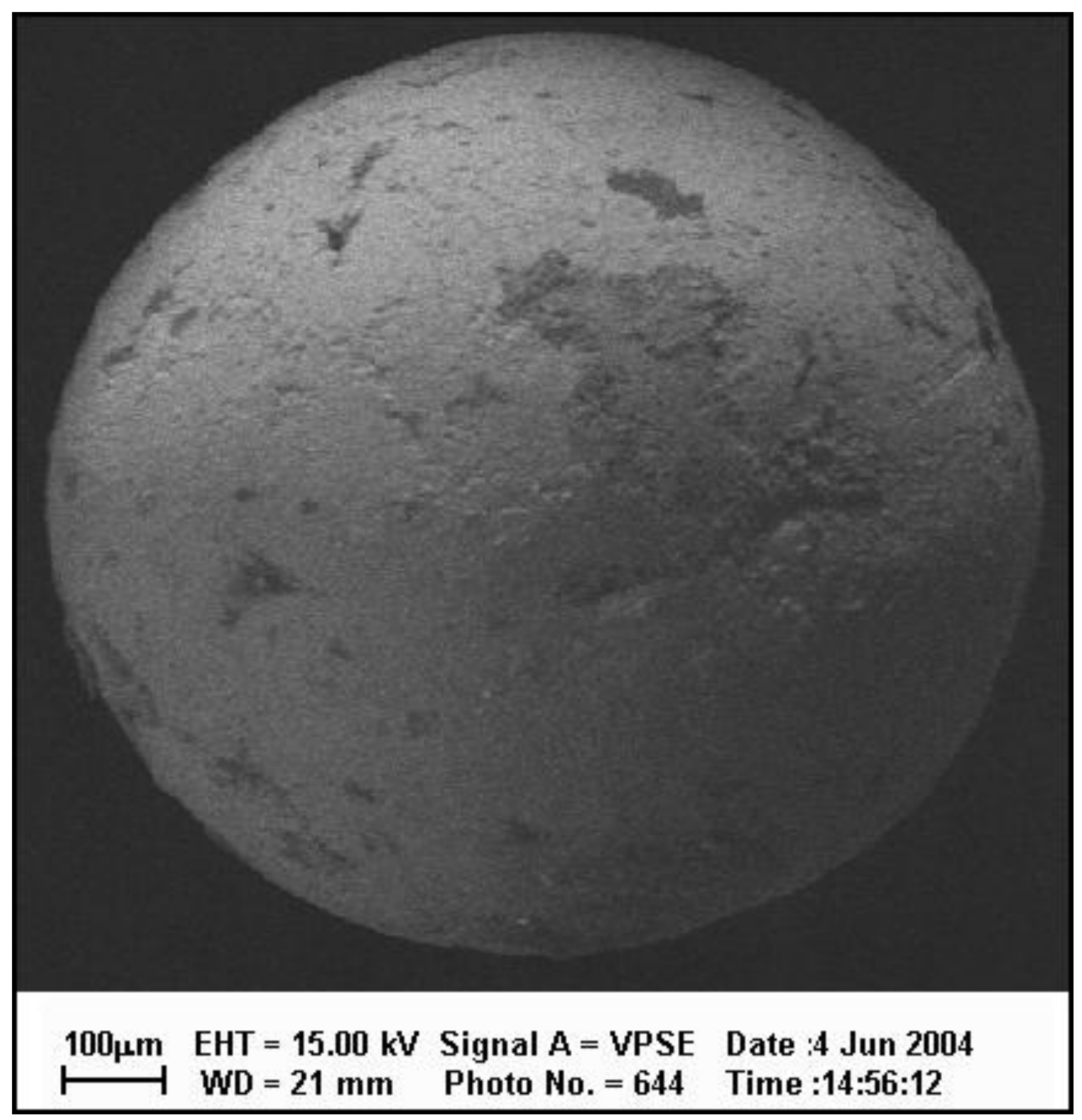

Figure 22. Large Gold Ball

An attempt to quantify the affect of gold ball formation was made. If the small gold grains and the large ball are assumed to be spheres of $1.5 \mu \mathrm{m}$ and $650 \mu \mathrm{m}$ respectively, than the numbers below are representative of the affect of gold ball formation. 
Table VIII. Quantification of Gold Ball Formation

\begin{tabular}{|c|c|c|c|}
\hline Particle & Diameter $(\mathrm{m})$ & Volume $\left(\mathrm{m}^{3}\right)$ & Surface Area $\left(\mathrm{m}^{2}\right)$ \\
\hline Gold Powder & 0.0000015 & $1.77 \mathrm{E}-18$ & 766.24 \\
\hline Gold Ball & 0.0006500 & $1.44 \mathrm{E}-10$ & 0.0041 \\
\hline \multicolumn{3}{|c|}{} \\
\hline \multicolumn{3}{|c|}{ Number of Gold Grains Needed to Make Ball = } & $\mathbf{8 1 , 3 0 0 , 0 0 0}$ \\
\hline \multicolumn{2}{|c|}{ Ratio of Surface Area (small : large) $=$} \\
\hline
\end{tabular}

Approximately 81 million small gold grains needed to come together to form the ball shown previously. The effective surface area has been reduced an astounding 187,000 times. Obviously the leaching kinetics for these leach tests will be lower than the tests where no gold balls formed; this was observed in the laboratory tests.

\subsubsection{Possible Reasons for Gold Ball Formation}

While the exact reason for gold ball formation is not clearly understood, a few theories have been developed based on laboratory experience. Specific cases where gold balls had formed were noted and whenever possible future tests were adjusted accordingly. The Teflon coated magnet is most likely the culprit in gold ball formation.

The most likely reason is the effect of the magnet continually pounding on the small gold grains. In the test that formed the both the most and the largest gold balls the flasks had shifted in the heat bath due to the circulating water. Once the flasks had shifted the magnetic stirrer no longer matched up well with the magnets in the flasks. Instead of spinning the magnets began to bounce around the base of the flasks, pounding upon the gold in the process. Balls may have formed as a result of the malfunctioning magnets.

It is also possible that a properly functioning magnet caused ball formation. The spinning magnet could have smeared gold grains together and over time rolled the smeared grains into balls.

In either case gold ball formation was probably aided by oxidized sulfur on the surface of the gold. Elemental analysis was attempted on the SEM but as expected the extremely high percentage of gold in the samples made it impossible to see a significant sulfur peak.

\subsubsection{High Temperature Leach Tests}

During the first year of research there were some high temperature $\left(>60^{\circ} \mathrm{C}\right)$ leach tests done. The tests were completed so that an idea of the limits of the leach reaction at high temperatures could be formed. Three of these tests will be detailed below. 


\subsubsection{High Temperature Autoclave Test}

A leach test was run in the autoclave to both see how the system acted at high temperature and how it acted in the autoclave. The test was set up according to the following parameters. Photographs of the autoclave are included in the section 5.0 Waste Removal.

Table IX. Autoclave Leach Test Parameters

\begin{tabular}{|c|c|l|}
\hline Free Hydroxide & 0 & $\mathrm{~g} / \mathrm{L}$ \\
\hline Temperature & 98 & $\left({ }^{\circ} \mathrm{C}\right)$ \\
\hline Au & 1.500 & $(\mathrm{~g})$ \\
\hline Volume & 1,000 & $(\mathrm{~mL})$ \\
\hline Sulfur & $75 \mathrm{~g} / \mathrm{L}$ & $2.34 \mathrm{~m} / \mathrm{L}$ \\
\hline Sulfide & $75 \mathrm{~g} / \mathrm{L}$ & $2.34 \mathrm{~m} / \mathrm{L}$ \\
\hline Total & $150 \mathrm{~g} / \mathrm{L}$ & $4.69 \mathrm{~m} / \mathrm{L}$ \\
\hline
\end{tabular}

Once the solution was poured into the autoclave the gold was added and the autoclave was bolted together. After the autoclave was placed into the heater the agitator was turned on to 300 RPM. A sample was taken after 5 days of leaching. Due to the high sulfur concentration the solution needed to be immediately diluted so that precipitates would not form. To get the solution in the $100 \mathrm{ppm}$ range of the ICP, the sample was finally diluted 10:1. Analysis on the ICP showed the solution contained $99.4 \mathrm{ppm}$ Au after dilution (nearly 1,000 ppm before dilution). According to those results essentially $100 \%$ of the gold that was placed in the autoclave had been leached over the 5 days.

\subsubsection{High Temperature Kettle Tests}

A pair of high temperature autoclave tests was completed to see if leach tests were reproducible with that set-up. Each test was set up according to the following parameters.

Table X. High Temperature Leach Test Parameters

\begin{tabular}{|c|c|ll|}
\hline Free Hydroxide & 0 & $\mathrm{~g} / \mathrm{L}$ \\
\hline Temperature & 75 & $\left({ }^{\circ} \mathrm{C}\right)$ \\
\hline Au & 0.204 & $(\mathrm{~g})$ \\
\hline Volume & 750 & $(\mathrm{~mL})$ & \\
\hline Sulfur & 10 & $\mathrm{~g} / \mathrm{L}$ & $0.31 \quad \mathrm{~m} / \mathrm{L}$ \\
\hline Sulfide & 65 & $\mathrm{~g} / \mathrm{L}$ & $2.03 \mathrm{~m} / \mathrm{L}$ \\
\hline Total Sulfur & 75 & $\mathrm{~g} / \mathrm{L}$ & $2.34 \mathrm{~m} / \mathrm{L}$ \\
\hline
\end{tabular}


Each test was done in the fume hood on a hot plate held at constant temperature. An external agitator was submerged into the leach vessel and it was set at full power. A cold thumb was placed on top of the kettle to keep the solution from dehydrating.

Results for both of the tests showed high \%-leach rates; however, the two tests had large variability (20\% after 2 days) between them. The results for these leach tests can be seen below. This method for leach tests was dropped because of the poor reproducibility and the difficulty in keeping the system the same test to test (temperature, agitation, and exposure to the atmosphere).

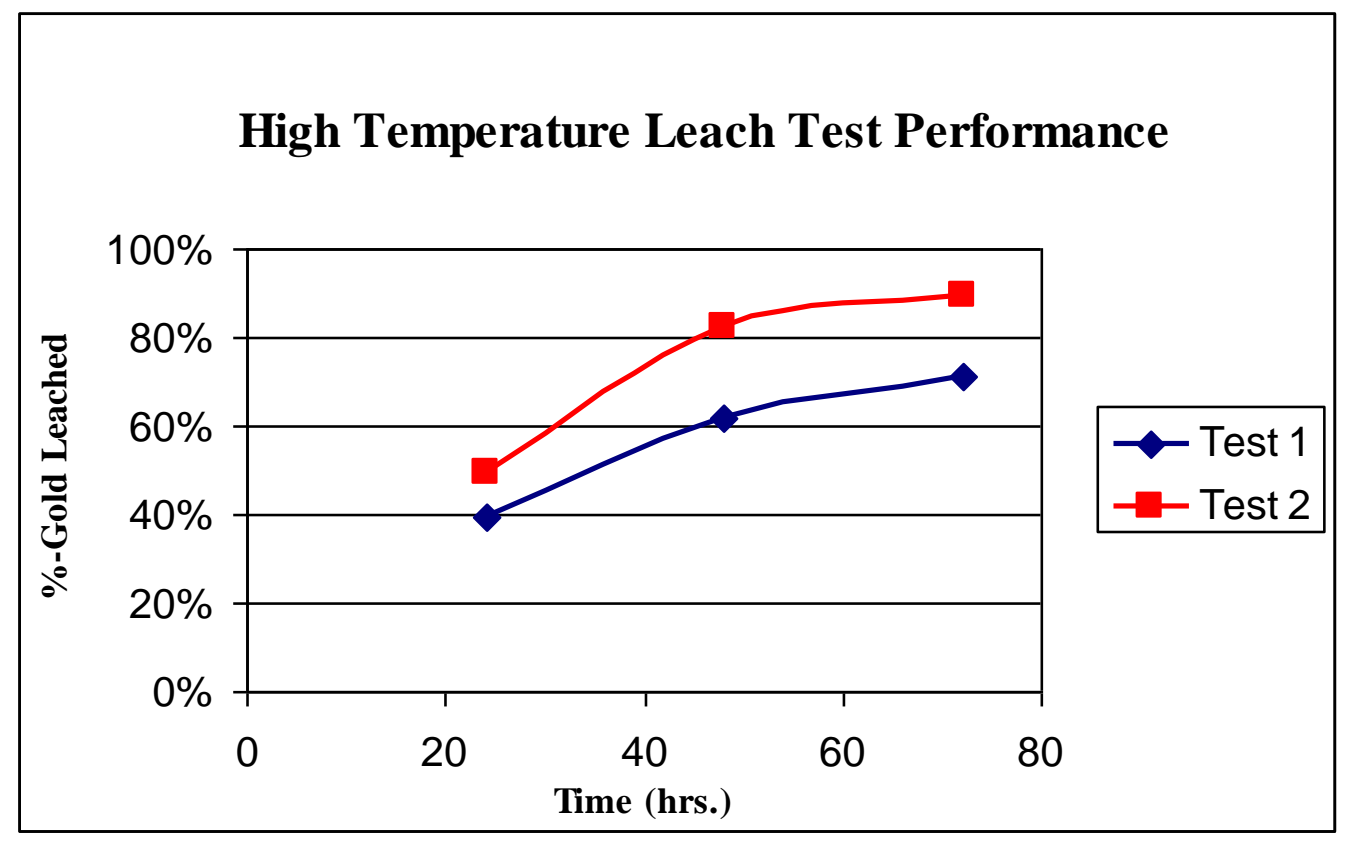

Figure 23. Identical $75^{\circ} \mathrm{C}$ Leach Tests Done with 999.9 Fine Gold

\subsubsection{Year 2 Leach Tests}

Year 2 leach tests were done with a procedure that was positively tested for experimental reproducibility. The test parameters were set up in accordance with phenomenon found during the electrochemical experiments conducted at Monash University. The laboratory procedure was developed with the notion that eventually the same procedure would be used with industrial ores. Steps were taken to insure that the procedure would be sufficient in agitating while denying the leach solution of oxygen from the atmosphere.

\subsubsection{Development of a Standard Operating Procedure for Leach Tests}

While large variability in leach results was incurred during the first year of leach tests, a standard operating procedure for leach tests was being developed. In the following section the 5-method evolution of the SOP will be detailed so that similar mistakes in future research can be avoided. 


\section{Method 1}

During the first series of tests chemicals were added in the order of 1) hydroxide, 2) sulfide, and 3) sulfur. Once chemicals were dissolved into solution it diluted to the required volume usually about 1 liter. A sample of the solution was taken for titration analysis on the auto titrator. Based on the free hydroxide number generated from the ' $\mathrm{ABC}$ ' titration, hydroxide was added to adjust the free hydroxide value to where it needed to be for the test. Gold was placed into a large based flask with a Teflon coated magnet. Next, $750 \mathrm{~mL}$ of solution was poured into the flask. After the flask was capped with a brushed glass topper the flask was placed into the heat bath and the magnetic agitator was turned on.

\section{Method 2}

The major problem with Method 1 was the lack of solution filtering prior to leaching. This problem was discovered when some dirtier sodium sulfide was used to make the leach solution. A decision was made to clean the solution by filtering. After filtering the solution it appeared to be much cleaner than solutions that were made with the 'clean' sodium sulfide.

\section{Method 3}

After running many tests with Method 2, gold balls began forming in the large bottom flasks. (See 2.3.3) It was determined that a different agitation method was required to continue the leach tests. The decision was made to go with an externally powered agitator. A switch was made from the large bottom flasks to the kettle so that the agitator could be immersed into the solution. A hole was punched through a rubber plug for the shaft connecting the agitator to the power source so that the system would be sealed off from the atmosphere. The kettle was placed into the heat bath while the agitator was set up so that it was suspended over the heat bath and kettle.

\section{Method 4}

After only a few tests with Method 3 it was determined that too much oxygen was being pulled through the seal from the atmosphere. Silicon sealer was added to help the problem but gold was observed to 'bury' itself in the silicon whenever it came in contact with it. Agitation rate also became a problem because to agitate the solution enough to move the gold cause the agitator to shake; the shaking agitator made the sealing problem worse.

Method 4 tried to handle the sealing problem by sparging the cell with oxygen free nitrogen gas. The rubber seal was left on over the agitator shaft. Also free hydroxide values were no longer taken because the electrochemical research showed only a relationship between leach rate and total hydroxide concentration.

\section{Method 5}

Method 5 is actually the final SOP for gold leaching tests. Even with the nitrogen sparge in Method 4 it was suspected that oxygen was still getting to the solution in destructive amounts. 
Also, the rubber plug around the agitator would often catch the shaft and slow agitation, or it would work its way up the agitator shaft away from the kettle leaving the solution very exposed to the atmosphere. See section 2.3.4.2 for the full SOP.

\subsubsection{Standard Operating Procedure for Leach Tests}

Both the metastable characteristics of the ASGLS and the physical properties of gold made it difficult to achieve reproducibility with tests conducted in the lab. To combat these phenomena, a strict Standard Operating Procedure (SOP) was created through laboratory experiences. All of the final tests were conducted under the same SOP. To set up test reproducible with the ones found in this thesis, follow the following twelve steps:

1. The final leach volume will be $750 \mathrm{~mL}$; however, reagent additions need to be calculated for a final volume of $1000 \mathrm{~mL}$. Solutions will be made larger than the final volume for two reasons: 1) there will be about a $50 \mathrm{~mL}$ volume loss after filtering the solution, and 2) there was only a $1000 \mathrm{~mL}$ volumetric flask available.

2. Obtain a $2000 \mathrm{~mL}$ flask and fill to about $600 \mathrm{~mL}$ with distilled water.

3. Weigh out sodium hydroxide to the nearest 0.01 grams and add directly to the flask under a fume hood. Gently shake the flask so that the hydroxide does not clog at the bottom of the flask. Let the flask sit while weighing out the sodium sulfide.

4. Weigh sodium sulfide out to the nearest 0.01 grams and add to the flask under the fume hood. By the time the sodium sulfide is added to the flask, all of the sodium hydroxide should be dissolved. Gently shake the flask to wet all of the sodium sulfide flakes.

5. While the sodium sulfide is dissolving, weigh out sulfur to the nearest 0.01 grams. Add the sulfur to the flask under the hood, and gently shake the flask to wet the sulfur flakes. Not all of the sodium sulfide flakes will be dissolved yet. Total solution volume should be closer to $750-800 \mathrm{~mL}$ now, depending on the amount of solid added to the solution.

6. Place the flask on a hot plate at medium temperature. Because of the dissolution of sodium hydroxide, the solution will have a temperature greater than room temperature. An elevated temperature will aid in the dissolution kinetics; however, it is important that the temperature be held similar to the other tests during dissolution of the sulfur and sodium sulfide. Below is a picture of partially dissolved sulfur and sodium sulfide flakes. 


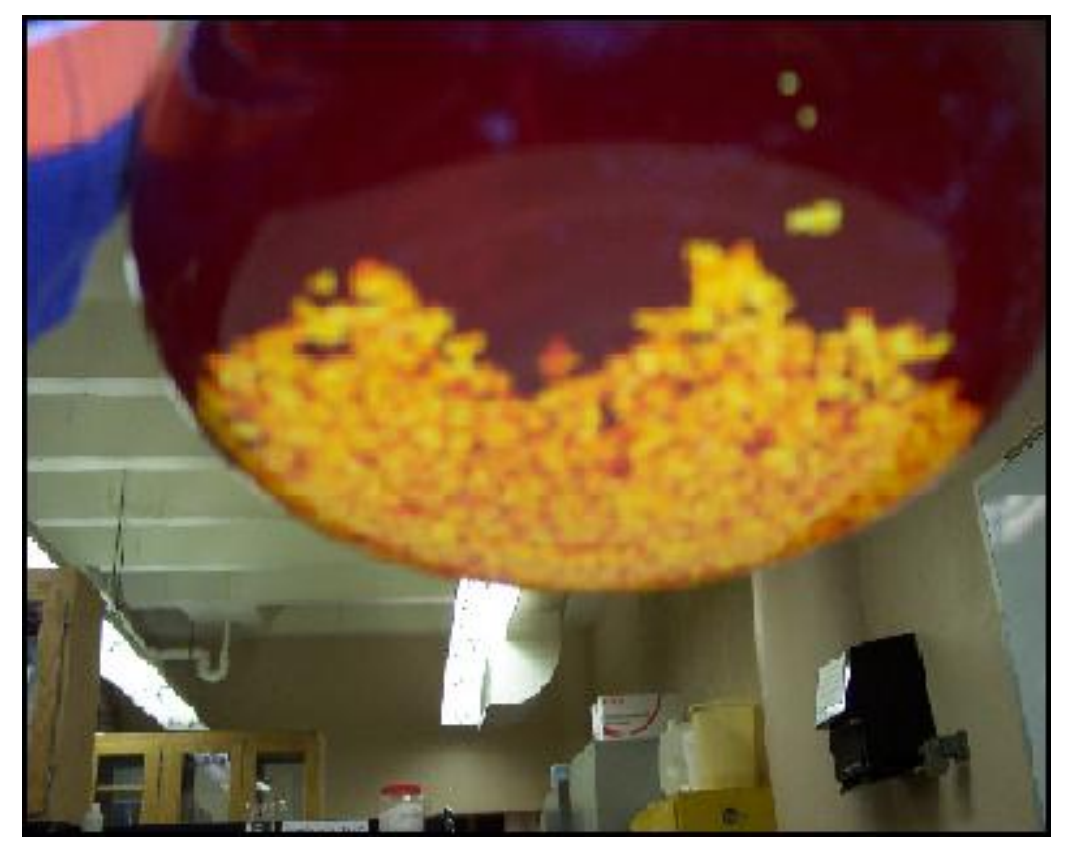

Figure 24. Partially Dissolved Sulfur \& Sulfide Flakes

7. Keep the solution temperature between $50-60^{\circ} \mathrm{C}$ and shake often during the dissolution process. Total dissolution time should not take longer than 30 minutes. If the heated solutions are kept longer on the hot plate open to the atmosphere it is suspected that the pick up of oxygen will change that chemistry of the solution.

8. As soon as the last solid has dissolved, add to the $1000 \mathrm{~mL}$ volumetric flask. With cold distilled water, wash any remaining solution from the walls of the empty flask and pour it into the volumetric flask until it is near the full line. Place the filled volumetric flask in a bucket of chilled water to bring the temperature back to room temperature. Once the solution has cooled, add distilled water until the proper volume is obtained. Pour the solution from the volumetric flask into a clean, dry flask so that the solution can be agitated to equilibrium.

9. Now the solution must be filtered to remove any solids that did not dissolve. Any colloidal solids remaining in the solution may deposit on the gold surface during the leach tests and alter the leach kinetics. It is suspected that most of the impurities come from the sodium sulfide. A Whatman $185 \mathrm{~mm} \mathrm{\# 3} \mathrm{filter} \mathrm{paper}$ should be placed onto a porcelain filter in the fume hood. To aid in the filtering process the porcelain filter is placed onto a $4000 \mathrm{~mL}$ Bukener flask and sealed with a rubber plug. A vacuum is pulled on the Bukener flask via flowing water passing quickly past an empty hose connected to the Bukener flask. The solution is now poured quickly onto the filter. While wearing a rubber glove, the experimenter may need to adjust the edges of the filter to avoid any short circuiting of solution. After filtering is done, pour the solution from the bunker flask into a clean, dry flask. Note the clarity of the solution; if it still looks cloudy there was a short circuit in the filtering, and it needs to be re-filtered. 
10. Obtain a $1000 \mathrm{~mL}$ kettle with a lid. Weigh out gold to the nearest 0.001 grams. Unless otherwise noted, all tests done in this thesis had 0.250 grams of gold added. Add a 2 inch Teflon coated magnet to the kettle with the gold.

11. It is optional to take an ORP of the solution now before gold is added. Into a 250 $\mathrm{mL}$ graduated cylinder, add clean leaching solution. Fill to $250 \mathrm{~mL}$ and add to the kettle with the magnet and gold. Repeat twice to obtain $750 \mathrm{~mL}$ total leach solution in the kettle.

12. Place the lid onto the kettle and plug the probe holes in the lid with rubber stoppers. Again, it is now optional to take an ORP before the solution is brought up to temperature. (See Figure 25.)

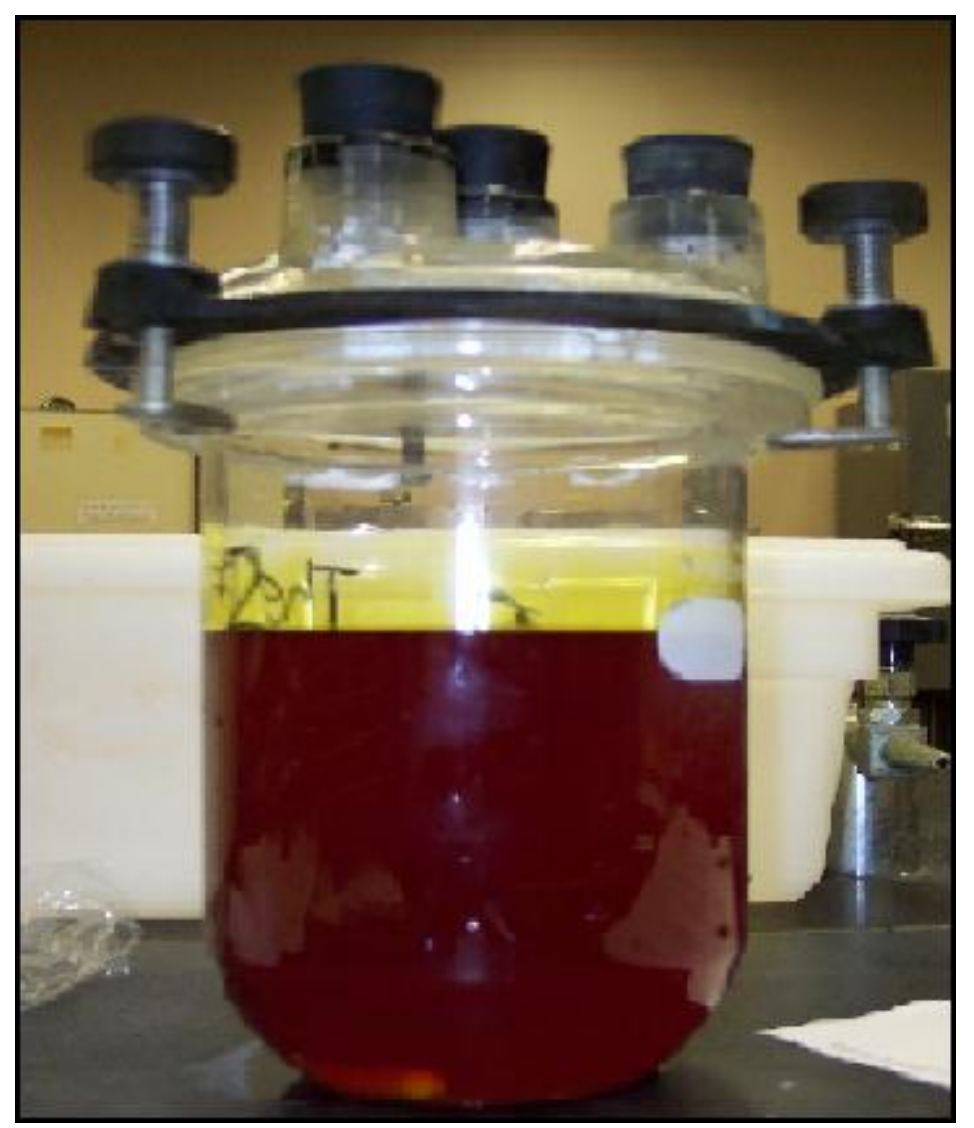

Figure 25. Kettle Test Outside of the Heat Bath

13. Place the filled kettle into a warm water bath at a specified temperature. A magnetic stirrer plate should be place underneath the warm water bath to provide agitation for the system. Start the stirrer 5 minutes after submerging the kettle so that it can be close to leach temperature before the agitation begins. Record to time that agitation starts as the start time for the leach test. Agitation should be held constant for each test at 330 RPM.

A typical test set up is seen below in Figure 4. In this particular test, three leach tests are being run at $60^{\circ} \mathrm{C}$. 


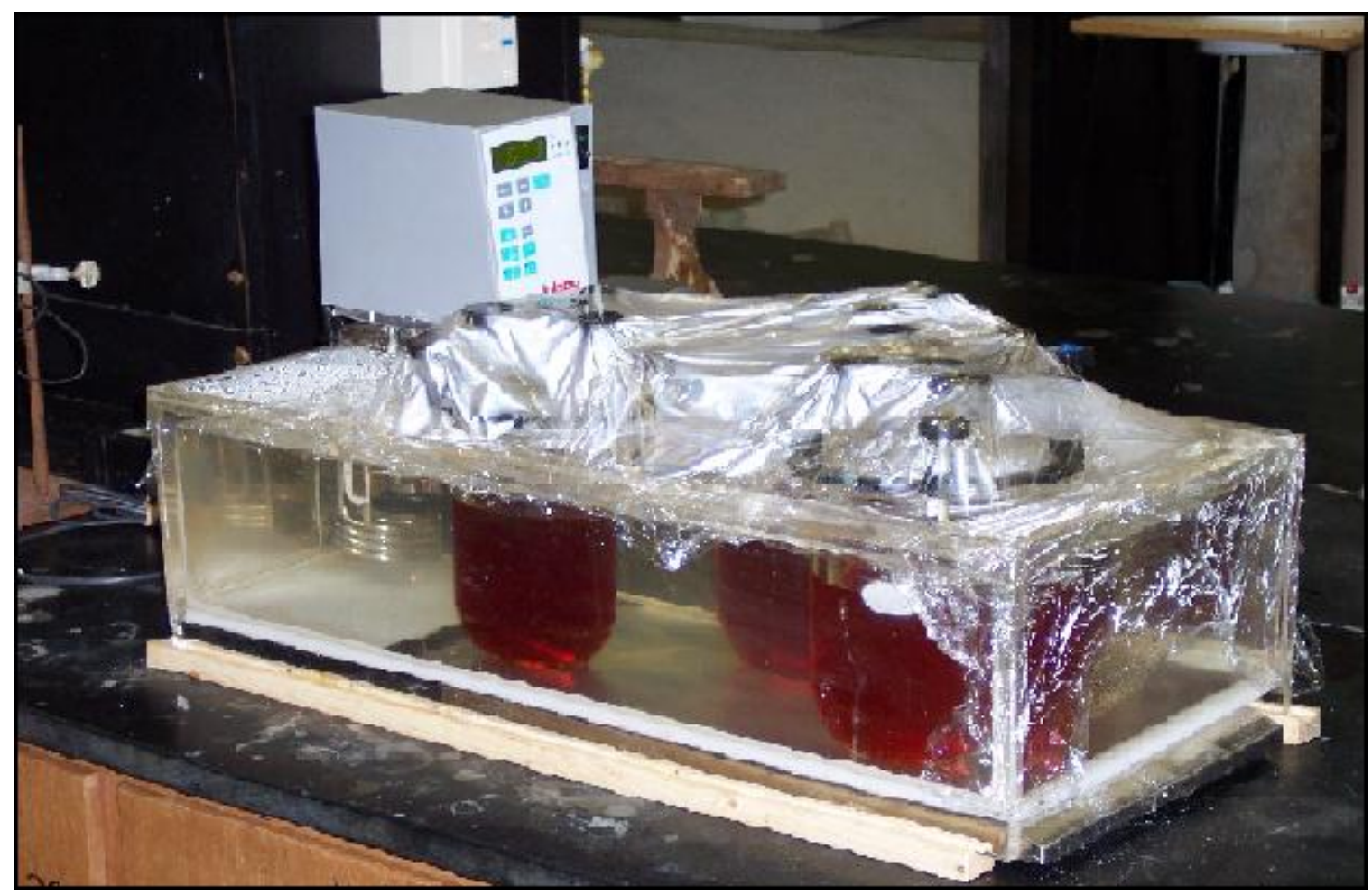

Figure 26. Typical Leach Test

Note the cellophane draped across the top of the heat bath; it is there because of the fast evaporation kinetics of water at high temperatures. Most of the evaporated water can be caught and drained back into the water bath. Without cellophane the bath would need to be watched hourly so that it did not reach critically low levels.

\subsubsection{Leach Matrix \#4}

Leach Matrix \#4 was set up to find the optimum operating conditions for leaching gold in a temperature range from $50-60^{\circ} \mathrm{C}$, a total sulfur concentration ranging from 3-5 molar, and a hydroxide concentration ranging from 2-4 molar. With the help of Stat-ease, the following test matrix was created. (See Table XI.) 
Table XI. Leach Matrix \#4 Reagent Additions

\begin{tabular}{|c|c|c|c|c|}
\hline Test & Sulfur Total & Sulfide (M) & Sulfur (M) & Hydroxide (M) \\
\hline 1 & 3.12 & 0.62 & 2.5 & 2 \\
\hline 2 & 3.12 & 0.62 & 2.5 & 4 \\
\hline 3 & 1.87 & 0.37 & 1.5 & 2 \\
\hline 4 & 1.87 & 0.37 & 1.5 & 2 \\
\hline 5 & 1.87 & 0.37 & 1.5 & 4 \\
\hline 6 & 3.12 & 0.62 & 2.5 & 4 \\
\hline 7 & 1.87 & 0.37 & 1.5 & 4 \\
\hline 8 & 3.12 & 0.62 & 2.5 & 4 \\
\hline
\end{tabular}

There are some important factors to note with Leach Matrix \#4. The total sulfur concentration is based off of a 50/50 mixture of sulfur to sulfide. However, due to an early calculation error there was not the full amount of sulfide added to each test. The actual ratio of sulfur to sulfide is near 5:1 for each of the tests in Matrix \#4. Since each test had the same relative error, the matrix was still accepted as a god model because the trends will be the same. Actual addition of chemicals to each test can be seen in Table IV.

Table XII. Actual Chemical Additions per Liter

\begin{tabular}{|c|c|c|c|}
\hline Test & $\mathrm{S}(\mathrm{g})$ & $\mathrm{Na}_{2} \mathrm{~S}(\mathrm{~g})$ & $\mathrm{NaOH}(\mathrm{g})$ \\
\hline 1 & 80 & 195 & 80 \\
\hline 2 & 80 & 195 & 80 \\
\hline 3 & 48 & 117 & 160 \\
\hline 4 & 48 & 117 & 80 \\
\hline 5 & 48 & 117 & 80 \\
\hline 6 & 80 & 195 & 160 \\
\hline 7 & 48 & 117 & 160 \\
\hline 8 & 80 & 195 & 160 \\
\hline
\end{tabular}

\subsubsection{Leach Matrix \#4 Experimental Analysis}

During each test the solution was periodically tested for solution potential and dissolved gold concentration. From the electrochemical work done at Monash University, it was shown that solution potential played a very critical part in the overall leach kinetics of gold. The leach kinetics for each test was measured by taking a solution sample and analyzing for gold concentration via Induction Coupled Plasma (ICP). 


\subsubsection{Measuring Solution Potential}

Potential readings were done on the system for two reasons: 1) to understand the effect of solution potential on leach rate; and, 2) to ensure that the leach solution was not being influenced by the atmosphere. For all the tests done in Matrix \#4 there were no major shifts in solution potential during the time the tests were run.

Solution potential was measured in $\mathrm{mV}$ with a calomel electrode. Potential readings were taken at the beginning of the leach test (the beginning of the leach test was assumed to be where the solution should be at equilibrium, or after about one hour), at 18 hours, and at 48 hours. For some of the tests additional potential reading were taken: prior to adding gold to the solution at room temperature, and after adding gold to the solution at room temperature.

Testing the solution potential was done by removing one of the rubber stoppers from the kettle and submerging the end of the calomel electrode into the solution. The magnetic stirrer was left on during the readings to assure equilibrium in the solution. Once the ORP had leveled off usually after five minutes in solution - the ORP value was recorded and the electrode was removed from the solution. Immediately after the test the rubber stopper was replaced back onto the kettle so that no more oxygen could enter the kettle.

\subsubsection{Analyzing for Dissolved Gold Concentration}

Two solution samples were taken during each test, the first at 18 hours and the second at 48 hours. While taking a sample the magnetic stirrer was turned off so that solid gold particles suspended in solution would be less likely to enter the sample. To take a sample, one of the rubber stoppers was removed and a $20 \mathrm{~mL}$ pipette was submerged about $5 \mathrm{~cm}$ into the solution. After $20 \mathrm{~mL}$ of warm solution was removed from the kettle it was transferred to a labeled $25 \mathrm{~mL}$ sample bottle. Since the solution was at the temperature of the leach test there was actually a little less than $20 \mathrm{~mL}$ of room temperature solution removed from the kettle. However, all samples were taken via the same method so that a minimal amount of error would be introduced. As soon as the sample taking was completed, the sample bottle was capped and the rubber stopper was replaced onto the kettle so that as little as possible oxygen could get into either the sample or the test.

\subsubsection{ICPStandard Analysis Procedure}

All solution analysis for dissolved gold was done with the ICP found in ELC room 107. The standards were prepared according to the following Table. Standards were stored in a dark cabinet to minimize the effect of ultraviolet rays causing the gold concentration to fade over time. The prepared standards were considered to be fresh for about a week; after this time new standards were prepared from the master standard. 
Table XIII. ICP Analysis Standard Solutions

\begin{tabular}{|c|c|}
\hline Solution & Solution Preparation Description \\
\hline Gold Standard & ASSURANCE; $1,000 \mathrm{mg} / \mathrm{L}(\mathrm{ppm}) ; 10 \%-\mathrm{HCl}$; lot 3-130AU \\
\hline $100 \mathrm{ppm}$ & $5 \mathrm{~mL}$ of standard (1000 ppm Au) diluted with $5 \%-\mathrm{HNO}_{3}$ to $50 \mathrm{~mL}$ \\
\hline $10 \mathrm{ppm}$ & $5 \mathrm{~mL}$ of $100 \mathrm{ppm} \mathrm{Au}$ diluted with $5 \%-\mathrm{HNO}_{3}$ to $50 \mathrm{~mL}$ \\
\hline $1 \mathrm{ppm}$ & $5 \mathrm{~mL}$ of $10 \mathrm{ppm} \mathrm{Au}$ diluted with $5 \%-\mathrm{HNO}_{3}$ to $50 \mathrm{~mL}$ \\
\hline
\end{tabular}

Each time the ICP was started it was recalibrated with the standards. Because the standards were dissolved in an acidic solution, prior to running a sample through the ICP a 1 molar $\mathrm{NaOH}$ solution was run to neutralize leftover solution in the tubes and nebulizer. By neutralizing the leftover solution the chance of a precipitate forming from the ASGLS samples was greatly reduced.

Once ASGLS solutions had made their way through the ICP the waste tube was removed from the normal waste bottle to a special ASGLS waste bottle. The normal waste bottle contains about 5\% nitric acid; if ASGLS solutions are exposed to acid they will form toxic $\mathrm{H}_{2} \mathrm{~S}$ gas. Once the ASGLS waste bottle had been mostly filled it the solution was disposed of in the same fashion as a fresh leach solution.

For each sample three ICP runs were completed to insure better accuracy. If the three solution analysis found similar levels of gold concentrations they were accepted as a good analysis. If there was any doubt in the sample concentrations found on the ICP.

\subsubsection{Test Duplication}

Most of the reason for duplicating any test had to do with the solid gold behaving strangely during the leach test. During some of the tests the solid gold began to clot and ball up. The gold that was purchased for this thesis was chosen partly because of the small uniform size of each gold crystal. To avoid error in leach rate from test to test a similar particle size range is critical. In tests where any clogging or balling was observed the results were tossed out and the test was rerun.

\subsubsection{Matrix \#4 Statistical Analysis}

The results for each of the eight tests in Matrix \#4 can be found in Table X. As expected the best performing leach solution was Test \#8, the high sulfur, high hydroxide, high temperature solution. The poorest performing solution was Test \#4 where all three variables were at there lowest. 
Table XIV. Matrix \#4 18 hrs. Leaching Results

\begin{tabular}{|c|c|c|c|c|c|c|}
\hline Test & Sample & Total Sulfur $(\mathrm{M})$ & $\mathrm{NaOH}(\mathrm{M})$ & $\mathrm{Au}(\mathrm{ppm})$ & \%-Leached & Temp. $\left({ }^{\circ} \mathrm{C}\right)$ \\
\hline 1 & $120104-1$ & 3.12 & 2 & 75.13 & $22.54 \%$ & 60 \\
\hline 2 & $120304-2$ & 3.12 & 2 & 50.89 & $15.27 \%$ & 50 \\
\hline 3 & $112804-3$ & 1.87 & 4 & 45.80 & $13.74 \%$ & 60 \\
\hline 4 & $120304-4$ & 1.87 & 2 & 23.69 & $7.11 \%$ & 50 \\
\hline 5 & $112804-5$ & 1.87 & 2 & 36.09 & $10.83 \%$ & 60 \\
\hline 6 & $120304-6$ & 3.12 & 4 & 52.51 & $15.75 \%$ & 50 \\
\hline 7 & $121504-7$ & 1.87 & 4 & 28.78 & $8.63 \%$ & 50 \\
\hline 8 & $111604-8$ & 3.12 & 4 & 102.77 & $30.83 \%$ & 60 \\
\hline
\end{tabular}

\subsubsection{Matrix \#4 ORP Analysis}

Solution potentials were measured often during the test; the 18 hour solution potentials are reported below in Table XV. Potentials are affected very little between identical 50 and $60^{\circ} \mathrm{C}$ tests.

Table XV. Solution Potentials After 18 hrs. Leaching

\begin{tabular}{|c|c|c|c|c|}
\hline Test & Total Sulfur $(\mathrm{M})$ & Hydroxide $(\mathrm{M})$ & Temperature & 18hr. ORP (mV vs. SCE) \\
\hline 1 & 3.12 & 2 & 50 & -762 \\
\hline 2 & 3.12 & 2 & 60 & -762 \\
\hline 3 & 1.87 & 4 & 50 & -763 \\
\hline 4 & 1.87 & 2 & 50 & -751 \\
\hline 5 & 1.87 & 2 & 60 & -747 \\
\hline 6 & 1.87 & 4 & 60 & -769 \\
\hline 7 & 3.12 & 4 & 50 & -770 \\
\hline 8 & 3.12 & 4 & 60 & -772 \\
\hline
\end{tabular}


Below is a graphical representation of the trends found in the solution potential. Data used for the figure is taken from the four tests done at $50^{\circ} \mathrm{C}$.

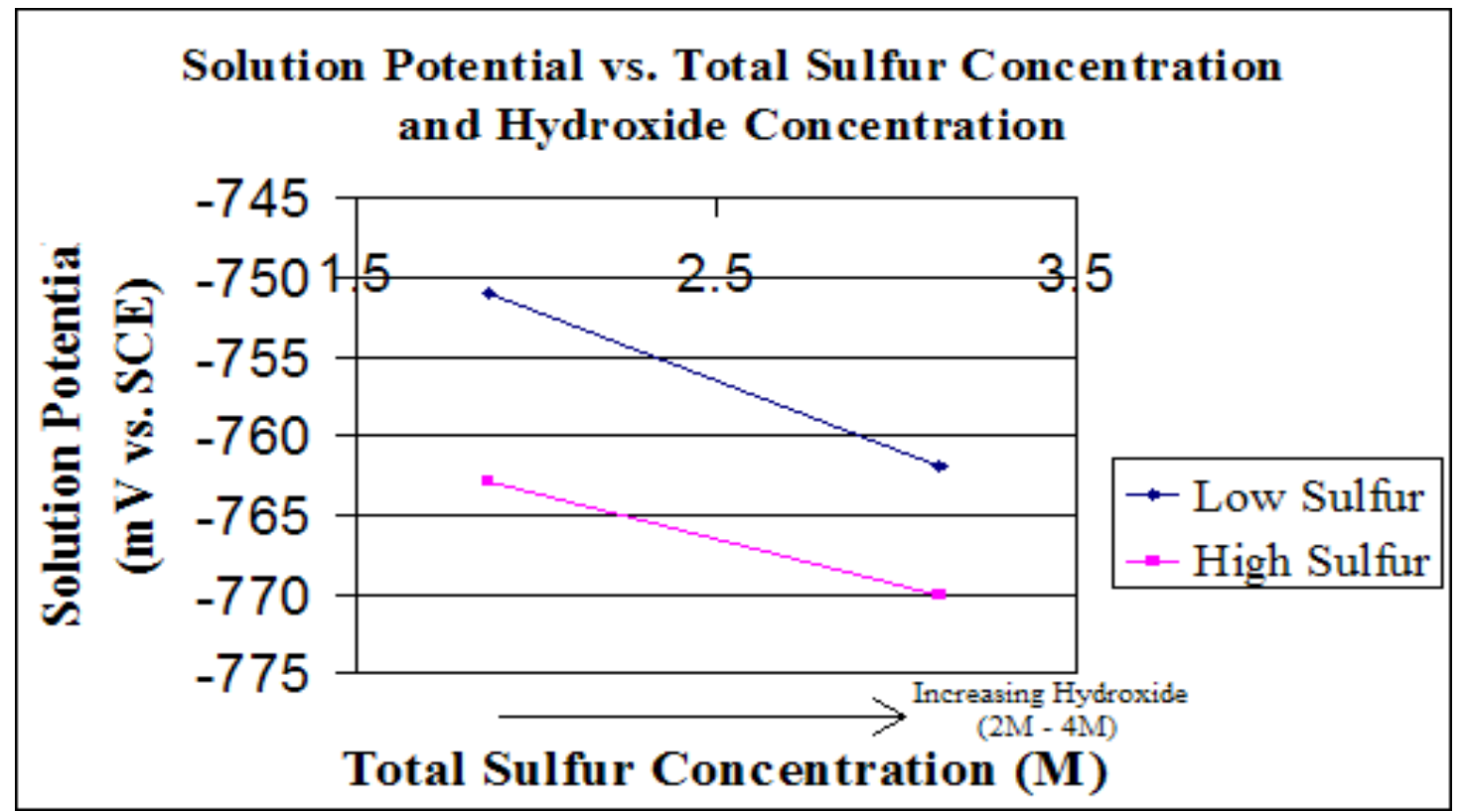

Figure 27. Solution Potential analysis at 18 Hours

From the above figure as sulfur and hydroxide concentration are increased the solution potential drops. Increasing sulfur concentration from $1.87 \mathrm{M}$ to $3.12 \mathrm{M}$ lowers the solution potential 8-10 $\mathrm{mV}$ in both cases. Doubling the hydroxide concentration from $2 \mathrm{M}$ to $4 \mathrm{M}$ also drops the potential 8-10 mV.

\subsubsection{Matrix \#4 Statistical Analysis}

Matrix \#4 was created on the program Stat-Ease. When all of the results were made available they were inserted back into the computer program so that a statistical analysis could be performed on the matrix. Upon entering the data, two of the least important factors were dropped from the model so that the adjusted R-squared value for the data set was 0.9385.

Below is a graph of the effect of sulfur versus temperature on the leachability of gold. Hydroxide concentration is held constant at 3 molar for this graph. The contours found on the graph represent the percent of gold that will leach under those conditions in 18 hours in the kettle. (See Figure 28.) 


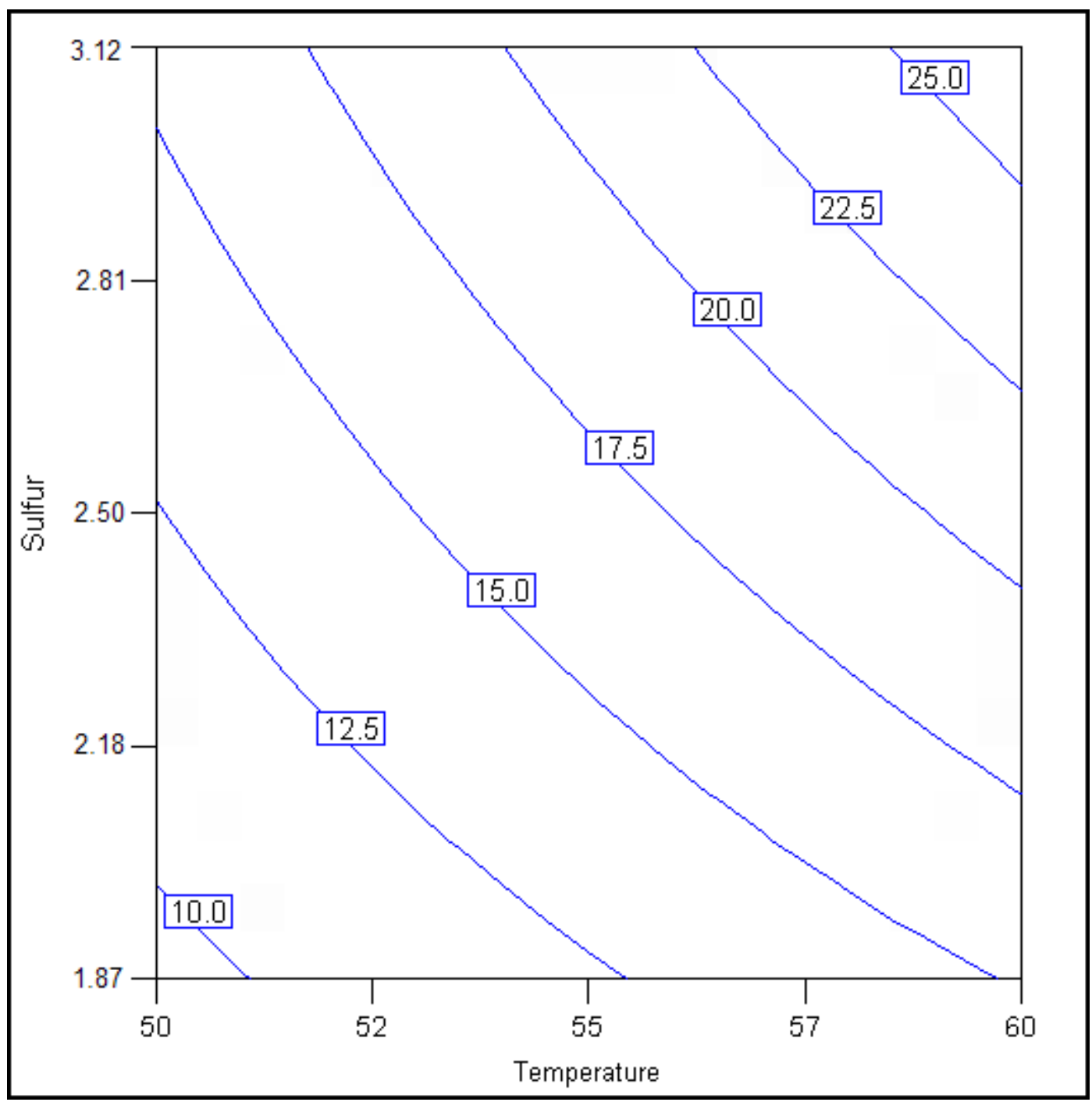

Figure 28. Effect of Sulfur Concentration and Temperature on Gold Leachability

The best performing solution had high total sulfur concentration at $60^{\circ} \mathrm{C}$. Sulfur concentration has a large affect on gold leaching; a 1 molar increase in total sulfur concentration - for instance, 2.12 to 3.12 - increases the total amount of gold leached about $8-10 \%$ at any given temperature. Increasing the temperature $10^{\circ} \mathrm{C}$ will increase the leachability 5-6\% for and given sulfur concentration.

In Figure 2 below, a 3-D model was created to show the effect of hydroxide concentration and sulfur concentration on gold leachability at $55^{\circ} \mathrm{C}$. 


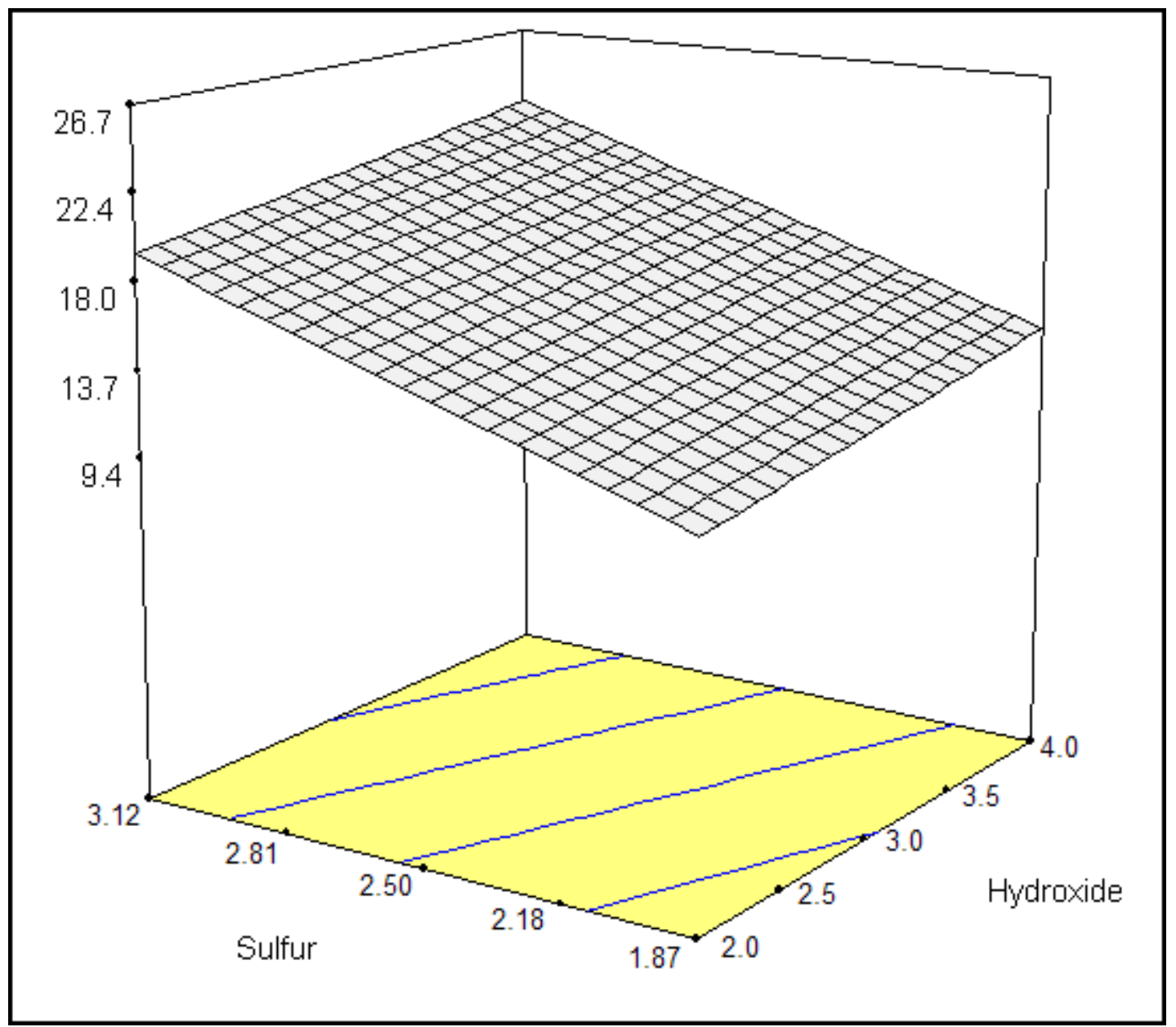

Figure 29. 3-D Model of Leaching Effects of Hydroxide and Sulfur Concentration

From the 3-D model, it is shown that increasing the sulfur concentration does more to help leaching than increasing hydroxide concentration. By increasing total sulfur concentration from 1.87 to $3.12 \mathrm{M}$, gold leaching is increased about $10 \%$ at all hydroxide concentrations. Although increasing hydroxide from 2 to $4 \mathrm{M}$ does speed up the leach rate, it only increases it 3-4\%. By increasing both sulfur and hydroxide concentrations the optimal leach conditions are achieved.

\subsubsection{Leach Matrix \#4 Extra Tests}

In addition to the tests done in matrix \#4 three tests were run to find actual optimum leaching conditions for industrial ores. Because of the calculation error prior to setting up the tests in matrix \#4 no data was available for a leach test deemed optimum from the electrochemical work done at Monash University. Of the three tests: one was done to see the 1:1 sulfur/sulfide leachability at 5 molar total sulfur; one was done to see the 1:1 sulfur/sulfide leachability for the best performing test in matrix \#4; and the final test was done to measure leachability at 5 molar total sulfur at the miscalculated ratio of sulfur to sulfide found in matrix \#4. All tests were run at $60^{\circ} \mathrm{C}$ and had a 4 molar hydroxide concentration. (See Table XVI.) 
Table XVI. Leach Matrix \#4 Extra Tests Parameters

\begin{tabular}{|c|c|c|c|c|}
\hline Test & Total Sulfur (M) & Sulfide (M) & Sulfur (M) & Hydroxide (M) \\
\hline 1 & 5 & 2.5 & 2.5 & 4 \\
\hline 2 & 3.54 & 1.77 & 1.77 & 4 \\
\hline 3 & 5 & 1.45 & 3.55 & 4 \\
\hline
\end{tabular}

\subsubsection{Matrix \#4 Extra Test Results}

Many difficulties occurred while setting up the Matrix \#4 extra tests. The high molar concentrations of sulfur greatly increased the amount of time required for dissolution of leaching chemicals. Once dissolved, test \#1 was supersaturated at room temperature and tests \#2 \& \#3 were near supersaturation at room temperature. This extreme concentration made filtering and handling the solutions before they were inserted in the heat bath difficult.

Sampling and analysis for the extra tests done for Matrix \#4 proved to be difficult because of the high molar concentrations, particularly that of Test \#1 where the sulfide concentration was 2.5 molar. Upon removing a sample from the $60^{\circ} \mathrm{C}$ heat bath precipitates of sodium, sulfur, and sulfide began to form. To combat the problem a $5 \mathrm{~mL}$ sample was taken and quickly diluted 5:1 in a $25 \mathrm{~mL}$ volumetric flask. Once the sample was analyzed on the ICP the gold concentration was multiplied by a factor of 5 .

For the other samples analysis was still difficult because of the relatively high concentrations. Some precipitates began to form during ICP analysis and began to clog the tubing. Tests run for each sample on the ICP had unusually high variance between samples. The final concentrations were chosen after a set of three analyses came between $\pm 5 \%$. Because of the difficulties incurred, and the knowledge that any stray away from the standard procedure can cause large leaching variances, Matrix \#4 extra tests the results should only lightly be compared to the rest of the tests in Matrix \#4. Results for the extra tests can be seen below in Table XVII.

Table XVII. Matrix \#4 Extra Tests After 18 hrs. Leaching

\begin{tabular}{|c|c|c|c|c|}
\hline Sample & Total Sulfur (M) & Hydroxide $(\mathrm{M})$ & $\mathrm{Au}(\mathrm{ppm})$ & \%-Leached \\
\hline $012105-1$ & 5 & 4 & 224 & $67.2 \%$ \\
\hline $012105-2$ & 3.53 & 4 & 93 & $27.8 \%$ \\
\hline $012105-3$ & 5 & 4 & 53 & $15.9 \%$ \\
\hline
\end{tabular}

\subsubsection{Matrix \#4 Extra Test Analysis}

Relative concentrations of sulfur to sulfide proved to be an important factor; when the ratio was decreased from $2.45: 1 \mathrm{~S}: \mathrm{S}^{2-}$ to $1: 1$ the percent leached after 18 hours increased from $15.9 \%$ to $67.2 \%$. Also increasing the total sulfur concentration from 3.53 M to $5 \mathrm{M}$ increased the percent gold leached from $27.8 \%$ to $67.2 \%$. The best solution for leaching at high temperatures should have high total sulfur concentrations with near $1: 1 \mathrm{~S}: \mathrm{S}^{2-}$. However, for laboratory purposes the 
high total sulfur concentration - particularly those with high sulfide concentrations - are difficult to set up, sample, and analyze with the methods detailed in this thesis.

\subsection{Gold Recovery Tests}

\subsection{Standard Operating Procedure for Recovery Tests}

Recovery tests are done to find how effectively gold can be deposited onto a certain solid. Solutions for these tests are leftovers from previous leach tests; they can be chosen by gold, sulfur, or hydroxide concentration. All of the recovery tests were done at room temperature on the shaking machine.

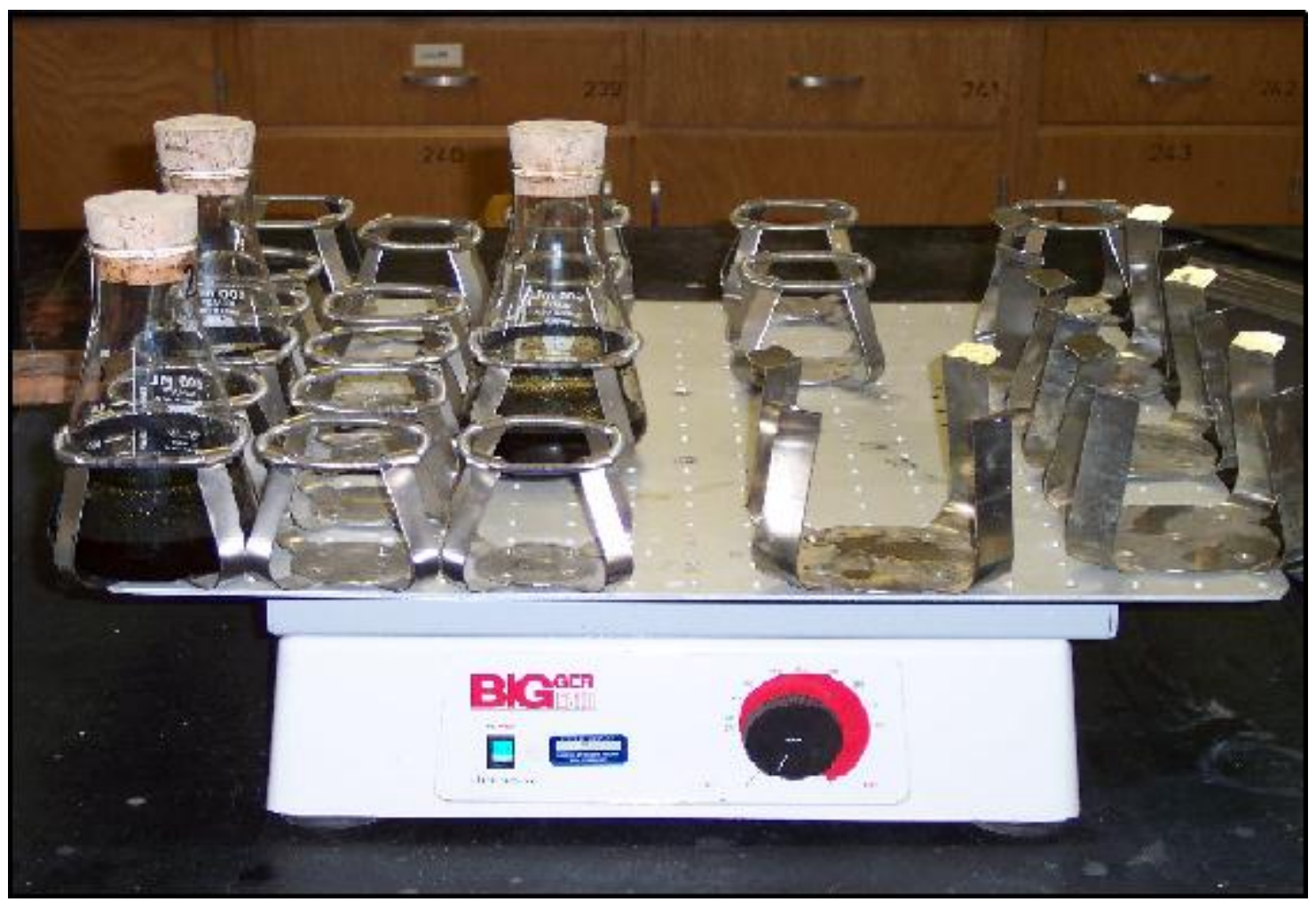

Figure 30. Laboratory ASGLS Recovery Test

The SOP for recovery tests is found below.

1. Weigh out the desired amount of recovery solid to the nearest 0.01 grams. Wash the solid with distilled water and allow it to dry on a paper towel for about an hour.

2. With the leach solution of interest, obtain a $20 \mathrm{~mL}$ sample. This sample will be the head sample for the recovery test.

3. Pour the solid into a $500 \mathrm{~mL}$ flask. Add $100 \mathrm{~mL}$ of the remaining leach solution. Plug the top of the flask with a cork and make sure the cork seals off any air from the atmosphere. (See Figure 31.) 


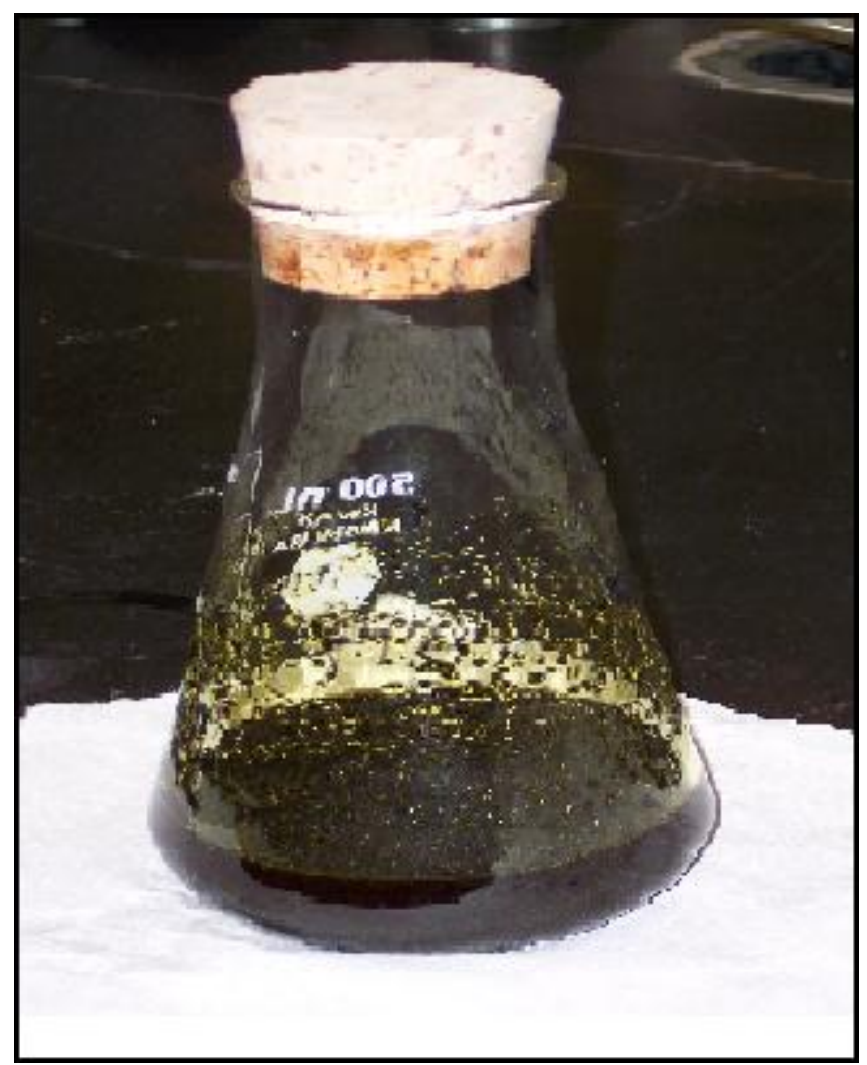

Figure 31. ASGLS Recovery Flask

4. $\quad$ Place the corked flask securely onto the shaking machine. Turn the machine to 250 RPM. Record the time that agitation begins as the start time for the experiment.

\subsection{Standard Operating Procedure for Analyzing Recovery Tests}

After the flask has been shaking for the specified amount of time, the solution needs to be filtered to remove the solid. Samples of solution should be analyzed with the same ICP SOP that the leach tests followed. The solids can be sent off for a fire assay analysis. All recovery test analysis followed the SOP below.

1. Stop the shaking machine and remove the flask from it.

2. Place a Whatman $185 \mathrm{~mm} \# 3$ filter into a clean, dry cone shaped filtering glass. Place a $20 \mathrm{~mL}$ labeled sample bottle underneath the glass.

3. Pour about $50 \mathrm{~mL}$ of solution into the filter. Make sure that no solution is short circuiting to the sample bottle. (See Figure 12) 


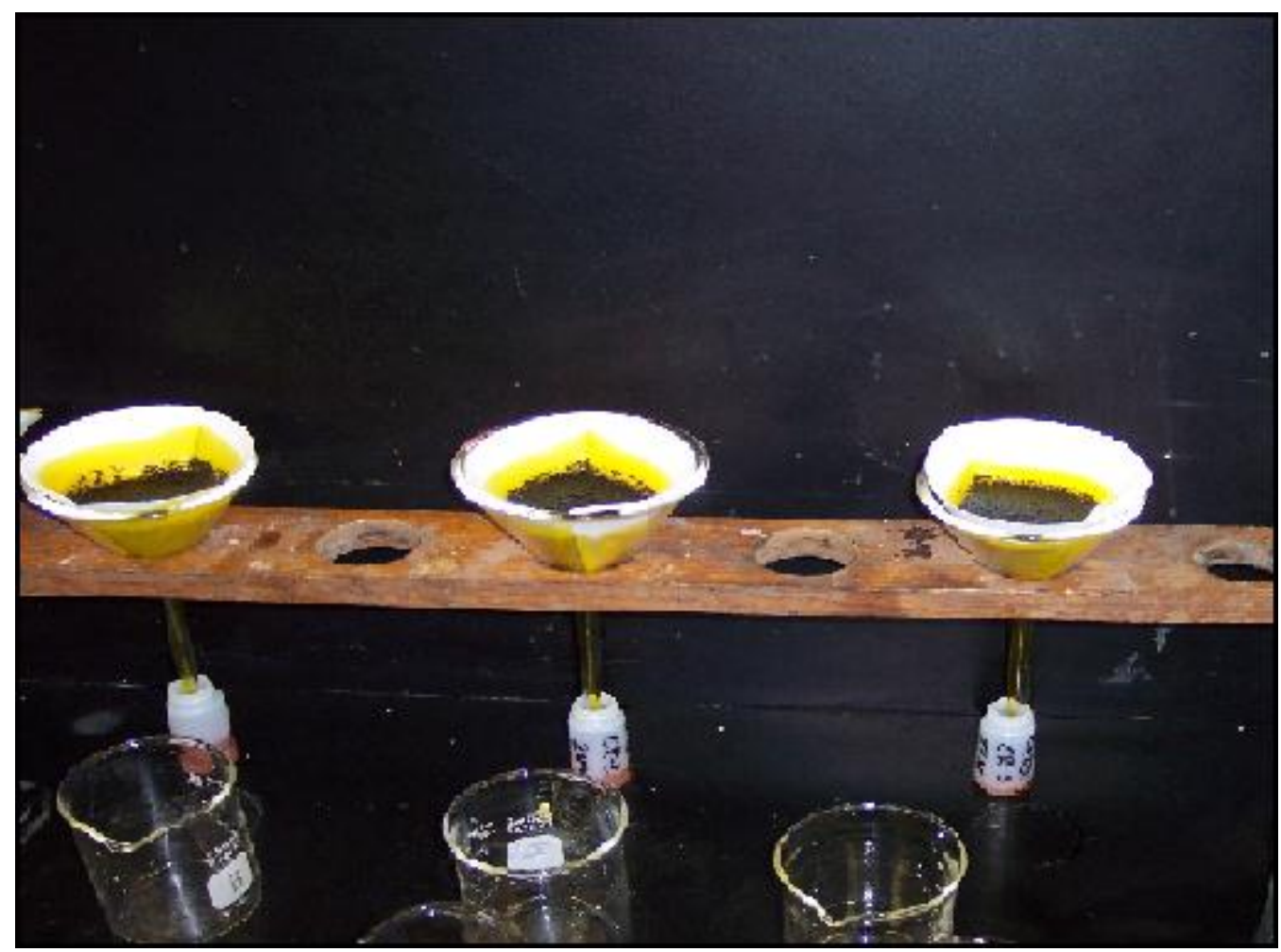

Figure 32. ASGLS Recovery Test Sample Collection

4. When the sample bottle is almost full, remove the sample bottle and put a lid on it. Place the filtering glass overtop of a $500 \mathrm{~mL}$ beaker to catch the remaining solution. Pour the rest of the solution from the 250 flask into the filtering glass.

5. Now the solution can be analyzed for gold concentration via the ICP. The same ICP procedure used for analyzing leach solutions for gold concentrations can be used. (See section

6. Wash any remaining solution and solid from the $250 \mathrm{~mL}$ flask with distilled water and pour the wash into the filtering glass. Allow all of the solution to drip through the filter.

7. Now the solids must be washed. Fill the filtering glass with distilled water and allow all of the wash solution to flow through the solid. Repeat this step once.

8. $\quad$ Dry the cleaned solids in the oven at $50^{\circ} \mathrm{C}$. Place them into a labeled bag.

\subsection{Carbon Recovery Matrix \#1}

The fourth carbon recovery matrix was set up to test the following two principles: 1) how the total amount of carbon affects the recovery efficiency; and, 2) how the relative strength (in terms of overall sulfur and hydroxide concentrations) of the solution affects the ability of gold to load onto carbon. 


\subsubsection{Experimental Set-Up}

Two different solutions were used, one high concentration gold and one low concentration gold. Each test required $100 \mathrm{~mL}$ of solution to be subjected to carbon treatment. The high concentration gold solution also had higher concentrations of both total sulfur and hydroxide. For each solution, two experiments were completed. One test had high carbon (25g) and the other had low carbon (10g). Coarse carbon was used for all tests. After 48 hours, the solution was filtered on an Whatman $185 \mathrm{~mm}$ \#3 filter paper to separate out the carbon. For each test there was a head sample and a post recovery sample taken. Each solution sample was subjected to ICP analysis (see ICP procedure). The four tests in the matrix were set up as follows. (See Table XVIII.)

\begin{tabular}{|c|c|c|c|c|c|}
\hline \multicolumn{7}{|c|}{ Table XVIII. Carbon Recovery Matrix \#1 } \\
\hline Test & Solution & Total Sulfur (M) & Hydroxide (M) & Gold (ppm) & Carbon (g) \\
\hline CR-1 & $111604-5$ & 1.87 & 2 & 45.1 & 10 \\
\hline CR-2 & $111604-5$ & 1.87 & 2 & 45.1 & 25 \\
\hline CR-3 & $111604-8$ & 3.12 & 4 & 223 & 10 \\
\hline CR-4 & $111604-8$ & 3.12 & 4 & 223 & 25 \\
\hline
\end{tabular}

\subsubsection{Results of Carbon Recovery Matrix \#1}

Since CR-3 and CR-4 had gold concentrations higher than 100 ppm both the head and post recovery samples were diluted 5:1. Samples associated with CR-1 and CR-2 were analyzed on the ICP without dilution. The results for the recovery matrix are as follows in Table XIX.

\begin{tabular}{|c|c|}
\hline \multicolumn{2}{|c|}{ Table XIX. Carbon Recovery Matrix \#1 Results } \\
\hline Test & Recovery to Carbon \\
\hline CR-1 & $89.88 \%$ \\
\hline CR-2 & $99.87 \%$ \\
\hline CR-3 & $-1.12 \%$ \\
\hline CR-4 & $6.55 \%$ \\
\hline
\end{tabular}

Increasing the amount of carbon from 10 to 25 grams increased the overall recovery of gold onto carbon, by $10 \%$ in the low concentration solution and by $5-7 \%$ in the high concentration sample. The reason for the negative loading of gold onto carbon in test CR-3 is most likely due to error in the ICP analysis. For CR-3 the overall gold loading can be assumed to be zero. By comparing the low concentration solutions to the high concentration samples it is easy to see that solution concentration plays a large factor in the effectiveness of carbon to remove gold from solution.

From an engineering standpoint, the results from Carbon Recovery Matrix \#1 are quite important. Gold is more stable on carbon in low sulfur/hydroxide concentration solutions, but in high sulfur/hydroxide solutions gold is more stable in solution. Much like in most cyanidization 
circuits, it is possible that an ore could be leached in a lower concentration sulfur/hydroxide solution, loaded onto carbon, and then stripped from the carbon in a lower volume, higher sulfur/hydroxide concentration solution.

\subsection{Carbon Recovery Matrix \#2}

The second carbon recovery matrix was set up to determine how the concentration of chemicals in solution affected the ability for carbon to remove gold from solution. Also, simultaneous experiments were to test whether the gold was preferentially loading onto the carbon or if it was possibly precipitating onto the solid substrate in the solution.

Three solutions were tested: one low sulfur, low hydroxide; one high sulfur, low hydroxide; and one low sulfur, high hydroxide. Two recovery tests were run per solution for a total of six tests. Fine carbon particles were used for all tests. Silica glass spheres (Tyler sieve \#18) were chosen as the added substrate because it was deemed to not affect the solution chemistry while still being large enough to easily separate from the carbon after the test was over in case a solid assay was required. The six tests were set up according to Table XX below.

Table XX. Carbon Recovery Matrix \#2 Test Description

\begin{tabular}{|c|c|c|c|c|c|}
\hline Test & Solution & Sulfur (M) & Hydroxide (M) & Glass & Carbon \\
\hline CR-1 & $112804-5$ & 1.87 & 2 & 0 & 25 \\
\hline CR-2 & $112804-5$ & 1.87 & 2 & 10 & 25 \\
\hline CR-3 & $120104-1$ & 3.12 & 2 & 0 & 25 \\
\hline CR-4 & $120104-1$ & 3.12 & 2 & 10 & 25 \\
\hline CR-5 & $112804-3$ & 1.87 & 4 & 0 & 25 \\
\hline CR-6 & $112804-3$ & 1.87 & 4 & 10 & 25 \\
\hline
\end{tabular}

\subsubsection{Carbon Recovery Matrix \#2 Results}

The three tests that had just carbon were analyzed to understand how solution chemistry affected the recoverability of carbon. All tests were run for 24 hours on the shaking machine at room temperature. Results for the tests can be seen below in Table XXI.

Table XXI. Effect of Concentration on Gold Recovery to Carbon

\begin{tabular}{|c|c|c|c|c|}
\hline Test & Total Sulfur (M) & Hydroxide (M) & Carbon $(\mathrm{g})$ & \%-Recovery To Carbon \\
\hline CR-1 & 1.87 & 2 & 25.0 & $97.6 \%$ \\
\hline CR-3 & 3.12 & 2 & 25.0 & $87.4 \%$ \\
\hline CR-5 & 1.87 & 4 & 25.0 & $66.5 \%$ \\
\hline
\end{tabular}

Judging from the three tests, the higher concentration of either total sulfur or of hydroxide lowers the recoverability of carbon. However, increasing hydroxide concentration has a much more 
detrimental affect to recoverability than increasing just sulfur concentration. From these results, a possible ASGLS strip carbon strip solution would need to be high in hydroxide concentration.

\section{Carbon with Glass Recovery Tests}

In the tests where glass was added the total amount of solids added to the solution was 35 grams. The 10 grams of glass was added to see how it would affect the recoverability of carbon. The results for the tests can be seen below in Table XXII. The \%-better with glass added calculation was done with the test having just carbon being the base line from which the calculation was done.

Table XXII. Results of Recovery Tests with Glass Added

\begin{tabular}{|c|c|c|}
\hline Test & Recovery to Solids & Percent Better with Glass Added \\
\hline CR-1 & $97.56 \%$ & $-0.29 \%$ \\
\hline CR-2 & $97.28 \%$ & \multirow{2}{*}{$2.24 \%$} \\
\hline CR-3 & $87.38 \%$ & \\
\hline CR-4 & $89.34 \%$ & $43.79 \%$ \\
\hline CR-5 & $66.50 \%$ & \\
\hline CR-6 & $95.63 \%$ & \\
\hline
\end{tabular}

Although there was little difference in the tests done on the first two solutions, the third solution showed nearly a $44 \%$ improvement when silica glass balls were added to the carbon. An improvement this large could be an error; but it is intriguing enough to run further recovery tests to investigate the phenomena.

\subsection{Industrial Ore Leaching}

\subsection{Greens Creek Gravity Middlings}

Kennecott Greens Creek Mining Company is located just southwest of Juneau, Alaska. A high grade sphalerite-galena ore is mined from underground and milled on site. The milling circuit consists of lead, zinc, and bulk flotation circuits; a gravity circuit is also set up to catch the appreciable amounts of electrum that does not respond to the flotation circuit well. The gravity circuit consists of rougher and cleaner spirals and finally a Gemini table. Concentrate from the Gemini table is processed on site and poured into dore bars. Middlings from the Gemini are collected in a barrel and shipped to an off-site facility for cyanide leaching to recovery precious metals. A sample of gravity middlings from Greens Creek mine was obtained to investigate its leachability in the ASGLS.

\subsubsection{Chemistry of Greens Creek Middlings}

A sulfur and carbon analysis was completed on the ore to help understand the matrix that the gold and silver was contained in. Three identical samples were tested to more accurately 
determine the carbon and sulfur concentrations. Results for the carbon/sulfur analysis can be seen below in Table XXIII. Note the high levels of weight percent sulfur while carbon contents are relatively low.

Table XXIII. Greens Creek Middling Carbon \& Sulfur Analysis

\begin{tabular}{|c|c|c|}
\hline Sample & \%-Sulfur by Weight & \%-Carbon by Weight \\
\hline Greens Creek Middling-1 & 15.78 & 0.16 \\
\hline Greens Creek Middling-2 & 15.86 & 0.21 \\
\hline Greens Creek Middling-3 & 15.47 & 0.17 \\
\hline Average & 15.70 & 0.18 \\
\hline
\end{tabular}

A pulverized sample of the Greens Creek Middling ore was taken for analysis on the X-ray Diffractometer (XRD). The results showed that the sample matrix was very high in galena ( $\mathrm{PbS}$ ) with trace amounts of pyrite $\left(\mathrm{FeS}_{2}\right)$. These results are intuitive because galena and pyrite are both heavy minerals that should show up in the gravity circuit. Native gold or electrum did not appear on the XRD scan probably because the concentrations of these minerals was about $1 \%$ for the sample, too low for conclusive XRD detection limits. The full XRD scan for the Greens Creek Middlings can be found in Appendix C.

\subsubsection{Greens Creek Middling Leach Test}

The Greens Creek Middling sample was leached under the following conditions (See Table XXIV) with the laboratory SOP found in section 2.3.4.1. Only ten grams of sample was leached because sample was limited and the high gold grades made it so that only a few grams were required for a fire assay after leaching. Below is a picture of the leach vessel with ore in it prior to leaching.

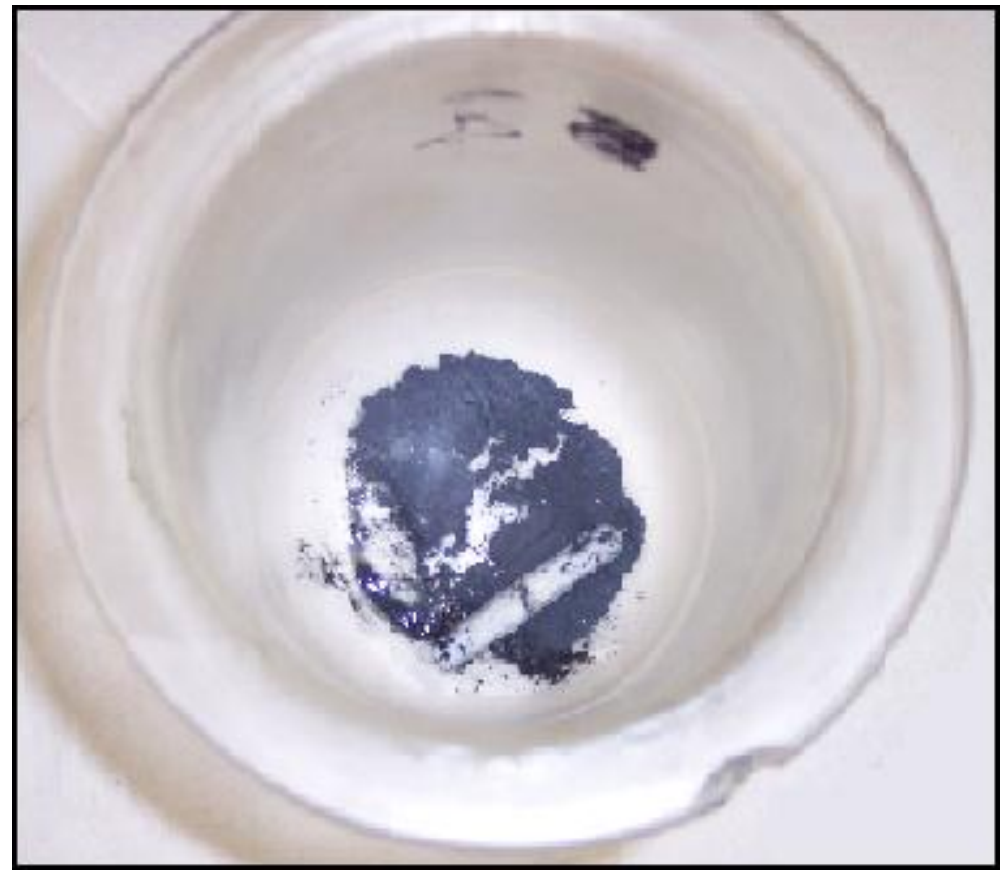


Figure 33. Greens Creek Middlings Prior to Leaching

Both a sample of the ore before leaching and after leaching was submitted for fire assay so that the performance for the leach test could be quantified.

Table XXIV. Greens Creek Middling Leach Test

\begin{tabular}{|c|c|c|c|c|}
\hline Sample Weight (g) & Sulfide (M) & Sulfur (M) & Temperature $\left({ }^{\circ} \mathrm{C}\right)$ & Volume (mL) \\
\hline 10 & 0.62 & 2.5 & 60 & 750 \\
\hline
\end{tabular}

Fire assays were completed by Wayne Olmstead at his Powell Mountain Analytical Laboratory in Deer Lodge, Montana. The leach test performed as seen below in Table XXV. About 93\% of the gold and $85 \%$ of the silver was leached after two days in the ASGLS. These are very good results considering the high head grades for the middling samples.

Table XXV. Greens Creek Middling Leach Test Results

\begin{tabular}{|c|c|c|}
\hline Description & $\mathrm{Ag}$ & $\mathrm{Au}$ \\
\hline Greens Creek Middlings Head (OPT) & 157 & 173 \\
\hline Greens Creek Middlings Tails (OPT) & 23.4 & 11.3 \\
\hline \%-Leached & $85.1 \%$ & \multicolumn{2}{|c|}{$93.5 \%$} \\
\cline { 3 - 3 } & & \\
\hline
\end{tabular}

\subsection{Newmont Roaster Calcine}

Ore obtained from Newmont Mining Company for this thesis is from the roaster found at the Carlin, Nevada mine site. Two ore samples were used: 1) ore that has been oxidized in the roaster but has not yet been leached with cyanide (head); and, 2) ore that has been oxidized and leached with cyanide (tails). Ore that has been roasted is likely to have appreciable amounts of sulfur from sulfide minerals that did not oxidize all of the way to sulfate. It is expected that sulfur in the ore sample will help ASGLS kinetics. The purpose of testing the tails sample is to see if the ASGLS will effectively leach the gold in the ore further than cyanide did.

\subsubsection{Newmont Roaster Calcine Leach Tests}

The Newmont roaster Calcine samples were leached under almost the same conditions as the Greens Creek Middling sample was. Forty grams of ore was used in these tests so that a full assay ton (about 30 grams) would be available for fire assay after the leach test was over.

Table XXVI. Newmont Roaster Calcine Leach Tests

\begin{tabular}{|c|c|c|c|c|c|}
\hline Sample & Weight (g) & Sulfide (M) & Sulfur $(\mathrm{M})$ & Temperature $\left({ }^{\circ} \mathrm{C}\right)$ & Volume $(\mathrm{mL})$ \\
\hline Head & 40 & 0.62 & 2.5 & 60 & 750 \\
\hline Tails & 40 & 0.62 & 2.5 & 60 & 750 \\
\hline
\end{tabular}


Below is a picture of the Newmont Roaster Calcine head before and after leaching. Notice that after leaching the ore has a darker color; this is probably due to the ore absorbing some sulfide during leaching.

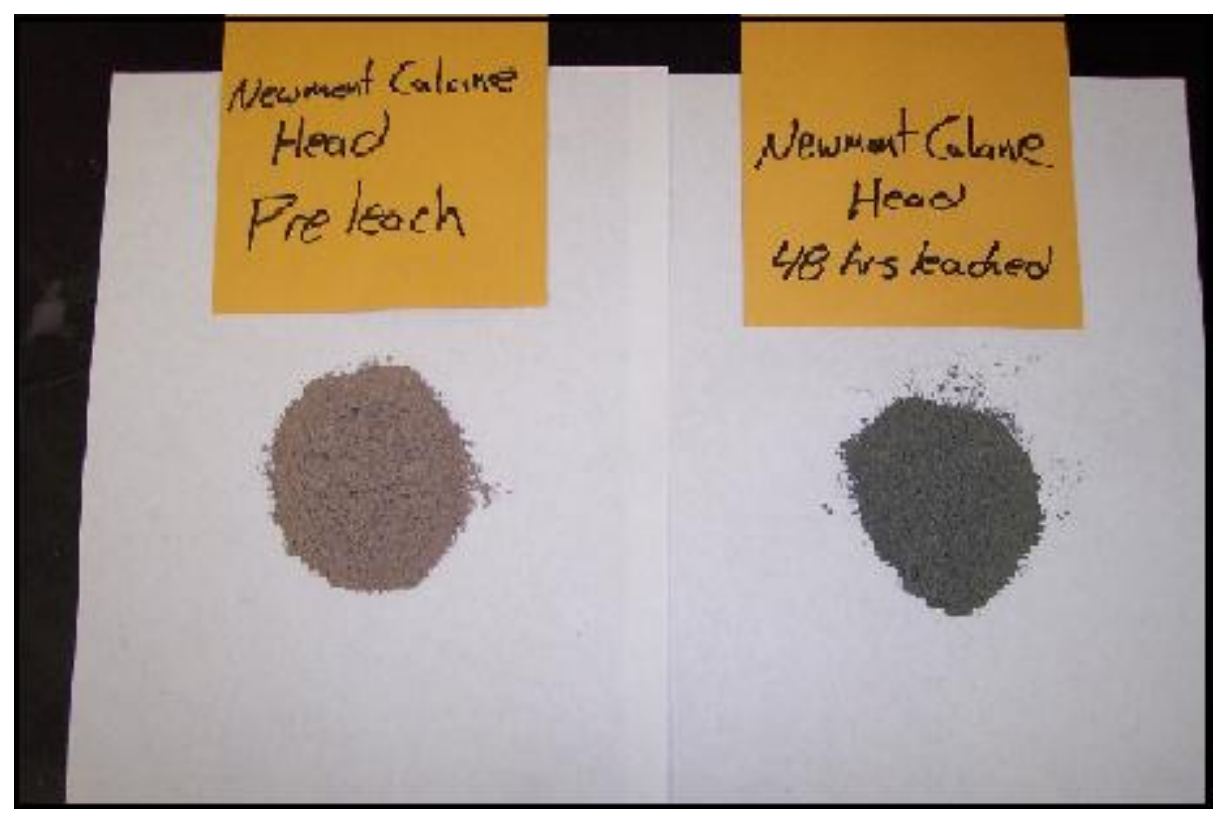

Figure 34. Newmont Calcine Heads Before and After Leaching

Again, all of the fire assays were completed by Wayne Olmstead; they can be seen in the table below. In the head sample, $84 \%$ of the gold and $68.8 \%$ of the silver was leached by the ASGLS in two days. For the tails sample and additional $10 \%$ of the gold and $33.3 \%$ of the silver was leached; these are very good results because the ore had already been treated with cyanide.

Table XXVII. Newmont Calcine Leach Test Results

\begin{tabular}{|c|c|c|}
\hline Description & $\mathrm{Ag}$ & $\mathrm{Au}$ \\
\hline Newmont Calcine Head Pre-leach (OPT) & 0.32 & 0.2120 \\
\hline Newmont Calcine Head Post-leach (OPT) & 0.10 & 0.0340 \\
\hline Newmont Calcine Tails Pre-leach (OPT) & 0.06 & 0.0200 \\
\hline Newmont Calcine Tails Post-leach (OPT) & 0.04 & 0.0180 \\
\hline \%-Leached Heads & $68.8 \%$ & $84.0 \%$ \\
\hline \%-Leached Tails & $33.3 \%$ & $10.0 \%$ \\
\hline
\end{tabular}

Industrial ores have been proven leachable by the ASGLS. Cyanide-like numbers for recovery were seen after two days in the leach system. 


\subsection{Waste Removal}

During waste removal the goal is to oxidize all of the sulfides and polysulfides to sulfate. The process requires sufficient oxidizer and temperature. On the laboratory scale waste removal tests were done in a stainless steel autoclave at elevated temperatures and applied oxygen pressure.

\subsection{Standard Laboratory Procedure for Waste Removal Tests}

For all of the waste removal tests a 2-liter Parr ${ }^{\mathrm{TM}} 316$ stainless steel was used as the reactor. An empty reactor can be seen below.

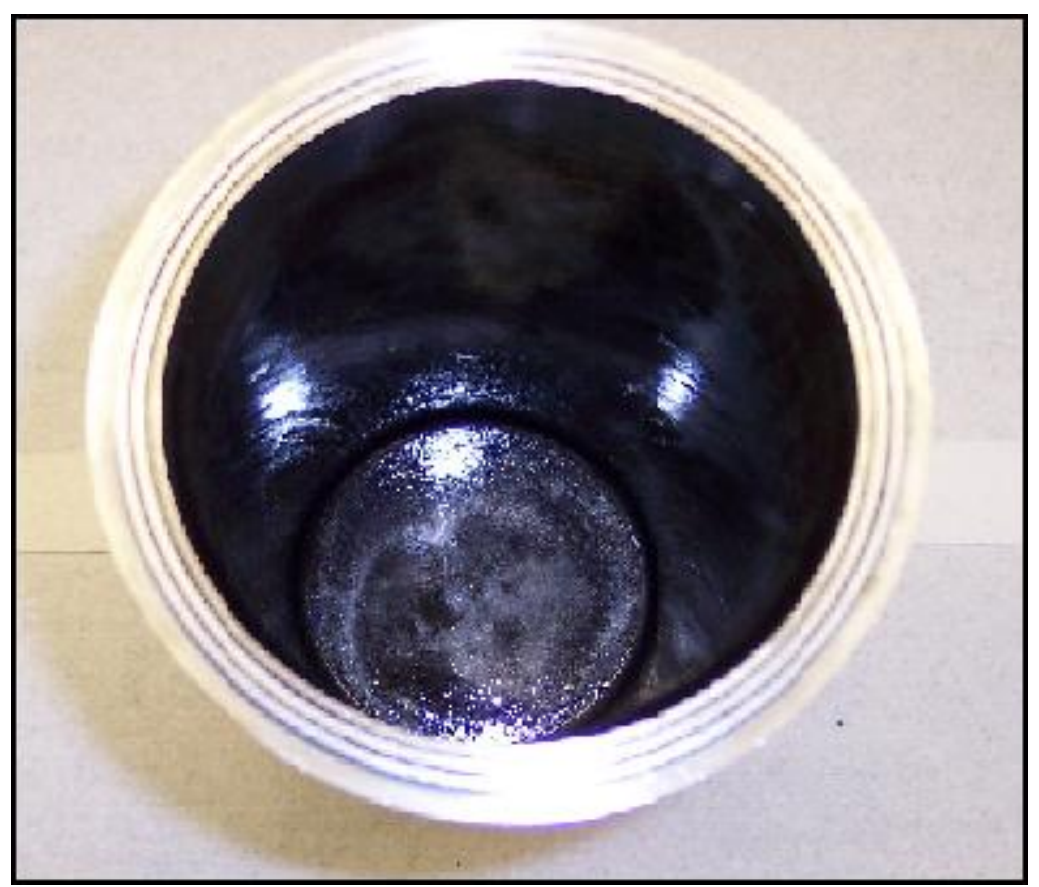

Figure 35. Top View of an Empty Autoclave

The insides of the autoclave can be seen below in Figure 36. Some of the important design features are labeled on the figure. Between each test the autoclave can be taken apart and cleaned before refilling it with new solution. 


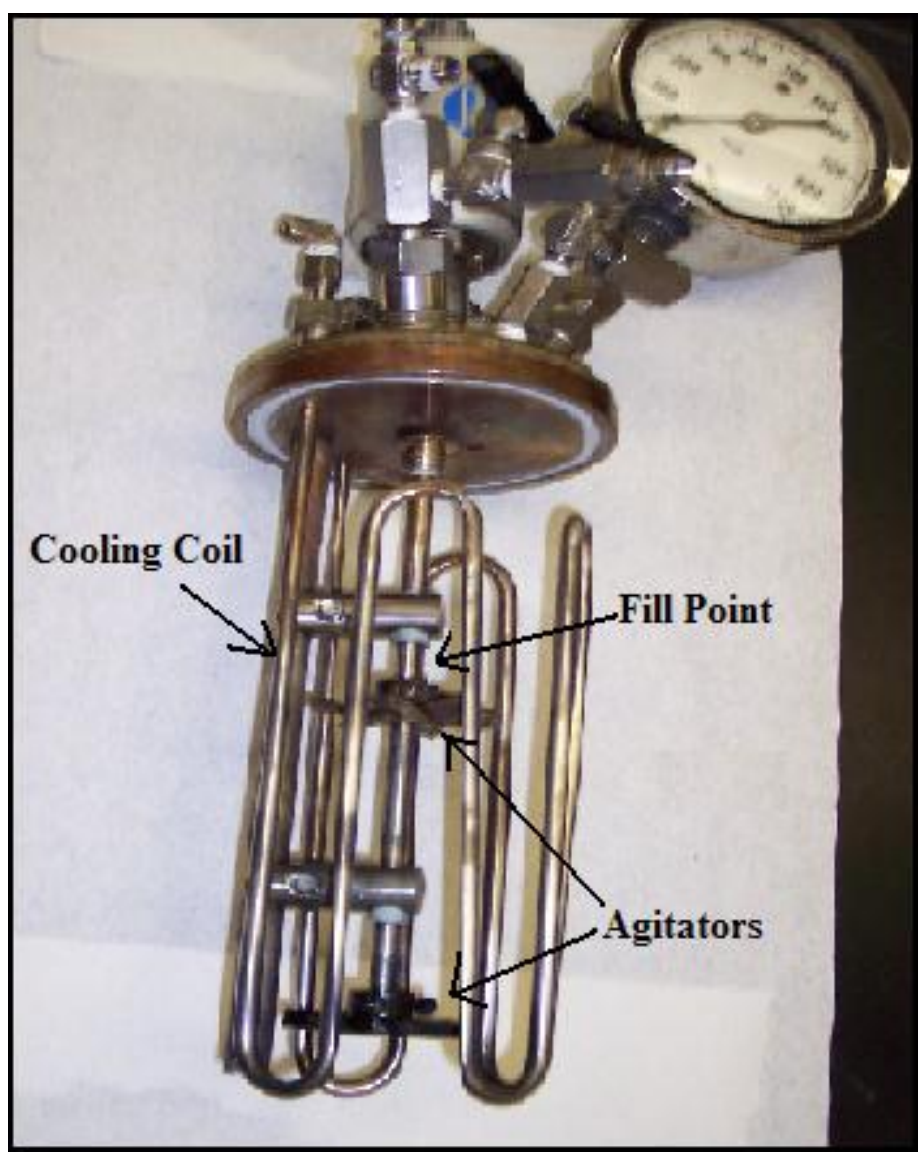

Figure 36. Inside View of the Autoclave

The cooling system is activated when a thermocouple placed in a tube that is in contact with the solution reaches the set temperature. Air is blown through the cooling coil to gently cool the solution back below the set point. For all waste removal tests in this thesis the set temperature was set at $170^{\circ} \mathrm{C}$.

When filling with solution, the autoclave should be filled to the point where the top agitator should be just submerged in the solution. (See Figure 35.) 


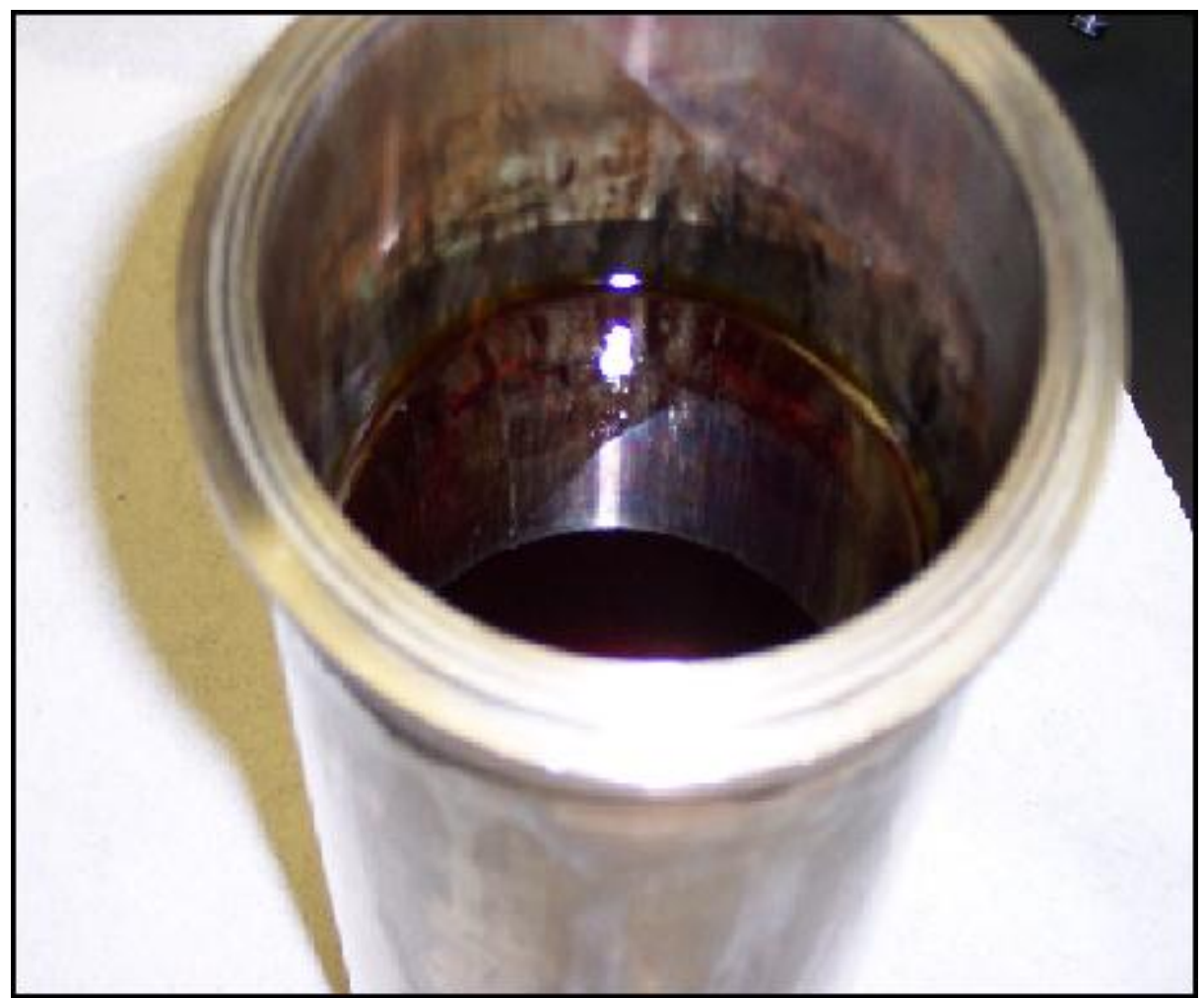

Figure 37 . Autoclave Filled With Waste ASGLS Solution

Finally the autoclave can be put back together. A stainless steel ring is clamped around the seam formed when the autoclave insides and vessel are put together. Figure 36 below shows the autoclave ready for service. 


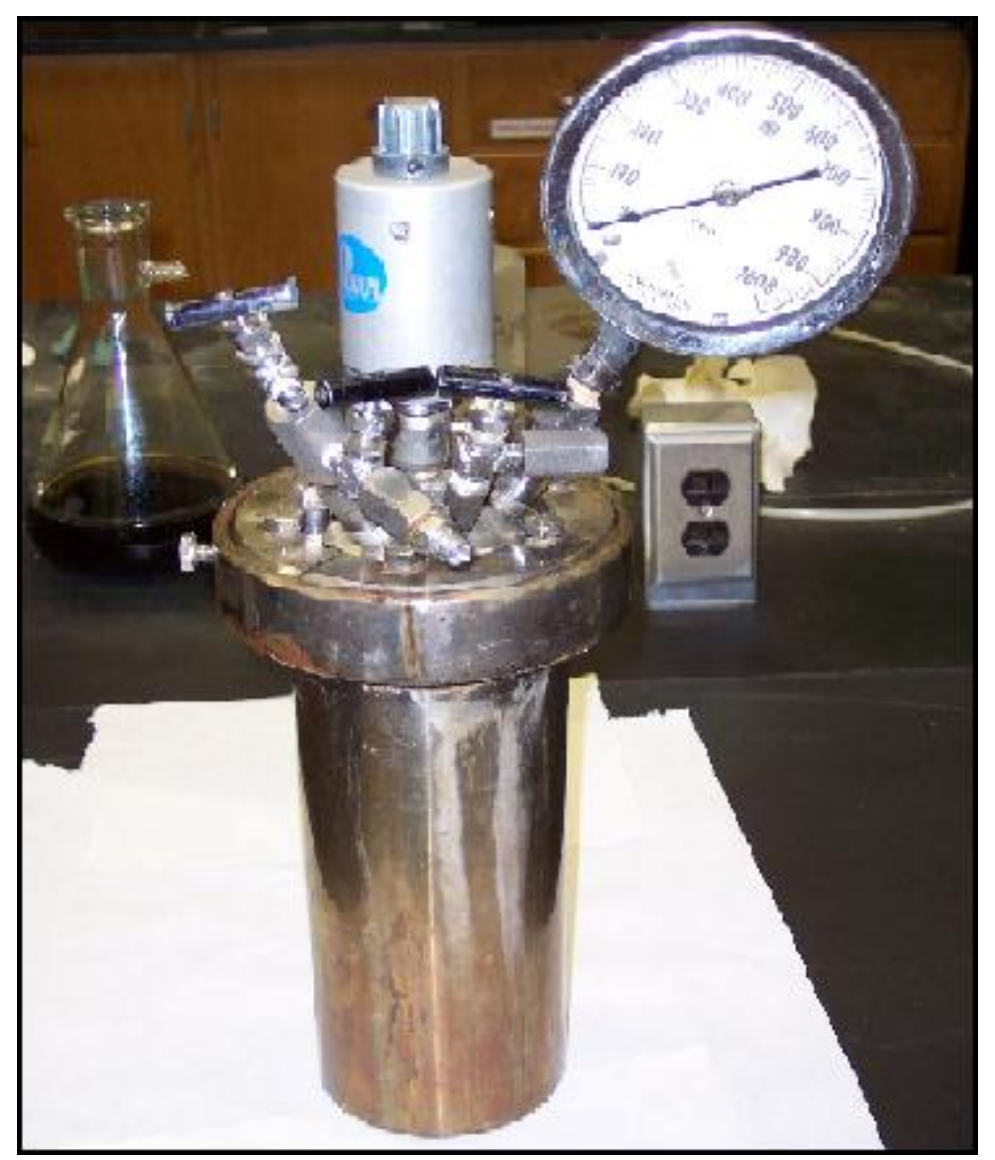

Figure 38. Autoclave Ready for Service

Figure 39 below shows the autoclave during a test. Note the heater and oxygen tube. Oxygen for the autoclave comes from a pressurized oxygen cylinder. For this thesis oxygen pressure was set at $40 \mathrm{psi}$ overpressure, or $140 \mathrm{psi}$ at $170^{\circ} \mathrm{C}$. 


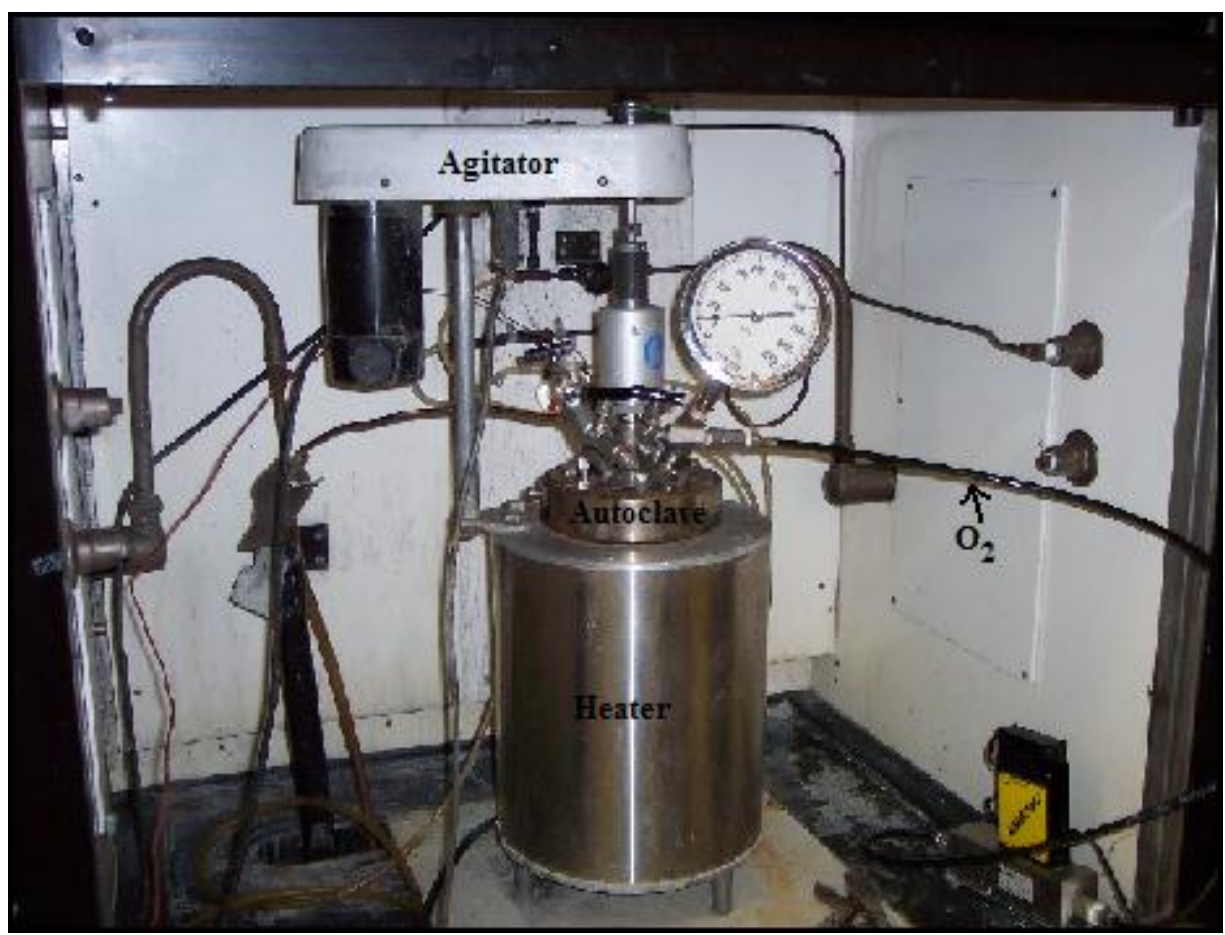

Figure 39 . Operating Autoclave

Results for waste removal are pending - I will have them to both Corby and Larry before they do a second revision.

\subsection{Future Work}

Work on the ASGLS will continue at Montana Tech at least through the next year (2005 - 2006). Work done on this thesis has defined the fundamentals of the ASGLS and it will provide an excellent base for future work. Future work should be focused in the following six areas: 1) Industrial ore leaching; 2) Removal of gold from solution; 3) Stripping gold from the gold removal substrate; 4) Cleaning of waste solutions; 5) Processing of waste solutions; and, 6) Building a pilot scale ASGLS. Each of these six areas will be discussed in further detail below.

\subsection{Industrial Ore Leaching}

Although some industrial ores were tested in this thesis, a wider range of ore should be acquired and tested. Perhaps the most critical type of ore that should be studied is highly refractory ores, especially those that are high in carbonaceous material. These ores should be tested for overall leachability and they should be investigated for preg-robbing characteristics. Also, these ore should be leach with a standard cyanide solution so that more weight can be given to the effectiveness of the ASGLS.

\subsection{Removal of Gold from Solution}

Carbon was shown to effectively remove gold from solution; however, the exact mechanism for carbon loading was left in doubt. The carbon loading mechanism should be defined. Also an ion exchange resin with a practical ASGLS $\mathrm{pH}$ range should be sought after and tested. 


\subsection{Stripping Gold from the Gold Removal Substrate}

If gold cannot be removed from the substrate that it has been loaded onto processing costs are likely to be very steep. Although stronger concentration solutions were found to hold gold in solution better, stripping gold from the carbon with stronger solutions was unsuccessful. An effective technique needs to be developed to make the ASGLS feasible on an industrial scale.

\subsection{Cleaning of Waste Solutions}

When removed from the autoclave the waste solutions are oftentimes quite impure and dirty. A technique should be developed to clean the solutions from precipitates formed during the oxidation process. Also a quality control method should be put into place to insure that the waste solutions have low chemical impurities.

\subsection{Processing of Waste Solutions}

An Electrodialysis (ED) unit has been purchased to process the waste solutions during the next phase of this project. The purpose is to convert the sodium sulfate solutions into one stream of sulfuric acid and one stream of sodium hydroxide. Theoretically the sodium hydroxide process stream should be able to be pumped back into the front end of the ASGLS as the polysulfide stabilizer. Process parameters for the ED waste solution processing should be defined.

\subsection{Building a Pilot Scale ASGLS}

Once the above objectives have been met a pilot scale leach plant with an industrial ore should be devised. The pilot plant should be able to: leach gold from ore; load aqueous gold onto a substrate; remove gold from the removal substrate; recirculate leach solution; treat solutions as waste; and convert waste streams into useful waste steams of sulfuric acid and sodium hydroxide.

\section{Thesis Conclusions}

Overall the research completed during this thesis provided valuable data as to the behavior of the ASGLS. Gold was successfully leached with the ASGLS; also, the parameters for leaching gold were optimized. A standard operating procedure for laboratory leach tests, gold recovery from solution tests, and waste removal tests was developed and practiced in the laboratory. Factors controlling electrochemical and electrokinetic phenomenon within the ASGLS are now understood. Industrial ores were leached with positive results. Waste solutions were successfully oxidized for disposal. With the help of this thesis future research in the area of the ASGLS will be much more productive. Conclusions for each of the areas covered in this thesis will be covered in greater detail below.

\subsection{Electrochemical \& Electrokinetic Conclusions}

By the reduction of polysulfides gold is oxidized. The oxidized gold may then be lixiviated by sulfide. Therefore solutions containing both sulfide and polysulfide will leach gold. Benefits to leach kinetics include the following: 1) increase the total sulfur concentration greater than 2 
molar sulfur; 2) add sulfur and sulfide at a ratio of $1.5-2: 1$; 3) increase the reaction temperature; 4) increase the hydroxide concentration; and, 5) decrease the purity of the gold with silver to at least $5 \%$ silver.

\subsection{Laboratory Leach Test \& Optimization Conclusions}

Mocking the ASLGLS on a laboratory scale is difficult but was completed with small experimental error between tests. Optimization results were similar to those found during the electrochemical \& electrokinetic phases of this thesis. By increasing total sulfur concentration, temperature, and hydroxide concentration gold leaching kinetics are increased.

\subsection{Gold Removal Conclusions}

Activated carbon was shown to successfully remove dissolved gold from solution. Some ion exchange resins were tested but failed to remove any gold from solution; this is likely due to the high $\mathrm{pH}$ range the ASGLS operates in. Although a window of opportunity for carbon stripping was spotted it was never tested positively. Also the actual mechanism for gold loading onto carbon is in question because in some solutions where silica glass was added to the solution gold removal kinetics were increased.

\subsection{Industrial Ore Leaching Conclusions}

Both the Greens Creek middlings and the Newmont calcine were leached very well by the ASGLS; 93.5\% and 84.0\% was leached, respectively. Also, a sample of Newmont calcine that had already been leached with cyanide had an additional $10 \%$ of the gold leached from it. All of these results are positive because these leach recoveries are on the level of cyanide recoveries.

\subsection{Waste Removal Conclusions}

Conclusions are pending - I will have them to both Corby and Larry before they do a second revision. 


\subsection{Bibliography}

Louw, N.J., Edwards, A.M., and Gussmann, H.W., A New Process to Extract Gold and Stibnite from Arsenical Concentrates, Chemical Engineering Conference

Jeffrey, M.I. and Anderson, C.G., A Fundamental Study of the Alkaline Sulfide Leaching of Gold,

Anderson, C.G., Applications of NSC Pressure Leaching

Jeffery, M.I., Zheng, J., and Richie, I.M. (2000) The Development of a rotating Electrochemical Quartz Crystal Microbalance for the Study of Leaching and Deposition of Metals, Measurement and Scientific Technology

Ward, M.D. (1995) Principles and Applications of the Electrochemical Quartz Crystal Microbalance, Physical electrochemistry: Principles, Methods and Applications, Edition I. Rubinstein (New York: Dekker) pgs. 293 - 338

Jeffery, M.I., Chapman, N., and Wall, J., Sulfide/Polysulfide Leaching of Gold

Hiskey, J.B., and Atluri, V.P., Dissolution Chemistry of Gold and Silver in Different Lixiviants, Mineral Processing and Extractive Metallurgy Review, 1988, Vol.4 pgs. 95-134

Power, G.P., and Richie, I.M., Metal Displacement Reactions. Modern Aspects of Electrochemistry, Plenum Press, 1975, London, pgs. 199-250

Jian Z., Xinzhe, L., Feng, D. and Zhichang, Y. Leaching Gold and Silver by Lime-SulfurSynthetic-Solution Part I

Jian Z., Xinzhe, L., Feng, D. and Zhichang, Y. Leaching Gold and Silver by Lime-SulfurSynthetic-Solution Part II

Hiskey, J.B., and Sanchez, V.M., Alkaline Pressure Oxidation of a Gold Bearing Arsenopyrite Concentrate, Mineral Processing and Extractive Metallurgy Review, 1995, Vol 15. pgs. 61-74

Stefansson, A., and Seward, T.M., Gold(I) Complexing by Sulfide Ligands in Hydrothermal Solutions to $450^{\circ} \mathrm{C}$ at 500 Bar, Goldschmidt 2000 Journal of Conference Abstracts. Volume 5(2), pg 953.

Behm, M., and Simonsson, D., Electrochemical Production of Polysulfides and Sodium Hydroxide from White Liquor Part I: Experiments with Rotating Disc and Ring-disc Electrodes, Journal of Applied Electrochemistry, 1997, Vol. 27, pgs. 507-518 
Behm, M., and Simonsson, D., Graphite as Anode Material for the Electrochemical Production of Polysulfide Ions in White Liquor, Journal of Applied Electrochemistry, 19997, Vol. 29, pgs. 321-324

Louw, N.J., Edwards, A.M., and Gussman, H.W., A New Process to Extract Gold and Stibnite from Arsenical Concentrates, CHEMSA, August, 1977

Linge, H.G., New Technology for Refractory Gold Ores, Extractive Metallurgy of Gold and Base Metals, Kalgoorlie, Oct. 26-28, 1992

Aylmore, M.G, and Muir, D.M. Thiosulfate Leaching of Gold - A Review, Minerals Engineering, Vol. 14, No. 2, pgs. 135-174, 2001

Anderson, C.G., Nordwick, S.M., and Krys, L.E. Processing of Antimony at the Sunshine Mine., Residues and Effluents - Processing and Environmental Considerations, The Minerals, Metals \& Materials Society, 1991, pgs. 349-366

Renders, P.J. and Seward, T.M., The Stability of Hydrosulfido- and Sulfido-Complexes of $\mathrm{Au}(\mathrm{I})$ and $\mathrm{Ag}(\mathrm{I})$ at $25^{\circ} \mathrm{C}$, Geochimica et Cosochemica Acta Vol. 53, pgs. 245-253. 1989

Shenberger, D.M., and Barnes, H.L., Solubility of Gold in Aqueous Sulfide Solutions from 150 to $350^{\circ} \mathrm{C}$, Geochimica et Cosochemica Acta Vol. 53, pgs. 269-278. 1989

Hayashi, K. and Ohmoto, H., Solubility of Gold in NaCl- and $\mathrm{H}_{2} \mathrm{~S}$-bearing Aqueous Solutions at 250-350 ${ }^{\circ} \mathrm{C}$, Geochimica et Cosochemica Acta Vol. 55, pgs. 2111-2126. 1991

Murray Jr., R.C., and Cubicciotti, D. Thermodynamics of Aqueous Sulfur Species to $300^{\circ} \mathrm{C}$ and Potential-pH Diagrams, Electrochemical Science and Technology, Vol. 130, No.4, 1983, pgs. 866-868

Seward, T.M., Thio Complexes of Gold and the Transport of Gold in Hydrothermal Ore Solutions, Geochimica et Cosochemica Acta Vol. 37, pgs. 379-399. 1973

Gilbert, F., Pascal, M.-L., and Pichavant, M. Gold Solubility and Speciation in Hydrothermal Solutions: Experimental Study of the Stability of the Hydrosulfide Complex of Gold (AuHSo) at 350 to 450oC and 500 Bars. Geochimica et Cosochemica Acta Vol. 62, pgs. 2931-2947. 1998 
During February 2002 and June-August 2004, a Rotating Electrochemical Quartz Crystal Microbalance (REQCM) was used to better understand the fundamental electrochemical and electrokinetic principals in the alkaline sulfide gold leaching system. All of the experiments were done on the REQCM located in the Chemical Engineering Department of Monash University, just southeast of Melbourne, Australia. Variables that were investigated in the system include:

- $\quad$ Effect of sulfide concentration $\left[\mathrm{S}^{2-}\right]$;

- $\quad$ Effect of hydrosulfide [HS ${ }^{-}$;

- $\quad$ Effect of polysulfide concentration $\left[\mathrm{S}_{\mathrm{x}}{ }^{2-}\right]$;

- $\quad$ Effect of $\mathrm{NaOH}$ concentration;

- $\quad$ Effect of temperature; and,

- $\quad$ Effect of gold purity.

\section{REQCM Background}

The REQCM is able to read both electrical and mass changes taking place on the metal surface. To make the working electrode, a thin, disc shaped quartz crystal has a platinum film sputtered onto it. Platinum is sputtered on to enable both current to pass through the electrode and so that metal can be deposited onto the surface prior to an experiment. Two wires are connected to the crystal; the first simply provides a path for electrical current to flow, the second sends a signal to a frequency reading device that can read what the resonant frequency of the crystal is, usually about $10 \mathrm{MHz}$. As the mass of the quartz crystal increases, the resonant frequency in the crystal decreases.

During the course of an experiment, mass (gold, silver, or alloy) is first deposited onto the platinum and then it is leached off. As mass is deposited the resonant frequency will go down; correspondingly, as mass is leached off of the surface the resonant frequency will go up. Resonant frequency changes can be related to changes in mass on the surface of the crystal. Mass changes in the range of \pm 1 nanogram $\left(10^{-9}\right.$ grams) can be accurately measured.

\section{Experimental Procedure}

During an experiment on the REQCM, the solid to be leached is electrically deposited onto the platinum surface until a desired solid mass has been plated, usually about $150 \mu$ g. All electrodes were plated with the same procedure; however, depending on the type of metal that was to be studied, different plating solutions are required. Plating solutions for $\mathrm{Au} / \mathrm{Ag}$ will have the concentrations found in Table I. Naturally, a pure Au solution will have no silver nitrate added. 


\begin{tabular}{|c|c|c|}
\hline \multicolumn{3}{|c|}{ TABLE I. GOLD/SILVER ALLOY SOLUTION } \\
\hline Chemical & Name & Concentration \\
\hline KAuCN & Potassium Dicyanoaurate & $0.02 \mathrm{M}$ \\
\hline $\mathrm{K}_{2} \mathrm{CO}_{3}$ & Potassium Carbonate & $0.086 \mathrm{M}$ \\
\hline $\mathrm{KCN}$ & Potassium Cyanide & $0.23 \mathrm{M}$ \\
\hline $\mathrm{AgNO}_{3}$ & Silver Nitrate & $*$ \\
\hline
\end{tabular}

Once the electrode is plugged in to the holder, the software will start to transfer resonant frequencies into mass. Because each crystal has a unique mass it is important to note the starting mass so that the counsel can be turned off once there has been about $150 \mu \mathrm{g}$ of metal plated. All plating is done while the electrode is spinning. Gold is plated onto the platinum flag at $25 \mathrm{~A} / \mathrm{m}^{2}$. The resulting gold surface is uniform and smooth. After $150 \mu \mathrm{g}$ have been plated the current and rotator are turned off and the electrode can be removed from the holder.

The freshly gold plated electrode will have gold plating solution on it after it is removed. All of the solution is washed off and the electrode is dried off with compressed air. Now the dry, gold-plated working electrode is ready to be plugged back into the holder for experiments.

The software package for the REQCM is called ___ . It has been set up to calculate the mass on the crystal with inputs of frequency. For the alkaline sulfide system, two different types of plots were obtained with the software: leach tests and potential scans.

During a leach test the electrode is simply submerged into the solution and the rate at which metal leaves the surface is recorded along with the Mixed Potential $\left(E_{m}\right)$, the potential at which leaching occurs. A typical leach test can be seen in Figure 1. 


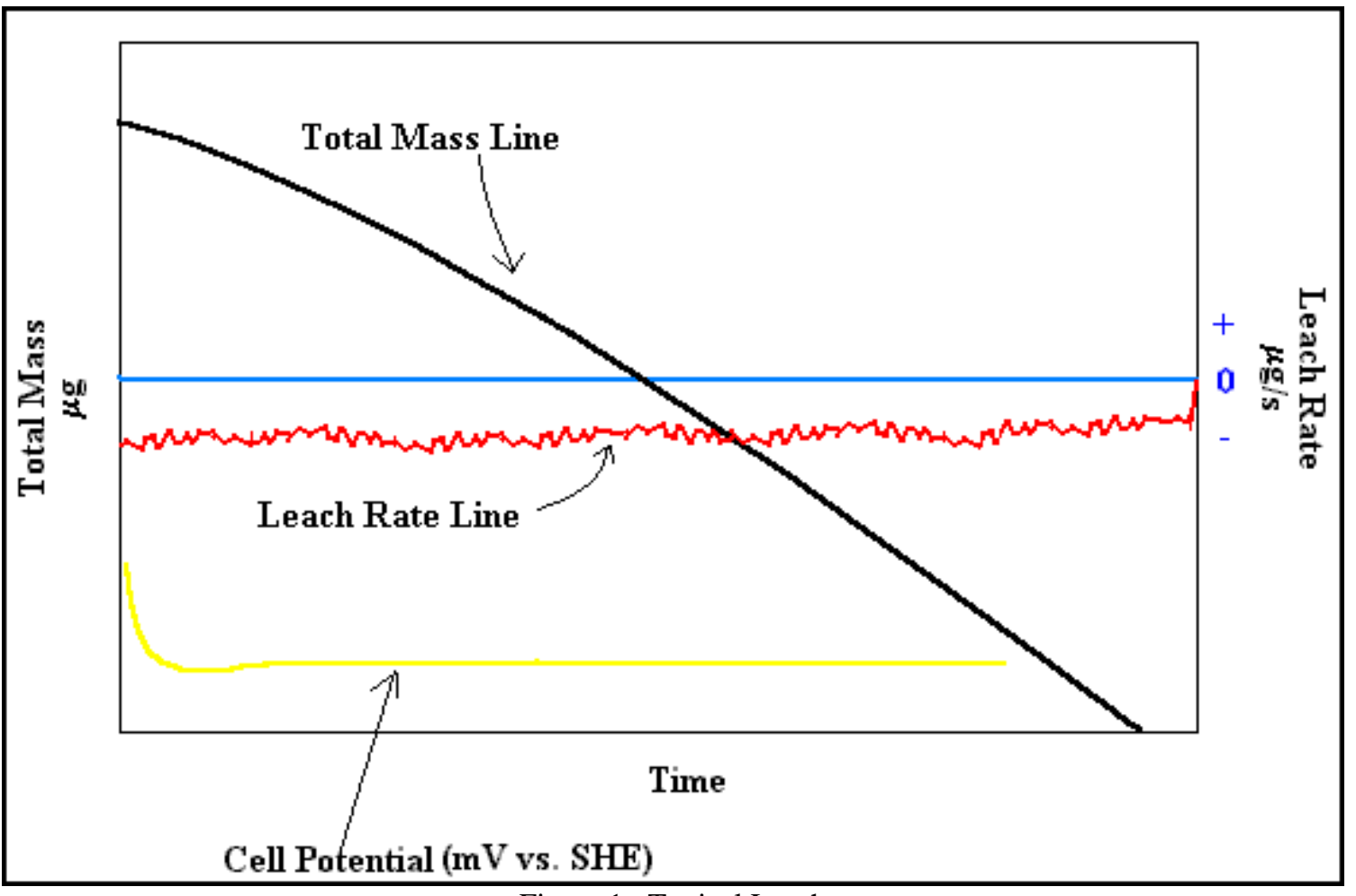

Figure 1. Typical Leach

Note that after the system reaches equilibrium the cell potential $\left(E_{m}\right)$ will level off. Also note the noise in the leach rate line. Even though it is relatively noisy, ( \pm 5 nanograms of the average leach rate) the overall mass loss line is smooth because with a properly working electrode the noise will average out to zero over time. Over a short time the total mass line will fall into a constant slope. The leach rate will be a negative value because mass is being lost off of the crystal.

During a potential scan, the cell potential is adjusted during the entire length of the experiment. Total current, leach rate, and cell potential are measured continuously during the scan. Before a scan is started the sell potential should be set at about $10 \mathrm{mV}$ below the $\mathrm{E}_{\mathrm{m}}$ so that the exact point where the total current goes from negative (reducing) to positive (oxidizing) can be seen on the graph.

Once the potential scan (forward scan) has reached a potential deemed high enough for the experiment the scan is reversed and potentials are allowed to return (back scan) to the starting point of the scan. Ideally the forward and back scans should be identical, but surface phenomena such as passivity often make the two scans different. Surface phenomena cause problems with potential scan reliability, so they should be avoided.

To avoid some of these surface phenomena on the forward scan, a leach test should be run first. If no leach test needs to be done, the electrode should sit in solution for about 5 minutes before starting the scan. For the sulfur/sulfide leaching system, passivity on the 
back scan can be avoided by reversing the scan before sulfide can be oxidized to sulfur on the electrode surface.

\section{Solution Preparation}

All solutions were prepared with solid reagent and de-ionized water. The solids used over the course of work were: sodium sulfide, sulfur, sodium hydroxide, and sodium hydrosulfide. Experimental solutions were prepared in $25 \mathrm{~mL}$ samples. Solids were weighed to $1 / 1000$ of a gram; once all solids were weighed, about $15 \mathrm{~mL}$ of water was added. A magnetic stir bar was dropped and the solution was placed on a hot plate to help in dissolution of solids. As soon as the last solid dissolved, the solution was removed from the heat and allowed to cool. Once cool, the solution was added to a volumetric flask, filled to volume, and added to the REQCM vessel.

\section{Effect of Sulfide $\left(\mathbf{S}^{2-}\right)$ Concentration}

Four potential scans were run on varying sulfide concentration solutions. No leach tests were run on the sulfide solutions because leach rates are effectively too slow to measure, even at the test temperature of $50^{\circ} \mathrm{C}$. From the graph in Figure 2, increases in sulfide concentration enhance gold oxidation. Also, gold oxidation occurs at lower potentials as sulfide concentration increases.

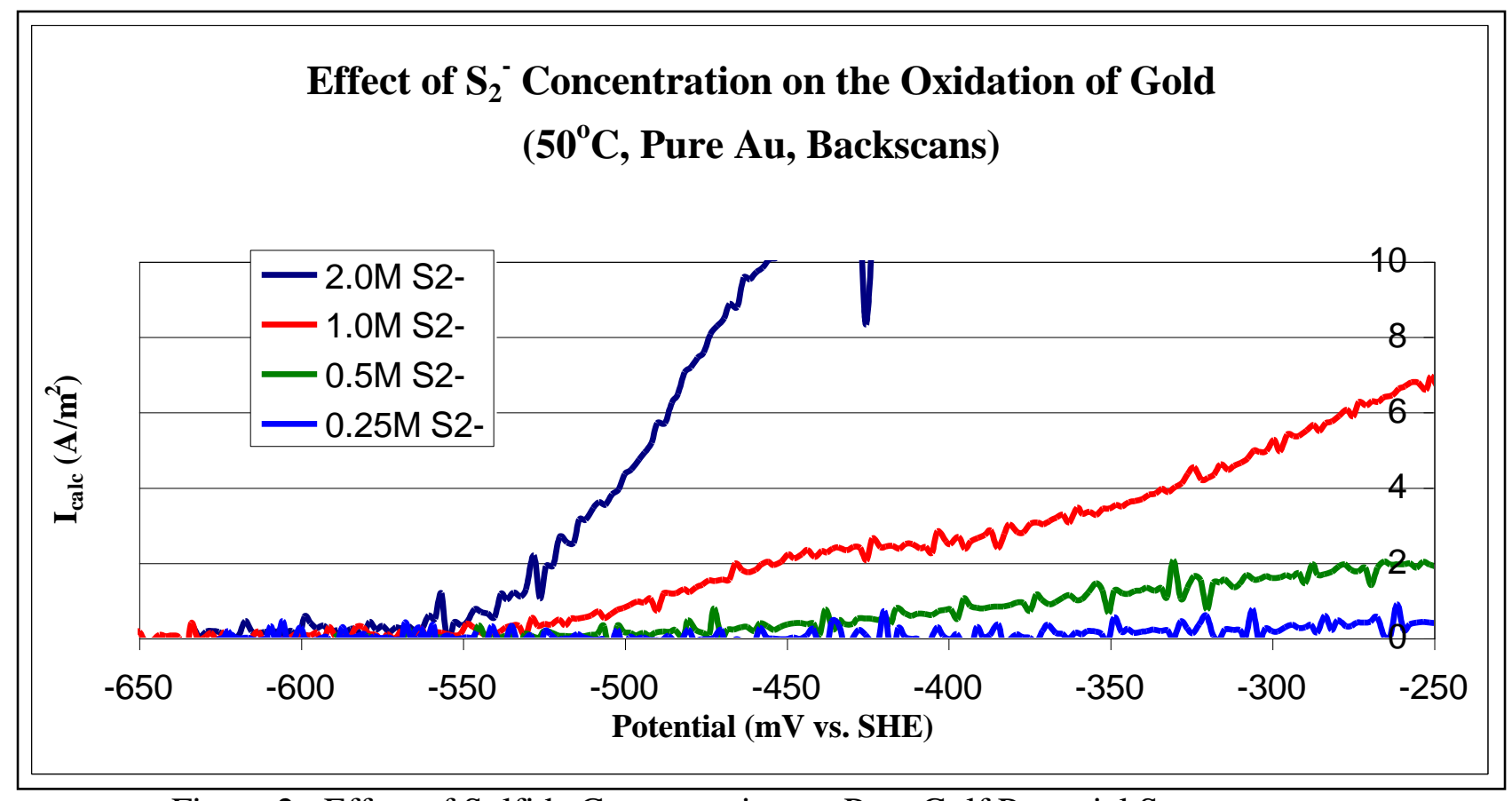

Figure 2: Effect of Sulfide Concentration on Pure Golf Potential Scans

\section{Effect of Hydrosulfide (HS ${ }^{-}$}


Tests were done to see if adding hydrosulfide rather than sulfide had an effect on the oxidation of gold. From Figure 3, it is shown that hydrosulfide has a negative impact on gold oxidation at higher potentials when compared to sulfide. However, it is important to

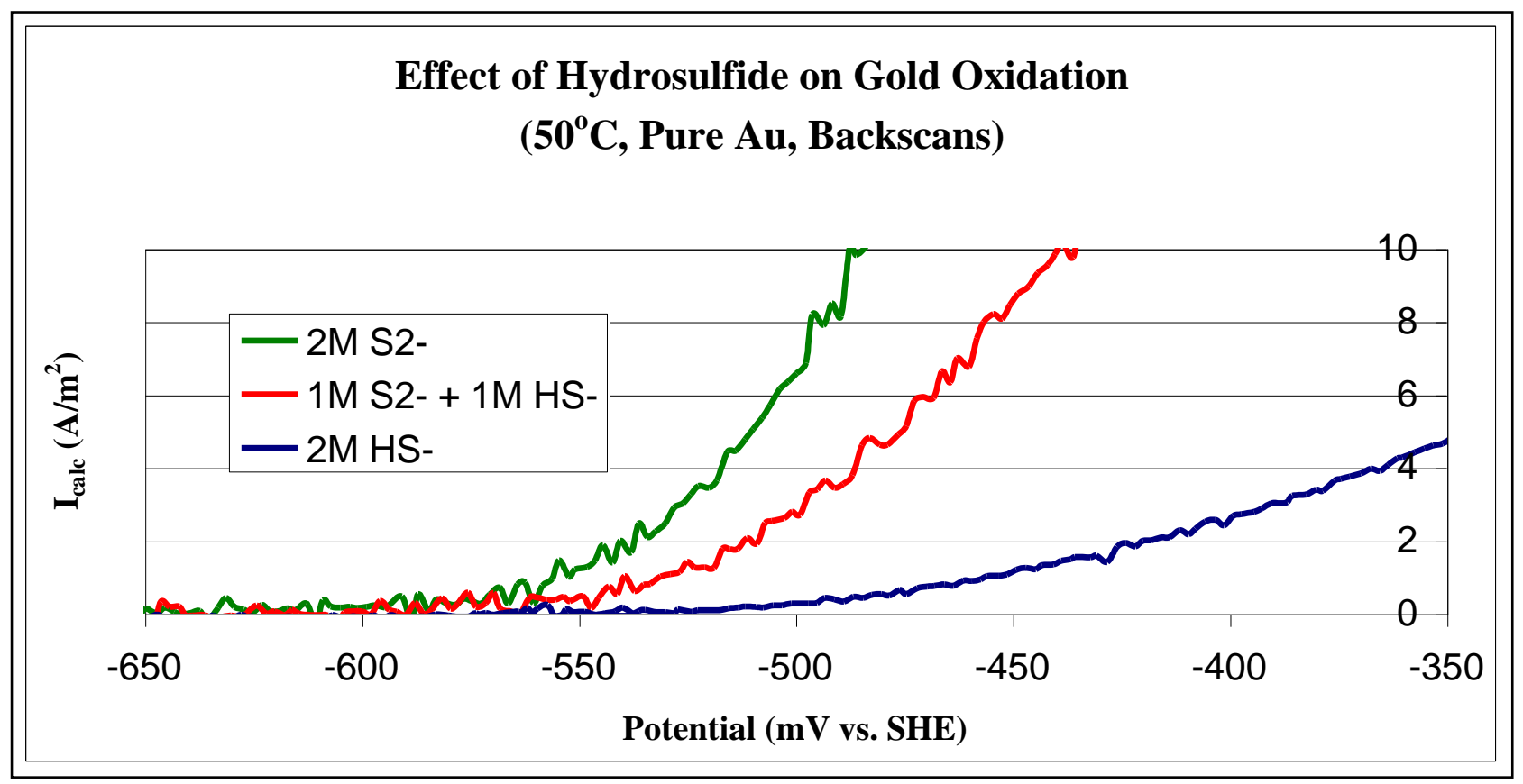

note that by adding hydrosulfide, the $\mathrm{E}_{\mathrm{m}}$ is raised.

Figure 3. Effect of Hydrosulfide on Gold Potential Scans

\section{Effect of Polysulfide}

To better understand the effects of polysulfide on gold oxidation two different tests were completed. In the first test the total sulfide concentration was held at 2 molar while the sulfur concentration was increased from 0-3 molar. During the second test the total sulfur (sulfide + sulfur) concentration was held at 2 molar and the mole-\% sulfur in the 2 molar solution was varied from 0-30 mole-\%.

$$
\mathrm{E}_{\mathrm{m}}=-720 \mathrm{mV}
$$$$
E_{m}=-660 \mathrm{mV}
$$

Figure 4 displays what happens with increasing sulfur concentration when sulfide concentration is held at 2 Molar. Notice there is little difference when potentials are lower than $-400 \mathrm{mV}$ vs. SHE; however, $\mathrm{E}_{\mathrm{m}}$ continues to get higher as sulfur concentration is increased. The effect of $E_{m}$ increasing can be seen in the leach tests in Figure 5. 


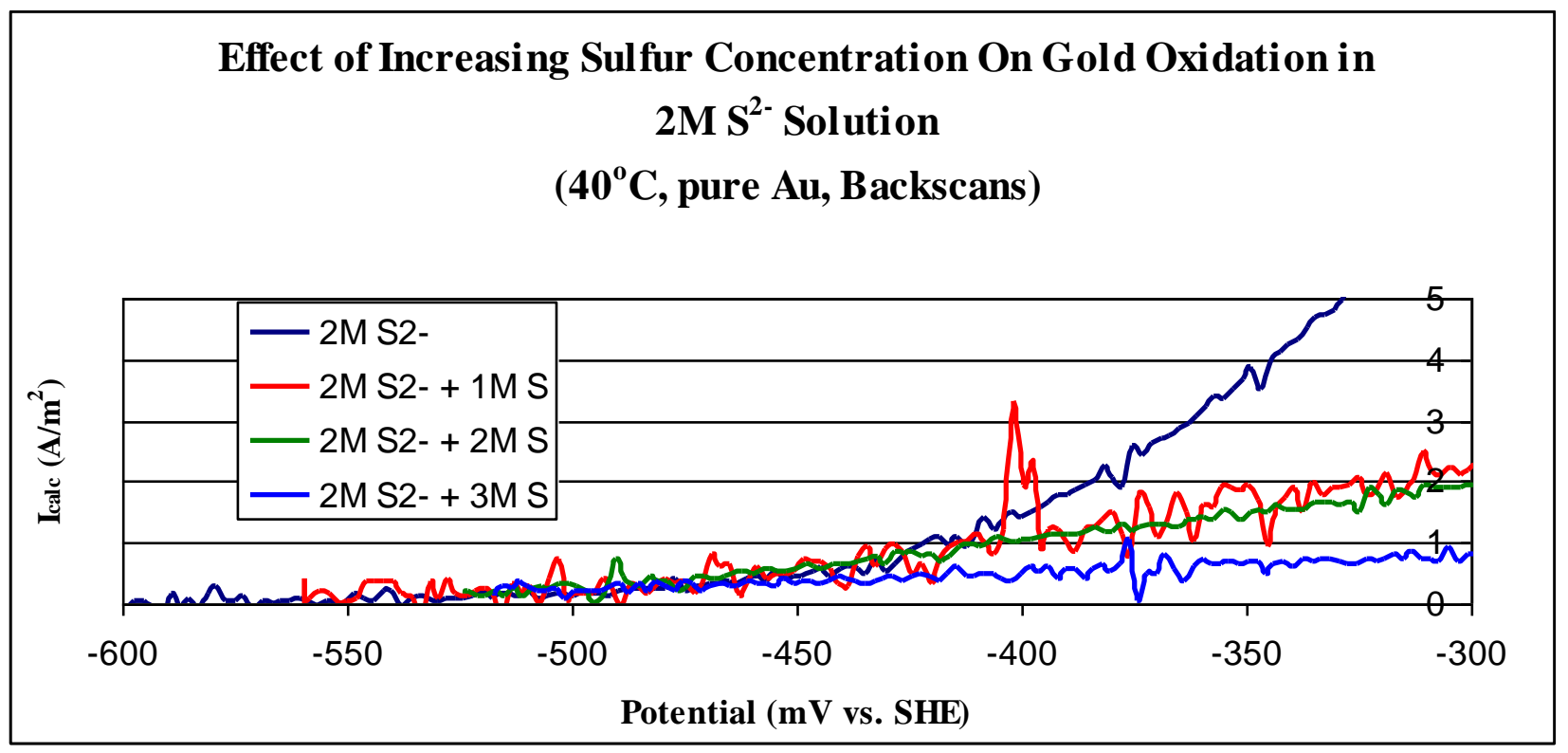

Figure 4. Effect of Increasing Sulfur Concentration on Gold Potential Scans

Solutions of $2 \mathrm{M} \mathrm{S}^{2-}$ essentially did not leach; in other words, the current flow due to the oxidation of gold is essentially zero at the solution potential $\left(E_{m}\right)$. As sulfur concentration increases, the leach rate also increases. Leach rates increased from 0.2 to $6.5,7.2$, and 8.3 nanograms/second respectively for $0,1,2$, and 3 molar sulfur additions to $2 \mathrm{M} \mathrm{S}^{2-}$.

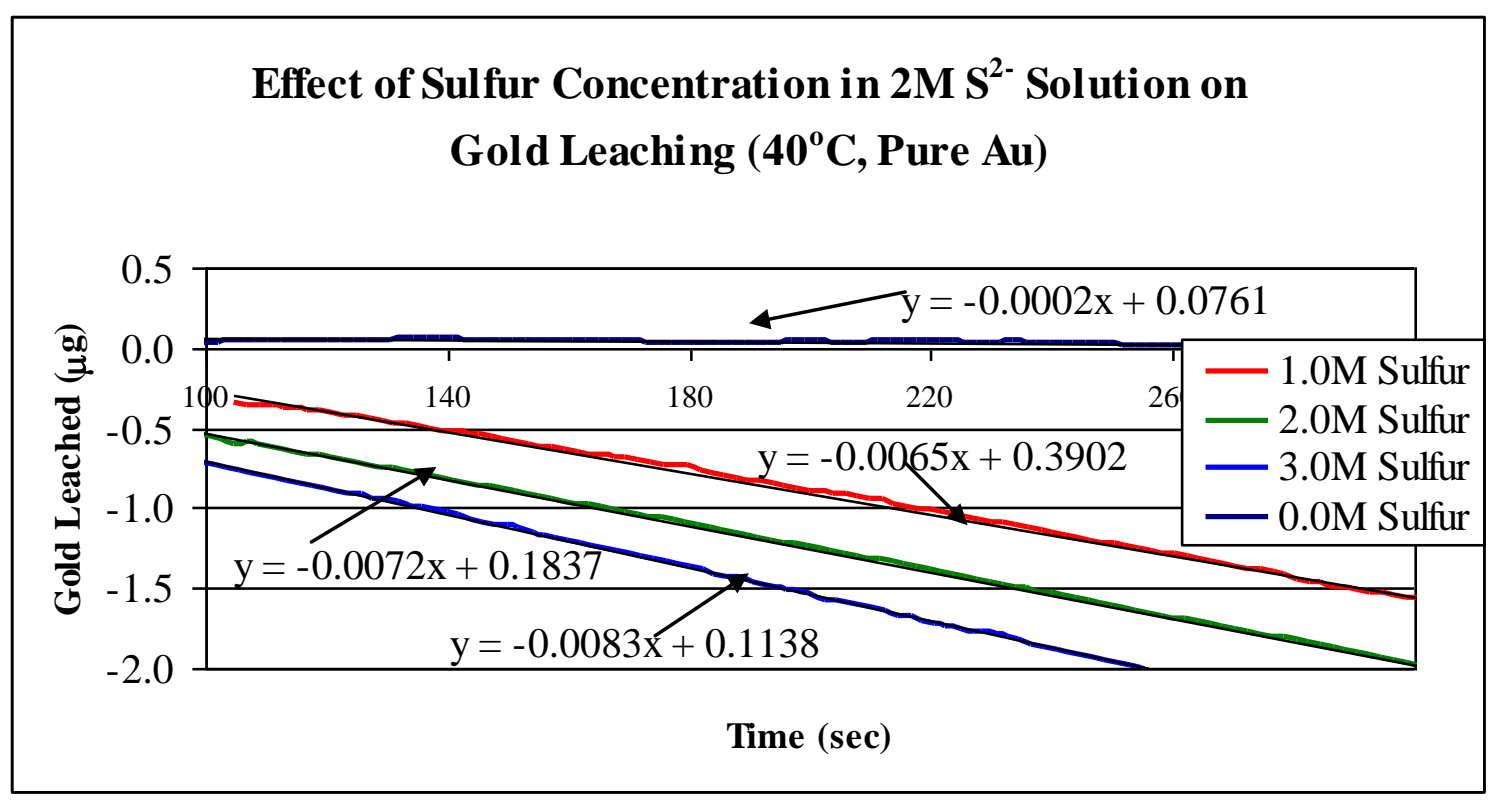

Figure 5. Corresponding Leach Tests to Solutions From Figure 6 
Holding the total sulfur concentration at 2 molar produced some informative results as to why leaching is difficult under these conditions. Even though the sulfur and sulfide concentrations are changing, there is still no real leaching occurring because $\mathrm{E}_{\mathrm{m}}$ potentials are occurring where gold oxidation is essentially zero (See Figure 6.) A small amount of leaching can be expected as sulfur concentration goes toward 100 mole-\%( $E_{m}$ increases to gold oxidation regions); however, the leach rates would most likely be insignificant.

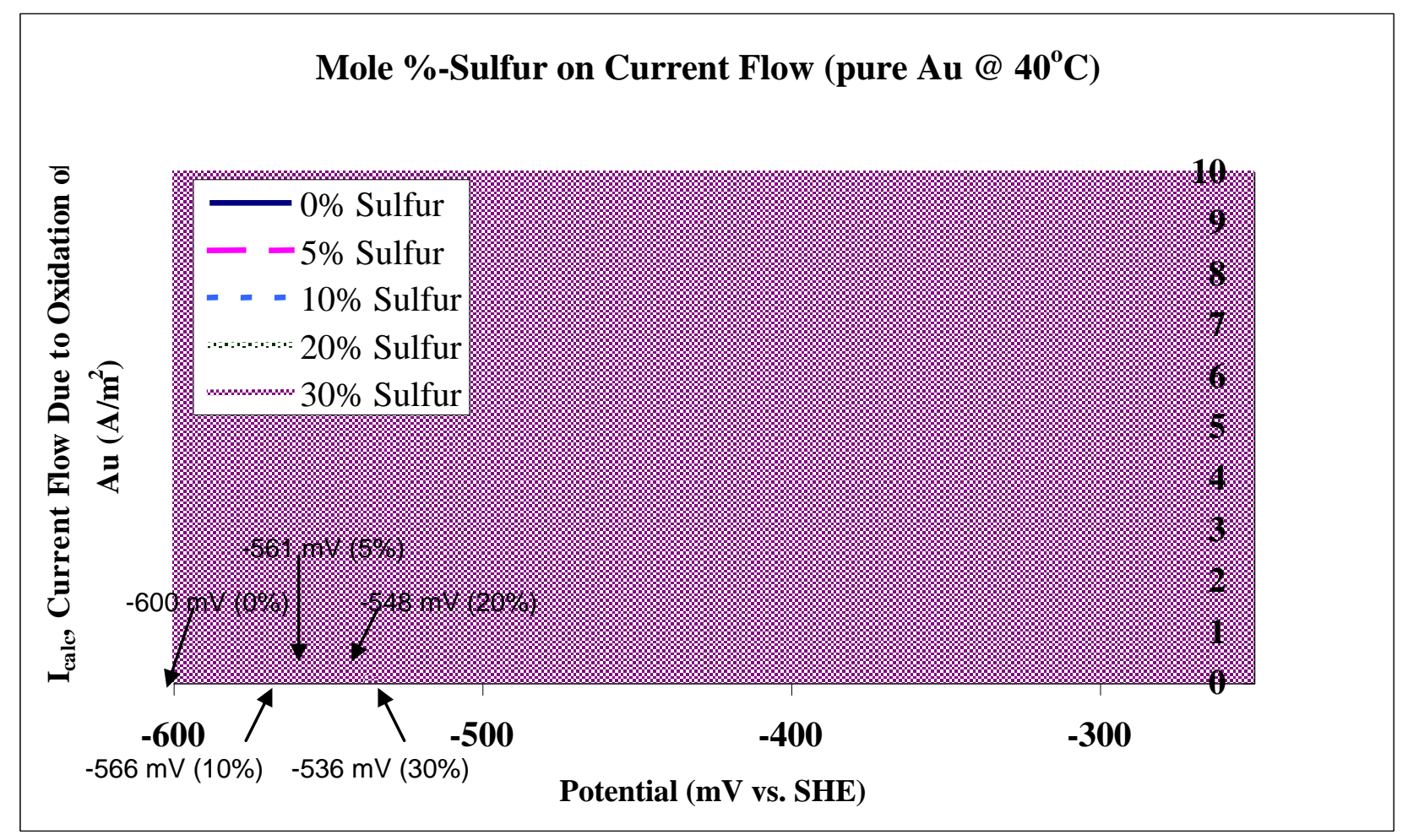

Figure 6. 2M Total Sulfur Pure Gold Potential Scans

\section{Effect of Hydroxide Addition}

Adding $\mathrm{NaOH}$ to the leaching system was tested to see the effect it had on gold oxidation. The first test that was run was done with $2 \mathrm{M} \mathrm{S}^{2-}$ with concentrations of hydroxide ranging from 0-3 molar (See Figure 7.) From Figure 7 it is shown that the addition of hydroxide increases the oxidation of gold to lower potentials. 


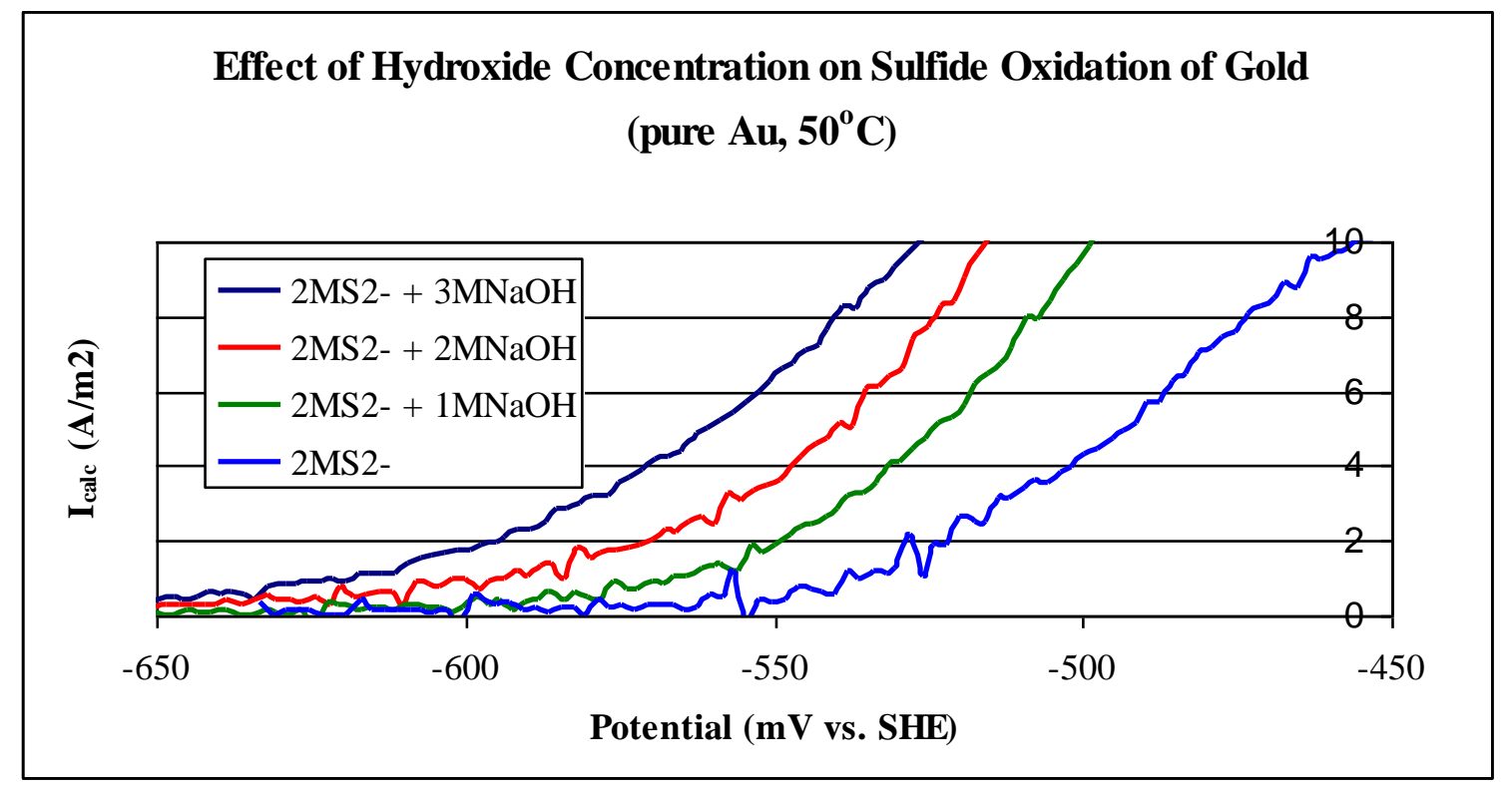

Figure 7. Effect of Increasing Hydroxide Concentration in $2 \mathrm{M} \mathrm{S}^{2-}$ Solution

Tests were also run to see effects of adding hydroxide to a polysulfide solution. During the polysulfide test work, the 1.2 gram rule was used on the solutions. Two different polysulfide solutions were prepared, one had $2 \mathrm{M} \mathrm{S}^{2-}$ with $2 \mathrm{M} \mathrm{S}$ while the second had $2 \mathrm{M}$ $\mathrm{S}^{2-}$ with $3 \mathrm{M} \mathrm{S}$. To each of these solutions, one had hydroxide added and one did not (See Figure 8.)

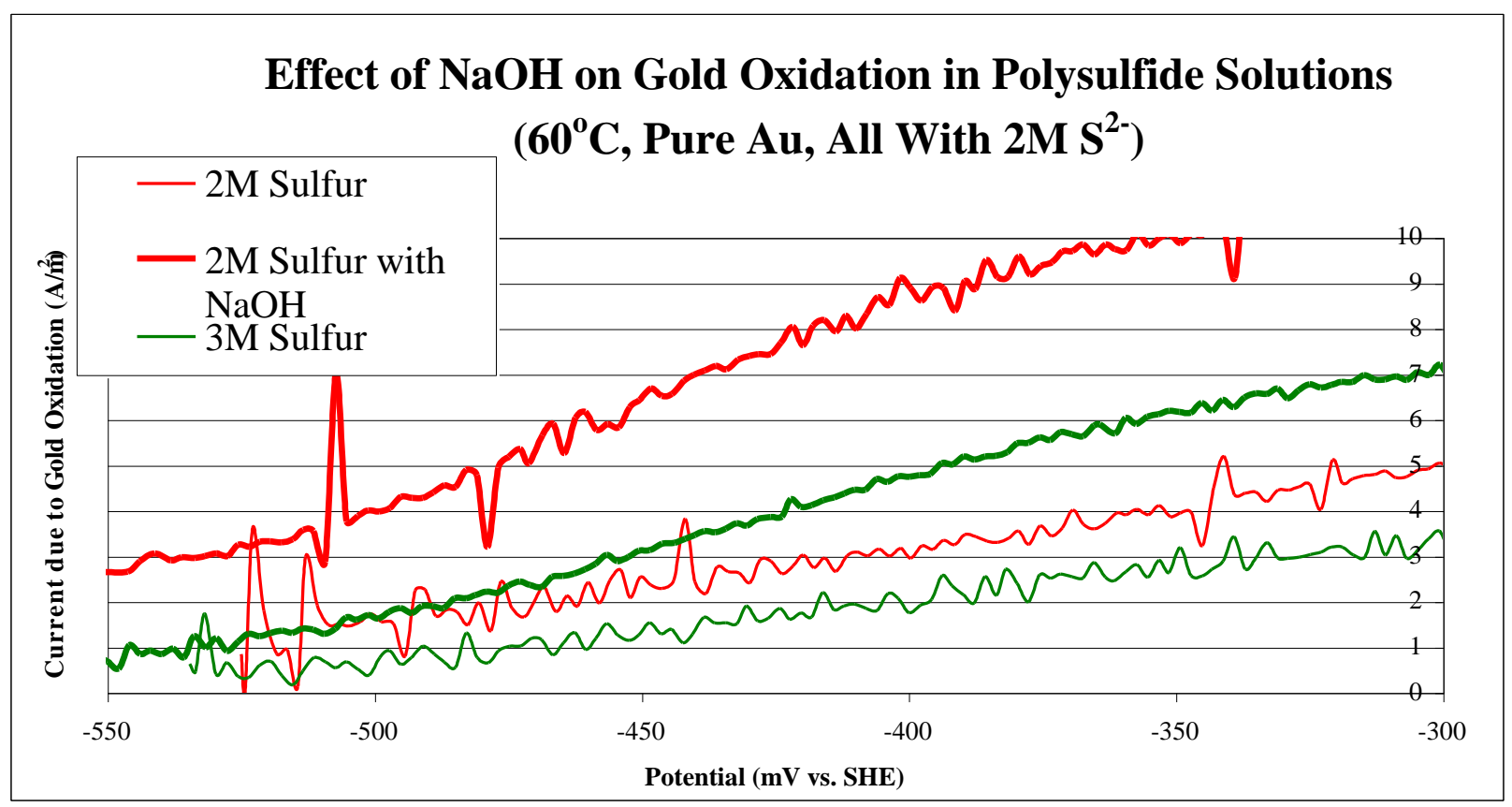

Figure 8. Effect of Hydroxide Addition on Gold Oxidation in Polysulfide 
From Figure 8, gold oxidizes more readily at lower potentials in solutions where hydroxide is added. To see the effect on gold oxidation at $\mathrm{E}_{\mathrm{m}}$, see Figure 9 below. For both solutions, by adding hydroxide the leach rate at $60^{\circ} \mathrm{C}$ is increased $20-25 \mathrm{ng} / \mathrm{s}$.

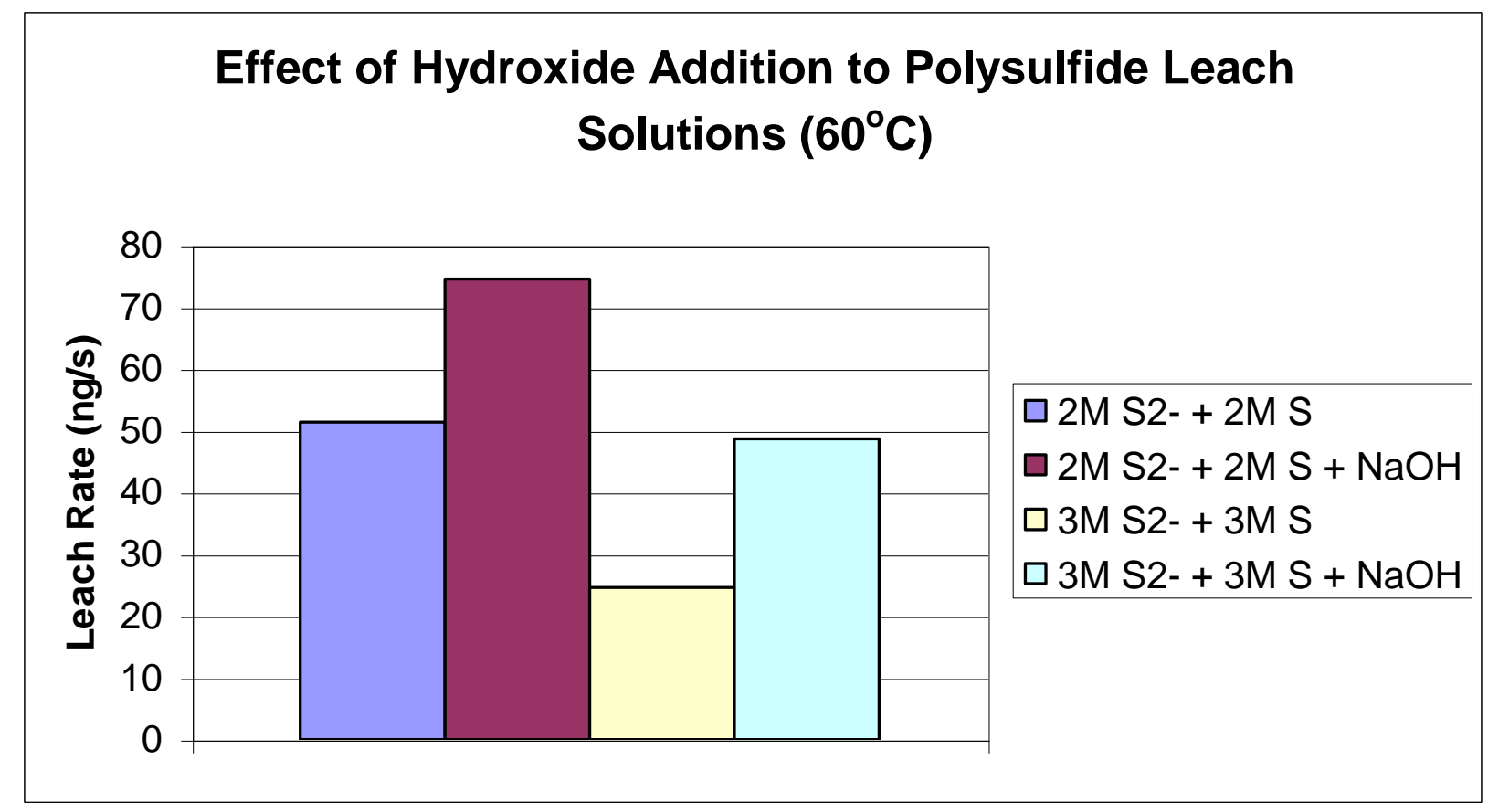

Figure 9. Effect of Hydroxide Addition on Gold Leach Kinetics

\section{Effect of Temperature}

In most cases, increasing the temperature of the solution will increase the reaction kinetics. Tests were run to understand how $2 \mathrm{M} \mathrm{S}^{2-}$ solutions and $2 \mathrm{M} \mathrm{S}^{2-}+1 \mathrm{M} \mathrm{S}$ polysulfide solutions were affected by temperature

Figure 10 shows the results for potential scans in $2 \mathrm{M} \mathrm{S}^{2-}$ solutions at temperatures from $30-60^{\circ} \mathrm{C}$. As temperature increases, gold oxidizes more readily at lower potentials. Notice the large jump in gold oxidation as the temperature goes from $50^{\circ} \mathrm{C}$ to $60^{\circ} \mathrm{C}$. 


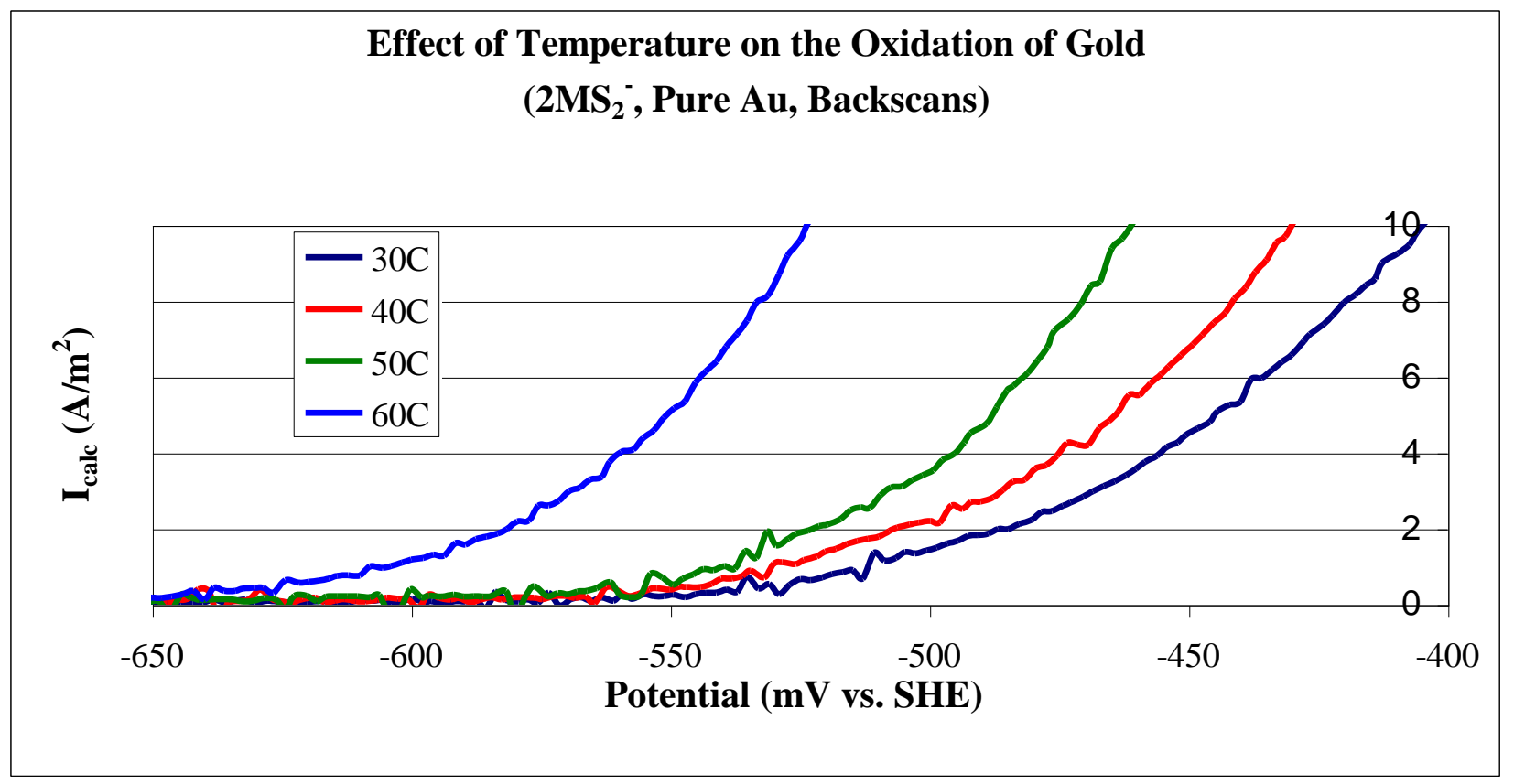

Figure 10. Temperature Effects on Gold Oxidation Potential Scans

In Figure 11, the effect of temperature on gold oxidation in polysulfide solutions is shown. Again, as temperature increases, gold will oxidize more readily at lower potentials. As with the $2 \mathrm{M} \mathrm{S}^{2-}$ solution above, there is a large jump as the temperature goes from $40^{\circ} \mathrm{C}$ to $50^{\circ} \mathrm{C}$.

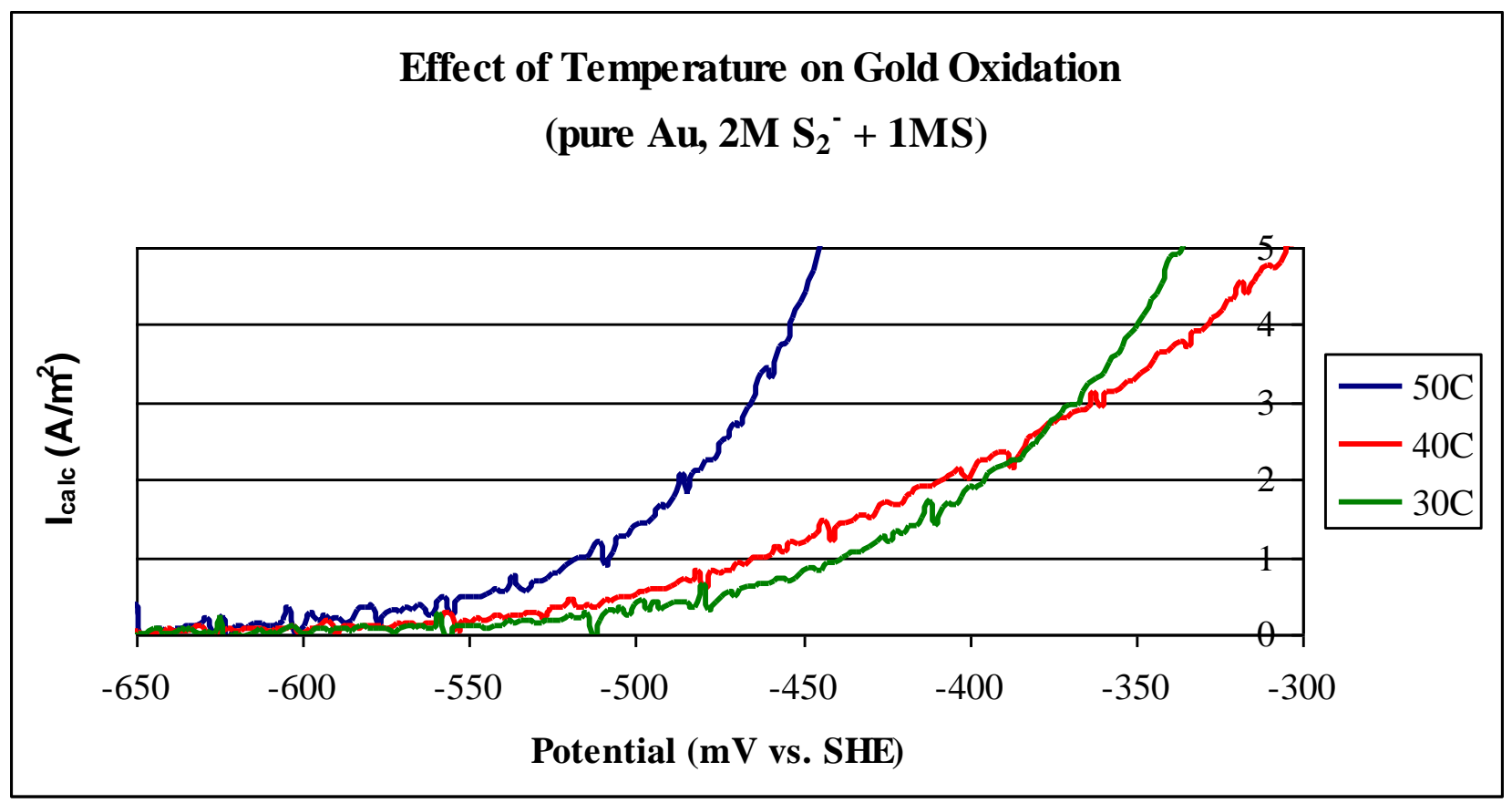

Figure 11. Effect of Temperature on Gold Oxidation in Polysulfide Solution. 
Leach tests were performed on the solutions found in Figure 11; they can be found in Figure 12. The same jump in oxidation kinetics from $40^{\circ} \mathrm{C}$ to $50^{\circ} \mathrm{C}$ can be seen in these leach tests. From $30^{\circ} \mathrm{C}$ to $40^{\circ} \mathrm{C}$ there is only a $0.5 \mathrm{ng} / \mathrm{s}$ rate increase while from $40^{\circ} \mathrm{C}$ to $50^{\circ} \mathrm{C}$ there is a $5.7 \mathrm{ng} / \mathrm{s}$ rate increase.

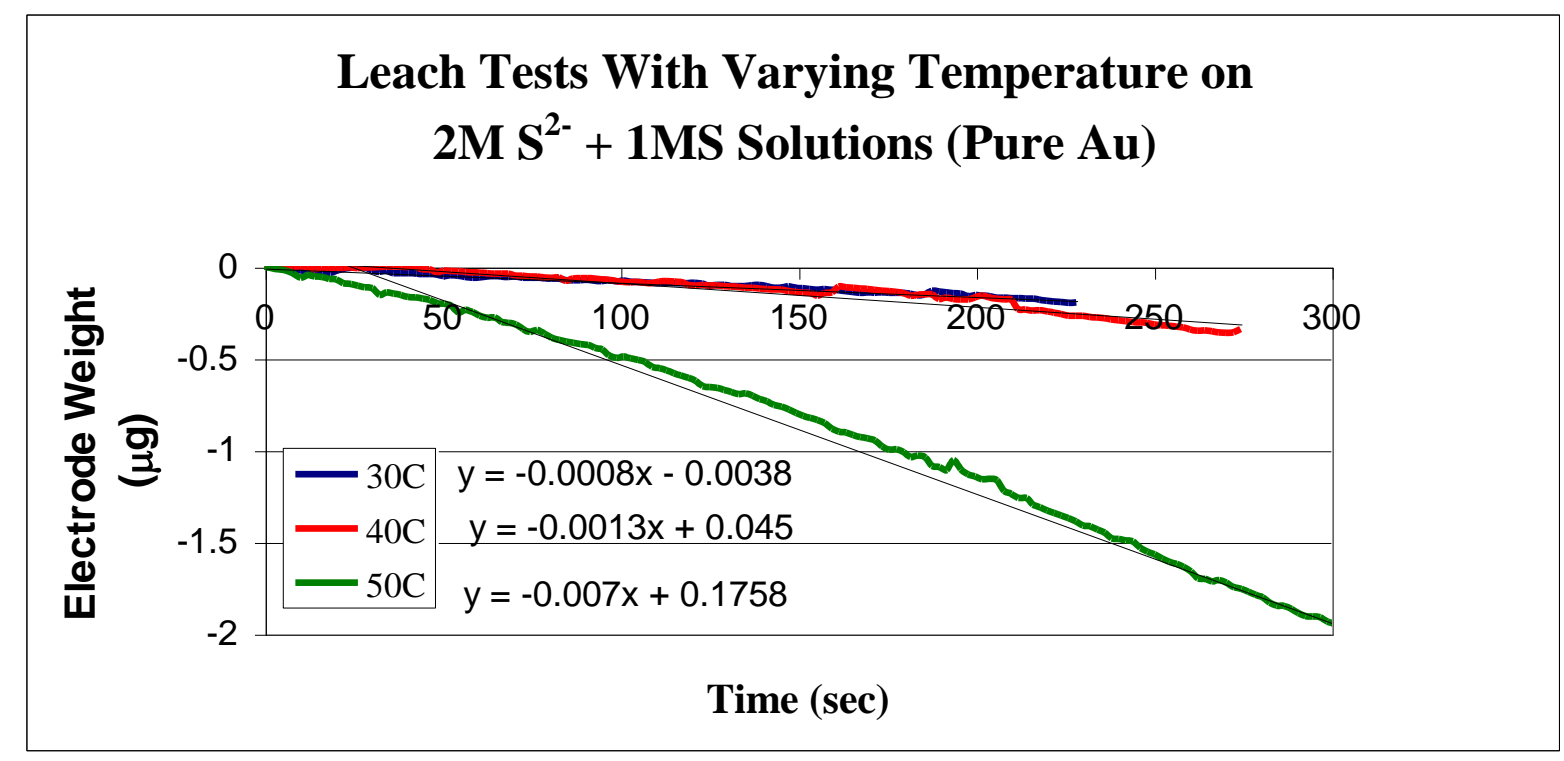

FigFigure 12. Leach Test Varying Temperature in Polysulfide Solutions

\section{Effect of Gold Purity}

Gold purity is a variable that needs to be studied because most natural gold deposits have at least 5\% Ag in them (Hurlbut and Klein, 1977). Two identical leach tests were run for three different solutions. The two tests contained one pure gold plate and one $5 \%$ silver plate (see Figure 13.) In the $2 \mathrm{M} \mathrm{S}^{2-}+1 \mathrm{M} \mathrm{S}$ solution, the $5 \%$ silver alloy leached slightly slower than its pure gold counterpart. However, for the more concentrated solutions (2 and $3 \mathrm{MS}$ ), the $5 \%$ enhanced the leach rate almost $100 \%$. 


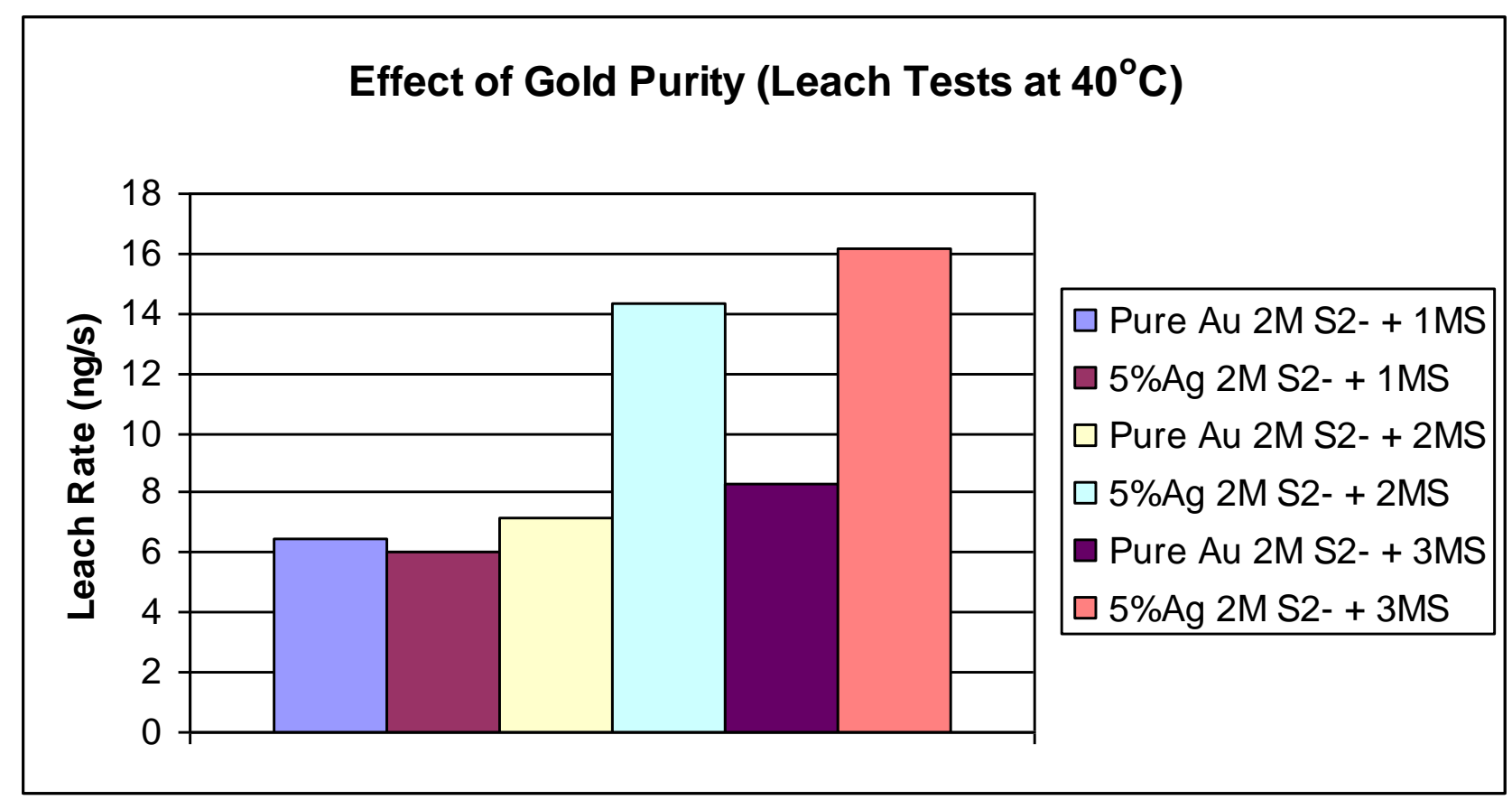

Figure 13. Effect of Gold Purity on Leach Rate

\section{Conclusions}

The first variable studied, effect of sulfide concentration, showed that increases in sulfide concentration help to speed the oxidation of gold at lower potentials. Hydrosulfide had two major effects on the system: 1) adding hydrosulfide slows gold leaching kinetics at a given potentials; and, 2) hydrosulfide additions raise the solution $\mathrm{E}_{\mathrm{m}}$. By adding sulfur to the system, the polysulfide concentration was increased. Higher polysulfide concentrations generally increased leach rates; however, more sulfur both slowed leach kinetics at given potentials and raised the $\mathrm{E}_{\mathrm{m}}$. Eventually the addition of more sulfur will slow the leach reaction. Hydroxide additions up to 3 molar were shown to increase leach kinetics at lower potentials in sulfide solutions. Also, hydroxide additions increased leach kinetics in polysulfide solutions. As expected, temperature increased leach kinetics; more importantly, there looks to be a large jump in leach kinetics around the 50$60^{\circ} \mathrm{C}$ temperature range. The purity of the gold effected leach kinetics positively for concentrated polysulfide solutions above $2 \mathrm{M} \mathrm{S}^{2-}+1 \mathrm{M} \mathrm{S}$. 


\title{
Fundamentals and applications of alkaline sulfide leaching and recovery of gold.
}

Corby Anderson, Eric Dahlgren, Hsin Huang, Paul Miranda, and Darby Stacey

The Center for Advanced Mineral and Metallurgical Processing, Montana Tech, Room

221, ELC Building, Butte, Montana, 59701 USA, Tel: 406-496-4794, Fax: 406-496-4512

E-mail: CAnderson@mtech.edu,WEB: WWW.MTECH.EDU/CAMP

Matthew Jeffrey and Irsan Chandra

Department of Chemical Engineering, Monash University, Clayton, Victoria, 3800,

Australia.

\begin{abstract}
$\underline{\text { ABSTRACT }}$
The latter part of the $20^{\text {th }}$ century saw great advances in the treatment of refractory gold ores coupled with increased reliance on the use of cyanide for gold processing. Now, in many parts of the world, there is social pressure to limit or eliminate the use of cyanide. As well, treatment of some refractory ores or concentrates which have excessive cyanide consumption, gold cyanide pregrobbing or significant sulfide content remain difficult. This paper will outline the history of the development of alkaline sulfide leaching as an ancillary process to nitrogen species catalyzed (NSC) pressure leaching. Electrochemical fundamentals and the applicable thermodynamics of the alkaline sulfide hydrometallurgical system will be outlined. As well, examples of refractory gold recovery with alkaline sulfide hydrometallurgy such as an arsenopyrite gold concentrate application, a chalcopyrite gold concentrate application, a pregrobbing gold ore application, and a cyanide consuming gold ore application will be delineated in this paper.
\end{abstract}




\section{INTRODUCTION}

As alkaline sulfide leaching of gold has its roots in the development and industrial application of nitrogen species catalyzed pressure leaching for partial oxidation of sulfides, a discourse in this topic is relevant. As well, the use of nitric acid in metal sulfide oxidation is not new. Many derivations of the technology have been researched and piloted $(1,2,3,4)$ However, as noted in Table I, only the nitrogen species catalyzed (NSC) acid pressure leach has ever been built and operated successfully on an industrial scale $(3,4)$. Table II illustrates the comparative operating criteria for these technologies as well as some selected conventional pressure oxidation gold plants. As seen, NSC is advantageous over the others in that it is faster, uses lower temperatures, uses lower pressures, uses a small amount of nitrogen species regenerated in-situ, and uses unlined 316L stainless steel autoclave.

Table I - Nitrogen species pressure leaching historical record

\begin{tabular}{clll}
\hline Process & Industrial & & \multicolumn{1}{c}{ Other } \\
Name & Application & Operation & Applications \\
\hline NSC & Ag, Cu & 11 Years & PGM's, Au, Ni, Co, Zn \\
REDOX & Au & Pilot 1 Year & None \\
NITROX & None & None & $\mathrm{Au}$ \\
\hline
\end{tabular}


Table II - Comparative nitrogen species catalyzed and selected conventional acid pressure leaching gold plant operating criteria

\begin{tabular}{|c|c|c|c|c|c|c|}
\hline Name & $\begin{array}{c}\text { Process } \\
\text { Temp }\end{array}$ & $\begin{array}{l}\text { Process } \\
\text { Pressure } \\
\end{array}$ & $\begin{array}{c}\text { Nitrogen } \\
\text { Species } \\
\text { Concentration } \\
\end{array}$ & $\begin{array}{c}\text { Nitrogen } \\
\text { Species } \\
\text { Regeneration } \\
\text { Process } \\
\end{array}$ & $\begin{array}{l}\text { Residence } \\
\text { time, min }\end{array}$ & $\begin{array}{l}\text { Materials of } \\
\text { Construction }\end{array}$ \\
\hline NSC & $125-$ & 625-975 kPag & $2-3 \mathrm{~g} / \mathrm{L}$ & In-situ & $15-45$ & $\begin{array}{l}\text { Stainless } \\
\text { steel }\end{array}$ \\
\hline REDOX & $85-95^{0}$ & Atmospheric & $140-180 \mathrm{~g} / \mathrm{L}$ & External & 60 & Titanium \\
\hline REDOX & 195- & $1950 \mathrm{kPag}$ & 70-110 g/L & External & 15 & Titanium \\
\hline NITROX & 85- & Atmospheric & $100-150 \mathrm{~g} / \mathrm{L}$ & External & 60 & $\begin{array}{l}\text { Stainless } \\
\text { steel }\end{array}$ \\
\hline McGlaughlin & $180^{\circ} \mathrm{C}$ & 2200 kPag & None just $\mathrm{O}_{2}$ & None just $\mathrm{O}_{2}$ & 90 & $\begin{array}{l}\text { Lead/acid } \\
\text { brick }\end{array}$ \\
\hline Sao Bento & $190^{\circ} \mathrm{C}$ & $1600 \mathrm{kPag}$ & None just $\mathrm{O}_{2}$ & None just $\mathrm{O}_{2}$ & 120 & $\begin{array}{l}\text { Lead/acid } \\
\text { brick }\end{array}$ \\
\hline Goldstrike & $225^{\circ} \mathrm{C}$ & 3000 kPag & None just $\mathrm{O}_{2}$ & None just $\mathrm{O}_{2}$ & 75 & $\begin{array}{l}\text { Lead/acid } \\
\text { brick }\end{array}$ \\
\hline Getchell & $210^{\circ} \mathrm{C}$ & 3200 kPag & None just $\mathrm{O}_{2}$ & None just $\mathrm{O}_{2}$ & 90 & $\begin{array}{l}\text { Lead/acid } \\
\text { brick }\end{array}$ \\
\hline
\end{tabular}




\section{Fundamentals of Nitrogen Species Catalyzed Pressure Leaching}

In order to understand the advantages of NSC pressure leaching, it is important to review the principles behind it. The commonly reported leach reaction of a sulfide mineral with nitric acid in conjunction with sulfuric acid is shown below.

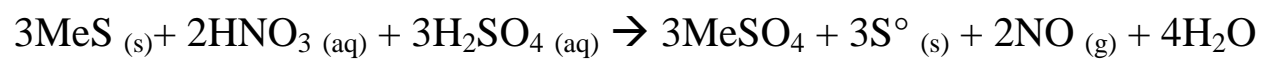

However, it is postulated that the actual reaction species is $\mathrm{NO}^{+}$and not $\mathrm{NO}_{3}{ }^{-}$ $(5,6,7)$. The addition of or presence of $\mathrm{NO}_{2}{ }^{-}$instead of $\mathrm{NO}_{3}{ }^{-}$accelerates the formation of $\mathrm{NO}^{+}$. As shown in Table III, the $\mathrm{NO}^{+} / \mathrm{NO}$ couple is capable of an extremely high redox potential (8). So, $\mathrm{NO}^{+}$is readily formed from nitrous rather than nitric acid. For example, a convenient source of nitrous acid can be sodium nitrite $(5,6,7)$. When it is added to an acidic solution, nitrous acid is readily formed.

$$
\mathrm{NaNO}_{2(\text { (aq) }}+\mathrm{H}^{+} \rightarrow \mathrm{HNO}_{2(\mathrm{aq})}+\mathrm{Na}^{+}
$$

Nitrous acid further reacts to form $\mathrm{NO}^{+}$.

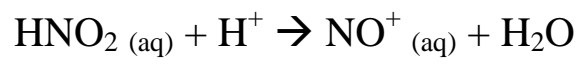

The $\mathrm{NO}^{+}$then reacts with the mineral and oxidizes the sulfide to sulfur.

$$
2 \mathrm{MeS}_{(\mathrm{s})}+4 \mathrm{NO}^{+}{ }_{(\mathrm{aq})} \rightarrow 2 \mathrm{Me}^{+2}(\mathrm{aq})+2 \mathrm{~S}^{\circ}+4 \mathrm{NO}_{(\mathrm{g})}
$$

Of course, at higher temperatures and/or nitrogen species concentrations, the sulfide can be fully oxidized to sulfate.

Table III - Relative potentials of hydrometallurgical oxidizers

\begin{tabular}{llc}
\hline Oxidant & \multicolumn{1}{c}{ Redox Equation } & $\begin{array}{c}\mathrm{E}_{\mathrm{h}}{ }^{\circ} \\
\left(\mathrm{pH}=0, \mathrm{H}_{2} \text { ref. }\right)\end{array}$ \\
\hline $\mathrm{Fe}^{+3}$ & $\mathrm{Fe}^{+3}+\mathrm{e}^{-} \rightarrow \mathrm{Fe}^{+2}$ & $0.770 \mathrm{~V}$ \\
$\mathrm{HNO}_{3}$ & $\mathrm{NO}_{3}^{-}+4 \mathrm{H}^{+}+3 \mathrm{e}^{-} \rightarrow \mathrm{NO}+2 \mathrm{H}_{2} \mathrm{O}$ & $0.957 \mathrm{~V}$ \\
$\mathrm{HNO}_{2}$ & $\mathrm{NO}_{2}^{-}+2 \mathrm{H}^{+}+\mathrm{e}^{-} \rightarrow \mathrm{NO}+\mathrm{H}_{2} \mathrm{O}$ & $1.202 \mathrm{~V}$ \\
$\mathrm{O}_{2(\mathrm{~g})}$ & $\mathrm{O}_{2}+4 \mathrm{H}^{+}+4 \mathrm{e}^{-} \rightarrow 2 \mathrm{H}_{2} \mathrm{O}$ & $1.230 \mathrm{~V}$ \\
$\mathrm{Cl}_{2}(\mathrm{~g})$ & $\mathrm{Cl}_{2(\mathrm{~g})}+2 \mathrm{e}^{-} \rightarrow 2 \mathrm{Cl}^{-}$ & $1.358 \mathrm{~V}$ \\
$\mathrm{NO}^{+}$ & $\mathrm{NO}^{+}+\mathrm{e}^{-} \rightarrow \mathrm{NO}$ & $1.450 \mathrm{~V}$ \\
\hline
\end{tabular}

As can be seen, nitric oxide gas, NO, is produced from the oxidation of sulfides. As this gas has a limited solubility in aqueous solutions, it tends to transfer out of solution. In the pressure leach system, a closed vessel with an oxygen overpressure is used. The nitric oxide gas emanating from the leach slurry accumulates in the headspace 
of the reactor where it reacts with the supplied oxygen to form nitrogen dioxide gas. The $\mathrm{NO}$ is then regenerated to $\mathrm{NO}^{+}$. Overall this can be viewed as:

$$
\begin{gathered}
\mathrm{NO}_{(\mathrm{g})}+\mathrm{O}_{2 \text { (g) }} \rightleftarrows 2 \mathrm{NO}_{2}(\mathrm{~g}) \\
2 \mathrm{NO}_{2 \text { (g) }} \rightleftarrows 2 \mathrm{NO}_{2}(\mathrm{aq}) \\
2 \mathrm{NO}_{2(\mathrm{aq})}+2 \mathrm{NO}(\text { (aq) } \\
\end{gathered}
$$

Since the nitrogen species is continuously regenerated, its role in the overall reaction as the actual oxidizer is not obvious. The net overall reaction has the sulfide mineral reacting with the acid solution and oxygen to solubilize the metal value into the sulfate solution and form some elemental sulfur. Of course, at higher temperatures and/or nitrous acid concentrations the sulfide would be fully oxidized to sulfate.

$$
2 \mathrm{MeS}_{(\mathrm{g})}+4 \mathrm{H}^{+}+\mathrm{O}_{2(\mathrm{~g})} \rightarrow 2 \mathrm{Me}^{+2}(\mathrm{aq})+2 \mathrm{~S}^{\circ}+2 \mathrm{H}_{2} \mathrm{O}
$$

Overall, the nitrogen intermediates serve as an expedient means to transport oxygen to the surface of the solid particle and allow the resulting reaction to take place at a heightened redox potential. This inherent asset of the unique system precludes the use of high temperatures and high pressures which lead to higher costs in other pressure leach processes. For example, commonly available stainless steel can be used for the reactor vessel, and complete oxidation of sulfide to sulfate can be achieved without the excessive conditions found in other pressure leach systems. Thus, the rapid kinetics of the system leads to smaller reactor volumes and higher unit throughputs. Finally, 99.9\% of the nitrogen species utilized in the leach system report to the gas phase when the pressure vessel is flashed, and they are readily destroyed and contained by commercially available scrubber systems. So, environmental impacts are minimized and the NSC leach plant solutions contain little or no nitrogen species.

\section{NSC Complete Sulfide Oxidation Application to an Arsenopyrite Gold Concentrate}

An example of the NSC complete sulfide oxidation process application to gold is listed in Tables IV and V. In this case, the sulfides are completely oxidized to sulfate due to high temperatures and long leaching times used. $94.2 \%$ of the gold was then recovered via conventional carbon in leach cyanidation of the leached solids. 
Table IV - Assay of gold concentrate treated with complete NSC sulfide oxidation

\begin{tabular}{lc} 
Element & Assay \\
\hline $\mathrm{Au}$ & $35.0 \mathrm{~g} / \mathrm{T}$ \\
$\mathrm{Fe}$ & $25.0 \%$ \\
$\mathrm{As}$ & $6.3 \%$ \\
Total S & $24.0 \%$ \\
\hline
\end{tabular}

Table V - NSC complete sulfide oxidation leach conditions

\begin{tabular}{ll}
\hline Operating Parameter & Level \\
\hline Initial Free Sulfuric Acid & $20 \mathrm{~g} / \mathrm{L}$ \\
Reactor Working Pressure & $975 \mathrm{kPag}$ \\
Slurry Solids Content & $100 \mathrm{~g} / \mathrm{L}$ \\
Solids Size & $80 \%$ minus 10 micron \\
Maximum Temperature & $170^{\circ} \mathrm{C}$ \\
Nitrogen Species Concentration & $2.0 \mathrm{~g} / \mathrm{L}$ \\
Reaction Time & 45 minutes \\
\hline
\end{tabular}

In this case fine grinding was utilized to accelerate the kinetics. This would have been the case with conventional pressure leaching as well. The key aspect here is the significantly lower temperatures and pressures utilized in NSC complete oxidation.

\section{FUNDAMENTALS OF ALKALINE SULFIDE HYDROMETALLURGY}

Another proven industrial methodology with NSC technology is the partial oxidation of the sulfide to elemental sulfur instead of sulfate. In this case, a lower temperature is used and subsequently less oxygen is consumed. As currently experienced in other industrial systems, the majority of the gold tends to accumulate in the elemental sulfur that is produced $(9,10,11)$. As practiced in industry, this product can be readily screened or floated away from the other leached solids. Then, the gold can be leached via alkaline sulfide lixiviation whereby the sulfur containing the gold is dissolved in an alkaline solution. To better illustrate this, Figure 1 (12) shows the classical equilibrium sulfur system while Figure 2 (13) shows the meta-stable species sulfur system. In reality, the species shown in Figure 2 dominate as the alkaline sulfide system is slow to reach equilibrium. Figure $3(14,15)$ shows the meta-stable region of sulfide species versus Eh at $\mathrm{pH}$ 13. Initially, the solution contained 3 moles/ $\mathrm{L} \mathrm{NaOH}$ with 0.0025 moles $/ \mathrm{L} \mathrm{Na}_{2} \mathrm{~S}$. The $\mathrm{pH}$ was 13 and Eh about $-0.5 \mathrm{~V}$. As seen, there is a optimal ratio between elemental sulfur and sulfide which ensures that the mixture produces enough of the correct amount and type of polysulfides without raising the Eh too high. After speciation, 0.005 moles of 
elemental sulfur were added into the solution in fifty 0.0001 molar increments. Figure 4 $(14,15)$ shows that the $\mathrm{pH}$ remains almost 


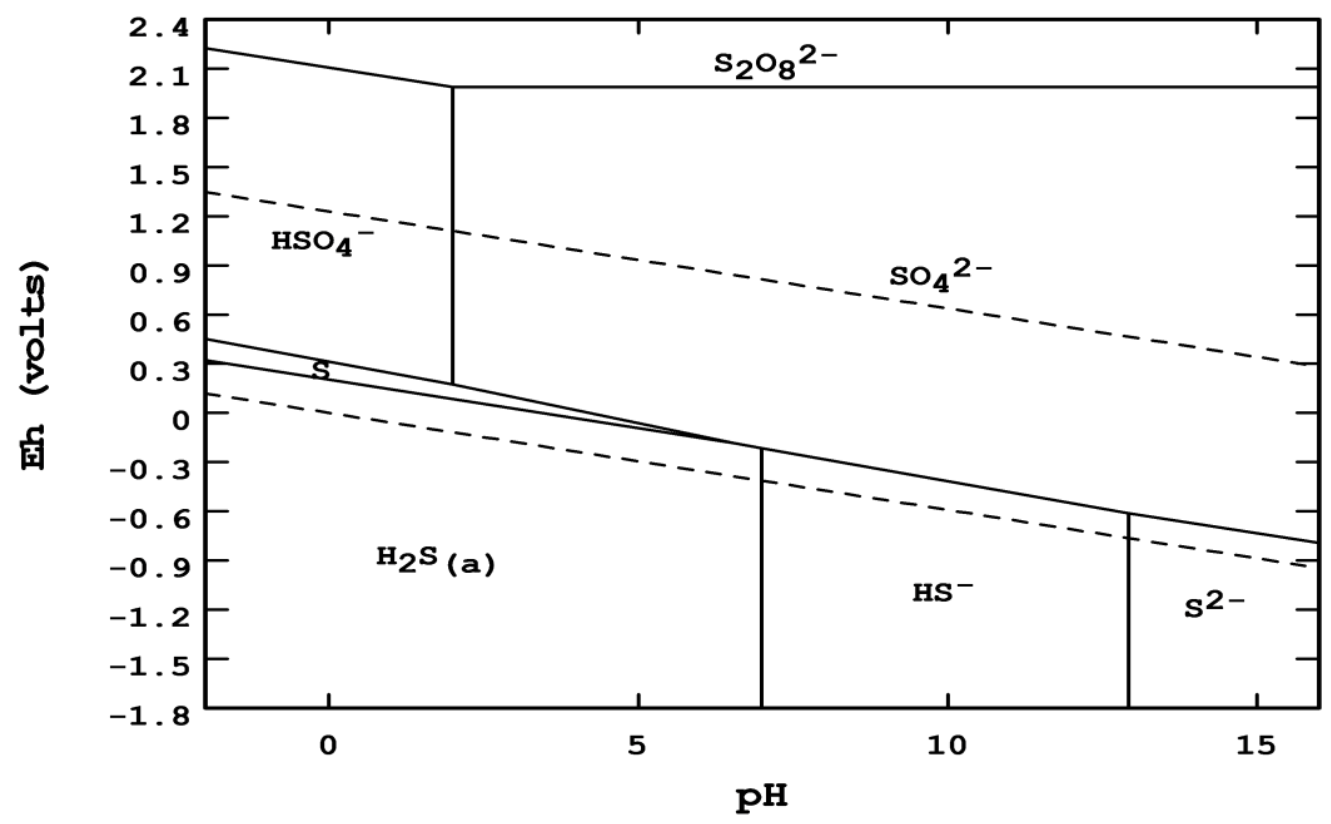

Figure 1 - Equilibrium Eh-pH diagram for sulfur

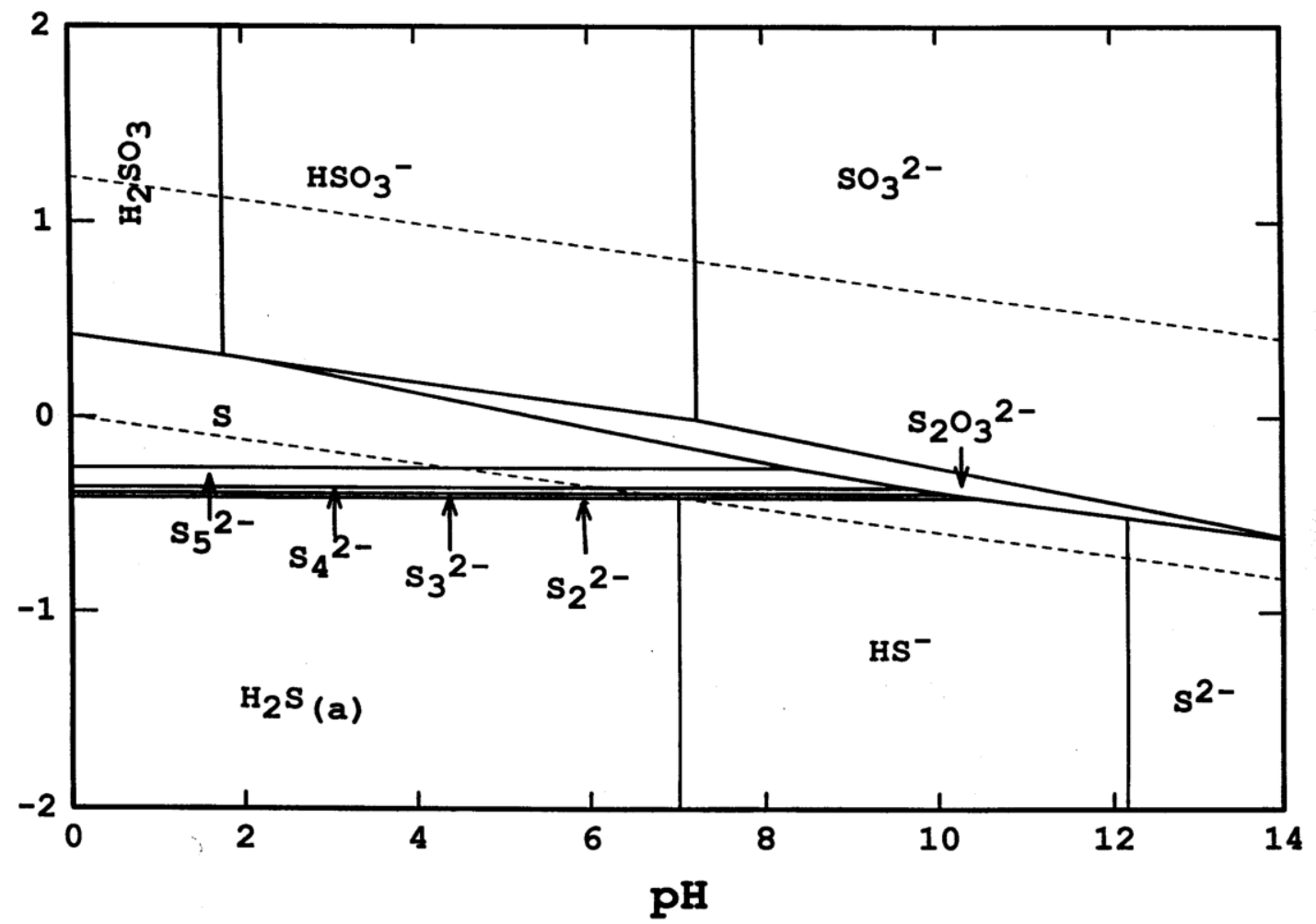

Figure 2 - Meta-stable species Eh-pH diagram for sulfur 


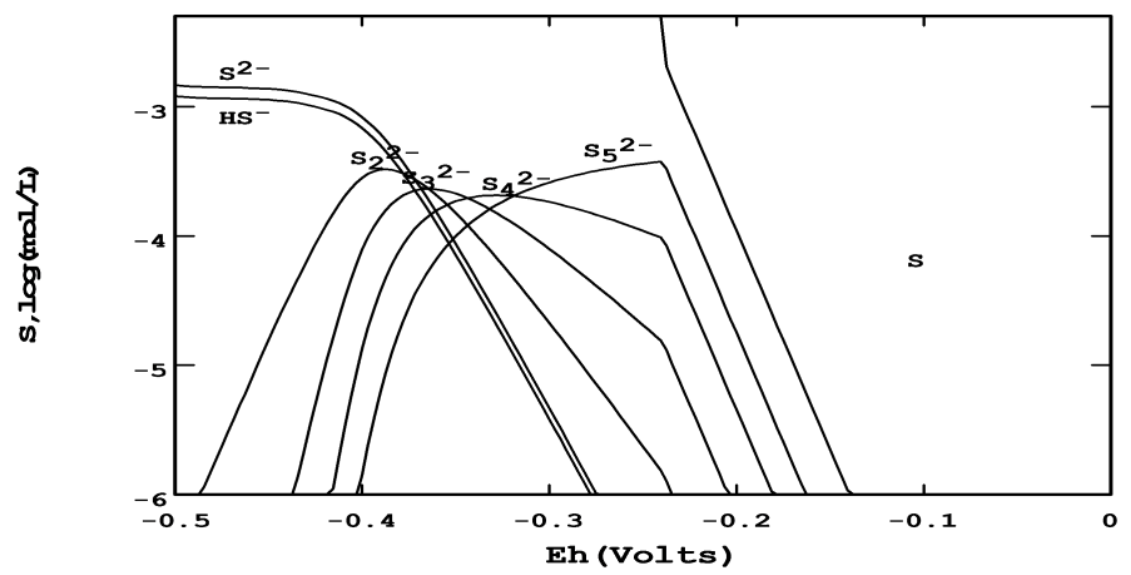

Figure 3 - Distribution of sulfide species in $\mathrm{S}, \log (\mathrm{mol} / \mathrm{L})$ versus Eh at $\mathrm{pH} 13$ and $25^{\circ} \mathrm{C}$

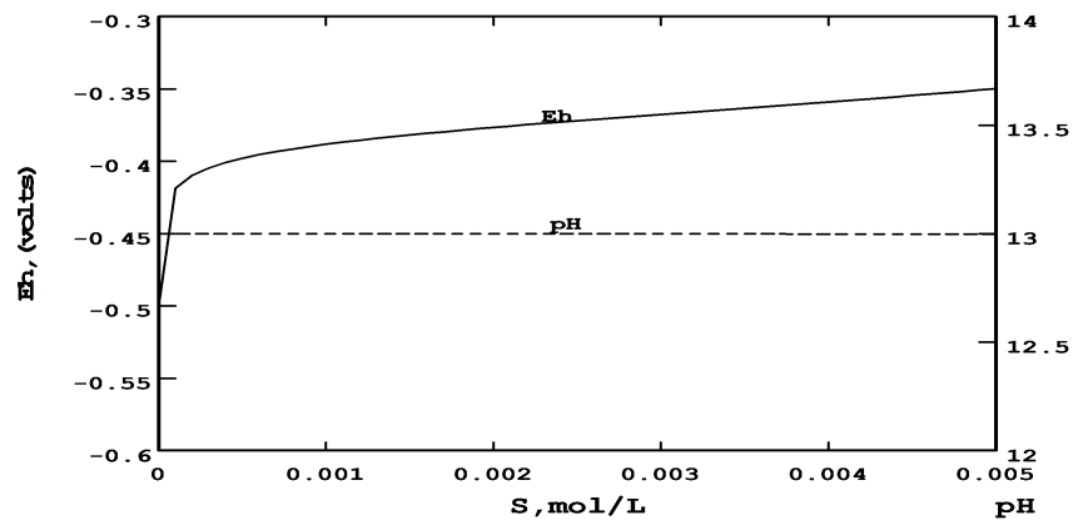

Figure 4. Alkaline sulfide $\mathrm{pH}$ and Eh responses versus S addition.

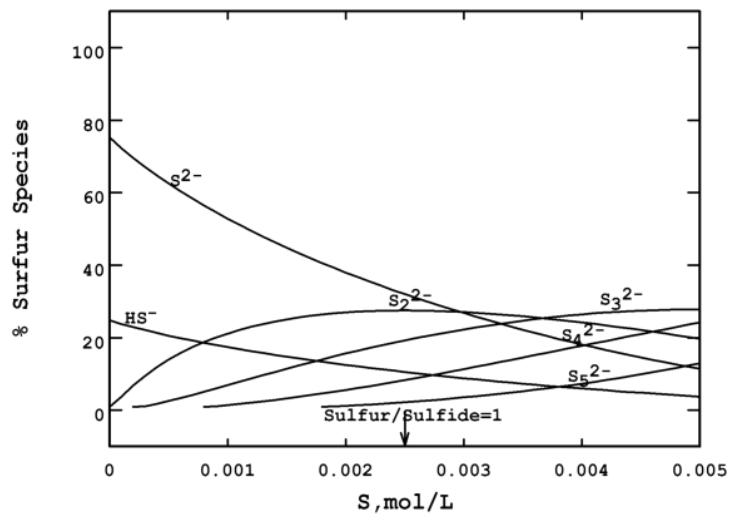

Figure 5. Percent distribution of sulfur species versus $S$ addition in alkaline sulfide.

The location where sulfur/sulfide $=1$ is also marked 


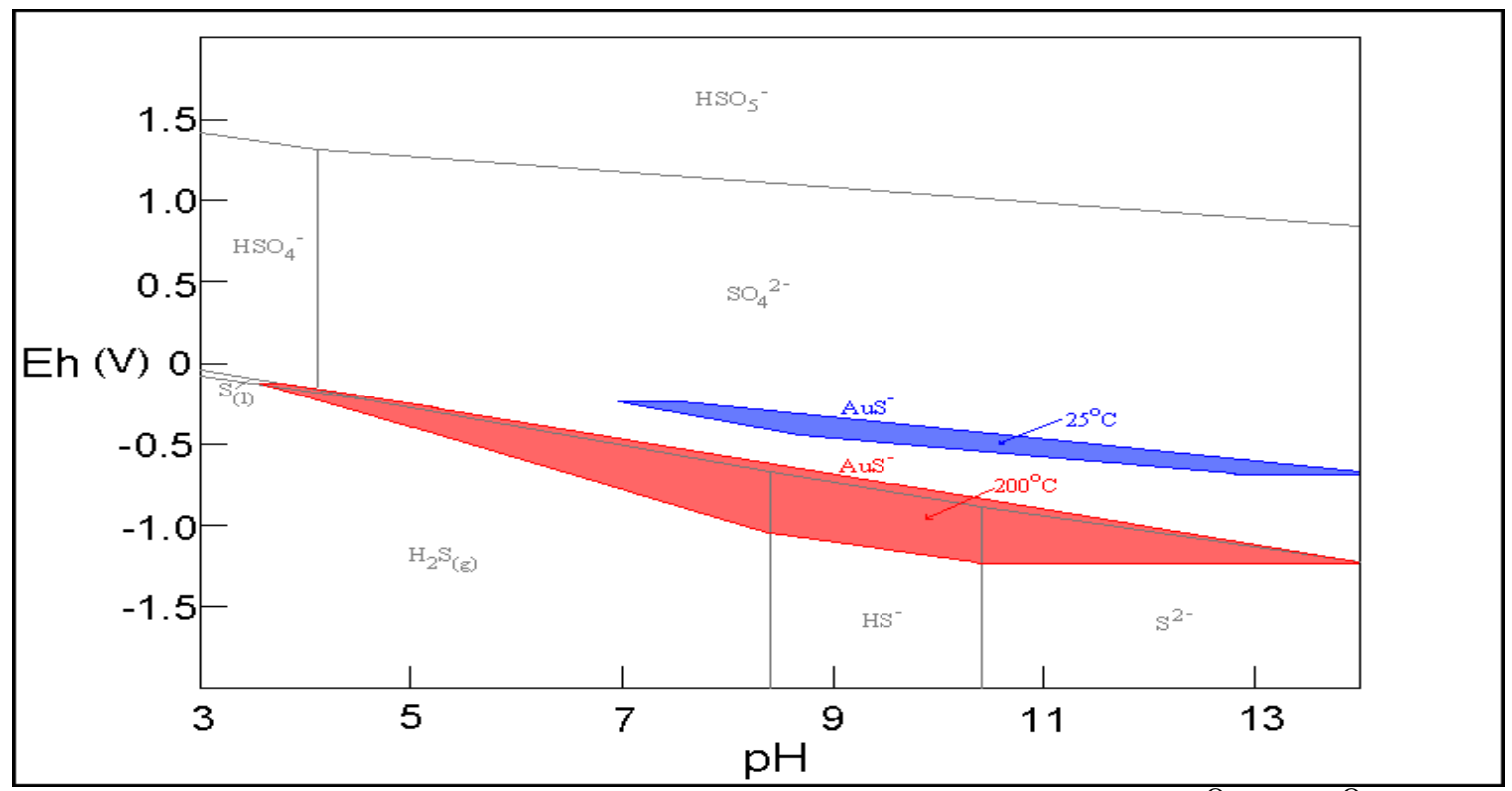

Figure 6 - Alkaline sulfide gold region as a function of temperature from $25^{\circ}$ to $200^{\circ} \mathrm{C}$.

constant at 13 and Eh increases with sulfur addition. Based on this, the resultant percent distributions of sulfur species are shown on Figure $5(14,15)$. Under this condition, $\mathrm{S}_{2}{ }^{2-}$ is maximum at $27.5 \%$, and the system still contains plenty of total sulfide at $42.5 \%$. Further, Figure 6illustrates the stability region for alkaline sulfide gold as a function of temperature from $25^{\mathrm{O}}$ to $200^{\circ} \mathrm{C}$. The gold sulfide region is larger at higher temperatures.

A combination of sodium hydroxide and elemental sulfur result in the formation of species other than just sulfide ( $\mathrm{S}^{-}$ ${ }^{2}$ ). Both sodium polysulfide $\left(\mathrm{Na}_{2} \mathrm{~S}_{\mathrm{X}}\right)$ and sodium thiosulfate $\left(\mathrm{Na}_{2} \mathrm{~S}_{2} \mathrm{O}_{3}\right)$ are created along with sulfide (16). This illustrated simplistically in the following scenario:

$$
\begin{aligned}
& 4 \mathrm{~S}^{\mathrm{O}}+6 \mathrm{NaOH} \rightarrow 2 \mathrm{Na}_{2} \mathrm{~S}+\mathrm{Na}_{2} \mathrm{~S}_{2} \mathrm{O}_{3}+3 \mathrm{H}_{2} \mathrm{O} \\
& (\mathrm{X}-1) \mathrm{S}^{\mathrm{O}}+\mathrm{Na}_{2} \mathrm{~S} \rightarrow \mathrm{Na}_{2} \mathrm{~S}_{\mathrm{X}}(\text { where } \mathrm{X}=2 \text { to } 5)
\end{aligned}
$$

Gold lixiviation has been confirmed to be the mechanistic result of leaching in the presence of both polysulfides and sulfides $(17,18)$ :

$$
2 \mathrm{Au}+\mathrm{S}_{2}^{2-}+2 \mathrm{~S}^{2-} \rightarrow 2 \mathrm{AuS}^{-}+2 \mathrm{~S}^{2-}
$$

Further, there is an optimal ratio between elemental sulfur and sulfide so that the mixture produces enough polysulfides without over oxidizing sulfide. Based on leaching optimization experiments, the best sulfur/sulfide ratio is around 1 which is indicated on Figure 5 . Under this condition, thermodynamically, $\mathrm{S}_{2}{ }^{2-}$ is maximum at $27.5 \%$, and the system still contains plenty of total sulfide at $42.5 \%$. This seems to suggest that $\mathrm{S}_{2}{ }^{2-}$ is largely responsible for oxidizing gold as listed equation 11. 
Also collaborative studies between The Department of Chemical Engineering at Monash University in Australia and the Center for Advanced Mineral and Metallurgical Processing have been completed on the actual kinetics and this mechanism of the alkaline sulfide system $(15,17,18)$. A rotating electrochemical quartz crystal microbalance (REQCM) was utilized to study the system and optimize it. An example of this work, as shown in Figure 3, confirms that complexation of gold is by sulfide after oxidation by polysulfide.

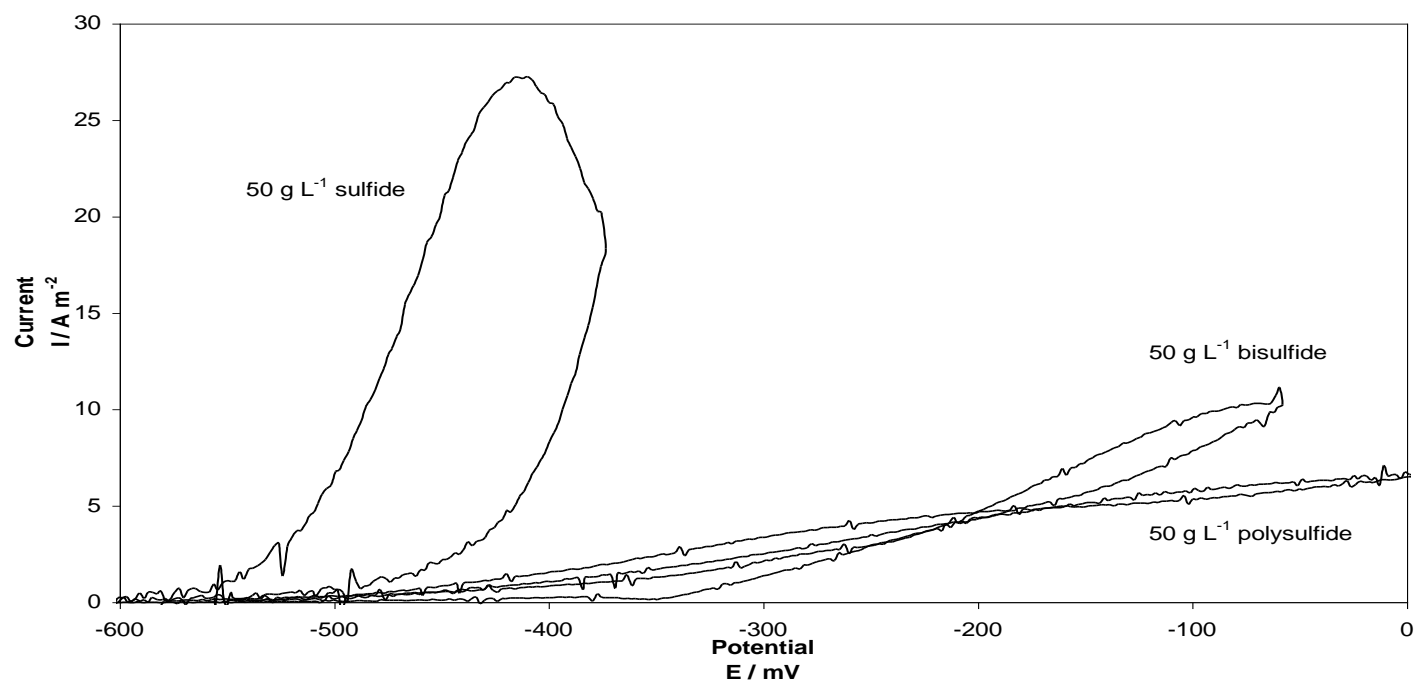

Figure 7 - REQCM reverse potential sweeps and calculated current for potential lixiviants at a concentration of $50 \mathrm{~g} / \mathrm{L}$ and at a temperature of $30^{\circ} \mathrm{C}(18)$.

Figure 7 shows a comparison of sulfide, bisulfide and polysulfide as potential lixiviants for the alkaline sulfide system. The large calculated current for sulfide in comparison to those of bisulfide and polysulfide, suggests that sulfide is the dominant lixiviant of the system, and that bisulfide and polysulfide are poor lixiviants. The actual leaching occurring in the polysulfide and bisulfide system may be attributable to the presence of some sulfide in equilibrium with the bisulfide and polysulfide. A number of ligands form stable compounds with the aurous cations, including sulfide and bisulfide. From the data in Table VI it can be seen that sulfide forms a more stable complex with gold than bisulfide as shown by the larger stability constant, confirming what was seen experimentally (18).

Table VI - Stability constants and standard reduction potentials for sulfur containing gold complexes

\begin{tabular}{ccc}
\hline Complex & Reaction & $\mathrm{E}^{0} / \mathrm{mV}$ \\
\hline $\mathrm{AuS}^{-}$ & $\mathrm{AuS} \mathrm{S}^{-}+\mathrm{e}^{-} \rightarrow \mathrm{Au}+\mathrm{S}^{2-}$ & -460 \\
$\mathrm{Au}(\mathrm{HS})_{2}{ }^{-}$ & $\mathrm{Au}(\mathrm{HS})_{2}^{-}+\mathrm{e}^{-} \rightarrow \mathrm{Au}+2 \mathrm{HS}^{-}$ & -90 \\
\hline
\end{tabular}

Thus, the reduction of polysulfide when acting as an oxidizer may be represented by the below half reaction: 


$$
\mathrm{S}_{\mathrm{x}}^{2-}+2(\mathrm{x}-1) \mathrm{e}^{-} \rightarrow \mathrm{xS}^{2-}
$$

For a polysulfide with $\mathrm{x}$ equals 2:

$$
\mathrm{S}_{2}{ }^{2-}+2 \mathrm{e}^{-} \rightarrow 2 \mathrm{~S}^{2-}
$$

Thus, assuming that sulfide is the complexing lixiviant and polysulfide $\left(\mathrm{S}_{2}{ }^{2-}\right)$ the oxidant, the overall reaction can be written as:

$$
2 \mathrm{Au}+\mathrm{S}_{2}{ }^{2-}+2 \mathrm{~S}^{2-} \rightarrow 2 \mathrm{AuS}^{-}+2 \mathrm{~S}^{2-}
$$

Gold leached by the alkaline sulfide system is readily recoverable by several means including electrowinning, gaseous precipitation, chemical precipitation, cementation, solvent extraction, and ion exchange. The use of electrowinning for recovery of gold leached in alkaline sulfide solutions is illustrated in Table VII.

Table VII - Gold electrowinning recovery from alkaline sulfide solutions

\begin{tabular}{ll}
\hline Operating Parameter & Level \\
\hline Initial gold tenor in solution & $0.983 \mathrm{~g} / \mathrm{L}$ \\
Initial total sulfur in solution & $52.3 \mathrm{~g} / \mathrm{L}$ \\
Initial free hydroxide in solution & $15 \mathrm{~g} / \mathrm{L}$ \\
Cathode current density & $600 \mathrm{~A} / \mathrm{m}^{2}$ \\
Anode current density & $1000 \mathrm{~A} / \mathrm{m}^{2}$ \\
Voltage & $4.1 \mathrm{Volts}$ \\
Temperature & $50^{\circ} \mathrm{C}$ \\
Final gold in tenor in solution & $0.010 \mathrm{~g} / \mathrm{L}$ \\
Gold recovery & $99.0 \%$ \\
\hline
\end{tabular}

Further, a proprietary gold recovery method has been adopted to quantitatively and selectively recover leached gold from complex alkaline sulfide solutions. This is illustrated by selectively removing gold from an alkaline sulfide leach solution doped with impurities such as $\mathrm{Hg}$, As, Sb and $\mathrm{Sn}$, which are the only other possible elements soluble in this lixiviant. The pertinent assays of the solution tested is shown in Table VIII. The gold recovery and selectivity of the gold sorbent, which assayed at $1561.4 \mathrm{~g} / \mathrm{T}$, is illustrated in Table IX. The treatment and final deportment of elements such as $\mathrm{As}, \mathrm{Hg}$ and $\mathrm{Sb}$ is beyond the scope of this paper and is the subject of other publications $(19,20,21)$.

Table VIII - Alkaline sulfide solution assay before and after sorbent treatment.

\begin{tabular}{ccc}
\hline & $\begin{array}{c}\text { Start } \\
\text { Liquid, }\end{array}$ & $\begin{array}{c}\text { Final } \\
\text { Liquid }\end{array}$ \\
\hline Gold & $88.7 \mathrm{ppm}$ & $1.5 \mathrm{ppm}$
\end{tabular}




$\begin{array}{llc}\text { Antimony } & 21.0 \mathrm{~g} / \mathrm{L} & 21.1 \mathrm{~g} / \mathrm{L} \\ \text { Arsenic } & 5.31 \mathrm{~g} / \mathrm{L} & 5.21 \mathrm{~g} / \mathrm{L} \\ \text { Tin } & 1.84 \mathrm{~g} / \mathrm{L} & 1.89 \mathrm{~g} / \mathrm{L} \\ \text { Mercury } & 274 \mathrm{ppm} & 274 \mathrm{ppm}\end{array}$

Table IX - Overall gold selectivity and recovery

\begin{tabular}{lll}
\hline & Liquid & Solid \\
\hline Gold & $1.7 \%$ & $98.3 \%$ \\
Antimony & $100.0 \%$ & $0.0 \%$ \\
Arsenic & $100.0 \%$ & $0.0 \%$ \\
Tin & $100.0 \%$ & $0.0 \%$ \\
Mercury & $100.0 \%$ & $0.0 \%$
\end{tabular}

Finally the alkaline sulfide solutions, which are barren of gold, can be recycled for further gold leaching or further processed with low temperature oxidation to sodium sulfate, $\mathrm{Na}_{2} \mathrm{SO}_{4}$. This has been routinely practiced in industry (22). The resultant sodium sulfate is further treated by purification and crystallization to produce high grade, marketable sodium sulfate. This process is illustrated simplistically in the following scenario:

$$
\begin{gathered}
2 \mathrm{O}_{2}+\mathrm{Na}_{2} \mathrm{~S} \rightarrow \mathrm{Na}_{2} \mathrm{SO}_{4} \\
3.5 \mathrm{O}_{2}+2 \mathrm{NaOH}+\mathrm{Na}_{2} \mathrm{~S}_{2} \rightarrow 2 \mathrm{Na}_{2} \mathrm{SO}_{4}+\mathrm{H}_{2} \mathrm{O} \\
8 \mathrm{O}_{2}+8 \mathrm{NaOH}+\mathrm{Na}_{2} \mathrm{~S}_{5} \rightarrow 5 \mathrm{Na}_{2} \mathrm{SO}_{4}+4 \mathrm{H}_{2} \mathrm{O} \\
2 \mathrm{NaOH}+2 \mathrm{O}_{2}+\mathrm{Na}_{2} \mathrm{~S}_{2} \mathrm{O}_{3} \rightarrow 2 \mathrm{Na}_{2} \mathrm{SO}_{4}+\mathrm{H}_{2} \mathrm{O}
\end{gathered}
$$

This versatile and environmentally benign chemical is then sold and utilized in industries such as pulp and paper, glass, ceramics, detergents, mineral feed supplements, textile dyes, bleach and photography. As such, there are no environmental or toxicological issues in the use of alkaline sulfide gold recovery because the waste products become value-added, marketable by-products.

The sodium sulfate produced can be used to regenerate the sodium hydroxide needed in the process in a manner analogous to industrial dual alkali scrubbing systems (23). This is as follows:

$$
2 \mathrm{Na}_{2} \mathrm{SO}_{4}+\mathrm{Ca}(\mathrm{OH})_{2} \rightarrow \mathrm{CaSO}_{4}+2 \mathrm{NaOH}
$$


The clean gypsum product can then be marketed and used in such applications as agricultural soil amendments or as an additive in primary cement manufacture. In addition, initial efforts are underway and have been successful in regenerating the necessary $\mathrm{H}_{2} \mathrm{SO}_{4}$ and $\mathrm{NaOH}$ reagents from the $\mathrm{Na}_{2} \mathrm{SO}_{4}$ by-product. The details of this process will be given in future publications and may be illustrated as follows:

$$
\mathrm{Na}_{2} \mathrm{SO}_{4}+2 \mathrm{H}_{2} \mathrm{O} \rightleftarrows \mathrm{H}_{2} \mathrm{SO}_{4}+2 \mathrm{NaOH}
$$

\section{APPLICATIONS OF ALKALINE SULFIDE HYDROMETALLURGY}

NSC partial sulfide oxidation and alkaline sulfide application to a gold bearing arsenopyrite concentrate.

An example of this application of NSC partial oxidation of sulfide to sulfur in the arsenopyrite gold concentrate of Table IV by NSC is illustrated in Table X .

Table X - Nitrogen species catalyzed partial sulfide oxidation leach conditions

\begin{tabular}{ll}
\hline Operating Parameter & Level \\
\hline Initial free sulfuric acid & $50 \mathrm{~g} / \mathrm{L}$ \\
Reactor working pressure & $620 \mathrm{kPag}$ \\
Slurry solids content & $100 \mathrm{~g} / \mathrm{L}$ \\
Solids size & $80 \%$ minus 10 micron \\
Maximum temperature & $125^{\circ} \mathrm{C}$ \\
Nitrogen species concentration & $2.0 \mathrm{~g} / \mathrm{L}$ \\
\hline
\end{tabular}

Gold is subsequently leached with alkaline sulfide hydrometallurgy recovering $93.3 \%$ of it.

NSC partial sulfide oxidation and alkaline sulfide application to a gold bearing chalcopyrite concentrate

Moreover, NSC partial sulfide oxidation pressure leaching followed by alkaline sulfide gold leaching and recovery is an advantageous application for treatment of copper sulfide gold ores and concentrates. First of all, primary sulfide copper ores and concentrates such as chalcopyrite can be effectively treated at low temperatures and pressures. Then, the sulfur produced can be used to directly leach and recover the gold. This is particularly advantageous in that it avoids the large cyanide consumptions often experienced when treating copper ores and concentrates. The following example illustrates the treatment of a gold bearing chalcopyrite ore (24).

500 tonnes per day of a gold bearing chalcopyrite ore has been proposed to be treated by NSC partial sulfide oxidation pressure leaching followed by SX-EW production of copper. The gold will be recovered via alkaline sulfide leaching followed by electrowinning. Table XI illustrates the plant conditions utilized while Table XII shows result of NSC partial sulfide oxidation on the chalcopyrite concentrate. Table XIII delineates the site-specific economics of the application for treatment of the chalcopyrite ore. Figure 4 illustrates the proposed flowsheet. A key aspect here is to maximize sulfide oxidation to elemental sulfur. This minimizes oxygen consumption, reduces in-situ acid production and the closed circuit process causes in-situ iron precipitation. 
This limits the amount of iron and acid build up in the process circuit thereby limiting amount of the circuit impurity bleed stream. As well, the elemental sulfur produced, acts as a non-cyanide lixiviant for gold in the alkaline sulfide recovery system. Another key aspect is that the solutions recycled do not buildup nitrates or nitrites, which could be damaging to the solvent extraction reagents. Continuous closed circuit testing and analysis of the flowsheet has consistently shown both nitrogen species solution concentrations of less than $0.1 \mathrm{ppm}$. Thus, by in-situ methods, the closed circuit NSC system does not allow any buildup of nitrogen species in the recycled solutions, which may be harmful.

Table XI - Nitrogen Species Catalyzed Partial Oxidation Leach Conditions with In-Situ Iron Precipitation

\begin{tabular}{ll}
\hline Operating Parameter & Level \\
\hline Initial free sulfuric acid & $15 \mathrm{~g} / \mathrm{L}$ \\
Reactor working pressure & $620 \mathrm{kPag}$ \\
Slurry solids content & $100 \mathrm{~g} / \mathrm{L}$ \\
Solids size & $80 \%$ minus 10 micron \\
Maximum temperature & $125^{\circ} \mathrm{C}$ \\
Nitrogen species concentration & $2.0 \mathrm{~g} / \mathrm{L}$ \\
\hline
\end{tabular}

Table XII - Summary of Nitrogen Species Catalyzed Partial Oxidation Leach

\begin{tabular}{cc}
\multicolumn{2}{c}{ Composition of Chalcopyrite Ore } \\
Element & Assay \\
$\mathrm{Cu}$ & $3.2 \%$ \\
$\mathrm{Fe}$ & $4.1 \%$ \\
$\mathrm{Total} \mathrm{S}$ & $5.7 \%$ \\
$\mathrm{Au}, \mathrm{g} / \mathrm{T}$ & $11.0 \mathrm{~g} / \mathrm{T}$
\end{tabular}

$\begin{array}{lcc}\text { Element } & \text { Solution } & \text { Solid } \\ \mathrm{Cu} & 99.0 \% & 1.0 \% \\ \mathrm{Fe} & 8.3 \% & 99.7 \% \\ \text { Total S } & 3.4 \% & 96.6 \% \\ \mathrm{Au} & 0.0 \% & 100.0 \%\end{array}$

Both sulfuric acid generation and iron dissolution are minimized along with maximum production of elemental sulfur to be utilized in non-cyanide gold recovery. Alkaline sulfide leaching coupled with electrowinning recovered $93.1 \%$ of the gold.

Table XIII - Summary of Site Specific Economics for NSC Partial Oxidation of a Gold Bearing Chalcopyrite Ore 


\begin{tabular}{lc}
\hline Economic Parameter & Cost \\
\hline NSC Chalcopyrite Ore Partial Oxidation Cost & $\$ 3,500,000.00$ \\
& USD \\
NSC Chalcopyrite Partial Oxidation Operating & $\$ 0.056$ USD/lb Cu \\
Cost & or \$ 0.36 USD/g \\
& $\mathrm{Au}$ \\
\hline
\end{tabular}

The costs shown in Table XIII are site specific for this particular project and represent the limited information allowed for publication on a client privileged basis.

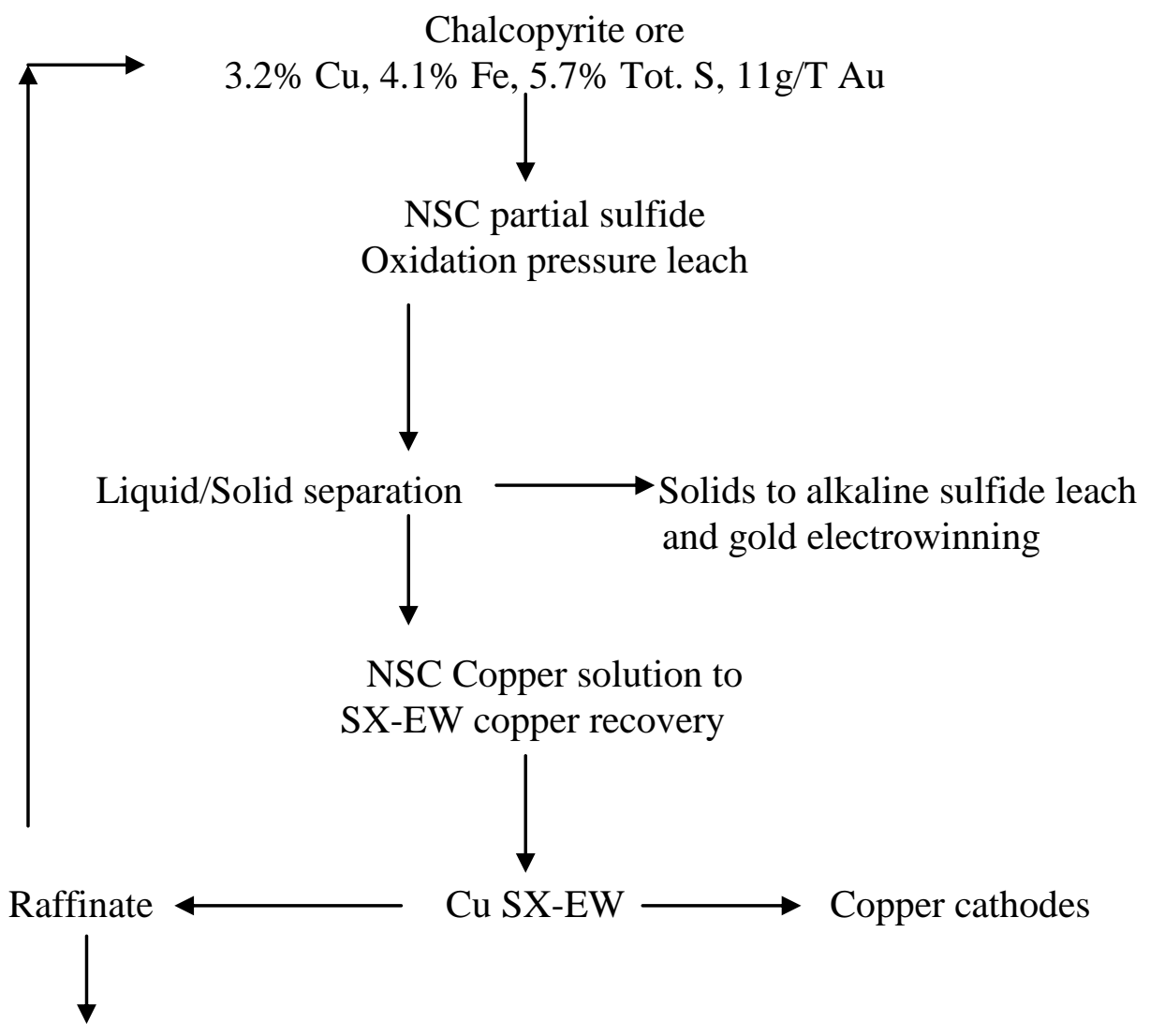

Copper circuit impurity bleedstream

Figure 4 - Flowsheet of NSC partial sulfide oxidation of a gold bearing chalcopyrite ore 


\section{Alkaline sulfide application to a pregrobbing carbonaceous ore}

As alkaline sulfide lixiviants are not pregrobbed of gold in the same manner as the cyanide system can be, many carbonaceous ores and concentrates can be more effectively leached with this system. A pregrobbing ore of the quality listed in Table XIV. was treated under the conditions listed in Table XV. As seen, the gold recovery to solution was $96.4 \%$, and it could be recovered from solution by the techniques previously outlined.

Table XIV - Assay of pregrobbing gold ore treated with alkaline sulfide

\begin{tabular}{lr}
\hline Element & \multicolumn{1}{c}{ Assay } \\
\hline $\mathrm{Au}$ & $7.0 \mathrm{~g} / \mathrm{T}$ \\
$\mathrm{Fe}$ & $2.1 \%$ \\
Carbon & $10.3 \%$ \\
Total S & $1.4 \%$ \\
\hline
\end{tabular}

Table XV - Alkaline sulfide baseline leach conditions and results

\begin{tabular}{ll}
\hline Operating Parameter & Level \\
\hline Free NaOH & $10 \mathrm{~g} / \mathrm{L}$ \\
Total sulfur content & $50 \mathrm{~g} / \mathrm{L}$ \\
Slurry solids content & $100 \mathrm{~g} / \mathrm{L}$ \\
Temperature & $50^{\circ} \mathrm{C}$ \\
Reaction Time & $24 \mathrm{Hours}$ \\
Gold Recovery to Solution & $96.4 \%$ \\
\hline
\end{tabular}

\section{Alkaline sulfide application to a cyanide consuming ore}

For many cyanide consuming elements and metals in gold ores and concentrates such as zinc and copper sulfides, the alkaline sulfide hydrometallurgical system does not suffer from this problem. Copper and zinc are insoluble in the alkaline sulfide system so there is little or no reagent 
consumption. A cyanide consuming ore of the quality listed in Table XVI. was treated under the conditions listed in Table XVII. As seen, the gold recovery to solution was 93.7\%, and it could be recovered from solution by the techniques previously outlined.

Table XVI - Assay of cyanide consuming gold material treated with alkaline sulfide

\begin{tabular}{lc}
\hline Element & Assay \\
\hline $\mathrm{Au}$ & $11.0 \mathrm{~g} / \mathrm{T}$ \\
$\mathrm{Zn}$ & $3.3 \%$ \\
$\mathrm{Cu}$ & $1.1 \%$ \\
Total S & $9.3 \%$ \\
\hline
\end{tabular}

Table XVII - Alkaline Sulfide Baseline Leach Conditions and Results.

\begin{tabular}{ll}
\hline Operating Parameter & Level \\
\hline Free NaOH & $10 \mathrm{~g} / \mathrm{L}$ \\
Total Sulfur Content & $50 \mathrm{~g} / \mathrm{L}$ \\
Slurry Solids Content & $100 \mathrm{~g} / \mathrm{L}$ \\
Temperature & $500 \mathrm{C}$ \\
Reaction Time & 24 hours \\
Gold Recovery to Solution & $93.7 \%$ \\
\hline
\end{tabular}

\section{Relative reagent costs of alkaline sulfide versus cyanide}

The relative reagent costs of sodium cyanide versus alkaline sulfide produced from sulfur are illustrated in Table XVIII. This does not include the by-product revenues or regeneration available when using the alkaline sulfide system or the costs associated with destruction of waste cyanide. Thus, as seen, there can be significant economic incentives with the use of alkaline sulfide leaching of gold instead of cyanide. This advantage was illustrated before in the treatment of gold bearing chalcopyrite where conventional pressure leaching followed by cyanidation is far more expensive.

Table XVIII- Comparative Cost of Gold Leaching Reagents

\begin{tabular}{cc}
\hline $\begin{array}{c}\text { Reagent } \\
\text { Type }\end{array}$ & Cost \\
\hline Sodium & $\$ 1.00 \$ 2.25 \mathrm{USD} / \mathrm{Kg}$ \\
cyanide & \\
Alkaline & $\$ 0.05-\$ 0.30 \mathrm{USD} / \mathrm{Kg}$ \\
sulfide & \\
\hline
\end{tabular}




\section{CONCLUSIONS}

This paper has outlined the development, fundamental basis and applications of alkaline sulfide hydrometallurgical recovery of gold. Now, that this industrially applied hydrometallurgical system is well understood, it will be applied on a macro scale at CAMP on many more complex gold ores and concentrates.

\section{ACKNOWLEDGEMENTS}

The authors would like to thank the Center for Advanced Separation Technology at Virginia Tech, The Montana Board of Research Commercialization Technology and the International Progam at Montana Tech for their support of some of this work.

\section{REFERENCES}

1. G. Van Weert, K. Fair, and J. Schneider, "The Nitrox Process for Treating Gold Bearing Arsenopyrites.”, Annual TMS/SME Proceedings, Denver, Colorado, USA, 1987.

2. M. Beattie and A. Ismay, "Applying the Redox Process to Arsenical Concentrates", Journal of Metals, Jan., pp 31-35, 1990.

3. C. G. Anderson, K. D. Harrison, and L. E. Krys, "Process Integration Of Sodium Nitrite Oxidation And Fine Grinding In Refractory Precious Metal Concentrate Pressure Leaching", Precious Metals 1993, Proceedings of the 17th IPMI Conference, June, 1993.

4. C.G. Anderson, "Applications of NSC Pressure Leaching”, Pressure Hydrometallurgy M. Collins and V. Papangelakis eds., $34^{\text {th }}$ Annual Hydrometallurgy Meeting of CIM, Banff, Alberta, Canada, 2004.

5. C. G Anderson, K. D. Harrison, and L. E., Krys, "Theoretical Considerations of Sodium Nitrite Oxidation and Fine Grinding In Refractory Precious Metals Concentrate Pressure Leaching", Minerals and Metallurgical Processing, AIME-SME, Volume 13, Number 1, February, 1996.

6. S. A., Baldwin and G. Van Weert, "On the Catalysis of Ferrous Sulfate Oxidation in Autoclaves by Nitrates and Nitrites", Hydrometallurgy, Elsevier Science, B.V., Vol. 42., 1996.

7. C.G. Anderson, L.E. Krys, and K. D., Harrison "Treatment of Metal Bearing Mineral Material", Sunshine Mining Co., U.S. Patent \#5,096,486, March 17, 1992.

8. E. Peters, Hydrometallurgical Process Innovation, Hydrometallurgy, 29, 1992, pp 431-459.

9. T.R. Barth, A.T.C. Hair, and T.P. Meier, "The Operation of the HBMS Zinc Pressure Leach Plant", Zinc and Lead Processing,Ed. Dutrizac, J.E., et. al., The Metallurgical Society of CIM, 1998. 
10. K. J. Fair, and G., Van Weert, "Optimizing the NITROX PROCESS Through Elemental Sulfur Formation", Precious Metals 1989, Ed. Harris, B., The International Precious Metals Institute, 305-317, Montreal, Quebec, Canada, 1989.

11. B. Krysa, B. Barlin, and D.Wittleton, "The Application of Zinc Pressure Leaching at the Hudson Bay Mining and Smelting Co. Limited", Projects '88, Paper \#8, $18^{\text {th }}$ Hydrometallurgical Meeting CIM, May, 1988.

12. M. Pourbaix, Atlas of Electrochemical Equilibria in Aqueous Solutions, Pergamon, Press, London, 1966.

13. H. H. Huang, StabCal Modeling Software, September, 2002.

14. H.H. Huang, L.G. Twidwell, C.G. Anderson and C.T. Young, "Chemical Titration Simulation - An Equilibrium Calculation Approach", The International Conference on Computational Analysis in Hydrometallurgy, $35^{\text {th }}$ Annual Hydrometallurgy Meeting of CIM, Calgary, Alberta, Canada, August, 2005.

15. D.L. Stacey, Development of the Alkaline Sulfide Gold Leaching Process, Metallurgical and Materials Engineering Master of Science Thesis, Montana Tech of the University of Montana, May, 2005.

16. C.G. Anderson, "The Chemical Analysis of Industrial Alkaline Sulfide Hydrometallurgical Processes", The Society of Mineral Analysts and the Canadian Mineral Analysts Annual Meeting, Spokane, Washington, April, 2002.

17. M. Jeffrey, and C.G. Anderson, "A Fundamental Study of the Alkaline Sulfide Leaching of Gold", The European Journal of Mineral Processing and Environmental Protection, Spring, 2003.

18. M. Jeffrey, N. Chapman, and J. Wall, "Sulfide/Polysulfide Leaching of Gold", Unpublished research report, Monash University, Department of Chemical Engineering, Melbourne, Australia, 2002.

19. C. G. Anderson, S.M. Nordwick and L.E. Krys, "Processing of Antimony at the Sunshine Mine", Residues and Effluents - Processing and Environmental Considerations, Edited by R.G. Reddy, W. P. Imrie, P.B. Queneau, AIME-TMS, San Diego, 1992, pp. 349-366.

20. C.G. Anderson, "The Hydrometallurgical Treatment of Mercury Bearing Ores and Concentrates", International Mercury Symposium, TMS Annual Meeting, San Diego, California, February 2003.

21. C.G. Anderson, "The Treatment of Arsenic Bearing Ores, Concentrates and Materials with Alkaline Sulfide Hydrometallurgy”, TMS International Arsenic Symposium, TMS Annual Meeting, San Francisco, 2005. 
22. C. R. Edwards, "Engineering the Equity Concentrate Leach Process",

Complex Sulfides: Processing of Ores, Concentrates and By-Products. Eds. Zunkel, A.D. et al, Proceedings of a symposium sponsored by the Metallurgical Society of

AIME and the CIMM, TMS-AIME Fall Extractive Meeting, San Diego CA, Nov.

10-13 1985, p. 197-219.

23. R.R Lunt, D.K. Modrow and G.K. Roset, "Adaption of Dilute Mold Lime Dual Alkali Scrubbing at Stillwater Mining Company's PGM Smelter", Hydrometallurgy 2003, Vancouver, B.C. October 2003.

24. C.G. Anderson, "The Application and Economics of Industrial Nitrogen Species Catalyzed Pressure Leaching and Non-Cyanide Precious Metals Recovery to Chalcopyrite Ores and Concentrates", COBRE 2003, Santiago,Chile. 
Appendix 21: Simultaneous Copper Cathode Production and Ferric Sulfate Regeneration (MT002) 


\title{
FINAL TECHNICAL REPORT
}

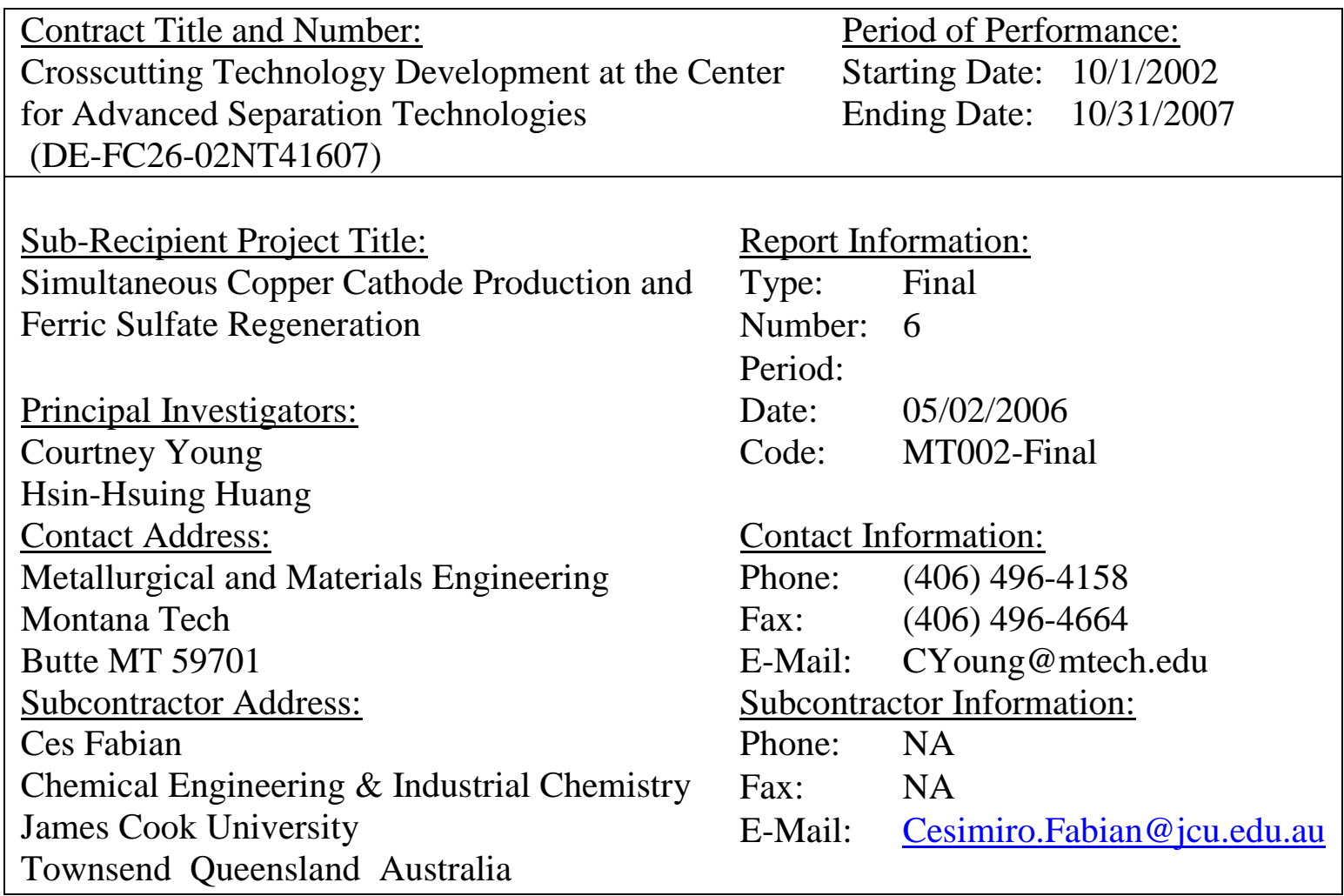

\section{DISCLAIMER}

This report was prepared as an account of work sponsored by an agency of the United States Government. Neither the United States Government nor any agency thereof, nor any of their employees, make any warranty, express or implied, nor assume any legal liability or responsibility for the accuracy, completeness, or usefulness of any information, apparatus, product, or process disclosed, or represents that its use would not infringe privately owned rights. Reference herein to any specific commercial product, process, or service by trade name, trademark, manufacturer, or otherwise does not necessarily constitute or imply endorsement, recommendation, or favoring by the United States Government or any agency thereof. The views and opinions of authors expressed herein do not necessarily state or reflect those of the United States Government or agency thereof.

\begin{abstract}
Various tests were conducted over a three-year period to investigate the feasibility of electrowinning copper $\left(\mathrm{Cu}^{0}\right)$ from cupric $\left(\mathrm{Cu}^{2+}\right)$ while simultaneously oxidizing ferrous $\left(\mathrm{Fe}^{2+}\right)$ to ferric $\left(\mathrm{Fe}^{3+}\right)$ using a membrane cell. Results indicated that several variables would be important to consider to minimize the applied voltage while keeping current density at a maximum. These variables included electrolyte concentrations $\left(\mathrm{Cu}^{2+}\right.$ and $\left.\mathrm{Fe}^{2+}\right)$, agitation/flow-rate, applied voltage, and temperature. Other variables were also tested: cathode type, anode type, membrane type, and cell design; however, certain ones showed
\end{abstract}


superior behavior. Ensuing factorial-design experiments were therefore conducted with these 4 variables fixed in order to investigate the effects of the other variables. For simplicity and to minimize the number of experiments, the temperature was held constant at $20^{\circ} \mathrm{C}$ (room temperature). Results were then statistically analyzed using StatEase software and yielded the following model:

$$
\begin{aligned}
\log _{10}(\text { Current Density })= & 0.97876+3.56^{*} 10^{-3} *\left[\mathrm{Cu}^{2+}\right]-3.95 * 10^{-4} *\left[\mathrm{Fe}^{2+}\right]+0.51 * \text { Volts } \\
& +8.13 * \text { Flowrate }-3.1 * 10^{-4} * \text { Volt*Flowrate }
\end{aligned}
$$

From the model, it can be concluded that the current density and therefore copper production will be increased by increasing the cupric concentration $(\mathrm{g} / \mathrm{L})$, applied voltage $(\mathrm{V})$, and/or flowrate (ml/min); however, there was a small but noticeable binary effect between flowrate and applied voltage that had a negative effect on the system. Furthermore, a negative effect on current density would also be realized by increasing the ferrous concentration. It appears that the applied voltage and the flowrate are the most significant variables due to the large values of their respective coefficients. Optimization of the model indicates that the voltage can be decreased from an industrial standard of approximately 2.0 Volts to between 1.5-1.7 Volts which equates to energy savings of $15-25 \%$. Furthermore, the model concurs with fundamental understanding of electrowinning principles.

Various tests were also conducted in collaboration with Montana Resources, a local Montana mining company in Butte, to see if ferric leaching could be used on their Central Zone Ore (CZO) and implemented along with the membrane electrowinning technology. CZO does not respond as well as the traditional East and West Zone Ores in their flotation operation: recoveries run low between $85-90 \%$ and should be in excess of $90 \%$ whereas grades run between $15-20 \%$ copper and should be in excess of $28 \%$. The poor metallurgical performance is attributed to the different mineralization. Instead of the major copper mineral being chalcopyrite $\left(\mathrm{CuFeS}_{2}\right)$, it is a coating of covellite $(\mathrm{CuS})$, digenite $\left(\mathrm{Cu}_{1.8} \mathrm{~S}\right)$ and chalcocite $\left(\mathrm{Cu}_{2} \mathrm{~S}\right)$ on pyrite $\left(\mathrm{FeS}_{2}\right)$. In order to recover these secondary copper minerals, the pyrite must be floated which, of course, dilutes the concentrate grade. Furthermore, because the copper minerals are present as coatings, comminution likely causes their fines and thus leads to a low recovery as well. On the other hand, the mineralogy is perfect for ferric leaching. In this regard, preliminary test were run to help establish important variables for leaching: particle size of the ore, percent solids by weight, temperature of the system, and duration of the leach. Factorial design experiments were therefore conducted to develop a model that would lead to optimal leaching conditions. Unfortunately, results indicate that no more than approximately 60\% extraction can be achieved. Diagnostic leach tests indicate that primary copper sulfide minerals must be present (i.e., chalcopyrite) in CZO because they are known not to leach well with ferric. An exhaustive literature search of the geology and mineralization concurs thereby concluding that ferric leaching is not a method to consider for copper extraction. However, as a result of this study, Montana Resources is exploring options for recovering the copper by leaching. It is recommended that they do so by processing the CZO through their current facilities and using an agitation leach on the resulting flotation concentrate to minimize the amount of material being contacted. This would also prevent problems from the clay content which is present in the CZO but is mostly removed from the flotation process. 


\section{TABLE OF CONTENTS}

DISCLAIMER

ABSTRACT

TABLE OF CONTENTS

iii

EXECUTIVE SUMMARY

INTRODUCTION

EXPERIMENTAL 3

RESULTS AND DISCUSSION 3

Task 1 - Cell Design and Selection 3

Suspended Cathode Compartment (SCC) 4

Slotted Design (SD)

Two-Half Cell (THC)

Task 2 - Cell Optimization

4

Task 3 - Ferric Leaching Application

5

Task 4 - Ferric Leaching Optimization

9

Task 5 - Diagnostic Leach

CONCLUSIONS

FUTURE WORK

REFERENCES

PUBLICATIONS AND PRESENTATIONS 


\section{EXECUTIVE SUMMARY}

Various tests were conducted over a three-year period to investigate the feasibility of electrowinning copper $\left(\mathrm{Cu}^{0}\right)$ from cupric $\left(\mathrm{Cu}^{2+}\right)$ while simultaneously oxidizing ferrous $\left(\mathrm{Fe}^{2+}\right)$ to ferric $\left(\mathrm{Fe}^{3+}\right)$ using a membrane cell with the goal of minimizing energy consumption. Several variables were found to be important: electrolyte concentrations $\left(\mathrm{Cu}^{2+}\right.$ and $\mathrm{Fe}^{2+}$ ), agitation/flow-rate, applied voltage, and temperature. Factorial-design experiments were conducted on these variables but, for simplicity, the temperature was held constant at room temperature. Concentrations of $\mathrm{Cu}^{2+}$ and $\mathrm{Fe}^{2+}$ were varied between 10 and $50 \mathrm{~g} / \mathrm{L}$, flowrates were varied between 0 and $540 \mathrm{~mL} / \mathrm{min}$, and the applied voltage was varied between 0.8-2.5 Volts. Results were then statistically analyzed using StatEase software to develop a model for optimizing. Optimization of the model indicates that the voltage could be decreased from an industrial standard of approximately 2.0 Volts to between 1.5-1.7 Volts which equates to energy savings of $15-25 \%$. Furthermore, the model concurs with fundamental understanding of electrowinning principles.

Various tests were also conducted in collaboration with Montana Resources, a local mining company in Butte, MT, to see if ferric leaching could be used on their Central Zone Ore (CZO) and implemented along with the membrane electrowinning technology. CZO cotains mineralogy that is perfect for ferric leaching. In this regard, preliminary test helped establish important variables for leaching and included particle size of the ore, percent solids by weight, temperature of the system, and duration of the leach. Factorial design experiments were conducted to develop a model that would lead to optimal leaching conditions. Unfortunately, results indicated that no more than approximately $60 \%$ extraction could be achieved. Diagnostic leach tests indicated that primary copper sulfide minerals must be present (i.e., chalcopyrite) in CZO because they do not leach well with ferric. A literature search of the geology and mineralization concurred and thereby concluded that ferric leaching is not a method to consider for copper extraction. However, as a result of this study, Montana Resources is exploring options for recovering the copper by leaching. It is recommended that they process $\mathrm{CZO}$ through their current flotation facilities but use an agitation leach on the resulting flotation concentrate to minimize the amount of material being contacted. This would also prevent problems from the clay content which is present in the CZO but would be mostly removed by the flotation process.

\section{INTRODUCTION}

An integrated closed-circuit process for treating copper sulfide minerals based on acidic ferric leaching and the subsequent electrolytic recovery of copper was proposed as an alternative low cost method for copper production (Young et al., 2002; Fabian et al., 2001). The process was based on the simultaneous recovery of copper and regeneration of ferric ions using a membrane-separated electrolytic cell divided into anolyte and catholyte compartments.

In conventional copper electrowinning, the cathodic reaction is the electrodeposition of copper on a stainless steel or copper starting sheet and the anodic reaction is the dissociation 
of water into hydrogen ions and oxygen resulting in the production of acid mist as described by the following electrochemical half-cell reactions:

$$
\begin{array}{lll}
\text { Cathodic Reaction: } & \mathrm{Cu}^{2+}+2 \mathrm{e}^{-} \rightarrow \mathrm{Cu}^{0} & \mathrm{E}_{\mathrm{c}}^{\mathrm{o}}=0.337 \mathrm{~V} \\
\text { Anodic Reaction: } & 2 \mathrm{H}_{2} \mathrm{O} \rightarrow 4 \mathrm{H}^{+}+\mathrm{O}_{2}+4 \mathrm{e}^{-} & \mathrm{E}_{\mathrm{a}}^{\mathrm{o}}=1.229 \mathrm{~V}
\end{array}
$$

From the half-cell reactions (Bard et al., 1985), an overall theoretical cell voltage of $0.892 \mathrm{~V}$ is calculated. However, this voltage is enough to barely get the reactions to occur and, as a result, applied voltages on an industrial scale are approximately 2 Volts. This is equivalent to an over-voltage of 1.1 Volts.

Alternatively, the electrochemical reactions occurring in the proposed electrolytic cell would result in the deposition of copper from cupric ions and the oxidation of ferrous ions to ferric ions:

$$
\begin{array}{lll}
\text { Cathodic Reaction: } & \mathrm{Cu}^{2+}+2 \mathrm{e}^{-} \rightarrow \mathrm{Cu}^{0} & \mathrm{E}_{\mathrm{c}}^{\mathrm{o}}=0.337 \mathrm{~V} \\
\text { Anodic Reaction: } & 2 \mathrm{Fe}^{2+} \rightarrow 2 \mathrm{Fe}^{3+}+2 \mathrm{e}^{-} & \mathrm{E}_{\mathrm{a}}^{\mathrm{o}}=0.771 \mathrm{~V}
\end{array}
$$

In this case, the difference in voltage for the half-cell reactions yields a theoretical cell voltage of $0.434 \mathrm{~V}$. Because the over-voltage of 1.1 Volts is expected to be the same, an overall applied voltage of approximately 1.5 Volts is estimated for this system. Thus, the proposed cell would offer a net reduction from 2 Volts to 1.5 Volts and thereby reduce energy consumption by approximately 0.5 Volts and save as much as $25 \%$ on electrical costs. Resulting ferric ions $\left(\mathrm{Fe}^{3+}\right)$ from the process would be recycled back to leaching:

$$
\begin{array}{ll}
\text { Metal Leach: } & \mathrm{Cu}^{0}+2 \mathrm{Fe}^{3+} \rightarrow \mathrm{Cu}^{2+}+2 \mathrm{Fe}^{2+} \\
\text { Sulfide Leach: } & \mathrm{Cu}_{\mathrm{x}} \mathrm{S}+2 \mathrm{xFe}^{3+} \rightarrow \mathrm{xCu}^{2+}+2 \mathrm{xFe}^{2+}+\mathrm{S}^{0} \quad \text { or } \\
& \mathrm{Cu}_{\mathrm{x}} \mathrm{S}+2 \mathrm{xFe}^{3+}+\mathrm{H}_{2} \mathrm{O}+3 / 2 \mathrm{O}_{2} \rightarrow \mathrm{xCu}^{2+}+2 \mathrm{xFe}^{2+}+\mathrm{SO}_{4}^{2-}+2 \mathrm{H}^{+}
\end{array}
$$

where, for example, $x=1$ (covellite), $x=2$ (chalcocite) and $x=1.8$ (digenite). The Mount Gordon process in Australia is based on this leaching technology and requires the solutions to be acidic ( $<\mathrm{pH} 2)$ to prevent iron precipitation (Baxter et al., 1999). Resulting cupric ions $\left(\mathrm{Cu}^{2+}\right)$ and ferrous ions $\left(\mathrm{Fe}^{2+}\right)$ can be electrowon directly (Marsden et al., 2005) but leads to poor quality of copper cathode or can be separated by conventional solvent extraction/electrowinning (SX/EW) technology (Twidwell et al., 1974) but can lead to high energy consumption as described earlier.

In this regard, a process was envisioned in which conventional SX is used to produce a pregnant solution of $\mathrm{Cu}^{2+}$ and a raffinate solution of $\mathrm{Fe}^{2+}$. These solutions are fed into the proposed membrane-separated electrolytic cell as catholyte and anolyte respectively such that the simultaneous electrowinning of copper and regeneration of ferric ions can be accomplished thereby reducing the energy consumption. Resulting copper is expected to be high-grade and resulting ferric ions would be recycled to leaching. Because oxygen evolution is eliminated, problems associated with acid-misting during electrowinning would also be prevented. 


\section{EXPERIMENTAL}

Project objectives were four-fold and included (1) developing an electrolytic cell with two compartments separated by a membrane to allow for the simultaneous regeneration of ferric ions and electrowinning of copper, (2) optimizing the electrowinning process to minimize electrical consumption with maximum current density, (3) developing a ferric leaching process for implementation in an industrial setting, and (4) optimizing the leaching process for implementing, along with the electrowinning process, in an industrial setting. To do this, a systematic approach was taken for both the electrowinning and leaching studies.

For the electrowinning study, several cell designs were drafted and, if accepted for experimentation, were constructed and tested. All designs were based on conventional cell geometry but many were eliminated based on the difficulty of including the membrane in the construction. Three designs were eventually tested and, from these, one was selected based on performance as measured by maximum current density. Preliminary tests on this design were run in order to determine ranges for a number of variables including flowrate, concentration, temperature, membrane type, and applied voltage. With variable ranges established, factorial-design experiments were conducted and evaluated using statistical modeling with StatEase software (Anderson and Whitcomb, 2005). The resulting model was then optimized with constraints of minimum voltage and maximum current density. Mr. Francis Dakubo (2006) did this thesis work.

For the leaching study, an ore from a local company (Montana Resources) was determined to be appropriate for examination based on discussions with them (Czehura, 2004). Because mineralogical analyses showed that the ore contained significant amounts of clay, heap leaching methods were ignored and preliminary tests were conducted using two-liter kettles and mixers for agitation leaching. Solution and solid analyses for copper content were performed by the company using proprietary techniques. Results were used to determine \% $\mathrm{Cu}$ extraction. Several variables were tested to establish their ranges as well: particle size, solids content, leach duration, and temperature. With variable ranges established, factorial design experiments were conducted and evaluated using StatEase software in hopes of developing a model for maximizing copper leaching. Mr. Thomas McIntyre (2006) did this thesis work.

\section{RESULTS AND DISCUSSION}

\section{$\underline{\text { Task } 1 \text { - Cell Design and Selection }}$}

Three electrolytic cells were constructed and tested. These designs included and named (1) Suspended Cathode Compartment (SCC), (2) Slotted Design (SD), and (3) Two-Half Cell (THC) and are shown in Figures 1-3, respectively. Each design consisted of the following parts a membrane (M), Cathode(C), Cathode Compartment (CC), Anode (A), Anode Compartment (AC), Overflow Weir (OW), and Feed Port (FP) and are appropriately labeled. Obviously, the membrane (M) separates the cathode compartment (CC) from the anode compartment (AC). In order to provide continuous flow, feed ports (FP) were needed to distribute each electrolyte and overflow weirs (OW) were needed to collect each after they 
passed through. Typically, the cells were set-up so that the overflow recirculated back to the feed reservoir via peristaltic pumps.

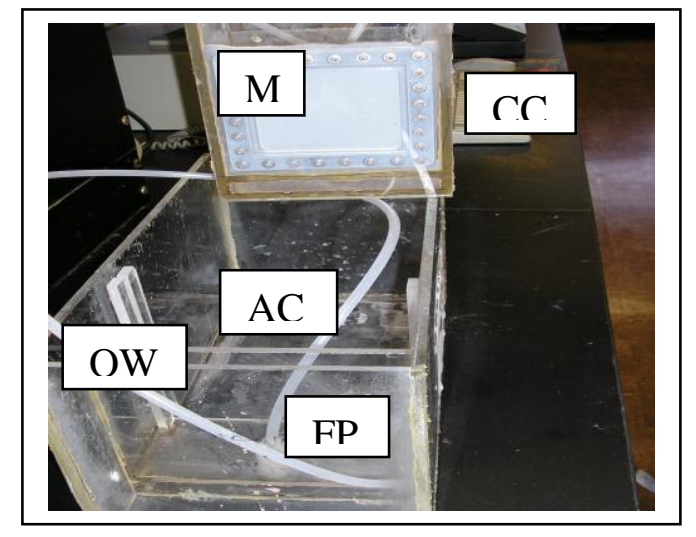

Figure 1. SCC electrolytic cell.

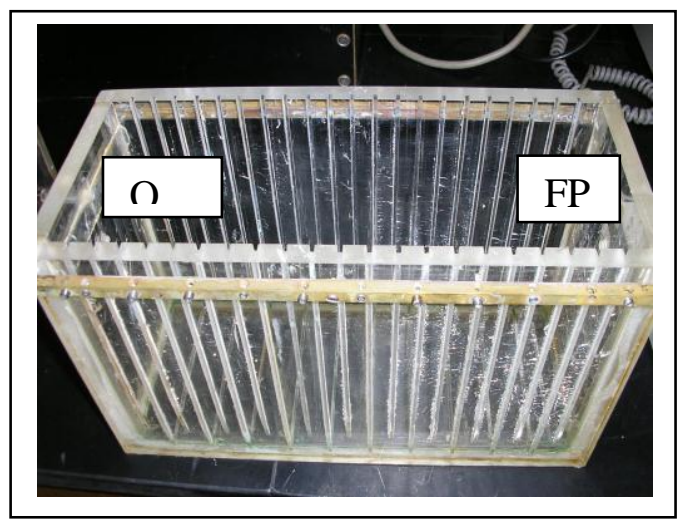

Figure 2. SD electrolytic cell.

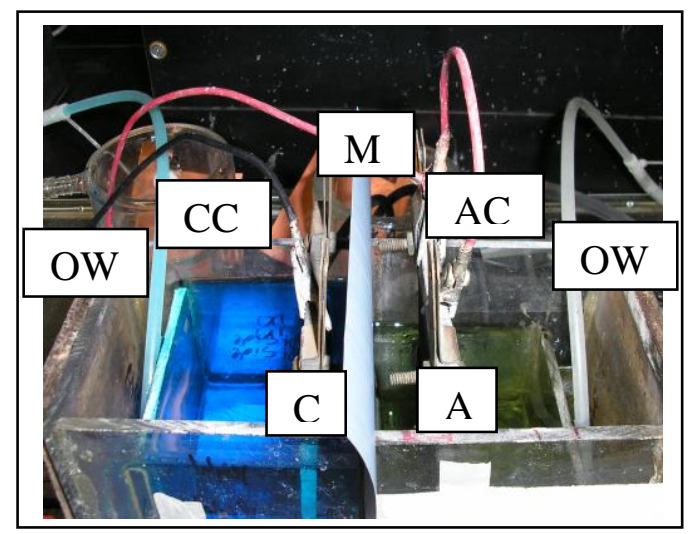

Figure 3. THC electrolytic cell.

\section{Suspended Cathode Compartment (SCC)}

Figure 1 shows the SCC electrolytic cell. After the membrane is attached to the cathode compartment (CC), the compartment is lowered into the anode compartment but can be easily raised if the membrane ever needed to be replaced. Consequently, the design was also referred to as "cell-within-a-cell" design but proved to be too cumbersome to attach the membrane.

\section{Slotted Design (SD)}

Figure 2 shows a photograph of the slotted electrolytic cell. The slots are equally spaced and are used to hold a membrane and any number of anodes and cathodes in alternate order which is in keeping with conventional cell designs. Electrolytes are introduced near the membrane and overflow a weir on the ends.

\section{Two-Half Cell (THC)}

In this design, the anode and cathode compartments are mirror images of each other and are sandwiched together with the membrane in between and two gaskets on each side of the membrane (see Figure 3). Each half-cell is fed through the base via an up-flow distributor to help move the electrolytes through. The half-cells are pressed together with a vice which allowed for easy assembly (and disassembly) of the membrane. In this illustration, a microporous ceramic membrane used in car batteries is shown. 
Each design was tested with fixed conditions: $30 \mathrm{~g} / \mathrm{L} \mathrm{Cu}^{2+}, 10 \mathrm{~g} / \mathrm{L} \mathrm{Fe}^{2+}, 150 \mathrm{~g} / \mathrm{L} \mathrm{H}_{2} \mathrm{SO}_{4}$ for both solutions, $100 \mathrm{ml} / \mathrm{min}$ flowrate for both solutions, 304 Stainless Steel as cathode with $10-\mathrm{cm}$ length and width dimensions yielding a surface area of $100 \mathrm{~cm}^{2}, \mathrm{~Pb}$ plate as anode with the same dimensions placed approximately $8 \mathrm{~cm}$ from cathode, a microporous ceramic membrane equidistant from the anode and cathode, room temperature, and an applied voltage of $2 \mathrm{~V}$. The THC design yielded the highest current density at $270 \mathrm{amps} / \mathrm{m}^{2}$ compared to the SCC design at $220 \mathrm{amps} / \mathrm{m}^{2}$ and the SD design at $100 \mathrm{amps} / \mathrm{m}^{2}$. In fact, each time other parameters were tested, the THC design was always found to be best and was attributed mostly to the improved flow through the compartments due to the up-flow distributor.

\section{Task 2 - Cell Optimization}

In order to optimize the operating conditions of the THC design, preliminary experiments were conducted to help determine what materials would be best as an anode, cathode and membrane and what range of conditions would be useful for factorial design testing. Using conditions similar to those noted above, it was determined that (1) either Copper or 304 Stainless Steel could be used as the cathode, (2) Carbon-cloth performed better as an anode material than Graphite-rods, Pb-plate and Pb-wool, and (3) microporous ceramic worked well and appeared to last indefinitely as compared to both cation and anion exchange membranes which decomposed under the conditions examined.

Using Stainless-Steel cathodes, Carbon-cloth anodes and microporous ceramic membranes, single-variable tests were performed in which all variables were held constant except one. This helped to establish operating ranges for some variables and determine which did not have a significant effect on current density. Some results are shown in Figures 4-7. As can be seen from each figure, the current densities start at some maximum value and eventually decay to a much lower value which occurs when all of the ferrous has been converted to ferric. When this happens, oxygen evolution begins and the electrowinning reaction slows considerably.

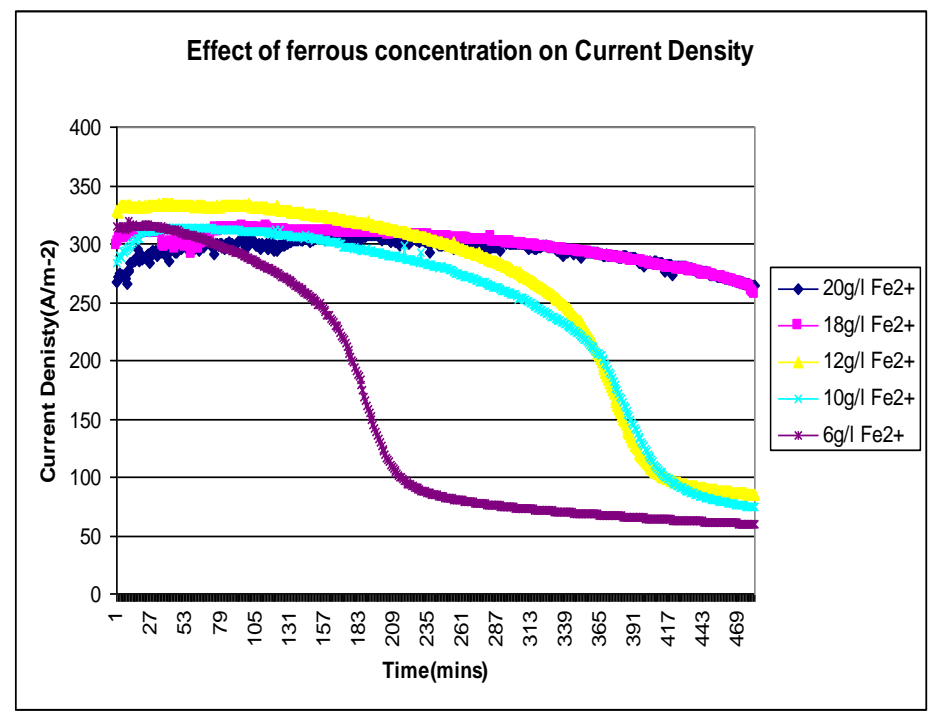

Figure 4. Effect of $\mathrm{Fe}^{2+}$ Concentration. 


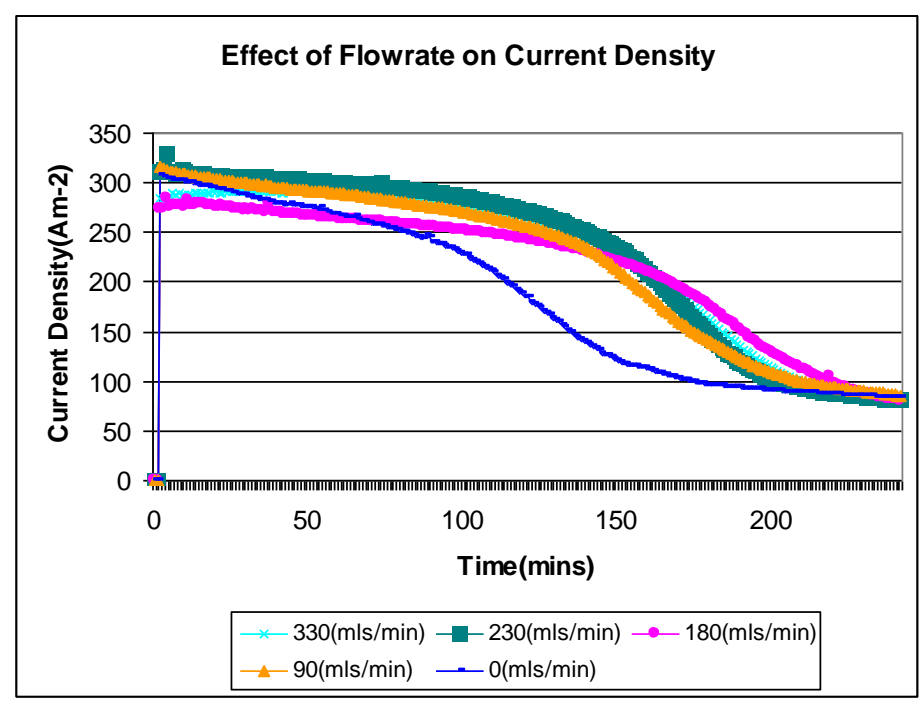

Figure 5. Effect of Flowrate

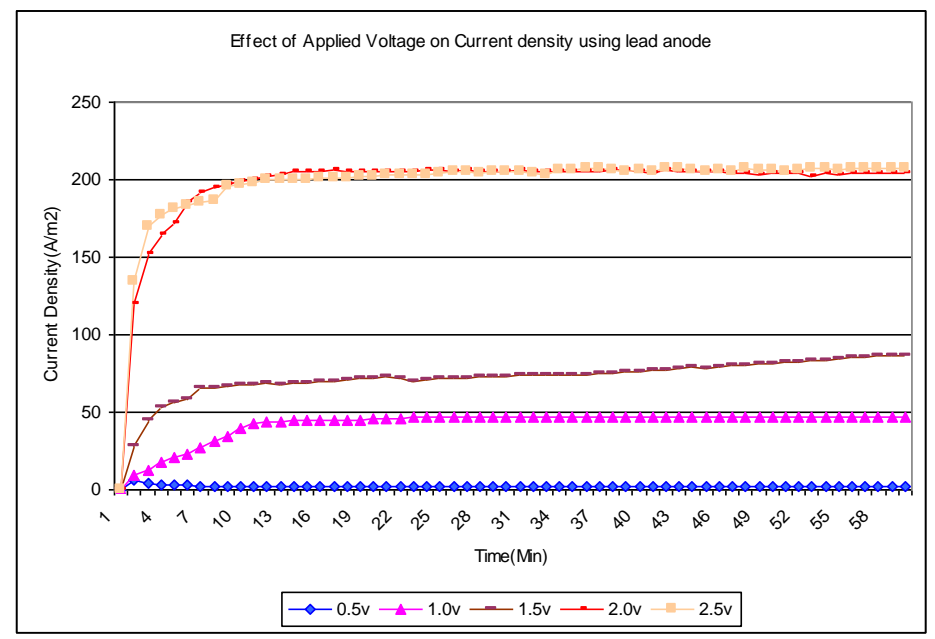

Figure 6. Effect of Applied Voltage

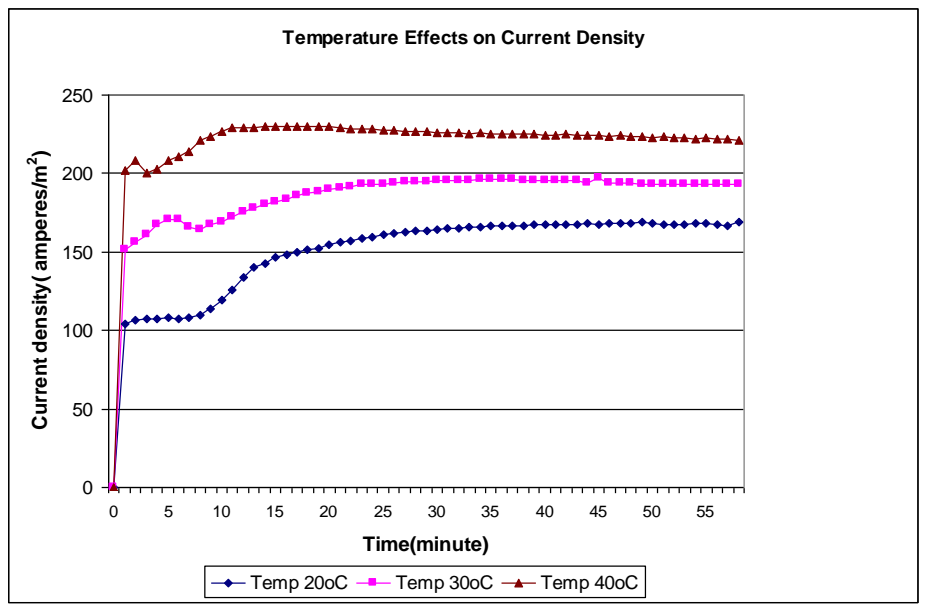

Figure 7. Effect of Temperature 
Because the solutions are being recirculated, higher ferrous concentrations simply allow the current density to be maintained longer as shown in Figure 4. In a real situation, this will depend on the residence time of the solution in the cell before it overflows and gets recycled to the leaching circuit. The same thing is observed in Figure 5 when flowrates are increased but is only apparent above stagnant. In this case, flow is needed to not only supply ferrous and cupric ions to their respective electrodes but also to remove ferric once it is produced. The slow step in these processes is envisioned to be supply ferrous to the anode because both are of the same charge. This explains why the high surface area electrodes were being considered and eventually the Carbon-cloth was selected. In regards to applied voltage, it is clear from Figure 6 that a voltage between 1.5-2 Volts is needed which is in agreement with expectations. Because an applied voltage of 2.5 Volts did not improve the results, it is evident that the system reached limiting current density. Furthermore, an examination of the copper cathode being produced showed that undesirable dendrites were beginning to form and would account for the low current efficiency of $90 \%$ as well as the noisy appearance of the plot. On the other hand, when voltages below 1.5 Volts were applied, current densities were too low to produce copper at an appreciable rate but yielded current efficiencies of 99.5\%. In comparison, the current efficiency at 2 Volts was 98\%. Finally, Figure 7 shows that current densities can be moderately increased by increasing temperatures from room temperature of 20 to 30 and $40^{\circ} \mathrm{C}$.

Based on these results, factorial-design experiments were conducted with the THC design using Stainless-Steel cathodes, Carbon-cloth anodes and microporous ceramic membranes as well as fixed conditions for temperature (ambient temperature near room temperature of $20^{\circ} \mathrm{C}$ ) and acidity $\left(100 \mathrm{~g} / \mathrm{L} \mathrm{H}_{2} \mathrm{SO}_{4}\right)$. It is worth noting that the acidity was never tested because it was decided to insure that iron precipitation as ferrihydrite would be avoided; furthermore, it was understood from the literature that such concentrations were common in leaching and SX circuits. Consequently, four variables were examined: cupric ion concentration from 10-50 g/L, ferric ion concentration from 10-50 g/L, applied voltage from 0.8-2.5 Volts, and solution flowrates from $0-540 \mathrm{~mL} / \mathrm{min}$.

In order to obtain the best results from a statistical study, a full factorial-design was completed in which mid-point values were also tested along with the end-points of the variable ranges. Because four variables were being tested (i.e, $n=4$ ), the study required $2^{n}$ or 16 experiments be conducted along with 5 duplicate mid-point experiments for a total of 21 . The conditions for each experiment are shown in the Appendix in Table A1 along with the results as measured by current density for each test (see Table A2). The ANOVA Table is shown in Table A3 and indicates that the four variables are significant because the values for "Prob $>F$ " are less than 0.05 which is equivalent to being greater than $95 \%$ confidence

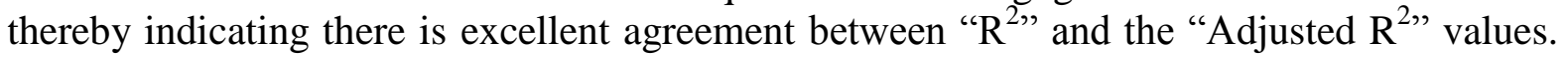
In this case, the significant variables are cupric ion concentration, applied voltage, and flowrate along with a binary involving both applied voltage and flowrate. Although the ANOVA Table shows that the ferrous concentration is not significant, it was decided that it should still be considered due to the fact that current densities drop substantially at long duration times. In this regard, it is important to note that the responses in Table A2 were determined after 30 minutes. In this regard, the following model was determined: 


$$
\begin{aligned}
\log _{10}(\text { Current Density })= & 0.97876+3.56 * 10^{-3} *\left[\mathrm{Cu}^{2+}\right]-3.95 * 10^{-4} *\left[\mathrm{Fe}^{2+}\right]+0.51 * \text { Volts } \\
& +8.13 * \text { Flowrate }-3.1 * 10^{-4} * \text { Volt*Flowrate }
\end{aligned}
$$

This model predicts that the current density and therefore copper production will be increased by increasing the cupric concentration $(\mathrm{g} / \mathrm{L})$, applied voltage $(\mathrm{V})$, and/or flowrate $(\mathrm{ml} / \mathrm{min})$. However, there is a small but noticeable binary effect between flowrate and applied voltage that has a negative effect on the system. Furthermore, a negative effect on current density is also realized by increasing the ferrous concentration. Applied voltage and flowrate are the most significant variables due to the large values of their respective coefficients.

Because the ANOVA Table also shows that the "model F-value" is of 37.06, it implies the model is also significant and suggests that there is only $0.01 \%$ chance that results are due to noise. This is illustrated in Figure 8 which shows a scatter plot between actual and predicted values has good linear agreement and additionally illustrates why the "Predicted $\mathrm{R}^{2 \text { " value of }}$

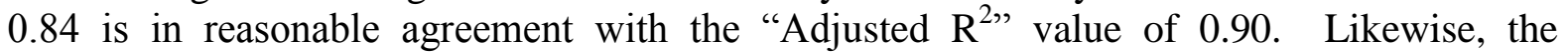
"Curvature F-value" of 24.71 implies there is significant curvature (as measured by difference between the average of the center points and the average of the factorial points) in the model and that there is only a $0.02 \%$ chance that results are due to noise. Similarly, because the "Adequate Precision" of 16.02 is greater than 4, the signal-to-noise ratio is adequate. Clearly, confidence in the results and significance of the the model are high. In this regard, the model can be used to adequately predict results and therefore can be used "to navigate the design space." Doing so suggests that optimum conditions can be obtained with an applied voltage near 1.60 Volts and electrolyte concentrations of $30 \mathrm{~g} / \mathrm{L}$.

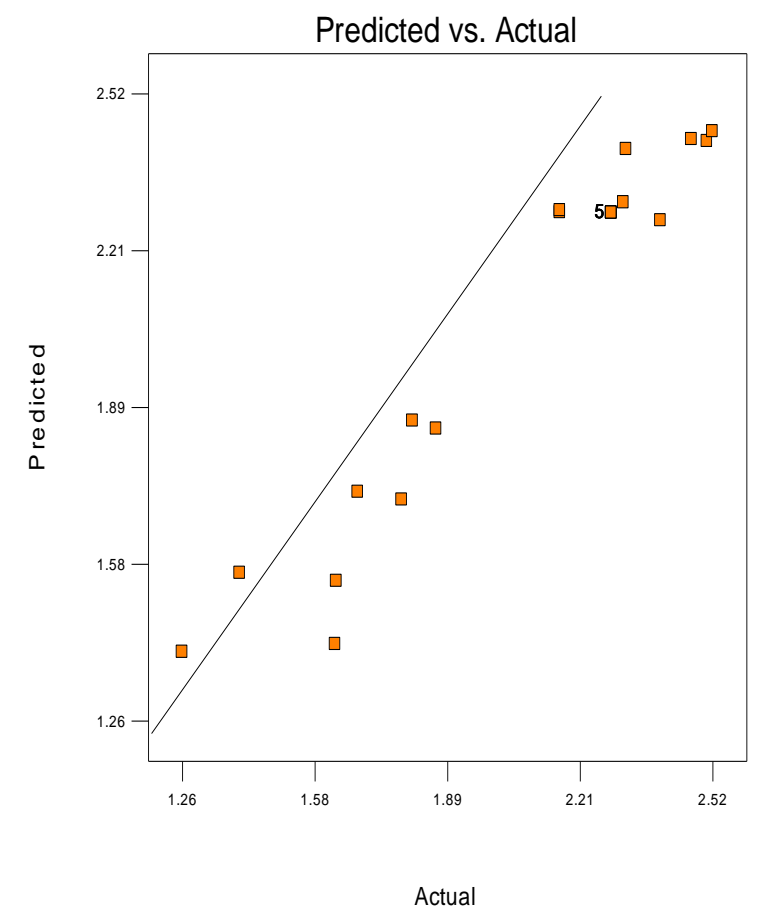

Figure 8. Scatter plot of predicted and actual electrowinning data. 
Finally, a critical analysis of the model shows that the results are also in agreement with phenomenological equations involving electrochemical and transport fundamentals (Maron and Prutton, 1974; Bard and Faulkner, 1980). For example, when two plates in an electrolytic cell are separated by a distance d, the current carried by the ions between the plates is represented by the equation, $\mathrm{I}=$ nvze/d, where $\mathrm{n}$ represents the concentration of ions, $\mathrm{v}$ is the velocity of the ions, $\mathrm{z}$ the valence of each ion and e represent the charge on an electron which clearly shows that current, and thus current density, are influenced by concentration and flowrate. Likewise, according to the Butler-Volmer and Fick Equations, current is related to both the voltage and temperature. Similarly, the Tafel equation and resulting Tafel plots illustrate that increasing cupric concentrations and mass flowrates will increase the limiting current density and simultaneously reduce the voltage that needs to be applied; however, they also illustrate that increasing ferrous concentrations will have the reverse effect. These items are illustrated by both the positive and negative influences predicted by the model.

\section{Task 3 - Ferric Leaching Application}

Montana Resources (MR), a copper mine and mill located in Butte, was contacted in 2004 to see if they had an interest in collaborating on this portion of the project. Ensuing discussions indicated they had a genuine interest in developing a leaching technology for their Central Zone Orebody (CZO) which contained mostly secondary copper sulfides (Czehura, 2004) such as covellite (CuS), digenite $\left(\mathrm{Cu}_{1.8} \mathrm{~S}\right)$ and chalcocite $\left(\mathrm{Cu}_{2} \mathrm{~S}\right)$ and consequently should therefore be amenable to ferric leaching (Baxter et al., 1999). Furthermore, when MR processes CZO through their mill, recoveries run between $85-90 \%$ and grades run between $15-20 \%$ copper. This low metallurgical performance is attributed to secondary copper minerals being present as coatings on pyrite $\left(\mathrm{FeS}_{2}\right)$ which, when ground, can produce copper fines that are difficult to recover; however, even when liberated, the pyrite ends up being recovered anyways which dilutes the concentrate. Poor recoveries and grades result. MR agrees that the only way that the CZO will be mined and processed is if a leaching technology can be employed on either the ore or a concentrate produced from the ore (Czehura, 2004). Furthermore, MR acknowledges that they would benefit greatly if they were to produce their own copper as opposed to selling their concentrate overseas to custom smelters. Currently, by comparison, MR processes their East and West Zone Ores and produces a chalcopyrite $\left(\mathrm{CuFeS}_{2}\right)$ concentrate with greater than $90 \%$ recovery and grades in excess of $28 \%$ copper. By processing their CZO, their mine life would be extended by at least 25 years.

To begin this portion of the study, MR delivered four ore samples in 55-gallon drums to Montana Tech. The samples were crushed and homogenized. Sample splits were then taken analyzed. Assays were determined to be $0.18,0.30,1.0$ and $1.5 \% \mathrm{Cu}$. The samples showed that the clay content was montmorillonite $\left(\mathrm{H}_{2} \mathrm{Al}_{2} \mathrm{Si}_{4} \mathrm{O}_{12} \bullet \mathrm{nH}_{2} \mathrm{O}\right)$ and was noted to generally increase with increasing copper concentration. Scoping tests were performed on each of samples to determine how effective ferric leaching was and thereby help identify conditions that would help optimize leaching performance. Variables included particle size, solids content, ferric ion concentration, sulfuric acid concentration, leach duration, and temperature. Because sulfuric acid concentrations between 50 and $150 \mathrm{~g} / \mathrm{L}$ did not effect the results, 
ensuing factorial-design experiments were fixed at the lower concentration of $50 \mathrm{~g} / \mathrm{L}$. On the other hand, because tests showed that increasing the ferric concentration from 5 to $30 \mathrm{~g} / \mathrm{L}$ also had relatively no effect on leaching, the high iron concentration of $30 \mathrm{~g} / \mathrm{L}$ was selected to follow the optimal results obtained by Dakubo (2006). As expected, smaller particles sizes improved leaching as did lower percent solids and, in general, increased leaching times. However, surprisingly, increasing temperature was often found to be decrease leaching performance. Depending on the ore, extraction efficiencies from these preliminary scoping tests ranged from $25-65 \%$.

These results suggested that optimal conditions in excess of $90 \%$ might be determined via factorial design experiments. For these studies, as just noted, the acid concentration was fixed at $50 \mathrm{~g} / \mathrm{L}$ and the ferric concentration was fixed at $30 \mathrm{~g} / \mathrm{L}$. Particle sizes were varied from 38 to 74 microns (with a midpoint of 56 microns), solids content from 10 to $30 \%$ solids by weight (with a midpoint of 20\%), leaching time from 1 to 4 hours (with a midpoint of 2.5 hours), and temperature from 25 to $90^{\circ} \mathrm{C}$ (with a midpoint of $60^{\circ} \mathrm{C}$ ). In this case, only a halffactorial design study was desired; thus, only 8 experiments were needed along with 2 midpoint experiments for a total of 10. Table A4 in the Appendix shows the experimental matrix. Results are presented in Figure 9 as well as Table A5 and include numbers not only for overall extraction but also for extraction of acid-soluble (AS) copper based on head and tail assays. AS-copper refers to the carbonates of malachite $\left(\mathrm{CuCO}_{3} \mathrm{Cu}(\mathrm{OH})_{2}\right)$ and azurite $\left(2 \mathrm{CuCO}_{3} \mathrm{Cu}(\mathrm{OH})_{2}\right)$; oxides of cuprite $\left(\mathrm{Cu}_{2} \mathrm{O}\right)$ and tenorite $(\mathrm{CuO})$; and silicates of chrysocolla $\left(\mathrm{CuSiO}_{3} * 2 \mathrm{H}_{2} \mathrm{O}\right)$. Non-AS soluble minerals would include the secondary sulfides such as covellite $(\mathrm{CuS})$, digenite $\left(\mathrm{Cu}_{1.8} \mathrm{~S}\right)$ and chalcocite $\left(\mathrm{Cu}_{2} \mathrm{~S}\right)$ as well as the primary sulfides such as chalcopyrite $\left(\mathrm{CuFeS}_{2}\right)$ and bornite $\left(\mathrm{Cu}_{5} \mathrm{FeS}_{4}\right)$.

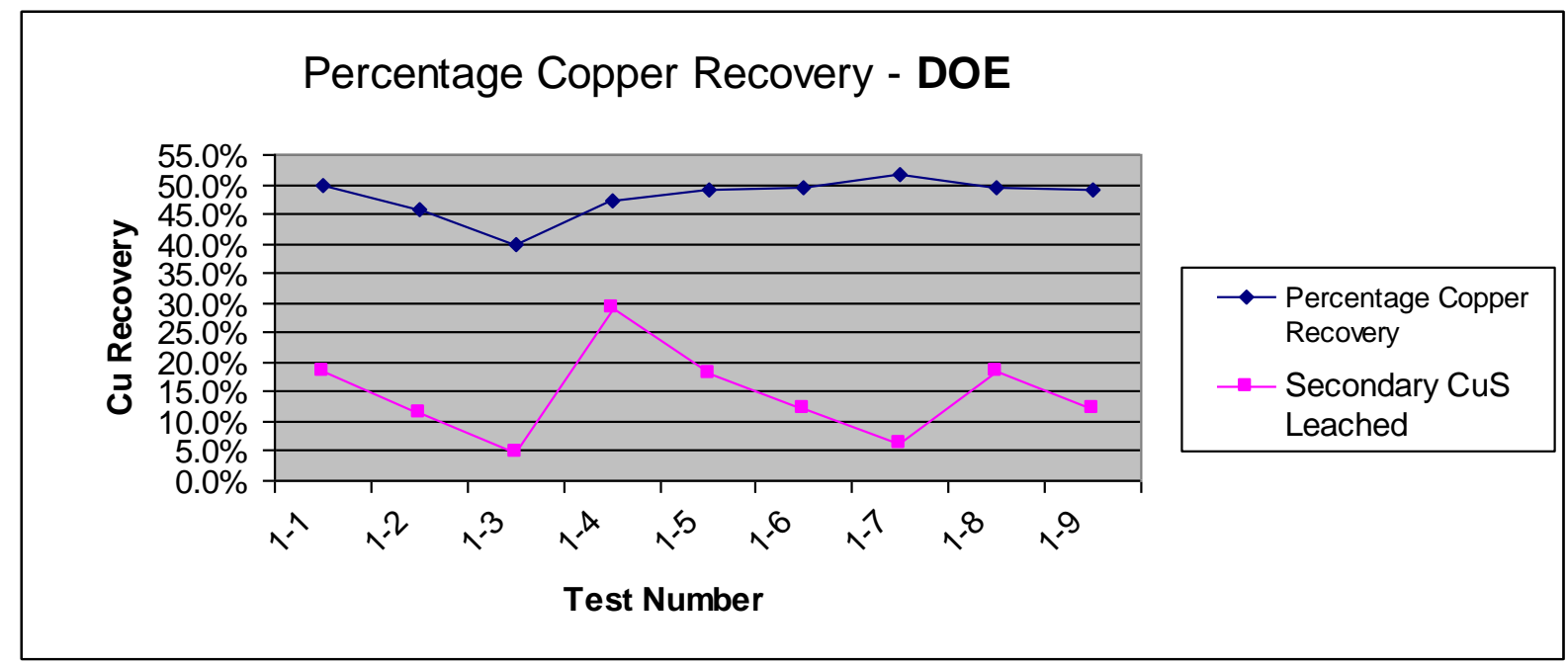

Figure 9. Total and secondary copper recovery as a function of test number.

As can be seen from the table and figure, the total copper recovery was near $50 \%$. The majority of the copper recovered was from AS-content of the ore with practically $85 \%$ (average of all tests) coming from that fraction. The copper that was leached from secondary copper sulfides is estimated to be only about $15 \%$ of the total copper. Clearly, ferric leaching 
is not performing as expected even though the results of the statistical analyis from StatEase were termed significant such that there was excellent correlation between the adjusted $\mathrm{R}^{2}$ value and the predicted $\mathrm{R}^{2}$ value. In this regard, the ANOVA Table for the model is shown in Table A6. The correlation is graphically shown in Figure 10 and indicates that only one data point fell any significant distance from the predicted line. Nevertheless, the "predicted R-squared" value of 0.9585 is in excellent agreement with the "adjusted R-squared" value of 0.9658. Furthermore, because the "adequate precision" of 17.439 is greater than 4 , the signal to noise ratio is good. In addition, the "F-values" are all much less than 0.1 which suggests that the coefficients determined for the model are signifcant. Consequently, the resulting model is acceptable.

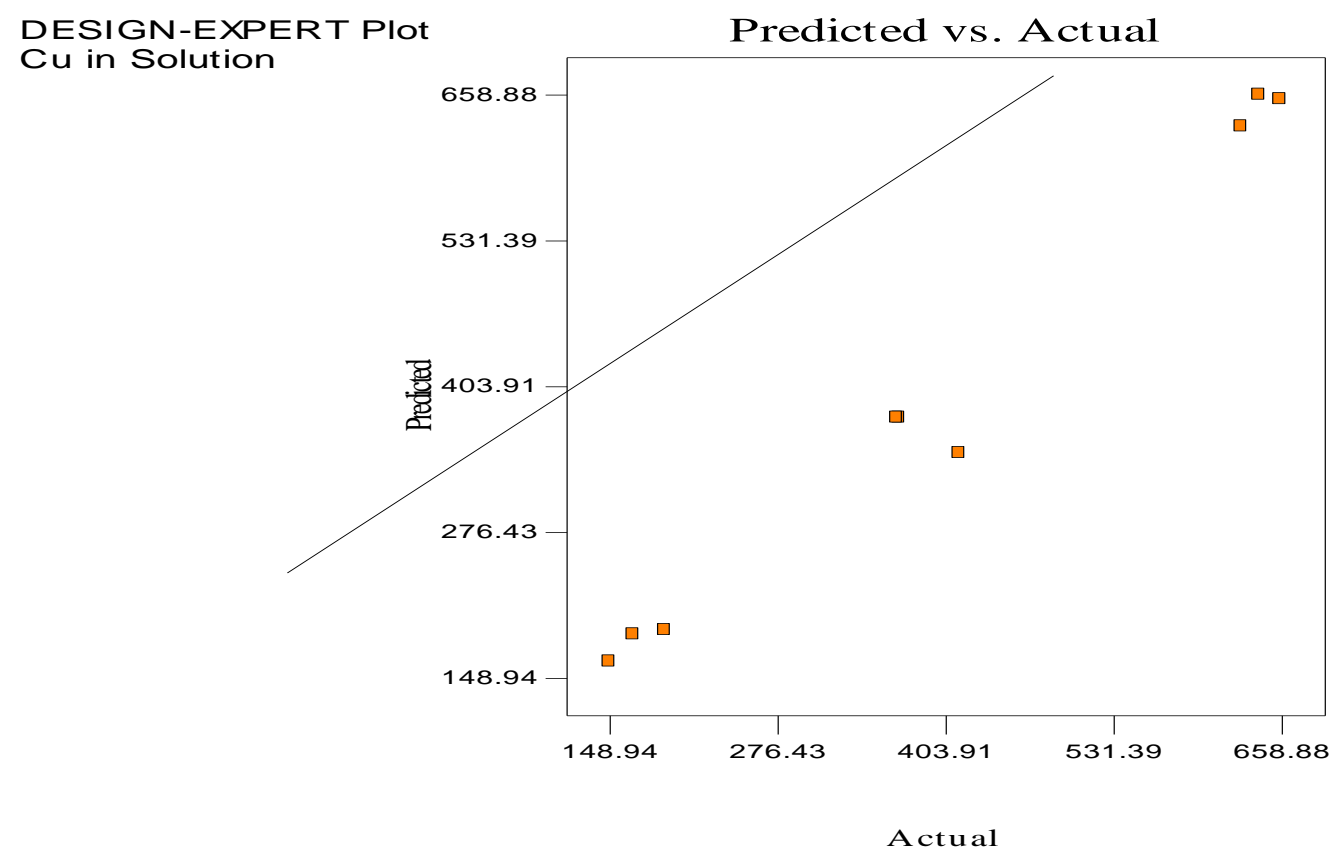

Figure 10. Scatter plot of predicted and actual leaching data.

Unfortunately, the model is not heirarchical and its equation cannot be shown but it still can be used to confidently navigate the design space. Examples are presented in Figures 11 and 12. The 3-dimensional plot in Figure 11 clearly shows that, at a fixed $\%$ solids of $30 \%$ and temperature of $25^{\circ} \mathrm{C}$, large particles and short durations do not leach significant amounts of copper but, as the particles become smaller, duration becomes less significant and more copper is leached. Similarly, Figure 12 shows that increasing the \% solids increases the amount of copper leached at a fixed temperature of $25^{\circ} \mathrm{C}$ and a long duration time of 4 hours but is relatively independent of particle size. Similar conclusions would be reached for other plots but, as already stated, can show that temperature has a negative impact on the system at 4 hours but not at 1 hour. This phenomenon can be attributed to the preg-robbing behavior of the clay and therefore its ability to adsorb copper as it is being leached. 
DESIGN-EXPERT PIOt

$\mathrm{Cu}$ in Solution

$X=A:$ Size

$\mathrm{Y}=\mathrm{C}:$ Time

Actual Factors

$\mathrm{B}$ : Solids $=30.00$

$\mathrm{D}$ : Temperature $=25.00$

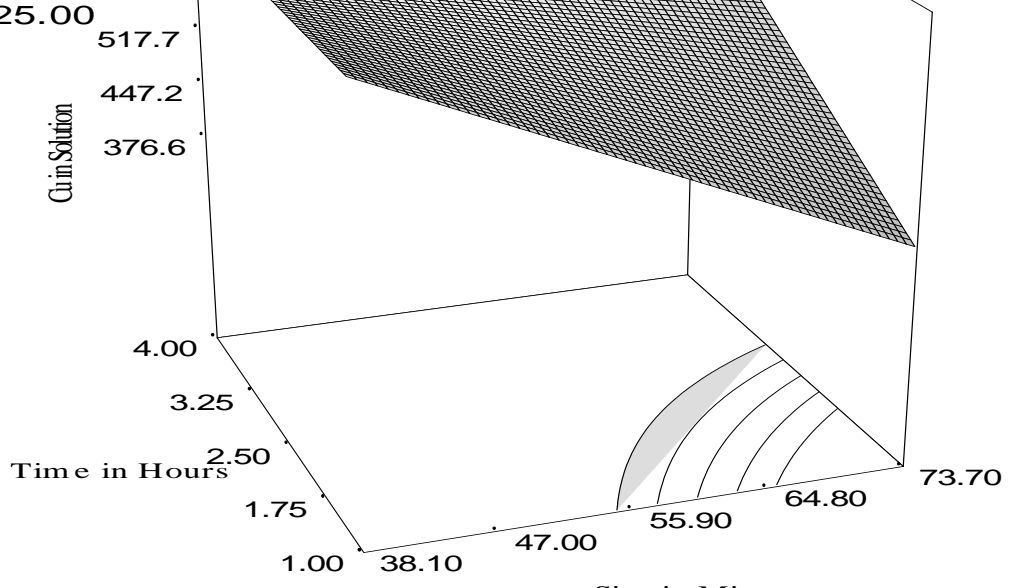

Size in Microns

Figure 11. Concentration of Copper in Solution as a function of time and size at a fixed \% solids of $30 \%$ and temperature of $25^{\circ} \mathrm{C}$.

DESIGN-EXPERT PIOT

$\mathrm{Cu}$ in Solution

- Design Points

$X=A:$ Size

$\mathrm{Y}=\mathrm{B}:$ Solids

Actual Factors

C: Time $=4.00$

D: Temperature $=25.00$

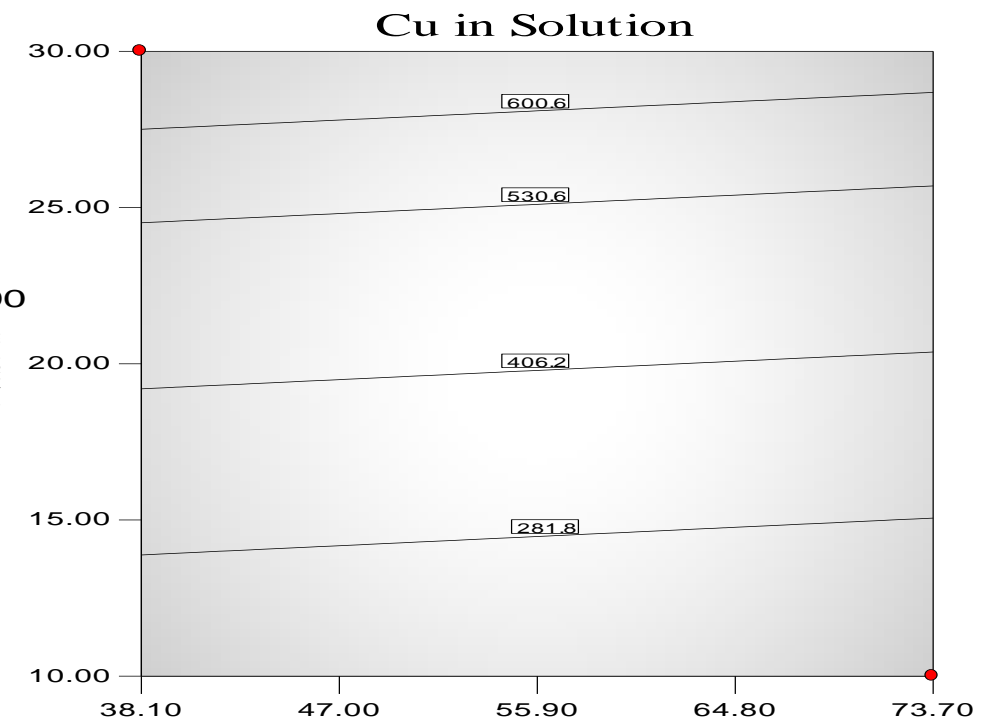

A: Size in microns

Figure 12. Concentration of copper in solution as a function of \% solids and size at fixed temperature of $25^{\circ} \mathrm{C}$ and a long duration time of 4 hours. 


\section{Task 4 - Ferric Leaching Optimization}

Because the ore samples gave similar scoping results, half-factorial design experiments were not conducted for the other ore samples. Furthermore, because only $15 \%$ of the secondary copper sulfide minerals were extracted, there also was no need to optimize the results. In this regard, Task 4 was not implemented due to the failure of Task 3 to yield total extraction efficiencies greater than $90 \%$ let alone for secondary minerals; however, Task 5 was created in order to explain the results.

\section{Task 5 - Diagnostic Leach}

In diagnostic leaching, samples are treated with increasing strengths of acid as well as oxidants to "quantitatively" determine the mineralogy that is present (Anderson, 1990). The 5-stage process determines the presence of (1) copper carbonate by an acetic acid leach, (2) copper oxides and silicates by a sulfuric acid/ sodium sulfite leach, (3) secondary copper sulfides by cyanide leach, (4) native copper by silver nitrate titration, and (5) primary copper sulfide by difference from total copper. Following each stage, the resulting solution is analyzed by AA or ICP and mass balances are conducted to determine the amount of each miner type present. Results are presented in Figure 13 with sample 1 being the high grade ore $(1.5 \% \mathrm{Cu})$ and decreasing to sample 4 which is the low grade ore $(0.2 \% \mathrm{Cu})$. As can be seen, AS-copper minerals account for $40-50 \%$ of the copper but is dependent on the sample. Likewise, the amount of secondary copper sulfides is has about the same range and are also sample-dependent. Nevertheless, the samples should have leached well with the exception of the two samples that unexpectantly showed significant significant amounts of primary copper minerals ranging between $5-15 \%$. Even in the case of sample 1, an $85 \%$ copper extraction should have been observed. It is critical to note that these numbers are similar to those determined microscopically by Guilbert and Zeihen (1964).

Mineralization of the Central Zone Orebody

$\%$ of Specific Mineral Type

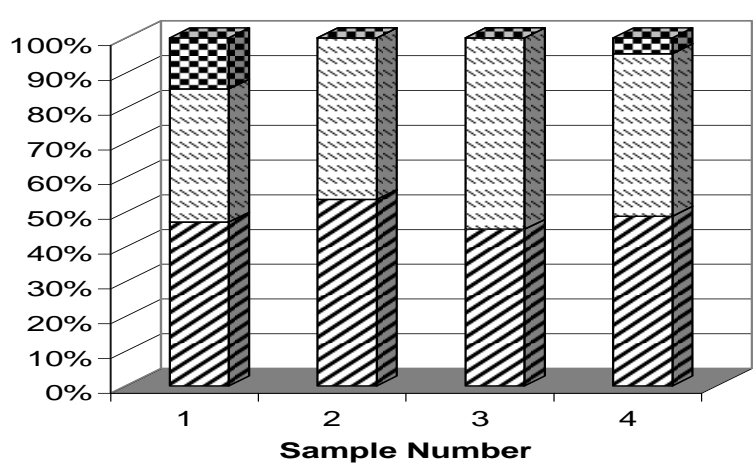

D Primary Copper Minerals (CuFes2)

$\checkmark$ Secondary Copper Minerals (CuS, Cu2s, etc..)

Acid Soluable Copper Minerals (oxides, silicates, carbonates,...)

Figure 13. Mineralization of the $\mathrm{CZO}$ as determined by diagnostic leaching. 
It is therefore summized that the ore samples were difficult to leach due to the presence of significant amounts of pyrite. As leaching occurs, the ferric concentration decreases and, of course, ferrous concentration increases. When this happens appreciably in the presence of significant amounts of pyrite $\left(\mathrm{FeS}_{2}\right)$, the solution potential can move from control by the $\mathrm{Fe}^{3+} / \mathrm{Fe}^{2+}$ redox couple at 0.77 Volts to control by the $\mathrm{Fe}_{2+} / \mathrm{FeS}_{2}$ redox couple near 0.35 Volts. These potentials are plotted on the $\mathrm{E}_{\mathrm{H}}-\mathrm{pH}$ diagram in Figure 14 and clearly indicate that, if control shifts to the lower potential, copper dissolution will be prevented. Furthermore, dissolved $\mathrm{Cu}^{2+}$ may precipitate out on the pyrite reminiscent of an activation process. This phenomenon is likely how the covellite/chalcocite coating formed geologically on the pyrite in the CZO and would also explain the decrease in copper concentration at longer leaching times.

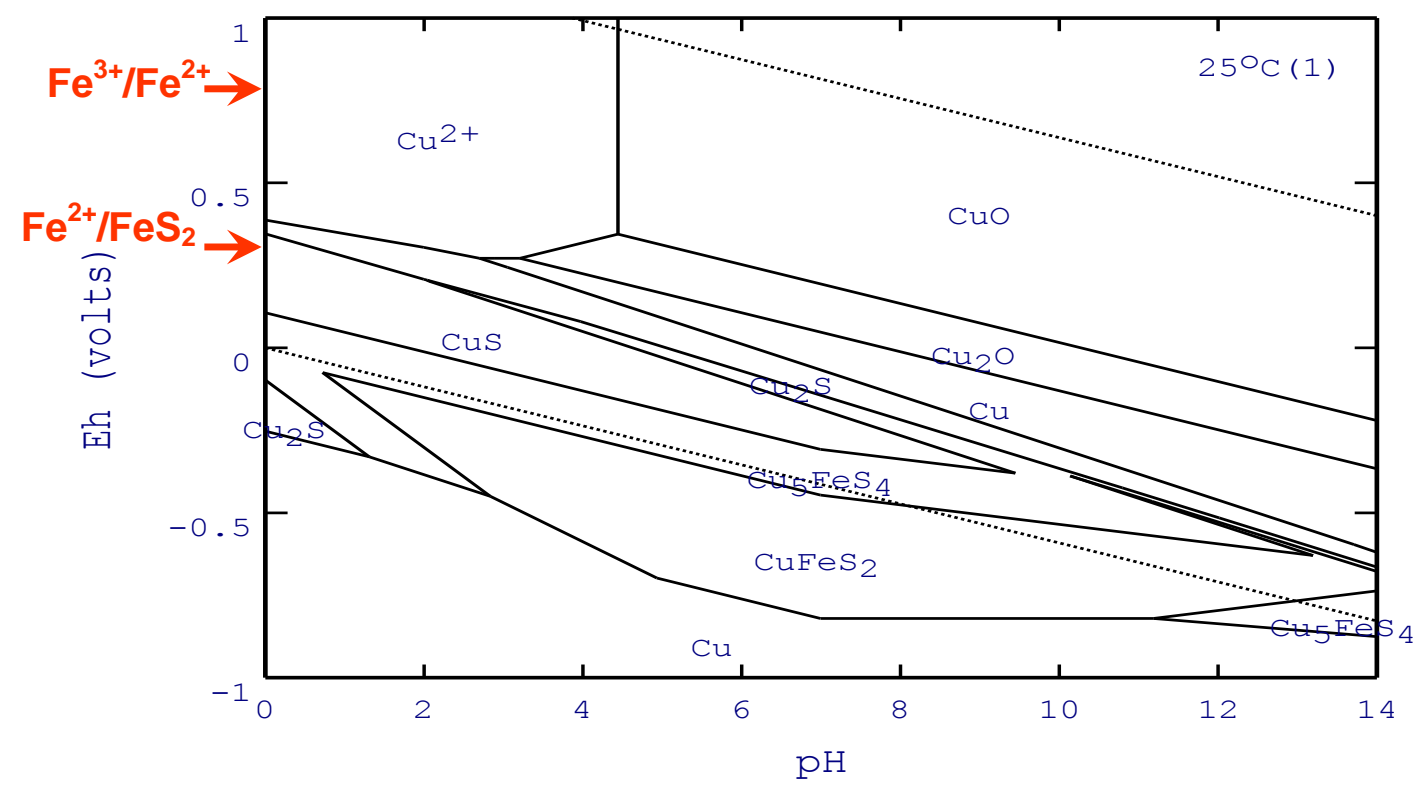

Figure 14. $\mathrm{E}_{\mathrm{H}}-\mathrm{pH}$ Diagram for $\mathrm{Cu}$-species in $\mathrm{Cu}-\mathrm{Fe}-\mathrm{S}-\mathrm{H}_{2} \mathrm{O}$ system with consideration of sulfur oxidation to sulfate $\left(\mathrm{SO}_{4}{ }^{2-}\right)$.

\section{CONCLUSIONS}

Various tests were conducted over a three-year period to investigate the feasibility of electrowinning copper $\left(\mathrm{Cu}^{0}\right)$ from cupric $\left(\mathrm{Cu}^{2+}\right)$ while simultaneously oxidizing ferrous $\left(\mathrm{Fe}^{2+}\right)$ to ferric $\left(\mathrm{Fe}^{3+}\right)$ using a membrane cell. Results indicated that several variables were important to consider to minimize the applied voltage while keeping current density at a maximum. These variables included electrolyte concentrations $\left(\mathrm{Cu}^{2+}\right.$ and $\left.\mathrm{Fe}^{2+}\right)$, agitation/flow-rate, applied voltage, and temperature. Other variables were also tested: cathode type, anode type, membrane type, and cell design; however, certain ones showed superior behavior and included carbon-cloth as the anode, microporous ceramic (used in car betteries) as the membrane, and the THC-design as the electrowinning cell. Although 304 Stainless Steel was selected for the cathode, copper starter sheet performed equally as well and could have been selected. Ensuing factorial-design experiments were therefore 
conducted with these 4 variables fixed in order to investigate the effects of the other variables. For simplicity and to minimize the number of experiments, the temperature was held constant at $20^{\circ} \mathrm{C}$ (room temperature). Concentrations of $\mathrm{Cu}^{2+}$ and $\mathrm{Fe}^{2+}$ were varied between 10 and $50 \mathrm{~g} / \mathrm{L}$, flowrates were varied between 0 and $540 \mathrm{~mL} / \mathrm{min}$, and the applied voltage was varied between $0.8-2.5$ Volts. Results were then statistically analyzed using StatEase software and yielded the following model:

$$
\begin{aligned}
\log _{10}(\text { Current Density })= & 0.97876+3.56 * 10^{-3} *\left[\mathrm{Cu}^{2+}\right]-3.95 * 10^{-4} *\left[\mathrm{Fe}^{2+}\right]+0.51 * \text { Volts } \\
& +8.13 * \text { Flowrate }-3.1 * 10^{-4} * \text { Volt*Flowrate }
\end{aligned}
$$

From the model, it was concluded that the current density and therefore copper production would be increased by increasing the cupric concentration, applied voltage, and/or flowrate; however, a small binary effect between flowrate and applied voltage was observed that had a negative effect on the system. Furthermore, a negative effect on current density was also realized by increasing the ferrous concentration. Applied voltage and flowrate are the most significant variables due to the large values of their respective coefficients. Optimization of the model indicates that the voltage can be decreased from an industrial standard of approximately 2.0 Volts to between 1.5-1.7 Volts which equates to energy savings of 15$25 \%$. Furthermore, the model concurs with fundamental understanding of electrowinning principles including equations for solution capacitance, Butler-Volmer, Fick's and Tafel.

Various tests were also conducted in collaboration with Montana Resources, a local Montana mining company in Butte, to see if ferric leaching could be used on their Central Zone Ore (CZO) and implemented along with the membrane electrowinning technology. It was noted that CZO does not respond as well as to their flotation operations as the traditional East and West Zone Ores do. Recoveries run low between $85-90 \%$ but should be in excess of $90 \%$ whereas grades run between $15-20 \%$ copper but should be in excess of $28 \%$. This poor metallurgical performance is attributed to the different mineralization. Instead of the major copper mineral being chalcopyrite $\left(\mathrm{CuFeS}_{2}\right)$, it is a coating of covellite (CuS), digenite $\left(\mathrm{Cu}_{1.8} \mathrm{~S}\right)$ and chalcocite $\left(\mathrm{Cu}_{2} \mathrm{~S}\right)$ on pyrite $\left(\mathrm{FeS}_{2}\right)$. In order to recover these secondary copper minerals, the pyrite must be floated which, of course, dilutes the concentrate grade. Furthermore, because the copper minerals are present as coatings, comminution likely causes their fines and thus leads to a low recovery as well.

On the other hand, the mineralogy is perfect for ferric leaching. In this regard, preliminary test helped establish important variables for leaching and included particle size of the ore, percent solids by weight, temperature of the system, and duration of the leach. Factorial design experiments were therefore conducted to develop a model that would lead to optimal leaching conditions. Unfortunately, results indicated that no more than approximately $60 \%$ extraction could be achieved. Diagnostic leach tests indicated that primary copper sulfide minerals must be present (i.e., chalcopyrite) in CZO because they do not leach well with ferric. A literature search of the geology and mineralization concurred and thereby concluded that ferric leaching is not a method to consider for copper extraction. However, as a result of this study, Montana Resources is exploring options for recovering the copper by leaching. It is recommended that they do so by processing the CZO through their current facilities and using an agitation leach on the resulting flotation concentrate to minimize the 
amount of material being contacted. This would also prevent problems from the clay content which is present in the CZO but would be mostly removed by the flotation process.

\section{FUTURE WORK}

This is the final report for this project. It is likely that MR and the PI's will team up again to write a follow-up proposal in the next CAST cycle pending everyone's availability. In this regard, future work is planned. Overall, the project met with many successes as noted by the Publications and Presentations list in the second to next section. It is particularly worthy to note that the project supported 3 graduate students instead of the the 1 that was originally proposed. Mr. Dave Douglas graduated in May of 2005 but, due to unfortunate circumstances, had to go non-thesis. This, together with the unfortunate loss of Eric Dahlgren as the postdoc, forced the project to be extended. Mr. Francis Dakubo graduated in May of 2006 with a thesis on the electrowinning work. Mr. Thom McIntyre also graduated in May of 2006 with a thesis on the ferric leaching work. Both were more than happy to be engaged in a fully funded project thanks to CAST.

\section{REFERENCES}

1. Young, C.A., Huang, H.H. and Fabian, C, "Simultaneous Electrolysis of Copper and Ferrous Ions to Produce Copper Cathode and Regenerate Ferric Sulfate," CAST Proposal, Montana Tech, Butte, MT (2002).

2. Fabian, C., Huang, H.H. and Young, C.A., "Copper Sulfide Hydrometallurgy: Plant Practice and Future Improvements," Preprint No. 02-202, SME, Littleton, CO (2001).

3. Bard, A.J., Parsons, R. and Jordan, J., Standard Potentials in Aqueous Solutions, Marcel Dekker, Inc., New York, NY (1985).

4. Baxter, K., Kaiser, C. and Richmond, G. "Design of Mt. Gordon Chalcocite Project," in: Proceedings of Alta Copper 1999 Sulfide Symposium, Sept. 5-10, pp. 1-21 (1999).

5. Marsden et al., "System for Direct Electrowinning of Copper," U.S. Patent No. 6,972,107 (2005).

6. Twidwell, L.G., Huang, H.H. and Miller, J.D., "Unit Operations in Extractive Metallurgy: Hydrometallurgy," Montana Tech, Butte, MT (1974).

7. Anderson. M.J. and Whitcomb, P.J., StateEase, V. 5.0, Minneapolis, MN (2004).

8. Dakubo, F. "Simultnaeous Electrowinning of Copper and Oxidation of Ferrous to Ferric Using Membrane Technology”, MS Thesis, Montana Tech, Butte, MT (2006).

9. Czehura, S., Personal Communications, Montana Resources, Butte, MT (2004).

10. McIntyre, T.F., "Ferric Leaching of Low Grade Secondary Copper Minerals", MS Thesis, Montana Tech, Butte, MT (2006).

11. Maron, S., Lando, J.B. and Prutton, C.F., Fundamentals of Physical Chemistry, Macmillan Publishing, New York, NY (1974).

12. Bard, A.J. and Faulkner, L.R., Electrochemical Methods, John Wiley \& Sons, Inc., New York NY (1980).

13. Anderson C. G., "The Application of Diagnostic Leaching to Copper and Gold Ores," CAMP, Montana Tech, Butte, MT (1990).

14. Guilbert J.M. and Zeihen L.G., "The mineralogy of the Butte District - Montana," presented at NWMA, Spokane, WA (1964). 


\section{PUBLICATIONS AND PRESENTATIONS}

The following publications and presentations were delivered as a direct result of this study.

1. Young, C.A. et al., "Simultaneous Electrolysis of Copper and Ferrous Ions," Poster Presentation, CAST 2003 Annual Workshop, Charleston, WV, Nov. 19-21 (2003).

2. Young, C.A., McIntyre, T.F., and Dakubo, F. "Application of Statistical Software for Hydrometallurgical Processing, Part I: Ferric Leaching," Poster Presentation, CAST 2005 Annual Workshop, Blacksburg, VA, July 28-30 (2005).

3. Young, C.A., McIntyre, T.F., and Dakubo, F. "Application of Statistical Software for Hydrometallurgical Processing, Part II: Electrowinning," CAST 2005 Annual Workshop, Blacksburg, VA, July 28-30 (2005).

4. Dakubo, F. "Simultaneous Electrowinning of Copper and Oxidation of Ferrous to Ferric Using Membrane Technology", MS Thesis, Montana Tech, Butte, MT (2006).

5. McIntyre, T.F., "Ferric Leaching of Low Grade Secondary Copper Minerals", MS Thesis, Montana Tech, Butte, MT (2006).

6. Young, C.A., McIntyre, T.F., and Dakubo, F. "Application of Statistical Software for Hydrometallurgical Processing, Part I: Ferric Leaching," Poster Presentation, NWMA Annual Meeting, December 3-8 (2006).

7. Young, C.A., McIntyre, T.F., and Dakubo, F. "Application of Statistical Software for Hydrometallurgical Processing, Part II: Electrowinning," NWMA Annual Meeting, December 3-8 (2006).

8. Young, C.A. and McIntyre, T.F., "Processing of CZO: Review and Recommendations," Montana Resources, Butte, January 19 (2007).

9. Dakubo, F. Huang, H.H., Fabian, C. and Young, C.A., "Optimization of a Membranic Copper-Electrowinning Technique," accepted for presentation at SME Annual Meeting, Denver, CO (2007). 


\section{APPENDICES}

Table A1. Conditions for Full-Factorial Design Electrowinning Study. \begin{tabular}{|c|c|c|c|c|c|} 
Std & Run & A: $\left[\mathrm{Cu}^{2+}\right], \mathrm{g} / \mathrm{L}$ & $\mathbf{B}:\left[\mathrm{Fe}^{2+}\right], \mathrm{g} / \mathrm{L}$ & $\mathbf{C}:$ Voltage, $\mathbf{V}$ & D: Flowrate, $\mathbf{m L} / \mathrm{min}$ \\
\hline
\end{tabular}

\begin{tabular}{|c|c|c|c|c|c|}
\hline 10 & 1 & 50 & 10 & 0.8 & 540 \\
\hline 6 & 2 & 50 & 10 & 2.5 & 0 \\
\hline 17 & 3 & 30 & 30 & 1.65 & 270 \\
\hline 12 & 4 & 50 & 50 & 0.8 & 540 \\
\hline 19 & 5 & 30 & 30 & 1.65 & 270 \\
\hline 9 & 6 & 10 & 10 & 0.8 & 540 \\
\hline 14 & 7 & 50 & 10 & 2.5 & 540 \\
\hline 11 & 8 & 10 & 50 & 0.8 & 540 \\
\hline 7 & 9 & 10 & 50 & 2.5 & 0 \\
\hline 5 & 10 & 10 & 10 & 2.5 & 0 \\
\hline 15 & 11 & 10 & 50 & 2.5 & 540 \\
\hline 2 & 12 & 50 & 10 & 0.8 & 0 \\
\hline 20 & 13 & 30 & 30 & 1.65 & 270 \\
\hline 3 & 14 & 10 & 50 & 0.8 & 0 \\
\hline 8 & 15 & 50 & 50 & 2.5 & 0 \\
\hline 4 & 16 & 50 & 50 & 0.8 & 0 \\
\hline 18 & 17 & 30 & 30 & 1.65 & 270 \\
\hline 21 & 18 & 30 & 30 & 1.65 & 270 \\
\hline 16 & 19 & 50 & 50 & 2.5 & 540 \\
\hline 13 & 20 & 10 & 10 & 2.5 & 540 \\
\hline 1 & 21 & 10 & 10 & 0.8 & 0 \\
\hline
\end{tabular}


Table A2. Response Table for Electrowinning Study.

\begin{tabular}{|c|c|c|c|}
\hline Std & \multicolumn{1}{|c|}{ Run } & Current, Amps & Current Density, Amps/m \\
\hline 10 & 1 & 0.604 & 64.7 \\
\hline 6 & 2 & 2.279 & 322.6 \\
\hline 17 & 3 & 1.846 & 191.5 \\
\hline 12 & 4 & 0.709 & 73.6 \\
\hline 19 & 5 & 1.846 & 191.5 \\
\hline 9 & 6 & 0.473 & 48 \\
\hline 14 & 7 & 2.641 & 332.5 \\
\hline 11 & 8 & 0.563 & 61 \\
\hline 7 & 9 & 2.425 & 250.5 \\
\hline 5 & 10 & 1.368 & 144.6 \\
\hline 15 & 11 & 1.368 & 144.6 \\
\hline 2 & 12 & 0.252 & 25.2 \\
\hline 20 & 13 & 1.846 & 191.5 \\
\hline 3 & 14 & 0.203 & 18.4 \\
\hline 8 & 15 & 2.72 & 207.6 \\
\hline 4 & 16 & 0.457 & 42.7 \\
\hline 18 & 17 & 1.846 & 191.5 \\
\hline 21 & 18 & 1.846 & 191.5 \\
\hline 16 & 19 & 2.981 & 296.5 \\
\hline 13 & 20 & 2.109 & 204.5 \\
\hline 1 & 21 & 0.408 & 42.4 \\
\hline & & & \\
\hline
\end{tabular}


Table A3. ANOVA Table for Electrowinning Study.

\begin{tabular}{|l|l|l|l|l|l|l|}
\hline Source & $\begin{array}{l}\text { Sum of } \\
\text { Squares }\end{array}$ & DF & $\begin{array}{l}\text { Mean } \\
\text { Squares }\end{array}$ & $\begin{array}{l}\text { F } \\
\text { Value }\end{array}$ & Prob $>$ F & \\
\hline Model & 2.36 & 5 & 0.47 & 37.06 & $<0.0001$ & Signnificant \\
\hline A & 0.081 & 1 & 0.081 & 6.39 & 0.0241 & \\
\hline B & $1.001 \times 10^{-}$ & 1 & $1.001 \times 10^{-0.003}$ & 0.079 & 0.7832 & \\
\hline C & 2.003 & 1 & 2.09 & 164.13 & $<0.0001$ & \\
\hline D & 0.11 & 1 & 0.11 & 8.30 & 0.0121 & \\
\hline CD & 0.081 & 1 & 0.081 & 6.39 & 0.241 & \\
\hline Curvature & 0.31 & 1 & 0.31 & 24.71 & 0.0002 & Significant \\
\hline Residual & 0.18 & 14 & 0.013 & & & \\
\hline Lack of fit & 0.18 & 10 & 0.018 & & & \\
\hline Pure Error & 0.000 & 4 & 0.000 & & & \\
\hline Cor Total & 2.85 & 20 & & & & \\
\hline & & & & & & \\
\hline Std Dev. & 0.11 & & R-squared & 0.9298 & & \\
\hline Mean & 2.06 & & $\begin{array}{l}\text { Adj.R- } \\
\text { squared }\end{array}$ & 0.9047 & & \\
\hline C.v. & 5.47 & & $\begin{array}{l}\text { Pred.R- } \\
\text { squared }\end{array}$ & 0.8400 & & \\
\hline PRESS & 0.46 & & $\begin{array}{l}\text { Adeq. } \\
\text { Precision }\end{array}$ & 16.022 & & \\
\hline
\end{tabular}

Table A4. Conditions for Half-Factorial Design Leaching Study.

\begin{tabular}{||c|c|c|c|c||}
\hline \hline RUN & $\begin{array}{c}\text { A: Size, } \\
\text { Microns }\end{array}$ & $\begin{array}{c}\text { B: \% Solids } \\
\text { by Weight }\end{array}$ & $\begin{array}{c}\text { C: Duration, } \\
\text { Hours }\end{array}$ & $\begin{array}{c}\text { D: Temperature, } \\
{ }^{0} \mathrm{C}\end{array}$ \\
\hline 1 & 73.7 & 30 & 4 & 25 \\
\hline 2 & 73.7 & 10 & 1 & 25 \\
\hline 3 & 38.1 & 30 & 1 & 25 \\
\hline 4 & 38.1 & 30 & 4 & 90 \\
\hline 5 & 55.9 & 20 & 2.5 & 60 \\
\hline 6 & 38.1 & 10 & 4 & 25 \\
\hline 7 & 38.1 & 10 & 1 & 90 \\
\hline 8 & 73.7 & 10 & 4 & 90 \\
\hline 9 & 73.7 & 30 & 1 & 90 \\
\hline 10 & 55.9 & 20 & 2.5 & 60 \\
\hline
\end{tabular}


Table A5. Response Table for Leaching Study. Summary Table of Results

DOE

\begin{tabular}{|c|c|c|c|c|c|c|c|c|c|}
\hline \multicolumn{10}{|c|}{$\% \mathrm{Cu}_{\text {total }}$ Recovery $=\left[\left(\mathrm{Wt} . \mathrm{Cu}_{\text {heads }}-\mathrm{Wt} . \mathrm{Cu}_{\text {tails }}\right) / \mathrm{Wt} . \mathrm{Cu}_{\text {heads }}\right]^{\star} 100$} \\
\hline & & & Total Wt $\%$ & Wt of Copper & $\% \mathrm{ASCU}$ & Wt of AS Cu & Wt of Sulfide Cu & Wt of available & WT \% Sulfide \\
\hline Test No. & Wt. Cuheads & Wt $\mathrm{Cl}_{\text {tails }}$ & Cu Recovery & Recovered & Recovered & Recovered & Recovered & Sulfide Cu & Cu Leached \\
\hline $1-1$ & 1.465 & 0.736 & $49.8 \%$ & 0.730 & $80.0 \%$ & 0.584 & 0.146 & 0.794 & $18.4 \%$ \\
\hline $1-2$ & 0.374 & 0.202 & $45.9 \%$ & 0.172 & $86.7 \%$ & 0.149 & 0.023 & 0.203 & $11.3 \%$ \\
\hline $1-3$ & 1.443 & 0.868 & $39.8 \%$ & 0.575 & $93.3 \%$ & 0.537 & 0.038 & 0.782 & $4.9 \%$ \\
\hline $1-4$ & 1.424 & 0.749 & $47.4 \%$ & 0.674 & $66.7 \%$ & 0.450 & 0.225 & 0.772 & $29.1 \%$ \\
\hline $1-5$ & 0.844 & 0.429 & $49.2 \%$ & 0.415 & $80.0 \%$ & 0.332 & 0.083 & 0.458 & $18.1 \%$ \\
\hline $1-6$ & 0.378 & 0.191 & $49.4 \%$ & 0.187 & $86.7 \%$ & 0.162 & 0.025 & 0.205 & $12.2 \%$ \\
\hline $1-7$ & 0.374 & 0.182 & $51.5 \%$ & 0.193 & $93.3 \%$ & 0.180 & 0.013 & 0.203 & $6.3 \%$ \\
\hline $1-8$ & 1.454 & 0.733 & $49.6 \%$ & 0.721 & $80.0 \%$ & 0.577 & 0.144 & 0.788 & $18.3 \%$ \\
\hline $1-9$ & 1.443 & 0.735 & $49.0 \%$ & 0.708 & $86.7 \%$ & 0.613 & 0.094 & 0.782 & $12.1 \%$ \\
\hline $1-10$ & 0.803 & \#VALUE! & \#VALUE! & \#VALUE! & $80.0 \%$ & \#VALUE! & \#VALUE! & 0.435 & \#VALUE! \\
\hline
\end{tabular}

Table A6. ANOVA Table for Leaching Study.

\begin{tabular}{|r|c|c|c|c|c||}
\hline SOURCE & $\begin{array}{l}\text { SUM OF } \\
\text { SQUARES }\end{array}$ & $\begin{array}{l}\text { DEGREES } \\
\text { FREEDOM }\end{array}$ & SQUARE & MEAN & F \\
\hline Model & $3.346 \mathrm{E}+5$ & 4 & 83641.53 & 57.56 & $\mathbf{0 . 0 0 0 9}$ \\
\hline $\mathbf{B}$ & $3.315 \mathrm{E}+5$ & 1 & $3.315 \mathrm{E}+5$ & 228.14 & 0.0001 \\
\hline AC & 29037.8 & 1 & 29037.8 & 19.98 & 0.0111 \\
\hline AD & 43230.02 & 1 & 43230.02 & 29.75 & 0.0055 \\
\hline $\mathbf{C D}$ & 30895.18 & 1 & 30895.18 & 21.26 & 0.0099 \\
\hline Residual & 5812.33 & 4 & 1453.08 & & \\
\hline Std. Dev. & 38.12 & $\mathbf{R}^{2}$ & $\mathbf{0 . 9 8 2 9}$ & & \\
\hline Mean & 398.37 & Adjusted $\mathbf{R}^{2}$ & $\mathbf{0 . 9 6 5 8}$ & & \\
\hline C.V. & 9.57 & Predicted $\mathbf{R}^{2}$ & $\mathbf{0 . 9 5 8 5}$ & & \\
\hline PRESS & 14116.03 & Adeq. Precision & $\mathbf{1 7 . 4 3 9}$ & & \\
\hline
\end{tabular}


Appendix 22: Recovery of Gold From Thiosulfate Leach Liquor Using Activated Carbon (MT005) 


\begin{tabular}{|c|c|}
\hline \multicolumn{2}{|c|}{ TECHNICAL PROGRESS REPORT } \\
\hline $\begin{array}{l}\text { Contract Title and Number: } \\
\text { Crosscutting Technology Development at the Center for } \\
\text { Advanced Separation Technologies } \\
\text { (DE-FC26-02NT41607) }\end{array}$ & 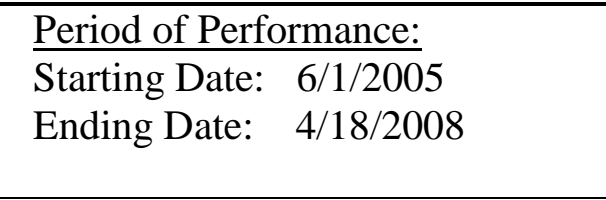 \\
\hline Sub-Recipient Project Title: & Report Information: \\
\hline Recovery Of Gold From Thiosulfate Leach Liquor & Type: $\quad$ Final \\
\hline Using Activated Carbon & $\begin{array}{ll}\text { Number: } & 5 \\
\text { Period: } & 5 / 31 / 06-4 / 18 / 08\end{array}$ \\
\hline Principal Investigators: & $4 / 18 / 08$ \\
\hline $\begin{array}{l}\text { Courtney A.Youmg } \\
\text { Larry G. Twidwell }\end{array}$ & MT005-R05 \\
\hline Contact Address: & Contact Information: \\
\hline Metallurgical and Materials Engineering & Phone: $\quad$ (406) 496-4158 \\
\hline Montana Tech & (406) 496-4664 \\
\hline Butte MT 59701 & CYoung@mtech.edu \\
\hline Subcontractor Address: & Subcontractor Information: \\
\hline Greg Hope, School of Biomolecular and Physical & Phone: $\quad$ (07) 37357550 \\
\hline Sciences, Nathan Campus, Griffith University, & (07) 37357656 \\
\hline 170 Kessels Road, Nathan, QLD 4111, Australia & G.Hope@griffith.edu.au \\
\hline
\end{tabular}

\section{DISCLAIMER}

This report was prepared as an account of work sponsored by an agency of the United States Government. Neither the United States Government nor any agency thereof, nor any of their employees, make any warranty, express or implied, nor assume any legal liability or responsibility for the accuracy, completeness, or usefulness of any information, apparatus, product, or process disclosed, or represents that its use would not infringe privately owned rights. Reference herein to any specific commercial product, process, or service by trade name, trademark, manufacturer, or otherwise does not necessarily constitute or imply endorsement, recommendation, or favoring by the United States Government or any agency thereof. The views and opinions of authors expressed herein do not necessarily state or reflect those of the United States Government or agency thereof.

\section{ABSTRACT}

This project was approved by CAST in mid-July of 2005 but official spending approval from Montana Tech was not received until November of 2005. A graduate student started working on the project in August of 2005 but, due to personal reasons, decided to leave campus in September at the start of the Fall 2005 Semester. His replacement, Mr. Nick Gow, was not found until May 2006. He will graduate in May of 2008. A second graduate student, Ms. Mariam Melashvilli, started in August of 2007 and is expected to graduate in December of 2008. This report therefore contains data that was generated essentially in the month of August of 2005 by the first student and since May 2006 by Mr. Gow and Ms. Melashvilli. 
In this study, a novel carbon adsorption technology for extracting gold from thiosulfate solutions is examined. Unless pretreated, activated carbons will not adsorb gold thiosulfate. However, by first adsorbing copper cyanide onto the carbon, gold thiosulfate can then be adsorbed in what appears to be an ion-exchange reaction. Previous results showed that copper cyanide was adsorbed at $\mathrm{pH} 10.5$ and was found to yield higher adsorption densities than at $\mathrm{pH}$ 11. The difference was attributed to solution speciation. When the Cu-pretreated carbon was then placed in gold thiosulfate solutions, gold take-up was observed with gold/copper ratios of 1:1 at $\mathrm{pH} 10.5$ for all concentrations; however, at $\mathrm{pH} 11$, ratios of 1:1 were observed only at low concentrations. Higher concentrations yielded a ratio of 0.6:1. Clearly, the results show that the envisioned process works but needs additional work to characterize as well as optimize.

Optimization studies have been conducted via statistical approaches with factorial-designed experiments using Stat-Ease software. Results were presented in the previous semi-annual report. In this report, four factors were identified and systematically varied between low and high values: initial $\mathrm{Cu}$ concentration $(0.001$ or $0.1 \mathrm{M}), \mathrm{pH}$ (9 or 12), time (1 or 5 hours) and temperature $\left(20\right.$ or $40^{\circ} \mathrm{C}$ ). All factors except time were shown to be important with the others yielding significantly better results at the higher values. Copper cyanide yielded metal loadings as high as 55,000 $\mathrm{g} \mathrm{Cu}$ per g of $\mathrm{C}$. Although gold exchange experiments have yet to be conducted at such a loading, it would be equivalent to approximately 5000 troy ounces of Au per ton of $\mathrm{C}(\mathrm{opt})$ assuming the Au from thiosulfate solution would exchange with the $\mathrm{Cu}$ at a 1:1 ratio. Because gold loadings from cyanide solutions typically average near 300 opt, this equivalent amount of 5000 opt would be more than enough to satisfy industrial purposes even if exchange ratios of 0.1:1 were observed.

Subsequent studies have therefore been conducted using Raman spectroscopy to characterize the adsorbed reactants and products to see if the proposed reaction mechanism can be confirmed. Tests were conducted at optimal copper cyanide concentrations based on the results of the factorial-designed experiments. Resulting copper-activated carbon was then contacted with gold thiosulfate solutions. Raman spectra collected during each step in the process showed copper cyanide was initially adsorbed but, following contact with aqueous gold thiosulfate, became gold cyanide thereby confirming the proposed reaction mechanism. By comparison, carbon nanotubes (CNTs) showed there was no affinity for copper cyanide and gold thiosulfate so their use as a non-resin adsorbent will be impossible.

Finally, elution tests were successfully conducted to illustrate that the resulting Au-loaded activated carbon could be stripped. This was accomplished by first preparing copperactivated carbon using optimal conditions from the statistical adsorption model and then contacting the pretreated carbon with gold-thiosulfate solutions. The resulting Au-loaded carbon was placed in a chromatographic column and eluted with a cyanide solution using traditional procedures. Preliminary studies showed that elution was best with high temperatures and high cyanide concentrations. Although high $\mathrm{Cu} / \mathrm{Au}$ ratios would mean high Au-loading, a ratio near 1.2 would be preferred to minimize the amount of $\mathrm{Cu}$ in the eluted solution and still obtain an elution efficiency of $100 \%$ as determined by Au recovery. 


\section{TABLE OF CONTENTS}

DISCLAIMER

ABSTRACT

TABLE OF CONTENTS

iii

INTRODUCTION 1

Background

Objective and Approach

PROJECT TASKS \& PROCEDURES 3

Initial Optimization Studies 3

Results and Discussion $\quad 5$

$\begin{array}{lr}\text { Factorial-Designed Optimization Studies } & 6\end{array}$

$\begin{array}{ll}\text { Results and Discussion } & 6\end{array}$

Adsorption on Carbon Nanotubes $\quad 9$

Characterization Studies by Raman Spectrscopy $\quad 9$

Results and Discussion $\quad 9$

Elution of Ion-Exchanged Au 12

Results and Discussion 12

$\begin{array}{ll}\text { CONCLUSIONS } & 14\end{array}$

$\begin{array}{ll}\text { FUTURE WORK } & 14\end{array}$

$\begin{array}{ll}\text { ACKNOWLEDGEMENTS } & 14\end{array}$

$\begin{array}{ll}\text { REFERENCES } & 15\end{array}$

PUBLICATIONS AND PRESENTATIONS 15 


\section{INTRODUCTION}

\section{Background}

In current commercial leaching of gold using cyanide $\left(\mathrm{CN}^{-}\right)$, gold $(\mathrm{Au})$ in an ore is oxidized and dissolves to form the cyano-aurous complex $\left[\mathrm{Au}(\mathrm{CN})_{2}{ }^{-}\right]$:

$$
\mathrm{Au}+2 \mathrm{CN}^{-} \rightarrow \mathrm{Au}(\mathrm{CN})_{2}^{-}+\mathrm{e}^{-}
$$

which requires oxygen $\left(\mathrm{O}_{2}\right)$ to be reduced to hydroxide $\left(\mathrm{OH}^{-}\right)$in the presence of water $\left(\mathrm{H}_{2} \mathrm{O}\right)$ :

$$
\mathrm{O}_{2}+2 \mathrm{H}_{2} \mathrm{O}+4 \mathrm{e}^{-} \rightarrow 4 \mathrm{OH}^{-}
$$

When electron-balanced, the overall reduction/oxidation (redox) reaction results:

$$
\mathrm{Au}+2 \mathrm{CN}^{-}+1 / 4 \mathrm{O}_{2}+1 / 2 \mathrm{H}_{2} \mathrm{O} \rightarrow \mathrm{Au}(\mathrm{CN})_{2}^{-}+\mathrm{OH}^{-}
$$

The process was patented by MacArthur and the Forrest (1887) and was conclusively shown to be electrochemical in nature by Kudryk and Kellogg (1954) but was not generally combined with carbon adsorption technology until about 1970 (Marsden and House, 2006):

$$
\mathrm{Au}(\mathrm{CN})_{2}^{-}+\mathrm{C}_{\text {site }} \rightarrow \mathrm{Au}(\mathrm{CN})_{2}{ }^{-} \text {ads }
$$

This concentration step is dependent on the complex chemistry of the aurous species.

The standard reduction potential for oxygen to water is $1.23 \mathrm{~V}$ vs SHE, and the standard reduction potential for the non-complexed aurous ions is $1.69 \mathrm{~V}$ vs SHE which means that, thermodynamically, free aurous ions are a stronger oxidizing agent than oxygen. However, in the presence of cyanide ions, the stability of the gold (I) cyanide complex lowers the standard reduction potential to $-0.57 \mathrm{~V}$ vs SHE thus enabling the univalent ions to be processed under ambient conditions. In practical gold cyanidation, copper competes for cyanide ions with gold and leads to high cyanide consumption rates. Because the copper in the ore is present in significantly larger amounts than the gold, the resulting copper cyanide is also at significantly higher concentrations and thereby competes with gold cyanide for the adsorption sites on the activated carbon (Jay, 2000).

Gold leaching with thiosulfate is a leading contender to replace cyanide in the extraction of gold from ore. In several respects, the process is similar to cyanidation. This is envisioned by comparing Reaction 5 with Reaction 3:

$$
\mathrm{Au}+2 \mathrm{~S}_{2} \mathrm{O}_{3}{ }^{2-}+1 / 4 \mathrm{O}_{2}+1 / 2 \mathrm{H}_{2} \mathrm{O} \rightarrow \mathrm{Au}\left(\mathrm{S}_{2} \mathrm{O}_{3}\right)_{2}{ }^{3-}+\mathrm{OH}^{-}
$$

Although the standard reduction potential of Reaction 5 is $0.153 \mathrm{~V}$ vs SHE and is not as low as Reaction 3 at $-0.57 \mathrm{~V}$, it still makes the reaction amenable for use with air oxidation. Various chemicals are added to help stabilize the thiosulfate including copper which also helps to catalyze the leaching reaction (Marsden and House, 2006). 
However, a major limitation for the development of thiosulfate leaching technology is the recovery of gold from leach liquors. Because activated carbon has a very low affinity for gold thiosulfate, it cannot be used like it is used with cyanide leach liquors:

$$
\mathrm{Au}\left(\mathrm{S}_{2} \mathrm{O}_{3}\right)_{2}{ }^{3-}+\mathrm{C}_{\text {site }} \rightarrow \text { No adsorption }
$$

In this regard, extensive research efforts reported in the literature have turned to developing resins and other technologies for gold recovery. However, resins suffer from fouling and, of course, are inherently expensive. An anti-fouling and cheaper alternative is therefore needed to make thiosulfate leaching technology competitive against cyanidation.

\section{Objective and Approach}

The main objective of this project is to develop a recovery technology for extracting gold thiosulfate from solution. It was therefore proposed (Young and Twidwell, 2005) that a novel non-resin technology be examined to assure that it works, does not suffer from the same limitations as resin, and will be compatible with existing gold cyanide production technology. An activated-carbon process was envisioned in which the carbon was pretreated with copper cyanide:

$$
\mathrm{Cu}(\mathrm{CN})_{2}^{-}+\mathrm{C}_{\text {site }} \rightarrow \mathrm{Cu}(\mathrm{CN})_{2}^{-} \text {ads }
$$

with the resulting pretreated carbon being used to extract the gold from thiosulfate solution:

$$
\mathrm{Au}\left(\mathrm{S}_{2} \mathrm{O}_{3}\right)_{2}{ }^{3-}+\mathrm{Cu}(\mathrm{CN})_{2}^{-} \text {ads } \rightarrow \mathrm{Au}(\mathrm{CN})_{2}^{-} \text {ads }+\mathrm{Cu}\left(\mathrm{S}_{2} \mathrm{O}_{3}\right)_{2}{ }^{3-}
$$

Assuming that the adsorbed cyano-aurous species produced by this "ion-exchange" method is the same as that produced by direct adsorption of the cyano-aurous complex (see Reaction 4), than current technology for carbon elution, electrowinning, and smelting can be used to produce gold bullion. To obtain these objectives, the approaches for the research are to (Task 1) verify that Reaction 8 occurs, (Task 2) determine parameters that critically affect Reaction 8, (Task 3) optimize Reaction 8 to maximize gold recovery from solution, (Task 4) spectroscopically and thermodynamically characterize the adsorbed species via comparison to direct adsorbed species, (Task 5) determine if conventional technology can be used to elute the adsorbed species, (Task 6) examine carbon nanotubes (CNT's) as a possible highsurface area adsorbent, (Task 7) develop a method for making CNT's from coal, and (Task 8) conduct a literature review throughout the project. 


\title{
PROJECT TASKS AND PROCEDURES
}

\author{
All Tasks have been completed. \\ $\underline{\text { Initial Optimization Studies }}$
}

Because the four identified variables did not include gas type which would control the amount of dissolved oxygen in solution and thereby the potential oxidation of the copper cyanide, kettle reactors were no longer used. In this regard, experiments were conducted on $100 \mathrm{~mL}$ solutions in $250 \mathrm{~mL}$ Erlenmeyer flasks. The solutions were brought to a specified $\mathrm{pH}$ and temperature; $\mathrm{K}_{2} \mathrm{CuCN}_{3}$ was then added at $0.001 \mathrm{M}$ concentrations. Copper was added to the solution as needed to help control the $\mathrm{Cu}: \mathrm{CN}$ ratio. Agitation was conducted at $300 \mathrm{rpm}$ with Teflon-coated magnetic stir-bars using temperature-controlled stir plates to obtain adequate mixing. After 1 gram of activated carbon was added, the flasks were then covered with parafilm to prevent evaporation. Samples measuring $20 \mathrm{~mL}$ at various times, acidified (under a hood) to $\mathrm{pH} 1$ using concentrated $\mathrm{HCl}$, diluted as needed to bring the concentrations of $\mathrm{Cu}$ below 100 ppm (i.e., operating window), and then analyzed by ICP.

However, it is important to note that several problems had to be overcome in order to establish what appears to be a simple procedure. First, attempts to use CuCN solid were unsuccessful due to the difficulties getting it to dissolve in solution. CuCN was preferred because its exact chemical make-up was known; whereas, that of $\mathrm{K}_{2} \mathrm{CuCN}_{3}$ contained various amounts of $\mathrm{KCuCN}_{2}$ but was not a lot of concern since one of the factors was addition of copper to help control $\mathrm{Cu}$ :CN ratio. Second, attempts to use an activated carbon obtained from a vendor also proved difficult since adsorption of $\mathrm{Cu}$ periodically showed that $\mathrm{Cu}$ adsorption was minimal at any $\mathrm{pH}$, particularly $\mathrm{pH}$ 9. This was rectified when a sample of activated carbon was obtained from Golden Sunlight, a cyanide leaching operation in Whitehall MT just 40 miles from Montana Tech.

\section{$\underline{\text { Results and Discussions }}$}

Results of experiments conducted at room temperature $\left(20^{\circ} \mathrm{C}\right)$ at a variety of pH's are shown and compared in Figure 1 to data previously collected. Adsorption is shown to be highest at $\mathrm{pH} 12$ and lowest at $\mathrm{pH} 9$ with $\mathrm{pH} 10.5$ in between. This trend conflicts with the literature (Marsden and House, 2006) and not readily explainable but could be attributed to the lack of $\mathrm{Ca}^{2+}$ in the system and thereby the inability for needed ion-pairs to form (McDougall et al., 1980). Although the data from the literature at $\mathrm{pH} 11$ does not follow this trend, the results are similar and may not agree due to different carbon types and surface areas being used.

Results of experiments conducted at elevated temperature $\left(40^{\circ} \mathrm{C}\right)$ at $\mathrm{pH} 9$ and 12 are shown in Figure 2. A comparison of the results in Figure 1 shows that, at constant $\mathrm{pH}$, increasing temperatures actually increases the amount of adsorption. Because this suggests that the copper cyanide adsorption process is endothermic, this does not follow gold cyanide adsorption mechanisms and therefore conflicts with expectations. This could also be attributed to the lack of $\mathrm{Ca}^{2+}$ in the system which would prevent ion-pair formation. 


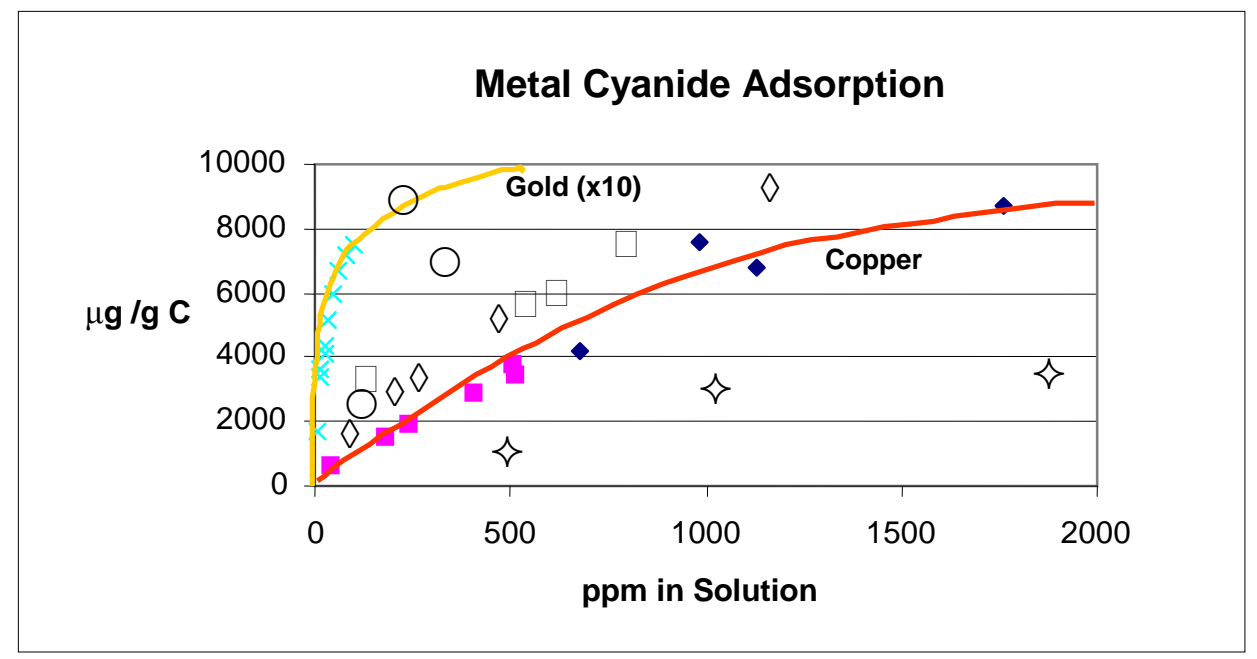

Figure 1. Adsorption isotherms of copper cyanide on activated carbon at room temperature $\left(20^{\circ} \mathrm{C}\right)$ compared to literature for gold cyanide (Marsden and House, 2006). Closed symbols are preliminary data conducted at $\mathrm{pH} 11$ for the proposal (Young and Twidwell, 2005) and open symbols are current data conducted by the first student at $\mathrm{pH} 10.5(\diamond)$ and by the second student at pH $9(\diamond), 10.5(\square)$, and $12(\bigcirc)$ for the project.

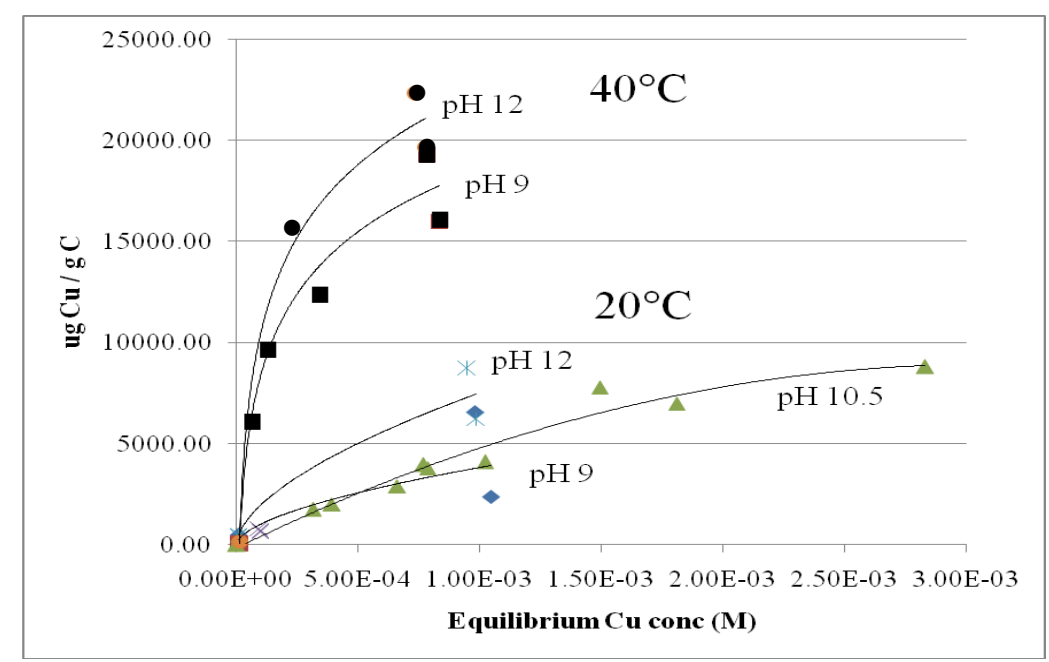

Figure 2. Adsorption isotherms of copper cyanide on activated carbon at elevated temperature $\left(40^{\circ} \mathrm{C}\right)$ at $\mathrm{pH} 9(\boldsymbol{\nabla})$ and $\mathrm{pH} 12(\bullet)$ compared to room temperature $\left(20^{\circ} \mathrm{C}\right)$..

From Figures 1 and 2, it can be seen that copper cyanide loaded as high as 9,000 and 22,000 $\mu \mathrm{g} \mathrm{Cu}$ per g of $\mathrm{C}(\mu \mathrm{g} / \mathrm{g} \mathrm{C})$ respectively which would be equivalent to between approximately 1,000 and 2,000 troy ounces of Au per ton of $\mathrm{C}$ (opt) assuming the Au from thiosulfate solution would exchange with the $\mathrm{Cu}$ at a 1:1 ratio. Previous results showed that the gold exchange reaction with copper was initially 1:1 but decreased to 0.6:1 at higher loadings. This was attributed to steric hindrance caused by the larger $\mathrm{Au}$ atom replacing the smaller $\mathrm{Cu}$ atom. However, because gold loadings from cyanide solutions typically average near 300 opt, the 1000-2000 opt equivalence would be more than enough to satisfy industrial needs even if exchange ratios one quarter that amount at 250-500 opt were observed. 
Regression analysis of the data in Figure 1 obtained at $\mathrm{pH} 12$ and 9 reveals that the maximum adsorption is approximately 12,000 and 10,000 $\mu \mathrm{g} / \mathrm{g} \mathrm{C}$, respectively. Dividing all of the adsorption amounts $(\Gamma)$ by these respective values $\left(\Gamma_{\mathrm{m}}\right)$ would therefore yield fractions ranging from 0 to 1 . Because it is desired to have this fraction be as close to 1 as possible, an indication that maximum adsorption is occurring, the term is herein called desirability; however, it is noted that it is the same as the term more commonly referred to as surface coverage $(\theta)$ as often used for adsorption isotherm studies such Langmuir, Freundlich, Frumkin, etc. (Young, 1995):

$$
\theta=\Gamma / \Gamma_{\mathrm{m}}
$$

Likewise, based on the Stern-Langmuir Equation:

$$
\mathrm{K}=\theta /[(1-\theta) \mathrm{C}]
$$

where $\mathrm{C}$ is the equilibrium concentration of the adsorbing species in solution and $\mathrm{K}$ is the adsorption reaction constant, the free energy of the adsorption reaction can be calculated for any temperature:

$$
\Delta \mathrm{G}_{\mathrm{ads}}=-2.303 \mathrm{RT} \log (55.55 \mathrm{~K})
$$

Furthermore, when the free energy is known for two different temperatures, the ClausiusClapeyron Equation can be used to estimate the enthalpy of adsorption:

$$
\Delta H_{a d s}=\frac{\Delta\left(\frac{\Delta G_{a d s}}{T}\right)}{\Delta\left(\frac{1}{T}\right)}
$$

which, in turn, can then be used to calculate the entropy of adsorption from the fundamental thermodynamic expression:

$$
\Delta S_{a d s}=\frac{\left(\Delta H_{a d s}-\Delta G_{a d s}\right)}{T_{a v g}}
$$

Based on Equations 11-13 and the data shown in Figures 1 and 2, the thermodynamic data presented in Table 1 were determined. As can be seen, the free energies of adsorption at $20^{\circ} \mathrm{C}$ and $40^{\circ} \mathrm{C}$ were found to average $-27.4 \mathrm{~kJ} / \mathrm{mol}$ and $-32.6 \mathrm{~kJ} / \mathrm{mol}$, respectively. Clearly, the free energies are negative indicating that the adsorption reaction is favorable but, because these values are significantly below $-20 \mathrm{~kJ} / \mathrm{mol}$, the reaction is chemisorption which would be expected of activated carbon surfaces. Furthermore, the free energies become more negative by approximately $-2 \mathrm{~kJ} / \mathrm{mol}$ with increasing $\mathrm{pH}$ which helps to explain the increased adsorption with increasing $\mathrm{pH}$. Table 1 also shows that the enthalpy of adsorption was determined to be 41.02 and $55.65 \mathrm{~kJ} / \mathrm{mol}$ at $\mathrm{pH} 9$ and 12, respectively; and the entropy of adsorption was calculated at 223.8 and $276.8 \mathrm{~J} / \mathrm{mol} /{ }^{\circ} \mathrm{K}$ at $\mathrm{pH} 9$ and 12 , respectively as well. 
The positive enthalpy indicates the adsorption reaction is endothermic which agrees with the conclusions noted earlier but are therefore in contrast with the literature (Marsden and House, 2006). It is further noted that, because the enthalpy is positive, the adsorption reaction must be entropically driven meaning that the system is becoming disordered. Normally, species that adsorb become ordered but, because the entropy values are significantly large, the disordering is likely due to water being displaced as the copper cyanide adsorbs. It is also likely that water disordering does not emanate so much from the activated carbon surface (which would tend to be hydrophobic and therefore already promote water displacement) but rather is caused mostly by the water sheath that surrounds the adsorbing species. In fact, if this disordering of water is similar to the melting of ice, then the enthalpy of melting, which is equal to $\sim 6 \mathrm{~kJ} / \mathrm{mol}$ (Robie et al., 1979), can be used to estimate the number of water molecules being displaced from approximately 7 at $20^{\circ} \mathrm{C}$ and 9 at $40^{\circ} \mathrm{C}$. These are reasonable numbers.

Table 1. Thermodynamic Data for Copper Cyanide Adsorption on Activated Carbon

\begin{tabular}{|c|c|c|c|}
\hline Temperature $\left({ }^{\circ} \mathrm{C}\right)$ & $\Delta \mathbf{G}_{\text {ads }}(\mathrm{kJ} / \mathrm{mol})$ & $\Delta H_{\text {ads }}(\mathrm{kJ} / \mathrm{mol})$ & $\Delta \mathrm{S}_{\mathrm{ads}}\left(\mathrm{J} / \mathrm{mol} /{ }^{0} \mathrm{~K}\right)$ \\
\hline $20 @$ pH 9 & -26.8 & \multirow{2}{*}{41.02} & \multirow{2}{*}{223.8} \\
\hline 40 @ pH 9 & -31.4 & & \\
\hline $20 @$ pH 12 & -28.1 & \multirow{2}{*}{55.65} & \multirow{2}{*}{276.8} \\
\hline $40 @ \mathrm{pH} 12$ & -33.8 & & \\
\hline
\end{tabular}

\section{$\underline{\text { Factorial-Designed Optimization Studies }}$}

Optimization studies have therefore been conducted via additional statistical approaches using factorial-designed experiments and was accomplished using Stat-Ease software. To do this, the same four factors were systematically varied between low and high values: initial $\mathrm{Cu}$ concentration ( 0.001 or $0.1 \mathrm{M})$, $\mathrm{pH}$ (9 or 12$)$, time ( 1 or 5 hours) and temperature (20 or $\left.40^{\circ} \mathrm{C}\right)$. A full two-level factorial study was completed and therefore involved $2^{\mathrm{n}}+(\mathrm{n}-1)$ experiments. Because $n=4$ factors, 19 experiments were conducted such that the 3 additional experiments included mid-point determinations at $0.01 \mathrm{M}, \mathrm{pH} 10.5,3$ hours and $30^{\circ} \mathrm{C}$. Results are shown in Figures 3-6.

\section{$\underline{\text { Results and Discussions }}$}

Figures 3 and 4 are referred to as Data Cubes and are used to get a quick assessment of the resulting model. As can be seen, the desirability response (i.e., surface coverage) after 1 hour of equilibration is maximum when all factors are at a maximum (see top right of Figure 3) and is minimum when the $\mathrm{pH}$ and temperature are low and the copper concentration is high (see top left of Figure 3). When equilibrium is allowed to take place for 5 hours, the respective maximum and minimum remain in the same two corners. For the most part, the other corners are relatively unchanged with the exception of the $\mathrm{pH}-\mathrm{Cu}$ Concentration face. This is the only situation where increasing time increased adsorption; otherwise, time had no noticeable effect on desirability. Of course, the models are better realized in the 3dimensional plot in Figure 5 and the contour plot in Figures 6. Adsorption amounts and 
desirability responses in this part of the study were similar to those obtained earlier. In this case, copper cyanide yielded metal loadings as high as 56,000 $\mu \mathrm{g}$ per $\mathrm{g} \mathrm{C}$ and the maximum loading was observed at conditions which did not favor $\mathrm{Cu}(\mathrm{CN})_{2}^{-}$in solution. According to the literature (Marsden and House, 2006), maximum adsorption should have been observed at $\mathrm{pH}$ 9, increased $\mathrm{Cu}$ concentrations, and lower temperatures.

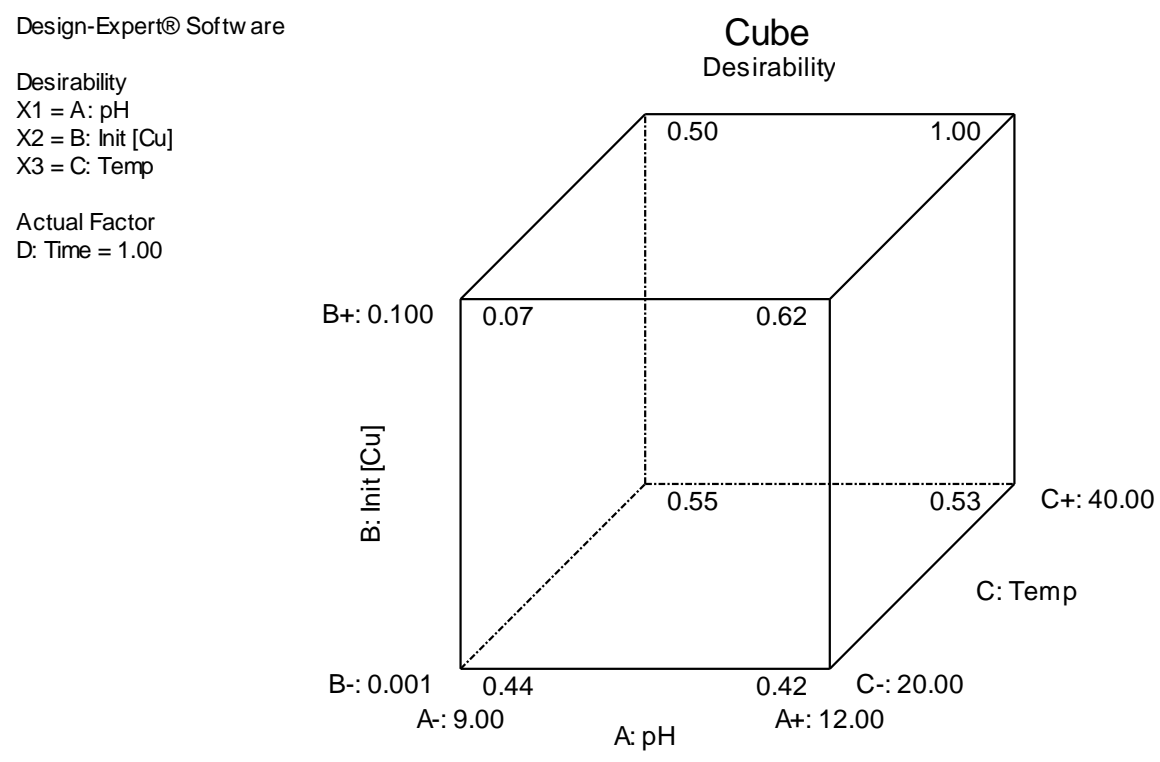

Figure 3. Data cube showing the effect of copper concentration, $\mathrm{pH}$ and temperature on desirability after 1 hour of adsorption.

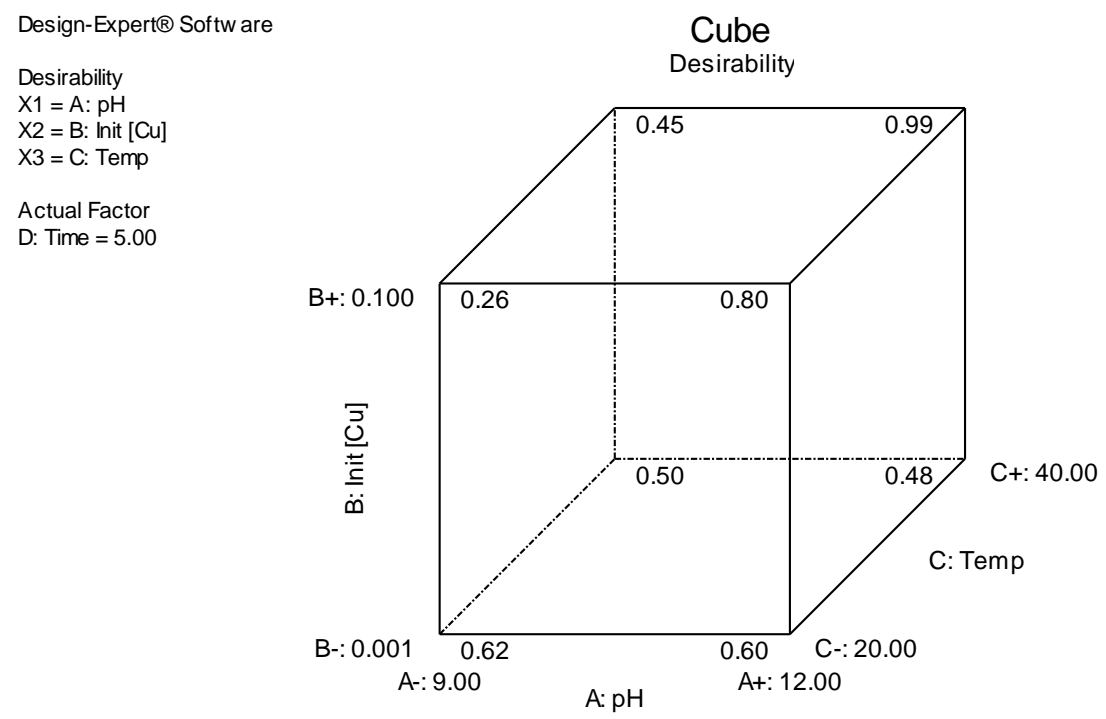

Figure 4. Data cube showing the effect of copper concentration, $\mathrm{pH}$ and temperature on desirability after 5 hours of adsorption. 
Design-Expert@ Softw are

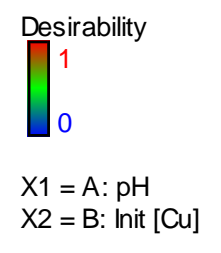

Actual Factors

C: Temp $=40.00$

D: Time $=1.00$

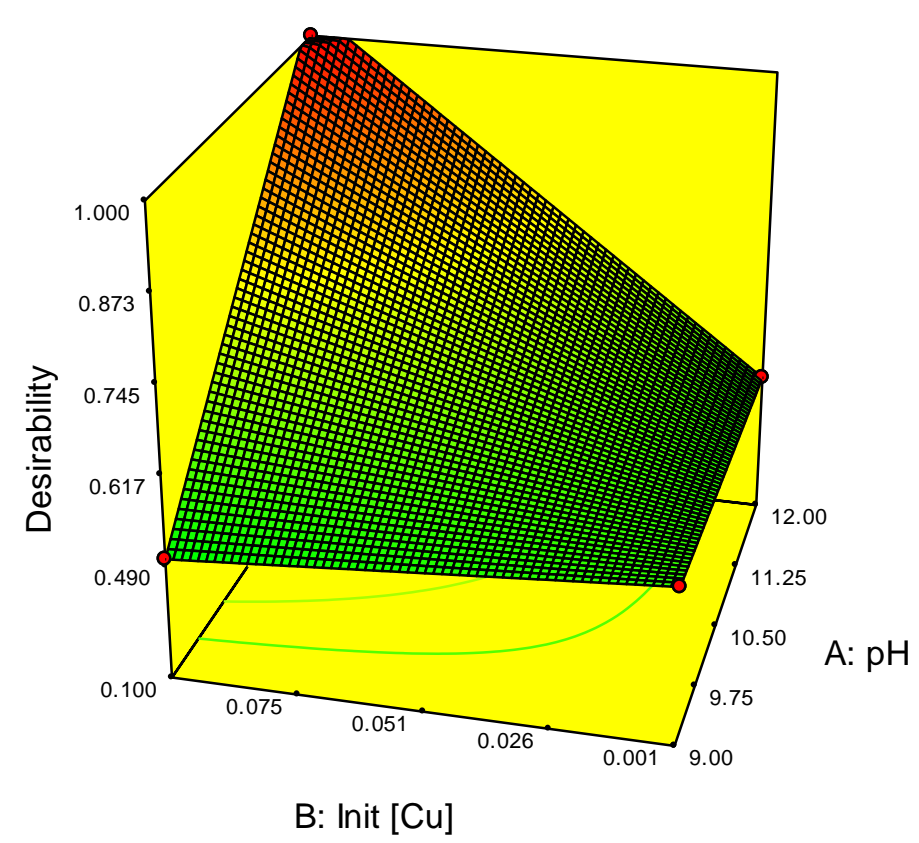

Figure 5. 3-dimensional plot showing the effect of copper concentration and $\mathrm{pH}$ on desirability at $40^{\circ} \mathrm{C}$ after 1 hour of adsorption.
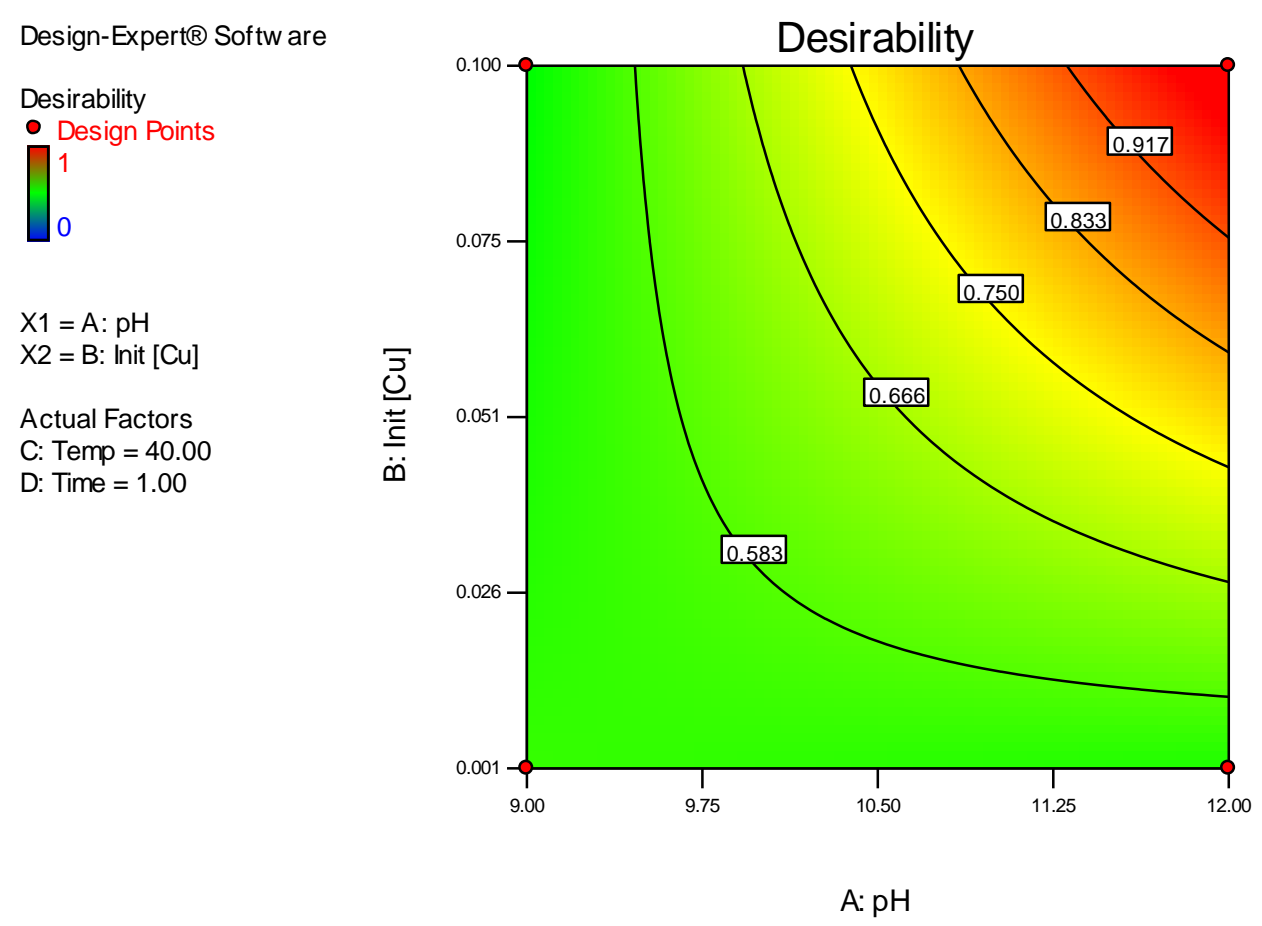

Figure 6. Contour plot showing the effect of copper concentration and $\mathrm{pH}$ on desirability at $40^{\circ} \mathrm{C}$ after 1 hour of adsorption. 


\section{Adsorption on Carbon Nantubes}

Adsorption studies of gold thiosulfate on untreated multi-walled carbon nanotubes (MWCNTs or simply CNTs) were conducted. As with untreated activated carbon, no adsorption was observed. Attempts were made to pretreat (i.e., functionalize) the CNTs using high $(0.1 \mathrm{M})$ and low $(0.001 \mathrm{M})$ concentrations of copper cyanide at $\mathrm{pH} 9$ and 12 as well as at room $\left(20^{\circ} \mathrm{C}\right)$ and elevated $\left(40^{\circ} \mathrm{C}\right)$ temperatures. Once again, adsorption was not observed. Clearly, CNTs do not have an affinity for either gold thiosulfate or copper cyanide and, in this regard, CNTs will need to be functionalized by some other method than copper cyanide adsorption. Because this process did not work, nanotechnology will have to wait another day for gold thiosulfate recovery from solution. However, no such methods will be explored further in this project and, in this regard, Task 7 was ignored in which a method for making CNT's from coal was to be explored.

\section{Characterization Studies by Raman Spectrscopy}

Subsequent studies have therefore been conducted using Raman spectroscopy to characterize the adsorbed reactants and products to see if the proposed reaction mechanism can be confirmed (see Reaction 8). Tests were conducted at optimal copper cyanide concentrations based on the results of the factorial-designed experiments. Resulting copper-activated carbon was then contacted with gold thiosulfate solutions. Raman spectra collected during each of these steps were compared to known spectra to help discern the actual reactions occurring. Results are presented in Figures 7-9. This work was conducted at Griffiths University in Brisbane, Australia.

\section{$\underline{\text { Results and Discussions }}$}

Raman spectra of known solid samples are shown in Figure 7. Clearly, peaks near Raman shifts of $2100 \mathrm{~cm}^{-1}$ are due to copper cyanide and those near $2250 \mathrm{~cm}^{-1}$ are due to gold cyanide. Clearly, it will be important to zoom into these regions to discern better what is happening.

In this regard, Figure 8 shows spectra of adsorbed copper cyanide on activated carbon. At $\mathrm{pH} 9$, the center peak at 2118 corresponds to copper dicyanide $\left[\mathrm{Cu}(\mathrm{CN})_{2}{ }^{-}\right]$whereas the peaks at 2096 and 2127 represent copper tricyanide $\left[\mathrm{Cu}(\mathrm{CN})_{3}{ }^{2-}\right]$. These peaks are slightly shifted from their aqueous counterparts at 2137 as well as 2094 and 2108, respectively, as observed by Lukey et al. (1999). At pH 10, there are only two peaks at 2090 and 2111 which match up quite well with copper tricyanide solid (see Figure 7) and aqueous (Lukey et al., 1999). Clearly, the adsorbed species is dependent on $\mathrm{pH}$. It can be concluded that, as the $\mathrm{pH}$ increases, the favored adsorbed species becomes the tricyanide species and may explain why adsorption increases accordingly (see Figure 1). 


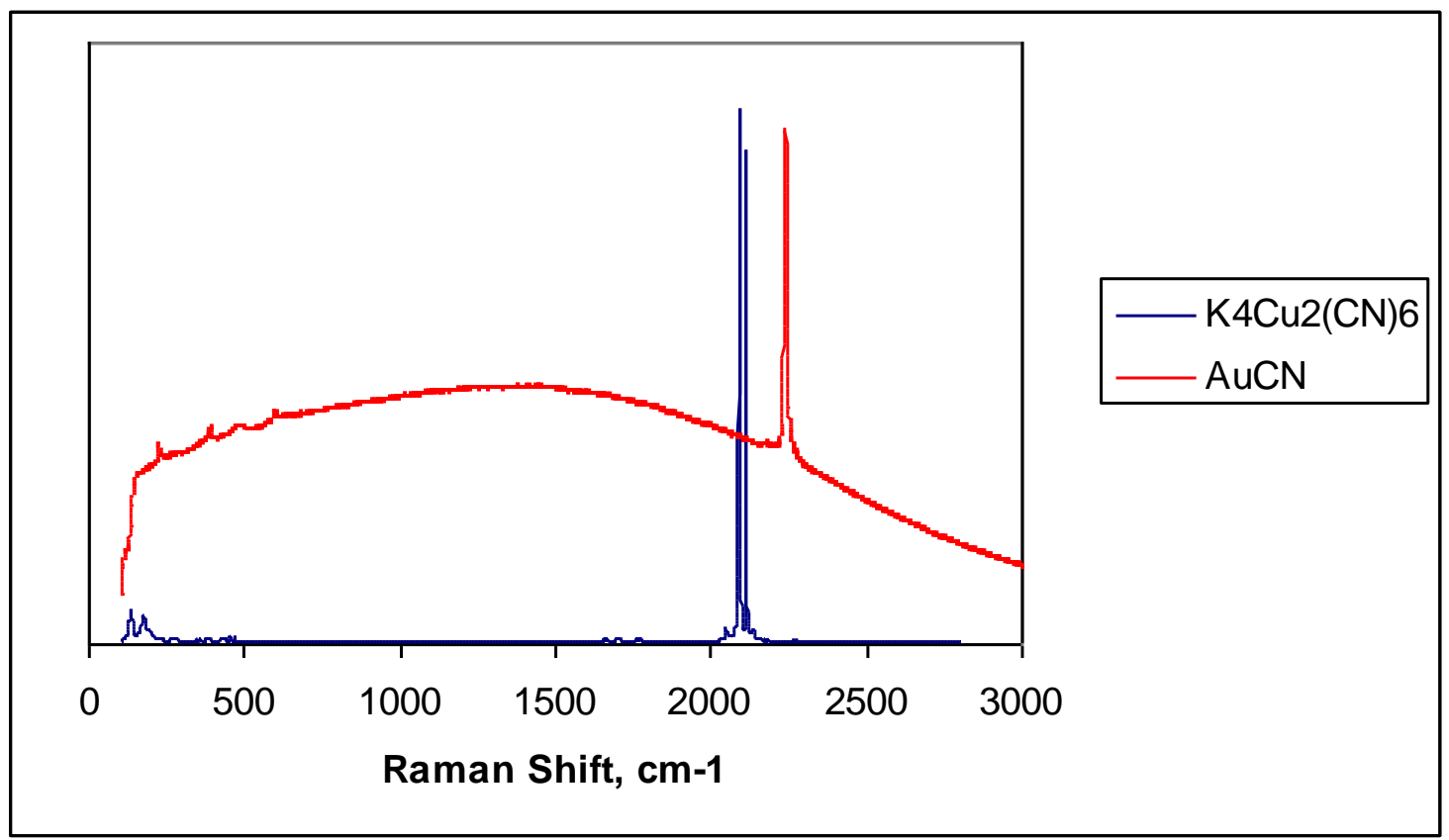

Figure 7. Raman spectra of solid cyanide species. Potassium-copper cyanide yield peaks near $2100 \mathrm{~cm}^{-1}$ (blue) whereas gold cyanide yields a peak near $2250 \mathrm{~cm}^{-1}$ (red).

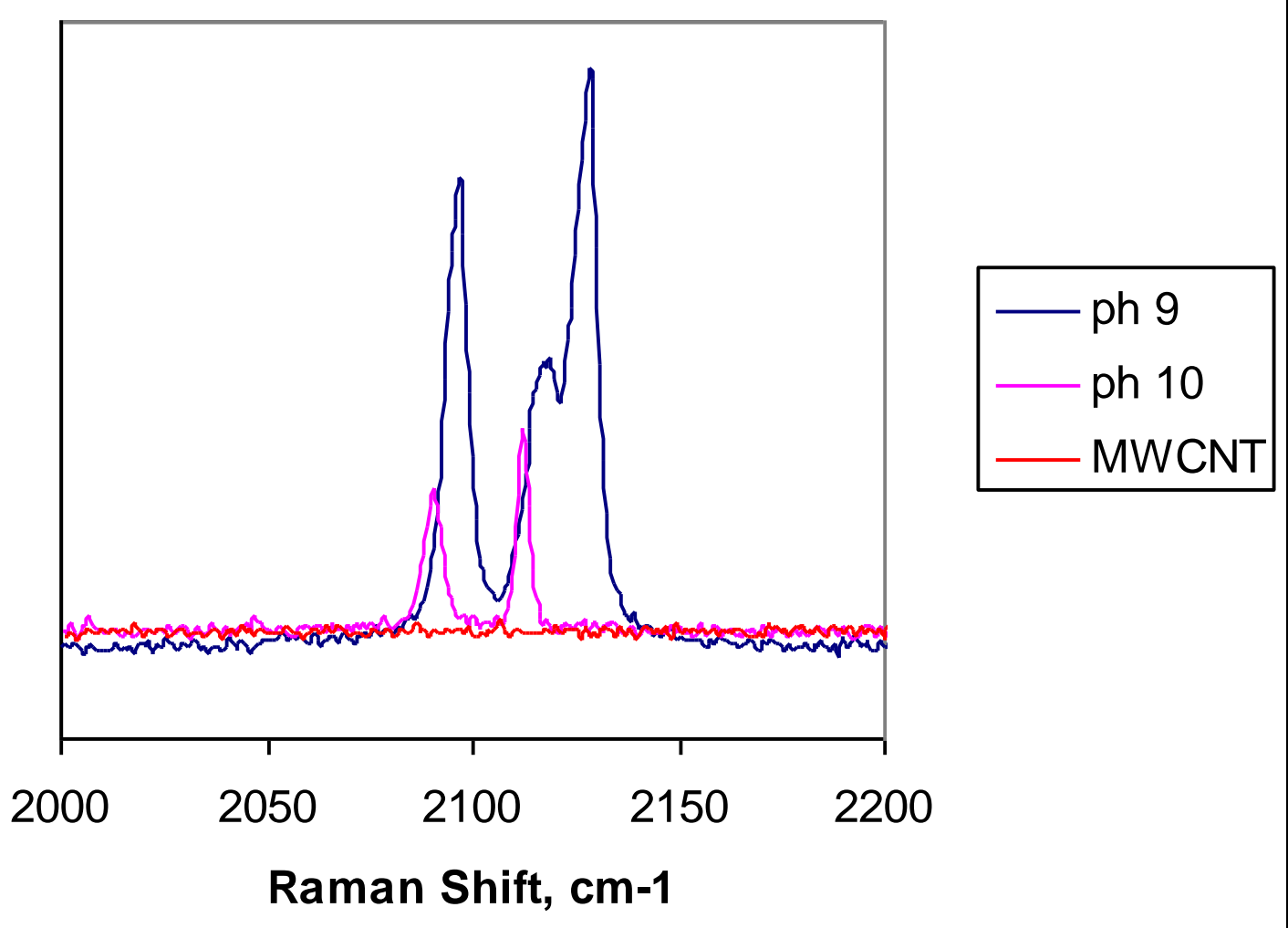

Figure 8. Raman spectra of copper cyanide adsorbed on activated carbon at pH 9 (blue) and pH 10 (pink). No adsorbed species are observed on carbon nanotubes (red). 


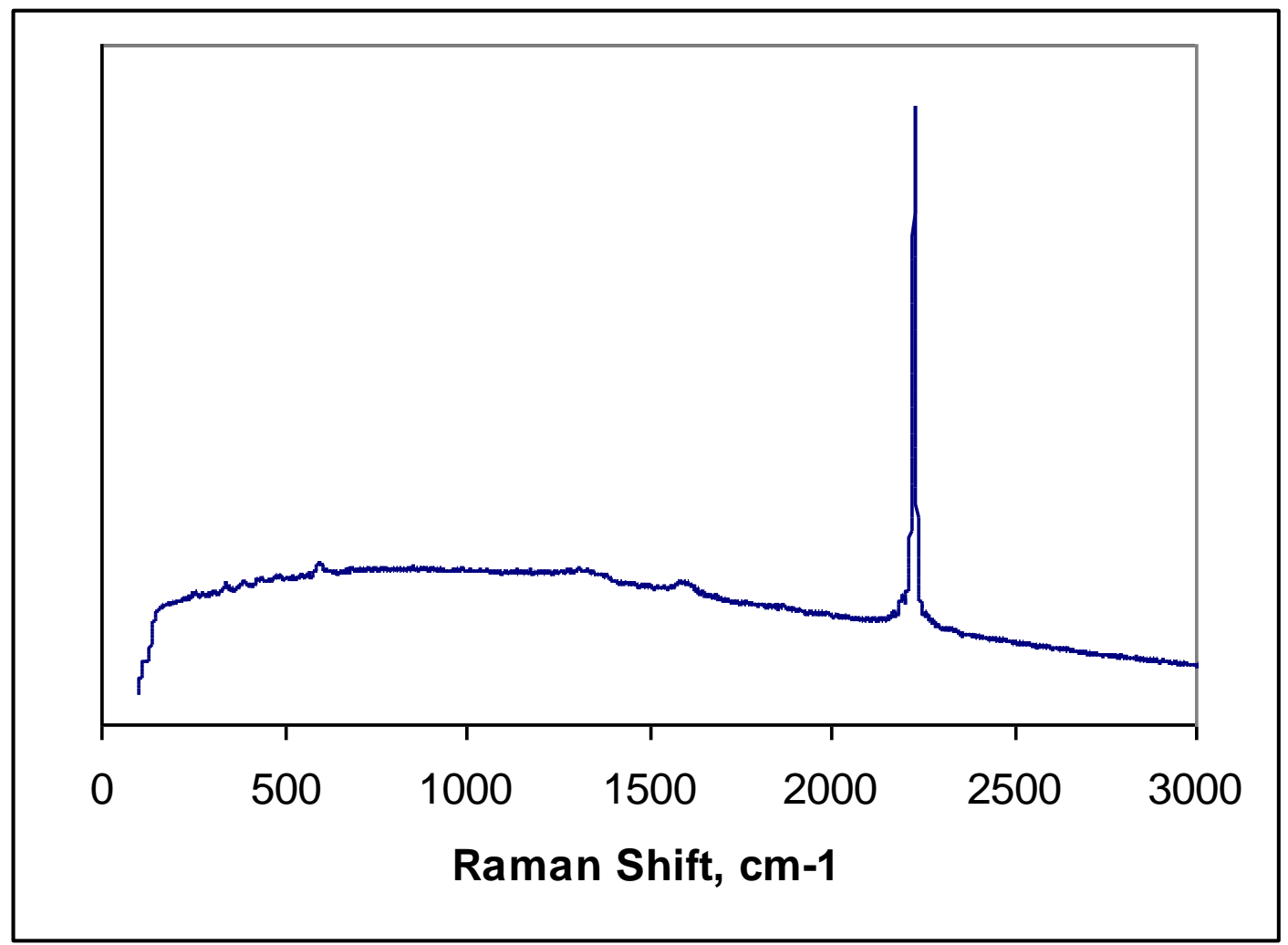

Figure 9. Raman spectra pretreated activated carbon with copper cyanide after being placed in gold thiosulfate solution. The strong peak is due to the formation of gold cyanide.

Figure 9 shows the Raman spectra of activate carbon was copper-treated at $\mathrm{pH} 10$ after contacting it with gold thiosulfate solution. No copper cyanide peaks are observed and only gold cyanide peaks are. This clearly indicates that the gold does ion-exchange with the copper thereby confirming Reaction 8; however, because the reaction was not observed for activated carbon pretreated at $\mathrm{pH} 9$, it may indicate that copper tricyanide plays a critical role. Reaction 8 may therefore be preferably written according to the following:

$$
\mathrm{Au}\left(\mathrm{S}_{2} \mathrm{O}_{3}\right)_{2}{ }^{3-}+\mathrm{Cu}(\mathrm{CN})_{3}{ }^{2-}{ }_{\text {ads }} \rightarrow \mathrm{Au}(\mathrm{CN})_{2}{ }_{\text {ads }}+\mathrm{CN}^{-}+\mathrm{Cu}\left(\mathrm{S}_{2} \mathrm{O}_{3}\right)_{2}{ }^{3-}
$$

It is important to note that the gold cyanide peak occurs at $2227 \mathrm{~cm}^{-1}$ which is slightly shifted from the $2239 \mathrm{~cm}^{-1}$ peak shown in Figure 7. The strength of the peak is likely due to a surface-enhanced Raman effect. This effect should also have enhanced copper cyanide spectra and therefore indicates that, under the conditions examined, all of the copper was ion exchanged with the gold. It is also important to note that the resulting activated carbon became a tinted yellow and could indicate that a surface precipitate of gold cyanide (AuCN) formed. In this case, Reaction 8 could be illustrated according to the following:

$$
\mathrm{Au}\left(\mathrm{S}_{2} \mathrm{O}_{3}\right)_{2}{ }^{3-}+\mathrm{Cu}(\mathrm{CN})_{3}{ }^{2-} \text { ads } \rightarrow \mathrm{AuCN}_{\mathrm{ads}}+2 \mathrm{CN}^{-}+\mathrm{Cu}\left(\mathrm{S}_{2} \mathrm{O}_{3}\right)_{2}{ }^{3-}
$$

It is possible that, in the presence of excess $\mathrm{Cu}$, that the resulting adsorbed Au species is a compound ranging from $\mathrm{Au}_{3} \mathrm{Cu}$ to $\mathrm{AuCu}_{3}$ (Hiskey and Lee, 2003) depending on the amount 
of excess copper (i.e. Cu-to-Au ratio). Either way, this reaction also confirms that the gold and copper are ion-exchanging.

\section{Elution of Ion-Exchanged Au}

The activated carbon obtained from Golden Sunlight in Whitehall was washed with 10\% $\mathrm{HCl}$, dried at $110^{\circ} \mathrm{C}$, reactivated in a furnace at $700^{\circ} \mathrm{C}$ for $15 \mathrm{~min}$, quenched in water, and then pretreated by copper cyanide adsorption. To do that, 10-gram portions of the carbon were weighed out and placed into 250 -ml flasks containing solutions of different copper cyanide concentrations at $\mathrm{pH} 12$ and $40^{\circ} \mathrm{C}$ in order to follow the optimal adsorption method determined above. After 5 hours of pretreatment, the carbon was then washed with distilled water and transferred into another 250-ml flask containing Au-thiosulfate of different concentrations near $\mathrm{pH}$ 11.5. After 1 hour, the carbon was transferred to another flask, pretreated with $2 \% \mathrm{NaCN}$ and $1 \% \mathrm{NaON}$ solution for another hour, transferred again to a water-jacketed chromatography column for temperature control, and eluted with distilled water. Eluant was collected as a function of time and reported as bed volumes. Following elution, the carbon was removed from the column, dried at $110^{\circ} \mathrm{C}$, and acid digested for ICP analysis.

\section{$\underline{\text { Results and Discussions }}$}

Figure 10 shows the recovery of gold from solution as a function of the molar ratio of $\mathrm{Cu}$ on the carbon to the $\mathrm{Au}$ in solution. When the ratio is 1.0, the gold recovery is $90 \%$. Tests were conducted at ratios greater than 1.8 and all were found to yield recoveries of $100 \%$. Extrapolating a line though the origin suggests that ratios in excess of 1.2 are needed to maintain recoveries at $100 \%$.

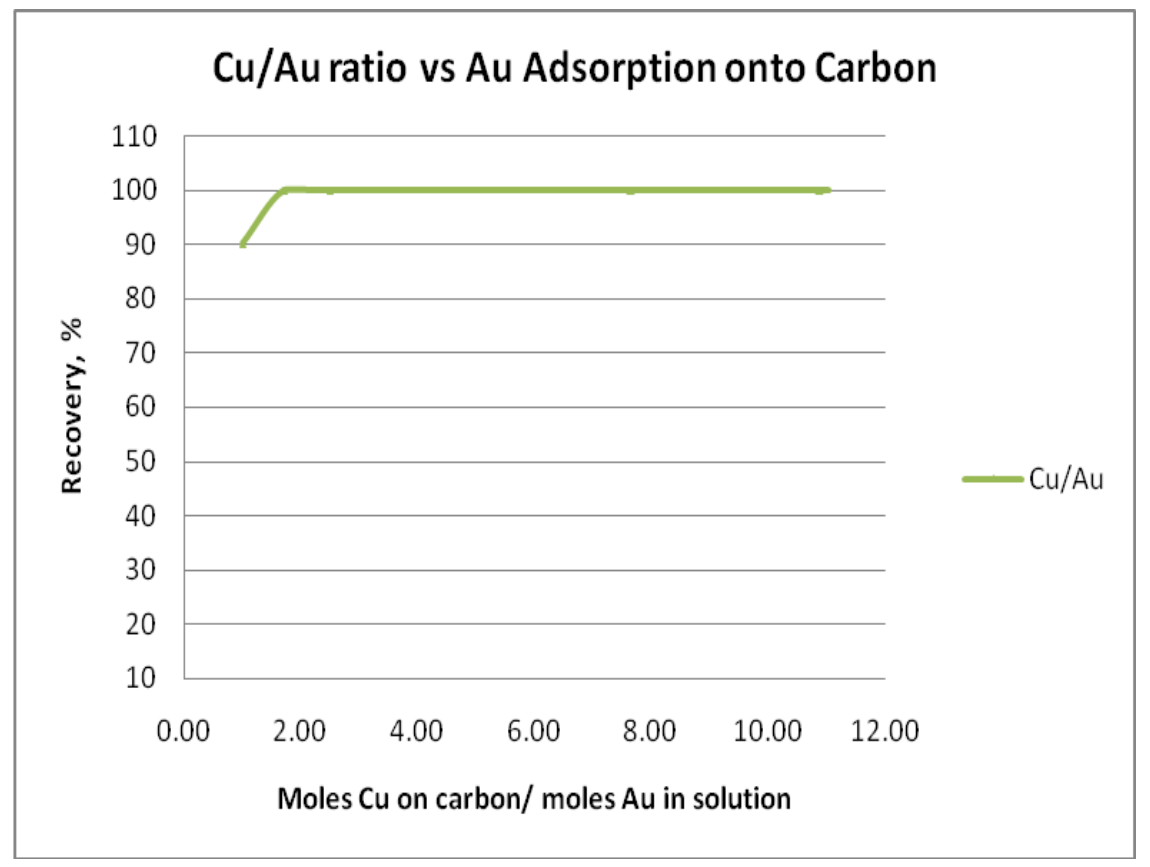

Figure 10. Recovery of Au from thiosulfate solution as a function of the molar ratio of $\mathrm{Cu}$ on the activated carbon to Au in solution. 


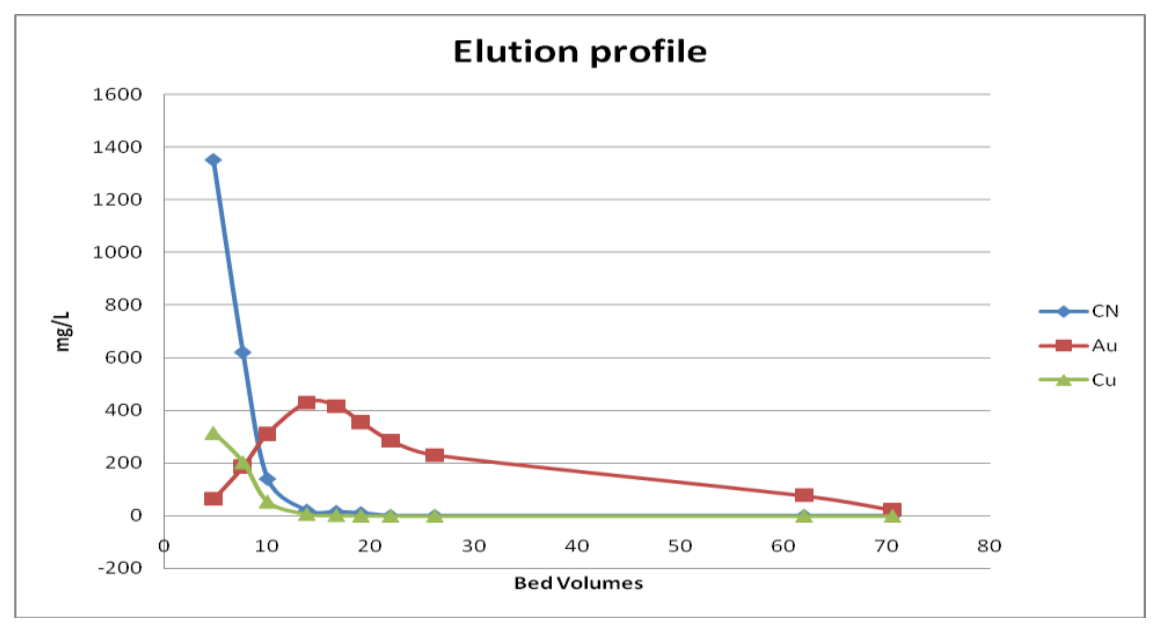

Figure 11. Elution of $\mathrm{Au}, \mathrm{Cu}$ and free $\mathrm{CN}$ as a function of time as measured by bed volume.

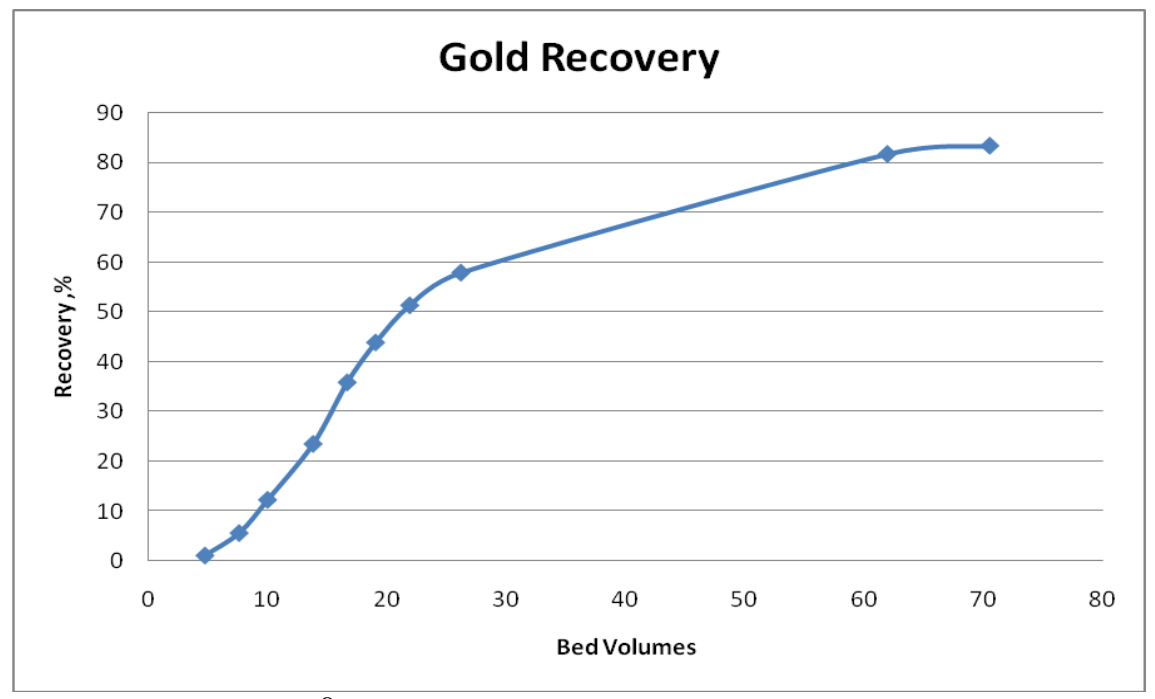

Figure 12. Elution of $\mathrm{Au}$ at $97^{\circ} \mathrm{C}$ from the activated carbon following its exchange with $\mathrm{Cu}$.

After soaking each of the resulting Au-loaded activated carbon in $\mathrm{NaCN} / \mathrm{NaOH}$ solution, the samples were placed in a chromatography column and eluted with distilled water at a controlled temperature. Example elution profiles and Au recovery curves as a function of bed volume (BV) are shown in Figures 11 and 12, respectively. Au recovery does not occur until 5 BVs have passed and reaches a maximum of $83 \%$ at $70 \mathrm{BVs}$. Initially, no $\mathrm{Au}$ is recovered because $\mathrm{Cu}$ is being eluted first which is observed in cyanidation circuits (Marsden and House, 2006). After approximately 15 BVs, Au concentrations reach a maximum of 420 $\mathrm{mg} / \mathrm{L}(\mathrm{ppm})$ which, in spite of being a batch measurement, is comparable to cyanidation circuits as well (Marsden and House, 2006). Based on these results, it is clear that Au-loaded activated carbon produced by exchange from Au-thiosulfate solution with Cu-cyanide pretreatment will behave similar to Au-cyanide adsorbed directly on activated carbon. It is therefore concluded that conventional elution technology can be used as part of this novel carbon adsorption method for extracting Au from thiosulfate solutions. 


\section{CONCLUSIONS}

A novel carbon adsorption technology for extracting gold from thiosulfate solutions has been developed and shows promise at becoming a cost effective method thereby allowing thiosulfate leaching to become economically competitive against cyanide leaching. Unless pretreated, activated carbons will not adsorb gold thiosulfate. However, by first adsorbing copper cyanide onto the carbon, gold thiosulfate can then be adsorbed in an ion-exchange reaction. CNT's will not adsorb either gold thiosufate or copper cyanide and will therefore some other method to be functionalized. Copper cyanide was adsorbed at $\mathrm{pH}$ 9, 10.5 and 12 with the majority of the work being done at $\mathrm{pH} 9$ and 12 . Contrary to the literature, maximum adsorption was observed at $\mathrm{pH} 12$ and increased temperatures (i.e., endothermic) but was explained due to the absence of $\mathrm{Ca}^{2+}$ in the system and therefore the ability for ionpairs to form. Thermodynamic analysis of the adsorption data showed that the adsorption process was endothermic. A detailed analysis of the free energy, enthalpy and entropy indicated the adsorption process was chemisorption but was entropically driven due to the displacement of water from the the activated carbon surface and the sheath of water around the adsorbing species. Factorially-designed experiments verified that maximum adsorption occurred at high $\mathrm{pH}$ and temperature and also produced a model that was used to plot results to conceptualize and visualize where maximum adsorption occurred. Raman spectroscopy was used to show that increased adsorption with increasing $\mathrm{pH}$ may be due to copper tricyanide $\left[\mathrm{Cu}(\mathrm{CN})_{3}{ }^{2-}\right]$; however, the resulting adsorbed gold species was likely gold dicyanide $\left[\mathrm{Au}(\mathrm{CN})_{2}{ }^{-}\right]$but could be a surface precipitate of AuCN and may very well involve $\mathrm{Cu}$ which, in excess, would allow $\mathrm{Cu}_{3} \mathrm{Au}$ to $\mathrm{Au}_{3} \mathrm{Cu}$ species to form as well. Clearly, the envisioned process appears to work well but needs additional work to understand.

\section{FUTURE WORK}

Optimization studies should be conducted with $\mathrm{Ca}^{2+}$ to ascertain the effect of ion-pair formation. Optimization studies should also be conducted to maximize gold elution as opposed to showing that stripping can be accomplished. Additional spectroscopic techniques need to be used to determine if $\mathrm{Cu}_{3} \mathrm{Au}$ and $\mathrm{Au}_{3} \mathrm{Cu}$ species form. In fact, Mariam Melashvilli will conduct these studies using separate but continuing funds from Newmont Mining Company.

\section{ACKNOWLEDGEMENTS}

Nick Gow completed the Raman spectroscopy studies in Brisbane, Australia, working with Dr. Greg Hope in the Department of Chemistry at Griffiths University with a matching grant from the U.S. Department of State that covered his travel and lodging costs. A subcontract has been issued through CAST to cover the additional non-per diem expenses of US\$10,000 incurred by Griffiths University and will be enacted in the next period pending the receipt of deliverables. 


\section{REFERENCES}

Burkin (1972), The Chemistry of Hydrometallurgical Processes, D. Van Nostrand, Inc., Princeton, NJ.

Jay, W.H. (2000), Copper Cyanidation Chemistry and the Application of Ion Exchange Resins and Solvent Extractants in Copper-Gold Cyanide Recovery Systems, In: Proceedings of Alta 2000 Conference, Adelaide, Australia.

Kudryk, V. and H.H. Kellogg, (1954), Mechanism and Rate-Controlling Factors in the Dissolution of Gold in Cyanide Solution, Trans. AIME J. of Metals, 541-548.

Hiskey, J.B. and Lee, J. (2003), Kinetics of Gold Cementation on Copper in Ammoniacal Thiosulfate Solutions, Hydrometallurgy 69: 45-56.

Lukey, G.C., van Deventer, J.S.J., Huntington, S.T., Chowdhury, R.L., Shallcross, D.C. (1999), Raman Study on the Speciation of Copper Cyanide Complexes in Highly Saline Solutions, Hydrometallurgy, 53:233-244.

MacArthur, J.S. (1916), Discovery of Cyanidation, Mining \& Scientific Press, London.

MacDougall, G.J. et al., (1980), The Mechanism of the Adsorption of Gold Cyanide on Activated Carbon, J. S. Afr. Inst. Min. \& Metall., 80: 344-356.

Marsden, J. and I. House (2006), The Chemisty of Gold Extraction, Ellis Horwood Publishers, New York.

Robie, R.A., Hemingway, B.S. and Fisher, J.R. (1979), Thermodynamic Properties of Minerals and Related Substances at 298.15 K and 1 Bar (10 5 Pascals) Pressure and at Higher Temperatures, USGS, Washington DC.

Young, C.A. and L.G. Twidwell (2005), Recovery of Gold from Thiosulfate Leach Liquor Using Activated Carbon, CAST Proposal, Montana Tech, Butte MT.

Young, C.A. (1995), Characterization of Adsorbed Oleate at Calcite and Flourite Surfaces by Infrared and Raman Spectroscopy, Dissertation, University of Utah, pp. 287.

\section{PUBLICATIONS AND PRESENTATIONS}

Preliminary Study of Carbon Adsorption for Gold Thiosulfate Recovery, Poster Presentation, C.A. Young, G.A. Hope, L.G. Twidwell, R.N. Gow and M. Melashvilli, CAST Workshop, Blacksburg, VA, July 24-26, 2007. 
Gold Thiosulfate Recovery from Solution by Carbon Adsorption, R.N. Gow, and C.A. Young, Precious Metals Processing, SME/TMS Fall Meeting, Tucson, AZ, October 3-7, 2007.

Recovery of Gold from Thiosulfate Solution Using Activated Carbon, R.N. Gow, and C.A. Young, SME Annual Meeting, Salt lake City, UT, February 27-29, 2008.

Gold Extraction by Pretreated Activated Carbon from Thiosulfate Liquors, R.N. Gow, Thesis, Montana Tech, Butte, MT, May, 2008.

Extraction of Au from Thiosulfate Liquors by Cu-Pretreated Carbon, G. Hope, G. Parker, R.N. Gow and C.A. Young, Proceedings of Hydro 2008, SME Fall Meeting, Phoenix, AZ, August 17-20, 2008.

Cu-Cyanide Adsorption on Activated Carbon, C.A. Young, R.N. Gow, G. Parker and G. Hope, Proceedings of Hydro 2008, SME Fall Meeting, Phoenix, AZ, August 17-20, 2008.

Optimization of Gold Ion-Exchange and Elution of Pretreated Activated Carbon, M. Melashvilli, Thesis, Montana Tech, Butte, MT, December, 2008. 
Appendix 23: Ion Exchange Recovery of Cobalt from Copper Leach Solutions (NM002) 


\section{TECHNICAL REPORT}

\begin{tabular}{ll|}
\hline Contract Title and Number: & Period of Performance: \\
\cline { 2 - 2 } $\begin{array}{l}\text { Crosscutting Technology Development at the Center for } \\
\text { (DE-FC26-02NT41607) }\end{array}$ & Endarting Date: 04/01/03 \\
& \\
\hline
\end{tabular}

Sub-Recipient Project Title:

Ion Exchange Recovery of Cobalt

From Copper Leach Solutions

Principal Investigators:

I. Gundiler, M. Hatch

Contact Address:

New Mexico Tech

Bureau of Geology \& Min. Res.

801 Leroy Place

Socorro, NM 87801

Subcontractor Address:

No subcontracts issued
Report Information:

Type: Final

Number:

Period:

Date: $\quad$ 03/15/07

Code: NM002-FINAL

Contact Information:

Phone: (505) 835-5730

Fax: (505) 835-6333

E-Mail: gundiler@gis.nmt.edu

Subcontractor Information:

Phone:

Fax:

E-Mail:

\section{$\underline{\text { Disclaimer }}$}

"This report was prepared as an account of work sponsored by an agency of the United States Government. Neither the United States Government nor any agency thereof, nor any of their employees, make any warranty, express or implied, nor assume any legal liability or responsibility for the accuracy, completeness, or usefulness of any information, apparatus, product, or process disclosed, or represents that its use would not infringe privately owned rights. Reference herein to any specific commercial product, process, or service by trade name, trademark, manufacturer, or otherwise does not necessarily constitute or imply endorsement, recommendation, or favoring by the United States Government or any agency thereof. The views and opinions of authors expressed herein do not necessarily state or reflect those of the United States Government or agency thereof." 


\begin{abstract}
Five new chelating ion exchange resins, a thiosuccinic acid (MH-13), a carboxymethlated diethylenetriamine (CM-DER), and three picolylated diethylenetriamine resins, a highly picolylated CHEL-38, less picolylated CHEL-40, and the CHEL-73 resin which is slightly more picolylated than CHEL-40, were synthesized and tested in small columns for absorption of cobalt, nickel, copper, iron, and zinc from acidic solutions and from copper leaching raffinate solution. Commercially available hydroxypropyl picolyl amine (HPPR) and dipicolylated polyamine (DPAR) resins were also tested under the same conditions and compared with the newly synthesized resins. The thiosuccinic acid resin (MH-13) absorbed slightly less copper than commercial iminodiacetic acid (IDA) resins, (Chelex-100), hence was not studied any further. Carboxymethylated polyamine (CM-DER) absorbed copper as effectively from $\mathrm{pH} 1.1$ to 1.7 sulfuric acid solutions as commercially available HPPR, but weakly absorbs cobalt from sulfuric acid solutions. Picolylated polyamine (CHEL) resins, showed the most promise. With appropriate raffinate feed volumes, CHEL-40 and CHEL-73 resins have a much higher absorption capacity for Co (II) in comparison with the commercially available resins. They also had as good or better capacity for $\mathrm{Cu}$ (II), Ni (II) and $\mathrm{Zn}$ (II). The CHEL-40 resin also had less capacity for Fe (III), which is a highly desirable advantage for recovery of copper and cobalt from leach solutions. Elution with 0.5$2 \mathrm{M} \mathrm{H}_{2} \mathrm{SO}_{4}$ of both the CHEL-40 and CHEL-73 resins which were loaded with appropriate volumes of raffinate feed, produced eluate fractions containing over 10 times the cobalt concentration and over 50 times the copper concentration of the original solutions.
\end{abstract}

\title{
TABLE OF CONTENTS
}

\begin{tabular}{|l|c|}
\hline \multicolumn{1}{|c|}{ Heading } & Page \\
\hline Title Page & 1 \\
\hline Disclaimer & 1 \\
\hline Abstract & 2 \\
\hline Table of Contents & 2 \\
\hline List of Tables & 3 \\
\hline List of Figures & 4 \\
\hline Introduction & 5 \\
\hline Executive Summary & 9 \\
\hline Experimental & 11 \\
\hline Results and Discussion & 15 \\
\hline Conclusion & 25 \\
\hline Acknowledgments & 27 \\
\hline References & 27 \\
\hline Bibliography & 28 \\
\hline List of Acronyms and Abbreviations & 29 \\
\hline
\end{tabular}




\section{LIST OF TABLES}

Table

Page

1. Absorption capacities of CM-DER and MH-13 resins for copper compared to two

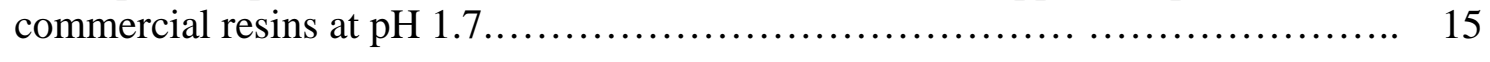

2. Absorption of copper on HPPR and CM-DER resins at various $\mathrm{pH}$ levels........... 16

3. Cobalt absorption on HPPR, CM-DER and CH-100 resins at $\mathrm{pH} 1.7$ and $1.1 \ldots \ldots . .16$

4. Ferric ion absorption on CM-DER, HPPR and CH-100 resins at $\mathrm{pH} 1.7 \ldots \ldots \ldots \ldots . .16$

5. Characterization of CHEL-38 and CHEL-40 resins ......................... 17

6. Absorption capacities of CHEL-38, CHEL-40, DPAR and HPPR resins for 38 ppm

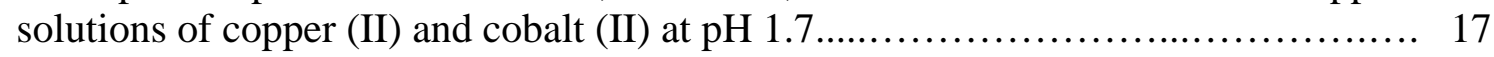

7. Absorption capacities of CHEL-38, CHEL-40, DPAR and HPPR resins for 190 ppm

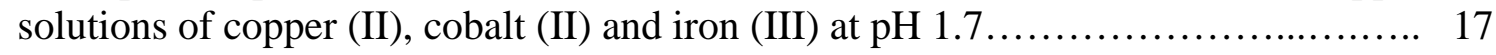

8. Absorption capacities of CHEL-38, CHEL-40 and HPPR resins at $\mathrm{pH} 1.1 \ldots \ldots \ldots . . .18$

9. Effect of $\mathrm{pH}$ on absorption capacities of CHEL-38, CHEL-40 and HPPR resins

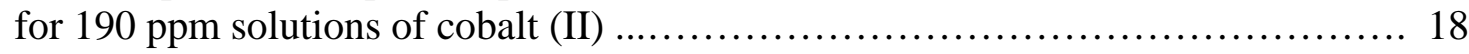

10. Absorption capacities for Cu (II), Co (II) and Ni (II) of CHEL-38, CHEL-40 and

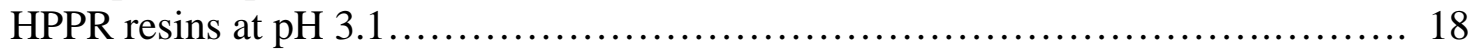

11. Effect of concentration on absorption capacities of CHEL-38, CHEL 40 and HPPR

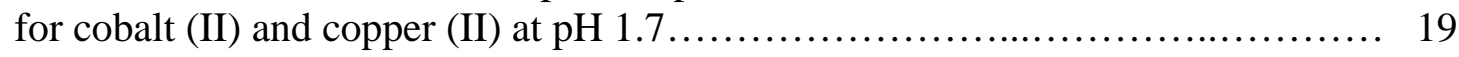

12. Absorption capacities of CHEL-38, CHEL 40 and HPPR for nickel (II) at pH 1.7... 19

13. Absorption capacities of CHEL-38, CHEL 40 and HPPR resins for 2000 ppm

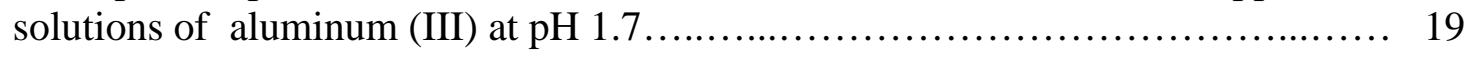

14. Copper heap-leach solvent extraction raffinate solution analysis $\quad \ldots \ldots \ldots \ldots \ldots \ldots . . .20$ 


\section{LIST OF FIGURES}

Figure Page

1. Synthesis of thiosuccinic acid resin $(\mathrm{MH}-13) \ldots \ldots \ldots \ldots \ldots \ldots \ldots \ldots \ldots \ldots \ldots \ldots \ldots \ldots \ldots \ldots$

2. Synthesis of diethylenetriamine resin (Intermediate)....................... 12

3. Synthesis of carboxymethylated diethylenetriamine resin (CM-DER).......... 13

4. Synthesis of highly picolylated diethylenetriamine resin (CHEL-38) ........ 13

5. Cobalt (II) absorption on various resins from fast-flow raffinate solution ...... 20

6. Nickel (II) absorption on various resins from fast-flow raffinate solution...... 21

7. Copper (II) absorption on various resins from fast-flow raffinate solution...... 22

8. Iron (III) absorption on various resins from fast-flow raffinate solution......... 22

9. Cobalt absorption on CHEL-40 from raffinate solution at slow flow rate........ 23

10. Copper absorption on CHEL-40 from raffinate solution at slow flow rate...... 23

11. Cobalt elution from CHEL-40.......................................... 23

12. Copper elution from CHEL-40...................................... 23

13. Cobalt absorption on CHEL-73 from raffinate solution at intermediate flow... 24

14. Copper absorption on CHEL-73 from raffinate solution at intermediate flow... 24

15. Cobalt elution from CHEL-73......................................... 25

16. Copper elution from CHEL-73...................................... 25 


\section{INTRODUCTION}

\section{$\underline{\text { Background }}$}

Cobalt is a strategic and critical metal, which is used in production of super alloys for gas turbine engines, corrosion and wear resistant alloys, cutting tools, magnets, electroplating, and in various chemicals. It is not mined or refined in the United States, although there are known reserves of cobalt ores and historical production from Idaho, Missouri, and Pennsylvania [1, 2]. Apparent consumption of cobalt in the U.S. in 2005 was 11,900 metric tons, of which 11,100 tons met by imports [1].

Cobalt occurs as arsenides, sulfides, and oxides; also found as substitutions in nickel and iron minerals. The vast majority of cobalt occurrences are in nickel-bearing laterite deposits, the rest occur in nickel-copper sulfide ores in mafic rocks in Australia, Canada, and Russia, and in sedimentary copper deposits in Congo (Zaire) and Zambia.

The richest deposits are in Congo and Zambia; cobalt sulfides occur mixed with chalcopyrite $\left(\mathrm{CuFeS}_{2}\right)$ and chalcocite $\left(\mathrm{Cu}_{2} \mathrm{~S}\right)$. Cobalt-copper flotation concentrates containing 1.5-4\% Co are roasted to convert sulfides to water-soluble sulfates. The calcine is leached in spent copper electrolyte, solution is purified to remove iron and aluminum, and copper is electrowon directly from the leach solution [3]. A portion of spent copper electrolyte is purified to remove remaining copper and iron, and cobalt is precipitated as hydroxide using lime. The precipitate is recovered and dissolved in sulfuric acid, and cobalt is recovered from concentrated solutions by electro-winning at $\mathrm{pH}$ 6.3-6.5. In Zaire, hydrated, black cobalt oxides also occur with hydrated copper carbonates, malachite $\left(\mathrm{CuCO}_{3} \cdot \mathrm{Cu}(\mathrm{OH})_{2}\right)$, which contains as much as $0.4 \%$ cobalt [4].

Processing of nickel-cobalt ores generally involves smelting sulfide concentrates, reduction roasting of lateritic ores, and either acidic or ammoniacal pressure leaching. Pressure leach slurries are washed by counter current decantation (CCD) circuits, purified, and neutralized. Nickel and cobalt are then recovered by solvent extraction [5, 6]. Cobalt is commonly recovered from nickel solutions as a mixed sulfide, hydroxide, or basic carbonate [3]. The mixed precipitate is then treated in cobalt refineries. The refining process generally includes dissolution of the interim product, solvent extraction separation of nickel and cobalt, purification of solutions by ion exchange, and recovery by electrowinning or hydrogen reduction to metal powder, or precipitation as a chemical compound.

There is, however, a readily accessible source for cobalt in the southwestern United States. It is widely known that significant cobalt values are present in the circulating solutions produced by the heap leaching operations of low-grade copper ores, and tankhouse electrolyte bleed [7]. The leach solutions generally contain $0.03-0.10 \mathrm{Kg} / \mathrm{m}^{3}$ concentrations (30-100 ppm) of cobalt, however, technology to recover cobalt from copper leach solutions economically has not been available. The U.S. Bureau of Mines (USBM) conducted extensive studies with spent copper leach solutions to recover cobalt, using ion exchange (IX) resins [8-10]. After screening over 200 resins, an experimental chelating resin XSF- 
4195, bis-picolylamine, $\left(-\mathrm{N}\left(\mathrm{CH}_{2}-\mathrm{C}_{5} \mathrm{H}_{4} \mathrm{~N}\right)_{2}\right)$, manufactured by the Dow Chemical Co., was selected. The resin was macro-porous styrene / divinylbenzene copolymers to which the chelating functional group was attached. Extensive studies have also been reported for characterization of this resin and for its hydrometallurgical applications [11-13]. Selectivity of this resin for the transition metals ions was found to be in the order $\mathrm{Cu}$ (II) $>>\mathrm{Ni}$ (II) $>\mathrm{Fe}$ (III) $>\mathrm{Zn}$ (II) $>$ Co (II) $>$ Cd (II) $>$ Fe (II). Due to its weakly basic character $\left(\mathrm{pK}_{\mathrm{a}}=3.5\right)$, the maximum absorption capacity for all metal ions is reached at $\mathrm{pH}$ about 3. Copper was much more strongly absorbed than other cations even at $\mathrm{pH} 1$, but required very strong sulfuric acid or ammonium hydroxide solution for elution.

Other applications of the XFS-4195 resin were found in cobalt and copper removal from nickel electrolytes in acidic solutions [14,15], and direct purification of solutions for battery grade $\mathrm{MnO}_{2}$ [17]. In general, electrolyte purification in cobalt refineries is carried out in near neutral solutions ( $\mathrm{pH} 6)$ using iminodiacetic acid $\left(-\mathrm{CH}_{2} \mathrm{NCH}_{2}(\mathrm{COOH})_{2}\right)$ resins [15] which costs about one-tenth of the picolylamine resin [16]. Recently there has been a renewed interest in ion-exchange recovery of nickel and cobalt directly from high-temperature acidic pressure leach slurries of laterite ores or CCD residues [17-20], using chelating resins.

The USBM studies [8-10] were conducted on recycle solutions, effluents from copper cementation on scrap iron, analyzing, in $\mathrm{Kg} / \mathrm{m}^{3}, 0.03 \mathrm{Co}, 0.06 \mathrm{Cu}, 2.0 \mathrm{Fe}, 0.2 \mathrm{Zn}$, and $4.5 \mathrm{Al}$, at $\mathrm{pH}$ 3. During the past two decades, however, cementation process was replaced with solvent extraction (SX) technology to recover copper. A chelating type copper selective organic reagent $(\mathrm{Cu} / \mathrm{Fe}>500 / 1)$ dissolved $(7-15 \%)$ in a suitable solvent, such as kerosene, is contacted with copper-bearing pregnant leach solution (PLS) in counter current reactors. Copper is then stripped from the loaded organic with spent electrolyte containing 35-40 $\mathrm{Kg} / \mathrm{m}^{3} \mathrm{Cu}$ and $180-220 \mathrm{Kg} / \mathrm{m}^{3}$ sulfuric acid $\left(\mathrm{H}_{2} \mathrm{SO}_{4}\right)$, and recovered in the electrowinning (EW) circuit. Product from the SX-EW process analyzes $99.99 \%$ copper, suitable for electrical applications.

Cost of copper extraction by the hydrometallurgical processes (heap leach, SX-EW) is half that of the conventional pyrometallurgical route, i.e., smelting and electro-refining, and it is free of $\mathrm{SO}_{2}$ emission which is a major concern for air polluting. Therefore, it is the preferred route when the mineralogy of the ore body, oxide minerals and some sulfides, i.e., chalcocite, is amenable for heap leaching. Chalcopyrite is not readily amenable to leaching under the heap leach conditions; it is concentrated by flotation, and the concentrates are treated by smelting to blister copper, and electrolytic refining to high-purity copper.

In recent years, copper mining operations in eastern Arizona and New Mexico were transformed into mine-for-leach operations. Five copper leaching operations in the southwest circulate a combined volume of leach solutions in excess of $22,700 \mathrm{~m}^{3} / \mathrm{h}(100,000 \mathrm{GPM})$ [21]. If only a fraction of cobalt contained in these solutions could be recovered, these five operations alone could supply a substantial portion of the U.S. imports.

Hydrometallurgical operations are not immune to operational and environmental problems. Large amounts of potentially combustible organic inventory in the plant, loss of expensive organic in the recycled aqueous effluent by entrainment or by evaporation, and potential for 
organic and heavy metal ion contamination of the ground water are a few of the concerns. Ion exchange recovery of copper from the leach solutions could alleviate some of these concerns.

Modifications of the "di-“" or bis-picolylamine resin (XFS-4195), XFS-4196, N-(2hydroxyethyl), and XFS-43084, N-(2-hydroxypropyl)-picolylamine resins were reported to be much more selective for copper separation at low $\mathrm{pH}$ [22]. A pilot plant study conducted with these resins demonstrated the possibility of IX recovery of copper directly from acidic leach solutions and electrowinning from the eluate [23]. Most of the iron was removed by scrubbing the resin with dilute electrolyte bleed before copper elution. There were, however, difficulties with the water balance. A recent study proposed an innovative process of scrubbing the loaded ferric ion from the resin with $\mathrm{SO}_{2}$ sparged PLS, and suggested the economic viability of the IX process versus SX, particularly for low $\mathrm{Cu}$ concentrations in PLS and low flow rates [24].

\section{Objectives and Approach}

Commercially available resins containing iminodiacetic acid $\left(-\mathrm{CH}_{2} \mathrm{~N}\left(\mathrm{CH}_{2} \mathrm{COOH}\right)_{2}\right)$, and aminophosphonic acid $\left(-\mathrm{CH}_{2} \mathrm{NHCH}_{2} \mathrm{PO}_{3} \mathrm{H}\right)$ functional groups are used in copper and nickel removal from cobalt electrolytes in near neutral solutions [14,15]. These resins, however, do not absorb copper from dilute $\mathrm{H}_{2} \mathrm{SO}_{4}$ very significantly at $\mathrm{pH}$ levels less than about $\mathrm{pH}=2$. Aminophosphonic acid types of chelating resins show a somewhat similar performance in dilute $\mathrm{H}_{2} \mathrm{SO}_{4}$ to the iminodiacetic acid resins, in selective absorption of $\mathrm{Cu}$ (II). Only at $\mathrm{pH}$ values of approximately 2.5 and above significant amounts of $\mathrm{Cu}$ (II) are absorbed.

The Dow Chemical Company also offers two experimental picolylamine resins, XFS-4196, $\mathrm{N}$-(2-hydroxyethyl), and XFS-43084, N-(2-hydroxypropyl) - picolylamine, designed for improved copper absorption and higher selectivity [13, 22]:

$$
\text { p- } \mathrm{CH}_{2} \mathrm{~N}\left(\mathrm{CH}_{2} \mathrm{CH}_{2} \mathrm{OH}\right) \mathrm{CH}_{2} \mathrm{C}_{5} \mathrm{H}_{4} \mathrm{~N} \text { and } \mathrm{p}-\mathrm{CH}_{2} \mathrm{NCH}_{2} \mathrm{CH}(\mathrm{OH}) \mathrm{CH}_{3} \mathrm{CH}_{2} \mathrm{C}_{5} \mathrm{H}_{4} \mathrm{~N}
$$

In all cases of the above resins the polymer backbone "p" is a crosslinked polystyrene network; they are either a "gel type" or a "macroporous type" resin. The morphological details can have a significant effect on the chelating and kinetics of exchange.

The first of these chelating resins (XSF-4196) absorbs significant amounts of $\mathrm{Cu}$ (II) from dilute $\mathrm{H}_{2} \mathrm{SO}_{4}$ at $\mathrm{pH}$ values from 0.4 to 4 . Thus, this resin is able to operate well at quite strongly acidic solutions of $\mathrm{H}_{2} \mathrm{SO}_{4}$. Also, selectivity for $\mathrm{Cu}$ (II) versus Fe (III) is quite favorable. However, these resins presently are quite expensive [20].

In the present research new chelating resins have been synthesized for screening and evaluation. The synthesis methods were limited to resin structures that would be favorable in potential costs and kinetic behavior. Most of the available resins have slow kinetics of ion exchange compared to the standard resin "workhorses", such as the sulfonic acid ( $\mathrm{p}-\mathrm{SO}_{3} \mathrm{H}$, pdenotes polymer) resins. This type of resin shows very poor selectivity for $\mathrm{Cu}$ (II), $\mathrm{Ni}$ (II), or Co (II), however. 
One approach in the synthesis of new or untested types of chelating resins are methods one of the authors (Dr. M.J. Hatch) developed (U.S. Patents 3,300,416, 3,277,023, and 3,277,024). These methods involve the nucleophilic displacement of tri-alkyl amines from quaternary ammonium resins using solutions of chelating molecules at elevated temperatures.

The quaternary ammonium resins are synthesized as shown in the following equation:

$$
\mathrm{p}-\mathrm{CH}_{2} \mathrm{C} 1+\mathrm{NR}_{3} \rightarrow \mathrm{p}-\mathrm{CH}_{2} \mathrm{NR}_{3} \mathrm{C} 1
$$

Resins of type p- $\mathrm{CH}_{2} \mathrm{NR}_{3} \mathrm{C} 1$, quaternary ammonium chloride resins $\left(\mathrm{R}=\mathrm{CH}_{3}\right)$, that are largescale commercial products for anion exchange. The "chloromethylated beads" $\left(\mathrm{p}-\mathrm{CH}_{2} \mathrm{C} 1\right)$ are made from a crosslinked styrene polymer by reaction with methyl chloromethyl ether $\left(\mathrm{CH}_{3} \mathrm{OCH}_{2} \mathrm{C} 1\right)$, using a Lewis acid catalyst such as $\mathrm{ZnC1}_{2}$ or $\mathrm{AlC1}_{3}$.

The quaternary ammonium chloride resin can be made to react with thiosuccinic acid in the presence of $\mathrm{NaOH}$ in diethehylene glycol solvent at 150-180C, and washed with an acid as needed to yield thio succinic acid resin:

$$
\mathrm{p}-\mathrm{CH}_{2} \mathrm{NR}_{3} \mathrm{Cl}+\mathrm{NaS}\left(\mathrm{CHCO}_{2} \mathrm{Na}\right) \mathrm{CH}_{2} \mathrm{CO}_{2} \mathrm{Na} \rightarrow \mathrm{p}-\mathrm{CH}_{2} \mathrm{~S}\left(\mathrm{CHCO}_{2} \mathrm{H}\right) \mathrm{CH}_{2} \mathrm{CO}_{2} \mathrm{H}(\mathrm{I})
$$

Chelating resin (I) does not have amino groups in it. Metal cations coordinate with carboxyl and thioether groups. These groups are not basic to protons as are amino groups, thus, coordination at the thioether groups will be essentially independent of $\mathrm{pH}$.

$$
\text { p- } \mathrm{CH}_{2} \mathrm{NR}_{3} \mathrm{C} 1+\mathrm{NH}_{2} \mathrm{CH}_{2} \mathrm{CH}_{2} \mathrm{NHCH}_{2} \mathrm{CH}_{2} \mathrm{NH}_{2} \rightarrow \text { Intermediate (II) }
$$

The Intermediate (II) resin would be reacted with $\mathrm{C}_{1} \mathrm{CH}_{2} \mathrm{CO}_{2} \mathrm{Na}$ to give a chelating resin which might have much stronger chelating abilities than the iminodiacetic acid resin. As a result, one would expect chelation should be more effective at low $\mathrm{pH}$ values than iminodiacetic acid resins. A final representative type-structure is given as:

$$
\text { p- } \mathrm{CH}_{2} \mathrm{~N}\left(\mathrm{CH}_{2} \mathrm{CO}_{2} \mathrm{H}\right) \mathrm{CH}_{2} \mathrm{CH}_{2} \mathrm{~N}\left(\mathrm{CH}_{2} \mathrm{CO}_{2} \mathrm{H}\right) \mathrm{CH}_{2} \mathrm{CH}_{2} \mathrm{~N}\left(\mathrm{CH}_{2} \mathrm{CO}_{2} \mathrm{H}\right)_{2} \text { (II) }
$$

The objectives of this research then can be summarized as synthesis and evaluation of new chelating ion exchange resins that could

- function in highly acidic (low pH) solutions

- have high capacity for copper and especially for cobalt

- have less affinity towards iron (III)

- have more favorable absorption and elution kinetics

- be synthesized more economically

than commercially available resins. 


\section{EXECUTIVE SUMMARY}

Cobalt, a critical and strategic metal, is not mined or refined in the U.S. as ore grade deposits are not present. However, a source exists in the southwestern U.S., since cobalt is present at low concentrations in the acidic solutions produced by heap leaching operations of low-grade copper ores but technology to recover the cobalt economically has not been available.

This research has focused on synthesizing five new resins on a laboratory scale and has screened, evaluated and compared them to some commercially available resins. Synthesis methods were limited to resin structures that could potentially be economically and kinetically favorable. Most of the available $\mathrm{Cu}-\mathrm{Co}-\mathrm{Ni}$ chelating resins have slow kinetics (exchange) compared to "standard" resins which are otherwise limited by poor selectivity for $\mathrm{Cu}$ (II), Ni (II) and Co (II) and generally do not function at low $\mathrm{pH}$. Many commercial resins also tend to absorb and concentrate Fe (III) along with the $\mathrm{Cu}$ and Co. The most widely used chelating resins contain either iminodiacetic acid $\left(-\mathrm{CH}_{2} \mathrm{NCH}_{2}(\mathrm{COOH})_{2}\right)$ or picolylamine, $\left(\mathrm{CH}_{2}-\mathrm{N}(\mathrm{R}) \mathrm{CH}_{2} \mathrm{C}_{5} \mathrm{H}_{4} \mathrm{~N}\right)$ functional groups. The iminodiacetic acid (IDA) resins are cheaper but their use is limited except in near neutral solutions and they absorb Fe (III) strongly; whereas dipicolyamine, DPAR $\left(-\mathrm{N}\left(\mathrm{CH}_{2}-\mathrm{C}_{5} \mathrm{H}_{4} \mathrm{~N}\right)_{2}\right)$ resins can work effectively in acidic solutions and they only weekly absorb Fe (III), but they are much more expensive than IDA resins, and can be regenerated effectively only with ammonia solutions.

The objective of this research then was lab-scale synthesis of new resins that would have a high capacity for cobalt as well as copper, a low capacity for Fe (III), have a more rapid rate of absorption and elution, function at low $\mathrm{pH}$, and that could potentially be synthesized as or more economically than the commercially available resins.

The approach used in synthesizing the new resins involved the high temperature (140-180C) nucleophilic displacement of somewhat labile quarternary ammonium resins with watersoluble chelating groups. The starting material in all cases was a readily available crosslinked, strong-base quaternary amine, anion exchange resin (Dowex-1, 2x). The synthesized resins were tested in small columns for absorption of copper, then with cobalt and other metals of interest at various concentrations and $\mathrm{pH}$.

The first resin, a thiosuccinic acid resin ( $\mathrm{MH}-13)$, was synthesized by reacting quaternary amine resin with metcaptosuccinic acid and sodium hydroxide. This resin showed less capacity for copper absorption from a $\mathrm{pH} 1.7$ solution than a commercial iminodiacetic acid (IDA) resin, (Chelex-100), therefore, it was not studied any further.

The second resin, a carboxymethylated diethylenetriamine (CM-DER), was prepared by first reacting quaternary amine resin with diethylenetriamine, and reacting the intermediate product with sodium choloroacetate. The performance of this resin was compared against an IDA resin (Chelex-100), and a commercially available (DOW XSF-43084) hydroxypropyl picolylamine resin (HPPR). Copper ion absorption capacity of these resins from $\mathrm{pH} 1.7$ solution was HPPR (28.6) >CM-DER (24.1) > IDA (19.7) $\mathrm{mg} / \mathrm{ml}$, and decreased to $<2 \mathrm{mg} / \mathrm{ml}$ 
as the $\mathrm{pH}$ was lowered to 0.5. Ferric ion absorption, however, was CM-DER (43.6), as compared to HPPR (7.8) and IDA (46.9) $\mathrm{mg} / \mathrm{ml}$, at $\mathrm{pH}$ 1.7, a typical $\mathrm{pH}$ for copper leaching and solvent extraction raffinate solutions.

On the other hand, cobalt absorption capacity of CM-DER resin $(0.54 \mathrm{mg} / \mathrm{ml})$, although it was an order of magnitude higher than both IDA and HPPR, was about 50 times lower than that of copper at the same $\mathrm{pH}$. Yet this resin can probably be produced much more cheaply than the picolylamine-based resins, and can be eluted and regenerated with $1 \mathrm{M}$ sulfuric acid solutions. This resin could find applications for copper recovery from low-grade solutions, such as acidic mine drainage (AMD) and dump-leach solutions, that are too dilute to be treated with solvent extraction, and have low concentrations of iron.

The next three resins were prepared by reacting the intermediate (diethylenetriamine) resin with picolylchloride $\left(\mathrm{C}_{5} \mathrm{H}_{4} \mathrm{~N}-\mathrm{CH}_{2} \mathrm{Cl}\right)$ to obtain highly picolylated CHEL-38, less picolylated CHEL-40, and the CHEL-73 resin which is slightly less picolylated than CHEL-40. All three resins showed comparable copper absorption capacity while only absorbing Fe (III) weakly. Cobalt absorption capacity of these resins, although higher by almost two orders of magnitude as compared to picolylamine resins, DPAR and HPPR, were about $4 \mathrm{mg} / \mathrm{ml}$ as compared to $26 \mathrm{mg} / \mathrm{ml}$ for copper from separate solutions containing $190 \mathrm{ppm}$ either copper or cobalt, at $\mathrm{pH} 1.7$.

Finally, absorption and elution characteristics of these resins were studied using actual heap leaching solution effluent from a copper mine, after copper recovery by solvent extraction. Using 200 bed volumes of feed solution applied at very fast flow rates, the picolylated polyamine resins had much higher capacities for cobalt than DPAR and HPPR resins, similar capacities for copper, nickel, zinc, and lower capacity for ferric iron. Elution with 0.5-2M sulfuric acid solutions yielded eluate fractions in which the cobalt concentrations were 10 times, and copper concentrations over 50 times more than present in the feed solutions at $\mathrm{pH}$ 2.1 .

Although picolylated polyamine (CHEL) resins have better capacity for cobalt than the currently available resins, their application for the intended purposes of this research, i.e., recovery of cobalt as by-product from hydrometallurgical copper extraction processes is not very likely, unless a cheaper and more economical method for synthesizing picolylchoride can be developed and implemented in industrial scale. However, these resins could find special applications, such as in purification of nickel electrolyte solutions for removing copper and cobalt impurities, and recovery of nickel and cobalt from acidic pressure leach slurries in processing lateritic nickel-cobalt ores. 


\section{EXPERIMENTAL}

\section{$\underline{\text { Synthesis of resins }}$}

In common with the more usual cation exchange resins, chelating resins are prepared as microporous (gel) or macroporous particles. Both types of resins are prepared by suspension polymerization in water of small droplets of a insoluble vinyl monomer plus some cross linking divinyl monomer. If the droplets do not contain any solvent, the usual type of microporous gel polymer results. If the droplets do contain a solvent for the monomers which is not a swelling solvent for the crosslinked polymer, the polymer bead that is formed has a macroporous structure. The microporous or macroporous crosslinking polymers are chemically modified to get desired metal bonding functional groups. As is customary , ion exchange capacities reported here in $\mathrm{meq} / \mathrm{ml}$ of resin, or $\mathrm{mg} / \mathrm{ml}$. These latter values are numerically equal to $\mathrm{Kg} / \mathrm{m}^{3}$.

\section{Synthesis of thiosuccinic acid resin (MH-13)}

MH-13 resin was prepared from 50/100 mesh, 2 per cent crosslinked, chloride form of quaternary ammonium ion exchange resin (Dowex-1, 2X). This low-crosslinked dry resin was added stepwise to a stirred solution made from hot ethylene glycol, excess mercaptosuccinic acid, and sodium hydroxide under a fume hood. The mixture was heated to $150-170^{\circ} \mathrm{C}$ and stirred for 20 hours. Well before that time, evolution of trimethylamine $\left.\left(\mathrm{CH}_{3}\right)_{3} \mathrm{~N}\right)$ had stopped. The light brown product was water-washed to give the water swollen di-sodium form. The resin had a wet-settled volume of about $30 \mathrm{ml}$. Its color was light brown, and its density was somewhat greater than that of water. It settled readily in water to form the resin bed. The synthesis of $\mathrm{MH}-13$ is depicted in Figure 1. It should be noted that all structural formulas are only representative type-structures.<smiles>CCC(C)c1ccc(C(C)CC)cc1</smiles>

Dowex-1, 2X<smiles>O=C(O)CC(S)C(=O)O</smiles>

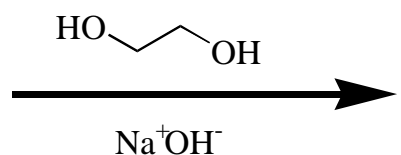

$\mathrm{Na}^{+} \mathrm{OH}^{-}$<smiles>CCC(C)c1ccc(CSC(CC(=O)O)C(=O)O)cc1</smiles>

Thiosuccinic Acid Resin

Mercaptosuccinic Acid

Figure 1. Synthesis of thiosuccinic acid resin (MH-13) 


\section{Synthesis of carboxymethylated diethylenetriamine resin (CM-DER)}

Carboxymethylated diethylenetriamine (CM-DER) was prepared in two steps. In the first step quarternary ammonium ion exchange resin (Dowex-1, 2x) was reacted with diethylene triamine in ethylene glycol at $150-180^{\circ} \mathrm{C}$ for about 20 hours to produce diethylene triamine resin, which was highly cross-linked (Figure 2). This resin would not chelate copper even from very mildly acidic solutions due to strong protonation of the amino groups.

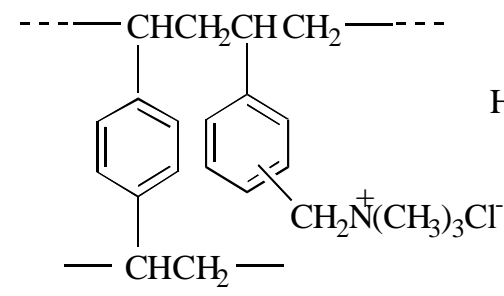

Quarternary ammonium resin

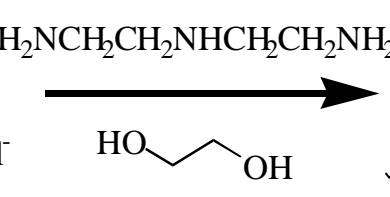

20 hrs.

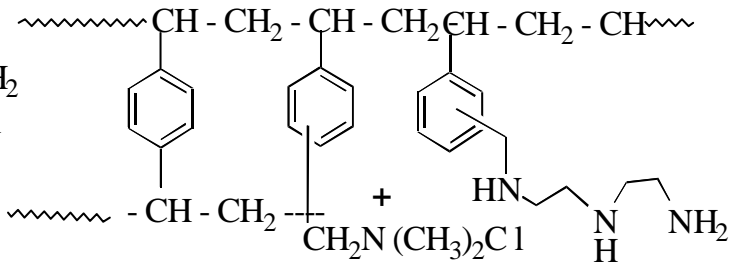

Diethylenetriamine resin

Figure 2. Synthesis of diethylenetriamine resin (Intermediate).

In the second step the diethylenetriamine resin was refluxed at about $100^{\circ} \mathrm{C}$ in an aqueous solution of excess sodium choloroacetate, $\mathrm{ClCH}_{2} \mathrm{CO}_{2} \mathrm{Na}$, buffered with sodium bicarbonate. This step introduced the carboxylmethylated groups on the nitrogen atoms of the diethylenetiramine resin to give the carboxymethylated diethylenetriamine structure in $\mathrm{Na}$ (I) form (Figure 3). Again all structural formulas re only representative type-structures. Because of structural nature of these reactions other closely related representative structures could be drawn, especially of the cross-linking reactions.

Diethylenetriamine resin
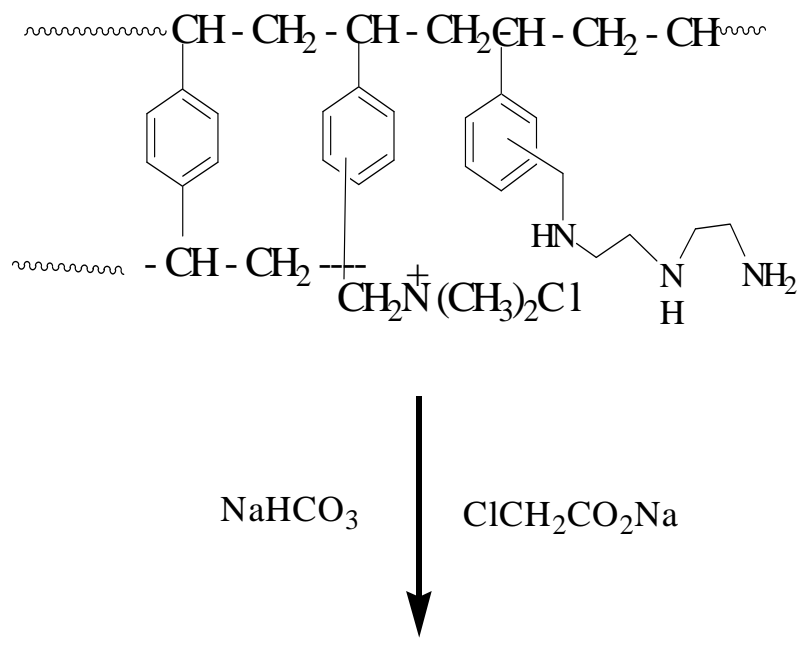


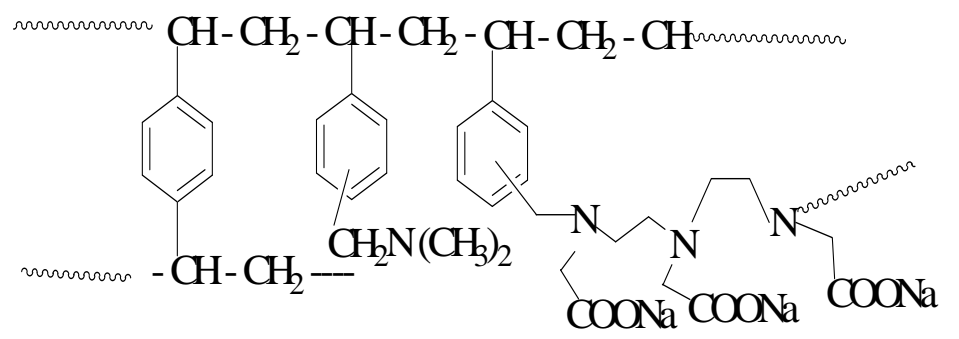

Carboxymethylated diethylenetriamine resin

Figure 3. Synthesis of carboxymethylated diethylenetriamine (CM-DER) resin

Synthesis of a highly picolylated diethylenetriamine resin (CHEL-38) and a less picolylated diethylenetriamine resin (CHEL-40)

Two more chelating ion exchange resins were synthesized with the objective of increasing cobalt selectively relative to copper and decreasing the $\mathrm{Fe}^{+3}$ selectivity. The first resin is a highly picolylated diethylenetriamine resin (CHEL-38). The second resin is a less picolylated diethylenetriamine resin (CHEL-40). These new resins have a somewhat macroporus gel type structures due to their highly non-uniform cross-linking.

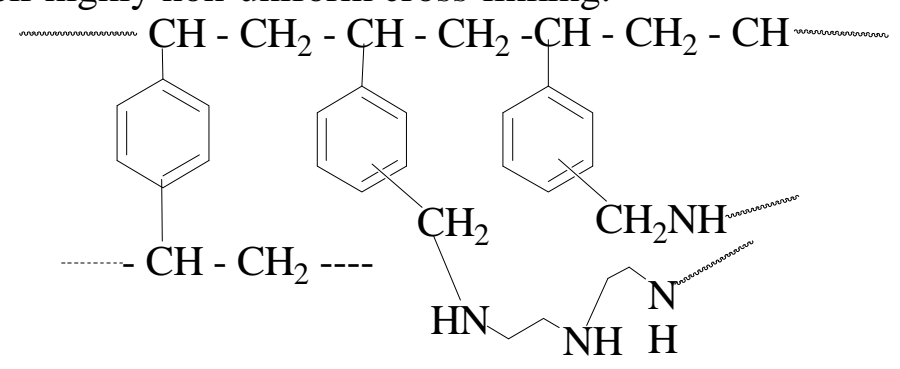

Diethylenetriamine Resin
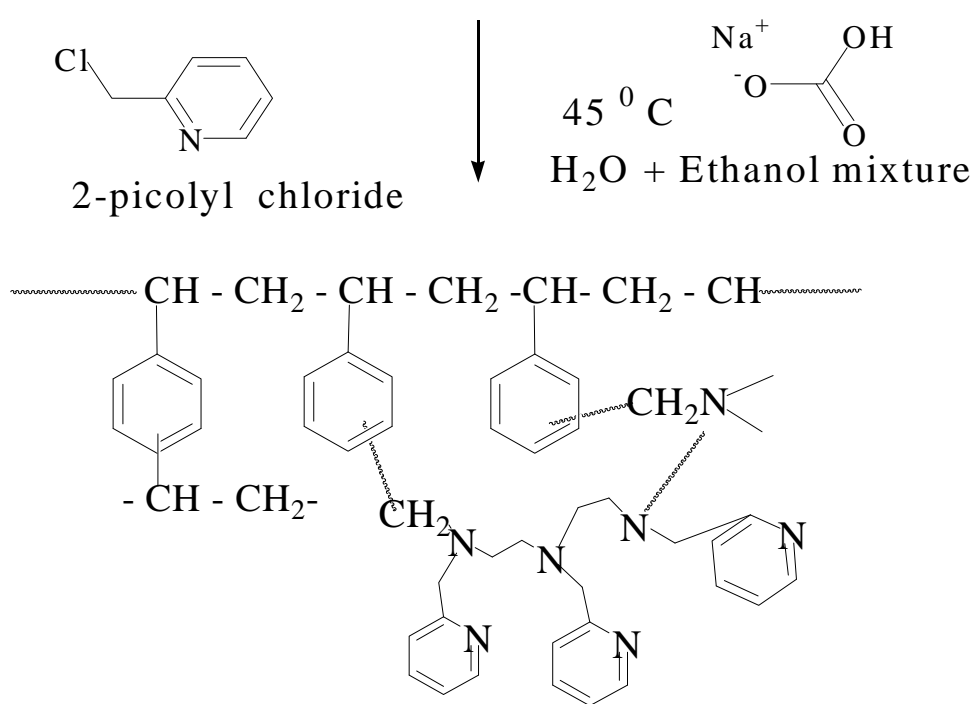

CHEL-38

Figure 4. Synthesis of highly picolylated diethylenetriamine resin (CHEL-38) 
These resins were synthesized from very finely divided samples of the diethylenetriamine resin, which was made by reacting a dry $2 \%$ cross-linked chloride form of quaternary ammonium ion exchange resin (Dowex $1-2 \mathrm{X}$ ) at $150-180^{\circ} \mathrm{C}$ with a stirred solution of diethylenetriamine in ethylene glycol for about 20 hours. The highly picolylated diethylenetriamine resin (CHEL-38) was prepared by overnight heating at about $45^{\circ} \mathrm{C}$ of a stirred slurry in water/ethanol mixture of filtered, water-swollen, finely divided diethylenetriamine resin, picolylchloride-hydrochloride salt and sodium bicarbonate in weight ratios of approximately 1.0 / 0.65 / 1.7. This reaction is depicted in Figure 4. The less picolyated diethylenetriamine resin (CHEL-40) was made in a similar procedure but using respective weight ratios of about 1.0 / 0.34 / 1.6 of the reactants as given above. Since the reaction is the same it is not illustrated separately. However, a better representative CHEL-40 resin type-structure would have one less picolyl $\left(-\mathrm{CH}_{2}-\mathrm{C}_{5} \mathrm{H}_{4} \mathrm{~N}\right)$ group on it.

\section{Characterization of CHEL-38 and CHEL-40 resins}

Moisture content was determined gravimetrically by weighing the filtered resin for wet weight and drying overnight to a constant weight at $105^{\circ} \mathrm{C}$.

Total base capacity was determined in the following manner [25]: About $5 \mathrm{ml}$ of wet swollen resin was placed in a small column. Then the $120 \mathrm{ml}$ of $5 \%$ hydrochloric acid was passed through the resins at a relatively constant flow rate during a period of one hour, and rinsed with $120 \mathrm{ml}$ of $0.001 \mathrm{~N} \mathrm{HCl}$ at the same flow rate. The resin was then removed from the column and the volume of the resins was measured by use of a graduate cylinder. The resin was placed back in columns, and was stripped of the acid adsorbed by the weak base functional groups by passing $20 \mathrm{ml} 1 \mathrm{~N} \mathrm{NH}_{4} \mathrm{OH}$ solution through the column, collecting the effluent, adjusting the $\mathrm{pH}$ to 4.5 and titrated for $\mathrm{Cl}^{-}$using standardized $\mathrm{AgNO}_{3}$ and potassium chromate as indicator. Contact with ammoniacal solution was allowed to occur over a 30 minute time period. The resin was rinsed with $25 \mathrm{ml}$ demineralized water, collecting the effluent in the same beaker. Chloride content was determined in the effluent by titration as described above and capacity was calculated.

\section{Synthesis of a picolated diethylenetriamine resin CHEL-73}

This resin was slightly less picolated than CHEL-40. It was prepared in the same manner as CHEL-40 except that slightly less picolyl chloride was used in preparation. The reaction is the same as that depicted in Figure 4, but one less picolyl group on the resin type-structure. 


\section{RESULTS AND DISCUSSION}

\section{$\underline{\text { MH-13 and CM-DER resin test results }}$}

The absorption capabilities of these resins was first investigated qualitatively by immersing a small quantity of the resin in a very dilute aqueous solution of $\mathrm{CuSO}_{4}$. The $\mathrm{MH}-13$ resin color became brownish green, which indicated that, the resin indeed absorbed $\mathrm{Cu}^{2+}$ and was coordinating (chelating) with the thioether, as well as the carboxylate groups of the resin. The total cation exchange capacity of the resin was determined. A measured quantity of the wetsettled resin in a small glass column was washed slowly with excess aqueous ammoniacal $\mathrm{CuSO}_{4}$ solution. It was then rinsed with dilute ammonia water, and finally, the resin was washed with 1.0 molar $\mathrm{H}_{2} \mathrm{SO}_{4}$ to elute all of the copper (II) cations. All the eluate was collected and it was titrated for $\mathrm{Cu}$ (II) by standard iodometric procedures [26].

The absorption capacity of the synthesized thiosuccinate resin from strongly acidic solutions was also investigated. A measured amount of resin was equilibrated with 20.0 bed volumes of 0.046 molar $\mathrm{CuSO}_{4}$ solution to which 4.0 bed volumes of 0.52 molar $\mathrm{H}_{2} \mathrm{SO}_{4}$ had been added. The remaining copper concentration was again determined by iodometric titration.

Equilibrium column studies were done by passing about 2000 bed volumes of a $\mathrm{pH}$ 1.7, 190 ppm $\mathrm{CuSO}_{4} / \mathrm{H}_{2} \mathrm{SO}_{4}$ solution through a small column of the resin, followed by $1 \mathrm{M} \mathrm{H}_{2} \mathrm{SO}_{4}$ elution and analysis of absorbed copper. The capacity of this resin was only $0.06 \mathrm{meq} \mathrm{Cu} / \mathrm{ml}$ compared with a capacity of $0.31 \mathrm{meq} \mathrm{Cu} / \mathrm{ml}$ of the commercial resin Chelex $100(\mathrm{CH}-100)$ shown in Table 1. This indicates, however, those thioether (sulfur) groups of the new resin coordinate less readily with $\mathrm{Cu}^{2+}$ than does the nitrogen in the imino groups of the IDA resin. This resin was not studied further, since its performance did not look promising even for $\mathrm{Cu}$ (II), which is expected to chelate Cu (II) more strongly than Co (II) on almost any chelating resin.

Small column (3 ml resin) equilibrium absorption tests were also conducted with (CM-DER) resin and compared with the thiosuccinate resin (MH-13), an iminodiacetic acid (IDA) resin (Chelex-100), and a hydroxypropyl picolylamine (HPPR) resin (Dow XFS-43084). The resins were then eluted with $1 \mathrm{M} \mathrm{H}_{2} \mathrm{SO}_{4}$ solution and total eluted copper ion concentrations were determined by iodometric titration [26]. These results are tabulated in Table-1.

Table 1. Absorption capacities of CM-DER and MH-13 resins for copper compared to two commercial resins at $\mathrm{pH} 1.7$.

\begin{tabular}{|l|c|c|c|c|c|c|}
\hline Resin & $\begin{array}{c}\mathrm{Cu}(\mathrm{II}) \\
p p m\end{array}$ & $\begin{array}{c}\mathrm{H}_{2} \mathrm{SO}_{4} \\
\mathrm{ml} / \mathrm{L}\end{array}$ & $\begin{array}{c}\mathrm{H}_{2} \mathrm{SO}_{4} \\
M\end{array}$ & $p H$ & $\begin{array}{c}\text { Capacity } \\
\text { meq Cu/ml }\end{array}$ & $\begin{array}{c}\text { Capacity } \\
\mathrm{mg} / \mathrm{ml}\end{array}$ \\
\hline HPPR & 38 & 1.0 & 0.018 & 1.7 & 0.45 & 14.3 \\
\hline CM-DER & 38 & 1.0 & 0.018 & 1.7 & 0.38 & 12.0 \\
\hline CH-100 & 38 & 1.0 & 0.018 & 1.7 & 0.31 & 9.8 \\
\hline MH-13 & 38 & 1.0 & 0.018 & 1.7 & 0.06 & 1.9 \\
\hline
\end{tabular}

Using the two higher capacity resins (HPPR and CM-DER), further column tests were conducted with more concentrated solutions and progressively more acidic solutions. Performance of both resins was comparable under these conditions (Table 2). 
Table 2. Absorption of copper on HPPR and CM-DER resins at various $\mathrm{pH}$ levels.

\begin{tabular}{|l|c|c|c|c|c|c|}
\hline \multicolumn{1}{|c|}{ Resin } & $\begin{array}{c}\mathrm{Cu}(\mathrm{II}) \\
\mathrm{mg} / \mathrm{L}\end{array}$ & $\begin{array}{c}\mathrm{H}_{2} \mathrm{SO}_{4} \\
\mathrm{ml} / \mathrm{L}\end{array}$ & $\begin{array}{c}\mathrm{H}_{2} \mathrm{SO}_{4} \\
M\end{array}$ & $p H$ & $\begin{array}{c}\text { Capacity } \\
\text { meq } \mathrm{Cu} / \mathrm{ml}\end{array}$ & $\begin{array}{c}\text { Capacity } \\
\mathrm{mg} / \mathrm{ml}\end{array}$ \\
\hline HPPR & 190 & 4.0 & 0.072 & 1.1 & 0.30 & 9.5 \\
\hline CM-DER & 190 & 4.0 & 0.072 & 1.1 & 0.32 & 10.1 \\
\hline HPPR & 190 & 8.0 & 0.144 & 0.8 & 0.11 & 3.5 \\
\hline CM-DER & 190 & 8.0 & 0.144 & 0.8 & 0.09 & 2.9 \\
\hline HPPR & 190 & 16.0 & 0.288 & 0.5 & 0.06 & 1.9 \\
\hline CM-DER & 190 & 16.0 & 0.288 & 0.5 & 0.05 & 1.6 \\
\hline
\end{tabular}

The relative column performance these two resins for cobalt absorption was determined from dilute sulfuric acid solutions. The columns were stripped with $1 \mathrm{M} \mathrm{H}_{2} \mathrm{SO}_{4}$ solution and the eluates were analyzed for cobalt by iodometric titration [27, 28]. The results (Table 3) show that the newly synthesized CM-DER resin has the highest capacity for cobalt under these conditions; however, cobalt absorption is 20 times less than that of copper (Table 2) at the same $\mathrm{pH}$.

Table 3. Cobalt absorption on HPPR, CM-DER and CH-100 resins at pH 1.7 and 1.1.

\begin{tabular}{|c|c|c|c|c|}
\hline Resin & $\begin{array}{c}\mathrm{Co}(\mathrm{II}) \\
\mathrm{mg} / \mathrm{L}\end{array}$ & $p \mathrm{H}$ & $\begin{array}{c}\mathrm{H}_{2} \mathrm{SO}_{4} \\
\mathrm{M}\end{array}$ & $\begin{array}{c}\text { Capacity } \\
\mathrm{mg} / \mathrm{ml}\end{array}$ \\
\hline HPPR & 38 & 1.7 & 0.018 & 0.04 \\
\hline CM-DER & 38 & 1.7 & 0.018 & 0.54 \\
\hline CH-100 & 38 & 1.7 & 0.018 & 0.13 \\
\hline HPPR & 190 & 1.1 & 0.072 & 0.02 \\
\hline CM-DER & 190 & 1.1 & 0.072 & 0.14 \\
\hline CH-100 & 190 & 1.1 & 0.072 & 0.05 \\
\hline
\end{tabular}

Note: probable errors are estimated to be $> \pm 10 \%$ for this low capacity values

Ferric ion absorption on CH-100, HPPR and CM-DER resins was also tested as was done for copper and cobalt absorption. Analysis of the $1 \mathrm{M} \mathrm{H}_{2} \mathrm{SO}_{4}$ stripping eluates was done by colorimetric determination of iron as thiocyanate complexes [28]. Ferric ion was analyzed by an oxidation reduction method [29] and the results tabulated in Table 4.

Table 4. Ferric ion absorption on CM-DER, HPPR and CH-100 resins at pH 1.7.

\begin{tabular}{|c|c|c|c|c|c|}
\hline Resin & $\begin{array}{c}\mathrm{Fe}(\mathrm{III}) \\
\mathrm{mg} / \mathrm{L}\end{array}$ & $\begin{array}{c}\mathrm{H}_{2} \mathrm{SO}_{4} \\
\mathrm{Ml} / \mathrm{L}\end{array}$ & $\begin{array}{c}\mathrm{H}_{2} \mathrm{SO}_{4} \\
M\end{array}$ & $p H$ & $\begin{array}{c}\text { Capacity } \\
\mathrm{mg} / \mathrm{ml}\end{array}$ \\
\hline CH-100 & 190 & 1.0 & 0.018 & 1.7 & 46.9 \\
\hline CM-DER & 190 & 1.0 & 0.018 & 1.7 & 43.6 \\
\hline HPPR & 190 & 1.0 & 0.018 & 1.7 & 7.8 \\
\hline
\end{tabular}

It is observed that the two resins which contain cation exchanging carboxymethyl groups CM-DER and $\mathrm{CH}-100$, have considerably higher capacity for $\mathrm{Fe}^{3+}$ than they do for $\mathrm{Cu}^{2+}$ absorption. However, HPPR has considerably lower capacity for ferric ion because it has only cation complexing amino groups. The latter resin is promoted by DOW as a potential alternative for use in extraction of copper from acidic leach solutions, instead of the solvent extraction process. 
Test results for CHEL-38 and CHEL-40

Results of characterization studies on CHEL-38 and CHEL-40 are presented in Table 5.

Table 5. Characterization of CHEL-38 and CHEL-40 resins.

\begin{tabular}{|c|c|c|}
\hline Properties & CHEL-38 & CHEL-40 \\
\hline Moisture Content & $52 \%$ & $60 \%$ \\
\hline Mesh Size & $50-110$ & $40-110$ \\
\hline Total Base Capacity & $1.27 \mathrm{meq} / \mathrm{ml}$ & $1.32 \mathrm{meq} / \mathrm{ml}$ \\
\hline
\end{tabular}

Very small column absorption tests for copper (II) and cobalt (II) were conducted in duplicate with finely divided CHEL-38 and CHEL-40 in comparison with finely divided commercially available Dow resins hydroxy-propyl picolyl amine resin (HPPR), and bispicolylated polyamine resin (DPAR). All the resins were water washed and then separately loaded with the test solutions, all the columns, except DPAR, were eluted with $1 \mathrm{M}$ or $2 \mathrm{M}$ $\mathrm{H}_{2} \mathrm{SO}_{4}$. The DPAR resin required aqueous ammonia solution for elution. Total absorbed copper ion and cobalt ion amounts [25, 27, 28] of all eluates were determined separately. These results are tabulated in Tables 6, 7 and 8. Table 6 shows the results for copper and cobalt feed solutions at $38 \mathrm{ppm}$ and $\mathrm{pH}$ 1.7. The feed flow rates were at 0.5 to 1.0 bed volumes per minute. The capacities include approximately $\pm 5 \%$ experimental error.

Table 6. Absorption capacities of CHEL-38, CHEL-40, DPAR and HPPR resins for $38 \mathrm{ppm}$ solutions of copper (II) and cobalt (II) at pH 1.7.

\begin{tabular}{|c|c|c|c|c|c|}
\hline Resin & $\begin{array}{c}\mathrm{Cu}(\mathrm{II}) \\
\mathrm{Co}(\mathrm{II}) \\
p p m\end{array}$ & $\begin{array}{c}\mathrm{H}_{2} \mathrm{SO}_{4} \\
\mathrm{ml} / \mathrm{L}\end{array}$ & $\begin{array}{c}\mathrm{H}_{2} \mathrm{SO}_{4} \\
M\end{array}$ & $\begin{array}{c}\text { Capacity } \\
\mathrm{mg} / \mathrm{ml} \\
\mathrm{Co}(\mathrm{II})\end{array}$ & $\begin{array}{c}\text { Capacity } \\
\mathrm{mg} / \mathrm{ml} \\
\mathrm{Cu}(\mathrm{II})\end{array}$ \\
\hline CHEL-38 & 38 & 1.0 & 0.018 & 3.8 & 14.4 \\
\hline CHEL-40 & 38 & 1.0 & 0.018 & 1.45 & 14.7 \\
\hline DPAR & 38 & 1.0 & 0.018 & 0.04 & 18.2 \\
\hline HPPR & 38 & 1.0 & 0.018 & 0.04 & 9.4 \\
\hline
\end{tabular}

Using the higher capacity resins (HPPR, CHEL-38, and CHEL-40), small column tests were

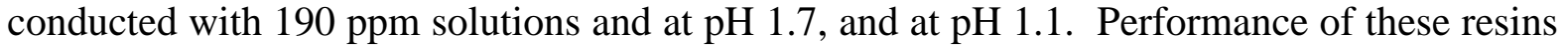
for copper, cobalt and iron are tabulated in Tables 7 and 8.

Table 7. Absorption capacities of CHEL-38, CHEL-40, DPAR and HPPR resins for $190 \mathrm{ppm}$ solutions of copper (II), cobalt (II) and iron (III) at pH 1.7.

\begin{tabular}{|c|c|c|c|c|c|}
\hline Resin & $\begin{array}{c}\mathrm{Cu}(\mathrm{II}), \mathrm{Co}(\mathrm{II}) \\
\& \mathrm{Fe}(\mathrm{III}) \\
p p m\end{array}$ & $\begin{array}{c}\mathrm{H}_{2} \mathrm{SO}_{4} \\
\mathrm{ml} / \mathrm{L}\end{array}$ & $\begin{array}{c}\text { Capacity } \\
\mathrm{mg} / \mathrm{ml} \\
\mathrm{Co}(\mathrm{II})\end{array}$ & $\begin{array}{c}\text { Capacity } \\
\mathrm{mg} / \mathrm{ml} \\
\mathrm{Fe}(\mathrm{III})\end{array}$ & $\begin{array}{c}\text { Capacity } \\
\mathrm{mg} / \mathrm{ml} \\
\mathrm{Cu}(\mathrm{II})\end{array}$ \\
\hline CHEL-38 & 190 & 1.0 & 4.9 & 2.1 & 25.2 \\
\hline CHEL-40 & 190 & 1.0 & 3.8 & 3.2 & 26.1 \\
\hline DPAR & 190 & 1.0 & 0.008 & 6.5 & 23.6 \\
\hline HPPR & 190 & 1.0 & 0.043 & 3.2 & 18.2 \\
\hline
\end{tabular}


Table 8. Absorption capacities of CHEL-38, CHEL-40 and HPPR resins at pH 1.1.

\begin{tabular}{|c|c|c|c|c|c|}
\hline Resin & $\mathrm{Cu}(\mathrm{II}) \mathrm{ppm}$ & $\mathrm{H}_{2} \mathrm{SO}_{4} \mathrm{ml} / \mathrm{L}$ & $\mathrm{H}_{2} \mathrm{SO}_{4} \mathrm{M}$ & $\mathrm{pH}$ & Capacity $(\mathrm{mg} / \mathrm{ml})$ \\
\hline CHEL-38 & 190 & 4.00 & 0.072 & 1.1 & 12.4 \\
\hline CHEL-40 & 190 & 4.00 & 0.072 & 1.1 & 13.4 \\
\hline HPPR & 190 & 4.00 & 0.072 & 1.1 & 8.8 \\
\hline
\end{tabular}

For the following very small column tests of various resins, the protonated hydrosulfate form resins were water washed and then separately loaded with the aqueous metal sulfate plus $\mathrm{H}_{2} \mathrm{SO}_{4}$ test solutions. After limited water washing, all the columns except dipicolylamine resin, which was tested for cobalt capacity, were eluted with $1 \mathrm{M}$ or $2 \mathrm{M} \mathrm{H}_{2} \mathrm{~S}_{4}$. Total absorbed metal ion amounts in all elutes were determined separately by the standard iodometric or chelometric methods. These results are tabulated in Tables 9, 10 and 11.

Table 9 shows capacity results for cobalt feed solutions at $190 \mathrm{ppm}$ for $\mathrm{pH} 1.7$ and $\mathrm{pH} 3.1$ for CHEL-38, CHEL-40, DPAR, and HPPR. Using the higher capacity resins (HPPR, CHEL-38, and CHEL-40), column tests were conducted with 1000 ppm solutions at pH 1.7 and pH 3.1. Results at pH 3.1 of these resins for copper, cobalt and nickel are tabulated in Table 10 . Table 11 summarizes the solution concentration effect for copper (II) and cobalt (II) capacities of several resins at $\mathrm{pH}$ 1.7. Nickel was analyzed by a chelometric method [30] and nickel capacities for these resins at 190 ppm and pH 1.7 are shown in Table 12. Finally, aluminum capacities were determined chelometrically [30] for these resins at $\mathrm{pH} 1.7$ with 2000 ppm Al solution. Results are shown in Table 13. As appropriate, the tables include and correlate earlier data.

Table 9. Effect of $\mathrm{pH}$ on absorption capacities for $190 \mathrm{ppm}$ solutions of cobalt (II) on CHEL-38, CHEL-40, DPAR and HPPR resins.

\begin{tabular}{|c|c|c|c|c|}
\hline Resin & $\mathrm{Co}^{2+} \mathrm{ppm}$ & $\mathrm{H}_{2} \mathrm{SO}_{4}$ elution & $\begin{array}{c}\mathrm{Co}^{2+} \text { capacity at } \\
p H=1.7,(\mathrm{mg} / \mathrm{ml})\end{array}$ & $\begin{array}{c}\mathrm{Co}^{2+} \text { capacity at } \\
p H=3.1,(\mathrm{mg} / \mathrm{ml})\end{array}$ \\
\hline CHEL-38 & 190 & $2 \mathrm{M}$ & 4.9 & 14.4 \\
\hline CHEL-40 & 190 & $2 \mathrm{M}$ & 3.8 & 13.8 \\
\hline DPAR & 190 & $2 \mathrm{M}$ & 0.08 & 11.6 \\
\hline HPPR & 190 & $2 \mathrm{M}$ & 0.04 & 2.4 \\
\hline
\end{tabular}

Table 10. Absorption capacities for copper (II), cobalt (II) and nickel (II) of CHEL-38, CHEL-40 and HPPR resins at $\mathrm{pH} 3.1$.

\begin{tabular}{|l|c|c|c|c|c|c|}
\hline \multicolumn{1}{|c|}{ Resin } & $p H$ & $\begin{array}{c}\text { Copper } \\
190 \mathrm{ppm}\end{array}$ & $\begin{array}{c}\text { Copper } \\
1000 \mathrm{ppm}\end{array}$ & $\begin{array}{c}\text { Cobalt } \\
190 \mathrm{ppm}\end{array}$ & $\begin{array}{c}\text { Cobalt } \\
1000 \mathrm{ppm}\end{array}$ & $\begin{array}{c}\text { Nickel } \\
190 \mathrm{ppm}\end{array}$ \\
\hline CHEL-38 & 3.1 & 28.3 & 37.2 & 14.4 & 17 & 9.3 \\
\hline CHEL-40 & 3.1 & 28.6 & 38.0 & 13.8 & 17 & 7.8 \\
\hline HPPR & 3.1 & 18.8 & 37.4 & 2.4 & 3.9 & 4.6 \\
\hline
\end{tabular}


Table 11. Effect of concentration on absorption capacities of CHEL-38, CHEL 40 and HPPR resins for cobalt (II) and copper (II) at pH 1.7.

\begin{tabular}{|c|c|c|c|c|c|}
\hline Resin & $\begin{array}{c}\mathrm{Cu}(\mathrm{II}) \\
\mathrm{Co}(\mathrm{II}) \\
p p m\end{array}$ & $\begin{array}{c}\mathrm{H}_{2} \mathrm{SO}_{4} \mathrm{M} \\
\text { Used for } \\
\text { elution }\end{array}$ & $p H$ & $\begin{array}{c}\text { Capacity } \\
\mathrm{mg} / \mathrm{ml} \\
\mathrm{Co}(\mathrm{II})\end{array}$ & $\begin{array}{c}\text { Capacity } \\
\mathrm{mg} / \mathrm{ml} \\
\mathrm{Cu}(\mathrm{II})\end{array}$ \\
\hline CHEL-38 & 1000 & $2 \mathrm{M}$ & 1.7 & 8.9 & 27.7 \\
& 190 & & & 4.9 & 25.2 \\
& 38 & & & 3.8 & 14.4 \\
\hline CHEL-40 & 1000 & $2 \mathrm{M}$ & 1.7 & 7.9 & 28.4 \\
& 190 & & & 4.2 & 26.1 \\
& 38 & & & 3.1 & 14.7 \\
\hline HPPR & 1000 & $2 \mathrm{M}$ & 1.7 & 1.25 & 23.9 \\
& 190 & & & 0.04 & 18.2 \\
& 38 & & & & 9.2 \\
\hline
\end{tabular}

For tests in Table 11, flow rates were at 0.5 to 1.0 bed volumes per minute. The feed volumes were approximately $1000 \mathrm{ppm}$ for $60 \mathrm{ml}, 190 \mathrm{ppm}$ for $200 \mathrm{ml}$ and $38 \mathrm{ppm}$ for $500 \mathrm{ml}$ bed volumes. The capacities were approximately $\pm 5 \%$ in experimental error.

Table 12. Absorption capacities of CHEL-38, CHEL 40, DPAR and HPPR resins for nickel (II) at $\mathrm{pH} 1.7$.

\begin{tabular}{|c|c|c|c|c|}
\hline Resin & $\mathrm{Ni}(\mathrm{II}) \mathrm{ppm}$ & $\mathrm{H}_{2} \mathrm{SO}_{4} \mathrm{M}$ & $\mathrm{pH}$ & $\begin{array}{c}\text { Capacity } \mathrm{mg} / \mathrm{ml} \\
\mathrm{Ni}(\mathrm{II})\end{array}$ \\
\hline CHEL-38 & 190 & 0.018 & 1.7 & 9.1 \\
\hline CHEL-40 & 190 & 0.018 & 1.7 & - \\
\hline HPPR & 190 & 0.018 & 1.7 & 2.4 \\
\hline DPAR & 190 & 0.018 & 1.7 & 15 \\
\hline
\end{tabular}

Feed volumes were about at least 200 bed volumes and flow rates were at 0.5 to 1.0 bed volumes per minute. The capacities were approximately $\pm 5 \%$ in experimental error

Table 13. Absorption capacities of CHEL-38, CHEL-40 and HPPR resins for 2000 ppm solutions of aluminum (III) at $\mathrm{pH} 1.7$.

\begin{tabular}{|c|c|c|c|}
\hline Resin & Al(III) $\mathrm{ppm}$ & Solution $\mathrm{pH}$ & Capacity $(\mathrm{mg} / \mathrm{ml})$ \\
\hline CHEL-38 & 2000 & 1.7 & 5.1 \\
\hline CHEL-40 & 2000 & 1.7 & 8.3 \\
\hline HPPR & 2000 & 1.7 & 4.9 \\
\hline
\end{tabular}

Feed flow rates were at 0.5 to 1.0 bed volumes per minute. Experimental error $\pm 5 \%$ 
Absorption capacities of these two promising new resins, CHEL-38 and CHEL-40, were evaluated from a solvent extraction raffinate of a copper leach solution sample, obtained from a large copper mine in the southwest, and their performance was compared to two commercially available resins, HPPR and DPAR, by loading small columns to equilibrium capacities and then eluting with $2 \mathrm{M} \mathrm{H}_{2} \mathrm{SO}_{4}$. The metals of interest were analyzed by atomic absorption spectroscopy (AAS). Metals in the $\mathrm{pH} 2.1$ raffinate feed were also analyzed and are listed in Table 14.

Table 14. Copper heap-leach solvent extraction raffinate solution analysis.

\begin{tabular}{|c|c|c|c|c|}
\hline Cu ppm & Co ppm & Fe ppm & Ni ppm & Zn ppm \\
\hline 42 & 18 & 420 & 60 & 214 \\
\hline
\end{tabular}

In addition large amounts of aluminum, sulfate, alkali, and alkaline earths were present.

About 3000 bed volumes of mining solution was passed through the small columns at fast flow rates, 2-5 bed volumes (BV) per minute, and the resins were then eluted with $2 \mathrm{M}$ $\mathrm{H}_{2} \mathrm{SO}_{4}$. Analyses of the sulfuric acid eluates by AAS indicated that the more strongly absorbing copper had eluted most of the iron, cobalt, nickel and zinc absorbed on the resin during the loading cycle. Therefore, a similar run was made in which small fractions were collected and analyzed individually. Cumulative amounts of various metals that were retained on resins as a function of the feed solution volume are shown in Figures 5-8.

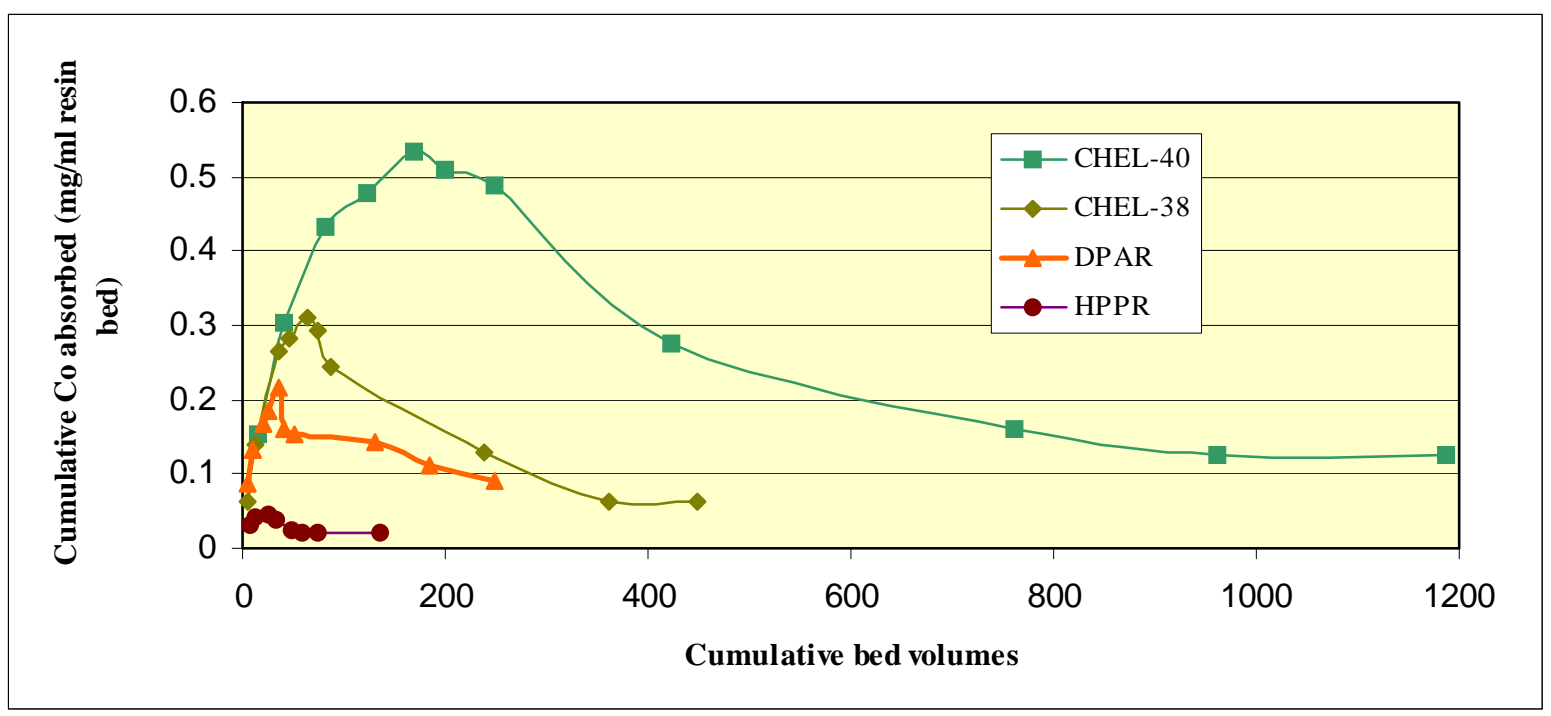

Figure 5. Cobalt (II) absorption on various resins from fast-flow raffinate solution. 


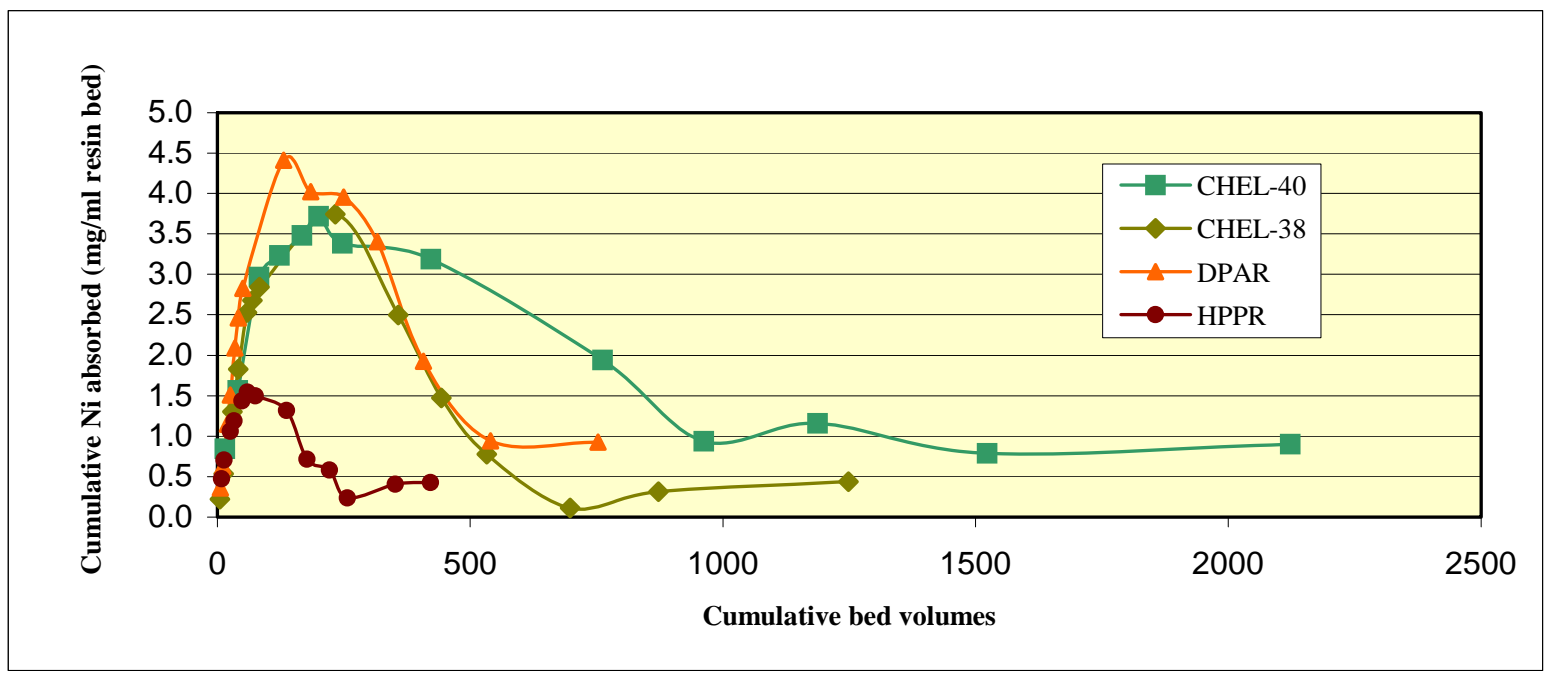

Figure 6. Nickel (II) absorption on various resins from fast-flow raffinate solution.

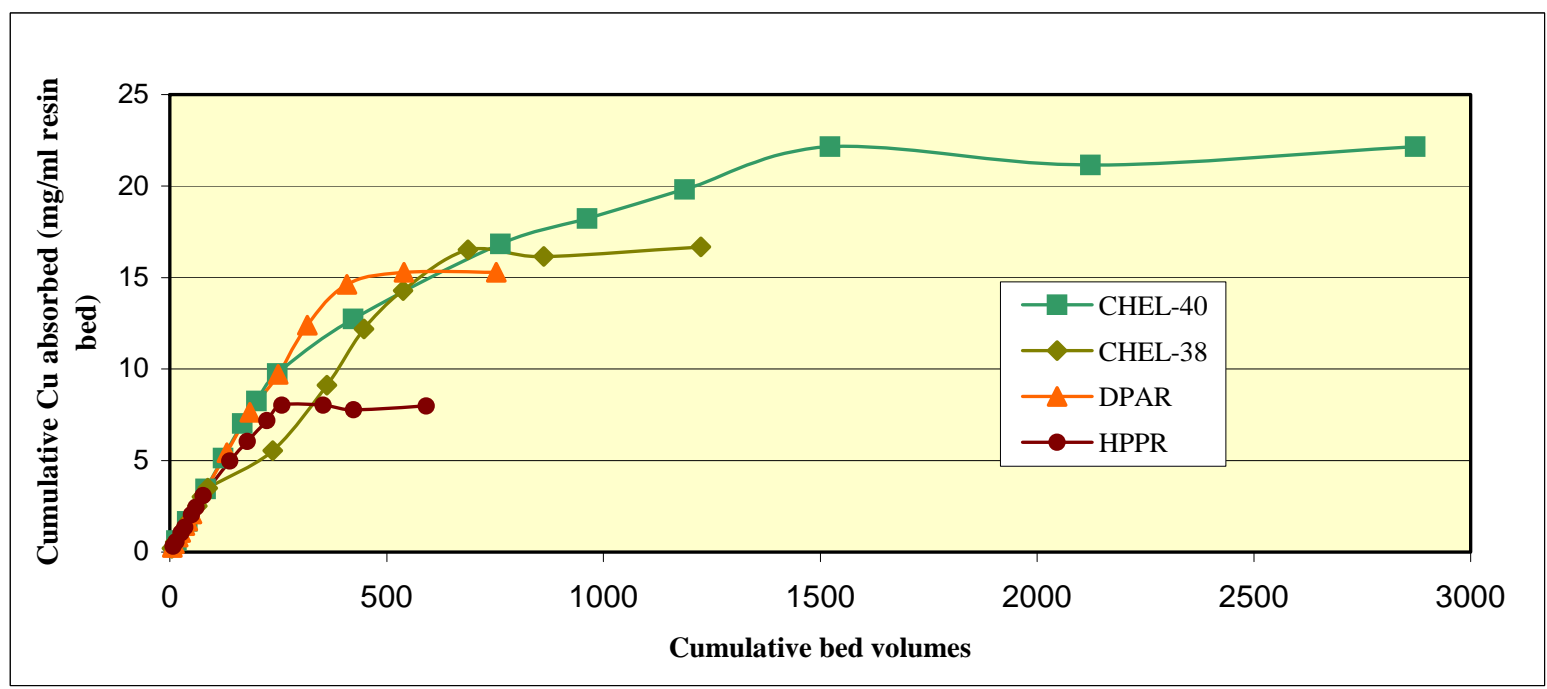

Figure 7. Copper (II) absorption on various resins from fast-flow raffinate solution. 


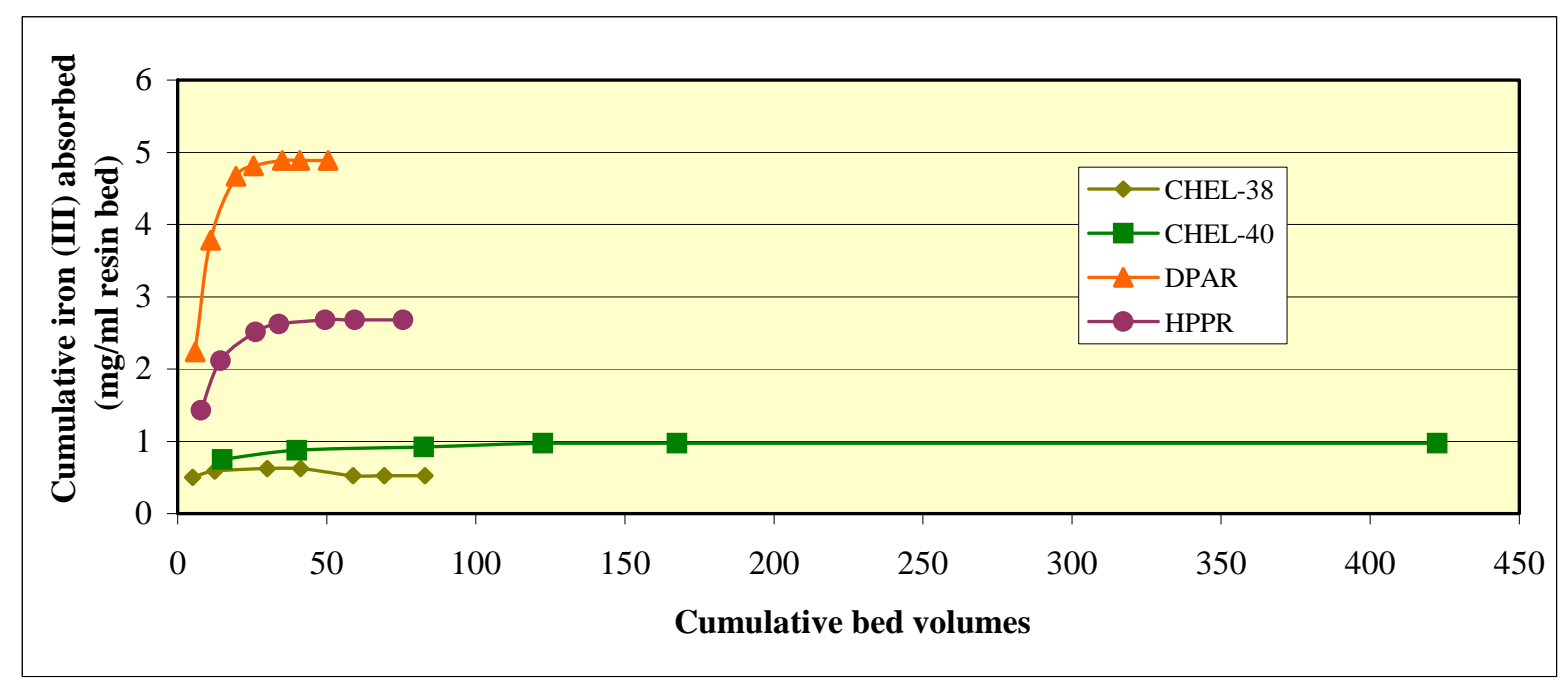

Figure 8. Iron (III) absorption on various resins from fast-flow raffinate solution.

At these high flow rates through small shallow beds, only a fraction of the amounts of metal cations fed to the resin was absorbed. Under these conditions, the maximum absorption capacities of all the resins or Co (II) and Ni (II) peaked at about $200 \mathrm{BV}$ or less, and capacity for Fe (III) leveled off at about $50 \mathrm{BV}$. The $\mathrm{Cu}$ (II) absorption capacity for Co (II) $(0.51 \mathrm{mg} / \mathrm{ml})$ was obtained by the CHEL-40 resin at $\sim 200 \mathrm{BV}$ of feed.

Another column run was made using 200 bed volumes of high-flow raffinate feed in order to optimize the capacity for cobalt on CHEL-40. The resin was eluted in large volume steps (at fast flow rates) with $\mathrm{H}_{2} \mathrm{O}(11 \mathrm{BV})$, followed by dilute $\mathrm{H}_{2} \mathrm{SO}_{4}$ solutions in step-wise increasing concentrations of $0.1 \mathrm{M}$ (28 BV), 0.5 M (58 BV), 1.0 M (58 BV), and 2.0 M (64 BV). The highest concentrations of cobalt, nickel, zinc, and iron were found in the water rinse, and in the $0.1 \mathrm{M} \mathrm{H}_{2} \mathrm{SO}_{4}$ elution steps, and only a very small portion of the copper was eluted. The copper concentration was very high in the $0.5 \mathrm{M}$, and moderately high in the $1.0 \mathrm{M} \mathrm{H}_{2} \mathrm{SO}_{4}$ elution steps.

The CHEL-40 resin was evaluated for the potential recovery of Co (II) by loading the resin bed with a limited amount of raffinate at a slow-flow rate, $2 \mathrm{BV} / \mathrm{hr}$. The studies were conducted on raffinate solution with somewhat lower copper (40 ppm) and cobalt (16 ppm) content. About 300 bed volumes (BV) of raffinate at a slow flow rate of $2 \mathrm{BV} /$ hour were applied to the small CHEL-40 column $(0.20 \pm 0.02 \mathrm{ml}$, bed depth $0.60 \pm 0.04 \mathrm{~cm})$. Effluent fractions were collected and analyzed by AAS for Co (II) and $\mathrm{Cu}$ (II) content. The cumulative amounts absorbed on the resin (Figures 9 and 10) were calculated by the difference from the amounts in the feed. After the resin was stripped with acid and washed with water, it was then loaded with approximately 225 bed volumes of raffinate at $2 \mathrm{BV} / \mathrm{hr}$ and slowly eluted stepwise with $\mathrm{H}_{2} \mathrm{O}(1 \mathrm{BV})$ and $0.5 \mathrm{M} \mathrm{H}_{2} \mathrm{SO}_{4}(2 \mathrm{BV})$ at a rate of $1 \mathrm{BV} / \mathrm{hr}$. Collected eluate fractions were analyzed for $\mathrm{Co}$ (II) and $\mathrm{Cu}$ (II) concentrations by AAS as before. 
The CHEL-40 capacity for absorbing cobalt at these slow flow rates increased over that found in earlier fast flow rates. However, the capacity for $\mathrm{Cu}$ (II) was the same as was determined previously for fast flow, since the stronger $\mathrm{Cu}$ (II) selectivity of the resin increases the rate of $\mathrm{Cu}$ (II) absorption over that of less strongly absorbed Co (II), so that a slower flow rate made little difference.

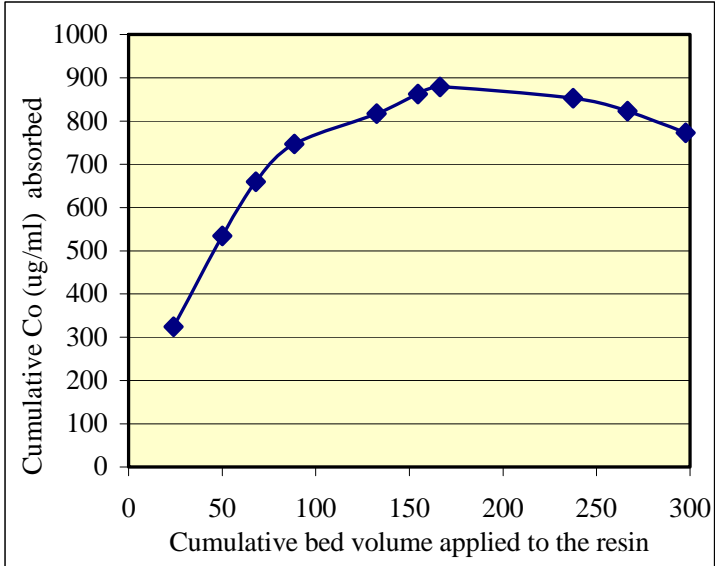

Figure 9. Cobalt absorption on CHEL-40 from raffinate solution at slow flow rate.

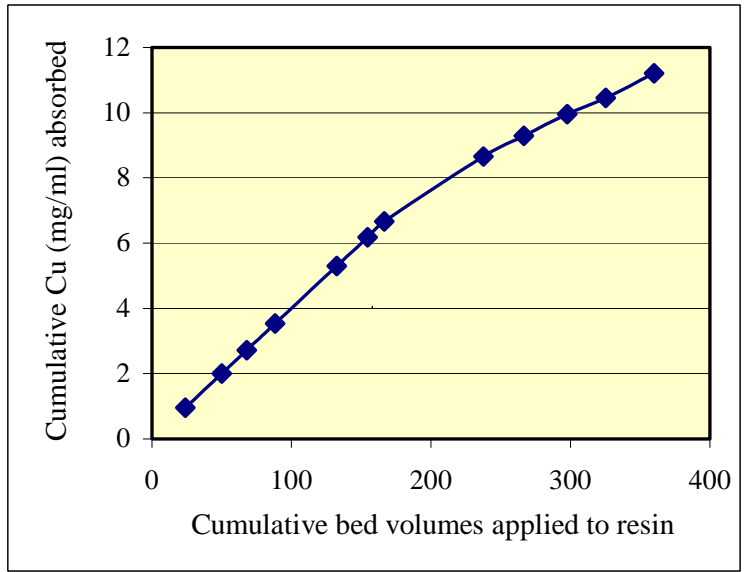

Figure 10. Copper absorption on CHEL-40 from raffinate solution at slow flow rate.

The regenerated resin was loaded with $\sim 225 \mathrm{BV}$ of raffinate at the slow flow rate of $2 \mathrm{BV} / \mathrm{hr}$. Slow flow step gradient elution with $\mathrm{H}_{2} 0,0.5,1$ and $2 \mathrm{M} \mathrm{H}_{2} \mathrm{SO}_{4}$ results for both Co (II) and $\mathrm{Cu}$ (II) were fairly similar within 6BV as shown in Figures 11 and 12. Most of the Co (II) was eluted at a concentration of about $200 \mathrm{ug} / \mathrm{ml}$ (ppm), over 10 times the concentration (16 ppm) in the feed solution; and most of the $\mathrm{Cu}$ (II) was eluted at an eluate concentration of about 3500 ppm, over 80 times the concentration (40 ppm) in the feed solution.

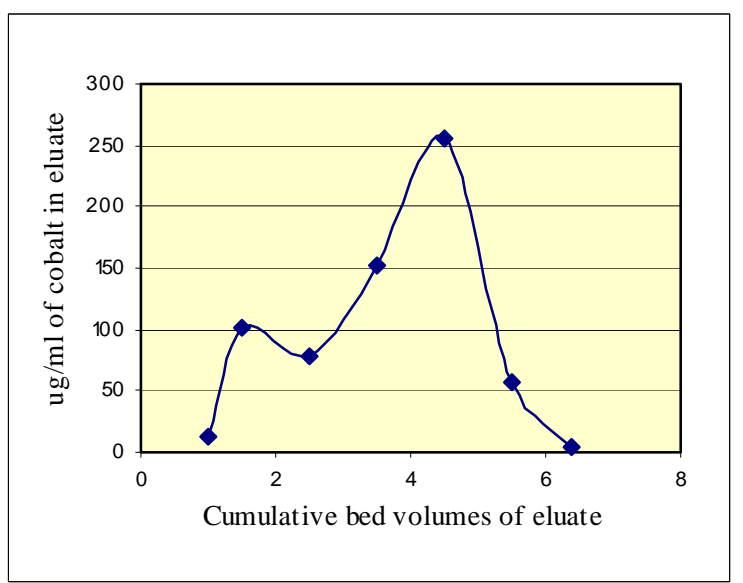

Figure 11. Cobalt elution from CHEL-40.

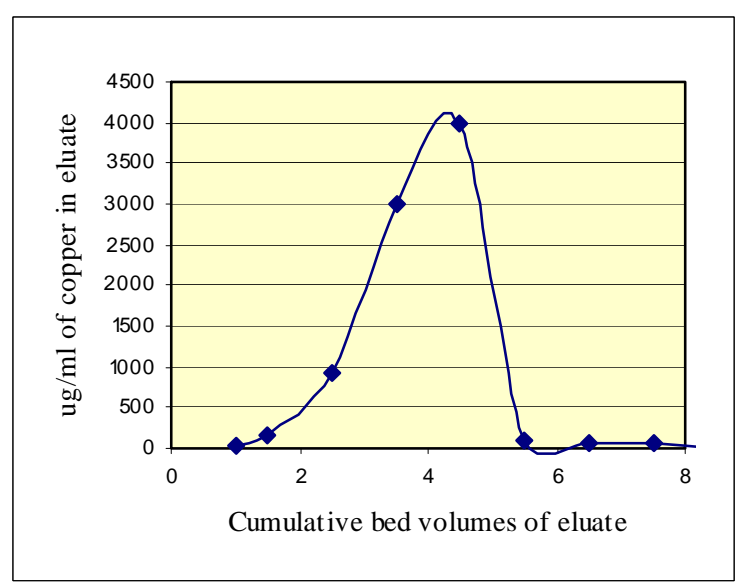

Figure 12. Copper elution from CHEL-40. 


\section{Column tests on CHEL-73}

The last picolylated diethylenetriamine resin CHEL-73, which was fairly equivalent in its preparation to that of the CHEL-40 resin, was also tested. A total of 300 bed volumes (BV) the copper leach raffinate solution (now at $10 \mathrm{ppm} \mathrm{Co}$, and $25 \mathrm{ppm} \mathrm{Cu}$ because of aging and metals absorption on sludge) was applied at a moderate flow rate of $\sim 8 \mathrm{BV} / \mathrm{hr}$ to a small column of CHEL-73 (bed volume $0.62 \pm 0.02 \mathrm{ml}$, bed depth $1.80 \pm 0.04 \mathrm{ml}$ ) which was about 3 times as deep as the CHEL-40 column. The amount of Co (II) absorbed on the resin was $\sim 0.9$ $\mathrm{mg} / \mathrm{ml}$ of resin, and the amount of $\mathrm{Cu}$ (II) about $5 \mathrm{mg} / \mathrm{ml}$, as shown in Figures 13 and 14 .

The effluent fractions from the feed were analyzed as above for Co (II) and $\mathrm{Cu}$ (II) content and the amounts absorbed on the resin were calculated. Elution of the loaded bed with $4 \mathrm{BV}$ of $\mathrm{H}_{2} \mathrm{O}$ at $2 \mathrm{BV} / \mathrm{hr}$ was followed by a total of $11.2 \mathrm{BV}$ of $2 \mathrm{M} \mathrm{H}_{2} \mathrm{SO}_{4}$. The eluted fractions were analyzed for Co (II) and $\mathrm{Cu}$ (II) [25, 27, 28].

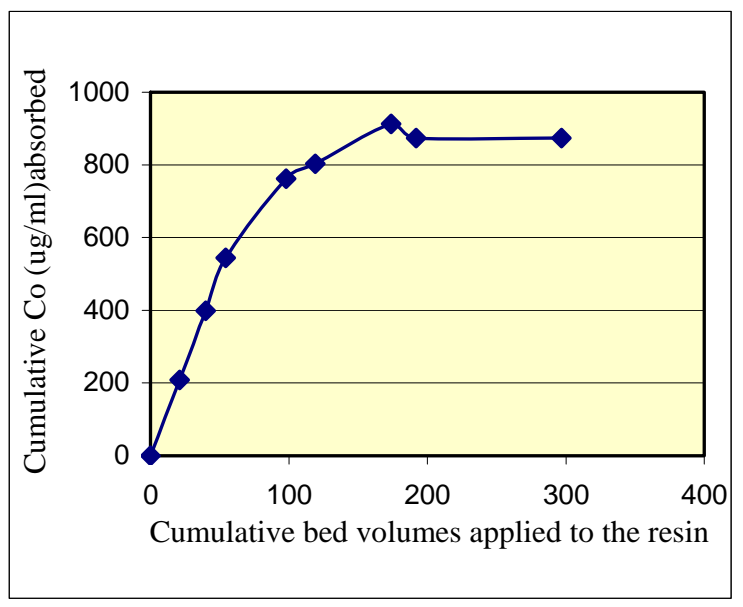

Figure 13. Cobalt absorption on CHEL-73 from raffinate solution at intermediate flow.

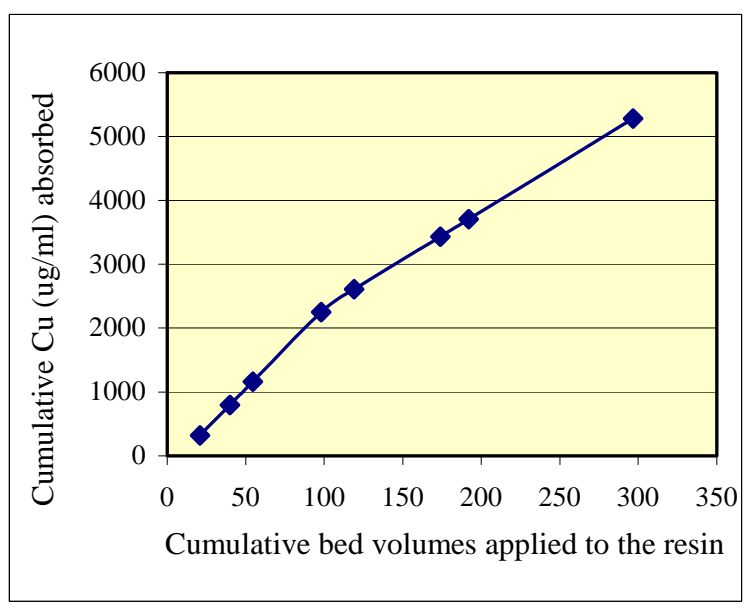

Figure 14. Copper absorption on CHEL-73 from raffinate solution at intermediate flow.

The decreased capacity of the resin for $\mathrm{Cu}$ (II) compared to that of CHEL-40 is explained by its smaller concentration in the raffinate feed ( $25 \mathrm{ppm}$ vs. the earlier 40 ). The equivalent Co (II) capacity is explained by the idea that the lesser amount of $\mathrm{Cu}$ (II) absorbed on the bed reduced the displacement of the Co (II) on the lower part of the bed. Slow flow (2BV $/ \mathrm{hr}$ ) elution of the loaded bed with $\mathrm{H}_{2} 0,2 \mathrm{M} \mathrm{H}_{2} \mathrm{SO}_{4}$ eluted both $\mathrm{Co}$ (II) and $\mathrm{Cu}$ (II) quite similarly. Essentially all the Co (II) and $\mathrm{Cu}$ (II) were eluted within 11BV. Most of the Co (II) was eluted by the $2 \mathrm{M} \mathrm{H}_{2} \mathrm{SO}_{4}$ at a concentration of $\sim 150 \mathrm{ppm}$, well over 10 times the concentration in the feed solution. Most of the $\mathrm{Cu}$ (II) was eluted at a concentration of $\sim 1500 \mathrm{ppm}$, about 60 times the feed concentration (Figures15 and 16). That the increase in $\mathrm{Cu}$ (II) concentration is less than attained with the CHEL-40 (slow flow) is explained by the lesser loading of $\mathrm{Cu}$ (II) on the CHEL-73 resin ( $\sim 5 \mathrm{~g} / \mathrm{ml}$ vs. $\sim 8 \mathrm{~g} / \mathrm{ml}$ ) due to the lower $(25 \mathrm{ppm}) \mathrm{Cu}$ ion concentration in the raffinate. 


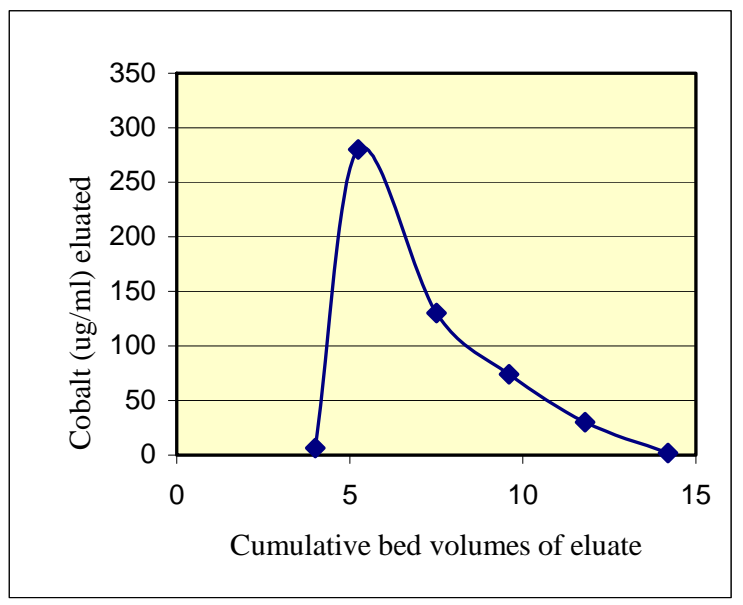

Figure 15. Cobalt elution from CHEL-73.

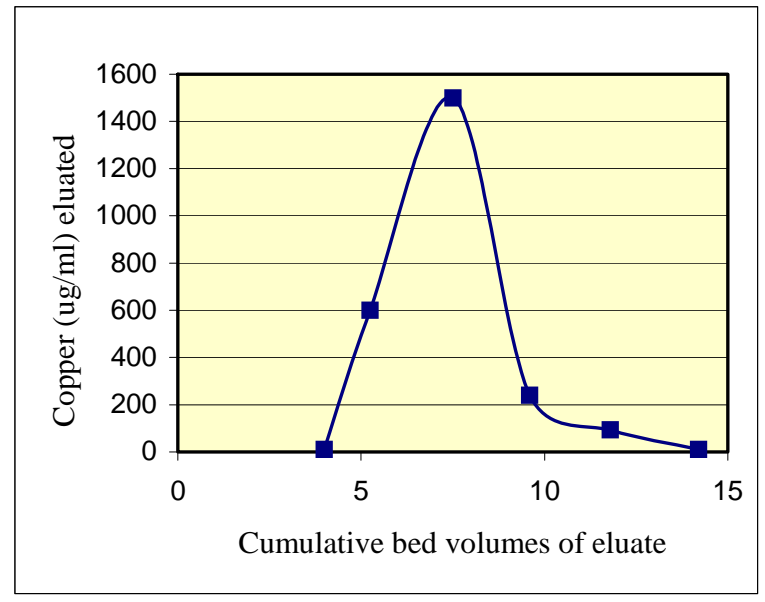

Figure 16. Copper elution from CHEL-73.

\section{CONCLUSIONS}

In search of new resins to recover copper and cobalt from highly acidic, dilute solutions, a small quantity of five cross linked gel-type chelating resins were synthesized and screening tests were conducted for the abilities of the resins to absorb copper, cobalt, and nickel ions from acidic $\left(\mathrm{H}_{2} \mathrm{SO}_{4}\right)$ solutions in comparison with three commercial chelating resins. A thiosuccinic acid resin (MH-13) was synthesized and tested with an acidic copper solution. The comparison tests were made a low cross linked, gel type iminoacetic acid resin (Chelex100) and a dipicolylamine resin. This resin had less capacity for $\mathrm{Cu}$ (II) than the iminodiacetic acid resin. Both the thiosuccinate and iminodiacetic acid resins had much less capacity in absorbing copper from acidic solutions than a commercial dipicolylamine (DOW M-4195) resin.

A carboxymethylated diethylenetriamine (CM-DER) resin was synthesized and evaluated. Small-scale equilibration tests were made by passing hundreds of bed volumes of acidic $\left(\mathrm{H}_{2} \mathrm{SO}_{4}\right)$ solutions containing $\mathrm{Cu}$, Co, and Fe (III) ions through the resin beds, eluting the resins with sulfuric acid, and analyzing the eluates for metal content. The CM-DER resin showed high capacity for copper and iron but only low capacity for cobalt. The comparison tests were made with commercial iminodiacetic acid (IDA) resin (Chelex-100) and an experimental hydroxypropyl picolylamine resin (Dow XFS-43084), HPPR. At pH 1.7 the CM-DER resin had copper and iron capacities about the same as Chelex-100. At pH 1.1, the copper capacity was about the same as that of HPPR resin and considerably better than that of the Chelex-100. At pH 1.7, the cobalt capacity, although low, was much better than that of the Chelex-100 or HPPR resins.

A highly-picolylated (CHEL-38) and a less-picolylated (CHEL-40) diethylenetriamine resins were synthesized and evaluated in comparison with the HPPR and the dipicolyamine (Dow M-4195) DPAR resins. The results showed that both the highly (CHEL-38) and (CHEL-40) resins were comparable to, or more effective, in absorbing $\mathrm{Cu}$ (II) from $\mathrm{pH} 1.1$ to 1.7 solutions then these commercial resins. In contrast to the DPAR resin, which required 
aqueous ammonium solutions for effective regeneration, the CHEL-38 and CHEL-40 resins were regenerated easily with $1 \mathrm{M} \mathrm{H}_{2} \mathrm{SO}_{4}$.

The CHEL-38 and the CHEL-40 resins had cobalt absorption capacities which were much better than those of the HPPR and DPAR commercial resins. Ferric ion absorption on CHEL38 and CHEL-40, moreover, were comparable to or less then that of the HPPR or the DPAR resins in the test made at $\mathrm{pH}$ 1.7. In addition, these new resins had somewhat better absorption capacities for $\mathrm{Cu}$ (II), while not absorbing Fe (III) as strongly, and were stripped and regenerated readily with 1 or $2 \mathrm{M} \mathrm{H}_{2} \mathrm{SO}_{4}$.

Using an actual copper solvent extraction raffinate solutions and very small short column tests at high flow rates were made with all the resins (except Chelex-100 and MH-13) at $\mathrm{pH}$ 2.1. With 200 bed volumes of feed solution applied at a very fast flow rate, the columns of the highly picolylated (CHEL-38) and less picolylated (CHEL-40) polyamine resins had much higher loading capacities then the commercial resins for cobalt; similar capacities for copper, nickel, zinc, and desirably lower capacities for iron. The CHEL-40 resin had the highest operating capacity for cobalt. The fast flow column tests indicate that, for recovering cobalt from $\mathrm{pH} 2.1$ mining raffinate solution, this resin should perform as well as, or better then the performance that was achieved with the DPAR resin for cobalt recovery from the effluent solutions (at $\mathrm{pH} 3$ ) of the now obsolete copper cementation recovery process [8-10].

The final resin synthesized was a less-picolylated diethylenetriamine resin (CHEL-73) which was compared with CHEL-40 resin. These resins were tested in small columns at slow (CHEL-40) and moderately fast (CHEL-73) flow rates for absorption of cobalt and copper at very low concentrations (16 and 16 ppm Co (II); 40 and 25 ppm Cu (II)) in a pH 2.1 copper raffinate solution. Tests at slow flow rates on the CHEL-40 resin showed that capacity for absorbing cobalt increased over that found in our earlier fast flow tests. However, the slow flow rates had little or no effect on the capacity for absorbing copper. The last resin, CHEL73 (which was slightly less picolylated than the CHEL-40 resin) absorbed cobalt at moderately high flow rates as well as did a lesser volume of CHEL-40 at a slow flow rate. Both resins had a much higher absorption capacity for cobalt than the commercially available chelating resin showed in our tests. Elution with $0.5-2 \mathrm{M} \mathrm{H}_{2} \mathrm{SO}_{4}$ of both the CHEL-40 and CHEL-73 resins which were loaded with appropriate volumes of raffinate feed gave main eluate fractions whose cobalt concentration was over 10 times, and the copper concentration of over 50 times those present in the raffinate feed solutions.

The carboxymethylated diethylenetriamine (CM-DER) resin showed comparable capacity for copper as did the commercial picolylamine resins. Although cobalt absorption capacity was low, this resin probably can be produced much more cheaply than the picolylamine-based resins, can be eluted and regenerated with $1 \mathrm{M}$ sulfuric acid solutions. This resin could be used for copper recovery from low-grade solutions, such as acidic mine drainage (AMD) and dump-leach solutions, that are too dilute to be treated with solvent extraction, provided iron concentration is low. 
Although picolylated polyamine (CHEL) resins have better capacity for cobalt than the currently available resins, their application for cobalt recovery as by-product from copper leach solutions is not very likely, unless a cheaper and more economical method for synthesizing picolylchoride can be developed and implemented in industrial scale. Also, these resins could find special applications, such as in purification of nickel electrolyte solutions for removing copper impurities, and recovery of nickel and cobalt from acidic pressure leach slurries in processing lateritic nickel-cobalt ores.

\section{ACKNOWLEDGMENTS}

The authors would like to acknowledge the research grant received through the Center for Advanced Separation Technologies (CAST). We are indebted to Premchendar Nandhikonda for conducting resin characterization and performance tests, and to Lynn Brandvold for her tireless efforts in editing this report.

\section{REFERENCES}

1. Shedd, K.B., Cobalt, in U.S. Geological Survey, Minerals Yearbook 2006.

2. Hodge, F.G., Cobalt and Cobalt Alloys, in Kirk \& Othmer Encyclopedia of Chemical Technology, Vol. 6, John Wiley \& Sons, Inc., 1994, pp. 760-777.

3. Kerfoot, G.E. and Weir, R.D., The Hydro and Electrometallurgy of Nickel and Cobalt, in Extractive Metallurgy of Nickel and Cobalt, Tyroler, G.P. and Landolt, C.A. (Eds.) TMS-AIME, 1988, pp.241-267.

4. Theys, L.F., Cobalt-Bearing Ores, in SME Mineral Processing Handbook, Zandon, V.A., SME-AIME, 1985, New York, NY, p.17-5.

5. Moskalyk, R.R., and Alfanzi, A.M., Review of Present Cobalt Recovery Practice, Minerals \& Metall. Process., 17 (4) 2000, pp. 205-216.

6. Wang, S., Cobalt-Its Recovery, Recycling, and Application, JOM, (10) 2006, 47-50.

7. Sole, K.C., and Hiskey, J.B., in Copper-Cobre 91, CIM, 1991, Vol. III, pp.229-243.

8. Jeffers, T.H. and Harvey, M.R., Cobalt Recovery from Copper Leach Solutions, U.S. Bureau of Mines, Report of Investigations 8927, 1985, 12 p.

9. Jeffers, T.H., Separation and Recovery of Cobalt from Copper Leach Solutions, Journal of Metals, Jan. 1985, pp. 47-50.

10. Bennet, P.G. and Jeffers, T.H., Recovery of Cobalt from Spent Copper Leach Solutions - improved Elution and Impurity Removal Techniques, Revised Process Economics, U.S. Bureau of Mines, Report of Investigations 9190, 1988, 9 p.

11. Grinstead, R.R., Selective Absorption of Copper, Nickel, Cobalt and Other Transition Metal Ions from Sufuric Acid Solutions with the Chelating Ion Exchange Resin XFS 4195, Hydrometallurgy, v. 12, 1984, pp. 387-400.

12. Grinstead, RR., Nasutavicus, W.A., Wheaton, R.M., and Jones, K.C., New Selective Ion Exchange Resins for Copper and Nickel, in Extractive Metallurgy of Copper, Yannopoulos, J.C. and Agarwal, J.C. (Eds.), TMS-AIME, 1976, vol. II, pp. 10091024.

13. Grinstead, R.R., New Developments in the Chemistry of XFS 4195 and XFS 43084 Chelating Ion Exchange Resins, in Ion Exchange Technology, Naden, D. and Streat, M. (Eds.) Soc. of Chemical Industry, Ellis Horwood Ltd., London, UK, 1984, pp. 509-518. 
14. Rosato, L., Harris, G.B. and Stanley, R.W., Separation of Nickel from Cobalt in Sulphate Medium by Ion Exchange, Hydrometallurgy, v.13, 1984, pp. 33-44.

15. Melling, J. and West, D.W., A Comparative Study of Some Chelating Ion Exchange Resins for Applications in Hydrometallurgy, in Reference 12, 1984, pp. 725-735.

16. John, L.W., Prefeasibility Process Flowsheets for Cobalt Recovery, Biometallurgical, Web Site (http://www.biomet.com.au/Extract/CoFS.htm).

17. Nicol, M.J., and Zainol, Z., The Development of a Resin-In-Pulp Process for the Recovery of Nickel and Cobalt from Leach Slurries, Int. J. Miner. Process, 72 (2003), pp. 407-415.

18. Mendes, F.D., and Martins, A.H., Selective Sorption of Nickel and Cobalt from Sulphate Solutions, ibid., 74(2004), pp. 359-371.

19. Mendes, F.D., and Martins, A.H., Selective Nickel And Cobalt Uptake From Pressure Sulfuric Acid Leach Solutions Using Column Resin Sorption, ibid., 77(2005), 53-63.

20. Mendes, F.D., and Martins, A.H.,Recovery of Nickel and Cobalt from Acid Leach Pulp by Ion Exchange using Chelating Resins, Minerals Engineering, 18 (2005), pp. 945-954.

21. Kennedy, B., Davenport, W.G., Jenkins, J., and Robinson, T., Electrolytic Copper Electrowinning and Solvent Extraction - World Operating Data, in Copper Leaching, Solvent Extraction, and Electrowinning Technology, Jergensen, G.W.II, (Ed.), SME, 1999, pp. 41-87.

22. Grinstead, R.R., Copper-Selective Ion-Exchange Resin with Improved Iron Rejection, Journal of Metals, March 1979, pp.13-16.

23. Jones, K.C., and Pyper, R.A., Copper Recovery from Acidic Leach Liquors by Continuous Ion-Exchange and Electrowinning, Journal of Metals, April, 1979, 19-25.

24. Rossiter, G.J., and Carey, K.C., Copper Recovery from Leach Liquors Using Continuous Ion Exchange, Proc. Copper Hydromet Roundtable '98, Randol Intern., Vancouver, 1998. Paper available at the Dow Chemical Co. Web site (http://dow.com/liquidpeps/pc/pt/cu.htm).

25. Harland, C.E., Ion Exchange Theory and Practice, $2^{\text {nd }}$ edition, 1994, pp. 78-79.

26. Young, R.S., Industrial Inorganic Analysis, London, Chapman and Hall, 1953.

27. Laitinen, H.A., and Burdett, L., Anal. Chem., 23 (1951) 1268.

28. Young, R. S., Chemical Analysis in Extractive Metallurgy, Barnes \& Noble, 1971. p178.

29. Mixer and Dubois, J. Am. Chem. Soc., 17 (1895) 405.

30. Mendham, J., Denny, R.C., Barnes, J. D., and Thomas, M/J.K., Vogel's Quantitative Chemical Analysis, $6^{\text {th }}$ edition, 2002, p.383.

\section{BIBLIOGRAPHY}

Premchendar Nandhikonda, M.Sc. Thesis, (Nov. 2005), "Preparation, Characterization, and Performance of Several New Chelating Resins for Recovery of Cobalt and Copper from Acidic Mining Solutions”, New Mexico Institute of Mining and Technology, Chemistry Dept, Socorro, New Mexico, 87801 USA 


\title{
LIST OF ACRONYMS AND ABBREVIATIONS
}

\author{
AAS - Atomic Absorption Spectrophotometry \\ AMD - Acid Mine Drainage \\ BV - Bed Volume \\ CCD - Counter Current Decantation \\ CHEL - Picolylated dithylenetriamine resin designation \\ Chelex-100- Commercial iminodiacetic ion exchange acid resin \\ CM-DER - Carboxymethylated dithylenetriamine resin \\ DPAR - Dipicolylated polyamine resin (bis-picolylamine) \\ Dowex-1 - Commercial quaternary amine ion exchange resin \\ Dowex-M4195 - Commercial bis-picolylamine ion exchange resin \\ EW - Electro-winning \\ GPM - Gallons per minute \\ HPPR - Hydroxypropyl picolylamine resin \\ IDA - Iminodiacetic acid \\ IX - Ion Exchange \\ MH-13 - Thiosuccinic acid resin \\ PLS - Pregnant leach solution \\ USBM - The U.S. Bureau of Mines
}


Appendix 24: Development of Enhanced Surfactants for the Potash Industry (NM003) 


\section{FINAL TECHNICAL REPORT}

Contract Title and Number:

Crosscutting Technology Development at the Center for

Advanced Separation Technologies

(DE-FC26-02NT41607)

Sub-Recipient Project Title:

Development of Enhanced Surfactants for the Potash Industry

Principal Investigators:

Bond, Hockensmith

Contact Address:

Department: Mat. \& Met. Engineering

New Mexico Tech

Socorro NM 87801

Subcontractor Address:

No subcontracts issued.
Period of Performance:

Starting Date: $4 / 1 / 03$

Ending Date: $11 / 30 / 07$
Report Information:

Type: Final

Number:

Period: $\quad 4 / 1 / 03-11 / 30 / 05$

Date: $\quad 9 / 21 / 06$

Code: NM003-Final

Contact Information:

Phone: (505) 835-5653

Fax: (505) 835-6333

E-Mail: gbond@nmt.edu

Subcontractor Information:

Phone:

Fax:

E-Mail: 


\section{DISCLAIMER}

This report was prepared as an account of work sponsored by an agency of the United States Government. Neither the United States Government nor any agency thereof, nor any of their employees, make any warranty, express or implied, nor assume any legal liability or responsibility for the accuracy, completeness, or usefulness of any information, apparatus, product, or process disclosed, or represents that its use would not infringe privately owned rights. Reference herein to any specific commercial product, process, or service by trade name, trademark, manufacturer, or otherwise does not necessarily constitute or imply endorsement, recommendation, or favoring by the United States Government or any agency thereof. The views and opinions of authors expressed herein do not necessarily state or reflect those of the United States Government or agency thereof.

\section{ABSTRACT}

Two alkyl diphenyl oxide (ADO) surfactants have been used to enhance the size, size distribution, and morphology of crystals of potassium sulfate $\left(\mathrm{K}_{2} \mathrm{SO}_{4}\right)$. The surfactants used are Calfax 16L-35 and Calfax DB-45 (Pilot Chemical Company). Calfax 16L-35 is a sodium hexadecyl diphenyl oxide disulfonate, while Calfax DB-45 is a sodium dodecyl diphenyl oxide disulfonate. The use of small concentrations of these surfactants added to mother liquor can improve both the crystal size and the crystal size distribution (CSD) with regard to the production of granular crystals for use in fertilizer. Both surfactants have demonstrated the ability to limit nucleation and increase crystal growth, thus narrowing the CSD during crystallization. A problematic variability in the results obtained has been addressed through careful study and refinement of the cooling regime during crystallization.

\section{TABLE OF CONTENTS}

\begin{tabular}{|l|c|}
\hline Heading & Page \\
\hline Title Page & 1 \\
\hline Disclaimer & 2 \\
\hline Abstract & 2 \\
\hline Table of Contents & 2 \\
\hline Introduction & 3 \\
\hline Executive Summary & 4 \\
\hline Experimental & 5 \\
\hline Results and Discussion & 8 \\
\hline Conclusion & 16 \\
\hline References & 17 \\
\hline List of Acronyms and Abbreviations & 17 \\
\hline
\end{tabular}




\section{INTRODUCTION}

Potassium sulfate $\left(\mathrm{K}_{2} \mathrm{SO}_{4}\right)$ is an important nutrient for plants because of the potassium content, and is essential for chloride-sensitive crops. It also provides soluble sulfur that is an essential secondary nutrient for improvement of root growth and seed production. More than $99 \%$ of the worldwide production of potassium sulfate is used in agriculture.

Potassium sulfate is produced from ores such as sylvinite $(\mathrm{KCl} \cdot \mathrm{NaCl})$, schoenite $\left(\mathrm{K}_{2} \mathrm{SO}_{4} \bullet \mathrm{MgSO}_{4} \bullet 6 \mathrm{H}_{2} \mathrm{O}\right)$, and langbeinite $\left(\mathrm{K}_{2} \mathrm{SO}_{4} \cdot 2 \mathrm{MgSO}_{4}\right)$ (Kirk and Othmer, 1991). Langbeinite ore is mined in New Mexico; it contains $18.8 \mathrm{wt} \%$ potassium, $11.7 \mathrm{wt} \%$ magnesium, and $23.0 \mathrm{wt} \%$ sulfur. Potassium chloride is reacted with hydrated langbeinite to produce potassium sulfate. The simplified reaction for this process is:

$$
\mathrm{K}_{2} \mathrm{SO}_{4} \cdot 2 \mathrm{MgSO}_{4}+4 \mathrm{KCl} \rightarrow 3 \mathrm{~K}_{2} \mathrm{SO}_{4}+2 \mathrm{MgCl}_{2}
$$

Pulverized langbeinite is hydrated at $50^{\circ} \mathrm{C}$ and hydrated langbeinite is reacted with aqueous potassium chloride solution. Potassium sulfate cake is removed from the process, filtered, and dried. Excess potassium chloride is crystallized, filtered, and then recycled back to the reaction tanks.

Potassium sulfate products are categorized as: "granular", with a size range of 0.85 $\mathrm{mm}$ to $3.35 \mathrm{~mm}$ (No. 20 to No. 6 screen); "standard" with a size range of $0.212 \mathrm{~mm}$ to 1.18 $\mathrm{mm}$ (No. 70 to No. 16 screen), and "special standard", with a size range of $0.075 \mathrm{~mm}$ to $0.425 \mathrm{~mm}$ (No. 200 to No. 40 screen). [The screen sizes refer to the ASTM standard sieve series and Tyler Equivalents Measurement E-11-70.] The small crystal fines of potassium sulfate produced during crystallization have low profitability, since they require further compaction and crystallization. For the fertilizer industry, coarse granular grades of potassium sulfate within the size range of $1.40 \mathrm{~mm}$ to $3.35 \mathrm{~mm}$ (No. 14 to No. 6 screen), hereinafter described as "granular crystals", command the highest premium. Multiple filtering steps are required to separate the fines from these coarser granular crystals. Profitability will be enhanced if the CSD (crystal size distribution formed during crystallization) can be narrowed to within this range of screen or "mesh" sizes. The goal of this research has been to increase the size and narrow the CSD of potassium sulfate crystals through the addition of surfactants to the mother liquor. Surfactants adsorb onto the surfaces of the newly formed crystals, affecting both nucleation and growth.

The addition of alkyl diphenyl oxide (ADO) surfactants to potassium sulfate mother liquor can reduce nucleation and increase crystal size (Gamble 2005). ADO surfactants are anionic surfactants, and both the linear-chain and branched-chain forms enhance crystal growth.

Aoun et al. (1999) describe a commonly used approach to define the crystal growth rate as the variation of the solution supersaturation with time. This technique is recommended when a rapid estimation of crystal growth rate is required. 


\section{EXECUTIVE SUMMARY}

The majority of the potassium sulfate presently produced is spread as fertilizer, and for this process farmers require a granular product with a suitable crystal size distribution. Coarse granular grades of potassium sulfate within the size range of $1.40 \mathrm{~mm}$ to $3.35 \mathrm{~mm}$ (No. 14 to No. 6 screen), hereinafter described as "granular crystals", command the highest premium. Multiple filtering steps are required to separate the fines from these coarser granular crystals. Hence profitability will be enhanced if the CSD can be narrowed to within this range of sizes. The present aim was to accomplish this through the use of surfactants, particularly alkyl diphenyl oxide surfactants.

It has been shown that both the crystal size and the CSD of potassium sulfate crystallized from potassium sulfate/magnesium sulfate brine at $40^{\circ} \mathrm{C}$ can be changed markedly by the addition of Calfax 16L-35 (a linear sodium hexadecyl diphenyl oxide disulfonate surfactant) and Calfax DB-45 (a branched sodium dodecyl diphenyl oxide disulfonate surfactant) at concentrations well below the critical micelle concentration (CMC).

Following some early modification of the experiments to reduce contamination, initial results indicated that the ratio of granular particles to fines is greatly improved by the surfactant additions. Crystals formed from the mother liquor with no surfactant at $40^{\circ} \mathrm{C}$ have a tendency to clump together, are very brittle, and have sizes ranging from $\sim 10 \mu \mathrm{m}$ to 2.5 $\mathrm{mm}$. Addition of Calfax $16 \mathrm{~L}-35$ at the CMC resulted in crystal sizes ranging from $\sim 6 \mu \mathrm{m}$ to $2 \mathrm{~mm}$, but the proportion of the mm-scale crystals to the $\mu \mathrm{m}$-scale fines was increased, an encouraging result. Lower concentrations of Calfax 16L-35 were tried. Crystals grown from solution containing $25 \%$ of the $\mathrm{CMC}$, for example, were much more uniform in size, (as seen in Figure $6 \mathrm{~b}$ below); in fact the size range obtained $(2 \mathrm{~mm}$ to $3 \mathrm{~mm})$ was that desired. The yield, however, was too low for industrial application. Encouraging early results were also obtained with Calfax DB-45.

Although the total crystal yield was reduced by the surfactant additions, appropriate amounts of surfactant appeared to result in very substantial increases in the yields of crystals in a desirable, marketable size range. However, in the course of experiments to demonstrate reproducibility and to optimize the surfactant concentrations, a problem was encountered. The results obtained showed unacceptable variability. The inconsistency of the data and inability to control reproducibly the shapes, sizes, and yields of the crystals grown led to the study of other parameters, in particular, the detailed characteristics of the cooling regime during crystallization. For similar nominal settings, variations could be observed in actual cooling rate, and also the extent and duration of "overshoot" at the end of the cooling process. Each of these factors could influence nucleation and growth. Experiments were therefore performed to find a cooling/heating process (with emphasis on the lowest temperature reached during cooling and the time spent at that temperature, as well as on cooling rate) that would reproducibly give granular crystals in the presence of the ADO surfactants. These experiments spanned both the end of the work performed under contract NM003, and the start of the work performed under the separate but related contract NM005. Thus the final solution to the problem appears in the report for NM005. 


\section{EXPERIMENTAL}

Dash and Rohani (1993) used a mixed-suspension, mixed-product removal (MSMPR) crystallizer in studies of crystal size and growth rate of $\mathrm{KCl}$. For the present work, a batch crystallizer has been developed from a modified version of their design. It is shown schematically in Figure 1.

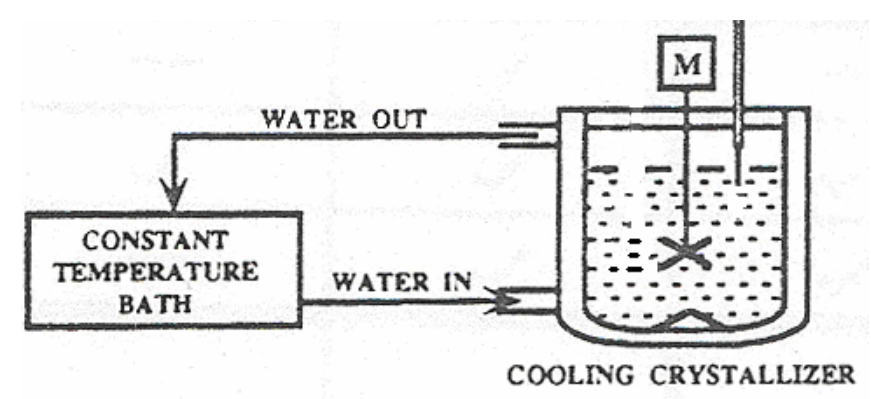

Figure 1: Schematic diagram of batch crystallizer.

In the crystallizer one liter of nanopure water is heated to $80^{\circ} \mathrm{C}$ in a Neslab RTE-7 Digital One Refrigerated cooling/heating bath. The crystallizer is a one-liter, glass-jacketed reactor with four baffles, (Wilmad-LabGlass). The four baffles located on the inside of the inner wall of the crystallizer improve the mixing of the solution by breaking up the flow of the solution inside the crystallizer. As the water is heating, a four-opening, flat flange closure is clamped on top of the unit and a three-bladed, 2.5-inch propeller is inserted through the top of the crystallizer. The propeller is turned by an Arrow 1750 (1/15 hp) electric motor. The impeller opening, located in the middle of the flange cover, is covered with aluminum foil to reduce the amount of vapor escaping. The speed of the electric motor is set to approximately $400 \mathrm{rpm}$, controlled by an integral power source. A thermometer is inserted into one of the four openings at the top of the crystallizer to measure the temperature of the solution. The remaining two openings are closed off with rubber stoppers and aluminum foil.

When the nanopure water has reached $80^{\circ} \mathrm{C}$, surfactant (when used, as described below) is added to the water, followed by 190.3 grams of $99 \%$ potassium sulfate $\left(\mathrm{K}_{2} \mathrm{SO}_{4}\right.$, $1.09 \mathrm{M}$ ) (from Sigma-Aldrich) and 9.9 grams of $99.5 \%$ magnesium sulfate heptahydrate $\left(\mathrm{MgSO}_{4} \bullet 7 \mathrm{H}_{2} \mathrm{O}, 0.04 \mathrm{M}\right)$ (from Fisher Scientific). The reagents are stirred in the crystallizer at $80^{\circ} \mathrm{C}$ for one hour to dissolve them completely. The solubility limit of potassium sulfate in water is $214 \mathrm{~g} / \mathrm{L}$ at $80^{\circ} \mathrm{C}$ (Linke 1965). The mother liquor becomes saturated with $\mathrm{K}_{2} \mathrm{SO}_{4}$ at $67^{\circ} \mathrm{C}$. The solubility limit of magnesium sulfate heptahydrate is $710 \mathrm{~g} / \mathrm{L}$ at $20^{\circ} \mathrm{C}$ and $910 \mathrm{~g} / \mathrm{L}$ at $40^{\circ} \mathrm{C}$. Hence the mother liquor remains undersaturated with respect to magnesium sulfate heptahydrate throughout the experiments, whereas it is supersaturated with respect to potassium sulfate at $40^{\circ} \mathrm{C}$.

After one hour at $80^{\circ} \mathrm{C}$, a $5 \mathrm{~mL}$ sample is removed from the crystallizer with a $10 \mathrm{~mL}$ graduated pipette. The sample is filtered into a $20 \mathrm{~mL}$ glass vial through a $12 \mathrm{~mL}$ Whatman autovial syringeless filter with a $0.2 \mu \mathrm{m}$ polytetrafluoroethylene filter. A $50 \mu \mathrm{L}$ sample is 
pipetted from the vial with an automatic pipetter, added to a volumetric flask, and diluted to $10 \mathrm{~mL}$ with nanopure water for later analysis by ion chromatography (IC). After the first sample is taken, the cooling/heating bath is lowered to $40^{\circ} \mathrm{C}$ (mean cooling rate of 0.65 ${ }^{\circ} \mathrm{C} / \mathrm{min}$ ). After the solution reaches $40^{\circ} \mathrm{C}$, another $5 \mathrm{~mL}$ sample is removed from the crystallizer and the filtering and dilution process described above is repeated. This procedure is continued for an hour and a half with samples taken every fifteen minutes. A total of eight samples are taken from each crystallizer batch.

In non-control experiments, various concentrations of Calfax surfactants from Pilot Chemical Company are added to the nanopure water at $80^{\circ} \mathrm{C}$ prior to the other reagents. The general structure of the Calfax surfactants is shown in Figure 2. Details of each surfactant, including the carbon chain length and chain type (linear or branched), are given in Table 1.

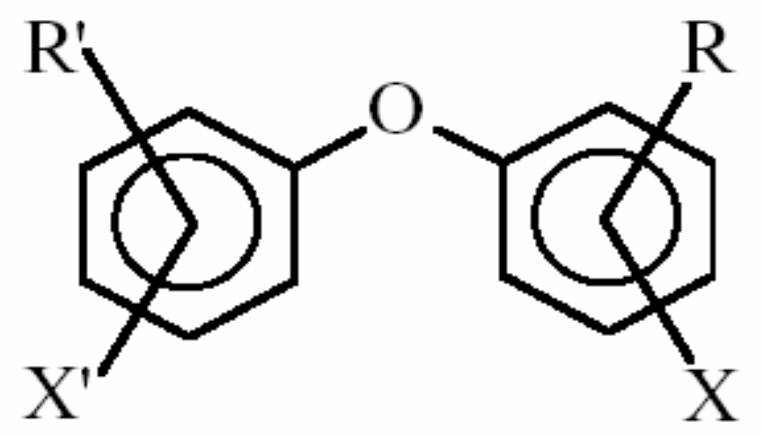

Figure 2: General chemical structure of Calfax surfactants (Pilot Chemical Company, 2005).

\begin{tabular}{|c|c|c|c|c|c|c|c|}
\hline $\begin{array}{c}\text { Surfactant } \\
\text { name }\end{array}$ & $\begin{array}{c}\text { Chemical name } \\
\text { of surfactant }\end{array}$ & $\mathbf{R}$ & $\mathbf{R}^{\prime}$ & $\begin{array}{c}\mathbf{X} \text { and } \\
\mathbf{X}^{\prime}\end{array}$ & $\begin{array}{c}\text { MW } \\
\text { (g/mol) }\end{array}$ & $\begin{array}{c}\text { Chain } \\
\text { length }\end{array}$ & $\begin{array}{c}\text { Chain } \\
\text { type }\end{array}$ \\
\hline $\begin{array}{c}\text { Calfax } \\
16 \mathrm{~L}-35\end{array}$ & $\begin{array}{c}\text { Sodium hexadecyl } \\
\text { diphenyl oxide } \\
\text { disulfonate }\end{array}$ & $\mathrm{Alkyl}$ & $\mathrm{H}$ & $\mathrm{SO}_{3} \mathrm{Na}$ & 608 & 16 & Linear \\
carbons & & $\mathrm{H}$ & $\mathrm{SO}_{3} \mathrm{Na}$ & 547 & $\begin{array}{c}12 \\
\text { carbons }\end{array}$ & Branched \\
\hline $\begin{array}{c}\text { Calfax } \\
\text { DB-45 }\end{array}$ & $\begin{array}{c}\text { Sodium dodecyl } \\
\text { diphenyl oxide } \\
\text { disulfonate }\end{array}$ & $\mathrm{Alkyl}$ & & & \\
\hline
\end{tabular}

Table 1: Table of Calfax surfactants (Pilot Chemical Company, 2005).

The ADO surfactants, Calfax 16L-35 and Calfax DB-45, were acquired from Pilot Chemical Company. The critical micelle concentration (CMC) of Calfax 16L-35 surfactant is $0.500 \times 10^{-3} \mathrm{~mol} / \mathrm{L}$ while that of Calfax DB-45 is $0.661 \times 10^{-3} \mathrm{~mol} / \mathrm{L}$ (Pilot Chemical Company, 2005). [The CMC is the concentration of a surfactant at which the surfactant molecules aggregate to form spherical micelle structures. At concentrations greater than the $\mathrm{CMC}$, not only does the amount of surfactant in free solution no longer increase with further additions but also surfactant aggregates are now present in the solution.] 
The surfactant and reagents are stirred in the crystallizer at $80^{\circ} \mathrm{C}$ for one hour to dissolve them. Sampling for IC is performed after one hour, in the same manner as for the surfactant-free mother liquor. After the first sample is taken, the cooling/heating bath is lowered to $40^{\circ} \mathrm{C}$ (mean cooling rate of $0.65{ }^{\circ} \mathrm{C} / \mathrm{min}$ ). As with the mother liquor, once the solution reaches $40^{\circ} \mathrm{C}$, another $5 \mathrm{~mL}$ sample is taken, and sampling continues to be performed every fifteen minutes for an hour and a half. Again, a total of eight samples are taken from each crystallizer batch. After the sampling is completed, the solution and crystals grown are filtered to measure the crystal yield and CSD.

At the end of each run, the crystals formed in the crystallizer are filtered through two Whatman \#2 filter papers in a $110 \mathrm{~mm}$ Buchner funnel. The crystals on the filter paper are then dried and weighed on an analytical scale. Once the crystal yield has been determined, the crystals are removed from the filter paper and placed in a sieve stack. The crystals that comprise the granular product most useful to the fertilizer industry are those retained in the sieves with mesh sizes of $1.4 \mathrm{~mm}$ (No. 14 screen), $2 \mathrm{~mm}$ (No. 10 screen), and $3.35 \mathrm{~mm}$ (No. 6 screen). The crystals are removed from each sieve and weighed on an analytical scale in order to find the CSD. The sieves, pan, and cover are then rinsed with nanopure water and put in an oven at $102^{\circ} \mathrm{C}$ for an hour to dry.

The diluted samples of mother liquor or mother liquor with surfactant, taken from each experiment, are analyzed for potassium and magnesium cations with an ion chromatograph (IC) (Dionex DX-500). The IC separation column is a Dionex CS12A (4 x $250 \mathrm{~mm}$ ) column. The software used to collect the output from the conductivity cell is Peaknet Version 5.1 from Dionex.

To determine the concentration of the diluted samples, standards are made for the potassium and magnesium ions with $\mathrm{K}_{2} \mathrm{SO}_{4}$ and $\mathrm{MgSO}_{4} \bullet 7 \mathrm{H}_{2} \mathrm{O}$ dissolved in nanopure water. The standard concentrations for the potassium ion are: $3.2 \times 10^{-4} \mathrm{M}, 6.4 \times 10^{-4} \mathrm{M}, 1.3 \times 10^{-3} \mathrm{M}$, $3.2 \times 10^{-3} \mathrm{M}, 6.4 \times 10^{-3} \mathrm{M}, 9.6 \times 10^{-3} \mathrm{M}$, and $1.2 \times 10^{-2} \mathrm{M}$. The standard concentrations for the magnesium ion are $4.1 \times 10^{-4} \mathrm{M}, 1.0 \times 10^{-3} \mathrm{M}, 1.4 \times 10^{-3} \mathrm{M}, 2.1 \times 10^{-3} \mathrm{M}, 2.7 \times 10^{-3} \mathrm{M}$, $3.3 \times 10^{-3} \mathrm{M}$, and $4.1 \times 10^{-3} \mathrm{M}$. These standards are measured on the IC, and the peak area for each standard is added to a Microsoft Excel spreadsheet and plotted as peak area versus concentration. From the plot, the best-fit line equation is calculated in Microsoft Excel, for each set of standards.

The diluted samples of mother liquor or mother liquor with surfactant from the crystallizer are measured on the IC. The peak area for each sample is put into the best-fit line equations from the standards to determine the concentration of the ions in the diluted samples. Once the concentrations of the ions are determined for the samples, they are plotted in Excel as a function of the time when the sample was removed from the crystallizer.

A field-emission scanning electron microscope (FESEM, Hitachi Model S-800) with a Crewe field-emission electron gun is used to obtain magnified images of crystals. The instrument is run at an accelerating voltage of $15 \mathrm{kV}$. The crystals are mounted on one-inchdiameter stainless-steel mounting posts with double-faced graphite tape. To produce the 
conductivity needed for the FESEM, the crystals, graphite tape, and mounting posts are sputtered with platinum.

X-ray diffraction (XRD) characterization was used in some of the experiments to confirm that similar phases are precipitated with or without the presence of surfactant. Crystals were ground to a fine powder, and examined with a standard powder diffractometer (Siemens Model D-500: Cu, $30 \mathrm{~mA}$ and $40 \mathrm{kV}$ ). All data were saved to JADE 3.1 for analysis of the peaks and d-spacings, and the spectra were identified from the powder diffraction files. The product was found to comprise arcanite $\left(\mathrm{K}_{2} \mathrm{SO}_{4}\right)$ and langbeinite.

\section{RESULTS AND DISCUSSION}

\section{$\underline{\text { Control runs }}$}

Control experiments were first completed, in which potassium sulfate crystals were grown without the addition of a surfactant. The mother liquor was cooled from $80^{\circ} \mathrm{C}$ to $40^{\circ} \mathrm{C}$ over a period of approximately sixty minutes. IC results showed a decrease in potassium ion concentration in the mother liquor by the end of this period (indicating that crystallization had already commenced), decreased for a further twenty minutes (until eighty minutes after the start of cooling), and then leveled out (Figure 3).

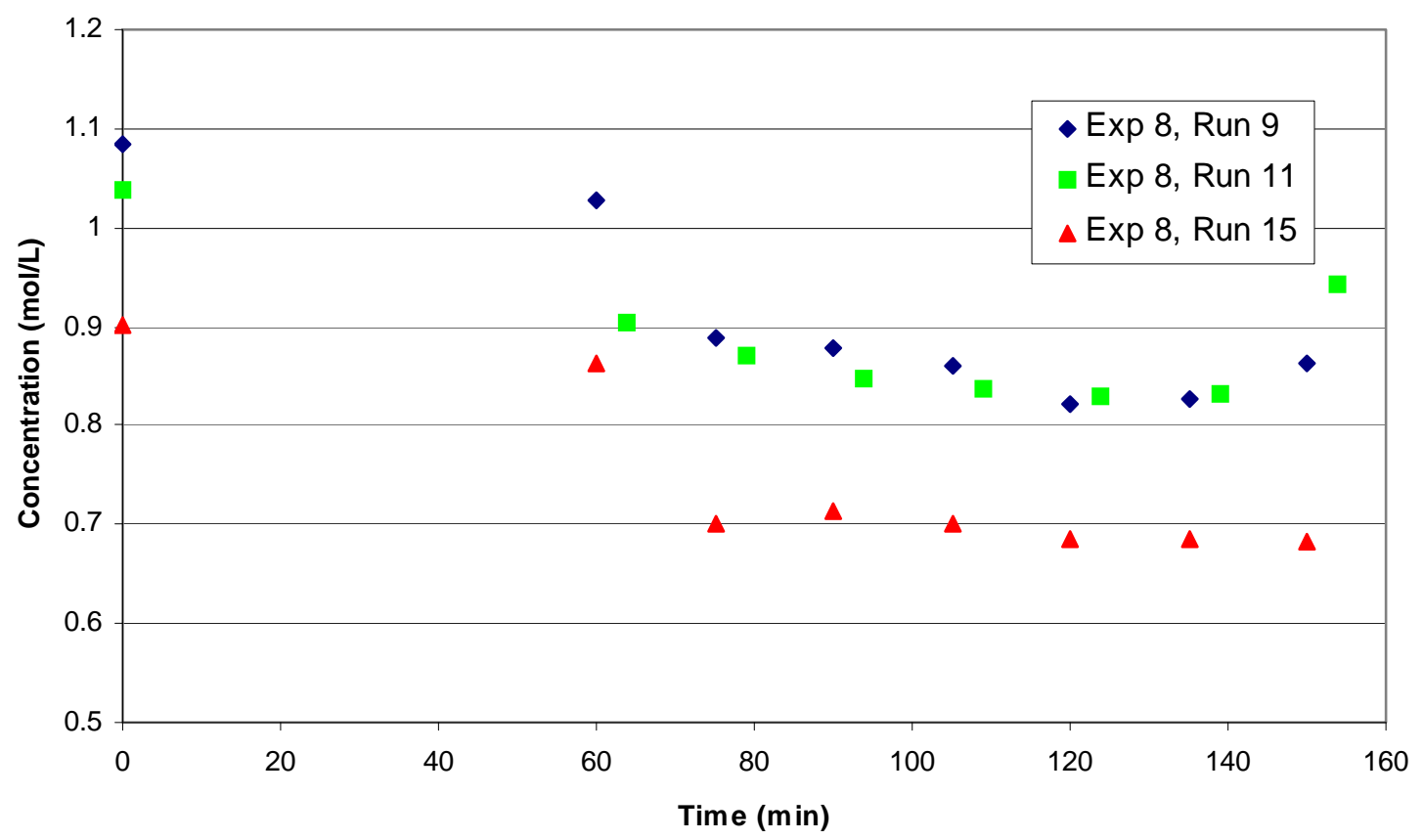

Figure 3: Potassium ion concentration as a function of time for three control runs.

In earlier runs, there was some variability in the height of the curves, with starting concentrations sometimes lower than the initial reagent concentration of $1.09 \mathrm{~mol} / \mathrm{L}$ (as seen in Figure 3). It is believed that this was caused by temperature drops during sampling that 
allowed the formation, prior to dilution of the solution, of crystals that were not included in the diluted "mother liquor" samples run on the IC. In order to avoid this temperature decrease and crystal growth in later experiments, the solution filters were preheated to the temperature of the solution, as was the nanopure water used to dilute the samples. This change in the method limited the amount of crystallization that occurred before IC analysis and resolved the problem. As expected, magnesium ion concentrations remained unchanged. The data indicated that the crystallization was always substantially complete at the end of eighty minutes from the start of cooling.

The theoretically possible yield from the potassium sulfate mother liquor without surfactant at $40^{\circ} \mathrm{C}$ was 42.30 grams. There was some variability in the crystal yield (and percent of theoretically possible yield obtained) from different experiments, as seen in Table 2 (which gives the data corresponding to the curves in Figure 3).

\begin{tabular}{|l|c|c|c|}
\hline $\begin{array}{c}\text { Experiment } \\
\text { number }\end{array}$ & $\begin{array}{c}\text { Theoretical } \\
\text { crystal yield } \\
\text { (g) }\end{array}$ & $\begin{array}{c}\text { Actual } \\
\text { crystal yield } \\
\text { (g) }\end{array}$ & $\begin{array}{c}\text { Percent } \\
\text { yield (\%) }\end{array}$ \\
\hline Exp. 8, run 9 & 42.30 & 39.36 & 93.05 \\
\hline Exp. 8, run 11 & 42.30 & 17.52 & 41.41 \\
\hline Exp. 8, run 15 & 42.30 & 28.40 & 67.14 \\
\hline
\end{tabular}

Table 2: Crystal yields of potassium sulfate crystal from runs without surfactant corresponding to the curves in Figure 3.

FESEM examination revealed faceted crystals with extensive agglomeration, as seen in Figures 4 and 5.

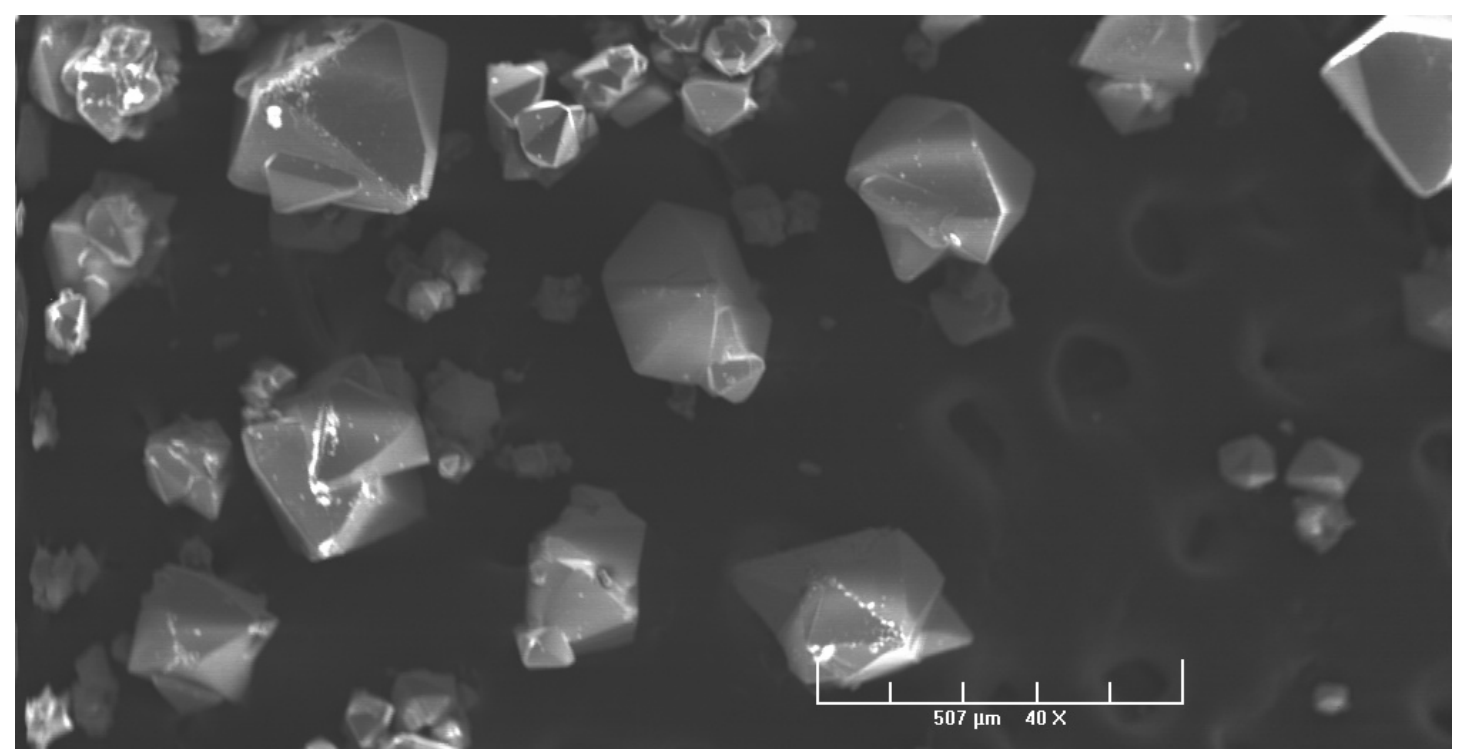

Figure 4: FESEM micrograph of potassium sulfate crystals grown without surfactant. 


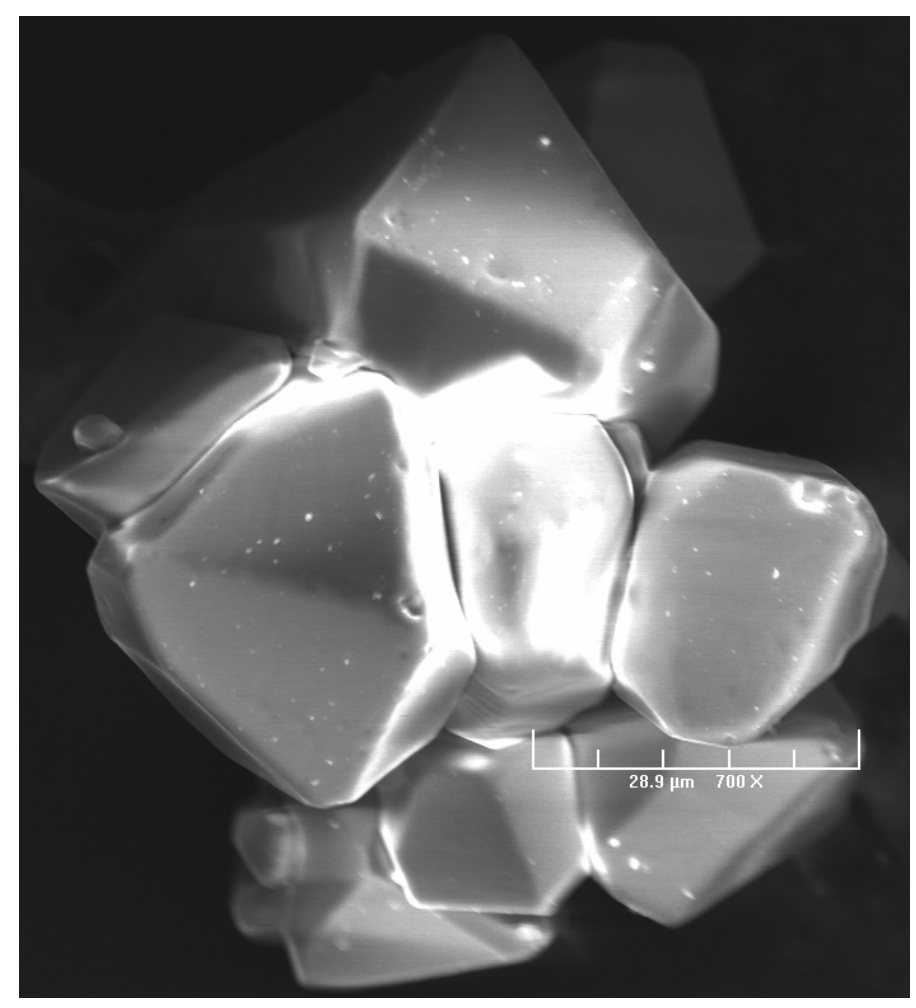

Figure 5: FESEM micrograph of a crystal agglomerate grown without surfactant.

\section{Crystallization with Calfax 16L-35}

Early experiments were performed with concentrations that were close to or larger than the CMC. At these higher concentrations, Calfax 16L-35 surfactant was found to inhibit crystallization considerably, producing very low yields of crystals. Experiments were then conducted with a range of lower concentrations. At $25 \%$ of the $\mathrm{CMC}$, a significant increase was observed in not only the uniformity but also the size of the crystals produced, but the crystal yield was markedly smaller than when no surfactant was added. IC data indicated that the crystallization at $40^{\circ} \mathrm{C}$ in the presence of the surfactant was essentially complete at a time of two hours after the start of cooling (compared to eighty minutes in the absence of surfactant).

The crystals produced from the mother liquor with no surfactant had a wide variation in sizes, were quite brittle, and tended to clump together into large aggregates. Some individual crystals measured $\sim 2 \mathrm{~mm}$, but there were many fines down to $\sim 10 \mu \mathrm{m}$ in width. Crystals grown in the presence of the Calfax 16L-35 surfactant, however, were much larger with a narrower CSD, and did not adhere to one another to form aggregates. The crystals are illustrated in Figure 6 . The results indicated that addition of suitable concentrations of Calfax 16L-35 surfactant held promise for the production of granular crystals. 


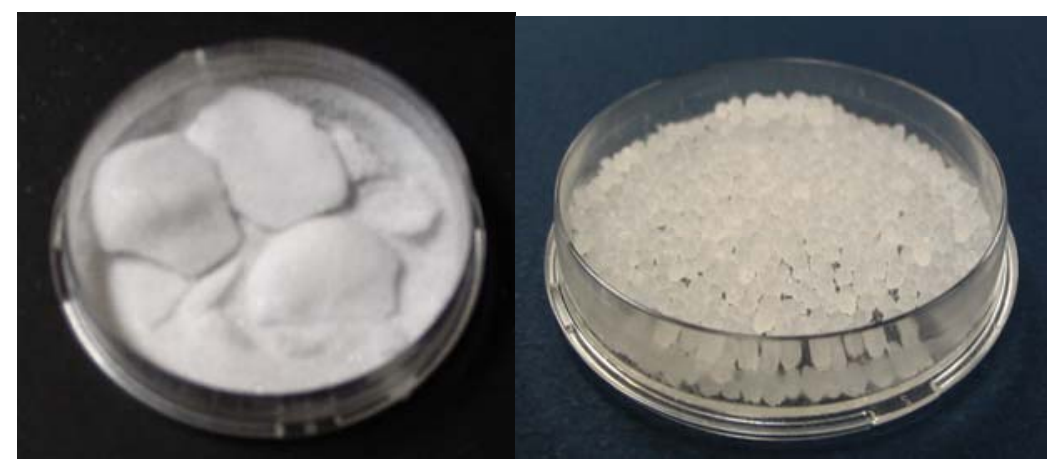

Figure 6: Potassium sulfate crystals grown a) without addition of surfactant, and $b$ ) in the presence of $25 \% \mathrm{CMC}$ of $16 \mathrm{~L}-35$.

The crystals formed with $25 \%$ CMC of surfactant were in the size range $2-3 \mathrm{~mm}$, with essentially no fines. Reduction of the surfactant concentration to $23 \%$ of the CMC increased the yield considerably. The crystal size distribution also became broader with sizes ranging from $\sim 0.5$ to $3 \mathrm{~mm}$, although still without the fines seen in the absence of surfactant. Typical crystals are shown in Figure 7.

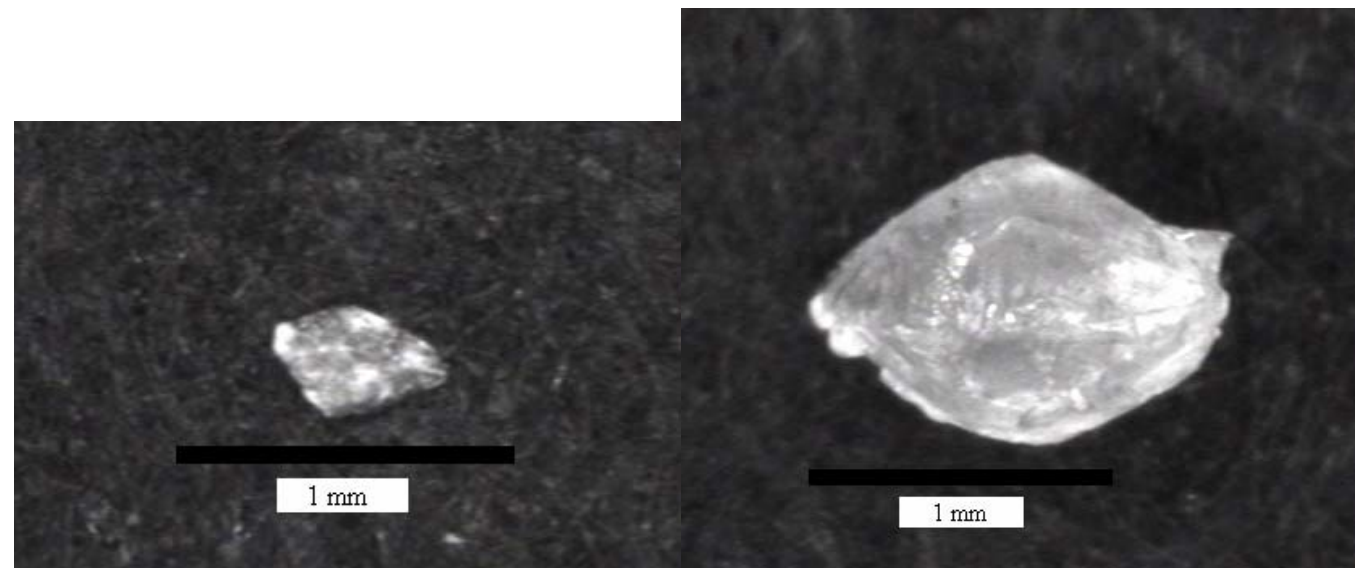

Figure 7: Potassium sulfate crystals grown in the presence of 23\% CMC of Calfax 16L-35.

Further experiments were performed with a range of modest surfactant concentrations in the hopes of optimizing the concentration and demonstrating repeatability. Unfortunately, while some runs produced larger amounts of granular crystals, the results were erratic. One run might produce a large crystal yield whereas the next run completed under the same nominal conditions might produce a much lower crystal yield, or a lower percentage of granular crystals. Some examples are given below of sets of results for surfactant concentrations that included the most promising results for some individual runs, but also exemplify the problem of variability in the results.

Five runs were completed with $31 \%$ of the CMC of Calfax $16 \mathrm{~L}-35(0.27 \mathrm{~g} / \mathrm{L})$. The crystals produced from these studies were more rounded in appearance than the faceted crystals from the mother liquor without surfactant. There was also no agglomeration of crystals. The crystal yields from these runs are shown in Table 3. The range of the total 
crystal yields was 8.75 grams to 11.78 grams. The range of crystal yields within the desired granular size range of 1.40 to $3.35 \mathrm{~mm}$ was 1.06 to 7.86 grams. The run that gave the lowest crystal yield within the granular size range (1.06 grams) also gave one of the higher total crystal yields (11.57 grams). This suggests that there was more extensive nucleation occurring during the early stages of this run, resulting in a larger number of smaller crystals. The percentage of the total crystal yields that was within the desired size range varied from $9.1 \%$ to $80.7 \%$.

\begin{tabular}{|c|c|c|c|}
\hline $\begin{array}{c}\text { \% of } \\
\text { CMC }\end{array}$ & $\begin{array}{c}\text { Total crystal } \\
\text { yield (g) }\end{array}$ & $\begin{array}{c}\text { Crystal yield in } \\
\text { size range of 1.40 } \\
\text { to } 3.35 \mathbf{~ m m ~ ( g ) ~}\end{array}$ & $\begin{array}{c}\text { Percent crystal yield } \\
\text { in size range of 1.40 } \\
\text { to 3.35 mm (\%) }\end{array}$ \\
\hline $31 \%$ & 8.75 & 5.58 & 63.8 \\
\hline $31 \%$ & 11.57 & 1.06 & 9.2 \\
\hline $31 \%$ & 9.74 & 7.86 & 80.7 \\
\hline $31 \%$ & 11.78 & 7.67 & 65.1 \\
\hline $31 \%$ & 11.62 & 4.38 & 37.7 \\
\hline
\end{tabular}

Table 3: Total crystal yields and crystal yields within the desired size range grown in the presence of $31 \%$ CMC of Calfax 16L-35.

Four runs were completed with $33 \%$ of the CMC of Calfax $16 \mathrm{~L}-35(0.28 \mathrm{~g} / \mathrm{L})$. The crystals produced from these runs were again more rounded, with no agglomeration in two of the runs and some agglomerates of smaller crystals in the other two runs. The crystal yields from the runs are shown in Table 4. The range of total crystal yields was 5.37 to 12.48 grams, while that of crystal yields within the desired granular size range was 4.80 grams to 8.83 grams. More than $70 \%$ of the crystals produced in each of these runs were within the desired size range, but the total yields were variable.

\begin{tabular}{|c|c|c|c|}
\hline $\begin{array}{c}\text { \% of } \\
\text { CMC }\end{array}$ & $\begin{array}{c}\text { Total crystal yield } \\
\text { (g) }\end{array}$ & $\begin{array}{c}\text { Crystal yield in size } \\
\text { range of 1.40 to 3.35 } \\
\mathbf{m m} \text { (g) }\end{array}$ & $\begin{array}{c}\text { Percent crystal yield } \\
\text { in size range of 1.40 } \\
\text { to } 3.35 \text { mm (\%) }\end{array}$ \\
\hline $33 \%$ & 12.48 & 8.83 & 70.8 \\
\hline $33 \%$ & 6.22 & 5.62 & 90.4 \\
\hline $33 \%$ & 5.37 & 4.80 & 89.4 \\
\hline $33 \%$ & 6.17 & 5.30 & 85.9 \\
\hline
\end{tabular}

Table 4: Total crystal yields and crystal yields within the desired size range grown in the presence of 33\% CMC of Calfax 16L-35. 
Six runs were completed with $34 \%$ of the CMC of Calfax 16L-35 (0.29 g/L). Three of these runs produced more rounded crystals with no agglomeration, and the crystal fines shared the same morphology. With regard to the other three runs: in one the crystals were somewhat less rounded, and agglomerates were also present; another resulted in both larger agglomerates and very small crystal fines; the other produced a mixture of larger, more rounded crystals and very small crystal fines. The crystal shown in Figure 8, which is approximately $1 \mathrm{~mm}$ in length and $0.6 \mathrm{~mm}$ in width, exemplifies the more rounded morphology. The yield data are summarized in Table 5.

\begin{tabular}{|c|c|c|c|}
\hline $\begin{array}{c}\text { \% of } \\
\text { CMC }\end{array}$ & $\begin{array}{c}\text { Total } \\
\text { crystal } \\
\text { yield (g) }\end{array}$ & $\begin{array}{c}\text { Crystal yield in } \\
\text { size range of 1.40 } \\
\text { to } 3.35 \text { mm (g) }\end{array}$ & $\begin{array}{c}\text { Percent crystal } \\
\text { yield in size } \\
\text { range of 1.40 to } \\
\mathbf{3 . 3 5} \mathbf{~ m m ~ ( \% )}\end{array}$ \\
\hline $34 \%$ & 14.51 & 3.44 & 23.7 \\
\hline $34 \%$ & 1.73 & 1.35 & 78.0 \\
\hline $34 \%$ & 12.23 & 1.23 & 10.1 \\
\hline $34 \%$ & 10.53 & 1.09 & 10.4 \\
\hline $34 \%$ & 14.19 & 3.56 & 25.1 \\
\hline $34 \%$ & 9.97 & 8.76 & 87.9 \\
\hline
\end{tabular}

Table 5: Total crystal yields and crystal yields within the desired size range grown in the presence of 34\% CMC of Calfax 16L-35.

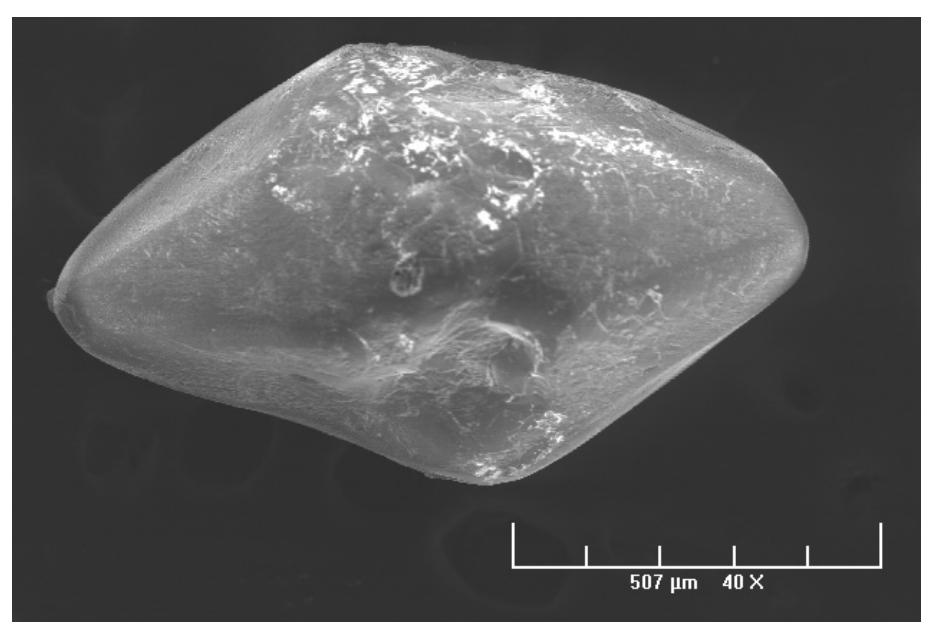

Figure 8: FESEM micrograph of a potassium sulfate crystal grown in the presence of $34 \%$ CMC of Calfax 16L-35.

The range of total crystal yields was 1.73 to 14.51 grams, while that of crystal yields within the desired granular size range was 1.09 grams to 8.76 grams. For four of the runs, 
the yield of granular crystals was less than $26 \%$ of the total crystal yield. For the other two runs, the percentage of granular crystals was much higher, although in one case this is misleading because the total crystal yield was very low in comparison with the other runs.

Three runs were completed with $35 \%$ of the CMC of Calfax $16 \mathrm{~L}-35(0.31 \mathrm{~g} / \mathrm{L})$. Crystals from these runs included both individual crystals of the more rounded morphology and agglomerates of smaller crystals (Table 6). The range of total crystal yields was 6.11 to 12.73 grams, while that of crystal yields within the desired granular size range was 5.20 to 6.75 grams. The percent of the total crystal yield within the granular size range varied from 50 to $87 \%$.

\begin{tabular}{|c|c|c|c|}
\hline $\begin{array}{c}\text { \% of } \\
\text { CMC }\end{array}$ & $\begin{array}{c}\text { Total crystal } \\
\text { yield (g) }\end{array}$ & $\begin{array}{c}\text { Crystal yield in size } \\
\text { range of 1.40 to } \\
3.35 \mathbf{~ m m ~ ( g ) ~}\end{array}$ & $\begin{array}{c}\text { Percent crystal } \\
\text { yield in size } \\
\text { range of 1.40 to } \\
\text { 3.35 mm (\%) }\end{array}$ \\
\hline $35 \%$ & 6.107 & 5.197 & 85.1 \\
\hline $35 \%$ & 12.728 & 6.404 & 50.3 \\
\hline $35 \%$ & 7.742 & 6.748 & 87.2 \\
\hline
\end{tabular}

Table 6: Total crystal yields and crystal yields within the desired size range grown in the presence of $35 \%$ CMC of Calfax 16L-35.

\section{Crystallization with Calfax DB-45}

Calfax DB-45 surfactant differs from Calfax 16L-35 surfactant in that it has a branched carbon chain instead of a linear carbon chain, and fewer carbons (12 rather than 16). Its $\mathrm{CMC}$ is $0.804 \mathrm{~g} / \mathrm{L}$. Experiments were performed with a range of surfactant concentrations from $26 \%$ to $36 \%$ of the $\mathrm{CMC}$, in an attempt to identify a concentration range that would produce a good crystal yield together with a CSD within the desired granular size range.

It was indicated above that the results with Calfax 16L-35 were erratic, in that the crystal yield and/or the percentage of granular crystals might vary considerably from one run to the next, even under the same nominal conditions. This tendency was again observed (in fact to a still greater extent) in the experiments with the Calfax DB-45. As for Calfax 16L-35 in the previous section, some examples are given below of sets of results for surfactant concentrations that included the most promising results for some individual runs, but also exemplify the problem of variability in the results.

Data from three runs performed with $32 \%$ CMC of Calfax DB-45 $(0.25 \mathrm{~g} / \mathrm{L})$ are shown in Table 7. The first of these runs produced 8.0 grams of granular crystals with rounded morphology, but also many crystal fines. The next two runs, however, produced very low yields and these consisted only of fines. 


\begin{tabular}{|l|l|l|l|}
\hline $\begin{array}{l}\text { \% of } \\
\text { CMC }\end{array}$ & $\begin{array}{l}\text { Total } \\
\text { crystal } \\
\text { yield (g) }\end{array}$ & $\begin{array}{l}\text { Crystal yield in } \\
\text { size range of 1.40 } \\
\text { to 3.35 mm (g) }\end{array}$ & $\begin{array}{l}\text { Percent crystal } \\
\text { yield in size } \\
\text { range of 1.40 to } \\
\mathbf{3 . 3 5} \text { mm (g) }\end{array}$ \\
\hline 32 & 16.41 & 8.00 & 48.8 \\
\hline 32 & 0.18 & 0.00 & 0.0 \\
\hline 32 & 0.41 & 0.00 & 0.0 \\
\hline
\end{tabular}

Table 7: Total crystal yields and crystal yields within the desired size range grown in the presence of $32 \%$ CMC of Calfax DB- 45 .

Table 8 shows data from four runs with $33 \%$ CMC of Calfax DB-45 $(0.26 \mathrm{~g} / \mathrm{L})$. The first of these runs produced only agglomerates, the second produced only individual crystals with rounded morphology, the third produced some rounded crystals, as well as many flakes and agglomerates, and the fourth produced only flakes and small fines. The total crystal yield ranged from 0.49 gram to 11.01 grams. The yield of granular crystals ranged from 0.73 gram to 9.12 grams.

\begin{tabular}{|c|c|c|c|}
\hline $\begin{array}{c}\text { \% of } \\
\text { CMC }\end{array}$ & $\begin{array}{c}\text { Total } \\
\text { crystal } \\
\text { yield (g) }\end{array}$ & $\begin{array}{c}\text { Crystal yield in } \\
\text { size range of 1.40 } \\
\text { to } 3.35 \text { mm (g) }\end{array}$ & $\begin{array}{c}\text { Percent crystal } \\
\text { yield in size range } \\
\text { of } \mathbf{1 . 4 0} \text { to } \mathbf{3 . 3 5} \\
\mathbf{m m} \text { (g) }\end{array}$ \\
\hline 33 & 1.27 & 0.73 & 57.5 \\
\hline 33 & 11.01 & 9.12 & 82.8 \\
\hline 33 & 6.70 & 4.37 & 65.2 \\
\hline 33 & 0.49 & 0.00 & 0.0 \\
\hline
\end{tabular}

Table 8: Total crystal yields and crystal yields within the desired size range grown in the presence of $33 \%$ CMC of Calfax DB- 45 .

Data for three runs with 34\% CMC of Calfax DB-45 $(0.27 \mathrm{~g} / \mathrm{L})$ are shown in Table 9. The first of these runs produced mostly individual rounded crystals with a small amount of agglomerates, while the second produced flakes and small agglomerates. The third produced many individual rounded crystals, but the majority of the crystals still appeared as either agglomerates or flakes. The total crystal yield ranged from 0.88 gram to 12.88 grams. The yield of granular crystals ranged from 1.98 grams to 8.29 grams. 


\begin{tabular}{|c|c|c|c|}
\hline $\begin{array}{c}\text { \% of } \\
\text { CMC }\end{array}$ & $\begin{array}{c}\text { Total } \\
\text { crystal } \\
\text { yield (g) }\end{array}$ & $\begin{array}{c}\text { Crystal yield in } \\
\text { size range of 1.40 } \\
\text { to } 3.35 \text { mm (g) }\end{array}$ & $\begin{array}{c}\text { Percent crystal } \\
\text { yield in size } \\
\text { range of 1.40 to } \\
\text { 3.35 mm (\%) }\end{array}$ \\
\hline 34 & 12.88 & 8.29 & 64.4 \\
\hline 34 & 0.88 & 0.03 & 3.4 \\
\hline 34 & 10.30 & 1.98 & 19.2 \\
\hline
\end{tabular}

Table 9: Total crystal yields and crystal yields within the desired size range grown in the presence of $34 \%$ CMC of Calfax DB- 45 .

The results obtained with the procedure described showed unacceptable variability. The inconsistency of the data and inability to control reproducibly the shapes, sizes, and yields of the crystals grown led to the study of other parameters, in particular, the detailed characteristics of the cooling regime during crystallization. For similar nominal settings, variations could be observed in actual cooling rate, and also the extent and duration of "overshoot" at the end of the cooling process. Each of these factors could influence nucleation and growth. Experiments were therefore performed to find a cooling/heating process (with emphasis on the lowest temperature reached during cooling and the time spent at that temperature, as well as on cooling rate) that would reproducibly give granular crystals in the presence of the ADO surfactants. These experiments encompassed both the end of the work performed under contract NM003, and the start of the work performed under the separate but related contract NM005. Thus the solution to the problem appears in the report for NM005.

\section{CONCLUSION}

The results of this research provided both challenges and encouragement. The profitability of the extraction and processing of langbeinite from potash deposits, for use in fertilizer, is heavily dependent on the ability to produce coarse granular crystals of potassium sulfate. Early results demonstrated the potential of alkyl diphenyl oxide (ADO) surfactants to provide considerable enhancement of both the crystal size and the crystal size distribution (CSD), but posed the question of how to obtain these benefits while retaining high crystal yield. Further experiments, however, revealed problems of reproducibility with the crystallization procedure being used. This problem has been addressed through careful study and refinement of the cooling regime during crystallization. However, these experiments spanned both the end of the work performed under contract NM003, and the start of the work performed under the separate but related contract NM005. Thus the solution to the problem appears in the report for NM005. 


\section{REFERENCES}

Aoun, M., E. Plasari, R. David, and J. Villermaux (1999). Chem. Eng. Sci. 54, 1161-1180.

Dash, S. R. and S. Rohani (1993). Chem. Eng. Comm. 125, 211-226.

Gamble, S., Mosaic Potash Carlsbad Inc, Carlsbad, NM， (2005). Personal communication, March 2005.

Kirk, R. E. and D.F. Othmer (1991). Encyclopedia of Chemical Technology. $4^{\text {th }}$ ed., Vol. 19, (Wiley: New York), pp. 1076-1080.

Linke, W. F. (1965). Solubilities: Inorganic and Metal-Organic Compounds. $4^{\text {th }}$ ed., Vol. 2, (American Chemical Society: Washington, D.C.), pp. 296-524.

Pilot Chemical Company (2005). A Guide to the Application of Calfax Diphenyl Oxide Disulfonates. Retrieved April 21, 2005 from: http://www.pilotchemical.com/pdf/CalfaxDipOxideDis85E.pdf

Pilot Chemical Company (2006). Emulsion Polymerization. Retrieved March 5, 2006 from: http://www.pilotchemical.com/emulsion.htm

Xie, Y., Q. Zhao, S. Bi, F. Chen, and Y. Yang. Research on the Mechanism and Optimum Adding Method of Additives in Seed Precipitation, ed.: P.N. Crepeau, Proceedings of the Light Metals 2003 Conference, San Diego, CA, March 2-6, 2003.

\section{LIST OF ACRONYMS AND ABBREVIATIONS}

ADO: alkyl diphenyl oxide

ASTM: American Society for Testing and Materials

CSD: crystal size distribution (formed during crystallization)

CMC: critical micelle concentration

FESEM: field-emission scanning electron microscope

IC: ion chromatography

M: molar

MSMPR: mixed-suspension, mixed-product removal (crystallizer)

XRD: X-ray diffraction 
Appendix 25: The Effect of Alkyl Diphenyl Oxide and Sulfonated Oleic Acid Surfactants on Nucleation and Growth of Potassium Sulfate Crystals: Optimization of Surfactants for the Potash Industry (NM005) 


\section{FINAL TECHNICAL REPORT}

Contract Title and Number:

Crosscutting Technology Development at the Center for Advanced Separation Technologies (DE-FC26-02NT41607)

Sub-Recipient Project Title:

The Effect of Alkyl Diphenyl Oxide and Sulfonated Oleic Acid Surfactants on Nucleation and Growth of Potassium Sulfate Crystals: Optimization of Surfactants for the Potash Industry

Principal Investigators:

Bond, Hockensmith

Contact Address:

Department: Mat. \& Met. Engineering

New Mexico Tech

Socorro NM 87801

Subcontractor Address:

No subcontracts issued.
Period of Performance:

Starting Date: 10/1/05

Ending Date: 9/30/07
Report Information:

Type: Final

Number:

Period: $\quad$ 10/1/05-9/30/06

Date: $\quad$ 9/24/06

Code: NM005-Final

Contact Information:

Phone: (505) 835-5653

Fax: (505) 835-6333

E-Mail: gbond@nmt.edu

Subcontractor Information:

Phone:

Fax:

E-Mail: 


\section{DISCLAIMER}

This report was prepared as an account of work sponsored by an agency of the United States Government. Neither the United States Government nor any agency thereof, nor any of their employees, make any warranty, express or implied, nor assume any legal liability or responsibility for the accuracy, completeness, or usefulness of any information, apparatus, product, or process disclosed, or represents that its use would not infringe privately owned rights. Reference herein to any specific commercial product, process, or service by trade name, trademark, manufacturer, or otherwise does not necessarily constitute or imply endorsement, recommendation, or favoring by the United States Government or any agency thereof. The views and opinions of authors expressed herein do not necessarily state or reflect those of the United States Government or agency thereof.

\section{ABSTRACT}

The objective of this work has been to improve both the crystal size and the crystal size distribution (CSD) of $\mathrm{K}_{2} \mathrm{SO}_{4}$ with regard to the production of granular crystals for use in fertilizer, while obtaining high crystal yields. Earlier work with two alkyl diphenyl oxide (ADO) surfactants (described in the report for the separate but related contract NM003) demonstrated that the surfactants (Calfax 16L-35 and Calfax DB-45) had the ability to narrow the CSD, but a problematic variability in the results obtained was encountered. An improved protocol for $\mathrm{K}_{2} \mathrm{SO}_{4}$ crystallization has been developed. Calfax DB-45, a branched $\mathrm{C}_{12}$ ADO surfactant, has been found to give excellent CSD enhancement without loss of crystal yield, with the best results obtained with $18 \%$ of the critical micelle concentration (CMC). Total crystal yields correspond to high percentages ( 81 to 93\%) of the maximum attainable given the solubility limit at the growth temperature. Of these total crystal yields, 81 to $85 \%$ are in the desired size range $(1.4$ to $4 \mathrm{~mm})$ when grown in the presence of $18 \%$ CMC surfactant, compared to less than $1 \%$ without surfactant. Initial studies with oleic acid surfactants resulted in smaller crystals than desired, but further work is recommended.

\section{TABLE OF CONTENTS}

\begin{tabular}{|l|c|}
\hline Heading & Page \\
\hline Title Page & 1 \\
\hline Disclaimer & 2 \\
\hline Abstract & 2 \\
\hline Table of Contents & 2 \\
\hline Introduction & 3 \\
\hline Executive Summary & 5 \\
\hline Experimental & 6 \\
\hline Results and Discussion & 10 \\
\hline Conclusion & 19 \\
\hline References & 19 \\
\hline List of Acronyms and Abbreviations & 20 \\
\hline
\end{tabular}




\section{INTRODUCTION}

In the United States, potash production during the year 2000 was valued at over $\$ 300$ million (Searls, 2001). The fertilizer industry utilizes $90 \%$ of the potash produced each year (Noyes, 1965; Searls, 2001). Potash is produced in New Mexico, Utah, and Michigan as well as in Canada. Much of the United States potash production comes from New Mexico, where both sylvinite and langbeinite ores are mined. These ores are the source materials for potassium chloride and potassium sulfate. Potassium sulfate, which is sold as a fertilizer for chloride-sensitive fruits and vegetables, accounts for 4-6\% of potassium sales (Searls, 2001).

Beneficiation of potash ores involves a number of processes including flotation, magnetic separation, crystallization and recrystallization, depending upon the individual producer and the source ores mined (Noyes, 1965). In New Mexico, potassium sulfate is derived from langbeinite that contains up to 18.8 weight percent of potassium (Kirk and Othmer, 1996). Langbeinite is a sulfate of potassium and magnesium with sodium chloride as the principal impurity. Some ores contain 30-45 weight percent of langbeinite. Processing of the ores begins with separation of the langbeinite from the chlorides by selective chloride dissolution. Potassium chloride is recovered from the solution by evaporation and crystallization, after prior removal of the sodium chloride. The langbeinite product is dewatered and dried. Potassium chloride brine may be applied to the langbeinite product in order to produce potassium sulfate. The eventual removal of chlorides and magnetic separation of ferrous materials delivers potassium sulfate filter cake that is dried and stored in three size grades: granular, standard and special standard.

Energy costs for crystallization processes, together with the capital and maintenance costs associated with the handling of hot brines, are important contributors to the costs of potassium sulfate production. Profitable sale of potassium sulfate requires the production of crystals in appropriate size ranges. The majority of the potassium sulfate produced is spread as fertilizer, and for this process farmers require a granular product with a suitable crystal size distribution (CSD). The crystals that comprise the granular product most useful to the fertilizer industry are those with mesh sizes in the range of $1.4 \mathrm{~mm}$ (No. 14 screen) to 4.00 mm (No. 6 screen, but smaller than No. 5 screen).

Surfactants may be added to influence crystal nucleation and growth, and hence the CSD, but nevertheless manufacturers are often plagued by inadequate control of crystal size. This leads to the necessity for continuous reprocessing of the unsaleable fines, which may be compacted if large enough or else fed through again. Improved control of the CSD will reduce beneficiation costs, lessen energy consumption for continued reprocessing, and lower environmental impact, while at the same time enhancing product recovery. This in turn improves the overall economic viability of the mine.

A detailed study of the influence of different surfactants on crystallization of potassium sulfate is an essential first step towards the improvement of surfactants for the control of crystallization. The broad range of parameters that influence crystallization of potassium sulfate is emphasized by the experimental results of Aoun et al. (1999), which show that the addition order of reagents and the stirring speed employed both have a significant impact on crystallization. Stirrer speed has a large effect on attrition (Mydlarz 
and Jones, 1989). When crystal size is considered in terms of mesh, crystal shape becomes an important issue particularly for production quality control. This concern stems from the loss of crystal size because of mechanical abrasion, breakage, and attrition due to other causes. The crystal shape does not depend on supersaturation (Meenan and Roberts, 1993), although CSD is supersaturation dependent (Chianese et al., 1993).

Mauri and Moret (2000) found significant impact on the step micromorphology and growth rate of potassium sulfate crystals when polysulfonated organic dyes (acid fuchsin and polysulfonated pyrene dyes) were added to saturated solutions of $\mathrm{K}_{2} \mathrm{SO}_{4}$. Acid fuchsin and polysulfonated pyrene are both strong polyelectrolytes that can adsorb on crystal terraces because of simultaneous strong interactions between their three or four sulfonate groups and the crystal surface via long-distance electrostatic forces. Once adsorbed, polyelectrolytes remain essentially immobile, provided that the distance between sulfonate groups effectively matches the distance between sulfate ions on the crystal surface.

Bujan et al. (2001) examined the effect that anionic surfactants have on the crystal growth rate of calcium hydrogen phosphate dihydrate crystals $\left(\mathrm{CaHPO}_{4} \bullet \mathrm{H}_{2} \mathrm{O}\right.$, DPCD). Depending on the type of anionic surfactant and its concentration, they observed a retardation of crystal growth below the critical micelle concentration (CMC), but also greater uniformity in crystal size and shape.

Alkyl diphenyl oxide (ADO) disulfonates are typical of the surfactants used in crystallization processes and they have been shown to slow down dissolution of langbeinite. We have been using a batch crystallizer based on a modified form of the mixed-suspension, mixed-product removal (MSMPR) crystallizer used by Dash and Rohani (1993), in order to study the effects of ADO disulfonate surfactants on the precipitation of potassium sulfate in the presence of magnesium sulfate. The focus of our earlier work (performed under contract NM003) was to investigate the crystallization of potassium sulfate from solutions containing different alkyl diphenyl oxide disulfonate surfactants. Crystals produced in the presence of Calfax 16L-35 during the course of that work excited the interest of Intrepid Mining Company, who had recently bought into the Carlsbad Potash Basin with plans to produce potassium sulfate.

Hence our intention at the start of the present work was twofold:

1. To optimize the yield obtainable with ADO disulfonate surfactants, while maintaining the desirable CSD, through studies with both a linear (Calfax 16L-35) and a branched (Calfax DB-45) ADO surfactant.

2. To study the performance of sulfonated oleic acid for comparison with a view to improving performance, following an initial examination of the effects of additions of oleic acid alone, and of oleic acid/potassium hydroxide and oleic acid/kerosene surfactants on crystallization of potassium sulfate. Calfax 16L-35 (sodium hexadecyl diphenyl oxide disulfonate) contains a closely similar number of carbon atoms to oleic acid $\left[\mathrm{CH}_{3}\left(\mathrm{CH}_{2}\right)_{7} \mathrm{CH}=\mathrm{CH}\left(\mathrm{CH}_{2}\right)_{7} \mathrm{COOH}\right]$ (Figure 3). It does not, of course contain the phenyl groups, but it is unsaturated, sulfonated, and has the kinked shape that results from the cis double bond. 


\section{EXECUTIVE SUMMARY}

Potassium sulfate is extracted from langbeinite-containing potash ores and sold as a fertilizer for chloride-sensitive fruits and vegetables. For this application, farmers require a granular product with a suitable crystal size distribution (CSD). Coarse granular grades of potassium sulfate within the size range of $1.40 \mathrm{~mm}$ to $4.00 \mathrm{~mm}$ (ASTM 14 mesh through 6 mesh, up to 5 mesh) have the highest market value. Smaller crystals down to $\sim 250 \mu \mathrm{m}$ (mesh size 60) may be used, but constitute a lower-grade, less desirable product. Smaller fines than this need to be separated through multiple filtering steps and then re-dissolved for subsequent crystallization.

The objective of this work has been to improve both the crystal size and the CSD of $\mathrm{K}_{2} \mathrm{SO}_{4}$ while obtaining high crystal yields. Work performed under the separate but related contract NM003 produced some encouraging results with Calfax 16L-35 (a linear sodium hexadecyl diphenyl oxide disulfonate surfactant) and Calfax DB-45 (a branched sodium dodecyl diphenyl oxide disulfonate surfactant) at concentrations well below the critical micelle concentration (CMC). However, a problematic variability in results was observed.

The original intention in this project was to optimize the yield obtainable with the alkyl diphenyl oxide (ADO) disulfonate surfactants, while maintaining the desirable CSD, and then to study the use of oleic acid surfactants, particularly sulfonated oleic acid. The problem of varying results, encountered in a time period spanning the end of NM 003 and the start of this project (NM 005) had, however, to be addressed first.

The effects of cooling rate, nucleation and growth temperatures, and stirring speeds have been investigated, and an improved protocol for $\mathrm{K}_{2} \mathrm{SO}_{4}$ crystallization has been developed. This entailed extensive experimentation, and hence there was little time for work on the oleic acid surfactants. Some initial tests were performed with unsulfonated oleic acid surfactants, with no enhancement of CSD observed. It is believed, however, that a satisfactory solution to the variability problem has been found, and excellent combinations of crystal yield and CSD have been obtained with Calfax DB-45.

Both ADO surfactants have demonstrated the ability to limit nucleation and increase crystal growth, thus narrowing the CSD during crystallization. In particular, with the improved crystallization protocol excellent CSD enhancement was observed with Calfax DB45 without loss of crystal yield, with the best results obtained for $18 \%$ of the critical micelle concentration (CMC). Total crystal yields correspond to high percentages ( 81 to 93\%) of the maximum attainable given the solubility limit at the growth temperature. Of these total crystal yields, 81 to $85 \%$ are in the desired size range (1.4 to $4 \mathrm{~mm}$ ) when grown in the presence of $18 \%$ CMC surfactant, compared to less than $1 \%$ without surfactant. Larger numbers of experiments are required in order to confirm reproducibility.

The major impact of this work is its potential to increase the ratio of granular particles to fines, and thus to improve the efficiency and lower the cost of production of potassium sulfate, improve the financial viability of the producer, and also benefit both consumers and the economy of the Carlsbad area where much of the langbeinite-containing ore is produced. 


\section{EXPERIMENTAL}

\section{$\underline{\text { Surfactants }}$}

The general structure of the Calfax surfactants is shown in Figure 1. Details of each surfactant, including the carbon chain length and chain type (linear or branched), are given in Table 1.

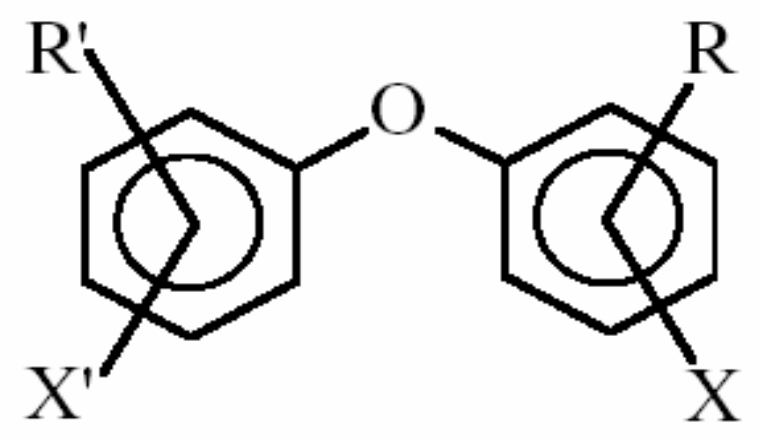

Figure 1: General chemical structure of Calfax surfactants (Pilot Chemical Company, 2005).

\begin{tabular}{|c|c|c|c|c|c|c|c|}
\hline $\begin{array}{c}\text { Surfactant } \\
\text { name }\end{array}$ & $\begin{array}{c}\text { Chemical name } \\
\text { of surfactant }\end{array}$ & $\mathbf{R}$ & $\mathbf{R}^{\prime}$ & $\begin{array}{c}\mathbf{X} \text { and } \\
\mathbf{X}^{\prime}\end{array}$ & $\begin{array}{c}\mathbf{M W} \\
\mathbf{( g / m o l})\end{array}$ & $\begin{array}{c}\text { Chain } \\
\text { length }\end{array}$ & $\begin{array}{c}\text { Chain } \\
\text { type }\end{array}$ \\
\hline $\begin{array}{c}\text { Calfax } \\
16 \mathrm{~L}-35\end{array}$ & $\begin{array}{c}\text { Sodium hexadecyl } \\
\text { diphenyl oxide } \\
\text { disulfonate }\end{array}$ & Alkyl & $\mathrm{H}$ & $\mathrm{SO}_{3} \mathrm{Na}$ & 608 & $\begin{array}{c}16 \\
\text { carbons }\end{array}$ & Linear \\
\hline $\begin{array}{c}\text { Calfax } \\
\text { DB-45 }\end{array}$ & $\begin{array}{c}\text { Sodium dodecyl } \\
\text { diphenyl oxide } \\
\text { disulfonate }\end{array}$ & Alkyl & $\mathrm{H}$ & $\mathrm{SO}_{3} \mathrm{Na}$ & 547 & $\begin{array}{c}12 \\
\text { carbons }\end{array}$ & Branched \\
\hline
\end{tabular}

Table 1: Table of Calfax surfactants (Pilot Chemical Company, 2005).

The ADO surfactants, Calfax 16L-35 and Calfax DB-45, were acquired from Pilot Chemical Company. The CMC of Calfax $16 \mathrm{~L}-35$ surfactant is $0.500 \times 10^{-3} \mathrm{~mol} / \mathrm{L}$ while that of Calfax DB-45 is $0.661 \times 10^{-3} \mathrm{~mol} / \mathrm{L}$ (Pilot Chemical Company, 2005). [The CMC is the concentration of a surfactant at which the surfactant molecules aggregate to form spherical micelle structures. At concentrations greater than the CMC, not only does the amount of surfactant in free solution no longer increase with further additions but also surfactant aggregates are now present in the solution.]

Priolene 6939 oleic acid was obtained from Uniqema (Chicago, IL). Experiments were performed with oleic acid/1M potassium hydroxide (1.25-250\% CMC) and oleic acid/kerosene oil (10-600\% CMC) (following the work of Ghazy et al., 2001), as well as 
small amounts (1-5\% CMC) of oleic acid alone. Kabil and Ghazy (1994) found the CMC of oleic acid to be $10^{-2} \mathrm{M}$.

\section{$\underline{\text { Procedure A }}$}

A batch crystallizer has been used, modified from the design of the MSMPR crystallizer used by Dash and Rohani (1993) in studies of crystal size and growth rate of KCl. It is shown schematically in Figure 2.

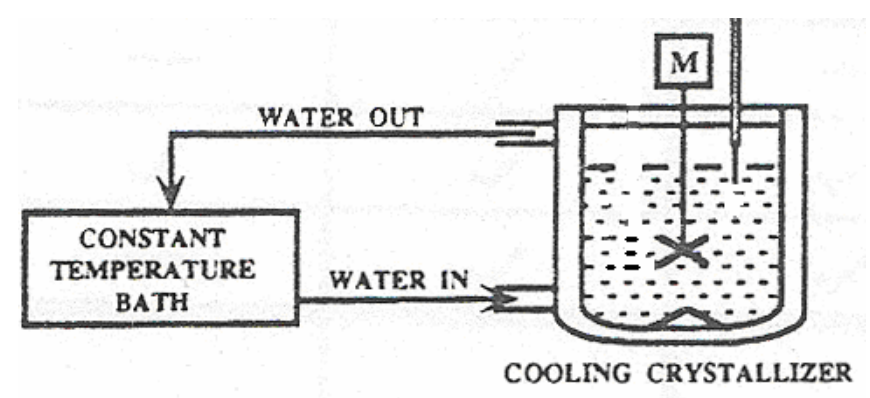

Figure 2: Schematic diagram of batch crystallizer.

The initial experiments, both to enhance crystal yield with ADO surfactants and to investigate the influence of oleic-acid surfactants, were performed as follows, according to the same procedure used in the experiments described in the report for contract NM003.

In the crystallizer one liter of nanopure water is heated to $80^{\circ} \mathrm{C}$ in a Neslab RTE-7 Digital One Refrigerated cooling/heating bath. The crystallizer is a one-liter, glass-jacketed reactor with four baffles, (Wilmad-LabGlass). The four baffles located on the inside of the inner wall of the crystallizer improve the mixing of the solution by breaking up the flow of the solution inside the crystallizer. As the water is heating, a four-opening, flat flange closure is clamped on top of the unit and a three-bladed, 2.5-inch propeller is inserted through the top of the crystallizer. The propeller is turned by an Arrow 1750 (1/15 hp) electric motor. The impeller opening, located in the middle of the flange cover, is covered with aluminum foil to reduce the amount of vapor escaping. The speed of the electric motor is set to approximately $400 \mathrm{rpm}$, controlled by an integral power source. A thermometer is inserted into one of the four openings at the top of the crystallizer to measure the temperature of the solution. The remaining two openings are closed off with rubber stoppers and aluminum foil.

When the nanopure water has reached $80^{\circ} \mathrm{C}$, surfactant (when used, as described below) is added to the water, followed by 190.3 grams of $99 \%$ potassium sulfate $\left(\mathrm{K}_{2} \mathrm{SO}_{4}\right.$, $1.09 \mathrm{M}$ ) (from Sigma-Aldrich) and 9.9 grams of $99.5 \%$ magnesium sulfate heptahydrate $\left(\mathrm{MgSO}_{4} \bullet 7 \mathrm{H}_{2} \mathrm{O}, 0.04 \mathrm{M}\right)$ (from Fisher Scientific). The reagents are stirred in the crystallizer at $80^{\circ} \mathrm{C}$ for one hour to dissolve them completely. The temperature of the cooling/heating bath is then lowered to $40^{\circ} \mathrm{C}$ at a mean cooling rate of $0.65{ }^{\circ} \mathrm{C} / \mathrm{min}$., and held for an hour and a half before the crystals are removed for examination. The solubility limit of potassium sulfate in water is $214 \mathrm{~g} / \mathrm{L}$ at $80^{\circ} \mathrm{C}$ (Linke 1965). The mother liquor becomes saturated with 
$\mathrm{K}_{2} \mathrm{SO}_{4}$ at $67^{\circ} \mathrm{C}$. The solubility limit of magnesium sulfate heptahydrate is $710 \mathrm{~g} / \mathrm{L}$ at $20^{\circ} \mathrm{C}$ and $910 \mathrm{~g} / \mathrm{L}$ at $40^{\circ} \mathrm{C}$. Hence the mother liquor remains undersaturated with respect to magnesium sulfate heptahydrate throughout the experiments, whereas it is supersaturated with respect to potassium sulfate at $40^{\circ} \mathrm{C}$.

At the end of each run, the crystals formed in the crystallizer are filtered through two Whatman \#2 filter papers in a $110 \mathrm{~mm}$ Buchner funnel. The crystals on the filter paper are then dried and weighed on an analytical scale. Once the crystal yield has been determined, the crystals are removed from the filter paper and placed in a sieve stack. The crystals that comprise the granular product most useful to the fertilizer industry are those retained in the sieves with mesh sizes of $1.4 \mathrm{~mm}$ (No. 14 screen), $2 \mathrm{~mm}$ (No. 10 screen), and $3.35 \mathrm{~mm}$ (No. 6 screen). The crystals are removed from each sieve and weighed on an analytical scale in order to find the CSD. The sieves, pan, and cover are then rinsed with nanopure water and put in an oven at $102^{\circ} \mathrm{C}$ for an hour to dry.

\section{Procedure B}

In the overlapping final phase of the previous work under contract NM003 and initial phase of this project (NM005), a problem of variable results emerged, (as described already in the final report for contract NM003). Numerous experiments have since been performed with different cooling regimes in order to improve the reproducibility of the results. The first procedure adopted as a result of these experiments is similar to Procedure A but with the following modification: the cooling rate of the solution is carefully controlled as the temperature is lowered from $80^{\circ} \mathrm{C}$ to $40^{\circ} \mathrm{C}$, to give a temperature profile for the "overshoot" below $40^{\circ} \mathrm{C}$ corresponding to that shown in Figure 3 (for runs 39 and 40) in which the solution is at temperatures below $40^{\circ} \mathrm{C}$ for approximately twenty-two minutes prior to crystal growth at $40^{\circ} \mathrm{C}$, which appears to allow for a desirable amount of nucleation to occur. The solution is then held at $40^{\circ} \mathrm{C}$ for approximately twenty hours while being stirred at approximately $400 \mathrm{rpm}$. After the twenty hours have passed, the solution and the crystals produced are filtered and the crystal yield and CSD are measured.

Further experiments have been performed in which the ADO surfactants are replaced by oleic acid/1M potassium hydroxide (1.25-250\% CMC), and oleic acid/kerosene oil (10$600 \%$ CMC), as well as small amounts (1-5\% CMC) of oleic acid alone. (The use of a $6.36 \times 10^{-2} \mathrm{M}$ oleic acid/kerosene oil solution follows the work of Ghazy et al., 2001.) 


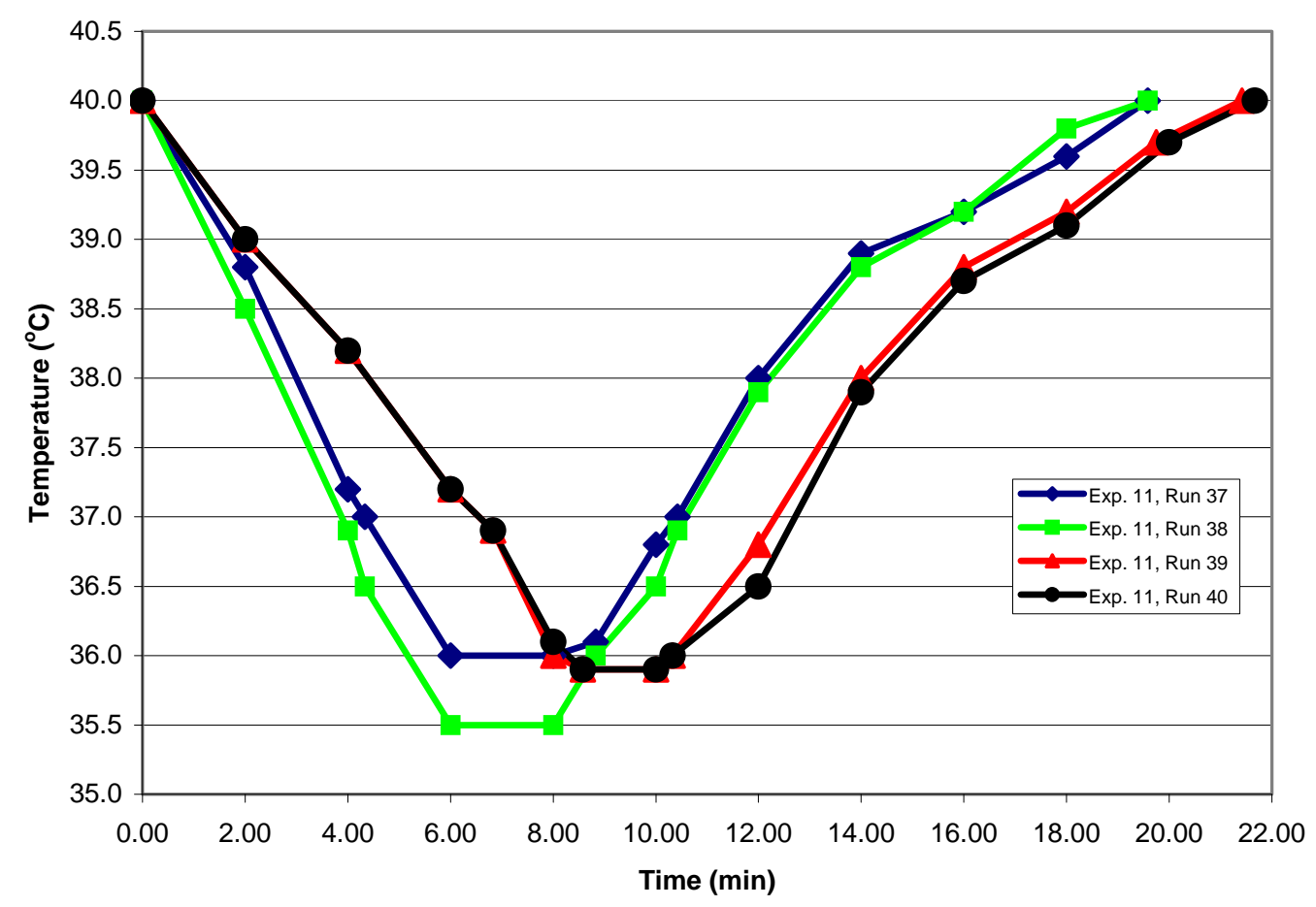

Figure 3: Temperature/time profiles for the solutions from runs 37 to 40. [Solution temperatures were lowered to a nominal $37^{\circ} \mathrm{C}$ and then increased back to $40^{\circ} \mathrm{C}$.]

\section{$\underline{\text { Procedure C }}$}

Procedure $\mathrm{C}$ is similar to Procedure $\mathrm{B}$, but with the following modifications. After the cooling regime of Procedure $\mathrm{B}$, the solution is held at $40^{\circ} \mathrm{C}$ for one hour while being stirred at approximately $400 \mathrm{rpm}$. The solution and the crystals that have formed are then allowed to cool to ambient temperature in one of three ways: 1) they are transferred into a covered beaker with a plastic impeller and allowed to stand (stirred or not) overnight at ambient temperature, or 2) they are left in the crystallizer as it cools to ambient temperature and are allowed to stand (stirred or not) overnight. Finally the solution and the crystals produced are filtered and the crystal yield and CSD are measured. In later experiments, an additional sieve (mesh size 5) has been added to the stack, in order to remove any large agglomerates.

\section{$\underline{\text { Characterization }}$}

A field-emission scanning electron microscope (FESEM, Hitachi Model S-800) with a Crewe field-emission electron gun is used to obtain magnified images of crystals. The instrument is run at an accelerating voltage of $15 \mathrm{kV}$. The crystals are mounted on one-inchdiameter stainless-steel mounting posts with double-faced graphite tape. To produce the conductivity needed for the FESEM, the crystals, graphite tape, and mounting posts are sputtered with platinum. 


\section{RESULTS AND DISCUSSION}

\section{$\underline{\text { Calfax 16L-35 ADO surfactant }}$}

The mother liquor is prepared at $80^{\circ} \mathrm{C}$. In the earlier tests (with Procedure A), the temperature was lowered to a nominal value of $40^{\circ} \mathrm{C}$ and held while crystallization occurred but, as indicated above, the results obtained were excessively variable. Studies of the influence of both cooling rate and nucleation temperature, prior to crystal growth at $40^{\circ} \mathrm{C}$, and closer control of these parameters (in procedure B) reduced the scatter in the data.

The goal was then to optimize the ADO surfactant concentration in order to produce as large a crystal yield as possible within the desired size range of $1.4 \mathrm{~mm}$ (14 mesh) to 3.35 $\mathrm{mm}$ (6 mesh). The crystal yields obtained in the desired size range with Procedure $\mathrm{B}$ and the addition of different percentages of the CMC of Calfax 16L-35 are shown in Figure 4 for the first exploratory runs with Procedure B. The addition of 28\% CMC of Calfax 16L-35 produced the highest total yield of crystals in the size range of $1.4 \mathrm{~mm}$ and above, and also produced the smallest proportion of fines (crystals of size $<1.4 \mathrm{~mm}$ ), as illustrated in Figures 5 (28\%CMC) and 6 (26\% CMC).

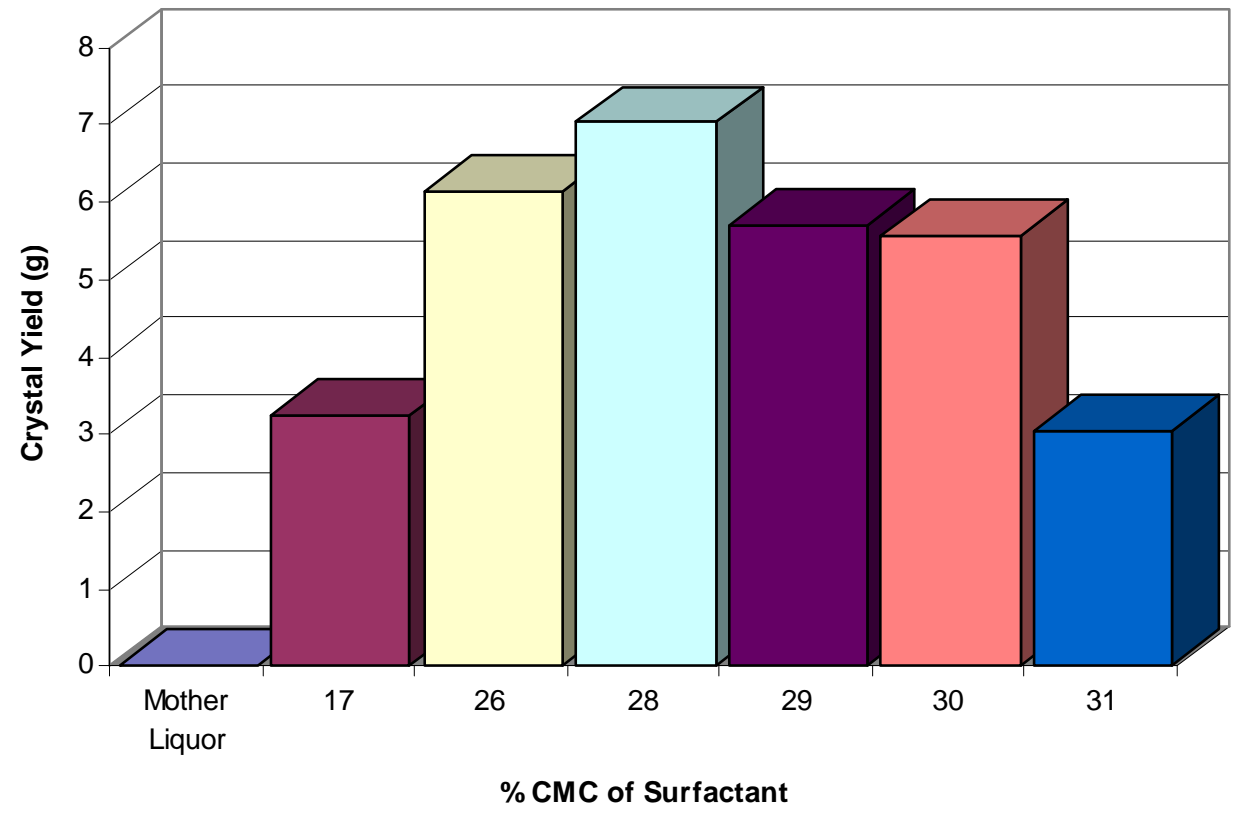

Figure 4: Total $\mathrm{K}_{2} \mathrm{SO}_{4}$ crystal yields in size range $>1.4 \mathrm{~mm}$, obtained with Procedure $\mathrm{B}$ and additions of Calfax 16L-35 surfactant. 


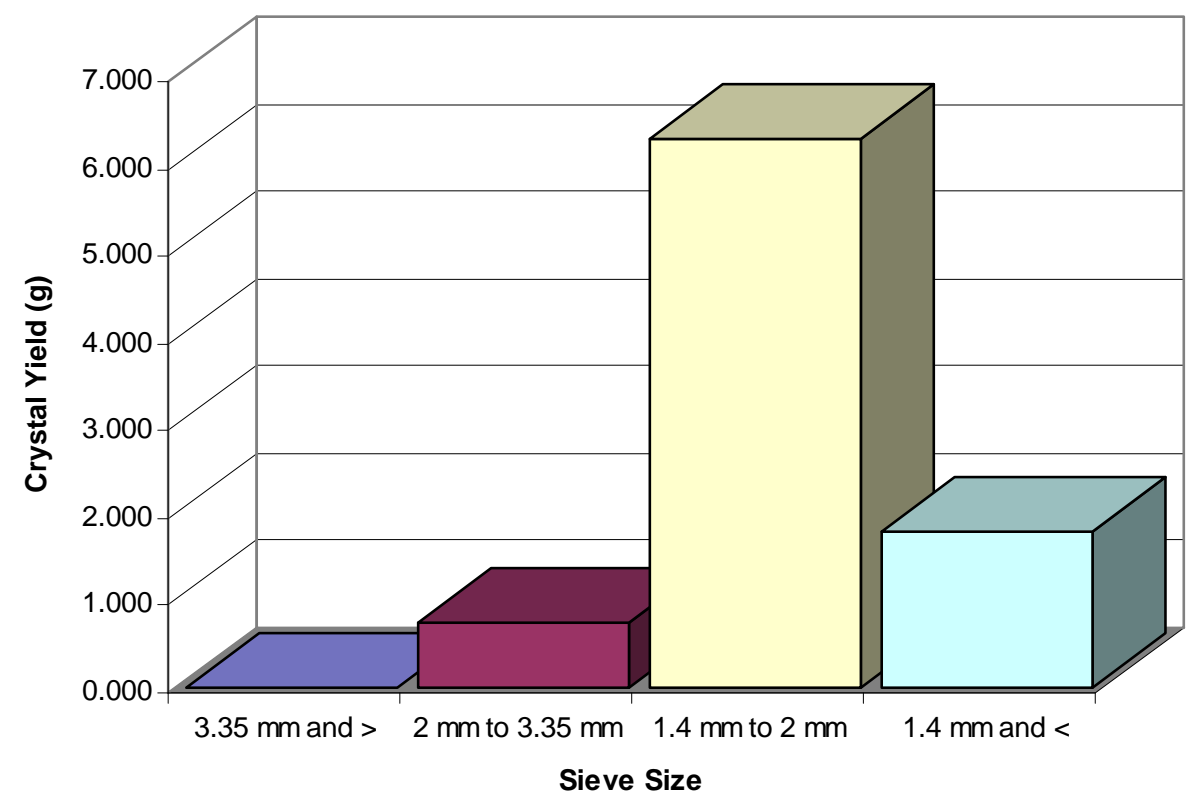

Figure 5: $\mathrm{K}_{2} \mathrm{SO}_{4}$ crystal yields by size range [ $>3.35 \mathrm{~mm}$ (6 mesh and larger), 2-3.35 $\mathrm{mm}$ (10 mesh), 1.4-2 mm (14 mesh), and < $1.4 \mathrm{~mm}$ (smaller than 14 mesh)], obtained with the addition of $28 \%$ CMC of Calfax 16L-35 surfactant.

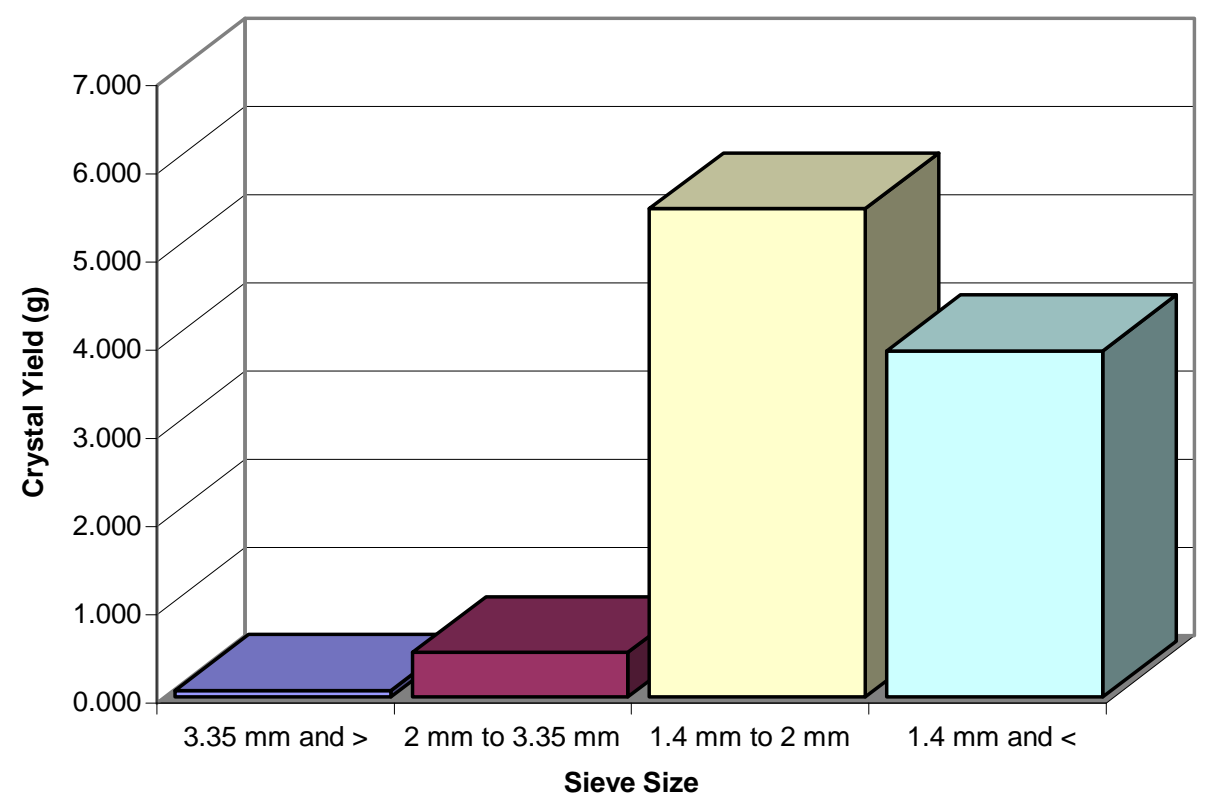

Figure 6: $\mathrm{K}_{2} \mathrm{SO}_{4}$ crystal yields by size range [ $>3.35 \mathrm{~mm}$ (6 mesh and larger), 2-3.35 $\mathrm{mm}$ (10 mesh), 1.4-2 mm (14 mesh), and < $1.4 \mathrm{~mm}$ (smaller than 14 mesh)], obtained with the addition of $26 \%$ CMC of Calfax 16L-35 surfactant. 
The use of 28\% of the CMC of Calfax 16L-35 produced the largest crystal yield of the desired sizes, while use of $17 \%$ of the CMC produced the largest crystal yield. Additions of $28 \%, 29 \%$, and $30 \%$ of the CMC of Calfax 16L-35 all produced more than $70 \%$ of the total crystal yield in the desired size range.

A pronounced change in crystal morphology from that obtained without surfactant was also observed (as shown in Figures 7 and 8), corresponding to the expected continual adsorption of surfactant on growing crystal faces. The presence of the surfactant reduced faceting and much reduced the formation of flakes and agglomerates.

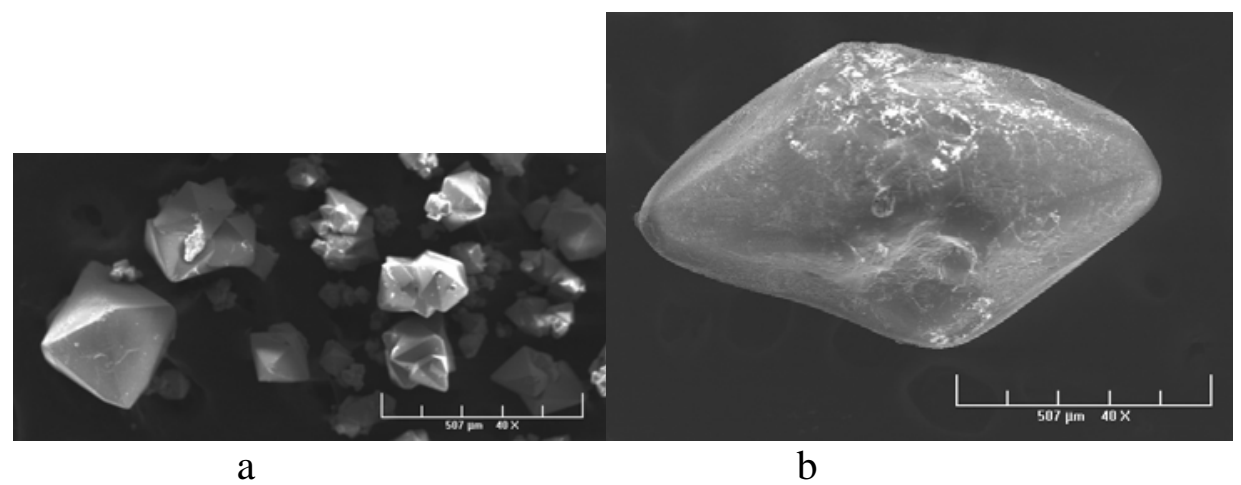

Figure 7: FESEM micrograph of $\mathrm{K}_{2} \mathrm{SO}_{4}$ crystals grown: a) without surfactant, b) with 34\% CMC of Calfax 16L-35 surfactant. [Scale bar: $\sim 0.5 \mathrm{~mm}$.]

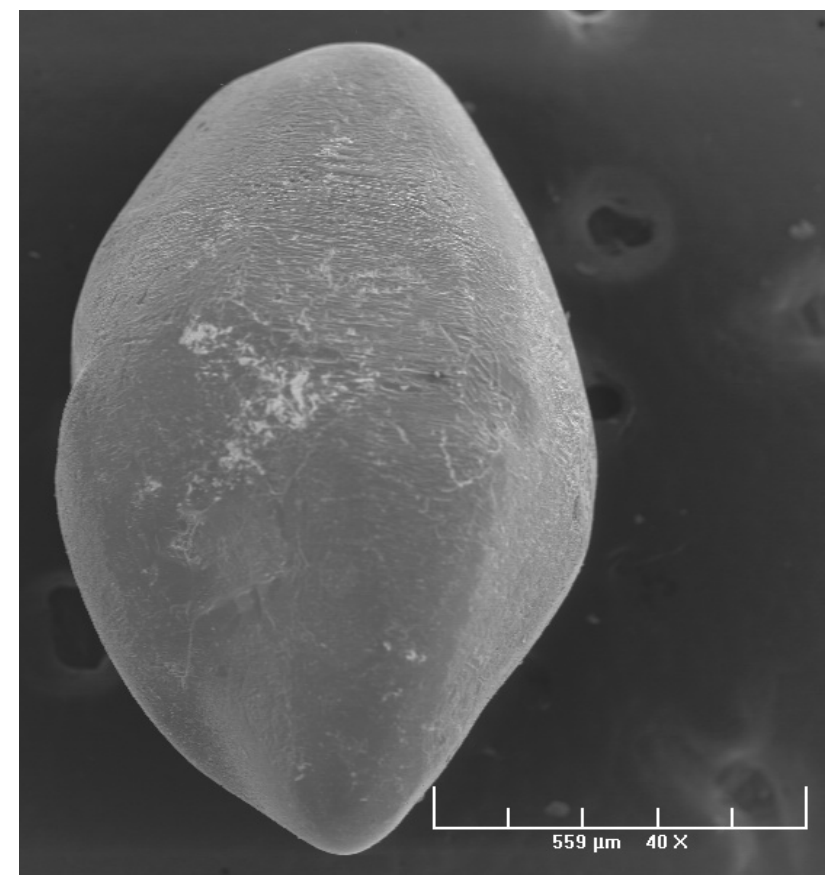

Figure 8: FESEM micrograph of a potassium sulfate crystal grown with 28\% of the CMC of Calfax 16L-35 added to the potassium sulfate mother liquor. [Scale bar: $\sim 0.56 \mathrm{~mm}$.] 
Results of early exploratory runs indicated that the Calfax DB-45 surfactant produced even greater CSD enhancement than Calfax 16L-35 with Procedure B, and so efforts with ADO surfactants were focused on the DB-45 surfactant as described below.

\section{$\underline{\text { Oleic acid }}$}

Studies were commenced on the use of oleic acid to enhance the CSD of potassium sulfate. A modified experimental setup, $20 \%$ of the size of that used for the ADO surfactants, was used so that multiple runs could be performed more easily. Priolene 6939 oleic acid was obtained from Uniqema (Chicago, IL). A value of $10^{-2} \mathrm{M}$ for the CMC of oleic acid was taken from the work of Kabil and Ghazy (1994).

The first experiments were focused on the effects of oleic acid/1M potassium hydroxide (1.25-250\% CMC) and oleic acid/kerosene oil (10-600\% CMC), as well as small amounts (1-5\% CMC) of oleic acid alone. (The use of a $6.36 \times 10^{-2} \mathrm{M}$ oleic acid/kerosene oil solution follows the work of Ghazy et al., 2001.) These experiments resulted in no enhancement of the CSD.

As discussed below, the focus then shifted to Calfax DB-45 ADO surfactant. Hence the effects of other parameters such as chemical modification of the oleic acid remain subjects of interest for future study.

\section{$\underline{\text { Calfax DB-45 ADO surfactant }}$}

The results of the first set of experiments performed according to Procedure B with Calfax DB-45 added to the potassium sulfate mother liquor are given in Table 2. Modest concentrations of the surfactant (18 and $23 \%$ CMC) produced higher crystal yields (both total and within the desired size range) than had been obtained with Calfax 16L-35. Individual granular crystals were produced, as shown in Figure 9, rather than agglomerates.

\begin{tabular}{|c|c|c|c|}
\hline $\begin{array}{c}\text { \% } \\
\text { CMC }\end{array}$ & $\begin{array}{c}\text { Total crystal } \\
\text { yield (g) }\end{array}$ & $\begin{array}{c}\text { Crystal yield in } \\
\text { size range } \\
\text { >1.4 } \mathbf{~ m m ~} \mathbf{( g )}\end{array}$ & $\begin{array}{c}\text { \% Crystal yield } \\
\text { in size range } \\
>\mathbf{1 . 4} \mathbf{~ m m}\end{array}$ \\
\hline 18 & 33.1 & 12.0 & 36.2 \\
\hline 23 & 12.3 & 9.0 & 72.9 \\
\hline 27 & 7.7 & 5.3 & 68.6 \\
\hline 32 & 3.8 & 2.3 & 61.3 \\
\hline
\end{tabular}

Table 2: $\mathrm{K}_{2} \mathrm{SO}_{4}$ crystal yields obtained with Procedure $\mathrm{B}$ and additions of Calfax DB-45 surfactant. 


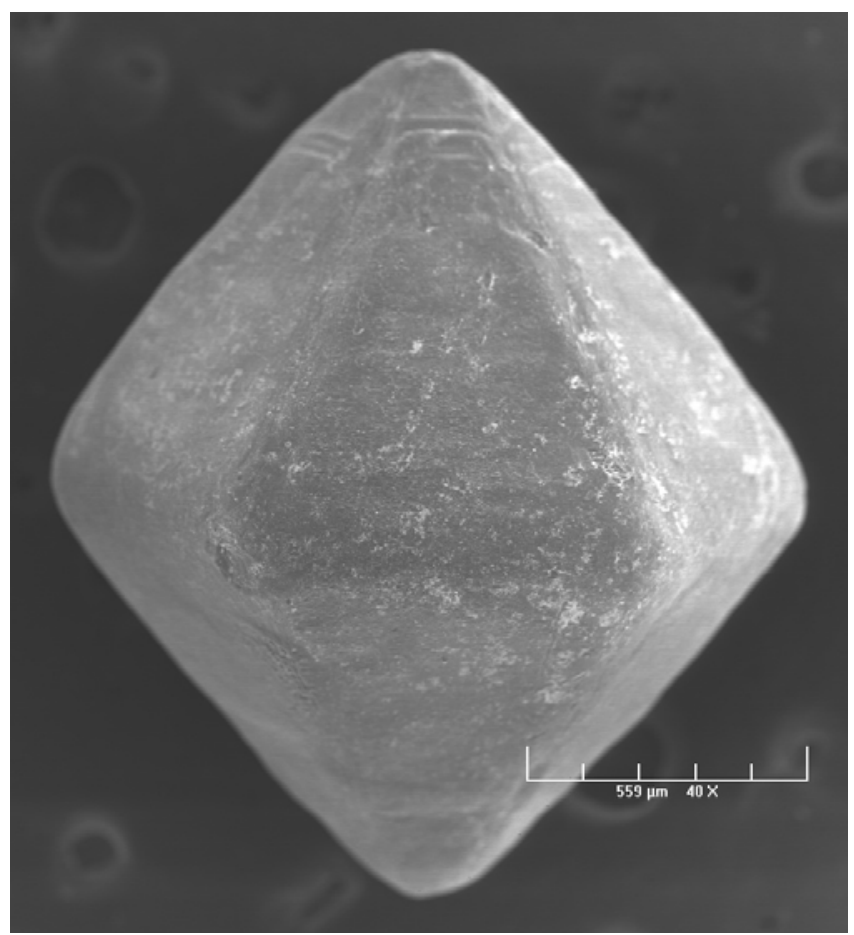

Figure 9: FESEM micrograph of a potassium sulfate crystal grown with 18\% of the CMC of Calfax DB-45 added to the potassium sulfate mother liquor. [Scale bar: $~ 0.56 \mathrm{~mm}$.]

The next goal was to repeat these results with Calfax DB-45 and select a concentration for further study, but a problem was immediately encountered. Table 3 shows the next set of data. It is clear that, while total crystal yields were reasonably repeatable, the yields in the desired size range varied considerably. For four runs at 18\% CMC, the total crystal yield ranged from $\sim 28$ to $34 \mathrm{~g}$, but the yield of crystals at least $1.4 \mathrm{~mm}$ in size varied from 0.5 to $19 \mathrm{~g}$.

\begin{tabular}{|c|c|c|c|}
\hline $\begin{array}{c}\text { \% } \\
\text { CMC }\end{array}$ & $\begin{array}{c}\text { Total crystal } \\
\text { yield (g) }\end{array}$ & $\begin{array}{c}\text { Crystal yield in } \\
\text { size range } \\
\mathbf{\text { 1.4 }} \mathbf{~ m m ~ ( g ) ~}\end{array}$ & $\begin{array}{c}\text { \% Crystal yield } \\
\text { in size range } \\
\mathbf{1 . 4} \mathbf{~ m m}\end{array}$ \\
\hline 18 & 29.3 & 8.9 & 30.4 \\
\hline 18 & 27.6 & 19.1 & 69.1 \\
\hline 18 & 28.5 & 2.3 & 8.2 \\
\hline 18 & 34.4 & 0.5 & 2.4 \\
\hline 32 & 1.2 & 0.8 & 65.3 \\
\hline 32 & 0.2 & 0.1 & 65.3 \\
\hline 15 & 39.1 & 0.1 & 0.3 \\
\hline 15 & 30.3 & 0.7 & 2.4 \\
\hline
\end{tabular}

Table 3: $\mathrm{K}_{2} \mathrm{SO}_{4}$ crystal yields obtained in further experiments with Procedure $\mathrm{B}$ and additions of Calfax DB-45 surfactant. 
Some of the $18 \%$ runs gave very encouraging results and so, at this point, the decision was made to focus attention on Calfax DB-45, to take account of two additional variables (stirrer speed, and the addition of a period of crystallization at ambient temperature), and to work thus to obtain good combinations of yield and CSD reproducibly. This focus, which proved productive and led finally to the development of Procedure C, occupied the remainder of the project. Hence no further experiments were performed on Calfax 16L-35 or oleic acid surfactants.

Procedure $\mathrm{C}$ allows for additional precipitation because of the time at lower temperature. It is also reasonable to assume a final cooling to ambient temperature in an industrial setting, and hence, if additional yield can be obtained with suitable CSD, this would be very helpful. Consideration was also given to the effects of stirring speed, already identified as an important parameter by, for example, Aoun et al. (1999). A $4.00 \mathrm{~mm}$ sieve (mesh size 5) was also added to separate out the occasional larger (less useful) agglomerates.

Table 4 shows the results of experiments in which the solution was either transferred to a covered beaker (b) and allowed to stand at ambient temperature overnight, while being stirred at $400 \mathrm{rpm}$, or left in the crystallizer when the heating/cooling bath was turned off, and allowed to stand overnight at ambient temperature in the crystallizer (c) while being stirred at $400 \mathrm{rpm}$.

\begin{tabular}{|c|c|c|c|c|}
\hline Experiment & \% CMC & $\begin{array}{c}\text { Total crystal } \\
\text { yield (g) }\end{array}$ & $\begin{array}{c}\text { Crystal yield in } \\
\text { size range 1.40 } \\
\text { to 4.00 mm (g) }\end{array}$ & $\begin{array}{c}\text { \% Crystal yield } \\
\text { in size range 1.40 } \\
\text { to 4.00 mm }\end{array}$ \\
\hline b & 0 & 59.1 & 0.1 & 0.2 \\
\hline b & 0 & 59.4 & 3.2 & 5.3 \\
\hline c & 0 & 67.5 & 0.1 & 0.2 \\
\hline c & 0 & 64.7 & 10.6 & 16.4 \\
\hline b & 18 & 61.4 & 12.42 & 20.2 \\
\hline b & 18 & 64.9 & 50.1 & 77.1 \\
\hline b & 18 & 60.0 & 9.1 & 15.2 \\
\hline c & 18 & 67.1 & 17.4 & 25.9 \\
\hline c & 18 & 55.4 & 38.0 & 68.6 \\
\hline c & 18 & 71.0 & 46.2 & 10.5 \\
\hline b & 50 & 13.2 & 1.4 & 58.4 \\
\hline c & 50 & 34.0 & 19.9 & 10.2 \\
\hline b & 60 & 8.6 & 0.9 & 53.0 \\
\hline c & 60 & 59.4 & 31.5 & 21.0 \\
\hline b & 80 & 0 & 0 & 26.6 \\
\hline c & 80 & 23.4 & 4.9 & 26.7 \\
\hline b & 90 & 5.1 & 1.4 & \\
\hline c & 90 & 33.3 & 8.9 & \\
\hline & & & & \\
\hline
\end{tabular}

Table 4: $\mathrm{K}_{2} \mathrm{SO}_{4}$ crystal yields obtained in experiments with Procedure $\mathrm{C}$ and additions of Calfax DB-45 surfactant. [b: Solution transferred to beaker, stirred at $400 \mathrm{rpm}$. c: Solution stood in crystallizer, stirred at $400 \mathrm{rpm}$. 
Clear and consistent trends are visible in the total crystal yield as a function of surfactant concentration, most interesting among which is the absence of any reduction in total crystal yield at $18 \%$ CMC. There is no apparent difference between crystal yields from experiment types (b) and (c) at 0 or 18\% CMC. At 50\% CMC or higher, however, crystallization appears to be much more inhibited by the surfactant in the beaker than in the crystallizer. This difference in behavior may be associated with the slightly lower and more variable stirring speed attainable with the impeller used in the beaker. Crystal yields in the desired size range show clear trends but are still quite variable.

Table 5 shows the much more encouraging results of experiments in which the solution was either transferred to a covered beaker (b) and allowed to stand at ambient temperature overnight, while being stirred at 500-600 rpm, or left in the crystallizer when the heating/cooling bath was turned off, and allowed to stand overnight at ambient temperature in the crystallizer (c) while being stirred at $600 \mathrm{rpm}$.

\begin{tabular}{|c|c|c|c|c|}
\hline Experiment & $\begin{array}{c}\text { \% } \\
\text { CMC }\end{array}$ & $\begin{array}{c}\text { Total crystal } \\
\text { yield (g) }\end{array}$ & $\begin{array}{c}\text { Crystal yield in } \\
\text { size range 1.40 } \\
\text { to 4.00 } \mathbf{~ m m ~ ( g ) ~}\end{array}$ & $\begin{array}{c}\text { \% Crystal yield } \\
\text { in size range } \\
\mathbf{1 . 4 0} \text { to 4.00 } \mathbf{~ m m ~}\end{array}$ \\
\hline c & 0 & 65.2 & 0.2 & 0.3 \\
\hline b & 15 & 47.7 & 0 & 0 \\
\hline c & 15 & 67.8 & 0.1 & 0.2 \\
\hline b & 18 & 56.9 & 48.2 & 84.7 \\
\hline c & 18 & 65.0 & 52.3 & 80.8 \\
\hline b & 32 & 59.2 & 43.1 & 72.8 \\
\hline c & 32 & 66.1 & 50.6 & 76.6 \\
\hline c & 32 & 67.3 & 51.4 & 76.4 \\
\hline b & 50 & 54.9 & 8.6 & 15.7 \\
\hline c & 50 & 60.6 & 25.7 & 42.4 \\
\hline
\end{tabular}

Table 5: $\mathrm{K}_{2} \mathrm{SO}_{4}$ crystal yields obtained in experiments with Procedure $\mathrm{C}$ and additions of Calfax DB-45 surfactant. [b: Solution transferred to beaker, stirred at 500-600 rpm. c: Solution stood in crystallizer, stirred at $600 \mathrm{rpm}$.]

The following points should be noted from these results:

1. The total crystal yield is retained in the presence of the surfactant, even at the highest concentration used (50\% CMC). This is different from the results obtained with a stirrer speed of $400 \mathrm{rpm}$ (Table 4), in which there is a reduction in total yield at 50\% CMC, particularly in the experiments in which the solution was transferred to a beaker. There is some indication (Table 5) of a reduction in total yield in which the solution was transferred to the beaker and stirred at 500-600 rpm, but more experiments would be needed to determine whether this difference was significant. It is, in any case, a much 
smaller effect than that observed at the slower stirring speed, and may be associated with the slightly lower and more variable stirring speed attainable with the impeller used in the beaker. No decrease in total yield is evident in the crystallizer.

2. Excellent CSD enhancement is obtained at 18 and 32\% CMC (as discussed further below). The enhancement was much less at 50\% CMC and was not observed at all at 15\% CMC. The uniformity of the crystals produced is seen in Figure 10.

3. The results appear reproducible. More experiments are required to confirm this. Many experiments had been performed to reach this stage, however, and the results shown in Table 5 were obtained at the end of the project. Hence further repetition of these results must remain part of the recommended future work.

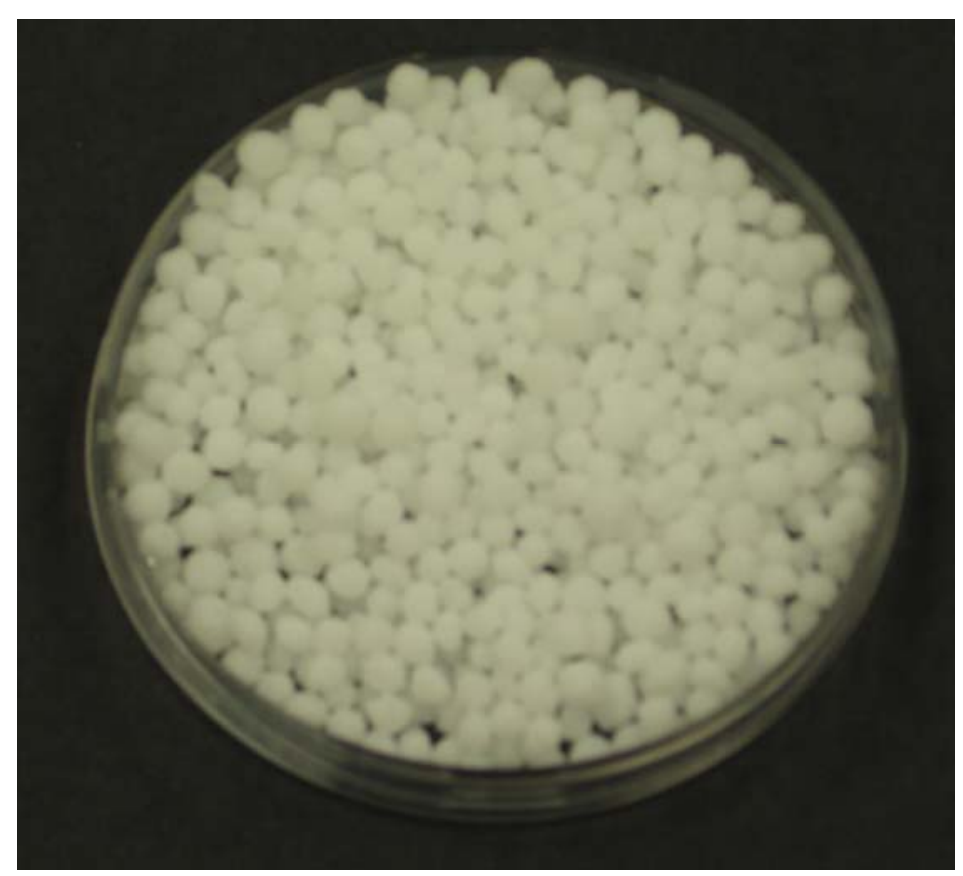

Figure 10: Potassium sulfate crystals grown with 18\% of the CMC of Calfax DB-45 added to the potassium sulfate mother liquor. [Solution stood in crystallizer, stirred at $600 \mathrm{rpm}$.]

Table 6 shows the crystal yields for 0 and 18 and $23 \%$ CMC (corresponding to those in Table 5) analyzed by size range. It can be seen that the majority of the crystals formed, particularly at $18 \%$ CMC, are in the 1.40 to $3.35 \mathrm{~mm}$ size range.

These results are particularly good when one considers the supersaturation and hence the total possible yield at ambient temperature. The solutions were prepared with $190.3 \mathrm{~g} / \mathrm{L}$ $\mathrm{K}_{2} \mathrm{SO}_{4}$. The solubility limit of $\mathrm{K}_{2} \mathrm{SO}_{4}$ at $25^{\circ} \mathrm{C}$ ( ambient temperature) is $120 \mathrm{~g} / \mathrm{L}$. Hence the maximum value of the total crystal yield that could be obtained in these experiments would be $70.3 \mathrm{~g}$ (assuming negligible evaporative water loss from the covered containers). Table 7 shows the crystal yields for 0 and $18 \%$ CMC as percentages of the maximum possible total yield. 


\begin{tabular}{|c|c|c|c|c|c|}
\hline $\begin{array}{c}\text { \% } \\
\text { CMC }\end{array}$ & $\begin{array}{c}\text { Crystal yield } \\
\mathbf{4 . 0 0} \mathbf{~ m m} \\
\text { (g) }\end{array}$ & $\begin{array}{c}\text { Crystal } \\
\text { yield, } \mathbf{4 . 0 0} \text { to } \\
\mathbf{3 . 3 5} \mathbf{~ m m} \text { (g) }\end{array}$ & $\begin{array}{c}\text { Crystal } \\
\text { yield, } \mathbf{1 . 4 0} \text { to } \\
\mathbf{3 . 3 5} \mathbf{~ m m} \text { (g) }\end{array}$ & $\begin{array}{c}\text { Crystal yield } \\
<\mathbf{1 . 4 0} \mathbf{~ m m} \\
\mathbf{( g )}\end{array}$ & $\begin{array}{c}\text { \% Crystal yield, } \\
\mathbf{1 . 4 0} \text { to 3.35 } \mathbf{~ m m}\end{array}$ \\
\hline 0 (c) & 1.7 & 0.1 & 0.1 & 63.3 & 0.15 \\
\hline 18 (b) & 0 & 0.2 & 48.0 & 8.8 & 84.4 \\
\hline 18 (c) & 0 & 0.2 & 52.1 & 12.5 & 80.2 \\
\hline 32 (b) & 0.3 & 1.8 & 41.3 & 15.1 & 69.8 \\
\hline 32 (c) & 0.6 & 0.8 & 49.8 & 14.9 & 75.3 \\
\hline 32 (c) & 1.4 & 3.7 & 47.7 & 14.6 & 70.9 \\
\hline
\end{tabular}

Table 6: $\mathrm{K}_{2} \mathrm{SO}_{4}$ crystal yields obtained in experiments with Procedure $\mathrm{C}$ and additions of Calfax DB-45 surfactant, analyzed by size range. [b: Solution transferred to beaker, stirred at 500-600 rpm. c: Solution stood in crystallizer, stirred at $600 \mathrm{rpm}$.

\begin{tabular}{|c|c|c|c|c|}
\hline $\begin{array}{c}\text { \% } \\
\text { CMC }\end{array}$ & $\begin{array}{c}\text { Total crystal } \\
\text { yield (g) }\end{array}$ & $\begin{array}{c}\text { Total crystal } \\
\text { yield as \% } \\
\mathbf{7 0 . 3} \mathbf{~ g}\end{array}$ & $\begin{array}{c}\text { Crystal yield } \\
\mathbf{( 1 . 4 0 ~ t o ~ 4 . 0 0} \\
\text { mm) in g }\end{array}$ & $\begin{array}{c}\text { Yield (1.40 } \\
\text { to 4.00 mm) } \\
\text { as \% 70.3 g }\end{array}$ \\
\hline 0 (c) & 65.2 & 92.7 & 0.2 & 0.3 \\
\hline 18 (b) & 56.9 & 80.9 & 48.2 & 68.6 \\
\hline 18 (c) & 65.0 & 92.5 & 52.3 & 74.4 \\
\hline
\end{tabular}

Table 7: $\mathrm{K}_{2} \mathrm{SO}_{4}$ crystal yields obtained in experiments with Procedure $\mathrm{C}$ and 0 or 18\% CMC of Calfax DB-45 surfactant, expressed as percentages of the maximum possible total yield. [b: Solution transferred to beaker, stirred at 500-600 rpm. c: Solution stood in crystallizer, stirred at $600 \mathrm{rpm}$.

The total crystal yields obtained are high, over $90 \%$ of the maximum attainable in the crystallizer (c) and $80 \%$ in the beaker (b). Crystals in the desired size range account for less than $1 \%$ of the maximum attainable crystal yield when grown with no surfactant at this stirring speed. The addition of 18\% CMC of Calfax DB-45, however, results in the production of $\sim 69$ (b) to 75 (c) \% of the maximum attainable total crystal yield having sizes in the desired range.

\section{$\underline{\text { Future work }}$}

It is suggested that future work include the following:

- Continue experiments with Calfax DB-45 to confirm reproducibility.

- Study the effects of addition of seed crystals both to the mother liquor alone and in the presence of surfactant. 
- Study the effects of high concentrations of magnesium chloride, in molar ratios of 3:1 to $3: 2\left(\mathrm{~K}_{2} \mathrm{SO}_{4}: \mathrm{MgCl}_{2}\right)$, with and without surfactant, to investigate the potential effect of recycled brines.

- Add potassium chloride to the potassium sulfate mother liquor to replicate more closely the potassium sulfate production process used by the New Mexico mining industry.

- Test the alkyl diphenyl oxide surfactants in a larger-scale apparatus or at a production facility.

- Conduct further experiments on oleic acid surfactants, particularly following sulfonation.

\section{CONCLUSION}

Substantial progress has been made in the use of alkyl diphenyl oxide (ADO) surfactants to enhance the crystal size distribution (CSD) of potassium sulfate $\left(\mathrm{K}_{2} \mathrm{SO}_{4}\right)$ crystals while obtaining high crystal yields. The effects of cooling rate, nucleation and growth temperatures, and stirring speed have been investigated, and an improved protocol for $\mathrm{K}_{2} \mathrm{SO}_{4}$ crystallization has been developed. The effects of oleic acid surfactants on the CSD of $\mathrm{K}_{2} \mathrm{SO}_{4}$ have been studied with no improvement in CSD being observed, but further experiments with sulfonated oleic acid are recommended.

The refined crystallization protocol has led to excellent results with Calfax DB-45 ADO surfactant. Under these conditions, there is little or no reduction in crystal yield in the presence of surfactant concentrations up to $50 \%$ of the critical micelle concentration (CMC). Total crystal yields with 18\% CMC of the surfactant correspond to high percentages ( $~ 81$ to $93 \%$ ) of the maximum attainable given the solubility limit at the growth temperature. Of these total crystal yields, 81 to $85 \%$ are in the desired size range (1.4 to $4 \mathrm{~mm}$ ), compared to less than $1 \%$ without surfactant. Larger numbers of experiments are required in order to confirm reproducibility.

\section{REFERENCES}

Aoun, M, E. Plasari, R. David, and J. Villermaux (1999). Chem. Eng. Sci. 54, 1161-1180.

Bujan, M., M. Sikiric, N. Filipovic-Vincekovic, N. Vdovic, N. Garti, and H. FurediMilhofer (2001). Langmuir 17, 6461-6470.

Chianese, A., F. Di Berardino, and A.G. Jones (1993). Chem. Eng. Sci. 48, 551-560.

Dash, S. R. and S. Rohani (1993). Chem. Eng. Comm. 125, 211-226.

Ghazy, S.E., M.A. Kabil, A.M. Shallaby, and N.S. Ammar (2001). Indian J. Chem. Technol. 8, 211-218.

Kabil, M. A. and S.E. Ghazy (1994). Sep. Sci. Technol. 29, 2533-2539. 
Kirk, R. E. and D.F. Othmer (1991). Encyclopedia of Chemical Technology. $4^{\text {th }}$ ed., Vol. 19, (Wiley: New York), pp. 1076-1080.

Linke, W. F. (1965). Solubilities: Inorganic and Metal-Organic Compounds. $4^{\text {th }}$ ed., Vol. 2, (American Chemical Society: Washington, D.C.), pp. 296-524.

Mauri, A. and M. Moret (2000). J. Crystal Growth 208, 599-614.

Meenan, P. and K.J. Roberts (1993). J. Mater. Sci. Letters 12, 1741-1744.

Mydlarz, J. and A.G. Jones (1989). Chem. Eng. Sci. 44, 1391-1402.

Noyes, R. (1965). Potash and Potassium Fertilizers 1966. Chemical Process Monograph No. 15, Noyes Development Corporation.

A Guide to the Application of Calfax Diphenyl Oxide Disulfonates. Accessed April 21, 2005 from Pilot Chemical Company Website: http://www.pilotchemical.com/pdf/CalfaxDipOxideDis85E.pdf

Searls, J.P. (2001). Potash. U.S. Geological Survey, Mineral Commodity Summaries, January 2001.

\section{LIST OF ACRONYMS AND ABBREVIATIONS}

ADO: alkyl diphenyl oxide

ASTM: American Society for Testing and Materials

CSD: crystal size distribution

CMC: critical micelle concentration

FESEM: field-emission scanning electron microscope

M: molar

MSMPR: mixed-suspension, mixed-product removal (crystallizer)

Rpm: revolutions per minute 
Appendix 26: Hydrometallurgical Processing of Chalcopyrite Concentrates (NV001) 


\section{FINAL TECHNICAL REPORT}

Contract Title and Number:

Crosscutting Technology Development at the Center

for Advanced Separation Technologies

(DE-FC26-02NT41607)

Sub-Recipient Project Title:

Hydrometallurgical Processing of Chalcopyrite

Concentrates

Principal Investigators:

M. C. Fuerstenau

M. Misra

Contact Address:

Chemical and Metallurgical Engineering

University of Nevada, Reno 89557

Subcontractor Address:

University of Nevada, Reno

Reno, NV 89557
Period of Performance:

Starting Date: 6/1/2005

Ending Date: 10/31/2007
Report Information:

Type: Final

Number:

Period:

Date: $\quad 1 / 16 / 07$

Code: NV003-Final

Contact Information:

Phone: (775) 784-4310

Fax: (775) 327-5059

E-Mail: mcf@unr.edu

Subcontractor Information:

Phone: (775) 784-4312

Fax: $\quad$ (775) 784-6680

E-Mail: jerry_best@vpaf.unr.edu 


\section{DISCLAIMER}

"This report was prepared as an account of work sponsored by an agency of the United States Government. Neither the United States Government nor any agency thereof, nor any of their employees, make any warranty, express or implied, nor assume any legal liability or responsibility for the accuracy, completeness, or usefulness of any information, apparatus, product, or process disclosed, or represents that its use would not infringe privately owned rights. Reference herein to any specific commercial product, process, or service by trade name, trademark, manufacturer, or otherwise does not necessarily constitute or imply endorsement, recommendation, or favoring by the United States Government or any agency thereof. The views and opinions of authors expressed herein do not necessarily state or reflect those of the United States Government or agency thereof." 


\begin{abstract}
Chalcopyrite leaching was studied with two techniques: (1) leaching experiments as a function of various leaching parameters: oxidant type and concentration, leaching time, particle size, temperature, pulp density, absence or presence of nanosize silica particles, absence or presence of nitrate, and absence or presence of ethylene glycol, and (2) electrochemical impedance spectroscopy (EIS) measurements. The effects of each of the leaching parameters on chalcopyrite dissolution is presented and discussed.

Elemental sulfur is formed as a reaction product of chalcopyrite leaching. This was verified with electron microscopy and x-ray fluorescence. Morphology of the adsorbed sulfur layer depends on oxidant and additives, such as nanosize silica particles, which, in turn, controls reaction kinetics. EIS was used to study the characteristics of the sulfur layer as well as reaction mechanisms.
\end{abstract}




\section{TABLE OF CONTENTS}

$\underline{\text { Page }}$

Disclaimer $\quad$ i

Abstract

Table of Contents

List of Tables

List of Figures

Executive Summary

Introduction

Experimental

Materials

iii

iv

Procedures

Leaching

Electrochemical Impedance Spectroscopy

Results and Discussion

Ferric as Oxidant

Particle Size

Pulp Density and Temperature

6

Nanosize Silica Addition

Nitrate Addition

$\begin{array}{ll}\text { Hydrogen Peroxide as Oxidant } & 11\end{array}$

Concentration $\quad 11$

Particle size $\quad 11$

Pulp Density $\quad 11$

$\begin{array}{ll}\text { Temperature } & 12\end{array}$

Ethylene Glycol $\quad 13$

Nanosize Silica Addition $\quad 14$

Reaction Mechanism $\quad 14$

$\begin{array}{ll}\text { Scanning Electron Microscopy } & 15\end{array}$

Electrochemical Impedance Spectroscopy $\quad 18$

Conclusion

References $\quad 22$

$\begin{array}{ll}\text { Appendix } & 24\end{array}$ 


\section{LIST OF TABLES}

$\underline{\text { Page }}$

Table 1. EIS Parameters for $\mathrm{H}_{2} \mathrm{SO}_{4}$ and $\mathrm{H}_{2} \mathrm{SO}_{4} / \mathrm{Fe}_{2}\left(\mathrm{SO}_{4}\right)_{3}$ Solutions .......... 20

Table 2. EIS Parameters for $\mathrm{H}_{2} \mathrm{SO}_{4}$ and $\mathrm{H}_{2} \mathrm{SO}_{4} / \mathrm{FeCl}_{3}$ Solutions ............. 21

Table 3. EIS Parameters for $\mathrm{H}_{2} \mathrm{SO}_{4} / \mathrm{H}_{2} \mathrm{O}_{2}$ Solutions ....................... 22 


\section{LIST OF FIGURES}

Figure 1. Experimental set-up for EIS studies

Figure 2. Dissolution of chalcopyrite concentrate $(-7 \mu \mathrm{m})$ with stoichiometric amounts of ferric sulfate and ferric chloride as a function of pulp density.

Figure 3. Electrochemical oxidation of chalcopyrite studied with Atomic Force Microscopy

Figure 4. Dissolution of chalcopyrite concentrate as a function of particle size and temperature with $\mathrm{Fe}_{2}\left(\mathrm{SO}_{4}\right)_{3}$ as oxidant

Figure 5. Dissolution of chalcopyrite as a function of leaching time and nanosize silica for 24 hours with $\mathrm{FeCl}_{3}$ as oxidant

Figure 6. Dissolution of chalcopyrite as a function of leaching time and nanosize silica for 72 hours with $\mathrm{FeCl}_{3}$ as oxidant

Figure 7. Dissolution of chalcopyrite as a function of leaching time and nanosize silica with $\mathrm{Fe}_{2}(\mathrm{SO})_{3}$ as oxidant

Figure 8. Dissolution of chalcopyrite concentrate as a function of sodium nitrate concentration with $\mathrm{Fe}_{2}\left(\mathrm{SO}_{4}\right)_{3}$ as oxidant

Figure 9. Dissolution of chalcopyrite as a function of $\mathrm{H}_{2} \mathrm{O}_{2}$ concentration and leaching time

Figure 10. Dissolution of chalcopyrite as a function of particle size and leaching time with $\mathrm{H}_{2} \mathrm{O}_{2}$ as oxidant

Figure 11. Dissolution of chalcopyrite as a function of pulp density with $\mathrm{H}_{2} \mathrm{O}_{2}$ as oxidant

Figure 12. Dissolution of chalcopyrite as a function of temperature and leaching time with $\mathrm{H}_{2} \mathrm{O}_{2}$ as oxidant

Figure 13. Dissolution of chalcopyrite as a function of ethylene glycol concentration and leaching time with $\mathrm{H}_{2} \mathrm{O}_{2}$ as oxidant

Figure 14. Hydrogen peroxide decomposition as a function of time

Figure 15. Dissolution of chalcopyrite as a function of leaching time and nanosize silica with $\mathrm{H}_{2} \mathrm{O}_{2}$ as oxidant 
Figure 16. Plot of $\left[1-(1-\alpha)^{1 / 3}\right]$ as a function of $\mathrm{H}_{2} \mathrm{O}_{2}$ concentration and leaching time

Figure 17. Electron micrograph of chalcopyrite surface prior to leaching ....... 16

Figure 18. Electron micrograph of chalcopyrite surface after leaching with

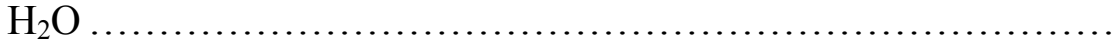

Figure 19. EDX spectrum of chalcopyrite surface after leaching with $\mathrm{H}_{2} \mathrm{O}_{2}$.

Figure 20. Electron micrograph of chalcopyrite surface after leaching with $\mathrm{H}_{2} \mathrm{O}_{2}$ in the presence of nanosize silica particles

Figure 21. EDX spectrum of chalcopyrite surface after leaching with $\mathrm{H}_{2} \mathrm{O}_{2}$ in the presence of nanosize silica particles

Figure 22. Equivalent electrical circuit of chalcopyrite leaching

Figure 23. Nyquist plot of chalcopyrite in $\mathrm{H}_{2} \mathrm{SO}_{4}$ solution

Figsure 24. Nyquist plot of chalcopyrite in $\mathrm{H}_{2} \mathrm{SO}_{4} / \mathrm{Fe}_{2}\left(\mathrm{SO}_{4}\right)_{3}$ solution 19

Figure 25. Nyquist plot of chalcopyrite in $\mathrm{H}_{2} \mathrm{SO}_{4} / \mathrm{FeCl}_{3}$ solution 20

Figure 26. Nyquist plot of chalcopyrite in $\mathrm{H}_{2} \mathrm{SO}_{4} / \mathrm{H}_{2} \mathrm{O}_{2}$ solution 


\section{EXECUTIVE SUMMARY}

It has been the desire of the metallurgical industry for many years to develop technology for processing chalcopyrite hydrometallurgically, and considerable study has been given this subject. In aqueous processing of chalcopyrite, a film of sulfur is formed on the chalcopyrite surface which impedes reaction rate. When ferric sulfate is used as oxidant, for example, the sulfur forms a compact layer, and the reaction is controlled by diffusion of reactants and products through this layer. The sulfur layer is more porous when ferric chloride and hydrogen peroxide are used as oxidants, and the reaction is controlled by chemical reaction at the surface. The objective of this research was to establish conditions under which the morphology of the sulfur layer can be altered to enhance leaching when modest temperature and pressure are employed. Two experimental approaches were involved: leaching and electrochemical impedance spectroscopy.

In the experimental work, sized samples of chalcopyrite mineral and chalcopyrite concentrate were investigated. In the leaching study, ferric iron, as sulfate and chloride, and hydrogen peroxide were used as oxidants. Other parameters investigated were: oxidant concentration, particle size, pulp density, temperature, nanosize silica addition, nitrate addition, and ethylene glycol addition. Of all of the parameters investigated, particle size is the most important.

Due to its effect on reaction rate, temperature of the system is also an important parameter. Virtually no leaching is possible at room temperature. As a result, most of the experiments were conducted at $65^{\circ}$ and $75^{\circ} \mathrm{C}$. Some experiments were conducted close to the boiling temperature of water, namely at $95^{\circ} \mathrm{C}$.

The addition of nanosize silica particles to the leaching system enhanced dissolution with ferric chloride, ferric sulfate and hydrogen peroxide as oxidants. Electron microscopy showed the presence of silica particles on the chalcopyrite surface, and x-ray fluorescence confirmed the presence of silica. These results suggest that the morphology of the sulfur layer is altered under these conditions, resulting in faster leaching kinetics.

The addition of sodium nitrate is also beneficial to chalcopyrite dissolution. When ferric sulfate was used as oxidant, dissolution of $-7 \mu \mathrm{m}$ particles was essentially complete in the presence of 0.1 molar nitrate, whereas only about 70 percent dissolution was obtained in its absence under these leaching conditions. Nitrate is a reasonably strong oxidant, and the oxidation potential of the system is raised in its presence which is probably responsible for the enhanced dissolution observed.

Pulp density is also important. As the pulp density is increased when oxidants are added in stoichiometric proportion, the effective concentration of oxidant per unit volume of solution is increased, and the reaction rate is increased. With hydrogen peroxide as oxidant, however, increasing the pulp density resulted in a decrease in dissolution. This is due to the fact that chalcopyrite functions as a catalyst for hydrogen peroxide decomposition. Further, 
cupric ion also functions as a catalyst for hydrogen peroxide decomposition, so as dissolution proceeds, greater decomposition of the oxidant occurs, and the reaction ceases.

To counter these phenomena, ethylene glycol was added to the leaching system. Ethylene glycol is known to stabilize hydrogen peroxide solution. In the absence of ethylene glycol, complete decomposition of hydrogen peroxide was noted after two hours in the chalcopyrite system. In the presence of $8 \mathrm{ml} / 1$ ethylene glycol, 80 percent of the hydrogen peroxide was still present after four hours of leaching.

Temperature is also an important parameter in hydrogen peroxide leaching of chalcopyrite. As the temperature is raised, the kinetics of reaction is increased, and greater dissolution of chalcopyrite is obtained. There is an optimum temperature, however. Above about $65^{\circ} \mathrm{C}$, the decomposition of hydrogen peroxide is greater than the increase in kinetics of chalcopyrite leaching, and dissolution is decreased.

The kinetics of chalcopyrite leaching with hydrogen peroxide are faster than with ferric sulfate and ferric chloride. This fact would seem to be related to the morphology of the sulfur formed on the chalcopyrite surface under these leaching conditions.

Electrochemical spectroscopy was also used to study these leaching systems. Simulation of measured impedance showed that reaction is mass transfer controlled when ferric sulfate is oxidant. With ferric chloride and hydrogen peroxide as oxidants, the kinetics is controlled by chemical reaction. 


\section{INTRODUCTION}

Chalcopyrite $\left(\mathrm{CuFeS}_{2}\right)$ is the most abundant and important source of copper metal available in the earth's crust. Over the years considerable research has been devoted to developing hydrometallurgical processes for leaching copper sulfide concentrates, especially chalcopyrite, at moderate temperature and pressure. Impetus for this research has been the desire for an economical alternative to smelting with its attendant generation of sulfur dioxide. Recent work has been directed to leaching at elevated temperature and pressure. This technology is now being commercialized (Marsden 2005). Considerable research has also been conducted utilizing bacteria for chalcopyrite oxidation.

Widely-used oxidants for the leaching of chalcopyrite are ferric chloride and ferric sulfate in an acidic medium. The ferric chloride leaching reaction can be written as (Dutrizac 1978):

$$
\mathrm{CuFeS}_{2}+3.5 \mathrm{FeCl}_{3} \rightarrow 0.5 \mathrm{CuCl}_{2}+0.5 \mathrm{CuCl}+4.5 \mathrm{FeCl}_{2}+\mathrm{S}^{\mathrm{o}}
$$

Lu et al. (2000) studied the effect of chloride ions on the dissolution of chalcopyrite in acidic media. They observed that the kinetics of dissolution was much faster in solutions containing chloride than in those that did not contain chloride. It is their contention that the role of chloride is to promote the formation of a more porous sulfur product.

Palmer et al. (1981) studied rate phenomena involved in the dissolution of chalcopyrite in chloride-bearing lixiviants. Kinetic observations indicated that the system is electrochemically controlled and that the anodic reaction products are $\mathrm{Cu}^{2+}, \mathrm{Fe}^{2+}$ and $\mathrm{S}^{\mathrm{o}}$, similar to what occurs with ferric sulfate.

In sulfuric acid solutions containing ferric sulfate, the reaction is (Miller et al. 1979):

$$
\mathrm{CuFeS}_{2}+4 \mathrm{Fe}^{3+} \rightarrow \mathrm{Cu}^{2+}+5 \mathrm{Fe}^{2+}+2 \mathrm{~S}^{\mathrm{o}}
$$

Hiroyoshi et al. (2000) observed that oxidative leaching of chalcopyrite with dissolved oxygen and/or ferric ions is promoted by high concentrations of ferrous ions in sulfuric acid solutions containing cupric ions. They consider that the reaction takes place in two steps: (1) reduction of chalcopyrite to $\mathrm{Cu}_{2} \mathrm{~S}$ by ferrous ions in the presence of cupric ions and (2) oxidation of the $\mathrm{Cu}_{2} \mathrm{~S}$ to cupric ions and elemental sulfur. $\mathrm{Cu}_{2} \mathrm{~S}$ is more amenable to oxidation than is chalcopyrite.

In ammoniacal solution the reaction is seen to produce thiosulfate and elemental sulfur (Reilly and Scott 1984):

$$
\mathrm{CuFeS}_{2}+4 \mathrm{NH}_{3}+6 \mathrm{OH}^{-} \rightarrow \mathrm{Cu}\left(\mathrm{NH}_{3}\right)_{4}{ }^{2+}+1 / 2 \mathrm{~S}_{2} \mathrm{O}_{3}{ }^{2-}+\mathrm{S}^{\mathrm{o}}+\mathrm{Fe}(\mathrm{OH})_{3}+3 / 2 \mathrm{H}_{2}+7 \mathrm{e}
$$

The sulfur layer that forms on the surface of the chalcopyrite in all of these reactions is passivating and highly protective. Hackl et al. (1995) suggest that this layer is composed of copper polysulfide, $\mathrm{CuS}_{\mathrm{n}}$. XPS analysis conducted by Balaz et al. (1996) revealed the 
existence of sulfur in three different chemical forms, $\mathrm{S}^{2-}, \mathrm{S}^{\circ}$ and $\mathrm{S}^{6+}$ when experiments were performed under combined bacterial and chemical leaching. Other investigators, such as Antonijevic et al. (1994) and Munoz et al. (1979), suggest that the sulfur is elemental in nature. Biegler and Swift (1976) observed the properties of sulfur to vary with experimental conditions. They suggest that the sulfur is amorphous and plastic. However, there is no real consistency among the various investigators as to the nature of the sulfur associated with the passive layer that is formed during chalcopyrite leaching. Nevertheless, it has been clearly established that the difficulty experienced in leaching chalcopyrite is due to the passive layer formation.

To increase recovery, investigators have tried a number of methods. One of the most successful techniques was developed by Miller et al. (1979) using silver catalysis. Recovery was almost $100 \%$, but the economics of using silver to extract copper precludes its use.

Ozone oxidation was tried by Halvik and Skrobian (1990). Again, this process has poor economics and has not been commercialized.

High oxidation potential offers a possibility of dissolving chalcopyrite. Chalcopyrite can be made the anode in an aqueous electrolyte with a counter electrode to complete the circuit. The anodic dissolution reaction (Ilangovan et al. 1975) can be written

$$
\mathrm{CuFeS}_{2} \leftrightarrows \mathrm{Cu}^{2+}+\mathrm{Fe}^{2+}+2 \mathrm{~S}^{\circ}+4 \mathrm{e}^{-} \text {(anodic) }
$$

The corresponding cathodic reaction is

$$
\mathrm{Cu}^{2+}+2 \mathrm{e}^{-} \leftrightarrows \mathrm{Cu} \text { (cathodic) }
$$

Various other studies have been conducted to dissolve chalcopyrite using an anodic potential; however, the sulfur-containing passive layer acts as an insulator between sulfide particles and increases the cell voltage tremendously with the reaction. This is a major problem which, with the low conductivity of ores, makes the electrochemical method impractical. As noted previously, the passive layer also exists in chemical leaching processing.

The objective of this investigation was to establish conditions under which chalcopyrite can be leached effectively at moderate temperature and pressure.

\section{EXPERIMENTAL}

Chalcopyrite ordered from Wards Earth Science and a chalcopyrite flotation concentrate were used in the study. Copper content of the mineral was $30.2 \%$ and that of the chalcopyrite concentrate was $28.0 \%$.

With the exception of nanosize silica $(50 \mathrm{~nm})$ which was a commercial product, reagentgrade chemicals were used in the study. The ferric chloride was anhydrous; the ferric sulfate 
was hydrated (average iron content, $22 \%$ ). The hydrogen peroxide was added as a solution $\left(30 \mathrm{wt} \% \mathrm{H}_{2} \mathrm{O}_{2}\right.$ ). De-ionized water was used.

Chalcopyrite mineral samples were prepared by dry grinding with a mortar and pestle and sized by screening. Chalcopyrite flotation concentrate samples of $-38 \mu \mathrm{m},-25 \mu \mathrm{m}$ and $-7 \mu \mathrm{m}$ were used.

The experimental work was divided into two areas: (1) leaching of sized particles of chalcopyrite and flotation concentrate, and (2) an electrochemical impedance investigation.

\section{Leaching}

Leaching was conducted in 500-ml or 1,000-ml reaction kettles. Sized chalcopyrite or concentrate samples of 1-50 grams were used in $200 \mathrm{ml}$ or $400 \mathrm{ml}$ of solution. The variables included: type and amount of oxidant, type and amount of nanosize silica, concentration of ethylene glycol, concentration of sodium nitrate, leaching time, and temperature.

The extent of chalcopyrite dissolution was established by measuring the copper concentration in solution with atomic absorption spectrometry and comparing that value with initial copper content in the solid.

\section{Oxidant}

The oxidants used were: ferric chloride, ferric sulfate and hydrogen peroxide. The amounts added varied from 0.01 to 0.5 molar.

\section{Temperature}

Temperature was varied from $25^{\circ}$ to $95^{\circ} \mathrm{C}$.

\section{Electrochemical Impedance Spectroscopic Characterization}

A Schlumberger Model SI 1286 electrochemical interface connected to a Schlumberger Model SI 12650 Gain Phase Analyzer, controlled by a computer, was used to study the electrochemical reaction in chalcopyrite leaching. Polished chalcopyrite samples were attached to a platinum wire using conductivity silver epoxy. Electrochemical experiments were conducted with various oxidants under various conditions. A photograph of the experimental cell and instrumentation is shown in Figure 1. 


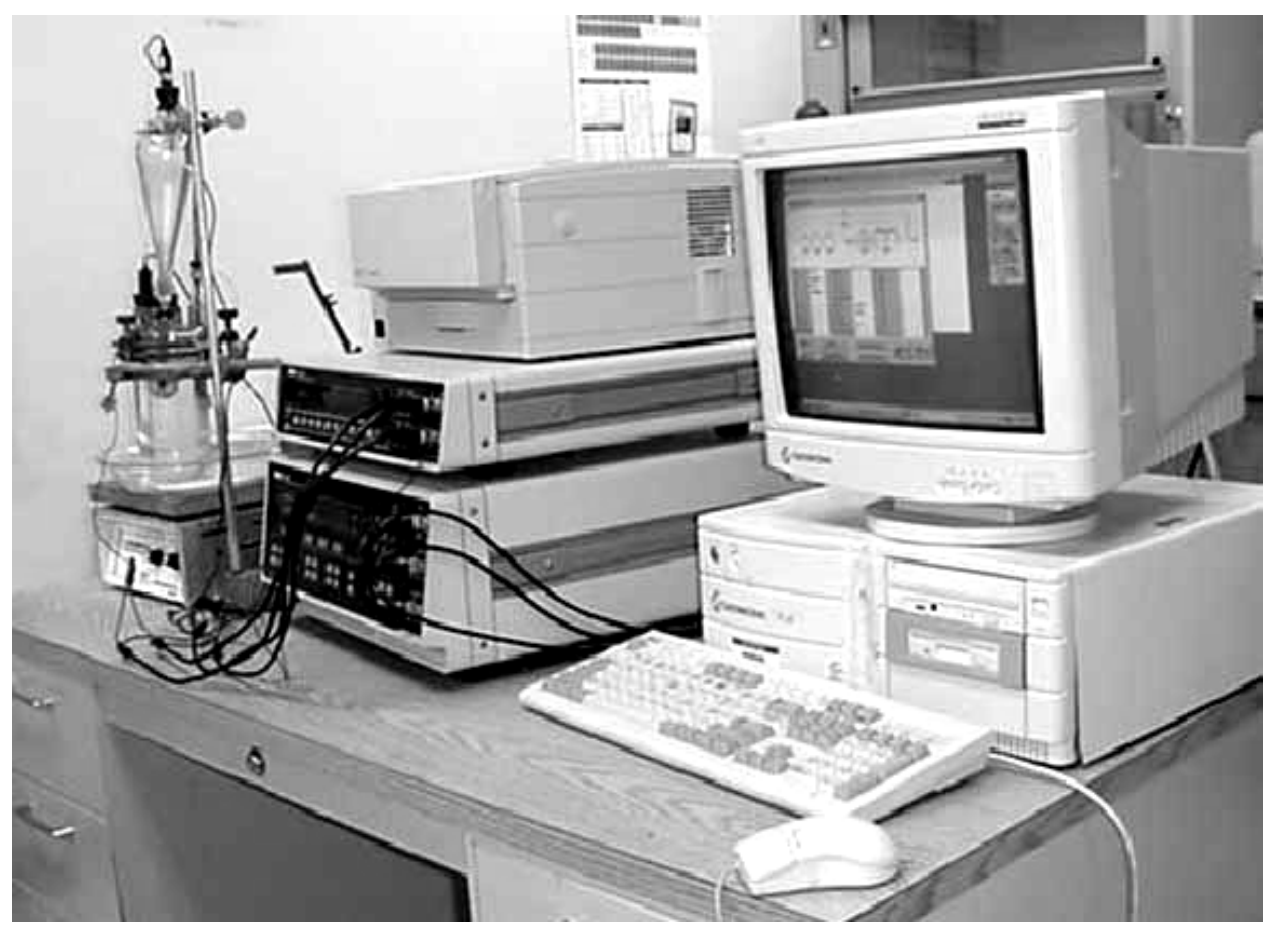

Figure 1. Electrochemical impedance spectroscopy.

\section{RESULTS AND DISCUSSION}

\section{Leaching Experiments}

\section{$\underline{\mathrm{Fe}^{3+} \text { as Oxidant }}$}

Leaching of chalcopyrite was conducted in the absence and presence of nanosize silica particles. Typical results obtained with $\mathrm{FeCl}_{3}$ and $\mathrm{Fe}_{2}\left(\mathrm{SO}_{4}\right)_{3}$ as oxidants are given in Figure 2 with stoichiometric ratios of oxidant to chalcopyrite. As can be noted, similarly to observations by others, $\mathrm{FeCl}_{3}$ is the more effective oxidant. This is attributed to the difference in adsorption characteristics of the sulfur produced during reaction. Sulfur is present as a compact layer on chalcopyrite when ferric sulfate is used, while sulfur is more porous in the case of ferric chloride. The kinetics of chalcopyrite dissolution with $\mathrm{FeCl}_{3}$ are controlled by chemical reaction of $\mathrm{Fe}^{3+}$ with the mineral surface, and in the case of $\mathrm{Fe}_{2}\left(\mathrm{SO}_{4}\right)_{3}$ as oxidant, are controlled by diffusion of reactants and products through the layer of sulfur. 


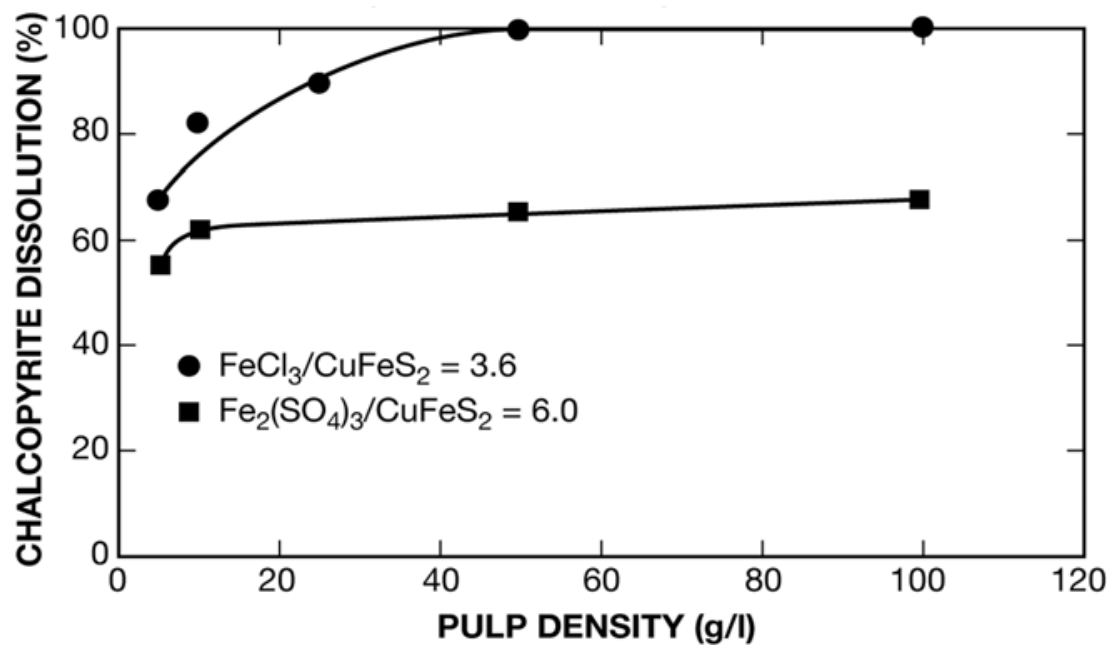

Figure 2. Dissolution of chalcopyrite concentrate $(-7 \mu \mathrm{m})$ with stoichiometric amounts of ferric sulfate and ferric chloride as a function of pulp density. Conditions: $0.5 \mathrm{~N} \mathrm{H}_{2} \mathrm{SO}_{4}, 75^{\circ} \mathrm{C}$, 8-hour leach.

The presence of sulfur on a chalcopyrite surface is readily apparent after oxidation. See Figure 3. These photographs were taken with an Atomic Force Microscope. Sulfur on the oxidized surface is 0.5 to 1.0 micron in height.

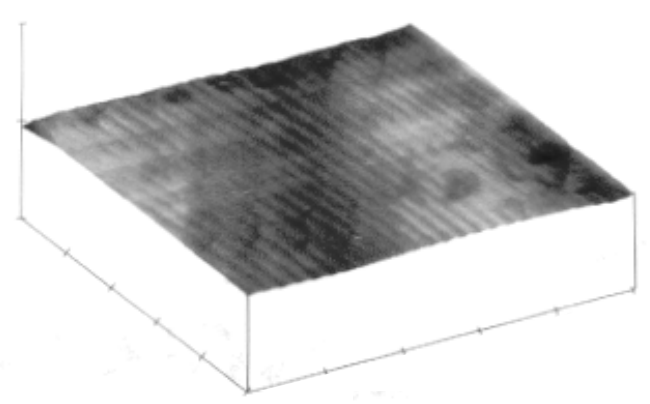

Initial surface of chalcopyrite

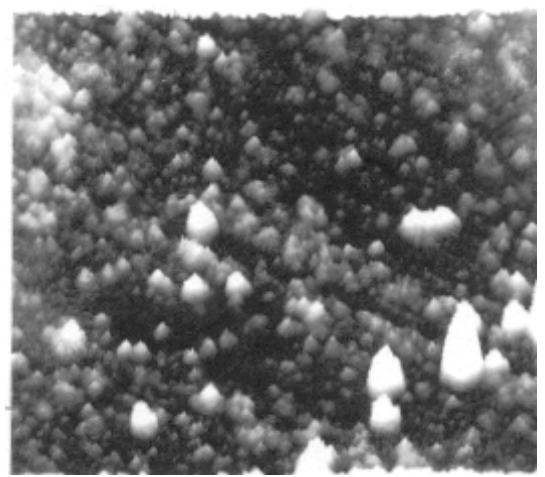

After 15 hours oxidation

Figure 3. Electrochemical oxidation of chalcopyrite studied with Atomic Force Microscopy.

\section{Effect of Particle Size}

The effect of particle size was examined with chalcopyrite which had been ground and sized to $-38 \mu \mathrm{m},-25 \mu \mathrm{m}$ and $-7 \mu \mathrm{m}$. Chalcopyrite dissolution was established at two temperatures in the presence of $0.5 \mathrm{~N} \mathrm{H}_{2} \mathrm{SO}_{4}, 300 \mathrm{~g} / \mathrm{L} \mathrm{Fe}_{2}\left(\mathrm{SO}_{4}\right)_{3}$ and pulp density of $50 \mathrm{~g} / \mathrm{L}$. As shown in Figure 4, about $65 \%$ of the $-7 \mu \mathrm{m}$ concentrate was dissolved after 8 hours of 
leaching, while only about $15 \%$ of the $-38 \mu \mathrm{m}$ concentrate was dissolved in that same time. Particle size is the most important parameter in chalcopyrite dissolution. With the very small particles sizes, as the reaction proceeds, the layer of sulfur will be smaller in thickness to impede transfer of reactants and products.

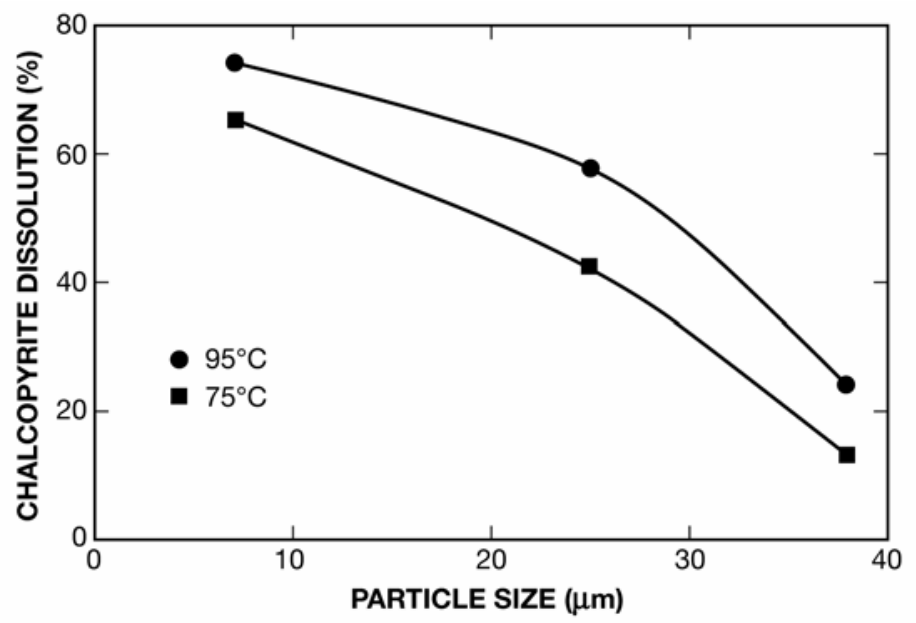

Figure 4. Dissolution of chalcopyrite concentrate as a function of particle size and temperature. Conditions: $300 \mathrm{~g} / 1 \mathrm{Fe}_{2}\left(\mathrm{SO}_{4}\right)_{3}, 0.5 \mathrm{~N} \mathrm{H}_{2} \mathrm{SO}_{4}$, pulp density $50 \mathrm{~g} / 1$, 8-hr leach.

\section{Effect of Pulp Density and Temperature}

The effect of pulp density was determined with $-7 \mu \mathrm{m}$ concentrate with ferric chloride and ferric sulfate in stoichiometric amounts. These results are given in Figure 2. As the pulp density was increased, the concentration of oxidant was increased accordingly. With increased level of oxidant, the kinetics of reaction increased. In the case of ferric chloride as oxidant, complete dissolution was noted after 8 hours of leaching with a pulp density of 50 $\mathrm{g} / \mathrm{L}$. The extent of dissolution with ferric sulfate as oxidant did not increase much with pulp density (or alternatively with increased level of oxidant). These results are also explained on the basis that sulfur forms as a compact passivating layer when ferric sulfate is used as oxidant, as opposed to a more porous and less coherent layer when ferric chloride is used.

Increasing the reaction temperature increases the reaction kinetics, and faster dissolution is experienced at elevated temperature (Figure 4).

\section{Effect of Nanosize Silica Addition}

The presence of nanosize silica increases the rate and extent of dissolution. This may be seen in Figures 5,6 and 7. Various amounts of nanosilica were added to a system containing $-38 \mu \mathrm{m}$ chalcopyrite particles in the presence of ferric chloride as oxidant. After 24 hours of leaching, the extent of chalcopyrite dissolution increased by a factor of about 1.5 when 17.5 $\mathrm{g} / \mathrm{L}$ nanosilica was added. The results obtained for a period of 72 hours are shown in Figure 6. The presence of silica on the surface of chalcopyrite will have a major effect on the 
modality of the sulfur layer and, hence, the dissolution of chalcopyrite. These effects are discussed in greater detail in the section, Scanning Electron Microscopy.

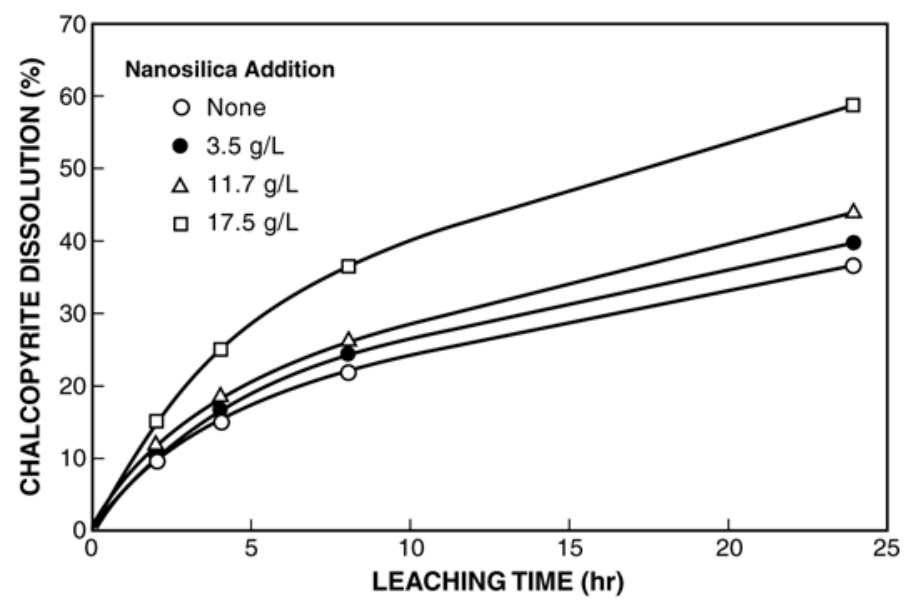

Figure 5. Dissolution of chalcopyrite $(-38 \mu \mathrm{m})$ as a function of leaching time and nanosize

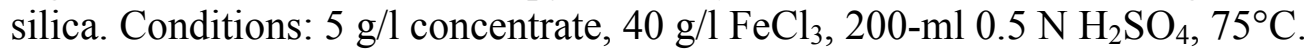

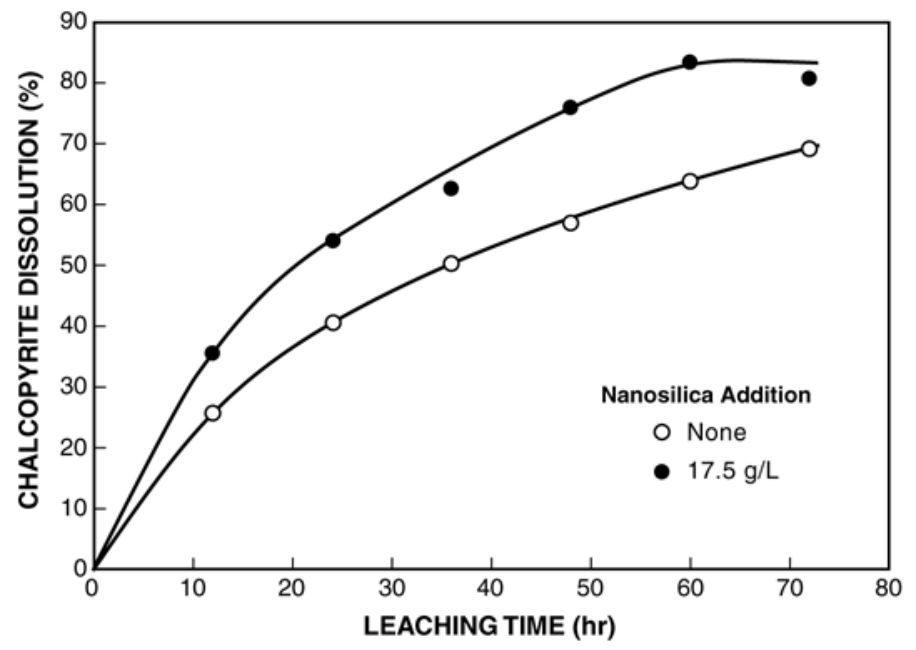

Figure 6. Dissolution of chalcopyrite $(-38 \mu \mathrm{m})$ as a function of leaching time in the absence and presence of nanosize silica. Conditions: $5 \mathrm{~g} / \mathrm{l}$ concentrate, $40 \mathrm{~g} / 1 \mathrm{FeCl}_{3}$, 200-ml $0.5 \mathrm{~N} \mathrm{H}_{2} \mathrm{SO}_{4}, 75^{\circ} \mathrm{C}$.

Similar experiments were conducted with ferric sulfate as oxidant. As can be noted in Figure 7, similar enhancement in leaching was obtained. 


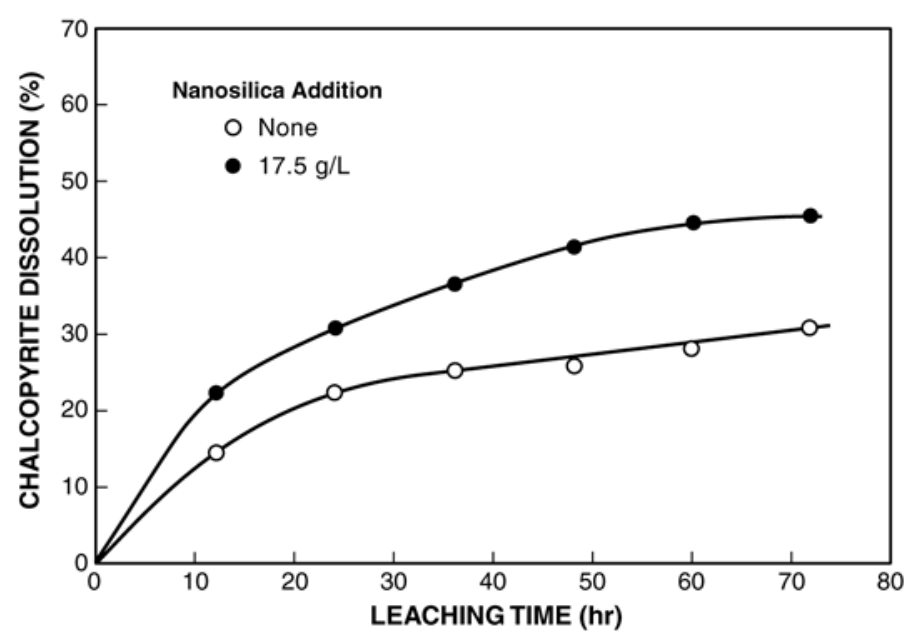

Figure 7. Dissolution of chalcpyrite $(-38 \mu \mathrm{m})$ as a function of leaching time in the absence and presence of nanosize silica. Conditions: $5 \mathrm{~g} / 1$ concentrate, $30 \mathrm{~g} / 1 \mathrm{Fe}_{2}\left(\mathrm{SO}_{4}\right)_{3}, 200-\mathrm{ml} 0.5 \mathrm{~N}$ $\mathrm{H}_{2}\left(\mathrm{SO}_{4}, 75^{\circ} \mathrm{C}\right.$.

\section{Effect of Sodium Nitrate Addition}

Nitrate is a relatively strong oxidant, and the addition of $\mathrm{NaNO}_{3}$ was examined in the presence of ferric sulfate. Leaching of $-7 \mu \mathrm{m}$ chalcopyrite concentrate as a function of sodium nitrate concentration is given in Figure 8 . When $30 \mathrm{~g} / \mathrm{L}$ ferric sulfate was added and the system was leached for 8 hours at $95^{\circ} \mathrm{C}$, dissolution of chalcopyrite of about $70 \%$ was achieved in the absence of nitrate. With an addition of $0.1 \mathrm{M}$ sodium nitrate, dissolution was essentially complete. The higher oxidation potential of the solution in the presence of nitrate is responsible for the enhanced dissolution kinetics.

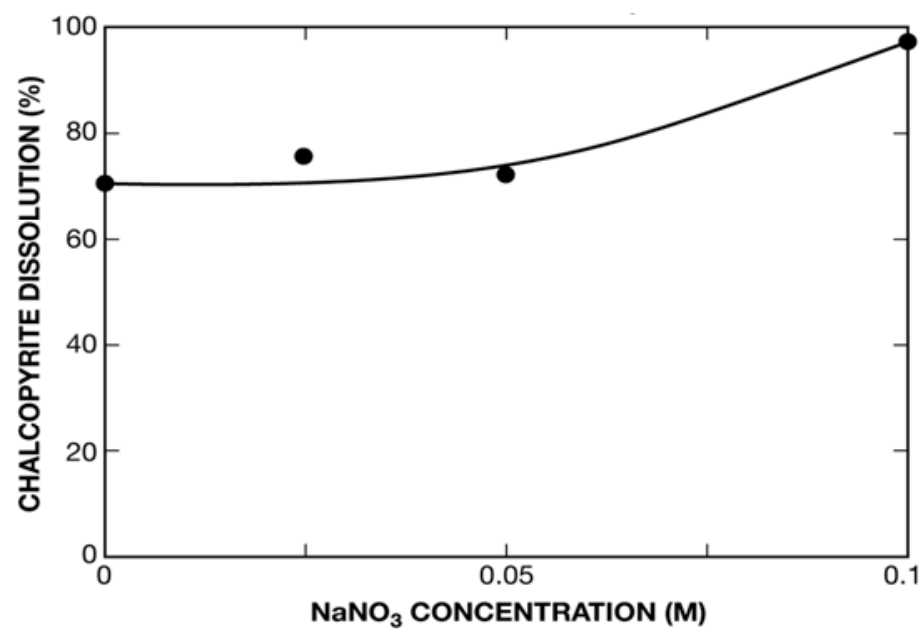

Figure 8. Dissolution of chalcopyrite concentrate $(-7 \mu \mathrm{m})$ as a function of sodium nitrate concentration. Conditions: $30 \mathrm{~g} / 1 \mathrm{Fe}_{2}\left(\mathrm{SO}_{4}\right)_{3}, 0.5 \mathrm{~N} \mathrm{H}_{2} \mathrm{SO}_{4}$, pulp density $5.0 \mathrm{~g} / 1,95^{\circ} \mathrm{C}, 8-\mathrm{hr}$ leach. 


\section{$\underline{\mathrm{H}}_{2} \underline{\mathrm{O}}_{2} \underline{\text { as Oxidant }}$}

\section{Effect of $\mathrm{H}_{2} \mathrm{O}_{2}$ Concentration}

Hydrogen peroxide is a strong oxidant, and the efficacy of using this reagent in dissolving chalcopyrite was also studied. Dissolution as a function of $\mathrm{H}_{2} \mathrm{O}_{2}$ concentration and leaching time is presented in Figure 9. With an addition of $0.048 \mathrm{M}$, about $20 \%$ of the chalcopyrite was dissolved after 4 hours of leaching. Dissolution was increased to about 70 $\%$ when $0.4 \mathrm{M}$ was added. These experiments were carried out at $65^{\circ} \mathrm{C}$ and $8 \mathrm{ml} / \mathrm{L}$ ethylene glycol. As expected, the kinetics of reaction was increased with higher concentrations of oxidant. The effect of ethylene glycol is discussed later.

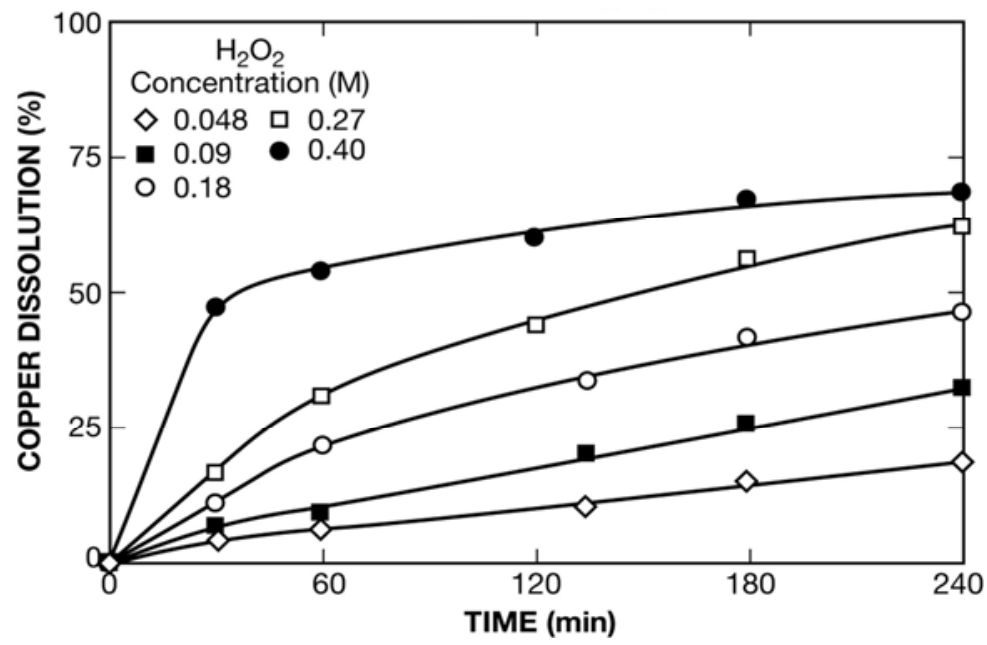

Figure 9. Dissolution of chalcopyrite $(-38 \mu \mathrm{m})$ as a function of hydrogen peroxide concentration and leaching time. Conditions: $1.25 \mathrm{~g} / 1$ chalcopyrite, $8 \mathrm{ml} / 1$ ethylene glycol, 400-ml $1.0 \mathrm{M} \mathrm{H}_{2} \mathrm{SO}_{4}, 65^{\circ} \mathrm{C}$.

\section{Effect of Particle Size}

The effect of particle size on the kinetics and extent of leaching was also examined. Chalcopyrite was hand ground and sized by screening. The results obtained when $1.25 \mathrm{~g} / \mathrm{L}$ chalcopyrite was leached with $0.26 \mathrm{M} \mathrm{H}_{2} \mathrm{O}_{2}$ in acidic medium at $65^{\circ} \mathrm{C}$ are shown in Figure 10. Since smaller particle size presents greater surface area for reactants, the kinetics was increased as the particle size was reduced.

\section{Effect of Pulp Density}

The stability of hydrogen peroxide is sensitive to cupric ion concentration and to chalcopyrite itself. As a result pulp density assumes a major role in this system. The dissolution of $-38 \mu \mathrm{m}$ chalcopyrite with stoichiometric amounts of hydrogen peroxide is shown in Figure 11. The extent of dissolution was reduced from about $75 \%$ at a pulp density of $2.5 \mathrm{~g} / \mathrm{L}$ to about $35 \%$ at $10 \mathrm{~g} / \mathrm{L}$. Higher pulp density is desirable and necessary, but the sensitivity of peroxide to both cupric ion and chalcopyrite rules out this possibility. 


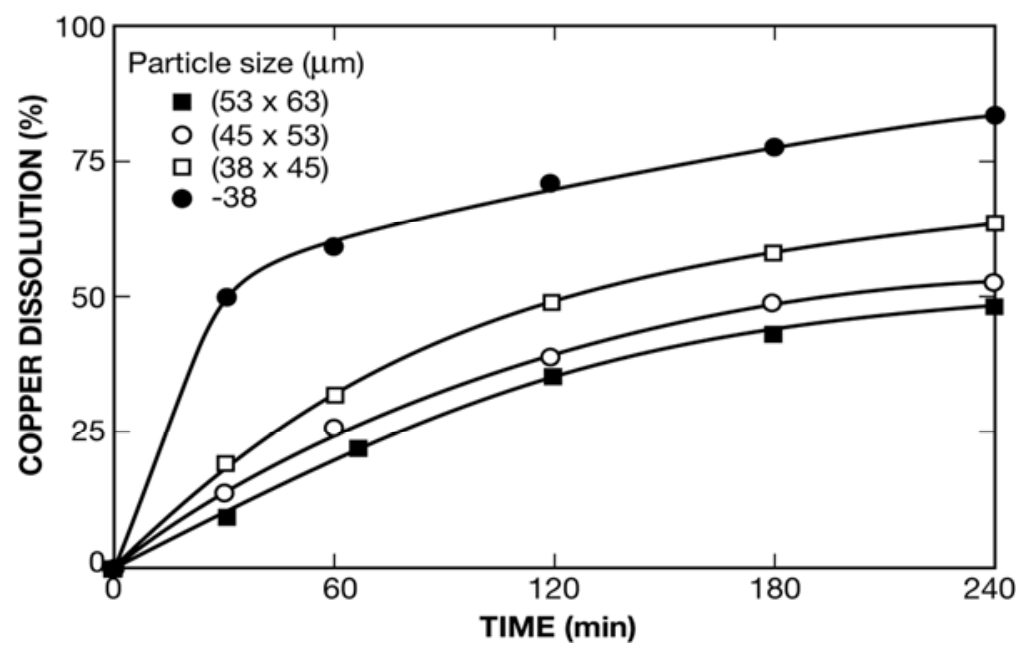

Figure 10. Dissolution of chalcopyrite as a function of particle size and leaching time. Conditions: $1.25 \mathrm{~g} / \mathrm{l}$ chalcopyrite, $0.26 \mathrm{M} \mathrm{H}_{2} \mathrm{O}_{2}, 8 \mathrm{ml} / \mathrm{l}$ ethylene glycol, $400-\mathrm{ml} 1.0 \mathrm{M} \mathrm{H}_{2} \mathrm{SO}_{4}$, $65^{\circ} \mathrm{C}$.

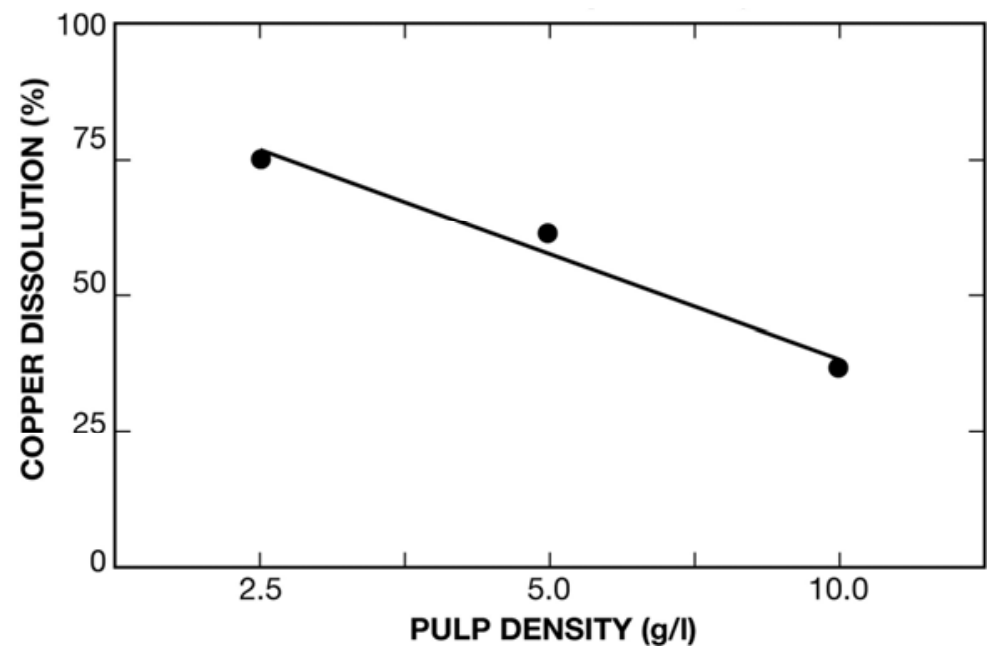

Figure 11. Dissolution of chalcopyrite $(-38 \mu \mathrm{m})$ as a function of pulp density. Conditions: stoichiometric $\mathrm{H}_{2} \mathrm{O}_{2}, 8 \mathrm{ml} / 1$ ethylene glycol, 400-ml $1.0 \mathrm{M} \mathrm{H}_{2} \mathrm{SO}_{4}, 65^{\circ} \mathrm{C}$, 4-hr leach.

\section{Effect of Temperature}

Temperature is always an important parameter in leaching systems, and chalcopyrite dissolution was determined at three temperatures, namely $25^{\circ}, 45^{\circ}$ and $65^{\circ} \mathrm{C}$. Normally, the higher is the temperature, the higher is the reaction rate. As shown in Figure 12, after 24 of hours of leaching, a temperature of $45^{\circ} \mathrm{C}$ appears to be optimum. This is due to the fact that hydrogen peroxide decomposes more rapidly at higher temperature. The optimum temperature will necessarily be between room temperature and some elevated temperature. The 'plateauing' after about five hours of leaching is due to the decomposition of peroxide. 
Obviously, at $45^{\circ} \mathrm{C}$ the kinetics of leaching is faster than the kinetics of peroxide decomposition.

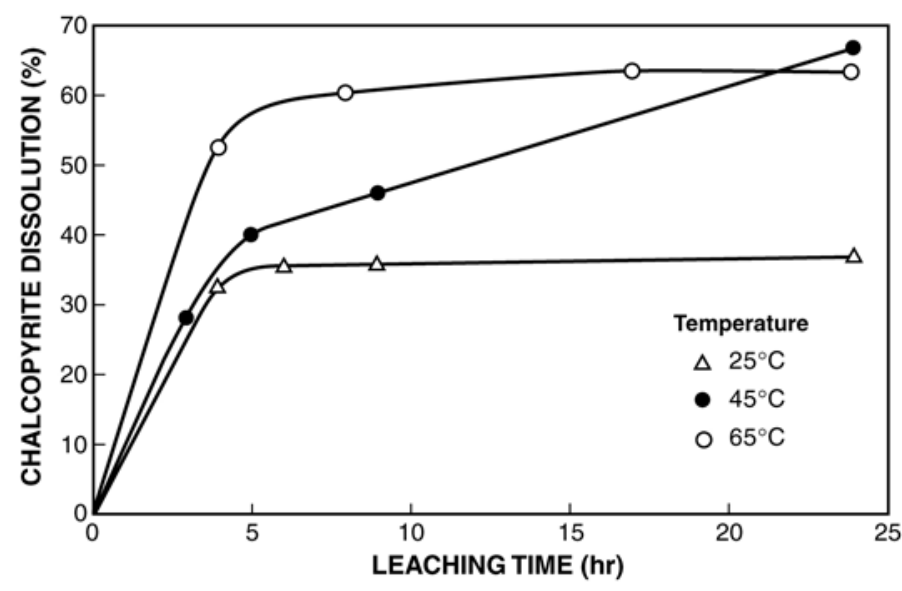

Figure 12. Dissolution of chalcopyrite $(-38 \mu \mathrm{m})$ as a function of temperature and leaching time. Conditions: $1.25 \mathrm{~g} / 1$ chalcopyrite, $3.0 \mathrm{~g} / 1 \mathrm{H}_{2} \mathrm{O}_{2}, 3.5 \mathrm{~g} / 1$ nanosilica, $400-\mathrm{ml} 0.5 \mathrm{~N} \mathrm{H}_{2} \mathrm{SO}_{4}$.

\section{Effect of Ethylene Glycol}

It is known that ethylene glycol stabilizes hydrogen peroxide, and experiments were undertaken to determine the effect of this reagent on leaching. The results presented in Figure 13 show the important effect that ethylene glycol has on chalcopyrite leaching. The addition of $8 \mathrm{ml} / \mathrm{L}$ glycol increased the extent of reaction by about a factor of four as compared with that obtained in its absence.

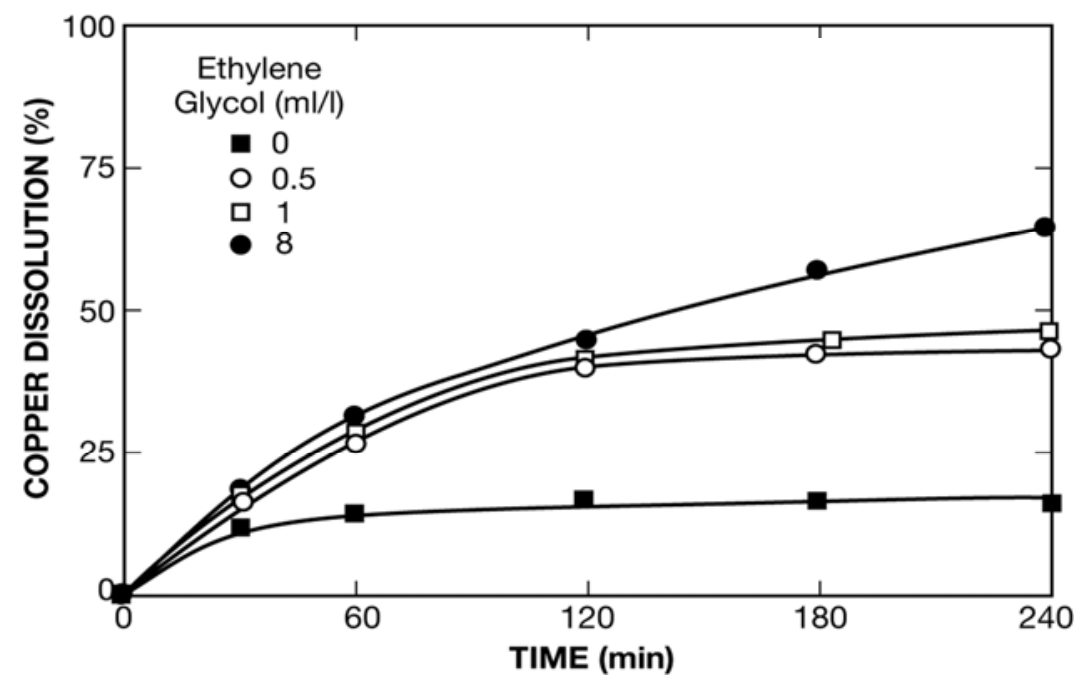

Figure 13. Dissolution of chalcopyrite $(-38 \mu \mathrm{m})$ as a function of ethylene glycol concentration and leaching time. Conditions: $1.25 \mathrm{~g} / \mathrm{l}$ chalcopyrite, $0.26 \mathrm{M} \mathrm{H}_{2} \mathrm{O}_{2}$, 400-ml $1.0 \mathrm{M} \mathrm{H}_{2} \mathrm{SO}_{4}, 65^{\circ} \mathrm{C}$. 
The stability of hydrogen peroxide in the absence and presence of ethylene glycol in a leaching system at $65^{\circ} \mathrm{C}$ was also examined. As can be noted Figure 14, in the absence of ethylene glycol, decomposition of hydrogen peroxide was essentially complete after 2 hours of leaching. In the presence of $8 \mathrm{ml} / 1$ ethylene glycol, only about $25 \%$ of the hydrogen peroxide initially present was decomposed after 4 hours.

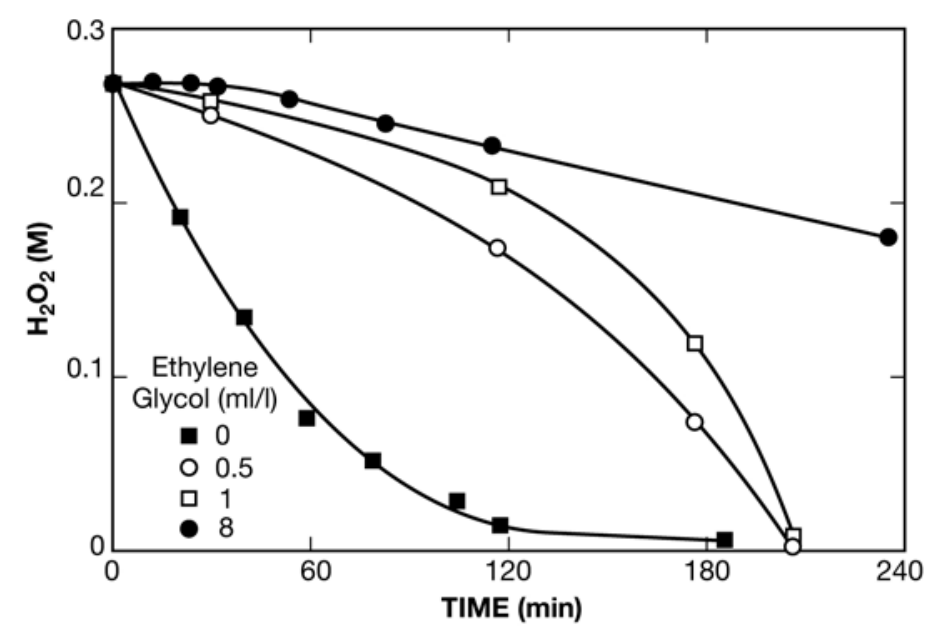

Figure 14. Hydrogen peroxide decomposition as a function of time. Conditions: $1.25 \mathrm{~g} / \mathrm{l}$ chalcopyrite $(-38 \mu \mathrm{m}), 0.26 \mathrm{M} \mathrm{H}_{2} \mathrm{O}_{2}, 400-\mathrm{ml} 1.0 \mathrm{M} \mathrm{H}_{2} \mathrm{SO}_{4}, 65^{\circ} \mathrm{C}$.

\section{Effect of Nanosize Silica Addition}

Nanosize silica additions were also made to these systems. These results are shown in Figure 15. There is approximately a three-fold increase in dissolution in the presence of 3.5 $\mathrm{g} / \mathrm{L}$ nanosize silica. Again, after about five hours of leaching, dissolution is essentially independent of time. This is due to the decomposition of hydrogen peroxide after this period of time. Since nanosize silica is placed in ethylene glycol for dispersion, some of the increase in dissolution can be attributed to the presence of ethylene glycol.

\section{Reaction Mechanism}

It is known that chalcopyrite dissolution with ferric chloride is controlled by chemical reaction at the chalcopyrite surface and that ferric sulfate oxidation is controlled by diffusion through the sulfur product layer. Experiments were run at various concentrations of hydrogen peroxide to establish the reaction mechanism in this system. Shrinking core models enable establishment of reaction mechanism. When a plot of $\left[1-(1-\alpha)^{1 / 3}\right]$ versus time yields a linear relationship, surface chemical reaction is controlling. Figure 16 shows this plot for various peroxide concentrations. Very good correlation can be noted indicating that chemical reaction between peroxide and the chalcopyrite surface is controlling the system. 


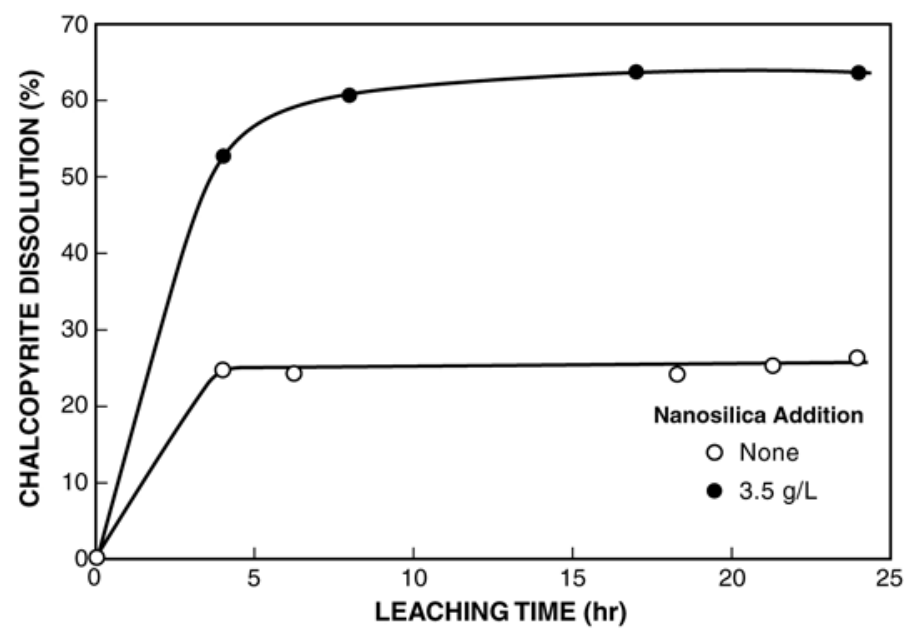

Figure 15. Dissolution of chalcopyrite $(-38 \mu \mathrm{m})$ as a function of leaching time in the absence and presence of nanosize silica. Conditions: $1.25 \mathrm{~g} / \mathrm{l}$ chalcopyrite, $3.0 \mathrm{~g} / \mathrm{l} \mathrm{H}_{2} \mathrm{O}_{2}, 400-\mathrm{ml} 0.5 \mathrm{~N}$ $\mathrm{H}_{2} \mathrm{SO}_{4}, 65^{\circ} \mathrm{C}$.

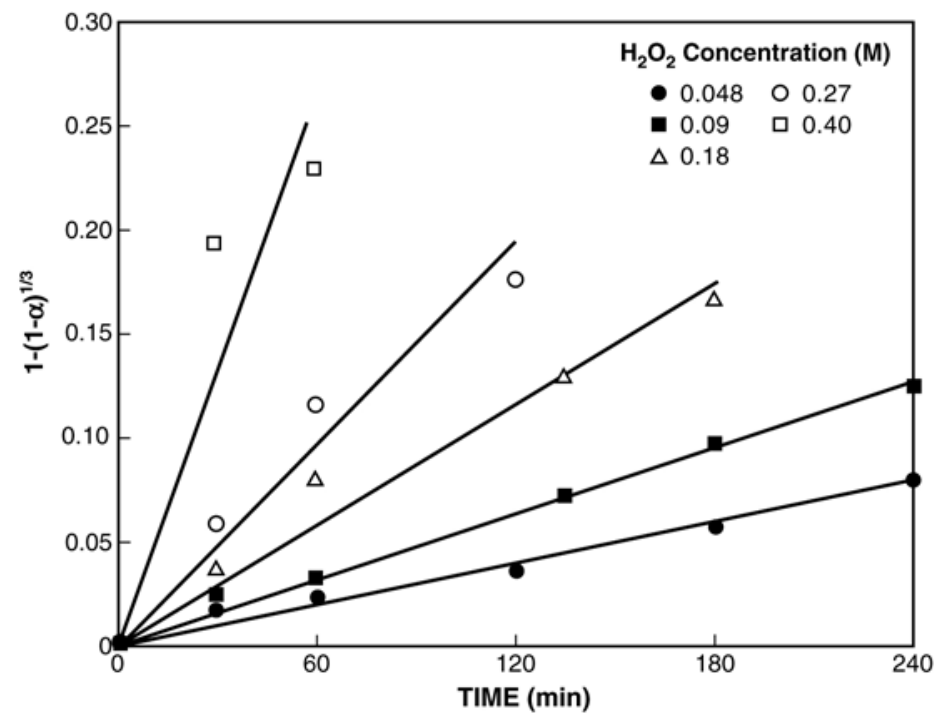

Figure 16. Plot of $\left[1-(1-\alpha)^{1 / 3}\right]$ as a function of hydrogen peroxide concentration and leaching time. Conditions: $1.25 \mathrm{~g} / \mathrm{lchalcopyrite}(-38 \mu \mathrm{m}), 8 \mathrm{ml} / \mathrm{l}$ ethylene glycol, $400-\mathrm{ml}$ $1.0 \mathrm{M} \mathrm{H}_{2} \mathrm{SO}_{4}, 65^{\circ} \mathrm{C}$.

\section{Scanning Electron Microscopy}

The surface of chalcopyrite was examined prior to and after leaching with ferric ion and hydrogen peroxide as oxidants in acidic medium in the absence and presence of nanosize silica. An electron micrograph of chalcopyrite prior to leaching is shown in Figure 17. A micrograph of chalcopyrite that was leached with hydrogen peroxide in a sulfuric acid solution at $\mathrm{pH} 1.5$ and $50^{\circ} \mathrm{C}$ for 24 hours is shown in Figure 18. The sulfur formed under 
these conditions is readily apparent. The light particles are sulfur particles on the dark background of chalcopyrite. An EDX spectrum of this surface shows a major sulfur peak (Figure 19). This peak includes the diffraction from the sulfur in the layer and the sulfur contained in the mineral.

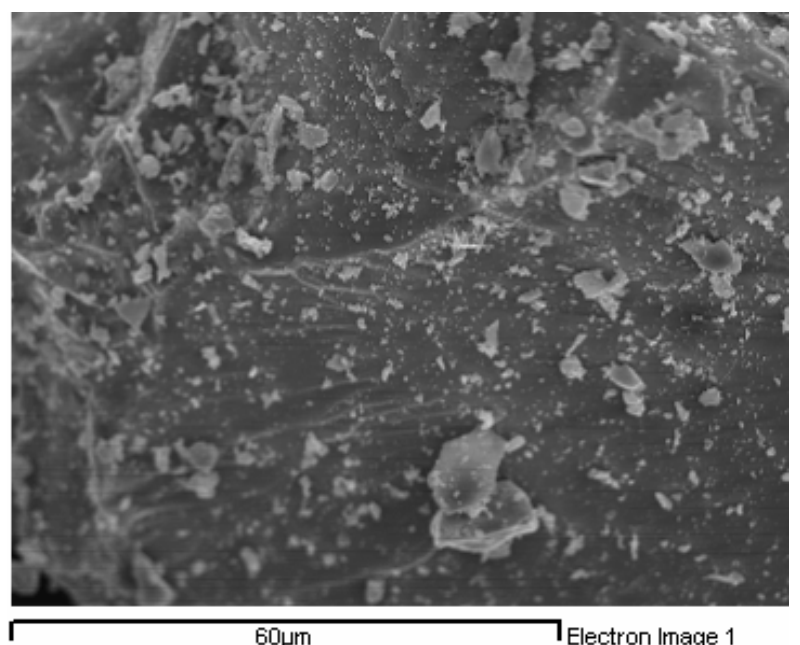

Figure 17. Electron micrograph of chalcopyrite particle prior to leaching.

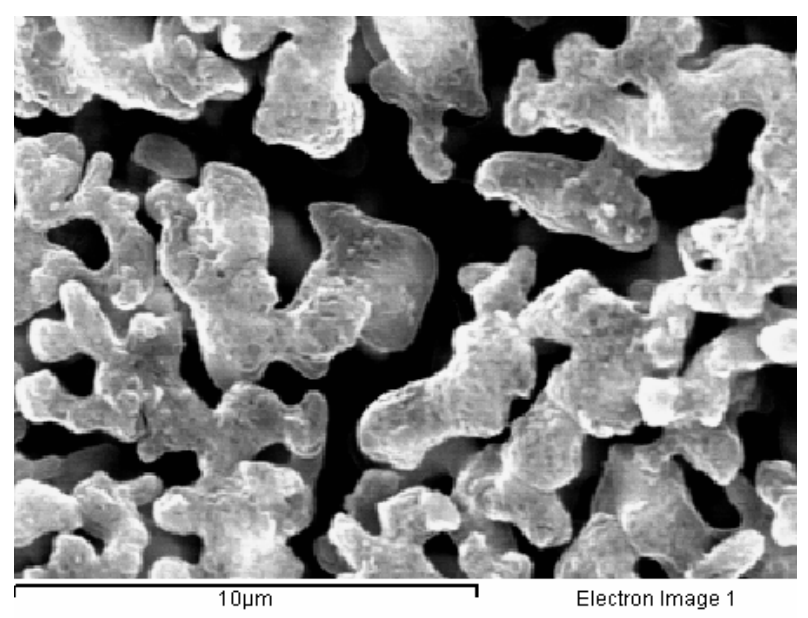

Figure 18. Electron micrograph of chalcopyrite surface after leaching with hydrogen peroxide.

When nanosize silica is added to the leaching system, crystals of $\mathrm{SiO}_{2}$ can be seen to cover the chalcopyrite surface (see Figure 20). The three clusters of light colored particles are sulfur. The EDX spectrum of this surface shows a silicon peak (Figure 21). 


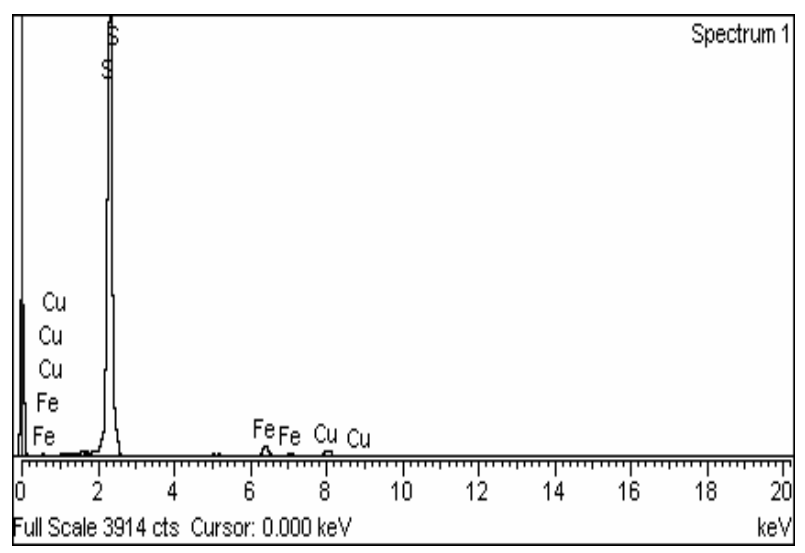

Figure 19. EDX spectrum of chalcopyrite surface after leaching with hydrogen peroxide.

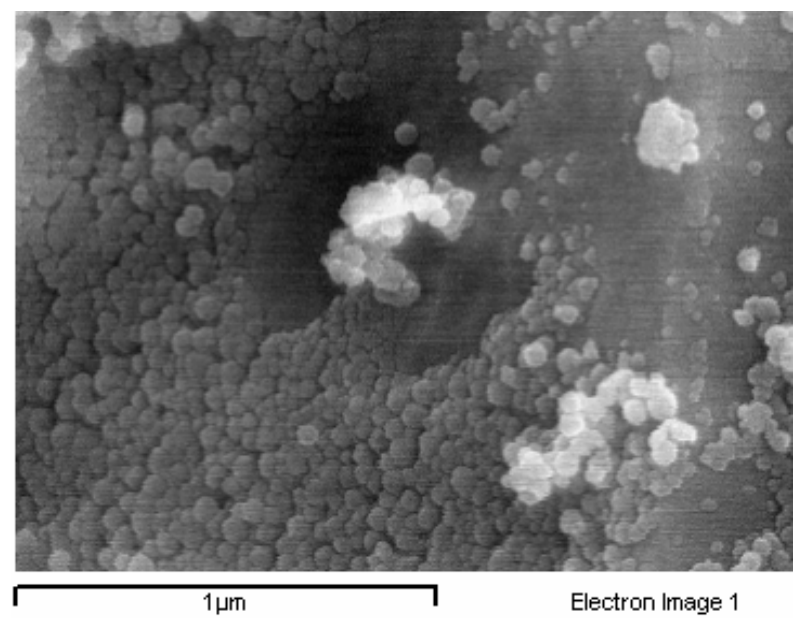

Figure 20. Electron micrograph of chalcopyrite surface after leaching with hydrogen peroxide in the presence of nanosize silica.

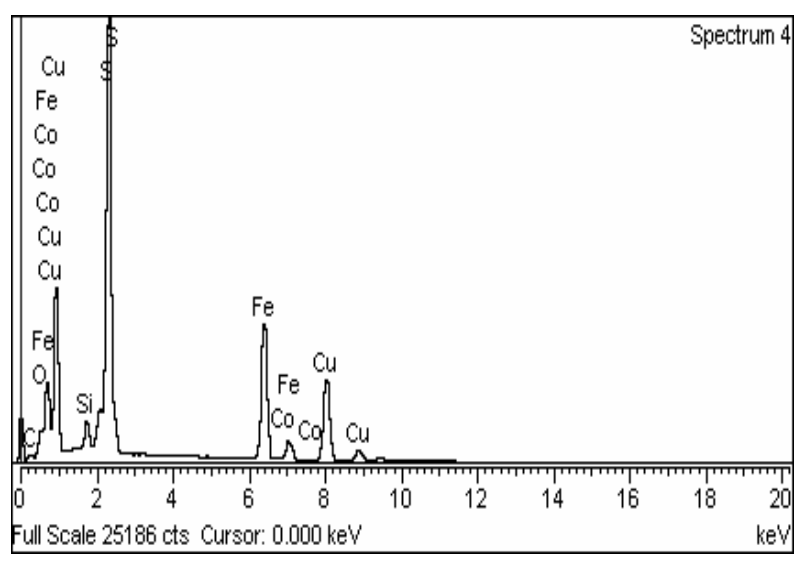

Figure 21. EDX spectrum of chalcopyrite surface after leaching with hydrogen peroxide in the presence of nanosize silica. 


\section{Electrochemical Impedance Spectroscopy}

Electrochemical impedance spectroscopy is a useful technique for studying the kinetics of chalcopyrite leaching. In these studies three electrodes are used: chalcopyrite as the working electrode, a saturated calomel electrode as reference, and a platinum electrode as a counter electrode. A voltage is impressed on the counter electrode and working electrode, and the frequency of the applied potential changes continuously. Impedance is then measured between the chalcopyrite surface and the counter electrode.

A schematic of the electrical circuit that simulates the chalcopyrite electrode layersolution is shown in Figure 22. The definitions of the electrical elements used to construct the equivalent circuit are listed in the Appendix. Values of these elements are assumed and adjusted until the electrical response fits the plot that is measured experimentally.

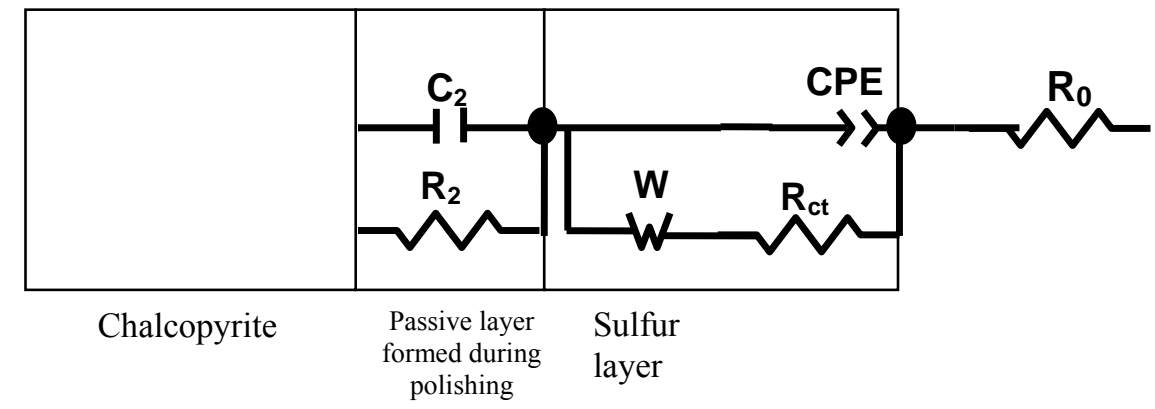

Figure 22. Equivalent electrical circuit of chalcopyrite in leaching system.

Three plots are obtained in this system; one of them, a Nyquist plot, is shown in Figure 23. In this plot real impedance, z', is plotted as a function of imaginary impedance, z'. The term, imaginary impedance, includes the capacitance and resistance of the oxidized layer on chalcopyrite formed from the electrode preparation, the capacitance and resistance of the electrical double layer and, also, those of the sulfur layer formed on the chalcopyrite surface during leaching.

Another element in the use of this technology is the Warburg element. This is needed because the capacitance and resistance are assumed to be that in a vacuum, and this parameter accounts for the solution and for the sulfur in the adsorbed layer. One component of the Warburg element, W-R, accounts for the difficulty in mass transfer through the sulfur layer.

Nyquist plots of chalcopyrite in sulfuric acid alone, ferric sulfate and ferric chloride, and hydrogen peroxide solutions have been determined. The data for sulfuric acid and ferric sulfate are shown in Figures 23 and 24. It is noted that the impedance of chalcopyrite surface increases with leaching time in both cases which indicates greater formation of elemental sulfur on the chalcopyrite surface as leaching proceeds. 


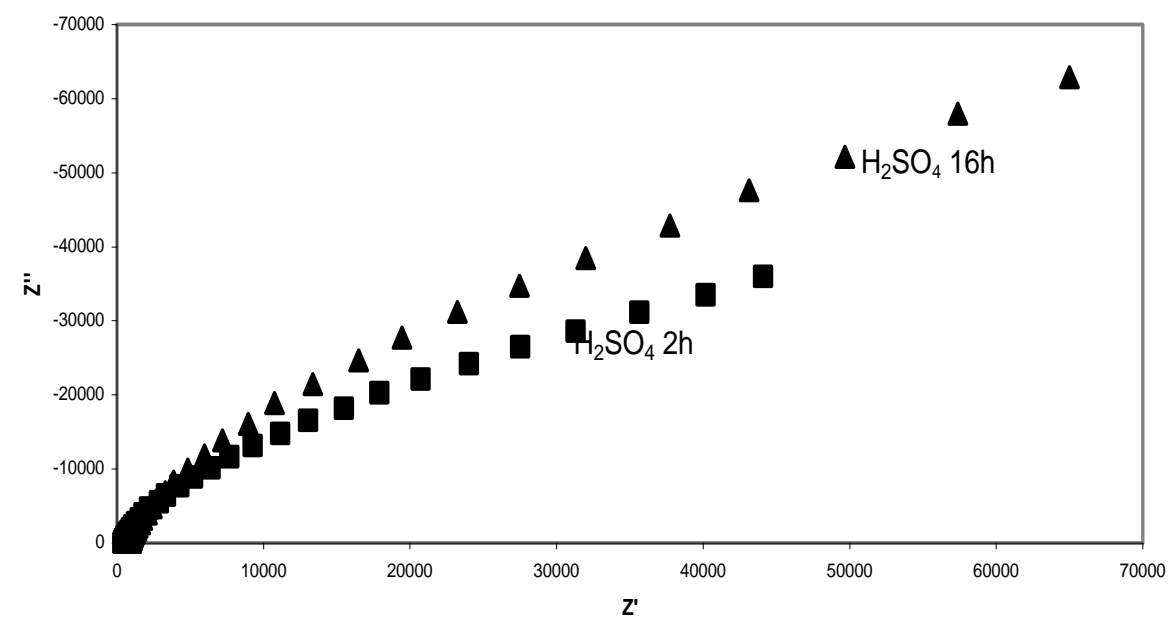

Figure 23. Nyquist plot of chalcopyrite in $0.5 \mathrm{~N} \mathrm{H}_{2} \mathrm{SO}_{4}$ after two periods of leaching. Ambient temperature.

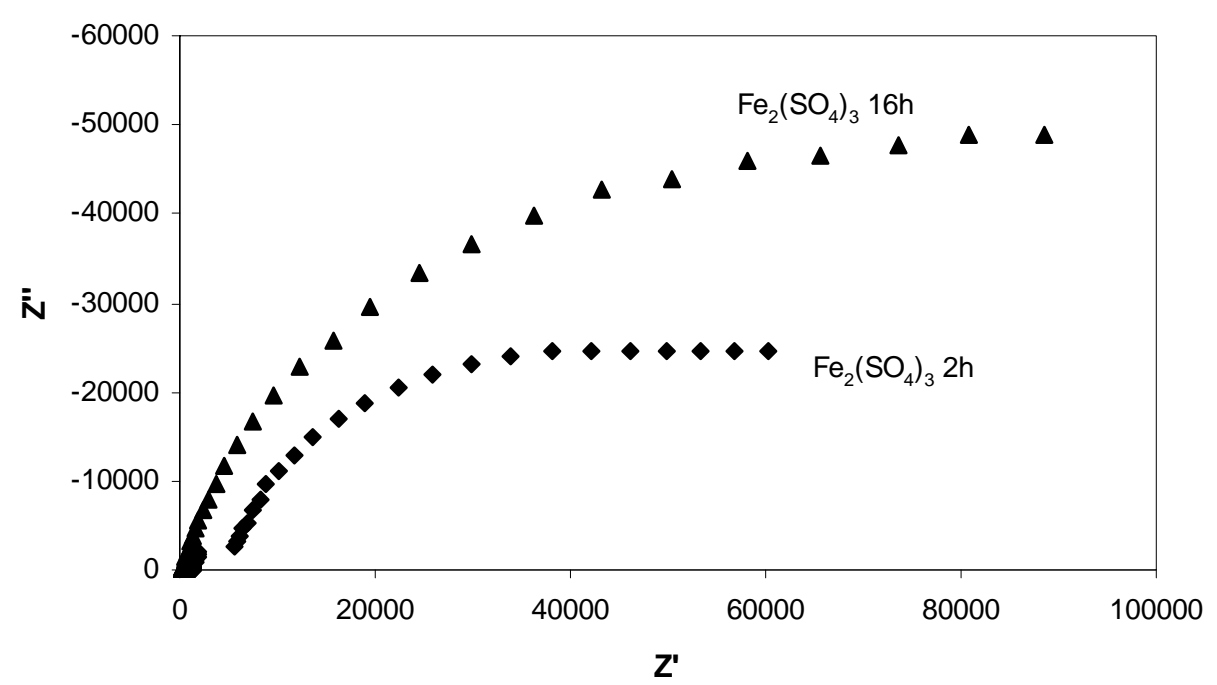

Figure 24. Nyquist plot of chalcopyrite after two periods of leaching in the presence of $30 \mathrm{~g} / 1$ $\mathrm{Fe}_{2}\left(\mathrm{SO}_{4}\right)_{3}$ and $0.5 \mathrm{~N} \mathrm{H}_{2} \mathrm{SO}_{4}$ after different periods of leaching. Ambient temperature.

The values assumed in simulating the circuits are given for two periods of leaching in Table 1. The electrode was leached for 24 hours in sulfuric acid solution. After this period of time, ferric sulfate was added, and the system was leached for another 24 hours. The values of most interest are Rct, the resistance to charge transfer, and W-R, the resistance to mass transfer. Comparison of these two values in both systems shows that the W-R is high which supports the premise that mass transfer of reactants and products is controlling in this system. 
Table 1. EIS parameters in $\mathrm{H}_{2} \mathrm{SO}_{4}$ and $\mathrm{Fe}_{2}\left(\mathrm{SO}_{4}\right)_{3}$ solutions.

\begin{tabular}{|c|c|c|c|c|c|c|c|c|c|}
\hline $\begin{array}{c}\text { Time, } \\
\text { h }\end{array}$ & $\begin{array}{l}\mathbf{R}_{\mathrm{ct}} \\
\mathbf{K} \Omega\end{array}$ & $\begin{array}{c}\text { CPE-T, } \\
10^{-6} \mathbf{F}\end{array}$ & $\begin{array}{c}\text { CPE- } \\
\mathbf{P}\end{array}$ & $\begin{array}{c}\mathbf{R}_{2}, \\
\Omega\end{array}$ & $\begin{array}{c}\mathrm{C}_{2}, \\
10^{-10} \mathrm{~F}\end{array}$ & $\begin{array}{c}\text { W-R, } \\
\text { K } \Omega\end{array}$ & W-T & W-P & $\begin{array}{c}\mathbf{R}_{\mathbf{0}}, \\
\Omega\end{array}$ \\
\hline \multicolumn{10}{|c|}{$\mathrm{H}_{2} \mathrm{SO}_{4}$ only } \\
\hline 2 & 12 & 8.0 & 0.86 & 400 & 7.4 & 240 & 32 & 0.43 & 1 \\
\hline 16 & 31 & 6.6 & 0.87 & 760 & 2.6 & 320 & 32 & 0.425 & 1 \\
\hline \multicolumn{10}{|c|}{$\mathrm{Fe}_{2}\left(\mathrm{SO}_{4}\right)_{3} / \mathrm{H}_{2} \mathrm{SO}_{4}$} \\
\hline 2 & 29 & 5.2 & 0.89 & 510 & 8.2 & 90 & 32 & 0.37 & 1 \\
\hline 16 & 51 & 4.7 & 0.876 & 575 & 3.6 & 158 & 32 & 0.30 & 1 \\
\hline
\end{tabular}

A Nyquist plot of chalcopyrite in a ferric chloride system is presented in Figure 25. In this case a chalcopyrite electrode was leached with sulfuric acid solution for one hour. Then, ferric chloride was added, and impedance measurements were made after one and two hours. It can be noted that impedance was reduced in the presence of ferric chloride and that the impedance was the same for one and two hours of leaching.

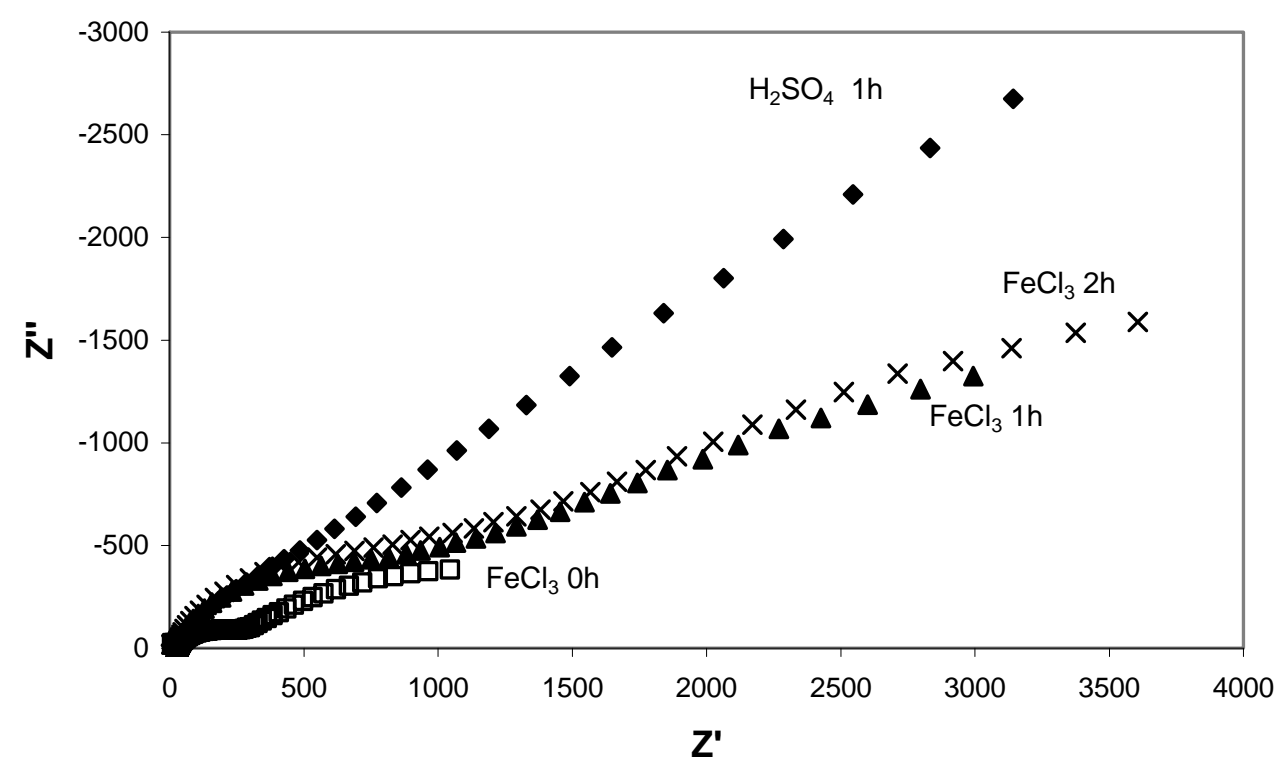

Figure 25. Nyquist plot of chalcopyrite in the presence of $19 \mathrm{~g} / 1 \mathrm{FeCl}_{3}$ and $0.5 \mathrm{~N} \mathrm{H}_{2} \mathrm{SO}_{4}$ after different periods of leaching. Ambient temperature.

EIS parameters are listed for this system in Table 2. It can be noted that values of W-R and Rct for sulfuric acid listed in Table 2 are different from those in Table 1.This is due to the fact that different electrodes were prepared for each of the systems. It can also be noted that the W-R is substantially lower in the presence of ferric chloride as opposed to sulfuric acid only. This fact in combination with real impedance measurements indicates that chalcopyrite leaching with ferric chloride is chemical reaction controlled. 
Table 2. EIS parameters in $\mathrm{H}_{2} \mathrm{SO}_{4}$ and $\mathrm{FeCl}_{3}$ solutions.

\begin{tabular}{|c|c|c|c|c|c|c|c|c|c|}
\hline $\begin{array}{c}\text { Time, } \\
\text { h }\end{array}$ & $\begin{array}{l}\mathbf{R}_{\mathrm{ct}}, \\
\mathbf{K} \boldsymbol{\Omega}\end{array}$ & $\begin{array}{c}\text { CPE-T, } \\
10^{-6} \mathrm{~F}\end{array}$ & $\begin{array}{c}\text { CPE- } \\
\text { P }\end{array}$ & $\begin{array}{l}\mathbf{R}_{\mathbf{2}}, \\
\mathbf{\Omega}\end{array}$ & $\begin{array}{c}\mathrm{C}_{2}, \\
10^{-10} \mathbf{F}\end{array}$ & $\begin{array}{l}\text { W-R, } \\
\text { K } \Omega\end{array}$ & W-T & W-P & $\begin{array}{l}\mathbf{R}_{\mathbf{0}}, \\
\mathbf{\Omega}\end{array}$ \\
\hline \multicolumn{10}{|c|}{$\mathrm{H}_{2} \mathrm{SO}_{4}$ only } \\
\hline 0.5 & 0.3 & 40 & 0.825 & 15 & 44 & 38 & 120 & 0.47 & 1 \\
\hline 1 & 0.32 & 34 & 0.825 & 17 & 36 & 31 & 120 & 0.435 & 1 \\
\hline \multicolumn{10}{|c|}{$\mathrm{FeCl}_{3} / \mathrm{H}_{2} \mathrm{SO}_{4}$} \\
\hline 0.5 & 0.42 & 8.4 & 0.87 & 25 & 35 & 5.1 & 20 & 0.32 & 1 \\
\hline 1 & 0.46 & 7.5 & 0.87 & 23 & 35 & 6.5 & 20 & 0.32 & 1 \\
\hline 2 & 0.52 & 6.9 & 0.87 & 22 & 45 & 6.8 & 12 & 0.33 & 1 \\
\hline
\end{tabular}

A Nyquist plot for the chalcopyrite - peroxide system after 2 and 16 hours of leaching is presented in Figure 26. In this case $0.5 \mathrm{~N} \mathrm{H}_{2} \mathrm{SO}_{4}$ and $0.09 \mathrm{M} \mathrm{H}_{2} \mathrm{O}_{2}$ were added together at the start of the experiment. It can be noted that impedance of the system is increased with leaching time, indicating that the sulfur layer is increased in thickness. The morphology of the sulfur layer must be different from that obtained when ferric sulfate is used as oxidant, however, since the leaching reaction is chemically controlled in the peroxide system (Figure $16)$.

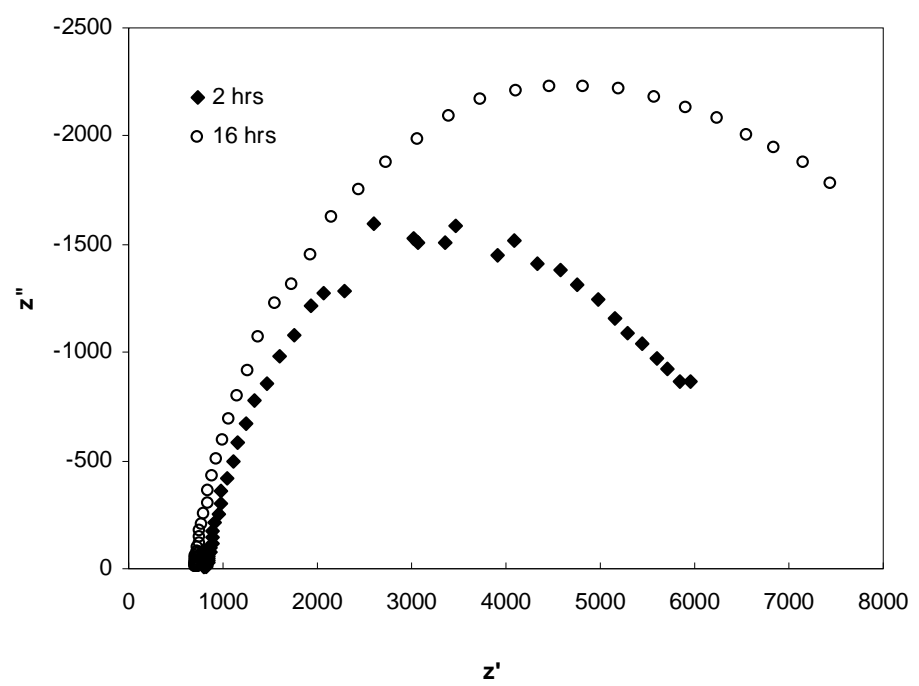

Figure 26. Nyquist plot of chalcopyrite in the presence of $0.5 \mathrm{~N} \mathrm{H}_{2} \mathrm{SO}_{4}$ and $0.09 \mathrm{M} \mathrm{H}_{2} \mathrm{O}_{2}$; after two periods of leaching. Ambient temperature.

Values assumed for the simulation of the equivalent electrical circuit are listed in Table 3. This simulation shows that W-R is relatively low as compared to the values obtained in the ferric sulfate system. This fact supports the premise that the leaching reaction is chemically controlled. 
Table 2. EIS parameters in $\mathrm{H}_{2} \mathrm{O}_{2} / \mathrm{H}_{2} \mathrm{SO}_{4}$ solution.

\begin{tabular}{|c|c|c|c|c|c|c|c|c|c|}
\hline $\begin{array}{c}\text { Time, } \\
\mathbf{h}\end{array}$ & $\begin{array}{c}\mathbf{R}_{\text {ct }} \\
\mathbf{K \Omega}\end{array}$ & $\begin{array}{c}\mathbf{C P E}-\mathbf{T}, \\
\mathbf{1 0}^{-6} \mathbf{F}\end{array}$ & $\begin{array}{c}\mathbf{C P E}- \\
\mathbf{P}\end{array}$ & $\begin{array}{c}\mathbf{R}_{\mathbf{2}}, \\
\boldsymbol{\Omega}\end{array}$ & $\begin{array}{c}\mathbf{C}_{\mathbf{2}} \\
\mathbf{1 0}^{-10} \mathbf{F}\end{array}$ & $\begin{array}{c}\mathbf{W}-\mathbf{R}, \\
\mathbf{K \Omega}\end{array}$ & $\mathbf{W}-\mathbf{T}$ & $\mathbf{W}-\mathbf{P}$ & $\begin{array}{c}\mathbf{R}_{\mathbf{0}}, \\
\boldsymbol{\Omega}\end{array}$ \\
\hline 2 & 1.4 & 22 & 0.83 & 825 & 35 & 7.5 & 130 & 0.15 & 1 \\
\hline 16 & 1.4 & 22 & 0.83 & 708 & 35 & 12 & 130 & 0.15 & 1 \\
\hline
\end{tabular}

\section{CONCLUSION}

A reaction product of leaching chalcopyrite in acidic medium is elemental sulfur which adheres to the mineral surface. Its presence has a major effect on dissolution and kinetics of reaction. The morphology of the sulfur layer is determined by the oxidant; a compact impervious layer is formed when ferric sulfate is used. A more porous layer is formed when ferric chloride and hydrogen peroxide are used. The morphology of the sulfur can also be altered by the presence of nanosize silica particles. Maximal enhancement of chalcopyrite dissolution by a factor of 1.5 was effected by its presence in some systems.

Sodium nitrate was added to the ferric sulfate system, and its presence is beneficial in leaching. Nitrate is a reasonably strong oxidant, and it apparently produces a change in the morphology of the sulfur layer. The concentration of sodium nitrate was large, and as a result, even though beneficial, its cost will probably preclude its use. Of the oxidants studied, ferric nitrate is the most effective oxidant of chalcopyrite. Oxidation systems more robust than ferric ion only are desirable.

Of all of the parameters investigated, particle size of the chalcopyrite is the most important. Chalcopyrite reduced in size to $-7 \mu \mathrm{m}$ can be effectively leached at moderate temperature and pressure. Further, it is also the most economic of the measures that can be used to enhance leaching that is the cost of grinding is less than that of the addition of nanosize silica particles and sodium nitrate. Future research should concentrate on developing technology for leaching chalcopyrite 10 microns and less in size.

Due to its instability in a chalcopyrite system, hydrogen peroxide will not find use in commercial processing. Chalcopyrite, itself, functions as a catalyst for the decomposition of chalcopyrite as does the reaction product, cupric ion. Significant stabilization of hydrogen peroxide can be obtained with the addition of ethylene glycol, however.

\section{REFERENCES}

Antonijevic, M. M., Z. Jankovic, and M. Dimitrijevic. 1994. "Investigations of the Kinetics of Chalcopyrite Oxidation by Potassium Dichromate.” Hydrometallurgy. 35:187.

Balaz, P., D. Kupka, Z. Bastl, and M. Achimovicova. 1996. "Combined Chemical and Bacterial Leaching of Ultrafine Ground Chalcopyrite. Hydrometallurgy. 42:237. 
Biegler, T., and D. A. Swift. 1976. "The Electrolytic Reduction of Chalcopyrite in Acid Solution.” Journal of Applied Electrochemistry. 6:229.

Dutrizac, J. E., 1978. "The Kinetics of Dissolution of Chalcopyrite in Ferric Ion Media." Metallurgical Transactions B. 9:431.

Hackl, R. P., D. B. Driesinger, E. Peters, and J. A. King. 1995. "Passivation of Chalcopyrite during Oxidative Leaching in Sulfate Media." Hydrometallurgy. 39:25.

Havlik, T., and M. Skrobian. 1990. "Acid Leaching of Chalcopyrite in the Presence of Ozone.” Can. Met. Quart. Vol. 29, p. 133.

Hiroyoshi, N., H. Miki, T. Hirajima, and M. Tsunekawa. 2000. "A Model for Ferrouspromoted Chalcopyrite Leaching.” Hydrometallurgy. 57:31.

Illingovan, S., D. R. Nagaraj, and K. I. Vasu. 1975. "Electrometallurgy and Chalcopyrite -II: Copper Powder from Slurry Anodes." J. of Electrochemical Society of India, vol. 24, p. 195.

Lu, Z. Y., M. L. Jeffrey, and F. Lawson. 2000. "An Electrochemical Study of the Effect of Chloride Ions on the Dissolution of Chalcopyrite in Acidic Solutions. Hydrometallurgy. $56: 145$.

Marsden, J. O. 2005. "Pressure Leaching of Chalcopyrite Concentrates -- Evolutionary or Revolutionary?" Robert H. Richards Lecture, Annual Meeting the Society for Mining, Metallurgy and Exploration.

Miller, J. D., and H. Q. Portillo. 1979. "Silver Catalysis in Ferric Sulfate Leaching of Chalcopyrite." Proceedings of the XIII International Mineral Processing Congress, J. Laskowski, ed. Warsaw, Poland, p. 691.

Munoz, P. B., J. D. Miller, and M. W. Wadsworth. 1979. "Reaction Mechanism for the Acid Ferric Sulfate Leaching of Chalcopyrite." Metallurgical Transactions B, 10:149.

Palmer, B. R., C. O. Nebo, M. F. Rau, and M. C. Fuerstenau. 1981. "Rate Phenomena Involved in Chalcopyrite Leaching with Chloride Lixiviants." Met. Trans. B: 595.

Reilly, I. G., and D. S. Scott. 1984. "Recovery of Elemental Sulfur during the Oxidative Ammoniacal Leaching of Chalcopyrite.” Metallurgical Transactions B. 15:726. 


\section{APPENDIX}

Definition of Symbols Used in Equivalent Circuit in Electrochemical Spectroscopy

Impedance

$\mathrm{R}_{\mathrm{ct}}$ is resistance of charge transfer.

(CPE-T) is capacitance of the electrical double layer between the surface and solution.

(CPE-P) is non-homogeneity factor of surface (roughness or porosity).

$R_{2}$ is resistance of initially-oxidized passive layer.

$\mathrm{C}_{2}$ is capacitance of initially-oxidized passive layer.

$\mathrm{W}$ is Warburg element, comprised of (W-R), (W-T), and (W-P).

(W-R) is resistance of mass transfer of diffusion layer.

(W-P) is non-homogeneity factor of surface.

$(\mathrm{W}-\mathrm{T})=\mathrm{L}^{2} / \mathrm{D}$ where $\mathrm{L}$ is effective diffusion thickness; $\mathrm{D}$ is effective diffusion coefficient. $R_{o}$ is resistance of solution and wire. 
Appendix 27: Thiosulfate as a Replacement For Cyanide In The Presence of Activators (NV003) 


\section{FINAL TECHNICAL REPORT}

Contract Title and Number: Crosscutting Technology Development at the Center for Advanced Separation Technologies (DE-FC26-02NT41607)
Period of Performance:

Starting Date: 6/1/2005

Ending Date: 09/30/2009
Sub-Recipient Project Title:

Thiosulfate as a Replacement for Cyanide in the Presence of Activators

Principal Investigators:

M. C. Fuerstenau and M. Misra

Contact Address:

Chemical and Metallurgical Engineering

University of Nevada, Reno 89557

Subcontractor Address:

University of Nevada, Reno

Reno, NV 89557
Report Information:

Type: Final

Number:

Period:

Date: $\quad 1 / 16 / 07$

Code: NV003-Final

Contact Information:

Phone: (775) 784-4310

Fax: (775) 327-5059

E-Mail: mcf@unr.edu

Subcontractor Information:

Phone: (775) 784-4312

Fax: (775) 784-6680

E-Mail: jerry_best@vpaf.unr.edu 


\section{DISCLAIMER}

“This report was prepared as an account of work sponsored by an agency of the United States Government. Neither the United States Government nor any agency thereof, nor any of their employees, make any warranty, express or implied, nor assume any legal liability or responsibility for the accuracy, completeness, or usefulness of any information, apparatus, product, or process disclosed, or represents that its use would not infringe privately owned rights. Reference herein to any specific commercial product, process, or service by trade name, trademark, manufacturer, or otherwise does not necessarily constitute or imply endorsement, recommendation, or favoring by the United States Government or any agency thereof. The views and opinions of authors expressed herein do not necessarily state or reflect those of the United States Government or agency thereof.” 


\section{ABSTRACT}

In the search for a replacement for cyanide as lixiviant for precious metals production, thiosulfate and other reagents have been suggested and studied. Although thiosulfate exhibits a number of positive attributes as a lixiviant for gold, consumption during processing is excessive. This study was undertaken to establish conditions under which the rat of gold dissolution could be increased substantially or thiosulfate consumption could be reduced significantly.

A rotating gold electrode was used to determine gold dissolution rates, and a flow-through electrochemical cell was used to measure thiosulfate concentrations. The inter-relationships among ammonium hydroxide, ammonium thiosulfate, and cupric sulfate concentrations, $\mathrm{pH}$ and temperature are complex. These inter-relationships were studied and related to the rate of gold dissolution. Several reagents were added to establish whether their presence will either increase the rate of gold dissolution or hinder the rate of thiosulfate decomposition. Of the reagents added, small additions of iodine and potassium permanganate only increased gold dissolution rate.

Elevated temperature and $\mathrm{pH}$ are required for effective leaching. Maximal dissolution of gold was determined at $\mathrm{pH} 11.5$ and $52^{\circ} \mathrm{C}$ in the presence of $0.2 \mathrm{M}\left(\mathrm{NH}_{4}\right)_{2} \mathrm{~S}_{2} \mathrm{O}_{3}, 0.2 \mathrm{M} \mathrm{NH}_{4} \mathrm{OH}$, and 0.008 $\mathrm{M} \mathrm{Cu}^{2+}$. Temperatures higher that $52^{\circ} \mathrm{C}$ result in precipitation of $\mathrm{CuO}$ which results in drastic reduction of gold dissolution. 


\section{TABLE OF CONTENTS}

Disclaimer $\quad$ i

Abstract $\quad$ ii

Table of Contents $\quad$ iii

List of Figures $\quad$ iv

List of Tables $\quad$ v

Executive Summary $\quad 1$

Introduction $\quad 3$

Experimental 6

Materials $\quad 6$

Equipment $\quad 6$

Leaching $\quad 6$

$\begin{array}{ll}\text { Chemical Analysis } & 7\end{array}$

Procedures

Gold Leaching $\quad 7$

$\begin{array}{ll}\text { Cupric Kinetic Study } & 7\end{array}$

$\begin{array}{ll}\text { Thiosulfate Measurement } & 7\end{array}$

Results and Discussion $\quad 8$

Interactions in Thiosulfate Leaching System $\quad 8$

Behavior of Cupric Ions $\quad 9$

Effect of $\mathrm{Cu}^{2+}$ on Gold Dissolution Rate 9

Kinetic Study of Cupric Decomposition $\quad 10$

Change of $\mathrm{Cu}^{2+}$ Concentration in Thiosulfate Solution $\quad 10$

The Rate Constant 14

Order of Cupric Decomposition Reaction $\quad 15$

Influence of Temperature $\quad 15$

Behavior of Thiosulfate in Leaching Solution $\quad 15$

Decomposition of Thiosulfate 15

Thiosulfate Decomposed by Cupric Ions 17

Effect of Ammonium Hydroxide 18

$\begin{array}{ll}\text { Effect of } \mathrm{pH} \text { and Temperature } & 20\end{array}$

Gold Dissolution Rates at Various Temperatures and pH 21

Effect of Additives 24

2-phenyl-2-Imidazoline $\quad 24$

Methyl Imidazoline $\quad 24$

Methylamine Hydrochloride $\quad 24$

Hydrogen Peroxide $\quad 24$

Sodium Sulfite $\quad 25$

EDTA 25

Iodine $\quad 25$

Potassium Permanganate $\quad 26$

Conclusions $\quad 26$

References $\quad 28$

Acknowledgments $\quad 29$ 


\section{LIST OF FIGURES}

Page

Fig. 1 Gold electrode used in study.............................. 6

Fig. 2 Apparatus constructed and used in thiosulfate determinations.... 7

Fig. $3 \quad$ Effect of cupric ion on gold dissolution rate................... 9

Fig. 4 Cupric-amine concentration $(\mathrm{Co} / \mathrm{C})$ as a function of time for various concentrations of ammonium thiosulfate................ 11

Fig. 5 Linearized plot of Co/C vs. time ......................... 12

Fig. $6 \quad$ Linearization of Co/C vs. time plot....................... 13

Fig. $7 \quad$ Linearization of Co/C vs. time plot......................... 13

Fig. 8 Regression of $\log \mathrm{k}_{1}$ vs. $\log \left[\mathrm{S}_{2} \mathrm{O}_{3}{ }^{2-}\right]$ plots for three temperatures of reaction................................... 14

Fig. $9 \quad$ Effect of thiosulfate on gold dissolution rate.................. 16

Fig. $10 \quad$ Eh/pHdiagram of gold in the presence of thiosulfate and ammonia................................................... 19

Fig. 11 Effect of ammonia on gold dissolution rate.................. 20

Fig. 12 Eh/pH diagram of copper in the presence of thiosulfate and ammonia............................................. 21

Fig. 13 Gold dissolution rate as a function of temperature and $\mathrm{pH}$........ 22

Fig. 14 Residual cupric concentration as the function of $\mathrm{pH} . \ldots \ldots \ldots \ldots . . .23$ 


\section{LIST OF TABLES}

Table 1 Lixiviants and oxidants in gold dissolution...........................

Table 2 Gold dissolution rate $\left(\mu \mathrm{g} / \mathrm{cm}^{2} \cdot \mathrm{h}\right)$ as a function of $\mathrm{NH}_{4} \mathrm{OH}$ and $\left(\mathrm{NH}_{4}\right)_{2} \mathrm{~S}_{2} \mathrm{O}_{3}$ concentrations.......................... 8

Table 3 Gold dissolution rate $\left(\mu \mathrm{g} / \mathrm{cm}^{2} \cdot \mathrm{h}\right)$ at various values of

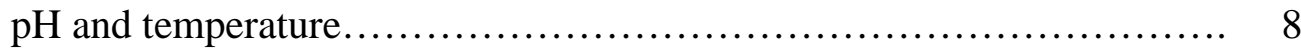

Table $4 \quad \mathrm{k}_{1}$ values for various concentrations of thiosulfate at $32^{\circ} \mathrm{C} \ldots \ldots \ldots \ldots \ldots . .12$

Table $5 \quad \mathrm{k}_{1}$ values for various concentrations of thiosulfate at $22^{\circ} \mathrm{C} \ldots \ldots \ldots \ldots \ldots . .14$

Table $6 \quad \mathrm{k}_{1}$ values for various concentrations of thiosulfate at $42^{\circ} \mathrm{C} \ldots \ldots \ldots \ldots \ldots . .14$

Table 7 Decomposition of thiosulfate in the absence of $\mathrm{Cu}^{2+} \ldots \ldots \ldots \ldots \ldots \ldots \ldots . . .17$

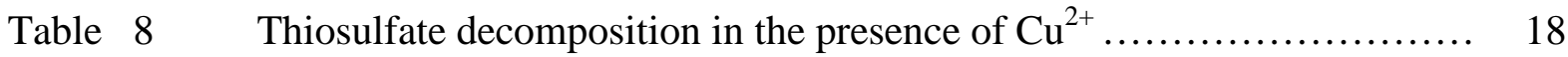

Table $9 \quad$ Regeneration of cupric-amine.................................... 18

Table 10 Effect of temperature on thiosulfate oxidation in the presence of cupric... 19

Table $11 \quad$ pK of water at various temperatures............................... 20

Table $12 \quad \mathrm{pH}$ as a function of temperature of thiosulfate leaching solution.......... 21

Table $13 \quad$ Effect of phenyl imidazoline..................................... 24

Table $14 \quad$ Effect of methyl imidazoline........................................ 24

Table $15 \quad$ Effect of methylamine hydrochloride.............................. 24

Table $16 \quad$ Effect of hydrogen peroxide..................................... 24

Table $17 \quad$ Effect of sodium sulfite........................................ 25

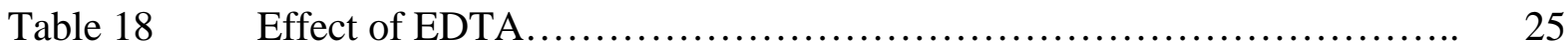

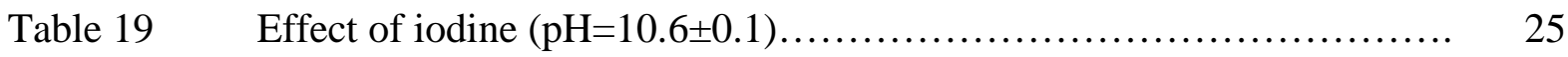

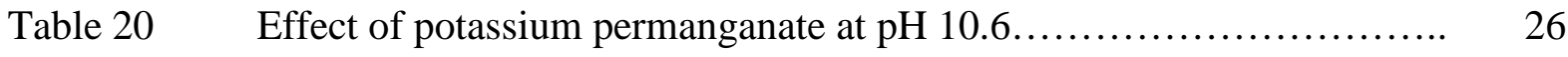

Table $21 \quad$ Effect of potassium permanganate at $\mathrm{pH}$............................ 26 


\section{EXECUTIVE SUMMARY}

Since its commercial implementation in 1889, cyanide has been used almost exclusively in the extraction of precious metals from ores. There are a number of reasons for this. Cyanide is very effective as a lixiviant for precious metals, and its cost is not excessive. As is well known, however, this reagent is extremely toxic, and considerable care must be exercised to prevent injury to personnel and damage to the environment during and after processing.

Another drawback to cyanide is the fact that many other toxic metals in the ores, including mercury, arsenic, selenium and antimony, are also dissolved with the gold. These dissolved secondary metals not only interfere with the extraction of gold but also pose an additional burden in the treatment of the liquid streams after extraction. Further, refractory carbonaceous ore 'rob' the pregnant leaching solution of gold values when cyanide is used as lixiviant.

For these reasons, considerable effort has been directed to developing other effective lixiviants to obviate the use of cyanide in precious metals processing. One of these reagents is ammonium thiosulfate. In general, researchers have found that ammonium thiosulfate is a good lixiviant for gold dissolution. One very positive aspect is that gold thiosulfate complexes do not adsorb on activated carbon. The problem with using this reagent, however, is the instability of thiosulfate in solution and excessive reagent loss during processing. Oxidative conditions are essential for the dissolution of gold, and under these conditions, thiosulfate is oxidized to tetrathionate. The development of reaction conditions under which gold oxidation is increased while keeping thiosulfate losses minimal was the objective of this investigation.

In the experimental work gold leaching studies were conducted electrochemically with a rotating gold electrode. Thiosulfate concentrations under a variety of leaching conditions were also measured electrochemically with a cell described by Breuer and associates.

The inter-relationships among ammonium hydroxide, ammonium thiosulfate, cupric ion, $\mathrm{pH}$ and temperature are complex. Virtually no leaching of gold with ammonium thiosulfate as lixiviant in the presence of oxygen only as oxidant can be effected. Cupric ion, stabilized with ammonium hydroxide forming $\mathrm{Cu}\left(\mathrm{NH}_{3}\right)_{4}{ }^{+}$, is added as oxidant.

The complexity of this system is demonstrated by the fact that at low ammonia addition, gold dissolution rate decreases with increased thiosulfate additions. At high ammonium hydroxide concentration, gold dissolution is increased with increased ammonium thiosulfate additions. An optimal ammonia concentration exists for maximal gold dissolution.

The status and concentration of cupric ion is determined by ammonia concentration, ammonium thiosulfate concentration, $\mathrm{pH}$ and temperature. Cupric decomposition is a pseudo second order reaction when the ammonia concentration, $\mathrm{pH}$ and temperature are kept constant. 
The reaction rate constant of cupric decomposition is greatly influenced by temperature. The rate constant increased by 17 times and the order of thiosulfate concentration increased from 1.09 to 2.17 when the temperature was increased from $22^{\circ}$ to $42^{\circ} \mathrm{C}$.

Temperature has a very important effect on the dissolution rate of gold. Higher temperature greatly accelerates the rate of gold dissolution, but it also decomposes thiosulfate faster and leads to greater precipitation of $\mathrm{CuO}$. Leaching temperatures greater than $52^{\circ} \mathrm{C}$ should not be used.

$\mathrm{pH}$ influences the stability of ammonium thiosulfate, cupric amine, and the status of cupric ion. The most favorable conditions for leaching of gold are $\mathrm{pH} 11.5$ and $52^{\circ} \mathrm{C}$. Higher $\mathrm{pH}$ is beneficial for the formation of more oxygen and thiosulfate from the polythionates, the decomposition products of thiosulfate. Too high a value of $\mathrm{pH}$ and/or temperature results in the precipitation of $\mathrm{CuO}$.

A number of reagents were added to the leaching systems to establish their effect on rate of gold dissolution. These reagents were phenyl imidazoline, methylamine hydrochloride, hydrogen peroxide, EDTA, sodium sulfite, iodine, and potassium permanganate. Of these reagents, only iodine and potassium permanganate increased the rate of gold dissolution. The rate of increase was about 50\% maximally. 


\section{INTRODUCTION}

Since its commercial implementation in 1889, cyanide has been used almost exclusively in the extraction of precious metals from ores. There are a number of reasons for this. Cyanide is very effective as a lixiviant for precious metals, and its cost is not excessive. As is well known, however, this reagent is extremely toxic, and considerable care must be exercised to prevent injury to personnel and damage to the environment during and after processing.

Another drawback to cyanide is the fact that many other toxic metals in the ores, including mercury, arsenic, selenium and antimony, are also dissolved with the gold. These dissolved secondary metals not only interfere with the extraction of gold but also pose an additional burden in the treatment of the liquid streams after extraction. Further, refractory carbonaceous ores 'rob' the pregnant leaching solutions of gold values when cyanide is used as lixiviant.

For these reasons, considerable effort has been directed to developing other effective lixiviants to obviate the use of cyanide in precious metals processing. Alternative non-cyanide lixiviants previously examined for gold dissolution are summarized in Table 1.

Table 1. Lixiviants and oxidants in gold dissolution. (Wan et al. 1993).

\begin{tabular}{|l|l|l|}
\hline \multicolumn{1}{|c|}{ Lixiviant } & \multicolumn{1}{c|}{ Complexing Ligand } & \multicolumn{1}{c|}{ Oxidants } \\
\hline Thiourea & $\mathrm{CS}\left(\mathrm{NH}_{2}\right)_{2}$ & $\mathrm{Fe}^{3+}, \mathrm{O}_{2}, \mathrm{H}_{2} \mathrm{O}_{2}$ \\
\hline Thiosulfate & $\mathrm{S}_{2} \mathrm{O}_{3}{ }^{2-}$ & $\mathrm{O}_{2}, \mathrm{H}_{2} \mathrm{O}_{2}, \mathrm{Cu}^{2+}, \mathrm{ClO}_{3}{ }^{-}$ \\
\hline Iodine & $\mathrm{I}^{-}$ & $\mathrm{I}_{2} / \mathrm{I}_{3}^{-}$ \\
\hline Polysulfides & $\mathrm{S}^{2-}$ & $\mathrm{S}^{2-} / \mathrm{S}_{2}{ }^{2-}$ \\
\hline Malononitriles & $\mathrm{CH}(\mathrm{CN})_{2}{ }^{-}$ & $\mathrm{O}_{2}$ \\
\hline
\end{tabular}

The drawbacks of using thiourea as lixiviant include high reagent consumption, the formation of passive surface coatings and low leaching efficiencies. Schulze (1984) has studied $\mathrm{SO}_{2} /$ redox couple control, while Little (1987) has studied ferric sulfate and lignin sulfonate additions to counter these drawbacks. Iodide is both expensive and corrosive. Polysulfides are environmentally unattractive, and malononitriles are expensive and generate free cyanide ions.

Gold and silver dissolution with ammonia as lixiviant has been reported by Han and Meng (1992, 1994). Jiang et al. (1993) and Wan et al. (1994) reported the enhancement in leaching of gold in the presence of cupric tetrammine in ammoniacal thiosulfate solutions. In general, researchers have found that ammonium thiosulfate is a good lixiviant for gold dissolution. The problem of using this reagent, however, is the instability of thiosulfate in solution and excessive reagent loss during processing. Oxidative conditions are essential for the dissolution of gold, and under these conditions, thiosulfate is oxidized to tetrathionate and, further, to polythiosulfate. Therefore, the development of reaction conditions is needed which can increase oxidation of gold while keeping thiosulfate losses minimal. 
The dissolution of gold involves anodic oxidation and complexation, while the cathodic reaction complementing this anodic reaction involves the reduction of an appropriate oxidant, which is cupric amine and/or oxygen in this system.

\section{Thiosulfate Solution Chemistry}

The unique chemistry of thiosulfate ion $\left(\mathrm{S}^{-} \mathrm{SO}_{3}\right)^{2-}$ is dominated by the sulfide-like sulfur atom which imparts reducing properties, strong complexing tendencies and sulfide forming capabilities. For effective gold dissolution with thiosulfate, three necessary components are required, namely ammonia, thiosulfate and copper ions. The reaction is described by the reaction,

$$
2 \mathrm{Au}+4 \mathrm{~S}_{2} \mathrm{O}_{3}{ }^{2-}+\mathrm{H}_{2} \mathrm{O}+1 / 2 \mathrm{O}_{2} \leftrightarrows 2 \mathrm{Au}\left(\mathrm{S}_{2} \mathrm{O}_{3}\right)_{2}{ }^{3-}+2 \mathrm{OH}^{-}
$$

Li et al. (1995) have modified this reaction to show the catalytic role of copper ions during dissolution.

$$
\left.\mathrm{Au}+\underline{5 \mathrm{~S}}_{2} \underline{\mathrm{O}}_{3} \underline{\mathrm{Cu}(\mathrm{NH}}_{3}\right)_{4} \leftrightarrows \mathrm{Au}\left(\mathrm{S}_{2} \mathrm{O}_{3}\right)_{2}{ }^{3-}+\mathrm{Cu}\left(\underline{\mathrm{S}}_{2} \underline{\mathrm{O}_{3}} \underline{3}_{3} \underline{3}^{5-}+4 \mathrm{NH}_{3}\right.
$$

Aylmore and Muir (2001) have proposed the following model for gold dissolution in ammoniacal thiosulfate systems.

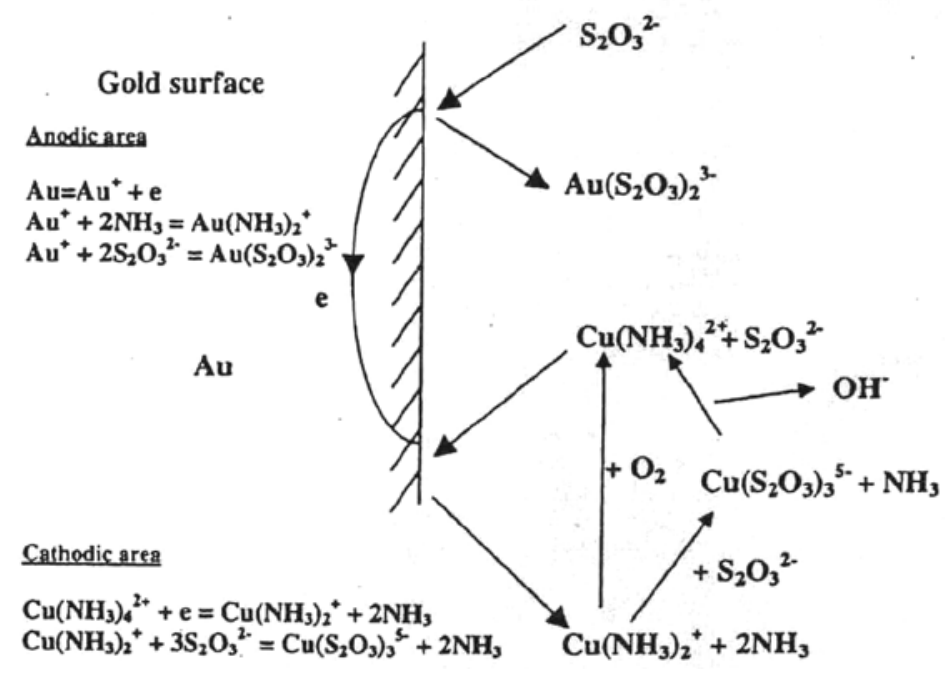

Ammonia or thiosulfate reacts with $\mathrm{Au}^{+}$ions at an anodic site forming either $\mathrm{Au}\left(\mathrm{NH}_{3}\right)_{2}{ }^{+}$or $\mathrm{Au}\left(\mathrm{S}_{2} \mathrm{O}_{3}\right)_{2}{ }^{3-}$ during oxidation. Depending on $\mathrm{S}_{2} \mathrm{O}_{3}{ }^{2-}$ concentration, $\mathrm{Cu}\left(\mathrm{NH}_{3}\right)_{2}{ }^{+}$can be converted to $\mathrm{Cu}\left(\mathrm{S}_{2} \mathrm{O}_{3}\right)_{3}{ }^{5-}$ ions. This is also the case for $\mathrm{Au}\left(\mathrm{NH}_{3}\right)_{2}{ }^{+}$. Both $\mathrm{Cu}\left(\mathrm{S}_{2} \mathrm{O}_{3}\right)_{3}{ }^{5-}$ and $\mathrm{Cu}\left(\mathrm{NH}_{3}\right)_{2}{ }^{+}$are oxidized to $\mathrm{Cu}\left(\mathrm{NH}_{3}\right)_{4}{ }^{2+}$ by dissolved oxygen (Jiang et al.1993, Li et al.1995, Wan 1997). Ammonia also has other important roles. In the absence of ammonia, $\mathrm{Cu}^{2+}$ oxidizes thiosulfate very rapidly to tetrathionate. In the presence of ammonia, however, this reaction occurs much more slowly. Further, ammonia stablilizes $\mathrm{Cu}^{2+}$ in solution. In its absence, $\mathrm{Cu}^{2+}$ would be hydrolyzed to $\mathrm{Cu}(\mathrm{OH})_{2}$ under the alkaline conditions used. 
The ratio of ammonia to thiosulfate is critical for preventing copper ions from remaining only as cuprous or as cupric ions. Excess $\mathrm{NH}_{3}$ over $\mathrm{S}_{2} \mathrm{O}_{3}{ }^{2-}$ favors the formation of $\mathrm{Cu}\left(\mathrm{NH}_{3}\right)^{2+}$. Similarly, excess $\mathrm{S}_{2} \mathrm{O}_{3}{ }^{2-}$ favors the formation of $\mathrm{Cu}\left(\mathrm{S}_{2} \mathrm{O}_{3}\right)_{3}{ }^{5-}$. An appropriate concentration ratio and Eh have to be maintained so that copper can easily transfer between the $\mathrm{Cu}^{2+}$ and $\mathrm{Cu}^{+}$states. Zipperian et al. (1988), Li et al. (1995) and Aylmore and Muir (2001) have shown that an oxidation potential of 0.25 volt is required for effective dissolution at $\mathrm{pH} 9-10$. The narrow range in $\mathrm{pH}(9-10)$ is that in which $\mathrm{Cu}\left(\mathrm{NH}_{3}\right)_{4}{ }^{2+}$ is stable with respect to $\mathrm{CuO}$ (Figure 12).

Thiosulfate oxidizes to tetrathionate between $\mathrm{pH}$ 9-10. Efforts have been made to reduce consumption by reducing the concentration of thiosulfate in solution so that leaching can be donduted for extended periods of time.e.g. 24 hours (Cao et al. 1992). In other cases sulfite has been added to stabilize thiosulfate (Kerley 1981).

The stability range (Eh) of thiosulfate is narrow in neutral to basic conditions. Since the oxidative degradation of thiosulfate to tetrathionate is promoted by cupric ion, reagent management of cupric ion and ammonia is critical to keeping thiosulfate losses to a minimum.

Another mechanism for keeping the thiosulfate oxidation losses to a minimum would be to increase the oxidation kinetics of gold dissolution and, hence, reduce reaction time.

Kristjansdottir and Thompson (1996) and Wadsworth et al. (1998) have shown that the addition of compounds based on nitrogen and sulfur-containing aromatic rings, such as thiophenol, pyridine and imidazole, function as activators in gold dissolution with cyanide and increase the rate of dissolution. In all cases Wadsworth et al. (1998) observed a 20-30\% increase in gold dissolution in the presence of activator. Activator additions of 3-5 mg/L were used. Interestingly, these authors found that an addition of $3 \mathrm{mg} / \mathrm{L}$ activator provided a higher rate of dissolution than doubling the addition of cyanide. This technology was implemented commercially at the Homestake Gold Mine, Lead, South Dakota. DuPont obtained the patent on this technology and has recently donated it to the University of Nevada, Reno.

In other aqueous systems, it has been shown that the addition of sucrose increases some rates of oxidation. In waste water treatment, for example, it has been shown that the addition of sucrose in the presence of $\mathrm{SO}_{4}{ }^{2-}$ results in an increase in the extent of oxidation of $\mathrm{Fe}(\mathrm{II})$ to $\mathrm{Fe}(\mathrm{III})$, i.e. $\mathrm{Fe}(\mathrm{OH})_{2}$ to $\mathrm{Fe}_{2} \mathrm{O}_{3}$ (Tamura et al. 1979). Sucrose apparently enhances charge transfer in these systems.

\section{Objective and Approach}

The objective of this work was to improve and optimize thiosulfate leaching of gold to establish whether this technology can be used to viably replace cyanide leaching in precious metals extraction. To realize this objective, the following areas were investigated:

- A study and development of chemical conditions under which the oxidation kinetics of gold dissolution can be increased.

- A study and development of chemical conditions under which the degradation of thiosulfate can be minimized. 


\section{EXPERIMENTAL}

\section{Chemicals}

All chemicals were reagent grade. Gold foil for use as a rotating electrode was purchased from Alfa Aesar with a purity of 99.9985\%. Water was first de-ionized and then distilled prior to use.

\section{Equipment}

Gold Leaching

Gold leaching studies were conducted in a 125-ml 5-neck flask with overhead stirring or with the gold disk rotating at $300 \mathrm{rpm}$. Temperature was controlled with a thermostat model LOTEMPTROL, 154. Area of the rotating gold electrode was $0.32 \mathrm{~cm}^{2}$. A diagram of the gold electrode prepared and used in this study is shown in Figure 1.

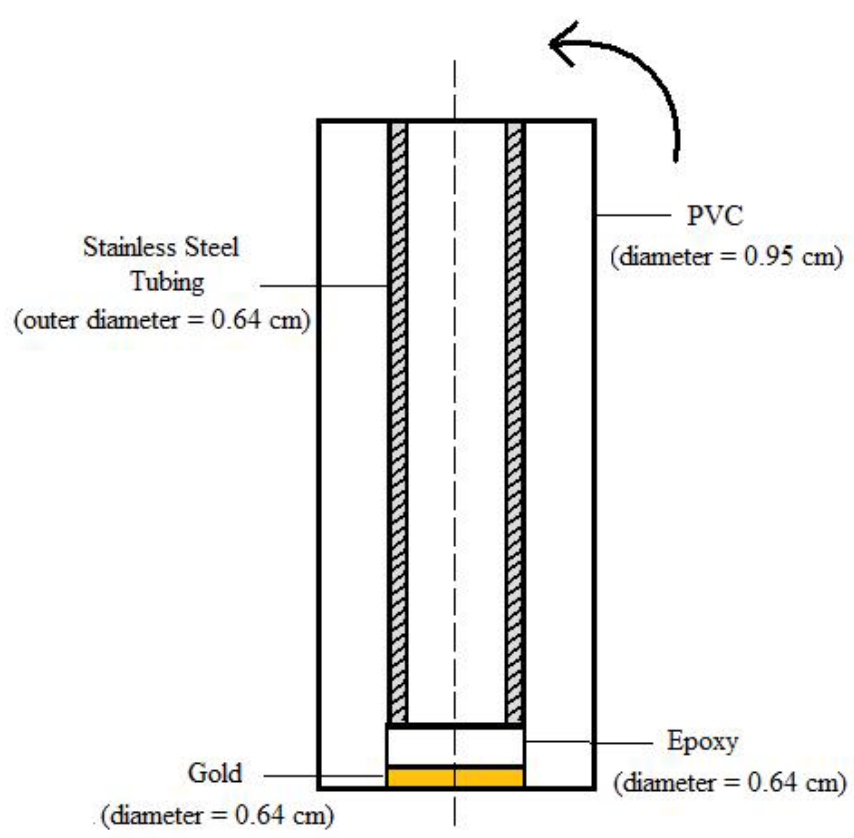

Figure 1. Gold electrode used in study.

\section{Eh/pH Measurement}

Both $\mathrm{pH}$ and Eh were measured with a Fisher Accumet 815MP meter. Adjustments in pH were made with $\mathrm{NaOH}$ or $\mathrm{HNO}_{3}$. 
Chemical Analysis

Gold concentrations were measured by atomic absorption spectrometry with a Varian SpectrAA-200. Concentration of $\mathrm{Cu}\left(\mathrm{NH}_{3}\right)_{4}{ }^{2+}$ was measured with a Bausch and Lomb SPECTRONIC 21 photometer. Thiosulfate was measured with a flow-through cell described by Breuer et al. (2004). The electric charge was measured with a Gamry potentiostat. Potentiostat sampling interval was 0.5 second.

\section{Procedures}

\section{Gold Leaching}

Selected amounts of cupric sulfate, ammonium hydroxide and water were placed into the flask and heated to the desired temperature. The rotating electrode was placed in the central neck, and the motor was turned on. A solution of thiosulfate and sodium hydroxide at the desired temperature was quickly transferred into the flask, and the leach was started.

\section{Cupric Kinetic Study}

Preparation of the leaching solution was the same as described above except an overhead stirrer was employed instead of the rotating electrode. The period of time required to take the sample and measure copper concentration was within 10 seconds.

\section{Thiosulfate Measurement}

Electrochemical determinations of thiosulfate concentration were made with equipment and techniques described by Breuer et al. (2004). A photograph of the flow-through apparatus that was constructed and used is shown in Figure 2.

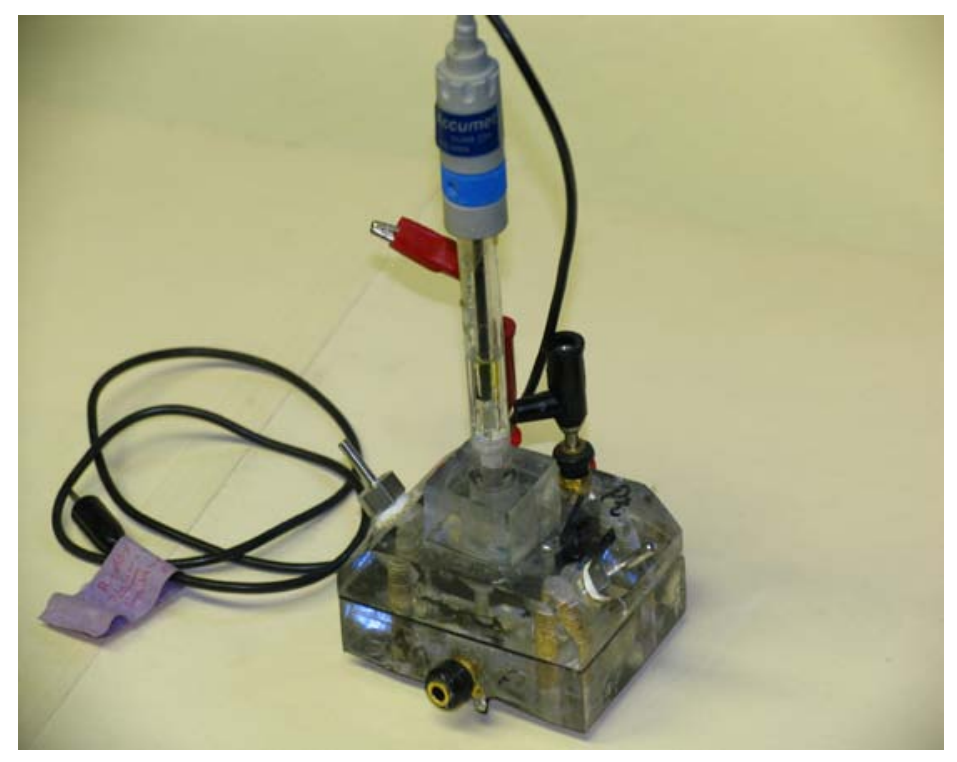

Figure 2. Apparatus constructed and used in thiosulfate determinations. 


\section{RESULTS AND DISCUSSION}

\section{Interactions in the Thiosulfate Leaching System}

In gold leaching experiments, significant interactions among lixiviants occur at various values of $\mathrm{pH}$ and temperature. Their effects on gold leaching rates can be seen in Table 2 .

Table 2.Gold dissolution rate $\left(\mu \mathrm{g} / \mathrm{cm}^{2} \cdot \mathrm{h}\right)$ as a function of $\mathrm{NH}_{4} \mathrm{OH}$ and $\left(\mathrm{NH}_{4}\right)_{2} \mathrm{~S}_{2} \mathrm{O}_{3}$ concentrations. Conditions: $\left[\mathrm{Cu}^{2+}\right]=0.0080 \mathrm{M} ; \mathrm{T}=22^{\circ} \mathrm{C}$; $\mathrm{rpm}=300$; leaching time $=2.0$ hours; $\mathrm{pH}$ not controlled (actual $\mathrm{pH}$ shown in parentheses).

\begin{tabular}{|c|c|c|c|c|}
\hline $\begin{array}{c}\left.\mathrm{NH}_{4}\right)_{2} \mathrm{~S}_{2} \mathrm{O}_{3}, \\
\mathrm{M}\end{array}$ & 0.1 & 0.2 & 0.3 & 0.5 \\
\hline 0.1 & $193(9.2)$ & $452(9.5)$ & $539(9.6)$ & $464(9.8)$ \\
\hline 0.2 & $126(9.0)$ & $383(9.4)$ & $536(9.5)$ & $555(9.7)$ \\
\hline 0.3 & $62(8.9)$ & $280(9.3)$ & $619(9.5)$ & $739(9.6)$ \\
\hline 0.5 & $35(8.8)$ & $98(9.1)$ & $285(9.3)$ & $869(9.5)$ \\
\hline
\end{tabular}

The data in Table 2 indicates that the concentration of ammonium thiosulfate exhibits different effects on the gold dissolution rate at different levels of ammonium hydroxide concentration. At low ammonium hydroxide concentration (0.1- 0.2M), elevation of ammonium thiosulfate concentration results in a significant decrease of gold dissolution rate, but at high ammonium hydroxide concentration $(0.5 \mathrm{M})$, the results reverse. That is, gold dissolution increases with increased levels of ammoniun thiosulfate.

Temperature also shows contradictive influences on the dissolution rate. See Table 3. At lower $\mathrm{pH}$ (9.5), increased temperature resulted in a decrease in gold dissolution. At higher $\mathrm{pH}$ (10.6), gold dissolution increased with elevated temperature.

Table 3. Gold dissolution rate $\left(\mu \mathrm{g} / \mathrm{cm}^{2} \cdot \mathrm{h}\right)$ at various values of $\mathrm{pH}$ and temperature. Conditions: $\left[\mathrm{Cu}^{2+}\right]=0.0080 \mathrm{M} ;\left[\mathrm{NH}_{4} \mathrm{OH}\right]=0.2 \mathrm{M} ;\left[\left(\mathrm{NH}_{4}\right)_{2} \mathrm{~S}_{2} \mathrm{O}_{3}\right]=0.20 \mathrm{M}$; leaching time $=2.0$ hours.

\begin{tabular}{|c|c|c|c|c|}
\hline \multicolumn{2}{|c|}{ Temperature } & $42^{\circ} \mathrm{C}$ & $52^{\circ} \mathrm{C}$ & $62^{\circ} \mathrm{C}$ \\
\hline \multirow{2}{*}{$\mathrm{pH}$} & 9.5 & 600 & 526 & 236 \\
\cline { 2 - 5 } & 10.6 & 1230 & 1219 & 1526 \\
\hline
\end{tabular}

The combinations of $\left[\mathrm{NH}_{4} \mathrm{OH}\right],\left[\left(\mathrm{NH}_{4}\right)_{2} \mathrm{~S}_{2} \mathrm{O}_{3}\right], \mathrm{pH}$ and temperature all influence the concentration and form of cupric ion. The relationship is complex, so these parameters were studied individually and interactively. 


\section{Behavior of Cupric Ions}

Effect of $\mathrm{Cu}^{2+}$ on Gold Dissolution Rate

Cupric ions play a crucial role in thiosulfate leaching of gold. Theoretically, gold can be oxidized by oxygen in the presence of thiosulfate according to the Equation 1 (Aylmore and Muir (2001):

$$
4 \mathrm{Au}+8 \mathrm{~S}_{2} \mathrm{O}_{3}{ }^{2-}+\mathrm{O}_{2}+2 \mathrm{H}_{2} \mathrm{O} \leftrightarrows 4\left[\mathrm{Au}\left(\mathrm{S}_{2} \mathrm{O}_{3}\right)_{2}\right]^{3-}+4 \mathrm{OH}^{-}
$$

Actually, however, this reaction occurs very slowly. Thus, cupric ions are always employed as a catalyst to accelerate the leaching process. While gold is oxidized, cupric ion is reduced. The cuprous ion formed is oxidized to cupric, again, by dissolved oxygen:

$$
\begin{aligned}
& \mathrm{Au}+5 \mathrm{~S}_{2} \mathrm{O}_{3}{ }^{2-}+\mathrm{Cu}\left(\mathrm{NH}_{3}\right)_{4}{ }^{2+} \leftrightarrows \mathrm{Au}\left(\mathrm{S}_{2} \mathrm{O}_{3}\right)_{2}{ }^{3+}+4 \mathrm{NH}_{3}+\mathrm{Cu}\left(\mathrm{S}_{2} \mathrm{O}_{3}\right)_{3}{ }^{5-} \\
& 2 \mathrm{Cu}\left(\mathrm{S}_{2} \mathrm{O}_{3}\right)_{3}{ }^{5-}+8 \mathrm{NH}_{3}+0.5 \mathrm{O}_{2}+\mathrm{H}_{2} \mathrm{O}=2 \mathrm{Cu}\left(\mathrm{NH}_{3}\right)_{4}{ }^{2+}+2 \mathrm{OH}^{-}+6 \mathrm{~S}_{2} \mathrm{O}_{3}{ }^{2-}
\end{aligned}
$$

Cupric ions can speed up gold dissolution by 18-20 times (Aylmore and Muir 2001); our experiments verified this.

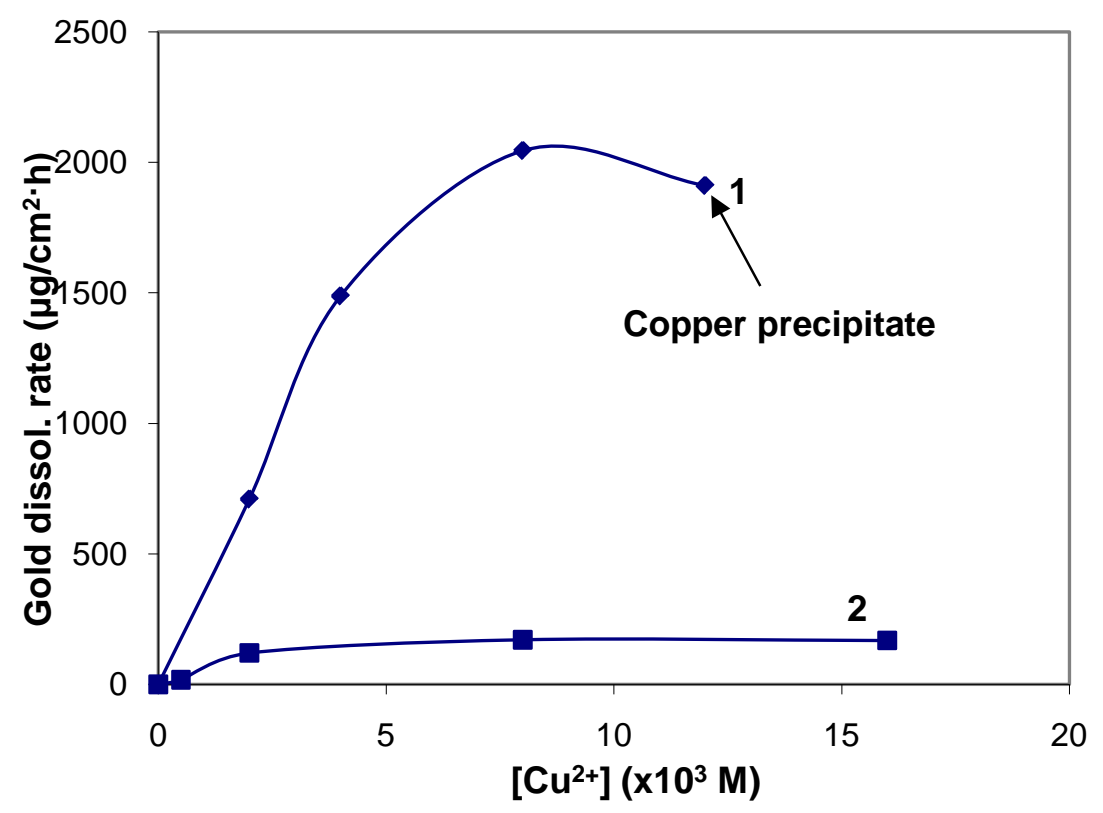

Figure 3. Effect of cupric ion on gold dissolution rate. Conditions: Curve 1, $\left[\mathrm{NH}_{4} \mathrm{OH}\right]=0.2 \mathrm{M}$; $\left[\left(\mathrm{NH}_{4}\right)_{2} \mathrm{~S}_{2} \mathrm{O}_{3}\right]=0.20 \mathrm{M} ; \mathrm{pH}=11.5 ; \mathrm{T}=42^{\circ} \mathrm{C}$. Curve 2, $\left[\mathrm{NH}_{4} \mathrm{OH}\right]=0.1 \mathrm{M} ;\left[\left(\mathrm{NH}_{4}\right)_{2} \mathrm{~S}_{2} \mathrm{O}_{3}\right]=0.10 \mathrm{M}$; $\mathrm{pH}=9.5 ; \mathrm{T}=22^{\circ} \mathrm{C}$; leaching time $=2.0$ hrs.

Figure 3 shows that for maximum dissolution rate, about $0.008 \mathrm{M}$ of cupric is necessary. On the other hand, cupric ions also oxidize and, consequently, consume the thiosulfate. When a cupric 
concentration of $1.2 \times 10^{-3} \mathrm{M}$ was used, a precipitate of cupric oxide was observed under these conditions. Too high dosage of cupric reduces leaching.

\section{Kinetic Study of Cupric Decomposition}

Decomposition of Cupric Ions

In an ammonium-hydroxide-thiosulfate solution, cupric-amine will oxidize thiosulfate and be reduced to cuprous thiosulfate as follows:

$$
2 \mathrm{Cu}\left(\mathrm{NH}_{3}\right)_{4}{ }^{2+}+8 \mathrm{~S}_{2} \mathrm{O}_{3}{ }^{2-} \leftrightarrows 2 \mathrm{Cu}\left(\mathrm{S}_{2} \mathrm{O}_{3}\right)_{3}{ }^{5-}+\mathrm{S}_{4} \mathrm{O}_{6}{ }^{2-}+8 \mathrm{NH}_{3}
$$

A kinetic study of how thiosulfate and temperature influence cupric concentration in the leaching system is necessary. The general form of the reaction rate of Equation 4 is:

$$
-\mathrm{d}\left[\mathrm{Cu}\left(\mathrm{NH}_{3}\right)_{4}{ }^{2+}\right] / \mathrm{dt}=\mathrm{k}\left[\mathrm{Cu}\left(\mathrm{NH}_{3}\right)_{4}{ }^{2+}\right]^{\mathrm{a}}\left[\mathrm{S}_{2} \mathrm{O}_{3}{ }^{2-}\right]^{\mathrm{b}}
$$

where $\mathrm{k}$ is the rate constant of reaction. Keeping the concentration of thiosulfate $>>\left[\mathrm{Cu}^{2+}\right]$, so that the change of the thiosulfate concentration can be neglected and regarded as constant, then :

where

$$
-\mathrm{d}\left[\mathrm{Cu}\left(\mathrm{NH}_{3}\right)_{4}{ }^{2+}\right] / \mathrm{dt}=\mathrm{k}_{1}\left[\mathrm{Cu}\left(\mathrm{NH}_{3}\right)_{4}{ }^{2+}\right]^{\mathrm{a}}
$$

$$
\mathrm{k}_{1}=\mathrm{k}\left[\mathrm{S}_{2} \mathrm{O}_{3}{ }^{2-}\right]^{\mathrm{b}}
$$

Here, 'a' in Equation 6 is the reaction order of the cupric decomposition. By means of measurements and regression analysis, the relationship of $\left[\mathrm{Cu}\left(\mathrm{NH}_{3}\right)_{4}{ }^{2+}\right]$ vs. time at various temperatures and $\mathrm{pH}$ values at various concentrations of thiosulfate, $\mathrm{k}_{1}$ and ' $\mathrm{a}$ ' in the Equation 6 can be determined.

Then, by taking the logarithm of Equation 7, a linear equation can be obtained:

$$
\log k_{1}=\log k+b \log \left[\mathrm{S}_{2} \mathrm{O}_{3}{ }^{2-}\right]
$$

In the $\log \mathrm{k}_{1}-\log \left[\mathrm{S}_{2} \mathrm{O}_{3}{ }^{2-}\right]$ plot, the slope of the line is equal to ' $\mathrm{b}$ ', and $\mathrm{k}$ can be calculated.

Derivation of Kinetic Equation

In all experiments of this section, $\mathrm{NH}_{4} \mathrm{OH}=0.5 \mathrm{M}$, initial $\left[\mathrm{Cu}^{2+}\right]=0.008 \mathrm{M}(510 \mathrm{mg} / \mathrm{L})$, and the concentrations of $\left(\mathrm{NH}_{4}\right)_{2} \mathrm{~S}_{2} \mathrm{O}_{3}$ varied from 0.10 to $0.20 \mathrm{M}$.

\section{Change of Cupric Concentration in Thiosulfate Solution}

The concentrations of cupric ion for various concentrations of thiosulfate at $32^{\circ} \mathrm{C}$ and $\mathrm{pH} 10.0$ are shown in Figure 4. It should be noted that the unit of cupric concentration is $\mathrm{C}_{\mathrm{o}} / \mathrm{C}$ instead of $\mathrm{C}$; 
here, $\mathrm{C}_{\mathrm{o}}$ is the original concentration of cupric-amine, $510 \mathrm{mg} / \mathrm{L}$, and $\mathrm{C}$ is the concentration at time, t.

Figure 4 shows that the concentration of cupric-amine decreases $\left(\mathrm{C}_{0} / \mathrm{C}\right.$ increases $)$ with increases in time and concentration of thiosulfate. The cupric concentration, expressed as $\mathrm{C}_{0} / \mathrm{C}$, as a function of time has characteristics of a second order polynomial. During the initial two hours of leaching, an excellent linear fit with a regression coefficient $\left(\mathrm{R}^{2}\right)$ larger than 0.99 can be seen in Figure 5. The linear relationship between $\mathrm{C}_{0} / \mathrm{C}$ and time is indicative of a second order reaction. The rate of a second order reaction can be expressed as:

$$
-\mathrm{dC} / \mathrm{C}^{2}=\mathrm{kdt}
$$

The decomposition of cupric amine within the initial two hours is described by a second order reaction. Because the linear relationship occurs just within the initial two hours and under a particular concentration range of thiosulfate, the cupric decomposition reaction might be defined as a pseudo second order reaction.

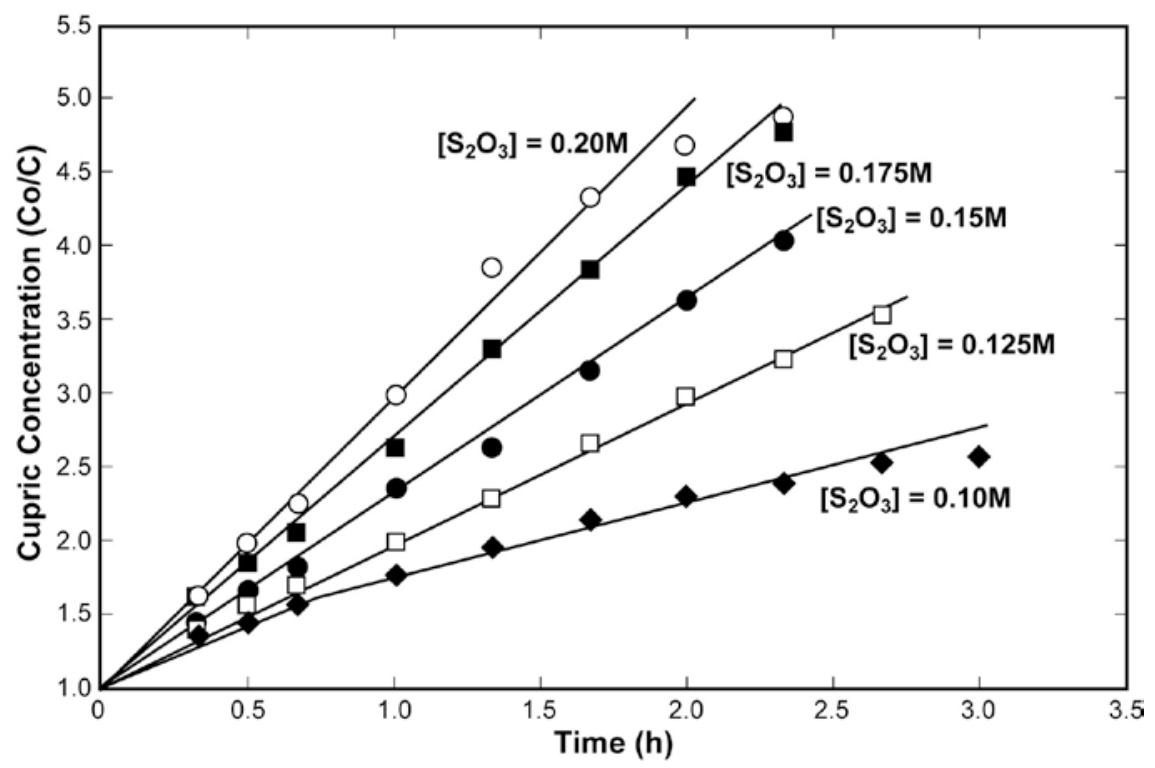

Figure 4. Cupric-amine concentration $\left(\mathrm{C}_{0} / \mathrm{C}\right)$ as a function of time for various concentrations of ammonium thiosulfate. $\mathrm{C}_{\mathrm{o}}=0.008 \mathrm{M}(510 \mathrm{mg} / \mathrm{L}), \mathrm{T}=32^{\circ} \mathrm{C} ; \mathrm{pH}=10.0$. 


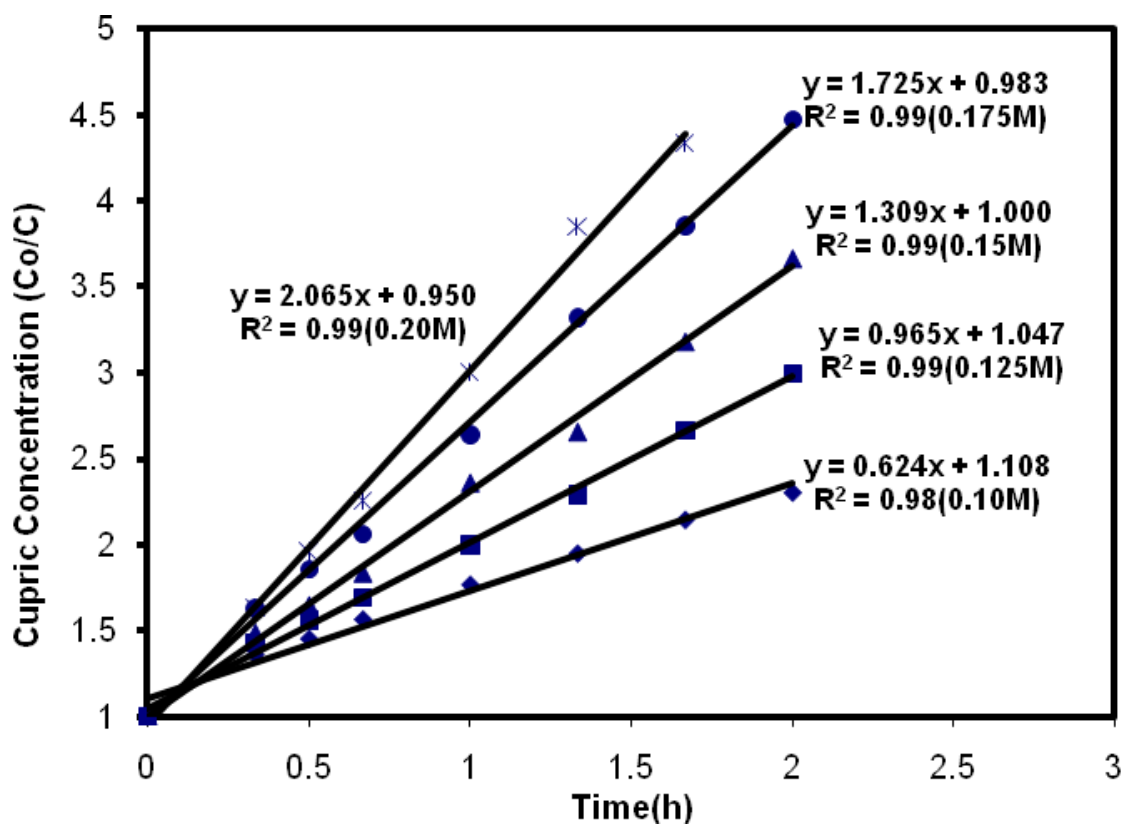

Figure 5. Linearized plot of $\mathrm{C}_{0} / \mathrm{C}$ vs. time. $\mathrm{C}_{\mathrm{o}}=0.008 \mathrm{M} ; \mathrm{T}=32.0^{\circ} \mathrm{C} ; \mathrm{pH}=10.0$. Numbers in parentheses are the molar concentrations of thiosulfate.

Figure 5 provides linearization of the data in Figure 4. Each line has a different slope, and each line corresponds to a particular concentration of thiosulfate.

As a pseudo second order reaction, Equation 6 may be expressed as

$$
-\mathrm{d}\left[\mathrm{Cu}\left(\mathrm{NH}_{3}\right)_{4}{ }^{2+}\right] / \mathrm{dt}=\mathrm{k}_{1}\left[\mathrm{Cu}\left(\mathrm{NH}_{3}\right)_{4}{ }^{2+}\right]^{2}
$$

Figure 5 yields $k_{1}$ and $b$ values in the form of $y=k_{1}+b x$ for various concentrations of thiosulfate. Those values are tabulated in Table 4.

Table 4. $\mathrm{k}_{1}$ values for various concentrations of thiosulfate at $32^{\circ} \mathrm{C}$.

\begin{tabular}{|c|c|c|c|c|}
\hline$\left[\mathrm{S}_{2} \mathrm{O}_{3}{ }^{2-}\right], \mathrm{M}$ & 0.125 & 0.15 & 0.175 & 0.20 \\
\hline $\mathrm{Log}\left[\mathrm{S}_{2} \mathrm{O}_{3}{ }^{2-}\right]$ & -0.903 & -0.824 & -0.757 & -0.699 \\
\hline $\mathrm{k}_{1}, \mathrm{~L} \mathrm{~mol}^{-1} \mathrm{~h}^{-1}$ & 0.9649 & 1.3086 & 1.7254 & 2.0649 \\
\hline $\mathrm{Log} \mathrm{k} \mathrm{k}_{1}$ & -0.0155 & 0.1168 & 0.2369 & 0.3149 \\
\hline
\end{tabular}

From these data, $\mathrm{k}_{32}{ }^{\mathrm{o}} \mathrm{C}=31.4$, and $\mathrm{b}=1.678$. The kinetic equation of cupric-amine decomposition at $32^{\circ} \mathrm{C}$ may be expressed as follows:

$$
-\mathrm{d}\left[\mathrm{Cu}\left(\mathrm{NH}_{3}\right)_{4}{ }^{2+}\right] / \mathrm{dt}=31.4\left[\mathrm{Cu}\left(\mathrm{NH}_{3}\right)_{4}{ }^{2+}\right]^{2}\left[\mathrm{~S}_{2} \mathrm{O}_{3}{ }^{2-}\right]^{1.678} \mathrm{~L}^{-1} \mathrm{~mol} \mathrm{~h}^{-1}
$$

In the same manner, the parameters at $22^{\circ} \mathrm{C}$ and $42^{\circ} \mathrm{C}$ can also be obtained. See Figures 6 and 7, and Tables 5 and 6. 


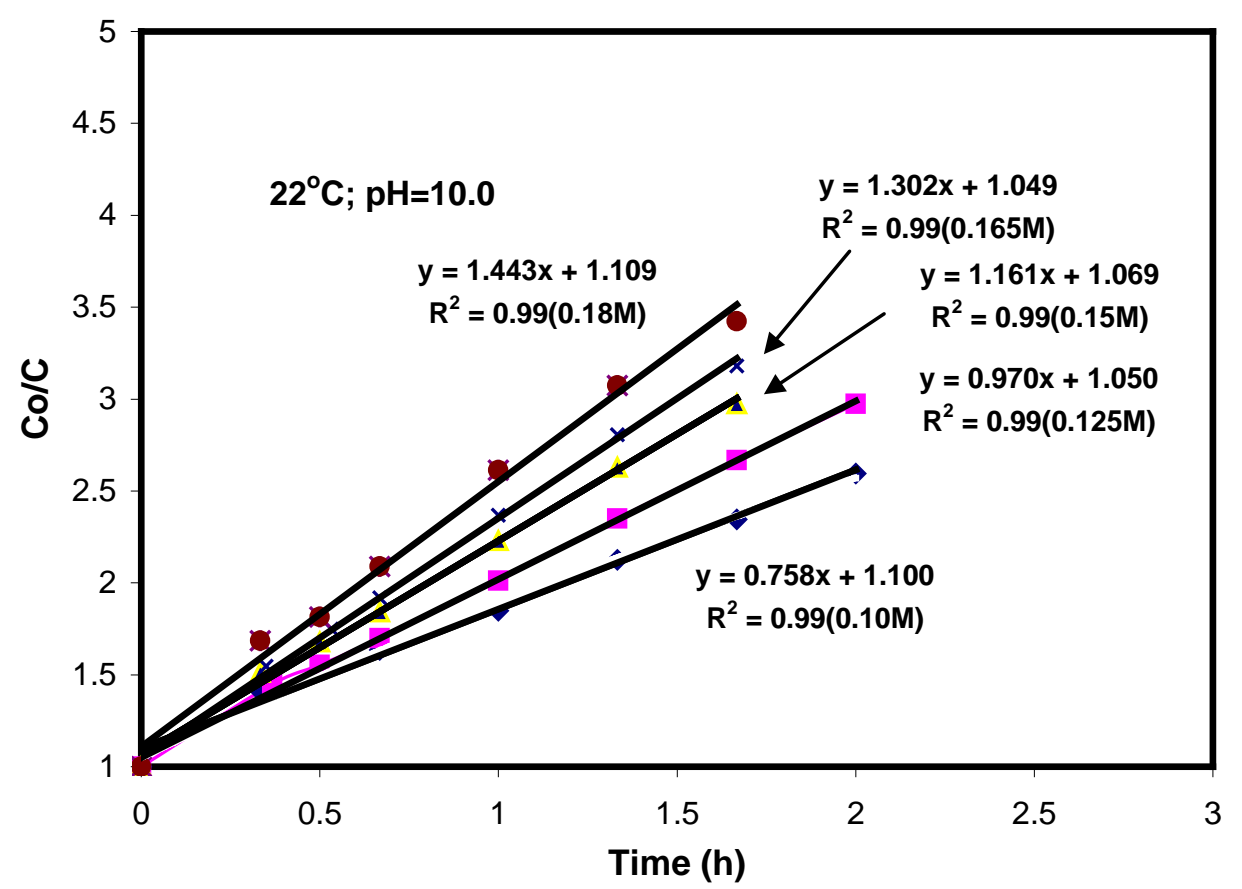

Figure 6. Linearization of $\mathrm{Co} / \mathrm{C}$ vs. time plot. $\mathrm{T}=22.0^{\circ} \mathrm{C} ; \mathrm{pH}=10.0$.

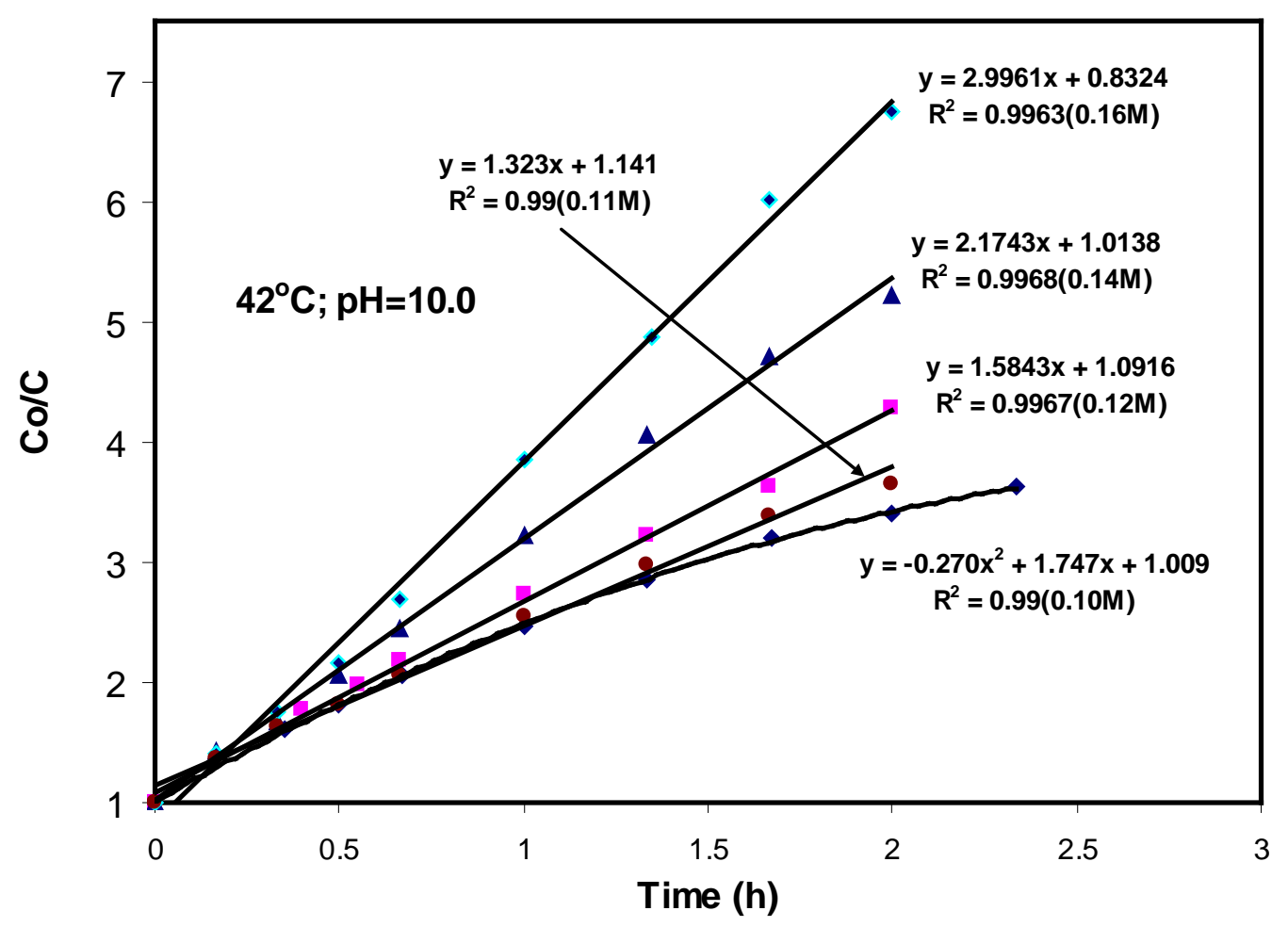

Figure 7. Linearization of $\mathrm{C}_{\mathrm{o}} / \mathrm{C}$ vs. time plot. $\mathrm{T}=42.0^{\circ} \mathrm{C} ; \mathrm{pH}=10.0$. 
Table 5. $\mathrm{k}_{1}$ values for various concentrations.of thiosulfate at $22^{\circ} \mathrm{C}$.

\begin{tabular}{|c|c|c|c|c|c|}
\hline$\left[\mathrm{S}_{2} \mathrm{O}_{3}{ }^{2-}\right], \mathrm{M}$ & $0.10 \mathrm{M}$ & $0.125 \mathrm{M}$ & $0.15 \mathrm{M}$ & $0.165 \mathrm{M}$ & $0.18 \mathrm{M}$ \\
\hline $\left.\mathrm{Log}^{2} \mathrm{~S}_{2} \mathrm{O}_{3}{ }^{2-}\right]$ & -1.0 & -0.903 & -0.824 & -0.783 & -0.7447 \\
\hline $\mathrm{k}_{1}, \mathrm{~L} \mathrm{~mol}^{-1} \mathrm{~h}^{-1}$ & 0.7579 & 0.9697 & 1.1609 & 1.3025 & 1.4429 \\
\hline $\log \mathrm{k}_{1}$ & -0.1204 & 0.0134 & 0.0648 & 0.1148 & 0.1592 \\
\hline
\end{tabular}

Table 6. $\mathrm{k}_{1}$ values for various concentrations of thiosulfate at $42^{\circ} \mathrm{C}$.

\begin{tabular}{|c|c|c|c|c|}
\hline$\left[\mathrm{S}_{2} \mathrm{O}_{3}{ }^{2-}\right], \mathrm{M}$ & 0.11 & 0.12 & 0.14 & 0.16 \\
\hline${\mathrm{Log}\left[\mathrm{S}_{2} \mathrm{O}_{3}{ }^{2-}\right]}$ & -0.959 & -0.921 & -0.854 & -0.796 \\
\hline $\mathrm{k}_{1}, \mathrm{~L} \mathrm{~mol}^{-1} \mathrm{~h}^{-1}$ & 1.323 & 1.584 & 2.174 & 2.996 \\
\hline $\mathrm{Log} \mathrm{k} \mathrm{k}_{1}$ & 0.122 & 0.200 & 0.337 & 0.477 \\
\hline
\end{tabular}

The Rate Constant

The linear relationships of $\log \mathrm{k}_{1}$ and $\log \left[\mathrm{S}_{2} \mathrm{O}_{3}{ }^{2-}\right]$ for all three temperatures, namely $22^{\circ} \mathrm{C}$, $32^{\circ} \mathrm{C}$ and $42^{\circ} \mathrm{C}$, are given in Figure 8 .

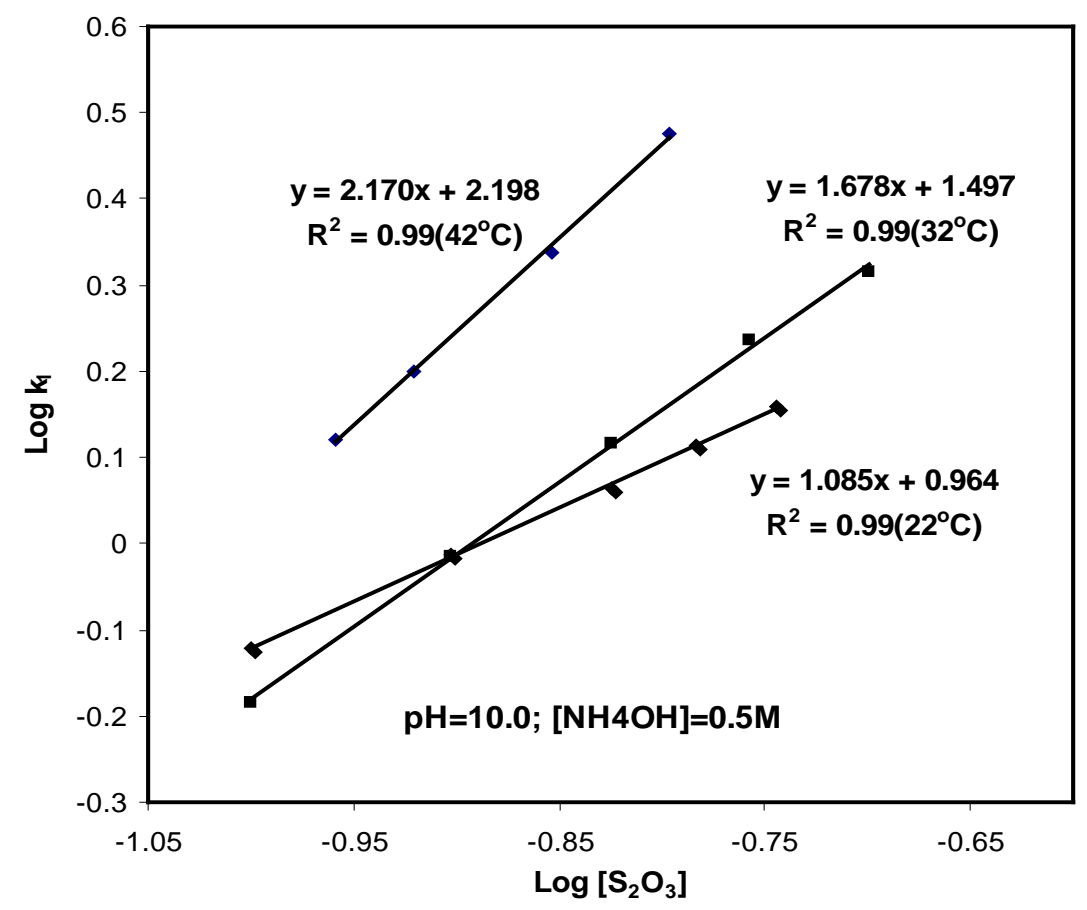

Figure 8. Regression of $\log \mathrm{k}_{1}$ vs. $\log \left[\mathrm{S}_{2} \mathrm{O}_{3}{ }^{2}\right]$ plots for three temperatures of reaction.

The values of ' $b$ ' and rate constant, $k$, at these temperatures are:

$$
\begin{aligned}
& \mathrm{T}=22^{\circ} \mathrm{C}, \mathrm{b}=1.085, \text { and } \mathrm{k}=9.23 . \\
& \mathrm{T}=32^{\circ} \mathrm{C}, \mathrm{b}=1.678, \text { and } \mathrm{k}=31.4 . \\
& \mathrm{T}=42^{\circ} \mathrm{C}, \mathrm{b}=2.170, \text { and } \mathrm{k}=158 .
\end{aligned}
$$


So far, the decomposition rate of cupric-amine may be expressed as follows:

$$
\begin{aligned}
&-\mathrm{d}\left[\mathrm{Cu}\left(\mathrm{NH}_{3}\right){ }_{4}{ }^{2+}\right] / \mathrm{dt}=(9.23-158)\left[\mathrm{Cu}\left(\mathrm{NH}_{3}\right)_{4}{ }^{2+}\right]^{2}\left[\mathrm{~S}_{2} \mathrm{O}_{3}{ }^{2-}\right]^{1.09-2.17} \mathrm{~L}^{-1} \mathrm{~mol} \mathrm{~h}^{-1} \\
&\left(\mathrm{~T}=22-42^{\circ} \mathrm{C} ;\left[\mathrm{NH}_{4} \mathrm{OH}\right]=0.5 \mathrm{M} ; \mathrm{pH}=10.0\right)
\end{aligned}
$$

Discussion

\section{Order of Cupric Decomposition Reaction}

The decomposition rate of cupric-amine complies with a pseudo second order reaction within the range of thiosulfate concentration from $0.10 \mathrm{M}$ to $0.20 \mathrm{M}$ within the first two hours of leaching.

\section{Influence of Temperature}

The decomposition rate of cupric-amine is almost proportional to the thiosulfate concentration at $22^{\circ} \mathrm{C}$; the power of thiosulfate concentration in Equation 12 is 1.09 . However, this power is increases significantly with increasing temperature. For instance, at $42^{\circ} \mathrm{C}$ the power is larger than 2 (2.17 in Equation 12). This means that the decomposition mechanism is changing with changing temperature. Simply speaking, thiosulfate decomposes cupric-amine faster the higher the temperature. The rate constant of cupric-amine decomposition is affected very significantly by temperature; the rate constant increases by a factor of 17 when the temperature is increased from $22^{\circ} \mathrm{C}$ to $42^{\circ} \mathrm{C}$.

\section{Interpretation of Results of Gold Dissolution}

This kinetic equation is very helpful in understanding and predicting the gold leaching results. For instance, elevation of leaching temperature will accelerate the dissolution of gold, but higher temperature also results in decomposition of cupric-amine. There is a temperature range, then, in which gold dissolution and sufficient concentration of cupric ion is optimized.

\section{Behavior of Thiosulfate in Leaching Solution}

Decomposition of Thiosulfate

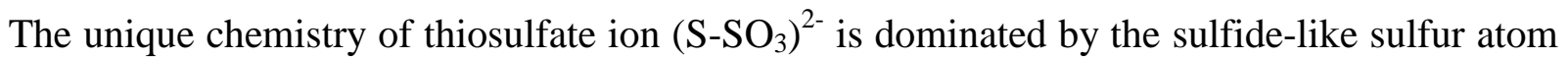
which imparts reducing properties, strong complexing tendencies, and sulfide forming capacities.

According to the Equation 2, thiosulfate should be a driving force for gold dissolution. However, the decomposition rate of cupric ions in thiosulfate solution (Equation 4) is proportional to the concentration of thiosulfate to the power of 2.17 at $42^{\circ} \mathrm{C}$ (see Equation 12). Too high a concentration of thiosulfate will hinder the gold leaching process because of the lack of cupricamine ions in the solution. The gold leaching rate at various concentrations of thiosulfate is shown in Figure 9. The results show that when the concentration of thiosulfate is increased from 0 to 0.20 $\mathrm{mol} / \mathrm{L}$ at $42^{\circ} \mathrm{C}$, gold dissolution rate increases rapidly. Above $0.20 \mathrm{~mol} / \mathrm{L}$, however, the gold 
dissolution rate plateaus. This is quite similar to the results of Jeffrey (2001) for the low concentration of cupric ion $(0.002 \mathrm{~mol} / \mathrm{L})$ and high concentration of ammonia $(0.84 \mathrm{M})$.

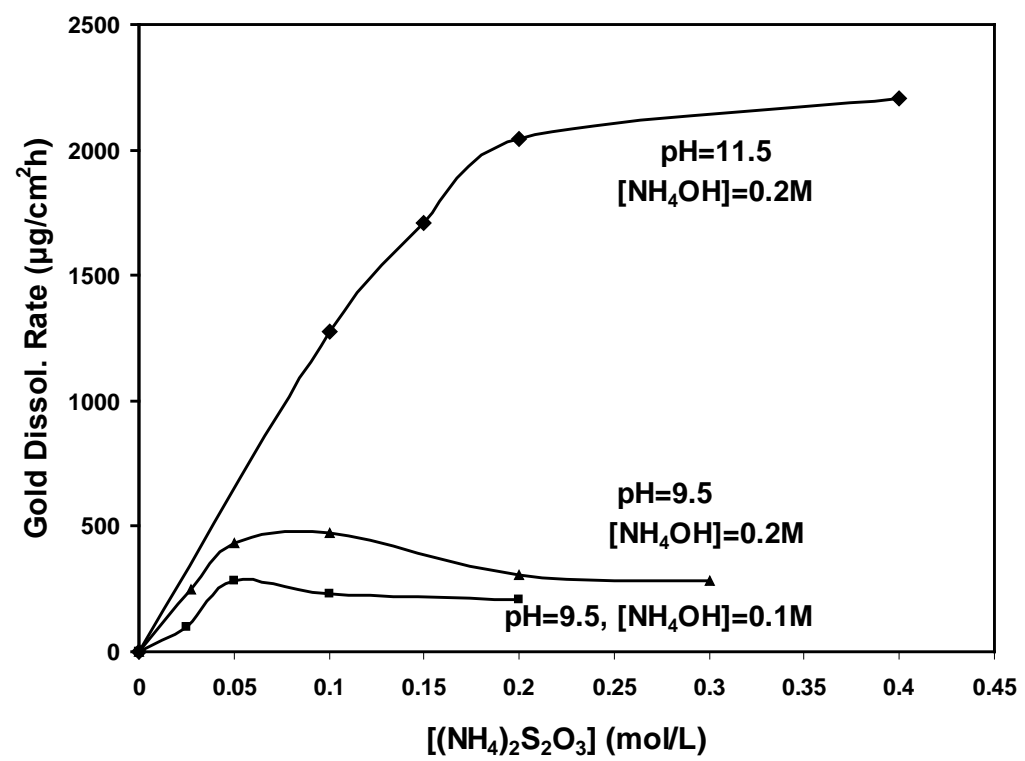

Figure 9. Effect of thiosulfate on gold dissolution rate. Conditions: $\left[\mathrm{Cu}^{2+}\right]=0.008 \mathrm{~mol} / \mathrm{L} ; \mathrm{T}=42^{\circ} \mathrm{C}$; leaching time $=2.0$ hours.

$\mathrm{pH}$ plays a very significant role in this system. Experiments were also conducted at $\mathrm{pH} 9.5$ at two concentrations of ammonium hydroxide. Higher concentrations of thiosulfate did not result in greater gold dissolution at $\mathrm{pH}$ 9.5. These phenomena are discussed later.

Thiosulfate oxidation by cupric amine is presented in Equation 4. Because the concentration of the thiosulfate is always much higher than that of cupric-amine in leaching solutions, it seems that the portion of thiosulfate consumed stoichiometrically by cupric should be neglectable. Many investigators have reported high consumption of thiosulfate in gold ore leaching; some efforts have been made in attempting to use chelating agents of copper to reduce thiosulfate oxidation by cupric ion Xia et al. (2003). Establishing the mechanisms of thiosulfate decomposition is a requisite to minimizing its losses during leaching.

Thiosulfate is consumed primarily in two ways:

i. Oxidation by oxidants (oxygen, cupric, etc.):

$$
\begin{gathered}
2 \mathrm{~S}_{2} \mathrm{O}_{3}{ }^{2-}+1 / 2 \mathrm{O}_{2}+\mathrm{H}_{2} \mathrm{O} \leftrightarrows \mathrm{S}_{4} \mathrm{O}_{6}{ }^{2-}+4 \mathrm{OH}^{-} \\
2 \mathrm{Cu}\left(\mathrm{NH}_{3}\right)_{4}{ }^{2+}+8 \mathrm{~S}_{2} \mathrm{O}_{3}{ }^{2-} \leftrightarrows 2 \mathrm{Cu}\left(\mathrm{S}_{2} \mathrm{O}_{3}\right)_{3}{ }^{5-}+\mathrm{S}_{4} \mathrm{O}_{6}{ }^{2-}+8 \mathrm{NH}_{3}
\end{gathered}
$$

ii. Disproportionation in aqueous solution: 


$$
\begin{aligned}
2 \mathrm{~S}_{2} \mathrm{O}_{3}{ }^{2-}+\mathrm{H}_{2} \mathrm{O} & \leftrightarrows 2 \mathrm{SO}_{4}{ }^{2-}+4 \mathrm{~S}^{\circ}+\mathrm{OH}^{-} \\
3 \mathrm{~S}_{2} \mathrm{O}_{3}{ }^{2-}+6 \mathrm{OH}^{-} & \leftrightarrows 4 \mathrm{SO}_{3}{ }^{2-}+2 \mathrm{~S}^{2-}+3 \mathrm{H}_{2} \mathrm{O} \\
\mathrm{S}_{2} \mathrm{O}_{3}{ }^{2-} \leftrightarrows & \mathrm{SO}_{3}{ }^{2-}+\mathrm{S}^{\mathrm{o}}
\end{aligned}
$$

On the other hand, polythionates, the products of decomposition of thiosulfate, also decompose in alkaline medium (Zhang et al. 2002, Molleman and Dreisinger 2002):

$$
\begin{aligned}
& 4 \mathrm{~S}_{4} \mathrm{O}_{6}{ }^{2-}+6 \mathrm{OH}^{-} \leftrightarrows 5 \mathrm{~S}_{2} \mathrm{O}_{3}{ }^{2-}+2 \mathrm{~S}_{3} \mathrm{O}_{6}{ }^{2-}+3 \mathrm{H}_{2} \mathrm{O} \\
& 2 \mathrm{~S}_{3} \mathrm{O}_{6}{ }^{2-}+6 \mathrm{OH}^{-} \leftrightarrows \mathrm{S}_{2} \mathrm{O}_{3}{ }^{2-}+4 \mathrm{SO}_{3}{ }^{2-}+3 \mathrm{H}_{2} \mathrm{O} \\
& \mathrm{S}_{4} \mathrm{O}_{6}{ }^{2-}+2 \mathrm{OH}^{-} \leftrightarrows 2 \mathrm{~S}_{2} \mathrm{O}_{3}{ }^{2-}+\mathrm{H}_{2} \mathrm{O}+1 / 2 \mathrm{O}_{2}
\end{aligned}
$$

Equations 18 and 20 imply that high $\mathrm{pH}$ is beneficial to the formation of thiosulfate from tetrathionate which initially comes from the thiosulfate oxidation by cupric ions (Byerley et al. 1973). The disproportionation of thiosulfate is relatively slow; so, the main mechanism of consumption of thiosulfate is apparently oxidation.

Equations 18 and 19 show that the increase of $\left[\mathrm{OH}^{-}\right]$should result in reversing the decomposition products of thiosulfate. High $\mathrm{pH}$, then, should prevent thiosulfate from decomposing and would generate oxygen in the regeneration of thiosulfate from tetrathionate.

\section{Thiosulfate Decomposed by Cupric Ions}

\section{Decomposition in the Absence of Cupric-Amine}

The concentrations of thiosulfate in a solution open to the atmosphere over 22 hours are listed in Table 7. Initial concentration of thiosulfate was $0.050 \mathrm{M}$. The results indicate that thiosulfate is quite stable at $42^{\circ} \mathrm{C}$ and $\mathrm{pH} 9.1$ for 22 hours. The kinetics of decomposition by oxygen is slow.

Table 7. Decomposition of thiosulfate in the absence of $\mathrm{Cu}^{2+}$. Conditions: $\mathrm{T}=42^{\circ} \mathrm{C}$; initial $\left[\left(\mathrm{NH}_{4}\right)_{2} \mathrm{~S}_{2} \mathrm{O}_{3}\right]=0.050 \mathrm{M} ;\left[\mathrm{NH}_{4} \mathrm{OH}\right]=0.2 \mathrm{M}, \mathrm{pH}=9.1$, No stirring.

\begin{tabular}{|c|c|c|c|c|c|c|}
\hline Time, h & 0 & 1 & 2 & 4 & 6 & 22 \\
\hline$\left.\left(\mathrm{NH}_{4}\right)_{2} \mathrm{~S}_{2} \mathrm{O}_{3}\right], \mathrm{M}$ & 0.050 & 0.0505 & 0.049 & 0.049 & 0.0495 & 0.0486 \\
\hline \% Decomposed & 0 & 0 & 2 & 2 & 1 & 3 \\
\hline
\end{tabular}

No decrease in thiosulfate concentration was observed either when an initial concentration was $0.20 \mathrm{~mol} / \mathrm{L}$.

\section{Decomposition in the Presence of Cupric-Amine}

The decomposition of thiosulfate was accelerated when cupric-amine was present at an initial concentration of $0.008 \mathrm{M}$ in the solution. See Table 8 . The results show that with an initial 
thiosulfate concentration of $0.050 \mathrm{M}$, about $30 \%$ of thiosulfate was decomposed after 24 hours of reaction at $42^{\circ} \mathrm{C}$ and $\mathrm{pH} 9.1$. A loss of $16 \%$ was noted when the solution $\mathrm{pH}$ was 11.4.

Table 8. Thiosulfate decomposition in the presence of $\mathrm{Cu}^{2+}$. Conditions: $\mathrm{T}=42^{\circ} \mathrm{C}$; initial $\left[\mathrm{CuSO}_{4}\right]$ $=0.008 \mathrm{M} ;\left[\mathrm{NH}_{4} \mathrm{OH}\right]=0.2 \mathrm{M} ; 24$ hrs reaction.

\begin{tabular}{|c|ll|ll|c|}
\hline \multicolumn{2}{|c|}{$\mathrm{pH}$} & \multicolumn{2}{c|}{9.1} & 11.4 \\
\hline$\left[\left(\mathrm{NH}_{4}\right)_{2} \mathrm{~S}_{2} \mathrm{O}_{3}\right], \mathrm{M}$ & Initial & 0.050 & Final & 0.035 & 0.0419 \\
\cline { 2 - 5 } & Initial & 0.20 & Final & 0.187 & 0.189 \\
\hline \multicolumn{2}{|c|}{$\left[\mathrm{Cu}^{2+}\right], \mathrm{M}$} & Final & 0.0065 & $\mathrm{NA}$ \\
& $\left(\right.$ Initial $\left.\left[\mathrm{Cu}^{2+}\right]=0.008 \mathrm{M}\right)$ & \\
\hline
\end{tabular}

For the initial $0.20 \mathrm{M}$ thiosulfate solution, the loss was $6.5 \%$ and $5.5 \%$ at $\mathrm{pH} 9.1$ and 11.4 , respectively.

From the stoichiometric oxidation reaction of thiosulfate and cupric amine (Equation 4), two moles of thiosulfate are consumed per mole of cupric amine. The data in Table 8 show that when $0.05 \mathrm{M}$ of thiosulfate was contacted with $0.008 \mathrm{M}$ of cupric amine, $0.015 \mathrm{M}$ thiosulfate was consumed. Only $0.0015 \mathrm{M}$ of cupric amine was consumed in this reaction. This amounts to two times more decomposition than stoichiometry would predict. These facts indicate that cupric ion accelerates the decomposition of thiosulfate and plays a role of catalyst not only in gold leaching but also in the decomposition of thiosulfate. Once tetrathionate is formed, this species can react further to form other polythionates, resulting in further decomposition of thiosulfate.

Although there are regenerations of $\mathrm{Cu}\left(\mathrm{NH}_{3}\right)_{4}{ }^{2+}$ and $\mathrm{S}_{2} \mathrm{O}_{3}{ }^{2-}$, some products like $\mathrm{SO}_{3}{ }^{2-}$ and $\mathrm{SO}_{4}{ }^{2-}$ are difficult to reverse to thiosulfate, and these sulfur species are regenerated to thiosulfate to only a very limited extent.

Table 9. Regeneration of cupric-amine. Conditions: $\mathrm{T}=42^{\circ} \mathrm{C}$; initial $\left[\mathrm{CuSO}_{4}\right]=0.008 \mathrm{M},\left[\mathrm{NH}_{4} \mathrm{OH}\right]$ $=0.2 \mathrm{M}$; initial $\left[\mathrm{S}_{2} \mathrm{O}_{3}{ }^{2-}\right]=0.050 \mathrm{M} ; \mathrm{pH}=9.1$.

\begin{tabular}{|c|c|c|c|c|c|}
\hline Time, $\mathrm{h}$ & 0 & 3 & 19 & 23 & 25 \\
\hline$\left[\mathrm{Cu}^{2+}\right], \mathrm{M}$ & 0.0080 & 0.0029 & 0.0060 & 0.0064 & 0.0065 \\
Decomposition & $0 \%$ & $64 \%$ & $25 \%$ & $20 \%$ & $19 \%$ \\
\hline
\end{tabular}

The results listed in Table 9 show that when thiosulfate and cupric-amine are first contacted, the concentration of cupric-amine decreases rapidly. That is, $64 \%$ of the cupric ion was lost during the first three hours; this represents the fast reaction of cupric ion and thiosulfate. However, the cupric concentration regenerated to $80 \%$ after 23 hours. This suggests that generated oxygen oxidized cuprous to cupric, although the regeneration rate is much slower than the decomposition by thiosulfate at initial contact.

Higher $\mathrm{pH}$ might slow the decomposition of thiosulfate (Table 5); but higher temperature could accelerate this process as shown in Table 10. 
Table 10. Effect of temperature on thiosulfate oxidation in the presence of cupric. Conditions: $\left[\mathrm{CuSO}_{4}\right]=0.008 \mathrm{M} ;\left[\mathrm{NH}_{4} \mathrm{OH}\right]=0.2 \mathrm{M}$; initial $\left[\left(\mathrm{NH}_{4}\right)_{2} \mathrm{~S}_{2} \mathrm{O}_{3}\right]=0.20 \mathrm{M} ; \mathrm{pH}=11.4 ; 24$ hours reaction.

\begin{tabular}{|l|c|c|}
\hline Temperature, ${ }^{\circ} \mathrm{C}$ & 42 & 52 \\
\hline$\left[\mathrm{S}_{2} \mathrm{O}_{3}\right], \mathrm{M}$ & 0.189 & 0.183 \\
\hline Decomposition, \% & 5.5 & 8.5 \\
\hline
\end{tabular}

\section{Discussion}

i) The thiosulfate decomposition rate in the presence of oxygen only is slow. Losses due to oxidation by oxygen are negligible.

ii) Cupric-amine acts as a catalyst: Cupric-amine will accelerate the decomposition of thiosulfate.

iii) High $\mathrm{pH}$ hinders the decomposition of thiosulfate: Higher values of $\mathrm{pH}$ should slow the decomposition of thiosulfate. Hydroxyl ion is beneficial in regenerating thiosulfate and cupric ions from polythionates, which are the products of decomposition of thiosulfate.

iv) Increase of temperature accelerates the decomposition of thiosulfate.

Effect of Ammonium Hydroxide

$\mathrm{NH}_{4} \mathrm{OH}$ forms the stable cupric complex: cupric-amine $\left[\mathrm{Cu}\left(\mathrm{NH}_{3}\right)_{4}{ }^{2+}\right]$ in alkaline media, and it is also a pH buffer (Aylmore and Muir 2001, Jeffrey 2001). An Eh-pH diagram for the $\mathrm{Au}-\mathrm{NH}_{3}-\mathrm{S}_{2} \mathrm{O}_{3}$ system is shown in Figure 10. Above about $\mathrm{pH} 9, \mathrm{Au}\left(\mathrm{NH}_{3}\right)_{4}{ }^{+}$is stable at moderate values of Eh.

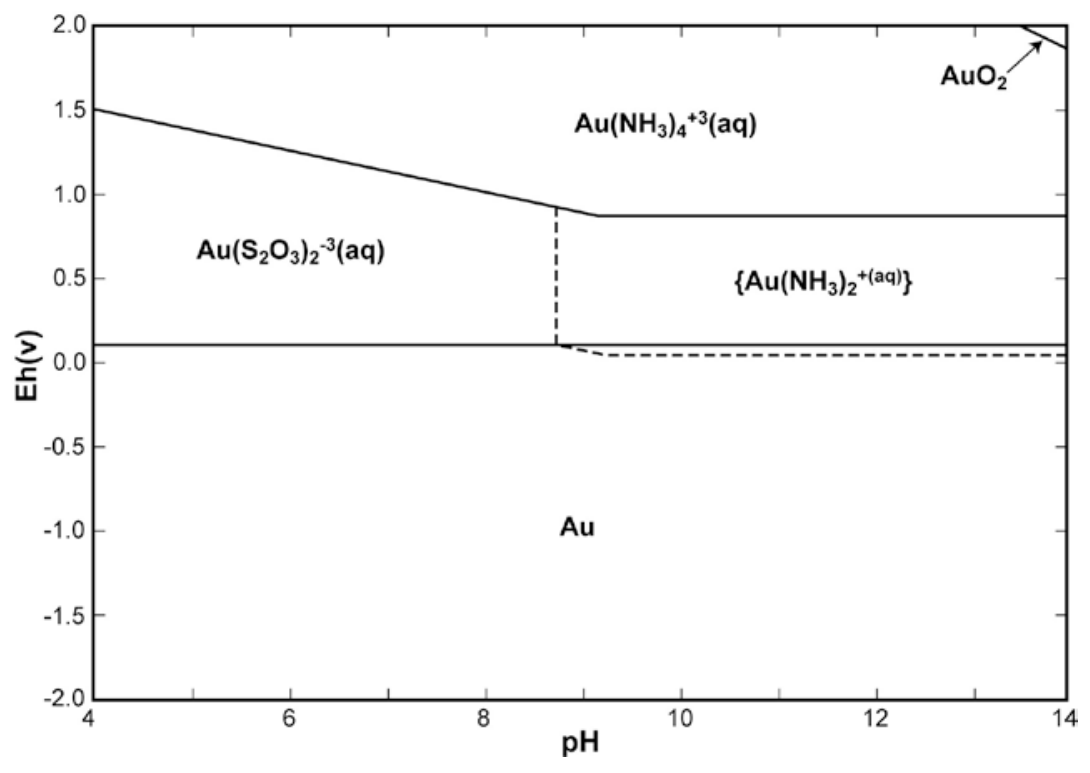

Figure 10. Eh/pH diagram of gold-ammonia-thiosulfate system. Conditions: $0.0005 \mathrm{M}$ Au, 0.1 M thioslfate, 0.1 M ammonia. (Aylmore and Muir 2001). 
Gold dissolution rate at various levels of ammonium hydroxide concentdastion at $\mathrm{pH} 9.6$ and 11.5 are shown in Figure 11.

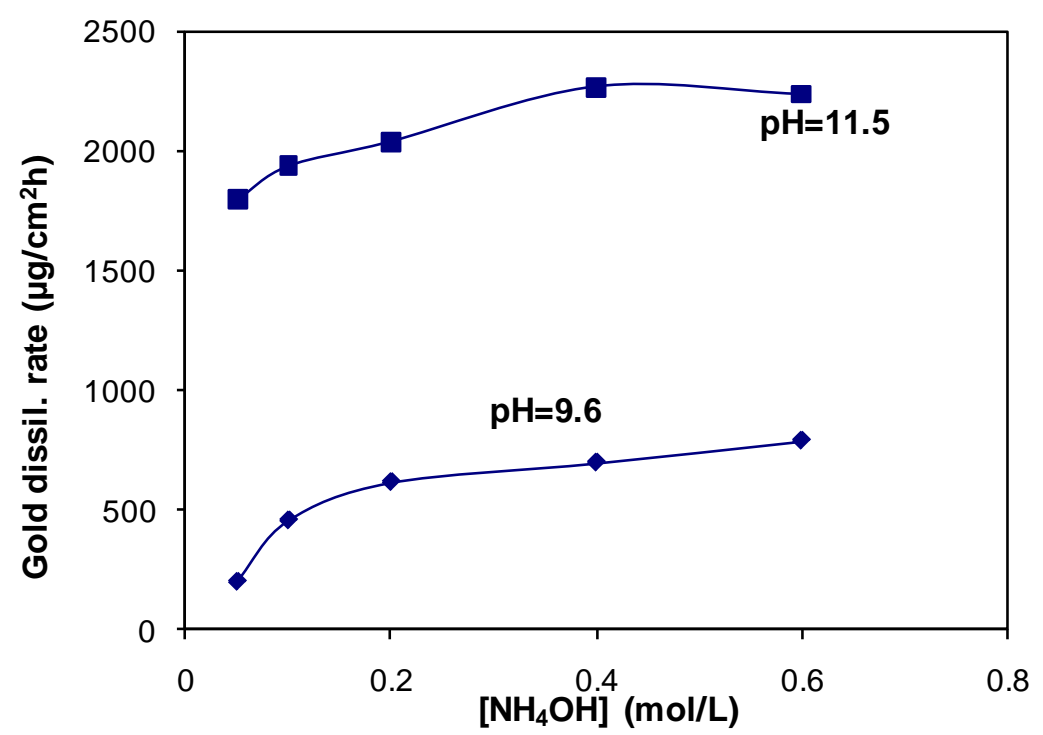

Figure 11 Effect of ammonia on gold dissolution rate. Conditions: $\left[\left(\mathrm{NH}_{4}\right)_{2} \mathrm{~S}_{2} \mathrm{O}_{3}\right]=0.20 \mathrm{M}$; $\left[\mathrm{CuSO}_{4}\right]=0.008 \mathrm{M} ; \mathrm{T}=42^{\circ} \mathrm{C}$; leaching time $=2.0$ hours.

The results indicate that when the concentration of ammonium hydroxide is within the range of 0.1 to $0.6 \mathrm{~mol} / \mathrm{L}$, there is no significant change in the gold dissolution rate. However, the solution $\mathrm{pH}$ does play an important role in that the dissolution rate was increased by a factor of four when the $\mathrm{pH}$ was increased from $\mathrm{pH} 9.6$ to 11.5.

These results would seem to contradict those listed in Table2, in which almost all of the results show the tendency for gold dissolution rate to increase significantly with an increase of ammonium hydroxide concentration. The difference is due to the fact that the experiments listed in Table 2 were performed without controlling solution $\mathrm{pH}$. Higher ammonium hydroxide concentrations will always result in higher $\mathrm{pH}$ values. So, the conclusion, then, is high concentrations of ammonium hydroxide are not necessary, but its concentration needs to match that of thiosulfate. An appropriate $\mathrm{pH}$ is even more important than the concentration of ammonium hydroxide.

\section{Effect of $\mathrm{pH}$ and Temperature}

The dissociation constant of water $\mathrm{K}_{\mathrm{w}}=\left[\mathrm{H}^{+}\right]\left[\mathrm{OH}^{-}\right]$is temperature dependent; the pKs of water at various temperatures are listed in Table 11.

Table 11. pK of water at various temperatures.

\begin{tabular}{|l|c|c|c|c|c|c|c|c|c|}
\hline $\mathrm{T},{ }^{\circ} \mathrm{C}$ & 0 & 10 & 18 & 25 & 30 & 50 & 60 & 70 & 80 \\
\hline $\mathrm{pK}_{\mathrm{w}}$ & 14.92 & 14.52 & 14.16 & 13.92 & 13.75 & 13.10 & 12.90 & 12.67 & 12.46 \\
\hline
\end{tabular}


Because $\mathrm{pK}_{\mathrm{w}}$ is reduced with increased temperature, the value of $\mathrm{pH}$ will be reduced with elevated temperature. See Table 12. To maintain the same $\mathrm{pH}$ at higher temperature, more hydroxide is needed.

Table 12. $\mathrm{pH}$ as a function of temperature of thiosulfate leaching solution. Conditions: $\left[\mathrm{NH}_{4} \mathrm{OH}\right]=$ $0.2 \mathrm{~mol} / \mathrm{L} ;\left[\left(\mathrm{NH}_{4}\right)_{2} \mathrm{~S}_{2} \mathrm{O}_{3}\right]=0.2 \mathrm{~mol} / \mathrm{L} ;\left[\mathrm{Cu}^{2+}\right]=0.008 \mathrm{~mol} / \mathrm{L}$.

\begin{tabular}{|c|c|c|c|c|c|}
\hline $\mathrm{T},{ }^{\circ} \mathrm{C}$ & 12 & 22 & 32 & 42 & 52 \\
\hline $\mathrm{pH}$ & 9.72 & 9.36 & 9.14 & 8.87 & 8.66 \\
\hline
\end{tabular}

The disproportionation reactions of thiosulfate and pH are related: See Equations 18, 19 and 20. Another reaction is given in Equation 21 (Molleman and Dreisinger 2002).

$$
\mathrm{HS}^{-}+\mathrm{SO}_{3}{ }^{2-}+\mathrm{OH}^{-} \leftrightarrows \mathrm{S}_{2} \mathrm{O}_{3}^{2-}+\mathrm{H}_{2} \mathrm{O}
$$

As to the status of copper, it may be seen from the Eh-pH diagram of a Cu- $\mathrm{NH}_{3}-\mathrm{S}_{2} \mathrm{O}_{3}{ }^{2-}$ system that the $\mathrm{pH}$ controls the status of cupric compounds: the cupric-amine, cupric thiosulfate, cupric oxide or cuprous/cupric sulfide See Figure 12.

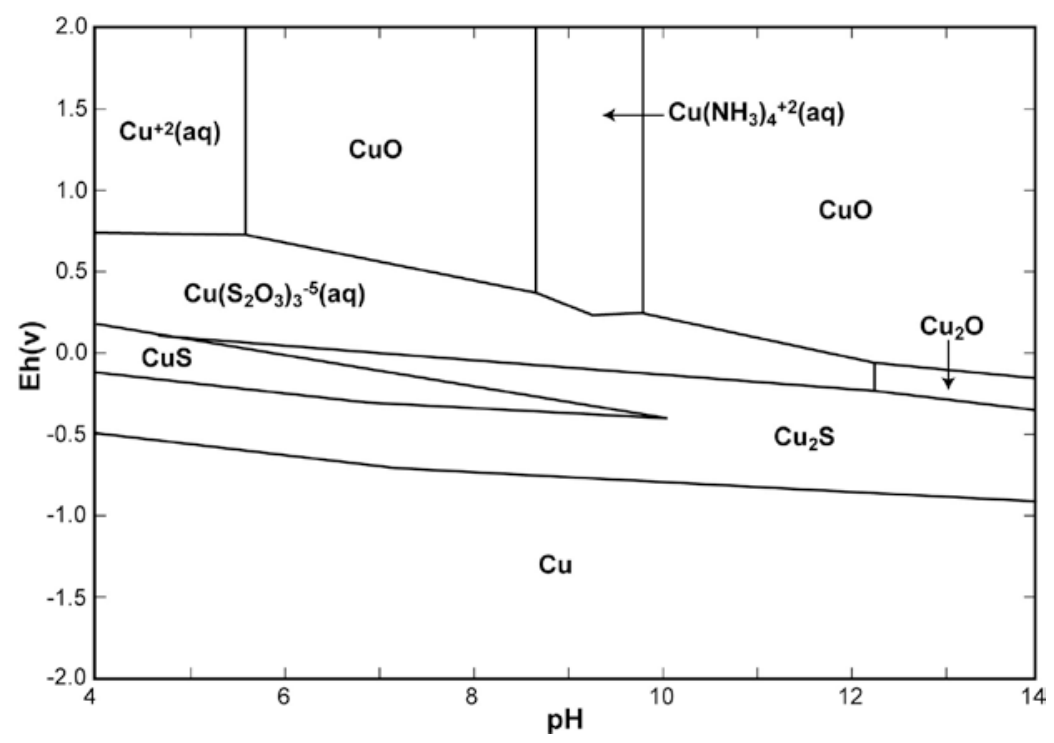

Figure 12. Eh/ph diagram of copper-ammonia-thiosulfate system. Conditions: ammonia $=0.1 \mathrm{M}$; thiosulfate $=0.1 \mathrm{M}$; cupric $=0.00005 \mathrm{M}$. (Li et al. 1995).

Gold Dissolution Rates at Various Temperatures and $\mathrm{pH}$

The results of gold dissolution rates at various temperatures and $\mathrm{pH}$ are shown in Figure 13. Under the experimental conditions involved: $\left(\left[\mathrm{NH}_{4} \mathrm{OH}\right]=0.2 \mathrm{M}\right.$; $\left[\left(\mathrm{NH}_{4}\right)_{2} \mathrm{~S}_{2} \mathrm{O}_{3}\right]=0.20 \mathrm{M}$; $\left[\mathrm{Cu}^{2+}\right]=$ $0.008 \mathrm{M})$, both $\mathrm{pH}$ and temperature are significant factors which affect gold dissolution rate greatly. The response curves of dissolution rate vs. $\mathrm{pH}$ reveal sharp peaks except for $22^{\circ} \mathrm{C}$. The optimal $\mathrm{pH}$ range is between $\mathrm{pH}$ 10.8-11.4 which depends on the temperature. This means that the $\mathrm{OH}^{-}$ 
concentration does promote the gold dissolution rate. At higher $\mathrm{pH}$ values, however, the stable species of cupric is cupric oxide at which point the dissolution rate drops sharply.

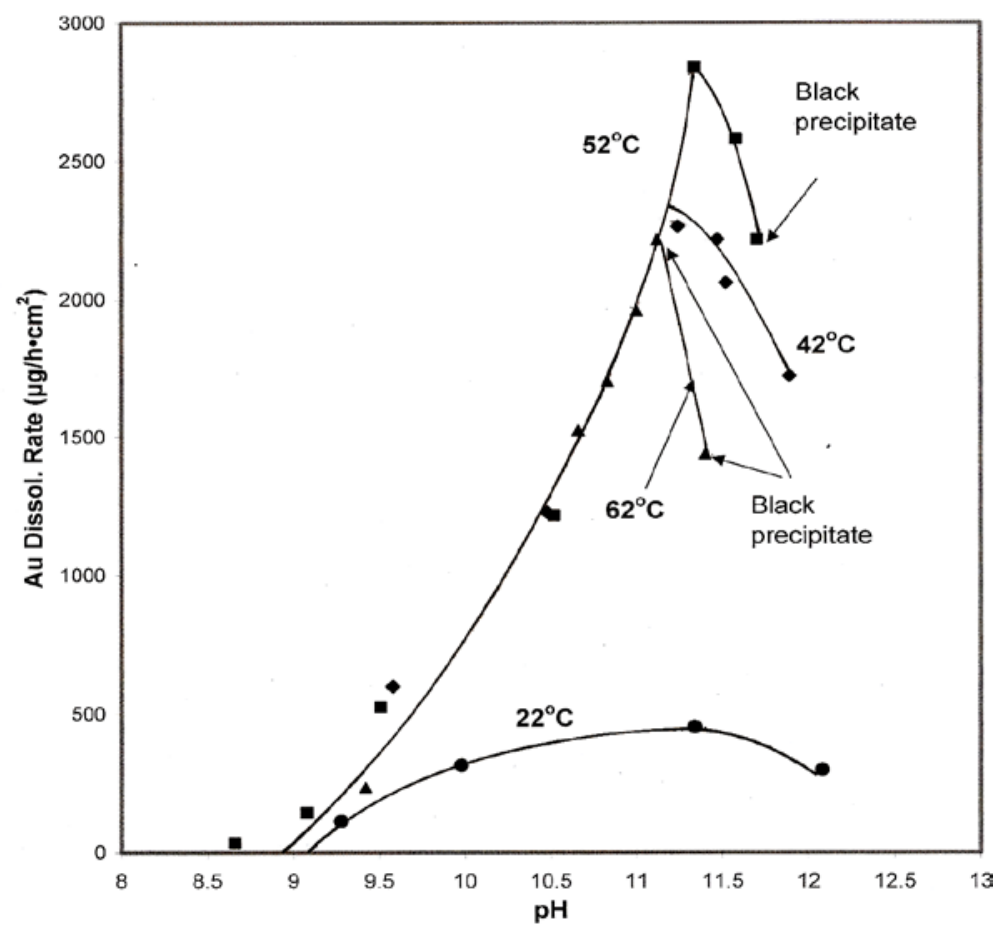

Figure 13. Gold dissolution rate as a function of $\mathrm{pH}$ and temperature. Conditions: $\left[\left(\mathrm{NH}_{4}\right)_{2} \mathrm{~S}_{2} \mathrm{O}_{3}\right]=0.2 \mathrm{M} ;\left[\mathrm{NH}_{4} \mathrm{OH}\right]=0.2 \mathrm{M} ;\left[\mathrm{Cu}^{2+}\right]=0.008 \mathrm{M}$; leaching time $2 \mathrm{hrs}$.

Figure 12 which represents equilibrium within this system indicates optimal $\mathrm{pH}$ for leaching should be between $\mathrm{pH}$ 9-10 where $\mathrm{Cu}\left(\mathrm{NH}_{3}\right)^{2+}$ is stable. Our results indicate that the system is not in an equilibrium condition, and high $\mathrm{pH}$ results in faster leaching kinetics. It should be mentioned that reactant concentrations differ between that in Figure 12 and our leaching data.

Aylmore and Muir (2001) reported that under conditions of $\left[\mathrm{NH}_{3} / \mathrm{NH}_{4}{ }^{+}\right]=1 \mathrm{M},\left[\mathrm{S}_{2} \mathrm{O}_{3}{ }^{2-}\right]=1 \mathrm{M}$, $\left[\mathrm{Cu}^{2+}\right]=0.05 \mathrm{M}$ and $\mathrm{Eh}>150 \mathrm{mV}$, the stable species of cupric will be cupric amine when $\mathrm{pH}<10.5$ and cupric oxide when $\mathrm{pH}>10.5$. Under our experimental conditions: $\left[\mathrm{NH}_{3} / \mathrm{NH}_{4}{ }^{+}\right]=0.2 \mathrm{M},\left[\mathrm{S}_{2} \mathrm{O}_{3}{ }^{2-}\right]$ $=0.2 \mathrm{M},\left[\mathrm{Cu}^{2+}\right]=0.008 \mathrm{M}$ and $\mathrm{Eh}=160-260 \mathrm{mV}$, cupric oxide was noted only above $\mathrm{pH} 11.1\left(62^{\circ} \mathrm{C}\right)$ and $11.7\left(52^{\circ} \mathrm{C}\right)$.

According to the Equation 22:

$$
2 \mathrm{Cu}\left(\mathrm{NH}_{3}\right)_{4}{ }^{2+}+\mathrm{bS}_{2} \mathrm{O}_{3}{ }^{2-} \leftrightarrows 2 \mathrm{Cu}\left(\mathrm{S}_{2} \mathrm{O}_{3}\right)_{3}{ }^{5-}+\mathrm{S}_{4} \mathrm{O}_{6}{ }^{2-}+8 \mathrm{NH}_{3}
$$

$\mathrm{NH}_{3}$ is produced during the leaching process. Ammonia does protonate slightly,

$$
\mathrm{NH}_{3}+\mathrm{H}_{2} \mathrm{O} \leftrightarrows \mathrm{NH}_{4}^{+}+\mathrm{OH}^{-}
$$

with $K_{b}=1.78 \times 10^{-5}$ (Butler 1964). 
Combining Equations (22) and (23), Equation (24) obtained:

$$
2 \mathrm{Cu}\left(\mathrm{NH}_{3}\right)_{4}{ }^{2+}+8 \mathrm{~S}_{2} \mathrm{O}_{3}{ }^{2-}+\mathrm{H}_{2} \mathrm{O} \leftrightarrows 2 \mathrm{Cu}\left(\mathrm{S}_{2} \mathrm{O}_{3}\right)_{3}{ }^{5-}+\mathrm{S}_{4} \mathrm{O}_{6}{ }^{2-}+\mathrm{NH}_{3} / \mathrm{NH}^{4+}+\mathrm{OH}^{-}
$$

If $\mathrm{pH}$ is elevated, for instance from $\mathrm{pH} 10$ to $11.3,\left[\mathrm{OH}^{-}\right]$is increased 20 -fold. The reaction shifts to the left, and the decomposition of cupric and thiosulfate will be hindered. This is beneficial to increase gold dissolution rate.

Figure 14 provides good evidence for explaining the effect of $\mathrm{pH}$. This figure indicates that the residual cupric-amine concentration at $\mathrm{pH} 11.4$ is about four times higher than that at $\mathrm{pH} 10$ at $42^{\circ} \mathrm{C}$ and $52^{\circ} \mathrm{C}$. This profile coincides with the gold dissolution curves shown in Figure 13. Above $\mathrm{pH}$ 11.4, cupric oxide precipitation occurs, which results in a significant decrease in concentration of cupric-amine and reduction in the dissolution of gold.

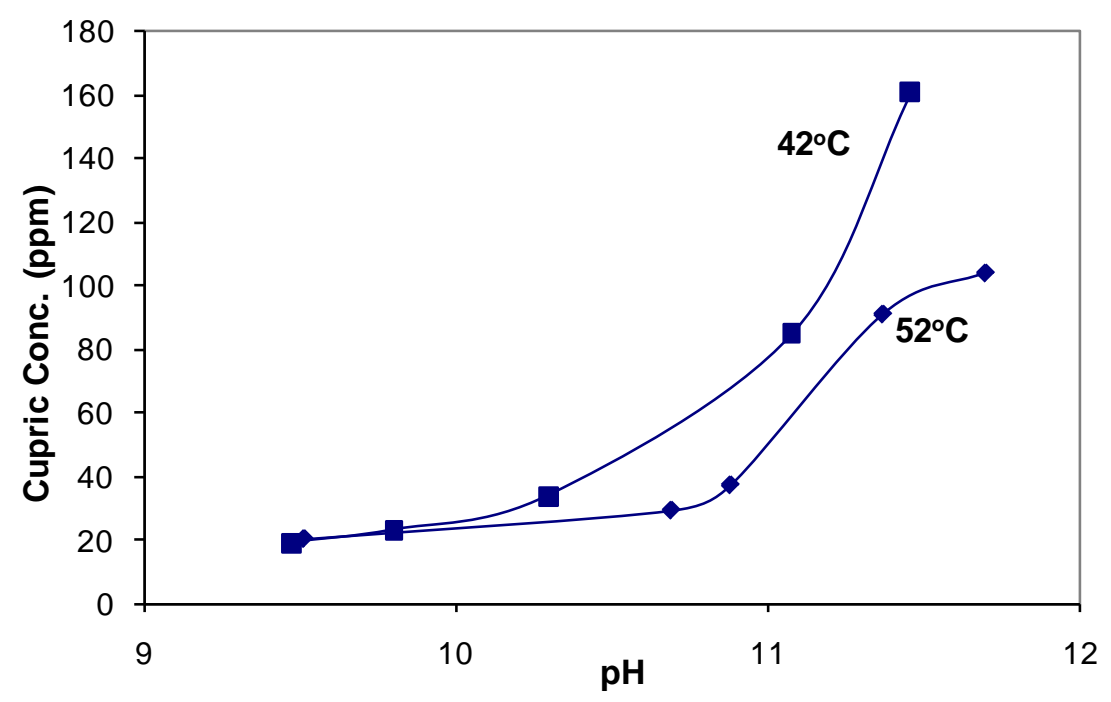

Figure 14. Residual cupric concentration as the function of $\mathrm{pH}$. Conditions: initial $\left[\mathrm{Cu}^{2+}\right]=$ $510 \mathrm{mg} / \mathrm{L},\left[\mathrm{NH}_{4} \mathrm{OH}\right]=0.2 \mathrm{M},\left[\left(\left[\mathrm{NH}_{4}\right)_{2} \mathrm{~S}_{2} \mathrm{O}_{3}\right]=0.2 \mathrm{M}\right.$, reaction time $=2.0 \mathrm{hrs}$.

Temperature is also a very important factor because it influences the rate of gold dissolution and the state of cupric ion. For example, as shown in Figure 13, the dissolution rate at $42^{\circ} \mathrm{C}$ is about four times higher than that at $22^{\circ} \mathrm{C}$ at $\mathrm{pH} 11.4$. It is even higher at $52^{\circ} \mathrm{C}$, although the cupric-amine concentration is lower because of decomposition. However, the dissolution rate decreases at $62^{\circ} \mathrm{C}$ because cupric oxide forms at lower values of $\mathrm{pH}$ under these conditions. Figure 13 shows that cupric oxide exists at $\mathrm{pH} 11.1$ at $62^{\circ} \mathrm{C}$, and this would reduce the cupric-amine concentration even further. Figure 13 also indicates that elevation of temperature changes the equilibrium between $\mathrm{Cu}\left(\mathrm{NH}_{3}\right)_{4}{ }^{2+}$ and $\mathrm{CuO}$ and that it lowers the threshold of $\mathrm{pH}$ at which the $\mathrm{CuO}$ forms. For instance, no $\mathrm{CuO}$ precipitated until $\mathrm{pH} 11.9$ at $42^{\circ} \mathrm{C}$, but it did precipitate at $\mathrm{pH} 11.7$ and 11.1 at $52^{\circ} \mathrm{C}$ and $62^{\circ} \mathrm{C}$, respectively. 


\section{Effect of Additives}

Experiments were conducted to study whether the addition of selected reagents would enhance the dissolution rate of gold. Phenyl imidazoline; methyl imidazoline; methylamine hydrochloride; hydrogen peroxide; iodine; EDTA; sodium sulfite and potassium permanganate were studied.

\section{2-phenyl-2-imidazoline}

Table.13. Effect of phenyl imidazoline. Conditions: $\left[\mathrm{NH}_{4} \mathrm{OH}\right]=0.2 \mathrm{M} ;\left[\left(\mathrm{NH}_{4}\right)_{2} \mathrm{~S}_{2} \mathrm{O}_{3}\right]=0.20 \mathrm{M}$; $\mathrm{T}$ $=42^{\circ} \mathrm{C} ;\left[\mathrm{Cu}^{2+}\right]=0.008 \mathrm{M} ; \mathrm{pH}=11.5$; leaching time $=2.0 \mathrm{hrs}$.

\begin{tabular}{|c|c|c|c|c|}
\hline Concentration, $\mathrm{g} / \mathrm{L}$ & 0 & 0.03 & 0.10 & 0.30 \\
\hline Dissol. Rate, $\mu \mathrm{g} / \mathrm{h} \cdot \mathrm{cm}^{2}$ & 2043 & 1276 & 1071 & 924 \\
\hline
\end{tabular}

As can be noted, the addition of phenyl imidazoline is detrimental to leaching rate. The greater is the amount added, the smaller rate is the dissolution of gold.

Methyl Imidazoline

Table 14. Effect of methyl imidazoline. Conditions: $\left[\mathrm{NH}_{4} \mathrm{OH}\right]=0.2 \mathrm{M} ;\left[\left(\mathrm{NH}_{4}\right)_{2} \mathrm{~S}_{2} \mathrm{O}_{3}\right]=0.20 \mathrm{M}$; $\mathrm{T}$ $=42^{\circ} \mathrm{C} ;\left[\mathrm{Cu}^{2+}\right]=0.008 \mathrm{M}, \mathrm{pH} 11.4$; leaching time $=2.0$ hrs.

\begin{tabular}{|c|c|c|}
\hline Concentration, $\mathrm{g} / \mathrm{L}$ & 0 & 0.84 \\
\hline Dissol. Rate, $\mu \mathrm{g} / \mathrm{h} \cdot \mathrm{cm}^{2}$ & 2043 & 1211 \\
\hline
\end{tabular}

The data show that the presence of methyl imidazoline is detrimental in the system.

Methylamine Hydrochloride

Table.15. Effect of methylamine hydrochloride. Conditions: $\left[\mathrm{NH}_{4} \mathrm{OH}\right]=0.2 \mathrm{M} ;\left[\left(\mathrm{NH}_{4}\right)_{2} \mathrm{~S}_{2} \mathrm{O}_{3}\right]=$ $0.20 \mathrm{M} ; \mathrm{T}=42^{\circ} \mathrm{C} ;\left[\mathrm{Cu}^{2+}\right]=0.008 \mathrm{M} ; \mathrm{pH} 11.5$; leaching time $=2.0$ hrs.

\begin{tabular}{|c|c|c|c|c|}
\hline Concentration, $\mathrm{M}$ & 0 & 0.008 & 0.02 & 0.04 \\
\hline Dissol. Rate, $\mu \mathrm{g} / \mathrm{h} \cdot \mathrm{cm}^{2}$ & 2043 & 1911 & 1495 & 1258 \\
\hline
\end{tabular}

Methylamine hydrochloride is an acidic substance, and greater amounts of sodium hydroxide are needed to maintain $\mathrm{pH}$ at 11.5.The presence of methylamine hydrochloride was detrimental to gold dissolution under these conditions.

Hydrogen Peroxide

Table16. Effect of hydrogen peroxide. Conditions: $\left[\mathrm{NH}_{4} \mathrm{OH}\right]=0.2 \mathrm{M} ;\left[\left(\mathrm{NH}_{4}\right)_{2} \mathrm{~S}_{2} \mathrm{O}_{3}\right]=0.20 \mathrm{M}$; $\mathrm{T}=42^{\circ} \mathrm{C} ;\left[\mathrm{Cu}^{2+}\right]=0.008 \mathrm{M}$; $\mathrm{pH} 11.5$; leaching time $=2.0$ hrs.

\begin{tabular}{|c|c|c|c|c|}
\hline$\left[\mathrm{H}_{2} \mathrm{O}_{2}\right], \mathrm{M}$ & 0 & 0.00075 & 0.003 & 0.006 \\
\hline Dissol. Rate, $\mu \mathrm{g} / \mathrm{h} \cdot \mathrm{cm}^{2}$ & 2043 & 2059 & 1913 & 1400 \\
\hline$\left[\mathrm{Cu}^{2+}\right], \mathrm{ppm}$ & 148 & 189 & 167 & 132 \\
\hline
\end{tabular}


Hydrogen peroxide is a strong oxidant, and it was thought that appropriate doses might increase oxidation of cuprous ion and results in an increase in cupric concentration. This fact was observed at lower additions of $\mathrm{H}_{2} \mathrm{O}_{2}$, but gold dissolution rate was noted to decrease.

\section{Sodium Sulfite}

Because sulfite is one of the products of thiosulfate decomposition, the addition of sulfite will reverse the reaction of thiosulfate decomposition. (EDTA) is a chelating agent of $\mathrm{Cu}^{2+}$, and chelated $\mathrm{Cu}^{2+}$ would be hardly to oxidize thiosulfate. Kerley reported that the addition of sulfite $(0.1 \mathrm{M})$ reduced the consumption of thiosulfate. We did not find this to be the case. See Table 17.

Table 17. Effect of sodium sulfite. Conditions: $\left[\mathrm{NH}_{4} \mathrm{OH}\right]=0.2 \mathrm{M} ;\left[\left(\mathrm{NH}_{4}\right)_{2} \mathrm{~S}_{2} \mathrm{O}_{3}\right]=0.20 \mathrm{M} ; \mathrm{T}=42^{\circ} \mathrm{C}$; $\left[\mathrm{Cu}^{2+}\right]=0.008 \mathrm{M} ; \mathrm{pH} 10.6$; leaching time $=2.0$ hrs.

\begin{tabular}{|c|c|c|c|c|}
\hline Dose of $\mathrm{Na}_{2} \mathrm{SO}_{3}, \mathrm{~g} / \mathrm{L}$ & 0 & 0.25 & 0.5 & 1.0 \\
\hline Dissol. Rate, $\mu \mathrm{g} / \mathrm{h} \cdot \mathrm{cm}^{2}$ & 1315 & 1293 & 754 & 369 \\
\hline Eh, (vs Ag-AgCl electrode) & -27 & -38 & -49 & -68 \\
\hline
\end{tabular}

Sodium sulfite is a reductant, and additions of the reagent decreased the solution potential and gold dissolution rate both.

\section{EDTA}

Table 18, Effect of EDTA. Conditions: $\left[\mathrm{NH}_{4} \mathrm{OH}\right]=0.2 \mathrm{M} ;\left[\left(\mathrm{NH}_{4}\right)_{2} \mathrm{~S}_{2} \mathrm{O}_{3}\right]=0.20 \mathrm{M} ; \mathrm{T}=42^{\circ} \mathrm{C}$; $\left[\mathrm{Cu}^{2+}\right]=0.008 \mathrm{M}$; $\mathrm{pH} 11.5$; leaching time $=2.0$ hrs.

\begin{tabular}{|c|c|c|c|c|}
\hline [ EDTA], M & 0 & 0.0008 & 0.002 & 0.005 \\
\hline Dissol. Rate, $\mu \mathrm{g} / \mathrm{h} \cdot \mathrm{cm}^{2}$ & 2043 & 1987 & 1557 & 693 \\
\hline
\end{tabular}

Ethylenediaminetetraacetic acid (EDTA) is a chelating agent of cupric ion. In its presence the free cupric ion concentration would be less and would be expected to oxidize thiosulfate to a lesser extent. Kerley (1981) reported that $0.03 \mathrm{M}$ of this reagent increaseed gold dissolution. Xia et al. (2002 reported a thiosulfate consumption of about 50\%. We observed a significant detrimental effect on gold leaching rate with its addition.

Iodine

Iodine is a strong oxidant, and a saturated aqueous solution of this reagent was tested.

Table 19, Effect of iodine (pH=10.6 \pm 0.1 ) Conditions: $\left[\mathrm{NH}_{4} \mathrm{OH}\right]=0.2 \mathrm{M}$; $\left[\left(\mathrm{NH}_{4}\right)_{2} \mathrm{~S}_{2} \mathrm{O}_{3}\right]=0.20 \mathrm{M}$; $\mathrm{T}=42^{\circ} \mathrm{C} ;\left[\mathrm{Cu}^{2+}\right]=0.008 \mathrm{M} ; \mathrm{pH} 10.6$; leaching time $=2.0 \mathrm{hrs}$.

\begin{tabular}{|c|c|c|c|c|c|}
\hline $\mathrm{I}_{2}$ saturated solution, $\mathrm{ml} / \mathrm{L}$ & 0 & 10 & 20 & 30 & 50 \\
\hline Dissol. Rate, $\mu \mathrm{g} / \mathrm{h} \cdot \mathrm{cm}^{2}$ & 1315 & 1316 & 1462 & 1440 & 1551 \\
\hline
\end{tabular}

As expected, iodine promoted the gold dissolution rate. Dissolution increased by about $20 \%$ when $50 \mathrm{ml}$ of saturated solution per liter was added at $\mathrm{pH} 10.6$ and $42^{\circ} \mathrm{C}$. 


\section{Potassium Permanganate}

Potassium permanganate is a commonly used oxidant, and experiments were run with relatively small additions of this reagent at two values of $\mathrm{pH}$. Dissolution rate was noted to increase by a factor of about 1.5 in its presence. See Tables 20 and 21.

Table 20. Effect of potassium permanganate at $\mathrm{pH}$ 10.6. Conditions: $\left[\mathrm{NH}_{4} \mathrm{OH}\right]=0.2 \mathrm{M}$; $\left[\left(\mathrm{NH}_{4}\right)_{2} \mathrm{~S}_{2} \mathrm{O}_{3}\right]=0.2 \mathrm{M}, \mathrm{T}=42^{\circ} \mathrm{C}$., $\left[\mathrm{Cu}^{2+}\right]=0.008 \mathrm{M}$, leaching time $=2.0 \mathrm{hrs}$.

\begin{tabular}{|l|c|c|c|c|c|}
\hline $\mathrm{KMnO}_{4}, \mathrm{mg} / \mathrm{L}$ & 0 & 10.0 & 20.0 & 40.0 & 80.0 \\
\hline Dissol. Rate, $\mu \mathrm{g} / \mathrm{h} \cdot \mathrm{cm}^{2}$ & 1315 & 1447 & 1677 & 1698 & 1743 \\
\hline
\end{tabular}

Table 21. Effect of potassium permanganate at $\mathrm{pH}$ 9.5. Conditions: $\left[\mathrm{NH}_{4} \mathrm{OH}\right]=0.2 \mathrm{M}$, $\left[\left(\mathrm{NH}_{4}\right)_{2} \mathrm{~S}_{2} \mathrm{O}_{3}\right]=0.2 \mathrm{M},\left[\mathrm{Cu}^{2+}\right]=0.008 \mathrm{M} ; \mathrm{T}=42^{\circ} \mathrm{C}$, leaching time $2.0 \mathrm{hrs}$.

\begin{tabular}{|c|c|c|c|c|c|c|}
\hline Concentration, $\mathrm{mg} / \mathrm{L}$ & 0 & 20 & 40 & 80 & 200 & 300 \\
\hline Dissol. Rate, $\mu \mathrm{g} / \mathrm{h} \cdot \mathrm{cm}^{2}$ & 438 & 488 & 537 & 584 & 618 & 667 \\
\hline
\end{tabular}

\section{CONCLUSIONS}

Experiments were conducted to establish the interrelationships of ammonium thiosulfate, cupric sulfate and ammonia concentrations, $\mathrm{pH}$ and temperature in this complex leaching system. Experiments were also conducted to establish if the kinetics of the leaching of gold or of the decomposition of thiosulfate could be affected with the addition of selected reagents.

- The most important conclusion of this study is that maximum gold dissolution is obtained at $\mathrm{pH} 11.4$ and $52^{\circ} \mathrm{C}$ in the presence of $0.2 \mathrm{M}\left(\mathrm{NH}_{4}\right)_{2} \mathrm{~S}_{2} \mathrm{O}_{3}, 0.2 \mathrm{M} \mathrm{NH}_{4} \mathrm{OH}$, and $0.008 \mathrm{M} \mathrm{Cu}^{2+}$.

- The kinetics of gold dissolution with thiosulfate with oxygen only as oxidant is too slow for practical use.

- Cupric-amine plays a very important role in thiosulfate leaching of gold. It greatly accelerates the gold dissolution rate as an oxidant of gold and catalyst in the reaction. It also oxidizes thiosulfate simultaneously.

- Ammonia is added to stabilize $\mathrm{Cu}^{2+}$ as $\mathrm{Cu}\left(\mathrm{NH}_{3}\right)_{4}{ }^{2+}$.

- An optimal ammonia concentration exists for maximal gold dissolution. At low ammonia addition, gold dissolution rate decreases with increased thiosulfate addition. At high ammonium hydroxide concentration, gold dissolution is increased with increased ammonium thiosulfate additions.

- A kinetic study of cupric amine shows that the cupric decomposition is a pseudo second order reaction when ammonium thiosulfate and ammonium hydroxide concentrations, $\mathrm{pH}$ and temperature are kept constant. The rate equation of cupric-amine decomposition is: 


$$
\begin{gathered}
-\mathrm{d}\left[\mathrm{Cu}\left(\mathrm{NH}_{3}\right)_{4}{ }^{2+}\right] / \mathrm{dt}=(9.23-158)\left[\mathrm{Cu}\left(\mathrm{NH}_{3}\right)_{4}{ }^{2+}\right]^{2}\left[\mathrm{~S}_{2} \mathrm{O}_{3}{ }^{2-}\right]^{1.09-2.17} \mathrm{~L}^{-1} \mathrm{~mol} \mathrm{~h}^{-1} \\
\left(\mathrm{~T}=22-42^{\circ} \mathrm{C} ;\left[\mathrm{NH}_{4} \mathrm{OH}\right]=0.5 \mathrm{M} ; \mathrm{pH}=10.0\right)
\end{gathered}
$$

- The rate constant and the order of thiosulfate concentration are greatly influenced by the temperature. The rate constant increased by 17 times, and the order of thiosulfate concentration increased from 1.09 to 2.17 when the temperature was increased from $22^{\circ}$ to $42^{\circ} \mathrm{C}$. While elevating the temperature and the concentration of thiosulfate in order to accelerate the dissolution rate, attention needs to be paid to monitor whether sufficient concentration of cupric-amine exists in the leaching solution. Concentration and status of cupric-amine are controlled by the interactions of ammonia-thiosulfate-temperature-pH in the leaching of gold.

- $\quad \mathrm{pH}$ influences the stability of thiosulfate, cupric-amine, and the status of cupric ion. Higher $\mathrm{pH}$ is beneficial for the formation of more oxygen and thiosulfate from the polythionates, the decomposition products of thiosulfate. On the other hand, too high of $\mathrm{pH}$ and/or temperature would result in cupric precipitation of $\mathrm{CuO}$, leading to a drastic decrease in gold dissolution rate.

- Elevation of temperature greatly accelerates the rate of gold dissolution. However, higher temperature also decomposes thiosulfate faster and leads to greater precipitation of $\mathrm{CuO}$. Experiments indicate that the temperature should not be higher than $52^{\circ} \mathrm{C}$.

- The addition of iodine (as expected) and potassium permanganate at relatively low concentration increases the rate of gold dissolution by a factor of $1.2-1.5$.

- The addition of phenyl imidazoline, methylamine hydrochloride, hydrogen peroxide, EDTA, and sodium sulfite are detrimental to gold dissolution. 


\section{REFERENCES}

Aylmore, M. G. and Muir, D. M. 2001.Thiosulfate Leaching of Gold—A Review. Minerals Engineering. 14(2):135-174.

Breuer, P. L, Jeffrey, M. I., Tan, E. H. K. and Bott, A. W. 2004. Design of a Flow-through Cell for Analysis of Thiosulfate in Solutions Containing Copper and Ammonia. Journal of the Electrochemical Society.151(7):D51-54.

Butler, J.N. 1964. Ionic Equilibrium. Addison-Wesley Publishing Company. 131.

Byerley, J. J., Fouda, S .A. and Rempel, G. L. 1973. Kinetics and Mechanism of the Oxidation of Thiosulfate Ions by Copper(II) Ions in Aqueous Ammonia Solution. J.C.S. Dalton Transactions. 889-893.

Cao, C., Hu, J. and Gong, Q. 1992. Randol Gold Forum ’92. 293.

Han, K. N. and Meng, X. 1992. U S. Patent 5,114,687. Ammonia Extraction of Gold and Silver from Ores and Other Materials.

Han, K. N. and Meng, X. 1992. U.S. Patent 5,308,381. Ammonia Extraction of Gold and Silver from Ores and Other Materials.

Jeffrey, M.I. 2001. Kinetic Aspects of Gold and Silver Leaching in Ammonia-Thiosulfate Solutions. Hydrometallurgy. 60:7-16.

Jiang, T., Chen, J. and Xu, S. 1993. Proceedings of XVIII International Mineral Processing Congress. 1141.

Kerley, B. J. Jr. 1981.U. S. Patent 4,269,622. Recovery of Precious Metals from Difficult Ores.

Kristjansdottir, S. S. and Thompson, J. S. 1996. U. S. Patent 5,484,470. Enhancement of Gold Lixiviation Using Nitrogen and Sulfur Heterocyclic Aromatic Compounds.

Li, J., Miller, J. D., Wan, R. Y. and LeVier, M. 1995. Proceedings of XIX International Mineral Processing Congress. 37.

Little, R. H. 1987. U. S. Patent 4,645,535. Method for the Recovery of Precious Metal from Ores.

Molleman, E and Dreisinger, D. 2002. The Treatment of Copper-Gold Ores by Ammonium Thiosulfate Leaching, Hydrometallurgy/ 66:1-21.

Schulze, R. G. 1984. New Aspects in Thiourea Leaching of Precious Metals. Journal of Metals. 36(6):62-65. 
Tamura, Y., Chyo, G. S. and Katsura, T. 1979. The $\mathrm{Fe}_{3} \mathrm{O}_{4}$ Formation by the 'Ferrite Process': Oxidation of the Reactive $\mathrm{Fe}(\mathrm{OH})_{2}$ Suspension Induced by Sucrose. Water Research. 13:21-31.

Wadsworth, M. E. Ximeng, Z., Thompon, J. S. and Pereira, C. J. 1998. Randol Gold Forum ’98. 299.

Wan, R.Y. 1997. Proceedings- World Gold '97 Conference, Singapore. 159-162.

Wan, R. Y., LeVier, K. M. and Miller, J. D. 1993. Fundamentals, Technology and Innovation. SME-TMS. Hydrometallurgy.415-436.

Wan, R. Y., LeVier, K. M. and Clayton, R. B. 1994. U. S. Patent 5,354,359.Hydrometallurgical Process for the Recovery of Precious Metal Ores with Thiosulfate Lixiviant.

Xia, C., Yen, W. T. and Deschenes, G. 2003. Improvement of Thiosulfate Stability in Gold Leaching. Minerals and Metallurgical Processsing.20(2):68-72.

Zhang, H. and Dreisinger, D. B. 2002. The Kinetics for the Decomposition of Tetrathionate in Alkaline Solutions. Hyedrometallrugy. 66: 59-65.

Zipperian, D., Raghavan, S. and Wilson, J. P. 1988. Gold and Silver Extraction by Ammoniacal Thiosulfate Leaching from a Rhyolite Ore. Hydrometallurgy.19:361-375.

\section{ACKNOWLEDGMENT}

The authors wish to acknowledge the experimental work performed by Kangnian Zhong on this project. 
Appendix 28: Bio-Assisted Heap Leaching of Nickel Laterites (UT002) 
FINAL TECHNICAL REPORT

Contract Title and Number:

Period of Performance:

Crosscutting Technology Development at the Center for

Advanced Separation Technologies

Starting 08/01/03

(DE-FC26-02NT41607)

Date:

Ending Date: 09/30/07

Sub-Recipient Project Title:

Report Information:

Bio-Assisted Heap Leaching of Nickel Laterites for the

Type: Final

Development of a Domestic Nickel Industry

Number: 3

Principal Investigators:

Period: $\quad$ 08/01/03-09/30/04

Saskia Duyvesteyn

Date: $\quad$ 01/15/05

Code: UT002-FINAL

Contact Address:

Contact Information:

135 S 1460 E Rm 412

Salt Lake City, UT 84112-0114

Phone: 775-753-1405

Fax:

E-Mail: saskia@barrick.com

Subcontractor Address:

Subcontractor Information:

"No subcontracts issued."

Phone:

Fax:

E-Mail: 


\section{DISCLAIMER}

“This report was prepared as an account of work sponsored by an agency of the United States Government. Neither the United States Government nor any agency thereof, nor any of their employees, make any warranty, express or implied, nor assume any legal liability or responsibility for the accuracy, completeness, or usefulness of any information, apparatus, product, or process disclosed, or represents that its use would not infringe privately owned rights. Reference herein to any specific commercial product, process, or service by trade name, trademark, manufacturer, or otherwise does not necessarily constitute or imply endorsement, recommendation, or favoring by the United States Government or any agency thereof. The views and opinions of authors expressed herein do not necessarily state or reflect those of the United States Government or agency thereof. ” 


\begin{abstract}
This research proposal involves a biotechnological approach for the production of nickel metal from low-grade laterite ore in a synergistic mode with co-production of citric acid called bio-assisted heap leaching (BAHL). There are two possible modes of operation of BAHL processes. The direct route involves combining the nickel laterite ore with an organic nutrient and placing it on heaps where fungi, such as Aspergillus niger, produce organic acids during their natural metabolic cycle. Recycle solution is sprayed over the heaps, and its percolation through the heaps results in the dissolution of nickel. The indirect route involves the separate production of a citric acid fermentation broth for leaching by fungi with the addition of growth medium. This organic acid broth is sprayed over the heaps and dissolves the nickel. In both cases the The resulting nickel-containing leach solution is collected and then the nickel and citrate values are recovered.

The solubilization of metal ions from ores by organic acids produced by microorganisms has been demonstrated to be a viable method for metal production with reduced environmental and remediation issues, as organic acids are readily biodegradable. Because many of these organic acids and other metabolites form strong chelating complexes with the base metal ions there can be a reduction in the acid requirements to achieve complete solubilization.
\end{abstract}




\section{TABLE OF CONTENTS}

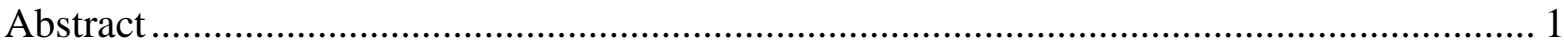

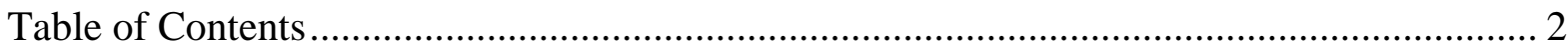

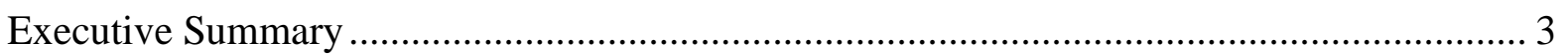

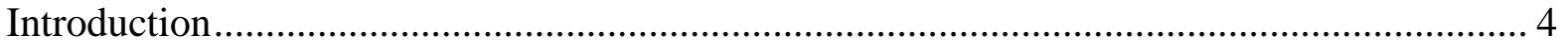

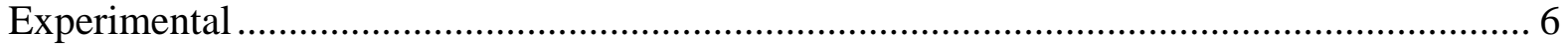

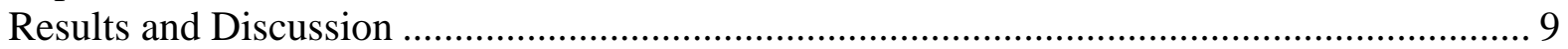

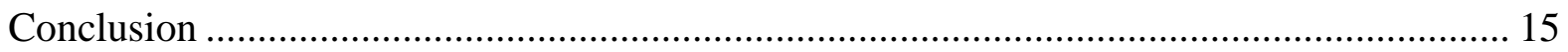

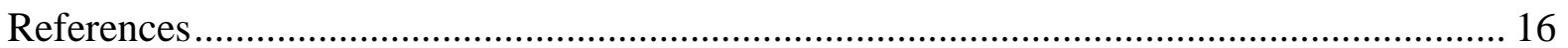




\section{EXECUTIVE SUMMARY}

The work discussed in this final report represents the groundwork involved in the investigation of the bio-assisted head leaching for the selective dissolution of nickel from low-grade laterite ores. The outlined objectives were not completed because the PI is no longer affiliated with the University of Utah. However, the results of the work that was completed is summarized.

This work has demonstrated the feasibility of utilizing fungi for the fungal heap leaching nickel laterite ores. It has been demonstrated that is it possible to produce viable fungi cultures that have been acclimated to conditions typical in heap leaching. The cultures have been able to maintain themselves both in the presence of laterite ore and high nickel concentrations.

From the current results it is impossible to determine the most effective process method: the direct approach, where the ore is contacted directly with nutrients and fungi in the heap, or the indirect approach, where the citric acid is fermented separately to produce a broth for leaching in a stirred reactor or a heap. The column leaching studies indicate that both methods result in the dissolution of nickel from the ore. Longer term studies will be required to determine the longevity of the fungi cultures. 


\section{INTRODUCTION}

\section{$\underline{\text { Background }}$}

The fungi typically used in bioleaching do not directly degrade the mineral, but rather degradation is achieved via the production of organic acid metabolites. The metal extraction by microbially-produced acids can occur through two possible mechanisms, which can take place separately or simultaneously. (O.H. Tuovinen and D.P. Kelly, 1974) First, metal cations may be directly displaced from the ore by hydrogen ions produced by the microorganisms resulting in a common acid leaching mechanism. (P.G. Tzeferis, 1994B) Second, compounds produced by the microorganism can bind metals into soluble complexes by chelation. (D.I. McKenzie, et al., 1987)

While leaching laterite ores with sulfuric acid releases substantial amounts of nickel, organic acids, especially hydroxy-carboxylic acids, can achieve similar or better nickel dissolution. (K.A.K. Alibhai, et al., 1993; P.G. Tzeferis, 1994A) While a variety of organic acids have given good results, citric acid, a well-established product of fungal metabolism, has been found to be the most effective mobilizer of nickel from laterites. When leaching is performed by combining organic acids with small amounts of sulfuric acid to maintain low pH's, there has shown to be a synergistic effect on leaching, such that the Ni dissolution is actually higher than simply a sum of the individual dissolutions.

Since citric acid production can be carried out in aerated, solid-substrate column reactors (Y.D. Hang, 1988), this project is really proposing to study citric acid production in a "heap leach" column reactor, where the acid is consumed by the ore as it is being leached. It has been shown that the citric acid production rate is not significantly affected by the amount of inoculum used (Y.D. Hang, 1988); thus unlike for other bio-leaching applications, blending of the inoculum and ore should not be problematic.

\section{Objective and Approach}

The specific objectives of the proposed research are to:

- demonstrate the feasibility of the bio-assisted heap leaching process for the selective dissolution of nickel from low-grade laterite ores;

- establish viable fungi cultures that have been acclimated to typical heap leaching conditions;

- determine the important process parameters, such as inoculum addition, acid addition composition(s), flowrate of recycle water, size distribution of agglomerated ore particles, effect of ions in recycle water, and initial and interim additions of the carbon source.

- develop methods for metal/citrate species analyses;

- determine the most effect process method: the direct approach, where the ore is contacted directly with nutrients and fungi in the heap, or the indirect approach, where the citric acid is fermented separately to produce a broth for leaching in a stirred reactor or a heap.

These objectives will be achieved by the following tasks. 
Modeling the equilibrium state of the organic acid-ore leaching system based on its thermodynamics can assist in understanding the organic leaching of laterites ores. Of particular importance is the type of the complexes that are formed, as many citrate complexes are polydentate in nature, which results in sequestration of metal ions. By studying the speciation of both synthetic and actual solutions and comparing the results to thermodynamic modeling studies, the influence of different parameters on the leaching can be understood.

The leaching studies will involve two types of tests. Simple diagnostic tests will be performed in shake flasks to determine ore leachability using a variety of acids and acid mixtures, including sulfuric acid, citric acid and oxalic acid, pure cultures vs. indigenous microorganisms and different types of organic media. As appropriate, speciation studies of the leachates produced will be performed and compared to the theoretical models. As the actual process will involve heap leaching, studying "typical" leaching parameters, such as temperature and solids concentration, is not applicable.

The issue of acid composition is very important. Sulfuric acid leaching is strongly $\mathrm{pH}$ dependent and essentially ceases above a $\mathrm{pH}$ of 2, while citric acid has a chelation formation mechanism occurring simultaneously with lattice destruction by hydrogen ions, making it less pH dependent. (P.G. Tzeferis and S. Agatzini-Leonardou, 1994) Sulfuric acid has also been reported to leach nickel twice as fast as citric acid, but this varies widely from study to study, indicating that leaching rates are ore specific. (K. Alibhai, et al., 1991) However, supplementing citric acid with sulfuric acid has been shown to increase the rate of leaching of nickel well beyond that of either single acid. (D.I. McKenzie, et al., 1987)

The other type of leaching test will be column tests and will be undertaken to further optimize the process once the key parameters are identified from the shake flask tests. As is common practice to simulate the leaching in heaps, the leaching studies will be performed in plexi-glass columns through which the recycle water is continually pumped. Heap leach cycles can range from a few weeks to many months. Important parameters to be studied will include the flowrate of recycle water, size distribution of agglomerated ore particles, effect of ions in recycle water, and initial and interim additions of the carbon source. Throughout the cycles samples will be taken to both determine the progress of the nickel leaching and provide samples for the speciation studies. As appropriate, speciation studies of the leachates produced will be performed and compared to the theoretical models. Once analyses of the leachates have been completed, it is likely that some of the thermodynamic parameters will not be available in the literature and will need to be determined to develop the thermodynamic model. 


\section{EXPERIMENTAL}

\section{Acquisition and characterization of ore samples}

Because of the very heterogeneous nature of laterite deposits, two ore samples have been acquired for use in the project activities. One ore is from an Australian laterite deposit, while the other is from a Latin American laterite deposit. Because of the chromite and other spinels present in the ore, special digestion procedures are required to ensure complete dissolution for accurate analyses. Free moisture and loss on ignition (LOI) were also performed on the samples.

\section{Establishment of microorganism cultures}

Aspergillus niger has been selected as the primary fungus of interest because of its ability to produced significant quantities of citric acid, which is thought to be the primary organic acid involved in nickel leaching. Aspergillus niger van Tieghem, anamorph (IMI 27809 [581; Clement A-1-215; NCTC 7193; NRRL 2322; NRRL 2354; QM 6906]) was ordered from ATCC (Catalogue number 10577). The fungus has been revived using the required potato dextrose agar medium, and fermentation will be allowed to occur until an initial culture of $500 \mathrm{~mL}$ is achieved. All subcultures for future work will stem from this original culture.

Three types of cultures methods were used. As the culture media for the leaching test is molasses based, the microorganisms have been acclimated to this media, which consists of molasses with essentials salts such as copper sulfate, zinc sulfate and ammonium phosphate. To acclimate the microorganisms to the ore, the higher nickel-grade ore (Latin American) ore was added to the potato dextrose-agar medium recipe. Because the fungi will be exposed to high concentrations of nickel in solution (>600 ppm), aliquots of a solution containing nickel were added to the potato dextrose-agar medium. The viable organisms have been established by alternating these methods. After the initial culture was established, a 1 inch by 1 inch square of the colony was removed and added to the molasses-based medium. Once this colony was established, a 1inch by 1 inch square of it was removed and added to a potatobased medium that contained either ore or nickel. Again once this colony was established, a 1inch by 1 inch square of it was removed and added to a molasses-based medium and so on. Table 1 shows the progression of the acclimation process. All of this was performed in duplicate. The two final cultures were used in the column leaching studies.

\section{$\underline{\text { Shake flask leaching studies }}$}

Preliminary shake flask leaching studies were completed using the Australian laterite ore. Leaching was performed on the two ore samples using the lixiviants $\mathrm{H}_{2} \mathrm{SO}_{4}$ at a concentration of $25 \mathrm{~g} / \mathrm{L}$ or citric acid, oxalic acid and glycine at a concentration of $0.5 \mathrm{M}$, a solids concentration of $20 \mathrm{~g} / \mathrm{L}$ and $28^{\circ} \mathrm{C}$. 
Table 1: Acclimation procedure

\begin{tabular}{|c|c|c|}
\hline Culture number & Medium & Additions \\
\hline 1 & Potato dextrose -agar & None \\
\hline 2 & Molasses & None \\
\hline 3 & Potato dextrose -agar & $0.5 \mathrm{~g}$ ore/L medium \\
\hline 4 & Molasses & None \\
\hline 5 & Potato dextrose -agar & $1.0 \mathrm{~g}$ ore/L medium \\
\hline 6 & Molasses & None \\
\hline 7 & Potato dextrose -agar & $2.5 \mathrm{~g}$ ore/L medium \\
\hline 8 & Molasses & None \\
\hline 9 & Potato dextrose -agar & $5.0 \mathrm{~g}$ ore/L medium \\
\hline 10 & Molasses & None \\
\hline 11 & Potato dextrose -agar & $10 \mathrm{~g}$ ore/L medium \\
\hline 12 & Molasses & None \\
\hline 13 & Potato dextrose -agar & $10 \mathrm{mg}$ Ni/L medium \\
\hline 14 & Molasses & None \\
\hline 15 & Potato dextrose -agar & $10 \mathrm{mg}$ Ni/L medium \\
\hline 16 & Molasses & None \\
\hline
\end{tabular}

\section{$\underline{\text { Column leaching studies }}$}

To be able to use the ore for column leach studies it was first necessary to agglomerate the ore. This procedure involved the addition of sulfuric acid to moist ore to create agglomerates. The sulfuric acid was added dropwise to a bucket containing the moist ore. The bucket was then placed at a $45^{\circ}$ angle and rotated to initiate the agglomeration process. Once there was no more agglomeration occurring, more acid was added and the bucket was rotated again. This process was repeated until the majority of the ore was agglomerated and an approximate top size of $1 / 2$ inch was achieved. All the column studies used the Australian ore because more of it was available of the two ore samples.

As shown in Figure 1, columns were constructed of 2" ID PVC piping with a length of approximately 20 inches. After packing a Plexiglas funnel with cheese cloth, it was attached to the bottom of the pipe. Approximately $1 \frac{1 / 2}{2}$ inches of glass beads were placed on top of the cheese cloth. Then approximately 600 grams of agglomerated ore was added to the column. The column was tapped gently on the side as the ore was added to minimize the formation of any air pockets that could lead to preferential channeling of the solution through the column. The ore was then covered with a $1 \frac{1 / 2}{2}$ inch layer of glass bead to improve the distribution of the lixiviant across the column cross-section. Multiple columns were prepared using this procedure, and a multi-channel peristaltic pump was used to pump the lixiviant from a reservoir into the top of the column. In some systems the resulting solution was collected in the same reservoir, such that the lixiviant could be continually recycled. 


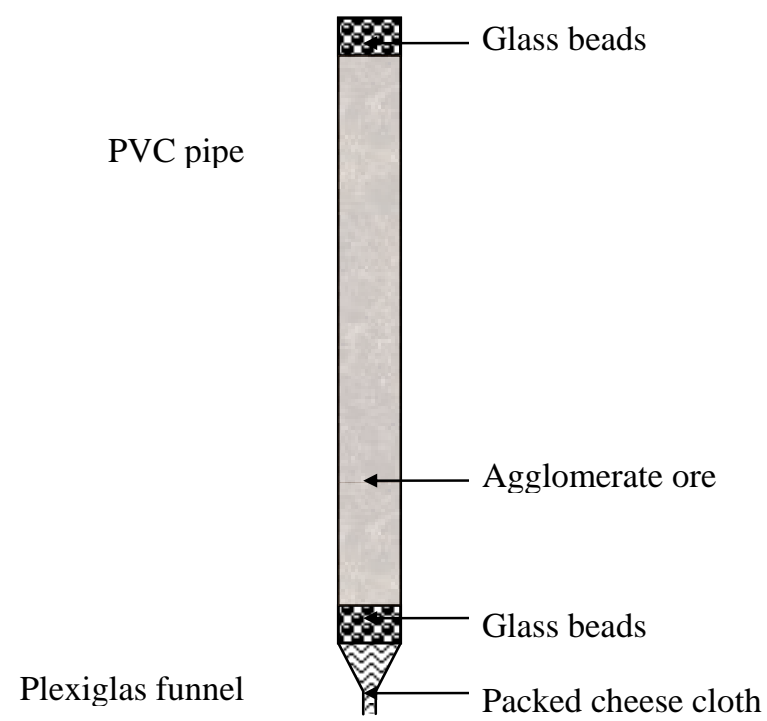

Figure 1: Schematic diagram of leaching column setup.

Four different lixiviant systems were used. One column was leached with fresh $0.5 \mathrm{M}$ citric acid that was continually added without any recycle of the resultant pregnant leachate. Two columns were inoculated with a fungi slurry. This was created using the "final" (Row 16 in Table 2) fungi that had been established on molasses by blending it into a slurry using a hand-mixer. Approximately $5 \mathrm{~mL}$ of this inoculum was dripped over the ore surface after every 2 inches of ore was added to the column. The reservoir was filled with the molasses solution used to grow the fungi culture, which was made up as necessary. For the fourth column, the container in which the fungi was grown was used as the reservoir. The lixiviant was continually recycled. 


\section{RESULTS AND DISCUSSION}

\section{$\underline{\text { Acquisition and characterization of ore samples }}$}

The chemical analyses of both the Australian and Latin American are shown in Table 2. The primary difference between the two ores is the iron (14.6\% vs. 16.1), magnesium (7.74\% vs. $8.42 \%$ ) and nickel (0.96\% vs. $1.41 \%)$, respectively.

\section{Establishment of microorganism cultures}

Aspergillus niger has been selected as the primary fungus of interest because of its ability to produced significant quantities of citric acid, which is thought to be the primary organic acid involved in nickel leaching. Aspergillus niger van Tieghem, anamorph (IMI 27809 [581; Clement A-1-215; NCTC 7193; NRRL 2322; NRRL 2354; QM 6906]) was ordered from ATCC (Catalogue number 10577). The fungus has been revived using the required potato dextrose agar medium, and fermentation will be allowed to occur until an initial culture of $500 \mathrm{~mL}$ is achieved. All subcultures for future work will stem from this original culture.

Figure 2 shows various images of a Aspergillus niger colonies established on a molassesbased medium. Figures $3-5$ show various images of Aspergillus niger colonies established on potato-based media that contain varying amounts of ore. Figure 5 clearly shows the spores and accompanying structures that are typical of fungi.

Table 2: Typical analyses of ore samples used in project activities

\begin{tabular}{|c|c|c|c|c|}
\hline Analyte & Sample A & Sample B & \multicolumn{2}{|c|}{ Composite sample } \\
\cline { 4 - 5 }$(\%)$ & Latin American ore & Australian ore & Laboratory results & Calculated results $^{*}$ \\
\hline $\mathrm{Co}$ & $0.037 \pm 0.003$ & $0.042 \pm 0.004$ & $0.030 \pm 0.009$ & 0.039 \\
\hline $\mathrm{Cr}$ & $0.278 \pm 0.009$ & $0.589 \pm 0.06$ & $0.262 \pm 0.06$ & 0.427 \\
\hline $\mathrm{Fe}$ & $16.1 \pm 0.9$ & $21.8 \pm 1$ & $14.6 \pm 1$ & 18.8 \\
\hline $\mathrm{Mg}$ & $8.42 \pm 0.1$ & $3.73 \pm 0.03$ & $7.74 \pm 0.6$ & 6.17 \\
\hline $\mathrm{Mn}$ & $0.246 \pm 0.02$ & $0.334 \pm 0.05$ & $0.215 \pm 0.05$ & 0.288 \\
\hline $\mathrm{Ni}$ & $1.41 \pm 0.04$ & $0.564 \pm 0.03$ & $0.96 \pm 0.04$ & 1.00 \\
\hline Si solids & $52.8 \pm 0.4$ & $52.9 \pm 1$ & $57.1 \pm 1$ & 52.8 \\
\hline LOI & $1.11 \pm 0.06$ & $0.81 \pm 0.06$ & $1.12 \pm 0.3$ & 0.963 \\
\hline Moisture & $23.5 \pm 2$ & $28.0 \pm 0.5$ & $31.7 \pm 5$ & 25.6 \\
\hline
\end{tabular}

\footnotetext{
* A composite sample of Samples A and B was analyzed. Based on the laboratory results for these two samples and the weighting of the mixture, the values were calculated for the anticipated results.
} 

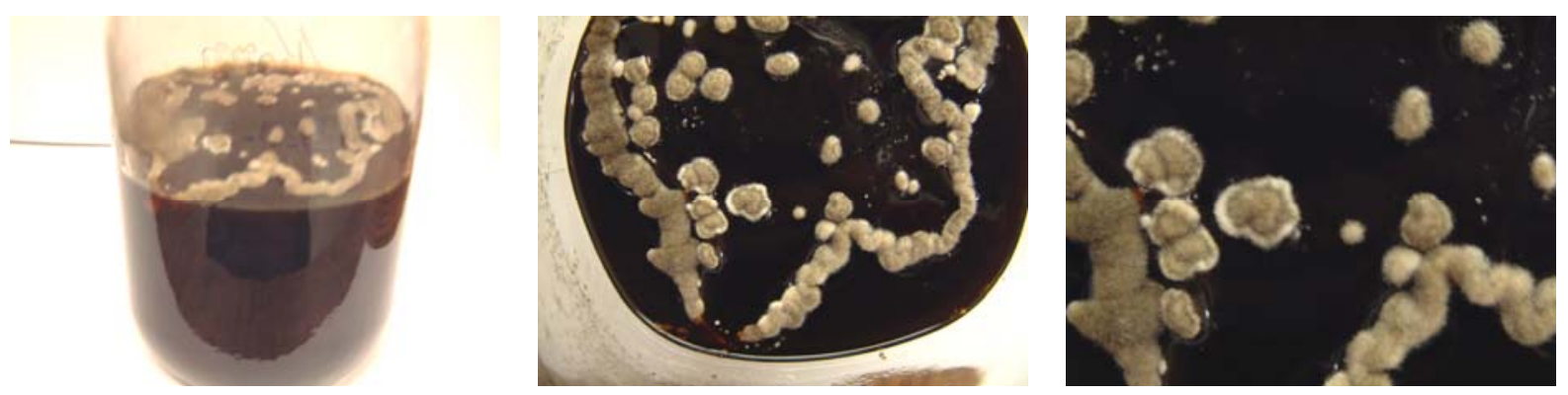

Figure 2: Images of Aspergillus niger colonies cultured on a molasses-based medium.
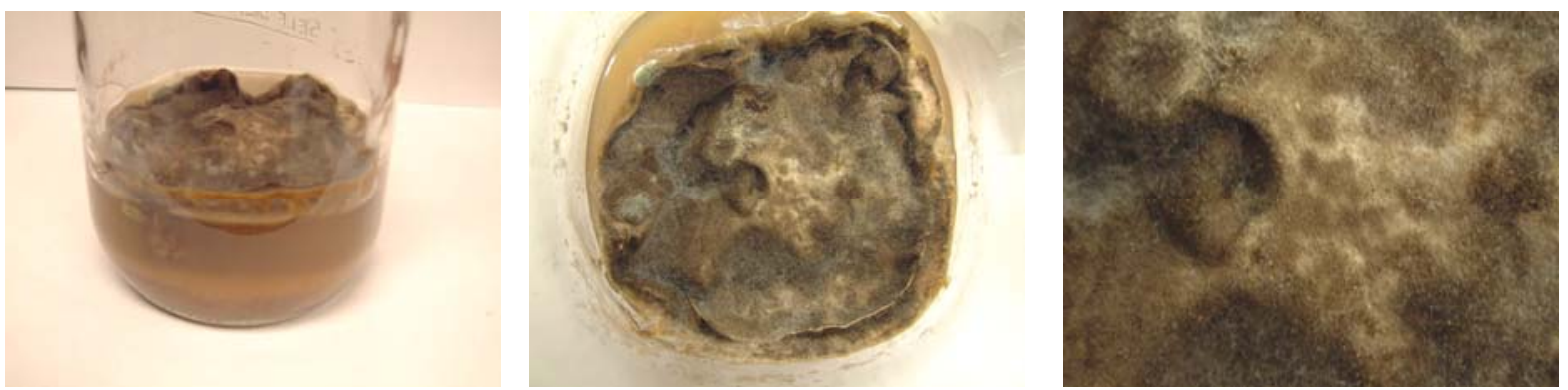

Figure 3: Images of Aspergillus niger colonies cultured on a potato-based medium.
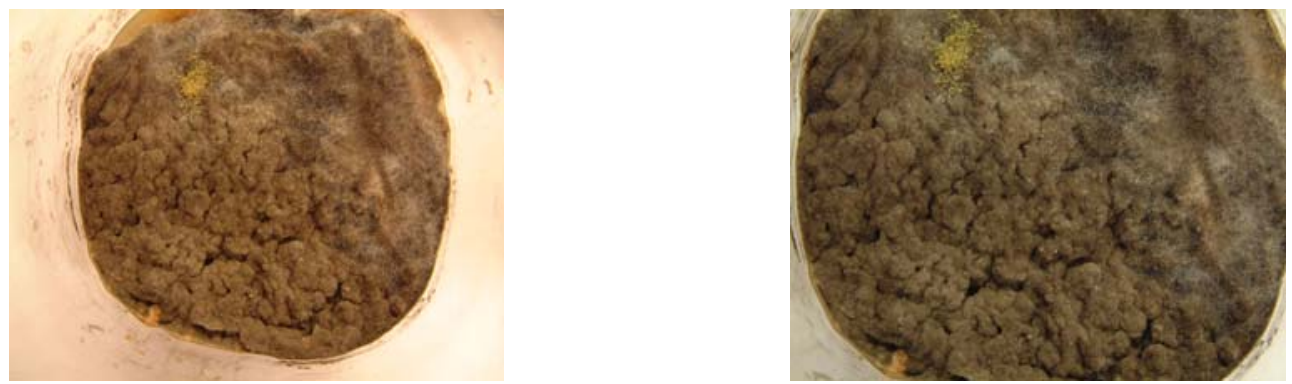

Figure 4: Images of Aspergillus niger colonies cultured on a potato-based medium.
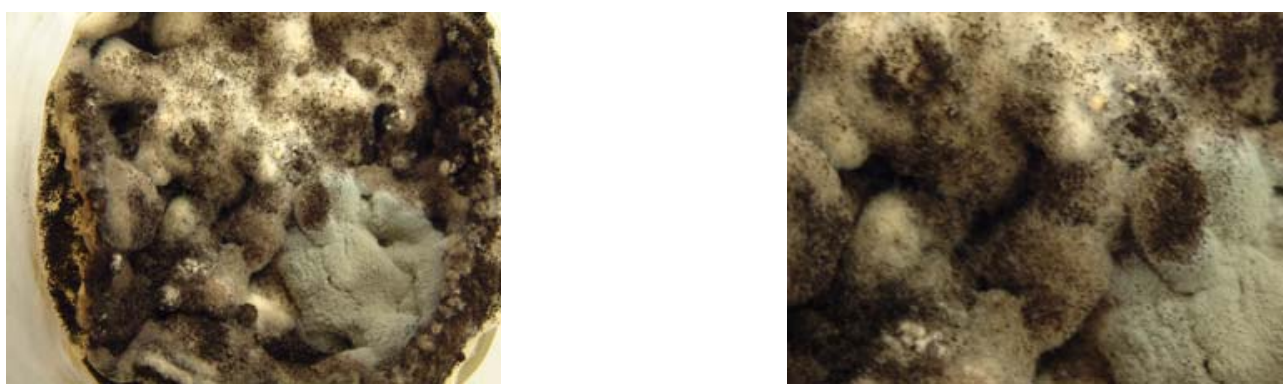

Figure 5: Images of Aspergillus niger colonies cultured on a potato-based medium. 


\section{Shake flask leaching studies}

The results for the dissolution of nickel during the shake flask studies are show in Figure 6, while Figure 6 shows the leaching of iron from the ores. The results in Figure 6 show that while oxalic acid is not the most efficient lixiviant in terms of nickel leaching, it is very effective for minimizing the leaching of iron from the ore. For shorter leaching times, glycine is also an effective lixiviant; however at longer times, the nickel precipitates out resulting in the reduction of the nickel concentration in the solution.

\section{Column leaching studies}

Figure 8 shows the nickel dissolution that occurred over approximately 20 days for each of the three lixiviant systems (4 columns) studied. The changes in $\mathrm{pH}$ that occurred during the 20-day leaching period for the 3 systems is shown Figure 9. Figure 10 is a superposition of Figure 9 on Figure 8.

When citric acid was used as the lixiviant, the concentration in the leachate increased from 750 and $1000 \mathrm{ppm}$ over the 20-day period. As the lixiviant concentration remained constant, it makes sense that the nickel concentration in the leachate remained about constant. The slight increase could be indicative to increased access to the nickel as the agglomerates break down. The $\mathrm{pH}$ also remained approximately constant at 0.65 with a slight increase over time, which would also indicate the breaking down of the agglomerates.

When the column as inoculated in-situ with the acclimated fungi, the initial concentration of nickel in the lixiviant ranged from 400 to $750 \mathrm{ppm}$. Over the 20 day leaching period, the $\mathrm{Ni}$ concentration decreased to about $100 \mathrm{ppm}$, although it remained constant at the initial level from about 10 days. The $\mathrm{pH}$ in both in-situ columns was very similar. Initially the $\mathrm{pH}$ was bout -0.6 and then increased gradually to 0 and finally to 0.5 . After 10 days, the $\mathrm{pH}$ in one column increased more than in the It is interesting to note that as the $\mathrm{pH}$ in the in-situ columns the nickel dissolution decreased. The increase in $\mathrm{pH}$ is indicative of a reduction in organic acid production, which could be due to a destruction of the fungi. Over a longer time period, the fungi may be able to re-establish itself, but the time period of the leaching was too short to determine this.

When the fungi were introduced ex-situ, the initial concentration was about $650 \mathrm{ppm}$. The concentration of nickel in the lixiviant then decreased to almost $0 \mathrm{ppm}$ by 10 days. As the solution was recycled, the decrease in concentration could be due to the entrainment of the lixiviant in the column. After 10 days the nickel concentration increased to a range of 400 to $750 \mathrm{ppm}$. The $\mathrm{pH}$ was initial about 0.75 , which then decreased to about -0.5 by about day 10 , after which it began to increase to 1.5 by day 20 . The trends in nickel concentration and $\mathrm{pH}$ were very similar. There is about a 5 day delay when the $\mathrm{pH}$ decreases, which would indicate an increase in organic acid production, and the nickel concentration increases. There is a similar delay once the $\mathrm{pH}$ increases above 0.6 and the nickel concentration decreases. 


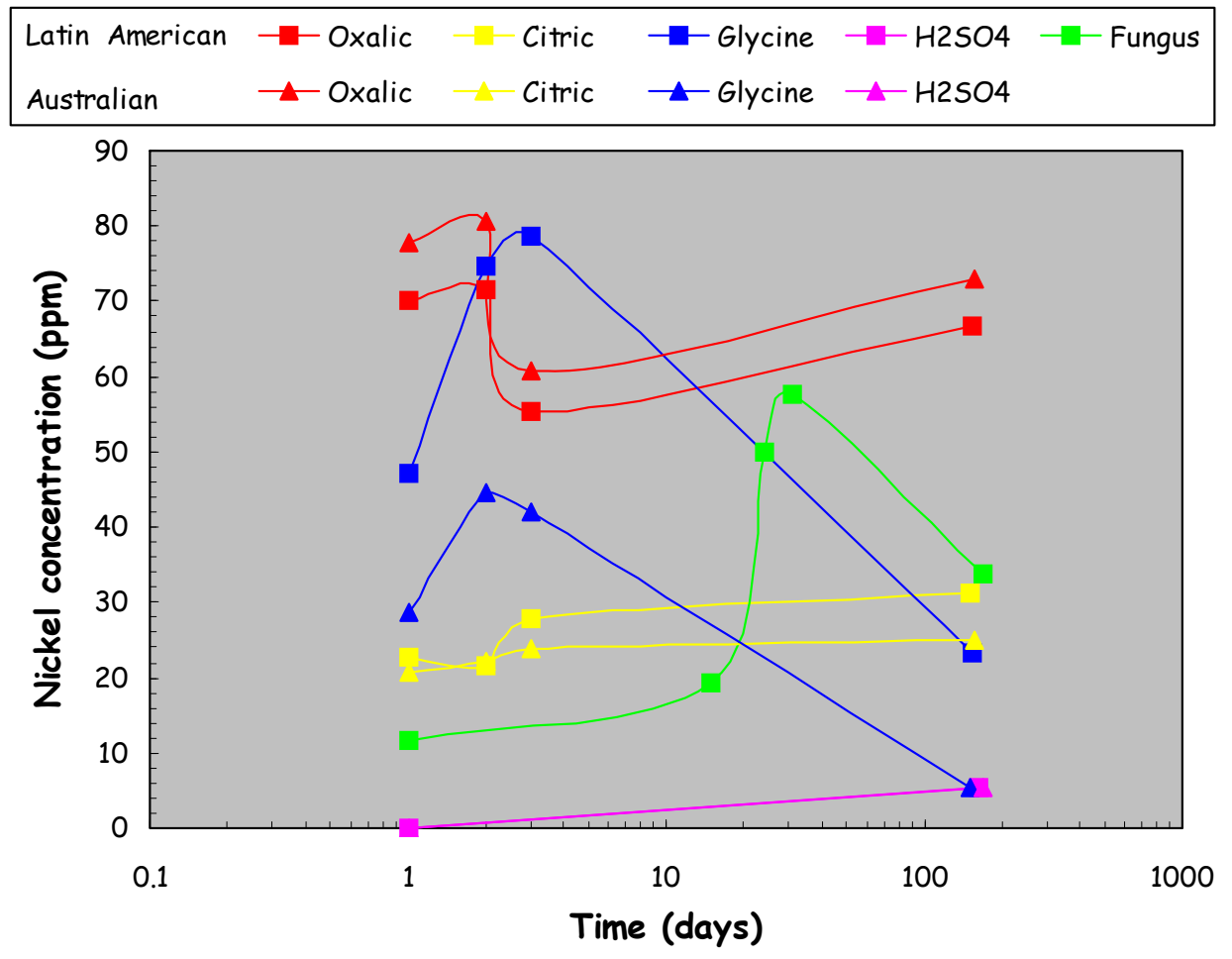

Figure 5: Flask leaching of laterite ores at an organic acid concentration of $0.5 \mathrm{M}$, a mineral acid concentration of $25 \mathrm{~g} / \mathrm{L}$, a solids concentration of $20 \mathrm{~g} / \mathrm{L}$ and $28{ }^{\circ} \mathrm{C}$.

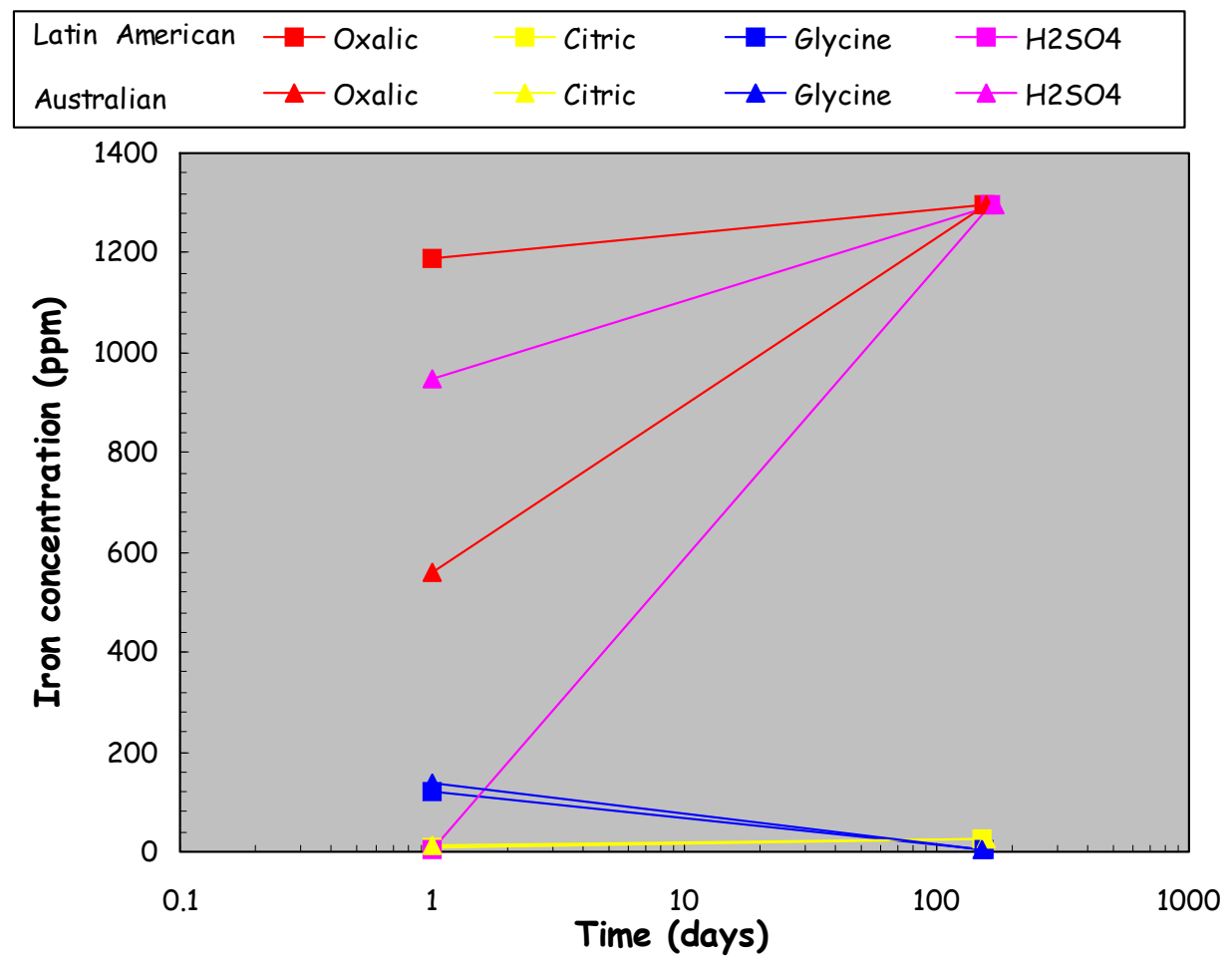

Figure 6: Flask leaching of laterite ores at an organic acid concentration of $0.5 \mathrm{M}$, a mineral acid concentration of $25 \mathrm{~g} / \mathrm{L}$, a solids concentration of $20 \mathrm{~g} / \mathrm{L}$ and $28{ }^{\circ} \mathrm{C}$. 


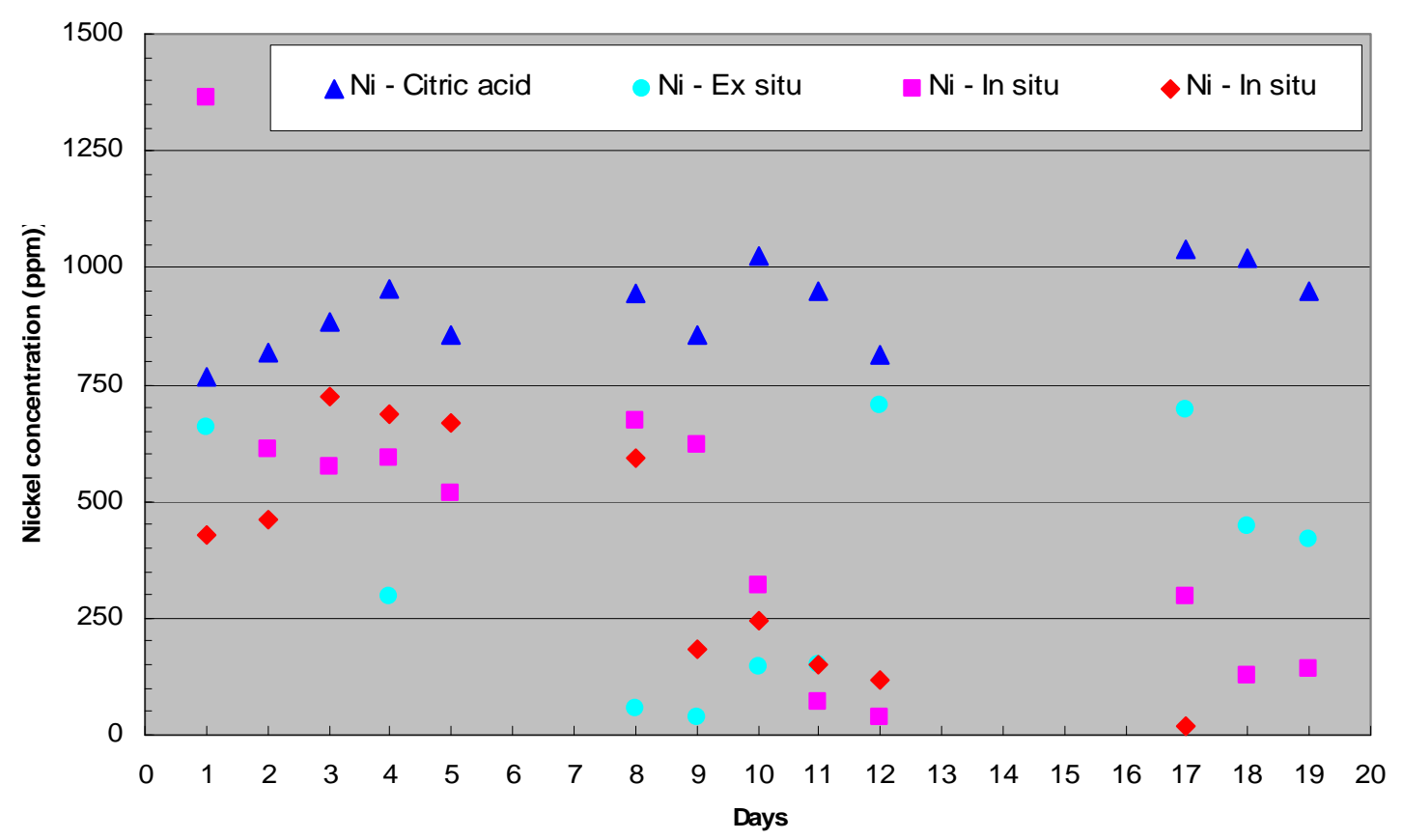

Figure 8: Nickel dissolution during column leaching of a laterite ore with a citric acid concentration of $0.5 \mathrm{M}$ and with molasses using in-situ inoculated fungi (2) and ex-situ inoculated fungi approximately $5 \mathrm{~mL} / \mathrm{hr}$ and $28^{\circ} \mathrm{C}$.

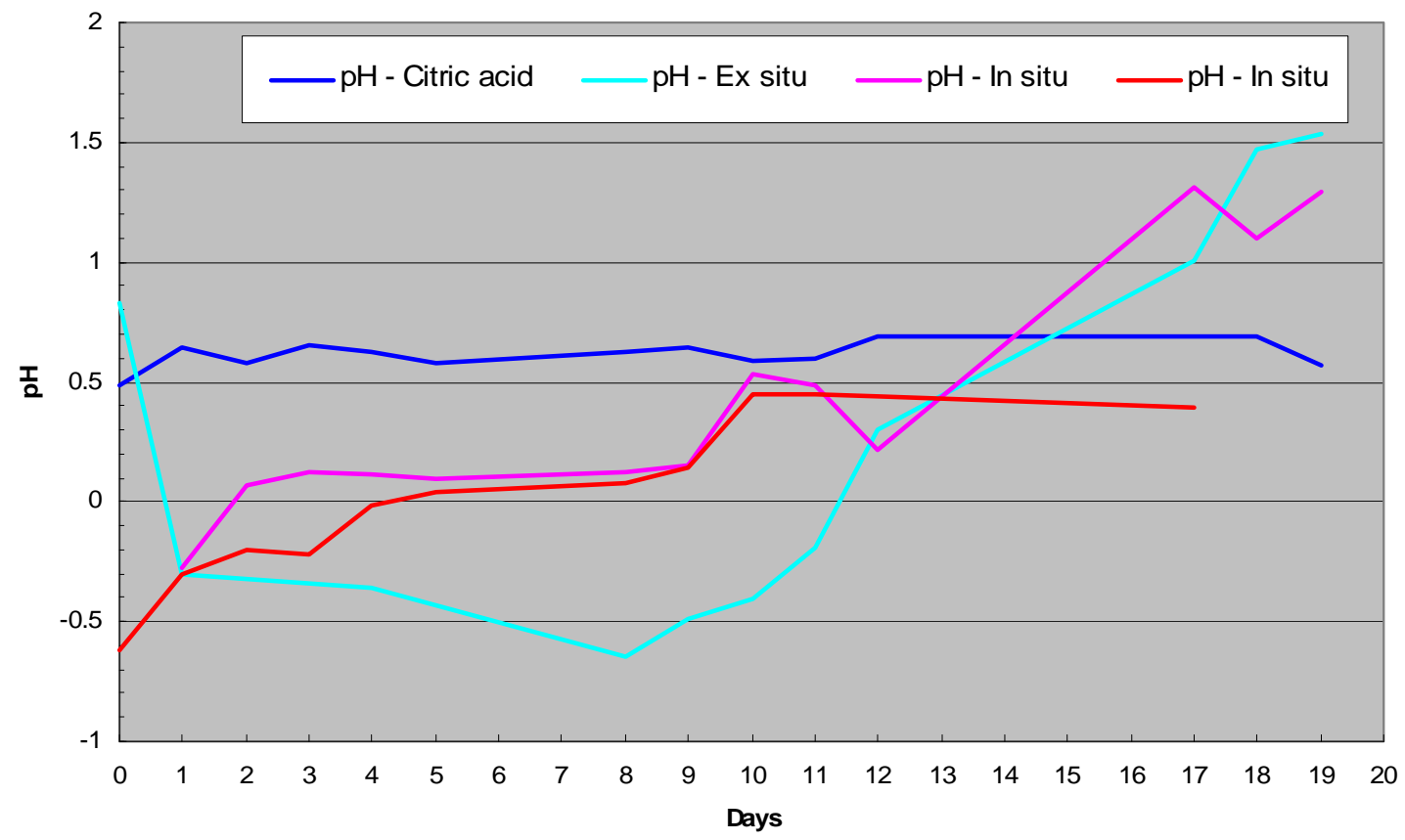

Figure 9: $\mathrm{pH}$ during column leaching of a laterite ore with a citric acid concentration of 0.5 $\mathrm{M}$ and with molasses using in-situ inoculated fungi (2) and ex-situ inoculated fungi approximately $5 \mathrm{~mL} / \mathrm{hr}$ and $28^{\circ} \mathrm{C}$. 


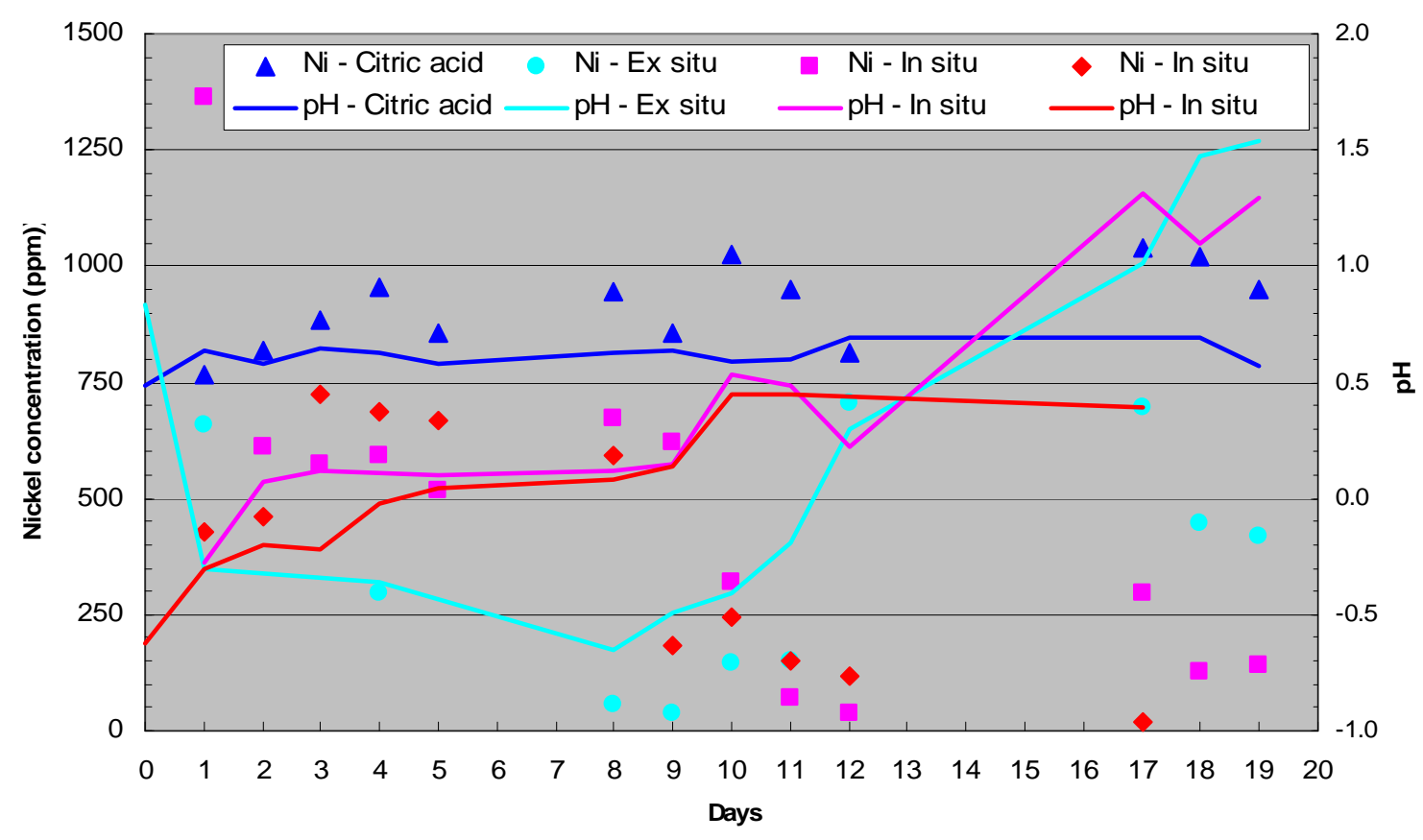

Figure 10: Superposition of Figures 8 and 9. 


\section{CONCLUSION}

The work discussed in this final report represents the groundwork involved in the investigation of the bio-assisted head leaching for the selective dissolution of nickel from low-grade laterite ores. The outlined objectives were not completed because the PI is no longer affiliated with the University of Utah. However, the results of the work that was completed is summarized.

This work has demonstrated the feasibility of utilizing fungi for the fungal heap leaching nickel laterite ores. It has been demonstrated that is it possible to produce viable fungi cultures that have been acclimated to conditions typical in heap leaching. The cultures have been able to maintain themselves both in the presence of laterite ore and high nickel concentrations.

From the current results it is impossible to determine the most effective process method: the direct approach, where the ore is contacted directly with nutrients and fungi in the heap, or the indirect approach, where the citric acid is fermented separately to produce a broth for leaching in a stirred reactor or a heap. The column leaching studies indicate that both methods result in the dissolution of nickel from the ore. Longer term studies will be required to determine the longevity of the fungi cultures. 


\section{REFERENCES}

K. Alibhai, D.J. Leak, A.W. Dudeney, S. Agatzini, and P. Tzeferis, 1991. "Microbial leaching of nickel from low grade Greek laterites," in Mineral Bioprocessing, ed. R.W. Smith and M. Misra. The Minerals, Metals, \& Materials Society: Warrendale, PA, pp. 191205.

K. Alibhai, A.W.L. Dudeney, D.J. Leak, S. Agatzini, 1993. "Bioleaching and bioprecipitation of nickel and iron from laterites,” FEMS Microbiology Reviews, 11, pp 8696.

D.I. McKenzie, L. Denys, and A. Buchanan, 1987. "The solubilization of nickel, cobalt and iron from laterites by means of organic chelating acids at low $\mathrm{pH}$," International Journal of Mineral Processing, 21, pp. 275-292.

O.H. Tuovinen and D.P. Kelly, 1974. "Use of microorganisms for the recovery of metals," International Metallurgy Review, 19, pp. 21-31.

P.G. Tzeferis, 1994A. "Fungal leaching of nickeliferous laterites,” Folia Microbiology, 39 (2), pp. 137-140.

P.G. Tzeferis, 1994B. "Mineral leaching of low-grade laterite ores using "bioacids" by molasses fungal metabolism,” Minerals and Metallurgical Processing, 11(2), pp. 125-129.

P.G. Tzeferis and S. Agatzini-Leonardou, 1994. "Leaching of nickel and iron from Greek non-sulfide nickeliferous ores by organic acids," Hydrometallurgy, 36, pp. 345-360. 
Appendix 29: Overcoming Technological Barriers to More Efficient Recovery of Copper from Chalcopyrite (UT005) 
FINAL TECHNICAL REPORT

Contract Title and Number: Crosscutting Technology Development at the Center for Advanced Separation Technologies (DE-FC26-02NT41607)

Sub-Recipient Project Title:

Overcoming technological barriers to more efficient recovery of copper from chalcopyrite

Principal Investigators:

Michael L. Free

Contact Address:

Michael L. Free

135 S. 1460 E. Room 412

Salt Lake City, UT 84112

Subcontractor Address:

No subcontracts issued.
Period of Performance:

Starting Date: $06 / 01 / 2004$

Ending Date: $\quad$ 10/31/2007
Report Information:

Type: Final

Number:

Period:

Date: Aug. 30, 2006

Code: UT005-FINAL

Contact Information:

Phone: 801-585-9798

Fax: $\quad$ 801-581-4937

E-Mail: mfree@mines.utah.edu

Subcontractor Information:

Phone:

Fax:

E-Mail:

\section{DISCLAIMER}

This report was prepared as an account of work sponsored by an agency of the United States Government. Neither the United States Government nor any agency thereof, nor any of their employees, make any warranty, express or implied, nor assume any legal liability or responsibility for the accuracy, completeness, or usefulness of any information, apparatus, product, or process disclosed, or represents that its use would not infringe privately owned rights. Reference herein to any specific commercial product, process, or service by trade name, trademark, manufacturer, or otherwise does not necessarily constitute or imply endorsement, recommendation, or favoring by the United States Government or any agency thereof. The views and opinions of authors expressed herein do not necessarily state or reflect those of the United States Government or agency thereof.

\section{ABSTRACT}

One of the important technological barriers affecting the efficient recovery of copper from chalcopyrite is the morphology of the copper electrodeposits, which restricts the use of traditional electroplating in cathode sheet form. This study identifies the effects of mass transport and different additives on the surface roughness of copper electrodeposits on a 
rotating disc electrode, obtained from a bath containing $0.1 \mathrm{~mol} / \mathrm{L} \mathrm{CuCl}, 4.0 \mathrm{~mol} / \mathrm{L} \mathrm{NaCl}$ and $0.01 \mathrm{~mol} / \mathrm{L} \mathrm{HCl}$ under controlled current conditions. It was found that the surface roughness of the deposit decreased with increase in rotational speed of the electrode. Pulsating currents also gave electrodeposits with surface roughness lower than those obtained under direct current conditions. It was found that gelatin gave more uniform deposits compared to those obtained without any additives. The effect of different inorganic additives on the surface roughness of copper electrodeposits was also studied for constant current conditions. The roughness values of electrodeposits with zinc ion additions were substantially less than electrodeposits without any inorganic additives. Results from this study indicate that copper electrowinning from halide media, which requires only half the energy of traditional sulfate media, can be performed at $250 \mathrm{~A} / \mathrm{m}^{2}$ using traditional electrowinning cells if mass transport levels are sufficient.

\section{TABLE OF CONTENTS}

DISCLAIMER . 1

ABSTRACT.

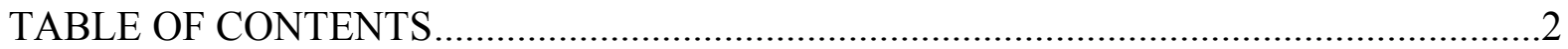

LIST OF GRAPHICAL MATERIALS ..................................................................... 3

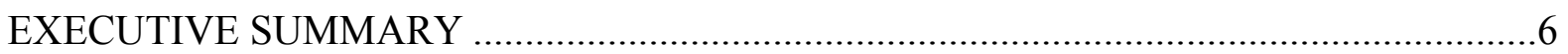

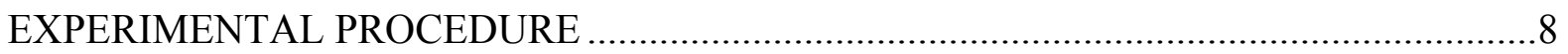

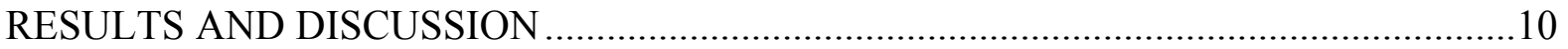

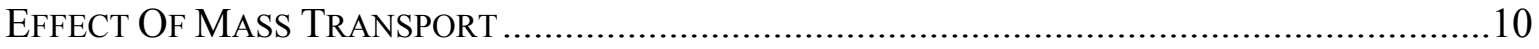

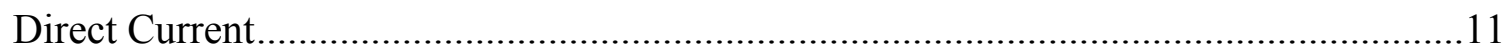

Direct Current - Effect Of Rotational Speed On Surface Roughness ..............................13

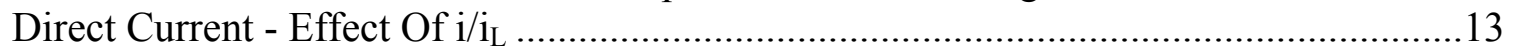

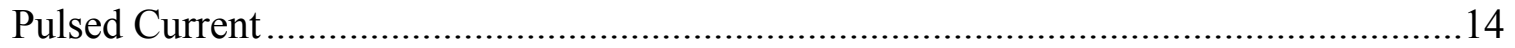

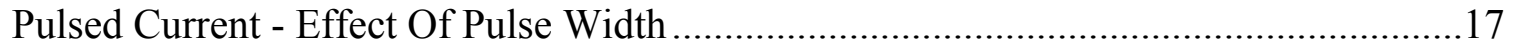

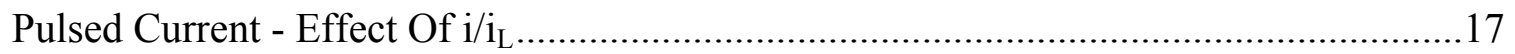

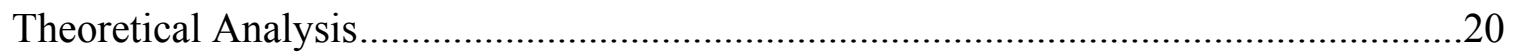

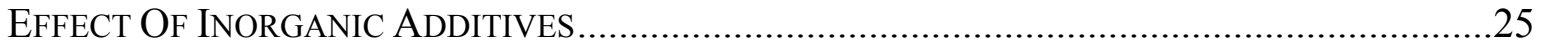

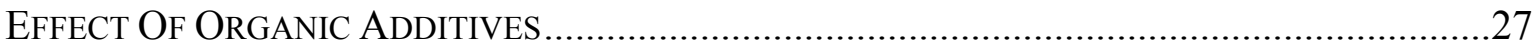

Effect Of Different Surfactants On Surface Roughness................................................27

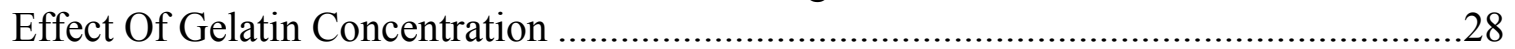

Effect Of Current Density In The Presence Of Organic Additives...................................28

Effect Of Electrodeposition Time ................................................................................. 30

Effect Of Combination Of Organic Additives On RMS Roughness .............................30

Steady-State Roughness Estimation And Evaluation........................................................ 31

Effect Of High Molecular Weight Organic Additives On Surface Roughness..................35

Effect Of Gelatin And Its Main Amino Acids (Glycine And Proline) On RMS

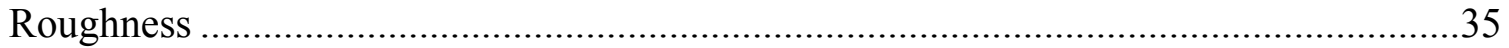

Effect Of Different Molecular Weights Of PEG And PEO On RMS Roughness ............35

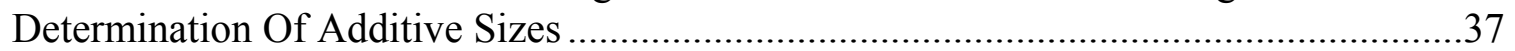




\section{LIST OF GRAPHICAL MATERIALS}

Figure 1: Experimental set up for electrodeposition tests (R: Reference electrode, W: Working electrode, C: Counter electrode, G: Gas vent).

Figure 2: Schematic of imaging system for topography measurement................................ 10

Figure 3: Eh-pH diagram for copper-chlorine-water system.

Figure 4: Potentiodynamic scans for argon purged electrolyte with $0.1 \mathrm{~mol} / \mathrm{L}$ $\mathrm{CuCl}, 4 \mathrm{~mol} / \mathrm{L} \mathrm{NaCl}$ and $0.01 \mathrm{~mol} / \mathrm{L} \mathrm{HCl}$ as function of rotational speed of electrode at a scan rate of $5 \mathrm{mV} / \mathrm{s}$

Figure 5: Limiting current densities under direct current conditions in a bath containing $0.1 \mathrm{~mol} / \mathrm{L} \mathrm{CuCl}, 4.0 \mathrm{~mol} / \mathrm{L} \mathrm{NaCl}$ and $0.01 \mathrm{~mol} / \mathrm{L} \mathrm{HCl}$.

Figure 6: Effect of rotational speed of the electrode on root mean square surface roughness of copper electrodeposits obtained from a bath containing $0.1 \mathrm{~mol} / \mathrm{L} \mathrm{CuCl}, 4.0 \mathrm{~mol} / \mathrm{L} \mathrm{NaCl}$ and $0.01 \mathrm{~mol} / \mathrm{L} \mathrm{HCl}$ under direct current conditions with cathodic current density of $25 \mathrm{~mA} / \mathrm{cm}^{2}$.

Figure 7: Effect of $\mathrm{i} / \mathrm{iL}$ on rms surface roughness of copper electrodeposits obtained from a bath containing $0.1 \mathrm{~mol} / \mathrm{L} \mathrm{CuCl}, 4.0 \mathrm{~mol} / \mathrm{L} \mathrm{NaCl}$ and $0.01 \mathrm{~mol} / \mathrm{L} \mathrm{HCl}$ under direct current conditions at various rotational speeds of copper working electrode.

Figure 8: Schematic of a typical pulse current transient in electrodeposition for different (A) pulse-on times and (B) pulse-off times.

Figure 9:Limiting current density for pulsating currents with pulse-on time of $5 \mathrm{~ms}$ for copper rotating disc in a bath containing $0.1 \mathrm{~mol} / \mathrm{L} \mathrm{CuCl}, 4 \mathrm{~mol} / \mathrm{L}$ $\mathrm{NaCl}$ and $0.01 \mathrm{~mol} / \mathrm{L} \mathrm{HCl}$ rotating at $100 \mathrm{rpm}$.

Figure 10: Limiting current density for pulsating currents with pulse-off time of $10 \mathrm{~ms}$ for copper rotating disc in a bath containing $0.1 \mathrm{~mol} / \mathrm{L} \mathrm{CuCl}, 4$ $\mathrm{mol} / \mathrm{L} \mathrm{NaCl}$ and $0.01 \mathrm{~mol} / \mathrm{L} \mathrm{HCl}$ rotating at $100 \mathrm{rpm}$.

Figure 11: Effect of pulse width on surface roughness of copper electrodeposits obtained from a bath containing $0.1 \mathrm{~mol} / \mathrm{L} \mathrm{CuCl}, 4.0 \mathrm{~mol} / \mathrm{L} \mathrm{NaCl}$ and $0.01 \mathrm{~mol} / \mathrm{L} \mathrm{HCl}$ under pulsed current conditions for $120 \mathrm{~min}$ at a rotational speed of 100 RPM for copper working electrode with peak cathodic current density of $45 \mathrm{~mA} / \mathrm{cm}^{2}$.

Figure 12: Surface topography map of 180 Coulombs per $\mathrm{cm}^{2}$ of copper deposited from $0.1 \mathrm{M} \mathrm{CuCl}, 4 \mathrm{M} \mathrm{NaCl}$ media using direct current (-25 $\mathrm{mA} / \mathrm{cm}^{2}$ at $\left.22^{\circ} \mathrm{C}\right)$. 
Figure 13: Surface topography map of 180 Coulombs per $\mathrm{cm}^{2}$ of copper deposited from $0.1 \mathrm{M} \mathrm{CuCl}, 4 \mathrm{M} \mathrm{NaCl}$ media using galvanostatic pulsing $\left(10 \%\right.$ duty cycle at Pulse width $\left.11 \mathrm{~ms},-25 \mathrm{~mA} / \mathrm{cm}^{2}, 22^{\circ} \mathrm{C}\right)$.

Figure 14: Effect of $\mathrm{i} / \mathrm{iL}$ on rms surface roughness of copper electrodeposits obtained from a bath containing $0.1 \mathrm{~mol} / \mathrm{L} \mathrm{CuCl}, 4.0 \mathrm{~mol} / \mathrm{L} \mathrm{NaCl}$ and $0.01 \mathrm{~mol} / \mathrm{L} \mathrm{HCl}$ under pulsed current conditions for $120 \mathrm{~min}$ at a rotational speed of 100 RPM for copper working electrode

Figure 15: Effect of $\mathrm{i} / \mathrm{i}_{\mathrm{L}}$ on root mean square surface roughness of copper electrodeposits obtained from a bath containing $0.1 \mathrm{~mol} / \mathrm{L} \mathrm{CuCl}, 4.0$ $\mathrm{mol} / \mathrm{L} \mathrm{NaCl}$ and $0.01 \mathrm{~mol} / \mathrm{L} \mathrm{HCl}$ under pulsed current conditions with pulse-on time of $1 \mathrm{~ms}$ and pulse-off time of $10 \mathrm{~ms}$.

Figure 16: Comparison of solution obtained by analytical and finite difference methods for concentration profile at different times after start of the electrodeposition process.

Figure 17: Finite difference modeling results for surface concentration of reacting ions obtained from a bath containing $0.1 \mathrm{~mol} / \mathrm{L} \mathrm{CuCl}, 4.0 \mathrm{~mol} / \mathrm{L} \mathrm{NaCl}$ and $0.01 \mathrm{~mol} / \mathrm{L} \mathrm{HCl}$ on a stationary electrode under pulsed current conditions with pulse-on time of $1 \mathrm{~ms}$ and pulse-off time of $10 \mathrm{~ms}$ with peak cathodic current density of $10 \mathrm{~mA} / \mathrm{cm}^{2}$ for pulsing and a constant cathodic current density of $10 \mathrm{~mA} / \mathrm{cm}^{2}$ for direct current transients.

Figure 18: Finite difference modeling results for surface concentration of reacting ions obtained from a bath containing $0.1 \mathrm{~mol} / \mathrm{L} \mathrm{CuCl}, 4.0 \mathrm{~mol} / \mathrm{L} \mathrm{NaCl}$ and $0.01 \mathrm{~mol} / \mathrm{L} \mathrm{HCl}$ on a disc electrode rotating at $100 \mathrm{rpm}$ under pulsed current conditions with pulse-off time of $10 \mathrm{~ms}$ with peak cathodic current density of $50 \mathrm{~mA} / \mathrm{cm}^{2}$.

Figure 19: Finite difference modeling results for surface concentration of reacting ions obtained from a bath containing $0.1 \mathrm{~mol} / \mathrm{L} \mathrm{CuCl}, 4.0 \mathrm{~mol} / \mathrm{L} \mathrm{NaCl}$ and $0.01 \mathrm{~mol} / \mathrm{L} \mathrm{HCl}$ on a disc electrode rotating at $100 \mathrm{rpm}$ under pulsed current conditions with pulse-on time of $1 \mathrm{~ms}$ with peak cathodic current density of $50 \mathrm{~mA} / \mathrm{cm}^{2}$.

Figure 20: Effect of different cationic additives $(\mathrm{A}=$ no additive, $\mathrm{B}=100 \mathrm{ppm}$ $\mathrm{Zn}^{+2}$ .26

Figure 21: Effect of time on surface roughness of copper electrodeposits obtained from an electrolyte containing $0.1 \mathrm{M} \mathrm{CuCl}, 4 \mathrm{M} \mathrm{NaCl}$ and $0.01 \mathrm{M} \mathrm{HCl}$ (base solution) at a cathodic current density of $25 \mathrm{~mA} / \mathrm{cm}^{2}$ at $1000 \mathrm{rpm}$ at a temperature of $60^{\circ} \mathrm{C}$.

Figure 22: Effect of different surfactants $(\mathrm{A}=$ no additive, $\mathrm{B}=0.1 \%$ by vol gelatin, $\mathrm{C}=0.001 \mathrm{M} \mathrm{C10TAB}, \mathrm{D}=0.001 \mathrm{M} \mathrm{C16TAB}, \mathrm{E}=0.001 \mathrm{M}$ thiourea, $\mathrm{F}=0.05 \%$ by volume triton $\mathrm{x} 100, \mathrm{G}=1 \%$ by volume $\mathrm{PEG}$ $\mathrm{MW} 200$, and $\mathrm{H}=1.2 \%$ by mass/volume $\mathrm{PEG} \mathrm{MW} 10,000)$ on surface roughness of copper electrodeposits obtained from an 
electrolyte containing $0.1 \mathrm{~mol} / \mathrm{L} \mathrm{CuCl}, 4 \mathrm{~mol} / \mathrm{L} \mathrm{NaCl}$ and $0.01 \mathrm{~mol} / \mathrm{L}$ $\mathrm{HCl}$ (base solution) at cathodic current density of $25 \mathrm{~mA} / \mathrm{cm}^{2}$ for 3 hours at $1000 \mathrm{rpm}$.

Figure 23: (a) Color map images of copper electrodeposits without surfactant (b). Color map images of copper electrodeposits with $0.1 \%$ by volume gelatin obtained from an electrolyte containing $0.1 \mathrm{~mol} / \mathrm{L} \mathrm{CuCl}, 4$ $\mathrm{mol} / \mathrm{L} \mathrm{NaCl}$ and $0.01 \mathrm{~mol} / \mathrm{L} \mathrm{HCl}$ (base solution) at cathodic current density of $25 \mathrm{~mA} / \mathrm{cm}^{2}$ for 3 hours at $1000 \mathrm{rpm}$.

Figure 24: Effect of concentration of gelatin $(0,0.01,0.1$, and $1 \%$ by volume in base solution on surface roughness of copper electrodeposits for cathodic current density of $25 \mathrm{~mA} / \mathrm{cm}^{2}$ for 3 hours at $1000 \mathrm{rpm}$.

Figure 25: Effect of DC current density on surface roughness of copper electrodeposits obtained from a base solution with and without $0.1 \%$ by volume gelatin for total amount of charge of $108 \mathrm{C} / \mathrm{cm}^{2}$......

Figure 26: Effect of DC current density on surface roughness of copper electrodeposits obtained from a base solution with and without $0.1 \%$ by volume gelatin for total amount of charge of $1,350 \mathrm{C} / \mathrm{cm}^{2}$.

Figure 27: Effect of electrodeposition time on surface roughness of copper electrodeposits obtained from a base solution with and without $0.1 \%$ by volume gelatin for cathodic current density of $25 \mathrm{~mA} / \mathrm{cm}^{2}$ at 1000 rpm.

Figure 28: Effect of $2.5 \mathrm{ppm}$ glycine (A), $2.5 \mathrm{ppm}$ thiourea (B), $7500 \mathrm{ppm}$ PEG (MW 200) (C), and their combinations on the surface roughness of copper electrodeposits obtained from an electrolyte containing 0.1 $\mathrm{mol} / \mathrm{L} \mathrm{CuCl}, 4 \mathrm{~mol} / \mathrm{L} \mathrm{NaCl}$ and $0.01 \mathrm{~mol} / \mathrm{L} \mathrm{HCl}$ (base solution) at cathodic current density of $25 \mathrm{~mA} / \mathrm{cm}^{2}$ for 3 hours at $1000 \mathrm{rpm}$..

Figure 29: Comparison of RMS roughness values after copper electrodeposition from $0.1 \mathrm{M} \mathrm{CuCl}, 4 \mathrm{M} \mathrm{NaCl}, 0.01 \mathrm{M} \mathrm{HCl}$ solution $\left(1000 \mathrm{RPM}, 25^{\circ} \mathrm{C}\right.$, $25 \mathrm{~mA} / \mathrm{cm}^{2}$ ).

Figure 30: Comparison of the natural logarithm of the ratio of RMS roughness to current density versus the inverse temperature multiplied by the inverse surface overpotential squared. Data based on galvanostatic copper electrodeposition from $0.1 \mathrm{M} \mathrm{CuCl}, 4 \mathrm{M} \mathrm{NaCl}, 0.01 \mathrm{M} \mathrm{HCl}$ solution (1000 RPM, $25^{\circ} \mathrm{C}, 180$ Coulombs $/ \mathrm{cm}^{2}$, and $-5,-10$, and -25 $\left.\mathrm{mA} / \mathrm{cm}^{2}\right)$.

Figure 31: Comparison of the natural logarithm of the ratio of RMS roughness to current density versus the inverse temperature multiplied by the inverse surface overpotential squared. Data based on galvanostatic copper electrodeposition from $0.1 \mathrm{M} \mathrm{CuCl}, 4 \mathrm{M} \mathrm{NaCl}, 0.01 \mathrm{M} \mathrm{HCl}$ solution containing 0.1 vol $\%$ gelatin $\left(1000 \mathrm{RPM}, 25^{\circ} \mathrm{C}, 180\right.$ Coulombs $/ \mathrm{cm}^{2}$, and $-5,-10$, and $-25 \mathrm{~mA} / \mathrm{cm}^{2}$ ) 
Figure 32: Comparison of the natural logarithm of the ratio of RMS roughness to current density versus the inverse temperature multiplied by the inverse surface overpotential squared. Data based on galvanostatic copper electrodeposition from $0.1 \mathrm{M} \mathrm{CuCl}, 4 \mathrm{M} \mathrm{NaCl}, 0.01 \mathrm{M} \mathrm{HCl}$ solution (1000 RPM, 30, 45, and $60^{\circ} \mathrm{C}, 180$ Coulombs $/ \mathrm{cm}^{2},-25$ $\left.\mathrm{mA} / \mathrm{cm}^{2}\right)$.

Figure 33: Effect of different surfactants $(\mathrm{A}=$ no additive, $\mathrm{B}=0.0127 \mathrm{~g} / \mathrm{L}$ gelatin, $\mathrm{C}=0.01 \mathrm{~g} / \mathrm{L}$ PEO $\mathrm{MW} 300,000$, and $\mathrm{D}=$ PAA $\mathrm{MW} 200,000)$ on surface roughness of copper electrodeposits obtained from an electrolyte containing $0.1 \mathrm{M} \mathrm{CuCl}, 4 \mathrm{M} \mathrm{NaCl}$ and $0.01 \mathrm{M} \mathrm{HCl}$ at cathodic current density of $25 \mathrm{~mA} / \mathrm{cm}^{2}$ for 3 hours at $1000 \mathrm{rpm}$.

Figure 34: Effect of concentration of glycine, proline, and gelatin on surface roughness of copper electrodeposits obtained from an electrolyte containing $0.1 \mathrm{M} \mathrm{CuCl}, 4 \mathrm{M} \mathrm{NaCl}$ and $0.01 \mathrm{M} \mathrm{HCl}$ at cathodic current density of $25 \mathrm{~mA} / \mathrm{cm}^{2}$ for 3 hours at $1000 \mathrm{rpm}$.

Figure 35: Effect of concentration of PEG MW 200, PEG MW 10,000, PEO MW 100,000, and PEO MW 300,000on surface roughness of copper electrodeposits obtained from an electrolyte containing $0.1 \mathrm{M} \mathrm{CuCl}, 4$ $\mathrm{M} \mathrm{NaCl}$ and $0.01 \mathrm{M} \mathrm{HCl}$ at cathodic current density of $25 \mathrm{~mA} / \mathrm{cm}^{2}$ for 3 hours at $1000 \mathrm{rpm}$.

Figure 36: Copper electrode with approximate 1 micron wide scratch in one direction.

Figure 37: Copper electrodeposit on approximate 1 micron wide scratched copper electrode in an electrolyte containing $0.1 \mathrm{M} \mathrm{CuCl}, 4 \mathrm{M} \mathrm{NaCl}$ and 0.01 $\mathrm{M} \mathrm{HCl}$ at cathodic current density of $25 \mathrm{~mA} / \mathrm{cm}^{2}$ for 20 seconds at $1000 \mathrm{rpm}$.

Figure 38: Copper electrodeposit on approximate 1 micron wide scratched copper electrode in an electrolyte containing $0.01 \mathrm{~g} / \mathrm{L}$ gelatin, $0.1 \mathrm{M} \mathrm{CuCl}, 4$ $\mathrm{M} \mathrm{NaCl}$ and $0.01 \mathrm{M} \mathrm{HCl}$ at cathodic current density of $25 \mathrm{~mA} / \mathrm{cm}^{2}$ for 20 seconds at $1000 \mathrm{rpm}$

Figure 39: Plot of $\ln \left(d N_{\text {formation }} / d t\right)$ vs. $1 / \eta_{s}^{2}$ from $0.1 \mathrm{M} \mathrm{CuCl}, 4 \mathrm{M} \mathrm{NaCl}, 0.01$ $\mathrm{M} \mathrm{HCl}$ solution at stationary copper electrode.

Figure 40: Plot of $\ln \left(d N_{\text {formation }} / d t\right)$ vs. $1 / \eta_{s}^{2}$ from $0.1 \%$ by volume gelatin, $0.1 \mathrm{M}$ $\mathrm{CuCl}, 4 \mathrm{M} \mathrm{NaCl}, 0.01 \mathrm{M} \mathrm{HCl}$ solution at stationary copper electrode.

\section{EXECUTIVE SUMMARY}

Traditional leaching, solvent extraction, and electrowinning, which is the dominant method for producing copper from other minerals, has not been successfully applied to recover copper from chalcopyrite on a large scale. One of the more promising alternative methods of recovering copper from chalcopyrite involves the use of halide solutions containing chloride and/or bromide ions and an oxidant such as cupric ions to leach the 
copper from the chalcopyrite. Many researchers have shown that use of halide media allows for hydrometallurgical extraction of copper from chalcopyrite and consequently, the elimination of the smelting process ${ }^{1-7}$. The dissolved copper is recovered from halide solution by electrowinning from the cuprous ion state, which requires only half of the energy needed for traditional electrowinning from the cupric ion $\left(\mathrm{Cu}^{2+)}\right.$ state, since the cuprous ion $\left(\mathrm{Cu}^{+}\right)$ needs only one electron. The dominant limitations to the implementation of halide leaching are associated with the fact that the electrodeposited copper product tends to be rough, granular, porous and non-adherent to starter sheets, rather than the traditional plate cathode copper. It is well known that electrodeposit morphology is controlled to a significant degree by chemistry, current density, additives, and mass transport. Consequently, the effect of mass transport, solution chemistry, current density, and additives were investigated in this study to determine appropriate combinations that can be used to achieve smooth high quality, cathode copper plate rather than rough cathode copper granules in halide-based copper-bearing solutions.

The importance of mass transport limitations on surface roughness of electrodeposits underscores the need to increase mass transport to the electrode surface. One way to enhance mass transport is to increase the bulk fluid velocity near the electrode. Another way of increasing the mass transport limiting current density is to apply a pulsed potential. If mass transport is controlling the current flow in electrodeposition, the limiting current density $\left(\mathrm{i}_{\mathrm{L}}\right)$ and the ratio of electrodeposition current to this limiting current density $\left(\mathrm{i} / \mathrm{i}_{\mathrm{L}}\right)$ are the important parameters that can be used to study these diffusion limited mass transfers. The bulk fluid velocity near the electrode can be controlled by using a rotating disc electrode. The effect of rotational speed of the electrode on surface morphology of copper electrodeposits was studied at 250,500 and $1000 \mathrm{rpm}$. The surface roughness of the electrodeposits decreased with the increase in the rotational speed of the electrode. This can be attributed to the improved mass kinetics facilitating the cuprous ions transport at the surface of the working electrode. Thus, improving electrolyte flow near the cathode improves the cathode quality. It was found that surface roughness increased when $\mathrm{i} / \mathrm{i}_{\mathrm{L}}$ ratio increased for direct as well as pulsed current transients. Finite difference approximation method was used to solve the diffusion-convection equation governing this mass transport. The surface concentration of the reacting species at the electrode surface was determined using this model under direct and pulsed current condition.

The reduction of the surface roughness in the plating processes can be accomplished by the addition of organic additives. Monomeric organic additives such as glycine amino acid, proline amino acid, C10TAB, C16TAB, triton $\mathrm{x} 100$, and thiourea and low molecular weight polymeric additives such as PEG MW 200, and PEG MW 10,000 do not reduce the surface roughness of the copper electrodeposits. However high molecular weight polymeric organic additives such as gelatin, PEO MW 300,000, and PAA MW 200,000 significantly reduce the surface roughness of the copper electrodeposits even though they consist of the same monomer units that do not reduce the roughness in their monomeric or short chain form. The model for estimating the surface roughness was developed and this proposed model provides a reasonable description of the effect of additives on electrodeposit roughness. The base line rate of nuclei from gelatin is higher than that of without adding additives. 
The industrial process for the electrowinning of copper contains some typical inorganic compounds. Inorganic ions possess the capacity to influence the electrochemical properties of the system. For this reason the effect of inorganic additives on the surface roughness of copper electrodeposits obtained from chloride media was investigated. The inorganic compounds used for investigation were chosen such that the cations present in the compounds would not get deposited along with copper and thereby won't influence the purity of electrodeposited copper. For this purpose only the cations having a lower standard reduction potential than cuprous ion were chosen. As a result of the investigation it was observed that zinc ions resulted in the highest improvement in the smoothness of copper electrodeposits. Results from this study indicate that copper electrowinning from halide media, which requires only half the energy of traditional sulfate media, can be performed at $250 \mathrm{~A} / \mathrm{m}^{2}$ using traditional electrowinning cells if mass transport levels are sufficient.

\section{EXPERIMENTAL PROCEDURE}

Electrochemical tests were performed using an EG\&G 273 potentiostat connected to an IBM compatible PC (operated using PowerSuite software made by Princeton Applied Research) and AFASR rotator from Pine Instruments for potentiostatic and galvanostatic current transients. For pulsed transients, electrochemical tests were performed using PCI4/750 potentiostat operated using Virtual Front Panel software, both made by Gamry Instruments, and an AFASR rotator from Pine Instruments. A three component cell as shown in figure 1 with a platinum counter electrode and a saturated calomel electrode (SCE), against which are potentials are reported, were used. The working electrode was a copper disc $\left(99.999 \%\right.$ pure) mounted in a Teflon holder with an area of $0.203 \mathrm{~cm}^{2}$ exposed to the electrolyte. It was polished with a 600 -grit polishing paper to remove the deposition products from the previous tests and then rinsed with pure water. This electrode was further polished with Micropolish alumina powder $(0.05 \mu \mathrm{m})$ to get a smooth clean defect free surface. Finally the electrode is cleaned in an ultrasonic water bath to remove any polishing particles on the surface. All solutions were prepared using reagent grade chemicals and ASTM Type I water. 


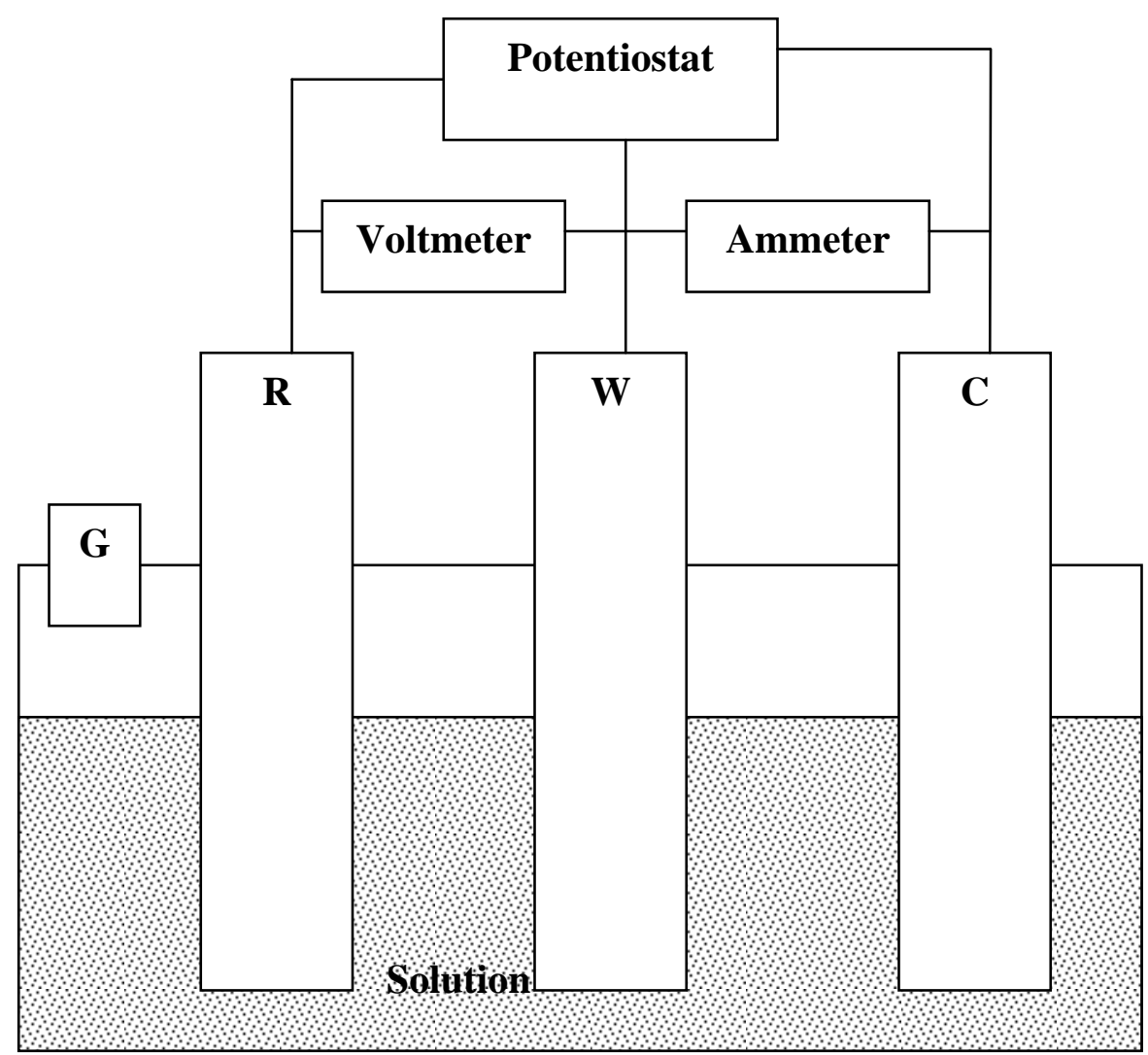

Figure 1: Experimental set up for electrodeposition tests (R: Reference electrode, W: Working electrode, C: Counter electrode, G: Gas vent).

Surface roughness was characterized by a CCD Iris camera by SONY with the help of LEAD CAPTURE and LEAD CONVERT software (by LEAD technologies) along with Microimage software (University of Utah). Surface roughness calculations were performed by MATLAB (Mathworks Inc.). A schematic of the imaging system is shown in figure 2 .

The working principle for this method can be explained as: The light beam ' $A$ ' from light source is split using a beam splitter arrangement as seen in figure 2 . Beam ' $\mathrm{B}$ ' gets reflected back as beam ' $D$ '. The interference between the beams ' $C$ ' and ' $D$ ' is recorded using a CCD camera and image analysis for topography measurements is done with the help of computer. 


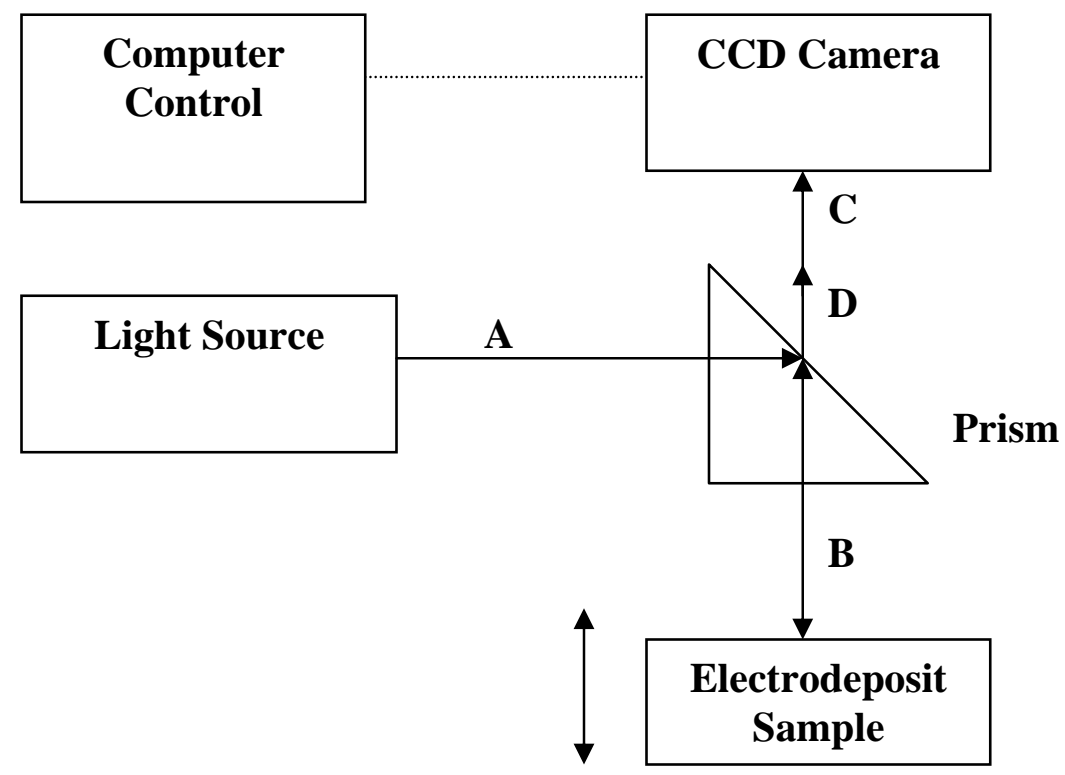

Figure 2: Schematic of imaging system for topography measurement.

\section{RESULTS AND DISCUSSION}

\section{Effect Of Mass Transport}

Possible species in copper-chlorine-water system were examined using Eh-pH diagram as shown in figure 7 obtained using HSC. According to the diagram seen in figure 3 , the cuprous ions predominance area is confined to a region between $\mathrm{pH} 0$ and 5 and a region between potentials of 0 and $+0.5 \mathrm{~V}$. The experiments in this research were performed at a $\mathrm{pH}$ of 2 . 


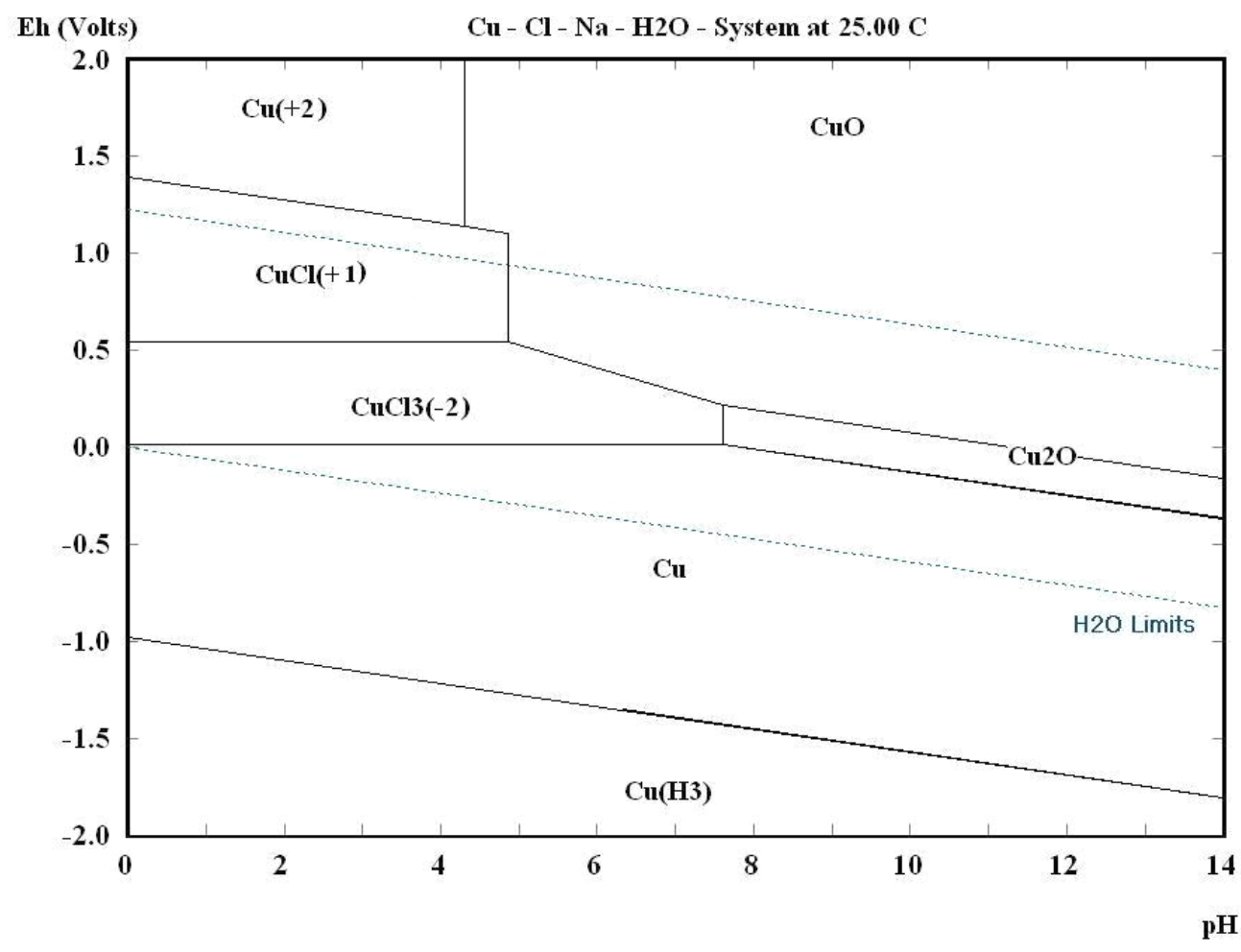

$\begin{array}{ll}\text { ELEMENTS } & \text { Molality } \\ \mathrm{Cu} & 1.000 \mathrm{E}-01 \\ \mathrm{Cl} & 4.100 \mathrm{E}+00 \\ \mathrm{Na} & 4.000 \mathrm{E}+00\end{array}$

\section{Figure 3: Eh-pH diagram for copper-chlorine-water system.}

\section{Direct Current}

If mass transport is controlling the current flow in electrodeposition, the limiting current density $\left(i_{L}\right)$ is an important parameter that can be used to study this diffusion limited mass transfer. Therefore, a series of experiments were carried out to study cathodic electrodeposition of copper under direct current conditions. For a given rotational speed of a rotating copper disc, a limiting current density was observed in the cathodic direction as seen in figure 4 and 5. 


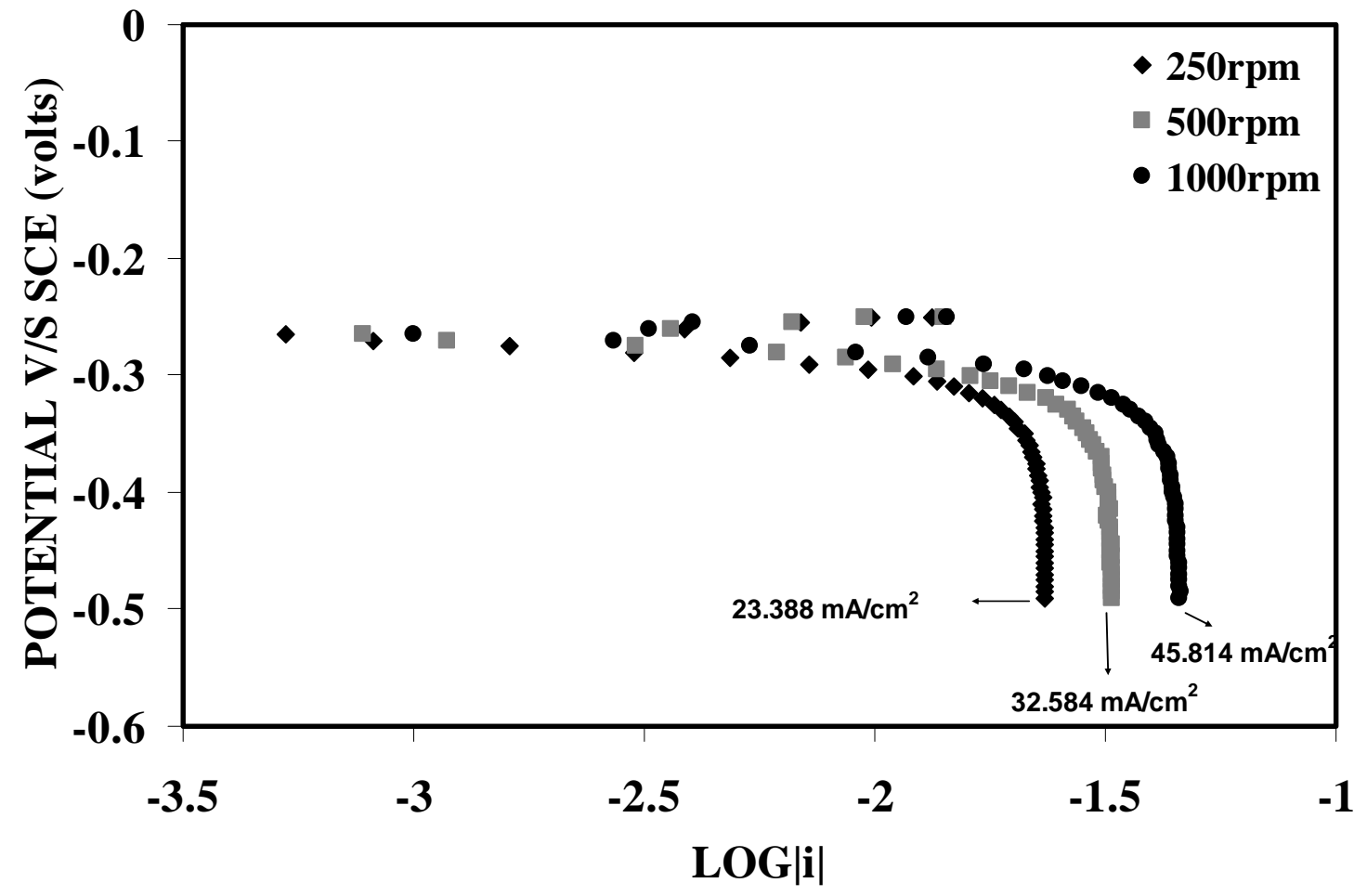

Figure 4: Potentiodynamic scans for argon purged electrolyte with $0.1 \mathrm{~mol} / \mathrm{L} \mathrm{CuCl}, 4$ $\mathrm{mol} / \mathrm{L} \mathrm{NaCl}$ and $0.01 \mathrm{~mol} / \mathrm{L} \mathrm{HCl}$ as function of rotational speed of electrode at a scan rate of $5 \mathrm{mV} / \mathrm{s}$.

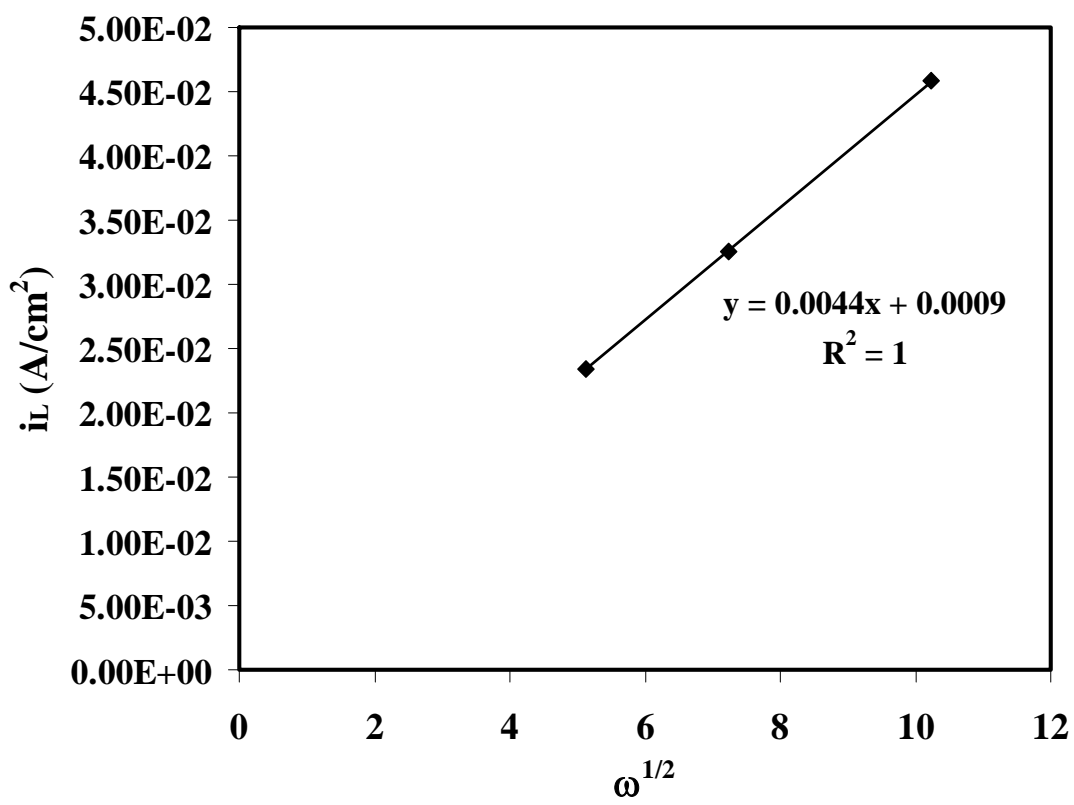

Figure 5: Limiting current densities under direct current conditions in a bath containing $0.1 \mathrm{~mol} / \mathrm{L} \mathrm{CuCl}, 4.0 \mathrm{~mol} / \mathrm{L} \mathrm{NaCl}$ and $0.01 \mathrm{~mol} / \mathrm{L} \mathrm{HCl}$. 
The effect of rotational speed of the electrode on surface morphology of copper electrodeposits was studied at 250, 500 and $1000 \mathrm{rpm}$. The surface roughness of the electrodeposits decreased with the increase in the rotational speed of the electrode as seen in figure 6 . This can be attributed to the improved mass transport of cuprous ions at the surface of the working electrode. Thus, increasing electrolyte flow near the cathode can reduce the cathode surface roughness at a constant current density.

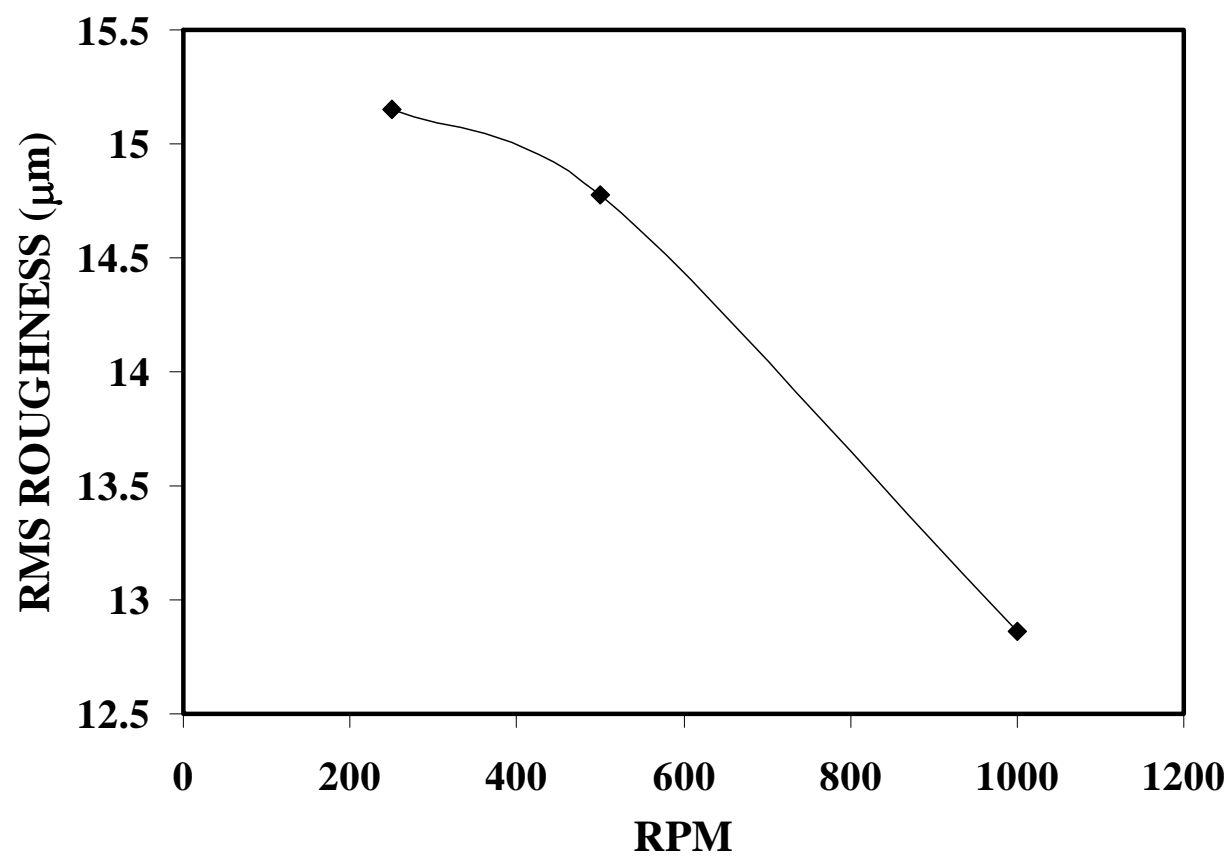

Figure 6: Effect of rotational speed of the electrode on root mean square surface roughness of copper electrodeposits obtained from a bath containing $0.1 \mathrm{~mol} / \mathrm{L} \mathrm{CuCl}$, 4.0 $\mathrm{mol} / \mathrm{L} \mathrm{NaCl}$ and $0.01 \mathrm{~mol} / \mathrm{L} \mathrm{HCl}$ under direct current conditions with cathodic current density of $25 \mathrm{~mA} / \mathrm{cm}^{2}$.

\section{Direct Current - Effect Of $i / i_{L}$}

As electrodeposit surfaces grow, the highest points on the growing surface receive greater access to incoming ions that diffuse to the electrode surface. Therefore, features extending the greatest distance from the surface grow at more rapid rates than features closest to the surface when growth is diffusion limited. Consequently, deposit growth is also a strong function of the extent to which the system approaches the mass transport limiting current density. The effect of $\mathrm{i} / \mathrm{i}_{\mathrm{L}}$ on root mean squared surface roughness of copper electrodeposits was studied. It was found that the surface roughness increased with increase in $\mathrm{i} / \mathrm{i}_{\mathrm{L}}$ for direct current conditions as seen in figure 7. 


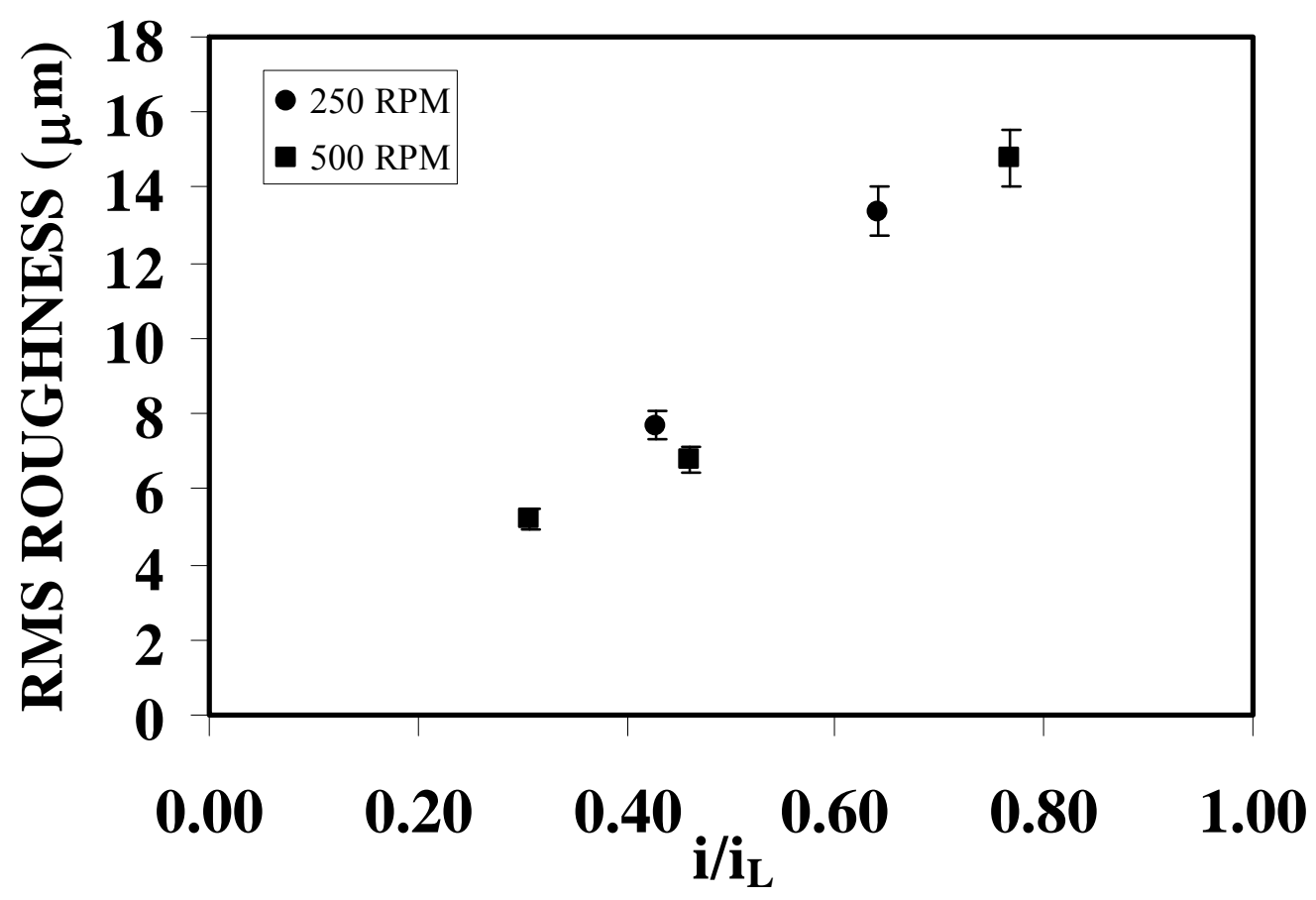

Figure 7: Effect of i/iL on rms surface roughness of copper electrodeposits obtained from a bath containing $0.1 \mathrm{~mol} / \mathrm{L} \mathrm{CuCl}, 4.0 \mathrm{~mol} / \mathrm{L} \mathrm{NaCl}$ and $0.01 \mathrm{~mol} / \mathrm{L} \mathrm{HCl}$ under direct current conditions at various rotational speeds of copper working electrode.

\section{Pulsed Current}

The application of a pulsed potential results in a short deposition time during which the depositing ions become depleted in the solution near the interface as seen in figure 8(A) and 8(B). This is followed by a longer rest interval during which the depositing ions are replenished near the interface. The act of replenishing the ions at the interface increases the average concentration of depositing ions at the interface, thereby increasing the effective limiting current density. A series of experiments were carried out to study cathodic electrodeposition of copper under pulsed current conditions. For pulsed current conditions, the magnitude of the peak current was changed and the potential response was measured. For a given pulse-on and pulse-off time, a limiting current density was observed in the cathodic direction and the results are shown in figure 9 and 10. 


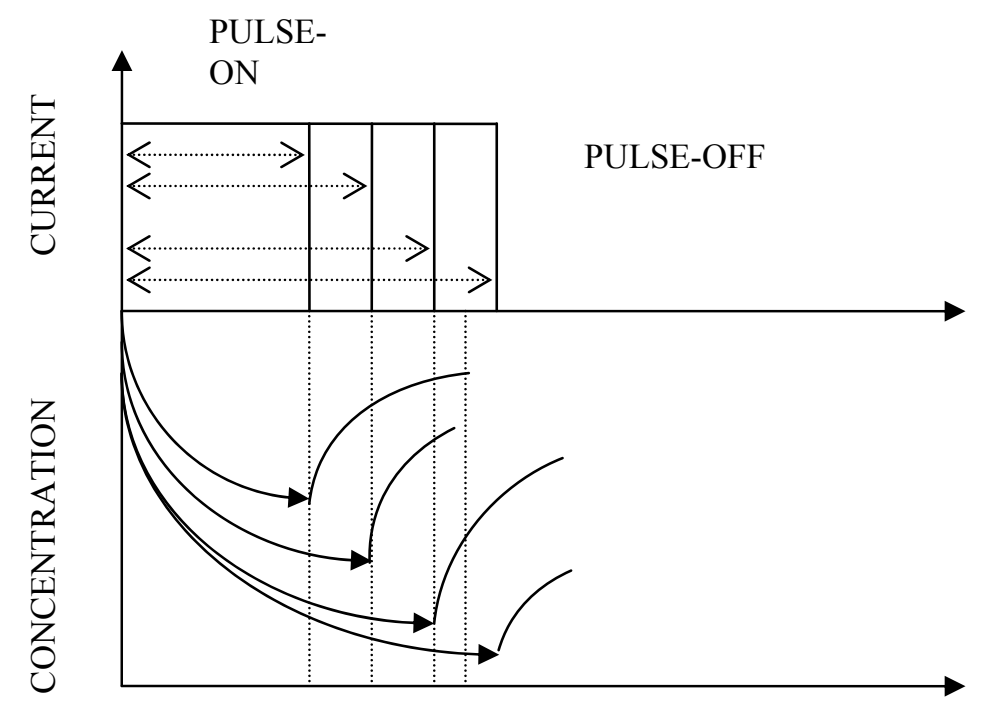

TIME

(A)

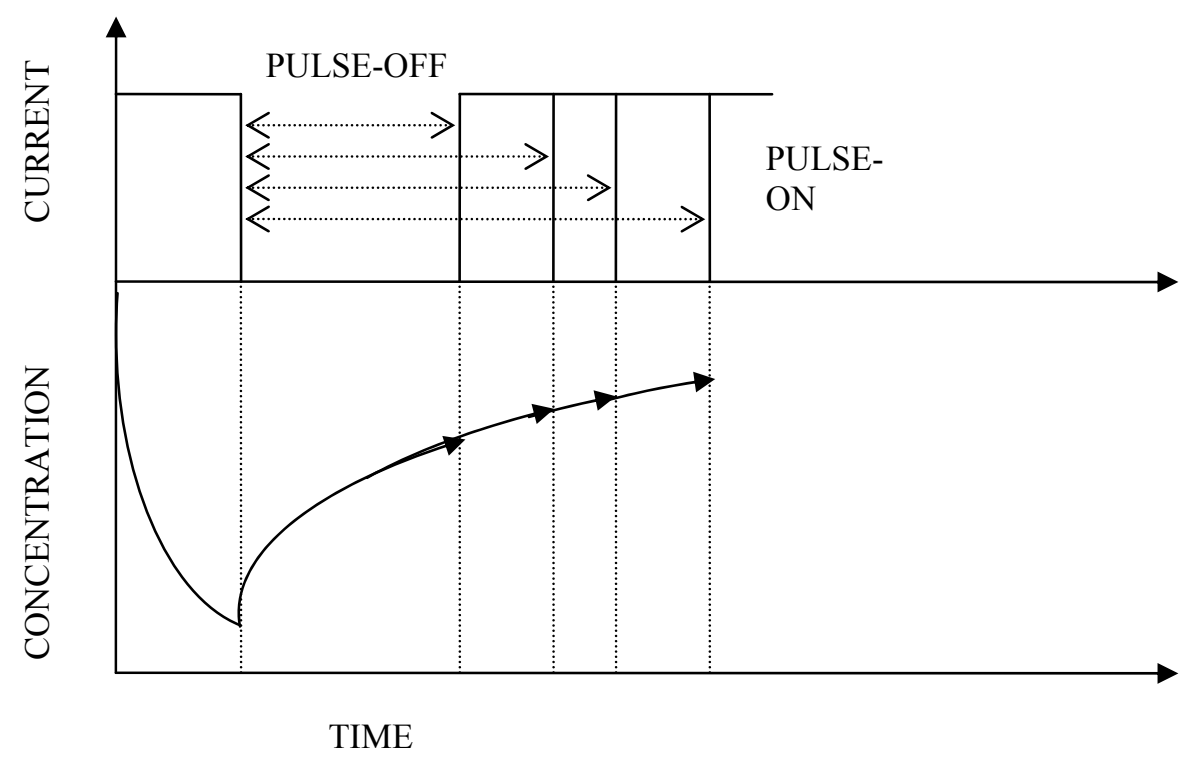

(B)

Figure 8: Schematic of a typical pulse current transient in electrodeposition for different (A) pulse-on times and (B) pulse-off times. 


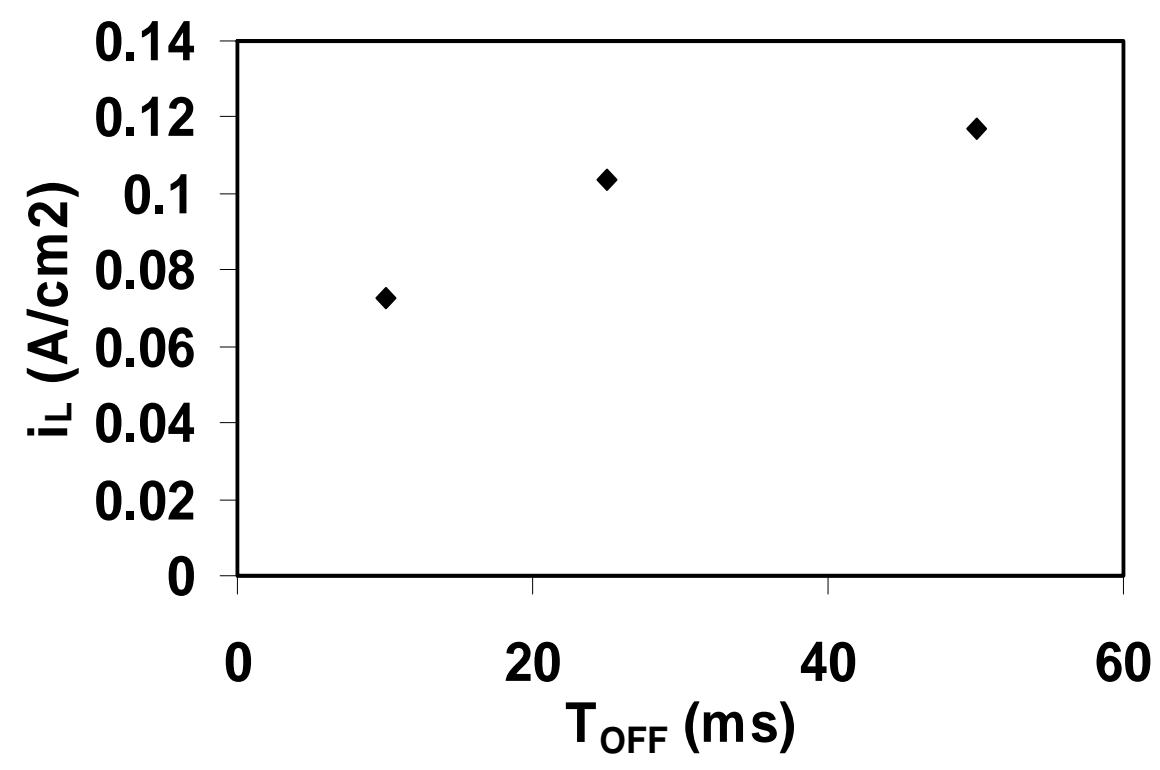

Figure 9:Limiting current density for pulsating currents with pulse-on time of $5 \mathrm{~ms}$ for copper rotating disc in a bath containing $0.1 \mathrm{~mol} / \mathrm{L} \mathrm{CuCl}, 4 \mathrm{~mol} / \mathrm{L} \mathrm{NaCl}$ and $0.01 \mathrm{~mol} / \mathrm{L}$ $\mathrm{HCl}$ rotating at $100 \mathrm{rpm}$.

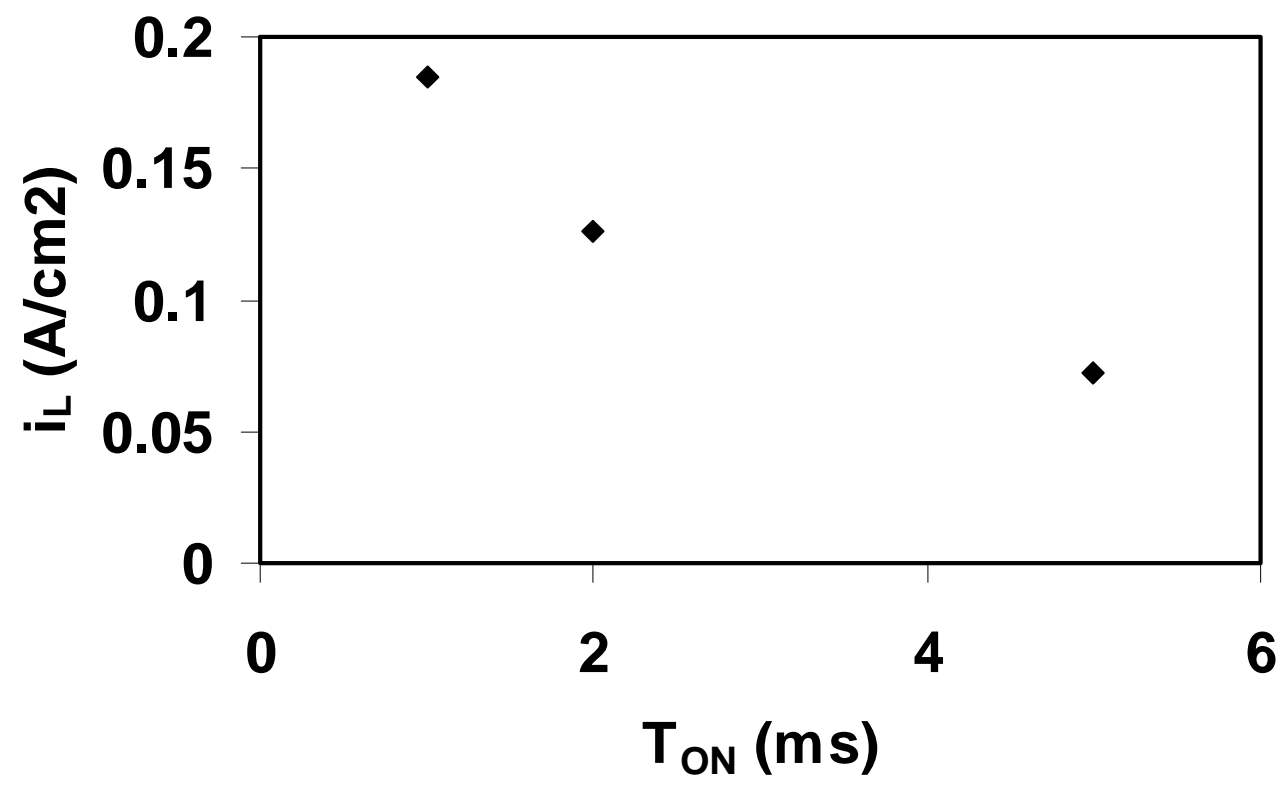

Figure 10: Limiting current density for pulsating currents with pulse-off time of 10ms for copper rotating disc in a bath containing $0.1 \mathrm{~mol} / \mathrm{L} \mathrm{CuCl}, 4 \mathrm{~mol} / \mathrm{L} \mathrm{NaCl}$ and 0.01 $\mathrm{mol} / \mathrm{L} \mathrm{HCl}$ rotating at $100 \mathrm{rpm}$. 


\section{Pulsed Current - Effect Of Pulse Width}

As seen from figure 11, as the pulse width increases the surface roughness decreases till a certain pulse width but after that it increases and at longer pulses e.g. $0.55 \mathrm{sec}$, it is very close to the DC current value of $25 \mu \mathrm{m}$. Thus, at longer pulse widths the electrodeposits grow in the same manner as under the DC current conditions.

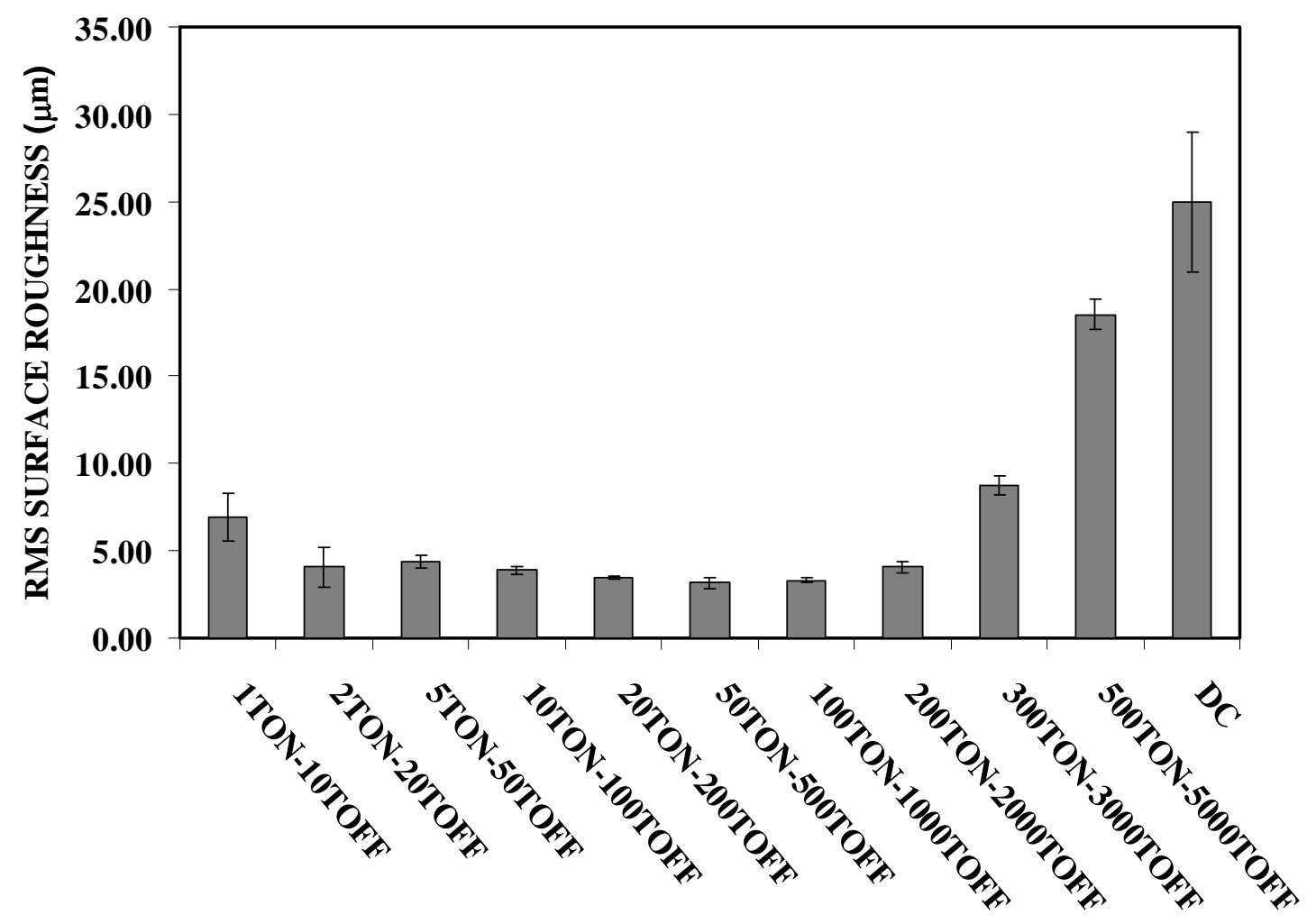

Figure 11: Effect of pulse width on surface roughness of copper electrodeposits obtained from a bath containing $0.1 \mathrm{~mol} / \mathrm{L} \mathrm{CuCl}, 4.0 \mathrm{~mol} / \mathrm{L} \mathrm{NaCl}$ and $0.01 \mathrm{~mol} / \mathrm{L} \mathrm{HCl}$ under pulsed current conditions for $120 \mathrm{~min}$ at a rotational speed of $100 \mathrm{RPM}$ for copper working electrode with peak cathodic current density of $45 \mathrm{~mA} / \mathrm{cm}^{2}$.

\section{Pulsed Current - Effect Of $i / i_{L}$}

One of the advantages of pulsing is the ability to apply a larger surface overpotential, which facilitates a smoother deposit as demonstrated by comparing figures 12 and 13 . 


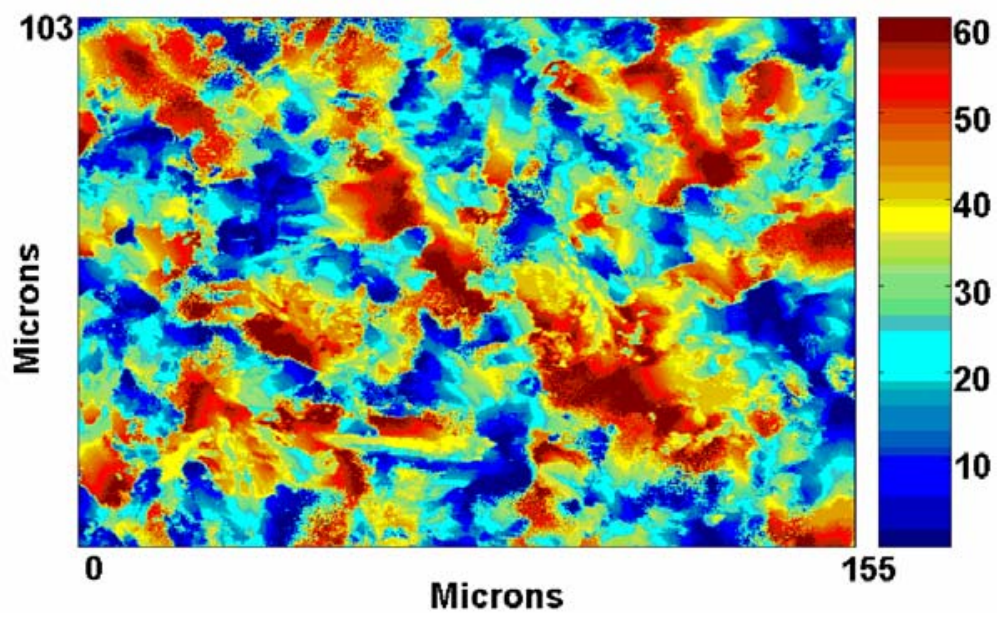

Figure 12: Surface topography map of 180 Coulombs per $\mathrm{cm}^{2}$ of copper deposited from $0.1 \mathrm{M} \mathrm{CuCl}, 4 \mathrm{M} \mathrm{NaCl,} \mathrm{0.01M} \mathrm{HCl} \mathrm{media} \mathrm{using} \mathrm{direct} \mathrm{current}\left(-25 \mathrm{~mA} / \mathrm{cm}^{2}\right.$ at $\left.22^{\circ} \mathrm{C}\right)$.

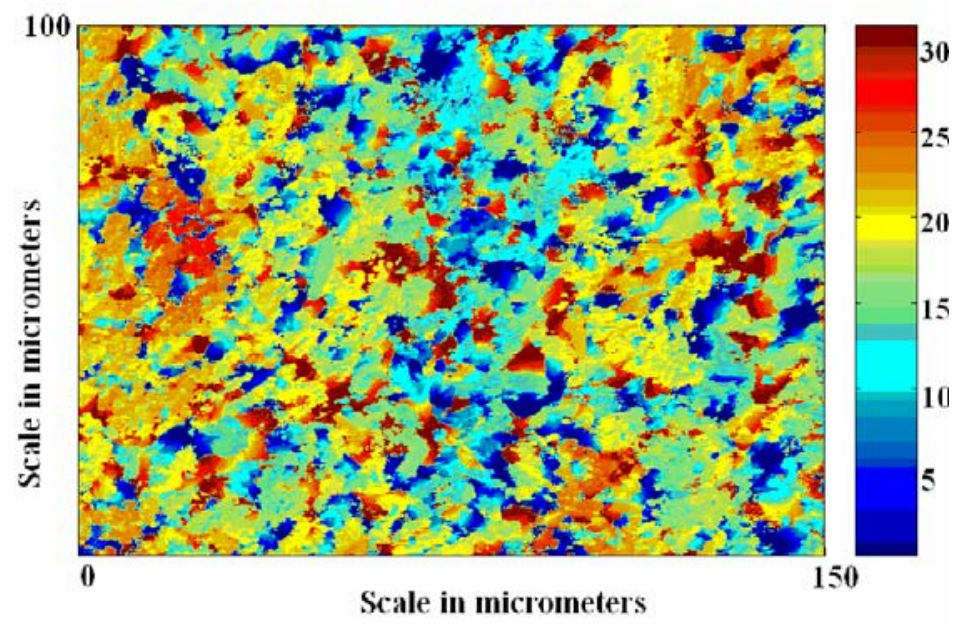

Figure 13: Surface topography map of 180 Coulombs per $\mathrm{cm}^{2}$ of copper deposited from $0.1 \mathrm{M} \mathrm{CuCl}, 4 \mathrm{M} \mathrm{NaCl,} \mathrm{0.01M} \mathrm{HCl} \mathrm{media} \mathrm{using} \mathrm{galvanostatic} \mathrm{pulsing} \mathrm{(10 \%} \mathrm{duty} \mathrm{cycle}$ at Pulse width $11 \mathrm{~ms},-25 \mathrm{~mA} / \mathrm{cm}^{2}, 22^{\circ} \mathrm{C}$ ).

As seen from figure 14 , the surface roughness of the electrodeposits increased as $\mathrm{i} / \mathrm{i}_{\mathrm{L}}$ ratio increased. This increase is found to be higher for longer pulse-on times as compared to shorter pulse-on times since the $i_{L}$ decreases with longer pulses. However, the surface roughness of the deposits obtained under pulsed current conditions was lower than those obtained under direct current conditions for higher values of $i / i_{L}$ as seen from figure 15 . It is clear that pulsing can decrease cathode surface roughness and help to facilitate the recovery of copper from chalcopyrite in chloride media. 


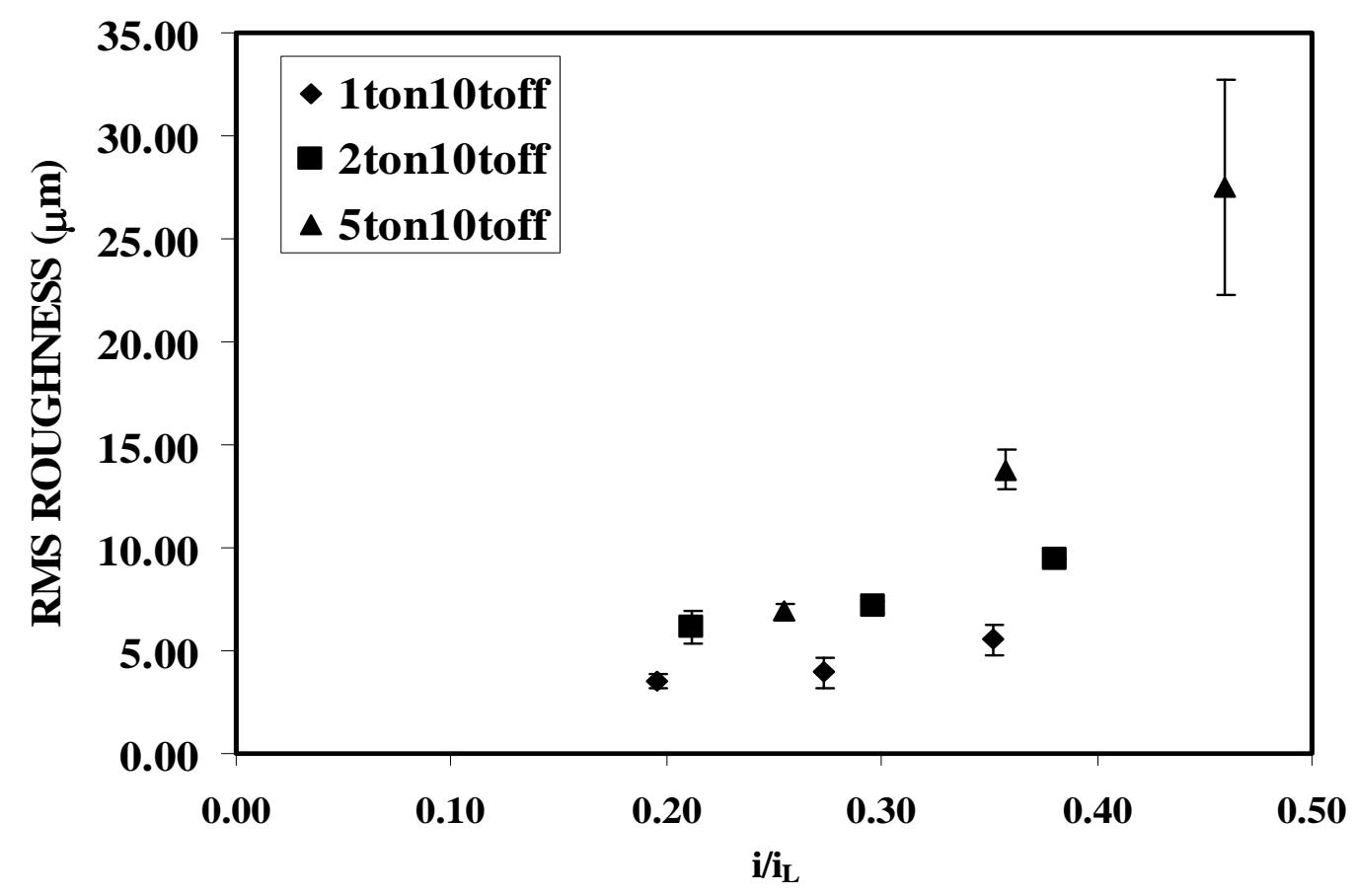

Figure 14: Effect of $\mathrm{i} / \mathrm{iL}$ on rms surface roughness of copper electrodeposits obtained from a bath containing $0.1 \mathrm{~mol} / \mathrm{L} \mathrm{CuCl}, 4.0 \mathrm{~mol} / \mathrm{L} \mathrm{NaCl}$ and $0.01 \mathrm{~mol} / \mathrm{L} \mathrm{HCl}$ under pulsed current conditions for 120 min at a rotational speed of 100 RPM for copper working electrode.

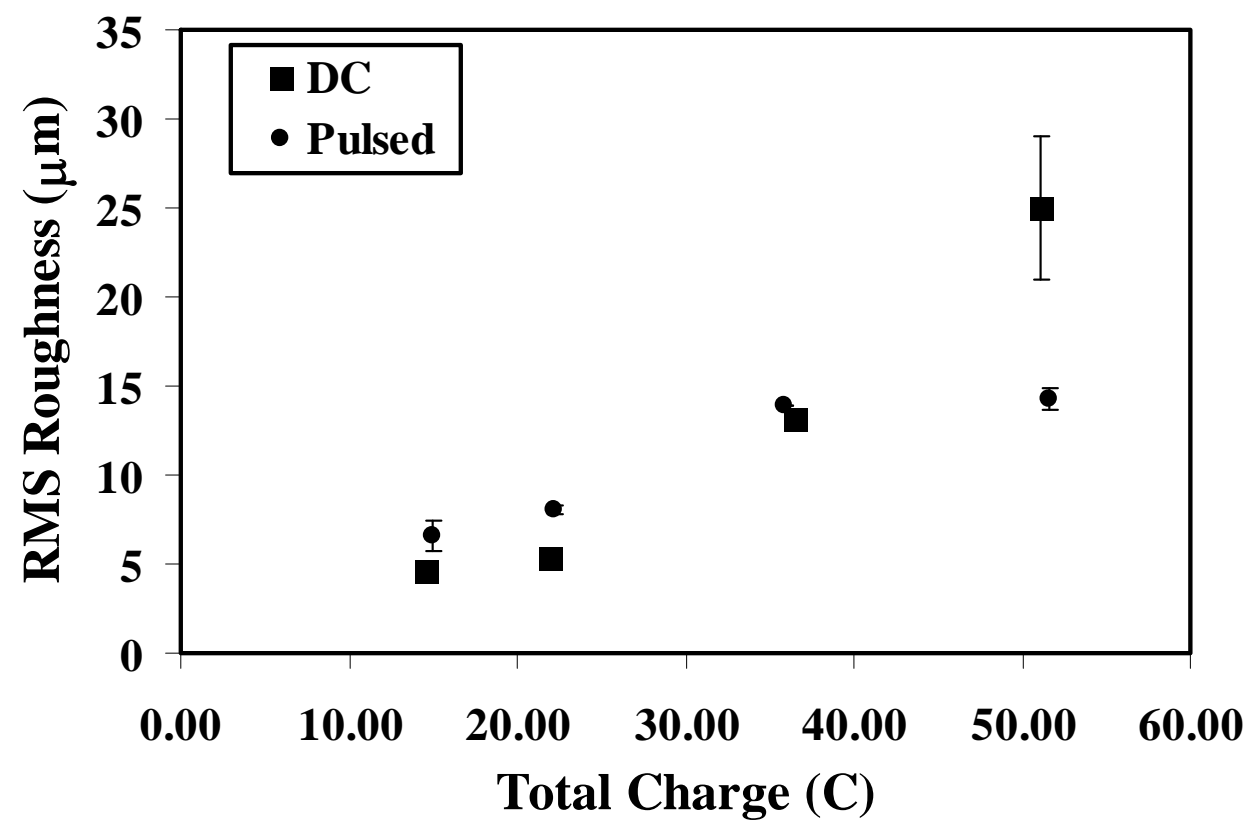

Figure 15: Effect of $i / i_{L}$ on root mean square surface roughness of copper electrodeposits obtained from a bath containing $0.1 \mathrm{~mol} / \mathrm{L} \mathrm{CuCl}, 4.0 \mathrm{~mol} / \mathrm{L} \mathrm{NaCl}$ and $0.01 \mathrm{~mol} / \mathrm{L} \mathrm{HCl}$ under pulsed current conditions with pulse-on time of $1 \mathrm{~ms}$ and pulseoff time of $10 \mathrm{~ms}$. 


\section{Theoretical Analysis}

The rate of electrodeposition reaction, characterized by the current density, depends on the electrode surface, composition of the electrolyte adjacent to the electrode. For electrodeposition, mass transfer in an electrolytic solution includes contributions due to movement of mobile ionic species, material balance, fluid flow and current flow. If the effect of double layer on capacitance is ignored then flux density of dissolved species $\mathrm{x}$ is given by

$$
N_{x}=-z_{x} u_{x} F c_{x} \nabla \Phi-D_{x} \nabla c_{x}+c_{x} V
$$

where $N_{x}$ is the flux density in mol per unit area of species $x, V$ is the bulk fluid velocity, $z_{x}$ is the charge of the ionic species $\mathrm{x}, \Phi$ is the electrostatic potential, $\mathrm{u}_{\mathrm{x}}$ is the mobility of the ionic species $\mathrm{x}, \mathrm{F}$ is faraday constant, $\mathrm{c}_{\mathrm{x}}$ is the concentration of ionic species $\mathrm{x}$ and $\mathrm{D}_{\mathrm{x}}$ is the diffusivity of the species $\mathrm{x}$. The three terms on the right side of above equation represent three mechanisms of mass transfer from left to right: migration of charged species in an electric field, molecular diffusion due to the concentration gradient and convection due to bulk motion of the aqueous medium. The material balance for the each species will give

$$
\frac{\partial c_{x}}{\partial t}=-\nabla \bullet N_{x}+R^{\prime}
$$

where $\mathrm{R}^{\prime}$ is the change in concentration due to the some reaction occurring in the bulk solution. When flux equation is introduced in the material balance, the result is

$$
\frac{\partial c_{x}}{\partial t}+V \bullet \nabla c_{x}=z_{x} F \nabla \bullet\left(u_{x} c_{x} \nabla \Phi\right)+\nabla \bullet\left(D_{x} \nabla c_{x}\right)+R^{\prime}
$$

If a supporting or indifferent electrolyte is dominant and no reaction occurs in the bulk medium, then mass transfer of minor species will be primarily due to convection and diffusion. The effect of migration will be small compared to diffusion for the depositing species in an electrolyte with a high ratio of supporting ions to depositing ions. Hence, for such a system with a constant diffusion coefficient, equation [3] can become

$$
\frac{\partial c_{x}}{\partial t}+V \bullet \nabla c_{x}=D_{x} \nabla^{2} c_{x}
$$

This second-order partial differential equation can be solved for concentration since the current in the electrodeposition process is known and is given by the Butler-Volmer equation. This equation describes the current density $i$ on a metal substrate as function of overpotential $\eta$ as:

$$
i=i_{0}\left[\exp \left(\frac{\phi z F \eta}{R T}\right)-\exp \left(\frac{-(1-\phi) z F \eta}{R T}\right)\right]
$$

where $i_{0}$ is the equilibrium exchange current density, $\phi$ is symmetry factor, $\mathrm{z}$ is the number of electrons transferred in the rate limiting step, F is Faraday constant, R is gas constant and T is 
absolute temperature. This equation is based on two main assumptions. First, the rate determining step is the charge transfer reaction. Second, the surface is homogenous so that current density is uniformly distributed over the entire solid surface.

For a given applied current, equation [4] reduces to

$$
\frac{\partial c}{\partial t}=D \nabla^{2} c-V \bullet \nabla c
$$

One way to solve this partial differential equation is to approximate the derivatives by finite differences. Using an explicit finite difference approach, a forward difference at time $t_{n}$ and a second order central difference for $1 \mathrm{D}$ space derivative at position $\mathrm{x}_{\mathrm{j}}$, the following equation results:

$$
\frac{c_{j}^{n+1}-c_{j}^{n}}{\Delta t}=D \frac{c_{j+1}^{n}-2 c_{j}^{n}+c_{j-1}^{n}}{(\Delta x)^{2}}-V \frac{c_{j+1}^{n}-c_{j}^{n}}{\Delta x}
$$

Thus knowing the values at time $n$, corresponding values at time $(n+1)$ can be determined.

The analytical solution of equation [6] in the absence of convection is

$$
c=c_{b}\left[1-\operatorname{erfc}\left(\frac{x}{2 \sqrt{D t}}\right)\right]
$$

for the following the boundary conditions ${ }^{8}$

$$
\begin{gathered}
c=c_{b} \text { at } x, y, z \geq 0, t=0 \\
c=c_{b} \text { at } x, y, z \rightarrow \infty, t>0 \\
c=0 \text { at } x, y, z=0, t>0
\end{gathered}
$$

where $c_{b}$ represents the bulk concentration in $\mathrm{mol} / \mathrm{cm}^{3}$. Figure 4 shows the comparison of this analytical solution with the solution obtained by the finite difference approach used in this research. 


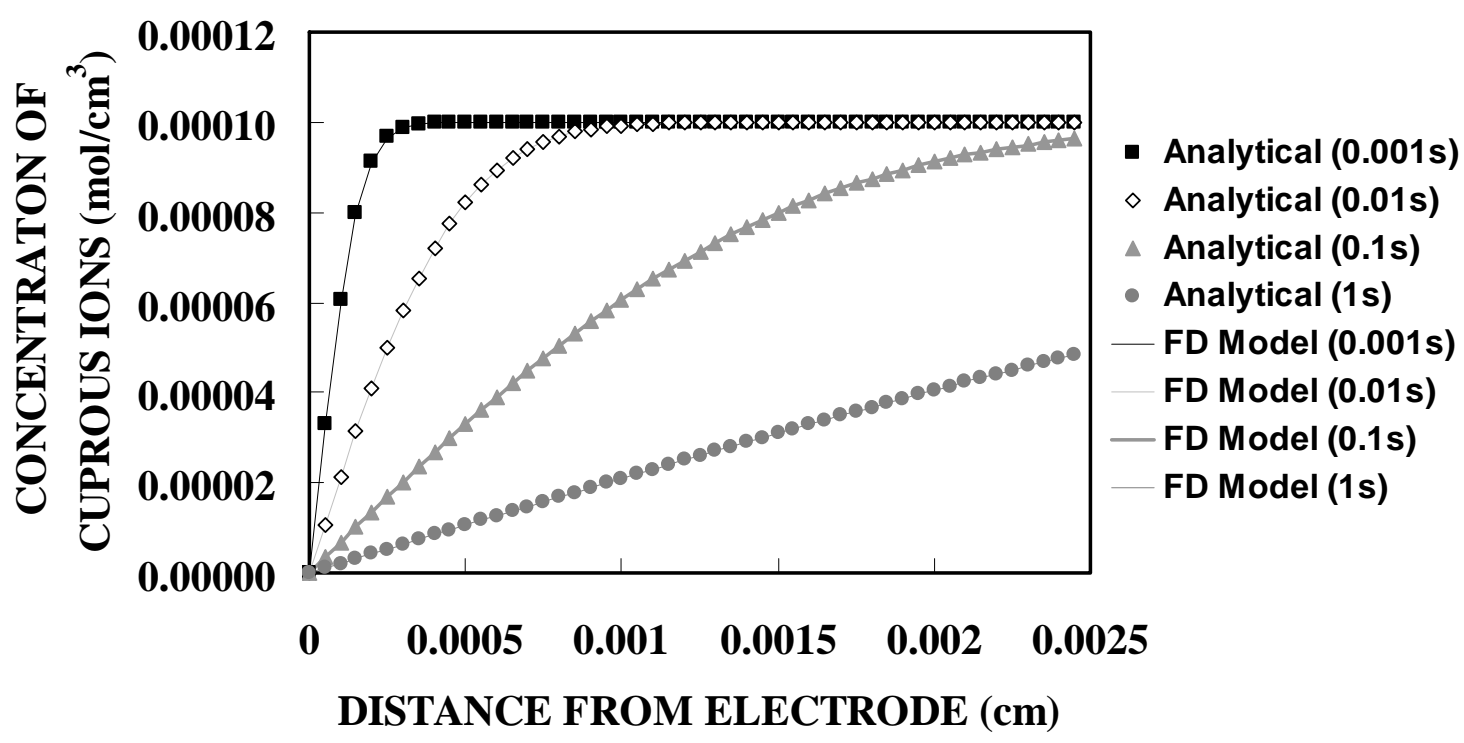

Figure 16: Comparison of solution obtained by analytical and finite difference methods for concentration profile at different times after start of the electrodeposition process.

During the actual electrodeposition process, the applied current is known but the concentration of ionic species at the surface is unknown. This is very important when the applied current is well below the mass limiting current. Hence the boundary conditions for solving equation [7] for an electrodeposition process carried out below the mass limiting current, in a solution with a large ratio of indifferent electrolyte to reacting ions, are

$$
\begin{aligned}
c & =c_{b} \text { at } x, y, z \geq 0, t=0 \\
c & =c_{b} \text { at } x, y, z \Rightarrow \infty, t \geq 0 \\
D \nabla c & =\frac{i}{n F} \text { at } x, y, z=0, t \geq 0
\end{aligned}
$$

Here, $\mathrm{n}$ is the charge number and $\mathrm{F}$ is the Faraday constant. $i$ is the applied current density. The solution of equation [6] using above boundary conditions will give the concentration of depositing ions at the surface which can be used to study the growth of electrodeposits on the surface. Figure 17 shows the variation of surface concentration with time under direct and pulsating current transients. As seen from figure, the application of pulsed potentials results in shorter deposition times followed by replenishing phase of the pulse. The act of replenishing the ions at the interface increases the average concentration of depositing ions at the interface as compared to that under direct current conditions 


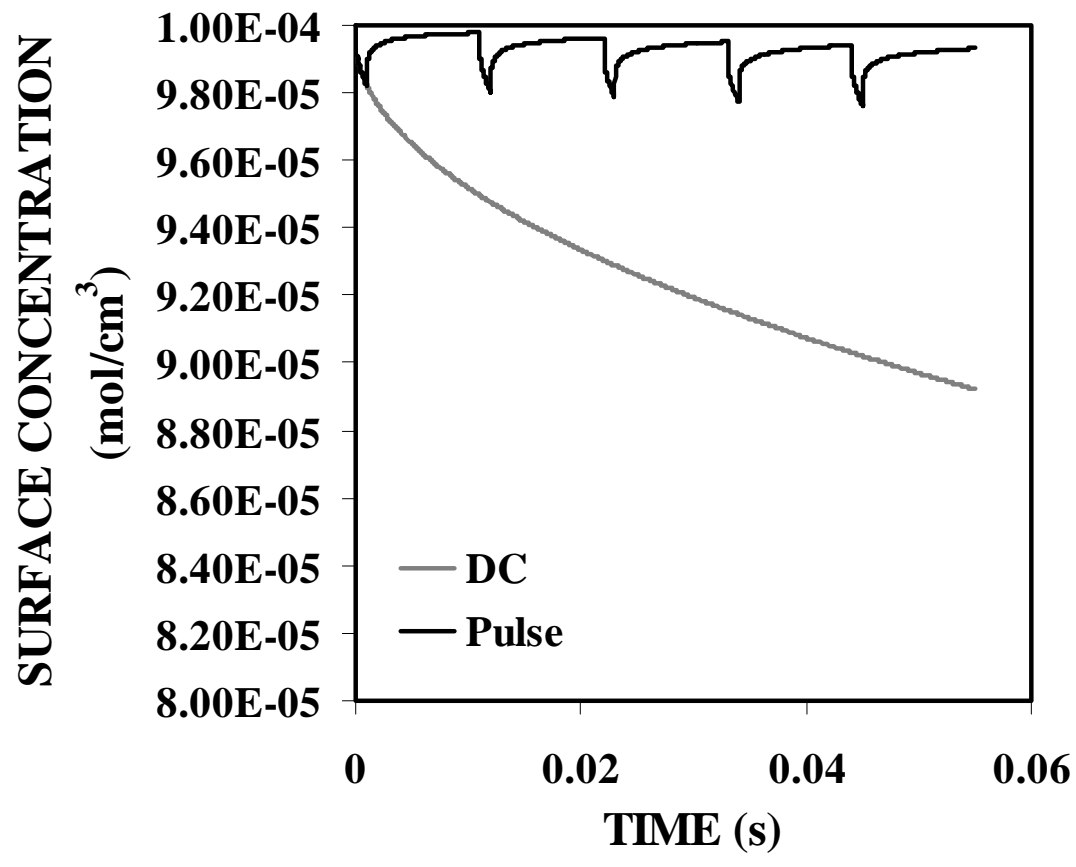

Figure 17: Finite difference modeling results for surface concentration of reacting ions obtained from a bath containing $0.1 \mathrm{~mol} / \mathrm{L} \mathrm{CuCl}, 4.0 \mathrm{~mol} / \mathrm{L} \mathrm{NaCl}$ and $0.01 \mathrm{~mol} / \mathrm{L} \mathrm{HCl}$ on a stationary electrode under pulsed current conditions with pulse-on time of 1 ms and pulse-off time of $10 \mathrm{~ms}$ with peak cathodic current density of $10 \mathrm{~mA} / \mathrm{cm}^{2}$ for pulsing and a constant cathodic current density of $10 \mathrm{~mA} / \mathrm{cm}^{2}$ for direct current transients.

Figure 18 shows the variation of surface concentration with different pulse-on times. As seen from figure 18, with longer pulse-on times, higher depletion of reacting ions occurs at the surface. Hence, as the pulse-on times increases, the average concentration at the surface decreases for a constant pulse-off time.

Figure 19 shows the variation of surface concentration with different pulse-on times. 


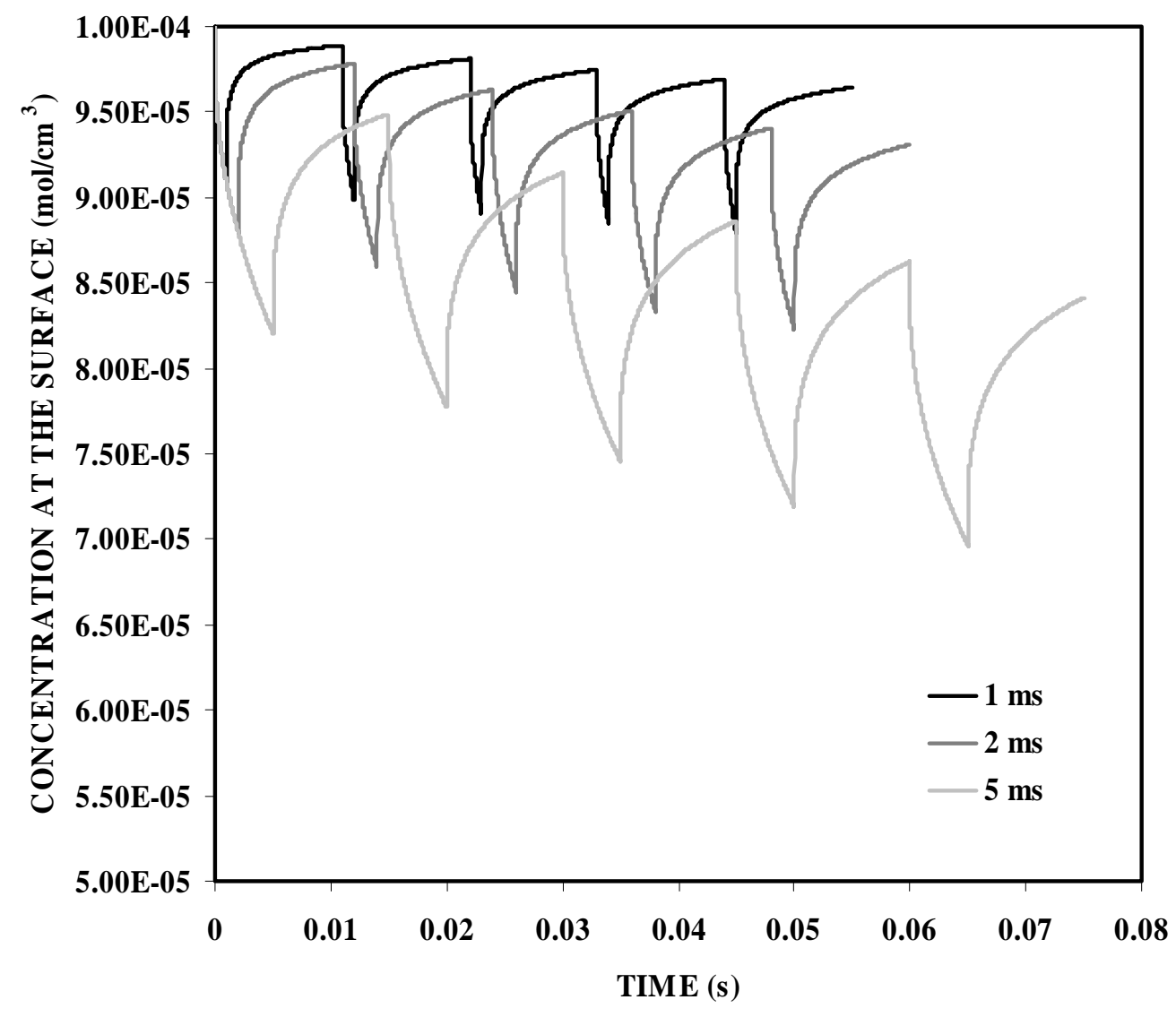

Figure 18: Finite difference modeling results for surface concentration of reacting ions obtained from a bath containing $0.1 \mathrm{~mol} / \mathrm{L} \mathrm{CuCl}, 4.0 \mathrm{~mol} / \mathrm{L} \mathrm{NaCl}$ and $0.01 \mathrm{~mol} / \mathrm{L} \mathrm{HCl}$ on a disc electrode rotating at $100 \mathrm{rpm}$ under pulsed current conditions with pulse-off time of $10 \mathrm{~ms}$ with peak cathodic current density of $50 \mathrm{~mA} / \mathrm{cm}^{2}$.

As seen from figure 19, with longer pulse-off times, higher replenishment of reacting ions occurs at the surface. Hence, as the pulse-off times increases, the average concentration at the surface increases for a constant pulse-on time. Thus, by knowing the concentration of depositing ions at the electrode surface, the electrodeposit growth with time can be modeled using these values. 


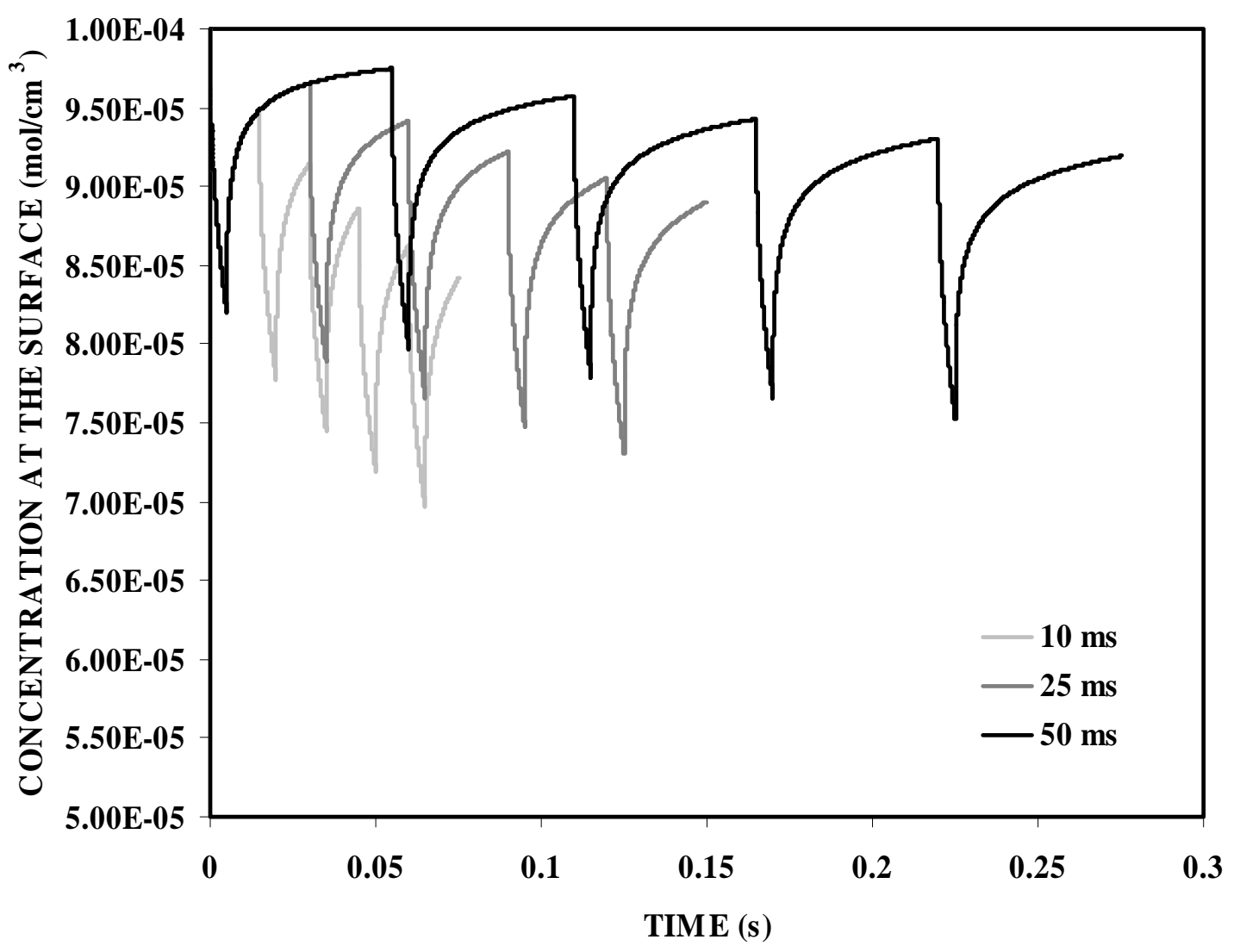

Figure 19: Finite difference modeling results for surface concentration of reacting ions obtained from a bath containing $0.1 \mathrm{~mol} / \mathrm{L} \mathrm{CuCl}, 4.0 \mathrm{~mol} / \mathrm{L} \mathrm{NaCl}$ and $0.01 \mathrm{~mol} / \mathrm{L} \mathrm{HCl}$ on a disc electrode rotating at $100 \mathrm{rpm}$ under pulsed current conditions with pulse-on time of $1 \mathrm{~ms}$ with peak cathodic current density of $50 \mathrm{~mA} / \mathrm{cm}^{2}$.

\section{Effect Of Inorganic Additives}

As seen from figure 20, it was observed that an addition of 100ppm zinc to the solution resulted in the highest improvement in smoothness of copper electrodeposits. This might be due to the change in the electrochemical properties of the system due to the addition of zinc ions. As is evident from figure 21, roughness of copper electrodeposits increases with time before attaining a constant value. 


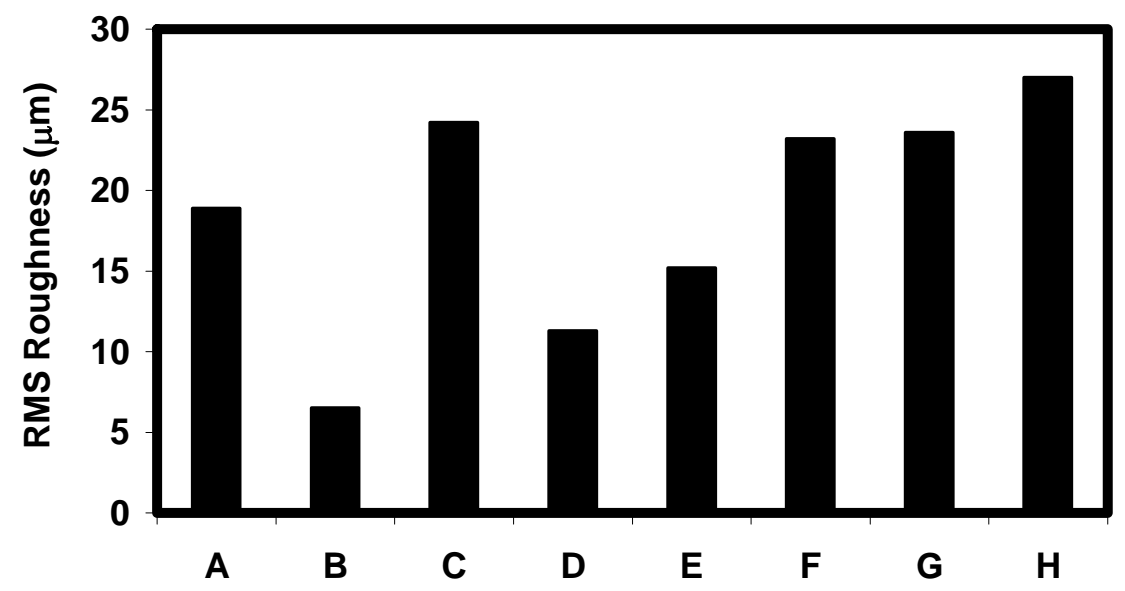

Figure 20: Effect of different cationic additives $\left(A=\right.$ no additive, $B=100 \mathrm{ppm} \mathrm{Zn}^{+2}$, $\mathrm{C}=100 \mathrm{ppm} \mathrm{Li}{ }^{+2}, \mathrm{D}=100 \mathrm{ppm} \mathrm{Mg}^{+2}, \mathrm{E}=100 \mathrm{ppm} \mathrm{Ni}{ }^{+2}, \mathrm{~F}=100 \mathrm{ppm} \mathrm{Ca}{ }^{+2}, \mathrm{G}=100 \mathrm{ppm}$ $\mathrm{Sn}^{+2}$, and $\mathrm{H}=100 \mathrm{ppm} \mathrm{Al}^{+3}$ ) on surface roughness of copper electrodeposits obtained from an electrolyte containing $0.1 \mathrm{~mol} / \mathrm{L} \mathrm{CuCl}, 4 \mathrm{~mol} / \mathrm{L} \mathrm{NaCl}$ and $0.01 \mathrm{~mol} / \mathrm{L} \mathrm{HCl}$ at a cathodic current density of $25 \mathrm{~mA} / \mathrm{cm}^{2}$ for 2 hours at $1000 \mathrm{rpm}$.

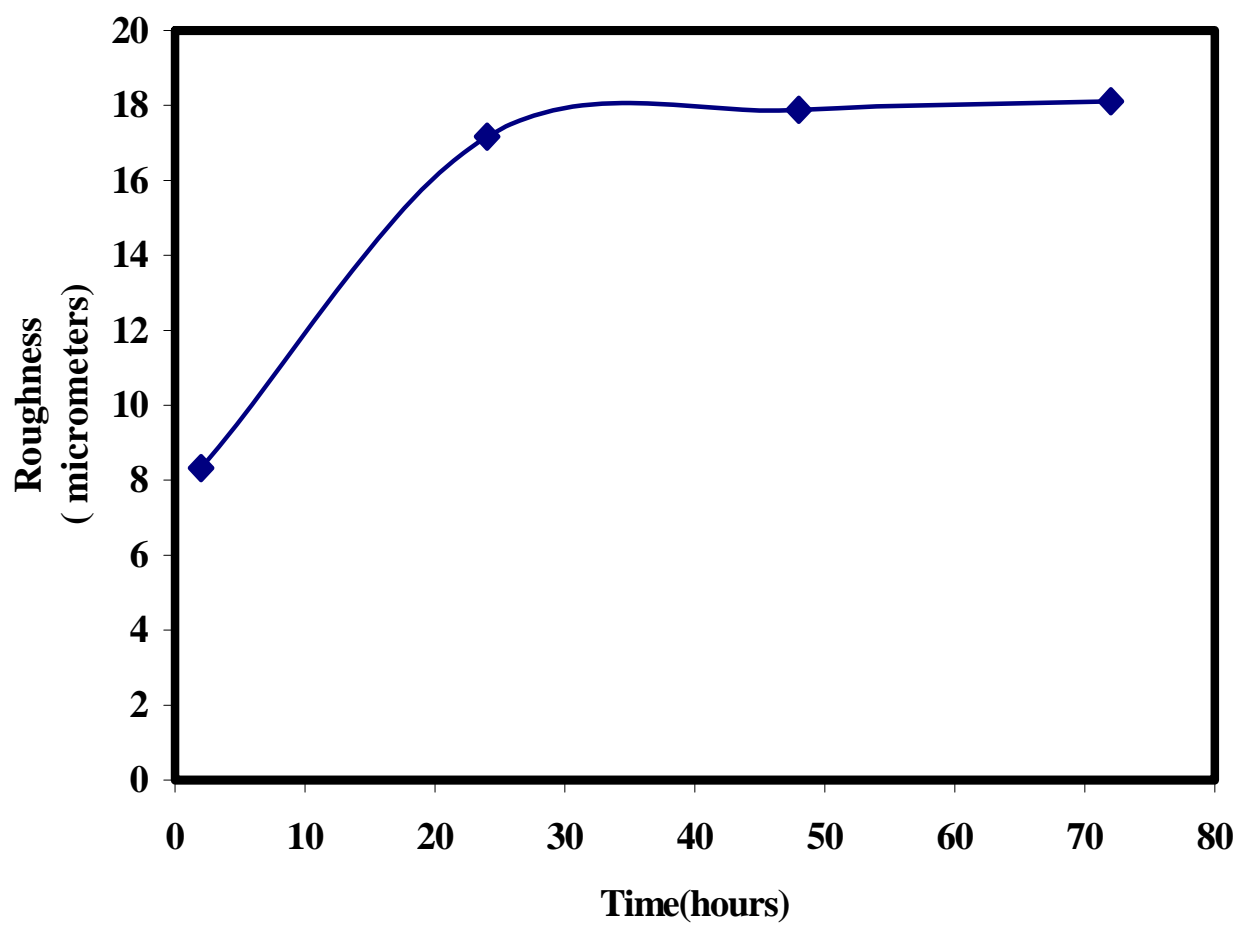

Figure 21: Effect of time on surface roughness of copper electrodeposits obtained from an electrolyte containing $0.1 \mathrm{M} \mathrm{CuCl}, 4 \mathrm{M} \mathrm{NaCl}$ and $0.01 \mathrm{M} \mathrm{HCl}$ (base solution) at a cathodic current density of $25 \mathrm{~mA} / \mathrm{cm}^{2}$ at $1000 \mathrm{rpm}$ at a temperature of $60^{\circ} \mathrm{C}$. 
Effect Of Organic Additives

Effect Of Different Surfactants On Surface Roughness

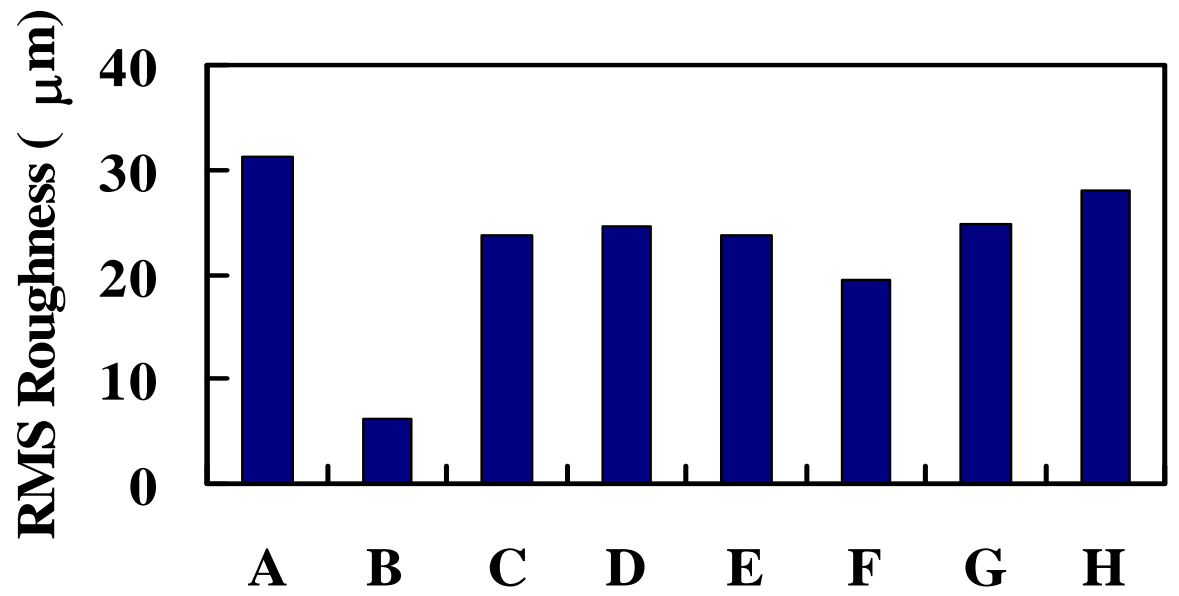

Figure 22: Effect of different surfactants $(A=$ no additive, $B=0.1 \%$ by vol gelatin, $C=$ $0.001 \mathrm{M}$ C10TAB, $\mathrm{D}=0.001 \mathrm{M}$ C16TAB, $\mathrm{E}=0.001 \mathrm{M}$ thiourea, $\mathrm{F}=0.05 \%$ by volume triton $x$ 100, $G=1 \%$ by volume PEG MW 200, and $H=1.2 \%$ by mass/volume PEG MW 10,000) on surface roughness of copper electrodeposits obtained from an electrolyte containing $0.1 \mathrm{~mol} / \mathrm{L} \mathrm{CuCl}, 4 \mathrm{~mol} / \mathrm{L} \mathrm{NaCl}$ and $0.01 \mathrm{~mol} / \mathrm{L} \mathrm{HCl}$ (base solution) at cathodic current density of $25 \mathrm{~mA} / \mathrm{cm}^{2}$ for 3 hours at $1000 \mathrm{rpm}$.

The effect of different surfactants such as C10TAB, C16TAB, thiourea, triton $\mathrm{x} 100$, PEG MW 200, PEG MW 10,000 and gelatin were analyzed on the surface roughness as shown in figure 22. As seen from this figure, gelatin gave smoother deposits relative to other additives. The root mean square roughness (RMS roughness) decreases significantly from approximately 32 microns to 6 microns with respect to control tests without additives. The color map contours for deposition tests without additives and with $0.1 \%$ by volume gelatin are shown in figure 23 (a) and (b).

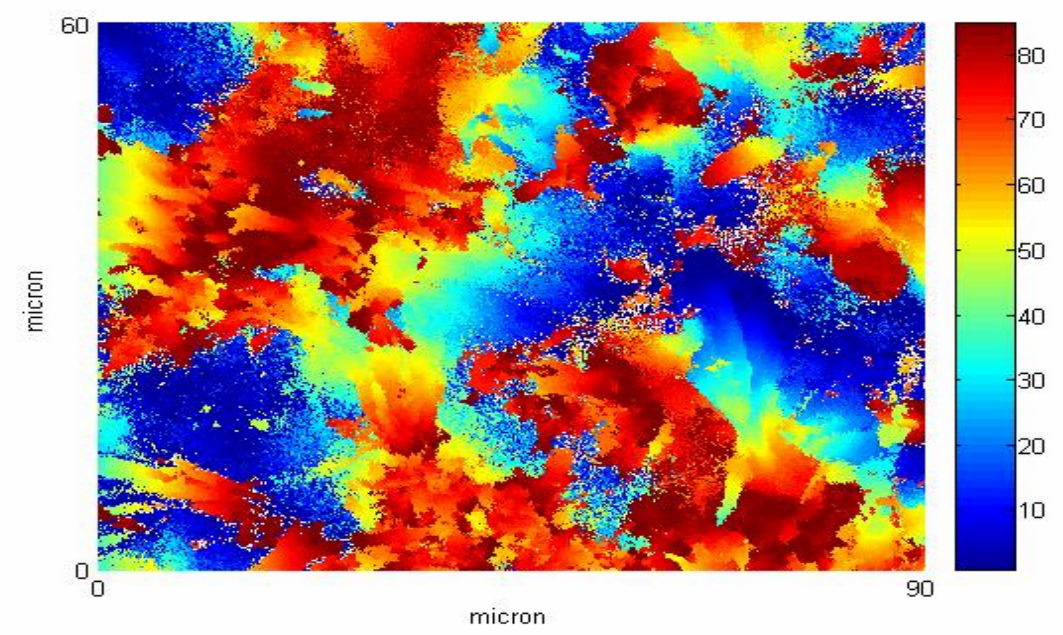

23 (a) 


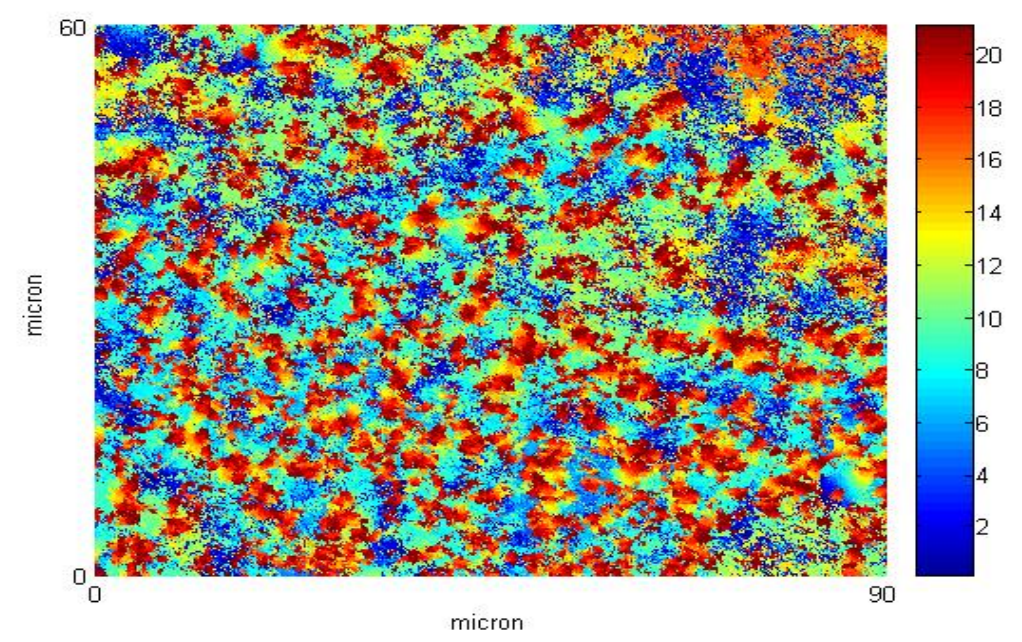

23 (b)

Figure 23: (a) Color map images of copper electrodeposits without surfactant (b). Color map images of copper electrodeposits with $0.1 \%$ by volume gelatin obtained from an electrolyte containing $0.1 \mathrm{~mol} / \mathrm{L} \mathrm{CuCl}, 4 \mathrm{~mol} / \mathrm{L} \mathrm{NaCl}$ and $0.01 \mathrm{~mol} / \mathrm{L} \mathrm{HCl}$ (base solution) at cathodic current density of $25 \mathrm{~mA} / \mathrm{cm}^{2}$ for 3 hours at $1000 \mathrm{rpm}$.

\section{Effect Of Gelatin Concentration}

The effect of gelatin concentration on the surface morphology was studied at $0.01,0.1$ and $1 \%$ by volume gelatin as given in figure 24 . The surface roughness value for each concentration of the gelatin remains the same.

\section{Effect Of Current Density In The Presence Of Organic Additives}

The effect of $\mathrm{i} / \mathrm{i}_{\mathrm{L}}$ on root mean squared surface roughness of copper electrodeposits was studied with the total charge supplied of $108 \mathrm{C} / \mathrm{cm}^{2}$ and $1,350 \mathrm{C} / \mathrm{cm}^{2}$ for each test as seen in figure 25 and figure 26, respectively. For the solution without gelatin, the surface roughness increased with increase in $\mathrm{i} / \mathrm{i}_{\mathrm{L}}$ for direct current conditions. However, the deposit morphology in solution containing gelatin was slightly rougher as the current density approaches the limiting value. 


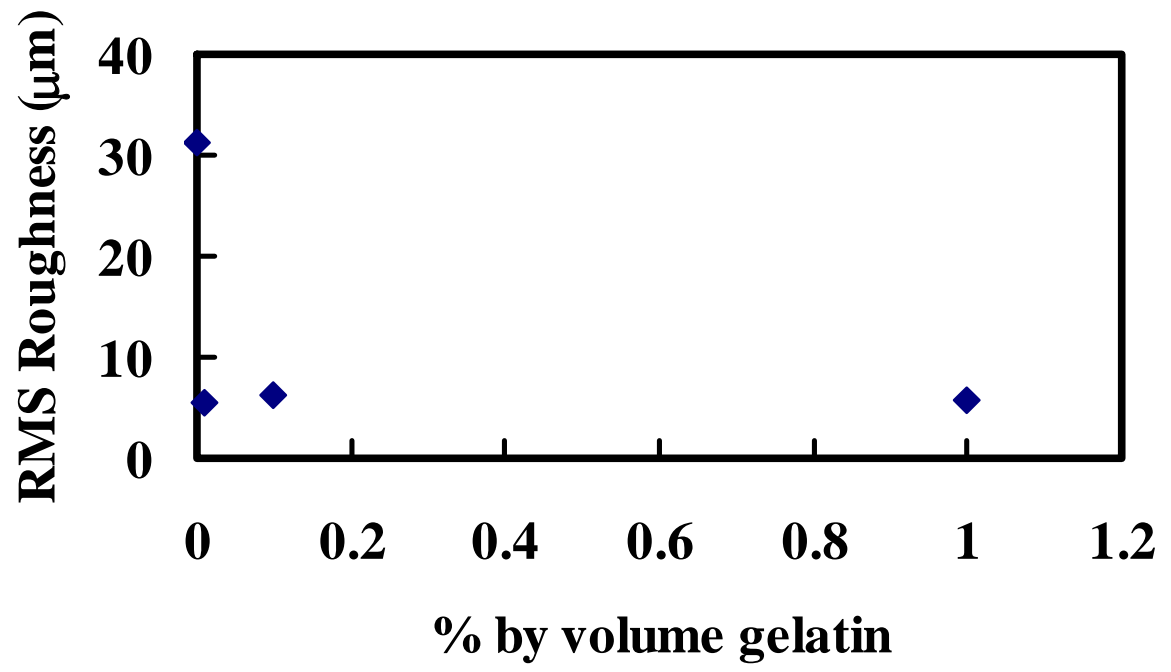

Figure 24: Effect of concentration of gelatin $(0,0.01,0.1$, and $1 \%$ by volume in base solution on surface roughness of copper electrodeposits for cathodic current density of $25 \mathrm{~mA} / \mathrm{cm}^{2}$ for 3 hours at $1000 \mathrm{rpm}$.

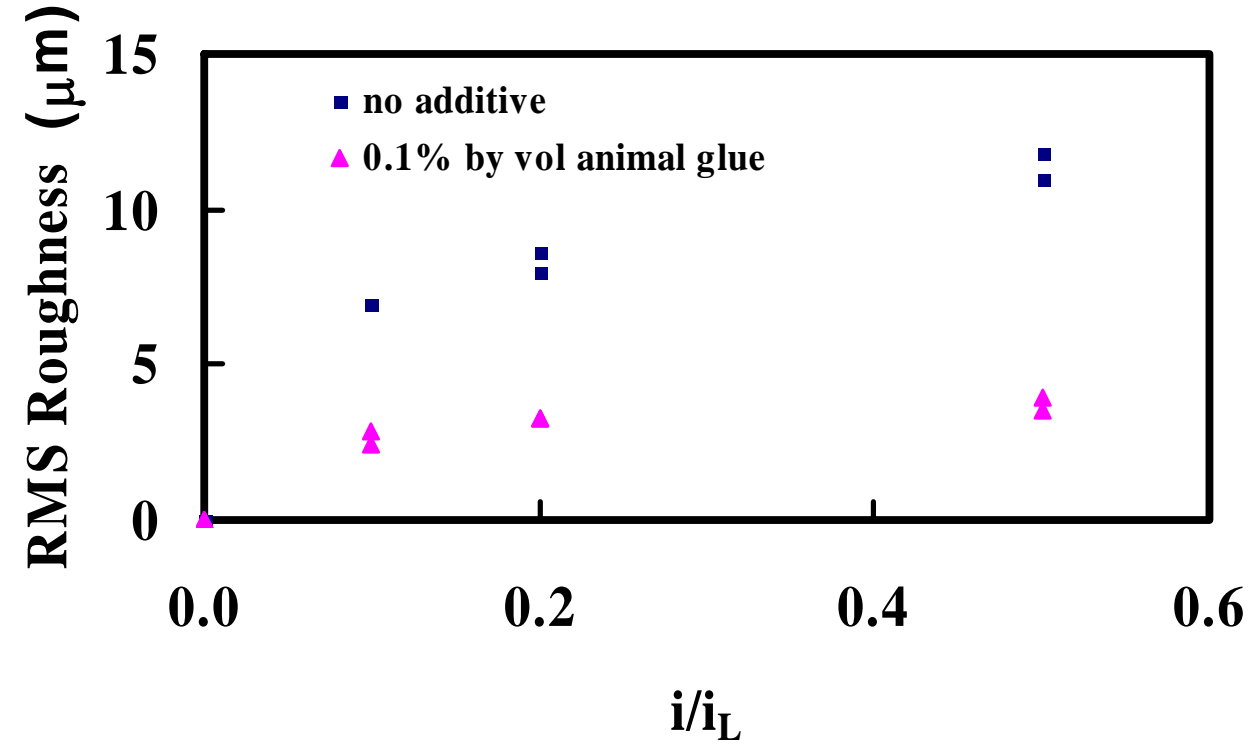

Figure 25: Effect of DC current density on surface roughness of copper electrodeposits obtained from a base solution with and without $0.1 \%$ by volume gelatin for total amount of charge of $108 \mathrm{C} / \mathrm{cm}^{2}$. 


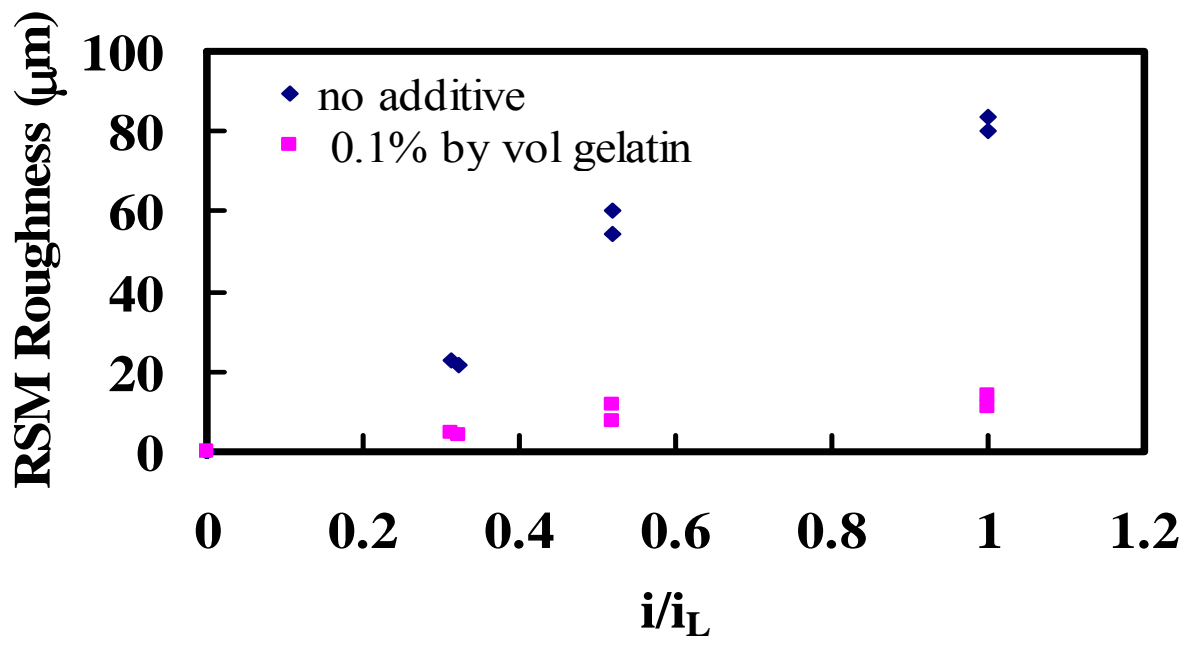

Figure 26: Effect of DC current density on surface roughness of copper electrodeposits obtained from a base solution with and without $0.1 \%$ by volume gelatin for total amount of charge of $1,350 \mathrm{C} / \mathrm{cm}^{2}$.

\section{Effect Of Electrodeposition Time}

The effect of electrodeposition time on the surface roughness in solution containing gelatin and without gelatin was studied. As seen in figure 27, gelatin improved the surface morphology and helped to achieve smooth deposits at long deposition time, unlike solution without gelatin.

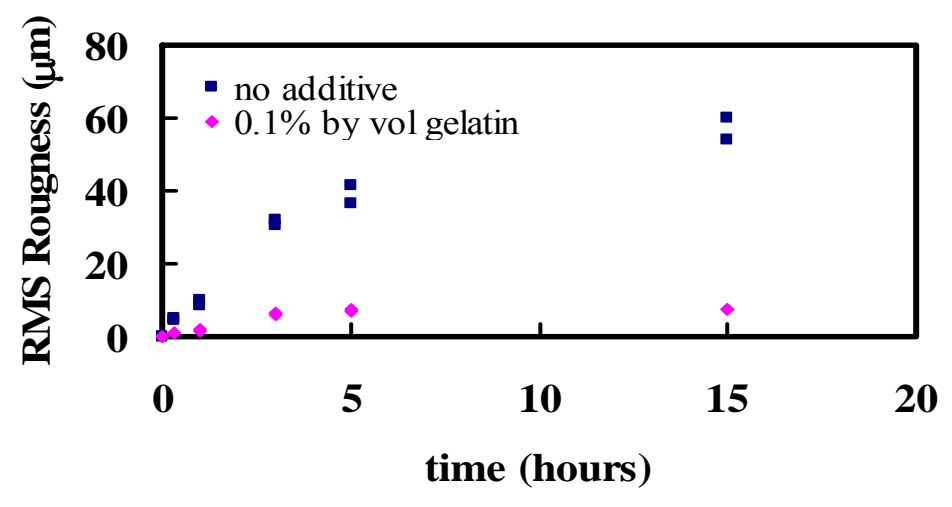

Figure 27: Effect of electrodeposition time on surface roughness of copper electrodeposits obtained from a base solution with and without $0.1 \%$ by volume gelatin for cathodic current density of $25 \mathrm{~mA} / \mathrm{cm}^{2}$ at $1000 \mathrm{rpm}$.

\section{Effect Of Combination Of Organic Additives On RMS Roughness}

The reduction of the surface irregularities in the plating processes can be accomplished by the addition of organic additives. The effects of combination of organic additives such as 
glycine, thiourea, PEG MW 200 on surface roughness were analyzed as shown in figure 28 . The combination of glycine, PEG, and thiourea reduces the surface roughness by a factor of two compared to baseline test with no organic additive.

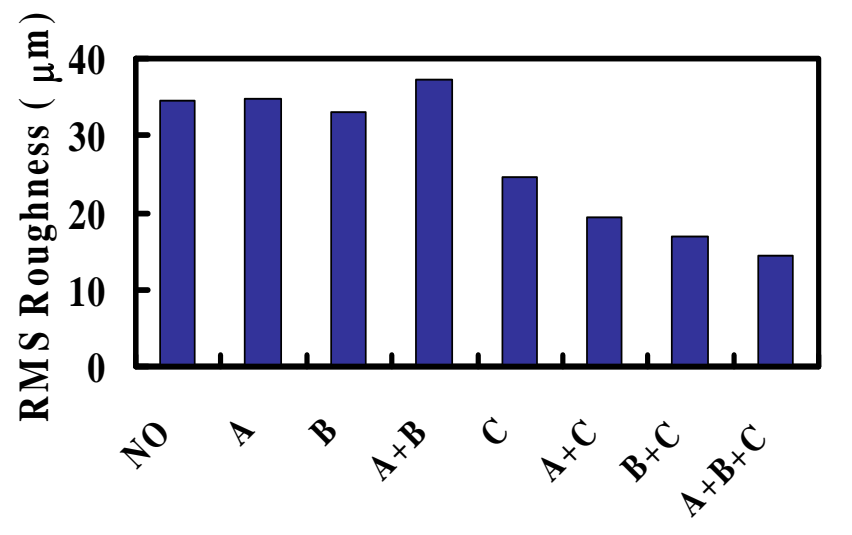

Figure 28: Effect of $2.5 \mathrm{ppm}$ glycine (A), $2.5 \mathrm{ppm}$ thiourea (B), $7500 \mathrm{ppm}$ PEG (MW 200) (C), and their combinations on the surface roughness of copper electrodeposits obtained from an electrolyte containing $0.1 \mathrm{~mol} / \mathrm{L} \mathrm{CuCl}, 4 \mathrm{~mol} / \mathrm{L} \mathrm{NaCl}$ and $0.01 \mathrm{~mol} / \mathrm{L} \mathrm{HCl} \mathrm{(base} \mathrm{solution)} \mathrm{at} \mathrm{cathodic} \mathrm{current} \mathrm{density} \mathrm{of} 25 \mathrm{~mA} / \mathrm{cm}^{2}$ for 3 hours at $1000 \mathrm{rpm}$.

\section{Steady-State Roughness Estimation And Evaluation}

If a steady-state roughness is achieved, the rate at which new nuclei are formed must be equivalent to the rate at which nuclei are destroyed due to the convergence or overlapping growth of adjacent nuclei.

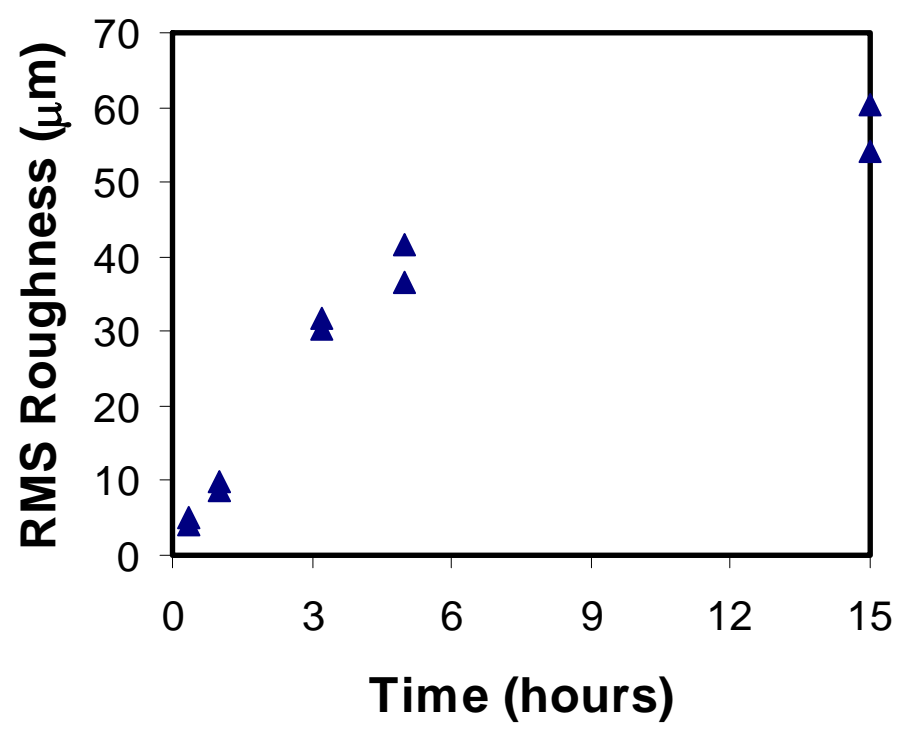

Figure 29: Comparison of RMS roughness values after copper electrodeposition from $0.1 \mathrm{M} \mathrm{CuCl}, 4 \mathrm{M} \mathrm{NaCl}, 0.01 \mathrm{M} \mathrm{HCl}$ solution (1000 RPM, $\left.25^{\circ} \mathrm{C},-25 \mathrm{~mA} / \mathrm{cm}^{2}\right)$. 
There are many factors that affect electrodeposit morphology as characterized by surface roughness. Some of the more influential factors are deposit thickness, current density, electrolyte composition, mass transport conditions, and temperature. In this study roughness of the surface is characterized by the Root Mean Squared (RMS) roughness which is defined mathematically as:

$$
\text { RMSroughness }=\sqrt{\frac{1}{l} \int_{0}^{l} y^{2}(x) d x}
$$

where $l$ is the length of the measured sequence, $y$ is the topographic height as a function of the x-position along the length of the surface. The steady-state nuclei formation rate can be mathematically described by the equation: ${ }^{9-10}$

$$
\frac{d N_{\text {formation }}}{d t}=N_{\infty} \exp \left(\frac{-\chi}{T \eta_{s}^{2}}\right)
$$

where $N_{\text {formation }}$ is the number density of new nuclei, $t$ is time, $N_{\infty}$ is the baseline rate of nuclei formation, $\chi$ is a constant ( $\chi$ and $N_{\infty}$ are functions of valence, geometry, surface energy, and frequency of attachment and detachment), $T$ is temperature, $\eta_{s}$ is the surface overpotential. The surface overpotential can be calculated using the measured overpotential and the individual components of the overpotential from the expression:

$$
\eta_{s}=E_{\text {applied }}-E_{\text {equil. }}-i R_{\text {solution }}-\frac{R T}{n F} \ln \left(1-\frac{i}{i_{L}}\right)
$$

in which $E_{\text {applied }}$ is the applied potential, $E_{\text {equil. }}$ is the equilibrium potential for the deposition half-cell reaction, $i$ is the current density of the deposition, $R_{\text {solution }}$ is the solution resistance, $R$ is the gas constant, $T$ is the absolute temperature, $n$ is the number of electrons transferred in the half-cell reaction, $F$ is the Faraday constant, and $i_{L}$ is the mass transport limiting current density. The associated area and mass balance based nuclei removal rate, which assumes an average radius of existing, growing nuclei, can be expressed as:

$$
\frac{d N_{\text {removal }}}{d t}=\frac{-2 k^{\prime}}{\pi r_{\text {ave }}} i
$$

where $k^{\prime}$ is a proportionality constant, $i$ is the current density, and $r_{\text {ave }}$ is the average radius of growing nuclei. Thus, the solution to the steady-state condition in which the rate of nuclei formation equals the rate of removal leads to an expression for the average radius of nuclei on a surface experiencing active growth and nucleation:

$$
r_{\text {ave }}=\frac{2 k i}{\pi N_{\infty}} \exp \left(\frac{-\chi}{T \eta_{s}^{2}}\right)
$$


If the nuclei are assumed to be hemispheres, the associated root-mean-squared roughness for adjacent nuclei of uniform radius (see Equation [7]) is equal to the average nucleus radius divided by the square root of three. Therefore, substitution for the average radius in terms of RMS roughness leads to:

$$
R M S_{\text {roughness }}=\frac{2 k i}{\sqrt{3} \pi N_{\infty}} \exp \left(\frac{-\chi}{T \eta_{s}^{2}}\right)
$$

Dividing by current density and taking the logarithm of both sides leads to:

$$
\ln \left(\frac{R M S_{\text {roughness }}}{i}\right)=\ln \left(\frac{2 k}{\sqrt{3} \pi N_{\infty}}\right)-\frac{\chi}{T \eta_{S}^{2}}
$$

Thus, the natural logarithm of the roughness divided by the current density should be proportional to the inverse of the square of the surface overpotential multiplied by the inverse of the temperature if the deposition achieves a steady-state roughness that can be modeling assuming an average nucleus radius. The data in Figures 30 to 32 show that the logarithm of the roughness divided by the current density is proportional to the inverse of the square of the surface overpotential multiplied by the inverse of the temperature with and without an organic additive.

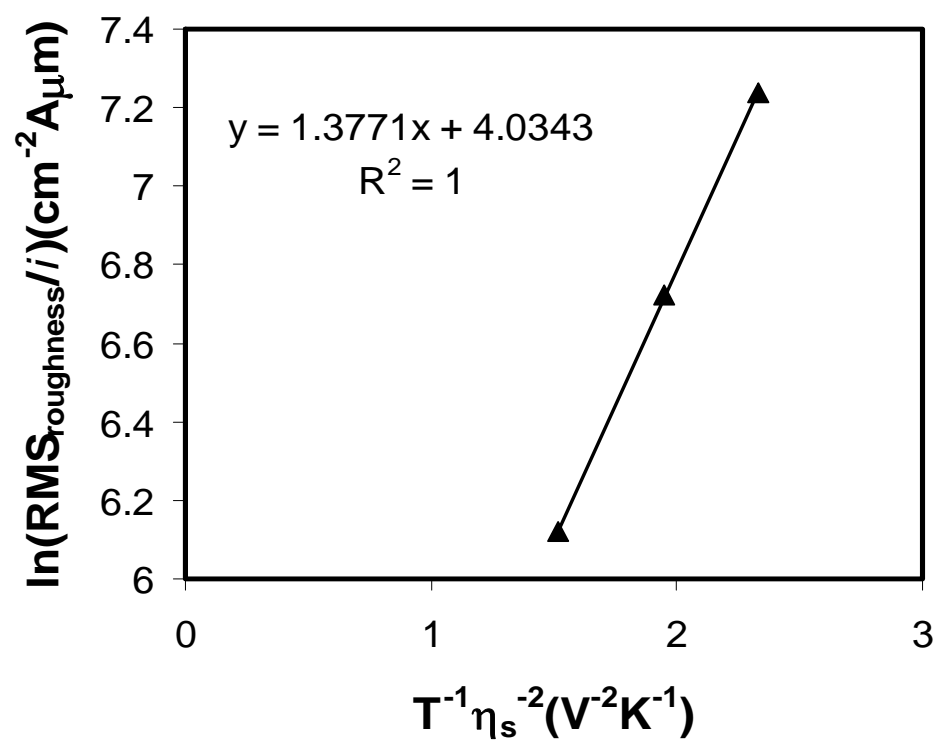

Figure 30: Comparison of the natural logarithm of the ratio of RMS roughness to current density versus the inverse temperature multiplied by the inverse surface overpotential squared. Data based on galvanostatic copper electrodeposition from 0.1 M CuCl, $4 \mathrm{M} \mathrm{NaCl,} \mathrm{0.01M} \mathrm{HCl} \mathrm{solution} \mathrm{(1000} \mathrm{RPM,} 25^{\circ} \mathrm{C}, 180$ Coulombs $/ \mathrm{cm}^{2}$, and -5, 10 , and $-25 \mathrm{~mA} / \mathrm{cm}^{2}$ ). 


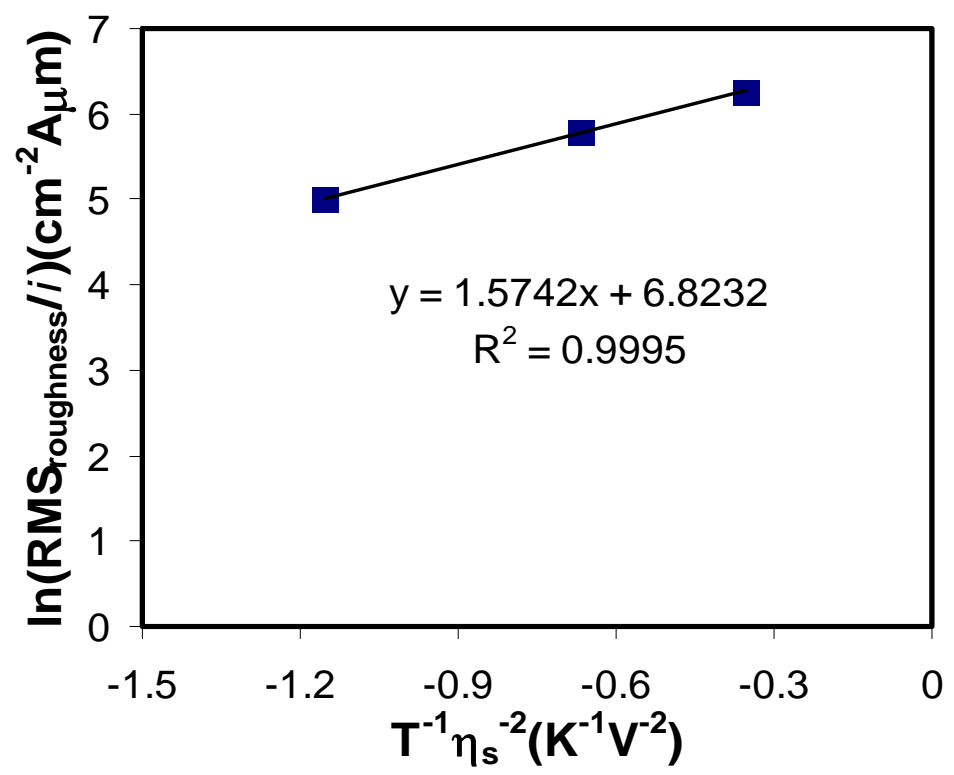

Figure 31: Comparison of the natural logarithm of the ratio of RMS roughness to current density versus the inverse temperature multiplied by the inverse surface overpotential squared. Data based on galvanostatic copper electrodeposition from 0.1 $\mathrm{M} \mathrm{CuCl,} 4 \mathrm{M} \mathrm{NaCl}, 0.01 \mathrm{M} \mathrm{HCl}$ solution containing 0.1 vol \% gelatin $\left(1000 \mathrm{RPM}, 25^{\circ} \mathrm{C}\right.$, 180 Coulombs $/ \mathrm{cm}^{2}$, and $-5,-10$, and $-25 \mathrm{~mA} / \mathrm{cm}^{2}$ ).

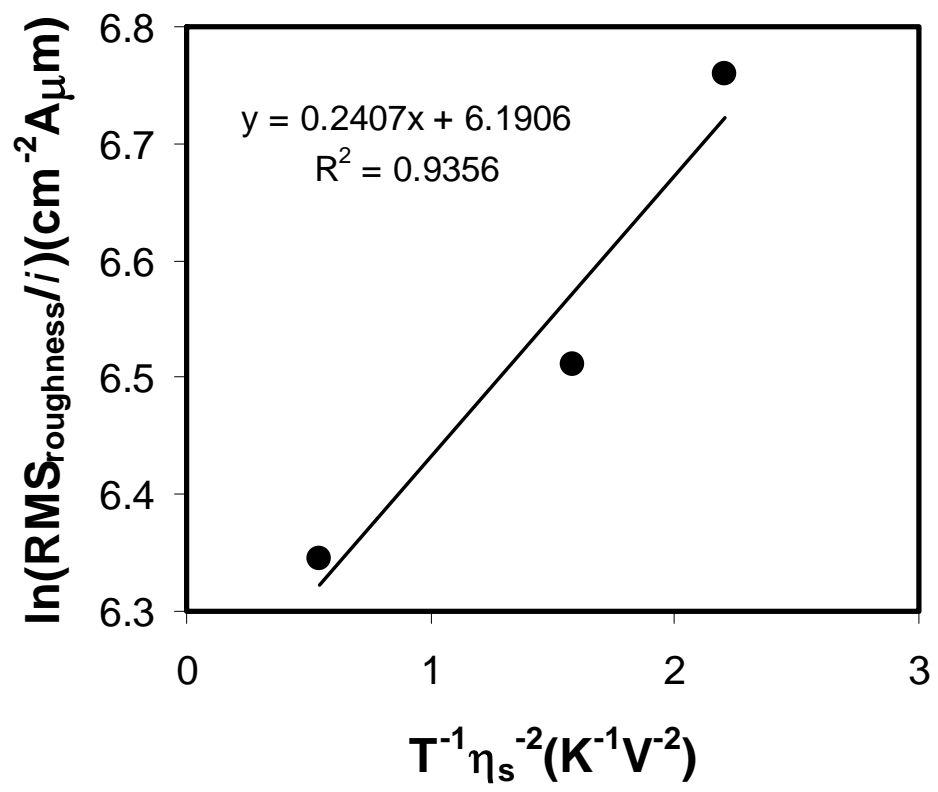

Figure 32: Comparison of the natural logarithm of the ratio of RMS roughness to current density versus the inverse temperature multiplied by the inverse surface overpotential squared. Data based on galvanostatic copper electrodeposition from 0.1 $\mathrm{M} \mathrm{CuCl}, 4 \mathrm{M} \mathrm{NaCl}, 0.01 \mathrm{M} \mathrm{HCl}$ solution (1000 RPM, 30, 45, and $60^{\circ} \mathrm{C}, 180$ Coulombs $/ \mathrm{cm}^{2},-25 \mathrm{~mA} / \mathrm{cm}^{2}$ ). 
Figure 32 shows data obtained at different temperatures $\left(30,45\right.$, and $\left.60^{\circ} \mathrm{C}\right)$ plotted in the same format. The data in Figures 30-32 show that this basic approach to estimating steady-state roughness has a reasonable range of applicability.

\section{Effect Of High Molecular Weight Organic Additives On Surface Roughness}

The reduction of the surface irregularities in the plating processes can be accomplished by the addition of organic additives. The effects of high molecular weight organic additives such as gelatin, PEO MW 300,000, and PAA MW 200,000 on surface roughness were analyzed as shown in figure 33. These high molecular weight organic additives significantly reduced the surface roughness compared to baseline test with no organic additive.

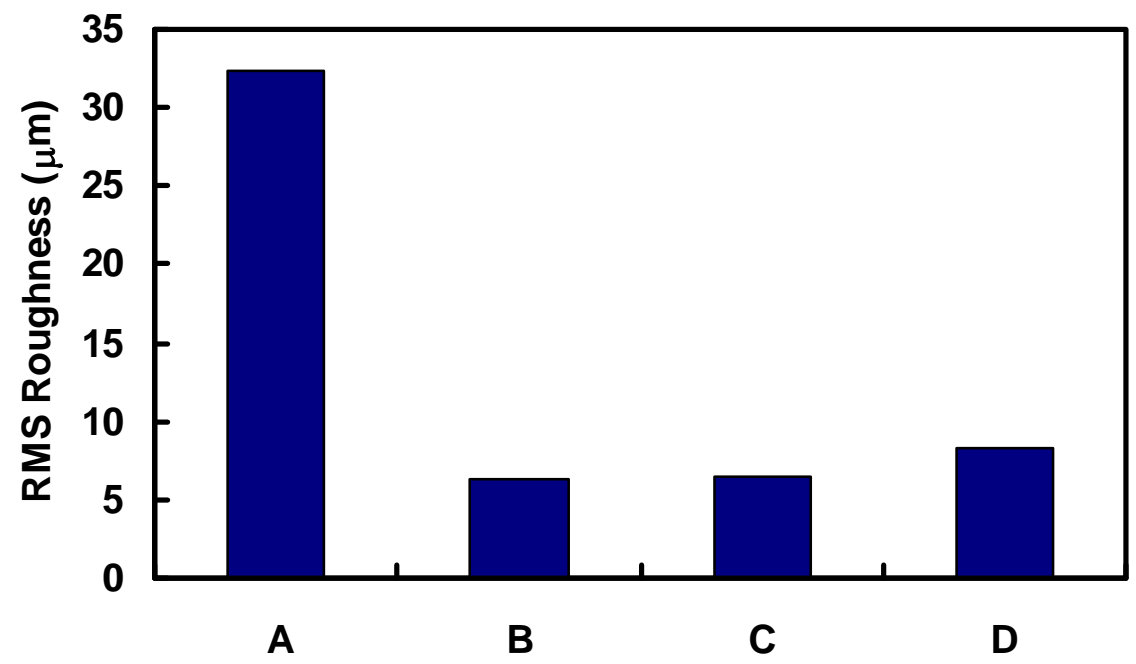

Figure 33: Effect of different surfactants $(A=$ no additive, $B=0.0127 \mathrm{~g} / \mathrm{L}$ gelatin, $\mathrm{C}=$ $0.01 \mathrm{~g} / \mathrm{L}$ PEO MW 300,000, and $\mathrm{D}=$ PAA $\mathrm{MW} 200,000)$ on surface roughness of copper electrodeposits obtained from an electrolyte containing $0.1 \mathrm{M} \mathrm{CuCl}, 4 \mathrm{M} \mathrm{NaCl}$ and $0.01 \mathrm{M} \mathrm{HCl}$ at cathodic current density of $25 \mathrm{~mA} / \mathrm{cm}^{2}$ for 3 hours at $1000 \mathrm{rpm}$.

\section{Effect Of Gelatin And Its Main Amino Acids (Glycine And Proline) On RMS Roughness}

The effect of gelatin and its main amino acid monomers (approximately $40 \%$ of glycine and $30 \%$ of praline found in gelatin in general) on surface morphology of copper electrodeposit was shown in figure 34. It was found that gelatin gave more uniform deposits compared to those obtained without any additives, yet its amino acid monomers (glycine and proline) did not significantly reduce the surface roughness as the gelatin did.

\section{Effect Of Different Molecular Weights Of PEG And PEO On RMS Roughness}

PEG and PEO have identical chemical structure except PEG has $\mathrm{H}$ atom attached to $\mathrm{O}$ at the end of the chain and PEG has shorter chain length ${ }^{11}$. The effect of different molecular weights of PEG and PEO on RMS roughness was shown in Figure 35. It found that Both 
PEOs (MW 100,000, and MW 300,000) significantly reduced the surface roughness compared to baseline test with no organic additive.

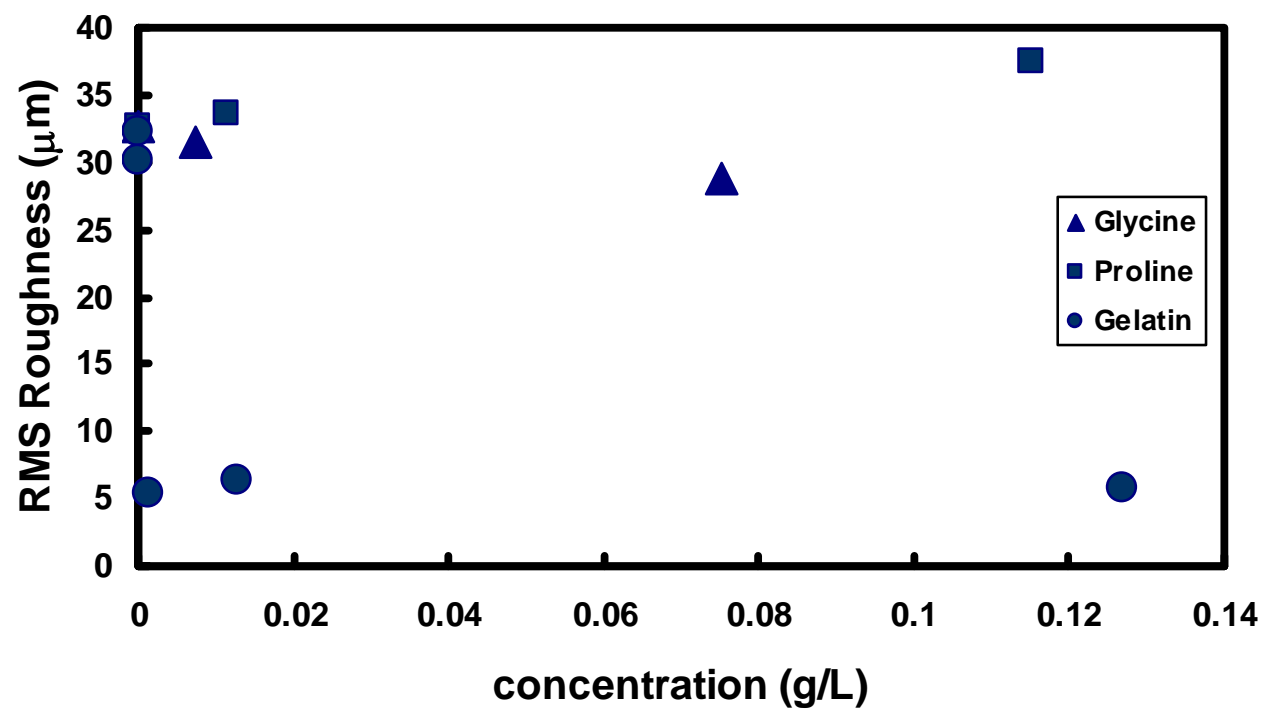

Figure 34: Effect of concentration of glycine, proline, and gelatin on surface roughness of copper electrodeposits obtained from an electrolyte containing $0.1 \mathrm{M} \mathrm{CuCl}, 4 \mathrm{M}$ $\mathrm{NaCl}$ and $0.01 \mathrm{M} \mathrm{HCl}$ at cathodic current density of $25 \mathrm{~mA} / \mathrm{cm}^{2}$ for 3 hours at 1000 rpm.

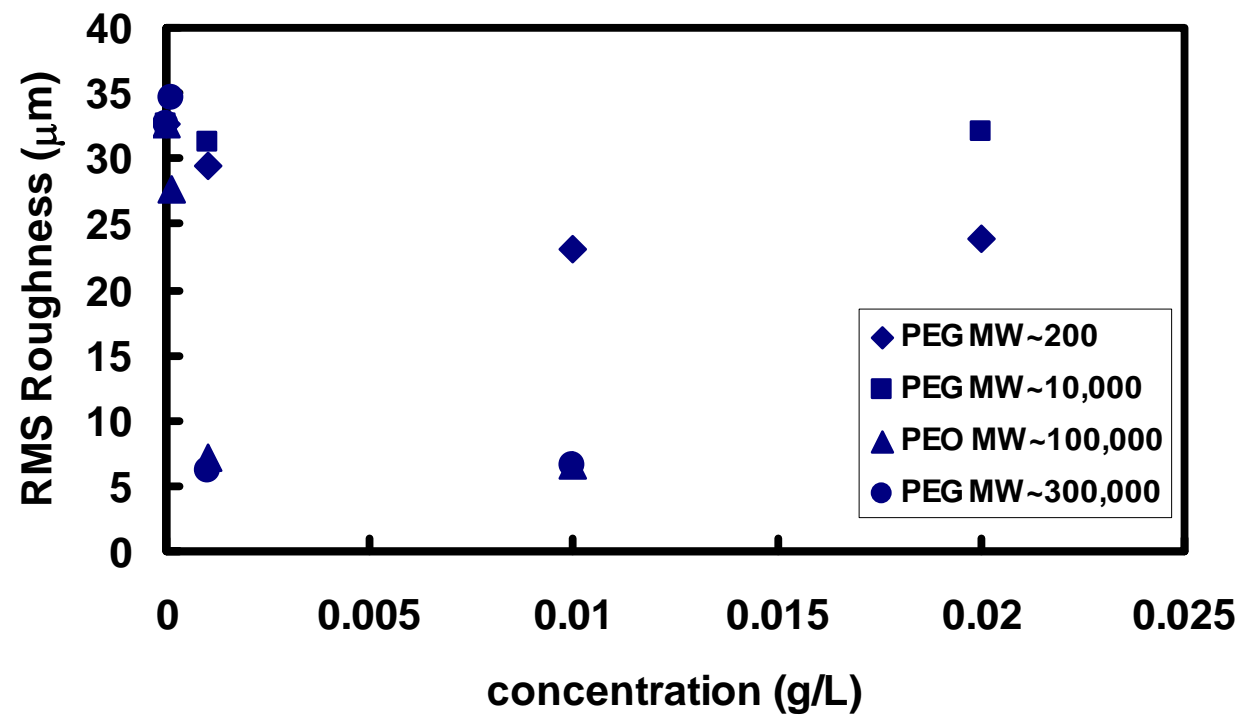

Figure 35: Effect of concentration of PEG MW 200, PEG MW 10,000, PEO MW 100,000, and PEO MW 300,000on surface roughness of copper electrodeposits obtained from an electrolyte containing $0.1 \mathrm{M} \mathrm{CuCl}, 4 \mathrm{M} \mathrm{NaCl}$ and $0.01 \mathrm{M} \mathrm{HCl}$ at cathodic current density of $25 \mathrm{~mA} / \mathrm{cm}^{2}$ for 3 hours at $1000 \mathrm{rpm}$. 
The diffusion coefficients can be used to find effective size using Stokes-Einstein Equation as shown below:

$$
D=\frac{R T}{6 \pi \mu R_{e f f}}
$$

where $D$ is diffusion coefficient, $R$ is gas constant, $T$ is temperature in Kelvin, $\mu$ is kinematic viscosity, and $\mathrm{R}_{\text {eff }}$ is effective radius.

According to Table 1, diffusion coefficients and approximate sizes for glucose, gelatin, and PAA were reported. The diffusivity of glucose was found in the literature. However, the diffusivities for both gelatin and PAA were determined by using deuterium light source ultraviolet spectroscopy. The value of the diffusivity was calculated by using the following relation:

$$
D=\frac{x^{2}}{2 t}
$$

where $x$ is distance which additive diffuses though, and $t$ is amount of time used to observe a peak.

Table 1: Diffusion coefficients and approximate sizes in distilled water.

\begin{tabular}{|c|c|c|}
\hline Compounds & $\mathbf{D}\left(\mathbf{c m}^{\mathbf{2}} / \mathbf{s}\right)$ & $\mathbf{R}_{\text {eff }}(\mathbf{n m})$ \\
\hline Glucose $^{12}$ & $1.40 \mathrm{E}-05$ & 0.12 \\
\hline gelatin $^{2}$ & $8.33 \mathrm{E}-09$ & 201 \\
\hline PAA & $1.04 \mathrm{E}-08$ & 161 \\
\hline
\end{tabular}

Figures 36 to 38 showed copper electrodeposits on copper electrode with approximate 1 micrometer wide trenches. This could mean that the gelatin could block the trenches or concentrate more at the edge of the trenches, which allowed only cuprous ions to diffuse to the bottom of the trenches. This may explain the smoothing of the electrodeposit. 


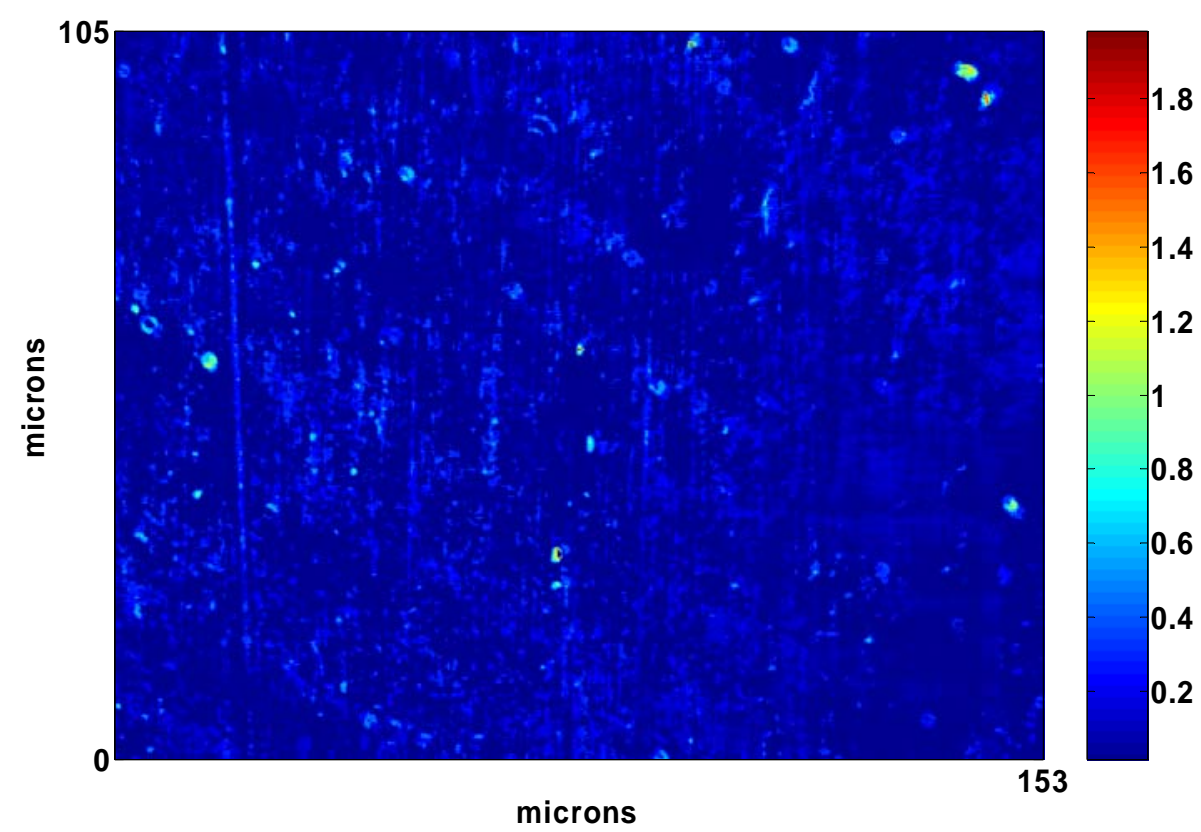

Figure 36: Copper electrode with approximate 1 micron wide scratch in one direction.

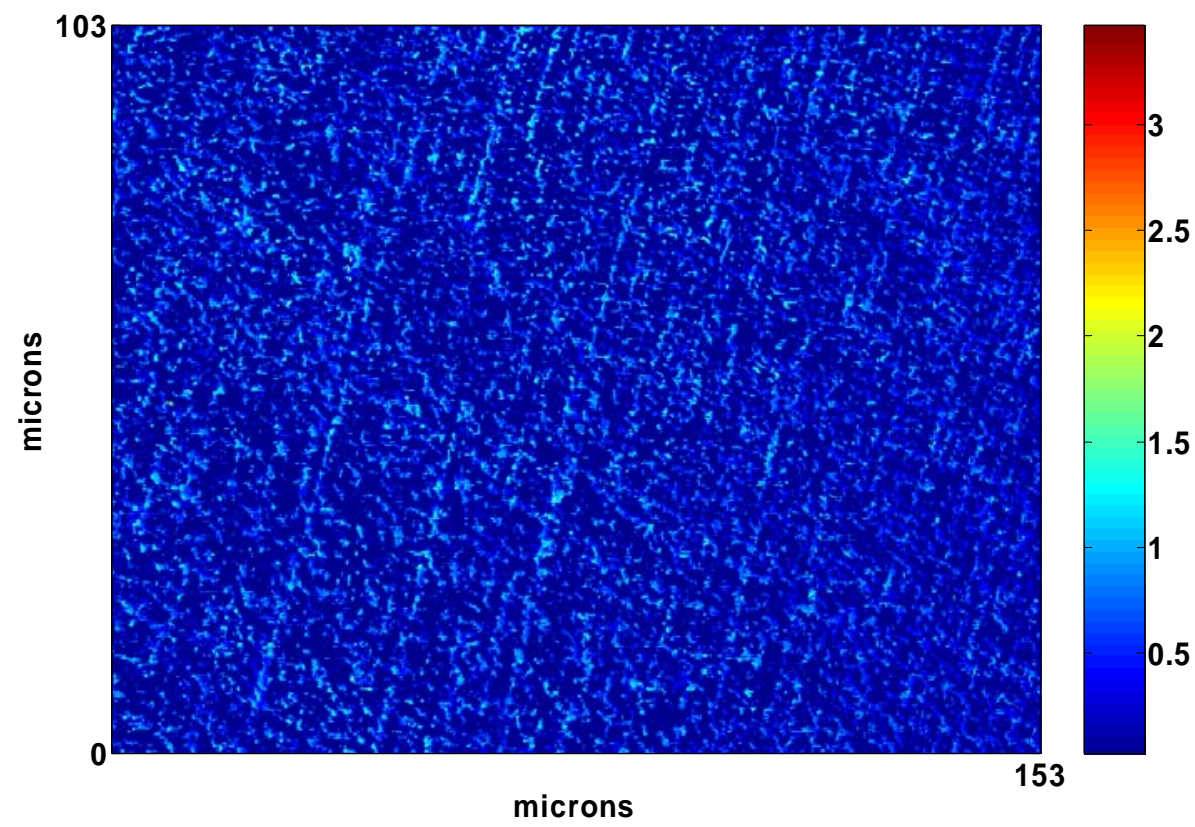

Figure 37: Copper electrodeposit on approximate 1 micron wide scratched copper electrode in an electrolyte containing $0.1 \mathrm{M} \mathrm{CuCl}, 4 \mathrm{M} \mathrm{NaCl}$ and $0.01 \mathrm{M} \mathrm{HCl}$ at cathodic current density of $25 \mathrm{~mA} / \mathrm{cm}^{2}$ for 20 seconds at $1000 \mathrm{rpm}$. 


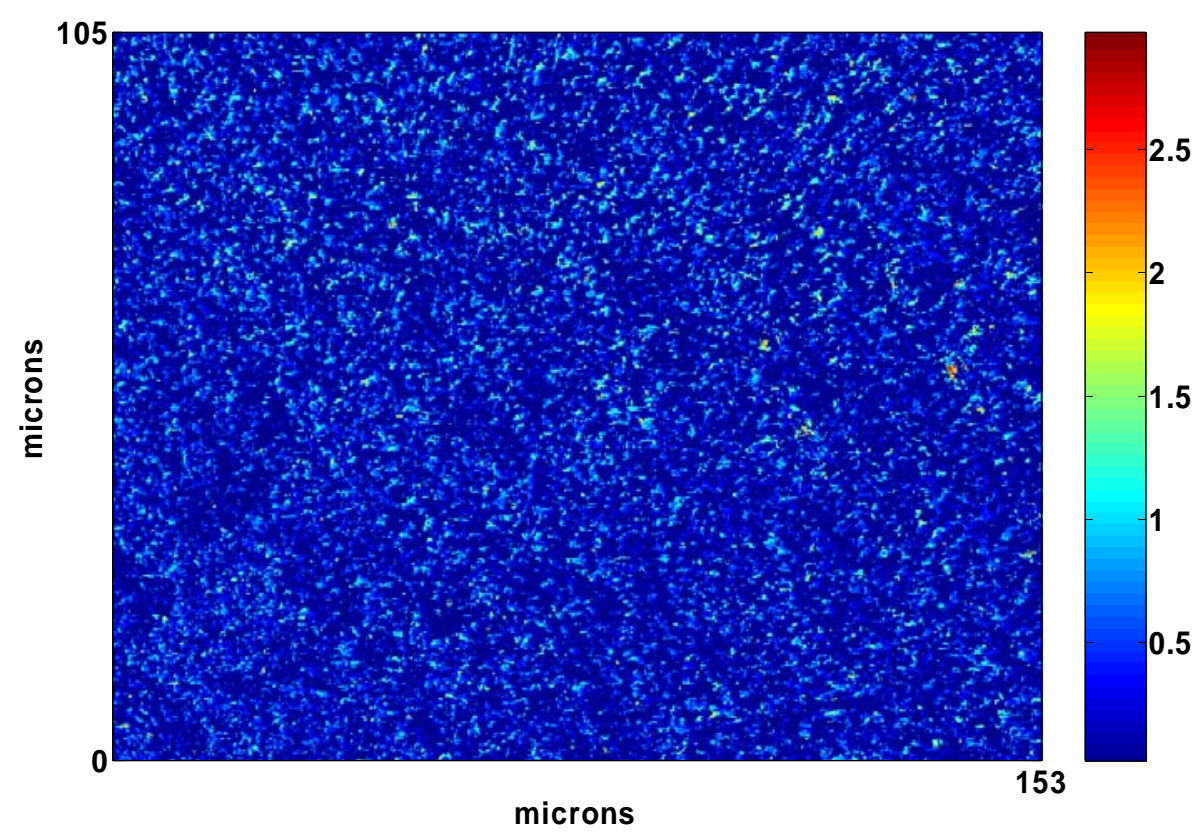

Figure 38: Copper electrodeposit on approximate 1 micron wide scratched copper electrode in an electrolyte containing $0.01 \mathrm{~g} / \mathrm{L}$ gelatin, $0.1 \mathrm{M} \mathrm{CuCl}, 4 \mathrm{M} \mathrm{NaCl}$ and 0.01 $\mathrm{M} \mathrm{HCl}$ at cathodic current density of $25 \mathrm{~mA} / \mathrm{cm}^{2}$ for 20 seconds at $1000 \mathrm{rpm}$.

Effect Of Additives On Base-line Nucleation Rate $N_{\infty}$

The steady-state nuclei formation rate is mathematically described by equation [8]. By plotting $\ln \left(d N_{\text {formation }} / d t\right)$ vs. $1 / \eta_{s}^{2}$, the value of $N_{\infty}$ can be obtained. Figures 39 and 40 show the plot of $\ln (\mathrm{dN} / \mathrm{dt})$ vs. $1 / \eta_{s}{ }^{2}$ from base solution containing no additives as well as with gelatin respectively. The value of $N_{\infty}$ from base solution without additives is 167,898 nuclei $/ \mathrm{mm}^{2}$. The value of $N_{\infty}$ from base solution containing additives is 250,475 nuclei $/ \mathrm{mm}^{2}$. 


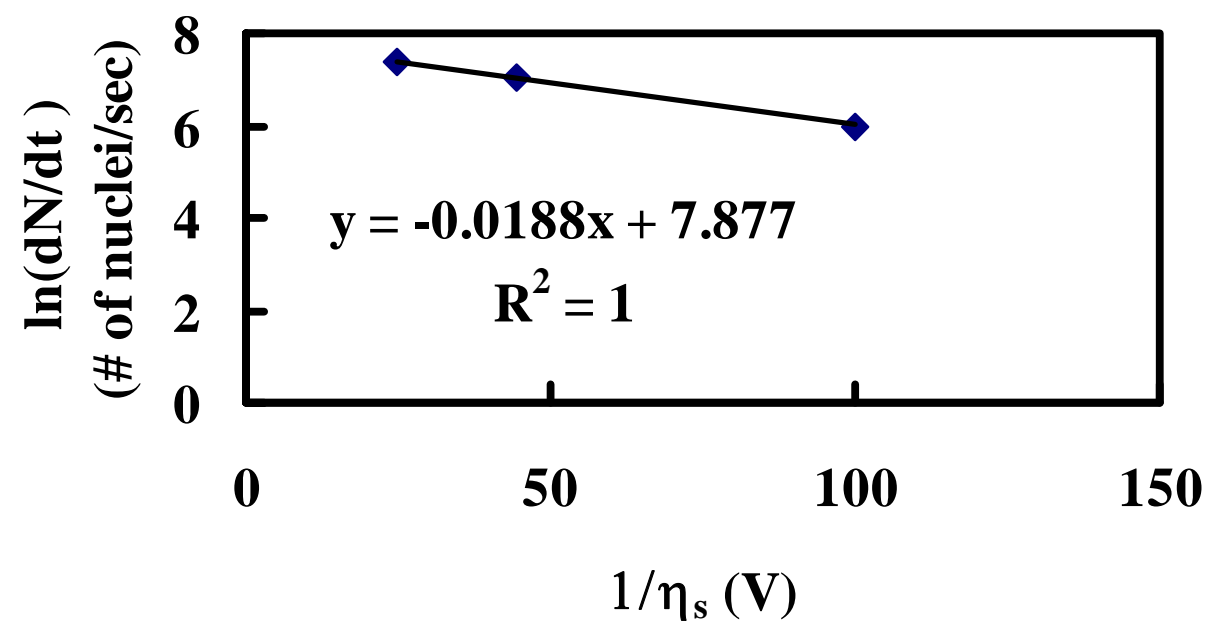

Figure 39: Plot of $\ln \left(d N_{\text {formation }} / d t\right)$ vs. $1 / \eta_{s}{ }^{2}$ from $0.1 \mathrm{M} \mathrm{CuCl}, 4 \mathrm{M} \mathrm{NaCl}$, and $0.01 \mathrm{M}$ HCl solution at stationary copper electrode.

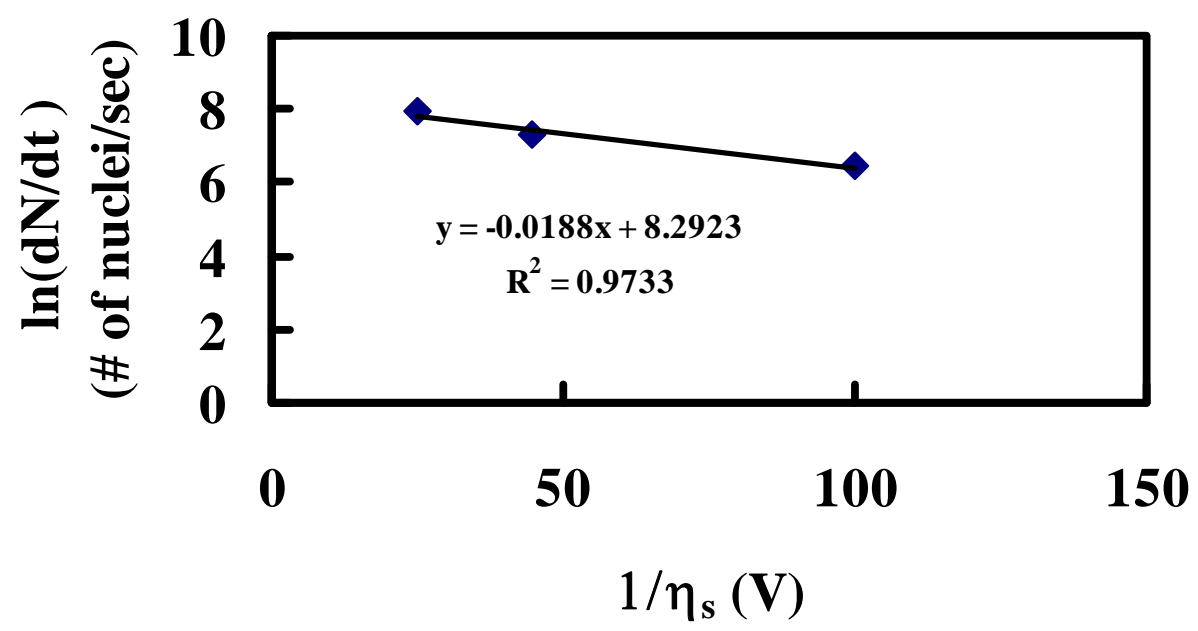

Figure 40: Plot of $\ln \left(d N_{\text {formation }} / d t\right)$ vs. $1 / \eta_{s}^{2}$ from $0.1 \%$ by volume gelatin, $0.1 \mathrm{M} \mathrm{CuCl,} 4$ $\mathrm{M} \mathrm{NaCl,} 0.01 \mathrm{M} \mathrm{HCl}$ solution at stationary copper electrode.

\section{CONCLUSIONS}

Results from this study indicate that copper electrowinning from halide media, which requires only half the energy of traditional sulfate media, can be performed at $250 \mathrm{~A} / \mathrm{m}^{2}$ or more using traditional electrowinning cells if mass transport levels are sufficient. Copper electrodeposit morphology, which is the primary factor in determining whether or not traditional electrowinning is possible, is controlled to a significant extent by mass transport. Improvement in the mass transport resulted in smoother deposits. It was also shown that 


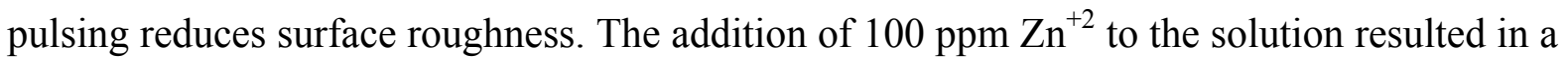
smoother copper electrodeposits than those obtained without any additive. The surface roughness of copper electrodeposits obtained from gelatin-containing solutions increased with increase in $\mathrm{i} / \mathrm{i}_{\mathrm{L}}$ ratio. Organic additives with high molecular weight such as gelatin, PEO, and PAA helped to reduce the surface roughness, yet monomer such as glycine and proline and low molecular weight molecules such as PEG did not significantly improve the surface roughness.

In halide technology, metallic copper is recovered from cuprous ion state in the aqueous solution instead of traditional cupric ion state. Since, cuprous ion needs one electron, this electrowinning reaction utilizes half of the voltage and half of the current of the traditional electrowinning reaction.

Thus, electrowinning limitations to the industrial implementation of halide technology can be overcome by increasing the bulk flow of electrolyte near the electrode surface or by using pulsating current. However, pulsating currents cannot achieve the same level of smoothing as direct current at comparable charge transfer levels. Addition of organic additives like gelatin, polyacrylamide (MW 200000), polyethylene oxide (MW 300000) can be used to obtain high quality smooth copper electrodeposits.

\section{REFERENCES}

1. M. L. Free, Electrometallurgy 2001, ed. J. A. Gonzales, J. Dutrizac, CIM, Montreal, QC, Canada, 235, 2001.

2. G. W. McDonald, H. Darus, S. H. Langer, Hydrometallurgy, v24, p291, 1990.

3. R. F. Dalton, G. Diaz, R. Price, and A. D. Zunkel, Journal of Metals, August, 51, 1991.

4. F. P. Haver, R. D. Baker, and M. M. Wong, Report of Investigations No. 8007, United States Department of the Interior Bureau of Mines, 1, 1975.

5. J. M. Demarthe, L. Gandon, and A. Georgeaux, United States Patent, No. 4288304, 8 September 1981.

6. C. Haakonsen, United States Patent, No. 4337128, 29 June 1982.

7. G. M. Ritcey, K. T. Price, and B. H. Lucas, United States Patent, No. 4362607, 7 December 1982.

8. A. J. Bard, Electrochemical Methods, John Wiley \& Sons Inc, p144, 1980.

9. R. T. C. Choo, J. M. Toguri, A. M El-Sherik, U. Erb, J. Applied Electrochemistry, 25, 384, 1995.

10. E. B. Budevski, Comprehensive Treatise of Electrochemistry, vol. 7, Ed. B. E. Conway, J. O’M. Bockris, E. Yeager, S. U. M. Khan, and R. E. White, Plenum, New York, 441, 1983.

11. http://en.wikipedia.org/wiki/Polyethylene_glycol

12. Ma, Y.; Zhu, C.; Ma, P.; Yu, K.T. J. Chem. Eng. Data., 2005, 50, 1192-1196. 
Appendix 30: Development of Chemical and Biochemical Techniques for the Extraction of Mercury from Fine Coal Particle Solutions (WV010) 
FINAL TECHNICAL REPORT

\begin{tabular}{|l|l|}
\hline Contract Title/Number: & Period of Performance: \\
\hline Crosscutting Technology Development at the Center for & Starting Date: 04/01/03 \\
Advanced Separation Technologies & Ending Date: 10/31/06 \\
(DE-FC26-02NT41607) & \\
\hline
\end{tabular}

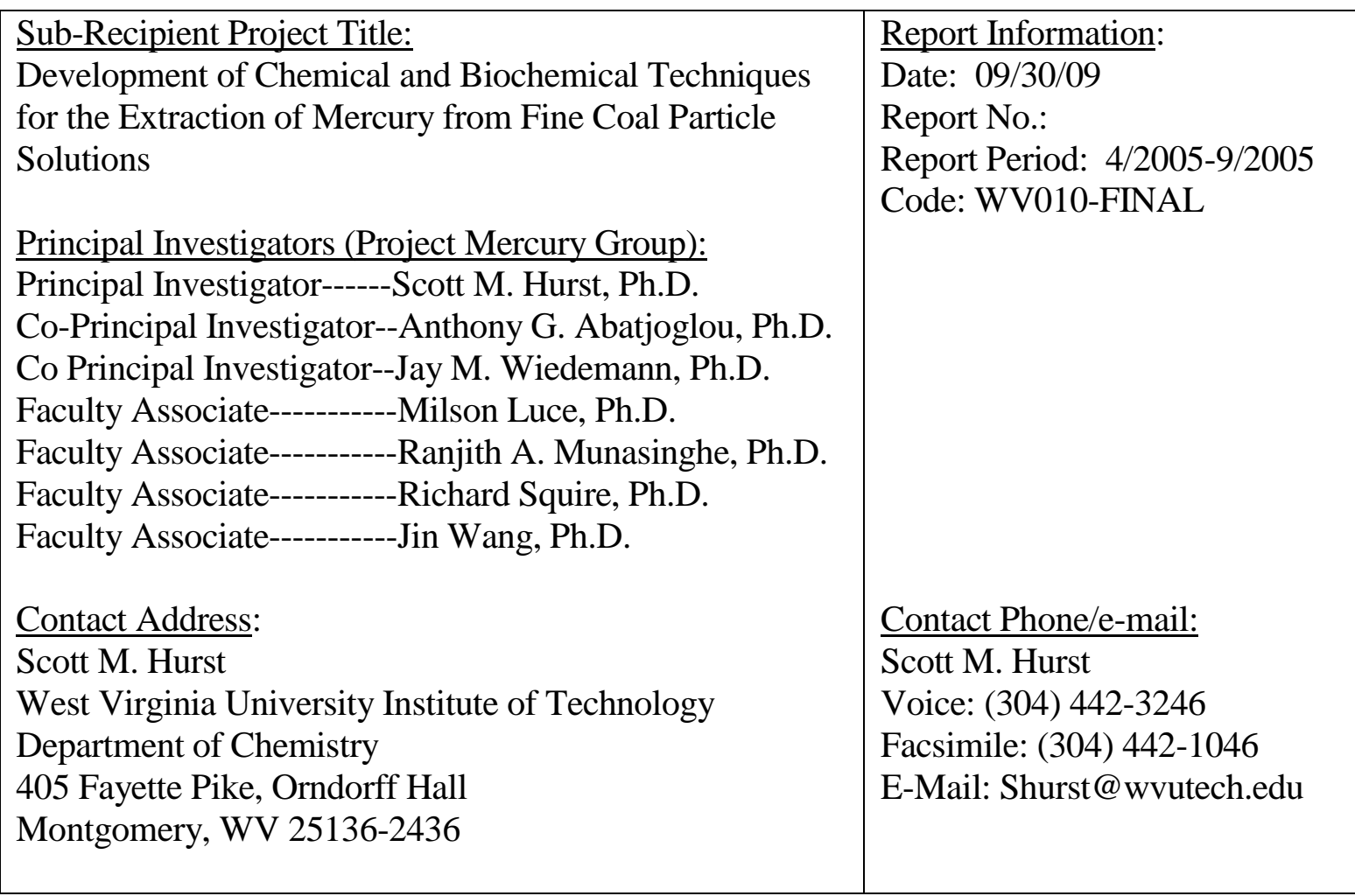




\begin{abstract}
The West Virginia University Institute of Technology (WVU Tech) Project, "Development of Chemical and Biochemical Techniques for the Extraction of Mercury from Fine Coal Particle Solutions", includes three objectives: development of a biochemical technique to facilitate the conversion of organically bound forms of mercury to water soluble mercury, development of a chemical technique using physically adsorbed ligands that selectively removes mercury from an aqueous solution that contains relatively large concentrations of other metals, and the development of a biochemical process for extracting liquid mercury from the ligands. A fourth objective of developing a novel process for extracting mercury from coal solutions in a "zero waste" manner based on the research results developed by meeting the first three objectives is the anticipated practical outcome of this work. The "Project Mercury Group" received the CAST Sub-Recipient Project Award on November 30, 2004. This report includes an account of progress the "Project Mercury Group" has made toward meeting these objectives from the effective start date of this period on April 1, 2005 through September 30, 2005.
\end{abstract}




\section{Technical Progress by the Project Mercury Group from April 1 to September 30, 2005}

The Project Mercury Group is composed of four collaborating subgroups:

1. Jay Wiedemann and Milson Luce oversee all work related to the biochemical processes: the production of partially purified bacterial metal oxidation enzymes (oxidases) that convert organic-bound mercury and mercury (I) to mercury (II); the production of partially purified bacterial metal reduction enzymes (reductases) and proteins that convert mercury(II) to mercury (0); and the isolation of specific genes so the relevant proteins may be overexpressed to produce the needed proteins in quantity at a low cost; in addition they are evaluating the potential impact the bacteria producing the oxidases and reductases may have on the environments where they may be practically introduced.

2. Anthony Abatjoglou oversees the laboratory scale work on adsorbents/ligands: the evaluation of extraction efficiency and other practical properties of novel mercury-scavenging adsorbents; and the assessment of synthetic pathways to develop novel mercury-scavenging adsorbents.

3. Scott Hurst, Richard Squire and Ranjith Munasinghe oversee computational and analytical support aspects of this project: the analysis of adsorbent properties reported in the literature to assess correlations between commonly measured properties and the selective properties sought; the evaluation of structural and electronic properties best associated with selectivity through molecular modeling; and more recently, the establishment of kinetics and mechanism of bonding and release of mercury by a selected protein, mer $\mathrm{p}$.

4. Jin Wang oversees the development of practical techniques for the coal mining industry that apply the results of the balance of the Project Mercury Group's results.

The principal investigator, the co- principal investigators and the faculty associates have focused their efforts on two general areas of concern to satisfy obligations included in the "Crosscutting Technology Development at the Center for Advanced Separation Technologies" sub-award, "Development of Chemical and Biochemical Techniques for the Extraction of Mercury from Fine Coal Particle Solutions:"

1. the acquisition of equipment, software, and supplies necessary to conduct the work required and

2. the education and training of undergraduate assistants to a level where they can truly begin assisting the senior personnel with the research.

\begin{tabular}{|l|l|rr|}
\hline Manufacturer & Description & \multicolumn{2}{|l|}{ Cost } \\
\hline Anasazi Instruments & NMR(Partly in Support of Project) & $\$ 79,900.00$ \\
\hline Dell & Computer for Molecular Modeling & $\$ 3,500.00$ \\
\hline Thermo Electron & Atomic absorption spectrometer & $\$ 56,743.00$ \\
\hline AH Systems & HyperChem Pro 7.5 book set & $\$$ & $1,395.00$ \\
\hline VWR & water purification system & $\$ 2,246.40$ \\
\hline Various Manufacturers & Total for Items Under $\$ 1000 /$ each & $\$ 5,507.66$ \\
\hline
\end{tabular}


The appropriate senior personnel have completed training of all undergraduate assistants in the use of the appropriate analytical equipment and in the use of the appropriate software to begin useful support of the project by April 15, 2005. Through the summer all of the current students have completed training. Equipment for which the assistants were trained are a ultra-violet visible spectrometer with sipper and translation stage, a Fourier-transform infra-red spectrometer, a Fourier-transform nuclear magnetic resonance spectrometer, a gas chromatograph with mass spectrometric detection, and the newly acquired atomic absorption spectrometer. The appropriate students have completed training for the use of HyperChem 7 Molecular Modeling software and Statistical Analysis System (SAS) software.

In addition seven members of the team participated in the CAST Technology Review Meeting held in Blacksburg, VA in July. There were three posters presented.

The following are brief descriptions of progress specific to each sub-group.

\section{Jay Wiedemann and Milson Luce Sub-Group}

The Wiedemann/Luce Sub-Group completed the following work during the term reviewed in this report.

Our efforts focus on the biological aspects of mercury (Hg) removal from coal. Specifically, we are studying the application of bacteria inherently resistant to mercury to the bioremediation of mercury-contaminated coal waste. As shown by others over the past decade or so, some bacteria are capable of thriving in the presence of mercury levels that are otherwise toxic. These bacteria achieve this by metabolizing mercury in the surrounding environment, transporting $\mathrm{Hg}^{2+}$ into the cell and subsequently reducing it to $\mathrm{Hg}^{0}$, a less toxic, volatile form which diffuses out of the cell. Such mercury-resistant bacteria bear a set of genes (the "mer" genes) which encode the highly-specific protein transporters and reductase enzymes involved in this metabolic pathway.

Our immediate goal is to identify and genetically characterize mercury-resistant bacteria native to West Virginia in order to incorporate these native bacteria, genetically engineered versions of these bacteria, and/or biochemical components of these bacteria into mercury bioremediation schemes. Toward this end, we have so far isolated and definitively identified 4 species of bacteria: 1 species (Citrobacter freundii) from a control sample (the Kanawha River), 1 species (Enterobacter asburiae) from a coal wastewater containment pond, and 2 species (Enterobacter asburiae and Klebsiella pneumoniae) from coal wastewater cultured in the presence of $1.5 \mathrm{mM} \mathrm{Hg}^{2+}$. We have isolated but not yet positively identified 9 additional species from the Kanawha River, 8 additional species from the coal wastewater pond, and 5 additional species from the coal wastewater cultured in the presence of mercury. These identifications were made based on a series of routine microbiological and biochemical assays carried out for each of the isolated species. This aspect of the project was completed over Summer, 2005 and the first 2 weeks of the Fall, 2005 semester.

Our next step is to genetically characterize these bacteria with respect to the $\mathrm{mer}$ genes. Because these genes have been shown, in general, to be highly diverse, we are 
likely to encounter different versions of these genes in each of the bacteria we have identified. We will thus isolate plasmid and chromosomal DNA separately in order to discover where the mer genes are located in each species. PCR assays using primer pairs designed to amplify conserved regions of known mer genes will allow us to ascertain which mer genes, if any, our bacteria carry. Conjugation experiments with non mercuryresistant $E$. coli will also be employed in order to demonstrate the presence of the mer genes on plasmids. Assuming mer genes are detected in our plasmid preparations, we will attempt to transform non-resistant $E$. coli with those plasmids

\section{Experiment}

Enzyme Preparation

Tubes containing litmus milk and $1 \mathrm{mM} \mathrm{HgCl}_{2}$ was inoculated with coal waste impoundment water $(\mathrm{pH}=6.5)$. The bacteria was allowed to grow for seven days at 37 ${ }^{\circ} \mathrm{C}$. The cells were disrupted by a homogenizer for 10 minutes. Cellular debris of broken cells were removed by centrifugation at 7,000 $\mathrm{x} g$ for 15 minutes. The supernatant (crude extract) was applied to a $20 \mathrm{ml}$ blue sepharose CL-6B column (an affinity column specific for the dinucleotide fold) equilibrated in buffer A (50 mM K $\mathrm{KPO}_{4}, \mathrm{pH} 7.3,0.5$ $\mathrm{mM}$ EDTA, and $2 \mathrm{mM}$ beta-mercaptoethanol) at a flow rate of $0.5 \mathrm{ml} / \mathrm{min}$. The column was washed with $150 \mathrm{ml}$ of buffer A. Mercuric reductase (Mer A) was then eluted by a 0 to 150 micoM gradient of NADPH. Fractions 5 to 10 were pooled and concentrated for future use.

Protein assay

Total protein concentration of each blue sepharose fraction was monitored by measuring the absorbance at $280 \mathrm{~nm}$. The NADPH concentration or gradient of each blue sepharose fraction was monitored by measuring the absorbance at $320 \mathrm{~nm}$.

\section{Enzyme Assay}

The activity of MerA was determined spectrophotometrically at $340 \mathrm{~nm}$ and $25^{\circ} \mathrm{C}$ in 50 $\mathrm{mM} \mathrm{K}_{2} \mathrm{HPO}_{4}$, pH 7.3, 0.5 mM EDTA, 2 mM beta-mercaptoethanol, $0.2 \mathrm{mM} \mathrm{NADPH}$ and $1 \mathrm{mM} \mathrm{HgCl}_{2}$ by continuously monitoring the oxidation of NADPH/min. One unit of an enzyme is defined as the amount of enzyme that catalyses the oxidation of 1 micromole of NADPH/min. The production of NADP+ was followed at $340 \mathrm{~nm}$ over a three min. time frame. The rate of production of NADP+ was calculated by using the molar extinction coefficient of NADPH $\left(6.22 \times 103 \mathrm{M}^{-1} \mathrm{~cm}^{-1}\right)$.

\section{Experimental Data and Conclusions}

MerA started to elute off the blue sepharose column at a NADPH concentration of 0.04 $\mathrm{mM}$ (Fractions 5-10). Figure One shows the elution of total protein (solid black dots), the NADPH gradient (open squares), and the activity of MerA (solid triangles). The concentrated fractions showed a 3 fold purification when compared to the crude extract (see Table One). 


$\begin{array}{lcc} & \text { Table One: Mercuric Reductase Purification } \\ \text { Fraction } & \text { Specific Activity (units/mg) } & \text { Purification (fold) } \\ \text { Crude extract } & 0.021 & 1 \\ \text { Blue Sepharose } & 0.063 & 3\end{array}$

Figure One: Relative Protein Concentration, NADPH Gradient, and MerA Activity

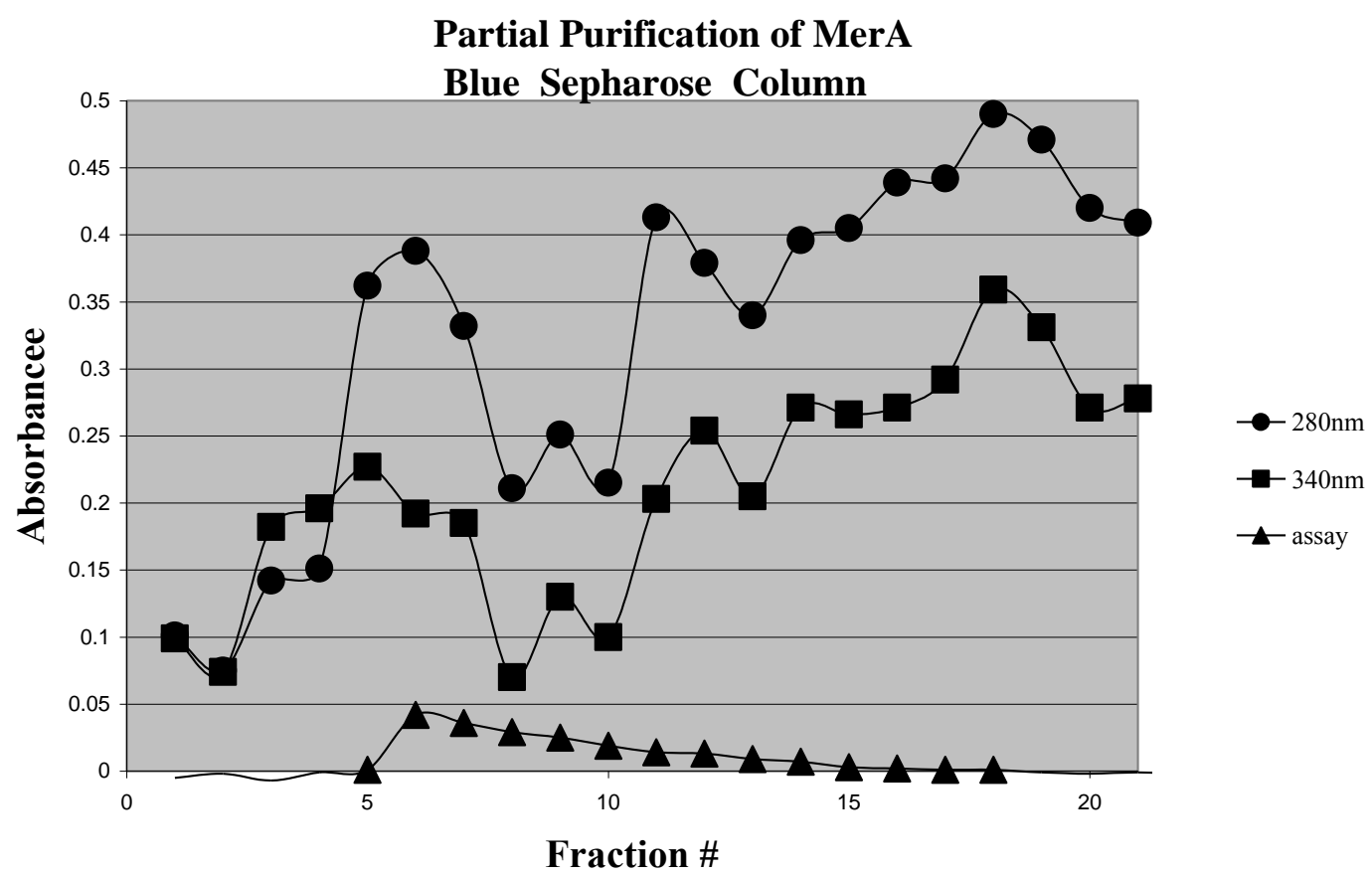




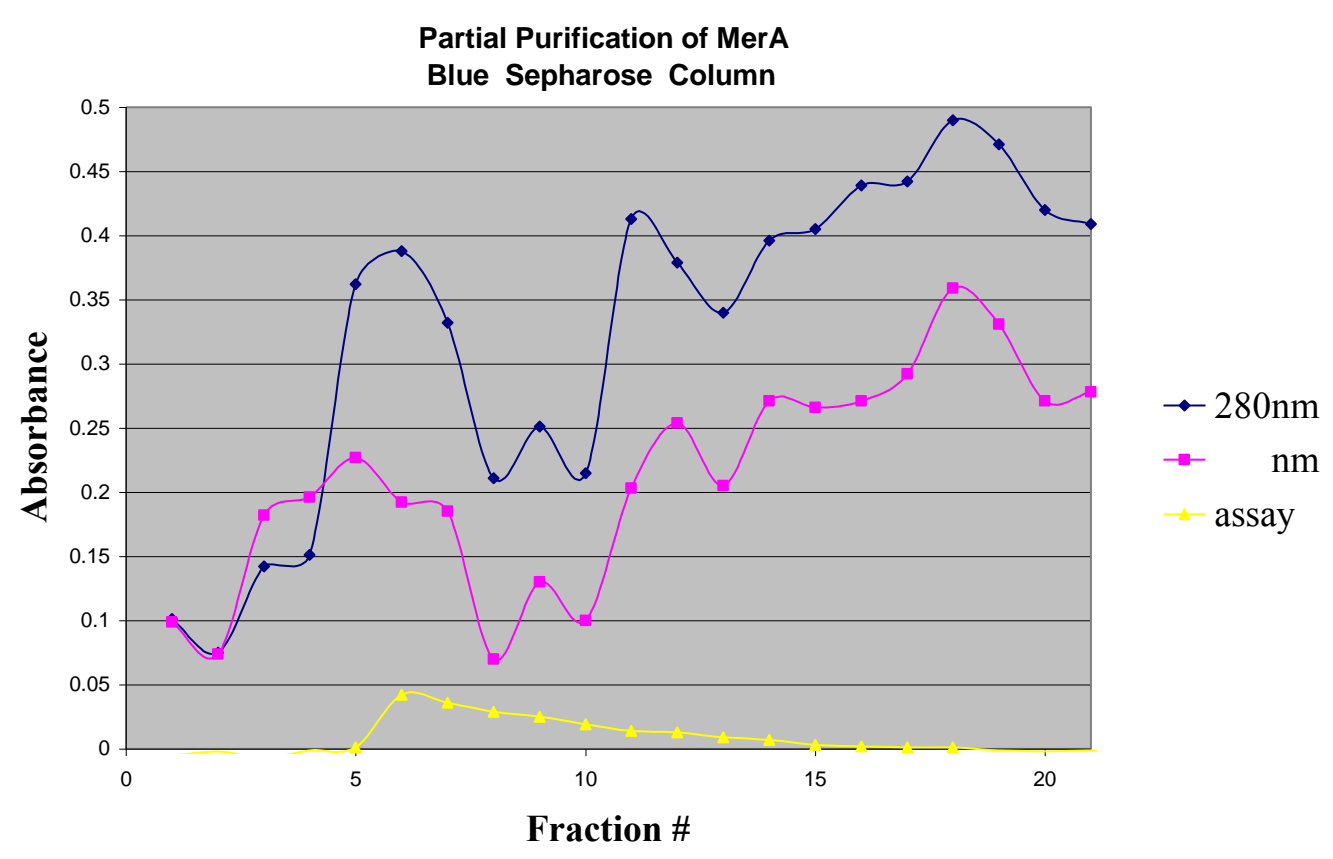

\section{Future Experiments}

More purification steps are required to isolate MerA from the crude bacterial extract. Hydrophobic chromatography or molecular weight chromatography techniques are being developed to better purify MerA. The MerA activity assessment will be followed by the volatilization of metallic mercury from the assay mixture by an atomic absorption spectrometer. The former experiment for the partial purification of MerA will be repeated and the volatilization of metallic mercury from the assay mixture will be monitored. Finally, we will directly analyze the rates of mercury metabolism by our various bacterial species using atomic absorption spectrometry. This aspect of the project is in its initial stages now and will continue through the Fall, 2005 semester.

\section{Anthony Abatjoglou Sub-Group}

The Abatjoglou Sub-Group completed the following work during the term reviewed in this report.

\section{"Novel Adsorbents for Trace Extraction Heavy Metals from Water"}

\section{Summary:}

We have prepared novel adsorbents for the removal of low concentrations of mercury and other metals from water. These adsorbents are prepared by physical adsorption of waterinsoluble ligands onto the surface of lipophilic supports such as high surface area, macroporous polystyrene beads, activated carbon spheres, as well as modified silicas. 
The novelty of these adsorbents relative to ones described before, is that unlike prior art adsorbents, the metal-complexing ligands are not chemically bonded onto the surface of the support, but are held in place by physical forces as a result of the similarity of their solubility and polarity properties with those of the support. This difference makes the adsorbents of this study easy to prepare and regenerate at low cost.

In the initial studies, we have tested lipophilic (water-insoluble) phosphines and thiols as ligands for mercury II extraction from water. For example an adsorbent made by depositing 1-octadecanethiol on macroporous polystyrene, was very effective for mercury removal from water solutions containing low concentrations of mercury. We have also demonstrated the effectiveness of this adsorbent for continuous extraction of mercury from water, and have preliminary results on adsorbent regeneration and mercury recovery.

This approach of making low-cost effective adsorbents, has been extended to ones that are practical for the extraction of metals that exist in water as oxoanions (such as for example, chromate, arsenate, selenate) anions. For example an adsorbent made by depositing octadecylpyridinium bromide on macroporous polystyrene, was effective for the removal of chromate anions from water.

\section{$\underline{\text { Experimental Results and discussion }}$}

\section{General Methods for the Preparation of Metal Scavenging Adsorbents.}

\section{a. Ligand Deposition by Solvent Evaporation}

A solution of water-insoluble metal-complexing ligand in a low-boiling organic solvent (e.g., dichloromethane), is mixed with the calculated amount of a polymeric support (e.g., high surface area macroreticular polystyrene), to provide a concentration of 1-2 mmoles ligand per gram of support. The solvent is then evaporated slowly on a rotary evaporator. The dried polymeric support which has now a surface coating of a metal-complexing ligand is suitable for use in metal extraction from water studies.

\section{b. Ligand Deposition by Incipient Wetness.}

A calculated amount of metal-complexing ligand which will provide a concentration of 12 mmoles ligand per gram of a polymeric support is dissolved in a specific solvent volume equal to the pore volume of the employed support. The ligand solution is then added to the polymeric support particles which are held in a container under vacuum. The solvent is then evaporated slowly on a rotary evaporator. The dried polymeric support now has a surface coating of a metal-complexing ligand which is uniformly deposited throughout the resin pore structure.

\section{Adsorbents for Extraction of Metal Cations.}

\section{i. Continuous mercury II extraction from water solutions:}

The adsorbent for this extraction was prepared as follows. A solution of 1.144 grams (4 mmoles) octadecanethiol (MW=286.57) in $12 \mathrm{ml}$ 1,2-dichloroethane was added to a 100 
$\mathrm{ml}$ round bottom flask containing 4 grams of macroreticular polystyrene (Aldrich catalog. \# 426989; mean pore size 46 Angstrom, 1,100 m²/g surface area). The solvent was slowly evaporated in a rotary evaporator under water aspirator vacuum and gentle heating to produce uniformly coated spheres containing 1 mmole octadecanethiol per gram of polymeric support.

The continuous mercury extraction was performed as follows. About $15 \mathrm{ml}$ of the adsorbent was slurry packed with the help of water in ca 0.5 " diameter glass column. A $200 \mathrm{ppm}$ solution of mercury II nitrate was eluted through the column at a flow rate of 1 $\mathrm{ml} / \mathrm{min}$. Samples of the eluent from the column as a function of time were analyzed for mercury concentration. The concentration of $\mathrm{Hg}^{2+}$ was less than the detection limit for all samples taken during this experiment. The results are shown in Table 1.

Table 1: Continuous mercury
extraction water using a
polystyrene/C-18 thiol-coated
adsorbent
\begin{tabular}{|c|c|}
\hline $\begin{array}{c}\text { Volume } \\
\text { of Eluted } \\
\text { water } \\
\text { (ml) }\end{array}$ & $\begin{array}{c}\text { Mercury } \\
\text { concentration } \\
\text { in eluted water } \\
\text { (ppm) }\end{array}$ \\
\hline 30 & Nil* \\
\hline 60 & Nil* \\
\hline 90 & Nil* \\
\hline 120 & Nil* \\
\hline 150 & Nil* \\
\hline 180 & Nil* \\
\hline 210 & Nil* \\
\hline 240 & Nil* \\
\hline * Below the detection limit $(0.6$ ppm) of \\
the employed UV method [M.J. Ahmed \\
and M.S. Alam Spectroscopy, 17,45 \\
$(2003)]$
\end{tabular}

\section{ii. Mercury recovery and adsorbent regeneration.}

The adsorbent from the mercury extraction experiment (see above), was used to test a method for mercury recovery, and adsorbent regeneration. In one experiment, we tested the effectiveness of a $5 \mathrm{wt}$. percent aqueous thiourea $\left(\mathrm{NH}_{2}\right)_{2} \mathrm{C}=\mathrm{S}$ solution as the eluent for mercury recovery and adsorbent regeneration. Preliminary results samples show that mercury is readily eluted from the adsorbent on the column. Some adsorbent discoloration (darkening) was observed and need further investigation. Mercury material balance tests as well as tests of the reuse of the resin after regeneration are planned. 


\section{Adsorbents for the Extraction of Complex Metal Anions.}

i. Adsorbent preparation:

A solution of 0.82 grams ( 2 mmoles) octadecyl pyridinium bromide (MW=412) in $7 \mathrm{ml}$ isopropanol was added to 4 grams of macroreticular polystyrene (Aldrich catalog. \# 426989; mean pore size 46 Angstrom, $1,100 \mathrm{~m}^{2} / \mathrm{g}$ surface area) contained in a $100 \mathrm{ml}$ round bottom flask under vacuum. The solvent was slowly evaporated in a rotary evaporator under water aspirator vacuum and gentle heating to produce uniformly coated spheres containing 0.5 mmoles octadecyl pyridinium bromide per gram of polymeric support.

A preliminary test of this adsorbent's effectiveness for chromate extraction, showed that it is capable of extracting chromate anions and its capacity is $2.8 \mathrm{mg}$ chromium per gram of adsorbent. This test was performed by work in collaboration with Dr. Dianchen Gang and Ravi Kumar of the WVU Tech department of Civil Engineering. The experimental protocol detailed below was followed the experimental protocol below.

The chromium stock solution (1000 mg/L) was prepared using potassium dichromate $\left(\mathrm{K}_{2} \mathrm{Cr}_{2} \mathrm{O}_{7}\right)$ and distilled water. All the working solutions were prepared by diluting the standard stock solution with distilled water. Batch adsorption experiments were carried out in 200-ml round bottom flasks immersed in a shaker. The concentration of $\mathrm{Cr}$ (VI) in solution before and after adsorption has been measured in according to the colorimetric methods described in standard methods. The measurements were made at wavelength $\lambda$ $=540 \mathrm{~nm}$ using Cary 50 Probe UV-Visible Spectrometer, which corresponds to the maximum absorbance. Standard chromium solutions from the potassium dichromate were prepared to obtain calibration curve for hexavalent chromium. The adsorbed amount of $\mathrm{Cr}$ (VI) was calculated from the concentrations in the solution before and after the adsorption using the formula

Where

$$
q_{e}=\frac{\left(C_{i}-C_{f}\right) V}{M} \mathrm{mg} / \mathrm{g}
$$

V- Volume of the solution taken in liters

$\mathrm{M}$ - Mass of Resin in grams.

$\mathrm{C}_{\mathrm{i}}$ - initial concentration of the solution (mg/L)

$\mathrm{C}_{\mathrm{f}}$ - final concentration of the solution $(\mathrm{mg} / \mathrm{L})$

$\mathrm{q}_{\mathrm{e}}$ - Adsorption capacity (mg/g)

$\mathrm{pH}$ adjustments have been done using solutions of concentrated $\mathrm{HCl}$ and $\mathrm{NaOH}$. As the maximum adsorption capacity or hexavalent chromium removal was noticed at $\mathrm{pH}=2$, experiment was conducted at $\mathrm{pH}=2$.

Prepare intermediate hexavalent chromium solution and then standard solution concentration of $5 \mathrm{mg} / \mathrm{L}$ from stock solution. Take $40 \mathrm{ml}$ of $5 \mathrm{mg} / \mathrm{L}$ solution in a flask and adjust the $\mathrm{pH}$ of the solution to 2 by adding $\mathrm{HCL}$ and $\mathrm{NaOH}$. Add $60 \mathrm{mg}$ of resin to the solution place it in a shaker and allow it to react with solution for 20 hrs and then 
filter the solution. Measure the final concentration of solution and calculate percentage removal and adsorption capacity of the hexavalent chromium solution.

Result and Calculations:

V- Volume of the solution taken in liters $=0.04$

$\mathrm{M}$ - Mass of Resin in grams. $=0.06$

$\mathrm{C}_{\mathrm{i}}$ - initial concentration of the solution $(\mathrm{mg} / \mathrm{L})=5$

$\mathrm{C}_{\mathrm{f}}$ - final concentration of the solution $(\mathrm{mg} / \mathrm{L})=0.8$

$\mathrm{q}_{\mathrm{e}}$ - Adsorption capacity (mg/g)

$$
\begin{aligned}
q_{e} & =\frac{\left(C_{i}-C_{f}\right) V}{M} \mathrm{mg} / \mathrm{g} \\
\Rightarrow \quad \mathrm{q}_{\mathrm{e}} & =2.8 \mathrm{mg} / \mathrm{g}
\end{aligned}
$$

Publications:

1. The initial results of work related to this invention were presented as a poster paper in the June 2005 DOE/NSF EPSCoR Conference in Morgantown, WV.

2. An invention disclosure has been submitted to the WVU Office of Technology Transfer to for evaluation

\section{Scott Hurst, Richard Squire and Ranjith Munasinghe Sub-Group}

The preliminary portion of the CAST research project by our subgroup in the previous report (03/30/05) consisted of two parts: first, a through literature search of various mercury ligands was begun and finished, and second, a good working knowledge of the software associated with HyperCube 7 Molecular Modeling programs was established.

We began and ended this period of performance (04/01/05 to 09/30/05) with two seminars that punctuate our research effort. The first was a general discussion of various organic and bio-organic ligands for mercury, hat the general applications might be, and finally, what specific tasks we needed to accomplish by the end of this next performance period. The second seminar focused on the specifics of coal slurry ponds, coal burning power plants, and a discussion of suitable locations therein to give our selective mercurycapturing bio-organic ligand the best opportunity to be successful. The impetus for this seminar was the DOE/National Energy Technology Laboratory's (NETL) Pittsburgh meeting on "Mercury Control Technology R\&D Program Review held from July 12 - 14, 2005. We are confident enough of our approach that we are currently writing a patent application and a non-disclosure agreement with AEP to cover possible applications. Below is a detailed description as to how we arrived at this conclusion.

Our survey of mercury organic ligands revealed three interesting facts: (1) almost all organic ligands that bound mercury did so in a non-discriminating fashion, i.e. they bound numerous other ions also. (2) They bound these ions very tightly with little or no possibility for re-use of the ligand. (3) They were quite expensive (\$200/gram). Our 
biology/biochemical subgroup continued to emphasize the use of a series of naturally occurring, "exclusively binding mercury" ligands, the so-named mer protein ligands. Utilizing the knowledge we had gained from our evaluations of organic ligands, we began to focus on one specific mercury bio-organic ligand, "mer P", a compound built from 72 amino acids, so we could try and understand how it functions. (Mer proteins fit into a class of bio-organic ligands called "metallothioenes" which are used to regulate metal ions in living organisms. Our focus was three-fold:

(1) Throughly understand the structure of this large (by our standards) molecule.

(2) Understand the nature of the specific bonding

(3) Devise a process where we could use the ligand numerous times.

(1) and (2) - Understanding the structure/nature of specific bonding: After considerable effort by a number of people (especially our undergraduates - Adam Broschinski, Matthew Naylor, Anna Bricker, and Shane Holt) we built a free-standing molecular model - 2 × 2 × 3 feet in dimensions. This enabled us to clearly identify the various substructures - alpha helices, beta-pleated sheets, and specifically the random coils associated with the active site (mercury binding position) and the conformational changes when mercury is bound and unbound. We matched our computational work with literature data such that we clearly identified the structural changes associated with the exclusive mercury binding site. There are numerous hydrogen bonds in the mer $\mathrm{p}$ structure with only 6 or 7 appearing directly related to mer p binding mercury. Further, these bonds and other structural features relegate the bind of only mercury as the binding is divalent and linear. We also found that upon adding denaturants, all of the mer $\mathrm{p}$ hydrogen bonds can be broken and the structure becomes somewhat random and importantly, releases any bound mercury. Upon removing the denaturants, the protein reassembles itself into its active conformation. More details about the molecular biology of this unusual protein can be found in the Luce/Wiedemann section of this report.

(3) The next step consisted of identifying locations in the processing of coal and/or it's burning where we could effectively utilize the mer group of proteins. We have several methods of accomplishing this; the simplest is to bind mer $\mathrm{p}$ to a substrate in a column and use it like an ion exchange column. The rate of mer p binding mercury is very fast; the formation constant is very large, but only for mercury. When the column is "exhausted" (all of the active sites become bound), denaturant is added, mer p loses its binding conformation and the bound mercury is released in a concentrated stream directed to a biological treatment pond containing another mer protein, mer a where the mercury is converted to its elemental form and recovered.

For future work we will complete a patent application, complete the AEP non-disclosure agreement, and assess the proper locations of mer p protein equipment to maximize its effectiveness. We will continue our quantitative evaluation of mer protein kinetics and binding in various mercury concentrations. 
I have developed a computer model of the MerP molecule using Matlab. Using HyperChem files my programs reads and stores the locations and charges of atoms and the chemical bonds.

I have a routine that identifies the backbone of the molecule and as necessary using the backbone simple routines can identify any atom in MerP.

The program can be used to perform any calculation related to the bonds, charges, and locations of atoms.

So far we used the program to compute the strengths of strong hydrogen bonds. We have also computed the difference between several MerP molecules (created under different conditions?) after and before it binds with Mercury.

The platform I have created includes more than 25 routines and can be used to analyze similar proteins. (The program can handle other proteins with a similar backbone without any modifications.) If you want me to describe these in more details please let me know. Ranjith

\section{Jin Wang Sub-Group}

The Wang sub-group recently began work on this project. This sub-group is currently learning about the current processes used for cleaning coal and how coal waste impoundments are developed. This sub-group will develop practical techniques from the results of the balance of the Project Mercury Group's findings. This sub-group provides

guidance about the direction the balance of the Project Mercury Group's research should take to most quickly lead to practical applications. 
Attachment 1: Project Mercury Group Undergraduate Research Assistants

Jay Wiedemann and Milson Luce Sub-Group

- Christian Robinson

- Anna Bricker

- Chad Davis

- Johnathan Craft

- Michael Brackman

- Daniel bailey

\section{Anthony Abatjoglou Sub-Group}

- Namon Crotty

- Jeremy Rader

\section{Scott Hurst, Richard Squire and Ranjith Munasinghe Sub-Group}

- Matthew Naylor

- Adam Broschinski

- Shane Holt

\section{Jin Wang Sub-Group}

- Samrawit Assefa

The West Virginia University Institute of Technology was fortunate to receive a grant from the West Virginia Higher Education Policy Commision and WV EPSCoR that provides funds for undergraduate research assistants that receive hourly wages to work with the Project Mercury Group. Currently, these undergraduate receive hourly wages from this funding source.

Grant No. EPS2005-24

Project Sponsor: West Virginia EPSCoR Research Challenge Fund

Project/Program Title: Summer/Semester Undergraduate Research Experience (SURE) Grant Program

Principal Investigator: Stephen Brown, Dean of WVU Tech BHS

Co- Principal Investigators: Scott Hurst

Jay Wiedemann

Dianchen Gang

Milson Luce

Ranjith Munasinghe

Anthony Abatjoglou

Grant Period: January 1, 2005 - December 31, 2006 (Two Years)

Total Award Increment Amount: \$35,000/year

Total Award Amount: \$35,000 


\section{Attachment 2: Expenditure Budget Detail Report}

Expenditure Budget Detail Report Pulled by Expenditure Date

FROM: 01-JUN-2004

TO: 31-OCT-2006

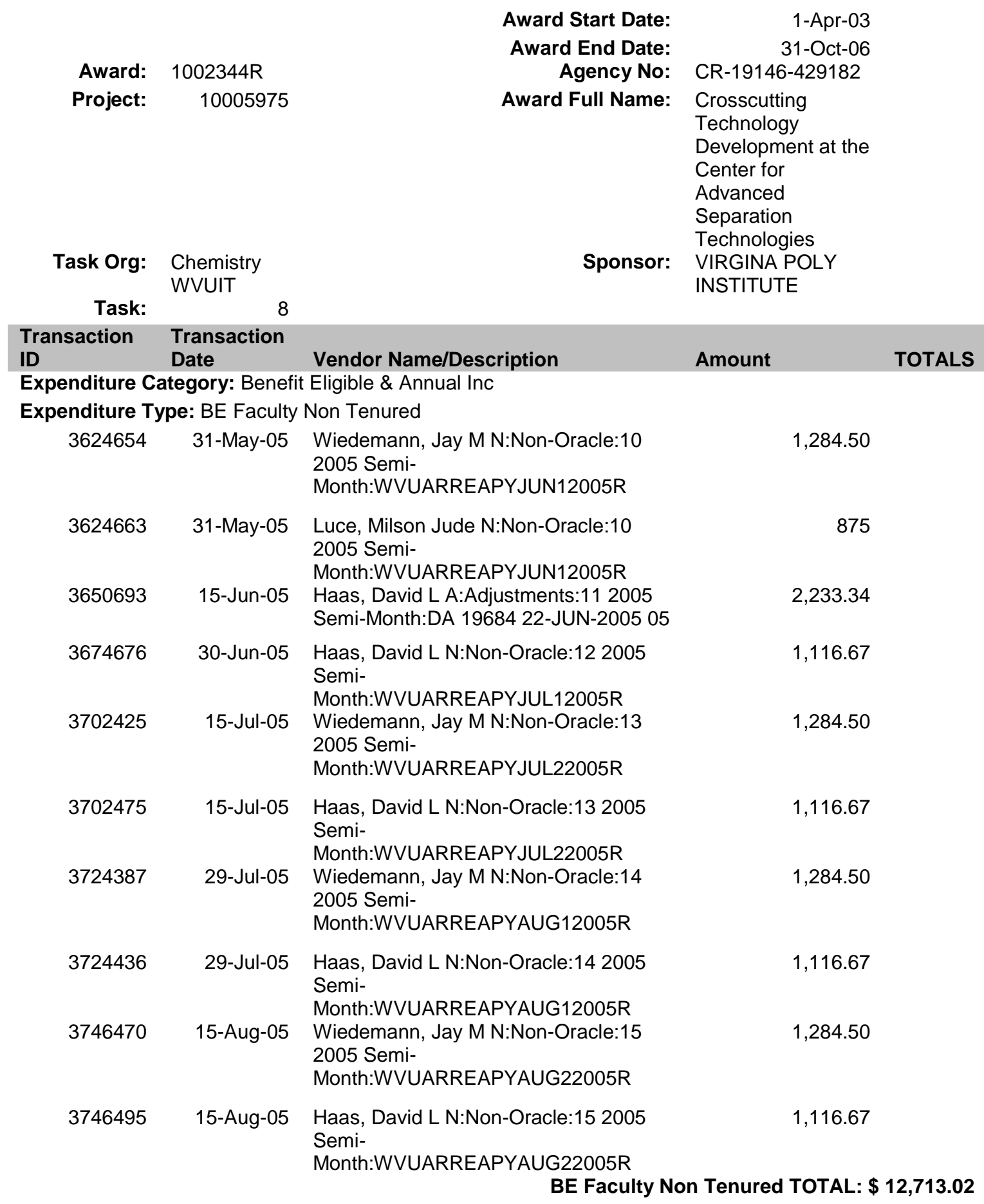

Expenditure Type: BE Faculty Tenured 


\begin{tabular}{|c|c|c|c|}
\hline 3676674 & 15-Jul-05 & $\begin{array}{l}\text { Munasinghe, Ranjith A N:Non- } \\
\text { Oracle:13 } 2005 \text { Semi- } \\
\text { Month:WVUSALARPYJUL12005R }\end{array}$ & $5,120.68$ \\
\hline 3701537 & 15-Jul-05 & $\begin{array}{l}\text { Wang, Jin N:Non-Oracle: } 132005 \text { Semi- } \\
\text { Month:WVUARREAPYJUL22005R }\end{array}$ & $1,250.00$ \\
\hline 3702637 & 29-Jul-05 & $\begin{array}{l}\text { Munasinghe, Ranjith A N:Non- } \\
\text { Oracle:14 } 2005 \text { Semi- } \\
\text { Month:WVUSALARPYJUL22005R }\end{array}$ & $1,280.17$ \\
\hline 3705639 & 30-Jun-05 & $\begin{array}{l}\text { Wang, Jin A:Adjustments:12 } 2005 \\
\text { Semi-Month:DA } 19684 \text { 20-JUL-2005 } 05\end{array}$ & $2,500.00$ \\
\hline 3723480 & 29-Jul-05 & $\begin{array}{l}\text { Wang, Jin N:Non-Oracle: } 142005 \text { Semi- } \\
\text { Month:WVUARREAPYAUG12005R }\end{array}$ & $1,250.00$ \\
\hline 3724209 & 15-Aug-05 & $\begin{array}{l}\text { Munasinghe, Ranjith A N:Non- } \\
\text { Oracle:15 } 2005 \text { Semi- } \\
\text { Month:WVUSALARPYAUG12005R }\end{array}$ & $1,280.17$ \\
\hline 3726180 & 15-Jul-05 & $\begin{array}{l}\text { Wiedemann, Jay M A:Adjustments:13 } \\
2005 \text { Semi-Month:DA } 19684 \text { 01-AUG- } \\
200508\end{array}$ & $2,569.00$ \\
\hline 3746015 & 15-Aug-05 & $\begin{array}{l}\text { Wang, Jin N:Non-Oracle:15 } 2005 \text { Semi- } \\
\text { Month:WVUARREAPYAUG22005R }\end{array}$ & $1,250.00$ \\
\hline
\end{tabular}

BE Faculty Tenured TOTAL: $\$ 16,500.02$

Expenditure Category: F\&A Reimb \& Aux Srvc Chrg

Expenditure Type: $F$ and $A$ Cost Reimbursement

$\begin{array}{rr}\text { 28-Feb-05 } & \\ \text { 28-Feb-05 } & 1,442.48 \\ \text { 15-Mar-05 } & 2,821.34 \\ \text { 30-Mar-05 } & 480.85 \\ \text { 31-Mar-05 } & 137.57 \\ \text { 31-Mar-05 } & 480.85 \\ \text { 6-Apr-05 } & 5.98 \\ \text { 15-Apr-05 } & 120.71 \\ \text { 18-Apr-05 } & 480.85 \\ \text { 28-Apr-05 } & 57.29 \\ \text { 29-Apr-05 } & 442.57 \\ \text { 6-May-05 } & 480.82 \\ \text { 13-May-05 } & 25.53 \\ \text { 27-May-05 } & 480.82 \\ \text { 30-May-05 } & 374.57 \\ \text { 31-May-05 } & 49.96 \\ \text { 6-Jun-05 } & 1,261.58 \\ \text { 15-Jun-05 } & 54.59 \\ \text { 24-Jun-05 } & 1,304.72 \\ \text { 30-Jun-05 } & 768.71 \\ \text { 30-Jun-05 } & 420.14 \\ \text { 30-Jun-05 } & 1,466.25 \\ \text { 4-Jul-05 } & 1,485.80 \\ \text { 15-Jul-05 } & 111.88 \\ \text { 15-Jul-05 } & 5,608.26 \\ & 753.36\end{array}$


$\begin{array}{rr}\text { 20-Jul-05 } & -103.5 \\ \text { 29-Jul-05 } & 2,788.90 \\ \text { 29-Jul-05 } & 2,260.08 \\ \text { 4-Aug-05 } & 151.98 \\ \text { 5-Aug-05 } & 287.29 \\ \text { 15-Aug-05 } & 2,788.90 \\ \text { 15-Aug-05 } & 2,038.08 \\ \text { 15-Aug-05 } & 753.36 \\ \text { 17-Aug-05 } & 26.86 \\ \text { 15-Sep-05 } & 63.71 \\ \text { 19-Sep-05 } & 192.93\end{array}$

F and A Cost Reimbursement TOTAL: \$ 32,366.07

Expenditure Category: Fringe Benefits

F\&A Reimb \& Aux Srvc Chrg TOTAL: \$ 32,366.07

Expenditure Type: FB Grant Fr Reim Benefit Elig

$$
\begin{array}{r}
\text { 31-May-05 } \\
\text { 15-Jun-05 } \\
\text { 30-Jun-05 } \\
\text { 15-Jul-05 } \\
\text { 15-Jul-05 } \\
29-J u l-05 \\
\text { 29-Jul-05 } \\
\text { 15-Aug-05 } \\
\text { 15-Aug-05 } \\
\text { 15-Aug-05 }
\end{array}
$$

$$
\begin{array}{r}
583.07 \\
603 \\
687.5 \\
1,715.27 \\
353.24 \\
1,002.88 \\
1,059.72 \\
1,002.88 \\
650.83 \\
353.24
\end{array}
$$

FB Grant Fr Reim Benefit Elig TOTAL: \$ 8,011.63

Expenditure Type: FB Grant Fr Reim Non Ben Elig

$$
\begin{array}{r}
\text { 28-Feb-05 } \\
\text { 15-Mar-05 } \\
\text { 31-Mar-05 } \\
\text { 15-Apr-05 } \\
\text { 29-Apr-05 } \\
\text { 13-May-05 } \\
\text { 30-Jun-05 } \\
\text { 15-Jul-05 } \\
\text { 29-Jul-05 } \\
\text { 15-Aug-05 }
\end{array}
$$

229.59

76.54

76.54

76.54

76.53

76.53

364.25

121.42

121.42

121.42

FB Grant Fr Reim Non Ben Elig TOTAL: \$ 1,340.78

Expenditure Type: FB Grant Fr Reim Undergrad Std

$$
\text { 28-Feb-05 }
$$

108.43

FB Grant Fr Reim Undergrad Std TOTAL: \$ 108.43

Expenditure Category: General Expenses

Expenditure Type: Ge Exp Bks \& Periodicals

6742107 
Ge Exp Bks \& Periodicals TOTAL: \$ 219.50

Expenditure Type: Ge Exp Com Ser \& Sup Other

6411459 31-Mar-05 WVU INSTITUTE OF TECHNOLOGY

13

Ge Exp Com Ser \& Sup Other TOTAL: $\$ 13.00$

Expenditure Type: Ge Exp Res \& Ed Sup \& Ser

\begin{tabular}{|c|c|c|c|}
\hline 6372737 & 30-Mar-05 & BANK ONE NA SIGMA-ALDRICH & 64.12 \\
\hline 6372737 & 30-Mar-05 & BANK ONE NA SIGMA-ALDRICH & 63.18 \\
\hline 6372737 & 30-Mar-05 & BANK ONE NA SIGMA-ALDRICH & 60.13 \\
\hline 6372737 & 30-Mar-05 & BANK ONE NA SIGMA-ALDRICH & 47.15 \\
\hline 6372737 & 30-Mar-05 & BANK ONE NA SIGMA-ALDRICH & 64.47 \\
\hline 6396126 & 6-Apr-05 & BANK ONE NA SIGMA-ALDRICH & 49.21 \\
\hline 6396126 & 6-Apr-05 & BANK ONE NA SIGMA-ALDRICH & 137.44 \\
\hline 6396126 & 6-Apr-05 & BANK ONE NA SIGMA-ALDRICH & 75.77 \\
\hline 6457069 & 18-Apr-05 & $\begin{array}{l}\text { BANK ONE NA CAROLINA BIOL } \\
\text { SUPPLY }\end{array}$ & 124.55 \\
\hline 6482941 & 26-Apr-05 & BANK ONE NA SIGMA-ALDRICH & 69.02 \\
\hline 6482941 & 26-Apr-05 & BANK ONE NA SIGMA-ALDRICH & 173.95 \\
\hline 6482941 & 26-Apr-05 & BANK ONE NA SIGMA-ALDRICH & 500.18 \\
\hline 6482941 & 28-Apr-05 & $\begin{array}{l}\text { BANK ONE NA CAROLINA BIOL } \\
\text { SUPPLY }\end{array}$ & 218.95 \\
\hline 6512151 & 6-May-05 & $\begin{array}{l}\text { BANK ONE NA CAROLINA BIOL } \\
\text { SUPPLY }\end{array}$ & 55.5 \\
\hline 6598665 & 23-May-05 & $\begin{array}{l}\text { BANK ONE NA VWR SCIENTIFIC } \\
\text { PROD VC }\end{array}$ & 82.38 \\
\hline 6598665 & 23-May-05 & $\begin{array}{l}\text { BANK ONE NA VWR SCIENTIFIC } \\
\text { PROD VC }\end{array}$ & 355.98 \\
\hline 6598665 & 23-May-05 & $\begin{array}{l}\text { BANK ONE NA VWR SCIENTIFIC } \\
\text { PROD VC }\end{array}$ & 10.44 \\
\hline 6598665 & 25-May-05 & BANK ONE NA SIGMA-ALDRICH & 197.14 \\
\hline 6598665 & 25-May-05 & BANK ONE NA SIGMA-ALDRICH & 79.52 \\
\hline 6598665 & 27-May-05 & $\begin{array}{l}\text { BANK ONE NA VWR SCIENTIFIC } \\
\text { PROD VC }\end{array}$ & 88.85 \\
\hline 6612480 & 30-May-05 & BANK ONE NA FISHER SCI ATL & 108.61 \\
\hline 6652741 & 6-Jun-05 & $\begin{array}{l}\text { BANK ONE NA VWR SCIENTIFIC } \\
\text { PROD VC }\end{array}$ & 38.19 \\
\hline 6652741 & 6-Jun-05 & $\begin{array}{l}\text { BANK ONE NA VWR SCIENTIFIC } \\
\text { PROD VC }\end{array}$ & 80.47 \\
\hline 6725740 & 27-Jun-05 & $\begin{array}{l}\text { BANK ONE NA VT OUTREACH } \\
\text { CONFERENCE }\end{array}$ & 260 \\
\hline 6725740 & 27-Jun-05 & $\begin{array}{l}\text { BANK ONE NA VT OUTREACH } \\
\text { CONFERENCE }\end{array}$ & 260 \\
\hline 6725740 & 28-Jun-05 & $\begin{array}{l}\text { BANK ONE NA VT OUTREACH } \\
\text { CONFERENCE }\end{array}$ & 175 \\
\hline 6725740 & 28-Jun-05 & $\begin{array}{l}\text { BANK ONE NA VT OUTREACH } \\
\text { CONFERENCE }\end{array}$ & 175 \\
\hline 6725740 & 28-Jun-05 & $\begin{array}{l}\text { BANK ONE NA VT OUTREACH } \\
\text { CONFERENCE }\end{array}$ & 260 \\
\hline
\end{tabular}




\begin{tabular}{|c|c|c|c|}
\hline 6725740 & 28-Jun-05 & $\begin{array}{l}\text { BANK ONE NA VT OUTREACH } \\
\text { CONFERENCE }\end{array}$ & 175 \\
\hline 6725740 & 28-Jun-05 & $\begin{array}{l}\text { BANK ONE NA VT OUTREACH } \\
\text { CONFERENCE }\end{array}$ & 175 \\
\hline 6725740 & 29-Jun-05 & $\begin{array}{l}\text { BANK ONE NA VWR SCIENTIFIC } \\
\text { PROD VC }\end{array}$ & 137.99 \\
\hline 6725740 & 29-Jun-05 & BANK ONE NA AWL*PRENTICE HALL & $1,272.00$ \\
\hline 6725740 & 30-Jun-05 & $\begin{array}{l}\text { BANK ONE NA VT OUTREACH } \\
\text { CONFERENCE }\end{array}$ & 85 \\
\hline 6725740 & 30-Jun-05 & $\begin{array}{l}\text { BANK ONE NA VT OUTREACH } \\
\text { CONFERENCE }\end{array}$ & 85 \\
\hline 6725740 & 30-Jun-05 & $\begin{array}{l}\text { BANK ONE NA VT OUTREACH } \\
\text { CONFERENCE }\end{array}$ & 85 \\
\hline 6725740 & 30-Jun-05 & $\begin{array}{l}\text { BANK ONE NA VT OUTREACH } \\
\text { CONFERENCE }\end{array}$ & 85 \\
\hline 6731922 & 30-Jun-05 & WVU INSTITUTE OF TECHNOLOGY & 913.34 \\
\hline 6736784 & 5-Jul-05 & WVU INSTITUTE OF TECHNOLOGY & $-1,820.00$ \\
\hline 6742107 & 4-Jul-05 & BANK ONE NA SIGMA-ALDRICH & 23.71 \\
\hline 6860247 & 5-Aug-05 & $\begin{array}{l}\text { BANK ONE NA VWR SCIENTIFIC } \\
\text { PRODUCT }\end{array}$ & 20.19 \\
\hline 6940514 & 17-Aug-05 & $\begin{array}{l}\text { BANK ONE NA VWR SCIENTIFIC } \\
\text { PRODUCT }\end{array}$ & 58.39 \\
\hline 7059970 & 15-Sep-05 & BANK ONE NA SIGMA-ALDRICH & 138.51 \\
\hline 7094101 & 19-Sep-05 & $\begin{array}{l}\text { BANK ONE NA VWR SCIENTIFIC } \\
\text { PROD VC }\end{array}$ & 419.42 \\
\hline
\end{tabular}

Ge Exp Res \& Ed Sup \& Ser TOTAL: \$ 5,728.75

Expenditure Type: Ge Exp T\&D Employee

$\begin{array}{lrlr}6736784 & \text { 5-Jul-05 } & \text { WVU INSTITUTE OF TECHNOLOGY } \\ 6795117 & \text { 20-Jul-05 } & \begin{array}{l}\text { BANK ONE NA VT OUTREACH } \\ \text { CONFERENCE }\end{array} & 1,820.00 \\ 6795117 & \text { 20-Jul-05 } & \begin{array}{l}\text { BANK ONE NA VT OUTREACH } \\ \text { CONFERENCE }\end{array} & -75 \\ 6795117 & \text { 20-Jul-05 } & \begin{array}{l}\text { BANK ONE NA VT OUTREACH } \\ \text { CONFERENCE }\end{array} \\ 6860247 & \text { 1-Aug-05 } & \text { BANK ONE NA RWW-MICROTEL } \\ 6860247 & \text { 1-Aug-05 } & \text { BANK ONE NA RWW-MICROTEL } \\ 6860247 & \text { 1-Aug-05 } & \text { BANK ONE NA RWW-MICROTEL } \\ 6860247 & \text { 1-Aug-05 } & \text { BANK ONE NA RWW-MICROTEL } & -75 \\ 6860247 & \text { 1-Aug-05 } & \text { BANK ONE NA RWW-MICROTEL } & 120.88 \\ & & \end{array}$

Ge Exp T\&D Employee TOTAL: \$ 2,199.40

Expenditure Category: Grad Assts Fellows Stud Emp

General Expenses TOTAL: \$ 8,160.65

Expenditure Type: STD Students

3651888 15-Sep-04 Rader, Jeremy Richard

119.33

A:Adjustments:17 2004 Semi-Month:DA 19684 14-JUL-2005 01 

19684 14-JUL-2005 01 19684 14-JUL-2005 01

3651891 15-Nov-04 Rader, Jeremy Richard A:Adjustments:21 2004 Semi-Month:DA

119.33 19684 14-JUL-2005 01

3651892

30-Nov-04 Rader, Jeremy Richard

A:Adjustments:22 2004 Semi-Month:DA 19684 14-JUL-2005 01

3651893

15-Dec-04 Rader, Jeremy Richard

A:Adjustments:23 2004 Semi-Month:DA 19684 14-JUL-2005 01

3651894

31-Jan-05 Rader, Jeremy Richard

A:Adjustments:2 2005 Semi-Month:DA 19684 14-JUL-2005 01

3651895

15-Feb-05 Rader, Jeremy Richard

A:Adjustments:3 2005 Semi-Month:DA 19684 14-JUL-2005 01

3651896

28-Feb-05 Rader, Jeremy Richard

A:Adjustments:4 2005 Semi-Month:DA 19684 14-JUL-2005 01

3651897 15-Jul-04 Henson, Mary F A:Adjustments:13 2004 Semi-Month:DA 19684 14-JUN-2005 02

3651898 31-Jul-04 Henson, Mary F A:Adjustments:14 2004 Semi-Month:DA 19684 14-JUN-2005 02

114.36

74.58

46.14

141.71

95.57

74.95

36.71

3651899 15-Aug-04 Henson, Mary F A:Adjustments:15 2004 Semi-Month:DA 19684 14-JUN-2005 02

242.49

3651900 31-Aug-04 Henson, Mary F A:Adjustments:16 2004 Semi-Month:DA 19684 14-JUN-2005 02

3651901 15-Sep-04 Henson, Mary F A:Adjustments:17 2004 Semi-Month:DA 19684 14-JUN-2005 02

3651902 30-Sep-04 Henson, Mary F A:Adjustments:18 2004 Semi-Month:DA 19684 14-JUN-2005 02

3651903 15-Jul-04 Chambers, Charis J A:Adjustments:13 2004 Semi-Month:DA 19684 14-JUN200503

3651904 31-Jul-04 Chambers, Charis J A:Adjustments:14 2004 Semi-Month:DA 19684 14-JUN200503

3651905

15-Aug-04 Chambers, Charis J A:Adjustments:15 2004 Semi-Month:DA 19684 14-JUN200503

3651906 31-Aug-04 Chambers, Charis J A:Adjustments:16 2004 Semi-Month:DA 19684 14-JUN200503

3651907 30-Jun-04 Davis, Chad G A:Adjustments:12 2004 Semi-Month:DA 19684 14-JUN-2005 04

296.74

207.4

169.11

602.16

481.73

433.55

457.64 


$\begin{array}{cclr}3651908 & \text { 15-Jul-04 } & \begin{array}{l}\text { Davis, Chad G A:Adjustments:13 2004 } \\ \text { Semi-Month:DA 19684 14-JUN-2005 04 }\end{array} & 371.24 \\ 3651909 & \text { 31-Jul-04 } & \begin{array}{l}\text { Davis, Chad G A:Adjustments:14 2004 } \\ \text { Semi-Month:DA 19684 14-JUN-2005 04 }\end{array} & 329.99 \\ 3651910 & \text { 15-Aug-04 } & \begin{array}{l}\text { Davis, Chad G A:Adjustments:15 2004 } \\ \text { Semi-Month:DA 19684 14-JUN-2005 } 04\end{array} & 300.53 \\ 3651911 & \text { 31-Aug-04 } & \begin{array}{l}\text { Davis, Chad G A:Adjustments:16 2004 } \\ \text { Semi-Month:DA 19684 14-JUN-2005 04 }\end{array} & 385.97 \\ 3651912 & \text { 15-Sep-04 } & \begin{array}{l}\text { Davis, Chad G A:Adjustments:17 2004 } \\ \text { Semi-Month:DA 19684 14-JUN-2005 04 }\end{array} & 152.88 \\ 3651913 & 30-S e p-04 & \begin{array}{l}\text { Davis, Chad G A:Adjustments:18 2004 } \\ \text { Semi-Month:DA 19684 14-JUN-2005 04 }\end{array} & 85.61\end{array}$

STD Students TOTAL: \$ 6,024.94

Expenditure Category: Non Benefits Eligible

Expenditure Type: NBE Faculty Non Tenured

\begin{tabular}{|c|c|c|c|}
\hline 3453665 & 28-Feb-05 & $\begin{array}{l}\text { Squire, Richard Huffman N:Non- } \\
\text { Oracle:4 } 2005 \text { Semi- } \\
\text { Month:WVUARREAPYMAR12005R }\end{array}$ & $2,906.24$ \\
\hline 3488212 & 15-Mar-05 & $\begin{array}{l}\text { Squire, Richard Huffman N:Non- } \\
\text { Oracle:5 } 2005 \text { Semi- } \\
\text { Month:WVUARREAPYMAR22005R }\end{array}$ & 968.8 \\
\hline 3516834 & 31-Mar-05 & $\begin{array}{l}\text { Squire, Richard Huffman N:Non- } \\
\text { Oracle:6 } 2005 \text { Semi- } \\
\text { Month:WVUARREAPYAPR12005R }\end{array}$ & 968.8 \\
\hline 3545092 & 15-Apr-05 & $\begin{array}{l}\text { Squire, Richard Huffman N:Non- } \\
\text { Oracle:7 } 2005 \text { Semi- } \\
\text { Month:WVUARREAPYAPR22005R }\end{array}$ & 968.8 \\
\hline 3574874 & 29-Apr-05 & $\begin{array}{l}\text { Squire, Richard Huffman N:Non- } \\
\text { Oracle:8 } 2005 \text { Semi- } \\
\text { Month:WVUARREAPYMAY12005R }\end{array}$ & 968.74 \\
\hline 3602174 & 13-May-05 & $\begin{array}{l}\text { Squire, Richard Huffman N:Non- } \\
\text { Oracle:9 } 2005 \text { Semi- } \\
\text { Month:WVUARREAPYMAY22005R }\end{array}$ & 968.74 \\
\hline 3674358 & 30-Jun-05 & $\begin{array}{l}\text { Squire, Richard Huffman N:Non- } \\
\text { Oracle:12 } 2005 \text { Semi- } \\
\text { Month:WVUARREAPYJUL12005R }\end{array}$ & $3,874.99$ \\
\hline 3701950 & 15-Jul-05 & $\begin{array}{l}\text { Squire, Richard Huffman N:Non- } \\
\text { Oracle:13 } 2005 \text { Semi- } \\
\text { Month:WVUARREAPYJUL22005R }\end{array}$ & $1,291.67$ \\
\hline 3723905 & 29-Jul-05 & $\begin{array}{l}\text { Squire, Richard Huffman N:Non- } \\
\text { Oracle:14 } 2005 \text { Semi- } \\
\text { Month:WVUARREAPYAUG12005R }\end{array}$ & $1,291.67$ \\
\hline 3746219 & 15-Aug-05 & $\begin{array}{l}\text { Squire, Richard Huffman N:Non- } \\
\text { Oracle:15 } 2005 \text { Semi- } \\
\text { Month:WVUARREAPYAUG22005R }\end{array}$ & $1,291.67$ \\
\hline
\end{tabular}

NBE Faculty Non Tenured TOTAL: \$ 15,500.12 
Expenditure Category: Repairs and Alterations

Expenditure Type: R\&A Routine Mnt of Bldgs $<25000$

7052398 24-Jun-05 CASTO TECHNICAL SERVICES INC

1,671.11

R\&A Routine Mnt of Bldgs

Expenditure Category: Travel
Expenditure Type: Travel Within USA Not WV
$6846313 \quad$ 4-Aug-05 Hurst, Scott M

Repairs and Alterations TOTAL: \$1,671.11

330.39

Travel Within USA Not WV TOTAL: \$ 330.39

Travel TOTAL: $\$ 330.39$

AWARD

TOTAL:

$\$ 102,727.16$ 
Appendix 31: Coal Desulfurization with Hypochlorite (WV011) 
Contact Title and Number

Crosscutting Technology Development at the Center for Advanced Separation Technologies (DE-FC26-02NT41607)
Period of Performance

Starting Date: 6/1/2004

Ending date: $\quad 6 / 30 / 2007$

\begin{tabular}{|c|c|}
\hline Sub-Recipient Project Title: & $\underline{\text { Report Information: }}$ \\
\hline Coal Desulfurization with Hypochlorite & Type: $\quad$ Final \\
\hline & Number: \\
\hline & $6 / 1 / 2004$ to $6 / 30 / 2006$ \\
\hline Principal Investigators: & $7 / 30 / 2006$ \\
\hline Eung Ha Cho & WV011 - FINAL \\
\hline Co-PI: $\quad$ Ray R-K Yang & \\
\hline & Contact Information: \\
\hline Contact Address: & Phone: $\quad 304-293-2111 \times 2433$ \\
\hline Department of Chemical Engineering & $304-293-4139$ \\
\hline West Virginia University & eung.cho@mail.wvu.edu \\
\hline P.O. Box 6102 & \\
\hline Morgantown, WV 26506-6102 & \\
\hline & Subcontractor Information: \\
\hline Subcontractor Address: & Phone: \\
\hline & Fax: \\
\hline No subcontracts issued & Email: \\
\hline
\end{tabular}




\section{DISCLAIMER}

This report was prepared as an account of work sponsored by an agency of the United States Government. Neither the United States Government nor any agency thereof, nor any of their employees, makes any warranty, express or implied, or assumes any legal liability, apparatus, product, or process disclosed, or represents that its use would not infringe privately owned rights. Reference herein to any specific commercial product, process, or service by trade name, trademark, manufacturer, or otherwise does not necessarily constitute or imply endorsement, recommendation, or favoring by the United States Government or any agency thereof. The views and opinions of authors expressed herein do not necessarily state or reflect those of the United States Government or any agency thereof. 


\begin{abstract}
Wet desulfurization of Upper Freeport coal and Illinois No. 6 coal (IBC 112) were conducted with sodium hypochlorite in laboratory. Upper Freeport coal was leached by hypochlorite under the various temperatures and $\mathrm{pHs}$. The temperature was varied from 60 to $90^{\circ} \mathrm{C}$, and the $\mathrm{pH}, 12.1$ to 13.5 . The hypochlorite concentration was 0.49 molar. On the other hand, the desulfurization of Illinois No. 6 coal (IBC 112) was conducted in one-cycle or three-cycle operation. In one-cycle operation, the coal was leached at $70^{\circ} \mathrm{C}$. In three-cycle operation, the coal was leached at room temperature and hydrolyzed in a sodium hydroxide solution at $90^{\circ} \mathrm{C}$, with this two-step operation repeated two more times.

The desulfurization method of Upper Freeport coal was found to reduce mainly pyritic sulfur. Approximately 59\% of pyritic sulfur was removed at the optimum conditions of 0.49 molar hypochlorite, 0.81 molar sodium hydroxide and $90^{\circ} \mathrm{C}$. The desulfurization methods for Illinois No. 6 coal (IBC 112) were not capable of reducing significant amounts of either pyritic sulfur or organic sulfur. The maximum pyritic sulfur reduction achieved was $37 \%$ and that of organic sulfur was only $18.5 \%$. This level of organic sulfur removal was much lower than that of Illinois No. 6 coal (IBC 101 ) being $37.8 \%$ which was found in the previous project funded by CAST. The large difference between these two values is considered to be due to the large difference in surface total acid concentration; that is, $0.88 \mathrm{vs} .3 .14 \mathrm{mmol} / \mathrm{g}$ coal.
\end{abstract}




\section{TABLE OF CONTENTS}

Disclaimer..............................................................

Abstract......................................................................

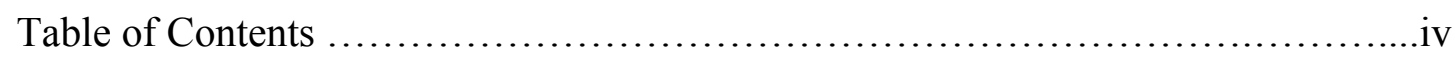

List of Tables.............................................................

Chapter 1. Introduction..................................................

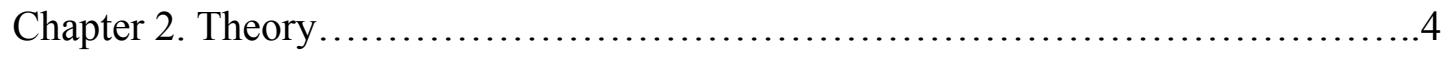

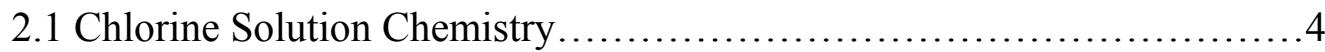

2.2 Hypochlorite Leaching........................................4

2.3 Coal Oxidation with Hypochlorite..................................6 6

Chapter 3. Experimental Methods...........................................

3.1 Materials........................................................

3.2 Experimental Apparatus............................................

3.3 Experimental Procedures........................................ 8

3.4 Analyses...................................................... 9

Chapter 4. Results and Discussion.........................................................13

4.1 Upper Freeport Coal............................................... 13

4.2 Illinois No. 6 Coal (IBC 112) ..................................... 14

Chapter 5. Conclusions................................................. 18

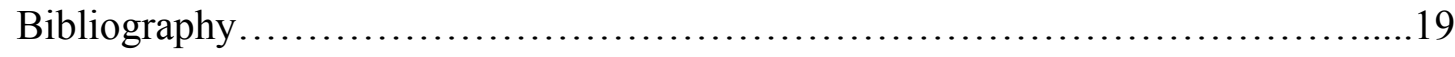

Acknowledgements...................................................21 


\section{LIST OF TABLES}

Table 1. Analytical Results for Untreated Coal....................................

Table 2. Effect of Temperature on the Desulfurization of Upper Freeport Coal

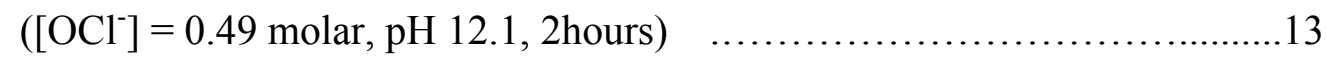

Table 3. Effect of $\mathrm{pH}$ on the Desulfurization of Upper Freeport Coal

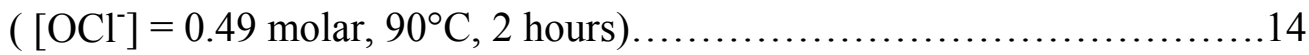

Table 4. Effect of Hypochlorite Concentration on the Desulfurization of Illinois No. 6 Coal (IBC 112) with One Cycle $\left(70^{\circ} \mathrm{C},[\mathrm{NaOH}]=0.4\right.$ molar,

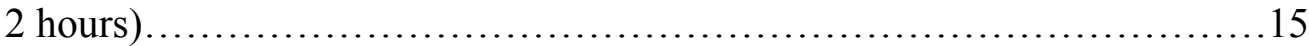

Table 5. Three-Cycle Desulfurization of Illinois No. 6 Coal (IBC 112)..............16

Table 6. Another Three-Cycle Desulfurization of Illinois No. 6 Coal (IBC 112).....17 


\section{CHAPTER 1}

\section{INTRODUCTION}

Coal is a very complex material and its chemical composition varies widely.

Of all the elements found in coal, sulfur is the single most important one which impedes the utilization of coal as a clean fuel. Many U.S. steam coals contain high percentages of sulfur which must be reduced as air pollution regulations become increasingly more stringent. According to Clean Air Acts of 1976 and 1990, sulfur dioxide emission from coal-fired power plants should be limited to $1.2 \mathrm{lb} / \mathrm{MBTU}$, which can be translated to a total sulfur content in normal coal below $0.8 \mathrm{wt} \%$.

Coal has three sources of sulfur: pyritic, organic and sulfate sulfur. The distribution of these sulfur contents and the total sulfur vary much from coal to coal produced in the U.S.. The total sulfur varies in the range of $2-4 \mathrm{wt} \%$ and the organic sulfur and pyritic sulfur contents are almost equally partitioned in many coals. The sulfate sulfur content is usually very small with its content being lower than $0.2 \%$.

Pyritic sulfur refers to ferrous disulfide $\left(\mathrm{FeS}_{2}\right)$. Much of the pyritic sulfur can be removed by physical separation methods such as gravity separation and froth flotation processes. The froth flotation process can remove up to $50 \%$ pyritic sulfur. However, the limitation of physical cleaning methods is that very fine pyrite particles are disseminated in the coal particles and those that are not exposed to the surface and not liberated are not amenable to physical separation.

Organic sulfur is part of, and chemically bonded to the coal matrix and it cannot be removed unless the chemical bonds holding it are broken. Meyers (Meyers, 1977) summarized the structure of the organic sulfur in the coal to include mercaptans 
(RSH), sulfides ( $\mathrm{R}-\mathrm{S}-\mathrm{R}$ '), disulfides ( $\mathrm{RS}-\mathrm{S}-\mathrm{R}^{\prime}$ ) and thiophenes where $\mathrm{R}$ and $\mathrm{R}$ ' stand for hydrocarbon groups. It is reported that certain forms of organic sulfur are amenable to removal by hypochlorite (Brubaker and Stoicos, 1985).

Some chemical methods were developed in the past; however, none has been commercialized. This may be largely because the organic sulfur cannot be removed easily. Chlorine and its derivatives are among a few regents that can remove organic sulfur from coal. The Chlorinolysis process was developed by Hsu et al. (1977). Chlorine gas and methyl chloroform were utilized to leach pyritic and organic sulfur at $74^{\circ} \mathrm{C}$. Methyl chloroform was used to solubilize bubbled chlorine gas. The coal matrix was chlorinated during the leaching. The chlorine content in the coal reached up to $25 \%$ in two hours. The coal was effectively dechlorinated by treating it with steam at a temperature of $500^{\circ} \mathrm{C}$. This process could remove up to $70 \%$ of organic sulfur and $77 \%$ of pyritic sulfur, and $76 \%$ of total sulfur.

Cho (1989) conducted a similar study in a system where Sewickley seam coal was leached in a 0.1-N hydrochloric acid solution through which chlorine gas was bubbled. The results showed that most of the pyritic sulfur and approximately $40 \%$ of the organic sulfur were removed at room temperature. It was observed that the chlorine content in the leach coal reached up to $31 \%$.

A similar desulfurization scheme with sodium hypochlorite (Brubarker and Stoicos, 1985) was utilized to remove substantial amounts of organic sulfur but not much pyritic sulfur from Illinois No. 6 coal. Further treatment with sodium carbonate at $80^{\circ} \mathrm{C}$ (hydrolysis) reduced the organic sulfur additionally. The organic sulfur was reduced by $62 \%$ when different coals were leached and subsequently hydrolyzed for 
one hour each, and this treatment was repeated twice more. The pyritic sulfur was reduced by only $23 \%$ under the same treatment. Another interesting result of this leaching scheme was that hypochlorite chlorinated the coal matrix only slightly, or much less severely, than aqueous chlorine. It was observed that the chlorine levels in the coal ranged between 2 and $2.7 \%$.

The disadvantage of the use of aqueous chlorine in the Chlorinolysis process is that it chlorinates the coal matrix extensively and that treatment at a high temperature $\left(500^{\circ} \mathrm{C}\right)$ is needed to restore the chlorinated coal. The cost of this treatment is high so that the entire desulfurization process with aqueous chlorine would not be feasible for commercialization. However, Brubaker and Stoicos (1985) have proven that desulfurization with hypochlorite does not readily chlorinate the coal structure, while it can reduce the organic sulfur as well as the aqueous chlorine process. However, they could not maintain the chlorine content in the treated coal under the threshold value.

The objectives of this study are:

(1) To determine the optimum conditions to maximize the sulfur removal from Upper Freeport coal and Illinois No. 6 coal (IBC 112) and at the same time to maintain the chlorine content under the threshold value;

(2) To determine the technical feasibility for commercialization of this technology. 


\section{CHAPTER 2}

\section{THEORY}

\subsection{Chlorine Solution Chemistry}

Sodium hypochlorite solution is not stable at low $\mathrm{pHs}$ due to the equilibrium reactions of chlorine (Garrels, 1965):

$$
\begin{array}{ll}
\mathrm{HClO}=\mathrm{H}^{+}+\mathrm{ClO}^{-} & \mathrm{K}=3.3 \times 10^{-8} \\
\mathrm{HClO}+\mathrm{Cl}^{-}+\mathrm{H}^{+}=\mathrm{Cl}_{2(\mathrm{aq})}+\mathrm{H}_{2} \mathrm{O} & \mathrm{K}=2.3 \times 10^{3} \\
\mathrm{Cl}_{2(\mathrm{~g})}=\mathrm{Cl}_{2(\mathrm{aq})} & \mathrm{K}=0.02 \\
\mathrm{Cl}_{2(\mathrm{aq})}+\mathrm{Cl}^{-}=\mathrm{Cl}_{3}^{-} & \mathrm{K}=0.2
\end{array}
$$

where $\mathrm{K}$ is the equilibrium constant at $25^{\circ} \mathrm{C}$. At high $\mathrm{pH}(>10)$, hypochlorite $\mathrm{ClO}^{-}$is predominant. Hypochlorous acid, $\mathrm{HClO}$, will become dominant when $\mathrm{pH}$ decreases. At $\mathrm{pH} 7.5$, the two species have the same concentration. Below 7.5, aqueous chlorine will form and escape as gaseous chlorine. So sodium hydroxide should be added to the hypochlorite solution to maintain high $\mathrm{pH}$ and stabilize the original hypochlorite concentration.

\subsection{Hypochlorite Leaching}

Some of the organic sulfur in coal may exist as carbon sulfide and disulfide. These sulfides react with hypochlorite to produce sulfonyl chloride ( $\mathrm{RSOCl}^{-}$) as shown (Hsu et al., 1977):

$$
\begin{aligned}
& \mathrm{R}-\mathrm{S}-\mathrm{R}^{\prime}+2 \mathrm{ClO}^{-}=\mathrm{RSClO}^{-}+\mathrm{R}^{\prime} \mathrm{ClO}^{-} \\
& \mathrm{RS}-\mathrm{S}-\mathrm{R}^{\prime}+2 \mathrm{ClO}^{-}=\mathrm{RSClO}^{-}+\mathrm{R}^{\prime} \mathrm{SClO}^{-}
\end{aligned}
$$

$\mathrm{R}$ and $\mathrm{R}^{\prime}$ stand for the hydrocarbon groups and $\mathrm{S}$ refers to sulfur. The sulfonyl chloride is further oxidized to sulfonate or sulfate: 


$$
\begin{aligned}
& 2 \mathrm{RSClO}^{-}+\mathrm{H}_{2} \mathrm{O}+3 \mathrm{ClO}^{-}=2 \mathrm{RSO}_{3} \mathrm{H}+5 \mathrm{Cl}^{-} \\
& 2 \mathrm{RSClO}^{-}+\mathrm{H}_{2} \mathrm{O}+5 \mathrm{ClO}^{-}=2 \mathrm{RCl}+2 \mathrm{H}^{+}+2 \mathrm{SO}_{4}{ }^{2-}+5 \mathrm{Cl}^{-}
\end{aligned}
$$

Reactions (7) and (8) are electrochemical reactions and their corresponding anodic reactions are:

$$
\begin{aligned}
& \mathrm{RSClO}^{-}+2 \mathrm{H}_{2} \mathrm{O}=\mathrm{RSO}_{3} \mathrm{H}+3 \mathrm{H}^{+}+\mathrm{Cl}^{-}+3 \mathrm{e} \\
& \mathrm{RSClO}^{-}+3 \mathrm{H}_{2} \mathrm{O}=\mathrm{RCl}+6 \mathrm{H}^{+}+\mathrm{SO}_{4}{ }^{2-}+5 \mathrm{e}
\end{aligned}
$$

And the cathodic reaction is:

$$
\mathrm{ClO}^{-}+2 \mathrm{H}^{+}+2 \mathrm{e}=\mathrm{Cl}^{-}+\mathrm{H}_{2} \mathrm{O} \quad \mathrm{E}^{0}\left(\text { at } 25^{\circ} \mathrm{C}\right)=1.72 \mathrm{eV}
$$

Pyritic sulfur also can be leached with hypochlorite. The anodic reaction is:

$$
\mathrm{FeS}_{2}+11 \mathrm{H}_{2} \mathrm{O}=\mathrm{Fe}(\mathrm{OH})_{3}(\mathrm{~s})+2 \mathrm{SO}_{4}{ }^{2-}+19 \mathrm{H}^{+}+15 \mathrm{e}
$$

$\mathrm{E}^{0}$ (given in cathodic reaction at $\left.25^{\circ} \mathrm{C}\right)=-0.4 \mathrm{eV}$

Then the overall reaction is:

$$
\begin{gathered}
2 \mathrm{FeS}_{2}+15 \mathrm{ClO}^{-}+7 \mathrm{H}_{2} \mathrm{O}=2 \mathrm{Fe}(\mathrm{OH})_{3}(\mathrm{~s})+15 \mathrm{Cl}^{-}+4 \mathrm{SO}_{4}{ }^{2-}+8 \mathrm{H}^{+} \\
\Delta \mathrm{G}^{\mathrm{o}}\left(\text { at } 25^{\circ} \mathrm{C}\right)=-1466.6 \mathrm{kcal} / \mathrm{mole}
\end{gathered}
$$

The magnitude of $\Delta \mathrm{G}^{\mathrm{o}}$ suggests that the overall reaction is thermodynamically very spontaneous under normal conditions.

The second step of the desulfurization is the hydrolysis step in which further removal of the organic sulfur and dechlorination will take place. In this step, the leach coal will be treated in a solution containing sodium hydroxide around $90^{\circ} \mathrm{C}$. The following reactions may take place in the hydrolysis step:

$$
\begin{aligned}
& \mathrm{RCl}+\mathrm{OH}^{-}=\mathrm{ROH}+\mathrm{Cl}^{-} \\
& \mathrm{RSO}_{3} \mathrm{H}+\mathrm{H}_{2} \mathrm{O}=\mathrm{ROH}+2 \mathrm{H}^{+}+\mathrm{SO}_{3}{ }^{2-}
\end{aligned}
$$




\subsection{Coal Oxidation with Hypochlorite}

It is known that coal is oxidized with hypochlorite, resulting in its weight loss. It is reported that sodium hypochlorite oxidizes Illinois No. 6 coal. The oxidation yields several products, depending on the $\mathrm{pH}$ and the kind of the coal (Mayo and Kirshen, 1979; Chakrabartty, 1978; Mayo, 1975). The products vary from black, highmolecular weight, bicarbonate-soluble acids to the benzene polycarboxylic acids and carbon dioxide. For example, at $\mathrm{pH} 13,96 \%$ of the coal was dissolved, and $80 \%$ of the carbon dissolved was found as the high-molecular-weight acids (Mayo and Kirshen, 1979). Between $\mathrm{pH} 9$ and 11, the production of soluble acids was lower while that of $\mathrm{CO}_{2}$ was higher. Below pH 9 more coal structure was destroyed - simple, oxidationresistant benzene and aliphatic carboxylic acids were the principle organic products in the solution. According to Chakrabartty (Chakrabartty, 1978), Mayo and Kirshen studied the oxidation of Illinois No. 6 coal with hypochlorite. It was found that in one experiment, $80 \%$ loss of the original carbon was accounted for as follows: $13.6 \%$ in undissolved residue, $59.4 \%$ in colored acids soluble in aqueous bicarbonate, $7.1 \%$ in lighter colored acid readily soluble in water and 19.9\% in carbon dioxide. 


\section{CHAPTER 3}

\section{EXPERIMENTAL METHODS}

\subsection{Materials}

Upper Freeport coal and Illinois No. 6 coal (IBC 112) were used in this study. Upper Freeport coal was obtained from the TJS Coal Mine, Shelocta, PA. Illinois No. 6 coal (IBC 112) was obtained from the Illinois Geological Survey. Each bulk coal sample was crushed and screened to produce a $65 \times 150$ mesh fraction which was used in this study. These fractions were analyzed and the results are given in Table 1.

Table 1. Analytical Results of Untreated Coal

\begin{tabular}{lcc}
\hline & Upper Freeport Coal & IBC 112 \\
\hline Ash, \% & 9.12 & 9.00 \\
Pyritic sulfur, \% & 0.88 & 0.46 \\
Organic sulfur, \% & 0.89 & 1.68 \\
Sulfate sulfur, \% & 0.01 & 0.01 \\
Total sulfur, \% & 1.78 & 2.15 \\
Total acid (mmol/g) & 0.45 & 0.88 \\
Chlorine content (\%) & 0.05 & 0.03 \\
\hline
\end{tabular}

Hypochlorite solutions to be used for the leaching experiments were prepared by diluting the hypochlorite stock solution which was purchased from a chemical supplier. It had about 2.14 molar hypochlorite concentration and $\mathrm{pH} 12.3-12.6$. This stock solution was kept in a refrigerator to minimize the loss due to evaporation.

\subsection{Experimental Apparatus}

The leaching experiments were conducted in a one-liter reactor placed in a constant-temperature paraffin-oil bath. The reactor had four necks. The central neck was equipped with a stirrer connected to a variable-speed motor. The stirring speed 
was $500 \mathrm{rpm}$ for all the experiments. One of the side necks was fitted with a reflux condenser which was used to prevent excessive evaporation at high temperatures. The next one was equipped with a thermometer which was used to measure the temperature of the solution. And the last one was used for charging the coal sample.

\subsection{Experimental Procedures}

\subsubsection{Upper Freeport Coal}

A 500-ml hypochlorite solution at the desired concentration was charged into the reactor. When the leach solution reached the desired temperature, fifty grams of coal sample was added to the reactor. The leaching continued for 2 hours. After leaching, the coal slurry was filtered on Whatman No.1 paper. The filtered coal was dried naturally in air for overnight, weighed and analyzed for total sulfur, pyritic sulfur, sulfate sulfur, chlorine content and ash content. Leaching conversions of pyritic sulfur and organic sulfur were determined using these data. The experiments were conducted at various temperatures $\left(60,75\right.$ and $\left.90^{\circ} \mathrm{C}\right)$ and $\mathrm{pHs}(12.1-13.5)$.

\subsubsection{Illinois No. 6 Coal (IBC 112)}

It was found that Illinois No 6 coal (IBC 112) could not be leached above $70^{\circ} \mathrm{C}$, mainly because of excessive loss of coal during leaching. The coal was leached at this temperature only in one cycle. The procedures were the same as for the Upper Freeport coal. Also, the coal was treated with two steps of leaching with hypochlorite at room temperature and hydrolysis at high $\mathrm{pH}$ and high temperature. This two-stage treatment was repeated two more times on the same sample.

In this latter option, fifty grams of coal was leached at room temperature with a $500 \mathrm{ml}$ hypochlorite solution in the reactor. The coal was stirred at $500 \mathrm{rpm}$ for one 
hour, then filtered and dried. The filtration was conducted on Whatman No.1 paper after decantation of the ultra-fine particles. The dried coal was weighed to determine the weight loss. About 2 grams of the dried coal was taken for analyses of total sulfur, pyritic sulfur and chlorine content. And the remaining coal sample was then hydrolyzed.

The hydrolysis was conducted in the reactor. A 500-ml sodium hydroxide solution was charged to the reactor and when the temperature reached $90^{\circ} \mathrm{C}$, the leach coal was added to start the hydrolysis. After hydrolysis for one hour, the coal was filtered and dried using the same method as mentioned previously. Again, about 2 grams of the dried coal was taken to be analyzed for total sulfur, pyritic sulfur and chlorine content. This is the end of the first cycle. The second and the third cycles were made in order to reduce more sulfur content by repeating the leaching and hydrolysis. The leaching was conducted with the dried coal which resulted from the hydrolysis step of the previous cycle. All the procedures were the same as in the first cycle.

\subsection{Analyses}

Total sulfur was analyzed according to ASTM method D3177-84, (Eschka Method). A weighed sample was well mixed with Eschka mixture and burned in a furnace at $800^{\circ} \mathrm{C}$. All the sulfur was transformed into sulfate and then dissolved into hot water. The sulfate was precipitated as barium sulfate, which was filtered, burned in a furnace and weighed.

Pyritic sulfur was analyzed according to ASTM method D2492-84. Coal was first leached with hydrochloric acid $(2+3-2$ volumes of concentrated $\mathrm{HCl}$ and 3 
volumes of water) to remove non-pyritic iron. Pyritic iron was again leached with nitric acid (1+7 - 1 volume of concentrated $\mathrm{HNO}_{3}$ and 7 volumes of water). This solution which contained the solubilized iron was analyzed by an atomic absorption spectrophotometer, which was stoichiometrically converted to pyritic sulfur.

Sulfate sulfur was analyzed according to ASTM method D2492-84. Sulfate sulfur was extracted together with non-pyritic iron using hydrochloric acid (2+3) solution and was precipitated as barium sulfate. The precipitate was filtered, burned in a furnace and weighed.

Organic sulfur was calculated by subtracting the combination of pyritic sulfur and sulfate sulfur from the total sulfur.

Ash content in coal was analyzed according to ASTM method D2795-85 and moisture content was analyzed according to ASTM method D3173-87.

Chlorine content in the coal was analyzed according to ASTM method D2361-85, (Eschka Method). A weighed sample was well mixed with Eschka mixture in a furnace at $675^{\circ} \mathrm{C}$. The chlorine content was dissolved as chloride ion with nitric acid solution (1+1 - 1 volume each of concentrated nitric acid and water). The chloride ion was analyzed by a back titration method using potassium thiocyanate with ferric ammonium sulfate as an indicator. In this method, an excessive amount of silver nitrate solution was added, chloride ion reacted with silver ion to silver chloride precipitate which was filtered out subsequently, and the unreacted silver ion in the filtrate was titrated with the titration reagent.

Chlorine concentration of the hypochlorite solution was analyzed according to ASTM method D2022-64. Solid potassium iodide was added to the chlorine- 
containing solution and dissolved. The solution was acidified by adding concentrated acetic acid. The aqueous chlorine oxidizes the iodide to iodine, which was titrated by sodium thiosulfate solution using starch as an indicator.

The concentration of the total acidic groups on the coal surface was analyzed in order to particularly explain the drastically different leaching behaviors between Upper Freeport coal and Illinois No. 6 (IBC 112) coal. The method adopted by Luo (Luo, 1994) was used for the determination of total acidic groups on the coal surface. The coal sample was contacted with barium ion in a solution. Then the barium ion exchanged with $\mathrm{H}^{+}$in the carboxylic and phenolic groups to form precipitates on the coal surface. The $\mathrm{H}^{+}$ions were produced. The following reactions were involved:

$$
\begin{aligned}
& 2 \mathrm{RCOOH}(\mathrm{s})+\mathrm{Ba}^{2+}=(\mathrm{RCOO})_{2} \mathrm{Ba}(\mathrm{s})+2 \mathrm{H}^{+} \\
& 2 \mathrm{ArOH}(\mathrm{s})+\mathrm{Ba}^{2+}=(\mathrm{ArO})_{2} \mathrm{Ba}(\mathrm{s})+2 \mathrm{H}^{+}
\end{aligned}
$$

where (s) denotes a solid species at the coal surface, $\mathrm{RCOOH}$ is carboxylic acid group and $\mathrm{ArOH}$ stands for phenolic group. The total acidic groups are taken as the combination of carboxylic and phenolic groups. The $\mathrm{H}^{+}$ions produced according to reactions 16 and 17 were titrated with sodium hydroxide solution.

For determination of the total acidic group content on coal, one gram of asreceived or oxidized coal sample was transferred into a $200 \mathrm{ml}$ plastic bottle. Before transferring the sample, the bottle was purged with nitrogen, and while still purging, $100 \mathrm{ml}$ of $0.3 \mathrm{~N} \mathrm{Ba}(\mathrm{OH})_{2}$ solution was pipetted into the bottle. The bottle was then sealed and agitated at room temperature on a shaker for 16 hours. The contents of each bottle with nitrogen purging was then vacuum-filtered using No. 1 Whatman filter paper. Immediately after this filtration step, $25 \mathrm{ml}$ of the filtrate was pipetted into the 
titrator cup, which already contained $30 \mathrm{ml}$ of $0.3 \mathrm{~N} \mathrm{HCl}$ and $10 \mathrm{ml}$ of $\mathrm{CO}_{2}$-free distilled water, and this solution was titrated with $0.2 \mathrm{~N} \mathrm{NaOH}$ using m-cresol purple as the indicator. A blank titration was also performed so that the content of total acidic groups in the coal (in meq/g coal) could be calculated. These procedures determined the concentrations of $\mathrm{Ba}(\mathrm{OH})_{2}$ in the blank test and the test with coal sample. The difference in concentrations of $\mathrm{Ba}(\mathrm{OH})_{2}$ in these two tests determined the concentration of the total acidic groups. 


\section{CHAPTER 4}

\section{RESULTS AND DISCUSSION}

\subsection{Upper Freeport Coal}

Two series of experiments were conducted; namely, those for temperature effect and $\mathrm{pH}$ effect. It was found throughout all the experiments that the loss of coal due to oxidation with hypochlorite was negligible.

Hypochlorite leaching was conducted at 60,75 , and $90^{\circ} \mathrm{C}$. No solid sodium hydroxide was added to the hypochlorite solution for this series of experiments. Table 2 presents the leaching results.

\section{Table 2. Effect of Temperature on the Desulfurization of Upper Freeport Coal} $\left(\left[\mathrm{OCl}^{-}\right]=0.49 \text { molar, } \mathrm{pH} \text { 12.1, } 2 \text { hours }\right)^{*}$

\begin{tabular}{|c|c|c|c|}
\hline Temperature, ${ }^{\circ} \mathrm{C}$ & \multicolumn{1}{|c|}{60} & \multicolumn{1}{c|}{75} & 90 \\
\hline $\mathrm{TS}, \%$ & $1.59(10.7)$ & $1.49(16.3)$ & $1.33(25.3)$ \\
\hline $\mathrm{PS}, \%$ & $0.67(23.9)$ & $0.59(32.9)$ & $0.48(45.5)$ \\
\hline $\mathrm{OS}, \%$ & $0.89(0)$ & $0.85(4.5)$ & $0.79(11.2)$ \\
\hline $\mathrm{Cl}, \%$ & 0.93 & 1.35 & 1.75 \\
\hline
\end{tabular}

*Values in parentheses are reduction percentages.

Table 2 shows that the reduction in each sulfur form increases as temperature increases. The total sulfur was reduced by only $25.3 \%$ at $90^{\circ} \mathrm{C}$. The reduction values in total sulfur are mainly attributed to the reductions in pyritic sulfur not by those in organic sulfur. The pyritic sulfur content was reduced by $45.5 \%$ at $90^{\circ} \mathrm{C}$. However, very small amounts of organic sulfur were reduced. The organic sulfur was reduced by $11.2 \%$ at $90^{\circ} \mathrm{C}$. Table 2 also shows that the chlorine content in the leach coal increases 
with increasing temperature. The chlorine content reaches $1.75 \%$ at $90^{\circ} \mathrm{C}$. This is well above the threshold value. The threshold value is generally accepted as a level of $0.3 \%$ chlorine in coal (Wandless, 1957).

Since the reduction in sulfur forms is at maximum at $90^{\circ} \mathrm{C}$ as shown in Table 2, this temperature was used for the next series of experiments. The series was conducted particularly in order to reduce the chlorine content in the leach coal by adding various amounts of solid sodium hydroxide, i.e., by changing the $\mathrm{pH}$ of the hypochlorite solution. The results are given in Table 3.

Table 3. Effect of pH on the Desulfurization of Upper Freeport Coal $\left(\left[\mathrm{OCl}^{-}\right]=0.49 \text { molar, } 90^{\circ} \mathrm{C}, 2 \text { hours }\right)^{*}$

\begin{tabular}{|c|c|c|c|c|}
\hline $\mathrm{pH}$ & 12.1 & 13.0 & 13.3 & 13.5 \\
\hline $\mathrm{NaOH}, \mathrm{M}$ & 0 & 0.2 & 0.4 & 0.81 \\
\hline $\mathrm{TS}, \%$ & $1.33(25.3)$ & $1.17(34.3)$ & $1.25(29.8)$ & $1.25(29.8)$ \\
\hline $\mathrm{PS}, \%$ & $0.48(45.5)$ & $0.36(59.1)$ & $0.36(59.1)$ & $0.36(59.1)$ \\
\hline $\mathrm{OS}, \%$ & $0.82(7.9)$ & $0.81(9.0)$ & $0.89(0)$ & $0.89(0)$ \\
\hline $\mathrm{Cl}, \%$ & 1.75 & 0.72 & 0.44 & 0.22 \\
\hline
\end{tabular}

*Values in parentheses are reduction percentages.

The $\mathrm{pH}$ was varied from 12.1 to 13.5 as shown in Table 3. The data in Table 3 show that the reduction of each sulfur form is at maximum at $\mathrm{pH} 13$ and further increase in $\mathrm{pH}$ does not increase the reduction. However, this $\mathrm{pH}$ is not an optimum condition because the chlorine content is above the threshold value $(0.3 \%)$. The optimum condition would rather be at $\mathrm{pH} 13.5$ in this respect.

\subsection{Illinois No. 6 Coal (IBC 112)}


Illinois No. 6 coal (IBC 112) was leached at $70^{\circ} \mathrm{C}$. This was the maximum temperature above which the coal reacted violently with hypochlorite to give a layer of bubbles on top of the solution believed to be carbon dioxide. It is suspected that the difference of leaching behavior between the two coals (Upper Freeport and Illinois No. 6 (IBC 112)) is due to the difference in surface total acid concentration, 0.45 vs. $0.88 \mathrm{mmol} / \mathrm{g}$. In other words, the Illinois No. 6 coal (IBC 112) which has higher surface total acid reacts more violently with hypochlorite, leading to much higher coal weight loss.

Table 4 shows the results under the various hypochlorite concentrations from 0.4 to 0.8 molar.

Table 4. Effect of Hypochlorite Concentration on the Desulfurization of Illinois No. 6 Coal (IBC 112) $\left(7^{\circ} \mathrm{C},[\mathrm{NaOH}]=0.4 \text { molar, } 2 \text { hours }\right)^{*}$

\begin{tabular}{|c|c|c|c|}
\hline$\left[\mathrm{OCl}^{-}\right], \mathrm{M}$ & 0.4 & 0.6 & 0.8 \\
\hline $\mathrm{TS}, \%$ & $1.89(12.1)$ & $1.73(19.5)$ & $1.86(13.5)$ \\
\hline $\mathrm{PS}, \%$ & $0.34(26.1)$ & $0.33(28.3)$ & $0.32(30.4)$ \\
\hline OS, \% & $1.57(6.5)$ & $1.56(7.1)$ & $1.54(8.3)$ \\
\hline $\mathrm{Cl}, \%$ & 0.15 & 0.25 & 0.49 \\
\hline Wt Loss, \% & 16.8 & 23.8 & 23.6 \\
\hline
\end{tabular}

${ }^{*}$ Values in parentheses are reduction percentages.

It can be seen that the sulfur reduction is generally low especially for the organic sulfur and the weight loss is exorbitantly high. Thus the coal was desulfurized in leach-andhydrolysis cycles under low concentrations of hypochlorite, and the results are presented in Tables 5 and 6. 
Table 5. Three-Cycle Desulfurization of Illinois No. 6 Coal (IBC 112)*

\begin{tabular}{|l|l|l|l|l|l|l|}
\hline \multirow{2}{*}{} & \multicolumn{2}{|l|}{ First Stage } & \multicolumn{2}{l|}{ Second Stage } & \multicolumn{2}{l|}{ Third Stage } \\
\cline { 2 - 6 } & $\begin{array}{l}\text { Leaching, } \\
25^{\circ} \mathrm{C}\end{array}$ & $\begin{array}{l}\text { Hydrolysis, } \\
90^{\circ} \mathrm{C}\end{array}$ & $\begin{array}{l}\text { Leaching, } \\
25^{\circ} \mathrm{C}\end{array}$ & $\begin{array}{l}\text { Hydrolysis, } \\
90^{\circ} \mathrm{C}\end{array}$ & $\begin{array}{l}\text { Leaching, } \\
25^{\circ} \mathrm{C}\end{array}$ & $\begin{array}{l}\text { Hydrolysis, } \\
90^{\circ} \mathrm{C}\end{array}$ \\
\hline$\left[\mathrm{OCl}^{-}\right], \mathrm{M}$ & 0.2 & & 0.2 & & 0.2 & \\
\hline$[\mathrm{NaOH}], \mathrm{M}$ & & 0.4 & & 0.4 & & 0.4 \\
\hline $\mathrm{TS}, \%$ & $\begin{array}{l}1.96 \\
(8.8)\end{array}$ & $\begin{array}{l}1.84 \\
(14.4)\end{array}$ & $\begin{array}{l}1.77 \\
(17.7)\end{array}$ & $\begin{array}{l}1.79 \\
(16.7)\end{array}$ & $\begin{array}{l}1.69 \\
(21.4)\end{array}$ & $\begin{array}{l}1.69 \\
(21.4)\end{array}$ \\
\hline $\mathrm{PS}, \%$ & 0.44 & 0.37 & 0.32 & 0.34 & 0.28 & 0.34 \\
$(4.3)$ & $(19.6)$ & $(30.4)$ & $(26.1)$ & $(39.1)$ & $(26.1)$ \\
\hline OS, \% & 1.52 & $\begin{array}{l}1.47 \\
(12.5)\end{array}$ & $\begin{array}{l}1.45 \\
(13.7)\end{array}$ & $\begin{array}{l}1.45 \\
(13.7)\end{array}$ & $\begin{array}{l}1.41 \\
(16.1)\end{array}$ & $\begin{array}{l}1.45 \\
(13.7)\end{array}$ \\
\hline Cl, \% & $(9.5)$ & 0.23 & 1.12 & 0.33 & 1.44 & 0.42 \\
\hline $\begin{array}{l}\text { Cum. Wt. } \\
\text { Loss, \% }\end{array}$ & 0.6 & 0.8 & 7.0 & 9.8 & 15.6 & 18.8 \\
\hline
\end{tabular}

* Values in parentheses are reduction percentages.

Table 5 shows the results of the experiments conducted with 0.2 molar hypochlorite for leaching and 0.4 molar sodium hydroxide for hydrolysis and repeated this twice more. It can be seen that the reductions in total sulfur and organic sulfur are more than those with one cycle as shown in Table 4. Also, it can be seen that the coal weight loss is lower than that with the one cycle. However, the level of reduction in sulfur forms does not show any effectiveness of this desulfurization method. The reduction in particularly organic sulfur is compared with that of different Illinois No. 6 coal (IBC 101) (Wendi Li and Eung Ha Cho, 2005). Its reduction of this coal (IBC 101) is much higher than that of IBC 112 . It is by $37.8 \%$ as opposed to $13.7 \%$ as shown in Table 5. This large difference may be attributed to the difference in surface total acid, with 3.14 (IBC 101) vs. $0.88 \mathrm{mmol} / \mathrm{g}$ (IBC 112). However, higher reactivity of the organic sulfur with higher surface total acid cannot be explained at this moment. 
The next series of experiments were conducted under high concentrations of hypochlorite and sodium hydroxide for the leaching and hydrolysis and reduced concentrations of them as the cycle increased. The results are shown in Table 6.

Table 6. Another Three-Cycle Desulfurization of Illinois No. 6 Coal (IBC 112)*

\begin{tabular}{|l|l|l|l|l|l|l|}
\hline \multirow{2}{*}{} & \multicolumn{2}{|l|}{ First Stage } & \multicolumn{2}{l|}{ Second Stage } & \multicolumn{2}{l|}{ Third Stage } \\
\cline { 2 - 7 } & $\begin{array}{l}\text { Leaching, } \\
25^{\circ} \mathrm{C}\end{array}$ & $\begin{array}{l}\text { Hydrolysis, } \\
90^{\circ} \mathrm{C}\end{array}$ & $\begin{array}{l}\text { Leaching, } \\
25^{\circ} \mathrm{C}\end{array}$ & $\begin{array}{l}\text { Hydrolysis, } \\
90^{\circ} \mathrm{C}\end{array}$ & $\begin{array}{l}\text { Leaching, } \\
25^{\circ} \mathrm{C}\end{array}$ & $\begin{array}{l}\text { Hydrolysis, } \\
90^{\circ} \mathrm{C}\end{array}$ \\
\hline$\left[\mathrm{OCl}^{-}\right], \mathrm{M}$ & 0.4 & & 0.2 & & 0.1 & \\
\hline$[\mathrm{NaOH}], \mathrm{M}$ & & 0.8 & & 0.4 & & 0.3 \\
\hline $\mathrm{TS}, \%$ & 1.90 & 1.82 & 1.76 & 1.76 & 1.73 & 1.66 \\
& $(11.6)$ & $(15.3)$ & $(18.1)$ & $(18.1)$ & $(19.5)$ & $(22.8)$ \\
\hline $\mathrm{PS}, \%$ & $\begin{array}{l}0.44 \\
(4.3)\end{array}$ & $\begin{array}{l}0.38 \\
(17.3)\end{array}$ & $\begin{array}{l}0.32 \\
(30.4)\end{array}$ & $\begin{array}{l}0.30 \\
(34.8)\end{array}$ & $\begin{array}{l}0.35 \\
(23.9)\end{array}$ & $\begin{array}{l}0.29 \\
(37)\end{array}$ \\
\hline $\mathrm{OS}, \%$ & 1.46 & 1.44 & $\begin{array}{l}1.44 \\
(14.3)\end{array}$ & $\begin{array}{l}1.46 \\
(13.1)\end{array}$ & $\begin{array}{l}1.38 \\
(17.9)\end{array}$ & $\begin{array}{l}1.37 \\
(18.5)\end{array}$ \\
\hline $\mathrm{Cl}, \%$ & 1.45 & 0.38 & 1.32 & 0.36 & 0.94 & 0.34 \\
\hline $\begin{array}{l}\text { Cum. Wt. } \\
\text { Loss, \% }\end{array}$ & 2.4 & 7.0 & 10.6 & 16.2 & 19.8 & 22.0 \\
\hline
\end{tabular}

* Values in parentheses are reduction percentages.

It is seen from Table 6 that the reduction of organic sulfur in this series is slightly more that that in the other series, and the chlorine content is lower than that also in the other series. However, the improvements are by slight margins. Thus this desulfurization method by leaching and hydrolysis is not particularly effective on IBC 112 coal.

As shown in Tables 5 and 6, the third cycle desulfurization does not add more benefit to the overall desulfurization particularly in terms of organic sulfur reduction and coal weight loss. In this respect, this coal desulfurization may well be carried out with two cycles. 


\section{CHAPTER 5}

\section{CONCLUSIONS}

1. The desulfurization of Upper Freeport coal with hypochlorite should be made in one cycle of leaching at $90^{\circ} \mathrm{C}$ while that of Illinois No. 6 coal (IBC 112) requires two consecutive cycles of leaching with hypochlorite at room temperature and hydrolysis at $90^{\circ} \mathrm{C}$.

2. The desulfurization method for upper Freeport coal can reduce mainly pyritic sulfur by $59.1 \%$ at the optimum condition of 0.49 molar hypochlorite and at $\mathrm{pH}$ 13.5.

3. The desulfurization method with Illinois No. 6 coal (IBC 112) cannot reduce significant amounts of pyritic sulfur or organic sulfur. The maximum organic sulfur reduction was only $18.5 \%$. This level of organic sulfur removal was much lower than that of Illinois No. 6 coal (IBC 101) being 37.8\% which was found in the previous project funded by CAST. The large difference between these two values is considered to be due to the large difference in surface total acid concentration; 0.88 vs. $3.14 \mathrm{mmol} / \mathrm{g}$ coal.

4. The desulfurization method with one cycle or two cycles does not seem to promise commercialization potential for the desulfurization of Upper Freeport coal or Illnois No. 6 coal (IBC 112). 


\section{Bibliography}

Brubaker, R. B. and Stoicos, T., 1985, "Precombustion Coal Desulfurization with Sodium Hypochlorite," Proceeding of the First International Conference on Processing and Utilization of High Sulfur Coal, Oct. 13-17, Columbus, Ohio, edited by Y. A. Attia, pp. 311-326.

Chakrabartty, Sujit K, 1978, “Oxidation of Coal by Alkaline Sodium Hypochlorite,” ACS Symposium Series, 71 (Org. Chem. Coal), 100-7.

Cho, E. H., 1989, “Coal Desulfurization with Aqueous Chlorine,” Met. Trans. B, vol. 20B, Oct., 567-571.

Garrels, R. M., 1965, "Solutions, Minerals and Equilibrium,” Freeman, Cooper and Company, San Francisco, p. 407.

Hsu, G. C., Kalvinskas, J. J., Ganguli, P. S. and Gavalas, G. R., 1977, “Coal Desulfurization by Low-Temperature Chlorinolysis," Coal Desulfurization, edited by Wheelock T. D., ACS Sym. Series 64, pp.207-217.

Luo, Qiuliang, 1994, “Coal Oxidation and Calcium Loading on Oxidized Coals,” Master of Science Thesis, West Virginia University.

Mayo, Frank R, 1975, “Application of Sodium Hypochlorite Oxidations to the Structure of Coal," Fuel, 54(4), 273-5.

Mayo, Frank R. and Kirshen, Norman A., 1979, “Oxidation of Coal by Aqueous Sodium Hypochlorite," Fuel, 58(10), 698-704.

Meyers, R. A., 1977, “Coal Desulfurization,” Marcel Dekker, New York, p.18.

Wandless, A. M., 1957, “British Coal Seams: A Review of the Properties with Suggestions for Research,” J. Inst. Fuel, 30, 545-8. 
Wendi, Li and Cho, Eung Ha, 2005, "Coal Desulfurization with Sodium Hypochlorite," Energy \& Fuels, 19, 499-507. 


\section{Acknowledgements}

The Upper Freeport coal sample provided by Mr. Jim Szalankiewicz, TJS Coal Mine, at Shelocta, PA is greatly appreciated. 
Appendix 32: Phyto-Extraction / Fabrication of Gold Nanoparticles (WV012) 
FINAL TECHNICAL REPORT

Contract Title and Number: Crosscutting Technology Development at the Center for Advanced Separation Technologies (DE-FC26-02NT41607)
Period of Performance:

Starting Date: 06/01/2004

Ending Date: 08/31/2009
Sub-Recipient Project Title:

Phyto-Extraction/Fabrication of Gold Nanoparticles

Principal Investigators:

Ray Y. K. Yang, Eung Ha Cho

Contact Address:

Ray Y. K. Yang

Chemical Engineering Department

West Virginia University

Morgantown WV 26506-6102

Subcontractor Address:

No subcontracts issued
Report Information:

Type: Final

Number:

Period: 06/01/04-08/31/09

Date: $\quad$ 08/28/09

Code: WV012-FINAL

Contact Information:

Phone: (304) 293 2111x2419

Fax:

E-Mail: ryang@mail.wvu.edu

Subcontractor Information:

Phone:

Fax:

E-Mail:

\section{DISCLAIMER}

This report was prepared as an account of work sponsored by an agency of the United States Government. Neither the United States Government nor any agency thereof, nor any of their employees, make any warranty, express or implied, nor assume any legal liability or responsibility for the accuracy, completeness, or usefulness of any information, apparatus, product, or process disclosed, or represents that its use would not infringe privately owned rights. Reference herein to any specific commercial product, process, or service by trade name, trademark, manufacturer, or otherwise does not necessarily constitute or imply endorsement, recommendation, or favoring by the United States Government or any agency thereof. The views and opinions of authors expressed herein do not necessarily state or reflect those of the United States Government or agency thereof. 


\begin{abstract}
This project investigated the extracellular biosynthesis of gold nanoparticles from the leaf extracts of ruptured cells of three cultivars of scented geranium (Pelargonium graveolens). It also studied the uptake of gold ions from gold solution by the seedlings of alfalfa (Medicago sativa) and mung bean (Vigna radiate) and by the suspended cells of stevia (Stevia rebaudiana), periwinkle (Catharanthus roseus), and carrot (Daucus carota) for their intracellular accumulations in the cells of these plant systems. Hopefully, the insight gathered from this research will contribute to future efforts in the manufacture of gold nanoparticles using more environmentally friendly approaches.

Gold nanoparticles were produced extracellularly from three cultivars of geranium: 'Rose' (R), 'Rober's Lemon Rose' (RR), and 'Lady Plymouth' (LP). The nanoparticle-formation reactions appeared to be very rapid, achieving high concentrations of gold nanoparticles in about one-half to one hour. Colors characteristic of gold nanoparticles in aqueous solution as a result of surface plasmon resonance were exhibited, and all the nanoparticles produced, though cultivar-dependent, were found to remain stable for several months. We also found that these bioreactions of geranium extract with potassium tetrachloroaurate solution to produce gold nanoparticles did not require light, and that oxygen appeared to have negligible effect on the progression of the reactions.

Uptake of gold ions from dilute potassium tetrachloroaurate solution to the seedlings of mung bean and alfalfa grown from seeds on two different types of sprouter was confirmed. Uptake of gold from the media of suspension cultures of periwinkle, stevia, and carrot (both light and dark), which contained chloroaurate ions, to the suspended plant cells was also confirmed.

Intracellular gold contents in the plant seedlings and the suspended cells were noticeably enhanced, overall, as the gold concentrations in contact with the suspended plant cells or the sprouts increased, indicating the extraction and transportation of gold ions into the cells of both plant systems studied. More importantly, the contents of gold in the cells cultivated in suspension cultures were, in general, two to several-fold higher compared to that in the sprouts, implying the long-term potential of using suspension culture of plant cells for more environmentally friendly large-scale production of gold nanoparticles.
\end{abstract}




\section{Table of Contents}

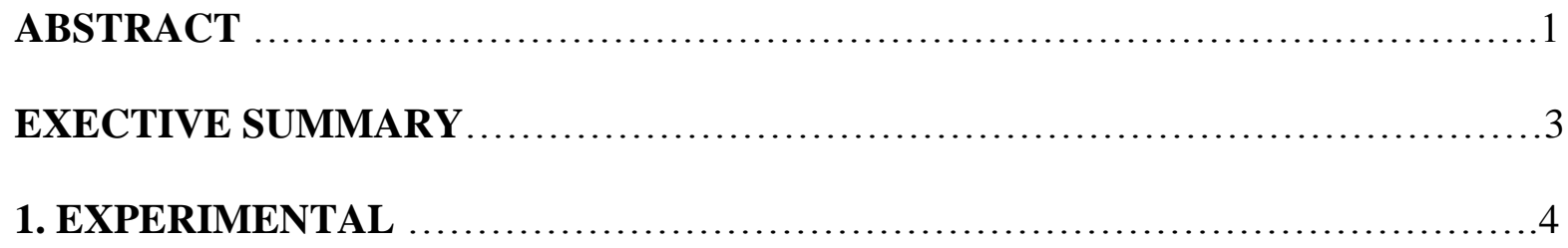

1.1 Gold Nanoparticles from Geranium Extract $\ldots \ldots \ldots \ldots \ldots \ldots \ldots \ldots \ldots \ldots \ldots \ldots \ldots \ldots$

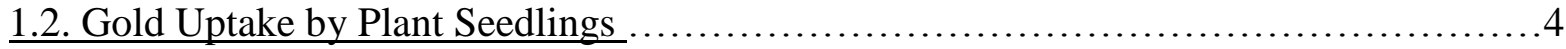

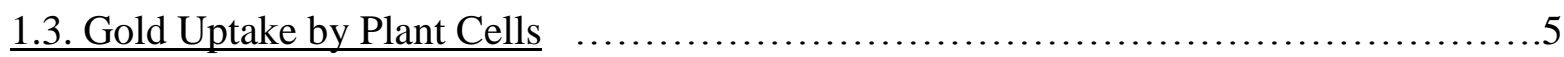

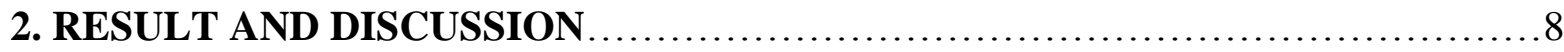

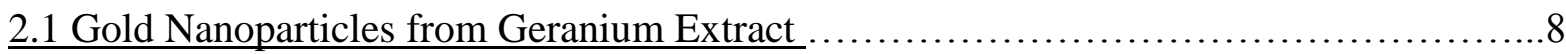

$\underline{2.2 \text { Gold Uptake by Plant Seedlings } \ldots \ldots \ldots \ldots \ldots \ldots \ldots \ldots \ldots \ldots \ldots \ldots \ldots \ldots \ldots \ldots \ldots \ldots \ldots \ldots \ldots \ldots}$

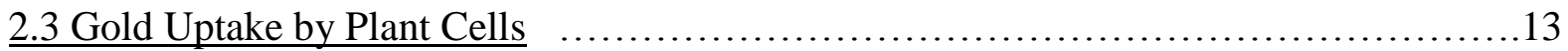

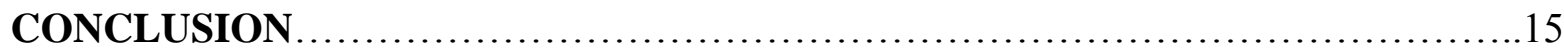

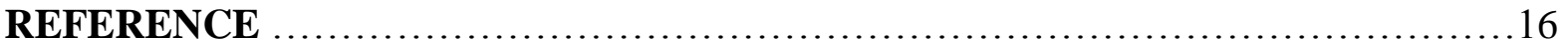




\section{EXECUTIVE SUMMARY}

Gold nanoparticles have typically been made by chemical methods using toxic compounds. Only recently has biological synthesis employing bacteria, fungi, and plants to produce gold nanoparticles been explored. This research encompasses topics involving biochemical processes that employ plants and plant systems. The project investigated the extracellular biosynthesis of gold nanoparticles from the leaf extracts of ruptured cells of three cultivars of scented geranium (Pelargonium graveolens). It also studied the uptake of gold ions from gold solutions by the seedlings of alfalfa (Medicago sativa) and mung bean (Vigna radiate) and by the suspended cells of stevia (Stevia rebaudiana), periwinkle (Catharanthus roseus), and carrot (Daucus carota) for their intracellular accumulations in the cells of these plant systems. Much of the research is intended to reveal more information about both extracellular and intracellular phyto-formations of gold nanoparticles. Hopefully, the insight gathered from this research will contribute to future efforts in the manufacture of gold nanoparticles using more environmentally friendly approaches.

Gold nanoparticles were produced extracellularly from three cultivars of geranium: 'Rose' (R), 'Rober's Lemon Rose' (RR), and 'Lady Plymouth' (LP). The nanoparticle-formation reactions appeared to be very rapid, achieving high concentrations of gold nanoparticles in about one-half to one hour. Colors characteristic of gold nanoparticles in aqueous solution as a result of surface plasmon resonance were exhibited, and the nanoparticles produced, though cultivar-dependent, were found to remain stable for several months. We found that these bioreactions of geranium extract with potassium tetrachloroaurate solution to produce gold nanoparticles did not require light, and that oxygen appeared to have negligible effect on the progression of the reactions. We noticed that the value of initial $\mathrm{pH}$ of the reaction mixture did have an impact on the morphology of the nanoparticles produced, as different $\mathrm{pH}$ values led to products that exhibited progressively different colors. Chemical simulation conducted using sodium borohydride to produce gold nanoparticles in aqueous solution, with trisodium citrate as a capping agent, showed the development of color very similar to that resulting from the reactions with the geranium extract.

Uptake of gold ions from $\mathrm{AuCl}_{4}{ }^{-}$- containing solution into the suspended cells of stevia, carrot, and periwinkle, as well as the sprouts of mung bean and alfalfa, was observed with no noticeable toxic effect on the plant systems used. We found that the intracellular contents of gold in the cells of carrot, stevia, and periwinkle in suspension cultures, and also in the sprouts of mung bean and alfalfa, were noticeably enhanced, overall, as the gold concentration in contact with the plant cells or the sprouts increased, confirming the extraction and transportation of gold ions into the cells of the plants tested. This observation was true for both types of sprouters used, though gold uptake in A-sprouter was several-fold higher than that in S-sprouter due to the fact that all parts (root, stem, and leaf) of the sprouts were exposed to the silver-containing sprouter solution in A-sprouter, while in S-sprouter only roots of the sprouters were in contact with the solution. More importantly, the contents of gold in the cells cultivated in suspension cultures were, in general, two to several-fold higher compared to that in the sprouts, implying the long-term potential of using suspension culture of plant cells for more environmentally friendly large-scale production of gold nanoparticles. 


\section{EXPERIMENTAL}

\subsection{Gold Nanoparticles from Geranium Extract}

Various experiments were performed using geranium extract as a reactant. Extract was prepared using leaves of three cultivars of scented geranium (Pelargonium graveolens): 'Rose' (R), 'Rober's Lemon Rose' (RR), and 'Lady Plymouth' (LP). The leaves were ground finely, water was added, and the mixture was then boiled for a short period of time. Afterward, the extracted leave mass was separated from the solution via filtration and discarded, while the extract, which was the pale yellow liquid filtrate, was collected. Then a small amount of the extract was mixed with different volumes of $1 \mathrm{mM}$ potassium tetrachloroaurate $\left(\mathrm{KAuCl}_{4}\right)\left(196.97 \mathrm{ppm} \mathrm{Au}^{3+}\right)$ solution to start the reactions. All reactions took place at approximately $25^{\circ} \mathrm{C}$ in clear $125 \mathrm{ml}$ glass jars or $30 \mathrm{ml}$ glass vials.

Several variables were tested to find factors that influence the reaction. In some situations, these samples were continuously exposed to fluorescent light. Other samples were kept in darkness. Exposure to air was also altered to determine if oxygen had any role in the reaction. Further studies involved examining the progress of the reaction with $\mathrm{KAuCl}_{4}$ in an environment in which $\mathrm{pH}$ had been adjusted immediately after reactants were mixed together, and the resulting products were analyzed. Initial $\mathrm{pH}$ values used range from 3.5 to 6.0 .

To provide more evidence that gold nanoparticles were present, the reactions were simulated chemically. Experiments were conducted using sodium borohydride $\left(\mathrm{NaBH}_{4}\right)$ to reduce gold ions in $1 \mathrm{mM} \mathrm{KAuCl}_{4}$ solution with no involvement of geranium extract. The reduction was performed in the presence of trisodium citrate, which acted as a capping agent to stabilize the nanoparticles. This chemical reduction allowed for comparison between the nanoparticle solutions produced chemically and those produced biologically using the geranium extract.

\subsection{Gold Uptake by Plant Seedlings}

The existence of gold nanoparticles in live alfalfa grown on agar-agar was observed using Xray absorption spectroscopy and transmission electron microscopy by Gardea-Torresdey et al. [1]. They reported that alfalfa sprouts grown in gold-rich agar media actively uptake $\mathrm{Au}^{\mathrm{O}}$ from the media and accumulate them as nanoparticles in their tissues. They thought that the $\mathrm{Au}^{3+}$ was reduced to $\mathrm{Au}^{\mathrm{o}}$ in the solid media and the latter was extracted by the roots of plant seedlings and transported to their shoots.

In this project we studied instead the uptake of gold ions from agar-free solutions by the seedlings of two plant species and by the suspended cells of stevia (Stevia rebaudiana), periwinkle (Catharanthus roseus), and carrot (Daucus carota) for intracellular accumulations of nanoparticles in these plant systems. The former is reported in this section and Section 2.2, while the latter in Sections 1.3 and 2.3. 
Two species of seedlings, mung bean (Vigna radiate) and alfalfa (Medicago sativa), were investigated to determine their abilities to facilitate intracellular gold uptake, a first step toward intracellular gold nanoparticle formation in living plant seedlings. The seeds were first treated with ZeroTol ${ }^{\circledR}$ solution (50 times dilution) for sterilization, and then soaked in deioned/distilled (DD) water for 24 hours, before being spread onto the screens inside a sprouter to start a sprouter run. Different dilute concentrations of potassium tetrachloroaurate $\left(\mathrm{KAuCl}_{4}\right)$ solution were provided to the seeds (before their germination) and the sprouts (after seed germination), without the addition of plant nutrients for the seeds and the seedlings.

Two types of sprouter were used: S-sprouter and A-sprouter, both were under $24 \mathrm{hr}$ fluorescent lighting at approximately $25^{\circ} \mathrm{C}$. In the S-sprouter, which had totally transparent cover, both mung bean and alfalfa were grown in the same container side by side and only the roots of the sprouts are in contact with the sprouter solution containing only dilute silver nitrate solution. On the other hand, the A-sprouter, which had partially transparent cover, periodically sprinkled seeds held on a screen (before seed germination) and all parts of the sprouts (after seed germination) with the same sprouter solution. The solution, after sprinkling over the entire plant seedlings, drained through the screen to the reservoir below, and was re-circulated with a pump to the top of the sprouter for periodic sprinkling.

After a one-week period of contact with dilute potassium tetrachloroaurate solution, starting from water-soaked seeds, the sprouts of each run were harvested. They were thoroughly washed several times, dried, ground, and burned to ash, known in phytomining as bio-ore. The ash, which included any gold nanoparticles or ions that had accumulated inside the seedlings, was dissolved in concentrated aqua regia and then diluted. Atomic absorption analysis was then conducted using a Perkin-Elmer atomic absorption spectrophotometer to find the concentration of gold formed inside the cells of the sprouts.

\subsection{Gold Uptake by Plant Cells}

Callus cultures of Catharanthus roseus (periwinkle), Stevia rebaudiana (stevia), Daucus carota (carrot), and Beta vulgaris (red beet) had all been established from their respective explants and maintained continuously by subculturing during the period of this task. The callus cultures of carrot, periwinkle, and stevia were initiated and maintained in Murashige and Skoog solid medium, while red beet was on solid agar with Gamborg's B5 medium. Two types of culture had been maintained for carrot since its initiation; one that had been kept in the dark all the time (designated as "carrot dark") and another in 12-hour light/dark (designated as "carrot light"). The cultures were kept in an incubator with controls of temperature and light cycles. Subculturing was performed at regular intervals ( $2-4$ weeks) in order to maintain a constant supply of nutrients for the established calluses. Shown in Figure 1.3.1 next page are the callus cultures of red beet, periwinkle, and carrot. 


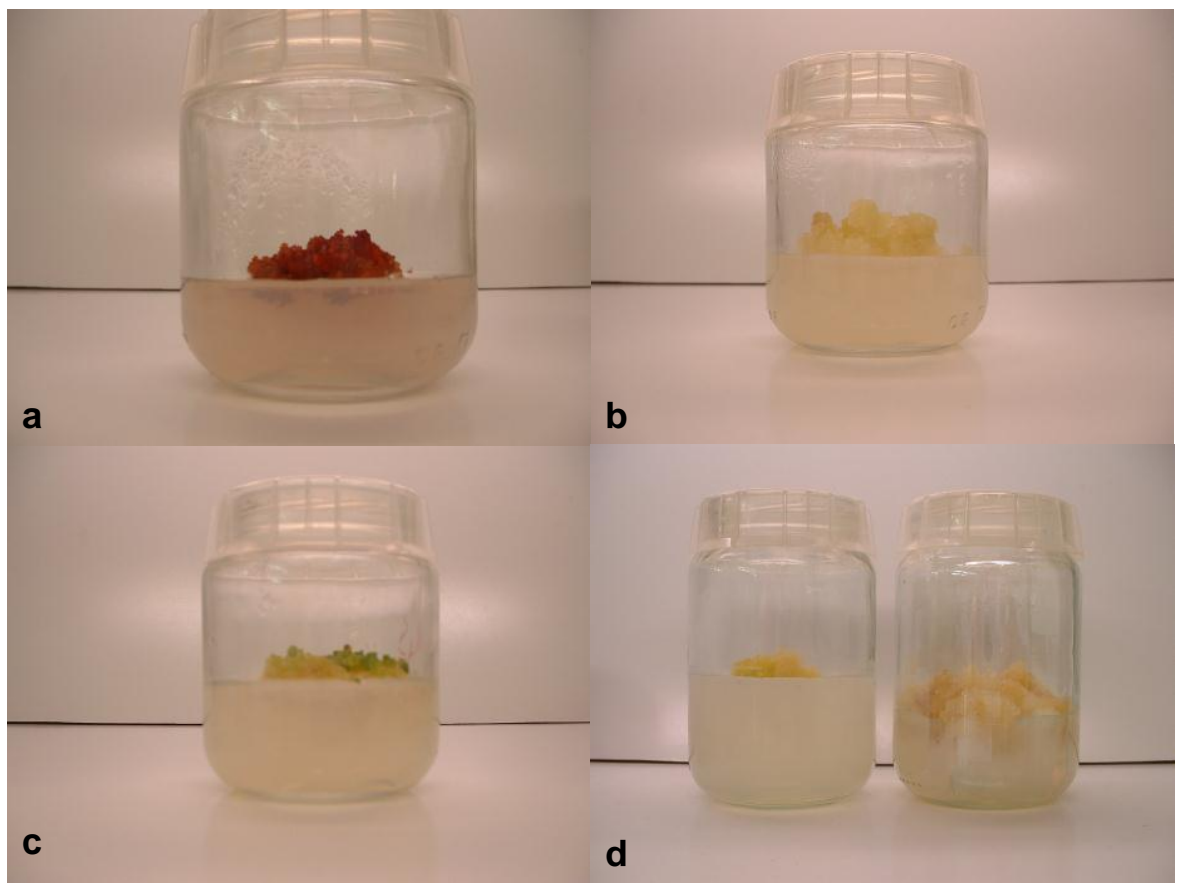

Figure 1.3.1. Callus cultures of (a) red beet, (b) periwinkle, (c) stevia, and (d) carrot, with the callus on the left grown in light and the callus on the right grown in dark.

Suspension cultures of $C$. roseus, $S$. rebaudiana, and D. carota (both carrot light and carrot dark) were also developed from the corresponding callus cultures. For suspension cultures, the medium formulation for each species is identical to that for the corresponding callus, only with agar omitted. Erlenmeyer flasks containing the suspension were agitated on an orbital shaker at 110 - 120 rpm to allow for maximum nutrient uptake by plant cells. Temperature and photoperiod were regulated; growth conditions were similar to that of the corresponding callus cultures. Subcultures were performed every 1 - 2 weeks. Hairy root cultures of stevia on agar medium and in liquid medium were also initiated and established during the period of this task; the latter is shown in Figure 1.3.2 next page. 


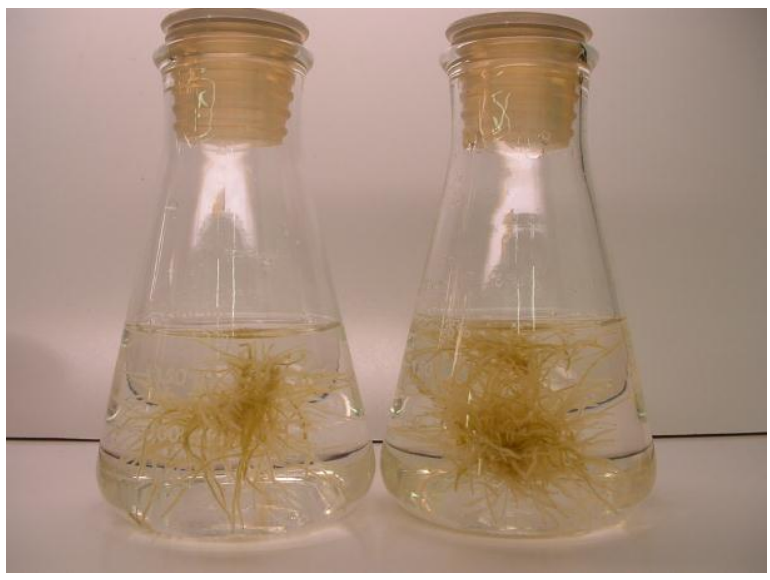

Figure 1.3.2 Stevia hairy root cultures grown under $18 \mathrm{hr}$ light, 8 days after subculturing from solid media to liquid media.

C. roseus, D. carota, and $S$. rebaudiana suspensions were studied for the uptake of gold from solution. Potassium tetrachloroaurate solution was added to the liquid media at the midpoint of subculture period. At the end of the period, cells and contents were washed six times with $200 \mathrm{ml}$ aliquots of distilled/deionized water, all under vacuum filtration. Then they were dried at $80^{\circ} \mathrm{C}$ overnight, burned in a muffle furnace for 3 hours at $700^{\circ} \mathrm{C}$ to produce a sootfree ash, dissolved in concentrated aqua regia and then diluted. Atomic absorption (AA) analysis was then conducted using a Perkin-Elmer atomic absorption spectrophotometer to find the concentration of gold formed inside the plant cells. Several concentrations of gold ions were tested for uptake by plant cells. 


\section{RESULTS AND DISCUSSION}

\subsection{Gold Nanoparticles from Geranium Extract}

Due to their nanoscale dimensions, gold nanoparticles possess surface plasmon resonances (SPR), which leads to their unique colors. The color in turn depends on the morphology of the gold nanoparticles, especially their aspect ratios - the ratio of the lengths of the largest dimension to the smallest dimension [2]. The spectra of anisotropic particles, such as nanorods, show two distinct peaks, corresponding to transverse and longitudinal oscillations. Shankar et al. [3] reported experiments in which geranium and chloroauric acid $\left(\mathrm{HAuCl}_{4}\right)$ were used to form gold nanoparticles, although the cultivar of geranium used was not mentioned. Their procedure led to the development of stable gold nanoparticles with great variability in size and morphology, displaying a number of shapes including rods, flat disks, and triangles.

As mentioned in Section 1.1, in this project we used three cultivars of scented geranium, 'Rose', 'Rober's Lemon Rose', and 'Lady Plymouth', for our investigation on extracellular formation of gold nanoparticles. Our results indicated that the bioreactions of these three cultivars of geranium with potassium tetrachloroaurate $\left(\mathrm{KAuCl}_{4}\right)$, as well as the nanoparticles produced, were cultivar-dependent. Obvious color changes of the reaction mixture were noted as soon as the reaction started and as the reaction progressed. The colors that developed were characteristic of gold nanoparticles in aqueous solution. Contrary to the formation of silver nanoparticles, this color transformation was more abrupt, and the time needed to reach its final state was much shorter.

The following definitions relating to time were used to describe the progress of the reactions more precisely. The time at which the reactants were combined is defined here as $t=0$. Immediately after this step, some reaction mixtures were placed in darkness. The period of time in which the mixture remains in darkness is termed $t_{d}$. These samples were eventually exposed to light for a period $t_{l}$, which takes place at $t>t_{d}$. Other samples had continuous exposure to light, and in this situation, $\mathrm{t}_{\mathrm{d}}=0$, and $\mathrm{t}_{\mathrm{l}} \neq 0$.

In contrast to the geranium reaction for producing silver nanoparticles, we found that this reaction did not require light. As seen in Figure 2.1.1 next page (using RR and LP as examples), reactions had no obvious difference between jars that were exposed to light and those shielded from light. Oxygen, as well, did not cause a significant visible impact on the progression of the reaction. Both early in the reaction and after ample time had passed, oxygen had no major effect, particularly with respect to LP and RR. All reactions began very quickly (colors were obvious after only 5 minutes) and lasted only about 1 hour or slightly less as indicated from the visible change in the intensity of color. From the difference in color intensity, it was observed that $\mathrm{R}$ reacted more rapidly than $\mathrm{RR}$. However, the difference became much smaller after time passed. 

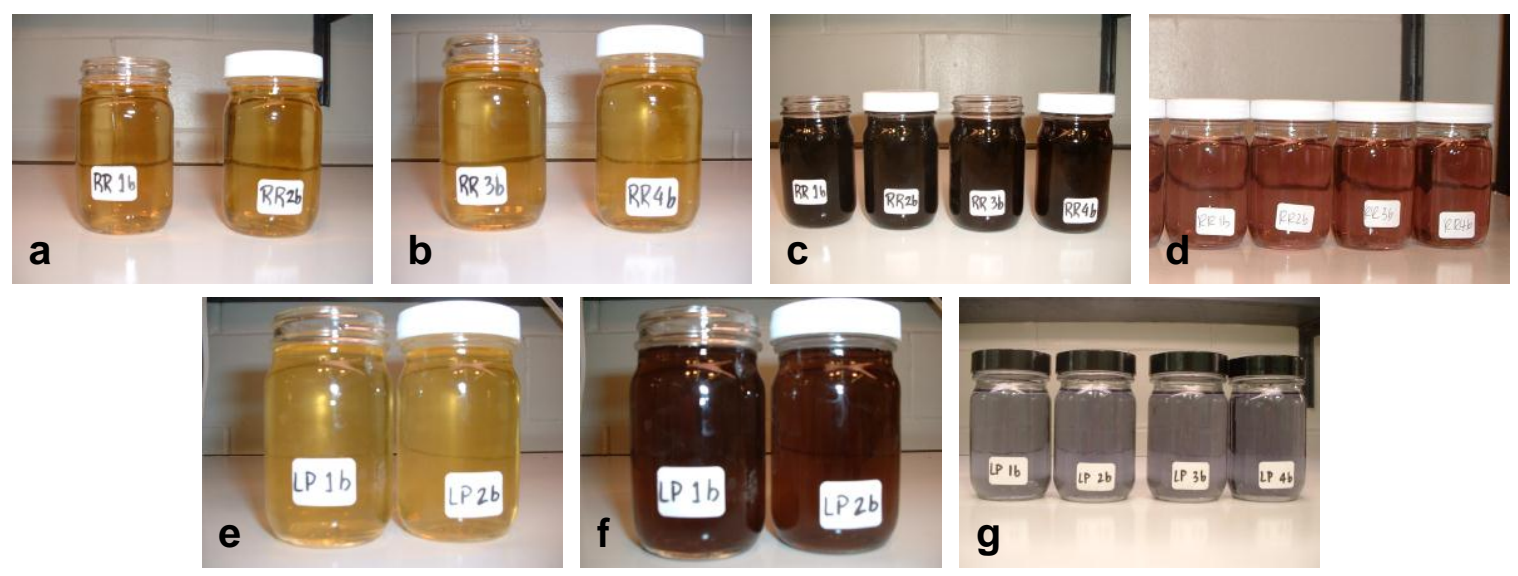

Figure 2.1.1 Effects of Light and Oxygen - Reactions of RR and LP extracts with $\mathrm{KAuCl}_{4}$ showing (a) $t_{d}=0, t_{l}=20$ min (b) $t_{d}=20$ min, $t_{l}=0$ (c) $t_{d}=0, t_{l}=65$ min for the two jars on the left, and $t_{d}=65 \mathrm{~min}, t_{1}=0$ for the two jars on the right (d) final RR products diluted 20X, (e) $t_{d}=0, t_{1}=5$ min (f) $t_{d}=0, t_{l}=30 \mathrm{~min}$, and (g) final LP products diluted 20X. (All final products were capped after $20 \mathrm{X}$ dilution).

The final results also revealed a noticeable difference in color between plant varieties. As can be seen in Figure 2.1.2 below, LP especially stood apart from the others, developing a light blue color, whereas both $\mathrm{R}$ and $\mathrm{RR}$ reacted to produce a pinkish red tint. It has been reported many times that spherical gold nanoparticles exhibit a ruby-red color in solution. Since gold nanoparticles can have a vast array of shapes and sizes, these findings suggest that LP may be forming anisotropic gold nanoparticles.
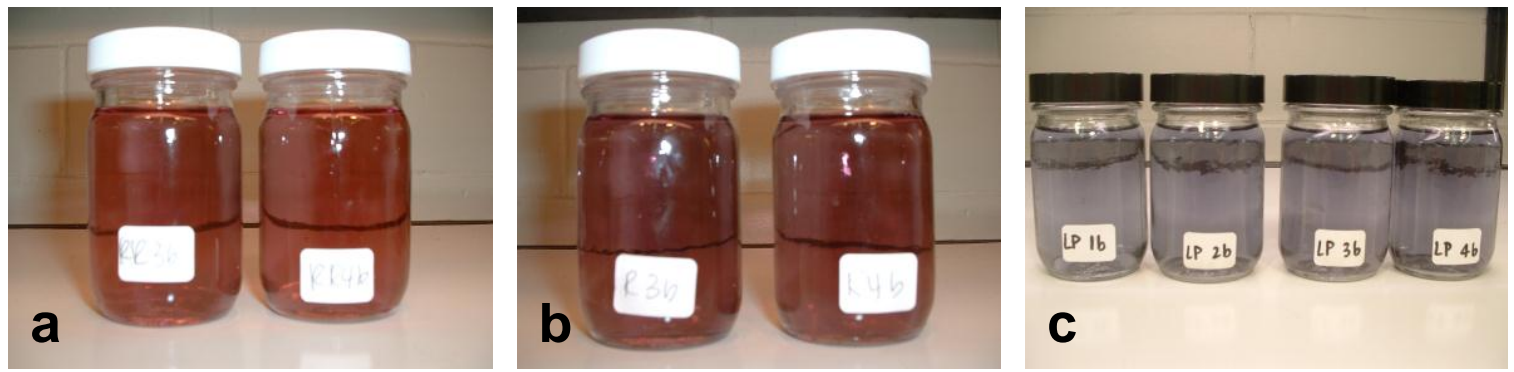

Figure 2.1.2 Final products from reactions of RR, R, and LP extracts with $\mathrm{KAuCl}_{4}$ after $20 \mathrm{X}$ dilution for (a) RR, (b) R, and (c) LP. (All final products were capped after 20X dilution).

Shown in Figure 2.1.3 below are the colors of the final mixtures with 20 time dilution so the color differences between cultivars, if any, may be detected. The three long vials shown in the picture on the left are the same vials shown in the photo on the right. In the latter, bottles having much larger diameters than the vials and containing exactly the same mixtures as in the corresponding vials are added to show the slight variations in colors due to different lengths of light paths. 

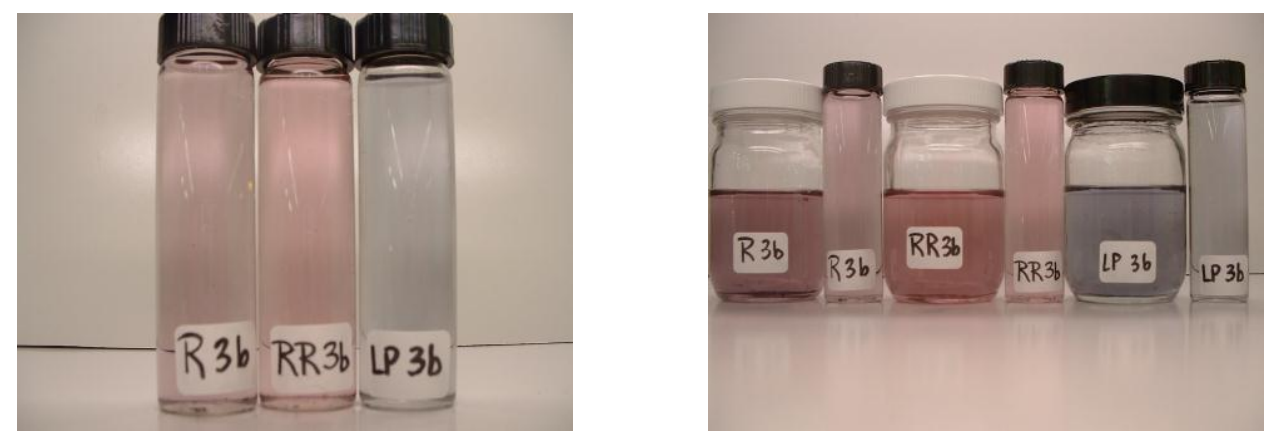

Figure 2.1.3 Colors of aqueous solution of gold nanoparticles produced from extracts of three cultivars of geranium: "Rober's Rose" (RR), “Rose”(R), and "Lady Plymouth" (LP)

To further support the assertion that formation of gold nanoparticles occurs due to the reduction of metal ions with geranium extract, sodium borohydride $\left(\mathrm{NaBH}_{4}\right)$ was used to chemically simulate the reaction with no involvement of geranium extract, as mentioned in Section 1.1. Pure $\mathrm{KAuCl}_{4}$ solutions can be seen in (a) of Figure 2.1.4 below. Addition of small amounts of $\mathrm{NaBH}_{4}$ powder to the gold solution resulted in immediate reduction of ions, causing striking displays of color. The colors produced, shown in the vial on the left in (b) of Figure 2.1.4, are typical of gold nanoparticles in solution, and are characteristic only of particles with nanoscale dimensions. It is of key importance that similar colors were obtained by the reduction of gold ions using geranium extract. Image (d) shows solutions containing gold nanoparticles reduced by $\mathrm{NaBH}_{4}$, as well as by $\mathrm{R}$ and LP extracts with no adjustment in $\mathrm{pH}$ for both. Thus, phyto-synthesis of silver nanoparticles using geranium extracts can achieve similar results as chemical-synthesis without the use of harsh chemical reagent such as $\mathrm{NaBH}_{4}$. Furthermore, no use of capping agent, such as trisodium citrate is required. Not only is this approach "greener", the nanoparticles produced remained stable for several orders of magnitude longer.

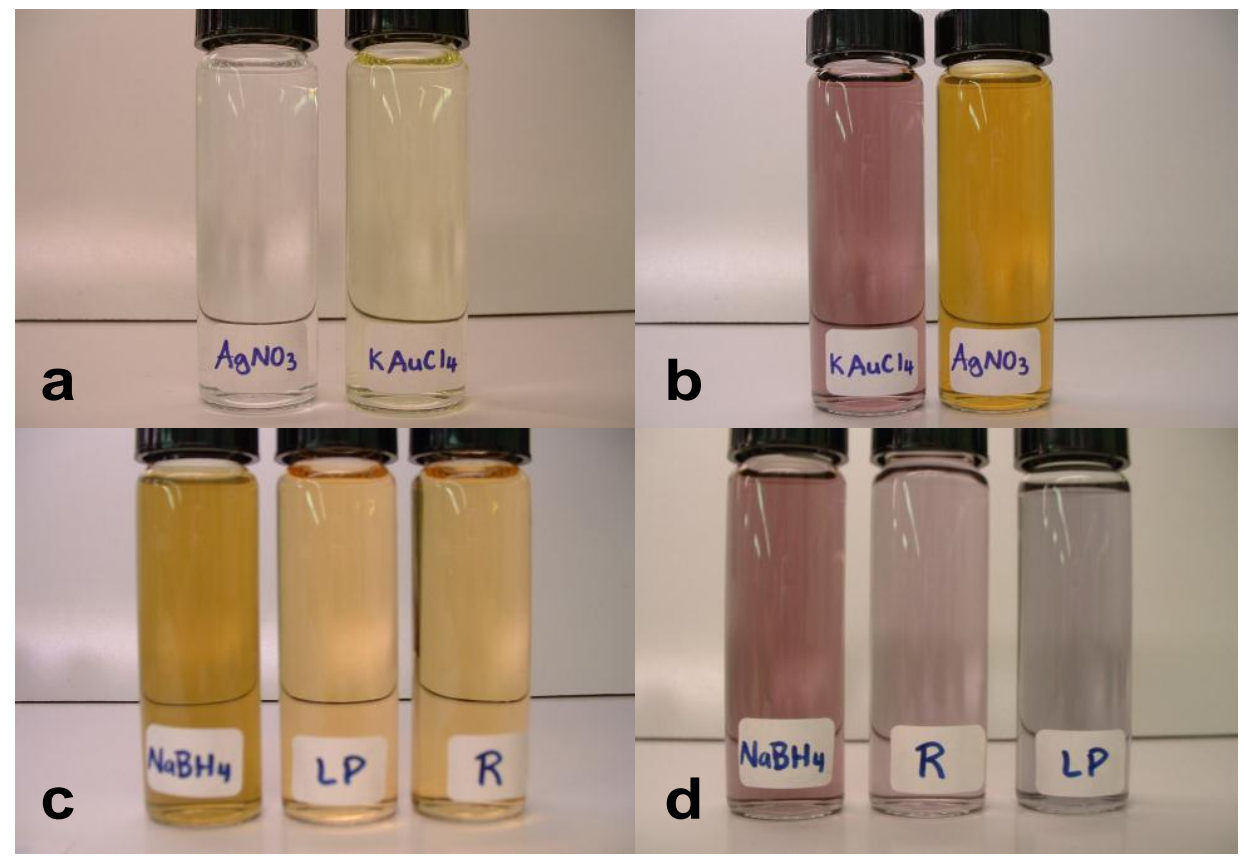


Figure 2.1.4 (Top) Solutions of $1 \mathrm{mM} \mathrm{AgNO}$ and $1 \mathrm{mM} \mathrm{KAuCl}_{4}$ shown (a) before and (b) after addition of a small amount of $\mathrm{NaBH}_{4}$; (Bottom) Solutions produced upon reaction of (c) $\mathrm{AgNO}_{3}$ and (d) $\mathrm{KAuCl}_{4}$ using either $\mathrm{NaBH}_{4}$, LP extract (initial $\mathrm{pH}$ 2.9), or $\mathrm{R}$ extract as the reducing agent.

Extracellular formation of gold nanoparticles using LP was also carried out under different initial $\mathrm{pH}$ environment. Figure 2.1.5 below shows the final solutions from these reactions. From the difference in colors, it is clear that the $\mathrm{pH}$ of the solution indeed affects the reaction. The products formed exhibit colors that change gradually in color, starting as dark blue and becoming more pinkish in shade as $\mathrm{pH}$ increases from 3.5 to 6.0. (See Figure 2.1.4 (d) for $\mathrm{pH}$ 2.9).

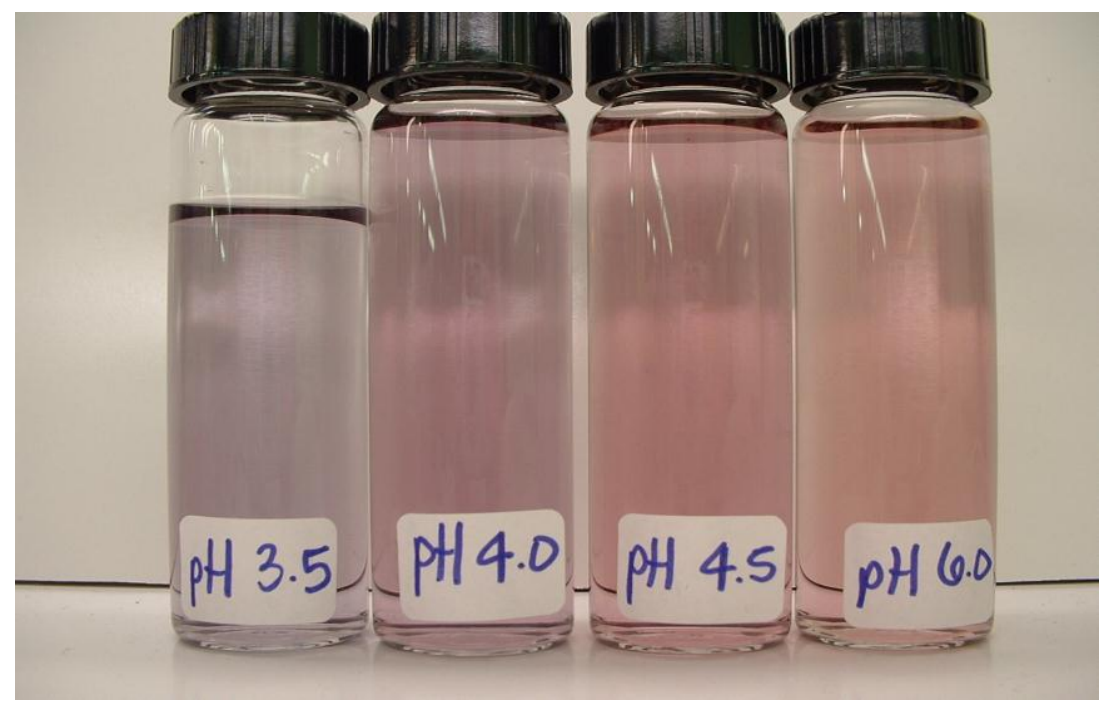

Figure 2.1.5 Effect of $\mathrm{pH}$ on the gold nanoparticles produced from geranium LP extract with $\mathrm{KAuCl}_{4}$ solution, with initial reaction $\mathrm{pH}$ adjusted (from left to right) to 3.5, 4.0, 4.5, and 6.0. Samples shown were diluted 20X following reaction.

The colors of RR and R are quite close, both pink-ruby red, considered as the typical color of gold nanoparticles in aqueous medium [3], but the color of LP is light blue. Spectrophtometer measurements of the samples of 20X diluted final mixtures show that the gold surface plasmon resonance bands occur at approximately 525, 526, and $533 \mathrm{~nm}$, respectively, for R, $\mathrm{RR}$, and LP. These values are quite close to the range of 510-560 nm reported in the literature for an unknown cultivar of geranium [3]. However, unlike that of RR and R, our spectrophotometer measurement of LP shows a second peak at $642 \mathrm{~nm}$, suggesting that gold nanoparticles formed with LP may be significantly anisotropic, leading to the distinctly different bluish color. On the other hand, gold nanoparticles formed with RR and R, having only one peak, may be predominantly more isotropic in shape. No observable changes in the colors of the samples of the 20X diluted final mixtures were detected even after several months of storage, indicating that the gold nanoparticles produced were very stable. 
Our major finding for this task is that exposure to either light or oxygen has no observable effects on the bio-formation of gold nanoparticles. The nanoparticle forming reaction is very rapid, as indicated by the very fast increase in the intensity of the color of the broth with time soon after the addition of dilute potassium tetrachloroaurate solution, and the reaction appears to be completed in about 35 to 60 minutes, much shorter than that for silver nanoparticles. The final color of the mixture indicates extremely high concentration of gold nanoparticles produced. We also observed that the gold nanoparticles produced were stable even after several months of storage.

\subsection{Gold Uptake by Plant Seedlings}

For this task, mung bean (Vigna radiate) and alfalfa (Medicago sativa) were grown from seeds on two different types of sprouter, A and S, using potassium tetrachloroaurate solution of different concentrations, as mentioned in Section 1.2. Difference in these two types of sprouters, as well as the procedures for the production of soot-free ash (bio-ore) from the sprouts and for the determination of gold contained therein using an atomic absorption spectrophotometer, has already been described in that section as well. Presented in Figures 2.1.1 below are the gold uptake data collected from A-sprouter with gold concentration in the sprouter solution being 10, 40, 80 to 160 ppm, with zero ppm as control.

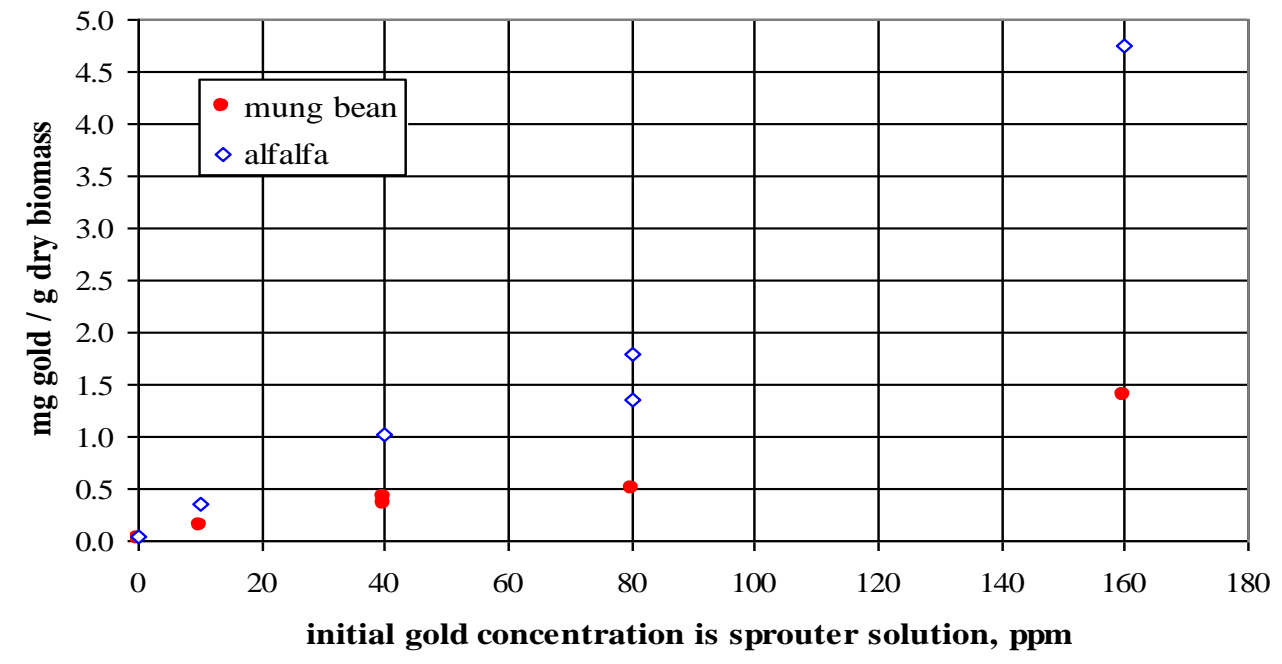

Figure 2.2.1 Gold uptake in mung bean and alfalfa sprouts versus gold concentration in sprouter solution for A-sprouter

The data indicate that, overall, as the concentration of gold in the potassium tetrachloroaurate solution used in the sprouter increases, the gold contents in the bio-ores also increase. In 
addition, overall, alfalfa sprout appear to extract and retain higher concentration of gold than mung bean sprout. The uptake data collected from S-sprouter are shown in Figure 2.2.2 below.

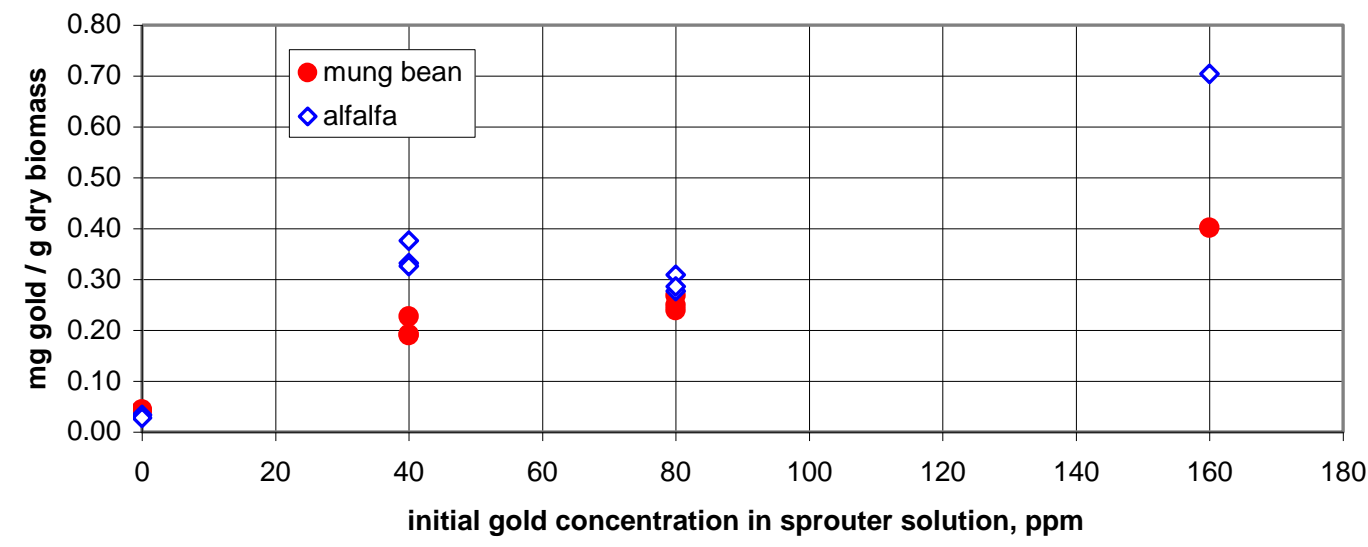

Figure 2.2.2 Gold uptake in mung bean and alfalfa sprouts versus gold concentration In sprouter solution for S-sprouter

Not surprisingly, the gold uptakes in both species of sprouts grown in S-sprouter were much lower than that grown in A-sprouter, since the latter allowed all parts (root, stem, and leaf) of the sprouts to be in contact with gold-containing sprouter solution. Uptake data from both species of plant and both types of sprouter confirm definitely the extraction and transportation of gold ions from the sprouter solution into the cells of the plants. These results also imply that all parts of the seedlings are capable of extracting and transporting gold from the gold solution.

Thus the major finding of this task is that intracellular gold contents in the plant seedlings were noticeably enhanced, overall, as the gold concentrations in contact with the plant cells or the sprouts increase, confirming the extraction and transportation of gold ions into the cells of the plant seedlings tested.

\subsection{Gold Uptake by Plant Cells}

Red beet (Beta vulgaris), carrot (Daucus carota), and mustard (Brassica junces) have been shown $[4,5]$ to be the plants with the best potential for phytomining for gold, if chemically induced by adding ammonium thiocyanate to the auriferous substrate to solubilize gold.

As mentioned in Section 1.3, suspension cell cultures of stevia, periwinkle, carrot light and carrot dark were developed from the corresponding callus cultures. They were used for study of gold uptake from these plant cells with initial gold concentrations of 10, 20, 30, and 40 $\mathrm{ppm}$ in the suspension culture media (with $0 \mathrm{ppm}$ as control). No toxic response as in silver 
uptake was observed and atomic absorption analysis indicated that gold was indeed taken up into viable plant cells.

Shown in Figure 2.3.1 below are the gold contents of cells of periwinkle, "carrot light" and "carrot dark" after exposing the cells to 10, 20,30, and $40 \mathrm{ppm}$ of gold ions in the suspension cultures for one week.

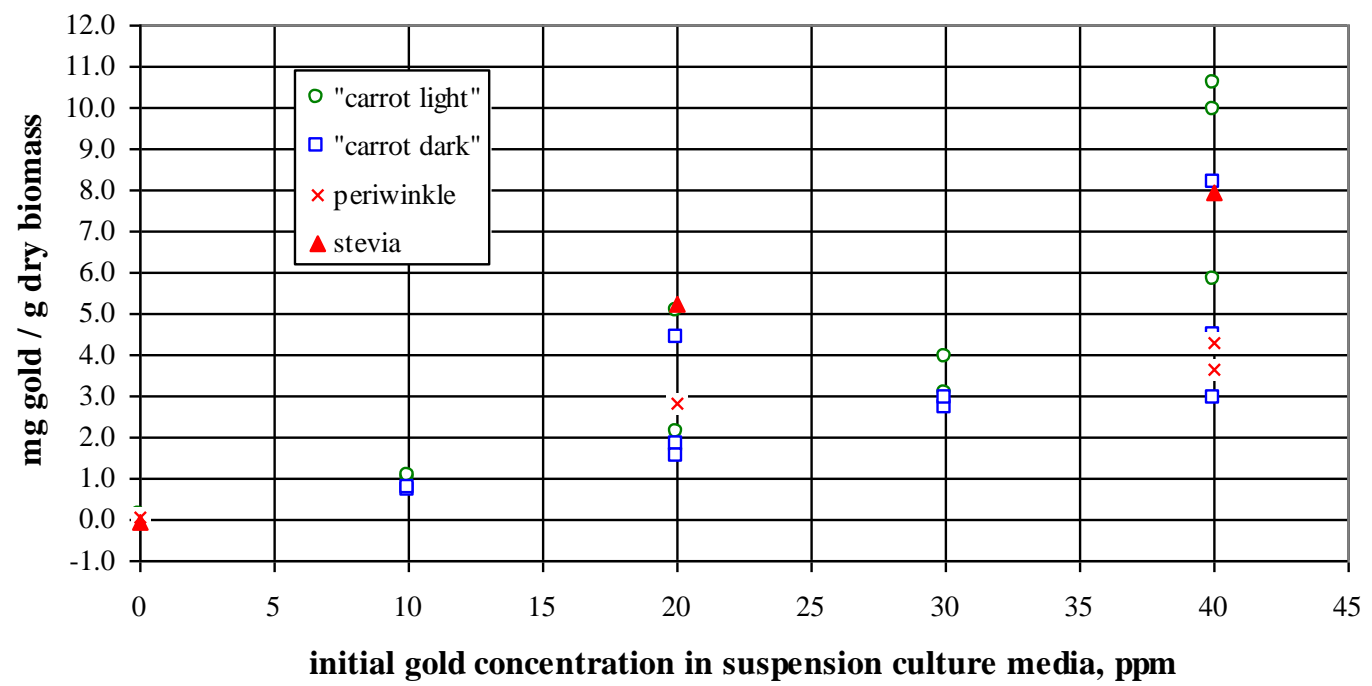

Figure 2.3.1. Gold uptake in carrot, periwinkle, and stevia cells versus initial gold concentration in suspension culture media.

The data in Figure 2.3.1 confirm the uptake of gold from the plant cells grown in suspension cultures after a period of exposure to $\mathrm{AuCl}_{4}{ }^{-}$- containing media. They also indicate that, overall, intra-cellular contents of gold in all species of cells increase with increasing concentration of gold in the suspension medium, confirming the extraction and transportation of gold ions into the cells of the plants tested. In addition, comparing the data in Figures 2.3.1 with that in Figures 2.2.2 (for S-sprouter) and 2.2.1 (for A-sprouter), one can also observe that, though plant species are different, the contents of gold in cells cultivated in suspension cultures are, in general, two to several-fold higher compared to that in sprouts. This result implies the long-term potential of using suspension culture of plant cells for more environmentally friendly large-scale production of gold nanoparticles. 


\section{CONCLUSION}

1. Gold nanoparticles were produced extracellularly from three cultivars of geranium: 'Rose' (R), 'Rober's Lemon Rose' (RR), and 'Lady Plymouth' (LP). The nanoparticle-formation reaction was very rapid, achieving high concentrations of gold nanoparticles in about onehalf to one hour after mixing geranium extract with dilute potassium tetrachloroaurate solution. Colors characteristic of gold nanoparticles in aqueous solution as a result of surface plasmon resonance were exhibited, and the nanoparticles produced, though cultivardependent, were found to remain stable for several months.

2. The bioreactions of geranium extract with potassium tetrachloroaurate solution to produce gold nanoparticles did not require light, and that oxygen appeared to have negligible effect on the progression of the reactions.

3. Uptake of gold ions from dilute potassium tetrachloroaurate solution to the seedlings of mung bean (Vigna radiate) and alfalfa (Medicago sativa) grown from seeds on two different types of sprouter was confirmed. Uptake of gold from the media of suspension culture of Catharanthus roseus (periwinkle), Stevia rebaudiana (stevia), and Daucus carota (carrot, both light and dark), which contained chloroaurate ions, to the suspended plant cells was also confirmed.

4. Intracellular gold contents in the plant seedlings and the suspended cells were noticeably enhanced, overall, as the gold concentrations in contact with the plant cells or the sprouts increased, indicating the extraction and transportation of gold ions into the cells of both plant systems studied. In addition, the contents of gold in plant cells cultivated in suspension cultures were, in general, two to several fold higher compared to that in the sprouts. 


\section{REFERENCES}

1. Gardea-Torresdey, J.L., et al., Nano Letters, 2, 397-401 (2002)

2. Murphy, C.J. and N.R. Jana, Advanced Materials, 14, 80-82 (2002)

3. Shankar, S. S. et al., J. Materials Chemistry, $\underline{13}$, 1822 (2003)

4. Anderson, C. W. N. et al., Nature, 395, 553 (1998).

5. Msuya, F. A., et al., Gold Bulletin, $\underline{33}, 134$ (2000). 
Appendix 33: Phytomining for Nickel and Silver Nanoparticles (WV016) 
FINAL TECHNICAL REPORT

Contract Title and Number: Crosscutting Technology Development at the Center for Advanced Separation Technologies (DE-FC26-02NT41607)
Period of Performance:

Starting Date: 06/01/2005

Ending Date: 08/31/2009
Sub-Recipient Project Title:

Phytomining for Nickel and Silver Nanoparticles

Principal Investigators:

Ray Y. K. Yang, Eung Ha Cho

Contact Address:

Ray Y. K. Yang

Chemical Engineering Department

West Virginia University

Morgantown WV 26506-6102

Subcontractor Address:

No subcontracts issued
Report Information:

Type: Final

Number:

Period: 06/01/05-08/31/09

Date: $\quad$ 08/28/09

Code: WV016-FINAL

Contact Information:

Phone: (304) 293 2111x2419

Fax:

E-Mail: ryang@mail.wvu.edu

Subcontractor Information:

Phone:

Fax:

E-Mail:

\section{DISCLAIMER}

This report was prepared as an account of work sponsored by an agency of the United States Government. Neither the United States Government nor any agency thereof, nor any of their employees, make any warranty, express or implied, nor assume any legal liability or responsibility for the accuracy, completeness, or usefulness of any information, apparatus, product, or process disclosed, or represents that its use would not infringe privately owned rights. Reference herein to any specific commercial product, process, or service by trade name, trademark, manufacturer, or otherwise does not necessarily constitute or imply endorsement, recommendation, or favoring by the United States Government or any agency thereof. The views and opinions of authors expressed herein do not necessarily state or reflect those of the United States Government or agency thereof. 


\begin{abstract}
Phytomining is the production of a metal by growing high-biomass plants that hyperaccumulate high concentrations of a target metal. A conventional phytomining operation would consist of planting a hyperaccumlator crop over a low-grade ore body or mineralized soil, followed by harvesting and incineration of the biomass to ash to produce "bio-ore". In this project, we have expanded conventional phytomining to include other forms of plant, notably plant seedlings and plant cells, and also different form of metal, i.e., metal nanoparticles.
\end{abstract}

The project investigated the extracellular biosynthesis of silver nanoparticles from the leaf extracts of ruptured cells of three cultivars of scented geranium (Pelargonium graveolens). It also studied the uptake of silver ions from silver solution by the seedlings of alfalfa (Medicago sativa) and mung bean (Vigna radiate) and also by the suspended cells of stevia (Stevia rebaudiana) for their intracellular accumulations the cells of these plant systems.

Silver nanoparticles were produced extracellularly from three cultivars of geranium: 'Rose' (R), 'Rober's Lemon Rose' (RR), and 'Lady Plymouth' (LP). Colors characteristic of silver nanoparticles in aqueous solutions as a result of surface plasmon resonance were exhibited and all the nanoparticles produced, though cultivar-dependent, were found to remain stable for several months. We also found that these bioreactions of geranium extract with silver nitrate solution to produce silver nanoparticles occurred only in the presence of light, but oxygen appeared to have negligible effect on the progression of the reactions. These reactions were found to be slower than their corresponding ones producing gold nanoparticles.

In spite of the toxic effect of silver ion, ability of the sprouts of mung bean and alfalfa germinated and grown in two different types of sprouter and the cells of stevia grown in suspension culture to uptake silver was observed. The intracellular contents of silver in the cells of stevia in suspension culture and in the sprouts of mung bean and alfalfa were noticeably enhanced, overall, as the silver concentrations in contact with the plant cells or the sprouts increased, confirming the extraction and transportation of silver ions into the cells of the plants studied. Due to the toxic effect of silver ions, it was observed that, at higher silver concentrations of the sprouter solution, part of the intracellular uptake of silver in the sprouts might be due to passive diffusion. The toxic effect of the silver ions on the suspended plant cells was more severe than that on the sprouts, causing cell death particularly when the silver ion concentration increased beyond $20 \mathrm{ppm}$. Hence the prospect of phytomining for silver nanoparticles is dim.

The nickel-uptake data obtained indicate that both alfalfa and brown mustard sprouts were capable of uptaking nickel from the sprouter solution into their plant tissue; the amounts of uptake increased with increasing nickel concentration in the sprouter solution. In addition, the amounts of uptake by alfalfa were in general larger than that by brown mustard. 


\title{
Table of Contents
}

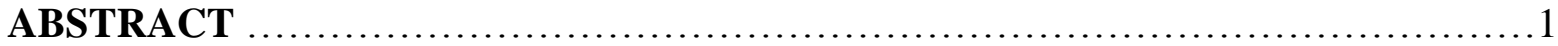

EXECTIVE SUMMARY ..................................................

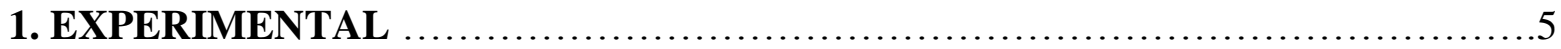

1.1 Silver Nanoparticles from Geranium Extract.....................................5

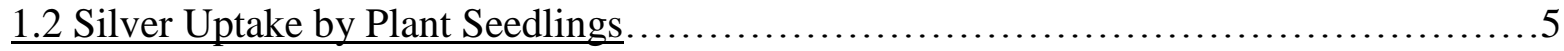

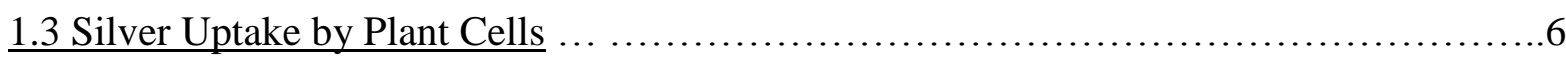

1.4 Nickel Uptake by Plant Seedlings............................................

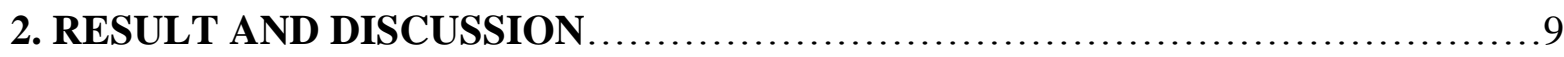

2.1 Silver Nanoparticles from Geranium Extract....................................9

2.2 Silver Uptake by Plant Seedlings..............................................

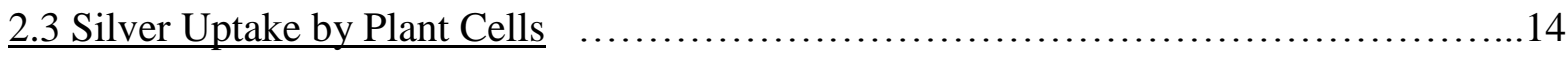

$\underline{2.4 \text { Nickel Uptake by Plant Seedlings } \ldots \ldots \ldots \ldots \ldots \ldots \ldots \ldots \ldots \ldots \ldots \ldots \ldots \ldots \ldots \ldots \ldots \ldots \ldots \ldots \ldots \ldots}$

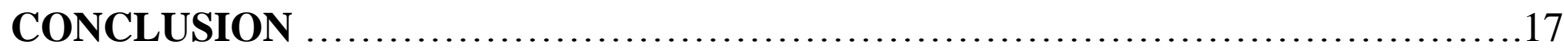

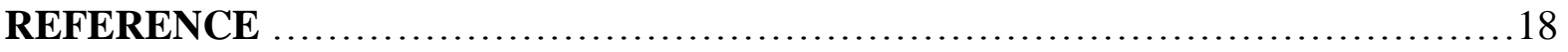




\section{EXECUTIVE SUMMARY}

Phytomining is the production of a metal by growing high-biomass plants that hyperaccumulate high concentrations of a target metal. A conventional phytomining operation would consist of planting a hyperaccumlator crop over a low-grade ore body or mineralized soil, followed by harvesting and incineration of the biomass to ash to produce "bio-ore". Some plants are natural hyperaccumulators, while others require induction for hyperaccumulation. For metals, except gold, the threshold concentration for hyperaccumulation is $1 \mathrm{mg} / \mathrm{g}$ dry biomass $[1,2]$. In this project, we have expanded conventional phytomining to include other forms of plant, notably plant seedlings and plant cells, and also different form of metal, i.e., metal nanoparticles.

Metal nanoparticles have typically been made by chemical methods using toxic compounds. Only recently has biological synthesis employing bacteria, fungi, and plants to produce silver nanoparticles been explored. This research encompasses topics involving biochemical processes that employ plants and plant systems. The project investigated the extracellular biosynthesis of silver nanoparticles from the leaf extracts of ruptured cells of three cultivars of scented geranium (Pelargonium graveolens). It also studied the uptake of silver ions from silver solution by the seedlings of alfalfa (Medicago sativa) and mung bean (Vigna radiate) and also by the suspended cells of stevia (Stevia rebaudiana) for their intracellular accumulations the cells of these plant systems. Much of the research is intended to reveal more information about the extracellular phyto-formations of silver nanoparticles and the intracellular phyto-uptake of silver and nickel.

Silver nanoparticles were produced extracellularly from three cultivars of geranium: 'Rose' (R), 'Rober's Lemon Rose' (RR), and 'Lady Plymouth' (LP). Colors characteristic of silver nanoparticles in aqueous solutions as a result of surface plasmon resonance were exhibited and the nanoparticles produced, though cultivar-dependent, were found to remain stable for several months. We found that the reaction of geranium extract with silver nitrate solution to produce silver nanoparticles occurred only in the presence of light, but oxygen appeared to have negligible effect on the progression of the reaction. These reactions were found to be slower than their corresponding ones producing gold nanoparticles. Chemical simulation conducted using sodium borohydride (with trisodium citrate as a capping agent) to reduce silver ions to silver nanoparticles in aqueous solution showed the development of color very similar to that resulting from the reactions with the geranium extract.

In spite of the toxic effect of silver ion, ability of the sprouts of mung bean and alfalfa germinated and grown in two different types of sprouter and the cells of stevia grown in suspension culture to uptake silver was observed. The intracellular contents of silver in the cells of stevia in suspension culture and in the sprouts of mung bean and alfalfa were noticeably enhanced, overall, as the silver concentrations in contact with the plant cells or the sprouts increased, confirming the extraction and transportation of silver ions into the cells of the plants tested. This observation was true for both types of sprouters used, though silver uptake in A-sprouter is several-fold higher than that in S-sprouter due to the fact that all parts (root, stem, and leaf) of the sprouts were exposed to the silver-containing sprouter solution in A-sprouter, while in S-sprouter only roots of the sprout were in contact with the solution. 
Due to the toxic effect of silver ions, it was observed that, at higher silver concentrations of the sprouter solution, part of the intracellular uptake of silver in the sprouts might be due to passive diffusion. The toxic effect of the silver ions on the suspended plant cells was more serious than that on the sprouts, causing cell death particularly when the silver ion concentration increased beyond $20 \mathrm{ppm}$.

The nickel-uptake data obtained indicate that both alfalfa and brown mustard sprouts were capable of uptaking nickel from the sprouter solution into their plant tissue; the amounts of uptake increased with increasing nickel concentration in the sprouter solution. In addition, the amounts of uptake by alfalfa were in general larger than that by brown mustard. These observations were true for both types of sprouter. It was also observed that in general uptakes in A-sprouter were hugely larger than that in S-sprouter indicating either significant nickel uptake from parts of seedlings other than roots or the significance of longer GG (germination and grow) time to allow further development of roots for sprouts grown in an A-sprouter. On the other hand, no conclusive statement could be made with regard to the relation between the amount of nickel uptake and the CC (chemical contact) time, due to the large experimental errors associated with the data. We also found that for brown mustard SS (seed soaking) time of one or two days had no impact on nickel uptake from brown mustard grown in A-sprouter. 


\section{EXPERIMENTAL}

\subsection{Silver Nanoparticles from Geranium Extract}

Various experiments were performed using geranium extract as a reactant. Extract was prepared using leaves of three cultivars of scented geranium (Pelargonium graveolens): 'Rose' (R), 'Rober's Lemon Rose' (RR), and 'Lady Plymouth' (LP). The leaves were ground finely, water was added, and the mixture was then boiled for a short period of time. Afterward, the extracted leave mass was separated from the solution via filtration and discarded, while the extract, which was the pale yellow liquid filtrate, was collected. Then a small amount of the geranium extract was mixed with different volumes of $1 \mathrm{mM}$ silver nitrate $\left(\mathrm{AgNO}_{3}\right)\left(107.9 \mathrm{ppm} \mathrm{Ag}^{+}\right)$solution to start the reactions. All reactions took place at approximately $25^{\circ} \mathrm{C}$ in clear $125 \mathrm{ml}$ glass jars or $30 \mathrm{ml}$ glass vials.

Several variables were tested to find factors that influence the reaction. In some situations, these samples were continuously exposed to fluorescent light. Other samples were kept in darkness. Exposure to air was also altered to determine if oxygen had any role in the reaction.

To provide more evidence that silver nanoparticles were present, the reactions were simulated chemically. Experiments were conducted using sodium borohydride $\left(\mathrm{NaBH}_{4}\right)$ to reduce silver ions in $1 \mathrm{mM} \mathrm{AgNO}_{3}$ solution with no involvement of geranium extract. The reduction was performed in the presence of trisodium citrate, which acted as a capping agent to stabilize the nanoparticles. This chemical reduction allowed for comparison between the nanoparticle solutions produced chemically and those produced biologically using geranium extract.

\subsection{Silver Uptake by Plant Seedlings}

Intracellular formation of silver nanoparticles by living plants grown in agar was reported by Gardea-Torresdey and co-workers [3]. They reported that alfalfa sprouts grown in silver-rich agar media actively extracted Ago from the media and accumulated them as nanoparticles in their tissues. They thought that the $\mathrm{Ag}^{+}$was reduced to $\mathrm{Ag}^{0}$ in the solid media and the latter was extracted by the roots of alfalfa seedlings, transported to their shoots, and then assembled into organized nanoparticles ranging from 2-20 nm in diameter.

In this project we studied instead the uptake of silver ions from agar-free solutions by the seedlings of two plant species and by the suspended cells of stevia (Stevia rebaudiana)) for intracellular accumulations of nanoparticles in these plant systems. The former is reported in this section and Section 2.2, while the latter in Sections 1.3 and 2.3. 
Two species of seedlings, mung bean (Vigna radiate) and alfalfa (Medicago sativa), were studied to determine their abilities to facilitate intracellular silver uptake, a first step toward intracellular silver nanoparticle formation in living plant seedlings. The seeds were first treated with ZeroTol ${ }^{\circledR}$ solution (50 times dilution) for sterilization, and then soaked in deioned/distilled (DD) water for 24 hours, before being spread onto the screens inside a sprouter to start a sprouter run. Different dilute concentrations of silver nitrate solution were provided to the seeds (before their germination) and the sprouts (after seed germination), without the addition of plant nutrients for the seeds and the seedlings.

Two types of sprouter were used: S-sprouter and A-sprouter, both were under $24 \mathrm{hr}$ fluorescent lighting at approximately $25^{\circ} \mathrm{C}$. In the S-sprouter, which had totally transparent cover, both mung bean and alfalfa were grown in the same container side by side and only the roots of the sprouts are in contact with the sprouter solution containing only dilute silver nitrate solution. On the other hand, the A-sprouter, which had partially transparent cover, periodically sprinkled seeds held on a screen (before seed germination) and all parts of the sprouts (after seed germination) with the same sprouter solution. The solution, after sprinkling over the entire plant seedlings, drained through the screen to the reservoir below, and was re-circulated with a pump to the top of the sprouter for periodic sprinkling.

After a one-week period of contact with dilute silver nitrate solution, starting from watersoaked seeds, the sprouts of each run were harvested. They were thoroughly washed several times, dried, ground, and burned to ash, known in phytomining as bio-ore. The ash, which included any silver nanoparticles or ions that had accumulated inside the seedlings, was dissolved in concentrated nitric acid and then diluted. Atomic absorption (AA) analysis was then conducted using a Perkin-Elmer atomic absorption spectrophotometer to find the concentration of silver formed inside the cells of the sprouts.

\subsection{Silver Uptake by Plant Cells}

Callus cultures of Catharanthus roseus (periwinkle), Stevia rebaudiana (stevia), Daucus carota (carrot), and Beta vulgaris (red beet) had all been established from their respective explants and maintained continuously by subculturing during the period of this task. The callus cultures of carrot, periwinkle, and stevia were initiated and maintained in Murashige and Skoog solid medium, while red beet was on solid agar with Gamborg's B5 medium. Two types of culture had been maintained for carrot since its initiation; one that had been kept in the dark all the time (designated as "carrot dark") and another in 12-hour light/dark (designated as "carrot light"). The cultures were kept in an incubator with controls of temperature and light cycles. Subculturing was performed at regular intervals ( $2-4$ weeks) in order to maintain a constant supply of nutrients for the established calluses. Shown in Figure 1.3.1 next page are the callus cultures of red beet, periwinkle, and carrot. 


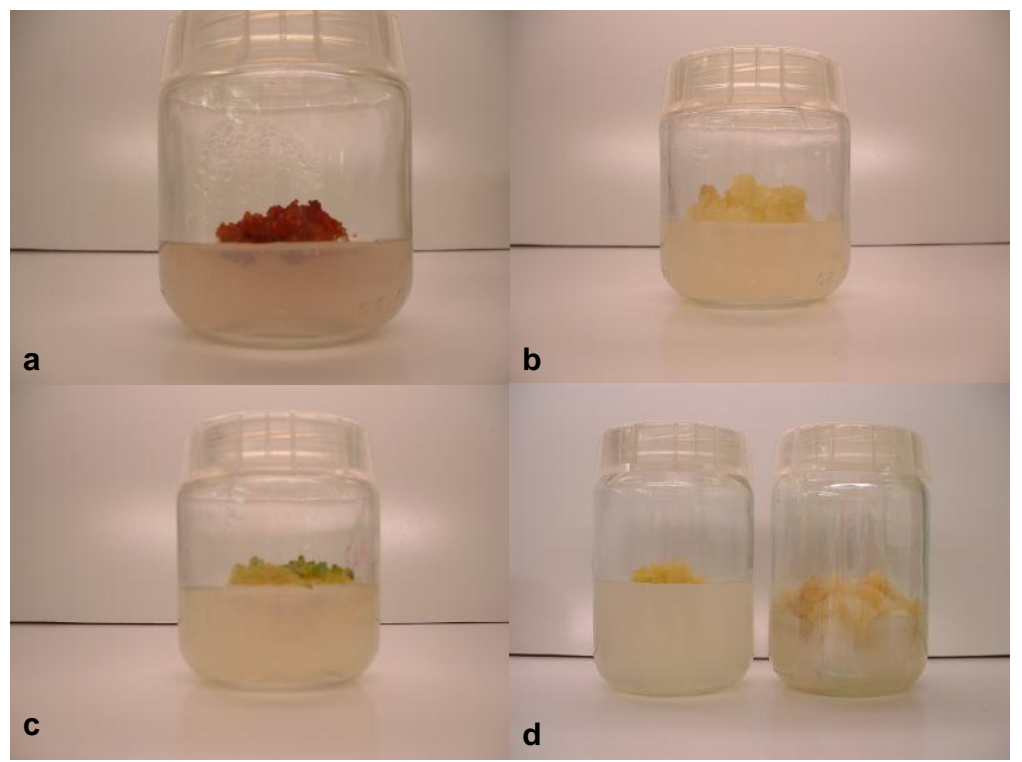

Figure 1.3.1. Callus cultures of (a) red beet, (b) periwinkle, (c) stevia, and (d) carrot, with the callus on the left grown in light and the callus on the right grown in dark.

Hairy root cultures of stevia on agar medium and in liquid medium were also initiated and established during the period of this task.

Suspension cultures of $C$. roseus, $S$. rebaudiana, and D. carota were also developed from the corresponding callus cultures. For suspension cultures, the medium formulation for each species is identical to that for the corresponding callus, only with agar omitted. Erlenmeyer flasks containing the suspension were agitated on an orbital shaker at $110-120$ rpm to allow for maximum nutrient uptake by plant cells. Temperature and photoperiod were regulated; growth conditions were similar to that of the corresponding callus cultures. Subcultures were performed every 1 - 2 weeks.

S. rebaudiana (stevia) suspensions were studied for the uptake of silver from solution. Silver nitrate solution was added to the liquid media at the midpoint of the subculture period. At the end of the period, cells and contents were washed six times with $200 \mathrm{ml}$ aliquots of distilled/deionized water, all under vacuum filtration. Then they were dried at $80^{\circ} \mathrm{C}$ overnight, burned for 3 hours at $700^{\circ} \mathrm{C}$, and dissolved in concentrated nitric acid. All the intracellular silver nanoparticles or ions were dissolved by the acid, and could be readily detected by atomic absorption analysis. Atomic absorption (AA) analysis was then conducted using a Perkin-Elmer atomic absorption spectrophotometer to find the concentration of silver formed inside the plant cells. Several concentrations of silver ions were tested for uptake by plant cells.

\subsection{Nickel Uptake by Plant Seedlings}

Two species of seedlings, alfalfa (Medicago sativa) and brown mustard (brassica juncea), were studied to determine their abilities to facilitate nickel nanoparticle formation. The seeds 
were first treated with ZeroTol ${ }^{\circledR}$ solution (50 times dilution) for sterilization, and then soaked in deioned distilled (DD) water for 24 hours, before being spread onto the screens inside a sprouter to start a sprouter run.

The sprouter runs conducted in this task are different from that described in Section 1.1 for silver uptake. Instead of contacting with solution containing metal ions for the whole period of experimental run, here the seeds germinated and grew first in contact only with DD water for a period of time, designated as GG (Germination and Grow) time. They then continued to grow in contact with nickel nitrate solution for an additional period of time, designated as CC (Chemical Contact) time. Total run time for each run is the sum of seed-soaking (SS) time, GG time, and CC time. During the CC period, different dilute concentrations of nickel nitrate solution containing no plant nutrients were provided to the seedlings.

Two types of sprouter were used: S-sprouter and A-sprouter, both were under $24 \mathrm{hr}$ fluorescent lighting at approximately $25{ }^{\circ} \mathrm{C}$. In the S-sprouter, which had totally transparent cover, both mung bean and brown mustard were grown in the same container side by side and only the roots of the sprouts are in contact with the sprouter solution containing only dilute nickel nitrate solution. On the other hand, the A-sprouter, which had partially transparent cover, periodically sprinkled seeds held on a screen (before seed germination) and all parts of the sprouts (after seed germination) with the same sprouter solution. The solution, after sprinkling over the entire plant seedlings, drained through the screen to the reservoir below, and was re-circulated with a pump to the top of the sprouter for periodic sprinkling.

The SS (seed-soaking) time for alfalfa was 24 hrs. But for brown mustard it was either 24 or $48 \mathrm{hrs}$. The CC time for the S-sprouter is two or three days, while that for the A-sprouter is one day. Thus the total run time for each run could be either 168 or 192 hrs. The initial concentration of nickel ion $\left(\mathrm{Ni}^{2+}\right)$ used in the sprouter solutions during the CC period were 3.21 or $6.42 \mathrm{ppm}$. For each concentration, at least three duplicate runs were carried out.

At the end of total run time, the sprouts were harvested. They were washed very thoroughly, dried, ground, and burned to ash. The ash, which included any nickel nanoparticles or ions that had accumulated inside the seedlings, was dissolved in concentrated nitric acid and then diluted. Atomic absorption analysis was used to find the concentration of nickel inside the cells of the seedlings. 


\section{RESULTS AND DISCUSSION}

\subsection{Silver Nanoparticles from Geranium Extract}

Due to their nanoscale dimensions, silver nanoparticles possess surface plasmon resonances (SPR) which leads to their unique colors. The color in turn depends on the morphology of the silver nanoparticles, especially their aspect ratios - the ratio of the lengths of the largest dimension to the smallest dimension [4]. The spectra of anisotropic particles, such as nanorods, show two distinct peaks, corresponding to transverse and longitudinal oscillations.

Shankar et al. [5] performed experiments using geranium (cultivar not specified) to produce silver nanoparticles, although the cultivar used was not mentioned in their publication. They observed mainly spherical nanoparticles ranging from 16-40 $\mathrm{nm}$ that remained stable even after 4 weeks.

As mentioned in Section 1.1, in this project we used three cultivars of scented geranium, 'Rose', 'Rober's Lemon Rose', and 'Lady Plymouth', for our investigation on the extracellular formation of silver nanoparticles. Our results indicated that the bioreactions of these three cultivars of geranium with silver nitrate, as well as the nanoparticles produced, were cultivardependent. Obvious color changes of the reaction mixture were noted as soon as the reaction started and as the reaction progressed. The colors that developed were characteristic of silver nanoparticles in aqueous solution.

The following definitions relating to time were used to describe the progress of the reactions more precisely. The time $t$ at which the reactants were combined is defined as $t=0$. Immediately after this step, some reaction mixtures were placed in darkness. The period of time in which the mixtures remain in darkness is termed $t_{d}$. These samples were eventually exposed to light for a period $t_{1}$, which took place at $t>t_{d}$. Other samples had continuous exposure to light, and in this situation, $\mathrm{t}_{\mathrm{d}}=0$, and $\mathrm{t}_{\mathrm{l}} \neq 0$.

The first aspect of the reaction that became apparent was that the reaction for formation of silver nanoparticles takes place only in lighted conditions, a fact that, to the best of our knowledge, has never been reported in the literature before. This is clearly seen to be true for each cultivar of geranium reacting with $\mathrm{AgNO}_{3}$. Figure 2.1.1 in the next page provides further support of this statement.

It was also found that after a length of time in darkness, the reaction would still begin upon exposure to light. Although postponed significantly, when the reaction was initiated, it proceeds at about the same reaction rate as if it had not been delayed. Shown in Figure 2.1.2 next page is an example of this observation. 

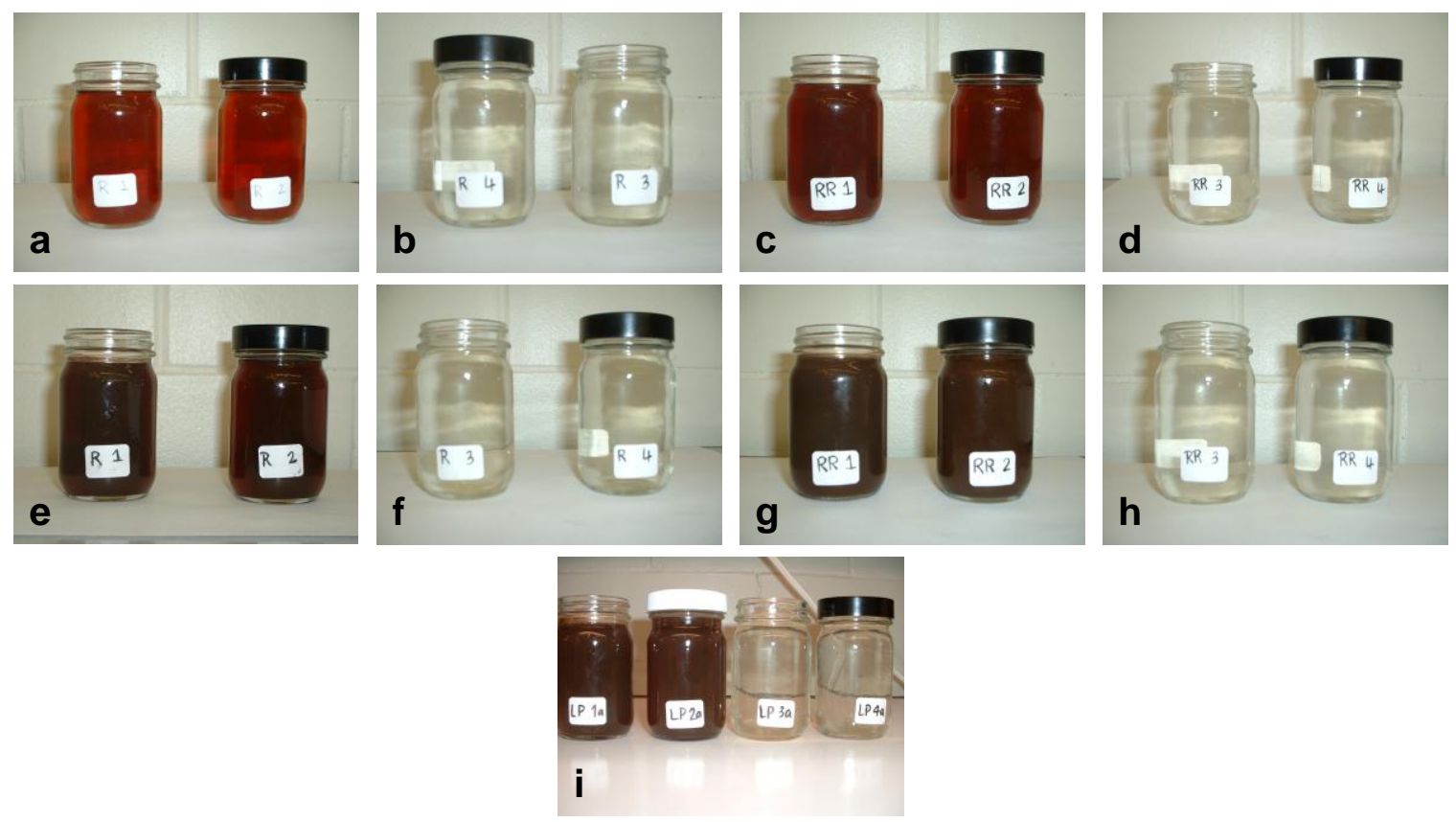

Figure 2.1.1 Effects of Light and Oxygen - Reactions of R (a, b, e, f), RR (c, d, g, h) and LP extracts with $\mathrm{AgNO}_{3}$ pictured at (a) $\mathrm{t}_{\mathrm{d}}=0, \mathrm{t}_{\mathrm{l}}=5 \mathrm{hr}$, (b) $\mathrm{t}_{\mathrm{d}}=5 \mathrm{hr}, \mathrm{t}_{\mathrm{l}}=0$, (c) $\mathrm{t}_{\mathrm{d}}=0, \mathrm{t}_{\mathrm{l}}=5 \mathrm{hr}$, (d) $\mathrm{t}_{\mathrm{d}}=5 \mathrm{hr}, \mathrm{t}_{\mathrm{l}}=0$, (e) $\mathrm{t}_{\mathrm{d}}=0, \mathrm{t}_{\mathrm{l}}=24 \mathrm{hr}$, (f) $\mathrm{t}_{\mathrm{d}}=24 \mathrm{hr}, \mathrm{t}_{\mathrm{l}}=0$, (g) $\mathrm{t}_{\mathrm{d}}=0, \mathrm{t}_{\mathrm{l}}=24 \mathrm{hr},(\mathrm{h}) \mathrm{t}_{\mathrm{d}}=24 \mathrm{hr}, \mathrm{t}_{\mathrm{l}}$ $=0$, and at (i) $t_{d}=0, t_{l}=26 \mathrm{hr}$ for the two jars on the left, and $t_{d}=26 \mathrm{hr}, t_{l}=0$ for the two jars on the right.
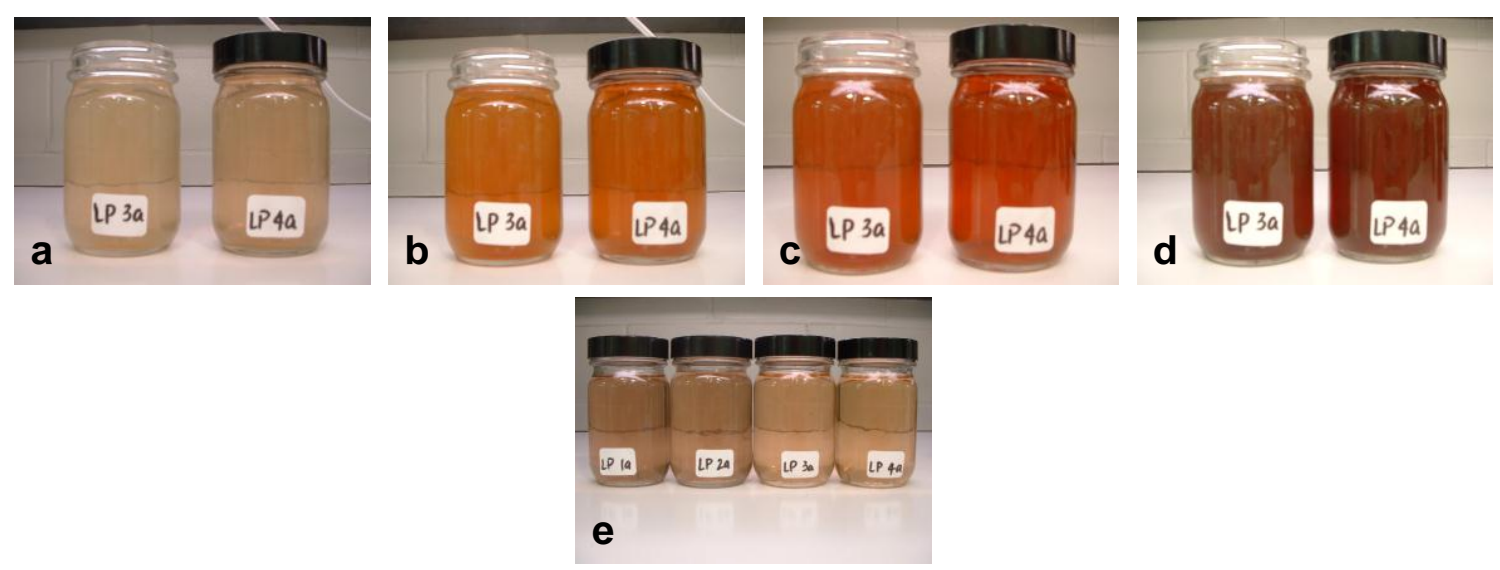

Figure 2.1.2 Effects of Delayed Light and Oxygen - Reaction of LP extract with $\mathrm{AgNO}_{3}, \mathrm{t}_{\mathrm{d}}=$ 13 days, shown at (a) $t_{1}=1 \mathrm{hr}$, (b) $t_{1}=4 \mathrm{hr}$, (c) $t_{1}=7 \mathrm{hr}$, and (d) $t_{1}=26 \mathrm{hr}$, and (e) final products after $20 \mathrm{X}$ dilution, with the two jars on the left having $t_{d}=0, t_{l}=14$ days and the two jars on the right having $t_{d}=13$ days, $t_{l}=27$ hr. (All final products were capped after $20 \mathrm{X}$ dilution). 
Regarding the exposure to oxygen, no significant or obvious effect could be identified. This is made evident from examination of images in Figures 2.1.1 and 2.1.2 showing jars 1 and 2, which are under equal constraints except uncapped jar 1 is open to air, and capped jar 2 has only approximately $12 \%(\mathrm{v} / \mathrm{v})$ air present in the tightly closed space right under the lid. Likewise, jars marked 3 and 4 are exactly alike except for contact with oxygen. These figures show that, based on color, reactions are identical regardless of the degree of presence of oxygen.

Among the cultivars, only a slight difference in reaction was noted. As seen in Figure 2.1.3 below, there is some variation in color of the solutions produced by different cultivars, but all colors were similar to the yellow-brown color characteristic of silver nanoparticles in aqueous solution.
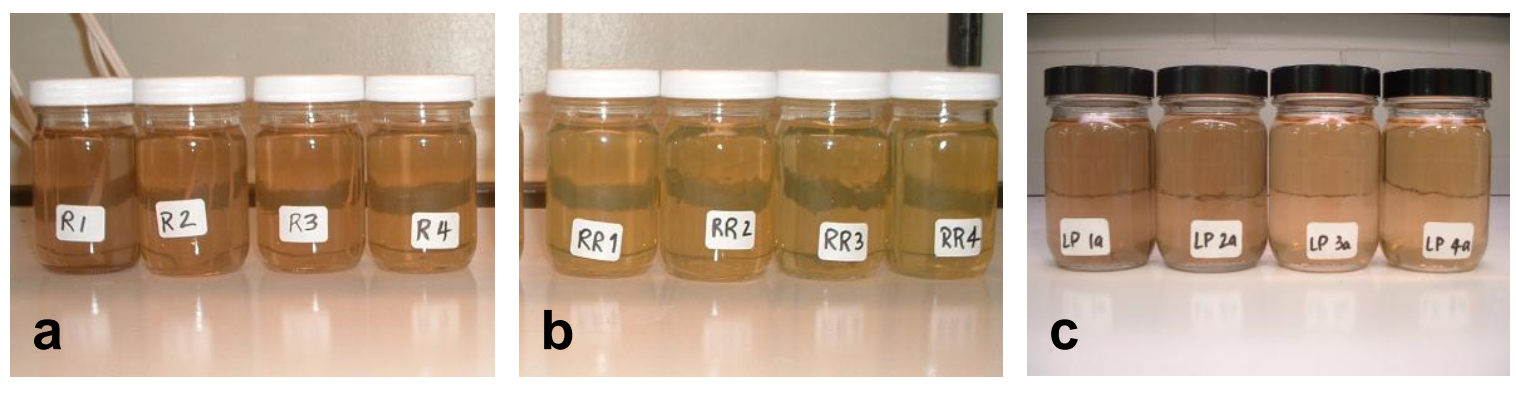

Figure 2.1.3 Final products from reactions of R, RR, and LP extracts with $\mathrm{AgNO}_{3}$ after 20X dilution. In (a), (b), and (c), the two jars on the left have $t_{d}=0$, and the two jars on the right have $t_{d} \neq 0$. (All final products were capped after 20X dilution).

To further support the assertion that formation of silver nanoparticles occurs due to the reduction of metal ions with geranium extract, sodium borohydride $\left(\mathrm{NaBH}_{4}\right)$ in the presence of trisodium citrate, which acts as a capping agent to stabilize the nanoparticles, was used to chemically simulate the reaction with no involvement of geranium extract, as mentioned in Section 1.1. Pure $\mathrm{AgNO}_{3}$ solution can be seen in (a) of Figure 2.1.4 next page. Addition of small amounts of $\mathrm{NaBH}_{4}$ powder to the silver solution resulted in immediate reduction of ions, causing striking displays of color. The color produced, shown in the vial on the right in (b) of Figure 2.1.4, is typical of mostly spherical silver nanoparticles in aqueous solution. It is of key importance that similar color was obtained by the reduction of silver ions using geranium extract. Image (c) shows solutions containing silver nanoparticles reduced by $\mathrm{NaBH}_{4}$, as well as by R and LP extracts with no adjustment in $\mathrm{pH}$ for both.

Thus, phyto-synthesis of silver nanoparticles using geranium extracts can achieve similar results as chemical-synthesis without the use of harsh chemical reagent such as $\mathrm{NaBH}_{4}$. Furthermore, no use of capping agent, such as trisodium citrate is required. All samples of silver nanoparticle solution produced from geranium extract remained homogeneous in appearance and appeared to be stable after approximately three months. They are also free of contaminations due probably to the well-known antibacterial property of silver nanoparticles. Not only is this approach "greener", the nanoparticles produced appeared to remain stable much longer. 


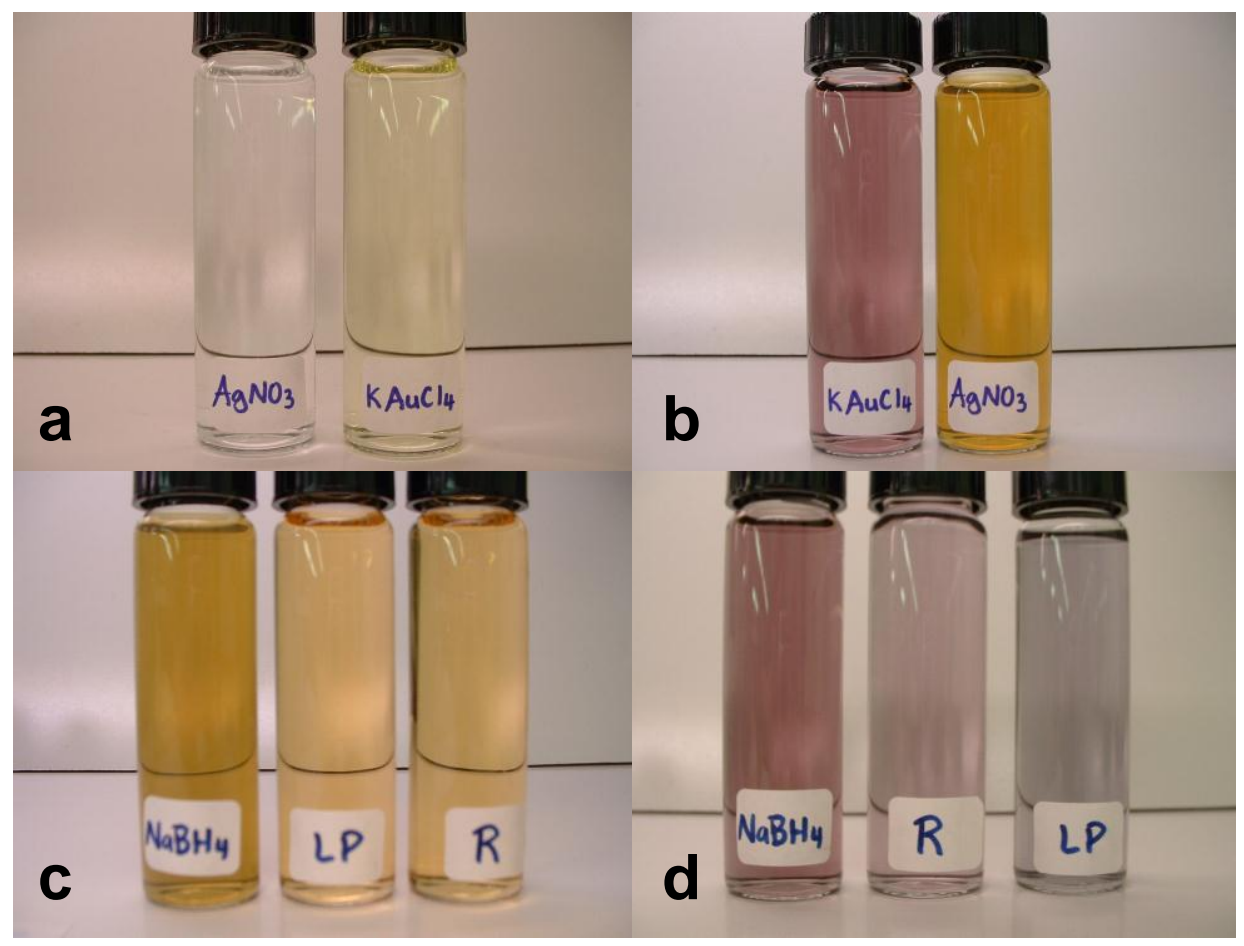

Figure 2.1.4 (Top) Solutions of $1 \mathrm{mM} \mathrm{AgNO}$ and $1 \mathrm{mM} \mathrm{KAuCl} 4$ shown (a) before and (b) after addition of a small amount of $\mathrm{NaBH}_{4}$; (Bottom) Solutions produced upon reaction of (c) $\mathrm{AgNO}_{3}$ and (d) $\mathrm{KAuCl}_{4}$ using either $\mathrm{NaBH}_{4}$, LP extract (initial $\mathrm{pH}$ 2.9), or $\mathrm{R}$ extract as the reducing agent.

Our major finding for this task is that the formation of silver nanoparticles took place only in lighted conditions. It was also found that after a length of time in darkness, the reaction would still begin upon exposure to light. Although postponed significantly, when the reaction was initiated, it proceeded at about the same reaction rate as if it had not been delayed. Regarding the exposure to oxygen, no significant or obvious effect could be identified. Though the bioreaction was cultivar-dependent, contrary to gold nanoparticles, only a slight difference in reaction was noted among the cultivars.

\subsection{Silver Uptake by Plant Seedlings}

Silver uptake data (in mg of silver per gram of dry biomass) of mung bean (Vigna radiate) and alfalfa (Medicago sativa) sprouts germinated and grown in different (initial) concentrations of sprouter solution for seven days in both types of sprouter, as described in Section 1.2, are presented in the following two figures. Shown in Figures 2.2.1 below are uptake data in A-sprouter for six different concentrations of sprouter solution: $3,5,10,20$, and 40 ppm of silver, plus zero as control. 


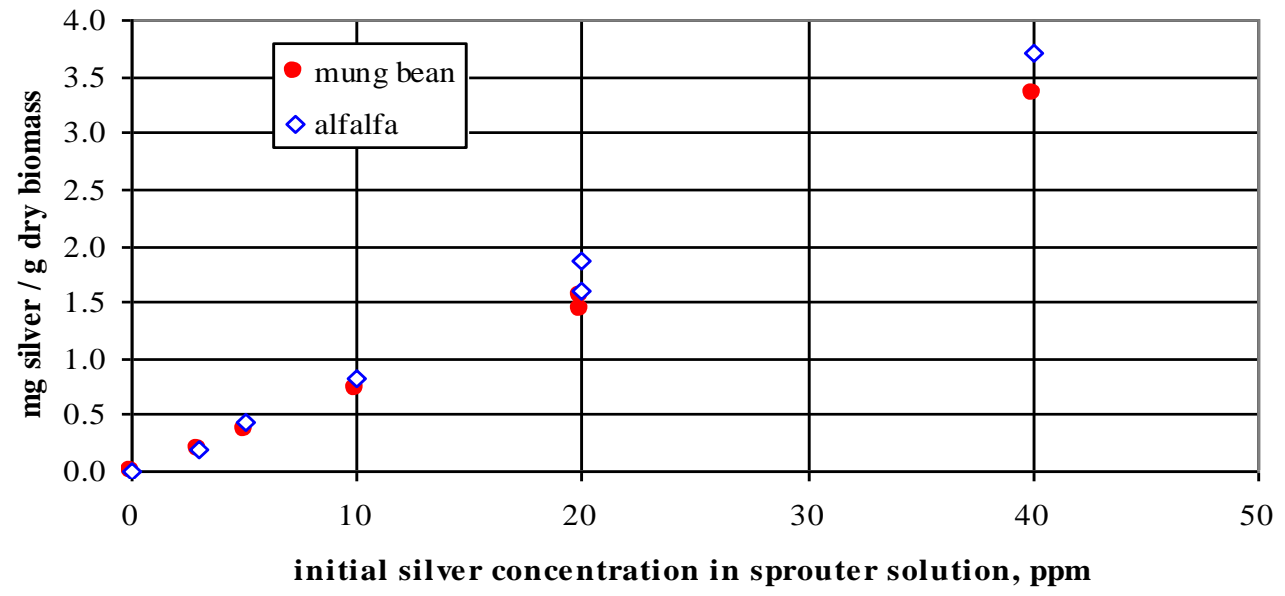

Figure 2.2.1 Dependence of silver uptake of mung bean and alfalfa sprouts in A-sprouter on initial silver concentration in sprouter solution

Uptake data in S-sprouter are given in Figure 2.2.2 below for seven different concentrations of sprouter solution: $3,5,10,20,40$, and $80 \mathrm{ppm}$ of silver, plus zero as control.

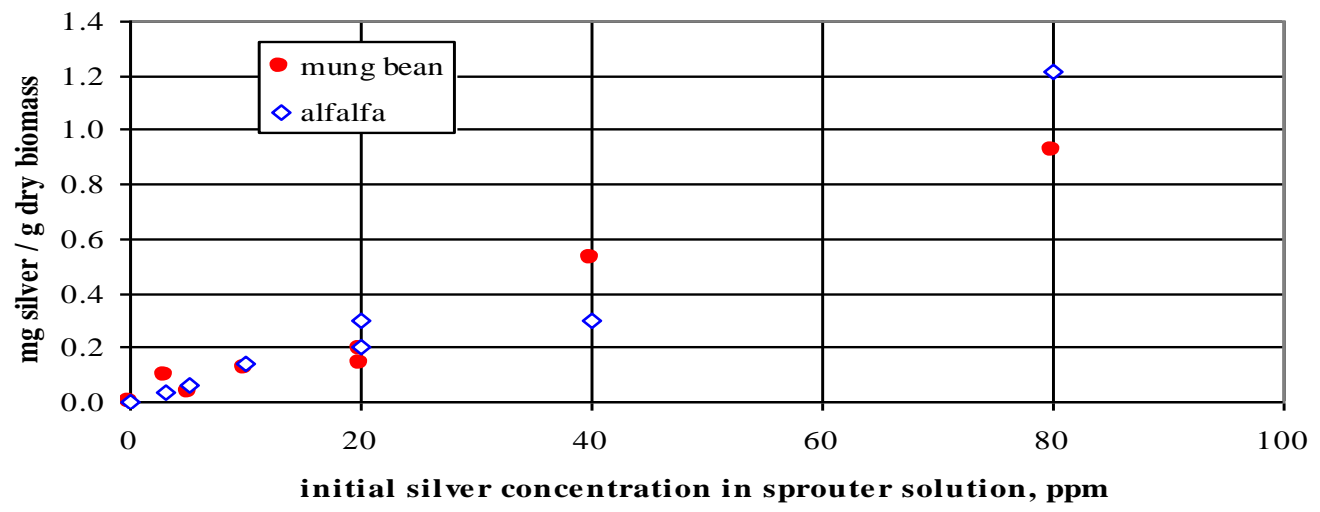

Figure 2.2.2 Dependence of silver uptake of mung bean and alfalfa sprouts in $\mathrm{S}$-sprouter on initial silver concentration in sprouter solution

Both figures indicate that, despite the toxic effects of $\mathrm{Ag}^{+}$, ability of both sprouts to uptake silver was observed. The uptake data indicate that the intra-cellular contents of silver in both types of seedlings increase with increasing concentrations of silver in the sprouter solution for both types of sprouter. Overall, silver uptake in A-sprouter is several-fold higher than that 
in S-sprouter. This is due to the fact that, in A-sprouter, all parts (root, stem, and leaf) of the sprouts were exposed to the silver-containing sprouter solution, while in S-sprouter only roots of the sprouts were in contact with the solution. It must be mentioned that, at higher silver concentrations, rate of seed germination reduced significantly and the growth of seedlings appeared to be stunted. Thus the intra-cellular accumulation of silver in the sprouts may not be completely due to active uptake, but may be due partially to passive diffusion.

\section{$\underline{2.3 \text { Silver Uptake by Plant Cells }}$}

Shown in Figure 2.3.1 below is the silver uptake in the cells versus the initial concentrations of silver ions (3, 5, 20, and $40 \mathrm{ppm}$ ) in the solution. A blank solution free of $\mathrm{Ag}^{+}$was chosen as control. The data confirm the uptake of silver from the plant cells grown in suspension after a period of exposure to silver nitrate solution. They also indicate that overall the intracellular content of silver increases with increasing concentration of silver in the medium. Although plant species are different, silver contents in plant cells grown in suspension culture are again higher than that in the sprouts, similar to the case of gold nanoparticles.

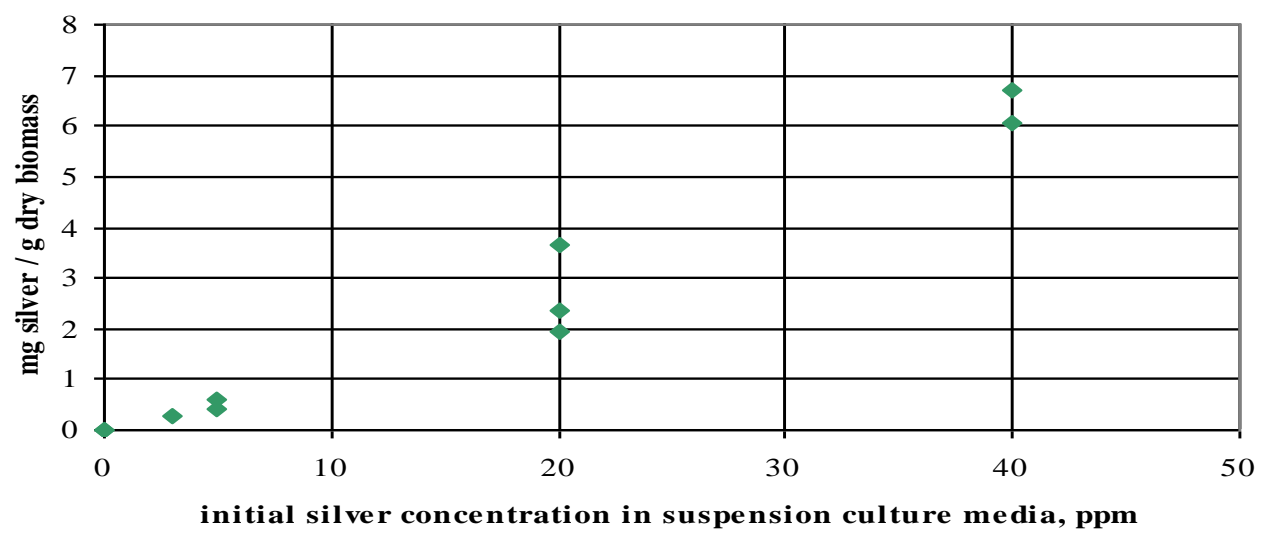

Figure 2.3.1 Dependence of silver uptake in stevia cells on initial silver concentration in sprouter solution

Normally the suspension culture is pale yellow in color at a healthy state. But when the (initial) concentration of silver ion is at 20 or $40 \mathrm{ppm}$, the toxic effect of the silver on the plant cells was obvious from the changes in the appearance of the suspension culture; the suspension turned dark-color after addition of the silver solution, indicating that the cells were no longer viable, and the uptake of silver might be due to passive diffusion. 


\subsection{Nickel Uptake by Plant Seedlings}

The nickel uptake data collected following the procedure described in Section 1.4 are presented in Figures 2.4.1, 2.4.2, and 2.4.3 below; the first two figures are for S-sprouter and the third one for A-sprouter. The nickel ion concentrations used in the sprouters were 3.21 and $6.42 \mathrm{ppm}$.

The data indicate that both alfalfa and brown mustard sprouts were capable of uptaking nickel from the sprouter solution into their plant tissue. In addition, all three figures show that the amounts of uptake increased with increasing nickel concentration in the sprouter solution. Furthermore, the amounts of uptake by alfalfa are in general larger than that by brown mustard. These observations are true for both types of sprouter. It was also observed that in general uptakes in A-sprouter are hugely larger than that in S-sprouter indicating either significant nickel uptake from parts of seedlings other than roots or the significance of longer GG time to allow further development of roots for sprouts grown in an A-sprouter. On the other hand, no conclusive statement can be made with regard to the relation between the amount of nickel uptake and the CC time, due to the large experimental errors associated with the data. We also found that for brown mustard SS time of one or two days had no impact on nickel uptake from brown mustard grown in an A-sprouter.

Nickel uptake vs. initial concentration in S-sprouter (Compare AS3d and BS3d)

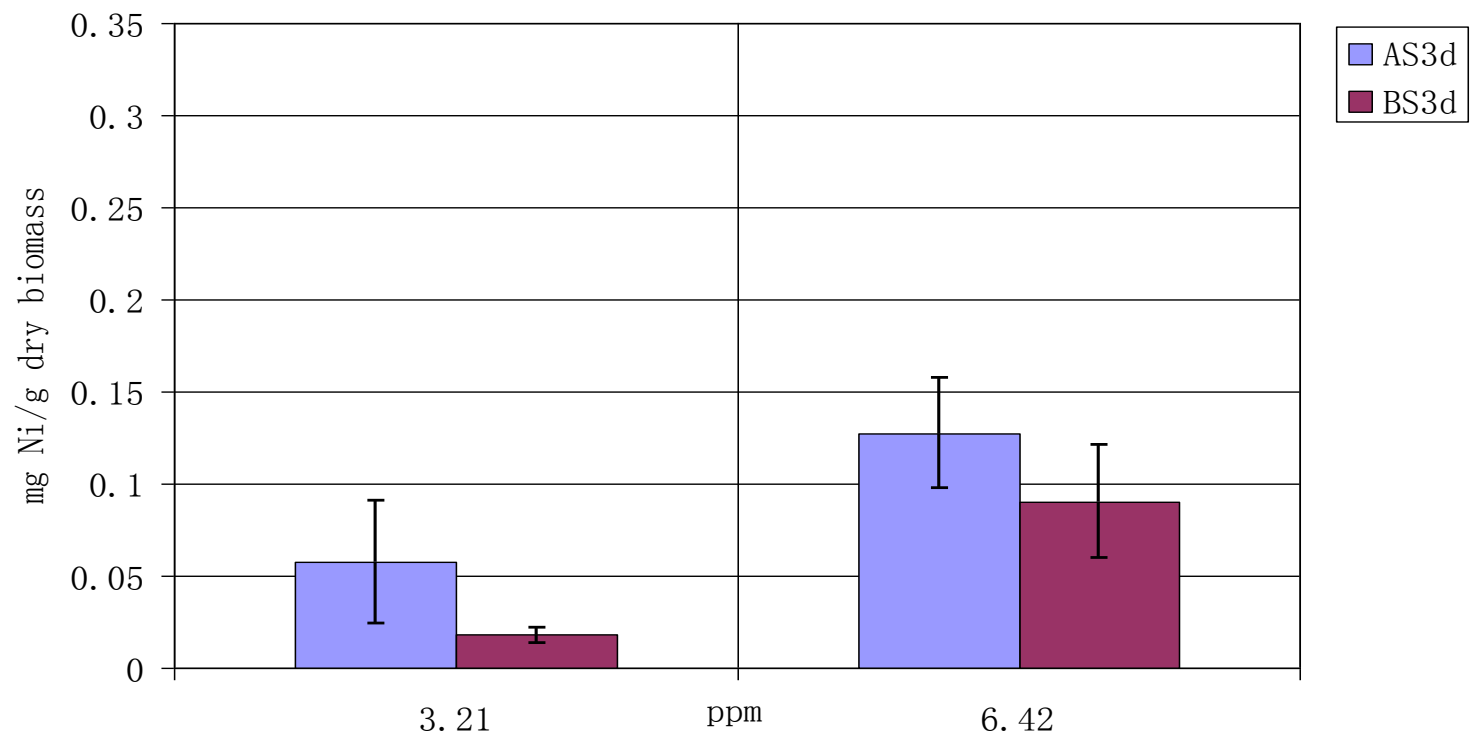

Figure 2.4.1 Dependence of nickel uptake of alfalfa and brown mustard sprouts in $\mathrm{S}$-sprouter on initial nickel ion concentration in sprouter solution (AS represents "alfalfa in S-sprouter"; BS represents "brown mustard in S-sprouter"; and 3d represents "CC time = 3 days") 
Nickel uptake vs. initial concentration in S-sprouter (Compare AS2d and BS2d)

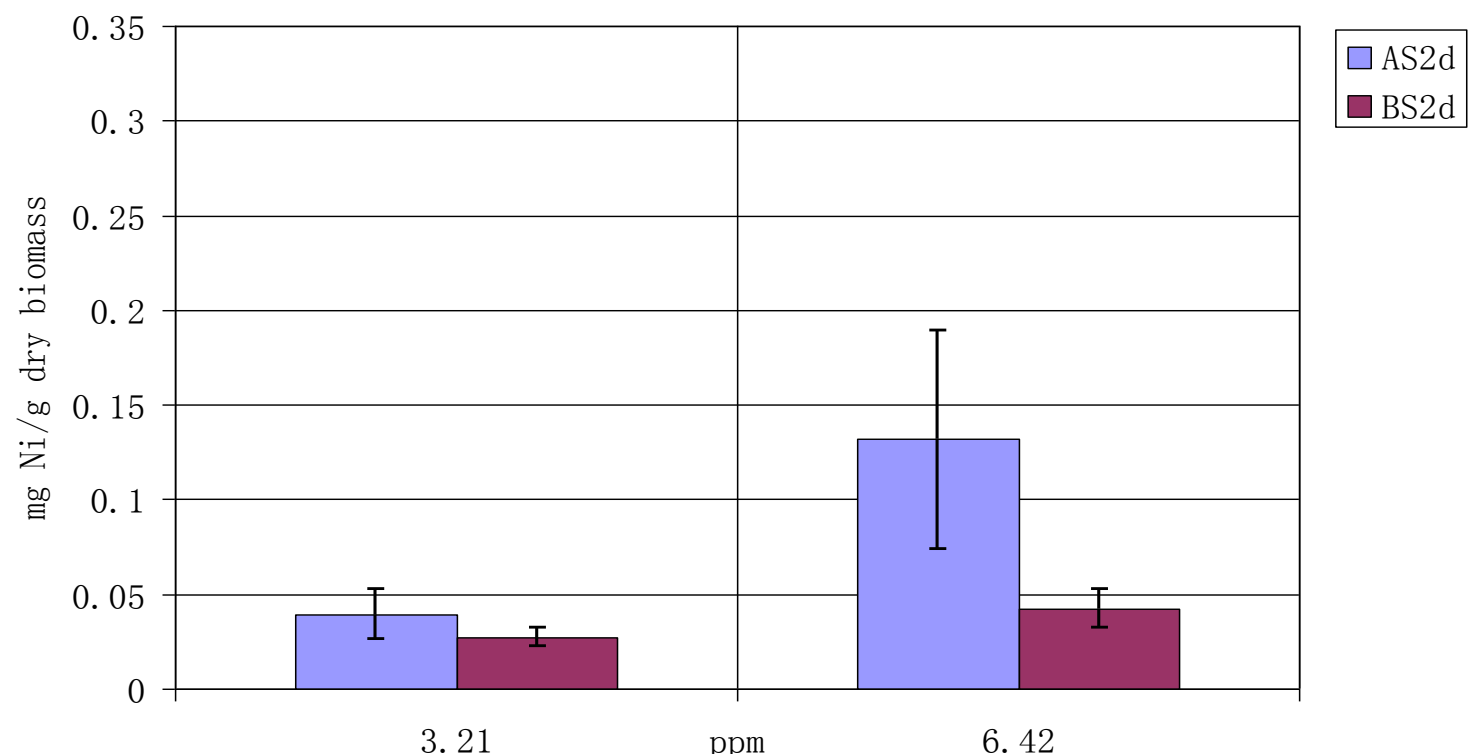

Figure 2.4.2 Dependence of nickel uptake of alfalfa and brown mustard sprouts in $\mathrm{S}$-sprouter on initial nickel ion concentration in sprouter solution (AS represents "alfalfa in S-sprouter"; BS represents "brown mustard in S-sprouter"; and 2d represents "CC time $=2$ days")

Nickel uptake vs. initial concentration in A-sprouter

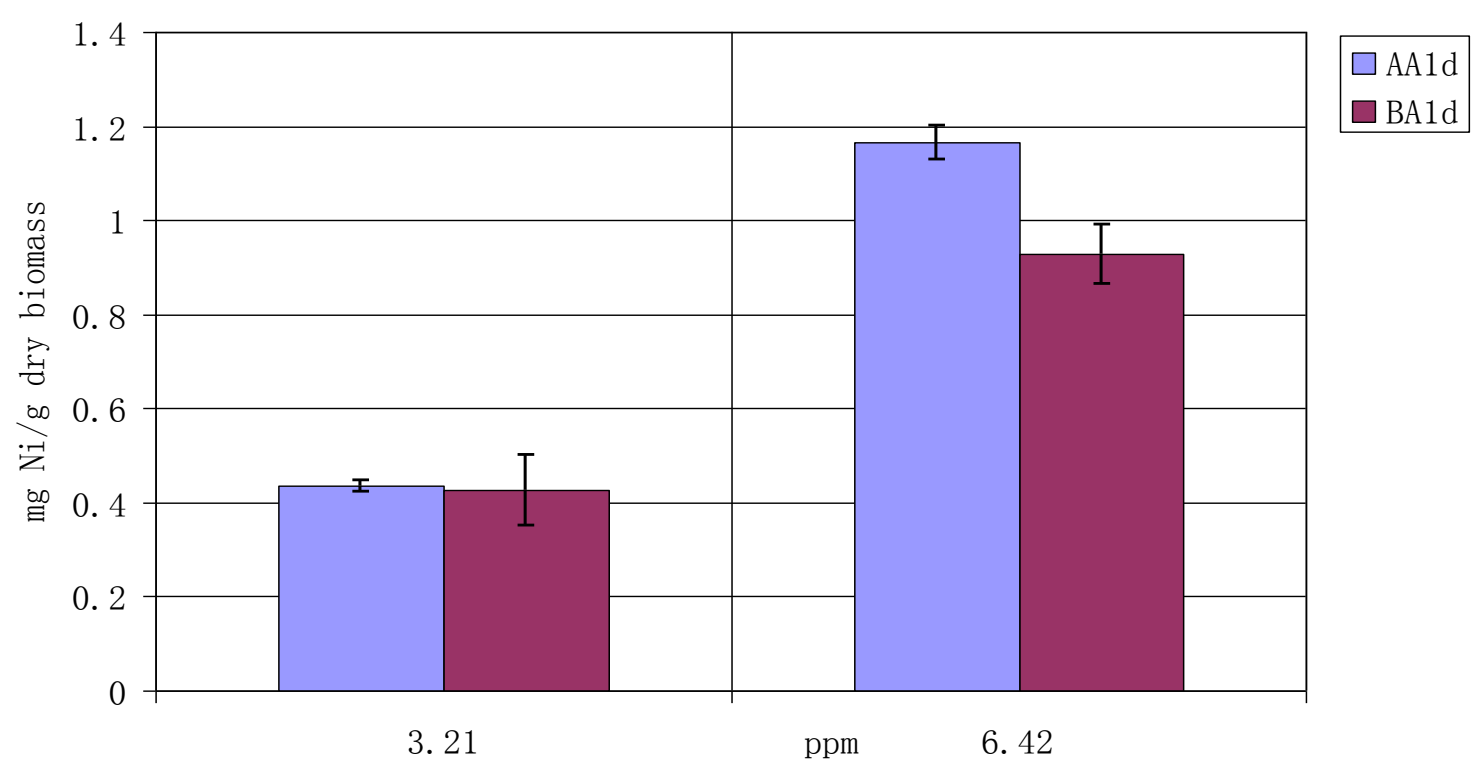

Figure 2.4.3 Dependence of nickel uptake of alfalfa and brown mustard sprouts in A-sprouter on initial nickel ion concentration in sprouter solution (AA represents "alfalfa in A-sprouter"; BA represents "brown mustard in A-sprouter"; and 1d represents "CC time $=1$ day") 


\section{CONCLUSION}

1. Silver nanoparticles were produced extracellularly from three cultivars of geranium: 'Rose' (R), 'Rober's Lemon Rose' (RR), and 'Lady Plymouth' (LP). The nanoparticleformation reaction as a result of mixing of the geranium extract with dilute silver nitrate was slower than the corresponding bioreaction for the formation of gold nanoparticles. Colors characteristic of silver nanoparticles in aqueous solution due to surface plasmon resonance were exhibited, and the nanoparticles produced were found to remain stable for several months.

2. The bioreaction associated with extracellular production of silver nanoparticles took place only in lighted conditions. Even after a length of time in darkness, the reaction would still begin upon exposure to light. Although postponed significantly, when the reaction was initiated, it proceeded at about the same reaction rate as if it had not been delayed. On the other hand, oxygen had no significant or obvious effect on the reaction. Though the bioreaction was cultivar-dependent, only a slight difference in reaction was observed among the three cultivars.

3. Despite the toxic effects of silver, ability of both alfalfa and mung bean sprouts to uptake silver was confirmed. The uptake data indicated that the intracellular contents of silver in both types of seedlings increased with increasing concentrations of silver in the sprouter solution for both types of sprouter. Overall, silver uptake in A-sprouter is several-fold higher than that in S-sprouter, due to the fact that in A-sprouter all parts of the sprouts were exposed to the silver-containing sprouter solution, but not in S-sprouter. Due to the toxic effect of silver ions, it was observed that, at higher silver concentrations of the sprouter solution, part of the intracellular uptake of silver in the sprouts might be due to passive diffusion.

4. Uptake of silver to the plant cells grown in suspension after a period of exposure to silver nitrate solution was also observed. Overall, the intracellular content of silver increased with increasing concentration of silver ions in the medium. Although plant species are different, silver contents in plant cells grown in suspension culture were much higher than that in the sprouts, similar to the case of gold nanoparticles. However, the toxic effect of the silver ions on the suspended plant cells was more severe than that on the sprouts, causing cell death particularly when the silver ion concentration increased beyond $20 \mathrm{ppm}$.

5. Both alfalfa and brown mustard sprouts were capable of uptaking nickel from the sprouter solution into their plant tissue. The amounts of uptake increased with increasing nickel concentration in the sprouter solution. Furthermore, the amounts of uptake by alfalfa are in general larger than that by brown mustard. These observations were true for both types of sprouter. 


\section{REFERENCES}

1. Brooks, R. R., et al., Trends in Plant Science, $\underline{3}$, 359-362 (1998).

2. Anderson, C. W. N., et al., Nature, 395, 553 (1998).

3. Gardea-Torresdey, J. L., et al., Langmuir, 19, 1357-1361 (2003).

4. Murphy, C.J. and N.R. Jana, Advanced Materials, 14, 80-82 (2002)

5. Shankar, S. S., et al., Biotechnology Progress, 19, 1627-1631 (2003). 
Appendix 34: Development of a Novel Optical Radiation Depolarization Technique for On-Line Measurements of Particle and Bubble Sizes (KY003) 


\section{FINAL TECHNICAL REPORT}

Contract Title and Number:
Crosscutting Technology Development at the Center for
Advanced Separation Technologies
(DE-FC26-02NT41607)

Contract Title and Number: Advanced Separation Technologies (DE-FC26-02NT41607)

Sub-Recipient Project Title:

Development of a Novel Optical Radiation

Depolarization Technique for On-Line Measurements of

Particle and Bubble Sizes

Principal Investigators:

Tao, Menguc, Crofcheck, Mustafa Aslan

Contact Address:

University of Kentucky

234 MMRB

Lexington KY 40506

Subcontractor Address:

No subcontracts issued.
Period of Performance:

Starting Date: 10/1/2003

Ending Date: 10/31/2007
Report Information:

Type: Final

Number:

Period: $\quad$ 10/1/03-10/31/06

Date: $\quad$ 8/11/2006

Code: $\quad$ KY003-FINAL

Contact Information:

Phone: (859) 257-2953

Fax: $\quad$ (859) 323-1962

E-Mail: dtao@engr.uky.edu

Subcontractor Information:

Phone:

Fax:

E-Mail:

Disclaimer

This report was prepared as an account of work sponsored by an agency of the United States Government. Neither the United States Government nor any agency thereof, nor any of their employees, make any warranty, express or implied, nor assume any legal liability or responsibility for the accuracy, completeness, or usefulness of any information, apparatus, product, or process disclosed, or represents that its use would not infringe privately owned rights. Reference herein to any specific commercial product, process, or service by trade name, trademark, manufacturer, or otherwise does not necessarily constitute or imply endorsement, recommendation, or favoring by the United States Government or any agency thereof. The views and opinions of authors expressed herein do not necessarily state or reflect those of the United States Government or agency thereof. 


\begin{abstract}
Grinding and froth flotation are the two most important processes for mineral beneficiation. The importance of grinding is well reflected in the fact that approximately $80 \%$ of beneficiation costs are for grinding, mainly due to high energy consumption. To reduce energy consumed by grinding, fines should be removed quickly from the grinding circuit. This requires a reliable on-line particle size analysis technique. Froth flotation is the most widely used solid-solid separation process for coal and minerals beneficiation and about $90 \%$ of mineral concentrates are produced from froth flotation. It is now recognized that air bubble size distribution plays an important role in flotation separation performance. Optimization of bubble size distribution is possible only if bubble size can be monitored online. Obviously, an on-line analysis technique for particle and bubble size is critical for enhanced grinding and flotation process efficiency.

The present project was aimed at the development of an optical radiation depolarization technique for on-line size analysis for grinding and flotation processes to minimize energy consumption during grinding and maximize separation efficiency of flotation. The technique is based on angular and radial profiles of reflection and transmittance of an object subjected to a collimated, polarized light beam. The size distribution was determined by best fitting experimentally determined vertical and horizontal polarization components of both radial and angular profiles of reflection and transmission. In addition to size analysis, the proposed technique can also determine the water film thickness and the bubble separation distance distribution in foams.
\end{abstract}

The two-phase GL (Gas-Liquid) results show that the present Elliptically polarized light scattering (EPLS) concept is very promising for characterization of optically thin columns and has many advantages over imaging techniques when in-situ measurement of bubble size are necessary at high flow rates with high surfactant concentrations. For optically-denser bubble laden media, the reliability of this technique can be improved by incorporating multiple scattering algorithms in data reduction.

The two-phase SL (Solid-Liquid) experiments indicate that the problem is more complex than GL phase measurements because of composite structure of the coal mixtures and wide range of size distribution. But it is still possible to sense the particle size distribution from the normalized scattering matrix elements. 


\section{TABLE OF CONTENTS}

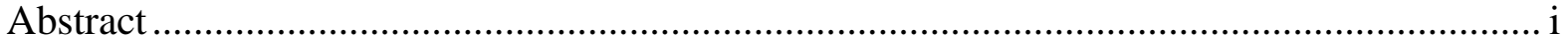

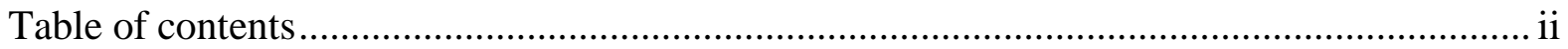

List of graphical materials ......................................................................................................

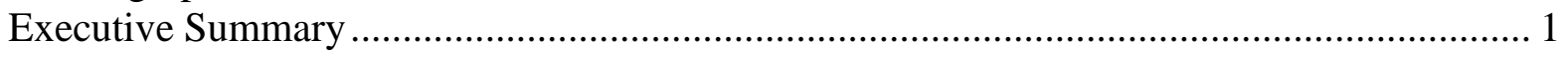

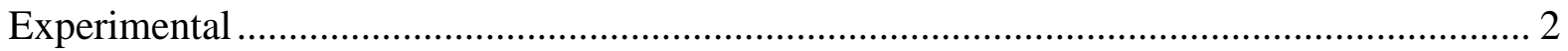

Customization of Algorithm ...................................................................................... 4

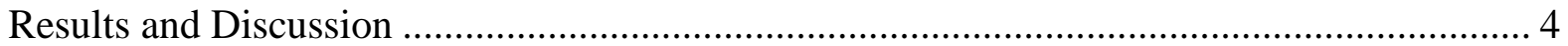

Relationship between Flow Rate and Scattered Light Intensity ............................................ 4

Determination of Size Distribution of the Bubbles............................................................... 5

Relationship between flow rate, volume fraction of bubbles and average bubble diameter 6

Two Phase Measurements -- Gas Bubbles in Liquid............................................................ 6

Two Phase (SL) Measurements Coal Particles in Liquid ....................................................... 8

Bubble size and Gas-hold up at GL phase: .................................................................. 10

Effect of Total Reflectance .................................................................................... 12

Coal Particle Size Characterization at SL phase:................................................................ 14

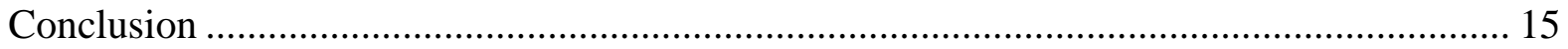

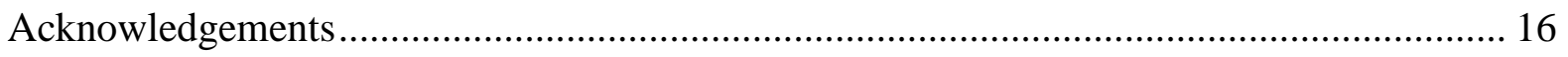

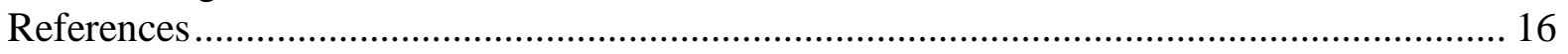

\section{LIST OF GRAPHICAL MATERIALS}

Figure 1. Gas-Liquid (GL) phase column and Optics used in the experimental system to obtain intensity and polarization information of the medium bubbles in the water. ........ 2

Figure 2. Gas-liquid (bubbles) and solid-liquid (coal particles) monitoring system................. 3

Figure 3. Sensor placement in the prototype on-line EPLS System .......................................... 4

Figure 4. Flow-rate regions based on scattered light intensity ………...................................... 5

Figure 5. Bubble size distribution for flow rate $Q=35.26 \mathrm{ml} / \mathrm{min}$ for the image shown. ....... 5

Figure 6. The relationship between flow rate, volume fraction of bubbles and average bubble

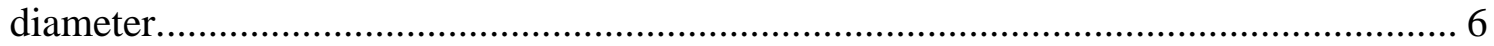

Figure 7. $S_{i j}$ 's at 120 degree as a function of mean bubble diameter....................................... 7

Figure 8. Scattering matrix elements $\left(M_{i j}\right)$ at scattering angle of $120^{\circ}$ as a function of gas

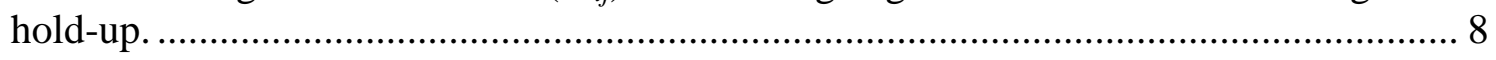

Figure 9. Comparison experiments with Lorenz-Mie model with a narrow size distribution.. 9

Figure 10. Effect real and imaginary parts of complex refractive index of the coal particles.10

Figure 11. Sensitivity of measured $M_{11}$ at $120^{\circ}$ on average bubble size................................ 11

Figure 12. Sensitivity of the $M_{22}$ signal at $120^{\circ}$ with varying gas-hold up............................. 11

Figure 13. Scattering matrix elements $\left(M_{\mathrm{ij}}\right)$ calculated from experimental intensity measurements between $90^{\circ}$ and $160^{\circ}$ at different superficial gas velocities and a surfactant concentration of $100 \mathrm{ppm}$.......................................................................... 12

Figure 14. Scattering matrix elements $\left(M_{i j}\right)$ at $120^{\circ}$ as a function of mean bubble diameter (mm). The solid lines indicate curve-fittings............................................................... 14

Figure 15. Real and imaginary parts of coal and ash........................................................... 15 


\section{EXECUTIVE SUMMARY}

Grinding and froth flotation are the two most important processes for mineral beneficiation. The importance of grinding is well reflected in the fact that approximately $80 \%$ of beneficiation costs are for grinding, mainly due to high energy consumption. To reduce energy consumed by grinding, fines should be removed quickly from the grinding circuit. This requires a reliable on-line particle size analysis technique. Froth flotation is the most widely used solid-solid separation process for coal and minerals beneficiation and about $90 \%$ of mineral concentrates are produced from froth flotation. It is now recognized that air bubble size distribution plays an important role in flotation separation performance (Tao, 2004). Optimization of bubble size distribution is possible only if bubble size can be monitored on-line. Obviously, an on-line analysis technique for particle and bubble size is critical for enhanced grinding and flotation process efficiency.

Light scattering is one of the most effective concepts for characterization of particles and bubbles. When either vertically or horizontally polarized light is incident on the medium, it goes through a number of scattering events. Bubbles are considered much larger than the wavelength of the incident light, which is a reasonable approximation even for bubble diameters as small as $5 \mu \mathrm{m}$.

The overall objective of the following project was to develop a concept based on optical radiation depolarization measurement technique for on-line real-time size analysis of grinding and flotation processes to minimize energy consumption during grinding and maximize flotation efficiency. The technique is based on use of angular and radial profiles of reflection and transmission of an object subjected to a collimated, polarized light beam.

The two-phase GL results show that the present EPLS concept is very promising for characterization of optically thin columns and has many advantages over imaging techniques when in-situ measurement of bubble size are necessary at high flow rates with high surfactant concentrations. For optically-denser bubble laden media, the reliability of this technique can be improved by incorporating multiple scattering algorithms in data reduction.

The two-phase SL experiments indicate that the problem is more complex than GL phase measurements because of composite structure of the coal mixtures and wide range of size distribution. But it is still possible to sense the particle size distribution from the normalized scattering matrix elements. 


\section{EXPERIMENTAL}

\section{Experimental setup:}

The bubble column setup employed for preliminary stages of this study is shown in Figure 1. The column was located on a tilting stage and two translation stages in order to align accurately in vertical direction. Nitrogen gas tank was used to provide constant pressure gas to generate bubble column in the water. Bel-Art Riteflow flowmeter model \# 40407-0075 was attached between gas tank and the column to control the flow-rate accurately between 3.5 and $522 \mathrm{ml} / \mathrm{min}$.

Elliptically polarized light scattering (EPLS) method was applied to measure two-phase flow (gas-liquid) in situ. Figure 1 also shows optical arrangement used in the experiments to modulate incident and scattered light to measure polarized light and scattered light by the bubbles. Polarization angle and type of incident light was modulated by retarder- 1 while the first polarizer ( $\mathrm{P}-1)$ is fixed at 45 degrees in the incident beam path. Scattered light from bubbles in the water was filtered by retarder-2 (R2) and polarizer-2 (P2). The setup was capable of taking measurements at different scattering angles $(\theta)$. A $20 \mathrm{~mW}$ HeNe laser $(\lambda=632 \mathrm{~nm})$ is employed as a light source. Scattered light that passes through R-2 and P-2 was detected by a photomultiplier tube (PMT; Hamamatsu R446) as a function of scattering angle, $\theta$.

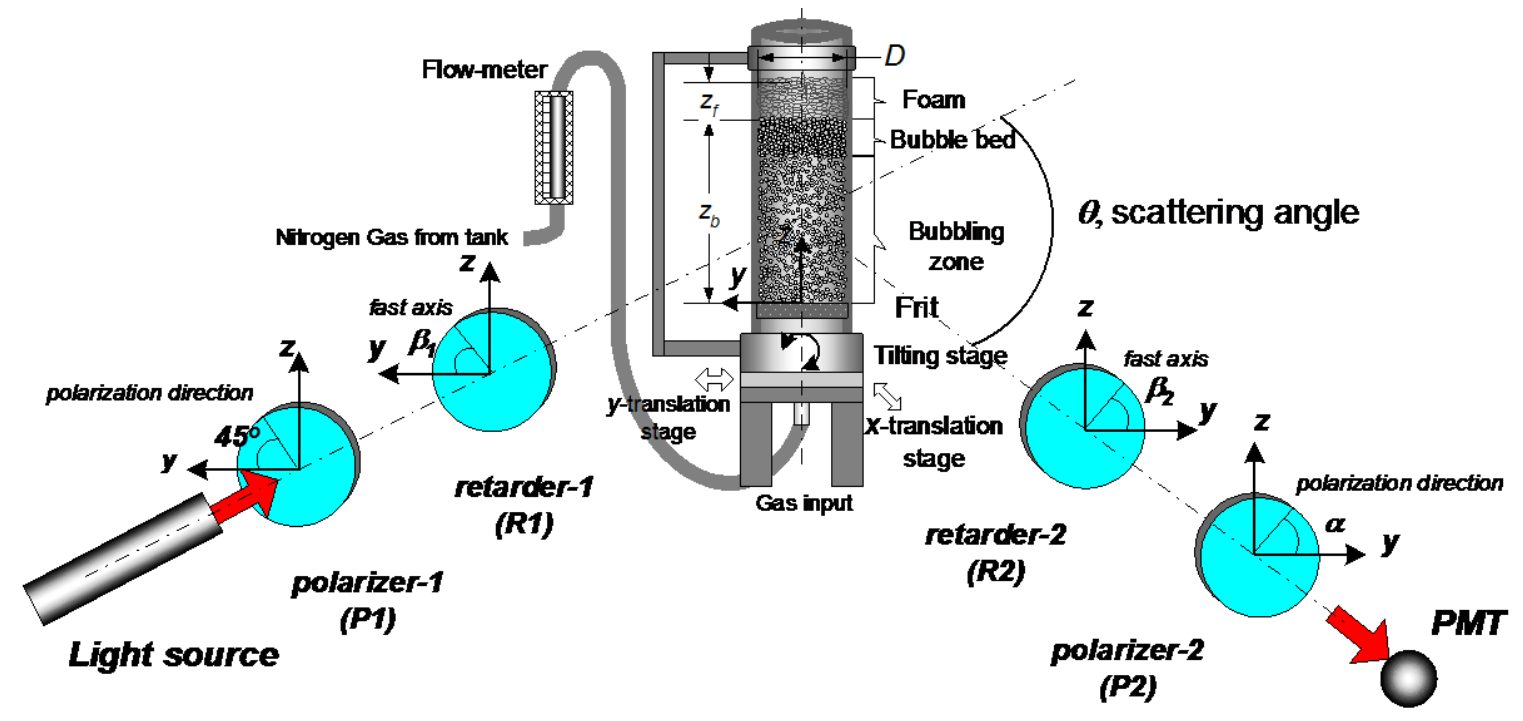

Figure 1. Gas-Liquid (GL) phase column and Optics used in the experimental system to obtain intensity and polarization information of the medium bubbles in the water. 
A prototype on-line EPLS System, shown in Figure 2 was fabricated in order to monitor the system at two different locations where the flow is essentially two- phase. With two sensors scattered light intensity and polarization information from bubbles and coal particles were monitored at optimum scattering angles. Based on the scattered light data, size distribution of both bubbles and coal particles and volume fractions of bubbles were obtained by using the developed algorithm. Two sensors allowed for in-situ process monitoring and control of the input parameters such as gas flow rate (gas pressure), surfactant concentration, and coal feeding rate.

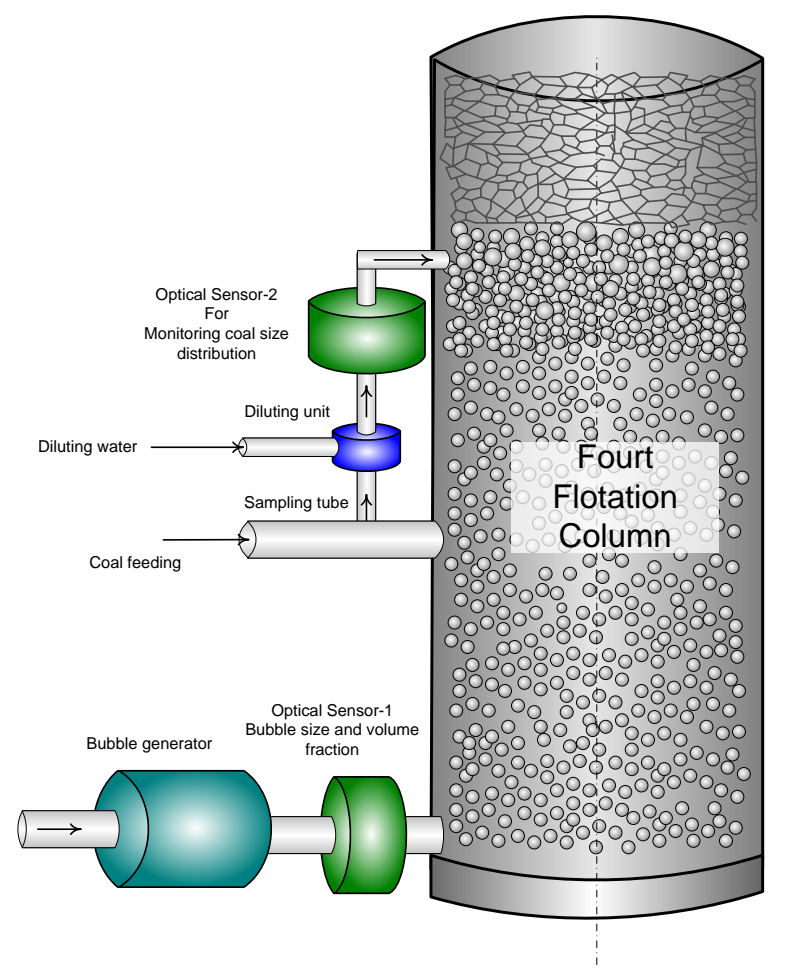

Figure 2. Gas-liquid (bubbles) and solid-liquid (coal particles) monitoring system.

Scattered light at two different scattering angles was collected by two polarizationmaintaining fiber-optic to the detector via polarization optics box where scattering light intensity, polarization state and degree were measured, shown in Figure 3. The data were collected using a data acquisition board on a PC. Scattering angles where the measurements were taken was varied to measure bubble size depending on flow-rate and surfactant ratio by two rotational disks. 


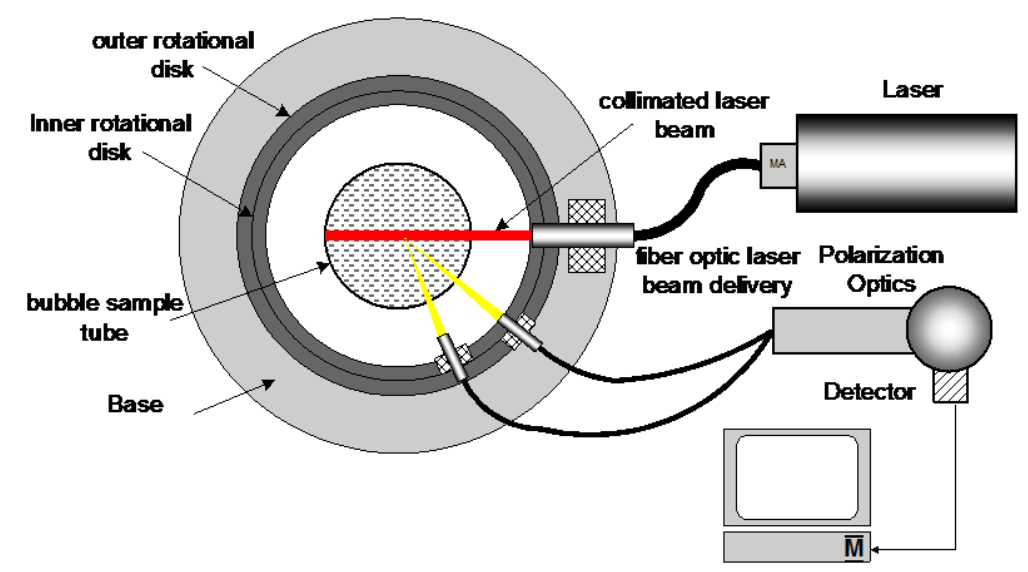

Figure 3. Sensor placement in the prototype on-line EPLS System

Customization of Algorithm

A detailed numerical study had been carried out (Wong and Mengüç, 2002) to understand the propagation of polarized light in a bubble-laden medium, which is similar in essence to froth flotation system. The potential use of polarized light for characterization of mono-dispersed air bubbles was investigated here using a vector Monte Carlo approach. The scattering matrix for the entire medium was constructed based on the Fresnel equations. Examination of the absorbing and multiple-scattering results showed that if circularly polarized light was used, the bubble size, the bubble separation, as well as the thickness of carbonaceous layer around bubbles can be determined, where circularly polarized light is a better choice than the linearly.

The theoretical models were mostly developed for single scattering. In the independent scattering regime for 2-phase GL flow, the separation distance was much larger than the particle diameter and therefore the interaction of the incident electromagnetic wave with an individual bubble is independent of other bubbles. For these conditions, the Lorenz-Mie solution can be used as a basis for theoretical predictions. However, if the bubbles were separated by smaller distances, the scattering may become dependent and the Lorenz-Mie solution may not be applicable. Therefore, experimental conditions over a wide range of scattering regime from single scattering to multiple scattering make it more difficult to compare the results with a single theoretical model.

\section{RESULTS AND DISCUSSION}

\section{Relationship between Flow Rate and Scattered Light Intensity}

In order to understand scattering light intensity behavior for different flow rates, scattering light intensity measurements were made at $\theta=90$ degree utilizing the first set-up (Figure 1). For each experiment at different flow rate, 2400 intensity measurements were taken during 120 seconds. The experiment was repeated for 7 different flow-rates. The average scattering light intensity is shown in Figure 4 as a function of flow rate and gas velocity. There are two regions in terms of the intensity of scattering light: in Region 1 while flow rate increases, volume fraction of bubble increases as bubble diameter increases slowly. However, in Region 2, while flow rate increases, small bubbles start coalescing with each other to form 
larger bubbles. This means that in Region 2, the number of bubbles is smaller than in Region 1 , but they were, on the average, larger.

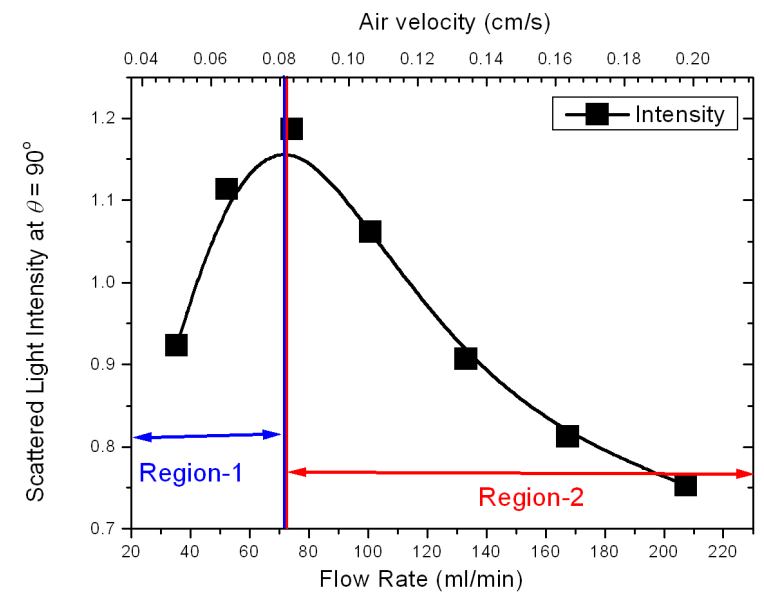

Figure 4. Flow-rate regions based on scattered light intensity

\section{Determination of Size Distribution of the Bubbles}

The digital images of bubbles were taken before and after each experiment for different flow rates. These images were taken by a Nikon D100 digital camera and they were image processed with Adobe PhotoShop and SIAMS-600 image processing software. The bubble size distribution was calculated based on total surface area method by SIAMS-600. As an example, the size distribution plot and corresponding processed image for $Q=35.26 \mathrm{ml} / \mathrm{min}$ are shown in Figure 5. Depending on the flow rate, location of the peak on size distribution curve changes. When the flow rate increases the major peak shifts to the right and the smaller peak disappears. The reasons for having a bimodal size distribution can be explained by noting that increasing aeration pressure increases the gas flow rate, which yields higher probability of bubble coalescence and presence of larger bubbles (Lain, 1999).

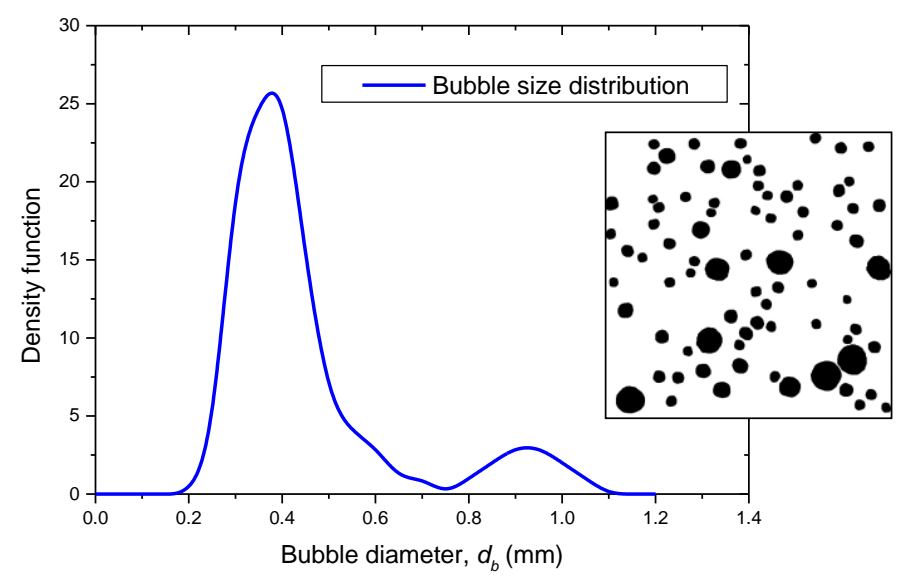

Figure 5. Bubble size distribution for flow rate $Q=35.26 \mathrm{ml} / \mathrm{min}$ for the image shown. 
Relationship between flow rate, volume fraction of bubbles and average bubble diameter

The frit pore sizes of the glass column were between $25 \mu \mathrm{m}$ and $50 \mu \mathrm{m}$. Pressure of $\mathrm{N}_{2}$ gas was kept at 5 psi. The experiments were conducted for five different flow rates: 35.26, 52.25, $74.28,100.77$, and $133.12 \mathrm{ml} / \mathrm{min}$. Close pictures of bubbles were taken before and after the measurement for each flow rate. Adobe Photoshop and SIAMS-600 image processing software were used to process the bubble pictures in order to find average bubble sizes for each flow rate. After image processing steps are given in (Aslan et al., 2003), the diameter of each bubble based on equivalent surface area of the bubble was calculated and for each picture, maximum, minimum, average bubble sizes were calculated by the software automatically. Figure 6 shows the relationship between flow rate, volume fraction of bubbles and average bubble diameter. Note that when the flow rate increases, average bubble diameter increases logarithmically, but volume fraction of bubbles increased linearly.

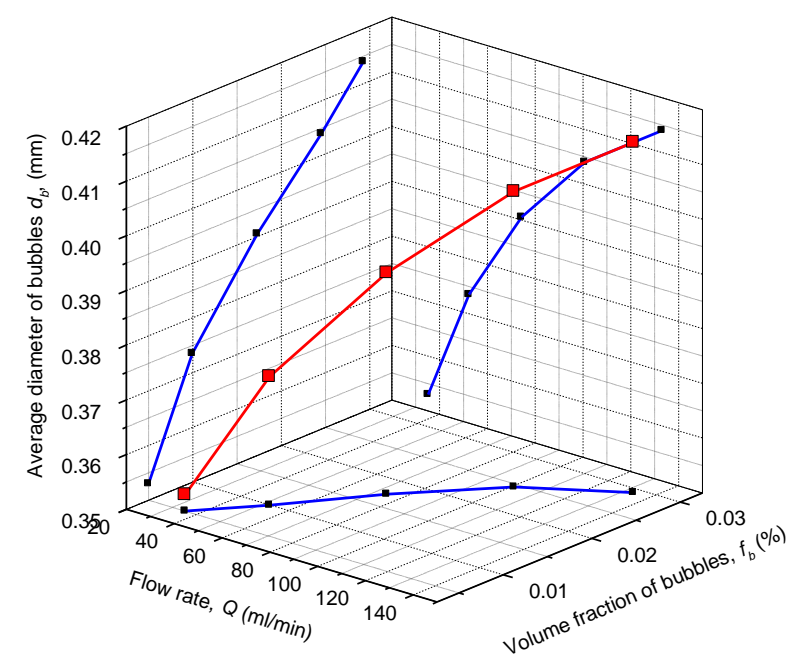

Figure 6. The relationship between flow rate, volume fraction of bubbles and average bubble diameter.

Two Phase Measurements -- Gas Bubbles in Liquid

A series of experiments were conducted to understand the relationship between input parameters such as flow rate and surfactant concentrations and scattering matrix elements $\left(M_{\mathrm{ij}}\right)$. Potential of using an elliptically polarized light scattering (EPLS) method to monitor both bubble size and gas hold-up in a bubble laden medium was explored. It is shown that with the use of EPLS, normalized scattering matrix elements $\left(M_{\mathrm{ij}}\right.$ 's) at different side and back scattering angles yielded the desired correlation between the bubble sizes and input parameters, including gas flow rate and surfactant concentrations, for the gas-liquid (GL) column. Experiments were carried out on gas-liquid columns containing different bubble sizes. The bubble size distributions were first evaluated using a digital image processing system at different gas flow rates and surfactant concentrations. Based on these images, bubbles were assumed to be spherical and their scattering matrix elements were calculated 
using the Lorenz-Mie theory. After the experiments were conducted, scattering matrix elements were determined using the intensity values measured for different polarization settings and the results were compared against the theoretical values. It was observed that the change in the bubble size yielded significant changes in $M_{11}, M_{33}, M_{44}$, and $M_{34}$ profiles at a single angle of $\theta=120^{\circ}$ for gas velocity range of 0.04 to $0.35 \mathrm{~cm} / \mathrm{s}(\mathrm{ID}=4.5 \mathrm{~cm})$. This optimum angle to conduct light scattering measurements depended on frit pore size, column diameter, gas pressure, and surfactant concentration. These results suggest that it is possible to design optical on-line sensors capable of monitoring bubble size and liquid hold-up based on EPLS.

In order to understand relationship between gas velocity and $M_{\mathrm{ij}}$ elements, additional single angle scattered measurements were conducted at $\theta=120^{\circ}$ where separation of gas velocity curves with most of scattering matrix elements is possible. Gas velocities were converted to mean bubble diameter and gas hold-up and plotted against the measured $M_{\mathrm{ij}}$ values, as shown in Figure 7 and Figure 8. Figure 7 depicts that it is possible to predict bubble diameter based on scattering measurements: $M_{11}, M_{22}, M_{33}, M_{44}$, and $M_{34}$ are sensitive to bubble diameter at low gas velocities with medium-low surfactant concentrations. At high superficial gas velocities $\left(v_{\mathrm{g}}>0.2 \mathrm{~cm} / \mathrm{s}\right)$, there is little change in the size of the bubbles as the flow rate increases.
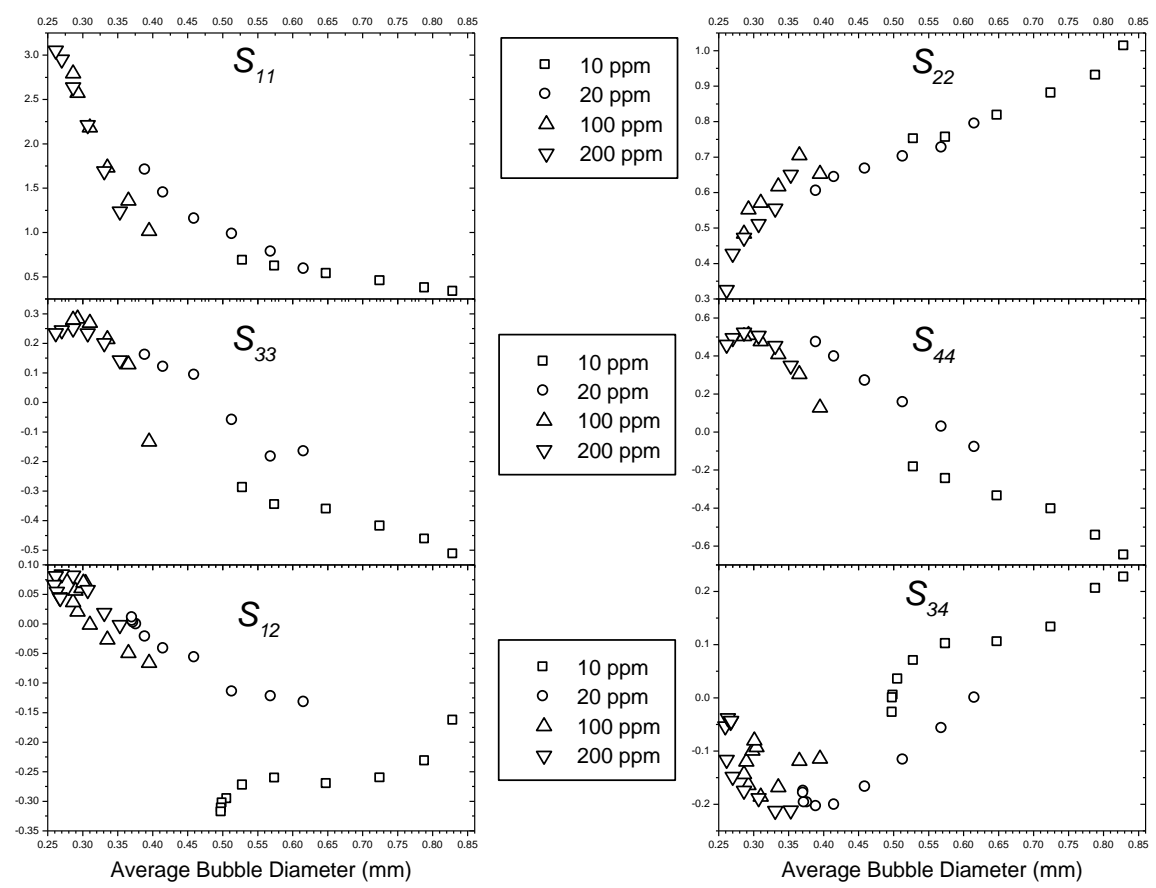

Figure 7. $S_{i j}$ 's at 120 degree as a function of mean bubble diameter. 

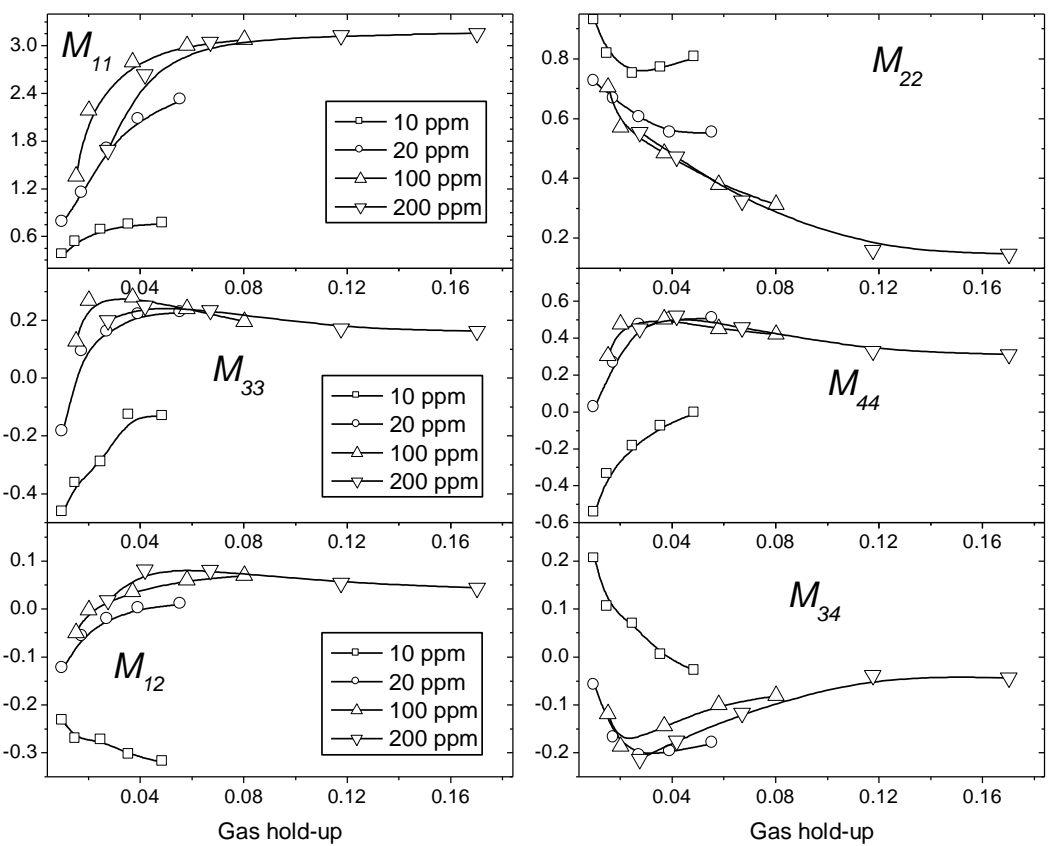

Figure 8. Scattering matrix elements $\left(M_{i j}\right)$ at scattering angle of $120^{\circ}$ as a function of gas hold-up.

Gas hold-up is the second parameter that can be related to the gas velocity. Figure 8 shows how the gas fraction in water affects the scattering at $120^{\circ}$. Even though the gas hold-up increases linearly with increasing gas velocity, effect of gas hold-up on $M_{\mathrm{ij}}$ depends on surfactant concentration. For a low-medium surfactant concentration, $M_{11}, M_{33}$, and $M_{44}$ increase as gas hold-up increases. $M_{12}$ decreases rapidly and $M_{34}$ increases when bubble size increases with increasing flow rate and surfactant concentration. $M_{22}$ changes drastically at higher surfactant concentrations, unlike other scattering matrix elements, because the shape factor of bubbles decreases with increasing gas velocity and the number of bubbles increases (multiple scattering regime). $M_{34}$ decreases at single scattering region but it increases in the multiple scattering zone. Monitoring gas hold-up based on $M_{\mathrm{ij}}$ measurements is possible at some gas velocities for known surfactant concentrations. Detailed results of GL-phase measurements are given elsewhere (Aslan et al., 2004; Aslan et al., 2006).

\section{Two Phase (SL) Measurements Coal Particles in Liquid}

There are many parameters that affect solid particle size measurement results in liquid using scattering light: carbon content of the coal mixture, optical property of the coal, shapes of the particles, volume fraction of particle in the water, etc. In this part of the study we addressed each of important parameters.

Sensor 2 in Figure 2 was designed to measure particle size in diluted solution. After the intensity values were measured, they were converted to scattering matrix elements. The corresponding $M_{i j}$ values are plotted in Figure 9 for three different volume fraction of coal particles. Solid curves are for Lorenz-Mie single scattering results obtained for a size 
distribution of $100 \%$ coal (red solid line) and 100\% mineral (sand \& clay) (black line) particles in water. It shows that pure carbon (coal) concentration in the coal mixture is important for predicting size distribution of particles. The second important parameter is refractive index of coal particles at visible wavelength range. In the literature there is a wide range of refractive index even at a fixed carbon concentration. Figure 10 shows how the normalized scattering matrix elements vary with different complex refractive index of coal particles. $M_{11}$ is sensitive to real part of refractive index and $M_{12}$ is more sensitive to imaginary part of refractive index. The imaginary part changes the type of $M_{34}$ curves significantly but its amplitude is still low.
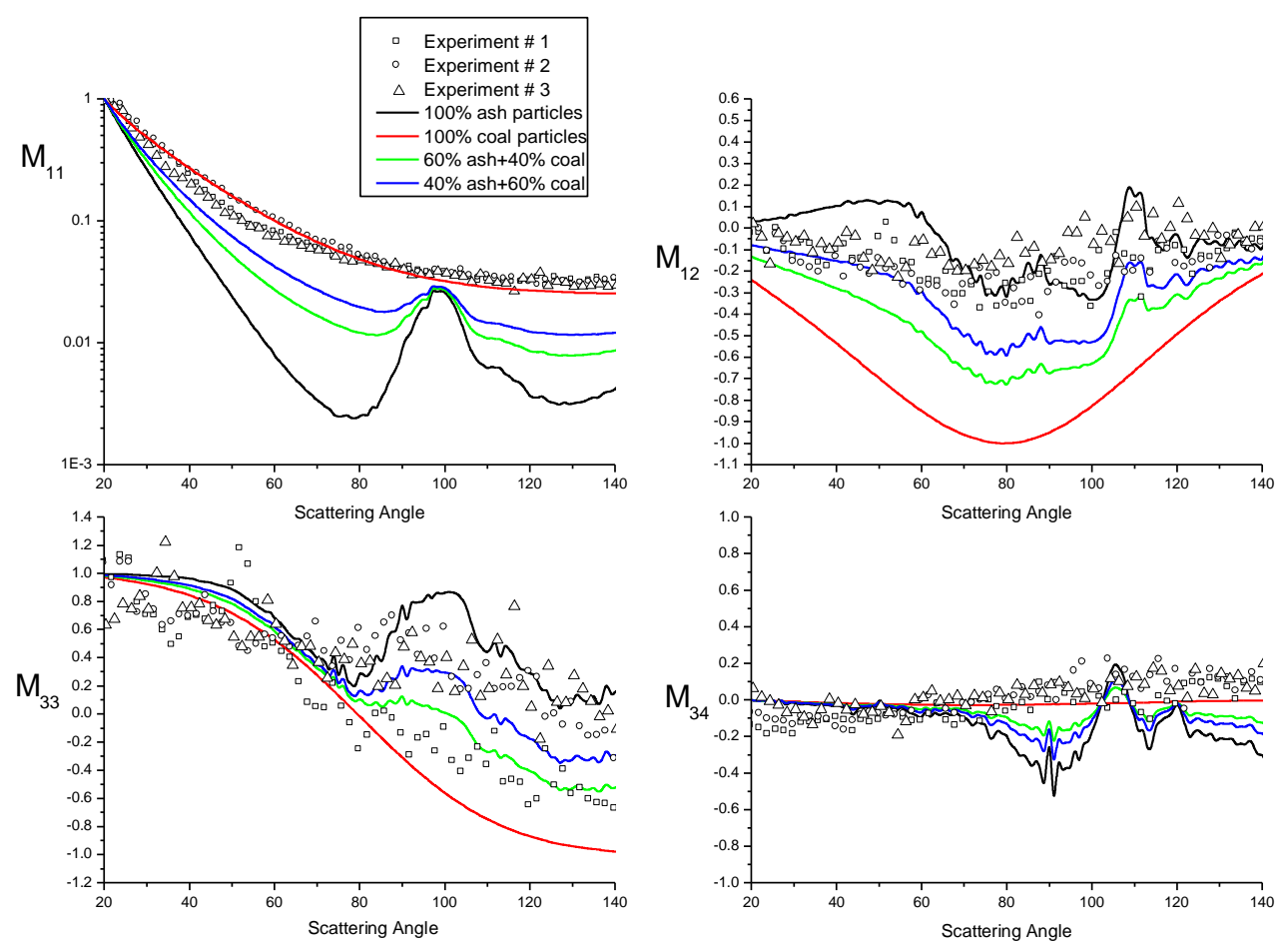

Figure 9. Comparison experiments with Lorenz-Mie model with a narrow size distribution. 

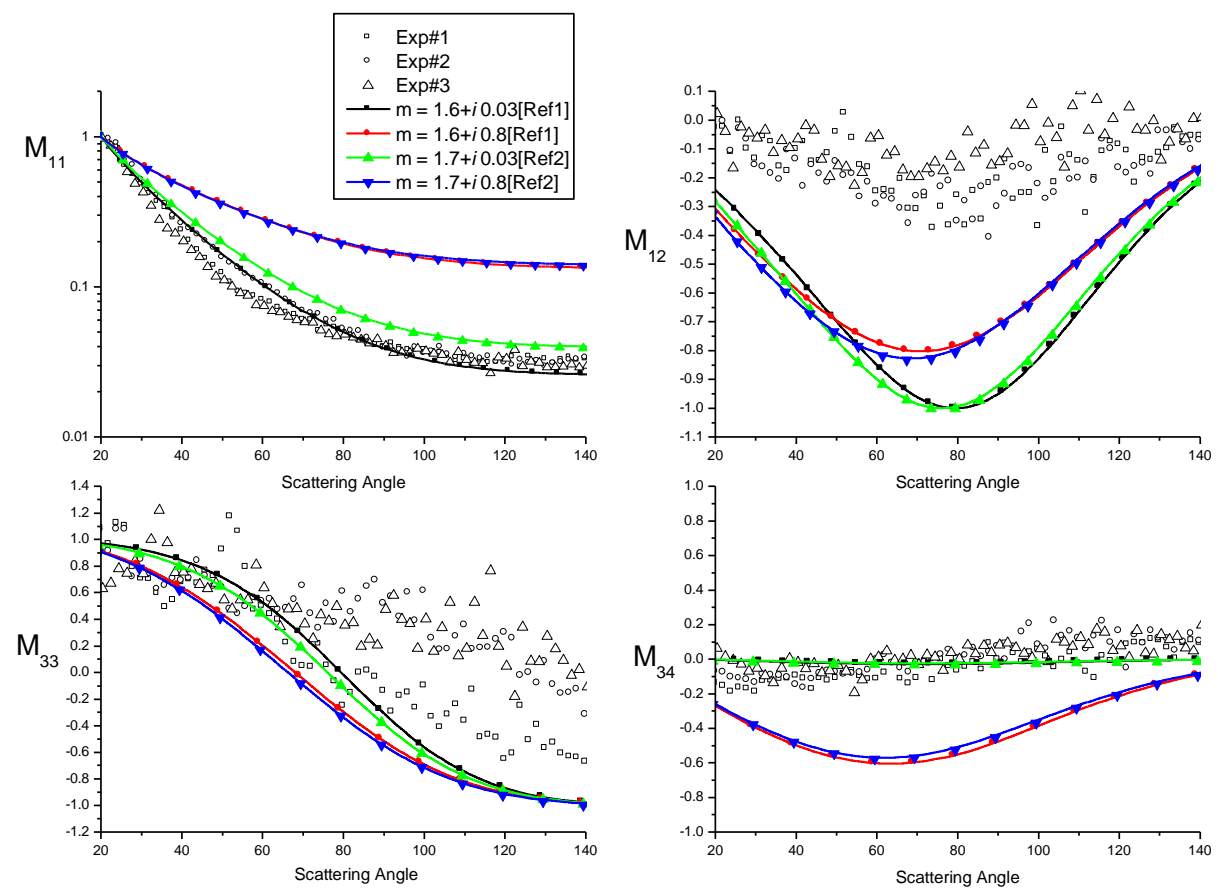

Figure 10. Effect real and imaginary parts of complex refractive index of the coal particles.

Bubble size and Gas-hold up at GL phase:

The normalized scattering matrix elements $\left(M_{11}\right.$ and $\left.M_{22}\right)$ that we use in this report can be calculated from the scattering amplitudes that relate two perpendicular components of the incident electromagnetic (EM) wave with two perpendicular components of the scattered EM wave for elliptical bubbles using the equations in Aslan et al. (2006). $M_{11}$ and $M_{22}$ are two major elements of $4 \times 4$ normalized scattering matrix, $\boldsymbol{M}$ that is function of scattered light intensity amplitude and polarization. They are not affected directly by surfactant ratio and gas flow rate.

The measured parameters $\left(M_{11}\right.$, and $\left.M_{22}\right)$ have different sensitivity to size of bubbles and to gas hold- up (Aslan et al., 2006). Sensitivity is also a function of scattering angle where the measurements were taken. Our results show that even though sensitivity of each scattering matrix elements to bubble size and gas hold-up, optimum location of the sensor can be 120 degree with low-to-medium flow rates and surfactant concentrations (Aslan et al., 2006). Another reason to choose 120 degree is that depending on flow rate and surfactant concentration bubbly medium becomes very optically thick where we can only measure back scattered light. Regardless of surfactant concentration average bubble size can be related with $M_{11}$ at 120 degree. Figure 11 shows that sensitivity of $M_{11}$ to bubble size is very high. Even though measurements' errors are higher for cases with smaller bubbles, it is clear to see exponential decay on $M_{11}$ when bubble size increases with decreasing numbers of bubbles. A 3 times change in bubble size causes a 6 fold change in $M_{11}$. 


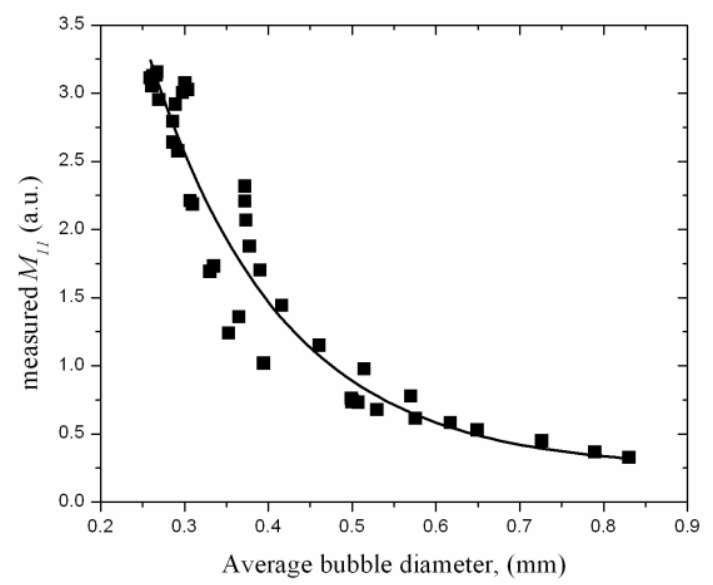

Figure 11 . Sensitivity of measured $M_{11}$ at $120^{\circ}$ on average bubble size.

The second parameter, gas-hold up can be directly related with $M_{22}$ at 120 degree with a second degree exponential decay curve. Figure 12 illustrates the relationship between $M_{22}$ and percentage gas-hold up. Even though $M_{22}$ decreases sharply at small amount of gas-hold up, it decreases slowly at large gas hold-up values. $M_{22}$ also shows how aspect ratio (circularity of bubble cross section or ratio of two major dimensions of bubble) changes: If $M_{22}$ is close to 1, it means bubble cross section is close to circular one. Figure 12 also shows when the gas-hold up increases, bubbles becomes more flat.

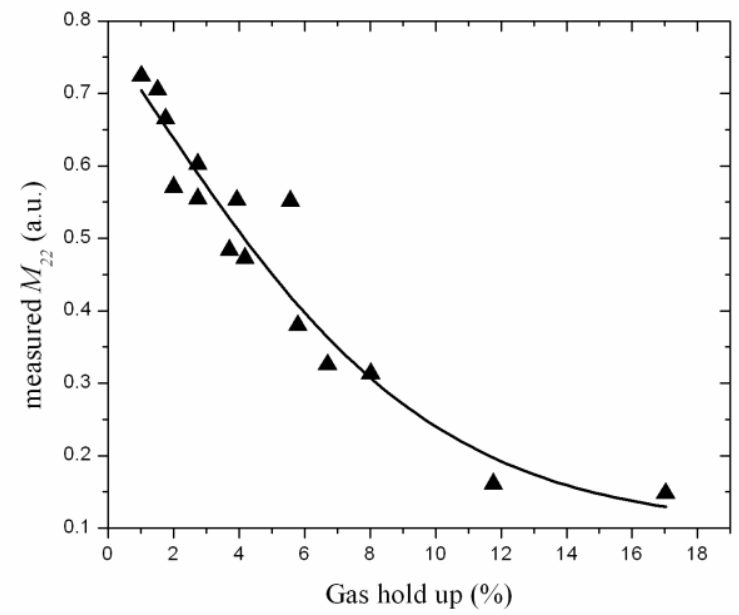

Figure 12 . Sensitivity of the $M_{22}$ signal at $120^{\circ}$ with varying gas-hold up.

The results show that the present EPLS concept is very promising for optically thin columns and has many advantages over imaging techniques when in-situ measurement of bubble size are necessary at high flow rates with high surfactant concentrations. For optically-denser bubble laden media, the reliability of this technique can be improved by incorporating multiple scattering algorithms (Vaillon, et al., 2004) in data reduction. 


\section{Effect of Total Reflectance}

Even though most light scattering particles that are optically more dense than the surrounding medium $\left(\mathrm{n}^{\prime}<1\right)$, relative refractive index for gas bubbles in the liquid is smaller than one $(n '=0.751$ : nitrogen bubbles in water). Therefore total reflection plays an important role on scattering and critical scattering angle creates two regions along scattering angle. Range of region-one where normal scattering occurs is from $\theta=0^{\circ}$ to $\theta_{\mathrm{c}}$ and region two from $\theta=\theta_{\mathrm{c}}$ to $180^{\circ}$. Definition of the critical scattering angle: $\theta_{c}=2 \cos ^{-1}\left(n^{\prime}\right) . \theta_{c}=82.8^{\circ}$ nitrogen gas bubbles in water. Scattering measurements taken in the region two indicate there is no total reflection effect between $\theta=90^{\circ}$ to $160^{\circ}$ (side to back scattering). Another reason to choose back scattering angles to take scattering measurements is that the medium (bubbles in water) become optically thick when gas velocity and surfactant concentrations are increased. After converting intensity values to scattering matrix elements, $M_{i j}$ values were plotted versus scattering angle for each flow rate (mean bubble size) as shown in Figure 13 for a surfactant concentration of $100 \mathrm{ppm}$.
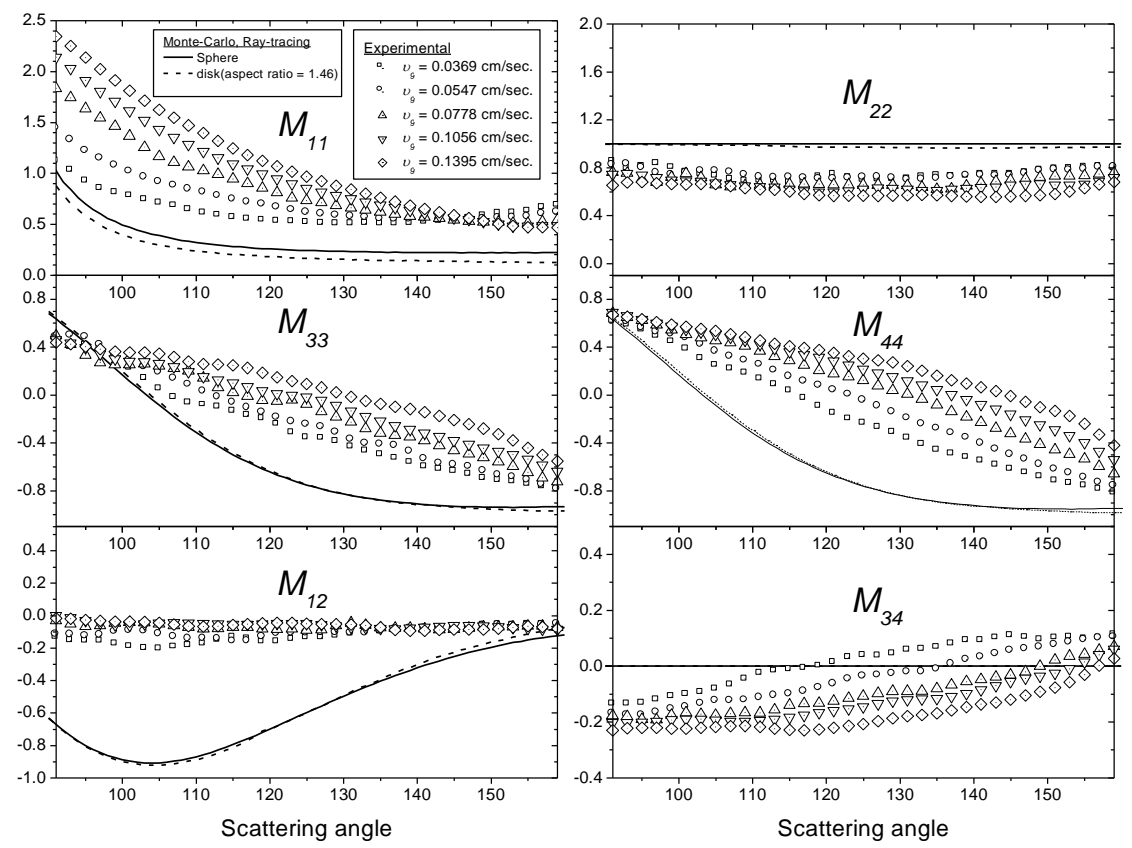

Figure 13. Scattering matrix elements $\left(M_{\mathrm{ij}}\right)$ calculated from experimental intensity measurements between $90^{\circ}$ and $160^{\circ}$ at different superficial gas velocities and a surfactant concentration of $100 \mathrm{ppm}$.

Solid curves in the Figure 13 show ray tracing results for a $0.5 \mathrm{~mm}$-diameter bubble in water. Details of the Ray-Tracing/Monte Carlo method are given in the literature (Macke, 2000). In the model calculations, the influence of possible non-spherical bubbles, light absorption, and multiple light scattering effects were neglected. Refractive index of nitrogen was assumed to be 1.000297 at $\lambda=632 \mathrm{~nm}$ for model calculations. Depending on surfactant concentration and gas velocity, bubble shape changes from sphere to disk with a maximum aspect-ratio of 1.46 (for large bubbles at high flow rates) were observed from the digital bubble pictures. 
Therefore, modeling results for two extreme shape cases (sphere and disk with aspect ration 1.46) are shown in Figure 13. Modeling results show shape effect on scattering radiation patterns are small because cross-section of bubbles in the plane where measurements taken are close to circular.

With the change mean bubble diameter, $M_{11}$ curves change as well, as expected. However, in back scattering angles, for example around $\square \theta=145^{\circ}$, the $M_{11}$ values were relatively less sensitive to bubble diameter. On the other hand, the profile of $M_{22}$ can reveal both the deviation of the bubble from presumed spherical shape and the demarcation zone between the single- and the multiple-scattering zones. When the flow rate increased, the bubble shape changed from sphere to oblate spheroid (Figure 13). The theoretical degree of linear polarization $\left(D L P=-M_{12}\right)$ that represents single scattering gives an upper border for light scattering measurements taken for a medium with many bubbles. $D L P\left(-M_{12}\right)$ does not show any trend to relate with gas velocity and $D L P$ is small for all gas velocities. Especially when gas velocity was high, $M_{12}$ information is disturbed by multiple scattering effects. The degree of circular polarization ( $D C P=M_{33}$ or $M_{44}$ ) increased when mean bubble size increased. The $M_{44}$ curve at lowest flow rate is close to curve from ray-tracing. Overall, $M_{33}$ and $M_{44}$ seem to be reasonable reliable parameters to monitor gas velocity between scattering angles 110 and $150^{\circ}$.

$M_{34}$ represents how much of linearly polarized light is converted to circularly polarized light by bubbles. The ray-tracing curve for $M_{34}$ was always zero between $\theta=90^{\circ}-160^{\circ}$, because the bubbles can not transform linearly polarized light to circularly polarized light by scattering, if the scattering angle is greater than the critical angle $\left(\theta>\theta_{\mathrm{c}}=82.8^{\circ}\right)$ (Kokhanovsky, 2003). However, experimentally measured $M_{34}$ values were not zero because increased gas velocity generated more bubbles, contributing to multiple scattering. The dynamic range of the experimentally measured $M_{34}$ is large enough to be used to monitor gas velocity.

In order to understand relationship between gas velocity and $M_{\mathrm{ij}}$, single angle scattered measurements were conducted at $\theta=120^{\circ}$ where separation gas velocity curves with most of scattering matrix elements is possible. Gas velocities were converted to mean bubble diameter and gas hold-up and plotted against the measured $M_{\mathrm{ij}}$ values, as shown in Figure 14.

Figure 14 shows that it is possible to predict bubble diameter based on scattering measurements: $M_{11}, M_{22}, M_{33}, M_{44}$, and $M_{34}$ are sensitive to bubble diameter at low gas velocities with medium-low surfactant concentrations. At high superficial gas velocities $\left(v_{\mathrm{g}}>\right.$ $0.2 \mathrm{~cm} / \mathrm{s}$ ), there are little to no changes in the size of the bubbles as the flow rate increases, which illicits small changes in the matrix elements. 

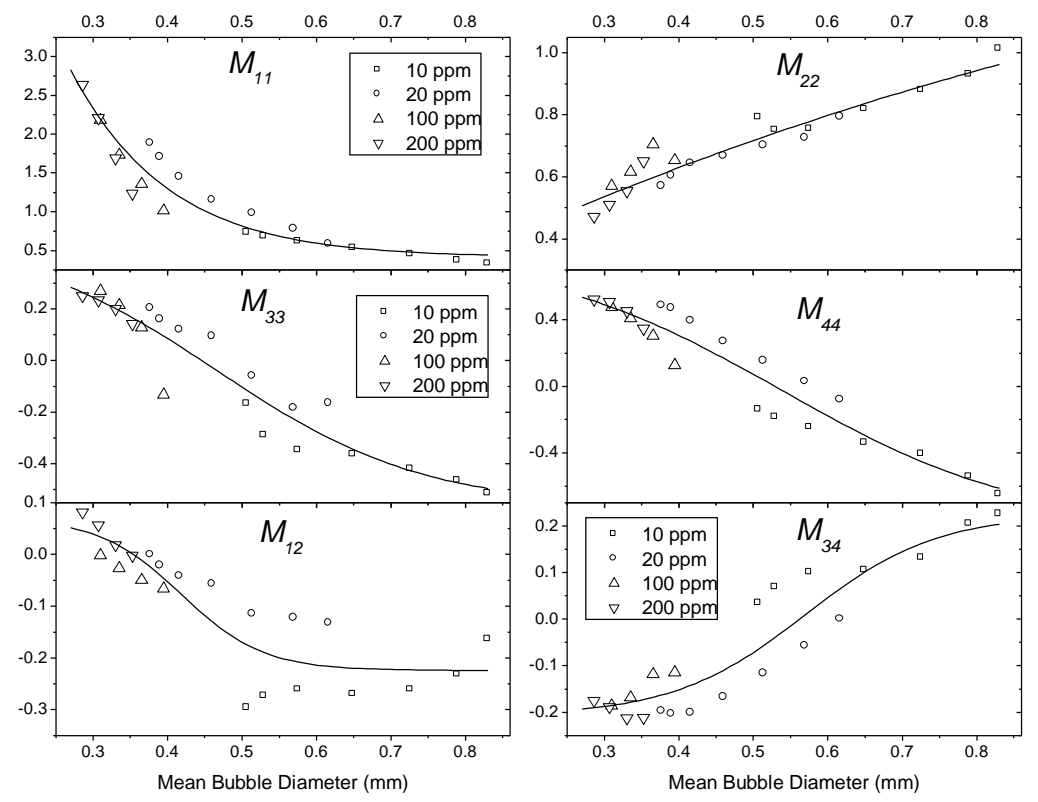

Figure 14. Scattering matrix elements $\left(M_{i j}\right)$ at $120^{\circ}$ as a function of mean bubble diameter (mm). The solid lines indicate curve-fittings.

Figure 14 also shows how the amount of the gas in the water affects the scattering at a scattering angle of $120^{\circ}$. Even though the gas hold-up increased linearly with increasing gas velocity, effect of gas hold-up on $M_{\mathrm{ij}}$ depends on surfactant concentration. For a lowmedium surfactant concentration, $M_{11}, M_{33}$, and $M_{44}$ increased as gas hold-up increased. $M_{12}$ decreased rapidly and $M_{34}$ increased when bubble size increased with increasing flow rate and surfactant concentration. $M_{22}$ changed drastically at higher surfactant concentrations, unlike other scattering matrix elements, because the shape factor of bubbles was decreasing with increasing gas velocity and the number of bubbles increased (multiple scattering regime). $M_{34}$ decreased at single scattering region but it decreased in the multiple scattering zone. Monitoring gas hold-up based on $M_{\mathrm{ij}}$ measurements is possible at some gas velocities for known surfactant concentrations.

Coal Particle Size Characterization at SL phase:

Mineral particle size distribution is strongly affected by the grinding process. The size distribution of particles can vary in a wide range from a few microns to several millimeters. Therefore, one of the major parameters that affect the efficiency of column froth flotation is the size distribution and concentration of mineral or coal particles. Even though the feed rate of coal particles can be well controlled, it is necessary to determine the size distribution of coal particles on line. Research efforts were made on the characterization of coal particles based on light scattering. Some difficulties were encountered such as optical thickness of the slurry, bimodal polydisperse characteristics of size distribution, different sizes and different optical properties of mineral and coal particles.

Major problems observed with characterization of coal particles with light scattering: 
1. Slurry to flotation column has three major types of particles: carbon, minerals, and composites. Unfortunately their optical properties are different. Therefore imaginary and real parts of coal given in the literature vary in wide range. Figure 15 shows the real part versus the imaginary part of refractive indices. In addition effective refractive index range calculated based on mixture of three types of materials in coal has wide range as well. Therefore scattering calculations with Lorentz-Mie model resulted in a wide range of variation.

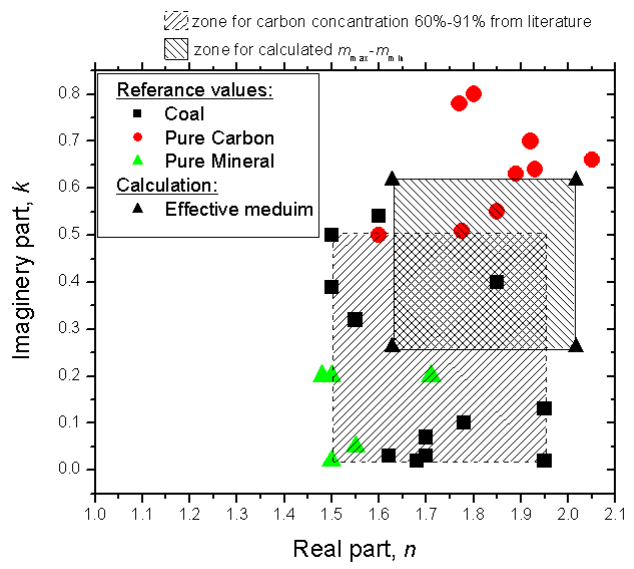

Figure 15. Real and imaginary parts of coal and ash.

2. Shapes of the three different types of particles in slurry are irregular. Because of larger size (microns to millimeters) of coal particle we can not assume the particles are spherical. That requires another approach (i.e. T-Matrix) to model scattering profiles from slurry.

3. In order to obtain scattering signals that provide information about particles in slurry, there is a need to dilute the slurry. So scattering problem needs to be simplified from dependent multiple scattering to independent multiple scattering. But when the slurry was dilute, in an attempt to do this, the particles may not have represented the exact size distribution of particles in the original slurry.

4. Size ranges of particles in slurry vary from nanometers to millimeters: Mineral particles such as clay may be in nanometers and carbon particles in microns to millimeters. Scattered light from slurry does not provide enough information about mineral particles and most of coal particle are too big for light scattering based characterization.

\section{CONCLUSION}

Elliptically scattered light by bubbles from a 2-phase gas-liquid medium was investigated for characterization of bubbles in floatation columns. Both experimental and theoretical analyses were performed.

The experimental results show that it is possible to monitor bubble size change in column by using scattering matrix elements of the bubbles. The best scattering angle should be around $\theta$ $=120 \mathrm{deg}$. where change in bubble size caused significant change in $S_{11}, S_{44} / S_{11}$, and $S_{34} / S_{11}$. 
However, the optimum angle to conduct the light scattering measurements depends on gas pressure, frit pore size range, and as well as column diameter.

Monte-Carlo model results show that if circularly polarized light is used, the bubble size, the bubble separation, as well as the thickness of carbonaceous layer around bubbles can be determined. It was also shown that it is possible to divide the flow characteristic to two zones, and carry out the experiments and correlations separately in each of these regimes.

A novel approach for measuring two major characteristics (bubble size and gas hold-up) of two phase column was developed and it was showed experimentally that elliptically polarized light scattering (EPLS) concept works to measure two characteristic from scattering matrix elements of GL phase medium.

Experiments show that some scattering matrix elements are sensitive to bubble size distribution parameters (mean bubble size, flow rate and gas hold-up). The scattering-matrix elements can be correlated with mean-bubble diameter and gas hold-up for known surfactant concentration even at high surfactant concentrations and high gas velocities. $S_{11}, S_{33}$, and $S_{44}$ at $\theta=120^{\circ}$ are good candidates at low-medium flow rate and surfactant concentration to monitor bubble size and gas hold-up, $S_{22}$ at $\theta=120^{\circ}$ is only scattering matrix element that can be used to monitor gas-hold-up at high flow rate and surfactant concentration. $S_{12}$ has two major problems not to be considered as a monitoring parameter: (1) It decreases at low surfactant concentration with increasing gas hold-up, but it increases when surfactant concentration medium-high. (2) Because of multiple scattering affect, it is a very small value and does not have a trend for different gas flow rates at medium-high flow rate and surfactant concentration.

The two-phase GL results show that the present EPLS concept is very promising for characterization of optically thin columns and has many advantages over imaging techniques when in-situ measurement of bubble size are necessary at high flow rates with high surfactant concentrations. For optically-denser bubble laden media, the reliability of this technique can be improved by incorporating multiple scattering algorithms in data reduction.

The two-phase SL experiments indicate that the problem is more complex than GL phase measurements because of composite structure of the coal mixtures and wide range of size distribution. But it is still possible to sense the particle size distribution from the normalized scattering matrix elements.

\section{ACKNOWLEDGEMENTS}

Special acknowledgements are made to Dr. Mustafa M. Aslan who performed most of the tests for this study with strong dedication and enthusiasm.

\section{REFERENCES}

Aslan, M., Yamada, J., Mengüç, M.P., Thomasson, A., "Characterization of Individual Cotton Fibers via Light Scattering: Experiments," AIAA Journal of Thermophysics and Heat Transfer Vol. 17, No. 4, pp. 442-449, 2003.

Aslan, M.M., Mengüç, M.P., Tao, D., and Crofcheck, C., "Bubble size and gas hold-up detection via Elliptically Polarized Light," International Symposium on Radiative Transfer, 2004, Istanbul-Turkey. (poster presentation). 
Aslan M.M., Crofcheck C., Tao D., and Mengüç, M.P., 2006 "Evaluation of Micro-Bubble Size and Gas Hold-Up in Two-Phase Gas-Liquid Columns via Scattered Light Measurements," Journal of Quantitative Spectroscopy and Radiative Transfer, Vol. 101

Kokhanovsky AA. Optical properties of bubbles. Journal of Optics-A 2003; 5: 47-52.

Lain, S., Broder, D., and Sommerfeld, M., 1999, "Experimental and numerical studies of the hydrodynamics in a bubble column," Chemical Engineering Science, 54, pp.4913-4920.

Macke A. Monte Carlo calculations of light scattering by large particles with multiple internal inclusions. Chapter 10 in Light Scattering by Nonspherical Particles. Ed. Mishchenko MI, Hovenier JW, and Travis LD. New York: Academic Press, 2000.

Tao D, "Role of bubble size in flotation of coarse and fine particles - A review," Separation Science and Technology 2004; 39: 741-760.

Vaillon R, Wong BT, and Mengüç MP. Polarized Radiative Transfer in a Particle Laden Transparent Medium via Monte Carlo Method. Journal of Quantitative Spectroscopy and Radiative Transfer, 2004; 84:383-394.

Wong, B. and Mengüç, M.P., 2002, "Depolarization of Radiation by Foams," Journal of Quantitative Spectroscopy and Radiative Transfer, 73, pp. 273-284. 
Appendix 35: Mineral Liberation Analysis in 3D by X-Ray MicroCT for the Evaluation of Particle Separation Efficiency (UT006) 


\section{FINAL TECHNICAL REPORT}

Contract Title and Number: Crosscutting Technology Development at the Center for Advanced Separation Technologies (DE-FC26-02NT41607)

Sub-Recipient Project Title: Mineral Liberation Analysis in 3D by X-Ray MicroCT for the Evaluation of Particle Separation Efficiency

Principal Investigators:

C.L. Lin

Contact Address:

University of Utah

135 South 1460 E. Room 412

Salt Lake City UT 84112

Subcontractor Address:

No subcontracts issued.
Period of Performance:

Starting Date: 6/1/2004

Ending Date: 8/31/2007
Report Information:

Type: Final

Number:

Period: 6/1/2004-8/31/2006

Date: $\quad$ 09/28/06

Code: UT006-Final

Contact Information:

Phone: (801) 581-5309

Fax: $\quad$ (801) 581-8119

E-Mail: cllin@mines.utah.edu

Subcontractor Information:

Phone:

Fax:

E-Mail: 


\title{
Disclaimer
}

The Disclaimer must follow the Title Page and must contain the following paragraph: "This report was prepared as an account of work sponsored by an agency of the United States Government. Neither the United States Government nor any agency thereof, nor any of their employees, make any warranty, express or implied, nor assume any legal liability or responsibility for the accuracy, completeness, or usefulness of any information, apparatus, product, or process disclosed, or represents that its use would not infringe privately owned rights. Reference herein to any specific commercial product, process, or service by trade name, trademark, manufacturer, or otherwise does not necessarily constitute or imply endorsement, recommendation, or favoring by the United States Government or any agency thereof. The views and opinions of authors expressed herein do not necessarily state or reflect those of the United States Government or agency thereof."

\begin{abstract}
This research project deals with the analysis and characterization of three-dimensional (3D) images of packed particle beds acquired with a one-of-a-kind X-ray microtomography facility located at the University of Utah. Methodologies for segmentation and mineral phase discrimination have been developed and applied in the analysis of coal and copper ore samples which are included in the study.

The segmentation image processing requires several steps and is based on a two-dimensional (2D) image processing procedure known as the watershed method. Different algorithms for $3 \mathrm{D}$ image segmentation have been implemented in $\mathrm{C}$ language.

The mineral phase discrimination methodology assumes that the histogram of a multiphase particle represents a given mixture of phases associated with the minerals present in the particle itself. If each mineral phase has a known attenuation coefficient distribution, then the composition can be determined based on the finite mixture distribution (FMD) model. The statistical analysis and FMD solver were carried out with ' $R$ ' free-licensing software.

The validity and applicability of these methodologies for particle analysis have been evaluated with packed beds of copper ore and coal particles. Two practical applications were demonstrated, including washability curves for the analysis of coal samples from plant operations and the mineralogical analysis of copper ore from industry operations.
\end{abstract}




\section{Table of contents}

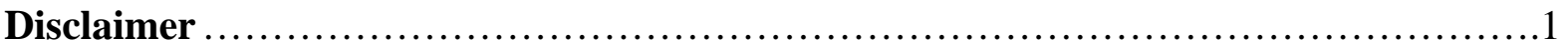

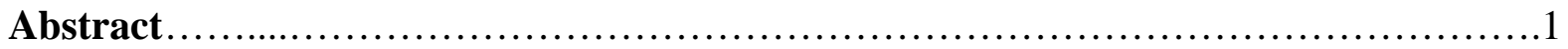

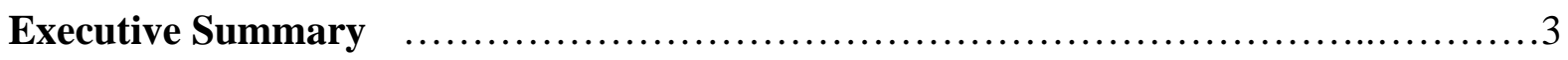

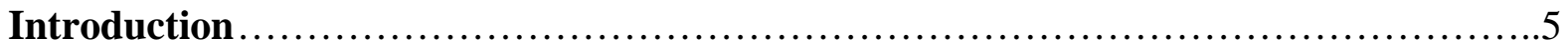

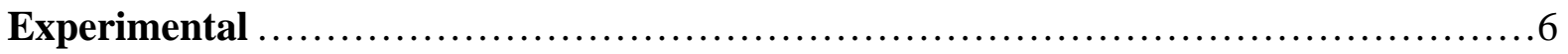

Particle Image Segmentation ....................................................

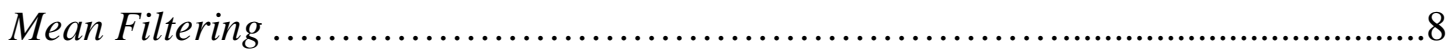

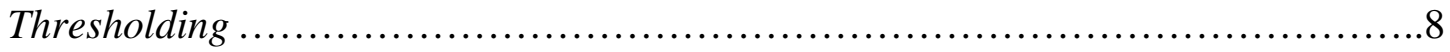

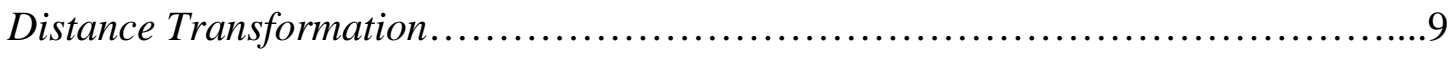

Local Maxima Markers...........................................................

The Semivariogram Operator....................................................

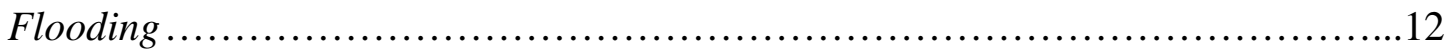

Particle Composition Analysis............................................................12

Finite Mixture Distribution (FMD) Models......................................13

Particle Histogram Analysis Process.............................................14

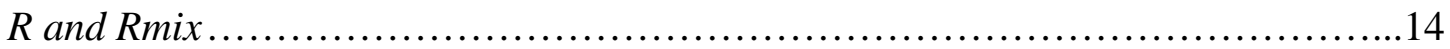

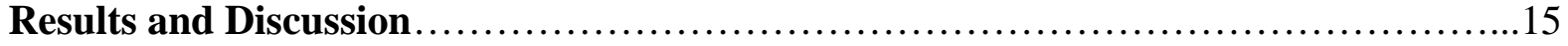

Linear Correlation of Density and Attenuation Coefficient ............................15

Segmentation of Packed Bed of Particles ...............................................................16

Mineral Composition Analysis by Finite Mixture Distribution Model ....................19

Practical Applications ................................................................20

XMT Washability Curves from a Packed Particle Bed..............................21

XMT Copper Mineral Analysis from a Packed Particle Bed............................24

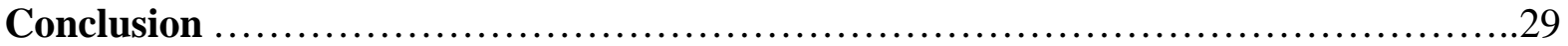

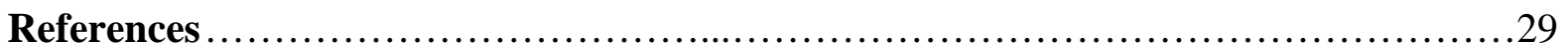




\section{Executive Summary}

For continued technological progress in the field of multiphase particulate separation processes, the need for quantitative spatial analysis of multiphase particles in threedimensions has increased significantly. Such quantitative information must be accurate enough so that the measured values can be used as parameters for simulation models, process design procedures, and control strategies. Cone-beam X-ray microtomography technology (XMT) offers a unique imaging capability that can produce high-resolution (a few micrometers) three-dimensional images of the internal structure of multiphase samples. We have adapted this new technology to the mining industry to provide for the advanced analysis of coal and ore samples; such analysis of on-line plant samples will allow for continuous control of particle separation processes. In this regard, The primary objective of the proposed project is the development of x-ray micorotomography technology (XMT) for detailed 3D liberation/exposure analysis of coal and mineral samples. The secondary objective is to evaluate the feasibility of the XMT technology for the control of separation processes. This new XMT technology combines the high-resolution 3-D XMT instrument and a classification algorithm in order to determine the sample composition and particle characteristics of plant samples. XMT will permit a fast, direct, and detailed 3-D analysis of particles with little sample preparation. Reconstruction of 3-D particle populations from XMT images not only provides sufficient information to construct the true liberation spectrum for the coal and ore samples in question, but also provides information on the particle size distribution, grain size distribution, and exposed surface area of mineral matter grains contained in each particle of the population sample.

XMT computer tools for 3D segmentation of packed particle bed images and for compositional analysis of individual particles have been developed. These tools have been shown to be reliable and applicable in various applications. The individual separation of the particles in a set allows for further individual compositional analysis of these particles and makes the process of sampling and analysis economically affordable because it allows for acquisition of individual particle information from XMT analysis over a group of particles instead of a single particle at a time basis.

The segmentation image processing requires several steps and is based on a two-dimensional (2D) image processing procedure known as the watershed method. Different algorithms for 3D image segmentation have been implemented in C language.

The mineral phase discrimination methodology assumes that the histogram of a multiphase particle represents a given mixture of phases associated with the minerals present in the particle itself. If each mineral phase has a known attenuation coefficient distribution, then the composition can be determined based on the finite mixture distribution (FMD) model. The statistical analysis and FMD solver were carried out with ' $\mathrm{R}$ ' free-licensing software.

The validity and applicability of these methodologies for particle analysis have been evaluated with packed beds of copper ore and coal particles. Two practical applications were demonstrated, including washability curves for the analysis of coal samples from plant operations and the mineralogical analysis of copper ore from industry operations.

The X-ray CT microtomography technique, joined to digital image processing tools, as the 3D segmentation developed in this research, and mineral phase analysis for particle 
characterization using the Finite Mixture Distribution analysis has provided important tools for mineral liberation analysis, fundamental information necessary to evaluate and control the efficiency of particle separations for both the coal and mineral industry. In addition the accomplishments from this research open a variety of other opportunities for further research and applications. For example, particle shape and grain shape distributions can be evaluated in 3D from packed particle beds. Also, internal phase distribution and continuity of multiphase particles may be evaluated for plant samples through the examination of XMT data.

Today's XMT technology has a limit on the order of 10 microns in resolution and the analyses of samples at such resolution requires long scanning times. However, the technology of microfocus x-ray generators and CD detectors improves every year, and it is expected that the utilization of XMT at high resolution will be achieved in the years to come. 


\section{Introduction}

New processing technology for improved productivity and efficiency is the key to success in today's highly competitive market place. It has been well established that appropriate analytical control systems can provide for such improvements. This is especially true of the particle separation processes used in the coal and mineral industries. Generally particulate materials are sampled from input and output streams and analyzed to evaluate the performance of the processing operations. In this regard, characterization and analysis of multiphase particles are of great technological importance. Most present methods to characterize and to analyze multiphase particles rely on heavy liquid fractionation (Leonard, 1979) or on microscopic observation of a series of thin or polished sections (Miller and Lin, 1988; King and Schneider, 1993) of the particulate sample. It is noted that these analytical procedures are time consuming and costly. The results are incomplete and cannot be obtained in a reasonable amount of time to provide a satisfactory feedback mechanism for the control of various unit operations in coal and mineral processing plants. In addition, the sectioning method is unsatisfactory for specific applications, for instance, to measure mineral exposure at the surface of locked particles which is most relevant for flotation separation processes. Even so, such traditional procedures are being used to obtain some limited information at great expense. For example automated instruments for polished section analysis which are currently being used in the mineral industry cost on the order of $\$ 1,000,000$.

These multiphase particulate assemblies are heterogeneous and have a wide variation of physical and/or chemical properties. Of course coal and mineral processing separation processes are based on the difference in the physical properties that influence the behavior of a particle inside the separation unit. In this regard, such heterogeneity can have a significant impact on the recovery and on the separation efficiency of concentration processes. In general, the separation efficiency for multiphase particulate systems depends on the statistical characteristics of particle microstructures, such as composition distribution, surface exposure of mineral grains, etc. For continued technological progress in the field of multiphase particulate separation processes, the need for quantitative spatial analysis of multiphase particles in three-dimensions has increased significantly. Such quantitative information must be accurate enough so that the measured values can be used as parameters for simulation models, process design procedures, and control strategies. Cone-beam x-ray microtomography (XMT) offers a quantitative imaging capability that can produce highresolution three-dimensional images of the internal structure of multiphase particulate samples at a resolution of 5 to 10 microns (Lin and Miller, 1996, 2002). We have adapted this new technology to the mining industry to provide for the advanced analysis of exploration samples, for on-line analysis of plant samples, and eventually for the control of separation processes.

As mention previously, cone-beam x-ray microtomography (XMT) offers a quantitative imaging capability that can produce high-resolution three-dimensional images of the internal structure of particulate samples. We have adapted this new technology to the mining industry to provide for the advanced analysis of ore samples and for the on-line analysis of plant samples in order to provide a liberation basis for control of separation processes. In this regard, the goal of this research is to provide the necessary technical information to use XMT for the measurement of mineral composition, and grain exposure. This new method combines the 3D high-resolution XMT technique and a classification algorithm for 
determining the sample composition and particle characteristics of plant samples. The XMT technique permits a fast, direct, and detailed 3D-liberation analysis of particles without sample preparation. Reconstruction of 3D particle populations from XMT images not only provides sufficient information to construct the true liberation spectrum for the particle samples in question, but also provides information on the particle size, grain size distribution, and exposed surface area of the mineral phases which constitute the particle population.

XMT technology will lead to improvements in the efficiency of particle separation by direct measurement of liberation and other particle characteristics in order to establish the necessary conditions for the desired separation efficiency. The XMT technique will be of great utility for the liberation analysis of a wide variety of mineral resources, including coal, industrial minerals, and metallic ores. In this regard, the XMT technique can be used to frequently monitor the size distribution of locked grains; this capability will make it possible to make continuous changes to maintain operations at a high level of efficiency. The results from this research can be easily incorporated into existing plant simulation software systems for convenient application in industrial practice. Development of such an analytical technique provides the basis for more rapid, detailed, and accurate liberation analysis in the $21^{\text {st }}$ century.

The objective of the research is to develop multiphase particle characterization and analysis technology for particle separation processes in the coal and mineral industries using x-ray microtomography. Importantly, the development and evaluation of XTM to establish the relationship between characteristics of multiphase particles and operation parameters allow for improved design and control of particle separation processes. The primary objective of the project is the development of x-ray micorotomography technology (XMT) for detailed 3D liberation/exposure analysis of coal and mineral samples, including software for mineral identification. The secondary objective is to evaluate the feasibility of the XMT technology for the control of separation processes.

\section{Experimental}

The main thrust of this research is the comparison of XMT liberation results in 3D with current assessment techniques for dissimilar mineral components of ore and coal samples. In this regard, copper ore and coal samples from Kennecott and Illinois No. 5 seams were selected for study. A high-resolution cone-beam microtomography system, installed at the University of Utah, is being used for detailed 3D analysis of these samples.

To effectively analyze a packed bed of multiphase particles for the determination of grain and particle size distributions, detection of particle/grain surface boundaries and classification of the particle population is critical. In this regard, implementation of the appropriate XMT software for size/composition classification of particles in ore and coal samples has been accomplished. Subsequent determination of the grain size distribution (including the exposed surface area) and liberation spectrum (coal washability analysis).

The major data processing steps and their corresponding algorithms for mineral compositional distribution are given in Figure 1. These algorithms include phase segmentation for data reduction, surface extraction to separate connected particles, labeling for particle classification, and mineral compositional classification for the three-dimensional CT image data set. 


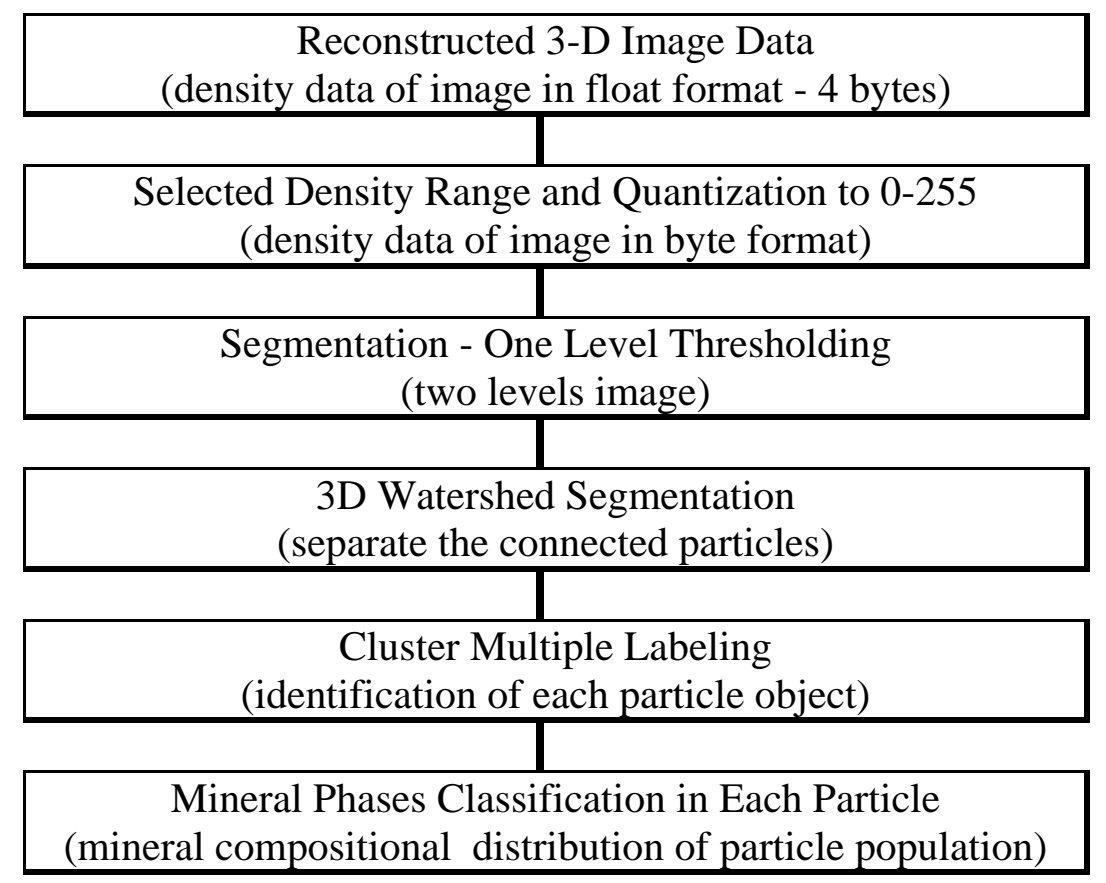

Figure 1. Major steps for the determination of mineral compositional distribution of particle population from 3-D image data.

Particle Image Segmentation

One of the problems faced for analysis of the three-dimensional spatial distribution of mineral phases in a multiphase particle population is the identification and separation of contacted particles. A watershed technique commonly used in 2D morphological analysis was extended to 3D and used in this study to separate these particles in contact with each other. The connected components labeling technique is widely used for applications involving images which consist of numerous objects. In this regard, the 26-connected component algorithm was used to label the separated particles and classify particle size by volume for the reconstructed three-dimensional particles. Details of the algorithm for 3D Watershed Segmentation is described as follows:

The process for segmentation of a three-dimensional image of a packed bed of particles requires dealing with particles of irregular shape. This condition makes the problem particularly challenging. Sequence of image processing operations are described that provide for improved segmentation of packed bed of irregularly shaped particles. First, noise in the back-projected image is reduced using a mean filter operator. Second, thresholding is applied to generate a binary image used in the third step to apply the distance transformation. Fourth, the application of the extended maxima transform gives the markers used to label the objects. Over-segmentation, usually encountered with watershed algorithms, is avoided by estimation of the H-extrema parameter using a semivariogram and by the merging of the region maxima with distances less than those given by the ellipsoid of influence calculated by the same semivariogram operator. Finally, flooding is carried out to establish spatial boundaries. Figure 2 illustrates the 3D watershed segmentation algorithm. 


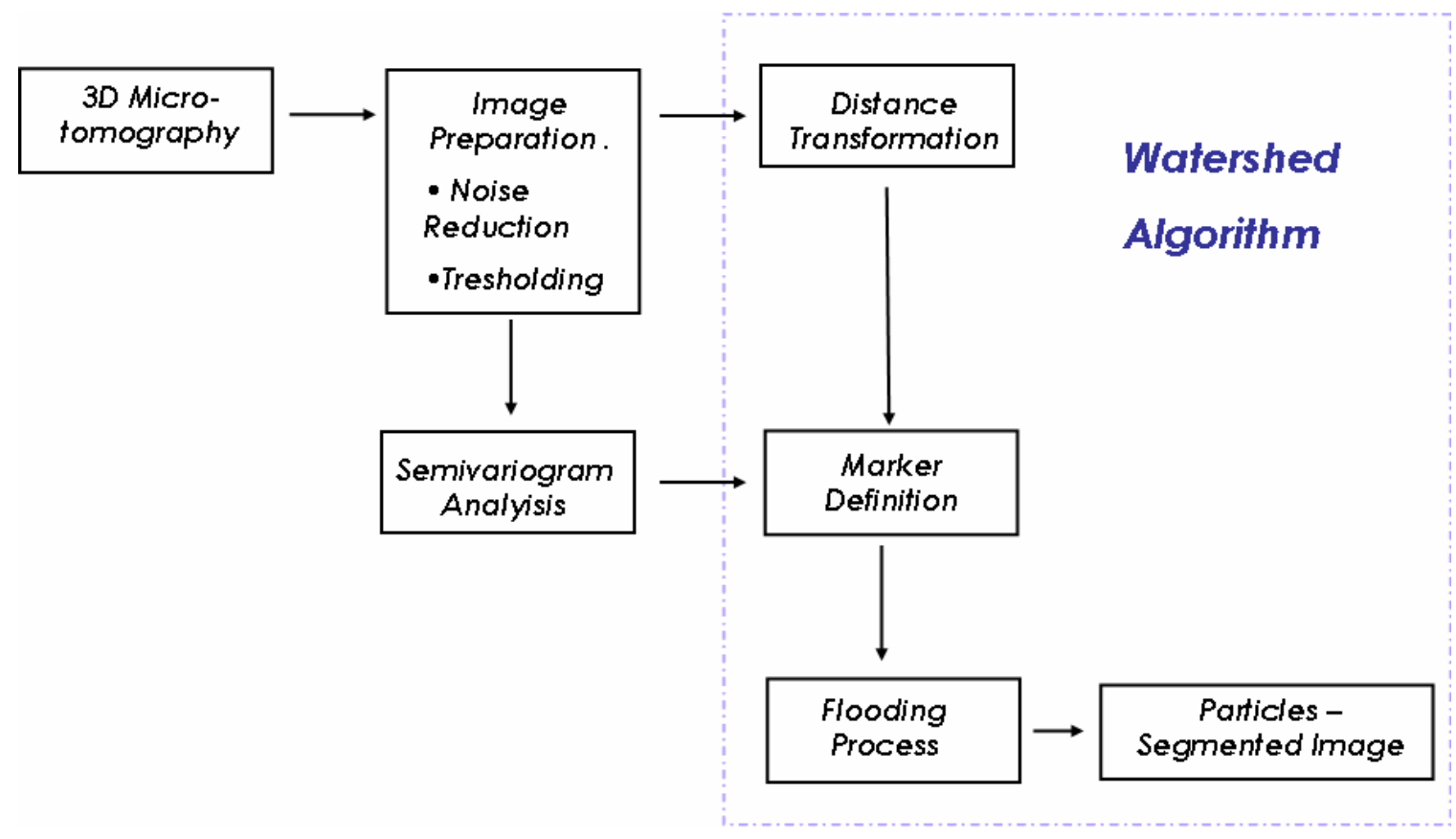

Figure 2. 3D watershed segmentation algorithm.

\section{Mean Filtering}

Due to statistical fluctuations in the photon emission, photon count and the finite size of a voxel in images obtained from XMT, the histogram of attenuation coefficients for each mineral phase is a normal distribution with a certain width. This peak width and subsequent overlap of the two attenuation signals in the image complicate the analysis of XMT images. In order to minimize this effect we use a simple $3 \times 3 \times 3$ mean filtering which changes the central pixel value for the average of the pixels inside the block. It is well known that this produces a bit of blurring of the image but our experience indicates that it significantly influence the shape of particles and tends to make a smoother topography of the image.

\section{Thresholding}

The threshold value defines the attenuation coefficient limit that separates background from foreground. The histogram of the image generally is a clear bi-modal distribution where the thresholding value can be defined by an interactive hand user definition. The image presented in Figure 3 shows one slice of the back-projected image from a bed of particles $2 \mathrm{~mm} \times 1.18$ $\mathrm{mm}$ in size. The noise is reduced by the use of a 3x3x3 mean filter. The histogram shows the total count of the number of pixels for the whole 512x512x256 image. Finally a binary image is obtained for a thresholding operation with a thresholding value of 30 . 

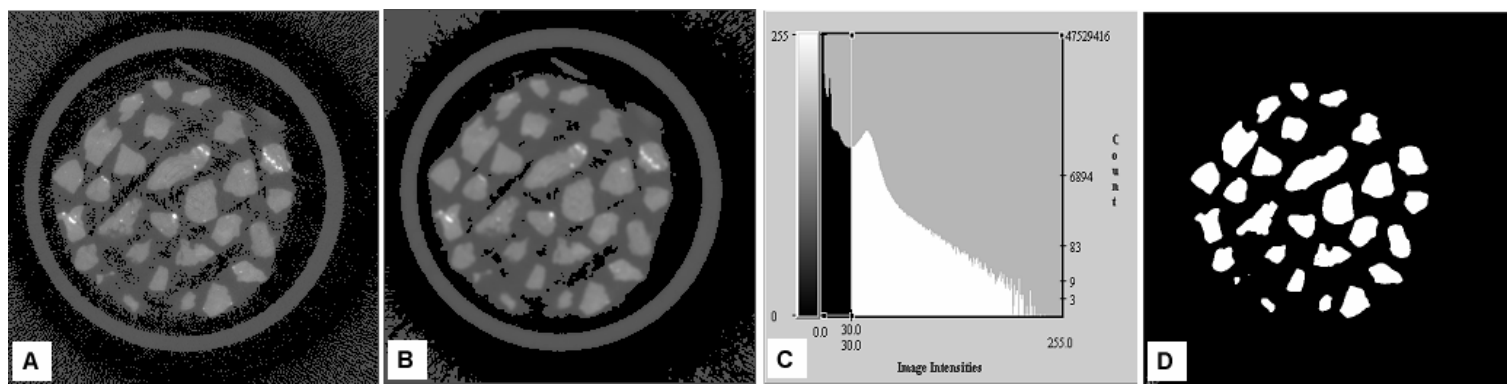

Figure 3. Examination of a bed of particles $2 \mathrm{~mm} \times 1.18 \mathrm{~mm}$ in size. A) The back projected image obtained from micro-tomographic analysis. B) Noise reduced image with a 3x3x3 mean filter. C) Histogram of the filtering image showing a threshold value of 30. D) The final binary image.

\section{Distance Transformation}

The distance transform is an operation that converts a binary image, consisting of feature and non-feature elements, to a picture where each element has a value that approximates the Euclidean distance to the nearest feature element. In our case the resulting image from the distance transform operation establishes for each voxel the distance from the white pixels to the nearest black pixel. The images shown in Figure 4 illustrate the distance transform operation applied to two overlapped circles for a two-dimensional image. The red colour in the right-hand image means a greater distance from the background. It is clear that the two circles are distinguished by the two local maxima in the distance values.

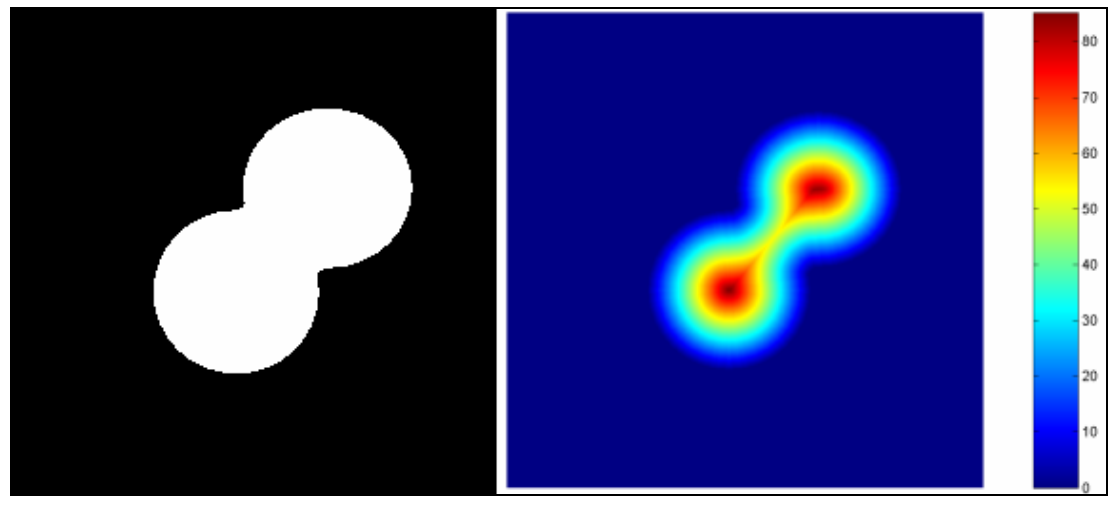

Figure 4. The left-hand image is a binary image of two overlapped circles. The right-hand image shows the result of the distance transformation operation applied to the binary image of the two overlapping circles.

Since the shape of the particles is a major issue in this analysis, it is most desirable to work with the Euclidean distance instead of other distance definitions as chess-board or city block. However, to find an acceptable balance between speed and accuracy we have implemented the three dimensional chamfer distance transform which is the closest approximation to the mathematical definition of distance having at the same time a good computational performance (Bougrfors, 1984). 


\section{Local Maxima Markers}

This distance transform image is a sort of surface with level lines mapping the distance of the interest object to the background and therefore it will present a local maxima value somewhere inside boundaries of the object. If the object of interest is a circle, as in the example of the two circles showed previously, we will have only one local maximum being in the center of the circle and in general this maximum can be used as a marker for the position of a particular particle in the image. Markers represent unique regions of space, which distinguish unique particle locations. To establish specific particle locations, we use the $\mathrm{H}$-extrema transform which requires the definition of a parameter $\mathrm{H}$ as described by Soille (1999) . The parameter $\mathrm{H}$ is related to the height of the maximum regions that will be extracted from the image to be used as markers (Vincent, 1993) The image presented in Figure 5 shows the markers used in the segmentation of a sample of particles $2.00 \mathrm{~mm} \times 1.18$ $\mathrm{mm}$ in size.

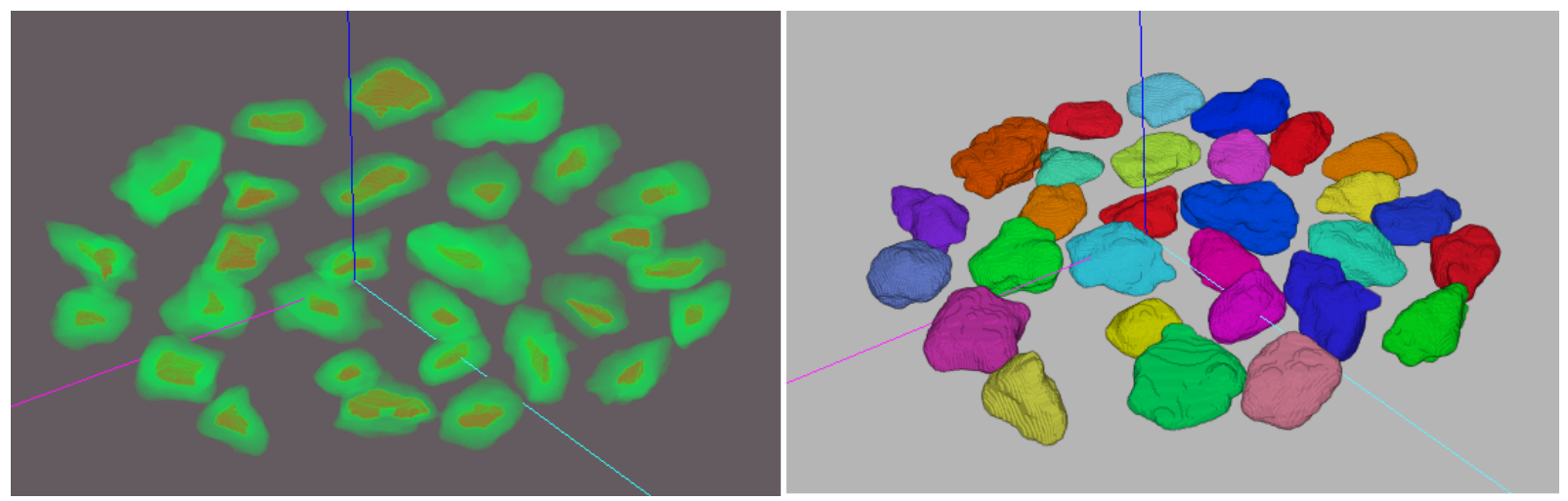

Figure 5. The left-hand image shows a transparent sample of particles $(2.00 \mathrm{~mm} \times 1.18 \mathrm{~mm})$ with markers inside each particle shape. The right-hand image shows the segmented image using the markers to label the particles with a different number.

At this point there are just two problems with this approach. First there is a problem of how to determine $\mathrm{H}$ correctly without having any previous information on the size of the particles before segmentation of the image. Second, because we are working with irregular particle shapes there may be problems with the number and locations of the maxima regions. If two maxima regions are found inside one particle and they are not connected this will lead to over-segmentation. Therefore, we will need an additional process in order to identify and merge maxima regions that may belong to the same particle. Both problems can be resolved using the semivariogram operator.

\section{The Semivariogram Operator}

In order to obtain information regarding the structure of the particles before image segmentation we make use of a geostatistic operator known as a semivariogram. The semivariogram operator is an estimator of the spatial correlation between two points separated by a distance " $h$ " in a given orientation. In our case it will be used to estimate the average size of the particles and the ellipsoid of influence of the pixels. The definition of the semivariogram is given by Journel and Huijbregts (1978): 


$$
\gamma(h)=\frac{1}{2 N(h)} \sum_{i=1}^{N(h)}(z(x)-z(x+h))^{2}
$$

$\gamma(h)$ : Semivariogram function.

$h: \quad$ Lag or distance between samples in a given orientation.

$z(x)$ : The value of the image in the position " $x$ ". In a binary image it can have a value of 1 if the voxel belongs to a particle or 0 otherwise.

$z(x+h)$ : The value of the image in the position " $x+h$ ".

$N(h)$ : Number of pairs of data separated by the vector $h$.

A 3D image and associated semivariograms are presented in Figure 6 to illustrate the analysis. In this image semivariograms were calculated in the $\mathrm{x}, \mathrm{y}$ and $\mathrm{z}$ orientation. It can be seen than beyond a certain distance the semivariograms in all the orientations achieve a plateau meaning that at that point the two quantities $z(x)$ and $z(x+h)$ are no longer correlated. These ranges represent the average size (in pixels) of the particles in the $\mathrm{x}, \mathrm{y}$, and $\mathrm{z}$ orientations respectively when we are working with binary images and also they represent the maximum distance for which two adjacent markers will belong to the same particle in each of the three orientations.
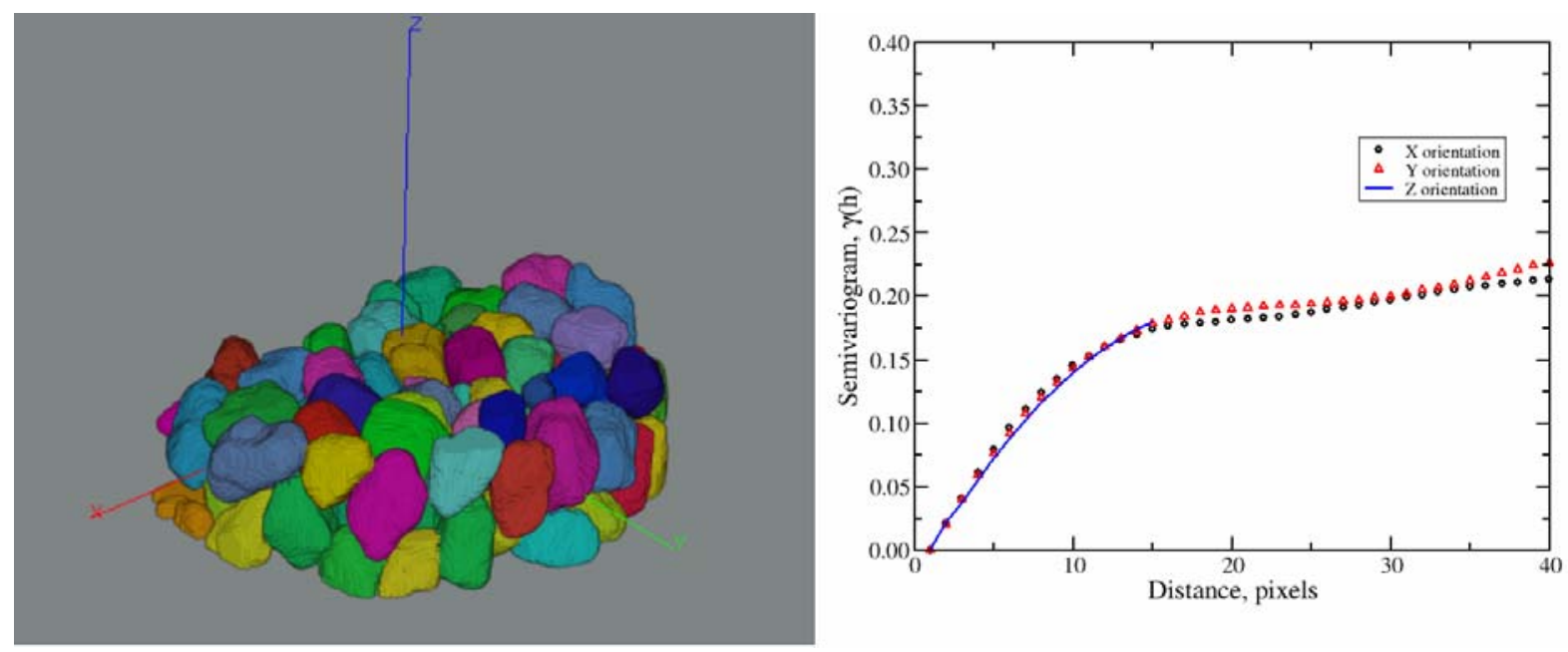

Figure 6. The left-hand image shows a 512x512x256 image of coal particles (19.1mm x $9.5 \mathrm{~mm})$ already segmented. The right-hand plot shows the semivariogram for the binary image in the $\mathrm{x}, \mathrm{y}$ and $\mathrm{z}$ orientations from the coal sample image. Because the size of the particles is relatively uniform and well distributed the resulting semivariogram is isotropic and shows a characteristic particle size of 15 voxels in all orientations.

Thus, once these ranges are calculated from the binary image half of the minimum range value will be used as the parameter $\mathrm{H}$ in the geodesic-reconstruction step. Also a merging of the labels of the markers is accomplished using these ranges and their respective orientations prior to the flooding step, i.e. if two markers are separated by a distance of 5 in the $x$ direction and the range values in this orientation is 10, both markers will have the same label number which is equivalent to saying that both spatial regions will be accommodated in one particle. 


\section{Flooding}

Once the markers are defined the process to label the particles with an index is carried out using the flooding model shown previously. The algorithm in this process doesn't need markers in the background because it will flood just the voxels "turned on" in the binary image.

\section{Particle Composition Analysis}

For the purpose of clarity we have to define the meaning of an overall and individual particle histogram. It has been seen that the attenuation coefficient histogram is a mathematical representation of the frequency of each attenuation coefficient value inside the sample under analysis. Until now, it has only been possible to make analysis in an overall base meaning that the whole 3D image of a particle packed bed sample is used to generate only one overall histogram. This histogram represents all the available sample information. With the development of the 3D watershed segmentation algorithm, now we are able to do a particle base analysis where the information of each particle inside the sample generates one histogram which characterizes just the material belonging to that particle (Figure 7).

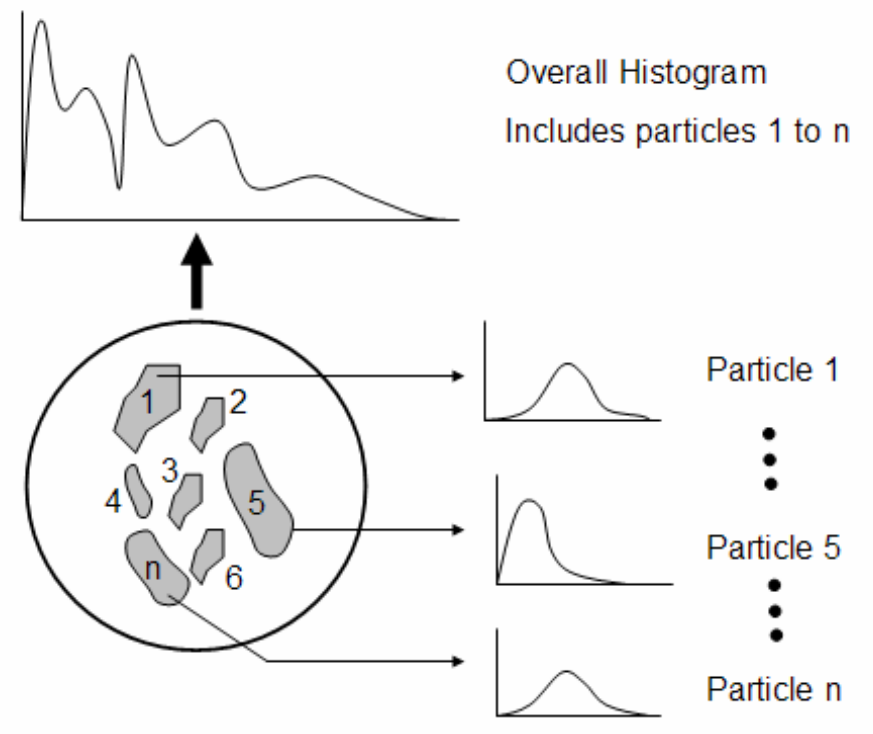

Figure 7. Example of an overall particle population histogram and an individual particle histograms.

Based on the tomographic image information (linear attenuation coefficient), the finite mixture model can be applied for the construction of the mineral composition inside each particle. The heterogeneous data coming from a XMT analysis can be modelled using finite mixture distributions to classify the composition of each voxel element inside each particle. The population in this case is the attenuation coefficient frequency and the problem to resolve is the estimation of the parameters that best fit the sampling data. 
Finite Mixture Distribution (FMD) Models

A mixture distribution is a compounding of statistical distributions, which arise naturally as densities associated with sampling of an inhomogeneous statistical population. A finite mixture has a finite number of components of densities and mixing proportions. The heterogeneous data coming from a XMT analysis can be modeled using finite mixture distributions to classify the composition of each voxel element. The population in this case is the attenuation coefficient frequency and the problem to resolve is the estimation of the parameters that best fit the sampling data.

Suppose that a random " $X$ " variable takes values in sample space $x_{\text {, }}$ and that its distribution can be represented by a probability density function of the form:

$$
g(x \mid \psi)=\pi_{1} f_{1}\left(x \mid \theta_{1}\right)+\pi_{2} f_{2}\left(x \mid \theta_{3}\right)+\pi_{3} f_{3}\left(x \mid \theta_{3}\right)+\ldots \ldots \ldots+\pi_{k} f_{k}\left(x \mid \theta_{k}\right)
$$

We say then that $\mathrm{X}$ has a finite mixture distribution and that $g(x)$ is a finite mixture density distribution characterized by all the collection of distinct parameters occurring in the mixed model $\psi=\left(\pi_{1}, \pi_{2}, \ldots, \pi_{k}, \theta_{1}^{T}, \theta_{2}^{T}, \ldots . ., \theta_{k}^{T}\right)$. The parameters $\pi_{1}, \pi_{2}, . ., \pi_{k}$ are called the mixing proportions and $f_{1}\left(x \mid \theta_{3}\right), \ldots, f_{k}\left(x \mid \theta_{k}\right)$ the component densities of the mixtures with parameters $\theta_{1}, \theta_{2}, \ldots ., \theta_{k}$ respectively. The component densities can be binomial distributions, normal distributions, lognormal distributions and so on.

There is no requirement that the component densities should all belong to the same parametric family. However, it is common practice to simplify the problem by assuming that they all have a common functional form but different parameters. The solution of this problem has been the subject of a large and diverse body of literature spanning nearly ninety years $^{(6)}$, therefore it is not the intention of this research to develop a full computational solution for each sample under analysis.

The most common algorithms used to resolve this kind of problems are the Newton methods, Maximum-Likelihood methods, and, recently, the Expectation-Maximization (EM) algorithm. The use and application of these algorithms depend of the particular problem to resolve; for example: smoothness of the sampling data distribution, conditions in parameters to be used, family of densities distributions, numerical stability, etc. Furthermore, there are many conditions which can be imposed to the parameters in order to resolve the problem which make certain algorithms more suitable for some conditions than for others conditions.

Usually the attenuation coefficients histograms are fitted adequately by a finite mixture of normal distributions or lognormal distributions (see Figure 8), therefore the number of parameters to be calculated in each mixture distribution will depend of the assumption of the total number of distributions. Each component will lead to two unknown parameters, mean and variance, and to one unknown proportion mixture. 


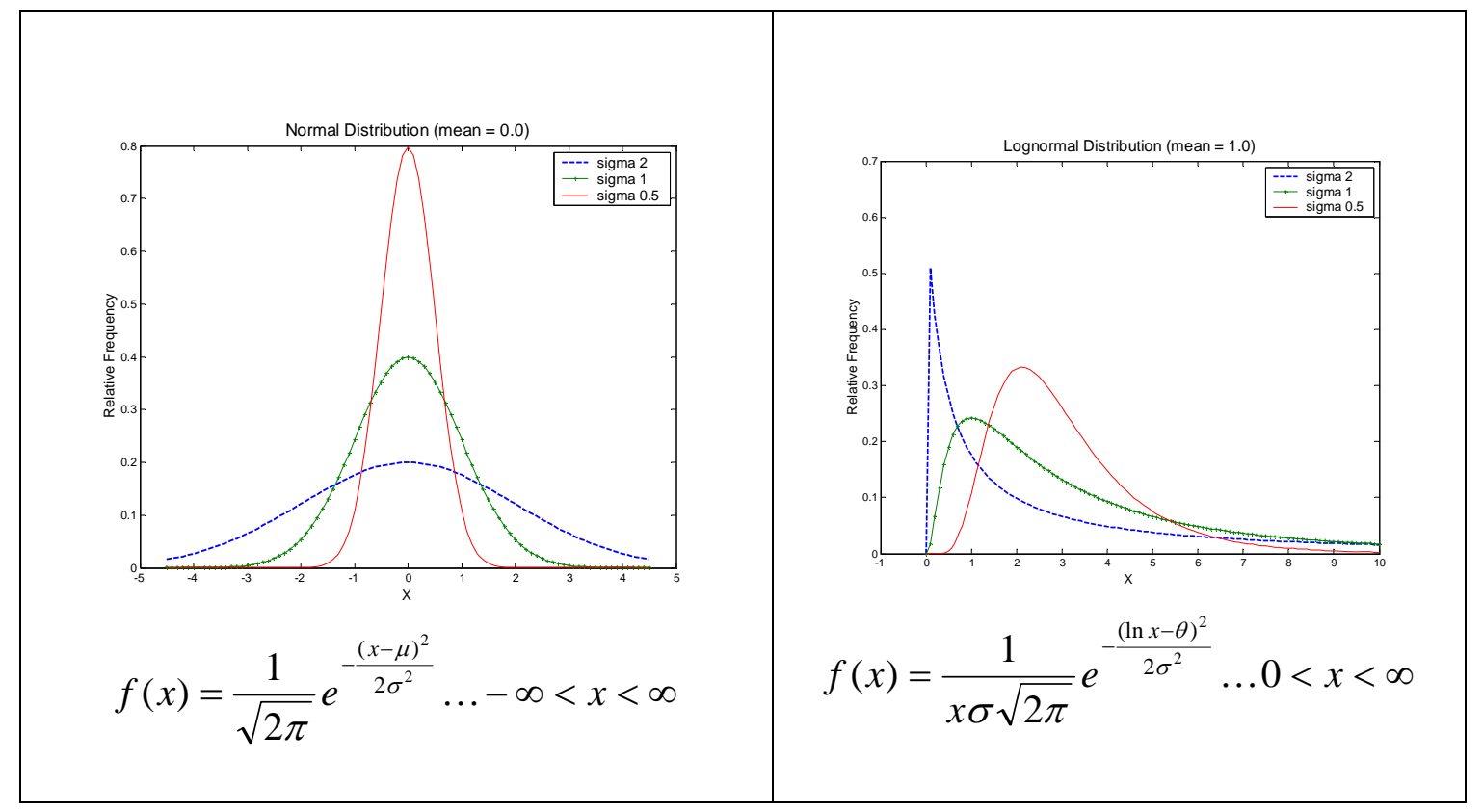

Figure 8. Normal and Lognormal Distribution Examples; left hand is the normal distribution; right hand is the lognormal distribution.

\section{Particle Histogram Analysis Process}

The analysis of particles can be carried out with many different objectives, for example shape characterization, size distribution, and compositional analysis. In this study, we are particularly interested in the development of a suitable technique to make a mineral composition analysis of a packed particle bed.

It was explained in the previous report that a suitable tool to separate touching particles in a particle packed bed has been developed. Once the particles have been labeled and identified, determination of the distributions of attenuation coefficients for each particle is relatively easy. The reconstructed image containing the attenuation coefficient data of the sample is scanned using the labeling in order to produce a histogram for each particle.

\section{$R$ and Rmix}

$\mathrm{R}$ is a language and environment for statistical computing and graphics developed as part of the GNU project sponsored by the free software foundation (FSF) which assures its availability in the future (R Project, 2005). Once the histogram for each particle has been generated then a finite mixture analysis is carried out using the free shareware software called R having a library known as Rmix (2005). The complete use and extension of the software is referenced to the user manual and help tutorial available with the software.

The basic procedure involving the finite mixture distribution analysis consists of the following three steps. First, data with the histogram information are stored in a text file which can be imported by $\mathrm{R}$ for its use. Second, data are graphically analyzed to determine visually the starting values and the number of components to be used. This information is determined using previous knowledge of the sample characteristics. Starting values for the means and sigmas (standard deviations) should be as good as possible, since the iteration 
algorithms will not converge if the starting value and constraints are not well chosen. Starting values for the proportions are less critical, since they can usually be improved very efficiently. Finally, the third and last step involves the application of one of the algorithms to resolve the finite mixture distribution problem.

\section{Results and Discussion}

\section{Linear Correlation of Density and Attenuation Coefficient}

It is known that the reconstruction algorithm applied to the data coming from a x-ray microtomography leads to the determination of attenuation coefficients in each voxel of the space domain. The space domain of measurements is divided in a given number of voxels based on the characteristic resolution of the test which could go up to $10 \mu \mathrm{m}$. Each one of these voxels will store a given attenuation coefficient which represents all the material inside of it. The attenuation coefficient assigned in a voxel is linearly related to the density of the material inside the voxel. A larger attenuation coefficient means that the material has a larger density which attenuates the pass of photons through the sample. This one-to-one relation allows us to match the attenuation coefficient of an unknown material sample with the density of a known material.

Figure 9 shows the result for four coal samples of known density scanned with the x-ray micro CT (XMT). It is clear that the mean of the attenuation coefficient is directly related to the density of the material; for a $1.3-1.5 \mathrm{~g} / \mathrm{cm}^{3}$ density range the attenuation coefficient distribution has a mean value of 0.02477 whereas for a $1.8-2.1 \mathrm{~g} / \mathrm{cm}^{3}$ density range the attenuation coefficient distribution has a mean value of 0.1128 . From this analysis we may see that a linear relation can be constructed between density and attenuation coefficient mean value. This relation is the starting point to classify elemental voxels in unknown samples. Figure 10 shows the relation between density and mean values for the four coal samples.

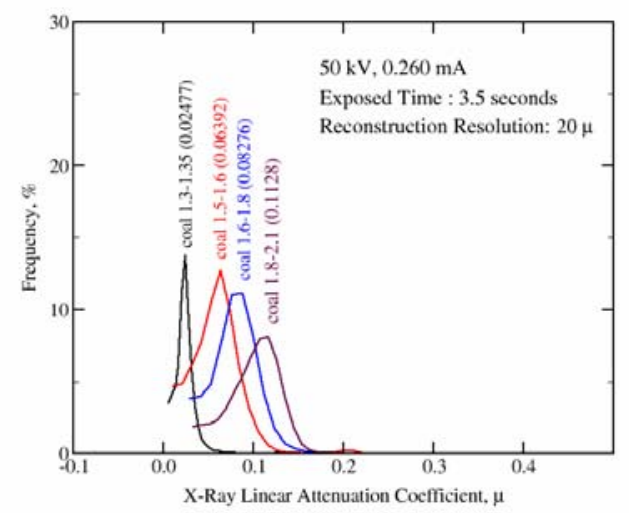

Figure 9. Histogram of four coal samples of known densities. Mean attenuation coefficients are given in parentheses.

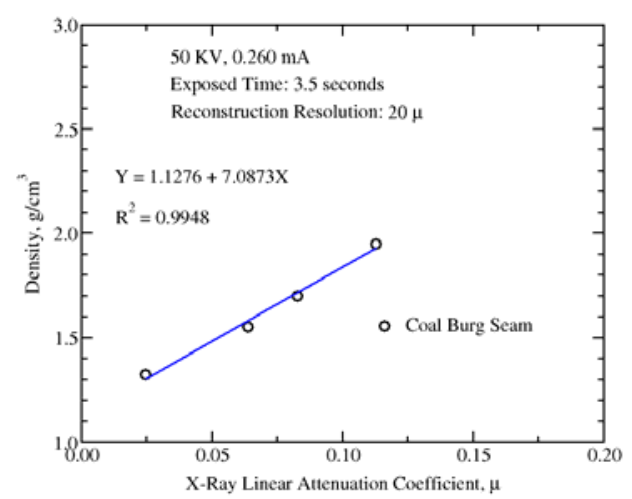

Figure 10. Relation between density and attenuation coefficient for four coal samples of known densities. 


\section{Segmentation of Packed Bed of Particles}

Several experiments were carried out in order to determine the applicability and confidence of this methodology in the analysis of real particle samples. Variations in particles shape and size have been tested to estimate their influence on the results. Glass beads, quartz sand and copper ore particles were used for this experiments. Based on the developed 3D watershed segmentation algorithm, original and resulting images for glass bead, and quartz sand particles are shown in Figures 11.
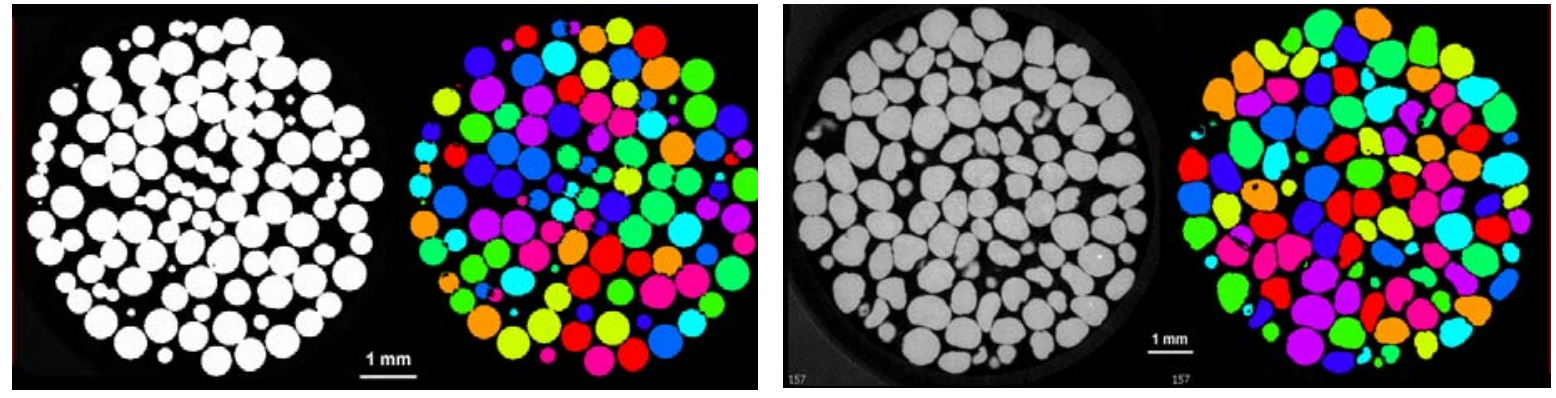

Figure 11. Comparison between original images and segmented images for a packed bed of glass-bead (left-hand side) and quartz-sand particles (right-hand side).

It is possible to observe a clear correspondence between the location of the original particles and the segmented particles; however, there is small percentage of oversegmentation due mainly to the presence of figure eight-shaped particles. In cases where this error level can be still considered high, it is suggested to scan the image for oversegmented particles and to merge the parts of particles by assignation of a same label number, however this process can be tedious and for the present research this level of error is considered acceptable.

In the case of copper ore particles the error during segmentation increases. Table 1 shows the results for segmentation of particle beds of different particles size and different resolution. Due to the highly nonspherical shape of these particles the error increases to around $10 \%$ for the coarser particle size classes.

Table 1. Particle segmentation for copper ore particles of different size and irregular shape.

\begin{tabular}{cccccc}
\hline $\begin{array}{c}\text { Particle } \\
\text { Size } \\
\text { (microns) }\end{array}$ & $\begin{array}{c}\text { Voxel } \\
\text { Resolution } \\
\text { (microns) }\end{array}$ & $\begin{array}{c}\text { Average } \\
\text { Diameter in } \\
\text { Voxels }\end{array}$ & $\begin{array}{c}\text { Particles } \\
\text { Over- } \\
\text { segmented }\end{array}$ & $\begin{array}{c}\text { Total } \\
\text { Number of } \\
\text { Particles }\end{array}$ & $\begin{array}{c}\text { Error } \\
\text { (\%) }\end{array}$ \\
\hline $6350-3175$ & 80 & 60 & 26 & 249 & 10.4 \\
$3175-1680$ & 40 & 61 & 18 & 283 & 6.4 \\
$1680-420$ & 20 & 53 & 19 & 213 & 8.9 \\
$420-149$ & 20 & 14 & - & - & High \\
\hline
\end{tabular}


The smaller particle size fraction, between 420 and 149 microns, has a very low resolution when compared to the rest of the samples. This is seen when we compare the average diameter of the particles expressed in number of voxels. Practice has shown that setting the $\mathrm{x}$-ray CT to get an average diameter of particles in fifty voxels or more assured good results for irregular particles. In this example, particles have only a few voxels per particle, which reduces the liberty to define the parameter for watershed segmentation algorithm and at the same time increases the volume effect at the surface of the particles which makes it extremely difficult in the initial thresholding process to separate air from particles.

Figure 12 shows the original image for the particles size fraction between 420-149 microns. In this figure, it is possible to see the problems of resolution mentioned before. Table 1 omits values for oversegmentation errors due to the impossibility of getting reasonably good segmentation results. In order to increase the reliability of the segmentation for such a small size of irregularly shaped particles it will be necessary to improve the resolution of the image such that each particle would have a diameter of around 50 voxels.

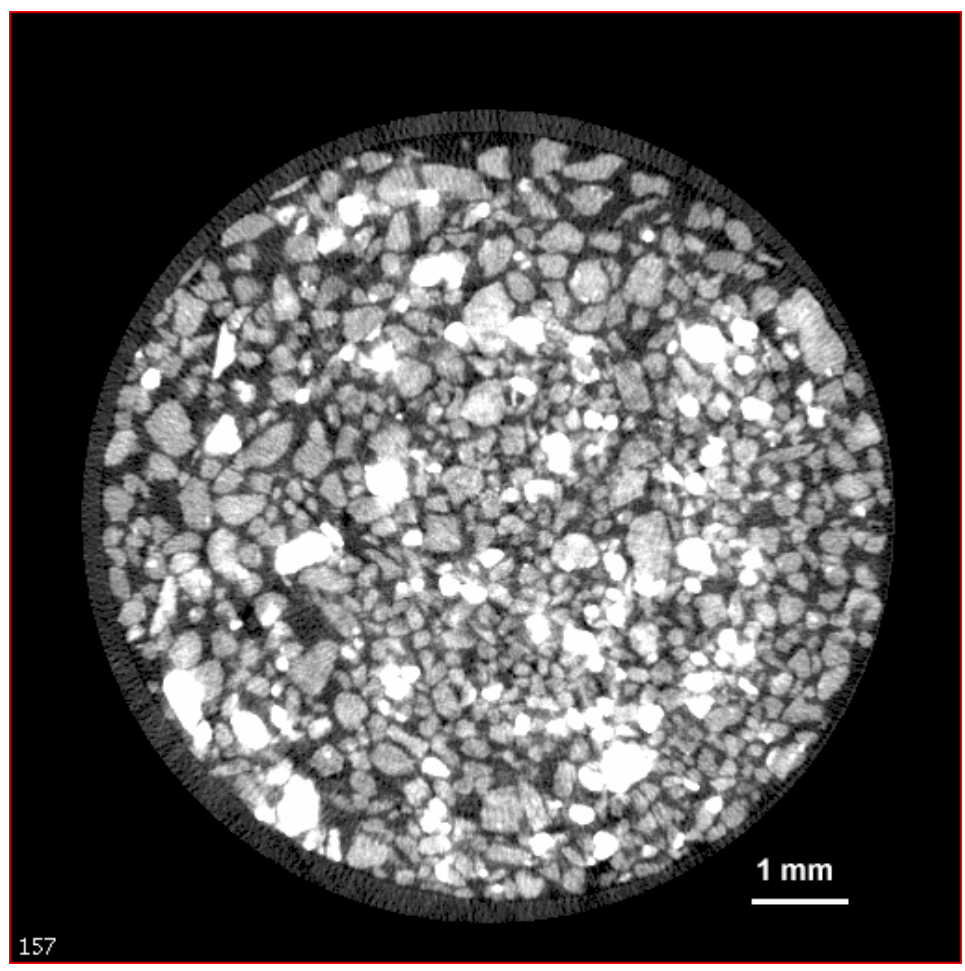

Figure 12. CT image for copper ore particles of size between 149 and 420 microns.

Figures 13 and 14 show 3D views of the segmented final images for copper ores with size of 6350-3175 microns and 3175-1680 microns, respectively. The different colors in the figures indicate the segmented particles. In each image different sectional views are shown in order to observe the total segmentation of the packed particle bed. Even when the tight packing and very irregular shape of these particles it is possible to observe that good segmentation can be obtained. 


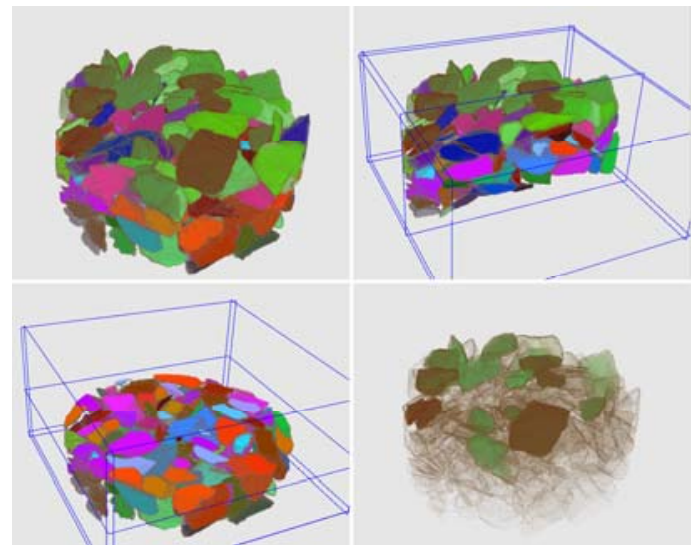

Figure 13. Segmented images for copper ore particles of size between 3175 to 6350 microns.

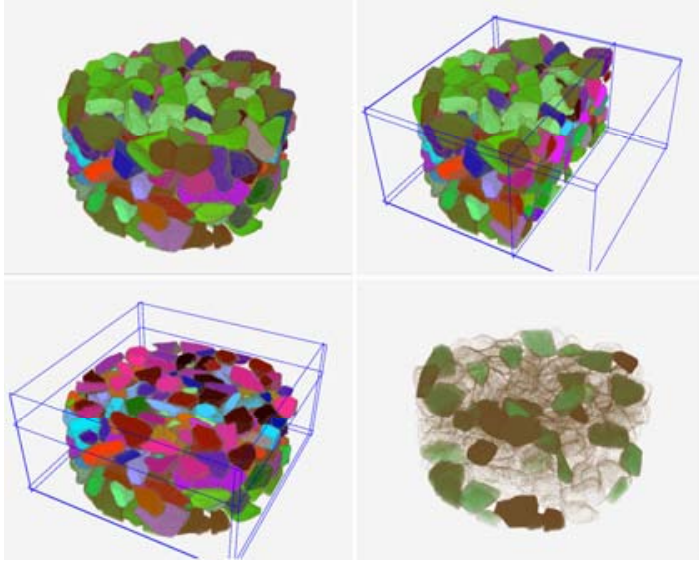

Figure 14. Segmented images for copper ore particles of size between 1680 to 3175 microns.

The segmentation of a wider particle size range in a single image decreases substantially the performance of the segmentation of particles in a 3D image because of the reduced freedom to set the watershed segmentation parameter. Further, an irregular shape reduces the performance of the segmentation process due to the creation of false markers. Finally and probably most important, voids inside the particles create problems for the application of the distance transform because such a condition will generate a maximum at the midway point between background and void hole and not next to center of the particle as expected. Figure 15 shows a comparison between one slice of the original copper ore image and its segmented image. In this case, oversegmentation (one particle has being separated in two smaller particles) and undersegmentation (two particles have not being separated) are present.

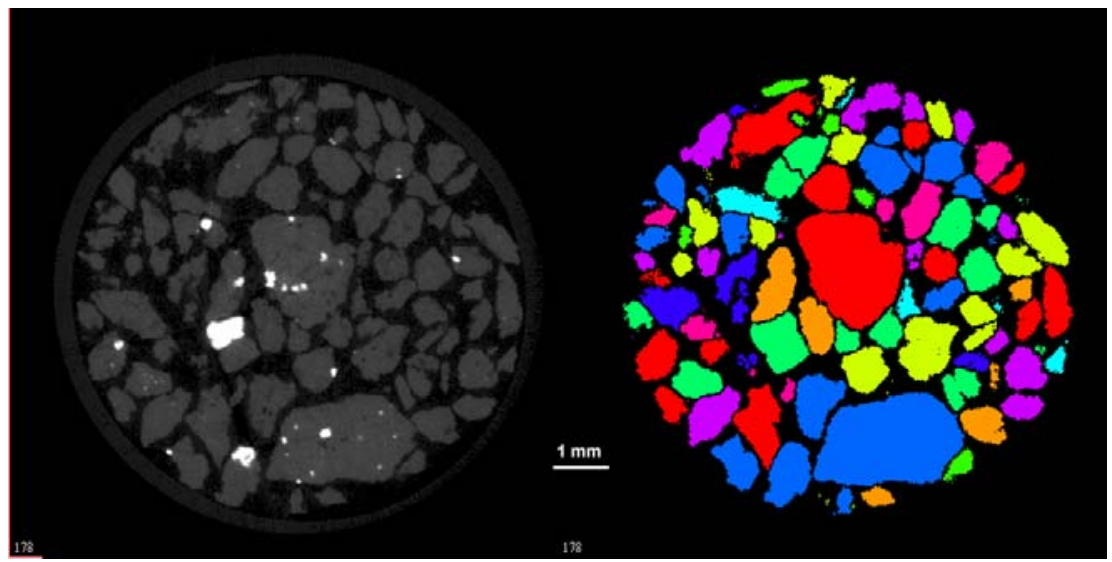

Figure 15. Comparison between original images and segmented images for a packed bed of copper ore particles with 500-1650 microns in size.

In summary, this methodology for 3D image segmentation allows for good results in those cases where the image is preprocessed in order to reduce holes and enhance contrast. It is clear, however, that due to the incredible variability of rock types and particle shapes from different operations that there is no such thing as a universal algorithm that can perform efficiently for all the cases. However, the software implementation developed in this research 
is intend to be of general applicability in all cases and a good starting point for new situations. A tune-up of the system will probably be required every time new samples are analyzed and in some cases further steps may be required in order to improve the results.

\section{Mineral Composition Analysis by Finite Mixture Distribution Model}

For detailed liberation analysis, the volumetric grade distribution of multiphase mineral particles can be measured directly by cone-beam x-ray microtomography. In order to illustrate the reliability of the XMT measurements a single particle of a copper ore sample of known composition was analyzed by scanning electron microscopy (SEM) and by XMT. A 3D XMT scan was initially run, and later the particle was sectioned, polished and a middle plane of the particle was analyzed by the SEM. The left-hand side image presented in Figure 16 is the 2D SEM image with 50X magnification. For comparison the same cross section (from a total of 240 sections) taken along the Z-direction from the three dimensional reconstruction of the copper ore particle is shown by the right-hand side image of Figure 16 at a resolution of 20 microns. The comparison clearly illustrates the ability of the high resolution x-ray microtomography system for quantitative analysis to determine the 3D spatial distribution of mineral phases in multiphase particles. Based on x-ray attenuation coefficients, differentiation of mineral phases within the sample is possible as indicated in Figure 16 for chalcocite and chalcopyrite.

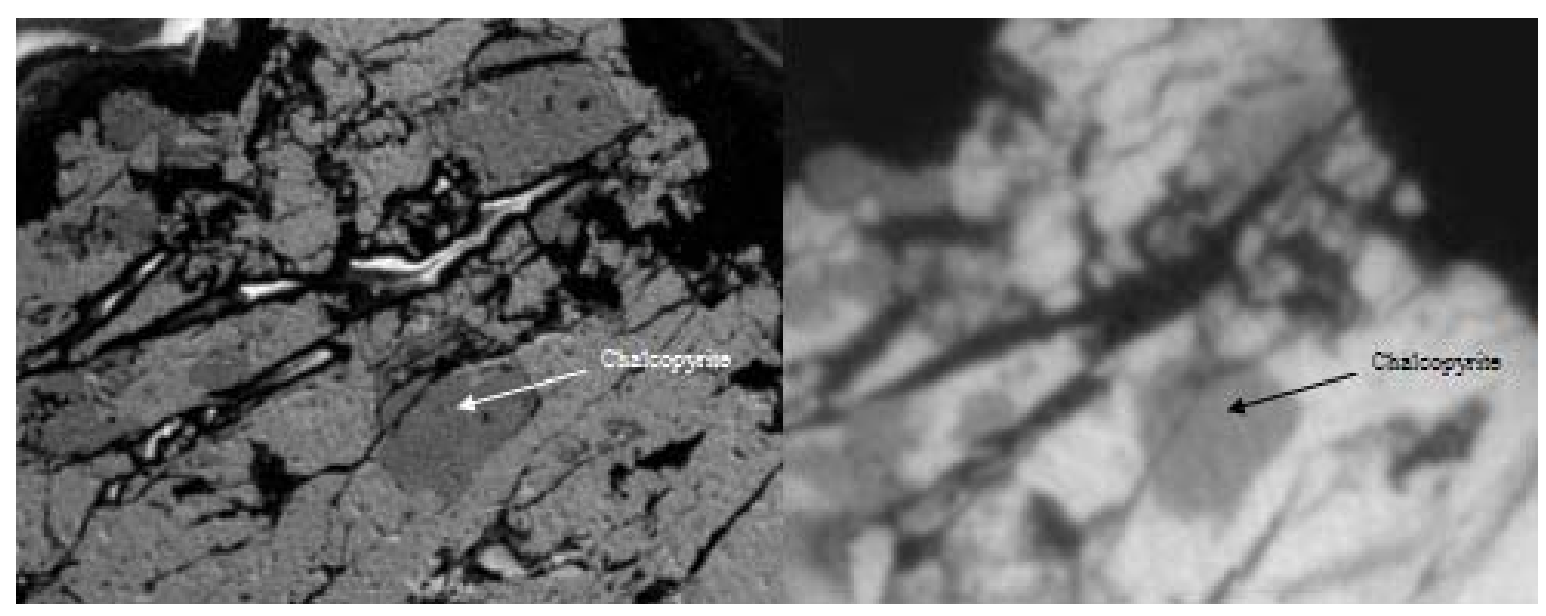

Figure 16. Images from a copper ore particle. Left-hand side shows the SEM 2D image (50X); Right-hand side shows the same cross section from the XMT 3D.

After analysis of the sample, a finite mixture distribution was fit to the particle composition data using the FMD model previously explained in Experimental section. Figure 17 shows the original histogram of the attenuation coefficient in blue and the final fit of the finite distribution model in green. The individual distributions or components are in red. The results are summarized in the Table 2 . 


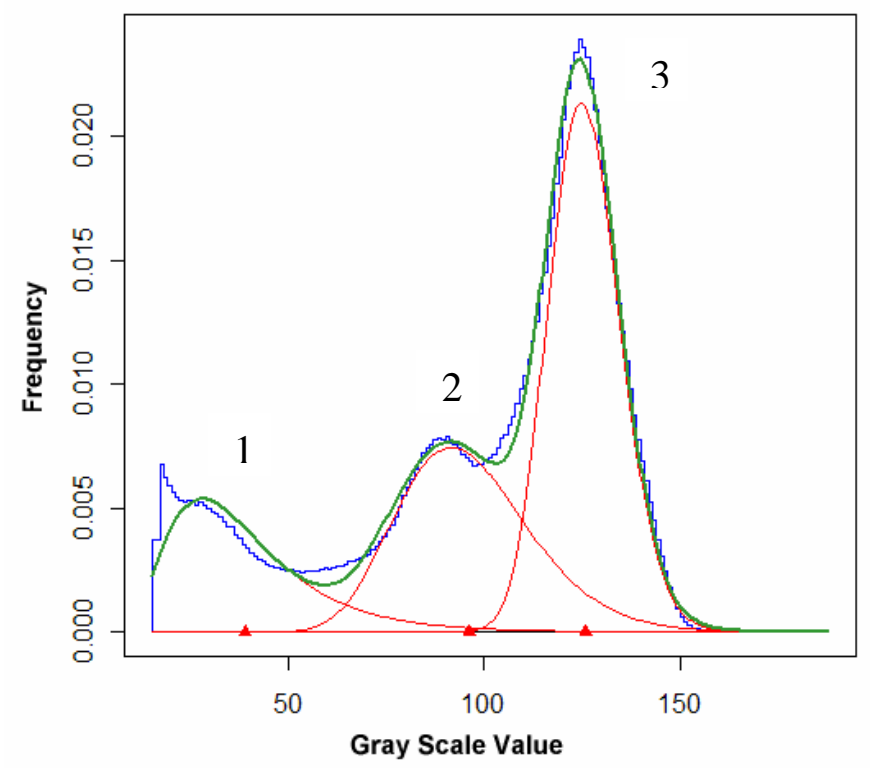

Figure 17. Single particle histogram fit by a finite mixture log-normal distribution model. Function curves 1, 2 and 3 are associated to Gangue, Chalcopyrite and Chalcocite, respectively

Table 2. Finite log-normal mixture distribution parameters.

\begin{tabular}{cccc}
\hline $\begin{array}{c}\text { Distribution } \\
\text { Function }\end{array}$ & Proportion & Mean & $\begin{array}{c}\text { Standard } \\
\text { Deviation }\end{array}$ \\
\hline 1 & 0.19 & 38.85 & 19.411 \\
2 & 0.313 & 96.2 & 17.456 \\
3 & 0.4892 & 125.88 & 9.196 \\
\hline
\end{tabular}

In summary, the SEM analysis confirms the 3D reconstruction analysis by XMT. Furthermore, the SEM analysis reveals the presence of chalcopyrite $\left(\mathrm{CuFeS}_{2}\right)$ and chalcocite $\left(\mathrm{C}_{2} \mathrm{~S}\right)$ which are also identified with the finite mixture model analysis based on the calibration curves. Together, XMT and FMD analysis provide the foundation for further study and mineralogical characterization of packed particle beds.

\section{Practical Applications}

Up to this point we have seen and implemented the fundamental computer tools to make a more detailed and better analysis of samples from XMT data. First of all, it has been demonstrated that the watershed segmentation process allows us to identify individual particles in packed particle beds by using a special segmentation procedure. This tool will reduce time and cost effort in the analysis of particles. Second it has been confirmed that the density and attenuation coefficients are related and that this relationship can be obtained through the experimental determination of calibration curves. It has been shown how a 
calibration curve associated with the use of FMD allows discrimination between different mineral phases. In this section both computer tools, 3D segmentation and FMD analysis, are used to show practical applications in the coal and mineral processing industries.

\section{XMT Washability Curves from a Packed Particle Bed}

In order to evaluate the ability to determine washability, curves from XMT analysis results were compared to the tradition sink-float results for a known sample. For this washability test, samples of coal were obtained from Illinois No. 5 seam and sink-float data for the traditional washability curves were provided by the South Illinois University (SIU). This data is shown in Table 3 and includes the percentage of weight sinking at 6 different density fractions for two different product streams; from now on they are identified as test 1 and test 5. From the data is possible observe that a few amount of the coal product has high density due to the presence of impurities.

Coal samples from these two different product streams were prepared for washability analysis using the 3-D x-ray microtomography technique. In each case particles were in the rage of 1000x420 microns, with variable density.

Table 3. Clean coal product samples test 1 and test 5.

\begin{tabular}{cccrr}
\hline & & & \multicolumn{2}{c}{ Product } \\
\cline { 3 - 5 } Relative & Mean & Test 1 & Test 5 \\
Density & Density & Wt. (\%) & Wt. (\%) \\
\hline $1.15-$ & 1.30 & 1.2250 & 73.91 & 61.78 \\
$1.30-$ & 1.40 & 1.3500 & 20.30 & 27.77 \\
$1.40-$ & 1.50 & 1.4500 & 3.85 & 7.46 \\
$1.50-1.625$ & 1.5625 & 1.27 & 2.14 \\
$1.625-2.00$ & 1.8125 & 0.63 & 0.79 \\
$2.00-2.80$ & 2.4000 & 0.04 & 0.06 \\
& & & & \\
\end{tabular}

Under carefully controlled laboratory conditions, coal particles from each density fraction were collected and mixed. Using a plastic container, $9 \mathrm{~mm}$ inside diameter and $20 \mathrm{~mm}$ in length, this mixed coal sample was scanned by 3D x-ray microtomography. The 3D reconstructed image set contains 512x512x220 voxels with a voxel resolution of 20 microns. Figure 18 shows some consecutive sectional slices from the reconstructed 3D image. Coal particles and pyrite and quartz grains are clearly distinguished in these images as dark gray, white and light gray respectively. The images reveal that test 1 and test 5 have same irregular shape type of particles and same particle size variation. 

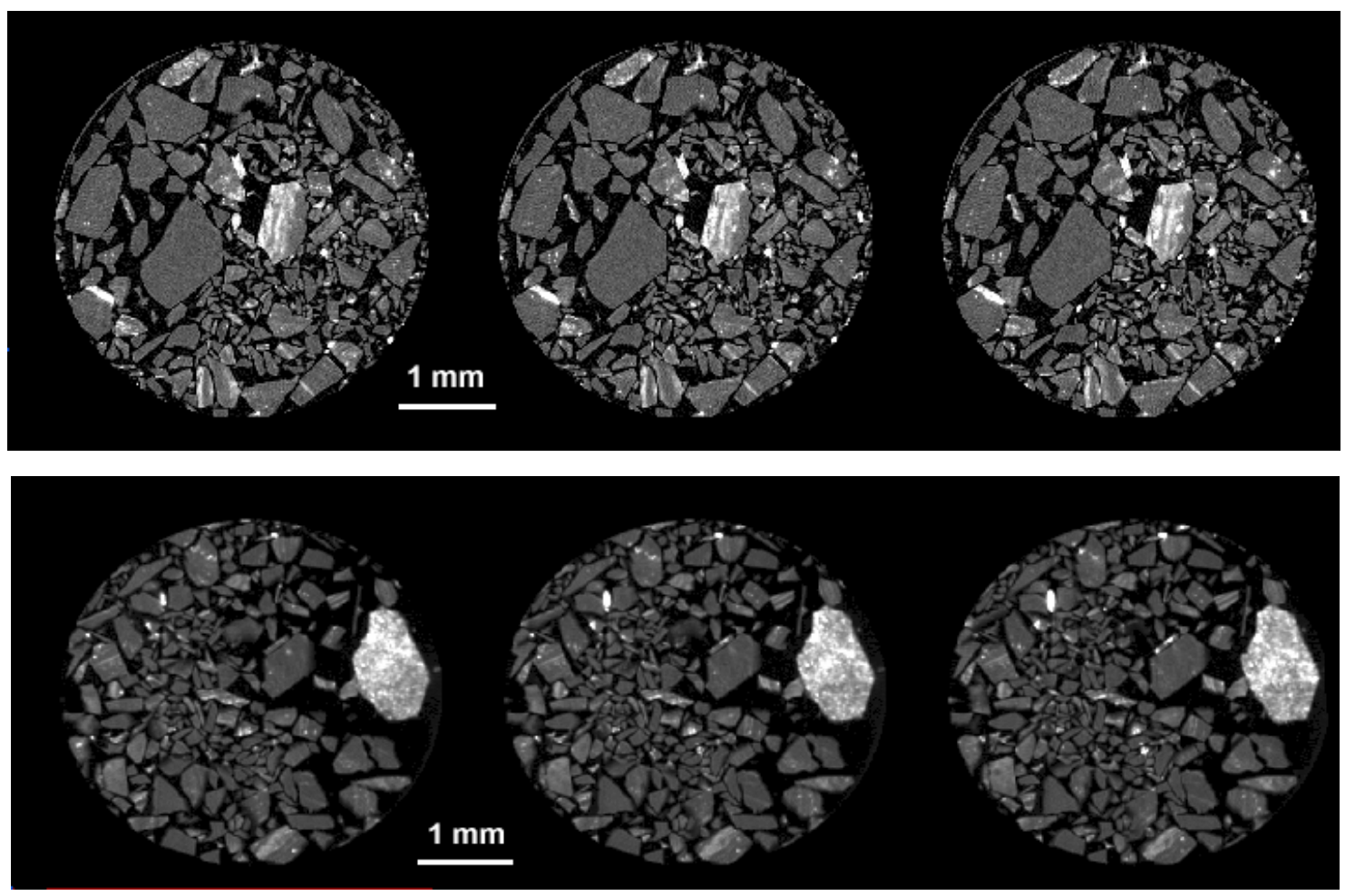

Figure 18. Sectional slices from reconstructed images for test 5 (top figure) and test 1 (bottom figure).

As well as for the determination of the calibration curve previously mentioned the XMT analysis for the coal samples were run at $50 \mathrm{kV}$ and with a 20 micron voxel resolution. Since the particles size varies between 1000 microns and 180 microns, the diameter per particle is expected to be around 50 voxels. As Figure 19 shows, the segmentation is good for such irregular shapes and particle sizes.

Once the particles have been segmented, the classification of the particles into the different density fractions is based on the apparent density. To do so, an algorithm has been developed that scans the segmented image, calculates the average density of each particle and assigns the volume of each particle to the respective density fraction. Because of density has been directly related to the attenuation coefficient value and because coal and contaminants attenuation values are easily distinguished, it is not necessary to use FMD analysis to determine phase composition in this case.

Figure 20 shows the washability curve from CT analysis of the clean coal product obtained for test 1 . The analysis reveals a good agreement between the results from sink-float analysis and the results from the XMT scan. In fact, for densities greater than $1.4 \mathrm{~g} / \mathrm{cm}^{3}$ the difference between the procedures is smaller than $3 \%$ of the total weight. For densities smaller than 1.3 $\mathrm{g} / \mathrm{cm}^{3}$ the difference between the measurements increases rapidly up to a value of $13 \%$. These differences are probably due to sampling errors associated with small particle sizes. 

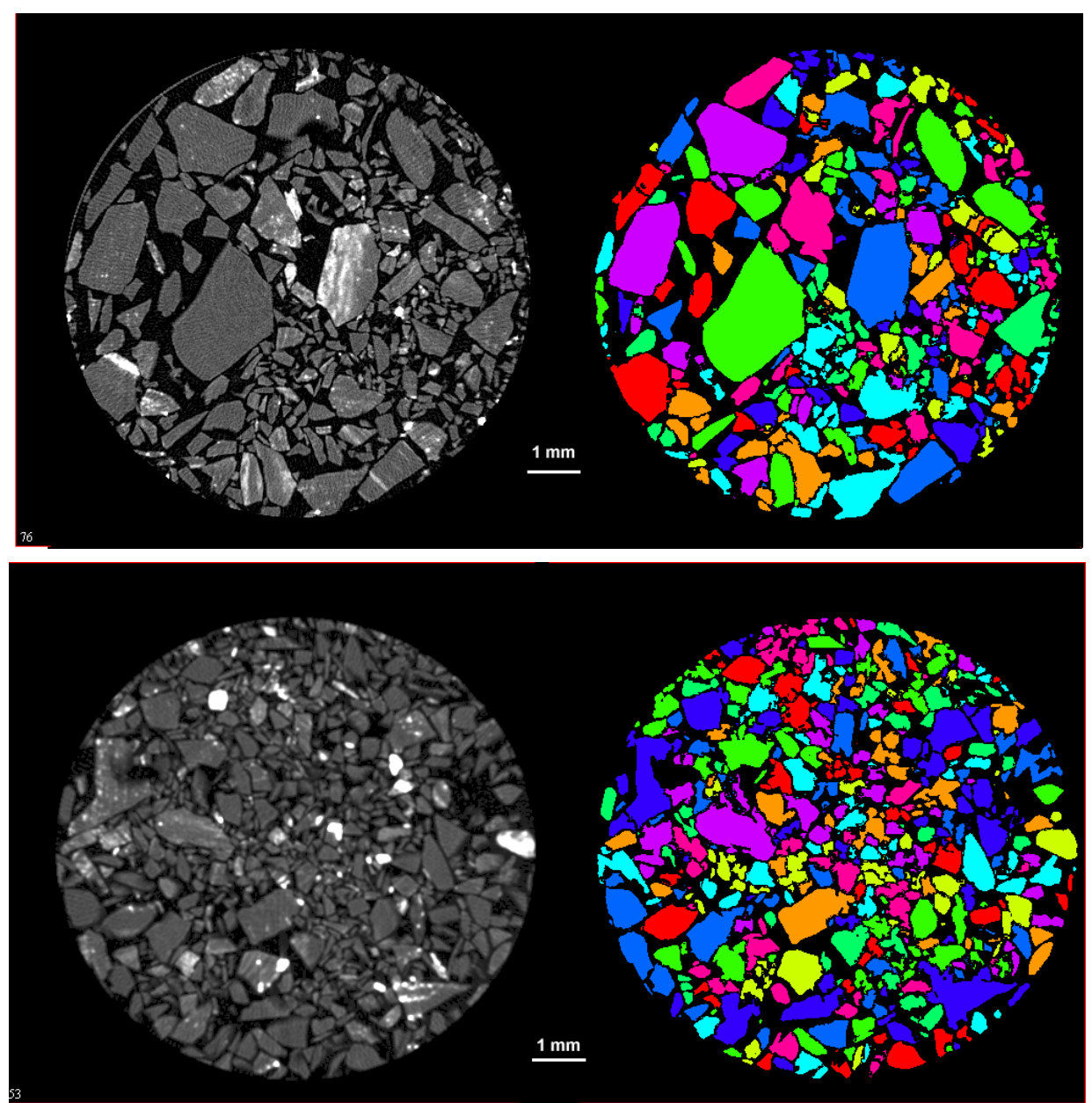

Figure 19. Segmentation of the reconstructed images of coal samples. Top shows one slice from test 5 and bottom shows one slice from test 1 .

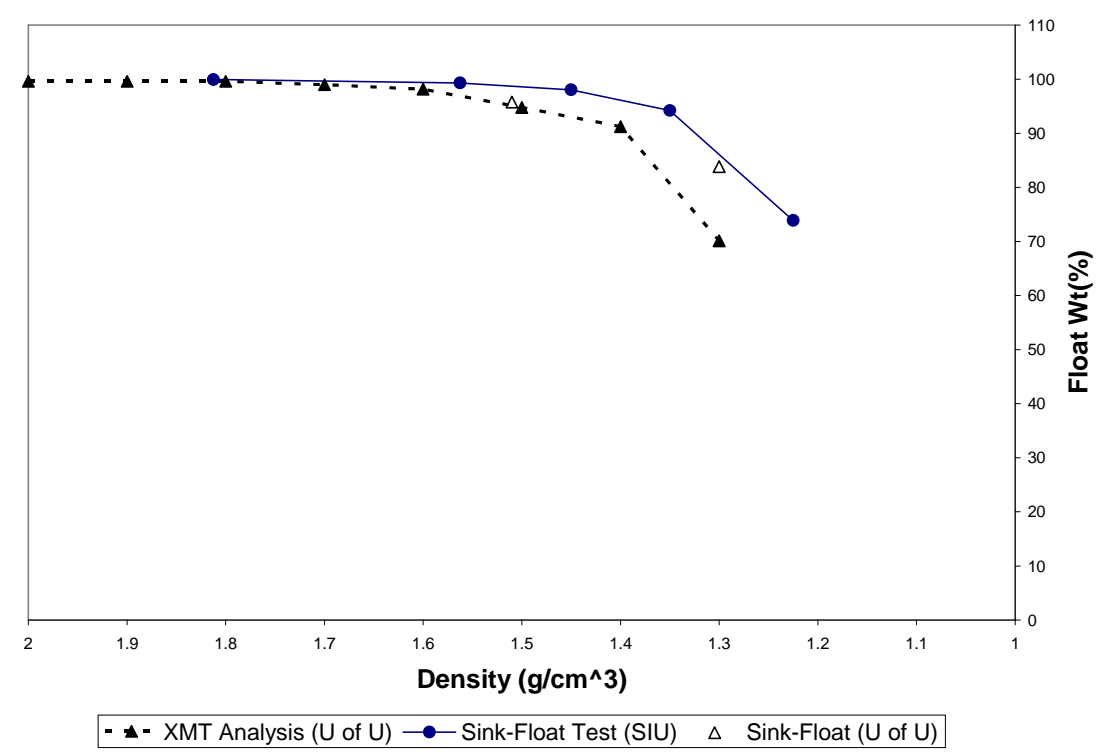

Figure 20. Washability curve for clean coal product from test 1. 
Figure 20 also includes data from a sink-float analysis carried out at the University of Utah ( $U$ of $U$ ) with the same sample used in the XMT analysis. The values are shown as unfilled triangles in the Figure 20. As Figure 20 shows, XMT washability curve is very close to the SIU washability curve and in the range of the $U$ of $U$ experimental data.

Difference at low densities is mainly due to sampling errors. In fact, whereas the SIU data are based in several experiments with hundreds of kilograms tested, the XMT analysis was carried out with a small sample of few grams. In fact, a good sampling improves drastically the results as it is shown in Figure 21. Figure 21 shows the washability for the test 5 product stream. We see an excellent correlation between XMT analysis and experimental sink-float data. In this case, the maximum difference in weight percentage is about $2 \%$ at a low density level of $1.3 \mathrm{~g} / \mathrm{cm}^{3}$.

As in test 1, the washability curve calculated with the XMT data follows very well the experimental sink-float data. However, in this case, the comparison between the SIU data with the experimental test carried out at the $U$ of $U$ with the same sample used in the XMT analysis shows important differences. This difference is explained because of the natural difficulties in running a sink-float test with a small sample of few grams.

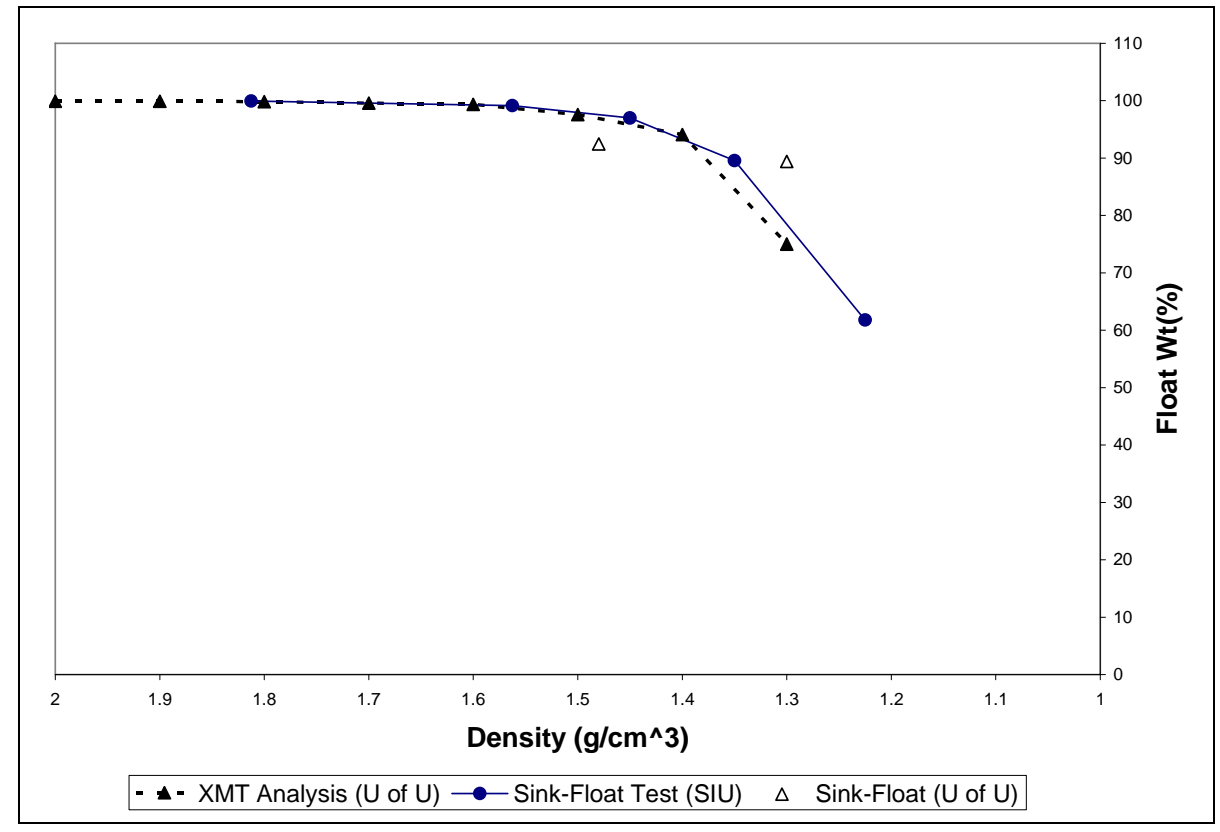

Figure 21. Washability curve for clean coal product from test 5.

\section{XMT Copper Mineral Analysis from a Packed Particle Bed}

In this experiment, a small sample of copper ore feed obtained from Kennecott at Utah was used for mineral composition analysis. The samples are known to be composed of pyrite, chalcopyrite and gangue. Particle size is between $2.00 \times 1.18 \mathrm{~mm}$ and the density of the material is between $2.65 \mathrm{~g} / \mathrm{cm}^{3}$ and $2.85 \mathrm{~g} / \mathrm{cm}^{3}$. Figure 22 shows a 3D transparent image of 
the packed bed of particles analyzed. In green we see the gangue or matrix and in black we can see some of the grains of chalcopyrite and pyrite. The 3D image captured with XMT was segmented using the watershed algorithm as explained before. After segmentation, the particles composition was determined for each particle using the FMD model explained previously.

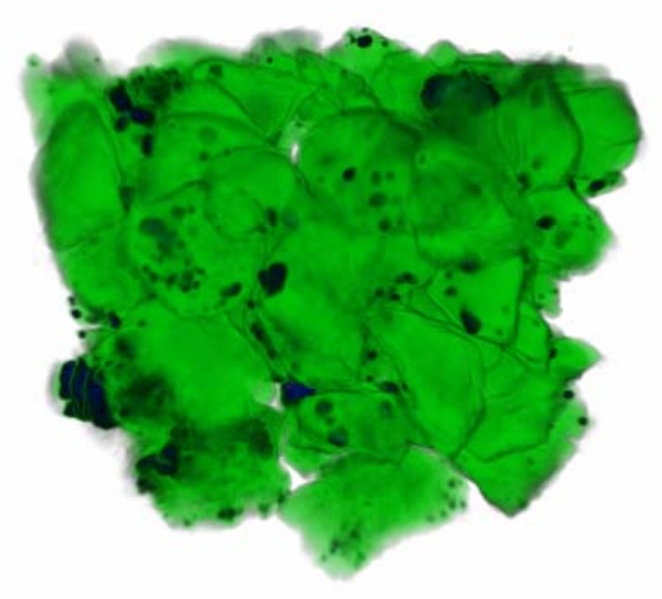

Figure 22. Transparent image of the packed particle bed of copper ore.

The segmentation process allows the determination of the attenuation coefficient histogram of each particle. In this particular packed particle 85 individual particles were found. From previous calibration work it is known that at an energy level of $130 \mathrm{kV}$ and using a 10 mil brass filter the attenuation coefficient histogram of the gangue, pyrite and chalcopyrite have a peak at a value of $0.01,0.03$ and 0.05 respectively. Using the knowledge about the attenuation coefficient peak values for gangue, pyrite and chalcopyrite we proceed to make the compositional analysis of each particle using the Rmix library for FMD analysis.

Figure 23 shows the histograms and volume rendering images of three selected particles labeled as numbers 10, 23 and 31. Red, blue and green colors represent the chalcopyrite, pyrite and silicate minerals (gangue) phases, respectively.

Tables 4 and 5 show a summary of the results for the 85 particles. In order to validate and probe the methodology the density for each particle was calculated using XMT data analysis. In fact, expected density of the particle is calculated based on the volume percentage distribution of each particle determined for the FMD model and assuming a mean density value for each phase.

Because the sample was selected belonging to the density fraction between $2.65 \mathrm{~g} / \mathrm{cm}^{3}$ and $2.85 \mathrm{~g} / \mathrm{cm}^{3}$, it is expected that each particle should have a density in this density interval. Based on the analysis from the FMD model, and assuming a density of $2.6 \mathrm{~g} / \mathrm{cm}^{3}$ for gangue, $4.2 \mathrm{~g} / \mathrm{cm}^{3}$ for chalcopyrite, and $5.0 \mathrm{~g} / \mathrm{cm}^{3}$ for pyrite, the density of each particle is calculated. The lines at the table highlighted in gray color show the particles which have a density greater than 2.85. Seven of the eighty five particles show this problem. An analysis of each one of these cases reveals two reasons for the unexpected values. The first reason, as is the 
case for particles 8, 12 and 30 is due to problems with the segmentation process which oversegmented some very complex shaped particles, characteristic of crushed material, leaving high densities phases in a high concentration in the smallest fragments. In the case of particles 10, 16, 42 and 72 the high density is due to the effective presence of high concentrations of pyrite and chalcopyrite. The presence of these particles may be due to sampling errors during sampling preparation. The error in the analysis represents an $8 \%$ of the total number of particles and a small percentage of the total volume analyzed because the problems are found in small irregular particles rather than in big particles. The present results are considered successful for the research.
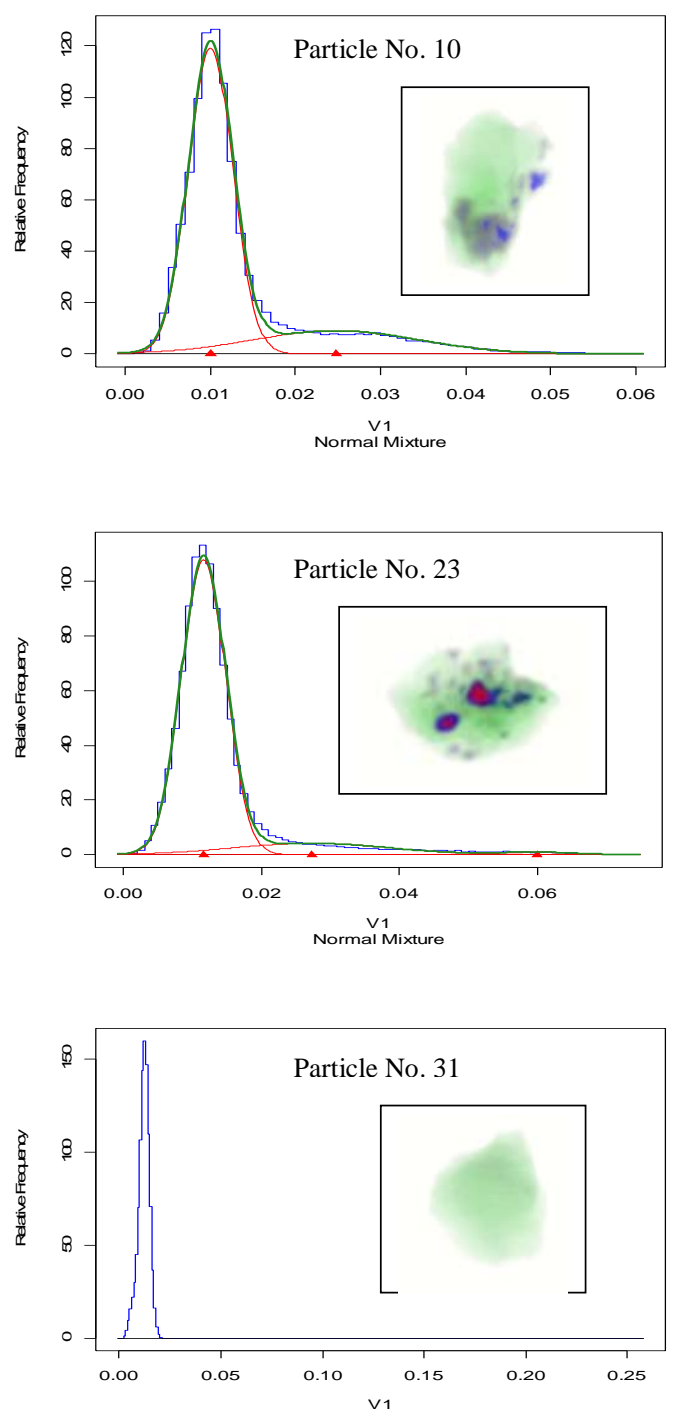

Figure 23. X-ray attenuation coefficient histogram and their best fit of finite mixture models of three selected particles and their corresponding volume rendering images. The chalcopyrite (in red), pyrite (in blue) and silicate minerals (in green) phases are clearly distinguished from the volume rendering images. 
Table 4. Average density for each particle in the packed particle bed of a copper sample $\left(2.00 \times 1.18 \mathrm{~mm}, 2.65 \times 2.85 \mathrm{~g} / \mathrm{cm}^{3}\right)$ as determined from XMT analysis using 3D image computer tools. Particles number 1 to 43.

\begin{tabular}{|c|c|c|c|c|c|}
\hline \multirow[b]{2}{*}{$\begin{array}{l}\text { Particle } \\
\text { Number }\end{array}$} & \multicolumn{4}{|c|}{ Vol \% } & \multirow[b]{2}{*}{$\begin{array}{c}\text { Density } \\
\mathrm{g} / \mathrm{cm}^{3}\end{array}$} \\
\hline & Chalcopyrite & Pyrite & Gangue & Total & \\
\hline$\overline{1}$ & & 0.3763 & 99.6237 & 100 & 2.61 \\
\hline 2 & 0.6527 & 4.0381 & 95.3092 & 100 & 2.71 \\
\hline 3 & 0.6824 & 5.0937 & 94.224 & 100 & 2.73 \\
\hline 4 & & & 100 & 100 & 2.60 \\
\hline 5 & 1.992 & 5.642 & 92.366 & 100 & 2.77 \\
\hline 6 & & 1.51 & 98.49 & 100 & 2.64 \\
\hline 7 & & 2.522 & 97.478 & 100 & 2.66 \\
\hline 8 & 19.29 & 17.59 & 63.13 & 100 & 3.33 \\
\hline 9 & 6.808 & 2.741 & 90.451 & 100 & 2.77 \\
\hline 10 & & 21.9 & 78.1 & 100 & 3.13 \\
\hline 11 & & & 100 & 100 & 2.60 \\
\hline 12 & 34.05 & 36.79 & 29.15 & 100 & 4.03 \\
\hline 13 & & & 100 & 100 & 2.60 \\
\hline 14 & & & 100 & 100 & 2.60 \\
\hline 15 & 1.153 & 4.48 & 94.367 & 100 & 2.73 \\
\hline 16 & 9.612 & 50.82 & 39.568 & 100 & 3.97 \\
\hline 17 & & & 100 & 100 & 2.60 \\
\hline 18 & 0.06529 & 0.66777 & 99.26695 & 100 & 2.62 \\
\hline 19 & 1.062 & 3.706 & 95.232 & 100 & 2.71 \\
\hline 20 & & 0.2487 & 99.7513 & 100 & 2.61 \\
\hline 21 & 0.07095 & 0.78838 & 99.14068 & 100 & 2.62 \\
\hline 22 & & 0.7858 & 99.2142 & 100 & 2.62 \\
\hline 23 & 1.292 & 8.893 & 89.815 & 100 & 2.83 \\
\hline 24 & & 2.636 & 97.364 & 100 & 2.66 \\
\hline 25 & 3.193 & 6.958 & 89.849 & 100 & 2.82 \\
\hline 26 & 0.8897 & 1.8854 & 97.2249 & 100 & 2.66 \\
\hline 27 & 1.536 & 2.748 & 95.717 & 100 & 2.69 \\
\hline 28 & 0.5913 & 3.4462 & 95.9625 & 100 & 2.69 \\
\hline 29 & 0.5081 & 3.0852 & 96.4067 & 100 & 2.68 \\
\hline 30 & 0.5915 & 17.9937 & 81.4149 & 100 & 3.04 \\
\hline 31 & & & 100 & 100 & 2.60 \\
\hline 32 & & 1.324 & 98.676 & 100 & 2.63 \\
\hline 33 & 0.705 & 2.508 & 96.787 & 100 & 2.67 \\
\hline 34 & 0.2315 & 1.5178 & 98.2507 & 100 & 2.64 \\
\hline 35 & & 1.228 & 98.772 & 100 & 2.63 \\
\hline 36 & & & 100 & 100 & 2.60 \\
\hline 37 & & & 100 & 100 & 2.60 \\
\hline 38 & & & 100 & 100 & 2.60 \\
\hline 39 & 1.184 & 5.202 & 93.615 & 100 & 2.74 \\
\hline 40 & 6.064 & 6.089 & 87.847 & 100 & 2.84 \\
\hline 41 & & 1.112 & 98.888 & 100 & 2.63 \\
\hline 42 & 11.31 & 10.62 & 78.08 & 100 & 3.04 \\
\hline 43 & & & 100 & 100 & 2.60 \\
\hline
\end{tabular}


Table 5. Average density for each particle in the packed particle bed of a copper sample $2.00 \times 1.18 \mathrm{~mm}, 2.65 \times 2.85 \mathrm{~g} / \mathrm{cm}^{3}$ ) as determined from XMT analysis using 3D image computer tools. Particles number 44 to 85 .

\begin{tabular}{|c|c|c|c|c|c|}
\hline \multirow[b]{2}{*}{$\begin{array}{l}\text { Particle } \\
\text { Number }\end{array}$} & \multicolumn{4}{|c|}{ Vol \% } & \multirow[b]{2}{*}{$\begin{array}{c}\text { Density } \\
\mathrm{g} / \mathrm{cm}^{3}\end{array}$} \\
\hline & Chalcopyrite & Pyrite & Gangue & Total & \\
\hline 44 & $\overline{0.2}$ & 0.2 & 99.6 & 100 & 2.61 \\
\hline 45 & & 0.3218 & 99.6782 & 100 & 2.61 \\
\hline 46 & 1.832 & 1.657 & 96.511 & 100 & 2.67 \\
\hline 47 & 2.838 & 1.575 & 95.587 & 100 & 2.68 \\
\hline 48 & 2.869 & 3.657 & 93.473 & 100 & 2.73 \\
\hline 49 & & 1.184 & 98.816 & 100 & 2.63 \\
\hline 50 & & 0.9797 & 99.0203 & 100 & 2.62 \\
\hline 51 & & 0.3509 & 99.6491 & 100 & 2.61 \\
\hline 52 & 0.825 & 2.022 & 97.153 & 100 & 2.66 \\
\hline 53 & 0.7747 & 2.737 & 96.4884 & 100 & 2.68 \\
\hline 54 & & 3.933 & 96.067 & 100 & 2.69 \\
\hline 55 & & 0.791 & 99.209 & 100 & 2.62 \\
\hline 56 & & 3.637 & 96.363 & 100 & 2.69 \\
\hline 57 & 0.8944 & 1.8752 & 97.2304 & 100 & 2.66 \\
\hline 58 & 0.2618 & 0.6567 & 99.0815 & 100 & 2.62 \\
\hline 59 & & 2.073 & 97.927 & 100 & 2.65 \\
\hline 60 & & 1.036 & 98.964 & 100 & 2.62 \\
\hline 61 & 1.046 & 6.311 & 92.644 & 100 & 2.77 \\
\hline 62 & 2.955 & 6.751 & 90.294 & 100 & 2.81 \\
\hline 63 & & 6.211 & 93.789 & 100 & 2.75 \\
\hline 64 & & 0.64 & 99.36 & 100 & 2.62 \\
\hline 65 & & 2.014 & 97.986 & 100 & 2.65 \\
\hline 66 & & 1.321 & 98.679 & 100 & 2.63 \\
\hline 67 & 0.251 & 2.342 & 97.407 & 100 & 2.66 \\
\hline 68 & & & 100 & 100 & 2.60 \\
\hline 69 & 0.4036 & 1.4639 & 98.1325 & 100 & 2.64 \\
\hline 70 & & & 100 & 100 & 2.60 \\
\hline 71 & & 0.7881 & 99.2119 & 100 & 2.62 \\
\hline 72 & 1.952 & 19.03 & 79.018 & 100 & 3.09 \\
\hline 73 & 3.967 & 7.578 & 88.456 & 100 & 2.85 \\
\hline 74 & & & 100 & 100 & 2.60 \\
\hline 75 & 1.225 & 7.932 & 90.843 & 100 & 2.81 \\
\hline 76 & 0.1523 & 2.1902 & 97.6575 & 100 & 2.66 \\
\hline 77 & & 7.697 & 92.303 & 100 & 2.78 \\
\hline 78 & & & 100 & 100 & 2.60 \\
\hline 79 & & 0.4244 & 99.5756 & 100 & 2.61 \\
\hline 80 & 0.9268 & 3.6941 & 95.3791 & 100 & 2.70 \\
\hline 81 & & 1.106 & 98.894 & 100 & 2.63 \\
\hline 82 & 0.1657 & 4.8115 & 95.0228 & 100 & 2.72 \\
\hline 83 & 0.6405 & 0.809 & 98.5505 & 100 & 2.63 \\
\hline 84 & & & 100 & 100 & 2.60 \\
\hline 85 & & 1.015 & 98.985 & 100 & 2.62 \\
\hline
\end{tabular}




\section{Conclusion}

XMT computer tools for 3D segmentation of packed particle bed images and for compositional analysis of individual particles have been developed. These tools have been shown to be reliable and applicable in various applications. The individual separation of the particles in a set allows for further individual compositional analysis of these particles and makes the process of sampling and analysis economically affordable because it allows for acquisition of individual particle information from XMT analysis over a group of particles instead of a single particle at a time basis.

Even though these tools have been found to be effective, due to the incredible variability of rock types and particle shapes from different operations, there is no universal procedure that can be expected to perform efficiently for all cases. However, the software developed in this research is intended to be of general application and is an excellent starting point when the analysis of new particle samples is required. It will probably be necessary to tune-up the software when new samples are analyzed and further steps may be necessary to improve the results in some circumstances. In particular, the results and application of the tools in coal washability analysis and mineral phase composition of residual copper ores from heap leaching are promising and further research is suggested.

The X-ray CT microtomography technique, joined to digital image processing tools, as the 3D segmentation developed in this research, and mineral phase analysis for particle characterization using the Finite Mixture Distribution analysis has provided important tools for mineral liberation analysis, fundamental information necessary to evaluate and control the efficiency of particle separations for both the coal and mineral industry. In addition the accomplishments from this research open a variety of other opportunities for further research and applications. For example, particle shape and grain shape distributions can be evaluated in 3D from packed particle beds. Also, internal phase distribution and continuity of multiphase particles may be evaluated for plant samples through the examination of XMT data.

Today's XMT technology has a limit on the order of 10 microns in resolution and the analyses of samples at such resolution require long scanning times. However, the technology of microfocus $\mathrm{X}$-ray generators and CD detectors improves every year, and it is expected that the utilization of XMT at high resolution will be achieved in the years to come.

\section{References}

Borgefors, G., 1984, “Distance transformations in arbitrary dimensions”, Computer Vision, Graphics, and Image Processing Vol.27, 321-345.

Journel, A.G., Huijbregts, CH.J., (1978), Mining Geostatistics, Academic Press.

Leonard J.W. (Ed.), 1979, Coal Preparation, 4th edition, AIME, New York.

Miller, J.D. and Lin, C.L., 1988, “Treatment of polished section data for detailed liberation analysis”, International Journal of Mineral Processing, Vol. 22, pp. 41-58.

King, R.P. and Schneider, C.L., 1993, "An effective SEM-based image analysis for quantitative mineralogy”, KONA, Vol. 11, pp. 165-177.

Lin, C.L. and Miller, J.D., 1996, “Cone beam x-ray microtomography for three-dimensional liberation analysis in the $21^{\text {st }}$ century”, International Journal of Mineral Processing, Vol. 47, pp. 61-73. 
Lin, C.L. and Miller, J.D., 2002, "Cone beam x-ray microtomography - a new facility for three-dimensional analysis of multiphase materials", Minerals \& Metallurgical Processing, Vol. 19, No. 2, pp. 65-71

Rmix, (2005) http://icarus.math.mcmaster.ca/peter/mix/mix.html

R Project, (2005) http://www.r-project.org.

Soille, P., 1999, Morphological Image Analysis: Principles and Applications, Springer.

Vincent, L., 1993, "Morphological Grayscale Reconstruction in Image Analysis: Applications and Efficient Algorithms", IEEE Transactions on Image Processing, Vol.2 No. 2, pp. $176-201$. 
Appendix 36: Development of a 3D Lattice-Boltzmann Model for Fluid Flow Simulation under Partially-Saturated Conditions in Packed Particle Beds (UT007) 


\section{FINAL TECHNICAL REPORT}

Contract Title and Number:

Crosscutting Technology Development at the Center for

Advanced Separation Technologies

(DE-FC26-02NT41607)

Sub-Recipient Project Title:

Development of a 3D Lattice-Boltzmann Model for

Fluid Flow Simulation under Partially-Saturated

Conditions in Packed Particle Beds

Principal Investigators:

J.D. Miller \& M. Sukop

Contact Address:

University of Utah

135 South 1460 E. Room 412

Salt Lake City UT 84112

Subcontractor Address:

Department of Earth Sciences

Florida International University

Office: 305-348-3117

Fax: 305-348-3877
Period of Performance:

Starting Date: 6/1/2005

Ending Date: 6/1/2006
Report Information:

Type: Final

Number:

Period:

Date: $\quad 3 / 31 / 06$

Code: UT007-Final

Contact Information:

Phone: (801) 581-5160

Fax: (801) 581-8119

E-Mail: jdmiller@mines.utah.edu

Subcontractor Information: Mike Sukop 


\title{
DISCLAIMER
}

"This report was prepared as an account of work sponsored by an agency of the United States Government. Neither the United States Government nor any agency thereof, nor any of their employees, make any warranty, express or implied, nor assume any legal liability or responsibility for the accuracy, completeness, or usefulness of any information, apparatus, product, or process disclosed, or represents that its use would not infringe privately owned rights. Reference herein to any specific commercial product, process, or service by trade name, trademark, manufacturer, or otherwise does not necessarily constitute or imply endorsement, recommendation, or favoring by the United States Government or any agency thereof. The views and opinions of authors expressed herein do not necessarily state or reflect those of the United States Government or agency thereof."

\begin{abstract}
The research has been designed to develop software capable of simulating the fluid flow of a leaching solution through a packed bed of particles under partially-saturated conditions. The results from the $1^{\text {st }}$ year of study are presented as a final report for the CAST II program. The $2^{\text {nd }}$ year of the research will be completed under the CAST III program. The software uses the Lattice Boltzmann Method (LBM), which has numerous advantages over other approaches. The research involves consideration of the mechanics of the heap leaching system as influenced by particle size distribution, pore-network structures, solution flow rate, oxygen flow rate, and moisture content. In a broader sense, this research is part of a long range program using X-Ray Microtomography (XMT) to determine to what extent the rate and practical recovery from heap leaching is limited by: (1) mineral exposure, (2) fluid flow/transport phenomena and (3) chemistry. It is expected that the results from the research will help to design heap leaching operations for more effective utilization of our mineral resources.
\end{abstract}

During this $1^{\text {st }}$ year, a three dimensional (3D) Lattice-Boltzmann Model (LBM) has been developed for one component fluid flow simulations through packed particle beds under totally saturated conditions. The software has been used to predict saturated permeability using real X-ray computed tomography (XMT) images of packed beds of sand, glass beads, and ore particles. Comparison with empirical equations as well as with experimental results has been done showing good agreement between theory and experiment. 


\section{TABLE OF CONTENTS}

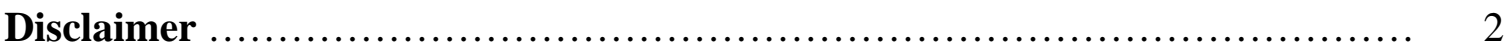

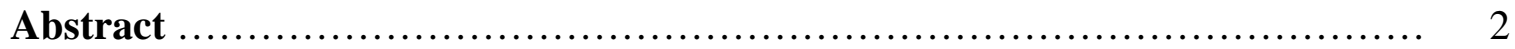

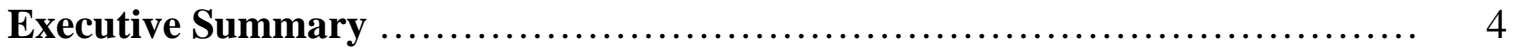

Introduction ................................................................. 5

Objectives and Approach................................................. 6

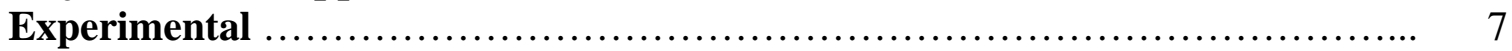

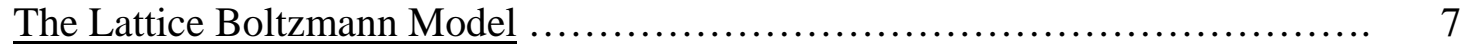

Overview of a Single Component LBM - The D3Q19 Model ................ 8

Software Development ................................................. $\quad 10$

Analytical Verification - Stokes Flow in a Pipe .......................... 13

Determination of Saturated Permeability using XMT Images $\ldots \ldots \ldots \ldots \ldots \ldots \ldots \ldots . . \ldots \ldots$

Darcy's Law .......................................................... 15

Experimental Set-Up ................................................ 16

LBM Permeability Estimation ........................................ 17

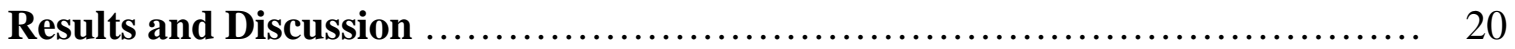

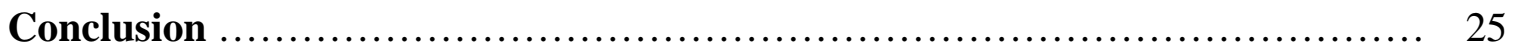

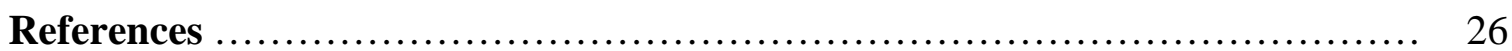

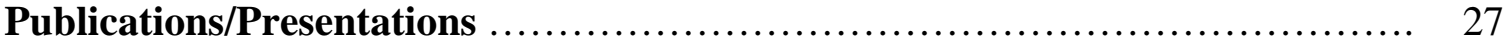




\section{EXECUTIVE SUMMARY}

The University of Utah in an effort to improve our understanding in the particle technology area and separation processes has developed an X-ray Microtomography facility in order to capture three dimensional (3D) images of real particle samples for further digital analysis. In particular, with the objective to improve the understanding for control of heap leaching operations, mathematical and physical modeling are indispensable for analysis of problems which can not be done on an experimental scale. In this regard, the 3D scientific visualization opens a complete new area of research where the porous structure of a packed particle bed of multiphase particles can be completely characterized and used for scaling up the micro phenomena that drive and control the whole leaching process of reactant transport, chemical reaction/dissolution and transport of solubilized products. In a broader sense, this research is part of a long range program using X-Ray Microtomography (XMT) to determine to what extent the rate and practical recovery from heap leaching is limited by: (1) mineral exposure, (2) fluid flow/transport phenomena and (3) chemistry. It is expected that the results from the research will help to design heap leaching operations for more effective utilization of our mineral resources.

A first step in this direction was to develop software capabilities of a complex model with mathematical and multi-physical phenomena such as being able to capture the basic phenomena of the whole process. In this regard, the Lattice Boltzmann Model (LBM) is an emergent mathematical technique able to handle the complex boundary conditions of flow in porous structures of actual ore samples and to simulate the process in a reasonable amount of time.

In fact, during this $1^{\text {st }}$ year of research under CAST II a single component model was constructed and the analytical verification with simple pipe flow was accomplished with excellent agreement between theory and experiment under laminar flow conditions. Familiarization with the method has been achieved and more complex tasks are about to start in the next phase of the research.

The same model has been modified and used for calculation and comparison of the permeability for packed beds of glass beads, sands and ore samples with different particle sizes. The results show again an excellent agreement between experimental results obtained from constant head experimental tests and those results obtained from LBM simulation of the flow using 3D images obtained from micro CT analysis of packed particle beds.

The next phase of the research during the $2^{\text {nd }}$ year under CAST III corresponds to the development of the multiphase Shan and Chen model. First, code parallelization will be required because intensive computing will need to be divised for several processors to make the memory requirements affordable and the time of simulation practical for the multiphase flow problem. Second, verification of the code with the analytical solution of Laplace's law will be done to verify the agreement with the surface tension model necessary to describe wetting and capillary filling of small pores. The verification of the interaction between solid and fluid phases will be done by verification with the analytical solution for the flow of two fluids of different viscosity/density in a pipe. And finally, the applicability of the method to analysis of actual packed particle beds will be determined by determination of a solid-water saturation curve for a simple sample of particles. 


\section{INTRODUCTION}

The fluid flow condition through the packed particle bed is an important consideration in the design and operation of the heap leaching process. In fact, for a given particle size distribution the moisture content, the geometry of the porous structure in the particle bed, and the local fluid velocity conditions will determine the extent of leaching and transport of solution from which the metal will be finally recovered.

Until recently it has not been possible to accomplish fluid flow analysis in such packed particle beds under partially-saturated conditions due to the lack of models to simulate multiphase (gas-liquid) flow behavior with very complex boundary conditions (pore network structure/geometry). Now such analysis may be possible by using X-Ray Micro CT (XMT) to capture the complex geometry involved in a particle bed structure and using Lattice Boltzmann methods (LBM) to simulate the fluid dynamic behavior of unsaturated flow.

The pore structure and the connectivity of the pore space are important features which determine fluid flow in a packed particle bed during heap leaching. In this regard, the X-Ray Microtomography technique is the best noninvasive nondestructive technology available today to characterize complex pore structures. XMT allows the capture of the shape and connectivity of the void space in three dimensions of a randomly organized particle bed through the 3D image reconstruction of the sample under analysis.

The image digitalization of the particle beds allows us to obtain the porous network structure characteristic of a given ore and particle size distribution. Computer simulation can then be used to calculate macro variables of the flow. However, in this regard the use of conventional FEM (Finite Element Method) or FDM (Finite Difference Method) for simulation of flow in the complex porous network structure of a porous media has not found practical application because the use of a small grid size is necessary making the computational time unacceptable.

In contrast to FEM or FDM methods, LBM has computational aspects that make it a particularly strong method for fluid flow simulations in complex pore structures due to its particular bottom-up approach. Also, it has recently been shown that LBM can simulate multi-phase (gas-solid-liquid) multi-component interactions with excellent results (Chen, 2003; Martys \& Chen, 1996; Shan \& Chen, 1993; Zhang \& Chen, 2003). However, so far applications have been largely limited to 2D problems or 3D fluid flow models under saturated conditions. This research will focus on the development and implementation of software able to handle 3D multiphase fluid flow under partially saturated conditions for use in the analysis and control of optimum conditions for recovery of copper and/or gold from heap leaching operations. It is expected that the software to be developed will have great utility and be extended to nano flow simulation, granular flow, and colloidal flow analysis. 


\section{OBJECTIVES AND APPROACH}

A two-year research program is in progress to develop a 3D Lattice Boltzmann Model for fluid flow simulation under partially-saturated conditions in packed particle beds in order to help identify optimum flow conditions for copper and/or gold recovery in heaps. This is the continuation of research developed by Dr. J.D. Miller and Dr. C.L. Lin using LBM in 3D problems in order to improve the understanding of the phenomena associated with fluid flow in packed particle beds (Lin \& Miller, 2004; Miller et al., 2003a; Miller et al., 2003b).

Development of the multiphase 3D LBM is a difficult and complex task. References and literature in this regard are limited. It is not expected to develop optimized and final-enduser software in the first approach. Instead, it is expected to develop as fast as possible a first prototype software to evaluate the capabilities and applicability of this technique for unsaturated flow assessment and characterization.

In order to accomplish the final goal the project has been divided into the following sequential steps: single component flow, surface interactions, and two-phase fluid flow. Each step consists of software development and experimental verification in order to gain confidence and assure reliability in the technique.

The first stage of the project involves the software development of a single component 3D flow model. This software will be the corner-stone for the development of the next stages and it will give us important insights about implementation issues. In this stage, the 3D simulation results will be compared to analytical solutions of Stokes-Flow in a pipe. Also, the 3D LBM simulation results through porous solids will be verified with Darcy's Law for flow in porous media. Real XMT image samples obtained from packed particle beds will be used in this regard.

The second stage will incorporate the wettability property of the fluid into the model. For this effect a fluid-solid interaction potential (Shan \& Chen, 1993) will be used. Verification with experimental data will be carried out.

The third stage of the research will involve the incorporation of an additional fluid into the model and description of the interface interactions. We need to simulate the interface between two fluids of significantly different viscosity as is the case of water and air. Up to this moment we are evaluating two different approaches: The multicomponent model as proposed by Shan and Chen (1993) and the Free Energy Method as explained by Swift et al. (1995, 1996). The final version of this software will be applied to complex 3D pore spaces in packed beds of irregularly shaped particles imaged from real samples using a unique highresolution cone-beam X-Ray Microtomography system (XMT) installed at the University of Utah. New insights regarding the relation between pore geometry, air ventilation, and water saturation are expected to be found. Further tests of the model will include verification of the ability to reproduce Darcy's Law in appropriate laminar flow regimes, saturated and unsaturated permeability measurements, and water potential/particle size/moisture content relationships. 


\section{EXPERIMENTAL}

Over the last few years, the Lattice Boltzmann Model (LBM) has become a promising numerical technique for the simulation of fluid flows under complex boundaries geometries. This property is making the LBM an important tool for research for the case of flow in porous media. It is also becoming popular for its capability to incorporate additional physical complexities, making multiphase flow problems computationally more feasible (Chen \& Doolen, 1998). Unlike the conventional Computer Fluid Dynamics (CFD), a 'top-down' approach based on discretization of macroscopic continuum equations, the LBM method is based on a 'bottom-up' approach where constructed kinetic models incorporate microscopic model interactions and mesoscopic kinetic equations so that the macroscopic averaged properties obey the desired macroscopic equations.

In the original lattice gas cellular automata (LGCA) model from which the LBM evolved, the mass and momentum are conserved locally in a discrete space-time model. The space is discretized in a lattice arrangement and particles are defined at each node of the lattice by a Boolean variable. During the streaming step, particles are only allowed to move one lattice unit along the lattice directions during each time step. During the collision step, two particles arriving at the same node collide and a collision rule is followed by the particles involved changing their directions but conserving local mass and momentum. Frish (Frish et al., 1986) found that sufficient symmetry of the lattice was necessary for the recovery of the Navier-Stokes equation at the macroscale. For example, in two dimensions a square lattice does not provide enough symmetry whereas hexagonal symmetry is sufficient to recover the Navier-Stokes equation.

In spite of the remarkable progress achieved in the LGCA, the difficulties related to lack of Galilean invariance, anomalous velocity dependency of the fluid pressure, statistical noise, exponential complexity and spurious invariants plus the interest to develop 3D models and high Reynolds numbers simulations led to the development of the LBM (Succi, 2001). The LBM evolved from the lattice gas cellular automata (LGCA) to overcome its difficulties but keeping many of the intrinsic advantages such as the locality equilibrium, space-time discretization, and the streaming and collision structure.

\section{The Lattice Boltzmann Model.}

The first LBM was developed by (Macnamara \& Zanetti, 1988). The fundamental difference between LGCA and LBM is that the later replaced the Boolean variable by a particle distribution function $f_{\alpha}$ which eliminates the statistical noise problem present at that moment in its ancestor. Later, Higuera and Jimenez (1989) made a great simplification when they linearized the collision term around the local equilibrium state by using a single relaxation term. The model using this type of relaxation term is known as the LBGK model for the Bhatnagar-Gross-Krook operator (Bhatnagar et al., 1954). The Bhatnagar-GrossKrook (BGK) approximation and discretization of the Boltzmann Equation leads to the following explicit equation: 


$$
f_{\alpha}\left(x+\vec{e}_{\alpha}, t+\Delta t\right)-f_{\alpha}(x, t)=-\frac{1}{\tau}\left[f_{\alpha}(x, t)-f_{\alpha}^{e}(x, t)\right]
$$

which is equivalent to:

$$
f_{\alpha}\left(x+\vec{e}_{\alpha}, t+\Delta t\right)=\left(1-\frac{1}{\tau}\right) f_{\alpha}(x, t)+\frac{1}{\tau} f_{\alpha}^{e}(x, t)
$$

where ,

$f_{\alpha}(x, t)$ : gives the probability of finding a particle with the velocity $\vec{e}_{\alpha}$ at lattice site $x$ at the time t.

$f_{\alpha}^{e}(x, t)$ : is the equilibrium distribution function.

$\tau \quad:$ is known as the relaxation time and controls the rate of approach to the dynamic equilibrium. Typical values are $0.5<\tau<2.0$. The lower limit is dictated by numerical stability conditions.

Currently, there are two different ways of showing that this discretization approximates the Navier-Stokes equations - either by the method of Chapman-Enskog expansion from statistical physics (Frisch et al., 1987), or by direct discretization of the Boltzmann equation as proposed by (He and Luo, 1997).

In the LBGK model the local equilibrium distribution is chosen in such a way that the micro-dynamics converge to the governing Navier-Stokes macroscopic equations. Qian (Qian et al., 1992) provided a whole family of solutions for this matter. We have selected the D3Q19 model for use in this research because it is a good compromise between stability and performance.

Overview of a Single Component LBM - The D3Q19 Model.

The D3Q9 model denomination comes from the three dimensional lattice representation and nineteen velocities according with Qian's notation. Depending on the dimensions and the number of velocity directions different models can be used. Figure 1 shows the sketch of a D3Q19 model.

The D3Q19 model consists of nineteen velocity vectors where one is in the center, six along the axis and twelve for all combinations of two axes. For each velocity vector a particle distribution function (DF) $f_{\alpha}$ is stored where the DF represents the amount of fluid molecules moving in the direction $e_{\alpha}$. Therefore, in the D3Q19 model there are particles not moving at all $\left(f_{0}\right)$, moving with speed $1\left(f_{2}, f_{3}, f_{4}, \ldots f_{7}\right)$ and moving with speed $\sqrt{2}$ $\left(f_{8}, f_{9}, f_{10}, \ldots f_{19}\right)$. This is particularly important in terms of computational requirements because each node of the lattice will need to store at least 19 float variables with the population moving in each velocity for each component, therefore a three dimensional problem is very computationally demanding. 


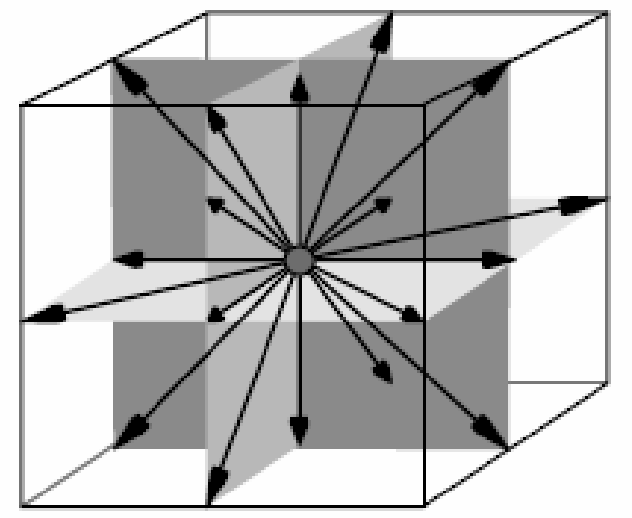

D3Q19
Velocity vectors:

$$
\begin{aligned}
& \mathrm{e}_{1}=(0,0,0) \\
& \mathrm{e}_{2,3}=(+/-1,0,0) \\
& \mathrm{e}_{4,5}=(0,+/-1,0) \\
& \mathrm{e}_{6,7}=(0,0,+/-1) \\
& \mathrm{e}_{8 . .11}=(+/-1,+/-1,0) \\
& \mathrm{e}_{12 . .15}=(+/-1,0,+/-1) \\
& \mathrm{e}_{16 . .19}=(0,+/-1,+/-1)
\end{aligned}
$$

Figure 1. Sketch of a three dimensional lattice with nineteen velocities ( D3Q19).

As mentioned above, the LBM consist of two basic steps, streaming and collision. The streaming step represents the advection of the particles in the fluid and if the size of a cell $\Delta x$ and the length of the time step $\Delta t$ are normalized to 1 (as is usually the case for computing practice), the streaming $\mathrm{DF}\left(f_{\alpha}^{\mid S}\right)$ can be easily represented by copying each DF to its adjacent cell along the corresponding velocity vector.

$$
f_{\alpha}^{\mid S}(x, t+\Delta t)=f_{\alpha}\left(x+e_{\alpha}, t\right)
$$

The collision step describes the evolution of a system towards equilibrium by relaxing the streamed DFs of a cell towards the equilibrium distribution function. It has been shown that LBGK approximation with a Maxwell-Boltzmann equilibrium distribution recovers the Navier-Stokes equations at low Mach number in the equilibrium limit (He \& Luo, 1997). For a D3Q19 lattice, a second order expansion of the equilibrium distribution function is commonly used:

$$
f_{\alpha}^{e q}(x)=w_{\alpha} \rho(x)\left[1+3 \frac{\vec{e}_{\alpha} \bullet \mathbf{u}}{c^{2}}+\frac{9}{2} \frac{\left(\vec{e}_{\alpha} \bullet \mathbf{u}\right)^{2}}{c^{4}}-\frac{3}{2} \frac{\mathbf{u}^{2}}{c^{2}}\right] \text {, for } \alpha 1,2,3 \ldots .19 . \quad \text { (equation 4) }
$$

Where,

$w_{\alpha} \quad$ : Weight values depending on the velocity direction. For the D3Q19 model they are defined as

$$
w_{1}=1 / 3
$$




$$
\begin{array}{ll}
w_{\alpha}=1 / 18 & \text { for } \alpha \mathrm{i}=2, . .7 \\
w_{\alpha}=1 / 36 & \text { for } \alpha=8, \ldots 19
\end{array}
$$

$c \quad$ : The lattice speed given by $\Delta x_{\text {lattice }} / \Delta t_{\text {lattice }}$. For simplicity it is defined as 1 .

$\rho(x)$ : The macroscopic fluid density at the cell $x$.

u : The macroscopic fluid velocity at the cell $x$.

This functional form of the equilibrium distribution assures that up to the fourth order momentum equations are identical to the respective velocity moments over the Maxwell distribution (Wolf-Gladrow, 1991). Also, the LBGK model defined as shown above assures mass and momentum conservation laws.

During iteration the macroscopic density and velocity fluid variables are computed at each node as the first and second momentum of the distribution functions. Then,

$$
\rho=\sum_{\alpha=1}^{19} f_{\alpha} \quad \text { and } \quad u=\sum_{\alpha=1}^{19} e_{\alpha} f_{\alpha}
$$

Moreover, the following relations are associated with the pressure $(P)$ and kinematic shear viscosity $(v)$ according with the following formulas:

$$
\begin{aligned}
& P=\frac{\rho}{3} \\
& v=\frac{1}{3}\left(\tau-\frac{1}{2}\right)
\end{aligned}
$$

More details about the LBM, treatment of boundary conditions, applications and techniques can be found at (Succi, 2001).

\section{Software Development}

3D software has been developed for analysis of fluid flow through porous media. The software is based on a single component Lattice Boltzmann Model as explained previously. The software is composed of two major structures: the 3D LBM software and a SILO translator. The former is the main core of the program and it performs the three dimensional Lattice Boltzmann simulation. The latter uses the output files of the 3D LBM to make a SILO format file which can be finally imported from the open source VISIT platform for Scientific Visualization. The major structure of the code is shown in Figure 2. 


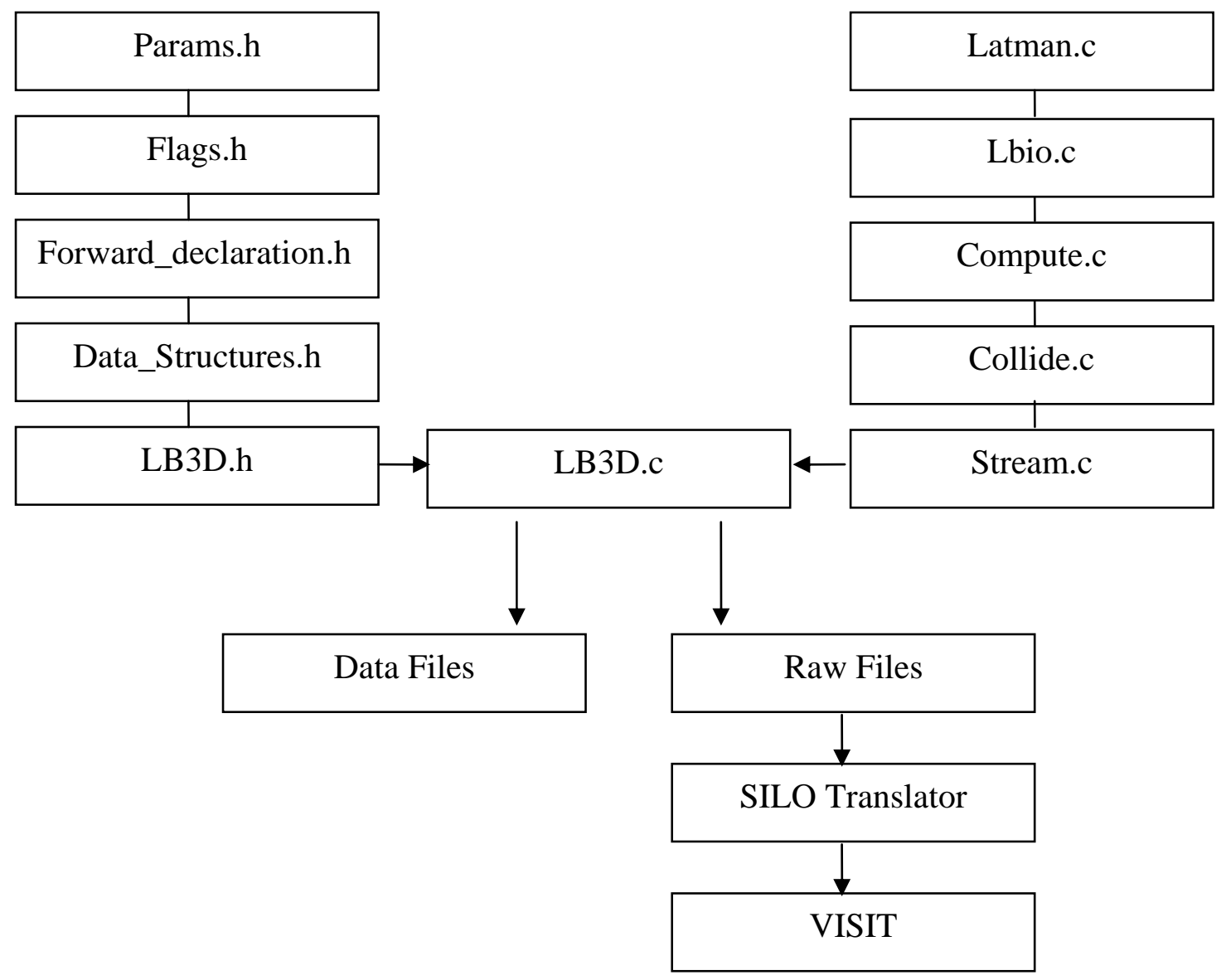

Figure 2. Software structure for flow simulation.

The sequential process for the iteration of the 3D LBM is shown in the following pseudo-code below. First the memory is allocated and the problem is initialized based on the initial condition of velocity and density which are evaluated in the equilibrium distribution functions for this matter. Then the iterative process of streaming and collision is executed until convergence or until the maximum step iteration is reached. Macro variables, such as velocity components and density, are computed based on the new values of the velocity distribution function after streaming during each time step. Then the resulting macro variables for each node in the lattice are saved in files from which the data can be visualized for further analysis. 
Pseudo Code for 3D LB Computation

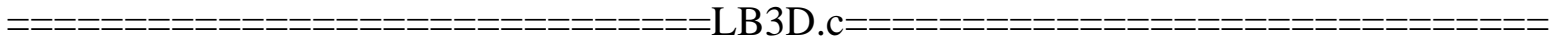
\#include "LB3D.h"

int main (int argc, char **argv)

\{

Locate memory and initialize variables.

Iteration: If maximum iteration or convergence has not been reached continue \{

Execute streaming step.

Compute macro variables such as density and velocity.

Execute collision step.

Add the volume -force to mimic pressure gradient.

Save outputs files.

\}

Free memory.

Return 0.

\}

Due to the collision step, the resulting distributions are modified with the volume force component so as to mimic the equivalent gradient pressure used to force the flow in a particular direction as shown in equation 8.

$$
\rho u=\sum_{i=1}^{19} f_{i} e_{i}+\tau g
$$




\section{Analytical Verification - Stokes Flow in a Pipe}

The Stokes Flow through a pipe has a well-known analytical solution. For this reason it was selected to verify the function of the software. The fluid enters the pipe from the left side and exits to the right under the effect of a constant pressure drop $(\Delta P)$ between the inlet and outlet section as shown in figure 3.
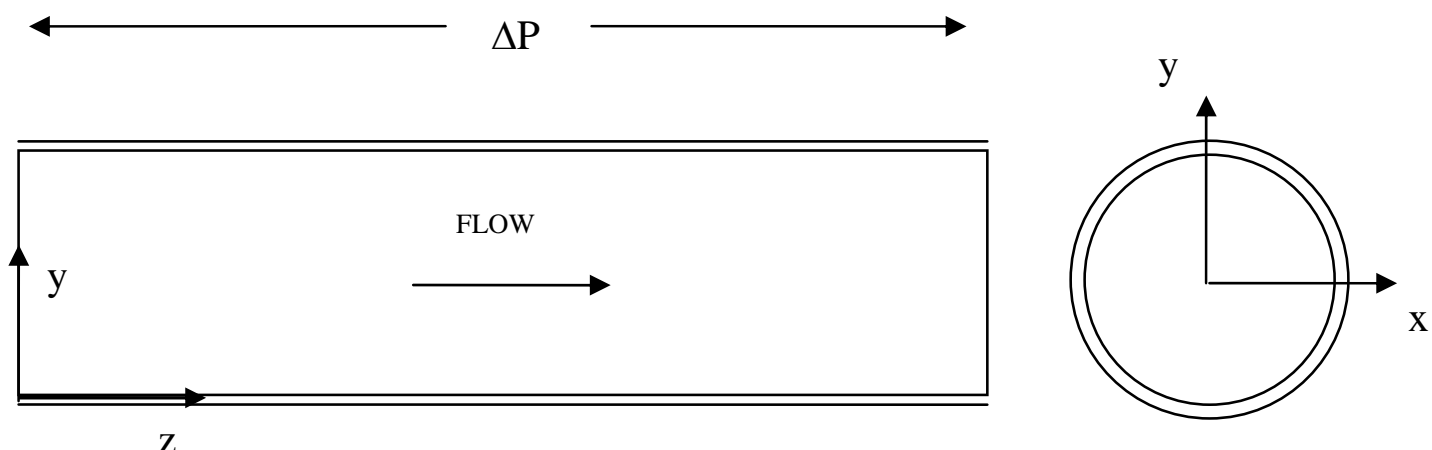

Figure 3. Laminar flow in a pipe.

A non-slip boundary condition is assumed at the walls. Therefore, the fluid velocity is assumed to be zero at the boundary walls because of the drag force of the wall exerted on the fluid. Under moderate-to-low Reynolds number flow conditions the solution is easily determined and it is given by (Bird et al., 2002):

$$
V_{x}(y)=V_{\max }\left[1-\left(\frac{y}{R}\right)^{2}\right]
$$

With,

$$
V_{\max }=\frac{R^{2} \Delta P}{4 \mu L}
$$

Where,

$$
\begin{array}{ll}
V_{x}(y) & : \text { Velocity in the } \mathrm{x} \text { direction at } \mathrm{y} \text { position. } \\
V_{\max } & : \text { Maximum velocity in the } \mathrm{x} \text { direction. } \\
R & \text { : Radius of the pipe. } \\
L & \text { : Length of the pipe } \\
\mu & \text { : Dynamic viscosity. }
\end{array}
$$

Since in the LBM presented above the pressure depends on the density (see equation 6) the model is considered a non exact incompressible method. To simulate a change in 
pressure requires a change in density. Therefore this LBM is known as a weakly compressible model. In this type of model, we can generate the gradient pressure with the application of an equivalent volume force added to the DFs designed in such a way to produce the same momentum input to the flow as the true gradient. This volume-force model does not allow us to recover an exact Reynolds number and/or the internal structure of the pressure field point-wise, however it is extremely efficient to recover the universal behavior of the fluid system in the range of the Reynolds numbers to be studied.

Figure 4 shows a comparison between the analytical solution of the pipe flow problem described above and the result for a simulation in a $64 \times 64 \times 64$ lattice. Because of the symmetry of the problem Figure 4 only shows the profile of the velocity along a line perpendicular to the flow. As is evident there is very good agreement between the analytical solution and the results from the 3D LBM simulation for low Mach number and low Reynolds number conditions.

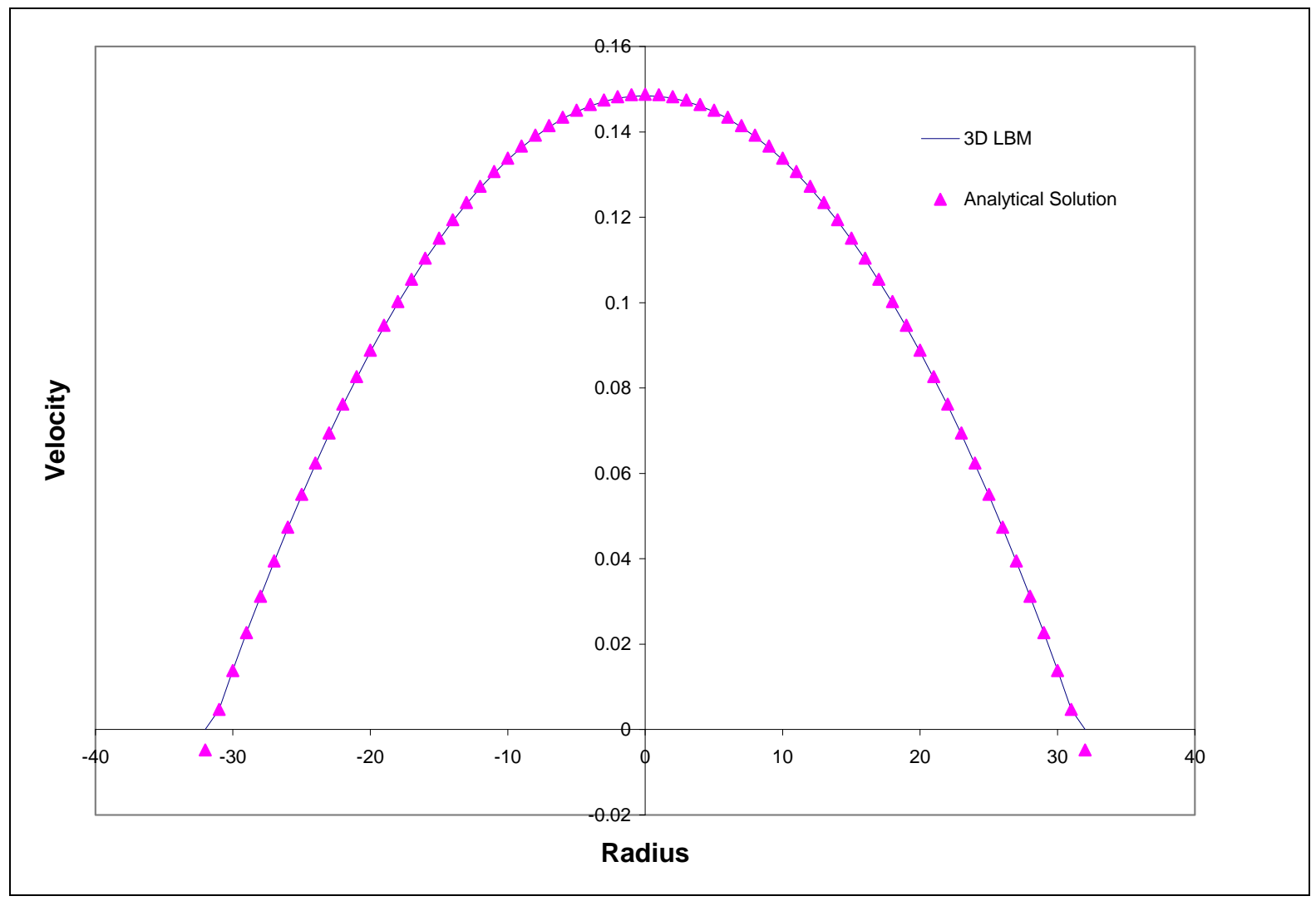

Figure 4. Comparison between analytical solution and a 3D LBM simulation for a 64x64x64 lattice. 


\section{$\underline{\text { Determination of Saturated Permeability using XMT Images }}$}

In this part of the research the utility of the 3D LBM to predict saturated permeability for packed particle beds is evaluated. In this regard, different particle samples of sand, glass beads, and ore (identified as C4) have been used for X-ray tomography analysis. The reconstructed 3D image is then used for LBM flow simulation to calculate the expected permeability based on the porous structure of the sample. The samples used for analysis have porosities from $25 \%$ to $40 \%$. Table 1 shows the particle size characteristics of the samples used in this study.

\begin{tabular}{cccc} 
Sample & $\begin{array}{c}\text { Dmin } \\
\mu \mathrm{m}\end{array}$ & $\begin{array}{c}\text { Dmax } \\
\mu \mathrm{m}\end{array}$ & $\begin{array}{c}\text { Diameter } \\
\mu \mathrm{m}\end{array}$ \\
\hline Filtersand & 300 & 425 & 357.07 \\
Limestone & 710 & 2000 & 1191.64 \\
Acusand & 420 & 595 & 499.90 \\
& & & \\
C4 & 2000 & 3175 & 2519.92 \\
C4 & 420 & 2000 & 916.52 \\
C4 & 149 & 420 & 250.16 \\
& & & \\
glass beads & 210 & 250 & 229.13 \\
glass beads & 300 & 420 & 354.96 \\
glass beads & 420 & 600 & 502.00
\end{tabular}

Table 1. Particle samples.

Darcy’s Law.

Darcy's law established that the flow rate passing through a granular material is proportional to the constant cross sectional area, proportional to the difference in piezometric head and inversely proportional to the length of the sample. A formal equation of Darcy's law is given by (Bear, 1972):

$$
\begin{aligned}
& Q=K A \frac{\left(\varphi_{1}-\varphi_{2}\right)}{L} \\
& \varphi_{i}=z_{i}+\frac{p_{i}}{\rho g}
\end{aligned}
$$

and,

$$
K=\frac{k g}{v}
$$

Where,

$Q$ is the flow rate in [cc/sec]. 
$A$ is the cross sectional area $\left[\mathrm{cm}^{2}\right]$.

$\frac{\left(\varphi_{1}-\varphi_{2}\right)}{L}$ is the hydraulic gradient [non dimensional].

$K$ is the hydraulic conductivity and has units [cm/sec].

$k$ is the permeability coefficient of the porous matrix $\left[\mathrm{cm}^{2}\right]$

\section{Experimental Set-Up}

The experimental determination of the coefficient of permeability is done by a constant-head method for laminar flow through granular samples. The experimental set-up follows the recommendations given in ASTM D 2434 "Standard Test Method for Permeability of Granular Soils".

A cylindrical container of around $10 \mathrm{~cm}$ length and $2.6 \mathrm{~cm}$ in diameter holds the sample which is fitted between two screens to contain the sample. A fiber of high porosity is attached to each screen to prevent movement of particles. The top screen has a spring attached for applying a light pressure load to hold the placement density and volume of the sample. The system consists of 60 cc syringes as containers and $\mathrm{CO}_{2}$ has been used for filling the void space before saturation with water. Figure 5 shows a diagram of the experiment.

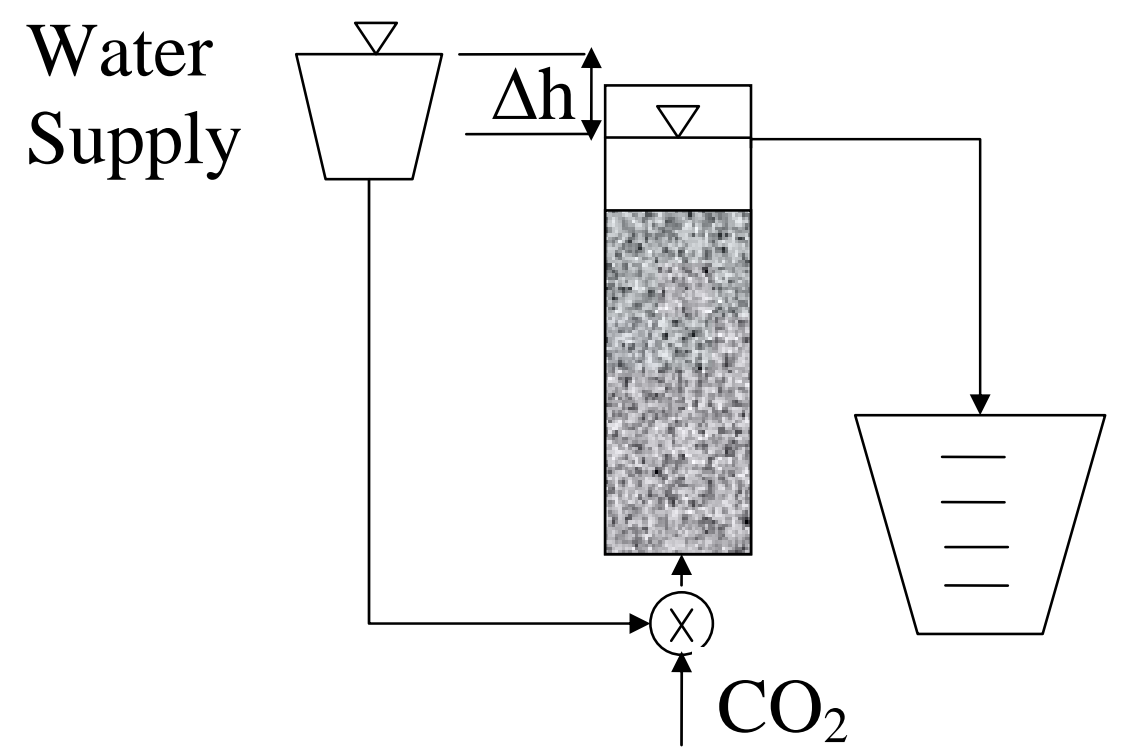

Figure 5. Test apparatus for permeability measurements. 
A constant head of water was kept on the inlet and the outlet of the sample so the head difference was constant during the experiment. The diameter and length of the sample were also measured. Experiments were run at different head differences but being careful to keep a laminar flow condition. The flow was driven with different hydraulic gradients, so a line could be fitted for the different flow rate v/s hydraulic gradient data. Once the hydraulic conductivity was calculated from the slope of the line, then the permeability was calculated from its relation with hydraulic conductivity according to equation 20. Because the fluid used was water at $20 \mathrm{C}$ degrees, the viscosity used for calculation was 1.0 cPoise.

\section{LBM Permeability Estimation}

Calculation of permeability from a 3D image captured with XMT analysis requires the simulation of fluid flow through the porous structure of the particle bed. In this regard, it is important to recall that the properties of the fluid are not important because the permeability is a characteristic of the tortuous path that the flow must follow through the particle bed and not a property which will depend on the fluid characteristics. Because of the latter, the flow simulation can be done with any type of fluid. Therefore in all our simulations the fluid properties are defined by a relaxation time of one given a lattice viscosity of 0.166 lattice units.

The flow is assumed to be vertical and it is forced for a constant pressure-model through the application of a volume force. Since the flow is at steady state and at a constant pressure, then the volume force can be balanced to the pressure according to the following equation:

$$
g_{x} \rho L^{3}=\left(P_{\text {in }}-P_{\text {out }}\right) * L^{2}=-\frac{\Delta P}{\Delta x} * L^{3}
$$

Then by substitution in equation (10) and (12) we get

$$
k=-\left(\frac{q \mu}{d P / d x}\right)=\frac{q v}{g}
$$

In LBM, the initialization is not critical for steady-state flows because the system will converge to that condition whatever the initial condition. Therefore, the initial conditions in all our simulations are set with velocities equal to zero and density equal to one in the whole domain.

The flow is constrained to the left and right sides with an artificial box, and the flow is only allowed in one direction, where the bottom and top are open. The flows in the inlet and outlet are treated with a periodic boundary condition to allow the departing particle to reenter the flow domain. The first four layers at the bottom and top of the flow direction are 
empty and they are initialized with fluid at the beginning of the simulation so the periodic boundary condition can be applied.

The standard bouncing back boundary condition is applied at the solid boundaries, including the artificial container around the porous matrix. The standard bounce-back scheme requires that when a particle distribution streams to a solid boundary node it is scattered back to the node from which it came. This treatment is independent of the direction of the particle distribution and is very easy to implement.

Once the flow reaches steady state, the flow rate $(q)$ can be calculated from the average of the flow velocity along the flow direction and then the permeability can be calculated according to equation 14 using the viscosity and gravity force values used for the simulation. Since the lattice permeability needs to be translated to physical units $\left[\mathrm{cm}^{2}\right]$, the calculated lattice permeability has to be scaled to physical units with a relationship given by the following equation

$$
k_{\text {physical }}=k_{\text {lattice }} * L_{x}^{2}
$$

Where,

$L_{x}$ is the voxel resolution of the image, usually $20 \mu \mathrm{m}$.

During the LB simulation, the particles of the single fluid enter the porous media from the inlet face and flow towards the outlet face. The flow occurs through the connected parts of the porous structure and the simulation is finished when the flow achieves steady state or the time of simulations reaches 5000 iterations, whichever happens first.

Figure 6 shows the results of a 128x128x128 glass bead sample used to calculate the permeability under saturated flow. The vectors shown in red display the path followed by the liquid around the solid particles. It is evident that the simulation is able to capture the underlying physics of the system. 

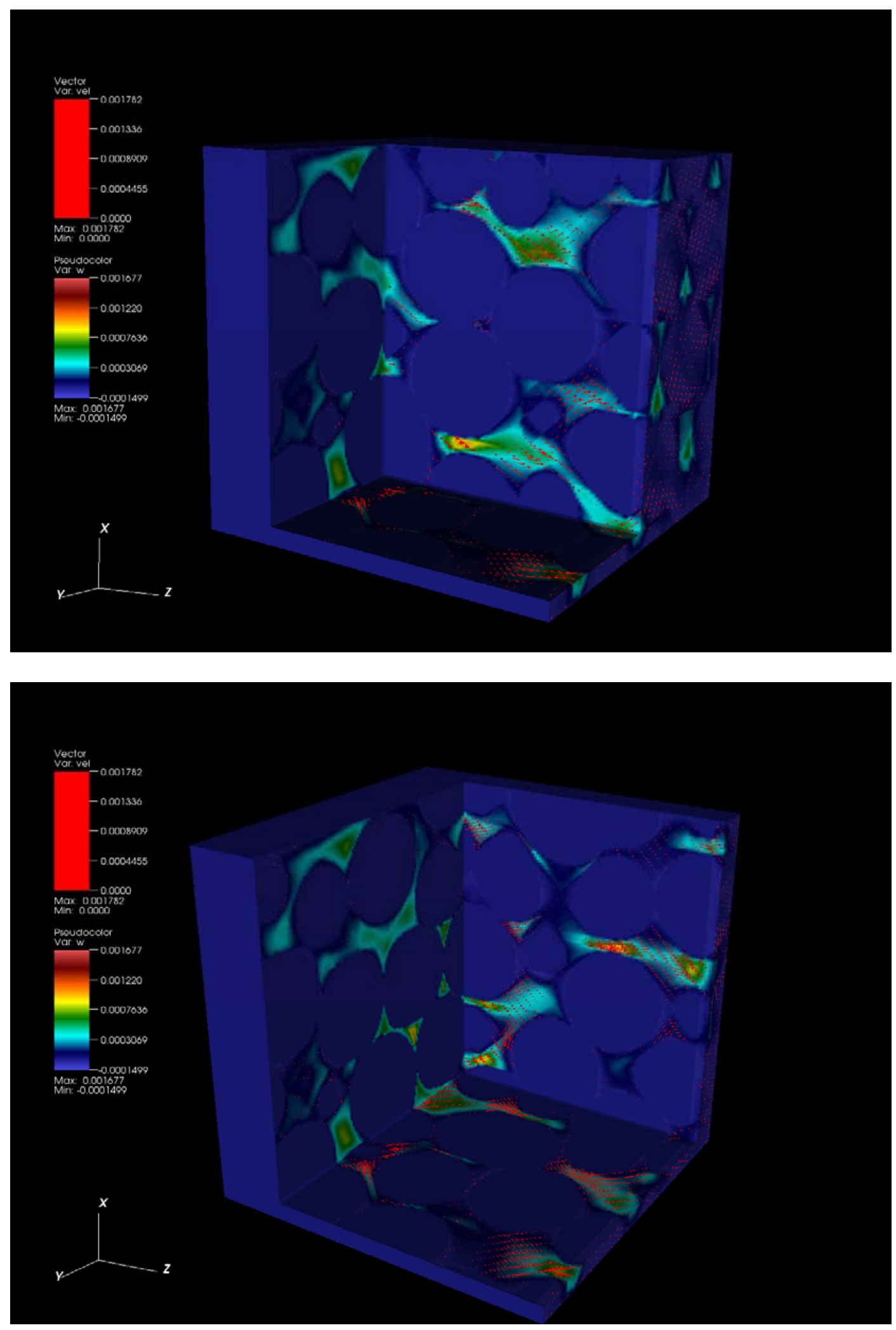

Fig 6. Velocity profiles along the flow direction (left to right) in a 3D XMT image. The vectors (in red) show the flow of the fluid through the pore network structure of glass beads. 


\section{RESULTS AND DISCUSSION}

We have made several permeability measurements from LBM simulations and from experimental tests. The methods have been explained above and here we will show a comparison between these results.

Figure 7, 8, 9 and 10 show the experimental results used for calculation of the hydraulic conductivity for Limestone, Acusand, Filtersand, and Ore. The slope corresponds to the hydraulic conductivity measured from different driving pressure gradients. The permeability is calculated using equation 12 for water at $20 \mathrm{C}$ degrees (viscosity equals 1 cPoise). The correlation value is excellent showing good agreement between theory (Darcy's Law) and experimental tests.

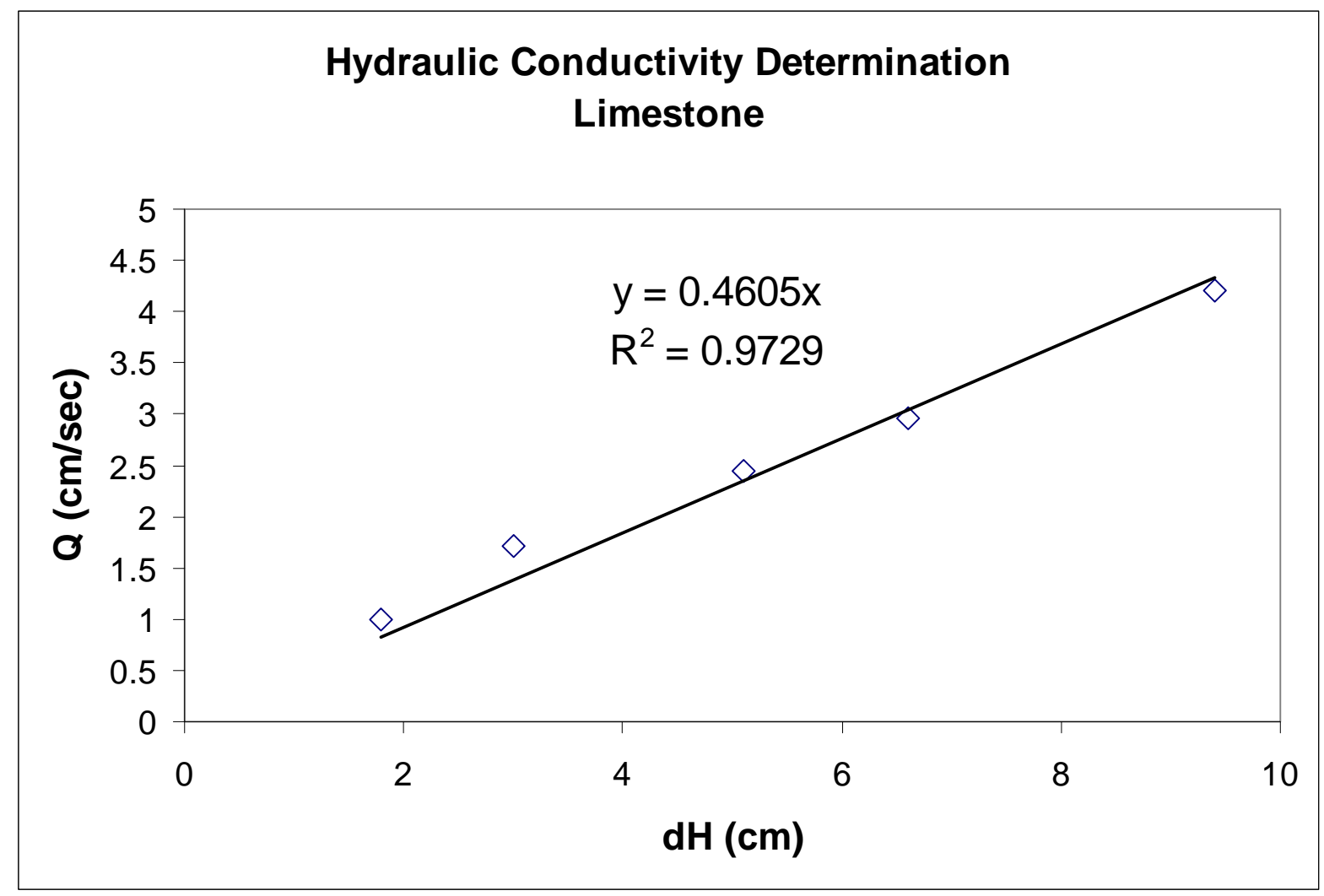

Figure 7. Relation between flow rate and pressure differential (cm of water) for Limestone. 


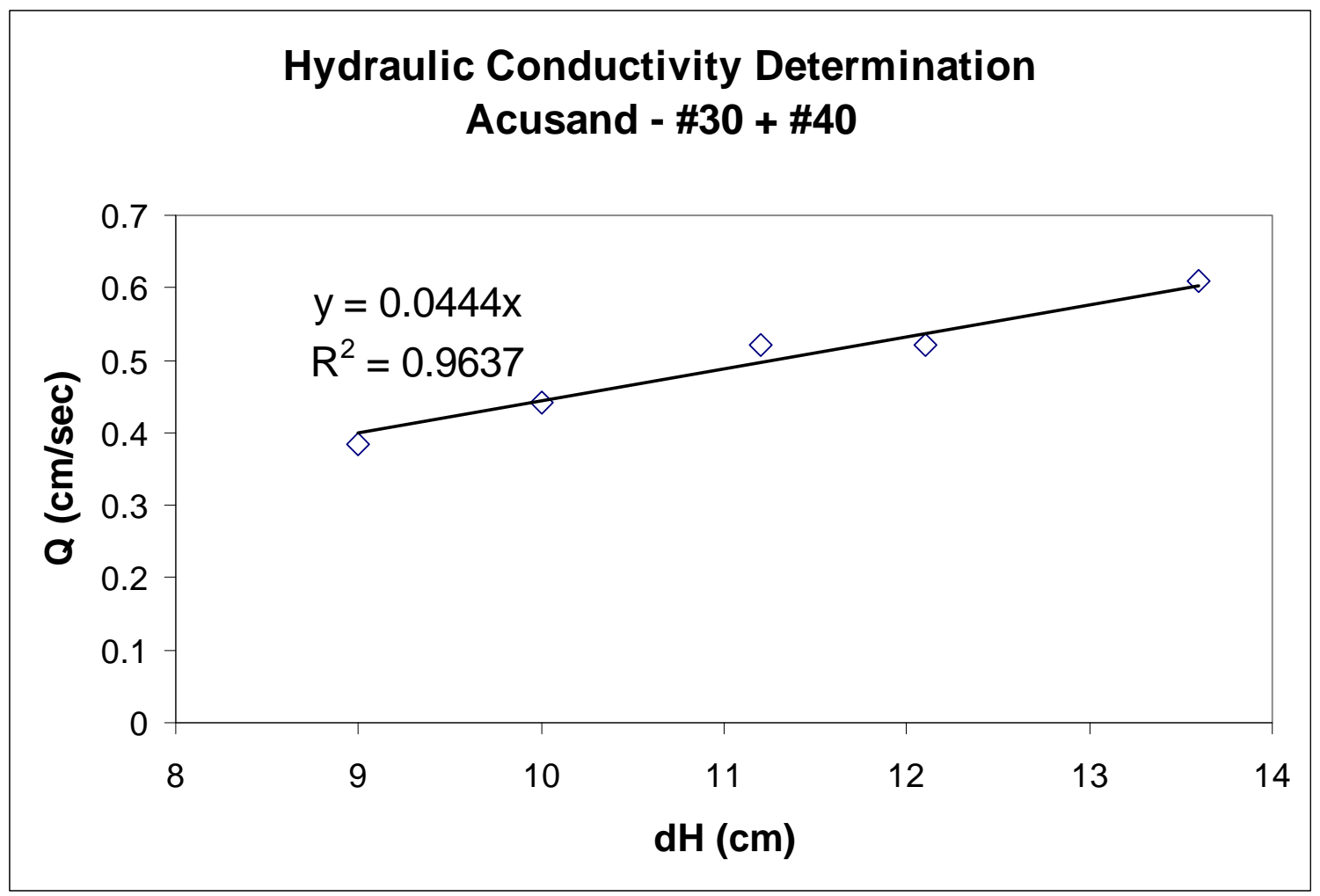

Figure 8. Relation between flow rate and pressure differential (cm of water) for Acusand.

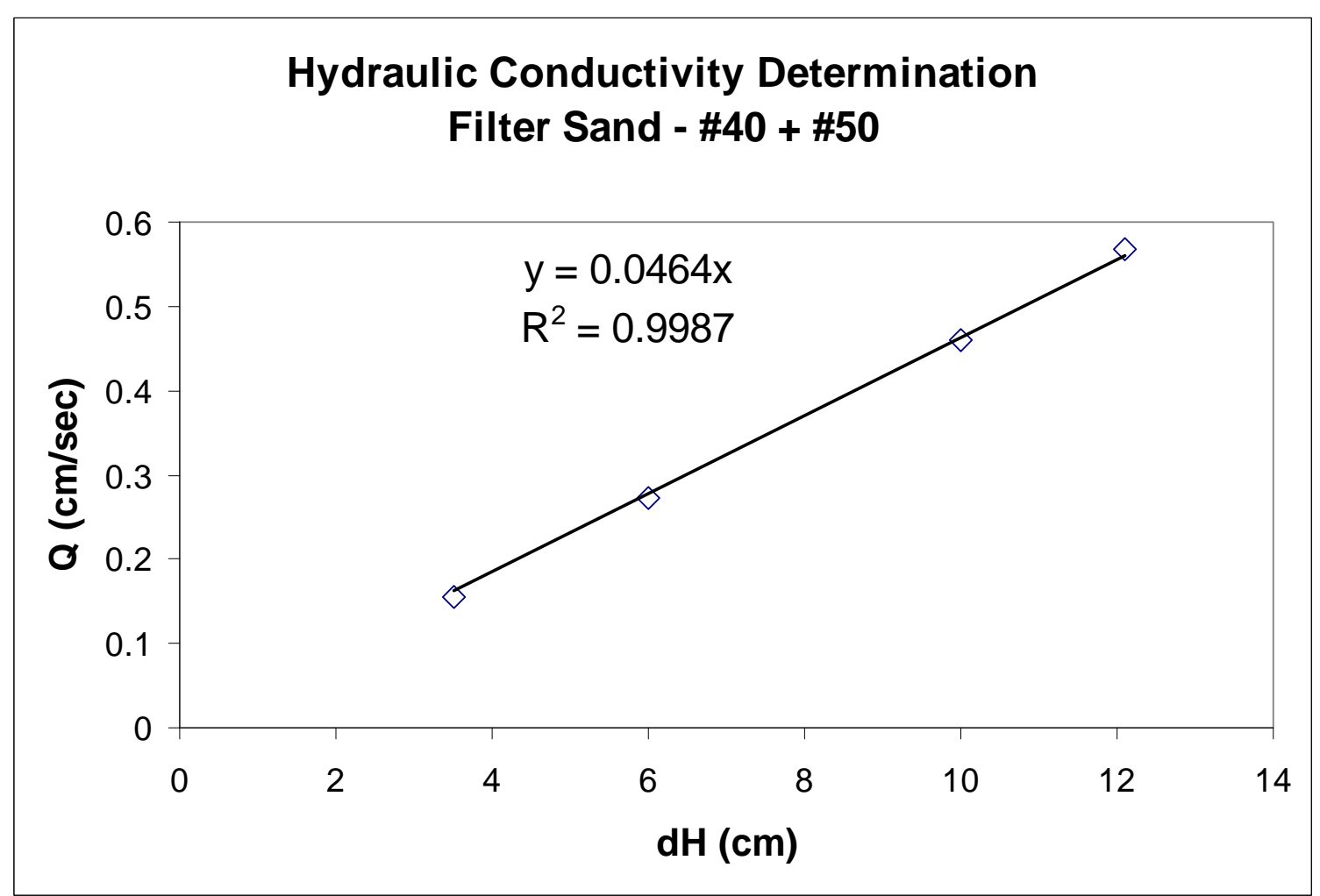

Figure 9. Relation between flow rate and pressure differential (cm of water) for Filter Sand. 

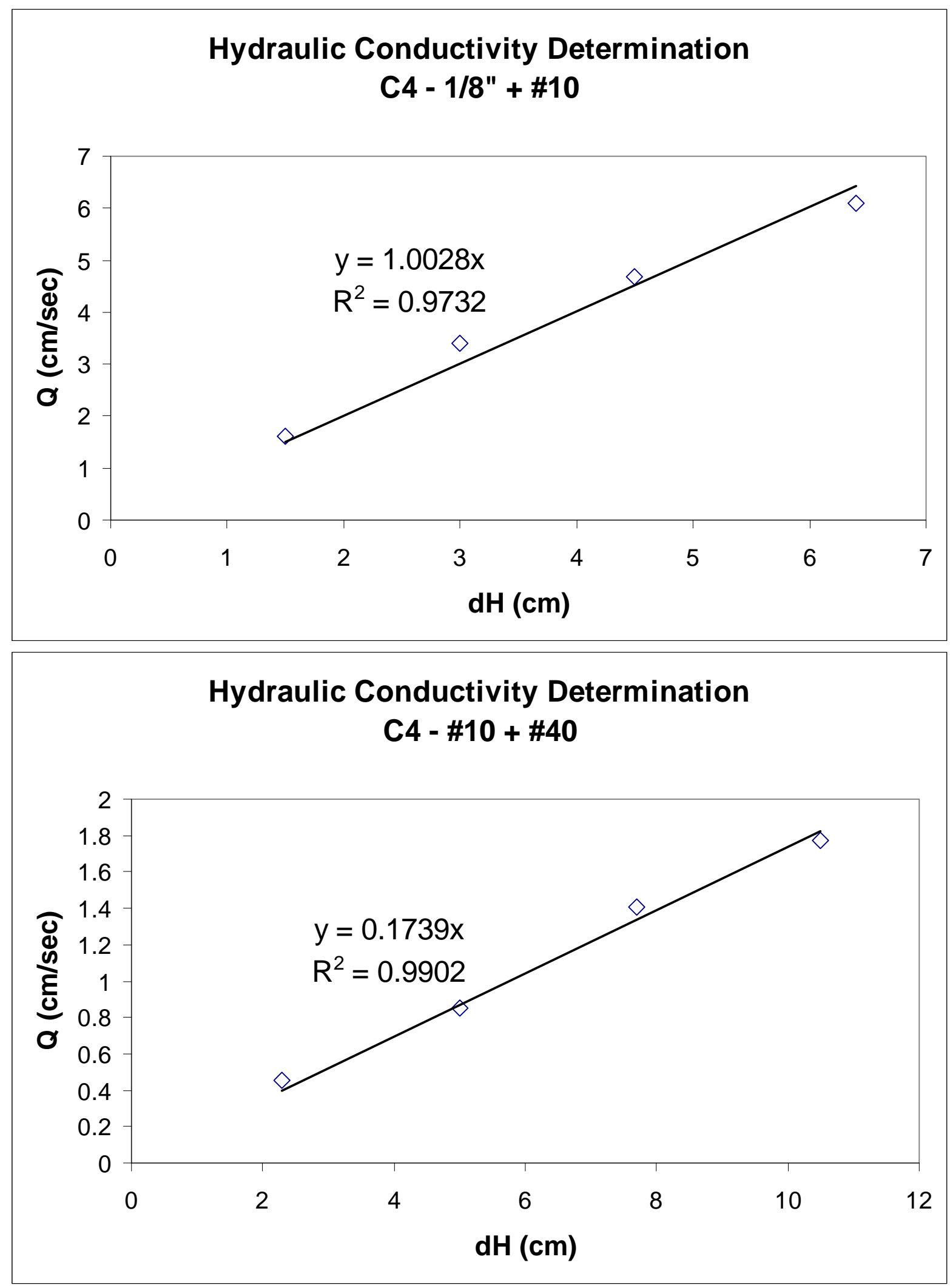

Figure 10. Relation between flow rate and pressure differential (cm of water) for different particle sizes of ore. 


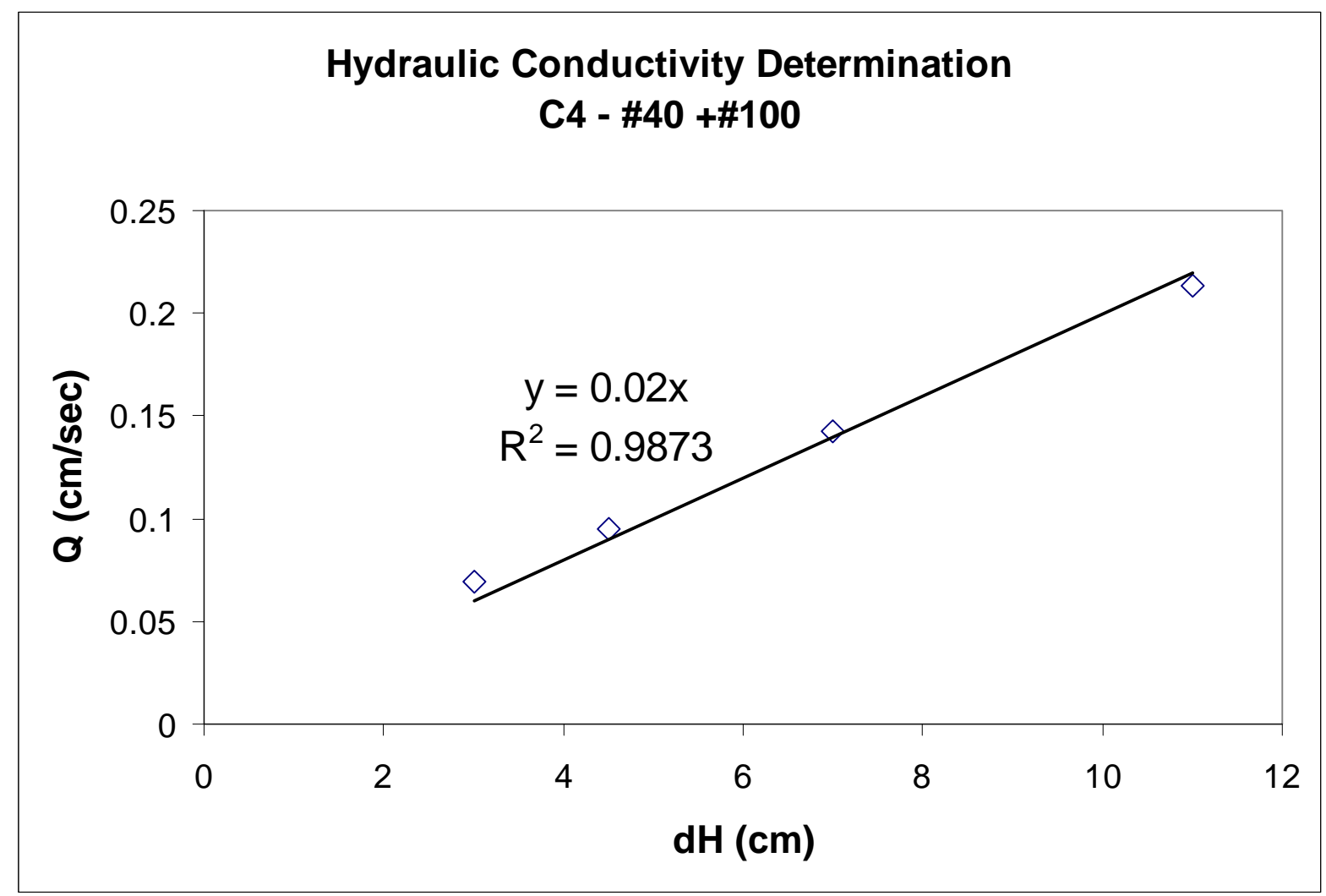

Figure 10 (continued). Relation between flow rate and pressure differential (cm of water) for different particle sizes of ore.

The permeability obtained from LBM simulation tends to change with the size of the sample and the resolution of the image. A compromise must be found between resolution and complexity, because a better resolution increases enormously the number of voxels to be used for getting a representative volume size of the sample. In this regard, a $20 \mu \mathrm{m}$ resolution has been a good compromise for most of the samples under analysis. When the amount of sample is very small as in the case of the glass beads which vary in size from 210 to $250 \mu \mathrm{m}$, then a $10 \mu \mathrm{m}$ resolution has been used.

Depending on the particle size, the size of the images used for simulation varies from $128 x 128 x 128$ voxels to $256 x 256 x 256$ voxels. To reduce the effect of the anisotropy, the permeability of the flow is simulated in the three major directions $\mathrm{x}, \mathrm{y}$ and $\mathrm{z}$. Table 2 shows a summary of the result of the simulations. The resolution of the voxels and the particle sizes are indicated for each sample. The porosity corresponds to the fraction of void space measured from the 3D image. 


\begin{tabular}{cccccccccc} 
Sample & $\begin{array}{c}\text { Resolution } \\
\mu \mathrm{m}\end{array}$ & $\begin{array}{c}\text { Dmin } \\
\mu \mathrm{m}\end{array}$ & $\begin{array}{c}\text { Dmax } \\
\mu \mathrm{m}\end{array}$ & $\begin{array}{c}\mathrm{K}_{\mathrm{x}} \\
\text { Porosity }\end{array}$ & $\begin{array}{c}\mathrm{K}_{\mathrm{y}} \\
\left(\mathrm{cm}^{2}\right)\end{array}$ & $\begin{array}{c}\mathrm{K} \\
\left(\mathrm{cm}^{2}\right)\end{array}$ & $\begin{array}{c}\left.\mathrm{cm}^{2}\right) \\
\left(\mathrm{cm}^{2}\right)\end{array}$ \\
\hline Filtersand & 20 & 300 & 425 & 0.421 & $1.393 \mathrm{E}-06$ & & $1.928 \mathrm{E}-06$ & $1.660 \mathrm{E}-06$ & $200 \times 200 \times 200$ \\
Acusand & 20 & 420 & 595 & 0.379 & $1.014 \mathrm{E}-06$ & $1.143 \mathrm{E}-06$ & $1.144 \mathrm{E}-06$ & $1.100 \mathrm{E}-06$ & $200 \times 200 \times 200$ \\
Limestone & 20 & 710 & 2000 & 0.392 & $8.300 \mathrm{E}-06$ & $9.570 \mathrm{E}-06$ & $1.020 \mathrm{E}-05$ & $9.357 \mathrm{E}-06$ & $256 \times 256 \times 256$ \\
C4 & 20 & 149 & 420 & 0.323 & $2.015 \mathrm{E}-07$ & $2.711 \mathrm{E}-07$ & $2.368 \mathrm{E}-07$ & $2.365 \mathrm{E}-07$ & $256 \times 256 \times 256$ \\
C4 & 20 & 420 & 2000 & 0.367 & $3.406 \mathrm{E}-06$ & $4.580 \mathrm{E}-06$ & $4.910 \mathrm{E}-06$ & $4.299 \mathrm{E}-06$ & $256 \times 256 \times 257$ \\
C4 & 20 & 2000 & 3175 & 0.407 & $2.133 \mathrm{E}-05$ & $2.078 \mathrm{E}-05$ & $1.767 \mathrm{E}-05$ & $1.993 \mathrm{E}-05$ & $256 \times 256 \times 256$ \\
glass beads & 10 & 210 & 250 & 0.363 & $4.288 \mathrm{E}-07$ & $4.515 \mathrm{E}-07$ & $4.556 \mathrm{E}-07$ & $4.453 \mathrm{E}-07$ & $256 \times 256 \times 256$ \\
glass beads & 20 & 300 & 420 & 0.293 & $6.630 \mathrm{E}-07$ & $6.954 \mathrm{E}-07$ & $6.849 \mathrm{E}-07$ & $6.811 \mathrm{E}-07$ & $128 \times 128 \times 128$ \\
glass beads & 20 & 420 & 600 & 0.388 & $2.275 \mathrm{E}-06$ & $2.425 \mathrm{E}-06$ & $2.459 \mathrm{E}-06$ & $2.386 \mathrm{E}-06$ & $128 \times 128 \times 128$
\end{tabular}

Table 2. Permeability obtained from the LB simulations in the $\mathrm{x}, \mathrm{y}$ and $\mathrm{z}$ directions. $\mathrm{K}$ is the average value obtained from the three major directions.

Table 3 shows a comparison between the average permeability calculated from LB simulations and the experimental value obtained from the tests as explained above. The relative error decreases for coarser particle sizes because the permeabilities are higher and the relative error is less sensitive for these differences. In general, good agreement is obtained between the LB simulation and the experimental values even for irregular particle shapes. Figure 11 shows the results as a function of particle size. The agreement is good for a wide spectrum of particle sizes.

\begin{tabular}{ccccc} 
Sample & $\begin{array}{c}\text { Average } \\
\text { Diameter } \\
\mu \mathrm{m}\end{array}$ & $\begin{array}{c}\mathrm{K}_{\mathrm{LB}} \\
\left(\mathrm{cm}^{2}\right)\end{array}$ & $\begin{array}{c}\mathrm{K}_{\text {Experimental }} \\
\left(\mathrm{cm}^{2}\right)\end{array}$ & $\begin{array}{c}\text { Relative } \\
\text { Error }\end{array}$ \\
\hline Filtersand & 357 & $1.660 \mathrm{E}-06$ & $9.449 \mathrm{E}-07$ & $76 \%$ \\
Acusand & 500 & $1.100 \mathrm{E}-06$ & $9.212 \mathrm{E}-07$ & $19 \%$ \\
Limestone & 1192 & $9.357 \mathrm{E}-06$ & $9.024 \mathrm{E}-06$ & $4 \%$ \\
C4 & 250 & $2.365 \mathrm{E}-07$ & $4.034 \mathrm{E}-07$ & $41 \%$ \\
C4 & 917 & $4.299 \mathrm{E}-06$ & $3.475 \mathrm{E}-06$ & $24 \%$ \\
C4 & 2520 & $1.993 \mathrm{E}-05$ & $2.023 \mathrm{E}-05$ & $1 \%$ \\
glass beads & 229 & $4.453 \mathrm{E}-07$ & $4.872 \mathrm{E}-07$ & $9 \%$ \\
glass beads & 355 & $6.811 \mathrm{E}-07$ & $1.101 \mathrm{E}-06$ & $38 \%$ \\
glass beads & 502 & $2.386 \mathrm{E}-06$ & $1.507 \mathrm{E}-06$ & $58 \%$
\end{tabular}

Table 3. Comparison between experimental and LB permeabilities. 


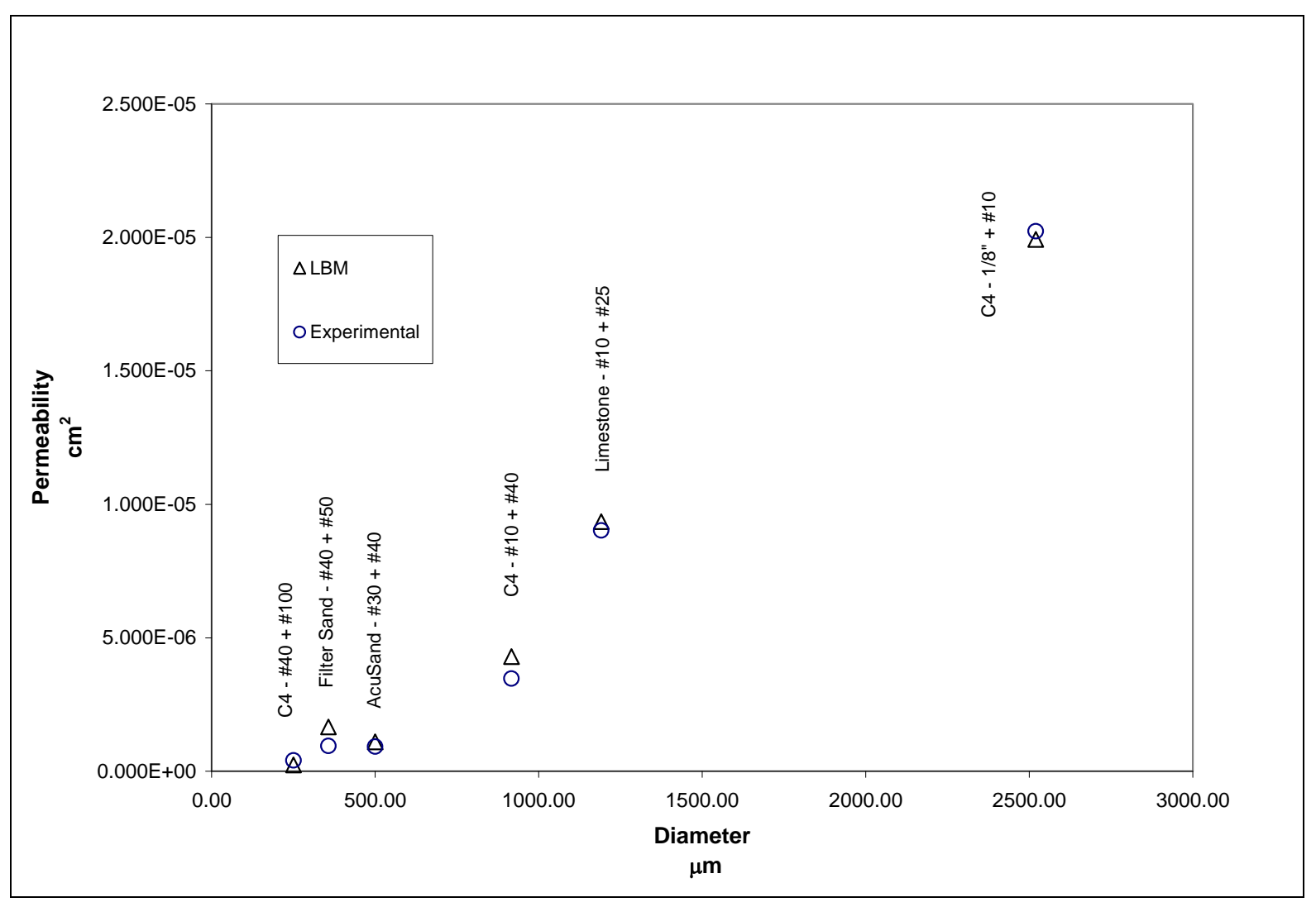

Figure 11. LB permeability and experimental permeability for irregularly shaped particles.

\section{CONCLUSION}

Up to this moment 3D LBM for saturated flow in packed particle beds has been developed. Comparison of the results with analytical solutions for pipe flow under laminar fluid flow conditions and with permeability measurements show good agreement. In fact, we are able to predict the permeability of the porous media with certain accuracy.

In general we will follow the approach explained before in the Objective and Approach section. We are now working on the parallelization of the model in order to make it scalable. This work is necessary in order to be able to handle development of a multiphase model in the next step of the research since this problem is at least twice as computer intensive as the single fluid component model.

In the near future we intend to implement the Shan and Chen model and evaluate the performance of the model with known analytical solutions. Two problems will be evaluated. One is the verification of the Law of Laplace and the second problem is the countercurrent flow of two fluids of different viscosity or density or both in pipe flow. 


\section{REFERENCES}

Bear J., Dynamics of Fluids in Porous Media, Dover Publications, New York, 1988.

Bhatnagar P., E. Gross, and M. Krook, A model for collision processes in gases. I: Small amplitude processes in charged and neutral one-component system. Phys. Rev., 94:511-525, 1954.

Chen H., Discrete Boltzmann systems and fluid flows. J. Comp. in Phys., Nov/Dec:632-637, 1993.

Chen S., G. Doolen, Lattice Boltzmann Method for fluid flows. Ann. Rev. Fluid. Mech., 30:329-64, 1998.

Frish U., B. Hasslacher, and Y. Pomeau, Lattice-gas automata for the Navier-Stokes equation, Phys. Rev. Lett., 56:1505-1508, 1986.

Frish U., D. d'Humieres, B. Hasslacher, P. Lallemand, Y. Pomeau, and J. Rivert, Lattice Gas Hydrodynamics in Two and Three Dimensions, Complex Syst., 1:649-707, 1987.

He X., L. Luo, A priori derivation of the Lattice Boltzmann equation, Phys. Rev. E, 55(60): R6333-R6336, 1997.

Higuera F., J. Jimenez, Boltzmann approach to lattice gas simulations, Europhys. Lett., 9:663-668, 1989.

Lin, C.L., J.D. Miller, Pore structure analysis of particle beds for fluid transport simulation during filtration, Int. J. Min. Proc., 73:281-294, 2004.

Macnamara G., G. Zannetti, Use of Boltzmann equation to simulate lattice-gas automata, Phys. Rev. Lett., 61:2332-2335, 1988.

Martys N., H. Chen, Simulation of multicomponent fluids in complex three-dimensional geometries by the Lattice Boltzmann Model, Phys. Rev. E, E 53:743-750, 1996.

Miller J.D., C.L. Lin, C. Roldan, and C. Garcia, Characterization and analysis of copper heap leaching by x-ray computed tomographic techniques, Proc. $3^{\text {nd }}$ World Congress on Industrial Process Tomography, Banff, Canada, 2-5 September 2003:707-712, 2003a.

Miller J.D., C.L. Lin, C. Roldan, and C. Garcia, Particle size distribution for copper heap leaching operations as established from 3D mineral exposure analysis by x-ray microCT, Copper 2003, Santiago, Chile, VI - Hydrometallurgy of Copper: 83-97, $2003 \mathrm{~b}$.

Qian Y., D d'Humieres, and P. Lallemand, Lattice BGK models for Navier-Stokes equation, Europhys. Lett., 17:479-484, 1992. 
Shan X., H. Chen, Lattice Boltzmann model for simulating flows with multiple phases and components, Phys. Rev. E, 47, 1993. Page Nos.

Succi S. The Lattice Boltzmann Equation for Fluid Dynamics and Beyond, Oxford University Press, New York, 2001.

Zhang R., H. Chen, Lattice Boltzmann method for simulations of liquid-vapor thermal flows, Phys. Rev. E, 67:066711, 2003.

\section{PUBLICATIONS/PRESENTATIONS}

X-ray microtomography and the LB method to measure permeability through particle packed beds, Presented at the International Conference of Mesoscopic Methods, Hampton, Virginia, 2006. Name of presenter? 
Appendix 37: On-Line Monitoring and Diagnosis of Coal Fines During Separation Process (WV008) 


\section{FINAL TECHNICAL REPORT}

Contract Title and Number:

Crosscutting Technology Development at the Center

for Advanced Separation Technologies

(DE-FC26-02NT41607)
Period of Performance:

Starting Date: 4/1/2003

Ending Date: 10/31/2008
Sub-Recipient Project Title:

On-line monitoring and diagnosing of coal fines

during separation process using LIBS

Principal Investigators:

Bruce S. Kang and Eric K. Johnson

Contact Address:

Mechanical \&Aerospace Engineering

P. O. Box 6106

West Virginia University

Morgantown, WV 26506

Subcontractor Address:

No subcontracts issued
Report Information:

Type: Final

Number:

Period: 4/1/2003-10/31/2008

Date: $\quad 3 / 22 / 2007$

Code: $\quad$ WV008-FINAL

Contact Information:

Phone: $\quad 304.293 .3111$ ext.2316

Fax: $\quad 304.293 .6689$

E-Mail: Bruce.Kang@mail.wvu.edu

Subcontractor Information:

Phone:

Fax:

E-Mail:

\section{DISCLAIMER}

This report was prepared as an account of work sponsored by an agency of the United States Government. Neither the United States Government nor any agency thereof, nor any of their employees, make any warranty, express or implied, nor assume any legal liability or responsibility for the accuracy, completeness, or usefulness of any information, apparatus, product, or process disclosed, or represents that its use would not infringe privately owned rights. Reference herein to any specific commercial product, process, or service by trade name, trademark, manufacturer, or otherwise does not necessarily constitute or imply endorsement, recommendation, or favoring by the United States Government or any agency thereof. The views and opinions of authors expressed herein do not necessarily state or reflect those of the United States Government or agency thereof. 


\begin{abstract}
A static and dynamic Laser Induced Breakdown Spectroscopy (LIBS) system to detect carbon, iron, and mercury in coal samples was developed. Significant information has been obtained in analyzing the LIBS signals. Linear correlation of LIBS signal intensity and concentration of selected trace elements has been established. Test results showed that carbon and mercury could be quantified using LIBS. However, due to the air quenching effect, sulfur was not detected using the current experimental apparatus. A unique scanning mirror apparatus was implemented in the LIBS system to observe spatial- and time-resolved dynamic LIBS signals of multiple emission lines. Based on qualitative and semi-quantitative analysis, dynamic LIBS testing provides basic information on the lifetime and temporal characteristics for individual emission lines of selected trace elements in coal samples.
\end{abstract}




\section{TABLE OF CONTENTS}

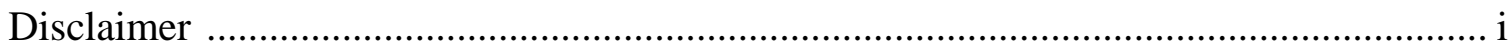

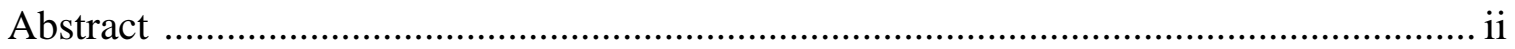

Table of Contents .....................................................................................................

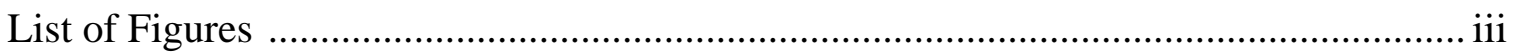

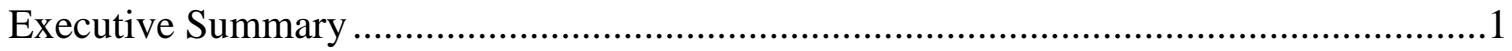

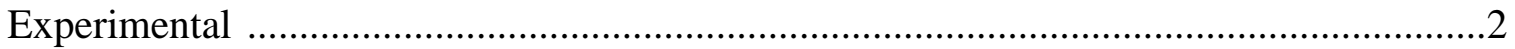

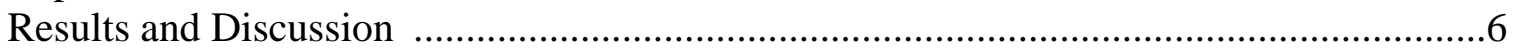

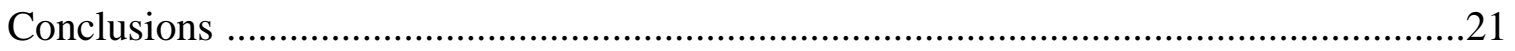

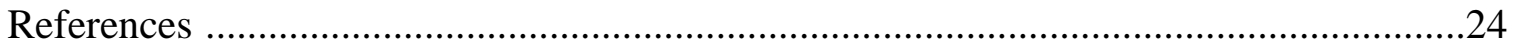

\section{List of Tables}

Table 1 - Mix Compositions in grams.......................................................................... 8

Table 2 - Elemental Concentrations of Synthetic Mixtures .............................................. 8

\section{List of Figures}

Figure 1 - Sample Preparation Apparatus.............................................. 2

Figure 2 - LIBS Testing Schematic.................................................

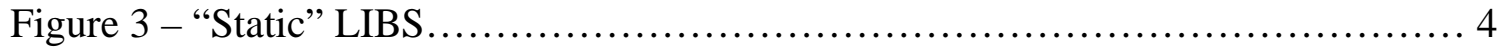

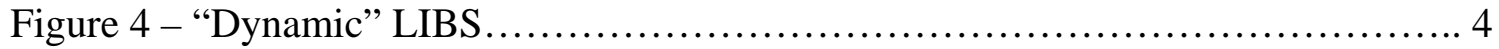

Figure 5 - LIBS Testing Apparatus..................................................

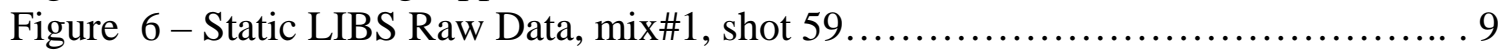

Figure 7 - 100 Shot Average of Mix 1 for qualitative and quantitative analyses...........10

Figure 8 - Correlation of the 445nm Carbon - related line........................... 10

Figure 9 - Correlation of the $447 \mathrm{~nm}$ Carbon - related line..............................11

Figure 10 - Correlation of the 436nm Mercury Emission Line...................... 12

Figure 11 - Correlation of the 432nm Iron Emission Line..............................12

Figure 12 - Correlation of the 439nm Iron Emission Line.......................... 13

Figure 13 - Correlation of the 441nm Iron Emission Line.............................. 13

Figure 14 - Modeling in OSLO....................................................

Figure 15- Traveling beam incident on the lens...................................16

Figure 16 - Eclipsing Function.................................................... 16

Figure 17 - Original and Background Corrected Signals............................. 17

Figure 18 - Background Corrected, Time Scaled Dynamic LIBS Signal.................18

Figure 19 - C 445nm temporal behavior............................................. 19

Figure 20 - C 447nm temporal behavior............................................. 19

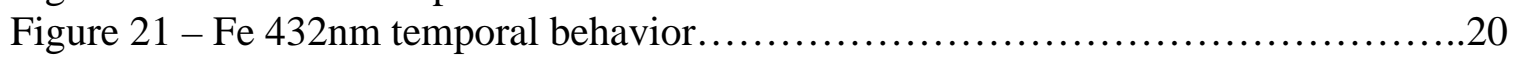

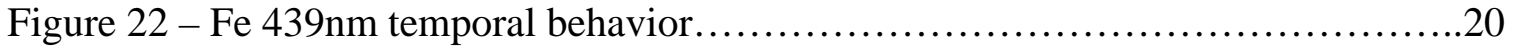

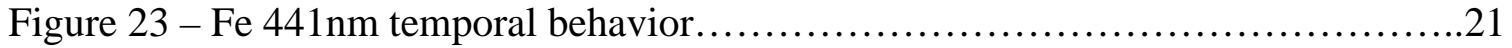

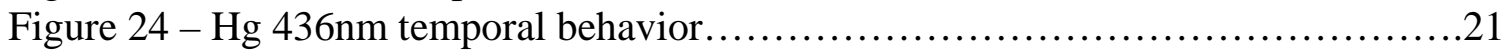




\section{EXECUTIVE SUMMARY}

This research investigated a fundamental study of laser-induced breakdown spectroscopy (LIBS) applied to coal samples with the unique capability of temporal resolution by means of a polygonal scanning mirror. Characteristic elemental emissions were observed and a method was established for quantification of the elements of interest. A method for determining temporal lifetime of the emission lines of interest was also developed.

Eleven synthetic mixtures of coal samples were made for the LIBS tests. Upon obtaining data, the strength of the modified intensity signal from the collected light was plotted with respect to known concentration in the specimen. The graph of relative intensity with respect to concentration resulted in a strong correlation within the carbon and mercury emission lines. Carbon, which ranged from $61-81 \%$, resulted in strong correlations between line intensities and signal strength. Of several LIBS trail runs on several selected spectrum lines, mercury was found to be detectable using the 436nm emission line However, due to limitation of the CCD detector ( 0.1 lux sensitivity) in the current LIBS system, the detection limit of mercury in coal samples can only be achieved to $800 \mathrm{ppm}$ in this study. A plot between the signal strength and the concentration yielded a strong correlation, with only one discrepancy point, possibly due to a mixing error. Sulfur could not be detected using this experimental apparatus. This may be due the air quenching effect. Iron emission lines showed poor correlation between signal strength and concentration. This could be due to the mixing of iron powder within the synthetic mixtures as opposed to iron oxide. 


\section{EXPERIMENTAL}

In the process of LIBS testing, there are two separate experimental apparatus. The first apparatus is the sample preparation laboratory where samples with known concentrations are prepared. In the second apparatus, the samples are subject to LIBS testing.

As shown in Figure 1, the sample preparation apparatus consisted of a scale for massing chemicals, a grinding mill for homogenization of the mixtures, and a pellet press and die for making pellets out of the synthetic coal mixtures. Chemicals used in making the mixtures were all in solid powder form and included graphite powder, coal powder (NIST sample 1635), iron powder and mercuric oxide. Powders were used because they could be homogenized by a grinding mill and pressed into solids.

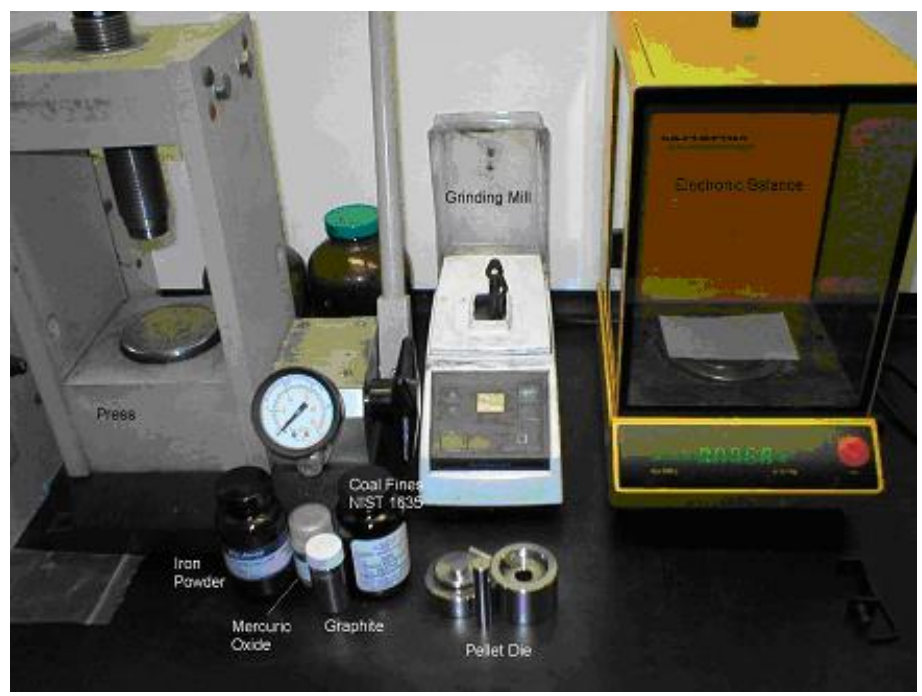

Figure 1 - Sample Preparation Apparatus

As shown schematically in Figure 2, the experimental LIBS apparatus allows optimum signal collection as well as temporal characteristics of the LIBS spark of coal. A Quanta Ray DCR 11 Q-switched Nd: YAG laser is fired, focused through a focusing lens (CVI Laser) and induces a plasma from a pellet of compressed material, which is held in place by a translatable sample holder by an air-tight sample chamber. 


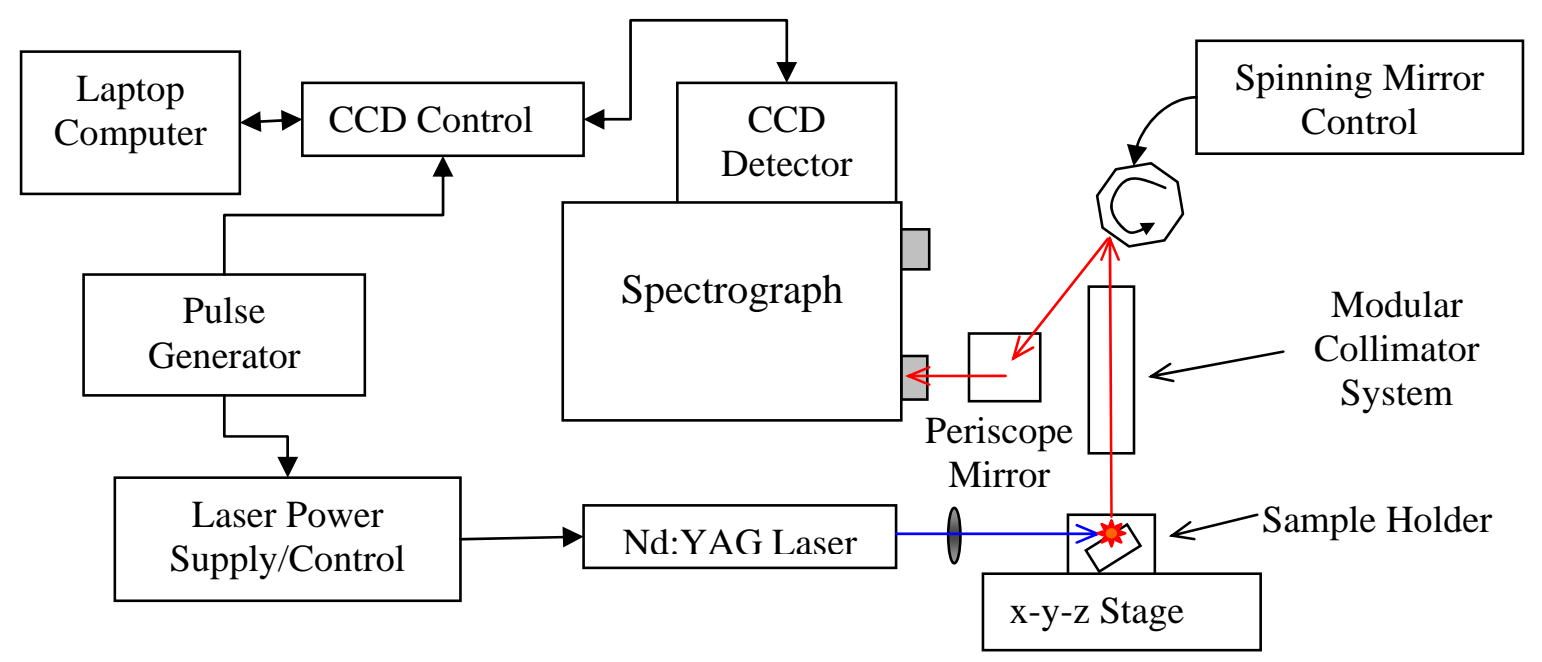

Figure 2 - LIBS Testing Schematic

Once the laser spark occurs, the light is collected and collimated by a modular collimator system (designed in house with parts purchased from Thor Labs). The modular collimator consists of a collection lens, a focusing lens, a pinhole, and a collimation lens. The collimated light travels to the polygonal scanning mirror (Lincoln Laser Company). The collimated light is reflected off one of the scanning mirror facets and goes a set of periscope mirrors, which reflects the light into a focusing lens. The lens focuses the collimated light down onto the entrance slit to the spectrograph (Horiba Jobin-Yvon HR640). Depending on whether the scanning mirror is enabled or disabled there is either a line or a focused spot on the entrance slit of the spectrometer.

When the scanning mirror is turned off, the LIBS spark is taken with no internal gating. Also, because of a lack of a shutter on the CCD camera, the CCD camera is fully exposed until the data is completely read out, which takes about 2.6 seconds. The image formed by the spark creates a spot on the entrance slit. This spot encompasses the entire lifetime of the laser-induced plasma, from the black body radiation in the beginning to the emission lines resulting from the recombination of atoms and molecules. This process was deemed “static LIBS”, as shown in Figure 3. 


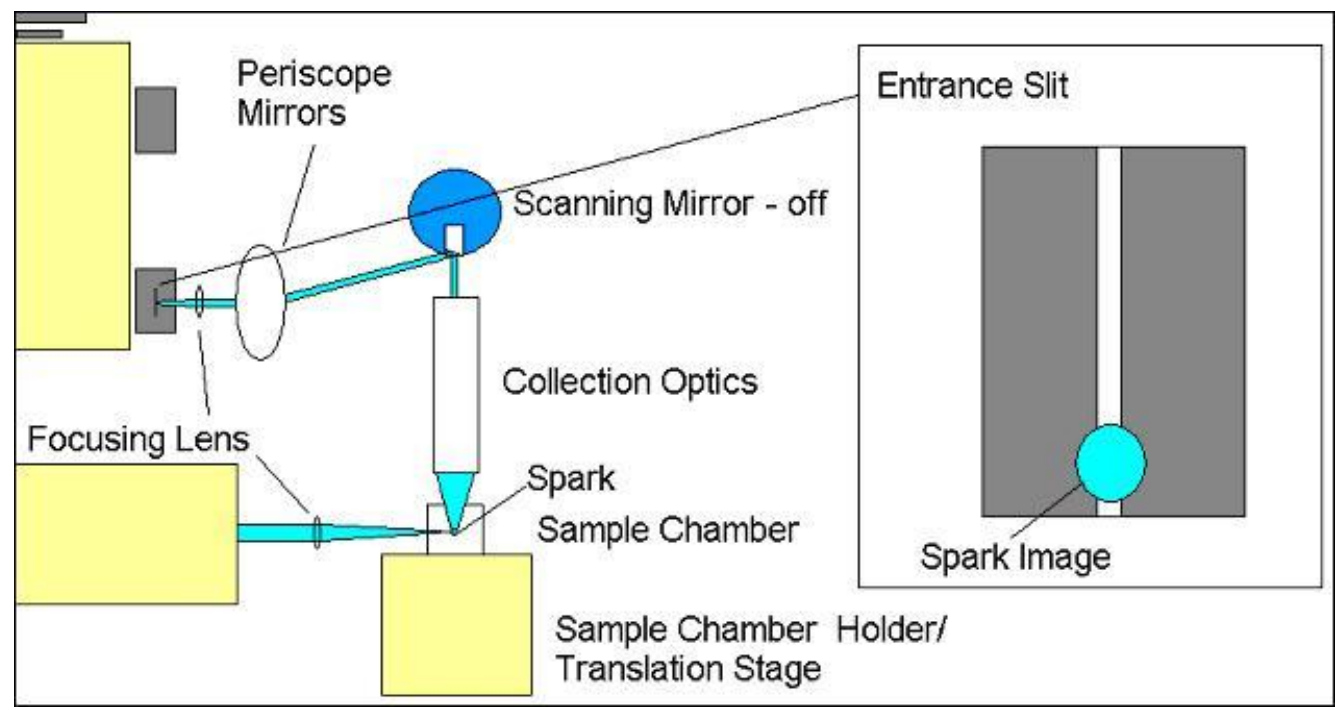

Figure 3 - "Static" LIBS

When the scanning mirror is enabled, the image of the spark is spread out across the entrance slit because of the mirror's motion. This creates a line on the entrance slit. Each point in the line represents a different time within the lifetime of the spark. This information provides us with an approximate lifetime of each emission line. This process was named “dynamic LIBS”, as shown in Figure 4. Figure 5 shows the LIBS testing apparatus.

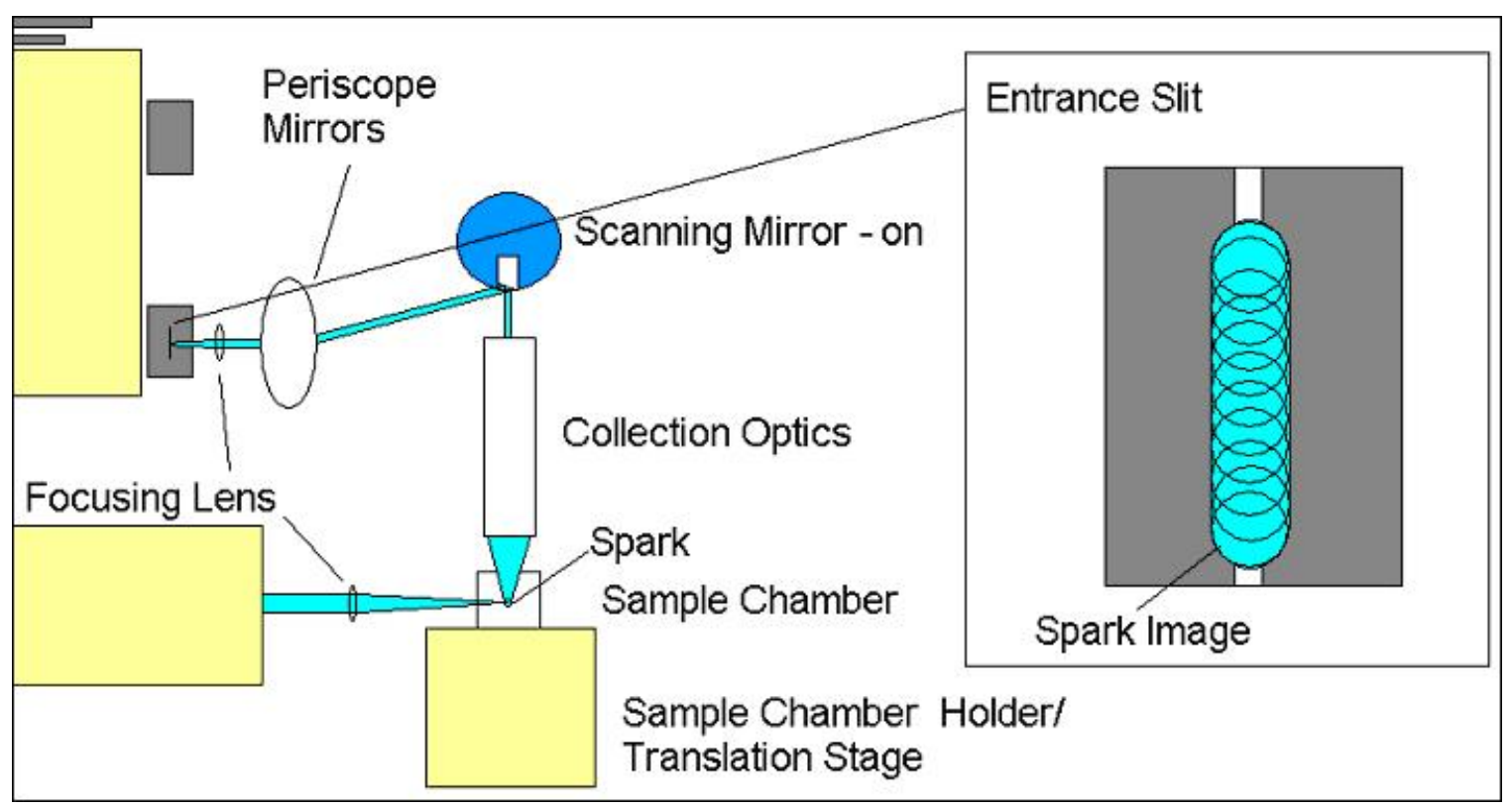

Figure 4 - “Dynamic” LIBS

11 synthetic mixtures of coal samples were made in order to test the LIBS process. Each mixture contained a homogenized blend of graphite powder, NIST 1635 (sub bituminous coal), iron powder, and mercuric oxide for elevating mercury levels in 
the mixtures. The concentration of each element varied with each mixture, creating various concentrations of $\mathrm{C}$, Fe, and $\mathrm{Hg}$ for each pellet.

A description of the operational procedure for the sample preparation is described in the following steps. Step 1 - each chemical was massed on a weighing dish, and transferred into a grinding capsule. Step 2 - the desired chemicals were massed and added into a labeled grinding capsule. Step 3 - the synthetic mix was homogenized through a ball miller. Step 4 - the mixture was then placed in a pellet die and pressed to form a pellet.

A description of the operational procedure of the LIBS testing is described in the following steps. Step1 - the laser, computer, detector controller and pulser are turned on and the software (Winspec32) is opened. Step 2 - the specimen holder is aligned to the optics via a translation stage. A mercury light source emits light through a pinhole at the approximate spark location. Step 3 - the pinhole is removed and replaced with the sample chamber that contains the calibration specimen. The spectrograph is then tuned to a selected wavelength range, and calibrated using a linear relationship between known and unknown emission lines. This step was only necessary when selecting the desired wavelength range to be observed. Step 4 - the calibration specimen is removed and the pinhole is placed over the lamp source, where step 2 is repeated. After step 2, the pinhole is removed and a sample chamber with a test specimen is placed in the sample holder. Step 5 - the spinning mirror is either turned on or left off, depending on the desired test. The laser is fired by the pulser, which also activates data acquisition for the CCD detector. If the user desires time resolved LIBS spectra (i.e. “dynamic LIBS”), then the spinning mirror is turned on. For LIBS data with no time resolution ("static LIBS"), the scanning mirror is left off. Step 6 - the laser is fired. The collected light should go through the pinhole in the modular collimated system. If the spark does not go through the pinhole, then the sample holder is appropriately translated. For dynamic LIBS testing, the spinning mirror is not synchronized, and a "brute force" method of testing is required to acquire data in which the end user fires the laser repeatedly until signal is collected. Step 7 - the collected signal is then saved to a file on the computer and step 6 is repeated until all the desired data is taken. 


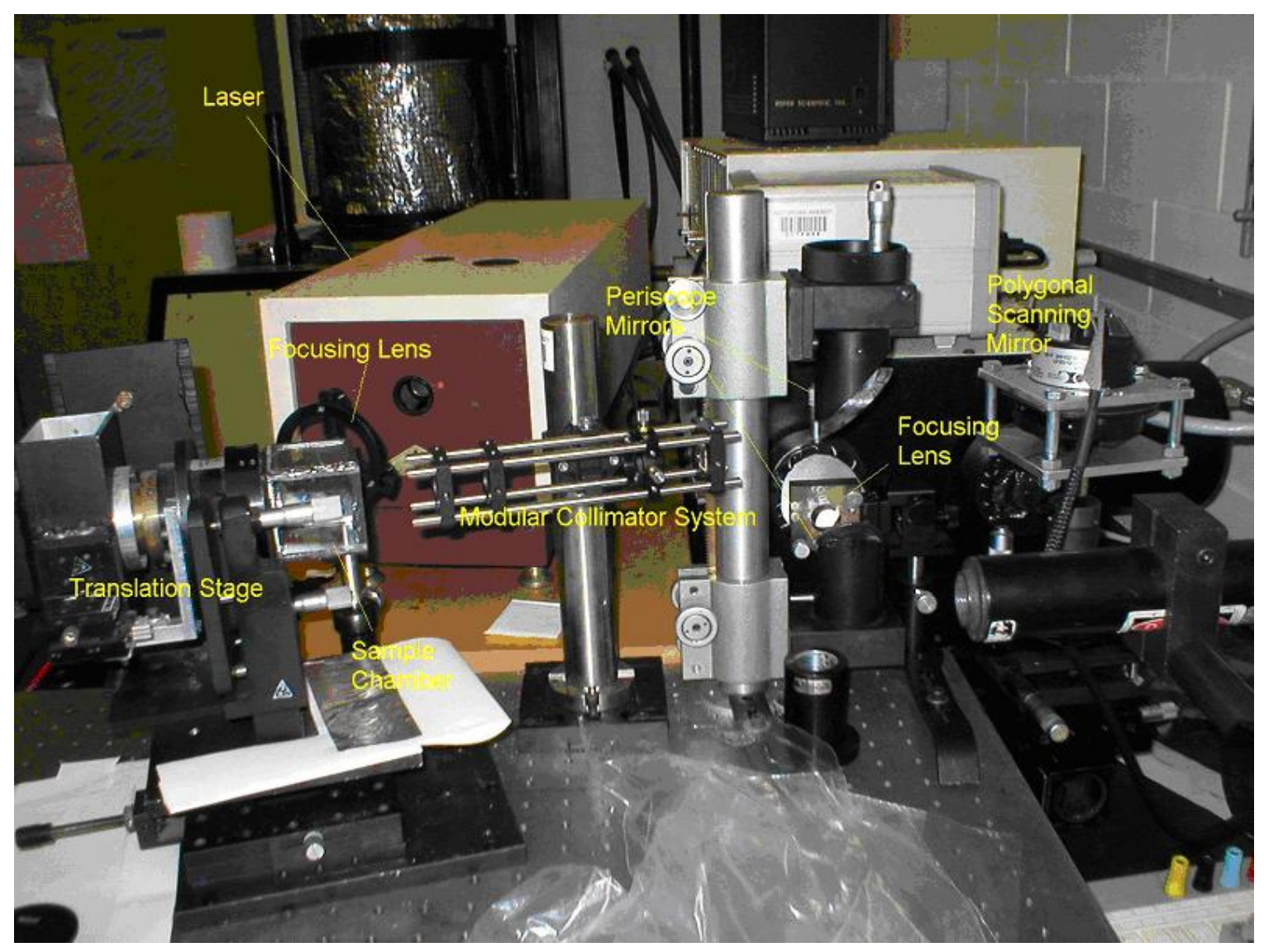

Figure 5 - LIBS Testing Apparatus

Before any data files can be analyzed, they have to be processed into a form that is useful for data processing. This procedure explains the file conversions from Winspec32 to Grams/AI and Microsoft Excel.

A description of the operational procedure of static data processing is described in the following steps. Step 1 - the raw data file is opened in Winspec32. Step 2 - the raw signal is then binned. Binning is a process in Winspec32 that sums the vertical pixels of the CCD and reduces the noise when compared to the signal of the emission lines. Step 3 - the binned signal is then converted into Grams/AI by a seamlessly integrated conversion. Step 4 - after the signal is converted to Grams/AI, it is then converted into a Microsoft Excel file through a program in Grams/AI called “Excel Exchange”. Details of this approach can be found in Ref. 6.

The data processing for dynamic LIBS is not necessary. The analysis for the dynamic LIBS signal only requires visual observations. 


\section{RESULTS AND DISCUSSION}

\section{Initial LIBS Testing}

Because LIBS observes emission lines, initial testing for signal acquisition and optimization began with steel. This choice was made because iron has over 4000 emission lines, and can be observed throughout the spectral regions of interest, making it an optimal candidate for spectrograph calibration. Both static and dynamic LIBS testing were applied to steel.

The first studies were observed in the wavelength range of $530-545 \mathrm{~nm}$. This wavelength range was chosen because it had an abundance of iron lines, and included at least one emission line from each of the elements of interest according to the MIT Wavelength Tables ( 3 sulfur lines, 1 carbon line and 1 mercury line). Static LIBS was applied to pyrite, sulfur powder, coal powder and graphite powder. No signals from carbon or sulfur were observed in this wavelength region. Pyrite testing only revealed iron emission lines. Mercury was not tested in this region.

The spectrograph was tuned to mercury's most sensitive line, $254 \mathrm{~nm}$. In this range, the spectrograph would encompass the $254 \mathrm{~nm}$ line from mercury as well as the $248 \mathrm{~nm}$ line in carbon. When LIBS testing was done on the coal sample from NIST, the expected carbon line was not observed in this range.

A list of the most sensitive emission lines for the elements of carbon, iron, mercury and sulfur was composed. The spectrograph was tuned to each one and LIBS testing was done on each respective element. In an attempt to find sulfur, the spectrograph was tuned to the most sensitive sulfur lines in the spectrum, and static LIBS testing was applied to a sulfur pellet. Sulfur showed no emission lines anywhere in the visible or infrared spectrum. It is hypothesized that this could be due to effects of an air environment that seem to quench sulfur emission lines.

Upon further examination of the wavelength tables, another decision was made to select the wavelength range of 431-452nm. The spectrograph was calibrated by a combination of the ceiling light containing mercury and a LIBS spark from iron. After calibration, static LIBS testing was done on coal powder, coal pellets, graphite powder, graphite flakes, a carbon rod, sulfur powder, sulfur pellets, and a mercury-copper amalgam. Upon testing the powders, no lines were observed in graphite or sulfur. Static LIBS testing applied to coal powder revealed emission lines at approximately $445 \mathrm{~nm}$ and $447 \mathrm{~nm}$, which were tested against a carbon rod and matched. The wavelengths were verified when LIBS was applied to pure carbon powder. The 436nm mercury emission line was observed in the testing, as well as several emission lines from the copper spectrum. After a consistent signal acquisition was obtained, the mercury-copper amalgam was subject to dynamic LIBS testing. An iron pellet and a small pyrite pebble were also subject to dynamic LIBS testing. Only iron emission lines appeared in the LIBS testing of the pyrite sample, no sulfur emission lines were observed. Since all 
carbon, iron and mercury were present within this wavelength range, this wavelength range was chosen.

\section{LIBS Testing of Synthetic Mixtures}

As shown in Tables 1 and 2, eleven synthetic mixtures of coal were made in order to test the LIBS process. Each mixture contained a homogenized blend of graphite powder, NIST 1635 (sub bituminous coal), iron powder, and mercuric oxide for elevating mercury levels in the mixtures. The concentration of each element varied with each mixture, creating various concentrations of $\mathrm{C}, \mathrm{Fe}$, and $\mathrm{Hg}$ for each pellet. Each chemical was massed on a weighing dish, and transferred into a grinding capsule. After the desired chemicals were massed and added into the capsule, the capsule was labeled, and ground together through a ball miller portable grinding unit. After grinding took place, the mixture was then placed in a pellet die and pressed to form a pellet.

\begin{tabular}{|l|l|l|l|l|l|l|l|l|l|l|l|}
\hline & Mix1 & Mix2 & Mix3 & Mix4 & Mix5 & Mix6 & Mix7 & Mix8 & Mix9 & $\begin{array}{l}\text { Mix1 } \\
0\end{array}$ & $\begin{array}{l}\text { Mix1 } \\
1\end{array}$ \\
\hline Graphite & 0.0770 & 0.3344 & $0 \mathrm{~g}$ & 0.0082 & 0.0936 & 0 & $\begin{array}{l}0.151 \\
9\end{array}$ & $\begin{array}{l}0.225 \\
9\end{array}$ & $\begin{array}{l}0.175 \\
7\end{array}$ & 0 & 0 \\
\hline NIST1635 & 0.0704 & 0 & 0.7575 & 0.3113 & 0.2985 & $\begin{array}{l}0.296 \\
7\end{array}$ & $\begin{array}{l}0.241 \\
5\end{array}$ & $\begin{array}{l}0.212 \\
1\end{array}$ & $\begin{array}{l}0.271 \\
5\end{array}$ & $\begin{array}{l}0.714 \\
1\end{array}$ & $\begin{array}{l}0.635 \\
7\end{array}$ \\
\hline $\begin{array}{l}\text { Iron } \\
\text { Powder }\end{array}$ & 0.0138 & 0.0060 & 0.0998 & 0.0199 & 0.0213 & $\begin{array}{l}0.009 \\
1\end{array}$ & 0 & $\begin{array}{l}0.001 \\
7\end{array}$ & $\begin{array}{l}0.007 \\
2\end{array}$ & $\begin{array}{l}0.414 \\
1\end{array}$ & 0.002 \\
7
\end{tabular}

Table 1 - Mix Compositions in grams

\begin{tabular}{|l|l|l|l|l|l|l|l|l|l|l|l|}
\hline & Mix1 & Mix2 & Mix3 & Mix4 & Mix5 & Mix6 & Mix7 & Mix8 & Mix9 & Mix10 & Mix11 \\
\hline $\begin{array}{l}\text { Carbon, } \\
\%\end{array}$ & 73.42 & 97.49 & 61.06 & 66.24 & 73.12 & 67.75 & 81.20 & 81.21 & 78.11 & 65.93 & 69.73 \\
\hline Iron, \% & 8.12 & 1.75 & 11.70 & 6.05 & 5.32 & 3.18 & 0.15 & 0.46 & 1.68 & 6.04 & 0.62 \\
\hline $\begin{array}{l}\text { Mercury, } \\
\%\end{array}$ & 5.81 & 0.70 & 1.18 & 0.52 & 0.08 & 0.25 & 0.31 & 2.77 & 2.74 & 0 & 0 \\
\hline
\end{tabular}

Table 2 - Elemental Concentration of Synthetic Mixtures

After the completion of sample preparation, the samples were subject to LIBS testing. Although the components to the synthetic mixtures were ground together, the final specimen was not a homogeneous pellet. To compensate for the lack of uniformity, 100 "static" LIBS shots were taken for each synthetic mixture. Standard deviation values of the shot intensities of each sample indicate that the mixtures were not thorough. After static testing of a specimen, the spinning mirror was then turned on for dynamic testing. Dynamic shots were collected from each sample, except for sample \#2, which was destroyed during static testing. 


\section{Static LIBS}

\section{Qualitative Analysis}

Qualitative analysis of the LIBS signals can be done with the data of the static LIBS shots, as typically shown in Figure 6. No processing of the data is necessary. Spectral lines are identified according to their wavelengths.

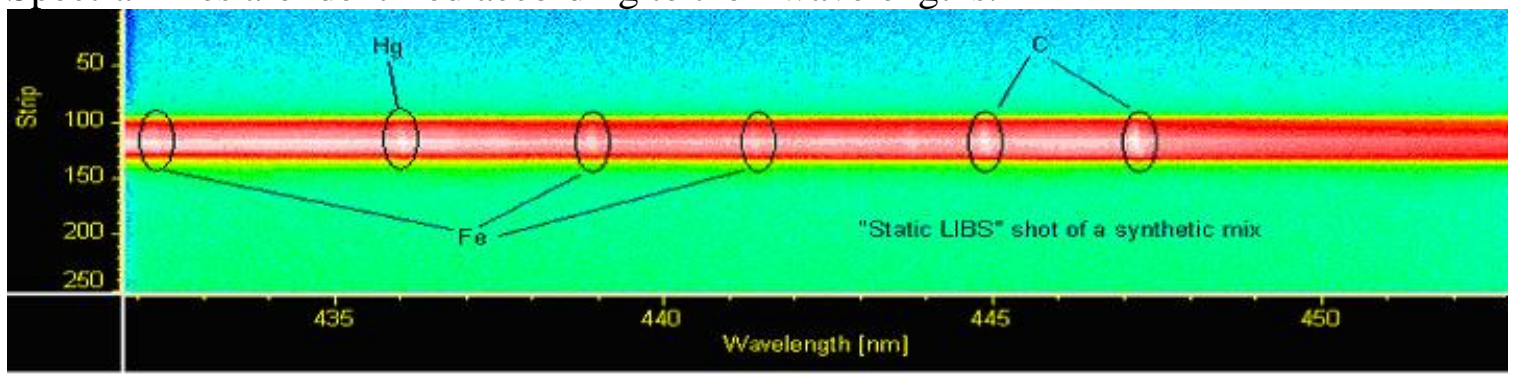

Figure 6 - Static LIBS Raw Data, mix\#1, shot 59

\section{Quantitative Analysis}

Quantitative analysis of the LIBS signal requires the data from the LIBS testing to be processed and converted into a spreadsheet. Each of the raw data files of the synthetic mixtures was converted and placed into Microsoft Excel. The data was then averaged, and analyzed for quantitative analysis. In this procedure, spectral signals were subtracted from the background (which included electronic response of the detector and the continuum radiation from the initial plasma formation). The intensity values were plotted and are expected to show a linear relationship with the concentration of the specified element.

$I_{\text {relative }}=I_{\text {peak }}-I_{\text {baseline }}$

Where $\mathrm{I}_{\text {peak }}$ is the intensity at the peak of the emission line, and $\mathrm{I}_{\text {baseline }}$ is the intensity at the baseline of the emission line. Because of the initial radiation resulting in a broad emission that interferes with the atomic and ionic emission signals, the baseline intensities to the left and right of the emission lines were averaged before subtracting the baseline value from the peak intensity. Figure 7 shows a typical plot using the described procedure. The intensity of the emission lines resulting from LIBS are expected to be linear with respect to the elemental concentration of the specie within the sample. 


\section{Shot Average - Mix 1}

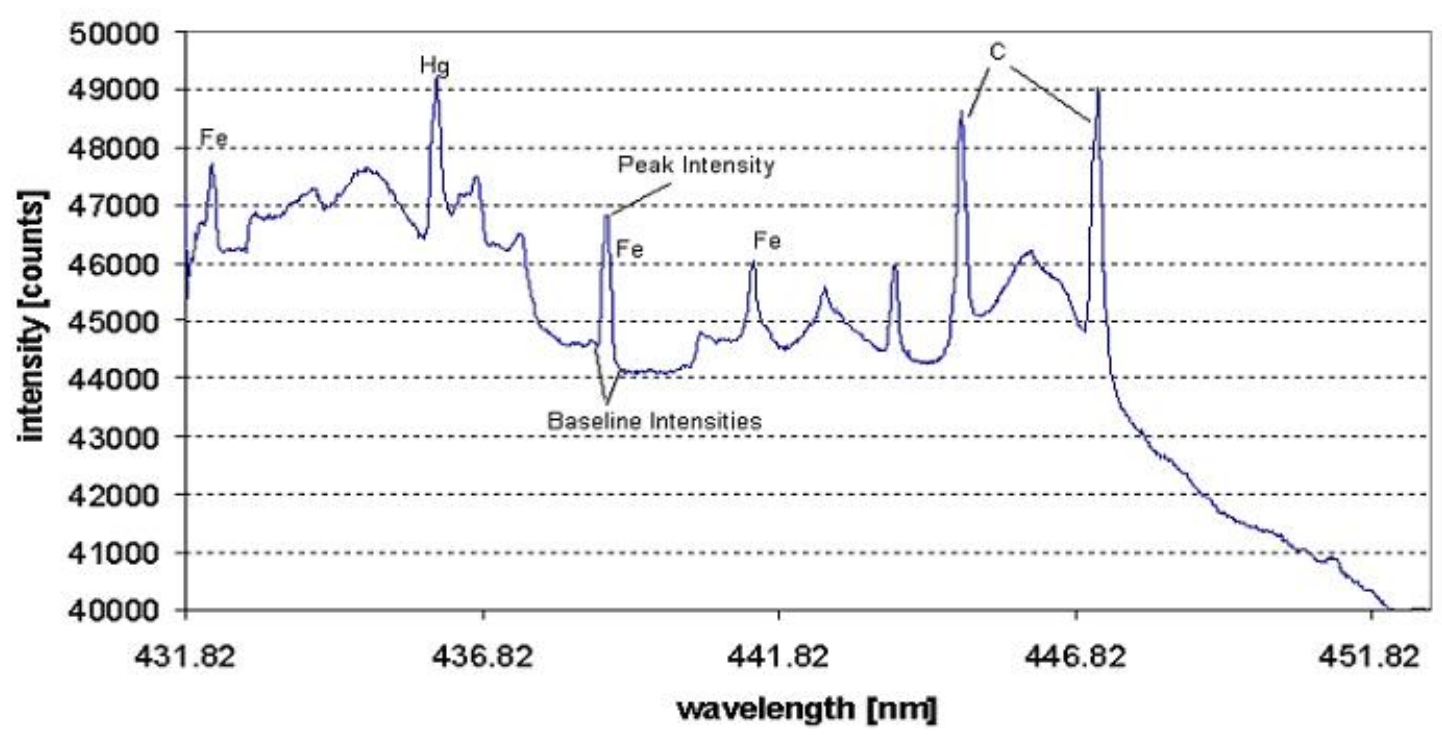

Figure 7 - 100 Shot Average of Mix 1 for qualitative and quantitative analyses

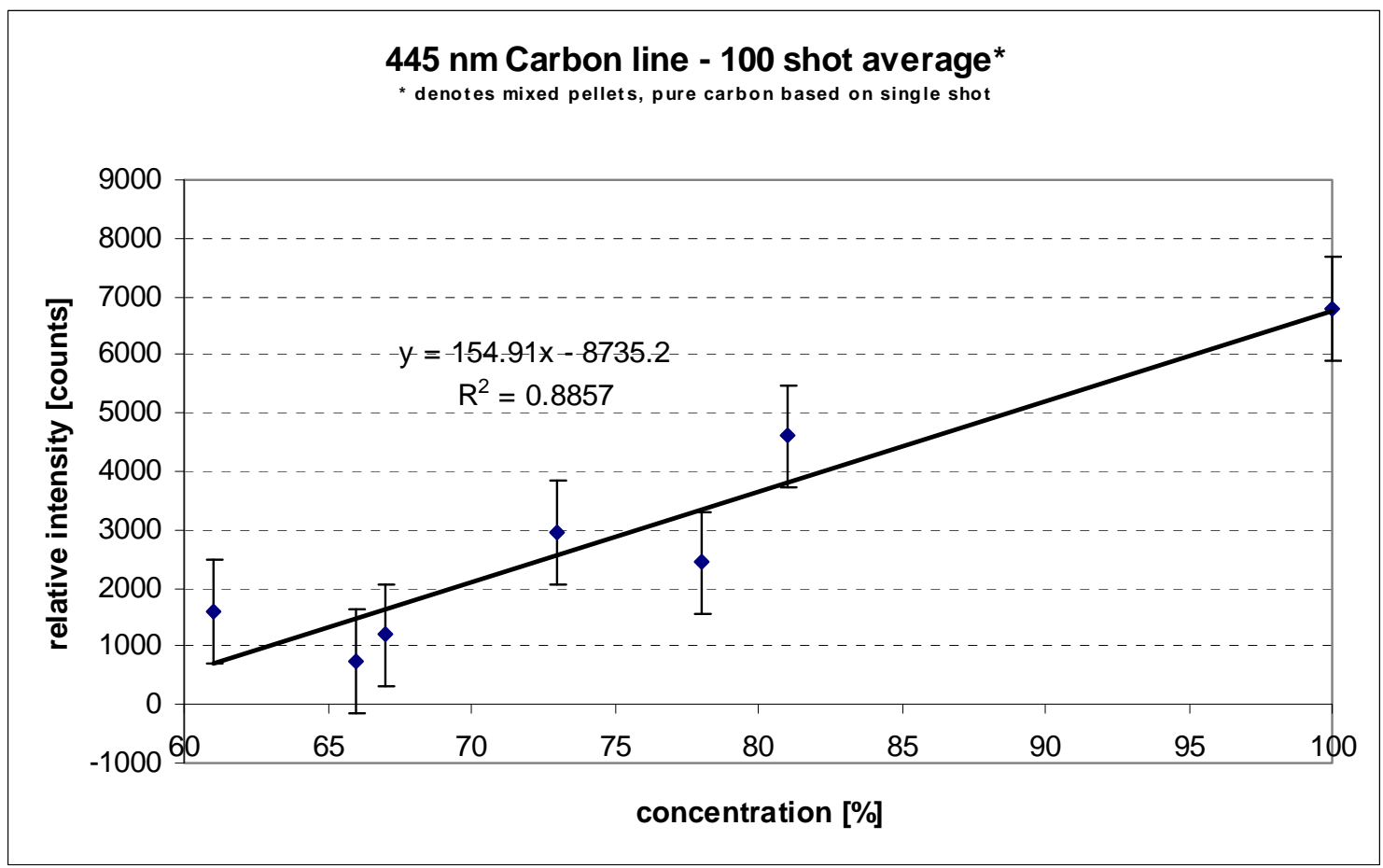

Figure 8 - Correlation of the $445 \mathrm{~nm}$ Carbon - related line 


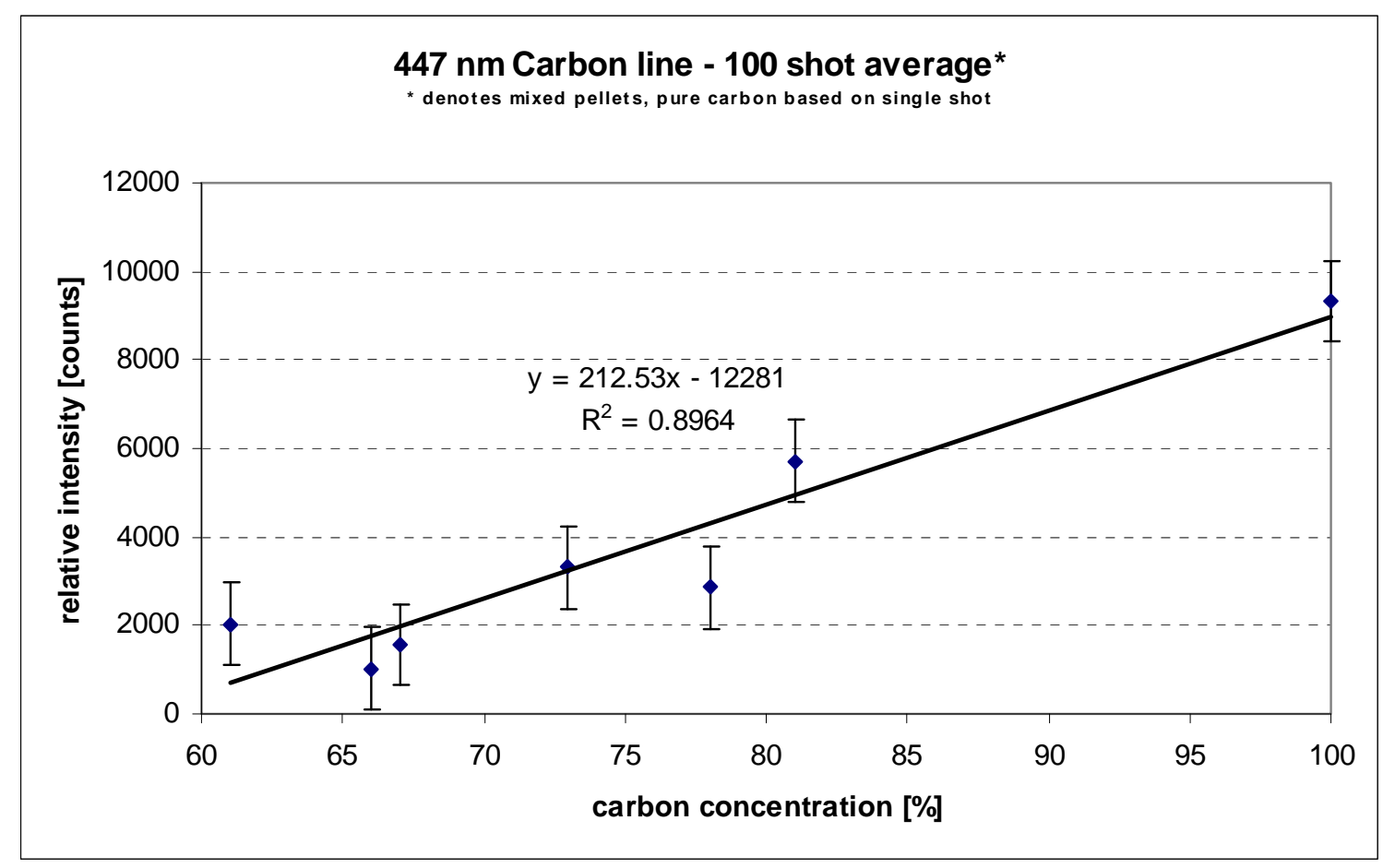

Figure 9 - Correlation of the $447 \mathrm{~nm}$ Carbon - related line

As shown in Figures 8 and 9, the linear fit observed in the 445 and $447 \mathrm{~nm}$ carbon-related lines showed a good correlation between elemental concentration and line intensity. Although these emission lines are not recorded in the MIT wavelength tables, they can be used as carbon-related lines to determine elemental concentration based on their intensity.

LIBS tests of mercury concentration ranged between 800 parts per million to 6 percent were conducted, as shown in Figures 10 and 11. While the strongest and most observed line is 253.6 [1-3], the $436 \mathrm{~nm}$ emission line was chosen because of its close proximity to the carbon-related and iron emission lines. The $436 \mathrm{~nm}$ emission line proved to have a linear relation between line intensity and elemental concentration. There was one discrepancy point within the data set, which is hypothesized to be a measurement error that occurred during the pellet formation process. 


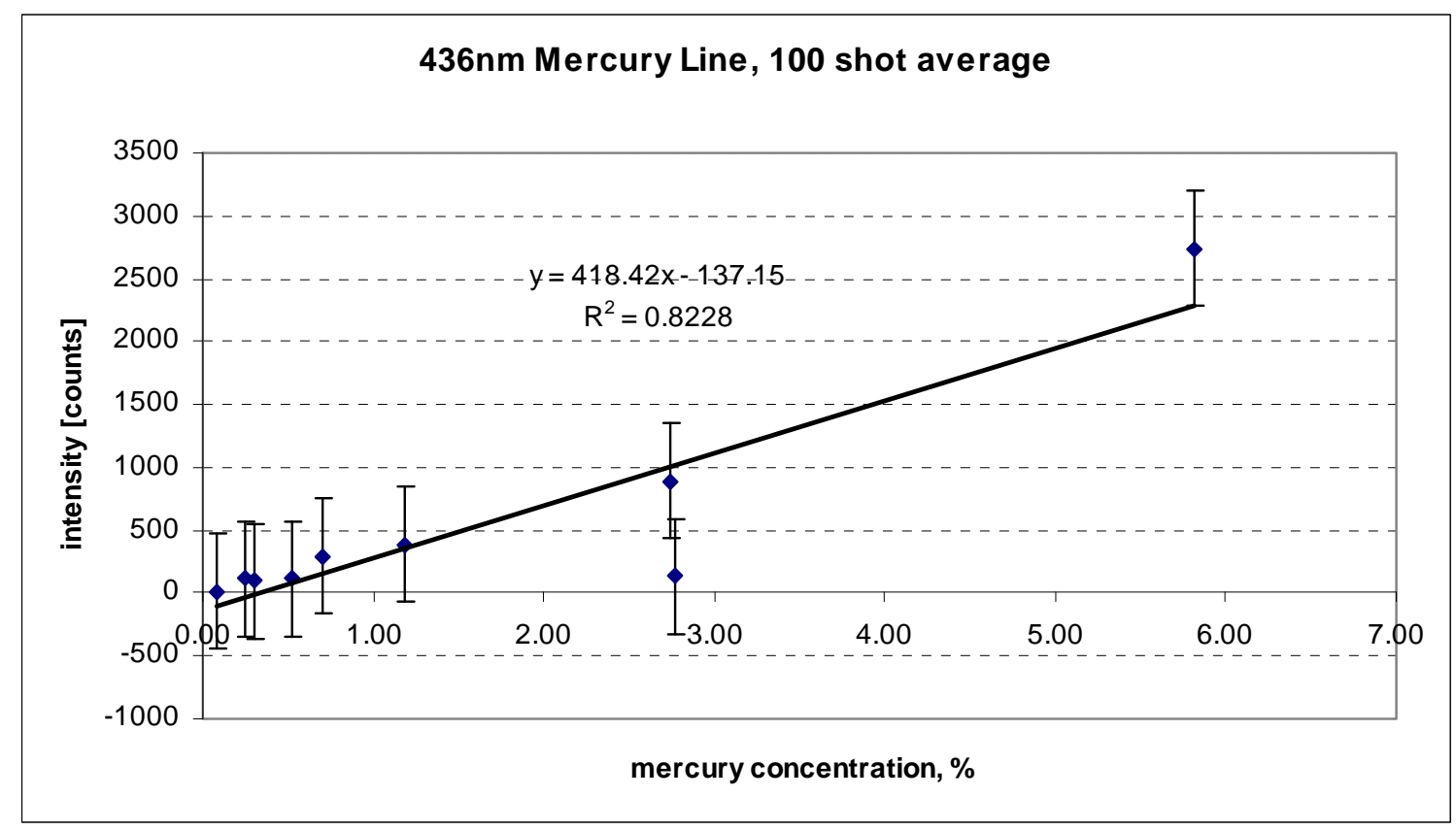

Figure 10 - Correlation of the 436nm Mercury Emission Line

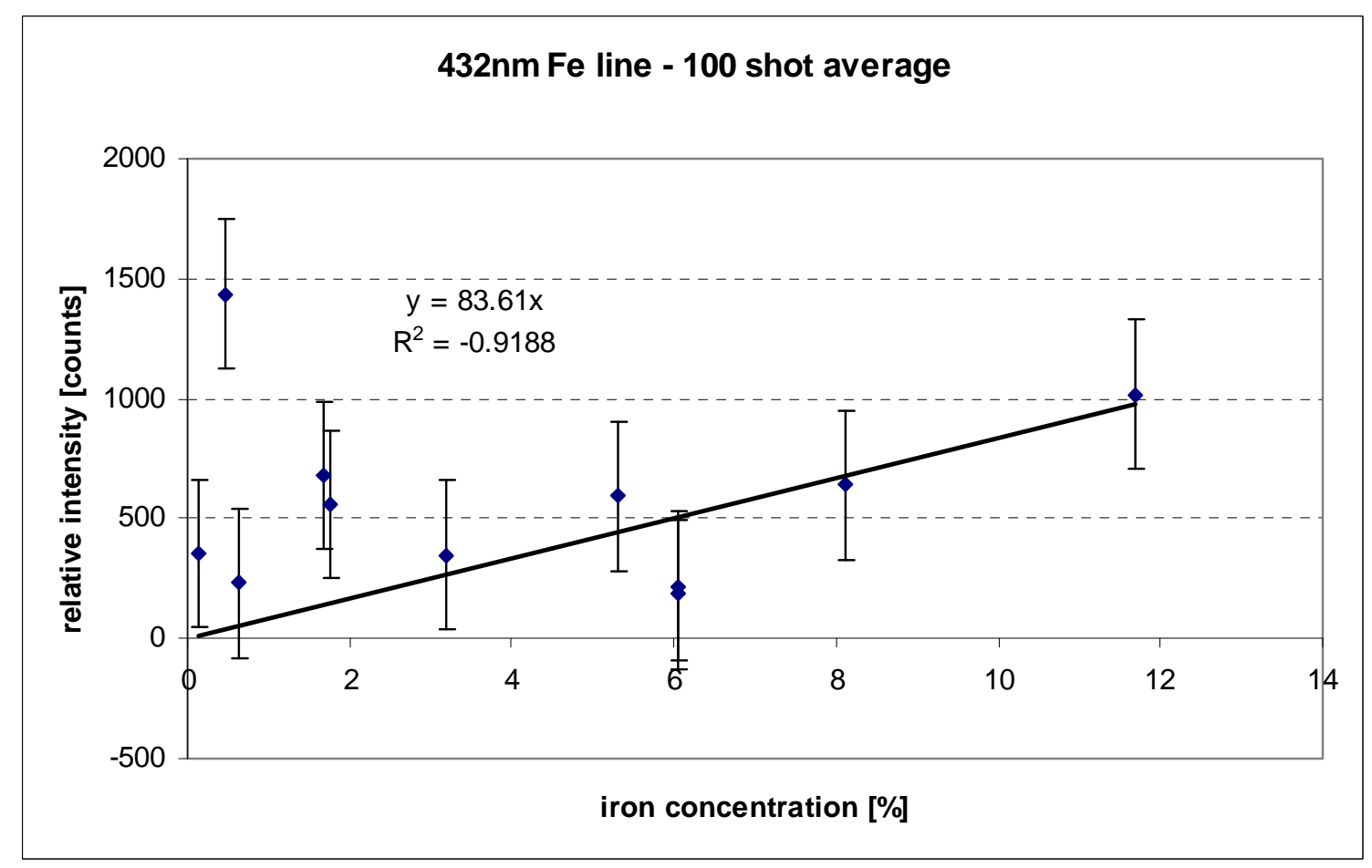

Figure 11 - Correlation of the 432nm Iron Emission Line 


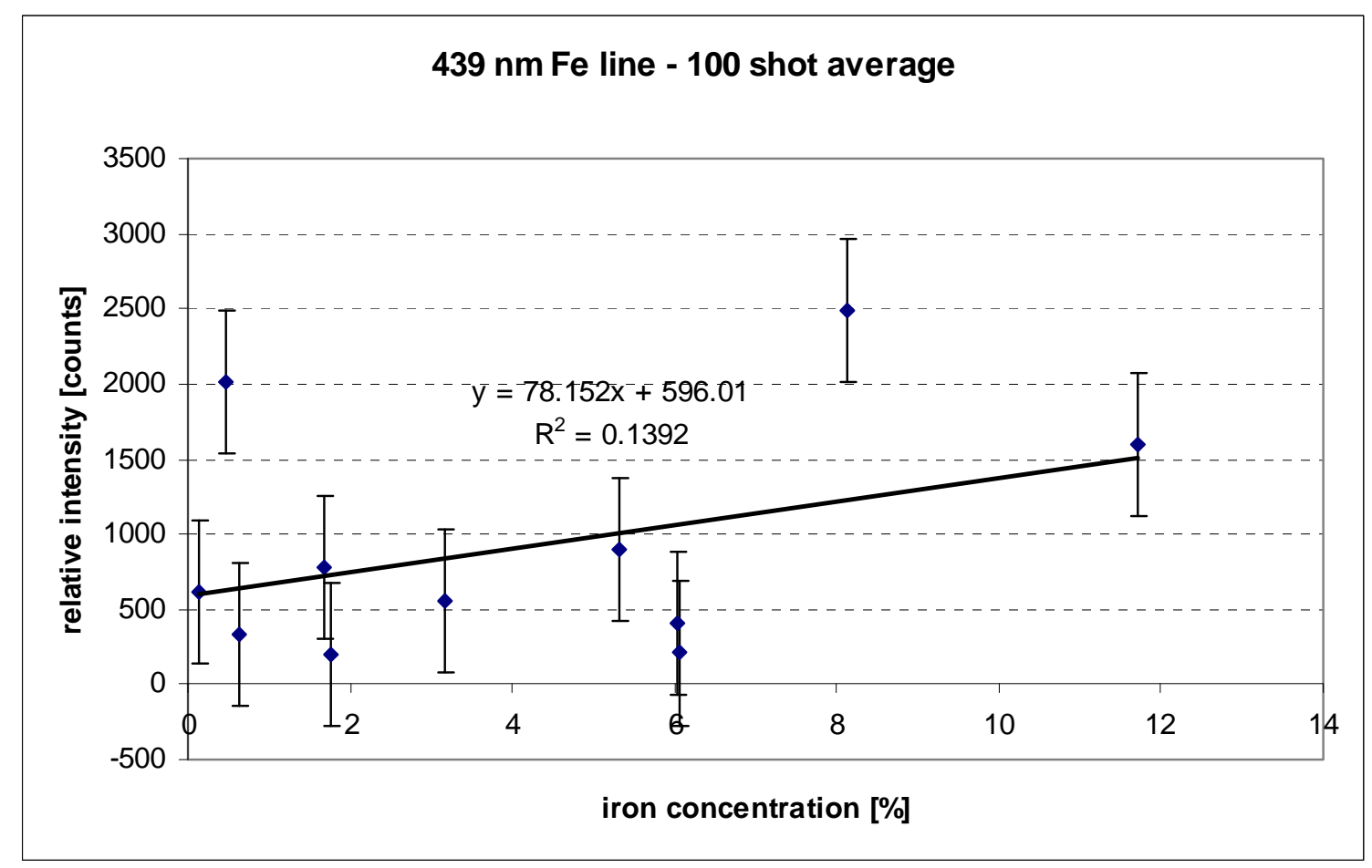

Figure 12 - Correlation of the 439nm Iron Emission Line

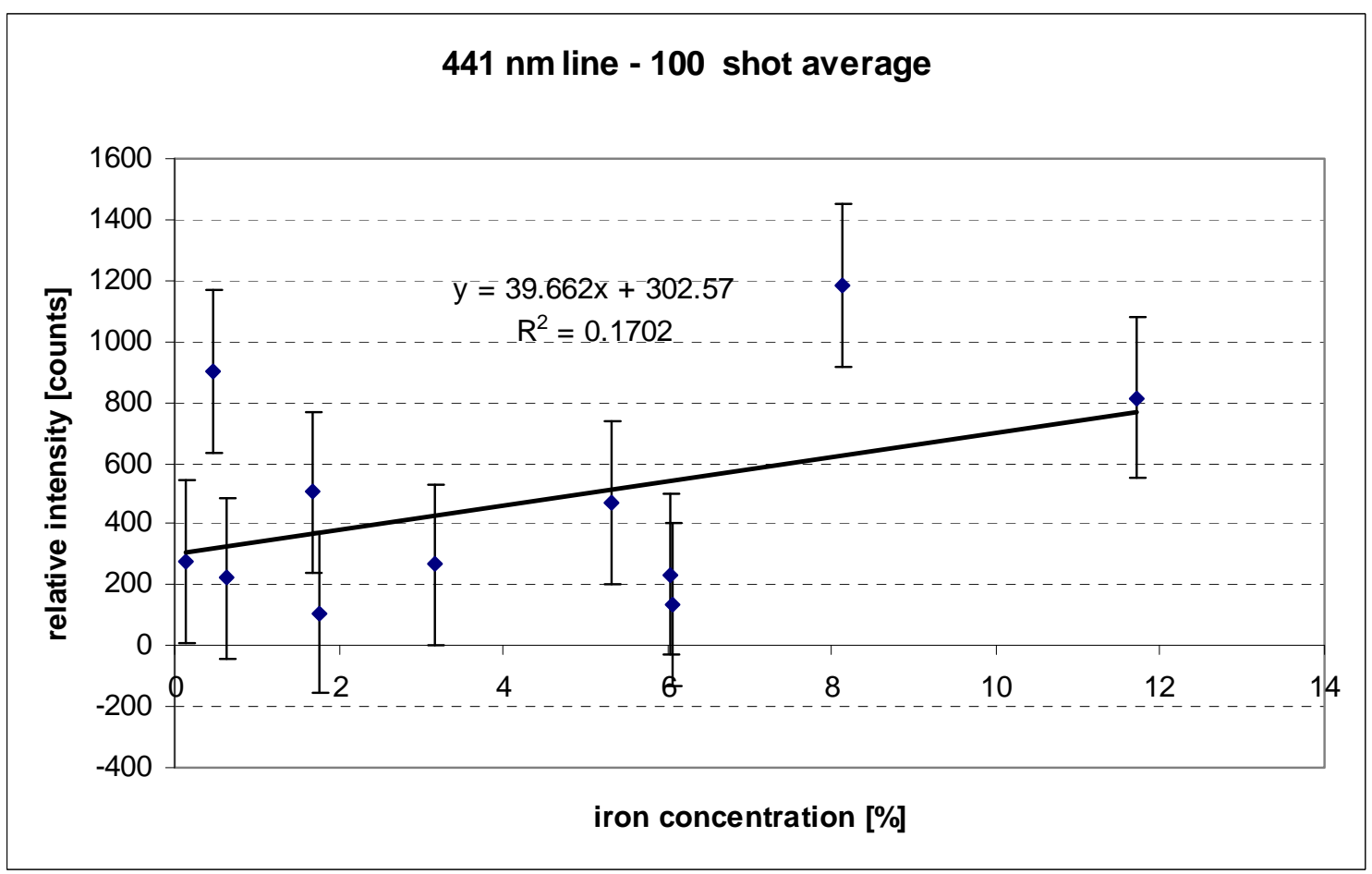

Figure 13 - Correlation of the 441nm Iron Emission Line 
As shown in Figures 12 and 13, the iron lines showed a poor correlation between emission line intensity and elemental concentration. The iron found in coal is mostly mineral forms of iron, such as iron oxide [2]. The iron used to make the synthetic mixtures was pure iron powder. It is hypothesized that the iron powder formed together when subject to the grinding unit instead of being dispersed.

\section{Dynamic LIBS}

\section{Theory of Dynamic LIBS}

Since the LIBS spark is brief, it is necessary to have a time frame in which the characteristic line spectra can be observed. In their review, "Quantitative micro-analysis by laser-induced breakdown spectroscopy: a review of the experimental approaches”, Tognoni states that early stages of the laser-induced plasma will contain black body continuum radiation [4]. The continuum correlates to a physical process of plasma formation and cannot be reduced through averaging, though it can be normalized though use of Wien's Law. The best way to compensate for the continuum emission is to activate the spectral acquisition with a proper delay after the laser-induced plasma has been produced. So far, LIBS experiments have been temporally resolved by the use of an Intensified CCD (or ICCD). The ICCD allows the option of temporal gating. This means that the end user of the equipment can set a delay time after the black body continuum has been reduced and during an optimal time for observing the characteristic line spectrum of the element in question.

The dynamic LIBS method was developed to observe temporal behaviors of multiple emission lines in a single LIBS spectrum. This system incorporated the use of a polygonal scanning mirror to achieve temporal differentiation within the emission spectrum. To current knowledge, this was the first time a polygonal scanning mirror was applied to a LIBS experiment for time-resolved studies. The polygonal scanning mirror reflected light off of one of its facets, creating a line image instead of a focused image of the LIBS spark. Each segment of the line will correspond to a different time. The scan line fell on the entrance slit of the spectrograph. The light fell on the CCD array in an area that correlated to a temporal profile, creating a temporally resolved, as well as a spatially resolved LIBS signal.

\section{Calculation of the Dynamic LIBS Time Frame}

The mirror used was a disk-shaped mirror with 30 facets around the circumference of the disk. When the mirror was turned on for dynamic LIBS testing, it spun at 12,000 rpm. The spinning of the scanning mirror allowed for a time-dependent signal to be observed. The dimensions for each facet were $9.8 \mathrm{~mm}$ high by $6.0 \mathrm{~mm}$ wide. The incident light on the facet was in the form of a circle with a diameter $8.9 \mathrm{~mm}$. The light filled up the spinning facet, which reflected a line on the entrance slit to the spectrograph. Each different position in the "line" corresponded to a different time of the spark life. The line was not made up of discrete spots, but rather an overlapping of the 
spots, which resulted in a convoluted signal, though time-dependent characteristics could still be observed.

The number of facets and the rotation speed in the scanning mirror determined the time frame in which the laser spark was observed. The scanning mirror had 30 facets around the circumference of a disk, which resulted in a 12 degree scan angle for each facet. The rotation speed of the scanning mirror was set at 200 revolutions per second. The value in revolutions per second was converted into degrees per second by multiplying by 360 . The scanning mirror had a rotation speed of 72,000 degrees per second. The full 12 degree scan of an individual mirror facet would take 167 microseconds. To encompass the 167 microsecond in a single scan, the full 12 degrees of the scan had to be used. The focusing lens did not include the full scan of the mirror facets. The optical path from the scanning mirror to the focusing lens was $330 \mathrm{~mm}$. For the full scan of a facet to be encompassed, the lens diameter would have to be $62.6 \mathrm{~mm}$. The diameter of the focusing lens was $25.4 \mathrm{~mm}$, resulting in a reduced scan angle, and therefore, a reduced time frame of observation.

To calculate the window of observation, the beam was modeled in OSLO. From the OSLO modeling, the length of the line incident on the entrance slit of the spectrometer was calculated to be 2 millimeters. The operational scan angle was calculated to be 2 degrees (Figure 14).

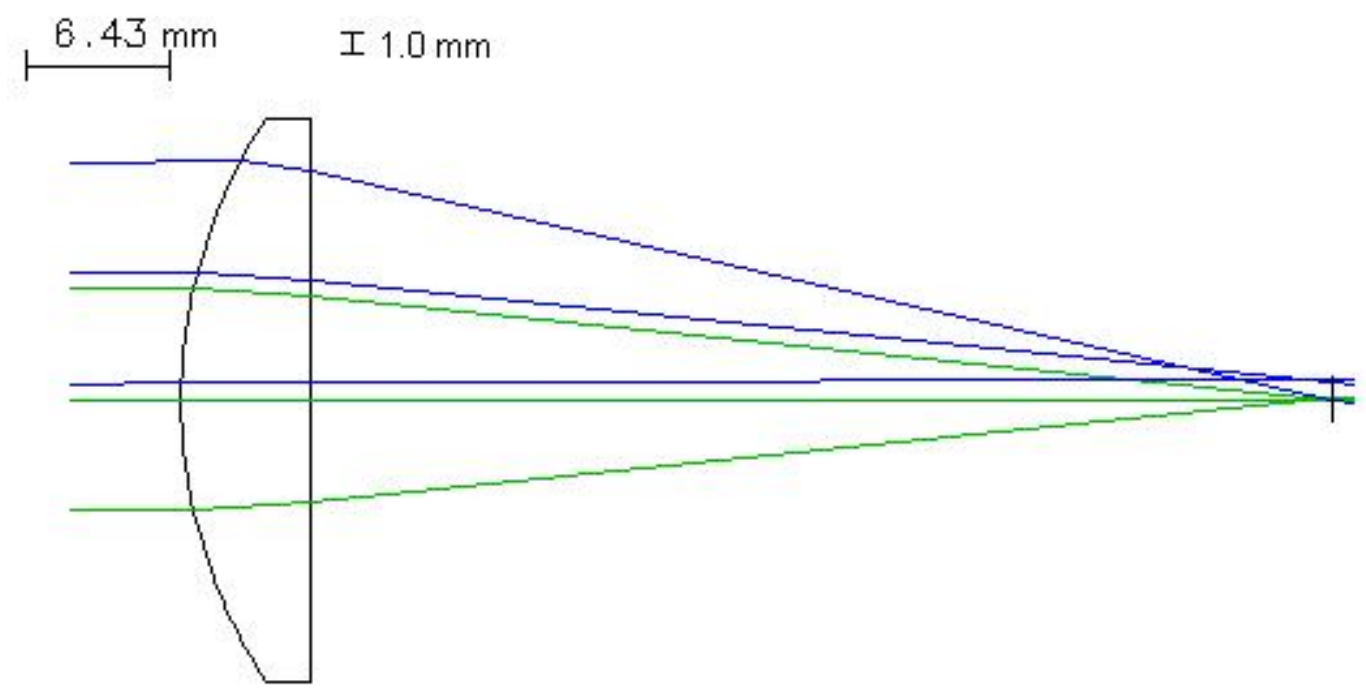

Figure 14 - Modeling in OSLO

From the rotation speed of the scanning mirror and the number of degrees per incident scan, the observation window was calculated at 27.8 microseconds. The size of the incident line (2mm) was used to calculate the region of the CCD used. The CCD has 256 pixels and is $6 \mathrm{~mm}$ high. The incident line from the laser spark was $2 \mathrm{~mm}$ high. Of the 256 pixels on the CCD, 86 pixels were actively collecting light from the laser spark. The time scale for the dynamic LIBS experiments was calculated to be 0.32 microseconds per pixel. 


\section{Signal Processing and Analysis}

The final focusing lens of the experimental set up eclipsed the dynamic LIBS signal. The signals of the true time-dependent characteristics of the emission lines were convoluted by the effects of the eclipsing. To recover this data, a function had to be formulated to de-convolute the effects of the eclipsing. The eclipsing of the beam and the lens was modeled in AUTOCAD. The modeling process calculated the areas of the incident beam of the laser spark as it moved off of the final focusing lens.

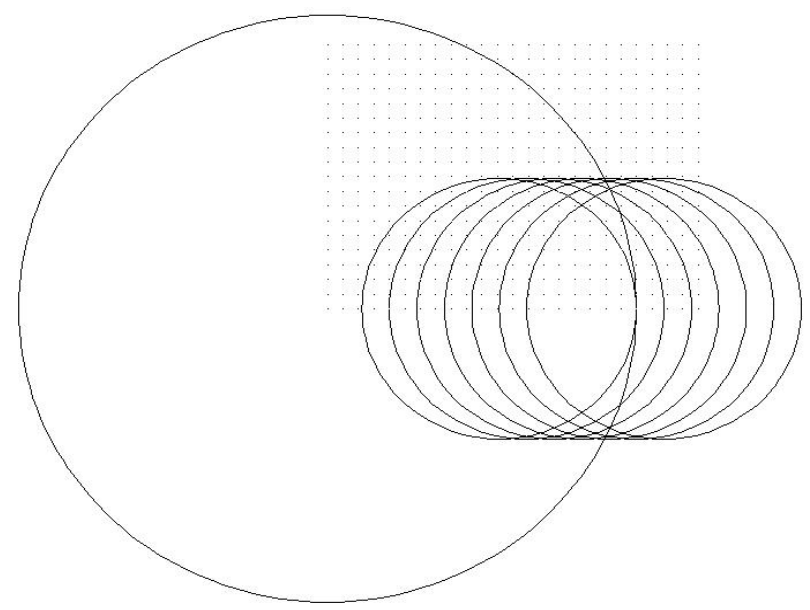

Figure 15- Traveling beam incident on the lens

The calculated areas were plotted with respect to center to center distance of the two circles (Figure 15). The larger circle modeled the clear aperture of the lens and the smaller circle modeled the incident light from the laser spark. A polynomial fit of the areas of the eclipsed smaller circle with respect to center to center distance was used to normalize the dynamic LIBS data. This equation best fit the trend in which the area of the eclipsed smaller beam decreased as the center to center distance of the two circles increased. The signal, $\mathrm{S}(\mathrm{x})$, from the dynamic LIBS test can be described as the time dependent behavior, which convoluted with the eclipsing function, as shown in Figure 16.

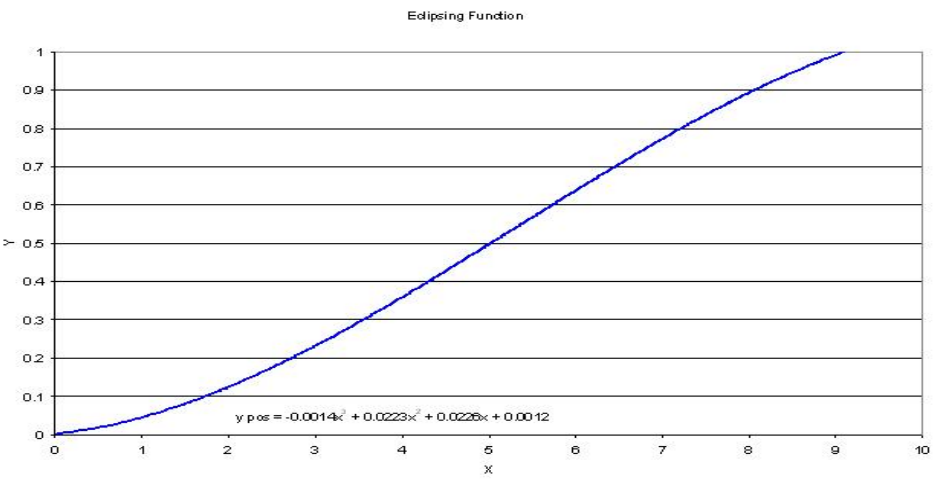

Figure 16 - Eclipsing Function 
Dynamic LIBS shows time-dependent characteristics of multiple emission lines within a single laser spark. Complications arise in signal analysis due to experimental conditions. The scanning mirror was not synchronized with the rest of the experimental set up. This results in a "brute force" testing method and random time intervals. Another complication was eclipsing, which was resolved by application of a normalizing function. The original dynamic LIBS signal was refined by background reduction and normalization before any trends in the data were observed, as shown in Figure 17.

HG 436

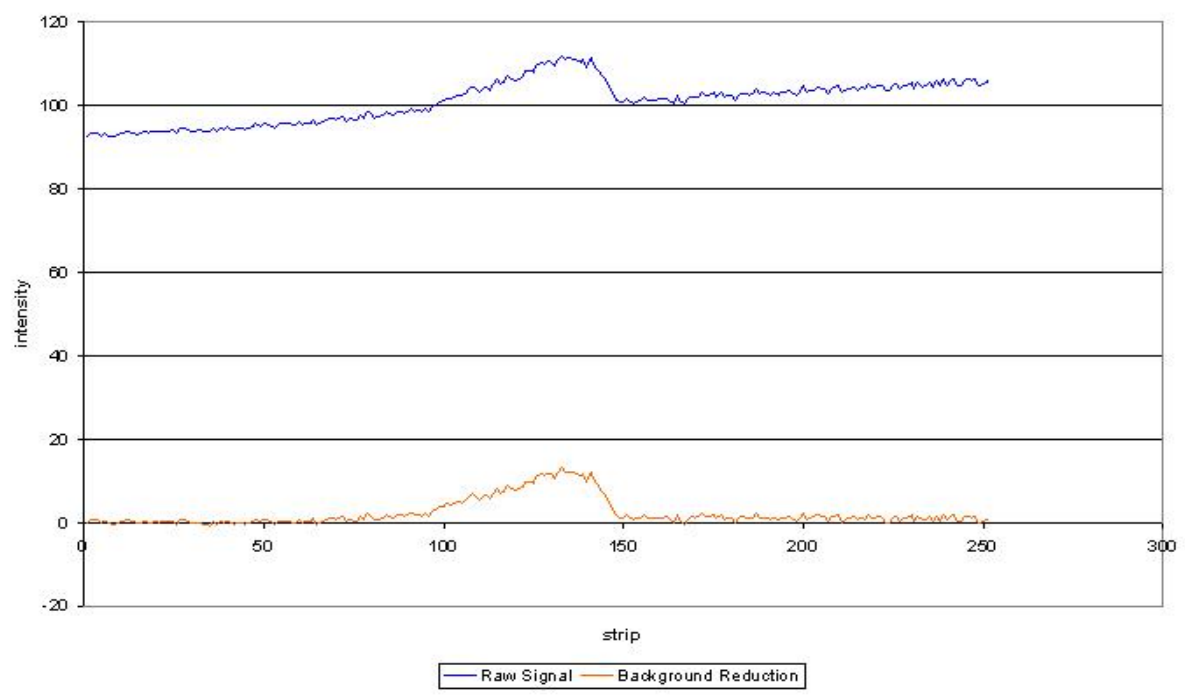

Figure 17 - Original and Background Corrected Signals

The application of a time scale and de-convolution of the eclipsing effect followed the initial background correction. Emission lines were plotted together to observe their temporal behavior simultaneously. Figure 17 shows processed data before the eclipsing effect was normalized. The solid line at $\mathrm{x}=0$ is approximately where the laser spark begins. A fast rise time is indicative of plasma formation. Decay times for the emissions of iron and mercury follow shortly after the plasma formation, while carbon decay seems to either be delayed or have a much slower decay rate, as indicated by the plateau shape following the plasma formation in the carbon related lines. 


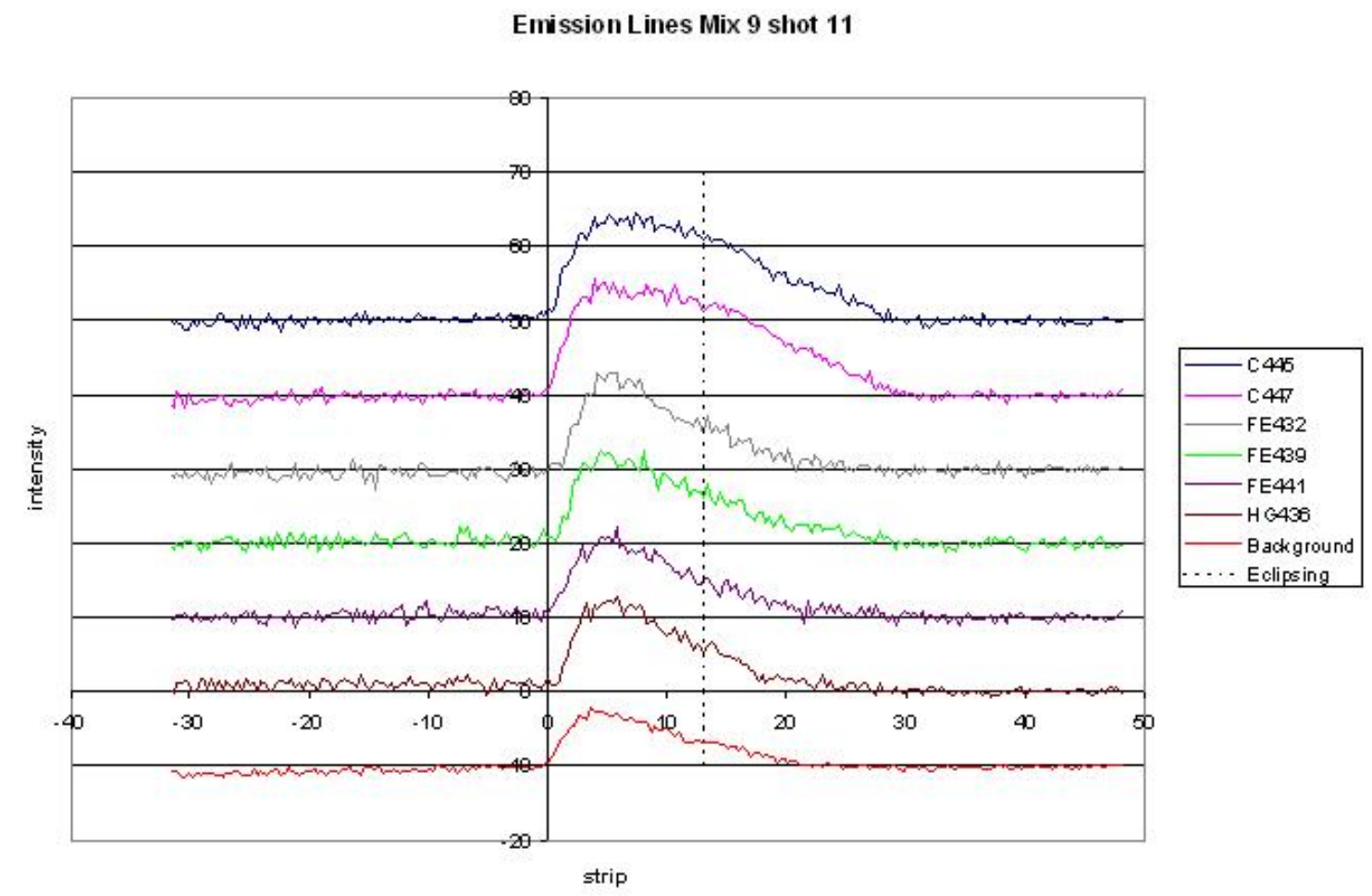

Figure 18 - Background Corrected, Time Scaled Dynamic LIBS Signal

The eclipsing effect, which takes place around ten to fifteen microseconds after spark formation, makes the remaining signal look linear. Normalization of the eclipsing effect revealed exponential decay in the emission lines, as shown in Figures 18. Figures 19 to 24 show details of the temporal plots of carbon, iron, and mercury. Figure 24 shows that the 436nm mercury line has the most immediate and fastest decay rate. Iron lines seemed to have immediate decay, but a slower decay time than the mercury decay (Figues 21 -23). All emission lines seem to show at least a 4 microsecond delay time before the decay begins. Carbon decay was the most difficult to observe. In some cases, carbon was observed to have a longer delay time before decay occurred. In other cases, carbon was shown to have a more gradual decay than iron or mercury. In either case, the carbon related emission lines showed drastically different characteristics than those of iron and mercury. 


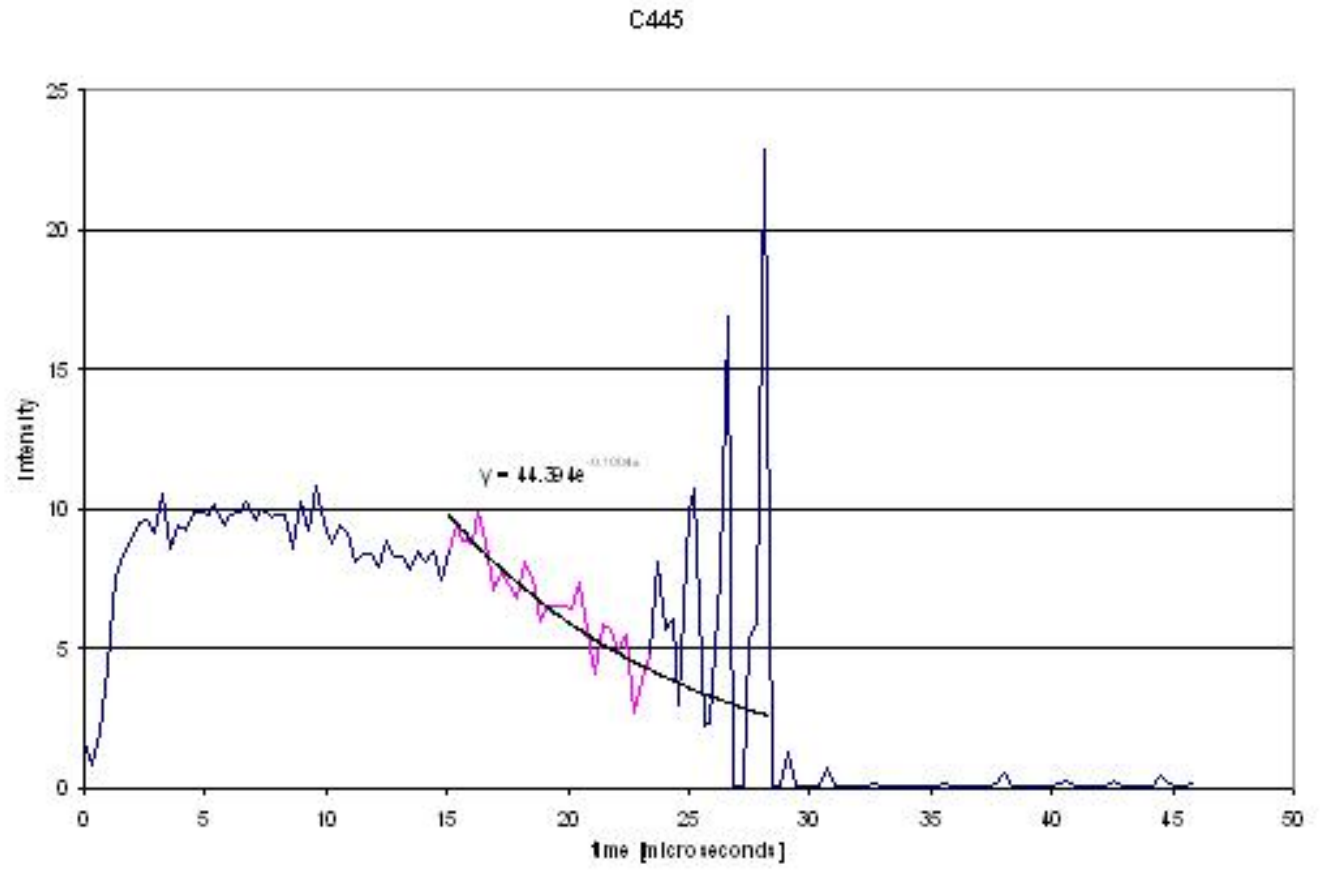

Figure 19 - C 445nm temporal behavior

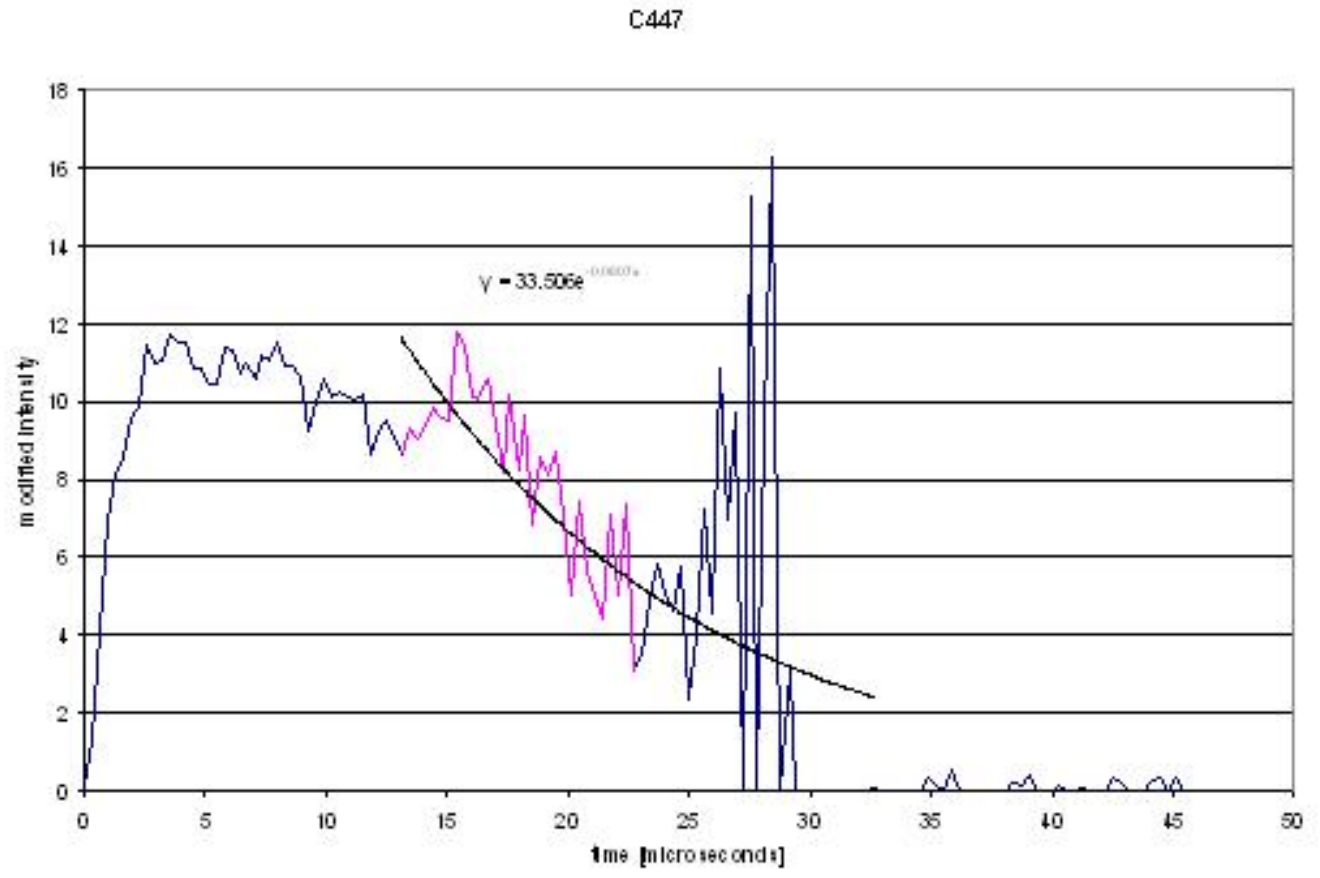

Figure 20 - C 447nm temporal behavior 


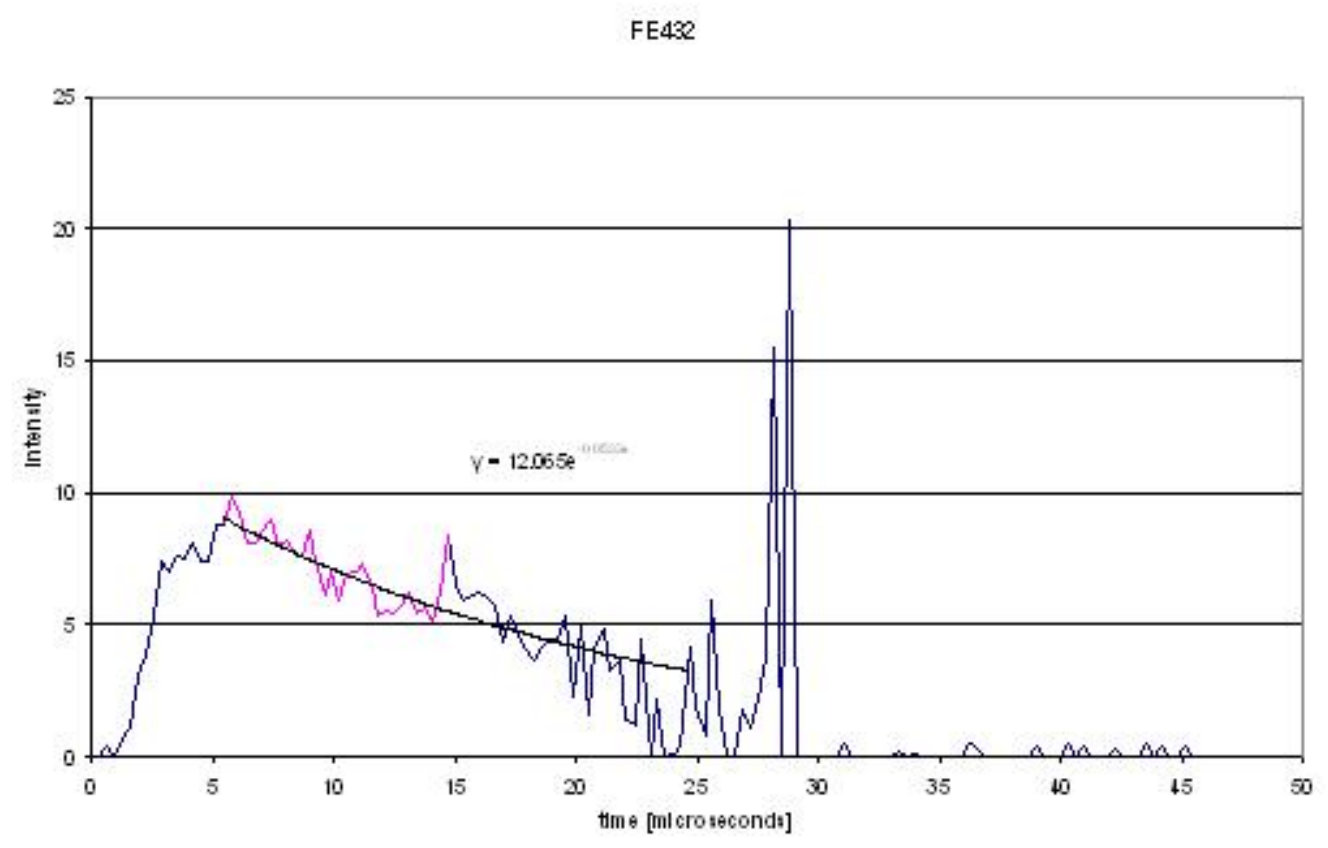

Figure 21 - Fe 432 nm temporal behavior

FE439

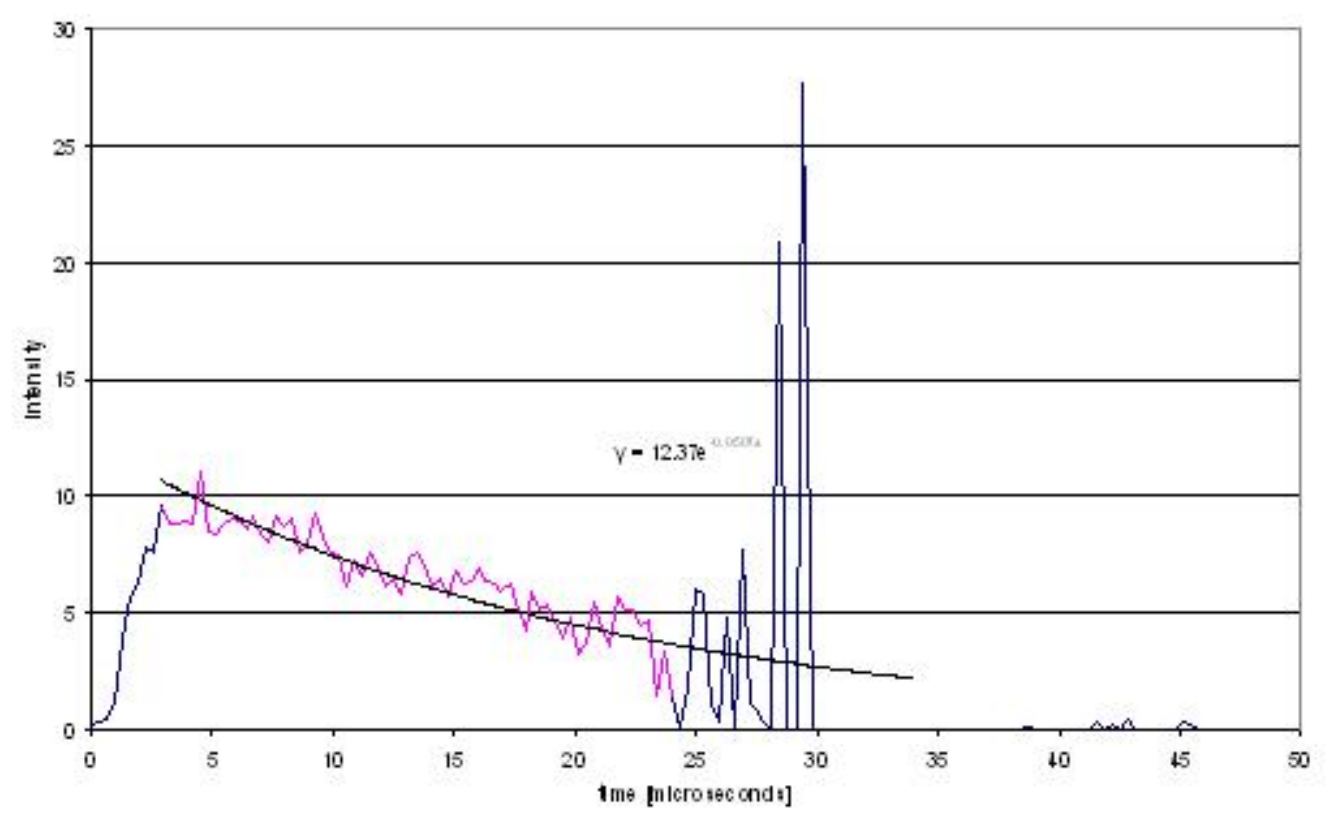

Figure 22 - Fe 439 nm temporal behavior 


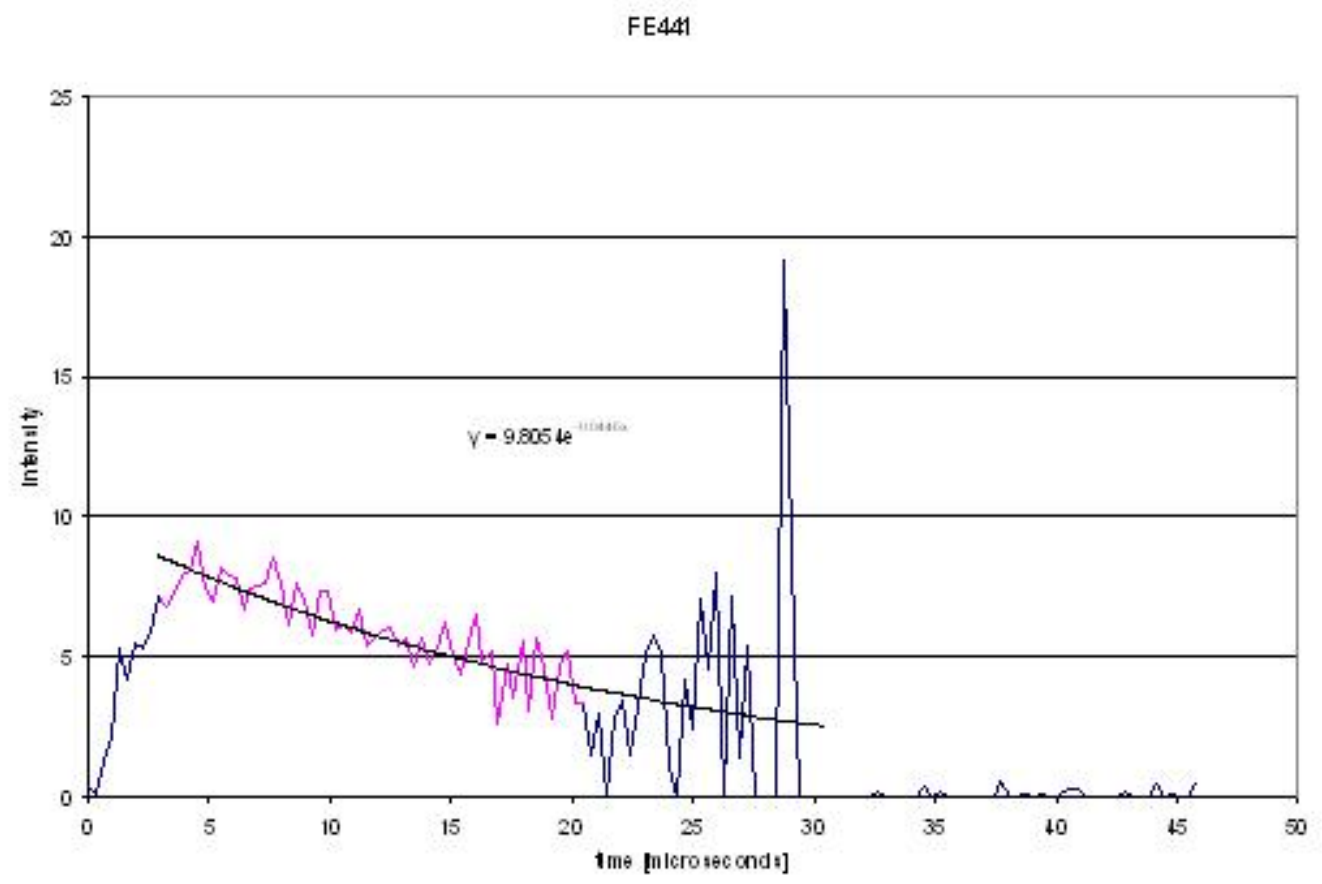

Figure 23 - Fe 441 nm temporal behavior

HG 436

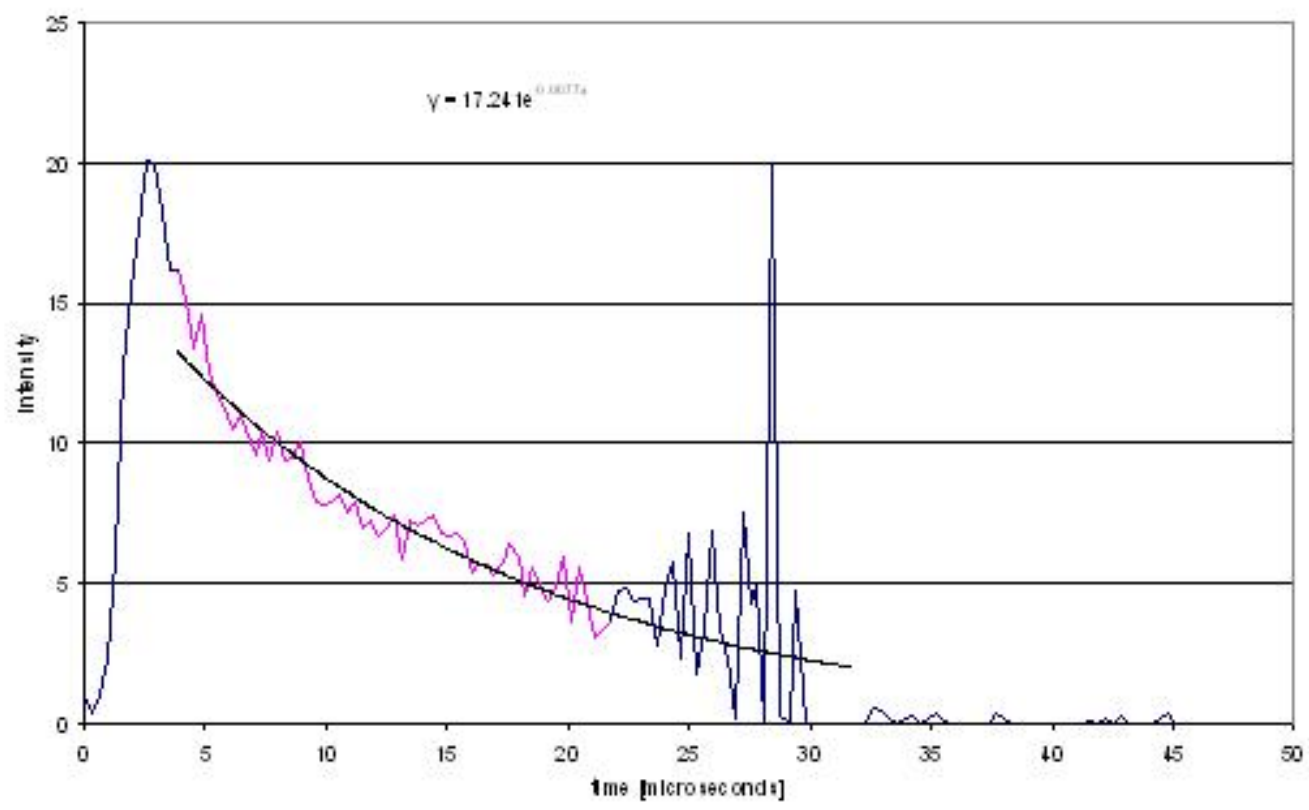

Figure 24 - Hg 436 nm temporal behavior 


\section{CONCLUSIONS}

A LIBS system has been developed to detect C, Fe, and Hg in coal. Significant information has been obtained in analyzing the signals. The ability to detect $\mathrm{C}, \mathrm{Fe}, \mathrm{S}$, and $\mathrm{Hg}$ by this system has produced mixed results. Upon obtaining data, the strength of the modified intensity signal from the collected light was plotted with respect to known concentration in the specimen. The graph of relative intensity with respect to concentration resulted in a strong correlation within the carbon and mercury emission lines. Carbon, which ranged from $61-81 \%$ mass within the synthetic coal mixture, resulted in strong correlations between line intensities and signal strength.

Use of the 436nm emission line as a means of detecting mercury was accurate to 800ppm. A plot between the signal strength and the concentration yielded a strong correlation, with only one discrepancy point, possibly due to a mixing error. The 800ppm limitation was due to the system hardware, i.e. limitation of the detection sensitivity of the CCD camera (0.1 Lux). A more sensitive detection instrument is required to attain lower detection limits.

Sulfur could not be detected using this experimental apparatus. This may be due the air quenching effect reported earlier [5]. Since the most sensitive emission lines in the sulfur spectrum are below 200nm, a combination of special optics and a vacuum environment are required to optimize the detection of sulfur.

Iron emission lines showed poor correlation between signal strength and concentration. This could be due to the mixing of pure iron in powder form within the synthetic mixtures as opposed to iron oxide. It is hypothesized that during the grinding and mixing process, the iron was drawn together instead of dispersed evenly, creating pockets of highly concentrated iron within the samples.

Based on qualitative and semi-quantitative analysis, dynamic LIBS testing provides basic information on the lifetime and temporal characteristics for individual emission lines. Mercury seems to have a more sudden decay, while carbon and iron show a slight delay before actual decay occurs. The data upon observation of emission lines of one specie compared to another suggests that emission lines posses differing temporal characteristics.

\section{Proposed Future Work}

\section{System Improvements}

The sensitivity and accuracy of the LIBS method developed within the scope of this research can be improved upon. This research has shown that all of the elements of interest have the possibility of being quantitatively analyzed much faster than the traditional ASTM methods. Because of the limited detection of mercury and sulfur within the synthetic coal mixtures, future work should be focused on the development of the sensitivity limits and the synchronization of the current LIBS system. 
Because of the effects of oxygen quenching mercury and sulfur emission lines, a vacuum environment or an inert gas environment could be created to enhance LIBS signals. To further enhance the signal, the CCD detector could be replaced by an Intensified CCD (ICCD) camera. An ICCD camera with time gating capabilities can be up to one thousand times more sensitive than a regular CCD camera (i.e. from 0.1 lux to 0.0001 lux). If an ICCD camera is used as a detector, and the samples are placed in a vacuum environment, the current LIBS apparatus could attain sensitivity in the part per billion range.

To improve upon the correlation of the iron signal, coal can be mixed with iron salts instead of pure iron. The iron salts should not be clumped together during grinding, and should produce a better distribution throughout the mixture. This would result in a better homogenization of iron throughout the synthetic mixtures, giving a more accurate representation of the iron concentration within the coal sample. Improvements within the pellet making procedure in this experiment can result in more accurate measurements and a better mixture for calibration of the LIBS system.

Dynamic LIBS testing gives us qualitative data of how different emission lines behave. The analysis of the dynamic LIBS signals allowed for observations of timedependent characteristics of different emission lines. Because the system was not synchronized, a "brute force" method was relied upon to obtain usable dynamic LIBS signals. Synchronization of the spinning mirror in conjunction with the laser and the CCD camera would be necessary for commercialization of this apparatus. Anomalies in the dynamic LIBS data were observed. A further study of these anomalies will allow for a better understanding of the temporal behavior of the emission lines and the laserinduced plasma.

\section{The Ideal LIBS Apparatus}

With all the recommended system improvements being implemented into one system, a significantly improved LIBS system can become a reality. The apparatus and procedure of this ideal apparatus is very similar to this experimental work. Differences between the ideal and current system would be the vacuum chamber in which LIBS testing can be done. Though the implementation of a vacuum chamber will result in the loss of instrument mobility and on-line measurements, ambient air that quenches sulfur and mercury emissions will be removed, resulting in a detectable sulfur signal and greater sensitivity to mercury. The ideal LIBS apparatus would have a high resolution spectrograph that interfaces with a specialized UV sensitive ICCD to select and tune the wavelength range, a vacuum chamber with specialized optics to tune in on the ultraviolet sulfur emission lines, synchronization between the laser and the spinning mirror. With these significant improvements to the system, an ideal LIBS apparatus can be built that can improve upon the existing results obtained in this research. 


\section{REFERENCES}

[1] Wiesburg, De Saro, Craparo; "Real Time, In-Situ Laser Sensor for Feedsock Monitoring in Gasifiers", Proceedings of the $29^{\text {th }}$ International Technical Conference on Coal Utilization \& Fuel Systems (2004), Vol. 1, 445-456.

[2] Harrison; Massachusetts Institute of Technology Wavelength Tables, 1969 M.I.T Press, Cambridge MA.

[3] Lazzari, De Rosa, Rastelli, Ciucci, Palleschi, Salvetti; "Detection of mercury in air by time-resolved laser-induced breakdown spectroscopy technique”, Laser and Particle Beams (1994), 12(3), 525-530.

[4] Tognoni, Palleschi, Corsi, Cristoforetti; “Quantitative micro-analysis by laser-induced breakdown spectroscopy: a review of the experimental approaches”, Spectrochimica Acta Part B (2002), 57, 1115-1130.

[5] Sturm, Noll; "Laser-induced breakdown spectroscopy of gas mistures of air, CO2, N2, and C3H8 for simultaneous C, H, O and N measurement”, Applied Optics (2003), 42(30) 6221-6225.

[6] Andrew Aurelio, "Laser-Induced Breakdown Spectroscopy as a Diagnostic Tool for Coal Fines”, MSME thesis, West Virginia University (2005). 
Appendix 38: Determination of Factors Affecting the Separation of Potentially Hazardous Trace Elements and their Behavior in Coal Tailings Impoundments (KY005) 


\section{TECHNICAL REPORT}

\begin{tabular}{lll}
\hline Contract Title and Number: & \multicolumn{2}{l}{ Period of Performance: } \\
\cline { 2 - 2 } Crosscutting Technology Development at the Center for & Starting Date: & $6 / 1 / 2005$ \\
Advanced Separation Technologies & Ending Date: & $10 / 31 / 2008$ \\
(DE-FC26-02NT41607) &
\end{tabular}

Sub-Recipient Project Title:

Determination of Factors Affecting the Separation of

Potentially Hazardous Trace Elements and their

Behavior in Coal Tailings Impoundments

Principal Investigators:

Huggins, Shah, Huffman

Contact Address:

University of Kentucky

533 S. Limestone Street

Lexington KY 40506

$\underline{\text { Subcontractor Address: }}$

No subcontracts issued.
Report Information:

Type: Final

Number:

Period: $\quad$ 10/1/06-9/30/08

Date: $\quad 9 / 30 / 07$

Code: KY005-Final

Contact Information:

Phone: (859) 257-4045

Fax: (859) 257-7215

E-Mail: fhuggins@engr.uky.edu

$\underline{\text { Subcontractor Information: }}$

Phone:

Fax:

E-Mail:

\section{DISCLAIMER}

"This report was prepared as an account of work sponsored by an agency of the United States Government. Neither the United States Government nor any agency thereof, nor any of their employees, make any warranty, express or implied, nor assume any legal liability or responsibility for the accuracy, completeness, or usefulness of any information, apparatus, product, or process disclosed, or represents that its use would not infringe privately owned rights. Reference herein to any specific commercial product, process, or service by trade name, trademark, manufacturer, or otherwise does not necessarily constitute or imply endorsement, recommendation, or favoring by the United States Government or any agency thereof. The views and opinions of authors expressed herein do not necessarily state or reflect those of the United States Government or agency thereof." 


\begin{abstract}
Conventional coal cleaning methods effectively reduce the concentrations of many trace elements in cleaned coals that are burnt in power plants. However, as a result, trace element concentrations in tailings from coal washing operations are enhanced and their behavior in tailings disposal may then become an environmental issue. Data on elemental concentrations alone do not provide sufficient insight to understand completely the behavior of trace elements during coal cleaning or in tailings disposal scenarios. In this project, modes of occurrence of trace elements in an Illinois \#6 coal, the resulting clean coal and tailings fractions, and leached tailings fractions were determined by XAFS spectroscopy to complement data obtained from conventional elemental (XRF, ICP-MS, etc.) and mineralogical (XRD) analyses. Two long-term leaching tests ( $\sim 150$ days $)$ were investigated because the 18-hour TCLP test was negative for all elements of interest. In one method, the tailings were stored continuously under water, while in the second (a variant of ASTM D5744) the tailings were cycled through dry and wet (submerged) periods on a weekly basis. Most elements of interest were found to be associated with either pyrite (As, Se, Hg) or with illite (Cr, V, Ni?), which largely dictated the behavior of these elements in the physical separation processes and in the leaching tests. Only $\mathrm{Ge}$ and to a lesser extent $\mathrm{Hg}$ and Se were noted to have significant affinity with the coal macerals and did not separate efficiently. The rapid mobilization of $\mathrm{Na}$ and alkaline earth elements $(\mathrm{Mg}, \mathrm{Ca}, \mathrm{Sr})$ was important in effectively buffering the $\mathrm{pH}$ during the alteration of pyrite and preventing or delaying the mobilization of arsenic. The formation of insoluble natrojarosite during the alteration of pyrite was also instrumental in sequestering arsenic.
\end{abstract}




\section{TABLE OF CONTENTS}

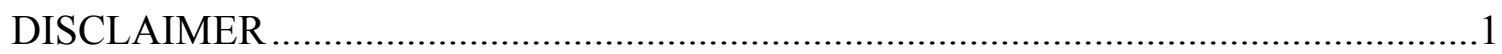

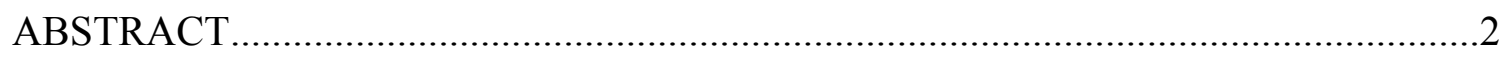

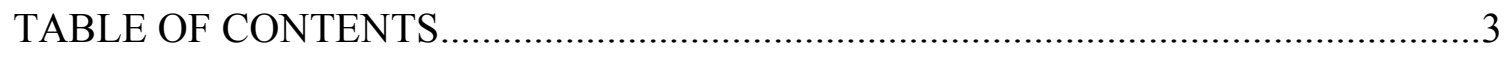

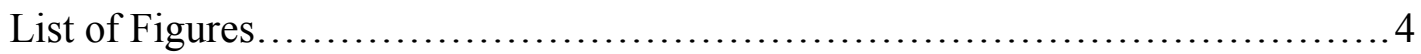

List of Tables..................................................................... 5

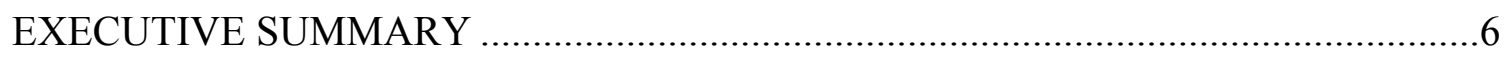

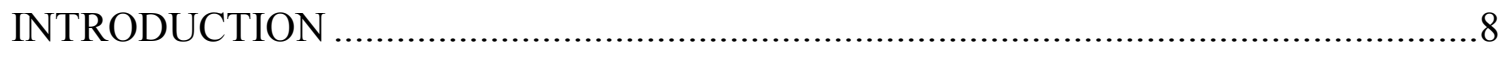

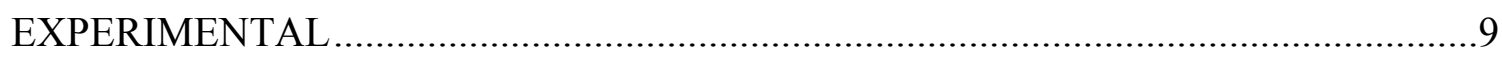

Collection and preparation of coal and tailings samples .........................................

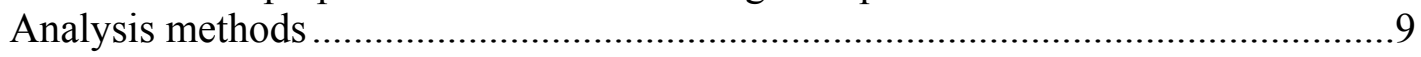

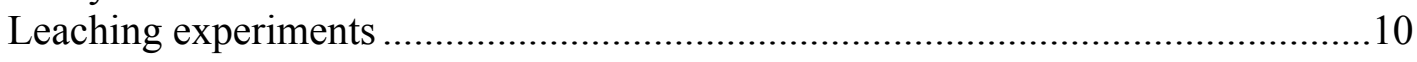

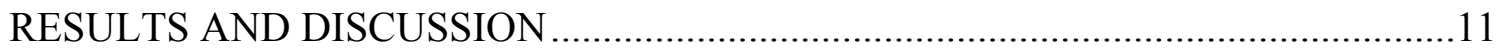

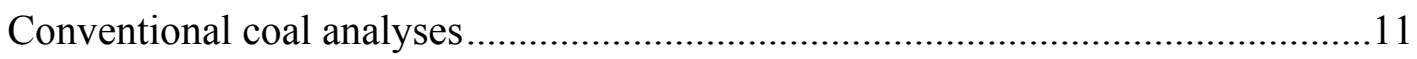

Partitioning of elements among coal fractions..........................................................11

Trace element mobility in leaching scenarios........................................................17

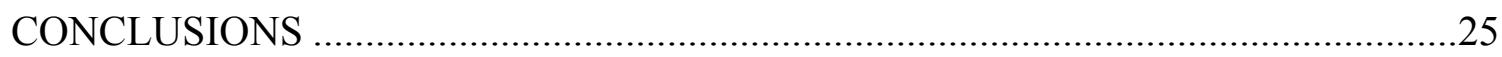

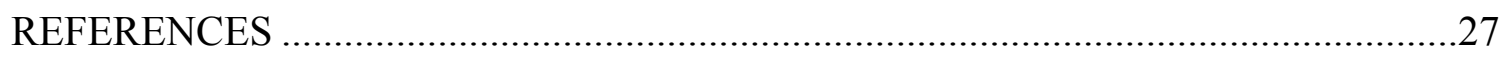

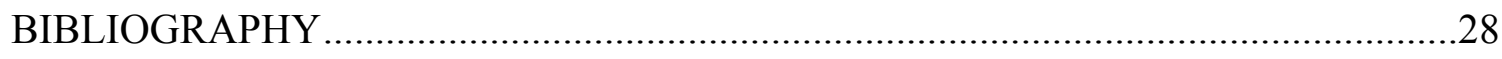

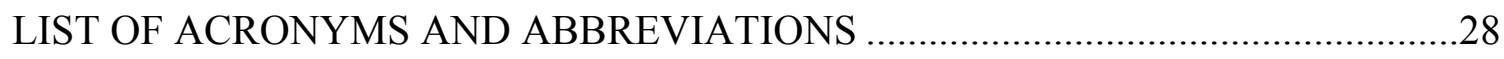




\section{List of Figures}

FIGURE 1: Diagram showing coal fractions generated at the preparation plant.

FIGURE 2: Schematic diagram for the cyclic leaching test of Illinois \#6 coal tailings fractions constructed for this project. The arrows indicate air flow paths.

FIGURE 3: Bar diagram summarizing the efficiency of separation of various elements between the CLN and PLR fractions during gravity cleaning methods applied to the feed (FEE) coal. Note the inverted nature of the bar for Ge (circled) and the short bar for $\mathrm{Hg}$.

FIGURE 4 (left): Arsenic XANES spectra of the four Illinois coal fractions. Distinct peaks due to As in pyrite and As as arsenate are indicated.

FIGURE 5 (right): Correlations between As and $\mathrm{Zn}$ concentrations and pyrite abundance in the four Illinois coal fractions. Note that the As contents are multiplied by a factor of 10 .

FIGURE 6 (left): Correlations between $\mathrm{V}, \mathrm{Cr}$ and $\mathrm{Ni}$ concentrations and illite abundance in the four Illinois \#6 coal fractions.

FIGURE 7 (right): Chromium XANES spectra of the four Illinois \#6 coal fractions.

FIGURE 8 (left): Summary of the distribution of iron among different minerals in the four coal fractions as determined by Mössbauer spectroscopy. The balance of the iron is present as pyrite, $\mathrm{FeS}_{2}$.

FIGURE 9 (right): Summary of the distribution of arsenic between pyrite and arsenate minerals in the four coal fractions measured about four months apart as determined by As XANES spectroscopy. The differences for each fraction indicate slow oxidation of arsenic associated with pyrite during sample storage. The balance of the arsenic is present as arsenic in pyrite.

FIGURE 10: Arsenic XANES spectra for the RBR and PLR tailings fractions before and after (leached) the TCLP test. Note the unchanged nature of the spectra before and after the test, indicating that there was no significant change in arsenic speciation as a result of the test, consistent with the lack of any As leached during the test.

FIGURE 11: Correlation between conductivity and sulfate anion concentration determined for the aqueous leachates generated in the cyclic test. Leachates for the two tailings fractions, PLR and RBR, are not differentiated in this plot.

FIGURE 12: Comparison of the cumulative amounts of leached elements from PLR tailings after weeks 22 and 23 of the cyclic testing and after week 20 of the baseline test. The data are presented as a percentage of the total amount of an element originally present 
in the tailings. Elements not shown were negligibly leached in all tests. Data for the RBR tailings were similar.

FIGURE 13: Cumulative \% extraction of alkali (Na) and alkaline earth $(\mathrm{Ca}, \mathrm{Mg}, \mathrm{Sr})$ elements from RBR (dashed lines) and PLR (solid lines) tailings fractions with length of time in the cyclic leaching test. Also shown is the variation of $\mathrm{pH}$ for the leachates.

FIGURE 14: Arsenic XANES spectra from samplings of the coal tailings fractions taken after 7 and 22 weeks into the cyclic test. Note the increasing intensity of the peak due to arsenate relative to that for As/pyrite.

\section{List of Tables}

TABLE 1: Heating Value (HV) and Proximate Analyses for Four Fractions of Illinois \#6 Coal.

TABLE 2: Ultimate Analyses of Different Streams at the Preparation Plant.

TABLE 3: Forms of Sulfur Analyses (wt \%) of Different Streams at the Preparation Plant.

TABLE 4: Major Element Analyses of $500^{\circ} \mathrm{C}$ Ash Prepared from Illinois \#6 Coal and Derived Fractions (wt $\%$ ).

TABLE 5: Trace Element Analyses of Illinois \#6 Coal and Derived Fractions (ppm).

TABLE 6: Major Mineral Data (wt\% of coal) for Illinois Coal and Derived Fractions.

TABLE 7: Summary of modes of occurrence for key trace elements in this sample of Illinois \#6 coal.

TABLE 8: Variation of conductivity, sulfate concentration, $\mathrm{pH}$, and Eh of leachate samples taken at different times during the cyclic and baseline leaching tests on the Illinois \#6 tailings fractions.

TABLE 9: ICP-MS Data for Leachates from Cyclic Testing on RBR Coal Tailings Fraction.

TABLE 10: ICP-MS Data for Leachates from Cyclic Testing on PLR Coal Tailings Fraction.

TABLE 11: ICP-MS Data for Leachates from Baseline Testing (Week 20) on Coal Tailings. 


\section{EXECUTIVE SUMMARY}

One of the perceived problems associated with contamination of local streams and rivers by waters from breached or failed impoundment dams is the possible introduction of potentially hazardous trace elements into surface waters and groundwater that might be used for public water supplies. Such elements are introduced into impoundment waters as a result of the aqueous leaching and alteration of pyrite and other minerals in coal tailings that can mobilize trace elements associated with them. How and in what quantity such elements are released depends on a number of factors, including not only how much of the element is present in the tailings but also how it occurs in the tailings. Whereas there have been many studies of the concentrations of trace elements in coals and coal tailings, there have been virtually no investigations of the modes of occurrence of trace elements in coal tailings and their significance in providing answers to questions relating to the mobility of trace elements in coal impoundments and similar disposal situations. In this project, we attempt to fill this knowledge gap by first determining the modes of occurrence of various key elements $(\mathrm{S}, \mathrm{Fe}$, $\mathrm{Cr}, \mathrm{V}, \mathrm{Mn}, \mathrm{Zn}, \mathrm{As}, \mathrm{Se}$ ) in clean coal and tailings fractions obtained from an Illinois \#6 coal by gravity separation methods employed at a commercial coal-cleaning plant in Southern Illinois. With this information on elements in the coal fractions, we then examine the leaching of arsenic and other trace metals from the tailings fractions in experiments that simulate disposal of tailings under water in coal impoundments. Such experiments included the 18-hour Toxicity Characteristic Leaching Procedure (TCLP), and two much longer term (up to 150 days) experiments. One of these tests, termed the baseline or static test, consisted of passively storing the tailings under water for the full length of the test; the other test, termed the cyclic test, was based on ASTM Standard Method D-5744 and was a much more aggressive test. This test was designed to accelerate the oxidation of pyrite and the mobilization of associated trace elements. It consisted of weekly cycles of submerging the tailings under water for 1 day, followed by 3 days' exposure to first dry air and then 3 days' exposure to humid air. These cycles were repeated for 22 weeks and the leachate obtained after each weekly submersion was collected for analysis.

Detailed information on trace elements in the Illinois \#6 coal and related fractions, as well as in the residues from the leaching tests, was obtained from (i) conventional coal analyses, (ii) multi-element analysis methods such as X-ray fluorescence (XRF) for major elements and ICP-MS for trace elements, (iii) X-ray diffraction to quantify the mineralogy of the coal fractions, and (iv) Mössbauer and X-ray absorption fine structure (XAFS) spectroscopic methods that provided direct information on elemental occurrences. A synthesis of the results from these analyses led to the following conclusions regarding the occurrence of key elements in this Illinois \#6 coal:

- Arsenic: $100 \%$ associated with pyrite in all fractions; increasing oxidation of arsenical pyrite to arsenate noted over time during sample storage.

- Chromium: Largely present in association with illite with remainder in organic association; $>98 \% \mathrm{Cr}$ in tailings fractions is associated with illite

- Germanium: Highly organically associated

- Iron $>90 \%$ associated with pyrite, $\sim 5 \%$ associated with illite and $\sim 3 \%$ with jarosite; slow conversion of pyritic iron to sulfate over time

- Manganese: Complex - appears to be at least 3 significant distinct species 
- Mercury: $\quad$ Some apparent association with pyrite; some with coal macerals

- Nickel: Similar to $\mathrm{Cr}$ and V?

- Selenium: Associated with pyrite, but some organic Se as well

- Vanadium: Similar to chromium, but with more of the element in organic association; $>95 \% \mathrm{~V}$ in tailings fractions is associated with illite

- Zinc: Complex - three distinct major species: sphalerite ( $\mathrm{ZnS})$; $\mathrm{Zn}$ assoc. w/ illite; and $\mathrm{Zn}_{3}\left(\mathrm{PO}_{4}\right)_{2} \cdot \mathrm{xH}_{2} \mathrm{O}$ (tentative).

Armed with this information on elemental modes of occurrence in the Illinois \#6 coal fractions, similar examination was undertaken of the leachates and residues obtained in the experiments designed to simulate leaching during tailings impoundments. The 18-hour TCLP test gave negative results for all RCRA elements indicating that these tailings were not an environmental hazard. Consequently, this test was not informative regarding trace element mobilities. In the longer term baseline and cyclic tests, it was observed that the $\mathrm{pH}$ of the leachates remained close to neutral until the very end of the test when more acidic values were encountered. The elements that were most mobile in these tests were sodium and the alkaline earth elements, $\mathrm{Mg}, \mathrm{Ca}$, and $\mathrm{Sr}$. Sulfate also showed significant mobility and exhibited a marked correlation $\left(\mathrm{r}^{2}>0.99,16\right.$ observations) with conductivity. However, neither iron nor arsenic showed any mobility although Mossbauer spectroscopy did indicate that a minor amount of iron as pyrite was oxidized to iron as jarosite and arsenic XAFS indicated that a considerable fraction of the arsenic was oxidized to arsenate $\left(\mathrm{AsO}_{4}{ }^{3-}\right)$ over the course of the leaching treatments. Elements primarily associated with illite, such as $\mathrm{K}, \mathrm{Cr}$ and $\mathrm{V}$, exhibited no significant mobility. Trace elements that did exhibit some mobility during these tests included $\mathrm{Mn}, \mathrm{Co}, \mathrm{Ni}$, and, to a lesser extent, $\mathrm{Zn}$.

To account for these observations, it is hypothesized that the rapid dissolution of $\mathrm{Na}$, followed by the slower dissolution of alkaline earth elements effectively buffers the $\mathrm{pH}$ of the leachates thereby minimizing the formation of acid $\left[\mathrm{H}^{+}\right]$that would accelerate the dissolution of pyrite and the release of arsenic and other associated elements. In addition, the presence of sodium at near-neutral $\mathrm{pH}$ values also promotes the formation of insoluble natrojarosite $\left(\mathrm{Na}(\mathrm{Fe})_{3}\left(\mathrm{SO}_{4}\right)_{2}(\mathrm{OH})_{6}\right)$ from any alteration of the pyrite that does occur. This sulfate mineral can also act as a sink for the arsenate anions as a result of their substitution for the sulfate anions thereby preventing the mobilization of As as arsenate. The following chemical equation effectively accounts for all of these observations:

$$
3 \mathrm{FeS}_{2}+3 \mathrm{H}_{2} \mathrm{O}+13.5 \mathrm{O}_{2}+\left[\mathrm{Na}^{+}\right]_{\mathrm{sol} ' \mathrm{n}} \rightarrow \mathrm{NaFe}_{3}\left(\mathrm{SO}_{4}\right)_{2}(\mathrm{OH})_{6}+4\left[\mathrm{SO}_{4}{ }^{2-}\right]_{\mathrm{sol} \text { 'n }}
$$

Not only does formation of insoluble jarosite by this reaction prevent the release of arsenic, but it also does not result in the formation of significant acid $\left[\mathrm{H}^{+}\right]$formation, while allowing for release of some $\left[\mathrm{SO}_{4}{ }^{2-}\right]$ anions, consistent with observation. It is clear that in these coal tailings, sodium plays an important role in preventing or delaying the dissolution of pyrite and the release of associated trace elements. These findings may have significant application to preventing and minimizing acid formation associated with disposal of coal tailings. 


\section{INTRODUCTION}

The major goal of separation technology is to increase the yield of valuable product by separating it from undesirable waste components. In the coal separation industry that serves the electrical power generation industry, the specific goal is to separate the incombustible mineral matter from the combustible macerals in order to develop a clean-coal product that has a higher calorific value and generates less combustion wastes and emissions. Such coal cleaning is especially valuable for coals from the bituminous coal regions of the eastern United States, since the mineralogy of the coals from these regions is generally dominated by quartz, clays and pyrite, which are minerals in coal that can be separated efficiently by conventional and/or advanced coal-cleaning methods. Such cleaning also removes certain trace elements designated as hazardous air pollutants (HAPs) by the 1990 Clean Air Act Amendments (CAAA). However, such HAP elements are then often concentrated in coal tailings and may become of concern to the 1976 Resource Conservation and Recovery Act (RCRA) during disposal of the mineral-rich tailings. The partitioning of a trace element between clean product and tailings is determined by the mineral phase in which the element occurs, its valence and magnetic states, and association with major inorganic or carbonaceous components of the coal. These mode-of-occurrence factors have only rarely been determined with respect to trace elements in coal cleaning operations and, as far as we can tell, never with respect to their behavior in coal tailings disposal options.

The first objective of this investigation was to determine why some hazardous air pollutant (HAP) elements separate well and others poorly during various coal cleaning processes. A second objective was then to investigate and interpret the behavior of the HAP elements in laboratory simulations of the disposal of tailings in ponds. To address these issues, basic information regarding the occurrence of trace elements and minerals in coal and their behavior in coal cleaning processes and tailings disposal scenarios has been obtained using a variety of techniques that enable us to determine the mineralogy of the coal and mode of occurrence of specific elements. Such techniques, supplemented by more conventional analysis methods, included X-ray diffraction (XRD) to determine the mineralogy and X-ray absorption fine structure (XAFS) spectroscopy to obtain direct data on the speciation of trace elements of interest in both clean and tailings fractions and in residues after leaching tests on the tailings fractions. 


\section{EXPERIMENTAL}

\section{Collection and preparation of coal and tailings samples}

Approximately $800 \mathrm{lbs}$ each of raw coal, the cleaned coal, and two tailings fractions were collected from a commercial preparation plant in Southern Illinois. Samples were collected incrementally over 30-minute intervals. The preparation plant treats feed from a single mine on the Illinois \#6 seam at a capacity of 1,000 tph. The overall separation scheme is shown in Figure 1. Reject from the plant is normally sent to a tailings impoundment.

The raw coal to the plant was screened after initial crushing in a rotary breaker. The coarse fraction of the raw coal screen, $2 \frac{1}{2} " \times 1 \mathrm{~mm}$, was fed to heavy media (HM) cyclones. The clean coal overflow from the HM cyclone separates from mineral-rich tailings due to the differences in density between coal, the mineral matter, and the magnetite heavy media. The minus $1 \mathrm{~mm}$ fine fraction of the raw coal screen was sent to classifying cyclones which in turn fed a spiral circuit. The spiral circuit also separates clean coal and mineral matter based on density differences. The combined clean product of the coarse and fine cleaning circuits has a size range of $2 \frac{1}{2} " \times 0$.

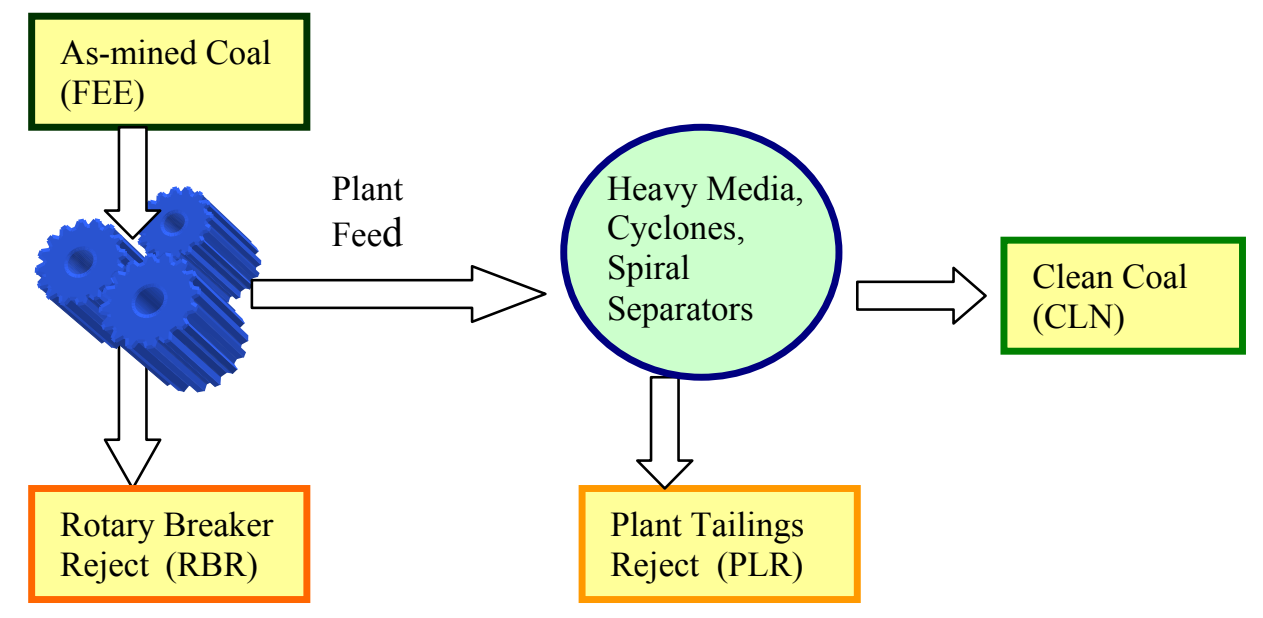

FIGURE 1: Diagram showing coal fractions generated at the preparation plant.

Samples of the raw feed, rotary breaker (RB) reject, the plant reject, and the clean coal were dried, crushed to $100 \%$ passing $1 / 4$ " mesh, and riffle split to obtain representative samples. Final samples for the analyses were further crushed to 100 mesh top size and split into various fractions, according to the mass needed for each type of analysis.

\section{$\underline{\text { Analysis methods }}$}

The following conventional analyses were carried out on each major coal fraction:

- Ultimate, proximate, forms of sulfur, heating value;

- X-ray fluorescence (XRF) of major elements in $500^{\circ} \mathrm{C}$ ash from each fraction;

- Trace element analyses by ICP-MS and individual analyses for $\mathrm{Hg}$ by mercury analyzer; 
- Mineral matter by X-ray diffraction (XRD) using Rietveld method.

Mode of occurrence analyses consisted of:

- Fe speciation and oxidation state determinations by Mössbauer spectroscopy;

- Speciation of sulfur and key trace elements by X-ray absorption fine structure (XAFS) spectroscopy.

\section{Leaching experiments}

To investigate the behavior and mobility of elements in simulated tailings disposal scenarios, the two tailings fractions have been subjected to three different leaching experiments. The first method attempted was the Toxicity Characteristic Leaching Procedure (TCLP). Samples were sent to a commercial laboratory for testing. All RCRA elements in both tailings fractions were found to be negligibly leached by the 18-hour TCLP test. Hence, two tests of longer duration were devised and constructed.

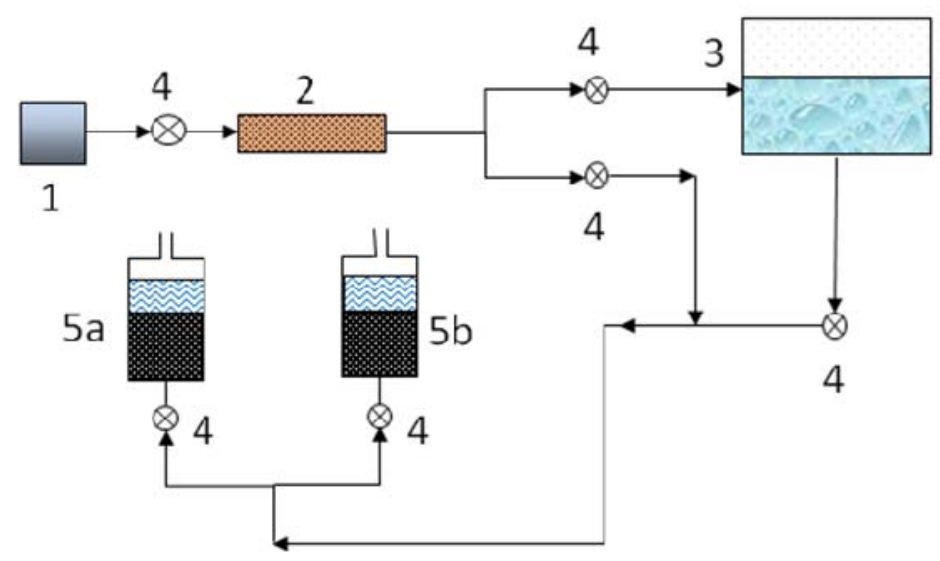

\section{Compressed Air 2. Desiccant 3. Humidifier 4. Valve 5. Leacr Vessel (a) RBR (b) PLR \\ Cyclicleaching consists of I day water submersion, 3 days dry air and 3 cays moist air, repeated weckly}

FIGURE 2: Schematic diagram for the cyclic leaching test of Illinois \#6 coal tailings fractions constructed for this project. The arrows indicate air flow paths.

One method, termed the baseline static test, consisted simply of storing $200 \mathrm{~g}$ of each tailings fraction under $200 \mathrm{ml}$ of de-ionized water and using $\mathrm{pH}$ to indicate any significant leaching activity. The second method, termed the cyclic test and shown schematically in Figure 2, was based on ASTM Method D-5744 (ASTM, 1996). This test operated on a 7-day cycle in which $200 \mathrm{~g}$ of tailings were soaked in $200 \mathrm{ml}$ of de-ionized water for one day, exposed to dry air for three days and then moist air for three days. For each cycle, the leachate was collected and a new cycle was started with fresh de-ionized water. The leachates were combined, as needed, to generate a multi-week sample, which was then sent for a battery of analyses consisting of measurements of $\mathrm{pH}, \mathrm{Eh}$, and sulfate, and ICP-MS for elemental analysis. Analyses were conducted after 0, 1, 3, 6, 7, 12, 15, 20 and 22 weeks. Small 
amounts of the solids were sampled after 7 and 22 weeks for Mössbauer and XAFS spectroscopy determinations. Both the static and cyclic tests were on-going for more than 150 days.

\section{RESULTS AND DISCUSSION}

\section{$\underline{\text { Conventional coal analyses }}$}

Data on the coal feed to the rotary breaker (FEE), the clean coal (CLN), and the tailings fractions generated by the rotary breaker (RBR) and gravity separation at the plant (PLR) are summarized in Table 1. Proximate analyses of the coal fractions are also shown in Table 1.

TABLE 1: Heating Value (HV) and Proximate Analyses for Four Fractions of Illinois \#6

\begin{tabular}{lcrccccc}
\hline \multicolumn{1}{c}{ Stream } & Wt\% & Coal & Moisture & Volatile & Ash & Fixed & VM \\
& Stream* & \multicolumn{1}{c}{ Btu/lb } & wt\% & Matter & wt\% & Carbon** & daf basis \\
\hline RB Feed (FEE) & 100 & 10,278 & 4.91 & 29.71 & 27.11 & 38.27 & 43.7 \\
RB Reject (RBR) & 1.5 & 1,869 & 5.70 & 10.83 & 76.83 & 6.65 & -- \\
Clean Coal (CLN) & 65 & 13,132 & 4.53 & 36.80 & 9.14 & 49.54 & 42.6 \\
Plant Reject (PLR) & 33.5 & 2,644 & 3.01 & 13.90 & 73.55 & 9.54 & -- \\
\hline
\end{tabular}

*based on plant historical data; ** by difference; daf - dry, ash-free

Ultimate analyses and forms of sulphur analyses, including estimates of pyritic sulphur from Mössbauer spectroscopy, are summarized in Tables 2 and 3, respectively.

TABLE 2: Ultimate Analyses of Different Streams at the Preparation Plant

\begin{tabular}{lrrrrrrr}
\hline \multicolumn{1}{c}{ Stream } & \%Ash & \%Moisture & $\% C$ & $\% H$ & $\% N$ & $\% O$ & $\% S$ \\
\hline RB Feed (FEE) & 27.66 & 5.15 & 52.52 & 4.10 & 0.98 & 10.65 & 4.09 \\
RB Reject (RBR) & 76.47 & 5.53 & 10.78 & 1.58 & 0.25 & 7.38 & 3.54 \\
Clean Coal (CLN) & 9.16 & 4.64 & 67.54 & 5.14 & 1.25 & 13.61 & 3.30 \\
Plant Reject (PLR) & 73.16 & 3.21 & 13.85 & 1.50 & 0.34 & 6.01 & 5.14 \\
\hline
\end{tabular}

TABLE 3: Forms of Sulfur Analyses (wt \%) of Different Streams at the Preparation Plant

\begin{tabular}{lcccc}
\hline \multicolumn{1}{c}{ Stream } & Pyritic & Sulfate & Organic & Pyr Mossb. $^{*}$ \\
\hline RB Feed (FEE) & 1.98 & 0.11 & 2.00 & 2.20 \\
RB Reject (RBR) & 2.88 & 0.10 & 0.56 & 3.59 \\
Clean Coal (CLN) & 0.86 & 0.11 & 2.33 & 1.05 \\
Plant Reject (PLR) & 4.67 & 0.19 & 0.28 & 4.46 \\
\hline
\end{tabular}

*estimated from Mössbauer spectroscopy

\section{Partitioning of elements among coal fractions}

Major elements and trace elements were determined in the different fractions using XRF on ash prepared at $500^{\circ} \mathrm{C}$ (Table 4) and ICP-MS on digested coal (Table 5), respectively. $\mathrm{Hg}$ was determined independently by CVAAS. The mineralogy of the different fractions was 
determined by X-ray diffraction using Rietveld analysis, supplemented by data from Mössbauer and chemical analyses (Table 6).

TABLE 4: Major Element Analyses of $500^{\circ} \mathrm{C}$ Ash Prepared from Illinois \#6 Coal and Derived Fractions (wt\%)

\begin{tabular}{ccccc}
\hline Oxide* & $C L N$ & FEE & RBR & PLR \\
\hline $\mathrm{SiO}_{2}$ & 52.62 & 56.49 & 63.17 & 57.81 \\
$\mathrm{TiO}_{2}$ & 1.02 & 0.85 & 0.85 & 0.83 \\
$\mathrm{Al}_{2} \mathrm{O}_{3}$ & 20.68 & 18.02 & 16.89 & 17.58 \\
$\mathrm{Fe}_{2} \mathrm{O}_{3}$ & 15.52 & 13.55 & 8.20 & 12.58 \\
$\mathrm{MgO}$ & 0.97 & 1.34 & 1.60 & 1.48 \\
$\mathrm{CaO}$ & 3.94 & 3.71 & 2.86 & 3.27 \\
$\mathrm{SrO}$ & 0.02 & 0.02 & 0.02 & 0.02 \\
$\mathrm{BaO}$ & 0.03 & 0.04 & 0.04 & 0.05 \\
$\mathrm{Na} 2 \mathrm{O}$ & 1.14 & 0.97 & 1.07 & 0.89 \\
$\mathrm{~K}_{2} \mathrm{O}$ & 2.17 & 2.57 & 2.71 & 2.87 \\
$\mathrm{SO}_{3}$ & 1.60 & 1.81 & 1.97 & 1.84 \\
$\mathrm{P}_{2} \mathrm{O}_{5}$ & 0.15 & 0.50 & 0.48 & 0.63 \\
\hline $\mathrm{Total}$ & 99.86 & 99.87 & 99.86 & 99.85 \\
\hline
\end{tabular}

*by X-ray fluorescence (XRF)

TABLE 5: Trace Element Analyses of Illinois \#6 Coal and Derived Fractions (ppm)

\begin{tabular}{ccccc}
\hline Element* & CLN & FEE & RBR & PLR \\
\hline${ }^{9} \mathrm{Be}$ & 1 & 1 & 2 & 1.8 \\
${ }^{31} \mathrm{P}$ & $<$ lod & 445 & 1900 & 1410 \\
${ }^{45} \mathrm{Sc}$ & $<$ lod & 5.5 & 12.5 & 12 \\
${ }^{47} \mathrm{Ti}$ & 552 & 1510 & 3900 & 3670 \\
${ }^{51} \mathrm{~V}$ & 29 & 55 & 116 & 154 \\
${ }^{52} \mathrm{Cr}$ & 23 & 68 & 161 & 230 \\
${ }^{55} \mathrm{Mn}$ & 24 & 81 & 180 & 170 \\
${ }^{59} \mathrm{Co}$ & 2.5 & 5.5 & 16 & 12 \\
${ }^{60} \mathrm{Ni}$ & 10 & 31 & 83 & 98 \\
${ }^{63} \mathrm{Cu}$ & 7 & 20 & 44 & 54 \\
${ }^{64} \mathrm{Zn}{ }^{66} \mathrm{Zn}$ & 20 & 52 & 165 & 300 \\
${ }^{72} \mathrm{Ge}$ & 2.6 & 2.2 & 1.8 & 0.7 \\
${ }^{75} \mathrm{As}$ & 1.2 & 4.2 & 8.7 & 11 \\
${ }^{85} \mathrm{Rb}$ & 11 & 11 & 30 & 22 \\
${ }^{88} \mathrm{Sr}$ & 21 & 51 & 113 & 73 \\
${ }^{118} \mathrm{Sn}$ & 0.55 & 1.3 & 2.8 & 2.7 \\
${ }^{121} \mathrm{Sb}$ & 0.6 & 1.2 & 1.75 & 2.3 \\
${ }^{133} \mathrm{Cs}$ & 1 & 2 & 3.5 & 3.4 \\
${ }^{137} \mathrm{Ba},{ }^{138} \mathrm{Ba}$ & 31 & 96 & 180 & 200 \\
${ }_{\mathrm{Hg}}^{208} \mathrm{~Pb}$ & 0.06 & 0.08 & 0.09 & 0.18 \\
\hline${ }^{61} \mathrm{~Pb}$ & 4 & 10 & 43 & 31 \\
\hline
\end{tabular}

*by ICP-MS on digested samples, except $\mathrm{Hg}$; <lod - below level of detection 
TABLE 6: Major Mineral Data (wt\% of coal) for Illinois Coal and Derived Fractions

\begin{tabular}{llcccc}
\hline Mineral & Method & CLN & FEE & RBR & PLR \\
\hline Quartz & XRD & 2.7 & 9.2 & 31.9 & 22.4 \\
Kaolinite & XRD & 1.3 & 3.7 & 9.1 & 5.3 \\
Illite & XRD & 2.6 & 9.7 & 26.5 & 35.0 \\
Pyrite & Mössbauer & 2.0 & 4.1 & 6.7 & 8.4 \\
Calcite & Ca in coal & 0.6 & 1.1 & 2.4 & 2.1 \\
Organic & $100-$ wt $\%$ Ash & 90.8 & 72.3 & 23.5 & 26.8 \\
\hline
\end{tabular}

The partitioning of various elements between the clean coal and tailings fractions is summarized in Figure 3. This plot shows the concentrations of the elements of interest in different gravity-separated fractions as a bar: the ends of the bars indicate the concentration of the element in the CLN and PLR fractions, while the concentration of the FEE coal is the intermediate value. The relative length of the bar is therefore a measure of the efficiency of the separation for that element. For this particular sample of the Illinois \#6 coal, most elements are separated with about the same efficiency as the overall ash, although some elements, notably $\mathrm{Zn}$, are more efficiently segregated. The major exceptions are $\mathrm{Hg}$ and $\mathrm{Ge}$; the relatively short length of the bar for $\mathrm{Hg}$ indicates that it has mixed inorganic and organic association, whereas the inverted nature of the bar for Ge indicates that it has an extremely strong organic association.

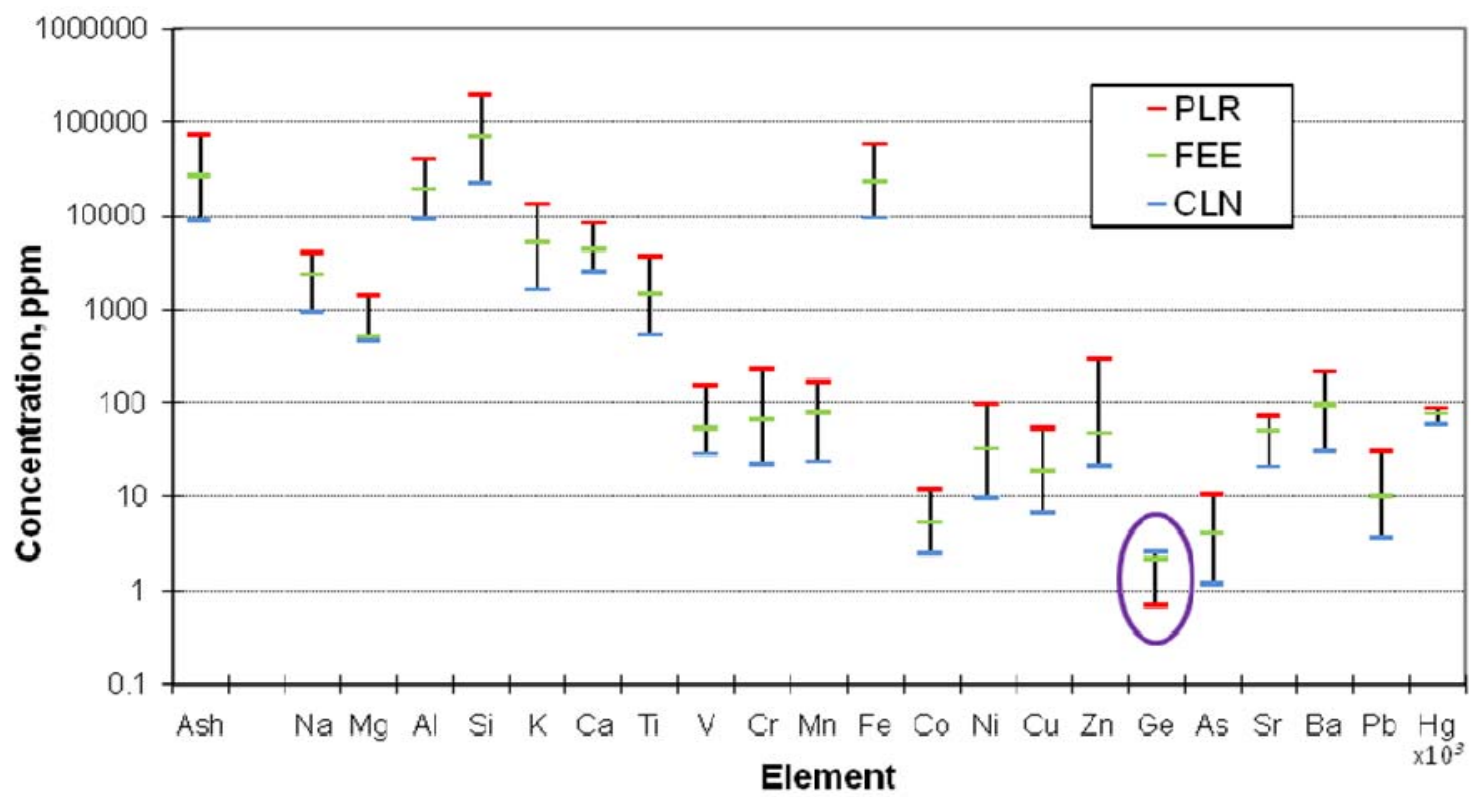

FIGURE 3: Bar diagram summarizing the efficiency of separation of various elements between the CLN and PLR fractions during gravity cleaning methods applied to the feed (FEE) coal. Note the inverted nature of the bar for Ge (circled) and the short bar for Hg.

Based on the data in Tables 4 and 6, the first separation by the rotary breaker clearly removed mineral bands enriched in quartz and kaolinite, but was less effective at removing pyrite. As 
can be seen from Table 6, illite and pyrite, which are the two major trace-element containing minerals in coal, exhibited relatively monotonic increases with ash across the four fractions. However, whereas pyrite as a relative fraction of the mineral matter decreased from $\sim 20 \%$ of the mineral matter in the CLN fraction to $\sim 8 \%$ in the RBR fraction, illite increased from $\sim 25 \%$ in CLN to almost $45 \%$ in PLR. Similar related variations can be seen in the wt $\%$ $\mathrm{Fe}_{2} \mathrm{O}_{3}$ and $\mathrm{K}_{2} \mathrm{O}$ trends in Table 4. The similarities in trend for these two minerals with ash limited the usefulness of correlating trace element concentrations with mineral abundances in the different fractions for inference of trace element association with specific minerals, but at the same time, increase the value of independent, direct determinations of elemental modes of occurrence that can be obtained from techniques like XAFS and Mössbauer spectroscopies. Two examples of using XAFS spectroscopy for determining trace element modes of occurrence are now presented, one for chalcophile (As, Zn) elements, the other for lithophile elements $(\mathrm{V}, \mathrm{Cr}, \mathrm{Ni})$, while a summary of the modes of occurrence for all key trace elements, based on both spectroscopic and chemical data, is presented in Table 7.

\section{Example 1: chalcophile elements, arsenic and zinc}

Arsenic and zinc are both thought to have a strong association with sulphide minerals in Illinois coals (Swaine, 1990; Finkelman, 1994). Arsenic XAFS spectra (Figure 4) of the Illinois coal fractions confirm that the principal occurrence of arsenic in this coal is its association with pyrite, based on their similarity to spectra in previous studies (Huggins et al., 1993; Kolker et al, 2000). Figure 5 shows the relationship between the concentrations of As and $\mathrm{Zn}$ and the abundance of pyrite for the four coal fractions. A highly correlated linear relationship $\left(\mathrm{R}^{2}>0.99\right)$ is revealed between As and pyrite and indicates that the pyrite in this Illinois \#6 coal contains on average approximately 154 ppm As. This amount is remarkably similar to that of $144 \mathrm{ppm}$ recently determined by microprobe for As in pyrite in a sampling of the Springfield (Illinois \#5) coal (Kolker and Huggins, 2007).
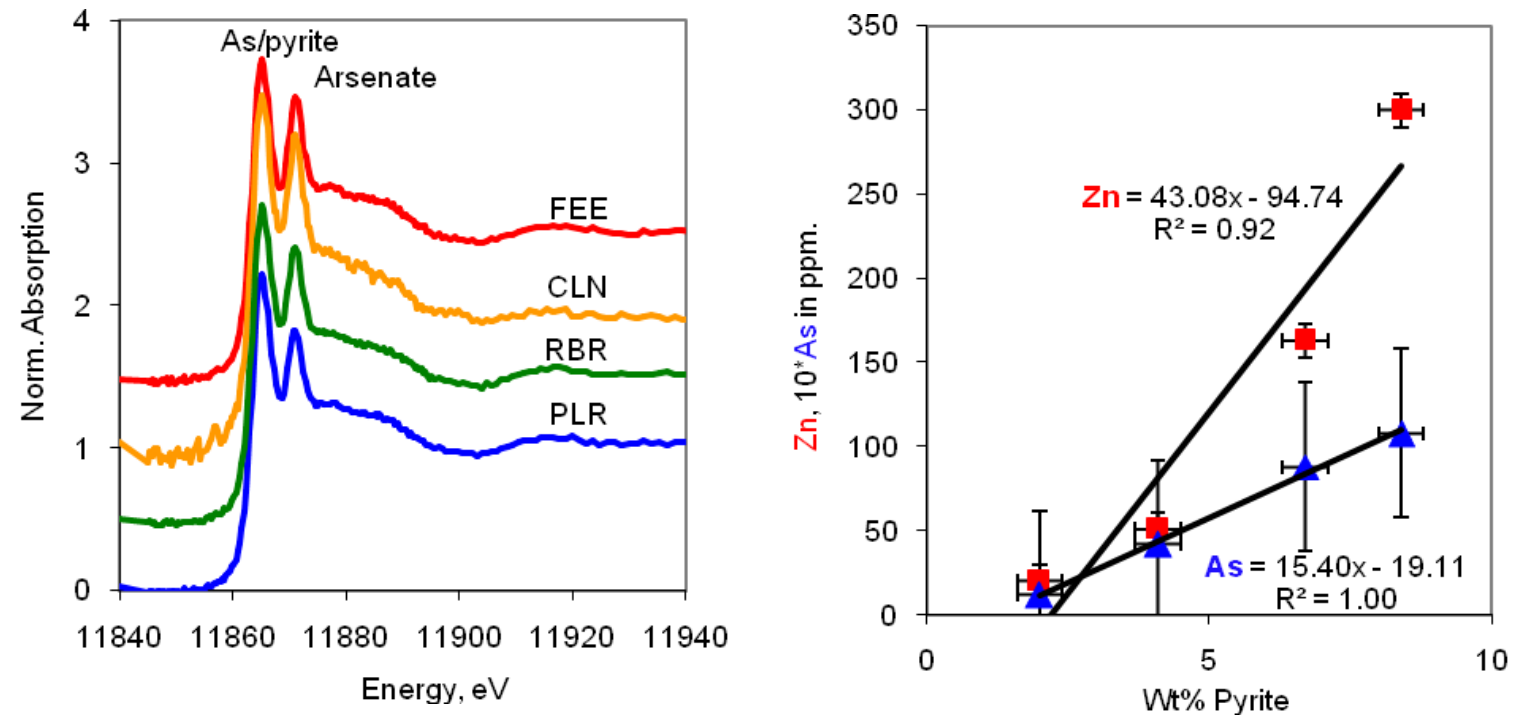

FIGURE 4 (left). Arsenic XANES spectra of the four Illinois coal fractions. Distinct peaks due to As in pyrite and As as arsenate are indicated.

FIGURE 5 (right). Correlations between As and $\mathrm{Zn}$ concentrations and pyrite abundance in the four Illinois coal fractions. Note that the As contents are multiplied by a factor of 10 . 
In contrast with arsenic, zinc does not exhibit nearly as strong a linear correlation with pyrite content. Further, zinc XAFS spectra (not shown) indicate that the zinc speciation is dominated by $\mathrm{ZnS}$; however, the presence of other $\mathrm{Zn}$ forms ( $\mathrm{Zn}$ /illite; $\mathrm{Zn}$ phosphate?) is also indicated. Consequently, it would appear that zinc in this coal is not associated strongly, if at all, with pyrite, but rather is present in independent forms.

\section{Example 2: lithophile elements, vanadium, chromium and nickel}

Figure 6 shows the correlations found between $\mathrm{V}, \mathrm{Cr}$, and $\mathrm{Ni}$ concentrations and the abundance of illite in the different coal fractions. For all three elements, the correlations with illite are highly significant $\left(\mathrm{R}^{2}>0.99\right)$. Evidence from previous XAFS investigations into the mode of occurrence of $\mathrm{V}$ and $\mathrm{Cr}$ in various Illinois basin coals indicates that both of these elements are likely to be distributed between illite and macerals (Maylotte et al., 1981; Huggins et al., 1997, 2000; Huggins and Huffman, 2004); however, less is established regarding the mode of occurrence of $\mathrm{Ni}$ and other mineral occurrences are certainly possible (Swaine, 1990; Finkelman, 1994). Based on the assumption that these three elements are distributed only between illite and the macerals in this coal, the average contents of these elements in illite are $400 \mathrm{ppm} \mathrm{V}, 625 \mathrm{ppm} \mathrm{Cr}$, and $280 \mathrm{ppm} \mathrm{Ni}$, while their contents in the macerals would average $21 \mathrm{ppm}, 7 \mathrm{ppm}$, and $6 \mathrm{ppm}$, respectively. From these figures, the maximum percentages of $\mathrm{V}, \mathrm{Cr}$, and $\mathrm{Ni}$ that can be removed from this particular coal by physical separation methods are estimated to be around $67 \%, 92 \%$, and $86 \%$, respectively.
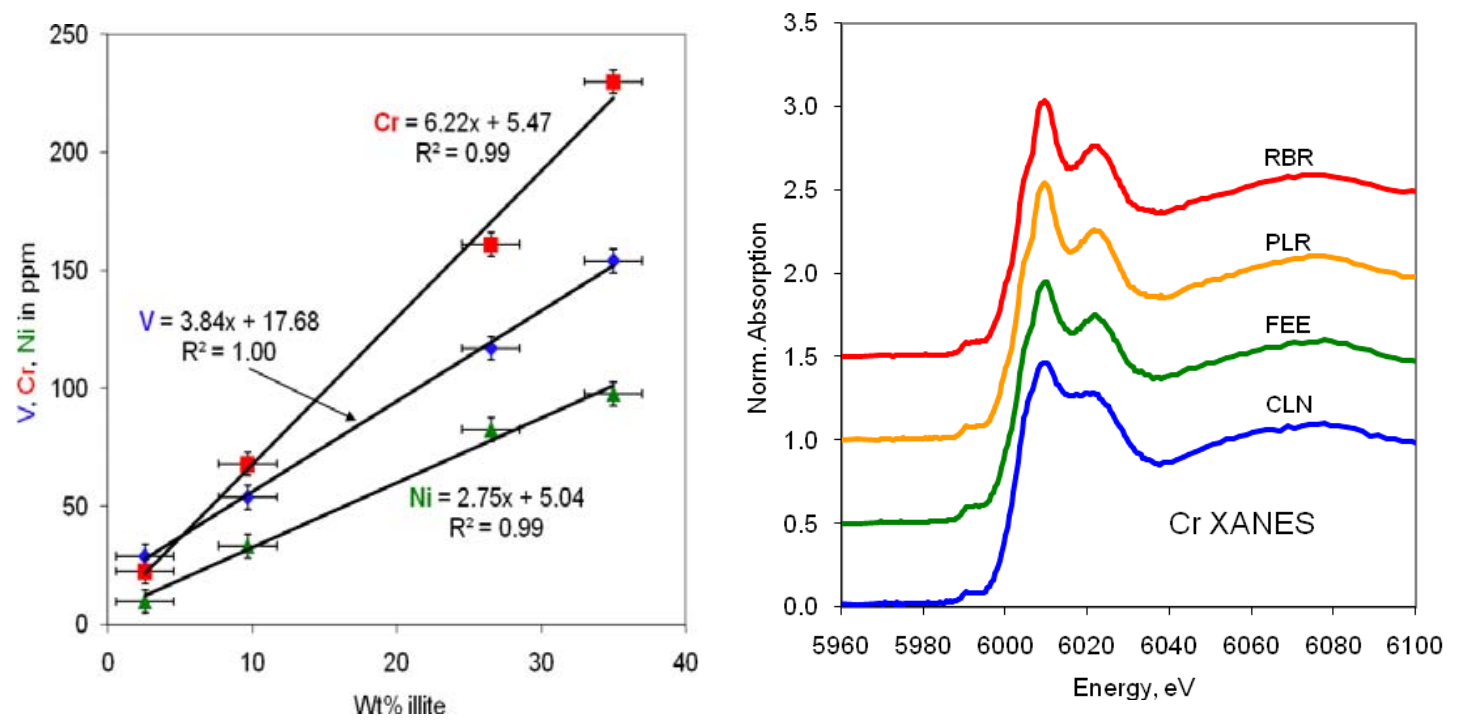

FIGURE 6 (left). Correlations between V, Cr and Ni concentrations and illite abundance in the four Illinois \#6 coal fractions.

FIGURE 7 (right). Chromium XANES spectra of the four Illinois \#6 coal fractions.

Direct evidence regarding the modes of occurrence of $\mathrm{Cr}$ and $\mathrm{V}$ was obtained by XAFS spectroscopy. Figure 7 shows Cr XAFS spectra obtained for the four fractions generated at the preparation plant. The corresponding V XAFS spectra were quite similar. The spectra exhibited by the tailings fractions, RBR and PLR, are clearly characteristic of the occurrence of $\mathrm{Cr}$ and $\mathrm{V}$ in illite, based on comparison with spectra presented for these elements in illite 
in previous studies (Maylotte, et al., 1981; Huggins, et al., 2000). The major difference between the $\mathrm{Cr}$ and $\mathrm{V}$ XANES spectra is the height of the pre-edge peak which is much larger for $\mathrm{V}$ than for $\mathrm{Cr}$, especially for the maceral-rich CLN fraction. Vanadium associated with the organic component in coal is mostly present in the $\mathrm{V}^{4+}$ oxidation state (Maylotte, et al., 1981), which results in a stronger pre-edge peak than that observed for $\mathrm{V}^{3+}$ or for $\mathrm{Cr}^{3+}$ in illite. From the height of the pre-edge peak and applying the correlation between peak-height and V valence state developed by Sutton et al. (2005), the average valence of V in illite is estimated to be about $3.3 \pm 0.2$, whereas it is $3.8 \pm 0.2$ in the form associated with the macerals. Based on the estimated $\mathrm{Cr}$ and $\mathrm{V}$ contents of the illite and the macerals, $\mathrm{Cr}$ and $\mathrm{V}$ in illite are calculated to represent more than $98 \%$ and $95 \%$, respectively, of the total $\mathrm{Cr}$ and $\mathrm{V}$ in these fractions. Corresponding estimates for the clean coal fraction suggest that about $60-70 \%$ of these elements in this fraction are also present as illite; however, the XANES spectra would appear to indicate a significantly smaller contribution from these elements in illite, no more than $25 \%$, indicating that the assumption of a constant content of these elements in illite may be an oversimplification and that the content of $\mathrm{Cr}$ and $\mathrm{V}$ in the macerals is underestimated by the intercept on the Cr trend in Figure 6. XAFS spectra for Ni have not been obtained.

\section{Summary of elemental modes of occurrence.}

The modes of occurrence, determined by a combination of both elemental and spectroscopic methods, of a number of important trace elements in the sample of Illinois \#6 coal, are summarized in Table 7. Additionally, iron Mössbauer spectroscopy revealed that iron in this coal was largely in the form of pyrite with relatively minor fractions $(2-10 \%)$ present as $\mathrm{Fe}^{2+}$ in clays and as iron sulfates (jarosite) (Figure 8). A minor fraction of magnetite was picked up in the CLN faction during the heavy media cleaning.

TABLE 7: Summary of modes of occurrence for key trace elements in this sample of Illinois \#6 coal

\begin{tabular}{|c|c|}
\hline Element & Comments on Mode of Occurrence in Illinois \#6 Coal (this sample) \\
\hline Arsenic & $\begin{array}{l}\text { Entirely associated with pyrite; increasing oxidation of arsenical pyrite to } \\
\text { arsenate noted over time during sample storage }\end{array}$ \\
\hline Chromium & $\begin{array}{l}\text { Largely present in association with illite with remainder in organic } \\
\text { association; }>98 \% \mathrm{Cr} \text { in tailings fractions is associated with illite }\end{array}$ \\
\hline Germanium & Highly organically associated \\
\hline Manganese & Complex - appears to be at least 3 significant distinct species \\
\hline Mercury & Some association with pyrite; some with macerals \\
\hline Nickel & Similar to $\mathrm{Cr}$ and V? \\
\hline Selenium & Also associated with pyrite, but some organic Se as well \\
\hline Vanadium & $\begin{array}{l}\text { Similar to chromium, but with more of the element in organic association; } \\
>95 \% \mathrm{~V} \text { in tailings fractions is associated with illite }\end{array}$ \\
\hline Zinc & $\begin{array}{l}\text { Complex - three distinct major species: sphalerite }(\mathrm{ZnS}) ; \mathrm{Zn} \text { assoc. w/ } \\
\text { illite; and } \mathrm{Zn}_{3}\left(\mathrm{PO}_{4}\right)_{2} \cdot \mathrm{xH}_{2} \mathrm{O} \text { (tentative) }\end{array}$ \\
\hline
\end{tabular}


Measurements of the coal fractions taken about 4 months apart by As XAFS spectroscopy (Figure 9) revealed that oxidation of As in pyrite had occurred in all fractions resulting in the increased formation of arsenic as arsenate. Over this period, the percentage of arsenic as arsenate increased by between $2 \%$ for the tailings fractions and $5 \%$ for the CLN fraction. This oxidized form of As is likely associated with the oxidation product of pyrite, viz. jarosite $\left(\mathrm{K}(\mathrm{Fe}, \mathrm{Al})_{3}\left(\mathrm{SO}_{4}\right)_{2}(\mathrm{OH})_{6}\right)$, in which $\mathrm{AsO}_{4}{ }^{3-}$ substitutes for $\mathrm{SO}_{4}{ }^{2-}$.
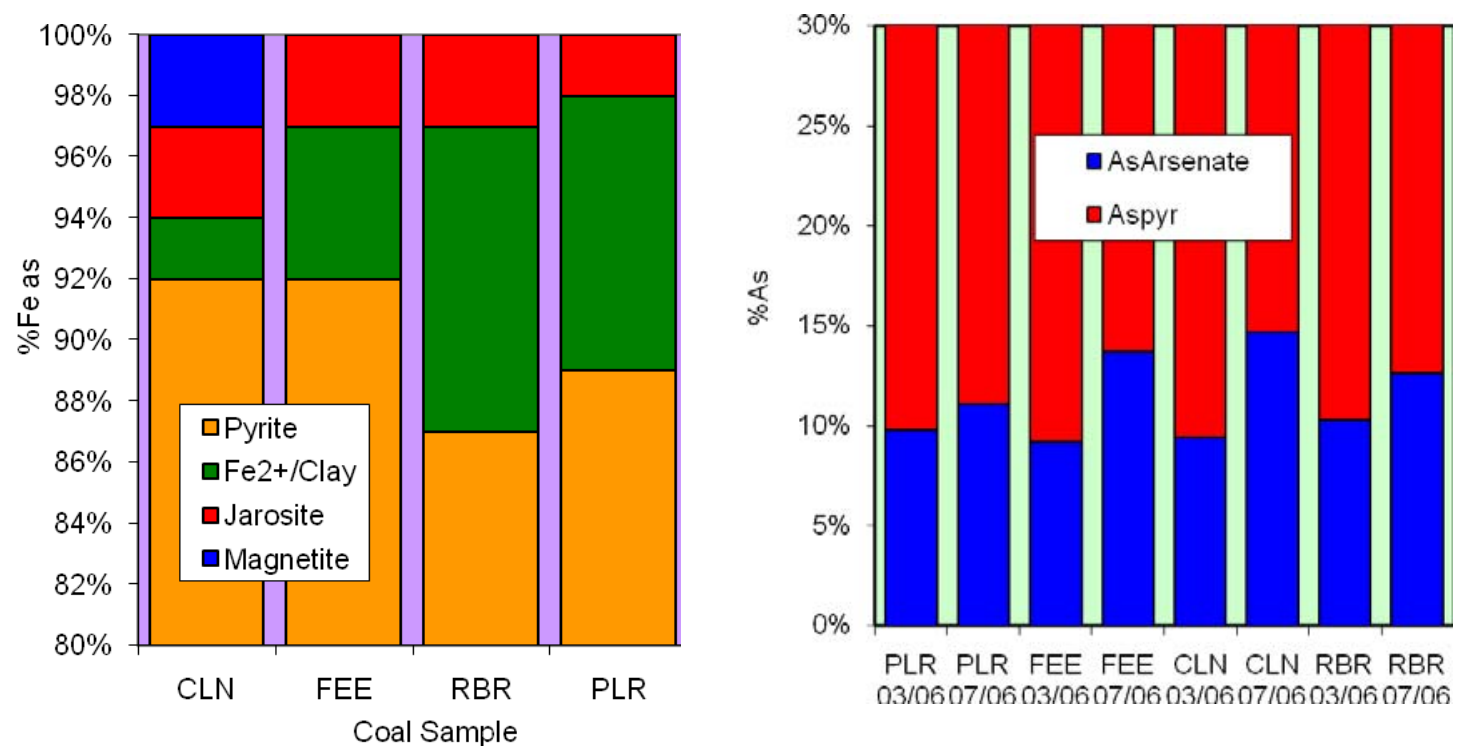

FIGURE 8 (left): Summary of the distribution of iron among different minerals in the four coal fractions as determined by Mössbauer spectroscopy. The balance of the iron is present as pyrite, $\mathrm{FeS}_{2}$.

FIGURE 9 (right): Summary of the distribution of arsenic between pyrite and arsenate minerals in the four coal fractions measured about four months apart as determined by As XANES spectroscopy. The differences for each fraction indicate slow oxidation of arsenic associated with pyrite during sample storage. The balance of the arsenic is present as arsenic in pyrite.

\section{$\underline{\text { Trace element mobility in leaching scenarios }}$}

With the modes of occurrence of key elements determined for the four coal fractions, the next stage of the research was to investigate their mobility in simulations of disposal of the tailings fractions in aqueous environments, such as tailings impoundments. The traditional way to do this is to subject the fractions to the Toxicity Characteristic Leaching Procedure (TCLP). However, according to the commercial testing laboratory that conducted the TCLP tests on the Illinois \#6 coal fractions, no RCRA element was leached to any significant extent. Although this result is good news for environmental risk assessments, it tells the scientist little or nothing about trace element mobility from these coal fractions.

The negative result from the TCLP test was also indicated by iron Mössbauer and arsenic XANES spectra (Figure 10) of fractions before and after the TCLP test. Each before and after pair of spectra were essentially identical, indicating that any dissolution of pyrite and release of any associated pyrite during the TCLP test was negligible. Hence, it was 
concluded that the duration and/or conditions of the TCLP test were not adequate to promote significant leaching of arsenic and other trace elements from the tailings fractions and therefore the TCLP test would not be informative for investigating trace element mobility. As a result, two longer-term methods of promoting trace element mobility from the tailings were constructed for such testing.

The baseline static test consisted of storing $200 \mathrm{~g}$ of tailings fraction under $200 \mathrm{ml}$ of deionized water for 20 weeks. It was designed to be the benchmark test for the more aggressive cyclic test based on ASTM D-5744 (ASTM, 1996) that involved a cycle of submerging $200 \mathrm{~g}$ of each tailings for 24 hours in $200 \mathrm{ml}$ of deionized water, draining off the water, followed by exposure of the tailings to first three days of dry air and then by three days of moist air. After a number of weekly cycles, the collected leachant waters were combined and submitted for determination of $\mathrm{pH}$, Eh, conductivity, sulphate concentration, and major and trace elements by ICP-MS. This cyclic testing was continued for 22 weeks. For the final week of the test, week 23, the $200 \mathrm{ml}$ of deionized water was replaced by an equivalent amount of dilute $(0.1 \mathrm{~N}) \mathrm{HNO}_{3}$ in order to make the final stage more acidic and aggressive.

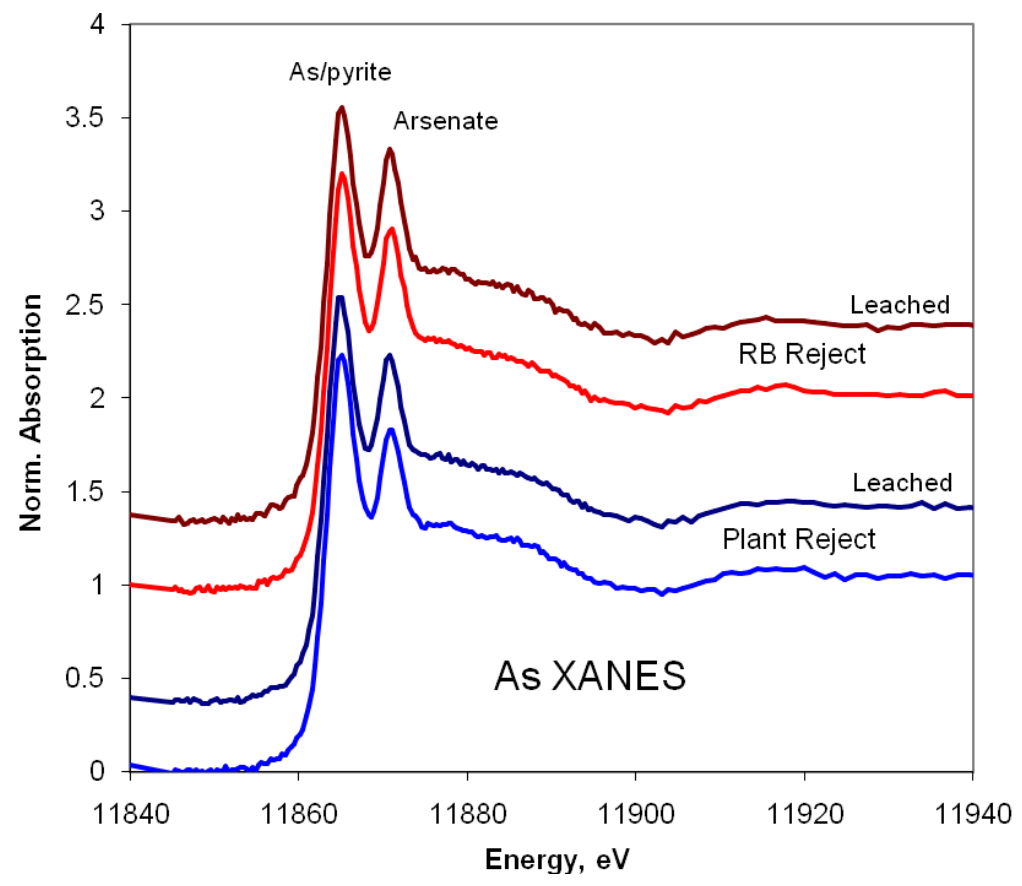

FIGURE 10: Arsenic XANES spectra for the RBR and PLR tailings fractions before and after (leached) the TCLP test. Note the unchanged nature of the spectra before and after the test, indicating that there was no significant change in arsenic speciation as a result of the test, consistent with the lack of any As leached during the test.

Data on the variation with time of conductivity, $\mathrm{Eh}, \mathrm{pH}$, and sulfate concentration are presented in Table 8 for the leachates from the rotary breaker reject (RBR) and plant reject (PLR) tailings fractions in the cyclic and baseline leaching tests. As documented in Figure 11 , there is a very strong correlation between sulfate concentration and conductivity. This relationship implies that sulfate anions are the only significant anions being generated as a 
result of the leaching conditions. As can also be seen from Table 8 , the variation in $\mathrm{pH}$ is relatively small and it remains approximately neutral even after 22 weeks' leaching, except possibly for the last week for the PLR sample, when the $\mathrm{pH}$ dropped below 5 . As would be expected, leaching with $0.1 \mathrm{~N} \mathrm{HNO}_{3}$ lowers the $\mathrm{pH}$ and increases conductivity significantly.

TABLE 8: Variation of conductivity, sulfate concentration, $\mathrm{pH}$, and Eh of leachate samples taken at different times during the cyclic and baseline leaching tests on the Illinois \#6 tailings fractions

\begin{tabular}{|c|cccc|cccc|}
\hline \multirow{2}{*}{ Week } & \multicolumn{4}{|c|}{ RB Reject } & \multicolumn{3}{c|}{ Plant Reject } \\
\cline { 2 - 9 } & $\begin{array}{c}\text { Conductivity } \\
\mu \Omega^{-1} . \mathrm{cm}^{-1}\end{array}$ & $\begin{array}{c}\text { Sulfate } \\
\mathrm{mg} / \mathrm{L}\end{array}$ & $\mathrm{pH}$ & Eh & $\begin{array}{c}\text { Conductivity } \\
\mu \Omega^{-1} . \mathrm{cm}^{-1}\end{array}$ & $\begin{array}{c}\text { Sulfate } \\
\mathrm{mg} / \mathrm{L}\end{array}$ & $\mathrm{pH}$ & Eh \\
\hline Cyclic 0 & 8644 & 1609 & 8.0 & 192.4 & 8377 & 1469 & 8.1 & 172.2 \\
1 & 6794 & 1268 & 7.3 & 164.4 & 5750 & 955 & 7.7 & 138.1 \\
$2-3$ & 3958 & 684 & 7.5 & 207 & 3086 & 501 & 7.6 & 215 \\
$4-6$ & 1688 & 292 & 7.3 & 175 & 1717 & 312 & 7.2 & 193 \\
7 & 1513 & 278 & 6.8 & 204 & 1158 & 195 & 6.8 & 194 \\
$8-12$ & 1887 & 337 & 7.0 & 191 & 2441 & 487 & 6.8 & 192 \\
$13-15$ & 1314 & 230 & 7.1 & 160.6 & 1718 & 344 & 6.8 & 245.9 \\
$16-20$ & 1475 & 289 & 7.0 & 220 & 1785 & 387 & 6.2 & 32 \\
$21-22$ & 832 & 145 & 6.6 & 156 & 1302 & 255 & 4.9 & 233 \\
\hline 23 (acid) & $>2000$ & 222 & 4.1 & 230 & $>2000$ & 477 & 2.4 & 354 \\
\hline Baseline 20 & $>2000$ & 7610 & & 154 & $>2000$ & 6410 & & 155 \\
\hline
\end{tabular}

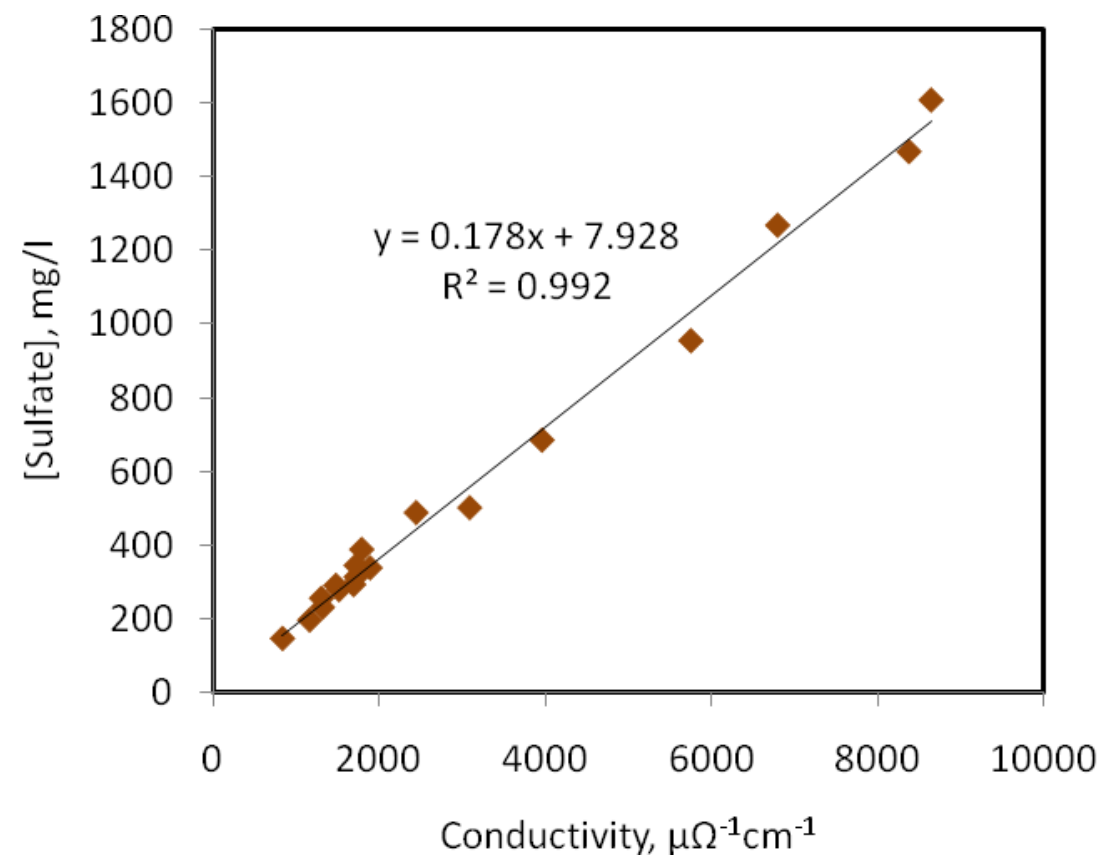

FIGURE 11: Correlation between conductivity and sulfate anion concentration determined for the aqueous leachates generated in the cyclic test. Leachates for the two tailings fractions, PLR and RBR, are not differentiated in this plot. 
TABLE 9: ICP-MS Data for Leachates from Cyclic Testing on RBR Coal Tailings Fraction

\begin{tabular}{|c|c|c|c|c|c|c|c|c|c|c|c|c|}
\hline \multirow{2}{*}{ Element } & \multicolumn{10}{|c|}{ Week } & \multirow{2}{*}{ Units } & \multirow{2}{*}{ MDL } \\
\hline & 0 & 1 & $2-3$ & $4-6$ & 7 & $8-12$ & $13-15$ & $16-20$ & $21-22$ & 23 & & \\
\hline Aluminum & $<\mathrm{MDL}$ & $<\mathrm{MDL}$ & $<\mathrm{MDL}$ & $<\mathrm{MDL}$ & $<\mathrm{MDL}$ & 0.23 & $<\mathrm{MDL}$ & $<\mathrm{MDL}$ & $<\mathrm{MDL}$ & 4.51 & $\mathrm{mg} / \mathrm{L}$ & 0.061 \\
\hline Antimony & $<\mathrm{MDL}$ & $<\mathrm{MDL}$ & $<\mathrm{MDL}$ & $<\mathrm{MDL}$ & $<\mathrm{MDL}$ & $<\mathrm{MDL}$ & $<\mathrm{MDL}$ & $<\mathrm{MDL}$ & $<\mathrm{MDL}$ & $<\mathrm{MDL}$ & $\mathrm{mg} / \mathrm{L}$ & 0.012 \\
\hline Arsenic & $<\mathrm{MDL}$ & $<\mathrm{MDL}$ & $<\mathrm{MDL}$ & $<\mathrm{MDL}$ & $<\mathrm{MDL}$ & $<\mathrm{MDL}$ & $<\mathrm{MDL}$ & $<\mathrm{MDL}$ & $<\mathrm{MDL}$ & $<\mathrm{MDL}$ & $\mathrm{mg} / \mathrm{L}$ & 0.014 \\
\hline Barium & 0.02 & 0.03 & 0.015 & 0.03 & 0.03 & 0.02 & 0.04 & 0.05 & 0.04 & 0.12 & $\mathrm{mg} / \mathrm{L}$ & 0.003 \\
\hline Beryllium & $<\mathrm{MDL}$ & $<\mathrm{MDL}$ & $<\mathrm{MDL}$ & $<\mathrm{MDL}$ & $<\mathrm{MDL}$ & $<\mathrm{MDL}$ & $<\mathrm{MDL}$ & $<\mathrm{MDL}$ & $<\mathrm{MDL}$ & 0.005 & $\mathrm{mg} / \mathrm{L}$ & 0.001 \\
\hline Boron & 1.07 & 0.74 & 0.52 & 0.33 & 0.22 & 0.36 & 0.23 & 0.39 & 0.12 & 1.32 & $\mathrm{mg} / \mathrm{L}$ & 0.008 \\
\hline Cadmium & $<\mathrm{MDL}$ & $<\mathrm{MDL}$ & $<\mathrm{MDL}$ & $<\mathrm{MDL}$ & $<\mathrm{MDL}$ & $<\mathrm{MDL}$ & $<\mathrm{MDL}$ & $<\mathrm{MDL}$ & $<\mathrm{MDL}$ & 0.05 & $\mathrm{mg} / \mathrm{L}$ & 0.001 \\
\hline Calcium & 315 & 325 & 279 & 231 & 277 & 386 & 257 & 321 & 172 & 1615 & $\mathrm{mg} / \mathrm{L}$ & 0.002 \\
\hline Chromium & $<\mathrm{MDL}$ & $<\mathrm{MDL}$ & $<\mathrm{MDL}$ & $<\mathrm{MDL}$ & 0.029 & 0.029 & $<\mathrm{MDL}$ & 0.05 & 0.05 & $<\mathrm{MDL}$ & $\mathrm{mg} / \mathrm{L}$ & 0.024 \\
\hline Cobalt & 0.15 & 0.005 & $<\mathrm{MDL}$ & 0.004 & 0.004 & 0.01 & $<\mathrm{MDL}$ & 0.02 & 0.01 & 0.63 & $\mathrm{mg} / \mathrm{L}$ & 0.001 \\
\hline Copper & $<\mathrm{MDL}$ & $<\mathrm{MDL}$ & $<\mathrm{MDL}$ & $<\mathrm{MDL}$ & 0.007 & 0.009 & $<\mathrm{MDL}$ & 0.005 & $<\mathrm{MDL}$ & 0.03 & $\mathrm{mg} / \mathrm{L}$ & 0.005 \\
\hline Gold & $<\mathrm{MDL}$ & $<\mathrm{MDL}$ & $<\mathrm{MDL}$ & $<\mathrm{MDL}$ & $<\mathrm{MDL}$ & $<\mathrm{MDL}$ & $<\mathrm{MDL}$ & $<\mathrm{MDL}$ & $<\mathrm{MDL}$ & $<\mathrm{MDL}$ & $\mathrm{mg} / \mathrm{L}$ & 0.034 \\
\hline Iron & 0.02 & $<\mathrm{MDL}$ & $<\mathrm{MDL}$ & 0.09 & 0.006 & 0.01 & $<\mathrm{MDL}$ & 0.02 & 0.03 & 6.02 & $\mathrm{mg} / \mathrm{L}$ & 0.002 \\
\hline Lead & $<\mathrm{MDL}$ & $<\mathrm{MDL}$ & $<\mathrm{MDL}$ & $<\mathrm{MDL}$ & $<\mathrm{MDL}$ & $<\mathrm{MDL}$ & $<\mathrm{MDL}$ & $<\mathrm{MDL}$ & $<\mathrm{MDL}$ & $<\mathrm{MDL}$ & $\mathrm{mg} / \mathrm{L}$ & 0.01 \\
\hline Lithium & 0.77 & 0.52 & 0.29 & 0.08 & 0.05 & 0.08 & 0.03 & 0.04 & 0.01 & 0.18 & $\mathrm{mg} / \mathrm{L}$ & 0.001 \\
\hline Magnesium & 59.7 & 48.8 & 34.5 & 21.2 & 23.3 & 30.6 & 17.3 & 20.8 & 8.97 & 86.3 & $\mathrm{mg} / \mathrm{L}$ & 0.001 \\
\hline Manganese & 1.46 & 1.61 & 0.82 & 0.43 & 0.49 & 0.77 & 0.46 & 0.88 & 0.41 & 8.76 & $\mathrm{mg} / \mathrm{L}$ & 0.001 \\
\hline Nickel & 0.79 & 0.07 & 0.03 & 0.01 & $<\mathrm{MDL}$ & $<\mathrm{MDL}$ & $<\mathrm{MDL}$ & 0.04 & 0.03 & 3.83 & $\mathrm{mg} / \mathrm{L}$ & 0.002 \\
\hline Phosphorus & $<\mathrm{MDL}$ & $<\mathrm{MDL}$ & $<\mathrm{MDL}$ & $<\mathrm{MDL}$ & $<\mathrm{MDL}$ & $<\mathrm{MDL}$ & $<\mathrm{MDL}$ & $<\mathrm{MDL}$ & $<\mathrm{MDL}$ & 39.3 & $\mathrm{mg} / \mathrm{L}$ & 0.009 \\
\hline Potassium & 23.9 & 28.7 & 23.9 & 18.8 & 18.8 & 25.3 & 14.4 & 19 & 9.55 & 59.7 & $\mathrm{mg} / \mathrm{L}$ & 0.191 \\
\hline Selenium & 0.45 & 0.24 & 0.05 & $<\mathrm{MDL}$ & 0.03 & 0.02 & $<\mathrm{MDL}$ & 0.04 & 0.014 & 0.09 & $\mathrm{mg} / \mathrm{L}$ & 0.011 \\
\hline Silicon & 1.86 & 2.01 & 2.17 & 1.65 & 1.58 & 2.57 & 1.55 & 3.38 & 1.4 & 73.9 & $\mathrm{mg} / \mathrm{L}$ & 0.009 \\
\hline Silver & $<\mathrm{MDL}$ & $<\mathrm{MDL}$ & $<\mathrm{MDL}$ & $<\mathrm{MDL}$ & $<\mathrm{MDL}$ & $<\mathrm{MDL}$ & $<\mathrm{MDL}$ & $<\mathrm{MDL}$ & $<\mathrm{MDL}$ & $<\mathrm{MDL}$ & $\mathrm{mg} / \mathrm{L}$ & 0.003 \\
\hline Sodium & 1600 & 1220 & 581 & 116 & 35.1 & 34.5 & 10.9 & 5.64 & 1.81 & 8.86 & $\mathrm{mg} / \mathrm{L}$ & 0.058 \\
\hline Strontium & 5.33 & 4.27 & 3.27 & 2.05 & 2.41 & 3.12 & 1.87 & 2.18 & 1.04 & 16.1 & $\mathrm{mg} / \mathrm{L}$ & 0.01 \\
\hline Sulfur & 1530 & 1310 & 742 & 284 & 277 & 368 & 232 & 290 & 152 & 216 & $\mathrm{mg} / \mathrm{L}$ & 0.014 \\
\hline Thalium & $<\mathrm{MDL}$ & $<\mathrm{MDL}$ & $<\mathrm{MDL}$ & $<\mathrm{MDL}$ & $<\mathrm{MDL}$ & $<\mathrm{MDL}$ & $<\mathrm{MDL}$ & $<\mathrm{MDL}$ & $<\mathrm{MDL}$ & $<\mathrm{MDL}$ & $\mathrm{mg} / \mathrm{L}$ & 0.041 \\
\hline Tin & $<\mathrm{MDL}$ & $<\mathrm{MDL}$ & $<\mathrm{MDL}$ & $<\mathrm{MDL}$ & $<\mathrm{MDL}$ & $<\mathrm{MDL}$ & $<\mathrm{MDL}$ & $<\mathrm{MDL}$ & $<\mathrm{MDL}$ & $<\mathrm{MDL}$ & $\mathrm{mg} / \mathrm{L}$ & 0.012 \\
\hline Vanadium & $<\mathrm{MDL}$ & $<\mathrm{MDL}$ & $<\mathrm{MDL}$ & $<\mathrm{MDL}$ & $<\mathrm{MDL}$ & $<\mathrm{MDL}$ & $<\mathrm{MDL}$ & $<\mathrm{MDL}$ & $<\mathrm{MDL}$ & $<\mathrm{MDL}$ & $\mathrm{mg} / \mathrm{L}$ & 0.008 \\
\hline Zinc & $<\mathrm{MDL}$ & 0.006 & $<\mathrm{MDL}$ & 0.11 & $<\mathrm{MDL}$ & $<\mathrm{MDL}$ & $<\mathrm{MDL}$ & 0.27 & 0.14 & 2.54 & $\mathrm{mg} / \mathrm{L}$ & 0.002 \\
\hline
\end{tabular}


TABLE 10: ICP-MS Data for Leachates from Cyclic Testing on PLR Coal Tailings Fraction

\begin{tabular}{|c|c|c|c|c|c|c|c|c|c|c|c|c|}
\hline \multirow{2}{*}{ Element } & \multicolumn{10}{|c|}{ Week } & \multirow{2}{*}{ Units } & \multirow{2}{*}{ MDL } \\
\hline & 0 & 1 & $2-3$ & $4-6$ & 7 & $8-12$ & $13-15$ & $16-20$ & $21-22$ & 23 & & \\
\hline Aluminum & $<\mathrm{MDL}$ & $<\mathrm{MDL}$ & $<\mathrm{MDL}$ & $<\mathrm{MDL}$ & 0.1 & 0.2 & $<\mathrm{MDL}$ & 0.17 & 1.07 & 58.1 & $\mathrm{mg} / \mathrm{L}$ & 0.061 \\
\hline Antimony & $<\mathrm{MDL}$ & $<\mathrm{MDL}$ & $<\mathrm{MDL}$ & $<\mathrm{MDL}$ & $<\mathrm{MDL}$ & $<\mathrm{MDL}$ & $<\mathrm{MDL}$ & $<\mathrm{MDL}$ & $<\mathrm{MDL}$ & 0.04 & $\mathrm{mg} / \mathrm{L}$ & 0.012 \\
\hline Arsenic & $<\mathrm{MDL}$ & $<\mathrm{MDL}$ & $<\mathrm{MDL}$ & $<\mathrm{MDL}$ & $<\mathrm{MDL}$ & $<\mathrm{MDL}$ & $<\mathrm{MDL}$ & $<\mathrm{MDL}$ & $<\mathrm{MDL}$ & $<\mathrm{MDL}$ & $\mathrm{mg} / \mathrm{L}$ & 0.014 \\
\hline Barium & 0.02 & 0.02 & 0.02 & 0.03 & 0.04 & 0.02 & 0.02 & 0.02 & 0.02 & 0.1 & $\mathrm{mg} / \mathrm{L}$ & 0.003 \\
\hline Beryllium & $<\mathrm{MDL}$ & $<\mathrm{MDL}$ & $<\mathrm{MDL}$ & $<\mathrm{MDL}$ & $<\mathrm{MDL}$ & $<\mathrm{MDL}$ & $<\mathrm{MDL}$ & $<\mathrm{MDL}$ & 0.001 & $<\mathrm{MDL}$ & $\mathrm{mg} / \mathrm{L}$ & 0.001 \\
\hline Boron & 1.64 & 1.18 & 0.77 & 0.41 & 0.27 & 0.37 & 0.27 & 0.43 & 0.15 & 0.5 & $\mathrm{mg} / \mathrm{L}$ & 0.008 \\
\hline Cadmium & $<\mathrm{MDL}$ & $<\mathrm{MDL}$ & $<\mathrm{MDL}$ & $<\mathrm{MDL}$ & $<\mathrm{MDL}$ & $<\mathrm{MDL}$ & 0.002 & 0.02 & 0.03 & 0.18 & $\mathrm{mg} / \mathrm{L}$ & 0.001 \\
\hline Calcium & 288 & 297 & 299 & 297 & 214 & 561 & 351 & 415 & 277 & 1579 & $\mathrm{mg} / \mathrm{L}$ & 0.002 \\
\hline Chromium & $<\mathrm{MDL}$ & $<\mathrm{MDL}$ & 0.025 & 0.025 & $<\mathrm{MDL}$ & 0.028 & $<\mathrm{MDL}$ & 0.06 & 0.05 & $<\mathrm{MDL}$ & $\mathrm{mg} / \mathrm{L}$ & 0.024 \\
\hline Cobalt & 0.1 & 0.01 & 0.02 & 0.027 & 0.004 & 0.03 & 0.03 & 0.22 & 0.18 & 1.13 & $\mathrm{mg} / \mathrm{L}$ & 0.001 \\
\hline Copper & $<\mathrm{MDL}$ & $<\mathrm{MDL}$ & $<\mathrm{MDL}$ & 0.007 & 0.01 & 0.009 & $<\mathrm{MDL}$ & $<\mathrm{MDL}$ & $<\mathrm{MDL}$ & 1.29 & $\mathrm{mg} / \mathrm{L}$ & 0.005 \\
\hline Gold & $<\mathrm{MDL}$ & $<\mathrm{MDL}$ & $<\mathrm{MDL}$ & $<\mathrm{MDL}$ & $<\mathrm{MDL}$ & $<\mathrm{MDL}$ & $<\mathrm{MDL}$ & $<\mathrm{MDL}$ & $<\mathrm{MDL}$ & $<\mathrm{MDL}$ & $\mathrm{mg} / \mathrm{L}$ & 0.034 \\
\hline Iron & 0.01 & $<\mathrm{MDL}$ & $<\mathrm{MDL}$ & $<\mathrm{MDL}$ & 0.007 & 0.07 & 0.008 & 1.44 & 11.1 & 95 & $\mathrm{mg} / \mathrm{L}$ & 0.002 \\
\hline Lead & $<\mathrm{MDL}$ & $<\mathrm{MDL}$ & $<\mathrm{MDL}$ & $<\mathrm{MDL}$ & $<\mathrm{MDL}$ & $<\mathrm{MDL}$ & $<\mathrm{MDL}$ & $<\mathrm{MDL}$ & $<\mathrm{MDL}$ & $<\mathrm{MDL}$ & $\mathrm{mg} / \mathrm{L}$ & 0.01 \\
\hline Lithium & 0.68 & 0.43 & 0.18 & 0.06 & 0.03 & 0.07 & 0.03 & 0.05 & 0.03 & 0.19 & $\mathrm{mg} / \mathrm{L}$ & 0.001 \\
\hline Magnesium & 53.5 & 44 & 36.9 & 28.9 & 19.7 & 44.3 & 21.1 & 25 & 11.5 & 63.9 & $\mathrm{mg} / \mathrm{L}$ & 0.001 \\
\hline Manganese & 1.53 & 1.53 & 1.64 & 1.65 & 1.44 & 2.82 & 2.06 & 4.4 & 2.92 & 14.4 & $\mathrm{mg} / \mathrm{L}$ & 0.001 \\
\hline Nickel & 0.43 & 0.14 & 0.15 & 0.09 & $<\mathrm{MDL}$ & 0.01 & 0.11 & 1.1 & 1.05 & 6.75 & $\mathrm{mg} / \mathrm{L}$ & 0.002 \\
\hline Phosphorus & $<\mathrm{MDL}$ & $<\mathrm{MDL}$ & $<\mathrm{MDL}$ & $<\mathrm{MDL}$ & $<\mathrm{MDL}$ & $<\mathrm{MDL}$ & $<\mathrm{MDL}$ & $<\mathrm{MDL}$ & $<\mathrm{MDL}$ & 20.1 & $\mathrm{mg} / \mathrm{L}$ & 0.009 \\
\hline Potassium & 27.1 & 29.7 & 23.2 & 16.9 & 13.1 & 23.3 & 14.8 & 17.3 & 10.8 & 55.1 & $\mathrm{mg} / \mathrm{L}$ & 0.191 \\
\hline Selenium & 0.45 & 0.36 & 0.2 & 0.1 & 0.1 & 0.07 & 0.02 & 0.21 & 0.12 & 0.25 & $\mathrm{mg} / \mathrm{L}$ & 0.011 \\
\hline Silicon & 3.23 & 2.8 & 2.85 & 2.21 & 2.13 & 2.58 & 2.12 & 5.35 & 4.46 & 53.6 & $\mathrm{mg} / \mathrm{L}$ & 0.009 \\
\hline Silver & $<\mathrm{MDL}$ & $<\mathrm{MDL}$ & $<\mathrm{MDL}$ & $<\mathrm{MDL}$ & $<\mathrm{MDL}$ & $<\mathrm{MDL}$ & $<\mathrm{MDL}$ & $<\mathrm{MDL}$ & $<\mathrm{MDL}$ & $<\mathrm{MDL}$ & $\mathrm{mg} / \mathrm{L}$ & 0.003 \\
\hline Sodium & 1510 & 978 & 348 & 57.2 & 16.7 & 26.2 & 9.56 & 5.92 & 2.22 & 7.7 & $\mathrm{mg} / \mathrm{L}$ & 0.058 \\
\hline Strontium & 5.11 & 3.71 & 2.94 & 2.05 & 1.38 & 2.94 & 1.66 & 1.73 & 1.05 & 10.2 & $\mathrm{mg} / \mathrm{L}$ & 0.01 \\
\hline Sulfur & 1400 & 1080 & 571 & 308 & 208 & 505 & 329 & 379 & 260 & 450 & $\mathrm{mg} / \mathrm{L}$ & 0.014 \\
\hline Thalium & $<\mathrm{MDL}$ & $<\mathrm{MDL}$ & $<\mathrm{MDL}$ & $<\mathrm{MDL}$ & $<\mathrm{MDL}$ & $<\mathrm{MDL}$ & $<\mathrm{MDL}$ & $<\mathrm{MDL}$ & $<\mathrm{MDL}$ & $<\mathrm{MDL}$ & $\mathrm{mg} / \mathrm{L}$ & 0.041 \\
\hline Tin & $<\mathrm{MDL}$ & $<\mathrm{MDL}$ & $<\mathrm{MDL}$ & $<\mathrm{MDL}$ & $<\mathrm{MDL}$ & $<\mathrm{MDL}$ & $<\mathrm{MDL}$ & $<\mathrm{MDL}$ & $<\mathrm{MDL}$ & 0.31 & $\mathrm{mg} / \mathrm{L}$ & 0.012 \\
\hline Vanadium & $<\mathrm{MDL}$ & $<\mathrm{MDL}$ & $<\mathrm{MDL}$ & $<\mathrm{MDL}$ & $<\mathrm{MDL}$ & $<\mathrm{MDL}$ & $<\mathrm{MDL}$ & $<\mathrm{MDL}$ & $<\mathrm{MDL}$ & 0.03 & $\mathrm{mg} / \mathrm{L}$ & 0.008 \\
\hline Zinc & $<\mathrm{MDL}$ & $<\mathrm{MDL}$ & $<\mathrm{MDL}$ & 0.1 & 0.002 & $<\mathrm{MDL}$ & 0.06 & 0.67 & 0.93 & 7.15 & $\mathrm{mg} / \mathrm{L}$ & 0.002 \\
\hline
\end{tabular}


Major and trace element concentrations for all leachates from the cyclic testing of the two tailings fractions are summarized in Tables 9 and 10. The corresponding data set for the baseline testing after 20 weeks is shown in Table 11. Data for all elements sought in the ICP-MS analyses are listed in these tables, even if their concentrations were below the minimum detection limit (MDL) in all samples.

TABLE 11: ICP-MS Data for Leachates from Baseline Testing (Week 20) on Coal Tailings

\begin{tabular}{lcccc}
\hline Element & RBR & PLR & Units & MDL \\
\hline Aluminum & 0 & 0.17 & $\mathrm{mg} / \mathrm{L}$ & 0.061 \\
\hline Antimony & 0 & 0.04 & $\mathrm{mg} / \mathrm{L}$ & 0.012 \\
\hline Arsenic & $<\mathrm{MDL}$ & $<\mathrm{MDL}$ & $\mathrm{mg} / \mathrm{L}$ & 0.014 \\
Barium & 0.01 & 0.01 & $\mathrm{mg} / \mathrm{L}$ & 0.003 \\
Beryllium & $<\mathrm{MDL}$ & $<\mathrm{MDL}$ & $\mathrm{mg} / \mathrm{L}$ & 0.001 \\
Boron & 0.44 & 0.78 & $\mathrm{mg} / \mathrm{L}$ & 0.008 \\
Cadmium & 0.003 & 0.003 & $\mathrm{mg} / \mathrm{L}$ & 0.001 \\
Calcium & 384 & 401 & $\mathrm{mg} / \mathrm{L}$ & 0.002 \\
Chromium & 0.029 & 0 & $\mathrm{mg} / \mathrm{L}$ & 0.024 \\
Cobalt & $<\mathrm{MDL}$ & $<\mathrm{MDL}$ & $\mathrm{mg} / \mathrm{L}$ & 0.001 \\
Copper & 0.01 & 0.006 & $\mathrm{mg} / \mathrm{L}$ & 0.005 \\
\hline Gold & $<\mathrm{MDL}$ & $<\mathrm{MDL}$ & $\mathrm{mg} / \mathrm{L}$ & 0.034 \\
Iron & 0.09 & 0.23 & $\mathrm{mg} / \mathrm{L}$ & 0.002 \\
Lead & $<\mathrm{MDL}$ & $<\mathrm{MDL}$ & $\mathrm{mg} / \mathrm{L}$ & 0.01 \\
\hline Lithium & 0.99 & 0.9 & $\mathrm{mg} / \mathrm{L}$ & 0.001 \\
Magnesium & 77.5 & 69.4 & $\mathrm{mg} / \mathrm{L}$ & 0.001 \\
Manganese & 1.41 & 1.62 & $\mathrm{mg} / \mathrm{L}$ & 0.001 \\
Nickel & 0.09 & 0.11 & $\mathrm{mg} / \mathrm{L}$ & 0.002 \\
Phosphorus & $<\mathrm{MDL}$ & $<\mathrm{MDL}$ & $\mathrm{mg} / \mathrm{L}$ & 0.009 \\
Potassium & 30.5 & 37.6 & $\mathrm{mg} / \mathrm{L}$ & 0.191 \\
Selenium & $<\mathrm{MDL}$ & $<\mathrm{MDL}$ & $\mathrm{mg} / \mathrm{L}$ & 0.011 \\
\hline Silicon & 1.59 & 2.38 & $\mathrm{mg} / \mathrm{L}$ & 0.009 \\
Silver & $<\mathrm{MDL}$ & $<\mathrm{MDL}$ & $\mathrm{mg} / \mathrm{L}$ & 0.003 \\
\hline Sodium & 1785 & 1606 & $\mathrm{mg} / \mathrm{L}$ & 0.058 \\
Strontium & 6.58 & 6.72 & $\mathrm{mg} / \mathrm{L}$ & 0.01 \\
\hline Sulfur & 1912 & 1750 & $\mathrm{mg} / \mathrm{L}$ & 0.014 \\
Thalium & $<\mathrm{MDL}$ & $<\mathrm{MDL}$ & $\mathrm{mg} / \mathrm{L}$ & 0.041 \\
Tin & $<\mathrm{MDL}$ & $<\mathrm{MDL}$ & $\mathrm{mg} / \mathrm{L}$ & 0.012 \\
Vanadium & $<\mathrm{MDL}$ & $<\mathrm{MDL}$ & $\mathrm{mg} / \mathrm{L}$ & 0.008 \\
Zinc & $<\mathrm{MDL}$ & $<\mathrm{MDL}$ & $\mathrm{mg} / \mathrm{L}$ & 0.002 \\
\hline & & & & \\
\hline
\end{tabular}

A comparison of the cumulative leached amounts for the cyclic testing after weeks 22 and 23 with the baseline data after week 20, as a percentage of the total amount of an element originally present in the tailings, is shown in Figure 12. These data confirm that the cyclic test was more aggressive than the baseline static test with respect to removal of elements from the tailings. In addition, these data show (Figure 13) that the elements most readily leached from the tailings were the alkali and alkaline earth metals ( $\mathrm{Na}, \mathrm{Mg}, \mathrm{Ca}$, and $\mathrm{Sr}$ ). Larger elements in these groups $(\mathrm{K}, \mathrm{Ba})$ were much more resistant to leaching. Among the trace elements, only $\mathrm{Mn}, \mathrm{Co}, \mathrm{Ni}, \mathrm{Zn}$, 
and Se were leached to any significant extent in these tests. Other key elements such as Al, Si, $\mathrm{V}, \mathrm{Cr}, \mathrm{Fe}$, and As were not significantly leached. It is clear from the lack of significant leaching of $\mathrm{K}, \mathrm{Al}$, and $\mathrm{Si}$, as well of $\mathrm{Cr}$ and $\mathrm{V}$, that illite is unaffected by the leaching treatments. It was found that more than $10 \%$ of the sulfur in the tailings is removed by leaching in the form of sulfate. However, there does not appear to be a corresponding fraction of iron leached to indicate significant alteration of pyrite. So where does the sulfur come from? The answer to this question was provided by spectroscopic examination of the tailings after leaching.

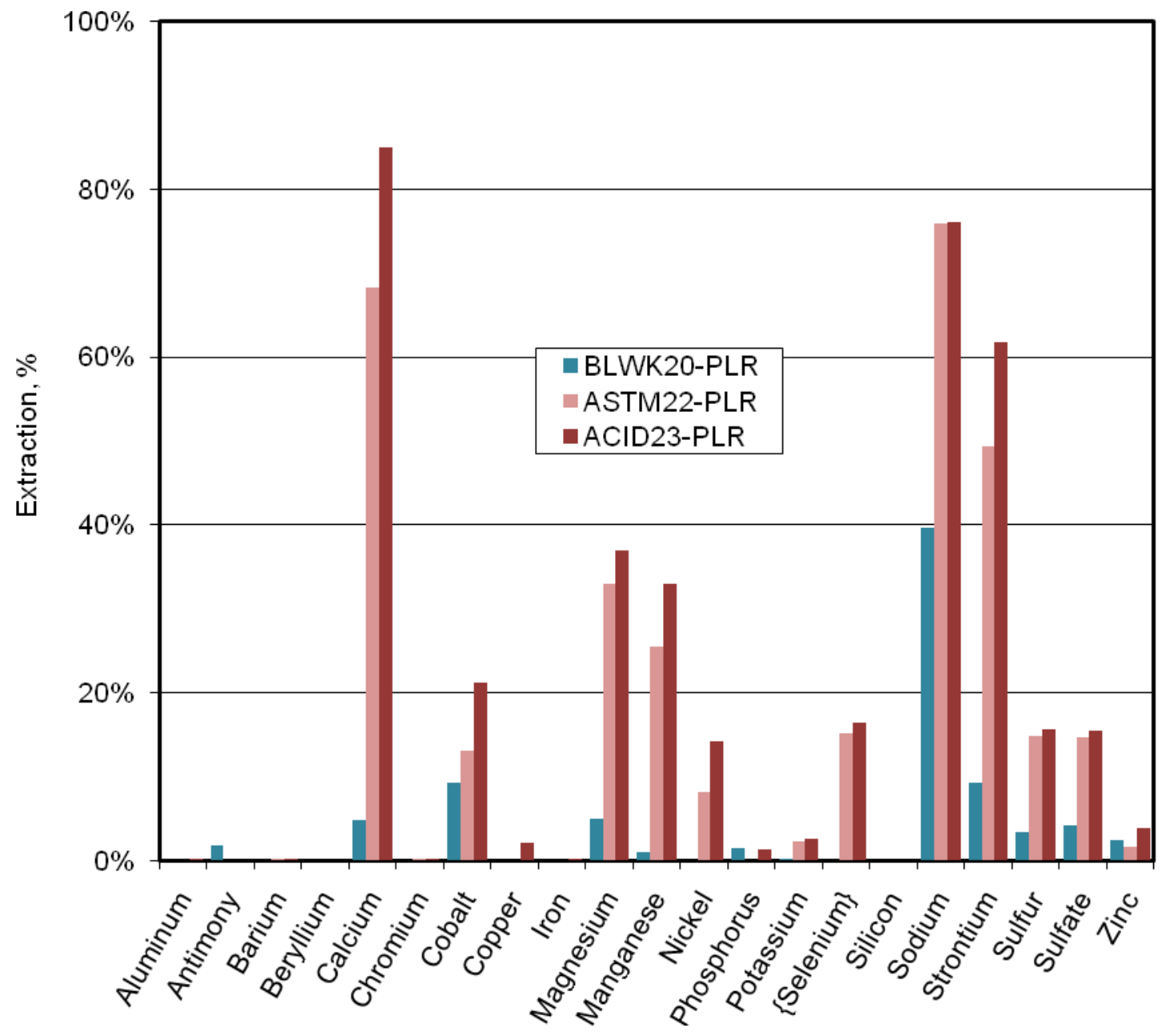

FIGURE 12: Comparison of the cumulative amounts of leached elements from PLR tailings after weeks 22 and 23 of the cyclic testing and after week 20 of the baseline test. The data are presented as a percentage of the total amount of an element originally present in the tailings. Elements not shown were negligibly leached in all tests. Data for the RBR tailings were similar.

After weeks 7 and 22 of the cyclic leaching treatment, small samples of both tailings fractions were separated and used for Fe Mössbauer and As XAFS speciation determinations. Both iron and arsenic showed significant increases in oxidized forms; specifically, about $8-10 \%$ of the iron in pyrite has been converted to iron as jarosite and a larger percentage of arsenic has been converted to arsenate forms (Figure 14). However, these oxidized forms clearly resist leaching since neither iron nor arsenic is detected to any significant extent in the leachates (q.v. Figure 12). 


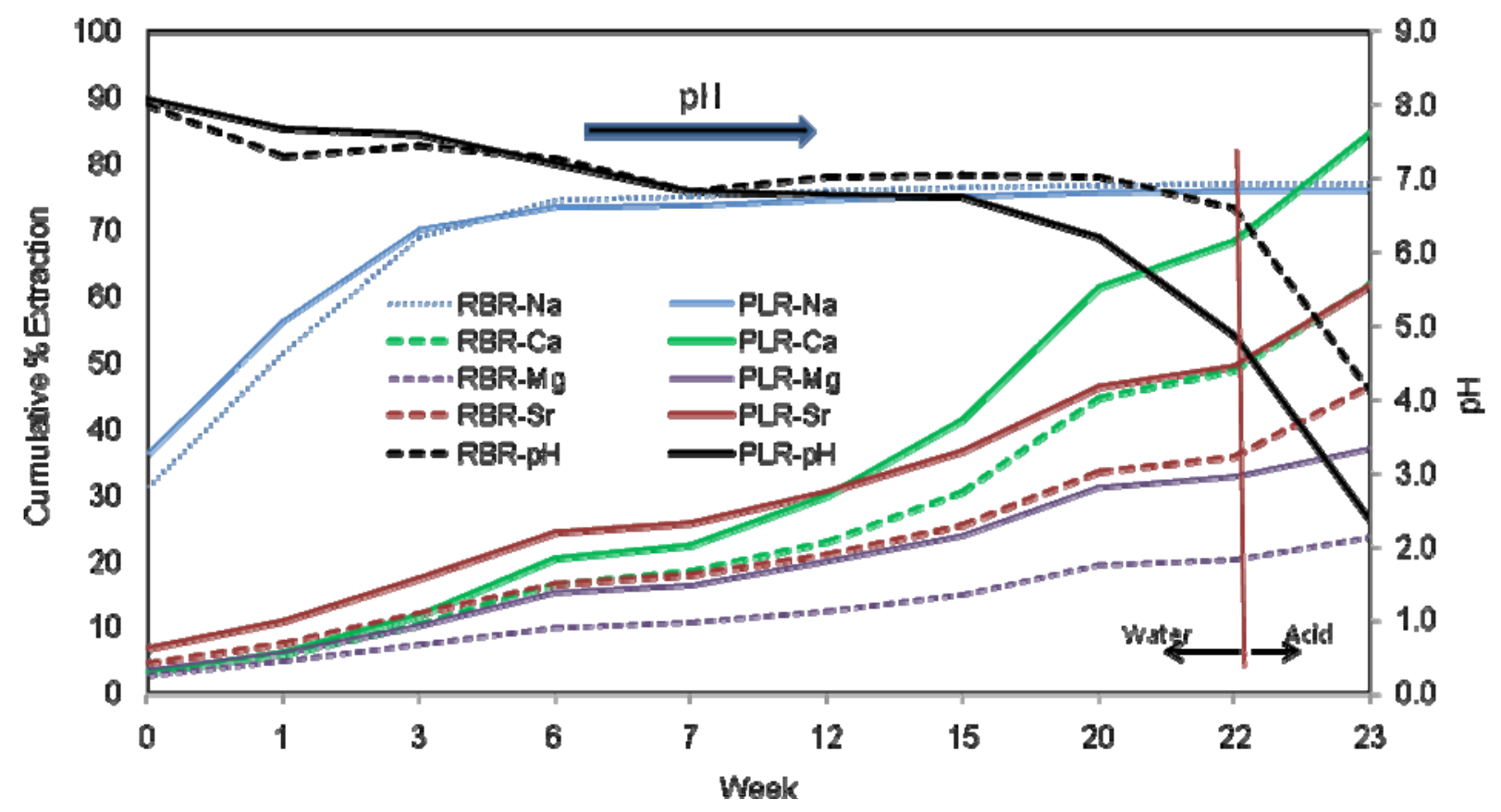

FIGURE 13: Cumulative \% extraction of alkali $(\mathrm{Na})$ and alkaline earth $(\mathrm{Ca}, \mathrm{Mg}, \mathrm{Sr})$ elements from RBR (dashed lines) and PLR (solid lines) tailings fractions with length of time in the cyclic leaching test. Also shown is the variation of $\mathrm{pH}$ for the leachates.

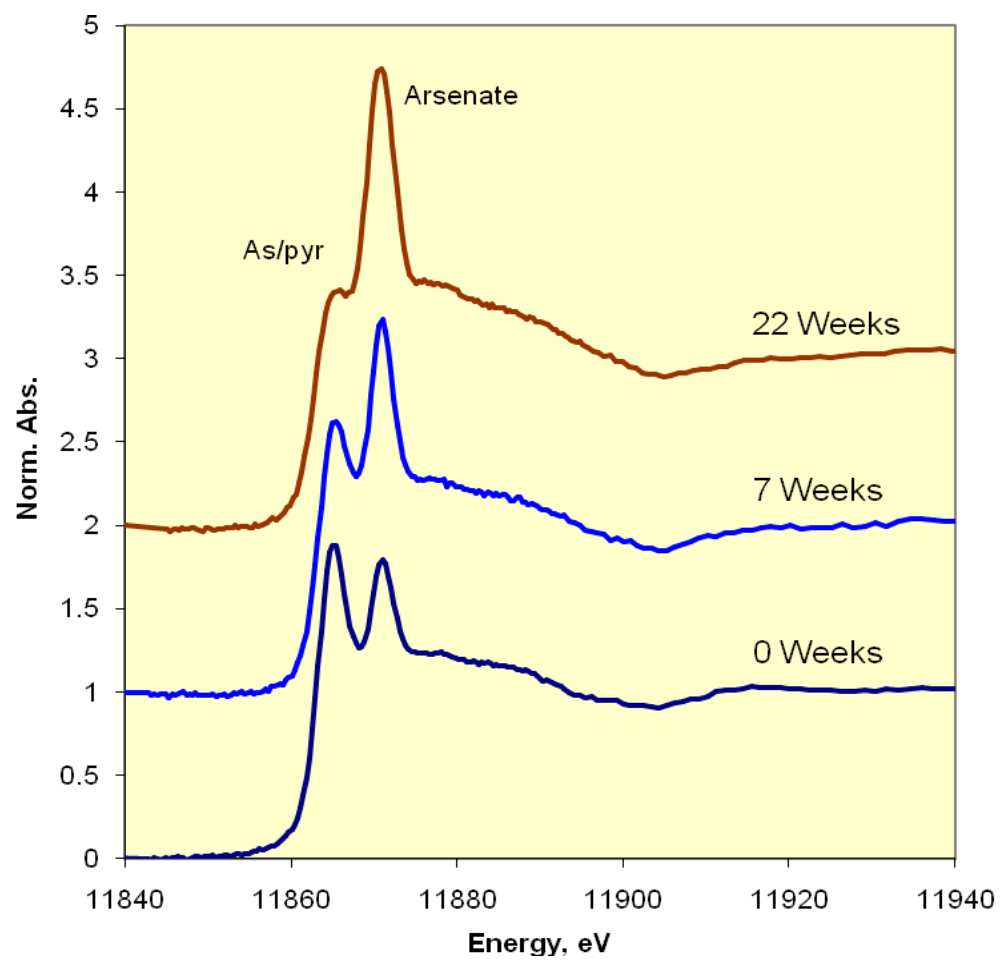

FIGURE 14: Arsenic XANES spectra from samplings of the coal tailings fractions taken after 7 and 22 weeks into the cyclic test. Note the increasing intensity of the peak due to arsenate relative to that for As/pyrite. 
The following scenario appears to account for the leaching behavior exhibited by these samples of tailings from coal from the Illinois \#6 seam. The first elements leached from the tailings in quantity are alkali and alkaline earth elements, including $\mathrm{Na}, \mathrm{Mg}, \mathrm{Ca}$, and $\mathrm{Sr}$. Sodium in particular is easily and rapidly leached from these tailings. It is followed by the slower release of $\mathrm{Ca}, \mathrm{Mg}$, and $\mathrm{Sr}$. The dissolution of these basic elements effectively buffers the $\mathrm{pH}$ of the leachate and delays it from becoming highly acidic, as would be anticipated for a coal so rich in pyrite. As long as the $\mathrm{pH}$ of the extract remains close to neutral, oxidation of pyrite, the associated release of arsenic, and, more importantly, the formation of acid, $\left[\mathrm{H}^{+}\right]_{\text {sol'n }}$, is minimal. Even though the pyrite and associated arsenic do show signs of significant oxidation, as demonstrated by the formation of jarosite and the large increase in arsenate relative to As associated with pyrite, these oxidized forms of these elements remain immobile. The following equation, which results in the formation of insoluble jarosite, is consistent with the observations made to date:

$$
3 \mathrm{FeS}_{2}+3 \mathrm{H}_{2} \mathrm{O}+13.5 \mathrm{O}_{2}+\left[\mathrm{Na}^{+}\right]_{\mathrm{sol} \text { 'n }} \rightarrow \mathrm{NaFe}_{3}\left(\mathrm{SO}_{4}\right)_{2}(\mathrm{OH})_{6}+4\left[\mathrm{SO}_{4}{ }^{2-}\right]_{\mathrm{sol} \text { 'n }}
$$

This reaction explains not only the release of some sulfate but also the lack of mobilization of iron and the lack of formation of acid $\left.\left(\left[\mathrm{H}^{+}\right]_{\text {sol'n }}\right]\right)$. Arsenic must remain predominantly with the jarosite, presumably as a result of the substitution of $\mathrm{AsO}_{4}{ }^{3-}$ for $\mathrm{SO}_{4}{ }^{2-}$ (Paktunc and Dutrizac, 2003). As long as insoluble jarosite formation is maintained and the acidity is buffered to near neutral $\mathrm{pH}$, mobilization of trace metals in pyrite such as arsenic can be avoided. The role of readily solublized $\mathrm{Na}^{+}$, which appears to act as an effective buffer to acid formation and is necessary for formation of jarosite, is clearly important and may lead to effective strategies to retard trace element mobilization from coal tailings.

\section{CONCLUSIONS}

The combination of multi-elemental analysis methods and techniques such as Mossbauer and XAFS spectroscopies, supplemented by conventional coal analyses, has been used to obtain detailed information regarding the modes of occurrence of sulphur, iron, and a number of key trace elements in an as-mined Illinois \#6 coal and in various clean coal and tailings fractions separated from the coal at a commercial preparation plant. For this coal, it was established that As is entirely associated with pyrite. Se and $\mathrm{Hg}$ are also associated with pyrite but to lesser extents. Conversely, $\mathrm{Cr}, \mathrm{V}$, and possibly $\mathrm{Ni}$ are principally associated with illite, while $\mathrm{Zn}$ exhibits a sulphide (as $\mathrm{ZnS}$ ) occurrence as well as a clay occurrence and possibly also a phosphate occurrence. The occurrence of $\mathrm{Mn}$ is also complex. Germanium was shown to be strongly associated with macerals and $\mathrm{Hg}$ also exhibited a significant organic association as well. By and large, the associations of trace elements with pyrite and illite determined to a large extent their separation efficiency in the coal cleaning processes.

With this framework established, the mobility of trace elements from leaching tests simulating tailings impoundments was then examined. Three different leaching tests were examined including the standard 18-hour TCLP test, which proved to be quite uninformative regarding trace element behaviour during leaching. Two longer-term ( $>150$ days) tests were then carried out, one of which was a variation of the cyclic test method, ASTM Standard Method D-5744, while the other was a baseline method involving storage of the tailings under water for the length 
of time of the test. Elements such as $\mathrm{Cr}$ and $\mathrm{V}$, which are associated $(>95 \%)$ with illite, exhibited no mobility whatsoever over the duration of the tests, no doubt reflecting the resistance of clay minerals to aqueous leaching at near-neutral $\mathrm{pH}$. Elements associated with pyrite, such as Fe and As, also showed little mobility. Elements that were mobilized included $\mathrm{Na}, \mathrm{Mg}, \mathrm{Ca}$, and Sr. The solubilisation of these alkali and alkaline earth elements effectively buffered the $\mathrm{pH}$ of the leachate solutions and prevented significant alteration of pyrite and the release of elements associated with it. What alteration of pyrite did occur was limited to minor formation of jarosite, which remained insoluble throughout the leaching tests and acted as a sink for arsenate anions formed during the alteration. The role of sodium in buffering the $\mathrm{pH}$ of the leachates and the formation of insoluble natrojarosite is clearly important for preventing release of potentially harmful trace elements from pyrite.

\section{ACKNOWLEDGEMENTS}

The experimental work described in this Report was largely carried out by Laal B. A. Seidu, a M. Sc. Candidate in the Department of Mining Engineering at the University of Kentucky. He also had the responsibility for the compilation and management of the analytical data. The cyclic leaching test was constructed by Mr. Seidu, with assistance from Nick Cprek.

Important advice on coal selection and the collection and preparation of samples for analysis was provided by Prof. Rick Honaker of the Department of Mining Engineering at the University of Kentucky.

The Principal Investigators acknowledge the following persons for their analytical skills and contributions to this study: G. Thomas, Center for Applied Energy Research, University of Kentucky, for proximate and ultimate analyses and $\mathrm{Hg}$ analyses of the Illinois coal fractions; $\mathrm{H}$. Francis and J. Backus, Kentucky Geological Survey, for X-ray fluorescence analyses of the ash and ICP-MS, pH, Eh, and sulfate analyses of the leachates from the cyclic and static leaching tests; J. Kyger, B. L. Higgins, and J. D. Robertson, University of Missouri at Columbia, for ICPMS determinations of trace metals in the coal fractions; and S. Pal, and M. Seehra, West Virginia University for Rietveld X-ray diffraction analyses of the mineralogy of the coal fractions.

This research was supported by a grant from the U.S. Department of Energy through the Center for Advanced Separation Technologies at Virginia Polytechnic Institute and State University, Blacksburg, VA. The Principal Investigators also acknowledge the U.S. Department of Energy for its support of the synchrotron facilities for XAFS spectroscopy at the National Synchrotron Light Source, Brookhaven National Laboratory, NY, and at the Stanford Synchrotron Radiation Laboratory, Stanford University, CA. 


\section{REFERENCES}

ASTM. (1996). Standard Method D-5744-96. Standard test method for accelerated weathering of solid waste materials using a modified humidity cell. American Society for Testing and Materials, Philadelphia, PA.

Finkelman, R. B. (1994). Mode of occurrence of potentially hazardous elements in coal: levels of confidence. Fuel Process. Technol. $\underline{39}, 21-34$.

Huggins, F. E. and Huffman, G. P. (2004). How do lithophile elements occur in organic association in bituminous coals? Int. J. Coal Geol., 58, [3], 193-204.

Huggins, F. E., Shah, N., Zhao, J., Lu, F. and Huffman, G. P. (1993). Nondestructive determination of trace element speciation in coal and ash by XAFS spectroscopy. Energy Fuels $\underline{7}, 482-489$.

Huggins, F. E., Srikantapura, S., Parekh, B. K., Blanchard, L. and Robertson, J. D. (1997). XANES spectroscopic characterization of selected elements in deep-cleaned fractions of Kentucky \#9 coal. Energy Fuels 11, 691- 701.

Huggins, F. E., Shah, N., Huffman, G. P., Kolker, A., Crowley, S., Palmer, C. A. and Finkelman, R. B. (2000). Mode of occurrence of chromium in four U.S. Coals. Fuel Process. Technol. $\underline{63}$, $79-92$.

Kolker, A. and Huggins, F. E. (2007). Progressive oxidation of pyrite in five bituminous coal samples: An As XANES and ${ }^{57} \mathrm{Fe}$ Mössbauer spectroscopic study. Applied Geochemistry, 22, [4], 778-787.

Kolker, A., Crowley, S., Palmer, C. A., Finkelman, R. B., Huggins, F. E., Shah, N. and Huffman, G. P., (2000). Mode of occurrence of arsenic in four U.S. coals. Fuel Process. Technol. $\underline{63}$, 167178.

Maylotte, D. H., Wong, J., St. Peters, R. L., Lytle, F. W. and Greegor, R. B. (1981). X-ray absorption spectroscopic investigation of trace vanadium sites in coal. Science 214, 554-556.

Paktunc, D. and Dutrizac, J. E. (2003). Characterization of arsenate-for-sulfate substitution in synthetic jarosite using X-ray diffraction and X-ray absorption spectroscopy. Canad. Mineral. $\underline{41}, 905-919$.

S.R. Sutton, J. Karner, J. Papike, J.S. Delaney, C. Shearer, M. Newville, P. Eng, M. Rivers, and M.D. Dyar, (2005). A new microscale oxybarometer for solar system basaltic glasses based on vanadium K edge XANES. Geochim. Cosmochim. Acta 69(9), 2333-2348.

Swaine, D. J. (1990). Trace Elements in Coal. Butterworths, Chichester, U.K.

U.S. Congress. (1976). Public Law 94-580, October 21 ${ }^{\text {st }}, 1976$. 
U.S. Congress. (1990). Amendments to the 1990 Clean Air Act, Federal Register.

\section{BIBLIOGRAPHY}

F. E. Huggins, L. B. A. Seidu, N. Shah, G. P. Huffman, and R. Q. Honaker, Behavior of arsenic and other trace elements during cleaning of an Illinois coal and simulated leaching of its tailings. Program and Full Papers, 2007 International Conference on Coal Science and Technology (Nottingham, U.K.), Paper 10D3, CD-ROM, 10 pp., (2007).

\section{LIST OF ACRONYMS AND ABBREVIATIONS}

$\begin{array}{ll}\text { ASTM } & \text { American Standards for Testing and Materials } \\ \text { CAAA } & \text { Clean Air Act Amendments } \\ \text { CLN } & \text { Clean (Coal) } \\ \text { FEE } & \text { Feed (Coal) } \\ \text { HAP } & \text { Hazardous Air Pollutant (Element) } \\ \text { ICP-MS } & \text { Inductively Coupled Plasma- Mass Spectroscopy } \\ \text { MDL } & \text { Minimum Detection Limit } \\ \text { PLR } & \text { Plant Reject (Tailings) } \\ \text { ppm } & \text { parts per million } \\ \text { RBR } & \text { Rotary Breaker Reject (Tailings) } \\ \text { RCRA } & \text { Resource Conservation and Recovery Act } \\ \text { TCLP } & \text { Toxicity Characteristic Leaching Procedure } \\ \text { tph } & \text { tons per hour } \\ \text { XAFS } & \text { X-ray Absorption Fine Structure (Spectroscopy) } \\ \text { XANES } & \text { X-ray Absorption Near-Edge Structure (Spectroscopy) } \\ \text { XRD } & \text { X-ray Diffraction } \\ \text { XRF } & \text { X-ray Fluorescence }\end{array}$


Appendix 39: Portable Sensor for Detecting Mercury and other Heavy Metals Encountered in Coal Processing and Utilization (WV013) 
TECHNICAL REPORT

Contract Title and Number:
Crosscutting Technology Development at the Center for
Advanced Separation Technologies
(DE-FC26-02NT41607)

Period of Performance:

Starting Date: 6/01/2004

Ending Date: 08/31/2009

Sub-Recipient Project Title:

Portable sensor for Detecting mercury and other

Heavy Metals Encountered in Coal Processing and

Utilization

Principal Investigator: M. S. Seehra

Report Information:

Type: Final

Number:

Period: $\quad$ 06/01/04 to 08/31/09

Date: $\quad$ 03/31/09

Code: WV013-Final

Contact Address:

Physics Department

Contact Information:

210 Hodges Hall

Phone: 304-293-3422 x1473

West Virginia University

Morgantown, WV 26506-6315

Subcontractor Address:

No subcontracts issued.

Fax: $\quad 304-293-5732$

E-Mail: mseehra@wvu.edu

Subcontractor Information: N/A

\section{DISCLAIMER}

This report was prepared as an account of work sponsored by an agency of the United States Government. Neither the United States Government nor any agency thereof, nor any of their employees, make any warranty, express or implied, nor assume any legal liability or responsibility for the accuracy, completeness, or usefulness of any information, apparatus, product, or process disclosed, or represents that its use would not infringe privately owned rights. Reference herein to any specific commercial product, process, or service by trade name, trademark, manufacturer, or otherwise does not necessarily constitute or imply endorsement, recommendation, or favoring by the United States Government or any agency thereof. The views and opinions of authors expressed herein do not necessarily state or reflect those of the United States Government or agency thereof. 


\begin{abstract}
The objectives of this project was research leading to development of sensor for detections of mercury and other ions such as $\mathrm{Pb}$ and $\mathrm{Cd}$ at the ppb levels in solutions using boron-doped electrochemistry. Dr. A. Manivannan was associated with this project as a coinvestigator till October 2005 when he resigned his position with WVU and joined NETL, Morgantown, WV. Since October 2005, co-investigator M. S. Seehra has been the Principal Investigator for the project.

The detection of mercury at ppb levels was successfully achieved in our initial studies [1-2]. However the issue of reproducibility from run to run with boron doped diamond still remained a major challenge. The problems encountered and steps taken to establish reproducible results are reviewed in this report. Also use of $\mathrm{GaN}$ electrodes in the place of BDD electrodes was also tested. The project was divided into seven different tasks to achieve the goals of the project. These tasks described in detail later included review of theoretical basis of electrochemical detection of $\mathrm{Hg}$, investigation of "deposition" and "stripping" potentials, re-examining the cleaning procedures of the electrode, use of GaN for mercury detection, detection of $\mathrm{Pb}$ and $\mathrm{Cd}$, role of gold and impurities and development of a portable sensor. The results of various tasks are provided in the forthcoming sections. All the above tasks were undertaken in an effort to establish reproducible and hence reliable sensor for mercury, with ppb detection limits in laboratory solutions. All the outlined objectives were achieved and results published in seven refereed papers and one M. S. thesis.
\end{abstract}




\section{TABLE OF CONTENTS}

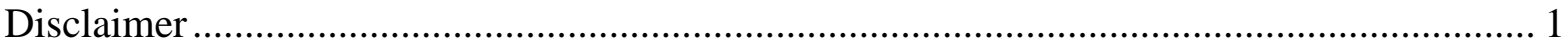

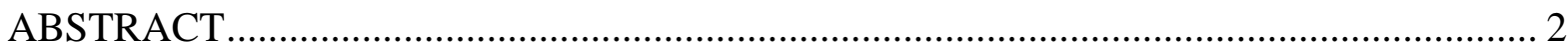

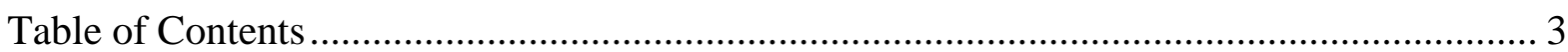

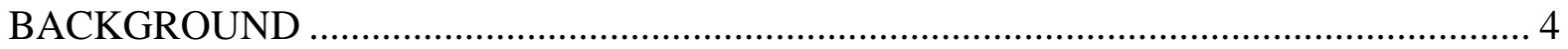

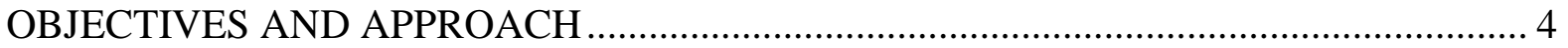

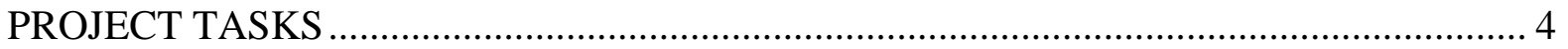

TASK 1: Review of theoretical basis for electrochemical detection ............................... 5

TASK 2: Investigation of parameters used in differential pulse voltammetry ................... 6

TASK 3: Ensuring complete recovery of the electrode surface with cleaning................... 8

TASK 4: Surface testing of various BDD electrodes .................................................. 12

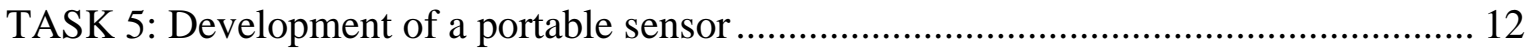

TASK 6: Testing GaN as a possible electrode for mercury detection .............................. 14

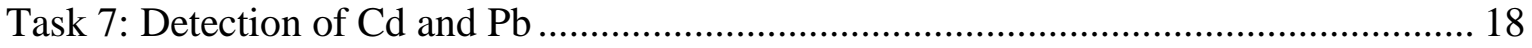

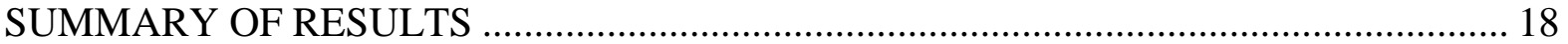




\section{BACKGROUND}

Detection of mercury with high accuracy and low detection limits were described in our paper published in 2005[3]. These results also agreed well with those obtained by CVAAS (Cold Vapor Atomic Absorption Spectroscopy) [3]. However the issue of reproducibility of the calibration curves, which required developing a new calibration curve for each new measurement was also noted in this paper [3]. Such procedure in practice was very time-consuming and hence not practical. Therefore development of procedures to overcome this problem and allow the use of a single calibration curve for determining an unknown concentration became a major focus of this research along with developing a portable sensor. To that end, rethinking of all aspect of the problem, both theoretical and experimental, was undertaken.

In experiments involving detection of $\mathrm{Hg}$, a gold solution is added in ppm concentrations in the electrolyte. This helps in capturing $\mathrm{Hg}$ from the solution since mercury easily forms an amalgam with $\mathrm{Au}$. This Au could be possible cause for not removing $\mathrm{Hg}$ from electrode completely for subsequent runs. Hence a theoretical understanding of the position of peaks becomes essential. Also a cleaning procedure to completely clean the electrode was highly desirable. The electrodes need to be examined periodically to ensure that the results are reliable. Hence some electrodes whose surface was found to be affected were replaced with new electrodes. The use of $\mathrm{GaN}$ as an electrode was tested, the results of which are also presented.

\section{OBJECTIVES AND APPROACH}

With the background clearly defined, our objective was to address the issue of reproducibility with BDD electrodes, investigate GaN for mercury detection as a possible alternative and to develop portable sensor with BDD electrodes. The reproducibility of the calibration curve from run to run was addressed by reexamining the parameters used in earlier work and by examining theoretical basis of the mechanism of electrochemical mercury detection. As a subsequent step the smaller BDD electrode is experimented with a portable potentiostat to develop a portable sensor. New GaN stationary electrodes (20mm x $20 \mathrm{~mm}$ ) were prepared and provided by Dr.Dimitris Korakakis of the Department of Computer Science and Electrical Engineering at WVU. Also rotating disk electrode experiments were prepared with GaN. Finally, the role of added gold and unavoidable impurities of $\mathrm{Cu}$ and $\mathrm{Ag}$ on the reproducibility problem was carefully analyzed and addressed.

\section{PROJECT TASKS}

To meet the objectives outlined above, the project was divided into the following seven tasks:

Task 1: Review of theoretical basis for electrochemical detection 
Task 2: Investigation of parameters used in differential pulse voltammetry

Task 3: Ensuring complete recovery of the electrode surface with cleaning

Task 4: Surface testing of various BDD electrodes

Task 5: Development of a portable sensor

Task 6: Testing GaN as a possible electrode for mercury detection

Task 7: Detection of $\mathrm{Cd}$ and $\mathrm{Pb}$

TASK 1: Review of theoretical basis for electrochemical detection

The understanding of theoretical concepts in electrochemical detection is essential to interpret the results. The Nernst equation can be used to interpret these results as shown:

$$
\mathrm{E}=\mathrm{E}^{\mathrm{o}}-\frac{0.05918}{n} \log \frac{1}{M}
$$

Here $\mathrm{M}$ is the molarity and $\mathrm{n}$ is the number of electrons involved in the reaction.

For $\mathrm{Hg}, 100 \mathrm{ppt}$ is equivalent to $4.9875 \times 10^{-10} \mathrm{M}$. For the reaction

$$
\mathrm{Hg}^{2+}+2 \mathrm{e}^{-} \Leftrightarrow \mathrm{Hg}(\mathrm{l}), \quad \mathrm{E}^{\mathrm{o}}=0.61 \mathrm{~V} \text { vs. SCE } \quad--- \text { (2) }
$$

Eqs. 1 and 2 yield $\mathrm{E}=0.35 \mathrm{~V}$. For gold two relevant reactions are

$$
\begin{array}{ll}
\mathrm{Au}^{3+}+2 \mathrm{e}^{-} \Leftrightarrow \mathrm{Au}^{+}, & \mathrm{E}^{0}=1.16 \text { vs. SCE } \\
\mathrm{Au}^{+}+\mathrm{e}^{-} \Leftrightarrow \mathrm{Eu}^{+}, & \mathrm{E}^{0}=1.16 \text { vs. SCE }
\end{array}
$$

For $\mathrm{Au}, 1.5 \mathrm{ppm}=0.51 \times 10^{-8} \mathrm{M}$. Substituting these values yields $\mathrm{E}^{0}=0.92 \mathrm{~V}$ for $\mathrm{n}=2$ in Eq. 3 and $\mathrm{E}^{0}=0.97 \mathrm{~V}$ using $\mathrm{n}=1$ for $\mathrm{Eq}(4)$. Since the final state must be $\mathrm{Au}(\mathrm{s})$, a broad peak centered on $0.95 \mathrm{~V}$ vs. SCE is predicted. For copper:

$$
\begin{array}{ll}
\mathrm{Cu}^{+2}+2 \mathrm{e}^{-} \Leftrightarrow \mathrm{Cu}(\mathrm{s}), & \mathrm{E}^{\mathrm{o}}=0.1 \mathrm{~V} \text { vs. SCE } \\
\mathrm{Cu}^{+}+\mathrm{e}^{-} \Leftrightarrow \mathrm{Cu}(\mathrm{s}), & \mathrm{E}^{0}=0.1 \mathrm{~V} \text { vs. SCE }
\end{array}
$$

Calculating the potential for eq.5 yields $\mathrm{E}=-0.23 \mathrm{~V}$ and for eq. 6 yields $-0.4 \mathrm{~V}$ for a copper concentration of $200 \mathrm{ppt}\left(3.5 \times 10^{-12} \mathrm{M}\right)$. The significance of $\mathrm{Au}$ and $\mathrm{Cu}$ peak calculations is explained in the later sections. A comparison of the Hg peaks calculated with the above equation and observed peak shifts are shown in Fig.1, providing a good semi-quantitative agreement.

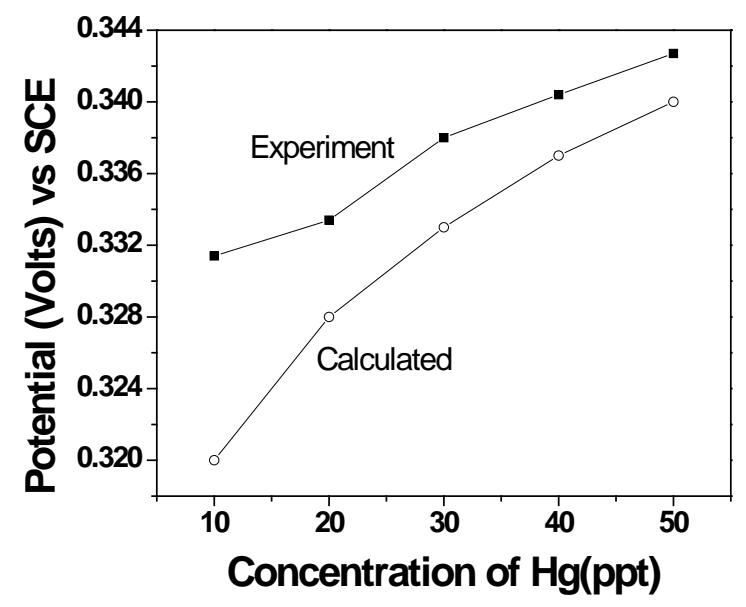

Fig. 1 Increase in peak voltage with increase in concentration for 10 to 50 ppt $\mathrm{Hg}^{2+}$ concentrations in $1 \mathrm{M} \mathrm{KCl}$ $(\mathrm{pH}=1)$ and comparison between calculated and observed values. 
TASK 2: Investigation of parameters used in differential pulse voltammetry

With the theoretical understanding from the Task 1 , the peaks from experiments could be clearly identified. Cu peak occurs at $-0.19 \mathrm{~V}, \mathrm{Hg}$ at $0.3 \mathrm{~V}$ and $\mathrm{Au}$ at $0.82 \mathrm{~V}$ with respect to $\mathrm{Ag} / \mathrm{AgCl}$ (fig.2). It is seen that with a deposition potential of $0.4 \mathrm{~V}$ which was

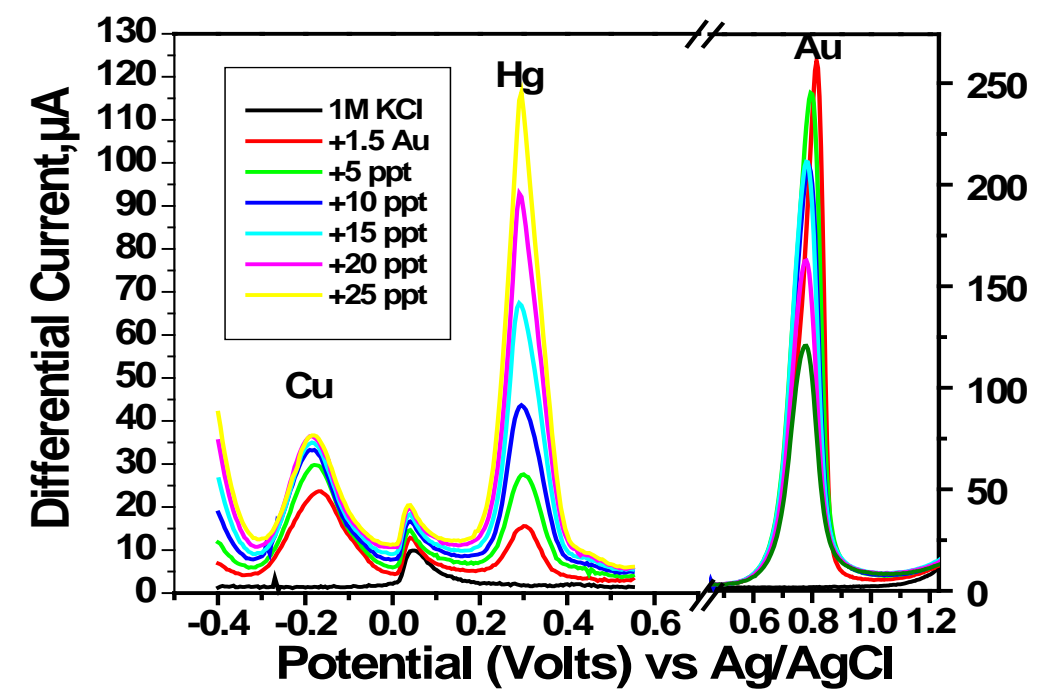

Fig. 2 DPV in the range of 5 to 25 ppt with gold added. Copper is an impurity in the gold standard solution.

used in earlier experiments, there is a good possibility of copper interference. It is believed that the possible source of copper is the gold standard solution. Hence experiments with various deposition potentials were tried. The plots for experiments with deposition potential $0.3 \mathrm{~V},-0.2 \mathrm{~V},-0.1 \mathrm{~V}$ and $-0.05 \mathrm{~V}$ were tested. The deposition potential $-0.05 \mathrm{~V}$ eliminates copper deposition completely and the plot is shown (Fig.3).

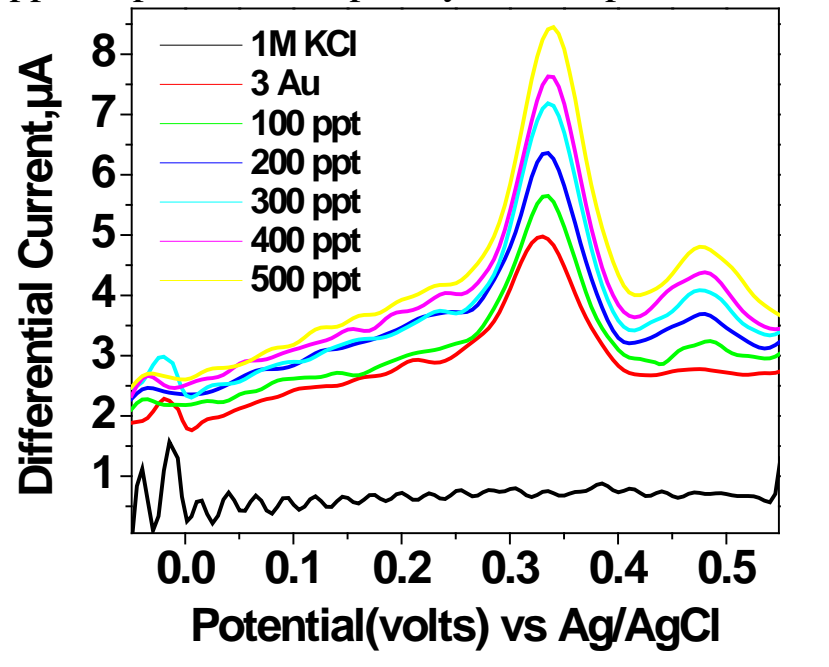

Fig. 3. DPV plot with a deposition potential of $-0.05 \mathrm{~V}$ in the range of $100-$ $500 \mathrm{ppt} \mathrm{Hg}^{2+}$

The mechanism of formation of mercury $1+$ and $2+$ states are understood by carrying out a series of experiments with increasing concentrations of mercury. At lower concentrations, $\mathrm{Hg}^{1+}$ states are more predominant. The formation of $\mathrm{Hg}^{2+}$ concentrations is significant after adding $100 \mathrm{ppb}$ of $\mathrm{Hg}$ (see Fig.4). The shoulder in most of the experiments conducted earlier is due to the interference of $\mathrm{Hg}^{2+}$ and $\mathrm{Hg}^{1+}$. 


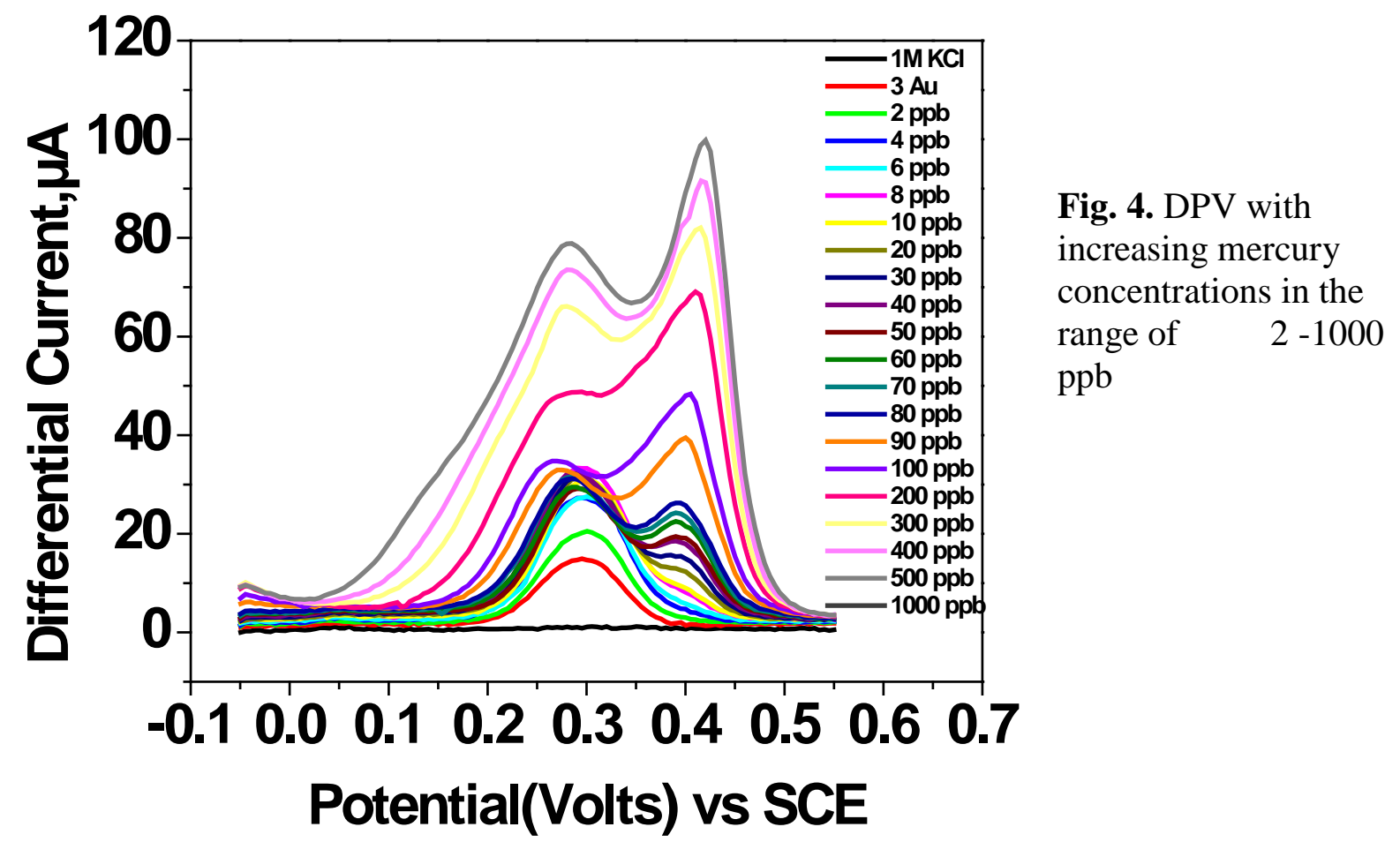

The

experiments with a deposition potential of $-0.4 \mathrm{~V}$ had significant copper peaks (see Fig.5) Also, these peaks remained constant with increasing Hg concentrations. This suggested that copper could also be used as an internal standard for mercury detection. The standard addition method used in earlier experiments can be replaced with the internal standard method if copper proves to be a good internal standard. The concentration of $\mathrm{Hg}$ (unknown)/Cu (internal standard) vs. concentration of $\mathrm{Hg}$ is plotted to determine the concentration of mercury already present in the solution (Fig.,6). These plots are from the experiments with gold solution added in ppm ranges, where the impurity in gold is the main source of copper.

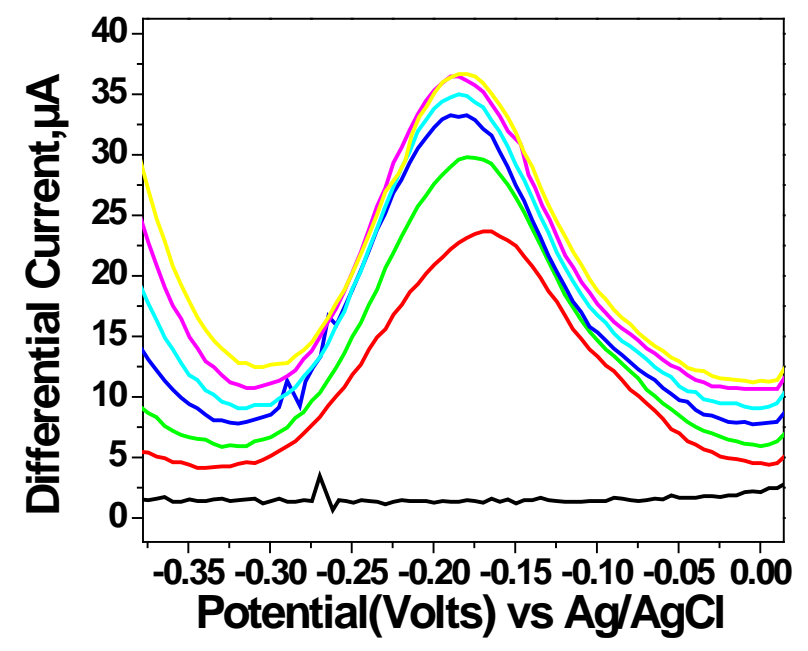

Figure 5 DPV scans revealing copper peaks for $5 p p t$ to 25ppt $\mathrm{Hg}^{2+}$ concentrations in $1 \mathrm{M} \mathrm{KCl}$ $(\mathrm{pH}$ 1). 1.5 ppm gold standard solution was added; deposition time = 300 sec; deposition potential $=-0.4 \mathrm{~V}$ vs. $\mathrm{Ag} / \mathrm{AgCl}$ 


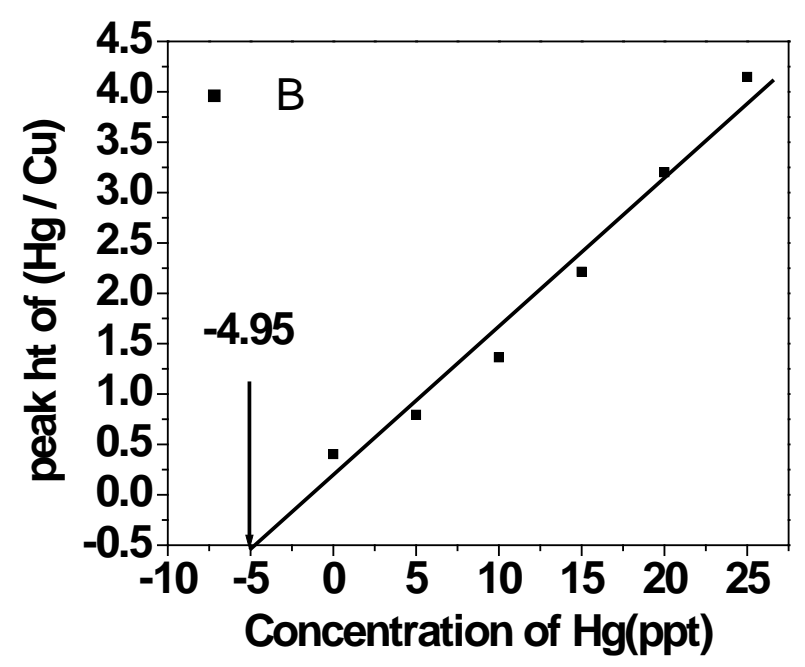

Figure 6 Calibration curve for internal standard. The standard addition plot extended to determine unknown mercury concentration

However for internal standard method when only copper was added, the peaks due to Hg were not clear (Fig.7). A 3 ppm copper solution was initially tested in our standard addition method. But the mercury peaks under same conditions (5-25 ppt range and 5 minute deposition time) are not clear with copper. Hence the idea to use copper as an internal standard was dropped.

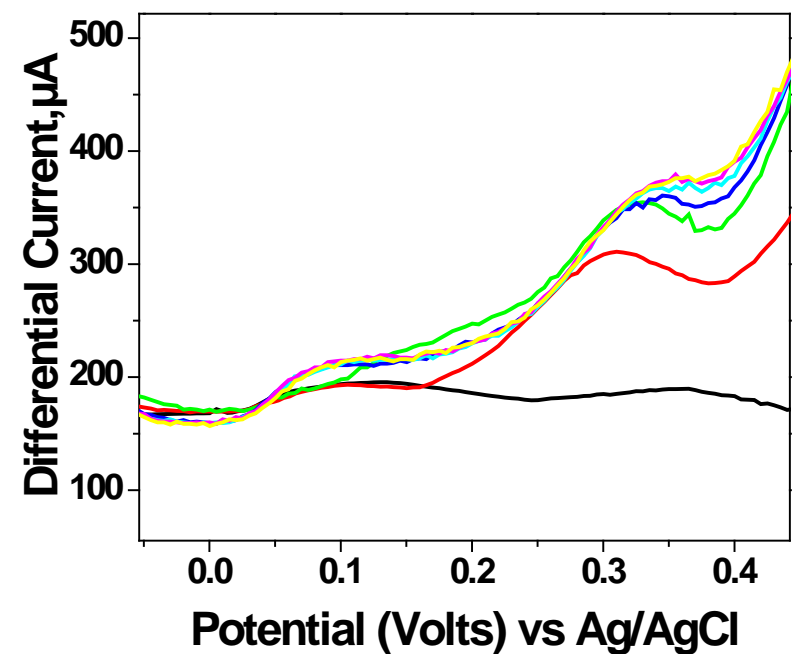

Figure 7 DPV scans for 5ppt to 25pt $\mathrm{Hg}^{2+}$ concentrations in $1 \mathrm{M} \mathrm{KCl}$ ( $\mathrm{pH}$ 1). 3 ppm copper standard solution was added; deposition time = 300 sec; deposition potential $=-0.4 \mathrm{~V}$ vs. $\mathrm{Ag} / \mathrm{AgCl}$

TASK 3: Ensuring complete recovery of the electrode surface with cleaning

The electrode cleaning procedures in the earlier work had to be re-evaluated in an effort to obtain reproducibility. The cleaning at $0.6 \mathrm{~V}$ after each run did not ensure removal of gold which could lead to error in measurements. After numerous trial and error procedures a set cleaning procedure was determined. Few of the procedures which led to the standard cleaning procedure, though not incorporated in the procedure itself are also worth mentioning. Initially cleaning the electrode free from any residual between successive mercury additions was tried. This way the electrode could be ensured to be free of mercury for subsequent addition. Various deposition times and deposition potentials were tried for 
cleaning between the runs. This method with the corresponding plots is given in detail. Another method tried was to deposit gold at a different potential and then mercury at other potential so that the peaks will be well separated and any interference due to impurity in gold could be overcome (Fig.8). However with this method also, the peaks were not reproducible and hence the procedure was not followed.

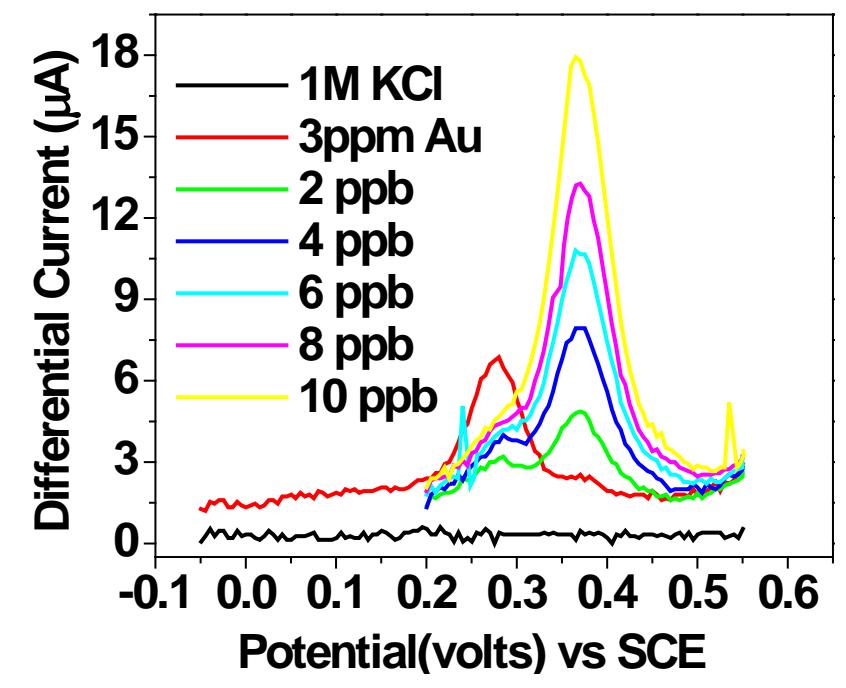

Figure 8.DPV plots from 2ppb $-10 \mathrm{ppb}$ with Au deposition potential:-0.05V and Hg deposition potential: $0.2 \mathrm{~V}$.

Next, we first applied a voltage of $1.2 \mathrm{~V}$ after each stripping of the added mercury with the idea that this voltage is sufficient to strip both mercury and gold. The differential current vs. potential for 10 to $50 \mathrm{ppb}$ of mercury is shown in Fig.9. Although the curve is linear for lower concentrations, the current gets saturated for higher concentrations of mercury as if the higher concentrations are not being detected. We believe this occurs because cleaning the electrode at $1.2 \mathrm{~V}$ removes not only $\mathrm{Hg}$ but also gold from the electrode. Not having enough gold on the electrode limits how much mercury can be efficiently detected giving the saturation behavior.

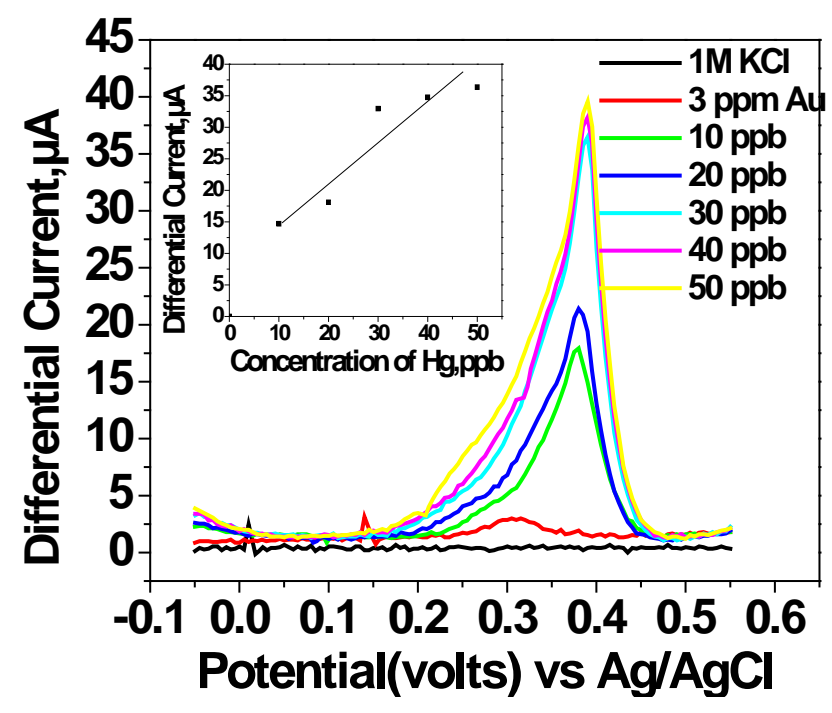

Figure 9.DPV curves for $10-50 \mathrm{ppb} \mathrm{Hg}^{2+}$ concentrations in $1 \mathrm{M}$ $\mathrm{KCl}(\mathrm{pH}$ 1).3 ppm Gold was added. Cleaning by applying constant potential (1.2 $\mathrm{V})$ at the electrode between $10 \mathrm{ppb}$ addition to remove mercurv and Gold 
To test the above hypothesis, we next chose 0.6 volts to clean the electrode between each concentration for the time equal to the deposition time. This voltage is sufficient to strip any residual Hg from the electrode but not Gold. The DPV curves in Fig.10, 11 and 12 (ppb to ppt range) show a good linear behavior of current versus concentration. However results were not reproducible and the linearity was affected after some runs.

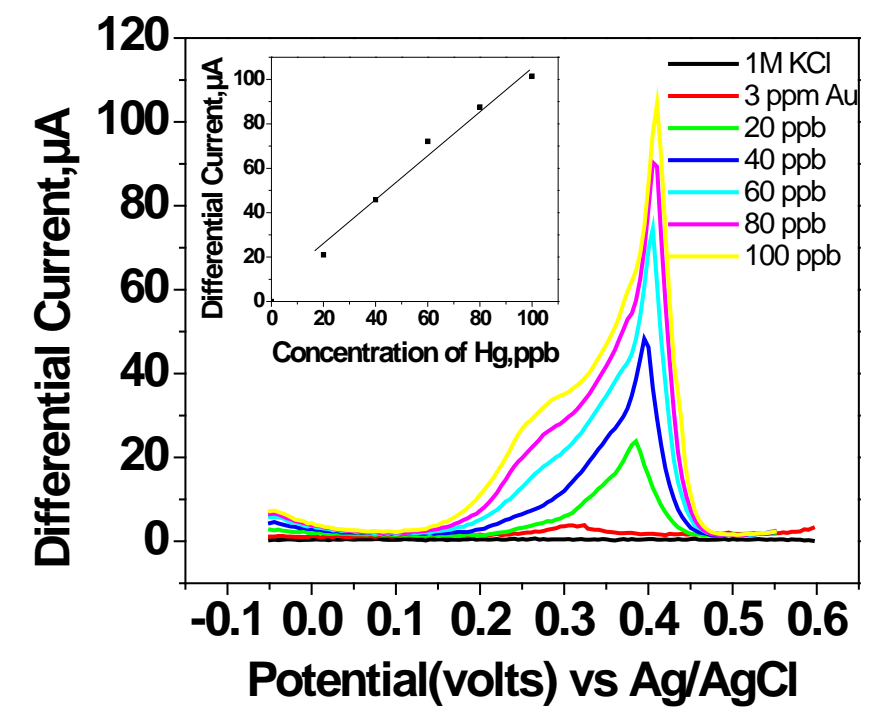

Figure 10. DPV curves for 20-100 ppb $\mathrm{Hg}^{2+}$ concentrations in $1 \mathrm{M} \mathrm{KCl}$ ( $\mathrm{pH}=1) .3 \mathrm{ppm}$ gold was added. Cleaning by applying constant potential $(0.6 \mathrm{~V})$ at the electrode between each $20 \mathrm{ppb}$ addition to remove mercury.

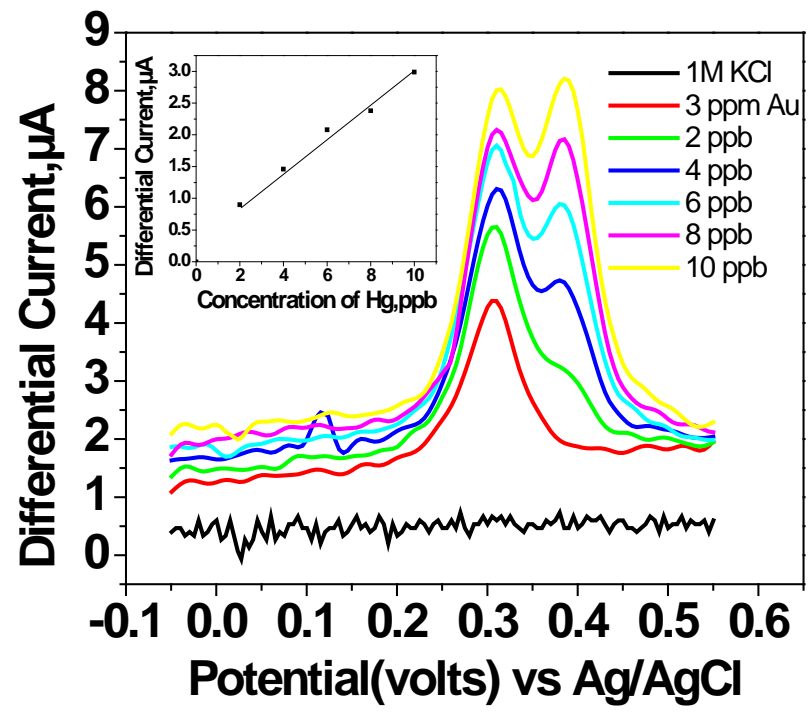

Fig.11. DPV curves for 2-10 $\mathrm{ppb} \mathrm{Hg}^{2+}$ concentrations in $1 \mathrm{M} \mathrm{KCl}(\mathrm{pH} 1) .3 \mathrm{ppm}$ Gold was added. Cleaning by applying constant potential $(0.6 \mathrm{~V})$ at the electrode between $2 \mathrm{ppb}$ addition to remove mercury

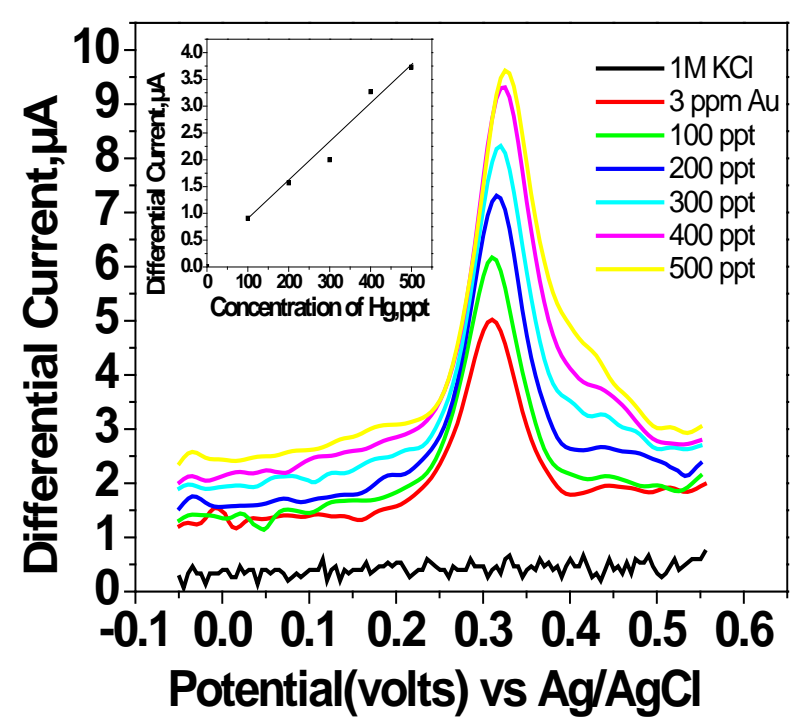

Fig.12.DPV curves for 100-500 ppt $\mathrm{Hg}^{2+}$ concentrations in $1 \mathrm{M} \mathrm{KCl}(\mathrm{pH}=1)$. 3ppm gold was added. Cleaning by applying constant potential $(0.6 \mathrm{~V})$ at the electrode between each $100 \mathrm{ppt}$ addition to remove mercury.

To clean the electrode after each run, the following procedure was developed. First we scan the electrode in a fresh $\mathrm{KCl}$ solution to determine what materials are present on the 
electrode. Curve 1 in Fig.13 shows the large peak near $0.9 \mathrm{~V}$ due to gold and the small peak near $0.35 \mathrm{~V}$ due to mercury. To strip these materials, the above linear scan is repeated four times, which completely eliminates the mercury peak and reduces the gold peak by a factor of about 20 (curve 2). The potential is then held at $1 \mathrm{~V}$ for 5 minutes which further reduces the peak height (curve 3). The final step is a cyclic voltammogram after which the peak is completely removed for the next 25 . This cleaning procedure takes about 20 minutes.

Fig. 13. Linear scan curves after each cleaning step

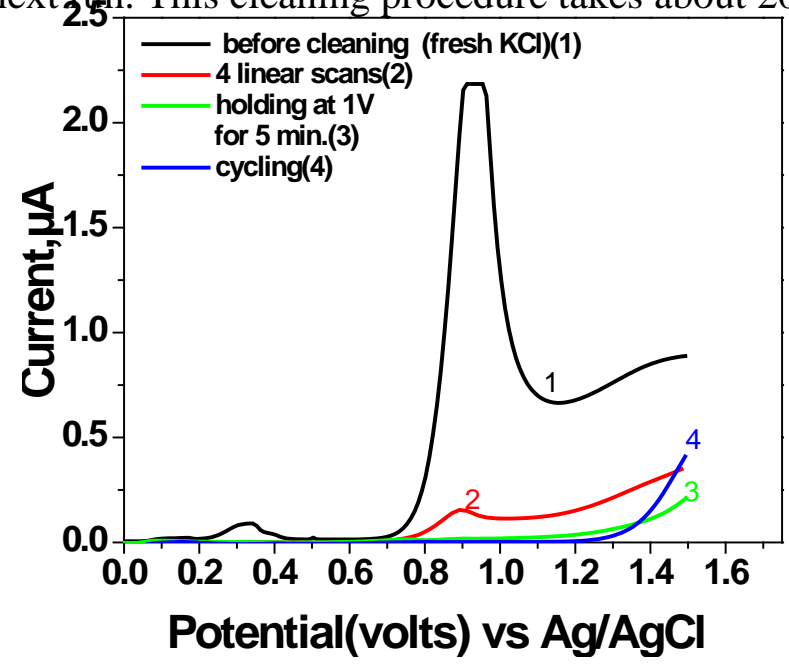

To test the above procedure, we prepared an "unknown sample" with Hg concentration of $23 \mathrm{ppb}$ and then determine its concentration in three successive runs using the standard addition method. In this method, DPV curves are obtained for the unknown mercury concentration followed by adding known concentrations of $\mathrm{Hg}$. With every mercury addition the electrodes were scanned to obtain DPV curves. Then current vs. concentration is plotted and the intercept for zero current yield yields the unknown concentration. The plots in Fig. 14 show the unknown concentration to be between 24 and 28ppb with a mean of 25ppb, in good agreement with $23 \mathrm{ppb}$ of $\mathrm{Hg}$ in the unknown. The deviation from the mean is within $10 \%$, a very respectable reliability.

We carried out a number of experiments using solutions of silver and copper solution instead of gold solution to capture mercury in our experiments. However, the results obtained from these experiments were not very reproducible suggesting that gold is superior to both silver and copper to capture Hg through amalgam formation.

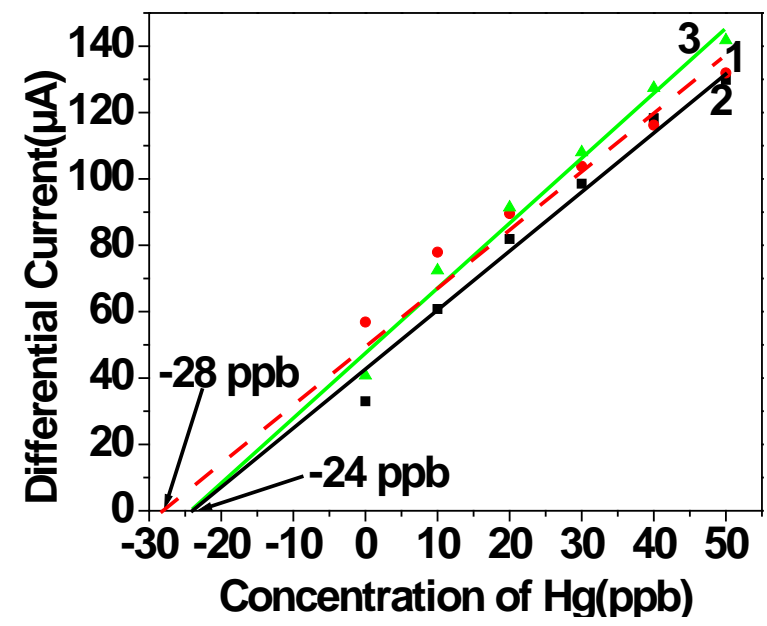

Figure 14 Standard addition method to determine $\mathrm{Hg}$ concentration in the "unknown"; 2 min deposition time; deposition potential $=-0.05 \mathrm{~V}$. 
TASK 4: Surface testing of various BDD electrodes

Thirteen experiments were conducted under the same conditions to test the reproducibility of one of the electrodes. The slope for each experiment is determined and plotted to see their variation. Of the thirteen experiments the slope for 8 runs is reproducible (Fig.15). A good linearity is observed in almost all the runs which can be seen from the calibration curves in fig.16. This way the surface of the electrode was tested. The electrodes which were not reproducible and which had huge slope variation were abandoned. A summary of these results were published in a recent paper [4].

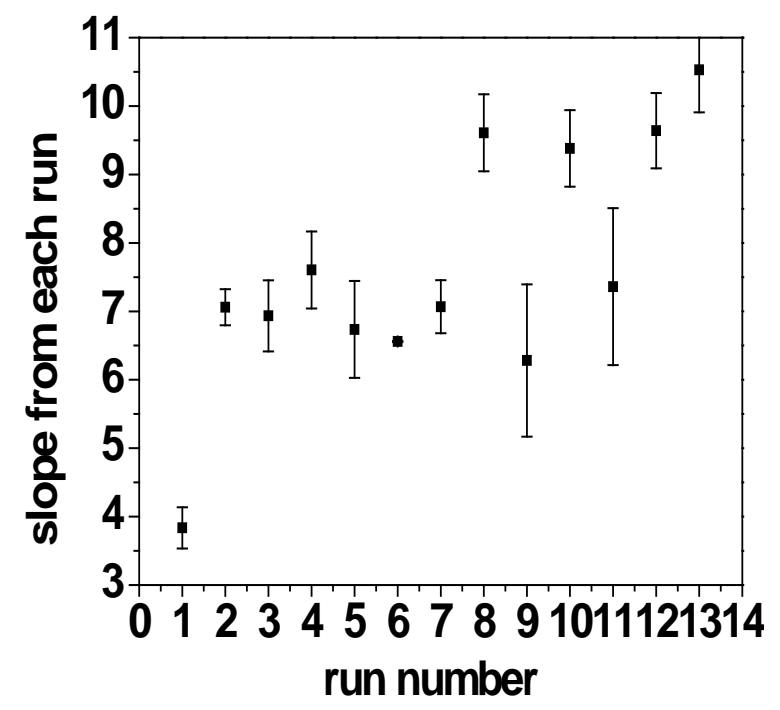

Fig. 15. Slope calculated from each run with the designated error values. All experiments conducted with $1 \mathrm{M} \mathrm{KCl}$ and in the same range ( 5 to $25 \mathrm{ppt}$ ) and 5 min dep. time; dep. pot $=$ $-0.4 \mathrm{~V}$

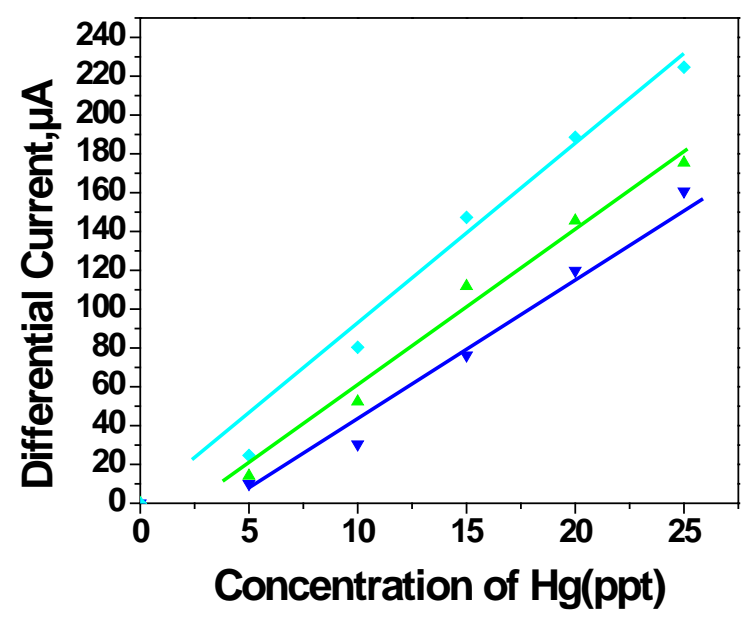

Fig.16. Calibration curves for experiments in the range ( 5 to 25 ppt ) and 5 min dep. time; dep. pot $=-0.4 \mathrm{~V}$

\section{TASK 5: Development of a portable sensor}

Having established good sensitivity and fairly good reproducibility with the lab potentiostat, our next goal was to develop a portable sensor. The parameters like scan rate, sensitivity, current range etc. had to be determined separately by trial and error for the portable potentiostat system. The $2 \mathrm{~mm}$ electrode was initially tested with lab potentiostat for sensitivity and then with the portable potentiostat. Both the results are presented here. It can 
be seen that the sensitivity and reproducibility are slightly lower compared to the bigger electrode (6mm) even with the lab potentiostat Fig.17 \& Fig.18.

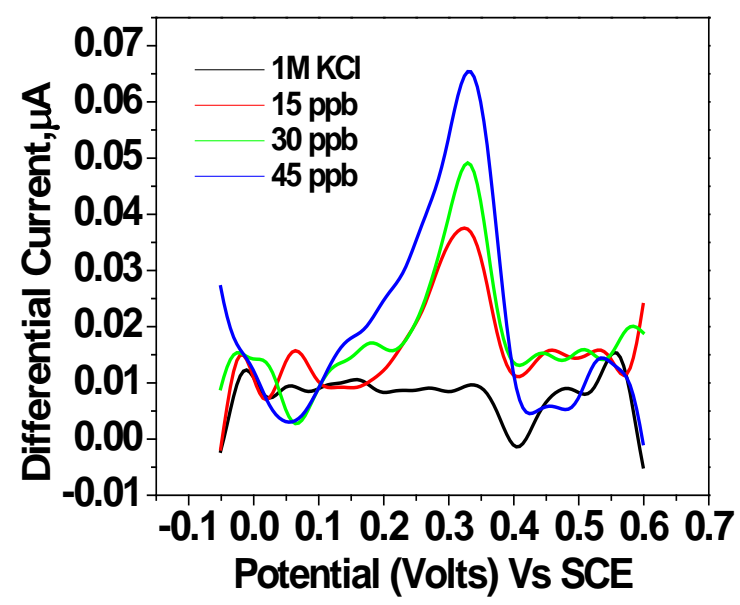

Fig.17 DPV curves are plotted for 10 to $50 \mathrm{ppb}$ of mercury with 3ppm Au. Deposition time $=2 \mathrm{~min}$.

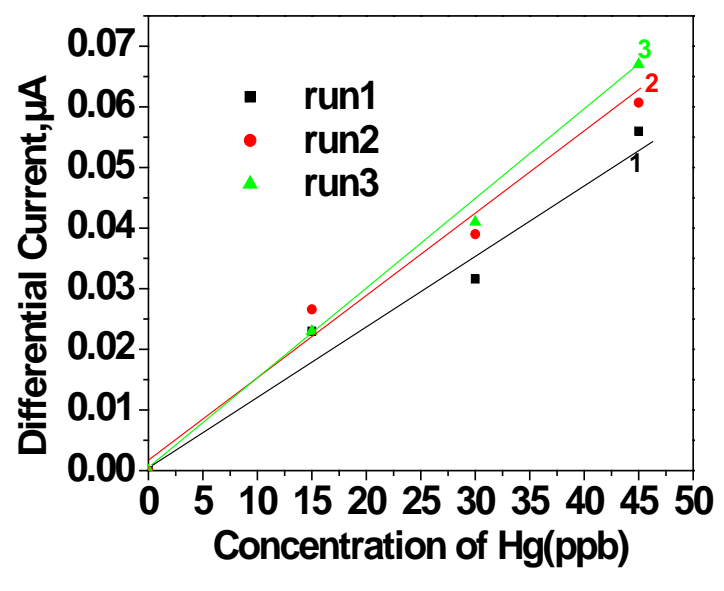

Fig.18. DPV curves for $10-50 \mathrm{ppb}$ mercury addition with $3 \mathrm{ppm}$ Au for three subsequent runs.

The linearity is slightly lower when the $2 \mathrm{~mm}$ electrode is used with the portable potentiostat instead of the lab potentiostat. It can be seen with the calibration plot of the below (Fig.20). So in summary, results with the portable potentiostat are similar to those obtained with the lab. potentiostat except for somewhat lower sensitivity.

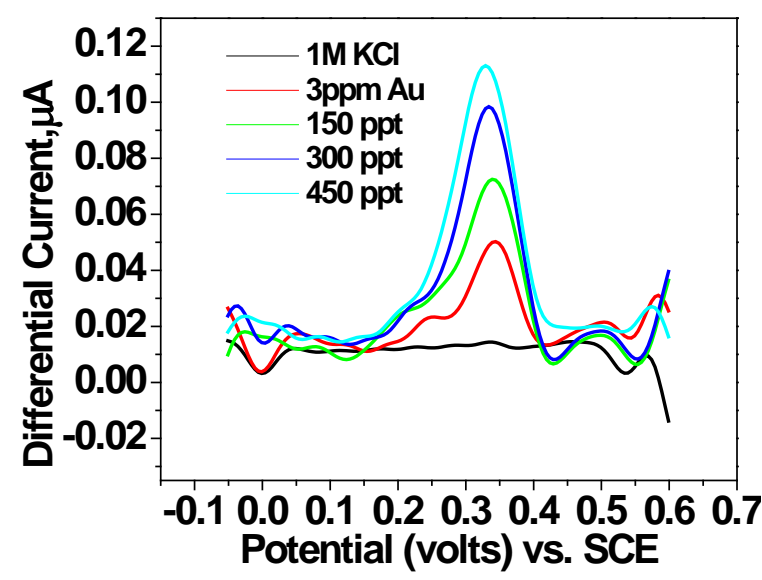

Fig.19. DPV curves for $100-500 \mathrm{ppt}$ mercury with 3 ppm gold. Deposition time $=5 \mathrm{~min}$.

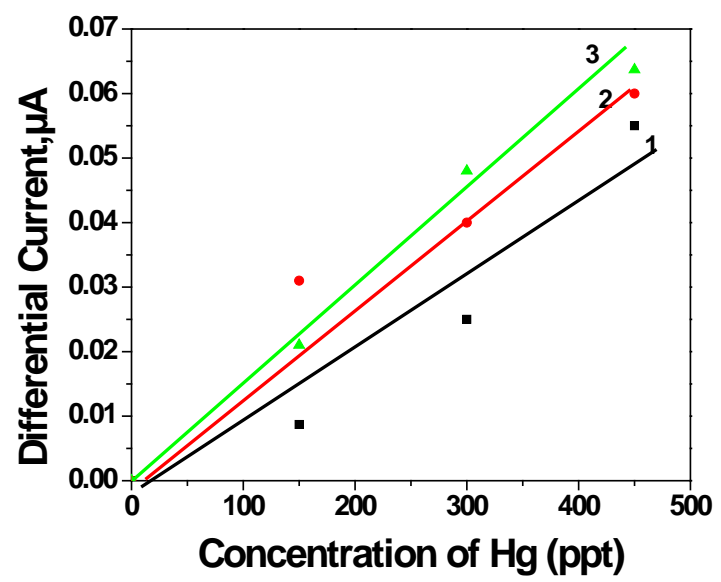

Fig. 20. Calibration curves for $100-500$ ppt mercury with 3 ppm Gold. 
TASK 6: Testing GaN as a possible electrode for mercury detection

Gallium Nitride electrodes were tested in the place of boron doped diamond electrodes. These are n-type materials grown on Sapphire substrates. Stationary electrodes of dimensions $20 \mathrm{~mm}$ x $20 \mathrm{~mm}$ with contacts of $1.7 \mathrm{~mm}$ x $4 \mathrm{~mm}$ were prepared for these studies. As with our previous studies with BDD electrodes, experiments were carried out in $1 \mathrm{M} \mathrm{KCl}$ medium with stationary electrodes with and without gold addition and with the Rotating Disk Electrode (RDE).Using the virgin GaN electrode and 5 minute deposition time, $\mathrm{Hg}$ in the ppb range could be detected; the DPV (Differential Pulse Voltammetry) scans for different concentrations are shown in Fig.21. The peak position for $\mathrm{Hg}^{2+}$ detection occurs near $-0.17 \mathrm{~V}$ vs. SCE (Standard Calomel Electrode) in contrast to the case for BDD (0.25 V). The peak height vs. concentration (Fig. 22) shows a non-linear behavior unlike the linear variation with the BDD electrode case.

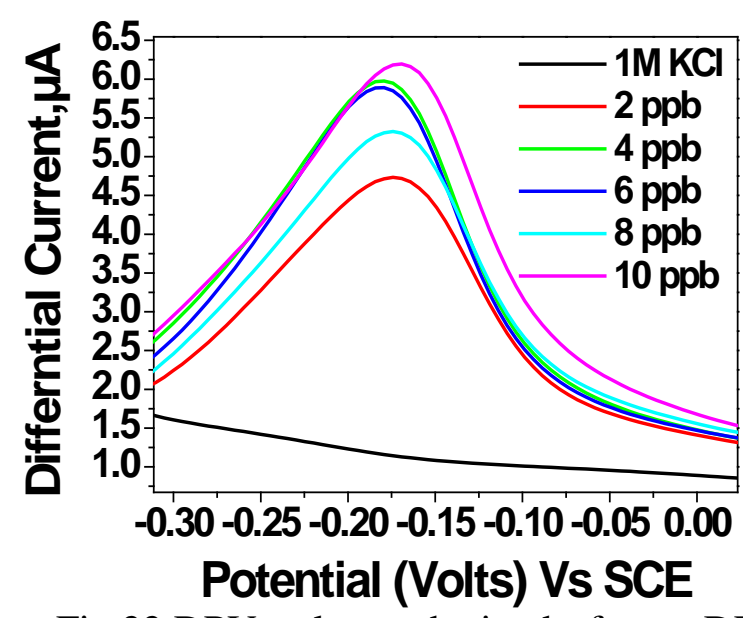

Fig.22.DPV plots obtained from DPV scans 2-10 ppb. Deposition time $=300$ s, deposition potential $=-0.4 \mathrm{~V}$ and scan rate $=50 \mathrm{mv} / \mathrm{s}$

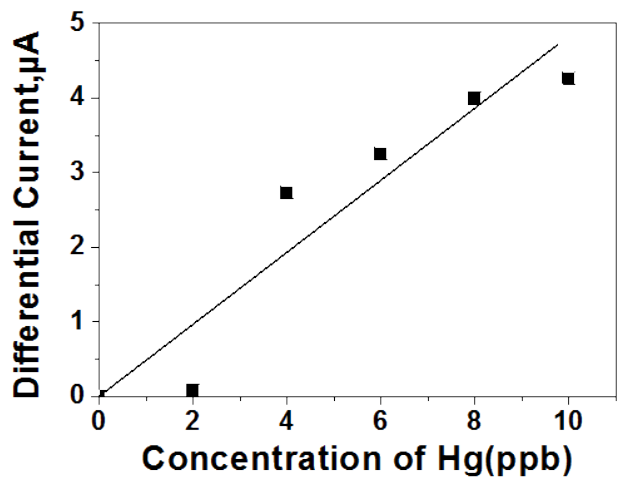

Fig. 21. Calibration plot for $2-10 \mathrm{ppb} \mathrm{Hg}^{2+}$ concentrations

With the addition of $3 \mathrm{ppm}$ of gold solution, the shape and the location of the peaks changes (fig.23), although the peak heights vs. concentration is more linear (Fig.24). A comparison of the two, with and without gold addition is shown in Fig.25. After the run with addition of gold, the lowest detection limit of the electrode changes whereas without gold addition the detection limit remains the same for four or five runs at least. So in contrast to the BDD case where Au was added to avoid the formation of calomel, the $\mathrm{Au}$ addition does not seem to provide any significant advantage for the GaN electrode. The experiment without gold addition was performed first, followed by the experiment with gold addition on the same electrode.

Another difficulty encountered was that different electrodes exhibit different behaviors. For example, a second electrode prepared from the same batch of $\mathrm{GaN}$ as the previous one detected peaks above $100 \mathrm{ppb}$ ranges only, but the linearity of the peaks is better than the previous electrode used. Also the deposition time needed for each run is 
reduced from 300s to 60s. The DPV scan results and the corresponding calibration plots are shown below. The reason for such behavior may be attributed to the possible contact difference during electrode preparation.

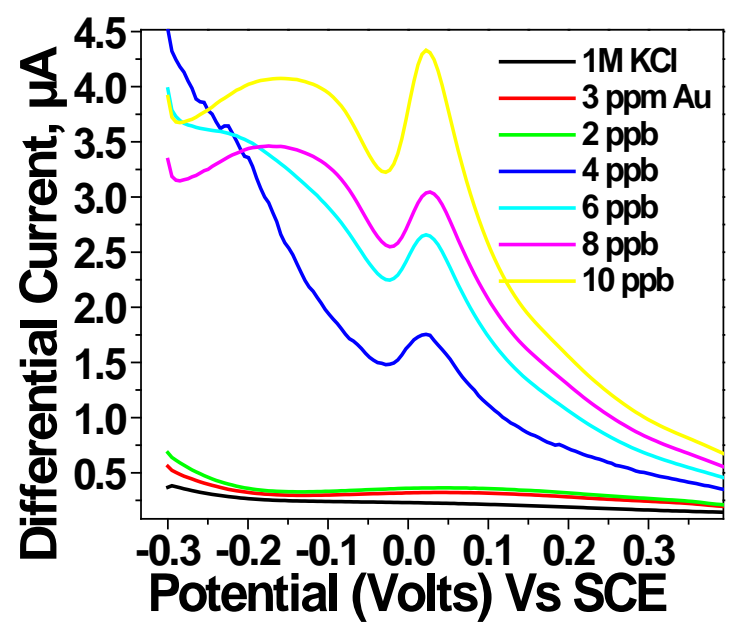

Fig.24. DPV scan for 2-10 $\mathrm{ppb} \mathrm{Hg}^{2+}$ concentrations in $1 \mathrm{M} \mathrm{KCl}$. Deposition time $=300 \mathrm{~s}$ and deposition potential $=-$ 0.4 V.3 ppm gold added to the analyte.

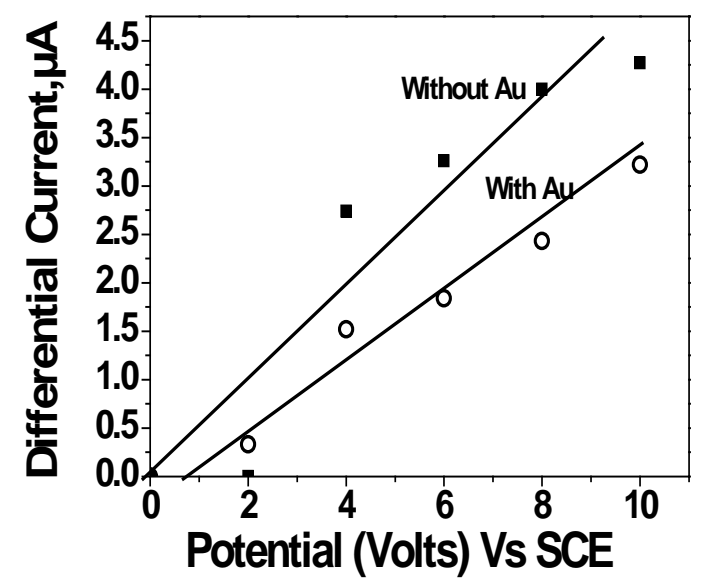

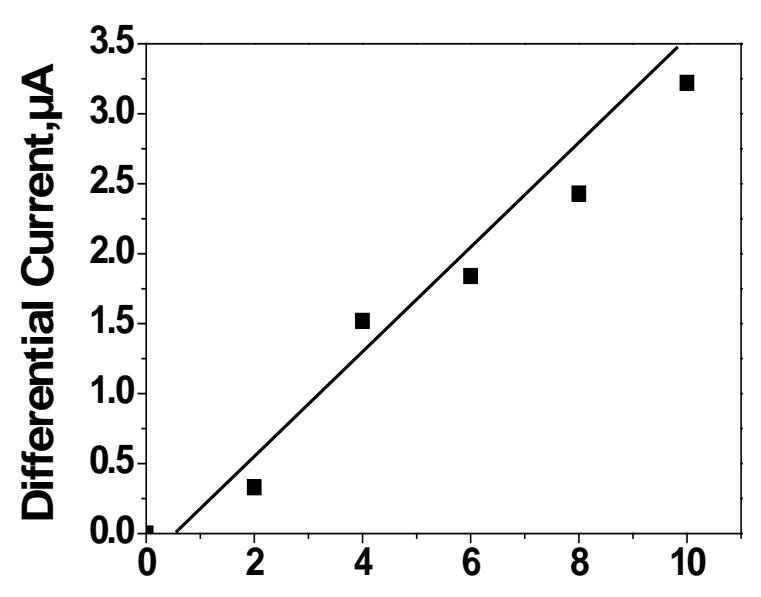

Fig.23. Calibration plots for 2-10 ppb $\mathrm{Hg}^{2+}$ concentrations in $1 \mathrm{M} \mathrm{KCl}$.

Fig.25. Calibration plots for 2-10 $\mathrm{ppbHg}^{2+}$ concentrations in $1 \mathrm{M}$ $\mathrm{KCl}$ with and without addition of Au. Deposition time $=300 \mathrm{~s}$ and deposition potential $=-0.4 \mathrm{~V}$. 


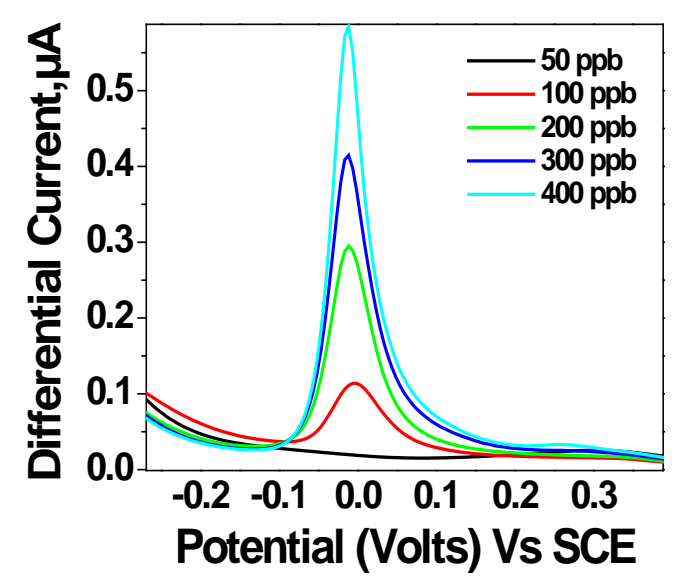

Figure 27.DPV scans for 50-400 ppb $\mathrm{Hg}^{2+}$ concentrations. Deposition time $=300 \mathrm{~s}$, deposition potential $=-0.4 \mathrm{~V}$ and scan rate $=50 \mathrm{mv} / \mathrm{S}$

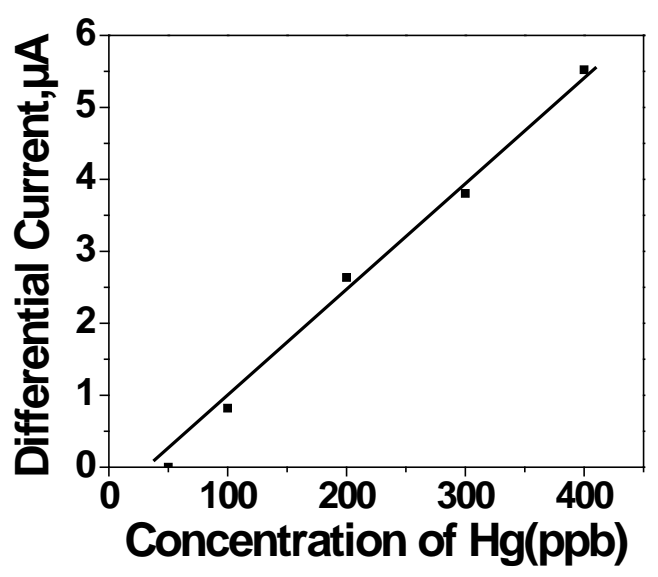

Fig. 26. Calibration plots from DPV scans for 50-400 pp $\mathrm{Hg}^{2+}$ concentrations.

Different analyte media were tested under the same conditions of deposition potential, deposition time, and scan rate and modulation interval as with chloride media. The peaks were obtained similar to the chloride media. Potassium iodate, potassium thiocyanate, and potassium nitrate were the other solutions used. The peaks are centered near $0.025 \mathrm{~V}$. DPV scans with thiocyanate media are as shown below. The peaks obtained for concentrations from 300 ppb to 800 ppb (Fig 28) and the corresponding calibration plots (Fig.29) are shown.

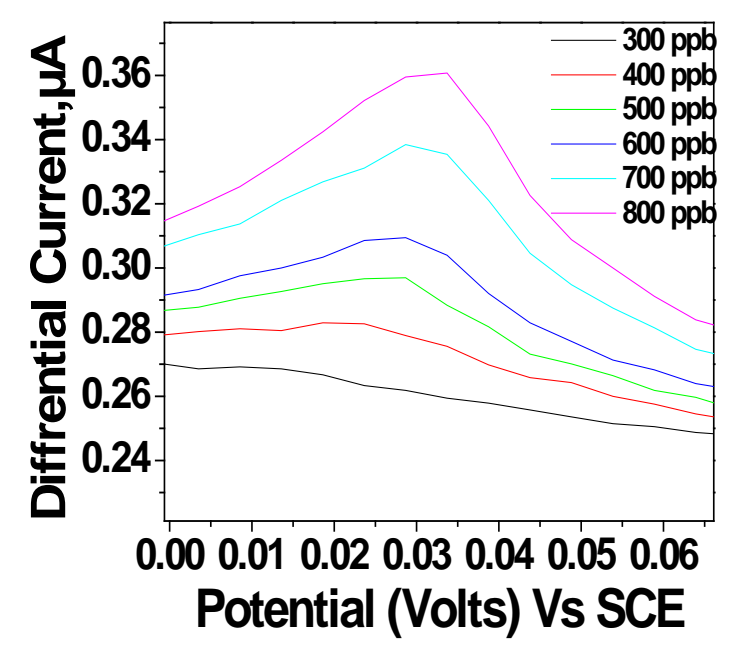

Fig. 29. DPV scans for $300-800 \mathrm{ppb} \mathrm{Hg}^{2+}$ concentrations in $1 \mathrm{M}$ KSCN. Deposition time $=300 \mathrm{~s}$; deposition potential=-0.4 V.

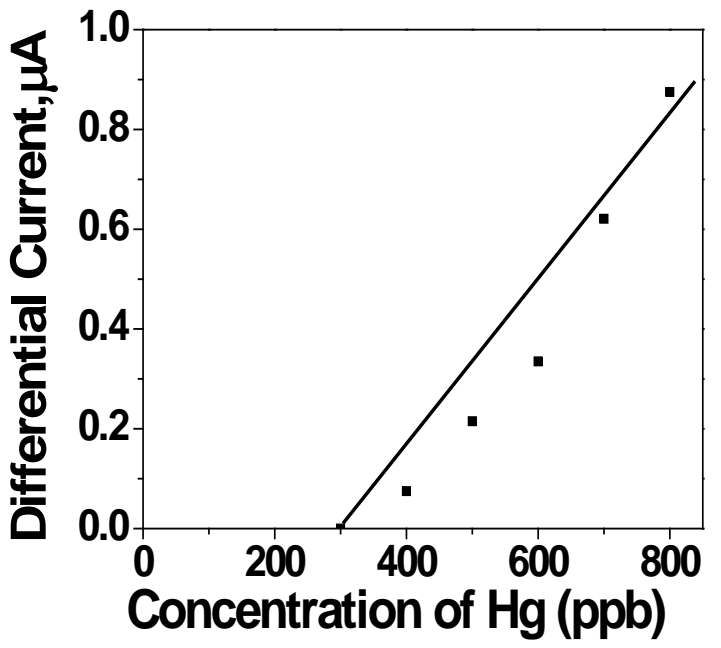

Fig. 28. Calibration plots from DPV scans for $300-800 \mathrm{ppb} \mathrm{Hg}^{2+}$ concentrations

The peaks with any of these GaN electrodes are not reproducible. The calibration plots obtained with the same electrode run at different times give different slopes (fig.30). 
The first run was performed the first day. The next three runs were performed consecutively on day2. All these runs were performed under identical conditions.

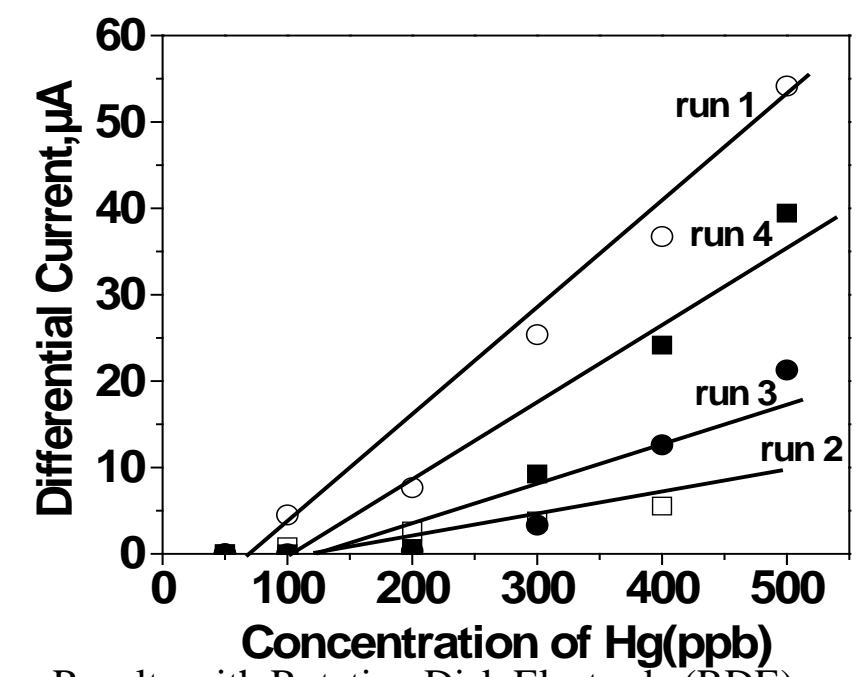

Figure 30.Comparison of calibration plots with the same electrode for 100-500ppb $\mathrm{Hg}^{2+}$ concentrations in $1 \mathrm{M} \mathrm{KCl}$. Deposition time $=300 \mathrm{~s}$ and deposition potential $=-0.4 \mathrm{~V}$.

Results with Rotating Disk Electrode (RDE)

Rotating Disk Electrodes were prepared similar to the BDD RDE's. The rotating disk electrode was used with a normal Autolab potentiostat. A rotation speed of $1500 \mathrm{rpm}$ was used. The same analyte $(1 \mathrm{M} \mathrm{KCl})$ was used for measurements. The peaks are centered on $0.01 \mathrm{~V}$ with the RDE setup and reasonably good linear variation is obtained. A deposition time of 60 secs is used for all measurements and the peaks obtained in the $100-500 \mathrm{ppb}$ ranges are shown in Fig.31 and Fig.32.

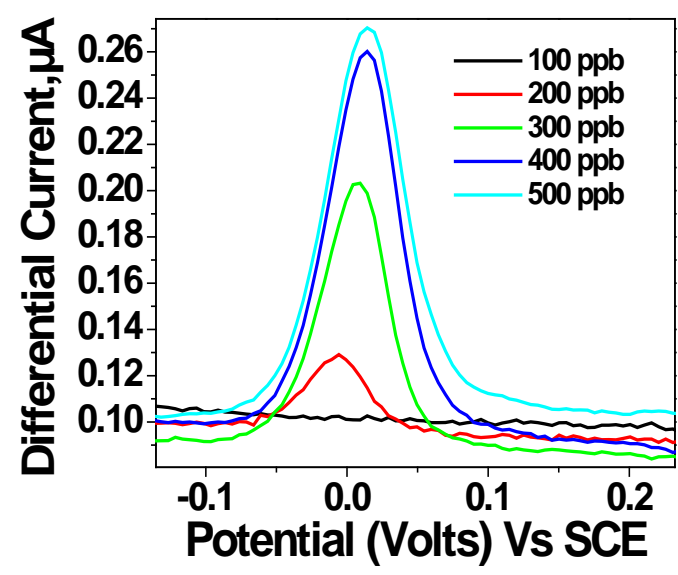

Figure 31.DPV scans with a RDE for 100

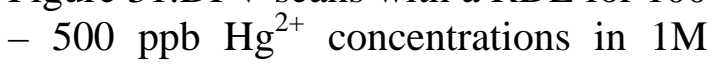
$\mathrm{KCl}$. Deposition time $=300 \mathrm{~s}$ and deposition potential $=-0.4 \mathrm{~V}$.

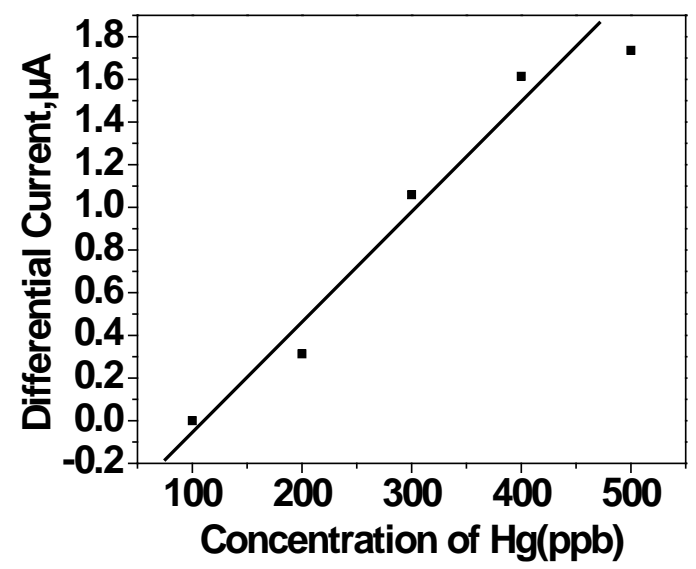

Figure 32.Calibration plots with a RDE for $100-500 \mathrm{ppb} \mathrm{Hg}^{2+}$ concentrations in $1 \mathrm{M} \mathrm{KCl}$. Deposition time $=300 \mathrm{~s}$ and deposition potential=-0.4 V.

1. Cleaning the electrode by applying $+1 \mathrm{~V}$ for 300 secs.

2. Cleaning the electrode with 1:1 solution of $\mathrm{HCl}: \mathrm{H}_{2} \mathrm{O}$.

3. Cleaning with strong acid (aqua regia, $\mathrm{HCl}, \mathrm{HNO}_{3}$ ). 
4. Cycling the electrode from $+1 \mathrm{~V}$ to $-1 \mathrm{~V}$ in Potassium Iodate.

5. Using $\mathrm{HCl}+\mathrm{SPM}$ (Sulphuric acid and Peroxide mixture) called piranha, used in semiconductor industry for cleaning GaN surfaces.

With all the above methods, the peak is removed as desired, but each procedure has certain problems like lowest detection limit changes after cleaning, the electrode becomes more sensitive or the electrode doesn't detect any concentration. Proper etching agents if found could make reproducibility of this electrodes possible. However, our experiments showed that the GaN we used is not as good as boron-doped diamond for detecting mercury in solutions. The electronic properties of the BDD electrode material were characterized and published in the journal Materials Characterization [5].

\section{Task 7: Detection of Cd and $\mathrm{Pb}$}

Experiments on the detection of trace levels of $\mathrm{Pb}$ and $\mathrm{Cd}$ in solutions using BDD electrodes were carried out by co-investigator Dr. A. Manivannan during his visits to Japan where he had an on-going collaboration. These results have been published in two papers [6, 7]. These studies showed that BDD electrodes are also excellent for detecting these heavy metal ions in solutions.

\section{SUMMARY OF RESULTS}

Seven different tasks described above were carried out to develop procedures for detecting $\mathrm{Hg}, \mathrm{Cd}$ and $\mathrm{Pb}$ at $\mathrm{ppb}$ levels in laboratory prepared solutions using BDD electrodes with both a laboratory potentiostat and a portable potentiostat. Results obtained by this technique for mercury detection were shown to agree very well with those obtained using the more cumbersome CVAAS technique [3]. The feasibility of a portable sensor based on these experiments has been demonstrated although no field tests were carried out. The initial task to understand the theoretical basis for electrochemical detection was performed effectively and the theoretical estimates provided a background for working with various electrolytes and electrodes. As a separate task, Gallium Nitride electrodes were tested in the place of BDD electrodes. Although, the GaN electrode could detect as low as 5ppb mercury concentration, $100 \mathrm{ppb}$ was more typical. Also the GaN electrodes need to be replaced after every 5 or 6 runs whereas BDD electrodes are active even after 100 runs. The issue of reproducibility which remained from earlier work was addressed by developing procedures for cleaning the BDD electrode after each run. These procedures resulted in significant improvements in reproducibility of the calibration curves by avoiding the deposition of any other impurity and hence the surface of the electrode could be cleaned more effectively. The results of these studies have been published in seven publications and one M. S. thesis listed below.

\section{PUBLICATIONS / PRESENTATIONS / THESIS}

1. A. Manivannan, M. S. Seehra, D.A. Tryk and A. Fujishima : Electrochemical detection of ionic mercury at BDD electrodes, Analytical Letters, 35 (2002) 355-368. 
2. A. Manivannan, M. S. Seehra, and A. Fujishima : Detection of mercury at the ppb level in solution using BDD electrode, Fuel Processing Technology, 85 (2004) 513-519.

3. A. Manivannan, L. Ramakrishnan, M. S. Seehra, E. Granite, J. E. Butler, D. A. Tryk and A. Fujishima: Mercury detection at BDD electrodes using rotating disk technique, J. Electroanalytical Technique, 577 (2005) 287- 293.

4. M. S. Seehra, S. Ranganathan and A. Manivannan: Electrochemical quantification of mercury in solutions using boron-doped diamond electrodes: Electrode regeneration and role of gold and impurities, Analytical Letters, 41(2008) 2162-2170.

5. A. Manivannan, S. Underwood, E. H. Morales and M. S. Seehra: Magnetic and electrical characterization of boron-doped diamond, Materials Characterization, 51(2004)329-333.

6. A. Manivannan, R. Kawasaki, D. A. Tryk and A. Fujishima: Interaction of $\mathrm{Pb}$ and $\mathrm{Cd}$ during anodic stripping voltammetric analysis at BDD electrodes, Electrochemica Acta, 49(2004), 3313-3318.

7. D. Drago, N. Spataru, R. Kawasaki, A. Manivannan, D. A. Tryk and Fujishima: Detection of $\mathrm{Pb} 2+$ in tap water at BDD electrodes with anodic stripping voltammetry, Electrochemica Acta, 51(2006) 2437-2441.

8. M.S. Thesis of L. Ramakrishnan: Electrochemical detection of mercury using BDD electrodes, West Virginia University, 2005.

9. A. Manivannan, L. Ramakrishnan, M. Seehra, E. Granite, J. Butler and A. Fujishima: PPT level detection of Mercury using diamond rotating electrodes and comparison with CVAAS, Podium presentation by A. Manivannan at the $8^{\text {th }}$ International Mini-Symposium on Diamond Electrochemistry held at Keio University, Hiyoshi, Yokohama, Japan, during March 4-5, 2005.

10. Half hour podium presentation entitled" Electrochemical quantification of mercury using boron-doped diamond electrodes” by M. S. Seehra (at the Workshop held at Virginia Tech, July 24- July 26, 2007) organized by CAST. 
Appendix 40: Gold Filters for Removing Mercury from Coal Fired Power Stations (MT003) 


\section{TECHNICAL REPORT}

Contract Title and Number:

Crosscutting Technology Development at the Center for Advanced Separation Technologies (DE-FC26-02NT41607)
Period of Performance:

Starting Date: 2003

Ending Date: 2008
Sub-Recipient Project Title:

DETERMING EFFECTIVENESS OF GOLD FILTER FOR REMOVING MERCURY FROM COAL FIRED POWER PLANTS

Principal Investigators:

Dr. Kumar Ganesan

$\underline{\text { Contact Address: }}$

Department of Environmental Engineering Montana Tech of The University of Montana 1300 West Park Street Butte, Montana 59701

$\underline{\text { Subcontractor Address: }}$

"No subcontracts issued."
Report Information:

Type: Final Report

Number: 6

Period:

Date: $\quad 11 / 12 / 07$

Code: MT003

Contact Information:

Phone: 406-496-4239

Fax: 406-496-4650

E-Mail: kganesan@mtech.edu

$\underline{\text { Subcontractor Information: }}$ 


\title{
DETERMING EFFECTIVENESS OF GOLD FILTER FOR REMOVING MERCURY FROM COAL FIRED POWER PLANTS
}

Final Report Submitted to:

The Center for Advanced Separation Technologies

Proposal Topic Area:

Solid-Solid Separation

$\square$ Solid-Liquid Separation

$\square$ Chemical/Biological Extraction

$\square$ Modeling and Control

$\underline{\mathbf{X}}$ Environmental Control

\author{
Submitted by: \\ Dr. Kumar Ganesan \\ Professor and Head \\ Department of Environmental Engineering \\ Montana Tech of the University of Montana Tech \\ 1300 West Park Street \\ Butte, Montana 59701
}

November 12, 2007

Principal Investigator Endorsement 


\section{$\underline{\text { Disclaimer }}$}

This report was prepared as an account of work sponsored by an agency of the United States Government. Neither the United States Government nor any agency thereof, nor any of their employees, make any warranty, express or implied, nor assume any legal liability or responsibility for the accuracy, completeness, or usefulness of any information, apparatus, product, or process disclosed, or represents that its use would not infringe privately owned rights. Reference herein to any specific commercial product, process, or service by trade name, trademark, manufacturer, or otherwise does not necessarily constitute or imply endorsement, recommendation, or favoring by the United States Government or any agency thereof. The views and opinions of authors expressed herein do not necessarily state or reflect those of the United States Government or agency thereof. 


\begin{abstract}
There is a clear need for devices to remove mercury cost effectively from power plant flue gases. The main objective of this research is to develop cost effective filters and to evaluate the effectiveness in removing mercury vapor from coal fired power plant's flue gas. Two different metallic filters were custom made out of fine metal threads and a disc with pinholes of less than one mm diameter. Tests were conducted under different flow rate and temperature conditions and the results showed very encouraging results. After the initial tests, commercially available metal plated beads were tested for the mercury removal efficiencies and the results showed efficiencies higher than $93 \%$. The next successful progress was electroplating metallic sponges at Montana Tech and test for mercury vapor removal efficiency. The test results showed higher than $80 \%$ efficiencies. The spent sponges were then heated to less than $100^{\circ} \mathrm{F}$ and it was determined they released over 99 percent of the absorbed mercury. Then the sponges were professionally plated with specific metals and were tested for its mercury removal efficiency. Finally one of the filters was field tested in a coal fired power plant at Colstrip, Montana. Over all, the performance of the metallic filter in real world condition was found to be as good as the laboratory results.
\end{abstract}

If the metallic filter developed in this research proves to be effective for removing mercury then it will be an environmentally friendly process. The metallic filter could be regenerated by a simple heating process and the mercury can be captured from the air stream without it getting into the environment in any form. The filtration process is not new for power plants, and with minimum modifications, the existing plants can use this technology if it is proven to be effective. Therefore the results of this research will benefit the coal industry and the power plants in dealing with the mercury issue. The environmental interest groups also will like it because it prevents the toxic mercury from getting into the environment rather than transferring it into another medium. 


\section{Table of Contents}

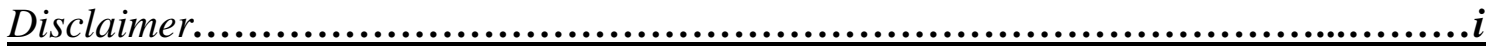

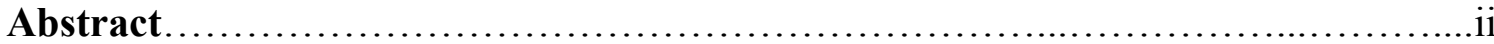

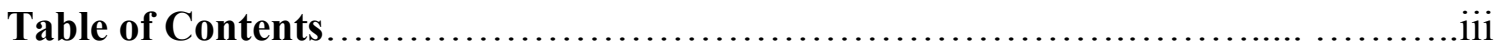

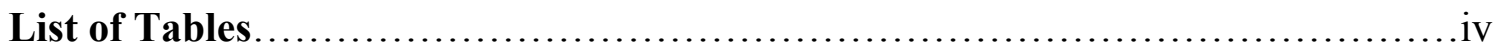

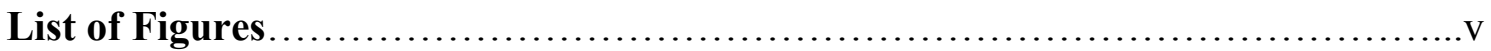

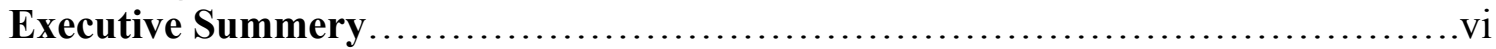

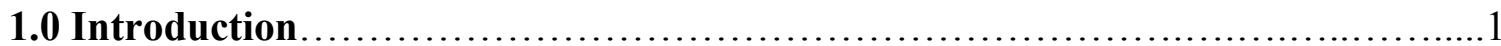

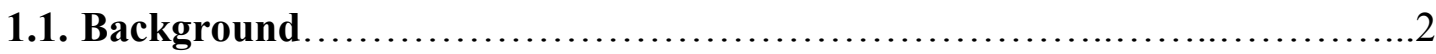

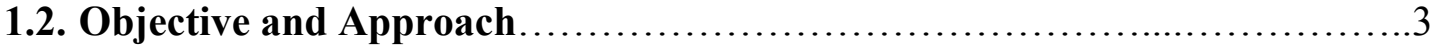

2.0 Experimental Procedures...................................................

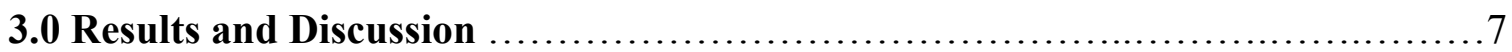

3.1. Metal Wire and Filter Test Results ...................................

3.2 Commercial Plated Metal Beads Test Results................................9

3.3 In-home Electroplated Metallic Filter Test Results..........................11

3.4 Professionally Electroplated Metallic Filter Test Results...................12

3.5 Field Experiment Results.......................................... 13

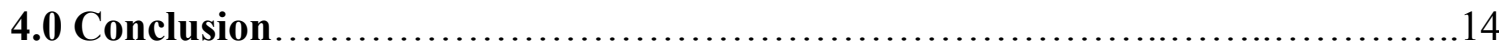

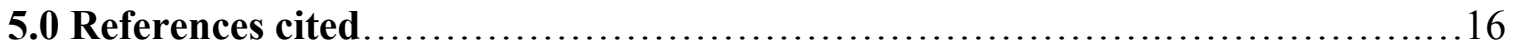




\section{List of Tables}

Number Title

Page

1. Metal \# 1 Wire and Filter @ 3 lpm flow rate test results.....................8

2. Metal \# 1 wire and filter@ 5 lpm flow rate test results.....................8

3. Metal \# 2 Wire Filter @ 5 lpm flow rate test results ..........................8

4. Metal \# 2 wire and filter @ 5 lpm flow rate test results .......................

5. Metal \# 2 wire and filter @ 3 lpm flow rate test results ........................ 9

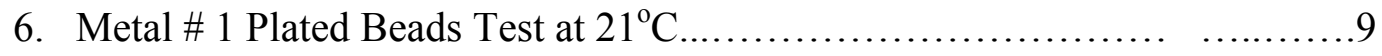

7. Metal \# 2 Plated Beads Test at $56^{\circ} \mathrm{C}$..................................... 10

8. (Test1) In-home Electroplated Metallic Sponge Test Results ...............11

9. (Test 2) In-home Electroplated metallic sponge test at $15 \mathrm{l} / \mathrm{m}$ flow rate .......11

10. Field Test Results ................................................. 14 


\section{List of Figures}

Number Title

Page

1. Anthropogenic Mercury Emissions ....................................1

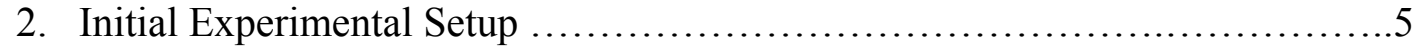

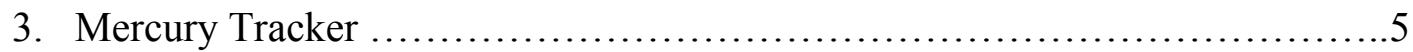

4. Metal Plated Beads......................................................

5. Metal Bead Filter Testing Setup......................................6

6. Metal Plated Bead Test Results.......................................... 10

7. Mercury removal efficiency of two different wires and beads.................11

8. In-house Electroplated Metallic Filter Test Results........................12

9. Professionally Electroplated Metallic Filter \# 1 Test Results..................13

10. Professionally Electroplated Metallic Filter \# 2 Test Results..................13 


\section{Executive Summary}

The CAST program funding was to develop and test metallic filters to remove mercury from gas streams. There is a clear need for cost effective control devices to remove mercury from power plant flue gases.

The main objective of this research is to develop cost effective metallic filters and to evaluate the effectiveness in removing mercury vapor from coal fired power plant's flue gas. Two different metallic filters were custom made out of fine metal threads and a disc with pinholes of less than one mm diameter. The initial tests were performed at room temperature conditions. Additional tests were performed at different flow rates and temperature conditions. The initial test results showed higher than $90 \%$ efficiency. Based on these preliminary test results, to increase the contact surface area, commercially available metal plated beads were tested for the mercury removal efficiencies. The results showed efficiencies higher than $93 \%$.

Since previous test results confirmed that the metallic filters can remove mercury effectively from gas streams, a substrate material with light weight, high surface area and low pressure drop was explored. A commercially available metallic sponge was chosen as the substrate. These metallic sponges were initially electroplated with the specific metals in house at Montana Tech's mercury laboratory. These sponges plated with precious metals were tested for mercury removal efficiency and showed over $90 \%$ efficiency for 30 hours or longer. The average mercury concentration used for the inlet was about $100 \mathrm{ug} / \mathrm{m} 3$. These metallic sponges were then sent to a commercial plating company for plating with the precious metals. This was done because it was hard to maintain the same quality control for each batch of plating in house. The sponges professionally plated were then tested in the same way and these filters showed over $90 \%$ efficiency continuously for over 42 hours. Two different precious metals were tried and both showed high efficiencies except that the one precious metal lasted for longer time relative to the other. Several laboratory tests were performed to confirm the efficiency of these sponges. The metallic sponges were heated after they were saturated with mercury vapor to recover mercury and simultaneously regenerate the sponge. Initial tests on regeneration of the filters were conducted at about $100 \mathrm{~F}$ and the de-sorption of mercury from the filter was measured through concentration in the air that passed through the heated filter in the opposite direction. It was determined that about $99 \%$ of the mercury vapor could be desorbed from the filters. Further increasing the temperature released the mercury as a puff and shortened the de-sorption time. It is planned to recover the mercury from this thermal de-sorption process in the future experiments. All these laboratory experiments were conducted at Montana Tech of the University of Montana's Mercury Laboratory and the laboratory test results indicated that the removal efficiency of the filters lasted longer even when exposed to lower mercury concentrations. Since these tests consistently showed promising results, the filters were field tested in a coal fired power plant's flue gas stream at Colstrip, Montana. Over all the performance of these filters were very similar to the laboratory test results. 


\subsection{INTRODUCTION}

Mercury is a naturally occurring element on earth's crust and is released to the atmosphere by surface evasion and volcanoes etc. However, anthropogenic emissions are believed to be the most significant source of mercury pollution to the environment. It is apparent that anthropogenic emissions of mercury into the atmosphere have significant environmental implications. Awareness of the mercury problem is increasing and in the United States, fossil fuel combustion is the most significant anthropogenic source of atmospheric mercury.

The US EPA stated that "Natural sources of mercury—such as volcanic eruptions and emissions from the ocean - have been estimated to contribute about one third of current worldwide mercury air emissions, whereas anthropogenic (human-caused) emissions account for the remaining two-thirds. These estimates are highly uncertain. Much of the mercury circulating through today's environment is mercury that was released years ago, when mercury was commonly used in many industrial, commercial, and residential products and processes. Land and water surfaces can repeatedly re-emit mercury into the atmosphere after its initial release into the environment. The pie chart below shows that anthropogenic emissions are roughly split between these natural emissions, re-emitted emissions from previous human activity, and direct emissions from current human activity.

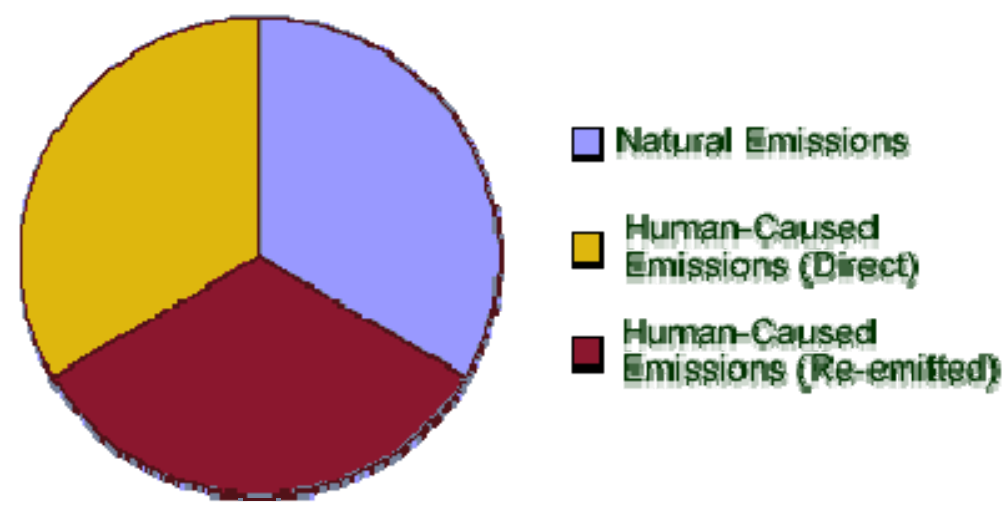

Figure 1: Anthropogenic Mercury Emissions

Source: Seingeur, 2004 and Mason and Sheu, 2002”.

Mercury has a very complex cycle in the environment and is a bio-accumulative hazardous air pollutant. Mercury cycles in the environment between air, water, sediments, soil and biota in various forms.

Since the 1990 Clean Air Act, the US EPA has researched mercury pollutant extensively, including how best to require reductions from power plants. According to EPA approximately 75 tons of mercury are found in the coal delivered to power plants each 
year and about two thirds, of this mercury (that is 50 tons of mercury) is emitted from coal fired power plants in the US.

Coal contains relatively low levels of mercury ranging from 0.010 to $3.5 \mathrm{ppmw}$ of mercury. However, the mercury emissions from coal combustion in power plants have received special attention because coal is burned in about 1700 power plants in the US. Mercury is classified as a hazardous air pollutant under the Clean Air Act Amendments (CAAA) of Title III. The EPA's 1998 Utility Air Toxics Report to the US Congress indicated that mercury posed the greatest concern of all the toxics emitted from power plants.

The US EPA announced its intention to rulemaking in 2003 to regulate power plant mercury emissions. The final regulations are expected in 2004, and compliance is projected for 2007. If EPA has its way, the new regulations will require $90 \%$ reduction in mercury emissions from power plants. This requirement will have significant financial implications for power plants. Therefore, developing cost effective control technologies to remove mercury will become increasingly critical.

Developing viable control technology to remove mercury from coal fired power plants is critical to meet the $90 \%$ mercury reduction proposed by the US EPA. Coal contains low levels of mercury, 0.1 to 3.5 ppmw, which is released in the flue gas during combustion. Mercury is present in flue gas in three different forms as elemental, ionic, and particulates. The amount of mercury in each of these forms is determined mainly by the type of coal being burned. It is reported that most of the mercury $(90 \%)$ from the combustion of western coal is released in vapor form mainly due to its low chloride content. The coal containing high chloride content tends to release mercury in ionic form $(40 \%)$ and vapor form $(60 \%)$. The ionic form of mercury is easy to collect on a conventional flue gas desulphurization system. The particulate form of mercury can be effectively captured by existing particulate control devices such as bag houses or electrostatic precipitators. However existing air pollution control devices in a coal fired power plant are very poor in capturing vapor form of mercury. Therefore developing cost effective and environmentally sound technologies to remove mercury vapor is critical.

The CAST program funding was used to test the effectiveness of metallic filters developed at Montana Tech to remove mercury vapor from coal fired power plant's flue gas. This report summarizes the research and tests conducted for the period from 2003 to 2004.

\section{$\underline{1.1 \text { Background }}$}

Several control technologies are in the developmental stage for controlling mercury. They mainly focus on pre-combustion and post combustion processes. Pre-combustion involves coal-cleaning technologies to reduce mercury burden at the source. Post combustion involves adsorption by carbon-based materials and conversion of mercury vapor to oxidized form for removal by wet system or particulate filters. 
It is reported that $90 \%$ of mercury emitted from burning western coal is in the form of mercury vapor (elemental) where as combustion of eastern coal generates $60 \%$ elemental mercury and $40 \%$ divalent mercury.

There is a clear need for devices to remove mercury cost effectively from power plant flue gases. Most of the current research has focused on removing mercury from the air stream and leaving it in the solid particles or converting elemental mercury to divalent mercury to absorb it in a wet scrubber. Here, the problem is that the mercury still enters the environment except that it is transferred from the air to water or to a solid form. The environmental benefit from the money spent is not realized. Thus, preventing mercury from getting into the environment will be the most effective way to spend the money.

This situation is mainly true for power plants that burn western US coal. This coal contains low levels of chloride in which $90 \%$ of the mercury exists as elemental mercury. Thus, to comply with the potential $90 \%$ mercury reduction required by EPA, one must oxidize the elemental mercury to mercuric chloride or to mercuric oxide form either to scrub it or to capture it as particulates. Power plants burning low chloride coals must inject additional chloride compounds or must use oxidizing agents such as ozone to oxidize the mercury to mercuric oxide as a way to collect particulates. In either case, mercury is merely transferred to another form and becomes expensive and complex to control. Therefore, it appears that removing mercury from the flue gas altogether will be the cost effective and environmentally friendly approach. If the metallic filter developed in this research works, it will be a very desirable and economic process. The metallic filter could be regenerated by a simple heating process and the mercury can be captured from the air stream without it getting into the environment in any form.

The filtration process is not new for power plants, and with minimum modifications, the existing plants can use this technology if it is proven to be effective. Therefore the results of this research will benefit the coal industry and the power plants in dealing with the mercury issue. The environmental interest groups also will like it because it prevents the toxic mercury from getting into the environment rather than transferring it into another medium.

\subsection{Objective and Approach}

The main objective of this research is to develop cost effective filters and to evaluate the effectiveness in removing mercury vapor from coal fired power plant's flue gas. The objective was modified to include another material to be tested along with first selected metal for mercury vapor removal, mainly due to its relatively low cost. Both of these filters were tested under different flow rates and temperature conditions. Similarly commercial metal plated beads were tested as filter materials.

A metallic sponge was electroplated at Montana Tech and tested for mercury vapor removal efficiency at a flow of 5 liters per minute. The test results showed higher than $80 \%$ efficiencies. The spent sponges were then heated to less than $100^{\circ} \mathrm{F}$ and they 
released over 99 percent of the mercury. After this initial test, the sponges were professionally plated with specific metals and were tested for its mercury removal efficiency at $5 \mathrm{l} / \mathrm{m}$ flow rate. Finally one of the filters was field tested in a coal fired power plant at Colstrip, Montana.

The flow rate of 5 liters per minute for these experiments was calculated based on 0.3 second residence time. The design of the test system is based on a proposed theory as follows. The filter efficiency could be assumed to follow a simple first order equation as follows:

$$
\mathbf{n}=\mathbf{1}-\mathbf{e}^{-\mathbf{A} / \mathbf{A m}}
$$

Where $\mathbf{n}=$ removal efficiency, $\mathbf{A}=$ available surface area $\mathbf{A m}=$ surface area for one mass transfer unit

This simple equation proposed above will be used as the starting point to evaluate the engineering design of mercury filters.

\subsection{EXPERIMENTAL PROCEDURES}

The metallic filters were custom made for this study with fine metal threads of less than one mm diameter in 30 centimeter lengths. Pinholes of less than one mm diameter were made to have the plates act as filters. The amount of filter volume required was based on a one second residence time which is a very conservative residence time. These filters and threads were also made out of two different metals, metal \# 2 was relatively cheaper than metal \# 1. Tests were performed in parallel with metal \# 1, to understand its effectiveness in removing mercury. The initial tests were performed at room temperature conditions. Then tests were conducted under different flow rate and temperature conditions.

The initial test results with the metallic wire and filters showed very encouraging results. The tests conducted with the alternative metal also had similar results. Then these tests were performed with two different metal plated bead filters which also showed efficiencies higher than $93 \%$.

The laboratory experiments were conducted at Montana Tech of the University of Montana's Mercury Laboratory. The experimental set up consisted of a mercury vapor source, connected to a pump to draw about $5 \mathrm{lpm}$ air containing mercury vapor(Figure 2). The entire experimental setup was under the fume hood. The mercury vapor was generated by passing room air through a closed box containing mercury contaminated soil. As air was passing through the mercury contaminated soil, the mercury from the soils volatilized in to the stream. The rate of airflow was controlled to generate the desired level of mercury vapor in the air stream. This seemed like a safe and easy way to 
generate mercury vapor. Since we had several mercury contaminated soil samples from mine sites, this worked well for us.

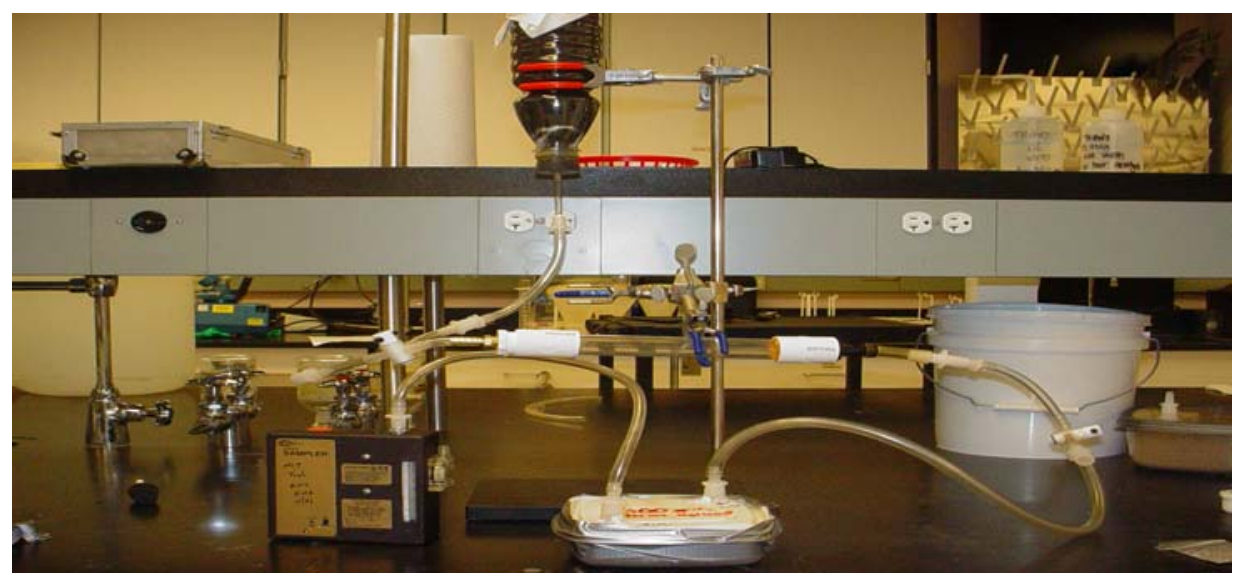

Figure 2: Initial Experimental Setup

During the efficiency testing, the mercury vapor was passed through the filter to be tested and the mercury vapor concentration levels at the inlet and outlet were measured by the Mercury Tracker (Figure 3 ) to evaluate the filter. This mercury analyzer has sensitivity to measure concentration of $0.01 \mu \mathrm{g} / \mathrm{m}^{3}$ of mercury. The filter's inlet and outlet mercury concentrations were used to calculate the mercury removal efficiency of the filters.

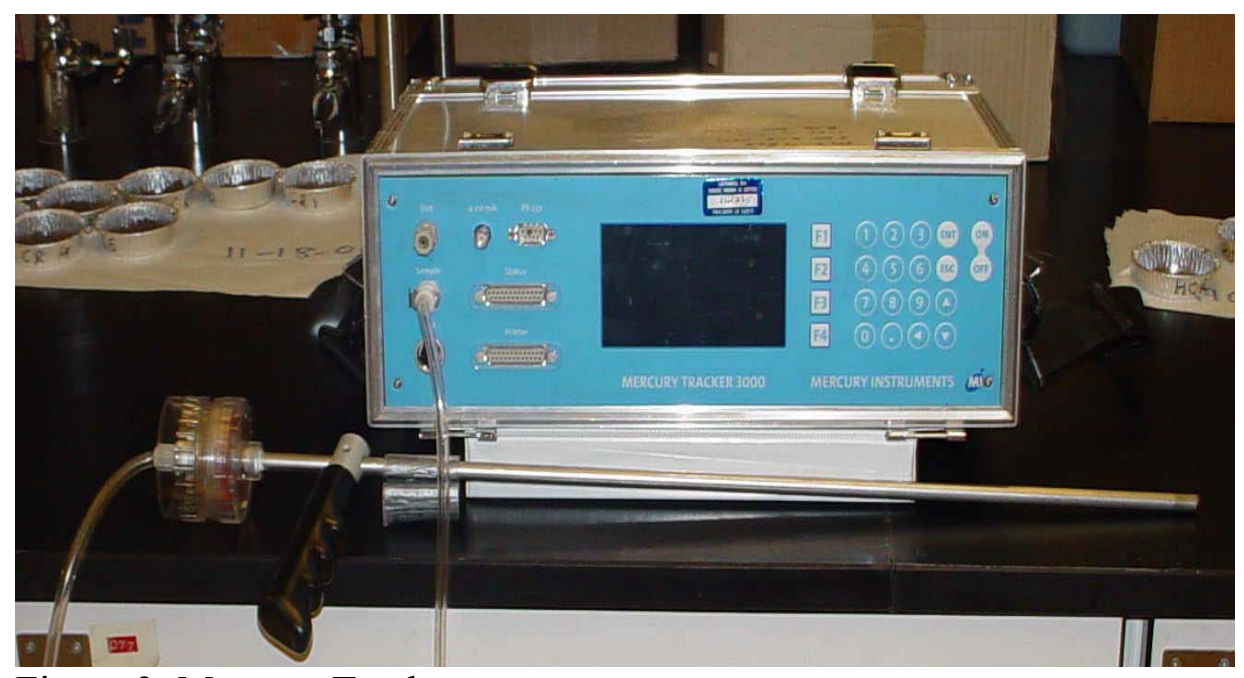

Figure 3: Mercury Tracker

The filters were filled in a $2.5 \mathrm{~cm}$ diameter and $15 \mathrm{~cm}$ long plastic tube. A flow rate of 5 liters per minute was maintained to provide a residence time of 0.3 seconds. The flow rate for these experiments was calculated based on 0.3 second residence time. 
After the initial tests, for cost effective purpose and also to increase the surface area of the filters, instead of solid metal, commercially available metal plated beads (Figure 4) were tested for the mercury removal efficiencies (Figure 5).

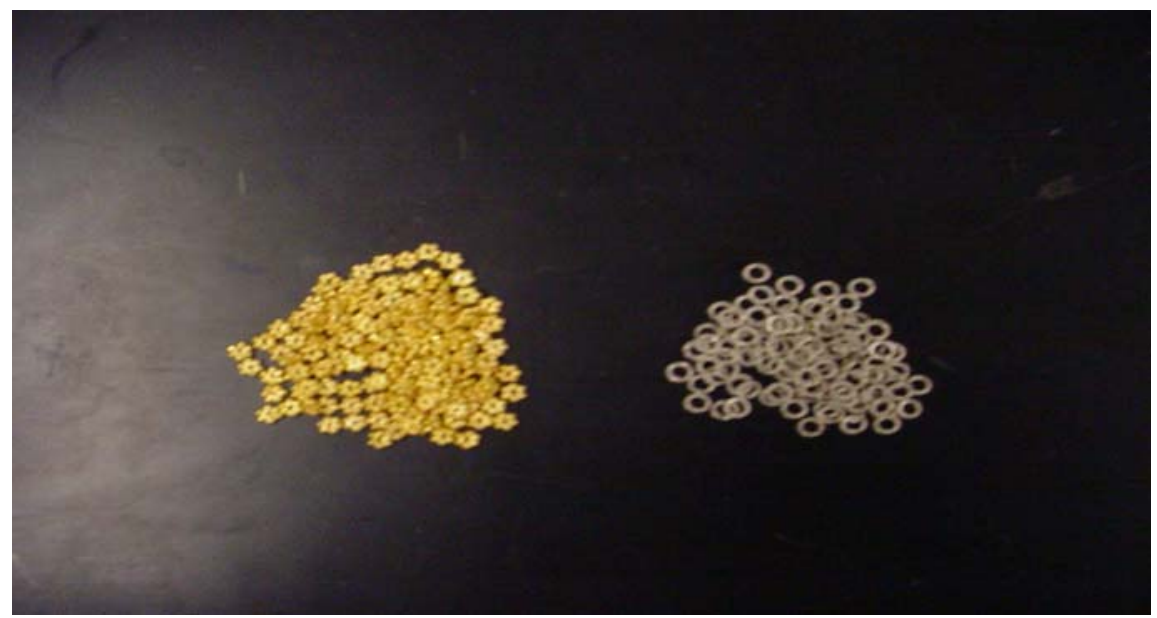

Figure 4: Metal Plated Beads

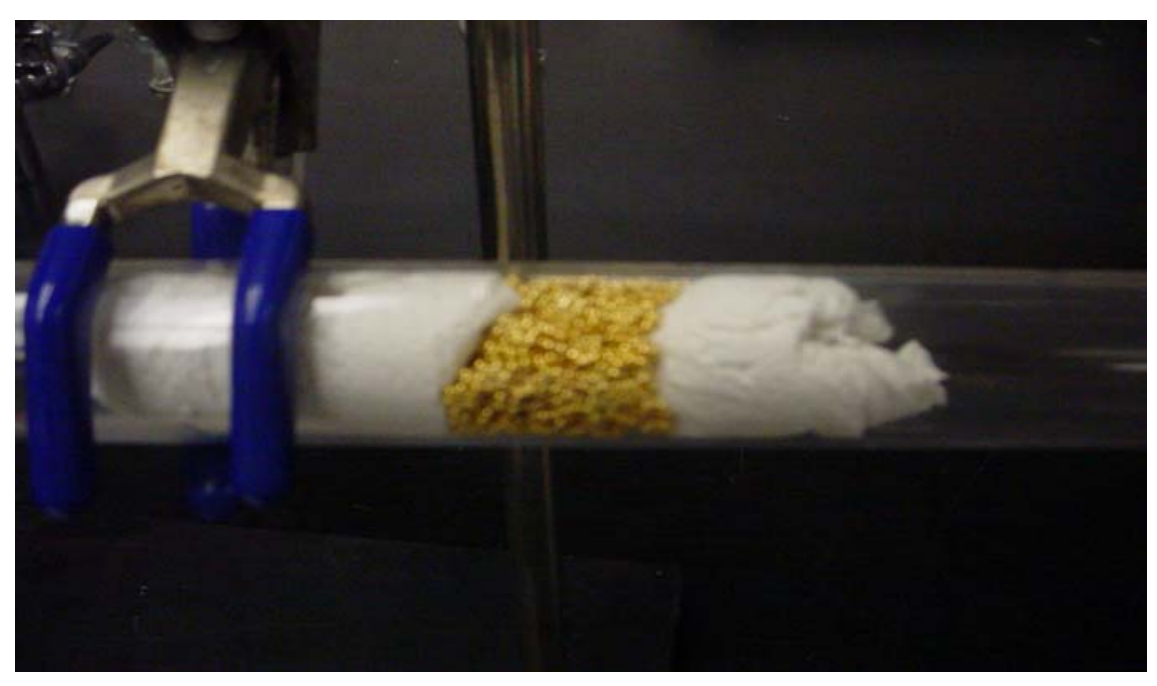

Figure 5: Metal Bead Filter Testing Setup

Because temperature of flue gas varies widely in the exhaust side of a power plant, these tests were conducted at three different temperatures of $70^{\circ} \mathrm{F}, 133^{\circ} \mathrm{F}$, and $163^{\circ} \mathrm{F}$. A $4 \mathrm{~cm}$ quartz tube was used as the reactor where preheated hot air augmented by mercury vapor was passed through the filter. 
To find cost effective solution for the filter material, metal coating was attempted on metallic wire mesh in the laboratory. These electroplated metallic filters were tested for mercury removal efficiency following the same procedures such as passing mercury contaminated air through the filter and measuring the concentration levels at the inlet and outlet of the filter and with these measured values the mercury removal efficiency was calculated. For couple of tests the flow rate was kept at $15 \mathrm{lpm}$. Then the flow through the filter was set at about $5 \mathrm{lpm}$ which is almost half the velocity that is normally used in a catalytic oxidizer.

Initial tests on regeneration of the filters were conducted at about $100 \mathrm{~F}$ and the desorption of mercury from the filter was measured through concentration in the air that passed through the heated filter in the opposite direction. It was determined that about $99 \%$ of the mercury vapor could be desorbed from the filters. After successfully completing these tests with home made filters, a professional plating company was asked to develop the filter according to the specifications given by the PI. Four filters were made by the professional company and then tested at the Montana Tech mercury laboratory for its mercury removal efficiency. Since the laboratory tests were successful, one of the professionally plated metallic filters was taken to a coal fired power plant at Colstrip, Montana and field tested under real world conditions. The laboratory and the field test results are reported here.

\subsection{RESULTS AND DISCUSSION}

The initial test results with custom made metallic wire and filters showed very encouraging results. Then for cost effective purpose tests were performed with an alternative metal and had similar test results. Tests were conducted with both metal \# 1 and metal \# 2 at two different flow rates of $3 \mathrm{lpm}$ and $51 / \mathrm{m}$ at room temperature conditions. After these tests, commercially plated metal beads were tested as filters. These tests were also performed under two different temperatures of $21^{\circ} \mathrm{C}$ and $56^{\circ} \mathrm{C}$. Further, these metal plated bead tests were also conducted at three different temperatures of $70^{\circ} \mathrm{F}, 133^{\circ} \mathrm{F}$, and $163^{\circ} \mathrm{F}$ with three different mercury concentration levels of $40 \mu \mathrm{g} / \mathrm{m}^{3}$, $42 \mu \mathrm{g} / \mathrm{m}^{3}$, and $59 \mu \mathrm{g} / \mathrm{m}^{3}$ respectively. Next metallic filters electroplated at Montana Tech laboratory were tested at two different $(15 \mathrm{lpm}$ and $5 \mathrm{lpm})$ flow rates in room temperature conditions. After successful tests with home made filters, professionally electroplated filters were tested. Finally one of the filters was field tested in a coal fired power plant at Colstrip, Montana.

\subsection{Metal Wire and Filter Test Results}

Tests with metal \# 1 wire and filter were performed at the flow rates of $3 \mathrm{lpm}$ at room temperature conditions. Table 1 shows the test results of metal \# 1, where the inlet mercury vapor concentrations ranged from $298 \mu \mathrm{g} / \mathrm{m}^{3}$ to $360 \mu \mathrm{g} / \mathrm{m}^{3}$ and the mercury removal efficiency was above $88 \%$. The total weight of the filter was about 15 grams. 
Table 1: Metal \#1 Wire and Filter@3 1pm flow rate test results

\begin{tabular}{|c|c|c|}
\hline \multicolumn{2}{|c|}{ Control of Mercury Vapor: Metal \# 1 Wire Filter*@ 3 lpm flow } \\
\cline { 1 - 2 } HG Vapor $\mu \mathrm{m}^{3} \mathbf{3}$ & \%Removal Efficiency \\
\hline 354 & Outlet & 89.3 \\
\hline 360 & 38 & 88.3 \\
\hline 298 & 42 & 89.3 \\
\hline
\end{tabular}

*Amount of metal \# 1 in wire: $8.05 \mathrm{~g}$.

**Amount of metal \# 1 in filter: $7.28 \mathrm{~g}$.

Then similar tests at $5 \mathrm{lpm}$ flow rate with metal \# 1 wire filter were performed at room temperature. Table 2 shows the test results. In this test the inlet mercury vapor concentrations ranged from $184 \mu \mathrm{g} / \mathrm{m}^{3}$ to $196 \mu \mathrm{g} / \mathrm{m}^{3}$ and the mercury removal efficiency was above $70 \%$. The total weight of the filter in this experiment was also about 15 grams.

Table 2: Metal \# 1 wire and filter@, 5 1/m flow rate test results

\begin{tabular}{|c|c|c|}
\hline \multicolumn{3}{|c|}{ Control of Mercury Vapor: Metal \# 1 Wire and Filter**@ 5 lpm flow } \\
\cline { 1 - 2 } HG Vapor $\mu \mathbf{m}^{3}$ & Outlet & \\
\hline 196 & 55 & 71.9 \\
\hline 184 & 54 & 70.7 \\
\hline 186 & 52 & 72.0 \\
\hline
\end{tabular}

*Amount of metal \# 1 in wire: $8.05 \mathrm{~g}$.

**Amount of metal \# 1 in filter: $7.28 \mathrm{~g}$.

Since metal \# 2 was relatively cheaper than metal \# 1, these filters and threads were made in metal \# 2 also. Similar tests were conducted with metal \# 2 wire filters at two different flow rates of $5 \mathrm{l} / \mathrm{m}$ and $3 \mathrm{l} / \mathrm{m}$. Tables 3 and 4 show the test results with $5 \mathrm{l} / \mathrm{m}$ flow rate. In the first test the mercury concentrations ranged from $34 \mu \mathrm{g} / \mathrm{m}^{3}$ to $117 \mu \mathrm{g} / \mathrm{m}^{3}$. The mercury removal efficiency was above $63 \%$.

Table 3: Metal \# 2 Wire Filter@ 5 lpm flow rate test results

\begin{tabular}{|c|c|c|}
\hline \multicolumn{3}{|c|}{ Metal \# 2 Wire Filter @ 5 l/m flow rate } \\
\cline { 1 - 2 } HG Vapor $\mu \mathrm{gg} / \mathrm{m}^{3}$ & \%Removal Efficiency \\
\hline 34.4 & 12.7 & 63.1 \\
\hline 66.8 & 18.8 & 71.9 \\
\hline 75 & 19.5 & 74.0 \\
\hline 117.2 & 13.2 & 88.7 \\
\hline
\end{tabular}

*Weight of metal \# 2 used: wire $-8.35 \mathrm{~g}$ 
Table 4 shows the second test results with $5 \mathrm{lpm}$ flow rate. In this test the mercury removal efficiency was above $76 \%$ where the inlet concentration ranged from $192 \mu \mathrm{g} / \mathrm{m}^{3}$ to $215 \mu \mathrm{g} / \mathrm{m}^{3}$. The weight of the filter was about 11 grams.

Table 4: Metal \# 2 wire and filter @ 5 lpm flow rate test results

\begin{tabular}{|c|c|c|}
\hline \multicolumn{3}{|c|}{ Metal \# 2 Wire* and Filter**@ 0 lpm flow rate } \\
\hline \multicolumn{2}{|c|}{ HG Vapor $\mu \mathrm{gm}^{3}$ \%Removal Efficiency } \\
\hline Inlet & Outlet & 78.1 \\
\hline 215 & 47 & 78.3 \\
\hline 198 & 43 & 76.6 \\
\hline 192 & 45 & \\
\hline
\end{tabular}

*Weight of metal \# 2 used: wire $-8.35 \mathrm{~g}$

**Amount of metal \# 2 in filters $-3.05 \mathrm{~g}$

In this test the flow rate was changed to $3 \mathrm{lpm}$. Table 5 shows the test results where the mercury removal efficiency was above $85 \%$. The inlet mercury concentration ranged from $252 \mu \mathrm{g} / \mathrm{m}^{3}$ to $263 \mu \mathrm{g} / \mathrm{m}^{3}$ and the total weight of the filter was about 13 grams.

Table 5: Metal \# 2 wire and filter @ 3 lpm flow rate test results

\begin{tabular}{|c|c|c|}
\hline \multicolumn{3}{|c|}{ Metal \# 2 Wire* and Filter**@3 lpm flow } \\
\hline \multicolumn{2}{|c|}{ HG Vapor $\mu \mathrm{g} / \mathrm{m}^{3}$} & \%Removal Efficiency \\
\hline Inlet & Outlet & \\
\hline 252 & 36 & 85.7 \\
\hline 263 & 27 & 89.7 \\
\hline 263 & 34 & 87.1 \\
\hline
\end{tabular}

*Weight of metal \# 2 used: wire $-8.35 \mathrm{~g}$.

$* *$ Amount of metal \# 2 in filter: $5.05 \mathrm{~g}$

\subsection{Test Results of Commercially Plated Metal Beads}

Commercially plated metal beads were purchased and tested as filters to remove mercury vapor. These tests were performed under different temperatures representing the flue gas temperature fluctuations. Table 6 shows the test results of the metal \#1 plated beads at $21^{\circ} \mathrm{C}$. The mercury removal efficiency was above $95 \%$ and the inlet mercury concentration level was about $28 \mu / \mathrm{m}^{3}$.

Table 6: Metal \# 1 Plated Beads Test at $21^{\circ} \mathrm{C}$

\begin{tabular}{|c|c|c|}
\hline \multicolumn{3}{|c|}{ Metal \# 1 Plated Beads @ 21.1 degrees C. } \\
\hline Inlet $\left(\boldsymbol{\mu} / \mathbf{m}^{3}\right)$ & Outlet $\left(\boldsymbol{\mu} / \mathbf{m}^{3}\right)$ & \%Removal Efficiency \\
\hline 28.0 & 1.3 & $95 \%$ \\
\hline 28.8 & 1.1 & $96 \%$ \\
\hline 28.9 & 1.5 & $95 \%$ \\
\hline
\end{tabular}


Table 7 shows the test results of the number 2 metal plated beads at $56^{\circ} \mathrm{C}$ temperature. Again the mercury removal efficiency stayed above 93\% where the mercury inlet concentrations ranged from $41 \mu \mathrm{g} / \mathrm{m}^{3}$ to $53 \mu \mathrm{g} / \mathrm{m}^{3}$.

Table 7: Metal \# 2 Plated Beads Test at $56^{\circ} \mathrm{C}$

\begin{tabular}{|c|c|c|}
\hline \multicolumn{3}{|c|}{ Metal \# 2 Plated Bead Test @ 56 degrees C. } \\
\hline Inlet $\left(\boldsymbol{\mu} / \mathbf{m}^{\mathbf{3}}\right)$ & Outlet $\left(\boldsymbol{\mu} / \mathbf{m}^{\mathbf{3}}\right)$ & \%Removal Efficiency \\
\hline 53.8 & 2.3 & 96 \\
\hline 42.8 & 2.1 & 95 \\
\hline 41 & 3 & 93 \\
\hline
\end{tabular}

Metal plated bead tests were also conducted at three different temperatures of $70^{\circ} \mathrm{F}$, $133^{\circ} \mathrm{F}$, and $163^{\circ} \mathrm{F}$ with three different mercury concentration levels of $40 \mu \mathrm{g} / \mathrm{m}^{3}, 42 \mu \mathrm{g} / \mathrm{m}^{3}$, and $59 \mu \mathrm{g} / \mathrm{m}^{3}$. Figure 6 shows the test results where the mercury removal efficiencies were consistently above $90 \%$.

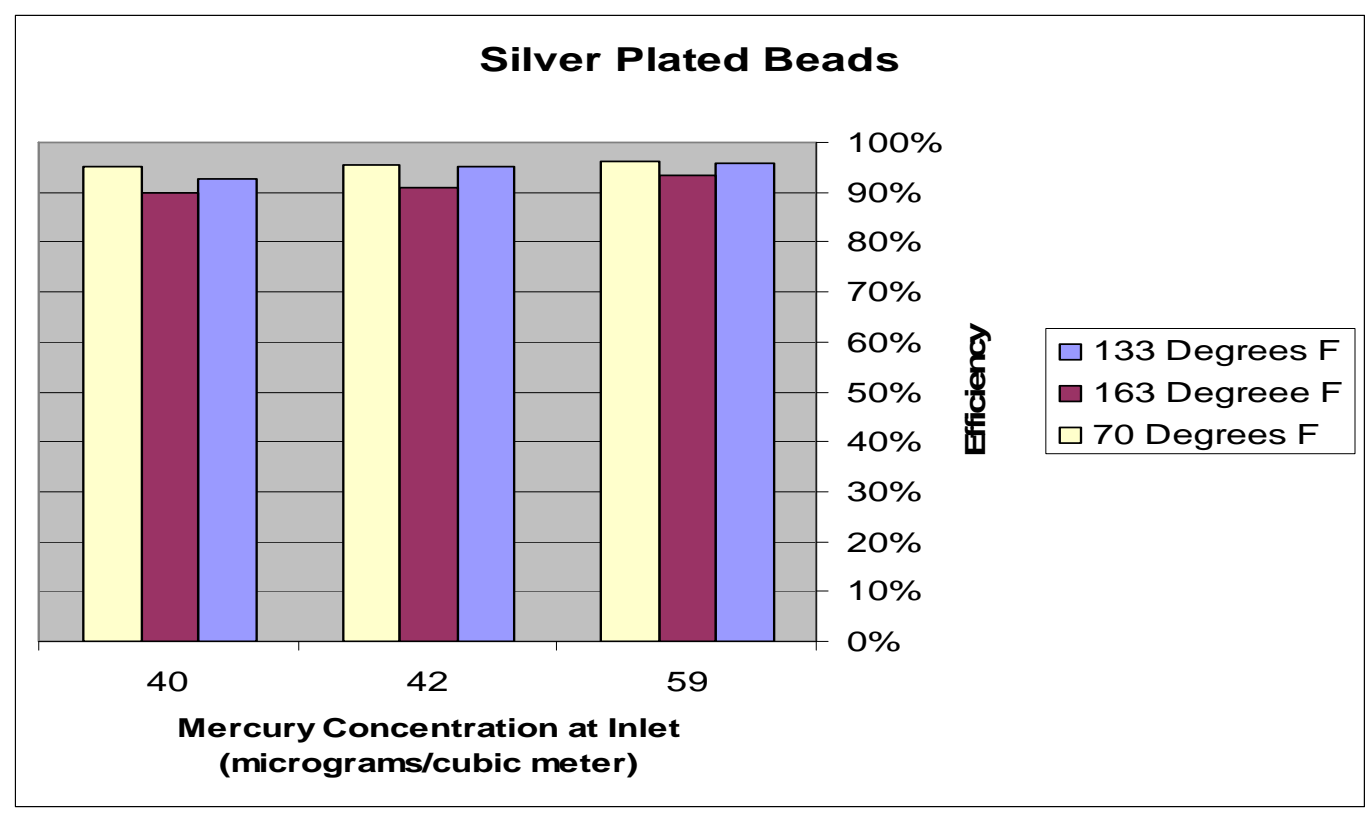

Figure 6: Metal Plated Bead Test Results

The following Figure 7 gives the relative efficiency of beads and the wire filters. Thus the preliminary results consistently showed that the mercury vapor could be removed effectively using metallic filters. 


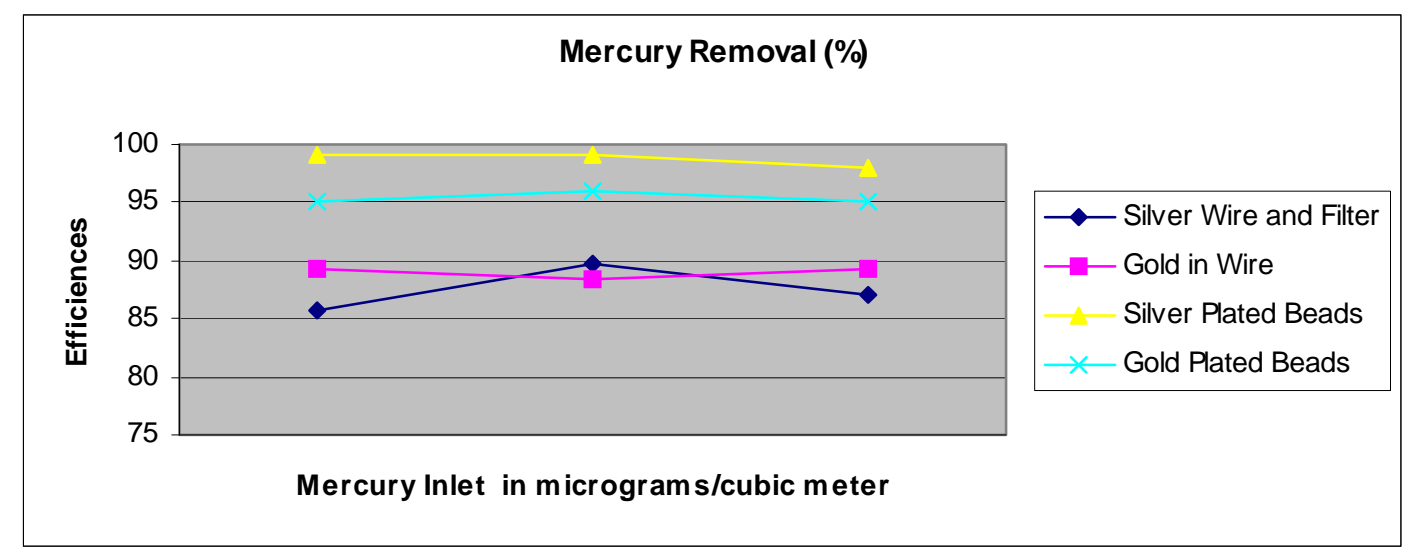

Figure 7: Mercury removal efficiency of two different wires and beads

\subsection{In-home Electroplated Metallic Filter Test Results}

Next step in developing the metallic filter was focused on electroplating the metallic sponges at Montana Tech laboratory. Since previous test results confirmed that the metallic filters can remove mercury effectively from gas streams, a substrate material with light weight, high surface area and low pressure drop was explored. A commercially available metallic sponge was chosen as the substrate. These metallic sponges were initially electroplated with the specific metals in house at Montana Tech's mercury laboratory. Initially, these in-home filters were tested for mercury vapor removal efficiencies at $15 \mathrm{lpm}$ flow rates. The results are given in Table 8 and 9 . The inlet mercury concentrations ranged from $89 \mu \mathrm{g} / \mathrm{m}^{3}$ to $432 \mu \mathrm{g} / \mathrm{m}^{3}$ and showed more than $80 \%$ mercury removal efficiencies.

Table 8: (Test1) In-home Electroplated Metallic Sponge Test Results

\begin{tabular}{|c|c|c|}
\hline \multicolumn{2}{|c|}{ Electroplated Metallic Sponge @ 15 lpm flow rate } \\
\hline \multicolumn{2}{|c|}{ HG Vapor $\mu \mathrm{g} / \mathrm{m}^{3}$} & \%Removal Efficiency \\
\hline 1 Inlet & Outlet & 88.3 \\
\hline 96.7 & 13.1 & 88.6 \\
\hline 90.2 & 11.0 & 89.1 \\
\hline 89.3 & 9.8 & 89.2 \\
\hline
\end{tabular}

Table 9: (Test 2) In-home Electroplated metallic sponge test at $15 \mathrm{lpm}$ flow rate

\begin{tabular}{|c|c|c|}
\hline \multicolumn{2}{|c|}{ Electroplated metallic Sponge $@ 15 \mathrm{lpm}$ flow rate } \\
\hline HG Vapor $\mu \mathrm{g} / \mathrm{m}^{3}$ & \%Removal Efficiency \\
\hline 432.15 & Outlet & 88.1 \\
\hline 334.30 & 51.28 & 85.4 \\
\hline 280.25 & 50.40 & 82.6 \\
\hline 233.15 & 48.83 & 80.2 \\
\hline
\end{tabular}


Further the metallic sponge filter that was electro plated at Montana Tech was tested at $5 \mathrm{lpm}$ flow rate in room temperature conditions. Since the previous test results confirmed that these metallic filters can remove mercury, next focus was to test the efficiency and longevity of these filters. Thus these tests were focused on looking at the efficiency and the time.

Figure 8 shows the test results of the in-house electroplated metallic filter. During this test the average inlet mercury concentration level ranged from 60 to $180 \mathrm{ug} / \mathrm{m}^{3}$ and most of the test period it was above $100 \mathrm{ug} / \mathrm{m}^{3}$ (Figure 8 ). The results showed $90 \%$ removal efficiency for about 30 hours after successfully completing this test.

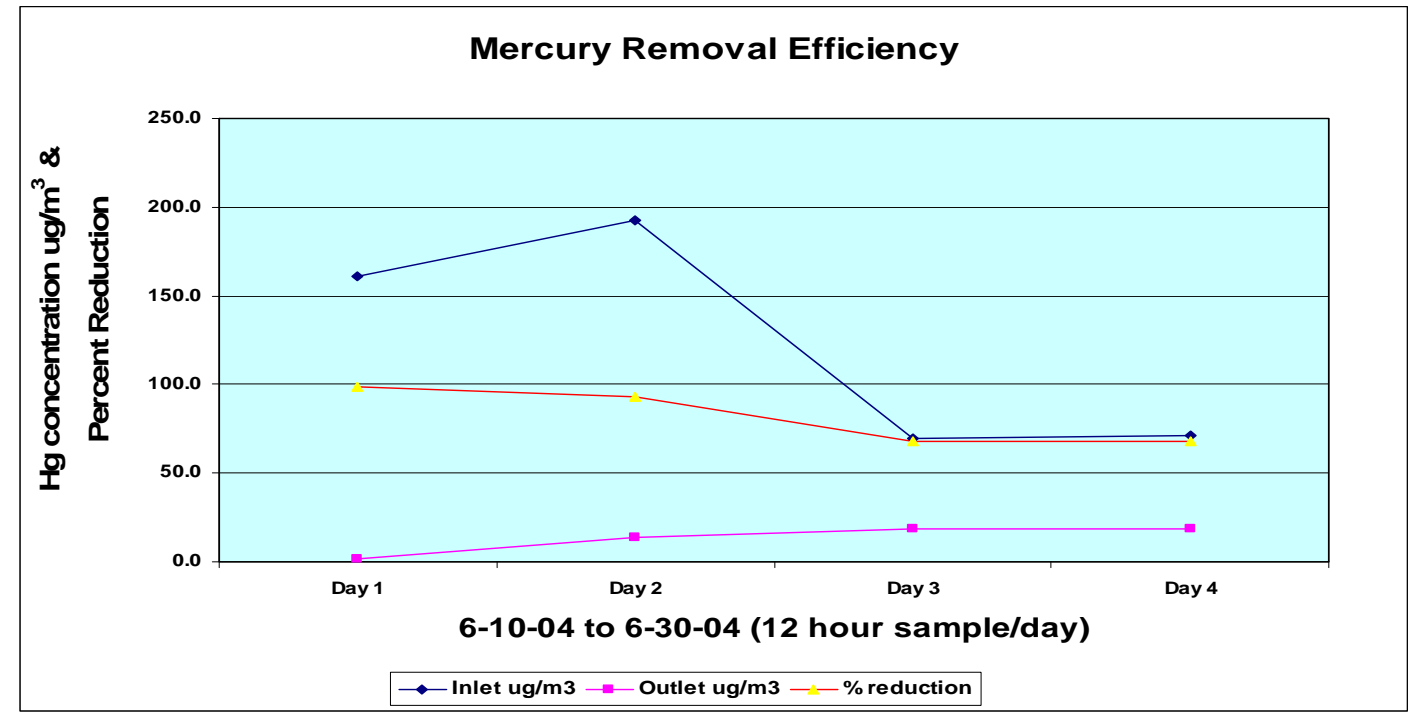

Figure 8: In-house Electroplated Metallic Filter Test Results

\section{$\underline{\text { 3.4 Professionally Electroplated Metallic Filter Test Results }}$}

Next the test was performed with the professionally electroplated metallic filter. During this test (Figure 9) the average inlet mercury concentration level ranged from $80 \mathrm{ug} / \mathrm{m}^{3}$ to $50 \mathrm{ug} / \mathrm{m}^{3}$. The $90 \%$ mercury removal efficiency of this filter lasted for about 42 hours compared to the in-house plated filter, where the $90 \%$ efficiency lasted only for 30 hours. 


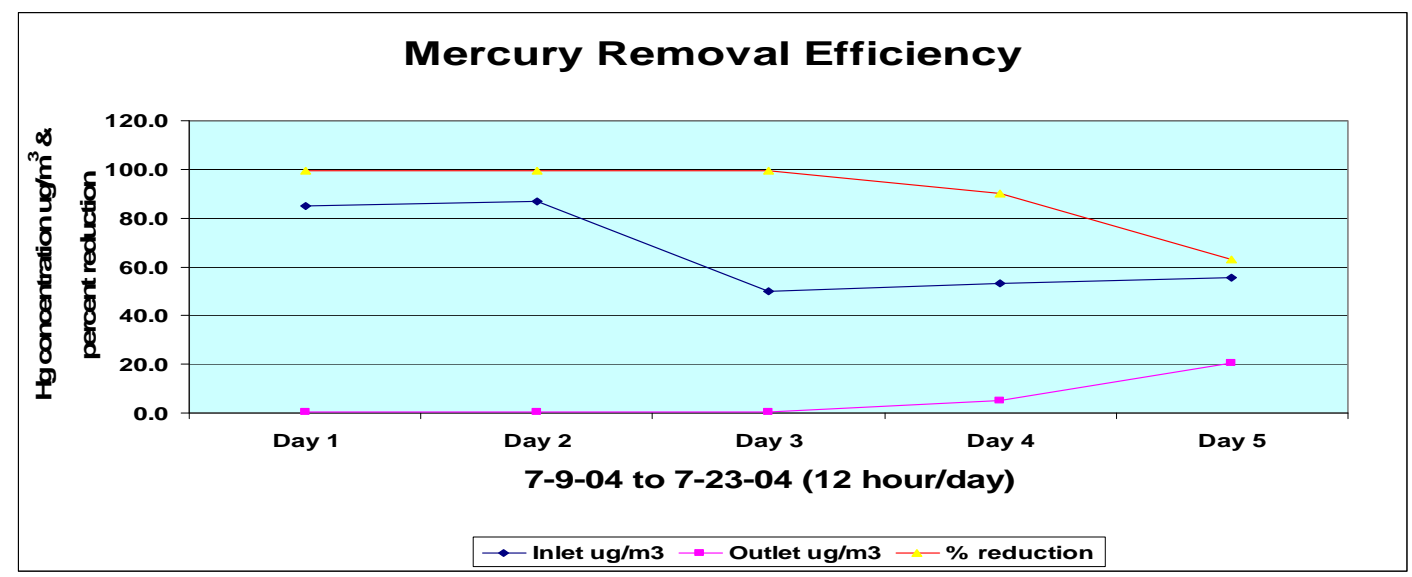

Figure 9: Professionally Electroplated Metallic Filter \# 1 Test Results

One more professionally electroplated filter was tested. The results show (Figure 10) that the $90 \%$ efficiency lasted for about 84 hours. During this test the inlet mercury concentration level ranged between 30 to $140 \mathrm{ug} / \mathrm{m}^{3}$ for most of the testing period. Thus the laboratory test results indicated that the removal efficiency of the electroplated metallic filters lasted longer with lower inlet mercury concentrations. Since these tests consistently showed promising results, we decided to field test this filter in a coal fired power plant's flue gas stream at Colstrip, Montana.

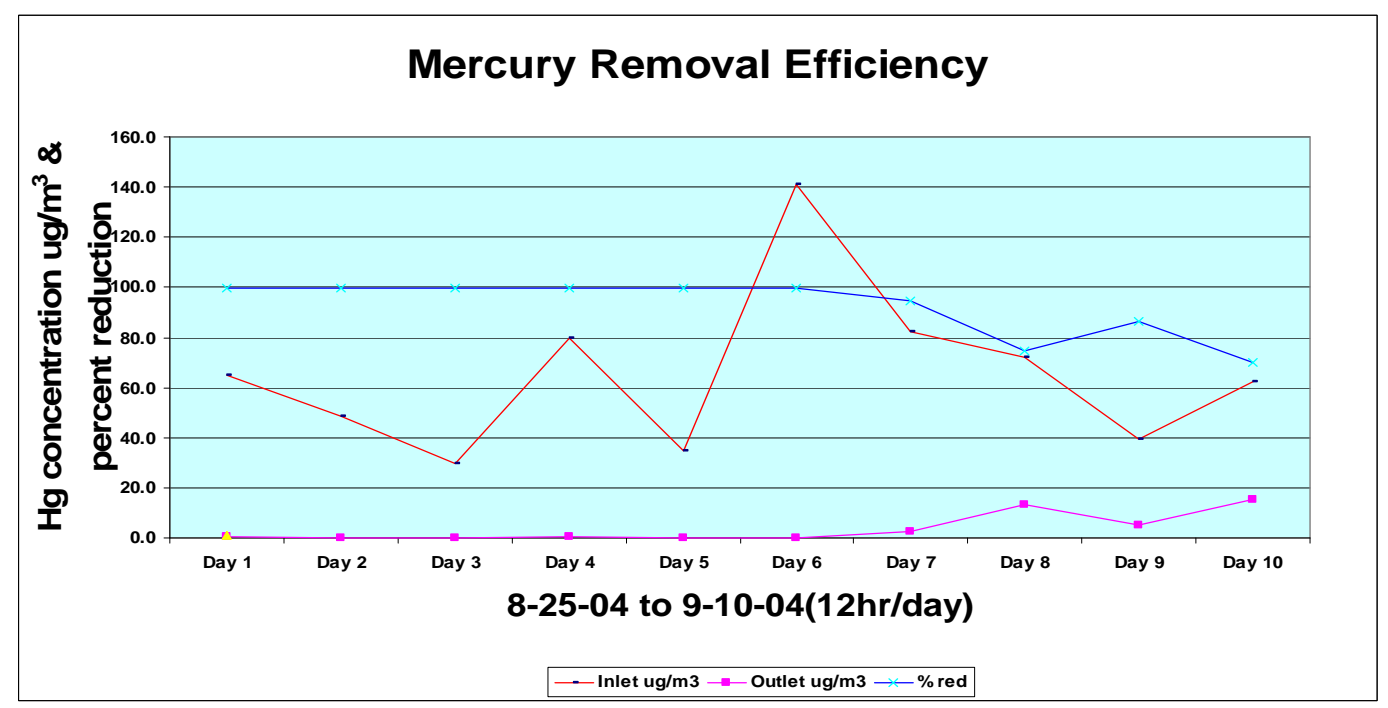

Figure 10: Professionally Electroplated Metallic Filter \# 2 Test Results

\subsection{Field Experiment Results:}

One of the filters was field tested in a coal fired power plant at Colstrip, Montana, that burns western coal. The selected metallic wire-mesh was professionally plated with metal \# 1. The filter was tested in one of the flue gas streams. Flue gas was drawn out of 
the stack just before it was emitted to the atmosphere. The objective of this initial field test was to evaluate the performance of the filter in the real world conditions.

Table 10 shows the field test results. The inlet mercury concentration levels at the flue gas fluctuated from $3.5 \mu \mathrm{g} / \mathrm{m}^{3}$ to $47.3 \mu \mathrm{g} / \mathrm{m}^{3}$. Mercury removal efficiency of the metallic filter was above $96 \%$ for the entire testing period.

Table 10: Field Test Results

\begin{tabular}{|l||l||l|l|l|}
\hline Date & Time & Inlet ug/m3 & Outlet ug/m3 & $\%$ red \\
\hline $9 / 21 / 04$ & $4: 15$ PM & 26.4 & 0.5 & 98.1 \\
\hline & $4: 30 \mathrm{PM}$ & 9.3 & 0.3 & 96.8 \\
\hline & $5: 00 \mathrm{PM}$ & 3.5 & 0.1 & 97.1 \\
\hline & $5: 20 \mathrm{PM}$ & 9.6 & 0.2 & 97.9 \\
\hline $9 / 22 / 04$ & $10: 35 \mathrm{AM}$ & 42.3 & 0.7 & 98.3 \\
\hline & $11: 00 \mathrm{AM}$ & 47.3 & 1.1 & 97.7 \\
\hline
\end{tabular}

Over all the performance of this filter in real world condition was determined to be as good as the laboratory results. The high relative humidity in the gas stream did not allow the testing to continue for longer period. Due to water condensation this field test was terminated in 19 hours.

The testing with stack gas needs further sample conditioning and long term testing to confirm the efficiency of the filter. Thus the preliminary field test results confirmed that these metallic filters are very effective in removing mercury vapor from coal fired flue gas streams.

\subsection{CONCLUSION}

In conclusion, this research project completed all the tasks mentioned in the proposal except one, but the tasks were slightly modified as the research progressed. This research focused on to develop and evaluate the effectiveness of metallic filters to remove mercury vapor cost effectively from coal fired power plant's flue gas. The test results with the metallic filters showed very encouraging results. Initial tests were completed experimenting with different metals including commercial metal plated beads as filter material. Tests were completed exposing the filters with different mercury concentration levels under various temperature and velocity conditions. The next successful progress was electroplating metallic sponges at Montana Tech and test for mercury vapor removal efficiency and the results showed higher than $80 \%$ mercury removal efficiencies. The spent sponges were then heated to less than $100^{\circ} \mathrm{F}$ and they released over 99 percent of the mercury. Then professionally plated metallic filter were tested at Montana Tech mercury laboratory for its mercury removal efficiency. Finally one of the filters was field tested in a coal fired power plant at Colstrip, Montana. Over all the performance of this filter in real world condition was determined to be as good as the laboratory results. However this field test was terminated after 19 hours, because of high relative humidity in the flue gas stream. 
In conclusion, unlike all other control systems where mercury is being transferred from a vapor phase to a solid phase, these metallic filters developed at Montana Tech will not only remove mercury from the flue gas effectively but mercury can be recovered and prevented from entering into the environment. This is a very desirable and environmentally friendly option over the technologies that are currently being evaluated. 


\section{References}

"Coal Chlorine Content Influences Mercury Removal", PRECIP Newsletter. January 2002, No. 312. www.mcilvainecompany.com/newsletters/precipn $1312 / \mathrm{Coal} \% 20$ Chlorine $\% 20$

Gorishi, B.; Gullet, B.K., "Sorption of Mercury Species by Activated Carbon and Calcium Based Sorbents: Effect of Temperature, Mercury Concentration and Acid Gases”, Waste Management \& Research, 1998, 6, 582-593.

Granite, E.J.; H.W., Hargis, R.A. “Carbon-Based Sorbents For the Removal of Mercury From Flue Gas", U.S. Department of Energy.

Guijarro, M.I.; Mendioroz, S.; Munoz, V., "Effect of Morphology of Sulfurized Materials in the Retention of Mercury from Gas Streams", Ind.Eng.Chem.Res.1998, 37, pg.1088-1094.

Hwang, J.Y., Huang, X., Tieder, R.E., Liu, X. "Control of Mercury Emissions from Coal Fired Power Plants Using Fly-Ash-Derived Carbon”, Michigan Technological University, EPA Grant Number R825370C028, www.es.epa.gov/ncer_abstracts/centers/cencitt/year3/material/hwang.html.

Ihle, J., “Mercury Emissions and Fuel Switching: What's in Your Coal?”, October 2002. www.coalage.com/ar/coal mercury emissions feul/.

Karatza, D.; Lancia, A.; Musmarra, D., "Fly Ash Capture of Mercuric Chloride Vapors From Exhaust Combustion Gas", Environ.Sci.Technol.1998, 32, pg.3999-4004.

Kloeppel, J., "Unlikely Mix- Used Tires, Pistachio Shells-Can Clean Up Pollution", News Bureau University of Illinois At Urbana-Champaign. (2000). www.news.uiuc.edu/scitips/00/08enviro.html.

Meij, R. "Distribution of Trace Species in Power Plant Streams: A European Perspective", American Power Conference, Chicago, 1994.

Mendioroz, S; Guijarro, M.I.; Bermejo, P.J.; Munoz, V., "Mercury Retrieval from Flue Gas by Monolithic Absorbents Based on Sulfurized Sepriolite", Environ.Sci.Technol. 1999, 33, pg.1697-1702.

Mercury Study Report to Congress, EPA-452/R-96-001g, 1996.

Miller E.L. and Dobb E. David, "Speciation of Mercury in Soils by Sequential Extraction" www.epa.gov/nerlesd1/pdf-ecb/542asd95.pdf 
Morency, J.R., Panagiotou, T., Senior, C.L. "Laboratory Duct Injection of a Elite-Based Mercury Sorbent”, Physical Sciences Inc.

Nordin, A.; Schager, P,; Hall, B., “ Mercury Speciation in Flue Gases: A Comparison of Results from Equilibrium Calculations with Results from Laboratory Experiments", Swedish Fame Days, Turkoo, Finland, 1990.

Nriagu, J.O. Environment 1990, 32,7-33.

Tumati, P.R.; DeVito, M.S., "Partitioning Behavior of Mercury During Coal Combustion", Joint ASME/IEEE Power Conference, Kansas City, KS, 1993.

U.S. EPA (1997), "Mercury Study Report to Congress EPA-425/R-97-003”, U.S. EPA Office of Air Quality Planning and Standards.

Young, B.C.; Miller, S.J.; Laudal, D.L., "Carbon Sorption of Trace Mercury Species”, Proceedings of the $11^{\text {th }}$ Annual Pittsburg Coal Conference, 1994, pg. 575-580. 
Appendix 41: Development of Metallic Filters to Control Mercury From Coal Fired Power Plant Flue Gas (MT004) 


\section{FINAL TECHNICAL REPORT}

Contract Title and Number: Crosscutting Technology Development at the Center for Advanced Separation Technologies (DE-FC26-02NT41607)

Sub-Recipient Project Title:

DEVELOPMENT OF METALLIC FILTERS TO CONTROL MERCURY FROM COAL FIRED POWER PLANT FLUE GAS

Principal Investigators:

Dr. Kumar Ganesan

Contact Address:

Department of Environmental Engineering Montana Tech of The University of Montana 1300 West Park Street Butte, Montana 59701

Subcontractor Address:

"No subcontracts issued."
Period of Performance:

Starting Date: 2004

Ending Date: 2008
Report Information:

Type: $\quad$ Final Report

Number: 6

Period: $\quad 2004-2008$

Date: $\quad 11 / 12 / 2007$

Code: $\quad$ MT004

Contact Information:

Phone: 406-496-4239

Fax: 406-496-4650

E-Mail: kganesan@mtech.edu

$\underline{\text { Subcontractor Information: }}$ 


\title{
DEVELOPMENT OF METALLIC FILTERS TO CONTROL MERCURY FROM COAL FIRED POWER PLANT FLUE GAS
}

\author{
Final Report Submitted to: \\ The Center for Advanced Separation Technologies \\ Proposal Topic Area: \\ Solid-Solid Separation \\ $\square$ Solid-Liquid Separation \\ $\square$ Chemical/Biological Extraction \\ $\square$ Modeling and Control \\ $\mathrm{X}$ Environmental Control \\ (Please check ONLY one)

\begin{abstract}
Submitted by:
Dr. Kumar Ganesan

Professor and Head

Department of Environmental Engineering

Montana Tech of the University of Montana Tech

1300 West Park Street

Butte, Montana 59701
\end{abstract}

November 12, 2007

\section{Principal Investigator Endorsement}




\section{DISCLAIMER}

This report was prepared as an account of work sponsored by an agency of the United States Government. Neither the United States Government nor any agency thereof, nor any of their employees, make any warranty, express or implied, nor assume any legal liability or responsibility for the accuracy, completeness, or usefulness of any information, apparatus, product, or process disclosed, or represents that its use would not infringe privately owned rights. Reference herein to any specific commercial product, process, or service by trade name, trademark, manufacturer, or otherwise does not necessarily constitute or imply endorsement, recommendation, or favoring by the United States Government or any agency thereof. The views and opinions of authors expressed herein do not necessarily state or reflect those of the United States Government or agency thereof. 


\begin{abstract}
The coal-fired power plant industry has a great need for effective mercury control devices to reduce mercury emissions and comply with EPA regulations. The focus of this project is to develop cost effective filter to remove mercury vapor from industrial plumes. A metallic (patent approved) filtering system has been developed and tested based on initial funding from the US Department of Energy (DOE CAST 03). This work was again funded by DOE and supported further by the Montana Board of Research and Commercialization Technology for testing and to evaluate its economic feasibility.

Two different types of metallic filters were tested at Montana Tech of the University of Montana's mercury laboratory for mercury removal efficiency. Both the metallic filters showed above $90 \%$ efficiencies. Spent filters saturated with mercury were thermally desorbed. The mercury was recovered and the filter was simultaneously regenerated. After the thermal desorption the filters went through a second round of mercury removal efficiency testing and were successful in removing mercury. Field testing in a coal fired power plant proved that the metallic filter has great potential as a mercury control device. Additionally, the two different metallic filters were tested in series in the laboratory, and the results confirmed that the combined mercury removal efficiency of the filters were cumulative. Then preliminary tests were conducted in a coal fired flue gas stack at the PPL's Colstrip power plant in Montana. The mercury removal efficiency was at or above $90 \%$ for the entire six test days.

Unlike all other mercury control systems under investigation, where mercury is being transferred from a vapor phase to a solid phase, this metallic filter will not only remove mercury from the flue gas effectively but mercury can be recovered and prevented from entering into the environment. This is a very environmentally desirable mercury control option.
\end{abstract}




\section{TABLE OF CONTENTS}

\section{DISCLAIMER}

..............

ABSTRACT

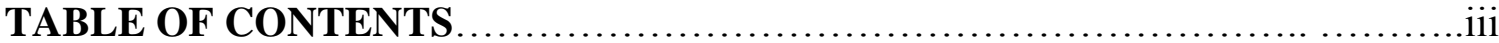

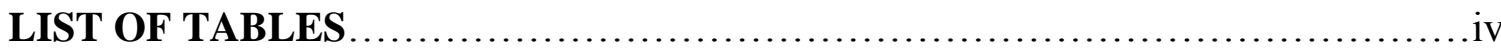

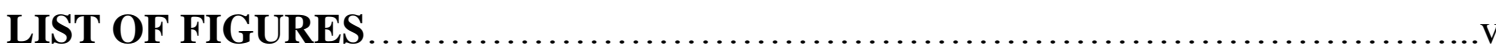

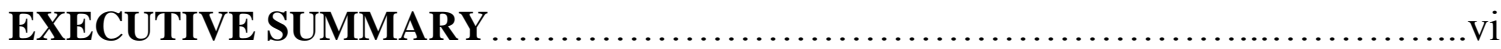

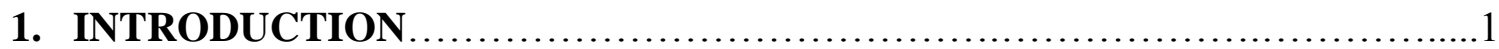

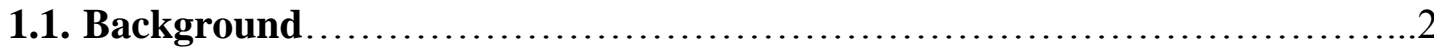

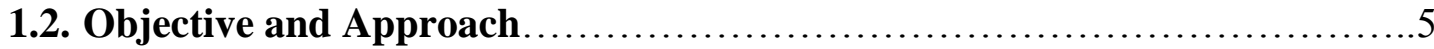

2. EXPERIMENTAL PROCEEDURES...........................................

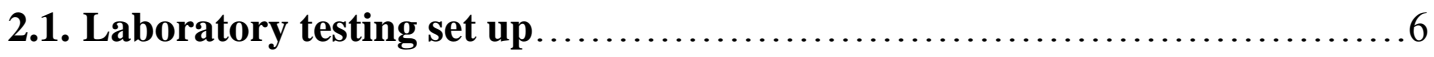

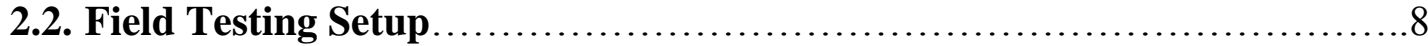

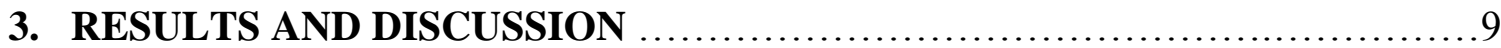

3.1. Laboratory Results - Metallic Filters in Series............................15

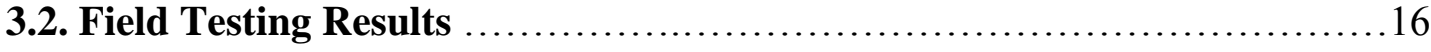

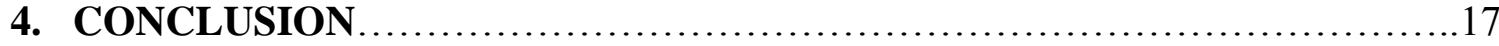

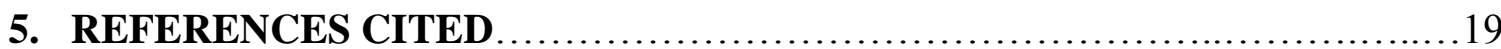




\section{LIST OF TABLES}

Number Title

Page

I. Mercury Control Option for Coal Fired Power Plants ........................4

II. Emerging Technologies and Mercury Removal Efficiency ....................4

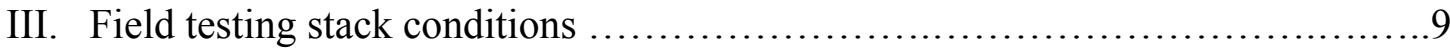




\section{LIST OF FIGURES}

Number

Title

Page

1. EPA- Sources of Human-Made Mercury in the U.S. (1995)....................1

2. Proposed Mercury Control Devices: Activated carbon injection ................ 3

3. Laboratory Experimental Setup ....................................6

4. Thermal de-sorption set up.........................................

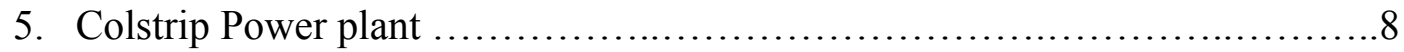

6. Mercury Removal Efficiency of Filter \# 1 ................................ 9

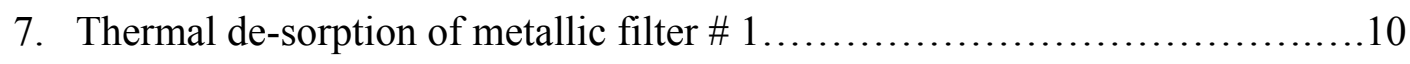

8. Mercury removal efficiency of filter $\# 1$ after thermal de-sorption .............. 10

9. Mercury removal efficiency of two different metallic filters ..................11

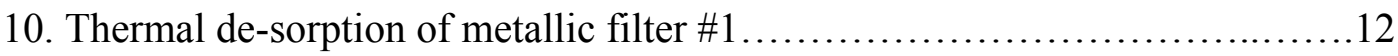

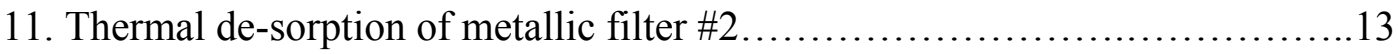

12. Mercury removal efficiency of metallic filters after $1^{\text {st }}$ thermal de-sorption....14

13. Mercury removal efficiency of metallic filters after $2^{\text {nd }}$ thermal de-sorption.... 15

14. Mercury removal efficiency of metallic filters in series (lab result............ 16

15. Mercury removal efficiency metallic filters in series at Colstrip............... 17 


\section{EXECUTIVE SUMMARY}

Montana Tech's patent pending mercury control device consists of metallic filters that have the potential to remove elemental mercury from flue gas of coal-fired power plants. Once the filter becomes saturated with mercury, the mercury can be easily removed through thermal de-sorption and collected in a mercury recovery system. This control device actually removes mercury from the system while regenerating the filters. The mercury removal efficiency of the metallic filters was evaluated in this project period.

The DOE's "CAST" 2003 project initially evaluated metallic wire, metal plated beads, and metallic sponge filters to remove mercury vapor and the experiments showed very encouraging results. Finally two different metallic filters, electroplated in-house as well as commercially metal plated were tested and the laboratory testing results consistently showed that the metallic filters remove elemental mercury from contaminated air with an efficiency greater than $90 \%$.

The goal of the mercury filter development was to achieve mercury removal efficiencies above $90 \%$ for the metallic filters, and all testing results were evaluated based on how well this $90 \%$ efficiency goal was met. Since previous test results confirmed that the metallic filters can remove mercury, next focus was to test the efficiency and longevity of these filters at their $90 \%$ efficiency.

All the laboratory experiments were conducted at Montana Tech of the University of Montana's Mercury Laboratory. The filters were exposed to between $20 \mu \mathrm{g} / \mathrm{m}^{3}$ and $30 \mu \mathrm{g} / \mathrm{m}^{3}$ mercury concentrations at $5 \mathrm{~L} / \mathrm{min}$ flow rate. Initial test with filter \# 1 showed above $90 \%$ mercury removal efficiency for 68 hours. Thermal de-sorption was performed to release the captured mercury and to regenerate the filter. The results showed that the thermal de-sorption process occurred at low temperatures $\left(150^{\circ} \mathrm{F}\right)$ and increasing the temperature further influenced the process.

After the first mercury removal efficiency tests, the metallic filters went through thermal de-sorption to release the mercury captured by the filters. After thermal de-sorption both the filters were retested separately for their mercury removal efficiency. The results again showed a mercury removal efficiency of above $90 \%$ for both the filters. The filters then went through a second round of thermal de-sorption and mercury removal efficiency testing. The $90 \%$ mercury removal efficiency of the filter \#1 and \#2 lasted about 27 hours and 3 hours instead of 68 hours and 20 hours respectively.

Because of the relatively high cost associated with the filter \#1, both the filters in series were investigated to determine their combined mercury removal efficiency. The results showed excellent mercury removal for the filters with the efficiency above $90 \%$ for 192 hours.

Then to assess the real world performance of the filters, the filters in series were field tested at the PPL's coal fired power plant in Colstrip, Montana. Tests were conducted for six consecutive days and the mercury removal efficiency was at or above $90 \%$ for the 
entire six days test in the field when exposed to actual power plant flue gas. These field test results again showed that the metallic filters in series are capable of removing mercury at low concentrations; and also indicated the $\mathrm{SO}_{2}, \mathrm{NOx}$, and other compounds that present in the flue gas have minimal, if any effect on the mercury removal efficiency of the metallic filters. 


\section{INTRODUCTION}

Based on 1995 preliminary data from the U.S. Environmental Protection Agency (EPA), the principal human-caused sources of mercury emissions in the United States are coalfired power plants (33 percent of the total emitted), municipal waste incinerators (19 percent), and medical waste incinerators (10 percent). (See Figure1)

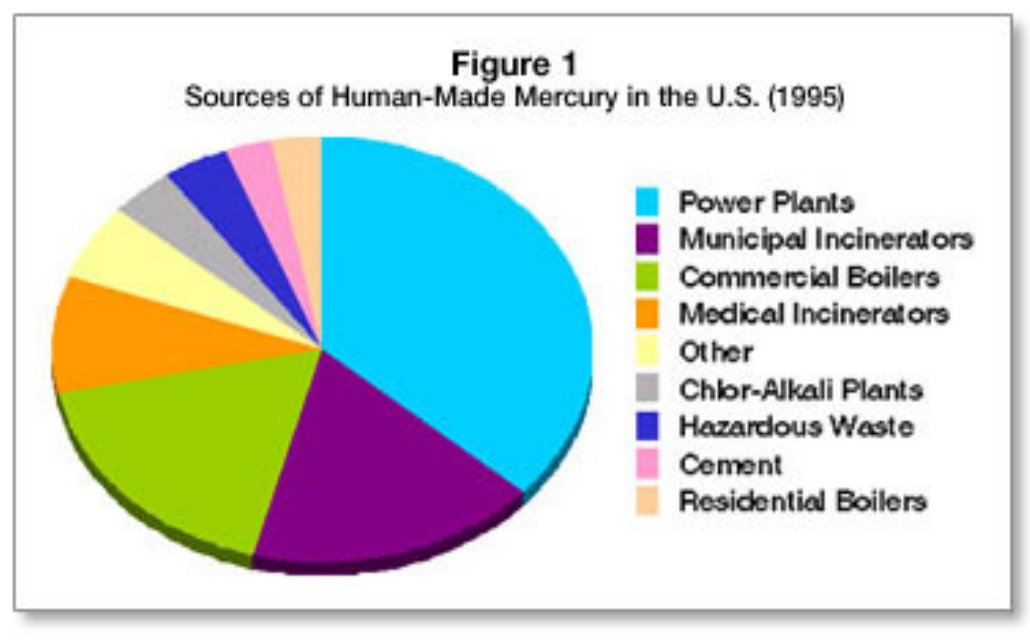

Figure 1: EPA- Sources of Human-Made Mercury in the U.S. (1995)

Mercury is at the top of the EPA's list among the air toxic metals addressed in the 1990 Clean Air Act Amendments. Mercury is a high-priority regulatory concern because of its persistence and bioaccumulation in the environment and its neurological health impacts. The largest anthropogenic source of mercury in the United States is coal-fired power plants, which release 50 tons of mercury annually or about one-third of the total anthropogenic emissions (EPA, 1997). As a result, coal-fired power plants are the focus of new EPA mercury regulations.

The US EPA announced its intention to rulemaking in 2003 to regulate power plant mercury emissions. The final regulations are expected in 2004, and compliance is projected for 2007. The US EPA is proposing two alternatives for controlling mercury emissions from coal-fired power plants, maximum achievable control technology (MACT) standards and a market-based cap-and-trade system. The MACT standards would reduce nationwide mercury emissions by $29 \%$ by the end of 2007 , while the capand-trade system will reduce mercury emissions by $69 \%$ by the end of 2018 (EPA, 2004). This requirement will have significant financial implications for power plants. Therefore, developing cost effective control technologies to remove mercury will become increasingly critical. 
Currently, there is no single best mercury control technology that can be broadly applied across the power plant industry. The proposed EPA mercury regulations for coal-fired power plants will have a significant financial impact on the industry; and there is a clear need for developing cost effective technologies to remove mercury from the flue gas not only to comply with the proposed standards, but also to protect the environment and human health from exposure to mercury.

\subsection{Background}

Mercury is present in three different species upon the combustion of coal: elemental mercury vapor, mercury (II), and particulate mercury. Elemental mercury vapor is the pure mercury gas released during combustion, mercury (II) results from the oxidation of elemental mercury by various elements in the flue gas, and particulate mercury forms when elemental mercury or mercury (II) attaches to solid particles (fly ash) in the flue gas.

The species of mercury present in the flue gas is determined by the chemical composition of the coal burned. Eastern bituminous coals have high mercury and chlorine contents and produce a flue gas predominately composed of mercury (II). On the other hand, western sub-bituminous and lignite coals have low mercury and chlorine contents and produce a flue gas with elemental mercury as the dominant species. Coal chlorine content is the main factor that affects the speciation of mercury upon combustion, due to its ability to oxidize elemental mercury to $\mathrm{HgCl}_{2}$. Other elements in the coal including sulfur, calcium, and iron impact the speciation of mercury, but to a lesser degree than chlorine. There is a great deal of research being conducted on the mechanisms of mercury-chlorine chemistry and other mercury speciation reactions that occur upon the combustion of coal.

There is little control over the amount of particulate mercury generated in the flue gas of coal-fired power plants (boiler operation has some effect). However, particulate mercury is easily removed in a bag-house or electrostatic precipitator (ESP) and is generally present in minimal amounts. Mercury (II) is also easily controlled if the power plant is equipped with a wet scrubber, because it is water-soluble. Therefore the major concern and challenge is to remove elemental mercury from the flue gas of coal-fired power plants that burn western sub bituminous and lignite coals.

Currently, there is no commercially available control technology to remove mercury from coal-fired power plants. Activated carbon injection is considered by most as the leading mercury control technology currently available for coal-fired power plants where mercury adsorbs to the carbon and is collected by an ESP or a baghouse (Figure 2). 


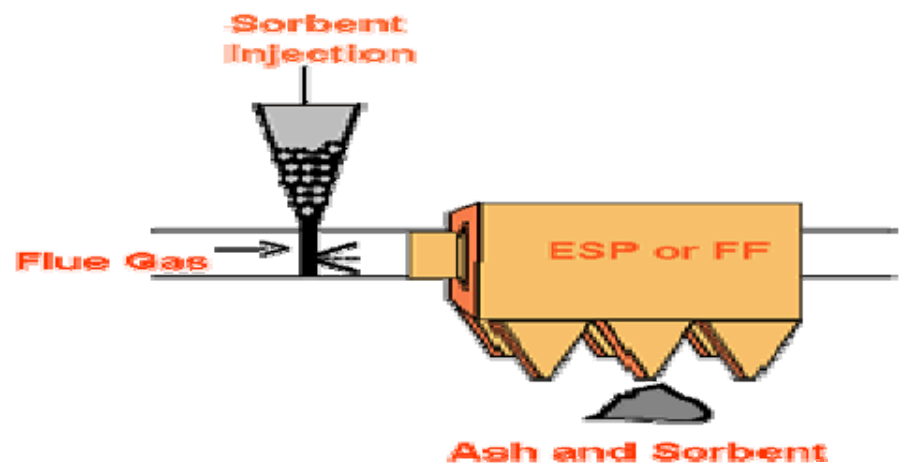

Figure 2: Proposed Mercury Control Devices: Activated carbon injection

Carbon injection may be effective, but mercury is not removed from the system; it is simply changed from the vapor form to being incorporated in the carbon. Having the fly ash with mercury mediated carbon can cause great problems for the power plant. The fly ash is a useful byproduct that can be utilized by other industries such as the cement industry. When the fly ash is contaminated with mercury, it can no longer be sold and now has to be disposed of in a proper manner. The same goes for technologies that try to oxidize elemental mercury to mercury (II) before the flue gas enters the wet scrubber. The mercury (II) removed by the wet scrubber is still present in the scrubber sludge. It is estimated that existing pollution control devices (scrubbers, ESP, bag-houses) capture about $40 \%$ of mercury emissions produced by coal-fired power plants (Pavlish et al., 2003).

Several control technologies are being studied. They mainly focus on pre-combustion and post combustion processes. Pre-combustion involves coal-cleaning technologies to reduce mercury burden at the source. Post combustion involves adsorption by carbonbased materials and conversion of mercury vapor to oxidized form for removal by wet system or particulate filters.

A pre-combustion technique such as coal cleaning is an excellent way to remove mercury from even getting into the combustion cycle. However, only about $20 \%$ of the mercury could be removed by this process. The economics of coal cleaning to get rid of the majority of mercury appears to be very unrealistic. Therefore other techniques must be explored to address the mercury removal problem.

It is reported that $90 \%$ of mercury emitted from burning western coal is in the form of mercury vapor (elemental) while for eastern coal it is $60 \%$ elemental mercury and $40 \%$ divalent mercury.

The traditional control devices like electrostatic precipitators (ESP), bag houses (BH), and flue gas desulphurization systems (FGD) are capable of removing mercury from the coal fired flue gas in varying amounts ranging from $24-34 \%$. However, only $15 \%$ of the plants have FGD systems, and $16 \%$ have $\mathrm{BH}$. The best control device that helps to oxidize mercury to ionic mercury form is the selective catalytic reduction system (SCR). 
Currently SCR systems are used for NOx control in power plants. Again only less than $40 \%$ of the plants in the US have SCR systems, refer to Table 1.

TABLE 1: Mercury Control Option for Coal Fired Power Plants

\begin{tabular}{|c|c|c|}
\hline Control Technique/Technology & $\begin{array}{c}\text { Potential } \\
\text { Removal }\end{array}$ & $\begin{array}{c}\text { Percentage of Utilities } \\
\text { Adapting the Technology }\end{array}$ \\
\hline Coal Cleaning (Pre-combustion & $21 \%$ & $77 \%$ of Eastern Coal \\
\hline Electrostatic Precipitator & $24 \%$ & $70 \%$ of Utilities \\
\hline Fabric Filter & $28 \%$ & $16 \%$ of Utilities \\
\hline Flue Gas Desulfurization & $34 \%$ & $15 \%$ \\
\hline $\begin{array}{c}\text { Selective Catalytic } \\
\text { Reduction(SCR) }\end{array}$ & $60-80 \%$ & $<40 \%$ \\
\hline
\end{tabular}

The potential techniques and technologies available and emerging for mercury reduction are summarized in Table II. The carbon technology works well, however, at elevated temperature and low mercury levels $\left(10-15 \mathrm{ug} / \mathrm{m}^{3}\right)$ the mass transfer rates will be significantly lower. In order to compensate this poor mass transfer rate, an excess amount of carbon is needed to get the mercury removal rate. It is reported in the mercury study report to Congress that this will be about $\$ 5,000$ to $\$ 70,000$ per pound of mercury removed. In addition, the carbon in the fly ash will reduce the marketability of fly ash.

\section{TABLE II: Emerging Technologies and Mercury Removal Efficiency}

\begin{tabular}{|c|c|}
\hline Technology & Potential Mercury Removal Efficiency \\
\hline Carbon Injection & $80-98 \%$ \\
\hline Carbon Filter Beds & $90 \%$ \\
\hline Condensing Heat Exchangers & $84 \%$ \\
\hline Fuel Switching & $>95 \%$ \\
\hline Selenium Filter & $>99 \% *$ \\
\hline Metallic Filters & $>90 \%$ \\
\hline *For High Concentrations of Mercury \\
\hline
\end{tabular}

The carbon filter beds are used in other countries for removing organic compounds that can also capture mercury vapor very effectively. But it is too expensive to implement in power plant systems. The condensing heat exchangers are not only expensive but not that efficient. In Sweden selenium filters have been tested to remove high concentrations 
of mercury and it may not work for low levels of mercury in the range of $15 \mu \mathrm{g} / \mathrm{m}^{3}$. Fuel switch, that is, switching to natural gas from coal will substantially eliminate the mercury problem but it is not realistic.

The most popular approach currently is to convert the elemental mercury to $\mathrm{Hg}^{2+}$ form which is much easier to collect than the mercury vapor. The EERC in Grand Forks, ND; DOE's NETL in Pittsburg, PA; EPRI, CA, and EPA RTP, NC have done studies to show that power plants with NOx control devices have converted $60-70 \%$ of elemental mercury to $\mathrm{Hg}^{2+}$ with and without ammonia injection. The power plants with FGD systems will be very effective in removing the $\mathrm{Hg}^{2+}$. However the amount being converted depends heavily on the type of coal burned. The most important drawback in this approach is that the mercury is still in the system. It is now in a form that is easily leachable and may cause problems in the future through waste sludge or by-products.

Montana Tech's patent pending mercury control device consists of metallic filters that have the potential to remove elemental mercury from the flue gas of coal-fired power plants. Once the filter becomes saturated with mercury, the mercury can be easily removed through thermal de-sorption and collected in a mercury recovery system. This control device actually removes mercury from the system with the end result being mercury concentrate, which can be utilized or sold by the power plant. The mercury removal efficiency of the metallic filters was evaluated in this project period.

The main advantage the metallic filters have over other proposed mercury control options is that the metallic filters completely remove mercury from the system. The mercury vapor is captured by the metallic filter, and then recovered through a thermal de-sorption system. There is no contaminated scrubber sludge or fly ash byproduct to dispose off after the mercury is captured. The end result is that mercury concentrate is recovered and prevented from further entering the environment.

The DOE's "CAST" program has funded the project in 2002 to test the effectiveness of metallic filters to remove mercury from coal fired power plants. The initial tests completed with gold wire, silver and gold plated beads showed very encouraging results.

Again because of funding from the DOE, this project continued to test filter efficiency for mercury removal. Two different metallic filters, electroplated in-house as well as commercially metal plated were tested and the laboratory testing results consistently showed that the metallic filters remove elemental mercury from contaminated air with an efficiency greater than $90 \%$. Further this work was supported by the Montana Board of Research and Commercialization Technology (MBRCT).

\subsection{Objective and Approach}

The main objective of this research project is to develop and test a bench scale metallic filter to remove mercury vapor cost effectively from coal fired flue gas. The goal of the laboratory testing was to achieve mercury removal efficiencies above $90 \%$ for the 
metallic filters, and all testing results were evaluated based on how well this $90 \%$ efficiency goal was met. Three types of metallic filters were tested individually. Then two different metal plated filters were selected and tested. Also two different filters in series proved their effectiveness in reducing mercury vapor from contaminated gas. Spent filters were successfully thermally desorbed and tested again for mercury removal efficiency. The testing of the filters in series indicated that the filters have the ability to remove mercury even at low concentrations. Finally the metallic filters were field tested in a flue gas stream of a coal fired power plant burning western coal.

\subsection{EXPERIMENTAL PROCEDURES}

The laboratory experiments were conducted at Montana Tech of the University of Montana Mercury Laboratory. The experimental set up consisted of a mercury vapor generator, a pump, the filter holder and the outlet set up. The required inlet mercury concentrations were produced by the mercury generator. The outlet air was sent through a carbon filter before released into the hood. The entire experimental setup was under the fume hood.

\subsection{Laboratory testing set up}

To perform the bench scale testing in the laboratory, each metallic filter was placed in a stainless steel tube, and mercury-contaminated air was passed through the filter at a flow rate of $5 \mathrm{~L} / \mathrm{min}$ (Figure 3). A mercury vaporizing system that produced a constant concentration of elemental mercury was used as the mercury source, and the mercury concentration was kept between $20 \mu \mathrm{g} / \mathrm{m}^{3}$ and $30 \mu \mathrm{g} / \mathrm{m}^{3}$. The mercury vaporizing system contains a mercury permeation tube located within a hot cell. By accurately controlling the temperature of the hot cell and permeation tube, a known and repeatable concentration of mercury vapor was generated.

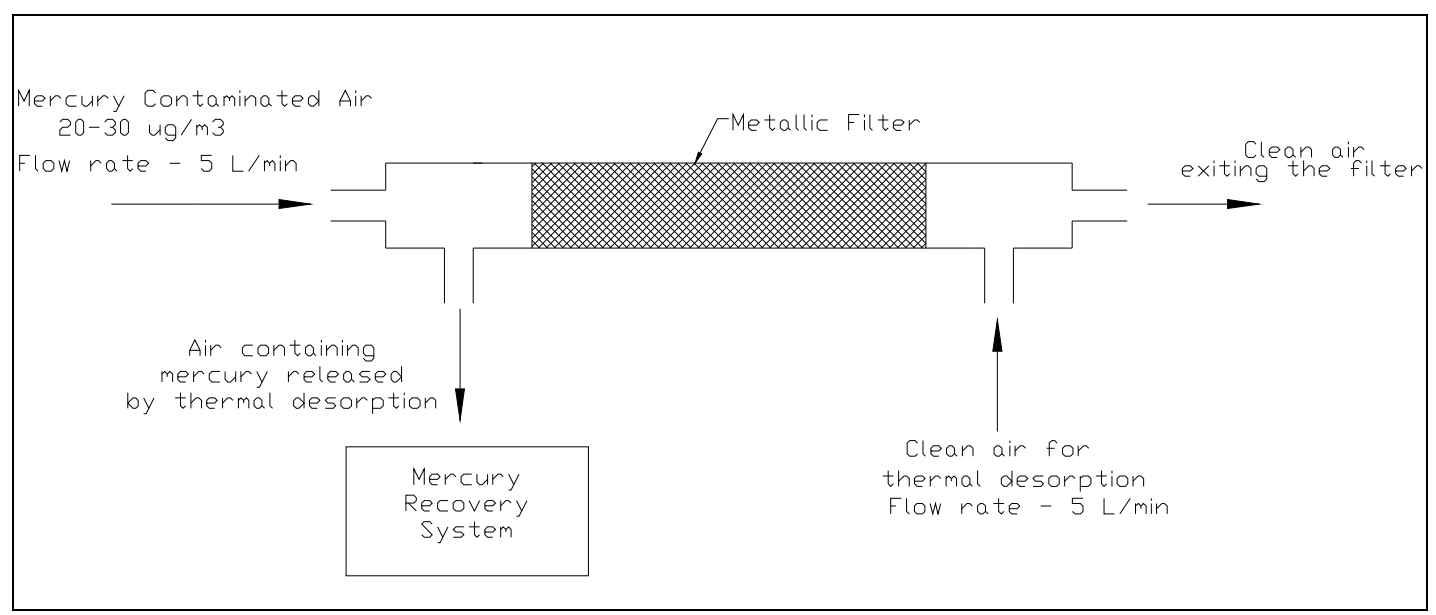

Figure 3: Laboratory Experimental Setup 
Throughout the test period, the filter's inlet and outlet mercury concentrations were measured with a portable mercury analyzer, Mercury Tracker, which is accurate down to $0.1 \mu \mathrm{g} / \mathrm{m}^{3}$. These measurements were used to calculate the mercury removal efficiency of the filters. Once the mercury removal efficiency of the metallic filter dropped to $50 \%$, the test was terminated and the filters went through a thermal de-sorption process. All testing results were evaluated based on how well the $90 \%$ efficiency goal was met. The mercury removal efficiency testing of the filters in series was also done with the same experimental setup except the filters were placed in a PVC pipe rather than the stainless steel pipe, due to the larger size requirement of the filters. The PVC pipe had the same diameter as the stainless steel pipe to maintain the same air velocity but the length of the PVC pipe was increased to hold few filters.

The thermal de-sorption process consisted of heating the saturated metallic filter to volatilize the captured mercury vapor, which was then collected in a mercury recovery system. To perform thermal de-sorption in the laboratory, the stainless steel tubing that holds the metallic filter was wrapped in heat tape, and clean air was passed through the metallic filter at a flow rate of $5 \mathrm{~L} / \mathrm{min}$ in the opposite direction. Figure 4 shows the thermal de-sorption set up.

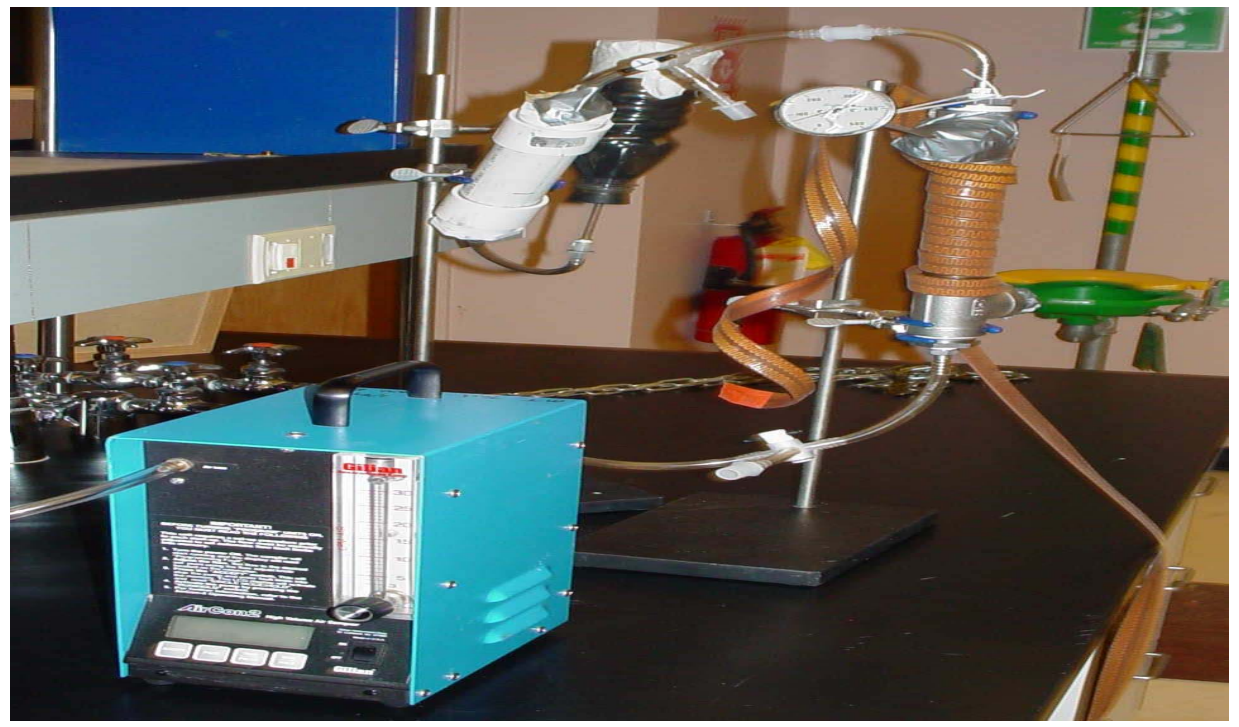

Figure 4: Thermal de-sorption set up

The temperature of the heat tape was gradually increased while the mercury concentration leaving the metallic filter was measured. The mercury recovery system used in the lab to capture the released mercury was a series of carbon filters. The entire experiment took place under a fume hood for added safety. The thermal de-sorption process was continued until the mercury concentration leaving the metallic filter was below $0.1 \mu \mathrm{g} / \mathrm{m}^{3}$. 


\section{$\underline{\text { 2.2 Field Testing Setup }}$}

The Colstrip powerplant (Figure 5) burns sub bituminous Powder River Basin coal, when combusted it results in $\mathrm{Hg}^{\circ}$ being the dominant species of mercury. The coal burned has an average moisture content of $26 \%$ and an average sulfur content of $0.72 \%$. The power plant is equipped with a wet scrubber, but has no mercury control devices in place.

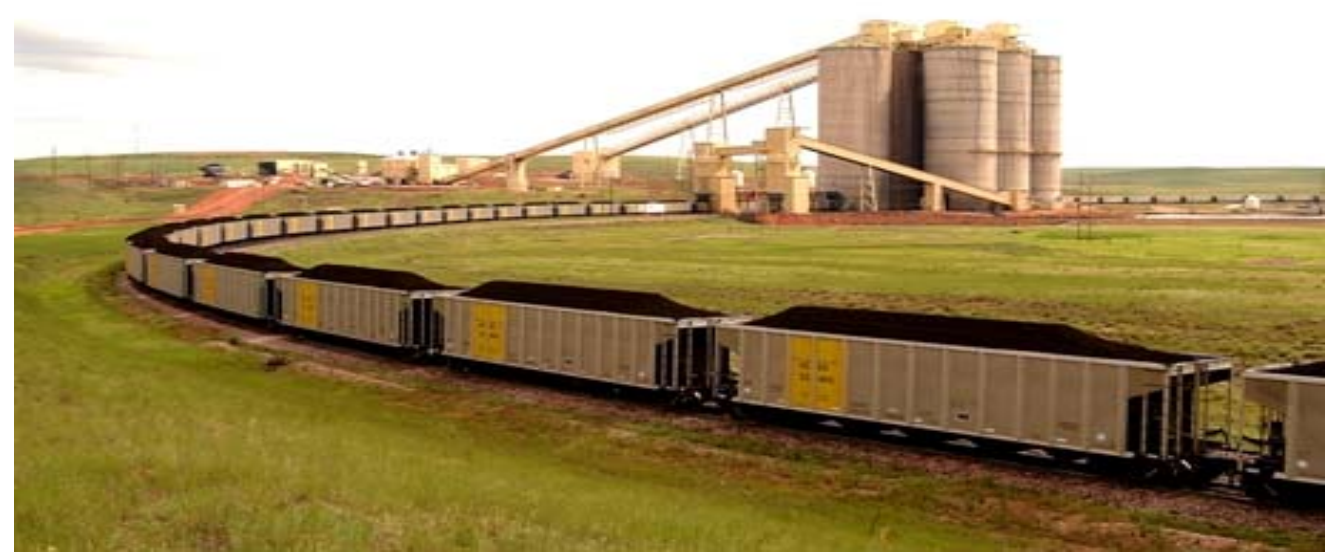

Figure 5 Colstrip Powerplant

Flue gas was drawn out of the stack (downstream of the wet scrubber and the reheater) just before it was emitted to the atmosphere. The sampling location greatly reduced the presence of undesirable gaseous compounds in the flue gas that may affect filter performance, mainly $\mathrm{SO}_{2}$. The flue gas was passed through a series of four impingers to condense and remove any water vapor before it entered the filters. The impingers were kept in an ice bath and the first three impingers had $100 \mathrm{ml}$ of water in them while the fourth was empty. The sample flow rate in the filter was $5 \mathrm{~L} / \mathrm{min}$.

In the field the filter's inlet and outlet mercury concentrations were measured with the Mercury Tracker throughout the test period to calculate the mercury removal efficiency of the filter. As recommended by the manufacturer, a gold trap filter was used in conjunction with the Mercury Tracker to eliminate any measurement errors caused by compounds in the flue gas (sulfur in particular). The mercury concentration readings taken with the gold trap filter were subtracted from the readings taken without the gold trap filter to get the true mercury concentration reading from the Mercury Tracker. Table III below shows the stack conditions during the field testing. 
Table III: Field testing stack conditions.

\begin{tabular}{|l|l|l|l|}
\hline \multicolumn{4}{|c|}{ Colstrip, MT Stack Properties } \\
\hline $\mathrm{SO}_{2}$ & $35.0 \mathrm{ppm}$ & Opacity & $15.50 \%$ \\
\hline $\mathrm{NOx}$ & $200 \mathrm{ppm}$ & Temperature & $190^{\circ} \mathrm{F}$ \\
\hline $\mathrm{CO}_{2}$ & $10.8 \%$ & Flow Rate & $1,925,000 \mathrm{scf} / \mathrm{min}$ \\
\hline
\end{tabular}

Based on the results of these tests the best performing metallic filter will be used to build a bench scale model to be tested in a flue gas stream of a coal fired power plant burning western coal.

\subsection{RESULTS AND DISCUSSION}

The preliminary mercury removal efficiency test of the metallic filter \# 1 was performed at Montana Tech Mercury laboratory. The inlet mercury concentration was held between $20 \mu \mathrm{g} / \mathrm{m}^{3}$ and $30 \mu \mathrm{g} / \mathrm{m}^{3}$ and the flow rate was kept at $5 \mathrm{~L} / \mathrm{min}$. The results showed a mercury removal efficiency of above $90 \%$ (Figure 6) for the metallic filter \#1.

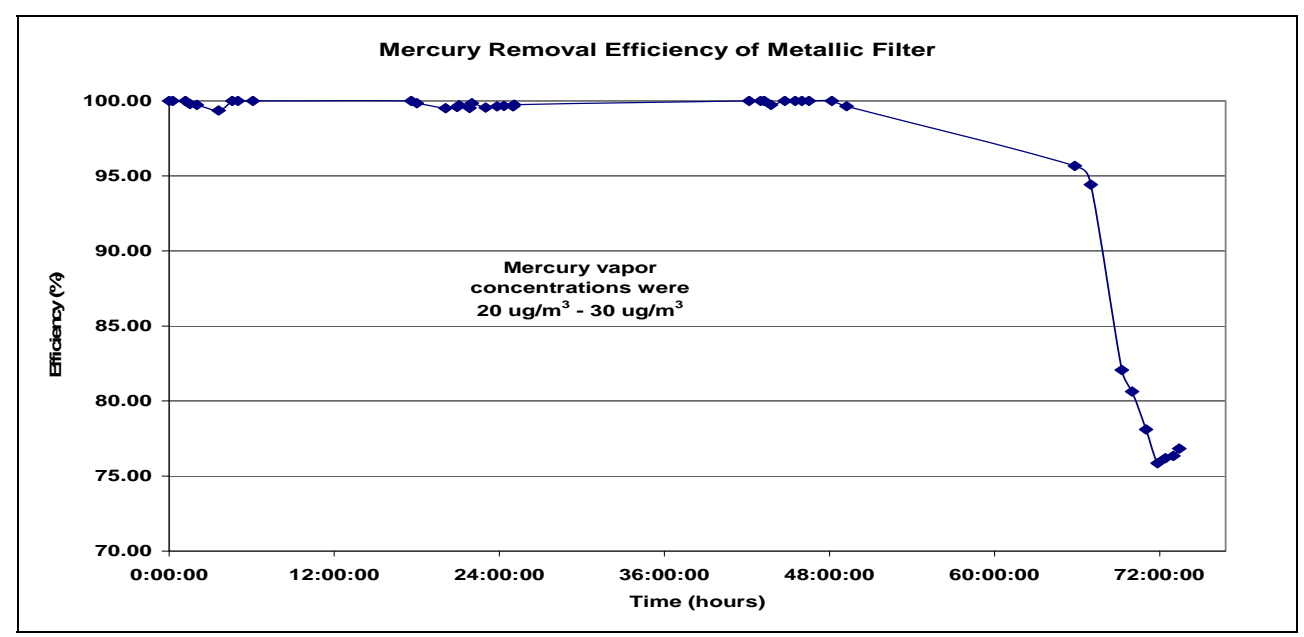

Figure 6: Mercury Removal Efficiency of Filter \# 1

As Figure 6 shows, the metallic filter \# 1 had a mercury removal efficiency of above $90 \%$ for nearly three days ( 68 hours) before gradually declining as the metallic filter became saturated with mercury.

After the first mercury removal efficiency test, the metallic filter went through thermal de-sorption to release the mercury captured by the filter. The airflow rate was kept at 
$5 \mathrm{~L} / \mathrm{min}$. The results showed that the thermal de-sorption process occurred at low temperatures $\left(150^{\circ} \mathrm{F}\right)$, but the rate at which mercury was released increased dramatically with increasing temperature (Figure 7).

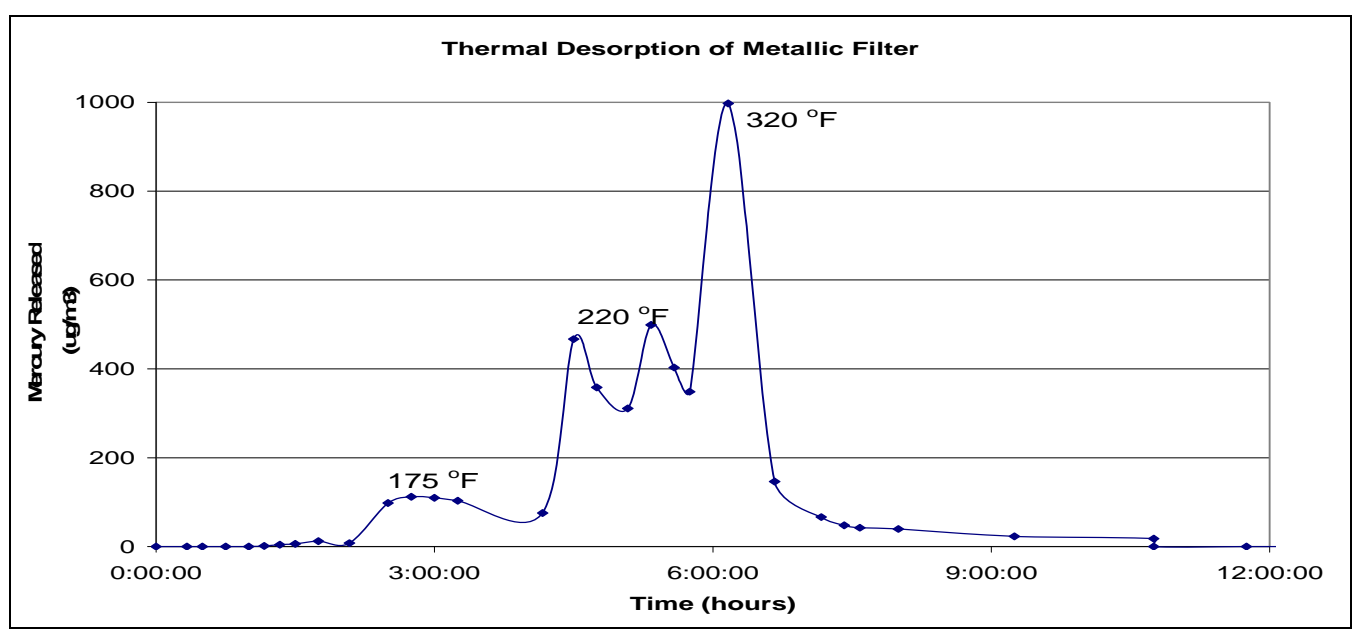

Figure 7: Thermal de-sorption cycle of metallic filter \# 1

After the thermal de-sorption was completed, the metallic filter \# 1 was tested for its mercury removal efficiency for the second time. As with the first test, the inlet mercury concentration was held between $20 \mu \mathrm{g} / \mathrm{m}^{3}$ and $30 \mu \mathrm{g} / \mathrm{m}^{3}$ and the flow rate was kept at $5 \mathrm{~L} / \mathrm{min}$. The results again showed a mercury removal efficiency of above $90 \%$ after the thermal de-sorption (Figure 8).

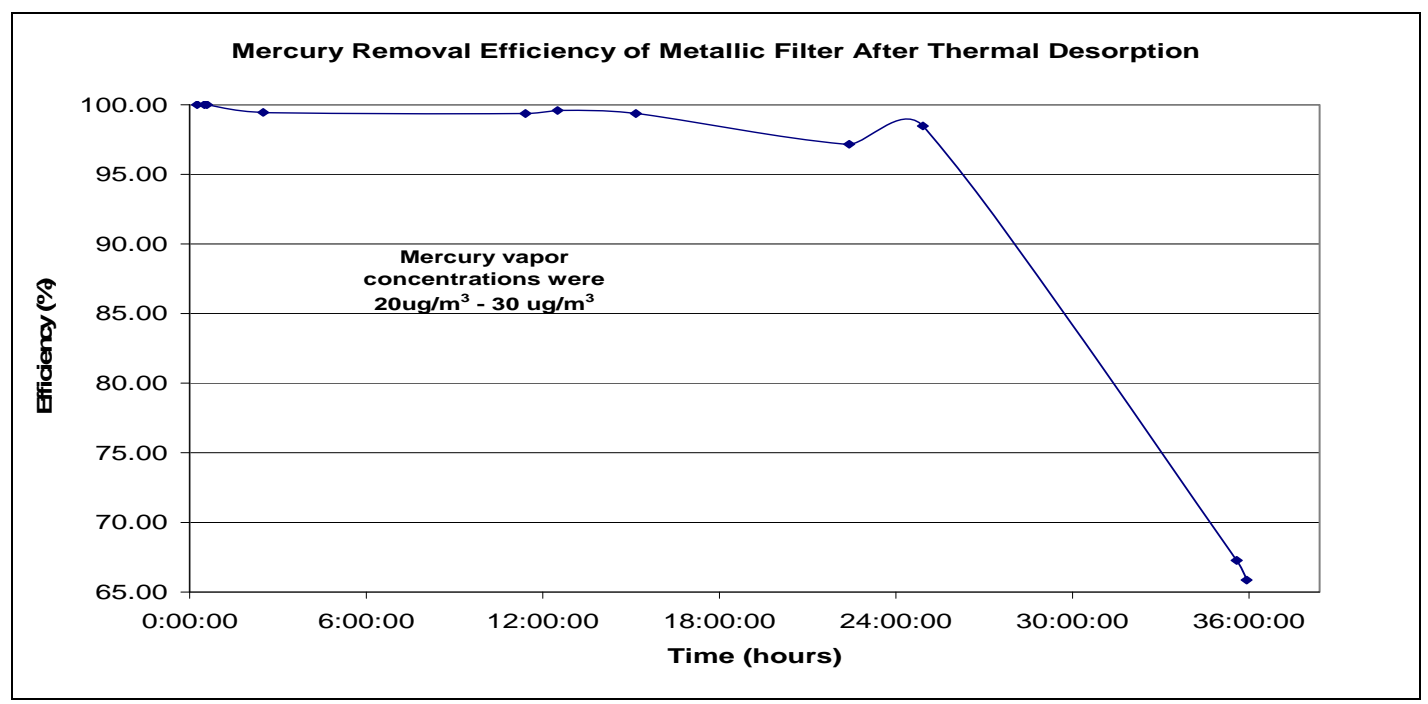

Figure 8: Mercury removal efficiency of filter \#1 after thermal de-sorption 
After thermal de-sorption, the mercury removal efficiency of the metallic filter \# 1 was still above $90 \%$, but the efficiency did not stay above $90 \%$ for as long ( 27 hours after thermal de-sorption versus 67 hours before thermal de-sorption).

Thus the preliminary laboratory results clearly indicated that the metallic filter removes elemental mercury from contaminated air with greater than $90 \%$ efficiency. Also the captured mercury can be released from the metallic filter through a simple thermal desorption process, after which the metallic filter can be used again to achieve greater than $90 \%$ mercury removal efficiency.

Since the metallic filter showed great promise as a mercury control device for coal fired power plants, again two different metallic filters were tested separately in the laboratory following the similar procedures with the inlet mercury concentration being held between $20 \mu \mathrm{g} / \mathrm{m}^{3}$ and $30 \mu \mathrm{g} / \mathrm{m}^{3}$ and at $5 \mathrm{~L} / \mathrm{min}$ flow rate. Also thermal de-sorption and retesting procedures were performed on these metallic filters.

Two different filters were professionally metal plated and tested separately for mercury removal efficiency. The results showed a mercury removal efficiency of above $90 \%$ for both of the metallic filters tested (Figure 9).

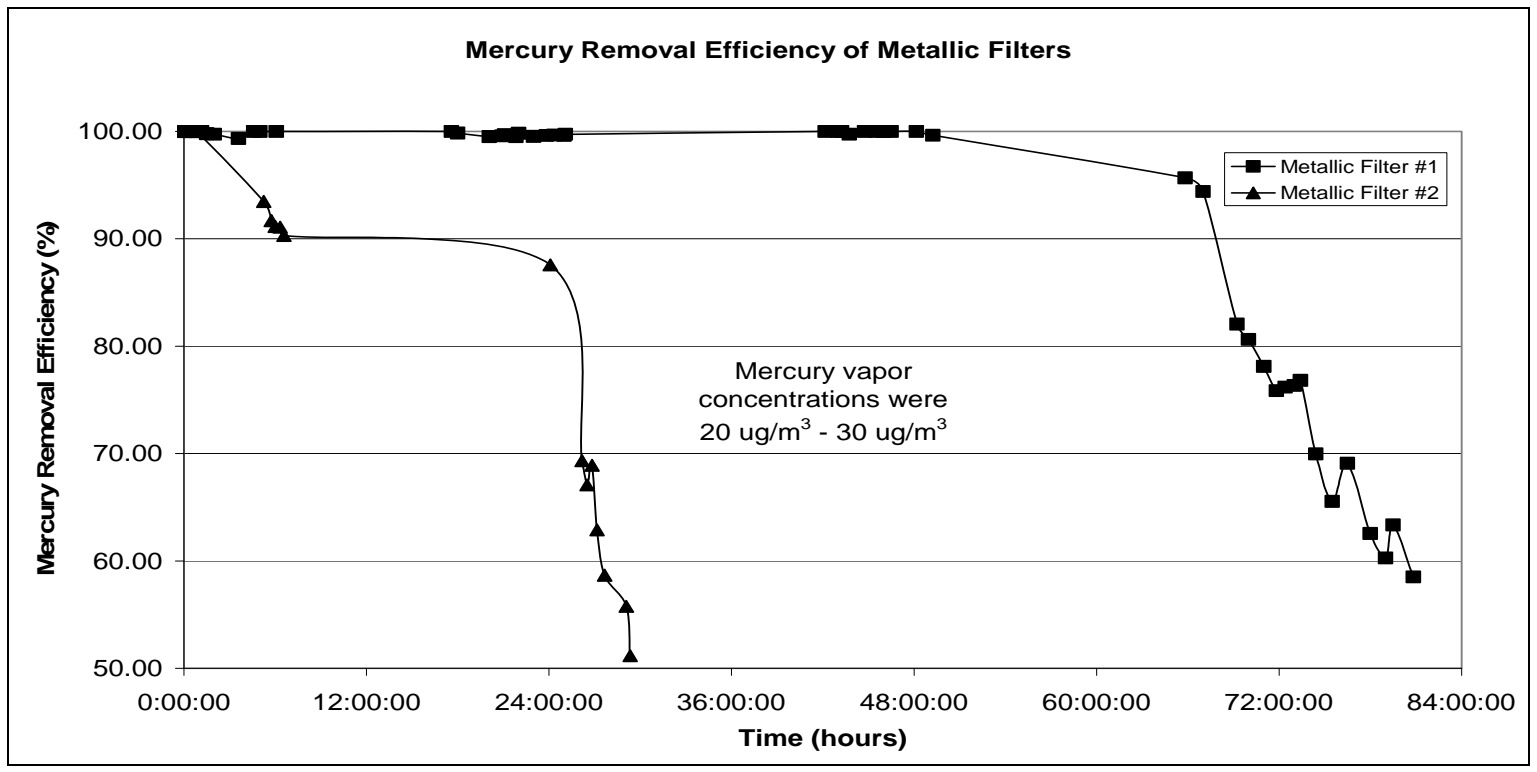

Figure 9: Mercury removal efficiency of two different metallic filters.

As Figure 9 shows, metallic filter \#1 had a mercury removal efficiency of above $90 \%$ for nearly three days ( 68 hours) before gradually declining as the filter became saturated with mercury. The mercury removal efficiency of metallic filter \#2 stayed above $90 \%$ for about 1 day (over 20 hours). Filter \#2's efficiency also declined more rapidly when 
compared to filter \#1's. It took filter \#1 nearly 90 hours to reach $50 \%$ mercury removal efficiency, whereas filter \#2's efficiency dropped to $50 \%$ in 30 hours. The faster decline in filter \#2's efficiency may be caused by the formation of oxides on the surface of the filter. Future work on the project includes determining an effective surface treatment for filter \#2 to prevent the formation of oxides.

After the first mercury removal efficiency tests, the metallic filters went through thermal de-sorption to release the mercury captured by the filters. The flow rate of the clean air was kept at $5 \mathrm{~L} / \mathrm{min}$. Figures 10 and 11 show the result of thermal de-sorption of each metallic filter to release the mercury captured by the filter. The results showed that the thermal de-sorption process occurred at low temperatures $\left(150^{\circ} \mathrm{F}\right)$, but the rate at which mercury released increased dramatically with increasing temperature.

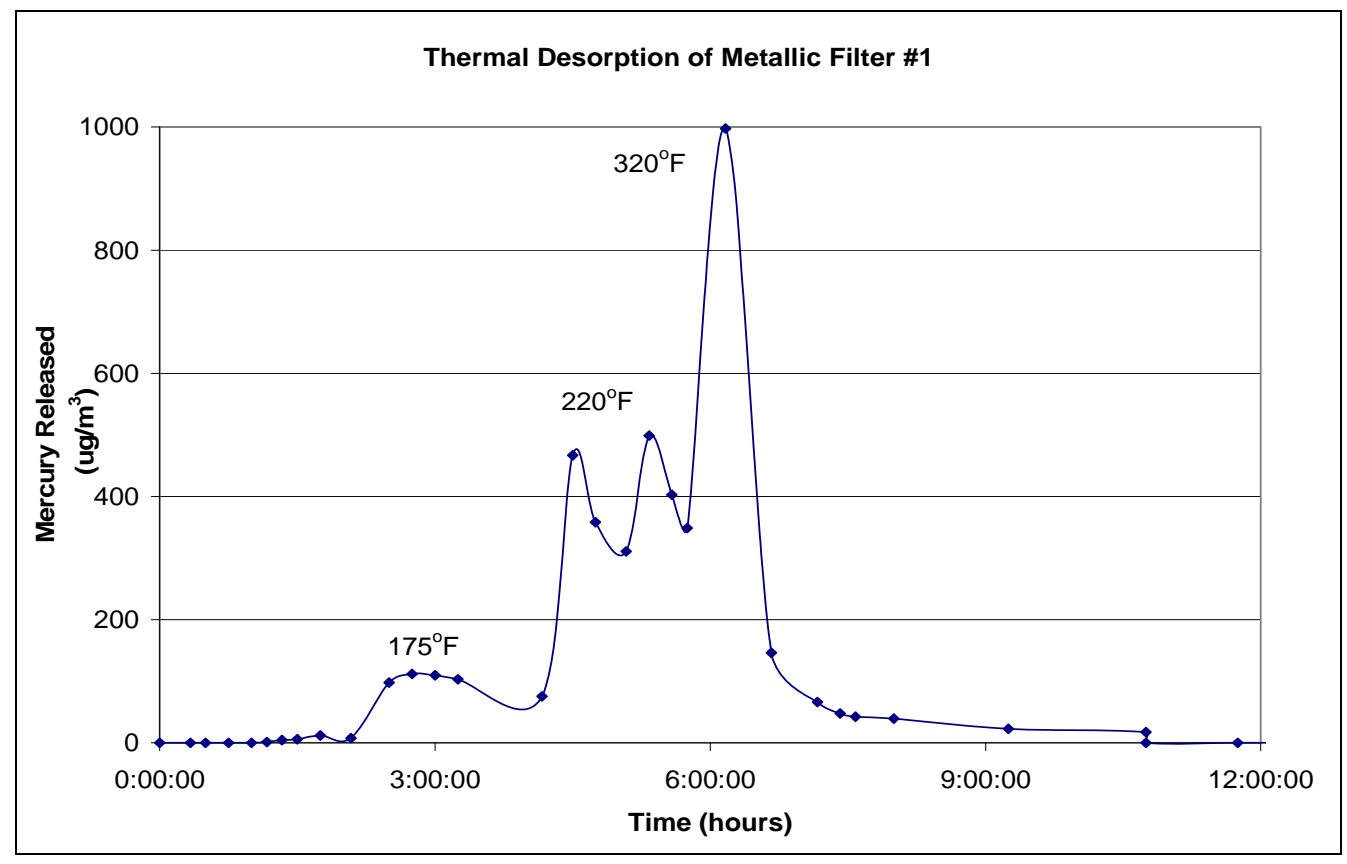

Figure 10: Thermal de-sorption of metallic filter \#1.

During the thermal de-sorption of metallic filter \#1, the captured mercury was released from the filter in highly concentrated puffs as the temperature was increased, but the mercury concentration being released would then decline as the temperature stabilized. When the temperature was raised above $300^{\circ} \mathrm{F}$, the concentration of mercury being released from filter \#1 peaked at $1,000 \mu \mathrm{g} / \mathrm{m}^{3}$ before declining. The duration of the 
thermal de-sorption process can be dramatically shortened by increasing the temperature and flow rate.

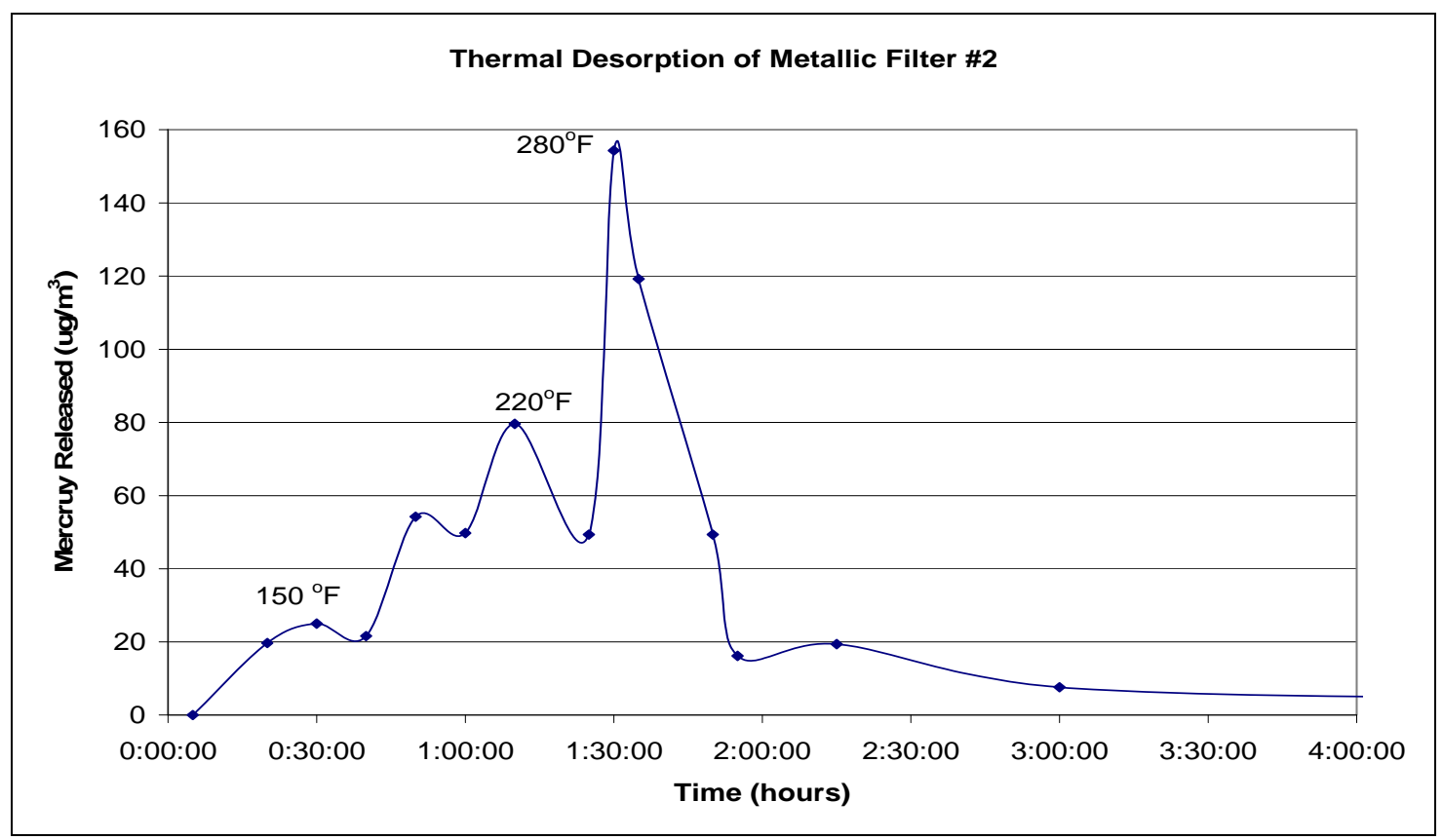

Figure 11: Thermal de-sorption of metallic filter \#2

The thermal de-sorption of metallic filter \#2 showed the same characteristics as did filter \#1 in that the mercury was released in concentrated puffs, but the concentration of the puffs was somewhat smaller for filter \#2. The smaller concentrations released could be due to the fact that filter \#2 captured less mercury than filter \#1 or it may be that the mercury concentration readings that were taken simply missed the big spikes in mercury being released from filter $\# 2$.

After thermal de-sorption was completed, the metallic filters were tested for their mercury removal efficiency for a second time. As with the first test, the inlet mercury concentration was held between $20 \mu \mathrm{g} / \mathrm{m}^{3}$ and $30 \mu \mathrm{g} / \mathrm{m}^{3}$, and the flow rate was kept at $5 \mathrm{~L} / \mathrm{min}$. The results again showed a mercury removal efficiency of above $90 \%$ for both filters (Figure 12). 


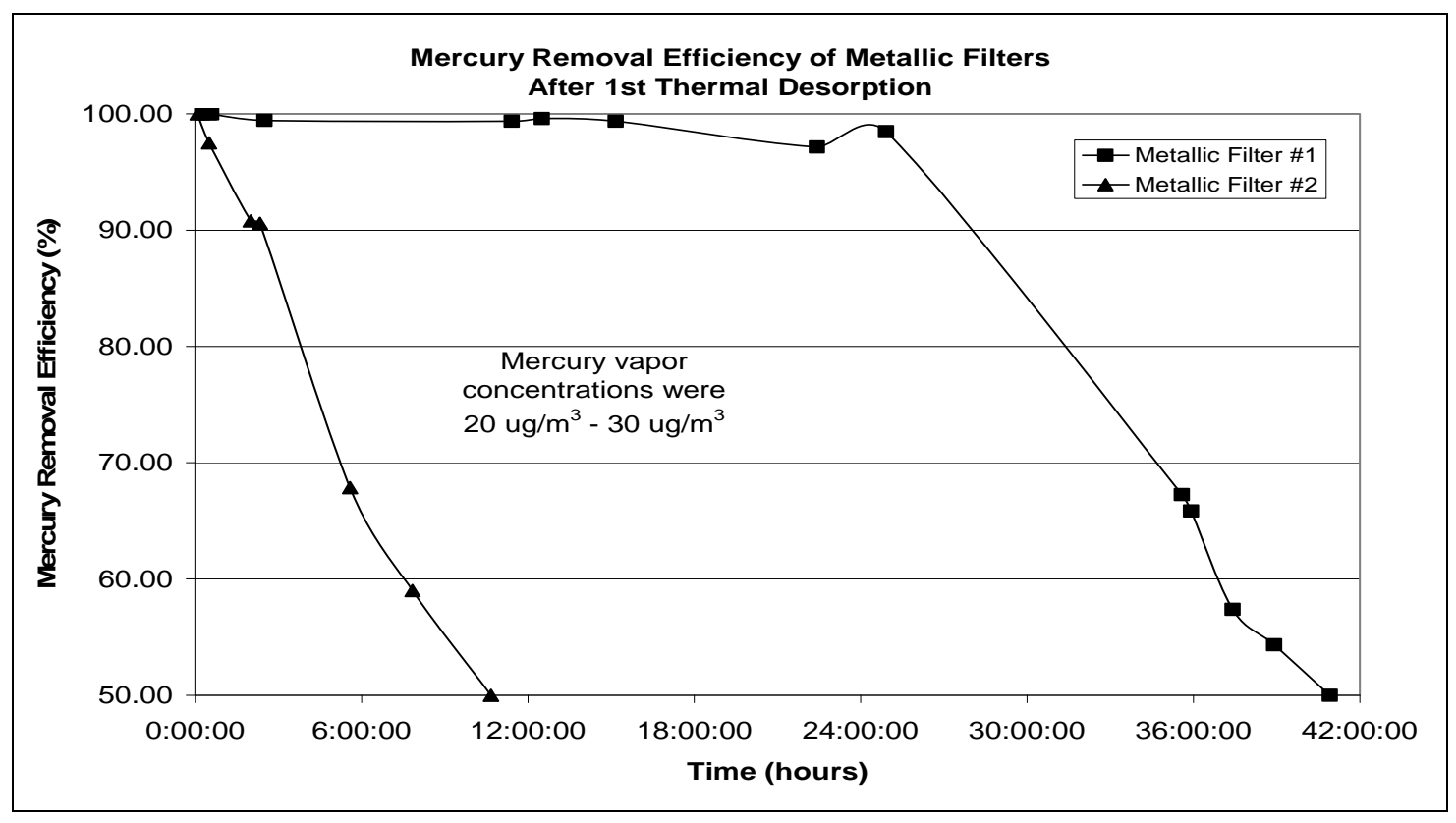

Figure 12: Mercury removal efficiency of metallic filters after $1^{\text {st }}$ thermal de-sorption.

After thermal de-sorption, there was a decrease in the time the mercury removal efficiency of the metallic filters was above $90 \%$. In comparison, filter $\# 1$ 's mercury removal efficiency stayed above $90 \%$ for 27 hours after thermal de-sorption versus 68 hours before thermal de-sorption, and filter \#2's efficiency was above $90 \%$ for 3 hours after thermal de-sorption compared to 20 hours before. After thermal de-sorption, it took filter \#1, 41 hours and filter \#2, 11 hours to reach 50\% efficiency.

The metallic filters then went through a second round of thermal de-sorption and mercury removal efficiency testing in the same manner as previously described. There was very little change in filter \#1's efficiency after the first and second thermal de-sorption, the mercury removal efficiency stayed above $90 \%$ for about 1 day (29 hours). After the second thermal de-sorption, the efficiency of filter \#2 was further reduced, with the efficiency staying above $90 \%$ for $1 \frac{1}{2}$ hours (as compared to 3 hours after the first thermal de-sorption) (Figure 13). 


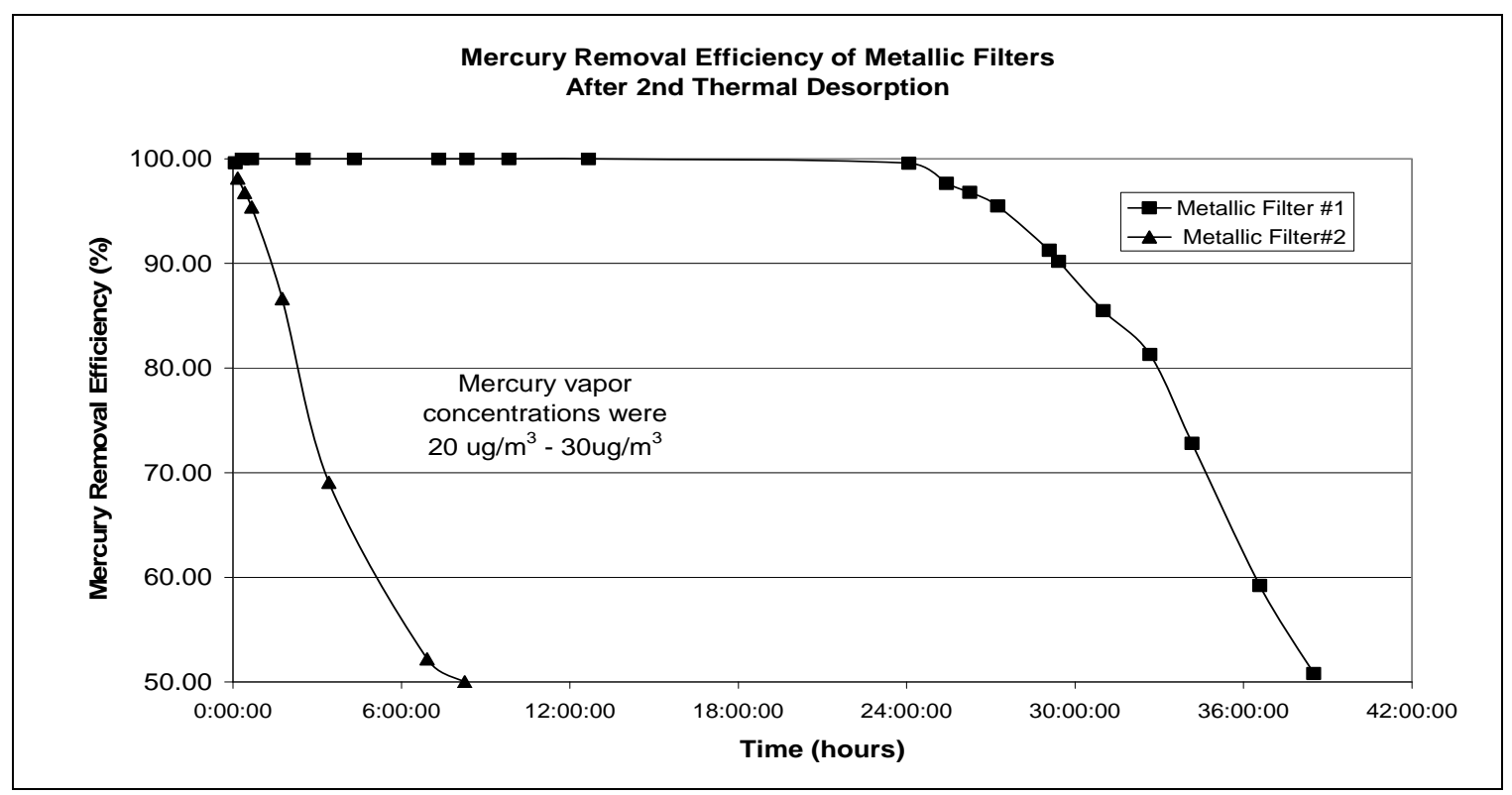

Figure 13: Mercury removal efficiency of metallic filters after $2^{\text {nd }}$ thermal de-sorption.

Thus the laboratory testing showed that the metallic filters had great potential to remove mercury from the flue gas of a coal-fired power plant. Thermal de-sorption did cause a slight decrease in the time period the filter's efficiency was above $90 \%$ ( 3 days versus 1 day for filter \#1 and 20 hours versus 3 hours for filter \#2), but continued thermal desorption did not appear to have a further effect on filter \#1's efficiency and only a slight reduction in filter \#2's efficiency. The one drawback associated with the filter \#1 was its relatively high cost, which led us to investigate the mercury removal ability of filter \#2 and filter \#1 in series.

\subsection{Laboratory Results - Metallic Filters in Series}

Two, \#2 filters in series with one, \#1 filter were tested to determine their combined mercury removal efficiency. The \#2 filters were placed at the inlet of the PVC pipe followed by the filter \#1. Again, the inlet mercury concentration was held between $20 \mu \mathrm{g} / \mathrm{m}^{3}$ and $30 \mu \mathrm{g} / \mathrm{m}^{3}$, and the flow rate was kept at $5 \mathrm{~L} / \mathrm{min}$. The results showed excellent mercury removal for the filters with the efficiency above $90 \%$ for 192 hours (Figure 14). 


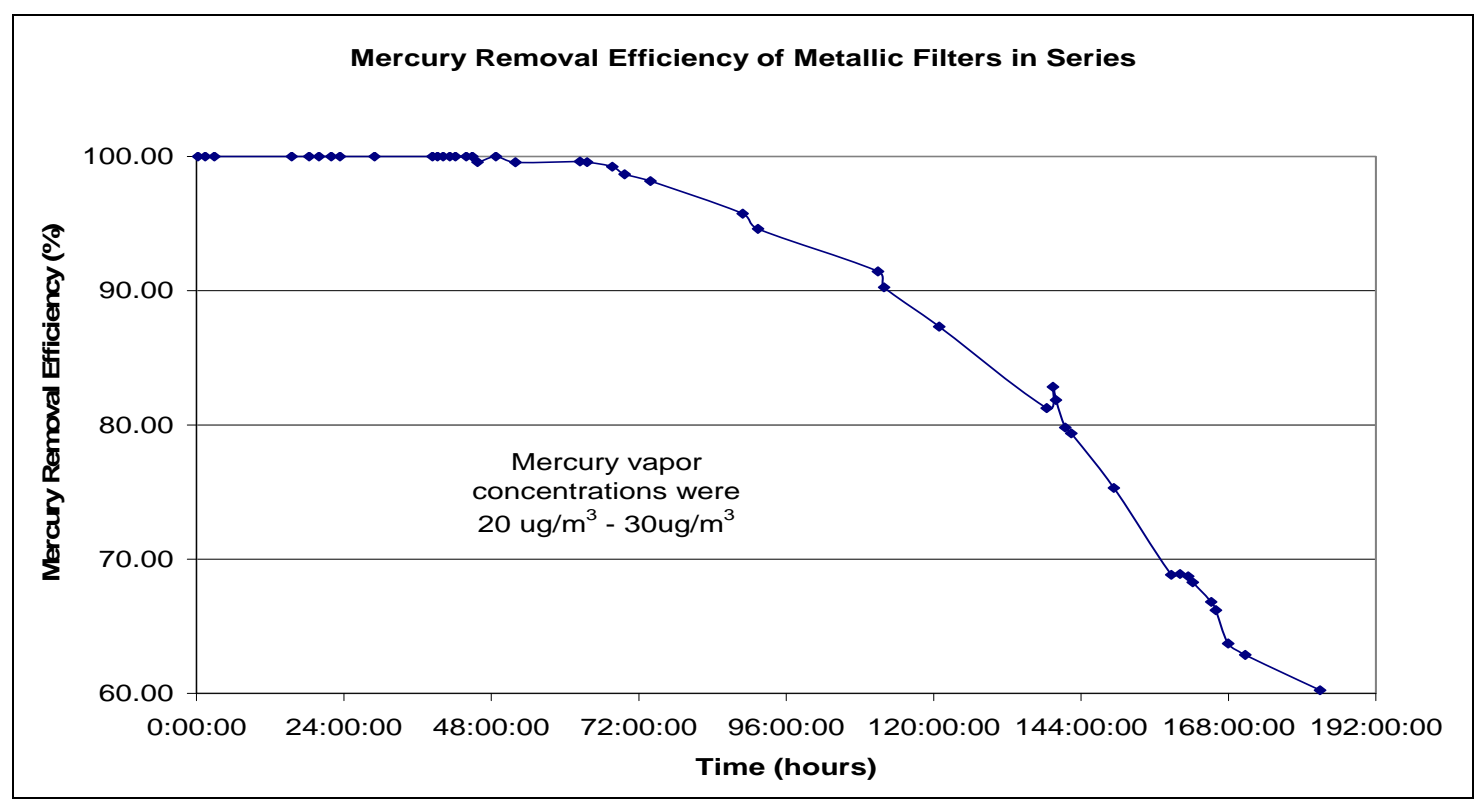

Figure 14: Mercury removal efficiency of metallic filters in series (lab result).

This test results showed that when run in series, the mercury removal efficiencies of the filters were cumulative. For example, the individual tests for filter \#1 and filter \#2 showed durations above $90 \%$ mercury removal efficiency to be 20 hours and 68 hours, respectively. When two \#2 filters and one \#1 filter were run in series, the duration above $90 \%$ mercury removal efficiency was simply the addition of each of the individual durations ( 20 hours +20 hours +68 hours $=108$ hours $)$. There was even a slight increase in the filter's mercury removal efficiency when they are run in series.

This result was very promising because the ability of the metallic filters to remove mercury at very low concentrations was unknown. When run in series, the first filter was exposed to the $20 \mu \mathrm{g} / \mathrm{m}^{3}-30 \mu \mathrm{g} / \mathrm{m}^{3}$ mercury concentration throughout the entire test. However, the second filter was exposed initially to $0 \mu \mathrm{g} / \mathrm{m}^{3}$, and then the concentration gradually increased as breakthrough occurred in the first filter. The mercury concentration in the air stream through the third filter occurred in the same manner; it was initially $0 \mu \mathrm{g} / \mathrm{m}^{3}$ and then gradually increased as breakthrough occurred in the second filter.

Then to assess the real world performance of the filters, the filters in series were field tested at the PPL's coal fired power plant in Colstrip, Montana.

\subsection{Field Testing Results}

Then preliminary tests were conducted for six consecutive days in a coal fired flue gas stack after the wet scrubber at the PPL's Colstrip power plant in Montana. The mercury 
removal efficiency was at or above $90 \%$ for the entire six days test in the field when exposed to actual power plant flue gas. In the power plant at Colstrip, Montana, the average mercury concentration was $4 \mu \mathrm{g} / \mathrm{m}^{3}-6 \mu \mathrm{g} / \mathrm{m}^{3}$. The mercury removal efficiency was at or above $90 \%$ for the entire six day test, with one exception at the end of day one (Figure 15).

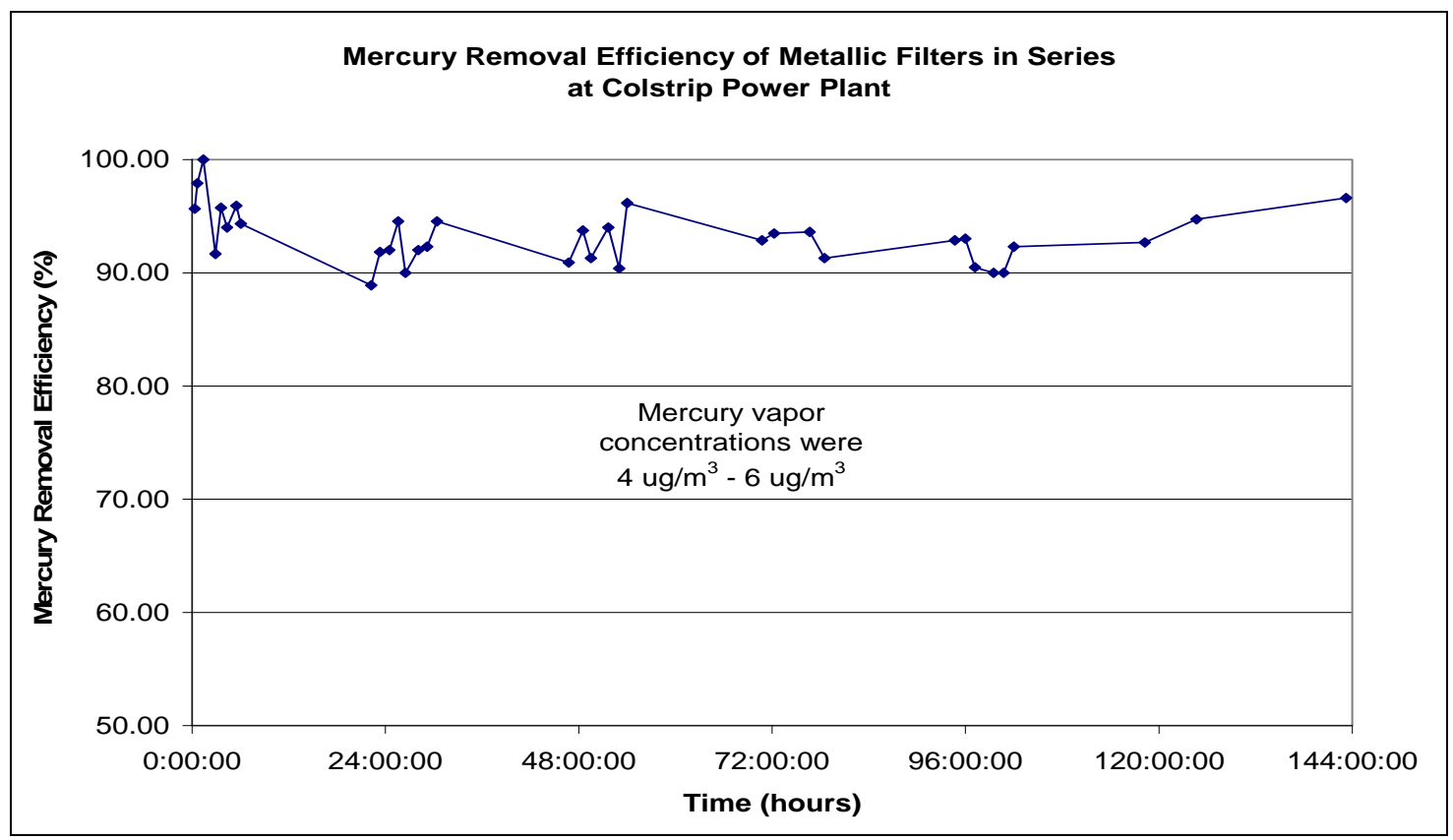

Figure 15: Mercury removal efficiency metallic filters in series at Colstrip.

These field test results again showed that the metallic filters in series are capable of removing mercury at low concentrations; the inlet mercury concentration entering the filter ranged between $4 \mu \mathrm{g} / \mathrm{m}^{3}$ and $6 \mu \mathrm{g} / \mathrm{m}^{3}$, which is five times lower than the concentrations used in the laboratory evaluations. The field testing also indicated the $\mathrm{SO}_{2}$, NOx, and other compounds present the flue gas have minimal if any effect on the mercury removal efficiency of the metallic filters. The condensate collected in the impingers during the field test was analyzed and mercury concentrations ranged from $0.1 \mu \mathrm{g} / \mathrm{L}-0.2 \mu \mathrm{g} / \mathrm{L}$. These low concentrations were expected since the main species of mercury present at the Colstrip power plant is elemental mercury, which is not water soluble.

\section{CONCLUSION}

The coal-fired power plant industry has a great need for effective mercury control devices to reduce mercury emissions and comply with upcoming EPA regulations. Two different 
metallic filters developed by Montana Tech showed great potential to remove mercury vapor from the flue gas of coal-fired power plants.

Laboratory tests showed that both metallic filters removed mercury vapor with efficiencies above $90 \%$. Additionally, the laboratory and field analysis of the filters in series indicated that the filters have the ability to remove mercury at low concentrations, which is very promising since the mercury concentrations at coal-fired power plants varies based on the mercury content of the coal burned. The field test at PPL's Colstrip, Montana power plant confirmed the practicality of the filters and showed that the mercury removal efficiency is still above $90 \%$ when exposed to actual power plant flue gas. Thus is conclusion, Montana Tech's patent pending mercury control device is the environmentally friendly control technology to remove mercury vapor from coal-fired power plants. 


\section{References}

"Coal Chlorine Content Influences Mercury Removal", PRECIP Newsletter. January 2002, No. 312. www.mcilvainecompany.com/newsletters/precipn $1312 / \mathrm{Coal} \% 20$ Chlorine $\% 20$

Gorishi, B.; Gullet, B.K., "Sorption of Mercury Species by Activated Carbon and

Calcium Based Sorbents: Effect of Temperature, Mercury Concentration and Acid Gases", Waste Management \& Research, 1998, 6, 582-593.

Granite, E.J.; H.W., Hargis, R.A. “Carbon-Based Sorbents For the Removal of Mercury From Flue Gas", U.S. Department of Energy.

Guijarro, M.I.; Mendioroz, S.; Munoz, V., "Effect of Morphology of Sulfurized Materials in the Retention of Mercury from Gas Streams", Ind.Eng.Chem.Res.1998, 37, pg.1088-1094.

Hwang, J.Y., Huang, X., Tieder, R.E., Liu, X. "Control of Mercury Emissions from Coal Fired Power Plants Using Fly-Ash-Derived Carbon”, Michigan Technological University, EPA Grant Number R825370C028, www.es.epa.gov/ncer_abstracts/centers/cencitt/year3/material/hwang.html.

Ihle, J., “Mercury Emissions and Fuel Switching: What's in Your Coal?”, October 2002. www.coalage.com/ar/coal mercury emissions feul/.

Karatza, D.; Lancia, A.; Musmarra, D., "Fly Ash Capture of Mercuric Chloride Vapors From Exhaust Combustion Gas", Environ.Sci.Technol.1998, 32, pg.3999-4004.

Kloeppel, J., "Unlikely Mix- Used Tires, Pistachio Shells-Can Clean Up Pollution", News Bureau University of Illinois At Urbana-Champaign. (2000). www.news.uiuc.edu/scitips/00/08enviro.html.

Meij, R. "Distribution of Trace Species in Power Plant Streams: A European Perspective", American Power Conference, Chicago, 1994.

Mendioroz, S; Guijarro, M.I.; Bermejo, P.J.; Munoz, V., "Mercury Retrieval from Flue Gas by Monolithic Absorbents Based on Sulfurized Sepriolite", Environ.Sci.Technol. 1999, 33, pg.1697-1702.

Mercury Study Report to Congress, EPA-452/R-96-001g, 1996.

Miller E.L. and Dobb E. David, "Speciation of Mercury in Soils by Sequential Extraction"

www.epa.gov/nerlesd1/pdf-ecb/542asd95.pdf 
Morency, J.R., Panagiotou, T., Senior, C.L. "Laboratory Duct Injection of a Elite-Based Mercury Sorbent”, Physical Sciences Inc.

Morris T., J. Sun, G. Szulczewski (2003), "Measurement of the chemical and morphological changes that occur on gold surfaces following thermal desorption and acid dissolution of adsorbed mercury", Analytica Chimica Acta, 496, pp. 279-287.

Nordin, A.; Schager, P,; Hall, B., “ Mercury Speciation in Flue Gases: A Comparison of Results from Equilibrium Calculations with Results from Laboratory Experiments", Swedish Fame Days, Turkoo, Finland, 1990.

Nriagu, J.O. Environment 1990, 32,7-33.

Pavlish J.H., E.A. Everett, A. Sondreal, M.D. Mann, E.S. Olson, K.C. Galbreath, D.L. Laudal, S.A. Benson (2003), "Status review of mercury control options for coal-fired power plants”, Fuel Processing Technology, 82, pp. 89-165.

Tumati, P.R.; DeVito, M.S., "Partitioning Behavior of Mercury During Coal Combustion”, Joint ASME/IEEE Power Conference, Kansas City, KS, 1993.

U.S. EPA (1997), "Mercury Study Report to Congress EPA-425/R-97-003", U.S. EPA Office of Air Quality Planning and Standards.

U.S. EPA (2005), "EPA Proposed Options for Significantly Reducing Mercury Emissions from Electric Utilities". http://www.epa.gov/mercury.

Young, B.C.; Miller, S.J.; Laudal, D.L., "Carbon Sorption of Trace Mercury Species", Proceedings of the $11^{\text {th }}$ Annual Pittsburg Coal Conference, 1994, pg. 575-580.

www.tva.gov/environment/air/ontheair/merc emis.html

Essortment Home $>>$ Science \& Technology $>>$ Science:General $>>$ Thermal desorption of gases

meme.essortment.com/thermaldesorpti_rsnb.htm 
Appendix: 42 Electrolytic Solution Purification and Metal Recovery from Toxic Waste Streams (UT003) 


\section{FINAL TECHNICAL REPORT}

Contract Title and Number:

Crosscutting Technology Development at the Center for

Advanced Separation Technologies

(DE-FC26-02NT41607)
Period of Performance:

Starting Date: June 1, 2003

Ending Date: Aug 30,2007
Sub-Recipient Project Title:

Electrolytic solution purification and metal recovery from metal-bearing toxic waste streams

Principal Investigators:

Jan D. Miller / Michael L. Free

Contact Address:

Michael L. Free

135 S. 1460 E. Room 412

Salt Lake City, UT 84112

Subcontractor Address:

No subcontracts issued.
Report Information:

Type: Final

Number:

Period:

Date: Aug. 30, 2006

Code:

Contact Information:

Phone: 801-585-9798

Fax: $\quad$ 801-581-4937

E-Mail: mfree@mines.utah.edu

Subcontractor Information:

Phone:

Fax:

E-Mail:

\section{DISCLAIMER}

This report was prepared as an account of work sponsored by an agency of the United States Government. Neither the United States Government nor any agency thereof, nor any of their employees, make any warranty, express or implied, nor assume any legal liability or responsibility for the accuracy, completeness, or usefulness of any information, apparatus, product, or process disclosed, or represents that its use would not infringe privately owned rights. Reference herein to any specific commercial product, process, or service by trade name, trademark, manufacturer, or otherwise does not necessarily constitute or imply endorsement, recommendation, or favoring by the United States Government or any agency thereof. The views and opinions of authors expressed herein do not necessarily state or reflect those of the United States Government or agency thereof.

\section{ABSTRACT}

In the research conducted for this project, a method for removing toxic metal ions from solution using a pulsed electrodeposition method was developed. In the development of the method, different potential pulse parameters including pulse on-time, off-time, and pulse potential were investigated to determine their effect on the performance of the pulsed electrodepositon process. Also investigated were the effects of solution parameters such as 
solution composition, ionic strength, and flow rate, along with investigation of the material of construction of the electrodes used to accomplish the pulsed electrodeposition. From this investigation it was shown that the method is a viable method for separating toxic metal ions in dilute aqueous solutions, and it was also shown in what way various parameters influence the performance of such a system.

\section{TABLE OF CONTENTS}

Title Page $\quad 1$

Disclaimer $\quad 1$

Abstract $\quad 1$

Introduction $\quad 2$

Executive Summary 3

Experimental $\quad 4$

Results and Discussion $\quad 12$

$\begin{array}{ll}\text { Conclusion } & 27\end{array}$

References $\quad 27$

$\begin{array}{ll}\text { Appendices } & 27\end{array}$

\section{INTRODUCTION}

The objective of this project is to provide a method for removing multiple metal ions from aqueous streams by selective pulse-plating with high surface area electrodes. This method can be used to recover metals individually as purified products rather than as toxic waste. This direct production of metal as a by-product rather than as a toxic waste contributes to better resource utilization and reduced toxic waste production. The use of pulsed electrodeposition maximizes mass transport and current efficiency (see Appendix A), and the sequential application of different electrical potentials allows for the recovery of high purity metal products. Because each metal has a unique electrochemical potential below which it will begin depositing, metals can often be separated effectively on the basis of their individual potentials (see Appendix B). Placement of a series of electrodes, each at different potentials, can be used to successively recover individual metals in a purified form. The use of a system in which cartridge type electrodes are used can facilitate recovery and recycling of individual metals rather than disposal of the metals as waste.

In this investigation, various parameters such as electrode composition and surface area, electrode pulse potential, solution composition, and pulse parameters are studied. These parameters are investigated in order to determine their effect both on the ability of the pulse plating apparatus to separate the metals, and on the rates at which the metals are removed from the solution. From this investigation a general set of guidelines can be developed for constructing pilot or industrial scale systems to recover dissolved toxic metal ions from dilute aqueous solutions in a more cost effective and environmentally sound manner than is currently practiced.

The specific problem to be solved in this investigation is the removal and separation of dissolved metal ions from dilute aqueous media. Waste streams composed of dilute aqueous solutions of toxic metal ions are produced via a number of industrial or natural 
processes. Acid mine drainage is one example of a natural process that produces these types of streams. Industrial processes include the waste streams from electroplating or metal finishing operations, or waste streams that can result from recycling solutions. These operations utilize the metals in the processes to the greatest extent possible for economic reasons, so the concentration of metals in waste streams from those processes is generally dilute, typically in the parts per million range. At such dilute concentrations, effective recovery of the metals can be difficult due to mass transport limitations. Despite the concentrations being relatively low in these solutions, the solutions still pose an industrial hazard and do not meet the limits set forth in government regulations concerning the maximum level of the metals discharged into the environment. What is needed is a method to effectively and economically recover the metals from dilute solutions to mitigate the environmental hazard posed by the waste streams. Because precipitation and ion exchange methods involve either the creation of non-recoverable toxic waste products, or the use of further processing steps, a new method is to be developed in order to avoid the drawbacks these methods present.

\section{EXECUTIVE SUMMARY}

Research has shown that using high surface area electrodes, toxic metal ion concentrations in aqueous media can be drastically reduced. This investigation has shown that copper, cadmium, mercury and lead can be reduced to less than $1 \mathrm{ppm}$ concentration using pulse plating schemes. The application of these pulse-plating schemes with high surface area electrodes for reducing the concentration of toxic metal ions has also been investigated. The use of such a system with multiple applied potentials and resistances for recovering purified metal products from a mixture of metal ions based on the difference in electrochemical potentials between different metals.

At the beginning of experimentation, various expectations as to the results were formulated.

It was first supposed that, because each element has a unique electrochemical potential below which the element will deposit itself onto the working electrode, it would be possible to effectively separate different dissolved metal ions from aqueous media. It was also theorized that various factors, such as electrode composition and surface area, dissolved metal ion concentration, flow rate through a device, applied potential, and solution ionic strength would influence the rate at which the various ions could be removed from the solution. Through experimentation, it was desirable to develop a characterization of the behavior of an electrode apparatus utilizing pulsed electrodeposition to remove dissolved metal ions from solution. This characterization has been accomplished through experimentation, and the results correspond well with the original expectations based upon electrochemical theory. Overall, it was found that the pulsed electrodeposition technique using flow through mesh electrodes is an effective method for removing dissolved metal ions from aqueous media. In a reasonable amount of time, the concentration of dissolved metals in dilute solutions is drastically reduced, despite the mass transport limitations present in working with dilute solutions. Pulsed electrodeposition is effective in overcoming these mass transport limitations to the extent necessary to achieve reasonable rates of removal. Characterization of the behavior of the electrode apparatus and the pulsed electrodeposition technique requires examination of a number of variables which can influence the behavior of such a deposition 
process. These variables include the pulse on-time, pulse off-time, flow rate of the solution through the apparatus, the material of construction of the electrode and that materials surface area, the concentration of the dissolved metal ions in the feed solution, the ionic strength of the solution, and the potential of the electrodes during the pulse on-time. An examination of the influence of these variables on the behavior of the system provided a useful characterization of the behavior of the system. Electrode potential during the pulse on-time was found to be a useful parameter for effecting the separation of metal ions from solution individually, as predicted from the outset. The pulse on-time, pulse off-time, solution flow rate, material surface area, solution ionic strength, and concentration of dissolved metal ions in the solution were all parameters that were found to have an effect on the rate of removal of the metal ions from the solution. These parameters also influence the amount of electrical energy required to remove the metal ions from solution, and so by examining the effect of those variables on the system, a useful compromise between removal rate and electrical efficiency can be reached. Overall, the use of a pulsed electrodeposition technique to separate dissolved metal ions from solution individually has been shown to be an effective method for reducing the toxic metal ion concentration of a solution, and the characterization of this type of system provides the information necessary to construct a pilot or industrial scale system with a reasonable expectation of successful operation.

\section{EXPERIMENTAL}

\section{Dilute Metal Detection Methods}

Detection of dilute amounts of dissolved metals in solution is no trivial matter in itself. Multiple methods were used to detect the dissolved metals in solution, both to adapt to the different solution types that were to be used and to perform a cross check of the accuracy of each method by analyzing the results using another technique.

\section{Ion Selective Electrodes:}

The initial tests of the electrodeposition method were performed using a single element in solution, which was selected to be copper. Copper does not pose a significant environmental or toxicity hazard, but it is convenient to use because successful plating can be easily verified by visual examination of the electrode for the copper color. Copper electroplating is also a widely used and fairly well understood process. In order to measure the copper content in solution, a copper ion selective electrode was used. Ion selective electrodes have the advantages of being inexpensive, easy to use, and reasonably accurate over a fairly broad range of concentrations as long as conditions are carefully controlled. These conditions include keeping temperature fairly constant, having a high enough ionic strength in the solution to get accurate results, and not having any ions in solution which can cause interferences with the electrode operation. Temperature changes can skew the results, and a low ionic strength can cause the slope of the potential vs. concentration line to become shallow such that an accurate reading is difficult to obtain. The ion selective electrode provides an inexpensive, easy to use, and reasonably accurate method of measuring the copper content of the solutions. However, conditions under which the electrode is used must be carefully controlled. Also, because other metal ions can cause interference with the 
operation of the electrode (lead and cadmium included), and because the electrode only detects copper, it is necessary to use other methods for detection in systems containing competitive elements.

\section{Inductively Coupled Plasma Spectroscopy:}

Emission spectroscopy is the detection and measurement of the content of an element by detecting the radiation emitted from the elements when they are excited into a more energetic state and then allowed to return to their ground state. In the case of inductively coupled plasma spectroscopy, also known as ICP, this excitation is achieved by passing an atomized solution through a plasma jet, then detecting the resulting radiation given off when the elements return to their ground state. Each element gives off light of a specific and unique wavelength, typically in the UV range. By detecting the intensity of the light at those unique wavelengths, the amount of an element present in the solution can be determined. A calibration must first be performed so that the detector can correlate the radiation intensity to the concentration of the element in solution. Because the instrument used to perform ICP is automated, a computer system automatically correlates the intensity data to the calibration curve and returns the content of the element in the solution with a high degree of accuracy down to levels of 1ppm or even lower. Because each element has unique wavelengths of radiation that are emitted, multiple wavelengths can be examined for intensity and in that way, multiple elements can be detected. This makes ICP useful for performing analysis on solutions containing many elements without having to conduct many tests for different elements. Calibration solutions are commercially available and can contain a large number of elements at once, making calibration and analysis for many elements simultaneously easily achievable. The disadvantages of ICP are the high capital and maintenance costs of the equipment, the less simplistic operation of the equipment compared to other methods, and the lack of an ability to detect elements accurately in the low parts per billion range. ICP was invaluable for performing the analysis on the more complicated solutions run during some of the removal analysis work, especially solutions containing mercury, lead, zinc, copper, iron, and cadmium simultaneously, which makes other methods difficult or impossible.

\section{Differential Pulse Stripping Voltametry:}

Differential pulse stripping voltametry is an electrochemical method of determining the content of dissolved metal ions in a solution. In order to perform the stripping voltametry test in a manner such that accurate results are obtained, a mercury thin film electrode should be used. The thin film is formed by rotating a glassy carbon working electrode in a mercury solution, with the required platinum counter electrode and calomel reference electrode, and applying a potential to the working electrode such that mercury will deposit on the surface. This method forms a mercury film electrode into which the dissolved metal ions can deposit without forming any alloys or oxidizing at higher potentials.

A sample of $10 \mathrm{~mL}$ was then placed in a beaker with a platinum counter electrode and a calomel reference electrode. It has also been found that these tests run best with $5 \mathrm{~mL}$ of a dilute mercury solution, approximately 30ppm, added to the test. This added mercury is believed to aid in keeping enough mercury in the system to allow all of the dissolved metal ions to be able to absorb into the mercury film, and also helps to regenerate the film during each test to compensate for any mercury that may have stripped off at the end of a previous 
test. The samples are then evaluated for metal content using a computer controlled potentiostat system that performs the differential pulse stripping voltametry.

Differential pulse stripping voltametry is a two-part process. First, the working electrode is set to a low potential, typically around $-1 \mathrm{~V}$, for about 6 minutes so that the dissolved metal ions in the solution are absorbed into the mercury thin film. Then, the potential is ramped linearly to a more positive potential while the potential is pulsed positive for a brief period of time. The current is measured at both the pulsed potential and at the baseline potential, and the difference between those two currents is known as the differential current. This differential current becomes relatively large at potentials where the baseline potential is lower than the electrochemical potential of one of the dissolved metal ions, and pulsed potential is higher than the electrochemical potential of one of the dissolved metal ions. When the differential current is plotted against the potential, peaks will form at the electrochemical potentials of the elements. The height of the peaks will also be proportional to the content of dissolved metal ions. In this manner, a plot can be developed showing which dissolved metal ions are present, and in what amount. Typically, the potential is elevated for 0.1 seconds, then the potential is returned to the baseline level is for 0.2 seconds. The current values are measured towards the end of each part of the cycle, so that capacity current effects are near zero. The amplitude of the potential pulse used was $25 \mathrm{mV}$, and the test was typically run with the baseline voltage ramped from $-1 \mathrm{~V}$ to $+0.2 \mathrm{~V}$. Theoretically, the peak height of the differential current can be found using equation (17)

$$
i_{p}=\frac{n F A D^{1 / 2} C}{\sqrt{\pi t}}\left[\frac{1-\exp \left(\frac{n F \Delta E}{2 R T}\right)}{1+\exp \left(\frac{n F \Delta E}{2 R T}\right)}\right]
$$

in which $\mathrm{i}_{\mathrm{p}}$ is the current peak height, $\mathrm{n}$ is the number of electrons transferred, $\mathrm{F}$ is Faraday's constant, $\mathrm{A}$ is the electrode surface area, $\mathrm{D}$ is the diffusivity of the dissolved metal ion, $\mathrm{t}$ is the measurement time during the pulse, typically near the end of the pulse at 20-45 milliseconds, $\mathrm{R}$ is the gas constant, $\mathrm{T}$ is the absolute temperature, and $\Delta \mathrm{E}$ is the potential pulse height above the baseline ramp potential, which is typically $25 \mathrm{mV}$ in these experiments. Note that the peak height follows a linear dependence on the concentration of the species in solution. It should also be noted that the peak height is dependent on the number of electrons required to perform the electrochemical reaction and the diffusivity of the species in the solution. Consequently, the peak height for each element at a given concentration is generally not the same as the peak height of another element that is at the same concentration. Figure 1 shows an example of data returned from an actual calibration test using differential pulse voltametry of a solution containing copper, lead, and cadmium. 


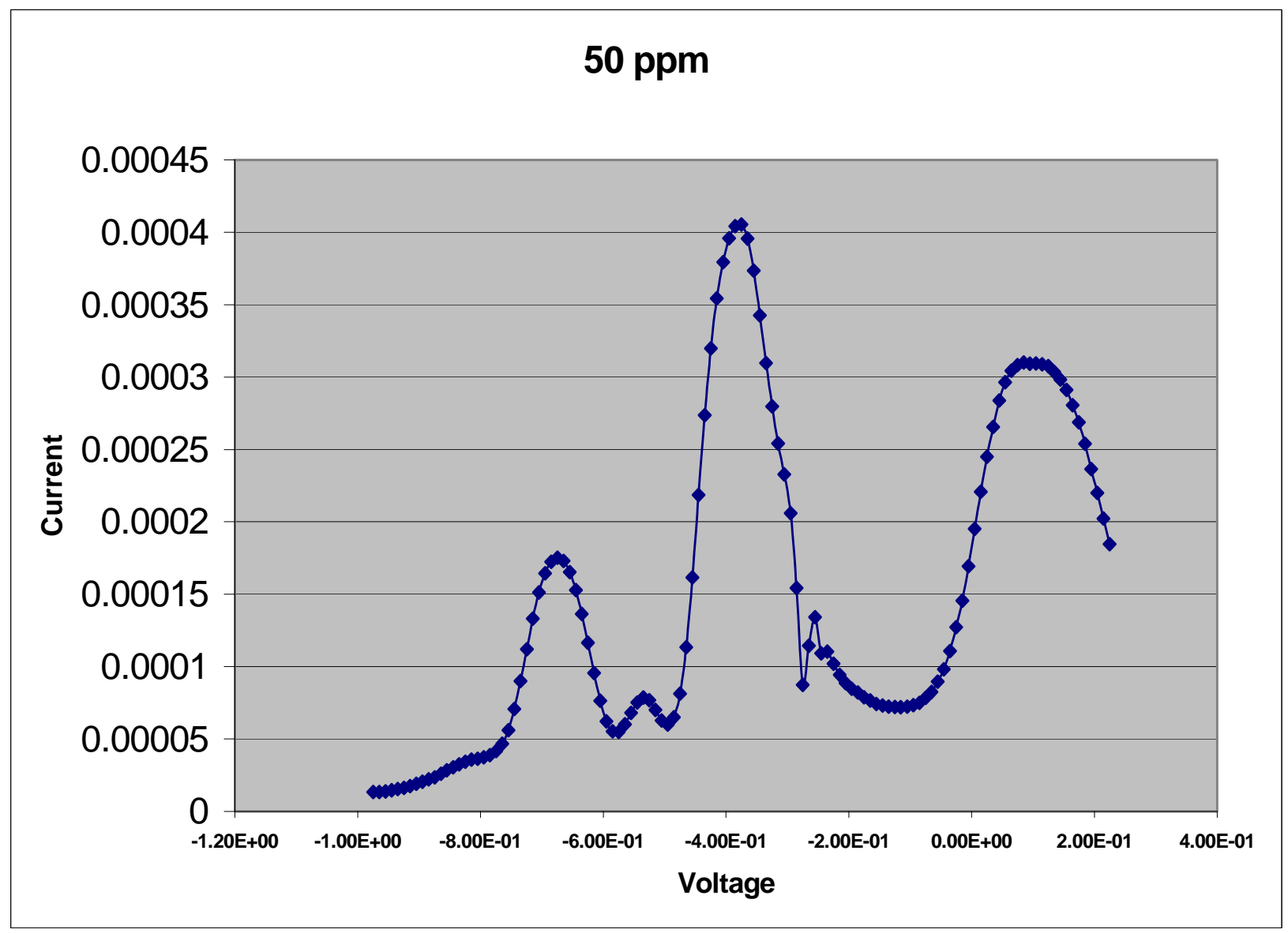

\section{Figure 1, Differential Pulse Voltametry Curve}

In Figure 1, distinct peaks can be seen for each of the three elements present in the test, with copper at the right, lead at the center, and cadmium at the left. These are in the order one would expect given their electrochemical potentials. The baseline current does exhibit a slope itself, increasing from left to right. In order to account for this, peak heights are taken relative to the baseline, not at their absolute values. Consistently performing the analysis in the same manner produces results with good accuracy.

In practice, in order to minimize the effects of experimental error, such as equipment resistances, a calibration curve is developed for each series of tests. This involves performing tests on solutions with known quantities of dissolved metal ions, formulating a concentration vs. peak height relationship. Then, solutions of unknown dissolved metal ion content can be evaluated for content based on peak height.

Solutions on Which the Tests Were Performed:

All testing was performed on solutions of dissolved metal nitrates, made either by dissolution of a metal nitrate salt or by the oxidation of the pure metal using nitric acid.

\section{Electrode Apparatus:}

In order to perform the electrodeposition process, it is necessary to have some kind of electrode system on which to plate out the dissolved metals, and to provide the counter 
reaction necessary for providing electrons to the reaction. The rate at which the process can occur is controlled by the surface area of the electrode to some extent, so it is also desirable to have as high a surface area as possible for the working electrode. This was accomplished by using a flow through electrode system consisting of conductive screens for electrodes. Platinum and carbon felt were examined for use in the electrodes. Platinum is an excellent electrode material because it is highly oxidation resistant, has good electrical conductivity, and is compatible as a surface onto which other metals can deposit themselves. Platinum was found to be useful both as the working and counter electrodes in the apparatus. The platinum wire mesh that was used in the construction of the experimental apparatus was a commercially available 100 mesh platinum gauze made from $0.0762 \mathrm{~mm}$ platinum wire and having an open area of approximately 49\%. Each platinum mesh electrode was approximately $25 \mathrm{~mm}$ by $25 \mathrm{~mm}$. Carbon felt is a material composed of closely packed carbon wires which are pressed together to form a $1 \mathrm{~cm}$ thick pad having a very high surface area, and approximately no open area of the facing surfaces due to close packing of the wires in the felt. Carbon is not as electrically conductive or oxidation resistant as platinum, but does provide a good surface for the metals to deposit on. Because of this, the carbon felt electrode is best suited for use as a working electrode, rather than a counter electrode, as oxidation and electrical resistance in the carbon felt pads can cause a loss of the electrical connection between the electrode and the source of electrical potential. In order to take advantage of the high surface area of the carbon felt without exposing the electrode to conditions that would cause it to degrade, each electrode apparatus was constructed using a carbon felt working electrode, and a platinum counter electrode. This type of electrode is referred to as the mixed material electrode, and this electrode takes advantage of each material's strengths and avoiding the material's weaknesses.

In order to connect the electrode screens to the potentiostat systems, contact wires must be attached to each electrode screen. In the case of the platinum electrodes, platinum wires were woven through each screen, and then doubled back and crimped, simply forming a physical connection between the wires and the screens. It is also possible to attach the wires using tack welding, provided the mesh wires are thick enough. This attachment approach provided the needed electrical contact and since only one material was used, there were no galvanic couples developed. In the case of the carbon felt electrodes, it was necessary to attach a wire to each electrode pad of different composition than the electrode. In this case, nickel coated copper electrical wire was used. Because the use of wire with different composition than the carbon electrodes can form a galvanic couple, it was necessary to isolate the wire from the fluid that would be passing through the system and to provide a good electrical connection between the contact wires and the wires of the carbon felt pad. Painting the wire with a colloidal silver paint and then inserting the wire into the pad while the paint was still wet formed a good electrical connection. This was then isolated from the fluid flow by submerging the electrode part containing the wire in a low viscosity epoxy and allowing the epoxy to harden and encase the wire and connection. The first carbon electrode was constructed without epoxy sealing, and it quickly failed due to galvanic corrosion of the contact wire inside the electrode. With the epoxy sealing, the carbon electrode was able to last for months of testing before failure occurred.

The electrodes must also be kept separate within the apparatus, as contact between the electrodes will cause a short circuit of the device. For this purpose, two 149 micron thick polypropylene mesh screens were used. The use of such thin polypropylene screens keeps 
the distance between the electrodes to a minimum, which helps to reduce the power necessary to overcome the resistance of the solution. The screens were cut to the same size as the electrodes, and inserted between the electrodes. Polypropylene screens were also inserted between the electrodes and the inlet and outlet of the housing in order to provide even distribution of the solution flow across the entire electrode surface.

In order to have the test solution flow through the electrodes, the electrodes must be sealed inside a watertight housing that can be attached to both input and output tubing. For this housing, a filter housing was modified to contain the electrodes. This involved grinding the filter housings down to a size appropriate for the electrodes, and then cutting grooves into the side of the housing so that the lead wires could run from the electrodes inside the housing to the potentiostat outside the housing. The housing was then sealed watertight using a caulking compound.

Initially, the tests were conducted without the use of a separate reference electrode. The counter electrode mesh was also used as the reference electrode in these tests. However, this arrangement proved to be problematic, as the counter electrode constantly varies in potential in order to provide the necessary electrons for the reaction to occur, and so the actual potential of the working electrode cannot be known accurately. While this arrangement can be used where electrodeposition of all species in the solution is desired, in situations where an accurate working electrode potential is needed to selectively electrodeposit a desired species from solution, a separate reference electrode is needed. To accomplish this, a saturated calomel electrode was attached to the apparatus by drilling a hole in the outside of the apparatus and inserting the tip of the reference electrode in close proximity to the working and counter electrodes, and in contact with the solution passing through the housing. The addition of the separate reference electrode provided the potentiostat system with a much more accurate reference on which to base the working electrode potential, which resulted in a much more accurate separation of the species present in the test solutions. 


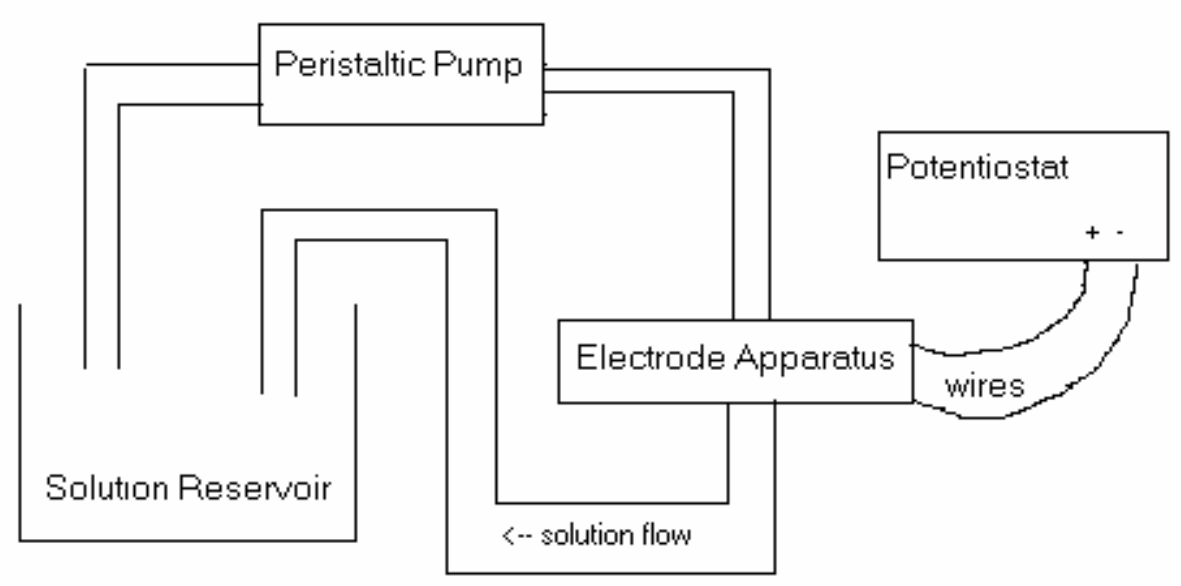

Figure 2: Diagram of the electrode apparatus system used.

The electrode housing was then mounted on a test stand, and tubing was connected to the inlet and outlet points on the filter housing. A diagram of the system is shown in Figure 2. Fluid was then circulated through the test apparatus using a pump with a variable flow rate. Typical flow rates used were around $2 \mathrm{~mL} / \mathrm{sec}$, but this was varied in order to examine the effect of flow rate on the removal rate of dissolved metal ions from the solution. Solution was provided to the apparatus from a $250 \mathrm{~mL}$ beaker, which was used in the circuit as a reservoir for the test solution. Solution was pumped out of the beaker using a peristaltic pump, through the electrode apparatus, and then back into the beaker, so that solution would be passed through the apparatus multiple times over the course of an experiment. Samples could then be withdrawn from the beaker at the desired intervals using a pipetor for later analysis of dissolved metal ion content.

\section{Potentiostat System:}

The potentiostat system used in the experiments was a Gamry potentiostat mounted into a PC as a pair of PCI boards, known as the Gamry PCI-4/750 potentiostat. The use of a computer controlled potentiostat allows for convenient data acquisition and monitoring of the processes. In order to perform the pulsing processes, a program is used in which the user specifies the pulse parameters and then signals the system to turn on power to the electrical connections. In the case of the pulsed electrodeposition process, a square wave pulsing scheme is used. Variables that can be specified include the number of cycles per second the potentiostat is to run, the percent on-time of the cycle peaks, and the baseline and peak potentials that will form the peaks. The system then displays the resultant potential at the working electrode relative to the reference electrode, and the current passing into the counter electrode as a function of time. This data can also be saved to a file, and an example of such a file is shown in Figure 2. 


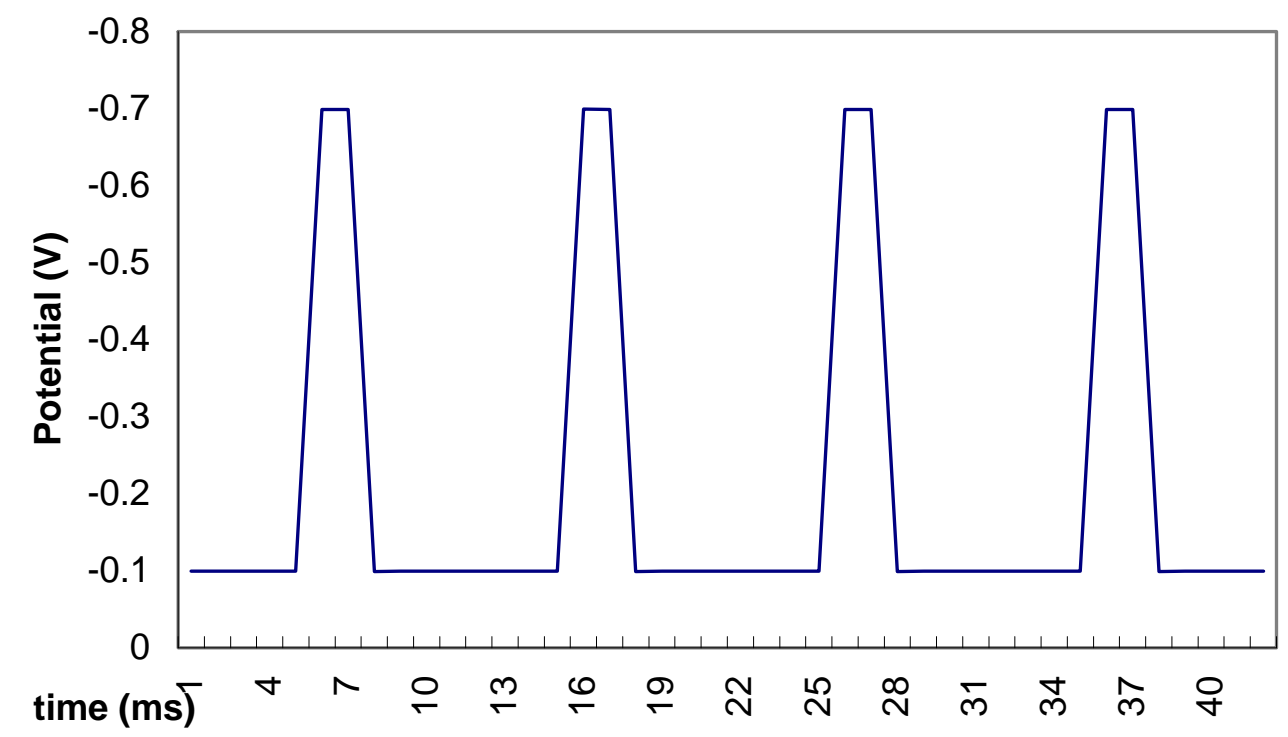

Figure 3: Potential pulse scheme for a $-0.7 \mathrm{~V}$ pulse potential, $-\mathbf{0 . 1 V}$ baseline potential, $2 \mathrm{~ms}$ on, $10 \mathrm{~ms}$ off pulse

Figure 3 shows the format of a $-0.7 \mathrm{~V}, 2 \mathrm{~ms}$ on, $10 \mathrm{~ms}$ off pulse scheme. Note that the pulse baseline is not set to 0 , but is still slightly negative, in this case $-0.1 \mathrm{~V}$. The baseline is typically set to $-0.1 \mathrm{~V}$ such that the current flow does not completely reverse itself during the pulse off-time and cause redissolution of some of the metal that has been deposited during the plating. The resulting current flow caused by the application of this pulsing scheme to a platinum electrode set with a solution containing 50ppm lead, copper, and cadmium is shown in Figure 4. 


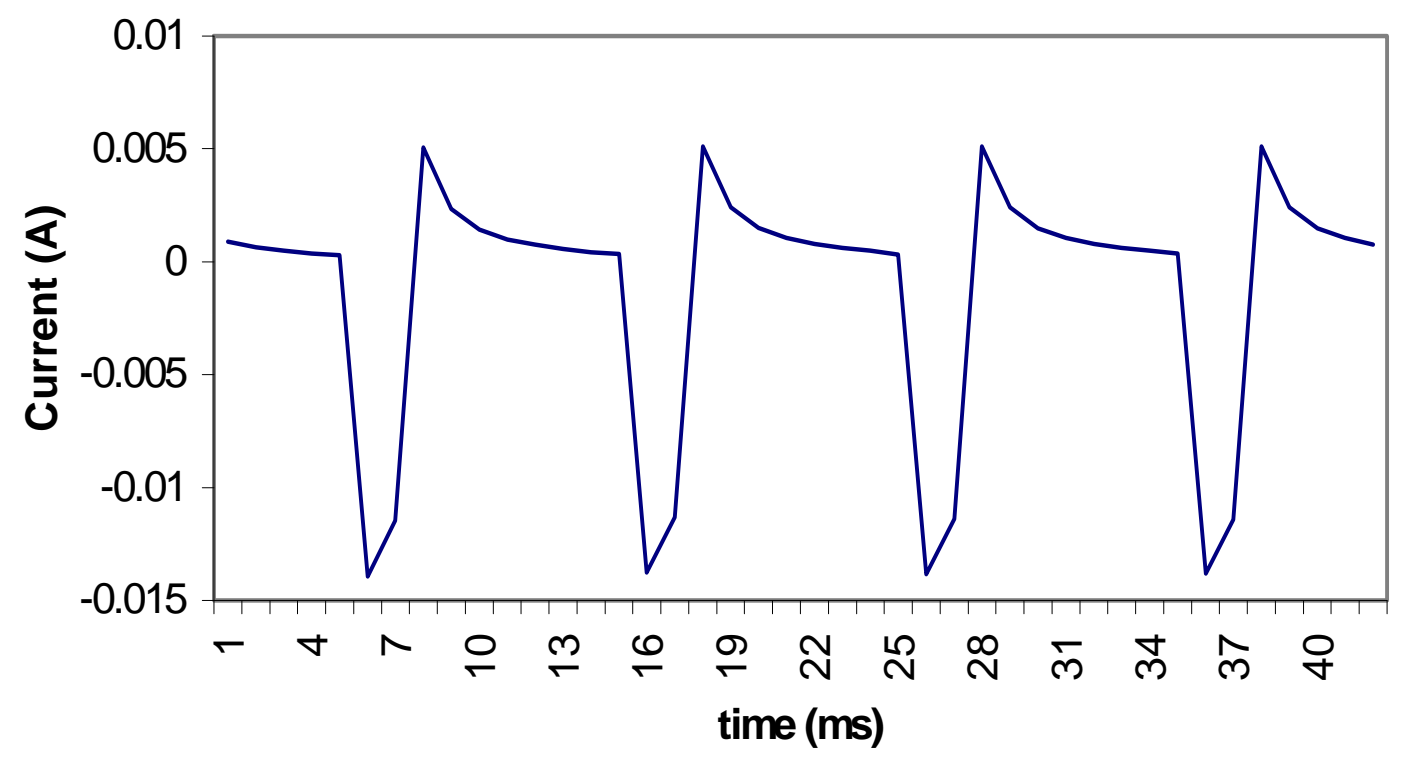

Figure 4: Current vs. time for a $-\mathbf{0 . 7 V}, 2 \mathrm{~ms}$ on, $10 \mathrm{~ms}$ off pulse scheme.

The resultant current shows an exponential decay when the voltage is changed both to the on cycle and to the off cycle. This is due to a capacitive charging effect that occurs when the charged water molecules are moved into and out of an electrical double layer that is formed. This capacitive charging effect creates an artificially high current in the direction the voltage is moved. When the capacitive decay is complete, it can be seen that the off cycle current is nearly zero. If the on cycle is allowed to continue on further the same effect of the current settling down to a value can be observed, and this value would be much closer to the true current due to electrodeposition in the system.

Using the potentiostat system, it is possible to vary a number of parameters, such as pulse on-time, off-time, potential peak height and potential baseline, and the effect of varying these variables can be analyzed to determine the behavior of the system.

\section{RESULTS AND DISCUSSION}

\section{General Results and System Performance:}

Overall, it was found that the pulsed electrodeposition technique using flow through mesh electrodes is an effective method for removing dissolved metal ions from aqueous media. The results obtained from experimentation correspond well with the results expected at the outset based upon electrochemical theory (see Appendices A and B). In a reasonable amount of time, the concentration of dissolved metals in dilute solutions is drastically reduced, despite the mass transport limitations present in working with dilute solutions. Pulsed electrodeposition is effective in overcoming these mass transport limitations to the extent necessary to achieve reasonable rates of removal. Characterization of the behavior of the electrode apparatus and pulsed electrodeposition technique requires examination of a number of variables which can influence the behavior of such a deposition process. These variables include the pulse on-time, pulse off-time, flow rate of the solution through the apparatus, the material of construction of the electrode and that materials surface area, the concentration of the dissolved metal ions in the feed solution, the ionic strength of the solution, and the potential of the electrodes during the pulse on-time. An examination of the 
influence of these variables on the behavior of the system will provide a useful characterization of the behavior of the system.

\section{Pulse Off-time:}

Variation of the pulse off-time is useful in the determining the most useful compromise between the rate at which the dissolved metal ions are removed from solution and the amount of electrical energy that must be spent to do so. Determination of an optimum pulse off-time can be seen as a balance between giving the solution enough relaxation time when the potential is not applied such that the boundary layer can be replenished with ions to plate out, and with having enough pulses such that the electrode is not idle too long and the rate of removal drops to a low level. When the potential is applied to the electrode in order to plate out the dissolved metal ions, the boundary layer is rapidly deleted of the dissolved metal ions and the rate of deposition drops of substantially. By removing the potential, the boundary layer is allowed to replenish the concentration of dissolved metal ions for deposition during the next pulse of potential. The limiting current density in a transient system is higher than that in a static system, so the pulses should be kept short enough that the static behavior is not reached, so that when the electrode is energized, the transient limiting current density is the result. It is therefore necessary to have a pulse off-time short enough to allow for the maximum amount of pulses in a period of time, but long enough to allow the boundary layers to replenish with dissolved metal ions. Figure 5 shows the variation of pulse on-time with the concentration of lead and copper after one hour of operation.

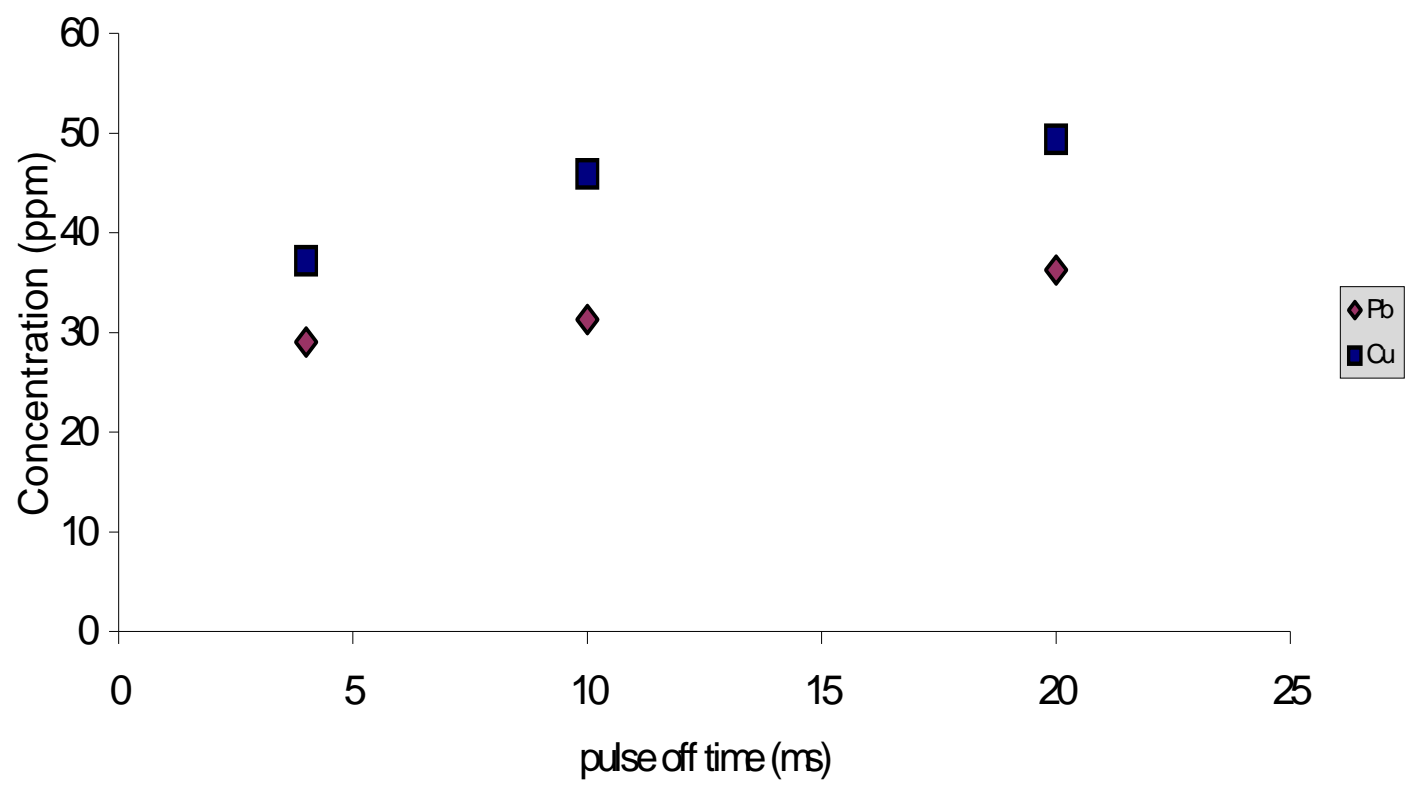

Figure 5: Pulse off-time vs. Concentration after 60 min of Plating, $-0.7 \mathrm{~V}$ pulse potential, $-\mathbf{0 . 1 \mathrm { V }}$ basline potential, $2 \mathrm{~ms}$ pulse on time, $10 \mathrm{~ms}$ pulse off time 
As can be seen from Figure 5, a shorter pulse off-time will lead to a reduced concentration at the end of a 60min test. However, the power consumption of the cycles having shorter pulse off-times is much higher due to the potential being applied to the electrode much more frequently. The rate increase is not proportional to the power expenditure increase, and so the efficiency of cycles having a shorter off-time is much lower than that of cycles having a longer off-time. A reasonable optimum then would be a compromise between an increased rate due to the increased number of pulses, and an increased efficiency due to decreased power consumption. A pulse off-time of $10 \mathrm{~ms}$ has been seen to be a useful compromise between the competing factors, and is the off-time that has been used for the majority of the tests in these experiments.

At the beginning of research it was hypothesized that electrode off-time would be optimized by finding a balance between the rate of removal and the efficiency of removal based on power consumption. This was in fact the case. A shorter off-time did lead to an increase in the rate of removal of dissolved metal ions from the aqueous solution. The decrease of the off-time from 10 milliseconds to 5 milliseconds brought about a rate increase of from $18.725 \mathrm{ppm} /$ hour to $20.977 \mathrm{ppm} /$ hour, a 12\% rate increase. However, this rate increase was brought about by energizing the electrode $28.6 \%$ of the time instead of $16.7 \%$ of the time, which is an increase of $71 \%$ in power consumption. When the pulse off-time was decreased from 20 milliseconds to 10 milliseconds, the removal rate was also improved by $36.5 \%$, with an attendant power consumption increase of $67 \%$. While the efficiency of the 20 millisecond off-time is still greater, the tradeoff between efficiency and rate in decreasing the off-time from 20 milliseconds to 10 milliseconds is not nearly as drastic as when decreasing the off-time from 10 milliseconds to 5 milliseconds. In most circumstances, the decreased power consumption will be worthwhile to achieve the higher rate, and so 10 milliseconds is considered a good compromise between rate and efficiency. This result is what was expected from the outset of development.

\section{Pulse On-time and Direct Current Plating vs. Pulse Plating Schemes:}

There are two different electrodeposition techniques that can be used to remove dissolved metal ions from the aqueous media, direct current plating and pulsed plating. While both methods can effectively remove dissolved metal ions from solution, each has advantages and disadvantages with respect to rate of removal, power consumption, and electrode reliability. 
Direct current electrodeposition offers the higher rate of the two methods, due to the fact that it is in an always on mode, whereas in using pulsed electrodeposition, the electrode is energized only $16.7 \%$ of the time (in using the typical $2 \mathrm{~ms}$ on, $10 \mathrm{~ms}$ off pulsing scheme). Therefore, as can be seen from Figure 6, the rate of the DC plating is somewhat higher than for the pulsed plating.

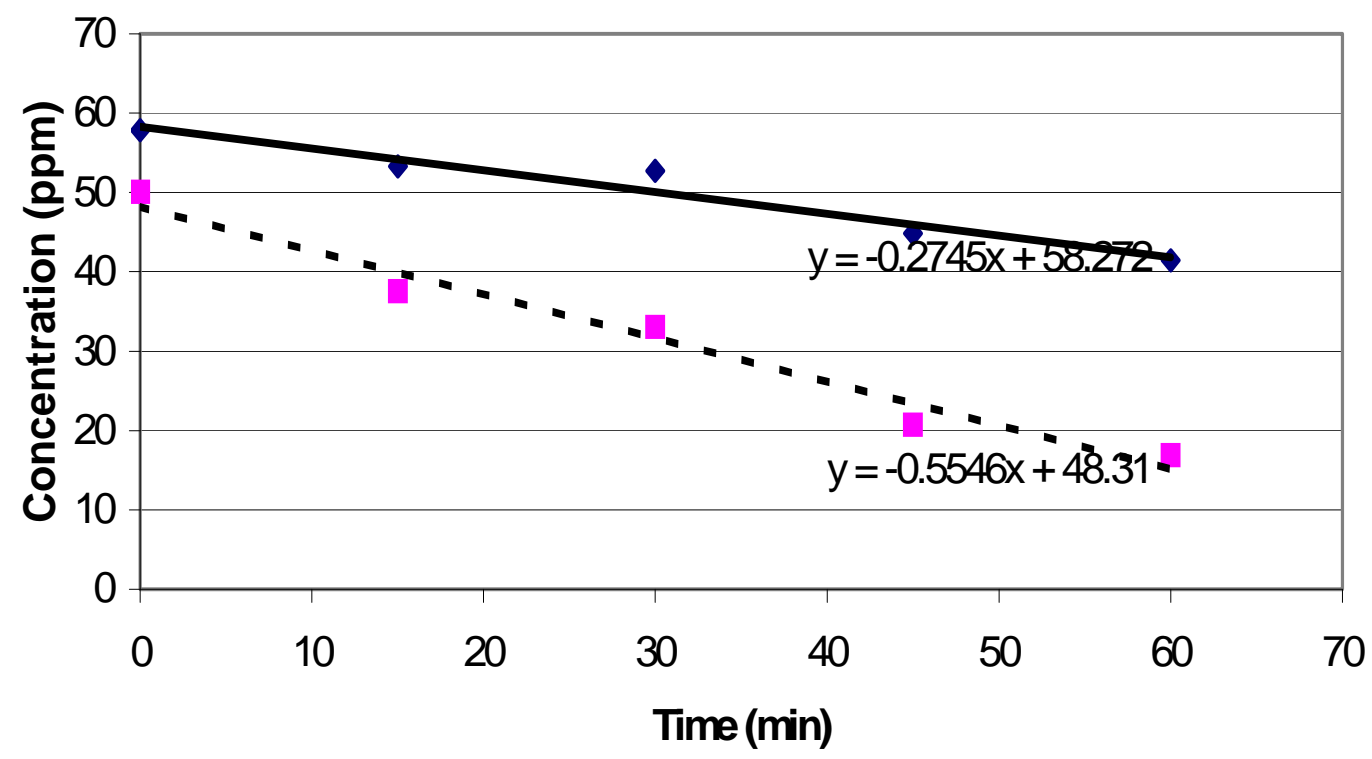

Figure 6: DC plating rate (square marks) vs. Pulse Plating rate (diamond marks), 2ms pulse on time, $10 \mathrm{~ms}$ pulse off time, $-0.5 \mathrm{~V}$ pulse potential, $-0.1 \mathrm{~V}$ baseline potential

The rate of removal shown for DC plating is nearly double the rate of pulse plating, $0.55 \mathrm{ppm} / \mathrm{min}$ versus $0.275 \mathrm{ppm} / \mathrm{min}$. However, in achieving these rates the DC electrodeposition requires that the working electrode be energized constantly, whereas the pulsed electrodeposition requires that the electrode only be energized $1 / 6$ of the time. In terms of the efficiency of the process, the pulsed electrodeposition is therefore 3 times more efficient than the direct current electrodeposition in terms of metal ions removed from solution for the amount of energy consumed, and this can make a large difference in the operating costs of such a system.

This increased efficiency is due to the fact that in pulsed electrodeposition, when the electrodes are energized the are of removal of the metal ions from solution is 3 times that of the rate of removal of metal ions from solution during direct current electroplating. This can be directly attributed to the fact that the transient limiting current density of a system is much higher than the steady state limiting current density of a system, and this increased efficiency due to pulsed electrodeposition is seen in the results of the experiments.

The structure of the deposited metal layers is also different for direct current plated layers versus pulse plated layers. Direct current electrodeposition tends to form a dendritic structure, as the layer grows from the working electrode towards the counter electrode in as much of a straight line as is possible. This can cause short-circuiting and failure of the electrodes in a relatively short period of time. Pulsed electrodeposition, on the other hand, tends to form a more dense, slab-like growth of the deposited metal, leading to electrodes 
that can be used longer and more reliably. This was in fact observed in the operation of the electrode apparatus, as the apparatus was able to be used for a number of weeks before failure during pulsed electrodeposition, but only a day or two when direct current electrodeposition was used.

From study of the theory of limiting current densities, as given with equations 1,2 , and 3 in Appendix A, it would seem that the pulsed electrodeposition would enjoy advantages in both power efficiency and removal rate when compared to direct current electrodeposition, in most circumstances. During these experiments, it was found that this was only partially true, with the direct current plating scheme providing a higher rate, but the pulsed electrodeposition providing a higher efficiency. Coupled with the increase in the quality of the deposited metal morphology and electrode reliability, the increased efficiency of the pulsed electrodeposition scheme provides a compelling reason for choosing to use that form of electrodeposition. The increase in rate from using D.C. plating rather than pulse plating is approximately $100 \%$, however, the increase in power consumption is nearly $500 \%$. Therefore, pulse plating is the obvious choice when taking power consumption into account. The reliability of the apparatus when pulse plating is also found to be greatly enhanced, providing many times the service life before failure than when using D.C. plating, leading to a more effective system for long term operation. This also corresponds to what was predicted.

\section{Flow Rate:}

The flow rate of solution through the plating apparatus was another factor investigated in this research. The flow rate of solution through the apparatus affects the residence time of the solution at the electrodes, and also the number of passes through the apparatus the fluid will make in a given period of time. These tests were run using the platinum electrode sets. Because the platinum electrodes are $25 \mathrm{~mm} \times 25 \mathrm{~mm}$ and constructed using $0.0762 \mathrm{~mm}$ wires, and assuming the mesh is twice the wire thickness due to the overlapping, the total volume of the working electrode area is approximately $95.25 \mathrm{~mm}^{3}$, or approximately $0.09525 \mathrm{~cm}^{3}$. For a $1 \mathrm{~cm}^{3} / \mathrm{sec}$ flow rate, this gives a residence time at the working electrode of approximately $.09525 \mathrm{sec}$, or about 95 milliseconds. Using a pulsing time of 2 milliseconds and a relaxation time of 10 milliseconds, and the average volume of fluid will experience 95/12, or 7.92, pulse cycles during each pass. Using a higher flow rate will lower the number of pulse cycles per pass acting on the solution, but will increase the number of passes the fluid makes through the system in a given period of time. Because of these competing factors, the flow rate had little effect on the rate of electrodeposition of the metal ions from the solution, as can be seen in figure 6 . 


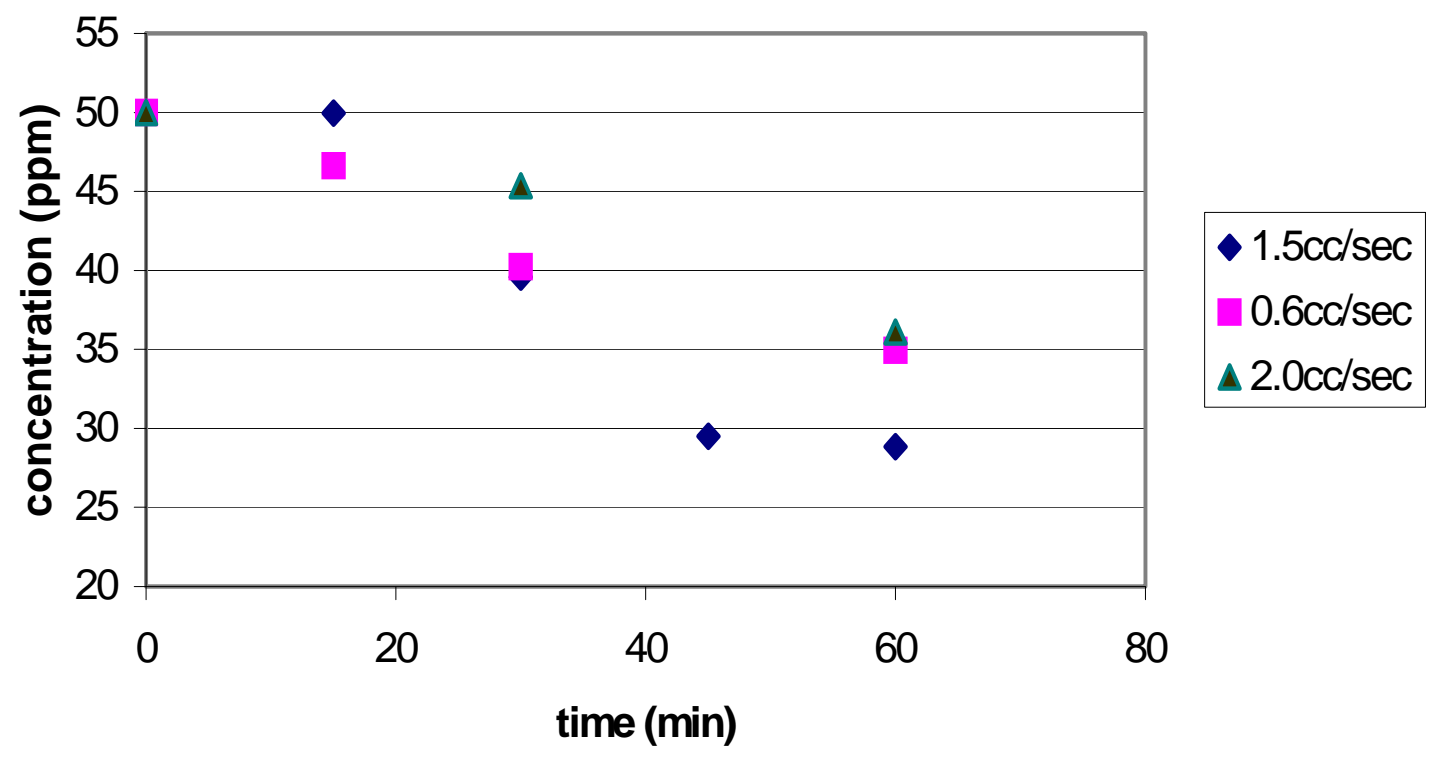

Figure 7: Pb Concentration vs. Time at different Flow Rates, $2 \mathrm{~ms}$ on time, 10ms off time, $-\mathbf{- 0 . 7 V}$ pulse potential, $-0.1 \mathrm{~V}$ baseline potential

The rates shown in Figure 7 show that the rate of removal of lead from solution is nearly exactly the same after 30 minutes of operation. While the rates of removal for the flow rates do not match up exactly at all times, they are close.

While the flow rates tested give approximately equal results, it is possible that this behavior could deviate greatly at very low or very high flow rates. However, it is impossible to test for these flow rates, as flow rates much higher than 2cc/sec will cause the electrode apparatus to fail, and rates much lower will cause the reference electrode to be out of contact with the fluid, and therefore the potentiostat will not function properly. In constructing a system where the fluid makes only one pass through the system, the flow rate could greatly influence the final concentration of metal ions in the solution, as the number of total pulse cycles acting to remove the metals from solution would be more greatly influenced.

Increases in flow rate did not substantially affect the rate of removal of dissolved metal ions from the solution using the pulsed electrodeposition apparatus. However, a number of experimental factors influenced the ability to examine the affect of flow rate on the system. Firstly, the range over which the pumping system could provide enough fluid to keep all electrodes, including the reference, in fluid contact necessitated a flow rate of at least about $0.5 \mathrm{cc} / \mathrm{sec}$ be provided to the system. Secondly, pumping rates over $2 \mathrm{cc} / \mathrm{sec}$ resulted in the failure of the caulking which held together the electrode apparatus, thereby destroying its ability to function properly. This meant that the relatively narrow range of $0.5 \mathrm{cc} / \mathrm{sec}$ to $2 \mathrm{cc} / \mathrm{sec}$ was the only range in which the flow rate could be tested, and this is a relatively small range. Also, because the solution was always ran through the apparatus for multiple passes, certain conclusions can be drawn about the effect of flow rate. In such a system, an increased flow rate will result in the residence time of the solution near the electrodes to be less during each pass, but the number of passes of the solution by the electrode will be increased. It can then be concluded that for a single pass through the system, lower flow rates and longer residence times in the apparatus will increase the removal of dissolved metal ions from the solution. This is what would be expected intuitively, as the longer the metal 
ions stay near the electrodes, the greater the chance they can undergo reduction to the solid metal form. Increasing the flow rate would require increasing the number of passes through the electrodes if the same removal of metal ions from solution is desired.

\section{Electrode Material of Construction and Surface Area:}

Two main materials of construction were examined for use in constructing the electrodes, platinum mesh and carbon felt. The platinum mesh electrode is composed of thin platinum wires woven in an interleaved pattern, forming a mesh approximately 2 wires thick. The platinum mesh electrode used in these experiments has an open area of approximately $49 \%$. The carbon felt electrode is composed of a tightly packed, small diameter carbon wires that form a porous pad approximately $1 \mathrm{~cm}$ thick. This creates an electrode with nearly no open area. Both types of electrodes are positioned in a housing such that the solution containing the dissolved metal ions flows perpendicularly through the mesh. The necessary working electrode properties are that they are good conductors of electricity with fairly low resistance, and that they are a compatible surface onto which the metal ions can plate out. For the counter electrodes, the electrodes must be able to provide a location for the water hydrolysis counter reaction to take place without the electrodes themselves becoming degraded by the oxidation. Both materials seem to perform well given those design parameters, although the carbon electrode can seem to have some degradation problems when used as the counter electrode. When used as a working electrode, both materials perform well, and the primary factor influencing the rate of deposition on each electrode is the surface area of the electrode. This surface area does not correspond to the overall surface are of the electrode bulk, but rather to the surface area at the edge of the electrode facing the counter electrode, as the area of the electrode on which the reaction will readily take place is the area closest to the opposite electrode. Because of this, the carbon electrode has a larger effective surface area then the platinum electrode, and as Figure 8 shows, the rate of removal using the carbon working electrode is higher than that using the platinum working electrode.

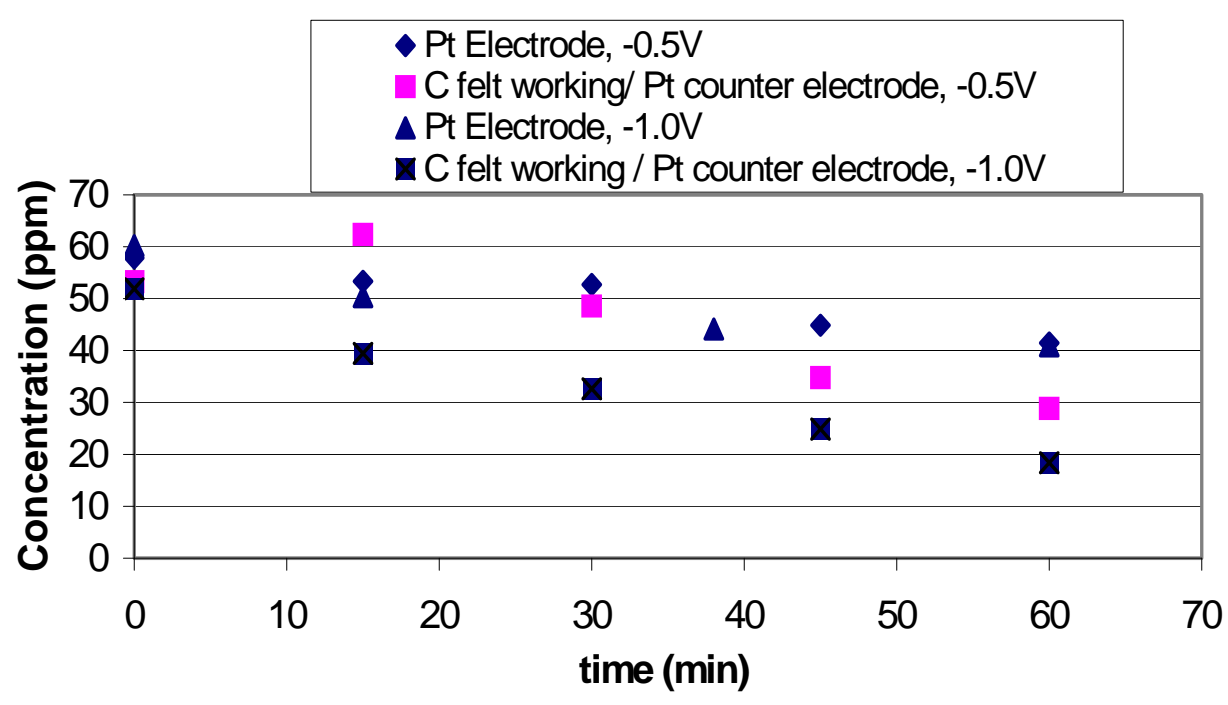


Figure 8: C felt vs. Pt electrode comparison, concentration vs. time, $2 \mathrm{~ms}$ pulse on time, $10 \mathrm{~ms}$ pulse off time

As can be seen from Figure 8, the carbon felt electrode has a higher surface area, and a corresponding higher removal rate of lead ions from the solution. Because the platinum mesh has a open area of approximately $49 \%$, the carbon felt has just under double the surface area of the platinum electrode. The rate of removal of the lead from solution using the carbon felt is also just under double that obtained using the platinum mesh, with the carbon felt removing lead from solution at a rate of $33.5 \mathrm{ppm} /$ hour and the platinum mesh removing the lead from solution at a rate of $19.23 \mathrm{ppm} /$ hour. The fact that the rate using carbon felt is not closer to double the rate using platinum can likely be attributed to the construction of the platinum electrode, as the epoxy sealing of the wire in the electrode reduces the surface area by isolating part of the electrode surface from the fluid flow. Overall, the surface area and removal rate correlate well.

It was originally hypothesized that increasing the surface area of the electrodes would have the effect of increasing the removal rate of dissolved metal ions from the solution. It was thought that the large increase in surface area obtained by using a carbon felt electrode would largely increase the removal rate, and while the removal rate was increased by the use of a larger surface area electrode, the result is not as dramatic as was expected. Rather than the entire surface area of the electrode being a factor, only the surface area that is near the opposite electrode has an effect. This is due to the tendency of the reaction to occur over the shortest distance possible for electron travel, which is between the closest points of the two electrodes. As such, the additional depth of the carbon felt electrode did not enhance the reaction rate, only the additional surface area presented at the very surface of the electrode. Thus, commercial systems would be more effective using a larger number of closely placed screens, rather than utilize fewer electrodes with higher surface areas. However, the increase in the surface area at the electrode surface does have a positive effect on the removal rate.

The material of construction of the electrode was not found to be critical as long as some basic requirements are met. The electrodes must have good electrical conductivity and must provide a good site on which the electrochemical reactions can occur. Additionally, the counter electrode must be able to provide a place for the electrochemical counter reaction to occur without being oxidized or degraded itself by the reaction. For these reasons, both platinum and carbon felt work well as working electrodes, and platinum worked best for the counter electrode. Other materials can be used effectively in such an electrode apparatus, so long as the aforementioned design parameters are met.

\section{Dissolved Metal Ion Concentration:}

Because the rate of process of electrodeposition from dilute aqueous media is primarily mass transport limited, it would be expected that the concentration of dissolved metal ions in the solution would influence the rate of removal of the metal ions from the solution. This is in fact the case. The rate of removal of metal ions from a more concentrated solution is correspondingly higher, as can be seen in Figure 9. 


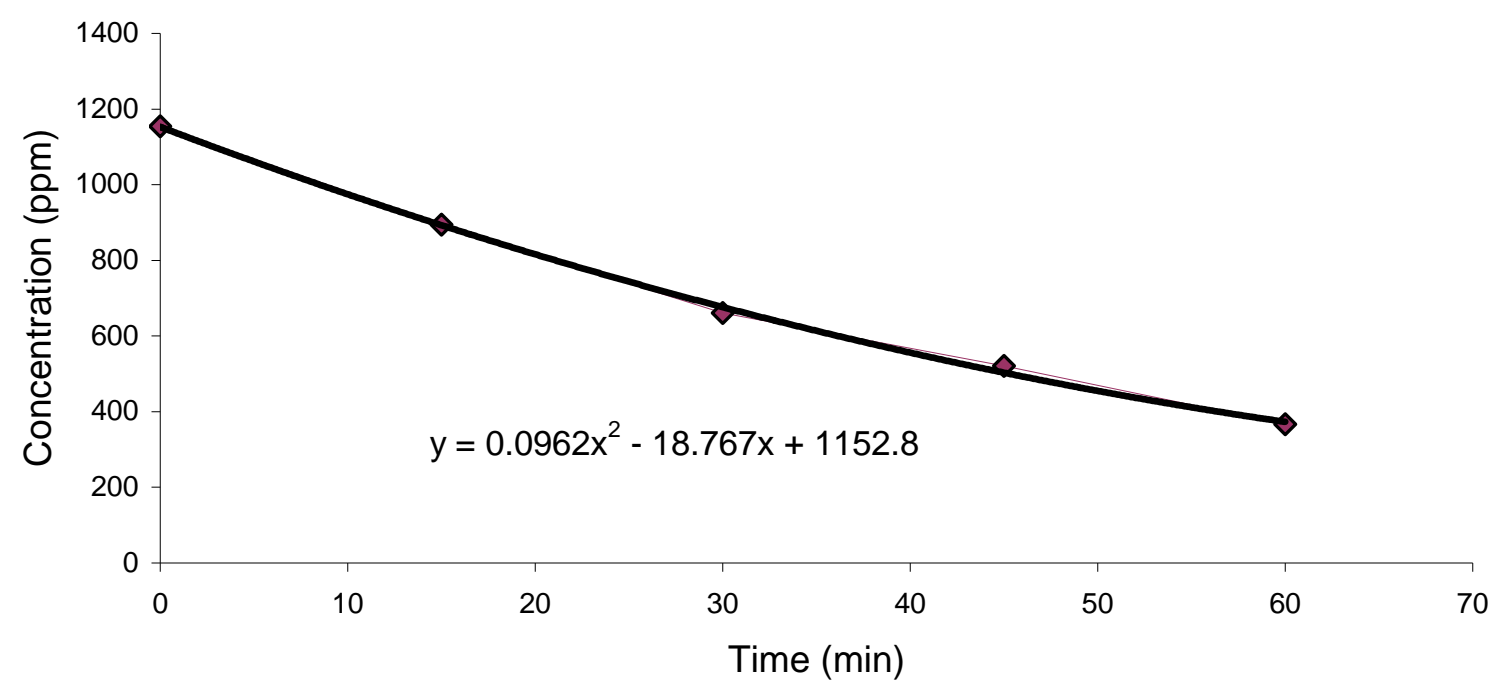

Figure 9: Lead Concentration vs. time in concentrated solution, Pt Electrode, -1V pulse potential, - -0.1V baseline potential, $2 \mathrm{~ms}$ pulse on time, $10 \mathrm{~ms}$ pulse off time.

In Figure 9, it can be seen more concentrated solutions experience a correspondingly higher removal rate than dilute solutions, simply because more material can be transported to the electrode surface to be plated out. In Figure 10, the more dilute solution is shown to have a much slower rate of removal of the dissolved metal ions, because the mass transport limited rate is much lower.

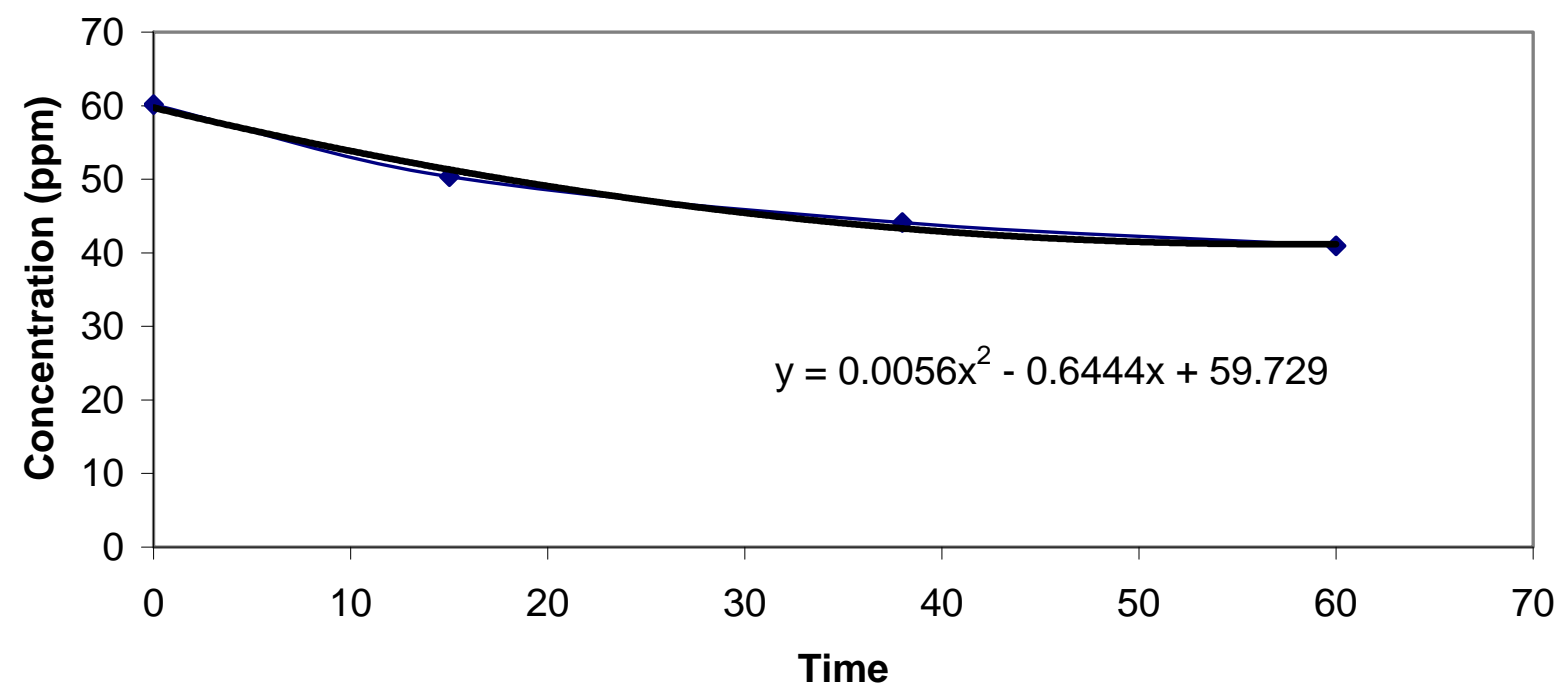

Figure 10: Lead Concentration vs. Time in dilute solution, Pt Electrode, $-\mathbf{- 1 . 0 V}$ pulse potential, $\mathbf{- 0 . 1 V}$ baseline potential, $2 \mathrm{~ms}$ pulse on time, $10 \mathrm{~ms}$ pulse off time 
It is therefore surmised that the rate of removal of the dissolved metal ions is dependent on the concentration of the dissolved metal ions in the solution. A curve fitting of the rates of removal of both the concentrated solution and dilute solution removal rates to one curve shows that the behavior follows a function, and in finding a function that fits well, a mathematical relationship between concentration and removal rate can be found. From the theory of transient limiting current density, the equation (Equation 2) shows the removal rate, which is the change in concentration with time, is a linear function of concentration and time, and so a second order polynomial would be expected to fit the data of concentration versus time well, and it indeed does. Therefore, it can be seen that the removal rate is of metal ions from solution is highly dependent on the concentration of dissolved metal ions in the solution.

Because the limiting current density equations (see Appendix A) for both the transient and steady states include a term for the bulk concentration of dissolved metal ions in the solution, it was expected that the rate of removal of the dissolved metal ions vary with the concentration of the metal ions in solution. This was in fact the case. The rate of removal of dissolved lead ions from a solution initially containing $1154 \mathrm{ppm}$ was $788 \mathrm{ppm} / \mathrm{hour}$, while the removal rate of dissolved lead ions from a solution initially containing 55.9ppm was $30.2 \mathrm{ppm}$ per hour. Increasing the concentration of lead in solution by 20.63 times lead to an increase in the removal rate of 26.05 times. This approximately follows the linear relationship expected from equation 2 , and the fact that the numbers do not match entirely can be attributed to random experimental variation and the influence of concentration on other factors, such as mass transport properties of the solution. The presence of other ions in the solutions, particularly the concentrated solutions, can exacerbate these effects. Overall, the feed dissolved metal ion concentration influences the system in practice the way it would be predicted from the theory.

\section{Ionic Strength of the Solution:}

The ionic strength of the solution determines the electrical resistance of the solution, which in turn determines how much power must be used to supply electrons to the reaction. A higher solution conductivity means that less potential must be applied at the counter electrode in order to overcome the resistance of the solution, and so power consumption is lower. The lower power consumption has little effect on the overall rate of the reaction, and it can be seen in Figure 11 that the rate of removal of dissolved metal ions from solution is not substantially influenced by the ionic strength of the solution. 


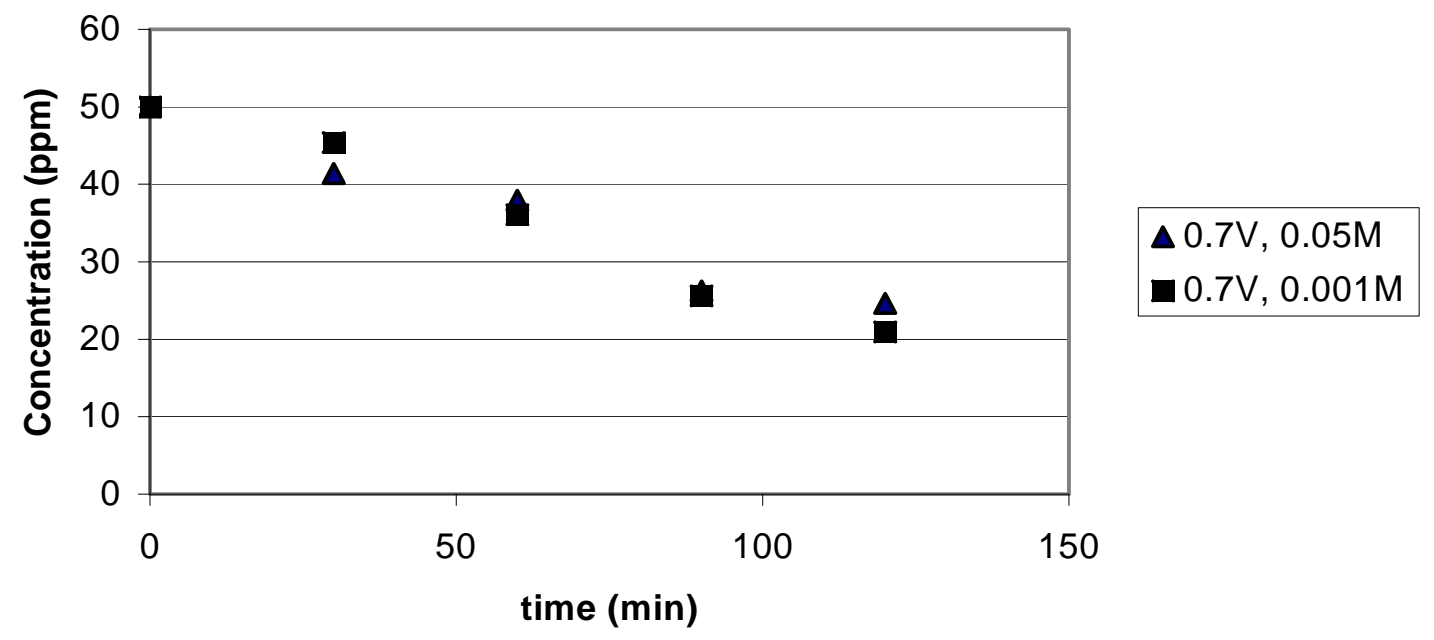

Figure 11: Pb Concentration vs. Time at different ionic strengths, $-0.7 \mathrm{~V}$ pulse potential, $-\mathbf{0 . 1 V}$ baseline potential, $2 \mathrm{~ms}$ pulse on time, $10 \mathrm{~ms}$ pulse off time.

As shown in Figure 11, the rate of removal of the lead ions from solution in solutions containing $0.05 \mathrm{M} \mathrm{NaNO}_{3}$ and $0.001 \mathrm{M} \mathrm{NaNO}_{3}$ are substantially the same. This would not be true of the power necessary to run the cell however, and the added electrical resistance in the $0.001 \mathrm{M}$ solution would require more power and a higher potential at the counter electrode than in the $0.05 \mathrm{M}$ solution.

It was originally hypothesized that the ionic strength of the solution would have little effect on the removal rate of ions from the solution, but would have an effect on the power required to do so. This is due to the fact that a solution with lower ionic strength will have a lower electrical conductivity, which is analogous to a higher electrical resistance, and the potentiostat must supply more power to the system in order to overcome that electrical resistance. The original hypothesis was found to be in fact true, with the rate of removal of metal ions from solution being little affected by the ionic strength of the solution.

\section{Electrode Potentials:}

Perhaps the most useful variable in determining the rate of removal of dissolved metal ions from the solution is the potential at which the electrodes are held during the pulse "on" cycle. Each element dissolved in solution has a potential below which it will plate out of the solution, and above which it will dissolve back into the solution. Above its plating potential, the rate of deposition of the dissolved metal ions onto the electrode is negative, as the metal will favor dissolution at those potentials. At potentials below the plating potential, the metal will plate out of the solution onto the electrode during the pulse plating cycles. The rate at which this plating occurs at or just below the plating potential of the element is low, however, the rate increases as the potential is lowered further from the plating potential of the element (known as using an over potential relative to the plating potential of the element). However, this influence of the over potential of the electrode on the rate is low except very close to the plating potential of the element when the concentration of the element in solution 
is low, due to the fact that the rate is so heavily mass transport limited. Figure 12 shows the removal rate of lead ions from solution vs. the pulse on-time potential of the electrode.

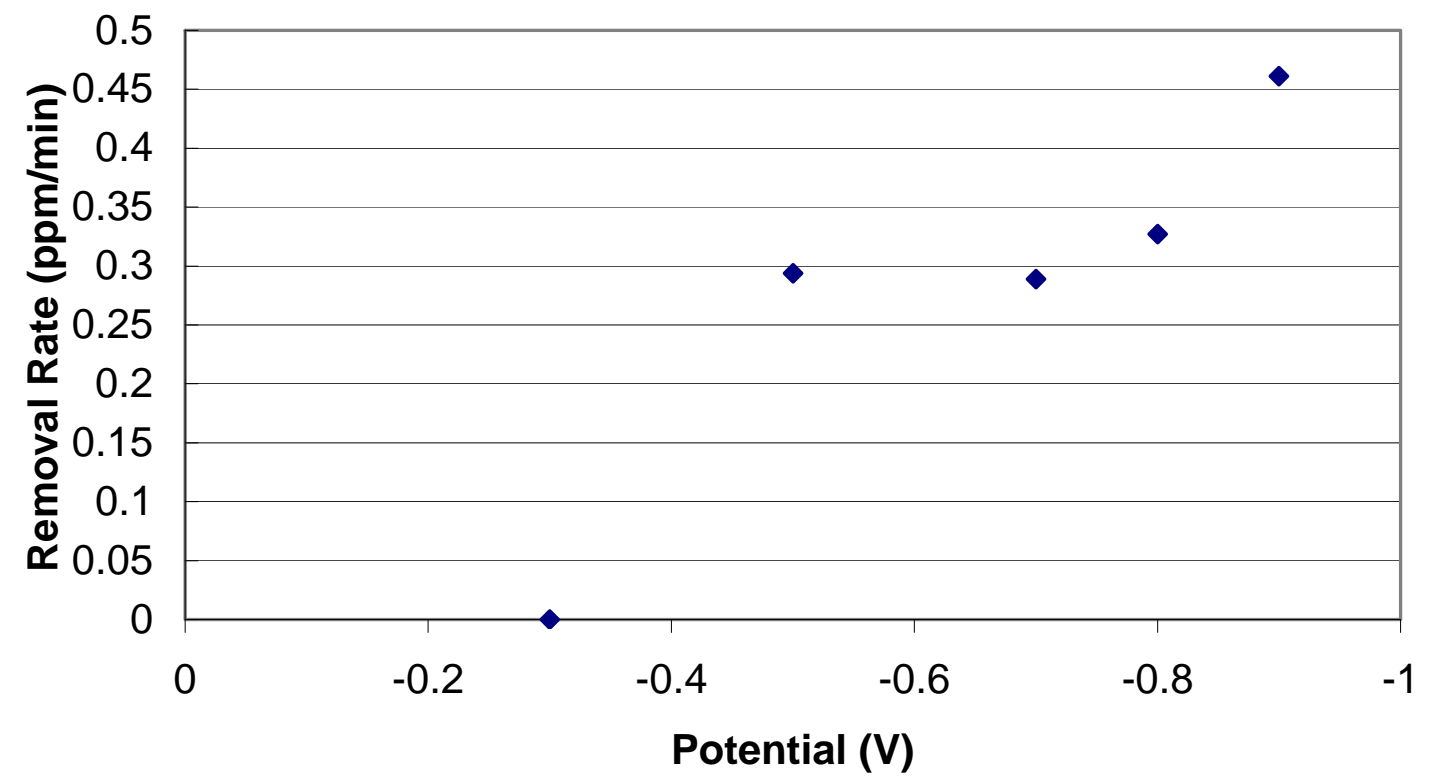

Figure 12: Removal Rate vs. Potential for $\mathrm{Pb}, 2 \mathrm{~ms}$ pulse on time, $10 \mathrm{~ms}$ pulse off time

In Figure 12, at $-0.3 \mathrm{~V}$ no lead removal takes place because the potential is above that necessary to remove lead from the solution, which is slightly above $-0.4 \mathrm{~V}$. At $-0.4 \mathrm{~V}$, lead is effectively removed from the solution, and decreasing the potential has a minimal effect on the lead removal rate. Figure 13 shows that a similar behavior is seen with cadmium, with the difference being that cadmium has a lower removal potential, and so the rate stays at 0 until that potential is reached at just below $-0.7 \mathrm{~V}$.

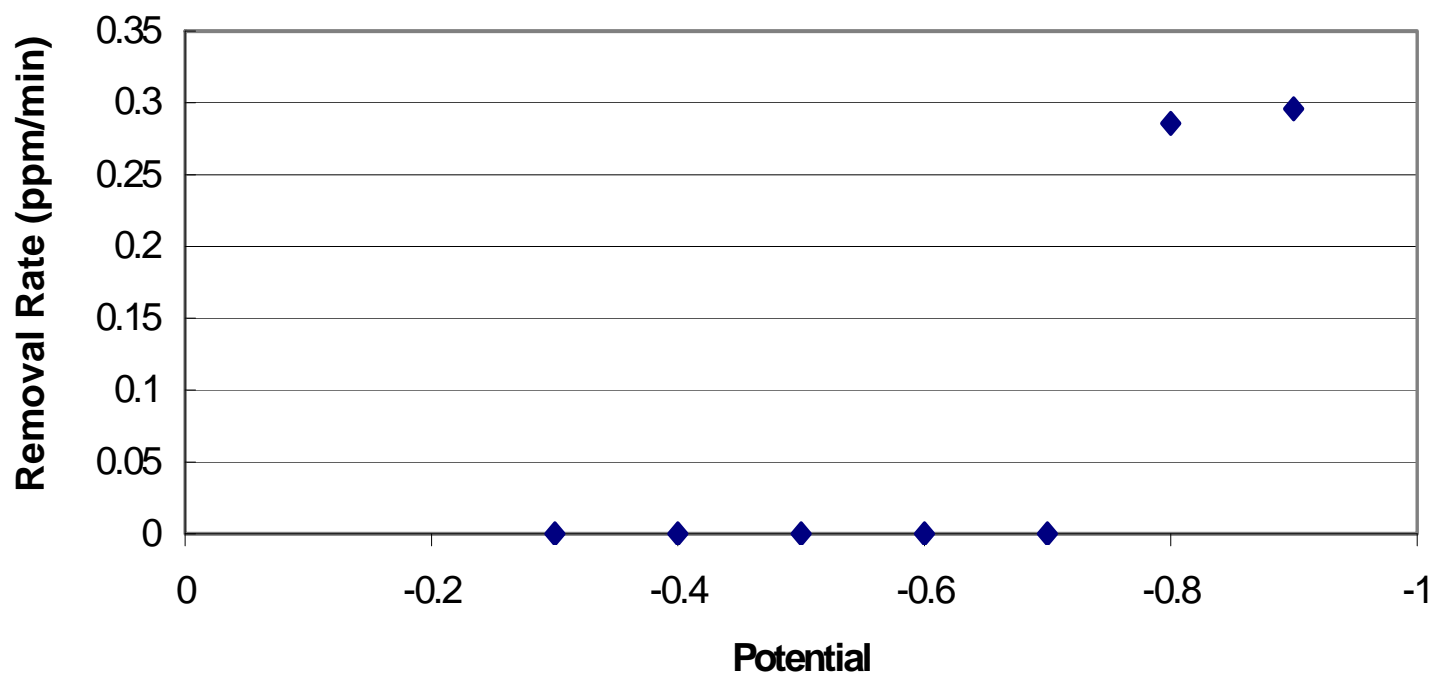

Figure 13: Removal Rate vs. Potential for Cd, $2 \mathrm{~ms}$ pulse on time, $10 \mathrm{~ms}$ pulse off time 
By using electrodes set at $-0.5 \mathrm{~V}$., lead can be removed from the solution, while the cadmium will remain in solution. In this manner, lead and cadmium can be separated from each other to create substantially pure metal products. In the case of the binary solution of lead and cadmium a pure lead product would be attainable. Because there would always be some amount of lead remaining in the solution, the cadmium product would contain some amount of lead. Therefore, it would be desirable to remove as much lead as possible by pulse plating the lead out at a sufficient rate for a sufficient amount of time such that the amount of lead remaining is minimal, before the cadmium plating is begun. In this manner, it is possible to separate a large number of metals from solution in a purified form, so long as the rate at which the metals are removed is reasonable, and the metals can be plated out in series based upon discernable plating potentials.

The removal rate versus potential can also be shown for copper, as in Figure 14. In this case, copper always comes out at some rate, because the electrochemical potential of copper is higher than for lead or cadmium. 


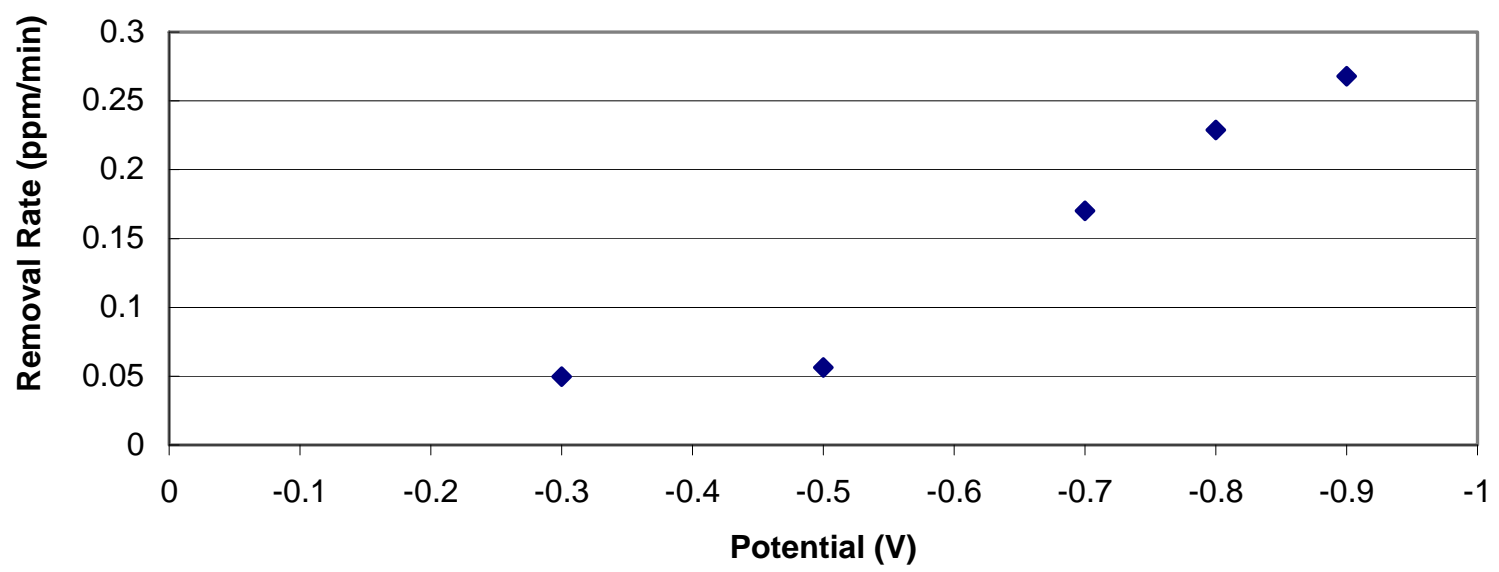

Figure 14: Removal Rate vs. Potential for $\mathrm{Cu}, 2 \mathrm{~ms}$ pulse on time, $10 \mathrm{~ms}$ pulse off time

Compared with lead or cadmium, copper has a slower removal rate, and this is only alleviated slightly by use of a higher overpotential. Therefore, in order to obtain similar removal of copper from a solution, a larger number of screens must be employed. The residual copper in any solution could also be higher because of this, and so obtaining a purified lead product from a solution containing copper, lead, and cadmium would be more difficult and expensive.

A plot of all three elements' removal rates versus potential shows that as the potential is made more negative, more elements will be removed from solution, as shown in Figure 15. Using such information, the potential necessary to obtain the desired product can be determined. 


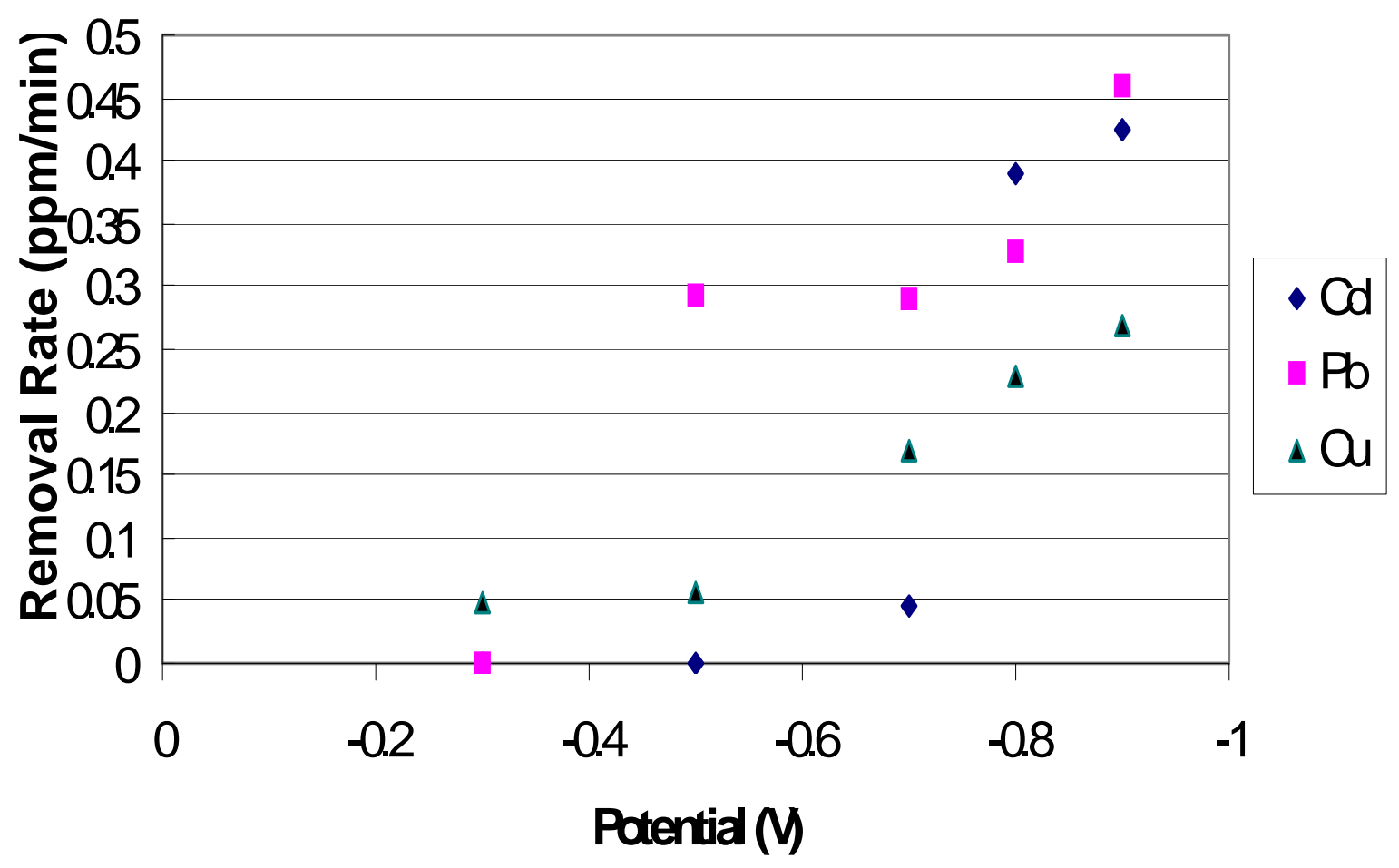

Figure 15: Removal Rate vs. Potential for $\mathrm{Cu}, \mathrm{Pb}$, and $\mathrm{Cd}, 2 \mathrm{~ms}$ pulse on time, $10 \mathrm{~ms}$ pulse off time

From Figure 15 it can be seen that different potentials will lead to a different product obtained by electrodeposition. At $-0.3 \mathrm{~V}$, for instance, a substantially pure copper product will be formed. At $-0.5 \mathrm{~V}$, a product containing mostly lead with some copper will be formed. At $-0.9 \mathrm{~V}$, a product containing large amounts of copper, lead, and cadmium will be formed.

It was originally hypothesized that individual metal ions could be recovered from the solution based on the unique electrochemical potential of the dissolved metal ion. This was found to be true in most circumstances, however, the rate of removal of the different ions tended to vary greatly. For example, it is difficult to recover a substantially pure product of lead from a lead-copper solution because the rate of copper removal is quite low, and the amount of time required to remove substantially all of the copper from solution would be quite large. However, recovery of lead and cadmium in from a binary solution of lead and cadmium would yield a highly pure lead product, as lead can be recovered from the solution at a reasonable rate, after which the cadmium can be recovered from the solution now depleted of almost all lead. In some circumstances involving complex solutions containing many species of dissolved metal ions, the rates of certain ions being removed from solution were negligible. This is thought to be due to a phenomenon known as anomalous plating. Certain combinations of metal ions are known to have some kind of interference reactions with one another, whereby ions will not be removed from solution even at potentials below their electrochemical potentials. In these experiments, a solution containing a mixture of dissolved mercury, copper, iron, lead, cadmium, and zinc would only show effective removal 
of lead and mercury from the system, regardless of the potential used. Some kind of anomalous plating phenomenon is thought to be responsible for the lack of any appreciable rate of reduction of copper, iron, cadmium, and zinc. Solutions containing only copper, lead, and cadmium alone showed reasonable reductions in the levels of copper and cadmium, so it is thought that the presence of either iron or zinc causes some kind of interference with the removal of either cadmium or copper. A more complex relationship could also be true, with the presence of all four ions simultaneously being the source of the problem.

Overall, in systems in which anomalous plating phenomena were not observed, the system behaved as predicted. While the rate of removal of each metal ion varies from one ion to the next, the rate of removal for any given ion is always zero when the working electrode potential is higher than the electrochemical potential of the given element. Using this property, it is possible to recover metals individually on the basis of their electrochemical potentials from the solution, as was initially predicted.

\section{CONCLUSION}

From the data obtained from experimentation, it can be concluded that the pulsed electrodeposition method and apparatus provide a useful way to recover dissolved metal ions from dilute solutions. The use of pulsed electrodeposition provides clear advantages over direct current electrodeposition. Optimal design parameters can also be developed by examining the behavior of the system under a variety of different conditions. It is also possible to construct a laboratory scale device to successfully remove metal ions from solution, and scale up to pilot or industrial scale systems is possible based upon information presented in this study. Such a system could be used for separating different dissolved metal ions from each other, as might be necessary in a recycling operation. Such a system might also be useful for mitigating water quality problems arising from the presence of dissolved toxic metal ions. This is an example of the successful application of electrochemical principles to the solving of real world problems.

\section{REFERENCES}

None cited

\section{APPENDICES}

\section{Appendix A - Pulsed Electrodeposition Theory}

The method investigated is the use of a pulsed electrodeposition technique and electrode system to remove dilute toxic metals from an aqueous solution. To be a viable method of metal recovery, the pulsed electrodeposition method must be able to remove the toxic metals from the dilute aqueous solution down to a concentration level where the hazard to the environment is substantially mitigated. This removal must be performed at a reasonable rate and in an economically competitive manner. The use of an electrodeposition method allows for the recovery of the metal in a purified form, from which the metal can be more easily sold or sent to a recycling operation. This also avoids the creation of a toxic 
waste precipitate that can only be disposed of by sending it to a toxic waste dump. The specific use of the pulsed electrodeposition method, as opposed to simply using a direct current electrodeposition method, offers numerous advantages for the removal of dissolved metal ions from dilute solutions. In pulsed electrodeposition, the metal tends to deposit in a dense plate, which allows for the electrodes to be used longer without short circuiting occurring than is allowed by the more dendritic growth deposits obtained from direct current plating. Pulsed electrodeposition can also result in a higher rate of deposition when the electrode is energized than direct current plating. The rate at which the dissolved metal ions can be removed from solution is controlled by the limiting current density of the electrochemical reaction. The limiting current density of the electrochemical reaction in a transient system, such as in pulsed electrodeposition, is much higher than that of the reaction in a more constant state, such as in direct current electrodeposition. While the electrodes are only energized for typically about $16.7 \%$ of the time, the rate of removal is $50 \%$ that of direct current plating, which is in a constantly energized state. Therefore, in terms of removal rate versus energy consumption, pulsed electrodeposition offers much higher efficiencies.

The controlling factor in the rate of deposition of the metal ions from solution in the electrodeposition process is the limiting current density, which is a measure of the rate of electron transfer to the reducing species from the counter reaction. In the case of direct current electrodeposition, this limiting current density can be expressed by equation (1):

$$
i_{l}=\frac{n F D C_{b u l k}}{z \delta}
$$

where, in equation (1), $i_{l}$ is the limiting current density, $\mathrm{n}$ is the number of electrons required to reduce the species from the dissolved state into the metal state, $\mathrm{F}$ is Faraday's constant $\left(96,485\right.$ Coulombs/mole), $\mathrm{D}$ is the diffusivity of the dissolved metal species, $\mathrm{C}_{\text {bulk }}$ is the concentration of the dissolved metal species, $\mathrm{z}$ is the number of electrons transferred in the rate limiting step, usually 1 , and $\delta$ is the boundary layer thickness of the solution at the interface. Equation (1) is for circumstances in which the rate is limited by diffusion, which is the case in dilute solutions of dissolved metal ions.

In the case of pulsed electrodeposition, the limiting current density can be expressed by equation (2):

$$
i_{l o T}=n F C_{b u l k} \sqrt{\frac{D}{\pi t}}
$$

in which $\mathrm{i}_{\mathrm{loT}}$ is the transient limiting current density, $\mathrm{n}$ is the number of electrons transferred to reduce the dissolved metal ions to pure metal, $\mathrm{F}$ is Faraday's constant, $\mathrm{C}_{\text {bulk }}$ is the concentration of the dissolved metal species, $\mathrm{D}$ is the diffusivity of the dissolved metal species, and $t$ is the time after the electrode has been energized.

If we rearrange equations (1) and (2) such that the terms common to each are on one side of the equation, then set the two equal, we can get ratio between the two limiting current densities, namely equation (3)

$$
\frac{i_{l o T}}{\sqrt{\frac{D}{\pi t}}}=\frac{i_{l} z \delta}{D}
$$

If we use some typical values for the unknowns of the equation, such as $\mathrm{z}=1, \mathrm{D}=$ $1 \times 10^{-8} \mathrm{~cm}^{2} / \mathrm{sec}$, the boundary layer thickness $\delta=1 \times 10^{-3} \mathrm{~cm}$ (10 microns), and looking at the 
rate at $\mathrm{t}=.002$ seconds ( 2 milliseconds), the ratios of the transient and steady state limiting current densities can be compared. In this case, the ratio is equation (4)

$$
\frac{i_{l o T}}{\sqrt{\frac{1 e-8}{3.14159 * .002}}}=\frac{i_{l o} * 1 * .001}{1 e-8}
$$

and so $792.67 * \mathrm{i}_{\text {loT }}=100,000 * \mathrm{i}_{\mathrm{lo}}$, or $\mathrm{i}_{\mathrm{loT}}=126.2 * \mathrm{i}_{\mathrm{lo}}$. Therefore, it can be seen that the transient limiting current density is over two orders of magnitude higher than the steady state limiting current density at the 2 millisecond point. This is just an estimate however, and changing the diffusivity or the boundary layer thickness around can give different results. For instance, if the diffusivity is much higher, but still reasonable at $1 \times 10^{-5} \mathrm{~cm}^{2} / \mathrm{sec}$, the ratio lowers down to $\mathrm{i}_{\mathrm{loT}}=3.99 * \mathrm{i}_{\mathrm{lo}}$. In realistic situations, transient limiting current density is significantly higher than the steady state limiting current density, and it desirable to use this to gain advantage in efficiency in addition to the advantages gained in reliability and the morphology of the deposited layers. It is the aim of this experimentation to show that a working laboratory scale device can be developed to utilize the pulsed electrodeposition method to recover dissolved metal ions from solution.

\section{Appendix B - Electrochemical Theory:}

In order to remove dissolved metal ions from solution, it is necessary to apply an appropriate potential to a working electrode such that the metal can electrodeposit from solution onto the electrode. It is also necessary to provide a potential at the counter electrode in order to provide the energy required for a counter reaction to occur, which is needed to supply the necessary electrons to the deposition reaction at the working electrode. The required potential for the electrodeposition of an element can be found in examining the underlying theory of electrochemical reactions.

In a solution, the dissolved metal ions present are in an electron deficient state, such as $\mathrm{Cu}^{2+}$ for copper or $\mathrm{Pb}^{2+}$ for lead. This is an oxidized state, as the oxidation number of the element $\left(2^{+}\right.$in this case), is higher than the natural state of the pure element, which is 0 . The oxidation of the element has ions the element by removing two electrons from each atom, leaving a net charge of $2^{+}$with each atom, which also serves to make the atoms soluble in water. In order to remove the dissolved metal ions from solution, it is necessary to provide two free electrons to the atom such that it will return to a neutral charge. This is shown for copper by the reaction

$$
\mathrm{Cu}^{2+}+2 \mathrm{e}^{-} \Leftrightarrow \mathrm{Cu}
$$

As shown by the double-sided arrow in the reaction, this reaction is reversible depending upon the reaction conditions. Although this reaction can go backward or forward, such reactions are written as reduction reactions by convention. This reaction is known as the half-cell reaction for copper, as it is only showing half of the total chemical reaction that is occurring. As written, electrons must be supplied to the reaction by another reaction, and this other reaction is known as the counter reaction. The counter reaction to the reaction as shown is an oxidation reaction, where the oxidation number of a species will be increased in the reaction, which signifies a positive increase in the net charge of the species involved. This positive increase in net charge is due to the removal of negative charge, which is accomplished by the release of electrons. Because it is not desirable to oxidize a solid material, and thus degrade or dissolve the species, the typical oxidation reaction that occurs 
in water for use as the counter reaction to a metal reduction is the oxidation of water, also known as water hydrolysis. This half-cell reaction is

$$
2 \mathrm{H}_{2} \mathrm{O} \Leftrightarrow \mathrm{O}_{2}+4 \mathrm{H}^{+}+4 \mathrm{e}^{-}
$$

For the reduction of copper, the overall reaction that occurs is therefore

$$
2 \mathrm{Cu}^{2+}+\mathrm{H}_{2} \mathrm{O} \Leftrightarrow 2 \mathrm{Cu}+\mathrm{O}_{2}+4 \mathrm{H}^{+}
$$

Note that for the overall reaction, there are no excess electrons on either side of the reaction, i.e. all electrons simply transfer from one species to another.

Thermodynamically, it is possible to examine the conditions under which this reaction will proceed either in the forward or backward direction by examining the free energy of the reaction. This is most easily done by examining the half-cell reactions for the change in free energy. The standard free energy of a reaction may be found using the standard free energy for each element in the reaction under standard conditions (1 bar pressure, 298K), and where the activity of the reactants is 1 (this is true where the reactant is a pure solid or liquid under standard conditions, where the reactant is a pure ideal gas with a pressure of 1 bar, where the reactant is a gas with a vapor pressure equal to that of the pure component vapor pressure just above the pure liquid at standard conditions, or where the reactant is a dissolved metal ion in an ideal solution where the molality of the ion is 1 ). The symbol for the change in standard free energy is $\Delta \mathrm{G}^{\circ}$, and so

$$
\Delta \mathrm{G}^{0} \text { reaction }=\Sigma \Delta \mathrm{G}_{\text {products }}^{0} \Sigma \Delta \mathrm{G}^{0} \text { reactants }
$$

The standard free energy of pure elements under standard conditions and the standard free energy of electrons are zero. So, for the example reaction (5)

$\Delta G^{0}$ reaction $=0-(0+65,520)=-65,520 \mathrm{~J} / \mathrm{mol}$. Because the overall free energy change of the reaction is negative, this reaction can occur without any input of energy. However, this only applies at standard conditions and standard activities.

Under conditions where the activities are not equal to 1 , the free energy of reaction must be adjusted by using equation (9)

$$
\Delta \mathrm{G}_{\text {reaction }}=\Delta \mathrm{G}_{\text {reaction }}^{\mathrm{O}}+\mathrm{RT} \ln \mathrm{Q}
$$

where $\mathrm{R}$ is the universal gas constant, $\mathrm{T}$ is the absolute temperature, and $\mathrm{Q}$ is the ratio of activities of the products over the reactants with appropriate stoichiometric consideration. So, for the example reaction involving copper, if the copper concentration is 50ppm, which is $7.87 \times 10^{-4}$ molar, and the reaction occurs at room temperature $(298 \mathrm{~K})$, then the free energy is

$$
\Delta G_{\text {reaction }}=-65,520 \mathrm{~J} / \mathrm{mol}+8.31 \mathrm{~J} / \mathrm{molK} * 298 \mathrm{~K} * \ln \left(\frac{1}{(1)^{2}\left(7.87 \times 10^{-4}\right)}\right)
$$

which is $-47,820 \mathrm{~J} / \mathrm{mol}$. For the reduction reaction of a metal, lowering the dissolved metal concentration lowers the free energy of the reaction. Equilibrium is achieved when the free energy of reaction is 0 , so there will reach a point in this reaction where a minimum copper concentration is reached, in this case at $3.23 \times 10^{-12}$ molar. This is only shown for the half cell reaction, and when the full reaction is taken into account the point at which that free energy is equal to 0 is the equilibrium point for the reaction.

In the case of electrochemical reactions, it can be more useful to look at the energy of the reaction in terms of the energy exchanged by the transfer of electrons. If the reaction free energy $\Delta \mathrm{G}$ is divided by the number of electron exchanges per reacting atom and the charge per mole of electrons, a measure of the amount of energy per electron transfer event is determined. The charge per mole of electrons is 96,485 Coulombs/mole, which is a quantity 
also known as Faraday's constant. This new quantity is known as the standard potential, $\mathrm{E}_{\mathrm{o}}$, and is given by equation (11)

$$
\mathrm{E}_{\mathrm{o}}=-\Delta \mathrm{G}^{\mathrm{o}} / \mathrm{nF}
$$

Where $\mathrm{n}$ is the number of electrons exchanged per atom reacted, and $\mathrm{F}$ is Faraday's constant. The units of E are in Joules/Coulomb, which is also known as Volts and is a measure of the energy per unit of charge. Combining this equation with the equation for non-standard free energy leads to equation (12)

$$
E=E_{o}-\frac{R T}{n F} \ln Q
$$

This expression is known as the Nernst Equation. For the example reaction of the reduction of copper from a 50ppm solution, this potential is $0.248 \mathrm{~V}$. The higher the potential of a reaction, the greater the driving force that reaction has towards acquiring electrons. Therefore, the reaction can proceed forward and undergo a reduction reaction by driving other reactions in the reverse direction, which causes the other species to be oxidized. By examining the potential of reactions, it is therefore possible to determine which reactions will be reducing and which reactions will be oxidizing in a given situation. By finding the concentrations of the species in the system at the point at which the net free energy change of the reaction is zero, it is possible to find the equilibrium concentration of the species in the final state. At this point, the potential of the forward proceeding half-cell reaction and the reverse proceeding half-cell reaction will be the equal to one another.

Examining the equilibrium potential of reactions can be useful in determining what state a system will reach if left under those conditions indefinitely. It does not give any information as to the amount of time it will take to reach that state. In order to determine the rate at which the reactions occur, the electrochemical kinetics of the reaction must be examined.

The rate at which an electrochemical half-cell reaction $\mathrm{M}^{2+}+2 \mathrm{e}^{-}=\mathrm{M}$ occurs is given by equation (13)

$$
R=k_{b} C_{M} \exp \left(\frac{\phi z F E}{R T}\right)-k_{f} C_{M 2+} \exp \left(\frac{-(1-\phi) z F E}{R T}\right)
$$

in which $\mathrm{R}$ is the rate, $\mathrm{k}_{\mathrm{b}}$ is the reverse reaction rate constant, $\mathrm{C}_{\mathrm{M}}$ is the metal concentration, $\phi$ is a symmetry factor that is assumed to be $0.5, \mathrm{z}$ is the number of electrons exchanged in the rate limiting step, usually $1, \mathrm{~F}$ is Faraday's constant, $\mathrm{E}$ is the potential, $\mathrm{R}$ is the gas constant, $\mathrm{T}$ is the temperature, $\mathrm{k}_{\mathrm{f}}$ is the forward rate constant, and $\mathrm{C}_{\mathrm{M} 2+}$ is the concentration of the $\mathrm{M}^{2+}$ dissolved metal ions species.

Because of the exponential term present in the equation, if the potential is positive and large, the first term dominates and the reaction rate is positive. If the potential is negative with large magnitude, the second term dominates and the reaction rate is negative. The sign of the reaction represents the electron flow direction with positive indicating an anodic or oxidizing reaction direction. If we combine two half-cell reactions, one reaction will have a dominant positive reaction rate term, and the other will have a dominant negative reaction rate term. Because the net reaction rate may be expressed in terms of the rate of electron exchange, the reaction rate may also be expressed as a current. Also, because the total reaction must consist of two combined half cell reactions, and these half cell reactions must have equal rates because the electrons consumed in one reaction must be generated by the other, an expression may be developed for the equilibrium exchange current density, 
which is a measure of the current due to electron exchange at the equilibrium potential for the reactions, known as $i_{0}$

$$
i_{o}=n F k_{b} C_{M} \exp \left(\frac{\phi z F E_{e q}}{R T}\right)=n F k_{f} C_{M 2+} \exp \left(\frac{-(1-\phi) z F E_{e q}}{R T}\right)
$$

Using the mathematical relationship of $\exp (\mathrm{Y}+\mathrm{x}-\mathrm{x})=\exp (\mathrm{x}) \exp (\mathrm{Y}-\mathrm{x})$, it is possible to rearrange the initial Bulter Volmer Equation (14) with $i_{o}$ to get a useful relationship between the reaction rate in terms of current and the overpotential of the reaction.

$$
i=i_{o} \exp \left(\frac{\phi z F\left(E-E_{e q}\right)}{R T}\right)-i_{o} \exp \left(\frac{-(1-\phi) z F\left(E-E_{e q}\right)}{R T}\right)
$$

where $i$ is the reaction rate for the half cell reaction and $\mathrm{E}$ is the potential at which the reaction is occurring. Equation (15) is known as the Butler-Volmer equation.

In order to remove dissolved metal ions from solution, it is necessary to have a negative current for the reaction so that electrons are provided to the dissolved metal ion and it can undergo the reduction to the pure metal form. This is simply due to the convention that the sign of the current is negative in the direction that negative charge is flowing. In order to generate this negative current, the potential at which the reaction occurs must be lower than the equilibrium potential of the reaction. If the potential is higher than the equilibrium potential, metal will dissolve into solution, or if it is already in solution, it will simply remain there. Because the equilibrium potential for the reaction of each element is based on the free energy of reaction for each element, each element has a different equilibrium potential below which this element will deposit from solution. This potential will also become lower as the concentration of the element in solution becomes lower. This potential is referred to as the electrochemical potential of the element, as is useful in determining the potential at which the working electrode should be set in order to recover the desired elements from solution.

Using the Butler-Volmer equation (15), it is also possible to estimate the rate at which the reaction will occur by combining the half-cell reactions present to find the overall rate of reaction. In the system, the current from oxidation reactions must equal the current going to the reduction reactions. If the working electrode is set to a known potential, it is possible to determine the current density at that electrode, which will equal the current density at the counter electrode, and knowing that current density it will also be possible to back calculate the potential needed to drive the reaction at the counter electrode. This only holds true if the rate is controlled by the reaction however, and the rate in the case of dilute solutions is typically controlled by mass transport limitations, as discussed earlier in the theory of pulsed electrodeposition.

With an understanding of electrochemical principles, it is possible to determine the potentials necessary to facilitate deposition of each element. This can be applied advantageously to recover elements individually in order to create a purified metal product. 
Appendix 43: Recovery of Chromium and Arsenic from Toxic Waste Streams by Reactive Polymer-Coated Absorbents (WV014) 


\section{FINAL TECHNICAL REPORT}

\begin{tabular}{|c|c|}
\hline Contract Title and Number: & Period of Performance: \\
\hline Crosscutting Technology Development at the Center for & Starting Date: \\
\hline Advanced Separation Technologies & Ending Date: \\
\hline
\end{tabular}

(DE-FC26-02NT41607)

Sub-Recipient Project Title:

Recovery of Chromium and Arsenic from Toxic

Waste Stream by Reactive Polymer-Coated

Absorbents

Principal Investigators:

Dianchen Gang, Baolin Deng

Contact Address:

Department of Civil Engineering

West Virginia University Institute of Technology

405 Fayette Pike, Montgomery, WV 25316

Subcontractor Address:

Baolin Deng

Department of Civil \& Environmental Engineering

University of Missouri-Columbia

Columbia, MO 65211
Report Information:

Type: Final

Number: Final

Period: $\quad 06 / 01 / 2004-10 / 31 / 2008$

Date: $\quad 10 / 31 / 2007$

Code: WV014-FINAL

Contact Information:

Phone: $\quad 304-442-3372$

Fax: $\quad 304-442-3391$

E-Mail: dianchen.gang@mail.wvu.edu

Subcontractor Information:

Phone: 573-882-0075

Fax: $\quad 573-882-4874$

E-Mail: DengB@missouri.edu

\section{$\underline{\text { Disclaimer }}$}

"This report was prepared as an account of work sponsored by an agency of the United States Government. Neither the United States Government nor any agency thereof, nor any of their employees, make any warranty, express or implied, nor assume any legal liability or responsibility for the accuracy, completeness, or usefulness of any information, apparatus, product, or process disclosed, or represents that its use would not infringe privately owned rights. Reference herein to any specific commercial product, process, or service by trade name, trademark, manufacturer, or otherwise does not necessarily constitute or imply endorsement, recommendation, or favoring by the United States Government or any agency thereof. The views and opinions of authors expressed herein do not necessarily state or reflect those of the United States Government or agency thereof." 


\begin{abstract}
The United States has an enormous untapped coal resource for energy production. However, coal is not widely used to alleviate the nation's dependency on foreign oil, in part, because of the environmental damage associated with the coal mining activities. Oxidation of pyrites and other metal sulfides in coal and metal mine tailings can generate high concentration of sulfuric acid, forming acid mine drainage (AMD) with $\mathrm{pH}$ as low as 2 to 4 . AMD may contain high concentrations of sulfate, iron, and many toxic elements existing in the original mineral assembly, including divalent heavy metals (e.g., $\mathrm{Pb}^{2+}, \mathrm{Cd}^{2+}, \mathrm{Cu}^{2+}$, and $\mathrm{Hg}^{2+}$ ) and oxyanions (e.g., $\mathrm{AsO}_{4}{ }^{3-} / \mathrm{AsO}_{3}{ }^{3-}$ and $\mathrm{CrO}_{4}{ }^{-}$). As a result, AMD frequently serves as a largescale and persistent source of pollution to surface and ground waters. Contaminants in their anionic forms (i.e., $\mathrm{AsO}_{4}{ }^{3-}$ and $\mathrm{CrO}_{4}{ }^{-}$) are highly soluble and are normally not removed by many treatment processes designed for divalent heavy metals such as lime neutralization and precipitation. Therefore, there is an urgent need for a cost-effective means of removing of anionic form contaminants. The new technology would increase the likelihood that a plentiful resource in this country, coal, could be utilized as an alternative to foreign oil without adverse environmental consequences.
\end{abstract}

The primary objective of the research is to remove and recover $\mathrm{Cr}(\mathrm{VI})$ and $\mathrm{As}(\mathrm{V} / \mathrm{III})$ from aqueous media by using a new polymer coated absorbent and iron-chitosan. The detailed objectives of this study include preparing the adsorbent, characterizing the sorbent, and evaluating the removal efficiencies of hexavalent chromium and arsenic from synthetic samples. The adsorption capacity of the adsorbent was determined by studying the equilibrium isotherm with batch experiments. Kinetics of the adsorption was evaluated using a pseudo-second-order reaction model. In addition, the effect of $\mathrm{pH}$, ionic strength, adsorbent dose and competing anions on the extent of adsorption were examined.

A composite sorbent for Cr(VI) adsorption, Quaternized poly(4-vinylpyridine) (QPVP) coated granular activated carbon (GAC), GAC-QPVP, was prepared by coating poly(4vinylpyridine) onto a commercial granular activated carbon (F400, Calgon), followed by cross-linking and quaternization processes. The sorbent was characterized by scanning electron microscopy, point of zero charge measurement, and BET analysis. The adsorption of chromium(VI) by GAC-QPVP was evaluated using batch adsorption techniques. The estimated maximum equilibrium uptake of chromium was $53.7 \mathrm{mg} / \mathrm{g}$ at $\mathrm{pH}=2.25,30.7 \mathrm{mg} / \mathrm{g}$ at $\mathrm{pH}=3.65$, and $18.9 \mathrm{mg} / \mathrm{g}$ at $\mathrm{pH}=6.03$. From kinetic data, it was found that sorption of chromium(VI) by GAC-QPVP was a rapid process. The results showed that Cr(VI) sorption rates could be described by a reversible second order kinetics, and equilibrium uptake of $\mathrm{Cr}(\mathrm{VI})$ increased with decreasing $\mathrm{pH}$, decreasing ionic strength, and increasing sorbent concentration. Adsorption capacity of chromium(VI) was also evaluated in the presence of sulfate, chloride, bicarbonate, and acetate ions.

The key component of the mass transfer operations and the diffusion coefficient was determined by a modified finite bath diffusion control model. Results indicated that the modified diffusion-control model fitted the experimental data well. The observed sorption kinetics was consistent with the finite bath diffusion, with a diffusion coefficient of 
$4.10 \times 10^{-6} \mathrm{~cm}^{2} / \mathrm{s}$ for the uptake of $\mathrm{Cr}(\mathrm{VI})$ by the Quaternized poly(4-vinylpyridine) (QPVP) coated activated carbon.

Desorption studies were also conducted to recover chromium(VI) using varying concentrations of sodium hydroxide and ammonium hydroxide. Maximum desorption efficiency of $80 \%$ was obtained by $1 \mathrm{M} \mathrm{NaOH}$. This desorbed adsorbent was again used to evaluate the efficiency for chromium(VI) removal. Column studies of chromium(VI) adsorption on GAC-QPVP were conducted using $5 \mathrm{mg} / \mathrm{L}$ chromium(VI) solution in a column with ID of $6 \mathrm{~mm}$ and $12 \mathrm{~mm}$. The break points were observed at 760 and 600 bed volumes with a flow rate of $20 \mathrm{ml} /$ day and $100 \mathrm{ml} /$ day, respectively. Overall, the study has demonstrated that GAC-QPVP can effectively remove $\mathrm{Cr}(\mathrm{VI})$ from aqueous solutions under a wide range of experimental conditions, without significant $\mathrm{Cr}(\mathrm{III})$ release associated with the virgin GAC treatment.

Iron-chitosan beads were prepared for arsenic removal. SEM micrograph of the iron-chitosan illustrated that the beads are porous in structure. XPS analysis showed the presence of iron and arsenic in the beads when exposed to arsenic. Adsorption experiments demonstrated that iron-chitosan could be used to remove arsenic from aqueous solution. Adsorption of arsenic(III) had no significant change in the $\mathrm{pH}$ range from 4 to 9.1. Arsenic(III) removal was drastically reduced after a $\mathrm{pH}$ of 9.1 . The maximum adsorption capacity increased from $1.95 \mathrm{mg} / \mathrm{g}$ to $5.97 \mathrm{mg} / \mathrm{g}$ and from $1.97 \mathrm{mg} / \mathrm{g}$ to $6.48 \mathrm{mg} / \mathrm{g}$ as the initial concentrations of arsenic(III) increased from $0.3 \mathrm{mg} / \mathrm{L}$ to $1 \mathrm{mg} / \mathrm{L}$ for $\mathrm{pH}=7$ and $\mathrm{pH}=8$ respectively. The adsorption isotherm fitted Freundlich model well and the maximum adsorption removal efficiency was observed at $\mathrm{pH}=9.1$. Column studies showed that the breakthrough point was at 768 empty bed volumes (EBV) when flow rate was $25 \mathrm{ml} / \mathrm{hr}$.

Results from the kinetic batch experiments indicated that over $60 \%$ of the arsenic were adsorbed by the iron-chitosan within 30 min of the adsorption and it takes about 2 hours to reach equilibrium. Pseudo-second-order model fitted the experimental data well with the reaction rate constants of $3.194 \times 10^{-2}$ and $1.151 \times 10^{-2} \mathrm{~g} \mathrm{mg}^{-1} \mathrm{~min}^{-1}$ for the initial arsenic(III) concentrations of 306 and $994 \mu \mathrm{g} / \mathrm{L}$, respectively. The initial rate $\left(k_{2} q_{e}^{2}\right)$ was increased from $8.48 \times 10^{-2}$ to $27.97 \times 10^{-2}$ with increasing initial arsenic(III) concentration.

The effects of anions (sulfates, phosphates, silicates) on As (V) and As (III) removal were studied with an initial concentration of $1000 \mu \mathrm{g} / \mathrm{L}$. The results showed that $\mathrm{SO}_{4}{ }^{2-}$ did not significantly affect arsenate and arsenite removal from DI water. The presence of phosphate and silicate decreased As (III) removal slightly in the $\mathrm{pH}$ range from 7.72 to 9.15 . But with further increase in $\mathrm{pH}$, a major decrease on arsenite removal was observed. At $\mathrm{pH}>9.87$ only $25 \%$ of arsenite could be adsorbed in the presence of phosphate and silicate. Regarding As (V) removal, at $\mathrm{pH}>9.8$ only $19 \%$ of arsenate could be adsorbed in the presence of phosphate and silicate. These indicate that phosphate and silicate compete with hydroxyl group for the iron surface in the alkaline solution and subsequently decrease arsenate adsorption. 


\section{TABLE OF CONTENTS}

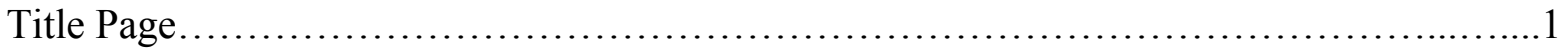

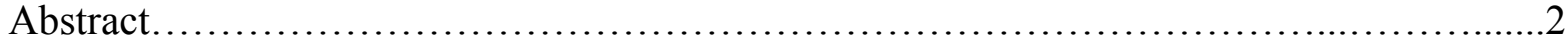

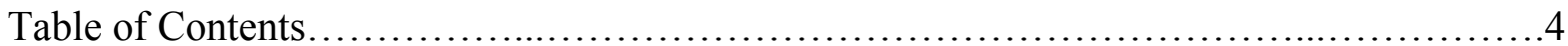

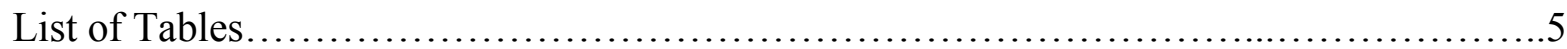

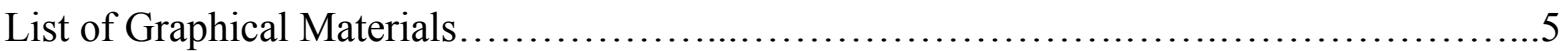

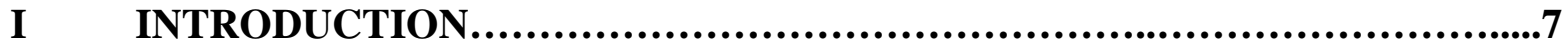

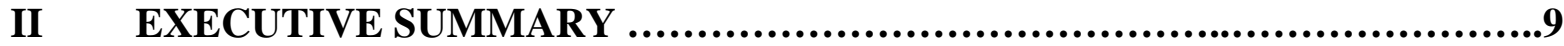

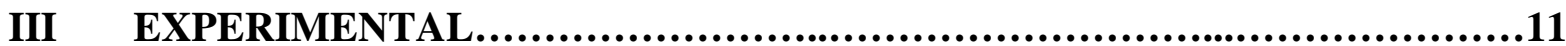

$3.1 \quad$ Materials................................................................... 11

3.2 Preparation of the Quaternized Polyvinylpyridine Coated Granular Activated Carbon

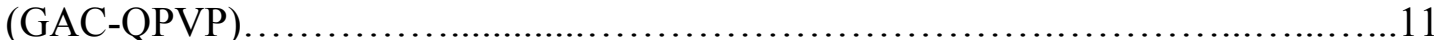

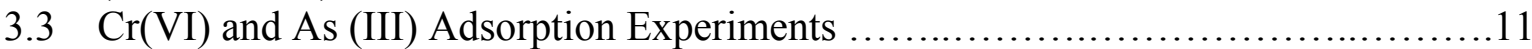

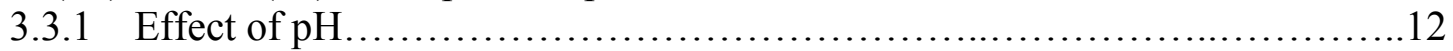

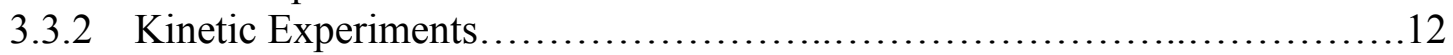

3.3.3 Adsorption Isotherms..................................................13

3.3.4 Adsorption of $\mathrm{Cr}(\mathrm{VI})$ by GAC-APVP in the Presence of Other

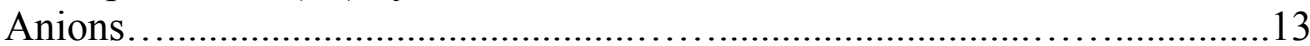

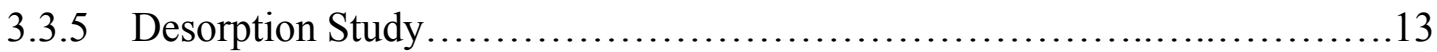

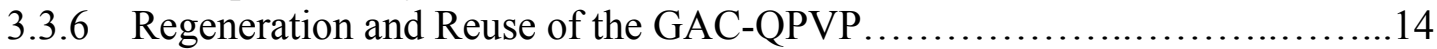

3.3.7 Column Study.............................................................

3.4 Preparation of Iron-chitosan Beads............................................

3.5 Arsenic Measurement Process.......................................................15

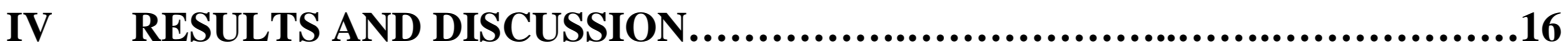

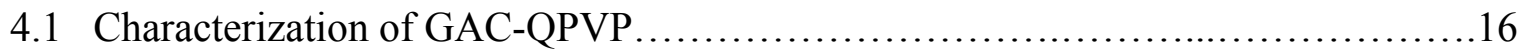

4.2 Structure Characterization of Iron-Chitosan Beads.................................. 18

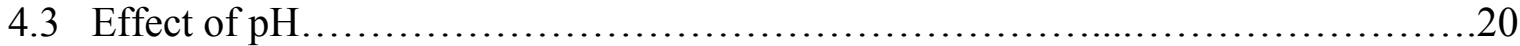

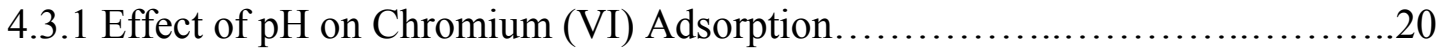

4.3.2 Effect of $\mathrm{pH}$ on Arsenic(III) Adsorption.......................................21

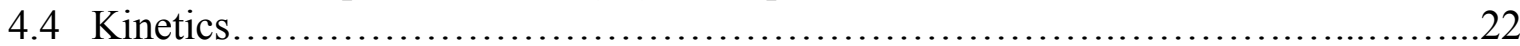

4.4.1 Chromium (VI) Adsorption Kinetics.....................................22

4.4.2 Chromium (VI) Adsorption Kinetics Modeling..............................23

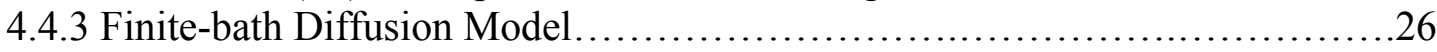

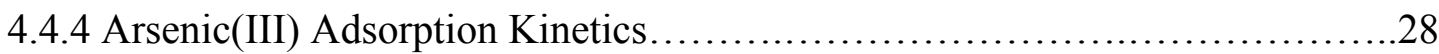

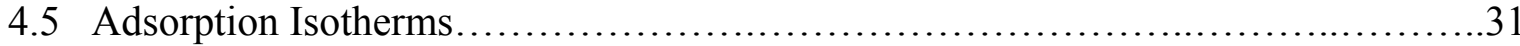

4.5.1 Chromium (VI) Adsorption Isotherms........................................ 31

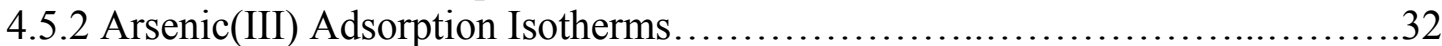

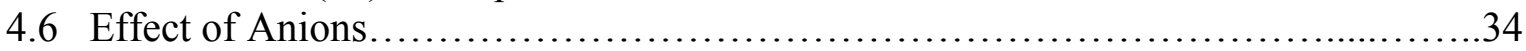

4.6.1 Effect of Anions on Chromium (VI) Removal by GAC-QPVP..................34

4.6.2 Effect of Anions on Arsenic Removal...........................................35

4.7 Effects of Ionic Strength and Modeling Cr(VI) sorption.............................37

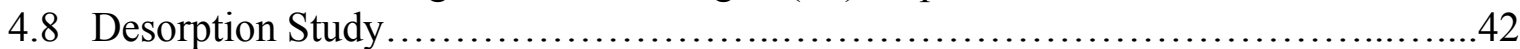

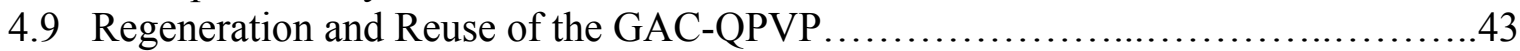

4.10 Column Operation................................................................... 44

4.10.1 Column Study of Chromium(VI) with GAC-QPVP .........................4 
4.10.2 Column Study of Arsenic(III) with Iron-chitosan.............................45

V CONCLUSIONS.............................................................45

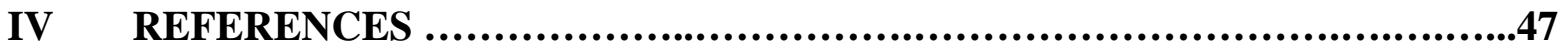

\section{LIST OF TABLES}

3.1 Atomic absorption spectrometer operating conditions …..........................15

4.1 Properties of the virgin GAC and GAC-QPVP..................................... 16

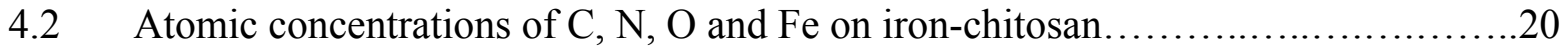

4.3 Atomic concentrations of As (III), C, N, O and Fe on iron-chitosan as obtained from

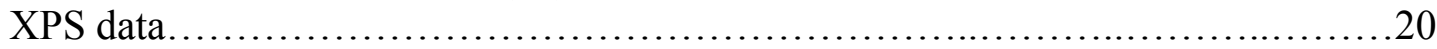

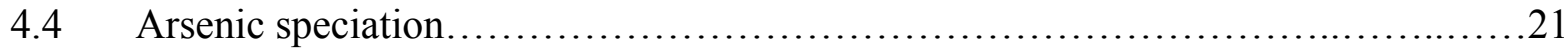

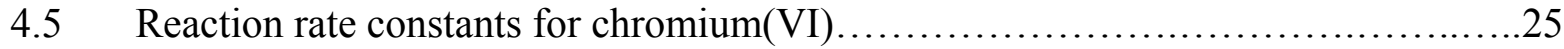

4.6 Values of $\lambda \bar{D}$ for adsorption of $\mathrm{Cr}(\mathrm{VI})$ on GAC-QPVP at different

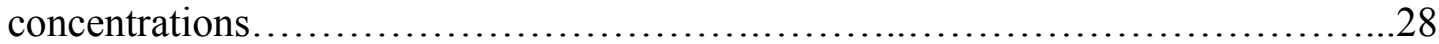

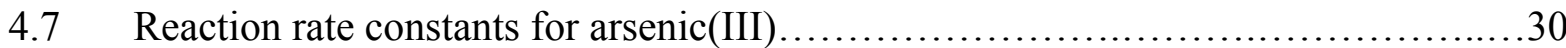

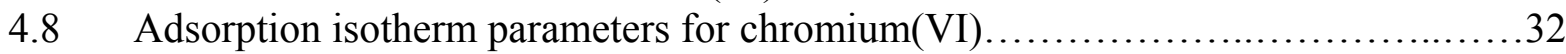

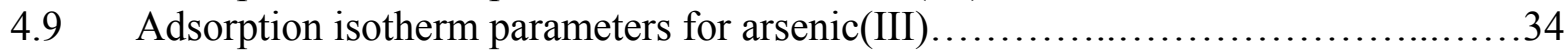

\section{LIST OF GRAPHICAL MATERIALS}

3.1 Schematic diagram: apparatus for preparing iron-chitosan beads ....................15

4.1 Scanning electron micrograph of the virgin GAC................................17

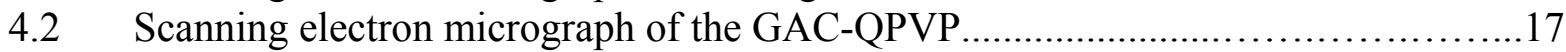

4.3 Oven dried iron-chitosan beads ............................................ 18

4.4 Scanning electron micrograph (SEM) of iron-chitosan bead...................... 18

4.5 XPS Spectrum of iron-chitosan bead............................................. 19

4.6 XPS Spectrum of arsenite uptake iron-chitosan bead............................... 19

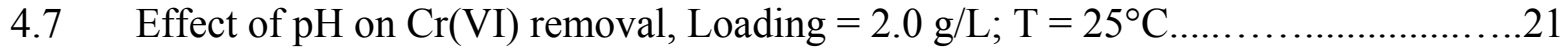

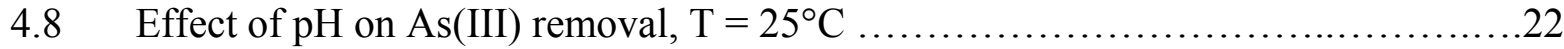

4.9 Rate of adsorption at different initial Concentrations, $\mathrm{pH}=2 ; \mathrm{T}=25^{\circ} \mathrm{C} \ldots \ldots \ldots \ldots \ldots . .22$

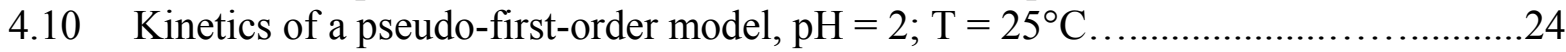

4.11 Kinetics of a pseudo-second-order model, $\mathrm{pH}=2 ; \mathrm{T}=25^{\circ} \mathrm{C} \ldots \ldots \ldots \ldots \ldots \ldots \ldots \ldots . .25$

4.12 Test of modified finite-bath-diffusion model (equation 4-7) .......................27

4.13 Test of modified finite-bath-diffusion model (equation 4-8).........................27

4.14 Rate of adsorption at different initial Concentrations, $\mathrm{pH}=9 ; \mathrm{T}=25^{\circ} \mathrm{C} \ldots \ldots \ldots \ldots \ldots .29$

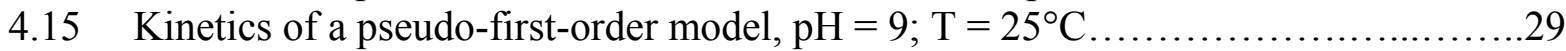

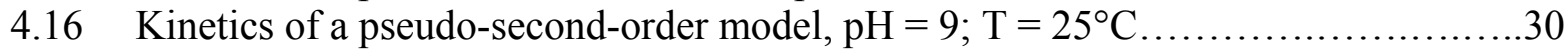

4.17 Adsorption isotherms at different $\mathrm{pH}$ and different initial concentrations...............32

4.18 Adsorption isotherms at different initial concentrations, $\mathrm{pH}=7.0 \ldots \ldots \ldots \ldots \ldots \ldots . .33$

4.19 Adsorption isotherms at different initial concentrations, $\mathrm{pH}=8.0 \ldots \ldots \ldots \ldots \ldots \ldots . .34$

4.20 Effect of anions on chromium removal, $\mathrm{pH}=2$; Loading $=1.5 \mathrm{~g} / \mathrm{L} ; \mathrm{T}=25^{\circ} \mathrm{C} \ldots \ldots .35$

4.21 Effect of anions on arsenate removal under various $\mathrm{pH}$ conditions....................36

4.22 Effect of anions on arsenate removal under various $\mathrm{pH}$ conditions...................37

4.23 (a) Experimental data and model prediction of sorption behavior of $\mathrm{Cr}(\mathrm{VI})$ on GACQPVP as a function of ionic strength...........................................

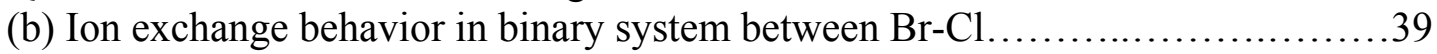


4.24 XPS spectra of GAC-QPVP sample after exposure to $\mathrm{Cr}(\mathrm{VI})$ solution for 24 hours.

Green lines represent the $\mathrm{Cr}(\mathrm{III})$ and $\mathrm{Cr}(\mathrm{VI})$ components.........................41

4.25 Effect of $\mathrm{pH}$ on the removal of $\mathrm{Cr}(\mathrm{VI})$ and total $\mathrm{Cr}$ on GAC and GAC-QPVP

$($ Sorbent $=1 \mathrm{~g} / \mathrm{L} ; \mathrm{I}=0.1 \mathrm{M}$; Initial $\mathrm{Cr}(\mathrm{VI})=10 \mathrm{mg} / \mathrm{L}) \ldots \ldots \ldots \ldots \ldots \ldots \ldots \ldots \ldots . \ldots 1$

4.26 Desorption of $\mathrm{Cr}(\mathrm{VI})$ with ammonium hydroxide and sodium hydroxide for different time periods..................................................................

4.27 Adsorption isotherms of original GAC-QPVP and regenerated GAC-QPVP. .........43

4.28 Column study of chromium(VI) with GAC-QPVP ..............................44

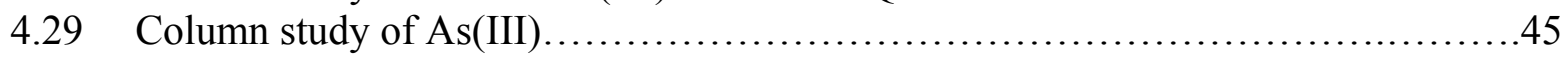




\section{INTRODUCTION}

One of the major challenges facing coal and metal mining industries today is to address public concerns of environmental damage associated with the mining activities. Oxidation of pyrites and other metal sulfides in coal and metal mine tailings can generate high concentration of sulfuric acid, forming acid mine drainage (AMD) with $\mathrm{pH}$ as low as 2 to 4 . AMD may contain high concentrations of sulfate, iron, and many toxic elements existing in the original mineral assembly, including divalent heavy metals (e.g., $\mathrm{Pb}^{2+}, \mathrm{Cd}^{2+}, \mathrm{Cu}^{2+}$, and $\mathrm{Hg}^{2+}$ ) and oxyanions (e.g., $\mathrm{AsO}_{4}{ }^{3-} / \mathrm{AsO}_{3}{ }^{3-}$ and $\mathrm{CrO}_{4}{ }^{-}$). As a result, $\mathrm{AMD}$ frequently serves as a large-scale and persistent source of pollution to surface and ground waters. Chromium, particularly it is in the hexavalent form, has been found to be highly toxic. Extensive information is available about the detrimental effects of chromium both to human beings and other biological systems (Costa, 2003). This study focuses on contaminants in their anionic forms (i.e., $\mathrm{AsO}_{4}{ }^{3-}$ and $\mathrm{CrO}_{4}{ }^{-}$), because these anions are highly soluble and are normally not removed by many traditional treatment processes designed for divalent toxic metals such as lime neutralization and precipitation.

Chromium has broad industrial applications mainly in metallurgical alloying, electroplating, refractoriness, tanning, corrosion inhibition, wood preservation, photographic sensitization, pigment and chromate preparation. Because of its broad industrial applications, chromium has been introduced into natural water bodies by industrial pollution. Over exposure of human beings to chrome dusts and mists causes irritation of skin and affects respiratory system leading possibly to a carcinoma. Ingestion causes epigastria pain, nausea, vomiting, severe diarrhea and hemorrhage. Because of these health hazards, the level of chromium in drinking water supplies and industrial effluents needs to be kept very low. According to the environmental protection agency (EPA), the effluent chromium(VI) concentration for the disposal of industrial wastes into natural waters is less than $0.05 \mathrm{mg} / \mathrm{L}$ (ATSDR, 2000).

Because of its toxilogical effects on both human beings and the aquatic environment, many researchers have tried to remove chromium(VI) from aqueous solutions. The major treatment methods existing or being studied for chromium(VI) removal are precipitation, ion exchange, and adsorption. Other processes capable of removing chromium(VI) from aqueous solutions include evaporation, concentration, electrolysis, reverse osmosis, and solvent extraction. The selection of any treatment method in particular depends upon effluent quality criteria in addition to economic factors and feasibility.

Recovery and recycling of heavy metals from the industrial wastewaters is definitely a wise dictum. Thus by adopting pollution control methods, the industries can not only recover costly metals but also conserve the environment. Adsorption is one of the major phenomena that were studied extensively for the removal of heavy metals. Adsorption process with proper adsorbents and under optimal operational conditions is a very effective method for removal of heavy metals. Considerable information is available in the literature about the removal of heavy metals in cationic forms such as $\mathrm{Hg}^{2+}, \mathrm{Cd}^{2+}, \mathrm{Cr}^{3+}$ etc., with various adsorbents such as activated carbon, coal, crushed coconut shell, chitosan and starch xanthate (Perez-Candela et al., 1995; Schmuhl et al., 2001). 
Arsenic is a metalloid element that has been notorious for its toxicity. Naturally arsenic is found as a main component of several minerals such as arsenopyrite. Throughout the ages, arsenic has used in medicine, cosmetics industry and agriculture. It was used as a cure for diseases such as syphilis and has been shown to assist in curing some leukemia. It has been used as an insecticide, and it is still used as a desiccant, rodenticide, and herbicide. Industrial uses of arsenic include doping of solid state devices, laser material, and bronzing etc. The metalloid, arsenic is also used as an alloying constituent in metallurgy for hardening copper and lead alloys (HSDB, 2005). It is used in glass manufacturing as component of electrical devices, in semiconductor industry, and as a catalyst in the production of ethylene oxide.

Traditional arsenic contaminated sites include areas of mining activities and smelters. Arsenic is also emitted into the atmosphere by high-temperature processes such as coal-fired power plants (Nikolaos and Jeffery, 1998). Arsenic is introduced into water through the dissolution of ores, and increasing concentrations in groundwater as a result of erosion from local rocks. Industrial effluents also contribute arsenic to water.

Drinking water poses the greatest threat to public health from arsenic. Long-term exposure to arsenic through drinking water causes cancer of the skin, lungs, urinary bladder, and kidney, as well as changes in skin pigmentation and hyperkeratosis. Increased risks of lung and bladder cancer and of arsenic-associated skin lesions have been observed at drinking water arsenic concentrations of less than $0.05 \mathrm{mg} / \mathrm{L}$. Due to the severe health effects, in 1993 WHO has re-evaluated the guideline value of arsenic in water and reduced from 0.05 to 0.01 $\mathrm{mg} / \mathrm{L}$ (Shrestha, 2004).

The cost and difficulty of reducing arsenic in water increases as the targeted concentration lowers. Available technologies for the arsenic(III) removal include coagulation/precipitation (McNeill and Edwards, 1997), filtration, lime softening, ion-exchange methods (Berdal et al., 2000), adsorption (Dambies, 2004), and membrane or reverse osmosis (Hering and Elimelech 1996). Among all the methods that are currently available, adsorption techniques have proven to be very effective. Adsorption (Huang and $\mathrm{Fu}, 1984$ ) tests demonstrated the potential for different types of media and resins to remove arsenic from liquid residuals. Due to the health impact of the contaminants and the high costs associated with the existing treatment technologies, there is a need to develop a low cost adsorbent for the cost effective removal of arsenic(III) from aqueous solutions.

The primary objective of the research is to develop effective sorbents for the removal of $\mathrm{Cr}(\mathrm{VI})$ and $\mathrm{As}(\mathrm{V} / \mathrm{III})$ from aqueous media. The detailed objectives include preparing the adsorbents, characterizing the sorbents, and evaluating the removal efficiencies of hexavalent chromium and arsenic from synthetic samples. The adsorption capacity of the adsorbent was determined by studying the equilibrium isotherm with batch experiments. Kinetics of the adsorption was evaluated using a pseudo-second-order reaction model. In addition, the effect of $\mathrm{pH}$, ionic strength, adsorbent dose and competing anions on the extent of adsorption were examined.

The contents of this work include:

- Effect of $\mathrm{pH}$ on chromium(VI) and arsenic(III) removal 
- Kinetics of chromium(VI) and arsenic(III) removal at different initial concentrations

- Adsorption isotherm experiments

- Effect of anions on chromium(VI) arsenic(III) removal

- Desorption of chromium(VI) using different bases

- Regeneration and reuse the sorbent (GAC-QPVP)

- Column study

\section{EXECUTIVE SUMMARY}

The United States has an enormous untapped coal resource for energy production. However, coal is not widely used to alleviate the nation's dependency on foreign oil, in part, because of the environmental damage associated with the coal mining activities. Oxidation of pyrites and other metal sulfides in coal and metal mine tailings can generate high concentration of sulfuric acid, forming acid mine drainage (AMD). AMD may contain high concentrations of sulfate, iron, and many toxic elements existing in the original mineral assembly, including divalent heavy metals (e.g., $\mathrm{Pb}^{2+}, \mathrm{Cd}^{2+}, \mathrm{Cu}^{2+}$, and $\mathrm{Hg}^{2+}$ ) and oxyanions (e.g., $\mathrm{AsO}_{4}{ }^{3-} / \mathrm{AsO}_{3}{ }^{3-}$ and $\mathrm{CrO}_{4}{ }^{-}$). As a result, AMD frequently serves as a large-scale and persistent source of pollution to surface and ground waters. AMD leaching from mining sites into creeks and rivers causes the rivers to be considered mining-influenced waters (MIW) and represents a significant environmental issue. Due to the health impact of the contaminants and the high costs associated with the existing treatment technologies, there is an urgent need for a costeffective means of removing trace levels of contaminants such as chromium and arsenic.

The study focuses on contaminants in their anionic forms (i.e., $\mathrm{AsO}_{4}{ }^{3-}$ and $\mathrm{CrO}_{4}{ }^{-}$), because these anions are highly soluble and are normally not removed by many treatment processes designed for divalent heavy metals such as lime neutralization and precipitation. Two novel adsorbents were developed and evaluated to remove $\mathrm{Cr}(\mathrm{VI})$ and As(III) from aqueous solutions. The new technology would increase the likelihood that a plentiful resource in this country, coal, could be utilized as an alternative to foreign oil without adverse environmental consequences. This would have a particularly important implication, because it would help to increase the United States' energy self-sufficiency and thereby to ensure the nation's energy security.

A composite sorbent (GAC-QPVP) was prepared by coating poly(4-vinylpyridine) onto a commercial granular activated carbon (F400, Calgon), followed by cross-linking and quaternization processes. The sorbent was characterized by scanning electron microscopy, point of zero charge measurement, and BET analysis. Batch experiments with variable $\mathrm{pH}$, ionic strength, and concentrations of $\mathrm{Cr}(\mathrm{VI})$, sorbent loading, and competing anions were conducted to evaluate the selective sorption of $\mathrm{Cr}(\mathrm{VI})$ from aqueous solutions. The results showed that $\mathrm{Cr}(\mathrm{VI})$ sorption rates could be described by a reversible second order kinetics, and equilibrium uptake of $\mathrm{Cr}(\mathrm{VI})$ increased with decreasing $\mathrm{pH}$, decreasing ionic strength, and increasing sorbent concentration. The estimated maximum equilibrium uptake of chromium (VI) was $53.7 \mathrm{mg} / \mathrm{g}$ at $\mathrm{pH}=2.25,30.7 \mathrm{mg} / \mathrm{g}$ at $\mathrm{pH}=3.65$, and $18.9 \mathrm{mg} / \mathrm{g}$ at $\mathrm{pH}=$ 6.03 , much higher than the maximum capacity of PVP-coated silica gel, an adsorbent for $\mathrm{Cr}$ removal examined previously (Gang et al., 2000). 
When compared with the untreated granular activated carbon, sorption onto GAC-QPVP resulted in much less $\mathrm{Cr}(\mathrm{VI})$ reduction and subsequent back release of $\mathrm{Cr}(\mathrm{III})$, which was significant at $\mathrm{pH}$ 2.0. The effect of phosphate, sulfate, and nitrate was minor on the selective sorption of $\mathrm{Cr}(\mathrm{VI})$. An ion exchange model that was linked with aqueous speciation chemistry described $\mathrm{Cr}(\mathrm{VI})$ sorption reasonably well as a function of $\mathrm{pH}$, ionic strength, and $\mathrm{Cr}(\mathrm{VI})$ concentration. Model simulations suggested that sorbed $\mathrm{Cr}(\mathrm{VI})$ was partially reduced to $\mathrm{Cr}$ (III) on the sorbent when $\mathrm{pH}$ was less than 4 . The presence of $\mathrm{Cr}$ (III) on the sorbent was confirmed by the X-ray photoelectron spectroscopic analysis of the reacted sorbent.

The key component of the mass transfer operations and the diffusion coefficient was determined by a modified finite bath diffusion control model. The finite bath diffusion control model with changing bulk concentrations was derived analytically and a constant related to radius of the absorbent particle and the fractional attainment of equilibrium adsorption was first introduced into this model. Results indicated that the adsorbent provided fast adsorption kinetics and the modified diffusion-control model fitted the experimental data reasonably well, with a diffusion coefficient of $4.10 \times 10^{-6} \mathrm{~cm}^{2} / \mathrm{s}$ for the adsorption of $\mathrm{Cr}(\mathrm{VI})$ onto the quaternized poly(4-vinylpyridine) (QPVP) coated activated carbon.

Overall, the study has demonstrated that GAC-QPVP can effectively remove Cr(VI) from aqueous solutions under a wide range of experimental conditions, without significant $\mathrm{Cr}$ (III) release associated with the virgin GAC treatment.

Iron-chitosan beads were prepared for arsenic removal. SEM micrograph of the iron-chitosan illustrated that the beads are porous in structure. XPS analysis showed the presence of iron and arsenic in the beads when exposed to arsenic. Adsorption experiments conducted on the removal of arsenic(III) using iron-chitosan lead to the following conclusions: Iron-chitosan could be used in removing arsenic(III), and removal of arsenic(III) depends on various factors, including $\mathrm{pH}$ of the solution, contact time, adsorbent loading, and the initial concentration of arsenic(III) in the solution. There was no significant change in the adsorption capacity under acidic conditions. But as the solution becomes basic, adsorption capacity was decreased.

Results from the kinetic experiments indicated that adsorption capacity at equilibrium increases from 1.51 to $4.60 \mathrm{mg} / \mathrm{g}$ with an increase in the initial arsenic(III) concentration from 306 to $994 \mu \mathrm{g} / \mathrm{L}$. More than $60 \%$ of the arsenic(III) was adsorbed by the iron-chitosan with in $30 \mathrm{~min}$ of the reaction and it takes about 2 hours to reach the equilibrium. Kinetic results fitted pseudo-second-order model well. Second order reaction rate was observed to decrease from $3.19 \times 10^{-2}$ to $1.15 \times 10^{-2} \mathrm{~g} \mathrm{mg}^{-1} \mathrm{~min}^{-1}$ as the initial arsenic(III) concentration increased from 306 to $994 \mu \mathrm{g} / \mathrm{L}$, respectively. It was noticed from the adsorption isotherms that maximum adsorption capacity increased from $1.95 \mathrm{mg} / \mathrm{g}$ to $5.97 \mathrm{mg} / \mathrm{g}$ and from 1.97 $\mathrm{mg} / \mathrm{g}$ to $6.48 \mathrm{mg} / \mathrm{g}$ as the initial concentration of arsenic(III) increased from $0.3 \mathrm{mg} / \mathrm{L}$ to 1 $\mathrm{mg} / \mathrm{L}$ for $\mathrm{pH}=7$ and $\mathrm{pH}=8$, respectively. The adsorption isotherms were fitted well to Freundlich model. The column studies of breakthrough curve indicated that the break point obtained after 768 bed volumes. 


\section{EXPERIMENTAL}

\section{$\underline{3.1 \text { Materials }}$}

The chromium stock solution $(1000 \mathrm{mg} / \mathrm{L})$ was prepared using potassium dichromate $\left(\mathrm{K}_{2} \mathrm{Cr}_{2} \mathrm{O}_{7}\right)$ and deionized water (DI, Millipore). All working solutions were prepared by diluting the standard stock solution with DI water. Hydrochloric acid, ammonium hydroxide, and sodium acetate were purchased from the Fisher scientific. The 4-vinylpyridine (95\%), cumene hydroperoxide (80\% purity), 1,4-dibromobutane, 1-bromohexadecane, potassium permanganate $\left(\mathrm{KMnO}_{4}\right)$ were from Aldrich-Sigma Chemical. Potassium dichromate and sodium sulfate were from MC\&B, Diphenylcarbazide (DPC) and sodium hydroxide were from VWR Company. All chemicals were of ACS reagent grade. Arsenic(III) stock solution (1000 mg/L) was prepared by dissolving $1.32 \mathrm{~g}$ of $\mathrm{As}_{2} \mathrm{O}_{3}$ (obtained from J. T. Baker), in water containing $4 \mathrm{~g} \mathrm{NaOH}$, which was then neutralized with $1 \% \mathrm{HCl}$ and dilute to $1 \mathrm{~L}$ with DI water. Sodium iodide $(\mathrm{NaI})$ and sodium borohydride $\left(\mathrm{NaHB}_{4}\right)$ were from Mallinckrodt. Hydrochloric acid $(\mathrm{HCl})$ was purchased from J. T. Baker.

\subsection{Preparation of the Quaternized Polyvinylpyridine Coated Granular Activated Carbon (GAC-QPVP)}

The GAC-QPVP was prepared using the following procedures (Fang et al. 2005): Polyvinylpyridine (PVP) was synthesized by bulk polymerization of purified 4-vinylpyridine initiated by cumene hydroxide $[0.5 \%(\mathrm{w} / \mathrm{v})]$ at $55^{\circ} \mathrm{C}$. PVP $(15 \mathrm{~g})$ was dissolved in $500 \mathrm{~mL}$ of chloroform, and $50 \mathrm{~g}$ GAC (F400) were added and agitated with a paddle stirrer for $12 \mathrm{~h}$. The GAC-PVP was dispersed in $300 \mathrm{ml}$ of methanol to which the cross-linking reagent, 1-4dibromobutane was added to the extent of $50 \%$ the theoretical amount required for polymer cross-linking $(15 \mathrm{~g})$, the mixture was stirred at $65^{\circ} \mathrm{C}$ for $48 \mathrm{~h}$ in a slow current of nitrogen. After washing with deionized water and methanol, the mixture were reacted with 1bromohexadecane in $300 \mathrm{ml}$ methanol at $65^{\circ} \mathrm{C}$ for $48 \mathrm{~h}$ to obtain long alkyl chain quaternary ammonium compound. The quaternized polyvinylpyridine coated GAC was then air dried and transferred to a plastic bottle for storage.

\section{$\underline{3.3 \mathrm{Cr}(\mathrm{VI}) \text { and As (III) Adsorption Experiments }}$}

Batch adsorption experiments were carried out in 200-mL conical flasks placed in a shaker. The concentrations of chromium(VI) in solution before and after adsorption were measured using an indirect, UV-Visible spectrophotometric method based on the reaction of chromium(VI) and diphenylcarbazide, which forms a red-violet colored complex. The absorbance of colored complex was measured in a double beam spectrophotometer at wavelength $(\lambda)$ of $540 \mathrm{~nm}$, and chromium(VI) concentration was determined by comparing absorbance to that of a calibration curve prepared using standardized concentrations of chromium(VI).

The adsorption studies were conducted at $25^{\circ} \mathrm{C}$. The $\mathrm{pH}$ of the chromium(VI) and As(III) solution was adjusted by adding $0.1 \mathrm{M} \mathrm{HCl}$ or $0.1 \mathrm{M} \mathrm{NaOH}$. One sample of the same concentration solution without the adsorbent was also prepared and treated under the same 
conditions as the solutions containing adsorbent. This blank was used as reference to establish the initial concentration for the solutions containing the adsorbent. These solutions after adding adsorbent were placed in a shaker. The conical flasks were then removed and solution filtered using filter paper. The filtered solution was analyzed for the final concentration of chromium(VI) and As(III). The amount chromium(VI) or As(III) adsorbed by adsorbents can be determined by subtracting the final concentration from the initial concentration. It can be obtained using the following formula:

$$
q=\frac{\left(C_{i}-C_{f}\right) V}{M}
$$

Where, $\mathrm{q}$ is the adsorption capacity $(\mathrm{mg} / \mathrm{g})$

$\mathrm{C}_{\mathrm{i}}$ - Initial concentration in solution $(\mathrm{mg} / \mathrm{L})$

$\mathrm{C}_{\mathrm{f}}-$ Final concentration in treated solution $(\mathrm{mg} / \mathrm{L})$

$\mathrm{V}$ - Volume of the solution taken (liters)

$\mathrm{M}$ - Weight of the adsorbent (grams)

\subsubsection{Effect of $p H$}

Adsorption of chromium(VI) from the aqueous solution onto GAC-QPVP was conducted at a $\mathrm{pH}$ range from 1 to 12 over a 20 hour period. Forty $\mathrm{ml}$ of a $5 \mathrm{mg} / \mathrm{L}$ chromium solution was placed in conical flask and $\mathrm{pH}$ of the solutions was adjusted by adding $0.1 \mathrm{M} \mathrm{HCl}$ or $0.1 \mathrm{M}$ $\mathrm{NaOH}$ solution. Then $60 \mathrm{mg}$ of GAC-QPVP was added to each solution. The conical flasks were covered with para film and placed in a shaker for 20 hours. The conical flasks were then removed and solution filtered. The filtered solution was analyzed for the final concentration of chromium(VI). The amount of chromium(VI) adsorbed per unit weight of adsorbent and percentage removal of chromium(VI) was calculated.

To analyze the role of $\mathrm{pH}$ on arsenic removal, adsorption experiments were conducted in a $\mathrm{pH}$ range of $4-12$ over $20 \mathrm{hrs}$. One hundred $\mathrm{mL}$ of $314 \mu \mathrm{g} / \mathrm{L}$ solution was prepared from arsenic stock solution in separate conical flasks. The $\mathrm{pH}$ of the solutions was also adjusted by adding $0.1 \mathrm{M} \mathrm{HCl}$ or $0.1 \mathrm{M} \mathrm{NaOH}$. Then, $15 \mathrm{mg}$ of iron-chitosan adsorbent was added to the conical flasks containing arsenic(III) solutions with different $\mathrm{pH}$. The conical flasks were sealed with para film and placed in shaker for $20 \mathrm{hrs}$. The conical flasks were removed and solution was filtered. The filtrate was analyzed for final concentration of arsenic using atomic absorption spectrometer. Removal efficiencies can be calculated from initial and final As(III) concentrations of solutions.

\subsubsection{Kinetic Experiments}

To evaluate the adsorption behavior, the adsorption rates of different initial concentrations of chromium(VI) were measured as a function of time ( 0 to $24 \mathrm{hrs}$ ) at $\mathrm{pH}=2.0$. Forty $\mathrm{ml}$ of a $2.5 \mathrm{mg} / \mathrm{L}, 15 \mathrm{mg} / \mathrm{L}$, and $43 \mathrm{mg} / \mathrm{L}$ chromium(VI) solution with desired amount of GAC-QPVP were added to the conical flasks. The conical flasks were sealed with para film and placed in shaker. The conical flasks were then periodically removed from 0 to 24 hours and solution 
was filtered. The filtered solution was analyzed to determine the adsorption capacity and rate of adsorption.

To determine the rate of adsorption of arsenic(III) on iron-chitosan, kinetics experiments were investigated at $25^{\circ} \mathrm{C}$. The experiments were performed with an initial concentration ranged from 300 to $1000 \mu \mathrm{g} / \mathrm{L}$ at $\mathrm{pH}=9$ for fixed adsorbent dose $(0.2 \mathrm{~g} / \mathrm{L})$. A desired amount of adsorbent $(20 \mathrm{mg})$ was added to the $100 \mathrm{ml}$ arsenic solution in a conical flask. Then conical flasks were placed in a shaker for mixing. The solution was sampled periodically by filtration and the filtrate analyzed for final arsenic concentration in the solution.

\subsubsection{Adsorption Isotherms}

Isotherm experiments were carried out by contacting predetermined amount of GAC-QPVP with a given volume $(40 \mathrm{~mL})$ of known chromium(VI) concentration solution. Here, adsorption isotherm experiments were conducted for three different initial concentrations (10 $\mathrm{mg} / \mathrm{L}, 26 \mathrm{mg} / \mathrm{L}$, and $65 \mathrm{mg} / \mathrm{L}$ ) by adding 5-200 $\mathrm{mg}$ of GAC-QPVP. After shaking for $20 \mathrm{hrs}$, samples were removed and solutions were filtered. The equilibrium chromium(VI) concentrations in the liquid phase were measured. The amount of chromium(VI) adsorbed was determined by performing mass balance.

Equilibrium adsorption isotherm studies for $\mathrm{As}(\mathrm{III})$ were conducted at different $\mathrm{pH}$ with the loading rate of adsorbent varied from 0.05 to $1.5 \mathrm{~g} / \mathrm{L}$. Arsenic(III) solutions $(100 \mathrm{~mL})$ with the iron-chitosan were placed in a shaker for $20 \mathrm{hrs}$. After equilibration, the solutions were filtered using filter paper and the filtrate was analyzed for equilibrium arsenic concentration $\left(\mathrm{C}_{\mathrm{e}}\right)$ and equilibrium adsorption capacity $\left(\mathrm{q}_{\mathrm{e}}\right)$ was calculated. The Freundlich and Langmuir isotherm models were used to analyze the data collected. The parameter values of two isotherm models were calculated using a nonlinear curve fitting technique (sigma plot).

\subsubsection{Adsorption of Cr(VI) by GAC-QPVP in the Presence of Other Anions}

To determine the effect of other anions on chromium(VI) removal from aqueous solution, experiments were conducted in the presence of different concentration of anions $\left(\mathrm{SO}_{4}{ }^{2-}, \mathrm{Cl}^{-}\right.$, $\mathrm{HCO}_{3}{ }^{-}$, and $\mathrm{CH}_{3} \mathrm{COO}^{-}$). Anion concentrations varying from 0.01 to $1.0 \mathrm{M}$ were prepared by adding required amount of sodium sulfate, sodium chloride, sodium carbonate, and sodium acetate to the $40 \mathrm{ml}$ of $5.1 \mathrm{mg} / \mathrm{L}$ chromium solution at $\mathrm{pH}=2.0$. Sixty milligrams of GACQPVP was added to these solutions and the conical flasks were placed in shaker for $20 \mathrm{hrs}$. Then solutions were filtered and filtrate was analyzed for the final concentration of chromium(VI).

\subsubsection{Desorption Study}

Chromium(VI) recovery from GAC-QPVP study was conducted in batch experiments. The study of the chromium(VI) adsorption was carried out in a series of $200 \mathrm{~mL}$ flasks, each one containing $60 \mathrm{mg}$ of GAC-QPVP and $40 \mathrm{~mL}$ of $1.5 \mathrm{~g} / \mathrm{L}$ chromium(VI) solution. The mixture was adjusted to $\mathrm{pH}$ of 2.0 and placed on shaker for $20 \mathrm{hrs}$. The solution was filtered and 
filtrate was analyzed for chromium(VI) concentration and amount of chromium(VI) adsorbed was determined. The adsorbed GAC-QPVP was collected and treated with $10 \mathrm{~mL}$ of varying concentrations of sodium hydroxide and ammonium hydroxide. It was again filtered and the desorbed chromium(VI) was determined in the filtrate. The desorption of chromium(VI) with varying concentrations of $\mathrm{NaOH}$ and $\mathrm{NH}_{4} \mathrm{OH}$ was also investigated for different desorption times (5 and $30 \mathrm{~min}$ ).

\subsubsection{Regeneration and Reuse of the GAC-QPVP}

To minimize the adsorbent usage, reuse experiments were investigated after regenerating the adsorbent. The desorbed GAC-QPVP was washed with $0.1 \mathrm{M} \mathrm{HCl}$ and distilled water to regenerate the adsorbent. The regenerated adsorbent was dried in an oven at $100^{\circ} \mathrm{C}$ for $6 \mathrm{hrs}$, and then the adsorbent was cooled and stored in a desiccator until reuse. Adsorption isotherm experiments were conducted using the regenerated GAC-QPVP following the procedures described in section 3.3.3.

\subsubsection{Column Study}

Column studies were conducted at $25^{\circ} \mathrm{C}$ using $300 \mathrm{mg}$ and $1,000 \mathrm{mg}$ of GAC-QPVP packed in a glass column with 6 and $12 \mathrm{~mm}$ internal diameter respectively. Chromium(VI) solution of $5 \mathrm{mg} / \mathrm{L}$ was allowed to pass-through the column at $\mathrm{pH}=2$. The solution was collected at regular intervals from the bottom of column to analyze the chromium(VI) concentration. The column studies were terminated when the adsorbent was exhausted.

Column study for arsenic (III) removal was investigated using $1.5 \mathrm{~g}$ ( $3 \mathrm{~mL}$ ) of iron-chitosan in a 12-mm-ID column. The adsorbent was packed and arsenic solution of initial concentration of $308 \mu \mathrm{g} / \mathrm{L}$ was passed through the packed column with flow rate of $25 \mathrm{ml} / \mathrm{hr}$. The solution was collected from the bottom of the column, and then amount of arsenic(III) present in the effluent was analyzed with atomic absorption spectrometer.

\section{$\underline{3.4 \text { Preparation of Iron-Chitosan Beads }}$}

About $10 \mathrm{~g}$ of chitosan was added to $0.5 \mathrm{~L}$ of $0.01 \mathrm{~N} \mathrm{Fe}\left(\mathrm{NO}_{3}\right)_{3} .9 \mathrm{H}_{2} \mathrm{O}$ solution under continuous stirring for $2 \mathrm{hrs}$ at $60^{\circ} \mathrm{C}$ temperature to form a viscous gel. Figure 3.1 shows the schematic diagram of apparatus for preparing iron-chitosan beads. The bead was formed by drop wise addition of chitosan gel with required viscosity into a $0.5 \mathrm{M}$ concentrated $\mathrm{NaOH}$ precipitation bath. The beads were then separated from $\mathrm{NaOH}$ solution, and washed several times with de-ionized water to a neutral $\mathrm{pH}$. The wet beads were dried in an oven under vacuum, and then in air. 


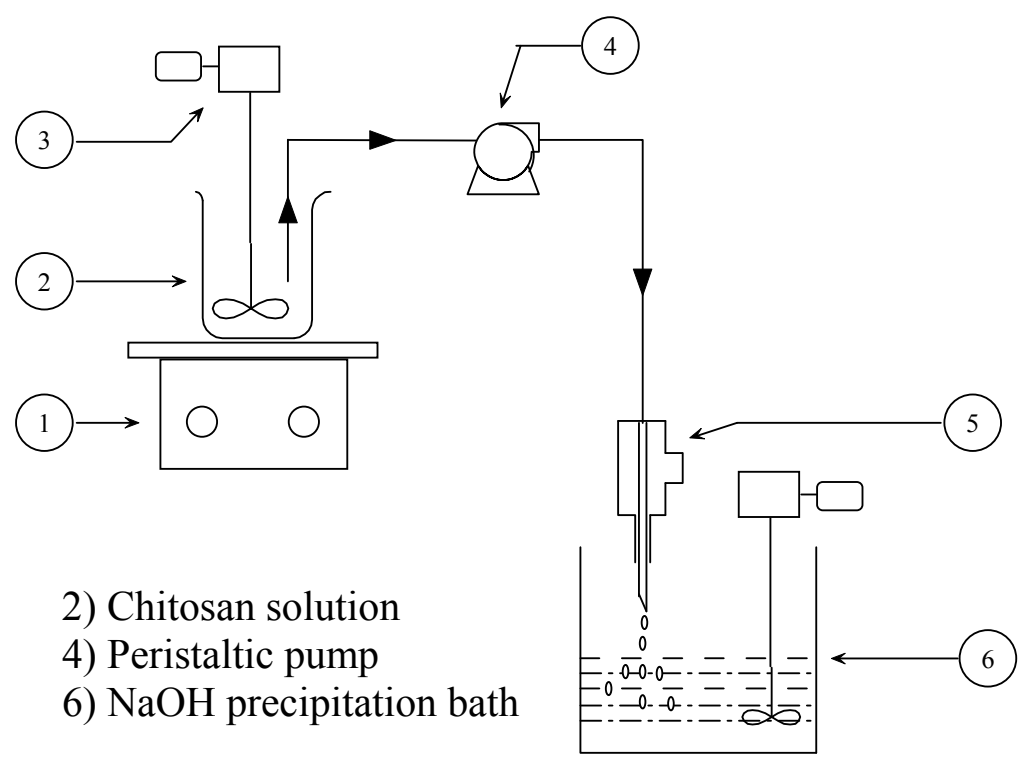

Fig. 3.1 Schematic diagram: apparatus for preparing iron-chitosan beads

\subsection{Arsenic Measurement Process}

For the determination of arsenic, many analytical methods are available such as atomic absorption spectrometry (AAS), inductively coupled plasma-atomic emission spectrometry (ICP-AES), and inductively coupled plasma-mass spectrometry (ICP-MS) and, atomic fluorescence spectrometry (AFS). In this study, $\mathrm{S}$ series system model atomic absorption spectrometer (AAS) (Thermo Electron Corporation) equipped with an arsenic hollow cathode lamp was employed for the measurements of arsenic concentration. An automatic intermittent hydride generation device was used to generate the arsenic hydride. Argon gas was used as the carrier gas for the transposition of arsenic hydride from the hydride generator to the atomizer. The atomic absorption operating conditions are listed in Table 3.1.

Table 3.1. Atomic absorption spectrometer operating conditions

\begin{tabular}{|c|c|}
\hline Parameter & Condition \\
\hline Wavelength $(\mathrm{nm})$ & 193.7 \\
Atomizer height $(\mathrm{mm})$ & 15.2 \\
Type of lamp & Hollow cathode \\
Lamp current $(\mathrm{mA})$ & 75 \\
Vapor mode & Flame Heating \\
Flame gases & Air-Acetylene \\
Flow rate of Acetylene $(\mathrm{L} / \mathrm{min})$ & 1 \\
Flow rate of Argon gas $(\mathrm{ml} / \mathrm{min})$ & 200 \\
\hline
\end{tabular}

Arsenous acid, the arsenic(III) oxidation state of arsenic is instantaneously converted by sodium borohydride reagent in acid solution to their volatile hydrides. The hydrides are 
purged continuously by argon into an atomizer of an atomic absorption spectrometer and converted to the gas-phase atoms. Rapid reaction of sodium borohydride with arsenic in an appropriate reaction cell minimizes dilution of the produced hydrides by the carrier gas..

Sodium borohydride reagent was prepared by dissolving $8 \mathrm{~g} \mathrm{NaBH}_{4}$ in $200 \mathrm{~mL}$ of $0.1 \mathrm{~N}$ $\mathrm{NaOH}$ daily just before use and $\mathrm{NaI}$ solution was obtained by dissolving $50 \mathrm{~g} \mathrm{NaI}$ in $500 \mathrm{~mL}$ deionized distilled water. Five $\mathrm{mL}$ of $\mathrm{NaI}$ solution was added to the sample, reagent blank, and standard solution as a stabilization reagent. All working solutions were prepared by using standard stock solution. To $50 \mathrm{~mL}$ of sample solution, $5 \mathrm{~mL} \mathrm{HCl}$ and $5 \mathrm{~mL} \mathrm{NaI}$ prereductant solution were added before analyzing the solution.

The inlet of reaction cell was connected to auxiliary purging gas controlled by flow meter. Then, samples were mixed with sodium borohydride solution and the generated arsenic hydride was transferred to the atomizer with argon gas and atomic absorption signal was measured under optimum conditions.

\section{RESULTS AND DISCUSSION}

\section{$\underline{4.1 \text { Characterization of GAC-QPVP }}$}

Since activated carbon has high specific surface area, hydrophobic nature and high chemical stability (USEPA, 1973) and polymers can be strongly coated onto it for enhanced metal binding, it was selected as supporting material.

In order to compare the surface characteristics of the virgin GAC and GAC-QPVP, scanning electron microscope (SEM) images of GAC before coating and after coating were obtained (Fang et al., 2005). The SEM images of the granular activated carbon in Figure 4.1 showed that there are considerable small cavities and attached fine particles over the activated carbon surface, forming a system of complicated pore networks. After coating and quaternization, the polymers have been deposited on the carbon surface and clogged the small cavities (Figure 4.2). This agrees with the results from BET surface area analysis, which showed that a reduction of the specific surface area from 902 to $68 \mathrm{~m}^{2} / \mathrm{g}$ after the coating and quaternization processes. Properties of the virgin GAC and GAC-QPVP are shown in Table 4.1

Table 4.1 Properties of the virgin GAC and GAC-QPVP

\begin{tabular}{|lcc|}
\hline & GAC (F400) & GAC-QPVP \\
\hline BET surface area, $\mathrm{m}^{2} / \mathrm{g}$ & 902 & 68 \\
Pore volume, $\mathrm{cm}^{3} / \mathrm{g}$ & 0.6845 & 0.0824 \\
Porosity & 0.4064 & 0.761 \\
Average pore diameter, $\mathrm{nm}$ & 3.035 & 4.849 \\
\hline
\end{tabular}




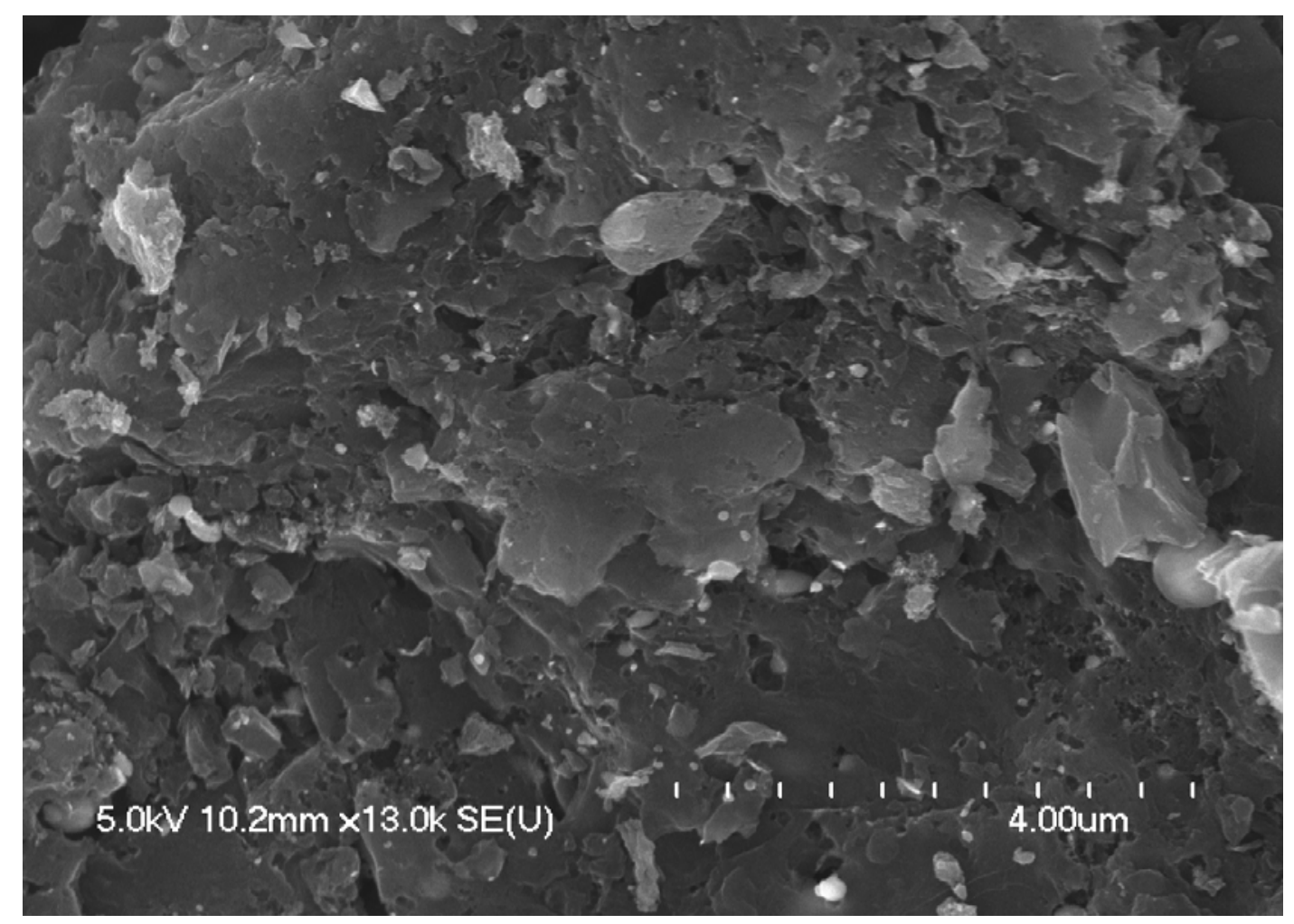

Fig. 4.1 Scanning electron micrograph of the virgin GAC.

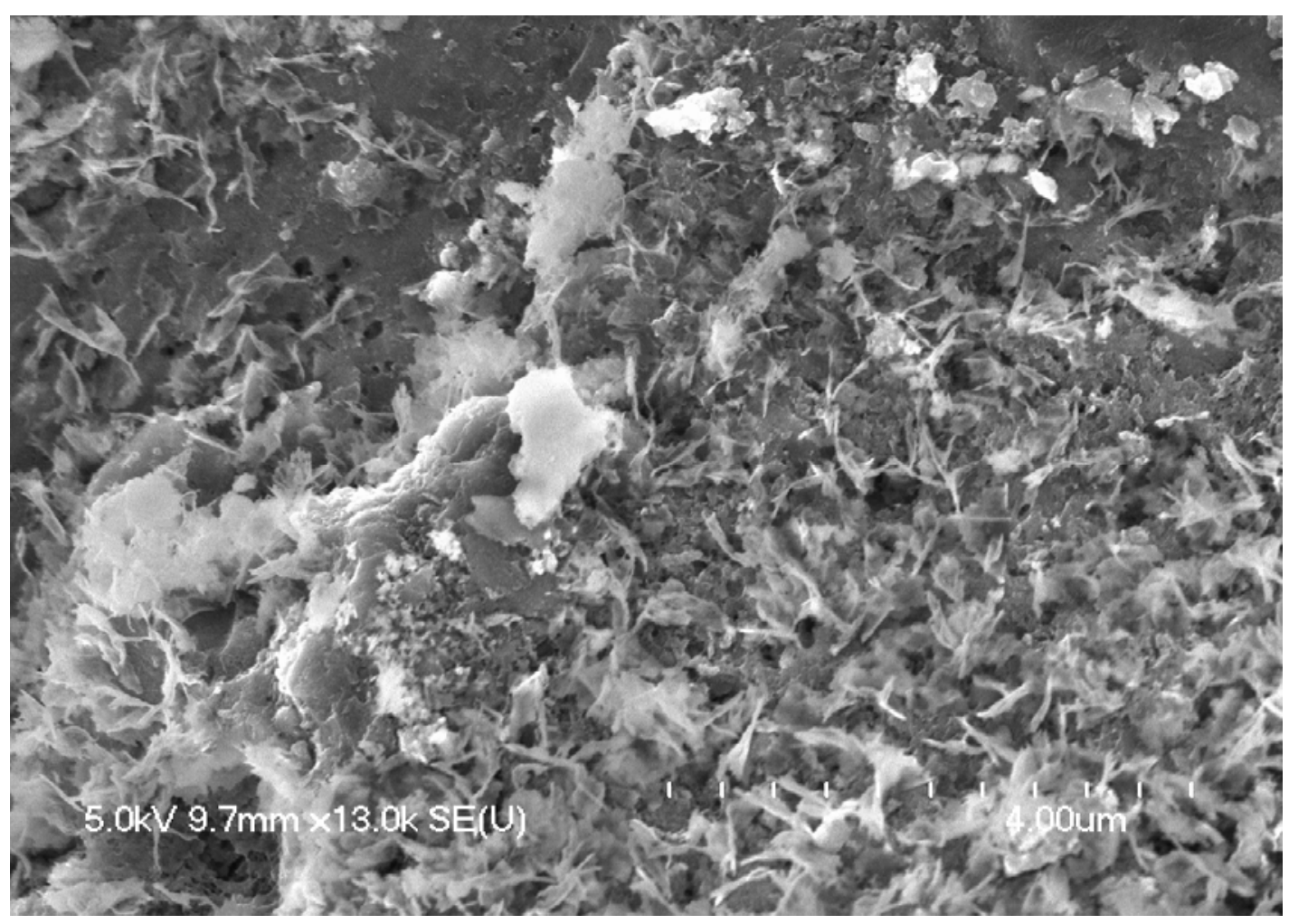

Fig. 4.2 Scanning electron micrograph of the GAC-QPVP. 
Oven dried beads of iron-chitosan are shown in Figure 4.3. Dried beads were characterized by SEM (Figure 4.4). From Figure 4.4 it is evident that the beads are porous in structure.

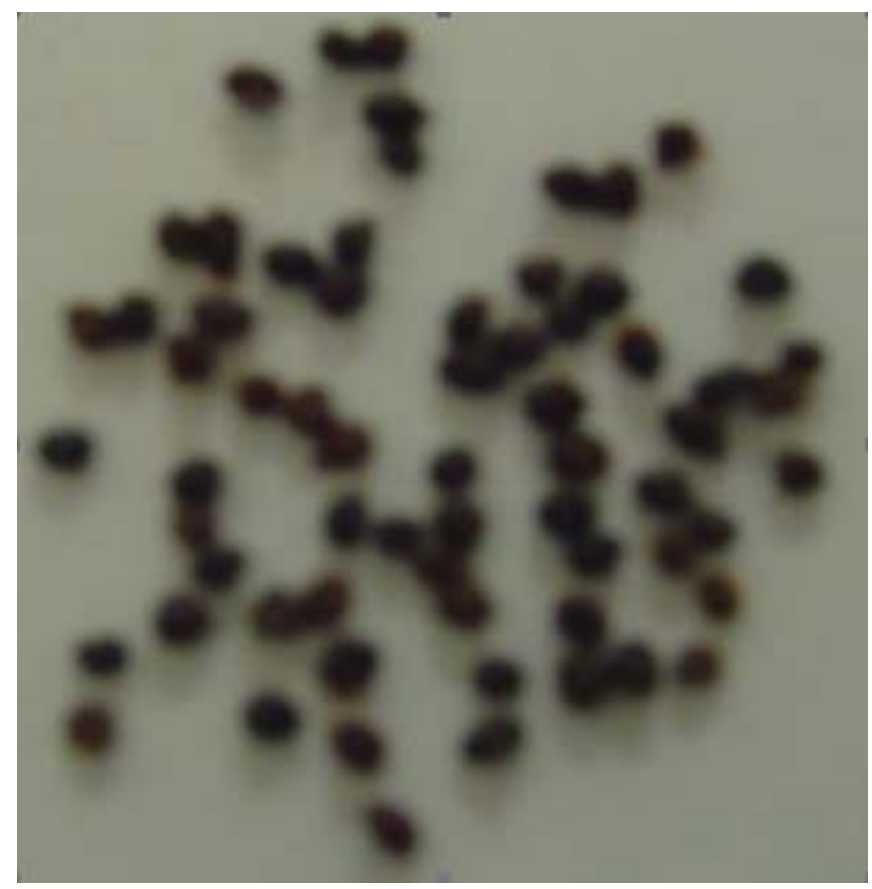

Fig. 4.3 Oven dried iron-chitosan beads

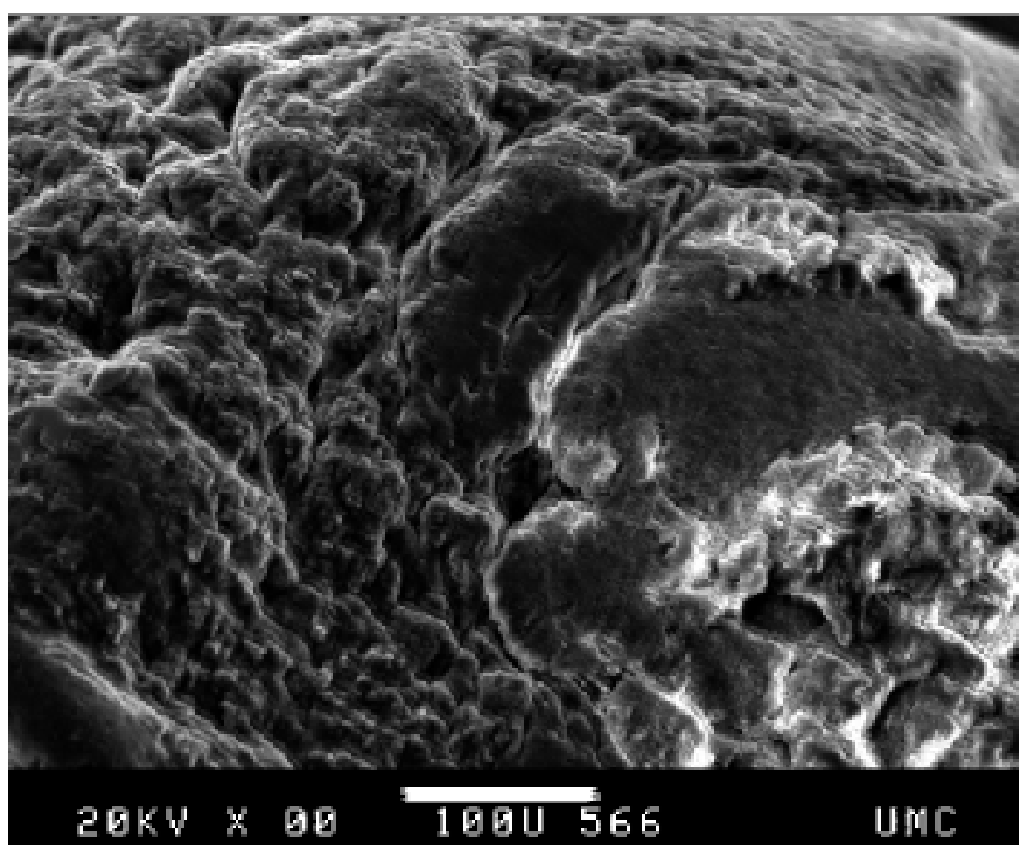

Fig. 4.4 Scanning electron micrograph (SEM) of iron-chitosan bead 
Further structural characterization of iron-chitosan beads includes X-Ray Photoelectron Spectroscopy (XPS) analysis. In XPS analysis, a survey scan was used to determine the elements existing on the surface. The high resolution utility scans were then used to measure the atomic concentrations of $\mathrm{Fe}, \mathrm{C}, \mathrm{N}$ and $\mathrm{O}$ in the sample. Figure 4.5 shows the peak positions of carbon, nitrogen, oxygen, and iron obtained by the XPS for pure iron-chitosan. In Fig. 4.5, the carbon 1s peak was observed at $283.0 \mathrm{eV}$ with a FWHM (full width at maximum height) of 2.015. The N-1s peak for iron-chitosan bead was found at $398.0 \mathrm{eV}$ (FWHM $2.00 \mathrm{eV}$ ), which can be attributed to the amino groups in chitosan. Table 4.2 shows the surface elemental concentration of $\mathrm{C}, \mathrm{N}, \mathrm{O}$ and $\mathrm{Fe}$ as determined from peak/area ratios, after correcting with the experimentally determined sensitive factor $( \pm 5 \%)$. The XPS analysis of iron-chitosan beads exposed to As (III) solutions show peaks of As (III), C-1s, N1s, O-1s and Fe as shown in Fig. 4.6. The binding energy and the atomic concentrations on the beads are given in Table 4.3.

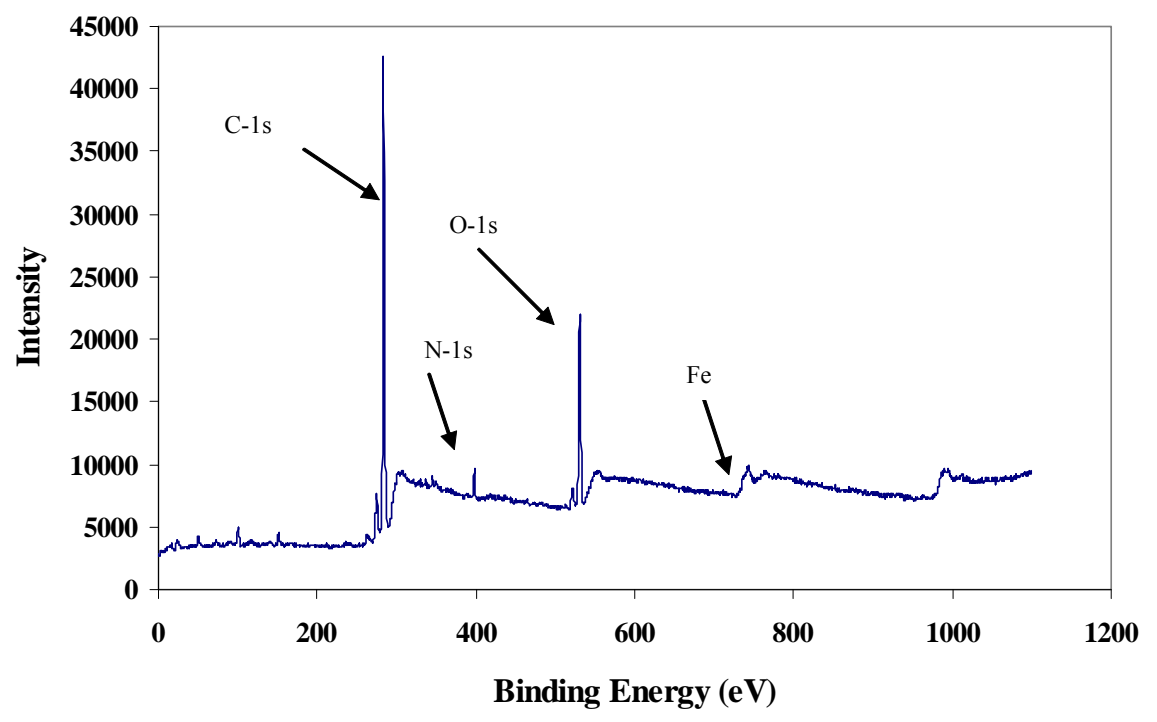

Fig. 4.5 XPS Spectrum of iron-chitosan bead

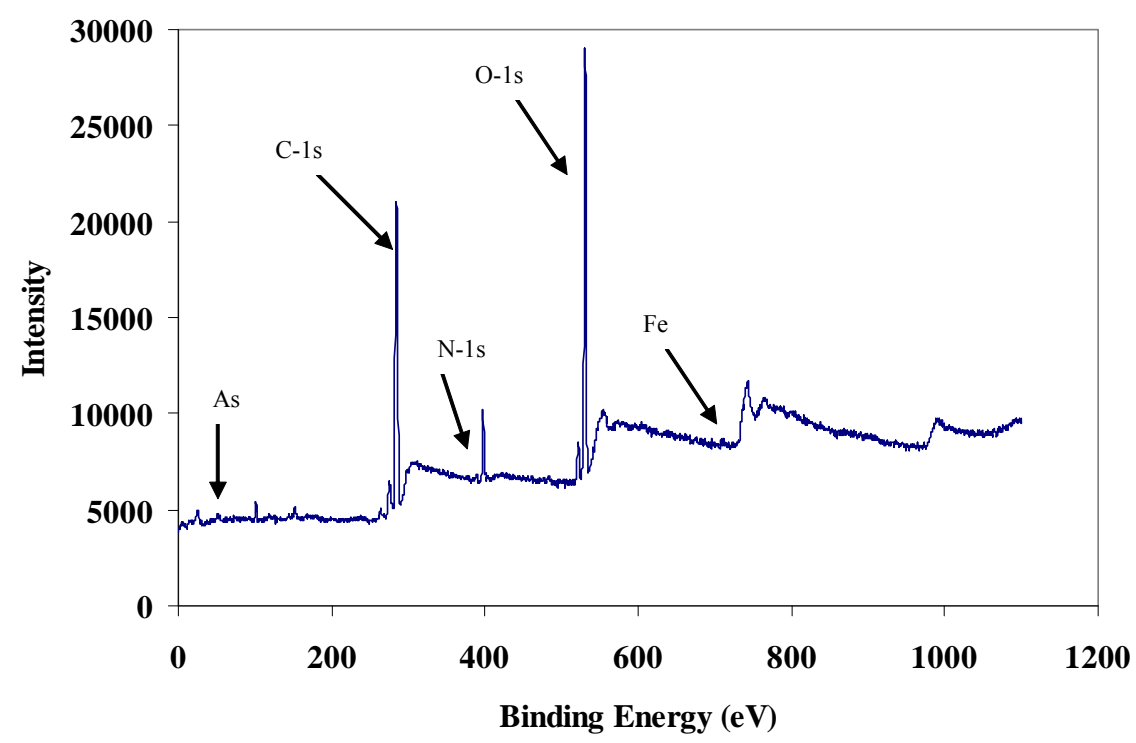

Fig. 4.6 XPS Spectrum of arsenite uptake iron-chitosan bead 
Table 4.2 Atomic concentrations of C, N, O and Fe on iron-chitosan

\begin{tabular}{|c|c|c|c|c|c|c|c|}
\hline \multicolumn{2}{|c|}{$\mathrm{C}$} & \multicolumn{2}{c|}{$\mathrm{N}$} & \multicolumn{2}{c|}{$\mathrm{O}$} & \multicolumn{2}{c|}{$\mathrm{Fe}$} \\
\hline $\begin{array}{c}\mathrm{BE} \\
(\mathrm{eV})\end{array}$ & $\begin{array}{c}\text { Atomic } \\
\text { Conc. } \\
(\%)\end{array}$ & $\begin{array}{c}\mathrm{BE} \\
(\mathrm{eV})\end{array}$ & $\begin{array}{c}\text { Atomic } \\
\text { Conc. } \\
(\%)\end{array}$ & $\begin{array}{c}\mathrm{BE} \\
(\mathrm{eV})\end{array}$ & $\begin{array}{c}\text { Atomic } \\
\text { Conc. } \\
(\%)\end{array}$ & $\begin{array}{c}\mathrm{BE} \\
(\mathrm{eV})\end{array}$ & $\begin{array}{c}\text { Atomic } \\
\text { Conc. } \\
(\%)\end{array}$ \\
\hline 283.0 & 77.04 & 398.0 & 2.62 & 530.5 & 14.33 & 711.0 & 0.36 \\
\hline
\end{tabular}

Table 4.3 Atomic concentrations of As (III), C, N, O and Fe on ironchitosan as obtained from XPS data

\begin{tabular}{|c|c|c|c|c|c|c|c|c|c|}
\hline \multicolumn{2}{|c|}{$\mathrm{C}$} & \multicolumn{2}{c|}{$\mathrm{N}$} & \multicolumn{2}{c|}{$\mathrm{O}$} & \multicolumn{2}{|c|}{$\mathrm{Fe}$} & \multicolumn{2}{c|}{ As } \\
\hline $\begin{array}{c}\mathrm{BE} \\
(\mathrm{eV})\end{array}$ & $\begin{array}{c}\text { Atomic } \\
\text { Conc. } \\
(\%)\end{array}$ & $\begin{array}{c}\mathrm{BE} \\
(\mathrm{eV})\end{array}$ & $\begin{array}{c}\text { Atomic } \\
\text { Conc. } \\
(\%)\end{array}$ & $\begin{array}{c}\mathrm{BE} \\
(\mathrm{eV})\end{array}$ & $\begin{array}{c}\text { Atomic } \\
\text { Conc. } \\
(\%)\end{array}$ & $\begin{array}{c}\mathrm{BE} \\
(\mathrm{eV})\end{array}$ & $\begin{array}{c}\text { Atomic } \\
\text { Conc. } \\
(\%)\end{array}$ & $\begin{array}{c}\mathrm{BE} \\
(\mathrm{eV})\end{array}$ & $\begin{array}{c}\text { Atomic } \\
\text { Conc. } \\
(\%)\end{array}$ \\
\hline 284.0 & 71.05 & 401.5 & 1.96 & 530.5 & 14.50 & 715.0 & 0.38 & 42.50 & 0.10 \\
\hline
\end{tabular}

\section{$\underline{4.3 \text { Effect of } \mathrm{pH}}$}

\subsubsection{Effect of pH on Chromium (VI) Adsorption}

The $\mathrm{pH}$ of a solution can have a significant effect on solute adsorption at the liquid-solid interface. Effect of $\mathrm{pH}$ on the chromium(VI) removal was investigated between a $\mathrm{pH}$ range of 1-12, with an initial concentration of $5 \mathrm{mg} / \mathrm{L}$, and the adsorbent loading rate was 2 grams of adsorbent/1 L of solution. Figure 4.7 shows that the maximum removal efficiency observed at $\mathrm{pH}=2.0$ and it decreases as the solution becomes basic. There was no apparent adsorption when the $\mathrm{pH}$ becomes greater than 11 . Adsorption capacity was reduced about 16 times when the $\mathrm{pH}$ was increased from 6 to 11 .

Levya et al. (1994) reported similar observations that the adsorption capacity of chromium(VI) was reduced about 17 times when the $\mathrm{pH}$ was increased from 6 to 10 using activated carbon. The reason behind this behavior may be explained by speciation of chromium in aqueous solution and chemical structure of adsorbent. Chromium(VI) is capable of existing in different forms including; $\mathrm{HCrO}_{4}{ }^{-}, \mathrm{Cr}_{2} \mathrm{O}_{7}{ }^{-2}$ and $\mathrm{CrO}_{4}{ }^{-2}$ depending on the conditions of the $\mathrm{pH}$ and total chromium concentration (Latimer, 1952). When $\mathrm{pH}<6.5$, chromium(VI) exists as $\mathrm{HCrO}_{4}{ }^{-}$or $\mathrm{Cr}_{2} \mathrm{O}_{7}{ }^{-2}$ complex, while at $\mathrm{pH}$ above 8 the dominant chemical form is $\mathrm{CrO}_{4}{ }^{-2}$. The molar fraction of $\mathrm{HCrO}_{4}{ }^{-}$complex was drastically diminished from 0.77 to 0.003 , when the $\mathrm{pH}$ was increased from 6 to 10 . This fact suggested that adsorption of chromium(VI) was mainly due to the anionic exchange of $\mathrm{HCrO}_{4}{ }^{-}$complex with bromide, since adsorption capacity was reduced 16 times as the $\mathrm{pH}$ varied from 6 to 11 .

$$
\begin{aligned}
& \mathrm{Cr}_{2} \mathrm{O}_{7}^{-2}+\mathrm{H}_{2} \mathrm{O} \Leftrightarrow 2 \mathrm{HCrO}_{4}^{-} \\
& \overline{G A C-\left(Q P y^{+}\right) B r^{-}}+\mathrm{HCrO}_{4}^{-} \Leftrightarrow \overline{G A C-\left(Q P y^{+}\right) \ldots H C r O_{4}^{-}}+\mathrm{Br}^{-}
\end{aligned}
$$


Where, $\overline{G A C-\left(Q P y^{+}\right) B r^{-}}$represents the quaternized PVP coated GAC.

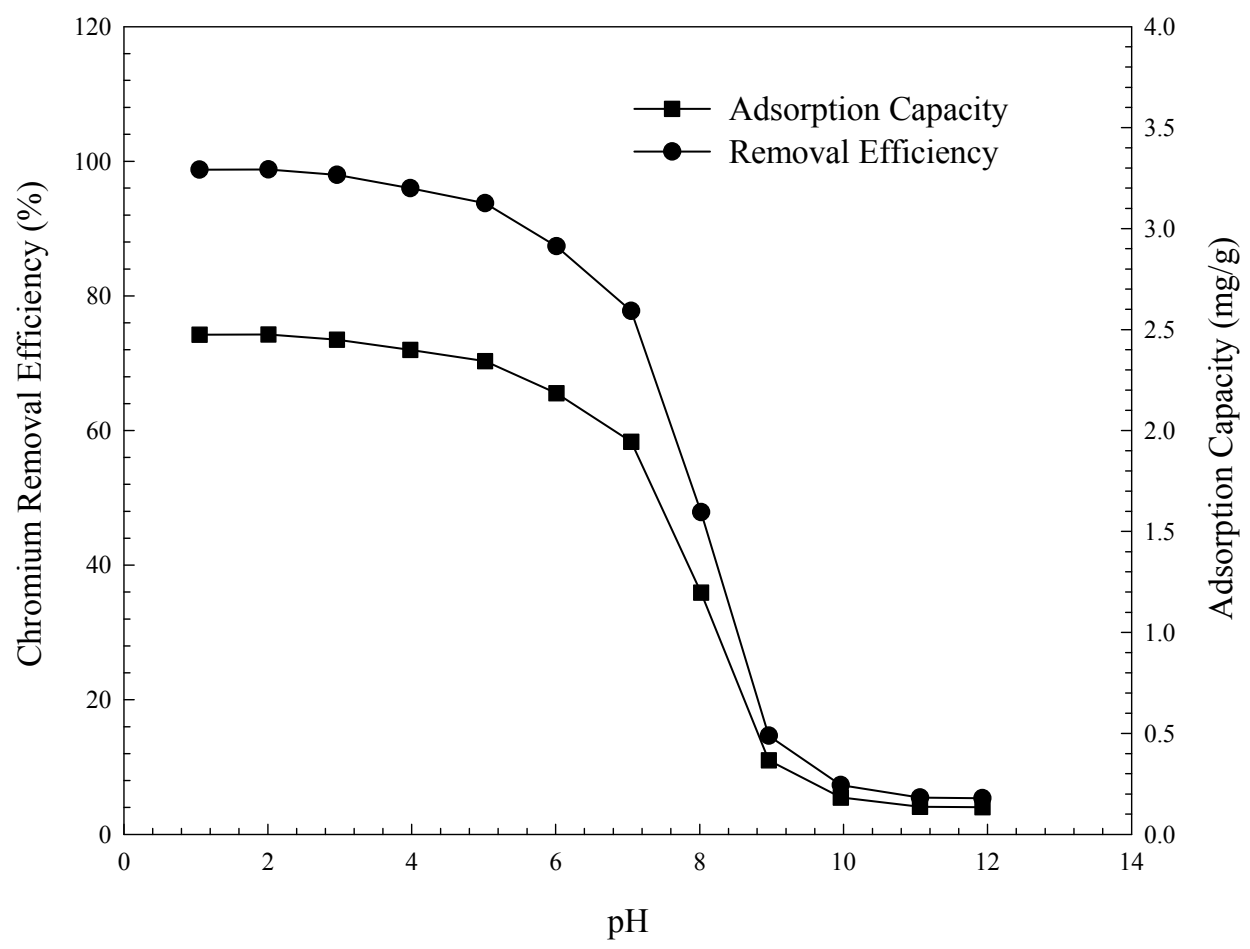

Fig. 4.7 Effect of $\mathrm{pH}$ on $\mathrm{Cr}(\mathrm{VI})$ removal, Loading $=2.0 \mathrm{~g} / \mathrm{L} ; \mathrm{T}=25^{\circ} \mathrm{C}$.

\subsubsection{Effect of pH on Arsenic(III) Adsorption}

Evaluation of arsenic(III) removal was conducted at different $\mathrm{pH}$ values varied from 4-12 at an initial concentration of $314 \mu \mathrm{g} / \mathrm{L}$ using iron-chitosan. $\mathrm{pH}$ in the solution was adjusted by $0.1 \mathrm{M} \mathrm{HCl}$ or $0.1 \mathrm{M} \mathrm{NaOH}$. The $\mathrm{pH}$ effect was started at $\mathrm{pH}=4$, since the iron-chitosan adsorbent was found to be unstable when the $\mathrm{pH}$ is less than 4. The amount of arsenic(III) adsorbed was calculated from the filtrate and representative results shown in Figure 4.8 indicated that $\mathrm{pH}$ had no significant effect on arsenic(III) removal from $\mathrm{pH}$ 4-9.1. However, when the $\mathrm{pH}$ was higher than 9.1, arsenic(III) removal was decreased with increasing $\mathrm{pH}$. This can be explained by using the speciation diagram of As in different $\mathrm{pH}$ of solution (Gupta and Chen, 1978). The chemical equilibrium of arsenic speciation is shown in Table 4.4.

Table 4.4 Arsenic speciation

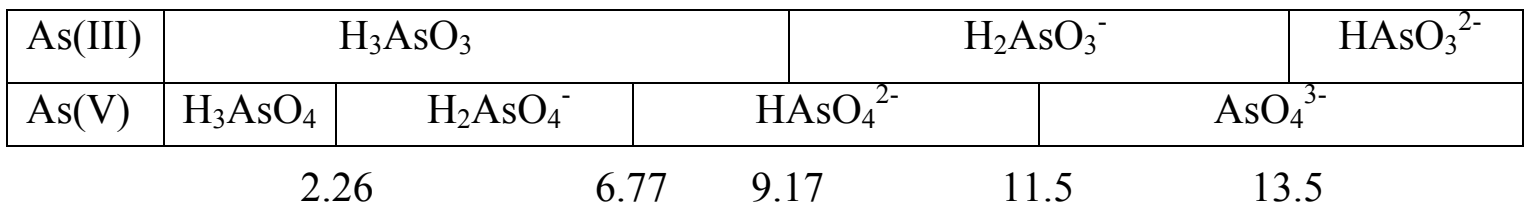


Both arsenite ( +3 oxidation) and arsenate ( +5 oxidation) occur in several protonate forms at near-neutral $\mathrm{pH}$. The predominant species are $\mathrm{H}_{3} \mathrm{AsO}_{3}$ for arsenic(III), $\mathrm{H}_{2} \mathrm{AsO}_{4}{ }^{-}$and $\mathrm{HAsO}_{4}{ }^{2-}$ for $\operatorname{arsenic}(\mathrm{V})$. Arsenic(V) exists as an anion at typical $\mathrm{pH}(5-8)$, while arsenic(III) remains as a neutral molecule at the same $\mathrm{pH}$ region. Arsenic(III) carries one negative charge at a $\mathrm{pH}$ over 9.2. As the arsenite exists as neutral molecule in $\mathrm{pH}$ range 4-9.1, it can be expected that arsenic(III) removal is due to chemical biding and/or hydrophobic forces, not electrostatic interactions.

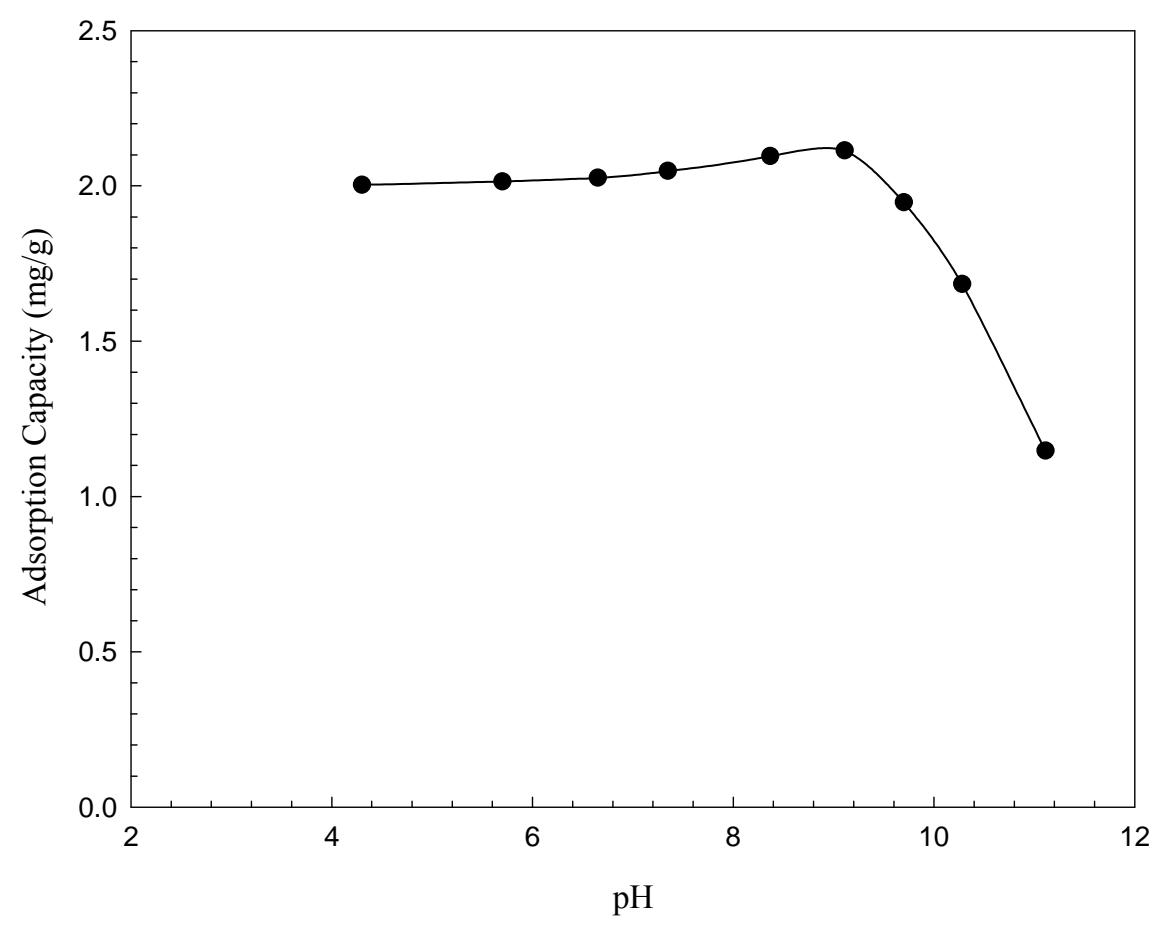

Figure 4.8 Effect of $\mathrm{pH}$ on $\mathrm{As}(\mathrm{III})$ removal, $\mathrm{T}=25^{\circ} \mathrm{C}$.

Studies on arsenic(III) removal by feerihydrite indicated that a maximum adsorption occurred at approximately pH 9.0 (Raven et al.,1997). Gu et al. (2005) examined the effect of $\mathrm{pH}$ on arsenic removal using iron impregnated GAC and reported that $\mathrm{pH}$ had no obvious effect on arsenic(III) removal from 4.4-9.0, with removal efficiency higher than 95\% under the experimental conditions. Another study indicated that the uptake of arsenic(III) by fresh and immobilized biomass was not greatly affected by solution $\mathrm{pH}$ with optimal biosorption occurring at pH 6-8 (Kamala et al., 2005). Another adsorption process for the removal of $\operatorname{arsenic}(\mathrm{V})$ and arsenic(III) was evaluated under various conditions using zirconium(IV) loaded chelating resin (Zr-LDA) (Balaji et al., 2005). Results show that arsenic(III) was adsorbed between $\mathrm{pH} 7$ and 10.5 .

\section{$\underline{4.4 \text { Kinetics }}$}

\subsubsection{Chromium (VI) Adsorption Kinetics}

To determine rate of adsorption and to find out the possibility of using this material as a lowcost adsorbent, the kinetics of the adsorption were investigated at various concentrations of 
chromium(VI). The $\mathrm{pH}$ of the solutions was maintained at 2, since maximum removal efficiency was obtained at $\mathrm{pH}=2$ and adsorbent loading for initial concentrations of $2.5,15$, and $43 \mathrm{mg} / \mathrm{L}$ was $0.375,1.5$, and $5 \mathrm{~g} / \mathrm{L}$, respectively. Representative results obtained from the kinetics study is shown in Figure 4.9. The adsorption capacity at equilibrium increases from 6.63 to $8.55 \mathrm{mg} / \mathrm{g}$ with an increase in the initial chromium(VI) concentration from 2.5 to $43 \mathrm{mg} / \mathrm{L}$.

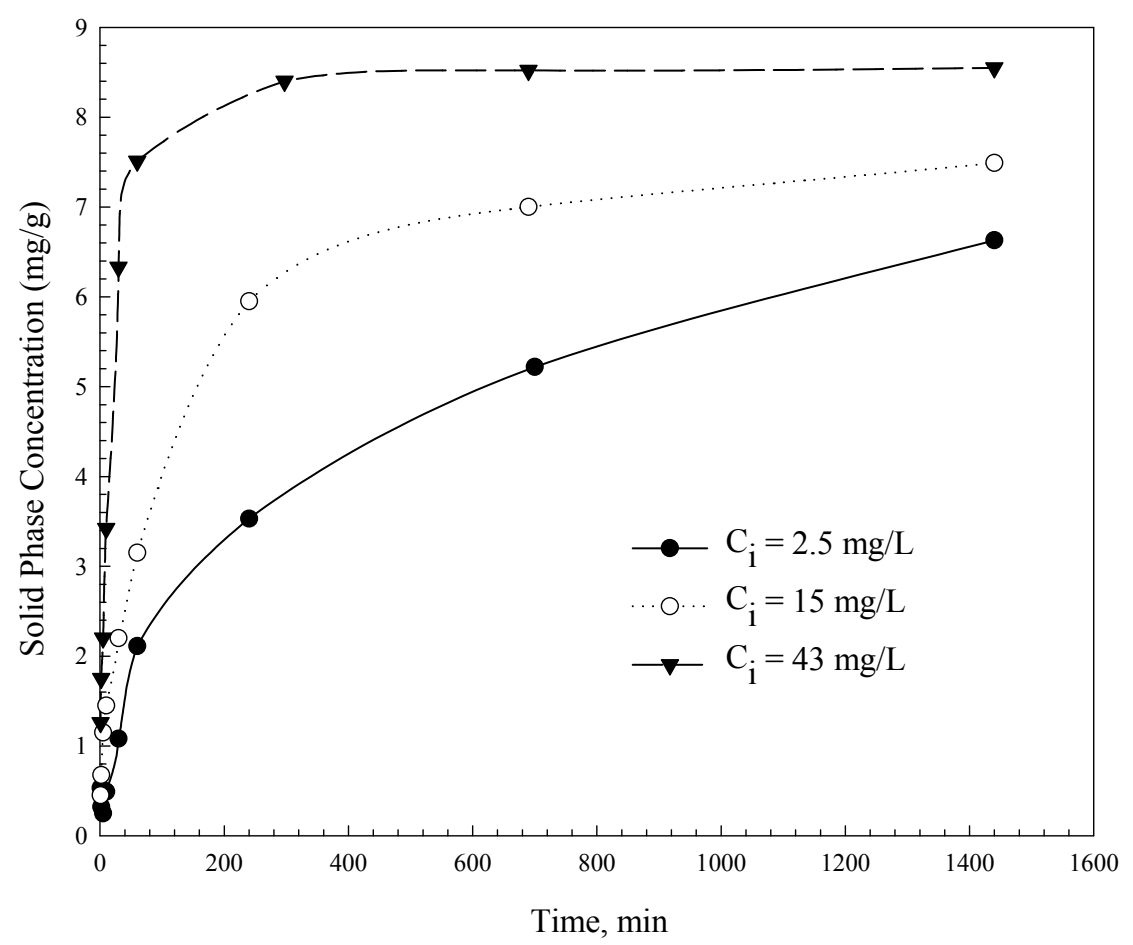

Figure 4.9 Rate of adsorption at different initial concentrations, $\mathrm{pH}=2 ; \mathrm{T}=25^{\circ} \mathrm{C}$.

The adsorption rate was fast at the beginning since there was more active sites to adsorb chromium(VI). As the initial chromium(VI) concentration increased, adsorption capacity was increased. The driving force which is the concentration gradient of chromium(VI) between solid phase and liquid phase was also an important factor. This driving force increases as adsorbate concentration increases, resulting in faster adsorption of chromium(VI).

\subsubsection{Chromium (VI) Adsorption Kinetics Modeling}

In order to determine rate constants, two different kinetic models were used, including pseudo-first-order model, and pseudo-second-order model. Kinetics of a pseudo-first-order model can be expressed as (Sarkar and Chattoraj, 1993)

$\frac{d q_{t}}{d t}=k_{1}\left(q_{e}-q_{t}\right)$

Where, $k_{1}$ is the rate constant of pseudo-first-order adsorption, $q_{t}(\mathrm{mg} / \mathrm{g})$ is the amount of chromium(VI) adsorbed at time $\mathrm{t}$, and $q_{e}$ is the amount of adsorption at equilibrium. After 
integration and applying the initial conditions $q_{t}=0$ at $t=0$ and $q_{t}=q_{t}$ at $t=t$, then Equation (4-3) becomes

$\ln \left(q_{e}-q_{t}\right)=\ln q_{e}-k_{1} t$

$k_{1}$ and $q_{e}$ can be obtained from the slope and intercept of the first order plot.

A pseudo-second-order equation can be represented as follows (Ho and Mckay, 1999)

$\frac{d q_{t}}{d t}=k_{2}\left(q_{e}-q_{t}\right)^{2}$

Where, $k_{2}$ is the pseudo-second-order adsorption rate constant. Integrating the Equation (45 ) and applying the initial conditions, we get

$\frac{t}{q_{t}}=\frac{t}{q_{e}}+\frac{1}{k_{2} q_{e}^{2}}$

$k_{2}$ and $q_{e}$ can be obtained from the intercept and slope of the plot of $\left(t / q_{t}\right)$ vs. $t$.

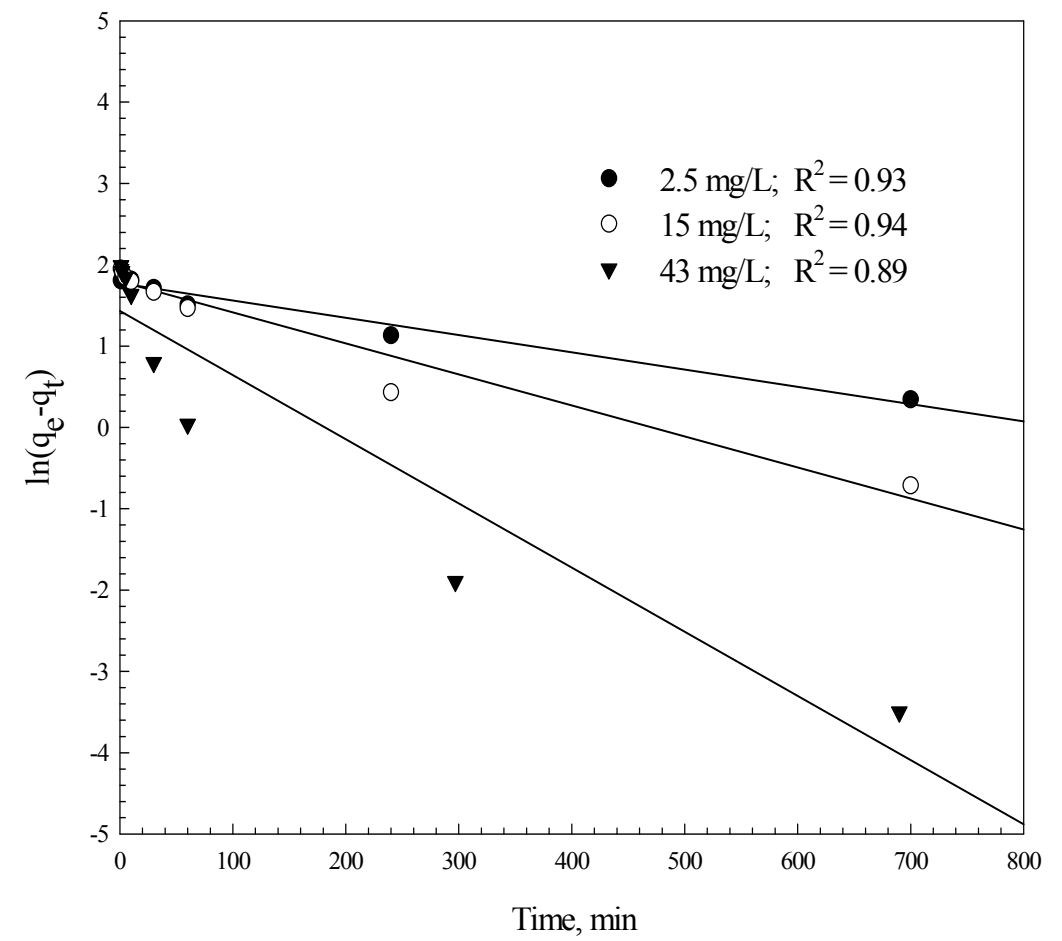

Figure 4.10 Kinetics of a pseudo-first-order model, $\mathrm{pH}=2 ; \mathrm{T}=25^{\circ} \mathrm{C}$. 


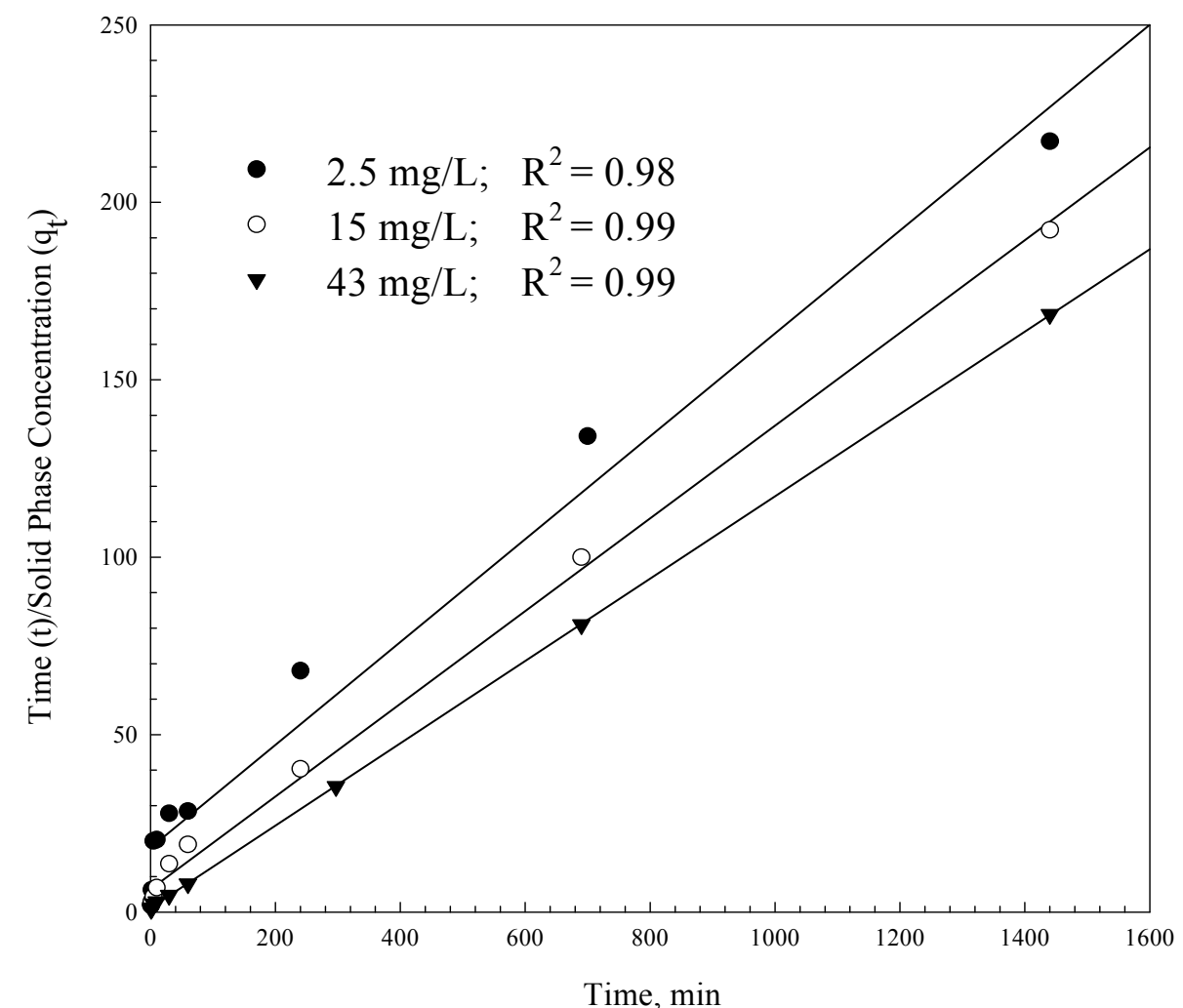

Figure 4.11 Kinetics of a pseudo-second-order model, $\mathrm{pH}=2 ; \mathrm{T}=25^{\circ} \mathrm{C}$.

The results in the Figures 4.10 and 4.11 indicated that the experimental data fit the pseudosecond-order model well. Table 4.5 lists the regression coefficients for the linear plots from the pseudo-first-order and pseudo-second-order equations. It was observed that second order rate constant increased from $1.16 \times 10^{-3}$ to $11.55 \times 10^{-3} \mathrm{~g} / \mathrm{mg} / \mathrm{min}$ and equilibrium adsorption capacity increased from 6.63 to $8.55 \mathrm{mg} / \mathrm{g}$ as the initial concentration increased from 2.5 to $43 \mathrm{mg} / \mathrm{L}$. It can be seen that $R^{2}$ values for the pseudo-second-order plots are greater than those obtained for the pseudo-first-order. This suggested that the chromate adsorption onto GAC-QPVP might follow the pseudo-second-order reaction. Karaca et al. (2004) demonstrated that kinetics of adsorption of phosphate onto dolomite also were pseudosecond-order, and concluded that adsorption would be controlled by combined chemisorption and intraparticle diffusion.

Table 4.5 Reaction rate constants for chromium(VI)

\begin{tabular}{|c|c|c|c|c|c|c|c|c|}
\hline \multirow{2}{*}{$\begin{array}{l}\text { Initial } \\
\begin{array}{l}\text { Concen- } \\
\text { tration } \\
(\mathrm{mg} / \mathrm{L})\end{array}\end{array}$} & $\begin{array}{l}k_{1} \times 10^{3} \\
\left(\mathrm{~min}^{-1}\right)\end{array}$ & $R_{1}^{2}$ & $\begin{array}{l}q_{e, \exp } \\
(\mathrm{mg} / \mathrm{g})\end{array}$ & $\begin{array}{l}q_{e, \text { cal }} \\
(\mathrm{mg} / \mathrm{g})\end{array}$ & $\begin{array}{l}k_{2} \times 10^{3} \\
\left(\mathrm{~g} \mathrm{mg}^{-1}\right. \\
\left.\mathrm{min}^{-1}\right)\end{array}$ & $R_{2}^{2}$ & $\begin{array}{l}q_{e, \exp } \\
(\mathrm{mg} / \mathrm{g})\end{array}$ & $\begin{array}{l}q_{e, \text { cal }} \\
(\mathrm{mg} / \mathrm{g})\end{array}$ \\
\hline 2.5 & 2.12 & 0.93 & 6.63 & 5.89 & 1.16 & 0.98 & 6.63 & 6.89 \\
\hline 12.3 & 3.81 & 0.94 & 7.49 & 6.02 & 2.64 & 0.99 & 7.49 & 7.65 \\
\hline 43 & 7.89 & 0.89 & 8.55 & 4.19 & 11.55 & 0.99 & 8.55 & 8.62 \\
\hline
\end{tabular}




\subsubsection{Finite-bath Diffusion Model}

Molecular diffusion determines the rate of most mass transfer processes. Determination of diffusion coefficient of the key component is very important for predicting rates of mass transfer, and many correlations. To find out the diffusion rate constant, a modified finitebath diffusion control model was used in this study (Gang et al., 2001a), which is:

$$
\bar{X}=\left(\frac{18 \overline{V D} \lambda}{V \bar{r}^{2}}\right)^{1 / 2} t^{1 / 2}+\bar{X}_{0}
$$

Where, $\bar{X}=$ Fractional attainment of equilibrium sorption

$\bar{X}_{0}=$ Fractional attainment constant

$t=$ Time, sec

$V=$ Volume of external solution, $\mathrm{L}$

$\bar{V}=$ Volume of adsorbent particles, $\mathrm{L}$

$\bar{r}=$ Initial radius of adsorbent particle, $\mathrm{cm}$

$\bar{D}=$ Effective diffusivity in reacted layer, $\mathrm{cm}^{2} \mathrm{~s}^{-1}$

$\lambda=$ Distribution coefficient

The plot of $\bar{X}$ verses $t^{1 / 2}$ should be linear, with constant $\bar{X}_{0}$ being determined from the intercept of the straight line. The fractional attainment data measured in the experiment is plotted in Figure 4.12 according to equation 4-7. The good linearity of data shows the validity of the model. The value of $\lambda \bar{D}$ was calculated from the slopes in Figure 4.12 and recorded in Table 4.6. Because the adsorbent used for rate measurement was a mixture of particles of different shapes, but with a narrow size range, the average radius $(0.40 \mathrm{~mm})$ was used for the calculation of $\lambda \bar{D}$.

According to the Gang et al. (2001a) simplification, another form of the modified finite-bath diffusion control model can be described as:

$$
\ln \left[1 /\left(1-\bar{X}+\bar{X}_{0}\right]-3\left[1-\left(1-\bar{X}+\bar{X}_{0}\right)^{1 / 3}\right]=\frac{3 \overline{V D} \lambda}{V^{2}} t\right.
$$

According to this model, a plot of $\ln \left[1 /\left(1-\bar{X}+\bar{X}_{0}\right]-3\left[1-\left(1-\bar{X}+\bar{X}_{0}\right)^{1 / 3}\right]\right.$ vs. $t$ should be linear. The data in the Figure 4.9 are again plotted in Figure 4.13 according to equation 4-8. The good linearity of the data indicates the validity of the model. The values of $\lambda \bar{D}$ calculated from the slopes of the linear plots of Figure 4.13 and given in Table 4.6 are found to be close to those obtained from equation 4-7. Experimental data reasonably fitted to the diffusion control model. The average value of the product of distribution coefficient and the effective diffusivity in the adsorption of chromium(VI) onto GAC-QPVP was $4.10 \times 10^{-6}$ $\mathrm{cm}^{2} / \mathrm{s}$. Chanda and Rampel (1993) also found that the sorption of uranyl sulfate on PVP coated silica gel correlated well with a similar model. They reported that the product of distribution coefficient and the effective diffusivity was $5.4 \times 10^{-6} \mathrm{~cm}^{2} / \mathrm{s}$. 


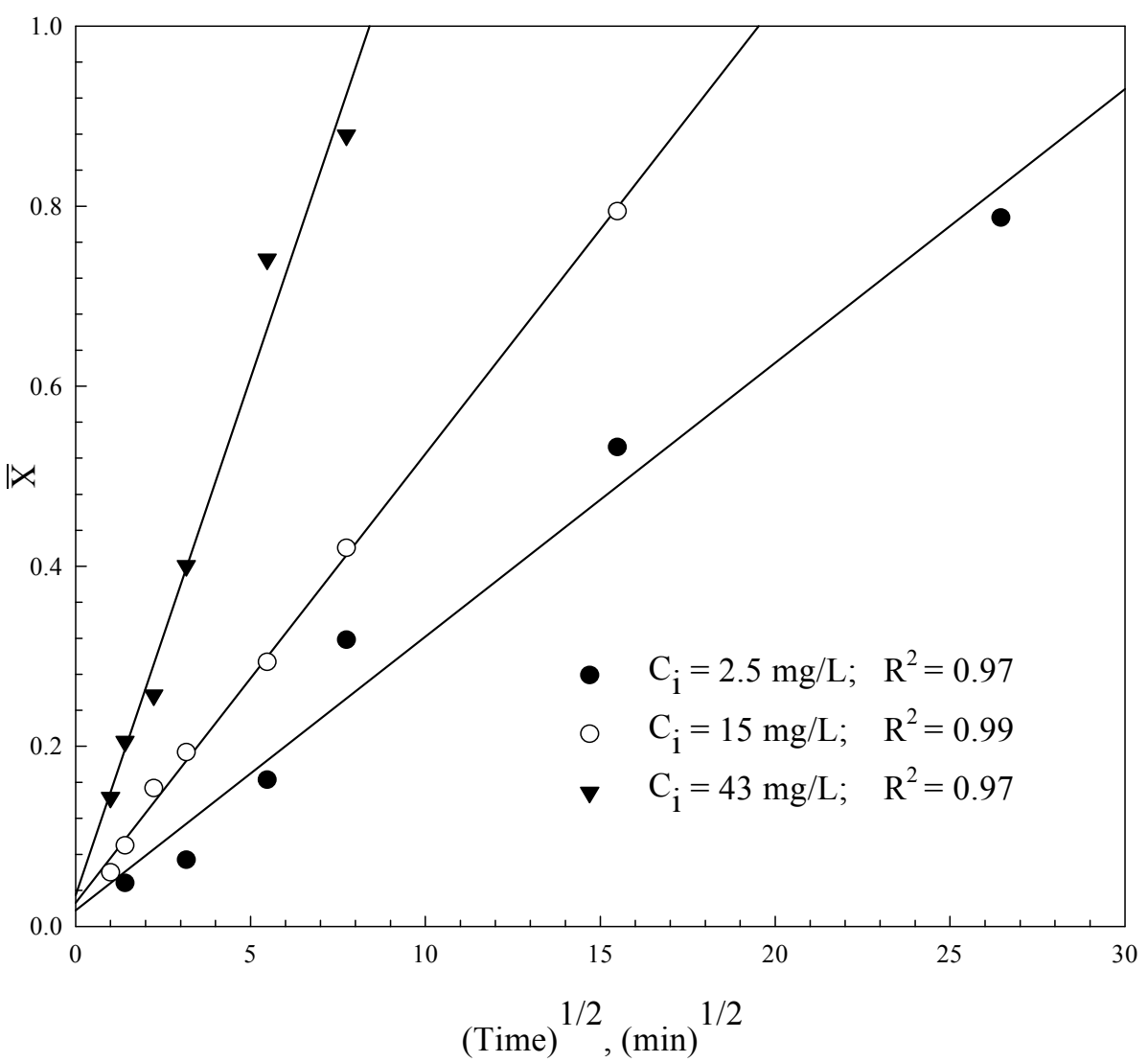

Figure 4.12 Test of modified finite-bath-diffusion model (equation 4-7)

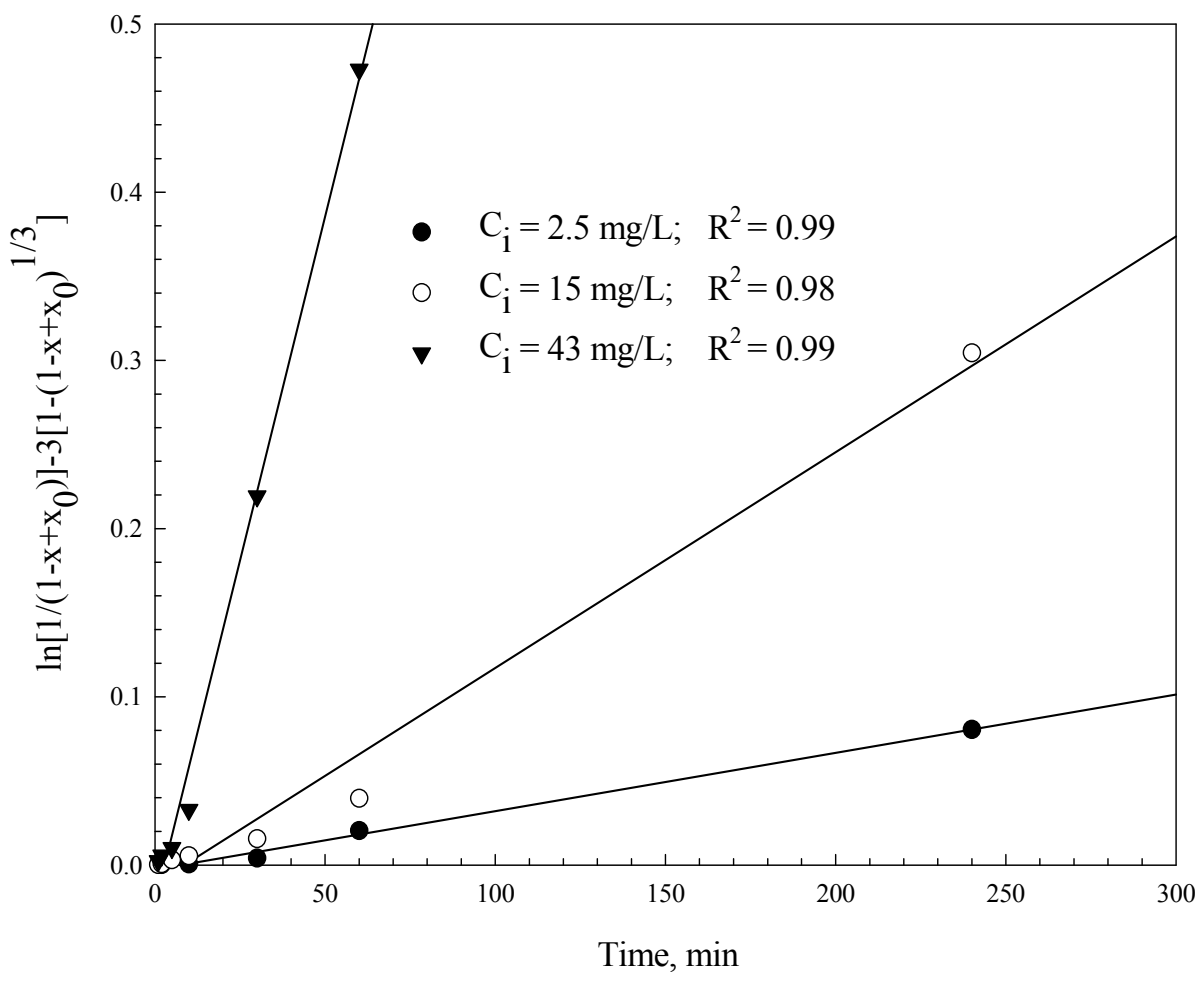

Figure 4.13 Test of modified finite-bath-diffusion model (equation 4-8) 
Table 4.6 Values of $\lambda \bar{D}$ for adsorption of Cr(VI) on GAC-QPVP at different concentrations

\begin{tabular}{|c|c|cc|}
\hline $\begin{array}{l}\text { Initial concentration } \\
\text { of } \mathrm{Cr}(\mathrm{VI}) \mathrm{in} \mathrm{mg} / \mathrm{L}\end{array}$ & $\bar{X}_{0}$ & \multicolumn{2}{|c|}{$\lambda \bar{D}\left(\mathrm{~cm}^{2} / \mathrm{s}\right)$ calculated from } \\
\cline { 3 - 4 } & & equation $4-7$ & equation $4-8$ \\
\hline 2.5 & 0.018 & $2.22 \times 10^{-6}$ & $5.00 \times 10^{-6}$ \\
\hline 15 & 0.026 & $1.50 \times 10^{-6}$ & $4.63 \times 10^{-6}$ \\
\hline 43 & 0.035 & $2.38 \times 10^{-6}$ & $8.85 \times 10^{-6}$ \\
\hline
\end{tabular}

\subsubsection{Arsenic(III) Adsorption Kinetics}

Kinetics of the arsenic(III) removal by iron-chitosan was conducted at various initial concentrations at $25^{\circ} \mathrm{C}$. The $\mathrm{pH}$ of the solutions was maintained at 9.0 because maximum removal of arsenic(III) was obtained near $\mathrm{pH}=9$. The adsorbent loading was $0.2 \mathrm{~g} / \mathrm{L}$ and initial arsenic(III) concentrations were 306, 584, and $994 \mu \mathrm{g} / \mathrm{L}$. A desired quantity of the adsorbent iron-chitosan $(20 \mathrm{mg})$ was placed in a separate conical flasks with $\mathrm{pH}$-adjusted arsenic(III) solution. Representative results from the kinetic batch experiments, presented in Figure 4.14, suggested that adsorption capacity at equilibrium increases from 1.51 to 4.60 $\mathrm{mg} / \mathrm{g}$ with an increase in the initial arsenic(III) concentration from 306 to $994 \mu \mathrm{g} / \mathrm{L}$. More than $60 \%$ of the arsenic was adsorbed by the iron-chitosan within 30 min of the reaction and it took about 2 hours to reach the equilibrium concentration. Adsorption rate was rapid at the beginning, since there was more active sites to adsorb the arsenic(III).

It has been observed that the sorption rate of arsenic(V) and arsenic(III) on minerals was initially rapid, followed by slow phase (Arai et al., 2004). Fuller et al. (1993) reported that As(V) adsorption on synthesized ferrihydrite had a rapid initial phase $(<5 \mathrm{~min})$ and adsorption continued for $182 \mathrm{~h}$. Fuller et al. (1993) proposed that rate limiting As(V) adsorption step was controlled by slow diffusion to adsorption sites within aggregates of ferrihydrite crystalline. Raven et al. (1997) studied the kinetics of arsenic(V) and arsenic(III) adsorption on ferrihydrite from $5 \mathrm{~min}$ to $96 \mathrm{~h}$ and it was found that most adsorption occurred within the first $2 \mathrm{~h}$. In studies of arsenic(III) adsorption kinetics $(0.5-24 \mathrm{~h})$ on surface and subsurface soils, Elkhatib et al. (1984) concluded that the initial reaction was rapid, with more than $50 \%$ of arsenic(III) adsorbed in soils in the first $0.5 \mathrm{~h}$.

In order to determine reaction rate constants of arsenic(III) adsorption onto iron-chitosan, pseudo-first-order model (4-4) and pseudo-second-order model (4-6) were used. The results shown in the Figures 4.15 and 4.16 indicated that the experimental data better fitted to the pseudo-second-order model with higher regression coefficient values. Table 4.7 lists the regression coefficients for the linear plots from the pseudo-first-order and pseudo-secondorder equations. It can be seen that $R^{2}$ values obtained from pseudo-second-order model are greater than those obtained from the pseudo-first-order model.

The second order rate constants $\left(k_{2}\right)$, and equilibrium adsorption capacities $\left(q_{e}\right)$ were calculated from the Figure 4.16 and given in the Table 4.7. It can be noticed that the rate constant was decreased from $3.19 \times 10^{-2}$ to $1.15 \times 10^{-2} \mathrm{~g} \mathrm{mg}^{-1} \mathrm{~min}^{-1}$ as the initial concentration increased from 306 to $994 \mu \mathrm{g} / \mathrm{L}$, where as the initial rate $\left(k_{2} q_{e}^{2}\right)$ was increased 
from $8.48 \times 10^{-2}$ to $27.97 \times 10^{-2}$ with increasing initial arsenic(III) concentration due to the increasing in driving force as the initial concentration increases.

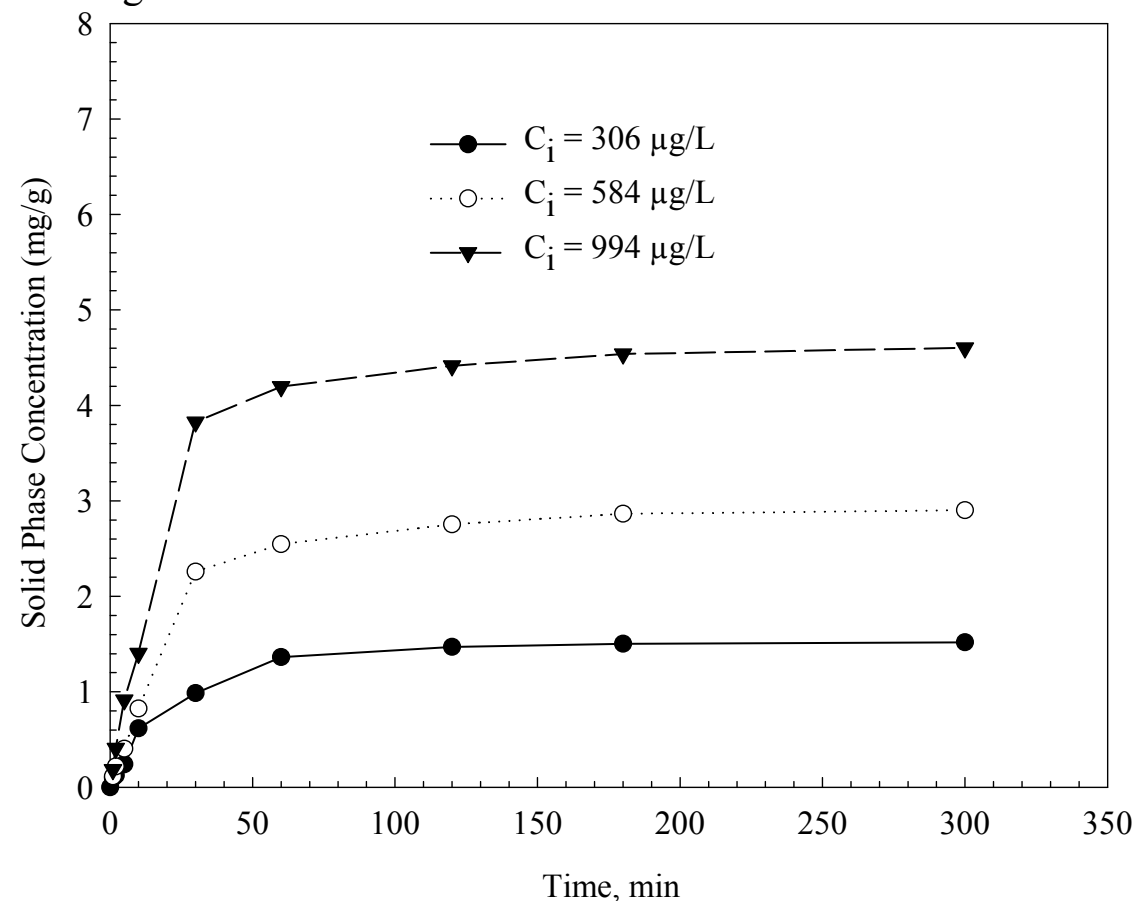

Figure 4.14 Rate of adsorption at different initial concentrations, $\mathrm{pH}=9 ; \mathrm{T}=25^{\circ} \mathrm{C}$.

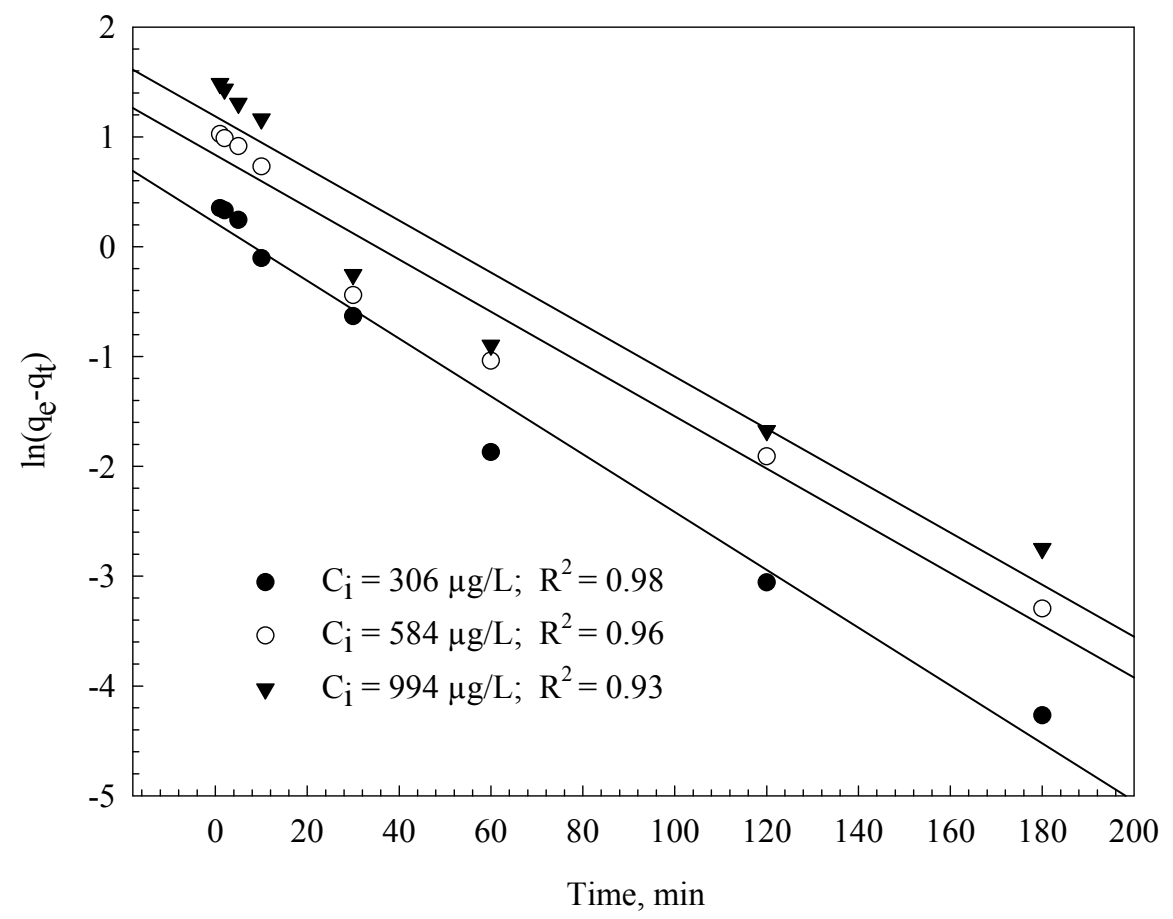

Figure 4.15 Kinetics of a pseudo-first-order model, $\mathrm{pH}=9 ; \mathrm{T}=25^{\circ} \mathrm{C}$. 


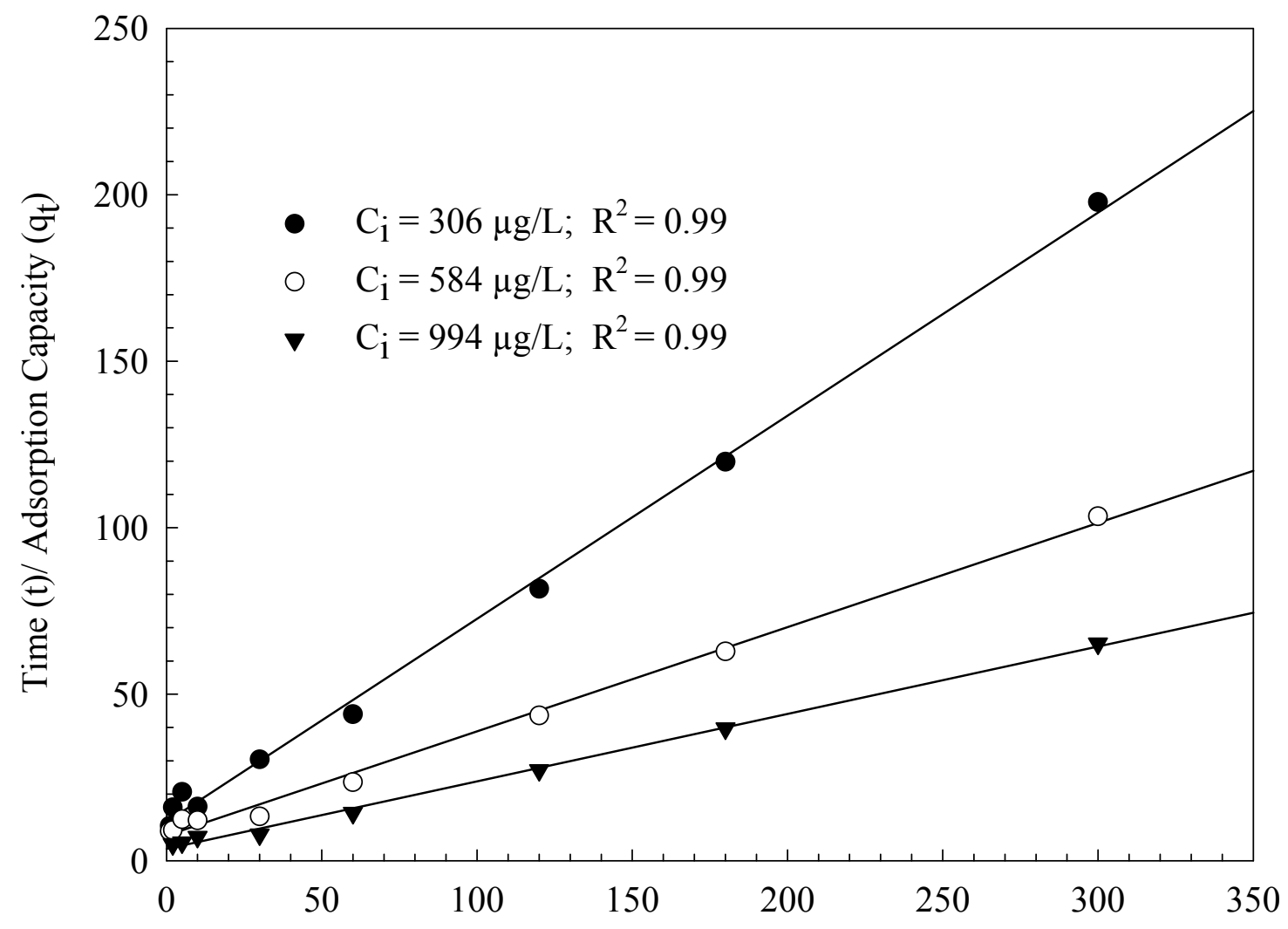

Time, $\min$

Figure 4.16 Kinetics of a pseudo-second-order model, $\mathrm{pH}=9 ; \mathrm{T}=25^{\circ} \mathrm{C}$.

Onyango et al. (2003) investigated the arsenic adsorption kinetics using chemically modified zeolite and results were fitted to these two models (pseudo first order and pseudo second order) and compared. It was concluded that sorption onto the active sites is consistent with a pseudo-second-order mechanism. Arsenic(III) adsorption onto granular ferric hydroxide (GFH) was analyzed by the Thirunavukkarasu et al. (2003) and it was found that most of arsenic(III) adsorption onto GFH occurred at $\mathrm{pH} 7.6$, with $68 \%$ of arsenic(III) removal within $1 \mathrm{~h}$ and $97 \%$ removal at the equilibrium time of $6 \mathrm{~h}$ and kinetic data were fitted to the pseudo-second-order reaction rate model.

Table 4.7 Reaction rate constants for arsenic(III)

\begin{tabular}{|c|c|c|c|c|c|c|c|c|c|}
\hline \multirow{2}{*}{$\begin{array}{l}\text { Initial } \\
\text { Concen } \\
\text { tration } \\
(\mu \mathrm{g} / \mathrm{L})\end{array}$} & \multicolumn{4}{|c|}{ Pseudo-first-order } & \multicolumn{5}{|c|}{ Pseudo-second-order } \\
\hline & $\begin{array}{l}k_{1} \times 10^{2} \\
\left(\min ^{-1}\right)\end{array}$ & $R_{1}^{2}$ & $\begin{array}{l}q_{e, \exp } \\
(\mathrm{mg} / \mathrm{g})\end{array}$ & $\begin{array}{l}q_{e, c a l} \\
(\mathrm{mg} / \mathrm{g})\end{array}$ & $\begin{array}{l}k_{2} \times 10^{2} \\
\left(\mathrm{~g} \mathrm{mg}^{-1}\right. \\
\left.\mathrm{min}^{-1}\right)\end{array}$ & $R_{2}^{2}$ & $\begin{array}{l}q_{e, \exp } \\
(\mathrm{mg} / \mathrm{g})\end{array}$ & $\begin{array}{c}q_{e, c a l} \\
(\mathrm{mg} / \mathrm{g})\end{array}$ & $\begin{array}{l}k_{2} q_{e}^{2} \\
\times 10^{2}\end{array}$ \\
\hline 306 & 2.63 & 0.98 & 1.51 & 1.24 & 3.19 & 0.99 & 1.51 & 1.63 & 8.48 \\
\hline 584 & 2.38 & 0.96 & 2.90 & 2.30 & 1.31 & 0.99 & 2.90 & 3.19 & 13.28 \\
\hline 994 & 2.37 & 0.93 & 4.60 & 3.26 & 1.15 & 0.99 & 4.60 & 4.93 & 27.97 \\
\hline
\end{tabular}




\subsection{Adsorption Isotherms}

\subsubsection{Chromium (VI) Adsorption Isotherms}

Isotherm experiments were conducted by adding a predetermined amount of GAC-QPVP $(0.5-5 \mathrm{~g} / \mathrm{L})$ into $40 \mathrm{ml}$ of chromium(VI) solution of known concentrations at different $\mathrm{pH}$ values. The solution and adsorbent were maintained in contact over fixed time period of 20 hrs, and then solutions were filtered. The filtrate was analyzed for the final concentration of chromium present in the solution (liquid-phase). The amount of chromium adsorbed by the adsorbent (solid-phase) was calculated using equation 3-1. To evaluate the adsorption of chromium(VI) onto GAC-QPVP, Freundlich and Langmuir isotherm models were used to fit the experimental data. The Freundlich isotherm model is represented mathematically as follows:

$q=k_{f} C^{1 / n}$

Where, $\mathrm{q}$ is the mass of chromium(VI) adsorbed per unit mass of adsorbent $(\mathrm{mg} / \mathrm{g}), \mathrm{C}$ is the concentration of chromium(VI) in solution $(\mathrm{mg} / \mathrm{L}), k_{f}$ and $1 / n$ are parameters of Freundlich isotherm, denote the adsorption capacity and intensity of adsorption, respectively. Langmuir model can be represented as follows:

$\frac{C_{e}}{q_{e}}=\frac{C_{e}}{q_{\max }}+\frac{1}{q_{\max } K_{L}}$

Where, $q_{e}=$ equilibrium adsorption capacity and $C_{e}=$ equilibrium concentration of $\mathrm{Cr}(\mathrm{VI})$. $q_{\max }$ and $K_{L}$ are Langmuir constants representing maximum adsorption capacity $(\mathrm{mg} / \mathrm{g})$ and binding energy.

It was noticed from the representative figure of adsorption isotherms (Figure 4.17) that the maximum adsorption capacity increased from 12.6 to $67.2 \mathrm{mg} / \mathrm{g}$ when the initial concentration increased from 10 to $65 \mathrm{mg} / \mathrm{L}$. The models' parameters of these isotherms were obtained through non-linear regression and given in Table 4.8. It can be seen that the adsorption capacity constant $k_{f}$ increased from 7.92 to 20.55 as the initial concentration increased from 10 to $65 \mathrm{mg} / \mathrm{L}$. Freundlich parameter adsorption intensity $(1 / n)$ values after the equilibrium batch studies $(20 \mathrm{~h})$ found to be varying from 0.22 to 0.34 for different initial concentrations. Similar results have been reported by Huang and Wu (1977) and Alaerts et al. (1989) in chromium(VI) removal by activated carbon. 


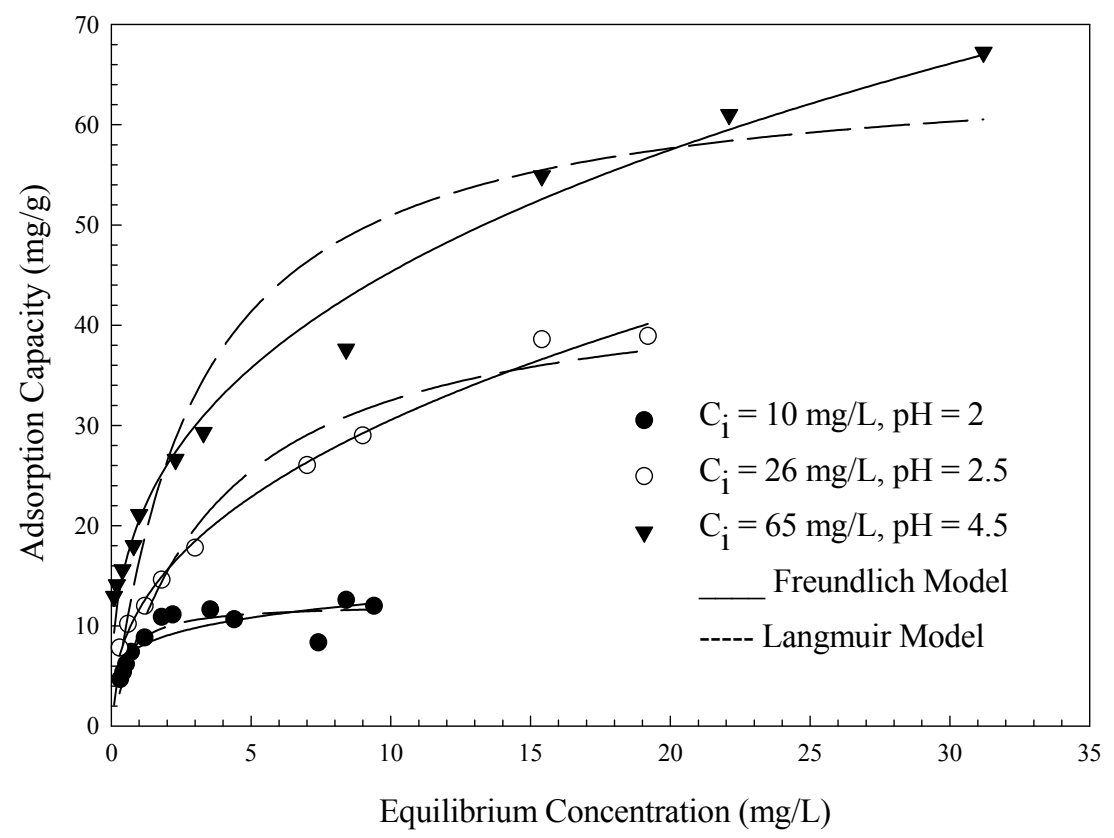

Figure 4.17 Adsorption isotherms at different $\mathrm{pH}$ and different initial concentrations. Data fitted to the Freundlich model (solid lines) and Langmuir model (dash lines).

Table 4.8 Adsorption isotherm parameters for chromium(VI)

\begin{tabular}{|c|l|ccc|ccc|}
\hline \multirow{2}{*}{$\begin{array}{c}\text { Initial concentration } \\
(\mathrm{mg} / \mathrm{L})\end{array}$} & & \multicolumn{3}{|c|}{ Freundlich parameters } & \multicolumn{3}{|c|}{ Langmuir constants } \\
\cline { 3 - 8 } & $\mathrm{pH}$ & $k_{f}$ & $1 / n$ & $R_{1}^{2}$ & $\begin{array}{c}q_{\max } \\
(\mathrm{mg} / \mathrm{g})\end{array}$ & $\begin{array}{c}K_{L} \\
\left(\mathrm{~L} \mathrm{mg}^{-1}\right)\end{array}$ & $R_{2}^{2}$ \\
\hline 10 & 2.0 & 7.92 & 0.22 & 0.71 & 11.54 & 2.20 & 0.94 \\
26 & 2.5 & 11.65 & 0.42 & 0.99 & 44.15 & 0.31 & 0.97 \\
65 & 4.5 & 20.55 & 0.34 & 0.99 & 68.66 & 0.37 & 0.98 \\
\hline
\end{tabular}

\subsubsection{Arsenic(III) Adsorption Isotherms}

Representative figures of adsorption isotherms describing the distribution between aqueous and adsorbed phases for arsenic(III) at different initial concentrations and different $\mathrm{pHs}$ are showed in the Figures 4.18 and 4.19. It can be observed that maximum adsorption capacity increased from $1.95 \mathrm{mg} / \mathrm{g}$ to $5.97 \mathrm{mg} / \mathrm{g}$ and from $1.97 \mathrm{mg} / \mathrm{g}$ to $6.48 \mathrm{mg} / \mathrm{g}$ as the initial concentration of arsenic(III) increased from $0.3 \mathrm{mg} / \mathrm{L}$ to $1 \mathrm{mg} / \mathrm{L}$ for $\mathrm{pH}=7$ and $\mathrm{pH}=8$ respectively. As the solution $\mathrm{pH}$ reduced from 8 to 7 , the adsorption capacity was reduced about 1-8 \%, because, as the solution became neutral from basic, the adsorption efficiency decreased slightly as shown in Figure 4.8. Ferguson and Anderson (1974) also reported that arsenic(III) adsorption on iron oxide decreased with decreasing $\mathrm{pH}$ from 8 to 6 .

Freundlich model (4-9) and Langmuir model (4-10) were used to fit the experimental data using the non-linear regression software (Sigma plot 2000). It was found that the experimental data fitted both Freundlich and Langmuir models as shown in Figures 4.18 and 4.19. However, Freundlich model had better agreement with experimental data, as indicated 
by the higher regression co-efficient values in Table 4.9 when compared to the Langmuir model. Freundlich parameter adsorption intensity $(1 / n)$ values after the equilibrium batch studies $(20 \mathrm{~h})$ found to be varying from 0.24 to 0.33 for different concentrations at different pHs.

It can be observed from the Freundlich parameters that adsorption intensity $(1 / n)$ and distribution coefficient $\left(k_{f}\right)$ were increased as the initial concentration of arsenic increased in the solution. This illustrates the dependence of the adsorption on initial concentration. Low $1 / n$ values $(<1)$ from the Freundlich isotherm suggested that any large change in the equilibrium concentration of arsenic would not result in a significant change in the amount of arsenic adsorbed.

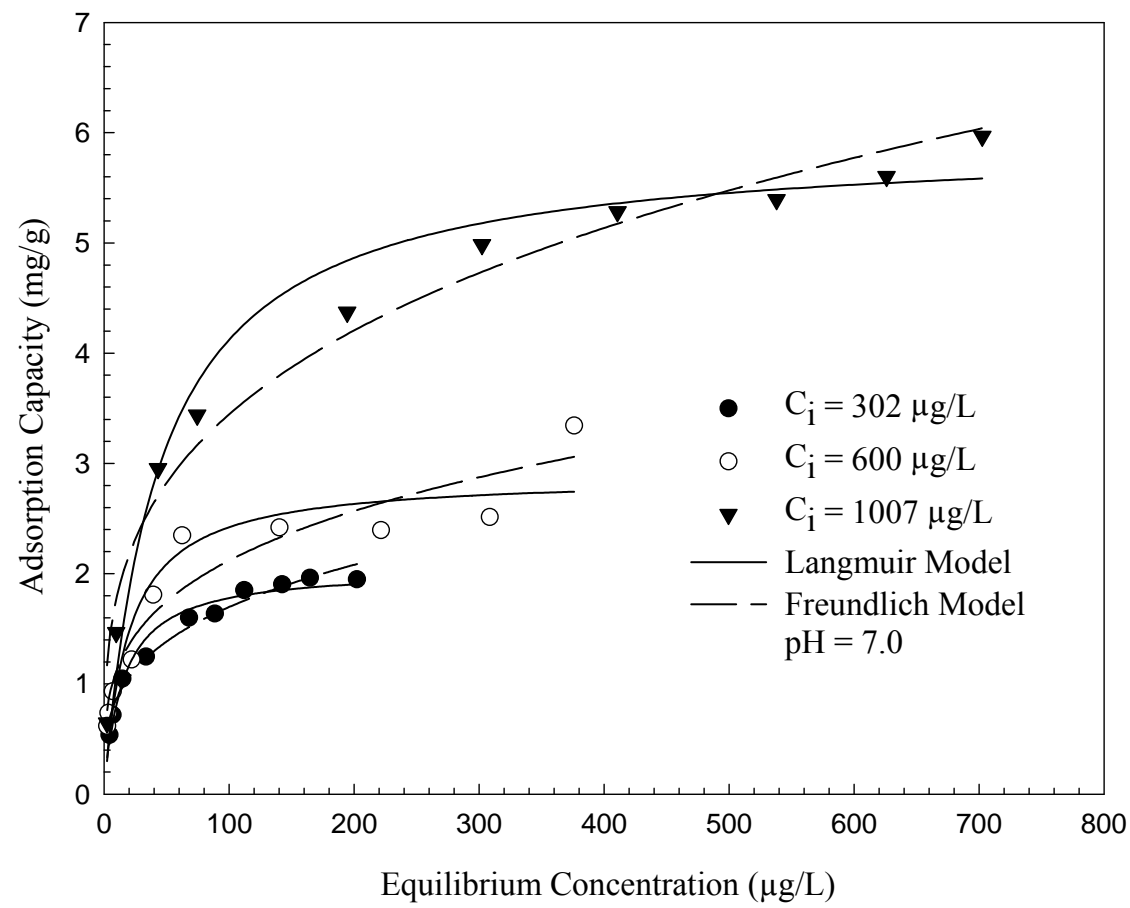

Figure 4.18 Adsorption isotherms at different initial concentrations, $\mathrm{pH}=7.0$ Data fitted to the Freundlich model (dash lines) and Langmuir model (solid lines)

Selim and Zhang (2005) reported that adsorption isotherms for arsenic(V) by different soils were better fit to the Freundlich model, and adsorption intensity values for $\operatorname{arsenic}(\mathrm{V})$ by different soils were found to be varying from 0.270 , to 0.340 . Similarly low $1 / n$ values for arsenic(V) adsorption have reported by others (Butcher et al.,1989; Manning and Goldberg, 1997). Other results were also obtained from the arsenic adsorption onto granular ferric hydroxide by Thirunavukkarasu et al. (2003). Langmuir adsorption maxima $q_{\max }$ increased significantly as the initial arsenic concentration increased. Where as the Langmuir coefficient $K_{L}$ exhibited a decreasing value as the initial concentration increased, which indicates that binding energy of arsenic(III) to the adsorbent is decreasing as the initial concentration increases. A study on removal of arsenic by iron coated GAC by Gu et al. 
(2005) reported similar results of decreasing binding energy as the initial concentration of arsenic increasing.

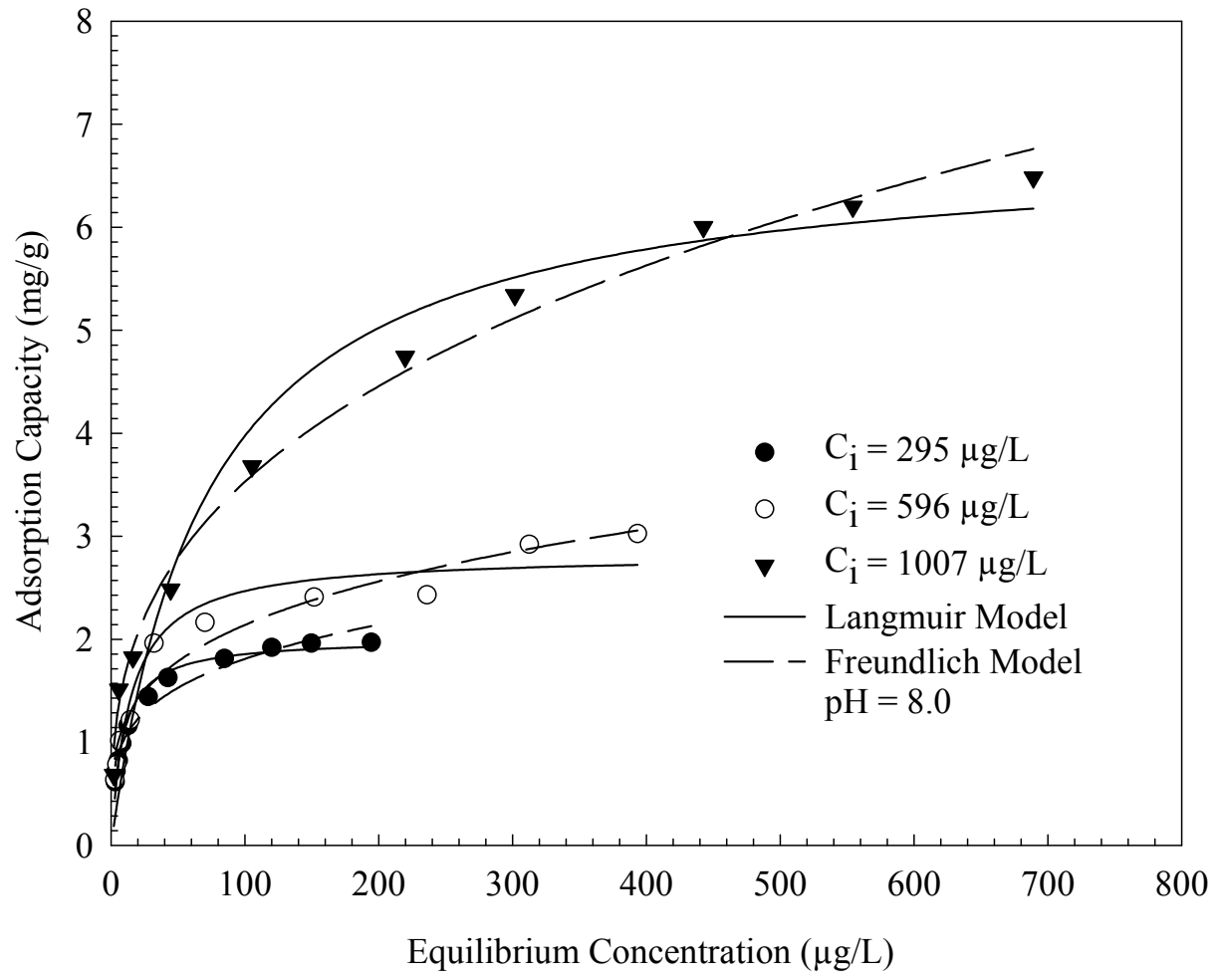

Figure 4.19 Adsorption isotherms at different initial concentrations, $\mathrm{pH}=8.0$ Data fitted to the Freundlich model (dash lines) and Langmuir model (solid lines).

Table 4.9 Adsorption isotherm parameters for arsenic(III)

\begin{tabular}{|c|c|cccccc|}
\hline \multirow{2}{*}{$\begin{array}{c}\text { Initial concentration } \\
(\mu \mathrm{g} / \mathrm{L})\end{array}$} & & \multicolumn{3}{|c}{ Freundlich parameters } & \multicolumn{3}{c|}{ Langmuir constants } \\
\cline { 3 - 9 } & $\mathrm{pH}$ & $\begin{array}{c}k_{f} \\
(\mathrm{~L} / \mathrm{g})\end{array}$ & $1 / n$ & $R_{1}^{2}$ & $\begin{array}{c}q_{\max } \\
(\mathrm{mg} / \mathrm{g})\end{array}$ & $\begin{array}{c}K_{L} \\
\left(\mathrm{~L} \mathrm{mg}^{-1}\right)\end{array}$ & $R_{2}^{2}$ \\
\hline 295 & 7.0 & 0.43 & 0.27 & 0.97 & 2.04 & 0.06 & 0.97 \\
596 & 7.0 & 0.59 & 0.27 & 0.91 & 2.88 & 0.05 & 0.89 \\
1007 & 7.0 & 0.91 & 0.28 & 0.97 & 5.89 & 0.02 & 0.97 \\
295 & 8.0 & 0.59 & 0.24 & 0.95 & 2.00 & 0.12 & 0.98 \\
600 & 8.0 & 0.64 & 0.26 & 0.95 & 2.82 & 0.07 & 0.94 \\
1007 & 8.0 & 0.74 & 0.33 & 0.99 & 6.82 & 0.01 & 0.95 \\
\hline
\end{tabular}

\subsection{Effect of Anions}

\subsubsection{Effect of Anions on Chromium (VI) Removal by GAC-QPVP}

Adsorption of chromium(VI) by GAC-QPVP was conducted in the presence of $\mathrm{SO}_{4}^{-2}, \mathrm{Cl}^{-}$, $\mathrm{CH}_{3} \mathrm{COO}^{-}$, and $\mathrm{HCO}_{3}{ }^{-}$ions to evaluate the effect of other anions on chromium(VI) sorption 
process. Due to the high concentrations of anions such as sulfate in AMD, it is important to evaluate role of anions on chromium(VI) removal. Adsorption capacity of GAC-QPVP was measured in a range of $0.01-1 \mathrm{M}$ ionic strength and at an initial concentration of $5.1 \mathrm{mg} / \mathrm{L}$.

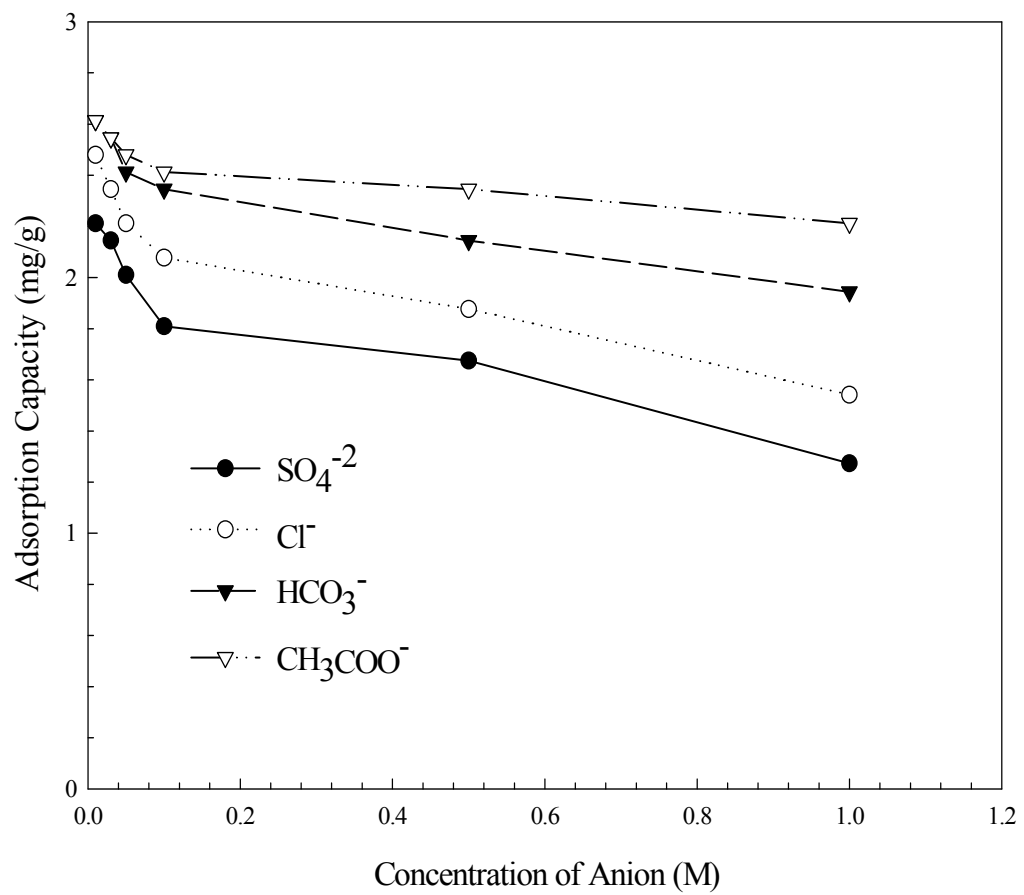

Figure 4.20 Effect of anions on chromium removal, $\mathrm{pH}=2$; Loading $=1.5 \mathrm{~g} / \mathrm{L} ; \mathrm{T}=25^{\circ} \mathrm{C}$.

As shown by Figure 4.20, increase in ionic concentration decreases the chromium(VI) adsorption capacity. Among the four anions, sulfate ion had the largest influence, followed by chloride, bicarbonate, and acetate ion. As the sulfate ion has high valance and chloride has smaller solvated volume, these two ions had greater influence on chromium(VI) removal. When the concentration of chromium(VI) ion is equal to that of sulfate ion there was no effect on the chromium removal by sulfate anion. When the anionic concentration was 0.01 $\mathrm{M}$, the effect of anions was very small. But when the concentration of sulfate is greater than 500 times the chromium(VI), adsorption capacity decreased about $25 \%$. This shows that GAC-QPVP had high affinity for chromium(VI) in the presence of other anions. Gang et al. (2000) noticed similar results in chromium(VI) removal by modified PVP coated on silica gel.

\subsubsection{Effect of Anions on Arsenic Removal}

Several anionic components might exist in groundwater sources that could compete with arsenic for the available adsorption sites (Katsoyiannis et al., 2002). Among the major coexisting anionic components, sulfate, phosphate, silicate, carbonate, and chloride are usually present in groundwater streams possibly inhibiting arsenic removal. In this study, effects of anions are coupled with $\mathrm{pH}$, since anionic hydroxyl group is known to interact strongly with (hydr)-oxide surfaces and also affects acid/base speciation of other anions (Gu et al., 2005). Different concentrations $(50-100 \mathrm{mg} / \mathrm{L})$ of anions $\left(\mathrm{SO}_{4}{ }^{2-}, \mathrm{PO}_{4}{ }^{3-}, \mathrm{SiO}_{3}{ }^{2-}\right)$ were selected on the basis of the concentrations of anions in natural water. In order to investigate the effect of 
these anions on As (V) and As (III) removal, arsenic solutions were spiked with sulfates, phosphates, silicates and assessed for their effects on the arsenate and arsenite removal under various $\mathrm{pH}$. Arsenic concentration was set at $1000 \mu \mathrm{g} / \mathrm{L}$. The results obtained are presented in Figures 4.21 and 4.22 .

Figures 4.21 and 4.22 show that $\mathrm{SO}_{4}{ }^{2-}$ did not significantly affect arsenate and arsenite removal. It can be noticed that in the presence of sulfates, the decrease in arsenate removal percentage was found to be higher at $\mathrm{pH}>9.0$ when compared to arsenite. Furthermore, it can be seen that the presence of phosphate and silicate decreased As (III) removal slightly in the $\mathrm{pH}$ range from 7.72 to 9.15 . But with further increase in $\mathrm{pH}$, a major decrease on arsenite removal was observed i.e., at $\mathrm{pH}>9.2$ (Fig.4.22). At $\mathrm{pH}>9.87$ only $25 \%$ of arsenite could be adsorbed in the presence of phosphate and silicate. Regarding As (V) removal, a slight decrease was observed in the $\mathrm{pH}$ range from 7.2 to 8.8 in the presence of phosphate and silicate. But with further increase in $\mathrm{pH}$, a considerable decrease on arsenate removal was observed. At $\mathrm{pH}>9.8$ only $19 \%$ of arsenate could be adsorbed in the presence of phosphate and silicate (Fig.4.21). These indicate that phosphate and silicate compete with hydroxyl group for the iron surface in the alkaline solution and subsequently decrease arsenate adsorption.

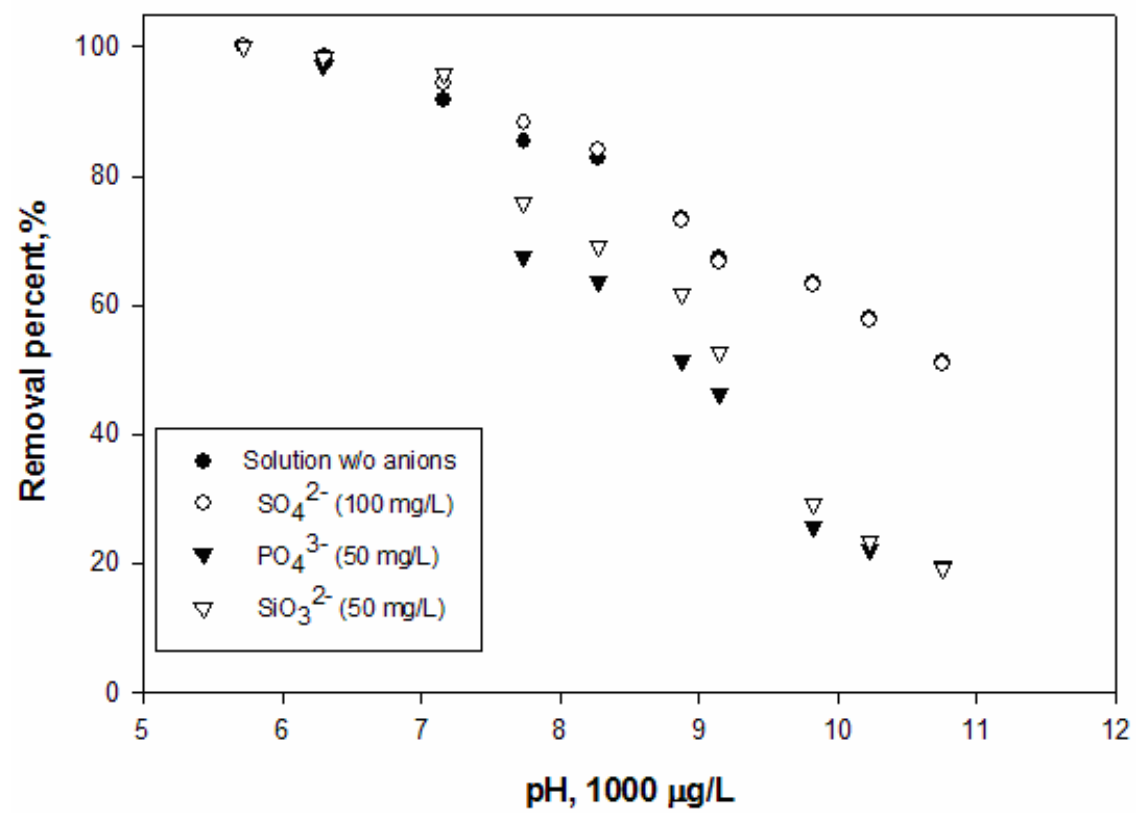

Fig. 4.21 Effect of anions on arsenate removal under various $\mathrm{pH}$ conditions. Initial As (V) concentration is $1.00 \mathrm{mg} / \mathrm{L}$ (Ionic strength controlled by $0.05 \mathrm{M}$ Nail)

The presence of the anions such as sulfates, phosphates, silicates in source water has been reported to interfere with arsenic removal (Hering et al., 1996; Meng et al., 2001, 2002; Holm, 2002). Genc and Tjell (2003) indicated that phosphates and silicates had major effect on arsenic removal than that of sulfates. The presence of sulfate in the influent water produces a slight reduction in As (V) removal, while silicate results in a much higher reduction due to competition (Ghosh et al., 1985; Gupta et al., 1978), supporting the results obtained in this study. Dissolved silicate is usually found in much higher concentrations than 
phosphate, and can interfere with removal of both arsenate and arsenite (Ghurye et al., 1999). Both arsenite and arsenate have a high affinity for Fe-oxides (Goldberg and Johnston, 2001), suggesting that the presence of Fe (III) could enhance the sorption properties of the adsorbent. Phosphates are adsorbed on iron hydroxides through the formation of surface complexes with the hydroxyl groups (Meng et al., 2001), decreasing greatly the efficiency of arsenic removal. Another study (Gu et al., 2005) on adsorption onto granulated activated carbon indicated that phosphates and silicates show great decrease in the removal of arsenic at $\mathrm{pH}>9.0$ when compared to the removal of arsenic in the presence of sulfates. This is consistent with the results obtained in this study.

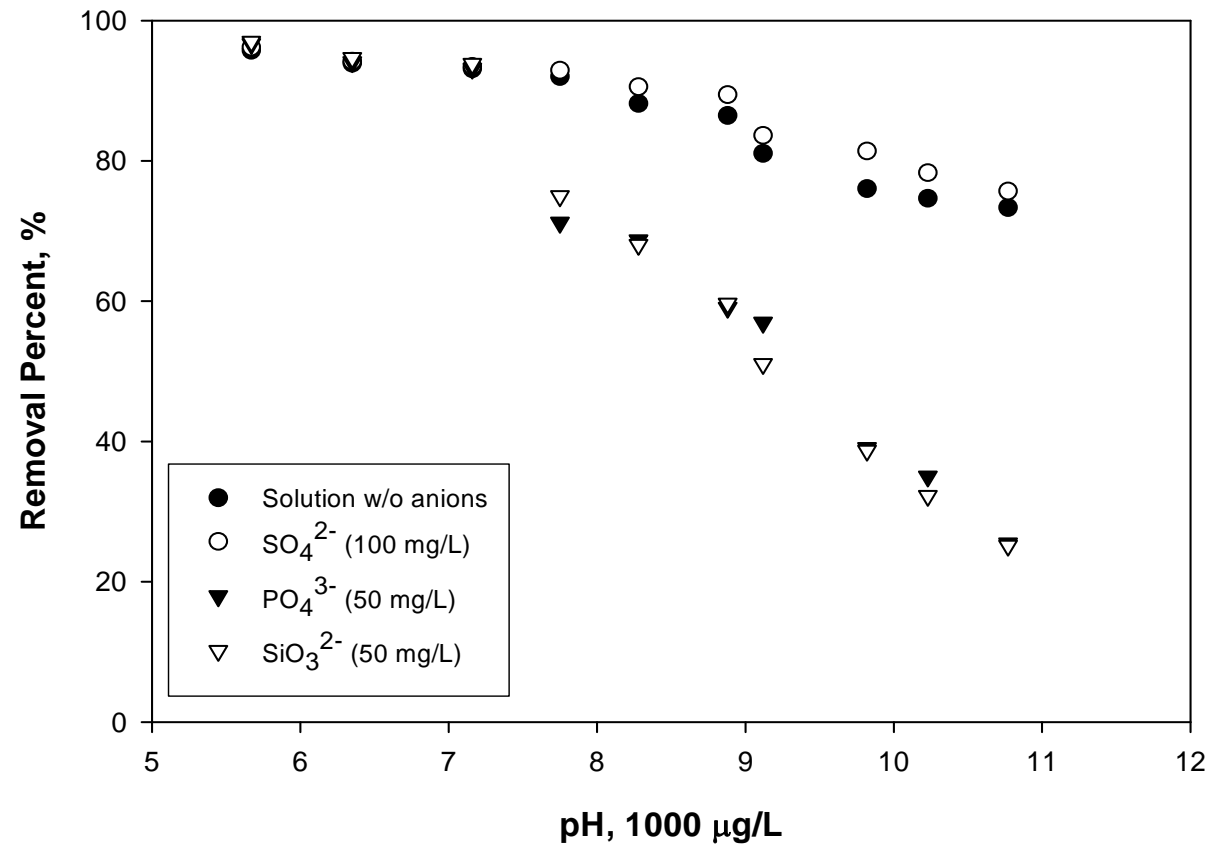

Fig. 4.22 Effect of anions on arsenite removal under various $\mathrm{pH}$ conditions. Initial As (III) concentration is $1.00 \mathrm{mg} / \mathrm{L}$ (Ionic strength controlled by $0.05 \mathrm{M}$ Nail)

\subsection{Effects of Ionic Strength and Modeling $\mathrm{Cr}(\mathrm{VI})$ sorption}

The effects of ionic strength (I) on Cr(VI) sorption on GAC-QPVP is illustrated in Figure 4.23a. Two different I values, 0.1 and $1.0 \mathrm{M} \mathrm{NaCl}$ were used, and the results were compared with those obtained for GAC-QPVP with no I control (where ionic strength varied from 0 to 0.01 due to the addition of $\mathrm{HCl}$ and $\mathrm{NaOH}$ for $\mathrm{pH}$ control). An increase in ionic strength markedly lowered $\mathrm{Cr}(\mathrm{VI})$ sorption and shifted the position of $\mathrm{pH}$ edge to lower $\mathrm{pH}$ values (Figure 4.23a). The results indicated that $\mathrm{Cr}(\mathrm{VI})$ sorption was affected by ion exchange processes. The fixed charges on the sorbent were most likely contributed from the electrondeficient pyridine rings (Gang, et al., 2000). Independent experiments were performed to investigate the exchange of $\mathrm{Cl}^{-}$for $\mathrm{Br}$ on the sorbent (Figure 4.23b) and results were consistent with the following ion exchange reaction:

$$
\mathrm{Cl}^{-}+\mathrm{X}-\mathrm{Br}=\mathrm{Br}^{-}+\mathrm{X}-\mathrm{Cl} \quad \mathrm{K}_{\mathrm{v}}(\mathrm{Br}-\mathrm{Cl})
$$


where $\mathrm{X}$ - denotes the sorbent site and $\mathrm{K}_{\mathrm{v}}$ is the Vanselow selectivity coefficient.

$\mathrm{Cr}(\mathrm{VI})$ sorption, similar to other metals, could usually be attributed to ion exchange, surface complexation, and precipitation. Our experimental observations that ionic strength significantly affected $\mathrm{Cr}(\mathrm{VI})$ sorption (Figure 4.23a), $\mathrm{Br}^{-}$release accompanied with the sorption of $\mathrm{Cl}^{-}$(Figure 4.23b) and $\mathrm{Cr}(\mathrm{VI})$, and electron-deficient properties of the pyridine rings on the QPVP polymers (Gang, et al., 2000) led us to construct an ionic exchange model to describe $\mathrm{Cr}(\mathrm{VI})$ sorption to GAC-QPVP. $\mathrm{Cr}(\mathrm{VI})$ exists in several species such as $\mathrm{HCrO}_{4}^{-}$, $\mathrm{CrO}_{4}{ }^{2-}$, and $\mathrm{Cr}_{2} \mathrm{O}_{7}{ }^{2-}$ with dominant species depending on $\mathrm{Cr}(\mathrm{VI})$ concentration and $\mathrm{pH}$ values (Selomulya, et al.; 1999). These species could all participate in the ion exchange process. To simply the ion exchange model and minimize the number of model parameters, we assumed a following reaction to represent the $\mathrm{Cr}(\mathrm{VI})$ exchange process:

$\mathrm{X}-\mathrm{Br}+\mathrm{HCrO}_{4}^{-}=\mathrm{X}-\mathrm{HCrO}_{4}+\mathrm{Br}^{-} \quad \mathrm{K}_{\mathrm{v}}\left(\mathrm{Br}-\mathrm{HCrO}_{4}\right)$

The Vanselow exchange coefficient $\left(\mathrm{K}_{\mathrm{v}}\right)$ in reactions 4-11 and 4-12 can be expressed as:

$$
\begin{aligned}
& \frac{N_{C l}\left\{\mathrm{Br}^{-}\right\}}{N_{B r}\left\{\mathrm{Cl}^{-}\right\}}=K_{v}(\mathrm{Br}-\mathrm{Cl}) \\
& \frac{N_{\mathrm{HCrO}}\left\{\mathrm{Br}^{-}\right\}}{N_{\mathrm{Br}}\left\{\mathrm{HCrO}_{4}^{-}\right\}}=K_{v}\left(\mathrm{Br}-\mathrm{HCrO}_{4}\right)
\end{aligned}
$$

where bracket \{\} denotes the activity in aqueous phase and $N_{A}$ the molar fraction of anion $A$ in the exchange phase. The molar fraction was defined as:

$$
N_{A}=\frac{[X-A]}{A E C}
$$

where $[\mathrm{X}-\mathrm{A}]$ is the molar concentration of $\mathrm{A}$ on the exchanger; and $\mathrm{AEC}$ is the anion exchange capacity. The reactions 4-11 and 4-12 were then built into an equilibrium speciation program, Minteqa2 (Allison, et al., 1991), and were linked with aqueous $\mathrm{Cr}(\mathrm{VI})$ speciation reactions built in the Minteqa2 database to formulate an ion exchange model to describe $\mathrm{Cr}(\mathrm{VI})$ sorption.

We first used the model to fit the binary exchange data between $\mathrm{Cl}^{-}$and $\mathrm{Br}^{-}$(Figure 4-23b) to determine $\mathrm{K}_{\mathrm{v}}(\mathrm{Cl}-\mathrm{Br})$ and $\mathrm{AEC}$. For the $\mathrm{Cl}-\mathrm{Br}$ binary system, we have

$[X-C l]+[X-B r]=A E C$

Combining Eq 4-16 with Eq 4-13 and after some algebraic manipulation, we obtain

$$
[X-C l]=A E C-\frac{1}{K_{v}(C l-B r)} \frac{[X-C l]\left\{B r^{-}\right\}}{\left\{C l^{-}\right\}}
$$

Parameters AEC and $\mathrm{K}_{\mathrm{v}}(\mathrm{Cl}-\mathrm{Br})$ were then be estimated by linear regression between $[\mathrm{X}-\mathrm{Cl}$ ] and $[\mathrm{X}-\mathrm{Cl}] /\left\{\mathrm{Cl}^{-}\right\}$(Figure 4.23b). The best fitted $\log \mathrm{K}_{\mathrm{v}}(\mathrm{Cl}-\mathrm{Br})$ was -1.07 and AEC $1.22 \mathrm{X}$ 
$10^{-3} \mathrm{~mol} / \mathrm{g}$. The estimated AEC was close to, but slightly larger than a value of $1.03 \times 10^{-3}$ $\mathrm{mol} / \mathrm{g}$, which was estimated from the Langmuir fit of the sorption isotherm at $\mathrm{pH} 2.25$. The $\mathrm{Cr}$ sorption profile as a function of $\mathrm{pH}$ without ionic strength control (Figure 4.23a) was used to estimate parameter $\mathrm{K}_{\mathrm{v}}\left(\mathrm{HCrO}_{4}-\mathrm{Br}\right)$. Because $\mathrm{HCl}$ or $\mathrm{NaOH}$ was used to adjust the $\mathrm{pH}$ in this experiment, a ternary exchange system $\left(\mathrm{HCrO}_{4}, \mathrm{Br}\right.$, and $\left.\mathrm{Cl}\right)$ was used to determine $\mathrm{K}_{\mathrm{v}}\left(\mathrm{HCrO}_{4}-\mathrm{Br}\right)$.

a) $\mathrm{Br}-\mathrm{Cl}-\mathrm{HCrO}_{4}$ Exchange

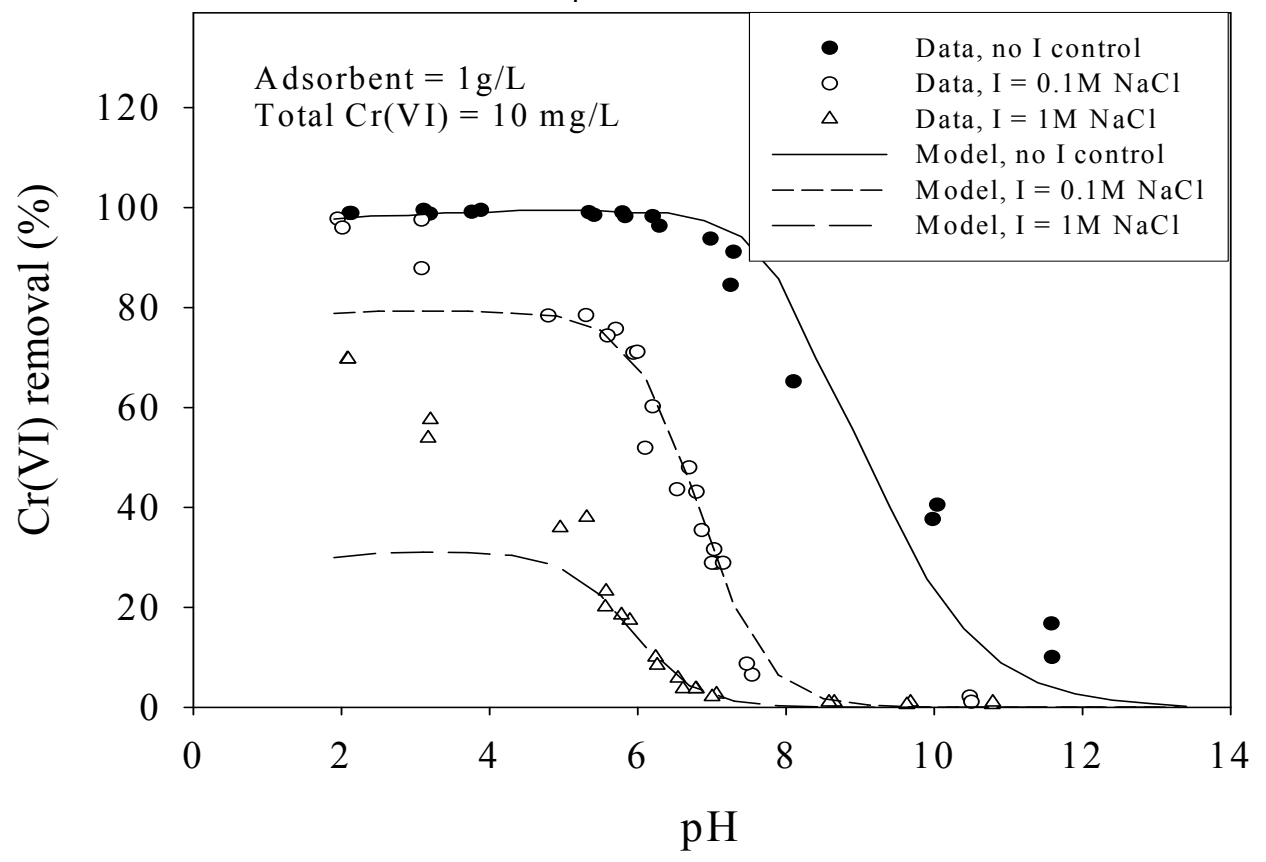

b) $\mathrm{Br}-\mathrm{Cl}$ Exchange

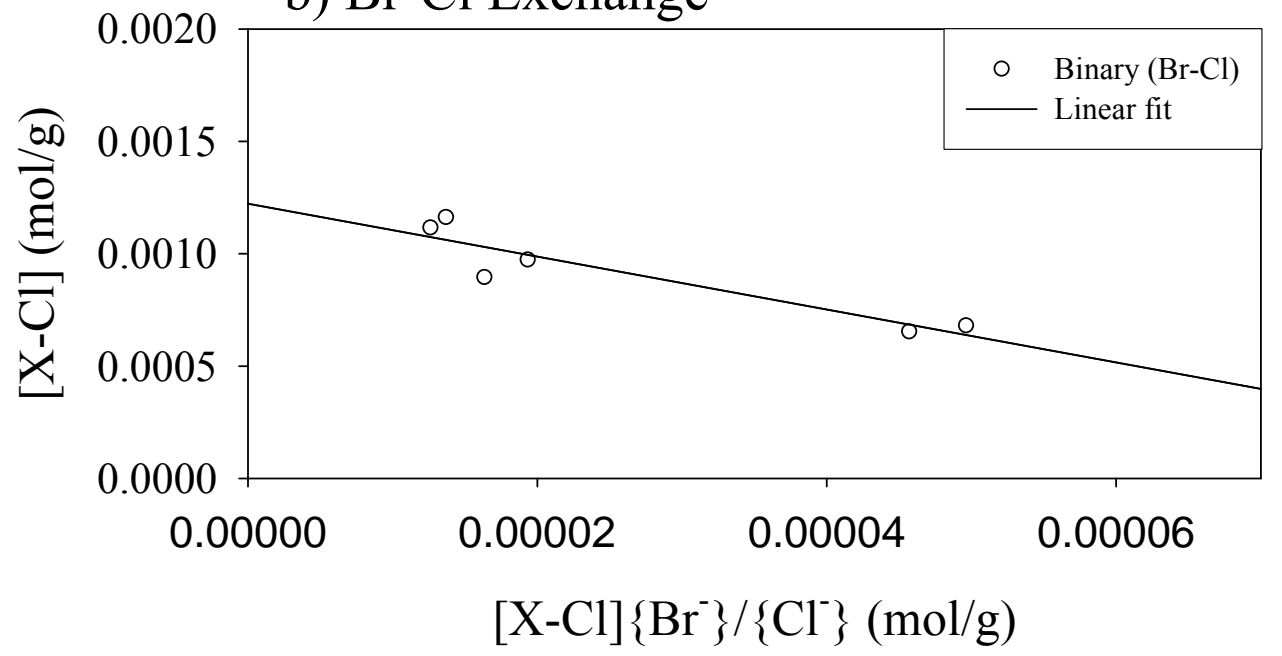

Figure 23. (a) Experimental data and model prediction of sorption behavior of $\mathrm{Cr}(\mathrm{VI})$ on GAC-QPVP as a function of ionic strength, (b) Ion exchange behavior in binary system between $\mathrm{Br}-\mathrm{Cl}$ 
Parameters $\mathrm{K}_{\mathrm{v}}(\mathrm{Cl}-\mathrm{Br})$ and $\mathrm{AEC}$ that were determined from the binary $\mathrm{Cl}-\mathrm{Br}$ exchange were fixed. A trial-and-error approach was used to fit $\mathrm{K}_{\mathrm{v}}\left(\mathrm{HCrO}_{4}-\mathrm{Br}\right)$. Specifically, we tried a value for $\mathrm{K}_{\mathrm{v}}\left(\mathrm{HCrO}_{4}-\mathrm{Br}\right)$, ran the Minteqa2 program to calculate $\mathrm{Cr}(\mathrm{VI})$ sorption, and estimated the errors between the calculated and measured $\mathrm{Cr}(\mathrm{VI})$ sorption. This process was repeated until the calculated best matched with the measured $\mathrm{Cr}(\mathrm{VI})$ sorption. The model well described the $\mathrm{Cr}(\mathrm{VI})$ sorption as a function of $\mathrm{pH}$ with a best fitted $\log \mathrm{K}_{\mathrm{v}}\left(\mathrm{HCrO}_{4}-\mathrm{Br}\right)=8.04$.

The model with the estimated parameters was then used to predict $\mathrm{Cr}$ sorption as a function of $\mathrm{pH}$ in 0.1 and $1 \mathrm{M} \mathrm{NaCl}$ solutions (Figure 4.23a). The predictions matched data well above $\mathrm{pH} 5$ at both ionic strength 0.1 and $1 \mathrm{M}$ (Figure 4.23a). Below $\mathrm{pH} 4$, however, the model under-predicted the observation uptake of $\mathrm{Cr}(\mathrm{VI})$ by the sorbent. The under-prediction increased with decreasing $\mathrm{pH}$ and increasing ionic strength with a maximum value of $18 \%$ at $\mathrm{I}=0.1 \mathrm{M}$, and $35 \%$ at $\mathrm{I}=1 \mathrm{M}$. The under-predictions suggested that the reactions other than reactions 1 and 2 on the sorbent also affected the $\mathrm{Cr}(\mathrm{VI})$ uptake.

To further understand the processes affecting the Cr(VI) sorption, the GAC-QPVP samples after reacted with $\mathrm{Cr}(\mathrm{VI})$ solution at $\mathrm{pH}$ around 2.0 for $24 \mathrm{~h}$ were analyzed with $\mathrm{X}$-ray photoelectron spectroscopy (XPS). XPS results (Figure 4.24) showed two Cr2 $\mathrm{p}_{3 / 2}$ intensity peaks at $577.1 \mathrm{eV}$ and $579.3 \mathrm{eV}$, which are consistent with $\mathrm{Cr}(\mathrm{III})$ and $\mathrm{Cr}(\mathrm{VI})$, respectively. Best fits to the data were obtained by using a Gaussian for $\mathrm{Cr}(\mathrm{III})$ and a Voigt for $\mathrm{Cr}(\mathrm{VI})$. Slow reduction of $\mathrm{Cr}(\mathrm{VI})$ occurred under the beam, where minor but noticeable reduction occurred after about 5-10 minutes of beam exposure. The mole fraction, $\mathrm{Cr}(\mathrm{III}) / \mathrm{Cr}$ (total), was calculated from spectra that were only exposed to the beam for 45 seconds. Curve fitting showed $37 \%$ of the sorbed chromium on the GAC-QPVP existed in its trivalent form.

The presence of $\mathrm{Cr}(\mathrm{III})$ on the sorbent was consistent with the under-predictions of $\mathrm{Cr}(\mathrm{VI})$ sorption (Figure 4.23a) because the ion exchange model only considers $\mathrm{Cr}(\mathrm{VI})$ species. The increasing differences between the model predictions and measurements with decreasing $\mathrm{pH}$ (Figure 4.23a) suggested $\mathrm{Cr}(\mathrm{VI})$ reduction was more favored at low $\mathrm{pH}$. This agreed with the facts that the rates of chromate reduction to $\mathrm{Cr}$ (III) increased with decreasing $\mathrm{pH}$ by many inorganic and organic reductants (Deng, 1995; Deng and Stone, 1996). The presence of $\mathrm{Cr}(\mathrm{III})$ also indicated that the activated carbon sites that were able to reduce $\mathrm{Cr}(\mathrm{VI})$ (Figure 4.25) were not completely blocked by the QPVP process, suggesting that the small pores in the polymer on the surface of GAC-QPVP were conduits for $\mathrm{Cr}(\mathrm{VI})$ to diffuse to the underneath reduction sites. After reduction, $\mathrm{Cr}$ was apparently occluded in the solid phase or slow to diffuse back to aqueous phase so that aqueous $\mathrm{Cr}$ (III) was undetectable within $24 \mathrm{~h}$ equilibration time (Figure 4.25). The $\mathrm{Cr}$ (III) was thermodynamically stable in aqueous phase below $\mathrm{pH}$ 4. This diffusion-reduction mechanism also explained the increasing underpredictions of $\mathrm{Cr}(\mathrm{VI})$ sorption with increasing ionic strength (Figure 4.23a) because in solutions with high ionic strength, $\mathrm{Cr}(\mathrm{VI})$ was less adsorbed and more available for diffusion into reduction sites.

Overall, under a wide range of experimental conditions examined in this study, GAC-QPVP is found to remove $\mathrm{Cr}(\mathrm{VI})$ efficiently from $\mathrm{pH} 2-6$ and in the presence of strong competing anions such as phosphate, sulfate, and nitrate. Its sorption capacity for $\mathrm{Cr}$ is much higher than 
that of PVP-coated silica gel. An ion exchange model is developed that well explains the dependence of $\mathrm{Cr}(\mathrm{VI})$ uptake on $\mathrm{pH}$, ionic strength, and $\mathrm{Cr}(\mathrm{VI})$ concentrations

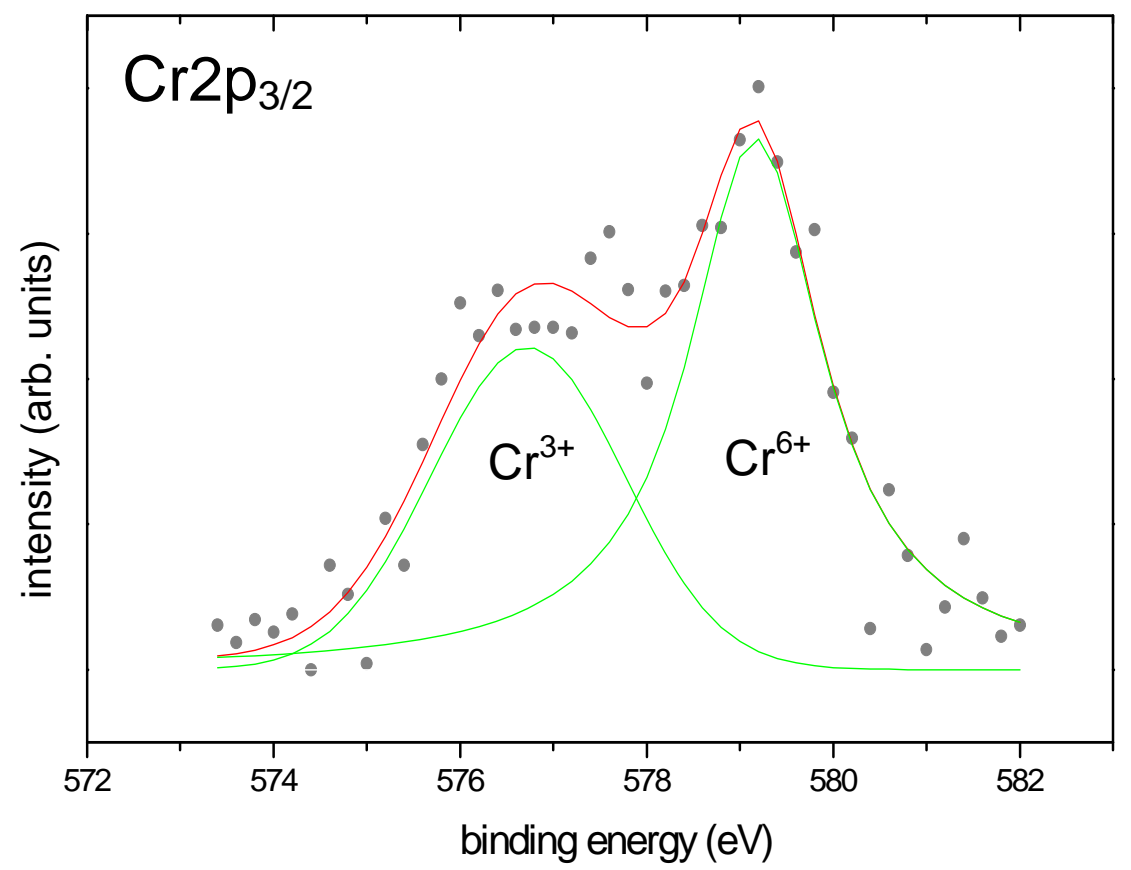

Figure 4.24. XPS spectra of GAC-QPVP sample after exposure to $\mathrm{Cr}$ (VI) solution for 24 hours. Green lines represent the $\mathrm{Cr}(\mathrm{III})$ and $\mathrm{Cr}(\mathrm{VI})$ components. The red line is the fit envelope, and the solid circles show the data.

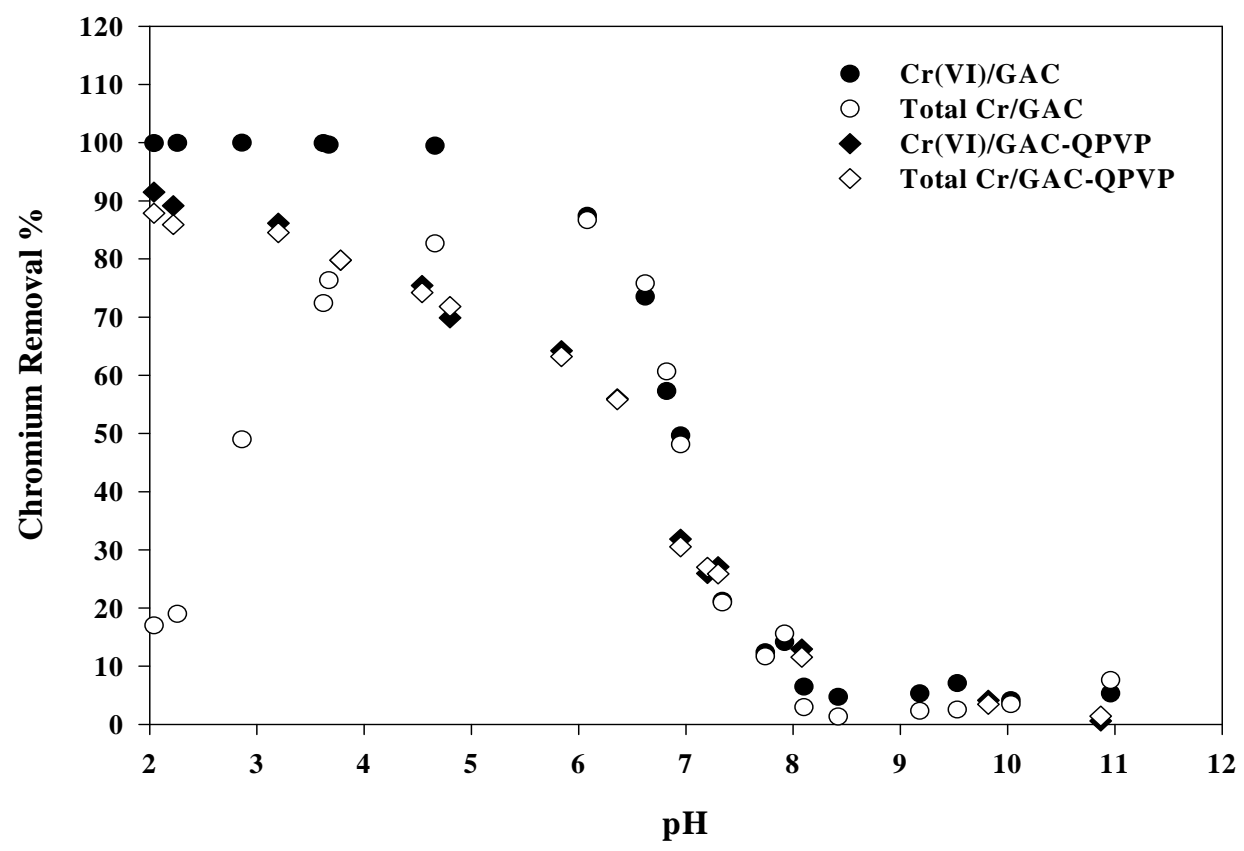

Figure 4.25. Effect of $\mathrm{pH}$ on the removal of $\mathrm{Cr}(\mathrm{VI})$ and total $\mathrm{Cr}$ on GAC and GAC-QPVP $($ Sorbent $=1 \mathrm{~g} / \mathrm{L} ; \mathrm{I}=0.1 \mathrm{M}$; Initial $\mathrm{Cr}(\mathrm{VI})=10 \mathrm{mg} / \mathrm{L})$ 


\section{$\underline{4.8 \text { Desorption Study }}$}

Desorption of chromium(VI) was studied with $\mathrm{NaOH}$ and $\mathrm{NH}_{4} \mathrm{OH}$ at various concentrations and for different time periods. Representation of the results in Figure 4.26 show that, increasing concentration of the base increased the desorption efficiency. But when the concentration of $\mathrm{NaOH}$ was greater than $0.1 \mathrm{M}$, there was only a small change in the chromium(VI) desorption.

It can be seen that maximum desorption by $1 \mathrm{M} \mathrm{NaOH}$ was $80 \%$, and by $1 \mathrm{M} \mathrm{NH}_{4} \mathrm{OH}$ (Figure 4.26) was 55\%. This is because of the strong basic nature of $\mathrm{NaOH}$. Desorption studies were also conducted for different time periods, 5 and 30 minutes. There was not much difference in the desorption efficiency between the two methods. This suggested that desorption rate was fast, due to the thin polymer layer on the surface of the activated carbon.

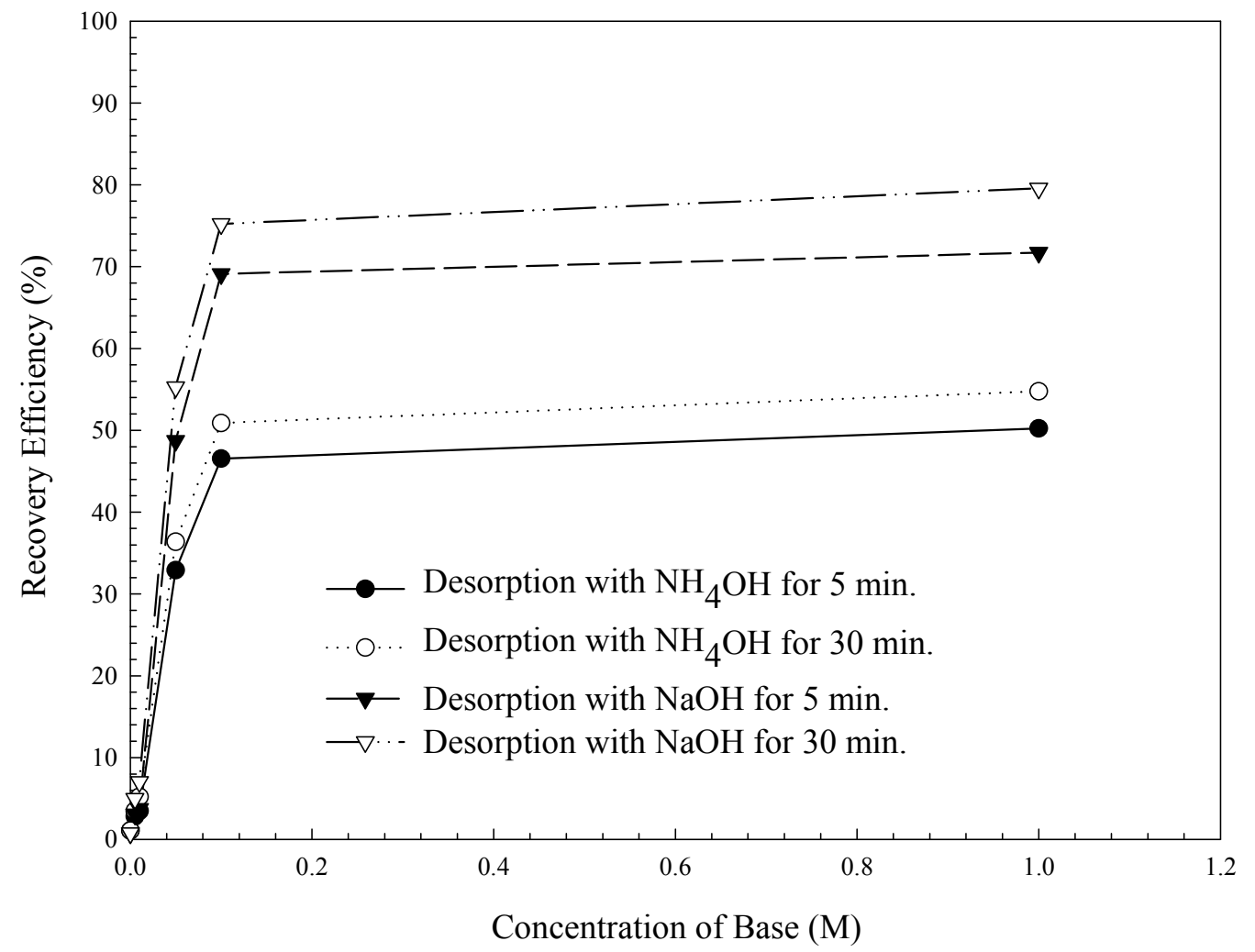

Figure 4.26 Desorption of Cr(VI) with ammonium hydroxide and sodium hydroxide for different time periods.

Ward (1990) used kaolinite and illite to remove chromium(VI) from the aqueous solution. Though chromium(VI) removal was $100 \%$ at low $\mathrm{pH}$, chromium(VI) was desorbed only about 25\%. Nishihama et al. (2001) reported that recovery of chromium(VI) from microcapsules containing quaternary ammonium salt extractant and phosphoric acid extractant using microcapsules containing tri-n-octylmethylammonium chloride (TOMAC) was less than $60 \%$ when $1 \mathrm{~g}$ of microcapsules containing TOMAC was used. 


\subsection{Regeneration and Reuse of the GAC-QPVP}

As there was not much difference in the recovery efficiency when base increased from $0.1 \mathrm{M}$ to $1 \mathrm{M}$ concentration, reuse experiments were conducted with $0.2 \mathrm{M} \mathrm{NaOH}$ to minimize the base usage. The reuse of the adsorbent, following adsorption-desorption process was also investigated. Batch adsorption isotherm experiments were conducted using regenerated GAC-QPVP following the similar experimental procedures described in section 3.3.3. Adsorption capacities of regenerated and original GAC-QPVP were compared in the Figure 4.27 .

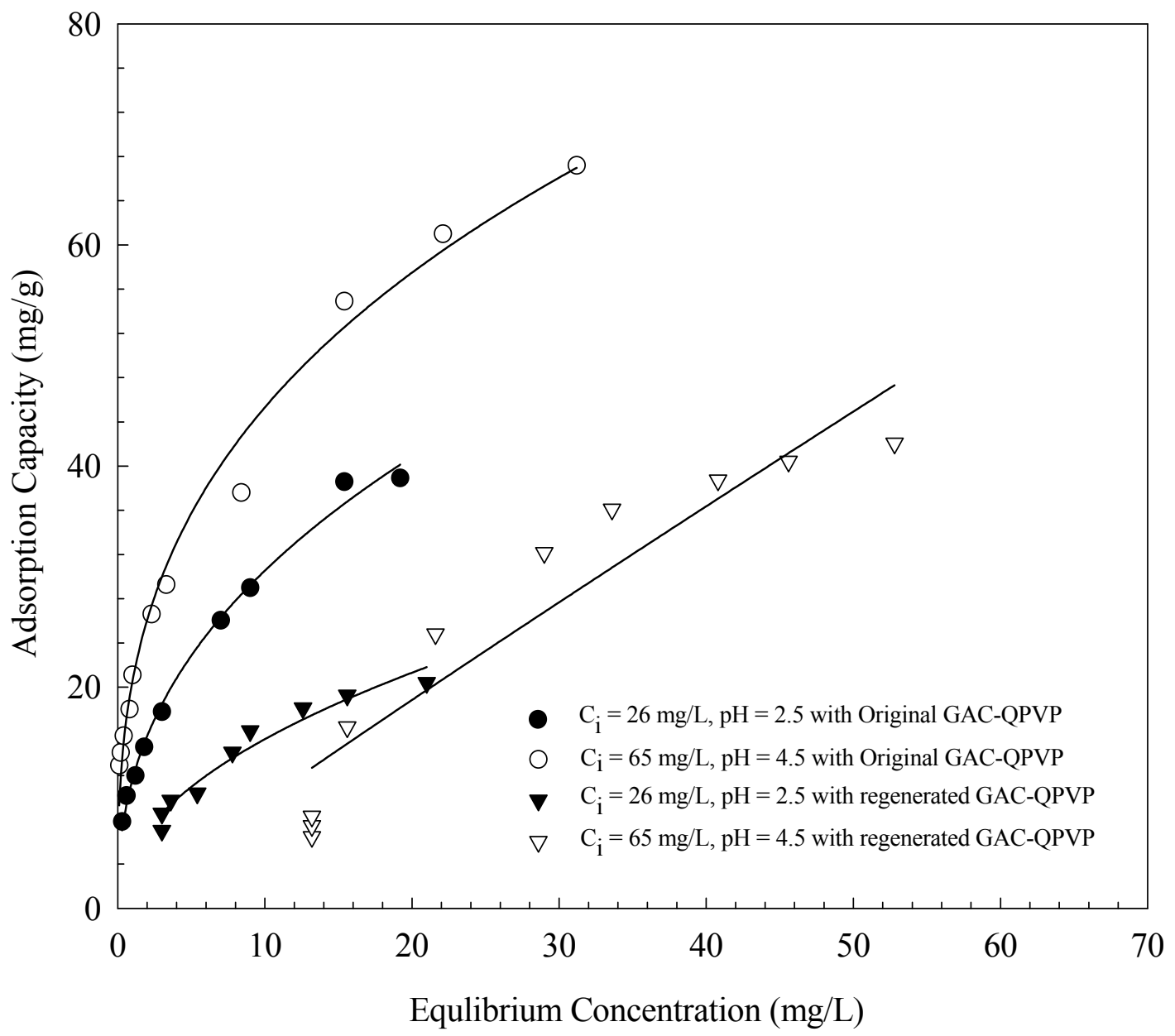

Figure 4.27 Adsorption isotherms of Original GAC-QPVP and regenerated GAC-QPVP.

It can be observed that adsorption capacities decreased by $35 \%-45 \%$. The maximum desorption efficiency was about $80 \%$ using $1 \mathrm{M} \mathrm{NaOH}$, and $20 \%$ sorption sites on GACQPVP losses were expected using the regenerated adsorbent. Gang et al. (2001b) observed that regenerated modified PVP coated silica gel adsorption capacity decreased about 10-20\%. These decreases in adsorption capacities were higher than expected. But the results indicate that GAC-QPVP does posses a sufficient loading capacity for use in repeated processing and therefore confirm the possible reuse of the GAC-QPVP in chromium(VI) removal from aqueous solutions. 


\section{$\underline{4.10 \text { Column Operation }}$}

\subsubsection{Column Study of Chromium(VI) with GAC-QPVP}

The column study of chromium(VI) with GAC-QPVP was conducted using $5 \mathrm{mg} / \mathrm{L}$ chromium(VI) solution in a column with ID of $6 \mathrm{~mm}$ and $12 \mathrm{~mm}$ with $300 \mathrm{mg}$ and $1000 \mathrm{mg}$ of the adsorbent respectively. Figure 4.28 shows the break through curves for different flow rates. It was found that the chromium(VI) concentration in the effluent steadily increases and finally equals to the feed concentration. As the flow rate increases, it can be seen that the breakthrough curve become steeper. The break point was reduced from 760 bed volumes to 600 bed volumes when the flow rate was increased from $20 \mathrm{ml} /$ day to $100 \mathrm{ml} /$ day respectively. Adsorbent has reached its saturation when number of bed volumes has reached 1800 and 2400 for $100 \mathrm{ml} /$ day and $20 \mathrm{ml} /$ day flow rates respectively.

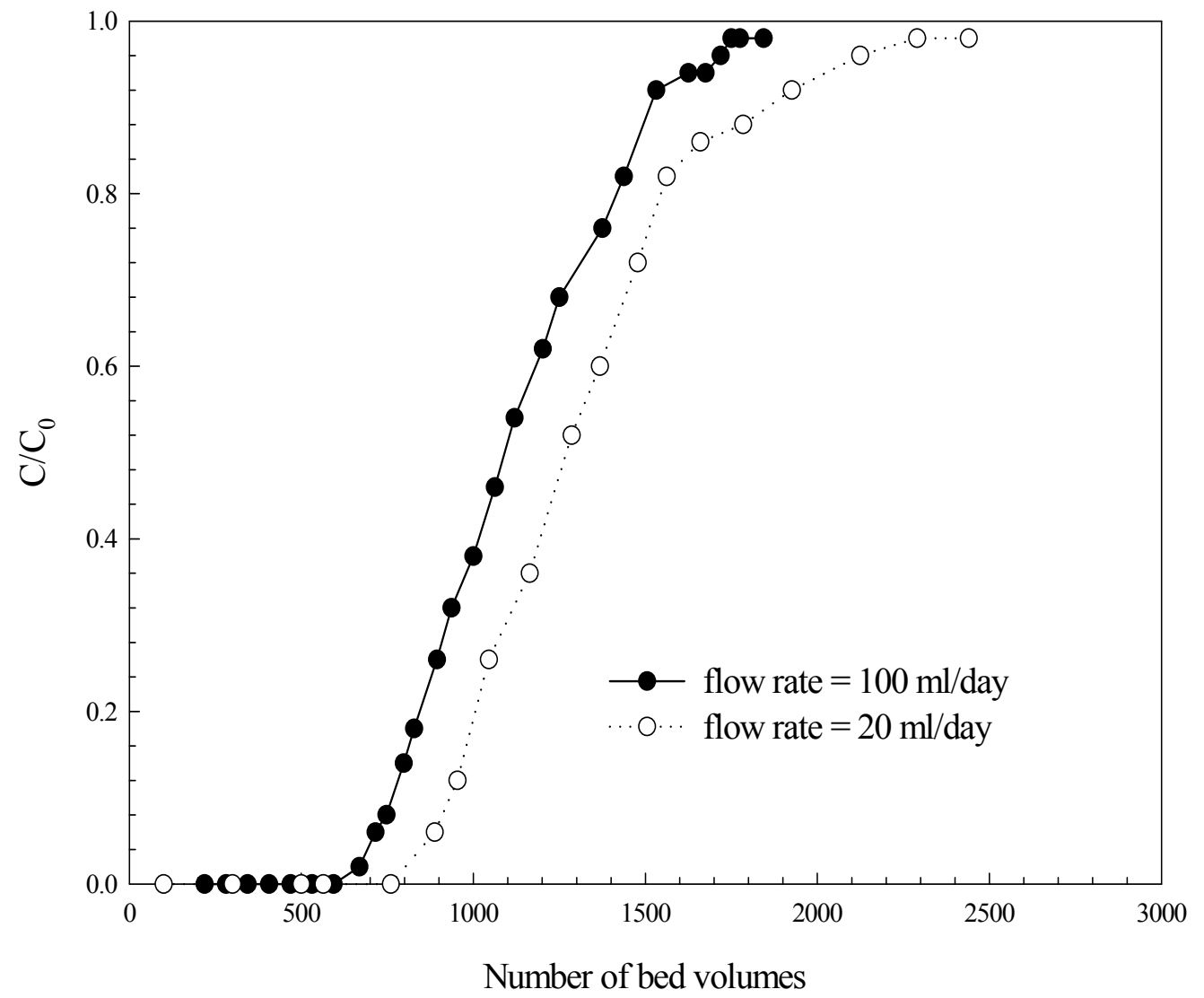

Figure 4.28 Column Study of Chromium(VI) with GAC-QPVP.

When we compare this column operation to the batch adsorption process, column operation has higher removal capacity. Adrian et al. (1993) reported that the metals were removed by passing a special leaching solution through the ore. In this study, the removal of chromium(VI) from four New Mexico soils (sand, sandy loam, and clay) using heap leaching was evaluated at a column scale. The heap leaching study demonstrated greater than $99 \%$ removal of chromium(VI). 


\subsubsection{Column Study of Arsenic(III) with Iron-chitosan}

Column tests were also conducted to investigate the use of iron-chitosan as a low-cost treatment technology for arsenic(III) removal. Experiments were conducted with 12-mm-ID column at a flow rate of $25 \mathrm{ml} / \mathrm{h}$ (empty bed contact time $2.5 \mathrm{~min}$ ) with an initial arsenic(III) concentration of $308 \mu \mathrm{g} / \mathrm{L}$. Iron-chitosan $(1.5 \mathrm{~g})$ was packed in column as fixed bed, then arsenic(III) solution was passed through the fixed bed and effluent solution was analyzed for arsenic concentration. The result of breakthrough curve was given in Figure 4.29. It can be seen that the break point obtained after 768 bed volumes and adsorbent was exhausted at 1400 bed volumes.

$\mathrm{Gu}$ et al. (2005) examined the arsenic breakthrough behaviors for an As-GAC sample prepared from Dacro $20 \times 40 \mathrm{LI}$ with an initial concentration of $56.1 \mu \mathrm{g} / \mathrm{L}$ arsenic(III). The duration of column studies ranged from 45 to 70 days with an empty bed contact time of 5 min. The results demonstrated that the adsorbent could effectively remove the arsenic from groundwater in the column setups. Sandy et al. (1973) also achieved high removal of arsenic using strong base anion exchange resins column.

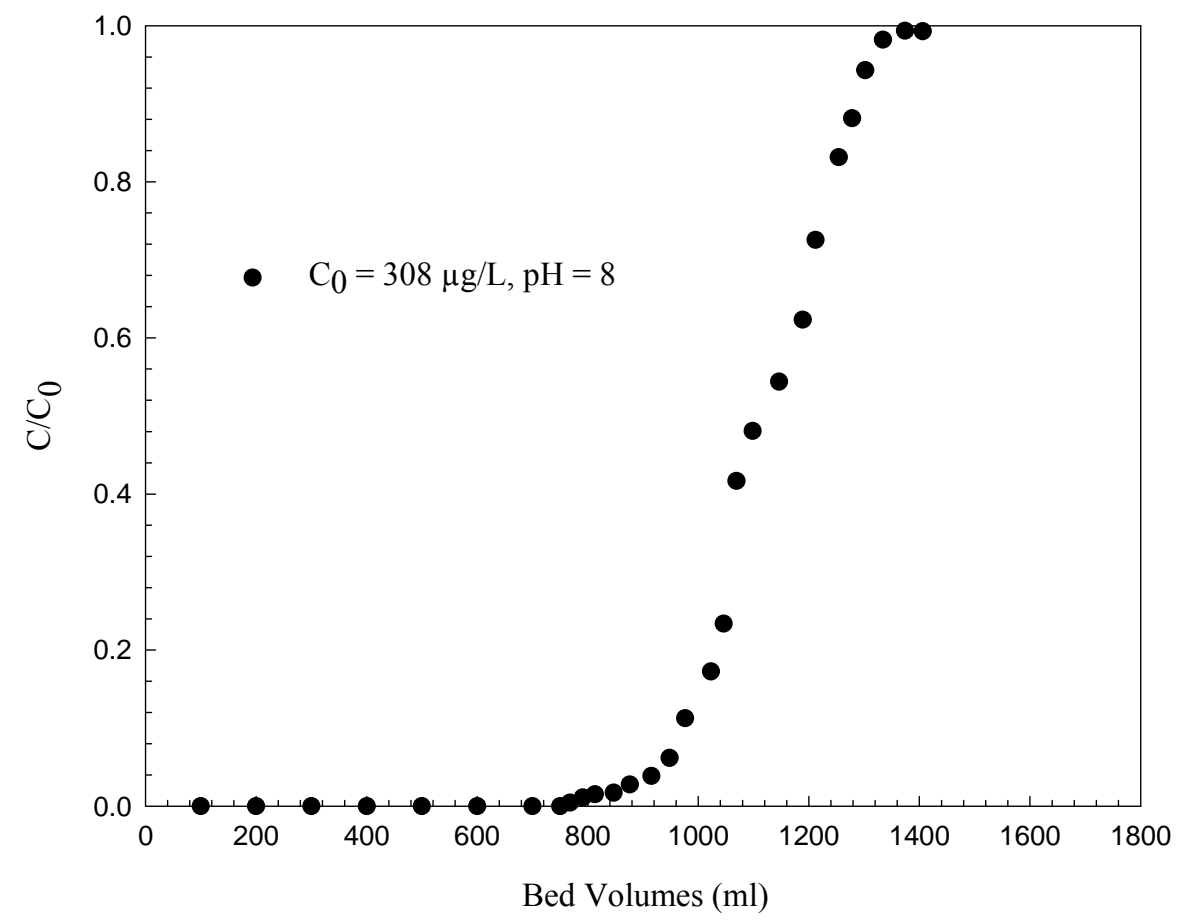

Figure 4.29 Column study

\section{CONCLUSIONS}

The present work has indicated that quaternized polyvinylpyridine coated granular activated carbon (GAC-QPVP) can be used to remove and recover chromium(VI) from aqueous solutions. The regenerated GAC-QPVP can also be reused to remove chromium(VI). Based on the results of this research following conclusions may be drawn: 
1. The adsorption of chromium(VI) depends on different factors such as $\mathrm{pH}$ of the solution, initial concentration of chromium(VI), time of contact with the adsorbent, and the concentrations of other ions such as $\mathrm{SO}_{4}^{-2}, \mathrm{Cl}^{-}, \mathrm{CH}_{3} \mathrm{COO}^{-}$, and $\mathrm{HCO}_{3}^{-}$. Increasing in anionic concentration decreases the chromium(VI) adsorption capacity. The effect of anions in decreasing order on the adsorption of chromium(VI) on GACQPVP was: $\mathrm{SO}_{4}^{-2}>\mathrm{Cl}^{-}>\mathrm{HCO}_{3}^{-}>\mathrm{CH}_{3} \mathrm{COO}^{-}$.

2. Sorption of chromium(VI) on GAC-QPVP was influenced by the $\mathrm{pH}$. Chromium(VI) removal decreased with increasing $\mathrm{pH}$ and maximum adsorption occurred at $\mathrm{pH}=$ 2.0. The mechanisms of chromium(VI) removal are anionic exchange with bromide ion on GAC-QPVP and reduction to $\mathrm{Cr}$ (III).

3. The kinetics experiments showed that $\mathrm{Cr}(\mathrm{VI})$ adsorption occurred with a fast initial rate and reached equilibrium within 6 hours. Pseudo second order kinetic model fitted the data well.

4. A finite-bath diffusion control model with changing bulk concentration could also describe the Cr(VI) adsorption kinetics. . The adsorption of chromium(VI) on GACQPVP was likely due to the finite bath diffusion, with a diffusion coefficient of $4.10 \times 10^{-6} \mathrm{~cm}^{2} / \mathrm{s}$.

5. The adsorption isotherm data fitted well to the Freundlich model with adsorption capacity increasing from 12.6 to $67.2 \mathrm{mg} / \mathrm{g}$ when the initial concentration was increasing from 10 to $65 \mathrm{mg} / \mathrm{L}$.

6. Desorption efficiency depends on the nature of bases used. Maximum desorption of chromium(VI) with $1 \mathrm{M} \mathrm{NaOH}$ was $80 \%$, and with $1 \mathrm{M} \mathrm{NH}_{4} \mathrm{OH}$ was $55 \%$. Desorption rate was fast, due to the larger surface area of the adsorbent. The regenerated GAC-QPVP was reused and adsorption capacity of regenerated adsorbent decreased by $35 \%-45 \%$ as compared to the fresh adsorbent.

7. Column study of chromium(VI) with GAC-QPVP was conducted using $5 \mathrm{mg} / \mathrm{L}$ chromium(VI) solution in a column with ID of $6 \mathrm{~mm}$ and $12 \mathrm{~mm}$. The break point was reduced from 760 bed volumes to 600 bed volumes when the flow rate was increased from $20 \mathrm{ml} /$ day to $100 \mathrm{ml} /$ day, respectively.

Adsorption experiments conducted on removal of arsenic(III) using iron-chitosan results can lead to the following conclusions:

1. Iron-chitosan can be used in removing arsenic(III), and removal of arsenic(III) depends on various factors include $\mathrm{pH}$ of solution, time of contact, adsorbent loading, initial concentration of arsenic(III) in the solution. There was no significant change in adsorption capacity when the solution is acidic. But as the solution becomes basic, adsorption capacity was decreased. 
2. Arsenic (III) adsorption capacity at equilibrium increases from 1.51 to $4.60 \mathrm{mg} / \mathrm{g}$ with an increase in the initial arsenic(III) concentration from 306 to $994 \mu \mathrm{g} / \mathrm{L}$. Kinetic batch experiments indicated that more than $60 \%$ of the arsenic(III) were adsorbed by the iron-chitosan with in $30 \mathrm{~min}$ and it takes about 2 hours to reach the equilibrium. Kinetic results fitted pseudo-second-order model well. Second order reaction rate was observed to be decreasing from $3.19 \times 10^{-2}$ to $1.15 \times 10^{-2} \mathrm{~g} \mathrm{mg}^{-1}$ $\min ^{-1}$ as the initial arsenic(III) concentration increased from 306 to $994 \mu \mathrm{g} / \mathrm{L}$, respectively.

3. It was noticed from the adsorption isotherms that maximum adsorption capacity increased from $1.95 \mathrm{mg} / \mathrm{g}$ to $5.97 \mathrm{mg} / \mathrm{g}$ and from $1.97 \mathrm{mg} / \mathrm{g}$ to $6.48 \mathrm{mg} / \mathrm{g}$ as the initial concentration of arsenic(III) increased from $0.3 \mathrm{mg} / \mathrm{L}$ to $1 \mathrm{mg} / \mathrm{L}$ for $\mathrm{pH}=7$ and $\mathrm{pH}=$ 8 , respectively. The adsorption isotherms were fitted well to Freundlich model. The results of breakthrough curve indicated that the break point obtained after 768 bed volumes.

\section{REFERENCES}

Adrian, T. H.; Brian, D.; Zohrab, A. S.; Don, Y. (1993). "Remediation of Chromiumcontaining soils by heap Leaching: Column Study.” J. Envi. Engg., 119(5), 825-841.

Alaerts, G. J., Jitjaturunt, V. and Kelderman, P. (1989). Use of Coconut Shell-Based Activated Carbon for Chromium(VI) Removal. Wat. Sci. Tech. 21(12), 1701-1704.

Allison, J. D., Brown, D. S., Novo-Gradac, K. J. (1991). "MINTEQ2/PRODEFA2, a geochemical assessment model for environmental systems: Version 3.0 user's manual," Environ. Res. Lab., Athens, GA, USA. FIELD URL.

Arai, Y.; Sparks, D. L.; Davis, J. A. (2004). "Effects of dissolved carbonate on arsenate adsorption and surface speciation at the hematite-water interface." Environ. Sci. Technol. 38, 817-824.

ATSDR, (2000). "Chromium Toxicity: U.S. Department of Health and Human Services, Agency for Toxic Substances and Disease Registry." Division of Toxicology and Environmental Medicine. 1-36.

Balaji, T.; Yokoyama, T.; Matsunaga, H. (2005). "Adsorption and removal of As(V) and As(III) using Zr-loaded lysine diacetic acid chelating resin." Chemosphere, 59(8), 11691174.

Berdal, A.; Verrie, D.; Zaganiaries, E. (2000). Removal of Arsenic from potable Water by Ion-exchange Resins. Proceedings of IEX 2000, Cambridge, U.K.

Butcher, B.; Davidoff, B.; Amacher, C.; Hinz, C.; Iskandar, I. K.; Selim, H. M. (1989). "Correlation of Freundlich Kd and n retention parameters with soils and elements." Soil Sci., $148,370-379$.

Chanda, M.; Rempel, G.L. (1993). "Poly(4-vinylpyridine) Gel Coated on Silica. High Capacity and Fast Kinetics in Uranyl Sulfate Recovery." Ind. Eng. Chem. Res. 32(4), 726732. 
Costa, M. (2003). "Potential hazards of hexavalent chromate in our drinking water." Reg. Toxicol. Pharmacol., 188, 1-5.

Dambies, L. (2004). Existing and prospective sorption technologies for the removal of arsenic in water. Sep. Sci. Technol., 39, 603-627.

Deng, B. Chromium(VI) reduction by naturally-occurring organic compounds: direct and surface-catalyzed reactions (metal pollutants, remediation). Johns Hopkins Univ., Baltimore, 1995.

Deng, B.; Stone, A. T. (1996) Surface-Catalyzed Chromium(VI) Reduction: Reactivity Comparisons of Different Organic Reductants and Different Oxide Surfaces. Environmental Science and Technology, 30, 2484-2494.

Elkhatib, E. A.; Bennett, O. L.; Wright, R. J. (1984). "Kinetics of arsenite adsorption in soils. Soil Sci. Am. J., 48, 758-762.

Fang, Jun; Gu, Zhimang; Gang, Dianchen; Deng, Baolin. (2005). "Evaluation of a Synthetic Adsorbent (GAC-QPVP) for Chromate Removal from Aqueous Solution.” Conference Paper: American Chemical Society, Washington, DC, Aug. 28 - Sep.1, 2005.

Ferguson, J. F.; Anderson, M. A. (1974). Chemical forms of arsenic in water supplies and their removal. In Chemistry of water supply, treatment, and distribution; Rubin, A.J.,E.D.; Ann Arbor Science, Ann Arbor, MI, 137-158.

Fuller, C. C.; Davis, J. A.; Waychunas, G. A. (1993). "Surface chemistry of ferrihydrite: Part 2. Kinetics of arsenate adsorption and coprecipitation." Geochim. Cosmochim. Acta., 32, 344-349.

Gang, D., Banerji, S.K, Clevenger, T.E. (2001b) Factors affecting chromium(VI) removal by modified poly(4-vinylpyridine) coated silica gel. Practice periodical of Hazardous, Toxic, and Radioactive Waste Management, 5(2): p.58-65.

Gang, D.; Banerji, S. K.; Clevenger, T. E. (2000) Chromium(VI) removal by modified PVPcoated silica gel. Pract. Periodical Hazard., Toxic, Radioact. Waste Manage. 4, (3), 105-110.

Gang, D.; Clevenger, T.E.; Banerji, S.K. (2001a) Modified poly (4-vinylpyridine) Coated Silica Gel. Fast Kinetics of Diffusion-Controlled sorption of Chromium(VI). Industrial \& Engineering Chemistry Research, 40(4), 1200-1204.

Genç, H.; Tjell, J. C. (2003) "Effect of phosphate, silicate, sulfate, and bicarbonate on arsenate removal using activated seawater neutralized red mud (Bauxsol)", J. de Physique IV, vol. 107, pp. 537-540.

Ghosh, M. M., and R. S. Teoh (1985) "Adsorption of Arsenic on Hydrous Aluminum Oxide" Seventh Mid-Atlantic Industrial Waste Conference.

Ghurye, G., Clifford, D. and Tripp, A.(1999) "Combined arsenic and nitrate removal by ion exchange" J. of the American Water Works Association, vol. 91(10), pp. 85-96.

Goldberg S., Johnston C. (2001) "Mechanisms of arsenic adsorption on amorphous oxides evaluated using macroscopic measurements, vibrational spectroscopy and surface complexation modeling", J. Colloid Interface Sci., vol. 234, pp. 204-16 
Gu, Zhimang; Fang, Jun; Baolin Deng. (2005). "Preparation and Evaluation of GAC-Based Iron-Containing Adsorbents for Arsenic Removal." Environ. Sci. Technol.,39(10), 38333843.

Gupta, S. K.; Chen, K. Y. (1978). “Arsenic removal by adsorption.” Journal of Water Pollution Control Federation, 50(3), 493-506.

Gupta, Shailendra K., and Kenneth Y. Chen (1978) "Arsenic Removal by Adsorption”, J. of water Pollution Control Federation, 50(3), pp. 493-506.

Hering, J. G.; Chen, P. Y.; Wilkie, J. A.; Elimelech, M.; Liang, S. (1996). “Arsenic removal by ferric chloride.” J. Am. Water Works Assoc., 88(4), 155-167.

Hering, J. G.; Elimelech, M. (1996). Arsenic removal from drinking water via membrane technology. Desalination, 117(1-3), 1-10.

Ho, Y.S.; Mckay, G. (1999) "Pseudo-second order model for sorption processes." Process Biochemistry, 34, 451-465.

Holm, T. R. (2002) "Effects of $\mathrm{CO}_{3}{ }^{2-}$ /bicarbonate, $\mathrm{Si}$, and $\mathrm{PO}_{4}{ }^{3-}$ on arsenic sorption to HFO”, J. of American water works association, vol. 94(4), pp. 174-181.

HSDB (2005). Hazardous Substance Data Bank. National Library of Medicine Records for element arsenic and arsenic compounds. Bethesada, HSDB.

Huang, C. P.; Fu, P. L. K. (1984). Treatment of Arsenate containing water by the activated carbon process." J. Water Pollution Control Federation. 56(3), 233-242.

Huang, C.P. and Wu, M.H. (1977). Removal of Chromium(VI) from Dilute Aqueous solution by Activated Carbon. Water Res. 11(8), 673-679.

Kamala, C. T.; Chu, K. H.; Chary, N. S.; Pandey, P. K.; Ramesh, S. L.; Sastry, A. R. K.; Sekhar, K. C. (2005). "Removal of arsenic(III) from aqueous solutions using fresh and immobilized plant biomass." Water Reasearch, 39(13), 2815-2826.

Karaca, S.; Gurses, A; Ejder, M; Acikyildiz, M. (2004) "Kinetic modeling of liquid-phase adsorption of phosphate on dolomite." Journal of Colloid and Interface Science, 277, 257263.

Katsoyiannis, Ioannis A. Anastasios I. Zouboulis (2002) "Removal of arsenic from contaminated water sources by sorption onto iron-oxide-coated polymeric materials', Water Research, vol. 36, pp. 5141-5155.

Latimer, W.M. (1952). "The Oxidation States of the Elements and their potentials in Aqueous Solution”. Prentice-Hall, Englewood Cliffs, N.J., USA.

Leyva, R. R.; A. Juarex Martinez and R. M. Gurerrero Coronado (1994). “Adsorption of Chromium(VI) from aqueous solutions on Activated Carbon.” Wat. Sci. Tech. 30(9), pp.191197.

Manning, B. A.; Goldberg, S. (1997). "Arsenic(III) and Arsenic(V) adsorption on three California soils." Soil. Sci., 162, 886-895.

McNeill, L. S.; Edwards, M. (1997) Predicting arsenate removal during metal hydroxide precipitation. J.Am. Water Works Assoc., 89(1), 75-86. 
Meng, X., Korfiatis G. P., Christisodoulatos, C., Bang, S. (2001) "Treatment of arsenic in Bangladesh well water using a household co-precipitation and filtration system", Water Research, vol. 35(12), pp. 2805-2810.

Meng, X., Korfiatis, G. P., Bang, S., Bang, K. W. (2002) "Combined effects of anions on arsenic removal by iron hydroxides", Toxicol. Lett., vol. 133 (1), pp. 103-111.

Nikolaos, P. N.; Jeffery, L. (1998). Arsenic Remediation Technology (AsRT). Environmental Research Institute, University of Connecticut and Gregory Dobbs.

Nishihama, S.; Hirai, T.; Komasawa, I. (2001). Review of Advanced Liquid-Liquid Extraction Systems for Separation of Metal Ions by a Combination of Conversion of the Metal Species with Chemical Reaction.” Ind. Eng. Chem. Res., 40, 3085-3091.

Onyango, M. S.; Kojima, Y.; Matsuda, H.; Ochieng, A. (2003). "Adsorption kinetics of arsenic removal from ground water by iron-modified zeolite." J. Chemical Engineering of Japan, 36(12), 1516-1522.

Perez-Candela, M.; Martin-Martinez; Jose, M.; (1995) Torregrosa M.; Rosa. "Chromium(VI) removal with activated carbons." Water Environmental Research, 72(1), 29-39.

Raven, K. P.; Amita Jain; Richard H. Loeppert. (1997). “Arsenite and Arsenate Adsorption on Ferrihydrite: Kinetics, Equilibrium, and Adsorption Envelopes." Environ. Sci. Technol., 32(3), 344-349.

Sandy, S. S., and Peter Nelson. (1973). "Concentration and separation of Arsenic from polluted Water by Ion Exchange." Environmental Science and Technology, 13(4), 476-478.

Sarkar, D.K.; Chattoraj, J. (1993) Colloid Interface Sci. 157, 219-226.

Schmuhl, R.; Krieg, H. M.; Keizer, K. (2001). "Adsorption of $\mathrm{Cu}$ (II) and $\mathrm{Cr}(\mathrm{VI})$ ions by chitosan: Kinetics and equilibrium studies." Water S. A. 27, 1-7.

Selim, H. M.; Zhang, H. (2005). "Kinetics of Arsenate Adsorption-Desorption in Soils." Environ. Sci. Technol., 39, 6101-6108.

Selomulya, C.; Meeyoo, V.; Amal, R. (1999) Mechanisms of Cr(VI) removal from water by various types of activated carbons. J. Chem. Technol. Biotechnol. 74, (2), 111-122.

Shrestha, P. K. (2004). Arsenic, Iron and Coliforms Removal Efficiency of Household Level Biosand Filters. M. S. Thesis, Tribhuva University, Nepal.

Thirunavukkarasu, O. S.; Viraraghavan, T.; Subramanian, K. S. (2003). "Arsenic removal from drinking water using granular ferric hydroxide.” Water S. A., 29(2), 161-170.

U.S. Environmental Protection Agency; Technology Transfer. (1973). "Process Design Manual for Carbon Adsorption”.

Ward, N. (1990). "Adsorption of hexavalent chromium on kaolinite and illite". MS thesis, The University of Arizona, Tucson, Ariz 
Appendix 44: Mercury Reduction From Coal Power Plant Emission Using Functionalized Ordered Mesoporous Carbons (FOMCs) (WV015) 


\section{FINAL TECHNICAL REPORT}

\begin{tabular}{|c|c|}
\hline Contract Title and Number: & Period of Performance: \\
\hline Crosscutting Technology Development at the Center for & Starting Date: $06 / 01 / 2005$ \\
\hline Advanced Separation Technologies & Ending Date: \\
\hline
\end{tabular}

(DE-FC26-02NT41607)

Sub-Recipient Project Title:

Mercury Reduction From Coal Power Plant

Emission Using Functionalized Ordered Mesoporous

Carbons (FOMCs)

Principal Investigators:

LianShin Lin, Dianchen Gang, Baolin Deng,

Contact Address:

Department of Civil \& Environmental Engineering

West Virginia University

Morgantown, WV 26506-6103

Subcontractor Address:

Baolin Deng

Department of Civil \& Environmental Engineering

University of Missouri-Columbia

Columbia, MO 65211
Report Information:

Type: Final

Number: Final

Period: $\quad 06 / 01 / 2005-10 / 31 / 2008$

Date: $\quad 04 / 30 / 2009$

Code: WV015-FINAL

Contact Information:

Phone: (304) 293-3031 x 2622

Fax: $\quad$ (304) 293-7109

E-Mail: lianshin.lin@mail.wvu.edu

Subcontractor Information:

Phone: 573-882-0075

Fax: $\quad 573-882-4874$

E-Mail: DengB@missouri.edu

\section{$\underline{\text { Disclaimer }}$}

"This report was prepared as an account of work sponsored by an agency of the United States Government. Neither the United States Government nor any agency thereof, nor any of their employees, make any warranty, express or implied, nor assume any legal liability or responsibility for the accuracy, completeness, or usefulness of any information, apparatus, product, or process disclosed, or represents that its use would not infringe privately owned rights. Reference herein to any specific commercial product, process, or service by trade name, trademark, manufacturer, or otherwise does not necessarily constitute or imply endorsement, recommendation, or favoring by the United States Government or any agency thereof. The views and opinions of authors expressed herein do not necessarily state or reflect those of the United States Government or agency thereof." 


\begin{abstract}
The objective of this project is to develop and evaluate functionalized ordered mesoporous carbons (FOMCs) for $\mathrm{Hg}$ removal from flue gas emissions. Highly ordered mesoporous carbons (OMCs) with uniform pore size were synthesized under defined conditions and their structures were optimized according to their capability toward $\mathrm{Hg}$ removal. OMCs were functionalized by elemental sulfur and other chemicals to enhance the $\mathrm{Hg}$ uptake. The study focuses on element mercury $\left(\mathrm{Hg}^{0}\right)$ removal, because it has very low water solubility and is normally not removed by water-based scrubbing processes. The modifications include adding sulfur to the surface of the ordered mesoporous carbons (OMCs). Sample characterizations with FT-IR, XRD, and TEM have been applied, which have confirmed the success of the functionalization procedures. The preliminary mercury uptake study results in gas phase showed that sulfur-impregnated ordered mesoporous carbon (SOMC-1) could adsorb Hg under the experimental conditions used in this study with an adsorption capacity of $200 \mu \mathrm{g} / \mathrm{g}$.

In an effort to enhance mercury $(\mathrm{Hg})$ removal from the aqueous solution, activated carbon (AC) was modified by a combined treatment of nitric acid and thionyl chloride, followed by the reaction with ethylenediamine to introduce $\mathrm{N}-$, S-, and Cl-containing functional groups. The modified activated carbon (MAC) was characterized by SEM-EDS, FT-IR, XPS, elemental analyzer, and potentiometry. Kinetics and equilibrium isotherms of $\mathrm{Hg}$ sorption by MAC were determined in batch experiments. Characterization indicated that additions of the organic-inorganic ligands onto AC surface were effective, likely through the acyl chloridecarbon reactions. Potentiometric titration showed that the modifications introduced more negative surface charges favoring cation sorption. Kinetics and isotherm studies demonstrated that $\mathrm{Hg}$ sorption by MAC was faster $(<30 \mathrm{~min})$ and higher $(>200 \%)$ than that by $\mathrm{AC}$, suggesting a high affinity of MAC for $\mathrm{Hg}$ ions. The sorption by MAC occurred in a wider $\mathrm{pH}$ range (4-10 vs. 5-7), and low ionic strength appeared to enhance $\mathrm{Hg}$ sorption. The sorption isotherms were best described by Freundlich model. The enhanced Hg sorption by MAC was primarily accomplished by the coordinations of ligand atoms $(\mathrm{O}, \mathrm{N}$ and $\mathrm{S})$ with $\mathrm{Hg}$ ions through the mechanisms of surface complexation, reduction, and ion exchange.
\end{abstract}




\section{TABLE OF CONTENTS}

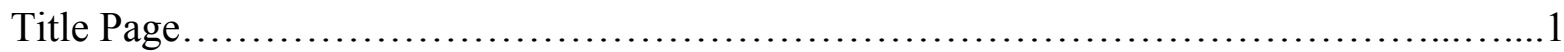

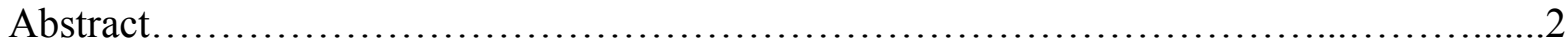

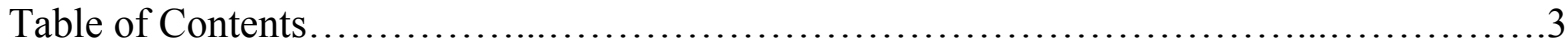

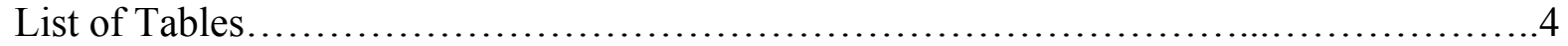

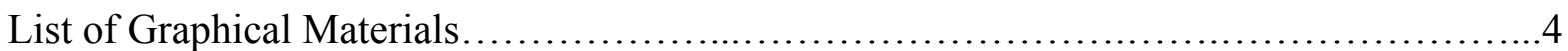

I INTRODUCTION.................................................................

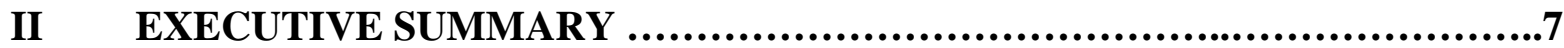

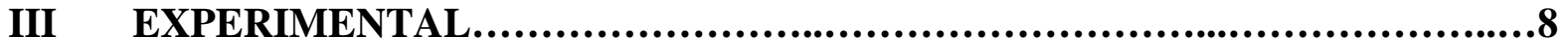

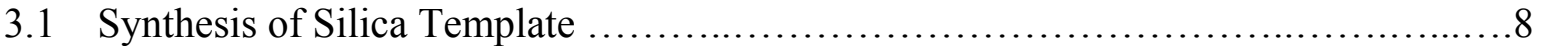

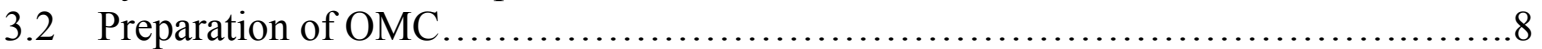

3.3 Functionalize the GAC and OMC with Different Chemicals to Enhance Hg Uptake...8

3.3.1 Addition of Amine Group onto the Activated Carbon Surfaces................ 9

3.3.2 Addition of Thiol Functional Groups onto the Activated Carbon Surfaces.....9

3.3.3 Functionalization of OMC Surfaces with Modification.........................10

3.3.4 Functionalization of OMC Surfaces with Elemental Sulfur...................10

$3.4 \mathrm{Hg}(\mathrm{II})$ Adsorption onto the Functionalized Activated Carbon.........................10

3.5 Mercury Sorption In Gas Phase ............................................... 11

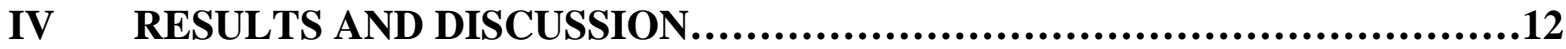

4.1 Characterization of OMCs.....................................................

4.2 Structure Characterization of Amine Group Functionalized GAC ...................13

4.3 Structure Characterization of Thiol Group Functionalized GAC ....................14

4.4 Structure Characterization of the Functionalized OMCs...........................14

4.5 Proton Binding Isotherm .........................................................

4.6 $\mathrm{Hg}(\mathrm{II})$ Adsorption onto the Activated Carbon..................................... 16

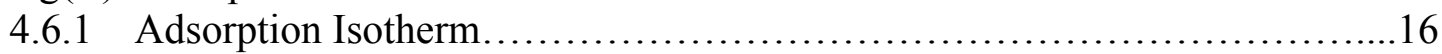

4.6.2 Effect of $\mathrm{pH}$ on $\mathrm{Hg}(\mathrm{II})$ Adsorption.....................................19

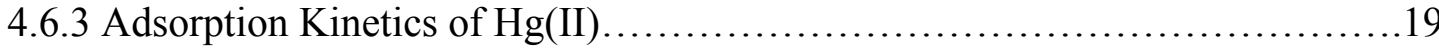

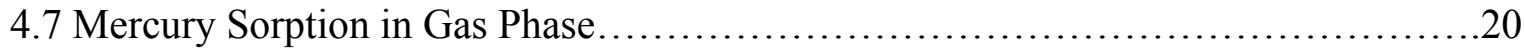

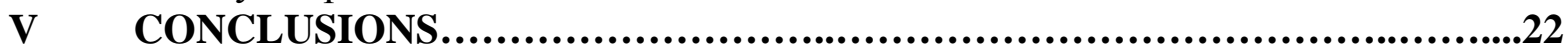

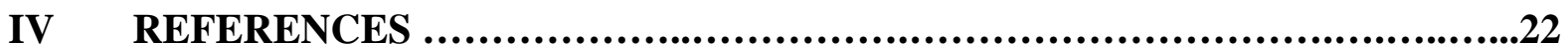




\section{LIST OF TABLES}

Table 1 Flue gas configurations 12

Table 2 Physicochemical properties of mesoporous silica templates and mesoporous carbons.......................................................... 13

Table 3 Equilibrium models for adsorption $\mathrm{Hg}^{2+}$ ion on carbon materials. 18

\section{LIST OF GRAPHICAL MATERIALS}

Figure 1 A two-step process for functionalization of amine group onto activated carbon (Yantasee et al., 2004)..........................................................................9

Figure 2 The process for functionalization of selected groups onto activated carbon....9 Figure 3 Procedure for functionalization of OMC surfaces (Jarrais et al., 2005).......10 Figure 4 Schematic representation of the experimental setup.......................11 Figure 5 TEM micrographs of silica templates and ordered mesoporous carbons (A-1, SBA-15 template (100); A-2, SBA-15 template (110); B-1, carbon (110) from SBA-15; B-2, carbon (110) from SBA-15)..............................13

Figure 6 Figure 7 FTIR spectra of the functionalized AC (top) and the virginal AC (bottom)...13 FTIR spectra of the functionalized activated carbons and un-treated activated carbon.............................................................. 14

Figure 8 FTIR spectra of the functionalized OMC (left, red) and un-treated OMC (right. purple)......................................................

Figure 9 X-ray diffraction patterns of OMC before and after surface functionalization................................................... 15

Figure 10 TEM micrographs of OMC before (left) and after (right) functionalization...15

Figure 11 Net binding proton curves of the AC and MAC.

Figure 12 Adsorption isotherms of adsorbents; buffer $\left(\mathrm{KH}_{2} \mathrm{PO}_{4}-\mathrm{NaOH}=0.01 \mathrm{M}\right.$; final solution pH $6.0( \pm 0.2)$; temperature $=25^{\circ} \mathrm{C}$.

Figure 13 Langmuir linear model for adsorption isotherms, buffer $\left(\mathrm{KH}_{2} \mathrm{PO}_{4}-\mathrm{NaOH}\right)=$

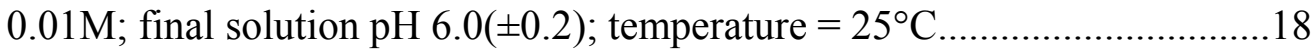

Figure 14 Frundlich linear model for adsorption isotherms of adsorbents conditions: buffer $\left(\mathrm{KH}_{2} \mathrm{PO}_{4}-\mathrm{NaOH}\right)=0.01 \mathrm{M}$; final solution $\mathrm{pH} 6.0( \pm 0.2)$; temperature $=25^{\circ} \mathrm{C}$.

Figure 15

Effect of $\mathrm{pH}$ on $\mathrm{Hg}(\mathrm{II})$ adsorption

Figure 16

Figure 17

Plots of removal versus time for the adsorption of mercury (II)

Effect of agitation time on removal of $\mathrm{Hg}^{2+}$ from aqueous solution.

Conditions: dosage of carbon $=100 \mathrm{mg}$ per $300 \mathrm{ml}$; buffer solution

$\left(\mathrm{KH}_{2} \mathrm{PO}_{4}-\mathrm{NaOH}\right)=0.01 \mathrm{M}$; final solution $\mathrm{pH} 6.0( \pm 0.2)$; temperature $=$ $25^{\circ} \mathrm{C}$.

Figure 18

Mercury $(\mathrm{Hg})$ uptake comparison between SSBA-15 and SOMC-1 ............21

Figure 19

Mercury $(\mathrm{Hg})$ uptake comparison between FOMC and AC. 


\section{INTRODUCTION}

Mercury is one of the toxic metals in the environment (William, 1991). Metal mercury and its compounds, especially methyl mercury, are neurotoxins and cause blockages of the enzyme binding sites and interfere with transport processes protein synthesis (Kim, 2004). Its harmful effect is due to the high affinity toward sulfur-containing biological molecules, once mercury enters to the food chain, large concentrations of mercury compounds accumulate in human and animals. With excessive exposure, individuals may develop symptoms such as tremors, loss of sensation in extremities, vision and hearing loss, and developmental and behavioral abnormalities (Baeyens, 1996).

Mercury may come from several sources, such as chloral kali, paint, pulp and paper, oil refining, electrical devices, rubber processing and coal-fired power plant emissions (Namasivayam, 1993; 1994; Lindberg, 1980). The permitted discharge EPA limit of wastewater for total mercury is $10 \mathrm{mg} / \mathrm{L}$, and the limit for drinking water is $2 \mathrm{mg} / \mathrm{L}$ (Nam, 2003). Related limits established by the Ministry of the Environment of Japan are, more stringent at 5 and $0.5 \mathrm{mg} / \mathrm{L}$, respectively (Takahashi, 2001). Meanwhile, the Word Health Organization (WHO) recommends a maximum uptake of $0.3 \mathrm{mg}$ per week and $1 \mathrm{mg} / \mathrm{L}$ as the maximum acceptable concentration in drinking water (Navasivayam, 1998).

Conventional mercury treatment techniques include sulfide precipitation, ion-exchange, alum and iron coagulation, and adsorption on activate carbon (Patterson, 1995). Technologies for mercury control from coal-fired power plants (CFPPs) have been well explored. The main processes include pre-combustion and post combustion. Pre-combustion involves coalcleaning technologies to reduce mercury burden at the source. Post combustion involves adsorption by carbon-based materials by wet system or particulate filters. Mercury emissions from CFPPs exist in various valence states: elemental $\mathrm{Hg}^{0}$ and oxidized $\mathrm{Hg}^{2+}$ forms. However, elemental mercury gas is the dominant form of mercury in the plume of a CFPP, ranging from $92 \%$ to $99 \%$ of the total mercury concentration in the flue gas (Lindberg, 1980). The chemical forms in the mercury emissions determine the effectiveness of controls to remove mercury because of the significant differences of their chemical and physical properties. For example, elemental mercury $\left(\mathrm{Hg}^{0}\right)$ should not be absorbed significantly by water-based scrubbing processes, because it has very low water solubility. Other exiting measures, such as electrostatic precipitators, have be demonstrated ineffective in removing volatile elements such as elemental mercury, allowing at least $90 \%$ of the mercury to be discharged into the atmosphere (Kaakinen, et al 1975).

Activated carbon (AC) is a common adsorbent for contaminant removal from water and air, because it has good porous structures, specific surface chemical properties, reusability, and minimal costs. Activated carbon adsorption has been demonstrated to be cable of controlling mercury emissions. These adsorption processes can be accomplished in two different ways: powdered activated carbon (PAC) injection and fixed-bed granular activated carbon (GAC) adsorption. PAC injection involves the injection of PAC directly into the plant's flue gas stream where it adsorbs gas-phase mercury and is collected in downstream particulate control 
devices, such as fabric filters. In the fixed-bed GAC adsorption situations, GAC is placed downstream of the flue gas desulfurization (FGD) units and particulate collectors, serving as the final treatment process before the flue gas is discharged into the atmosphere. The regular activated carbons with large fraction of micropores $(<2 \mathrm{~nm})$ are not suitable for large-sized contaminants and their applications can be limited by slow diffusion kinetics, especially in the high particulate environment. Another study demonstrated that regular activated carbon showed little adsorptive capacity for elemental mercury when temperature is over $90^{\circ}$ because of the physical adsorption mechanism between the $\mathrm{Hg}$ and virgin GAC (Vidic and McLaughlin, 1996).

Surface modification of activated carbons is recognized as an attractive approach for enhancing heavy metal removal. Different heavy metal ions have different affinities for different surface functional groups. According to the hard and soft acid - base (HSAB) theory, mercury ions (II) are classified as a soft acid, which forms strong bonds with groups containing nitrogen and sulphur atoms (e.g., - $\mathrm{CN}$, - $\mathrm{RS}$, - $\mathrm{SH}$, and $-\mathrm{NH}_{2}$ ). In recent years, various sulphur and nitrogen containing reagents, such as calixarenes, crown ethers, dipyridylamide, and polythiourea on charcoal have been used for the extraction of mercury from aqueous solutions. The introduction of chemical functional groups onto carbon surfaces was carried out by various methods, e.g., reduction of a nitro group formed by a $\mathrm{HNO}_{3} / \mathrm{H}_{2} \mathrm{SO}_{4}$ mixture and then reduced by iron powder and distilled in hydrochloric acid. Reaction of carboxyl groups was introduced by acid treatment with ethylenediamine compounds.

To develop sorbent with high mercury adsorption capacity, we focused our effort on multifunctional surface ligands, i.e., sorbent possessing several "active" groups toward mercury ions. The surface functionalization of activated carbons was investigated in terms of the amount of groups immobilized. The carboxyl groups introduced by nitric acid were activated by thionyl chloride treatment. Nitrogen, sulfur and chlorine groups grafted and immobilized on activated carbon through alkyl chains were useful as functional groups, since they had a high reactivity. When the groups of mercuric affinity were introduced to activated carbon (AC) with thionyl, the novel activation carbon with sulfur, nitrogen and chlorine atoms would be expected to have excellent adsorption ability for $\mathrm{Hg}^{2+}$

In recent couple years, ordered mesoporous carbons (OMCs) have attracted much attention because OMCs could be widely utilized in industries as catalyst supports and gas separation media, etc. There is also a great potential to use OMCs for environmental improvement, including the removal of inorganic and organic contaminants from liquid and gas phases. Although functionalized regular thermal activated carbons have been prepared using sulfur, salt, and other chemicals, to remove mercury from flue gas of CFPPs, no research work has been started in functionalized OMCs. The investigators believe that functionalized OMCs would have great potential in the elemental mercury removal from flue gas because of their desirable properties, such as high surface area, controlled pore size, and elemental mercury reactive function groups. 


\section{EXECUTIVE SUMMARY}

Risk assessment under the 1990 Clean Air Act Amendments (CAAAs) demonstrated that mercury exposure had been associated with both neurological and developmental damage in humans. To reduce the health risks caused by mercury emissions, the United States Environmental Protection Agency (USEPA) has recently issued the Clean Air Mercury Rule (CAMR) to permanently cap and reduce mercury emissions from coal-fired power plants. Based on the new regulation, a first phase cap of 38 tons per year (tpy) will become effective in 2010 and a second phase cap of 15 tpy will become effective in 2018. This new regulation has significant financial implications for the CFPPs, because of the high costs associated with mercury removal. Therefore, developing innovative technologies for cost effective mercury emission control is critical to sustain and promote coal usage as an integrated component of the Nation's energy policy and to ensure the Nation's energy security.

To develop sorbent with high mercury adsorption capacity, we focused our effort on development of multifunctional surface ligands, i.e., sorbent possessing several "active" groups toward mercury ions. The surface functionalization of activated carbons was investigated in terms of the amount of groups immobilized. The carboxyl groups introduced by nitric acid were activated by thionyl chloride treatment. Nitrogen, sulfur and chlorine groups grafted and immobilized on activated carbon through alkyl chains were useful as functional groups, since they had a higher reactivity with mercury. When the groups of mercuric affinity were introduced to activated carbon (AC) with thionyl, the novel activation carbon obtained containing simultaneously sulfur, nitrogen and chlorine atoms would be expected to have excellent adsorption ability for $\mathrm{Hg}^{2+}$. In this study, activated carbon (AC) was modified by a combined treatment of nitric acid and thionyl chloride to introduce amine, amide, thiol, sulfurous, chlorine, and carboxylic functional groups.

Surface chemistry of the modified activated carbon (MAC) was characterized by SEM-EDS, FT-IR, potentiometry and XPS. Elements in samples were analyzed with an elemental analyzer. Mercury sorption kinetics and equilibrium isotherm by MAC were determined in batch experimental systems. Surface characterization indicated that additions of the organicinorganic ligands to $\mathrm{AC}$ by the treatment were effective, likely through the acyl chloridecarbon surface reactions. Potentiometric titration showed the modifications introduced more negative surface charges favoring cation sorption. Kinetics studies demonstrated that the $\mathrm{Hg}$ (II) sorption by MAC was faster ( $<30 \mathrm{~min})$ and higher $(>200 \%)$ than by $\mathrm{AC}$, suggesting a high affinity of the modified material for $\mathrm{Hg}$ (II) ions. The sorption occurred in a wider $\mathrm{pH}$ range (4-10 vs. 5-7), and low ionic strength appeared to enhance the Hg (II) sorption. Sample analysis by X-ray photoelectron spectroscopy showed that the sorption of the MAC toward $\mathrm{Hg}$ (II) was accomplished mainly by the chemical coordination of the ligand atoms $(\mathrm{O}, \mathrm{N}$ and $\mathrm{S}$ ) of the functional groups with the $\mathrm{Hg}$ ions. The sorption isotherms could be best fitted by the Freundlich model and accounted for by mechanisms of surface complexation, reduction, and ion exchange.

The other objective of this project is to develop and evaluate a novel method for removing elemental mercury by using functionalized ordered mesoporous carbons (FOMCs). Ordered mesoporous carbons (OMCs) were synthesized under defined conditions and their structures 
were optimized according to their capability toward mercury removal. Then, OMCs were functionalized by sulfur and other chemicals to enhance the $\mathrm{Hg}$ uptake. Advantages of using FOMCs for mercury removal include: (1) fast kinetics, (2) large capacity per unit weight, (3) tolerant for particulate environment and high temperature. The preliminary mercury uptake study results in gas phase showed that sulfur-impregnated ordered mesoporous carbon (SOMC-1) could adsorb Hg under the experimental conditions used in this study with an adsorption capacity of about $200 \mu \mathrm{g} / \mathrm{g}$.

\section{EXPERIMENTAL}

\subsection{Synthesis of Silica Template}

One important type of silica hosts, hexagonal SBA-15, was synthesized. In a typical synthesis, $100 \mathrm{ml}$ of concentrated hydrochloride acid $(\mathrm{HCl}, 37 \%)$ was added into $525 \mathrm{ml}$ of distilled water with stirring, and then $20 \mathrm{~g}$ of triblock copolymer Pluronic P123 $\left(\mathrm{EO}_{20} \mathrm{PO}_{70} \mathrm{EO}_{20}, \mathrm{BASF}\right)$ was added. After Pluronic P123 was completely dissolved with stirring for $1 \mathrm{hrs}, 46.5 \mathrm{ml}$ of tetraethylorthosilicate (TEOS, 98\%, Aldrich) was added to the homogenous solution with vigorous stirring for $10 \mathrm{~min}$. The resulting mixture was left for 4 hrs at $40^{\circ} \mathrm{C}$ and subsequently for $24 \mathrm{hrs}$ at $90^{\circ} \mathrm{C}$. The solid product obtained was washed with $80 \sim 90^{\circ} \mathrm{C}$ hot distilled water, and dried in an oven at $105^{\circ} \mathrm{C}$ overnight. After drying, product was calcined at $550^{\circ} \mathrm{C}$ for $8 \mathrm{hrs}$. The white silica template SBA-15 was stored for the next preparation of $\mathrm{OMC}$.

\subsection{Preparation of OMC}

The synthesis of ordered mesoporous carbon (OMC) was accomplished by in situ polymerization of monomeric acrylic acid in the porous structure of silica template SBA-15 (hexagonal structure) in a basic aqueous solution. Ninety (90) $\mathrm{ml}$ of monomer acrylic acid $(>99 \%$, Aldrich) was added into $180 \mathrm{ml}$ of distilled water with stirring, and then $9 \mathrm{~g}$ of SBA15 host was added and suspended in acrylic acid solution. After the mixture was stirred for $30 \mathrm{~min}, 0.02 \mathrm{~g}$ of 2,2-azobisisobutyronitrile (AIBN) was added as a free radical initiator. The mixture was then heated to $60^{\circ} \mathrm{C}$ for the in situ polymerization. The polyacrylic acid (PAA) with the silica template was dried by oven at $200^{\circ} \mathrm{C}$ overnight. Afterwards, the sample composite was heated under $\mathrm{N}_{2}$ flow at a temperature ramp rate of $5^{\circ} \mathrm{C} \min ^{-1}$ to $700^{\circ} \mathrm{C}$ and held for $8 \mathrm{hrs}$ for carbonization. OMC was recovered by the dissolution of silica template using aqueous hydrofluoric acid (HF, 48\%, Aldrich) for $15 \mathrm{hrs}$. After etching, the product was washed and dried in oven at $90 \sim 100^{\circ} \mathrm{C}$ overnight. SBA-15 silicate templates and ordered mesoporous carbon prepared following the above procedure have highly ordered porous structure.

\section{$\underline{\text { 3.3 Functionalize the GAC and OMC with Different Chemicals to Enhance Hg Uptake. }}$}

Three procedures are conducted for adding different functional groups onto activated carbon or OMC. Activated carbon is used in some experiments as preliminary materials for procedure development. 


\subsubsection{Addition of Amine Group onto the Activated Carbon Surfaces}

This procedure is adapted from the work by Yantasee et al. (2004) as illustrated by the reactive scheme presented in Figure 1.

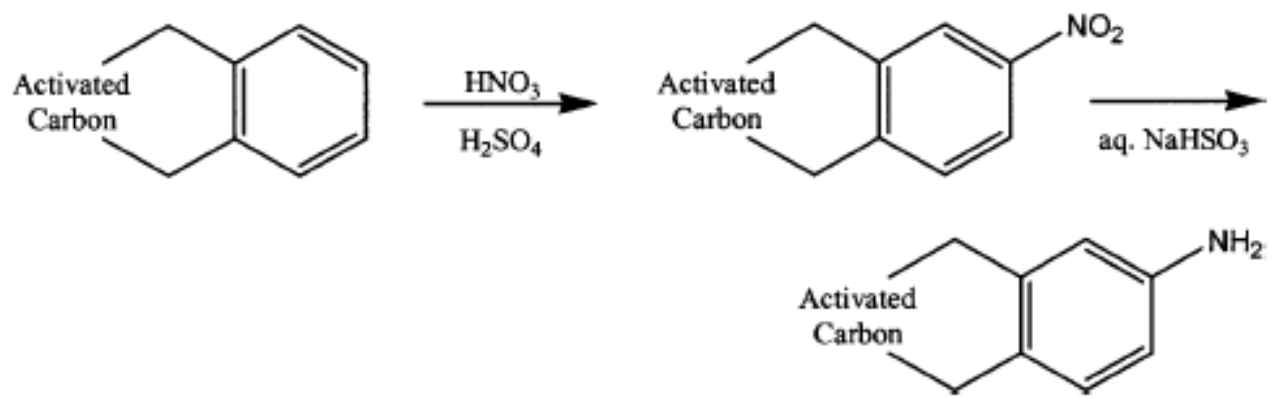

Figure 1. A two-step process for functionalization of amine group onto activated carbon (Yantasee et al., 2004).

This is a two-step process. The first step involves an electrophilic substitution for the nitration of activated carbon. At room temperature, Twenty-five $(250 \mathrm{~mL}$ of concentrated sulfuric acid $(18 \mathrm{M})$ was added slowly to $25 \mathrm{~mL}$ of concentrated $(15.7 \mathrm{M})$ nitric acid $\left(\mathrm{HNO}_{3}\right)$. Then, a $4.485 \mathrm{~g}$ of as-received activated carbon was slowly added to the acid mixture and stirred for $50 \mathrm{~min}$. The mixture was filtered and washed with deionized water and subsequently with ethanol. The filtrate was then air-dried at ambient temperature. The resulting product from the first step was nitrobenzene. The second step is the conversion of nitroarene to aniline. A $2.53 \mathrm{~g}$ of treated activated carbon, $25 \mathrm{~mL}$ of water, $10 \mathrm{~mL}$ of concentrated ammonium hydroxide, and a stir bar were placed in a $250 \mathrm{~mL}$ round-bottom flask and stirred for $30 \mathrm{~min}$. A $25.5 \mathrm{~g}$ of sodium hydrosulfite $\left(\mathrm{Na}_{2} \mathrm{~S}_{2} \mathrm{O}_{3}\right)$ was added to this solution slowly and allowed to stir overnight at room temperature. A reflux condenser was fitted on the flask to avoid solvent evaporation due to the increase in the solution temperature. A $10 \mathrm{~mL}$ of $17.5 \mathrm{M}$ glacial acetic acid was diluted in $100 \mathrm{~mL}$ of water and was then added to the solution and stirred for $5 \mathrm{~h}$ at reflux $\left(100^{\circ} \mathrm{C}\right)$. The solution was then cooled to room temperature, filtered, washed with a copious amount of water, followed by ethanol, and finally air-dried.

\subsubsection{Addition of Thiol Functional Groups onto the Activated Carbon Surfaces}

This procedure is adapted from the work by Jarrais et al (2005) and Silva et al. (2002) as illustrated by the reactive scheme presented in Figure 2.

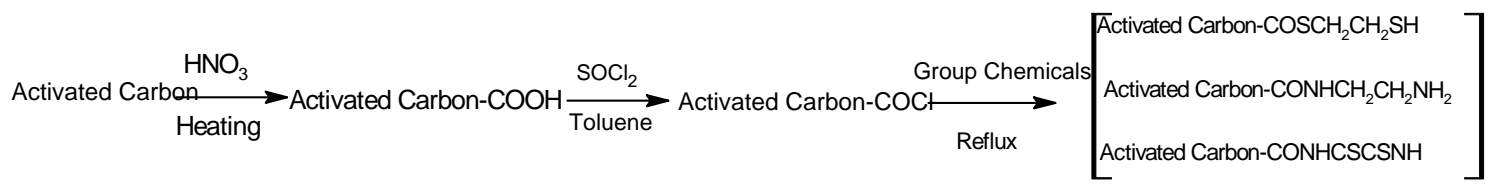

Figure 2. The process for functionalization of selected groups onto activated carbon.

The process involves in the following several steps: (1) AC oxidation: a $5 \mathrm{~g}$ of activated carbon (AC) was slowly added into $50 \mathrm{ml}$ of concentrated nitric acid $\left(\mathrm{HNO}_{3}\right)$, followed by heating at $70{ }^{\circ} \mathrm{C}$ for 120 min while stirring. The acid-treated $\mathrm{AC}$ was filtered and washed 
with deionized water, then air dried at $60{ }^{\circ} \mathrm{C}$. (2) Reaction with thionyl chloride: the obtained products were refluxed in $50 \mathrm{ml}$ of $5 \% \mathrm{SOCl}_{2}$ dry toluene solution at $70{ }^{\circ} \mathrm{C}$ for 24 hours, the solid was separated by filtration and washed with anhydrous toluene, dried in an oven at 40 ${ }^{\circ} \mathrm{C}$ for overnight under vacuum, obtaining the ACs with carbonyl chloride groups (ACCOCls). (3) Functionalization of $\mathrm{AC}$ : $\mathrm{AC}-\mathrm{COCl}(3.0 \mathrm{~g})$ was refluxed with a solution containing several chemicals (ethylenediamine; 1,3-propanedithiol, and dithiooxamide) in 40 $\mathrm{mL}$ of dry toluene at $90^{\circ} \mathrm{C}$ for $24 \mathrm{~h}$, and then distilled 2 hours. The solid was collected by filtration and cleaned by extensively washing with toluene and anhydrous ethanol. (4) Purification: the obtained products were sonicated for 10 minutes in anhydrous ethanol, washing with DI and dried in an oven at $40{ }^{\circ} \mathrm{C}$ for overnight under vacuum, resulting in functionalized -ACs.

\subsubsection{Functionalization of OMC Surfaces with Modification}

There is no reported procedure for surface functionalization of OMC with amine group, so a procedure is developed based on some studies by Jarrais et al (2005) and Silva et al. (2002) (Figure 3). It involves: (1) OMC oxidation: a $2.5 \mathrm{~g}$ of $\mathrm{OMC}$ was slowly added to $25 \mathrm{~mL}$ of concentrated nitric acid $\left(\mathrm{HNO}_{3}\right)$ with heating, and stirred for $120 \mathrm{~min}$. The mixture was filtered and washed with deionized (DI) water, then air-dried at $40^{\circ} \mathrm{C}$. (2) Reaction with thionyl chloride: The treated OMC (B1) was refluxed in $5 \% \mathrm{SOCl}_{2}$ in dry toluene solution at $70^{\circ} \mathrm{C}$ for 4 hours, then filtered and extensively washed with toluene, dried in an oven at $40^{\circ} \mathrm{C}$ for overnight under vacuum. (3) Fictionalization of OMC: the treated OMC (B2) was refluxed with a solution containing amine group compound bis(3-aminopropyl)amine in 40 $\mathrm{mL}$ of dry toluene for $4 \mathrm{~h}$, then filtered and extensively washed with toluene. The products were also extracted for $2 \mathrm{~h}$ with toluene and dried in an oven at $150{ }^{\circ} \mathrm{C}$ for $13 \mathrm{~h}$ under vacuum $(\mathrm{B} 3)$.

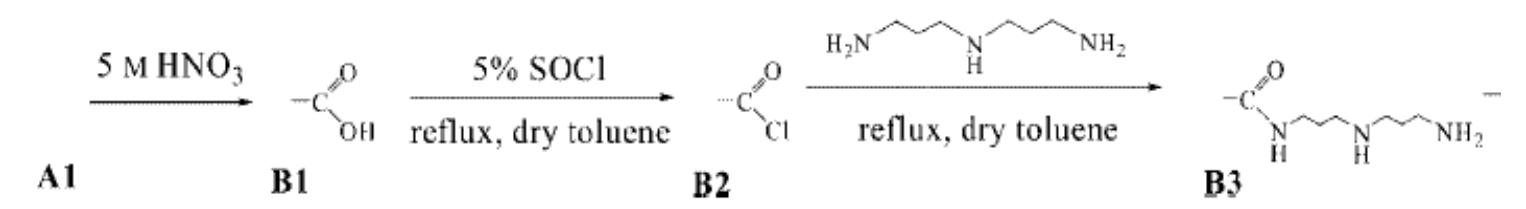

Figure 3. Procedure for functionalization of OMC surfaces (Jarrais et al., 2005)

\subsubsection{Functionalization of OMC surfaces with elemental sulfur}

Eight (8) $\mathrm{g}$ of $\mathrm{OMC}$ and $8 \mathrm{~g}$ elemental sulfur were granulated into fine powder and thoroughly mixed. Then the mixture was heated under $\mathrm{N}_{2}$ flow at a temperature ramp rate of $5^{\circ} \mathrm{C} \mathrm{min}^{-1}$ to $600^{\circ} \mathrm{C}$ and held for $4 \mathrm{hrs}$ for vulcanization.

\section{$\underline{3.4 \mathrm{Hg}(\mathrm{II}) \text { Adsorption onto the Functionalized Activated Carbon }}$}

All the adsorption experiments were carried out in batch systems. The amount of $\mathrm{Hg}(\mathrm{II})$ adsorption was determined by measuring the loss of aqueous $\mathrm{Hg}(\mathrm{II})$ in the suspension with ACs. $\mathrm{Hg}(\mathrm{II})$ concentration was analyzed by inductively coupled plasma (ICP) atomic emission spectrometry. 


\subsection{Mercury Sorption in Gas Phase}

One to five grams of FOMCs will be placed in a stainless steel column of $1.0 \mathrm{~cm}$ inner diameter to form a 2 to $10 \mathrm{~cm}$ bed depth. Since the expected adsorption capacity is quite high, a smaller amount may have to be used to complete the experiments in a reasonable time period. The column will be operated in down flow mode to minimize the potential for fluidization of the packed bed. The columns will be placed in a temperature-controlled oven in order to regulate the temperature of the influent gas. Influent concentration of $\mathrm{Hg}^{\mathrm{O}}$ can be controlled by a mercury permeation device (Vidic and McLaughlin, 1996) or by a contact bed of liquid mercury under controlled temperature and carrier gas flow rate. The effluent mercury concentration was monitored using on-line double beam, automatic atomic absorption spectrometer as well as a semi-continuous Hg analyzer (PSA Sir Galahad), and the dynamic adsorptive capacity of the FOMCs was computed by integrating the area above the breakthrough curve. Exhaust gas was scrubbed by permanganate solution to remove any residual metals before it was released into the atmosphere. Tested variables included temperature and influent mercury concentration. A control at room temperature was included to compare the results with regular activated carbon reported in the literatures. The kinetics of trace metal adsorption was assessed based on experiments with the initial concentration of $20 \mu \mathrm{g} / \mathrm{m}^{3}$, and varying carrier gas flow rates. A schematic representation of the experimental setup is shown in Figure 4.

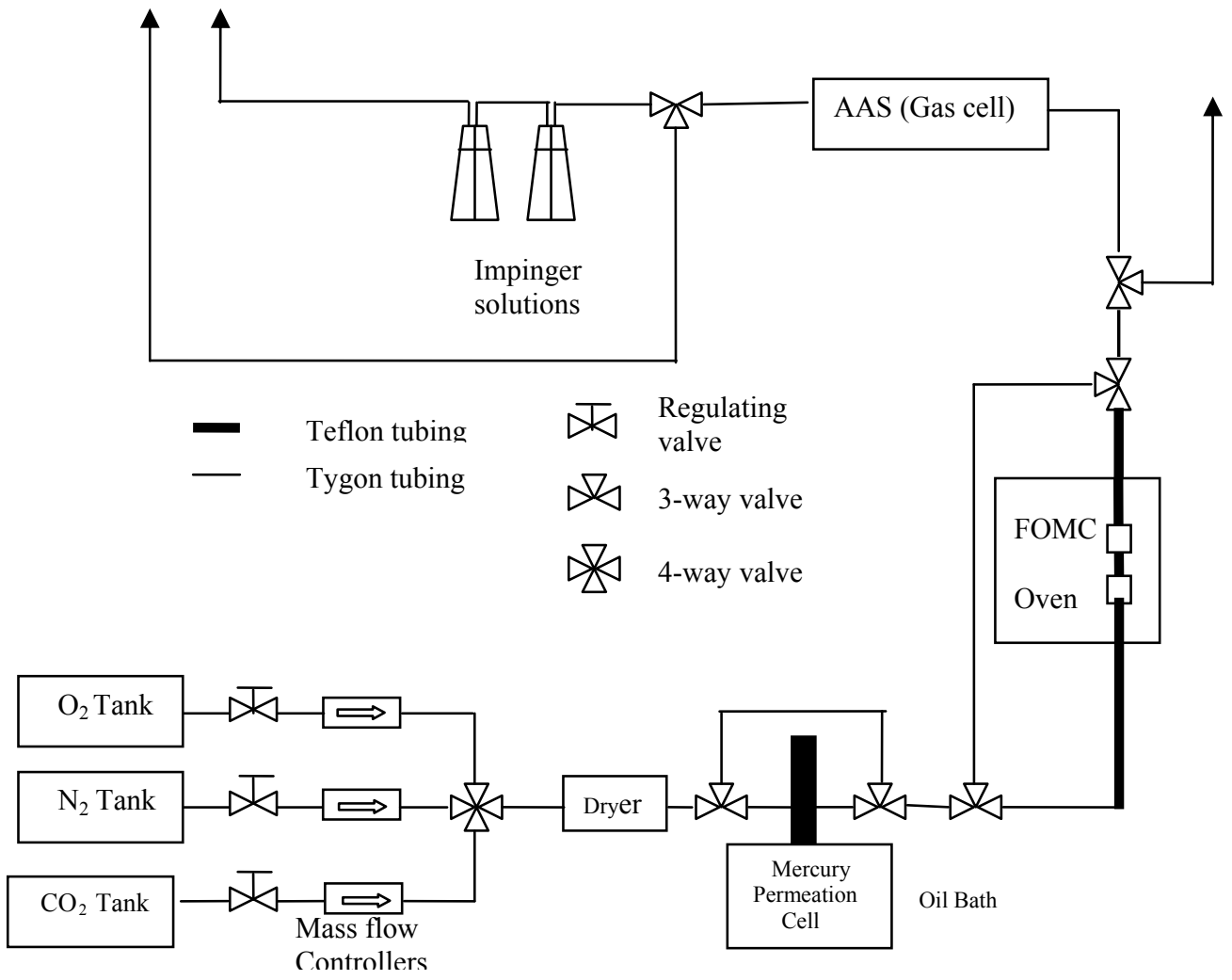

Figure 4. Schematic representation of the experimental setup 
The preliminary mercury uptake studies were conducted using a bench-scale packed bed reactor which contains a system for mixing gases to represent different types of flue gases resulting from coal combustion. The gas stream from this system is routed through a reactor (where samples are placed) in a furnace that provides controlled temperatures from 20 to over $1000^{\circ} \mathrm{C}$. In this study, 40 milligrams of adsorbent was packed in the reactor and the simulated flue gas with an initial $\mathrm{Hg}$ concentration of $18.5 \mu \mathrm{g} / \mathrm{m}^{3}$ was routed through the reactor at $140^{\circ} \mathrm{C}$. The flue gas configuration used in this study is shown in Table 1 .

Table 1. Flue gas configuration

\begin{tabular}{cc}
\hline \hline Gas Component & Concentration \\
\hline $\mathrm{NO}$ & $300 \mathrm{PPM}$ \\
$\mathrm{NO}_{2}$ & $20 \mathrm{PPM}$ \\
$\mathrm{SO}_{2}$ & $300 \mathrm{PPM}$ \\
$\mathrm{HCl}$ & $5-10 \mathrm{PPM}$ \\
$\mathrm{CO}_{2}$ & $13.5 \%$ \\
$\mathrm{O}_{2}$ & $6.0 \%$ \\
$\mathrm{H}_{2} \mathrm{O}$ & $6.0 \%$ \\
$\mathrm{~N}_{2}$ & Balance \\
\hline \hline
\end{tabular}

\section{RESULTS AND DISCUSSION}

\subsection{Characterization of OMCs}

The TEM micrographs of silica templates in Figure 5 clearly show the long range orders of uniform mesoporous structures of SBA-15 (Fig.5. A-1 and A-2). OMCs (Fig.5. B-1 and B2) derived from polyacrylic acid (PAA) impregnation into the SBA-15 template maintain highly ordered two-dimensional hexagonal symmetry to the parent SBA-15. The center-tocenter distance of adjacent channels is about $10 \mathrm{~nm}$. The average pore size of SBA-15 (Table 1) is $6.6 \mathrm{~nm}$. With the template dissolution by acid etching, the pore left in the carbon is expected to be $3-4 \mathrm{~nm}$. This is only slightly smaller than the average pore size of $4.1 \mathrm{~nm}$ determined by Barrett-Joyner-Halenda (BJH) analysis (Table 2).
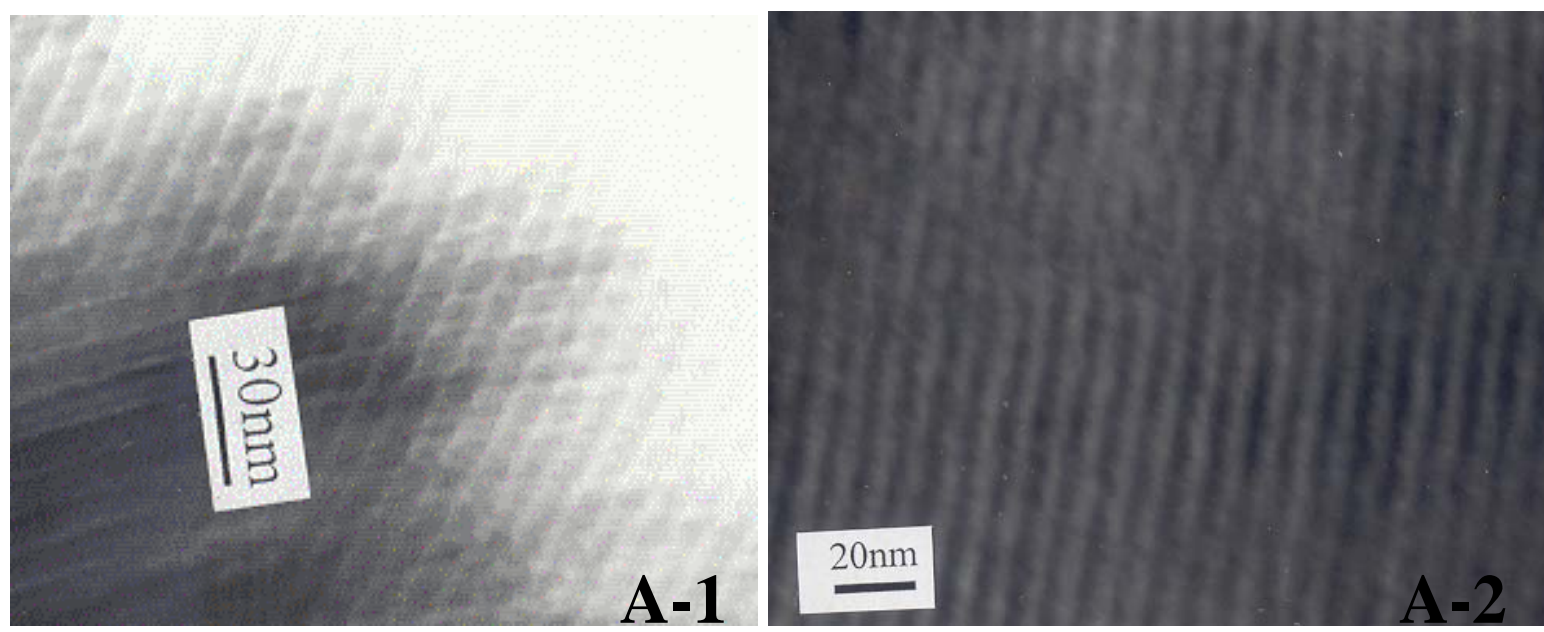


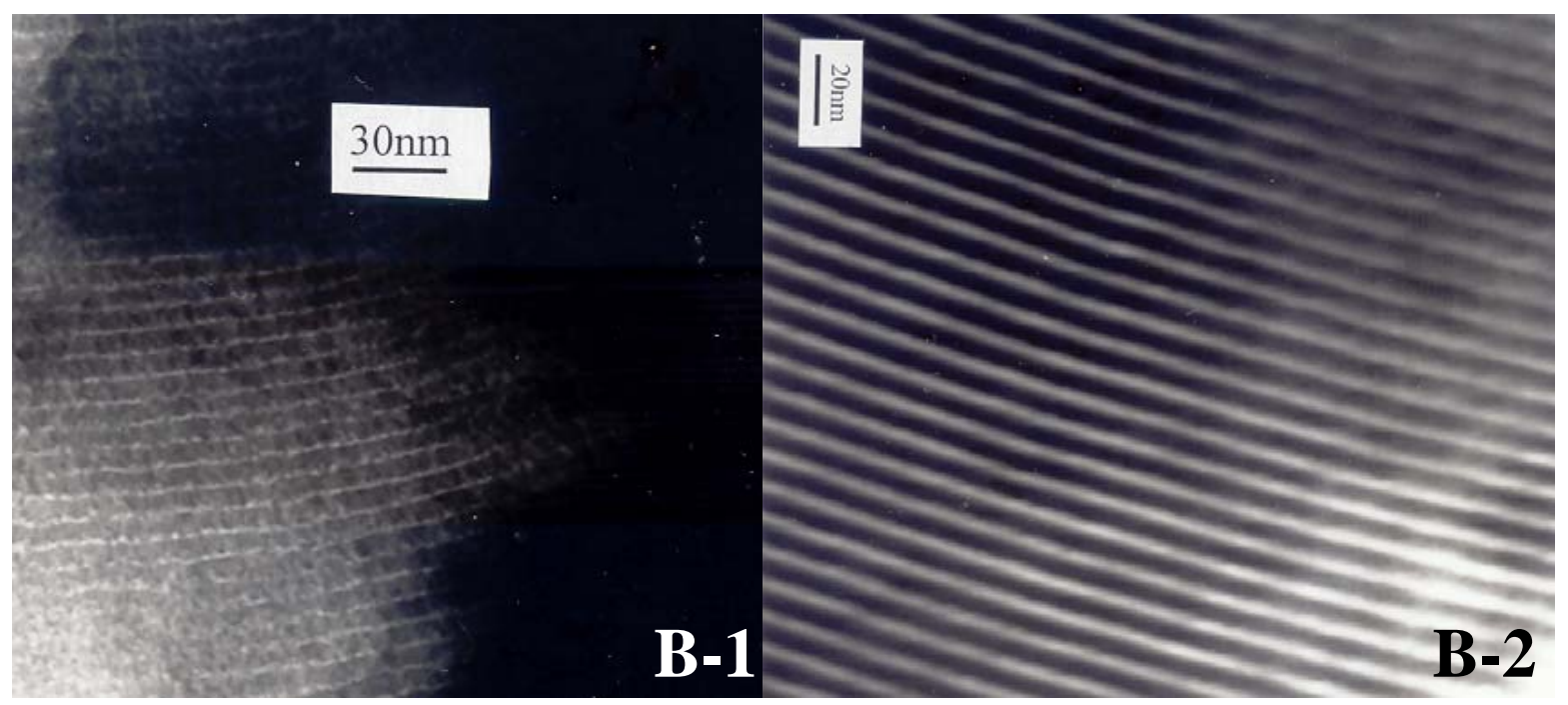

Figure 5. TEM micrographs of silica templates and ordered mesoporous carbons (A-1, SBA-15 template (100); A-2, SBA-15 template (110); B-1, carbon (110) from SBA-15; B2, carbon (110) from SBA-15)

Table 2. Physicochemical properties of mesoporous silica templates and mesoporous carbons

\begin{tabular}{cccccc}
\hline \hline & $\begin{array}{c}\text { BET Surface } \\
\text { area }\left(\mathrm{m}^{2} / \mathrm{g}\right)\end{array}$ & $\begin{array}{c}\text { Average Pore } \\
\text { Diameter }(\mathrm{nm})\end{array}$ & $\begin{array}{c}\text { Median Pore } \\
\text { Diameter }(\mathrm{nm})\end{array}$ & Porosity & $\begin{array}{c}\text { Volume of } \\
\text { pore }\left(\mathrm{cm}^{3} / \mathrm{g}\right)\end{array}$ \\
\hline SBA-15 & 609 & 6.6 & 7.8 & 0.50 & 1.01 \\
OMC & 607 & 4.1 & 3.7 & 0.38 & 0.62 \\
\hline \hline
\end{tabular}

\subsection{Structure Characterization of Amine Group Functionalized GAC}

It is important to verify whether the functionalization process is successful. Figure 6 is the FTIR spectra of the functionalized activated carbon (top) as compared with the un-treated sample (bottom). Comparing their patterns, after the functionalization with amine, there is a new peak, it is probably amine group.

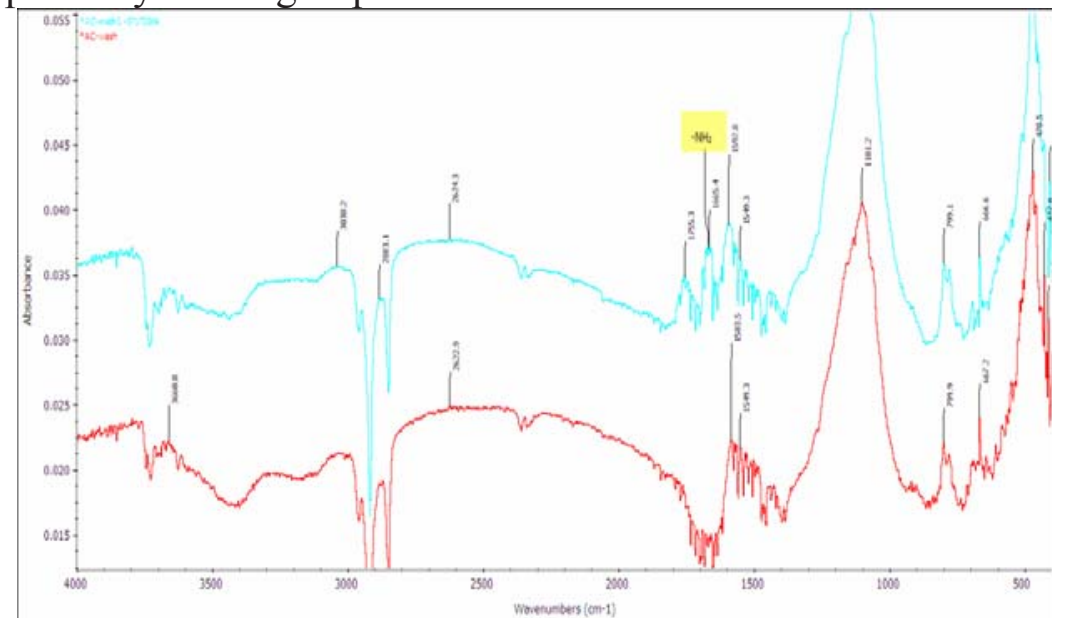

Figure 6. FTIR spectra of the functionalized AC (top) and the virginal AC (bottom) 


\subsection{Structure Characterization of Thiol Group Functionalized GAC}

Figure 7 is the FTIR spectra of the functionalized activated carbon as compared with the untreated sample. FTIR spectrum of modified ACs exhibit new peaks around $1642 \mathrm{~cm}^{-1}$ (CONH-), $1465 \mathrm{~cm}^{-1}$ due to CON stretching, $610 \mathrm{~cm}^{-1}$ due to the $\mathrm{N}$ distortion, and around $1409 \mathrm{~cm}^{-1}(-\mathrm{SH})$. The features of spectra indicate that the groups had been attached onto ACs successfully.

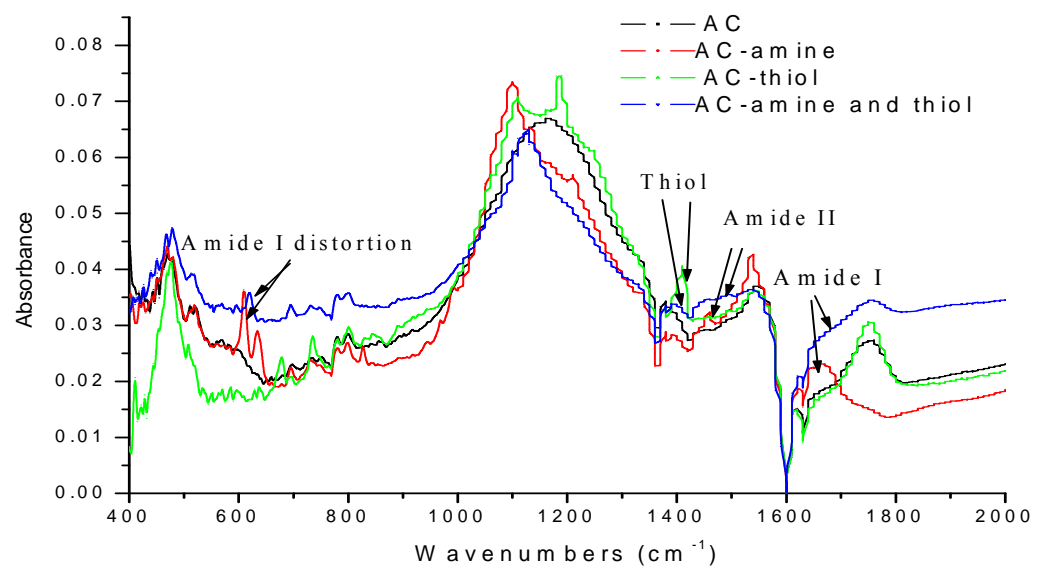

Figure 7. FTIR spectra of the functionalized activated carbons and un-treated activated carbon.

\subsection{Structure Characterization of the Functionalized OMCs}

FTIR spectra of the functionalized OMC (left, red) and original OMC (right, purple) are shown in Figure 8. After the functionalization there are two new peaks that are assigned to amine groups.

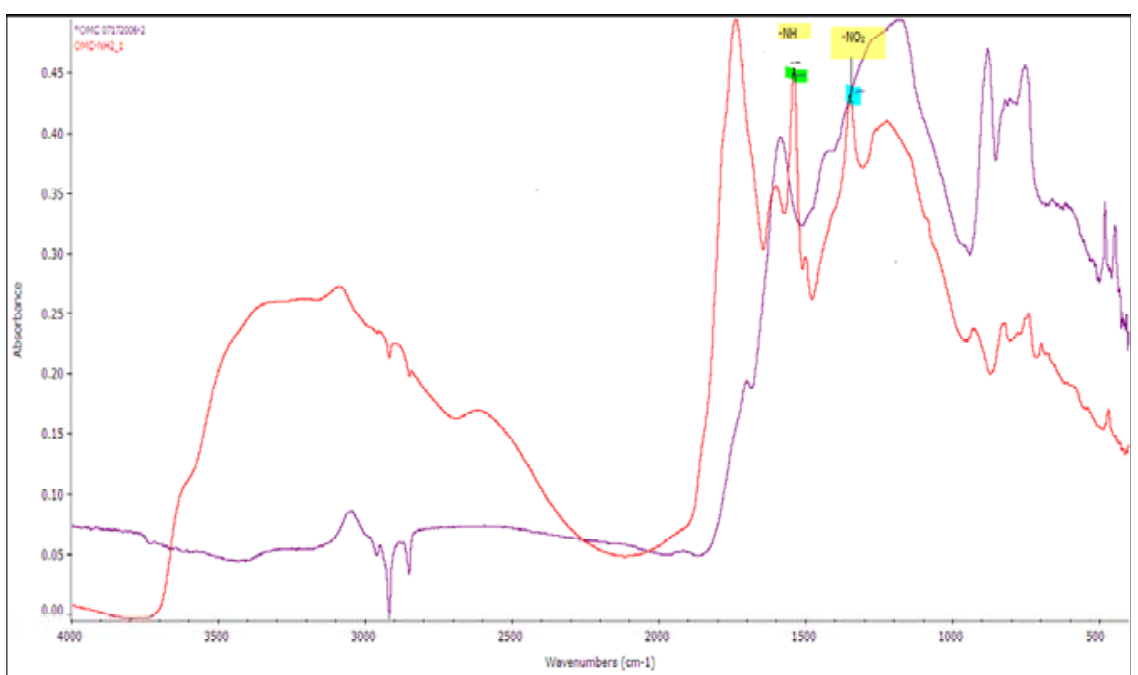

Figure 8. FTIR spectra of the functionalized OMC (left, red) and un-treated OMC (right, purple). 
This process has significant modified the mesoporous structure, as indicated by XRD analysis (Figure 9). The original peaks in unmodified OMC have replaced with a single peak. The modification is confirmed by TEM micrographs (Figure 10).

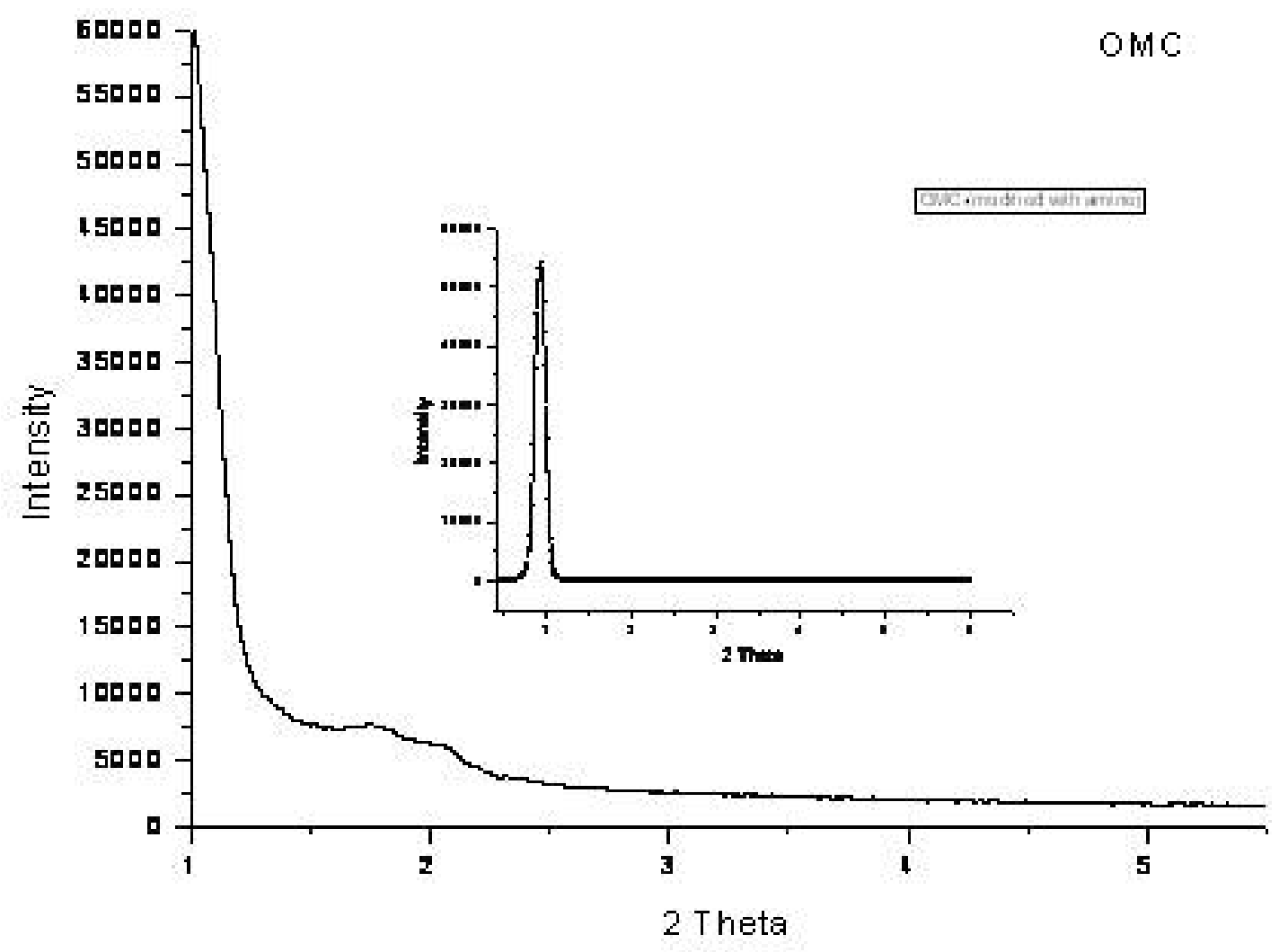

Figure 9. X-ray diffraction patterns of OMC before and after surface functionalization
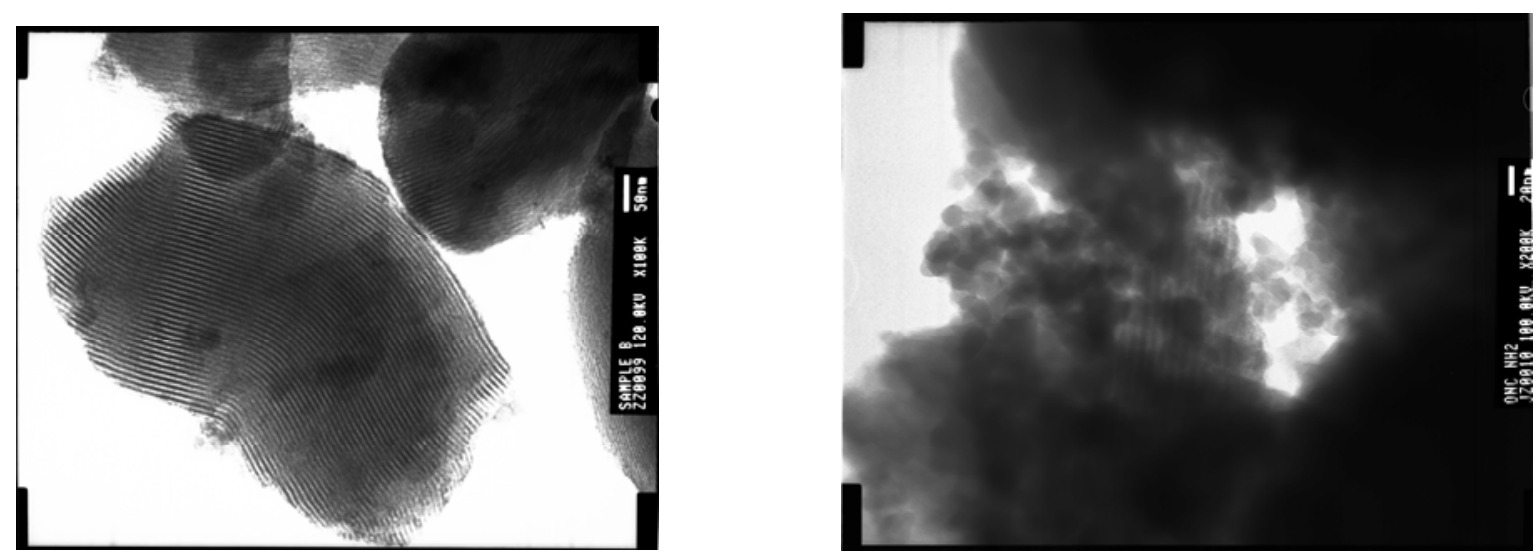

Figure 10. TEM micrographs of OMC before (left) and after (right) functionalization. 


\section{$\underline{4.5}$ Proton Binding Isotherm}

Proton binding isotherm of original AC, MAC is shown in Fig.11. The investigation $\mathrm{pH}$ range is between $\mathrm{pH} 2.0 \sim 11.00$. For different samples, it was observed that the intersection points of proton binding isotherm curves with the $\mathrm{pH}$ axis were different. Functionalization makes activated carbons more hydrophilic, decreases its $\mathrm{pH}$ of the point of zero charge, and increases the negative surface charge density. Results suggest that MAC have more acidic surface groups than original activated carbon. The points of intersection of the Q vs. $\mathrm{pH}$ curves show that the $\mathrm{pH}_{\mathrm{zpc}}$ 's of $\mathrm{AC}$ and $\mathrm{MAC}$ are found to be 9.6 down to around 2.50 2.60 respectively. The amount of protons adsorbed was calculated using the following Eq. (1) (Puziy and Alexander, 2001; Shameem Hasan, 2006):

$$
\mathrm{Q}=\frac{\mathrm{V}_{0+} \mathrm{Vt}}{\mathrm{m}}\left([\mathrm{H}]_{\mathrm{i}}-[\mathrm{OH}]_{i}+[\mathrm{H}]_{\mathrm{e}}+[\mathrm{OH}]_{\mathrm{e}}\right)
$$

Where $\mathrm{V}$ are the volumes of background electrolyte and titrant added, and $\mathrm{m}$ is the mass of adsorbent. Subscripts i and e refer to initial and equilibrium concentrations. The initial proton concentration was calculated from the amount of titrant added. The equilibrium proton concentration was calculated from the measured $\mathrm{pH}$ using an activity coefficient correction from Davis equation (Zhimang Gu, 2006).

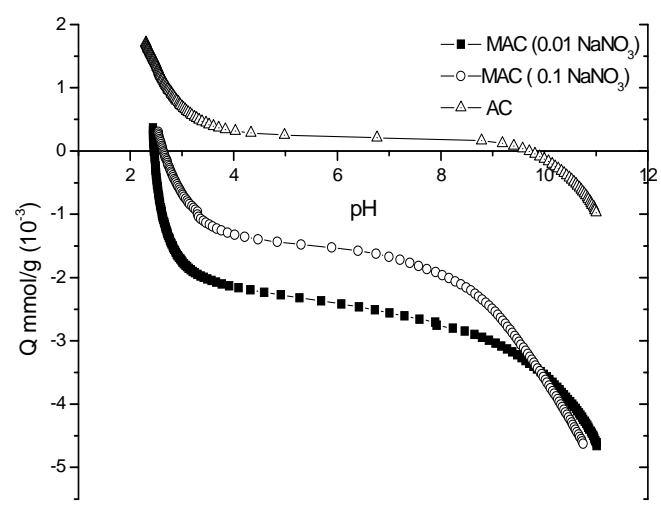

Figure. 11 Net binding proton curves of the AC and MAC

\section{$\underline{4.6 \mathrm{Hg}(\mathrm{II}) \text { Adsorption onto the Activated Carbon }}$}

All the adsorption experiments were carried out in batch systems. The amount of $\mathrm{Hg}(\mathrm{II})$ adsorption was determined by measuring the loss of aqueous $\mathrm{Hg}$ (II) in the suspension with ACs. $\mathrm{Hg}(\mathrm{II})$ concentration was analyzed by inductively coupled plasma (ICP) atomic emission spectrometry.

\subsubsection{Adsorption Isotherm}

To determine the adsorption capacity of $\mathrm{Hg}$ (II), fifteen (15) milligrams of the adsorbent were added to $30.0 \mathrm{ml} \mathrm{Hg}$ (II) solution, allowing the system to equilibrate for $24 \mathrm{~h}$ at the room temperature. The test was conducted at $\mathrm{pH} 2.0$ controlled by $\mathrm{HNO}_{3}$. Under this condition, 
dominant $\mathrm{Hg}$ (II) species is $\mathrm{Hg}^{2+}$. The initial $\mathrm{Hg}$ (II) concentrations ranged from 10 to $40 \mathrm{mg} / \mathrm{l}$, prepared from $\mathrm{Hg}\left(\mathrm{NO}_{3}\right)_{2} \cdot 2 \mathrm{H}_{2} \mathrm{O}$. Final concentration in the filtrate was determined by ICP. As shown by Fig.12, functionalized-ACs have higher adsorptive capacities than the untreated AC.

For isotherm studies, a series of $60 \mathrm{ml}$ glass bottles were used. Each test bottle was filled with a mercury solution with concentration varying from 20 to $80 \mathrm{mg} / \mathrm{l}$ and adjusted to the desired $\mathrm{pH}$ and temperature. A known amount of carbon was added into each test bottle. Mercury concentration retained in the adsorbent phase was calculated by using Eq. (2).

$Q_{e}=\frac{\left(C_{0}-C_{e}\right) V}{W}$

Where $C_{\mathrm{o}}$ and $C_{\mathrm{e}}$ are the initial and equilibrium concentrations of mercury ion in solution, $V$ is the volume and $W$ is the weight of the adsorbent. The study of adsorption isotherm is helpful to determine the adsorption capacity of adsorbate for the given adsorbent. Fig. 12 shows the adsorptive isotherms of mercury removal onto carbon adsorbents. It can be sure that the $Q_{\mathrm{e}}$ of the MAC for $\mathrm{Hg}^{2+}$ is much higher than the capacity of AC. This suggests that the surface modification approach used in this study have significantly increased the adsorption capacity of the activated carbon.

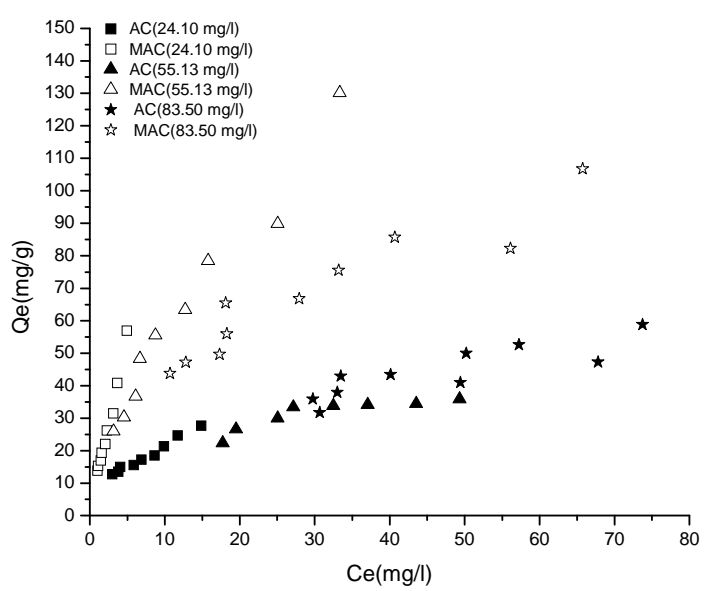

Figure 12. Adsorption isotherms of adsorbents; buffer $\left(\mathrm{KH}_{2} \mathrm{PO}_{4}-\mathrm{NaOH}=0.01 \mathrm{M}\right.$; final solution pH $6.0( \pm 0.2)$; temperature $=25^{\circ} \mathrm{C}$.

The Langmuir isotherm and the Freundlich isotherm model were applied to analyze the adsorption equilibrium of $\mathrm{Hg}^{2+}$ onto carbon. Langmuir and Freundlich linear forms are represented by Eqs. (3), (4):

$$
\begin{aligned}
& \frac{C_{e}}{Q_{e}}=\left(\frac{1}{Q^{0} b}\right)+\left(\frac{1}{Q^{0}}\right) C_{e} \\
& \log _{10} Q_{e}=\log _{10} K_{f}+1 / n \log _{10} C_{e}
\end{aligned}
$$


Where, Ce is the equilibrium concentration ( $\mathrm{mg} / \mathrm{l})$, Qe is the amount adsorbed ( $\mathrm{mg} / \mathrm{g}$ ), and $\mathrm{Q}^{\mathrm{o}}$ and $\mathrm{b}$ are Langmuir constants related to adsorption capacity and rate of adsorption, respectively. In the Freundlich equation, $K_{\mathrm{f}}$ and $n$ are constants incorporating all factors affecting the adsorption capacity and an indication of the favorability of $\mathrm{Hg}^{2+}$ adsorption onto the adsorbents, $1 / n$ and $b$ are related to enthalpy and intensity of adsorption. Solid lines in Fig. 13, Fig.14 represent the Langmuir and Frundlich isotherm linear models. Results of the modeling of the isotherms of $\mathrm{Hg}^{2+}$ adsorption by carbon adsorbents, according to Langmuir and Freundlich models, are summarized in Table 3.

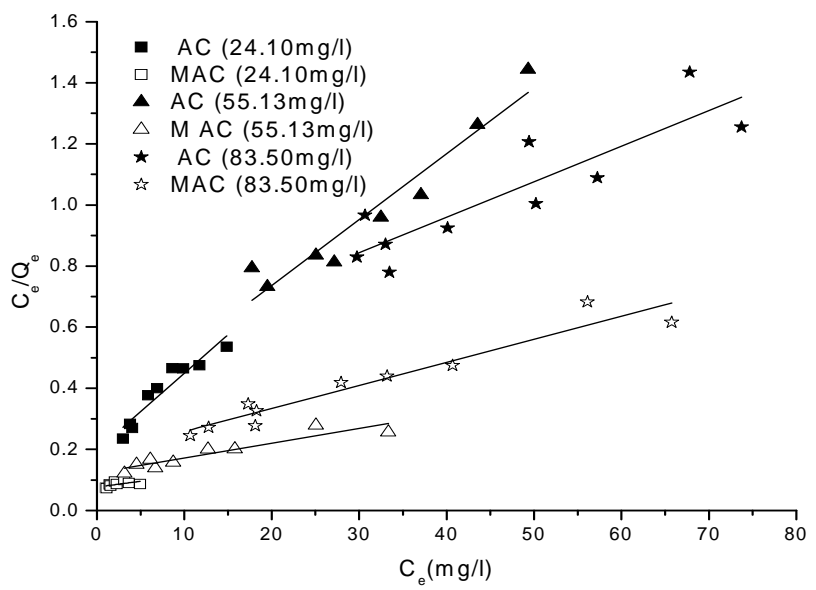

Figure 13. Langmuir linear model for adsorption isotherms Conditions: Buffer $\left(\mathrm{KH}_{2} \mathrm{PO}_{4}-\mathrm{NaOH}\right)=0.01 \mathrm{M}$; Final solution $\mathrm{pH} 6.0( \pm 0.2)$; temperature $=25^{\circ} \mathrm{C}$.

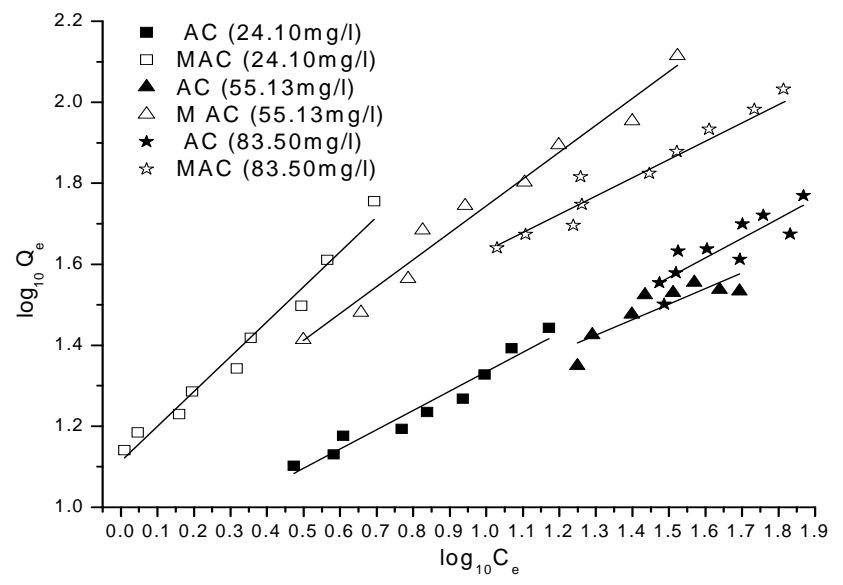

Figure14. Frundlich linear model for adsorption isotherms of adsorbents Conditions: Buffer $\left(\mathrm{KH}_{2} \mathrm{PO}_{4}-\mathrm{NaOH}\right)=0.01 \mathrm{M}$; Final solution $\mathrm{pH} \mathrm{6.0}( \pm 0.2)$; temperature $=25^{\circ} \mathrm{C}$.

Tab.3 Equilibrium models for adsorption $\mathrm{Hg}^{2+}$ ion on carbon materials

\begin{tabular}{|c|c|c|c|c|c|c|c|}
\hline Materials & Conc.(mg/l) & \multicolumn{3}{|c|}{ Langmuir parameters } & \multicolumn{3}{|c|}{ Freundlich parameters } \\
\hline & & $\mathrm{b}$ & $\mathrm{Q}_{0}$ & $r^{2}$ & $\mathrm{~K}_{\mathrm{f}}\left(\mathrm{mg}\left({ }^{1-1 / n}\right) 1^{1 / n} \mathrm{~g}^{-1}\right)$ & $\mathrm{n}$ & $r^{2}$ \\
\hline $\mathrm{AC}$ & 24.10 & 0.128 & 40.000 & 0.902 & 7.194 & 2.096 & 0.951 \\
\hline MAC & & 0.039 & 333.333 & 0.323 & 12.972 & 1.160 & 0.977 \\
\hline $\mathrm{AC}$ & 55.13 & 0.069 & 47.619 & 0.931 & 8.414 & 2.604 & 0.745 \\
\hline
\end{tabular}




\begin{tabular}{cccccccc}
\hline MAC & & 0.033 & 250.000 & 0.864 & 12.647 & 1.508 & 0.973 \\
AC & \multirow{2}{*}{83.50} & 0.031 & 77.909 & 0.816 & 6.934 & 2.070 & 0.752 \\
MAC & & 0.036 & 159.857 & 0.938 & 13.347 & 2.001 & $\underline{\underline{0.9790}}$ \\
\hline
\end{tabular}

\subsubsection{Effect of $\mathrm{pH}$ on $\mathrm{Hg}(\mathrm{II})$ Adsorption}

The influence of $\mathrm{pH}$ on $\mathrm{Hg}(\mathrm{II})$ adsorption onto $\mathrm{FACs}$ and $\mathrm{AC}$ was examined at $\mathrm{pHs}$ ranging from 1 to 12, with a $12 \mathrm{~h}$ equilibration time. As shown in Fig. 15 and Fig 16, for all four adsorbents examined, percent adsorption of $\mathrm{Hg}$ (II) increased with increasing $\mathrm{pH}$ from 1 to 5, reached maximum from $\mathrm{pH} 5$ to 8 , then decreased significantly with further $\mathrm{pH}$ increases. The optimal $\mathrm{pH}$ value for $\mathrm{Hg}$ (II) was around 6.0. The amounts of $\mathrm{Hg}(\mathrm{II})$ adsorbed were higher for FACs than for AC.

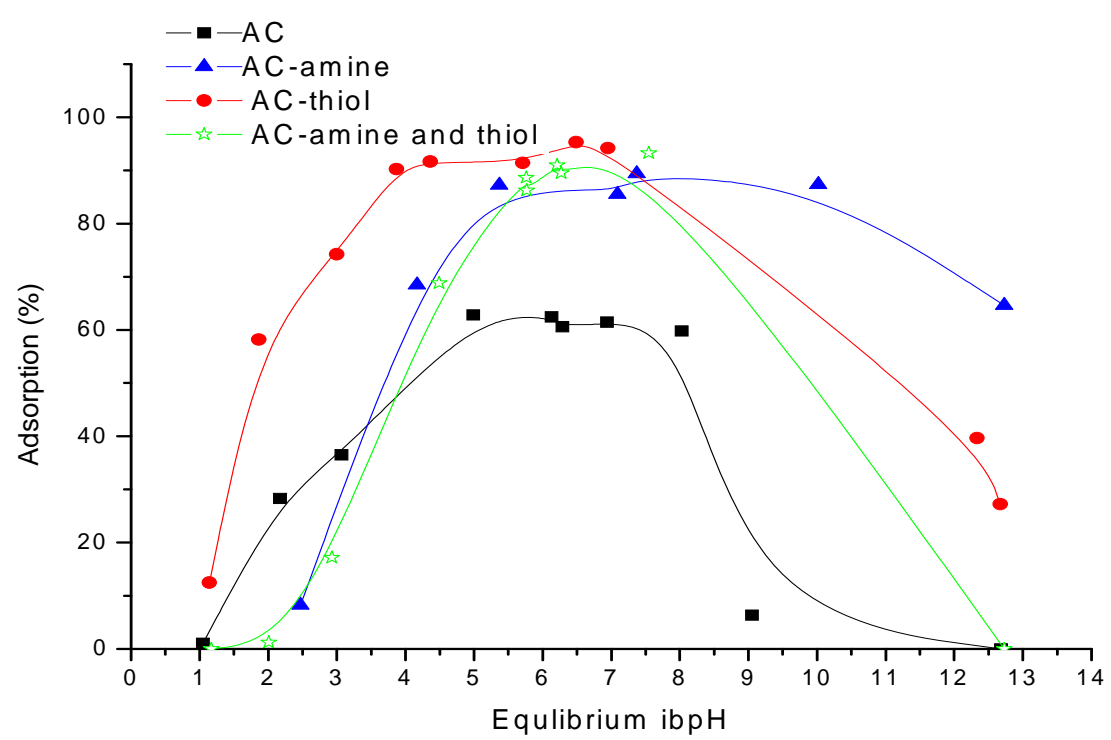

Figure 15. Effect of pH on $\mathrm{Hg}(\mathrm{II})$ adsorption

\subsubsection{Adsorption Kinetics of Hg(II)}

The adsorption kinetics of $\mathrm{Hg}(\mathrm{II})$ was examined near $\mathrm{pH} 3.0$. A $0.1000 \mathrm{~g}$ of $\mathrm{AC}$ or functionalized $\mathrm{AC}$ was dispersed in a $400 \mathrm{ml}$ of solution containing $6 \mathrm{mg} / \mathrm{l}$ of $\mathrm{Hg}$ (II). Sample aliquots were removed for ICP analysis at 0, 10, 20, 30, 40, 50, 60, 120, 240, 480 and 1200 min. Experimental results (Fig.16) show that functionalized AC has a faster adsorption kinetics and higher removal efficiency for $\mathrm{Hg}(\mathrm{II})$ than un-treated AC.

Batch tests were performed to determine kinetics of adsorption. Glass flasks (500 ml) with initial concentration of $20 \sim 50 \mathrm{mg} / \mathrm{l}$ were placed in a shaker. A known amount of carbon $(0.1000 \mathrm{~g})$ was added into the flask. At pre-decided time intervals, the solutions were taken and separated from the adsorbent and analyzed to determine the uptake of $\mathrm{Hg}^{2+}$. Results of effect of agitation time vs. $\mathrm{Hg}^{2+}$ removal rate in the solutions are presented in Fig.17-A, percentage removals of $\mathrm{Hg}^{2+}$ for all the carbons used increase with the increase in contact time at $\mathrm{pH}$ 6.0. Most Hg-ion adsorption took place in the first hour (Fig.17-B) followed by a slower process. 


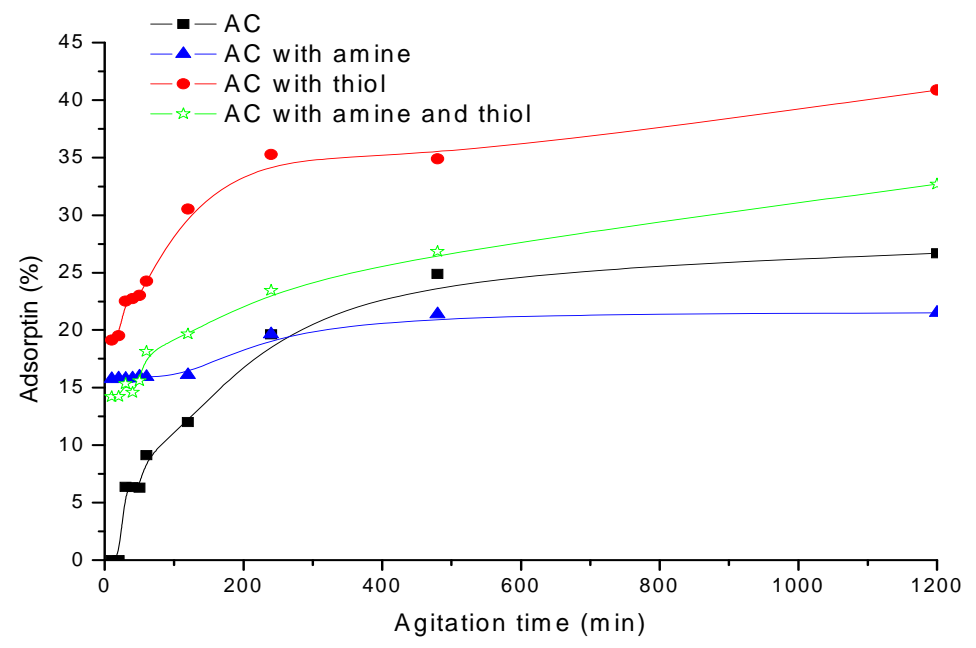

Figure 16. Plots of removal versus time for the adsorption of mercury (II)

Adsorption kinetic curves indicate that MAC adsorption is faster than $\mathrm{AC}$ adsorption, the MAC ligand-Hg-ion equilibrium was achieved during the first $30 \mathrm{~min}$. This behavior demonstrates that the ligand groups have a high affinity for the mercury ions. The data from different initial concentration reveal that the mercury adsorption capacity increased when the initial mercury concentration increases.
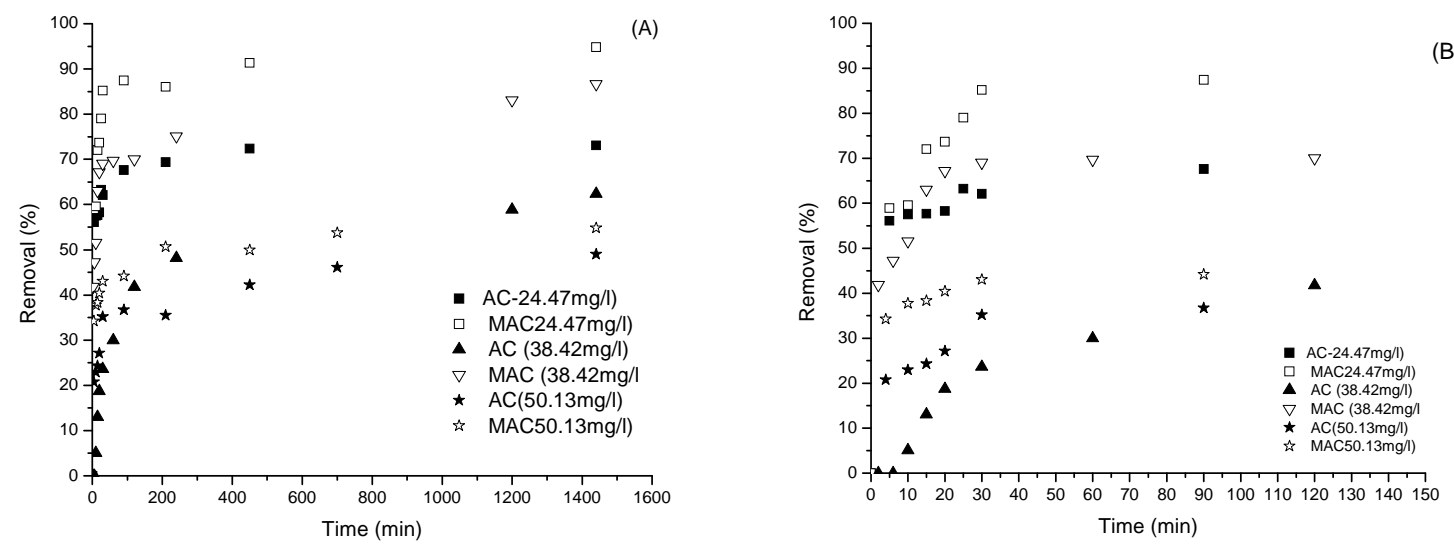

Figure17 (A), (B). Effect of agitation time on removal of $\mathrm{Hg}^{2+}$ from aqueous solution. Conditions: dosage of carbon $=100 \mathrm{mg}$ per $300 \mathrm{ml}$; buffer solution $\left(\mathrm{KH}_{2} \mathrm{PO}_{4}-\mathrm{NaOH}\right)=0.01 \mathrm{M}$; Final solution $\mathrm{pH} 6.0( \pm 0.2)$; temperature $=25^{\circ} \mathrm{C}$

\section{$\underline{4.7 \text { Mercury Sorption in Gas Phase }}$}

The preliminary study results in Figure 18 showed that sulfur-impregnated ordered mesoporous carbon (SOMC-1) could adsorb $\mathrm{Hg}$ under the experimental conditions used in this study with an adsorption capacity of about $200 \mu \mathrm{g} / \mathrm{g}$. On the other hand, sulfurimpregnated ordered mesoporous silica dioxide (SSBA-15) showed no ability to remove 
mercury from the simulated flue gas. The results demonstrated that the composition of the nano-carrier plays an important role in the functionalization processes, Although OMC and SBA-15 have the similar structure and they were sulfur-impregnated under identical conditions, the adsorption behaviors were totally different. The possible reason is that the silica dioxide mesoporous structure cannot be impregnated with sulfur at $700{ }^{\circ} \mathrm{C}$. Another study results in Figure 19 showed that sulfur-impregnated ordered mesoporous carbon (FOMC) could adsorb $\mathrm{Hg}$ under the experimental conditions with an adsorption capacity of $0.65 \mu \mathrm{g} / \mathrm{g}$. On the other hand, activated carbon (AC) showed an adsorption capacity of 0.35 $\mu \mathrm{g} / \mathrm{g}$. The preliminary finding verified our first working hypothesis: ordered mesoporous carbon is one of the most versatile nano-carriers that could be functionalized for contaminant removal.

\section{Comparison between SSBA-15 and SOMC-1}

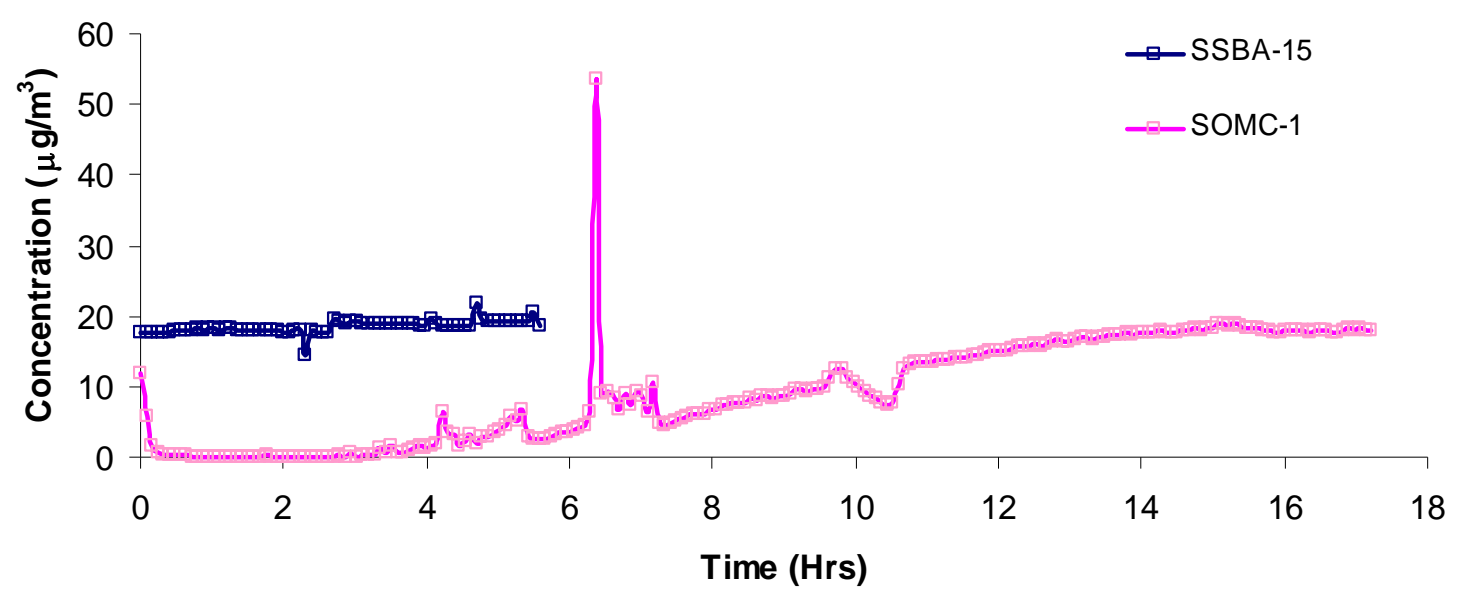

Figure 18. Mercury (Hg) uptake comparison between SSBA-15 and SOMC-1

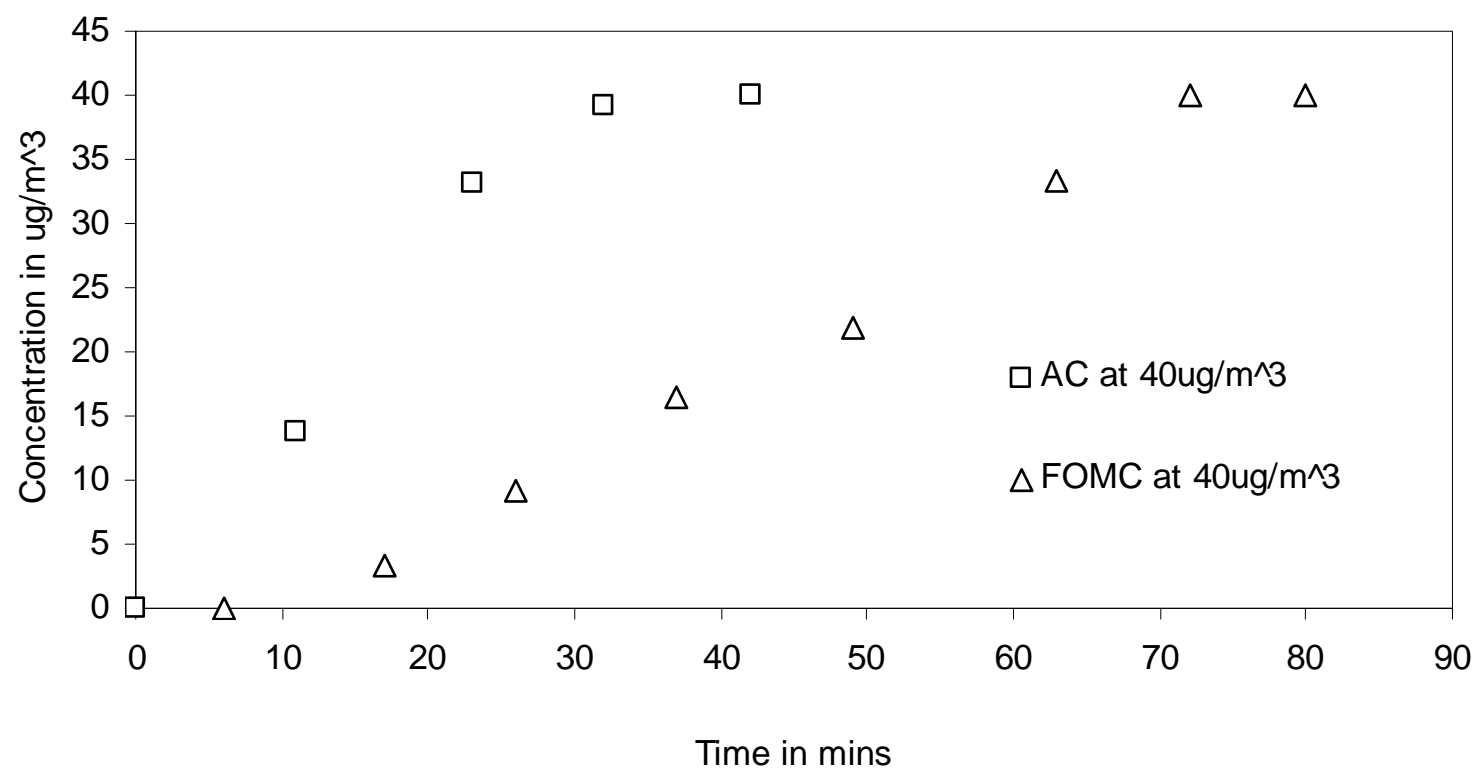

Figure 19. Mercury (Hg) uptake comparison between FOMC and AC 


\section{CONCLUSIONS}

Activated carbon (AC) was modified by a combined treatment of nitric acid and thionyl chloride to introduce amine, amide, thiol, sulfurous, chlorine, and carboxylic functional groups. Surface chemistry of the modified activated carbon (MAC) was characterized by SEM-EDS, FT-IR, potentiometry and XPS. Elements in samples were analyzed with an elemental analyzer. Mercury sorption kinetics and equilibrium isotherm by MAC were determined in batch experimental systems. Surface characterization indicated that additions of the organic-inorganic ligands to AC by the treatment were effective, likely through the acyl chloride-carbon surface reactions. Potentiometric titration showed the modifications introduced more negative surface charges favoring cation sorption. Kinetics studies demonstrated that the $\mathrm{Hg}$ (II) sorption by MAC was faster ( $<30 \mathrm{~min})$ and higher $(>200 \%)$ than by AC, suggesting a high affinity of the modified material for $\mathrm{Hg}$ (II) ions. The sorption occurred in a wider $\mathrm{pH}$ range (4-10 vs. 5-7), and low ionic strength appeared to enhance the $\mathrm{Hg}$ (II) sorption. Sample analysis by X-ray photoelectron spectroscopy showed that the sorption of the MAC toward $\mathrm{Hg}$ (II) was accomplished mainly by the chemical coordination of the ligand atoms $(\mathrm{O}, \mathrm{N}$ and $\mathrm{S})$ of the functional groups with the $\mathrm{Hg}$ ions. The sorption isotherms could be best fitted by the Freundlich model and accounted for by mechanisms of surface complexation, reduction, and ion exchange. The preliminary mercury uptake study results in gas phase showed that sulfur-impregnated ordered mesoporous carbon (SOMC-1) could adsorb $\mathrm{Hg}$ under the experimental conditions used in this study with an adsorption capacity of about $200 \mu \mathrm{g} / \mathrm{g}$.

\section{REFERENCES}

Baeyens, R., Ebinghous, R., Vasilev, O. (Eds.), 1996. Global and Regional Mercury Cycles: Sources, Fluxes and Mass Balances. Kluwer Academic Publishers.

Jarrais, Bruno; Silva, Ana Rosa; Freire, Cristina. Anchoring of vanadyl acetylacetonate onto amine-functionalized activated carbons: Catalytic activity in the epoxidation of an allylic alcohol. European Journal of Inorganic Chemistry (2005), (22), 4582-4589.

Kaakinen, J. W.; Jorden, R. M.; Lawasani, M. H.; West, R. E. Environ. Sci. Technol. 1975, 9,862 .

Kim, C. H.; Kim, S.-S.; Guo, F.; Hogan, T. P.; Pinnavaia, T. Communication Polymer Intercalation in Mesostructured Carbon. J. Adv. Mater. 2004, 16, 736-739

Lindberg, S. E. Atmos. Environ. 1980, 14, 227.

Nam, K.H., Gomez-Salazar, S., Tavlarides, L.L. Mercury (II) adsorption from wastewater using a thiol functional adsorbent. Ind. Eng. Chem. Res. 2003. 42, 1955-1964

Namasivayam C, Periasamy K. Bicarbonate treated peanut hull carbon for mercury (II) removal from aqueous solution. Wat. Res 1993;27:1663-8.

Namasivayam, C.; Periasamy, K. Bicarbonate-treated peanut hull carbon for mercury (II) removal from aqueous solution. Water Res. 1994, 27, 1663.

Navasivayam, C. Biosorbents for metal ions. Edited by J. Wase and C. Forster. Bioresource Technology (1998), 64(2), 161. 
Patterson JW. Wastewater treatment technology. Ann Arbor, MI: Ann Arbor Science, 1975.

Puziy, Alexander M.; Poddubnaya etc. Elucidation of the ion binding mechanism in heterogeneous carbon-composite adsorbents. Carbon (2001), 39(15), 2313-2324.

Shameem Hasan, Abburi Krishnaiah and Dabir S. Viswanath. Adsorption of Divalent Cadmium (Cd(II)) from Aqueous Solutions onto Chitosan-Coated Perlite Beads Ind. Eng. Chem. Res. 2006, 45, 5066-5077

Silva, A. R.; Martins, M.; Freitas, M. M. A.; Valente, A.; Freire, C.; de Castro, B.; Figueiredo, J. L. (2006) Immobilization of amine-functionalized nickel(II) Schiff base complexes onto activated carbon treated with thionyl chloride. Microporous and Mesoporous Materials (2002), 55(3), 275-284.

Takahashi et al., 2001 In: Y. Takahashi, K. Watanuki, S. Kubota, O. Wada, Y. Arikawa, S. Monma and T. Hirano, Editors, An Encyclopedia of Water, Maruzen, Tokyo (2001) p. 798.

Vidic, Radisav D.; McLaughlin, J. Brendan. Journal of the Air \& Waste Management Association (1996), 46(3), 241-50.

William F. Fitzgerald; Thomas W. Clarkson Mercury and Monomethylmercury: Present and Future Concerns. Environmental Health Perspectives, Vol. 96. (Dec., 1991), pp. 159-166

Yantasee, Wassana; Lin, Yuehe; Fryxell, Glen E.; Alford, Kentin L.; Busche, Brad J.; Johnson, Christian D. Selective Removal of Copper(II) from Aqueous Solutions Using Fine-Grained Activated Carbon Functionalized with Amine. Industrial \& Engineering Chemistry Research (2004), 43(11), 2759-2764.

Zhimang Gu. Development and Evaluation of Innovative Iron-Containing Porous Carbon Adsorbents for Arsenic Removal. Ph.D Dissertation. University of Missouri-Columbia, DECEMBER, 2006 
Appendix 45: Removal of Metal Ions from Acid Mine Drainage using a Novel Low-Cost, Low Technology (WV017) 


\title{
FINAL REPORT
}

\begin{tabular}{ll|}
\hline Contract Title and Number: & Period of Performance: \\
\cline { 2 - 2 } Crosscutting Technology Development at the Center for & Starting Date: 01/01/2006 \\
Advanced Separation Technologies & Ending Date: \\
(DE-FC26-02NT41607) & $08 / 31 / 09$ \\
\hline
\end{tabular}

\begin{tabular}{|c|c|}
\hline Sub-Recipient Project Title: & Report Information: \\
\hline Removal of metal ions from Acid Mine & Type: Final \\
\hline Drainage using a Novel Low-Cost, Low & Number: \\
\hline Technology & Period: \\
\hline Principal Investigators: & Date: 09/02/09 \\
\hline Benjamin Dawson-Andoh & Code:WV017-Final \\
\hline Contact Address: & Contact Information: \\
\hline 206D Percival Hall & Phone:304-293-8750 \\
\hline Division of Forestry and Natural Resources & Fax:304-293-2441 \\
\hline West Virginia University & E- \\
\hline Morgantown, WV. 26506-6125 & Mail:bdawsona@wvu.edu \\
\hline Subcontractor Address: & $\underline{\text { Subcontractor Information: }}$ \\
\hline Insert address of subcontractor. If none & Phone: \\
\hline awarded, insert "No subcontracts issued." & Fax: \\
\hline & E-Mail: \\
\hline
\end{tabular}

\begin{abstract}
This study evaluated the potential of a low-cost, low-technology to remediate Acid Mine Drainage (AMD) through the reduction of its iron content and acidity. The bark, wood and fungal inoculated wood of three wood species, northern red oak (Quercus rubra), soft maple (Acer saccharinum) and yellow-poplar (Liriodendron tulipifera L.) were evaluated. AMD pH was increased by the bark, wood and fungal inoculated wood of all three wood species. The highest increase in AMD $\mathrm{pH}$ was recorded for yellow-poplar bark where a $\mathrm{pH}$ increase from 2.72 to 4.2 (56 \% increase) was observed. The bark, wood and fungal inoculated wood of all three wood species reduced the AMD iron content. The highest reduction in iron content was exhibited by fungal inoculated northern red oak wood.
\end{abstract}




\section{INTRODUCTION}

\section{1. $\quad$ Background}

The overburden of coal mining operations, contain iron disulfide which on coming into contact with water, is oxidized to produce sulfuric acid and ferric hydroxide ("yellowboy"). The resulting water leachate, Acid Mine Drainage (AMD), is replete with iron minerals, iron pyrites and other metals such as cadmium, copper, zinc and selenium. $\mathrm{pH}$ of the water is subsequently low further enhancing leaching of more metal ions from the coal refuse or overburden. Acid Mine Drainage requires treatment to remove metal ions and increase $\mathrm{pH}$ before discharge into local rivers, streams or public water systems.

In West Virginia, AMD discharges from coal mines are regulated by the West Virginia Department of Environmental Protection through the issue of permits. In addition to permitted coal mines, AMD are also discharged from abandoned old mines. The U.S. Federal Protection Agency (EPA) use levels of only iron and manganese to monitor the efficacy of various remedial treatments. Other important parameters include $\mathrm{pH}$ and turbidity. In this study, only iron concentrations and $\mathrm{pH}$ of AMD were monitored in the treatments evaluated in this study.

All coal mining operations in West Virginia negatively impact the environment notably rivers and streams because of the presence of metallic ions in the discharges of AMD and its associated low pH. Today, polluted discharges from mining operations are treated by collection in earthen sediments where sediments and pollutants are allowed to settle to the bottom.

Current technologies to remove metal ions from AMD include activated carbon, ion exchange resins or zeolites, reverse osmosis, distillation/filtration/ultrafiltration, etc. Such technologies are relatively expensive making mine operators reluctant to properly implement them. The research work proposed here represents a novel, low-cost, low-technology which has great potential for removing metal ions from AMD. We propose to use two types of biomaterials: lignocellulosic biomass and lignocellulosic biomass modified by fungal biomass, to remove metal ions from AMD discharged from coal mines. Modification of lignocellulosic biomass is effected by inoculation with two microfungi. 
Biomaterials such as lignocellulosic biomass are potential materials for the effective removal of heavy metal ions especially from dilute solutions. This attribute of biomass is attributed to the presence of chemical moieties which act as adsorbent sites. Adsorption of metal ions from solution can occur by either passive or active. Sorption of metal ions from dilute solutions occurs by passive process (Davis et al. 2008). They are cheaper than existing traditional technologies and more efficient in the case of dilute solutions of metal ions. .

Recent studies have demonstrated the capability of lignocellulosic biomass to remove metal ions from solution and its potential for the remediation of industrial discharges containing metal ions (Minn 2000, USDA Forest Products Lab. 2004, Basso et al. 2002). Likewise, fungal biomass has also been shown to remove metal ions from solution by both active (living fungi) and passive (non-living) processes respectively. Both processes have been proposed for the detoxification of industrial waster effluents (Volesky 1990b, Rae et al. 2003). Passive biosorption of metal ions by wood or microbial biomass occurs by many mechanisms including complexation, coordination, chelation, ion exchange, and adsorption (Antsuki et al. 2003). This process is modulated by chemical functional groups such as carboxyl, hydroxyl, sulfhydryl, and phosphoryl groups present in the wood or fungal biomass. Additionally, counter ions in polysaccharides in wood biomass also undergo ion exchange with bivalent metal ions.

Filters made from lignocellulosic materials (wood) removed metal ions solution and also improved the $\mathrm{pH}$ (Basso et al. 2002). Carboxylic acid and other functional moieties such phenolic $\mathrm{OH}$, carbonyl in lignocellulosic materials, can chelate polluting metals from solution. This chelating capacity of lignocellulosic materials can be further enhanced by chemical or biological treatment. Studies at the Forest Products Laboratory in Madison, WI, have demonstrated that metal polluting cleaning capacities of Douglas-fir (Pseudotsuga menziesii) fibers can be greatly increased by treatment with nitric acid (USDA Forest Products Lab. 2004). Treatment of lignocellulosic materials with acids is an oxidative process that produces functional groups such as carboxylic acid and carbonyl functional groups. These groups act as chelants. Anions such as phosphorus and possible sulfates may also be removed using biomass (Shin et al. 2004). Lignocellulose mat-fiber made from Juniper (Juniperus monoperma) removed metals and non-metals from AMD in the Wayne National Forest in Ohio (Shin et al. 2004). 


\subsection{Objective and Approach}

The objectives of this study were to evaluate the efficacy of two types of waste streams to:

a. Reduce the iron content of AMD and

b. Reduce the $\mathrm{pH}$ of AMD

Three types of materials were evaluated:

a. Ground bark of northern red oak (Quercus rubra), soft maple (Acer saccharinum) and yellow-poplar (Liriodendron tulipifera L.),

b. Ground wood of northern red oak, soft maple and yellow-poplar, and

c. Ground wood of northern red oak, soft maple and yellow-poplar inoculated with the microfungus, Aspergilus niger

Null Hypotheses:

a. Mean initial iron content of AMD = Final iron content of AMD after treatment ground bark of three types of lignocellulosic biomass,

b. Mean initial iron content of AMD = Final iron content of AMD after treatment three types of ground wood biomass and

c. Mean initial $\mathrm{pH}$ of $\mathrm{AMD}=$ Final $\mathrm{pH}$ of $\mathrm{AMD}$ after treatment with ground wood inoculated with the microfungus, Aspergilus niger

\subsection{Brief Overview of Scientific and/technical approach}

The lignocellulosic biomass (bark wood or wood inoculated with A. niger) of each wood species was air-dried and ground in a Wiley Mill to pass through 100 mesh sieve. Ground lignocellulosic biomass or biomass inoculated with A. niger was oven-dried at $103 \pm 2{ }^{0} \mathrm{C}$. Five (5) g of biomass or biomass inoculated with A. niger was weighed into a $250 \mathrm{~mL}$ Erlenmeyer flask and $50 \mathrm{~mL}$ of AMD was added using a pipette (Figure 7). All Erlenmeyer flasks were shaken on an orbital shaker for different time periods (0, 2,4,6,8 hours; 0, 30, 60, 90, 120 minutes; 0, 15, 30, 45, 60 minutes). These represented different treatments. At the end of each treatment period, the contents of Erlenmeyer flasks 
were filtered through a 0.42 micron membrane filter. All filtrates, controls and initial AMD solution were analyzed for iron content using induced coupled plasma- atomic emission spectroscopy (ICP-AES). pH of initial AMD, controls and filtrates were measured using a $\mathrm{pH}$ meter (Oakton P110, Singapore). Reduction of iron concentration and $\mathrm{pH}$ were determined

All sample flasks and containers used in this study were soaked for 24 hours in acid liquid detergent (Citrad, Decon Labs, King of Prussia, PA) and then rinsed three times with deionized water prior to use in study.

\subsection{PROJECT TASKS}

The tasks reported here is an overall view of all studies carried out in this project. The project was divided into two tasks. In task 1, ground bark or wood lignocellulosic biomass was evaluated for its ability to reduce iron content of AMD and increase its $\mathrm{pH}$. In task 2, ground wood was modified by inoculation with fungal (A. niger) mycelia under solid state fermentation conditions to produce a material which contains both lignocellulosic and fungal biomass.

\subsection{Experimental design}

This experiment was a completely random design with time of exposure to AMD as treatment. Different time scales were used as treatment. There were five replicates for each treatment.

\subsection{Materials for Tasks 1 and 2:}

a. Acid Mine Drainage

b. Ground (to pass 35 mesh) air-dried soft maple, northern red oak and yellowpoplar bark or wood

c. Ground wood of soft maple, northern red oak and yellow-poplar inoculated with A. niger 
The AMD was collected from Scotts Run in the Monongahela County of West Virginia and stored in a Carboy at $5{ }^{0} \mathrm{C}$ until used.

\subsection{Task 1:}

\subsubsection{Method:}

Air-dried lignocellulosic material was ground in a Wiley-Mill to pass 35 mesh and retained by 16 mesh screen. Milled bark was oven-dried at $103 \pm 2{ }^{0} \mathrm{C}$ and kept in desiccators until use.

Five grams of oven-dried Wiley-milled bark of each wood species were weighed into a 250 ml Erlenmeyer flask using an analytical balance. Fifty mL of AMD was pipetted into each Erlenmeyer flask. All Erlenmeyer flasks used in this studies were first washed in an acid-bath detergent (Citrad, Decon Labs, King of Prussia, PA) followed by rinsing three times in deionized water.

All Erlenmeyer flasks were shaken on a orbital shaker operating at $50 \mathrm{rpm}$ at room temperature $\left(24{ }^{0} \mathrm{C}\right)$. Two sets of experiments with corresponding time exposure treatments of (1): 0, 2, 4, 6, and 8 hours and (2): 0, 30, 60, and 90 minutes were used. At the end of each time exposure to AMD, all replicates Erlenmeyer flasks were removed from the rotary shaker and their contents harvested by first filtering through a filter paper and then through a 0.45 $\mu \mathrm{m}$ membrane filter. All filtrates including the controls were analyzed by plasma-inducedatomic emission spectroscopy (ICP-AES). Concentrations of iron metal ions in control and treatment filtrates were reported in $\mathrm{mg} / \mathrm{ml}$. The $\mathrm{pH}$ of each filtrate was also measured using a $\mathrm{pH}$ meter. The $\mathrm{pH}$ meter was standardized before measurements.

\subsection{Task 2:}

\subsubsection{Method:}

\subsubsection{Solid state inoculation of ground wood}

Shallow rectangular aluminum pans [102 X 305 mm (4 X 12 inches) were filled with ground wood of each of the three wood species. Each aluminum pan was wrapped with aluminum foil and autoclaved at $123{ }^{0} \mathrm{C}$ for 45 minutes to sterilize the wood. At the end of the autoclave, pans were allowed to cool under a laminar flow hood. Sterilized wood in each aluminum pan was inoculated with inoculi of A. niger. To prepare the inoculum of $A$. niger 
(Figure 9), $50 \mathrm{~mL}$ sterilized liquid media (0.5\% weight by volume of malt) was inoculated with three $5 \mathrm{~mm}$ discs taken from a malt-agar (1.5\% agar, 2\% malt) A. niger grown media. Liquid cultures were incubated at $25{ }^{0} \mathrm{C}$ for two weeks; filtered through a sterile filter paper; rinsed once with $10 \mathrm{~mL}$ of sterile deionized water and blended in a sterile stainless steel blender containing $250 \mathrm{~mL}$ sterile deionized water under a laminar flow hood.

$10 \mathrm{~mL}$ of blended mycelia of $A$. niger was added to each sterile ground wood in aluminum pan under aseptic conditions. Inoculated ground wood was wrapped with aluminum foil and inoculated at $25{ }^{0} \mathrm{C}$ for 6 weeks. Growth of fungi in wood was confirmed microscopically (Figure 8). At the end of this period, the inoculate ground wood was oven-dried at $103 \pm 2{ }^{0} \mathrm{C}$ for 48 hours and kept in desiccators until use.

\subsection{Results and discussion}

The effect of Wiley-milled bark of the three wood species on the $\mathrm{pH}$ and reduction of iron in AMD is shown in Figures 1 and 2. The AMD used in this study was stored at $3{ }^{0} \mathrm{C}$ and $\mathrm{pH}$ was measured for each study using a pH meter (Oakton P110, Singapore). pH meter was calibrated using $\mathrm{pH}$ solutions before each measurement. Overall, initial $\mathrm{pH}$ of AMD varied between 2.50 to 2.72 .

\section{Effect of bark of three wood species on $\mathrm{pH} A M D$}

The bark of all three wood species increased the $\mathrm{pH}$ of AMD from 2.50-2.72 to 3.48-4.20, an increase of approximately 40-68\%. Increase in $\mathrm{pH}$ of AMD occurred rapidly in the first time window, 2 hours (Figures 1). For the three wood species, the highest and lowest $\mathrm{pH}$ increases for the AMD were produced by the yellow-poplar and soft maple barks respectively.

\section{Effect of bark of three wood species on iron contents of AMD}

The iron content of AMD solution was reduced by the barks of all three wood species. The efficiency of iron removal as defined by the percentage of iron removed (amount of iron removed/initial iron content of AMD X 100) varied 72, 65 and $63 \%$ for the barks of northern red oak, soft maple and yellow-poplar respectively (Fig. 2.). Northern red oak bark was the most efficient and yellow-poplar bark was the least efficient in the reduction of iron in AMD solution. Like the observed effect on $\mathrm{pH}$, rapid reduction in iron content of AMD solution 
occurred in the first two hour window. Overall, northern red oak (72 \%) was most efficient in the reduction of iron content and yellow-poplar (63\%) was the least efficient in reducing the iron content of the AMD solution. Maximum reduction in AMD iron content was 83 \% (6 hours) and 76 \% (8 hours) for northern red oak and soft maple respectively.

\section{Effect of wood of three wood species on $\mathrm{pH}$ of $A M D$}

The wood of all three wood species like the bark also increased the pH of AMD from approximately 2.80 to 3.45 for northern red oak and soft maple and to 4.00 for yellow-poplar (Figure 3). Although a different time scale was used, the observed increase in $\mathrm{pH}$ occurred in the first time window, 30 minutes. The behavior of the wood of the three wood species was similar to their bark.

\section{Effect of bark of three wood species on iron contents of AMD}

The iron content of AMD was also reduced by the wood of all three wood species. Iron reduction occurred primarily in the first 30 minute window (Figure 4). For northern red oak and soft maple, further reduction in iron concentration occurred in the 60 and 90 minute time windows and appeared to have attained equilibrium by the 60 minute window. For yellowpoplar, reduction in iron content continued to decrease up to the 90 minute window.

\section{Effect of fungal inoculated wood of three wood species on $\mathrm{pH}$ of AMD}

Fungal inoculated wood of northern red oak and soft maple increased $\mathrm{pH}$ of AMD from approximately 2.50 to 3.2 and 3.2 respectively in the first 30 minute window Figure 5). The amount of increase in $\mathrm{pH}$ was less than exhibited by non-inoculated bark or wood of the same wood species. While the $\mathrm{pH}$ for soft maple attained equilibrium within 30 minutes, $\mathrm{pH}$ for northern red oak increased slightly to the end of experiment, 120 minutes. However, this slight increase in $\mathrm{pH}$ might not be statistically significantly different from the first from the initial increase in pH (30 minutes). Fungal inoculated wood of yellow-poplar wood exhibited the highest increase in $\mathrm{pH}$ of AMD (from 2.50 to 3.50). 
Effect of fungal inoculated wood of three wood species on iron content of AMD

Iron content of AMD was reduced by fungal inoculated wood of all three wood species (Figure 6). The greatest reduction (85\%) was exhibited by fungal inoculated northern red oak. For soft maple and yellow-poplar, reductions in iron contents of yellow-poplar were 74 and $43 \%$ respectively. Thus, for fungal inoculated wood, yellow-poplar was the least efficient in the reduction of iron content of AMD. Inoculation of northern red oak and soft maple enhanced the iron reduction capability for these two wood species while it reduced that for yellow-poplar.

\subsection{SUMMARY}

In conclusion, AMD pH was increased by the bark, wood and fungal inoculated wood of all three wood species. The highest increase in AMD pH was recorded for yellow-poplar bark where a pH increase from 2.72 to 4.2 (56 \% increase) was observed. The bark, wood and fungal inoculated wood of all three wood species reduced the AMD iron content. The highest reduction in iron content was exhibited by fungal inoculated northern red oak wood.

Overall, the bark, wood and fungal inoculated wood of all three wood species have the potential to be used for the remediation of AMD. Wood residues are abundant in West Virginia and future studies should further explore the implementation of the results of this study. AMD from existing and defunct coal mining operations represent major threat to the environment in West Virginia.

\subsection{FUTURE WORK}

Future studies should also evaluate the sorption kinetics of iron in AMD by the substrates used in this study. Further, modification of wood using other commonly occurring microfungi should also be investigated. Our studies indicated that fungi grew poorly on wood bark. Finally, pilot scale implementation of the results of this study should be evaluated. 


\subsection{REFERENCES}

Antsuki, T., D. Sano and T. Onumra. 2003. Functional metal-binding proteins by metalstimulated bacteria for the development of an innovative metal removal technology. Water Science and Technology. 47 (10): 109-115.

Basso, M. C., E. G. Cerrella,and A. L. Cukierman. 2002. Lignocellulosic materials as potential biosorbents of trace toxic metals from waste water. Industrial Engineering and Chemical Research. 41: 3580-3585.

Davis A., A. Bhoval and S. Datta. 2008. Continuous biosorption in rotation packed-bed contactor. Ind. Eng. Chem. Res. 47:4230-4235

Min, S. H. 2000. Mechanisms of cadmium removal by base-treated juniper fiber, M.S.

Thesis. Dept. of Civil and Environmental Engineering, University of Wisconsin, Madison.

Rae, I. B. and S. W. Gibb. 2003. Removal of metals from aqueous solutions using natural chitinous materials. Water Science and Technology. 47 (10): 189-196.

Using wood and Agricultural fibers to clean water. Forest Products Laboratory. USDA Forest and Service, Madison, WI. http://www.fpl.fs.us/init_biobased.htm

Shin, E. W., J. S. Han, and S. H. Min. 2004. Removal of phosphorus from water using lignocellulosic material modified with iron species from acid mine drainage. Environmental Technology.

Volesky. 1990b. Bisorption by fungal biomass. In "Bisorption of Heavy Metals". Ed: CRC Press, Boca Raton. 139-172.

\subsection{PUBLICATIONS/PRESENTATIONS}

1. Dawson-Andoh, B. 2009. Remediation of Acid Mine Drainage using lignocellulosic biomass. Forest Products Journal. In Preparation.

2. Dawson-Andoh, B and J. Slahor. 2007. Removal of metal ions from Acid Mine Drainage using lignocellulosic biomass. Virginia Tech., Blacksburg, July 2007. Presentation

\subsection{APPENDICES (IF ABSOLUTELY NECESSARY)}

None 
AMD pH Over Time

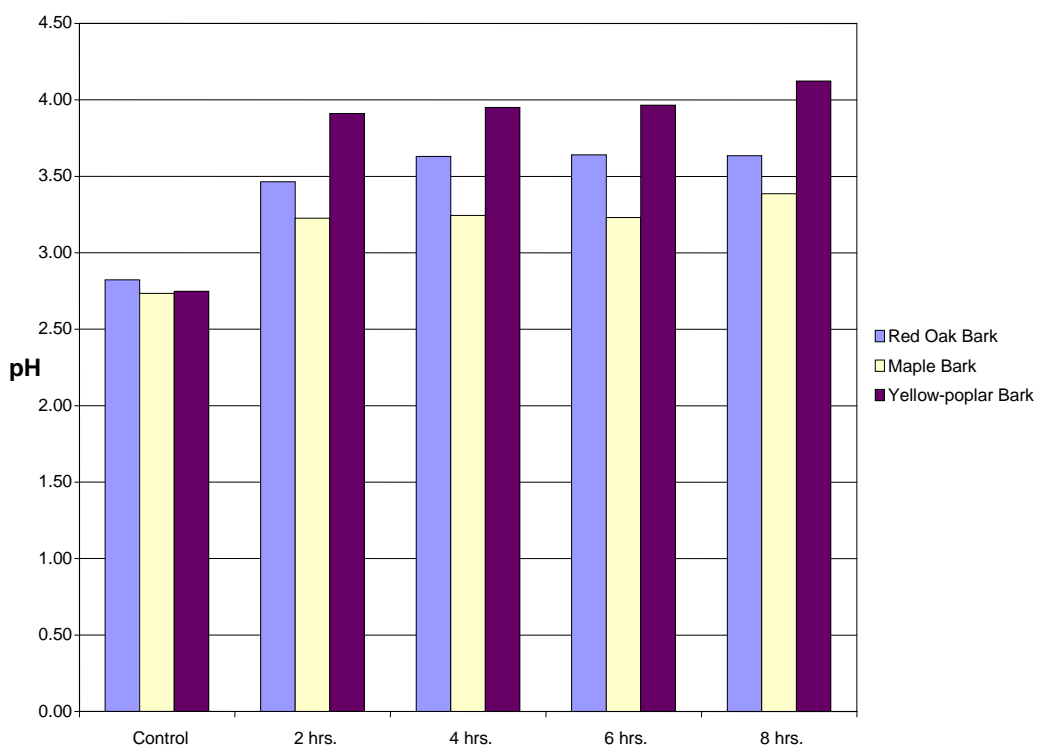

Figure 1.Effect of wood bark on AMD pH 


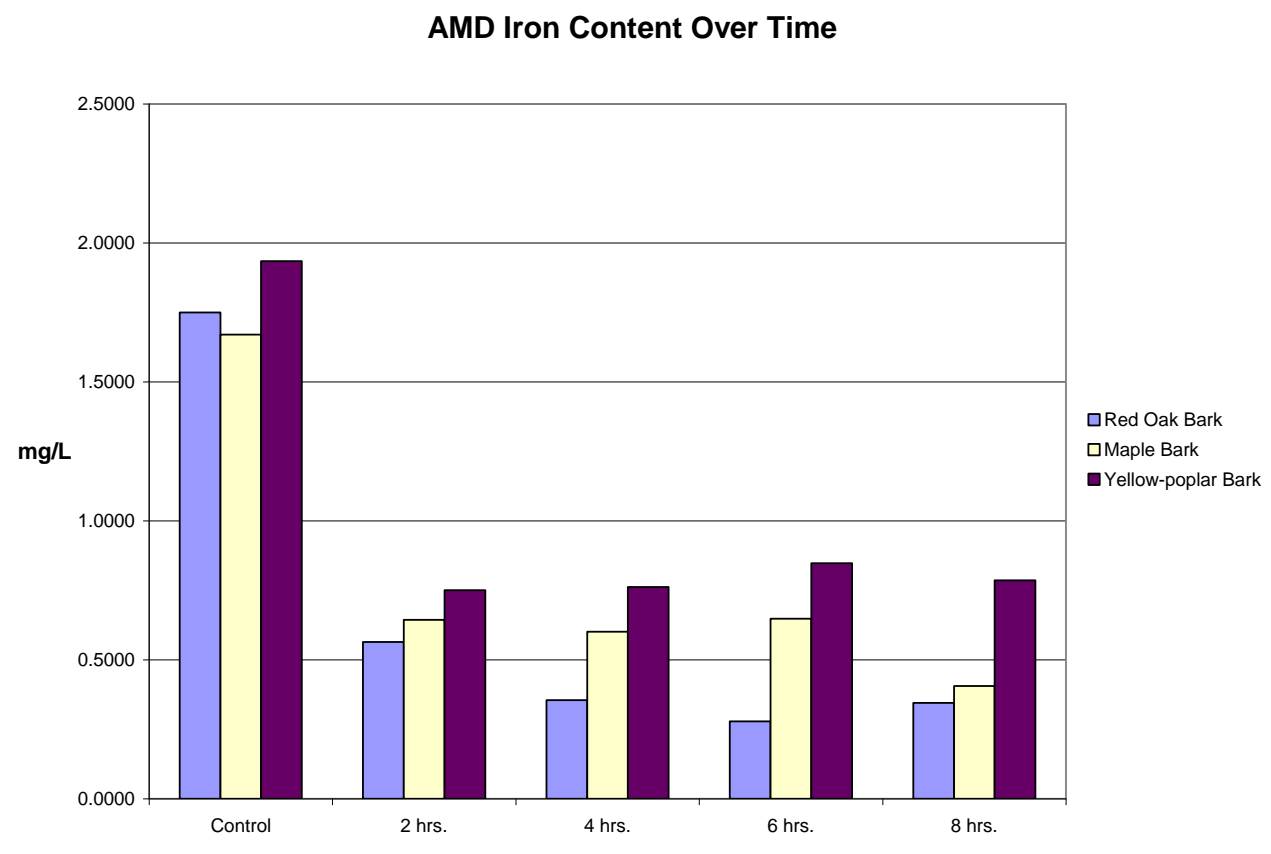

Figure 2. Effect of wood bark on iron content of AMD 
$\mathrm{pH}$ of AMD and Wood Over Time

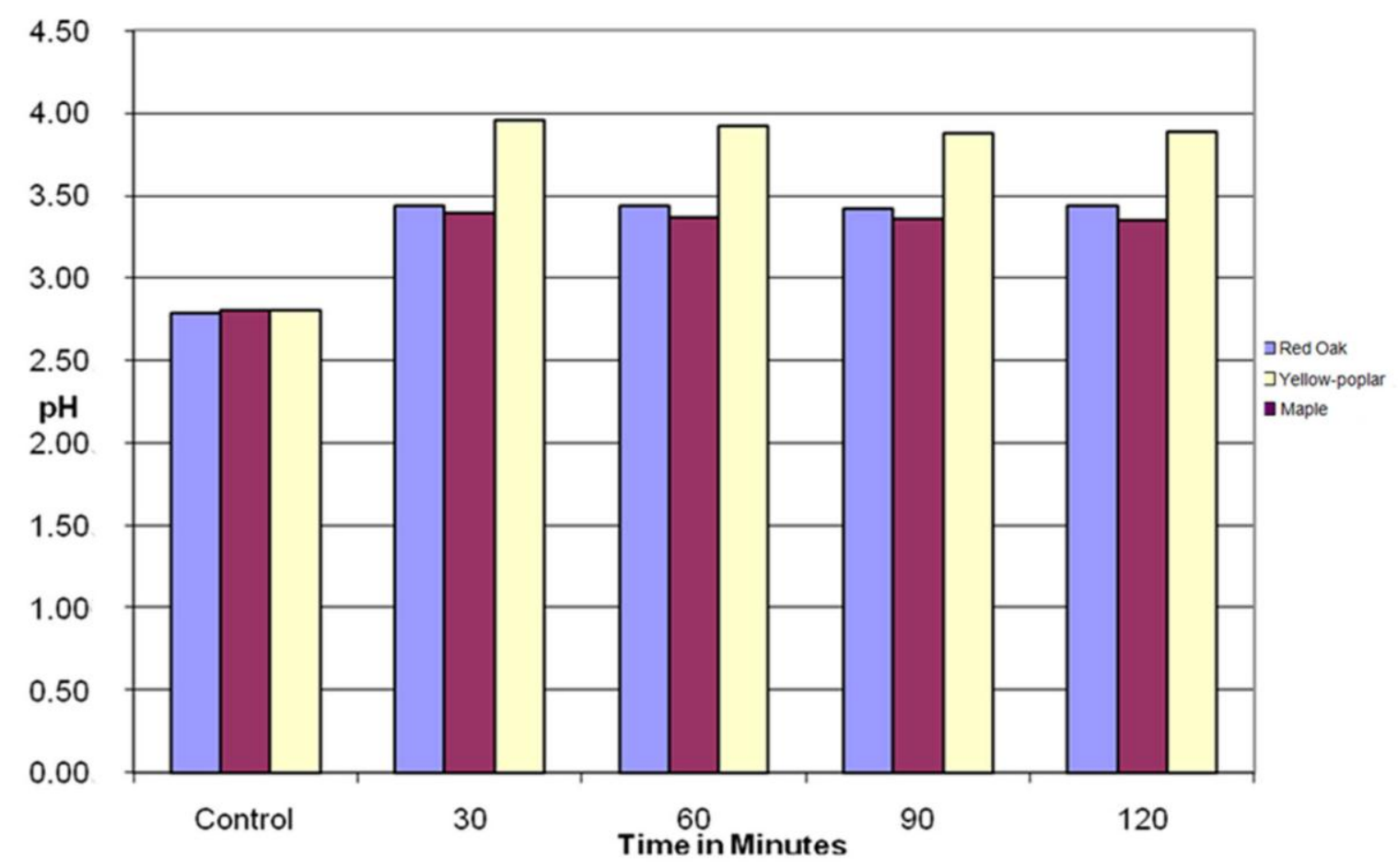

Figure 3. Effect of wood on AMD pH 


\section{Iron Concentration of AMD and Wood Over Time}

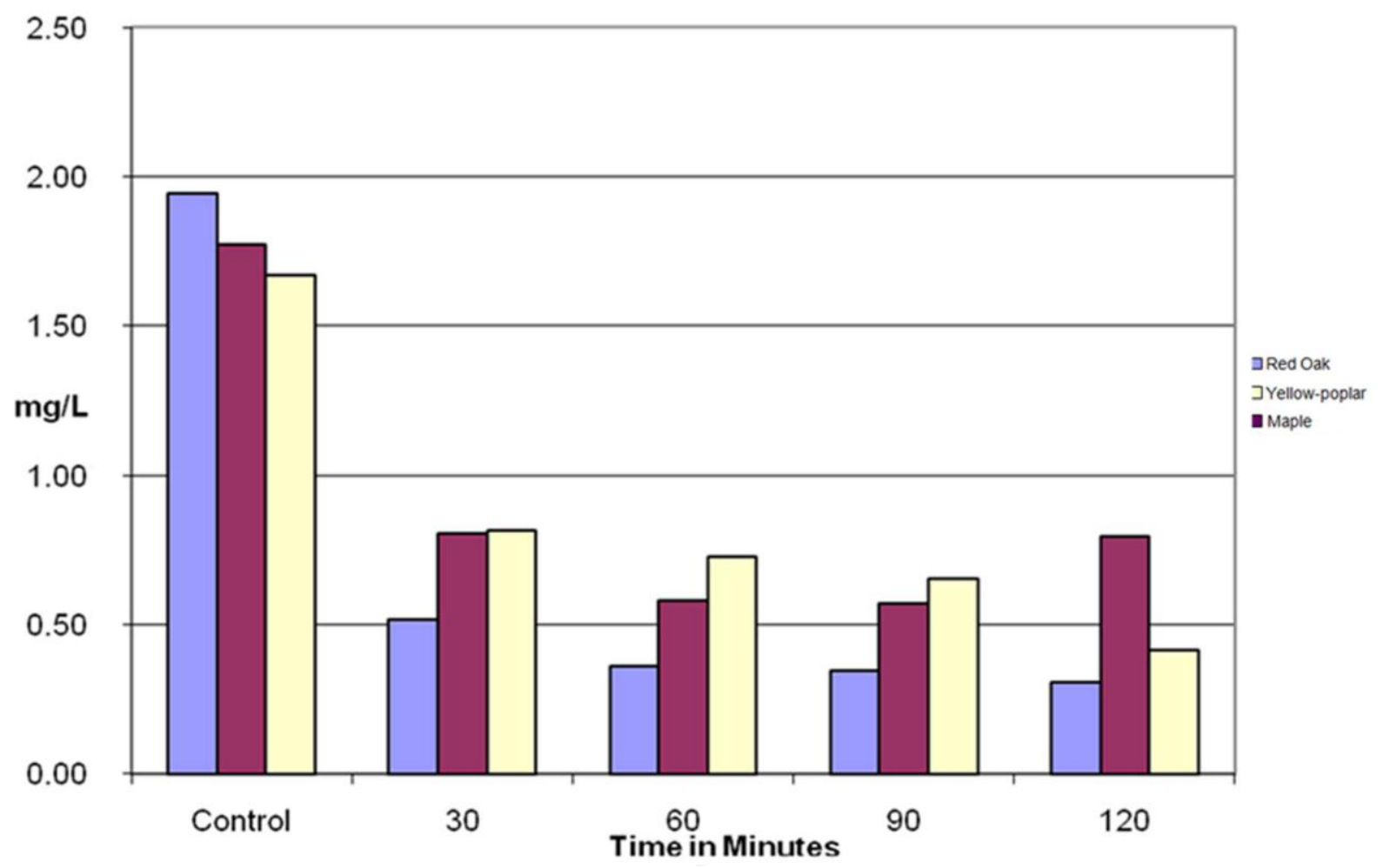

Figure 4. Effect of wood on AMD iron content 
pH of AMD and Wood Over Time for Fungi 1

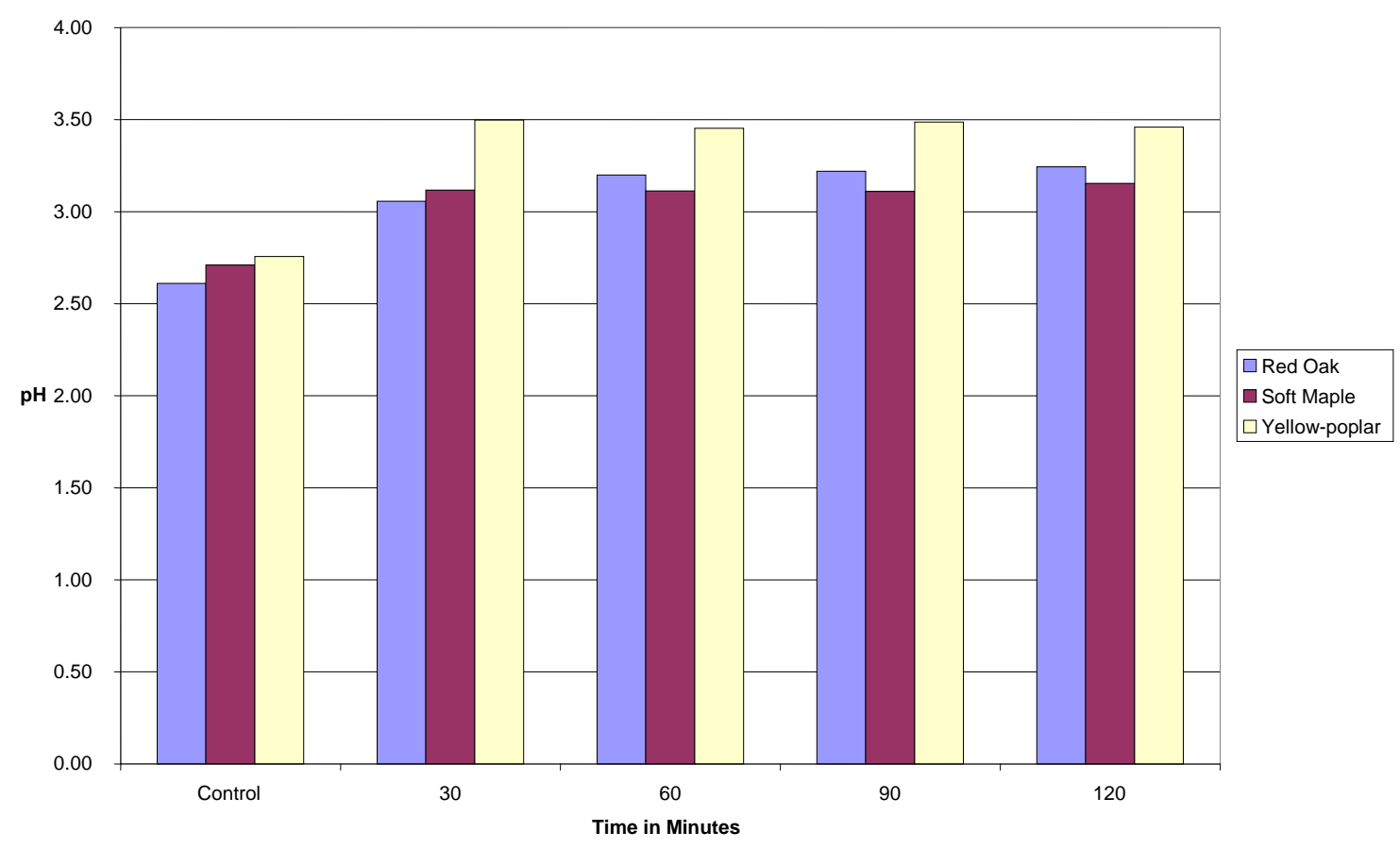

Figure 5. Effect of fungal inoculated wood on AMD pH 
Iron Concentration of AMD and Wood Over Time for Fungi 1

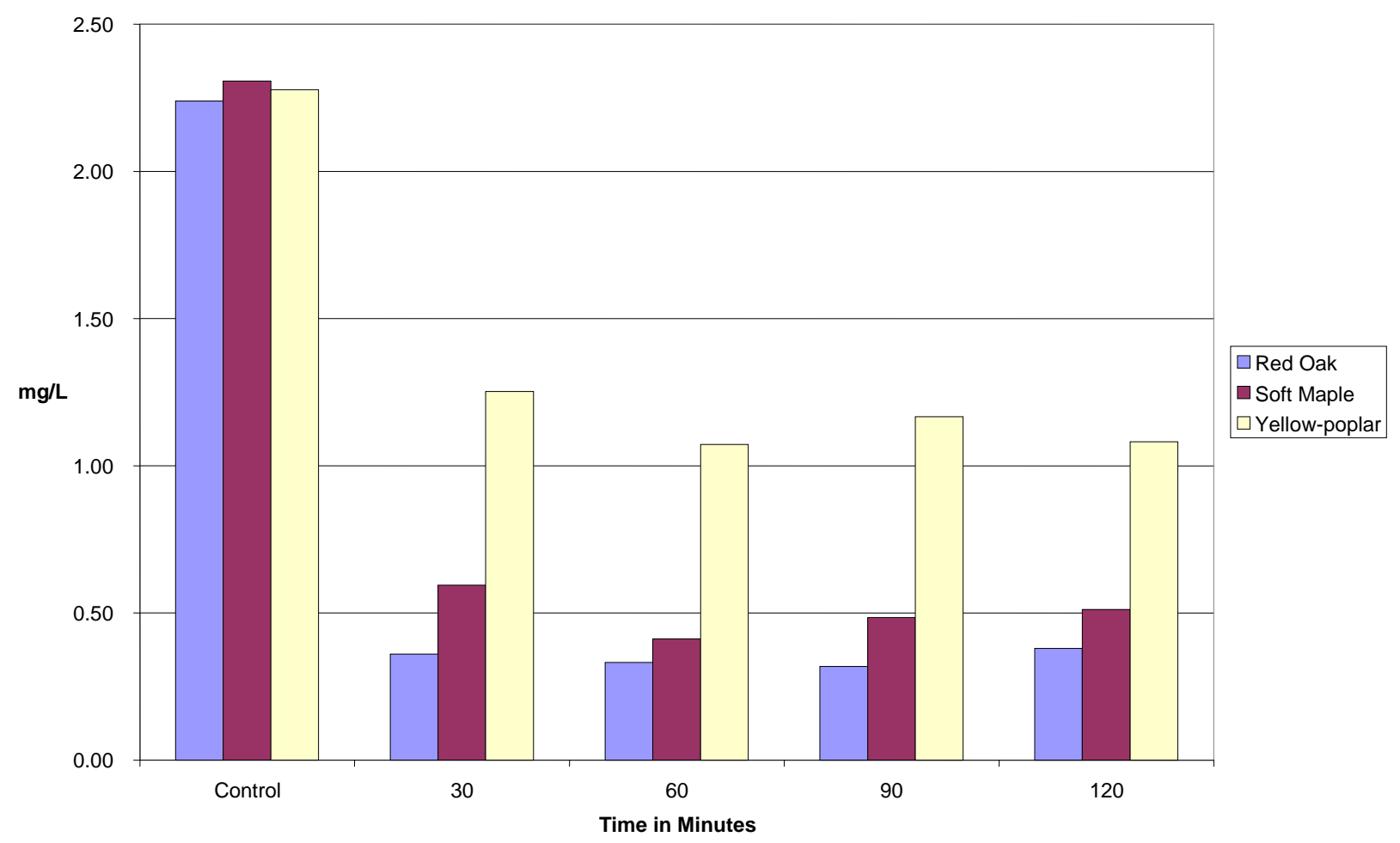

Figure 6. Effect of fungal inoculated wood on AMD content 


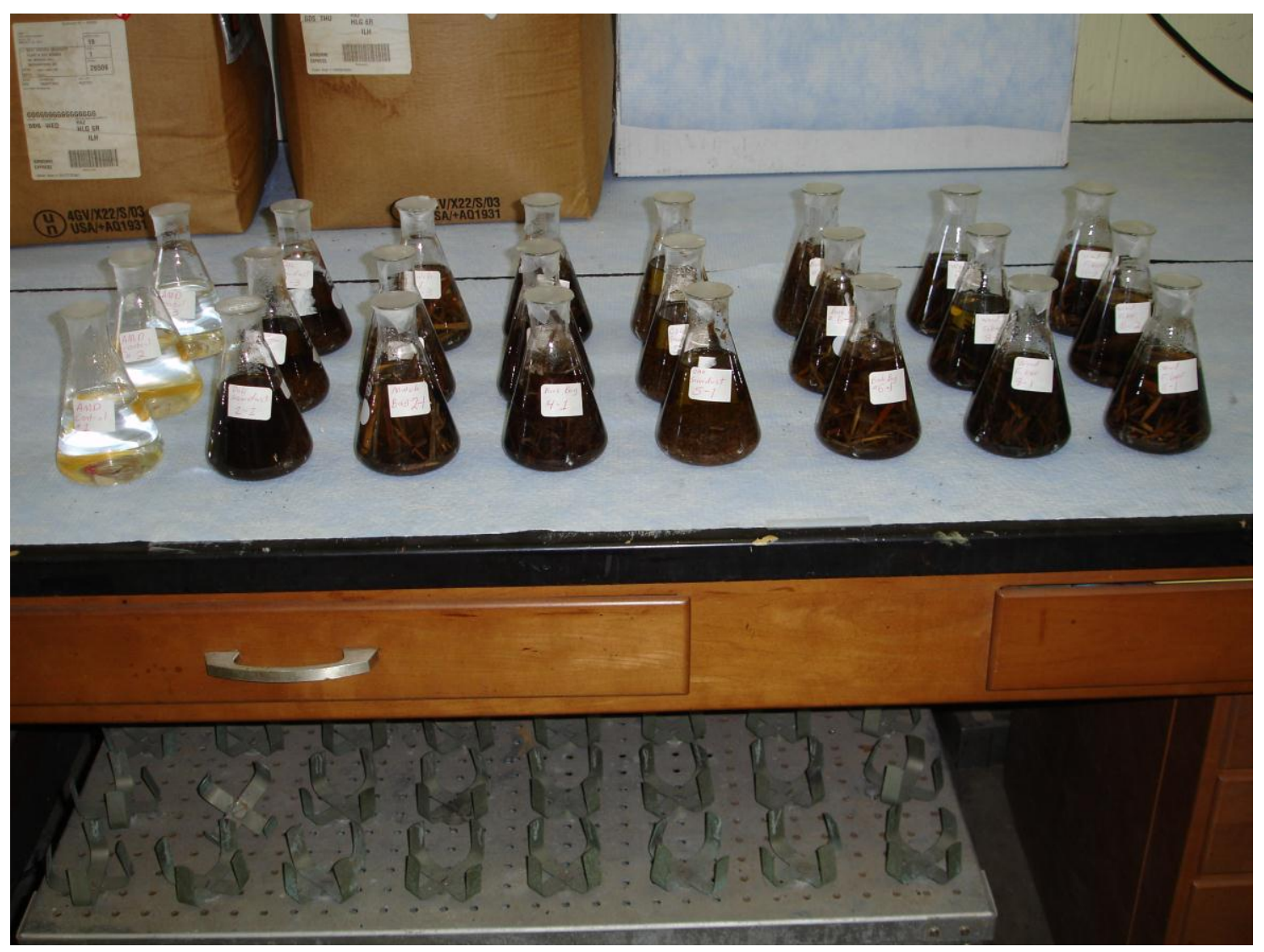

Figure 7. Erlenmeyer flasks containing ground wood and AMD 


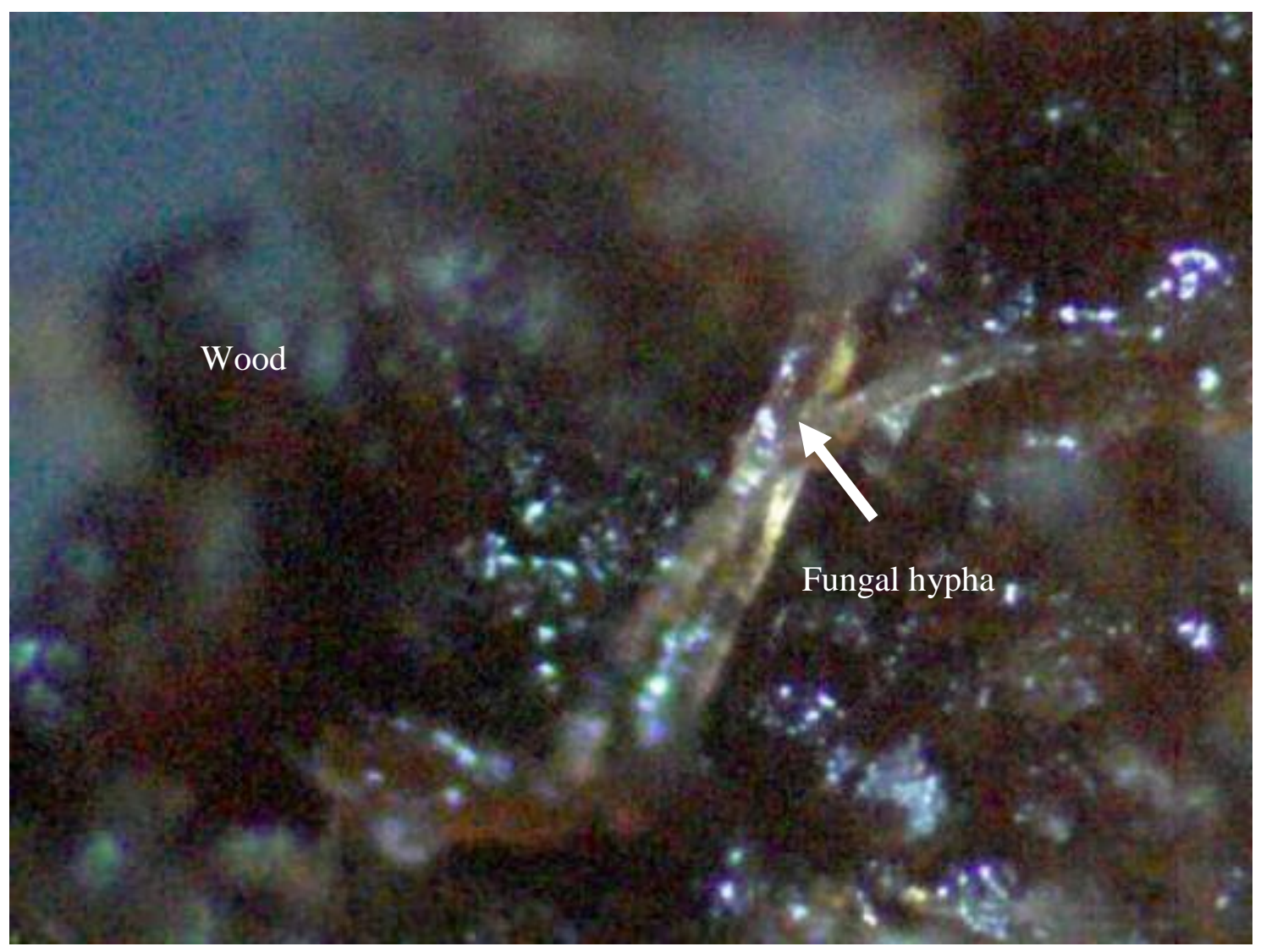

Figure 8. A micrograph of solid wood inoculated with Aspergilus niger 


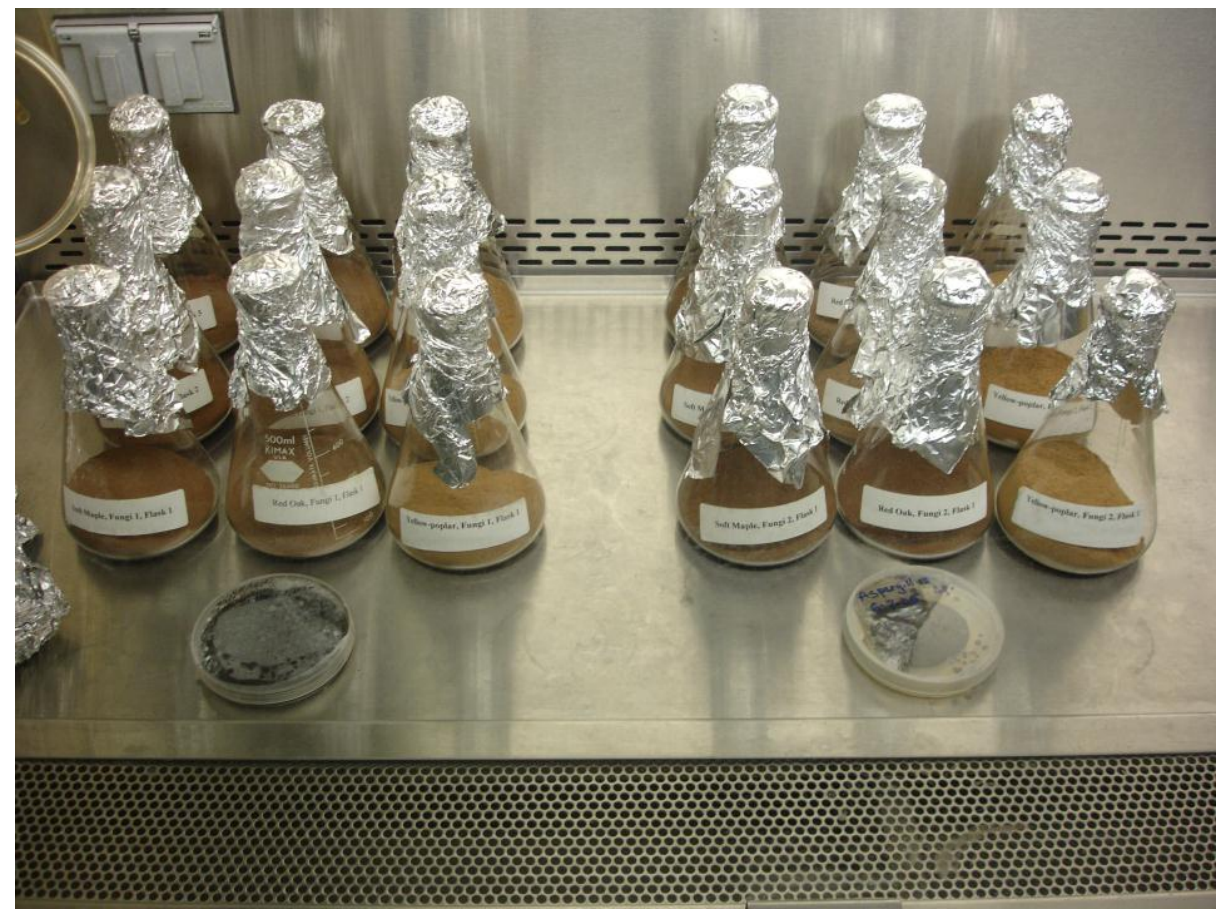

Figure 9. Preparation of fungal inoculi for inoculation of wood 